\title{
2014 NIST Assessment of Phasor Measurement Unit Performance
}

\author{
Allen Goldstein \\ This publication is available free of charge from \\ http://dx.doi.org/10.6028/NIST.IR.8106
}

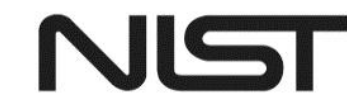

National Institute of Standards and Technology U.S. Department of Commerce 


\title{
2014 NIST Assessment of Phasor Measurement Unit Performance
}

\author{
Allen Goldstein \\ Physical Measurement Laboratory
}

This publication is available free of charge from:

http://dx.doi.org/10.6028/NIST.IR.8106

February 2016

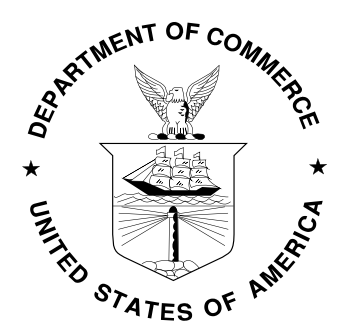

U.S. Department of Commerce Penny Pritzker, Secretary

National Institute of Standards and Technology Willie May, Acting Under Secretary of Commerce for Standards and Technology and Acting Director 


\section{Foreword}

In 2005, the Institute of Electrical and Electronic Engineers Standards Association (IEEE SA) published IEEE Std. C37.118.1-2005 Standard for Synchrophasors for Power Systems. This standard replaced IEEE Std. 1344-1995. These standards define a Phasor Measurement Unit (PMU) as a device that produces synchronized phasor, frequency, and rate of change of frequency (ROCOF) estimates from voltage and/or current signals and a time synchronizing signal. The 1995 standard required that a PMU have less than $1 \mu$ s error in timing. The 2005 standard removed the timing error limit and instead set amplitude and phase angle limits using a combined metric called Total Vector Error (TVE). The limits were placed only on the performance of the phasor measurements and not on frequency or rate of change of frequency (ROCOF) measurements. Also the performance limits were only given for "steady state" power system conditions: conditions where the magnitude and frequency of the power system signal were unchanging but not for "dynamic" conditions where frequency and/or magnitude were changing.

In 2005, NIST began to develop test methods for synchrophasors and in 2006, NIST began to measure the performance of PMUs sponsored with supplemental funding from the Department of Energy (DOE). Working closely with DOE, the IEEE working group responsible for the synchrophasor standard (IEEE Power and Energy Society (PES), Power System Relaying Committee (PSRC) Working Group H11), and with the Bonneville Power Administration's (BPA's) PMU lab, NIST developed tests for PMUs. In 2007, NIST began to offer special test services for PMUs.

In 2009, NIST was contracted to create a Report of Special Test for Operador Nacional do Sistema Electrica (ONS), Brazil. The contract required that PMUs be tested for accuracy of frequency and ROCOF measurements as well as performance under some dynamic system conditions. As a result of this testing, NIST made proposals to IEEE PSRC H11 for dynamic tests to be performed on PMUs.

In 2010, NIST awarded an American Recovery and Reinvestment Act (ARRA) grant to Fluke Calibration ${ }^{1}$ to develop a commercially available PMU calibration system. A prototype of this system is on loan to the NIST Power System Synchrometrology Lab. It is one of four PMU test systems in operation at NIST today.

In 2011, IEEE SA published C37.118.1-2011 IEEE Standards for Synchrophasor Measurements for Power Systems which incorporated many of the dynamic tests developed by NIST and BPA. The standard eliminated the PMU "levels" and implemented $\mathrm{M}$ and $\mathrm{P}$ classes of PMU. It revised the steady state performance limits, added performance limits for frequency and ROCOF measurements, and added a set of dynamic performance limits. Many of the limits chosen were based on extensive testing of actual and simulated PMUs performed at NIST.

In 2012, NIST invited all known PMU manufacturers to participate in a performance assessment of their PMU products. Tests were performed and the data were provided to the vendors. When the performance data were shared with the PMU vendors many of them upgraded their PMU hardware and/or software, and NIST re-tested them. Some of the vendors provided prototype equipment that has not been released to production at the time of this publication.

${ }^{1}$ Certain commercial equipment, instruments, or materials are identified in this paper to foster understanding. Such identification does not imply recommendation or endorsement by the National Institute of Standards and Technology, nor does it imply that the materials or equipment identified are necessarily the best available for the purpose 
In 2014, acting upon lessons learned through PMU testing at NIST, BPA, and other locations, IEEE SA amended the synchrophasor standard with IEEE Std. C37.118.1a-2014, clarifying some tests, resolving some ambiguities, and adjusting some performance limits.

This NISTIR reports on the performance of ten prototype and commercially available PMUs, as well as the C37.118.1a2014 amended Annex C Reference Signal Processing Model against the limits of the 2014 amended IEEE synchrophasor standard. The purpose of this study was to establish a baseline range of performance for commercial PMUs and to provide generic PMU performance information to the IEEE PSRC H11 Working Group to support further improvement of the synchrophasor standards. The identities of the PMU models and manufacturers are therefore withheld.

NIST continues to work closely with IEEE and with IEC, who are now working to create a joint synchrophasor standard. NIST is also working closely with IEEE SA to create a Synchrophasor Conformity Assessment Program which will use third-party independent laboratories to test PMUs for compliance certification to meet power industry needs. NIST also continues to test PMUs from manufacturers to help them prepare for certification of compliance against the synchrophasor standards. 


\section{Table of Contents}

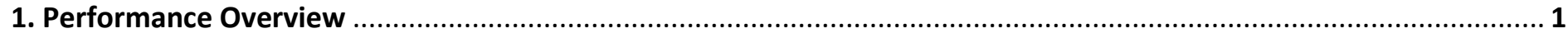

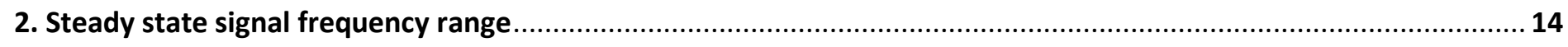

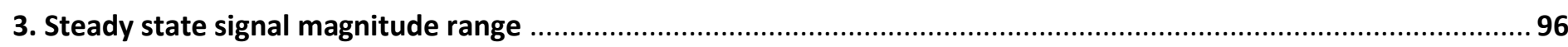

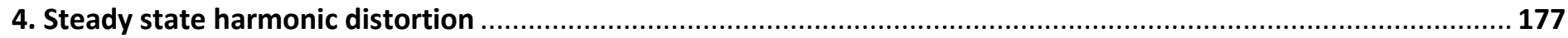

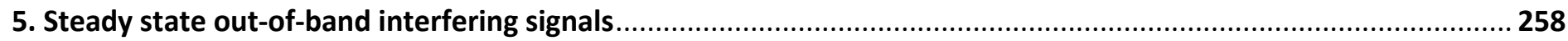

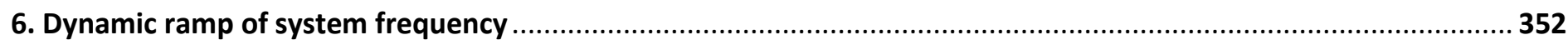

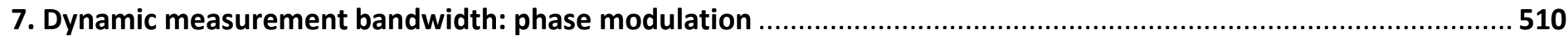

8. Dynamic measurement bandwidth: amplitude modulation ........................................................................ 592

9. Dynamic step change in phase

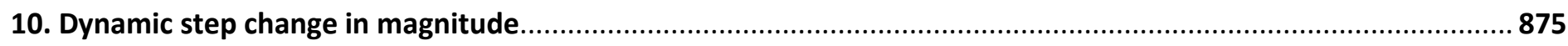

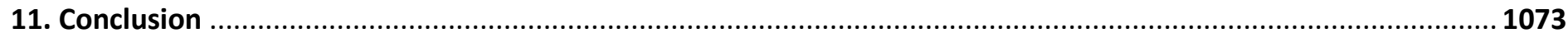




\section{Performance overview}

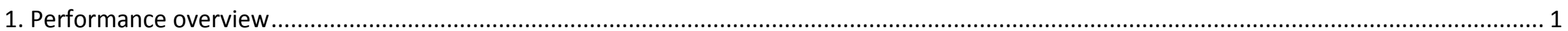

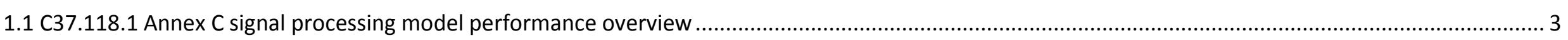

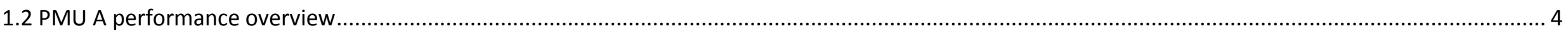

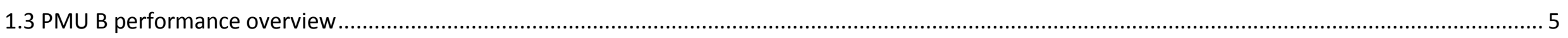

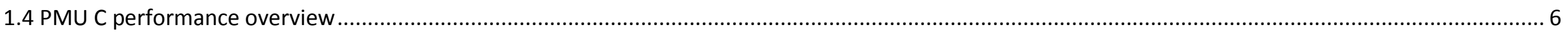

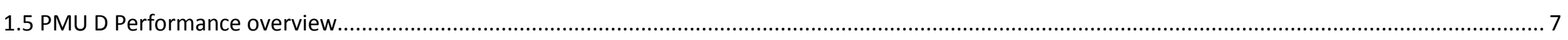

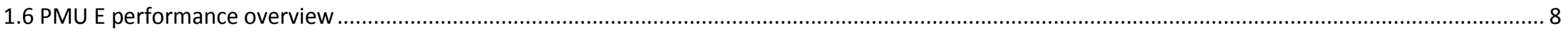

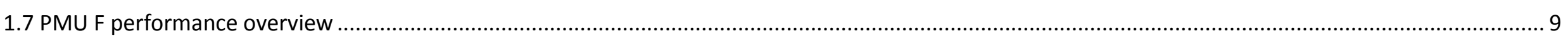

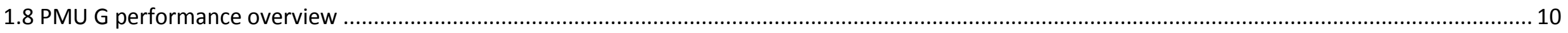

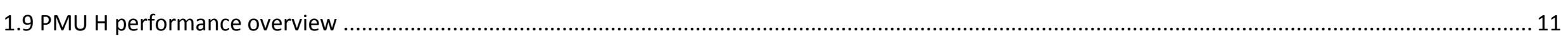

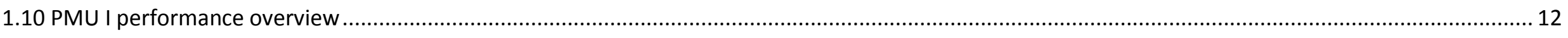

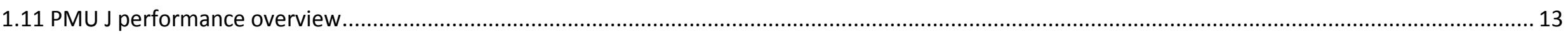


The sections of the overview provide tables of pass, fail, or indeterminate results for each of the PMUs in the assessment. These results are for IEEE ${ }^{(T M)}$ Std. C37.118.1-2011 amended by IEEE Std. C37.118.1a-2014.

The rows of the tables provide results for each of the test types required by the synchrophasor standard. The tests are:

- Steady state frequency range tests

- Steady state harmonic distortion tests

- Steady state out-of-band interfering signals tests

- Steady state magnitude tests

- Dynamic test of ramp of system frequency

- Dynamic bandwidth modulation tests

- Phase modulation tests

- Magnitude modulation tests

- Dynamic step tests

○ Phase step tests

- Magnitude step tests

The columns of the tables show pass/fail/indeterminate results for Total Vector Error (TVE), Frequency Error (FE) and Rate of Change of Frequency Error (RFE) for each of the reporting rates required for $60 \mathrm{~Hz}$ nominal system frequency. Fifty $\mathrm{Hz}$ nominal system frequency was not tested for this assessment.

- $\quad$ indicates the PMU passes the test by performing within the limits set by the standard.

- F indicates the PMU fails the test by performing outside the limits set by the standard.

- I indicates the result is indeterminate because the PMU performance is close enough to the limits to be within the test system's range of uncertainty.

- - (dash) indicates that a test was not required or that performance limits have not been established for the test type, class, and reporting rate. 


\subsection{C37.118.1 Annex C signal processing model performance overview}

Table 1: Annex C signal processing model TVE, FE, and RFE

\begin{tabular}{|c|c|c|c|c|c|c|c|c|c|c|c|c|c|c|c|c|c|c|c|c|c|c|c|c|c|c|c|c|c|c|c|c|c|c|c|c|}
\hline Fs (FPS) & \multicolumn{3}{|c|}{$10 \mathrm{M}$} & \multicolumn{3}{|c|}{$10 P$} & \multicolumn{3}{|c|}{$12 \mathrm{M}$} & \multicolumn{3}{|c|}{$12 \mathrm{P}$} & \multicolumn{3}{|c|}{$15 \mathrm{M}$} & \multicolumn{3}{|c|}{$15 \mathrm{P}$} & \multicolumn{3}{|c|}{$20 M$} & \multicolumn{3}{|c|}{$20 P$} & \multicolumn{3}{|c|}{$30 M$} & \multicolumn{3}{|c|}{$30 P$} & \multicolumn{3}{|c|}{$60 M$} & \multicolumn{3}{|c|}{$60 P$} \\
\hline & $\mathrm{T}$ & $\mathrm{FE}$ & $\mathrm{RF}$ & $T$ & $\mathrm{~F}$ & $\mathrm{R}$ & $T$ & $\mathrm{~F}$ & $\mathrm{R}$ & $T$ & $\mathrm{~F}$ & $\mathrm{R}$ & $T$ & $\mathrm{~F}$ & $\mathrm{R}$ & $T$ & $\mathrm{~F}$ & $\mathrm{R}$ & $T$ & $\mathrm{~F}$ & $\mathrm{R}$ & $\mathrm{T}$ & $F$ & $\mathrm{R}$ & $\mathrm{T}$ & $\mathrm{F}$ & $\mathrm{R}$ & $T$ & $\mathrm{~F}$ & $\mathrm{R}$ & $\mathrm{T}$ & $\mathrm{F}$ & $R$ & $\mathrm{~T}$ & $\mathrm{~F}$ & $\mathrm{R}$ \\
\hline Test & V & & $E$ & V & $E$ & $\mathrm{~F}$ & V & $E$ & $\mathrm{~F}$ & V & $E$ & $\mathrm{~F}$ & V & $E$ & $\mathrm{~F}$ & $\mathrm{~V}$ & $E$ & $F$ & V & $E$ & $\mathrm{~F}$ & $\mathrm{~V}$ & $E$ & $\mathrm{~F}$ & $\mathrm{~V}$ & $E$ & $\mathrm{~F}$ & V & $E$ & $F$ & V & $E$ & $\mathrm{~F}$ & V & $E$ & $F$ \\
\hline & $\mathrm{E}$ & & & $\mathrm{E}$ & & $E$ & $\mathrm{E}$ & & $\mathrm{E}$ & $E$ & & $E$ & $E$ & & $E$ & $\mathrm{E}$ & & $E$ & $E$ & & $E$ & $\mathrm{E}$ & & $E$ & $\mathrm{E}$ & & $E$ & $E$ & & $E$ & $\mathrm{E}$ & & $E$ & $\mathrm{E}$ & & $\mathrm{E}$ \\
\hline SS Freq. Range & $\mathrm{P}$ & $\mathrm{P}$ & $\mathrm{P}$ & $\mathrm{P}$ & $\mathrm{P}$ & $\mathrm{P}$ & $\mathrm{P}$ & $\mathrm{P}$ & $\mathrm{P}$ & $\mathrm{P}$ & $\mathrm{P}$ & $P$ & $\mathrm{P}$ & $\mathrm{P}$ & $\mathrm{P}$ & $\mathrm{P}$ & $P$ & $P$ & $P$ & $\mathrm{P}$ & $P$ & $P$ & $P$ & $\mathrm{P}$ & $\mathrm{P}$ & $P$ & $\mathrm{P}$ & $\mathrm{P}$ & $\mathrm{P}$ & $\mathrm{P}$ & $\mathrm{P}$ & $\mathrm{P}$ & $\mathrm{P}$ & $P$ & $P$ & $\mathrm{P}$ \\
\hline SS Harmonic & $P$ & $P$ & - & $P$ & $P$ & $P$ & $P$ & $P$ & - & $P$ & $P$ & $P$ & $\mathrm{P}$ & $\mathrm{P}$ & - & $\mathrm{P}$ & $\mathrm{P}$ & $P$ & $\mathrm{P}$ & $\mathrm{P}$ & - & $\mathrm{P}$ & $P$ & $\mathrm{P}$ & $\mathrm{P}$ & $\mathrm{P}$ & - & $P$ & $P$ & $P$ & $P$ & $P$ & - & $\mathrm{P}$ & $P$ & $P$ \\
\hline SS OOB Interf. & $\mathrm{P}$ & $\mathrm{P}$ & - & - & - & - & $\mathrm{P}$ & $\mathrm{P}$ & - & - & - & - & $\mathrm{P}$ & $\mathrm{P}$ & - & - & - & - & $\mathrm{P}$ & $\mathrm{P}$ & - & - & - & - & $\mathrm{P}$ & $\mathrm{P}$ & - & - & - & - & $\mathrm{P}$ & $\mathrm{P}$ & - & - & - & - \\
\hline SS Magnitude & $P$ & - & - & $\mathrm{P}$ & - & - & $P$ & - & - & $P$ & - & - & $\mathrm{P}$ & 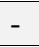 & - & $\mathrm{P}$ & - & - & $\mathrm{P}$ & - & - & $P$ & - & - & $\mathrm{P}$ & - & - & $P$ & - & - & $\mathrm{P}$ & - & & $\mathrm{P}$ & - & - \\
\hline Dyn Ramp & $P$ & $P$ & $P$ & $P$ & $P$ & $P$ & $P$ & $P$ & $\mathrm{P}$ & $P$ & $P$ & $P$ & $P$ & $P$ & $P$ & $\mathrm{P}$ & $\mathrm{P}$ & $P$ & $\mathrm{P}$ & $\mathrm{P}$ & $P$ & $P$ & $P$ & $\mathrm{P}$ & $\mathrm{P}$ & $\mathrm{P}$ & $P$ & $P$ & $P$ & $P$ & $\mathrm{P}$ & $\mathrm{P}$ & $P$ & $\mathrm{P}$ & $\mathrm{P}$ & $P$ \\
\hline Dyn Phase Mod & $P$ & $\mathrm{P}$ & $P$ & $\mathrm{P}$ & $\mathrm{P}$ & $\mathrm{P}$ & $\mathrm{P}$ & $\mathrm{P}$ & $\mathrm{P}$ & $\mathrm{P}$ & $\mathrm{P}$ & $P$ & $\mathrm{P}$ & $\mathrm{P}$ & $\mathrm{P}$ & $\mathrm{P}$ & $\mathrm{P}$ & $\mathrm{P}$ & $P$ & $\mathrm{P}$ & $P$ & $P$ & $P$ & $\mathrm{P}$ & $\mathrm{P}$ & $P$ & $\mathrm{P}$ & $\mathrm{P}$ & $\mathrm{P}$ & $\mathrm{P}$ & $\mathrm{P}$ & $\mathrm{P}$ & $\mathrm{P}$ & $\mathrm{P}$ & $P$ & $\mathrm{P}$ \\
\hline Dyn Ampl Mod & $P$ & $P$ & $P$ & $P$ & $P$ & $P$ & $P$ & $P$ & $P$ & $P$ & $P$ & $P$ & $P$ & $P$ & $P$ & $\mathrm{P}$ & $P$ & $P$ & $P$ & $P$ & $P$ & $P$ & $P$ & $\mathrm{P}$ & $P$ & $P$ & $P$ & $P$ & $P$ & $P$ & $P$ & $P$ & $P$ & $P$ & $P$ & $P$ \\
\hline
\end{tabular}

Table 2: Annex C signal processing model response time, delay time, and overshoot pass/fail

\begin{tabular}{|c|c|c|c|c|c|c|c|c|c|c|c|c|c|}
\hline \multicolumn{2}{|r|}{ Fs (FPS) } & $10 \mathrm{M}$ & $10 P$ & $12 \mathrm{M}$ & $12 \mathrm{P}$ & $15 \mathrm{M}$ & $15 P$ & $20 \mathrm{M}$ & $20 P$ & $30 \mathrm{M}$ & $30 P$ & $60 \mathrm{M}$ & $60 P$ \\
\hline \multirow{5}{*}{ Phase Step } & Phasor Resp. Time & $\mathrm{P}$ & $\mathrm{P}$ & $\mathrm{P}$ & $P$ & $P$ & $\mathrm{P}$ & $\mathrm{P}$ & $\mathrm{P}$ & $P$ & $\mathrm{P}$ & $P$ & $\mathrm{P}$ \\
\hline & Phasor Delay Time & $P$ & $P$ & $P$ & $P$ & $P$ & $P$ & $P$ & $P$ & $P$ & $P$ & $P$ & $P$ \\
\hline & Phasor Overshoot & $\mathrm{P}$ & $\mathrm{P}$ & $\mathrm{P}$ & $\mathrm{P}$ & $\mathrm{P}$ & $\mathrm{P}$ & $\mathrm{P}$ & $\mathrm{P}$ & $\mathrm{P}$ & $\mathrm{P}$ & $\mathrm{P}$ & $\mathrm{P}$ \\
\hline & Freq Resp. Time & $P$ & $\mathrm{P}$ & $\mathrm{P}$ & $\mathrm{P}$ & $\mathrm{P}$ & $\mathrm{P}$ & $\mathrm{P}$ & $\mathrm{P}$ & $\mathrm{P}$ & $\mathrm{P}$ & $\mathrm{P}$ & $\mathrm{P}$ \\
\hline & ROCOF Resp. Time & $P$ & $\mathrm{P}$ & $\mathrm{P}$ & $P$ & $P$ & $\mathrm{P}$ & $\mathrm{P}$ & $P$ & $\mathrm{P}$ & $\mathrm{P}$ & $P$ & $\mathrm{P}$ \\
\hline & & & & & & & & & & & & & \\
\hline \multirow{3}{*}{$\begin{array}{c}\text { Magnitude } \\
\text { Step }\end{array}$} & Magnitude Overshoot & $\mathrm{P}$ & $\mathrm{P}$ & $\mathrm{P}$ & $P$ & $\mathrm{P}$ & $\mathrm{P}$ & $\mathrm{P}$ & $\mathrm{P}$ & $\mathrm{P}$ & $\mathrm{P}$ & $\mathrm{P}$ & $\mathrm{P}$ \\
\hline & Freq Resp. Time & $\mathrm{P}$ & $\mathrm{P}$ & $\mathrm{P}$ & $\mathrm{P}$ & $\mathrm{P}$ & $\mathrm{P}$ & $\mathrm{P}$ & $\mathrm{P}$ & $\mathrm{P}$ & $\mathrm{P}$ & $\mathrm{P}$ & $\mathrm{P}$ \\
\hline & ROCOF Resp. Time & $\mathrm{P}$ & $\mathrm{P}$ & $\mathrm{P}$ & $\mathrm{P}$ & $\mathrm{P}$ & $\mathrm{P}$ & $\mathrm{P}$ & $\mathrm{P}$ & $\mathrm{P}$ & $\mathrm{P}$ & $\mathrm{P}$ & $\mathrm{P}$ \\
\hline
\end{tabular}




\subsection{PMU A performance overview}

PMU A is a commercially available PMU.

Table 3: PMU A TVE, FE, RFE pass/fail

\begin{tabular}{|c|c|c|c|c|c|c|c|c|c|c|c|c|c|c|c|c|c|c|c|c|c|c|c|c|c|c|c|c|c|c|c|c|c|c|c|c|}
\hline Fs (FPS) & \multicolumn{3}{|c|}{$10 M$} & \multicolumn{3}{|c|}{$10 \mathrm{P}$} & \multicolumn{3}{|c|}{$12 \mathrm{M}$} & \multicolumn{3}{|c|}{$12 \mathrm{P}$} & \multicolumn{3}{|c|}{$15 \mathrm{M}$} & \multicolumn{3}{|c|}{$15 \mathrm{P}$} & \multicolumn{3}{|c|}{$20 \mathrm{M}$} & \multicolumn{3}{|c|}{$20 P$} & \multicolumn{3}{|c|}{$30 \mathrm{M}$} & \multicolumn{3}{|c|}{$30 P$} & \multicolumn{3}{|c|}{$60 M$} & \multicolumn{3}{|c|}{$60 P$} \\
\hline & $T$ & $\mathrm{FE}$ & $\mathrm{RF}$ & $T$ & $\mathrm{~F}$ & $\mathrm{R}$ & $T$ & $\mathrm{~F}$ & $\mathrm{R}$ & $T$ & $\mathrm{~F}$ & $\mathrm{R}$ & $T$ & $\mathrm{~F}$ & $\mathrm{R}$ & $T$ & $\mathrm{~F}$ & $\mathrm{R}$ & $\mathrm{T}$ & $\mathrm{F}$ & $\mathrm{R}$ & $\mathrm{T}$ & $\mathrm{F}$ & $\mathrm{R}$ & $T$ & $\mathrm{~F}$ & $\mathrm{R}$ & $T$ & $\mathrm{~F}$ & $\mathrm{R}$ & $T$ & $\mathrm{~F}$ & $\mathrm{R}$ & $T$ & $\mathrm{~F}$ & $\mathrm{R}$ \\
\hline Test & V & & $E$ & V & $E$ & $\mathrm{~F}$ & $\mathrm{~V}$ & $E$ & $\mathrm{~F}$ & V & $E$ & $\mathrm{~F}$ & V & $\mathrm{E}$ & $\mathrm{F}$ & $\mathrm{V}$ & $\mathrm{E}$ & $\mathrm{F}$ & V & $\mathrm{E}$ & $F$ & $\mathrm{~V}$ & $E$ & $\mathrm{~F}$ & $\mathrm{~V}$ & $E$ & $\mathrm{~F}$ & $\mathrm{~V}$ & $\mathrm{E}$ & $\mathrm{F}$ & $\mathrm{V}$ & $\mathrm{E}$ & $\mathrm{F}$ & V & $E$ & $\mathrm{~F}$ \\
\hline & $E$ & & & $E$ & & $E$ & $E$ & & $E$ & $E$ & & $E$ & $\mathrm{E}$ & & $E$ & $E$ & & $E$ & $\mathrm{E}$ & & $\mathrm{E}$ & $\mathrm{E}$ & & $E$ & $E$ & & $\mathrm{E}$ & $\mathrm{E}$ & & $E$ & $E$ & & $\mathrm{E}$ & $E$ & & $E$ \\
\hline SS Freq. Range & $\mathrm{P}$ & $\mathrm{P}$ & $P$ & $P$ & $\mathrm{~F}$ & $\mathrm{~F}$ & $\mathrm{P}$ & $\mathrm{P}$ & $\mathrm{P}$ & $\mathrm{P}$ & $\mathrm{F}$ & $\mathrm{F}$ & $\mathrm{P}$ & $\mathrm{P}$ & $\mathrm{P}$ & $\mathrm{P}$ & $\mathrm{F}$ & $\mathrm{F}$ & $\mathrm{P}$ & $\mathrm{P}$ & $P$ & $P$ & $\mathrm{~F}$ & $\mathrm{~F}$ & $\mathrm{P}$ & $P$ & $\mathrm{~F}$ & $\mathrm{P}$ & $\mathrm{F}$ & $\mathrm{F}$ & $\mathrm{P}$ & $\mathrm{F}$ & $F$ & $P$ & $\mathrm{~F}$ & $\mathrm{~F}$ \\
\hline SS Harmonic & $P$ & $\mathrm{P}$ & - & $\mathrm{P}$ & $\mathrm{P}$ & $\mathrm{F}$ & $\mathrm{P}$ & $\mathrm{P}$ & - & $\mathrm{P}$ & $\mathrm{P}$ & $\mathrm{F}$ & $\mathrm{P}$ & $\mathrm{P}$ & - & $\mathrm{P}$ & $P$ & $\mathrm{~F}$ & $\mathrm{P}$ & $\mathrm{P}$ & - & $\mathrm{P}$ & $\mathrm{P}$ & $\mathrm{F}$ & $\mathrm{P}$ & $\mathrm{P}$ & - & $\mathrm{P}$ & $\mathrm{P}$ & $\mathrm{F}$ & $\mathrm{P}$ & $\mathrm{P}$ & - & $\mathrm{P}$ & $\mathrm{P}$ & $\mathrm{F}$ \\
\hline SS OOB Interf. & $\mathrm{P}$ & $\mathrm{P}$ & - & - & - & - & $\mathrm{P}$ & $\mathrm{P}$ & - & - & - & - & $\mathrm{P}$ & $\mathrm{P}$ & - & - & - & - & $\mathrm{P}$ & $\mathrm{F}$ & - & - & - & - & $\mathrm{P}$ & $\mathrm{F}$ & - & - & - & - & $\mathrm{P}$ & $\mathrm{F}$ & - & - & - & - \\
\hline SS Magnitude & $P$ & - & - & $P$ & $P$ & $P$ & $P$ & - & - & $P$ & - & - & $P$ & - & - & $\mathrm{P}$ & - & - & $P$ & - & - & $P$ & - & - & $\mathrm{P}$ & - & - & $P$ & - & - & $P$ & - & - & $P$ & - & - \\
\hline Dyn Ramp & $P$ & $\mathrm{P}$ & $\mathrm{P}$ & $\mathrm{P}$ & $\mathrm{P}$ & $\mathrm{F}$ & $\mathrm{P}$ & $\mathrm{P}$ & $\mathrm{P}$ & $\mathrm{P}$ & $\mathrm{P}$ & $\mathrm{F}$ & $\mathrm{P}$ & $\mathrm{P}$ & $\mathrm{P}$ & $\mathrm{P}$ & $P$ & $\mathrm{~F}$ & $\mathrm{P}$ & $\mathrm{P}$ & $\mathrm{P}$ & $\mathrm{P}$ & $\mathrm{P}$ & $\mathrm{F}$ & $\mathrm{P}$ & $P$ & $\mathrm{P}$ & $\mathrm{P}$ & $\mathrm{P}$ & $\mathrm{F}$ & $\mathrm{P}$ & $\mathrm{P}$ & $\mathrm{F}$ & $\mathrm{P}$ & $\mathrm{P}$ & $\mathrm{F}$ \\
\hline Dyn Phase Mod & $\mathrm{P}$ & $P$ & $\mathrm{~F}$ & $\mathrm{P}$ & $\mathrm{P}$ & $\mathrm{F}$ & $\mathrm{P}$ & $\mathrm{P}$ & $\mathrm{F}$ & $\mathrm{P}$ & $\mathrm{P}$ & $\mathrm{F}$ & $\mathrm{P}$ & $\mathrm{P}$ & $\mathrm{F}$ & $\mathrm{P}$ & $P$ & $\mathrm{~F}$ & $\mathrm{P}$ & $\mathrm{P}$ & $\mathrm{F}$ & $\mathrm{P}$ & $\mathrm{P}$ & $\mathrm{P}$ & $\mathrm{P}$ & $P$ & $\mathrm{~F}$ & $\mathrm{P}$ & $\mathrm{P}$ & $\mathrm{P}$ & $\mathrm{P}$ & $\mathrm{P}$ & $\mathrm{P}$ & $\mathrm{P}$ & $\mathrm{P}$ & $\mathrm{P}$ \\
\hline Dyn Ampl Mod & $\mathrm{P}$ & $P$ & $\mathrm{P}$ & $\mathrm{P}$ & $\mathrm{P}$ & $\mathrm{F}$ & $\mathrm{P}$ & $\mathrm{P}$ & $\mathrm{P}$ & $P$ & $\mathrm{P}$ & $\mathrm{F}$ & $\mathrm{P}$ & $P$ & $\mathrm{P}$ & $\mathrm{P}$ & $P$ & $\mathrm{~F}$ & $\mathrm{P}$ & $\mathrm{P}$ & $\mathrm{P}$ & $\mathrm{P}$ & $\mathrm{P}$ & $\mathrm{P}$ & $\mathrm{P}$ & $P$ & $\mathrm{P}$ & $\mathrm{P}$ & $\mathrm{P}$ & $\mathrm{P}$ & $\mathrm{P}$ & $\mathrm{P}$ & $P$ & $\mathrm{P}$ & $\mathrm{P}$ & $\mathrm{P}$ \\
\hline
\end{tabular}

Table 4: PMU A response time, delay time and overshoot pass/fail

\begin{tabular}{|c|c|c|c|c|c|c|c|c|c|c|c|c|c|}
\hline & Fs (FPS) & $10 \mathrm{M}$ & $10 P$ & $12 \mathrm{M}$ & $12 \mathrm{P}$ & $15 \mathrm{M}$ & $15 P$ & $20 \mathrm{M}$ & $20 P$ & $30 M$ & $30 P$ & $60 M$ & $60 P$ \\
\hline \multirow{5}{*}{ Phase Step } & Phasor Resp. Time & $\mathrm{P}$ & $\mathrm{P}$ & $\mathrm{P}$ & $\mathrm{P}$ & $\mathrm{P}$ & $\mathrm{P}$ & $\mathrm{P}$ & $\mathrm{P}$ & $\mathrm{P}$ & $\mathrm{P}$ & $\mathrm{P}$ & $P$ \\
\hline & Phasor Delay Time & $\mathrm{P}$ & $P$ & $P$ & $\mathrm{P}$ & $\mathrm{P}$ & $P$ & $P$ & $P$ & $P$ & $P$ & $P$ & $P$ \\
\hline & Phasor Overshoot & $\mathrm{P}$ & $P$ & $\mathrm{P}$ & $P$ & $P$ & $P$ & $\mathrm{P}$ & $P$ & $\mathrm{P}$ & $P$ & $\mathrm{P}$ & $P$ \\
\hline & Freq Resp. Time & $\mathrm{P}$ & $\mathrm{P}$ & $\mathrm{P}$ & $\mathrm{P}$ & $\mathrm{P}$ & $\mathrm{P}$ & $\mathrm{P}$ & $\mathrm{P}$ & $\mathrm{P}$ & $\mathrm{P}$ & $\mathrm{P}$ & $\mathrm{P}$ \\
\hline & ROCOF Resp. Time & $P$ & $\mathrm{~F}$ & $\mathrm{P}$ & $\mathrm{F}$ & $P$ & $\mathrm{~F}$ & $\mathrm{P}$ & $\mathrm{F}$ & $\mathrm{P}$ & $\mathrm{F}$ & $\mathrm{F}$ & $\mathrm{F}$ \\
\hline & & & & & & & & & & & & & \\
\hline \multirow{3}{*}{$\begin{array}{c}\text { Magnitude } \\
\text { Step }\end{array}$} & Magnitude Overshoot & $\mathrm{P}$ & $\mathrm{P}$ & $\mathrm{P}$ & $\mathrm{P}$ & $\mathrm{P}$ & $\mathrm{P}$ & $\mathrm{P}$ & $\mathrm{P}$ & $\mathrm{P}$ & $\mathrm{P}$ & $\mathrm{P}$ & $\mathrm{P}$ \\
\hline & Freq Resp. Time & $\mathrm{P}$ & $\mathrm{P}$ & $\mathrm{P}$ & $\mathrm{P}$ & $\mathrm{P}$ & $\mathrm{P}$ & $\mathrm{P}$ & $\mathrm{P}$ & $\mathrm{P}$ & $\mathrm{P}$ & $\mathrm{P}$ & $\mathrm{P}$ \\
\hline & ROCOF Resp. Time & $\mathrm{P}$ & $\mathrm{F}$ & $\mathrm{P}$ & $\mathrm{F}$ & $\mathrm{P}$ & $\mathrm{F}$ & $\mathrm{P}$ & $\mathrm{F}$ & $\mathrm{P}$ & $\mathrm{F}$ & $\mathrm{F}$ & $\mathrm{F}$ \\
\hline
\end{tabular}




\subsection{PMU B performance overview}

PMU B is a commercially available PMU.

Table 5: PMU B TVE, FE, RFE pass/fail

\begin{tabular}{|c|c|c|c|c|c|c|c|c|c|c|c|c|c|c|c|c|c|c|c|c|c|c|c|c|c|c|c|c|c|c|c|c|c|c|c|c|}
\hline Fs (FPS) & \multicolumn{3}{|c|}{$10 \mathrm{M}$} & \multicolumn{3}{|c|}{$10 \mathrm{P}$} & \multicolumn{3}{|c|}{$12 \mathrm{M}$} & \multicolumn{3}{|c|}{$12 \mathrm{P}$} & \multicolumn{3}{|c|}{$15 \mathrm{M}$} & \multicolumn{3}{|c|}{$15 \mathrm{P}$} & \multicolumn{3}{|c|}{$20 \mathrm{M}$} & \multicolumn{3}{|c|}{$20 P$} & \multicolumn{3}{|c|}{$30 \mathrm{M}$} & \multicolumn{3}{|c|}{$30 P$} & \multicolumn{3}{|c|}{$60 M$} & \multicolumn{3}{|c|}{$60 P$} \\
\hline Test & $\begin{array}{l}\mathrm{T} \\
\mathrm{V} \\
\mathrm{E}\end{array}$ & $\mathrm{FE}$ & $\begin{array}{c}\text { RF } \\
E\end{array}$ & $\begin{array}{l}T \\
V \\
E\end{array}$ & $\begin{array}{l}F \\
E\end{array}$ & $\begin{array}{l}\text { R } \\
F \\
E\end{array}$ & $\begin{array}{l}\mathrm{T} \\
\mathrm{V} \\
\mathrm{E}\end{array}$ & $\begin{array}{l}F \\
E\end{array}$ & $\begin{array}{l}\text { R } \\
F \\
E\end{array}$ & $\begin{array}{l}T \\
V \\
E\end{array}$ & $\begin{array}{l}F \\
E\end{array}$ & $\begin{array}{l}\mathrm{R} \\
\mathrm{F} \\
\mathrm{E}\end{array}$ & $\begin{array}{l}T \\
V \\
E\end{array}$ & $\begin{array}{l}F \\
E\end{array}$ & $\begin{array}{l}\text { R } \\
F \\
E\end{array}$ & $\begin{array}{l}T \\
V \\
E\end{array}$ & $\begin{array}{l}F \\
E\end{array}$ & $\begin{array}{l}R \\
F \\
E\end{array}$ & $\begin{array}{l}T \\
V \\
E\end{array}$ & $\begin{array}{l}\mathrm{F} \\
\mathrm{E}\end{array}$ & $\begin{array}{l}R \\
F \\
E\end{array}$ & $\begin{array}{l}\mathrm{T} \\
\mathrm{V} \\
\mathrm{E}\end{array}$ & \begin{tabular}{l|}
$F$ \\
$E$
\end{tabular} & $\begin{array}{l}\mathrm{R} \\
\mathrm{F} \\
\mathrm{E}\end{array}$ & $\begin{array}{l}\mathrm{T} \\
\mathrm{V} \\
\mathrm{E}\end{array}$ & $\begin{array}{l}\mathrm{F} \\
\mathrm{E}\end{array}$ & $\begin{array}{l}\mathrm{R} \\
\mathrm{F} \\
\mathrm{E}\end{array}$ & $\begin{array}{l}\mathrm{T} \\
\mathrm{V} \\
\mathrm{E}\end{array}$ & $\begin{array}{l}\mathrm{F} \\
\mathrm{E}\end{array}$ & $\begin{array}{l}\mathrm{R} \\
\mathrm{F} \\
\mathrm{E}\end{array}$ & $\begin{array}{l}\mathrm{T} \\
\mathrm{V} \\
\mathrm{E}\end{array}$ & $\begin{array}{l}\mathrm{F} \\
\mathrm{E}\end{array}$ & $\begin{array}{l}\mathrm{R} \\
\mathrm{F} \\
\mathrm{E}\end{array}$ & $\begin{array}{l}\mathrm{T} \\
\mathrm{V} \\
\mathrm{E}\end{array}$ & $\begin{array}{l}F \\
E\end{array}$ & $\begin{array}{l}\mathrm{R} \\
F \\
\mathrm{E}\end{array}$ \\
\hline SS Freq. Range & $\mathrm{P}$ & $\mathrm{P}$ & $\mathrm{P}$ & $P$ & $P$ & $P$ & $P$ & $\mathrm{P}$ & $\mathrm{P}$ & $\mathrm{P}$ & $\mathrm{P}$ & $P$ & $P$ & $\mathrm{P}$ & $\mathrm{P}$ & $P$ & $\mathrm{P}$ & $\mathrm{P}$ & $P$ & $\mathrm{P}$ & $P$ & $\mathrm{P}$ & $P$ & $\mathrm{P}$ & $\mathrm{P}$ & $\mathrm{P}$ & $\mathrm{P}$ & $\mathrm{P}$ & $P$ & $\mathrm{P}$ & $\mathrm{P}$ & $P$ & $\mathrm{P}$ & $P$ & $\mathrm{P}$ & $P$ \\
\hline SS Harmonic & $\mathrm{P}$ & $P$ & - & $P$ & $\mathrm{P}$ & $\mathrm{P}$ & $\mathrm{P}$ & $P$ & - & $\mathrm{P}$ & $\mathrm{P}$ & $\mathrm{P}$ & $P$ & $\mathrm{P}$ & - & $P$ & $P$ & $P$ & $P$ & $\mathrm{P}$ & - & $\mathrm{P}$ & $\mathrm{P}$ & $\mathrm{P}$ & $\mathrm{P}$ & $\mathrm{P}$ & - & $\mathrm{P}$ & $\mathrm{P}$ & $\mathrm{P}$ & $\mathrm{P}$ & $\mathrm{P}$ & - & $\mathrm{P}$ & $\mathrm{P}$ & $\mathrm{P}$ \\
\hline SS OOB Interf. & $F$ & $\mathrm{~F}$ & - & - & - & - & $\mathrm{F}$ & $\mathrm{F}$ & - & - & - & - & $\mathrm{F}$ & $\mathrm{F}$ & - & - & - & - & $\mathrm{F}$ & $\mathrm{F}$ & - & - & - & - & $P$ & $\mathrm{~F}$ & - & - & - & - & $P$ & $\mathrm{~F}$ & - & - & - & - \\
\hline SS Magnitude & $\mathrm{P}$ & - & - & $P$ & - & - & $P$ & - & - & $P$ & - & - & $P$ & - & - & $P$ & - & - & $P$ & - & - & $\mathrm{P}$ & - & - & $\mathrm{P}$ & - & - & $P$ & - & - & $P$ & - & - & $P$ & - & - \\
\hline Dyn Ramp & $P$ & $\mathrm{~F}$ & $\mathrm{P}$ & $P$ & $\mathrm{~F}$ & $\mathrm{P}$ & $\mathrm{P}$ & $\mathrm{F}$ & $\mathrm{P}$ & $\mathrm{P}$ & $\mathrm{F}$ & $P$ & $P$ & $\mathrm{~F}$ & $\mathrm{P}$ & $P$ & $\mathrm{~F}$ & $P$ & $P$ & $\mathrm{~F}$ & $P$ & $\mathrm{P}$ & $\mathrm{F}$ & $\mathrm{P}$ & $\mathrm{P}$ & $\mathrm{F}$ & $\mathrm{P}$ & $\mathrm{P}$ & $\mathrm{F}$ & $\mathrm{P}$ & $\mathrm{P}$ & $\mathrm{F}$ & $P$ & $\mathrm{P}$ & $\mathrm{F}$ & $\mathrm{P}$ \\
\hline Dyn Phase Mod & $\mathrm{P}$ & $P$ & $\mathrm{P}$ & $P$ & $\mathrm{P}$ & $\mathrm{P}$ & $\mathrm{P}$ & $\mathrm{P}$ & $\mathrm{P}$ & $\mathrm{P}$ & $\mathrm{P}$ & $\mathrm{P}$ & $P$ & $\mathrm{~F}$ & $\mathrm{P}$ & $P$ & $P$ & $P$ & $P$ & $\mathrm{~F}$ & $\mathrm{~F}$ & $\mathrm{P}$ & $\mathrm{P}$ & $\mathrm{P}$ & $\mathrm{P}$ & $\mathrm{F}$ & $\mathrm{F}$ & $\mathrm{P}$ & $\mathrm{P}$ & $\mathrm{P}$ & $\mathrm{P}$ & $\mathrm{F}$ & $\mathrm{F}$ & $\mathrm{P}$ & $\mathrm{P}$ & $\mathrm{P}$ \\
\hline Dyn Ampl Mod & $\mathrm{P}$ & $\mathrm{P}$ & $\mathrm{P}$ & $P$ & $P$ & $P$ & $P$ & $\mathrm{P}$ & $P$ & $P$ & $\mathrm{P}$ & $\mathrm{P}$ & $P$ & $P$ & $P$ & P & $P$ & $P$ & $P$ & $\mathrm{P}$ & $\mathrm{P}$ & $\mathrm{P}$ & $P$ & $\mathrm{P}$ & $\mathrm{P}$ & $\mathrm{P}$ & $\mathrm{P}$ & $\mathrm{P}$ & $\mathrm{P}$ & $\mathrm{P}$ & $\mathrm{P}$ & $P$ & $P$ & $P$ & $P$ & $P$ \\
\hline
\end{tabular}

Table 6: PMU B response time, delay time and overshoot pass/fail

\begin{tabular}{|c|c|c|c|c|c|c|c|c|c|c|c|c|c|}
\hline \multicolumn{2}{|r|}{ Fs (FPS) } & $10 \mathrm{M}$ & $10 \mathrm{P}$ & $12 \mathrm{M}$ & $12 \mathrm{P}$ & $15 \mathrm{M}$ & $15 P$ & $20 M$ & $20 P$ & $30 \mathrm{M}$ & $30 P$ & $60 M$ & $60 P$ \\
\hline \multirow{5}{*}{ Phase Step } & Phasor Resp. Time & $\mathrm{P}$ & $\mathrm{F}$ & $\mathrm{P}$ & $\mathrm{F}$ & $\mathrm{P}$ & $\mathrm{F}$ & $\mathrm{P}$ & $\mathrm{F}$ & $\mathrm{P}$ & $\mathrm{F}$ & $\mathrm{P}$ & $\mathrm{F}$ \\
\hline & Phasor Delay Time & $\mathrm{P}$ & $P$ & $\mathrm{P}$ & $P$ & $P$ & $P$ & $\mathrm{P}$ & $\mathrm{P}$ & $\mathrm{P}$ & $P$ & $\mathrm{P}$ & $\mathrm{P}$ \\
\hline & Phasor Overshoot & $\mathrm{F}$ & $\mathrm{F}$ & $\mathrm{F}$ & $\mathrm{F}$ & $\mathrm{F}$ & $\mathrm{F}$ & $\mathrm{F}$ & $\mathrm{F}$ & $\mathrm{F}$ & $\mathrm{F}$ & $\mathrm{F}$ & $\mathrm{F}$ \\
\hline & Freq Resp. Time & $\mathrm{P}$ & $\mathrm{F}$ & $\mathrm{P}$ & $\mathrm{F}$ & $\mathrm{P}$ & $\mathrm{F}$ & $\mathrm{P}$ & $\mathrm{F}$ & $\mathrm{P}$ & $\mathrm{F}$ & $\mathrm{P}$ & $\mathrm{F}$ \\
\hline & ROCOF Resp. Time & $P$ & $\mathrm{~F}$ & $\mathrm{P}$ & $\mathrm{F}$ & $\mathrm{P}$ & $\mathrm{F}$ & $\mathrm{P}$ & $\mathrm{F}$ & $\mathrm{P}$ & $\mathrm{F}$ & $P$ & $\mathrm{~F}$ \\
\hline & & & & & & & & & & & & & \\
\hline \multirow{3}{*}{$\begin{array}{l}\text { Magnitude } \\
\text { Step }\end{array}$} & Magnitude Overshoot & $\mathrm{F}$ & $\mathrm{F}$ & $\mathrm{F}$ & $\mathrm{F}$ & $\mathrm{F}$ & $\mathrm{F}$ & $\mathrm{F}$ & $\mathrm{F}$ & $\mathrm{F}$ & $\mathrm{F}$ & $\mathrm{F}$ & $\mathrm{F}$ \\
\hline & Freq Resp. Time & $\mathrm{P}$ & $\mathrm{P}$ & $\mathrm{P}$ & $P$ & $\mathrm{P}$ & $\mathrm{P}$ & $\mathrm{P}$ & $P$ & $\mathrm{P}$ & $P$ & $\mathrm{P}$ & $\mathrm{P}$ \\
\hline & ROCOF Resp. Time & $P$ & $P$ & $\mathrm{P}$ & $P$ & $P$ & $P$ & $\mathrm{P}$ & $\mathrm{P}$ & $P$ & $P$ & $P$ & $P$ \\
\hline
\end{tabular}




\subsection{PMU C performance overview}

PMU C is a commercially available PMU.

Table 7: PMU C TVE, FE, RFE pass/fail

\begin{tabular}{|c|c|c|c|c|c|c|c|c|c|c|c|c|c|c|c|c|c|c|c|c|c|c|c|c|c|c|c|c|c|c|c|c|c|c|c|c|}
\hline Fs (FPS) & \multicolumn{3}{|c|}{$10 \mathrm{M}$} & \multicolumn{3}{|c|}{$10 P$} & \multicolumn{3}{|c|}{$12 \mathrm{M}$} & \multicolumn{3}{|c|}{$12 \mathrm{P}$} & \multicolumn{3}{|c|}{$15 \mathrm{M}$} & \multicolumn{3}{|c|}{$15 \mathrm{P}$} & \multicolumn{3}{|c|}{$20 \mathrm{M}$} & \multicolumn{3}{|c|}{$20 P$} & \multicolumn{3}{|c|}{$30 \mathrm{M}$} & \multicolumn{3}{|c|}{$30 P$} & \multicolumn{3}{|c|}{$60 \mathrm{M}$} & \multicolumn{3}{|c|}{$60 P$} \\
\hline Test & $\begin{array}{l}\mathrm{T} \\
\mathrm{V} \\
\mathrm{E}\end{array}$ & $\mathrm{FE}$ & $\begin{array}{c}\text { RF } \\
E\end{array}$ & $\begin{array}{l}\mathrm{T} \\
\mathrm{V} \\
\mathrm{E}\end{array}$ & $\begin{array}{l}F \\
E\end{array}$ & $\begin{array}{l}\mathrm{R} \\
\mathrm{F} \\
\mathrm{E}\end{array}$ & $\begin{array}{l}\mathrm{T} \\
\mathrm{V} \\
\mathrm{E}\end{array}$ & $\begin{array}{l}F \\
E\end{array}$ & $\begin{array}{l}R \\
F \\
E\end{array}$ & $\begin{array}{l}\mathrm{T} \\
\mathrm{V} \\
\mathrm{E}\end{array}$ & $\begin{array}{l}F \\
E\end{array}$ & $\begin{array}{l}R \\
F \\
E\end{array}$ & $\begin{array}{l}\mathrm{T} \\
\mathrm{V} \\
\mathrm{E}\end{array}$ & $\begin{array}{l}F \\
E\end{array}$ & $\begin{array}{l}R \\
F \\
E\end{array}$ & $\begin{array}{l}\mathrm{T} \\
\mathrm{V} \\
\mathrm{E}\end{array}$ & $\begin{array}{l}F \\
E\end{array}$ & $\begin{array}{l}\mathrm{R} \\
\mathrm{F} \\
\mathrm{E}\end{array}$ & $\begin{array}{l}\mathrm{T} \\
\mathrm{V} \\
\mathrm{E}\end{array}$ & $\begin{array}{l}F \\
E\end{array}$ & $\begin{array}{l}R \\
F \\
E\end{array}$ & $\begin{array}{l}\mathrm{T} \\
\mathrm{V} \\
\mathrm{E}\end{array}$ & $\begin{array}{l}F \\
E\end{array}$ & $\begin{array}{l}\mathrm{R} \\
\mathrm{F} \\
\mathrm{E}\end{array}$ & $\begin{array}{l}\mathrm{T} \\
\mathrm{V} \\
\mathrm{E}\end{array}$ & $\begin{array}{l}F \\
E\end{array}$ & $\begin{array}{l}\mathrm{R} \\
\mathrm{F} \\
\mathrm{E}\end{array}$ & $\begin{array}{l}\mathrm{T} \\
\mathrm{V} \\
\mathrm{E}\end{array}$ & $\begin{array}{l}F \\
E\end{array}$ & $\begin{array}{l}\mathrm{R} \\
\mathrm{F} \\
\mathrm{E}\end{array}$ & $\begin{array}{l}\mathrm{T} \\
\mathrm{V} \\
\mathrm{E}\end{array}$ & $\begin{array}{l}\mathrm{F} \\
\mathrm{E}\end{array}$ & $\begin{array}{l}\mathrm{R} \\
\mathrm{F} \\
\mathrm{E}\end{array}$ & $\begin{array}{l}\mathrm{T} \\
\mathrm{V} \\
\mathrm{E}\end{array}$ & $\begin{array}{l}F \\
E\end{array}$ & $\begin{array}{l}R \\
F \\
E\end{array}$ \\
\hline SS Freq. Range & $\mathrm{P}$ & $\mathrm{F}$ & $\mathrm{F}$ & $\mathrm{P}$ & $\mathrm{P}$ & $\mathrm{P}$ & $\mathrm{P}$ & $\mathrm{F}$ & $\mathrm{F}$ & $P$ & $P$ & $\mathrm{P}$ & $\mathrm{P}$ & $\mathrm{F}$ & $\mathrm{F}$ & $\mathrm{P}$ & $\mathrm{P}$ & $\mathrm{P}$ & $P$ & $\mathrm{~F}$ & $\mathrm{~F}$ & $\mathrm{P}$ & $\mathrm{P}$ & $\mathrm{P}$ & $\mathrm{P}$ & $\mathrm{F}$ & $\mathrm{F}$ & $\mathrm{P}$ & $\mathrm{P}$ & $\mathrm{P}$ & $\mathrm{P}$ & $\mathrm{F}$ & $\mathrm{F}$ & $\mathrm{P}$ & $\mathrm{F}$ & $\mathrm{F}$ \\
\hline SS Harmonic & $\mathrm{P}$ & $\mathrm{P}$ & - & $\mathrm{P}$ & $\mathrm{P}$ & $\mathrm{P}$ & $\mathrm{P}$ & $\mathrm{P}$ & - & $P$ & $P$ & $\mathrm{P}$ & $\mathrm{P}$ & $\mathrm{P}$ & - & $\mathrm{P}$ & $\mathrm{P}$ & $\mathrm{P}$ & $\mathrm{P}$ & $\mathrm{P}$ & - & $\mathrm{P}$ & $\mathrm{P}$ & $\mathrm{P}$ & $\mathrm{P}$ & $\mathrm{P}$ & - & $\mathrm{P}$ & $\mathrm{P}$ & $\mathrm{P}$ & $\mathrm{P}$ & $\mathrm{P}$ & - & $\mathrm{P}$ & $\mathrm{F}$ & $\mathrm{F}$ \\
\hline SS OOB Interf. & $\mathrm{F}$ & $\mathrm{F}$ & - & - & - & - & $\mathrm{F}$ & $\mathrm{F}$ & - & - & - & - & $\mathrm{F}$ & $\mathrm{F}$ & - & - & - & - & $\mathrm{F}$ & $\mathrm{F}$ & - & - & - & - & $\mathrm{F}$ & $\mathrm{F}$ & - & - & - & - & $P$ & $\mathrm{~F}$ & - & - & - & - \\
\hline SS Magnitude & $\mathrm{P}$ & - & - & $\mathrm{P}$ & - & - & $P$ & - & - & $P$ & - & - & $\mathrm{P}$ & - & - & $\mathrm{P}$ & - & - & $P$ & - & - & $\mathrm{P}$ & - & - & $\mathrm{P}$ & - & - & $\mathrm{P}$ & - & - & $\mathrm{P}$ & - & - & $\mathrm{P}$ & - & - \\
\hline Dyn Ramp & $\mathrm{P}$ & $\mathrm{F}$ & $\mathrm{P}$ & $\mathrm{P}$ & $\mathrm{F}$ & $\mathrm{P}$ & $\mathrm{P}$ & $\mathrm{F}$ & $P$ & $P$ & $\mathrm{~F}$ & $\mathrm{P}$ & $\mathrm{P}$ & $\mathrm{F}$ & $\mathrm{F}$ & $\mathrm{P}$ & $\mathrm{F}$ & $\mathrm{F}$ & $\mathrm{P}$ & $\mathrm{F}$ & $\mathrm{F}$ & $\mathrm{P}$ & $\mathrm{F}$ & $\mathrm{P}$ & $\mathrm{P}$ & $\mathrm{F}$ & $\mathrm{F}$ & $\mathrm{P}$ & $\mathrm{F}$ & $\mathrm{P}$ & $\mathrm{P}$ & $\mathrm{F}$ & $\mathrm{F}$ & $\mathrm{P}$ & $\mathrm{F}$ & $\mathrm{F}$ \\
\hline Dyn Phase Mod & $\mathrm{P}$ & $\mathrm{P}$ & $\mathrm{F}$ & $\mathrm{P}$ & $\mathrm{P}$ & $\mathrm{P}$ & $\mathrm{P}$ & $\mathrm{P}$ & $\mathrm{F}$ & $\mathrm{P}$ & $\mathrm{P}$ & $\mathrm{P}$ & $\mathrm{P}$ & $\mathrm{P}$ & $\mathrm{F}$ & $\mathrm{P}$ & $\mathrm{P}$ & $\mathrm{P}$ & $\mathrm{P}$ & $\mathrm{P}$ & $\mathrm{F}$ & $\mathrm{P}$ & $\mathrm{P}$ & $\mathrm{P}$ & $\mathrm{P}$ & $\mathrm{P}$ & $\mathrm{F}$ & $\mathrm{P}$ & $\mathrm{P}$ & $\mathrm{P}$ & $\mathrm{P}$ & $\mathrm{P}$ & $\mathrm{P}$ & $\mathrm{P}$ & $\mathrm{P}$ & $\mathrm{P}$ \\
\hline Dyn Ampl Mod & $\mathrm{P}$ & $\mathrm{P}$ & $\mathrm{P}$ & $\mathrm{P}$ & $P$ & $\mathrm{P}$ & $P$ & $\mathrm{P}$ & $P$ & $P$ & $P$ & $P$ & $\mathrm{P}$ & $\mathrm{P}$ & $P$ & $\mathrm{P}$ & $\mathrm{P}$ & $\mathrm{P}$ & $P$ & $\mathrm{P}$ & $\mathrm{P}$ & $\mathrm{P}$ & $\mathrm{P}$ & $\mathrm{P}$ & $\mathrm{P}$ & $P$ & $\mathrm{P}$ & $\mathrm{P}$ & $\mathrm{P}$ & $\mathrm{P}$ & $\mathrm{P}$ & $\mathrm{P}$ & $P$ & $P$ & $P$ & $\mathrm{P}$ \\
\hline
\end{tabular}

Table 8: PMU C response time, delay time and overshoot pass/fail

\begin{tabular}{|c|c|c|c|c|c|c|c|c|c|c|c|c|c|}
\hline & Fs (FPS) & $10 \mathrm{M}$ & $10 P$ & $12 \mathrm{M}$ & $12 \mathrm{P}$ & $15 \mathrm{M}$ & $15 P$ & $20 M$ & $20 P$ & $30 \mathrm{M}$ & $30 P$ & $60 M$ & $60 P$ \\
\hline \multirow{5}{*}{ Phase Step } & Phasor Resp. Time & $\mathrm{P}$ & $\mathrm{P}$ & $\mathrm{P}$ & $\mathrm{P}$ & $\mathrm{P}$ & $\mathrm{P}$ & $\mathrm{P}$ & $\mathrm{P}$ & $\mathrm{P}$ & $\mathrm{P}$ & $\mathrm{P}$ & $\mathrm{P}$ \\
\hline & Phasor Delay Time & $\mathrm{P}$ & $\mathrm{P}$ & $\mathrm{P}$ & $\mathrm{P}$ & $\mathrm{P}$ & $\mathrm{P}$ & $\mathrm{P}$ & $\mathrm{P}$ & $\mathrm{P}$ & $\mathrm{P}$ & $\mathrm{P}$ & $\mathrm{P}$ \\
\hline & Phasor Overshoot & $\mathrm{P}$ & $\mathrm{P}$ & $\mathrm{P}$ & $\mathrm{P}$ & $\mathrm{P}$ & $\mathrm{P}$ & $\mathrm{P}$ & $\mathrm{P}$ & $\mathrm{P}$ & $\mathrm{P}$ & $\mathrm{P}$ & $\mathrm{P}$ \\
\hline & Freq Resp. Time & $\mathrm{P}$ & $\mathrm{F}$ & $\mathrm{P}$ & $\mathrm{F}$ & $\mathrm{P}$ & $\mathrm{F}$ & $\mathrm{P}$ & $\mathrm{F}$ & $\mathrm{P}$ & $\mathrm{P}$ & $\mathrm{P}$ & $\mathrm{F}$ \\
\hline & ROCOF Resp. Time & $\mathrm{P}$ & $\mathrm{F}$ & $\mathrm{P}$ & $\mathrm{F}$ & $\mathrm{P}$ & $\mathrm{F}$ & $\mathrm{P}$ & $\mathrm{F}$ & $\mathrm{P}$ & $\mathrm{F}$ & $\mathrm{P}$ & $\mathrm{F}$ \\
\hline \multirow{4}{*}{$\begin{array}{l}\text { Magnitude } \\
\text { Step }\end{array}$} & & & & & & & & & & & & & \\
\hline & Magnitude Overshoot & $\mathrm{P}$ & $\mathrm{P}$ & $\mathrm{P}$ & $\mathrm{P}$ & $\mathrm{P}$ & $\mathrm{P}$ & $\mathrm{P}$ & $\mathrm{P}$ & $\mathrm{P}$ & $\mathrm{P}$ & $\mathrm{P}$ & $\mathrm{P}$ \\
\hline & Freq Resp. Time & $\mathrm{P}$ & $\mathrm{F}$ & $\mathrm{P}$ & $\mathrm{F}$ & $\mathrm{P}$ & $\mathrm{F}$ & $\mathrm{P}$ & $\mathrm{F}$ & $\mathrm{P}$ & $\mathrm{P}$ & $\mathrm{P}$ & $\mathrm{F}$ \\
\hline & ROCOF Resp. Time & $P$ & $\mathrm{P}$ & $P$ & $P$ & $\mathrm{P}$ & $\mathrm{P}$ & $P$ & $\mathrm{~F}$ & $P$ & $P$ & $P$ & $\mathrm{P}$ \\
\hline
\end{tabular}




\subsection{PMU D Performance overview}

PMU D is a prototype PMU and is not yet commercially available

\section{Table 9: PMU D TVE, FE, RFE pass/fail}

\begin{tabular}{|c|c|c|c|c|c|c|c|c|c|c|c|c|c|c|c|c|c|c|c|c|c|c|c|c|c|c|c|c|c|c|c|c|c|c|c|c|}
\hline Fs (FPS) & \multicolumn{3}{|c|}{$10 \mathrm{M}$} & \multicolumn{3}{|c|}{$10 \mathrm{P}$} & \multicolumn{3}{|c|}{$12 \mathrm{M}$} & \multicolumn{3}{|c|}{$12 \mathrm{P}$} & \multicolumn{3}{|c|}{$15 \mathrm{M}$} & \multicolumn{3}{|c|}{$15 \mathrm{P}$} & \multicolumn{3}{|c|}{$20 \mathrm{M}$} & \multicolumn{3}{|c|}{$20 P$} & \multicolumn{3}{|c|}{$30 \mathrm{M}$} & \multicolumn{3}{|c|}{$30 P$} & \multicolumn{3}{|c|}{$60 M$} & \multicolumn{3}{|c|}{$60 P$} \\
\hline & $\mathrm{T}$ & $\mathrm{FE}$ & $\mathrm{RF}$ & $T$ & $\mathrm{~F}$ & $R$ & $\mathrm{~T}$ & $F$ & $\mathrm{R}$ & $\mathrm{T}$ & $F$ & $\mathrm{R}$ & $T$ & $F$ & $\mathrm{R}$ & $\mathrm{T}$ & $F$ & $\mathrm{R}$ & $T$ & $\mathrm{~F}$ & $\mathrm{R}$ & $\mathrm{T}$ & $F$ & $\mathrm{R}$ & $\mathrm{T}$ & $F$ & $\mathrm{R}$ & $\mathrm{T}$ & $\mathrm{F}$ & $\mathrm{R}$ & $\mathrm{T}$ & $F$ & $R$ & $T$ & $\mathrm{~F}$ & $\mathrm{R}$ \\
\hline Test & V & & $E$ & V & $E$ & $\mathrm{~F}$ & V & $E$ & $\mathrm{~F}$ & V & $\mathrm{E}$ & $\mathrm{F}$ & V & $E$ & $\mathrm{~F}$ & V & $E$ & $\mathrm{~F}$ & V & $\mathrm{E}$ & $\mathrm{F}$ & $\mathrm{V}$ & $\mathrm{E}$ & $\mathrm{F}$ & $\mathrm{V}$ & $E$ & $\mathrm{~F}$ & V & $\mathrm{E}$ & $\mathrm{F}$ & $\mathrm{V}$ & $\mathrm{E}$ & $\mathrm{F}$ & V & $E$ & $\mathrm{~F}$ \\
\hline & $E$ & & & $E$ & & $E$ & $E$ & & $\mathrm{E}$ & $E$ & & $E$ & $E$ & & $E$ & $\mathrm{E}$ & & $\mathrm{E}$ & $\mathrm{E}$ & & $\mathrm{E}$ & $E$ & & $\mathrm{E}$ & $E$ & & $\mathrm{E}$ & $E$ & & $E$ & $\mathrm{E}$ & & $E$ & $E$ & & $\mathrm{E}$ \\
\hline SS Freq. Range & $P$ & $\mathrm{P}$ & $\mathrm{P}$ & $P$ & $\mathrm{P}$ & $\mathrm{P}$ & $\mathrm{P}$ & $\mathrm{P}$ & $P$ & $\mathrm{P}$ & $\mathrm{P}$ & $\mathrm{P}$ & $\mathrm{P}$ & $\mathrm{P}$ & $\mathrm{P}$ & $\mathrm{P}$ & $\mathrm{P}$ & $\mathrm{P}$ & $\mathrm{P}$ & $\mathrm{P}$ & $\mathrm{P}$ & $\mathrm{P}$ & $\mathrm{P}$ & $\mathrm{P}$ & $\mathrm{P}$ & $\mathrm{P}$ & $\mathrm{P}$ & $\mathrm{P}$ & $\mathrm{P}$ & $\mathrm{P}$ & $\mathrm{P}$ & $\mathrm{P}$ & $\mathrm{P}$ & $\mathrm{P}$ & $\mathrm{P}$ & $\mathrm{P}$ \\
\hline SS Harmonic & $\mathrm{P}$ & $P$ & - & $P$ & $P$ & $P$ & $P$ & $\mathrm{P}$ & - & $P$ & $\mathrm{P}$ & $\mathrm{P}$ & $\mathrm{P}$ & $\mathrm{P}$ & - & $\mathrm{P}$ & $P$ & $\mathrm{P}$ & $P$ & $\mathrm{P}$ & $\mathrm{P}$ & $P$ & $\mathrm{P}$ & $\mathrm{P}$ & $\mathrm{P}$ & $\mathrm{P}$ & - & $\mathrm{P}$ & $\mathrm{P}$ & - & $\mathrm{P}$ & $\mathrm{P}$ & - & $P$ & $\mathrm{P}$ & $\mathrm{P}$ \\
\hline SS OOB Interf. & $P$ & $P$ & - & - & - & - & $P$ & $P$ & - & - & - & - & $P$ & $P$ & - & - & - & - & $P$ & $\mathrm{P}$ & - & - & - & - & $\mathrm{P}$ & $\mathrm{P}$ & - & - & - & - & $\mathrm{P}$ & $P$ & - & - & - & - \\
\hline SS Magnitude & $\mathrm{P}$ & - & - & $P$ & - & - & $\mathrm{P}$ & - & - & $\mathrm{P}$ & - & - & $\mathrm{P}$ & - & - & $\mathrm{P}$ & - & - & $P$ & - & - & $P$ & - & - & $\mathrm{P}$ & - & - & $\mathrm{P}$ & - & - & $\mathrm{P}$ & - & - & $\mathrm{P}$ & - & - \\
\hline Dyn Ramp & $P$ & $P$ & $\mathrm{P}$ & $P$ & $P$ & $\mathrm{P}$ & $\mathrm{P}$ & $P$ & $P$ & $\mathrm{P}$ & $\mathrm{P}$ & $P$ & $\mathrm{P}$ & $P$ & $\mathrm{P}$ & $\mathrm{P}$ & $\mathrm{P}$ & $\mathrm{P}$ & $P$ & $P$ & $\mathrm{P}$ & $P$ & $P$ & $\mathrm{P}$ & $P$ & $\mathrm{P}$ & $\mathrm{P}$ & $\mathrm{P}$ & $\mathrm{P}$ & $P$ & $\mathrm{P}$ & $P$ & $P$ & $P$ & $P$ & $\mathrm{P}$ \\
\hline Dyn Phase Mod & $\mathrm{P}$ & $\mathrm{P}$ & $\mathrm{P}$ & $\mathrm{P}$ & $\mathrm{P}$ & $P$ & $P$ & $\mathrm{P}$ & $P$ & $P$ & $\mathrm{P}$ & $\mathrm{P}$ & $\mathrm{P}$ & $\mathrm{P}$ & $P$ & $\mathrm{P}$ & $\mathrm{P}$ & $\mathrm{P}$ & $\mathrm{P}$ & $\mathrm{P}$ & $\mathrm{P}$ & $\mathrm{P}$ & $\mathrm{P}$ & $\mathrm{P}$ & $\mathrm{P}$ & $\mathrm{P}$ & $\mathrm{P}$ & $\mathrm{P}$ & $\mathrm{P}$ & $P$ & $\mathrm{P}$ & $P$ & $\mathrm{P}$ & $\mathrm{P}$ & $P$ & $\mathrm{P}$ \\
\hline Dyn Ampl Mod & $P$ & $P$ & $P$ & $P$ & $P$ & $P$ & $P$ & $P$ & $P$ & $\mathrm{P}$ & $P$ & $P$ & $P$ & $P$ & $P$ & $\mathrm{P}$ & $P$ & $\mathrm{P}$ & $P$ & $P$ & $\mathrm{P}$ & $\mathrm{P}$ & $P$ & $\mathrm{P}$ & $\mathrm{P}$ & $\mathrm{P}$ & $P$ & $P$ & $\mathrm{P}$ & $P$ & $P$ & $P$ & $P$ & $P$ & $P$ & $\mathrm{P}$ \\
\hline
\end{tabular}

Table 10: PMU D response time, delay time and overshoot pass/fail

\begin{tabular}{|c|c|c|c|c|c|c|c|c|c|c|c|c|c|}
\hline & Fs (FPS) & $10 \mathrm{M}$ & $10 P$ & $12 \mathrm{M}$ & $12 \mathrm{P}$ & $15 \mathrm{M}$ & $15 \mathrm{P}$ & $20 \mathrm{M}$ & $20 P$ & $30 \mathrm{M}$ & $30 P$ & $60 \mathrm{M}$ & $60 P$ \\
\hline \multirow{5}{*}{ Phase Step } & Phasor Resp. Time & $\mathrm{P}$ & 1 & $\mathrm{P}$ & 1 & $\mathrm{P}$ & 1 & $\mathrm{P}$ & 1 & $\mathrm{P}$ & 1 & $\mathrm{P}$ & 1 \\
\hline & Phasor Delay Time & $\mathrm{P}$ & $\mathrm{P}$ & $\mathrm{P}$ & $\mathrm{P}$ & $\mathrm{P}$ & $\mathrm{P}$ & $\mathrm{P}$ & $\mathrm{P}$ & $\mathrm{P}$ & $\mathrm{P}$ & $\mathrm{P}$ & $\mathrm{P}$ \\
\hline & Phasor Overshoot & $P$ & $P$ & $P$ & $P$ & $P$ & $P$ & $P$ & $P$ & $P$ & $P$ & $P$ & $P$ \\
\hline & Freq Resp. Time & $\mathrm{P}$ & $\mathrm{P}$ & $\mathrm{P}$ & $\mathrm{P}$ & $\mathrm{P}$ & $\mathrm{P}$ & $\mathrm{P}$ & $\mathrm{P}$ & $\mathrm{P}$ & $\mathrm{P}$ & $\mathrm{P}$ & $\mathrm{P}$ \\
\hline & ROCOF Resp. Time & $\mathrm{P}$ & $\mathrm{P}$ & $\mathrm{P}$ & $\mathrm{P}$ & $\mathrm{P}$ & $\mathrm{P}$ & $\mathrm{P}$ & $\mathrm{P}$ & $\mathrm{P}$ & $\mathrm{P}$ & $\mathrm{P}$ & $\mathrm{P}$ \\
\hline & & & & & & & & & & & & & \\
\hline \multirow{3}{*}{$\begin{array}{l}\text { Magnitude } \\
\text { Step }\end{array}$} & Magnitude Overshoot & $\mathrm{P}$ & $\mathrm{P}$ & $P$ & $\mathrm{P}$ & $\mathrm{P}$ & $\mathrm{P}$ & $\mathrm{P}$ & $P$ & $\mathrm{P}$ & $P$ & $\mathrm{P}$ & $\mathrm{P}$ \\
\hline & Freq Resp. Time & $\mathrm{P}$ & $\mathrm{P}$ & $\mathrm{P}$ & $\mathrm{P}$ & $\mathrm{P}$ & $\mathrm{P}$ & $\mathrm{P}$ & $\mathrm{P}$ & $\mathrm{P}$ & $\mathrm{P}$ & $\mathrm{P}$ & $\mathrm{P}$ \\
\hline & ROCOF Resp. Time & $\mathrm{P}$ & $\mathrm{P}$ & $\mathrm{P}$ & $\mathrm{P}$ & $\mathrm{P}$ & $\mathrm{P}$ & $\mathrm{P}$ & $\mathrm{P}$ & $\mathrm{P}$ & $P$ & $\mathrm{P}$ & $\mathrm{P}$ \\
\hline
\end{tabular}




\subsection{PMU E performance overview}

PMU E is a commercially available PMU. PMU E does not support P class. Data for reporting rate Fs = 12 FPS, M class was corrupted and could not be recovered.

Table 11: PMU E TVE, FE, RFE pass/fail

\begin{tabular}{|c|c|c|c|c|c|c|c|c|c|c|c|c|c|c|c|c|c|c|c|c|c|c|c|c|c|c|c|c|c|c|c|c|c|c|c|c|}
\hline Fs (FPS) & \multicolumn{3}{|c|}{$10 \mathrm{M}$} & \multicolumn{3}{|c|}{$10 \mathrm{P}$} & \multicolumn{3}{|c|}{$12 \mathrm{M}$} & \multicolumn{3}{|c|}{$12 \mathrm{P}$} & \multicolumn{3}{|c|}{$15 \mathrm{M}$} & \multicolumn{3}{|c|}{$15 \mathrm{P}$} & \multicolumn{3}{|c|}{$20 \mathrm{M}$} & \multicolumn{3}{|c|}{$20 P$} & \multicolumn{3}{|c|}{$30 \mathrm{M}$} & \multicolumn{3}{|c|}{$30 P$} & \multicolumn{3}{|c|}{$60 \mathrm{M}$} & \multicolumn{3}{|c|}{$60 P$} \\
\hline Test & $\begin{array}{l}\mathrm{T} \\
\mathrm{V} \\
\mathrm{E}\end{array}$ & $\mathrm{FE}$ & $\begin{array}{c}\text { RF } \\
E\end{array}$ & $\begin{array}{l}\mathrm{T} \\
\mathrm{V} \\
\mathrm{E}\end{array}$ & $\begin{array}{l}\mathrm{F} \\
E\end{array}$ & $\begin{array}{l}R \\
F \\
E\end{array}$ & $\begin{array}{l}\mathrm{T} \\
\mathrm{V} \\
\mathrm{E}\end{array}$ & $\begin{array}{l}F \\
E\end{array}$ & $\begin{array}{l}\mathrm{R} \\
\mathrm{F} \\
\mathrm{E}\end{array}$ & $\begin{array}{l}T \\
V \\
E\end{array}$ & $\begin{array}{l}F \\
E\end{array}$ & $\begin{array}{l}R \\
F \\
E\end{array}$ & $\begin{array}{l}\mathrm{T} \\
\mathrm{V} \\
\mathrm{E}\end{array}$ & $\begin{array}{l}F \\
E\end{array}$ & $\begin{array}{l}R \\
F \\
E\end{array}$ & $\begin{array}{l}\mathrm{T} \\
\mathrm{V} \\
\mathrm{E}\end{array}$ & $\begin{array}{l}F \\
E\end{array}$ & $\begin{array}{l}\mathrm{R} \\
\mathrm{F} \\
\mathrm{E}\end{array}$ & $\begin{array}{l}\mathrm{T} \\
\mathrm{V} \\
\mathrm{E}\end{array}$ & $\begin{array}{l}\mathrm{F} \\
\mathrm{E}\end{array}$ & $\begin{array}{l}\text { R } \\
F \\
E\end{array}$ & $\begin{array}{l}T \\
V \\
E\end{array}$ & $\begin{array}{l}F \\
E\end{array}$ & $\begin{array}{l}\mathrm{R} \\
\mathrm{F} \\
\mathrm{E}\end{array}$ & $\begin{array}{l}\mathrm{T} \\
\mathrm{V} \\
\mathrm{E}\end{array}$ & $\begin{array}{l}F \\
E\end{array}$ & $\begin{array}{l}\mathrm{R} \\
\mathrm{F} \\
\mathrm{E}\end{array}$ & $\begin{array}{l}\mathrm{T} \\
\mathrm{V} \\
\mathrm{E}\end{array}$ & $\begin{array}{l}F \\
E\end{array}$ & $\begin{array}{l}\mathrm{R} \\
\mathrm{F} \\
\mathrm{E}\end{array}$ & $\begin{array}{l}\mathrm{T} \\
\mathrm{V} \\
\mathrm{E}\end{array}$ & $\begin{array}{l}F \\
E\end{array}$ & $\begin{array}{l}\text { R } \\
F \\
E\end{array}$ & $\begin{array}{l}\mathrm{T} \\
\mathrm{V} \\
\mathrm{E}\end{array}$ & $\begin{array}{l}F \\
E\end{array}$ & $\begin{array}{l}\text { R } \\
F \\
E\end{array}$ \\
\hline SS Freq. Range & $\mathrm{P}$ & $P$ & $\mathrm{P}$ & - & - & - & - & - & - & - & - & - & $P$ & $P$ & $\mathrm{P}$ & - & - & - & $\mathrm{P}$ & $P$ & $\mathrm{P}$ & - & - & - & $P$ & $\mathrm{P}$ & $P$ & - & - & - & $P$ & $\mathrm{P}$ & $\mathrm{P}$ & - & - & - \\
\hline SS Harmonic & $P$ & $P$ & - & - & - & - & - & - & - & - & - & - & $P$ & $P$ & - & - & - & - & $\mathrm{P}$ & $P$ & - & - & - & - & $P$ & $P$ & - & - & - & - & $P$ & $P$ & - & - & - & - \\
\hline SS OOB Interf. & $\mathrm{F}$ & $\mathrm{F}$ & - & - & - & - & - & - & - & - & - & - & $\mathrm{F}$ & $\mathrm{F}$ & - & - & - & - & $\mathrm{F}$ & $\mathrm{F}$ & - & - & - & - & $\mathrm{F}$ & $\mathrm{F}$ & - & - & - & - & $P$ & $\mathrm{~F}$ & - & - & - & - \\
\hline SS Magnitude & $\mathrm{P}$ & - & - & - & - & - & - & - & - & - & - & - & $\mathrm{P}$ & - & - & - & - & - & $\mathrm{I}$ & - & - & - & - & - & $\mathrm{P}$ & - & - & - & - & - & $\mathrm{F}$ & - & - & - & - & - \\
\hline Dyn Ramp & $\mathrm{P}$ & $\mathrm{F}$ & $\mathrm{P}$ & - & - & - & - & - & - & - & - & - & $\mathrm{P}$ & $\mathrm{F}$ & $\mathrm{P}$ & - & - & - & $\mathrm{P}$ & $\mathrm{F}$ & $\mathrm{P}$ & - & - & - & $\mathrm{P}$ & $\mathrm{F}$ & $\mathrm{P}$ & - & - & - & $\mathrm{P}$ & $\mathrm{F}$ & $\mathrm{P}$ & - & - & - \\
\hline Dyn Phase Mod & $\mathrm{P}$ & 1 & $\mathrm{P}$ & - & - & - & - & - & - & - & - & - & $\mathrm{P}$ & $\mathrm{F}$ & $\mathrm{F}$ & - & - & - & $\mathrm{F}$ & $\mathrm{F}$ & $\mathrm{F}$ & - & - & - & $\mathrm{F}$ & $\mathrm{F}$ & $\mathrm{F}$ & - & - & - & $\mathrm{F}$ & $\mathrm{F}$ & $\mathrm{F}$ & - & - & - \\
\hline Dyn Ampl Mod & $\mathrm{P}$ & $\mathrm{P}$ & $\mathrm{P}$ & - & - & - & - & - & - & - & - & - & $\mathrm{F}$ & $\mathrm{P}$ & $\mathrm{P}$ & - & - & - & $\mathrm{F}$ & $\mathrm{P}$ & $\mathrm{P}$ & - & - & - & $\mathrm{F}$ & $\mathrm{P}$ & $\mathrm{P}$ & - & - & - & $\mathrm{F}$ & $\mathrm{P}$ & $\mathrm{P}$ & - & - & - \\
\hline
\end{tabular}

Table 12: PMU E response time, delay time and overshoot pass/fail

\begin{tabular}{|c|c|c|c|c|c|c|c|c|c|c|c|c|c|}
\hline \multicolumn{2}{|r|}{ Fs (FPS) } & $10 \mathrm{M}$ & $10 P$ & $12 \mathrm{M}$ & $12 \mathrm{P}$ & $15 \mathrm{M}$ & $15 P$ & $20 M$ & $20 P$ & $30 M$ & $30 P$ & $60 M$ & $60 P$ \\
\hline \multirow{5}{*}{ Phase Step } & Phasor Resp. Time & $\mathrm{P}$ & - & - & - & $\mathrm{P}$ & - & $\mathrm{P}$ & - & $\mathrm{P}$ & - & $\mathrm{F}$ & - \\
\hline & Phasor Delay Time & $\mathrm{P}$ & - & - & - & $\mathrm{P}$ & - & $\mathrm{P}$ & - & $P$ & - & $\mathrm{P}$ & - \\
\hline & Phasor Overshoot & $\mathrm{P}$ & - & - & - & $\mathrm{P}$ & - & $\mathrm{P}$ & - & $\mathrm{P}$ & - & $\mathrm{P}$ & - \\
\hline & Freq Resp. Time & $\mathrm{P}$ & - & - & - & $\mathrm{P}$ & - & $\mathrm{P}$ & - & $\mathrm{P}$ & - & $\mathrm{P}$ & - \\
\hline & ROCOF Resp. Time & $\mathrm{P}$ & - & - & - & $\mathrm{P}$ & - & $\mathrm{P}$ & - & $\mathrm{P}$ & - & $\mathrm{P}$ & - \\
\hline & & & & & & & & & & & & & \\
\hline \multirow{3}{*}{$\begin{array}{l}\text { Magnitude } \\
\text { Step }\end{array}$} & Magnitude Overshoot & $\mathrm{P}$ & - & - & - & $\mathrm{P}$ & - & $\mathrm{P}$ & - & $\mathrm{P}$ & - & $\mathrm{P}$ & - \\
\hline & Freq Resp. Time & $\mathrm{P}$ & - & - & - & $\mathrm{P}$ & - & $\mathrm{P}$ & - & $\mathrm{P}$ & - & $\mathrm{P}$ & - \\
\hline & ROCOF Resp. Time & $\mathrm{P}$ & - & - & - & $\mathrm{P}$ & - & $\mathrm{P}$ & - & $\mathrm{P}$ & - & $\mathrm{P}$ & - \\
\hline
\end{tabular}




\subsection{PMU F performance overview}

$\mathrm{PMU} F$ is a prototype PMU and is not yet commercially available.

\section{Table 13: PMU F TVE, FE, RFE pass/fail}

\begin{tabular}{|c|c|c|c|c|c|c|c|c|c|c|c|c|c|c|c|c|c|c|c|c|c|c|c|c|c|c|c|c|c|c|c|c|c|c|c|c|}
\hline Fs (FPS) & \multicolumn{3}{|c|}{$10 \mathrm{M}$} & \multicolumn{3}{|c|}{$10 P$} & \multicolumn{3}{|c|}{$12 \mathrm{M}$} & \multicolumn{3}{|c|}{$12 \mathrm{P}$} & \multicolumn{3}{|c|}{$15 \mathrm{M}$} & \multicolumn{3}{|c|}{$15 \mathrm{P}$} & \multicolumn{3}{|c|}{$20 \mathrm{M}$} & \multicolumn{3}{|c|}{$20 P$} & \multicolumn{3}{|c|}{$30 \mathrm{M}$} & \multicolumn{3}{|c|}{$30 P$} & \multicolumn{3}{|c|}{$60 M$} & \multicolumn{3}{|c|}{$60 P$} \\
\hline & $T$ & $\mathrm{FE}$ & $\mathrm{RF}$ & $\mathrm{T}$ & $F$ & $\mathrm{R}$ & $\mathrm{T}$ & $\mathrm{F}$ & $R$ & $\mathrm{~T}$ & $\mathrm{~F}$ & $\mathrm{R}$ & $\mathrm{T}$ & $F$ & $R$ & $\mathrm{~T}$ & $F$ & $R$ & $\mathrm{~T}$ & $F$ & $R$ & $T$ & $F$ & $\mathrm{R}$ & $\mathrm{T}$ & $F$ & $R$ & $\mathrm{~T}$ & $F$ & $R$ & $\mathrm{~T}$ & $F$ & $\mathrm{R}$ & $T$ & $F$ & $R$ \\
\hline Test & $\mathrm{V}$ & & $E$ & V & $E$ & $\mathrm{~F}$ & V & $\mathrm{E}$ & $\mathrm{F}$ & V & $E$ & $\mathrm{~F}$ & V & $E$ & $\mathrm{~F}$ & V & $E$ & $\mathrm{~F}$ & V & $E$ & $\mathrm{~F}$ & V & $E$ & $\mathrm{~F}$ & V & $E$ & $\mathrm{~F}$ & V & $E$ & $\mathrm{~F}$ & V & $\mathrm{E}$ & $\mathrm{F}$ & $\mathrm{V}$ & $E$ & $\mathrm{~F}$ \\
\hline & $E$ & & & $E$ & & $E$ & $\mathrm{E}$ & & $E$ & $E$ & & $E$ & $E$ & & $E$ & $E$ & & $E$ & $E$ & & $E$ & $\mathrm{E}$ & & $E$ & $E$ & & $E$ & $E$ & & $\mathrm{E}$ & $E$ & & $\mathrm{E}$ & $E$ & & $\mathrm{E}$ \\
\hline SS Freq. Range & $P$ & $\mathrm{P}$ & 1 & $\mathrm{P}$ & $\mathrm{P}$ & $\mathrm{F}$ & $\mathrm{P}$ & $\mathrm{P}$ & $\mathrm{F}$ & $\mathrm{P}$ & $\mathrm{P}$ & $\mathrm{F}$ & $\mathrm{P}$ & $\mathrm{P}$ & $\mathrm{F}$ & $\mathrm{P}$ & $\mathrm{P}$ & $\mathrm{F}$ & $\mathrm{P}$ & $\mathrm{P}$ & $\mathrm{F}$ & $\mathrm{P}$ & $\mathrm{P}$ & $\mathrm{F}$ & $\mathrm{P}$ & $P$ & I & $\mathrm{P}$ & $\mathrm{P}$ & $\mathrm{F}$ & $\mathrm{P}$ & $\mathrm{P}$ & $\mathrm{F}$ & $\mathrm{P}$ & $\mathrm{P}$ & $\mathrm{P}$ \\
\hline SS Harmonic & $\mathrm{P}$ & - & - & $P$ & - & - & $\mathrm{P}$ & - & - & $\mathrm{P}$ & - & - & $P$ & - & - & $P$ & - & - & $\mathrm{P}$ & - & - & $\mathrm{P}$ & - & - & $P$ & - & - & $P$ & - & - & $\mathrm{P}$ & - & - & $\mathrm{P}$ & - & - \\
\hline SS OOB Interf. & $\mathrm{P}$ & $\mathrm{P}$ & - & $\mathrm{P}$ & $\mathrm{P}$ & $\mathrm{P}$ & $\mathrm{P}$ & $\mathrm{P}$ & - & $\mathrm{P}$ & $P$ & $\mathrm{P}$ & $\mathrm{P}$ & $\mathrm{P}$ & - & $P$ & $\mathrm{P}$ & $\mathrm{P}$ & $\mathrm{P}$ & $\mathrm{P}$ & - & $\mathrm{P}$ & $\mathrm{P}$ & $\mathrm{P}$ & $\mathrm{P}$ & $\mathrm{P}$ & - & $\mathrm{P}$ & $P$ & $\mathrm{P}$ & $\mathrm{P}$ & $P$ & - & $\mathrm{P}$ & $\mathrm{P}$ & $P$ \\
\hline SS Magnitude & $P$ & $\mathrm{P}$ & - & - & - & - & $\mathrm{P}$ & 1 & - & - & - & - & $\mathrm{P}$ & 1 & - & - & - & - & $\mathrm{P}$ & $\mathrm{F}$ & - & - & - & - & $\mathrm{P}$ & $\mathrm{F}$ & - & - & - & - & $\mathrm{P}$ & $\mathrm{F}$ & - & - & - & - \\
\hline Dyn Ramp & $\mathrm{P}$ & $\mathrm{P}$ & $\mathrm{F}$ & $\mathrm{P}$ & $\mathrm{P}$ & $\mathrm{P}$ & $\mathrm{P}$ & $\mathrm{P}$ & $\mathrm{P}$ & $\mathrm{P}$ & $\mathrm{P}$ & $\mathrm{P}$ & $\mathrm{P}$ & $\mathrm{P}$ & $\mathrm{F}$ & $\mathrm{P}$ & $\mathrm{P}$ & $\mathrm{P}$ & $\mathrm{P}$ & $\mathrm{P}$ & $\mathrm{F}$ & $\mathrm{P}$ & $\mathrm{P}$ & $\mathrm{P}$ & $\mathrm{P}$ & $\mathrm{P}$ & $\mathrm{F}$ & $\mathrm{P}$ & $\mathrm{P}$ & $\mathrm{P}$ & $\mathrm{P}$ & $\mathrm{P}$ & $\mathrm{F}$ & $\mathrm{P}$ & $\mathrm{P}$ & $\mathrm{P}$ \\
\hline Dyn Phase Mod & $\mathrm{P}$ & $\mathrm{P}$ & $\mathrm{F}$ & $P$ & $\mathrm{P}$ & $P$ & $\mathrm{P}$ & $\mathrm{P}$ & $\mathrm{F}$ & $P$ & $P$ & $P$ & 1 & $\mathrm{P}$ & $\mathrm{F}$ & $P$ & $\mathrm{P}$ & $P$ & $P$ & $\mathrm{P}$ & $\mathrm{P}$ & $\mathrm{P}$ & $\mathrm{P}$ & $\mathrm{P}$ & $P$ & $P$ & $\mathrm{P}$ & $P$ & $P$ & $\mathrm{P}$ & $\mathrm{P}$ & $\mathrm{P}$ & $\mathrm{P}$ & $\mathrm{P}$ & $\mathrm{P}$ & $P$ \\
\hline Dyn Ampl Mod & $P$ & $\mathrm{P}$ & $P$ & $P$ & $\mathrm{P}$ & $P$ & $\mathrm{~F}$ & $\mathrm{P}$ & $P$ & $P$ & $P$ & $P$ & $\mathrm{~F}$ & $P$ & $P$ & $P$ & $P$ & $P$ & $P$ & $\mathrm{P}$ & $P$ & $\mathrm{P}$ & $P$ & $\mathrm{P}$ & $P$ & $P$ & $\mathrm{P}$ & $\mathrm{P}$ & $P$ & $P$ & $\mathrm{P}$ & $P$ & $\mathrm{P}$ & $P$ & $\mathrm{P}$ & $P$ \\
\hline
\end{tabular}

Table 14: PMU F response time, delay time and overshoot pass/fail

\begin{tabular}{|c|c|c|c|c|c|c|c|c|c|c|c|c|c|}
\hline \multicolumn{2}{|r|}{ Fs (FPS) } & $10 \mathrm{M}$ & $10 \mathrm{P}$ & $12 \mathrm{M}$ & $12 \mathrm{P}$ & $15 \mathrm{M}$ & $15 P$ & $20 \mathrm{M}$ & $20 P$ & $30 \mathrm{M}$ & $30 P$ & $60 \mathrm{M}$ & $60 P$ \\
\hline \multirow{4}{*}{ Phase Step } & Phasor Resp. Time & $\mathrm{P}$ & $P$ & $P$ & $P$ & $P$ & $P$ & $P$ & $P$ & $\mathrm{P}$ & $P$ & $P$ & $P$ \\
\hline & Phasor Delay Time & $\mathrm{P}$ & $\mathrm{P}$ & $P$ & $\mathrm{P}$ & $\mathrm{P}$ & $P$ & $P$ & $P$ & $P$ & $P$ & $\mathrm{P}$ & $\mathrm{P}$ \\
\hline & Freq Resp. Time & $\mathrm{P}$ & I & $\mathrm{P}$ & $\mathrm{P}$ & $\mathrm{P}$ & $\mathrm{F}$ & $\mathrm{P}$ & $\mathrm{F}$ & $P$ & $\mathrm{~F}$ & $\mathrm{P}$ & $\mathrm{F}$ \\
\hline & ROCOF Resp. Time & $P$ & $\mathrm{~F}$ & $\mathrm{P}$ & I & $P$ & $\mathrm{~F}$ & $P$ & $\mathrm{~F}$ & $\mathrm{P}$ & $\mathrm{F}$ & $\mathrm{P}$ & $\mathrm{F}$ \\
\hline \multirow{4}{*}{$\begin{array}{l}\text { Magnitude } \\
\text { Step }\end{array}$} & Phasor Delay Time & $P$ & $\mathrm{P}$ & $P$ & $P$ & $P$ & $P$ & $P$ & $P$ & $P$ & $P$ & $P$ & $\mathrm{P}$ \\
\hline & Magnitude Overshoot & $P$ & $\mathrm{P}$ & $\mathrm{P}$ & $P$ & $P$ & $\mathrm{P}$ & $P$ & $\mathrm{P}$ & $\mathrm{P}$ & $P$ & $P$ & $P$ \\
\hline & Freq Resp. Time & $P$ & $P$ & $P$ & $P$ & $P$ & $\mathrm{~F}$ & $P$ & 1 & $P$ & I & $P$ & $\mathrm{~F}$ \\
\hline & ROCOF Resp. Time & $P$ & $P$ & $P$ & $\mathrm{P}$ & $P$ & $\mathrm{~F}$ & $P$ & & $P$ & $P$ & $P$ & $\mathrm{~F}$ \\
\hline
\end{tabular}




\subsection{PMU G performance overview}

PMU G is a commercially available PMU. PMU G does not support P class. PMU G ROCOF measurements are always equal to 0.

Table 15: PMU G TVE, FE, RFE pass/fail

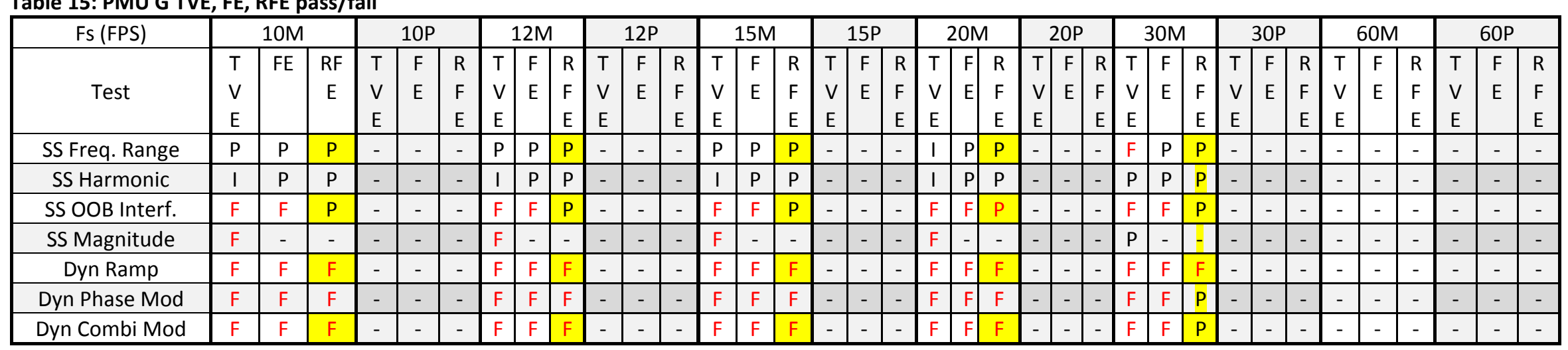

Table 16: PMU G response time, delay time and overshoot pass/fail

\begin{tabular}{|c|c|c|c|c|c|c|c|c|c|c|c|c|c|}
\hline \multicolumn{2}{|r|}{ Fs (FPS) } & $10 \mathrm{M}$ & $10 P$ & $12 \mathrm{M}$ & $12 \mathrm{P}$ & $15 \mathrm{M}$ & $15 P$ & $20 M$ & $20 P$ & $30 \mathrm{M}$ & $30 P$ & $60 M$ & $60 P$ \\
\hline \multirow{5}{*}{ Phase Step } & Phasor Resp. Time & $\mathrm{F}$ & - & $P$ & - & $P$ & - & $\mathrm{F}$ & - & $\mathrm{F}$ & - & - & - \\
\hline & Phasor Delay Time & $P$ & - & $P$ & - & $P$ & - & $P$ & - & $P$ & - & - & - \\
\hline & Phasor Overshoot & $\mathrm{F}$ & - & $\mathrm{F}$ & - & $\mathrm{F}$ & - & $\mathrm{F}$ & - & $\mathrm{F}$ & - & - & - \\
\hline & Freq Resp. Time & $\mathrm{P}$ & - & $\mathrm{P}$ & - & $\mathrm{P}$ & - & $\mathrm{P}$ & - & $\mathrm{P}$ & - & - & - \\
\hline & ROCOF Resp. Time & $P$ & - & $P$ & - & $P$ & - & $P$ & - & $P$ & - & - & - \\
\hline & & & & & & & & & & & & & \\
\hline \multirow{3}{*}{$\begin{array}{c}\text { Magnitude } \\
\text { Step }\end{array}$} & Magnitude Overshoot & $\mathrm{F}$ & - & $\mathrm{F}$ & - & $\mathrm{F}$ & - & $P$ & - & $\mathrm{F}$ & - & - & - \\
\hline & Freq Resp. Time & $\mathrm{P}$ & - & $\mathrm{P}$ & - & $\mathrm{P}$ & - & $\mathrm{P}$ & - & $\mathrm{P}$ & - & - & - \\
\hline & ROCOF Resp. Time & $P$ & - & $P$ & - & $P$ & - & $P$ & - & $P$ & - & - & - \\
\hline
\end{tabular}




\subsection{PMU H performance overview}

$\mathrm{PMU} \mathrm{H}$ is a prototype PMU and is not yet commercially available.

Table 17: PMU H TVE, FE, RFE pass/fail

\begin{tabular}{|c|c|c|c|c|c|c|c|c|c|c|c|c|c|c|c|c|c|c|c|c|c|c|c|c|c|c|c|c|c|c|c|c|c|c|c|c|}
\hline Fs (FPS) & \multicolumn{3}{|c|}{$10 \mathrm{M}$} & \multicolumn{3}{|c|}{$10 \mathrm{P}$} & \multicolumn{3}{|c|}{$12 \mathrm{M}$} & \multicolumn{3}{|c|}{$12 \mathrm{P}$} & \multicolumn{3}{|c|}{$15 \mathrm{M}$} & \multicolumn{3}{|c|}{$15 P$} & \multicolumn{3}{|c|}{$20 \mathrm{M}$} & \multicolumn{3}{|c|}{$20 P$} & \multicolumn{3}{|c|}{$30 M$} & \multicolumn{3}{|c|}{$30 P$} & \multicolumn{3}{|c|}{$60 M$} & \multicolumn{3}{|c|}{$60 P$} \\
\hline & $\mathrm{T}$ & FE & RF & $T$ & $\mathrm{~F}$ & $\mathrm{R}$ & $\mathrm{T}$ & $\mathrm{F}$ & $\mathrm{R}$ & $T$ & $\mathrm{~F}$ & $R$ & $\mathrm{~T}$ & $\mathrm{~F}$ & $\mathrm{R}$ & $\bar{T}$ & $\mathrm{~F}$ & $\mathrm{R}$ & $T$ & $\mathrm{~F}$ & $\mathrm{R}$ & $\mathrm{T}$ & $F$ & $R$ & $\mathrm{~T}$ & $\mathrm{~F}$ & $\mathrm{R}$ & $\mathrm{T}$ & $F$ & $\mathrm{R}$ & $T$ & $\mathrm{~F}$ & $\mathrm{R}$ & $\mathrm{T}$ & $\mathrm{F}$ & $\mathrm{R}$ \\
\hline Test & V & & $E$ & V & $E$ & $\mathrm{~F}$ & $\mathrm{~V}$ & $E$ & $\mathrm{~F}$ & V & $E$ & $\mathrm{~F}$ & V & $E$ & $\mathrm{~F}$ & V & $E$ & $\mathrm{~F}$ & $\mathrm{~V}$ & $E$ & $\mathrm{~F}$ & $\mathrm{~V}$ & $E$ & $\mathrm{~F}$ & $\mathrm{v}$ & $\mathrm{E}$ & $\mathrm{F}$ & $\mathrm{V}$ & $E$ & $\mathrm{~F}$ & V & $E$ & $\mathrm{~F}$ & V & $\mathrm{E}$ & $\mathrm{F}$ \\
\hline & $E$ & & & $E$ & & $E$ & $\mathrm{E}$ & & $E$ & $E$ & & $E$ & $E$ & & $E$ & $E$ & & $E$ & $E$ & & $E$ & $E$ & & $\mathrm{E}$ & $E$ & & $E$ & $E$ & & $E$ & $E$ & & $E$ & $E$ & & $E$ \\
\hline SS Freq. Range & $P$ & $P$ & $P$ & $\mathrm{P}$ & $P$ & $P$ & $\mathrm{P}$ & $P$ & $P$ & $\mathrm{P}$ & $P$ & $P$ & $P$ & $P$ & $P$ & $P$ & $P$ & $P$ & $P$ & $P$ & $P$ & $P$ & $\mathrm{P}$ & $\mathrm{P}$ & $P$ & $\mathrm{P}$ & $\mathrm{P}$ & $P$ & $P$ & $P$ & $\mathrm{P}$ & $P$ & $P$ & $P$ & $P$ & $P$ \\
\hline SS Harmonic & $\mathrm{P}$ & $P$ & - & $\mathrm{P}$ & $\mathrm{P}$ & $\mathrm{P}$ & $\mathrm{P}$ & $\mathrm{P}$ & - & $\mathrm{P}$ & $\mathrm{P}$ & $P$ & $\mathrm{P}$ & $P$ & - & $\mathrm{P}$ & $P$ & $\mathrm{P}$ & $\mathrm{P}$ & $\mathrm{P}$ & - & $\mathrm{P}$ & $\mathrm{P}$ & $\mathrm{P}$ & $\mathrm{P}$ & $\mathrm{P}$ & - & $\mathrm{P}$ & $\mathrm{P}$ & $\mathrm{P}$ & $\mathrm{P}$ & $\mathrm{P}$ & - & $P$ & $\mathrm{P}$ & $\mathrm{P}$ \\
\hline SS OOB Interf. & $\mathrm{P}$ & $P$ & - & - & - & - & $\mathrm{P}$ & $\mathrm{P}$ & $P$ & - & - & - & $\mathrm{P}$ & $P$ & - & - & - & - & $\mathrm{P}$ & $\mathrm{P}$ & - & - & - & - & $\mathrm{P}$ & $\mathrm{P}$ & - & - & - & - & $\mathrm{P}$ & $\mathrm{P}$ & - & - & - & - \\
\hline SS Magnitude & $P$ & - & - & $P$ & - & - & $P$ & - & - & $P$ & - & - & $P$ & - & - & $P$ & - & - & $P$ & - & - & $P$ & - & - & $\mathrm{P}$ & - & - & $\mathrm{P}$ & - & - & $P$ & - & - & $P$ & - & - \\
\hline Dyn Ramp & $\mathrm{P}$ & $\mathrm{P}$ & $\mathrm{P}$ & $\mathrm{P}$ & $\mathrm{P}$ & $\mathrm{P}$ & $\mathrm{P}$ & $\mathrm{P}$ & $\mathrm{P}$ & $\mathrm{P}$ & $\mathrm{P}$ & $\mathrm{P}$ & $\mathrm{P}$ & $P$ & $\mathrm{P}$ & $\mathrm{P}$ & $\mathrm{P}$ & $\mathrm{P}$ & $\mathrm{P}$ & $\mathrm{P}$ & $\mathrm{P}$ & $\mathrm{P}$ & $\mathrm{P}$ & $\mathrm{P}$ & $\mathrm{P}$ & $\mathrm{P}$ & $\mathrm{P}$ & $\mathrm{P}$ & $\mathrm{P}$ & $\mathrm{P}$ & $\mathrm{P}$ & $\mathrm{P}$ & $\mathrm{P}$ & $P$ & $\mathrm{P}$ & $\mathrm{P}$ \\
\hline Dyn Phase Mod & $\mathrm{P}$ & $P$ & $P$ & $P$ & $\mathrm{P}$ & $P$ & $\mathrm{P}$ & $\mathrm{P}$ & $P$ & $\mathrm{P}$ & $\mathrm{P}$ & $P$ & $P$ & $P$ & $\mathrm{P}$ & $P$ & $P$ & $P$ & $P$ & $P$ & $P$ & $\mathrm{P}$ & $\mathrm{P}$ & $\mathrm{P}$ & $P$ & $\mathrm{P}$ & $\mathrm{P}$ & $\mathrm{P}$ & $\mathrm{P}$ & $\mathrm{P}$ & $\mathrm{P}$ & $\mathrm{P}$ & $P$ & $P$ & $P$ & $\mathrm{P}$ \\
\hline Dyn Ampl Mod & $P$ & $P$ & $P$ & $P$ & $P$ & $P$ & $\mathrm{P}$ & $P$ & $\mathrm{P}$ & $P$ & $P$ & $P$ & $P$ & $P$ & $P$ & $P$ & $P$ & $P$ & $\mathrm{P}$ & $\mathrm{P}$ & $P$ & $\mathrm{P}$ & $\mathrm{P}$ & $\mathrm{P}$ & $\mathrm{P}$ & $\mathrm{P}$ & $\mathrm{P}$ & $P$ & $\mathrm{P}$ & $P$ & $\mathrm{P}$ & $P$ & $\mathrm{P}$ & $P$ & $P$ & $P$ \\
\hline
\end{tabular}

Table 18: PMU H response time, delay time and overshoot pass/fail

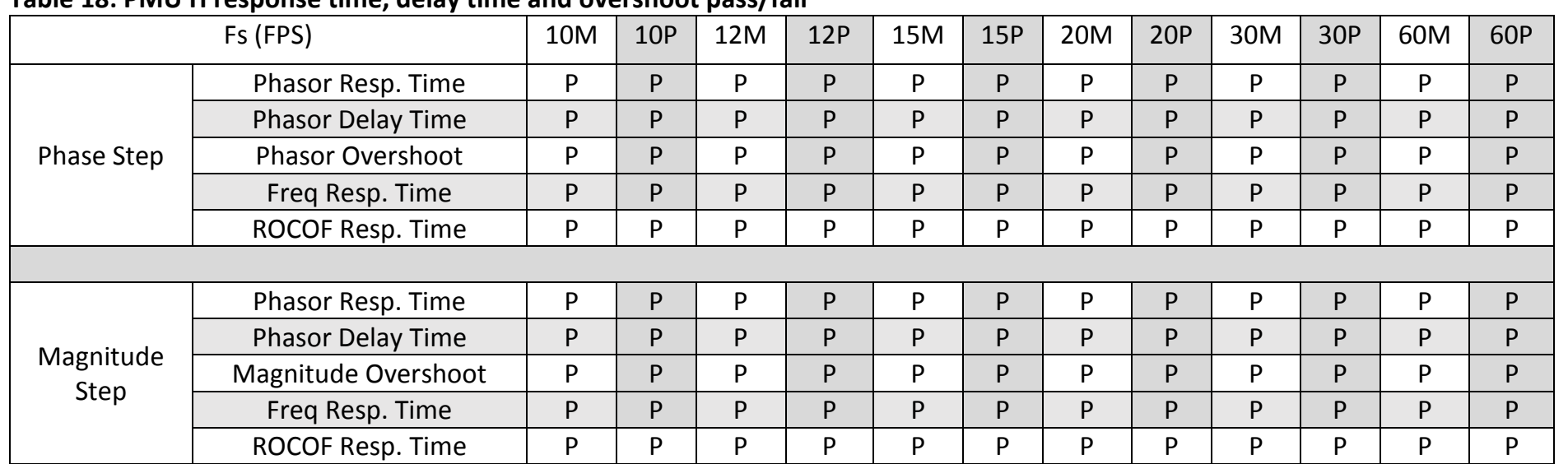




\subsection{PMU I performance overview}

PMI I is a commercially available PMU. PMU I does not support P class.

Table 19: PMU I TVE, FE, RFE pass/fail

\begin{tabular}{|c|c|c|c|c|c|c|c|c|c|c|c|c|c|c|c|c|c|c|c|c|c|c|c|c|c|c|c|c|c|c|c|c|c|c|c|c|}
\hline Fs (FPS) & \multicolumn{3}{|c|}{$10 \mathrm{M}$} & \multicolumn{3}{|c|}{$10 \mathrm{P}$} & \multicolumn{3}{|c|}{$12 \mathrm{M}$} & \multicolumn{3}{|c|}{$12 \mathrm{P}$} & \multicolumn{3}{|c|}{$15 \mathrm{M}$} & \multicolumn{3}{|c|}{$15 \mathrm{P}$} & \multicolumn{3}{|c|}{$20 \mathrm{M}$} & \multicolumn{3}{|c|}{$20 P$} & \multicolumn{3}{|c|}{$30 \mathrm{M}$} & \multicolumn{3}{|c|}{$30 P$} & \multicolumn{3}{|c|}{$60 M$} & \multicolumn{3}{|c|}{$60 P$} \\
\hline Test & $\begin{array}{l}\mathrm{T} \\
\mathrm{V} \\
\mathrm{E}\end{array}$ & $\mathrm{FE}$ & $\begin{array}{c}\text { RF } \\
E\end{array}$ & $\begin{array}{l}\mathrm{T} \\
\mathrm{V} \\
\mathrm{E}\end{array}$ & $\begin{array}{l}F \\
E\end{array}$ & $\begin{array}{l}R \\
F \\
E\end{array}$ & $\begin{array}{l}T \\
V \\
E\end{array}$ & $\begin{array}{l}F \\
E\end{array}$ & $\begin{array}{l}R \\
F \\
E\end{array}$ & $\begin{array}{l}T \\
V \\
E\end{array}$ & $\begin{array}{l}F \\
E\end{array}$ & $\begin{array}{l}R \\
F \\
E\end{array}$ & $\begin{array}{l}\mathrm{T} \\
\mathrm{V} \\
\mathrm{E}\end{array}$ & $\begin{array}{l}F \\
E\end{array}$ & $\begin{array}{l}R \\
F \\
E\end{array}$ & $\begin{array}{l}\mathrm{T} \\
\mathrm{V} \\
\mathrm{E}\end{array}$ & $\begin{array}{l}F \\
E\end{array}$ & $\begin{array}{l}R \\
F \\
E\end{array}$ & $\begin{array}{l}\mathrm{T} \\
\mathrm{V} \\
\mathrm{E}\end{array}$ & $\begin{array}{l}F \\
E\end{array}$ & $\begin{array}{l}R \\
F \\
E\end{array}$ & $\begin{array}{l}\mathrm{T} \\
\mathrm{V} \\
\mathrm{E}\end{array}$ & $\begin{array}{l}F \\
E\end{array}$ & $\begin{array}{l}\mathrm{R} \\
\mathrm{F} \\
\mathrm{E}\end{array}$ & $\begin{array}{l} \\
V \\
E\end{array}$ & $\begin{array}{l}F \\
E\end{array}$ & $\begin{array}{l}\mathrm{R} \\
\mathrm{F} \\
\mathrm{E}\end{array}$ & $\begin{array}{l}\mathrm{T} \\
\mathrm{V} \\
\mathrm{E}\end{array}$ & \begin{tabular}{|l|}
$F$ \\
$E$
\end{tabular} & $\begin{array}{l}\mathrm{R} \\
\mathrm{F} \\
\mathrm{E}\end{array}$ & \begin{tabular}{|c|}
$\mathrm{T}$ \\
$\mathrm{V}$ \\
$\mathrm{E}$ \\
\end{tabular} & $\begin{array}{l}F \\
E\end{array}$ & $\begin{array}{l}\mathrm{R} \\
\mathrm{F} \\
\mathrm{E}\end{array}$ & $\begin{array}{c}\mathrm{T} \\
\mathrm{V} \\
\mathrm{E}\end{array}$ & $\begin{array}{l}F \\
E\end{array}$ & $\begin{array}{l}R \\
F \\
E\end{array}$ \\
\hline SS Freq. Range & $\mathrm{P}$ & $\mathrm{P}$ & $\mathrm{P}$ & - & - & - & $\mathrm{P}$ & $\mathrm{P}$ & $\mathrm{P}$ & - & - & - & $\mathrm{P}$ & $\mathrm{P}$ & $\mathrm{P}$ & - & - & - & $\mathrm{P}$ & $\mathrm{P}$ & $\mathrm{P}$ & - & - & - & $\mathrm{P}$ & $\mathrm{P}$ & $\mathrm{F}$ & - & - & - & $\mathrm{P}$ & $\mathrm{P}$ & & - & - & - \\
\hline SS Harmonic & $\mathrm{F}$ & $\mathrm{P}$ & - & - & - & - & $\mathrm{F}$ & 1 & - & - & - & - & $\mathrm{P}$ & $\mathrm{P}$ & - & - & - & - & $\mathrm{P}$ & $\mathrm{P}$ & - & - & - & - & $\mathrm{P}$ & $\mathrm{P}$ & - & - & - & - & $P$ & $\mathrm{P}$ & - & - & - & - \\
\hline SS OOB Interf. & $\mathrm{P}$ & $\mathrm{F}$ & - & - & - & - & $P$ & $\mathrm{~F}$ & - & - & - & - & $P$ & $\mathrm{~F}$ & - & - & - & - & $\mathrm{P}$ & $\mathrm{F}$ & - & - & - & - & $\mathrm{P}$ & $\mathrm{F}$ & - & - & - & - & $P$ & $\mathrm{~F}$ & - & - & - & - \\
\hline SS Magnitude & $\mathrm{P}$ & - & - & - & - & - & $P$ & - & - & - & - & - & $P$ & - & - & - & - & - & $\mathrm{P}$ & - & - & - & - & - & $\mathrm{P}$ & - & - & - & - & - & $\mathrm{P}$ & - & - & - & - & - \\
\hline Dyn Ramp & $\mathrm{F}$ & $\mathrm{F}$ & $\mathrm{P}$ & - & - & - & $\mathrm{F}$ & $\mathrm{F}$ & $\mathrm{P}$ & - & - & - & $\mathrm{F}$ & $\mathrm{F}$ & $\mathrm{P}$ & - & - & - & $\mathrm{F}$ & $\mathrm{F}$ & $\mathrm{F}$ & - & - & - & $\mathrm{P}$ & $\mathrm{F}$ & $\mathrm{P}$ & - & - & - & $P$ & $\mathrm{~F}$ & $\mathrm{~F}$ & - & - & - \\
\hline Dyn Phase Mod & $\mathrm{F}$ & 1 & 1 & - & - & - & $\mathrm{F}$ & 1 & I & - & - & - & $\mathrm{F}$ & I & 1 & - & - & - & $\mathrm{F}$ & 1 & 1 & - & - & - & $\mathrm{F}$ & $\mathrm{P}$ & 1 & - & - & - & $\mathrm{P}$ & $\mathrm{P}$ & $\mathrm{P}$ & - & - & - \\
\hline Dyn Combi Mod & $\mathrm{F}$ & $\mathrm{P}$ & $\mathrm{P}$ & - & - & - & $\mathrm{F}$ & $\mathrm{P}$ & $\mathrm{P}$ & - & - & - & $\mathrm{F}$ & $\mathrm{P}$ & $\mathrm{P}$ & - & - & - & $\mathrm{F}$ & $\mathrm{P}$ & $\mathrm{P}$ & - & - & - & $\mathrm{F}$ & $\mathrm{P}$ & $\mathrm{P}$ & - & - & - & $\mathrm{P}$ & $\mathrm{P}$ & $\mathrm{P}$ & - & - & - \\
\hline
\end{tabular}

Table 20: PMU I response time, delay time and overshoot pass/fail

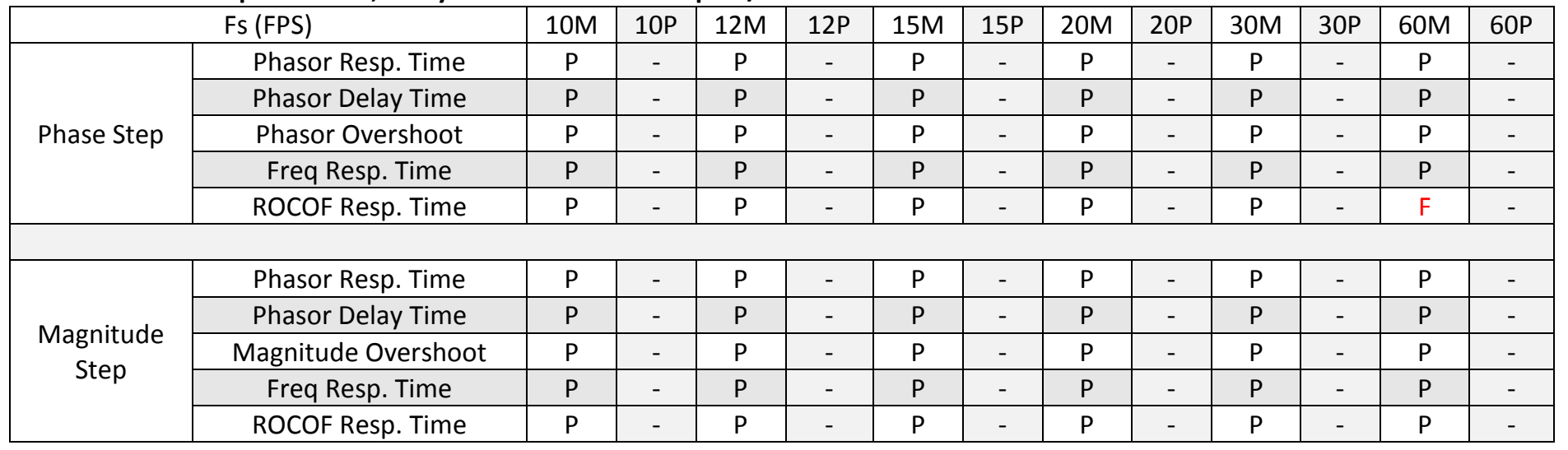




\subsection{PMU J performance overview}

PMU J is a prototype PMU and is not yet commercially available.

Table 21: PMU J TVE, FE, RFE pass/fail

\begin{tabular}{|c|c|c|c|c|c|c|c|c|c|c|c|c|c|c|c|c|c|c|c|c|c|c|c|c|c|c|c|c|c|c|c|c|c|c|c|c|}
\hline Fs (FPS) & \multicolumn{3}{|c|}{$10 \mathrm{M}$} & \multicolumn{3}{|c|}{$10 P$} & \multicolumn{3}{|c|}{$12 \mathrm{M}$} & \multicolumn{3}{|c|}{$12 \mathrm{P}$} & \multicolumn{3}{|c|}{$15 \mathrm{M}$} & \multicolumn{3}{|c|}{$15 \mathrm{P}$} & \multicolumn{3}{|c|}{$20 M$} & \multicolumn{3}{|c|}{$20 P$} & \multicolumn{3}{|c|}{$30 \mathrm{M}$} & \multicolumn{3}{|c|}{$30 P$} & \multicolumn{3}{|c|}{$60 \mathrm{M}$} & \multicolumn{3}{|c|}{$60 P$} \\
\hline & $T$ & FE & $\mathrm{RF}$ & $T$ & $\mathrm{~F}$ & $\mathrm{R}$ & $T$ & $\mathrm{~F}$ & $\mathrm{R}$ & $\mathrm{T}$ & $\mathrm{F}$ & $\mathrm{R}$ & $\mathrm{T}$ & $\mathrm{F}$ & $\mathrm{R}$ & $\mathrm{T}$ & $\mathrm{F}$ & $\mathrm{R}$ & $\mathrm{T}$ & $\mathrm{F}$ & $\mathrm{R}$ & $\mathrm{T}$ & $\mathrm{F}$ & $\mathrm{R}$ & $\mathrm{T}$ & $\mathrm{F}$ & $\mathrm{R}$ & $T$ & $\mathrm{~F}$ & $\mathrm{R}$ & $\mathrm{T}$ & $\mathrm{F}$ & $\mathrm{R}$ & $\mathrm{T}$ & $\mathrm{F}$ & $\mathrm{R}$ \\
\hline Test & $\mathrm{V}$ & & $\mathrm{E}$ & V & $\mathrm{E}$ & $\mathrm{F}$ & V & $\mathrm{E}$ & $\mathrm{F}$ & V & $\mathrm{E}$ & $\mathrm{F}$ & V & $E$ & $\mathrm{~F}$ & $\mathrm{~V}$ & $E$ & $\mathrm{~F}$ & $\mathrm{~V}$ & $\mathrm{E}$ & $\mathrm{F}$ & $\mathrm{V}$ & $E$ & $\mathrm{~F}$ & $\mathrm{~V}$ & $E$ & $\mathrm{~F}$ & $\mathrm{~V}$ & $E$ & $\mathrm{~F}$ & $\mathrm{~V}$ & $E$ & $\mathrm{~F}$ & $\mathrm{~V}$ & $\mathrm{E}$ & $\Gamma$ \\
\hline & $\mathrm{E}$ & & & $\mathrm{E}$ & & $E$ & $\mathrm{E}$ & & $E$ & $\mathrm{E}$ & & $E$ & $E$ & & $E$ & $\mathrm{E}$ & & $E$ & $\mathrm{E}$ & & $\mathrm{E}$ & $\mathrm{E}$ & & $E$ & $\mathrm{E}$ & & $\mathrm{E}$ & $\mathrm{E}$ & & $\mathrm{E}$ & $\mathrm{E}$ & & $E$ & $E$ & & $E$ \\
\hline SS Freq. Range & $P$ & $P$ & $P$ & $P$ & $P$ & $P$ & $P$ & $P$ & $P$ & $P$ & $P$ & $P$ & $P$ & $P$ & $P$ & $\mathrm{P}$ & $P$ & $P$ & $P$ & $P$ & $P$ & $P$ & $P$ & $P$ & $P$ & $P$ & $P$ & $P$ & $P$ & $\mathrm{P}$ & $P$ & $P$ & $P$ & $P$ & $P$ & $P$ \\
\hline SS Harmonic & $\mathrm{P}$ & $P$ & $\mathrm{P}$ & $\mathrm{P}$ & $P$ & $P$ & $\mathrm{P}$ & $\mathrm{P}$ & $P$ & $P$ & $P$ & $P$ & $P$ & $\mathrm{P}$ & - & $\mathrm{P}$ & $\mathrm{P}$ & $P$ & $\mathrm{P}$ & $P$ & - & $\mathrm{P}$ & $\mathrm{P}$ & $P$ & $\mathrm{P}$ & $P$ & - & $\mathrm{P}$ & $P$ & $\mathrm{P}$ & $P$ & $P$ & - & $\mathrm{P}$ & $\mathrm{P}$ & $\mathrm{P}$ \\
\hline SS OOB Interf. & - & - & - & - & - & - & - & - & - & - & - & - & $P$ & $P$ & - & - & - & - & $P$ & $P$ & - & - & - & - & $P$ & $P$ & - & - & - & - & $P$ & $P$ & - & - & - & - \\
\hline SS Magnitude & $\mathrm{P}$ & - & - & $\mathrm{P}$ & - & - & $\mathrm{P}$ & - & - & $P$ & - & - & $P$ & - & - & $\mathrm{P}$ & - & - & $\mathrm{P}$ & - & - & $\mathrm{P}$ & - & - & $\mathrm{P}$ & - & - & $\mathrm{P}$ & - & - & $P$ & - & - & $\mathrm{P}$ & - & - \\
\hline Dyn Ramp & $\mathrm{P}$ & $P$ & $\mathrm{P}$ & $P$ & $P$ & $P$ & $\mathrm{P}$ & $P$ & $\mathrm{P}$ & $P$ & $P$ & $P$ & $\mathrm{P}$ & $\mathrm{P}$ & $P$ & $\mathrm{P}$ & $P$ & $P$ & $\mathrm{P}$ & $P$ & $P$ & $P$ & $\mathrm{P}$ & $\mathrm{P}$ & $\mathrm{P}$ & $P$ & $P$ & $\mathrm{P}$ & $\mathrm{P}$ & $P$ & $P$ & $P$ & $P$ & $P$ & $\mathrm{P}$ & $P$ \\
\hline Dyn Phase Mod & $\mathrm{P}$ & $P$ & $\mathrm{P}$ & $\mathrm{P}$ & $\mathrm{P}$ & $P$ & $\mathrm{P}$ & $\mathrm{P}$ & $P$ & $P$ & $P$ & $P$ & $P$ & $\mathrm{P}$ & $P$ & $\mathrm{P}$ & $\mathrm{P}$ & $\mathrm{P}$ & $\mathrm{P}$ & $\mathrm{P}$ & $P$ & $\mathrm{P}$ & $P$ & $P$ & $\mathrm{P}$ & $P$ & $\mathrm{P}$ & $\mathrm{P}$ & $P$ & $P$ & $\mathrm{P}$ & $P$ & $P$ & $P$ & $\mathrm{P}$ & $\mathrm{P}$ \\
\hline Dyn Combi Mod & $\mathrm{P}$ & $\mathrm{P}$ & $\mathrm{P}$ & $\mathrm{P}$ & $\mathrm{P}$ & $\mathrm{P}$ & $\mathrm{P}$ & $\mathrm{P}$ & $P$ & $\mathrm{P}$ & $\mathrm{P}$ & $P$ & $P$ & $\mathrm{P}$ & $\mathrm{P}$ & $\mathrm{P}$ & $\mathrm{P}$ & $\mathrm{P}$ & $\mathrm{P}$ & $\mathrm{P}$ & $P$ & $P$ & $P$ & $P$ & $\mathrm{P}$ & $P$ & $P$ & $\mathrm{P}$ & $P$ & $\mathrm{P}$ & $\mathrm{P}$ & $P$ & $P$ & $\mathrm{P}$ & $\mathrm{P}$ & $P$ \\
\hline
\end{tabular}

Table 22: PMU J response time, delay time and overshoot pass/fail

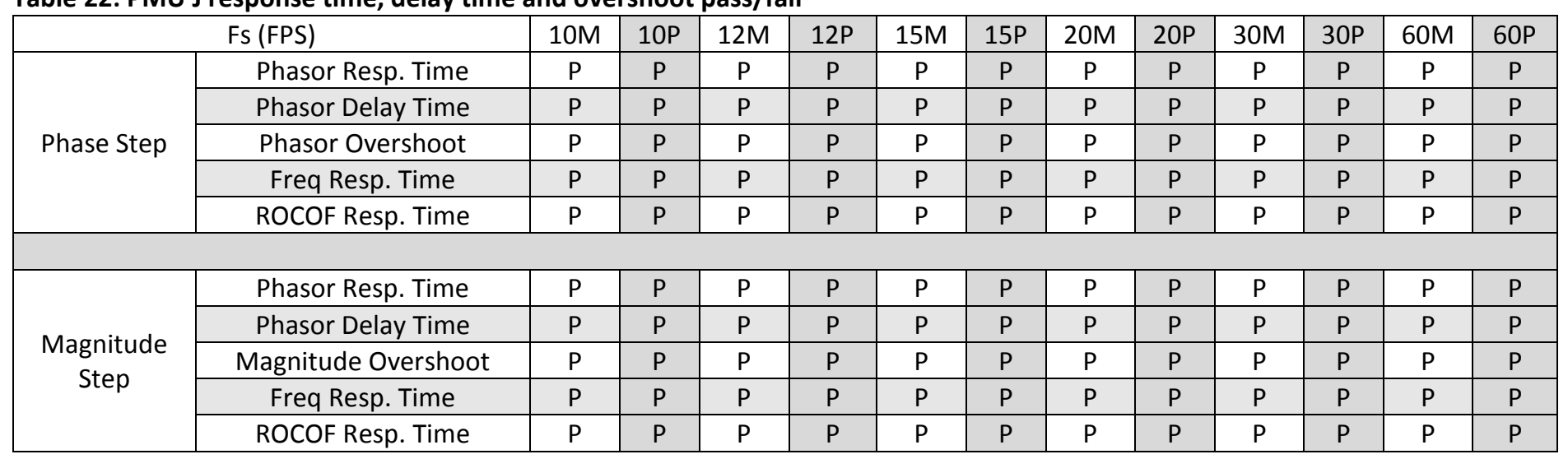




\section{Steady state frequency range}

2. Steady state frequency range. 14

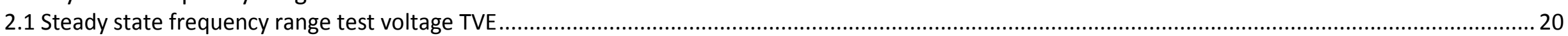

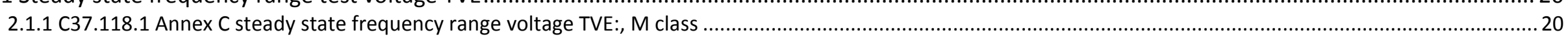

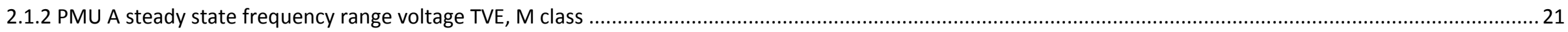

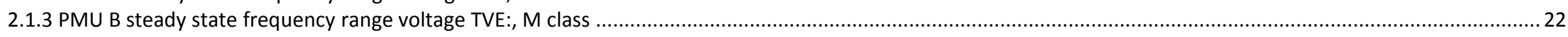

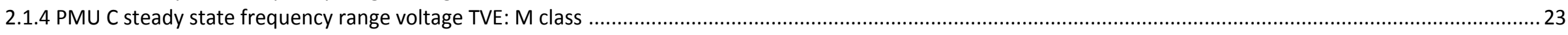

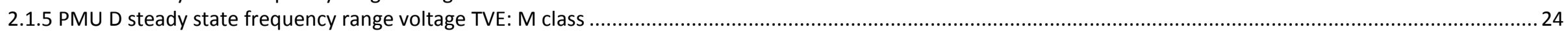

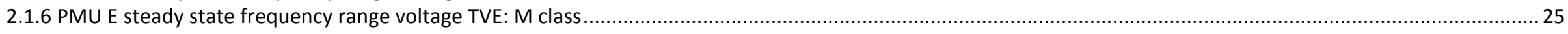

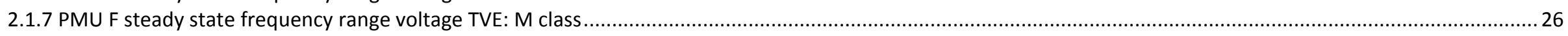

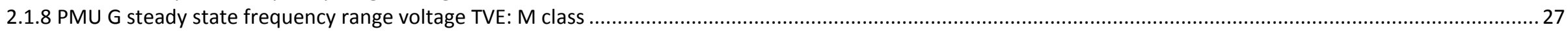

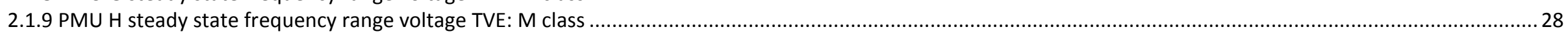

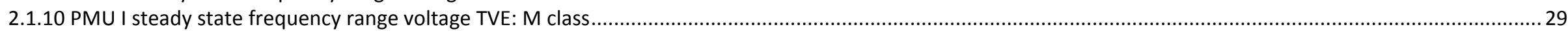

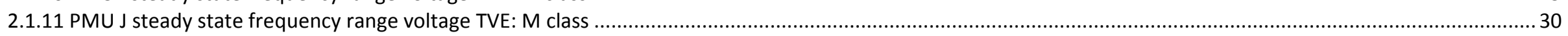

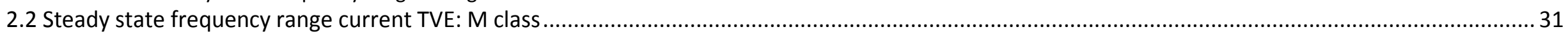

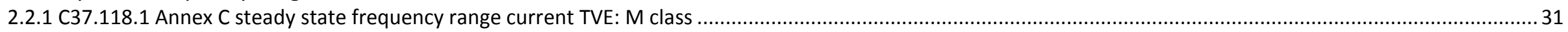

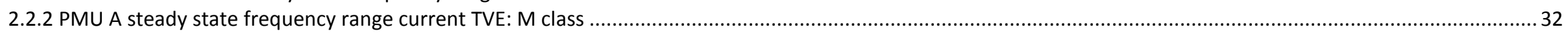

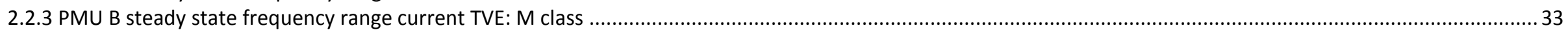

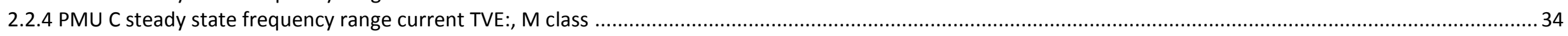

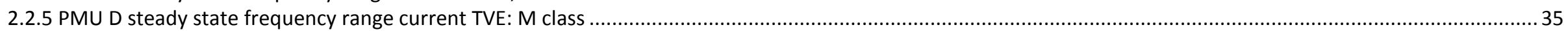

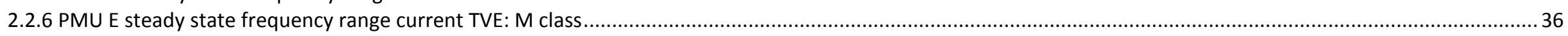

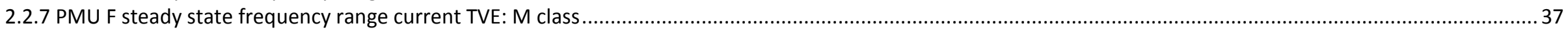

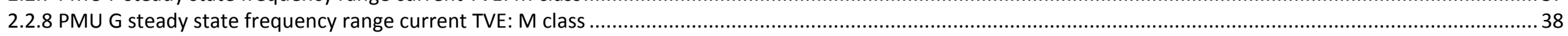

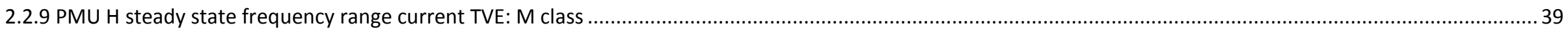

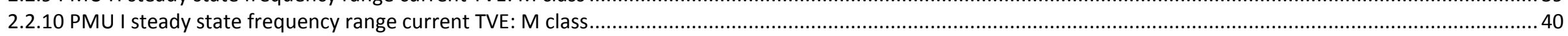

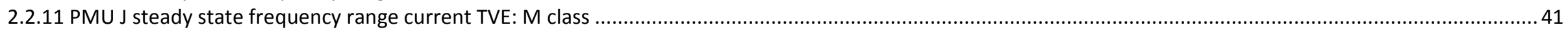

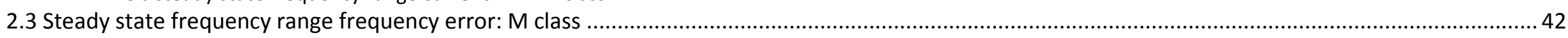

2.3.1 C37.118.1 Annex C steady state frequency range frequency error: M class ........................................................................................................................... 42

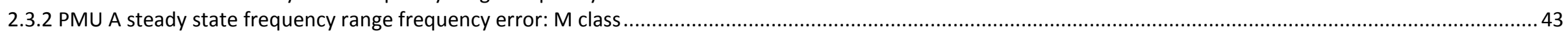

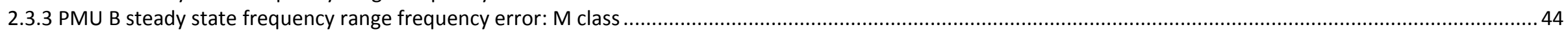

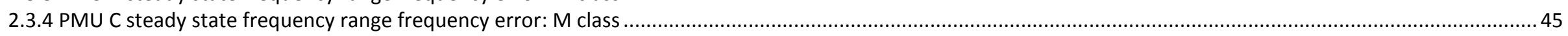

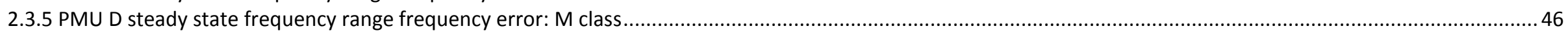

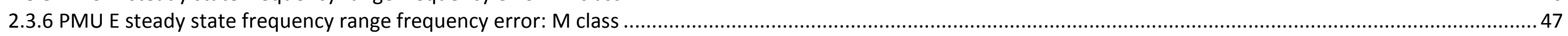

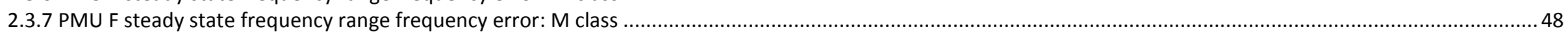

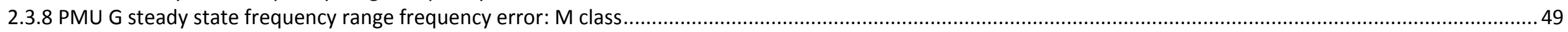

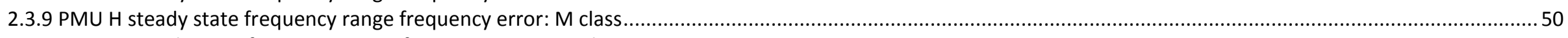

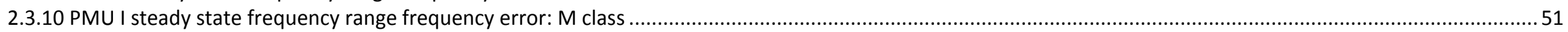

2.3.11 PMU J steady state frequency range frequency error: M class ...................................................................................................................................... 52

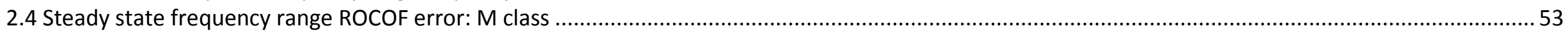

2.4.1 C37.118.1 Annex C steady state frequency range ROCOF error: M class ....................................................................................................................... 53

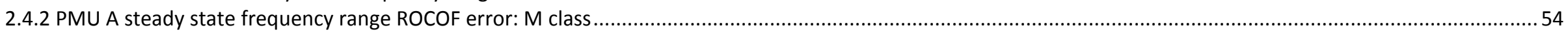


2.4.3 PMU B steady state frequency range ROCOF error: $M$ class 55

2.4.4 PMU C steady state frequency range ROCOF error: $M$ class

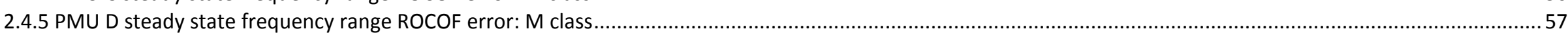

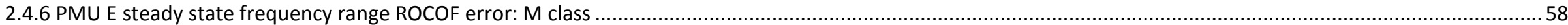

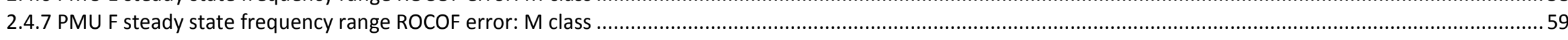

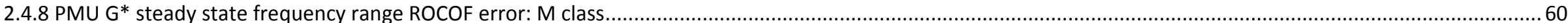

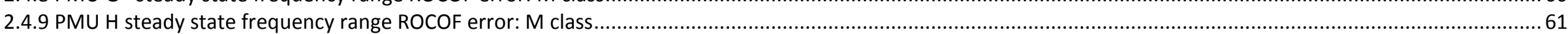

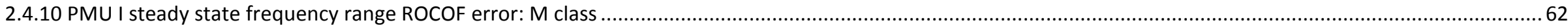

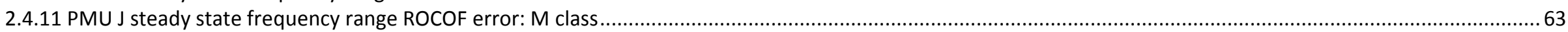

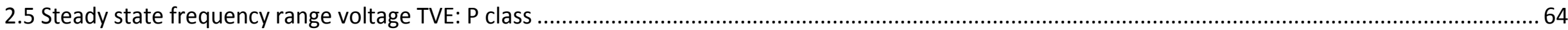

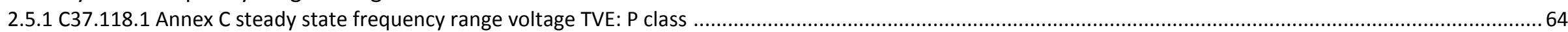

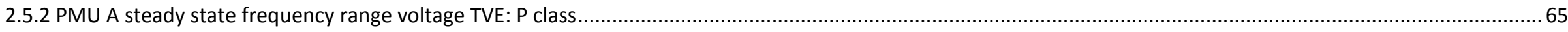

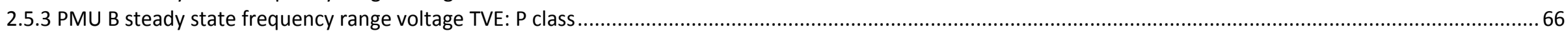

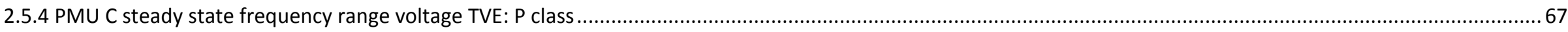

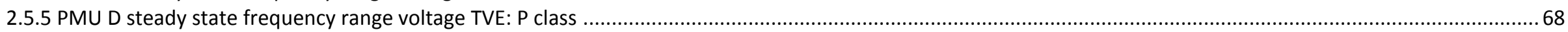

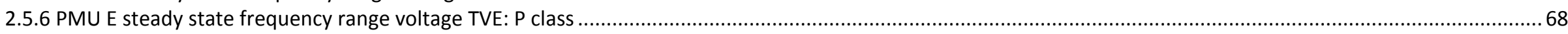

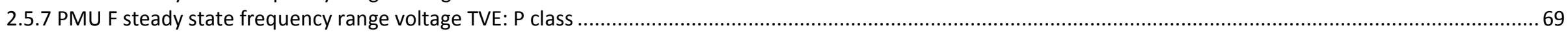

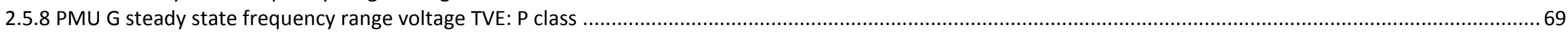

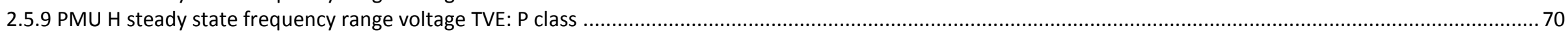

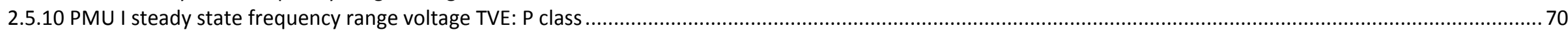

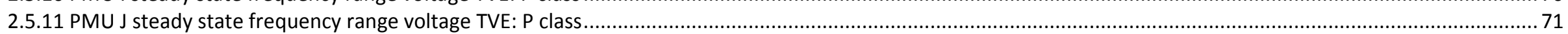

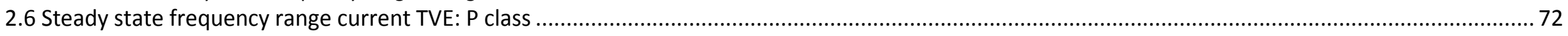

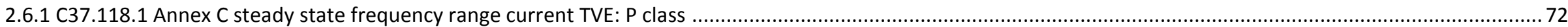

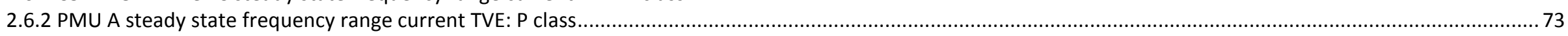

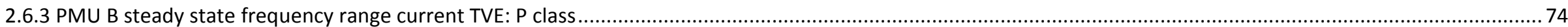

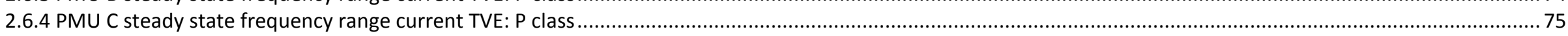

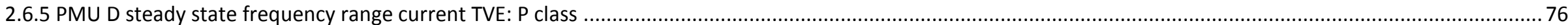

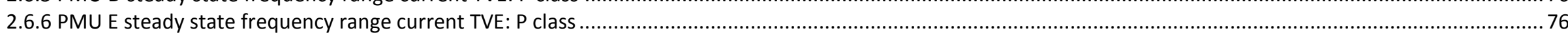

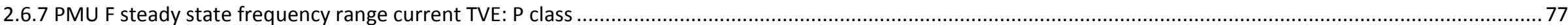

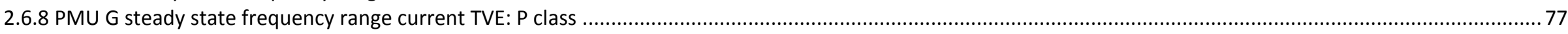

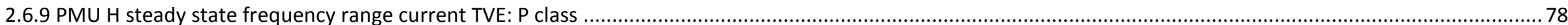

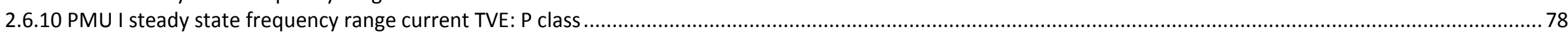

2.6.11 PMU J steady state frequency range current TVE: P class

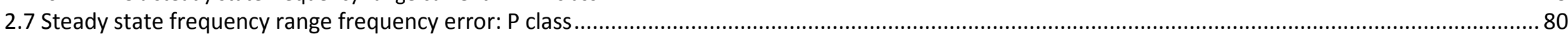

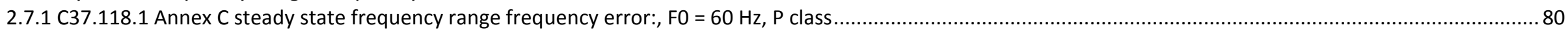

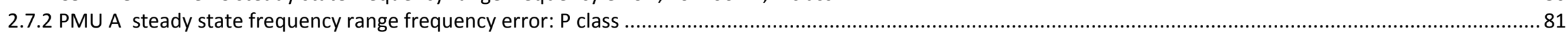

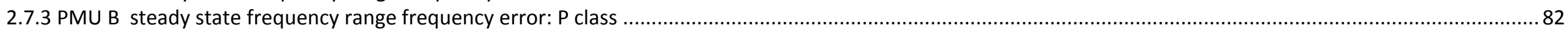

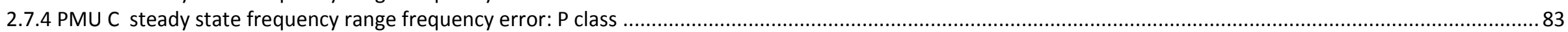

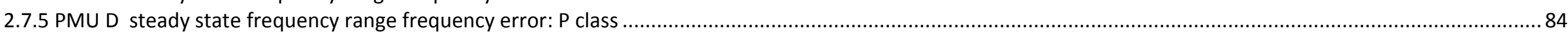

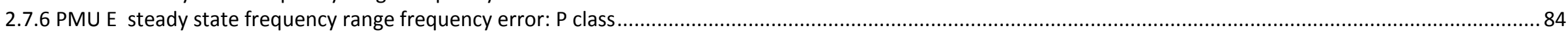

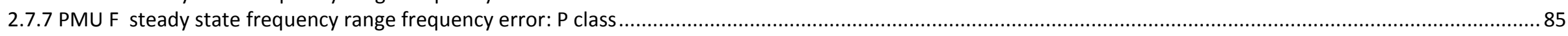

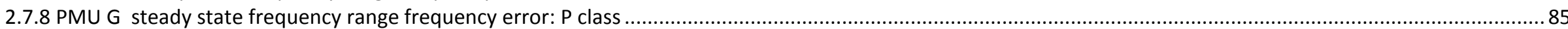

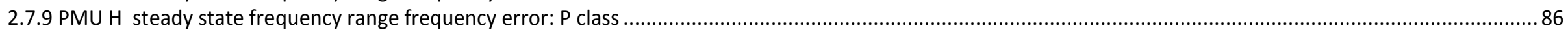


2.7.10 PMU I steady state frequency range frequency error: $P$ class

2.8 Steady state frequency range ROCOF error: P class.

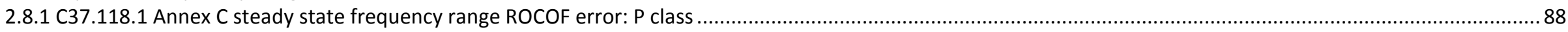

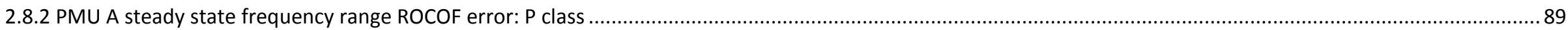

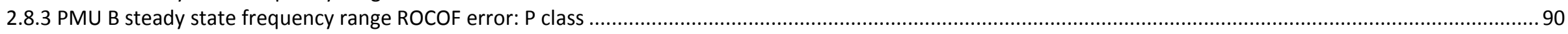

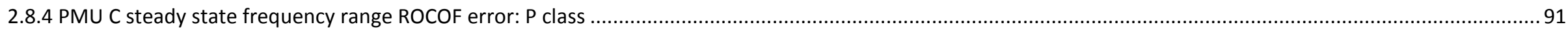

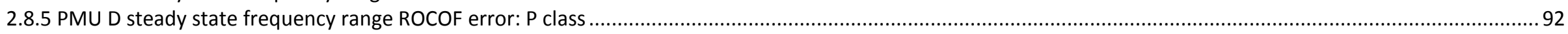

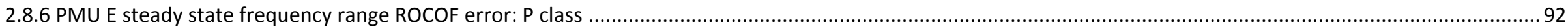

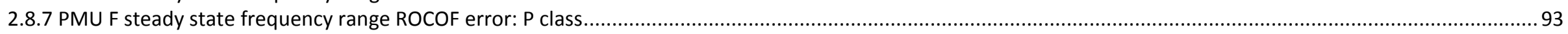

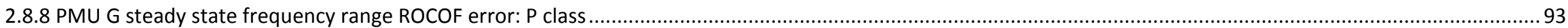

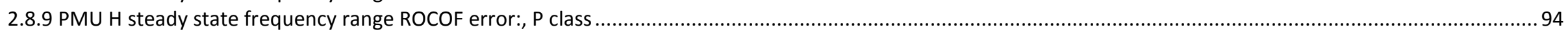

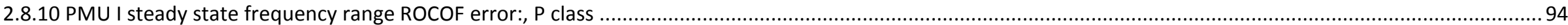

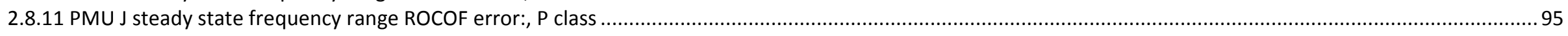


The steady state frequency range is tested by a series of individual steady state tests where the input signal magnitude and frequency are fixed for duration of 5 seconds. During this period, the total vector error (TVE), frequency error (FE) and rate of change of frequency error (RFE) are measured. A series of individual tests are run at $0.1 \mathrm{~Hz}$ increments across the signal frequency range which is determined by the PMU's reporting rate (Fs) given in frames per second (FPS) and class. The maximum TVE, FE and RFE of all the test runs is compared against the limits for TVE, FE and RFE to determine if the unit passes or fails the test.

a) Apply input signals at nominal magnitude at the frequency range limit: nominal frequency minus the lesser of $\mathrm{F}_{\mathrm{s}} / 5 \mathrm{or} 5 \mathrm{~Hz}$.

b) Wait for the system to settle.

c) Capture the PMU output for $5 \mathrm{~s}$.

d) Calculate the errors: ME, PE, FE and RFE for each report.

e) Calculate the Max TVE, FE and RFE.

f) Increase the frequency by $0.1 \mathrm{~Hz}$.

g) Repeat step b) through step g) until the upper frequency range limit is reached.

For M class:

- signal frequency range is $\pm \mathrm{Fs} / 5 \mathrm{~Hz}$ for $10 \leq \mathrm{Fs} \leq 25 \mathrm{FPS}$ and $\pm 5 \mathrm{~Hz}$ for Fs $>25 \mathrm{FPS}$.

- TVE limit is $1 \%$

- FE limit is $0.005 \mathrm{~Hz}$

- RFE limit is $0.1 \mathrm{~Hz} / \mathrm{s}$

For $P$ class:

- signal frequency range is $\pm 2 \mathrm{~Hz}$

- TVE limit is $1 \%$

- FE limit is $.005 \mathrm{~Hz}$

- RFE limit is $0.4 \mathrm{~Hz} / \mathrm{s}$

To illustrate an example of one PMU's response to one test run, the below plots of maximum TVE, FE and RFE are shown from a single test run on PMU E at Fs = $30 \mathrm{FPS}$ at $63.3 \mathrm{~Hz}$ input frequency: 


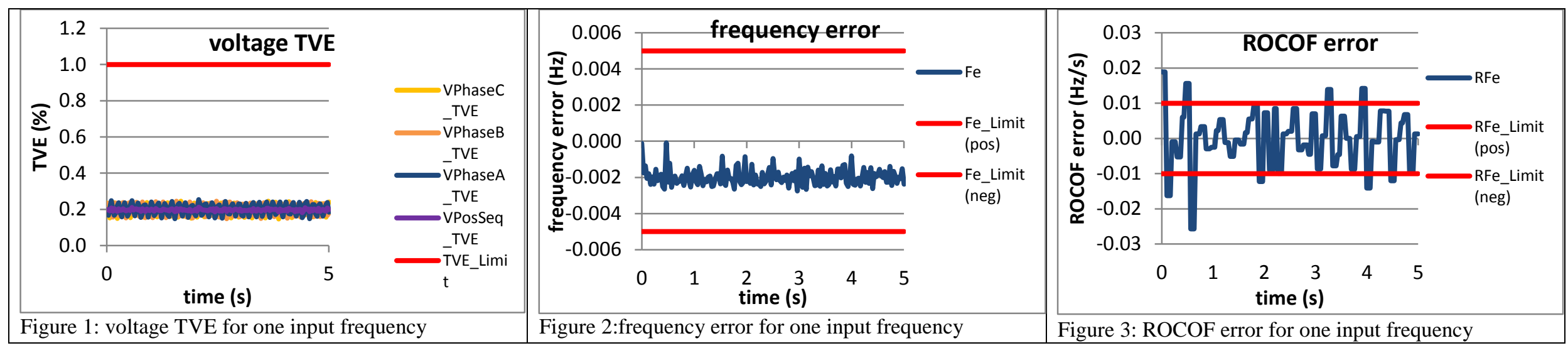

The plots in the body of this section are made from maximum values of TVE, FE and RFE from each test run. The tests are run at $0.1 \mathrm{~Hz}$ intervals across the signal frequency range. The $x$-axis shows the test input signal frequency and the $Y$ axis shows the maximum value for each tests. Red lines show the limits. The $y$-axis of the frequency error and ROCOF error plots show the maximum and minimum errors reached over all test runs. The FE and RFE limits for both the positive and negative errors are shown as red horizontal lines. An arrow shows the maximum value data point from the above example at $63.3 \mathrm{~Hz}$.
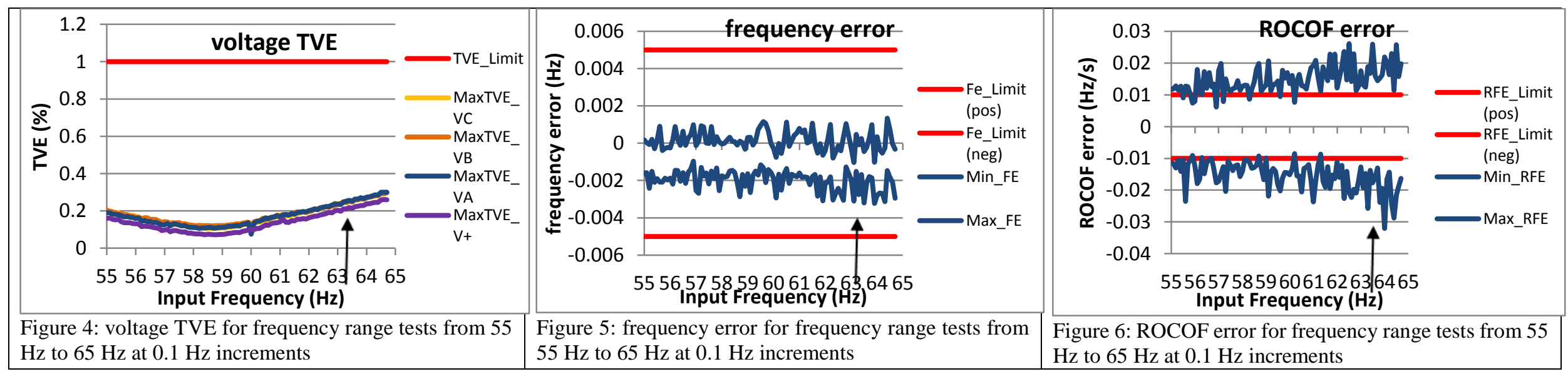

For Frequency and ROCOF error, graphing the maximum and minimum errors along with positive and negative limit lines allows us to see if the error is symmetrical or has a mean offset from 0 and whether the offset is positive or negative; this is important for frequency ramp and modulation tests to be shown later in this report. C37.118.1-2011 specifies that frequency and ROCOF errors be expressed as absolute numbers, meaning that the absolute value of FE and RFE shall not exceed a single limit. However showing the maximum and minimum errors and limits can provide useful information as explained above. 
Steady state frequency range test results

\begin{tabular}{|c|c|c|c|c|c|c|c|c|c|c|c|c|c|c|c|c|c|c|c|c|c|c|c|c|c|c|c|c|c|c|c|c|c|c|c|c|}
\hline \multirow{4}{*}{$\begin{array}{l}\text { Fs (FPS) } \\
\text { Test }\end{array}$} & \multicolumn{3}{|c|}{$10 \mathrm{M}$} & \multicolumn{3}{|c|}{$10 \mathrm{P}$} & \multicolumn{3}{|c|}{$12 \mathrm{M}$} & \multicolumn{3}{|c|}{$12 \mathrm{P}$} & \multicolumn{3}{|c|}{$15 \mathrm{M}$} & \multicolumn{3}{|c|}{$15 \mathrm{P}$} & \multicolumn{3}{|c|}{$20 \mathrm{M}$} & \multicolumn{3}{|c|}{$20 P$} & \multicolumn{3}{|c|}{$30 \mathrm{M}$} & \multicolumn{3}{|c|}{$30 P$} & \multicolumn{3}{|c|}{$60 \mathrm{M}$} & \multicolumn{3}{|c|}{$60 P$} \\
\hline & $\mathrm{T}$ & $\mathrm{F}$ & $\mathrm{R}$ & $T$ & $F$ & $R$ & $T$ & $\mathrm{~F}$ & $\mathrm{R}$ & $T$ & $\mathrm{~F}$ & $\mathrm{R}$ & $\mathrm{T}$ & $\mathrm{F}$ & $R$ & $T$ & $F$ & $R$ & $T$ & $\mathrm{~F}$ & $\mathrm{R}$ & \begin{tabular}{l|l}
$T$ \\
\end{tabular} & $\mathrm{~F}$ & $\mathrm{R}$ & $\mathrm{T}$ & $F$ & $\mathrm{R}$ & $\mathrm{T}$ & $\mathrm{F}$ & $\mathrm{R}$ & $T$ & $\mathrm{~F}$ & $\mathrm{R}$ & $T$ & $F$ & $R$ \\
\hline & V & $E$ & $\mathrm{~F}$ & V & $\mathrm{E}$ & $\mathrm{F}$ & V & $\mathrm{E}$ & $\mathrm{F}$ & $\mathrm{V}$ & $\mathrm{E}$ & $\mathrm{F}$ & $\mathrm{V}$ & $\mathrm{E}$ & $\mathrm{F}$ & V & $\mathrm{E}$ & $\mathrm{F}$ & V & $\mathrm{E}$ & $\mathrm{F}$ & $\mathrm{V}$ & $\mathrm{E}$ & $\mathrm{F}$ & $\mathrm{V}$ & E & $\mathrm{F}$ & V & $E$ & $\mathrm{~F}$ & $\mathrm{~V}$ & $E$ & $\mathrm{~F}$ & $\mathrm{~V}$ & $E$ & $\mathrm{~F}$ \\
\hline & $\mathrm{E}$ & & $E$ & E & & $E$ & $E$ & & $E$ & $\mathrm{E}$ & & $\mathrm{E}$ & $\mathrm{E}$ & & $E$ & $E$ & & $\mathrm{E}$ & $\mathrm{E}$ & & $\mathrm{E}$ & $\mathrm{E}$ & & $\mathrm{E}$ & $\mathrm{E}$ & & $E$ & $\mathrm{E}$ & & $E$ & $\mathrm{E}$ & & $\mathrm{E}$ & $\mathrm{E}$ & & $E$ \\
\hline C37.118.1 Annex C & $\mathrm{P}$ & $\mathrm{P}$ & $\mathrm{P}$ & $\mathrm{P}$ & $\mathrm{P}$ & $\mathrm{P}$ & $\mathrm{P}$ & $\mathrm{P}$ & $\mathrm{P}$ & $\mathrm{P}$ & $\mathrm{P}$ & $\mathrm{P}$ & $\mathrm{P}$ & $\mathrm{P}$ & $\mathrm{P}$ & $\mathrm{P}$ & $\mathrm{P}$ & $\mathrm{P}$ & $\mathrm{P}$ & $\mathrm{P}$ & $\mathrm{P}$ & $\mathrm{P}$ & $\mathrm{P}$ & $\mathrm{P}$ & $\mathrm{P}$ & $\mathrm{P}$ & $\mathrm{P}$ & $\mathrm{P}$ & $\mathrm{P}$ & $\mathrm{P}$ & $\mathrm{P}$ & $\mathrm{P}$ & $\mathrm{P}$ & $\mathrm{P}$ & $\mathrm{P}$ & $\mathrm{P}$ \\
\hline PMU A & $P$ & $P$ & $\mathrm{~F}$ & $P$ & $\mathrm{~F}$ & $\mathrm{~F}$ & $P$ & $\mathrm{P}$ & $\mathrm{F}$ & $P$ & $\mathrm{~F}$ & $\mathrm{~F}$ & $P$ & $P$ & $\mathrm{~F}$ & $P$ & $\mathrm{~F}$ & $\mathrm{~F}$ & $P$ & $\mathrm{P}$ & $\mathrm{F}$ & $P$ & $\mathrm{~F}$ & $\mathrm{~F}$ & $\mathrm{P}$ & $P$ & $\mathrm{~F}$ & $P$ & $\mathrm{~F}$ & $\mathrm{~F}$ & $P$ & $\mathrm{~F}$ & $\mathrm{~F}$ & $P$ & $\mathrm{~F}$ & $\mathrm{~F}$ \\
\hline PMU B & $\mathrm{P}$ & $P$ & $P$ & $P$ & $\mathrm{P}$ & $P$ & $P$ & $P$ & $\mathrm{P}$ & $P$ & $P$ & $\mathrm{P}$ & $P$ & $P$ & $P$ & $P$ & $P$ & $P$ & $P$ & $\mathrm{P}$ & $\mathrm{P}$ & $P$ & $P$ & $\mathrm{P}$ & $\mathrm{P}$ & $P$ & $P$ & $\mathrm{P}$ & $\mathrm{P}$ & $\mathrm{P}$ & $P$ & $P$ & $\mathrm{P}$ & $P$ & $P$ & $P$ \\
\hline PMU C & $P$ & $\mathrm{~F}$ & $\mathrm{~F}$ & $P$ & $P$ & $P$ & $P$ & $\mathrm{~F}$ & $\mathrm{~F}$ & $P$ & $P$ & $P$ & $P$ & $\mathrm{~F}$ & $\mathrm{~F}$ & $P$ & $P$ & $P$ & $P$ & $\mathrm{~F}$ & $\mathrm{~F}$ & $P$ & $P$ & $\mathrm{P}$ & $P$ & $\mathrm{~F}$ & $\mathrm{~F}$ & $P$ & $P$ & $P$ & $P$ & $\mathrm{~F}$ & $\mathrm{~F}$ & $P$ & $\mathrm{~F}$ & $\mathrm{~F}$ \\
\hline PMU D & $P$ & $P$ & $P$ & $P$ & $P$ & $P$ & $P$ & $\mathrm{P}$ & $\mathrm{P}$ & $\mathrm{P}$ & $P$ & $P$ & $\mathrm{P}$ & $P$ & $P$ & $P$ & $P$ & $P$ & $\mathrm{P}$ & $\mathrm{P}$ & $\mathrm{P}$ & $\mathrm{P}$ & $\mathrm{P}$ & $\mathrm{P}$ & $\mathrm{P}$ & $P$ & $P$ & $P$ & $\mathrm{P}$ & $\mathrm{P}$ & $\mathrm{P}$ & $P$ & $\mathrm{P}$ & $P$ & $P$ & $P$ \\
\hline PMU E & $P$ & $P$ & $P$ & - & - & - & - & - & - & - & - & - & $P$ & $P$ & $P$ & - & - & - & $P$ & $P$ & $P$ & - & - & - & $P$ & $P$ & $P$ & - & - & - & $P$ & $P$ & $P$ & - & - & - \\
\hline PMU F & $\mathrm{P}$ & $\mathrm{P}$ & $\mathrm{F}$ & $\mathrm{P}$ & $P$ & $\mathrm{~F}$ & $P$ & $\mathrm{P}$ & $\mathrm{F}$ & $P$ & $P$ & $\mathrm{~F}$ & $P$ & $P$ & $\mathrm{~F}$ & $P$ & $P$ & $\mathrm{~F}$ & $P$ & $\mathrm{P}$ & $\mathrm{F}$ & $\mathrm{P}$ & $\mathrm{P}$ & $\mathrm{F}$ & $\mathrm{P}$ & $P$ & $\mathrm{~F}$ & $P$ & $P$ & $\mathrm{~F}$ & $P$ & $P$ & $\mathrm{~F}$ & $P$ & $P$ & $P$ \\
\hline PMU G* & $P$ & $P$ & $P$ & - & - & - & $P$ & $P$ & $P$ & - & - & - & $P$ & $P$ & $P$ & - & - & - & I & $\mathrm{P}$ & $\mathrm{P}$ & - & - & - & $\mathrm{F}$ & $P$ & $P$ & - & - & - & - & - & - & - & - & - \\
\hline PMU H & $P$ & $P$ & $P$ & $P$ & $P$ & $P$ & $P$ & $P$ & $P$ & $P$ & $P$ & $P$ & $P$ & $P$ & $P$ & $P$ & $P$ & $P$ & $P$ & $P$ & $P$ & $P$ & $\mathrm{P}$ & $\mathrm{P}$ & $P$ & $P$ & $P$ & $P$ & $P$ & $P$ & $P$ & $P$ & $P$ & $P$ & $P$ & $P$ \\
\hline PMU I & $P$ & $P$ & $P$ & - & - & - & $P$ & $\mathrm{P}$ & $P$ & - & - & - & $P$ & $P$ & $P$ & - & - & - & $P$ & $\mathrm{P}$ & $P$ & - & - & - & $\mathrm{P}$ & $P$ & $\mathrm{~F}$ & - & - & - & $P$ & $P$ & $P$ & - & - & - \\
\hline PMU J & $P$ & $P$ & $P$ & $P$ & $P$ & $P$ & $P$ & $\mathrm{P}$ & $P$ & $P$ & $P$ & $P$ & $P$ & $P$ & $P$ & $P$ & $P$ & $P$ & $P$ & $\mathrm{P}$ & $P$ & $\mathrm{P}$ & $\mathrm{P}$ & $\mathrm{P}$ & $\mathrm{P}$ & $P$ & $P$ & $P$ & $P$ & $P$ & $P$ & $P$ & $P$ & $P$ & $P$ & $P$ \\
\hline
\end{tabular}

$\mathrm{P}=$ pass (measurement is within the limit), $\mathrm{F}=$ fail (measurement is outside the limit), I = indeterminate (measurement is within the test system uncertainty of the limit)

* PMU G always outputs ROCOF $=0$. 


\subsection{Steady state frequency range test voltage TVE}

2.1.1 C37.118.1 Annex $C$ steady state frequency range voltage TVE:, M class

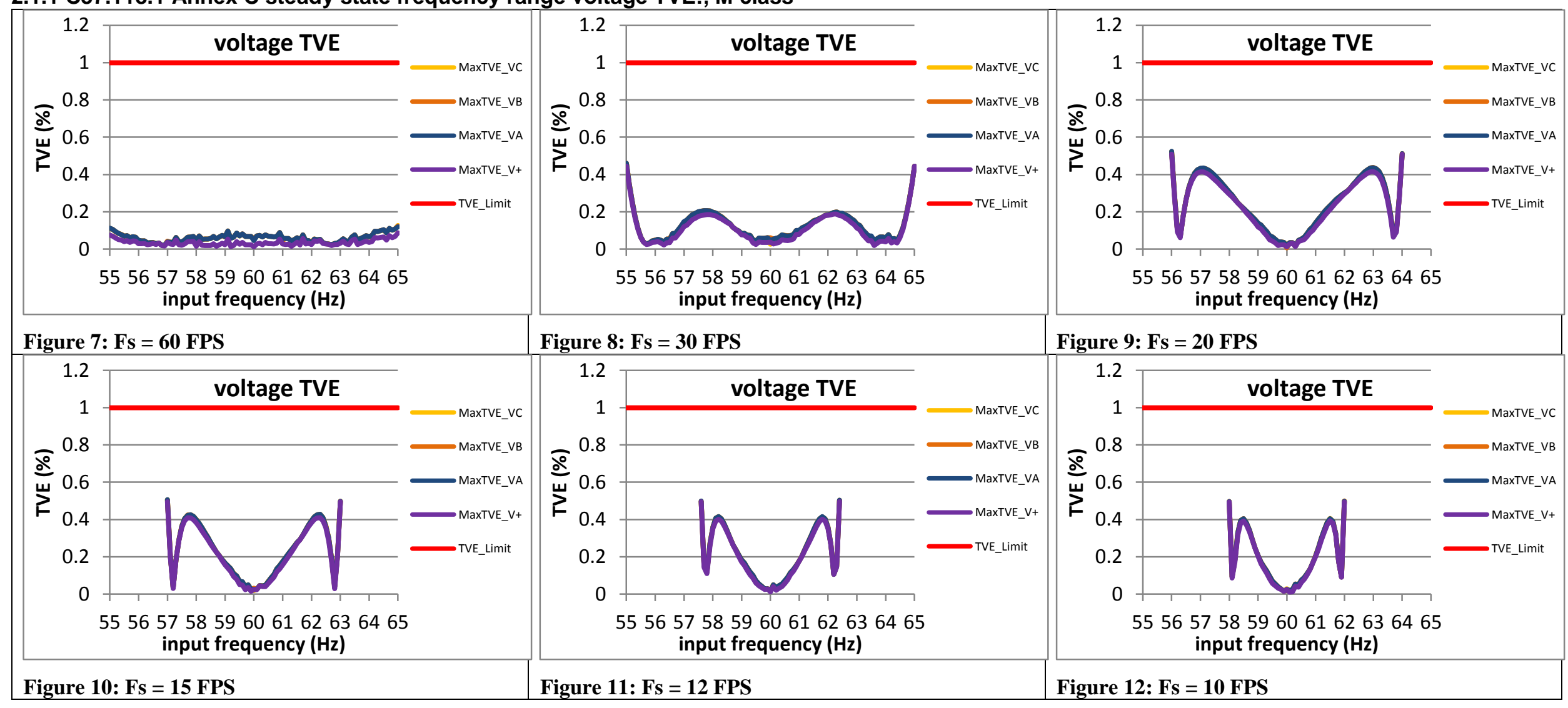


2.1.2 PMU A steady state frequency range voltage TVE, M class

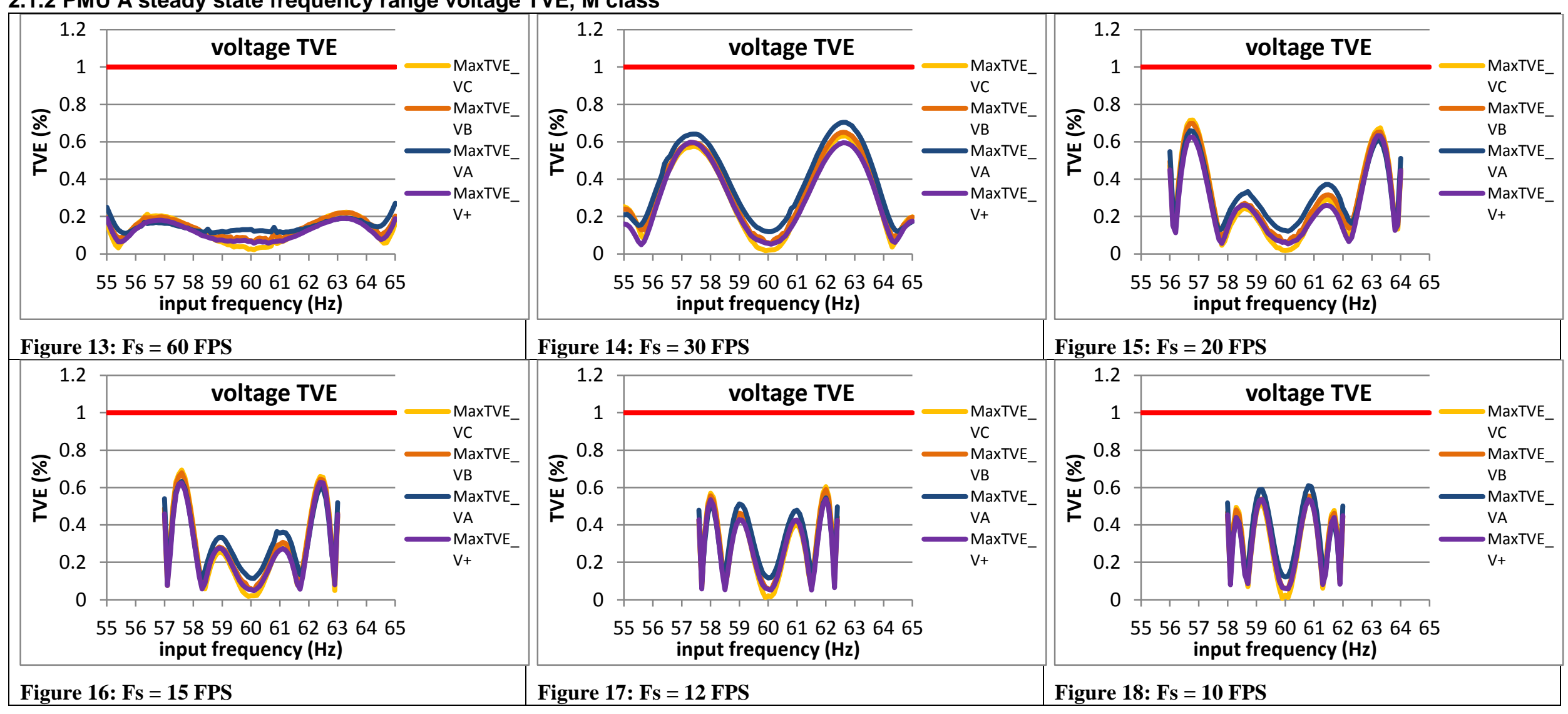


2.1.3 PMU B steady state frequency range voltage TVE:, M class

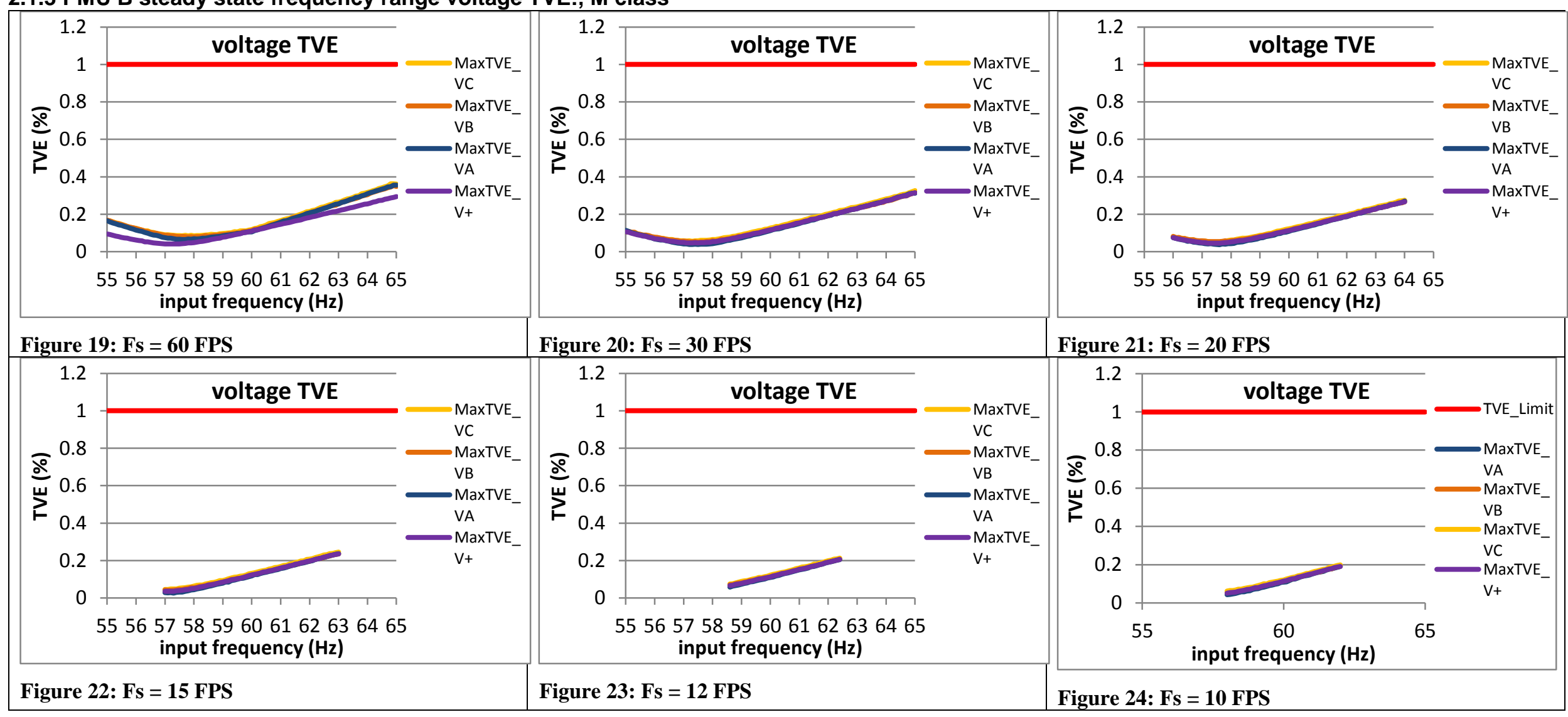


2.1.4 PMU C steady state frequency range voltage TVE: M class

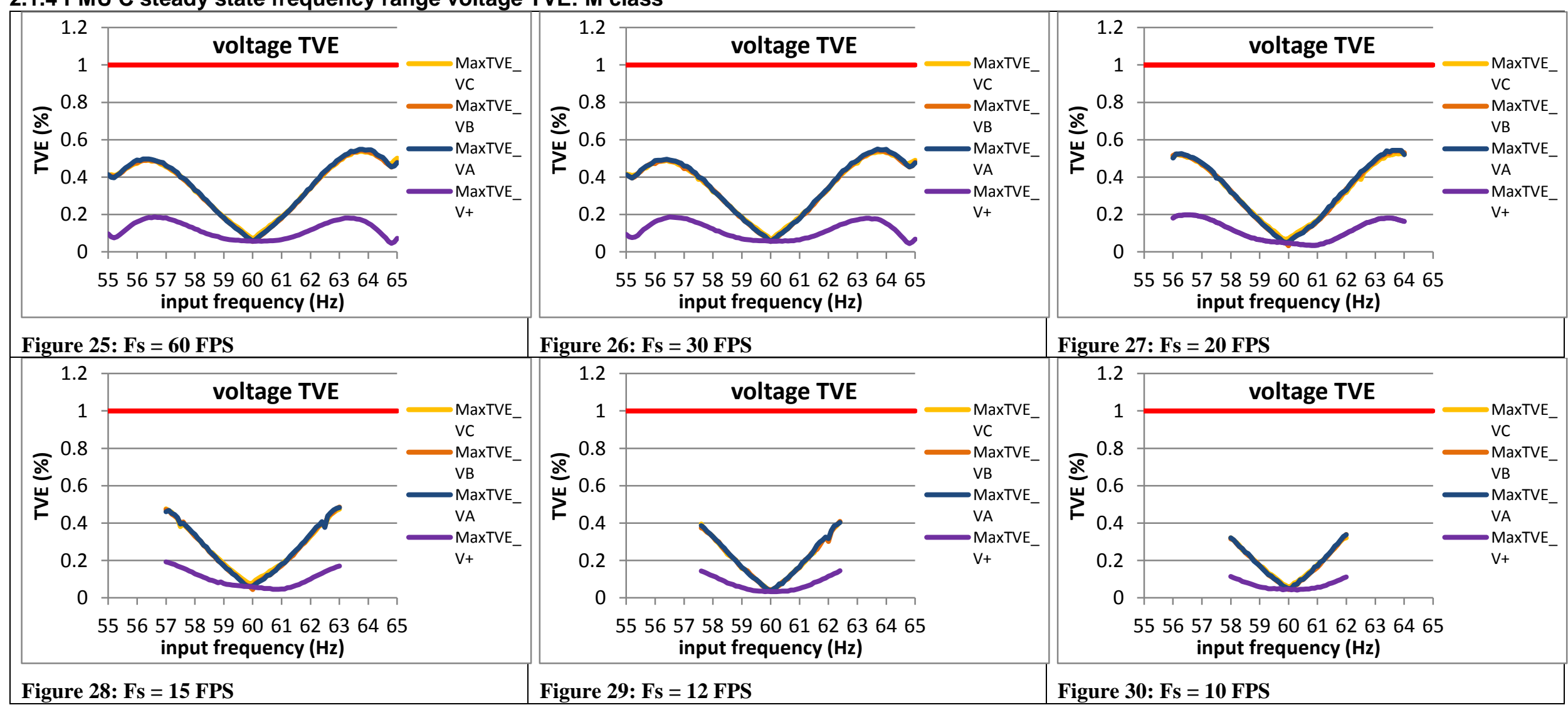




\subsubsection{PMU D steady state frequency range voltage TVE: M class}
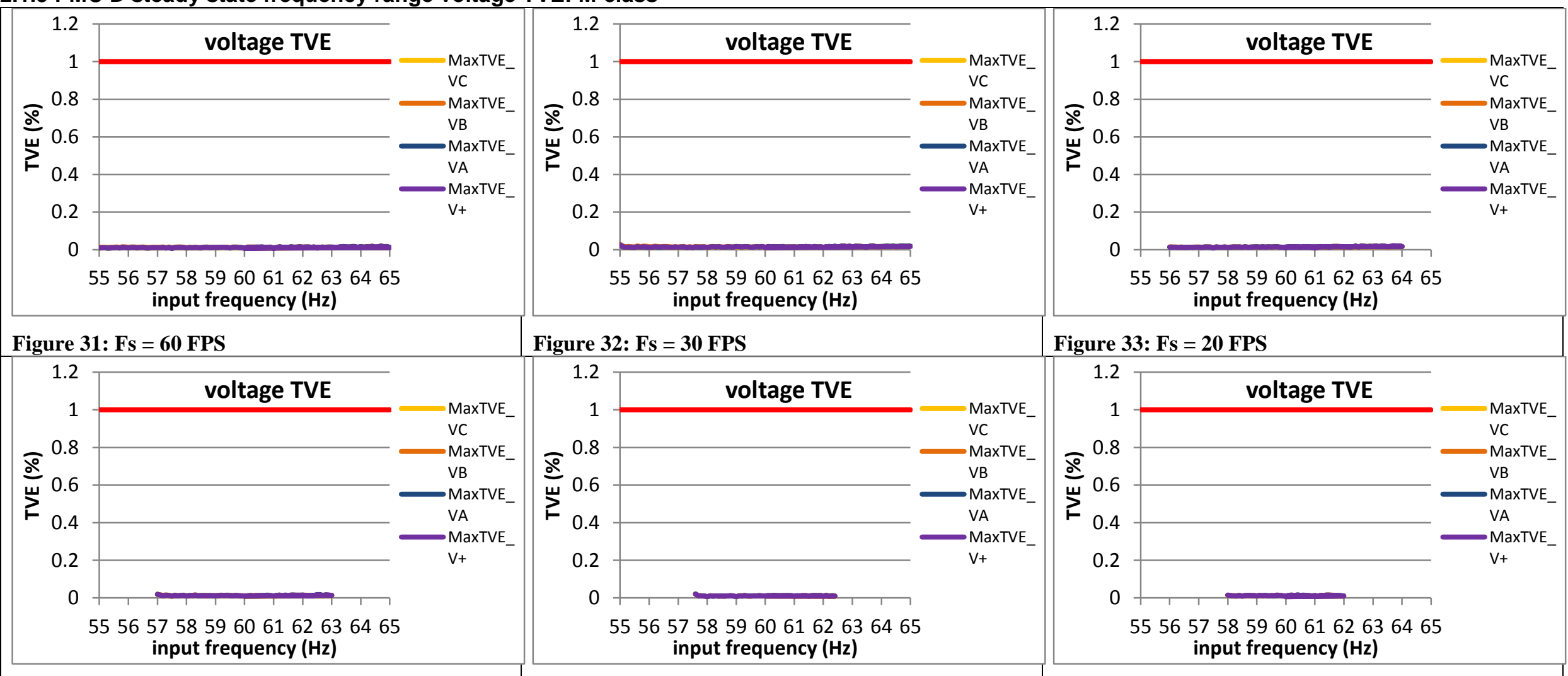

Figure 32: Fs = 30 FPS

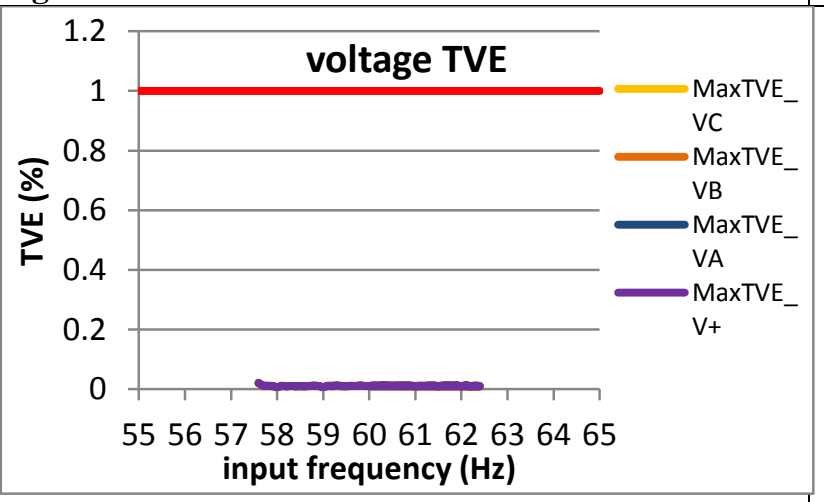

Figure 33: Fs = 20 FPS

Figure 34: Fs = 15 FPS

Figure 35: Fs = 12 FPS

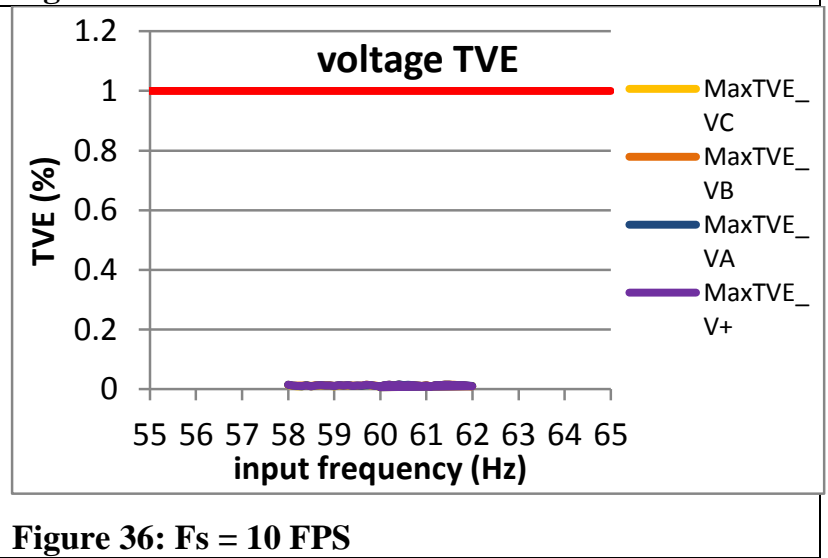




\subsubsection{PMU E steady state frequency range voltage TVE: M class}

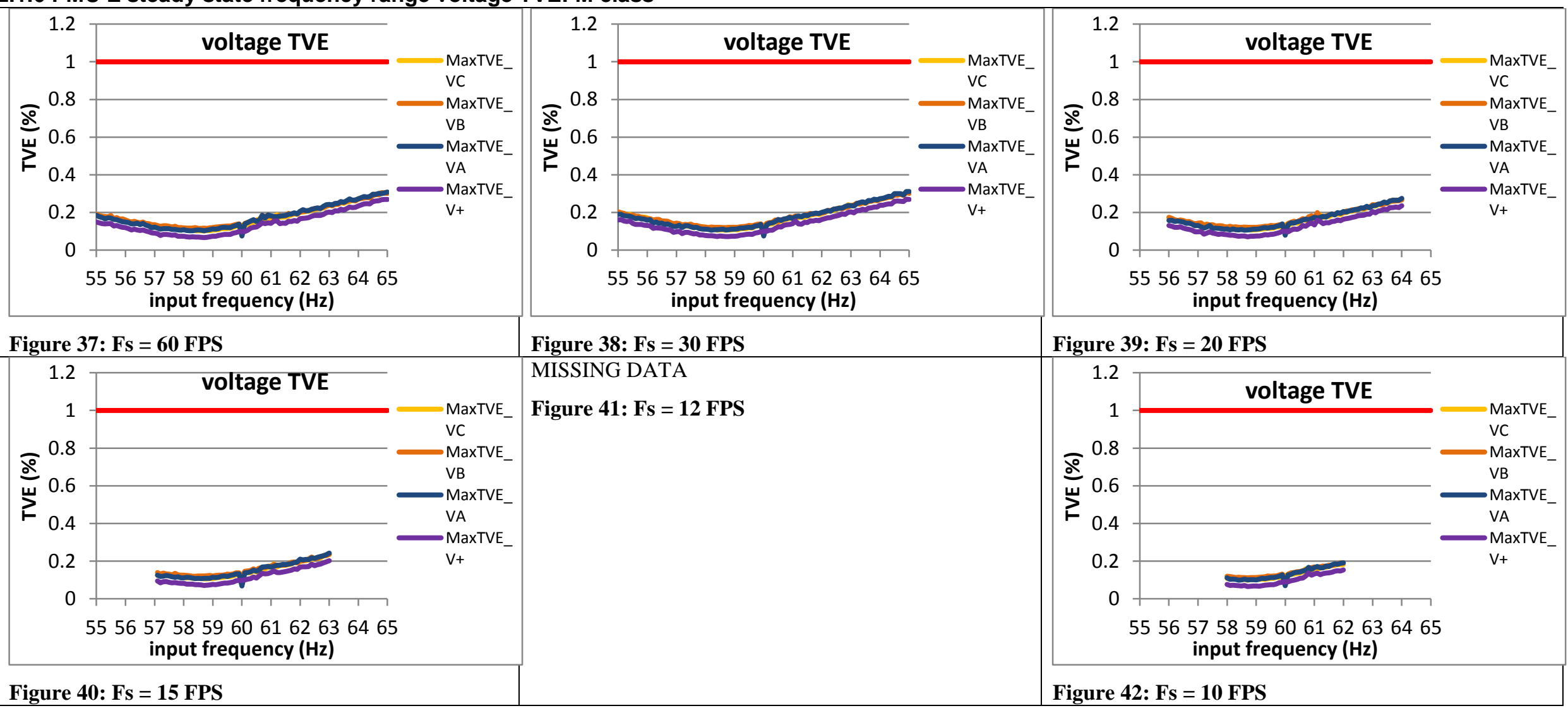


2.1.7 PMU F steady state frequency range voltage TVE: M class

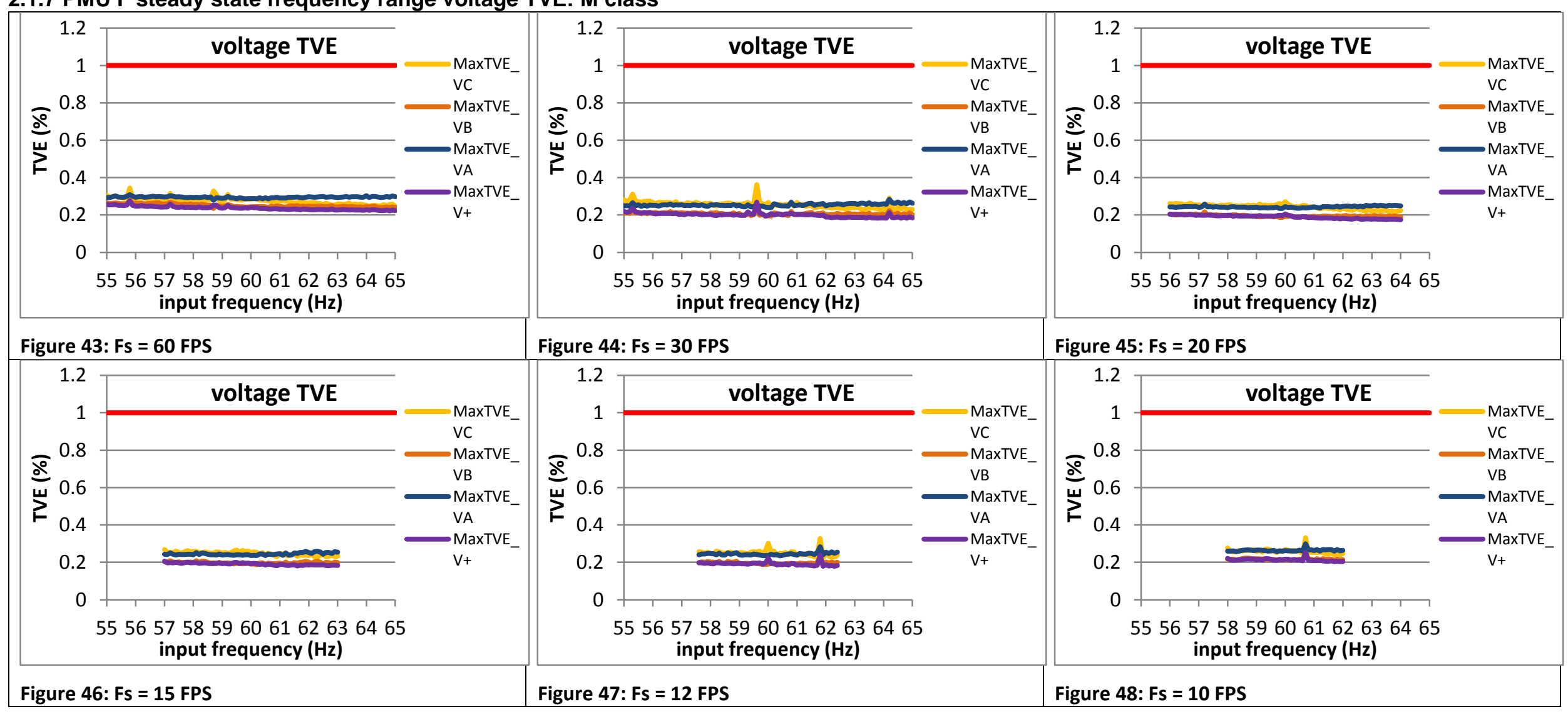


2.1.8 PMU G steady state frequency range voltage TVE: M class

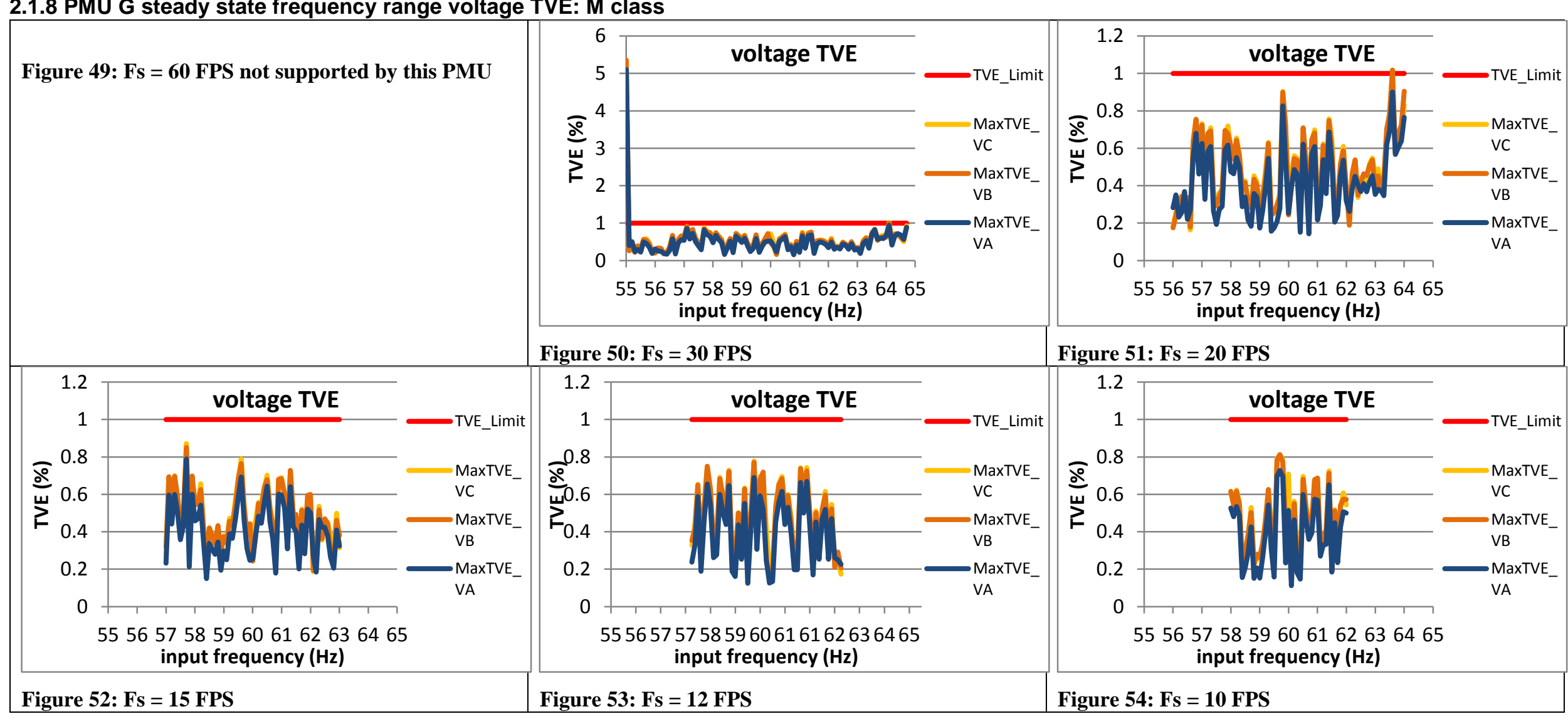




\subsubsection{PMU H steady state frequency range voltage TVE: M class}

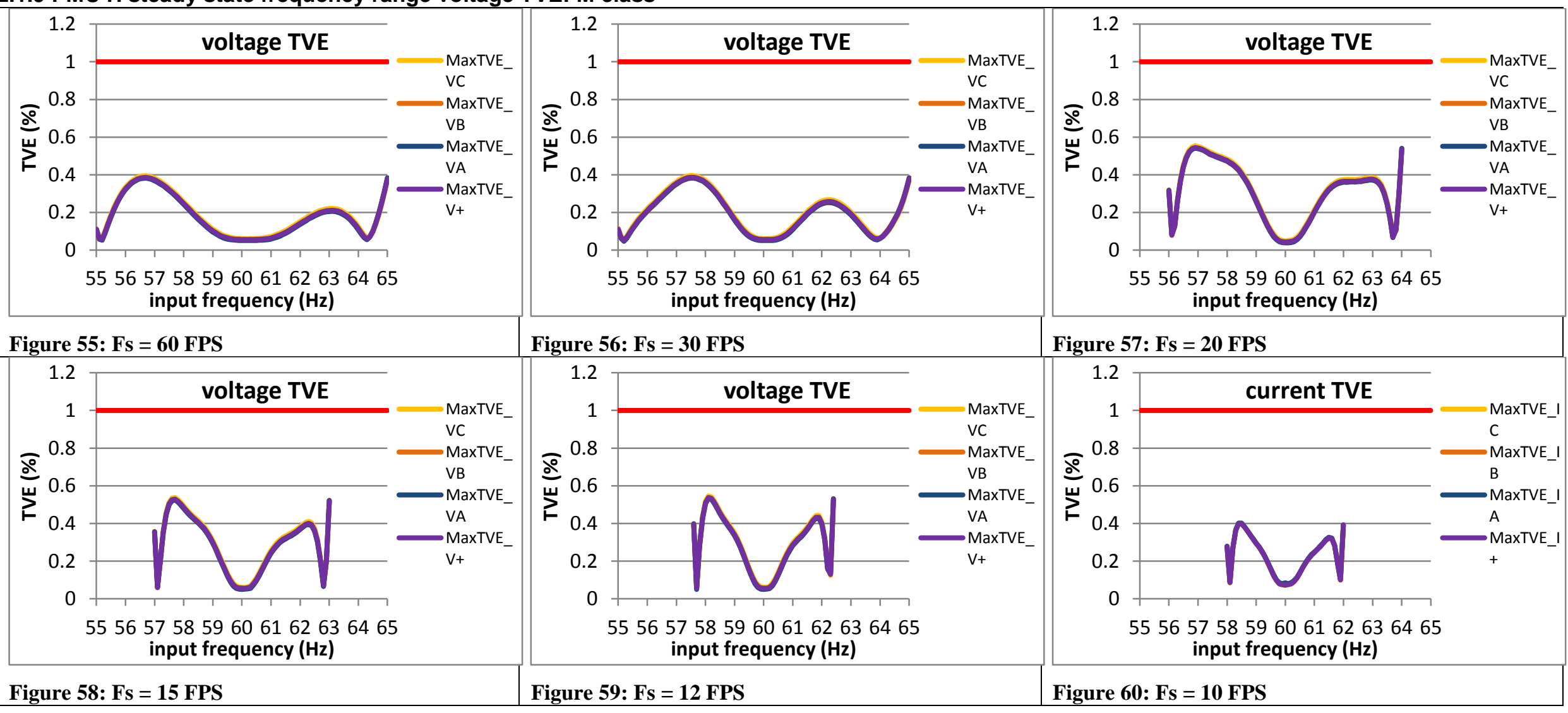




\subsubsection{PMU I steady state frequency range voltage TVE: M class}

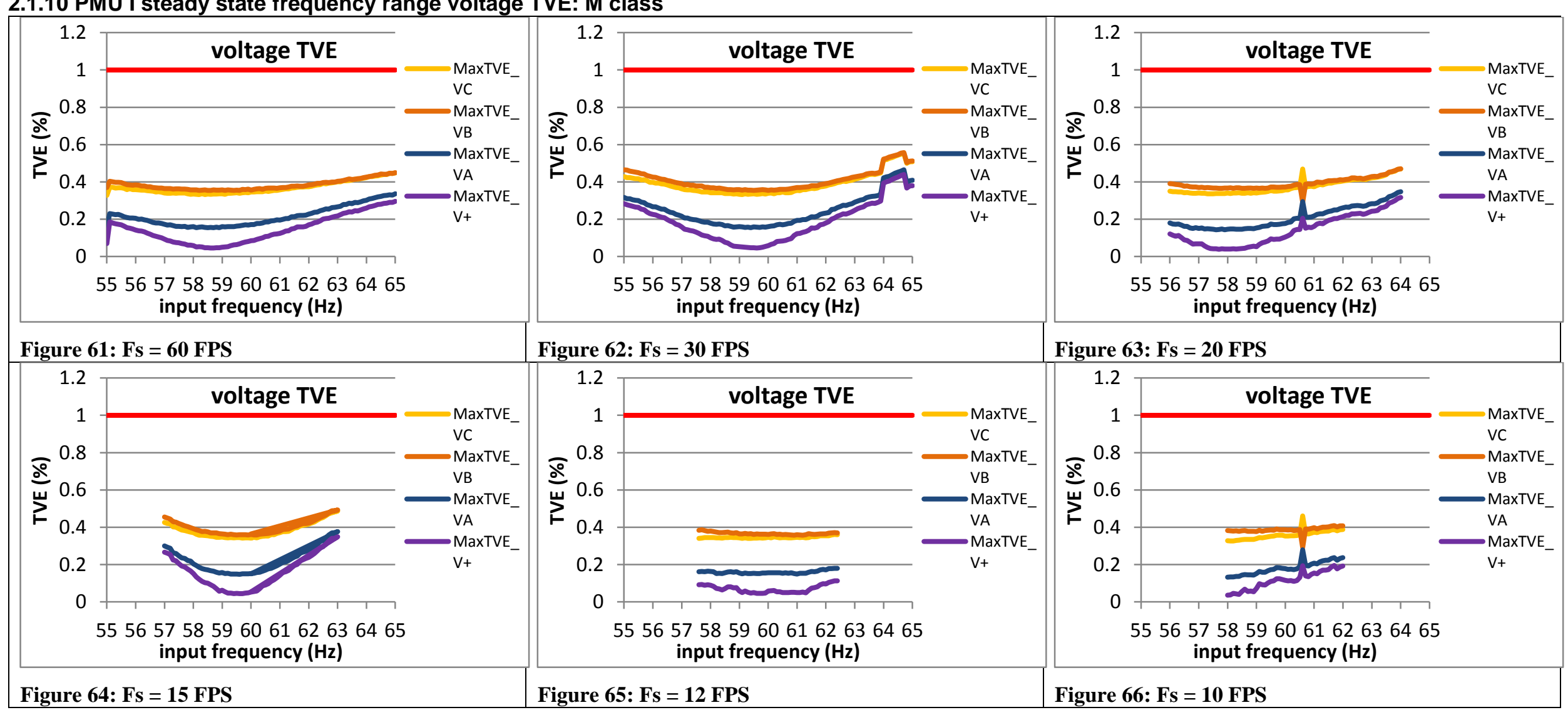


2.1.11 PMU J steady state frequency range voltage TVE: M class

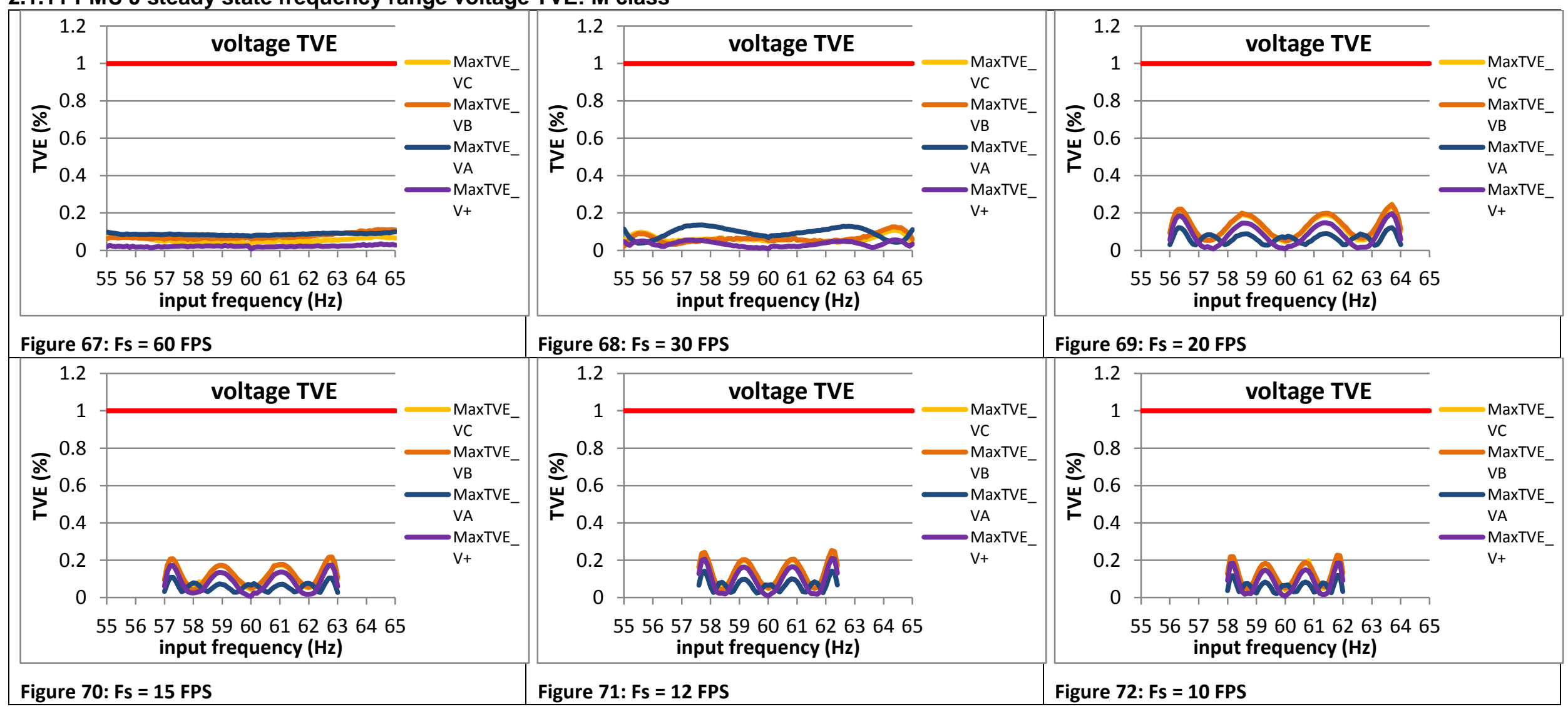




\subsection{Steady state frequency range current TVE: M class}

\subsubsection{C37.118.1 Annex C steady state frequency range current TVE: M class}
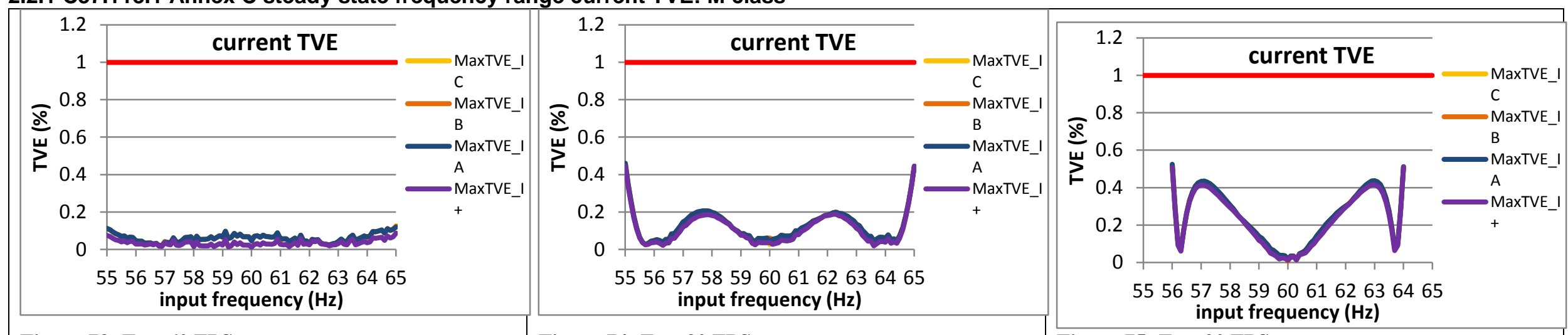

Figure 73: Fs = 60 FPS

Figure 74: Fs = 30 FPS
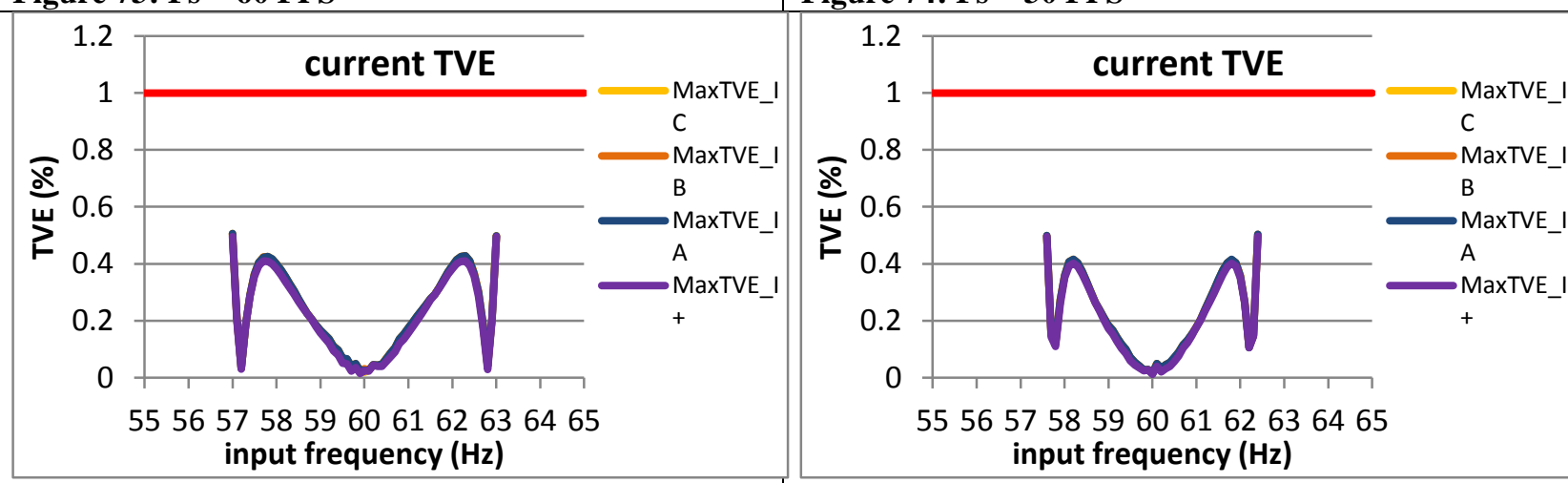

Figure 75: Fs = 20 FPS

Figure 76: Fs = 15 FPS

Figure 77: Fs = 12 FPS

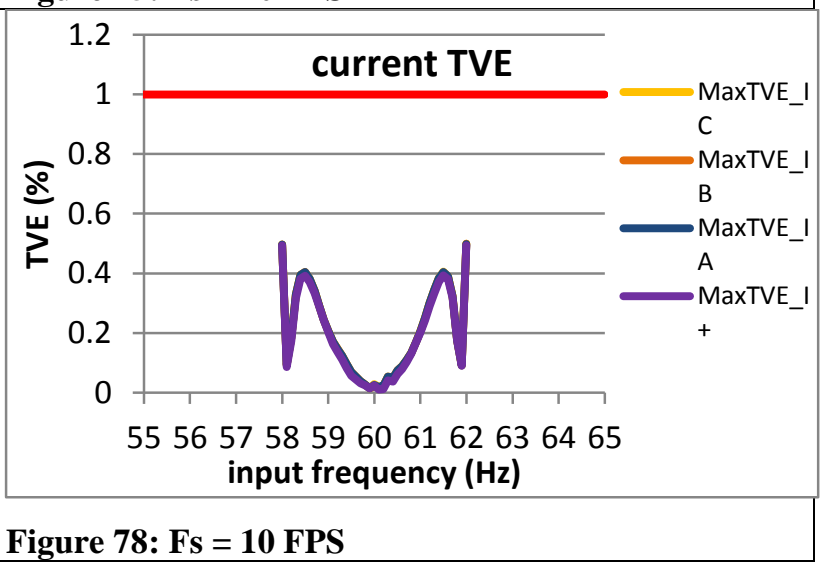


2.2.2 PMU A steady state frequency range current TVE: M class

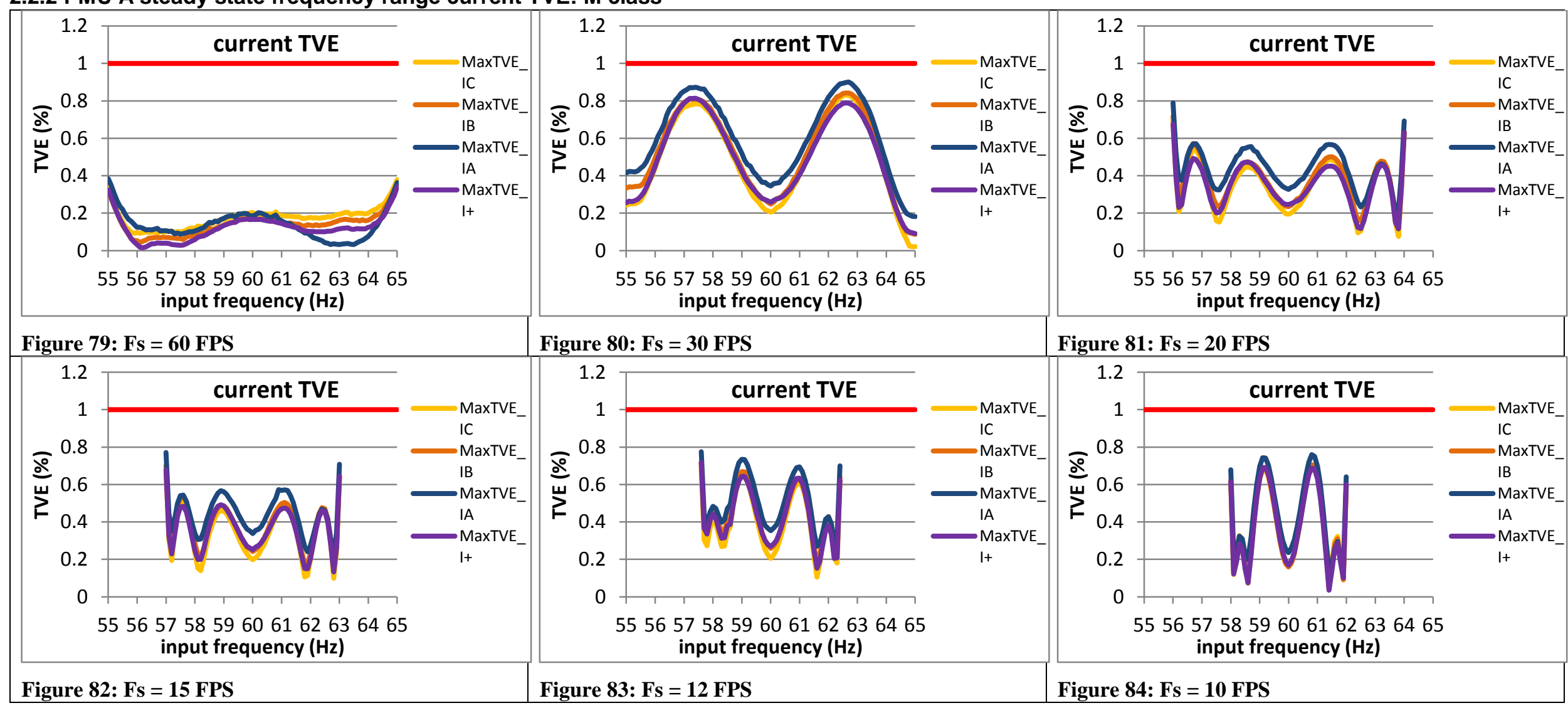




\subsubsection{PMU B steady state frequency range current TVE: M class}
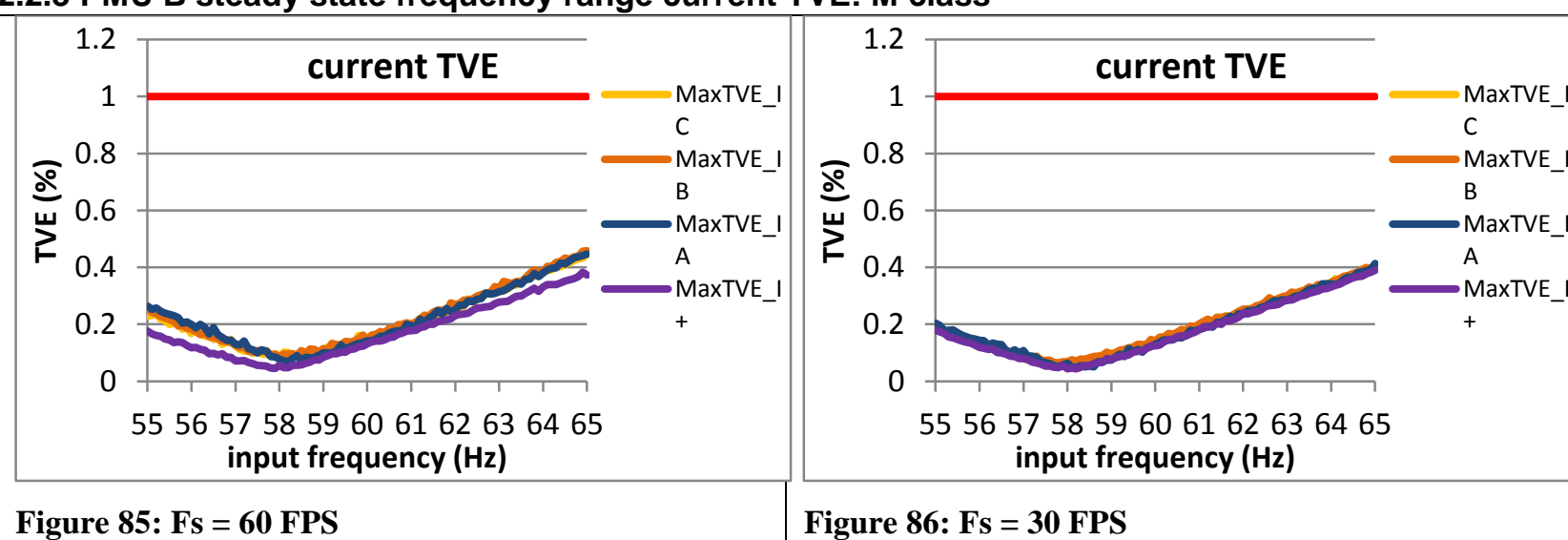

Figure 86: Fs = 30 FPS

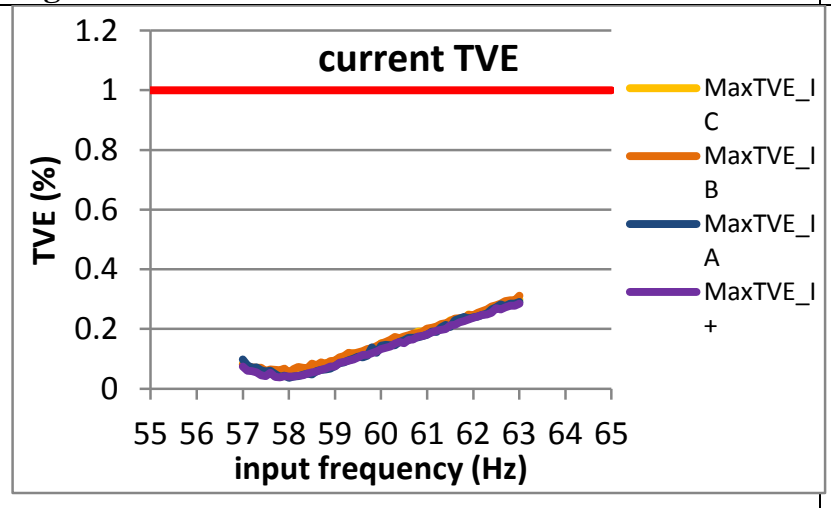

Figure 88: Fs = 15 FPS

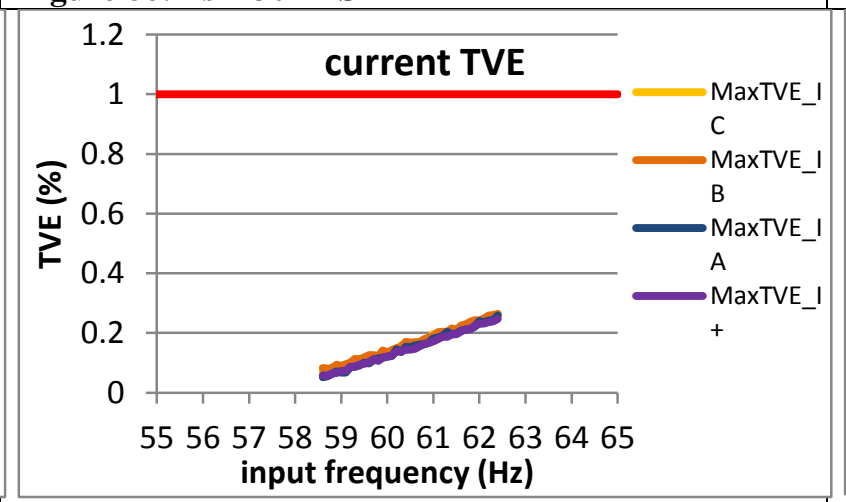

Figure 89: Fs = 12 FPS

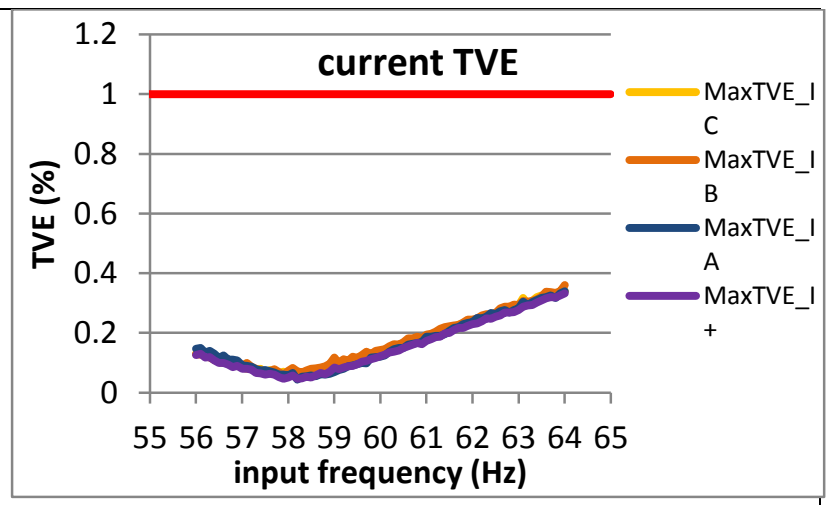

Figure 87: Fs = 20 FPS

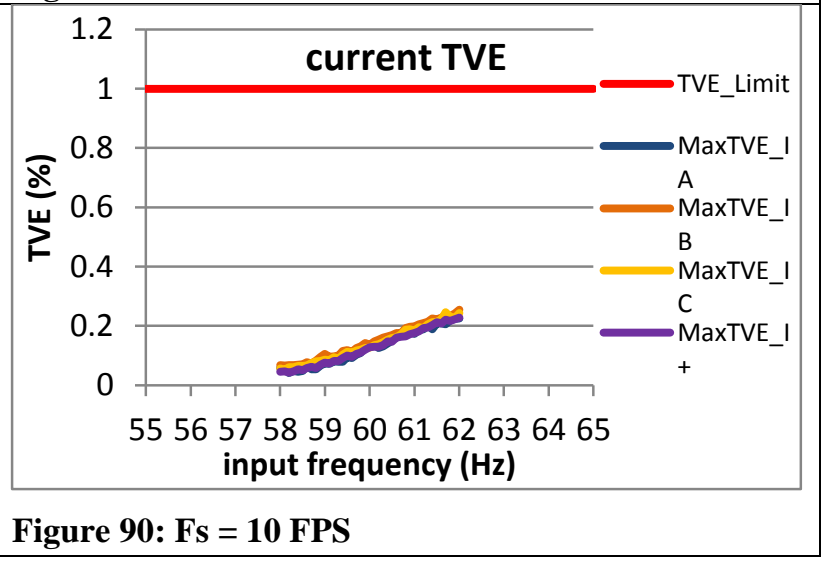




\subsubsection{PMU C steady state frequency range current TVE:, M class}
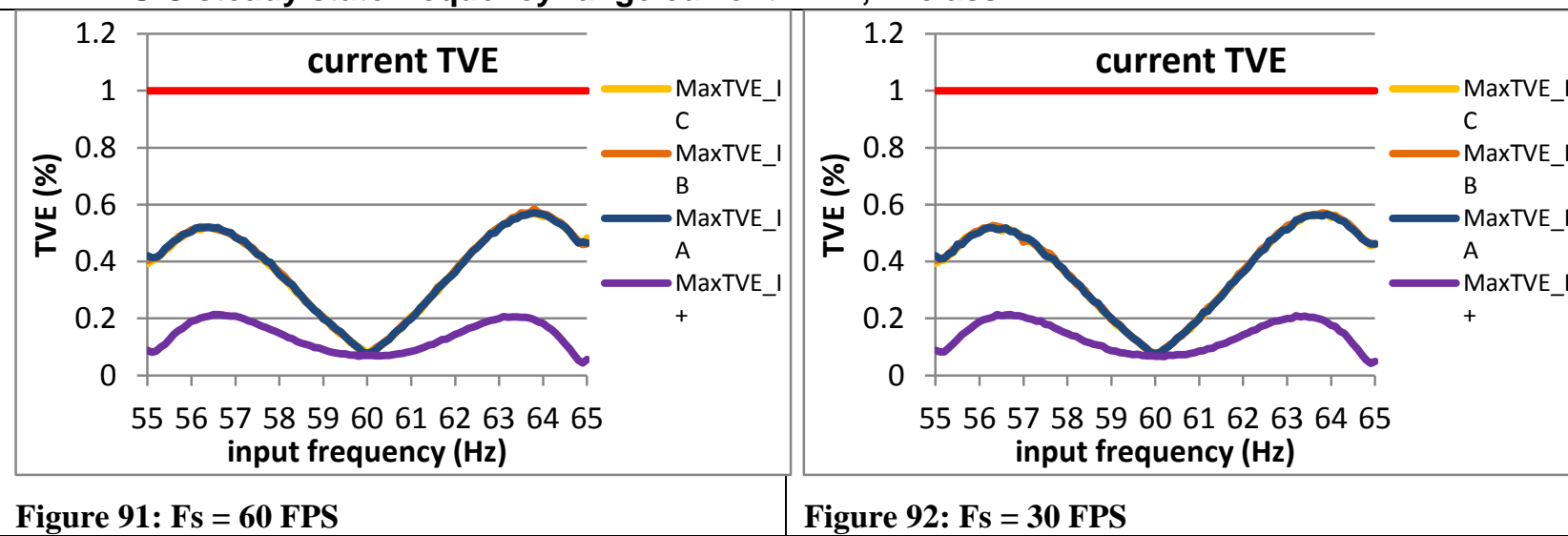

Figure 92: Fs = 30 FPS

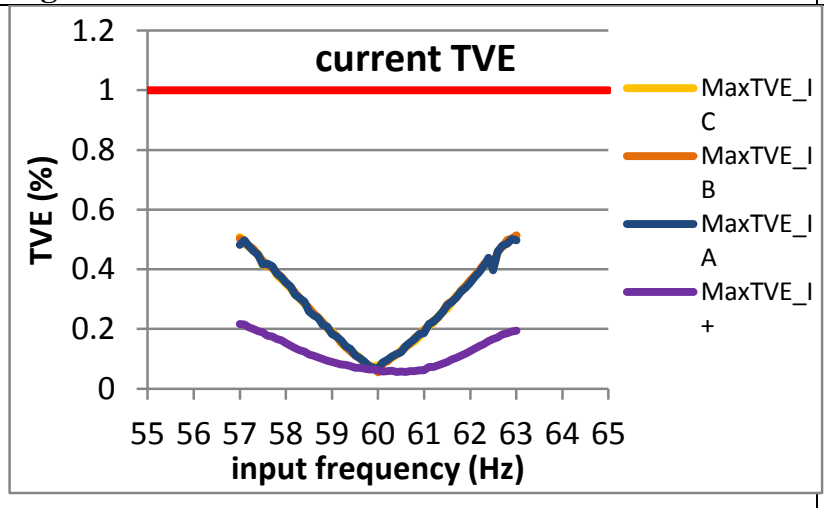

Figure 94: Fs = 15 FPS

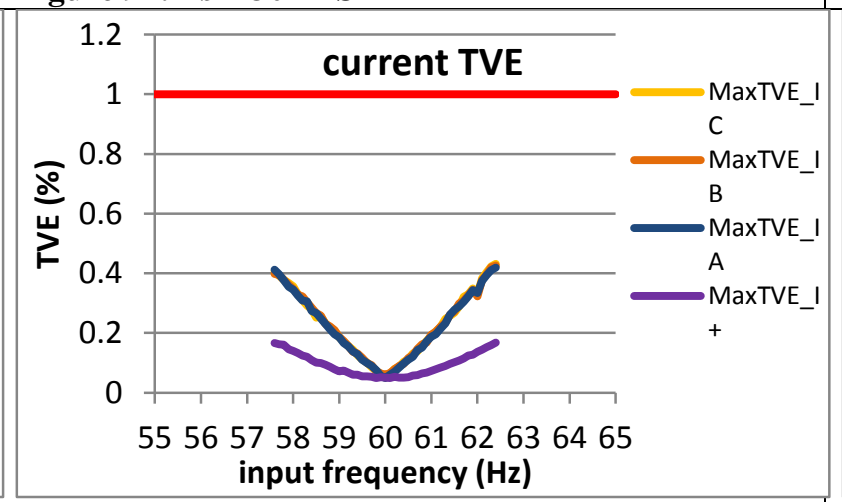

Figure 95: Fs = 12 FPS

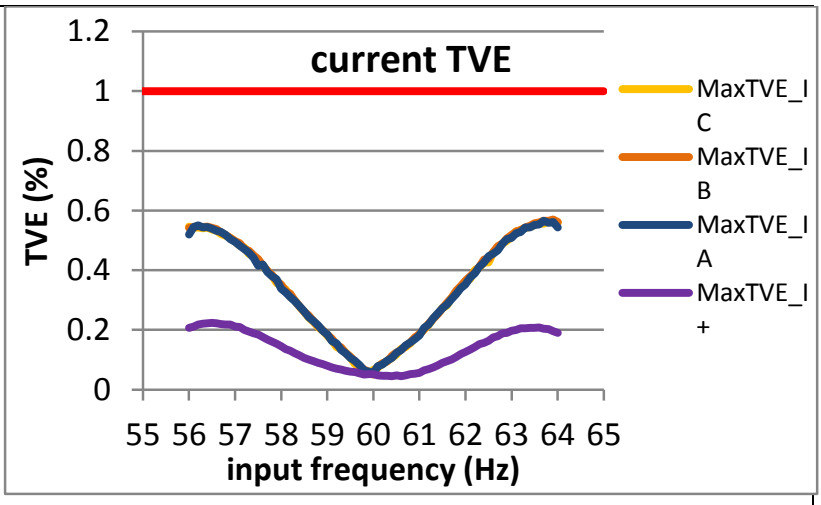

Figure 93: Fs = 20 FPS

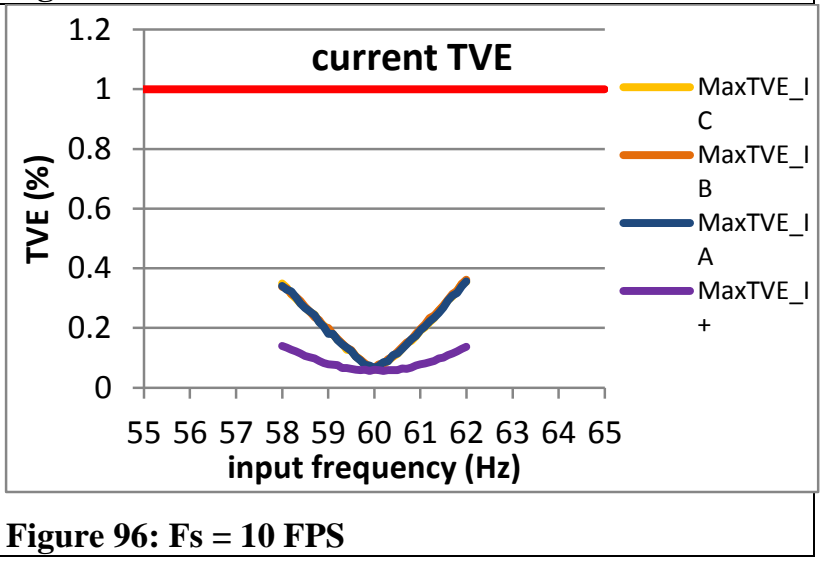




\subsubsection{PMU D steady state frequency range current TVE: M class}

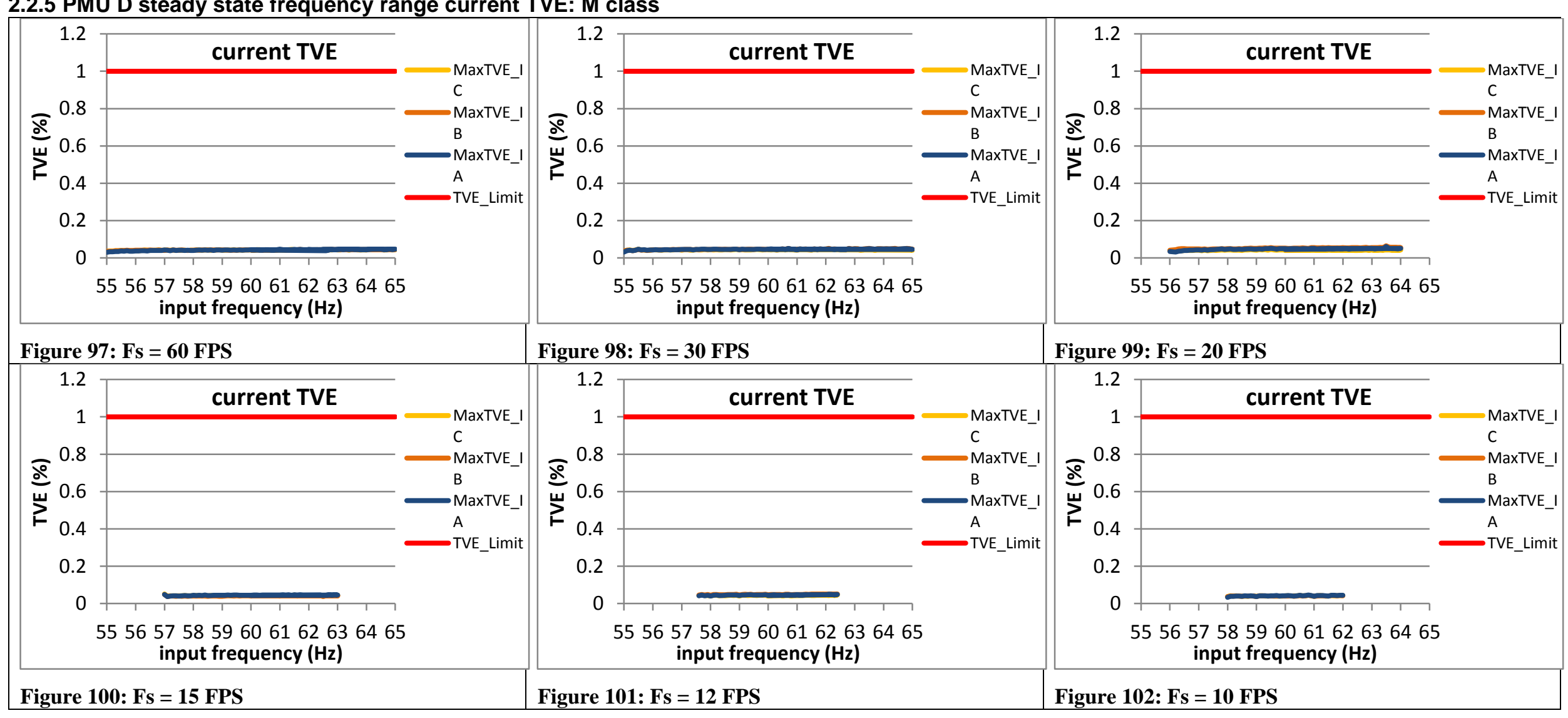




\subsubsection{PMU E steady state frequency range current TVE: M class}

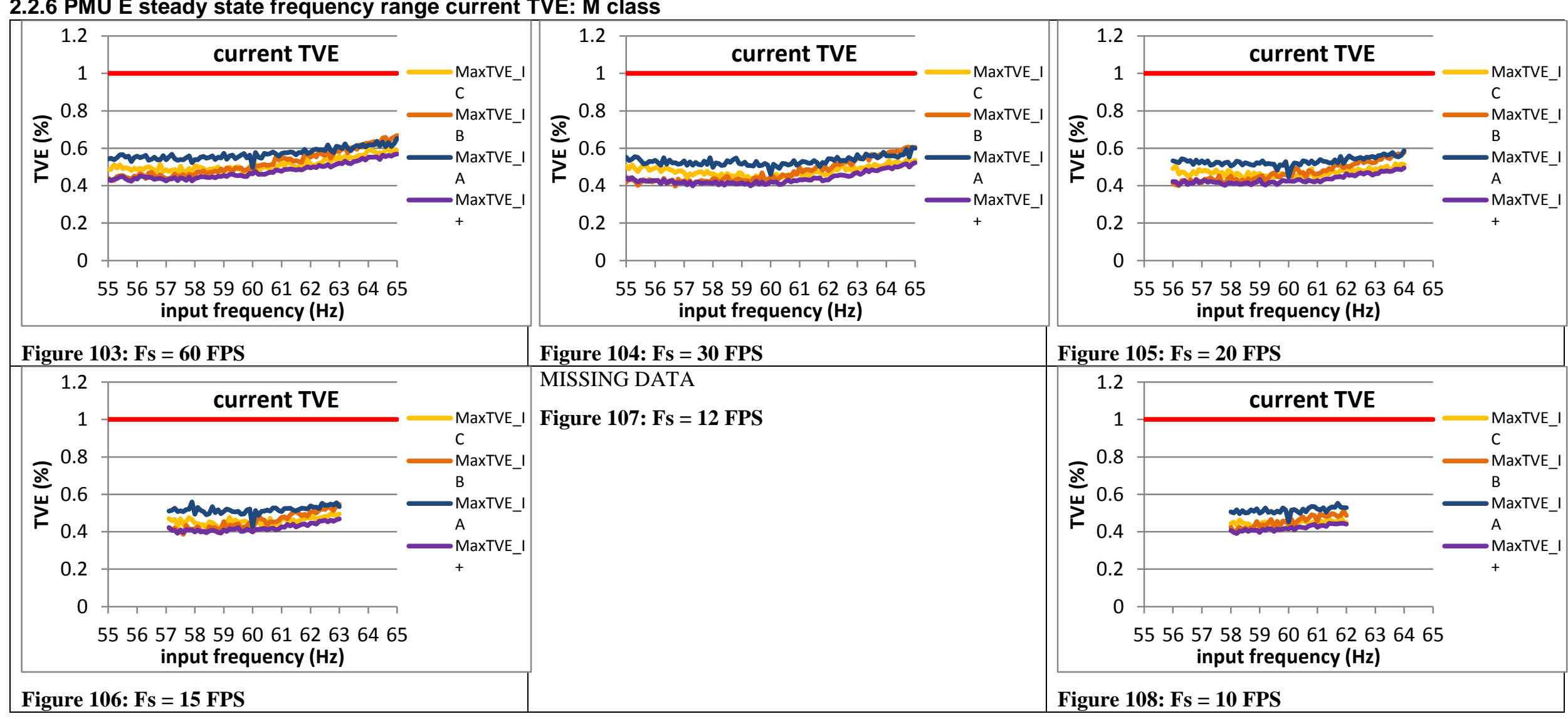




\subsubsection{PMU F steady state frequency range current TVE: M class}
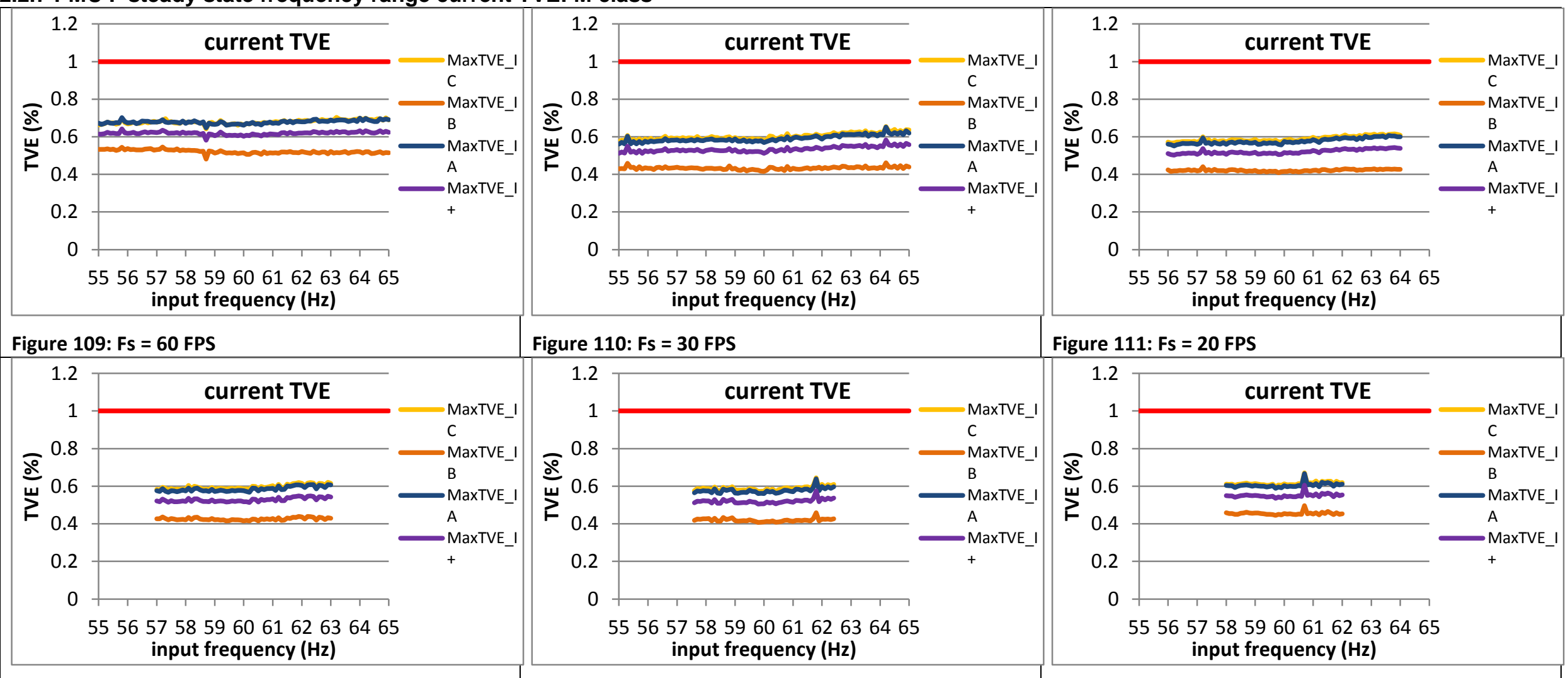

Figure 110: $\mathrm{Fs}=30 \mathrm{FPS}$
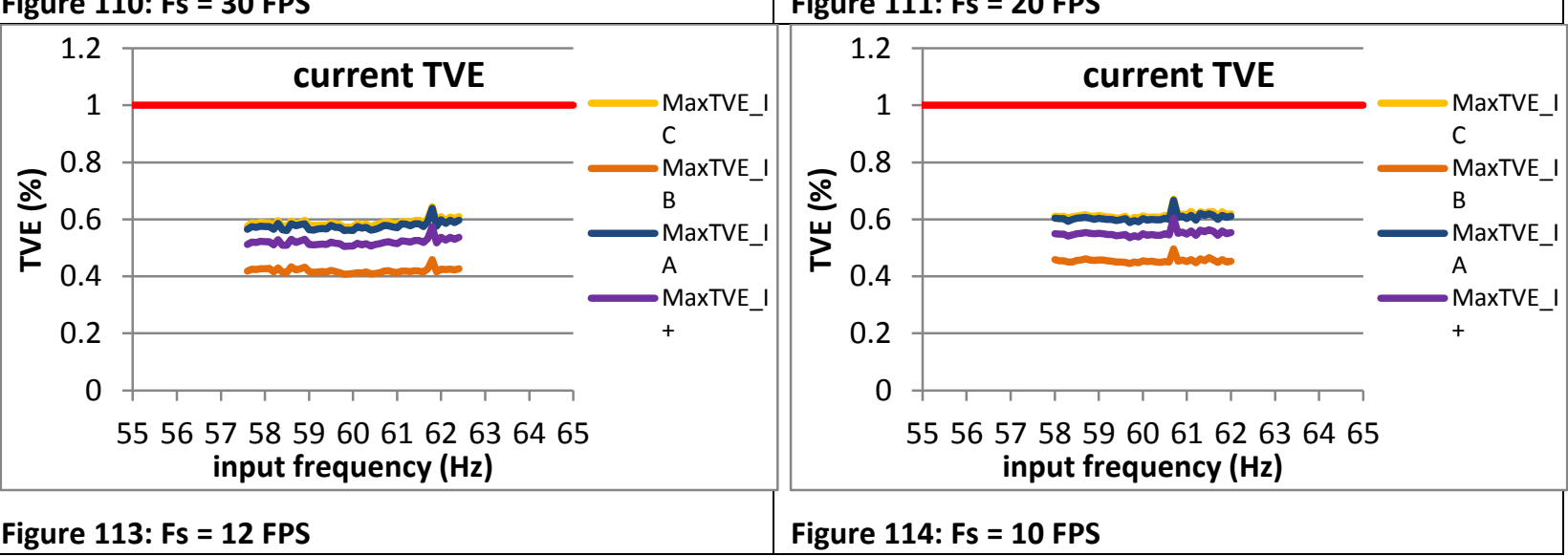

Figure 112: Fs = 15 FPS

Figure 113: Fs = 12 FPS

Figure 114: Fs = $10 \mathrm{FPS}$ 
2.2.8 PMU G steady state frequency range current TVE: M class

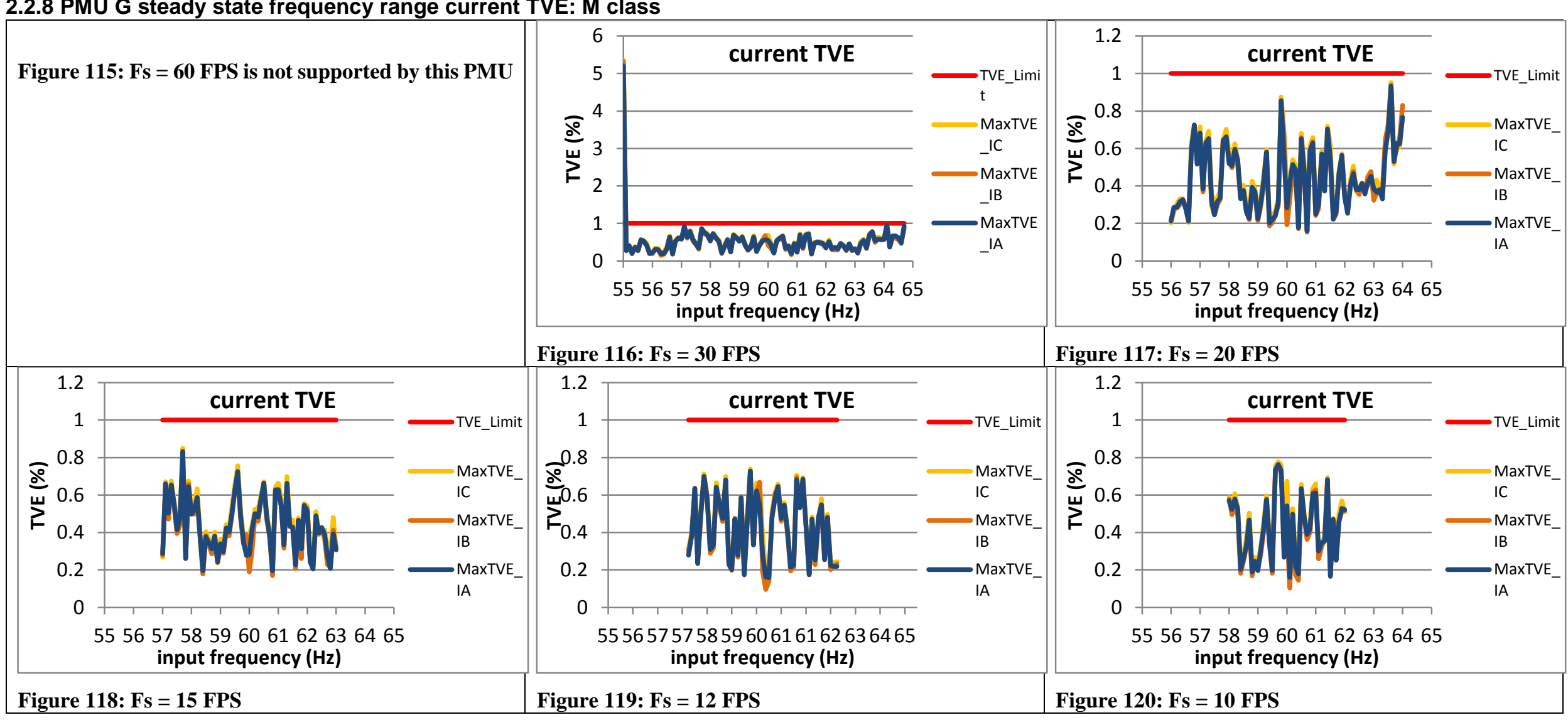


2.2.9 PMU H steady state frequency range current TVE: M class

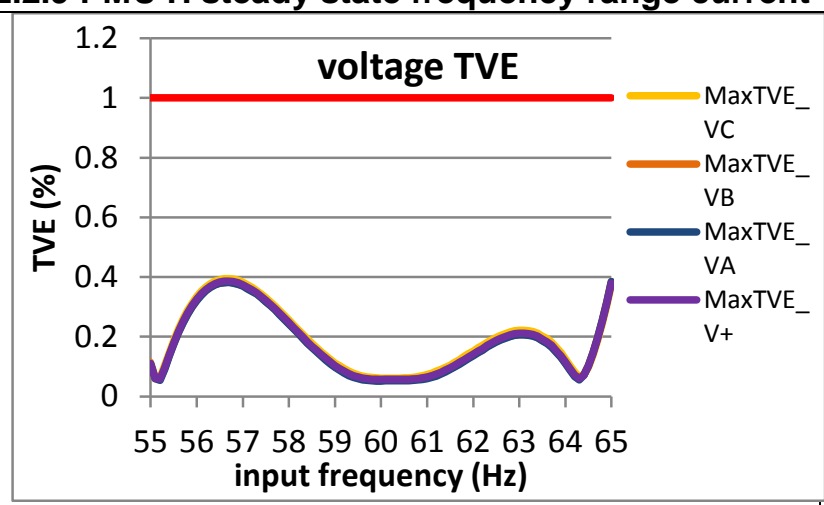

Figure 121: Fs = 60 FPS

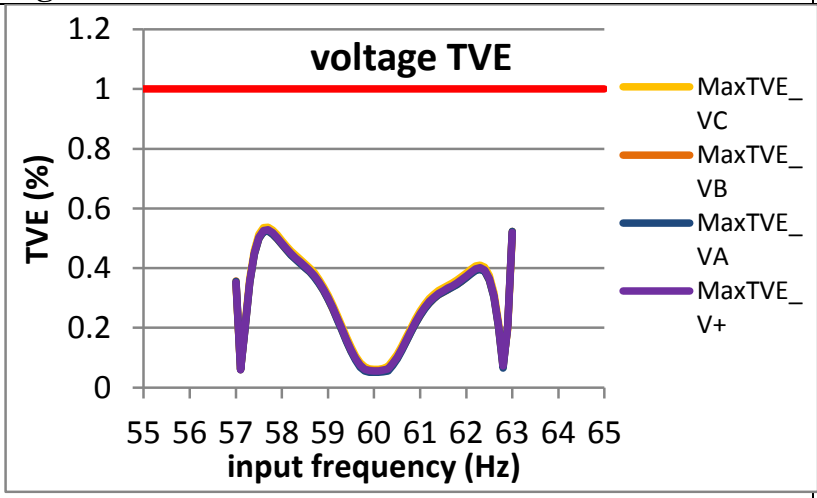

Figure 124: Fs = 15 FPS

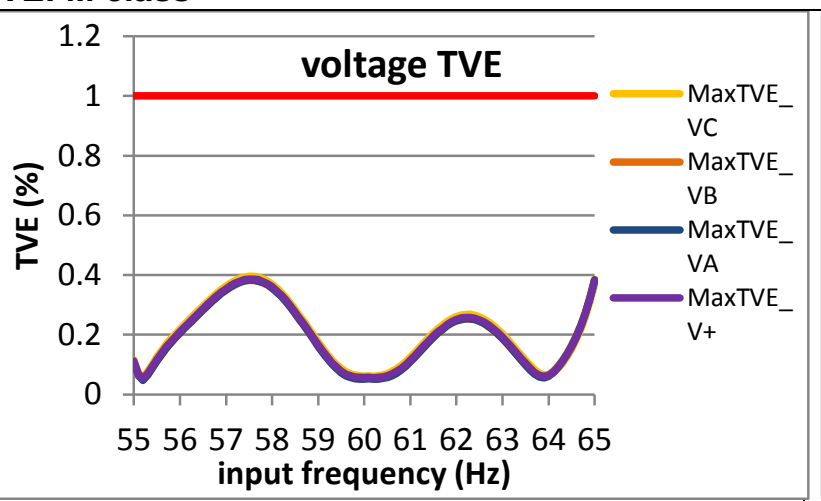

Figure 122: Fs = 30 FPS

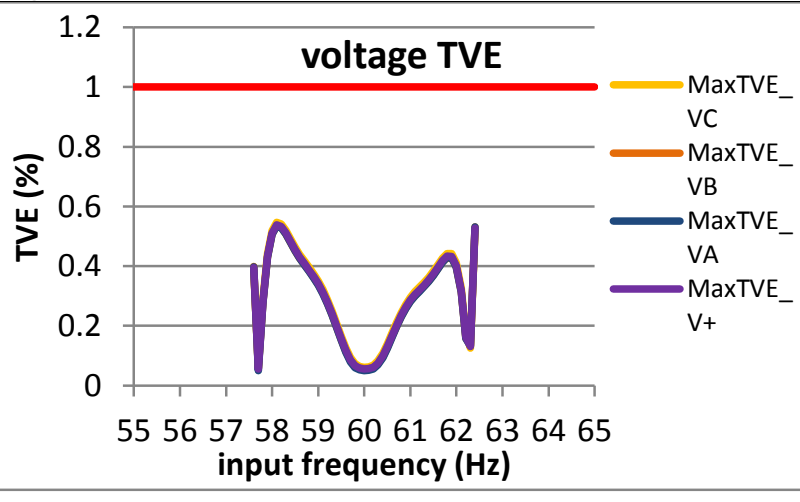

Figure 125: Fs = 12 FPS

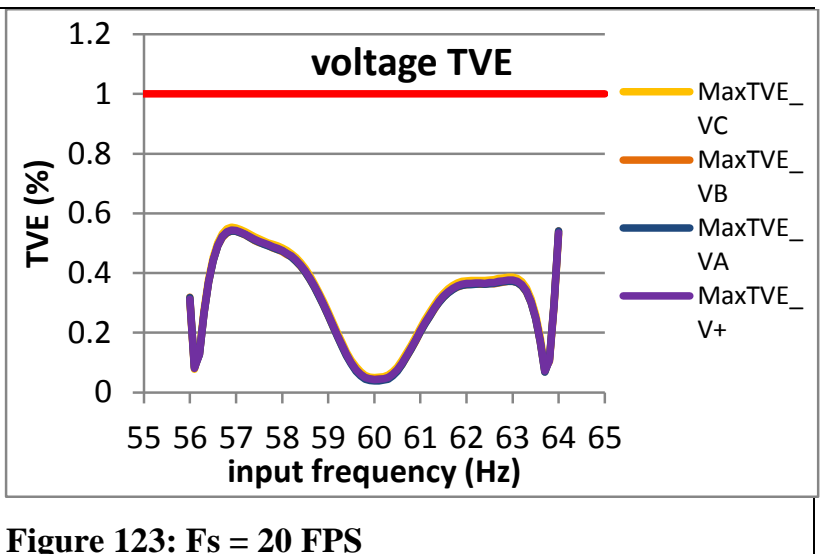

Figure 123: Fs = 20 FPS

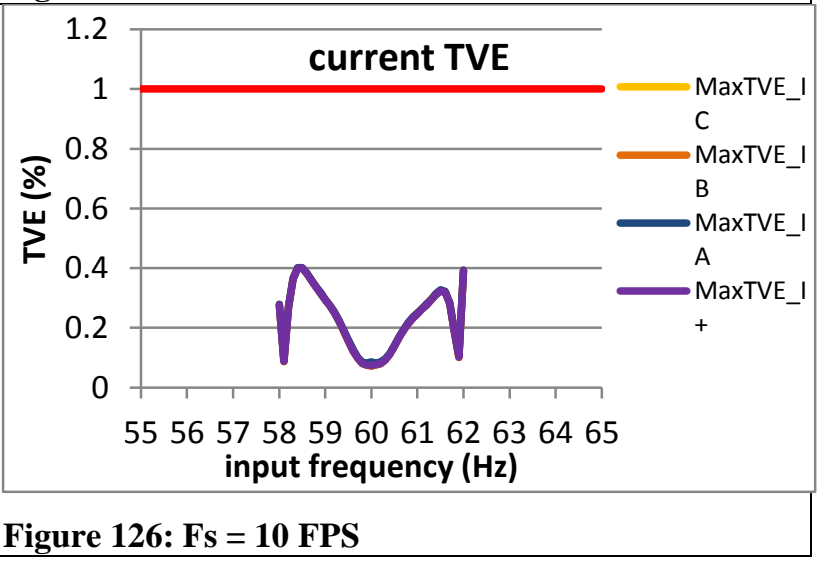




\subsubsection{PMU I steady state frequency range current TVE: M class}
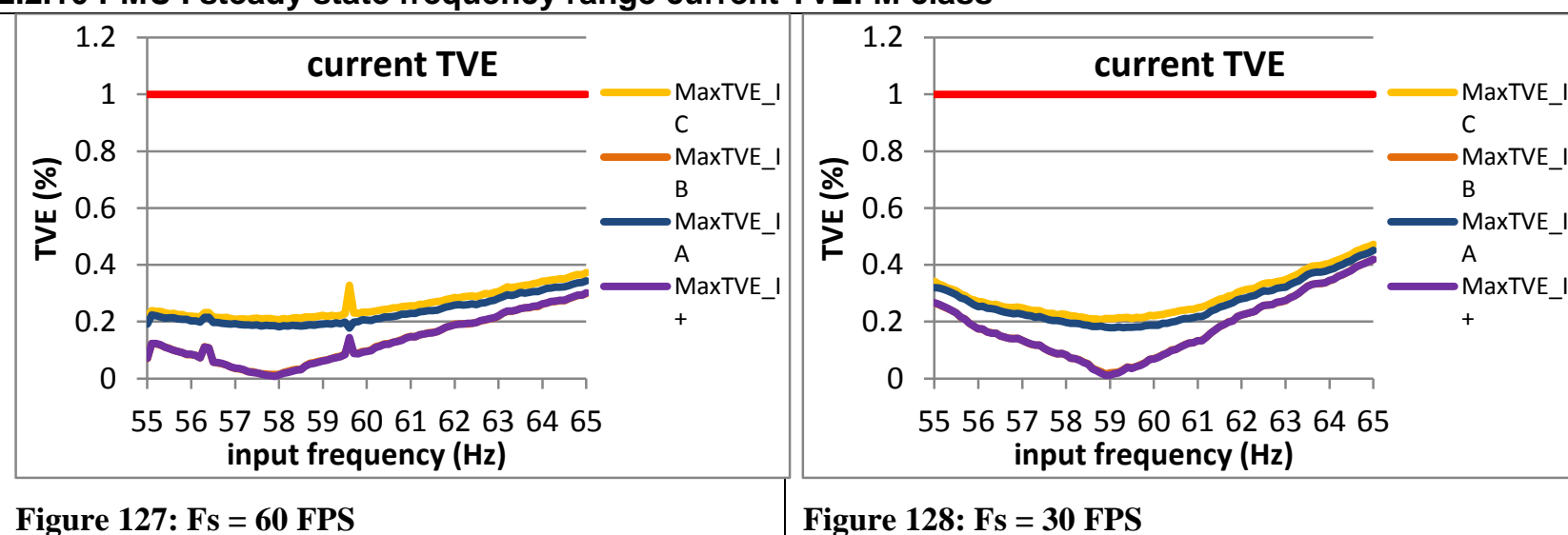

Figure 128: Fs = 30 FPS

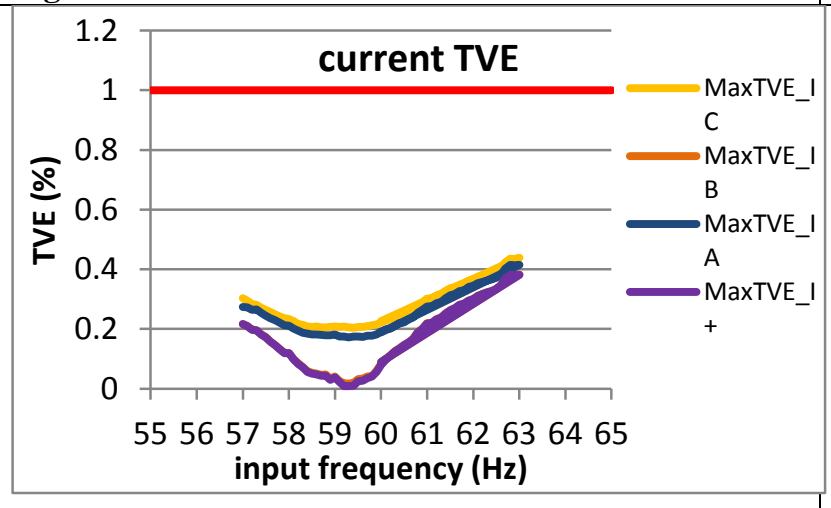

Figure 130: Fs = 15 FPS

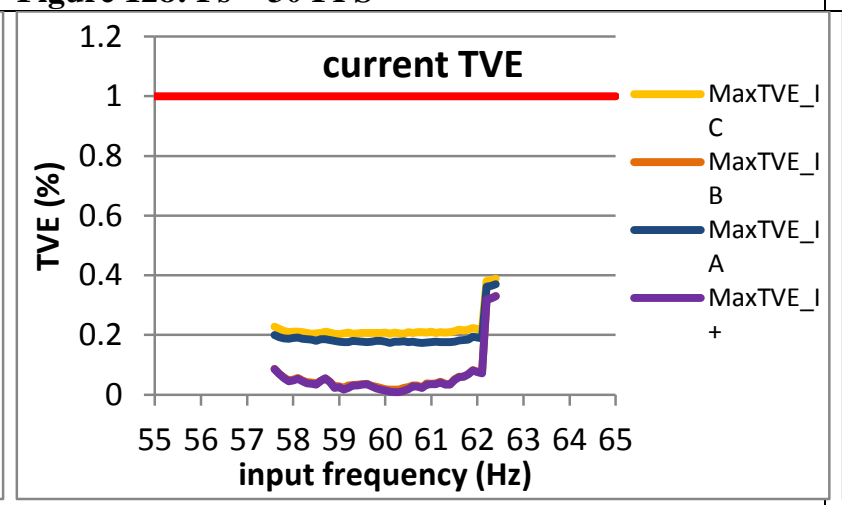

Figure 131: Fs = 12 FPS

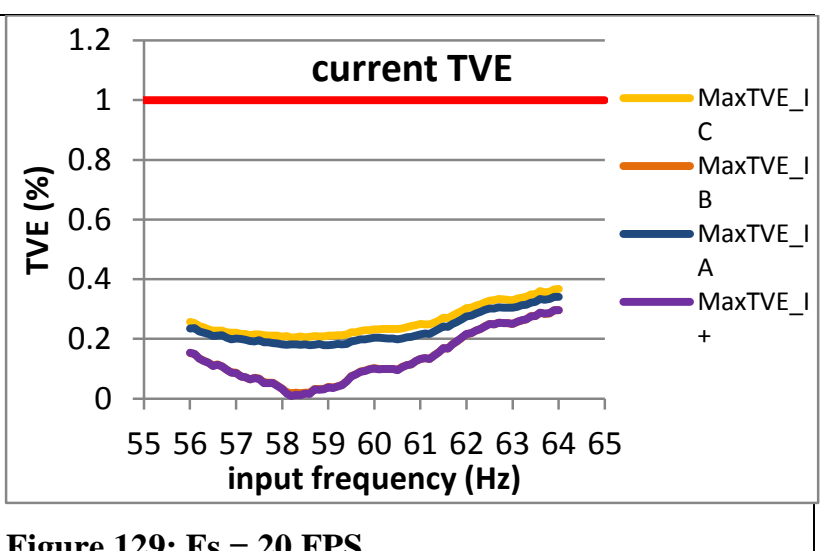

Figure 129: Fs = 20 FPS

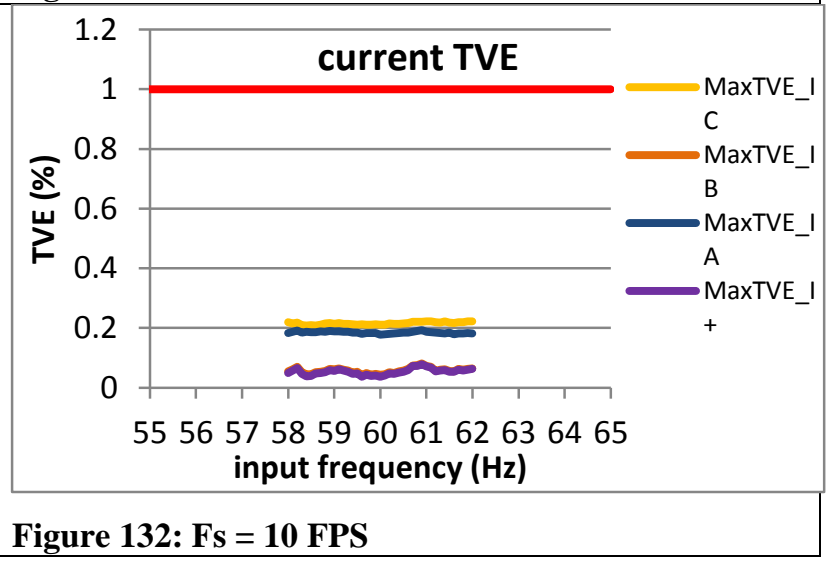


2.2.11 PMU J steady state frequency range current TVE: M class

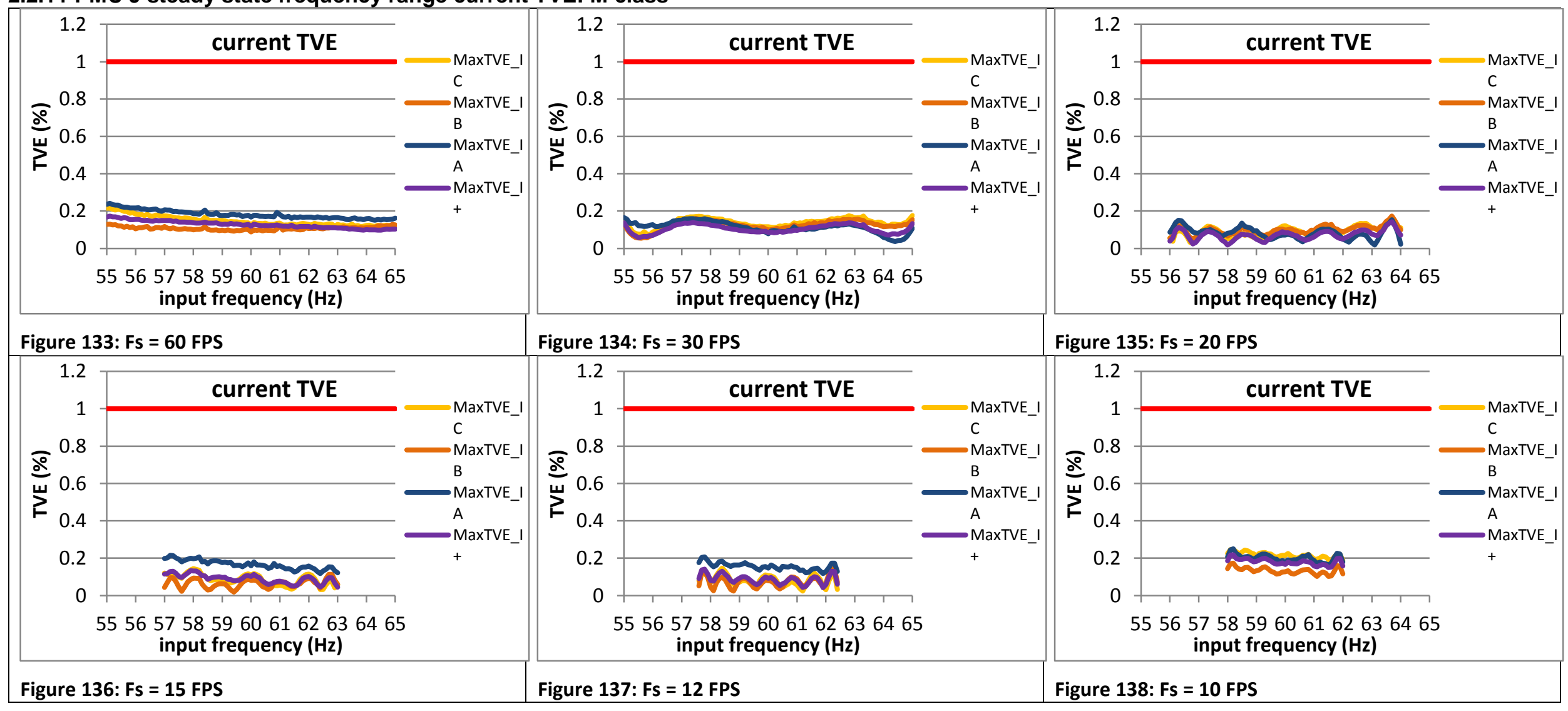




\subsection{Steady state frequency range frequency error: $M$ class}

\subsubsection{C37.118.1 Annex $C$ steady state frequency range frequency error: $M$ class}

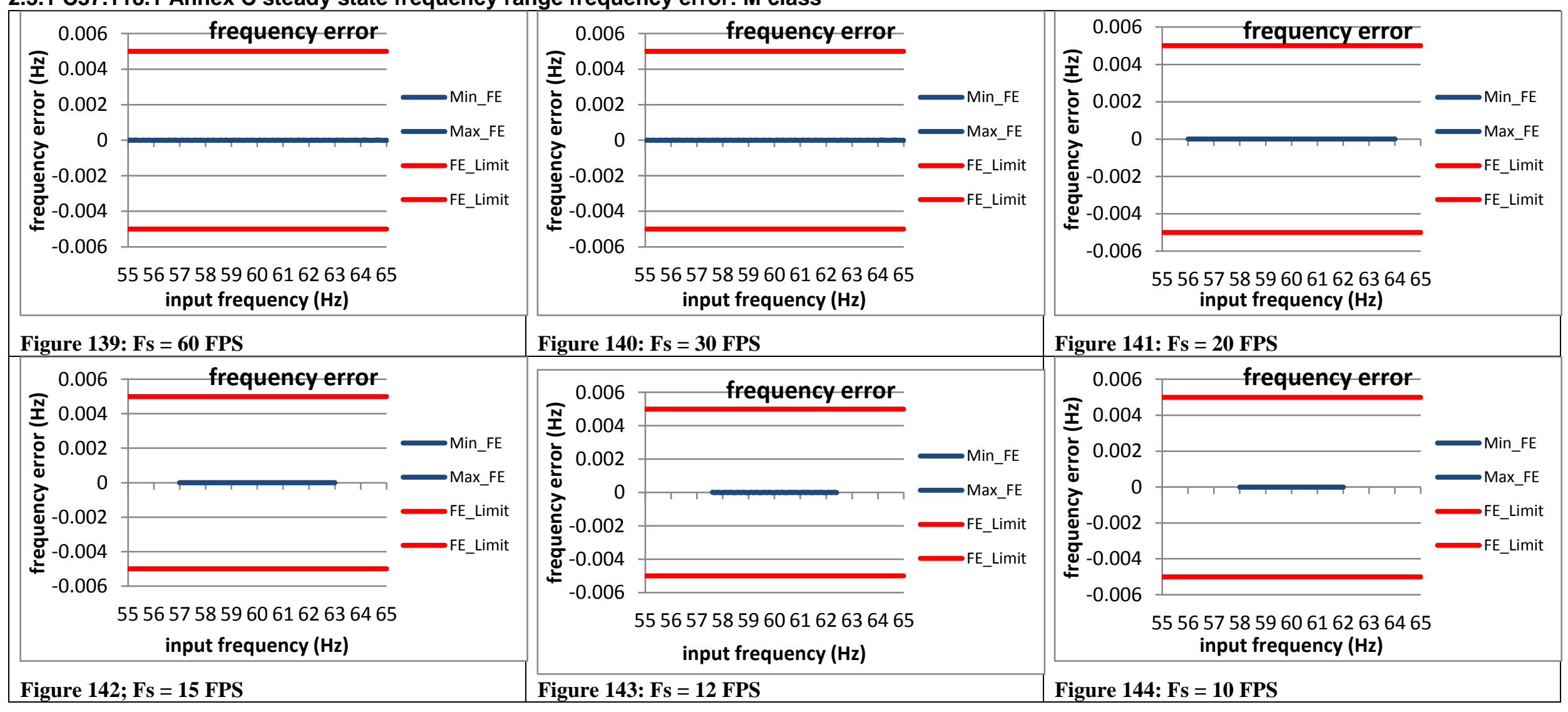




\subsubsection{PMU A steady state frequency range frequency error: $M$ class}

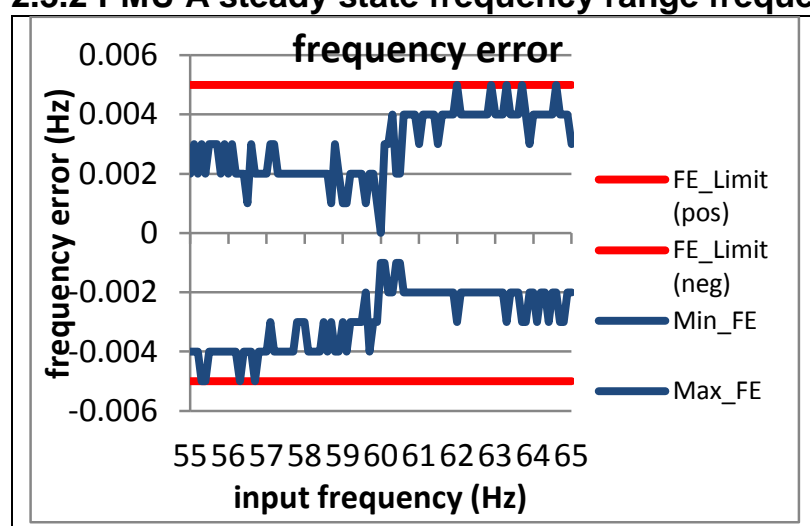

Figure 145: Fs = 60 FPS

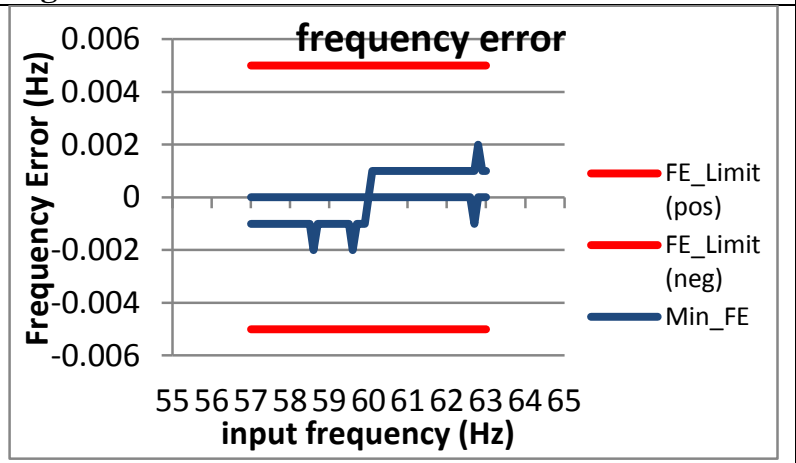

Figure 148: Fs $=15$ FPS
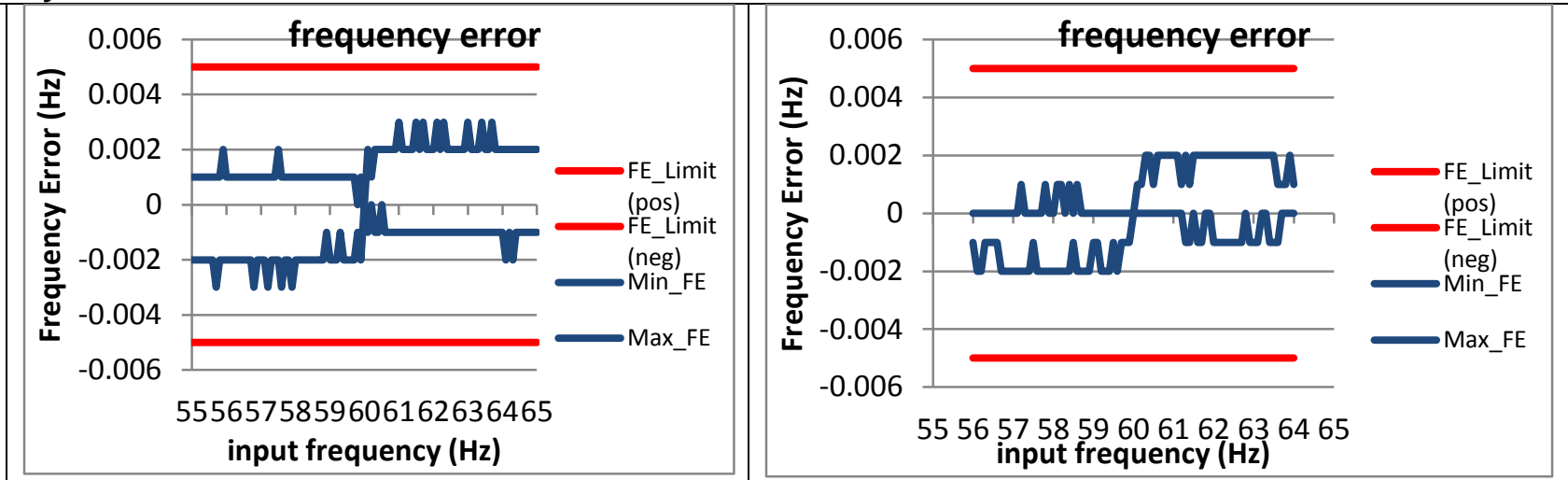

Figure 146: Fs = 30 FPS

MISSING DATA
Figure 147: Fs = 20 FPS

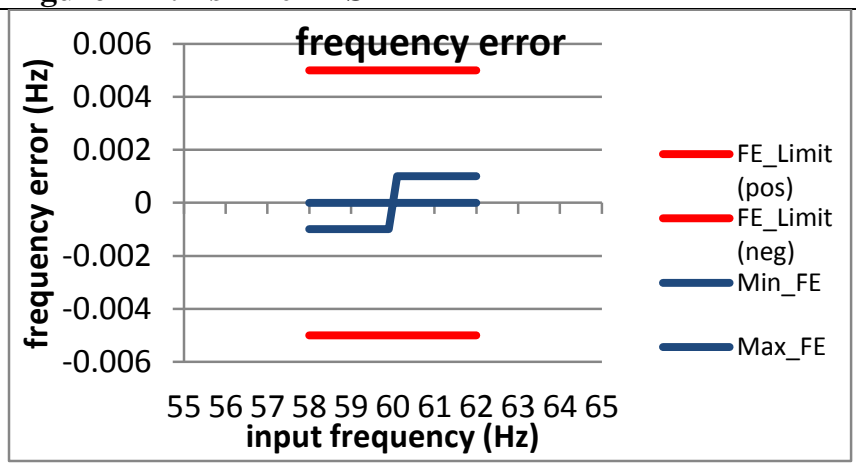

Figure 149: Fs = 10 FPS 
2.3.3 PMU B steady state frequency range frequency error: $M$ class

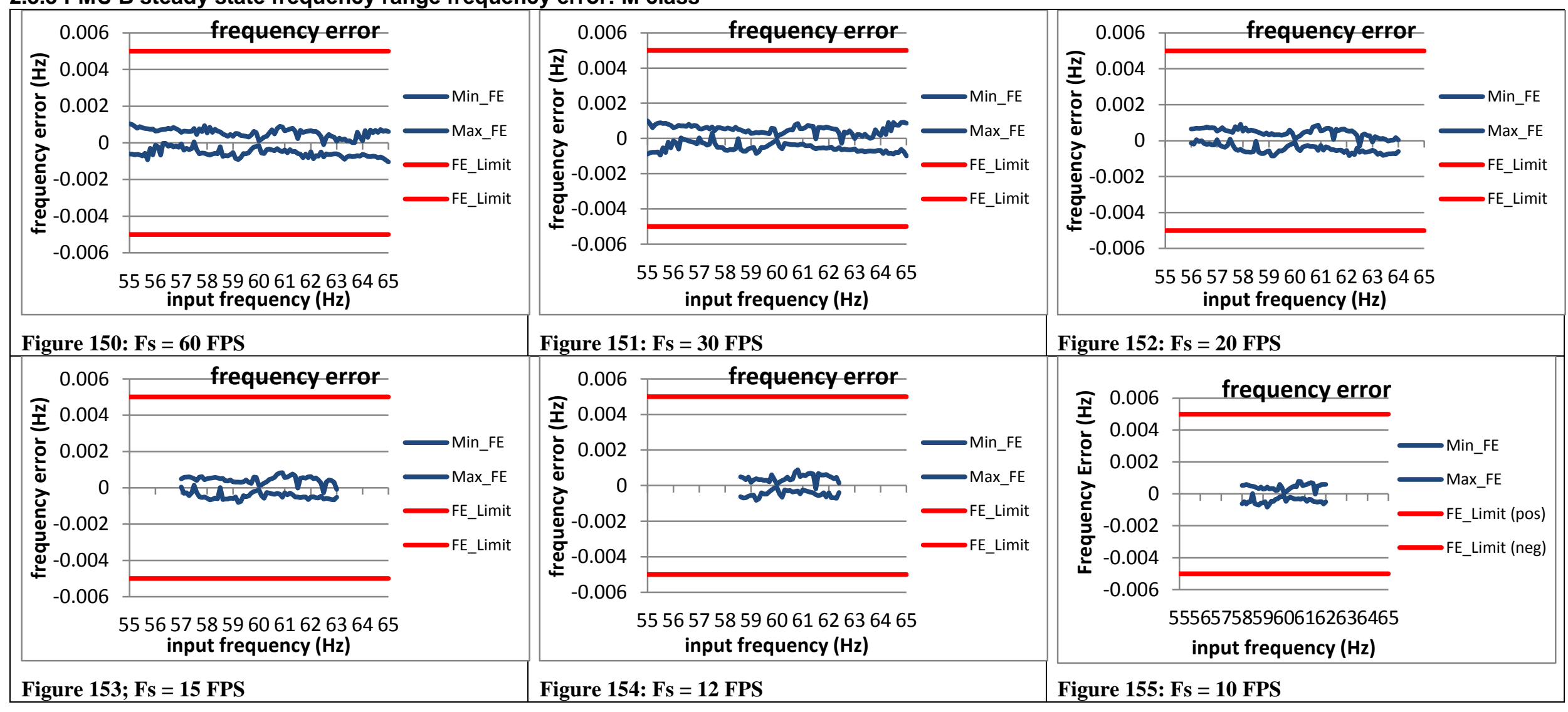




\subsubsection{PMU C steady state frequency range frequency error: M class}

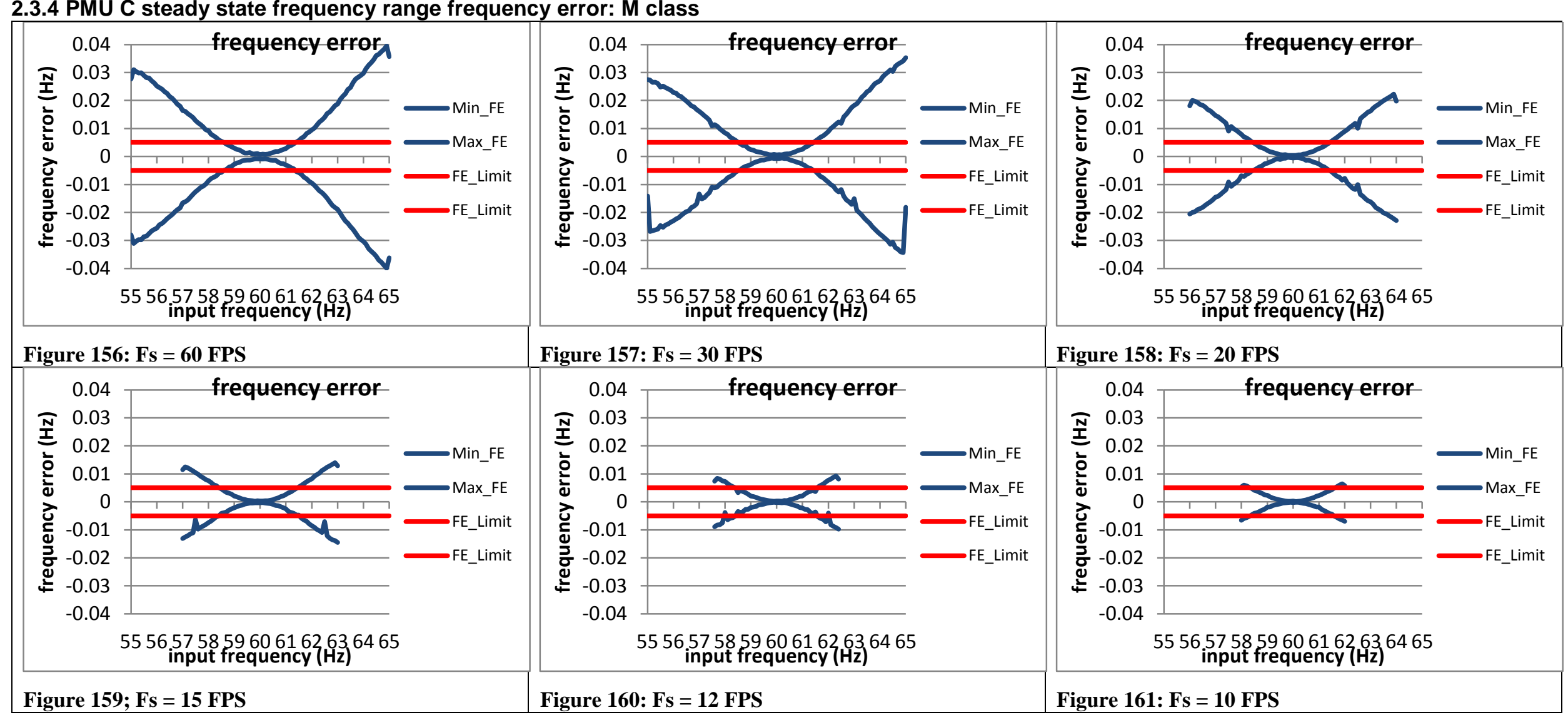




\subsubsection{PMU D steady state frequency range frequency error: M class}

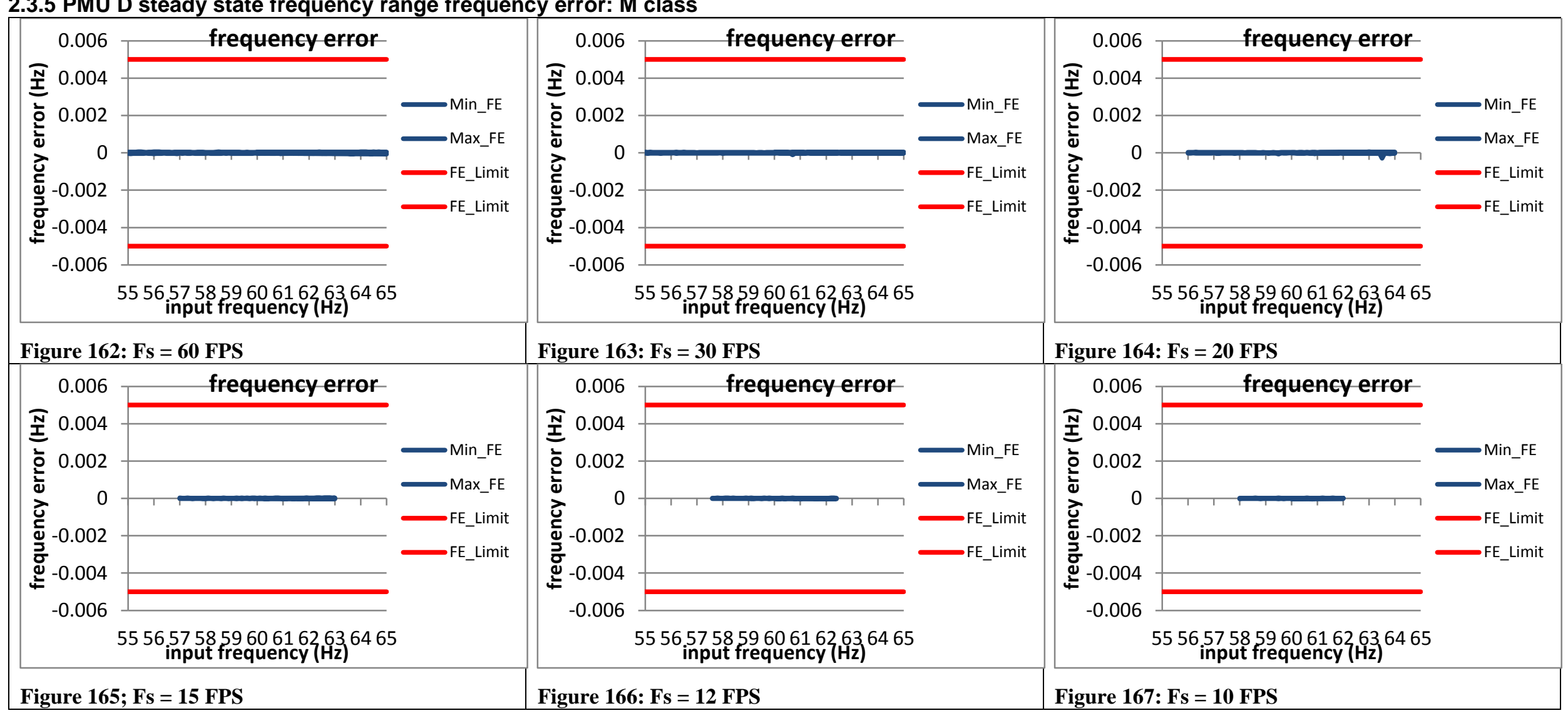


2.3.6 PMU E steady state frequency range frequency error: $M$ class

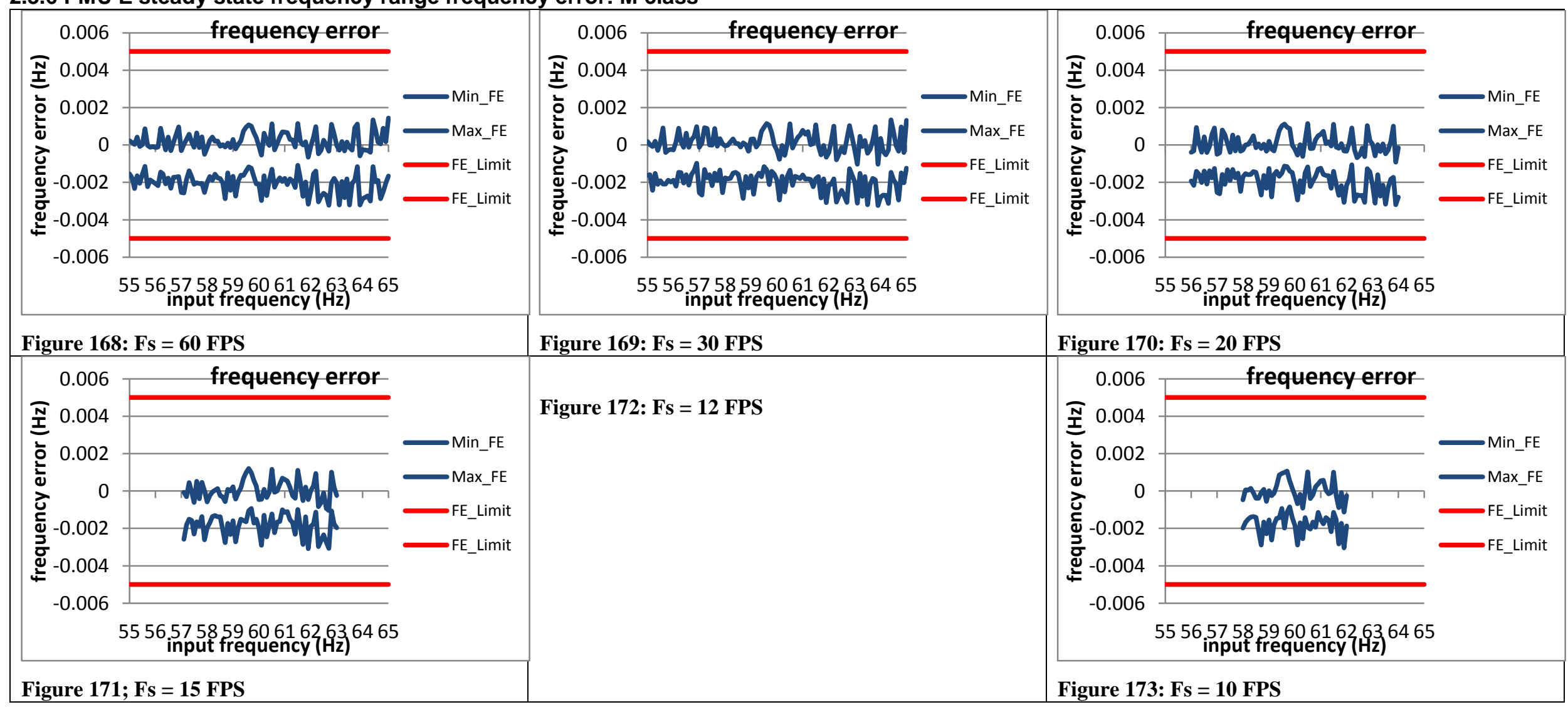


2.3.7 PMU F steady state frequency range frequency error: $M$ class

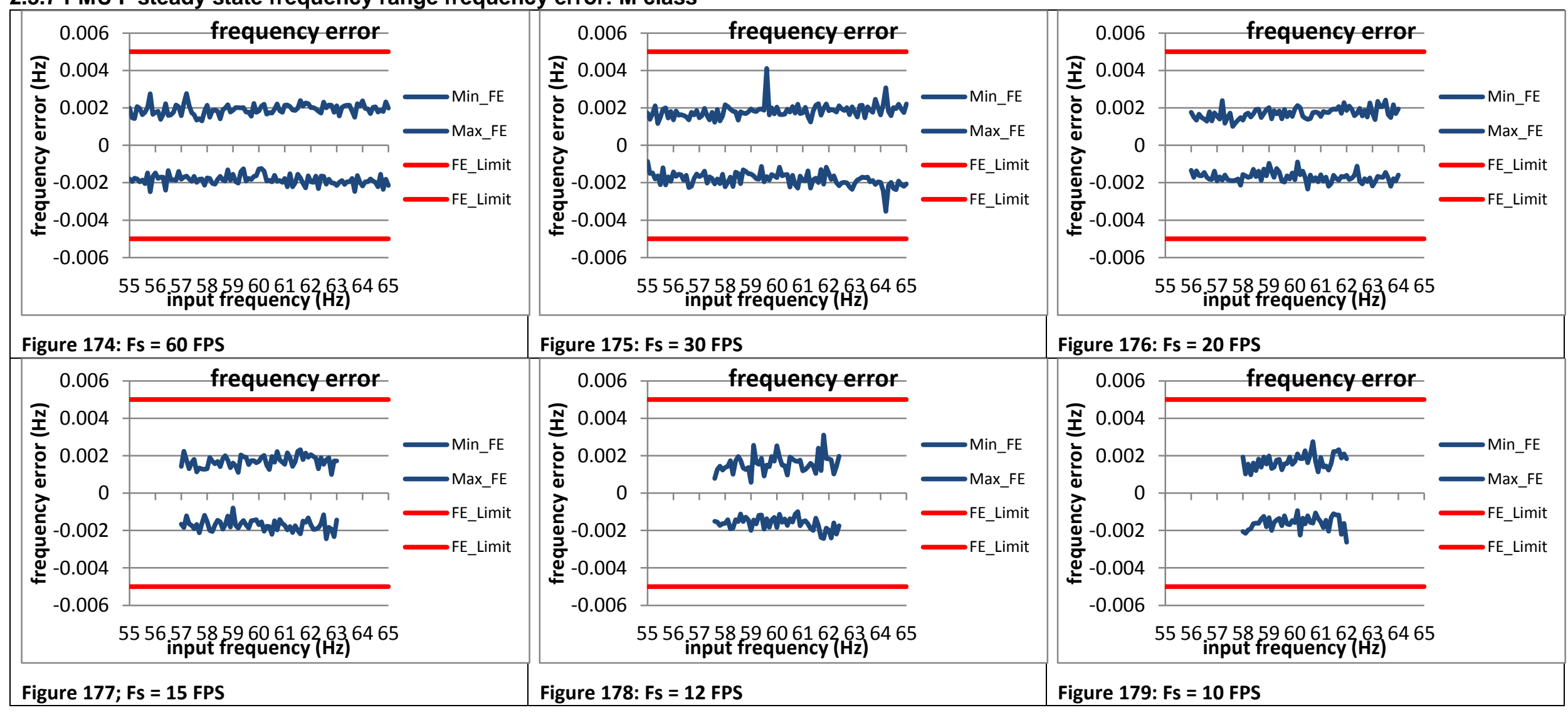


2.3.8 PMU G steady state frequency range frequency error: M class

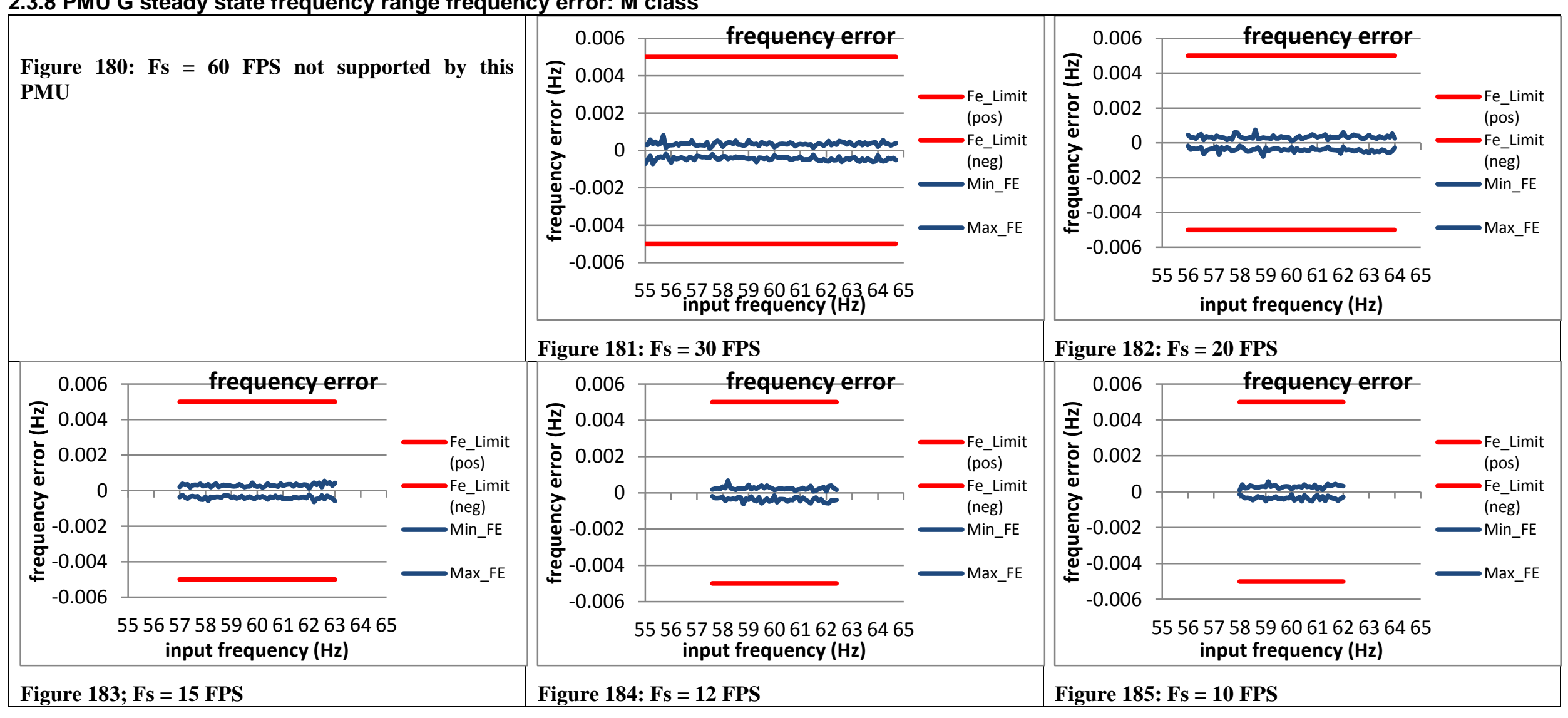


2.3.9 PMU H steady state frequency range frequency error: $M$ class

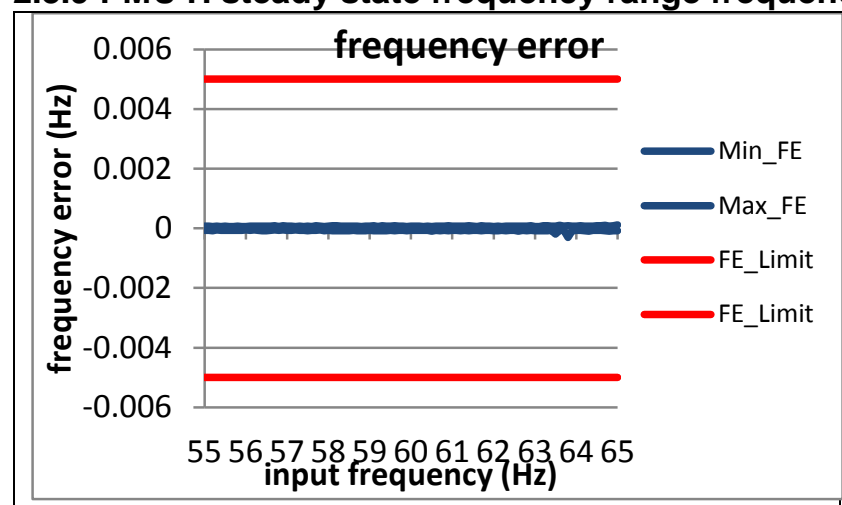

Figure 186: Fs = 60 FPS

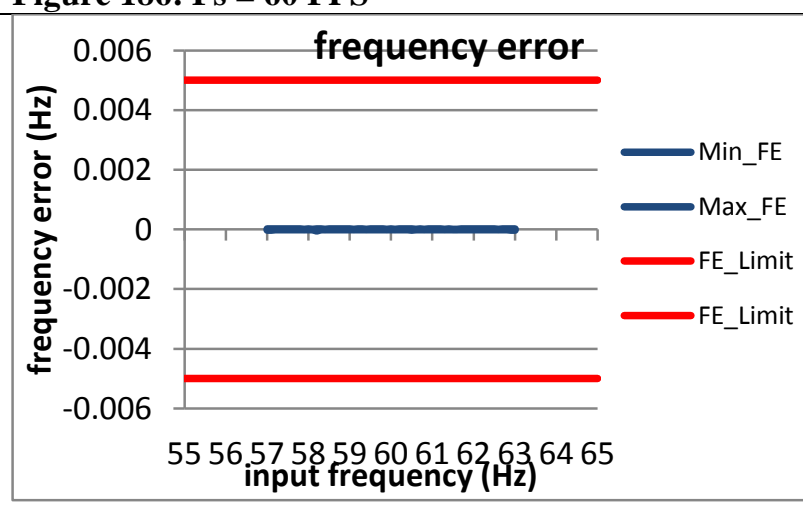

Figure 189; Fs = 15 FPS
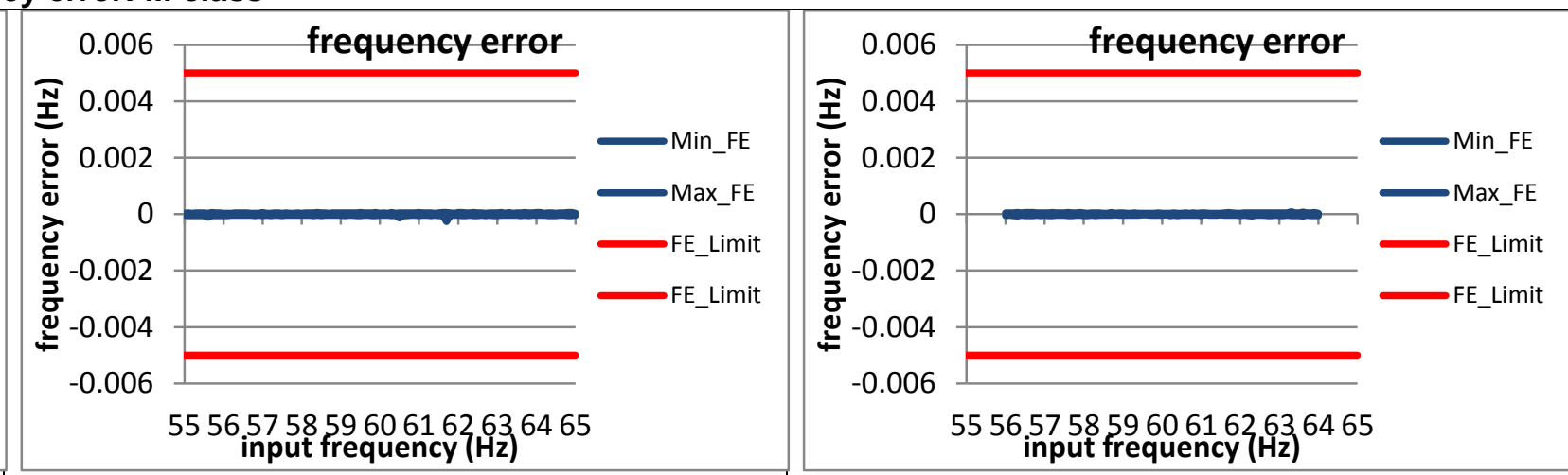

Figure 187: Fs = 30 FPS

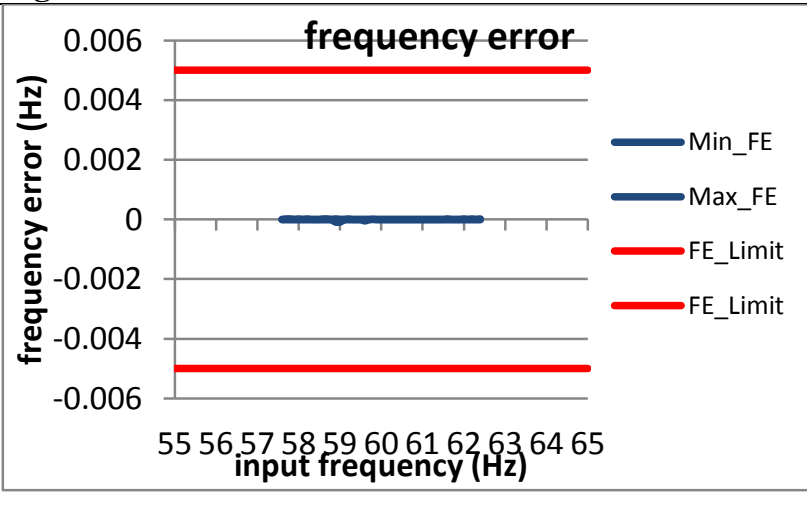

Figure 190: Fs = 12 FPS
Figure 188: Fs = 20 FPS

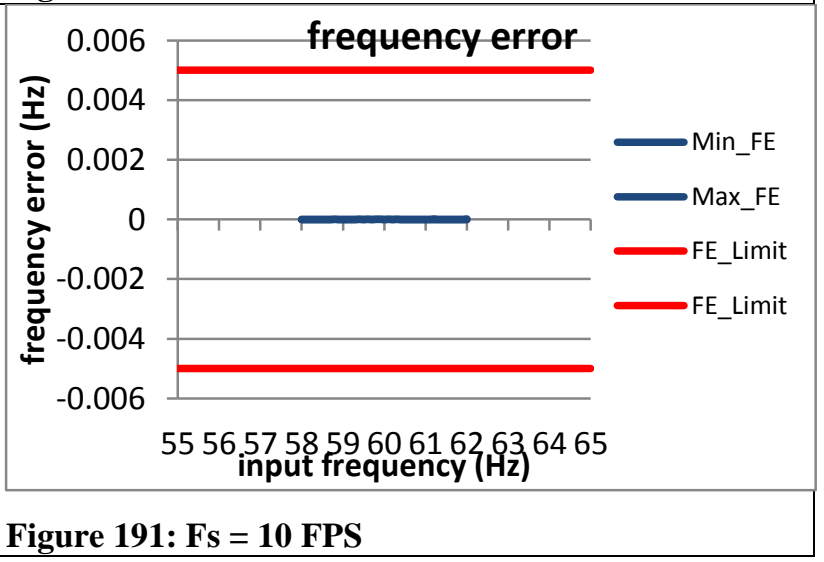


2.3.10 PMU I steady state frequency range frequency error: M class

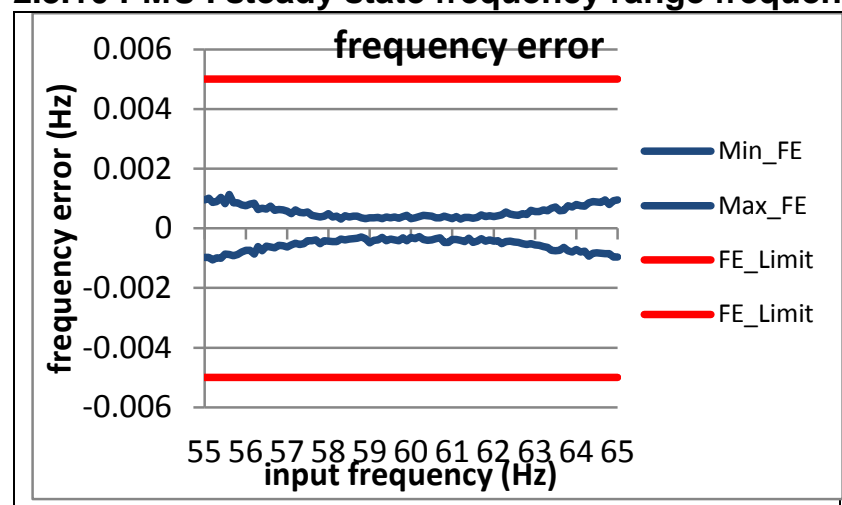

Figure 192: Fs = 60 FPS

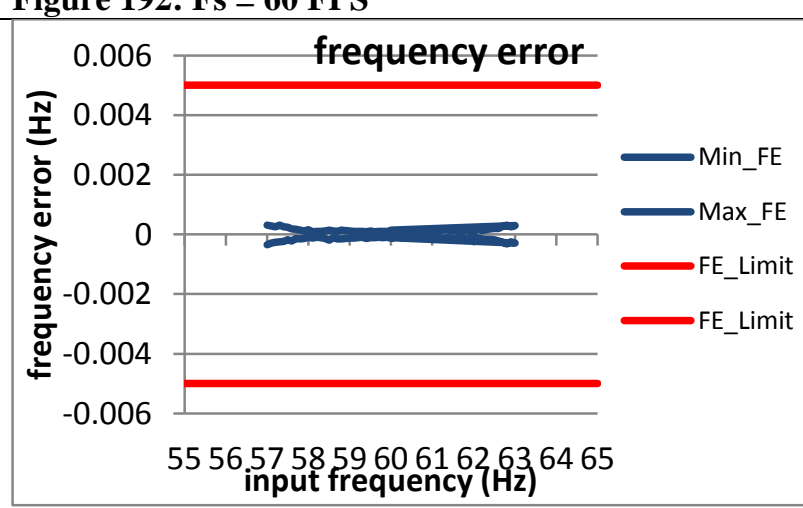

Figure 195; Fs = 15 FPS

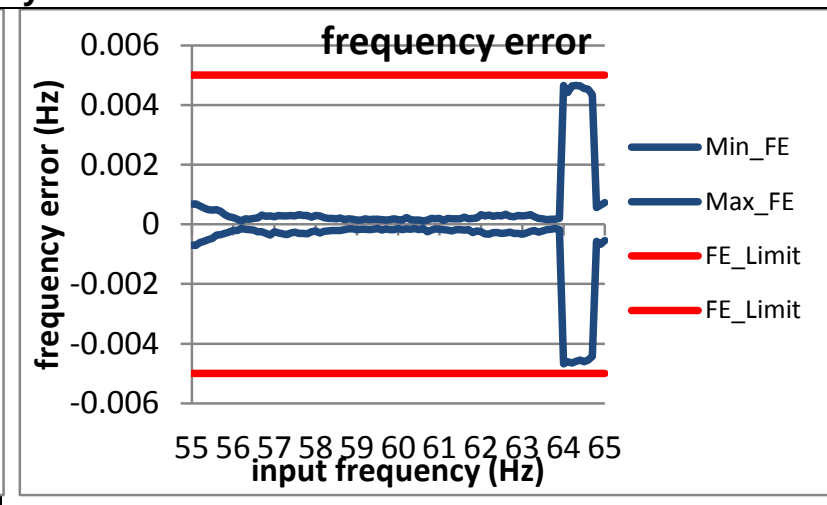

Figure 193: Fs = 30 FPS

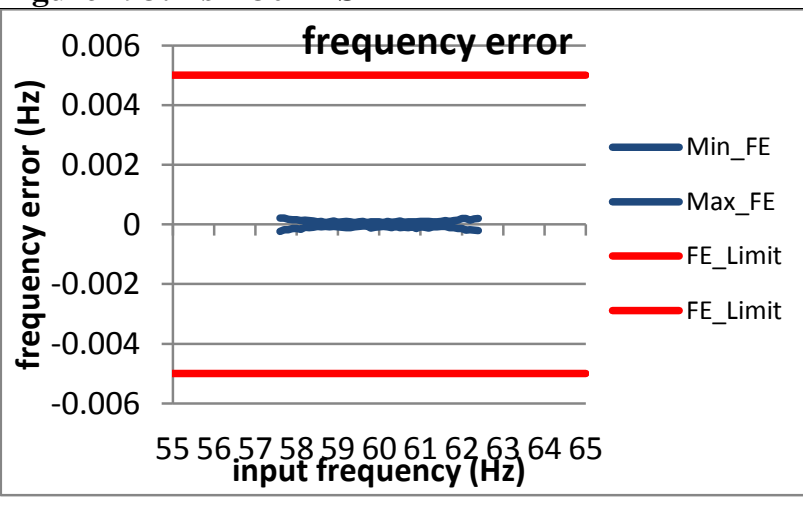

Figure 196: Fs = 12 FPS

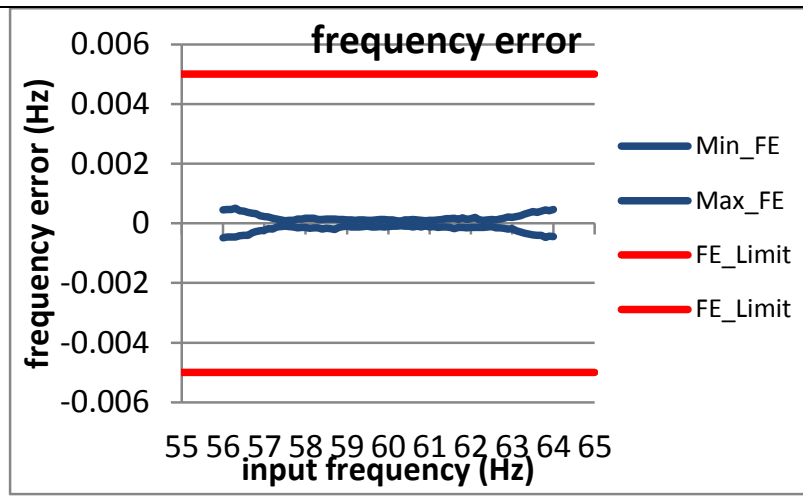

Figure 194: Fs = 20 FPS

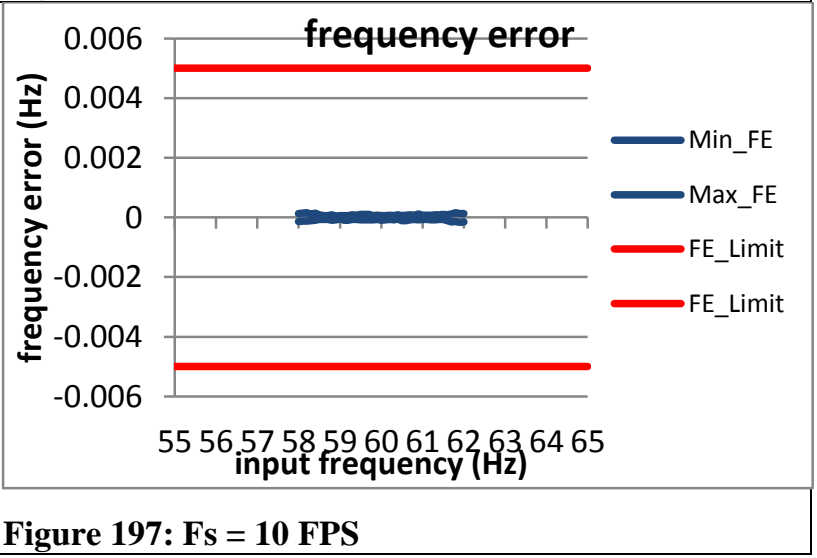


2.3.11 PMU J steady state frequency range frequency error: $M$ class

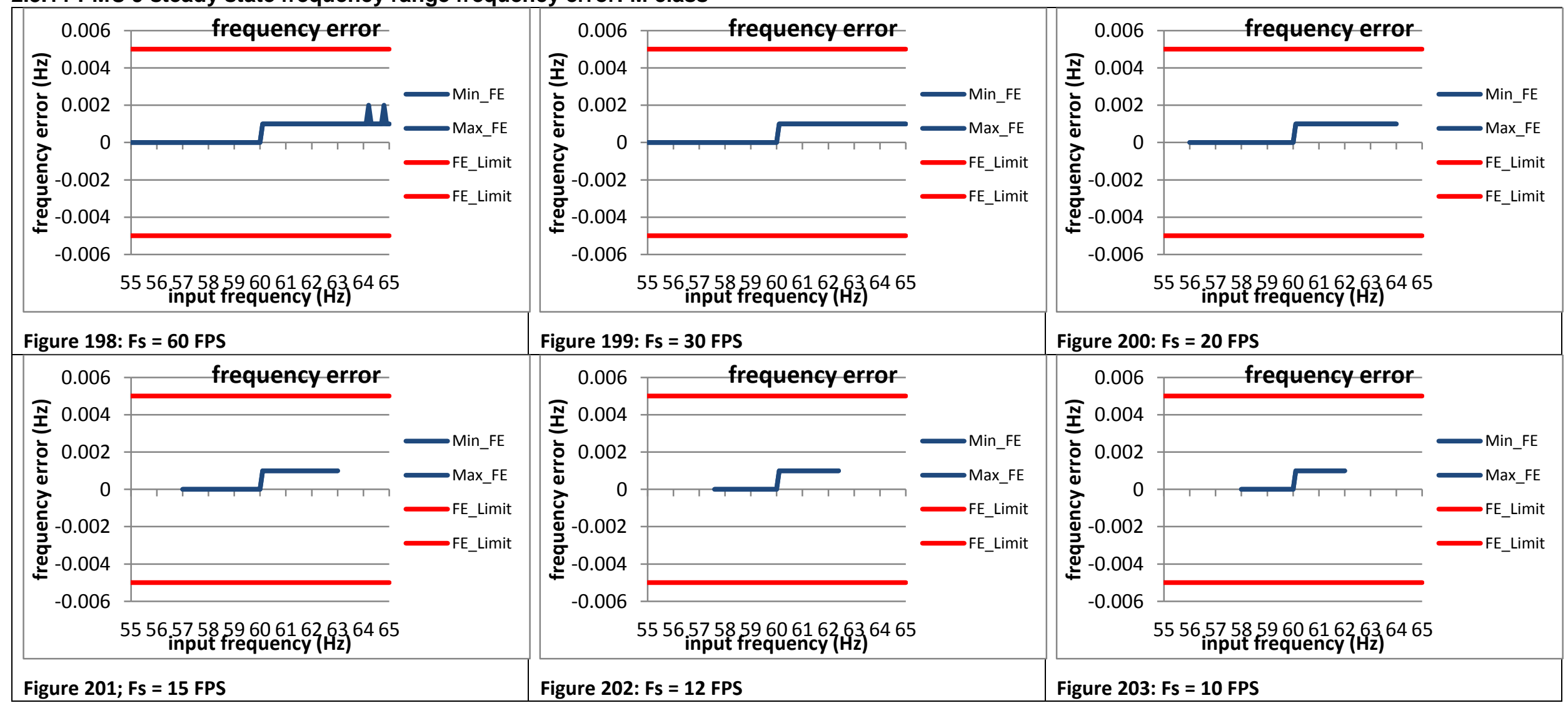




\subsection{Steady state frequency range ROCOF error: M class}

\subsubsection{C37.118.1 Annex $C$ steady state frequency range ROCOF error: M class}

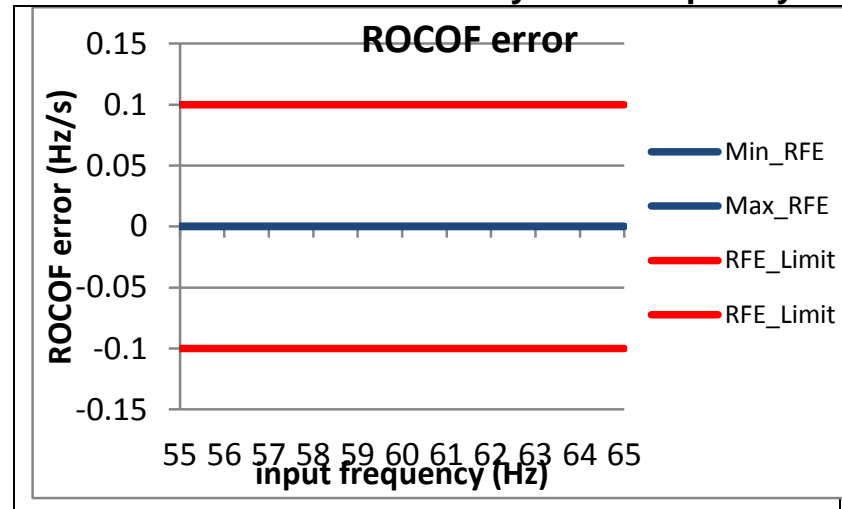

Figure 204: Fs = 60 FPS

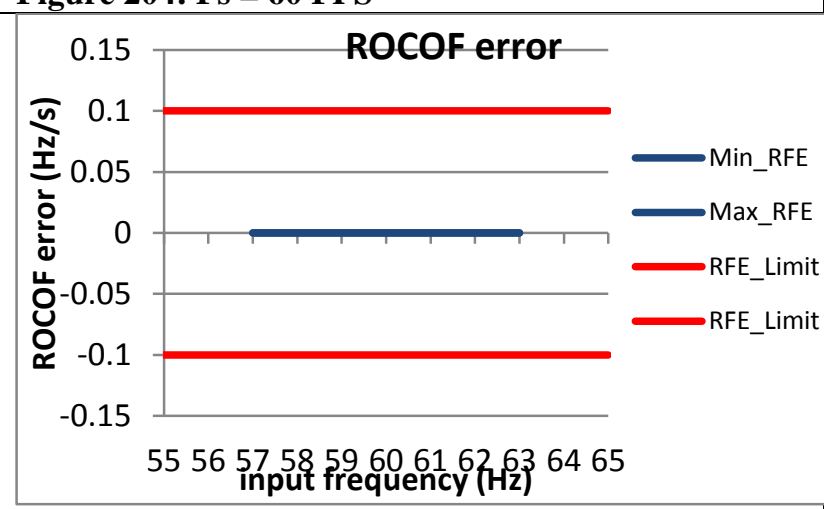

Figure 207: Fs = 15 FPS

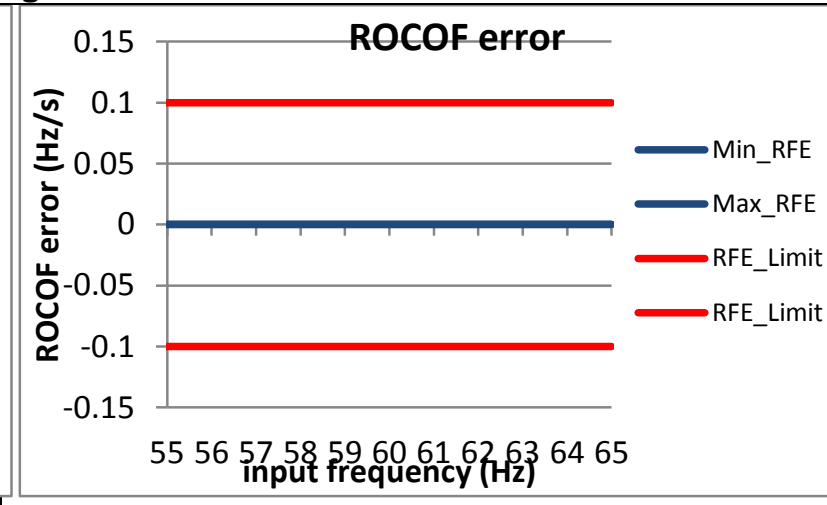

Figure 205: Fs = 30 FPS

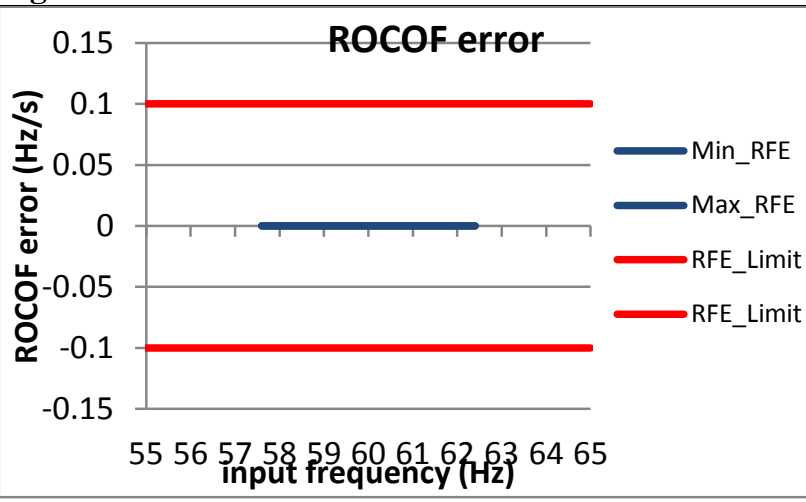

Figure 208: Fs = 12 FPS

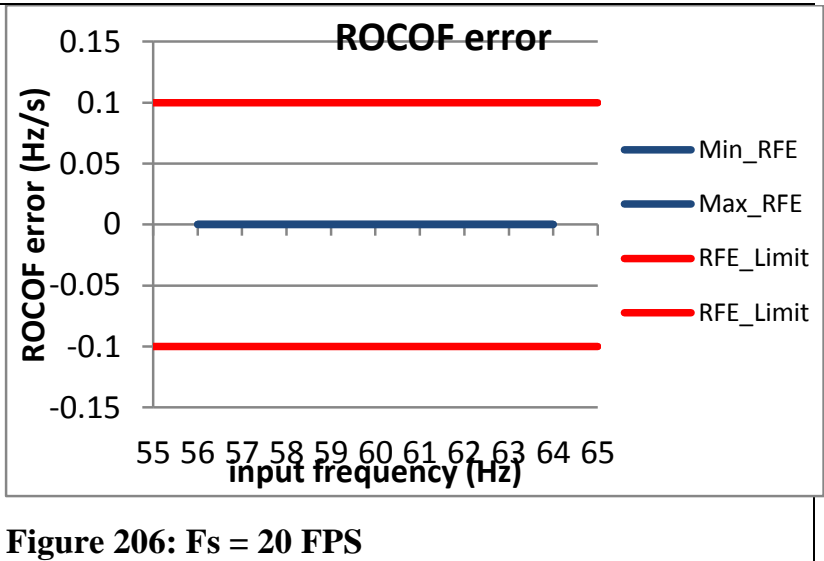

Figure 206: Fs $=20$ FPS

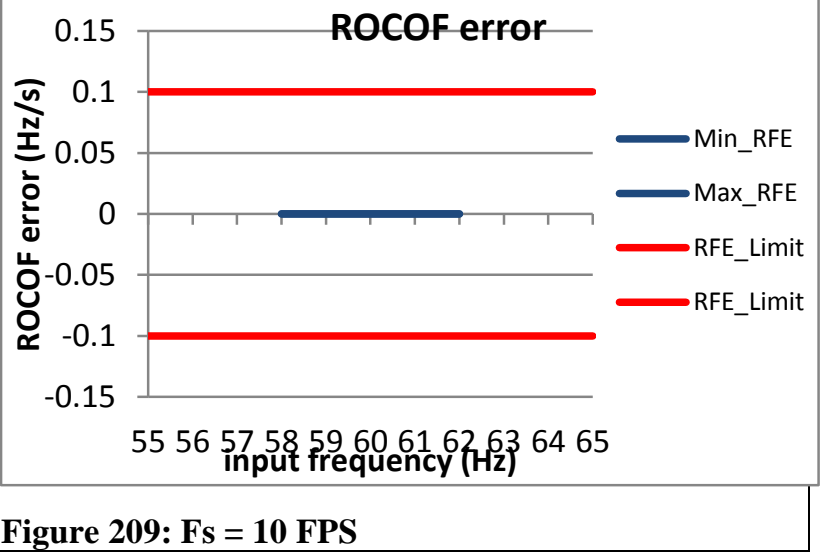




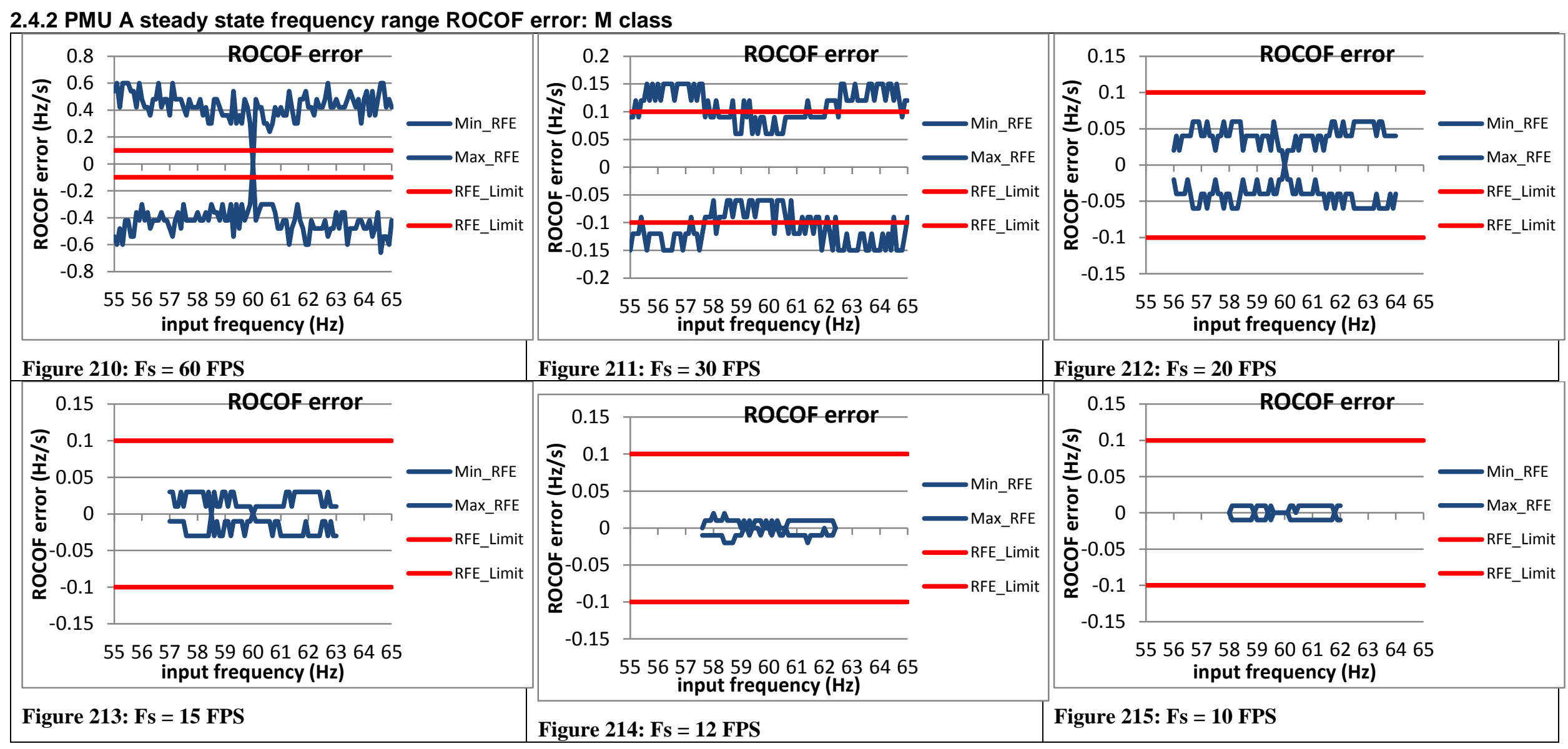




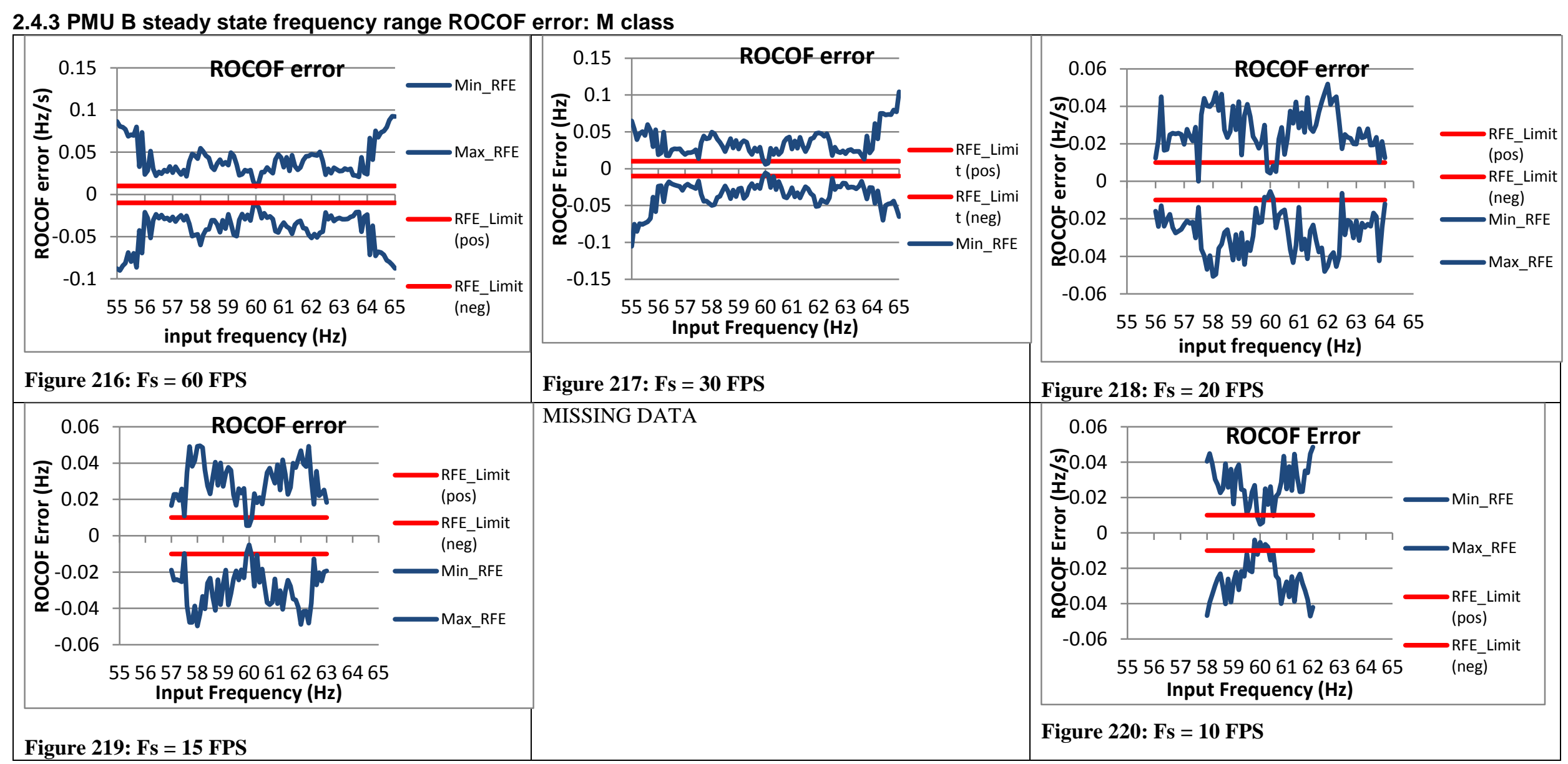




\subsubsection{PMU C steady state frequency range ROCOF error: M class}

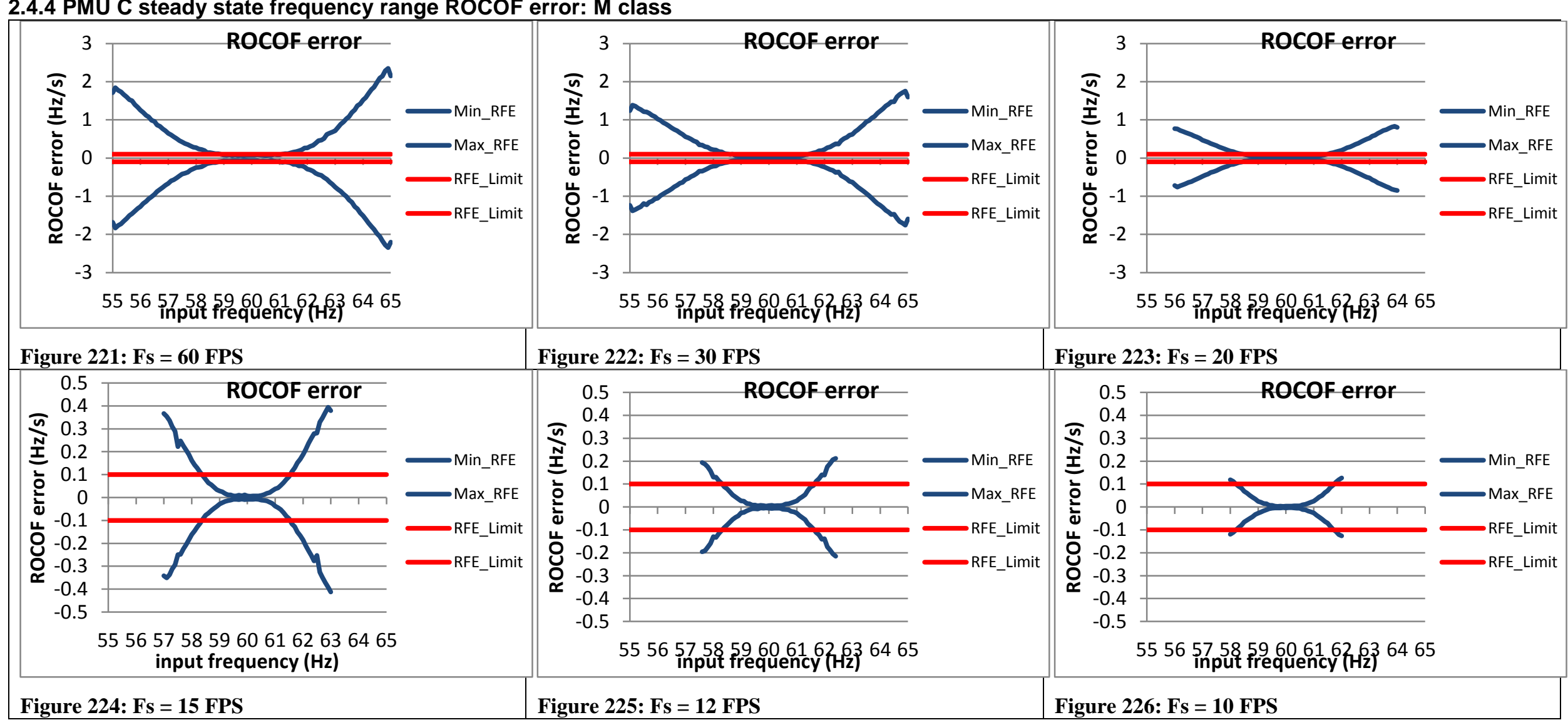


2.4.5 PMU D steady state frequency range ROCOF error: M class

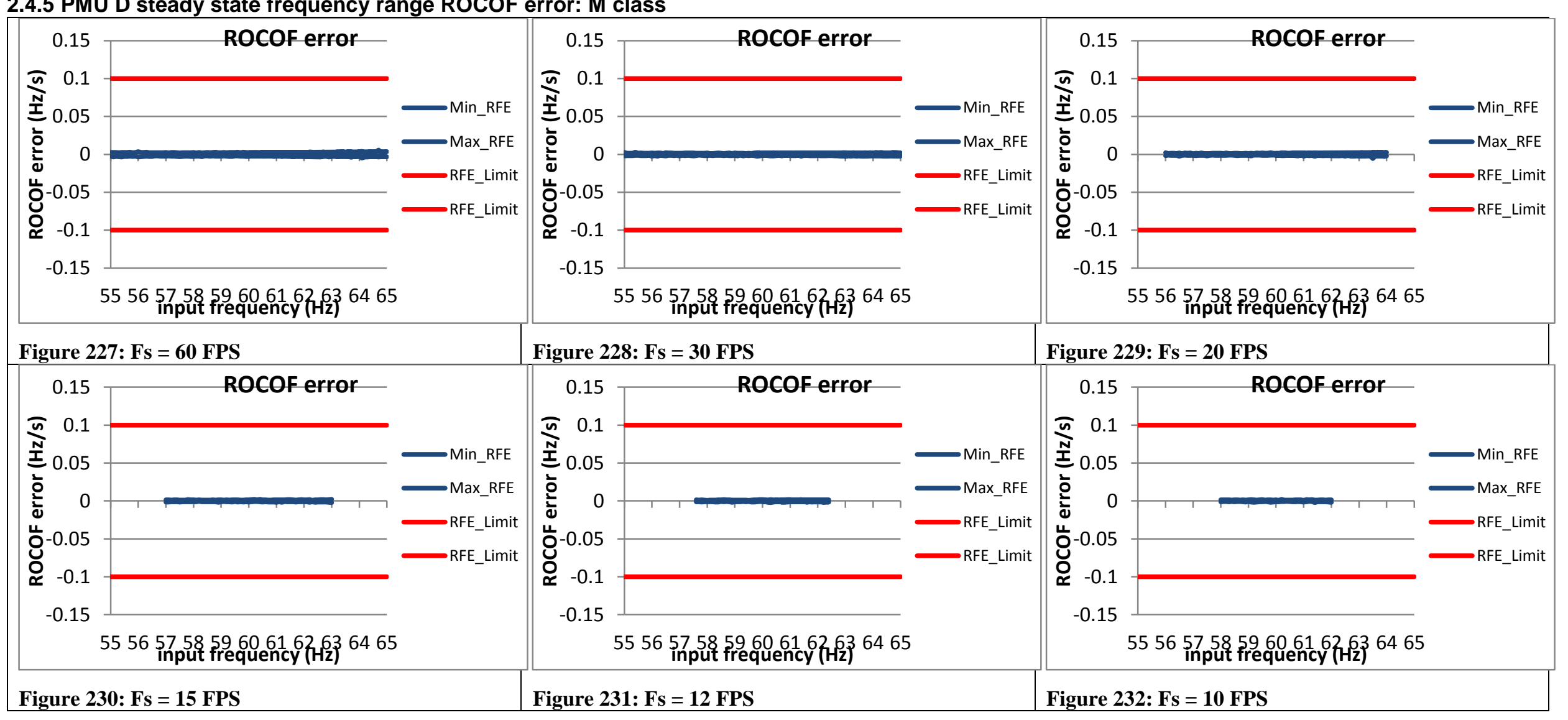


2.4.6 PMU E steady state frequency range ROCOF error: M class

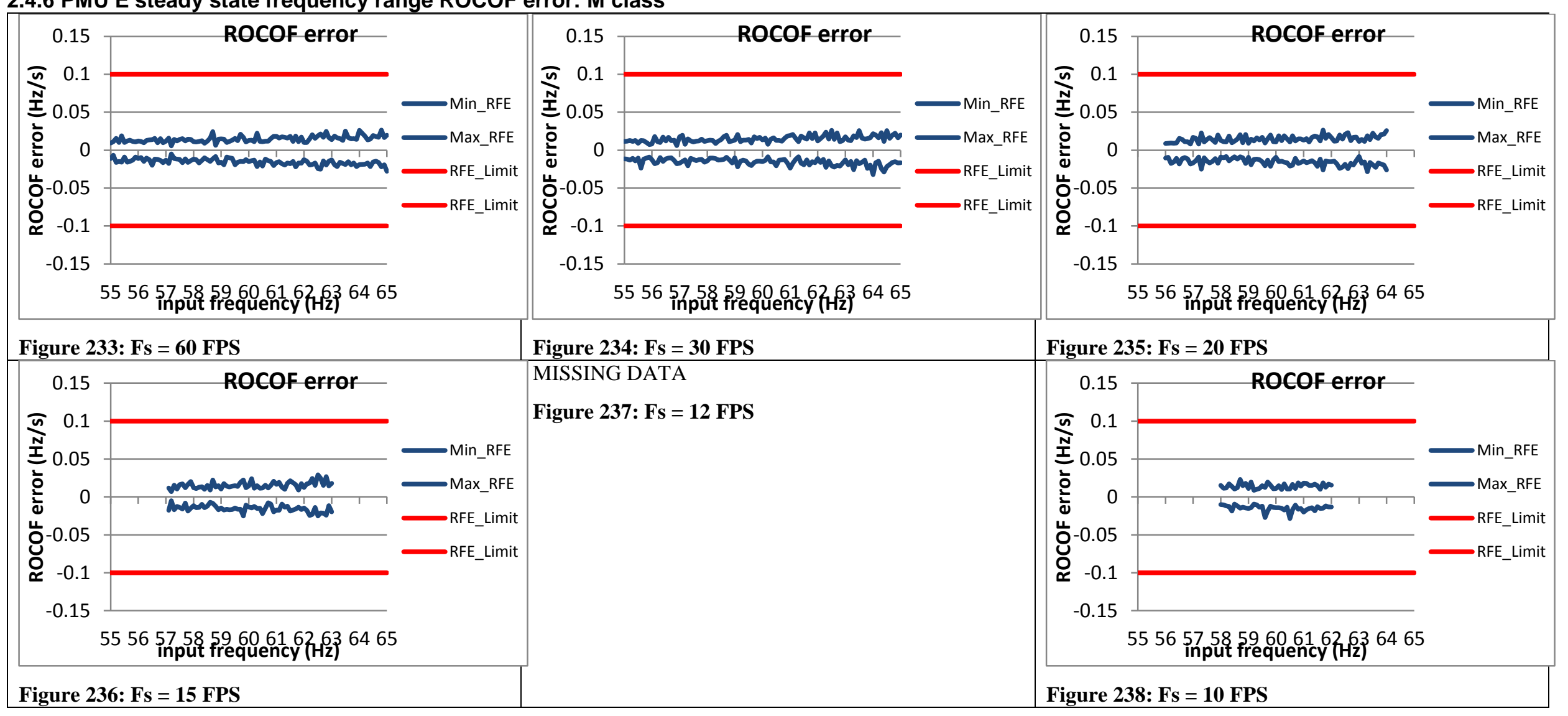


2.4.7 PMU F steady state frequency range ROCOF error: M class

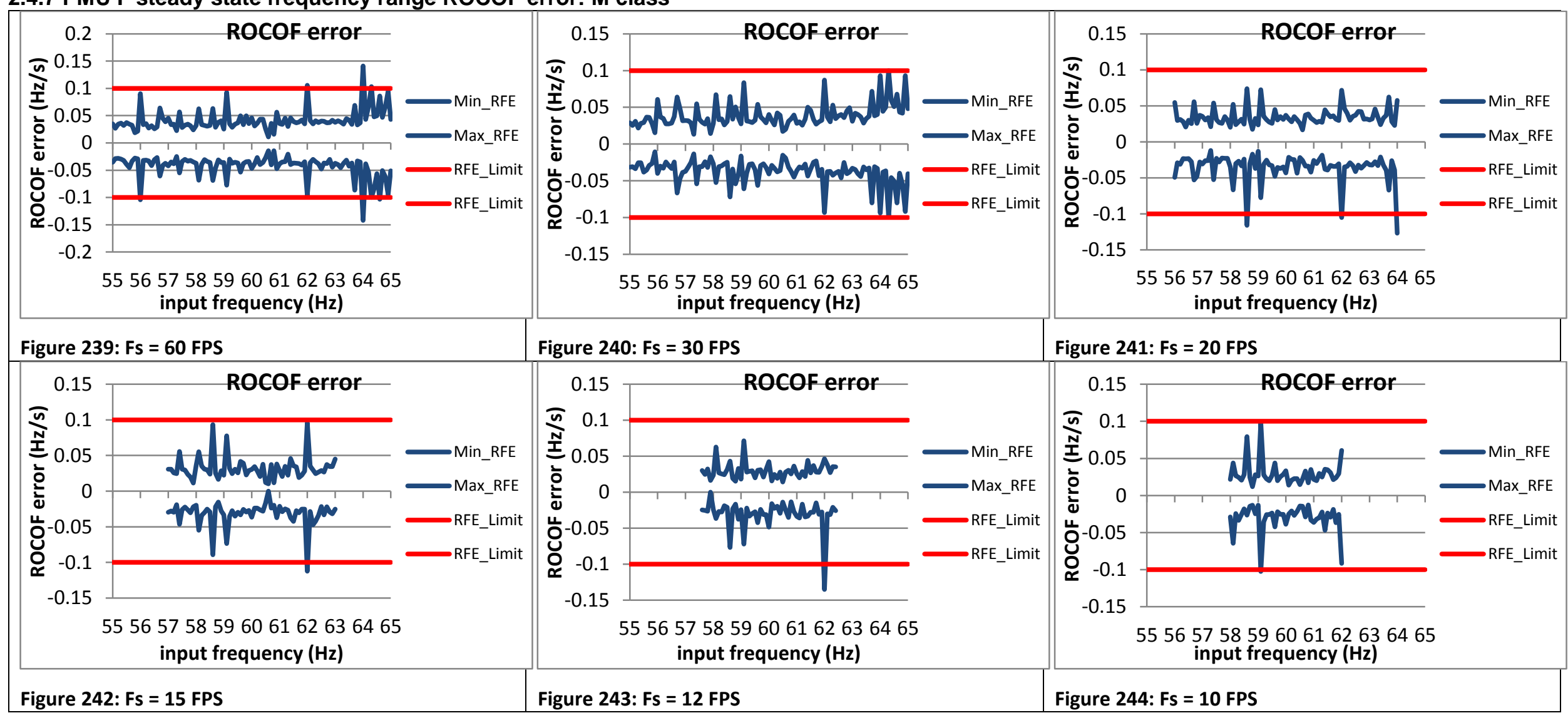




\subsubsection{PMU G* steady state frequency range ROCOF error: M class}

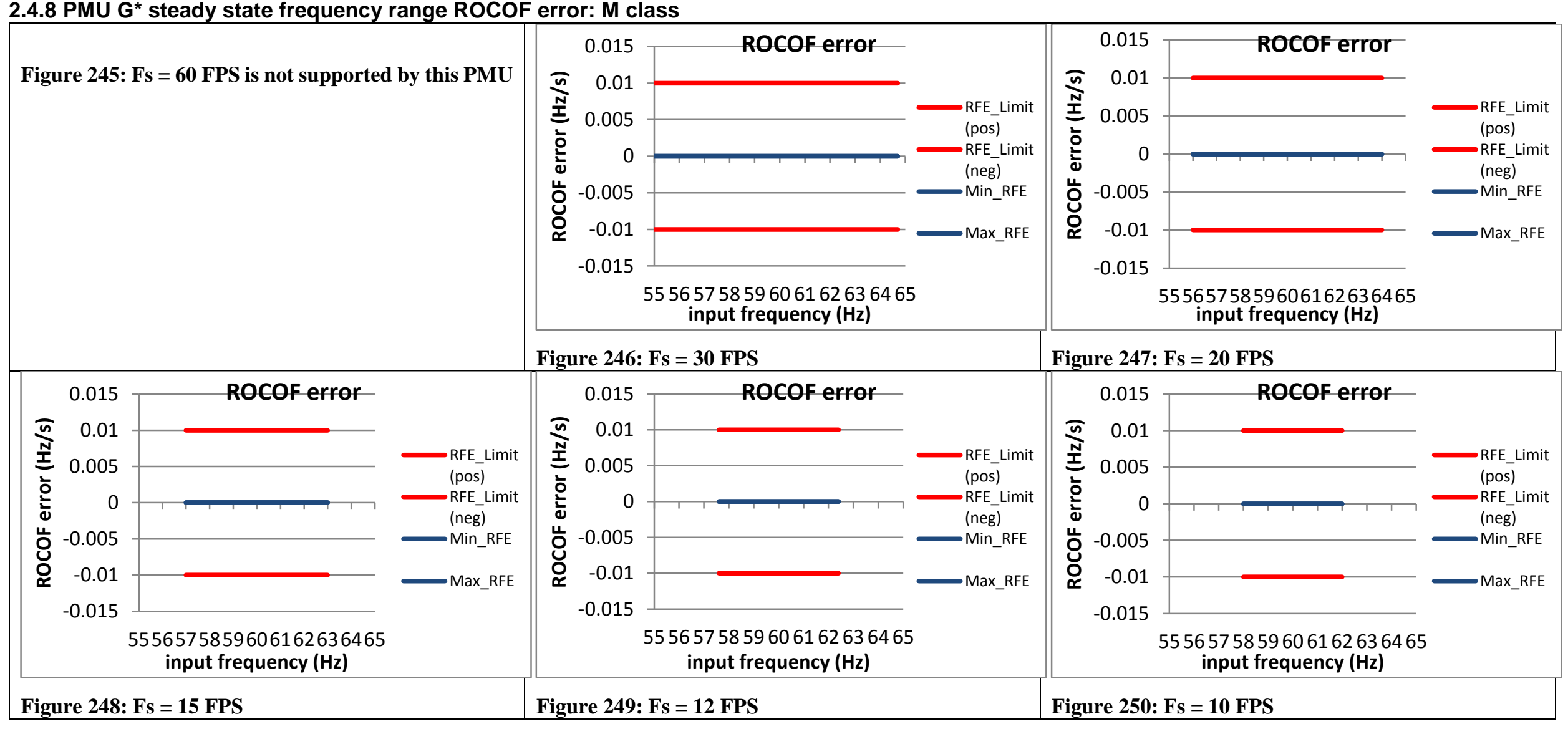


2.4.9 PMU H steady state frequency range ROCOF error: M class

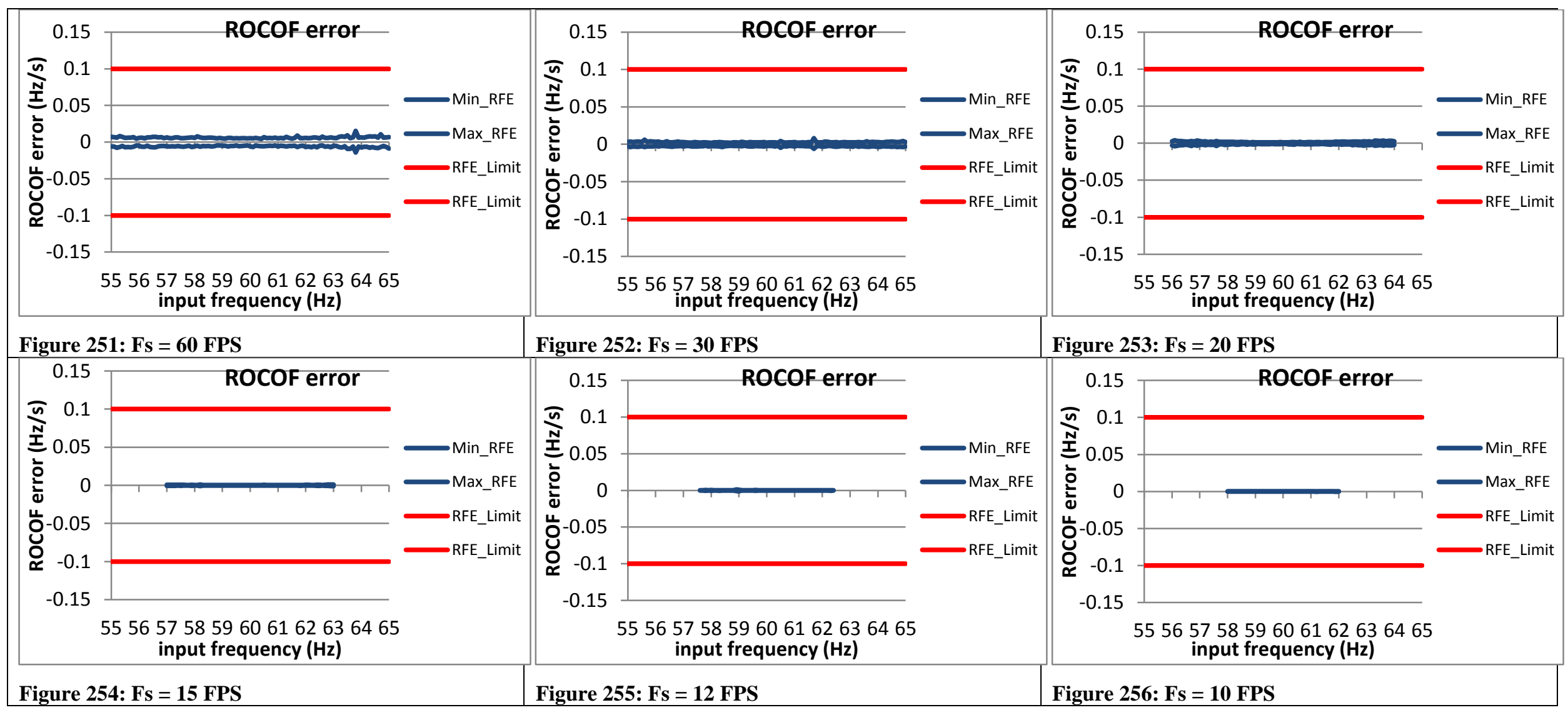




\subsubsection{PMU I steady state frequency range ROCOF error: M class}

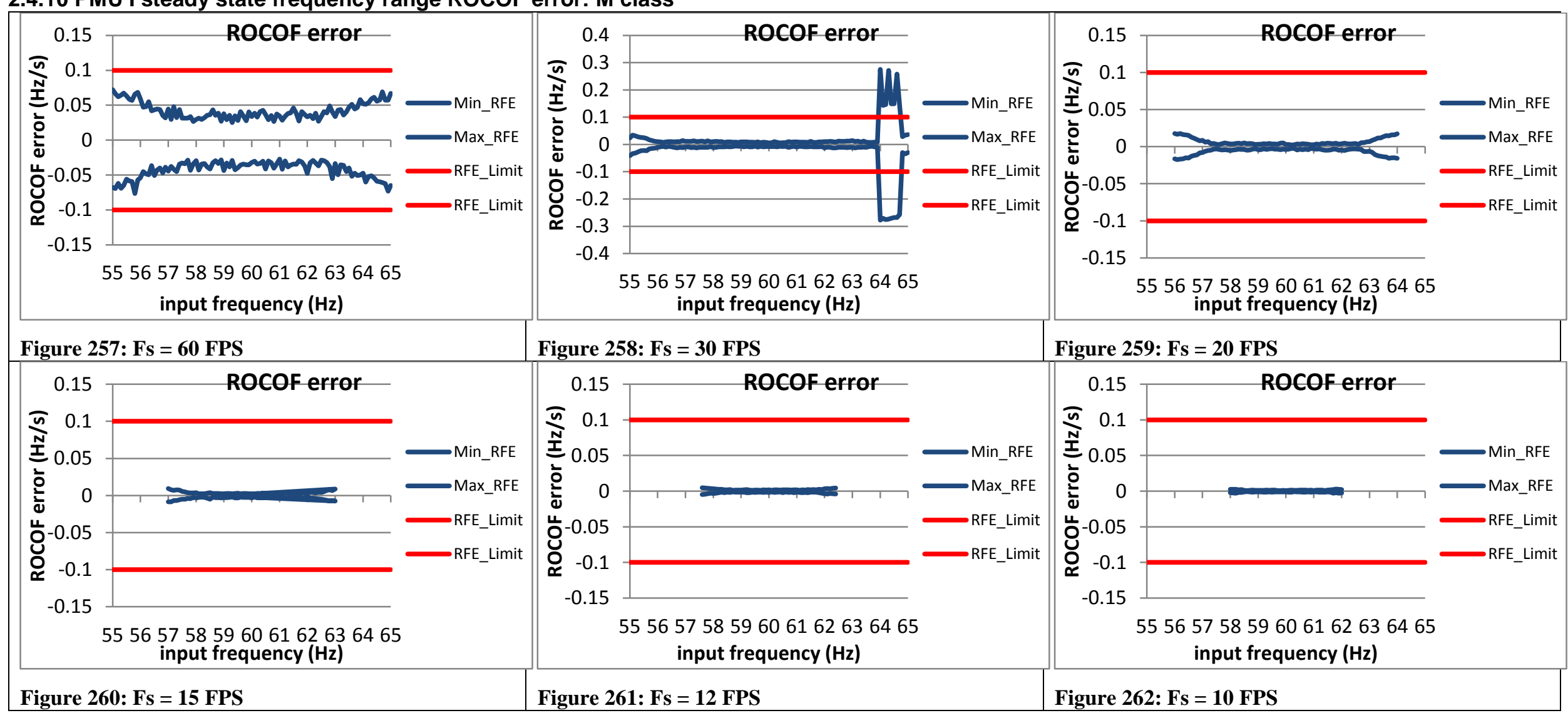


2.4.11 PMU J steady state frequency range ROCOF error: M class

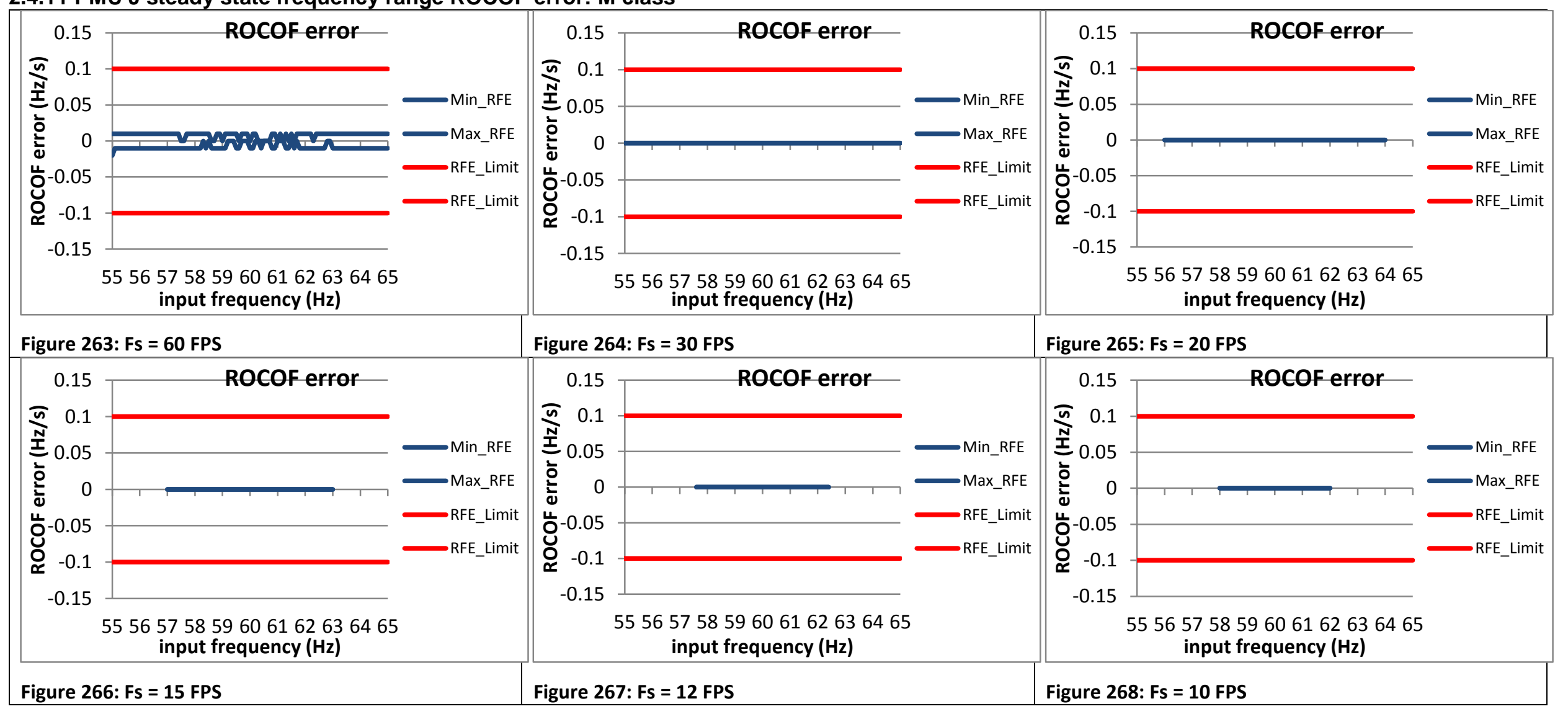




\subsection{Steady state frequency range voltage TVE: P class}

2.5.1 C37.118.1 Annex C steady state frequency range voltage TVE: P class

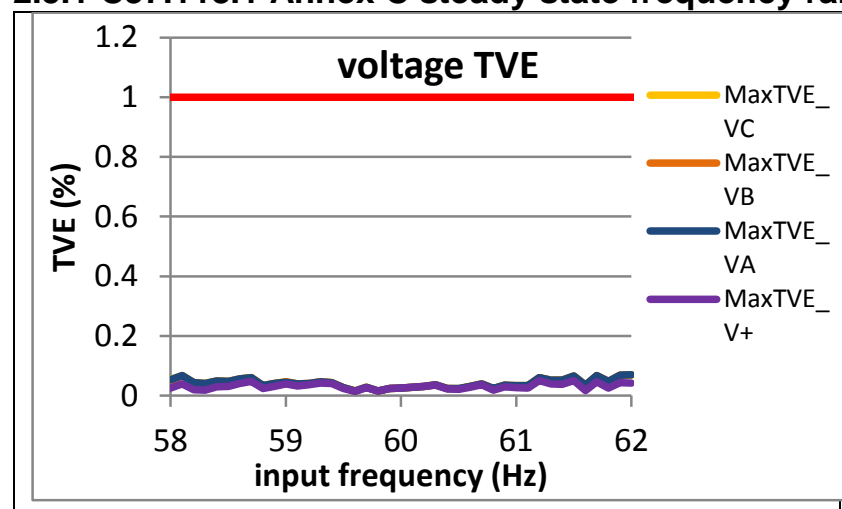

Figure 269: Fs = 60 FPS

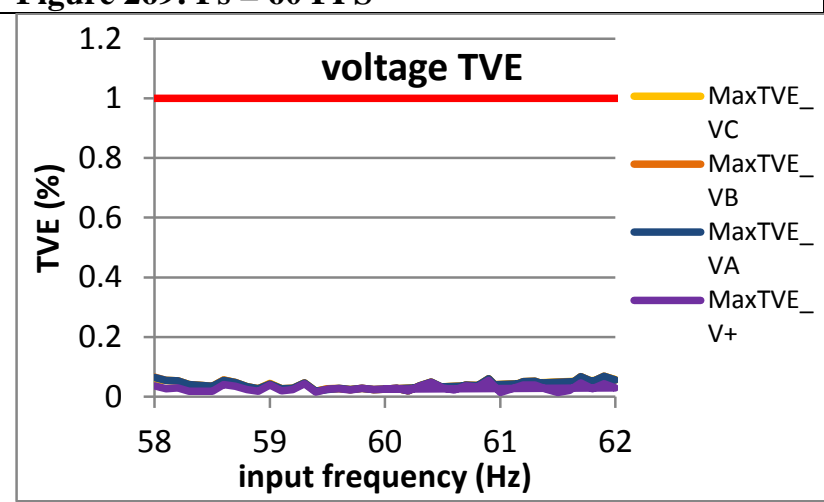

Figure 272: Fs = 15 FPS

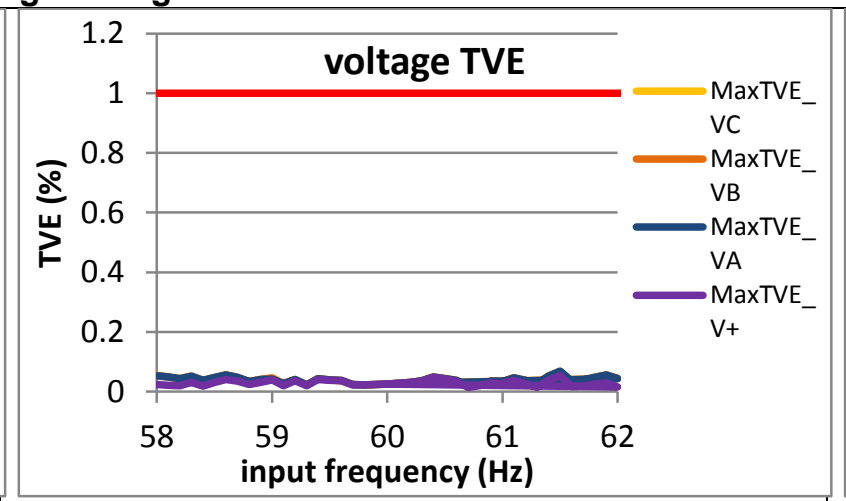

Figure 270: Fs = 30 FPS

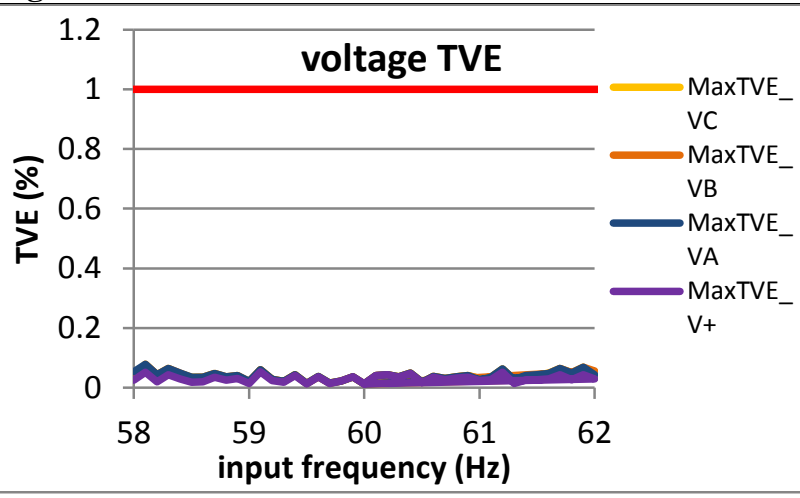

Figure 273: Fs = 12 FPS

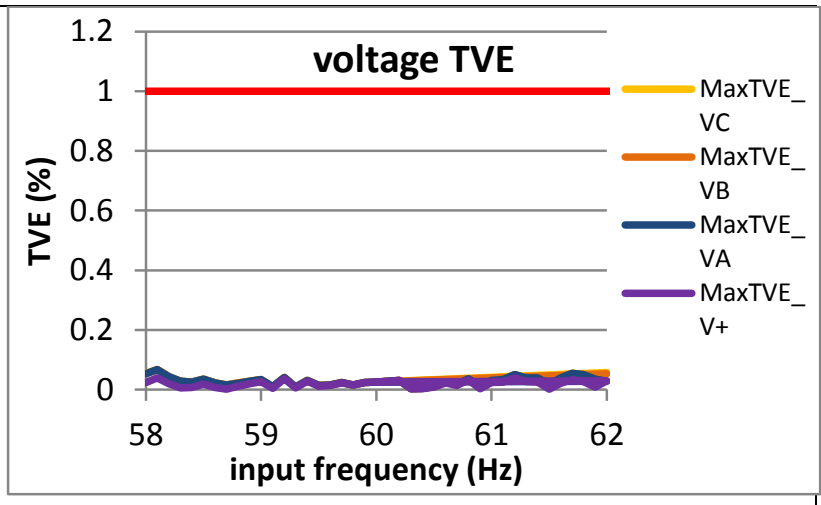

Figure 271: Fs = 20 FPS

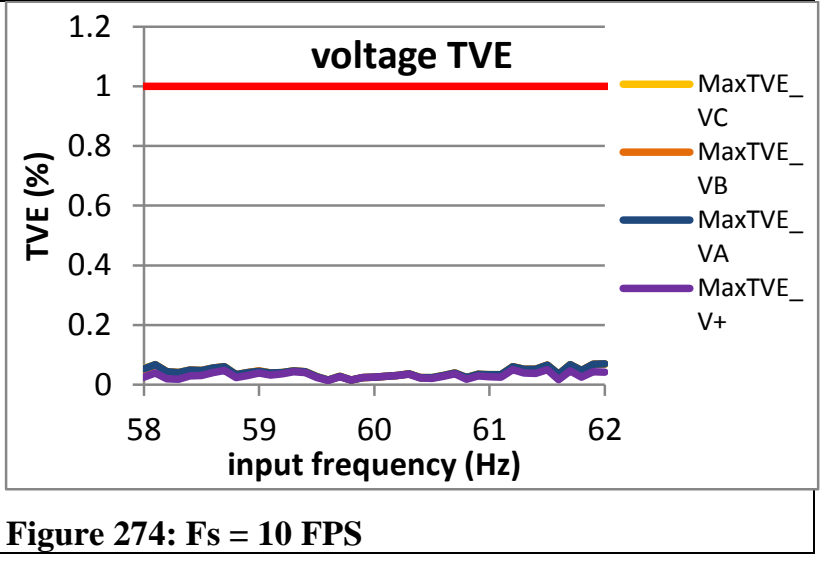




\subsubsection{PMU A steady state frequency range voltage TVE: P class}
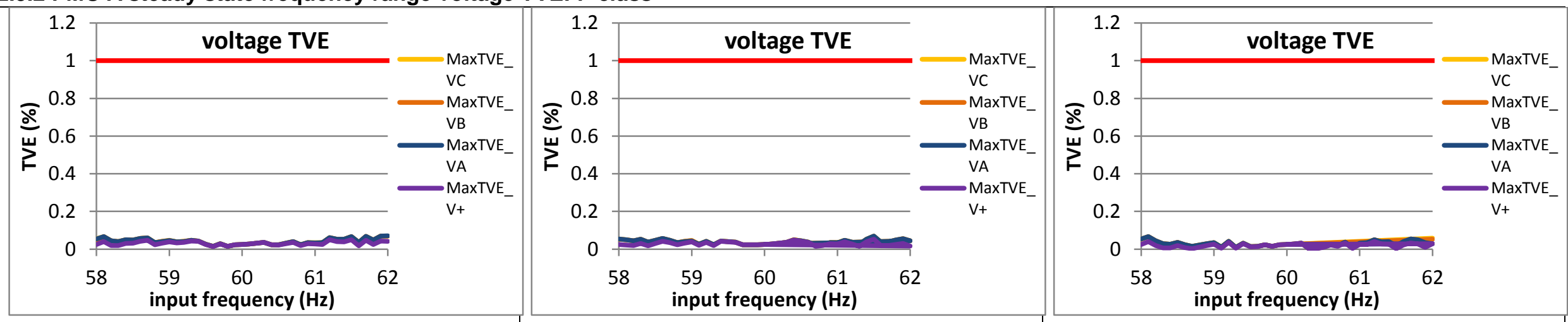

Figure 275: Fs = 60 FPS

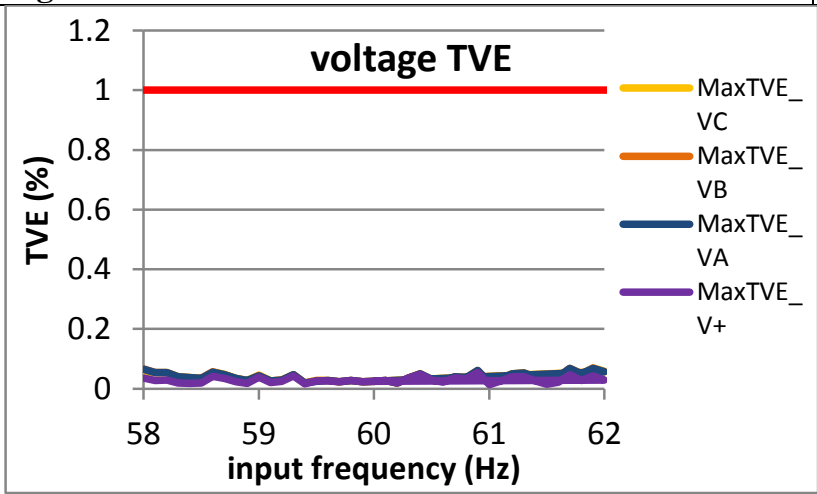

Figure 276: Fs = 30 FPS

Figure 277: Fs = 20 FPS

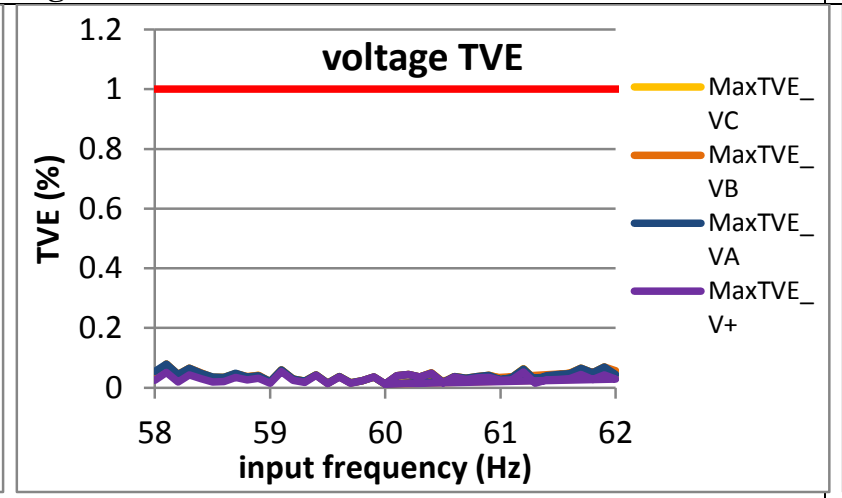

\begin{tabular}{|c|c|c|c|}
\hline 1.2 & voltage TVE & & \\
\hline 1 & & & $\begin{array}{l}\text { MaxTVE } \\
\text { VC }\end{array}$ \\
\hline$\overbrace{}^{0.8}$ & & & $\begin{array}{l}\text { MaxTVE } \\
\text { VB }\end{array}$ \\
\hline$\sum_{0.4}^{0.6}$ & & & $\begin{array}{l}\text { MaxTVE } \\
\text { VA }\end{array}$ \\
\hline 0.2 & & & $\begin{array}{l}\operatorname{maxTVE}_{-} \\
\mathrm{V}_{+}\end{array}$ \\
\hline 0 & $N_{1} n_{1}$ & & \\
\hline & $\begin{array}{ccc}59 & 60 & 61 \\
\text { input frequency } & (\mathrm{Hz})\end{array}$ & 62 & \\
\hline
\end{tabular}

Figure 278: Fs = 15 FPS

Figure 279: Fs = 12 FPS

Figure 280: Fs = 10 FPS 


\subsubsection{PMU B steady state frequency range voltage TVE: P class}
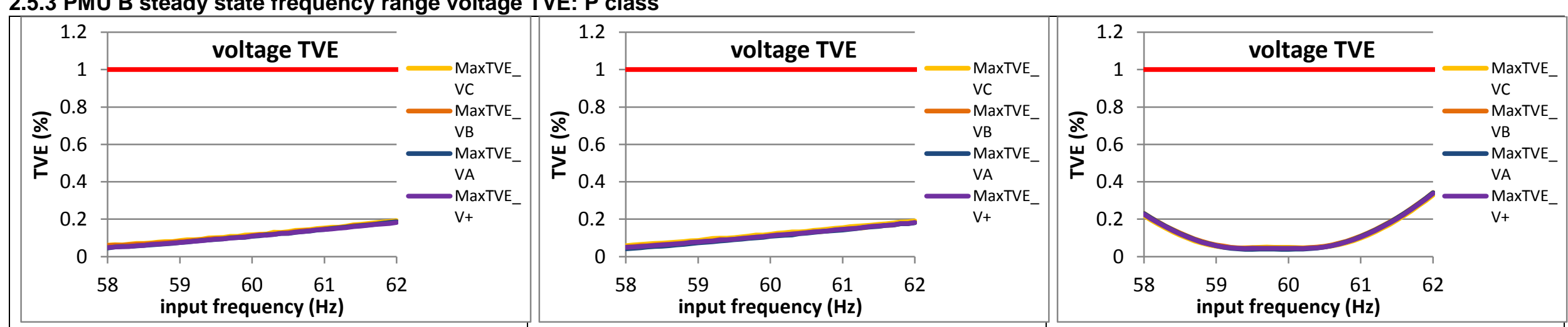

Figure 281: Fs = 60 FPS

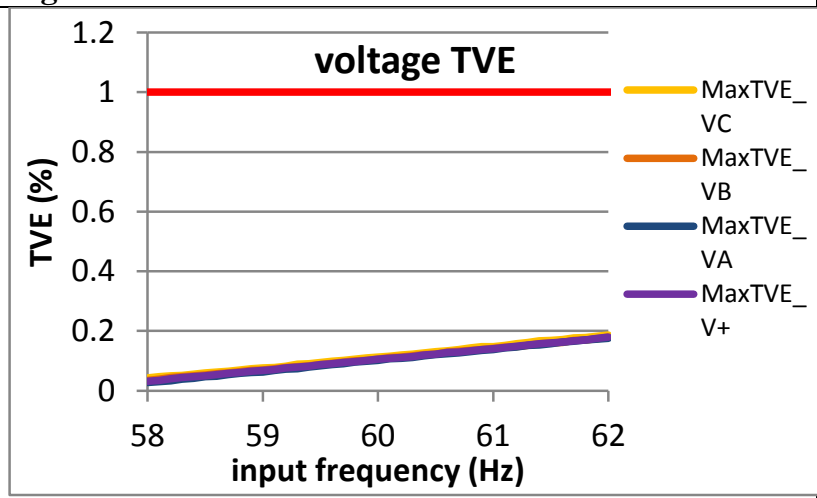

Figure 282: Fs = 30 FPS

Figure 283: Fs = 20 FPS
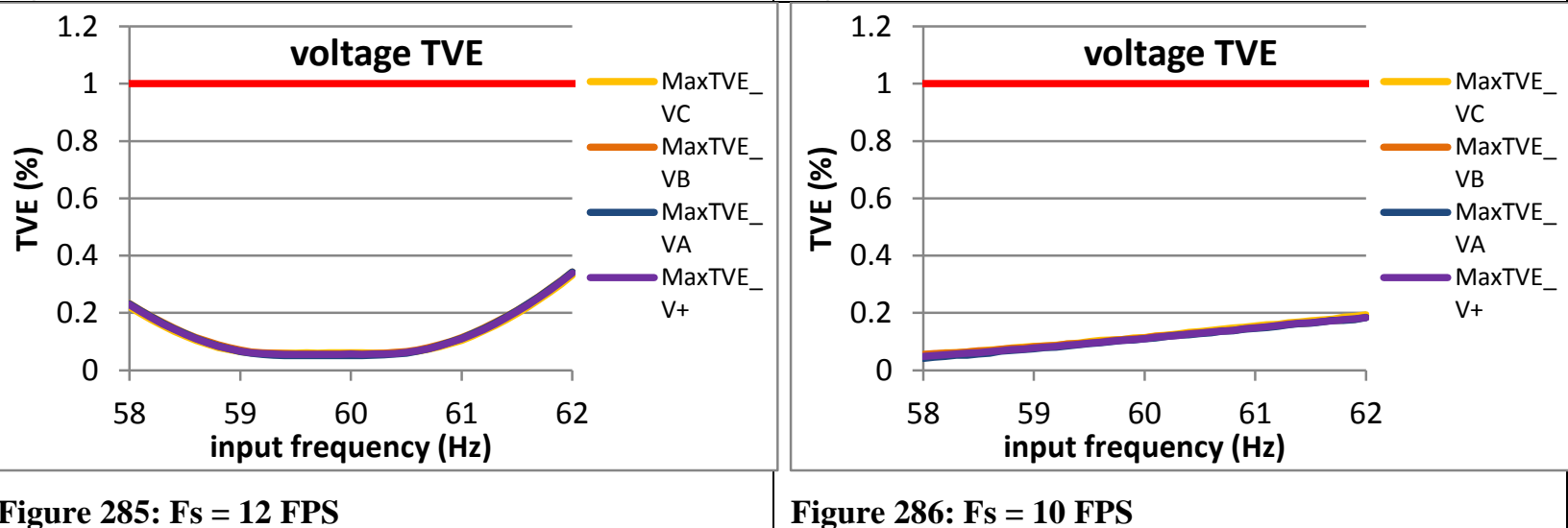

Figure 284: Fs $=15$ FPS

Figure 285: Fs = 12 FPS

Figure 286: Fs = 10 FPS 
2.5.4 PMU C steady state frequency range voltage TVE: P class
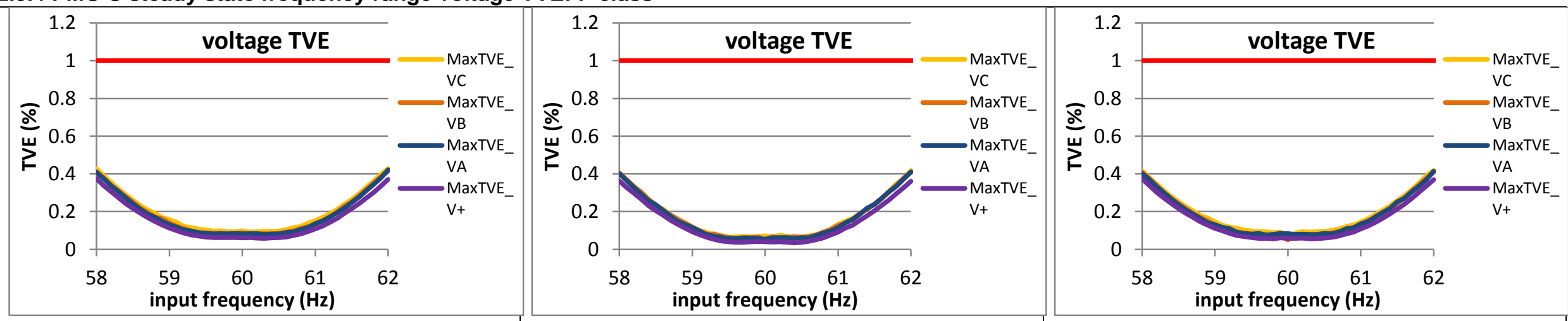

Figure 287: Fs = 60 FPS

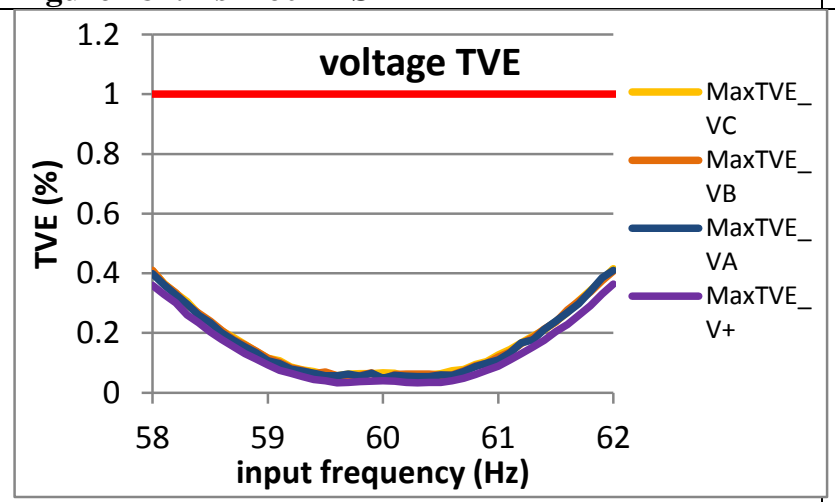

Figure 288: Fs = 30 FPS

Figure 289: Fs $=20$ FPS
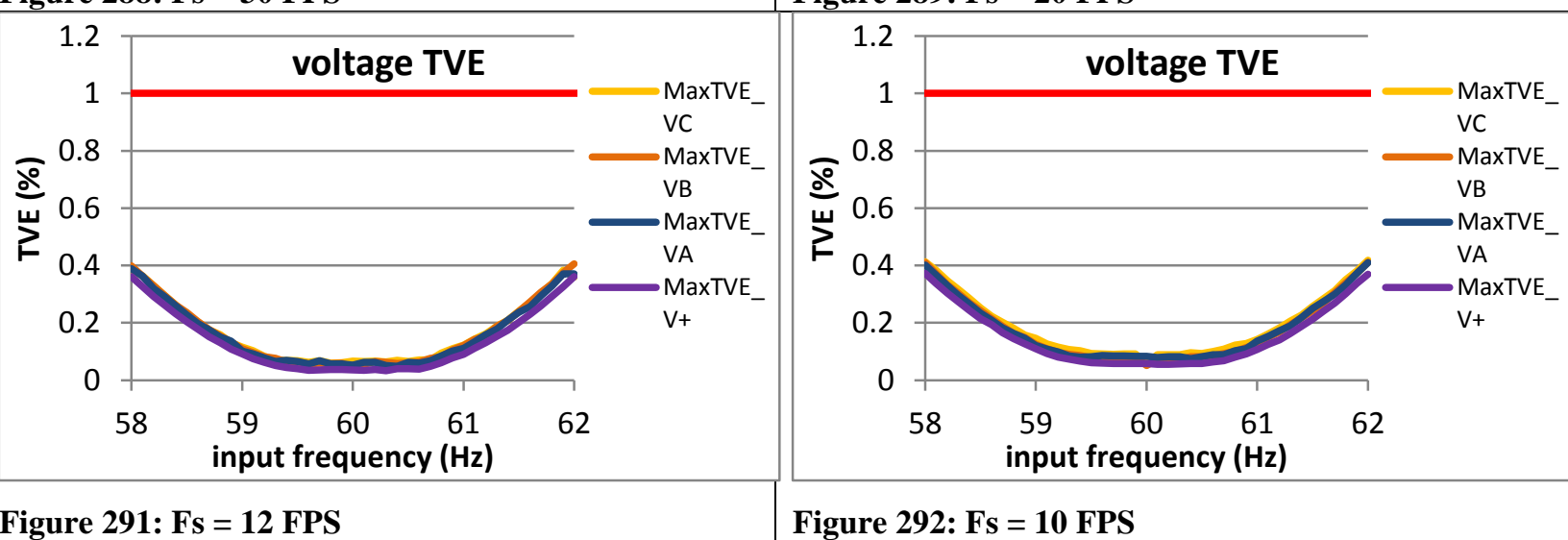

Figure 290: Fs = 15 FPS

Figure 291: Fs = 12 FPS

Figure 292: Fs = 10 FPS 
2.5.5 PMU D steady state frequency range voltage TVE: P class

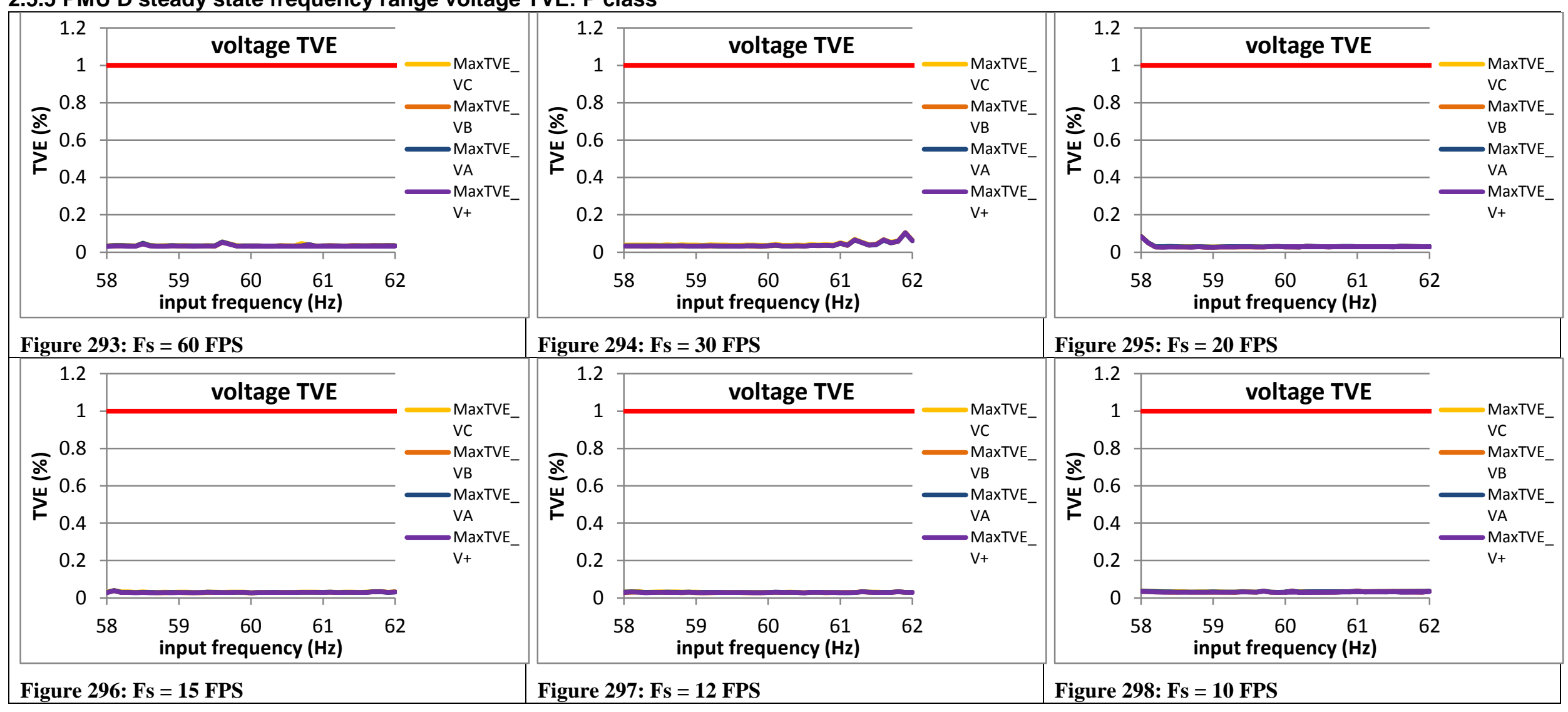

2.5.6 PMU E steady state frequency range voltage TVE: $P$ class

This PMU does not support $P$ class 
2.5.7 PMU F steady state frequency range voltage TVE: P class

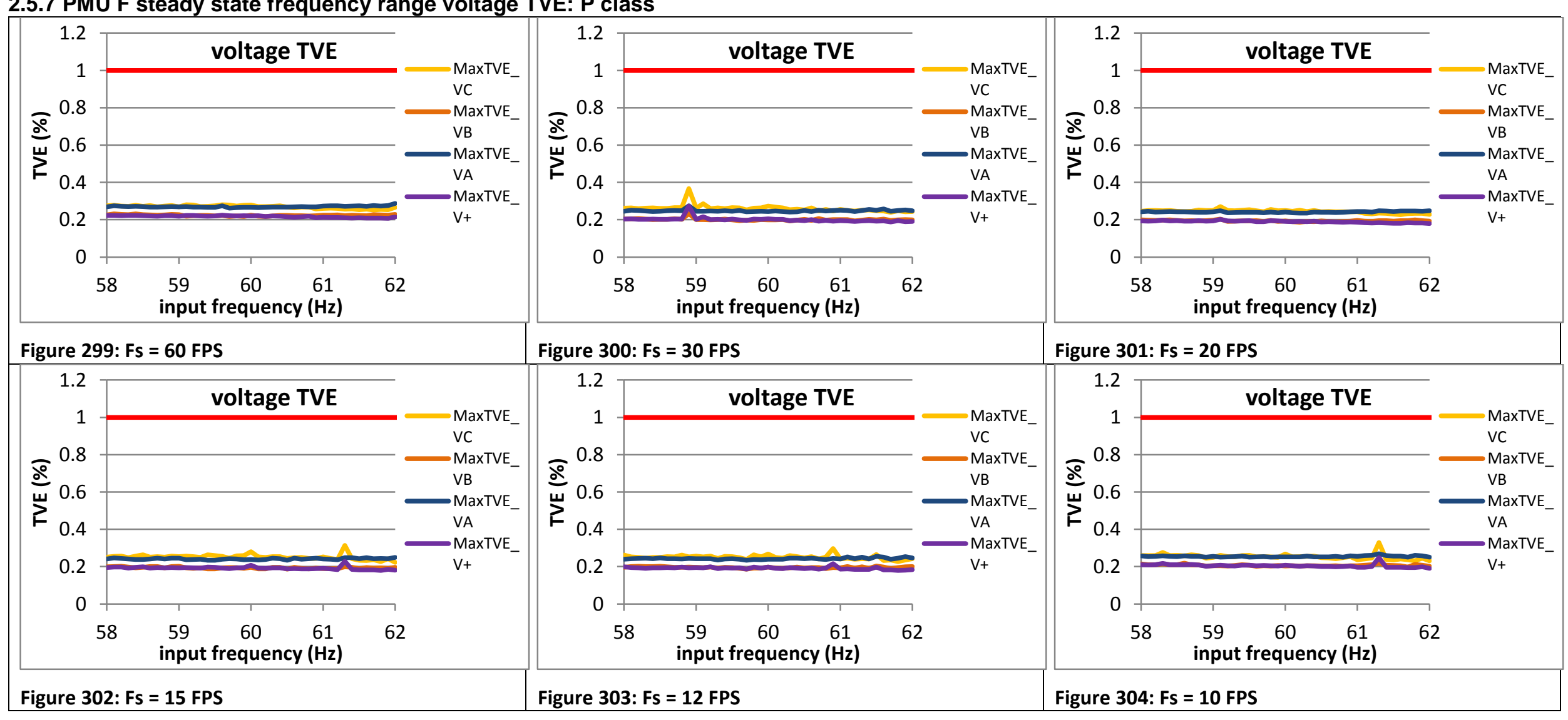

2.5.8 PMU G steady state frequency range voltage TVE: $P$ class

This PMU does not support P class 
2.5.9 PMU H steady state frequency range voltage TVE: P class

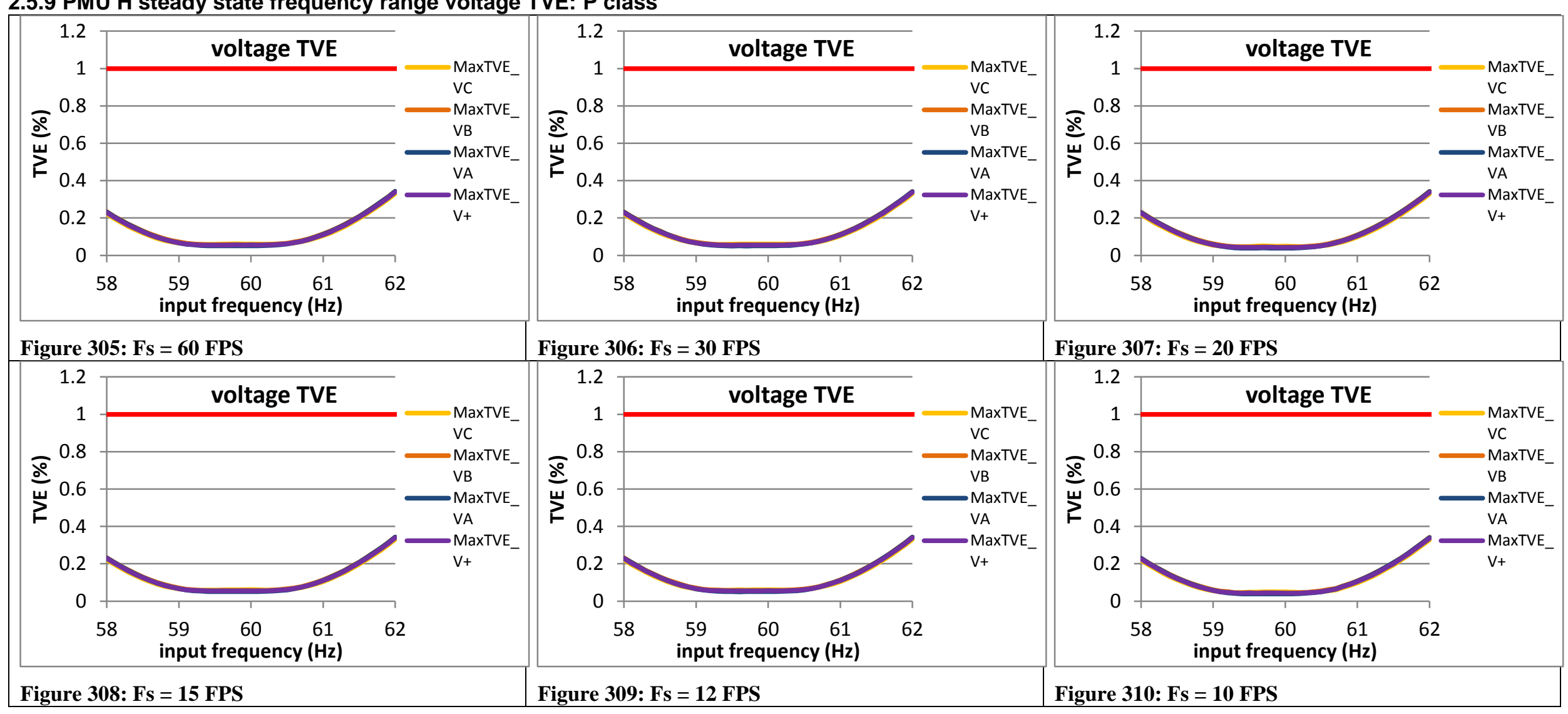

2.5.10 PMU I steady state frequency range voltage TVE: P class

PMU I does not support P class 
2.5.11 PMU J steady state frequency range voltage TVE: P class
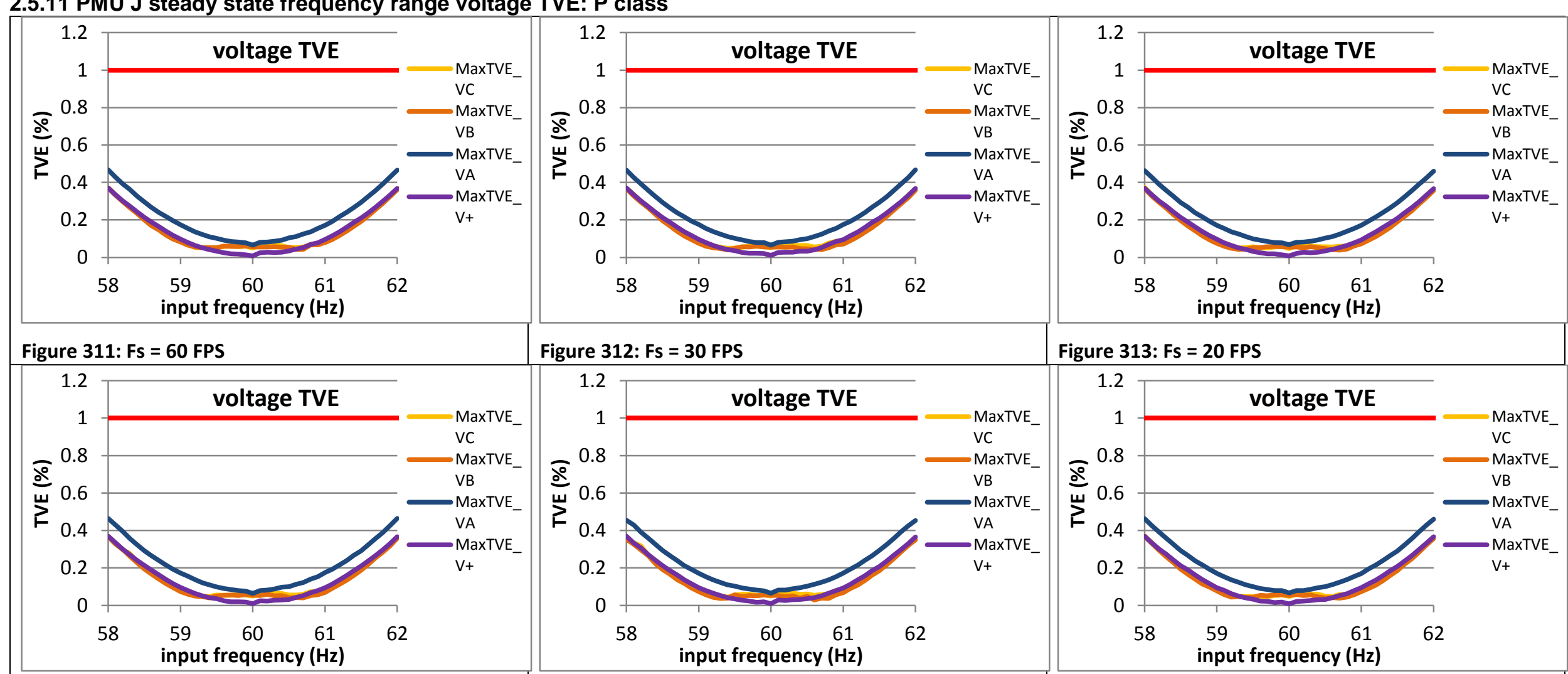

Figure 312: Fs $=30 \mathrm{FPS}$

Figure 313: Fs = 20 FPS
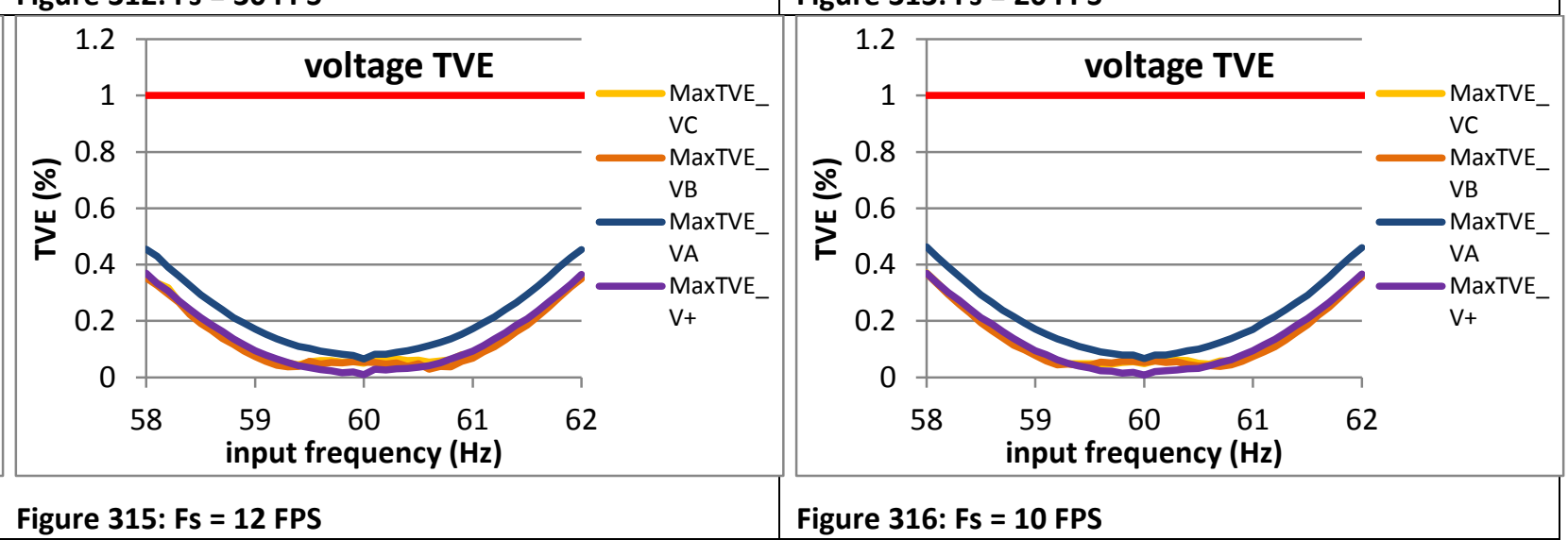

Figure 314: $\mathrm{Fs}=15 \mathrm{FPS}$

Figure 315: Fs = 12 FPS

Figure 316: Fs = $10 \mathrm{FPS}$ 


\subsection{Steady state frequency range current TVE: P class}

2.6.1 C37.118.1 Annex $C$ steady state frequency range current TVE: P class

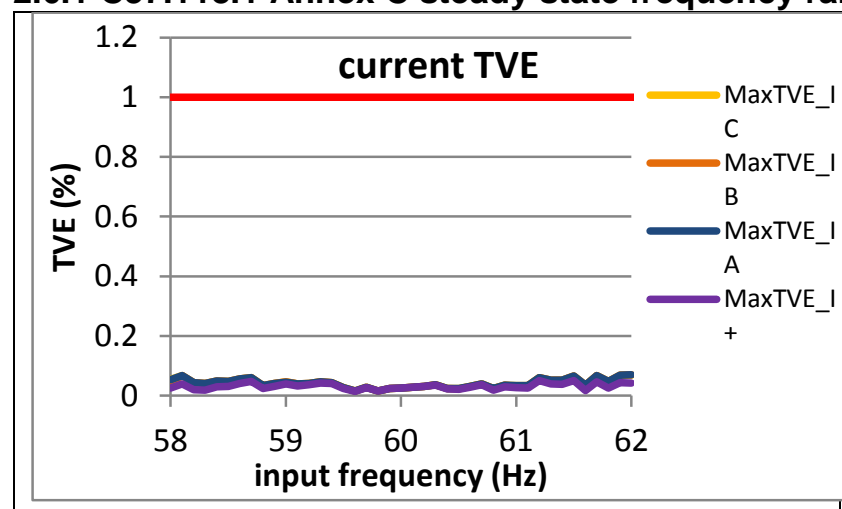

Figure 317: Fs = 60 FPS

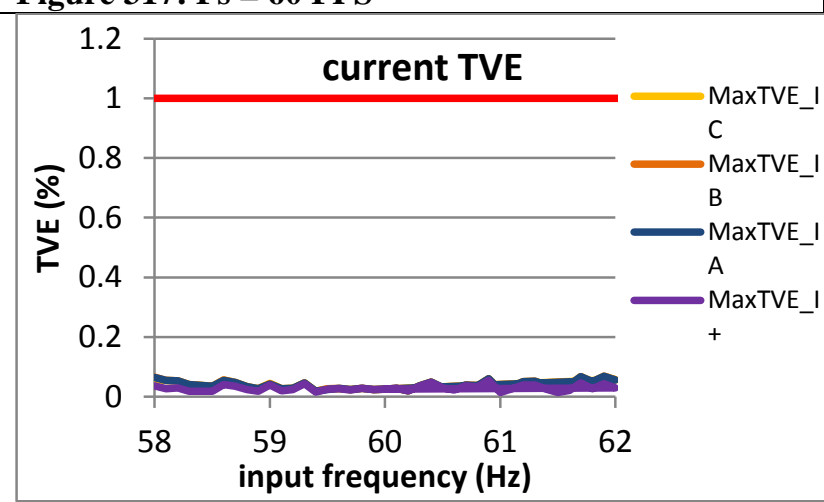

Figure 320: Fs = 15 FPS
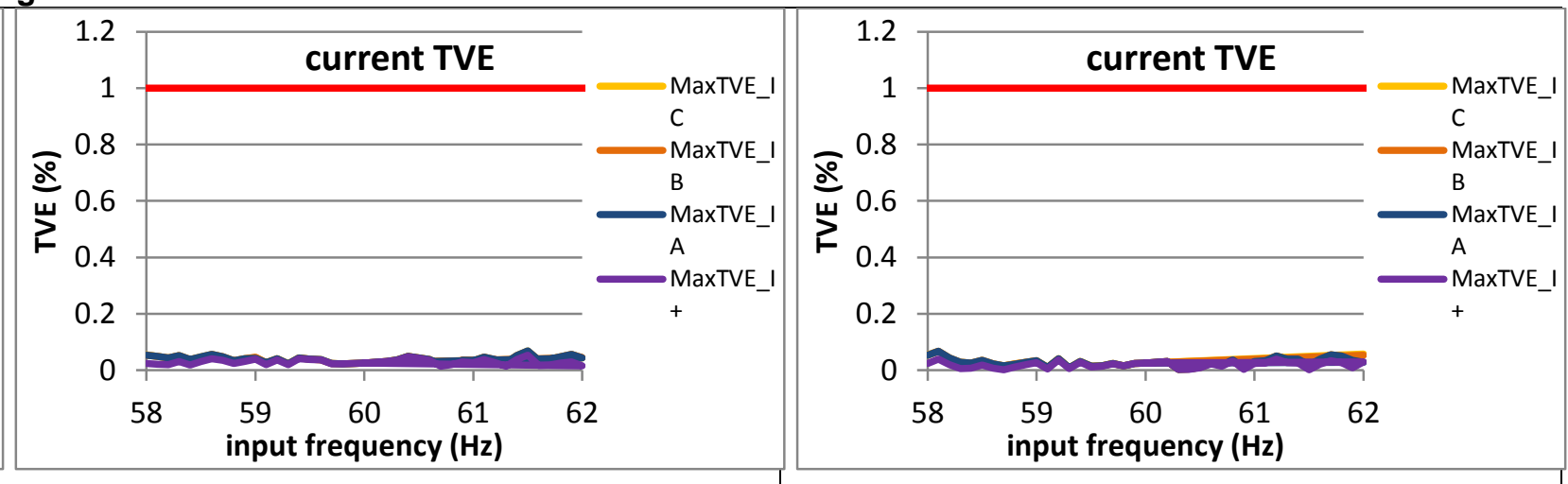

Figure 318: Fs = 30 FPS

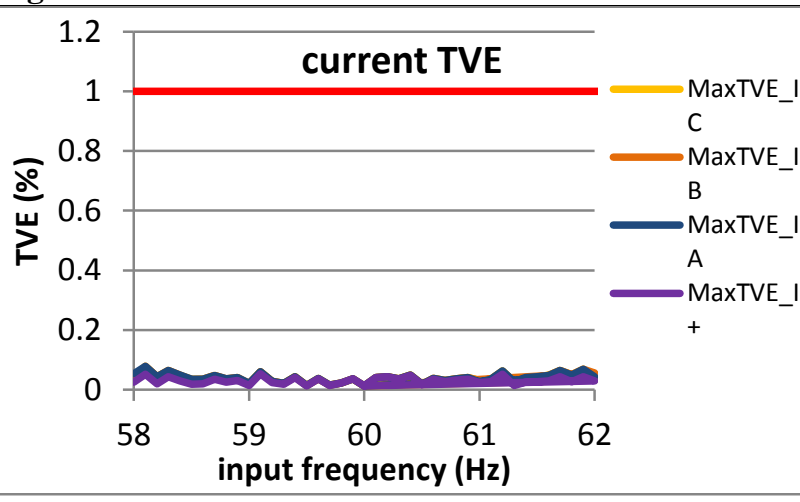

Figure 321: Fs = 12 FPS
Figure 319: Fs = 20 FPS

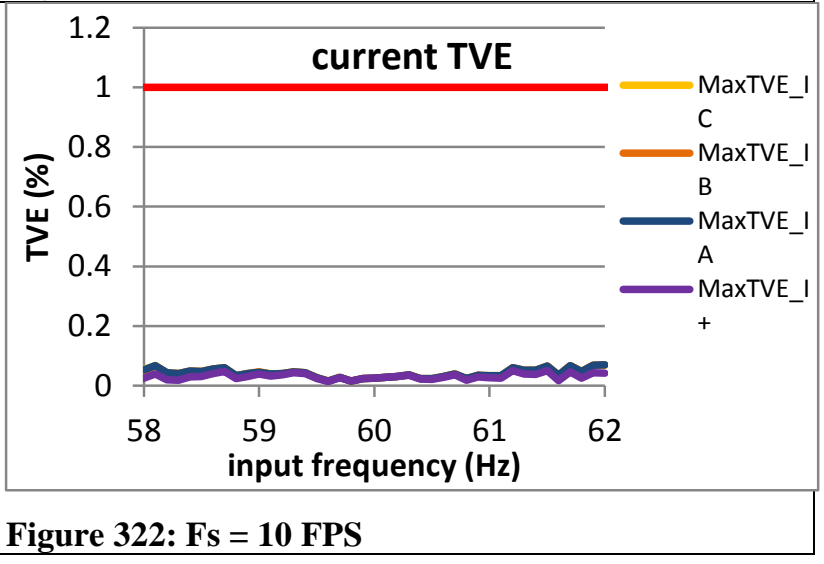


2.6.2 PMU A steady state frequency range current TVE: P class
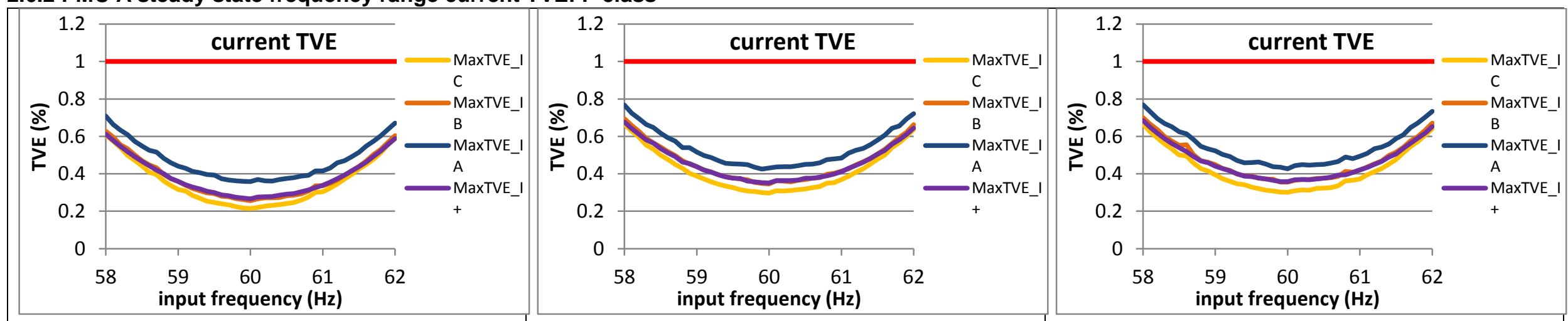

Figure 323: Fs = 60 FPS

Figure 324: Fs = 30 FPS
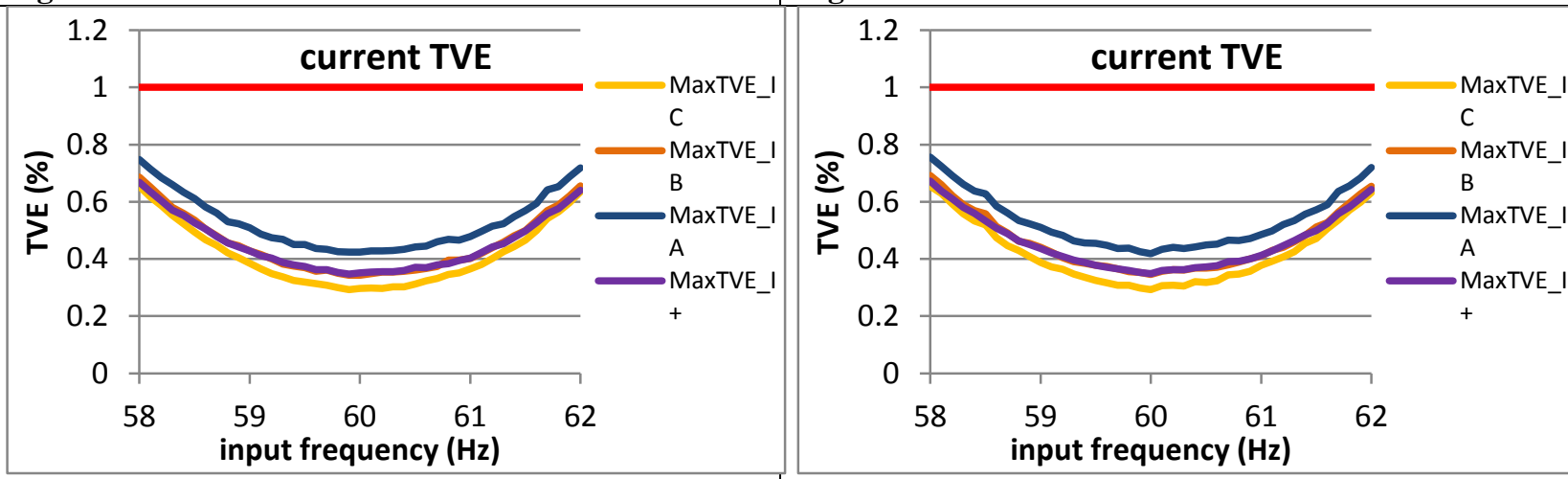

Figure 325: Fs = 20 FPS

Figure 326: Fs = 15 FPS

Figure 327: Fs = 12 FPS

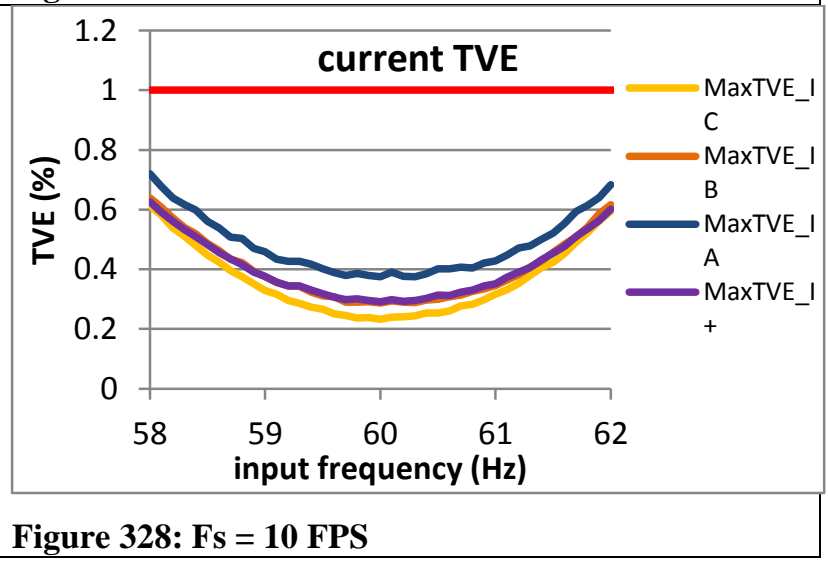




\subsubsection{PMU B steady state frequency range current TVE: P class}
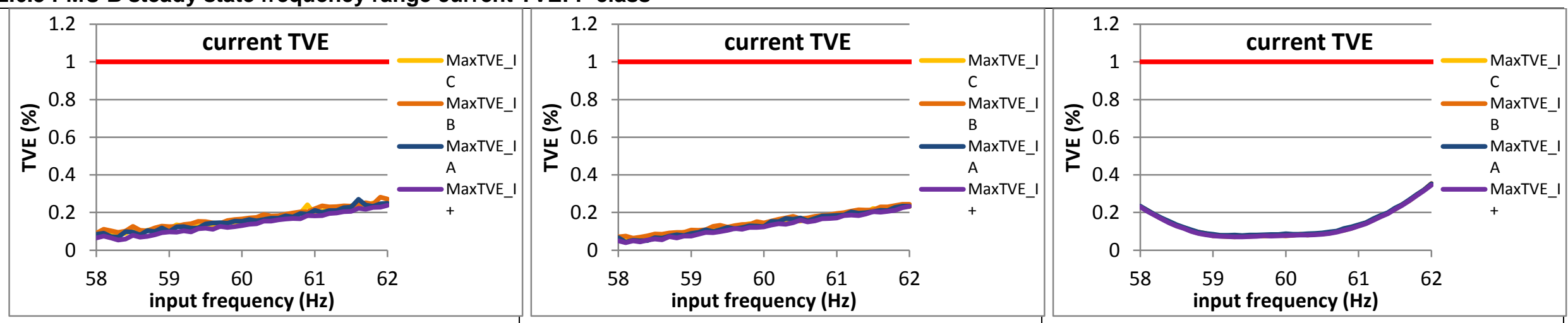

Figure 329: Fs = 60 FPS

Figure 330: Fs = 30 FPS

Figure 331: Fs = 20 FPS
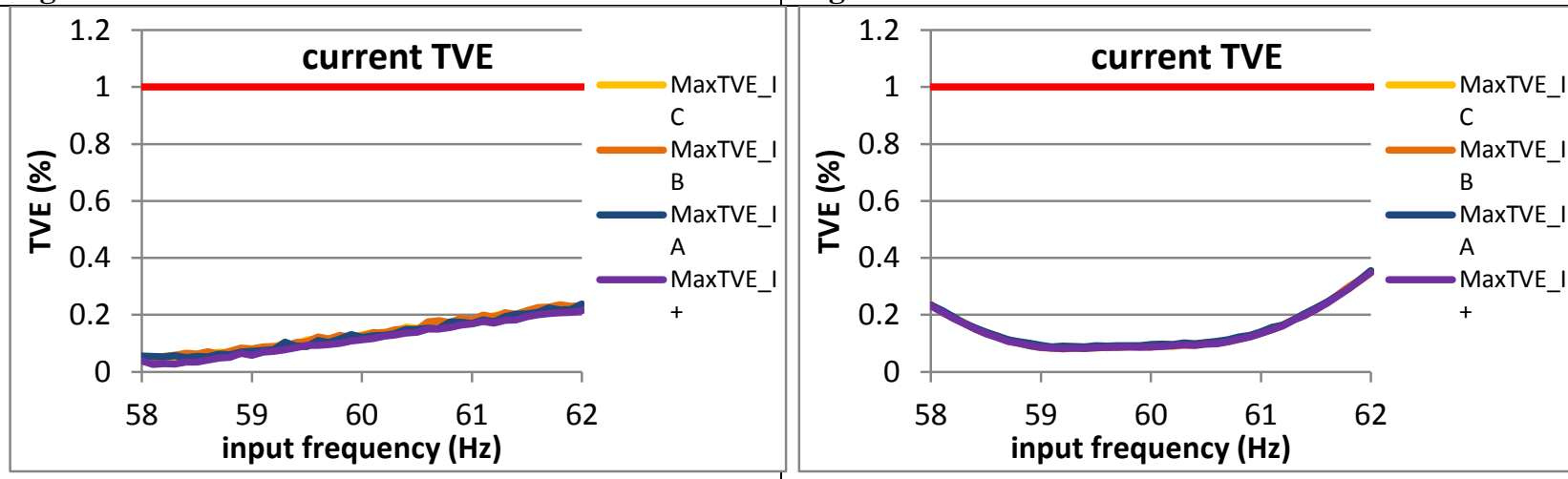

1.2 (2)

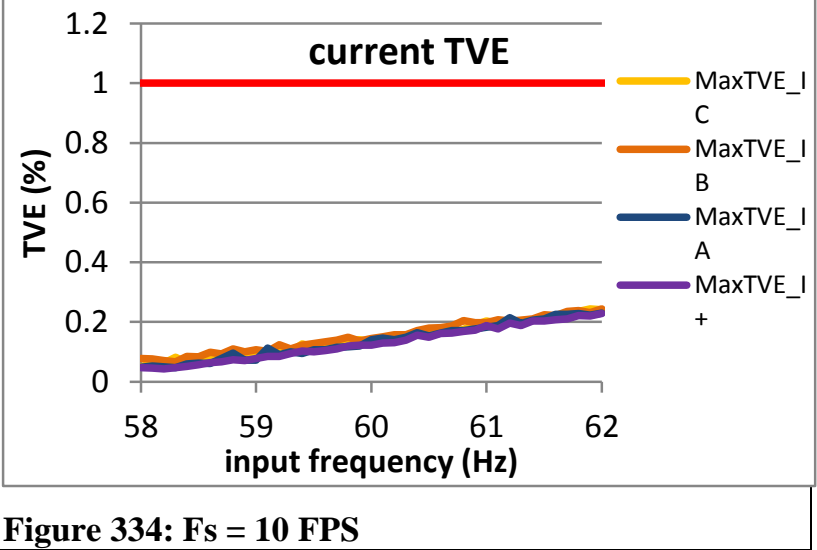

Figure 332: Fs = 15 FPS

Figure 333: Fs = 12 FPS

Figure 334: Fs = 10 FPS 


\subsubsection{PMU C steady state frequency range current TVE: P class}
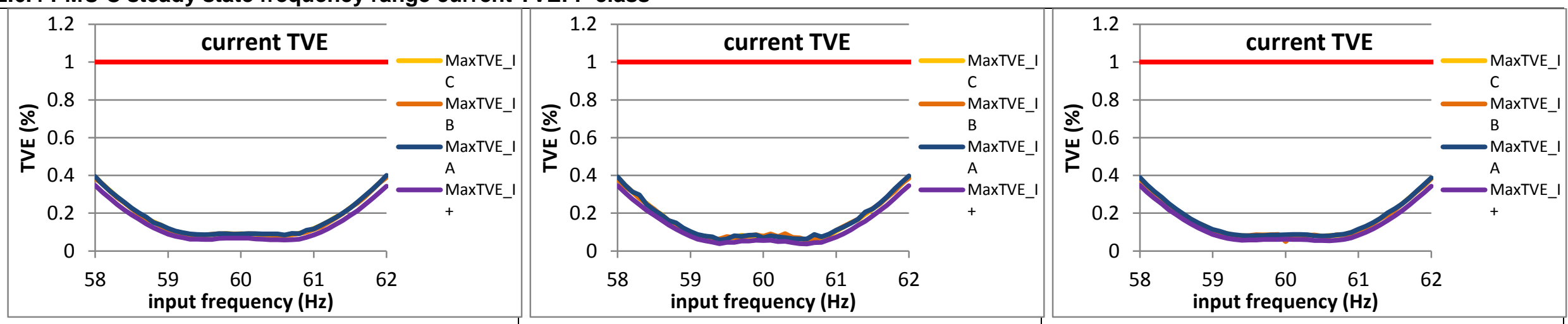

Figure 335: Fs = 60 FPS

Figure 336: Fs = 30 FPS

Figure 337: Fs = 20 FPS
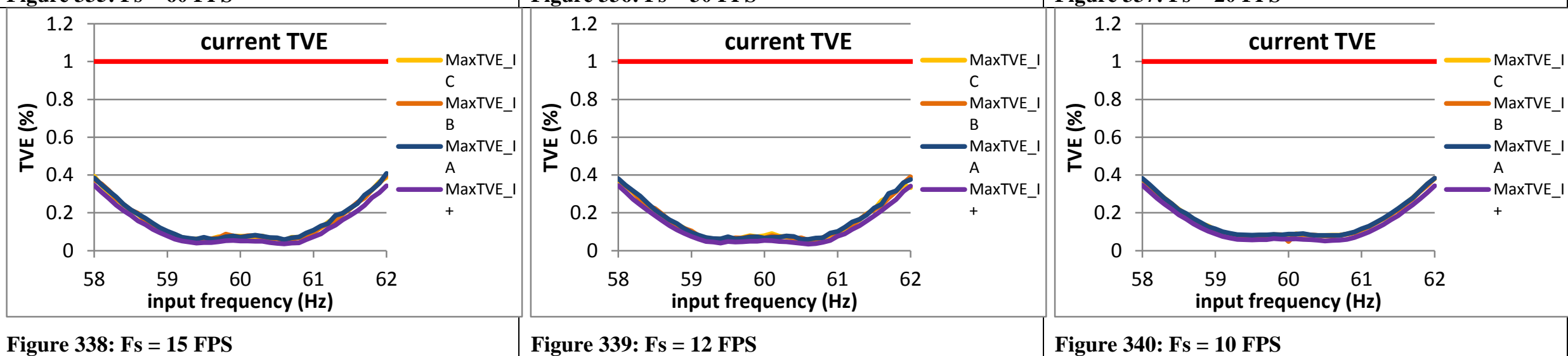

Figure 338: Fs = 15 FPS

Figure 339: Fs = 12 FPS

Figure 340: Fs = 10 FPS 
2.6.5 PMU D steady state frequency range current TVE: P class

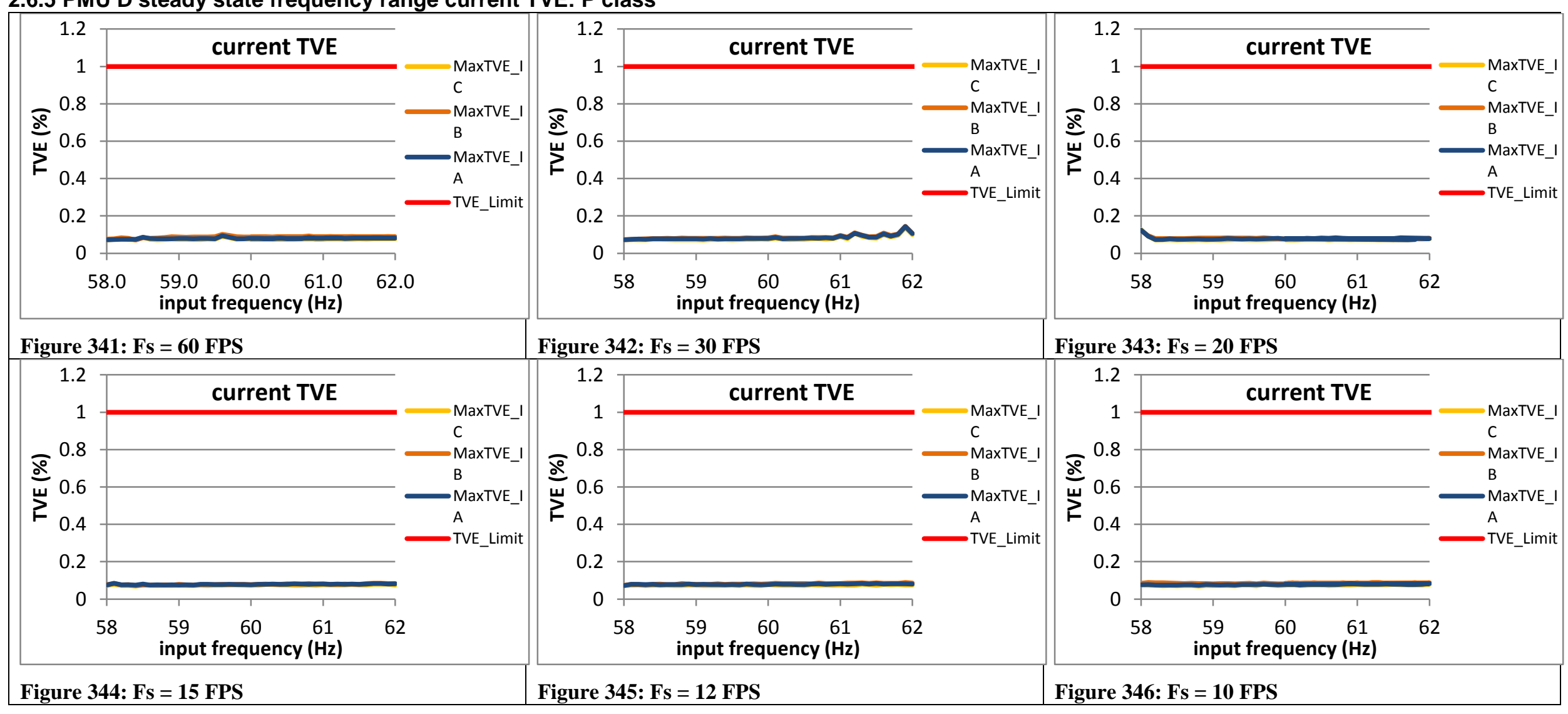

\subsubsection{PMU E steady state frequency range current TVE: P class}

This PMU does not support P class 
2.6.7 PMU F steady state frequency range current TVE: P class

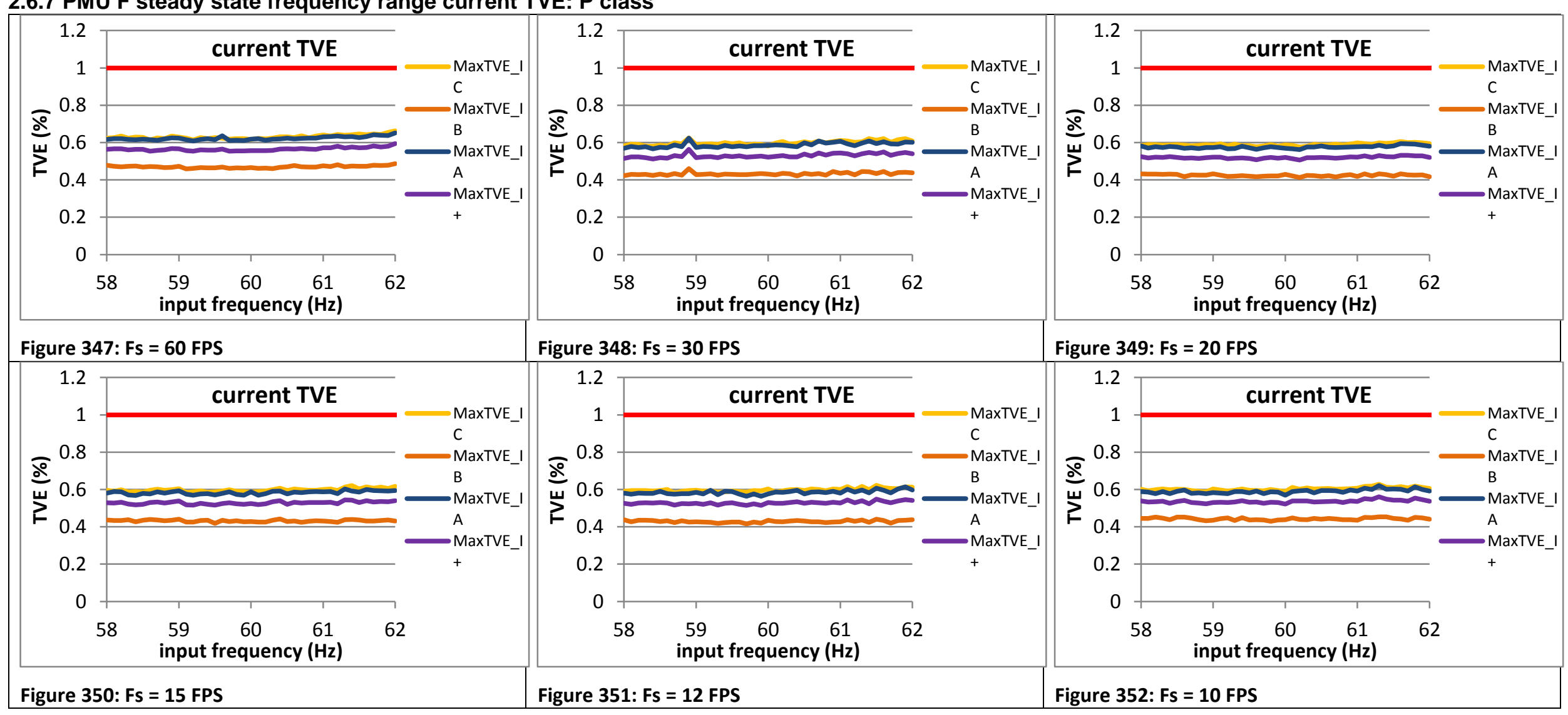

2.6.8 PMU G steady state frequency range current TVE: P class

This PMU does not support P class 
2.6.9 PMU H steady state frequency range current TVE: P class

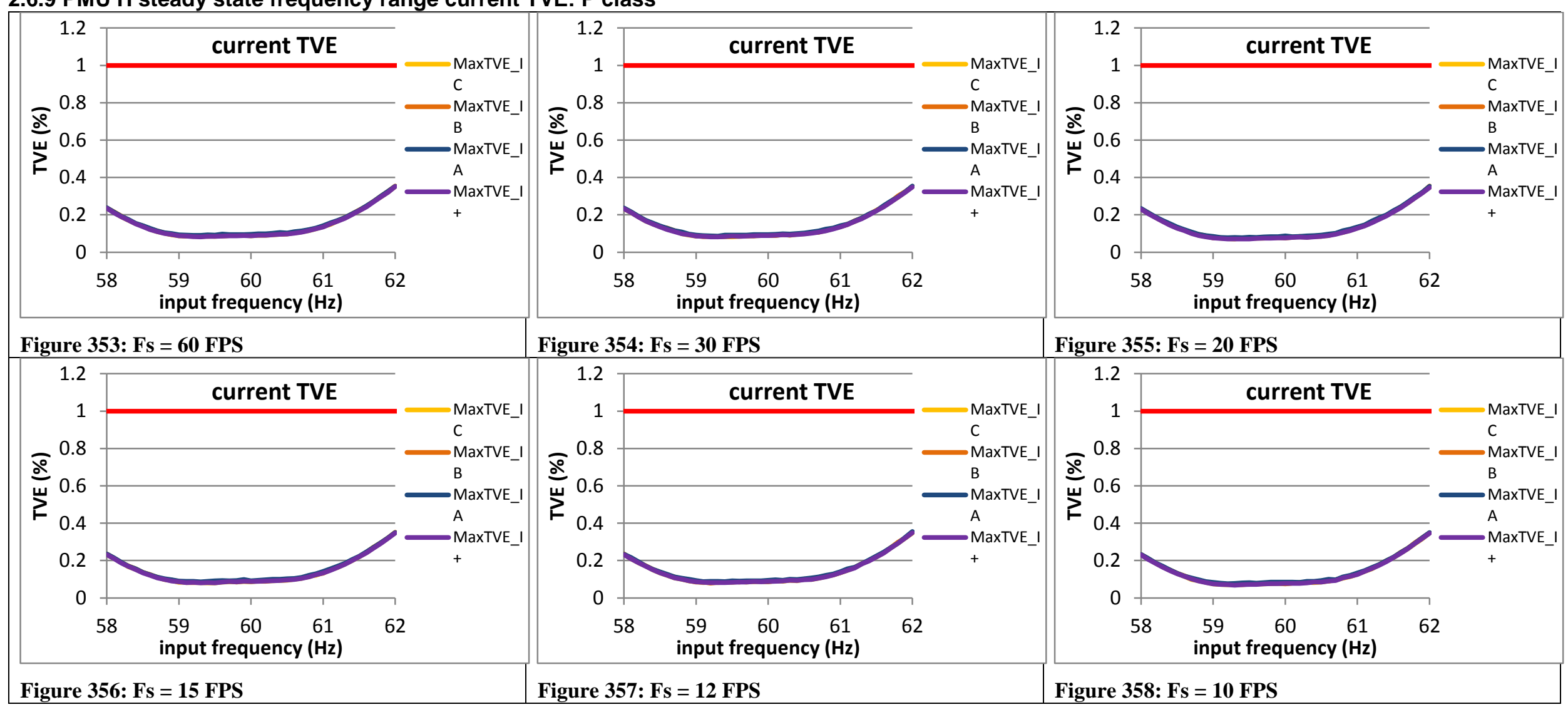

2.6.10 PMU I steady state frequency range current TVE: P class

PMU I does not support P class 


\subsubsection{PMU J steady state frequency range current TVE: P class}

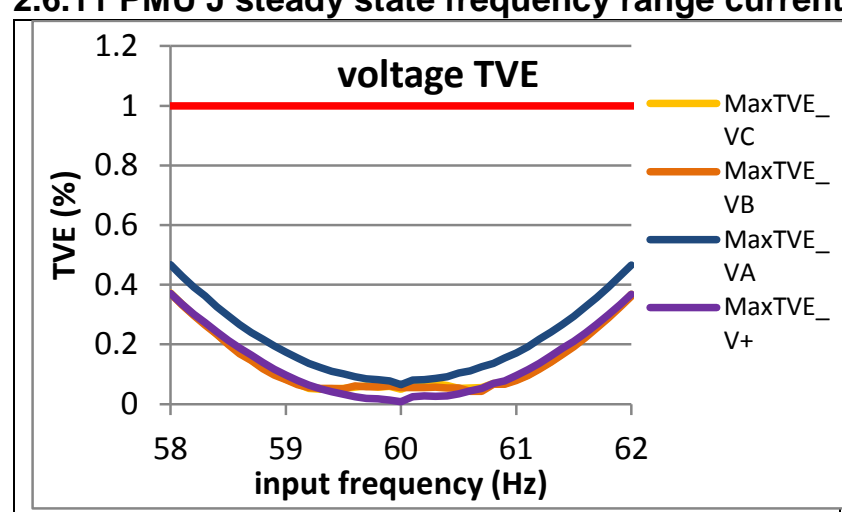

Figure 359: Fs $=60$ FPS

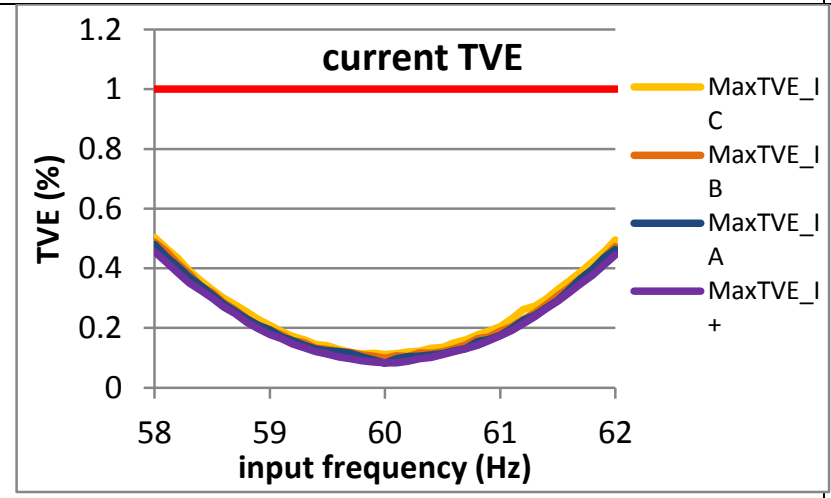

Figure 362: Fs = 15 FPS

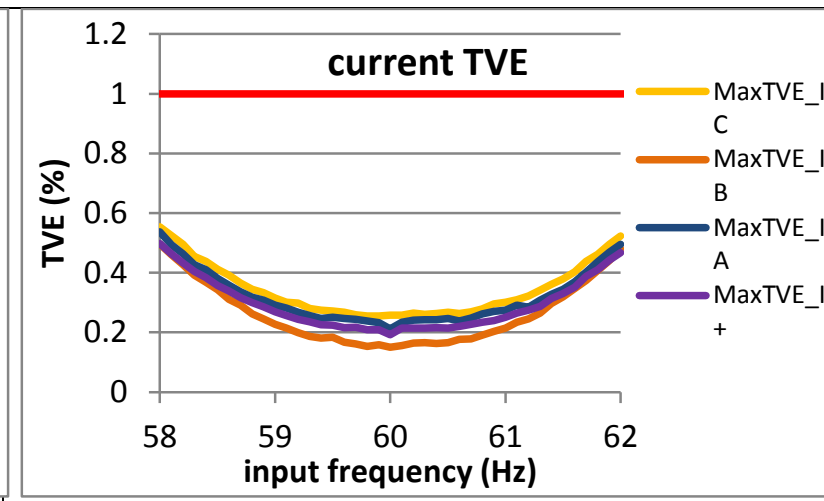

Figure 360: $\mathrm{Fs}=30 \mathrm{FPS}$

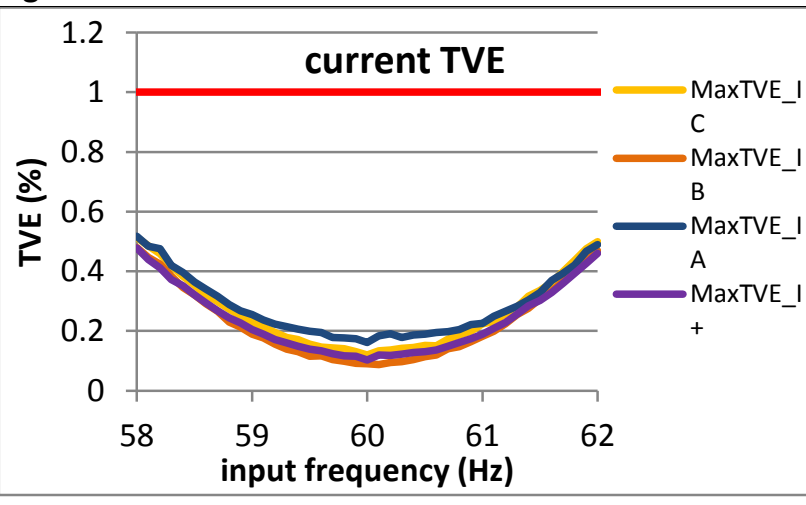

Figure 363: Fs = 12 FPS

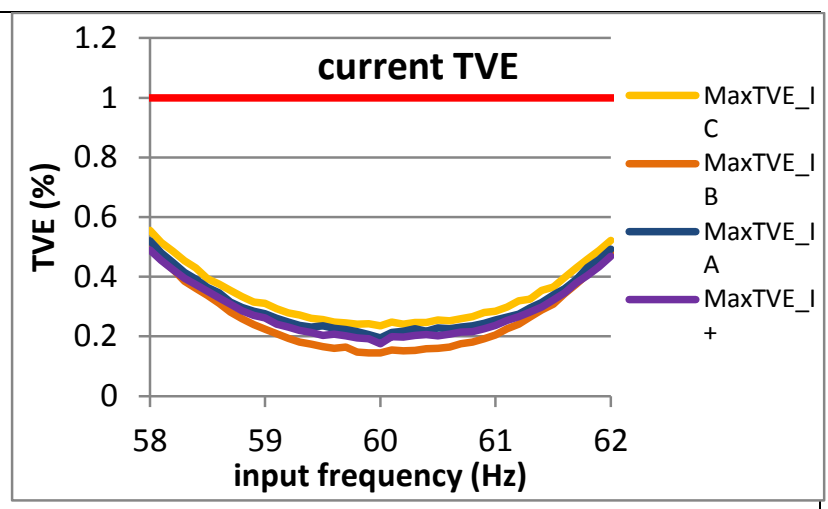

Figure 361: Fs $=20$ FPS

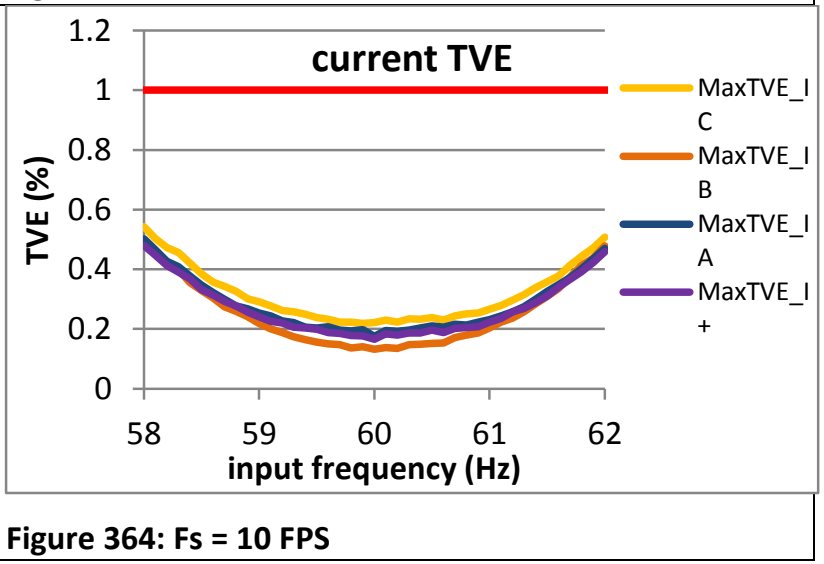




\subsection{Steady state frequency range frequency error: P class}

\subsubsection{C37.118.1 Annex C steady state frequency range frequency error:, $F 0=60 \mathrm{~Hz}, \mathrm{P}$ class}
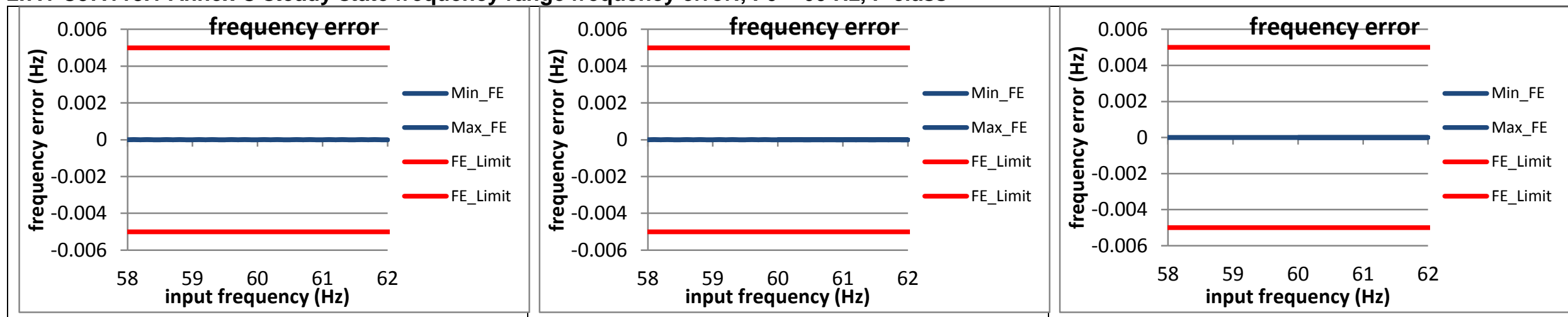

Figure 365: Fs = 60 FPS

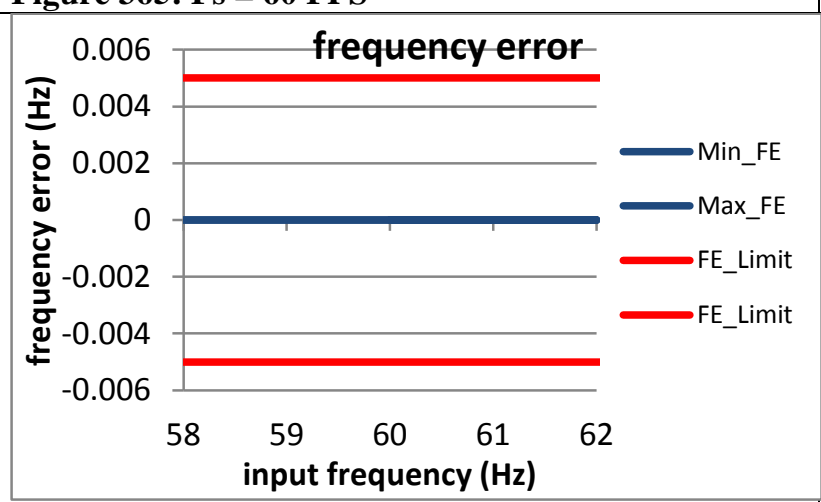

Figure 366: $\mathrm{Fs}=30$ FPS

Figure 367: Fs = 20 FPS
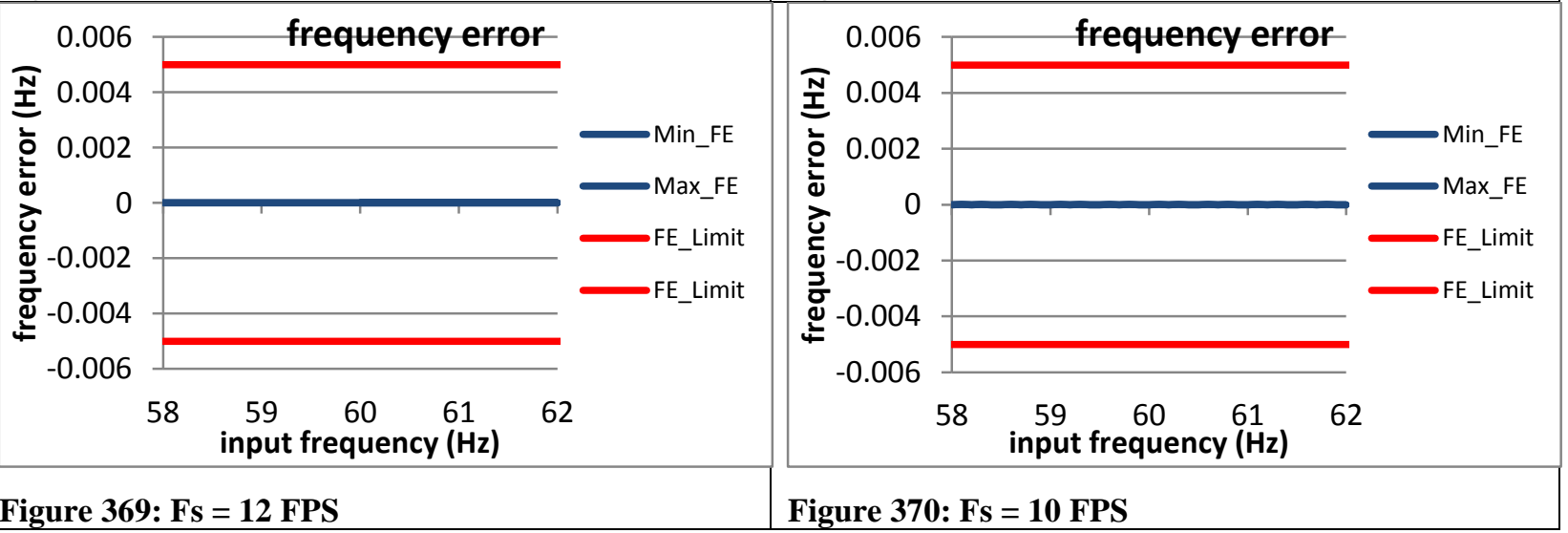

Figure 368: Fs = 15 FPS

Figure 369: Fs = 12 FPS

Figure 370: Fs = 10 FPS 
2.7.2 PMU A steady state frequency range frequency error: $P$ class

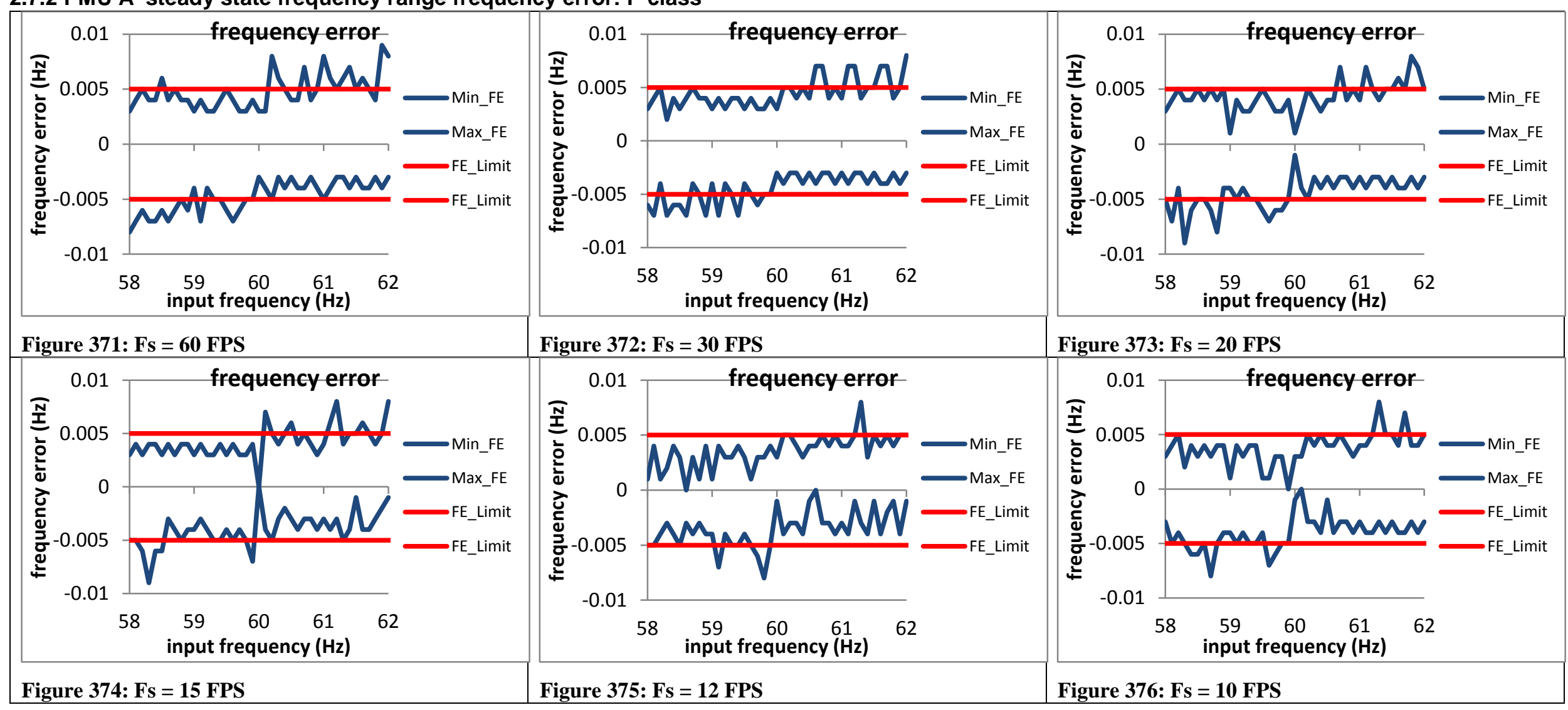




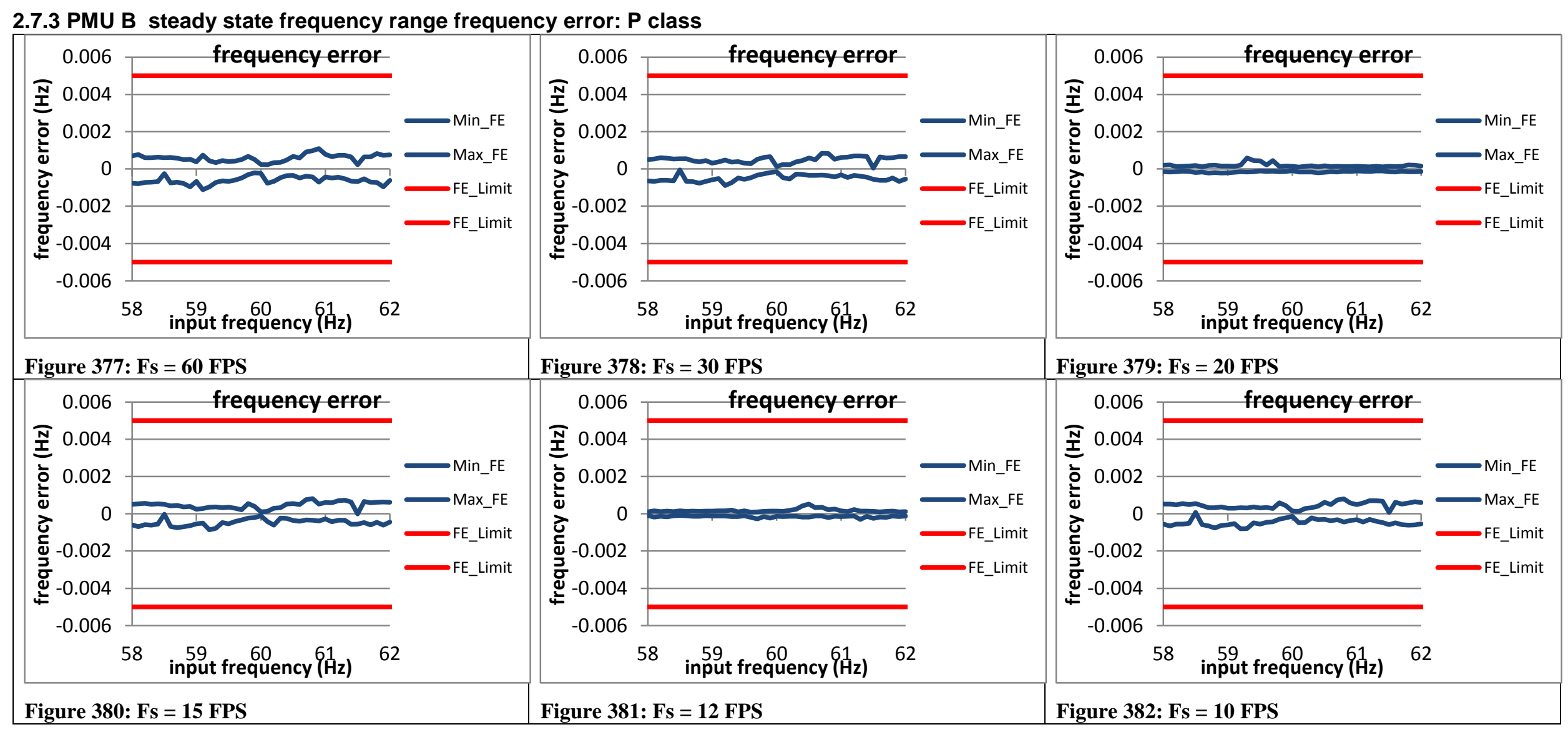




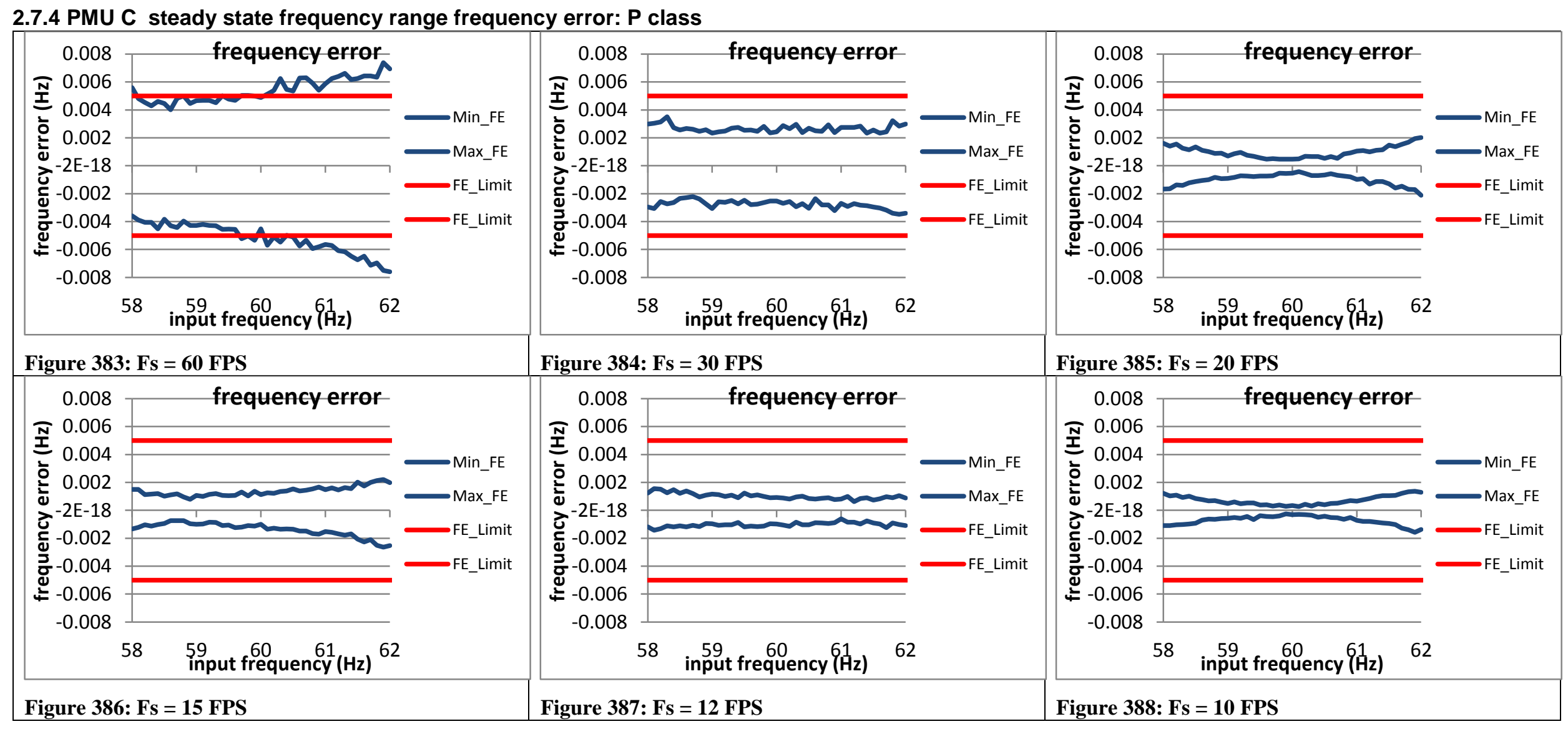




\subsubsection{PMU D steady state frequency range frequency error: $P$ class}

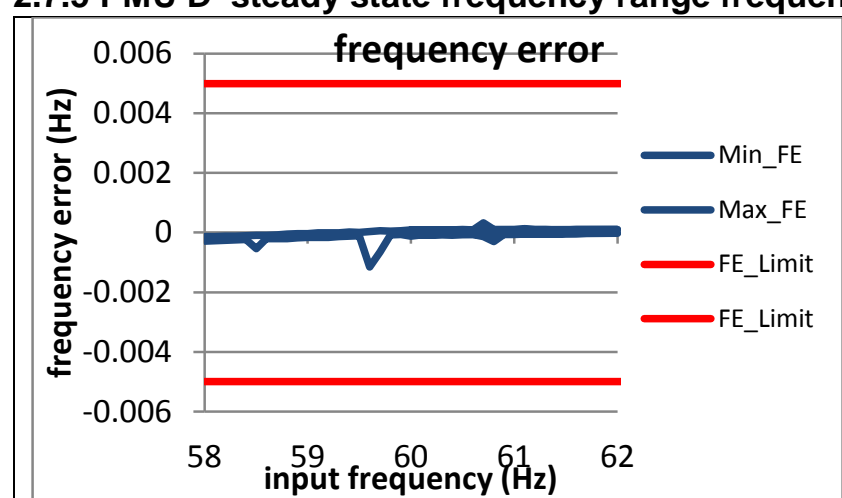

Figure 389: Fs = 60 FPS

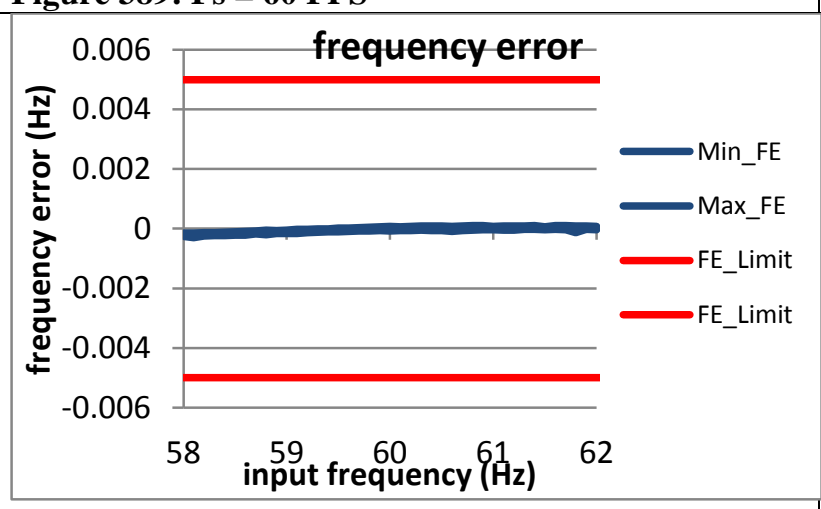

Figure 392: Fs = 15 FPS

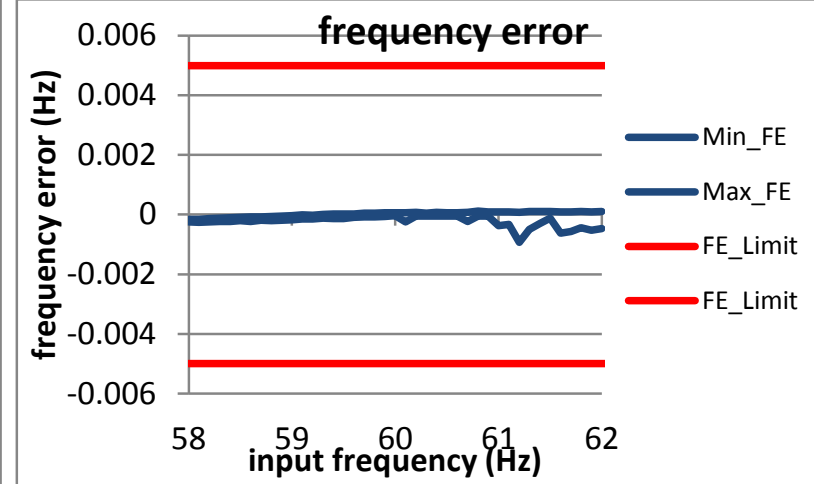

Figure 390: Fs = 30 FPS

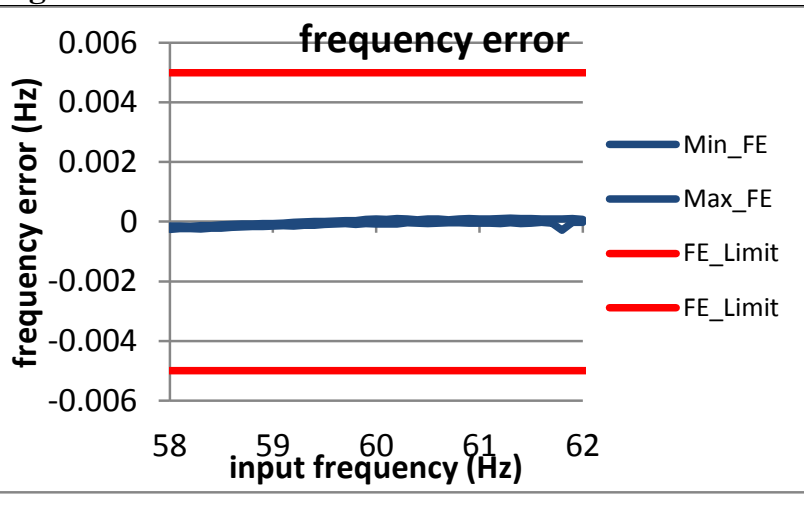

Figure 393: Fs = 12 FPS

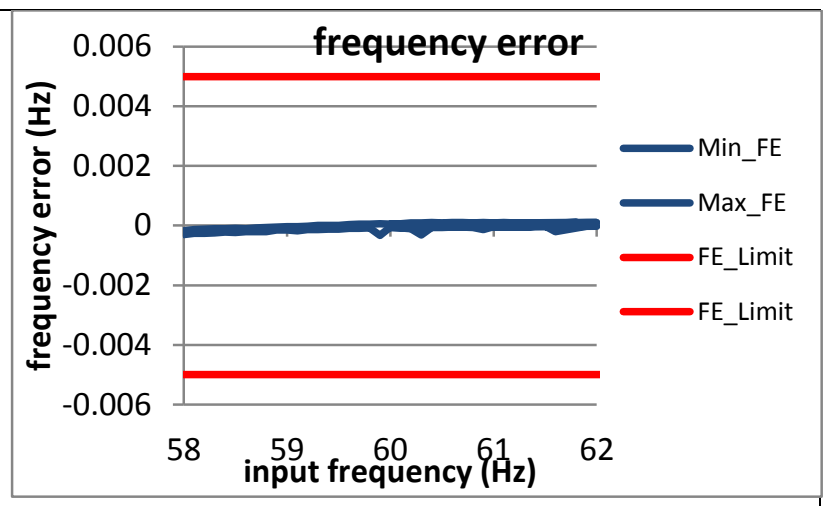

Figure 391: Fs = 20 FPS

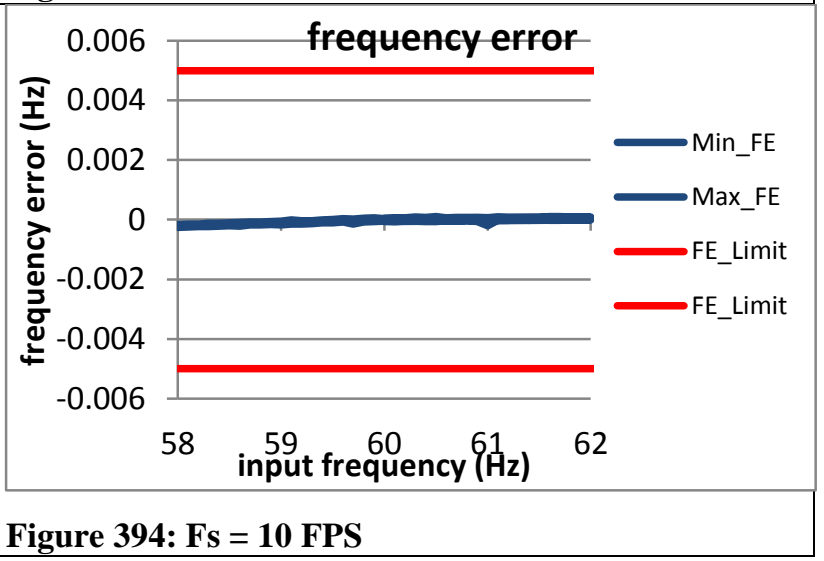

2.7.6 PMU E steady state frequency range frequency error: $P$ class

This PMU does not support P class 
2.7.7 PMU F steady state frequency range frequency error: $P$ class

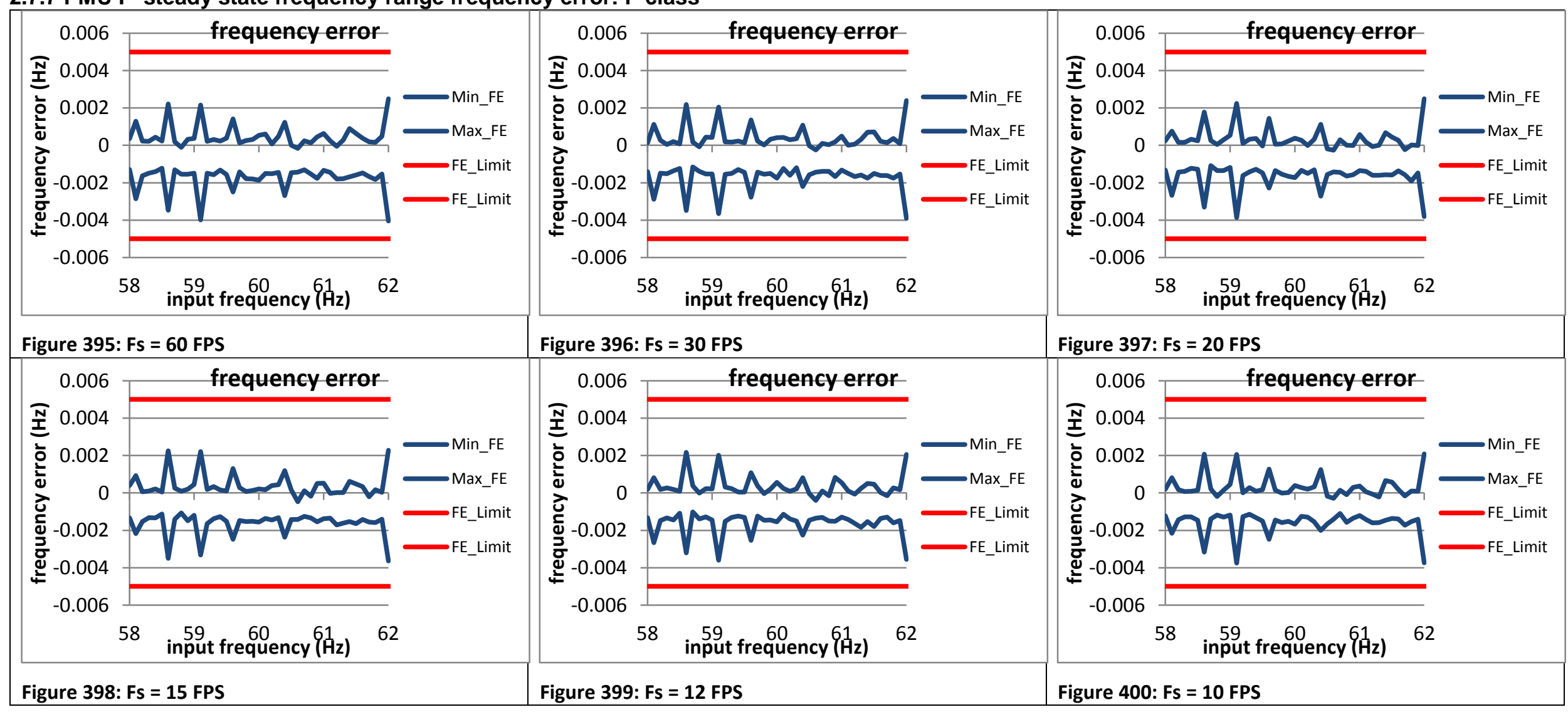

2.7.8 PMU G steady state frequency range frequency error: $P$ class

This PMU does not support P class 
2.7.9 PMU H steady state frequency range frequency error: $\mathrm{P}$ class

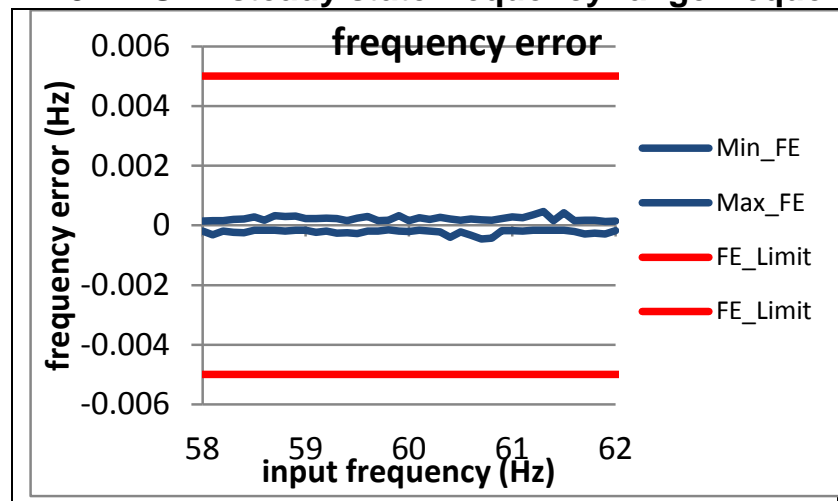

Figure 401: Fs = 60 FPS

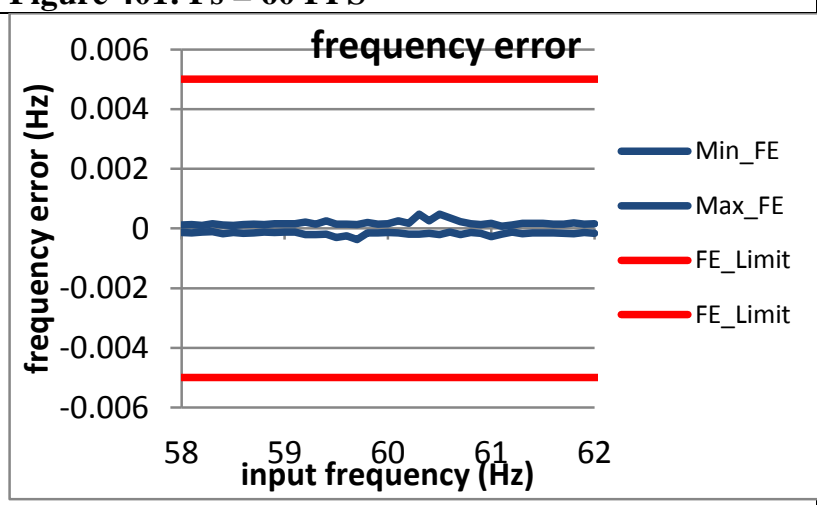

Figure 404: Fs = 15 FPS

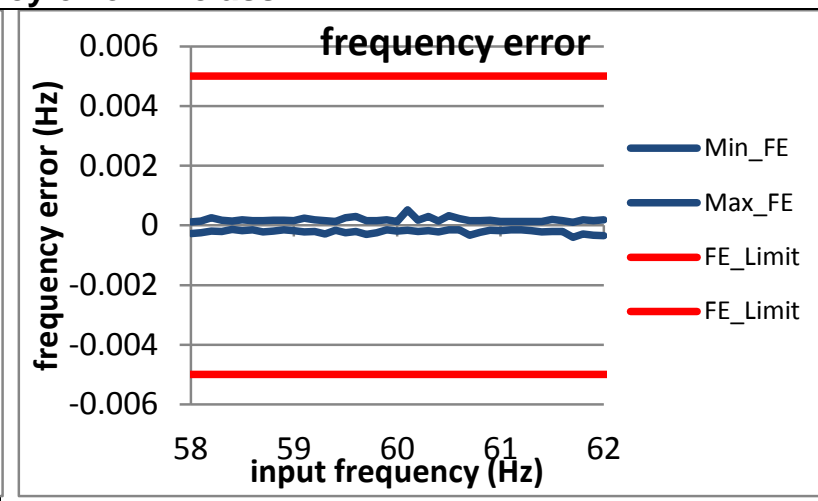

Figure 402: Fs = 30 FPS

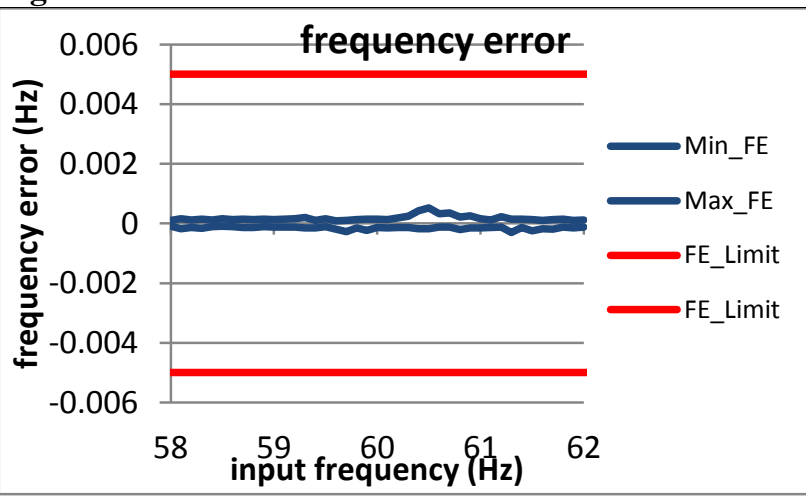

Figure 405: Fs = 12 FPS

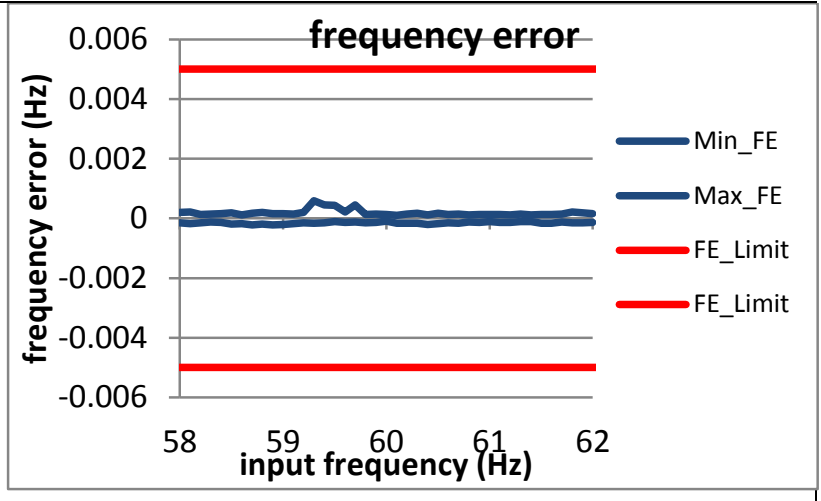

Figure 403: Fs = 20 FPS

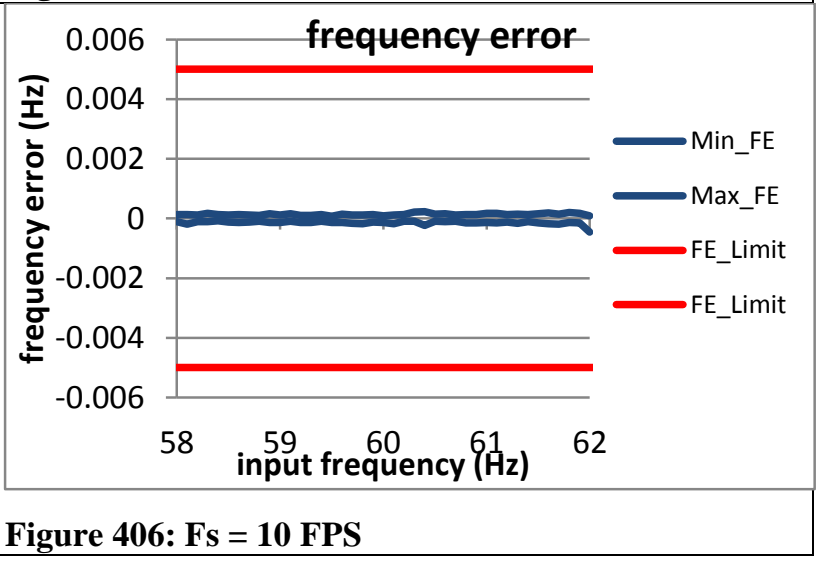

2.7.10 PMU I steady state frequency range frequency error: $P$ class

PMU I does not support P class 


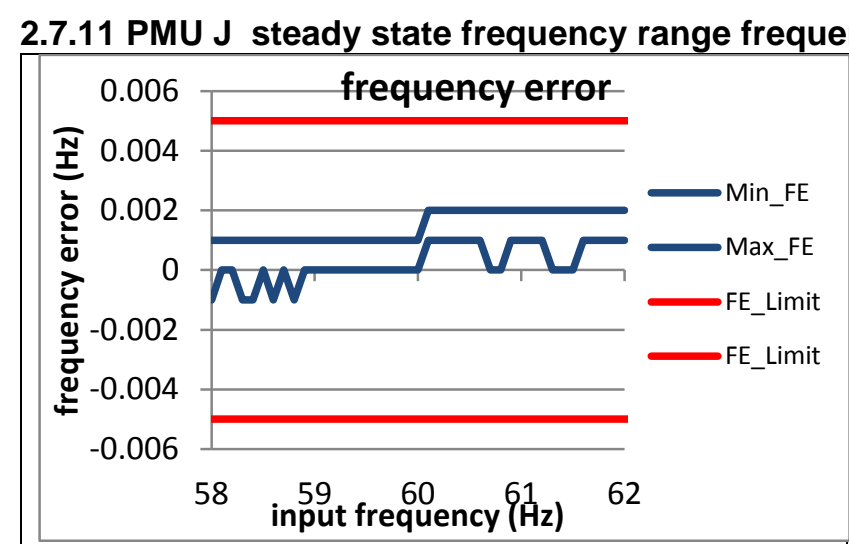

Figure 407: Fs = 60 FPS
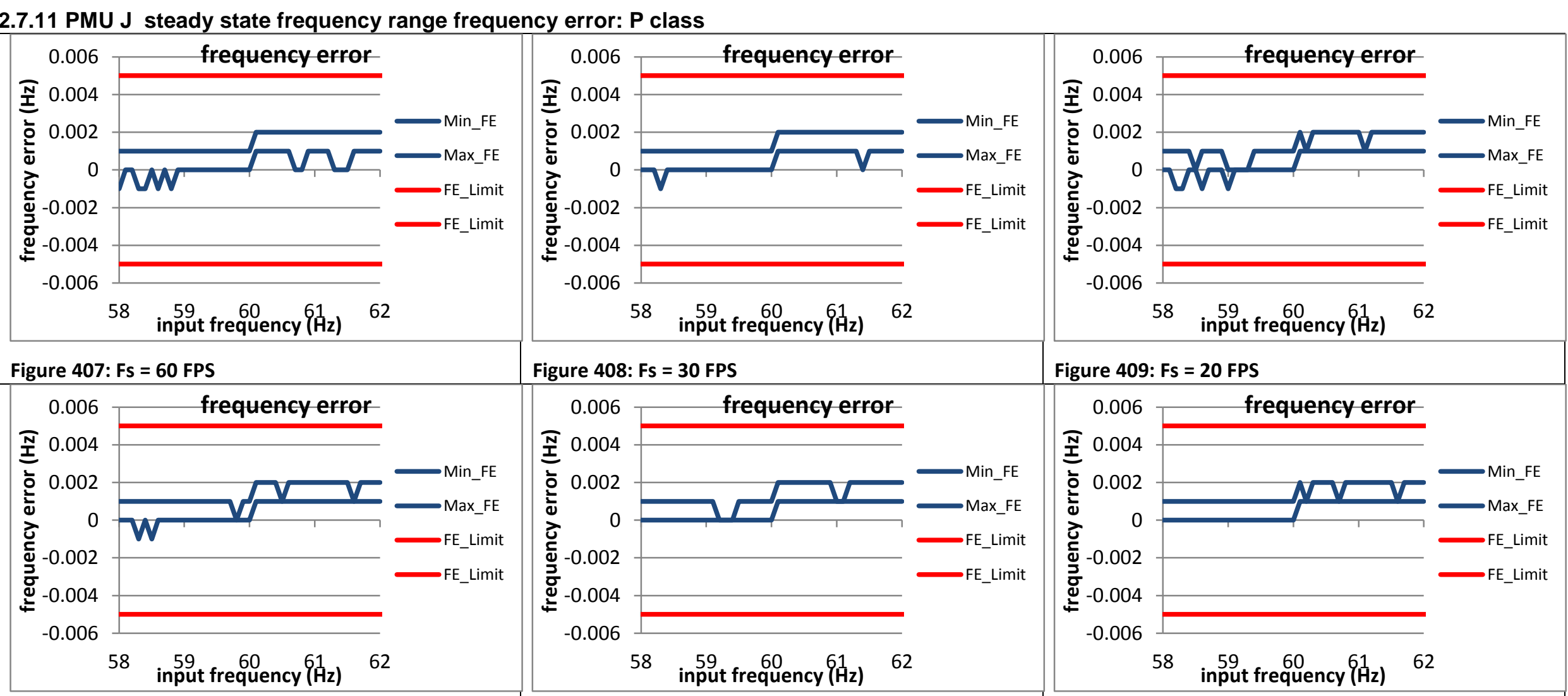

Figure 408: $\mathrm{Fs}=30 \mathrm{FPS}$

Figure 409: Fs = 20 FPS
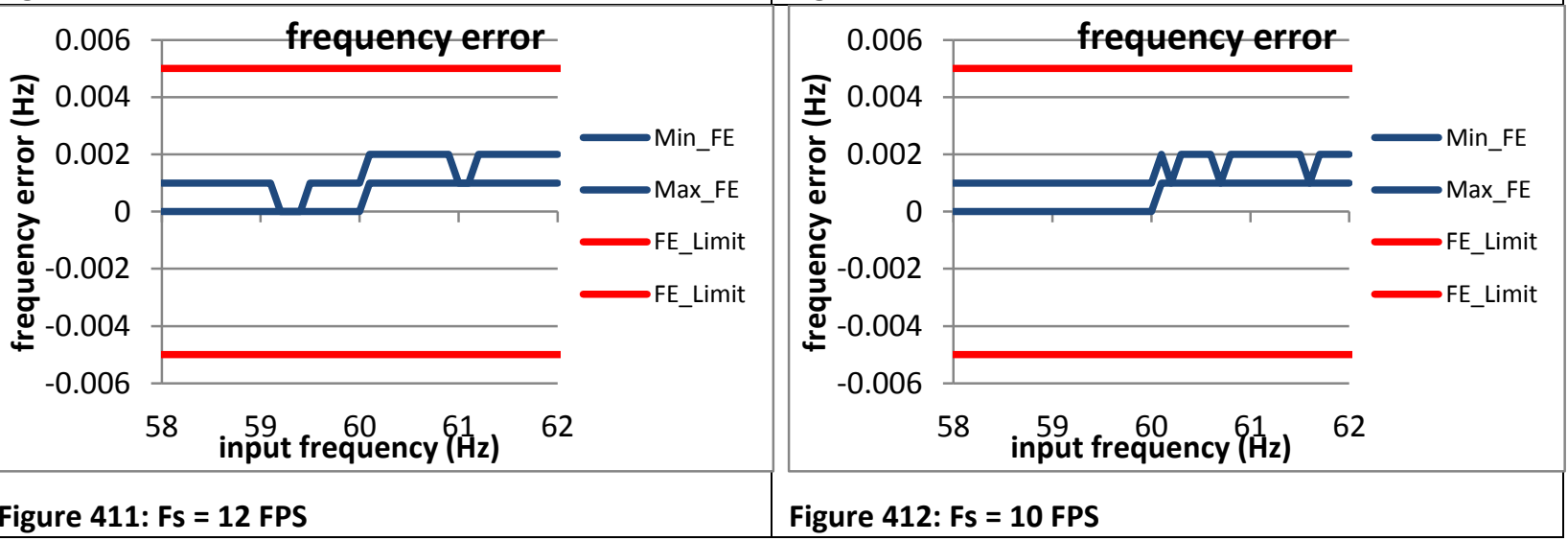

Figure 410: Fs = 15 FPS

Figure 411: Fs = 12 FPS

Figure 412: Fs $=10 \mathrm{FPS}$ 


\subsection{Steady state frequency range ROCOF error: P class}

\subsubsection{C37.118.1 Annex $C$ steady state frequency range ROCOF error: $P$ class}

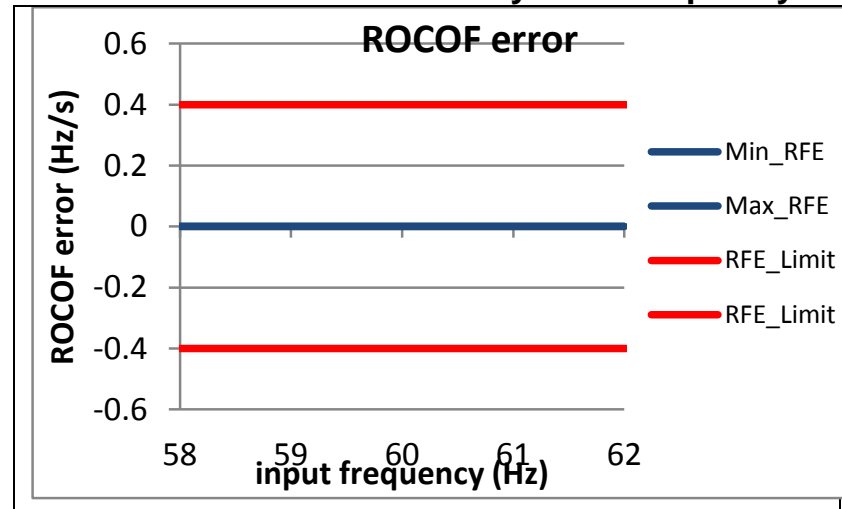

Figure 413: Fs = 60 FPS

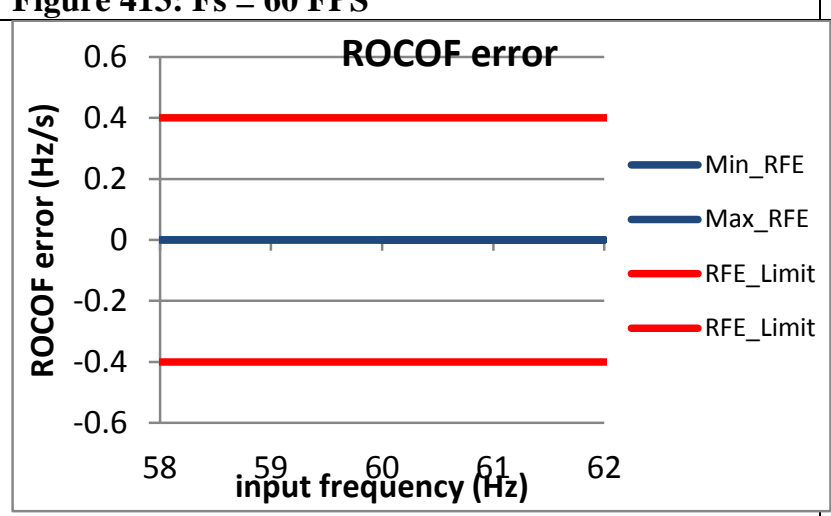

Figure 416: Fs = 15 FPS

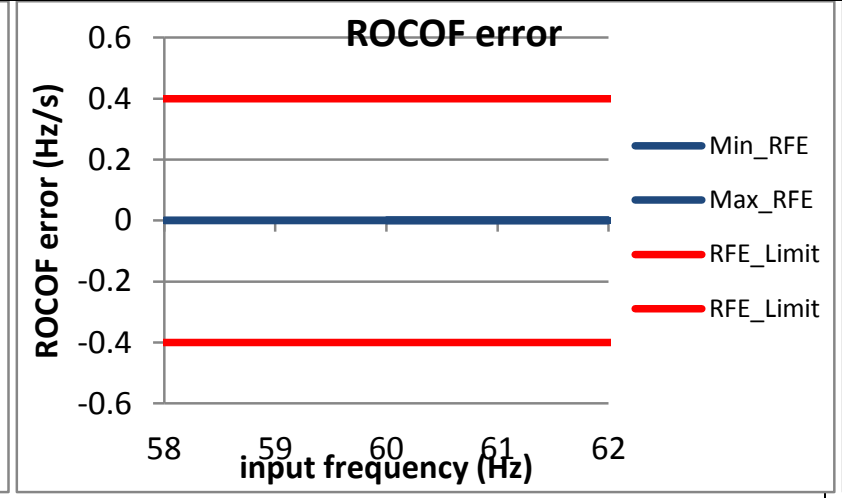

Figure 414: Fs = 30 FPS

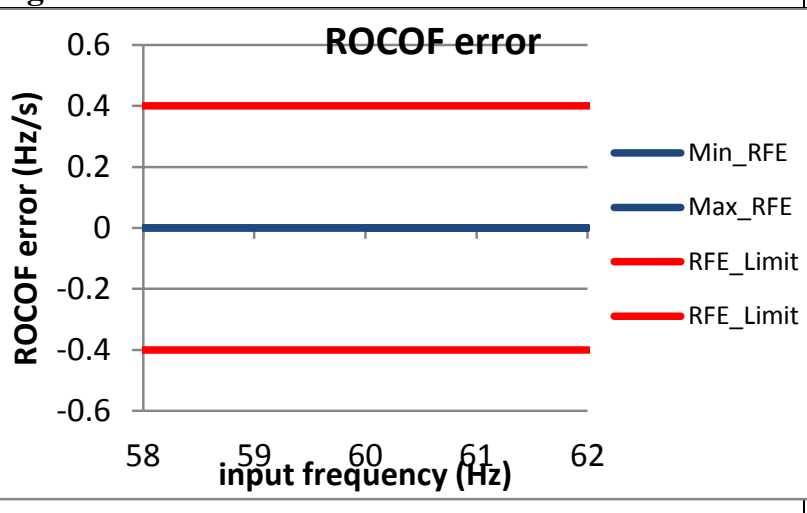

Figure 417: Fs = 12 FPS

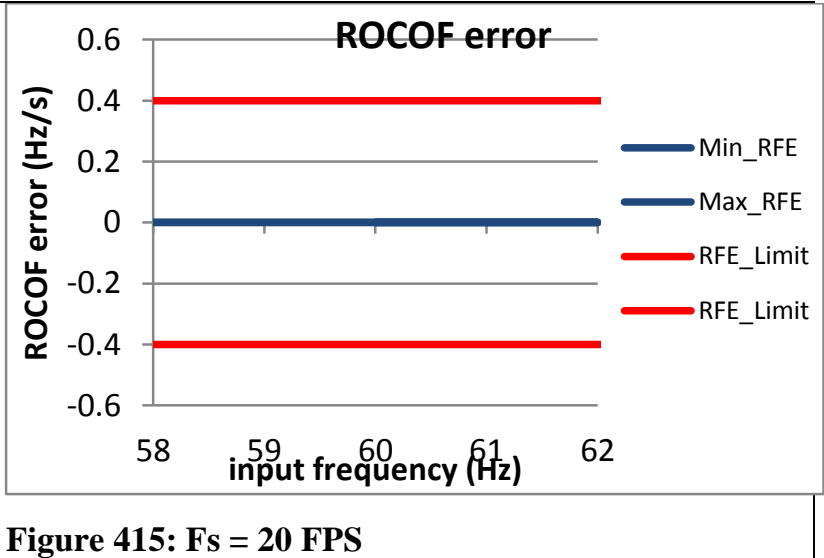

Figure 415: Fs = 20 FPS

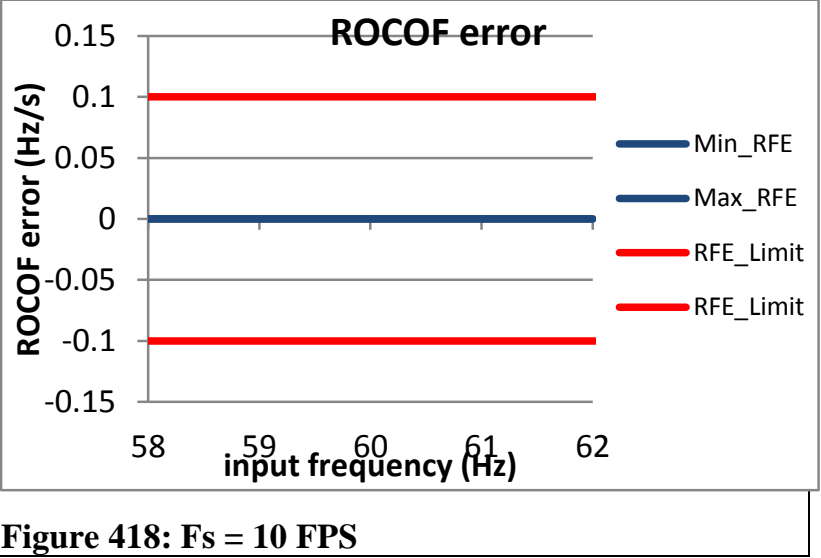


2.8.2 PMU A steady state frequency range ROCOF error: P class

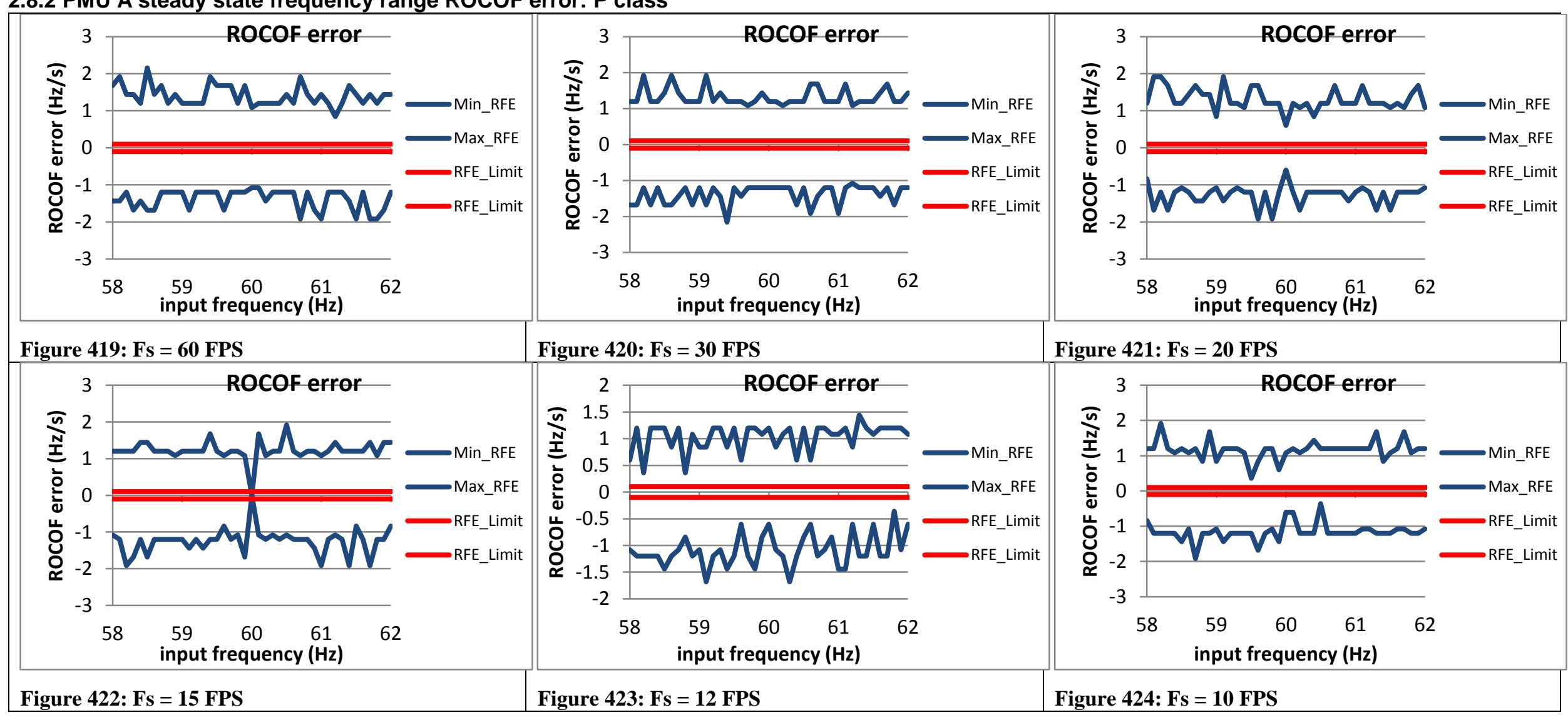


2.8.3 PMU B steady state frequency range ROCOF error: P class

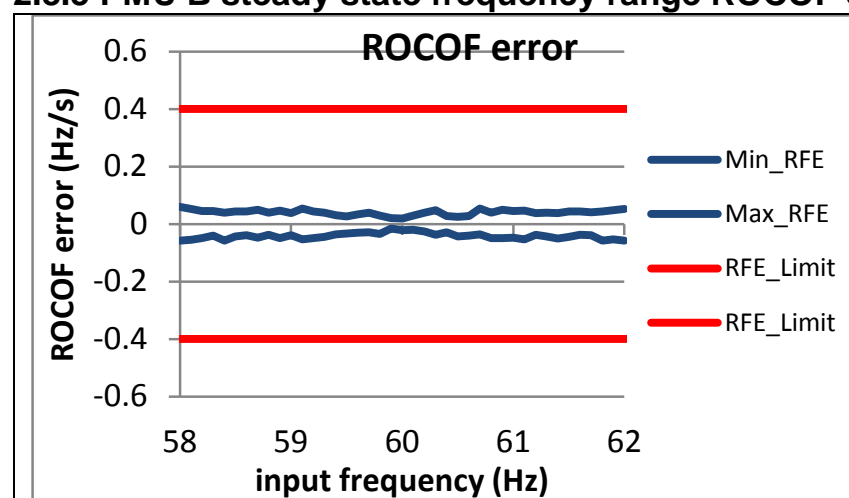

Figure 425: Fs = 60 FPS
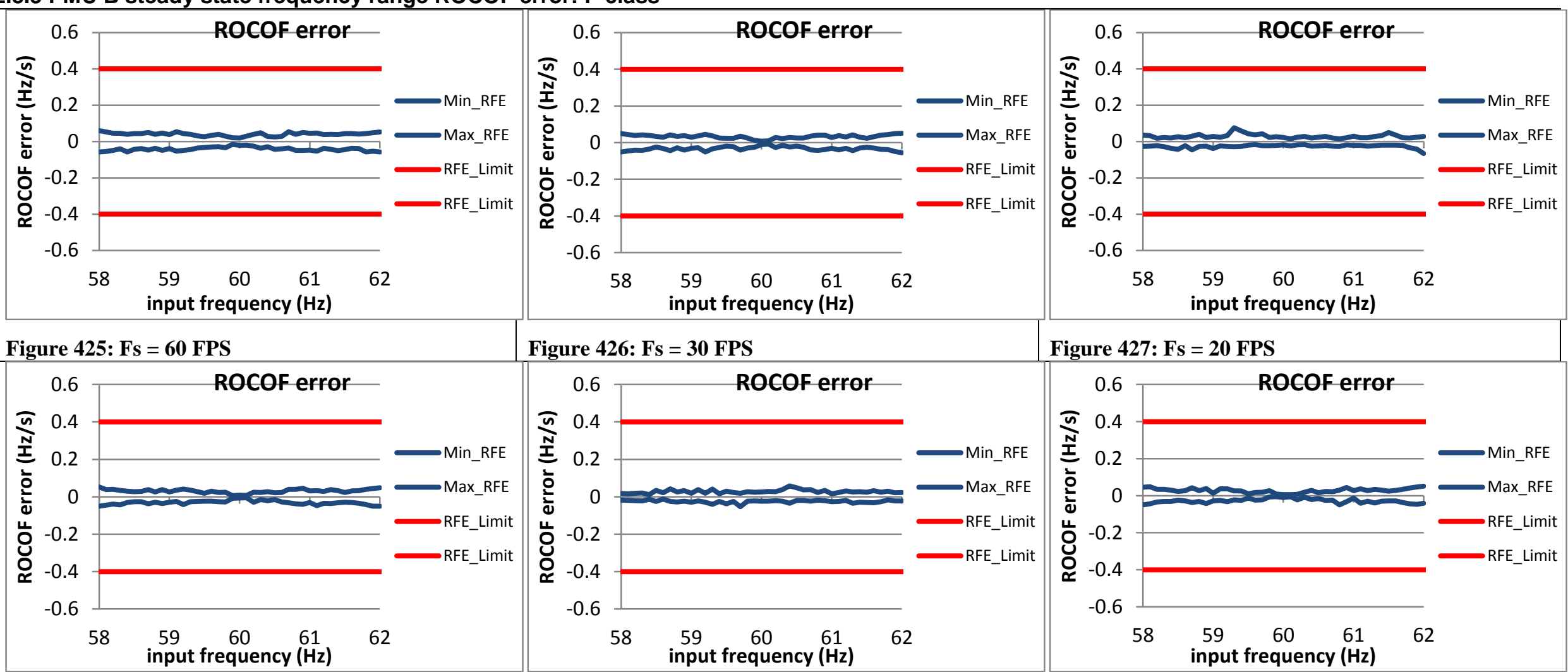

Figure 426: Fs = 30 FPS

Figure 427: Fs $=20$ FPS
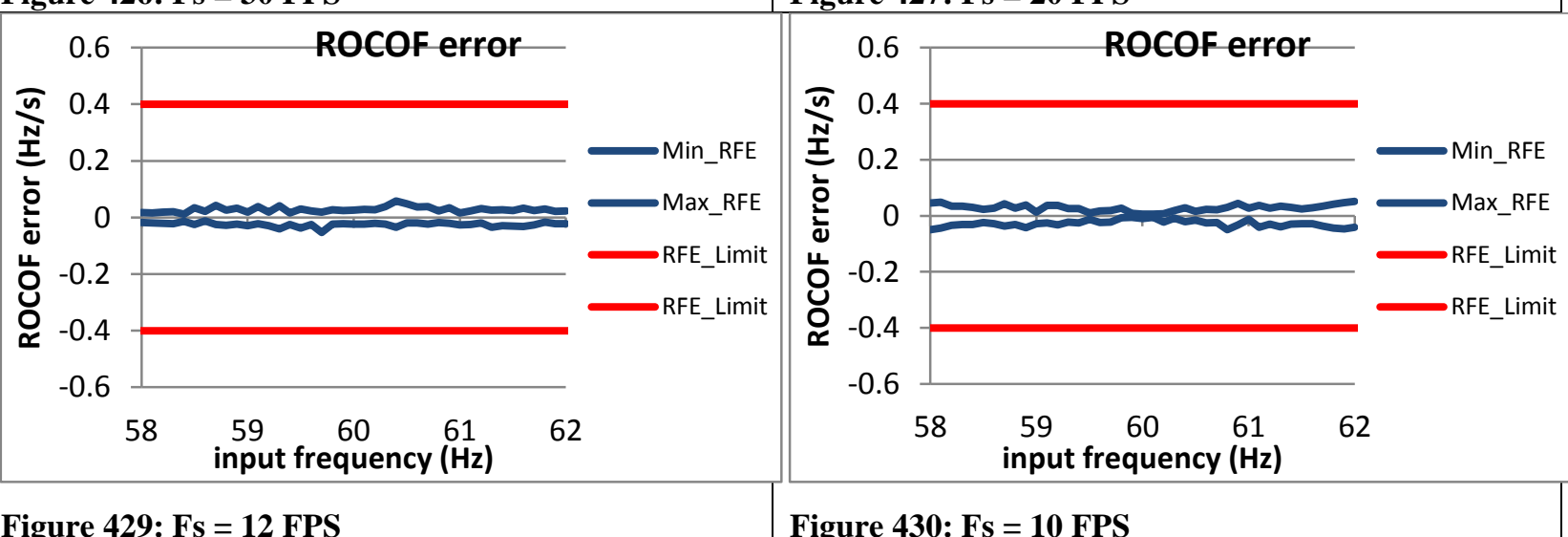

Figure 428: Fs = 15 FPS

Figure 429: Fs = 12 FPS

Figure 430: Fs = 10 FPS 
2.8.4 PMU C steady state frequency range ROCOF error: P class

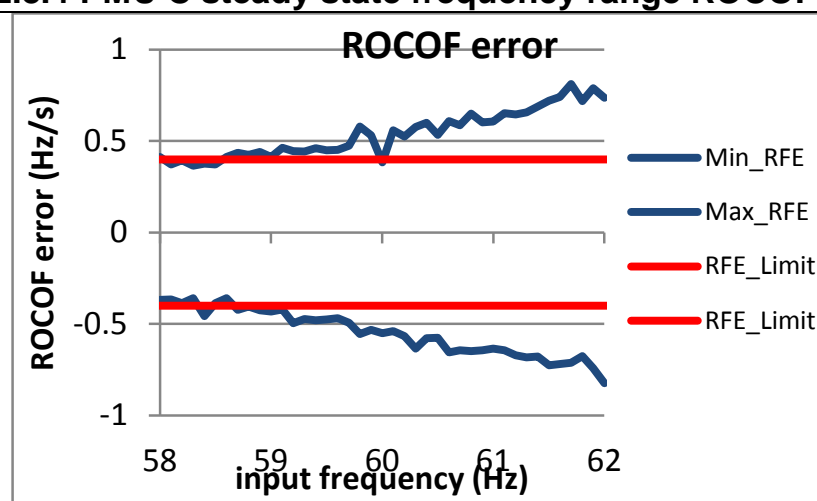

Figure 431: Fs = 60 FPS

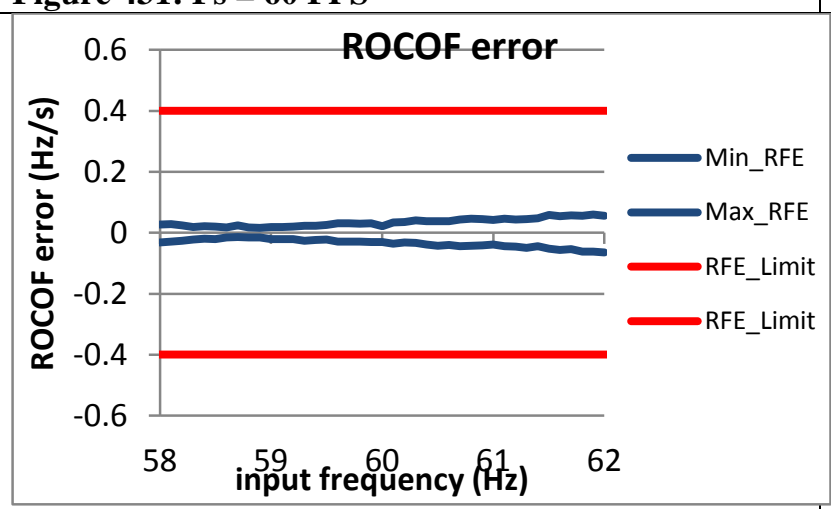

Figure 434: Fs = 15 FPS

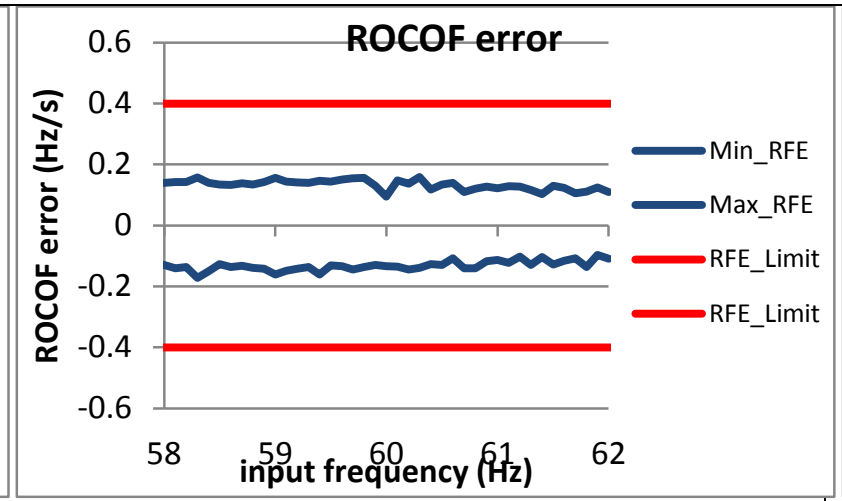

Figure 432: Fs = 30 FPS

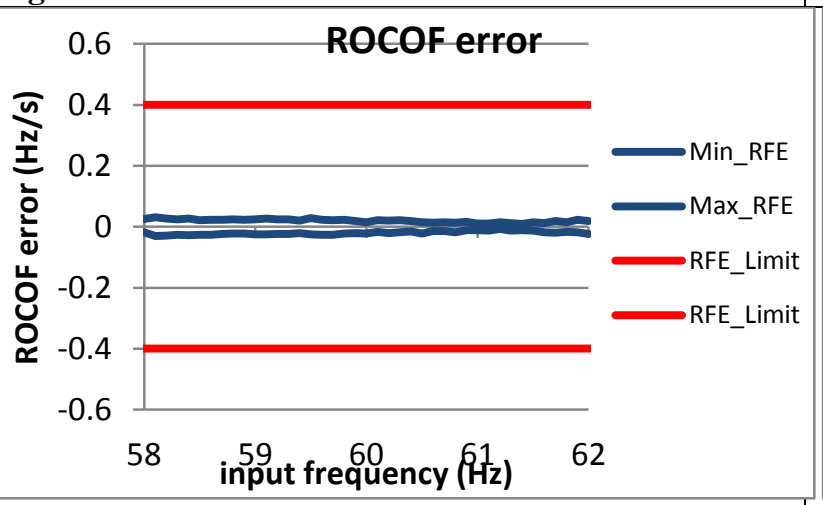

Figure 435: Fs = 12 FPS

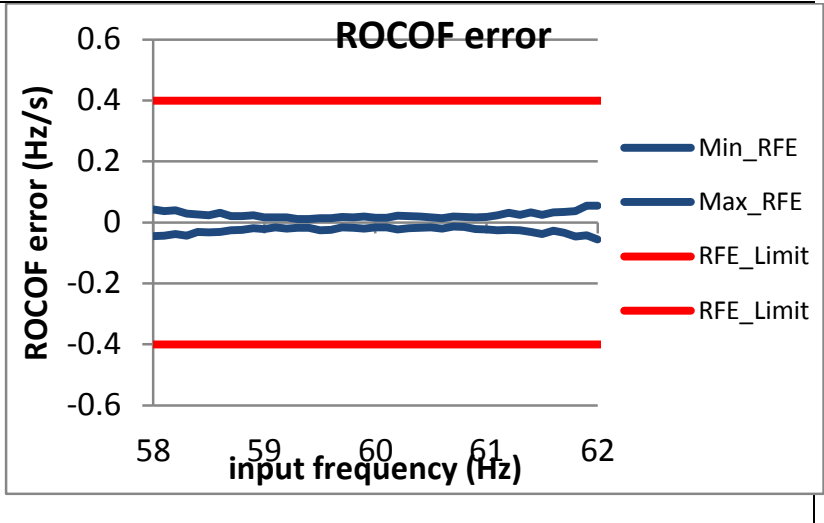

Figure 433: Fs = 20 FPS

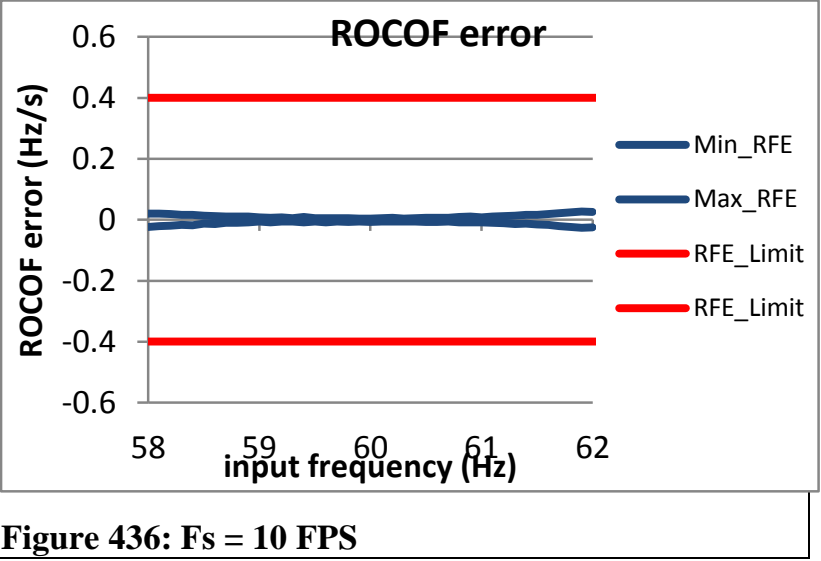


2.8.5 PMU D steady state frequency range ROCOF error: $P$ class

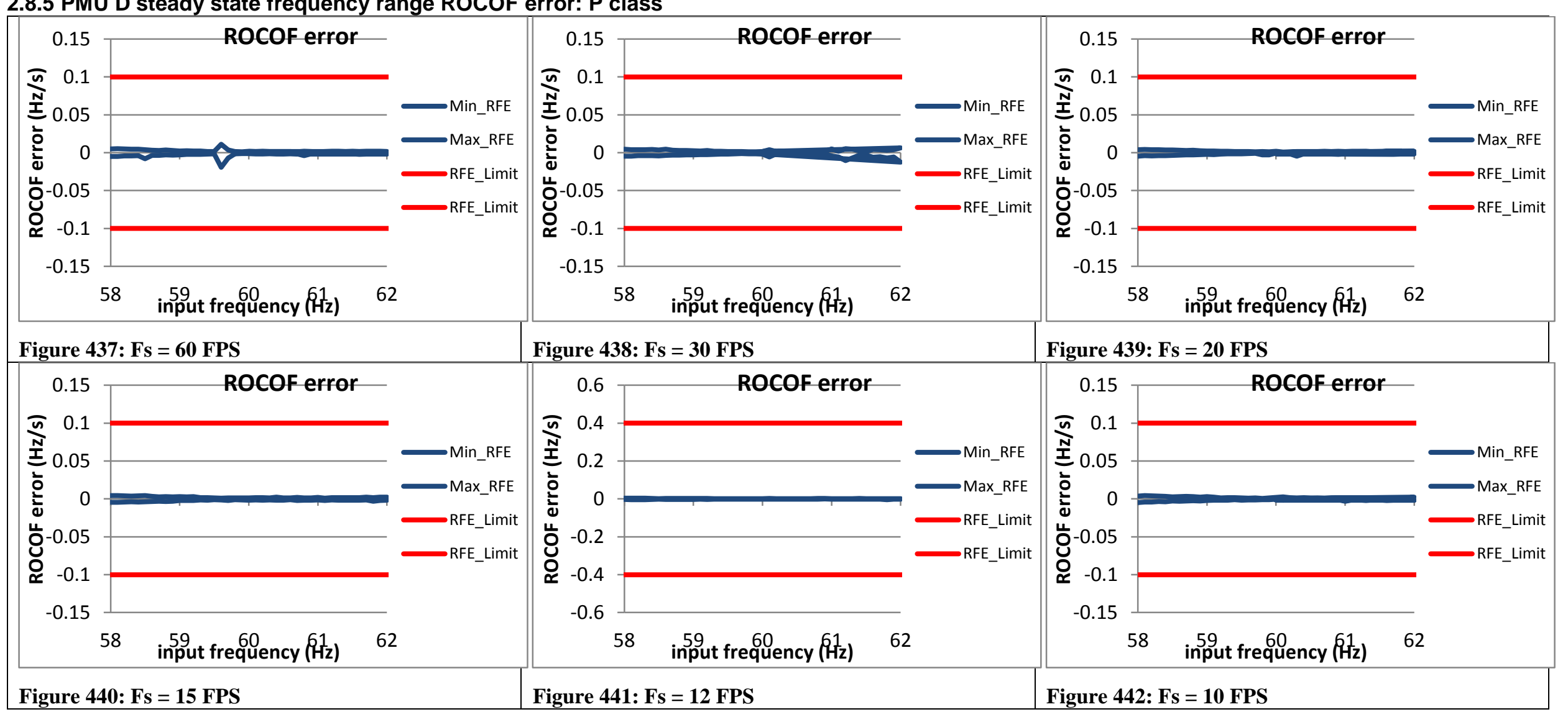

2.8.6 PMU E steady state frequency range ROCOF error: $P$ class

PMU E does not support P class 
2.8.7 PMU F steady state frequency range ROCOF error: $P$ class

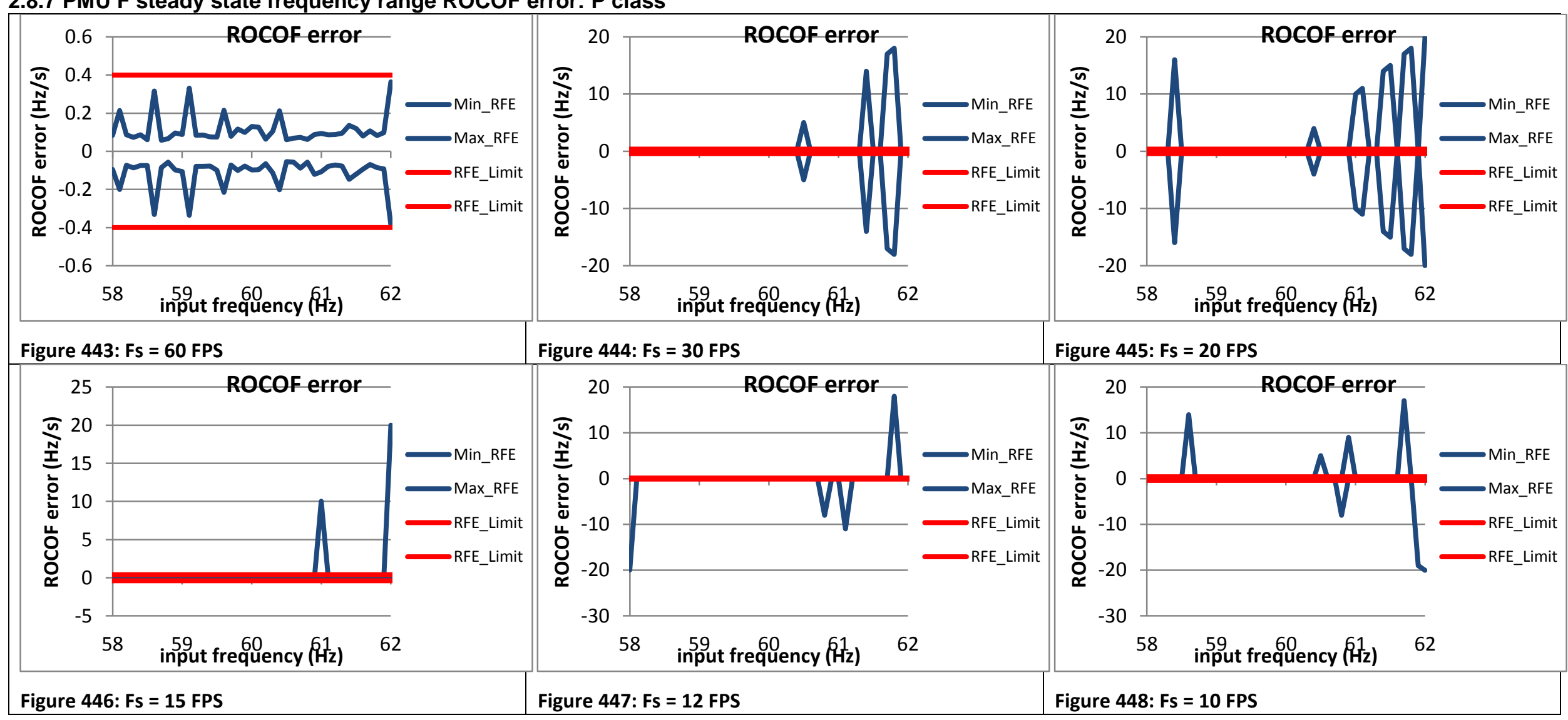

2.8.8 PMU G steady state frequency range ROCOF error: P class

PMU G does not support P class 
2.8.9 PMU H steady state frequency range ROCOF error:, P class

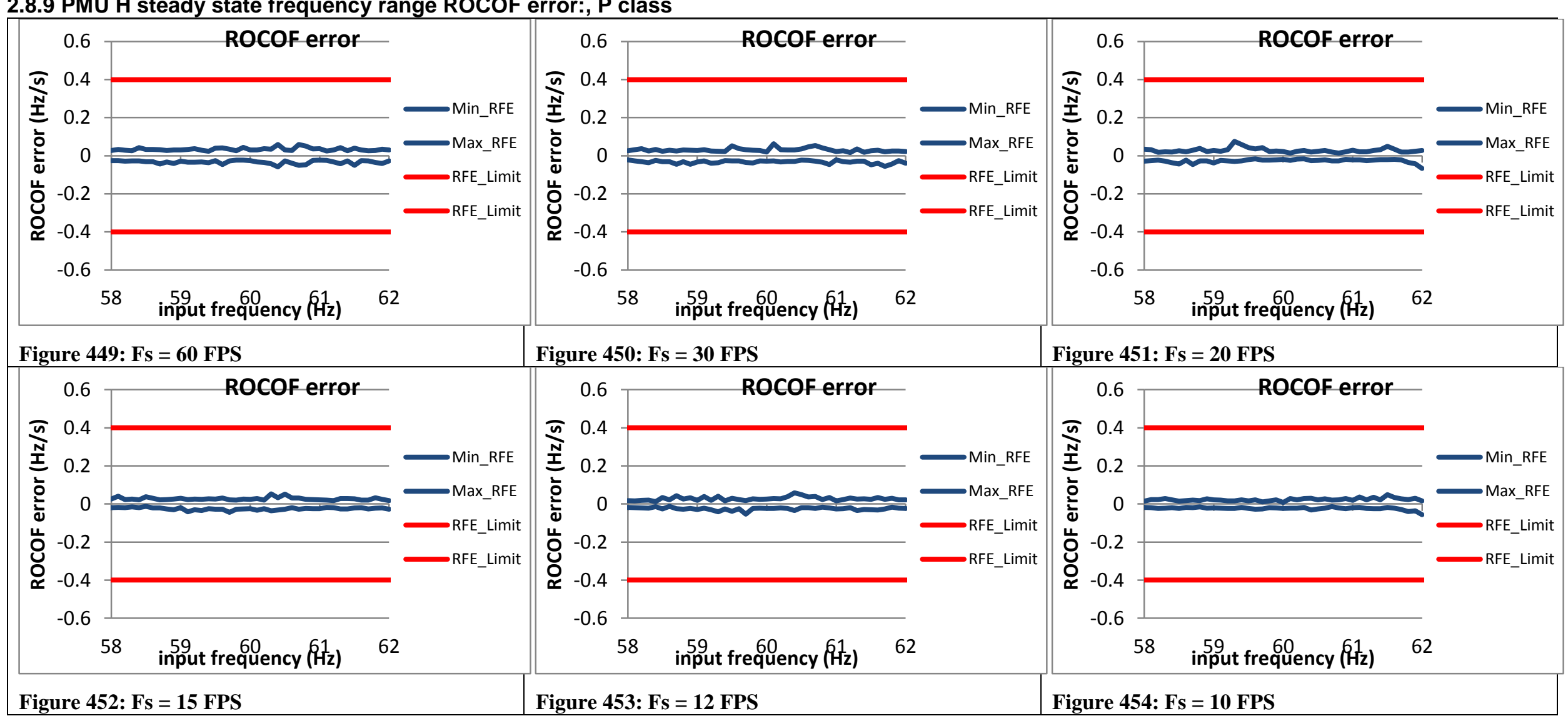

2.8.10 PMU I steady state frequency range ROCOF error:, $P$ class

PMU I does not support P class 
2.8.11 PMU J steady state frequency range ROCOF error:, P class

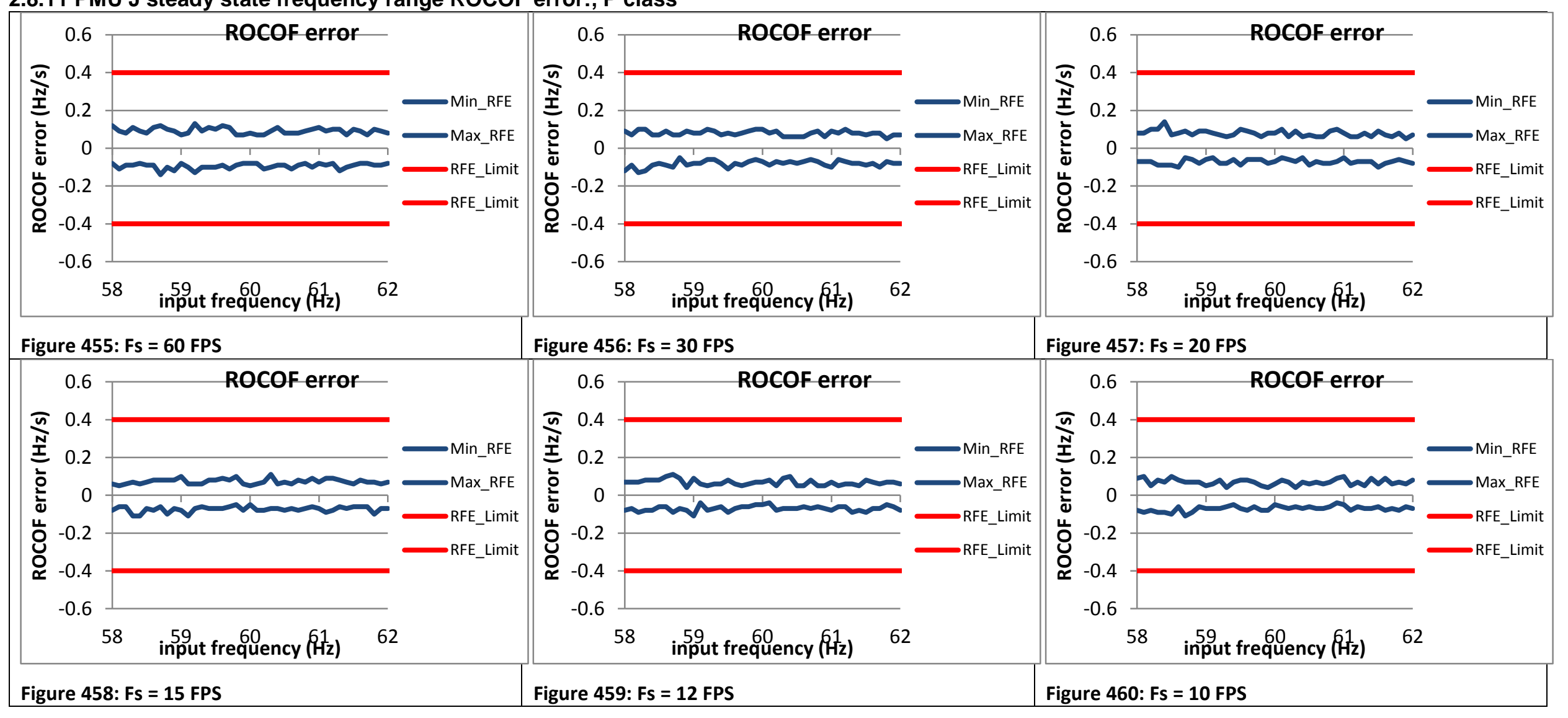




\section{Steady state signal magnitude}

3. Steady state signal magnitude...... 96

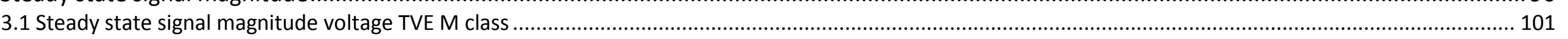

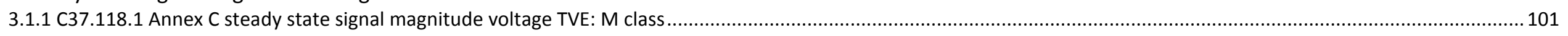

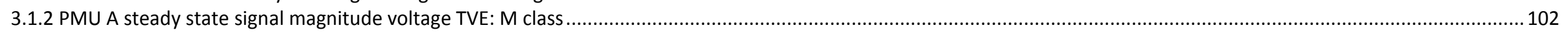

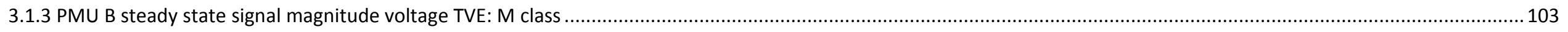

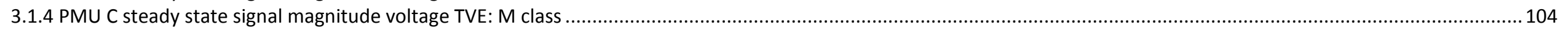

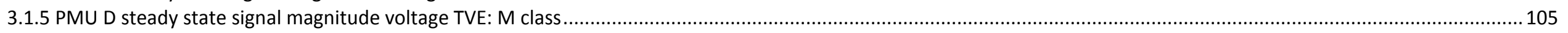

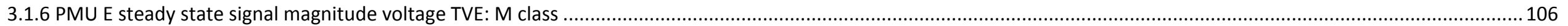

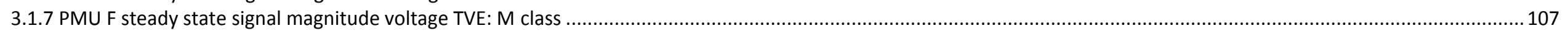

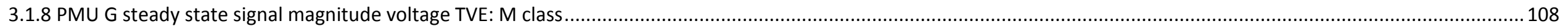

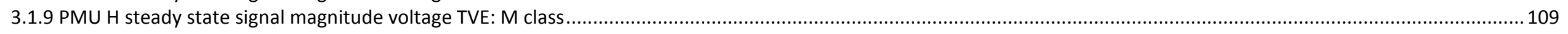

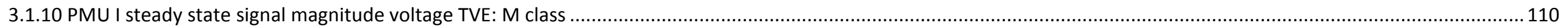

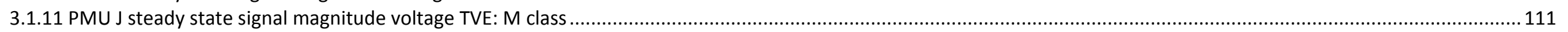

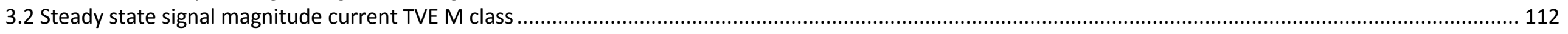

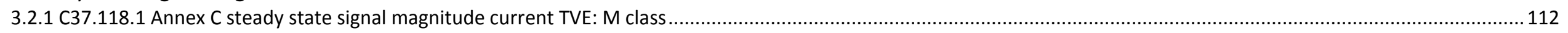

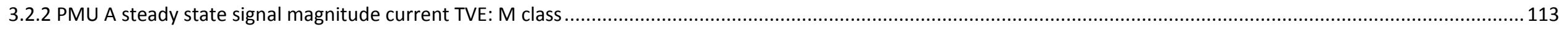

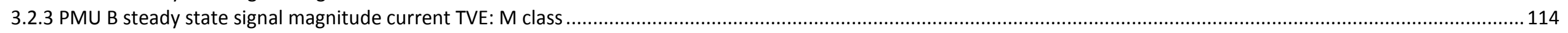

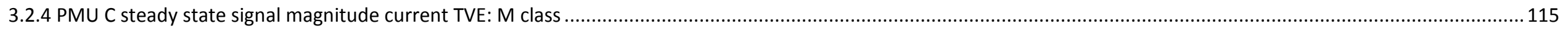

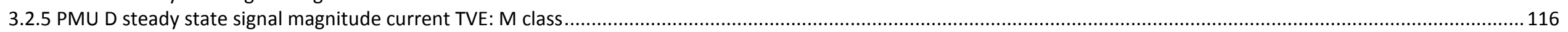

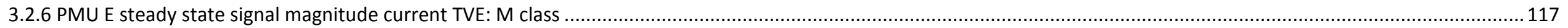

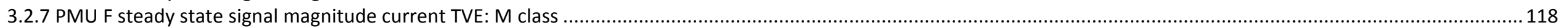

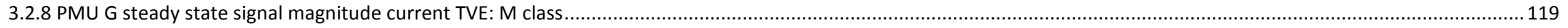

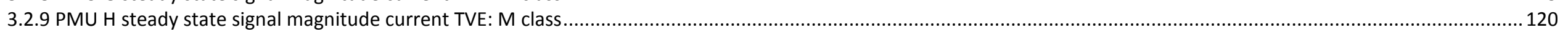

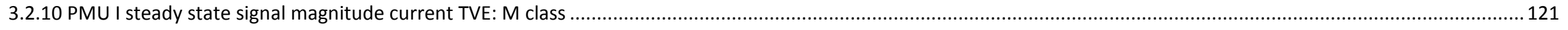

3.2.11 PMU J steady state signal magnitude current TVE: M class .................................................................................................................................................... 122

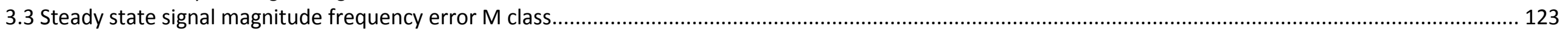

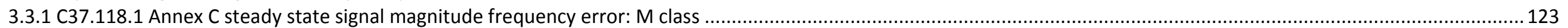

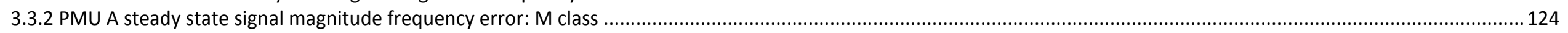

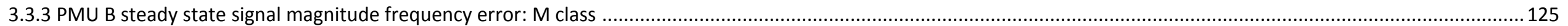

3.3.4 PMU C steady state signal magnitude frequency error: M class ................................................................................................................................................... 126

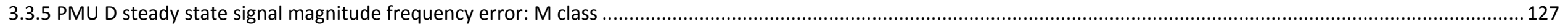

3.3.6 PMU E steady state signal magnitude frequency error: M class ..................................................................................................................................................... 128

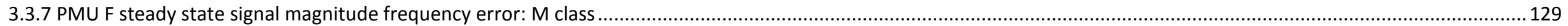

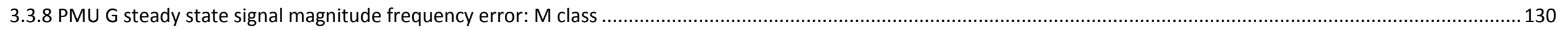




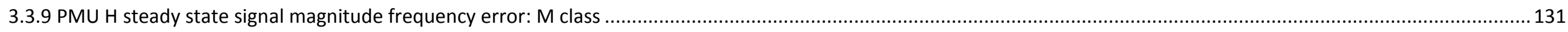

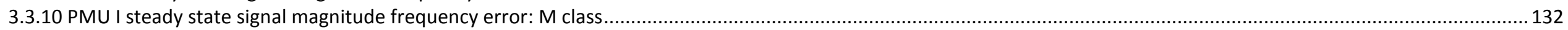

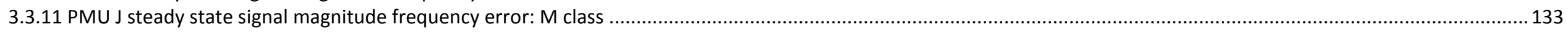

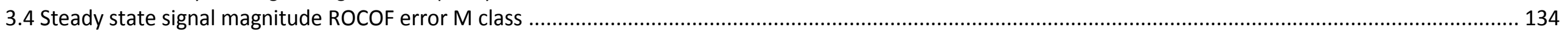

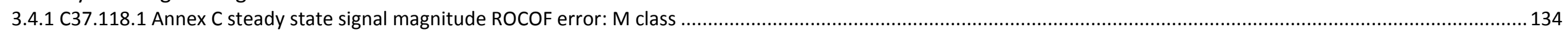

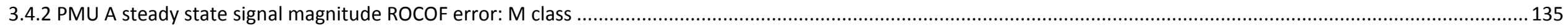

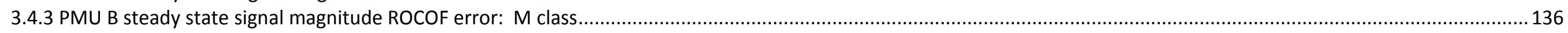

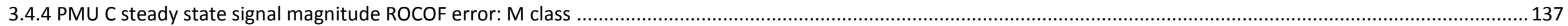

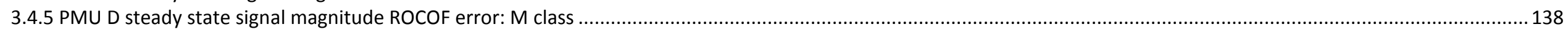

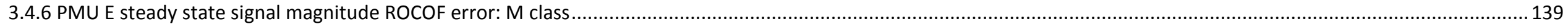

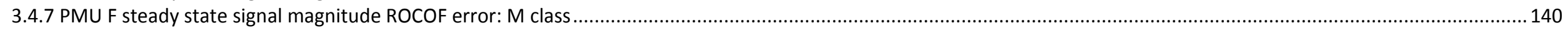

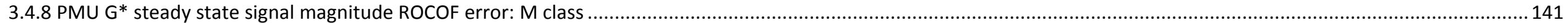

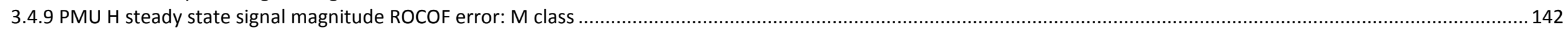

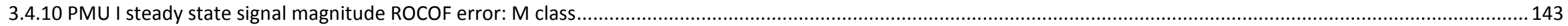

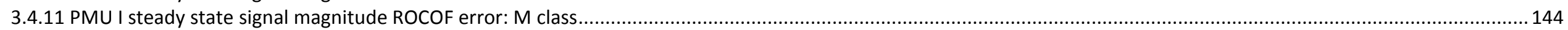

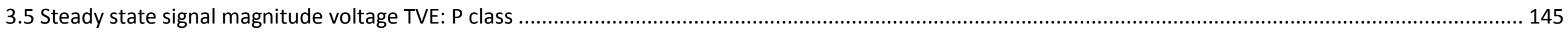

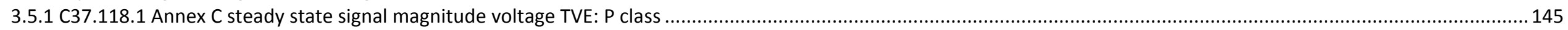

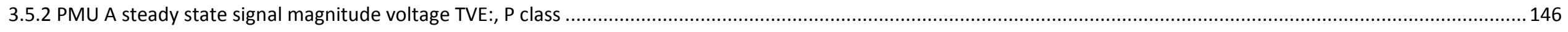

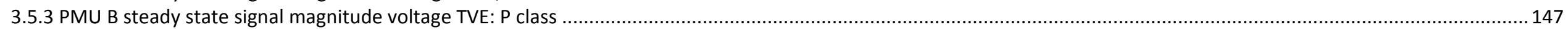

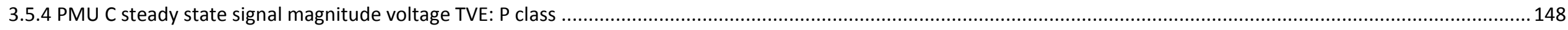

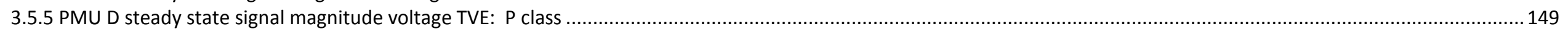

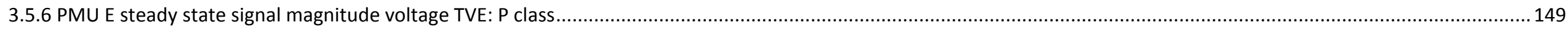

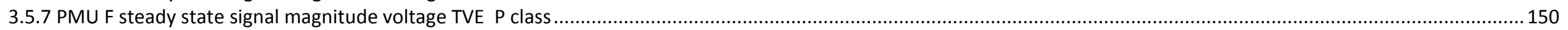

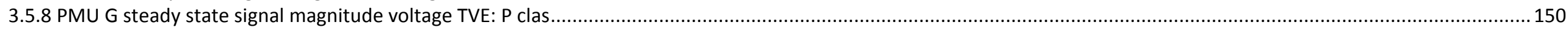

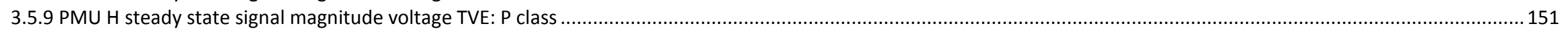

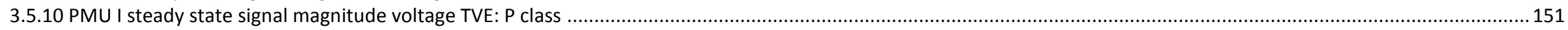

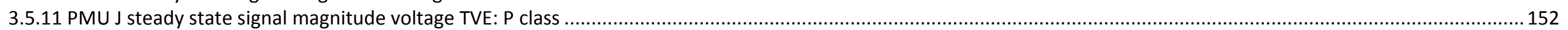

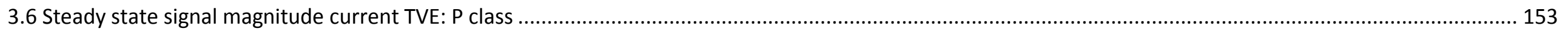

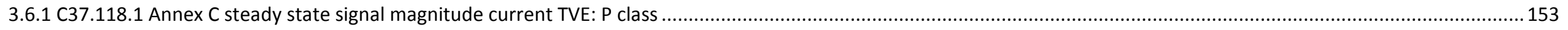

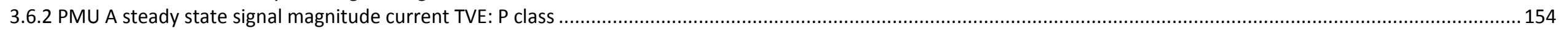

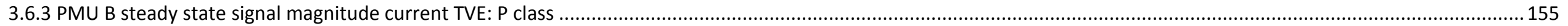

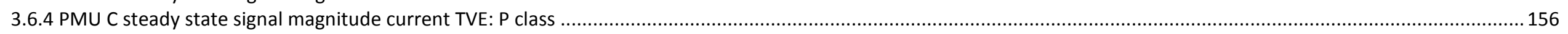

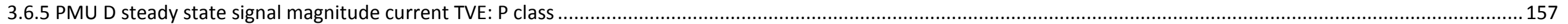

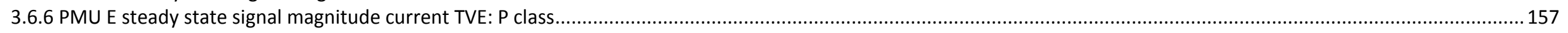

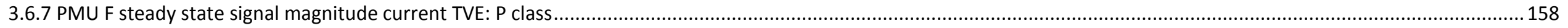

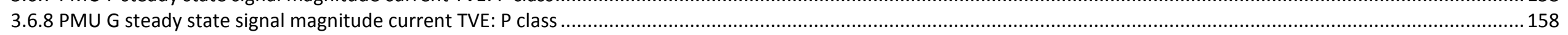

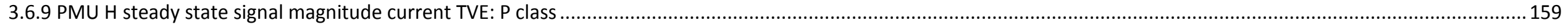

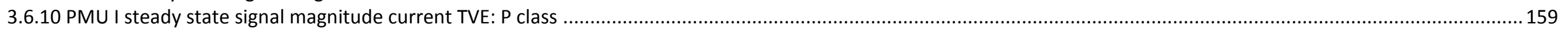


3.6.11 PMU J steady state signal magnitude current TVE: P class

3.7 Steady state signal magnitude frequency error: P class......

3.7.1 C37.118.1 Annex C steady state signal magnitude frequency error:, F0 $=60 \mathrm{~Hz}$, P class ….............................................................................................161

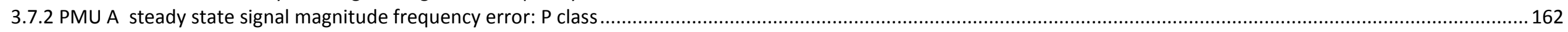

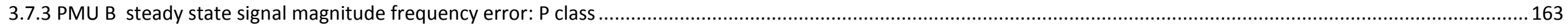

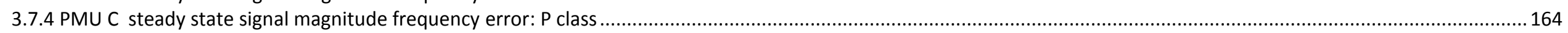

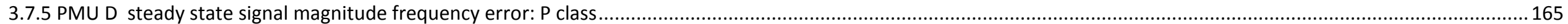

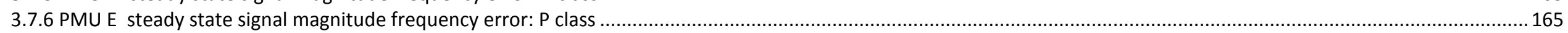

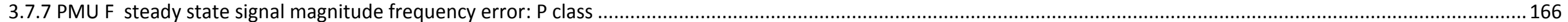

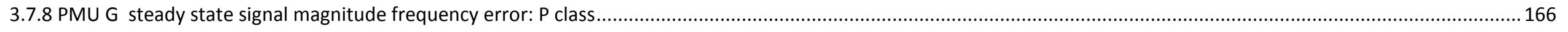

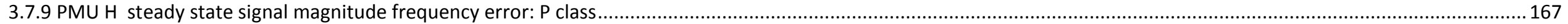

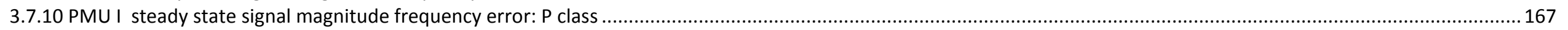

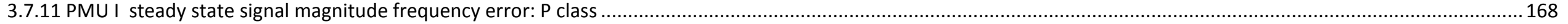

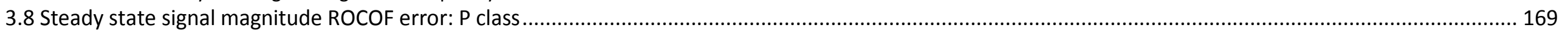

3.8.1 C37.118.1 Annex C steady state signal magnitude ROCOF error:, FO = 60 Hz, P class ...............................................................................................................

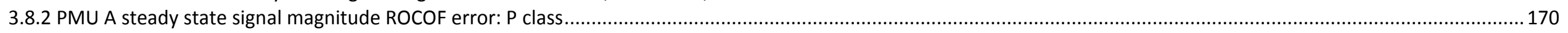

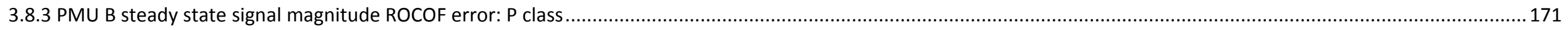

3.8.4 PMU C steady state signal magnitude ROCOF error: P class ..................................................................................................................................................... 172

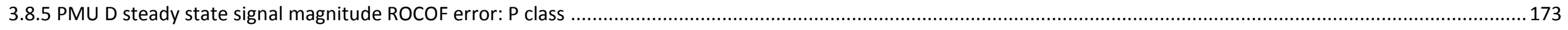

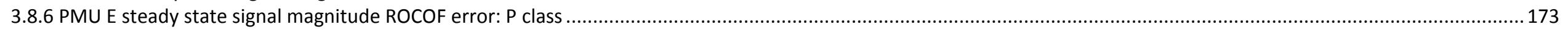

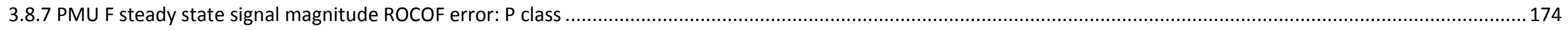

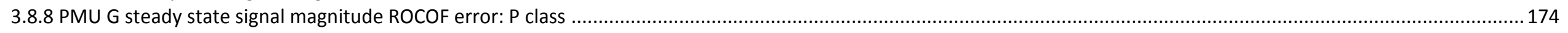

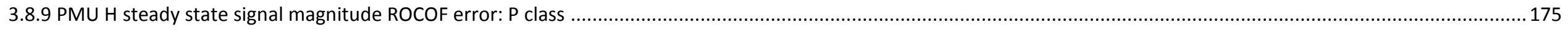

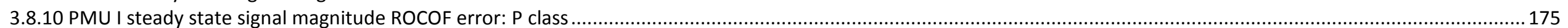

3.8.11 PMU J steady state signal magnitude ROCOF error: P class ......................................................................................................................................................... 


\section{Reference 5.5.5 of IEEE Std C37.118.1-2011 and C37.118.1a-2014}

The steady state signal magnitude is tested by a series of individual steady state tests where the input signal magnitude and frequency is fixed for duration of 5 seconds. Total Vector Error (TVE), Frequency Error (FE) and Rate of Change of Frequency Error (RFE) are measured.

A series of individual tests are run at 10\% signal magnitude increments across the C37.118.1-2011 required magnitude ranges determined by the PMU class:

- The magnitude range for $\mathrm{M}$ class begins at $10 \%$ of nominal input magnitude and ends at $120 \%$ nominal voltage magnitude and $200 \%$ nominal current magnitude.

- The magnitude range for $\mathrm{P}$ class begins at $80 \%$ of nominal input magnitude and ends at $120 \%$ nominal voltage magnitude and $200 \%$ nominal current magnitude.

The maximum TVE of all the test runs is shown along with the limit for TVE to determine if the unit passes or fails the test. There is no limit for FE or RFE but the results are plotted in this report.

Test plan:

For both current and voltage input ranges, apply steady state, nominal frequency, balanced three-phase inputs.

a) Begin at the low magnitude limits:

- P class: voltage $=80 \%$ of nominal, current $=10 \%$ of nominal.

- M class: voltage $=10 \%$ of nominal, current $=10 \%$ of nominal.

b) Wait for the system to settle.

c) Capture the PMU output for 5 seconds.

d) Calculate the errors: TVE, FE, RFE for each report.

e) Calculate the Max TVE.

f) Increase the input magnitudes by $10 \%$ of the nominal value (not a percentage of the actual value).

g) Repeat step b) through step f) until the upper magnitude limits are reached: $120 \%$ of nominal voltage, $200 \%$ of nominal current (P and $\mathrm{M}$ class). 
Steady state signal magnitude test results

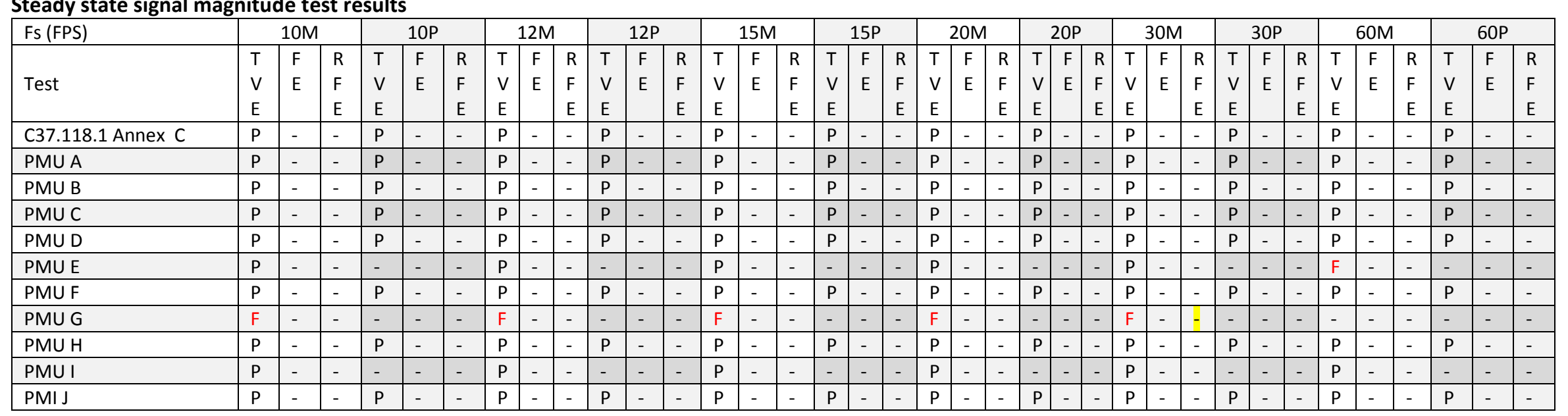

$\mathrm{P}=$ pass (results are within the limits), $\mathrm{F}=$ fail (results are outside the limits), I = indeterminate (results are within the test instrument's uncertainty of the limits) Dash (-) indicates that there is no performance limit for frequency and ROCOF tests.

The PMU standard does not specify performance limits for frequency and ROCOF error. In the table above, this is specified as a dash (-). Frequency and ROCOF is plotted below for information purposes. There are no limit lines shown. 


\subsection{Steady state signal magnitude voltage TVE M class}

\subsubsection{C37.118.1 Annex C steady state signal magnitude voltage TVE: M class}

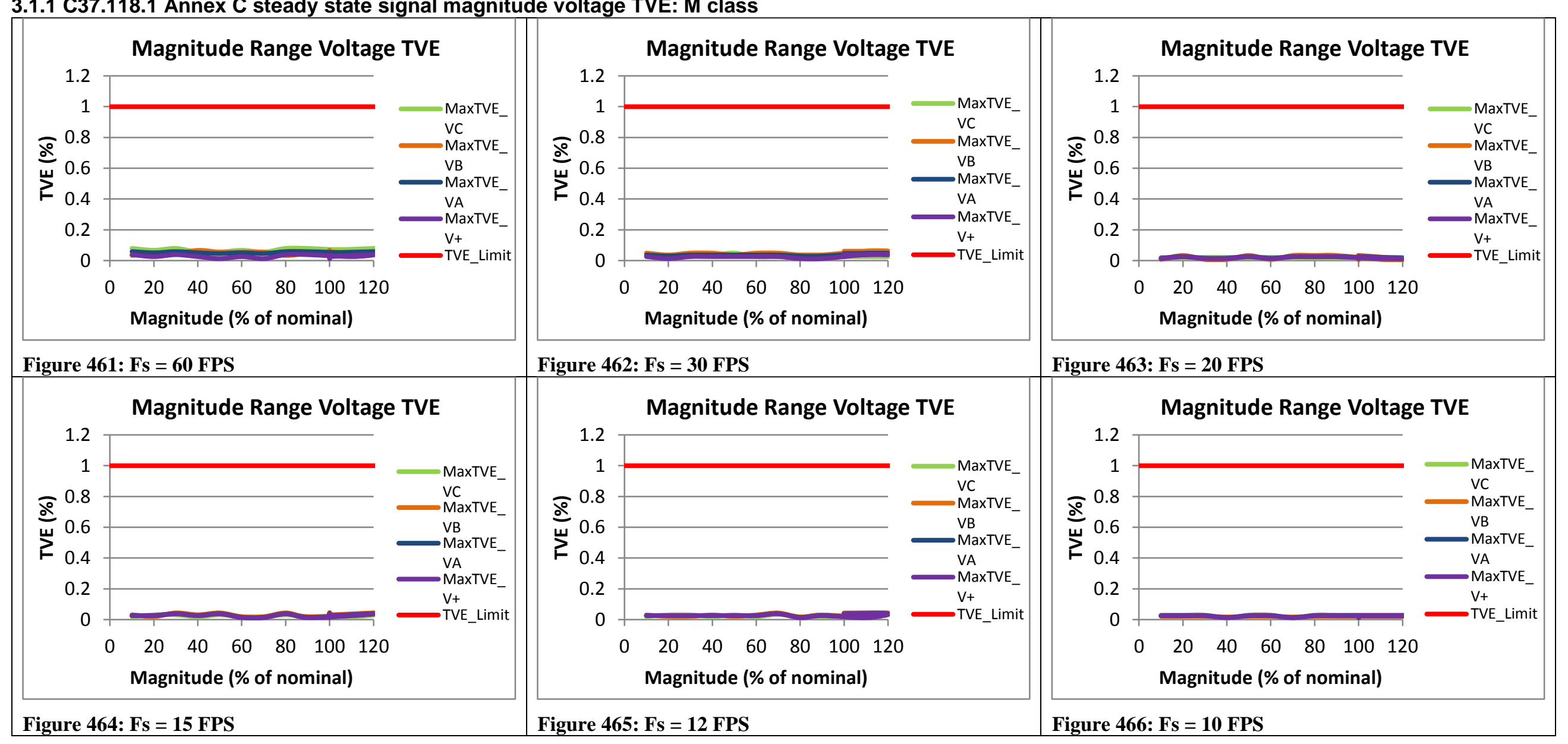


3.1.2 PMU A steady state signal magnitude voltage TVE: M class

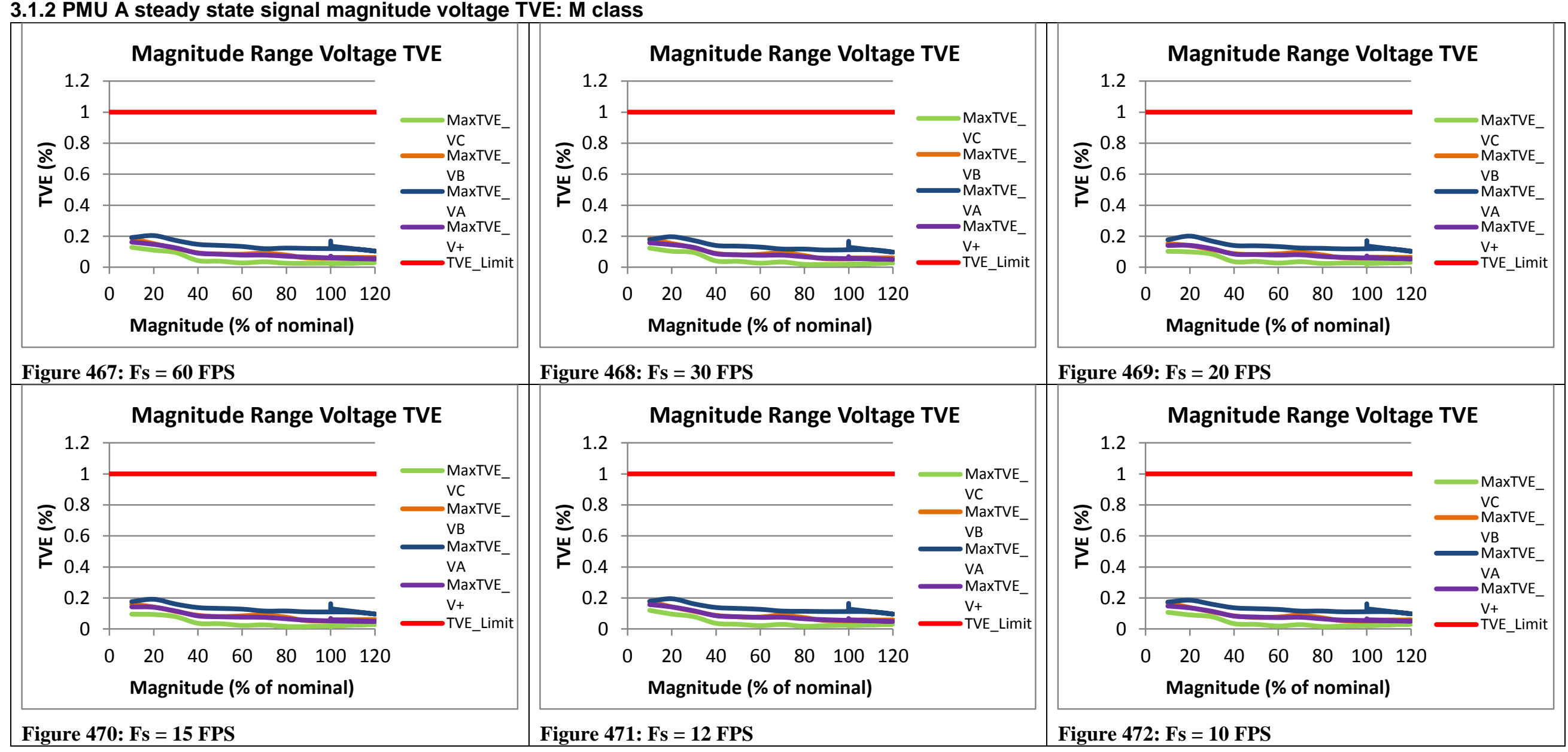


3.1.3 PMU B steady state signal magnitude voltage TVE: M class

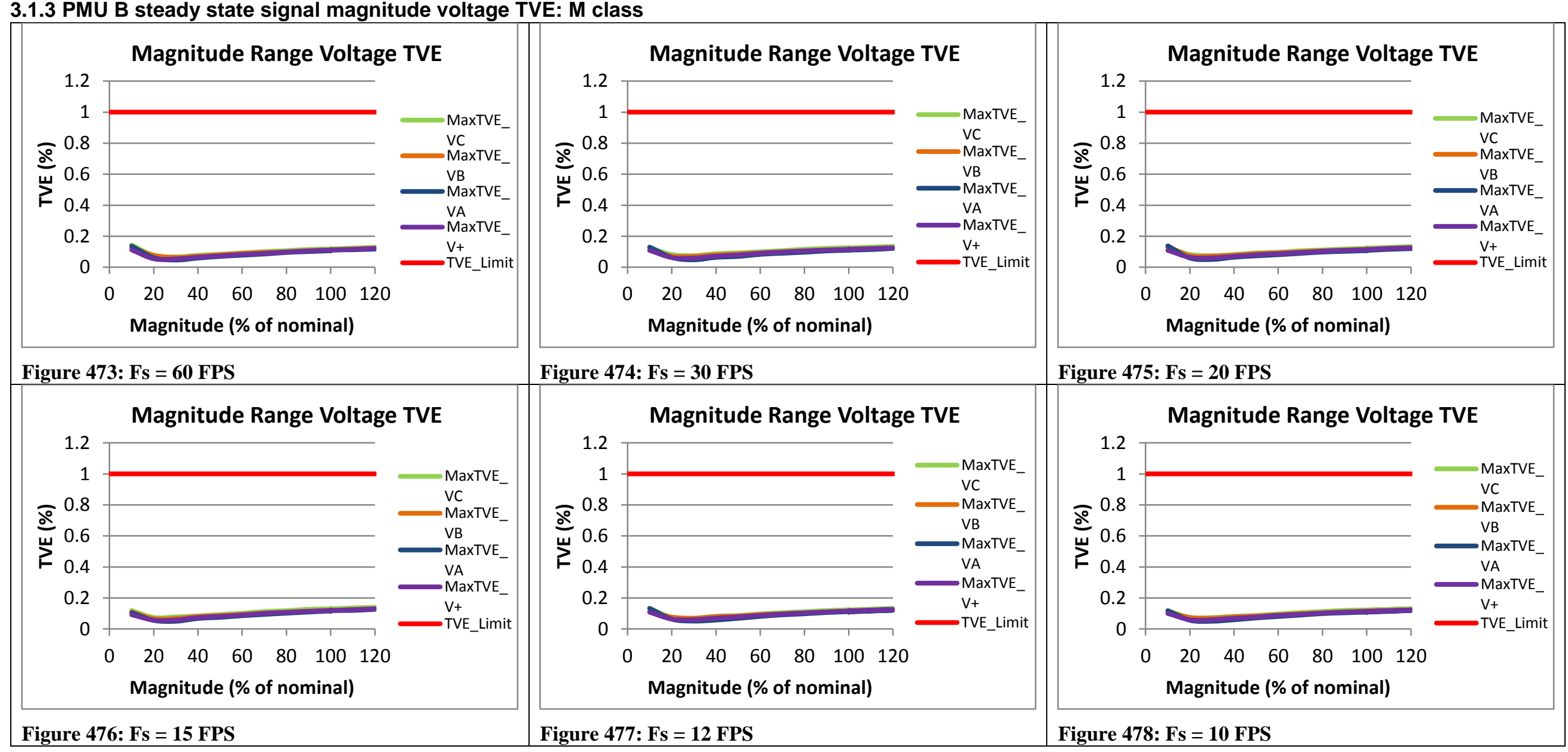


3.1.4 PMU C steady state signal magnitude voltage TVE: M class

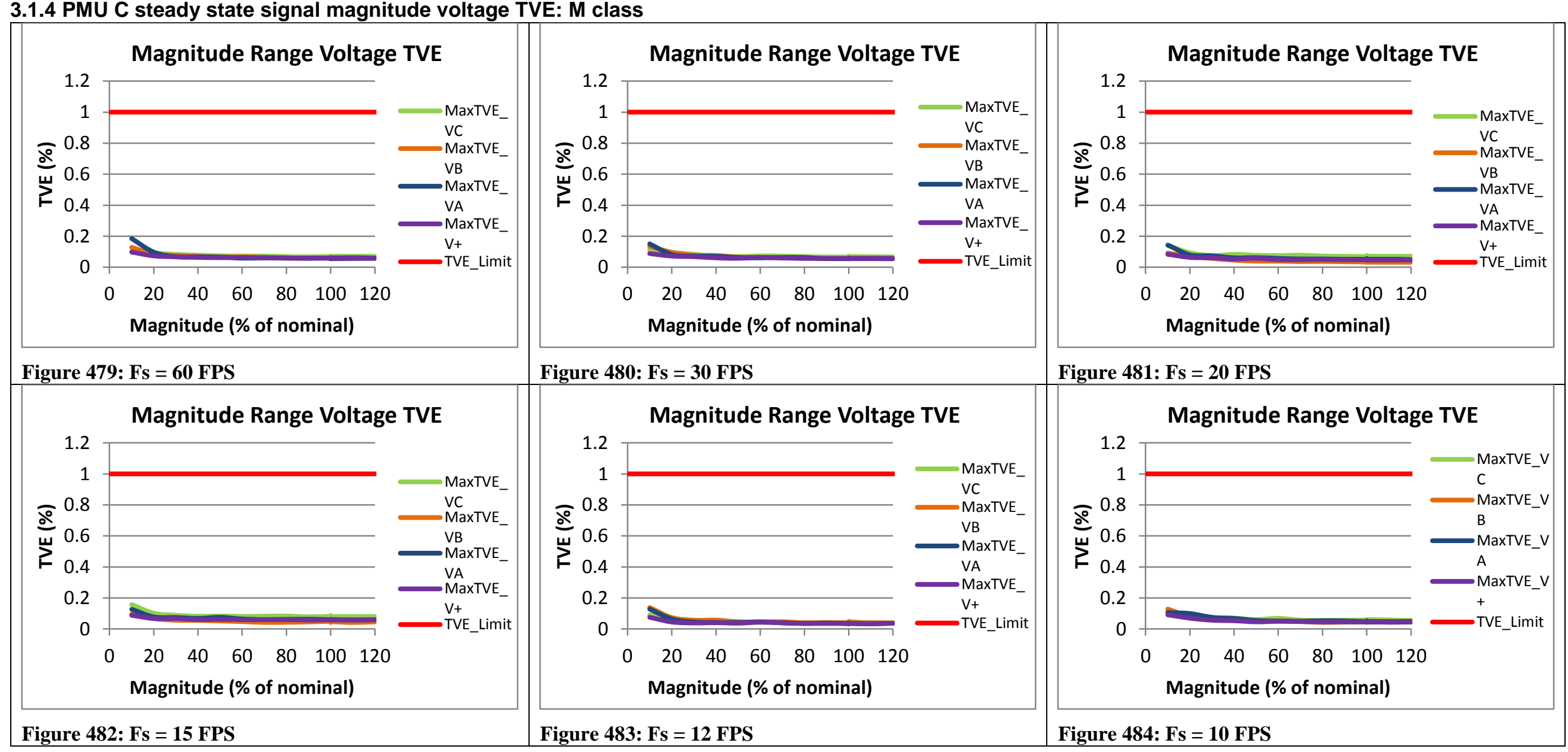


3.1.5 PMU D steady state signal magnitude voltage TVE: M class

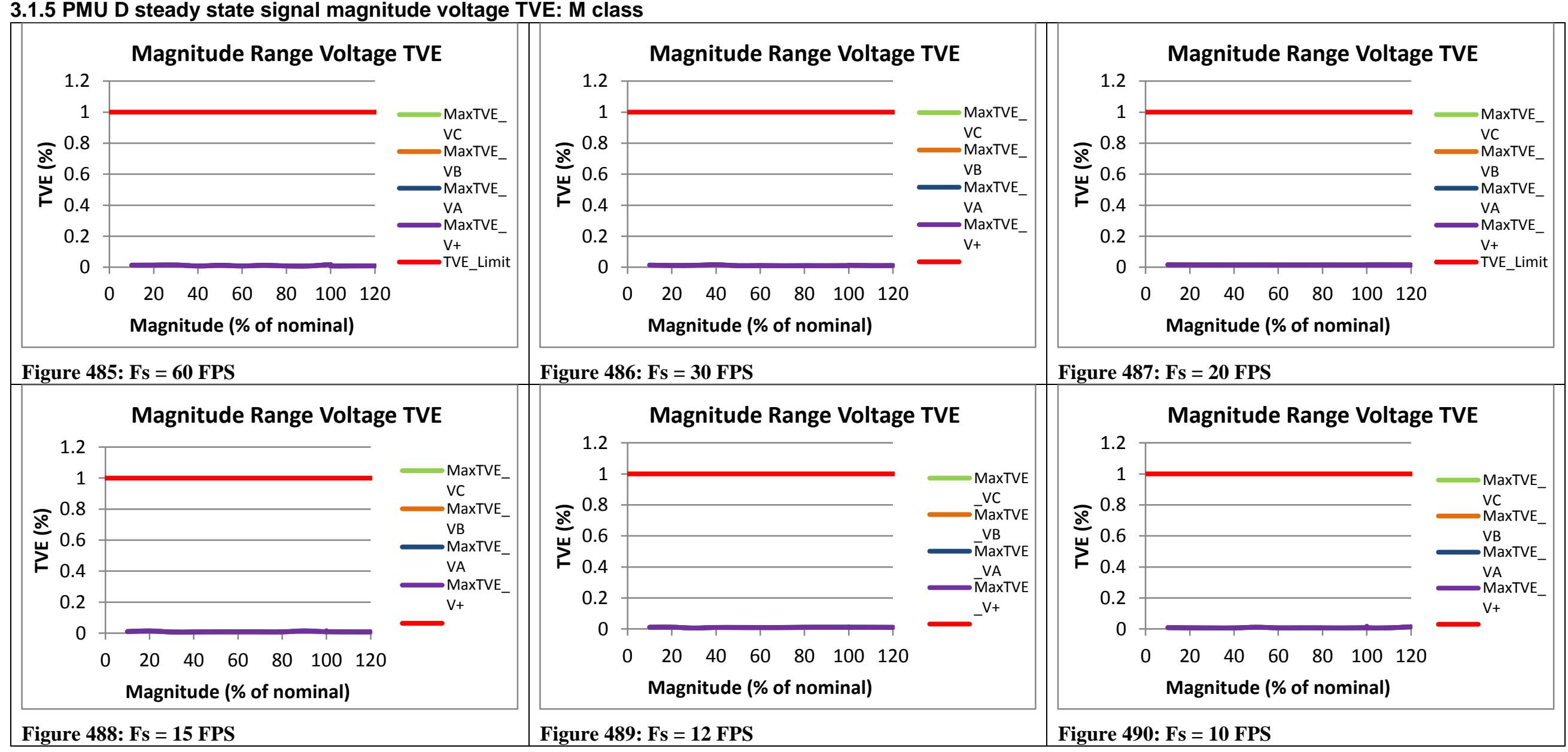


3.1.6 PMU E steady state signal magnitude voltage TVE: M class

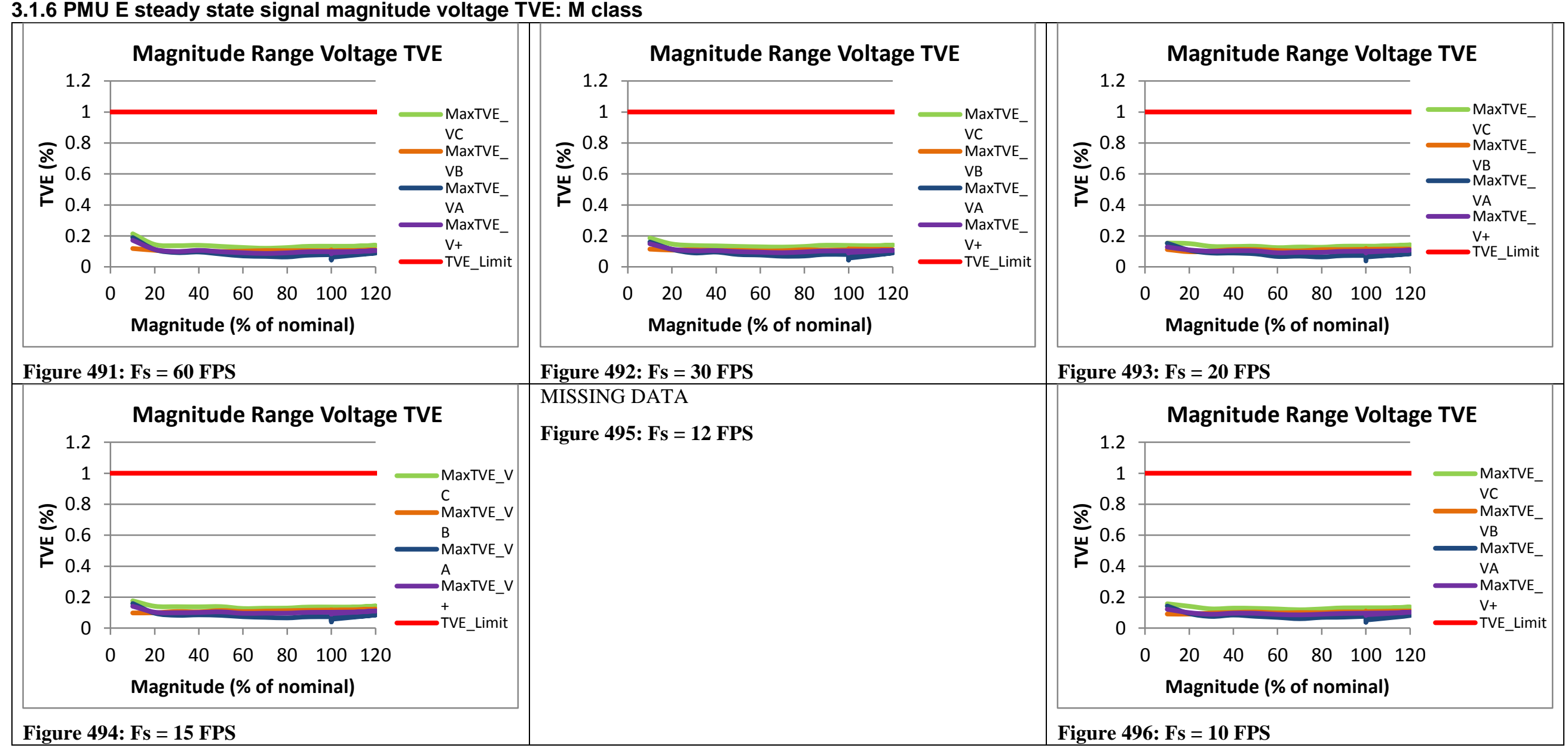


3.1.7 PMU F steady state signal magnitude voltage TVE: M class

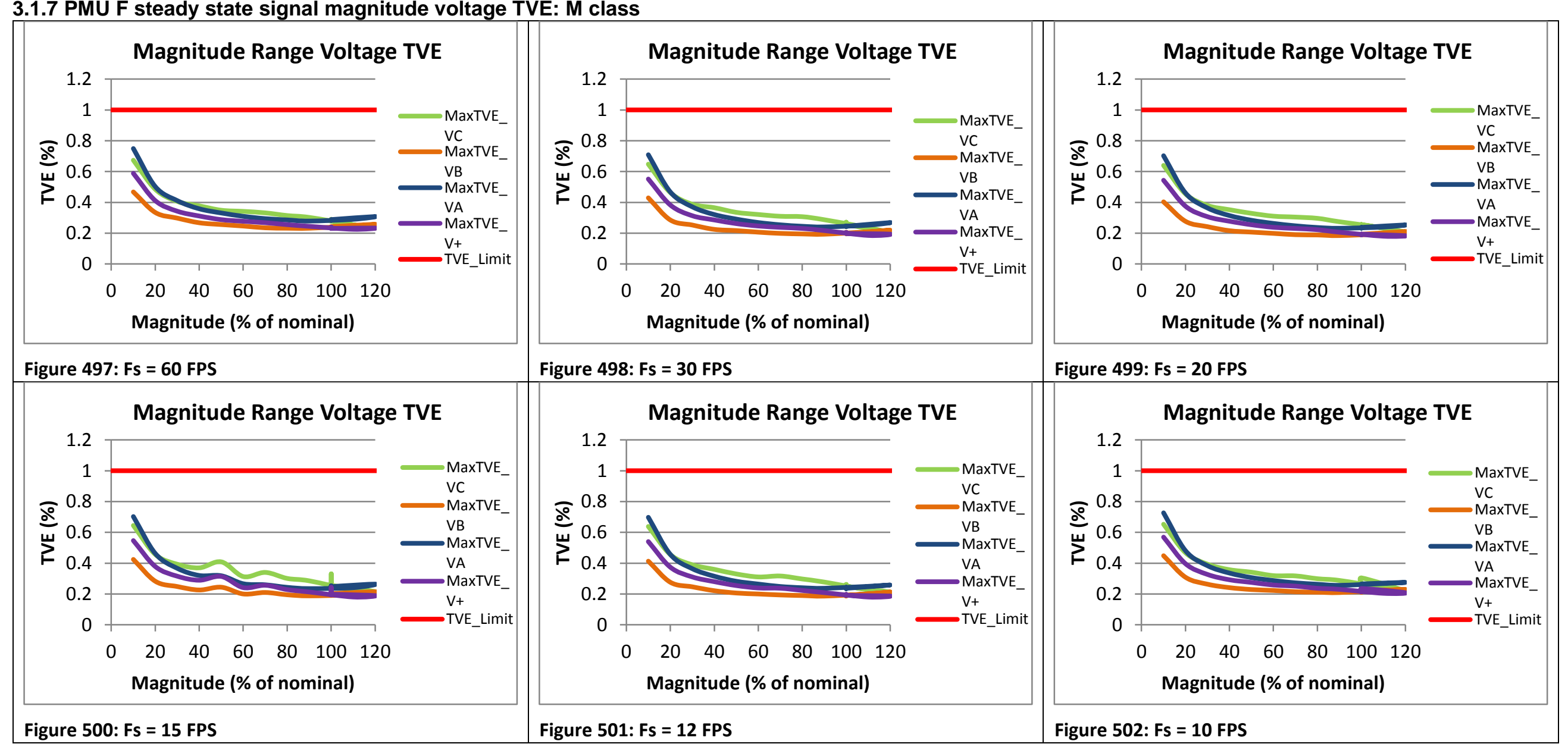


3.1.8 PMU G steady state signal magnitude voltage TVE: M class

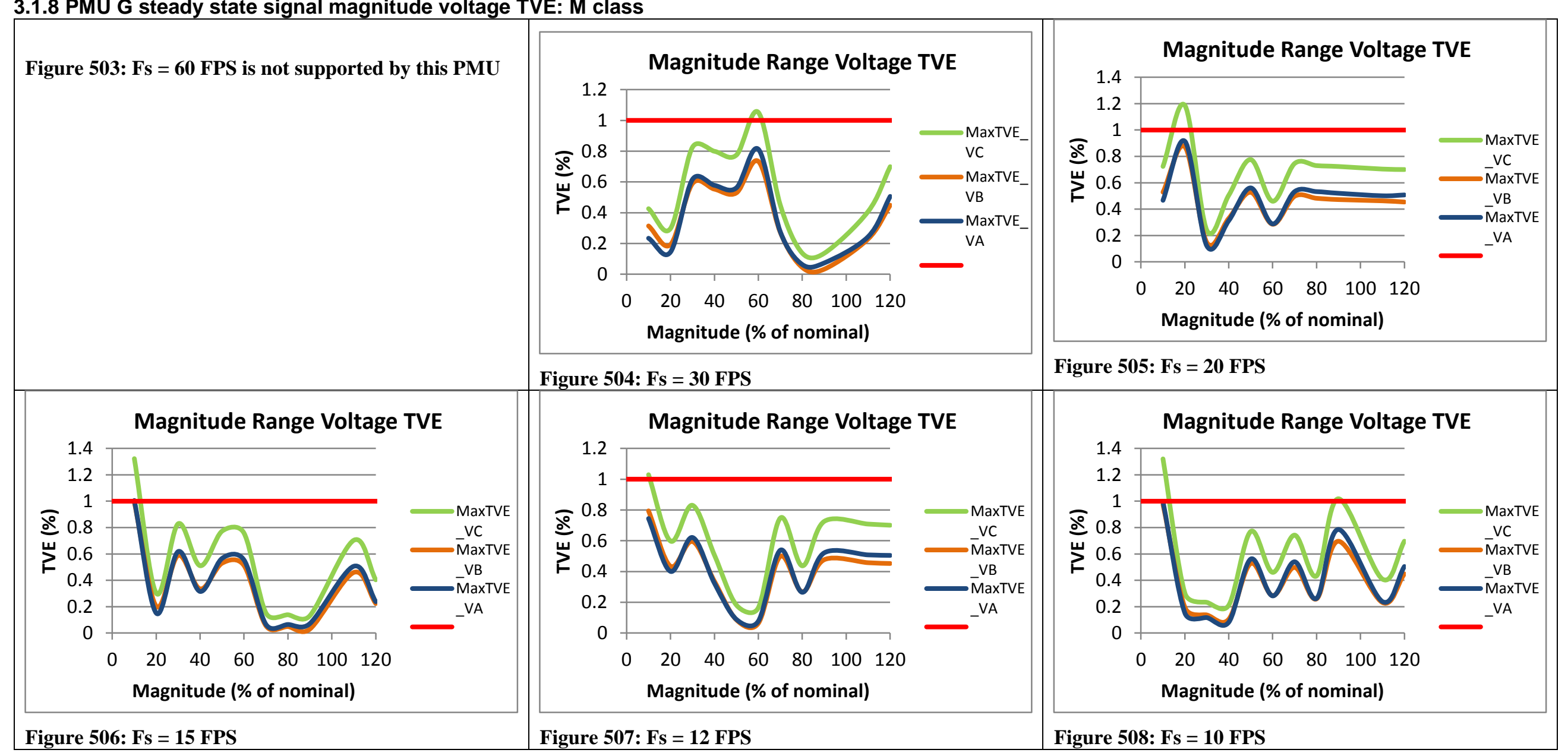


3.1.9 PMU H steady state signal magnitude voltage TVE: M class

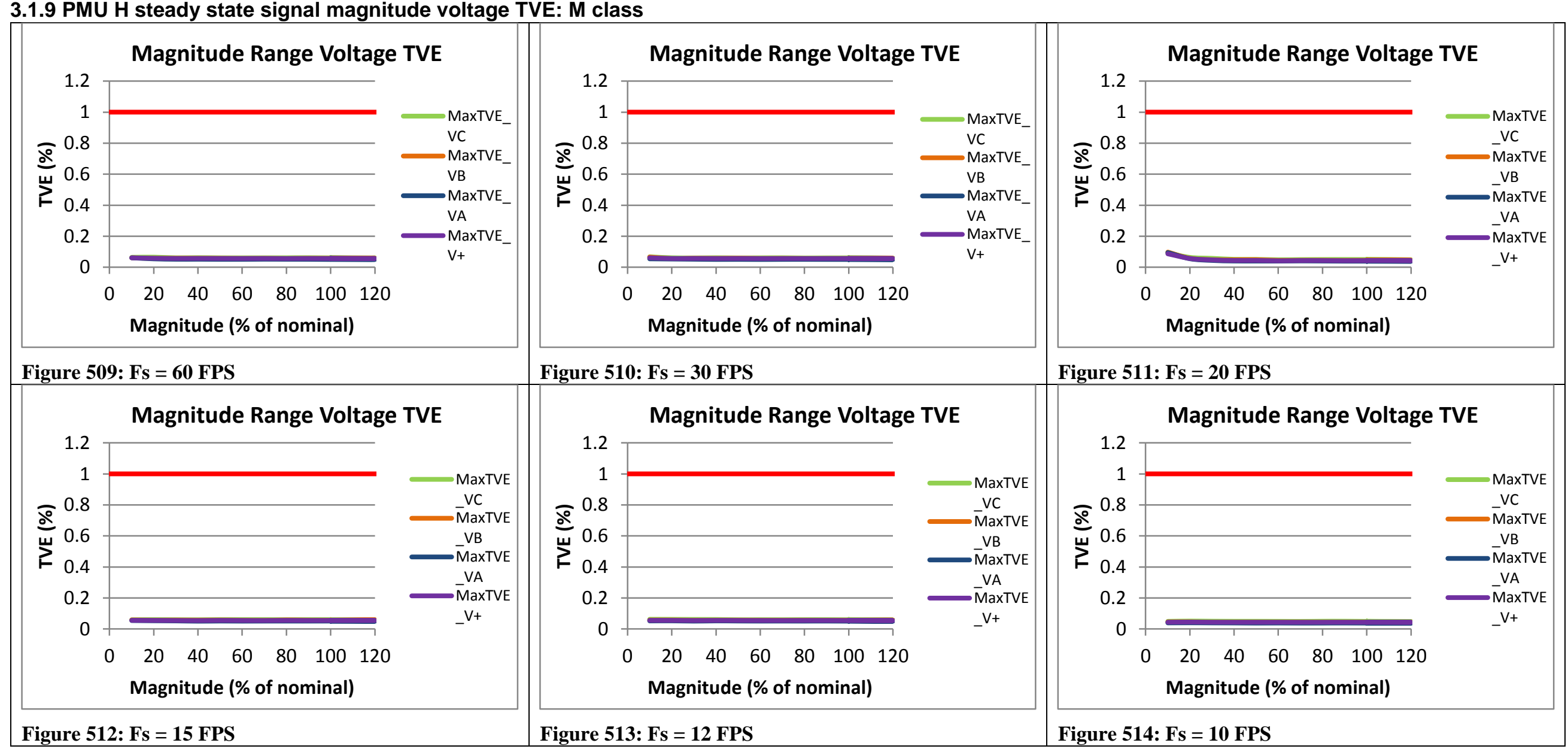


3.1.10 PMU I steady state signal magnitude voltage TVE: M class

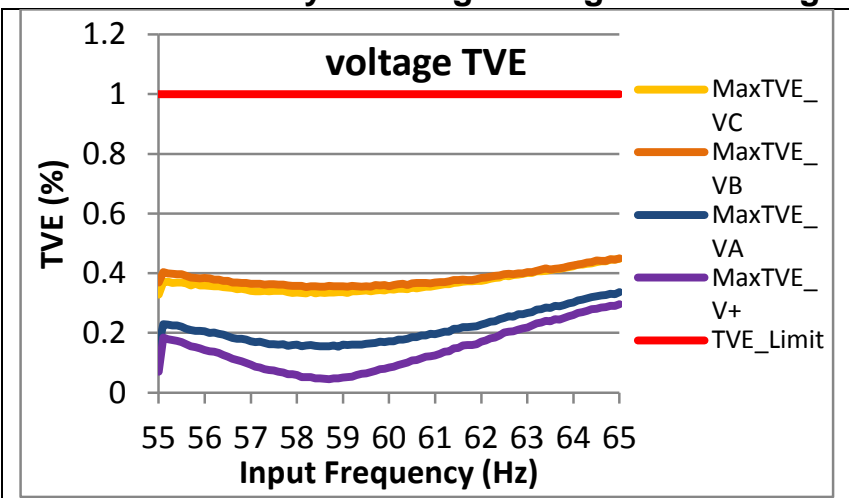

Figure 515: Fs = 60 FPS

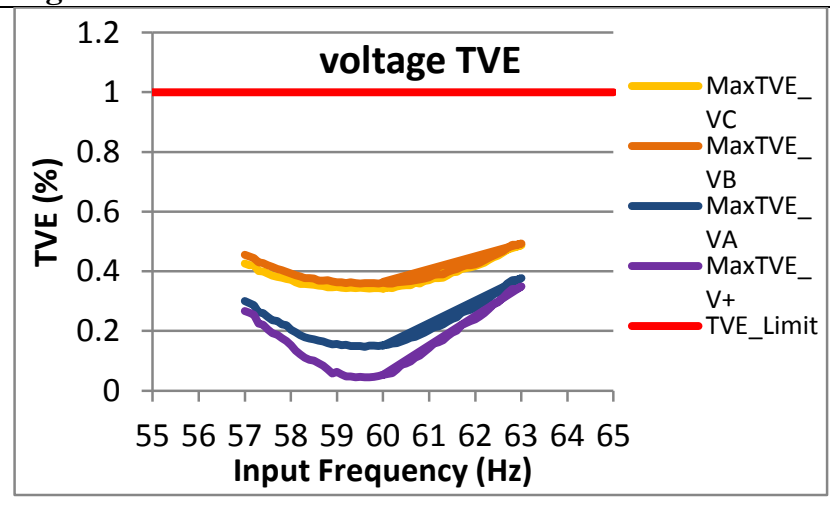

Figure 518: Fs = 15 FPS

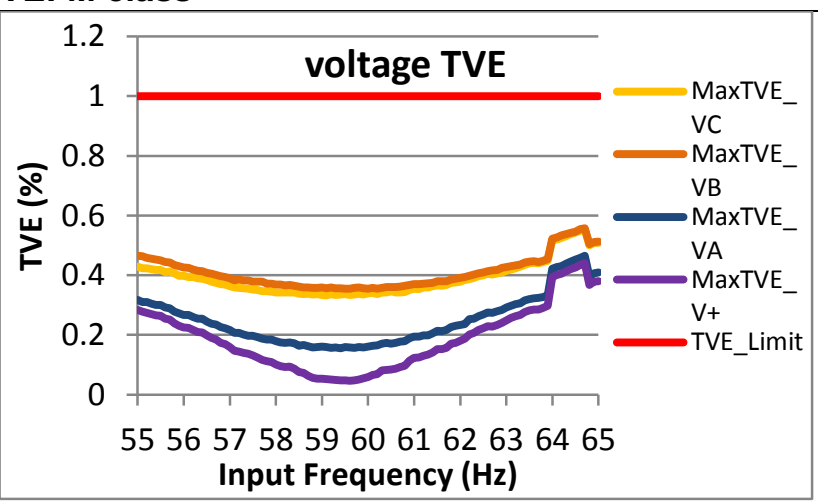

Figure 516: Fs $=30$ FPS

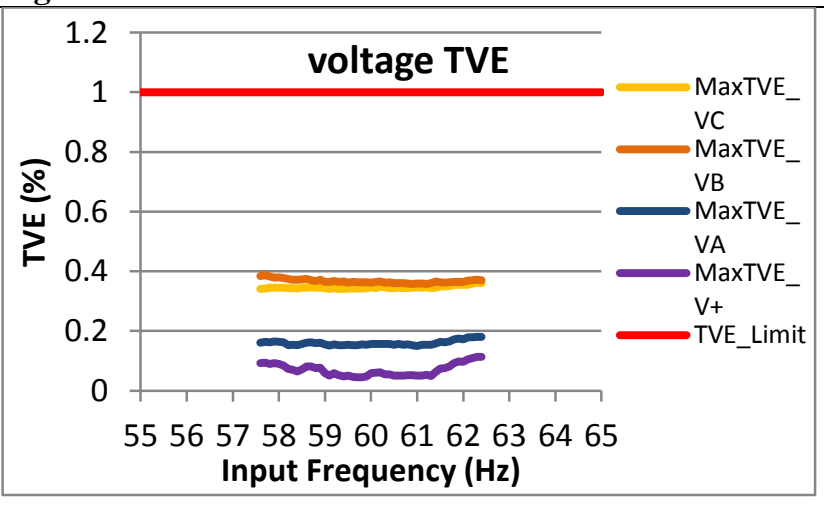

Figure 519: Fs = 12 FPS

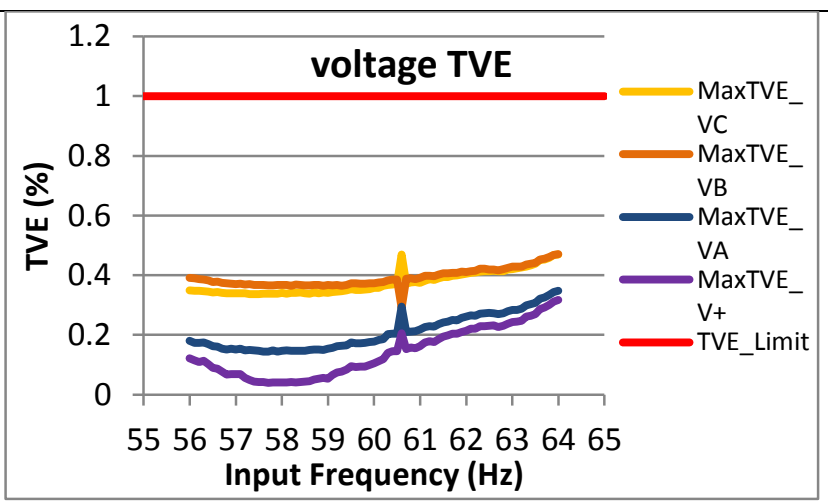

Figure 517: Fs $=20$ FPS

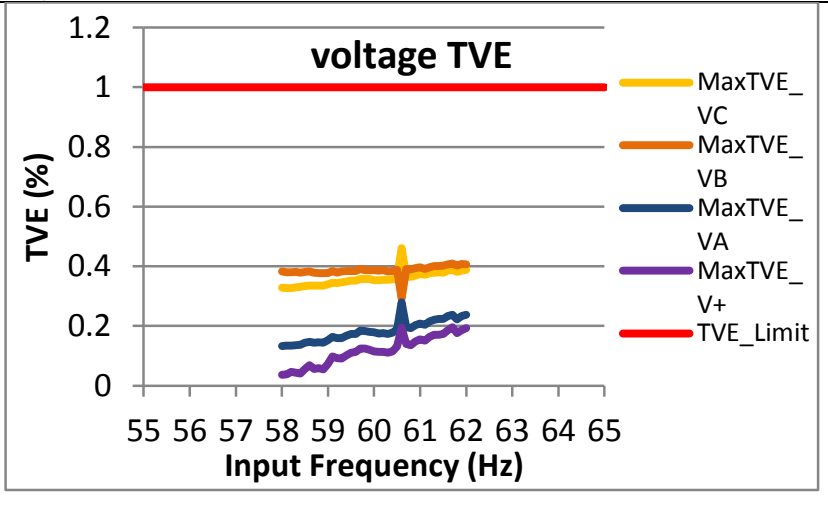

Figure 520: Fs = 10 FPS 
3.1.11 PMU J steady state signal magnitude voltage TVE: M class

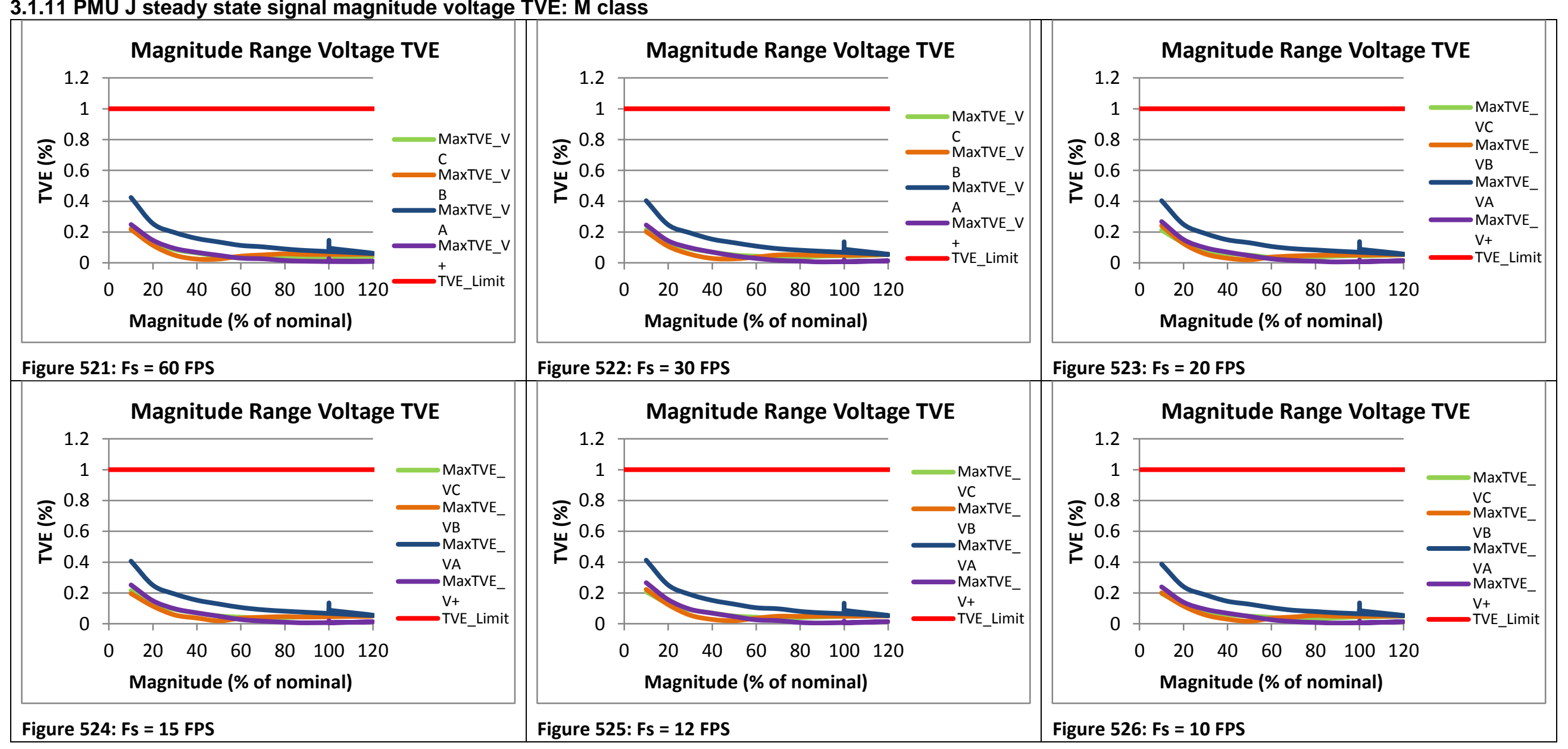




\subsection{Steady state signal magnitude current TVE M class}

\subsubsection{C37.118.1 Annex $C$ steady state signal magnitude current TVE: M class}

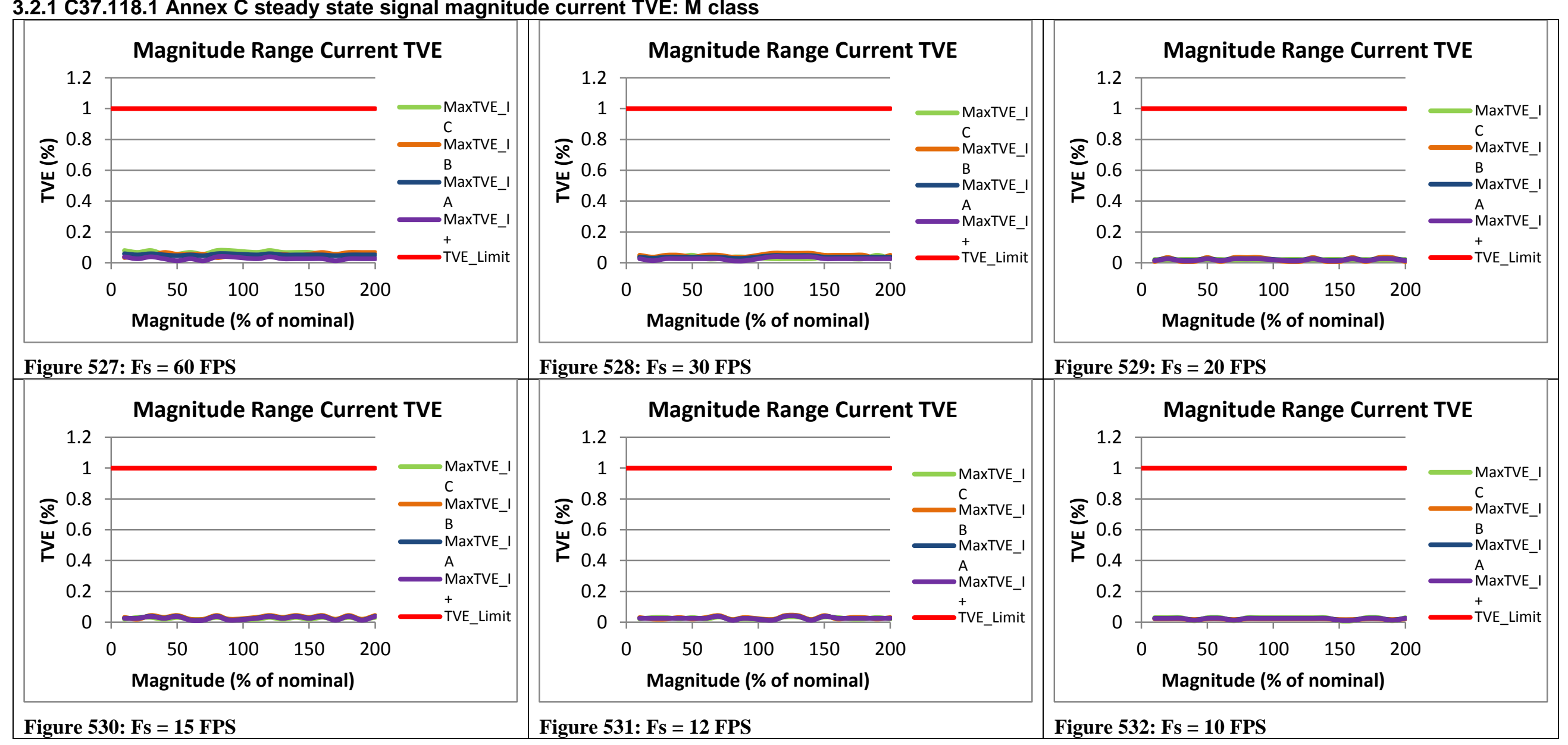


3.2.2 PMU A steady state signal magnitude current TVE: M class

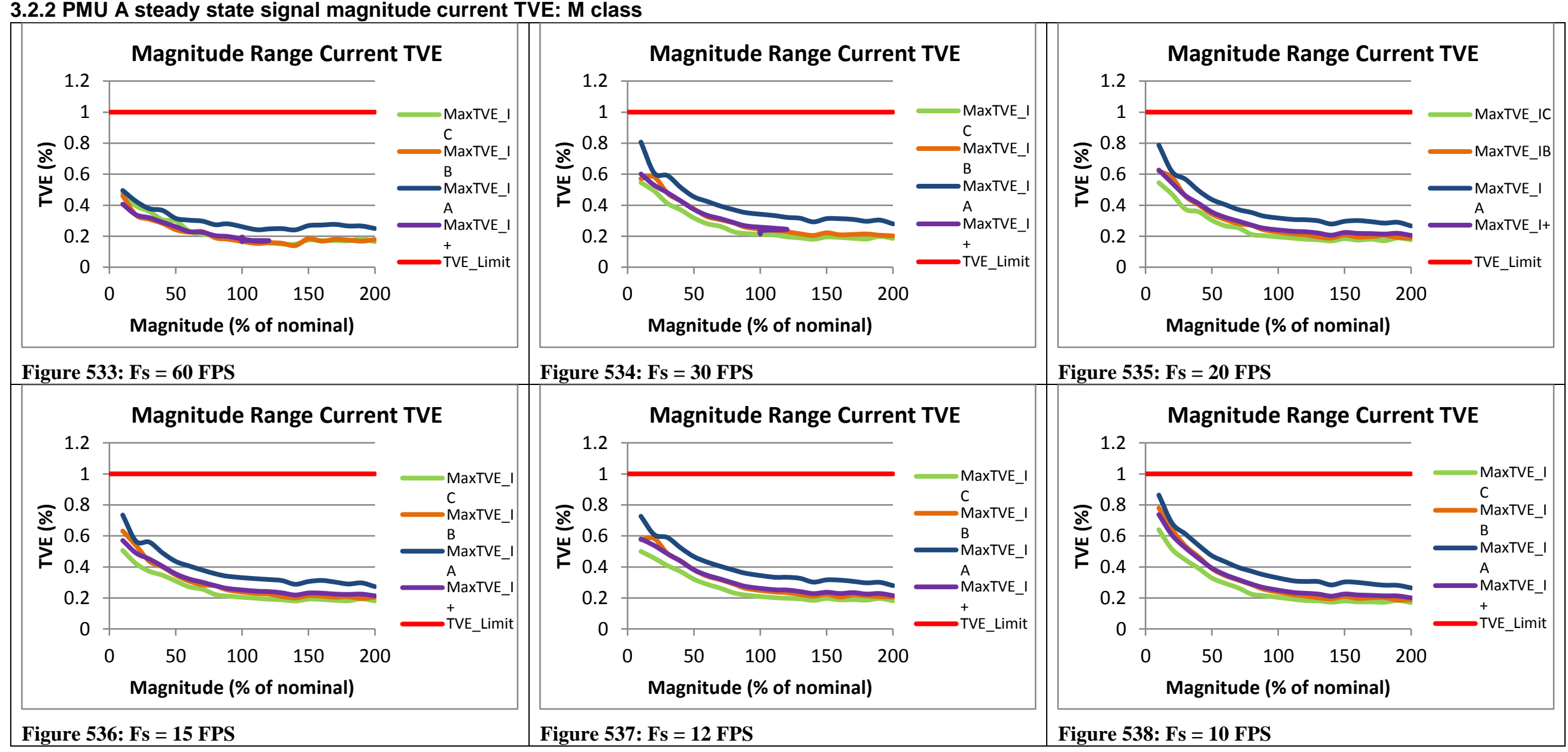


3.2.3 PMU B steady state signal magnitude current TVE: M class

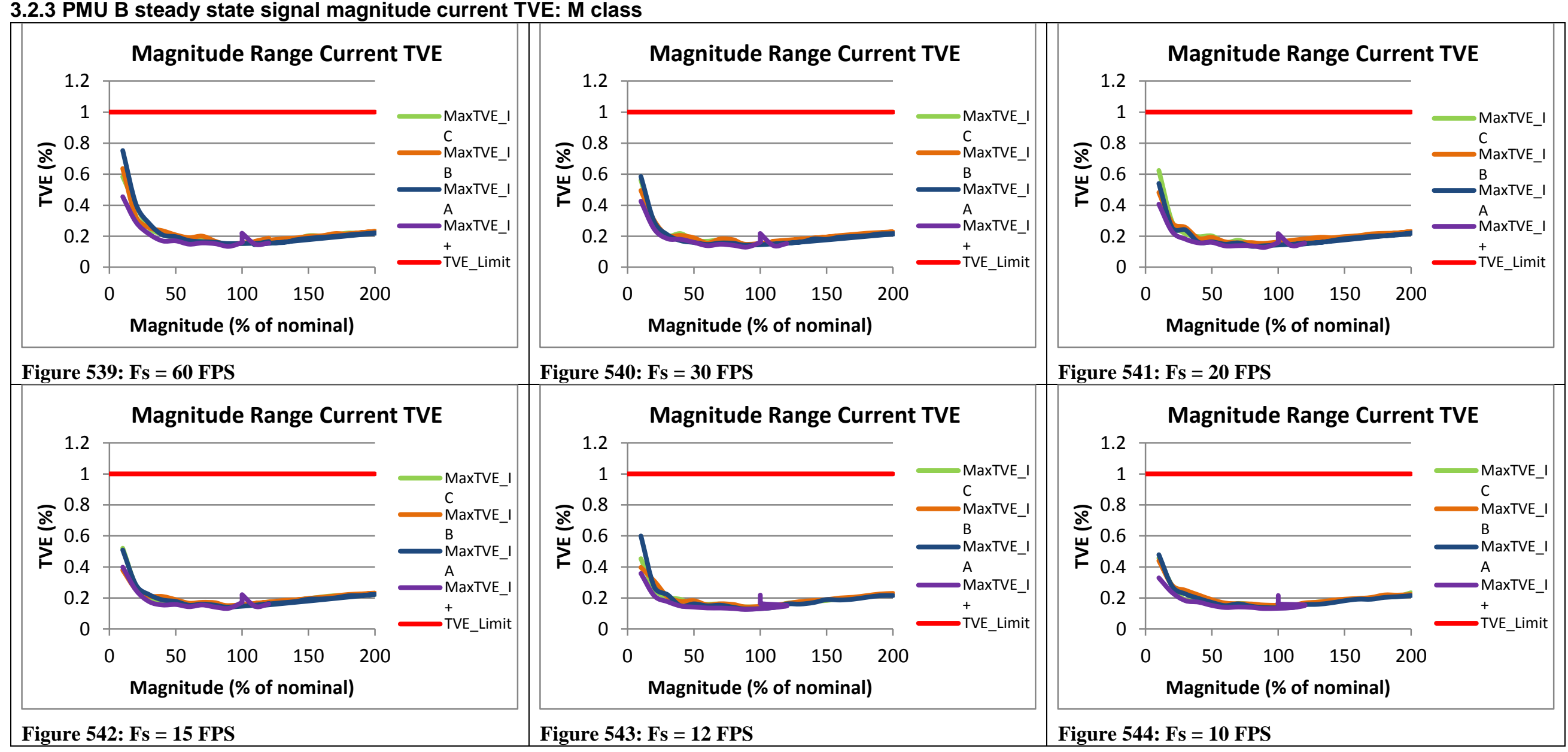


3.2.4 PMU C steady state signal magnitude current TVE: M class

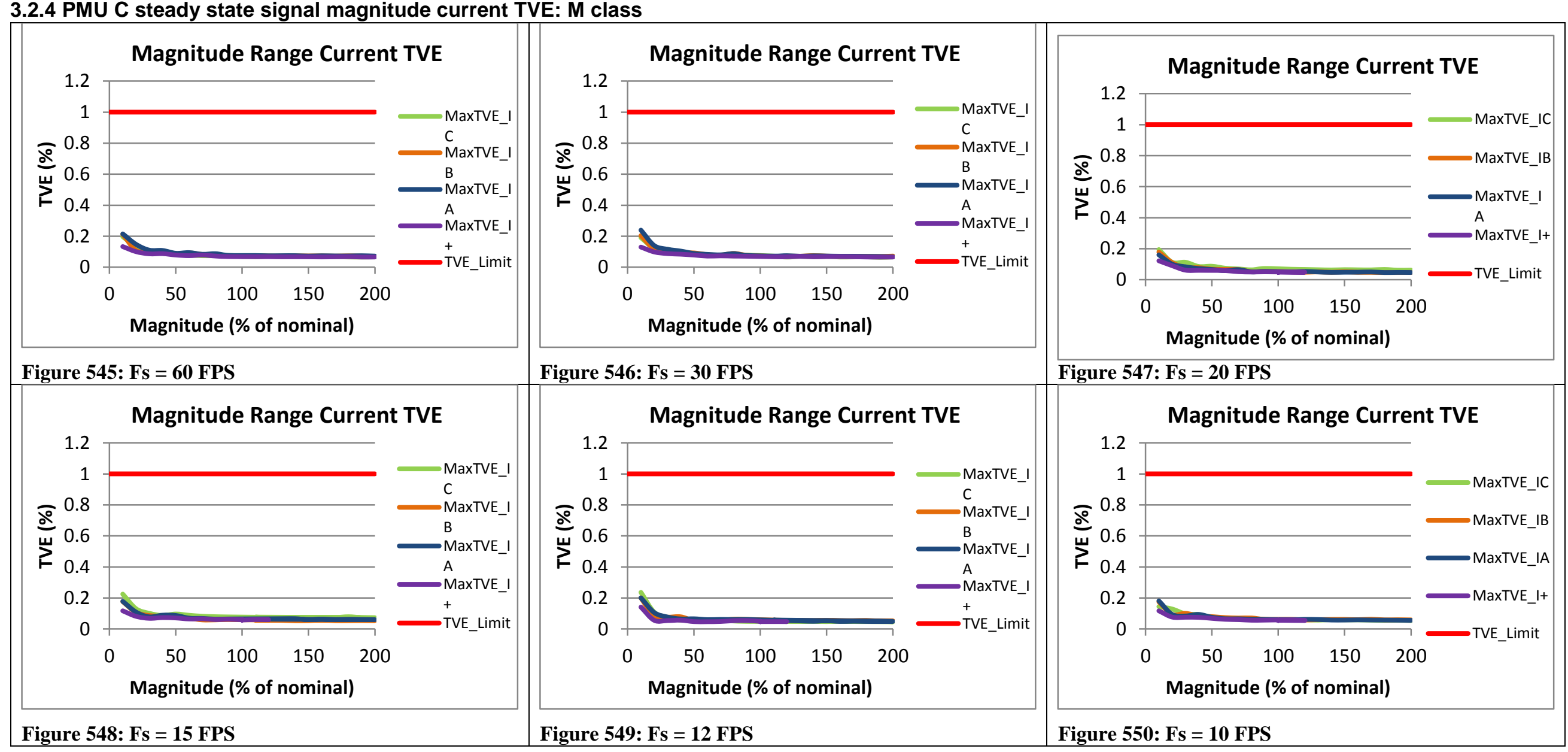


3.2.5 PMU D steady state signal magnitude current TVE: M class

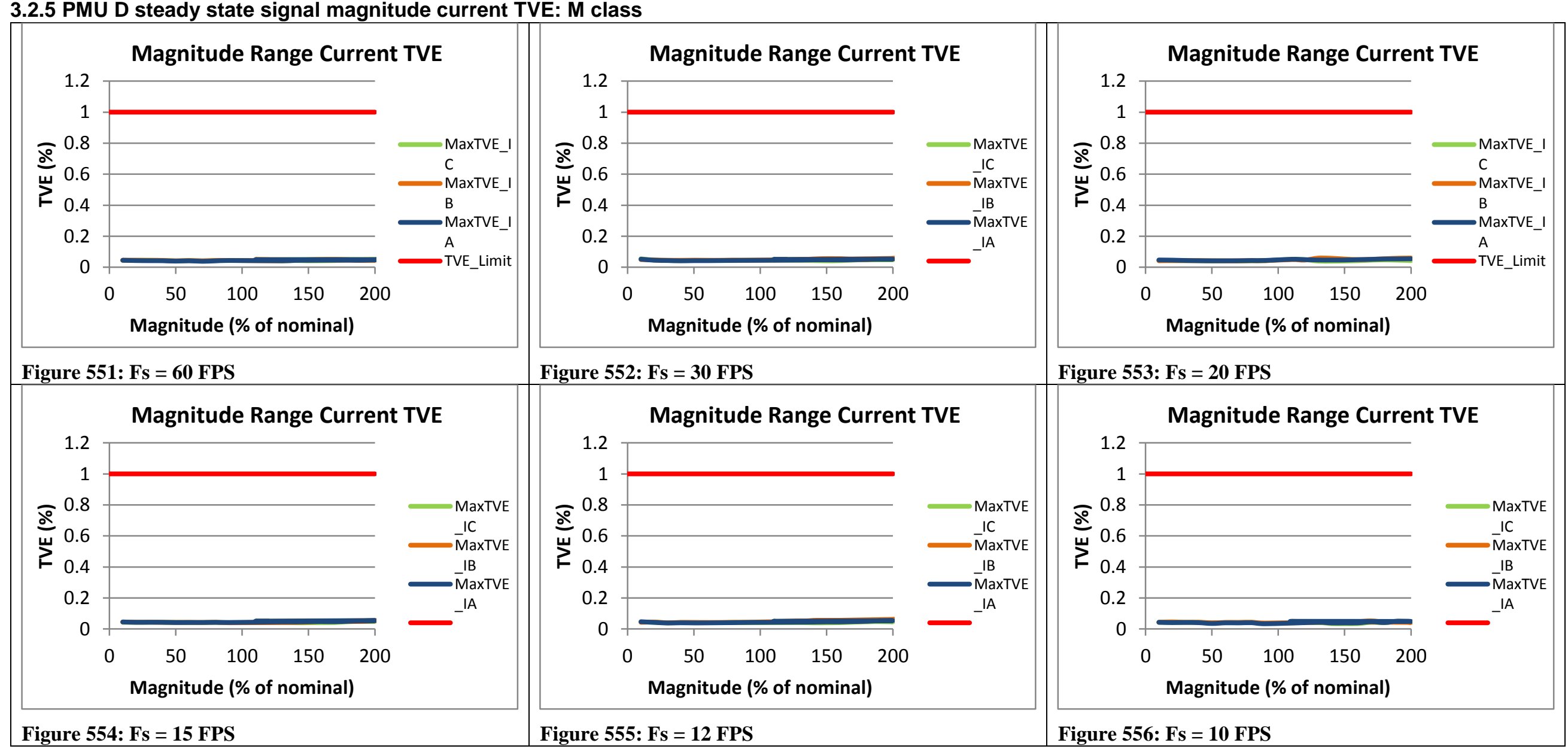


3.2.6 PMU E steady state signal magnitude current TVE: M class

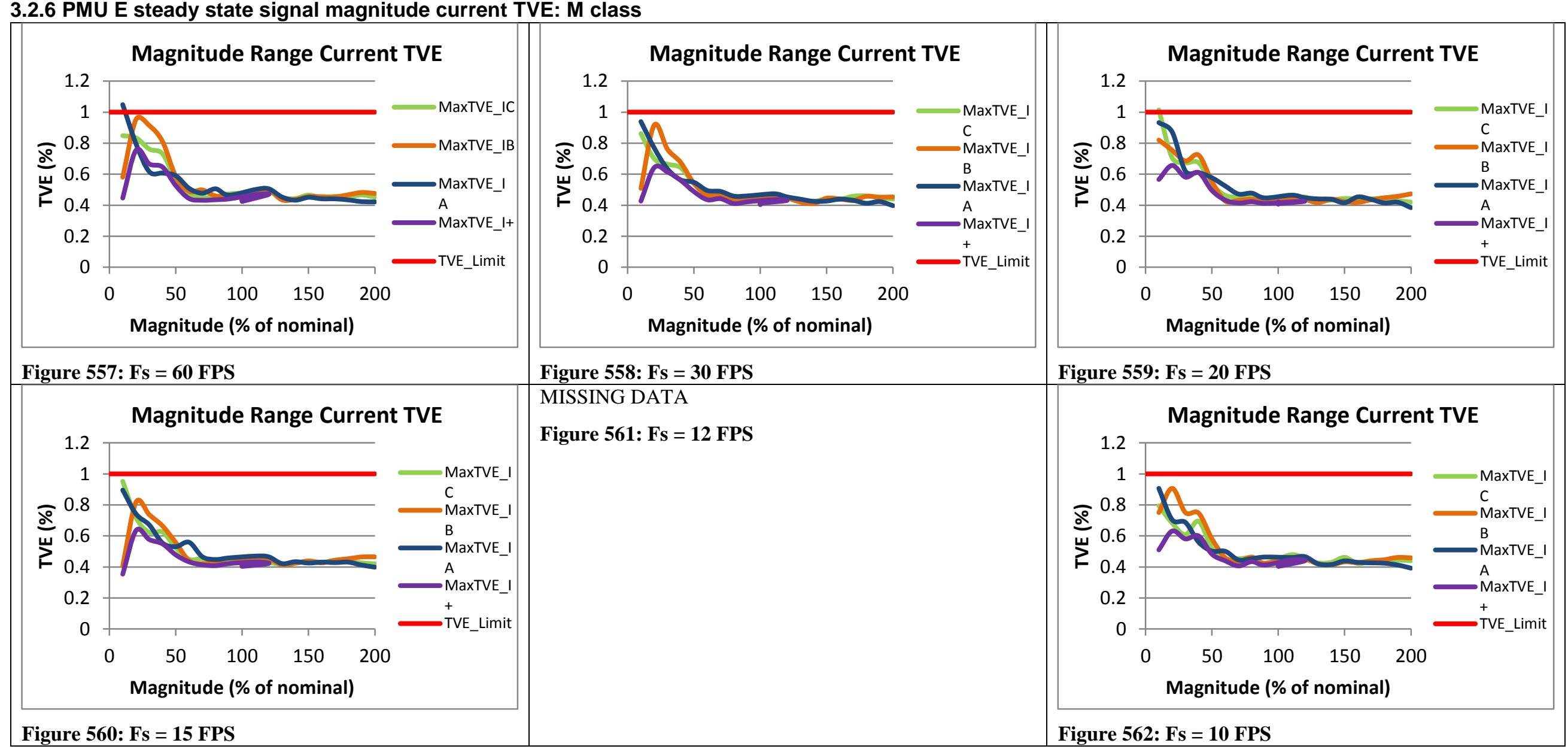


3.2.7 PMU F steady state signal magnitude current TVE: M class

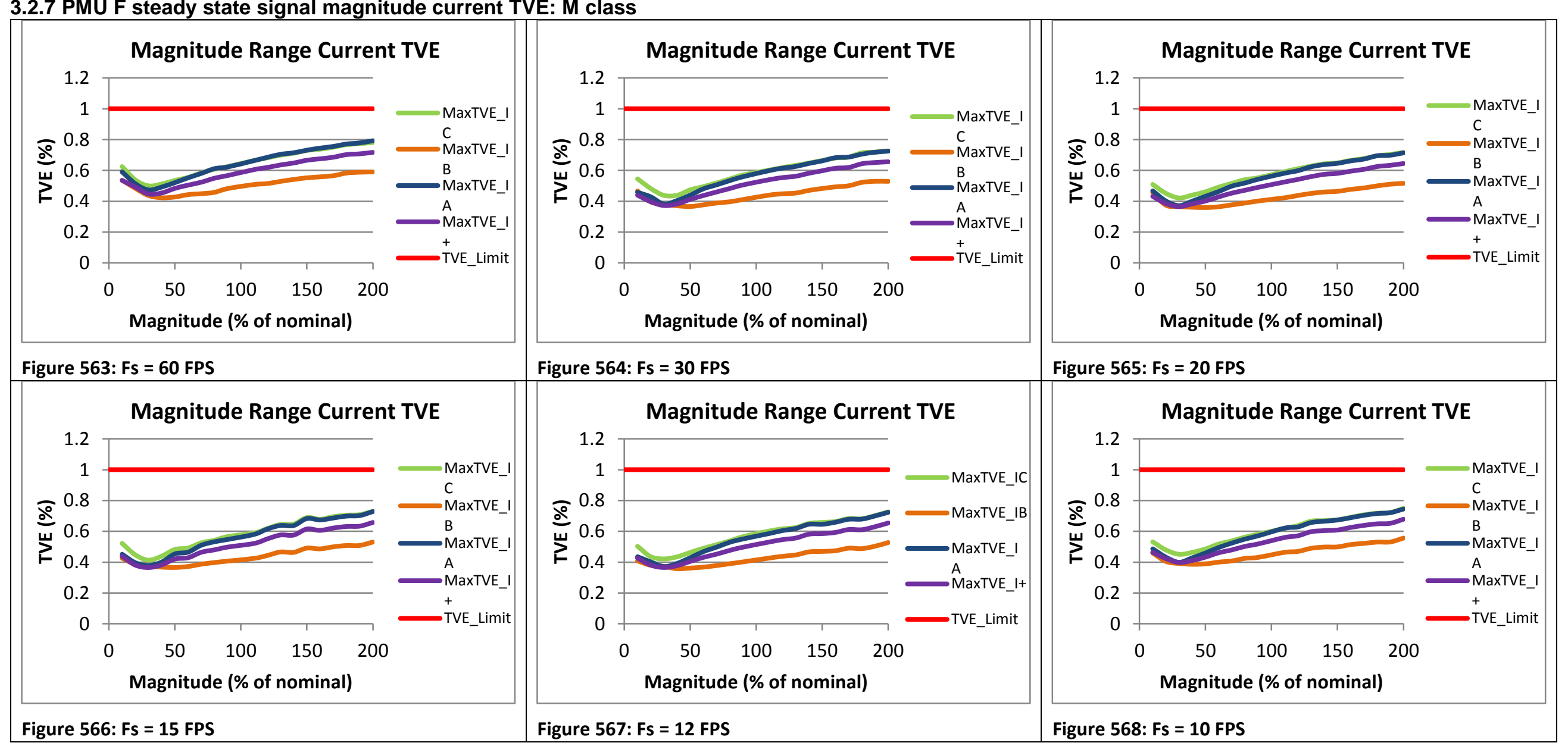


3.2.8 PMU G steady state signal magnitude current TVE: M class

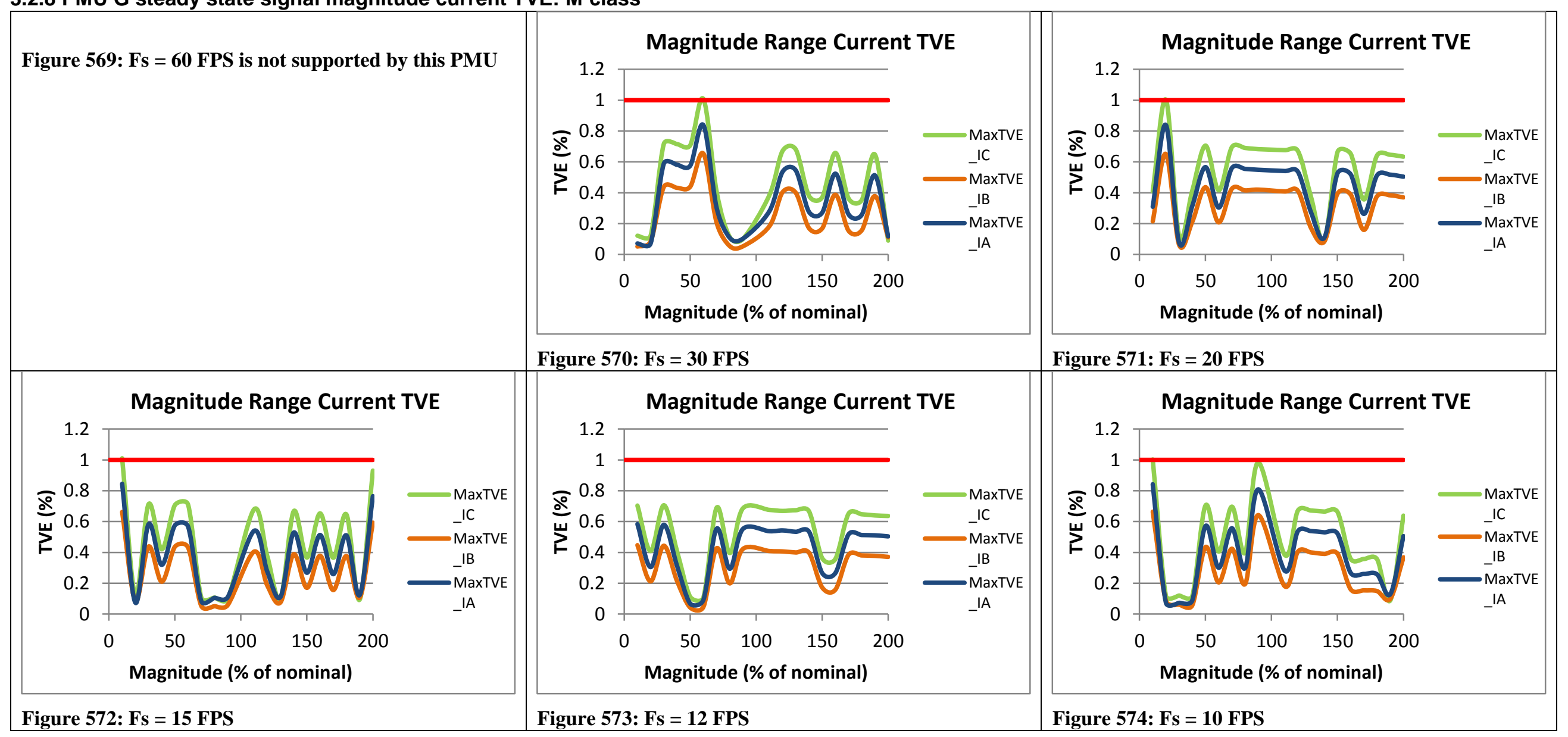


3.2.9 PMU H steady state signal magnitude current TVE: M class

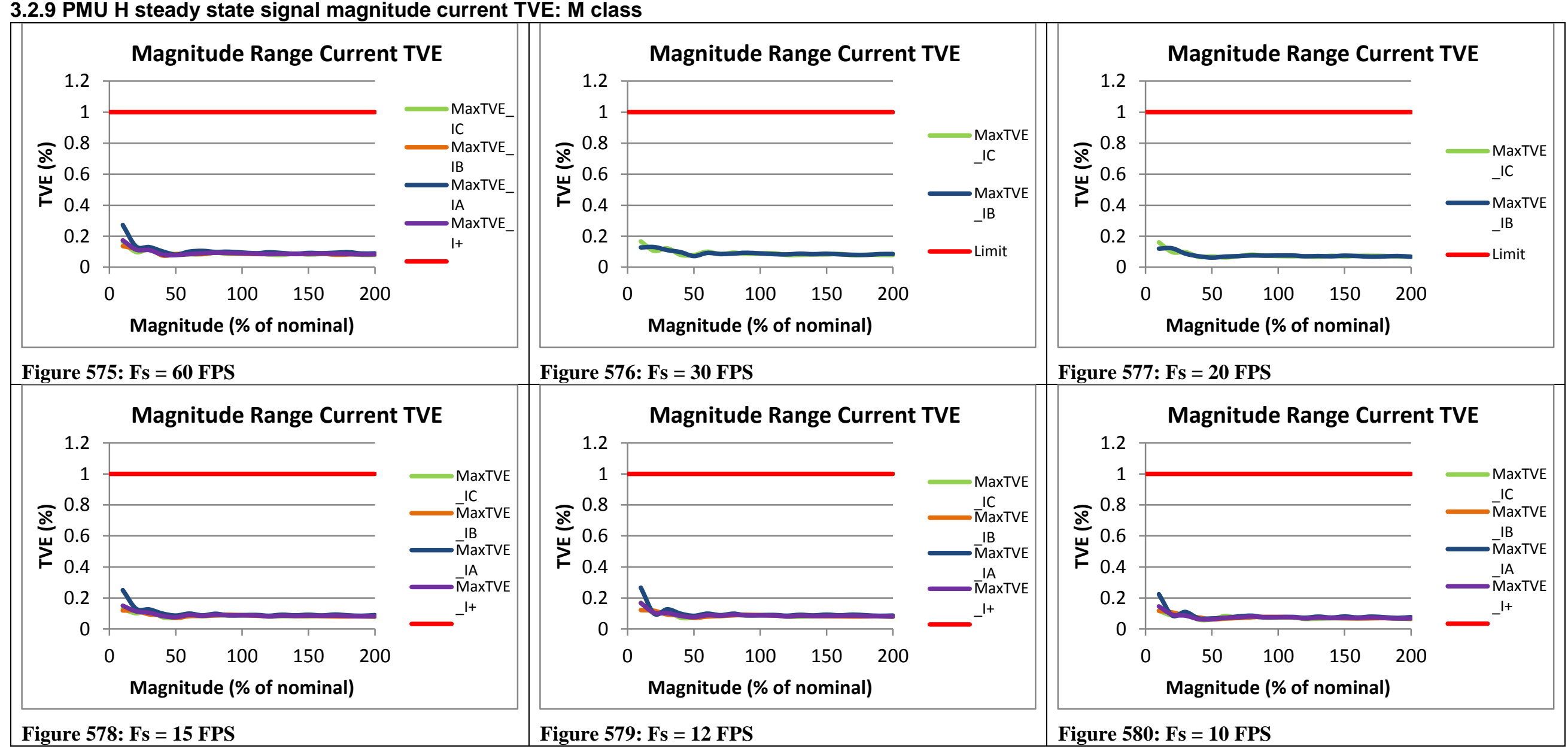


3.2.10 PMU I steady state signal magnitude current TVE: M class

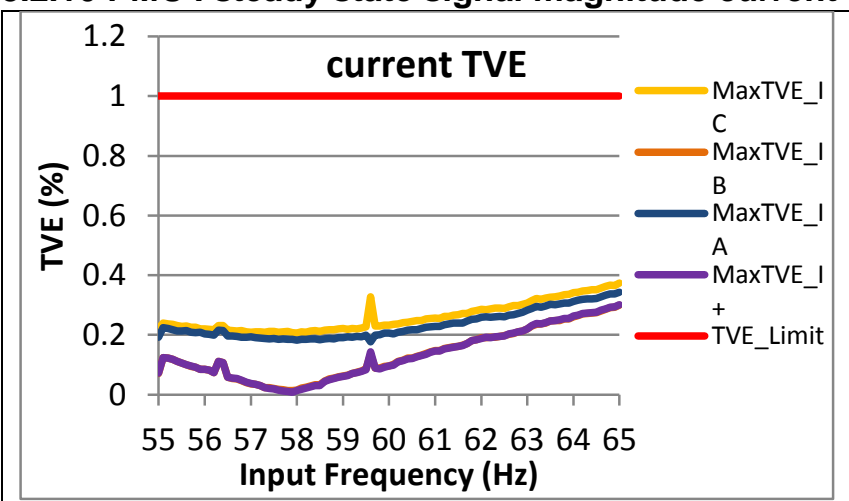

Figure 581: Fs = 60 FPS

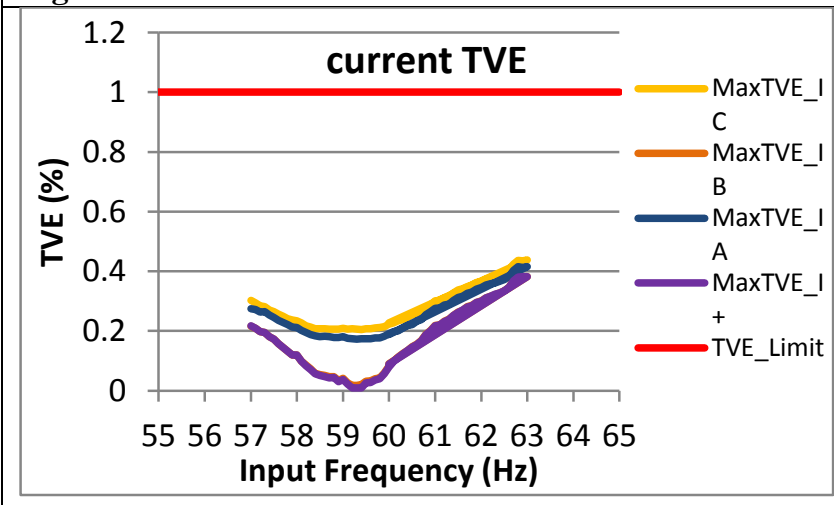

Figure 584: Fs = 15 FPS

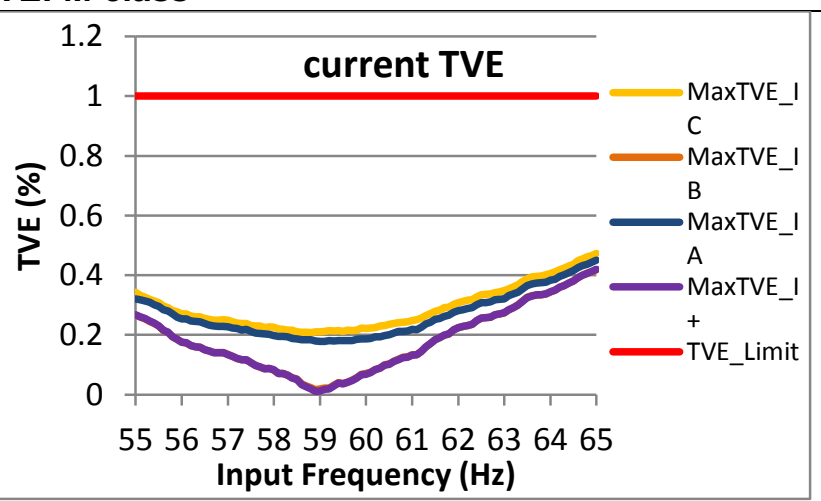

Figure 582: Fs = 30 FPS

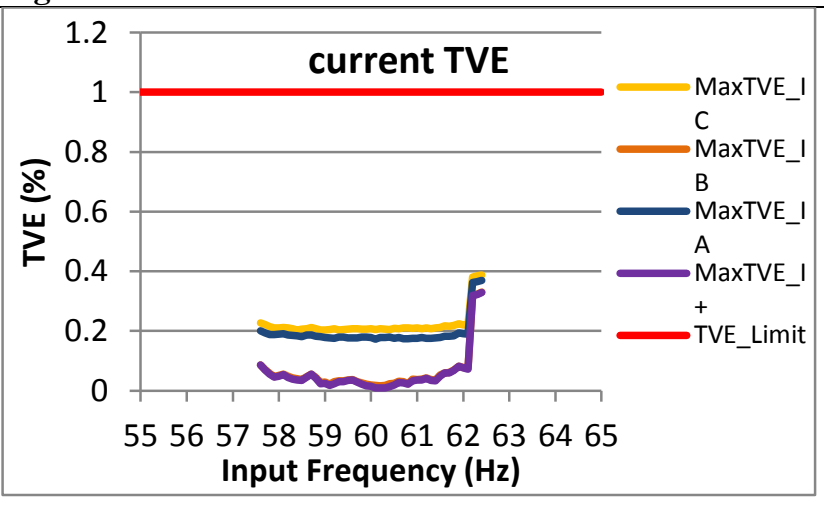

Figure 585: Fs = 12 FPS

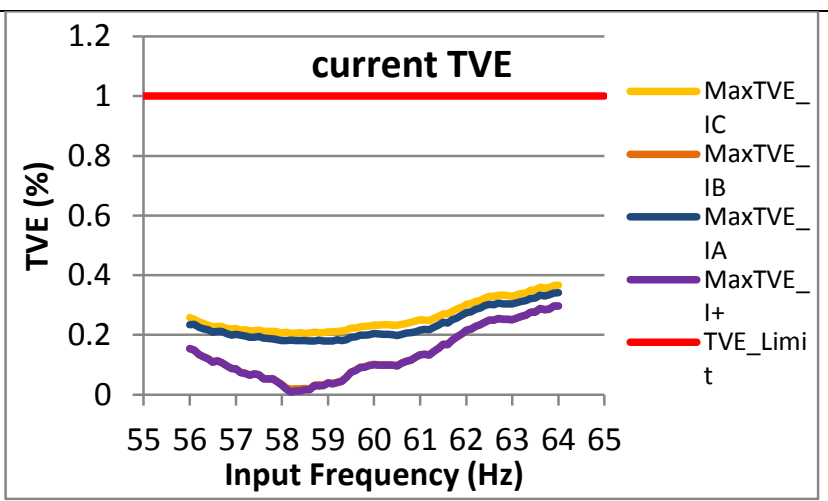

Figure 583: Fs = 20 FPS

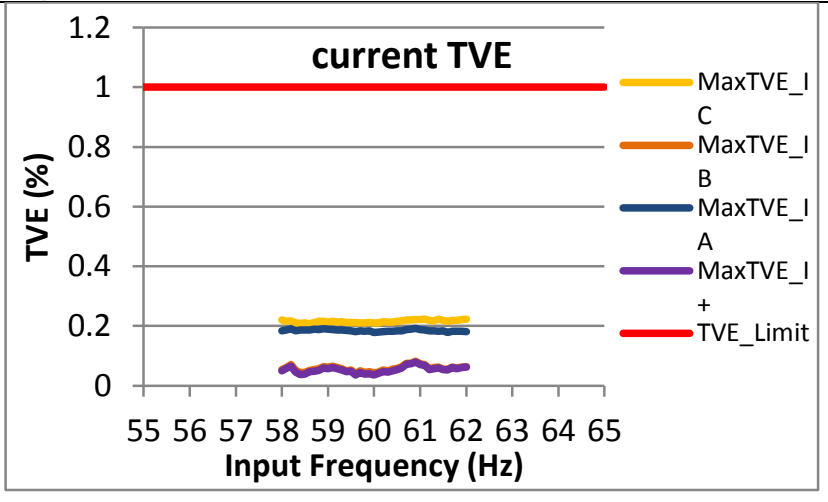

Figure 586: Fs = 10 FPS 
3.2.11 PMU J steady state signal magnitude current TVE: M class

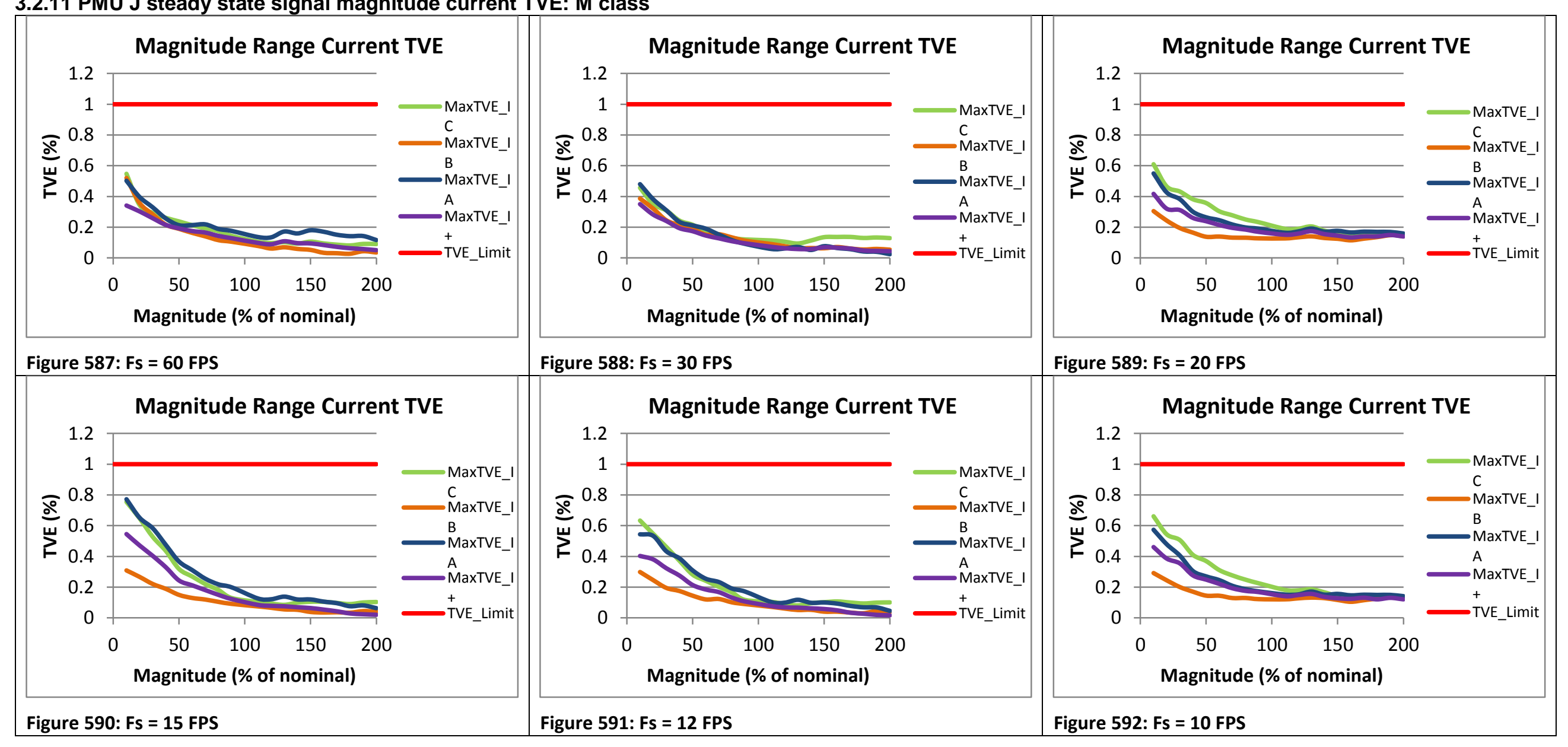




\subsection{Steady state signal magnitude frequency error $M$ class}

No performance limits are shown in the plots below because the steady state magnitude range tests do not require frequency error to meet performance limits

\subsubsection{C37.118.1 Annex $C$ steady state signal magnitude frequency error: $M$ class}

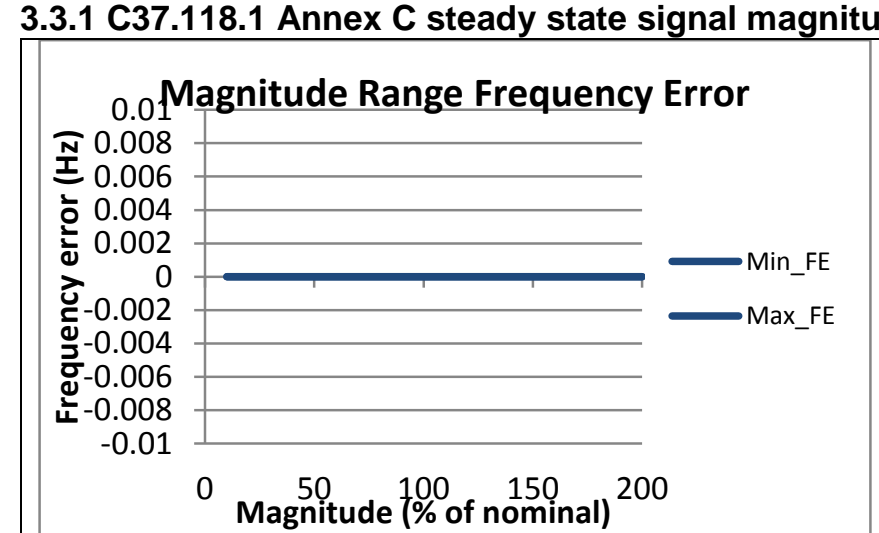

Figure 593: Fs = 60 FPS

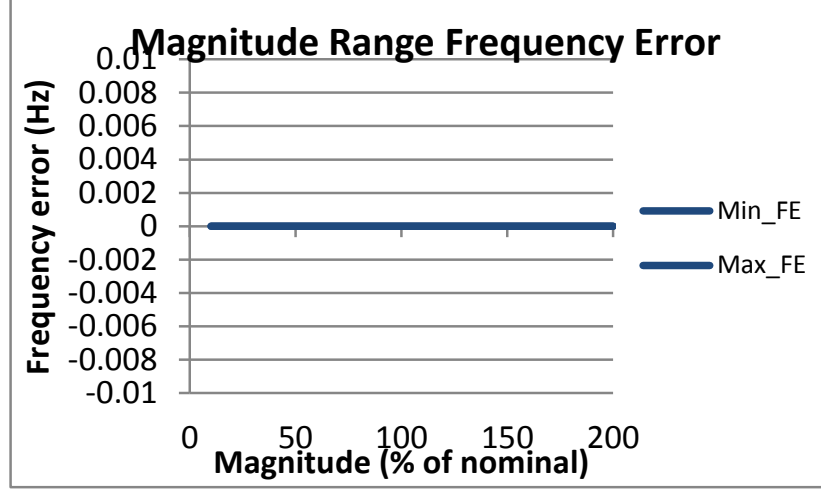

Figure 596; Fs = 15 FPS

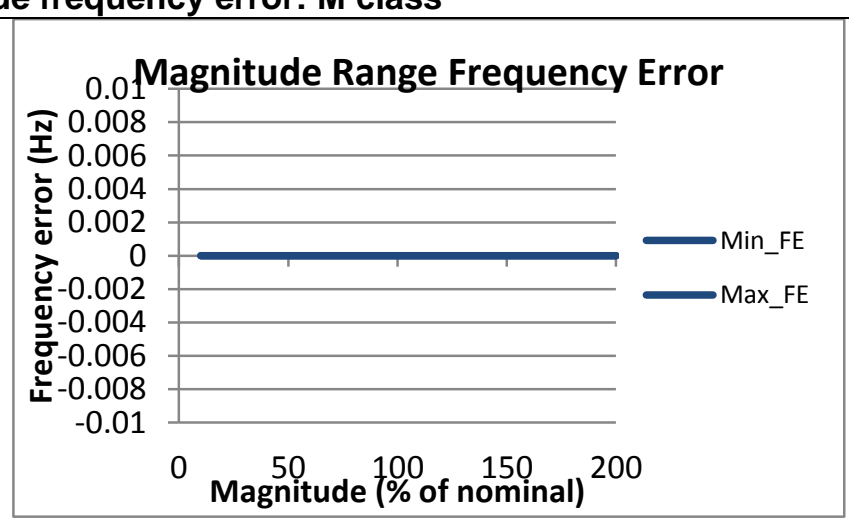

Figure 594: Fs = 30 FPS

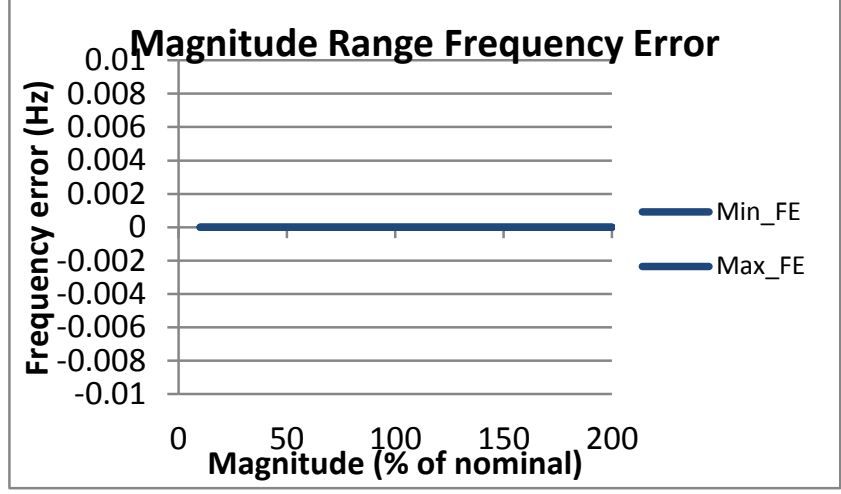

Figure 597: Fs = 12 FPS

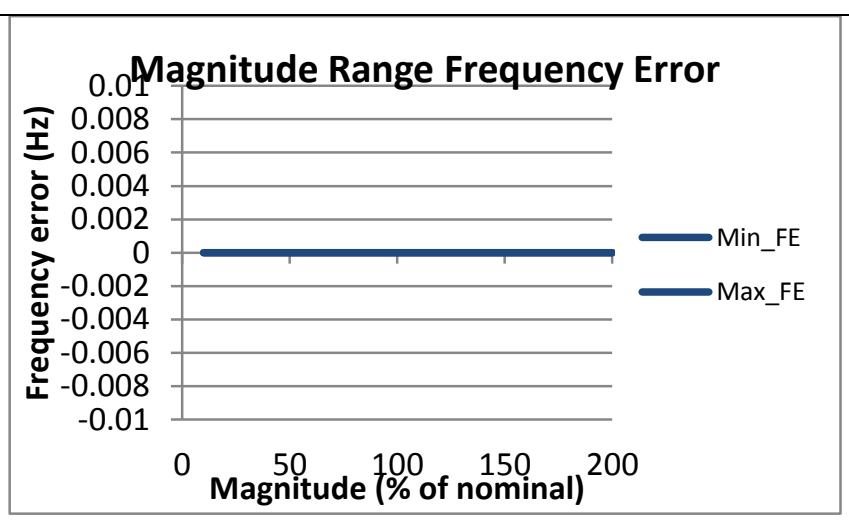

Figure 595: Fs = 20 FPS

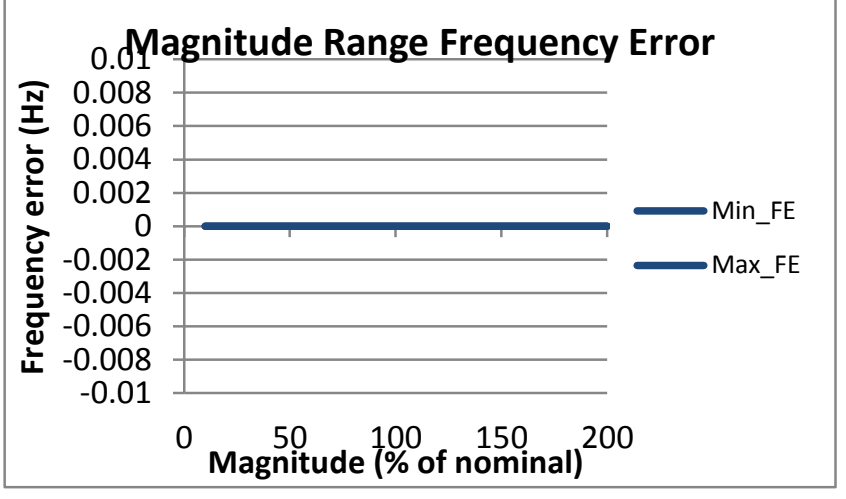

Figure 598: Fs = 10 FPS 
3.3.2 PMU A steady state signal magnitude frequency error: $M$ class

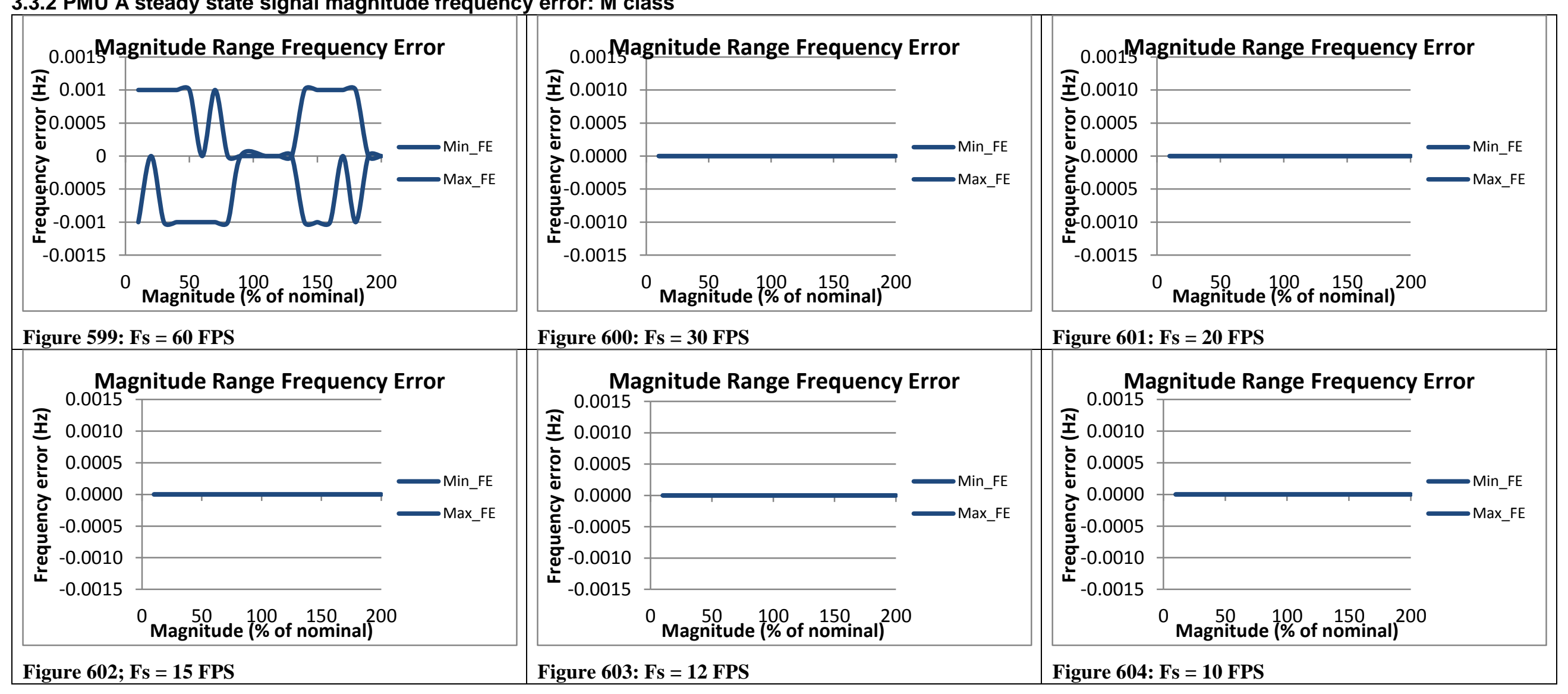


3.3.3 PMU B steady state signal magnitude frequency error: $M$ class

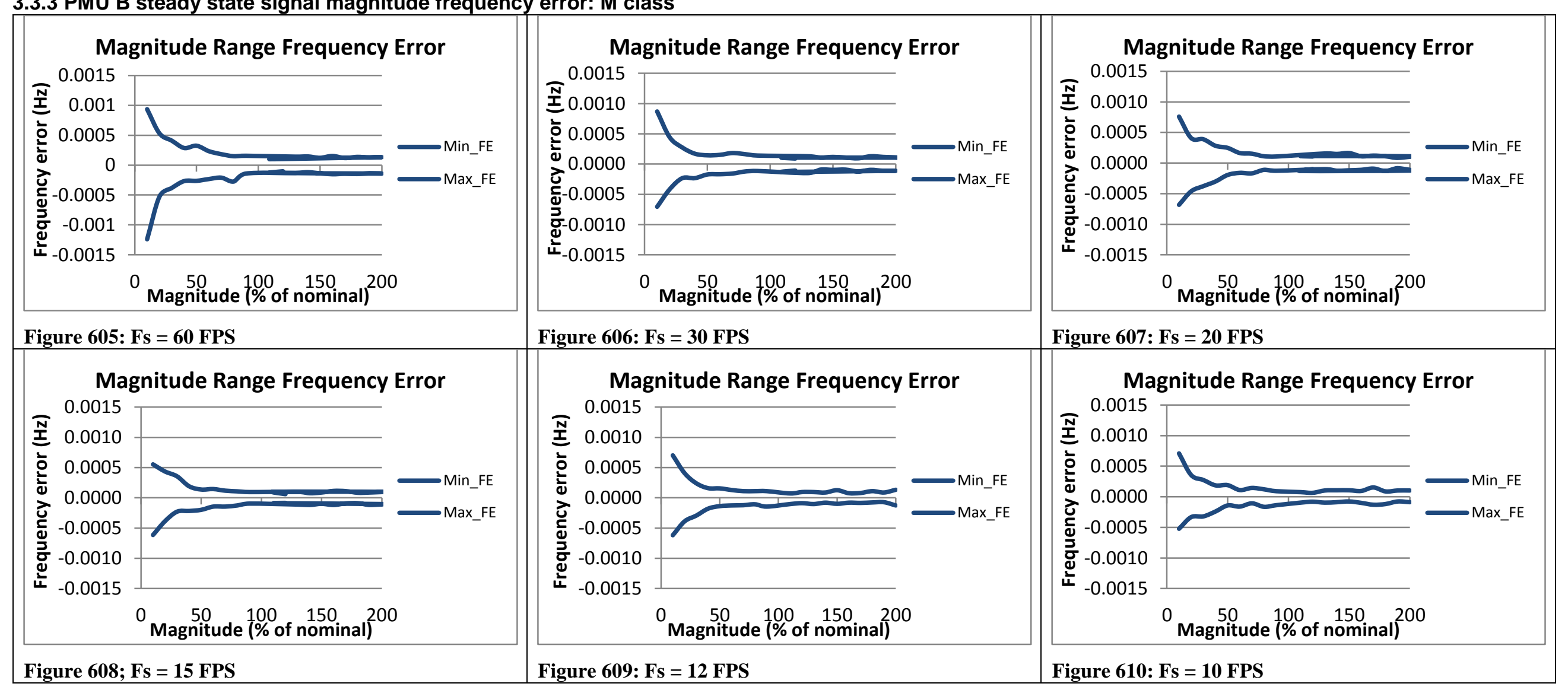


3.3.4 PMU C steady state signal magnitude frequency error: $M$ class

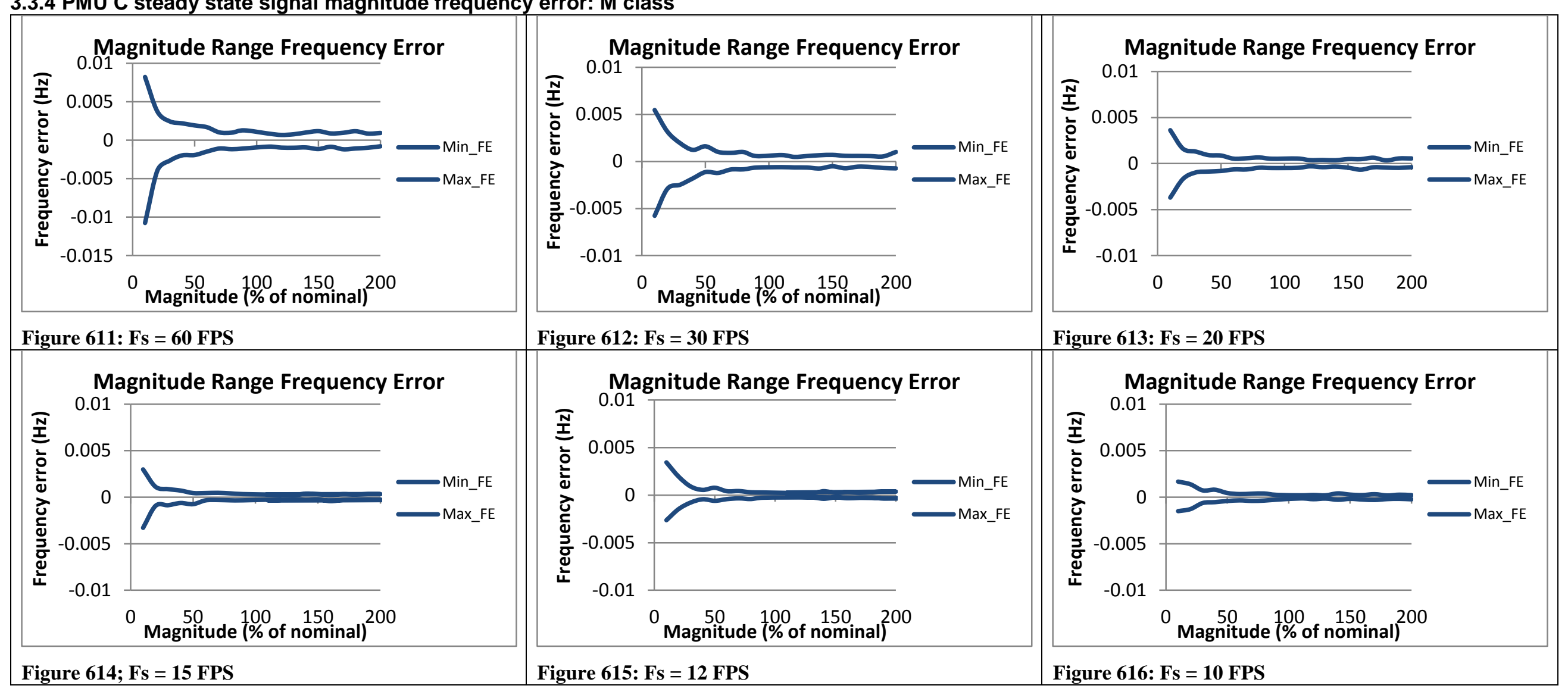


3.3.5 PMU D steady state signal magnitude frequency error: $M$ class

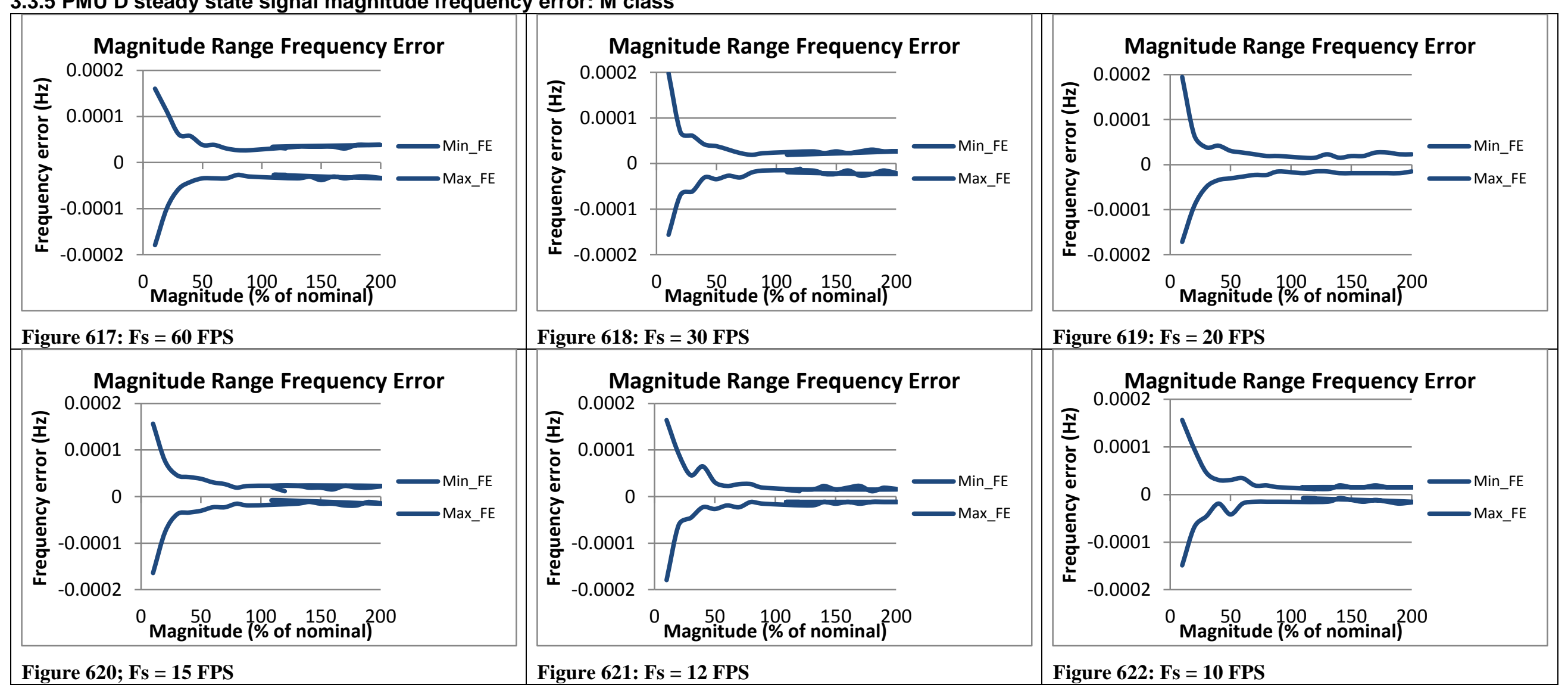


3.3.6 PMU E steady state signal magnitude frequency error: $M$ class

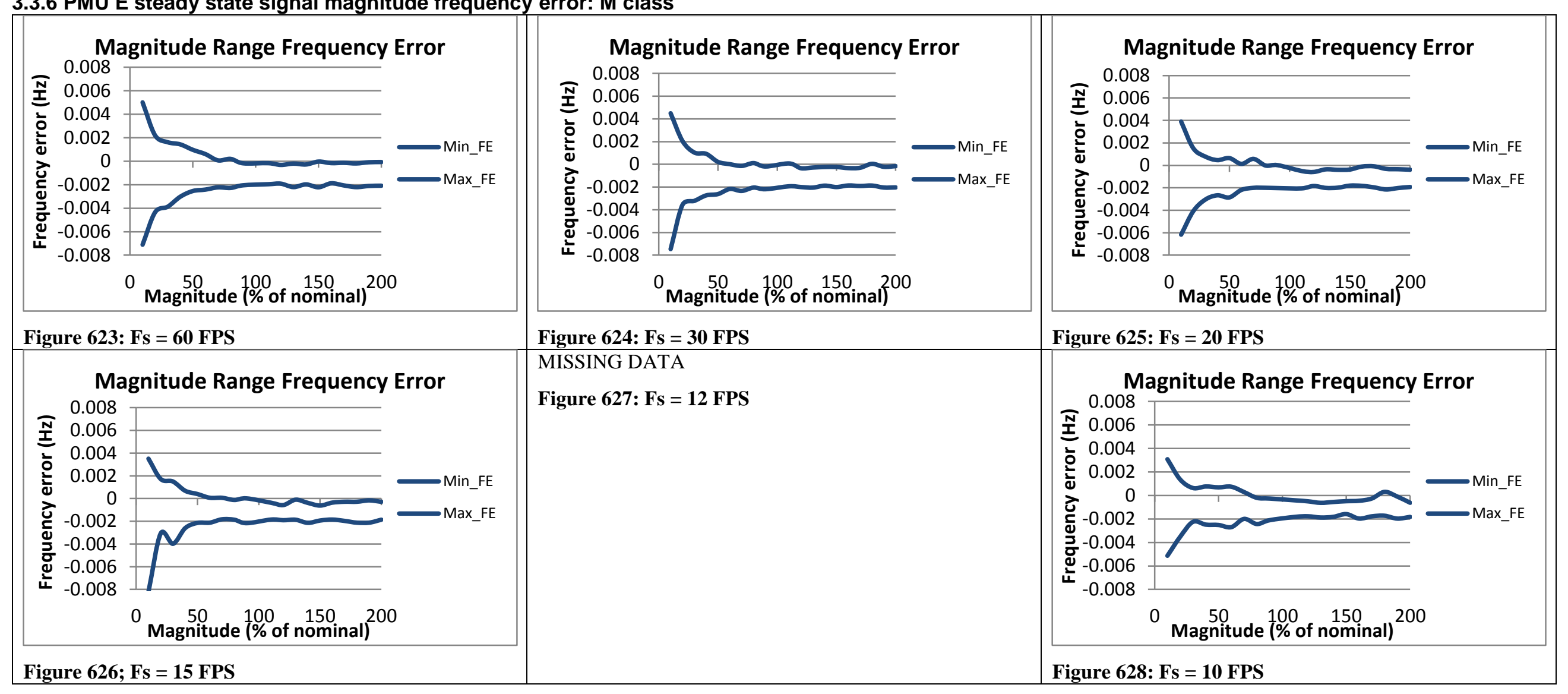


3.3.7 PMU F steady state signal magnitude frequency error: $M$ class

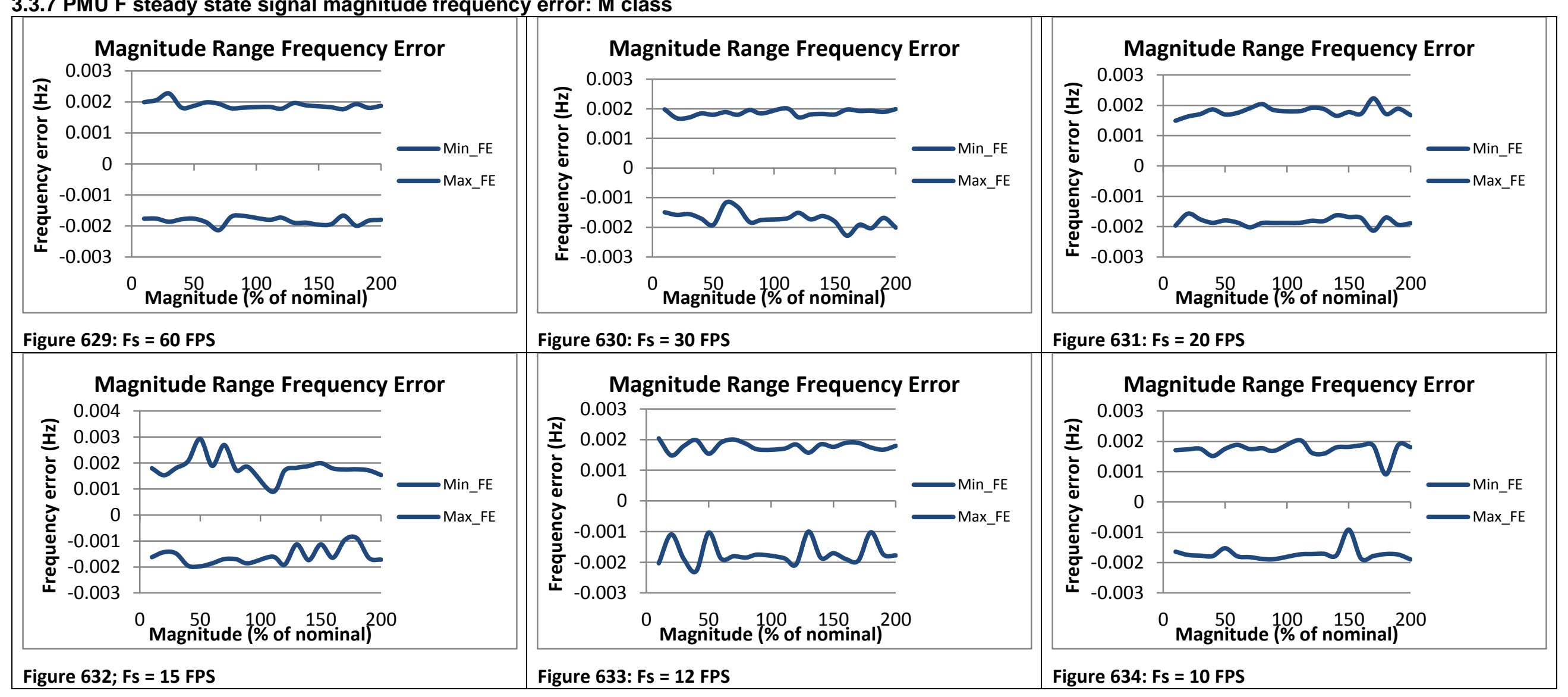


3.3.8 PMU G steady state signal magnitude frequency error: M class

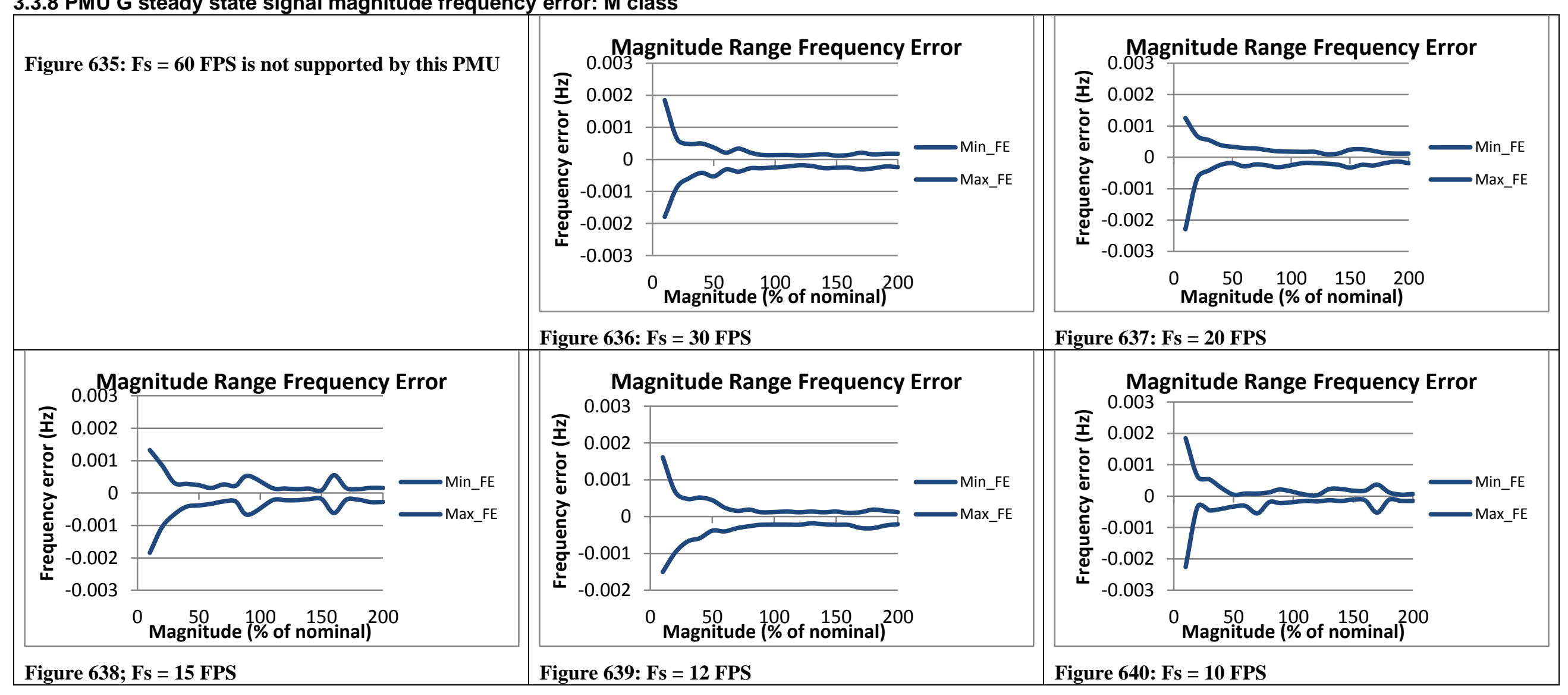


3.3.9 PMU H steady state signal magnitude frequency error: $\mathrm{M}$ class

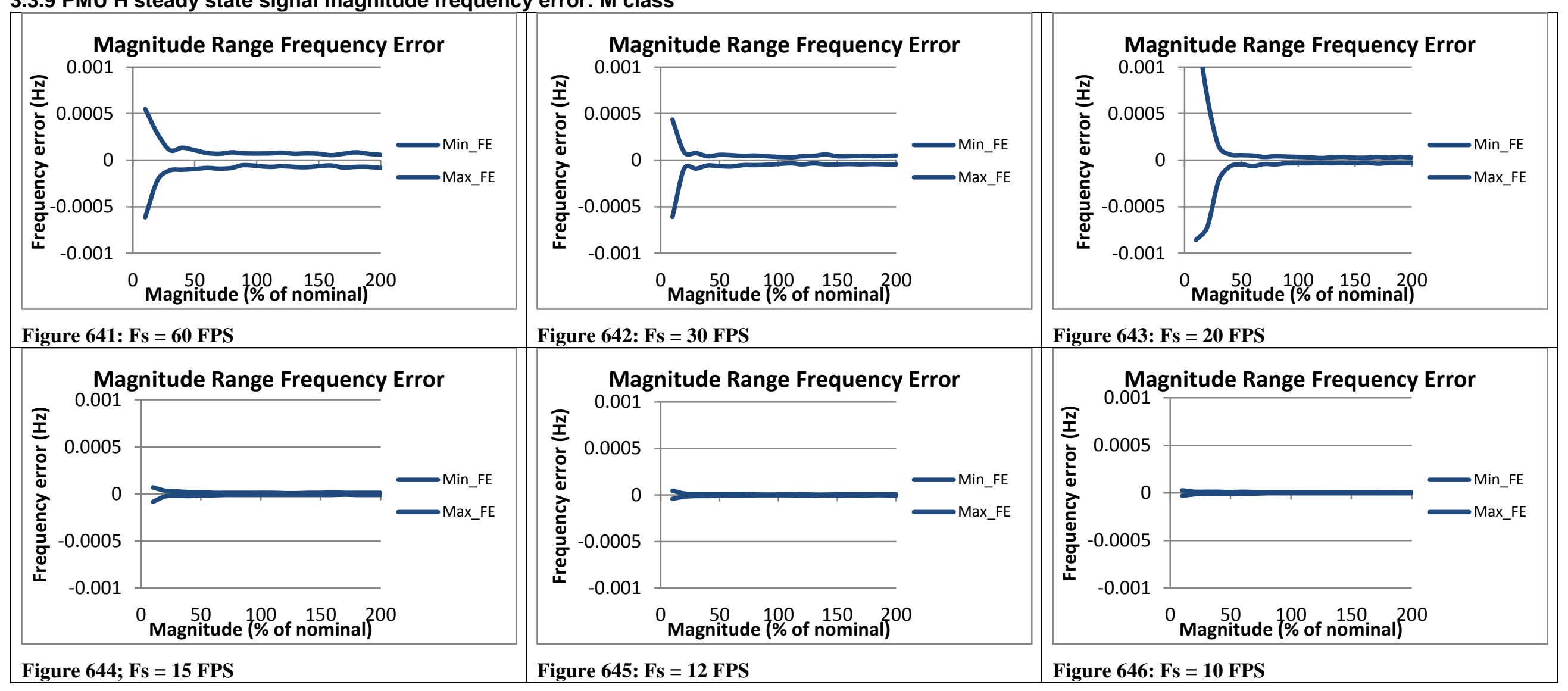


3.3.10 PMU I steady state signal magnitude frequency error: M class

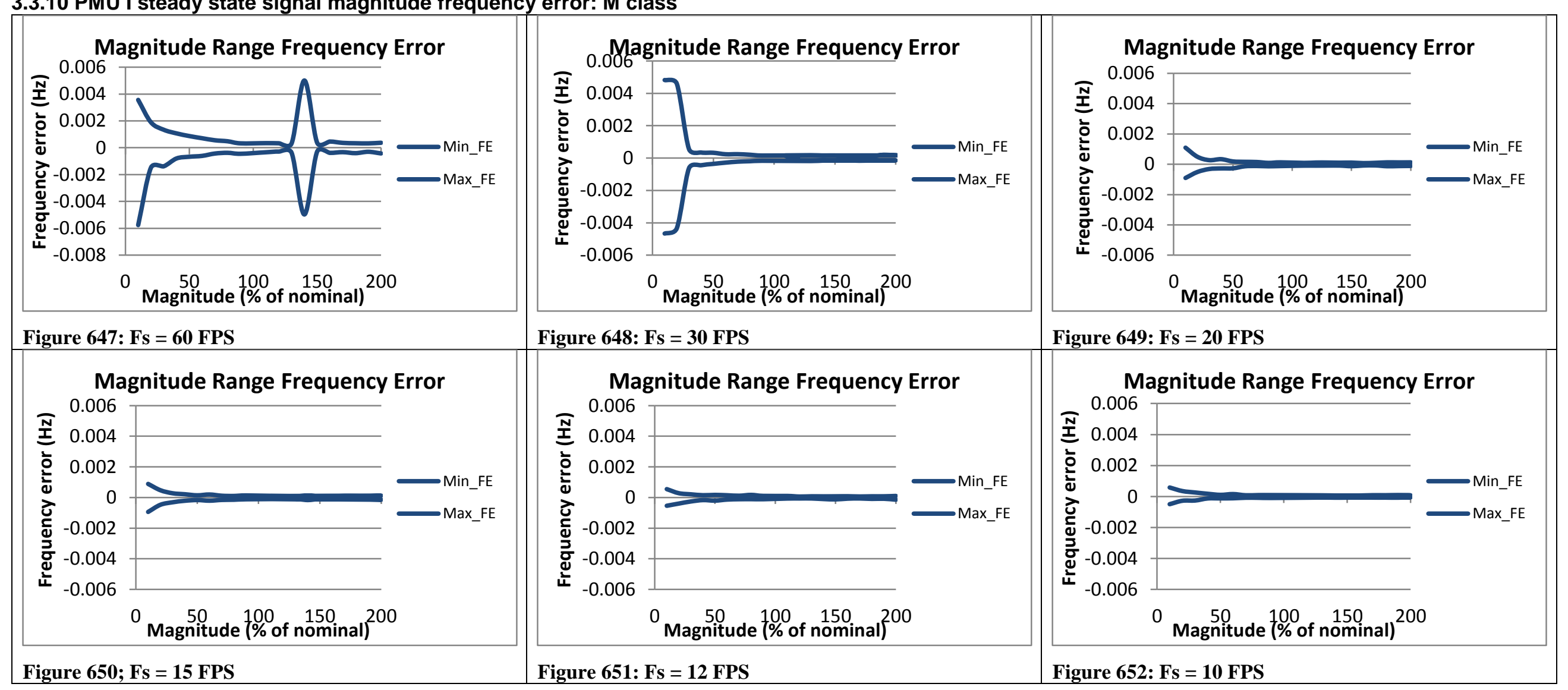


3.3.11 PMU J steady state signal magnitude frequency error: $M$ class

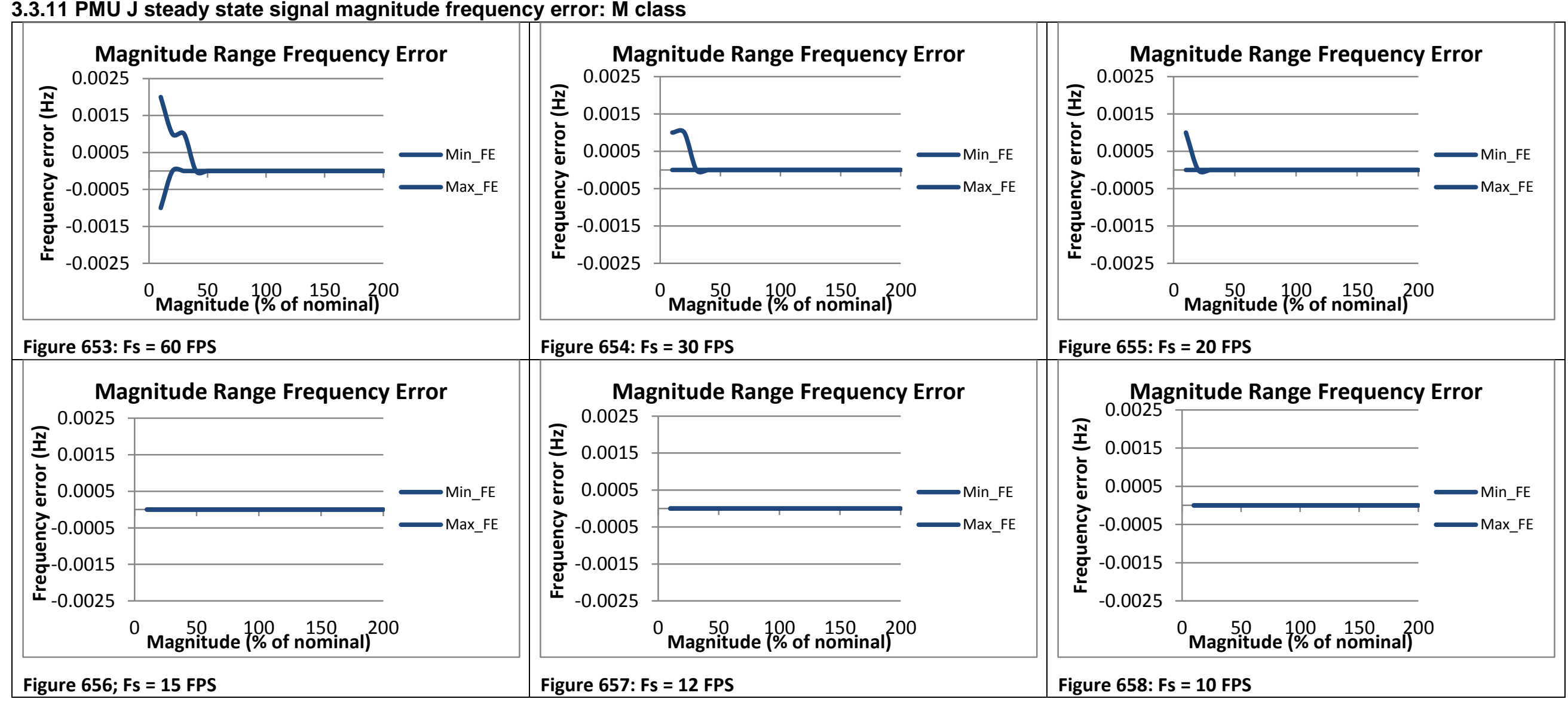




\subsection{Steady state signal magnitude ROCOF error M class}

No limits are shown in the plots below because the steady state magnitude range tests do not require ROCOF error to meet performance limits

\subsubsection{C37.118.1 Annex C steady state signal magnitude ROCOF error: M class}
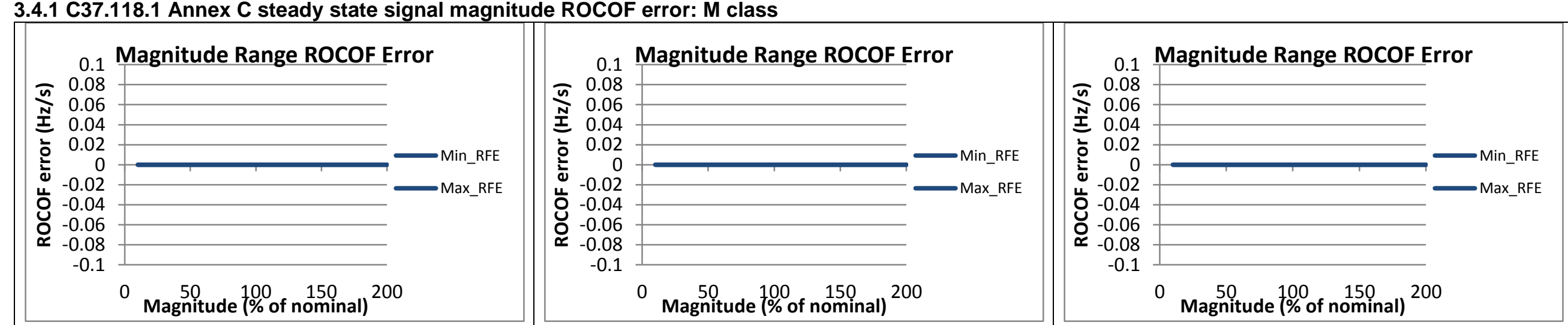

Figure 659: Fs = 60 FPS

Figure 660: Fs = 30 FPS

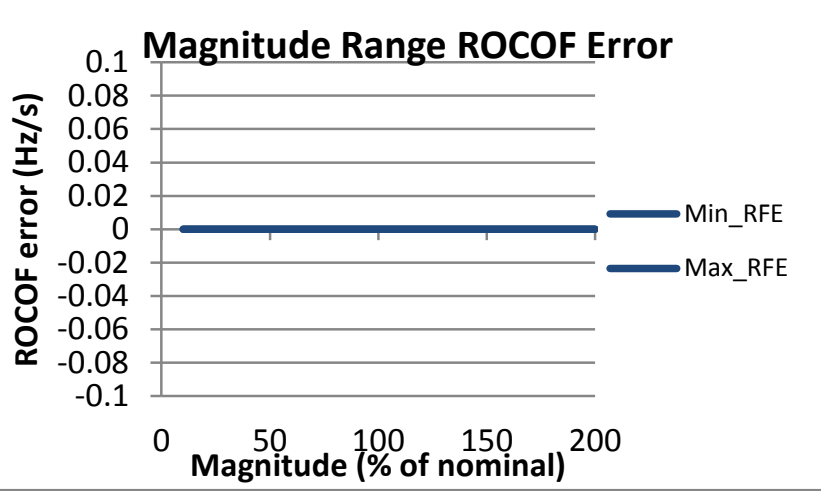

Figure 662: Fs = 15 FPS

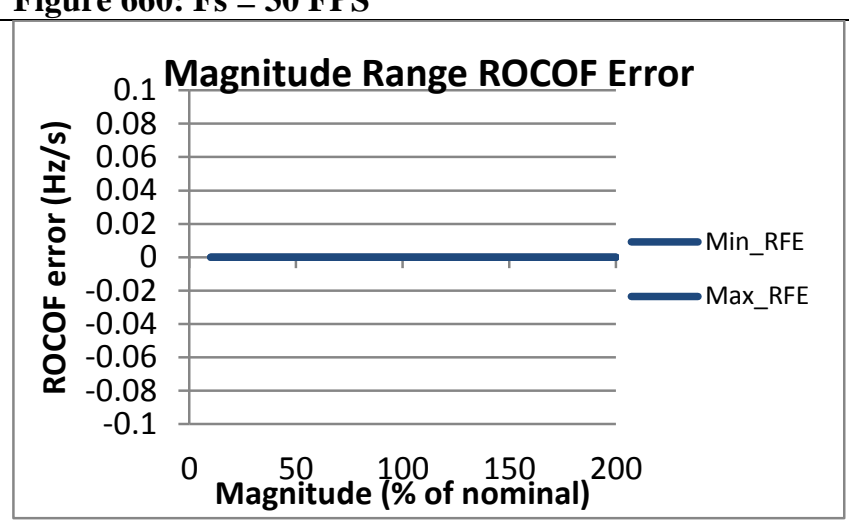

Figure 663: Fs = 12 FPS

\section{Figure 661: Fs = 20 FPS}

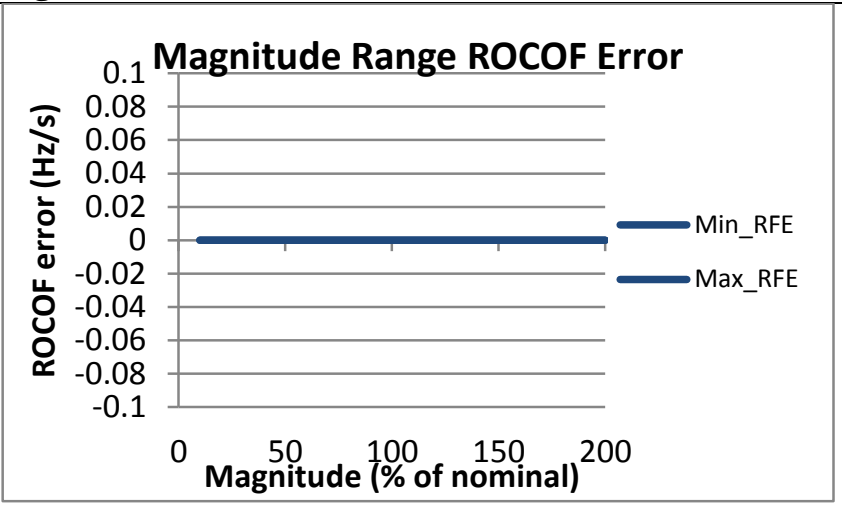

Figure 664: Fs = 10 FPS 
3.4.2 PMU A steady state signal magnitude ROCOF error: M class

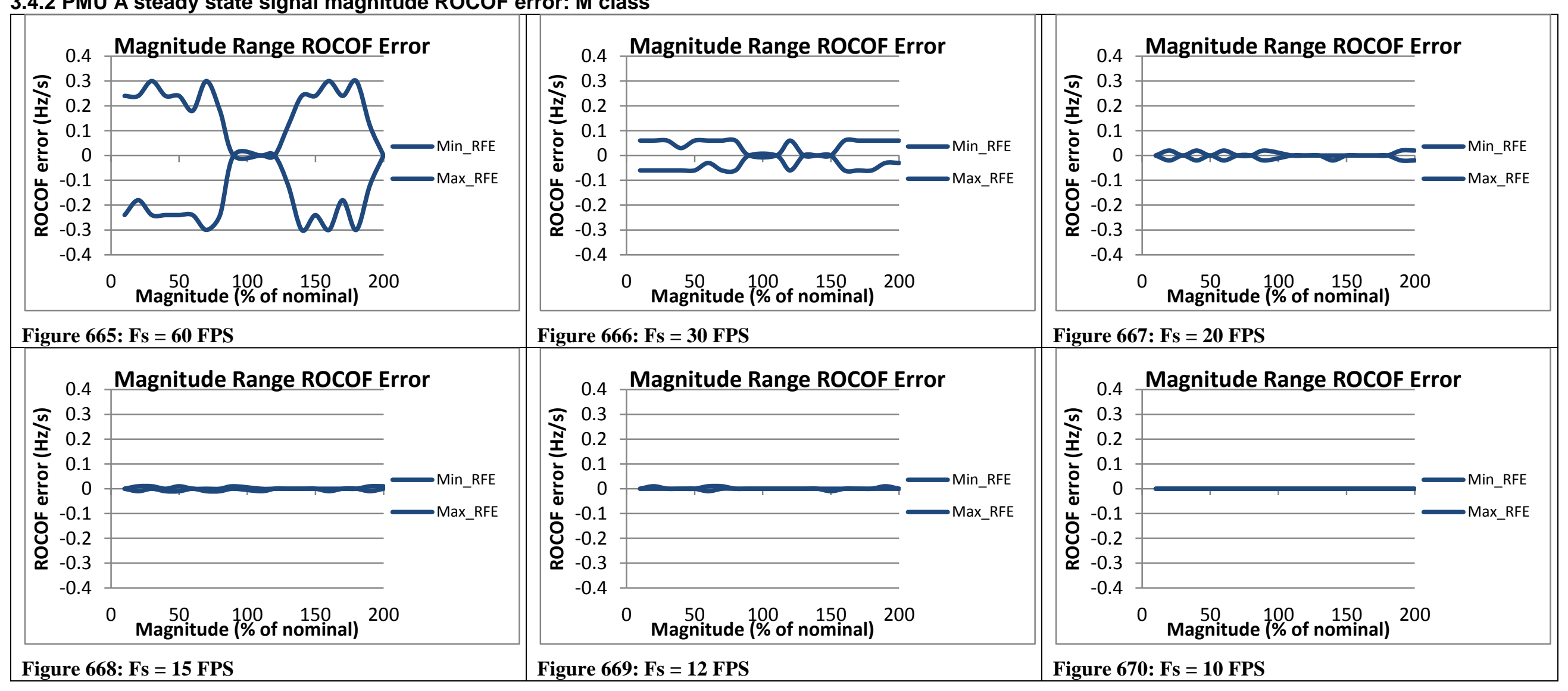


3.4.3 PMU B steady state signal magnitude ROCOF error: M class

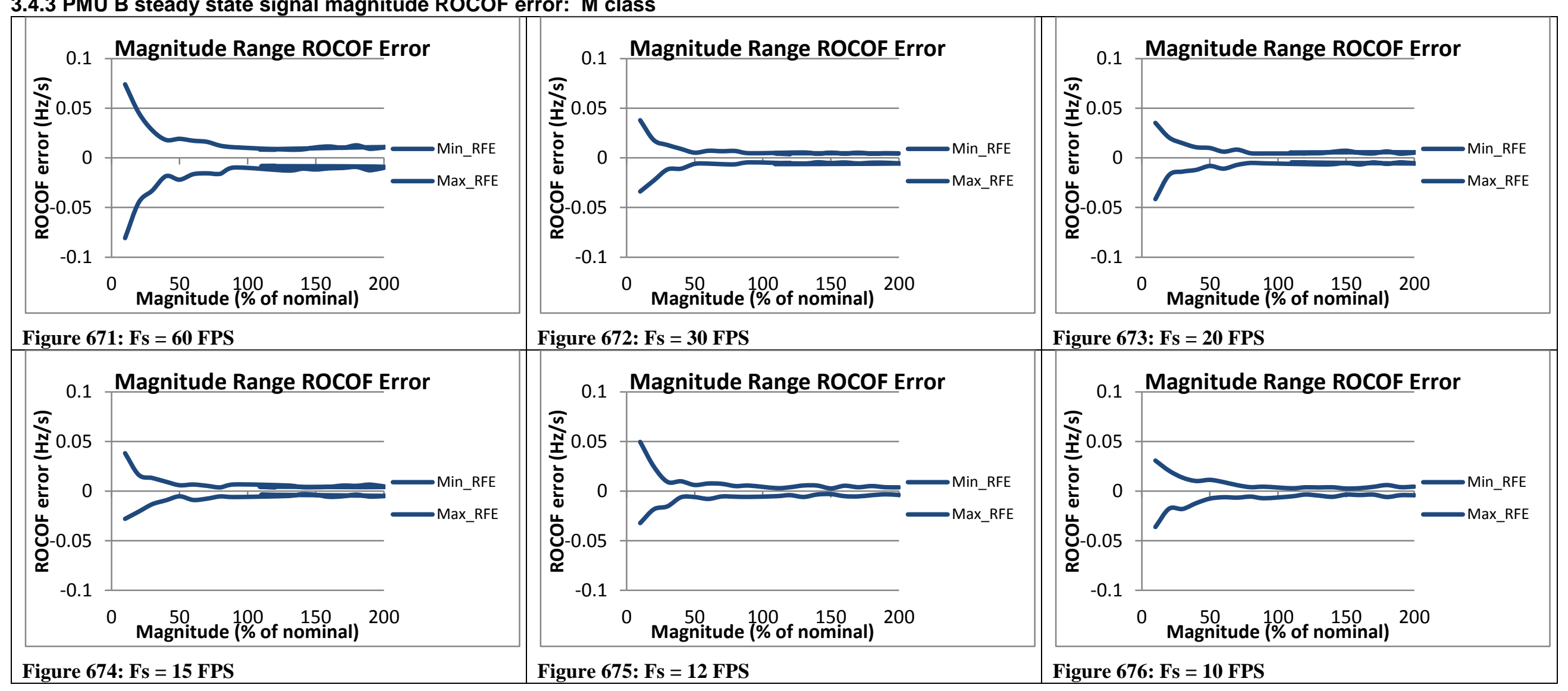


3.4.4 PMU C steady state signal magnitude ROCOF error: M class

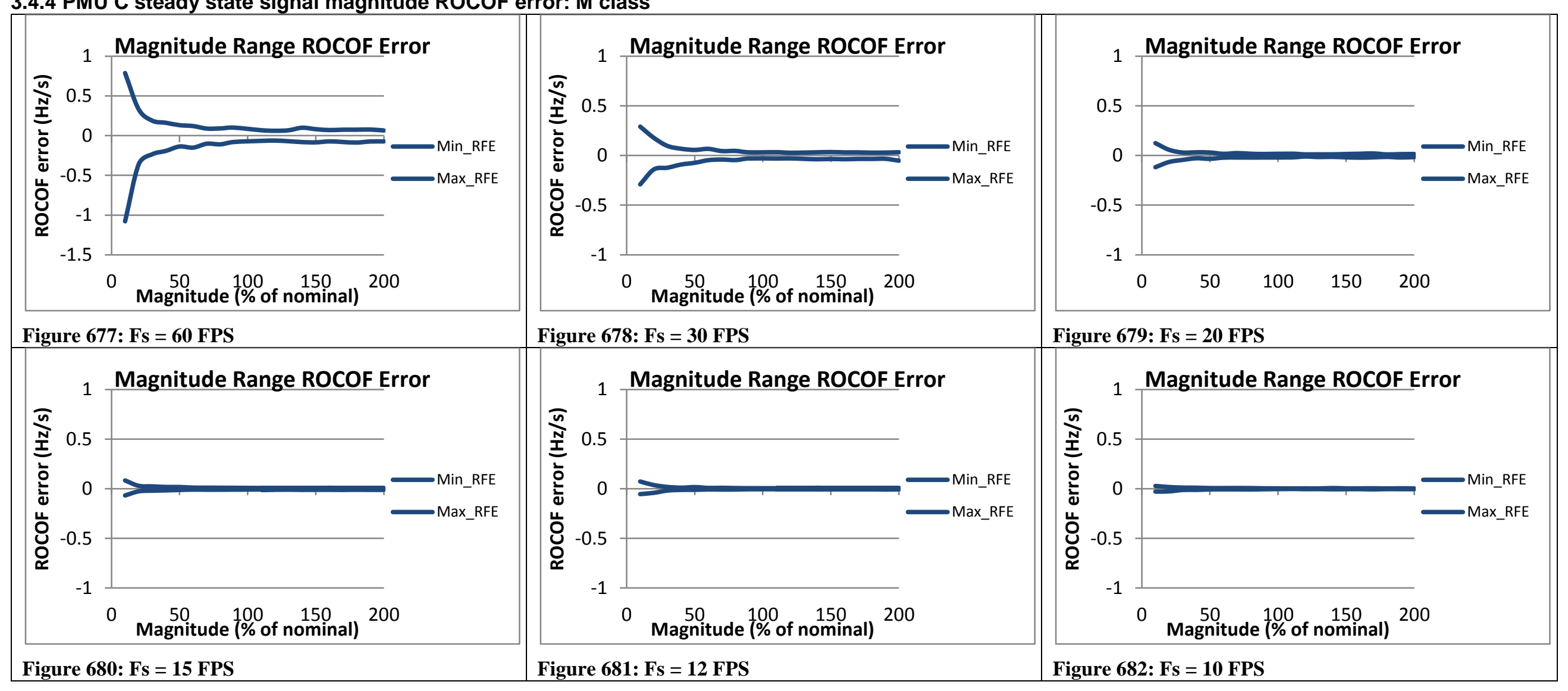


3.4.5 PMU D steady state signal magnitude ROCOF error: M class

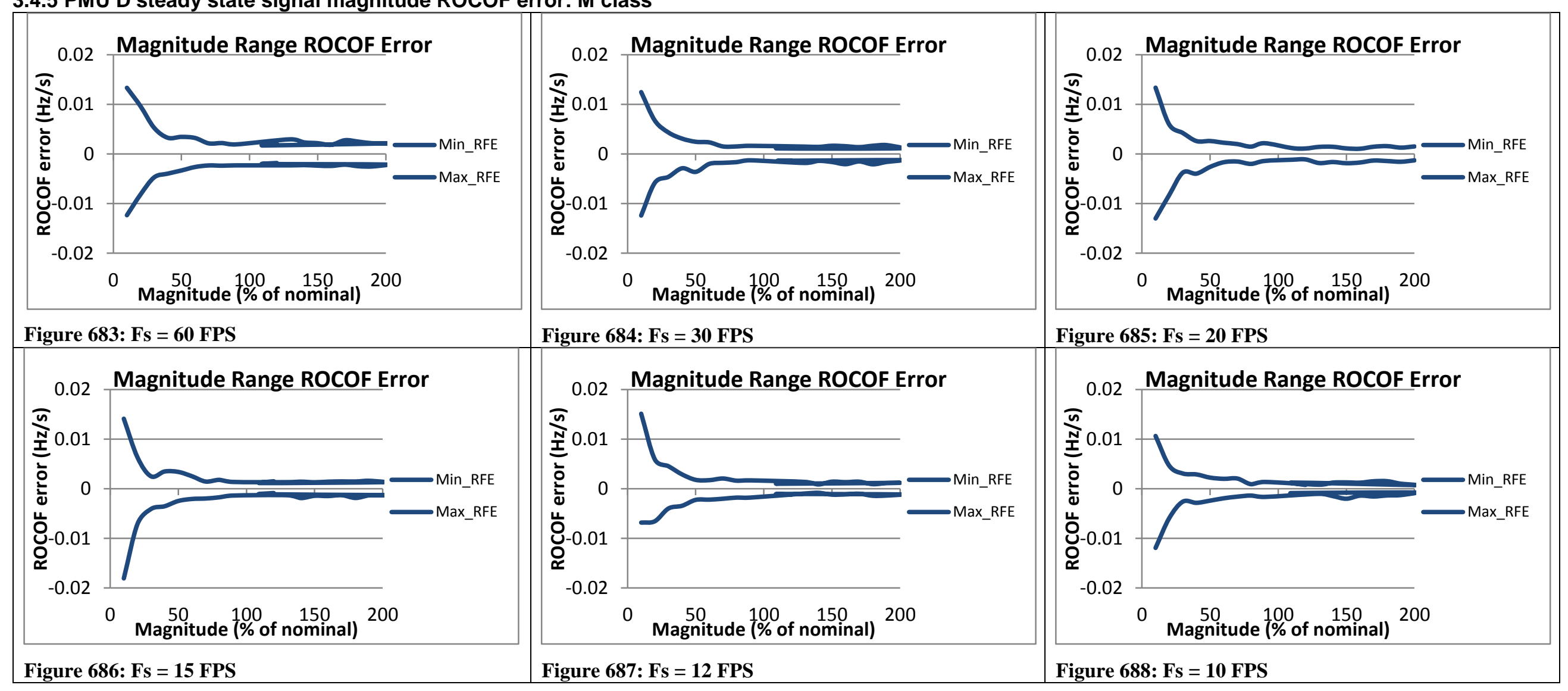


3.4.6 PMU E steady state signal magnitude ROCOF error: M class

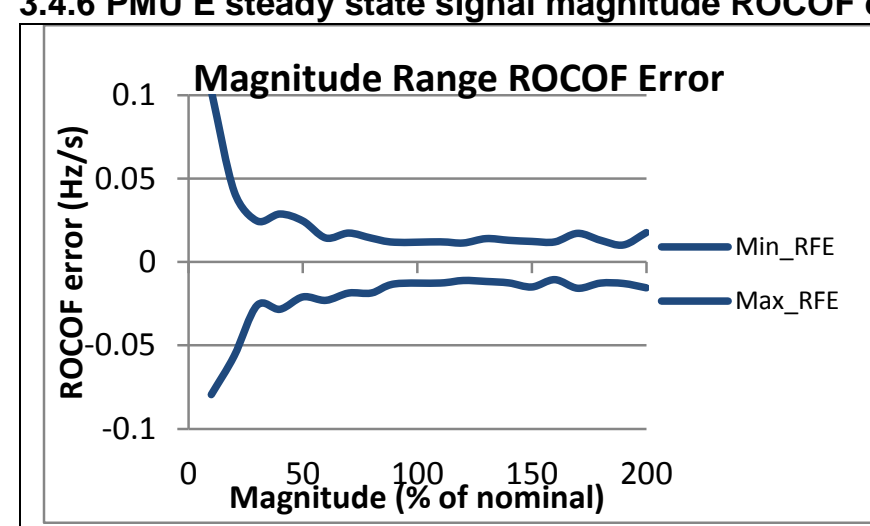

Figure 689: Fs = 60 FPS

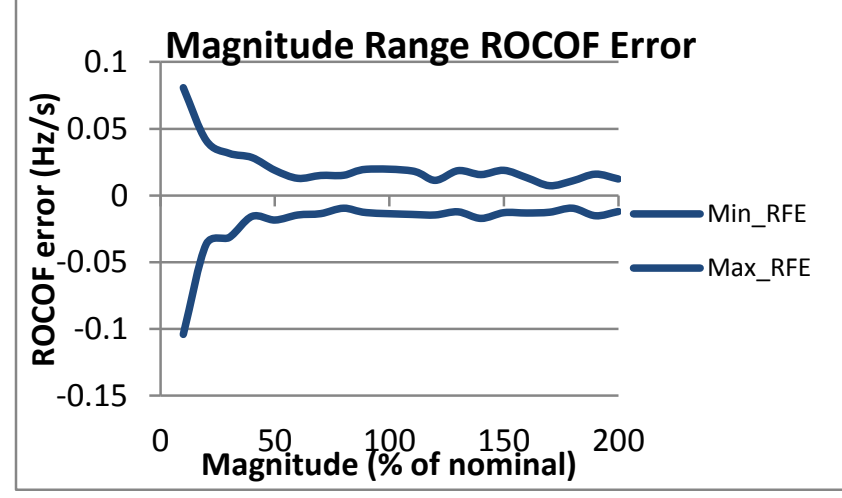

Figure 692: Fs = 15 FPS

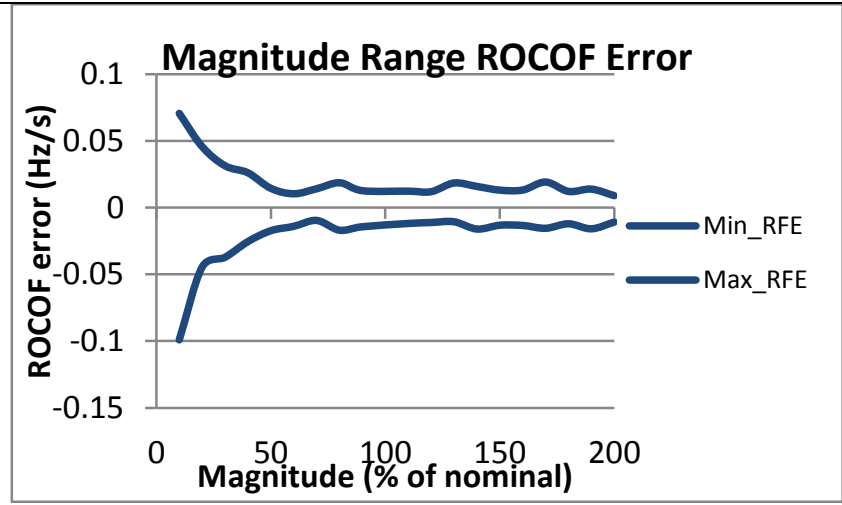

Figure 690: Fs = 30 FPS

MISSING DATA

Figure 693: Fs = 12 FPS

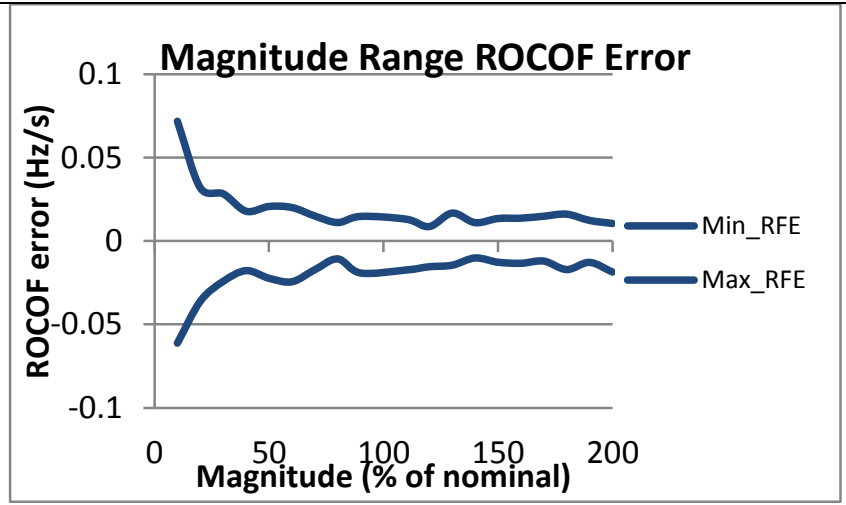

Figure 691: Fs = 20 FPS

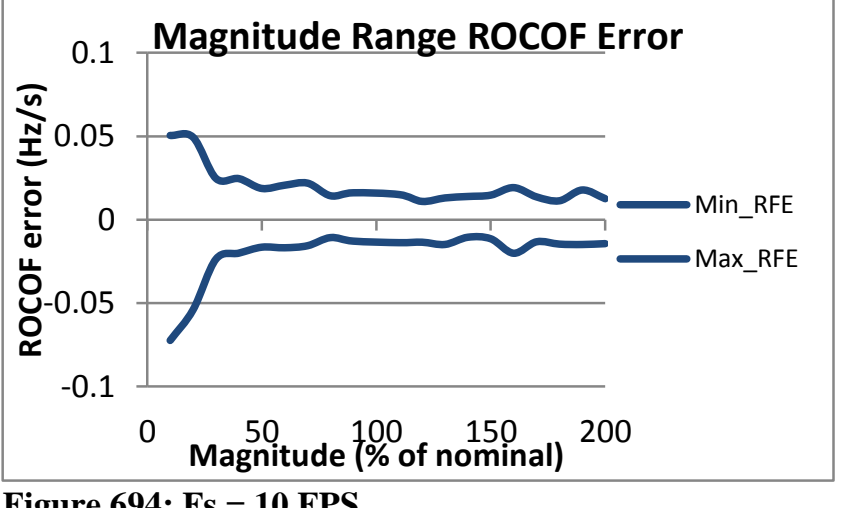

Figure 694: Fs = 10 FPS 
3.4.7 PMU F steady state signal magnitude ROCOF error: M class

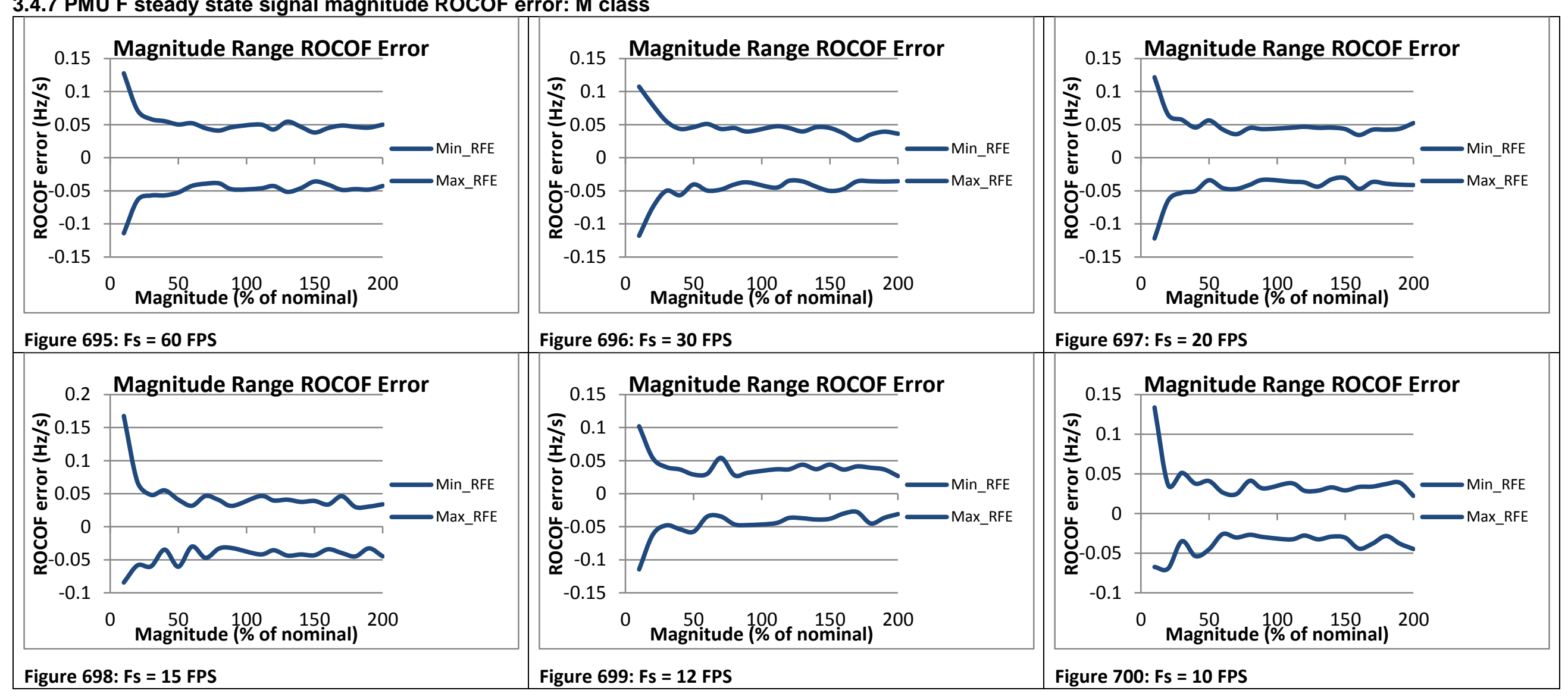


3.4.8 PMU G* steady state signal magnitude ROCOF error: M class

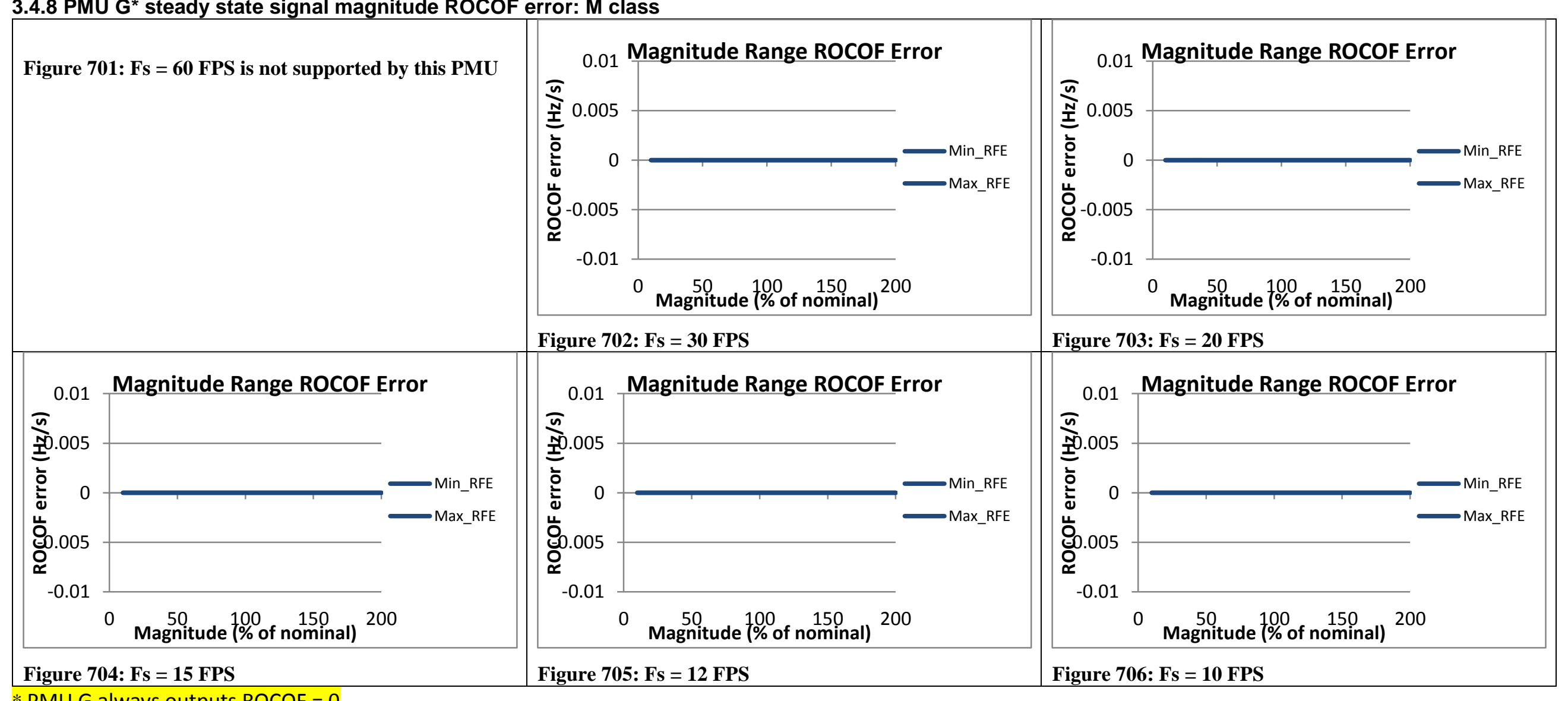

* PMU G always outputs ROCOF $=0$ 
3.4.9 PMU H steady state signal magnitude ROCOF error: M class

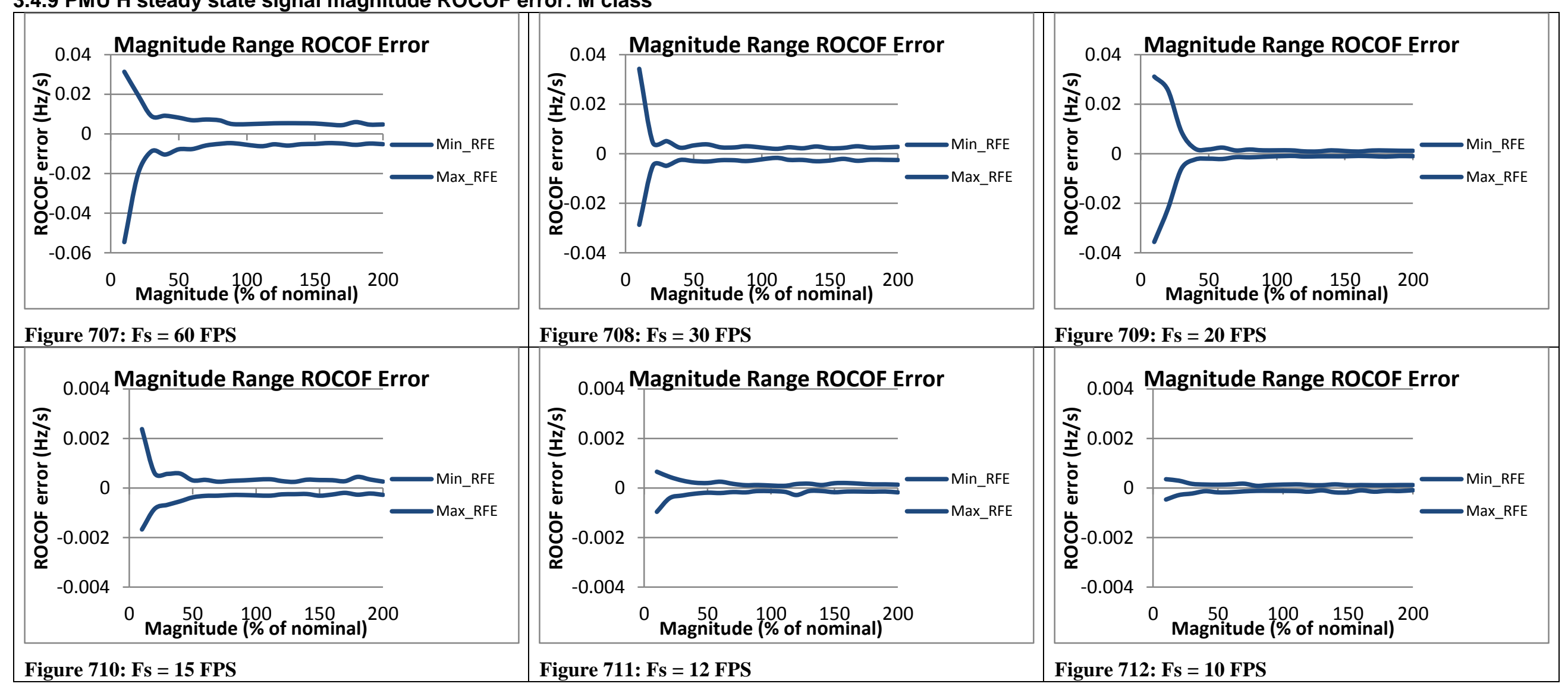


3.4.10 PMU I steady state signal magnitude ROCOF error: M class

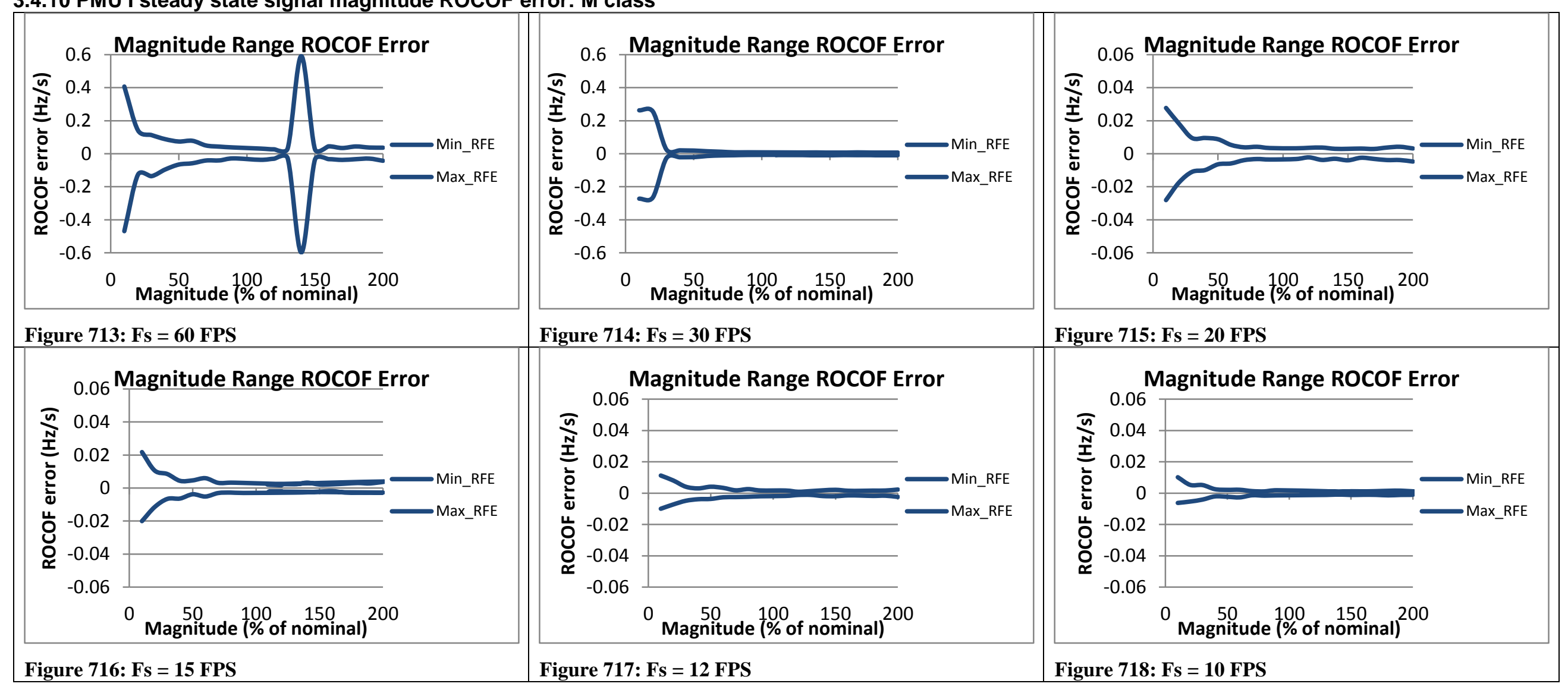


3.4.11 PMU I steady state signal magnitude ROCOF error: M class

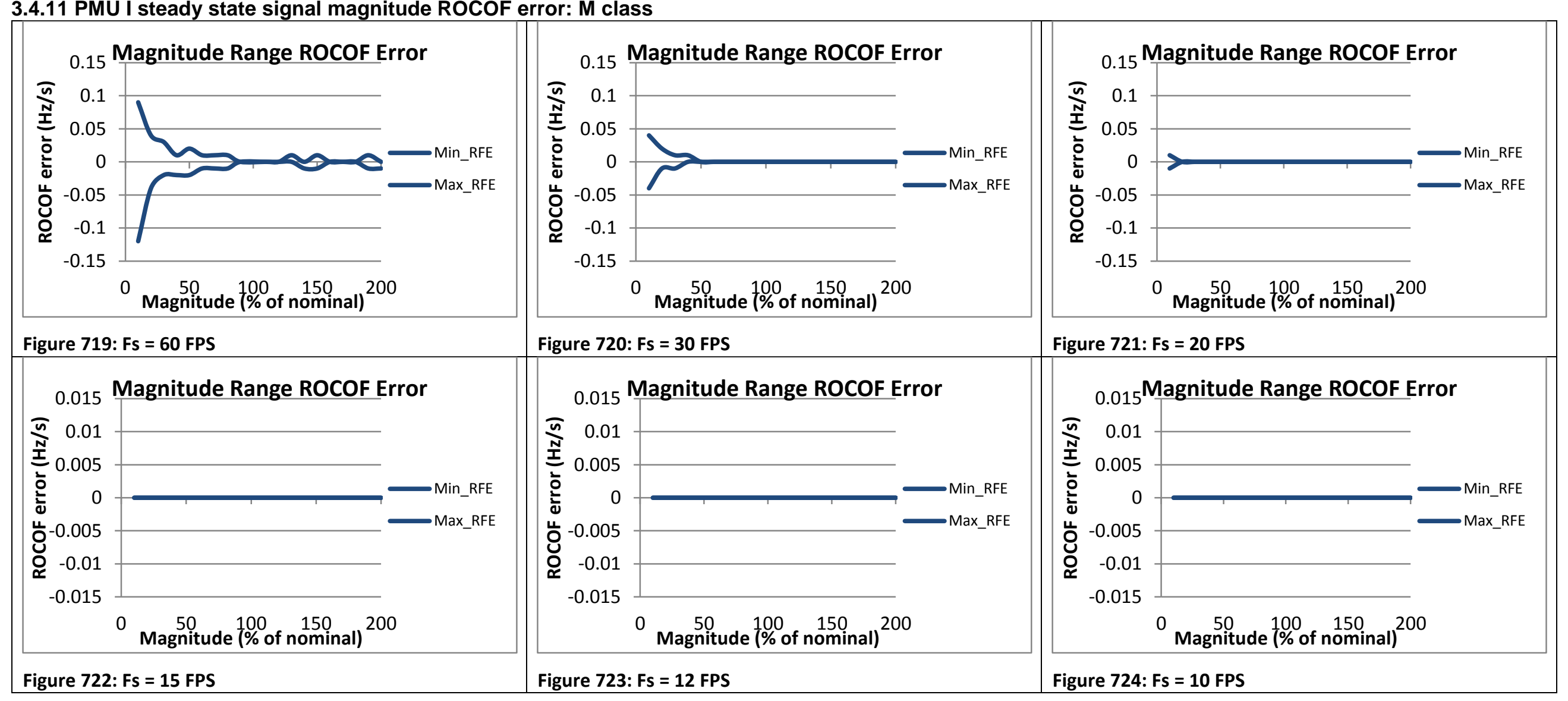




\subsection{Steady state signal magnitude voltage TVE: P class}

\subsubsection{C37.118.1 Annex C steady state signal magnitude voltage TVE: P class}

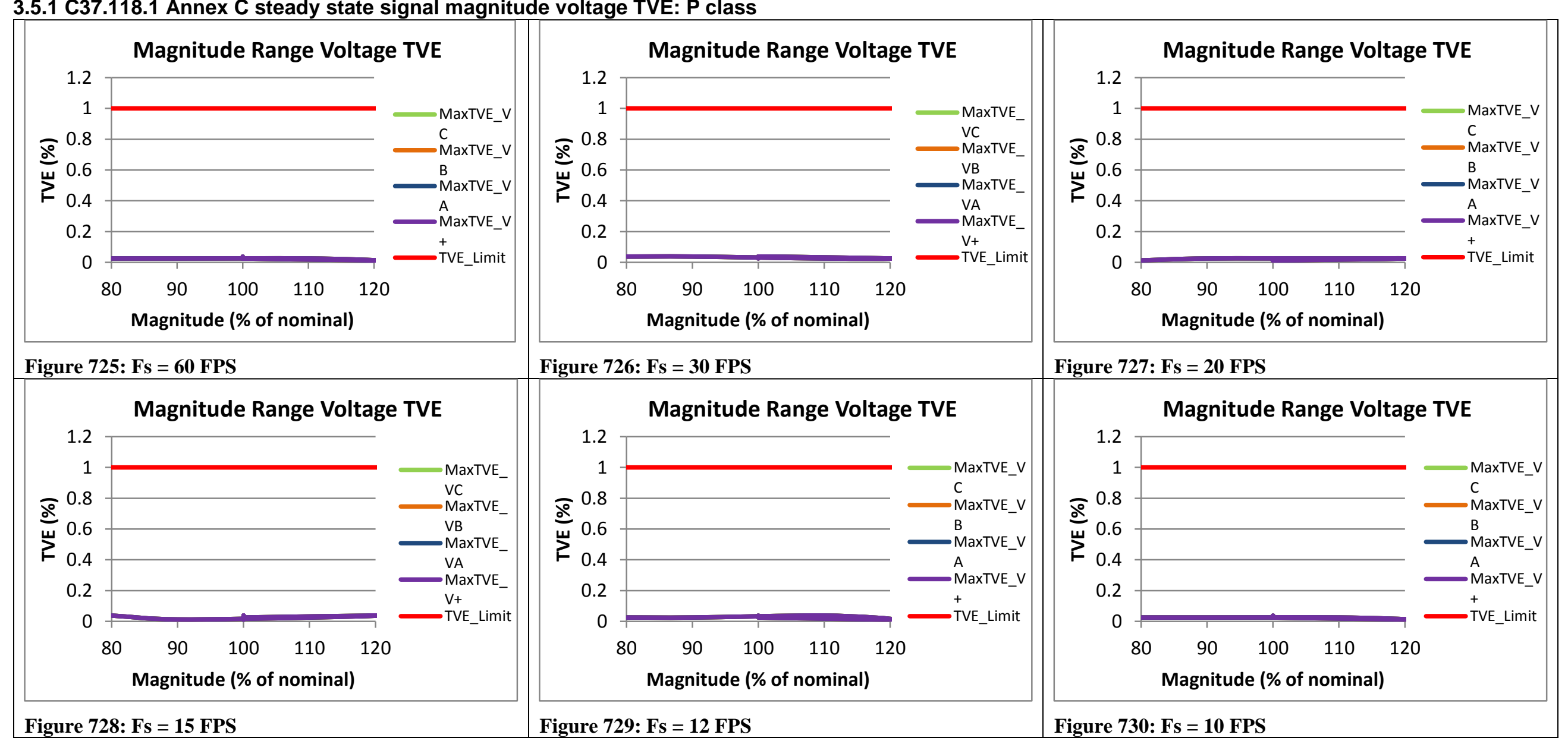


3.5.2 PMU A steady state signal magnitude voltage TVE:, P class

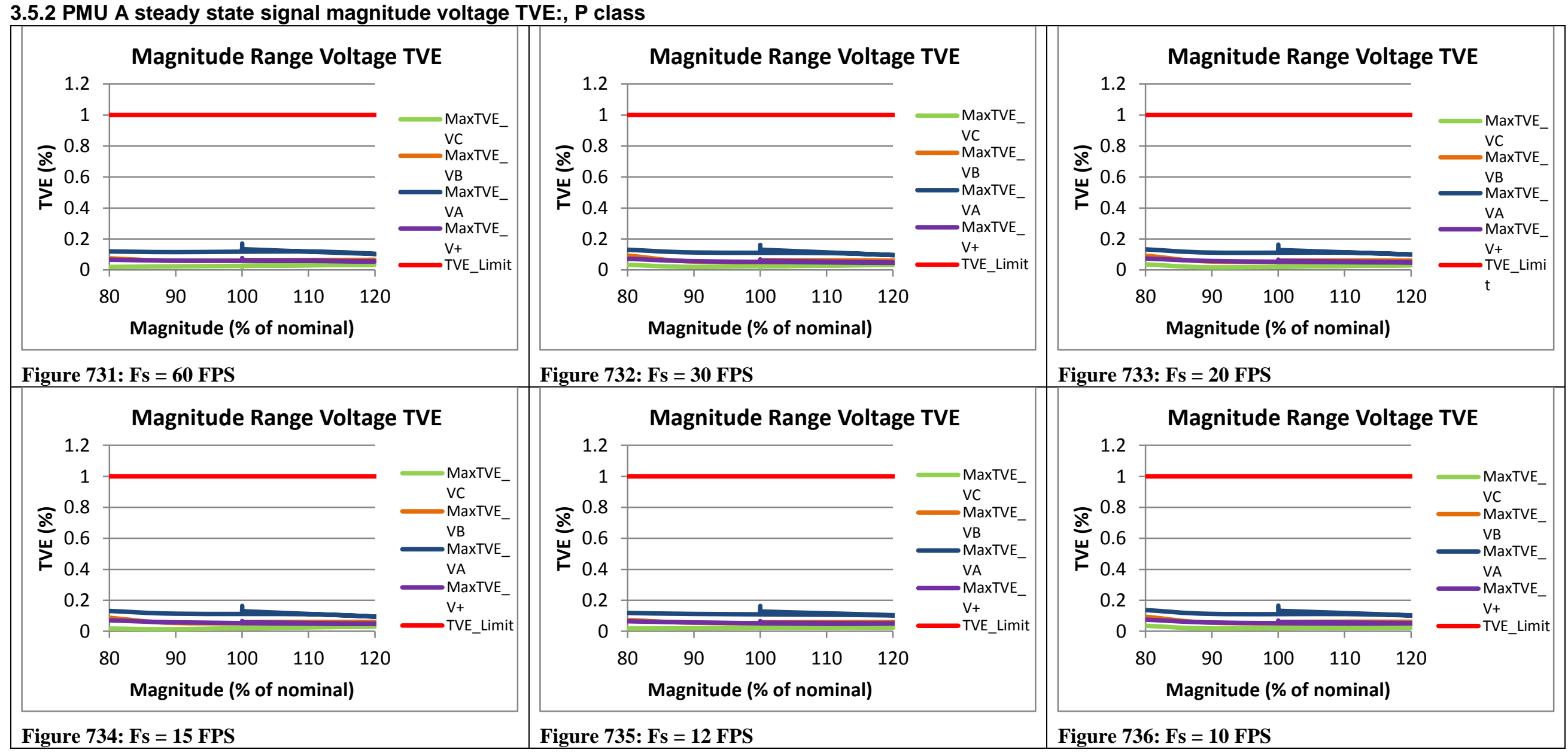


3.5.3 PMU B steady state signal magnitude voltage TVE: P class

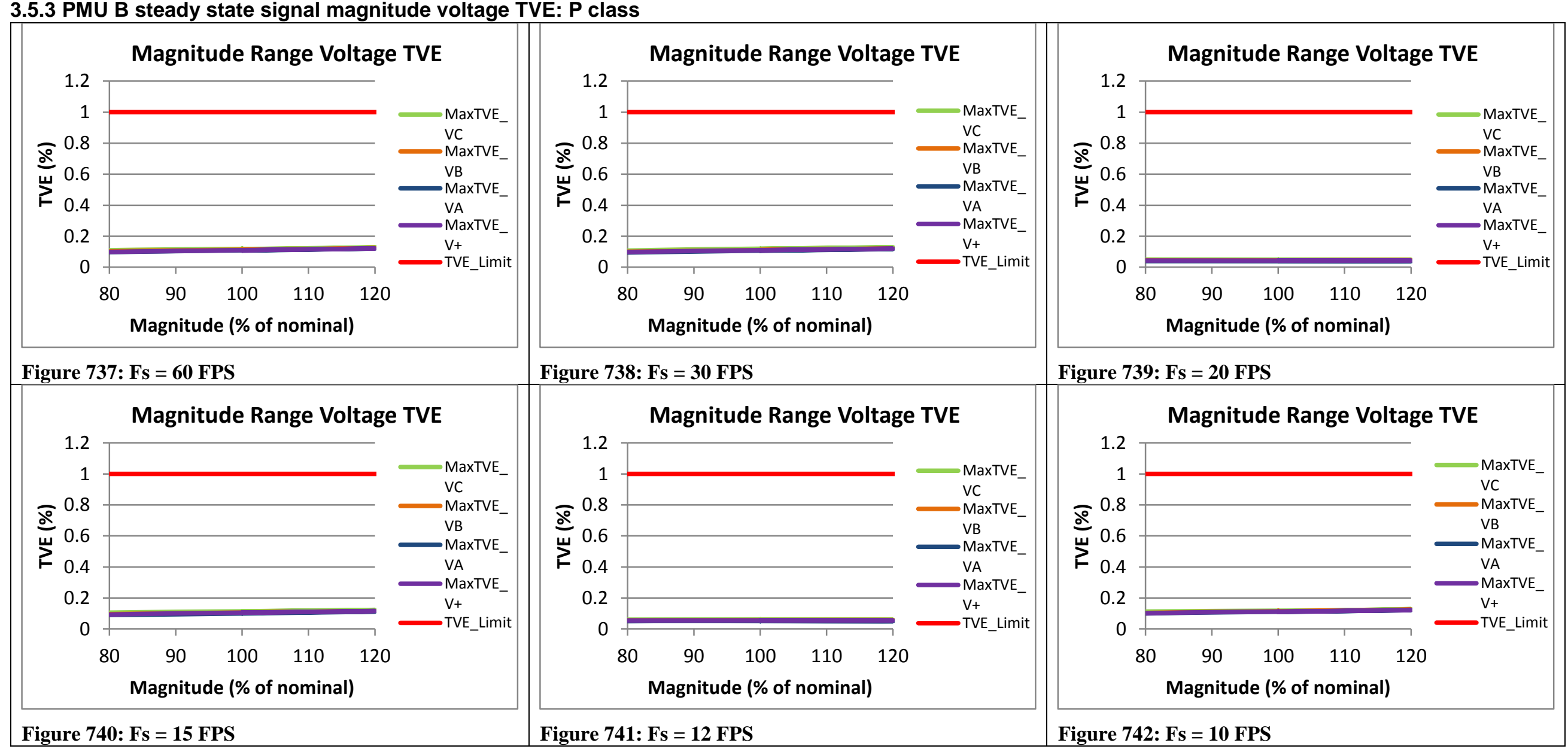


3.5.4 PMU C steady state signal magnitude voltage TVE: P class

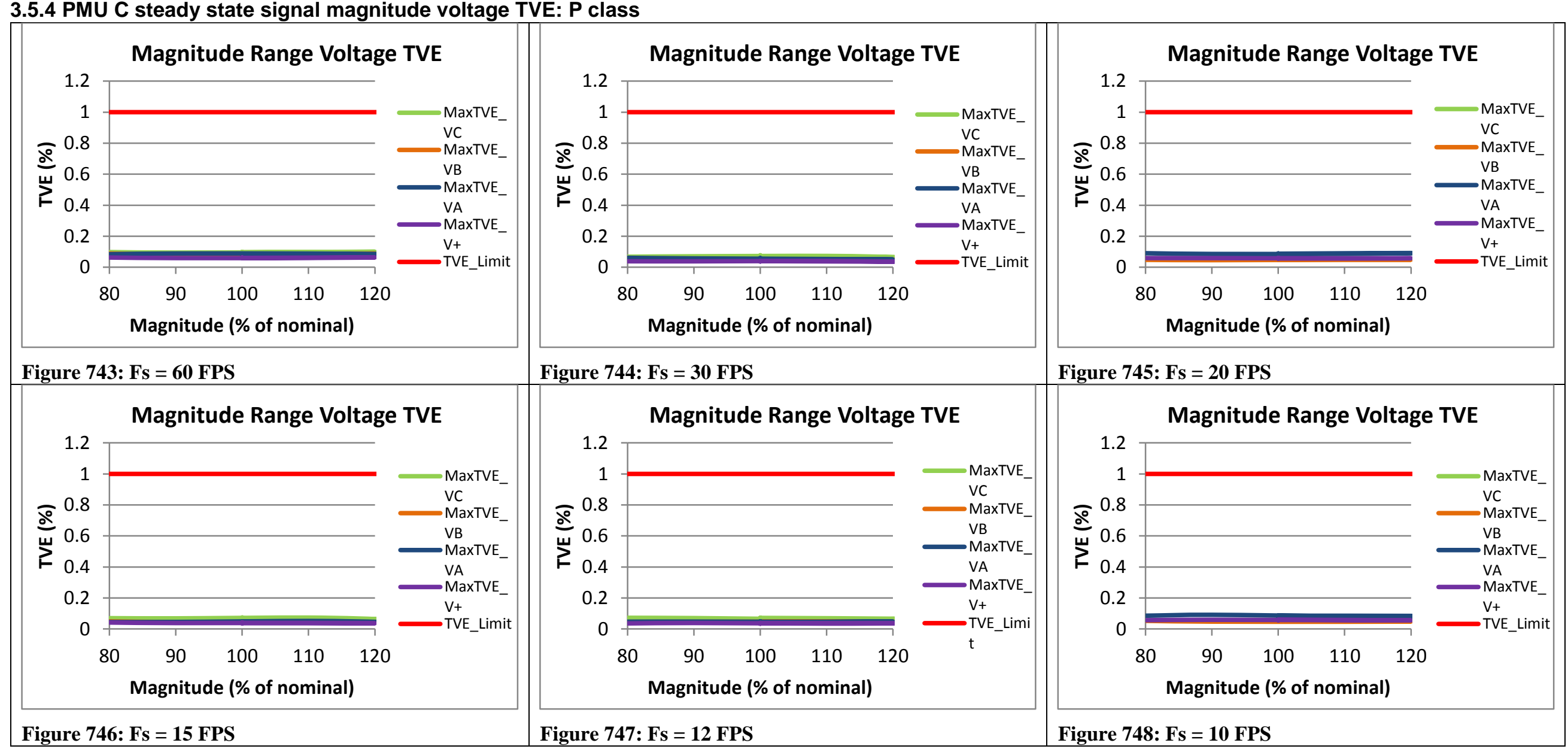


3.5.5 PMU D steady state signal magnitude voltage TVE: P class

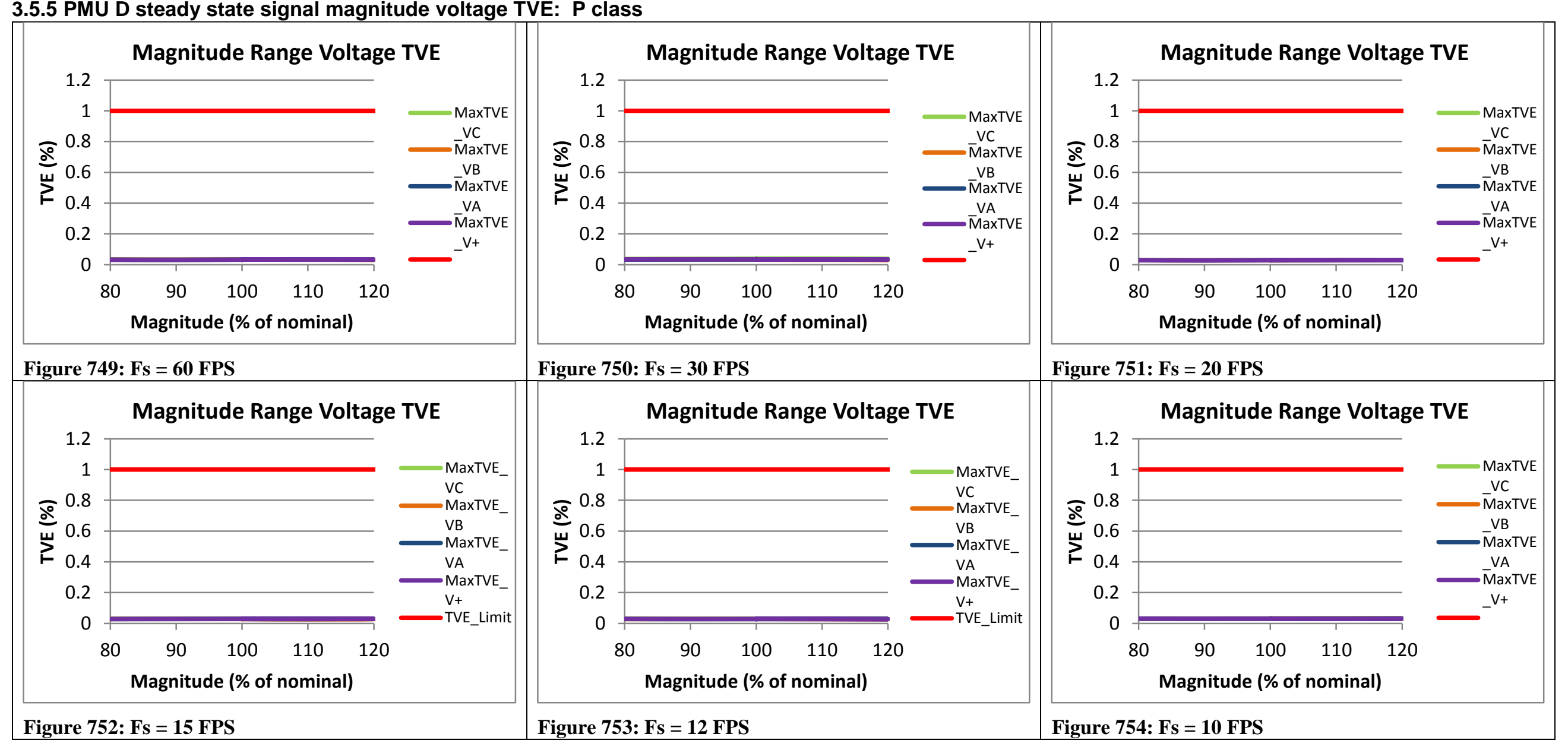


3.5.7 PMU F steady state signal magnitude voltage TVE P class

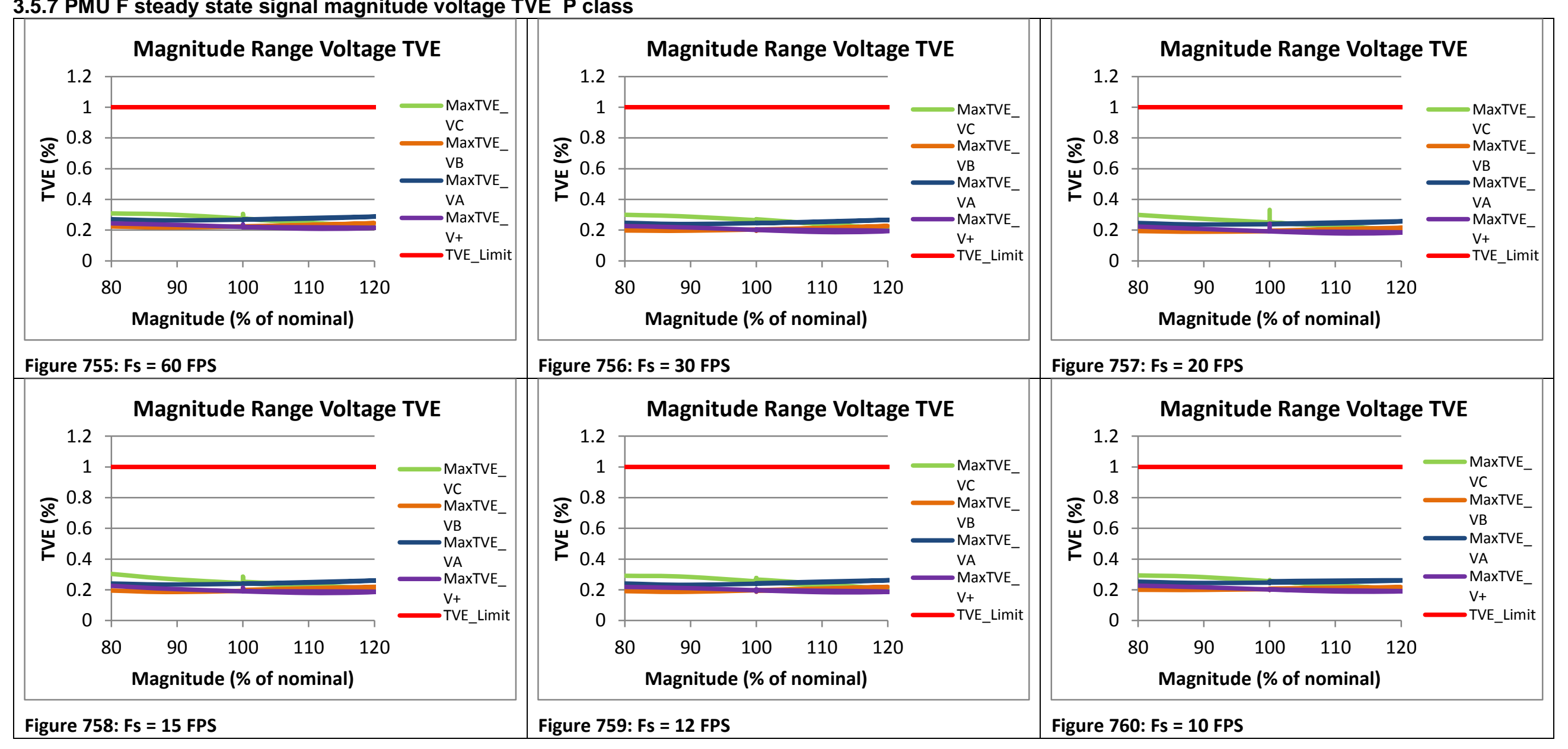

3.5.8 PMU G steady state signal magnitude voltage TVE: P class

PMU G does not support P class 


\subsubsection{PMU H steady state signal magnitude voltage TVE: P class}

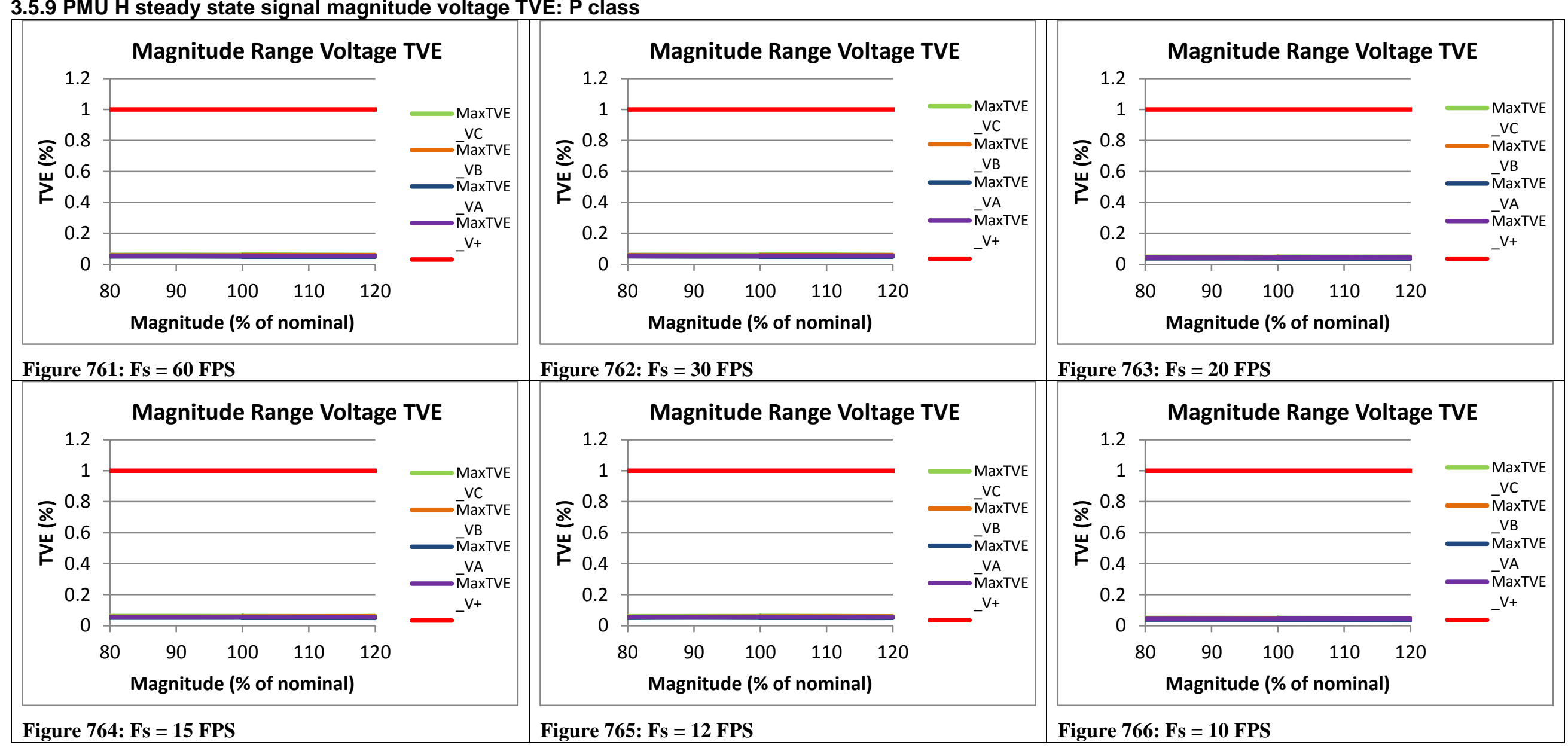

3.5.10 PMU I steady state signal magnitude voltage TVE: P class

PMU I does not support P Class 
3.5.11 PMU J steady state signal magnitude voltage TVE: P class

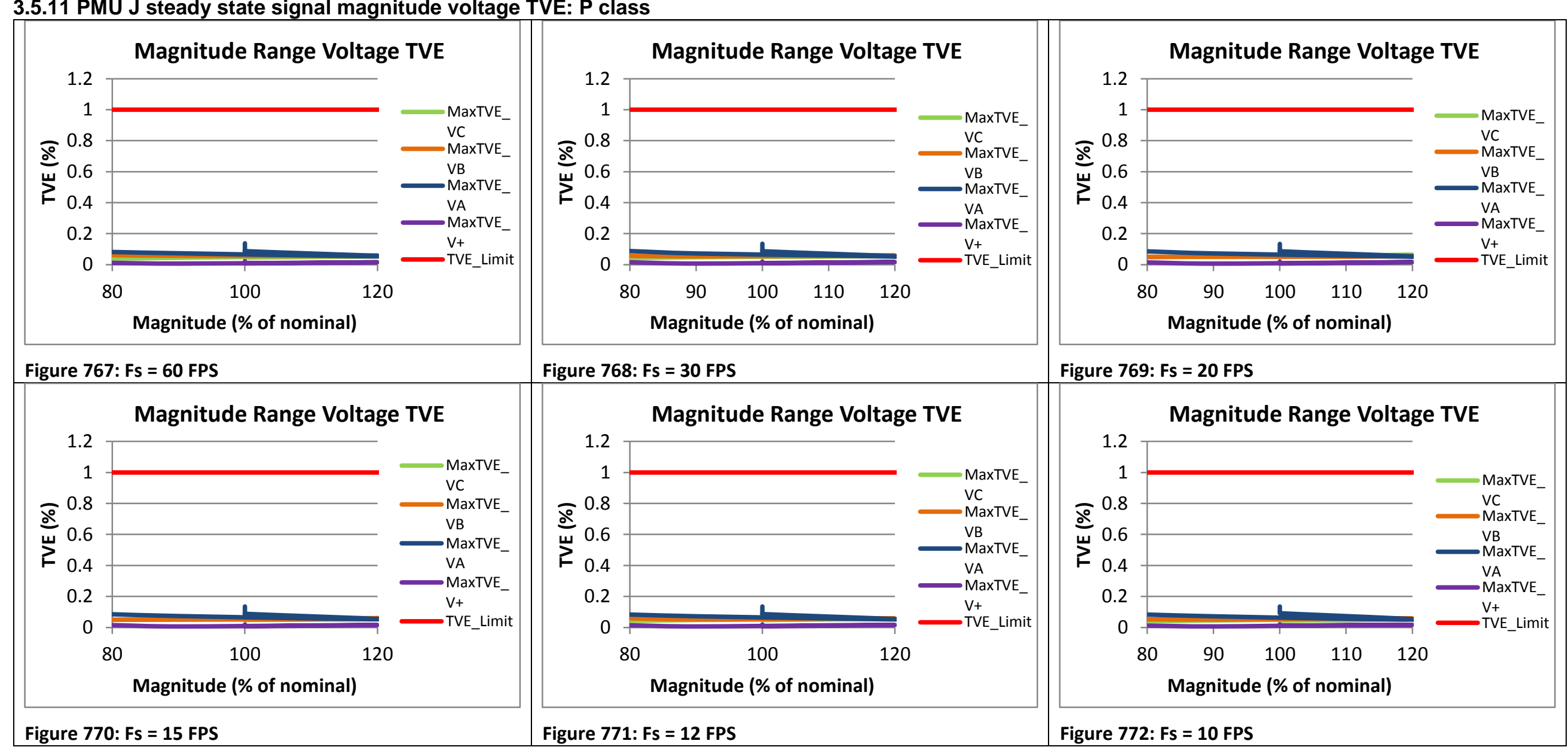




\subsection{Steady state signal magnitude current TVE: P class}

\subsubsection{C37.118.1 Annex C steady state signal magnitude current TVE: P class}

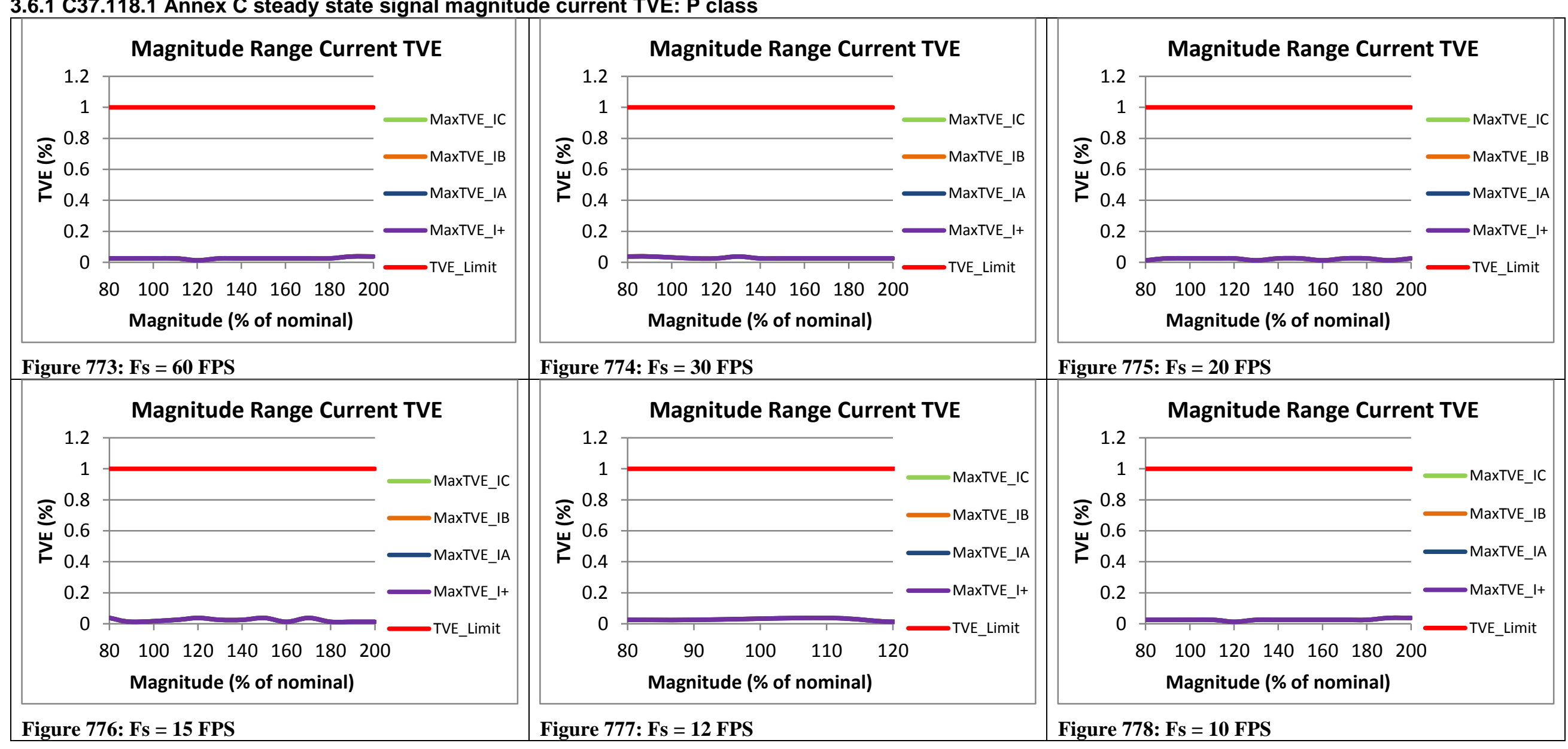


3.6.2 PMU A steady state signal magnitude current TVE: P class

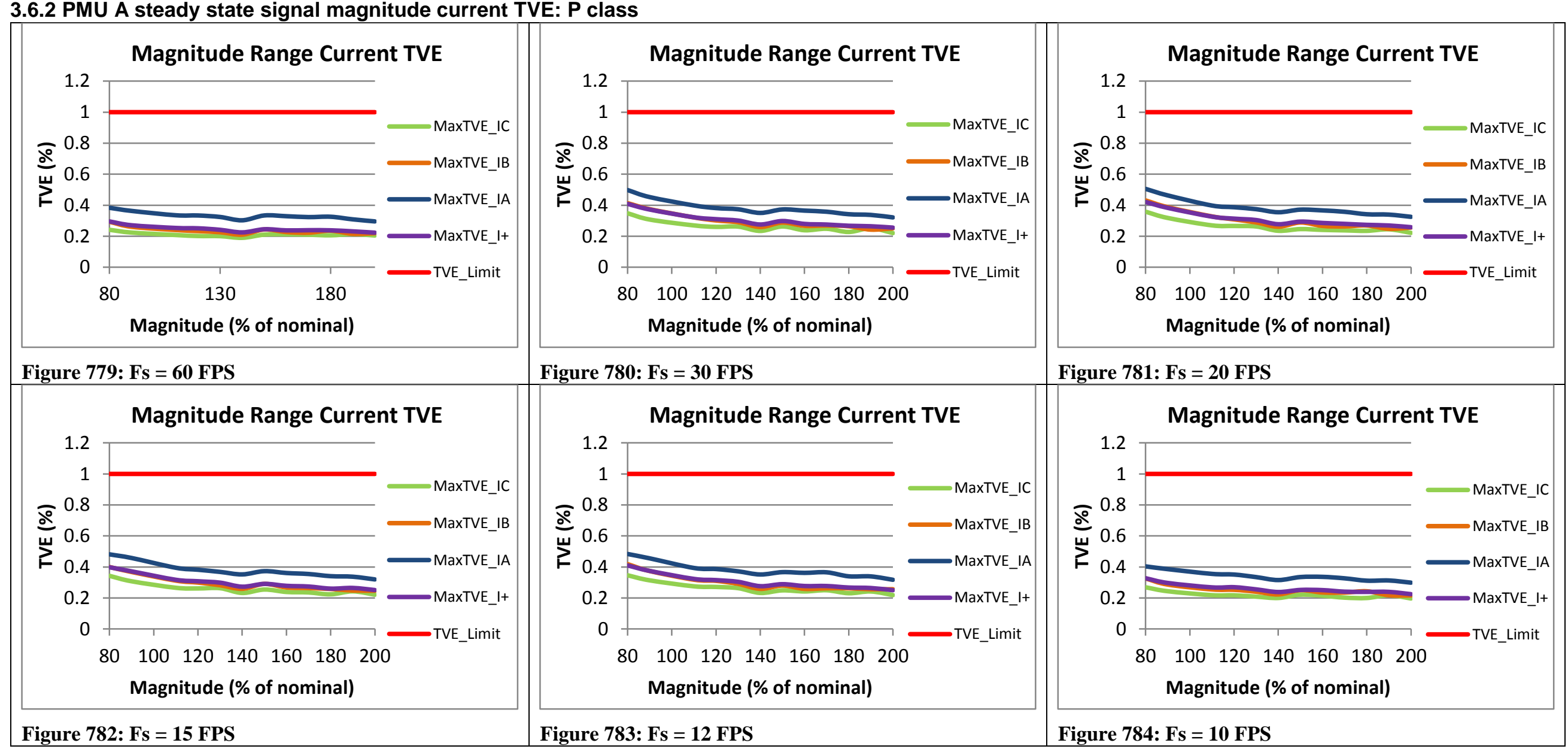


3.6.3 PMU B steady state signal magnitude current TVE: P class

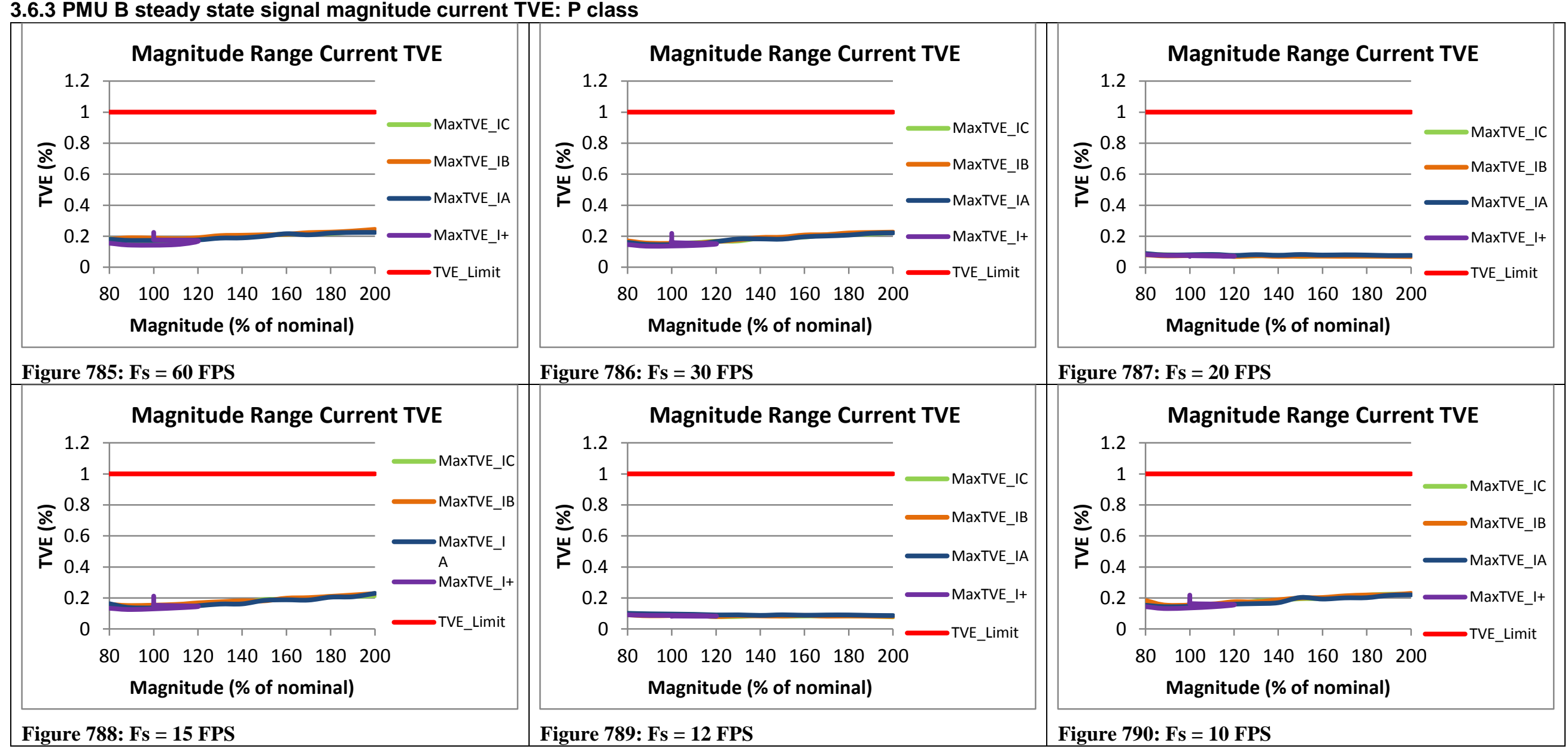


3.6.4 PMU C steady state signal magnitude current TVE: P class

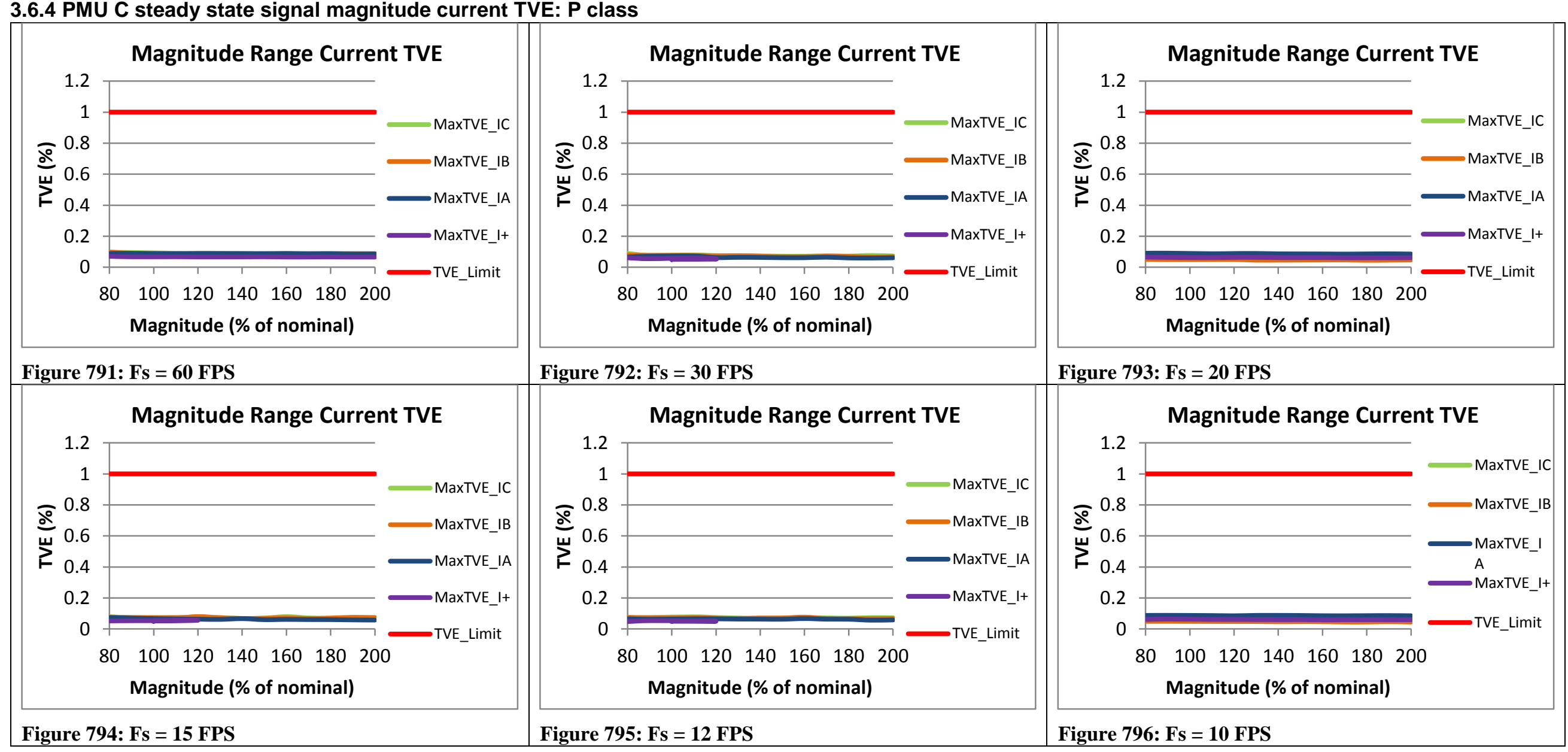


3.6.5 PMU D steady state signal magnitude current TVE: P class

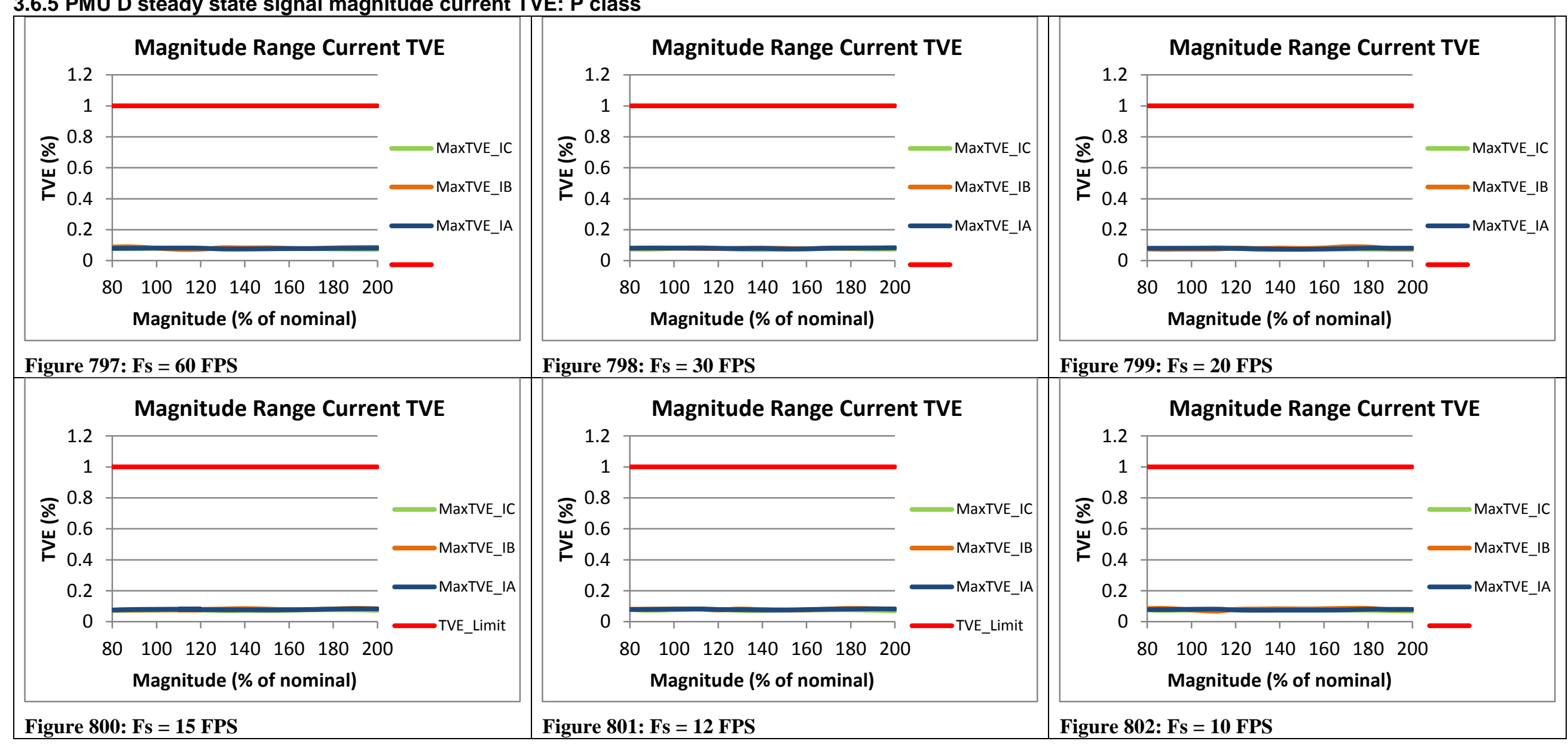

3.6.6 PMU E steady state signal magnitude current TVE: P class

PMU E does not support P class 
3.6.7 PMU F steady state signal magnitude current TVE: P class

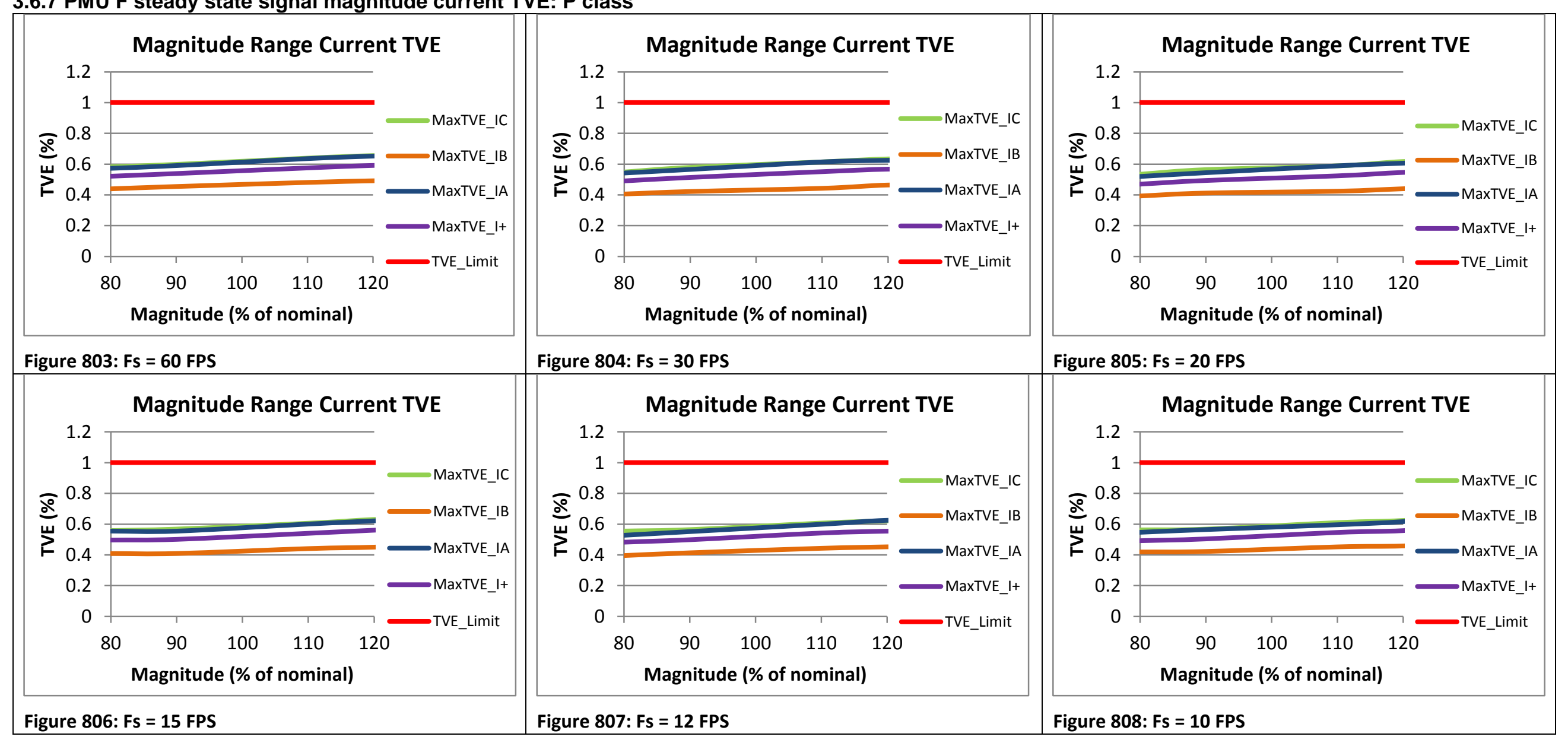

3.6.8 PMU G steady state signal magnitude current TVE: P class

PMU G does not support P class 
3.6.9 PMU H steady state signal magnitude current TVE: P class

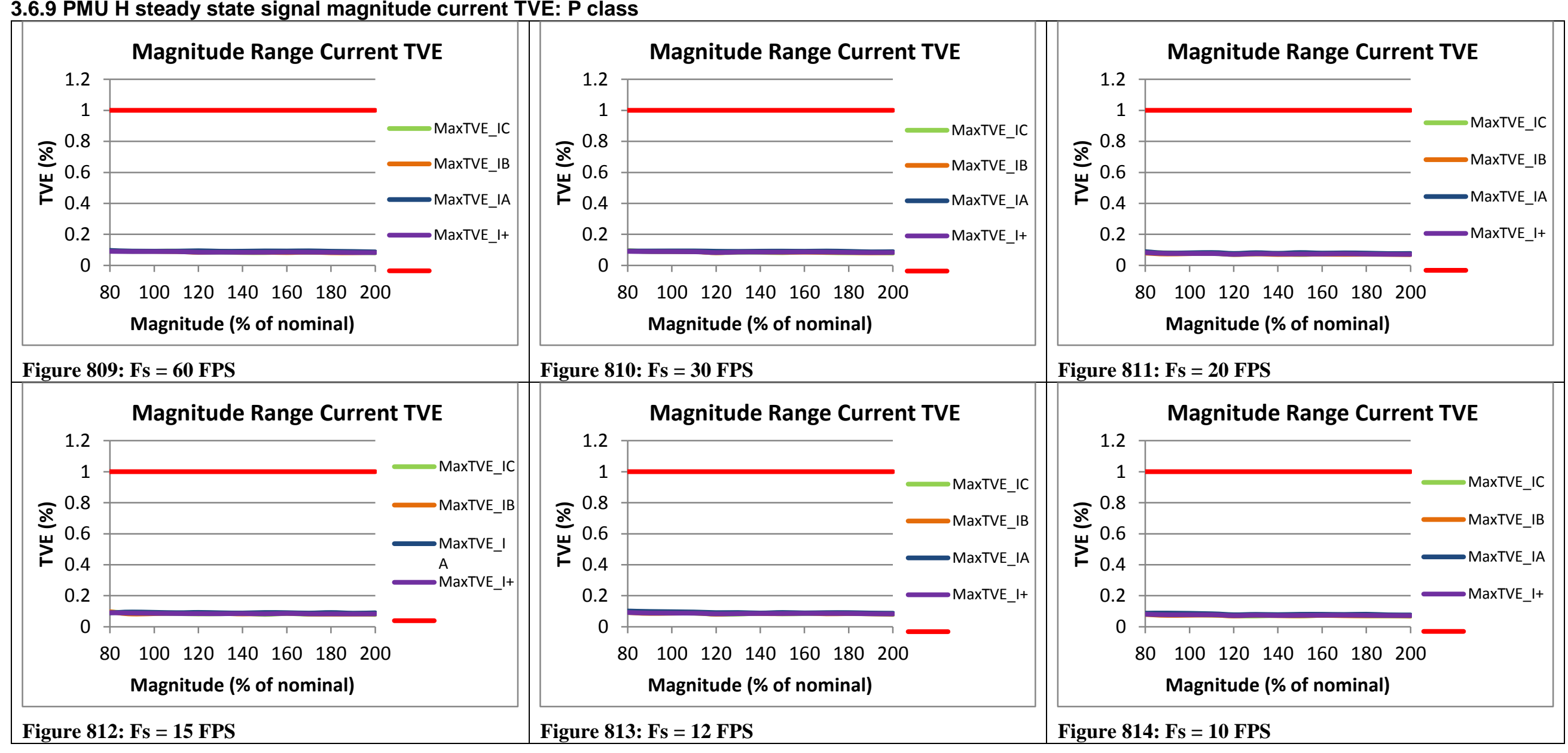

3.6.10 PMU I steady state signal magnitude current TVE: P class

PMU I does not support P class 
3.6.11 PMU J steady state signal magnitude current TVE: P class

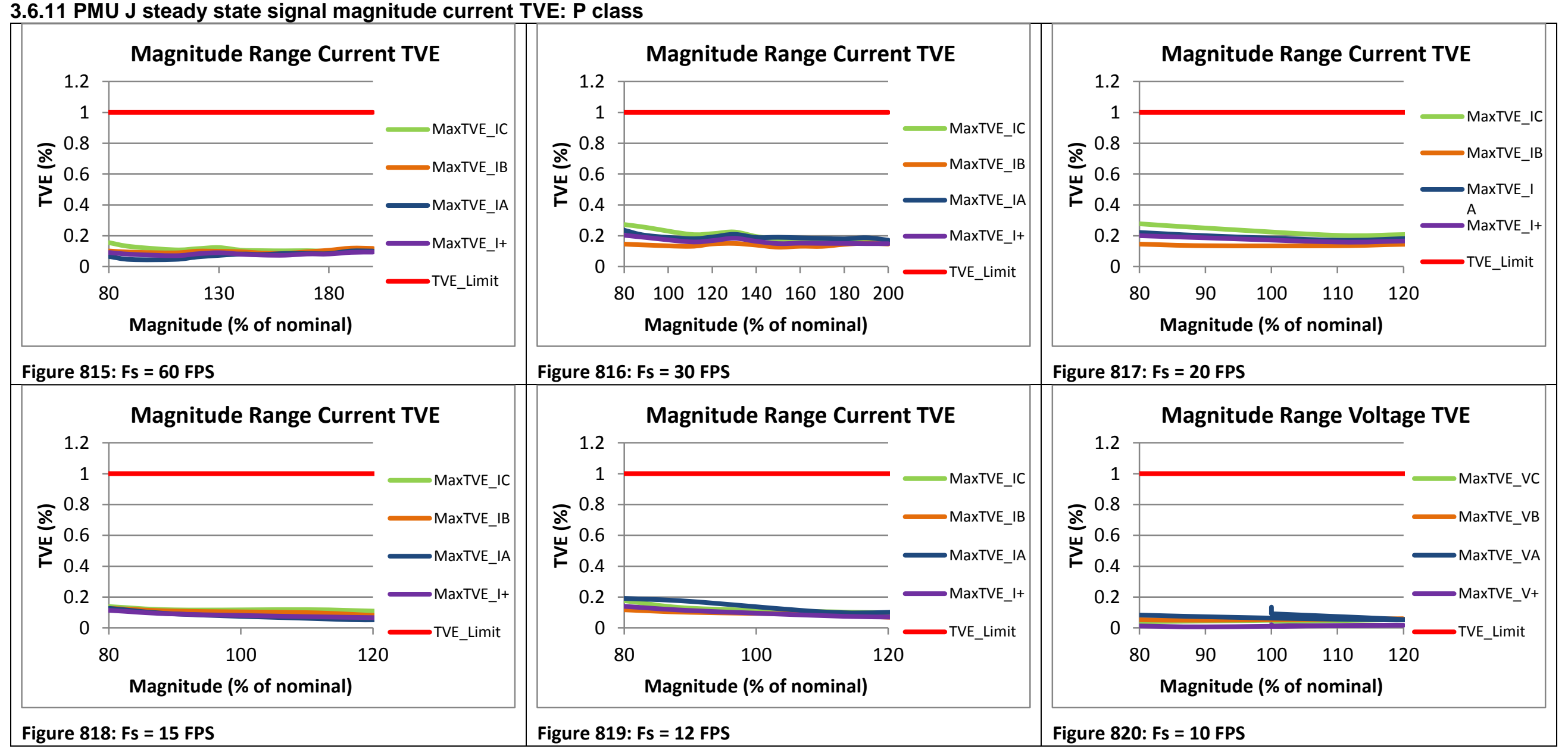




\subsection{Steady state signal magnitude frequency error: $P$ class}

No performance limits are shown in the plots below because the steady state magnitude range tests do not require frequency error to meet performance limits

\subsubsection{C37.118.1 Annex C steady state signal magnitude frequency error:, F0 $=60 \mathrm{~Hz}, \mathrm{P}$ class}

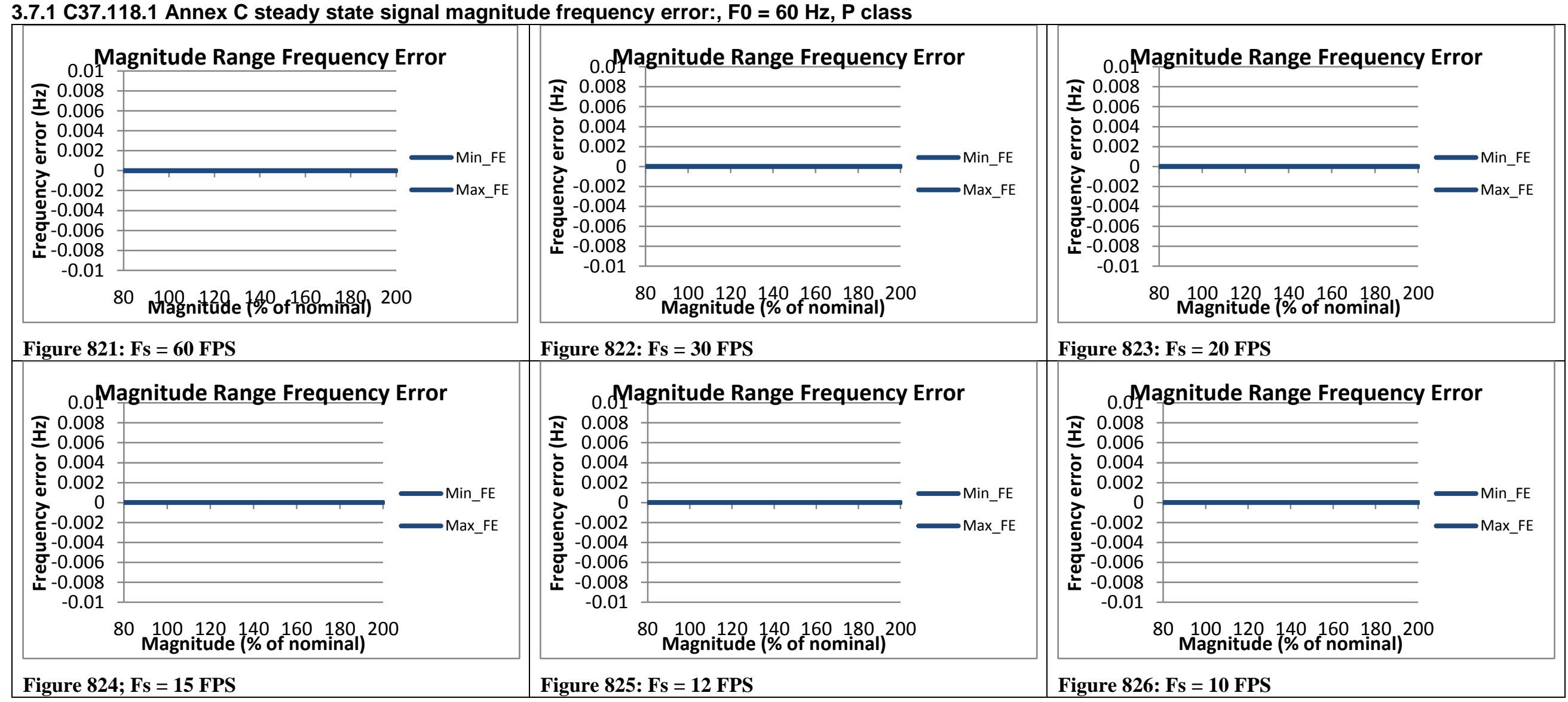


3.7.2 PMU A steady state signal magnitude frequency error: $P$ class

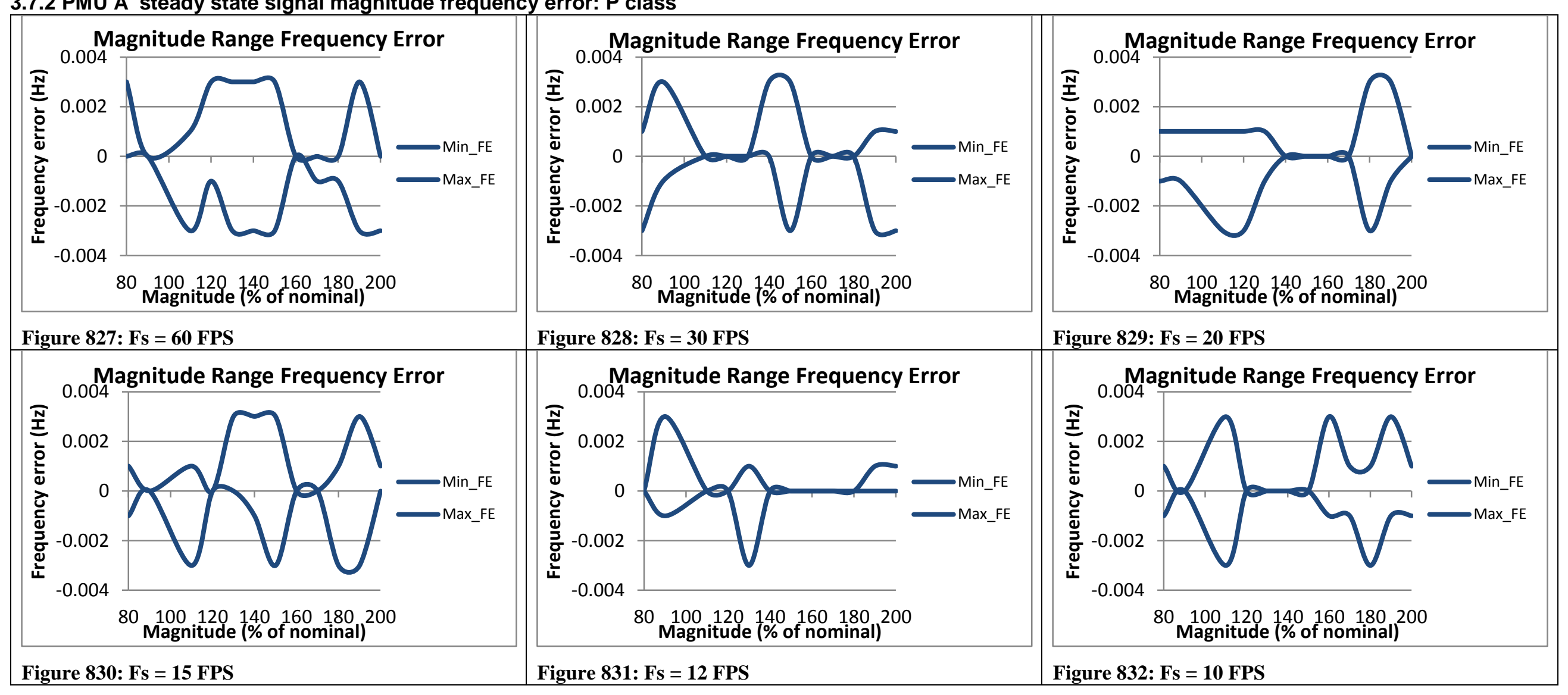


3.7.3 PMU B steady state signal magnitude frequency error: $P$ class

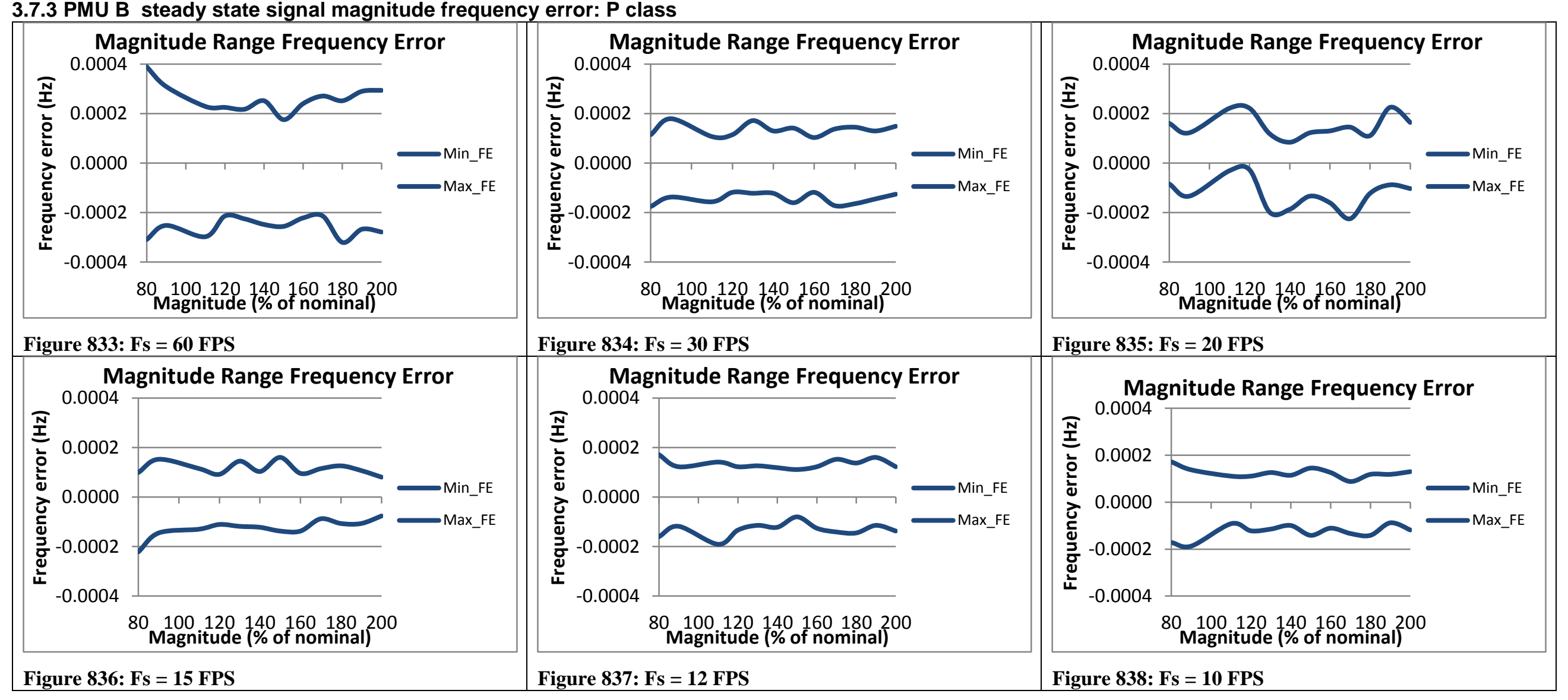


3.7.4 PMU C steady state signal magnitude frequency error: $P$ class

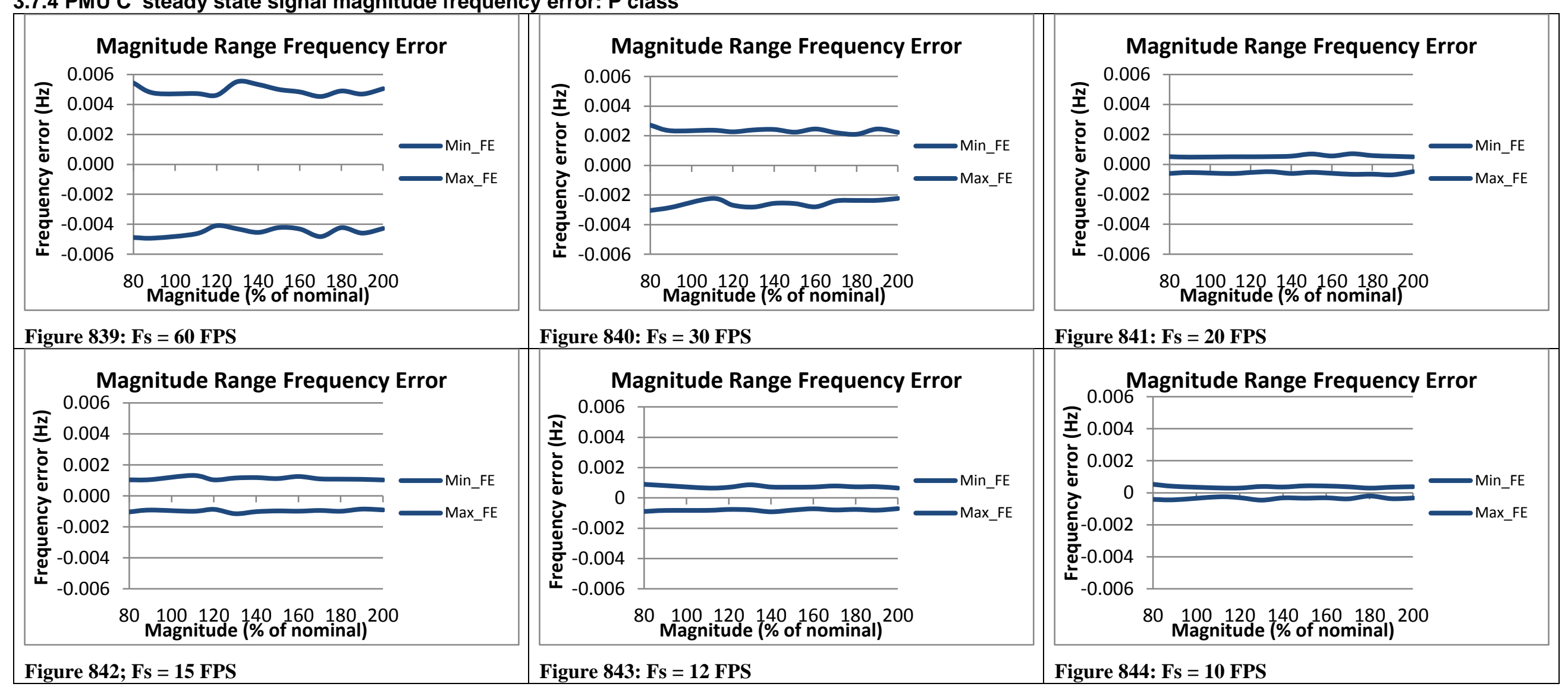


3.7.5 PMU D steady state signal magnitude frequency error: $P$ class

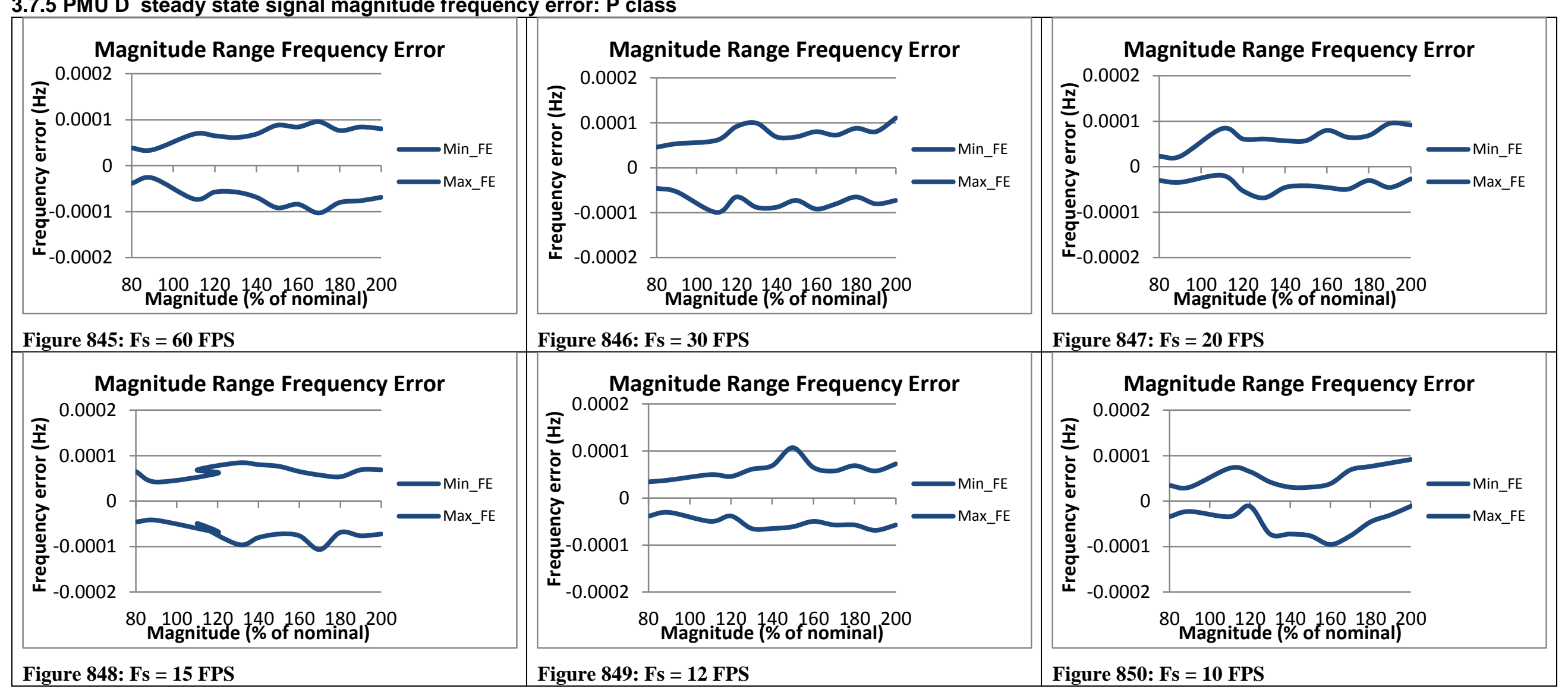

3.7.6 PMU E steady state signal magnitude frequency error: P class

PMU E does not support P class 
3.7.7 PMU F steady state signal magnitude frequency error: $P$ class

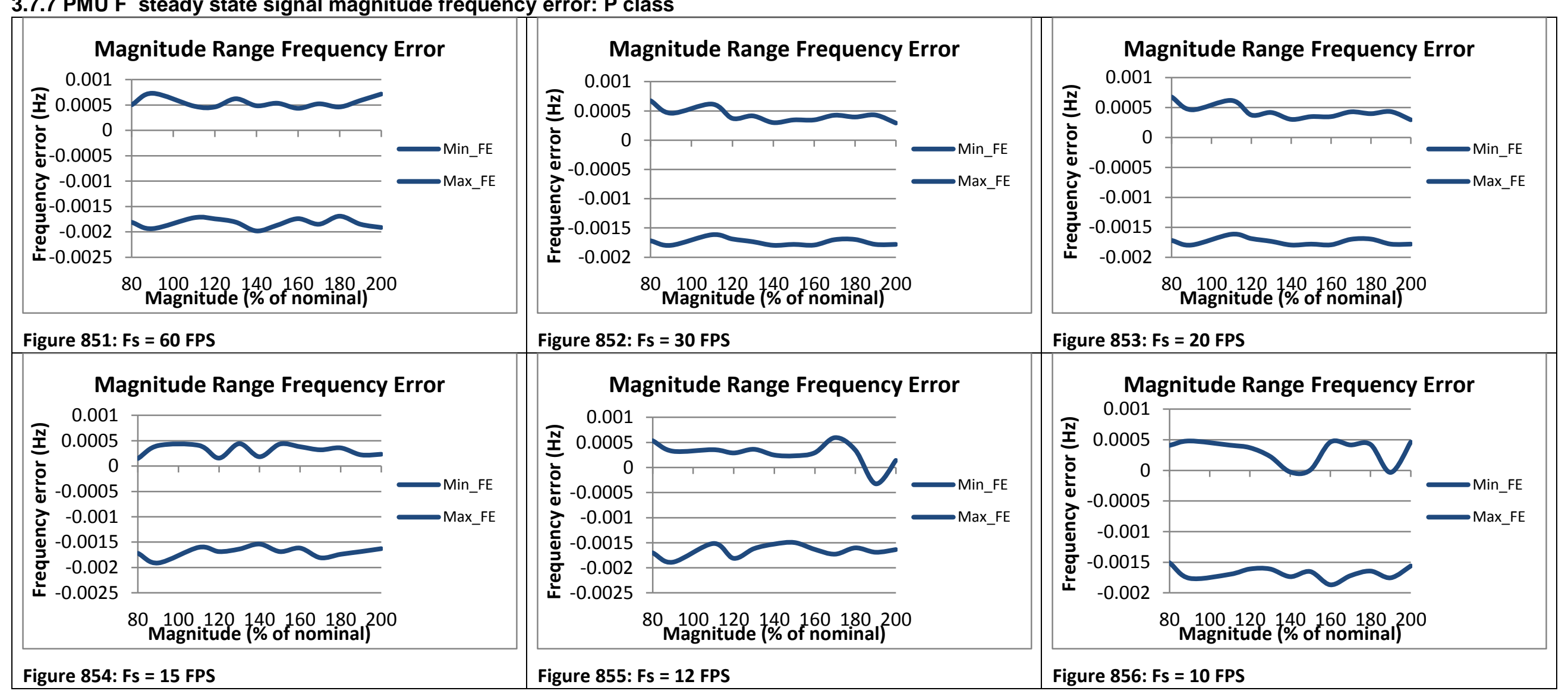

3.7.8 PMU G steady state signal magnitude frequency error: $P$ class

PMU G does not support P class 


\subsubsection{PMU H steady state signal magnitude frequency error: P class}

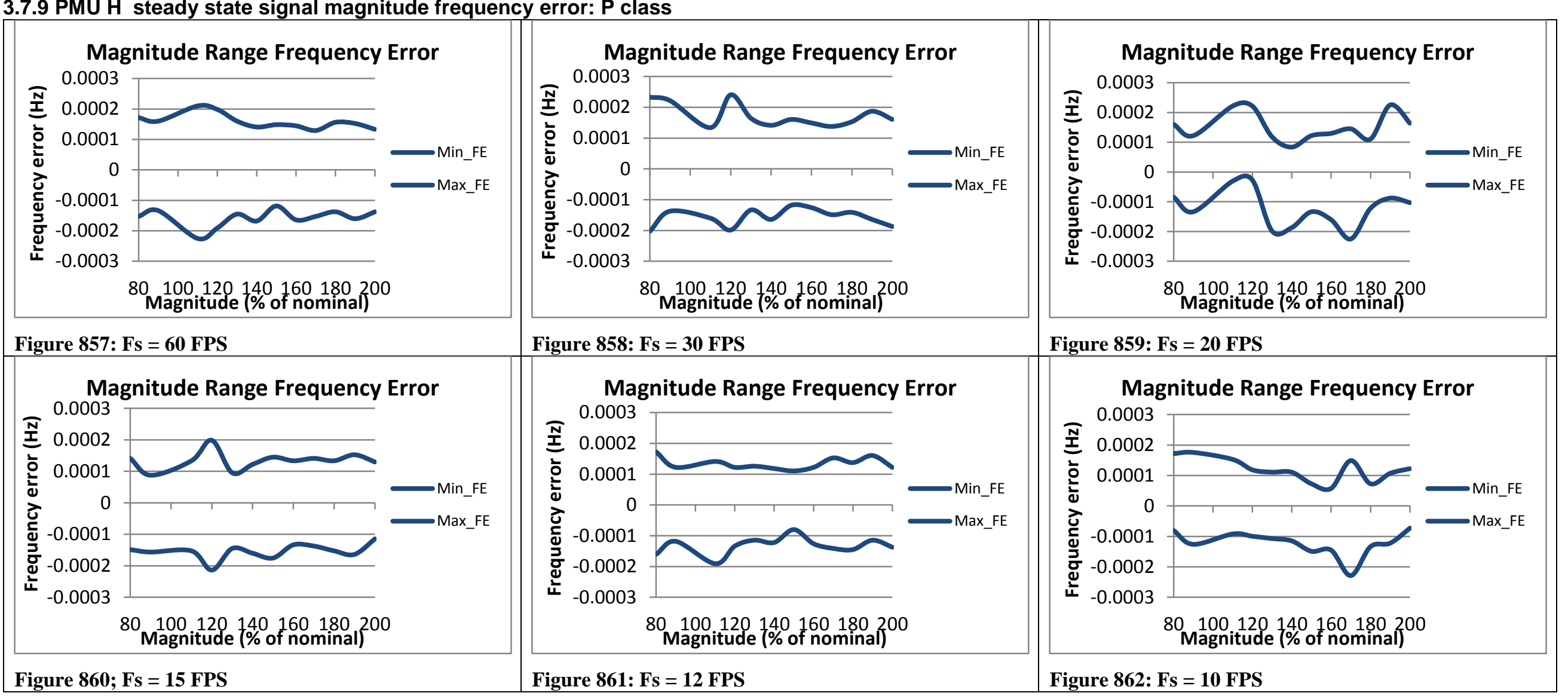

3.7.10 PMU I steady state signal magnitude frequency error: P class

PMU I does not support P class 


\subsubsection{PMU I steady state signal magnitude frequency error: $P$ class}

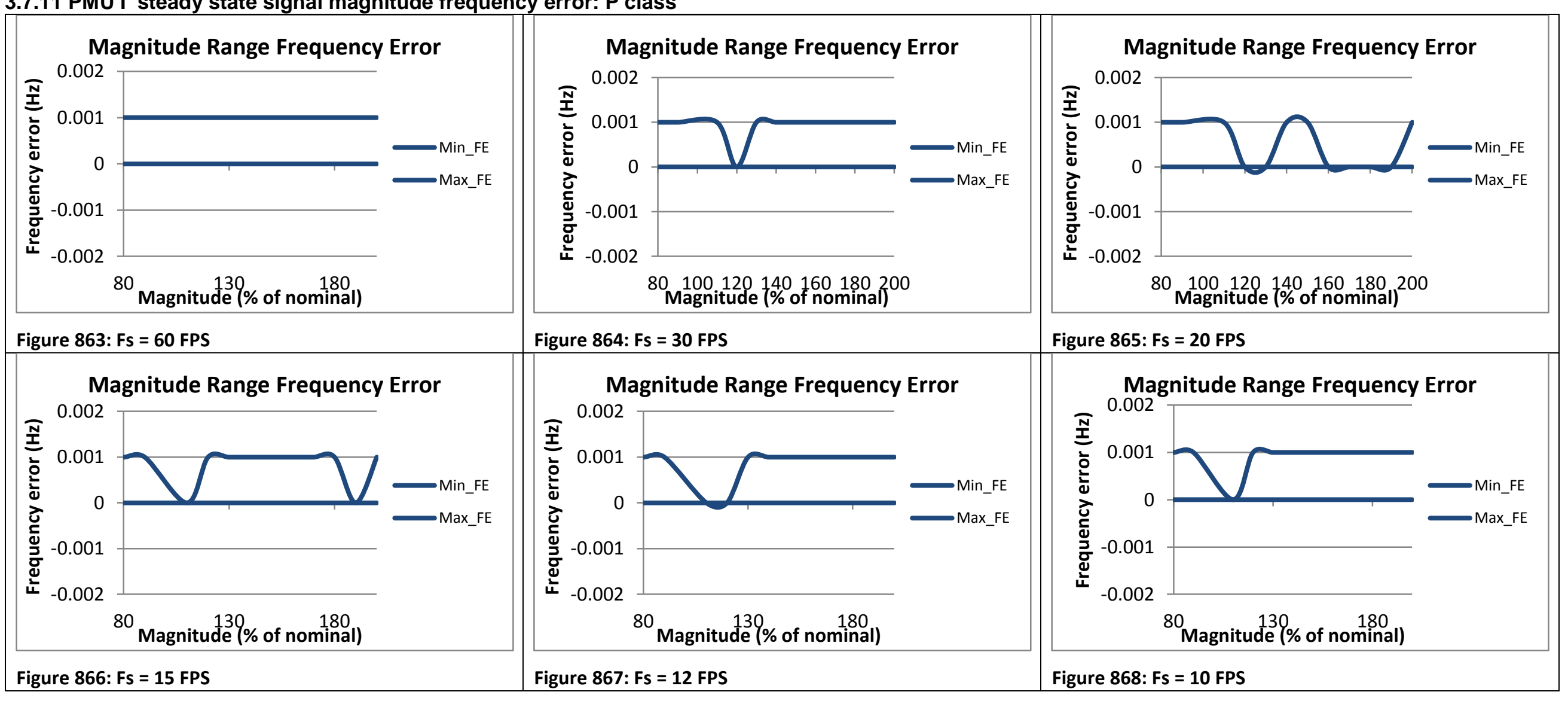




\subsection{Steady state signal magnitude ROCOF error: P class}

No performance limits are shown in the plots below because the steady state magnitude range tests do not require frequency error to meet performance limits

3.8.1 C37.118.1 Annex C steady state signal magnitude ROCOF error:, F0 $=60 \mathrm{~Hz}$, P class

\begin{tabular}{|c|c|c|}
\hline 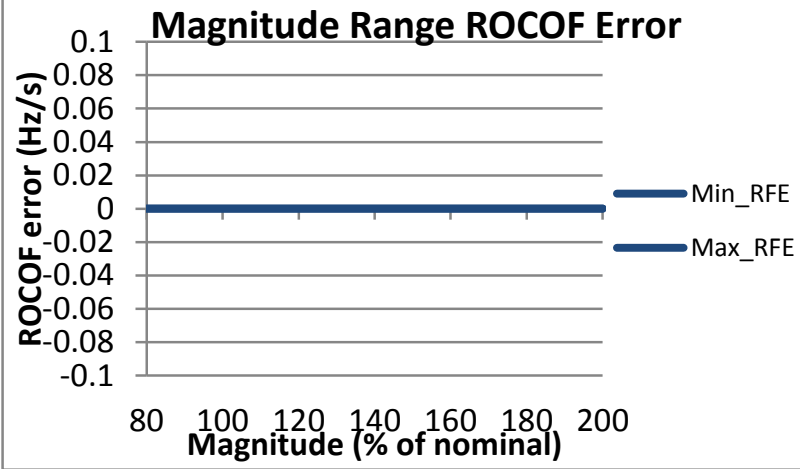 & 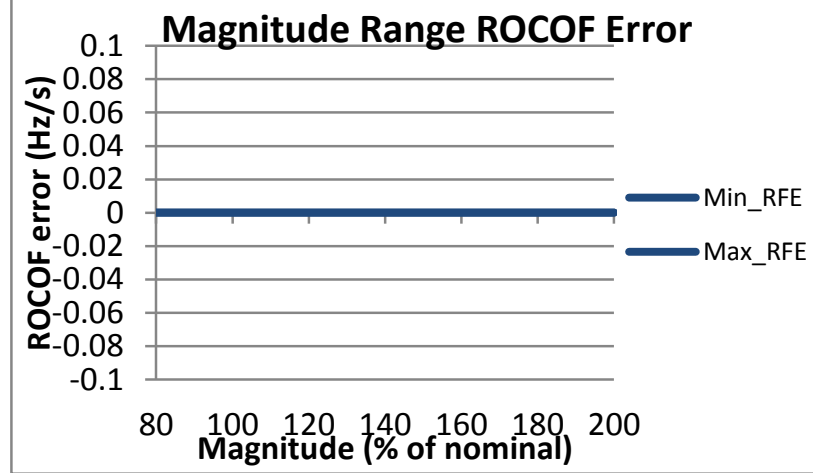 & 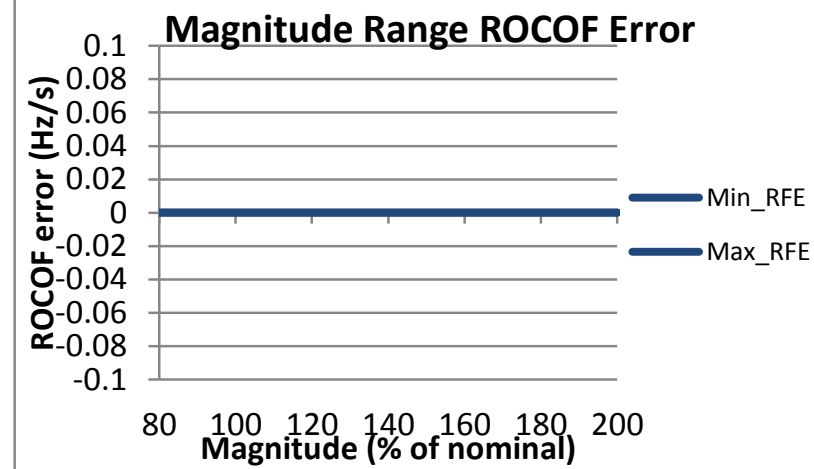 \\
\hline Figure 869: Fs $=60 \mathrm{FPS}$ & Figure 870: $\mathrm{Fs}=30 \mathrm{FPS}$ & Figure 871: Fs = 20 FPS \\
\hline 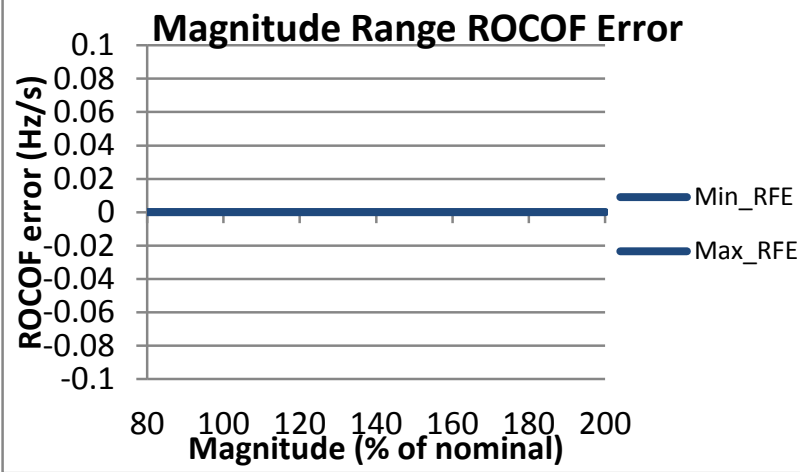 & 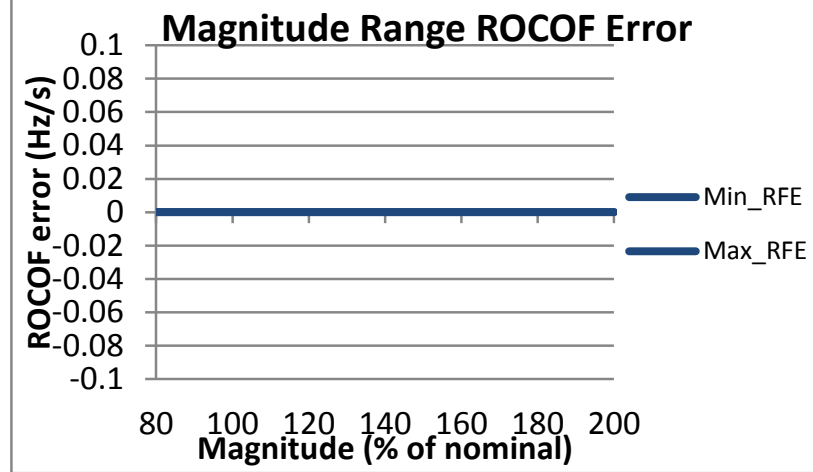 & 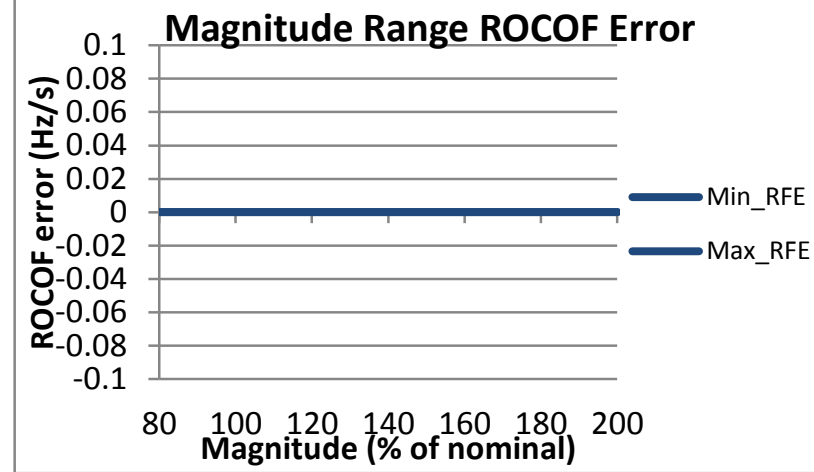 \\
\hline Figure 872: Fs $=15$ FPS & Figure 873: Fs = 12 FPS & Figure 874: $\mathrm{Fs}=10 \mathrm{FPS}$ \\
\hline
\end{tabular}


3.8.2 PMU A steady state signal magnitude ROCOF error: P class

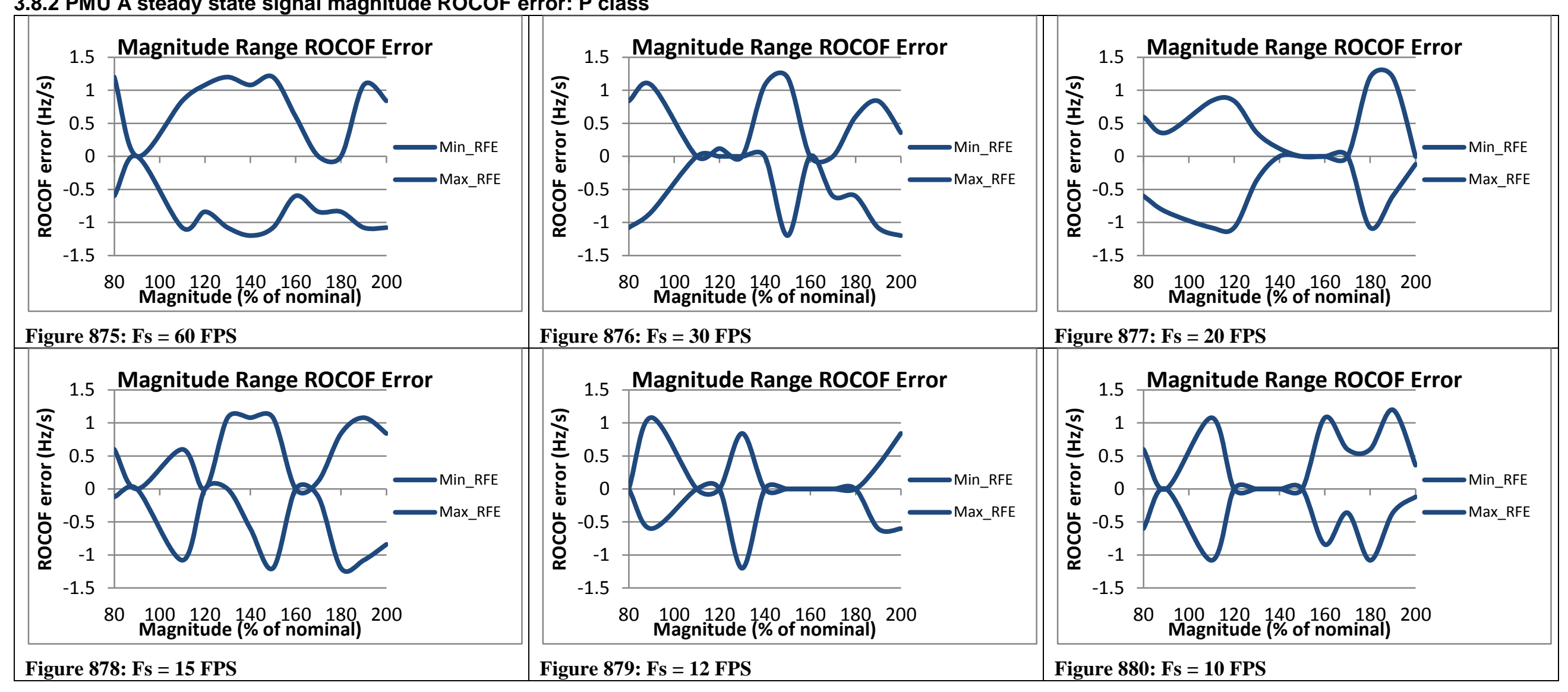


3.8.3 PMU B steady state signal magnitude ROCOF error: P class

\begin{tabular}{|c|c|c|}
\hline 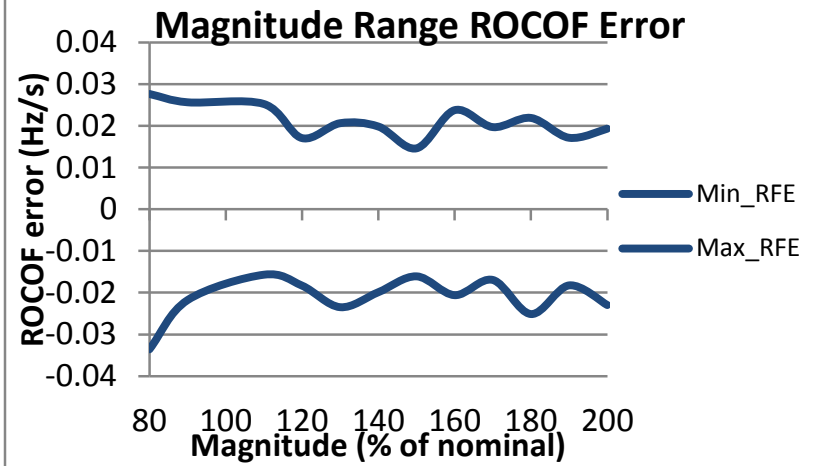 & 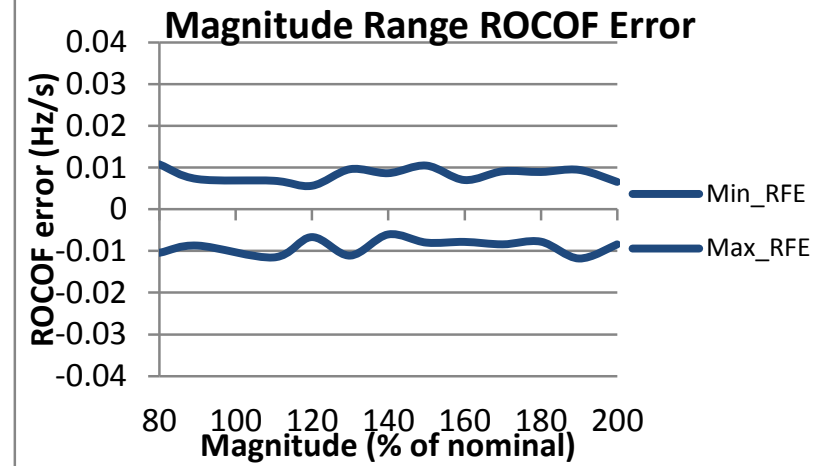 & 80 100 120 12 \\
\hline Figure 881: Fs = 60 FPS & Figure 882: Fs = 30 FPS & Figure 883: Fs = 20 FPS \\
\hline 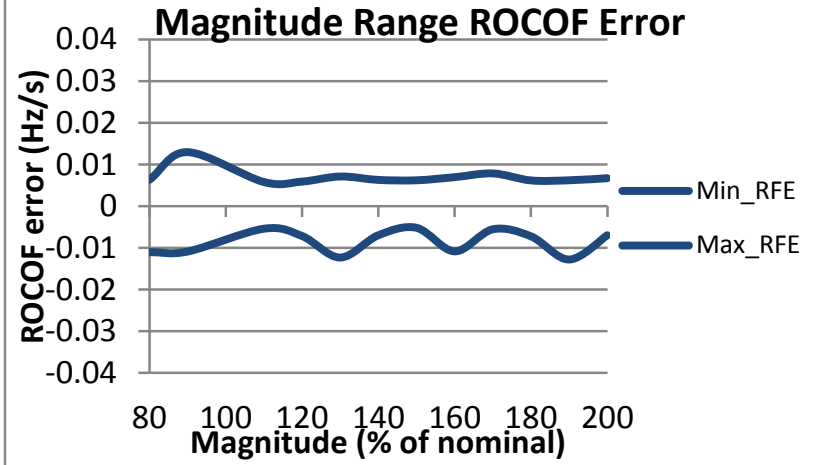 & 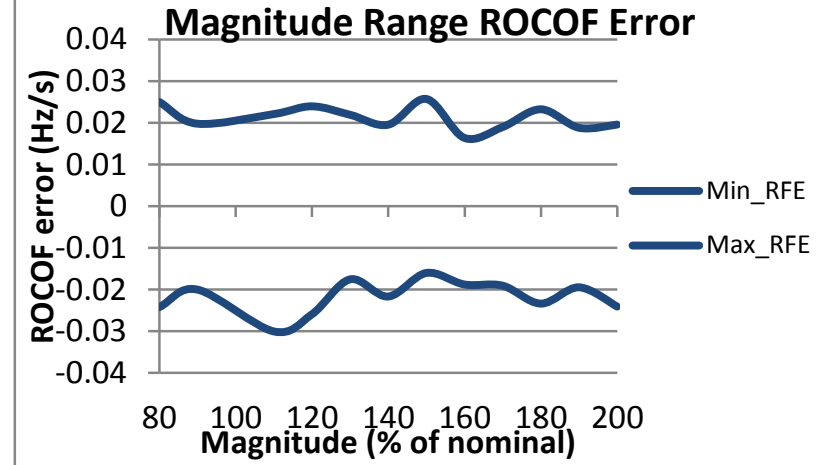 & 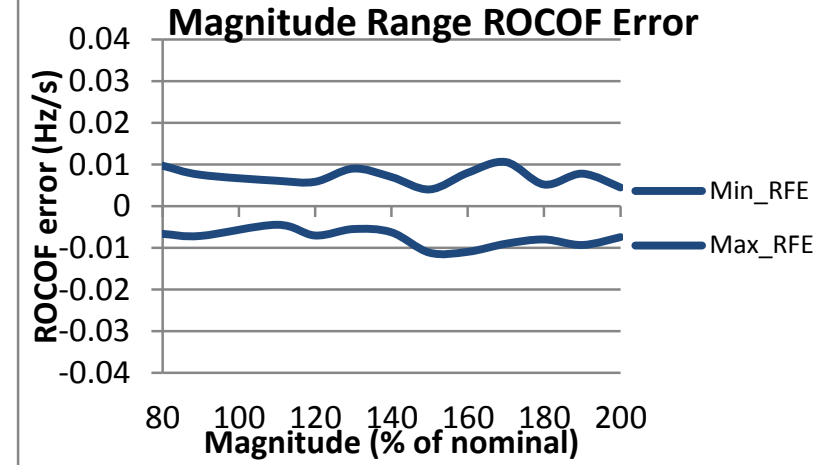 \\
\hline Figure 884: Fs = 15 FPS & Figure 885: Fs = 12 FPS & Figure 886: Fs = 10 FPS \\
\hline
\end{tabular}


3.8.4 PMU C steady state signal magnitude ROCOF error: $P$ class

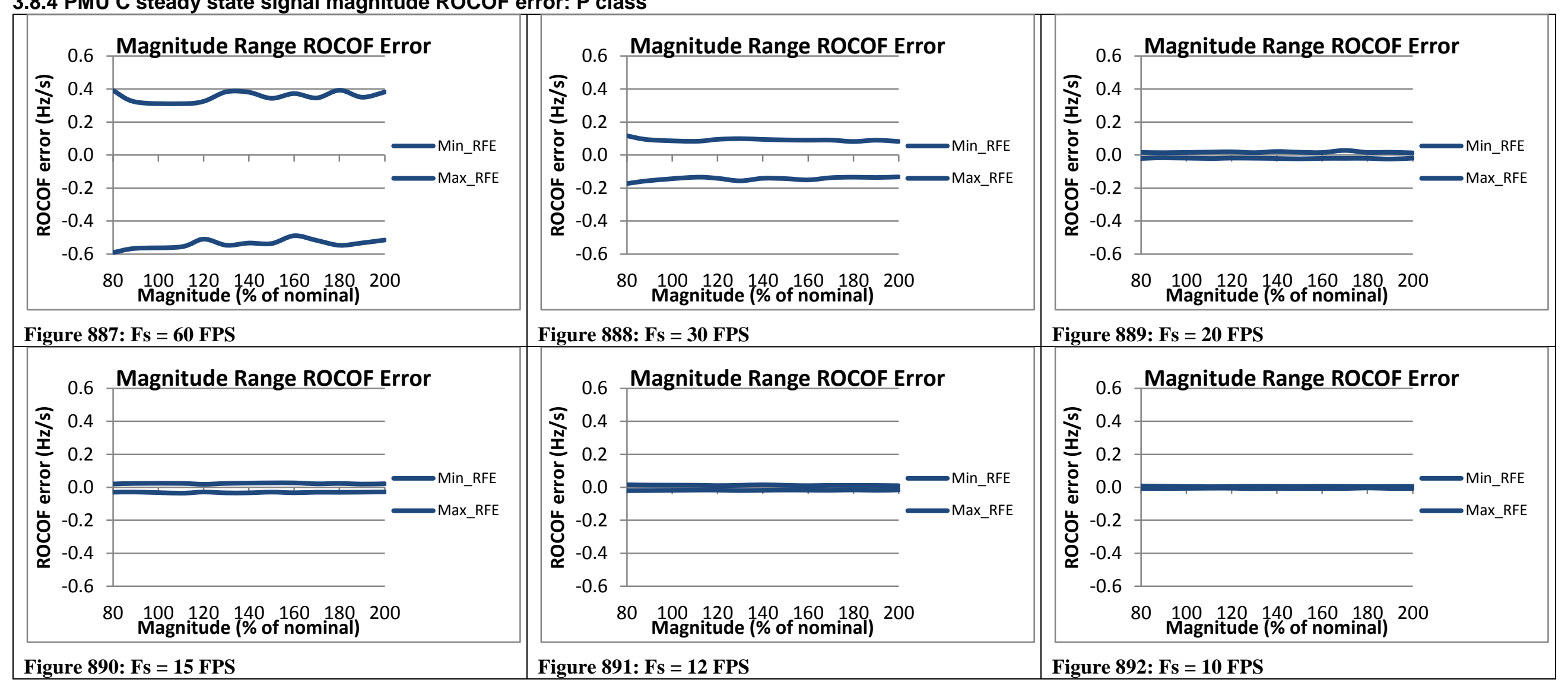




\subsubsection{PMU D steady state signal magnitude ROCOF error: $P$ class}
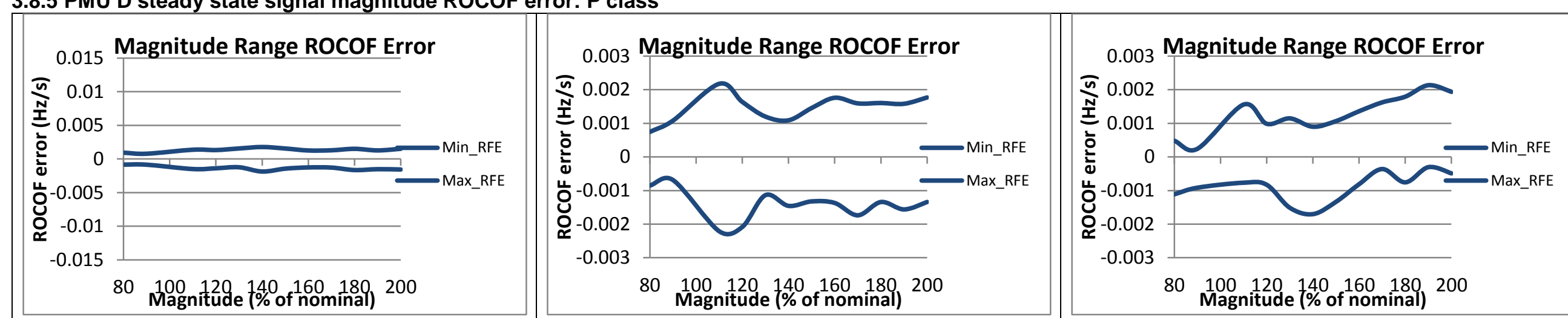

Figure 893: Fs = 60 FPS

Figure 894: Fs $=30$ FPS

Figure 895: Fs = 20 FPS

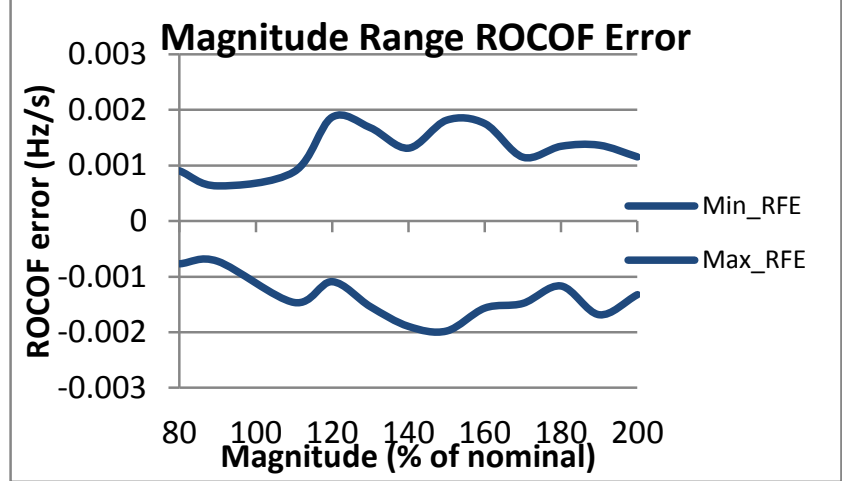

Figure 896: Fs = 15 FPS

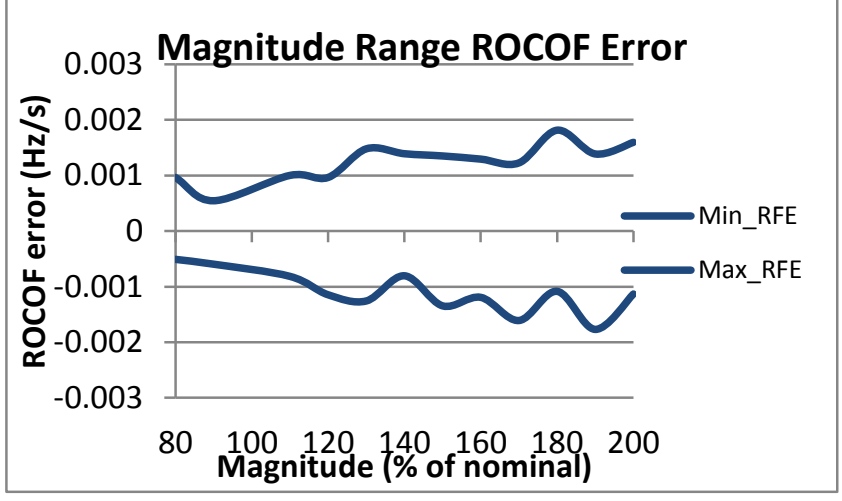

Figure 897: Fs = 12 FPS

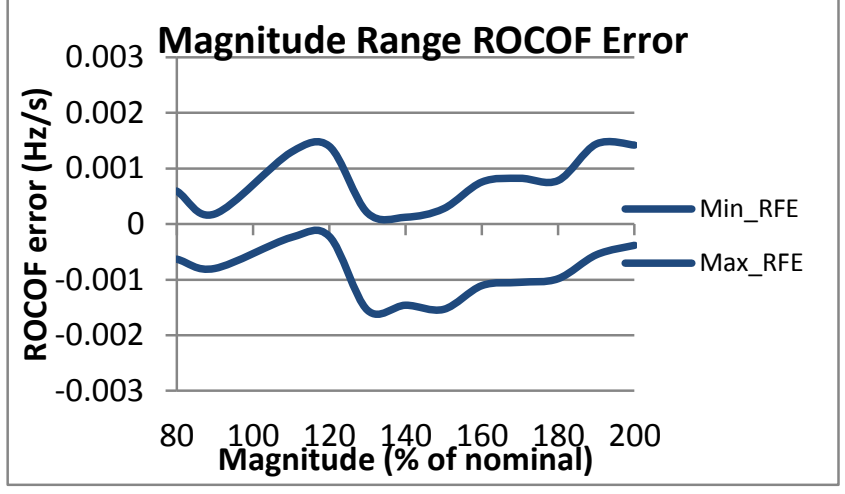

Figure 898: Fs = 10 FPS

3.8.6 PMU E steady state signal magnitude ROCOF error: $P$ class

PMU E does not support $P$ class 


\subsubsection{PMU F steady state signal magnitude ROCOF error: $P$ class}

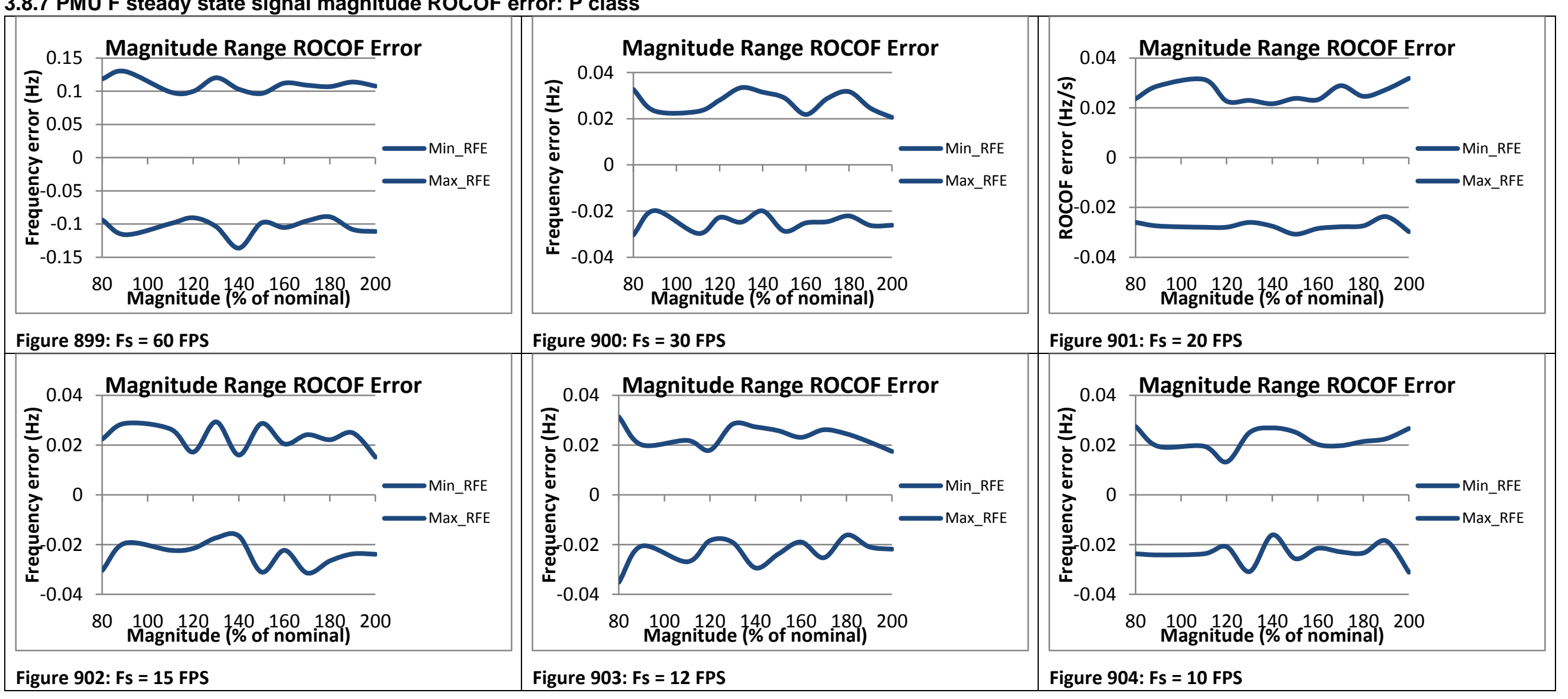

3.8.8 PMU G steady state signal magnitude ROCOF error: P class

PMU $G$ does not support $P$ class 


\subsubsection{PMU H steady state signal magnitude ROCOF error: P class}
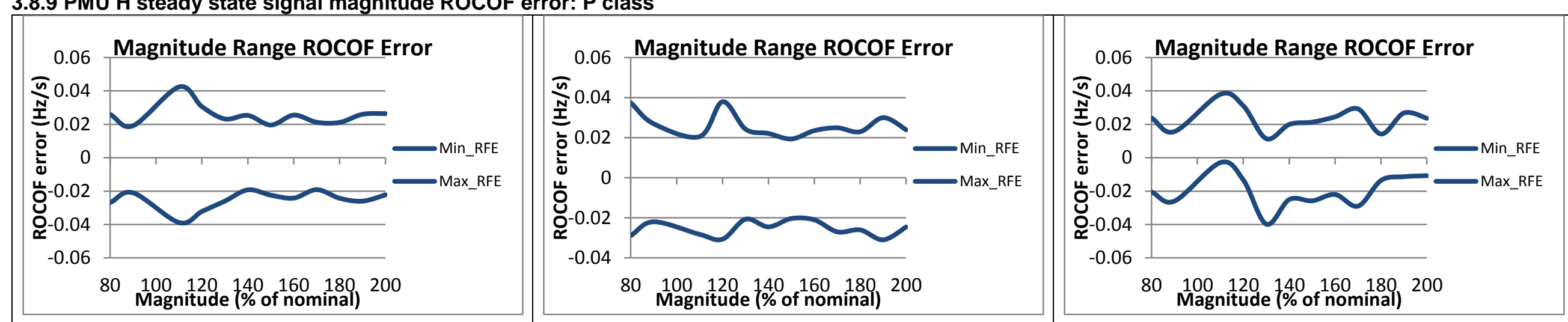

Figure 905: Fs = 60 FPS

Figure 906: Fs = 30 FPS

Figure 907: Fs = 20 FPS
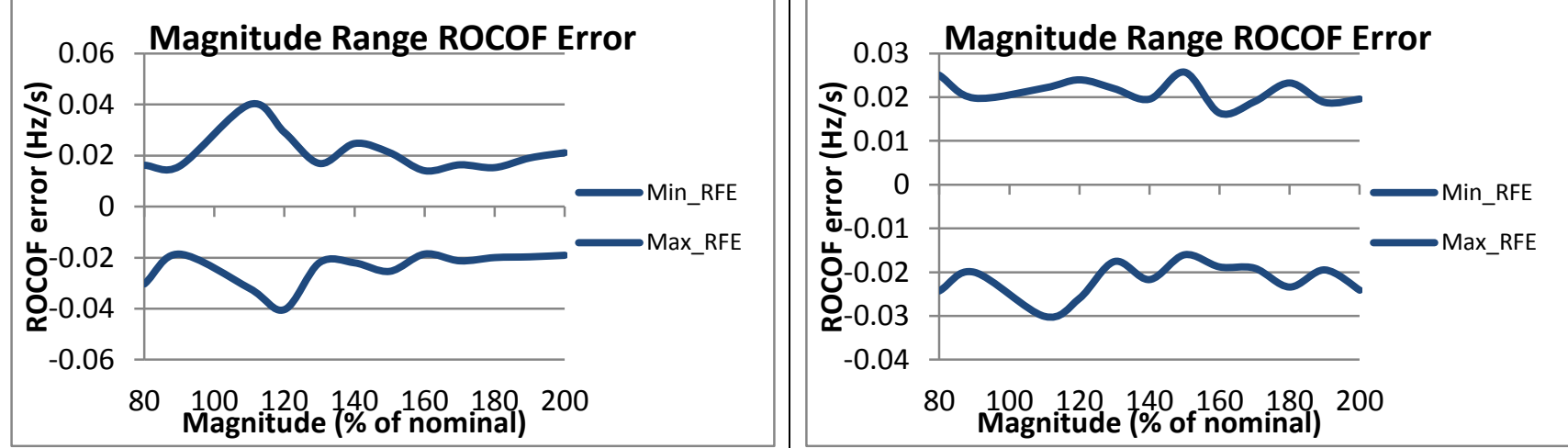

Figure 908: Fs = 15 FPS

Figure 909: Fs = 12 FPS

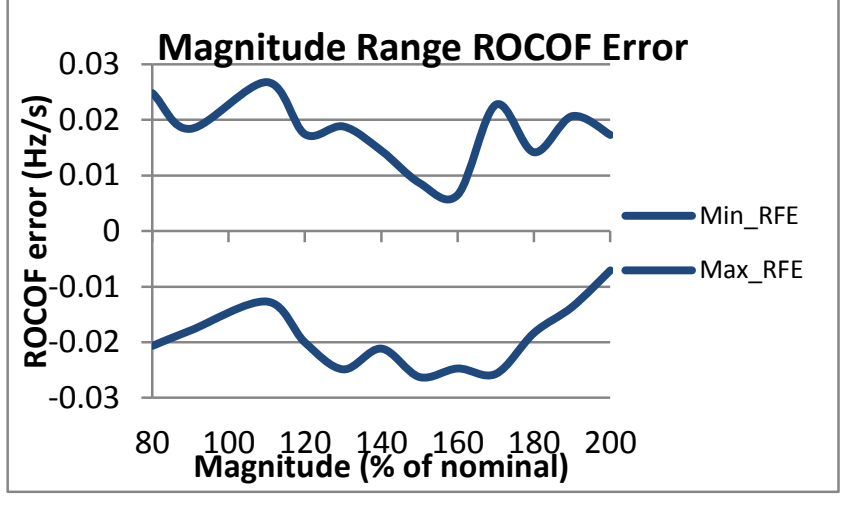

Figure 910: Fs = 10 FPS

3.8.10 PMU I steady state signal magnitude ROCOF error: P class

PMU I does not support P class 
3.8.11 PMU J steady state signal magnitude ROCOF error: P class

\begin{tabular}{|c|c|c|}
\hline \begin{tabular}{|l|l} 
& \\
\end{tabular} & 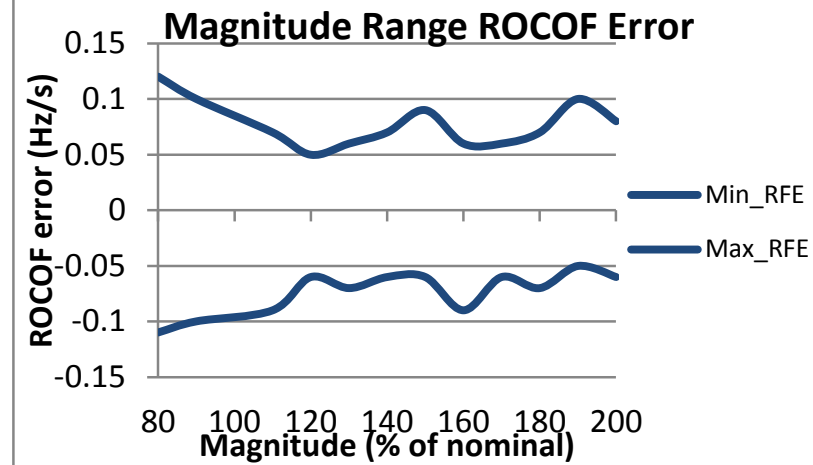 & \begin{tabular}{|l|l|l|l|l|l|} 
& \\
\end{tabular} \\
\hline Figure 911: Fs = 60 FPS & Figure 912: Fs = $30 \mathrm{FPS}$ & Figure 913: $\mathrm{Fs}=20 \mathrm{FPS}$ \\
\hline \begin{tabular}{|l|l|l|} 
& \\
\end{tabular} & 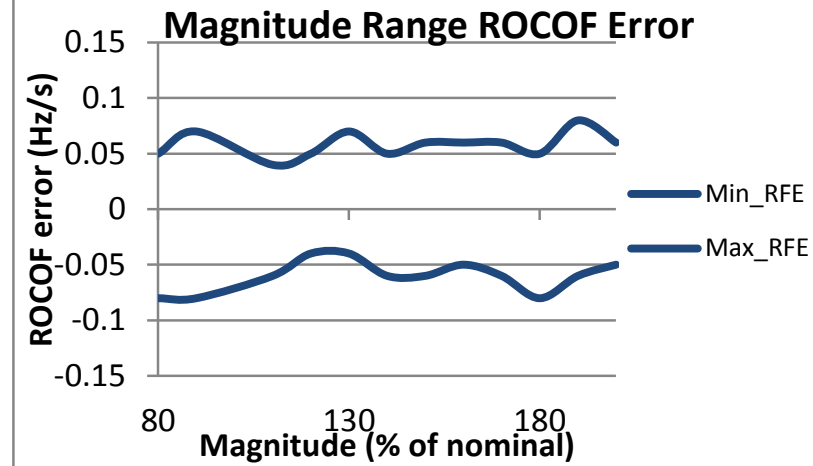 & \begin{tabular}{|l|l|l|} 
& \\
\end{tabular} \\
\hline Figure 914: Fs = $15 \mathrm{FPS}$ & Figure 915: $\mathrm{Fs}=12 \mathrm{FPS}$ & Figure 916: $\mathrm{Fs}=10 \mathrm{FPS}$ \\
\hline
\end{tabular}




\section{Steady state harmonic distortion}

\section{Table of Contents}

4. Steady state harmonic distortion

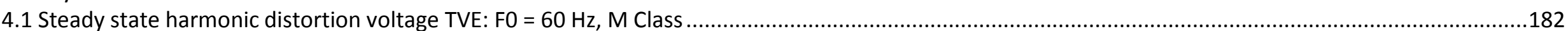

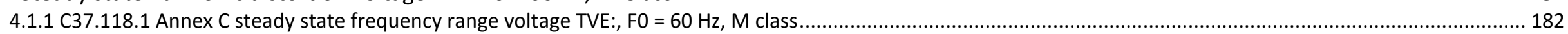

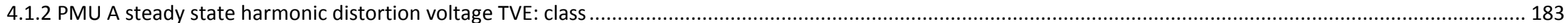

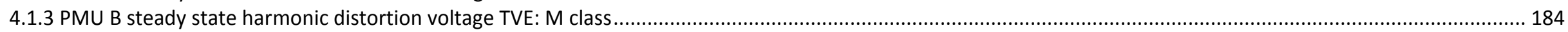

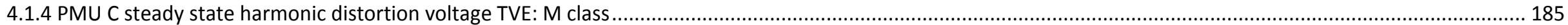

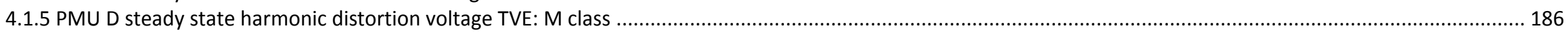

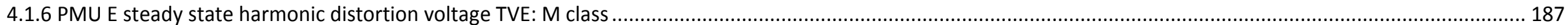

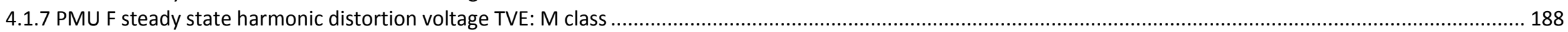

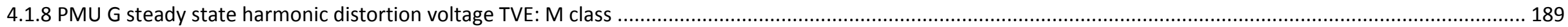

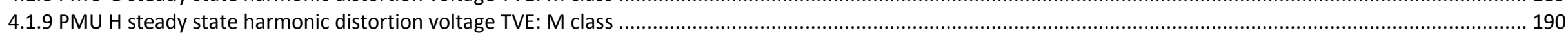

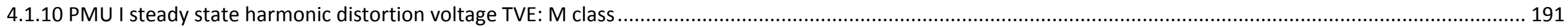

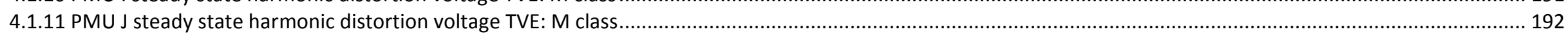

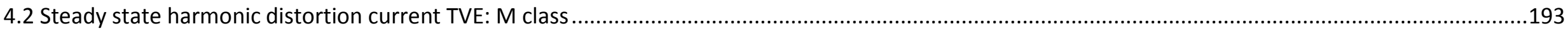

4.2.1 C37.118.1 Annex C steady state harmonic distortion current TVE: M class ……........................................................................................................... 193

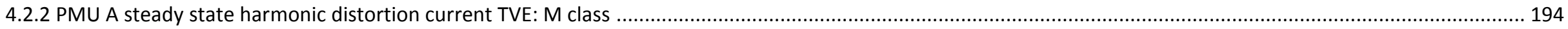

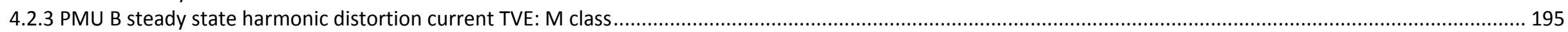

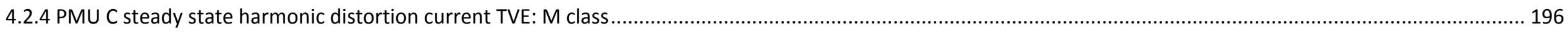

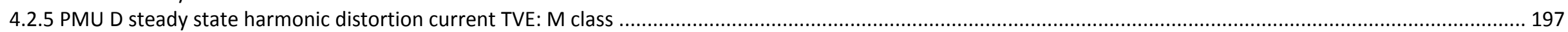

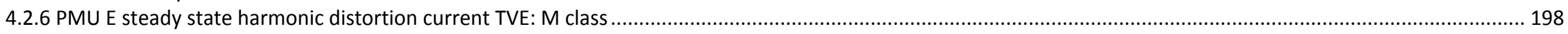

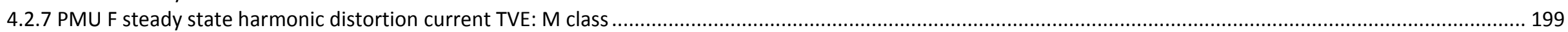

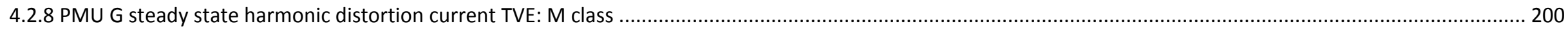

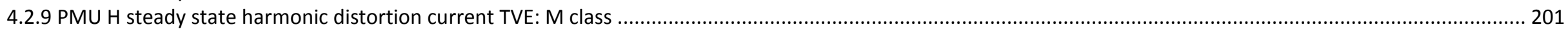

4.2.10 PMU I steady state harmonic distortion current TVE: M class ....................................................................................................................................... 202

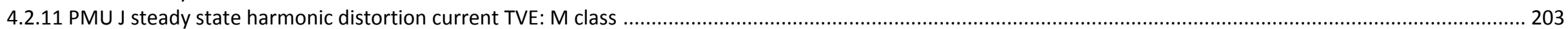

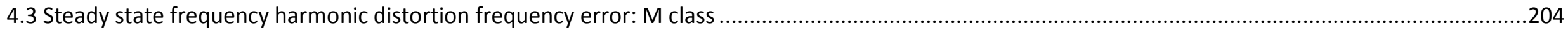

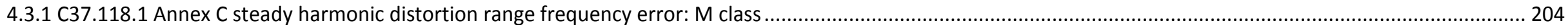

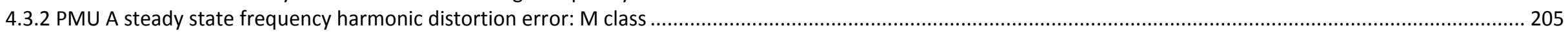

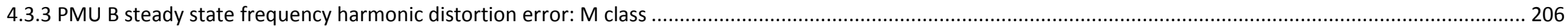

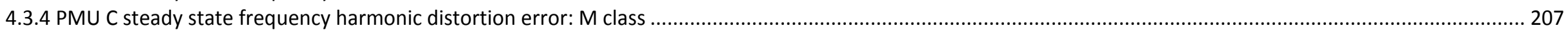

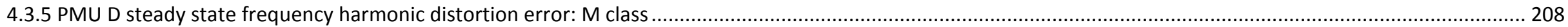

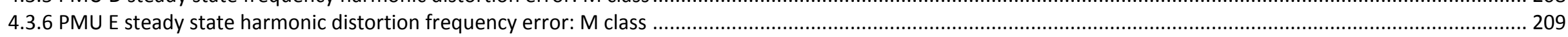

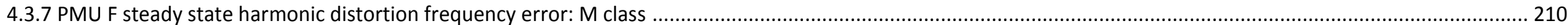

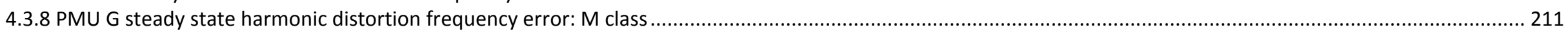


4.3.9 PMU H steady state harmonic distortion frequency error: $\mathrm{M}$ class

4.3.10 PMU I steady state harmonic distortion frequency error: $\mathrm{M}$ class

4.3.11 PMU J steady state harmonic distortion frequency error: $\mathrm{M}$ class

4.4 Steady state harmonic distortion ROCOF error: M class ....

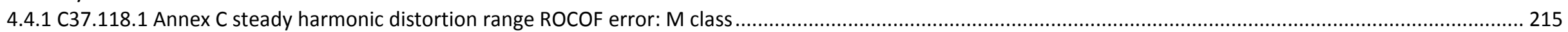

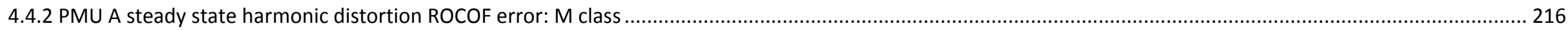

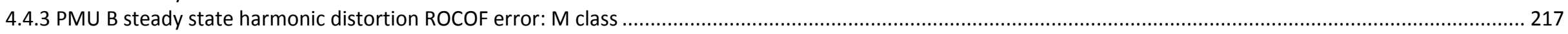

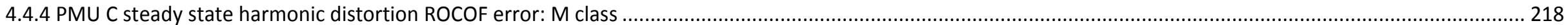

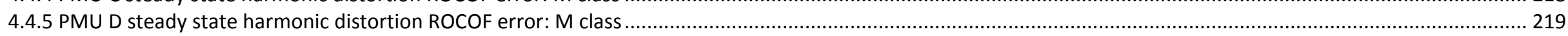

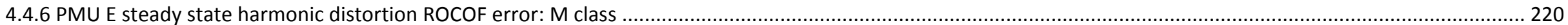

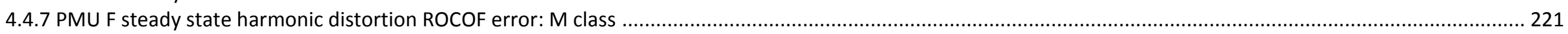

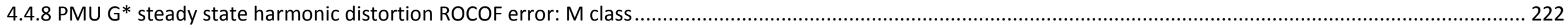

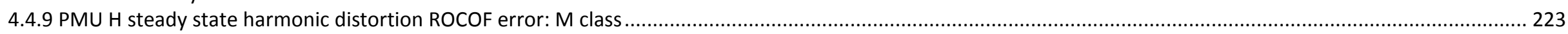

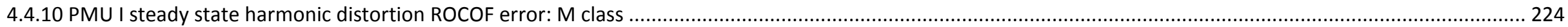

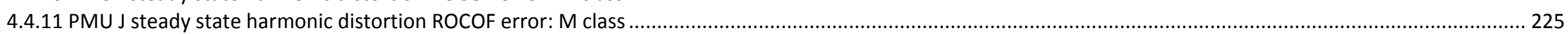

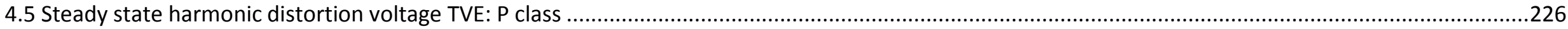

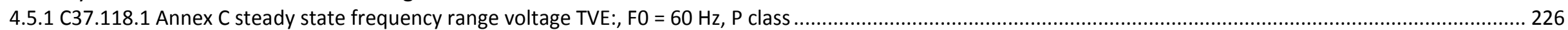

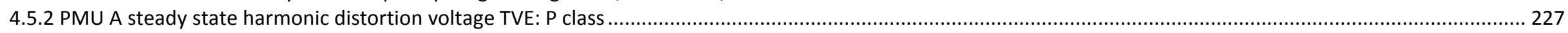

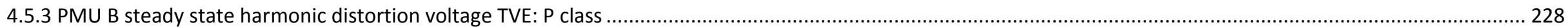

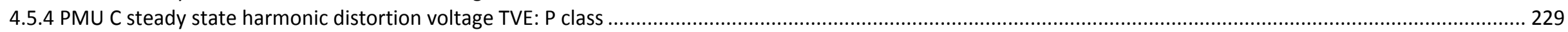

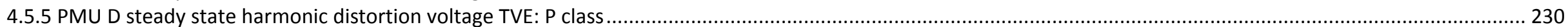

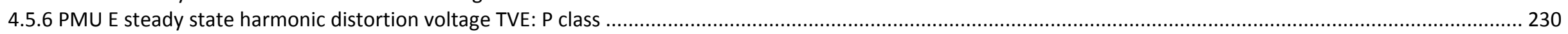

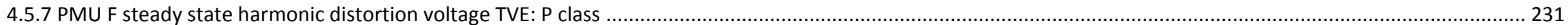

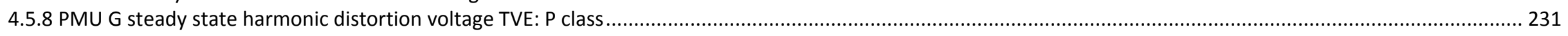

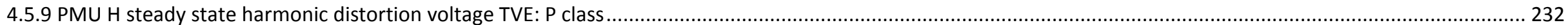

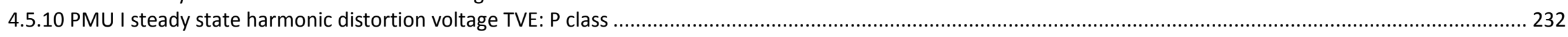

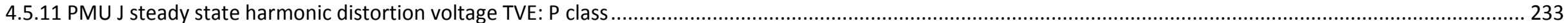

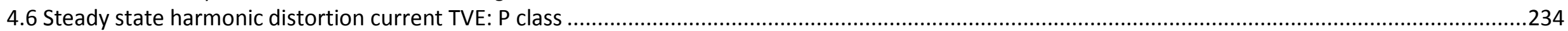

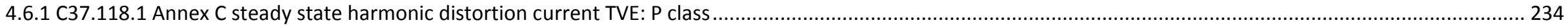

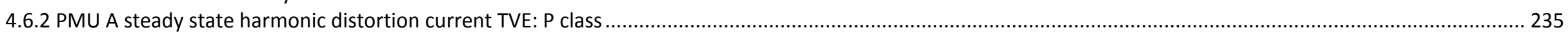

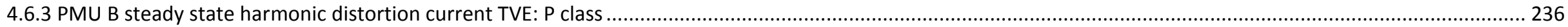

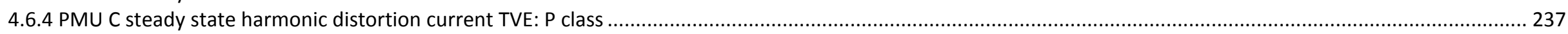

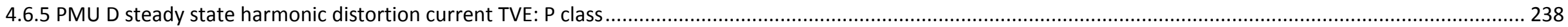

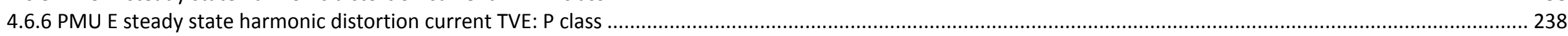

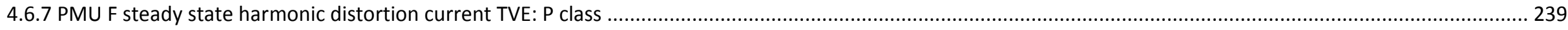

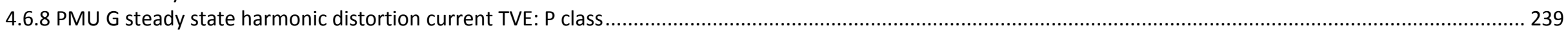

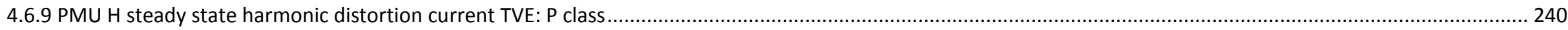

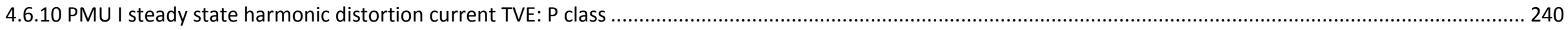

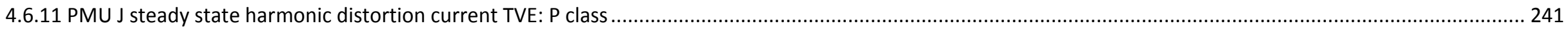

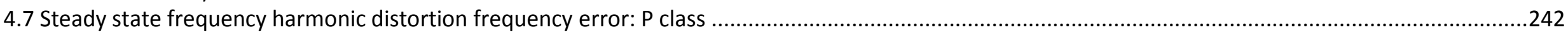

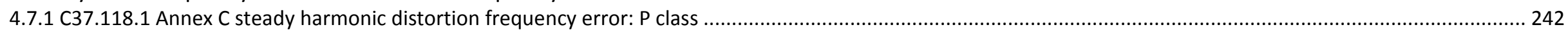


4.7.2 PMU A steady state harmonic distortion frequency error: $\mathrm{P}$ class

4.7.3 PMU B steady state harmonic distortion frequency error: $P$ class

4.7.4 PMU C steady state harmonic distortion frequency error: $P$ class.

4.7.5 PMU D steady state harmonic distortion frequency error: $P$ class

4.7.6 PMU E steady state harmonic distortion frequency error: $P$ class.

4.7.7 PMU F steady state harmonic distortion frequency error: P class

4.7.8 PMU G steady state harmonic distortion frequency error: P class

4.7.9 PMU H steady state harmonic distortion frequency error: P class

4.7.10 PMU I steady state harmonic distortion frequency error: $P$ class

4.7.11 PMU J steady state harmonic distortion frequency error: $P$ class

4.8.1 C37.118.1 Annex C steady harmonic distortion ROCOF error: P class

250

4.8.2 PMU A steady state harmonic distortion ROCOF error: P class

4.8.3 PMU B steady state harmonic distortion ROCOF error: P class

4.8.4 PMU C steady state harmonic distortion ROCOF error: $P$ class

252

4.8.5 PMU D steady state harmonic distortion ROCOF error: $P$ class

4.8.6 PMU E steady state harmonic distortion ROCOF error: P class

4.8.7 PMU F steady state harmonic distortion ROCOF error: P class

4.8.8 PMU G* steady state harmonic distortion ROCOF error: P class

255

4.8.9 PMU H steady state harmonic distortion ROCOF error: P class

4.8.10 PMU I steady state harmonic distortion ROCOF error: P class

256

4.8.11 PMU J steady state harmonic distortion ROCOF error: P class...... 
The steady state response to harmonic distortion is tested by a series of individual steady state tests where the input signal magnitude and frequency is fixed for duration of 5 seconds and a single harmonic is added with the magnitude of the harmonic dependent on the PMU class. The maximum TVE, FE and RFE of all the test runs is compared against the limits for TVE, FE and RFE to determine if the unit passes or fails the test.

The relative phase of the harmonic is not specified by C37.118.1-2011 or by the amendment C37.118.1a-2014. However the relative phase of the harmonic affects the PMU measurement, especially ROCOF, therefore the IEEE Conformance Assessment Program (ICAP) PMU Test Suite Specification requires that the harmonic be in-phase with the fundamental signal by crossing zero in the positive-going direction at the same time the fundamental signal crosses zero in the positive-going direction. That means the second harmonic will be negative sequence, the third harmonic will be zero sequence and the fourth harmonic will be positive sequence, repeating the cycle every three harmonics.

M class limits:

- TVE: $1 \%$

- FE:

- for Fs > 20 FPS: $0.025 \mathrm{~Hz}$

- for Fs $\leq 20$ FPS: $0.005 \mathrm{~Hz}$

- RFE: Limit suspended

P class limits:

- TVE: $1 \%$

- FE: $0.005 \mathrm{~Hz}$

- RFE: $0.4 \mathrm{~Hz} / \mathrm{s}$

Test Plan:

a) Apply input signals at nominal magnitude with the addition of one harmonic, starting with the second harmonic, with the magnitude set to the level specified by IEEE Std C37.118.1-2011 for Class P or M PMUs:

- $1 \%$ of nominal magnitude for $P$ class.

- $10 \%$ of nominal magnitude for $M$ class.

b) Wait for the system to settle.

c) Capture the PMU output for 5 seconds.

d) Calculate the errors: $\mathrm{ME}, \mathrm{PE}, \mathrm{FE}, \mathrm{RFE}$ for each report.

e) Calculate the Max TVE, FE and RFE.

f) Change to injecting the next harmonic.

g) Repeat step b) through step f) until all harmonics between 2 nd harmonic and 50th harmonic have been tested. 
Steady state harmonic distortion test results

\begin{tabular}{|c|c|c|c|c|c|c|c|c|c|c|c|c|c|c|c|c|c|c|c|c|c|c|c|c|c|c|c|c|c|c|c|c|c|c|c|c|}
\hline \multirow{4}{*}{$\begin{array}{l}\text { Fs (FPS) } \\
\text { Test }\end{array}$} & \multicolumn{3}{|c|}{$10 \mathrm{M}$} & \multicolumn{3}{|c|}{$10 P$} & \multicolumn{3}{|c|}{$12 \mathrm{M}$} & \multicolumn{3}{|c|}{$12 \mathrm{P}$} & \multicolumn{3}{|c|}{$15 \mathrm{M}$} & \multicolumn{3}{|c|}{$15 P$} & \multicolumn{3}{|c|}{$20 \mathrm{M}$} & \multicolumn{3}{|c|}{$20 P$} & \multicolumn{3}{|c|}{$30 \mathrm{M}$} & \multicolumn{3}{|c|}{$30 P$} & \multicolumn{3}{|c|}{$60 \mathrm{M}$} & \multicolumn{3}{|c|}{$60 P$} \\
\hline & $T$ & $F$ & $\mathrm{R}$ & $T$ & $F$ & $\mathrm{R}$ & $T$ & $\mathrm{~F}$ & $R$ & $T$ & $\mathrm{~F}$ & $\mathrm{R}$ & $\mathrm{T}$ & $\mathrm{F}$ & $R$ & $T$ & $F$ & $\mathrm{R}$ & $\mathrm{T}$ & $\mathrm{F}$ & $\mathrm{R}$ & $T$ & $\mathrm{~F}$ & $\mathrm{R}$ & $\mathrm{T}$ & $\mathrm{F}$ & $\mathrm{R}$ & $T$ & $\mathrm{~F}$ & $\mathrm{R}$ & $\mathrm{T}$ & $F$ & $R$ & $T$ & $F$ & $R$ \\
\hline & $\mathrm{V}$ & $E$ & $\mathrm{~F}$ & V & $E$ & $\mathrm{~F}$ & V & $\mathrm{E}$ & $\mathrm{F}$ & V & $\mathrm{E}$ & $\mathrm{F}$ & V & $\mathrm{E}$ & $\mathrm{F}$ & V & $\mathrm{E}$ & $\mathrm{F}$ & $\mathrm{V}$ & $\mathrm{E}$ & $\mathrm{F}$ & $\mathrm{V}$ & $\mathrm{E}$ & $\mathrm{F}$ & $\mathrm{V}$ & $\mathrm{E}$ & $\mathrm{F}$ & $\mathrm{V}$ & $\mathrm{E}$ & $\mathrm{F}$ & V & $\mathrm{E}$ & $\mathrm{F}$ & V & $\mathrm{E}$ & $\mathrm{F}$ \\
\hline & $\mathrm{E}$ & & $E$ & $\mathrm{E}$ & & $\mathrm{E}$ & $\mathrm{E}$ & & $\mathrm{E}$ & $E$ & & $\mathrm{E}$ & $E$ & & $\mathrm{E}$ & $E$ & & $\mathrm{E}$ & $\mathrm{E}$ & & $\mathrm{E}$ & $\mathrm{E}$ & & $\mathrm{E}$ & $E$ & & $\mathrm{E}$ & $\mathrm{E}$ & & $E$ & $E$ & & $\mathrm{E}$ & $E$ & & $\mathrm{E}$ \\
\hline C37.118.1 Annex C & $P$ & $\mathrm{P}$ & - & $\mathrm{P}$ & $\mathrm{P}$ & $\mathrm{P}$ & $\mathrm{P}$ & $\mathrm{P}$ & - & $\mathrm{P}$ & $P$ & $P$ & $\mathrm{P}$ & $P$ & - & $\mathrm{P}$ & $\mathrm{P}$ & $\mathrm{P}$ & $\mathrm{P}$ & $\mathrm{P}$ & - & $\mathrm{P}$ & $\mathrm{P}$ & $\mathrm{P}$ & $\mathrm{P}$ & $\mathrm{P}$ & - & $\mathrm{P}$ & $\mathrm{P}$ & $\mathrm{P}$ & $\mathrm{P}$ & $P$ & - & $\mathrm{P}$ & $\mathrm{P}$ & $\mathrm{P}$ \\
\hline PMU A & $P$ & $\mathrm{P}$ & - & $P$ & $P$ & $\mathrm{~F}$ & $P$ & $P$ & - & $P$ & $P$ & $\mathrm{~F}$ & $P$ & $P$ & - & $P$ & $P$ & $\mathrm{~F}$ & $P$ & $\mathrm{P}$ & - & $P$ & $P$ & $\mathrm{~F}$ & $\mathrm{P}$ & $P$ & - & $P$ & $\mathrm{P}$ & $\mathrm{F}$ & $P$ & $P$ & - & $P$ & $P$ & $\mathrm{~F}$ \\
\hline PMU B & $P$ & $\mathrm{P}$ & - & $P$ & $\mathrm{P}$ & $P$ & $P$ & $P$ & - & $P$ & $P$ & $P$ & $\mathrm{P}$ & $P$ & - & $P$ & $P$ & $P$ & $\mathrm{P}$ & $\mathrm{P}$ & - & $P$ & $P$ & $\mathrm{P}$ & $\mathrm{P}$ & $P$ & - & $P$ & $P$ & $P$ & $P$ & $P$ & - & $P$ & $P$ & $P$ \\
\hline PMU C & $P$ & $P$ & - & $P$ & $P$ & $P$ & $P$ & $P$ & - & $P$ & $P$ & $P$ & $P$ & $P$ & - & $P$ & $P$ & $P$ & $P$ & $P$ & - & $P$ & $P$ & $\mathrm{P}$ & $P$ & $P$ & - & $P$ & $P$ & $P$ & $P$ & $P$ & - & $P$ & $\mathrm{~F}$ & $\mathrm{~F}$ \\
\hline PMU D & $P$ & $\mathrm{P}$ & - & $P$ & $\mathrm{P}$ & $P$ & $P$ & $\mathrm{P}$ & - & $P$ & $P$ & $P$ & $P$ & $P$ & - & $P$ & $P$ & $P$ & $P$ & $\mathrm{P}$ & - & $\mathrm{P}$ & $\mathrm{P}$ & $\mathrm{P}$ & $\mathrm{P}$ & $\mathrm{P}$ & - & $\mathrm{P}$ & $\mathrm{P}$ & $P$ & $P$ & $P$ & - & $P$ & $P$ & $P$ \\
\hline PMU E & $P$ & $P$ & - & - & - & - & & & & - & - & - & $P$ & $P$ & - & - & - & - & $P$ & $\mathrm{P}$ & - & - & - & - & $P$ & $\mathrm{P}$ & - & - & - & - & $P$ & $P$ & - & - & - & - \\
\hline PMU F & $P$ & $P$ & - & $P$ & $P$ & $P$ & $P$ & $\mathrm{P}$ & - & $P$ & $P$ & $P$ & $P$ & $P$ & - & $P$ & $P$ & $P$ & $P$ & $\mathrm{P}$ & - & $\mathrm{P}$ & $\mathrm{P}$ & $\mathrm{P}$ & $P$ & $\mathrm{P}$ & - & $P$ & $P$ & $P$ & $P$ & $P$ & - & $P$ & $P$ & $P$ \\
\hline PMU G & I & $P$ & - & - & - & - & I & $P$ & - & - & - & - & I & $P$ & - & - & - & - & I & $P$ & - & - & - & - & I & $P$ & - & - & - & - & I & $P$ & - & - & - & - \\
\hline PMU H & $P$ & $\mathrm{P}$ & - & $P$ & $P$ & $P$ & $P$ & $P$ & - & $P$ & $P$ & $P$ & $\mathrm{P}$ & $P$ & - & $P$ & $P$ & $P$ & $\mathrm{P}$ & $\mathrm{P}$ & - & $P$ & $P$ & $\mathrm{P}$ & $\mathrm{P}$ & $P$ & - & $P$ & $P$ & $P$ & $P$ & $P$ & - & $P$ & $P$ & $P$ \\
\hline PMU I & $\mathrm{F}$ & $P$ & - & - & - & - & $\mathrm{F}$ & I & - & - & - & - & $P$ & $P$ & - & - & - & - & $P$ & $P$ & - & - & - & - & $P$ & $P$ & - & - & - & - & $P$ & $P$ & - & - & - & - \\
\hline PMU J & $P$ & $P$ & - & $P$ & $P$ & $P$ & $P$ & $P$ & - & $P$ & $P$ & $P$ & $P$ & $P$ & - & $P$ & $P$ & $P$ & $P$ & $\mathrm{P}$ & - & $P$ & $P$ & $P$ & $P$ & $\mathrm{P}$ & - & $P$ & $P$ & $P$ & $P$ & $P$ & - & $P$ & $P$ & $P$ \\
\hline
\end{tabular}

$\mathrm{P}=$ Pass (does not exceed the limit), $\mathrm{F}=$ Fails (exceeds the limit), I = Indeterminate within the test equipment uncertainty of the limit. 


\subsection{Steady state harmonic distortion voltage TVE: $F 0=60 \mathrm{~Hz}$, M Class}

\subsubsection{C37.118.1 Annex C steady state frequency range voltage TVE:, F0 $=60 \mathrm{~Hz}, \mathrm{M}$ class}

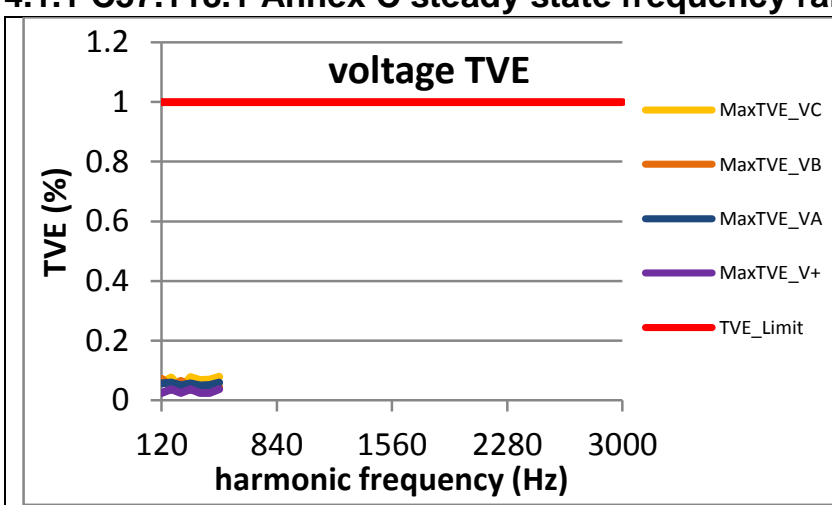

\section{Figure 1: Fs = 60 FPS}
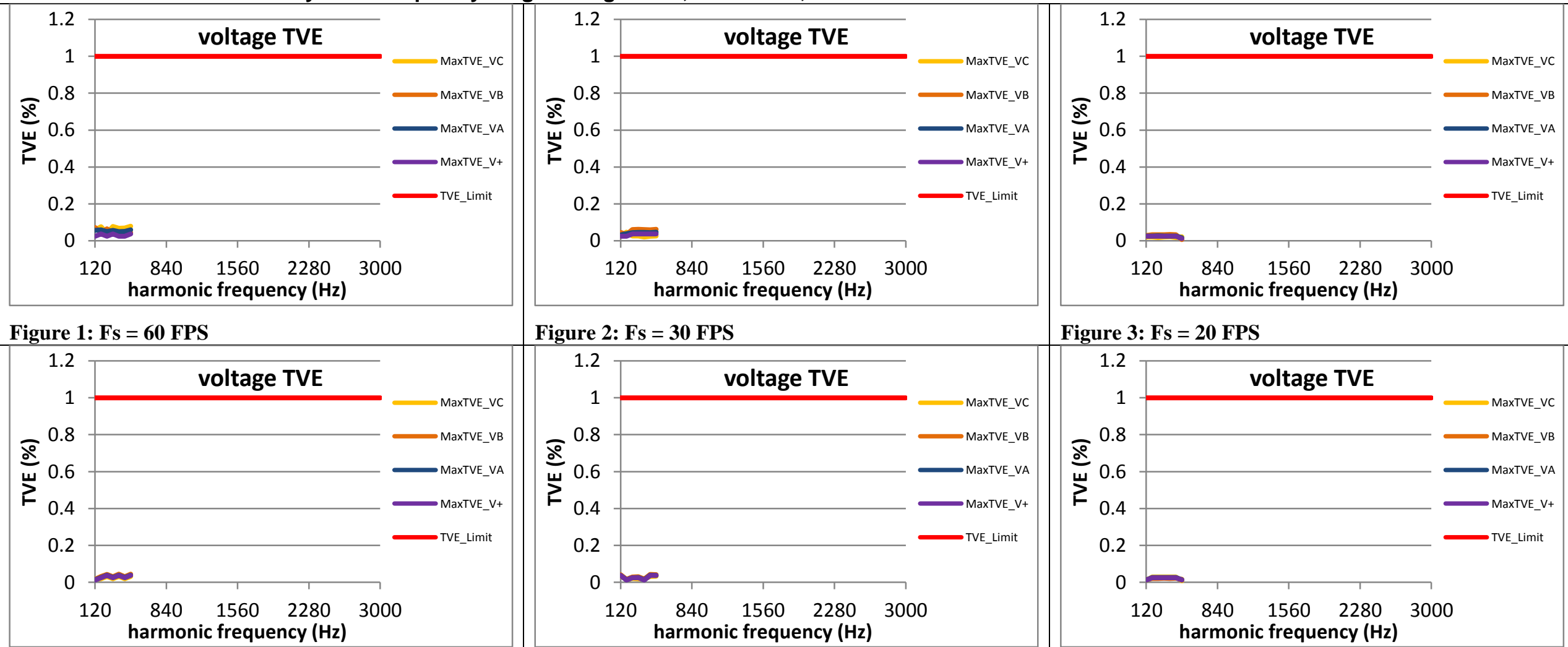

Figure 2: Fs = 30 FPS

\section{Figure 3: Fs = 20 FPS}

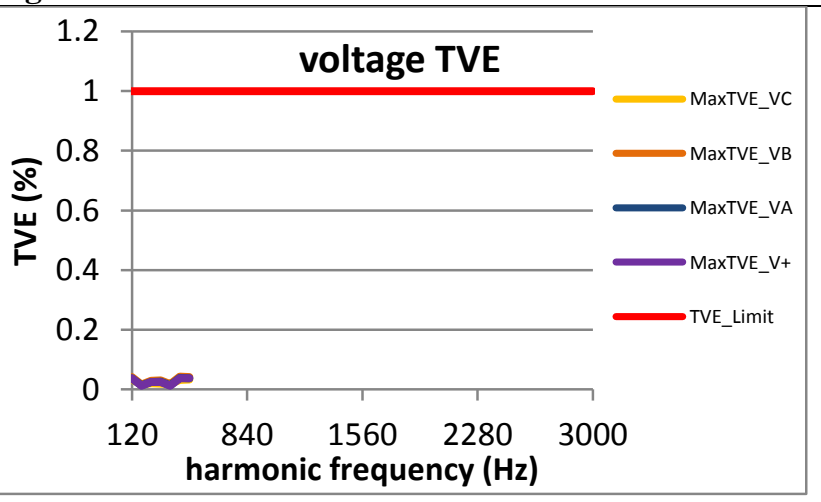

Figure 5: Fs = 12 FPS

\section{Figure 4: Fs = 15 FPS}

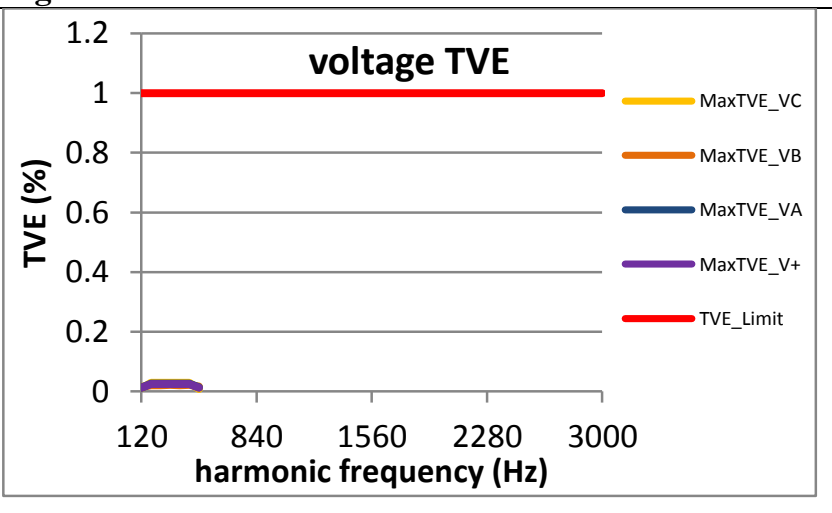

Figure 6: Fs = 10 FPS

The C37.118.1 Annex C model is a digital simulation with an internal sampling rate of 960 samples per second and so can only be tested with a harmonic frequency up to $420 \mathrm{~Hz}$. 
4.1.2 PMU A steady state harmonic distortion voltage TVE: class

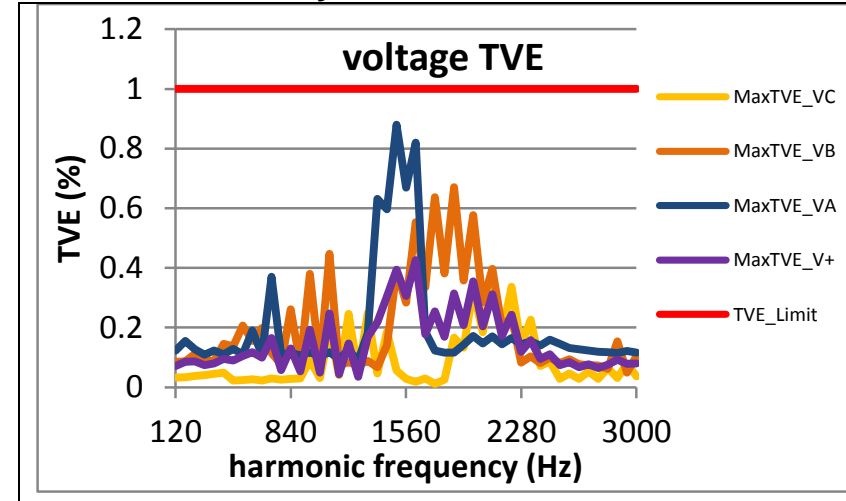

Figure 7: Fs = 60 FPS

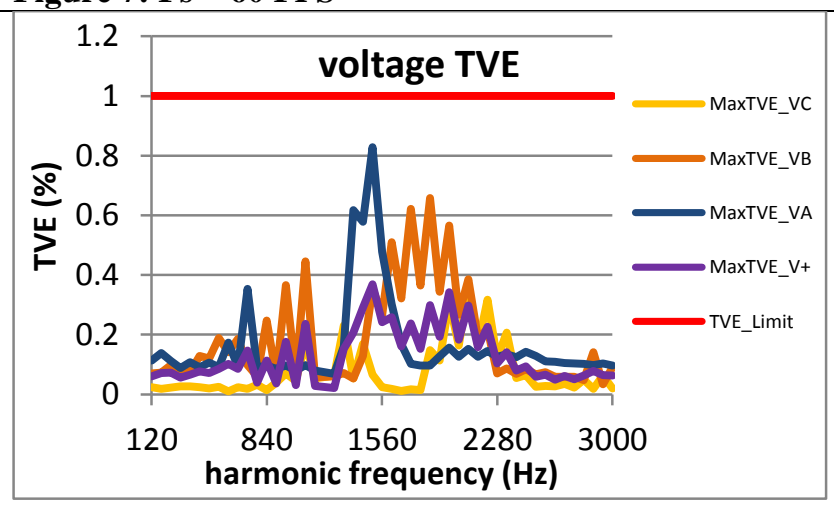

Figure 10: Fs = 15 FPS

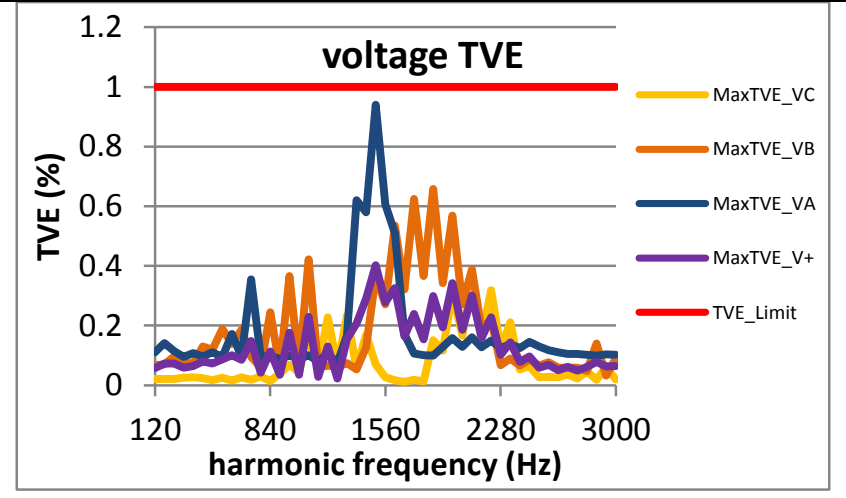

Figure 8: Fs = 30 FPS

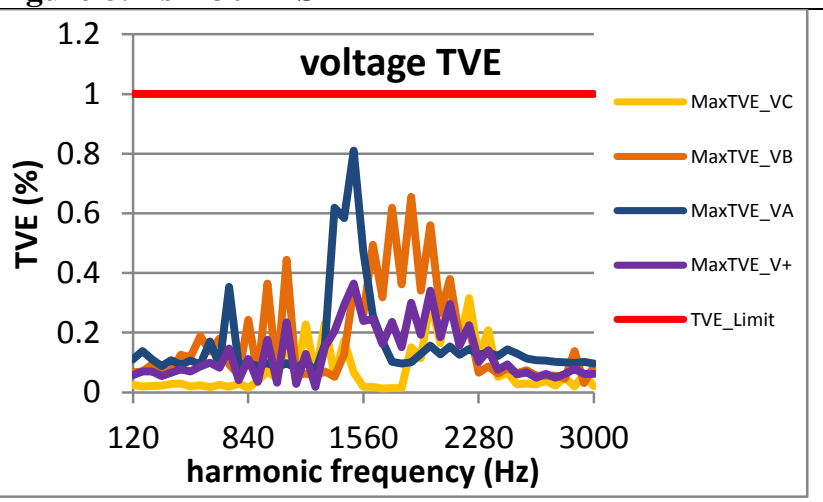

Figure 11: Fs = 12 FPS

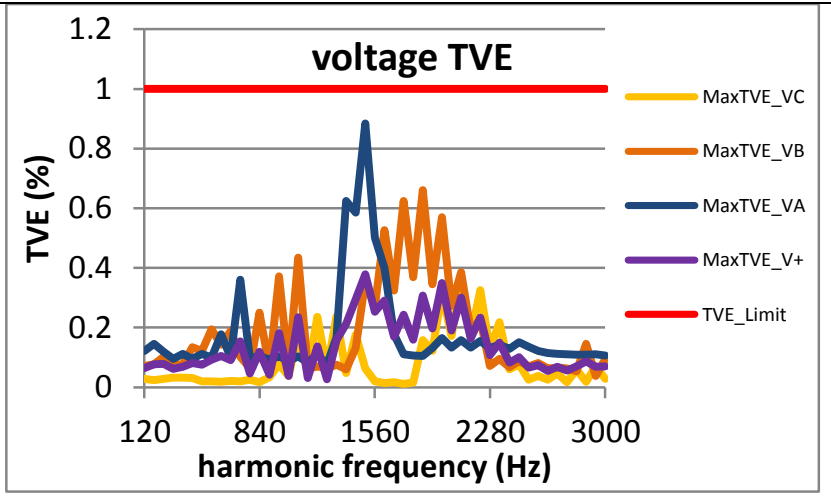

Figure 9: Fs = 20 FPS

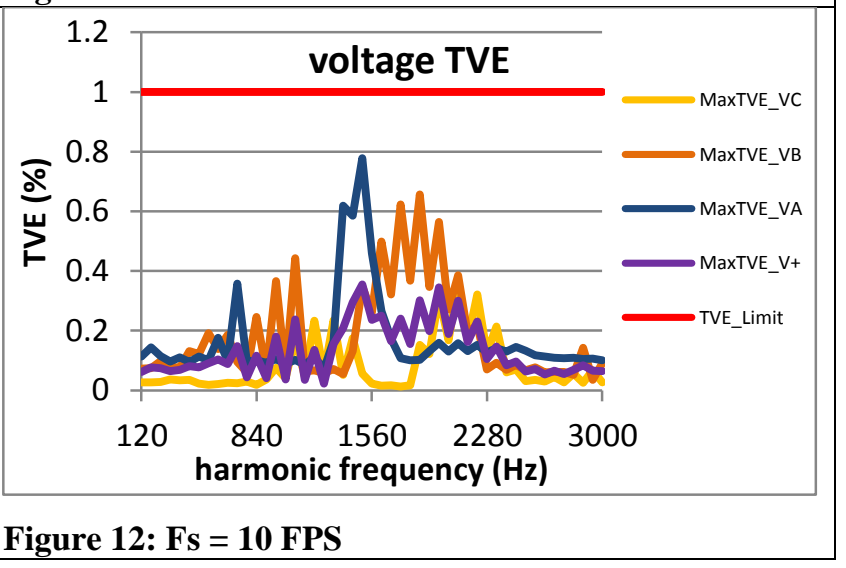


4.1.3 PMU B steady state harmonic distortion voltage TVE: M class
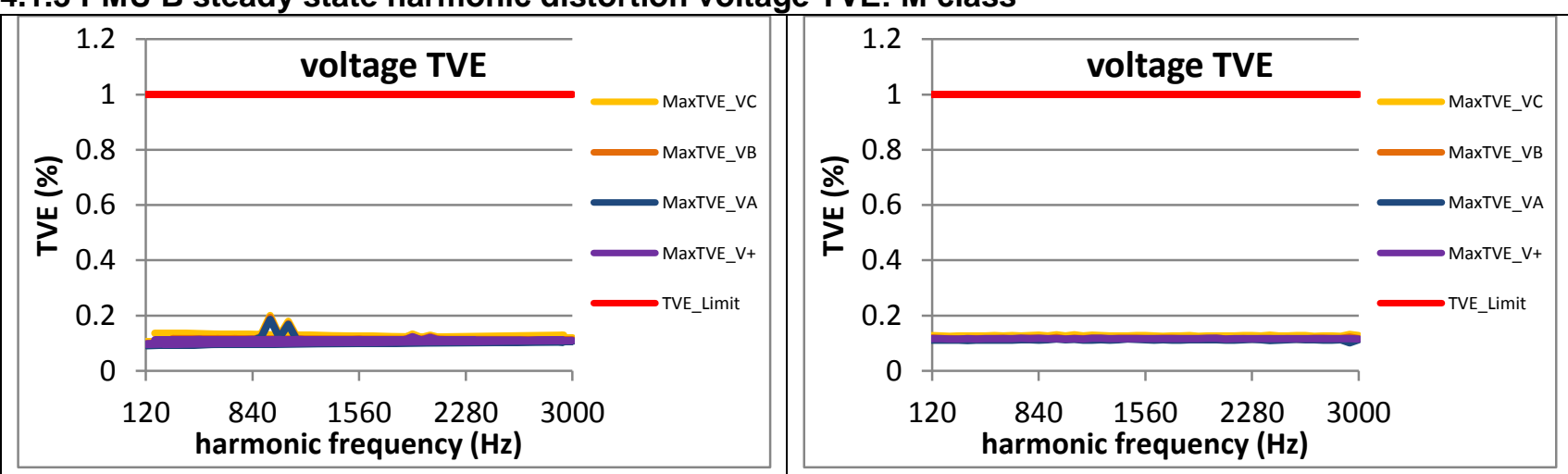

Figure 14: Fs = 30 FPS

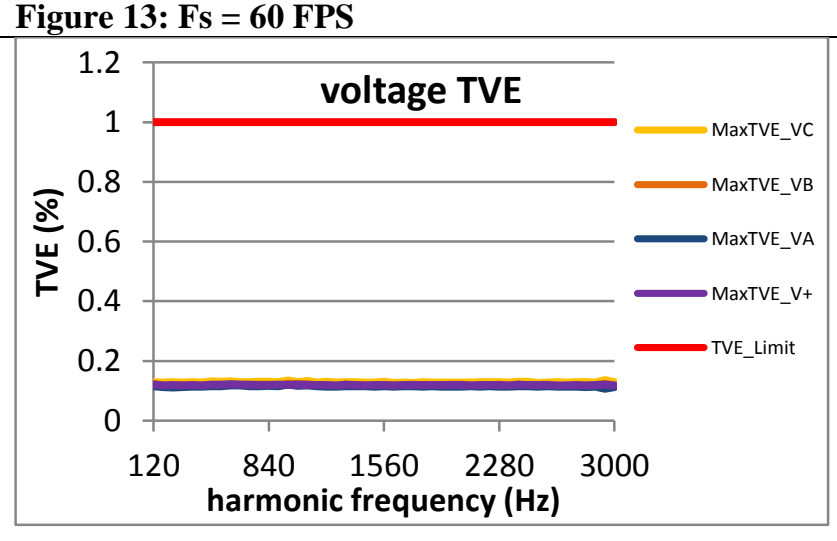

Figure 16: Fs = 15 FPS

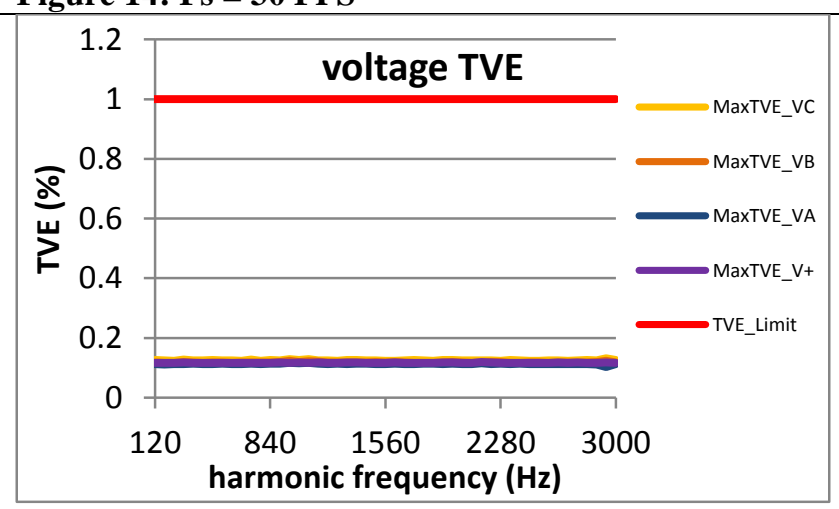

Figure 17: Fs = 12 FPS

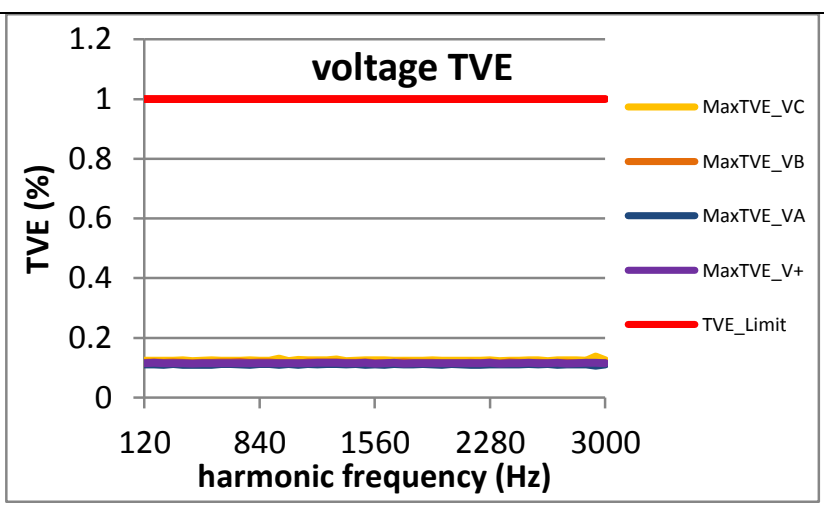

Figure 15: Fs = 20 FPS

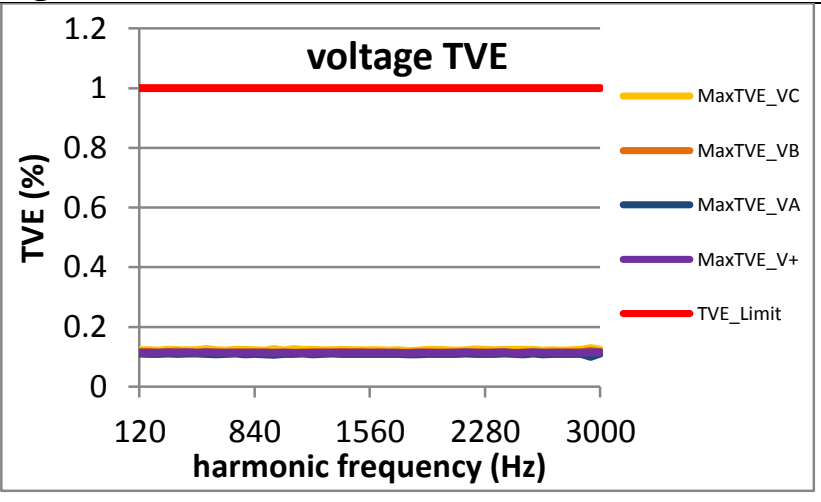

Figure 18: Fs = 10 FPS 
4.1.4 PMU C steady state harmonic distortion voltage TVE: M class
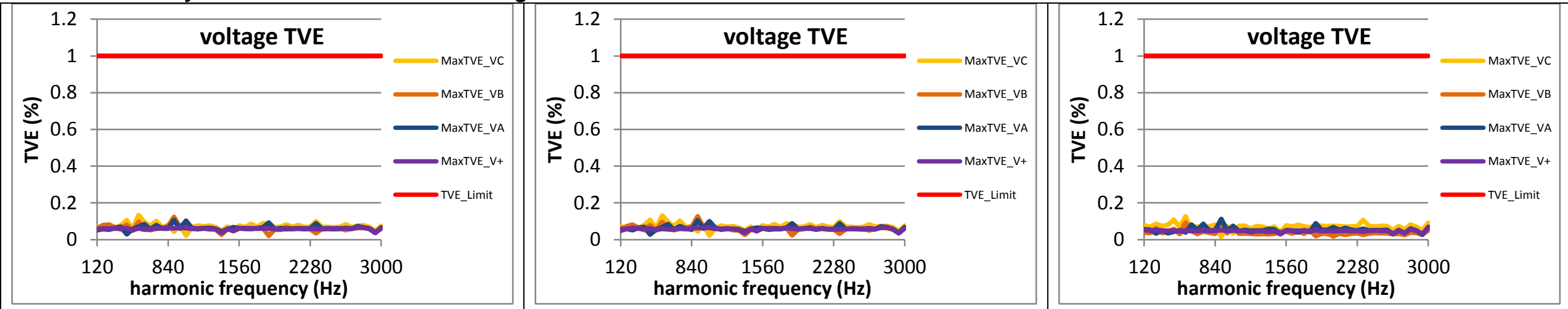

Figure 19: Fs = 60 FPS

\section{Figure 20: Fs = 30 FPS}

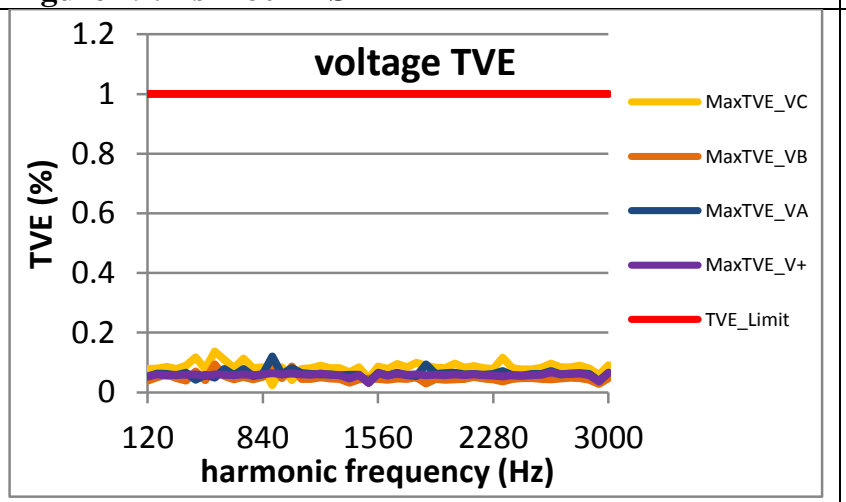

Figure 22: Fs = 15 FPS

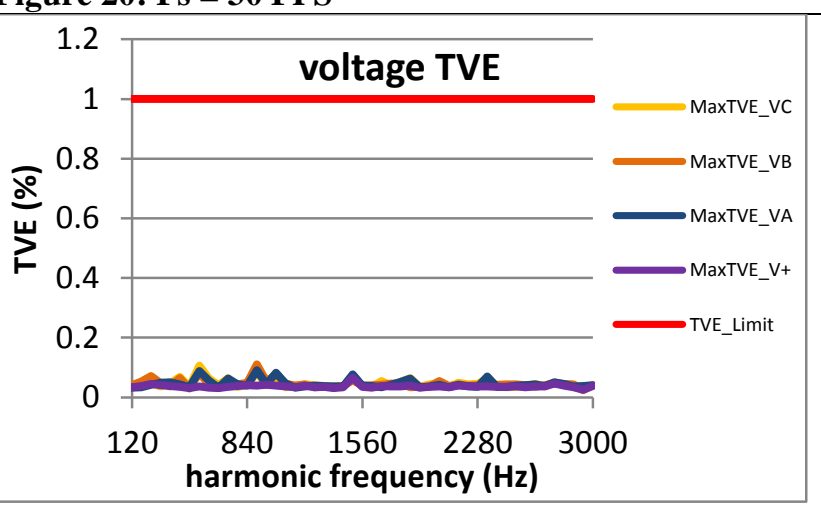

Figure 23: Fs = 12 FPS
Figure 21: Fs = 20 FPS

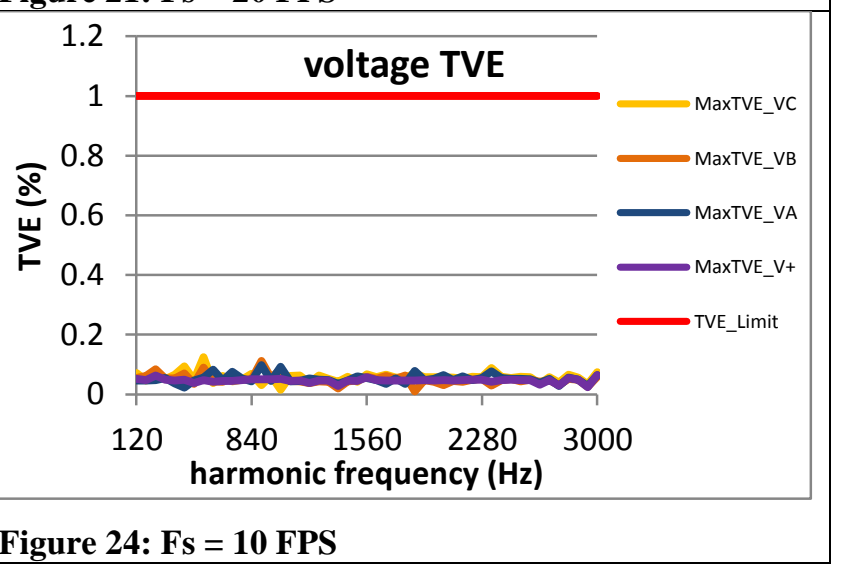


4.1.5 PMU D steady state harmonic distortion voltage TVE: M class
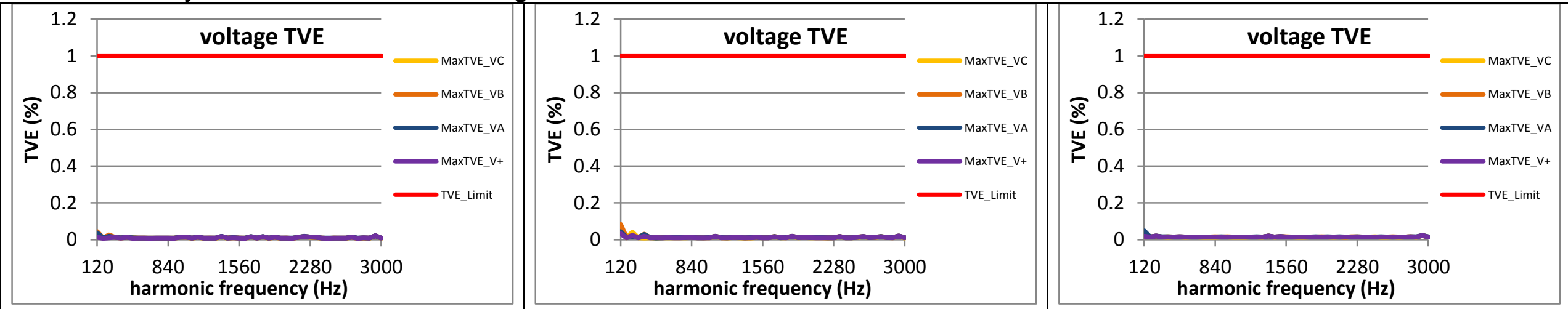

Figure 25: Fs = 60 FPS

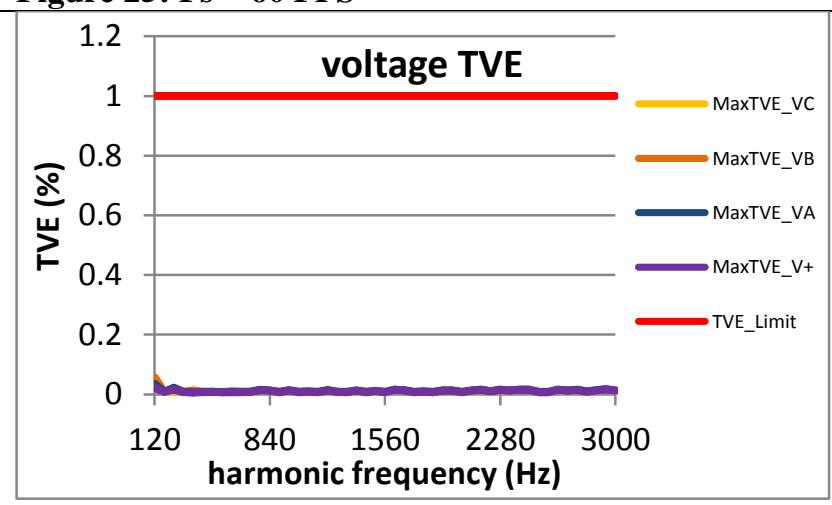

Figure 26: Fs = 30 FPS

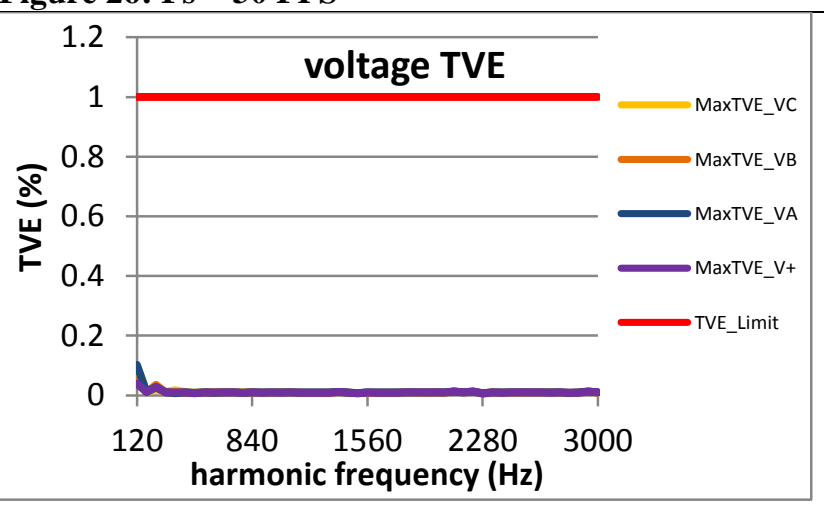

Figure 27: Fs = 20 FPS

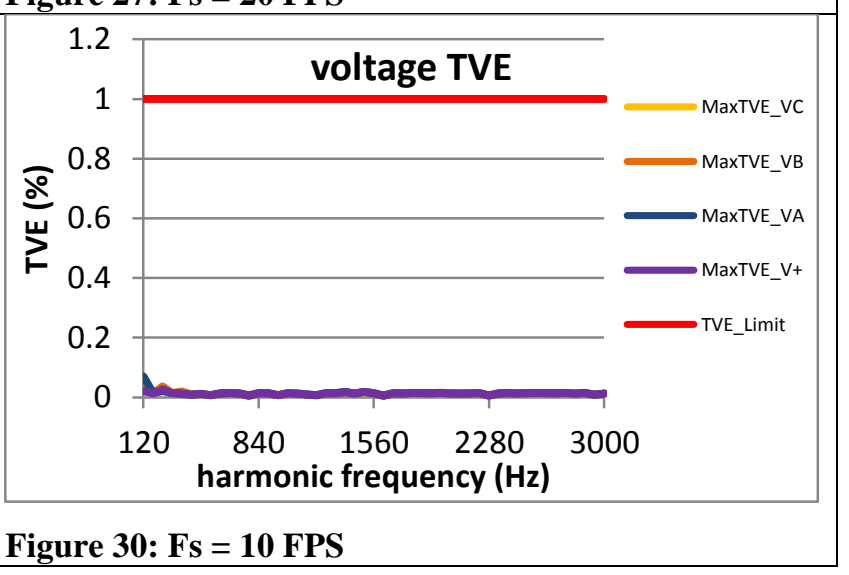

Figure 28: Fs = 15 FPS

Figure 29: Fs = 12 FPS 
4.1.6 PMU E steady state harmonic distortion voltage TVE: M class

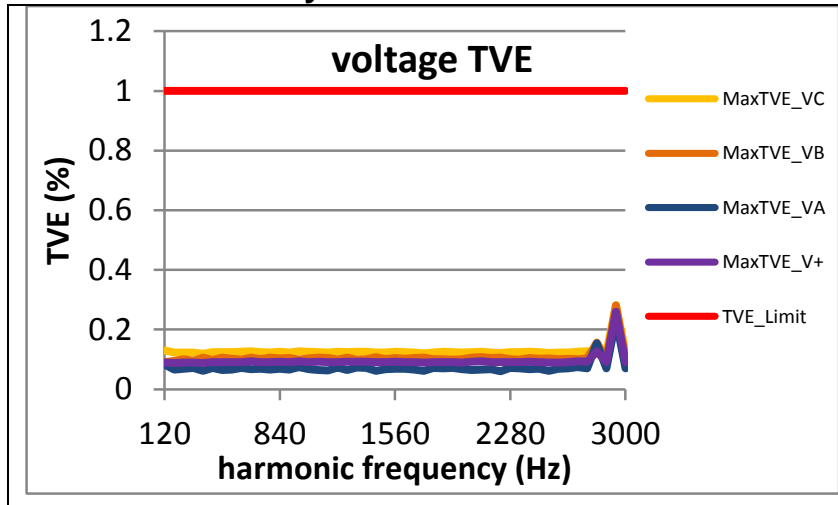

\section{Figure 31: Fs = 60 FPS}

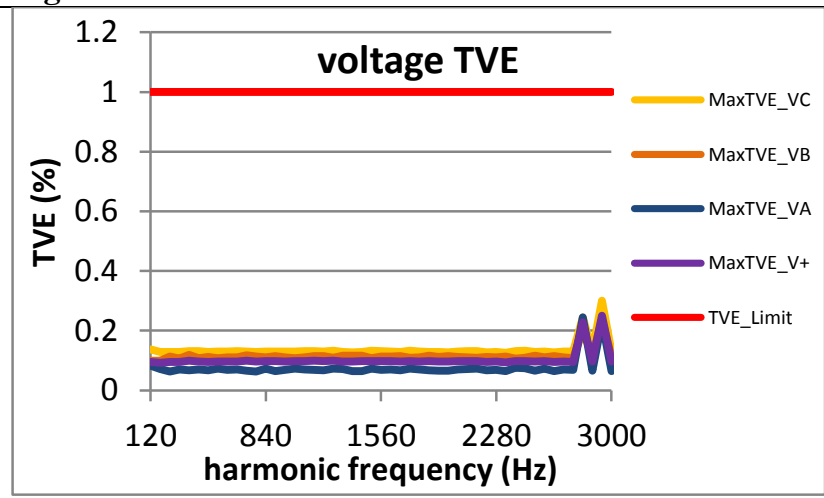

Figure 34: Fs = 15 FPS

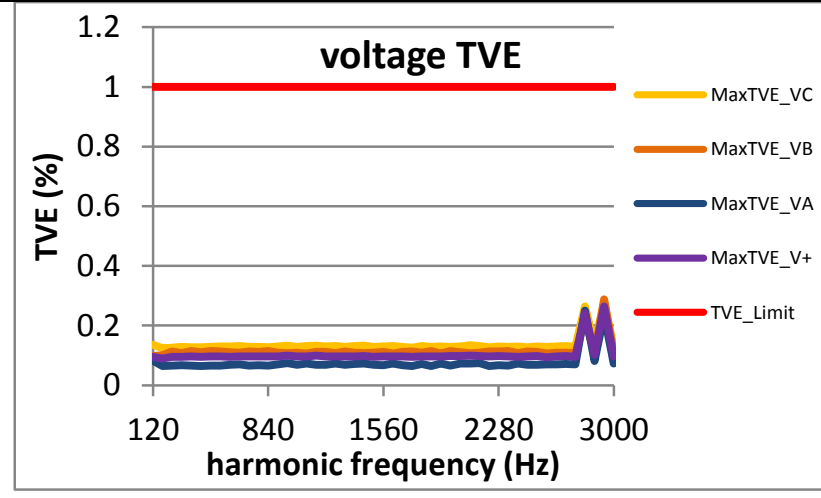

Figure 32: Fs = 30 FPS

Data was lost

Figure 35: Fs = 12 FPS

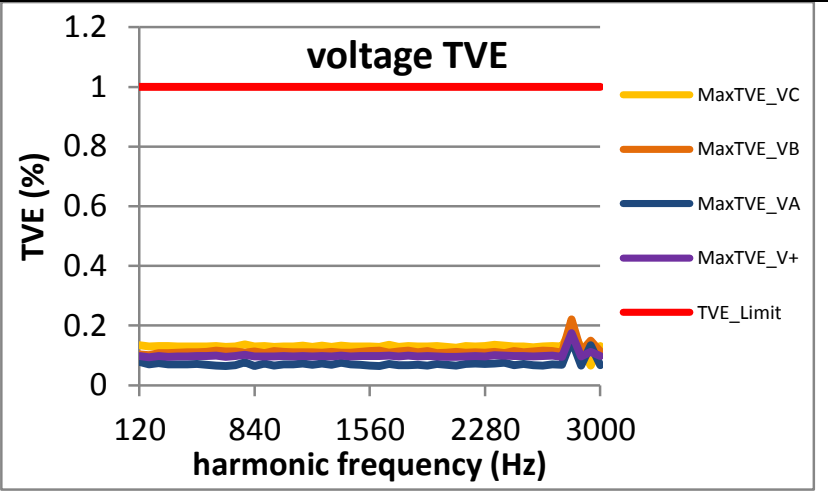

Figure 33: Fs = 20 FPS

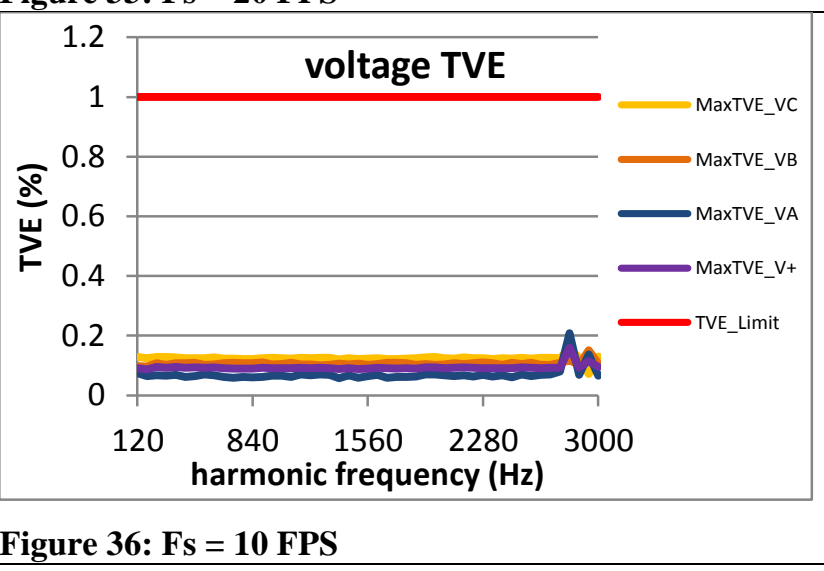


4.1.7 PMU F steady state harmonic distortion voltage TVE: M class
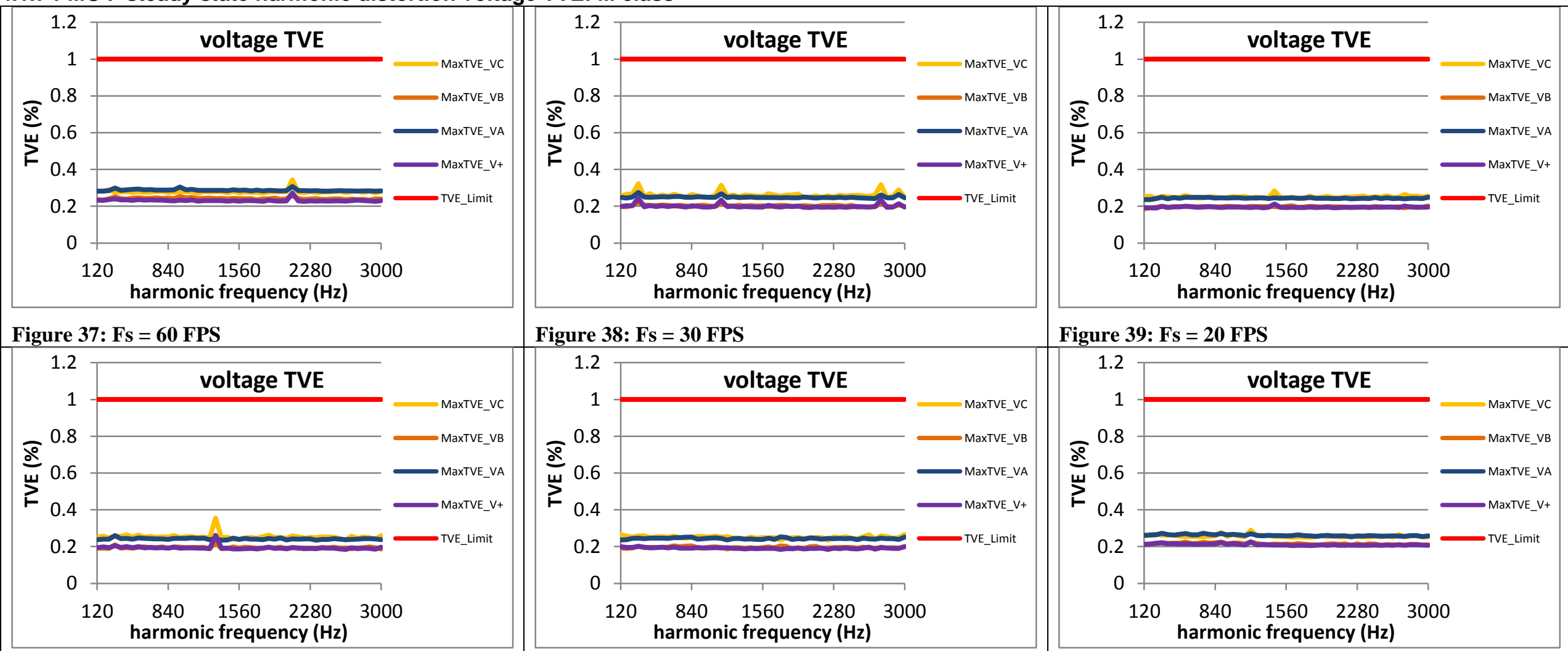

\section{Figure 38: Fs = 30 FPS}

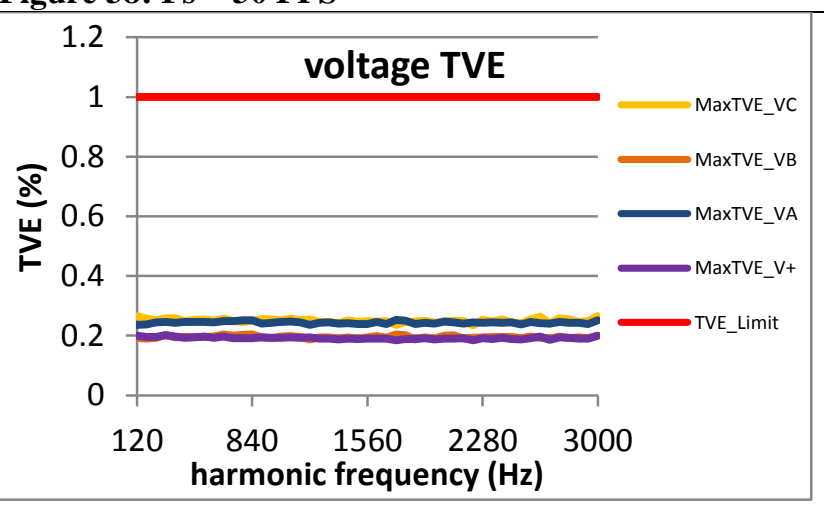

Figure 39: Fs = 20 FPS

Figure 40: Fs = 15 FPS

Figure 41: Fs = 12 FPS 


\subsubsection{PMU G steady state harmonic distortion voltage TVE: M class}

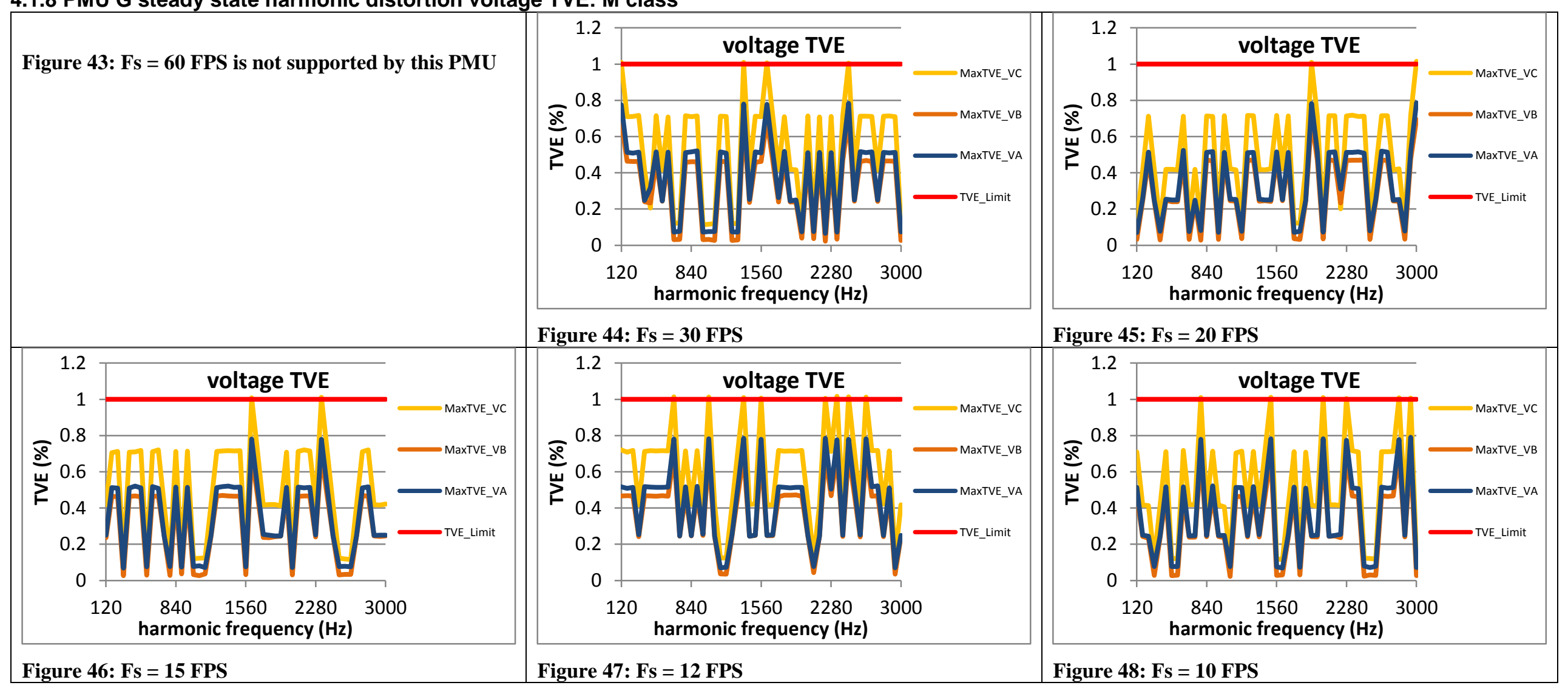


4.1.9 PMU H steady state harmonic distortion voltage TVE: M class
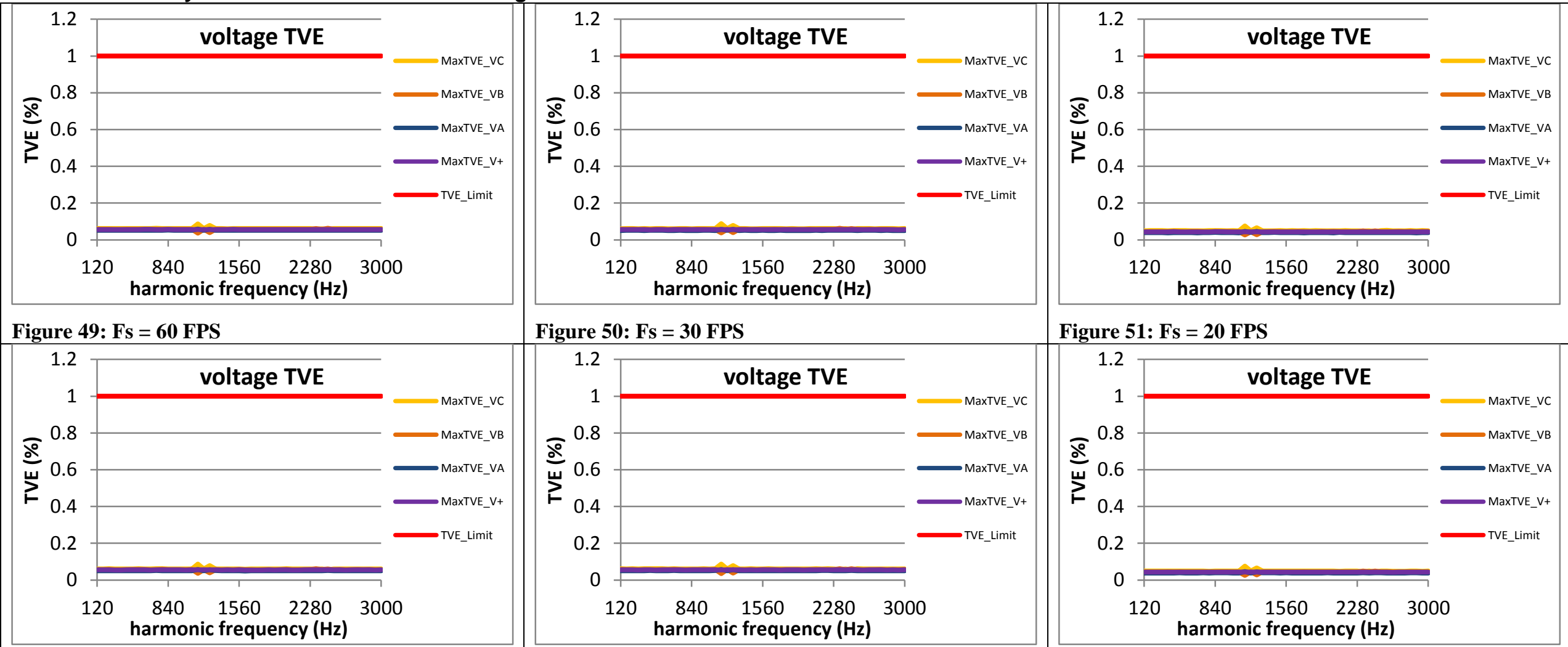

\section{Figure 50: Fs = 30 FPS}

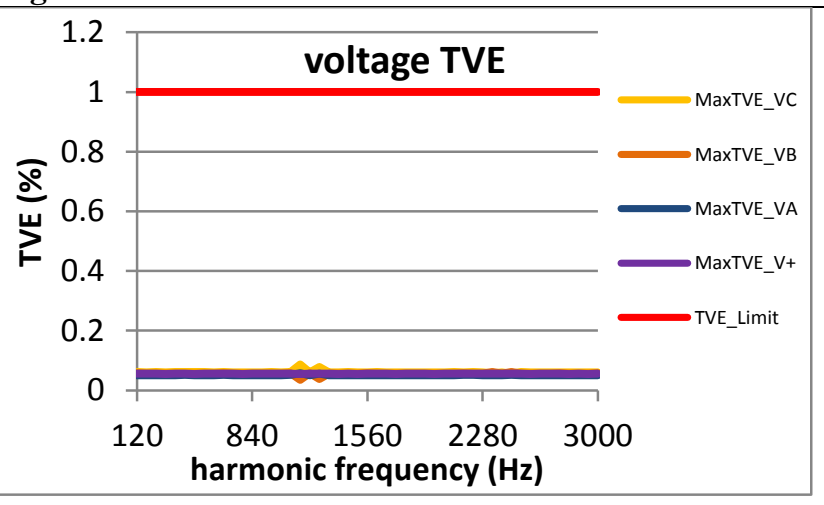

Figure 51: Fs = 20 FPS

Figure 52: Fs = 15 FPS

Figure 53: Fs = 12 FPS

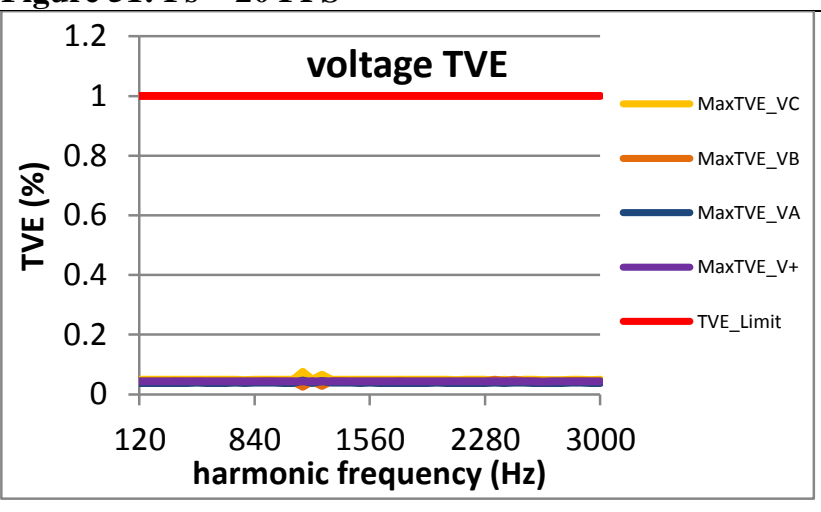

Figure 54: Fs = 10 FPS 


\subsubsection{PMU I steady state harmonic distortion voltage TVE: M class}

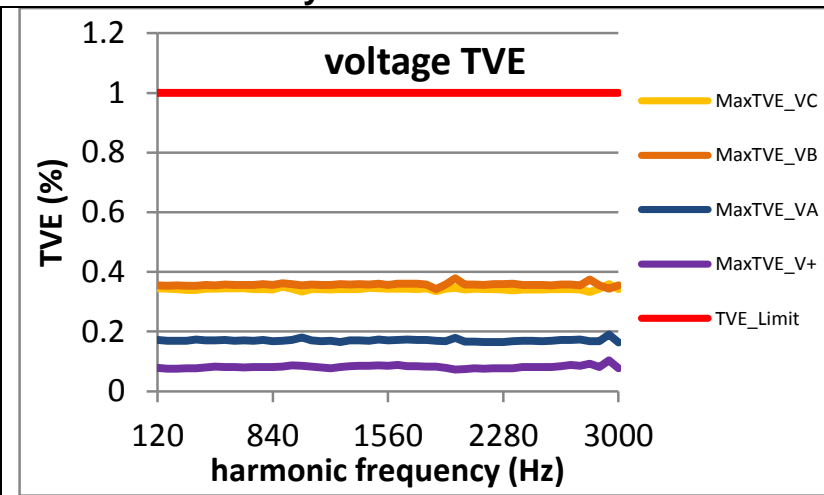

\section{Figure 55: Fs = 60 FPS}

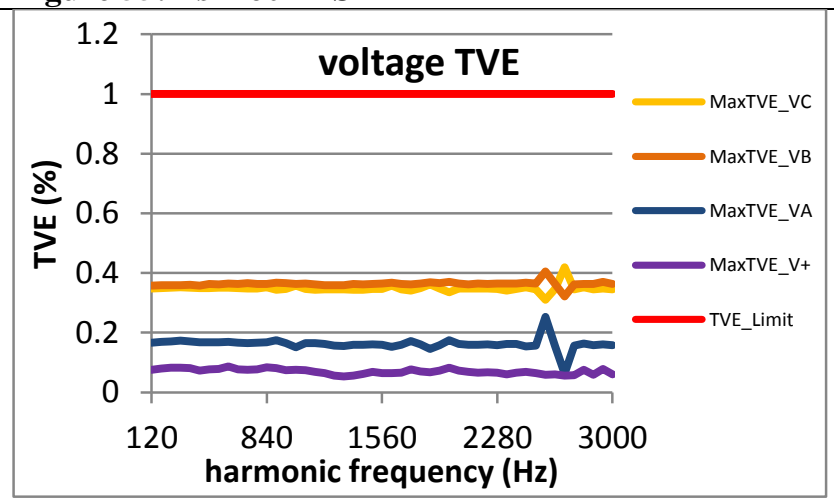

Figure 58: Fs = 15 FPS

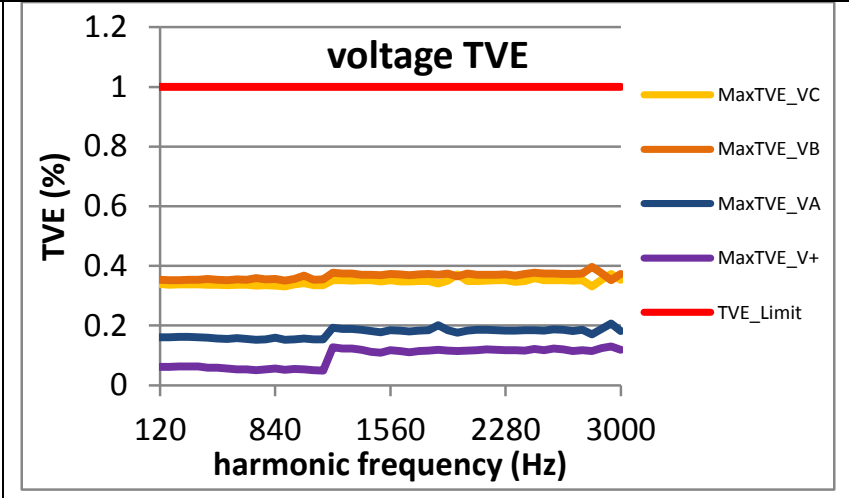

Figure 56: $\mathrm{Fs}=30$ FPS

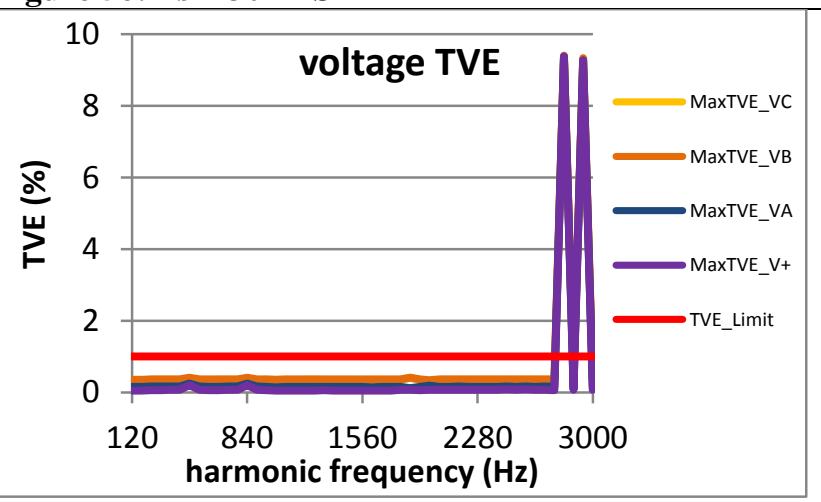

Figure 59: Fs = 12 FPS

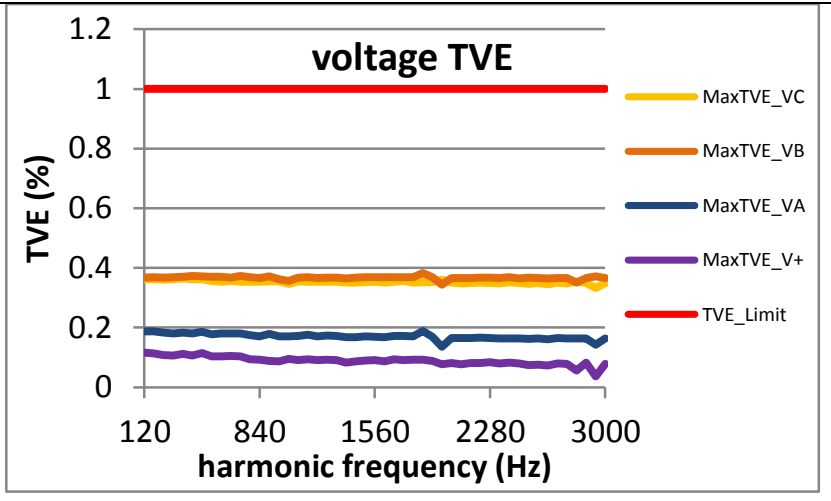

Figure 57: Fs = 20 FPS

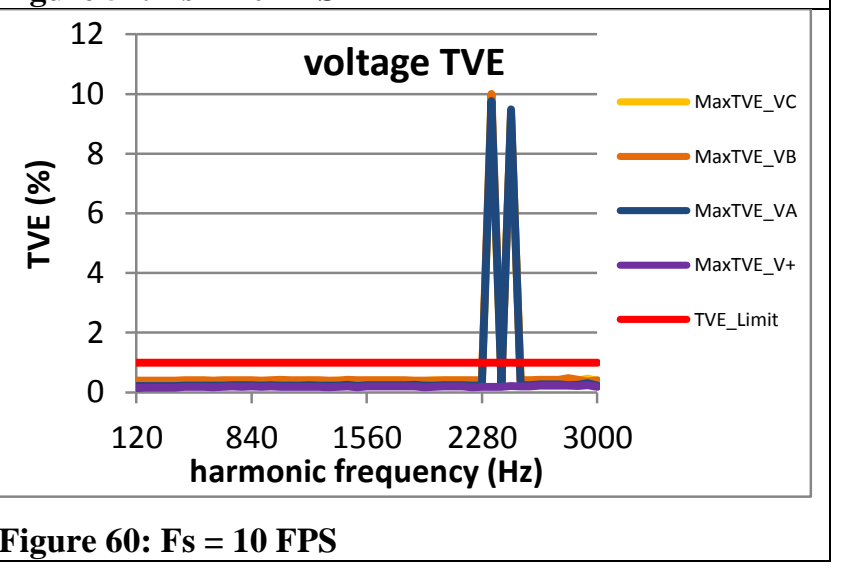


4.1.11 PMU J steady state harmonic distortion voltage TVE: M class
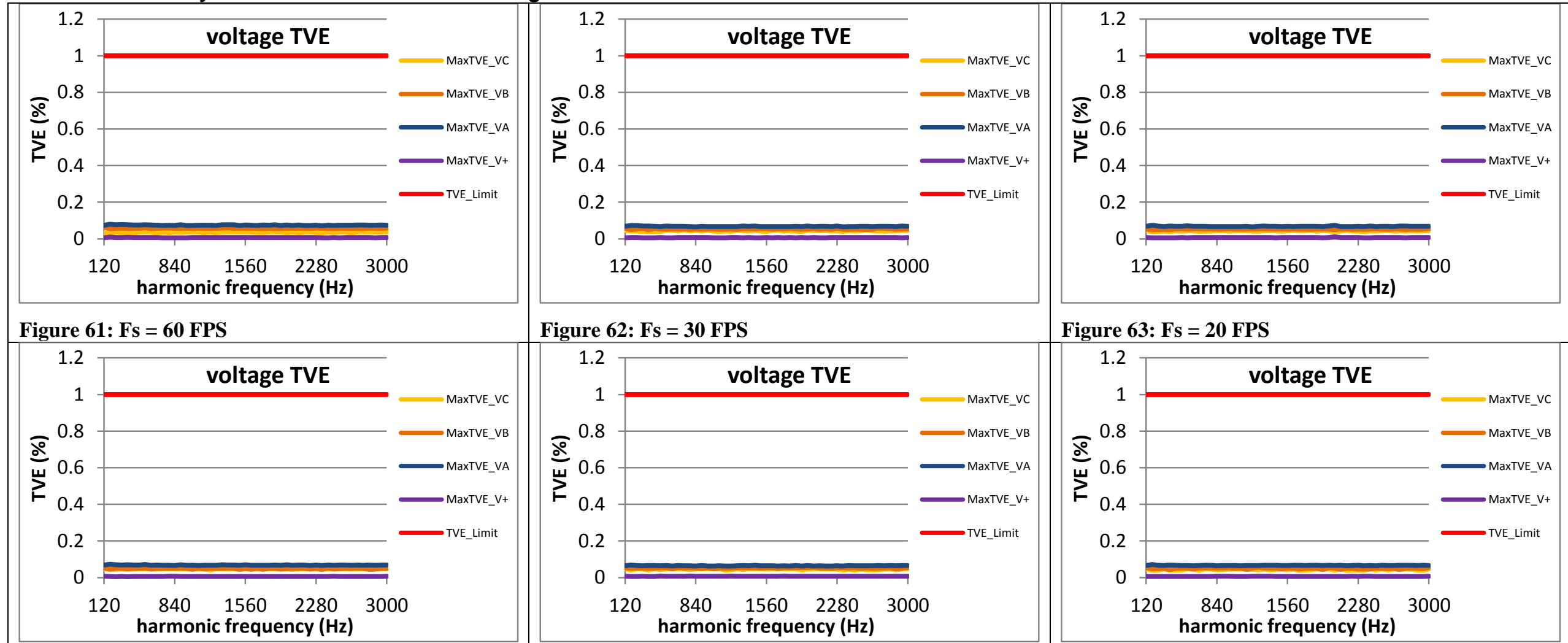

\section{Figure 62: Fs = 30 FPS}

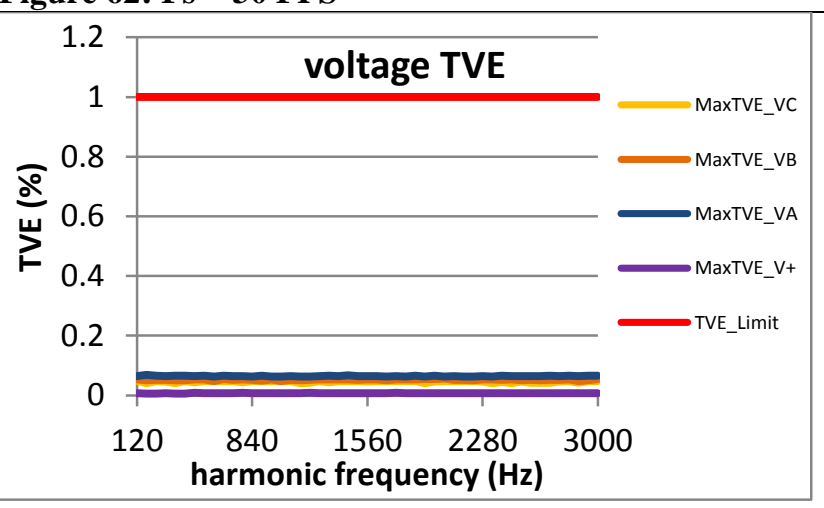

Figure 63: Fs = 20 FPS

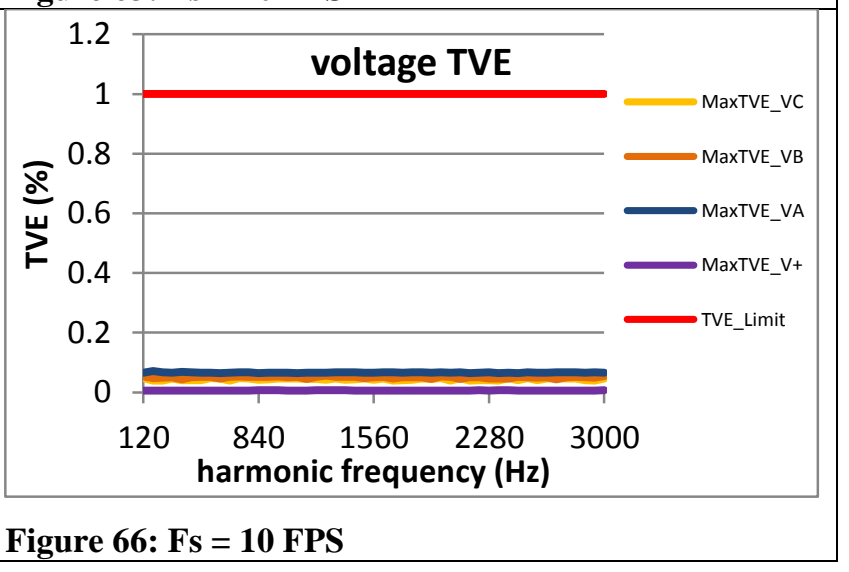

Figure 64: Fs = 15 FPS

Figure 65: Fs = 12 FPS 


\subsection{Steady state harmonic distortion current TVE: M class}

4.2.1 C37.118.1 Annex C steady state harmonic distortion current TVE: M class

The C37.118.1 Annex C model is a digital simulation with an internal sampling rate of 960 samples per second and so can only be tested with a harmonic frequency up to $420 \mathrm{~Hz}$.

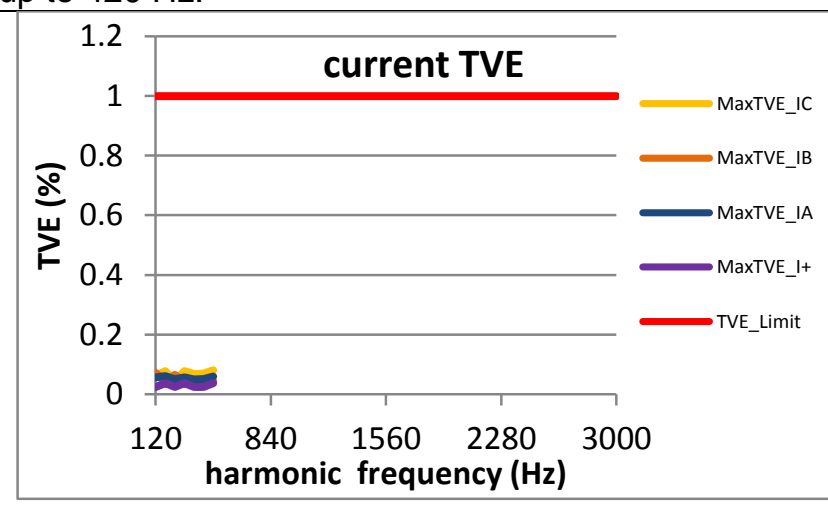

\section{Figure 67: Fs = 60 FPS}

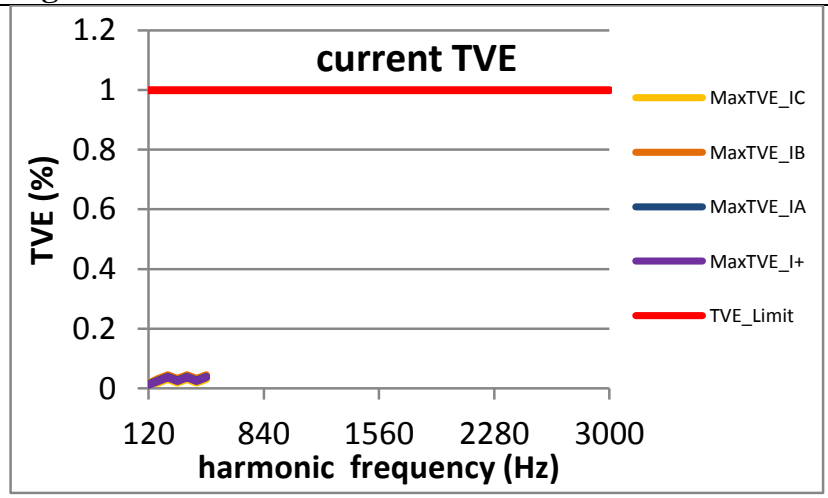

Figure 70: Fs = 15 FPS

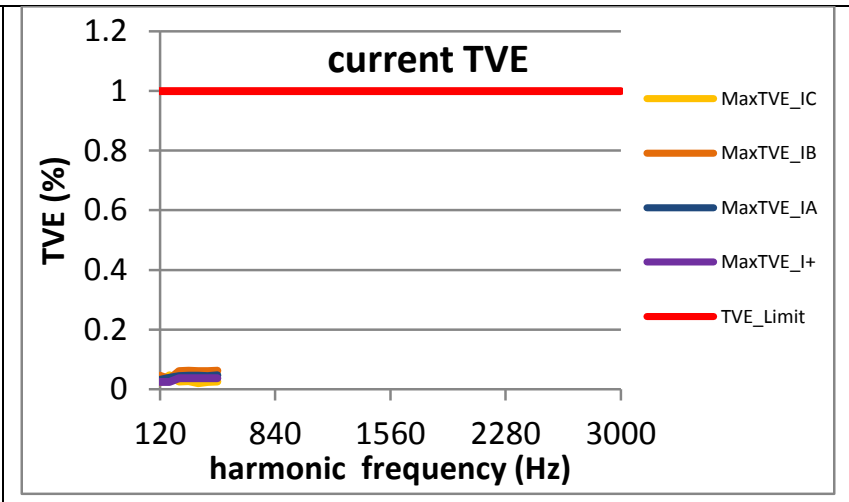

Figure 68: Fs = 30 FPS

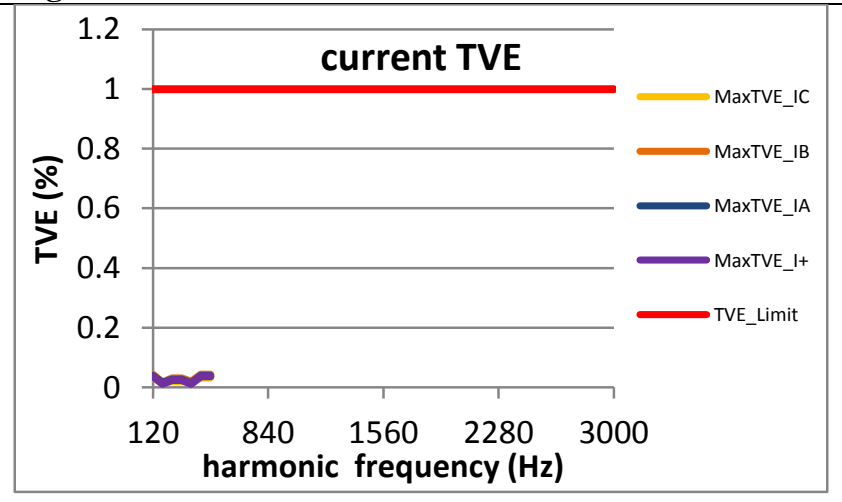

Figure 71: Fs = 12 FPS

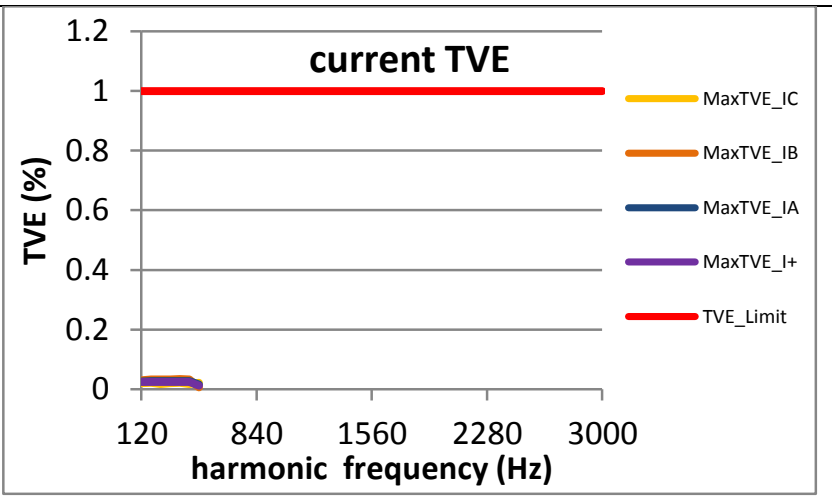

Figure 69: Fs = 20 FPS

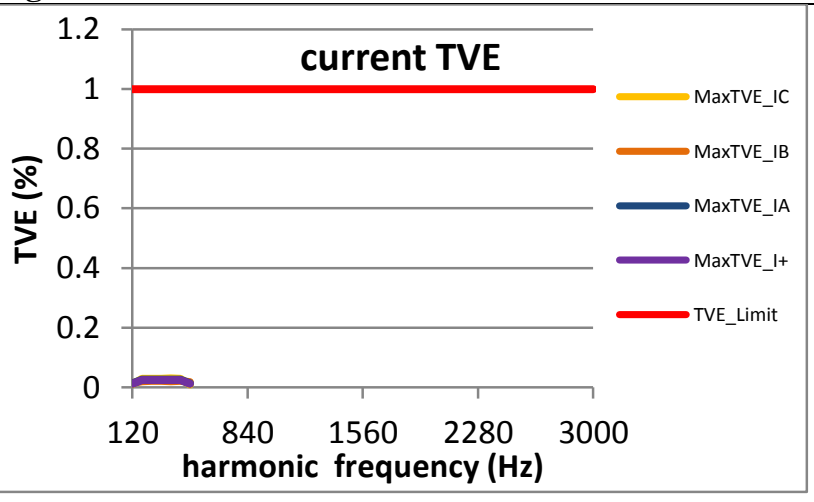

Figure 72: Fs = 10 FPS 
4.2.2 PMU A steady state harmonic distortion current TVE: M class
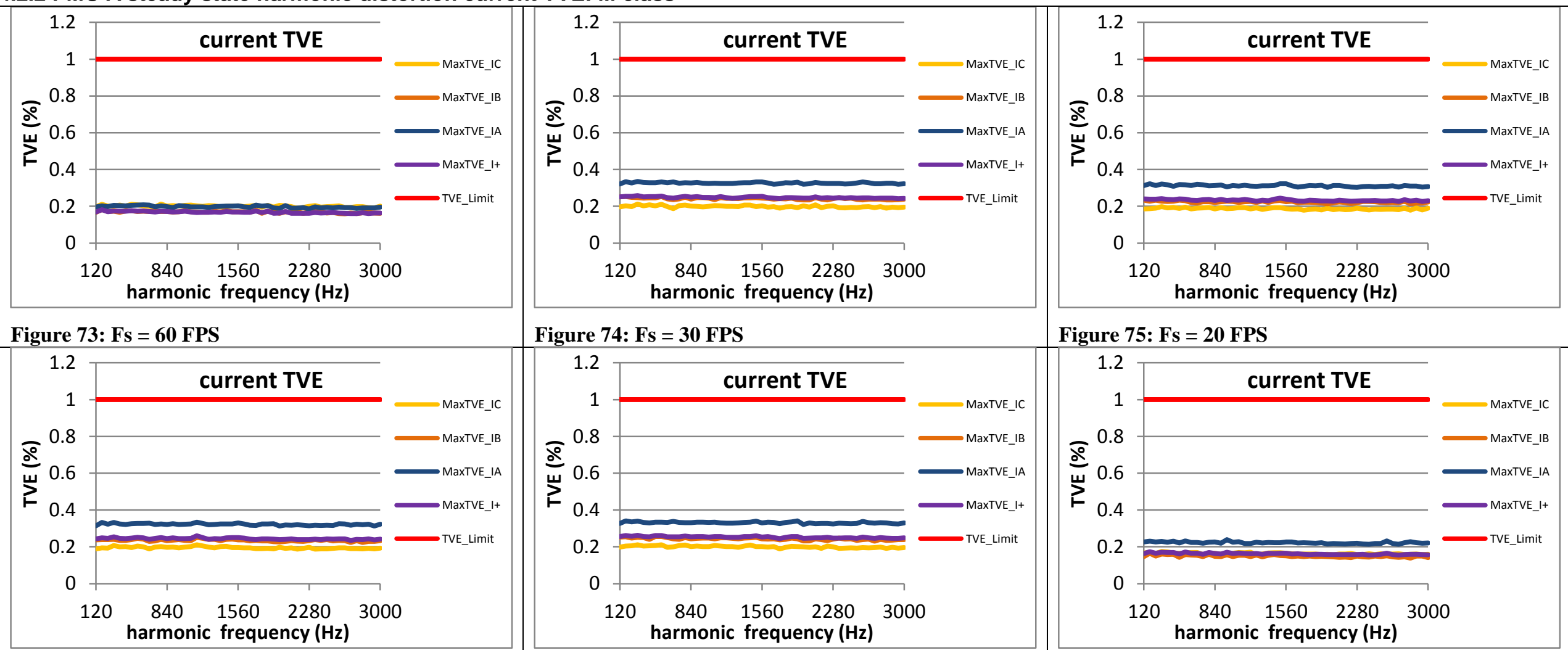

\section{Figure 74: Fs = 30 FPS}

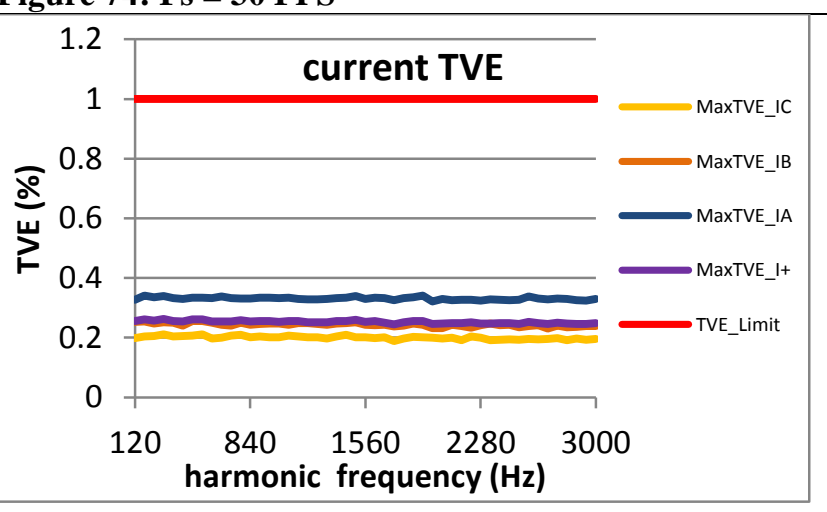

Figure 75: Fs = 20 FPS

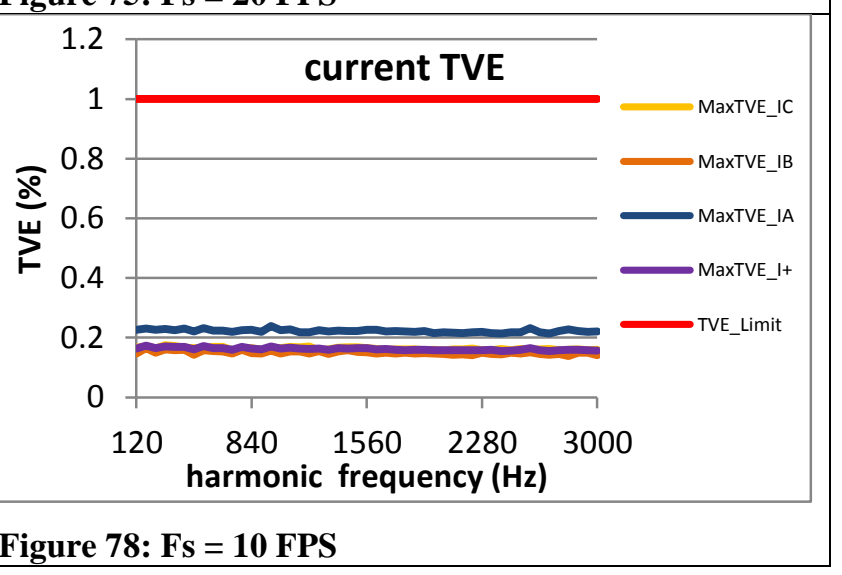

Figure 76: Fs = 15 FPS

Figure 77: Fs = 12 FPS 
4.2.3 PMU B steady state harmonic distortion current TVE: M class
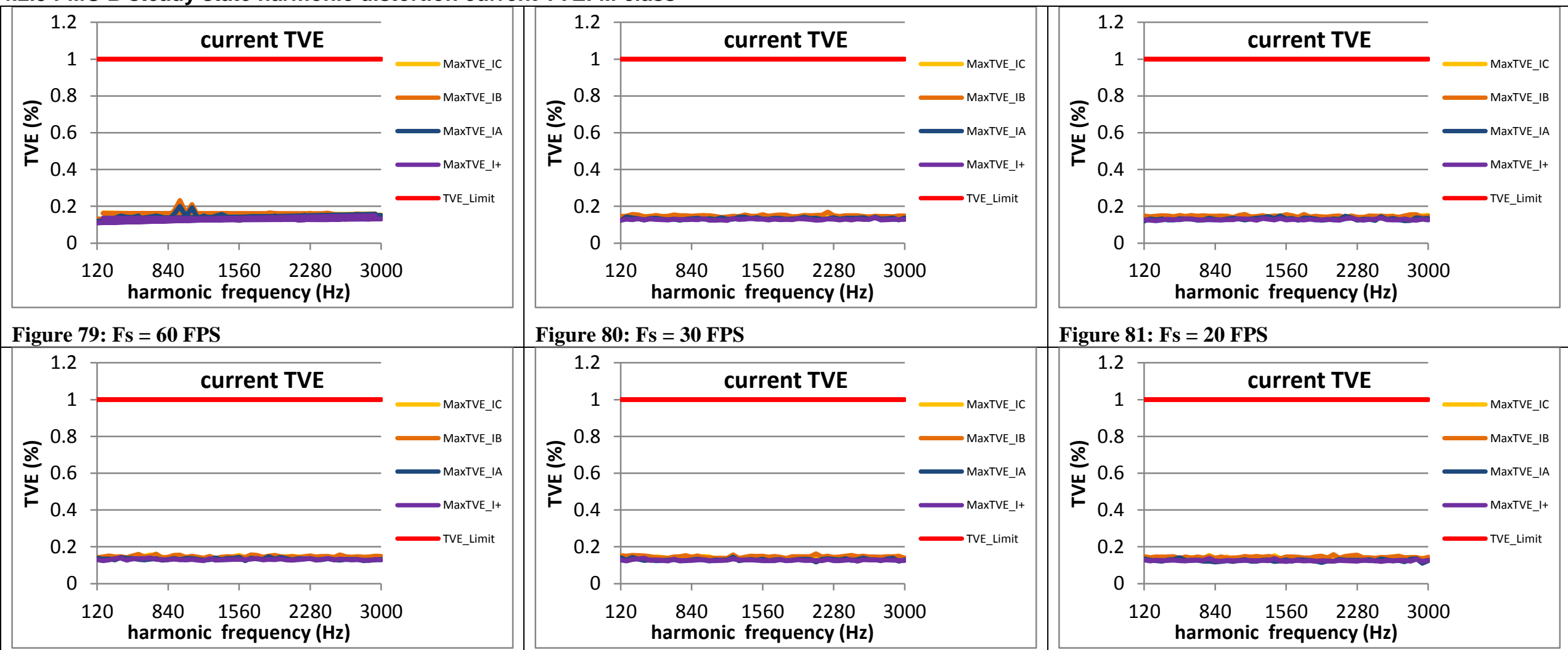

\section{Figure 80: Fs = 30 FPS}

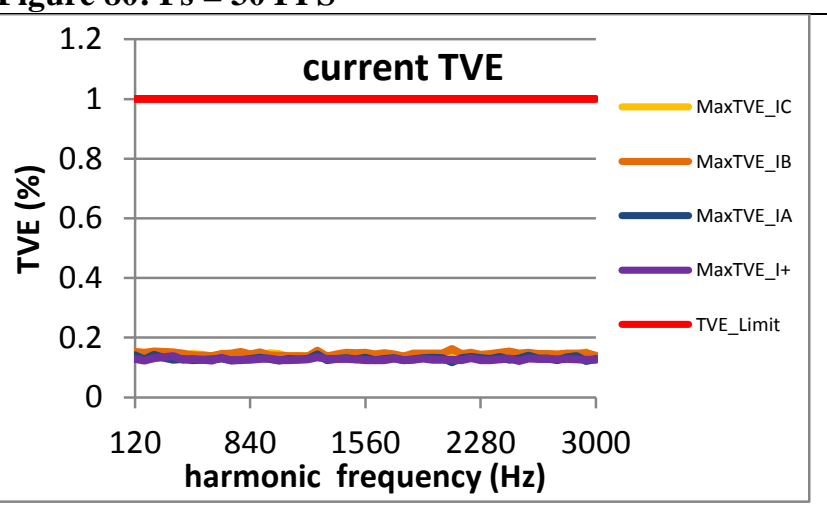

Figure 81: Fs = 20 FPS

Figure 82: Fs = 15 FPS

Figure 83: Fs = 12 FPS 
4.2.4 PMU C steady state harmonic distortion current TVE: M class
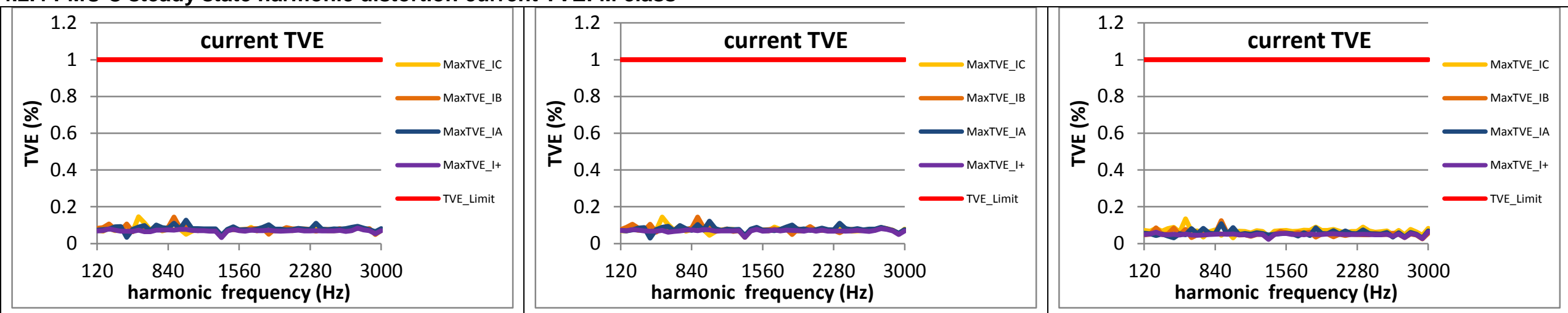

Figure 85: Fs = 60 FPS

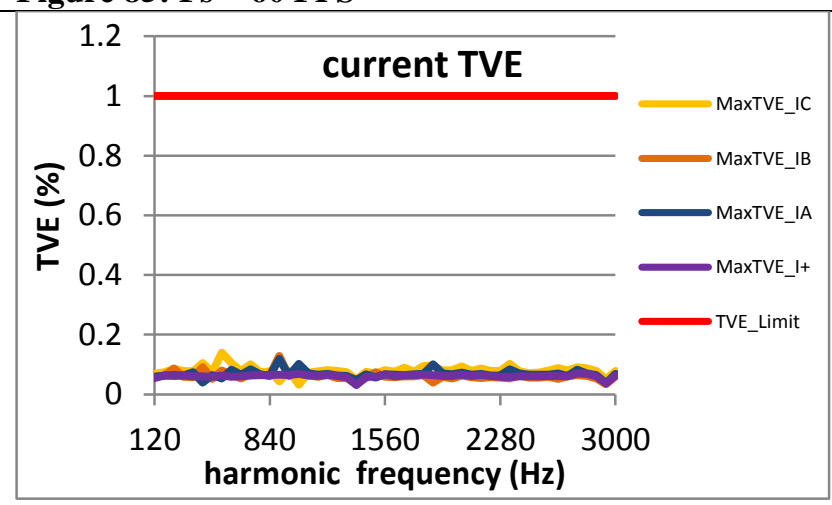

Figure 86: Fs = 30 FPS

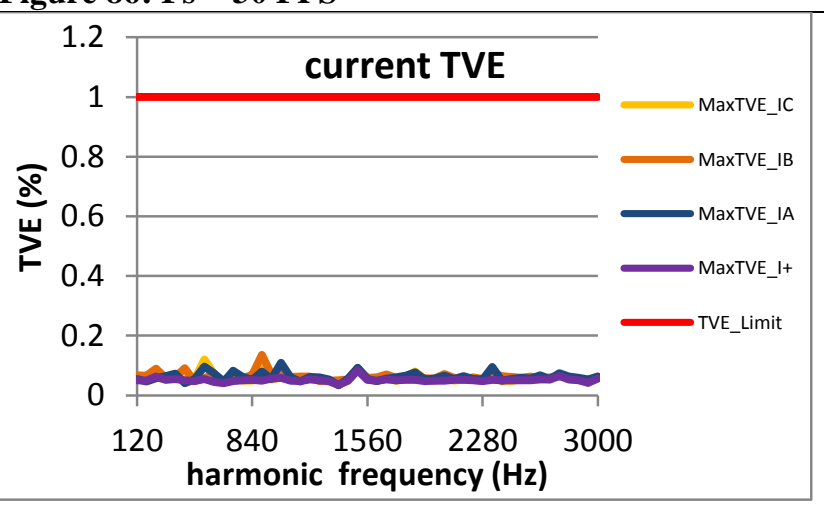

Figure 87: Fs = 20 FPS

Figure 88: Fs = 15 FPS

Figure 89: Fs = 12 FPS 
4.2.5 PMU D steady state harmonic distortion current TVE: M class
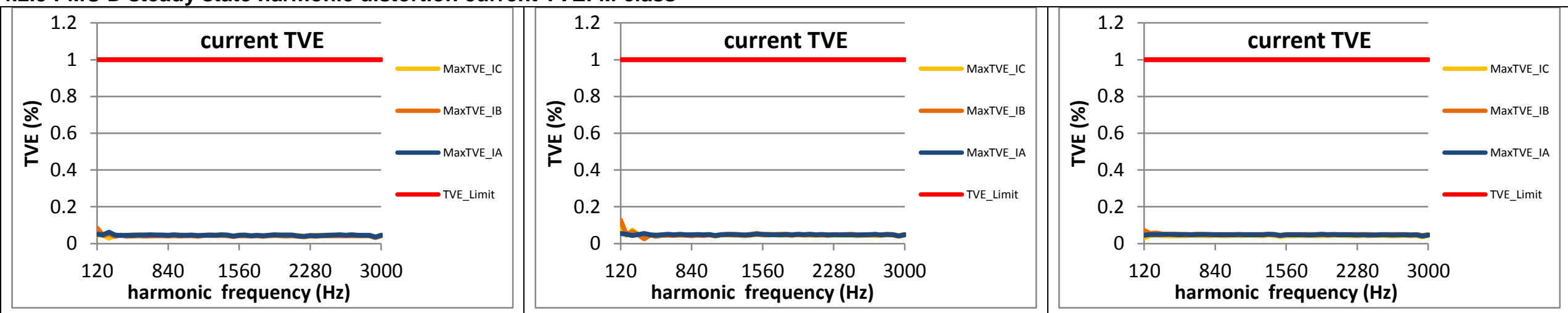

Figure 91: Fs = 60 FPS

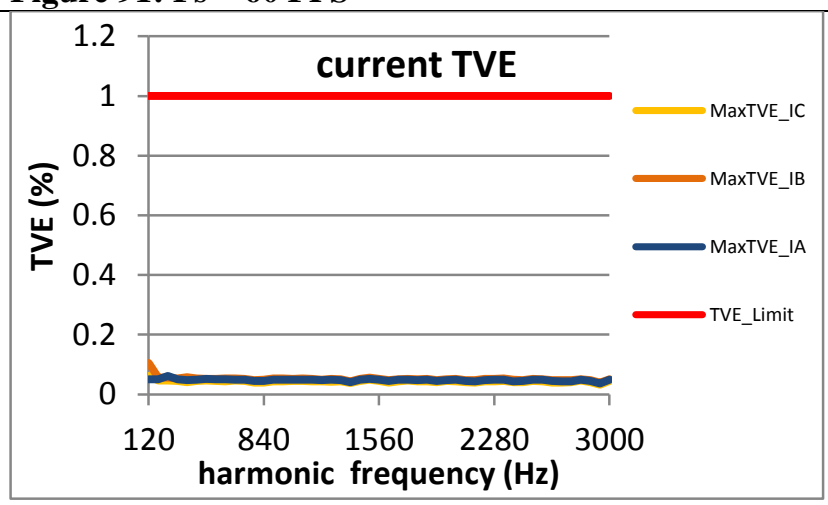

Figure 92: Fs = 30 FPS

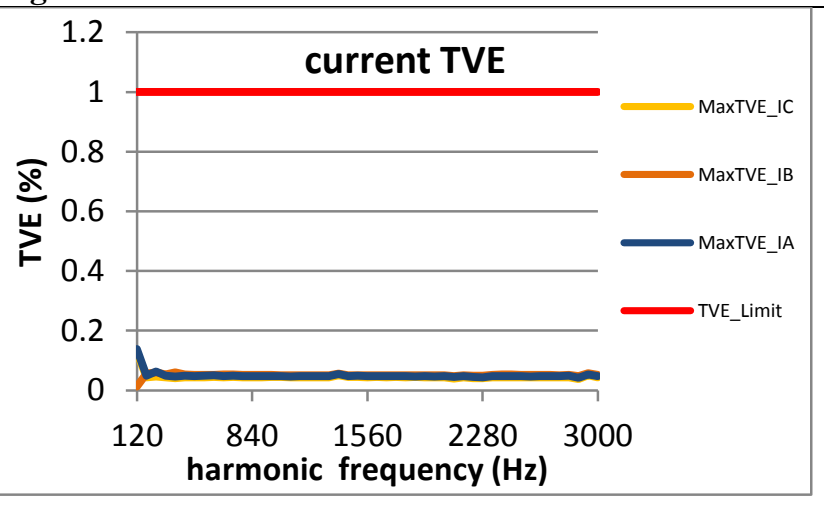

Figure 93: Fs = 20 FPS

Figure 94: Fs = 15 FPS

Figure 95: Fs = 12 FPS 
4.2.6 PMU E steady state harmonic distortion current TVE: M class

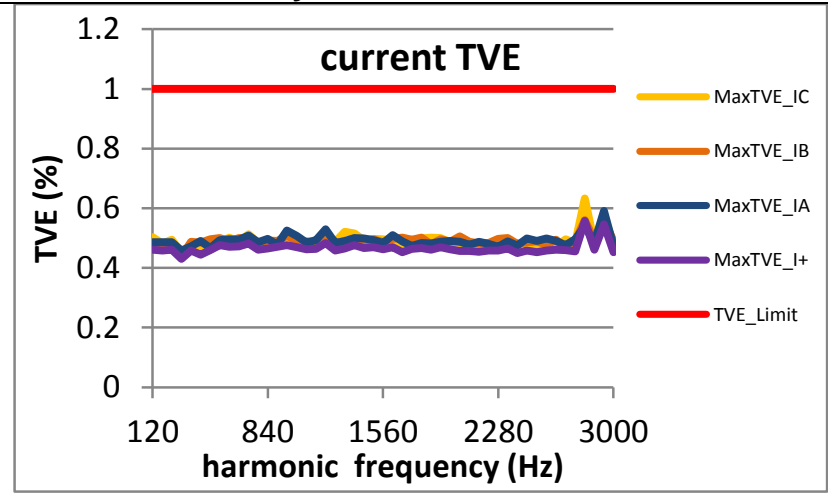

Figure 97: Fs = 60 FPS

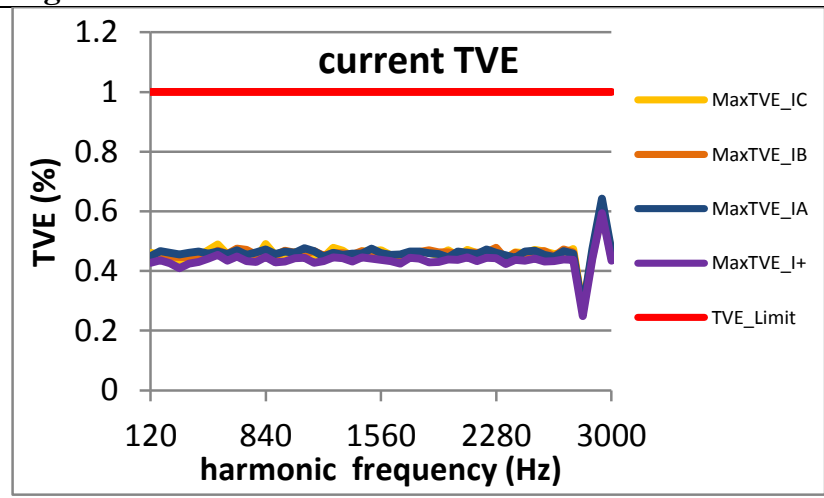

Figure 100: Fs = 15 FPS

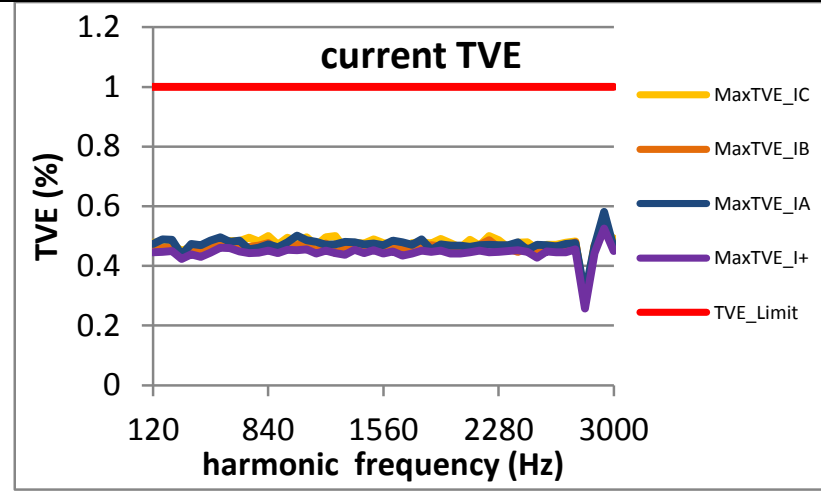

Figure 98: Fs = 30 FPS

data was lost

Figure 101: Fs = 12 FPS

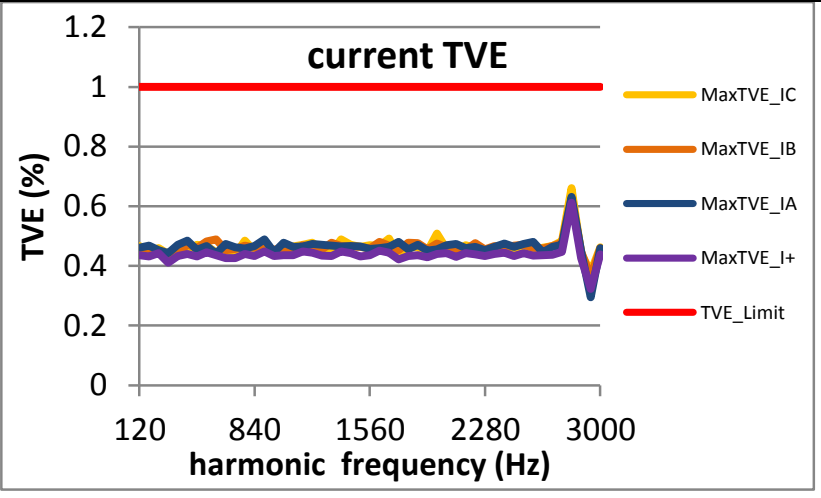

Figure 99: Fs = 20 FPS

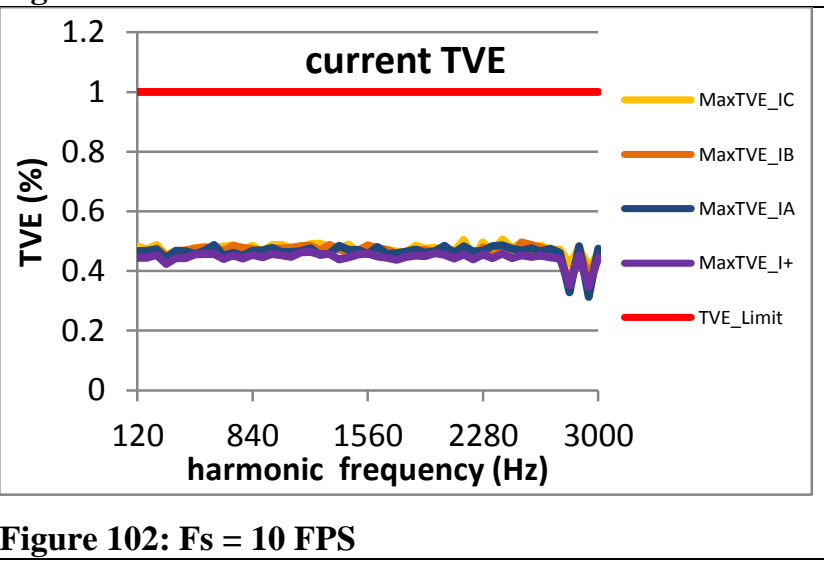


4.2.7 PMU F steady state harmonic distortion current TVE: M class
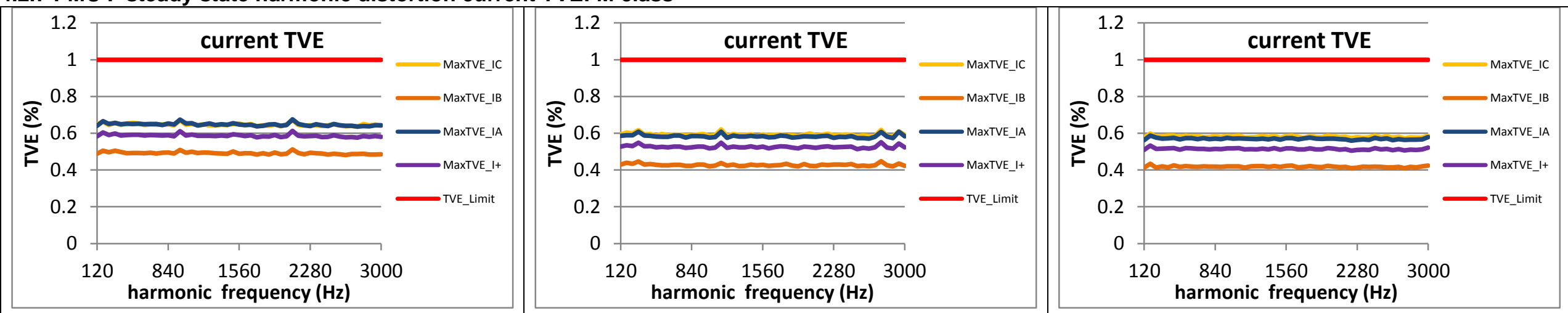

Figure 103: Fs = 60 FPS

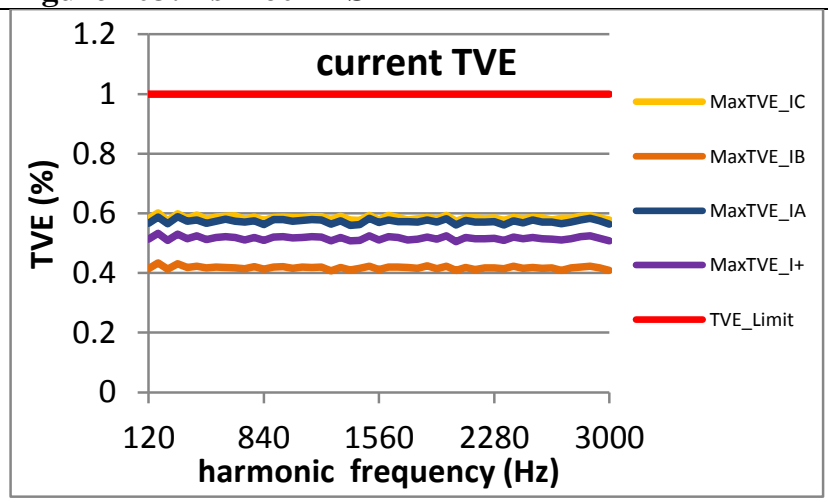

Figure 104: Fs = 30 FPS

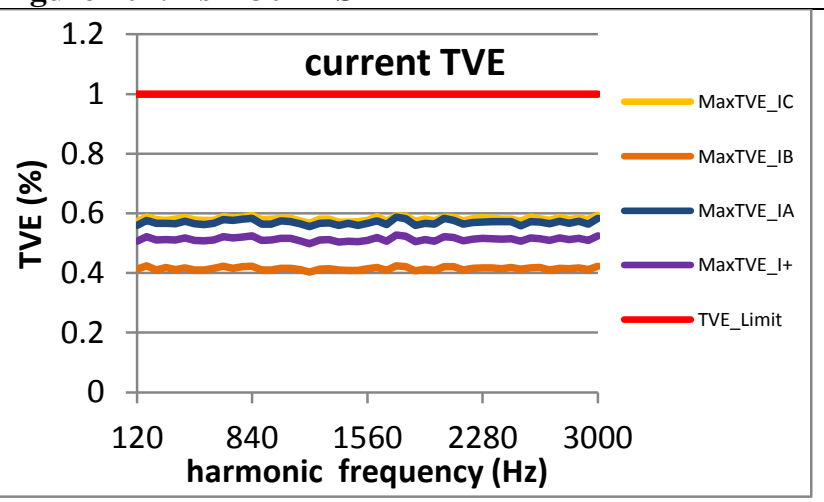

Figure 105: Fs = 20 FPS

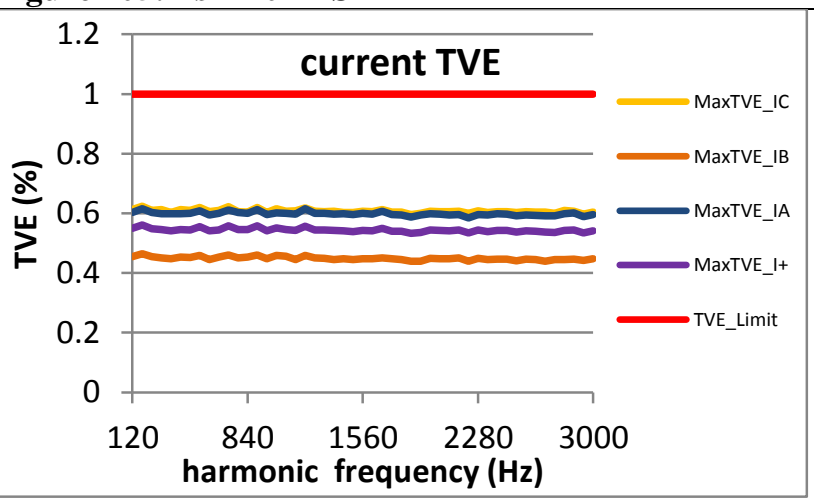

Figure 106: Fs = 15 FPS

Figure 107: Fs = 12 FPS 


\subsubsection{PMU G steady state harmonic distortion current TVE: M class}

Figure 109: Fs = 60 FPS is not supported by this PMU

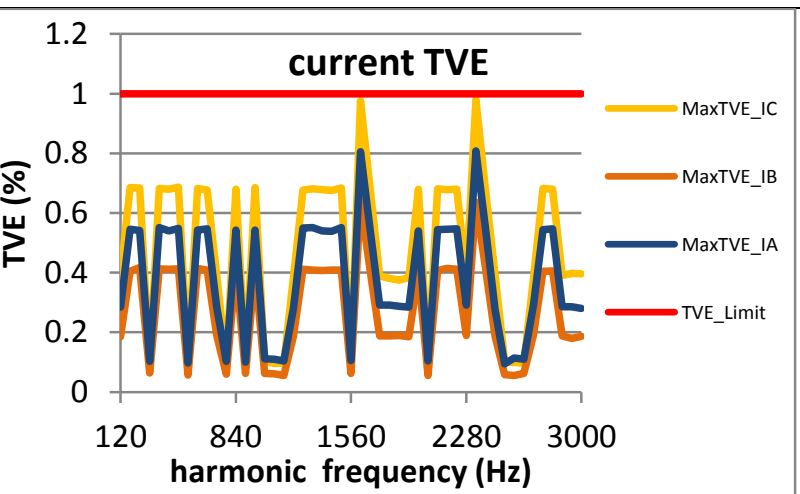

Figure 112: Fs = 15 FPS

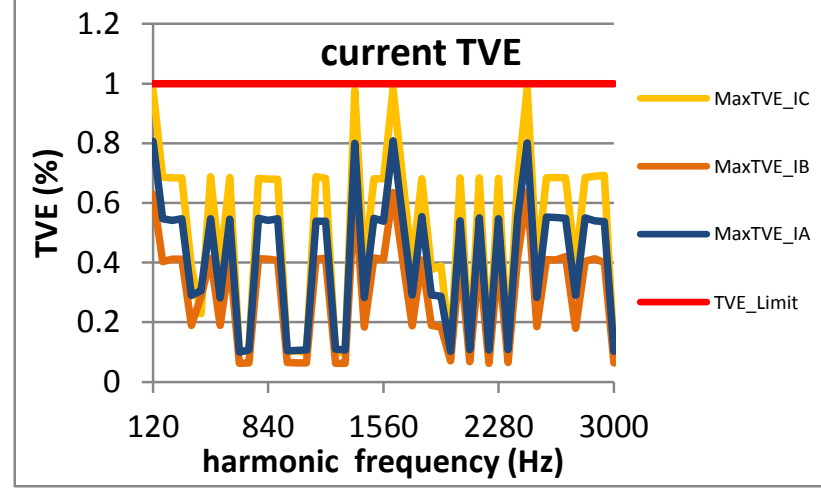

Figure 110: Fs = 30 FPS

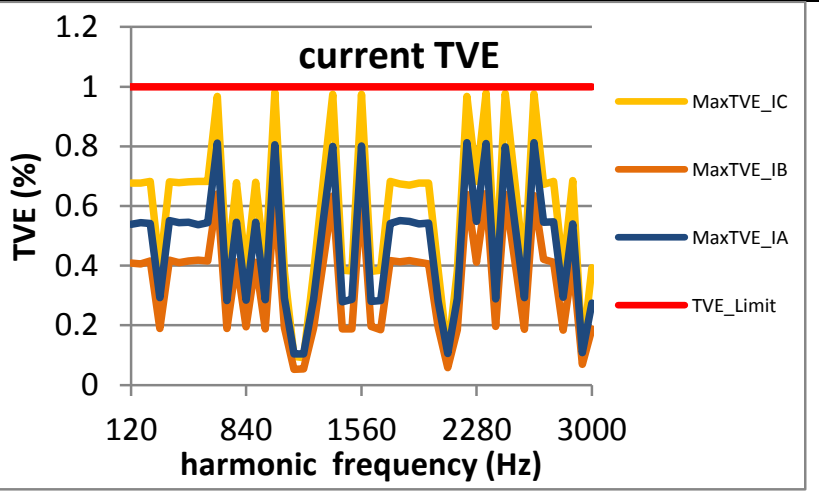

Figure 113: Fs = 12 FPS

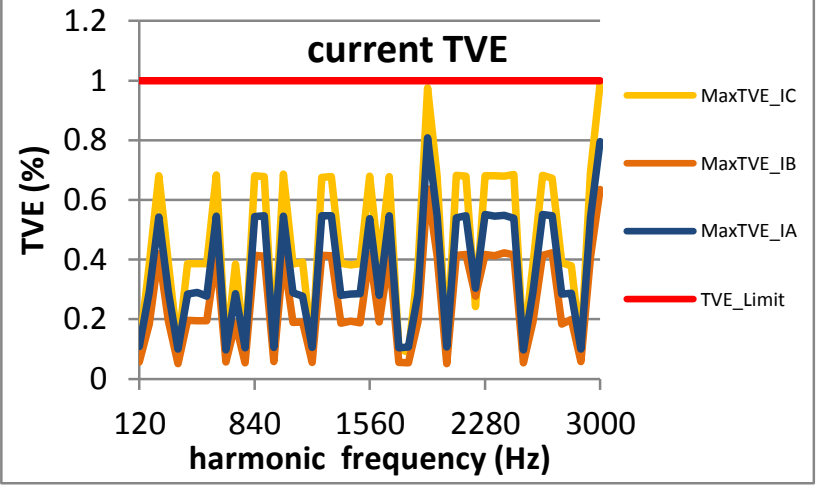

Figure 111: Fs = 20 FPS

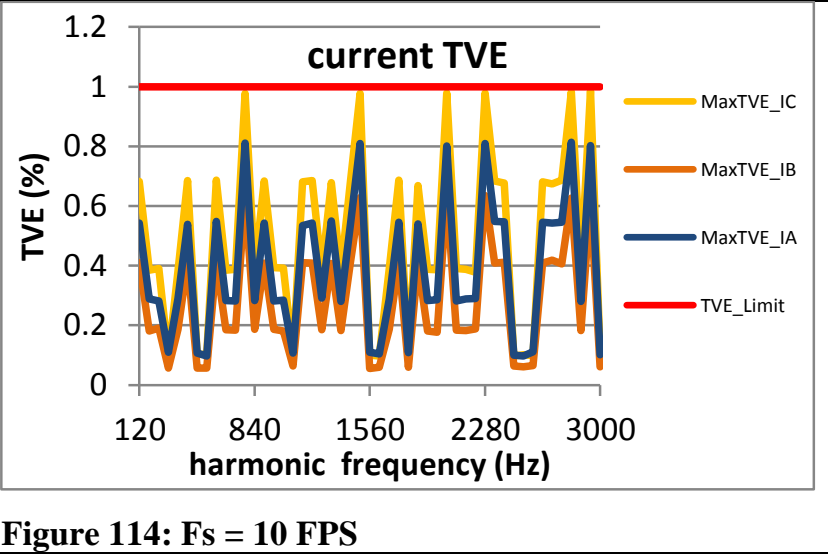


4.2.9 PMU H steady state harmonic distortion current TVE: M class

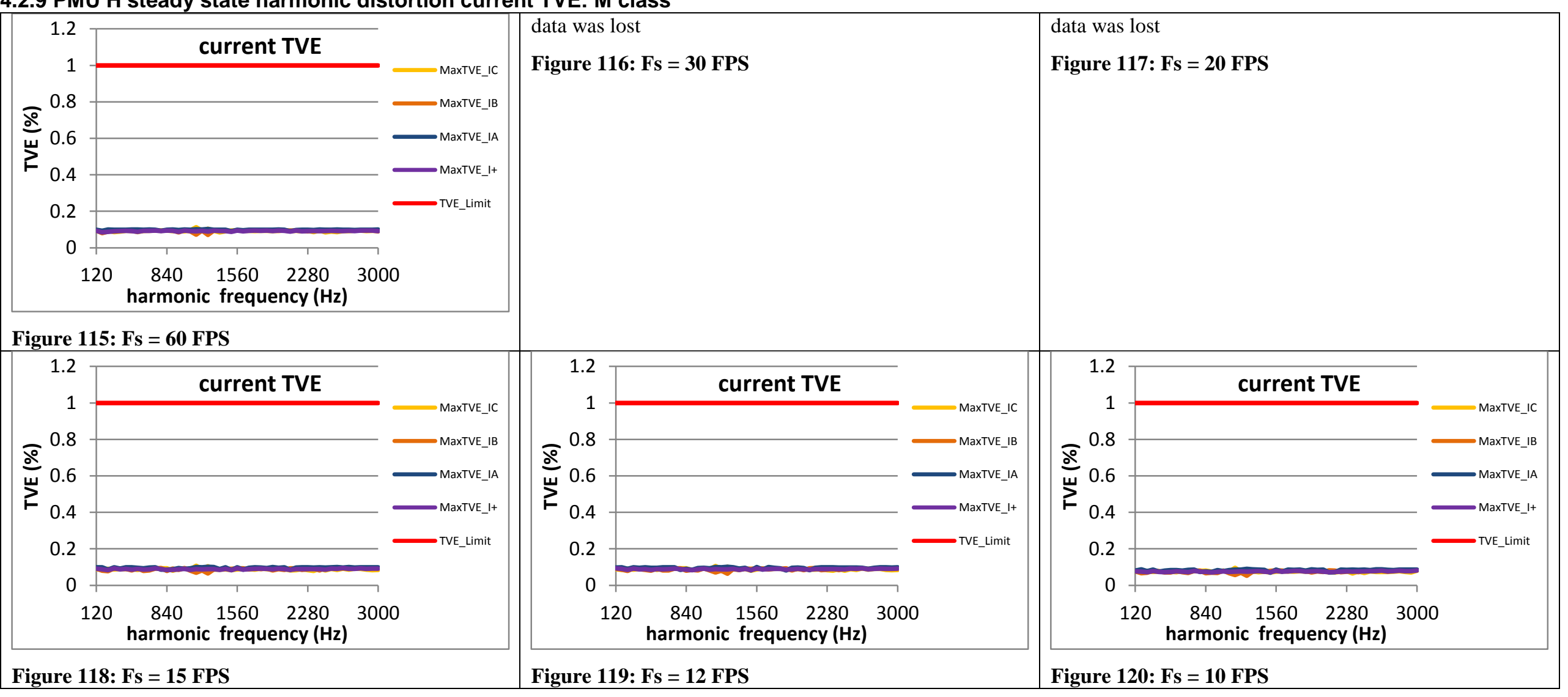




\subsubsection{PMU I steady state harmonic distortion current TVE: M class}
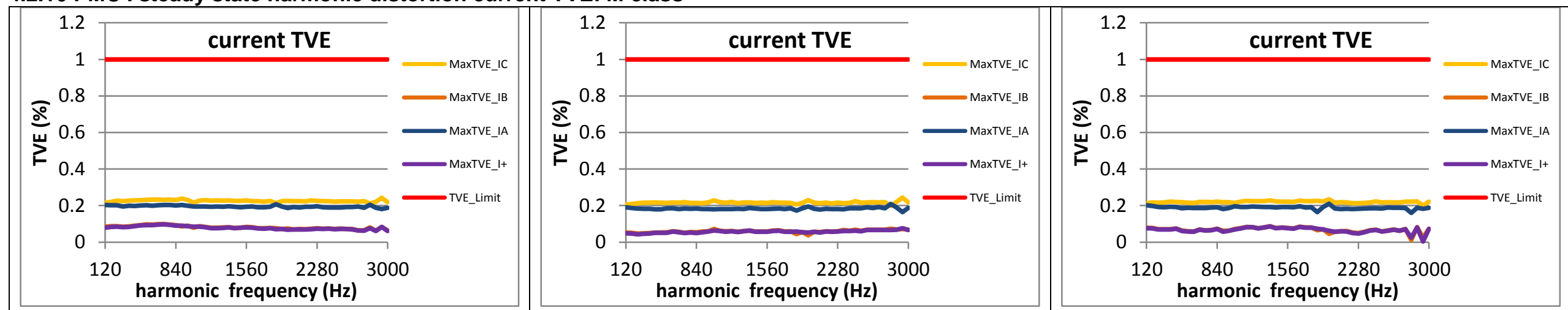

Figure 121: Fs = 60 FPS

Figure 122: Fs = 30 FPS
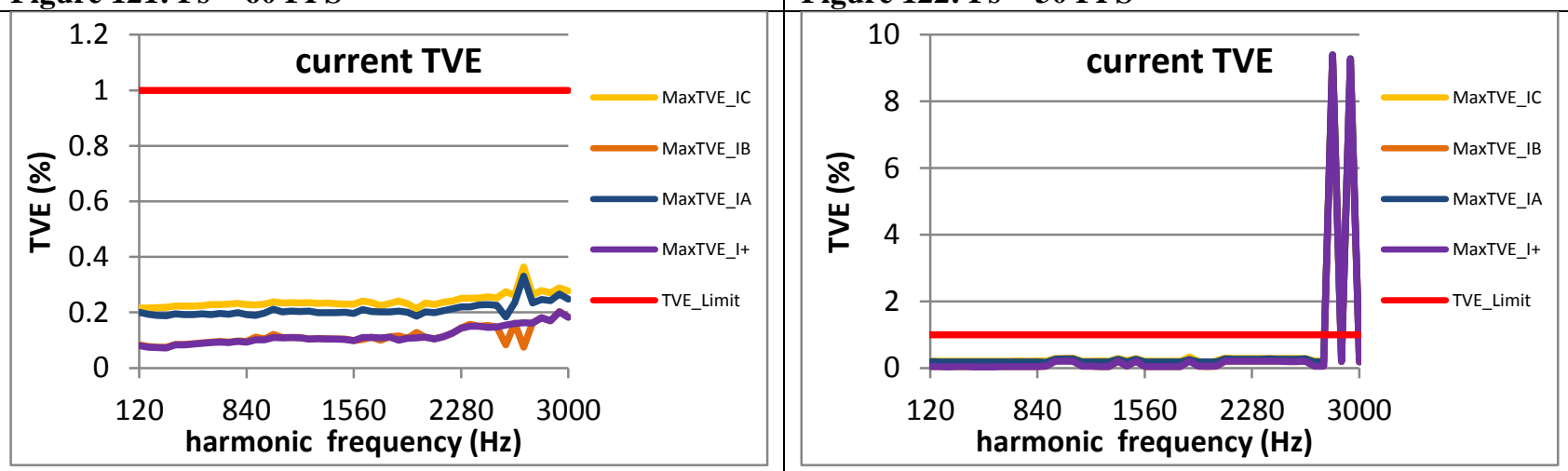

Figure 123: Fs = 20 FPS

Figure 124: Fs = 15 FPS

Figure 125: Fs = 12 FPS

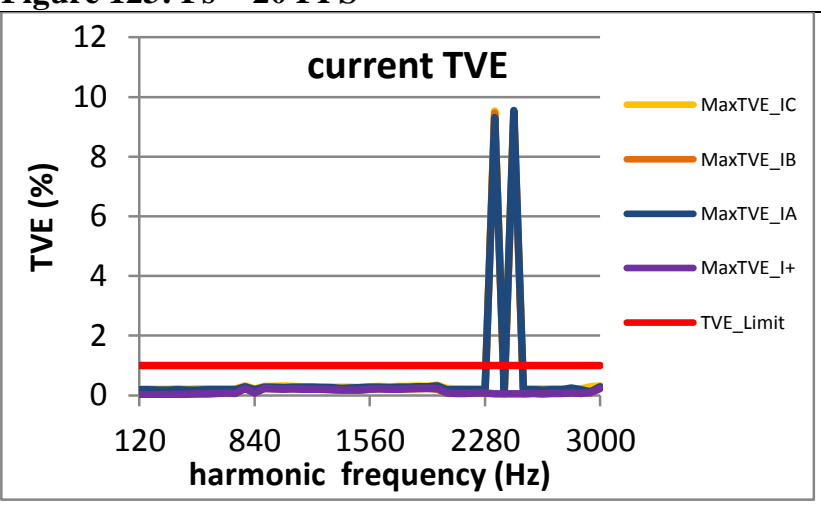

Figure 126: Fs = 10 FPS 
4.2.11 PMU J steady state harmonic distortion current TVE: M class
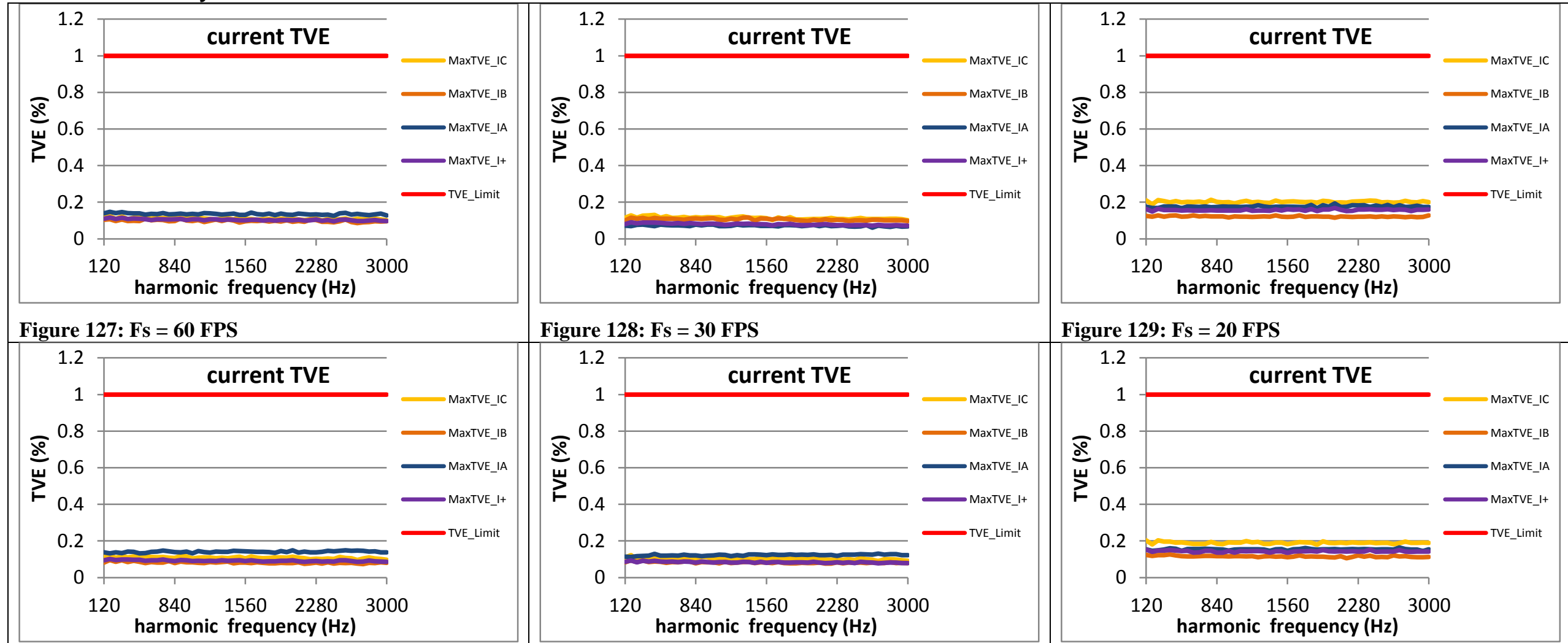

\section{Figure 128: Fs = 30 FPS}

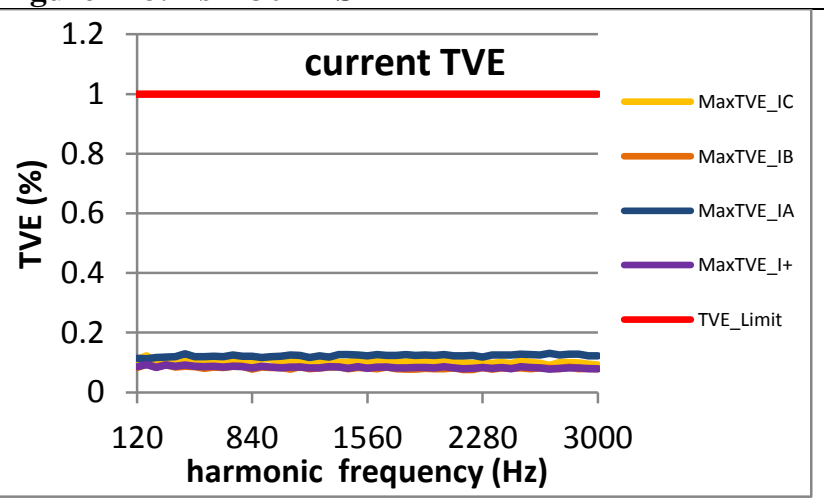

Figure 129: Fs = 20 FPS

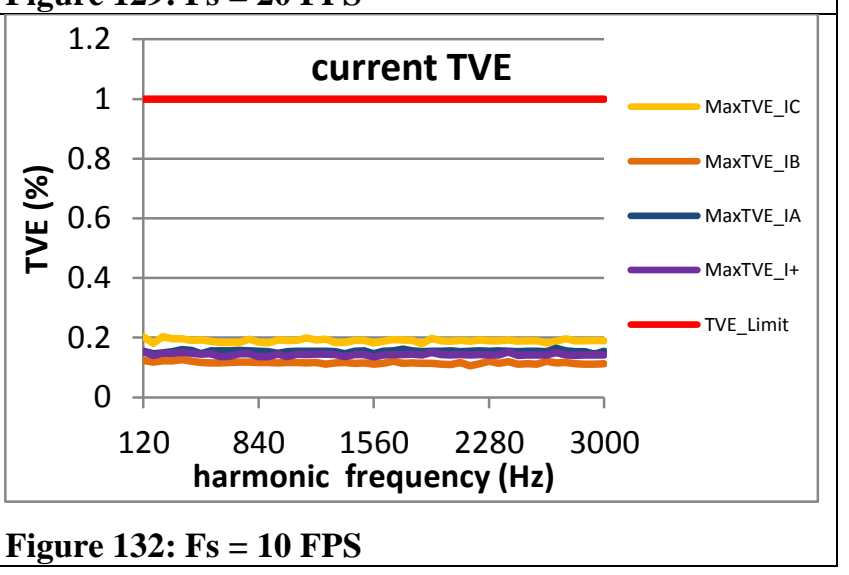




\subsection{Steady state frequency harmonic distortion frequency error: M class}

\subsubsection{C37.118.1 Annex C steady harmonic distortion range frequency error: $M$ class}

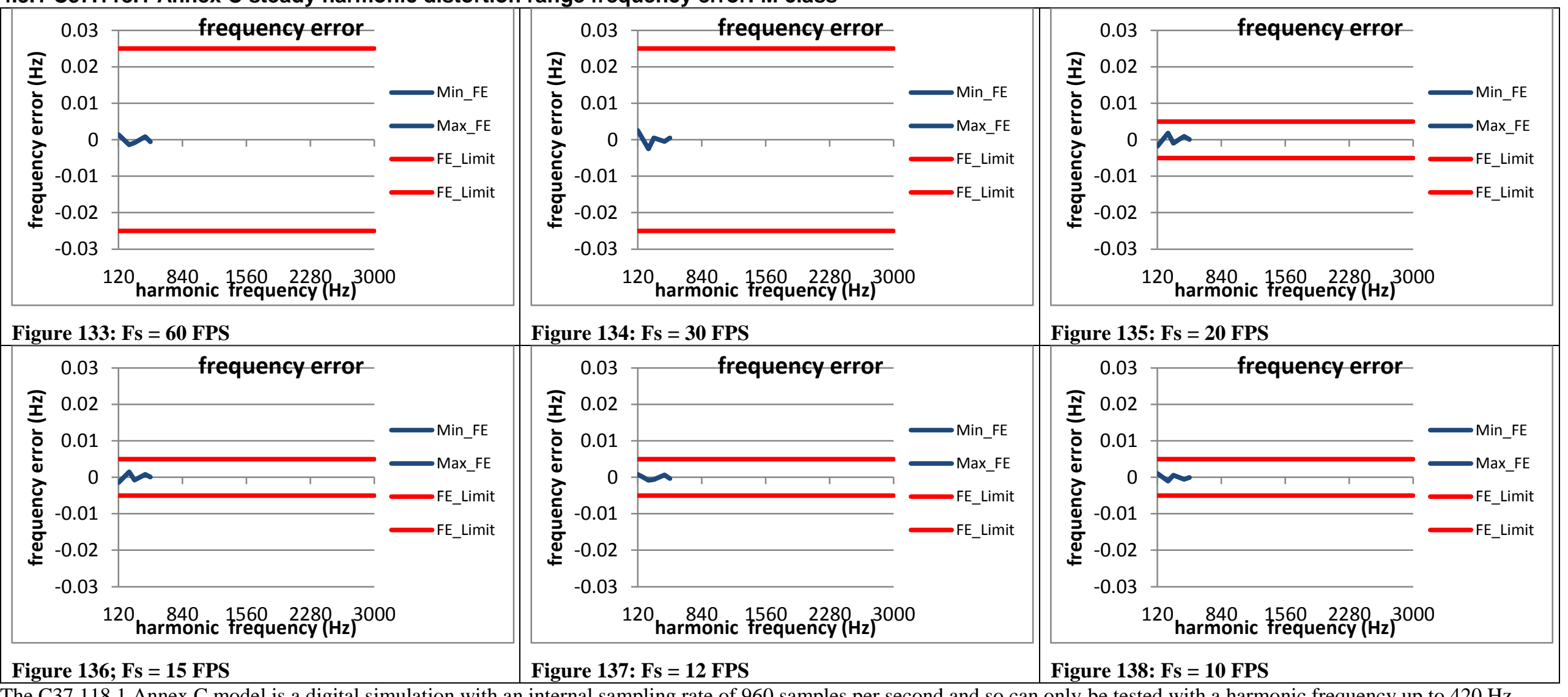

The C37.118.1 Annex C model is a digital simulation with an internal sampling rate of 960 samples per second and so can only be tested with a harmonic frequency up to $420 \mathrm{~Hz}$. 
4.3.2 PMU A steady state frequency harmonic distortion error: $M$ class

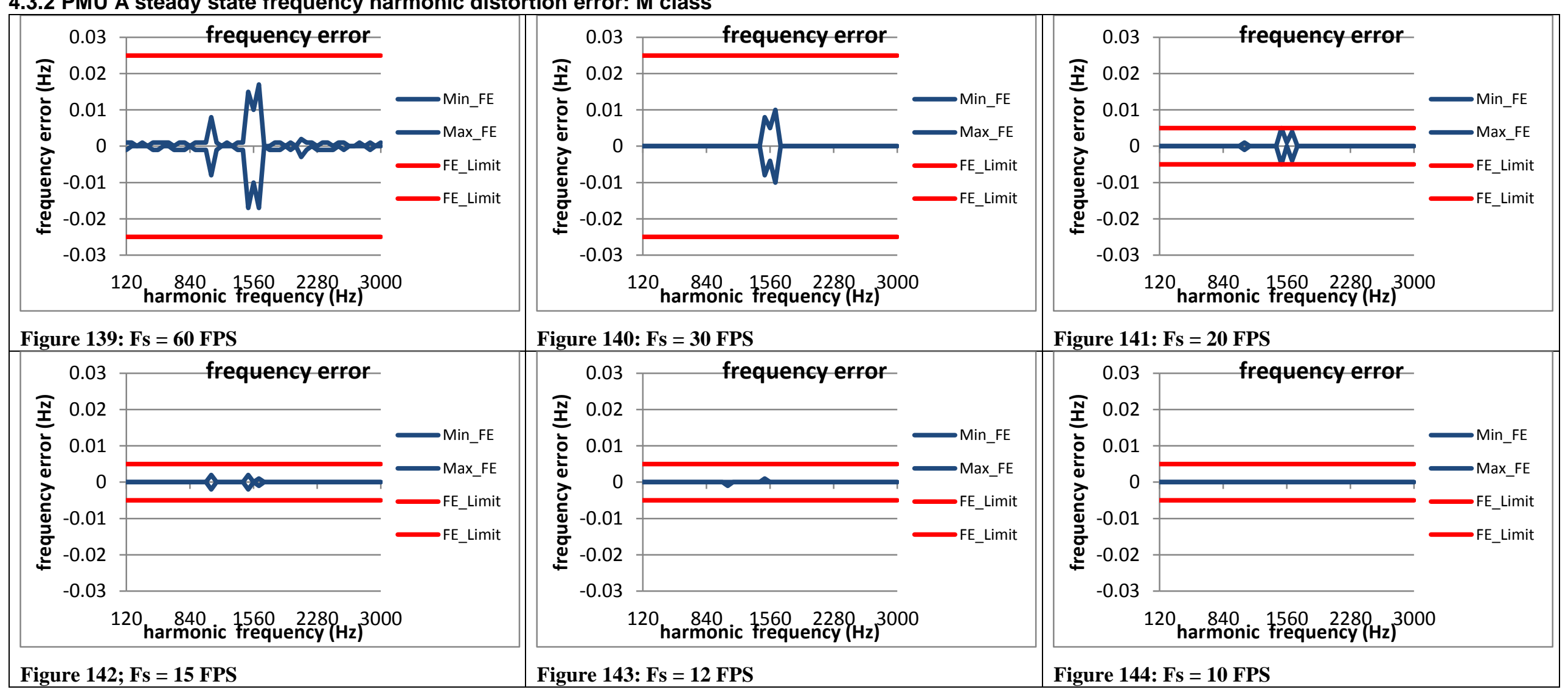


4.3.3 PMU B steady state frequency harmonic distortion error: $M$ class

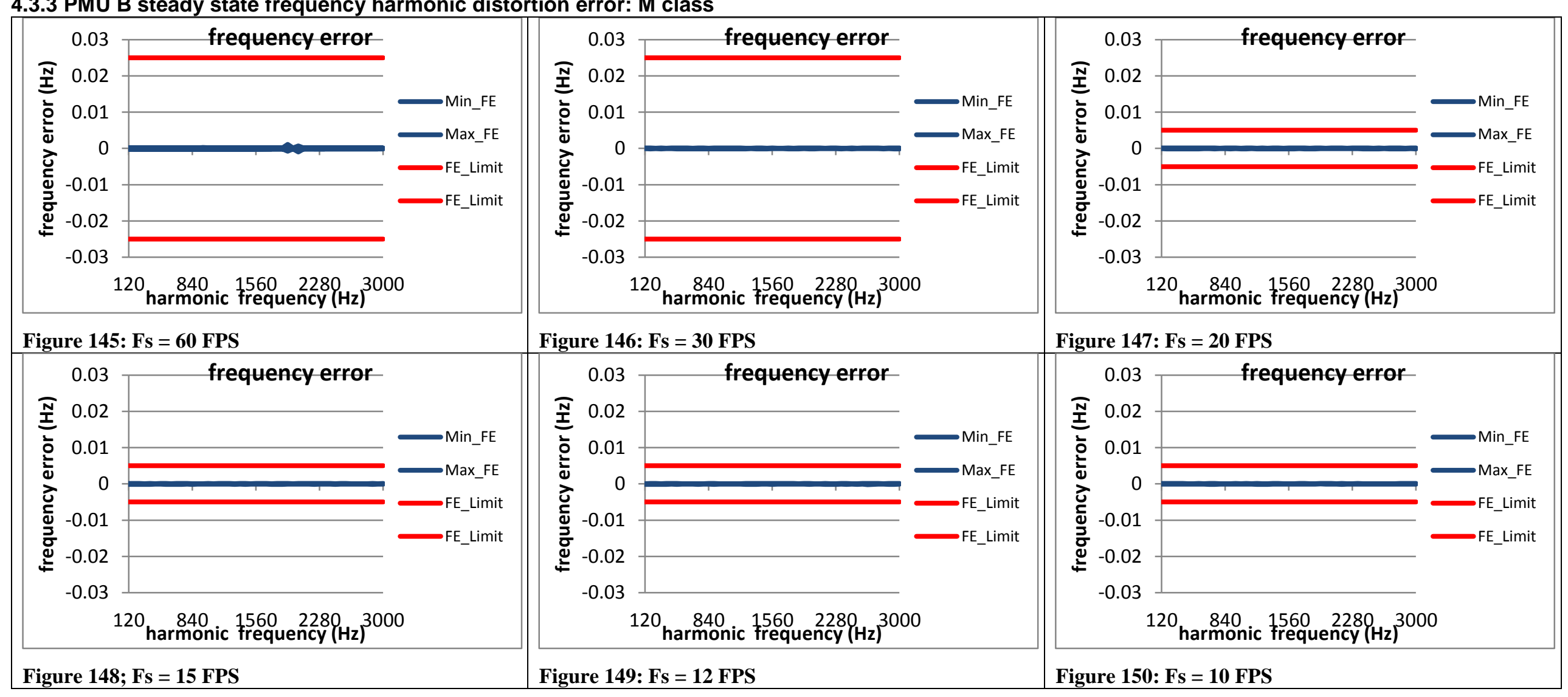


4.3.4 PMU C steady state frequency harmonic distortion error: M class

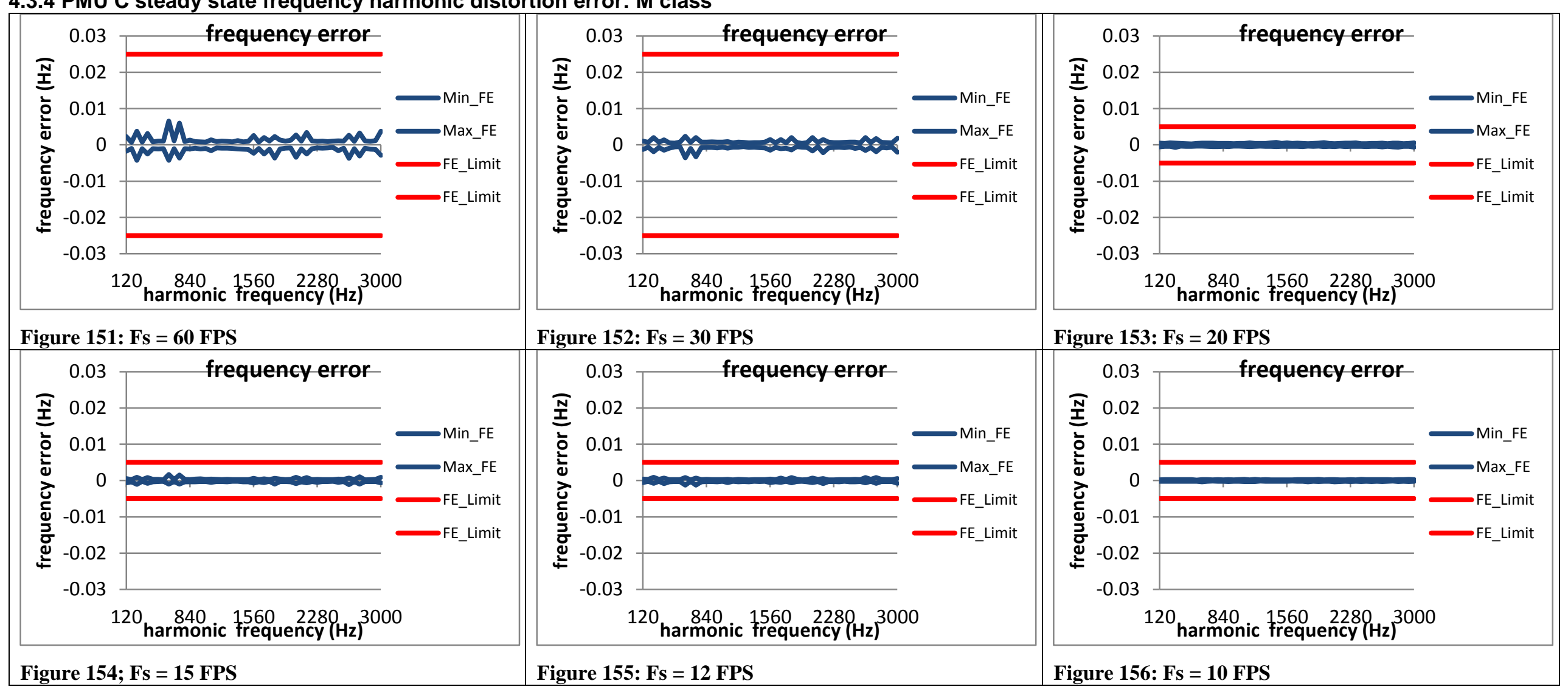


4.3.5 PMU D steady state frequency harmonic distortion error: $M$ class

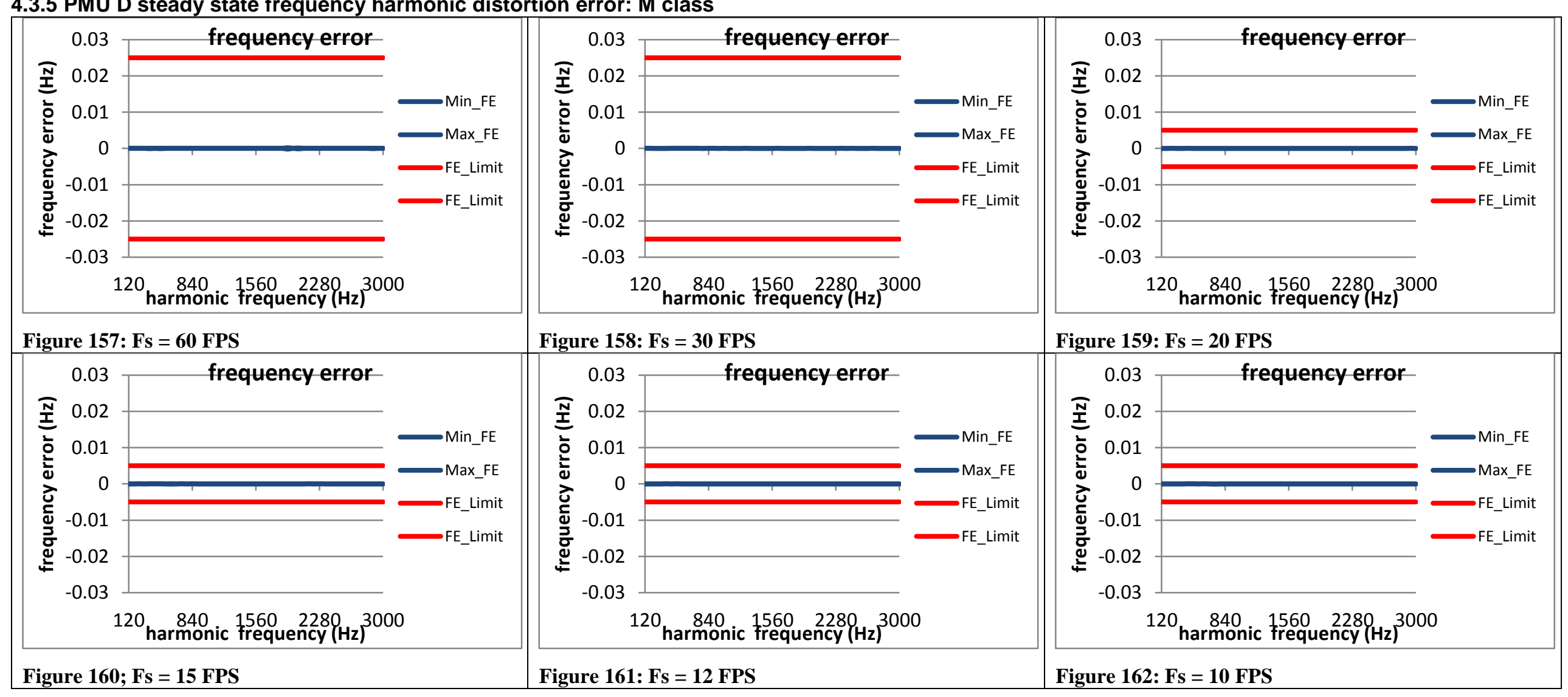


4.3.6 PMU E steady state harmonic distortion frequency error: M class

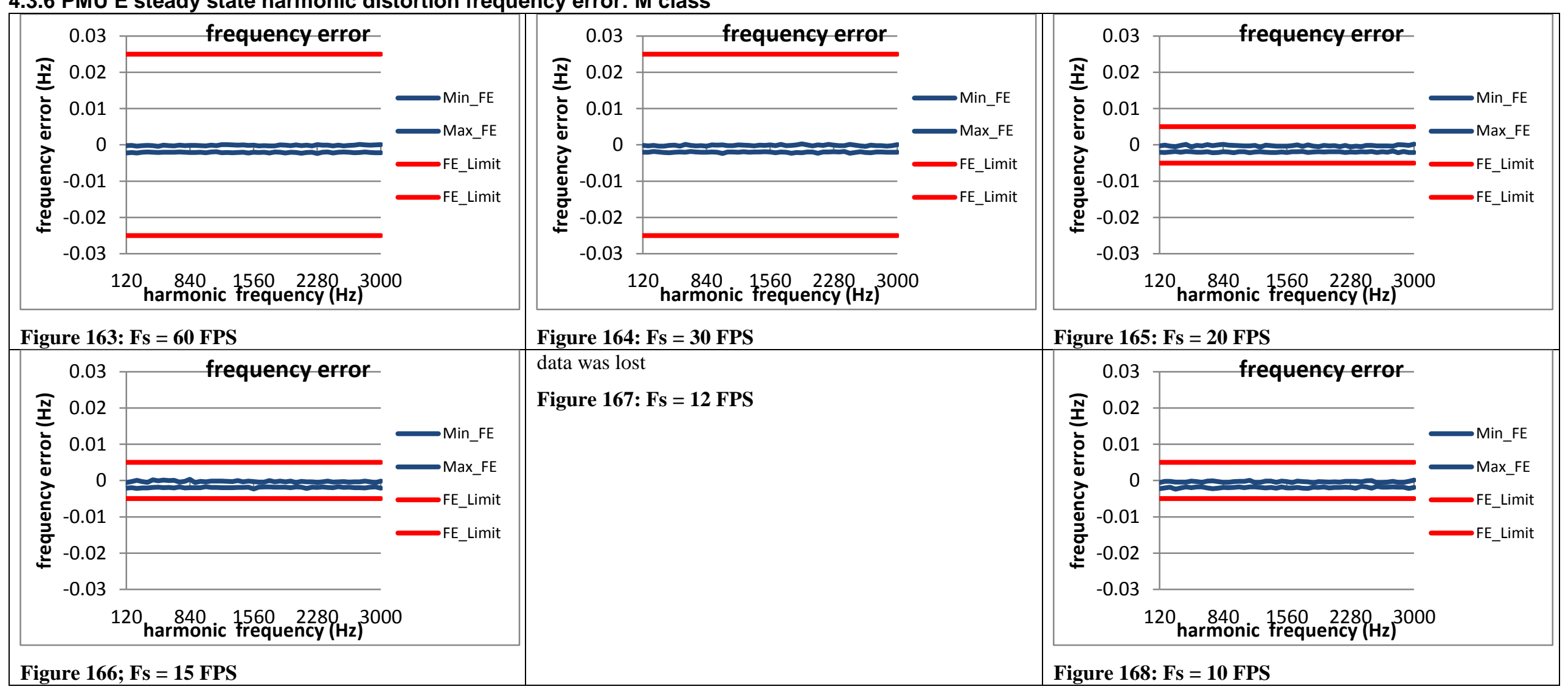


4.3.7 PMU F steady state harmonic distortion frequency error: M class

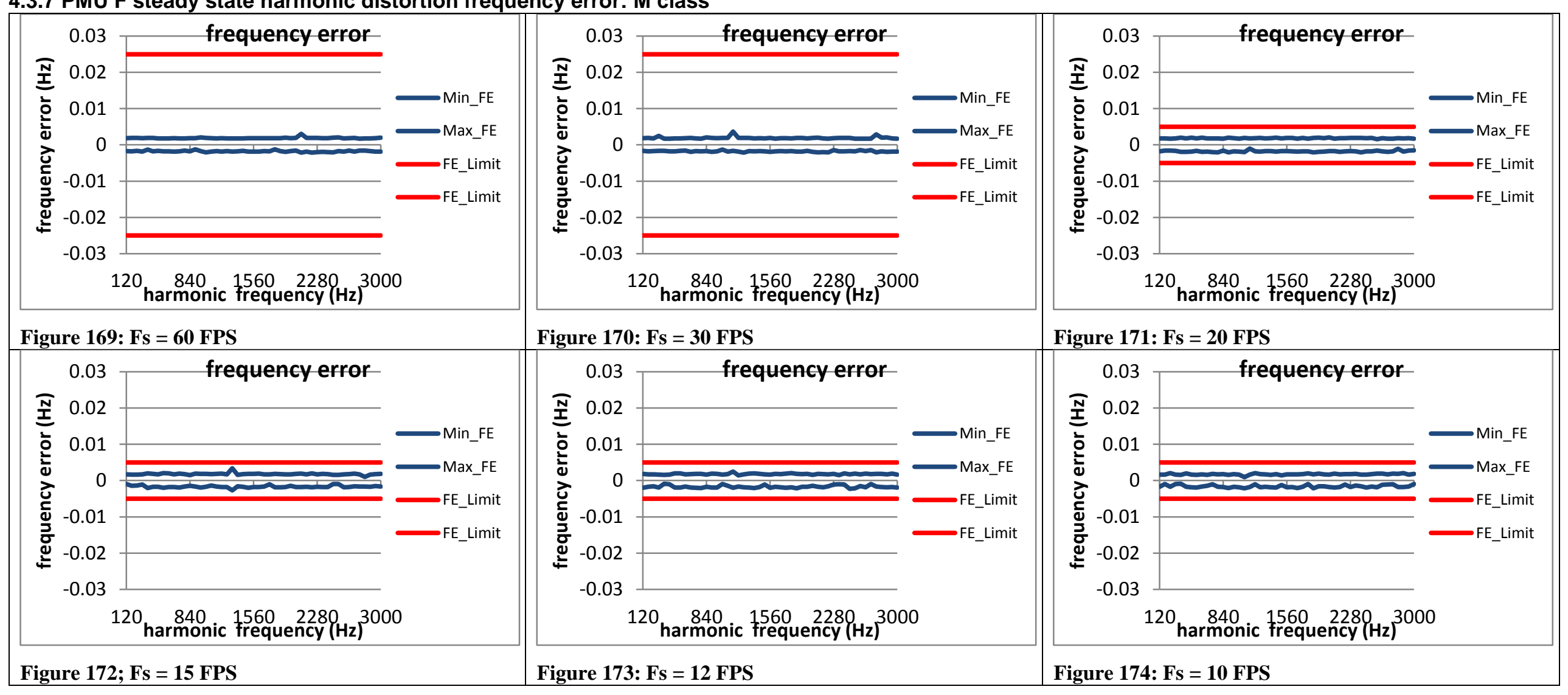


4.3.8 PMU G steady state harmonic distortion frequency error: M class

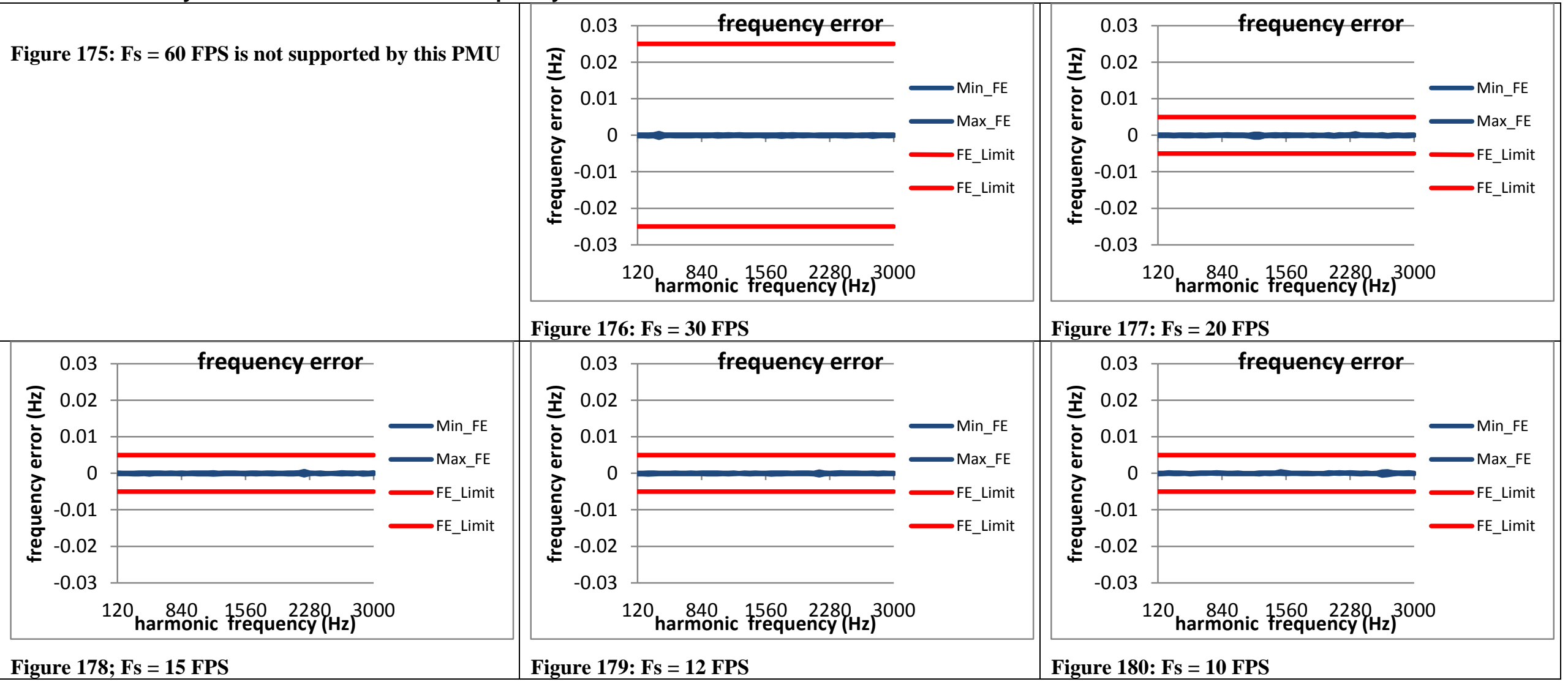


4.3.9 PMU H steady state harmonic distortion frequency error: $M$ class

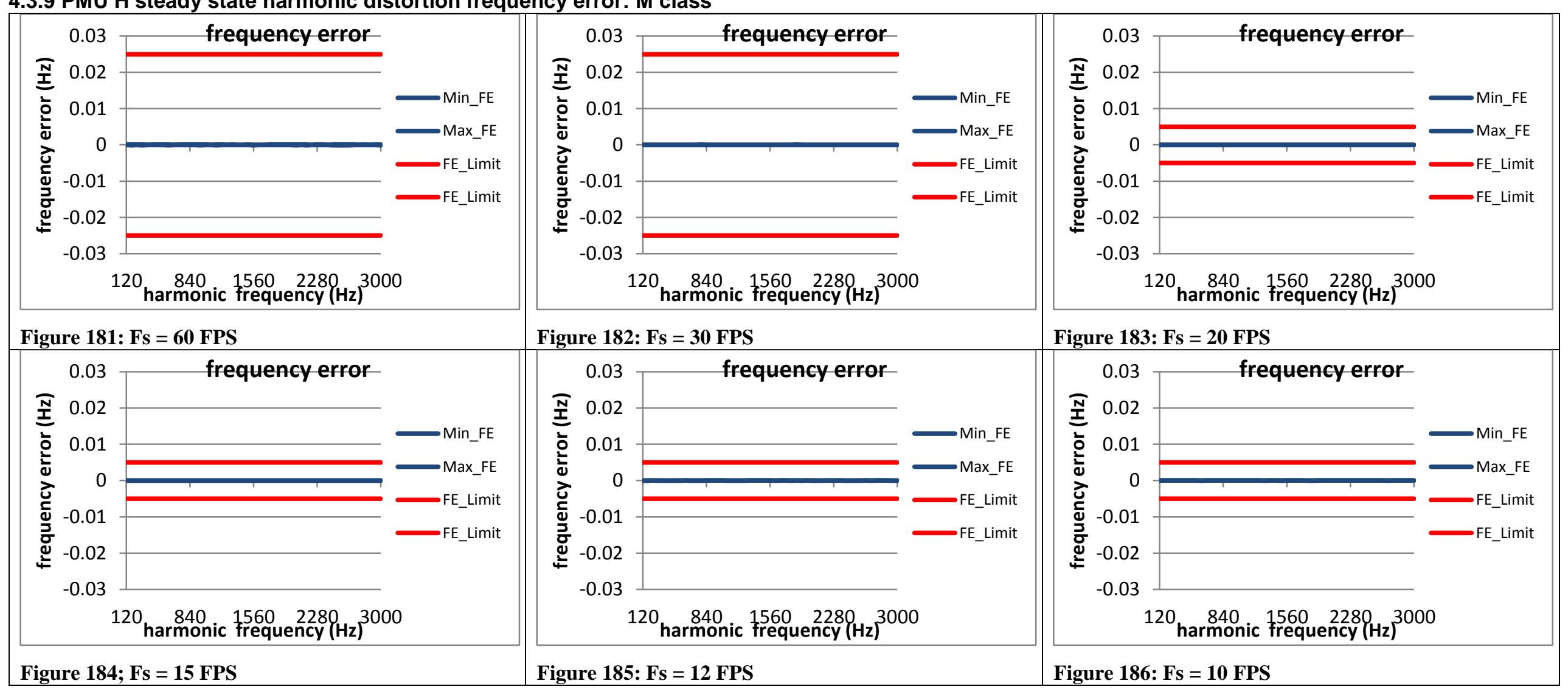


4.3.10 PMU I steady state harmonic distortion frequency error: M class

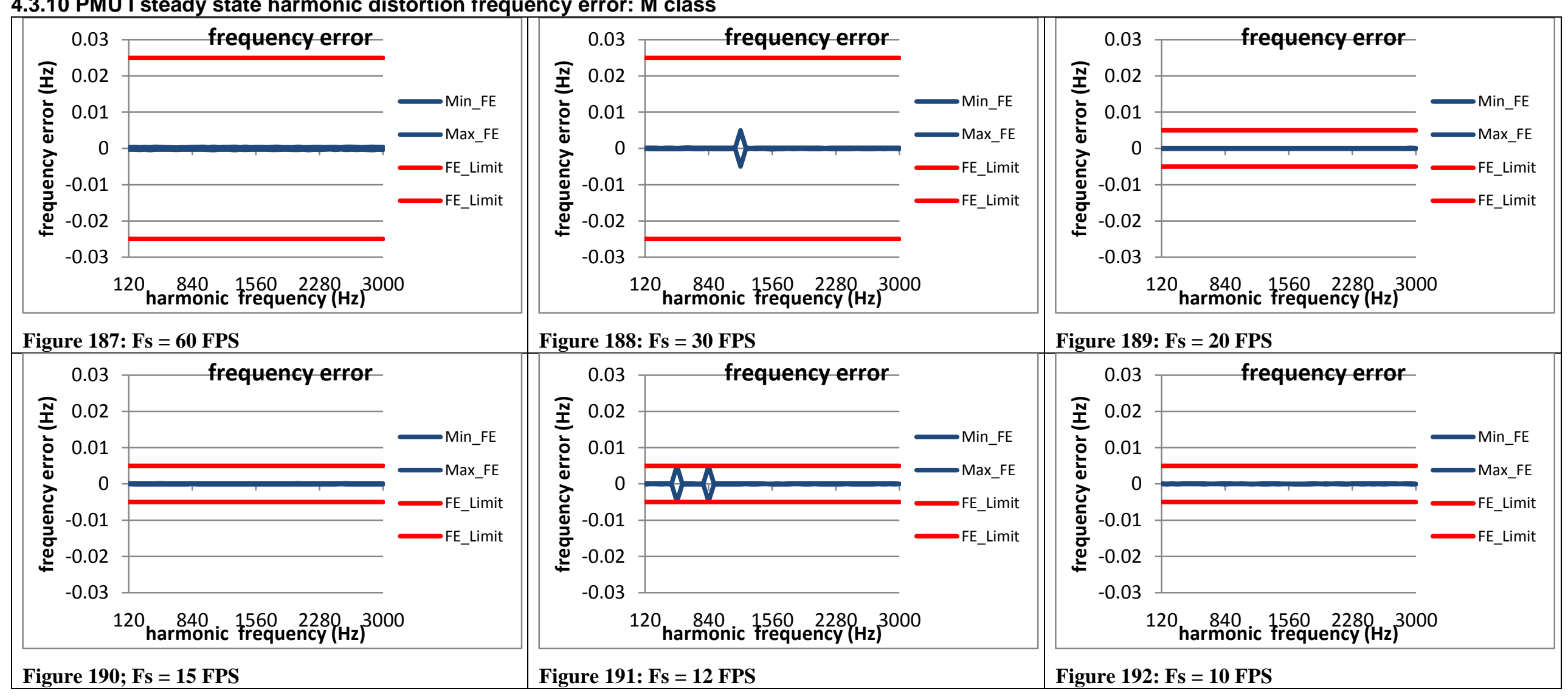


4.3.11 PMU J steady state harmonic distortion frequency error: M class

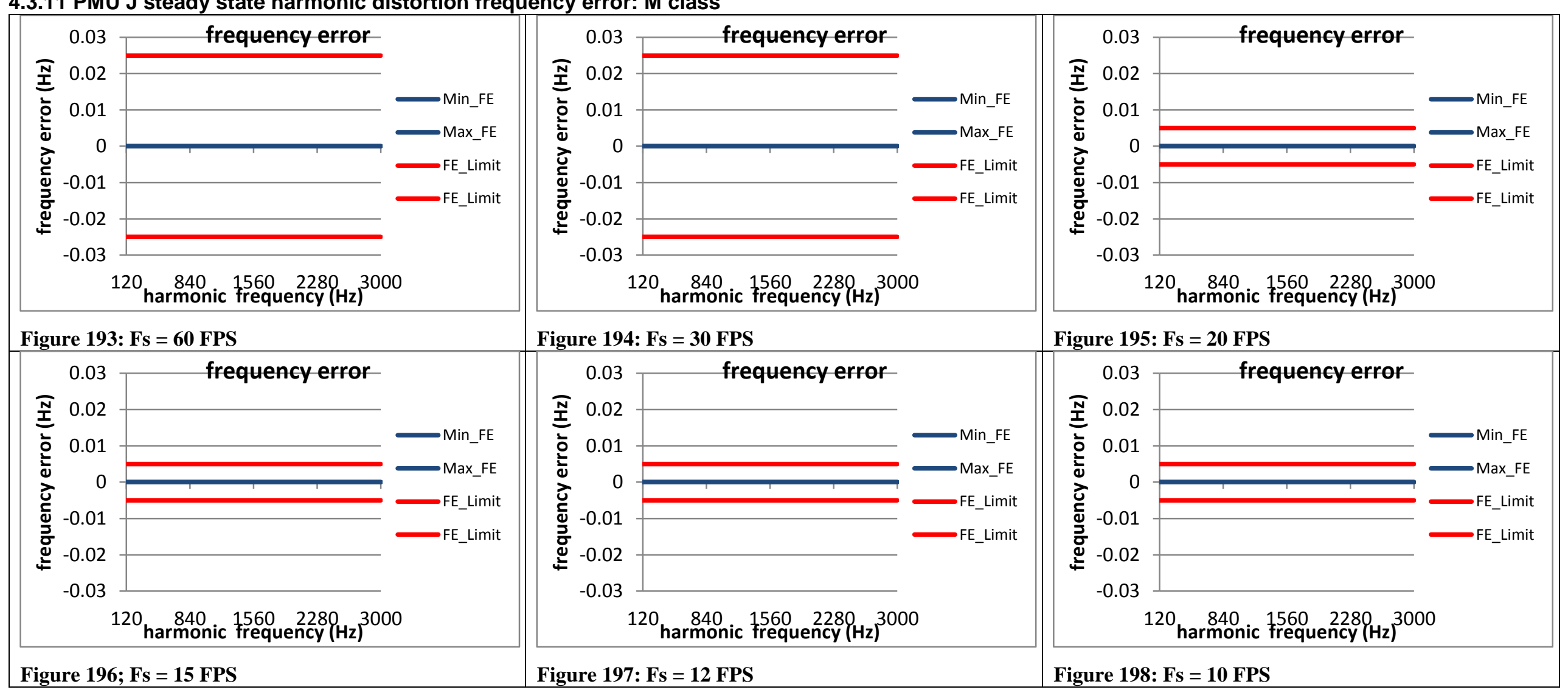




\subsection{Steady state harmonic distortion ROCOF error: M class}

\subsubsection{C37.118.1 Annex C steady harmonic distortion range ROCOF error: M class}

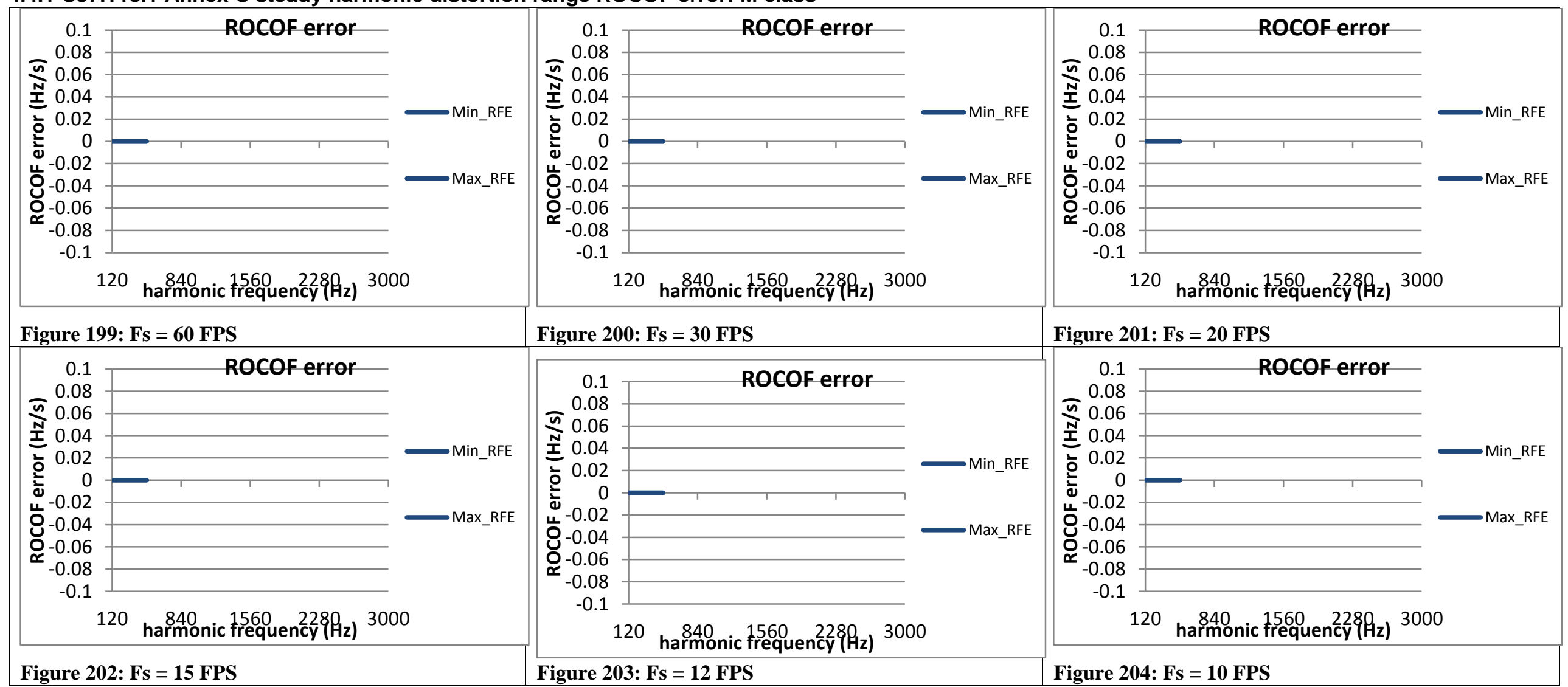

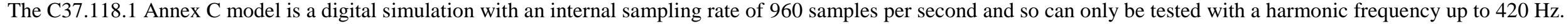


4.4.2 PMU A steady state harmonic distortion ROCOF error: M class

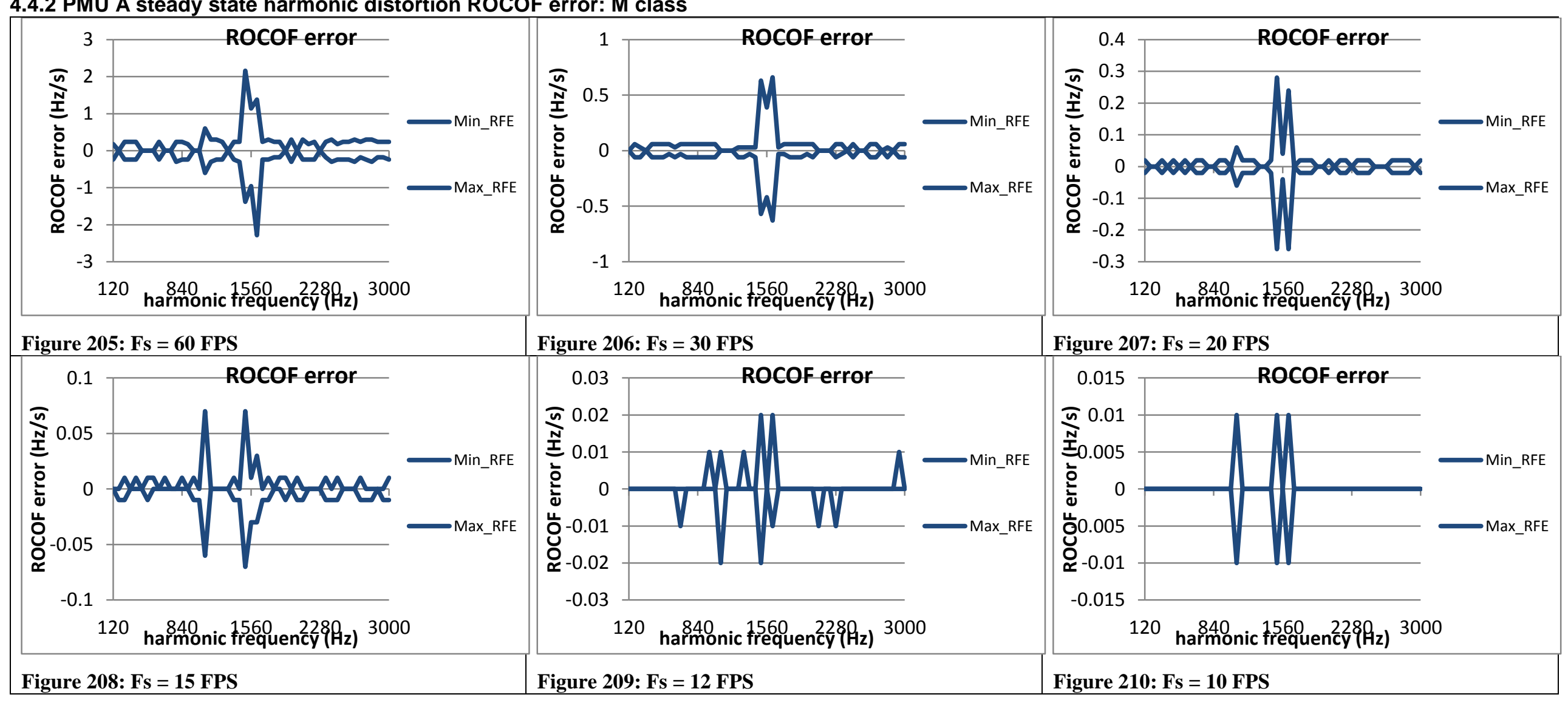


4.4.3 PMU B steady state harmonic distortion ROCOF error: M class

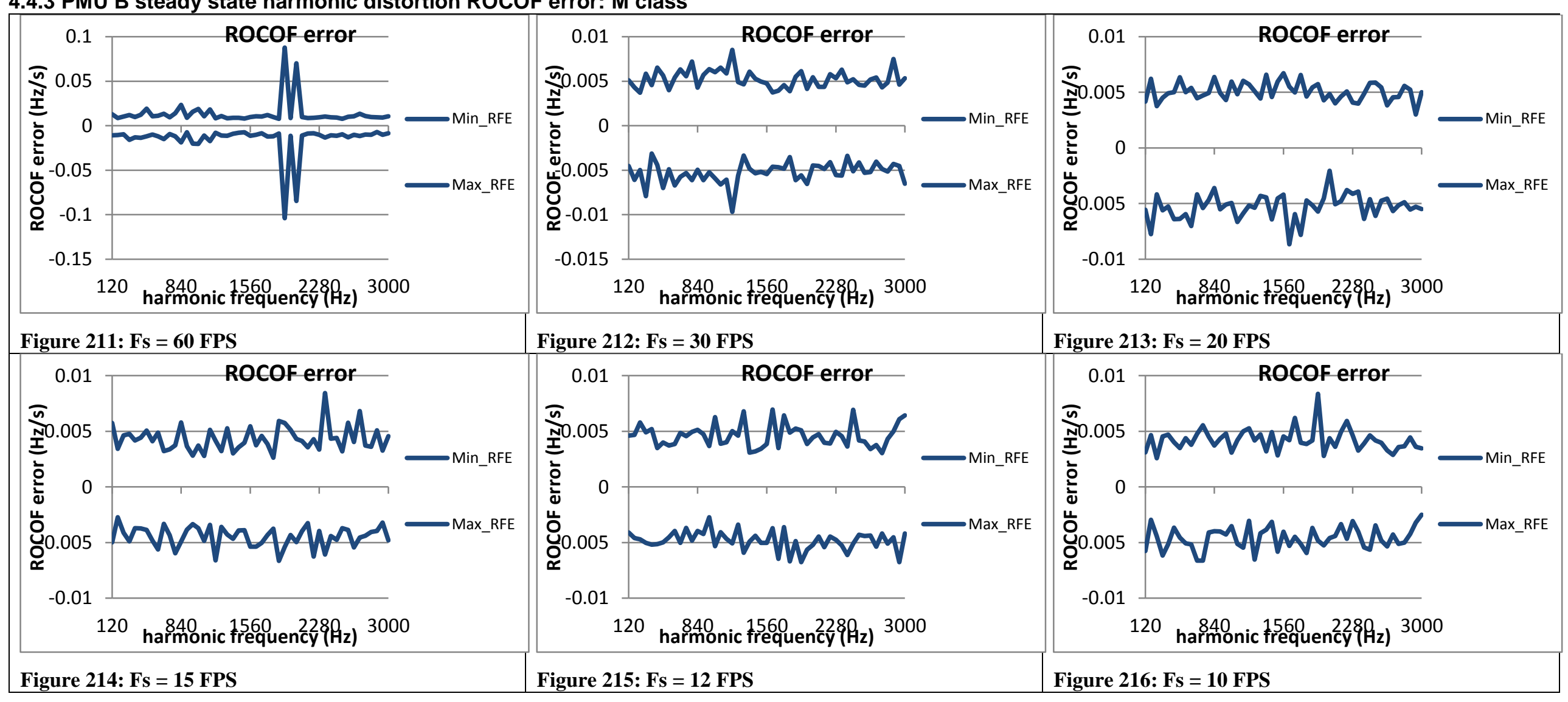


4.4.4 PMU C steady state harmonic distortion ROCOF error: M class

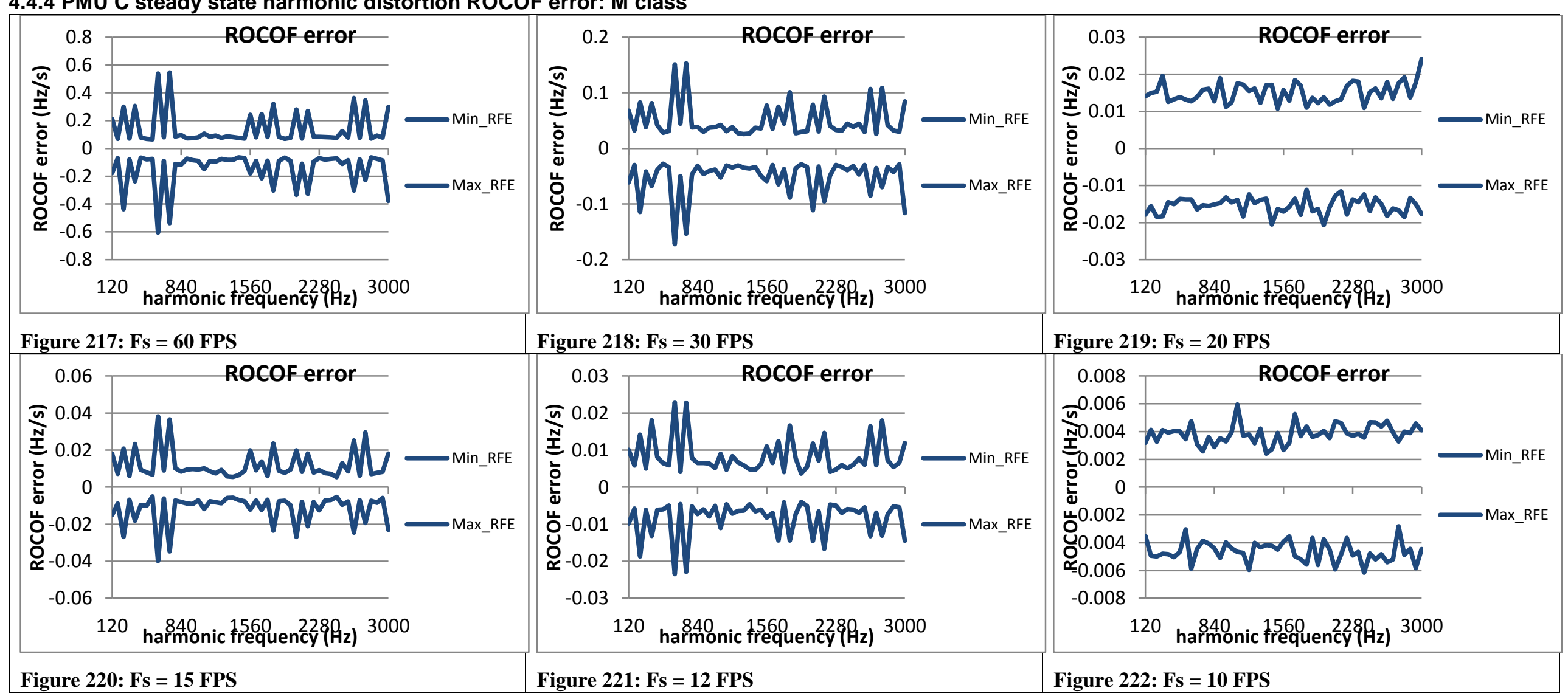


4.4.5 PMU D steady state harmonic distortion ROCOF error: M class

\begin{tabular}{|c|c|c|c|}
\hline 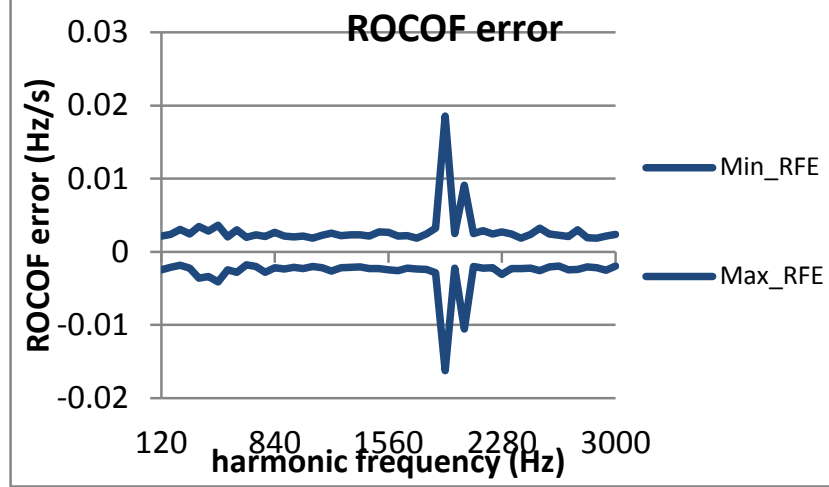 & 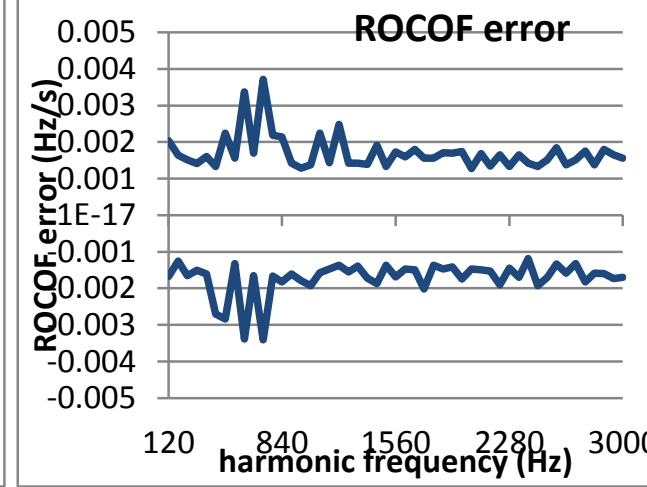 & -Max_RFE & 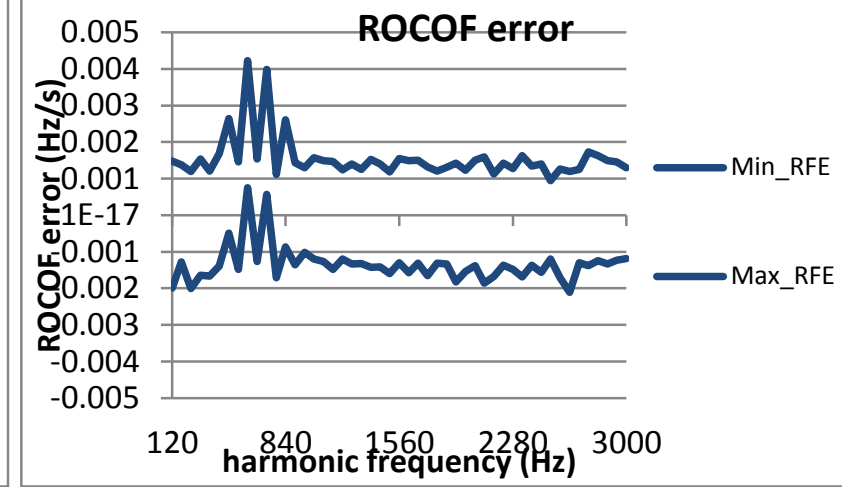 \\
\hline Figure 223: Fs = 60 FPS & Figure 224: Fs = 30 FPS & & Figure 225: $\mathrm{Fs}=20 \mathrm{FPS}$ \\
\hline 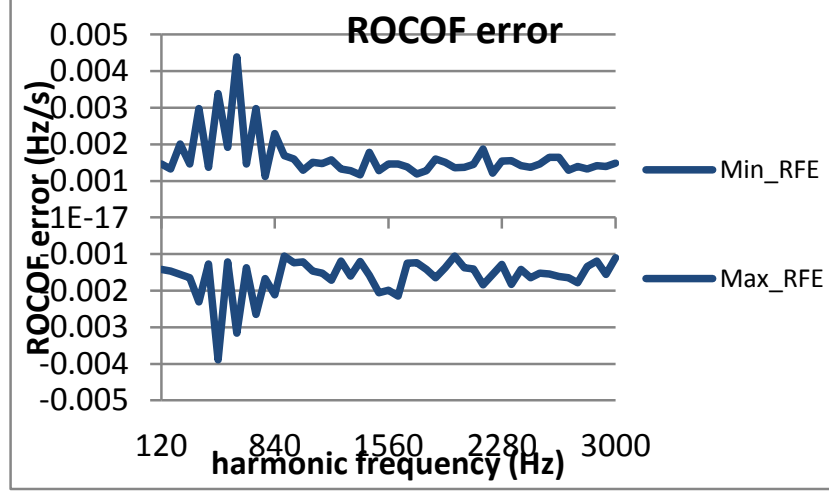 & 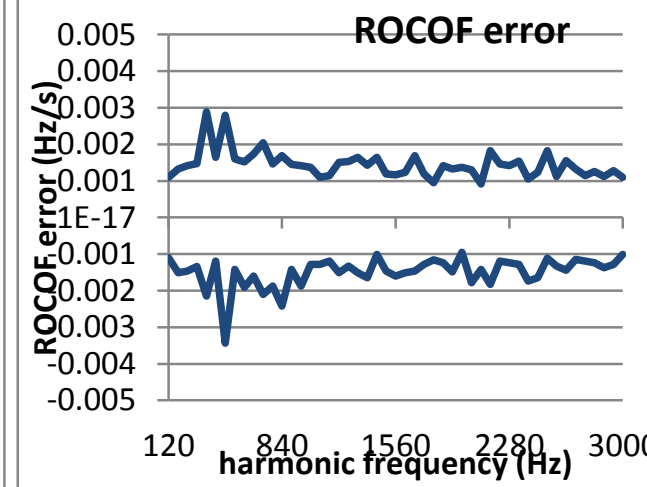 & Min_R & 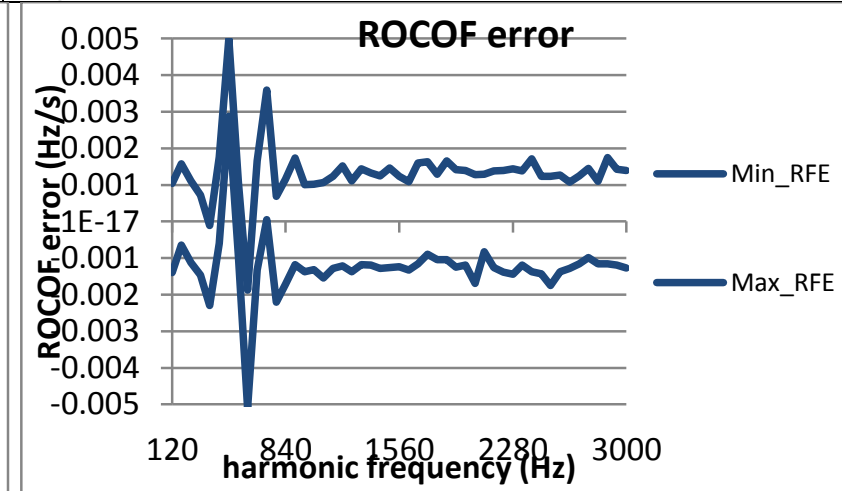 \\
\hline Figure 226: Fs = 15 FPS & Figure 227: Fs = 12 FPS & & Figure 228: Fs = 10 FPS \\
\hline
\end{tabular}


4.4.6 PMU E steady state harmonic distortion ROCOF error: M class

\begin{tabular}{|c|c|c|}
\hline 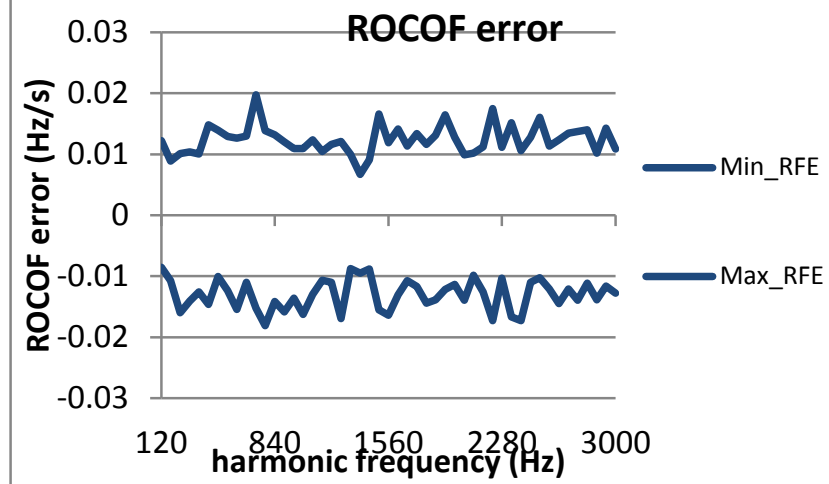 & 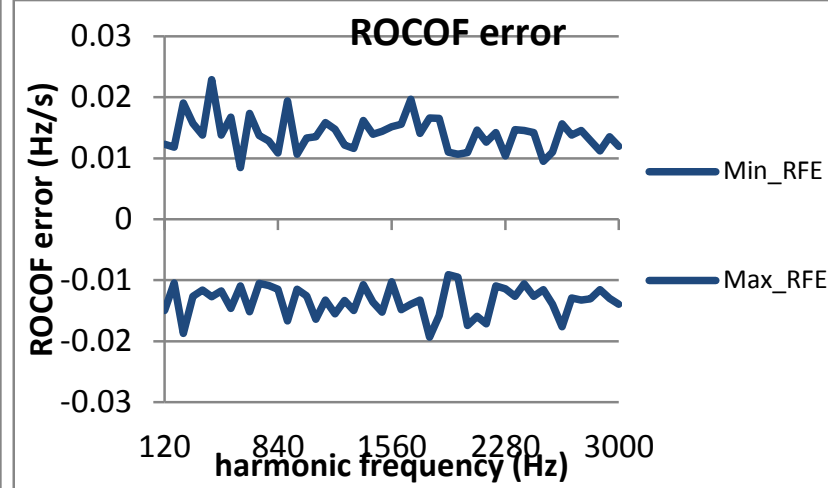 & 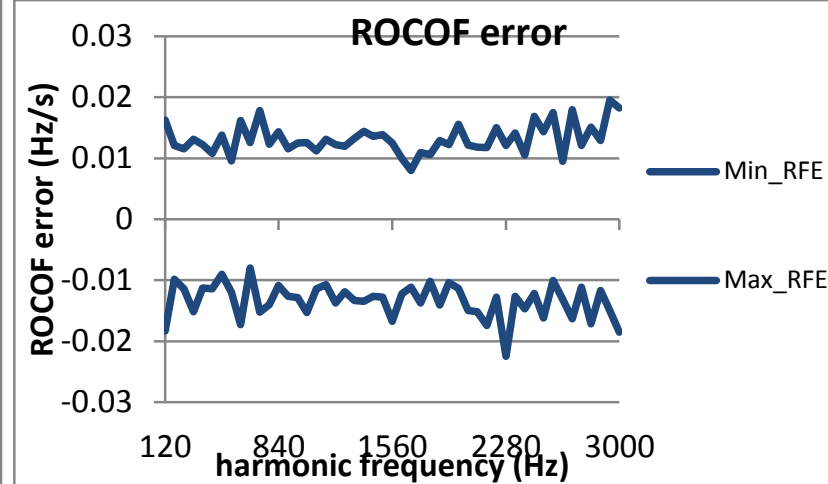 \\
\hline Figure 229: Fs = 60 FPS & Figure 230: Fs = 30 FPS & Figure 231: Fs = 20 FPS \\
\hline 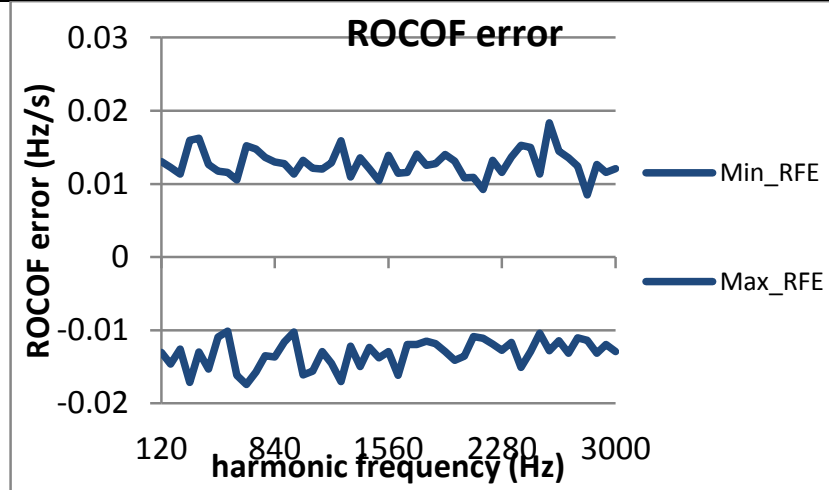 & $\begin{array}{l}\text { data was lost } \\
\text { Figure 233: Fs = 12 FPS }\end{array}$ & 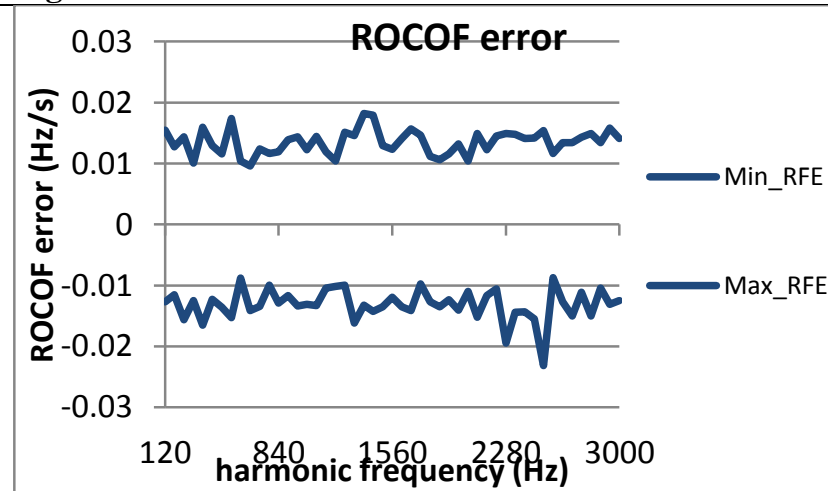 \\
\hline Figure 232: Fs = 15 FPS & & Figure 234: Fs = 10 FPS \\
\hline
\end{tabular}


4.4.7 PMU F steady state harmonic distortion ROCOF error: M class

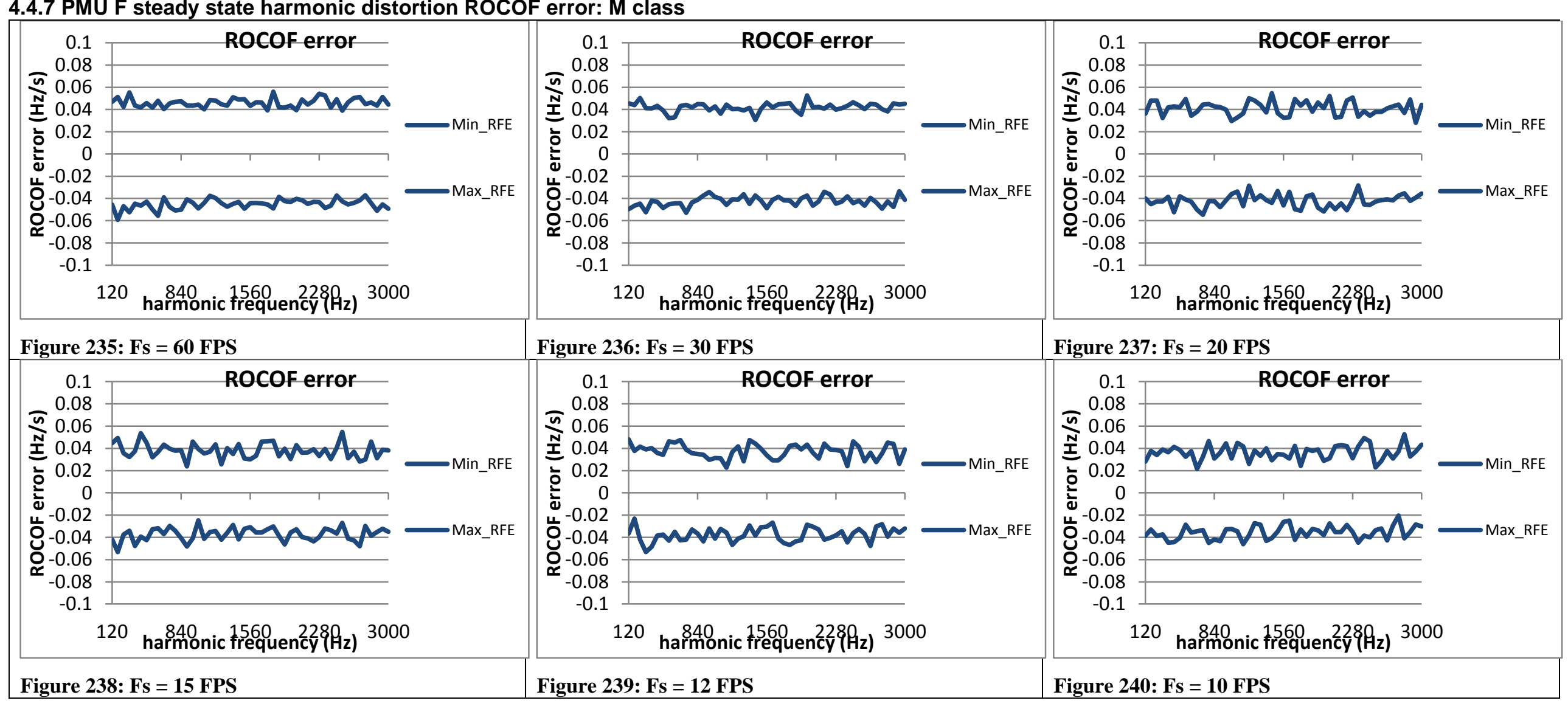


4.4.8 PMU G* steady state harmonic distortion ROCOF error: M class

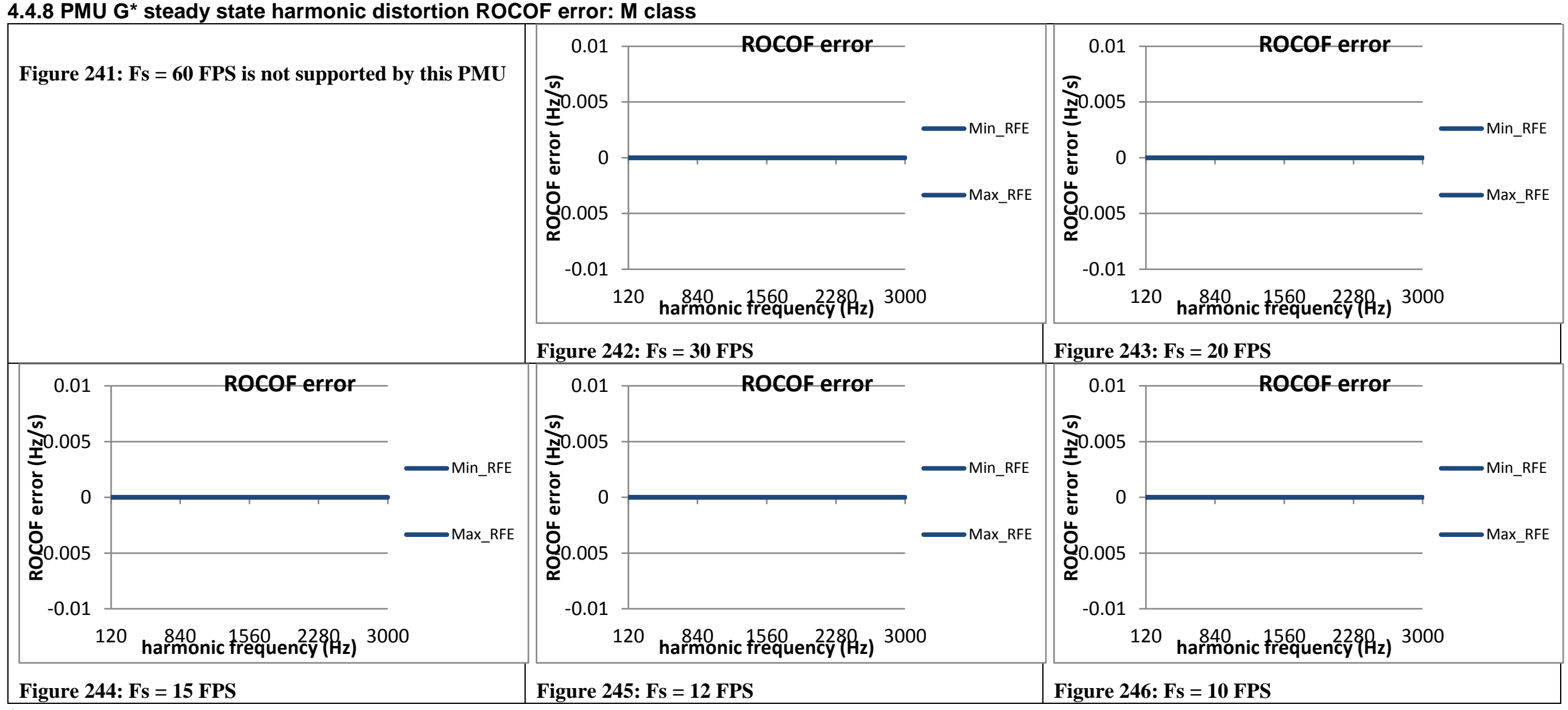

* PMU G always outputs ROCOF $=0$ 
4.4.9 PMU H steady state harmonic distortion ROCOF error: M class

\begin{tabular}{|c|c|c|}
\hline 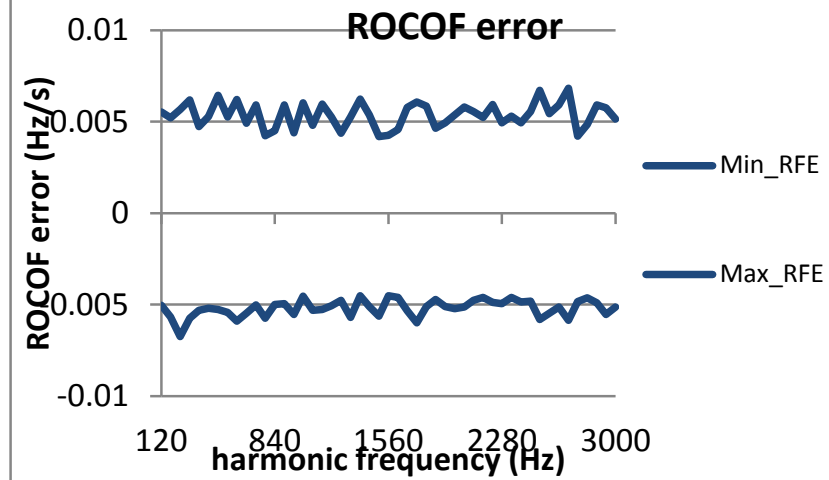 & & 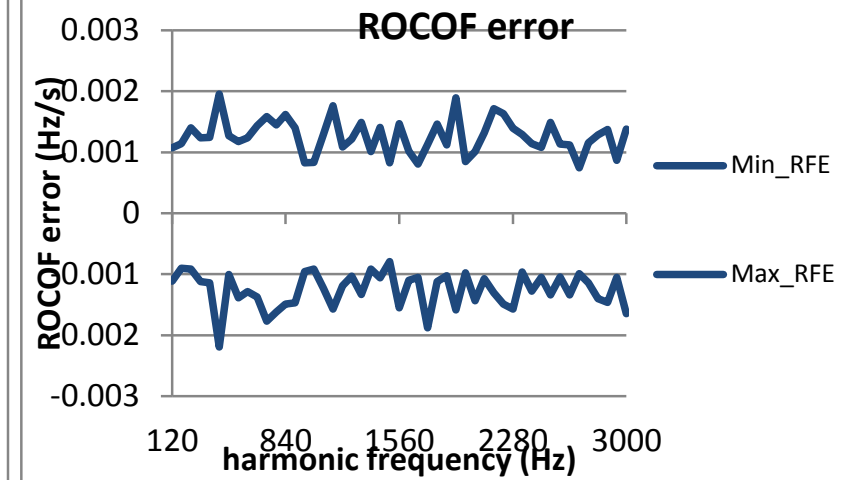 \\
\hline Figure 247: Fs = 60 FPS & Figure 248: Fs & Figure 249: \\
\hline 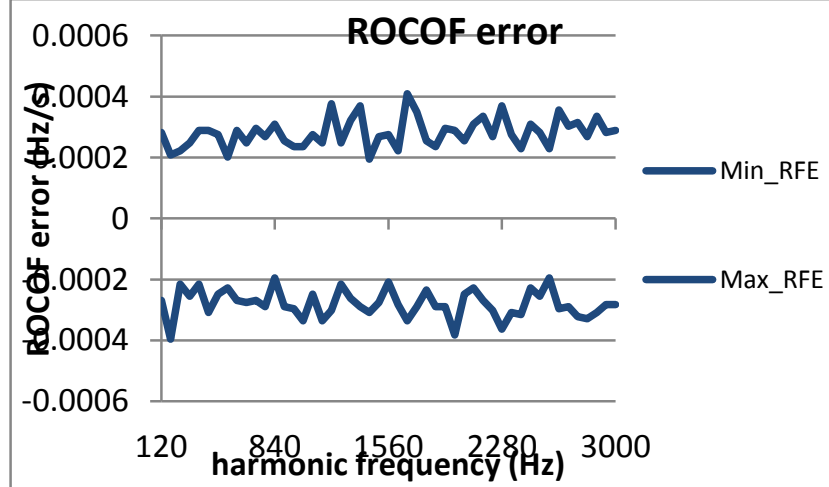 & 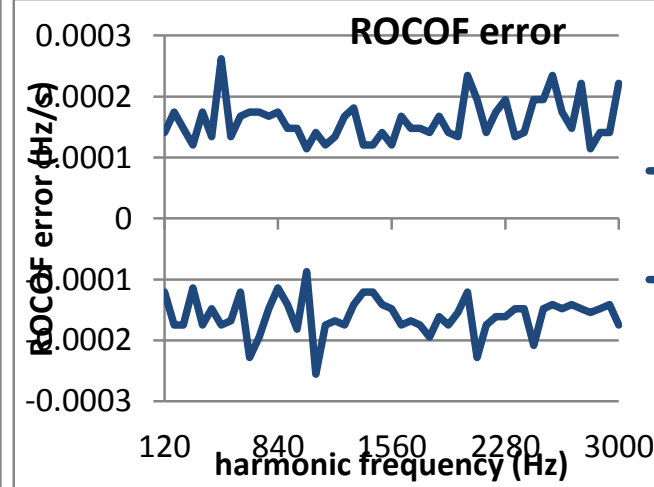 & 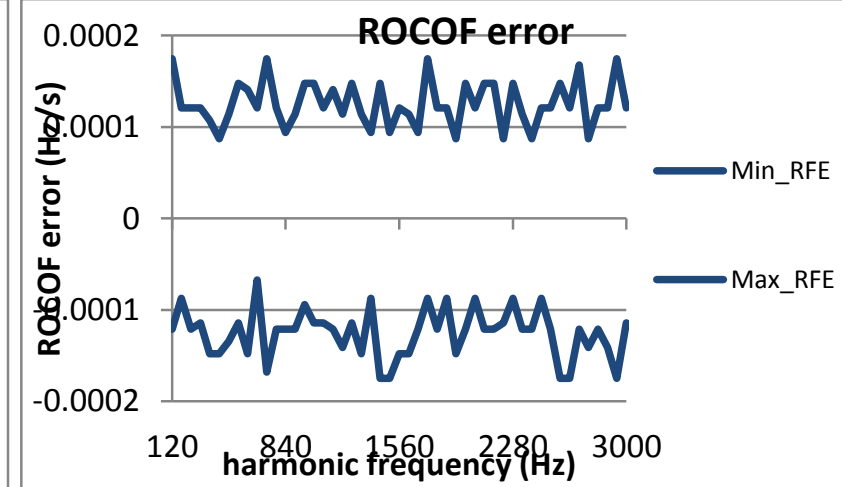 \\
\hline Figure 250: Fs = 15 FPS & Figure 251: Fs = 12 FPS & Figure 252: Fs = \\
\hline
\end{tabular}


4.4.10 PMU I steady state harmonic distortion ROCOF error: M class

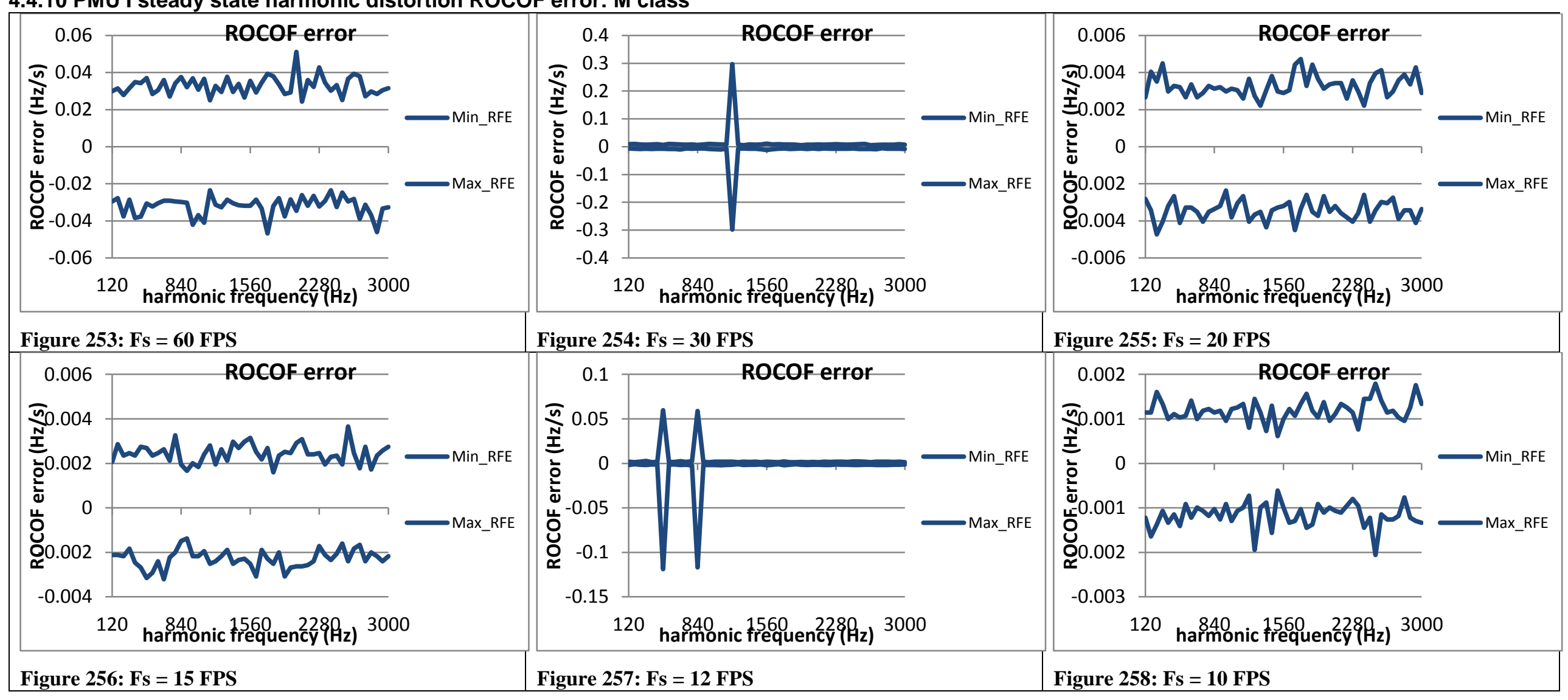


4.4.11 PMU J steady state harmonic distortion ROCOF error: M class

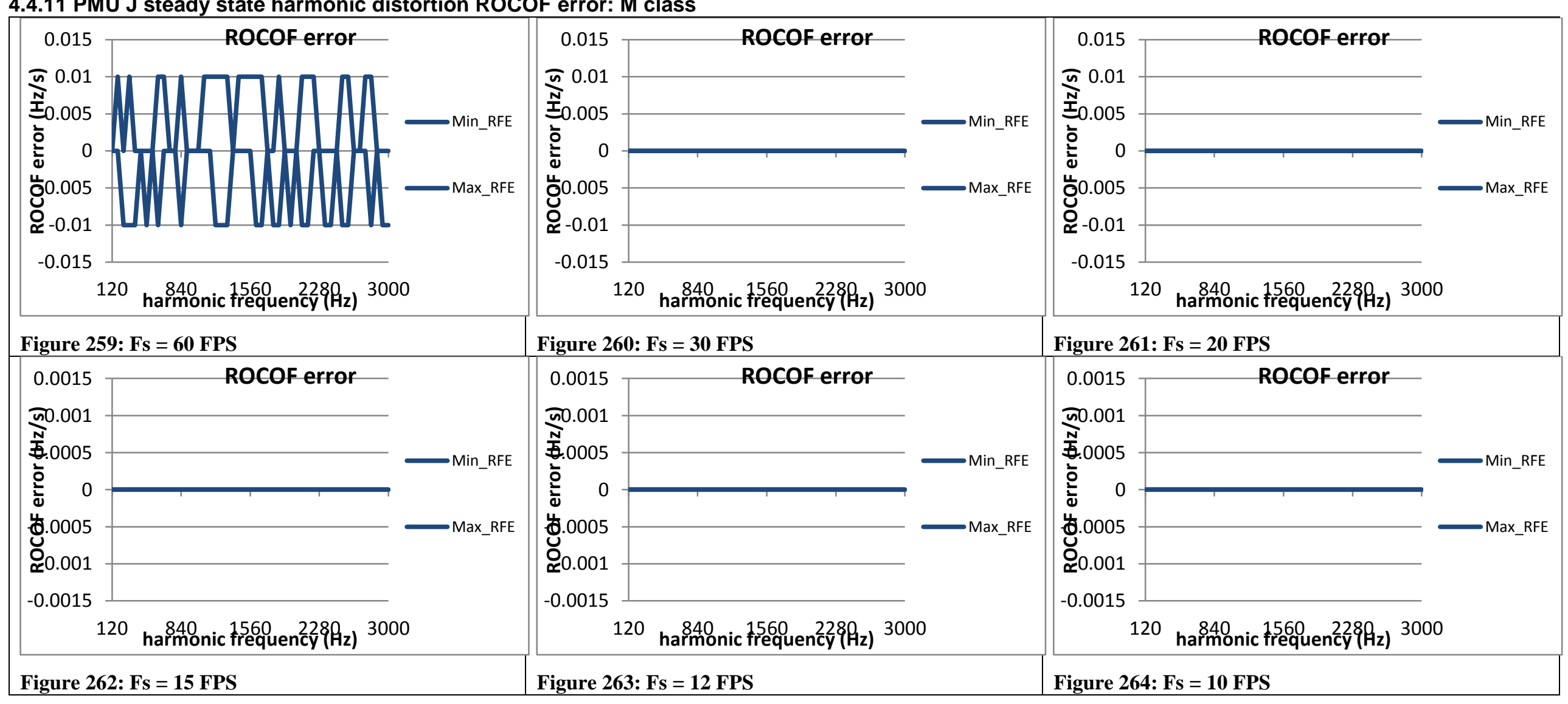




\subsection{Steady state harmonic distortion voltage TVE: P class}

\subsubsection{C37.118.1 Annex $C$ steady state frequency range voltage TVE:, F0 $=60 \mathrm{~Hz}, \mathrm{P}$ class}

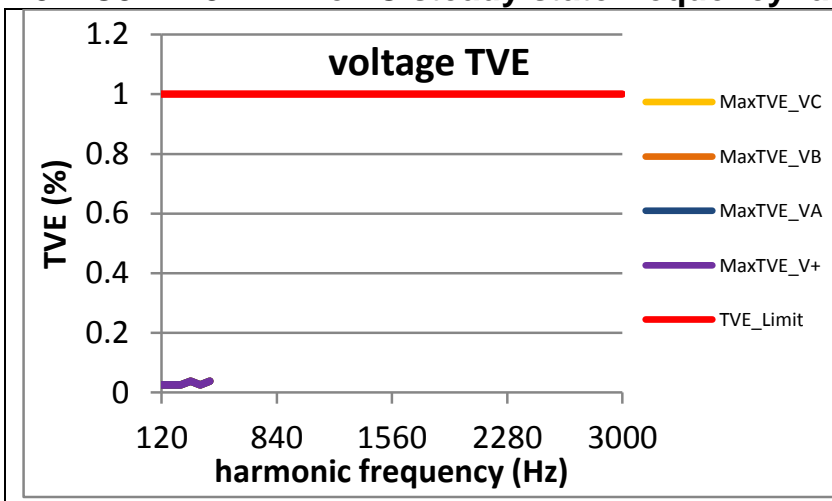

Figure 265: Fs = 60 FPS
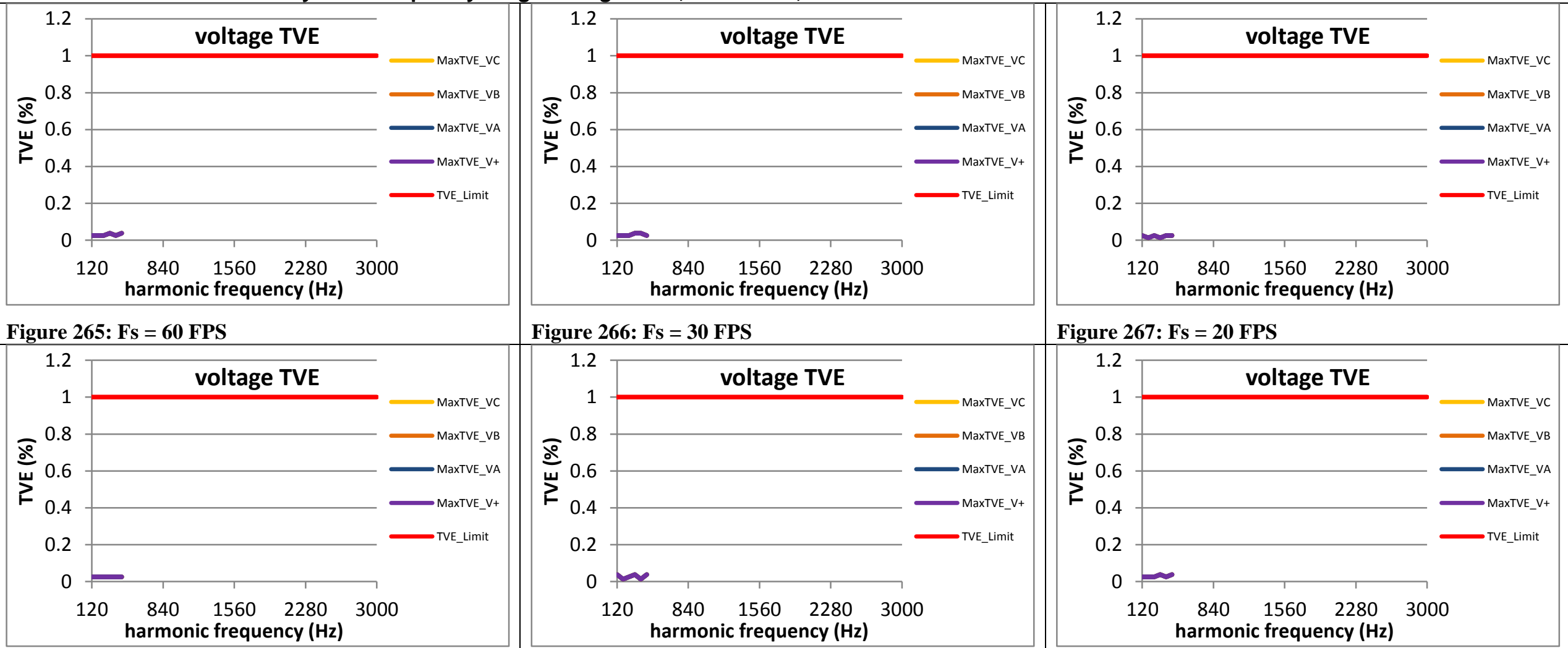

Figure 266: Fs = 30 FPS

Figure 267: Fs = 20 FPS
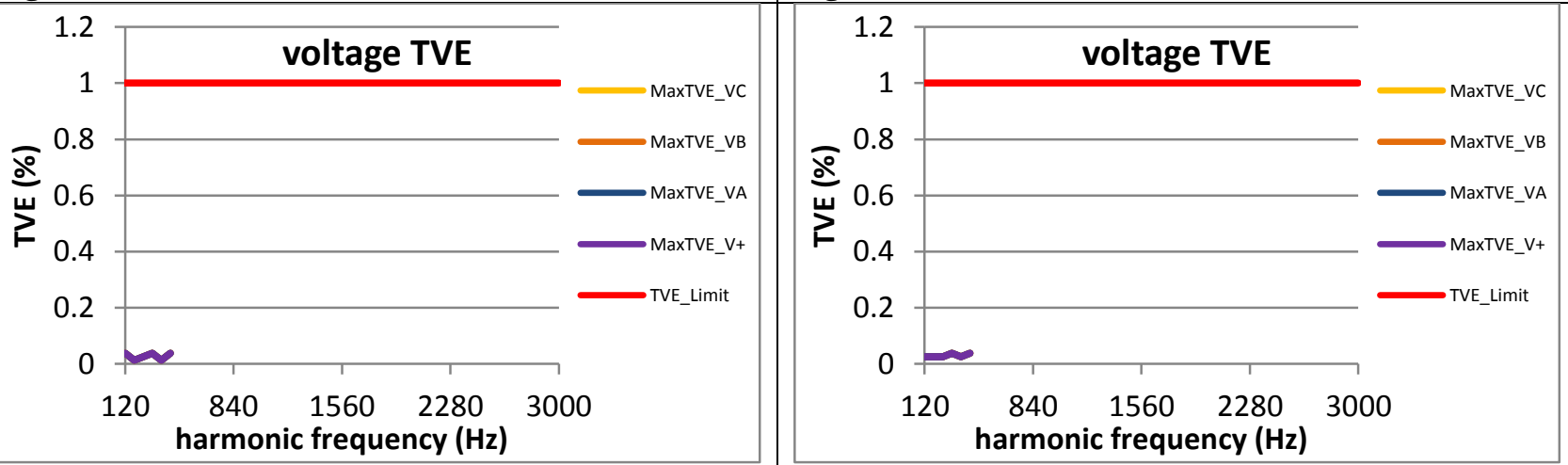

Figure 268: Fs $=15$ FPS

\section{Figure 269: Fs = 12 FPS}

Figure 270: Fs = 10 FPS

The C37.118.1 Annex C model is a digital simulation with an internal sampling rate of 960 samples per second and so can only be tested with a harmonic frequency up to $420 \mathrm{~Hz}$. 
4.5.2 PMU A steady state harmonic distortion voltage TVE: P class
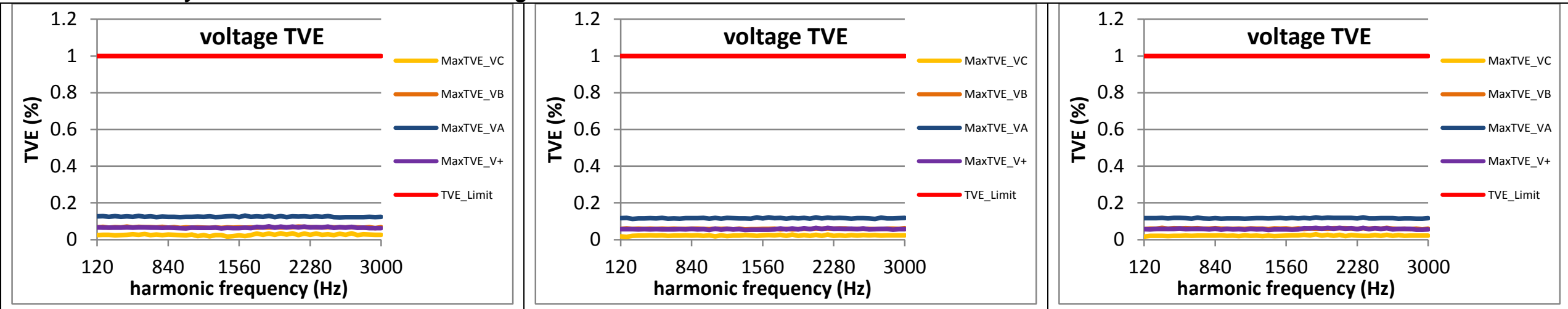

Figure 271: Fs = 60 FPS

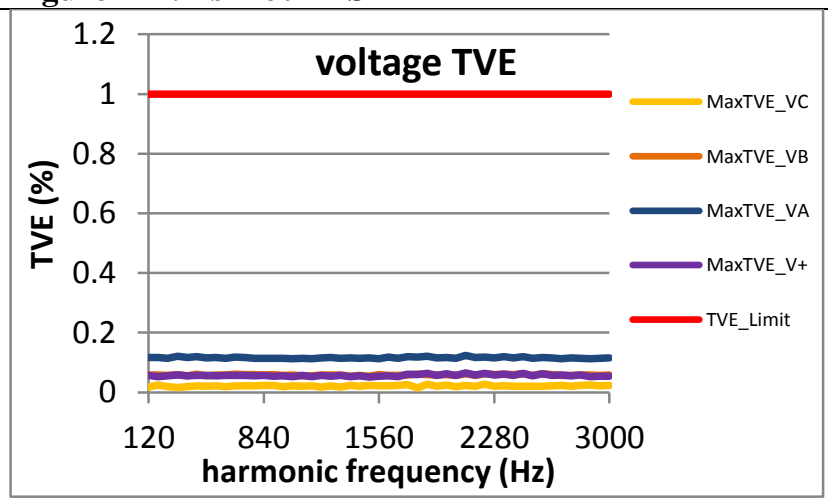

Figure 272: Fs = 30 FPS

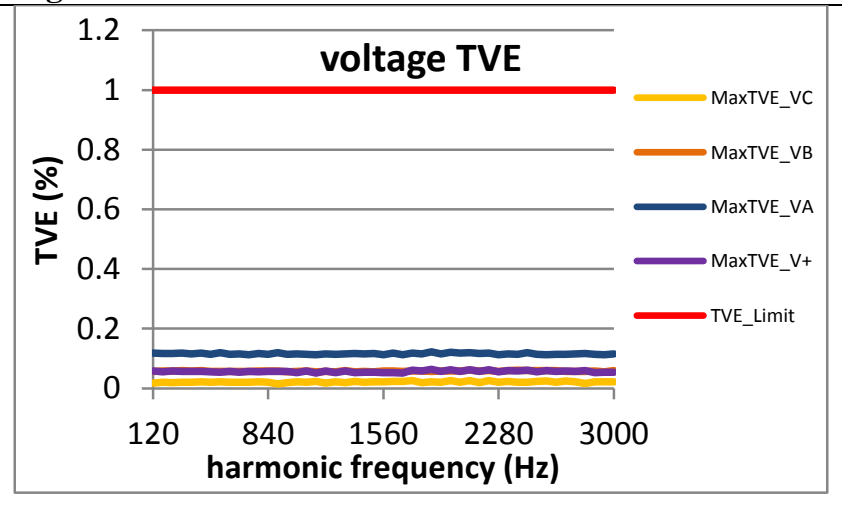

Figure 273: Fs = 20 FPS

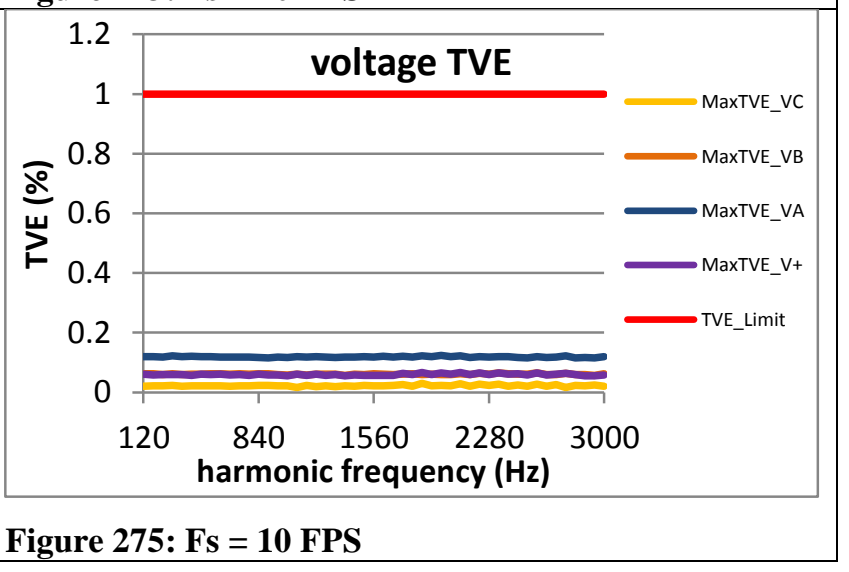

Figure 274: Fs = 15 FPS 
4.5.3 PMU B steady state harmonic distortion voltage TVE: P class
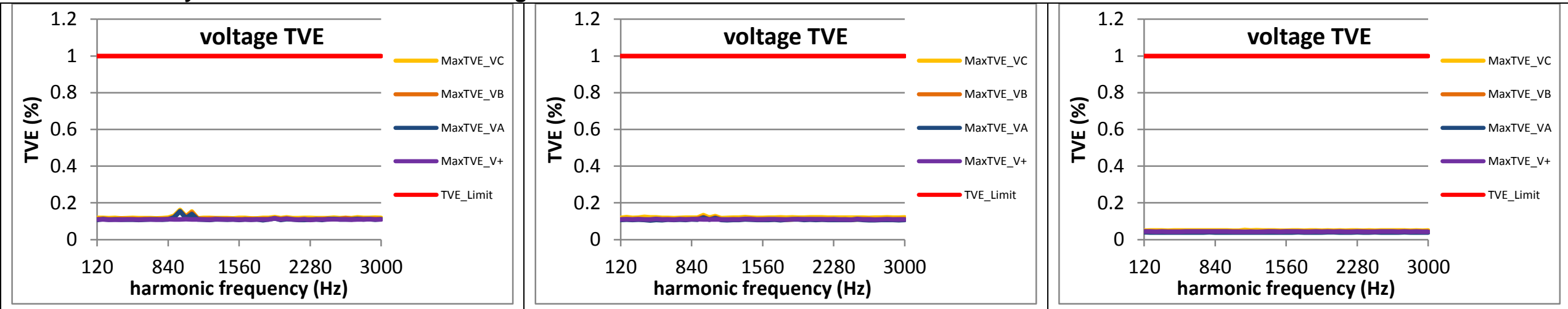

Figure 276: Fs = 60 FPS

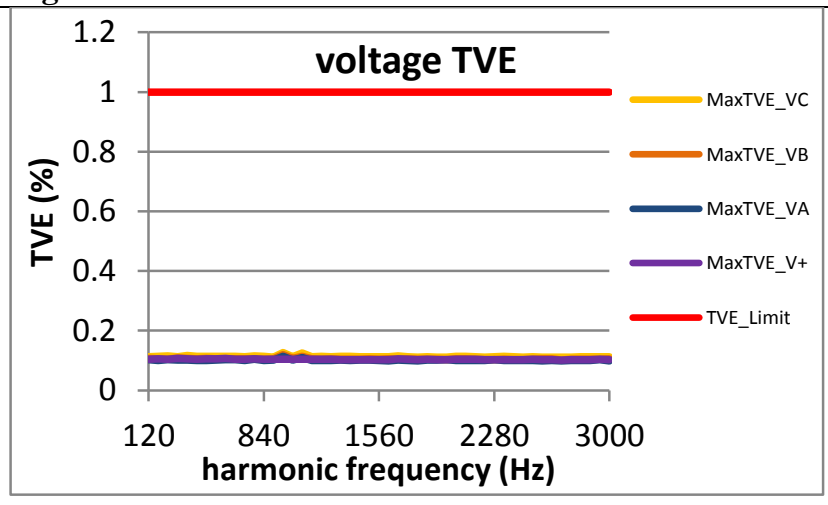

Figure 277: Fs = 30 FPS

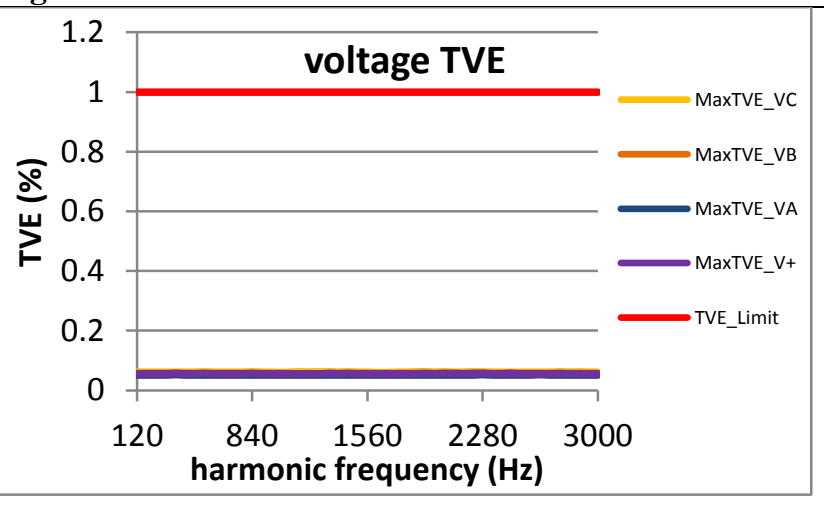

Figure 278: Fs = 20 FPS

Figure 279: Fs = 15 FPS

Figure 280: Fs = 12 FPS

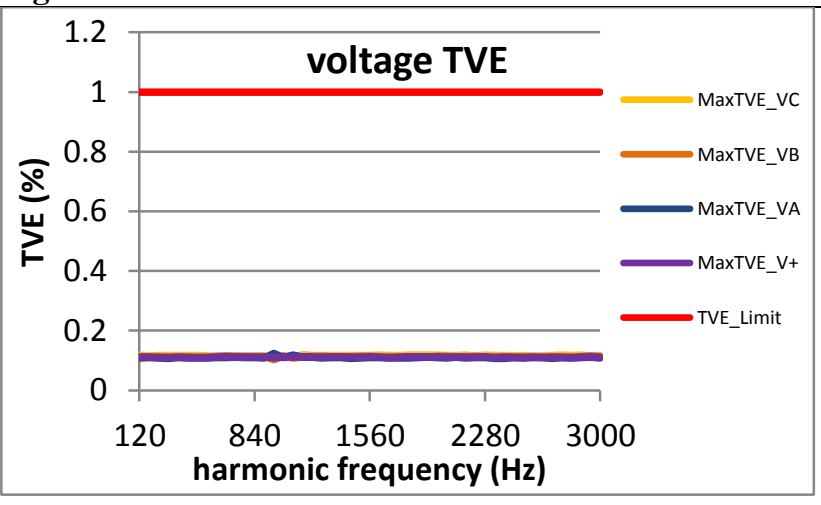

Figure 281: Fs = 10 FPS 
4.5.4 PMU C steady state harmonic distortion voltage TVE: P class
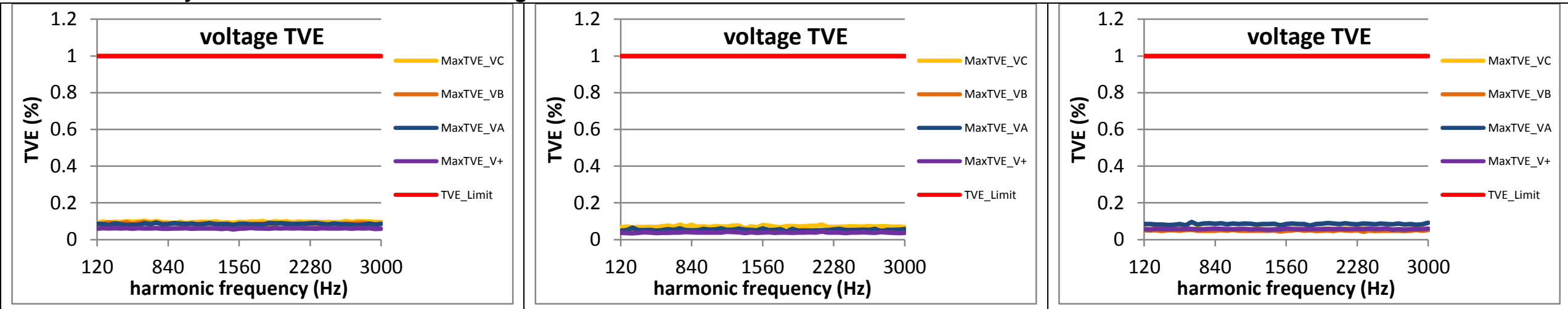

Figure 282: Fs = 60 FPS

\section{Figure 283: Fs = 30 FPS}

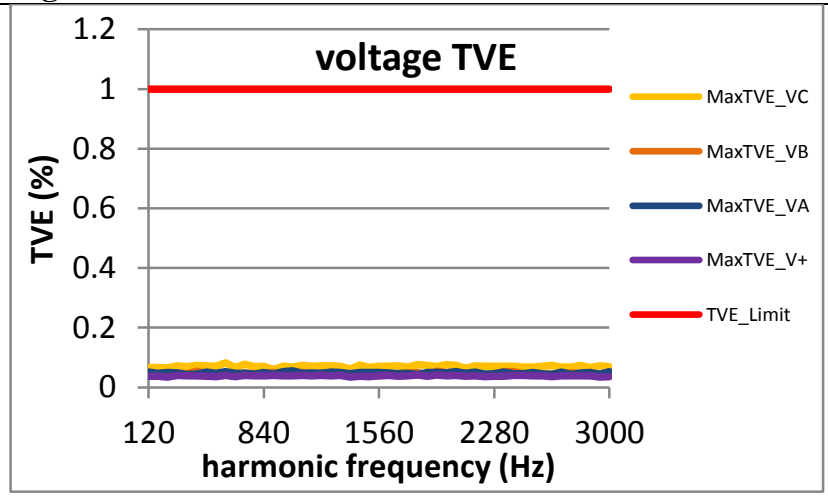

Figure 285: Fs = 15 FPS

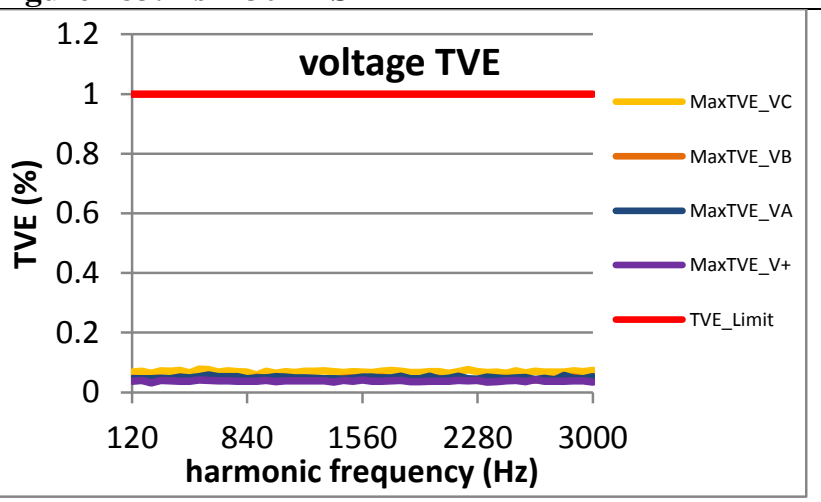

Figure 286: Fs = 12 FPS
Figure 284: Fs = 20 FPS

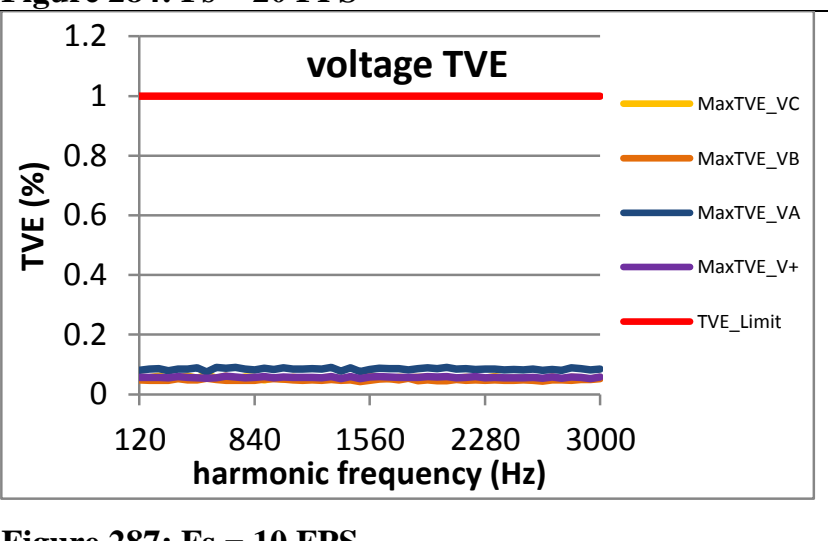

Figure 287: Fs = 10 FPS 
4.5.5 PMU D steady state harmonic distortion voltage TVE: P class
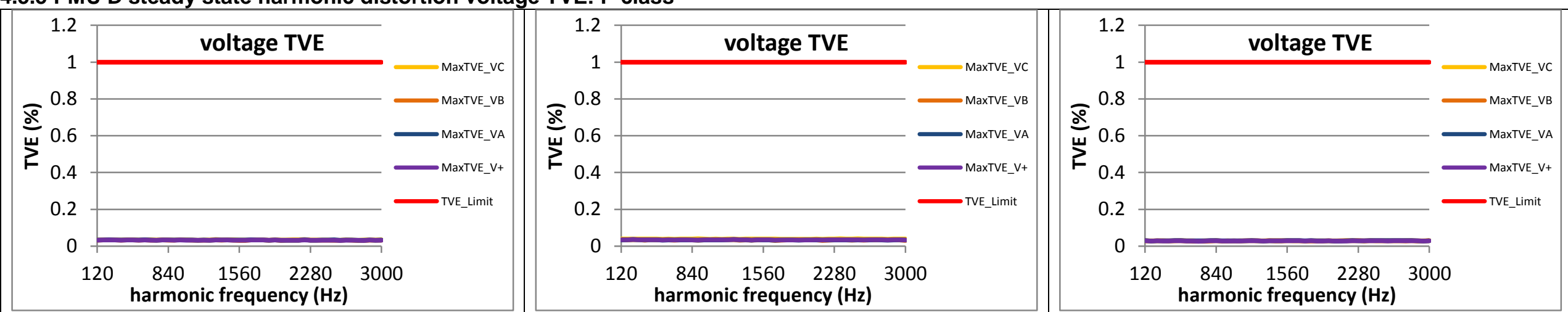

Figure 288: Fs = 60 FPS

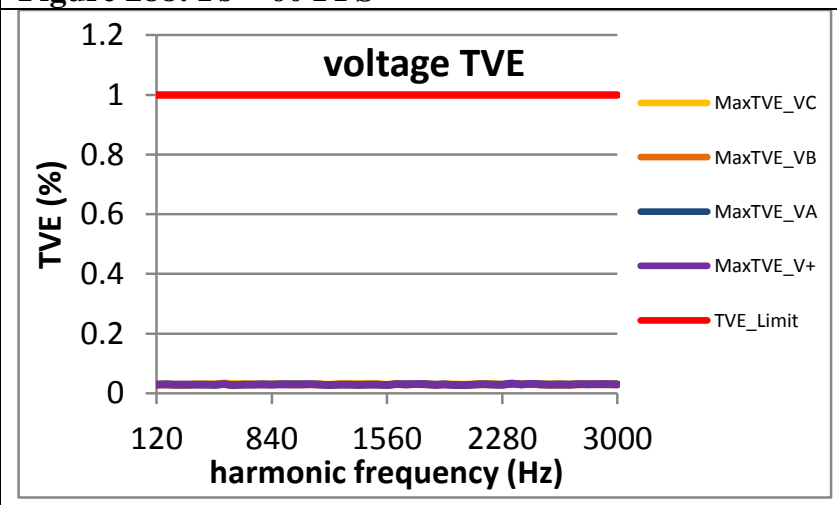

\section{Figure 289: Fs = 30 FPS}

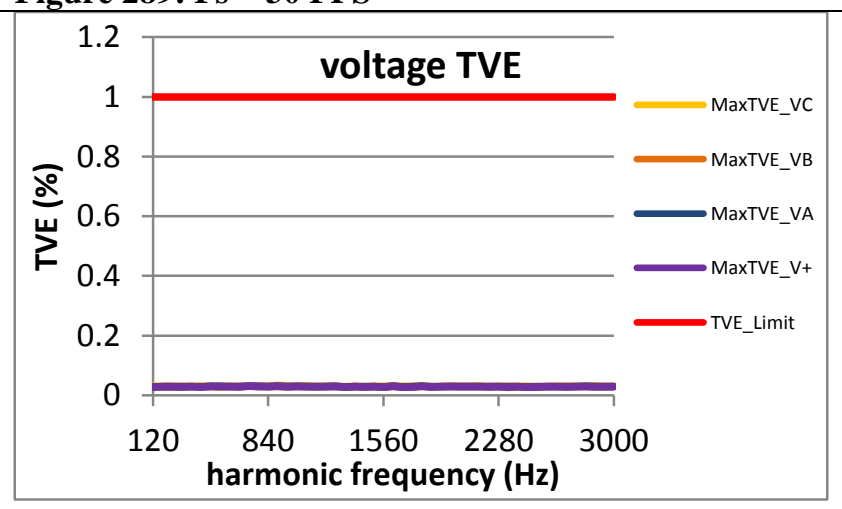

Figure 290: Fs = 20 FPS

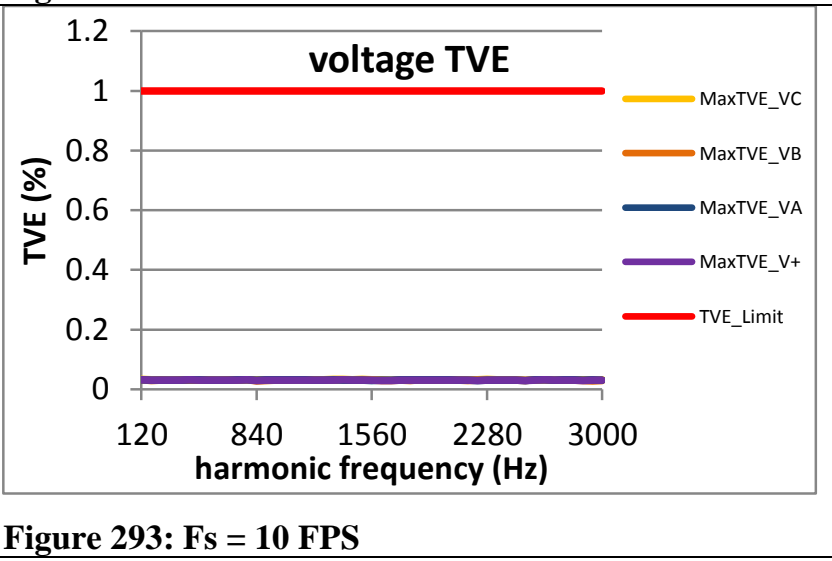

4.5.6 PMU E steady state harmonic distortion voltage TVE: P class

PMU E does not support P Class 
4.5.7 PMU F steady state harmonic distortion voltage TVE: P class
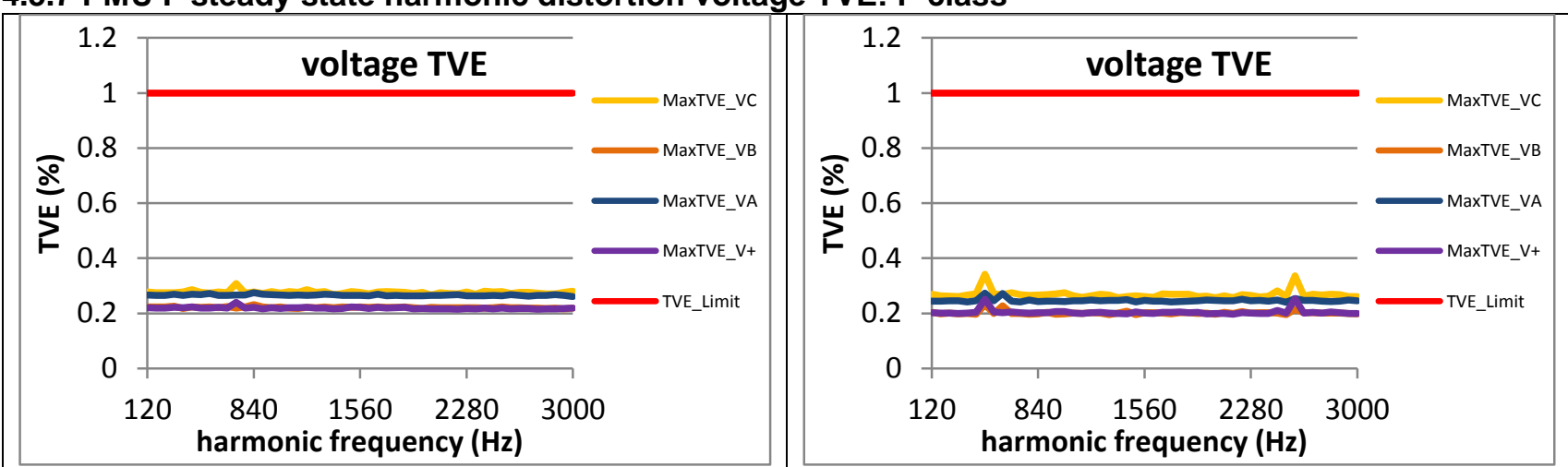

Figure 294: Fs = 60 FPS

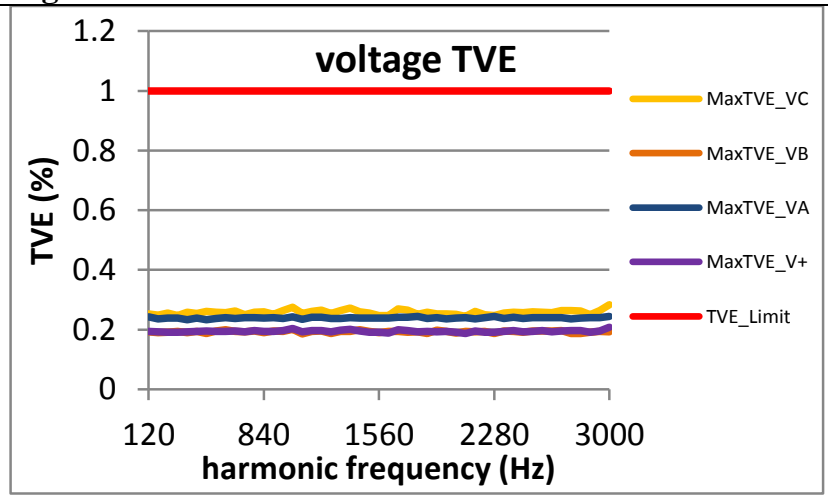

Figure 297: Fs $=15$ FPS

\section{Figure 295: Fs = 30 FPS}

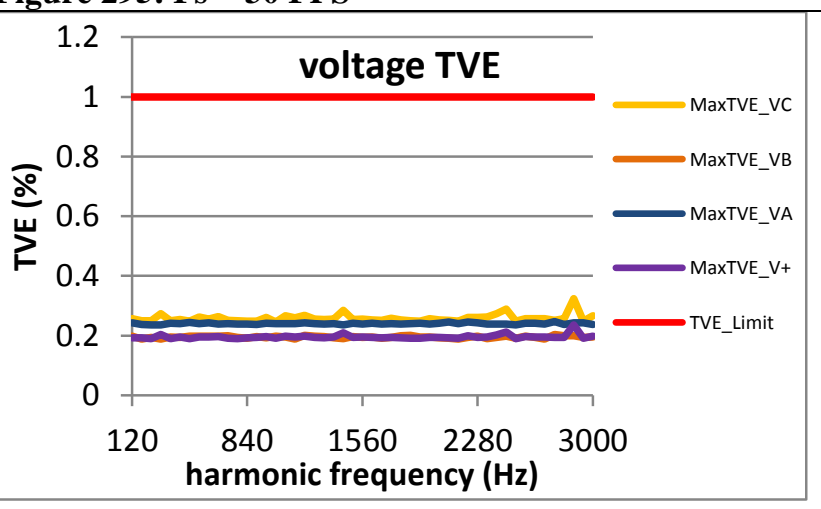

Figure 298: Fs $=12$ FPS

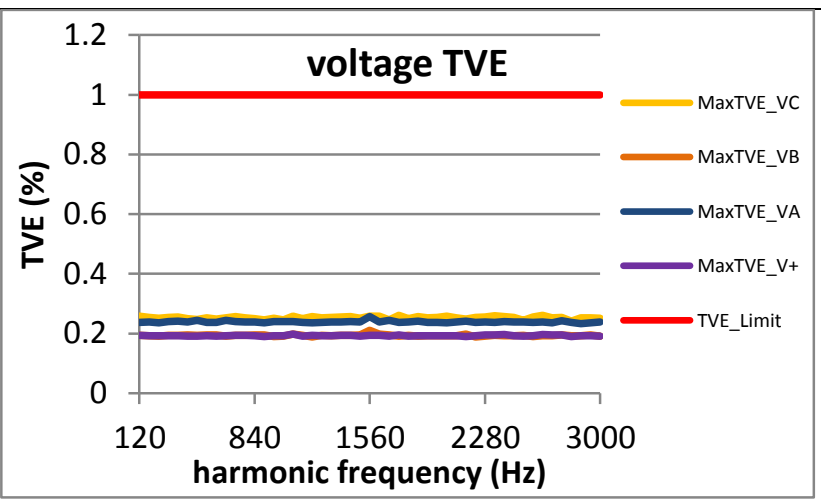

Figure 296: Fs = 20 FPS

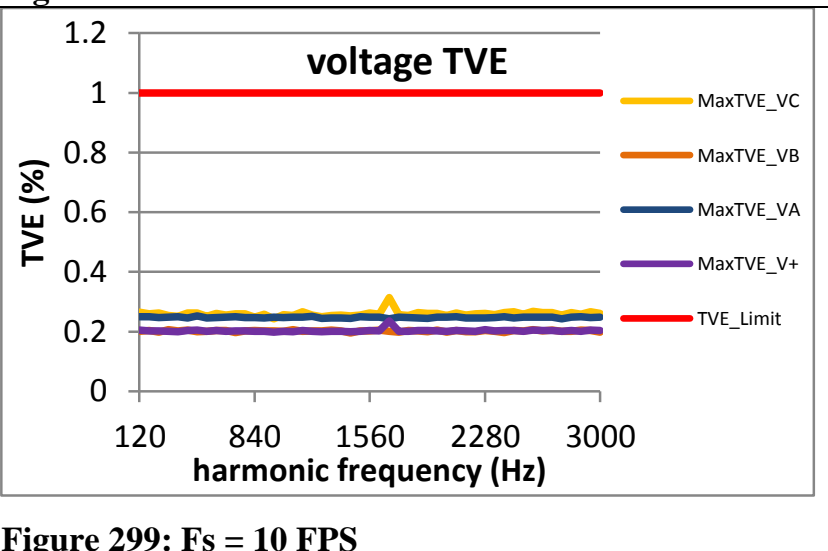

4.5.8 PMU G steady state harmonic distortion voltage TVE: P class

PMU G does not support P class 
4.5.9 PMU H steady state harmonic distortion voltage TVE: P class
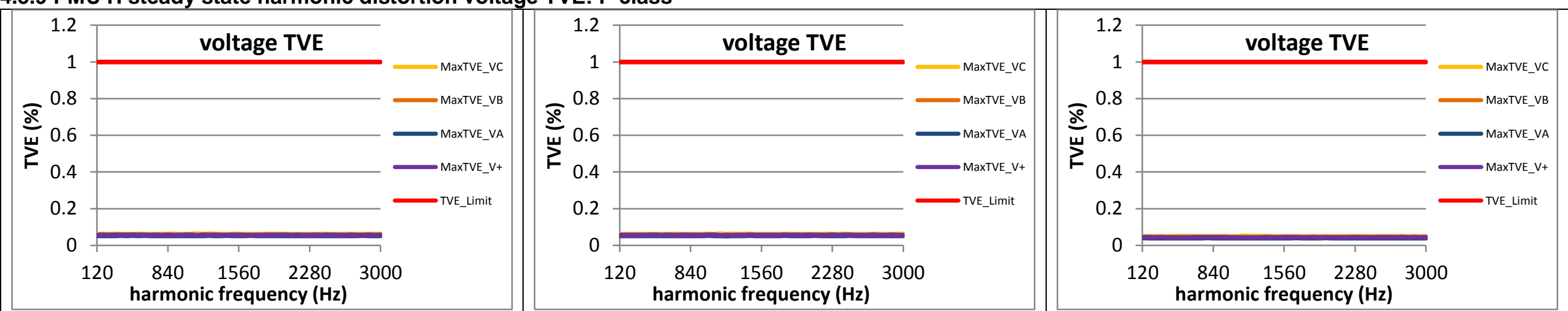

Figure 300: Fs = 60 FPS

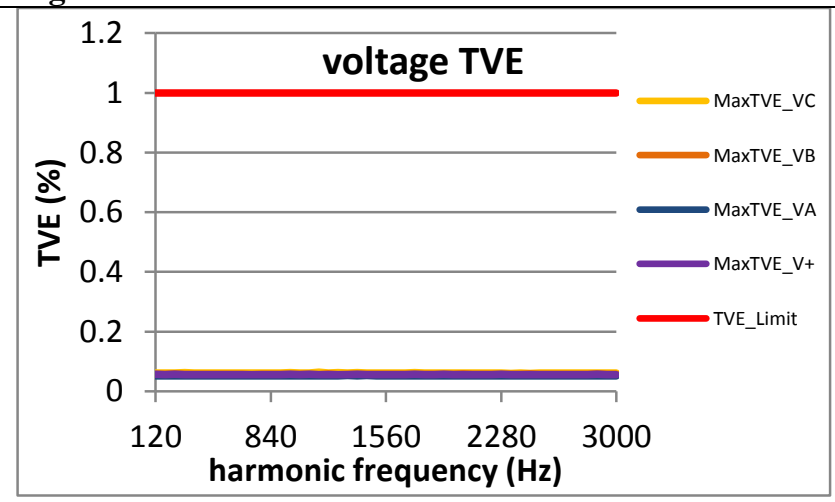

\section{Figure 301: Fs = 30 FPS}

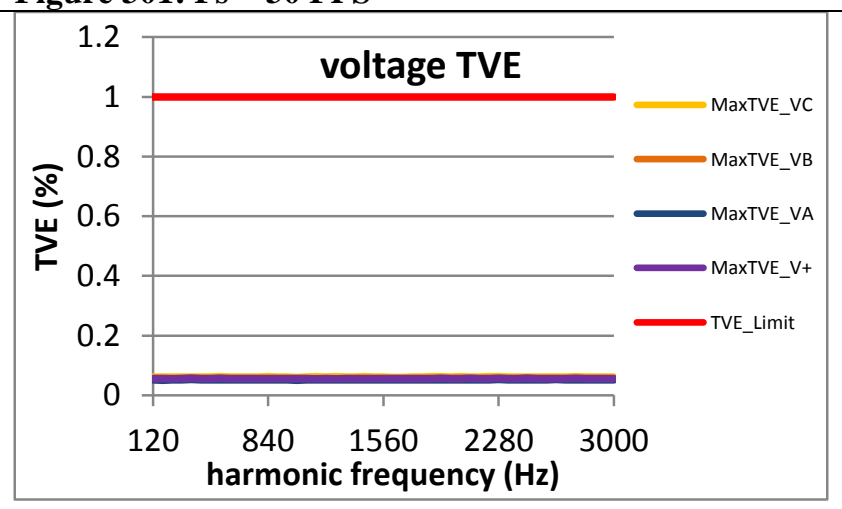

Figure 302: Fs = 20 FPS

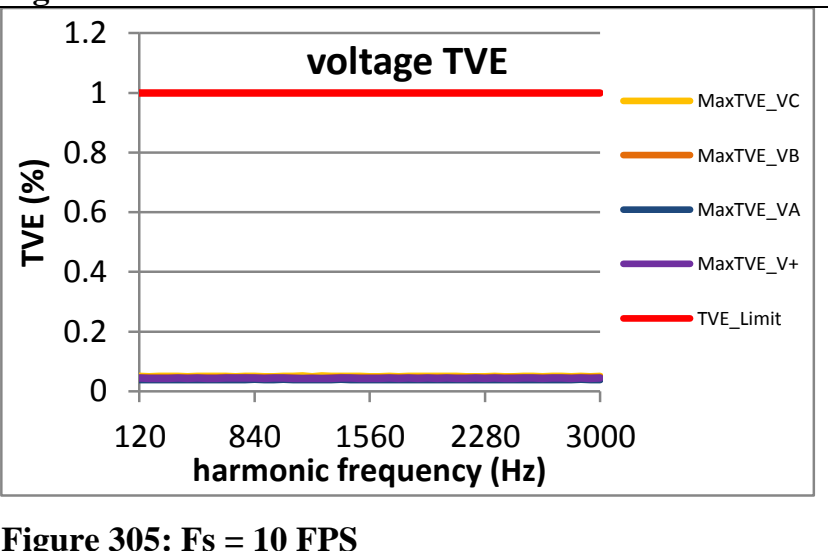

Figure 304: Fs = 12 FPS

Figure 305: Fs = 10 FPS

4.5.10 PMU I steady state harmonic distortion voltage TVE: P class

PMU I does not support P class 
4.5.11 PMU J steady state harmonic distortion voltage TVE: P class
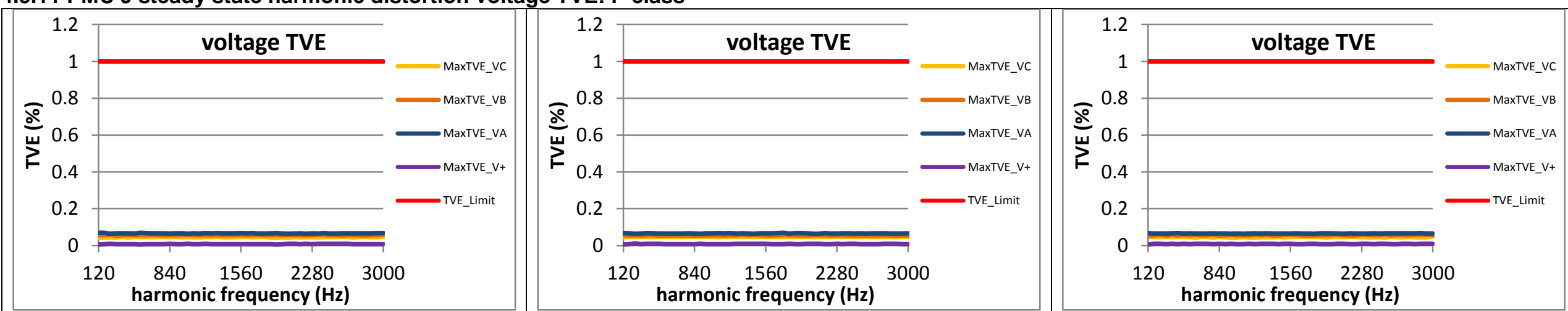

Figure 306: Fs = 60 FPS

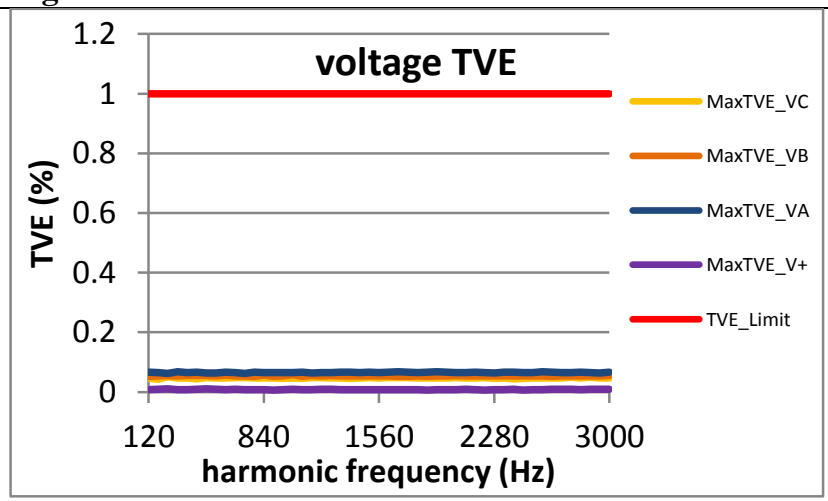

Figure 307: Fs = 30 FPS

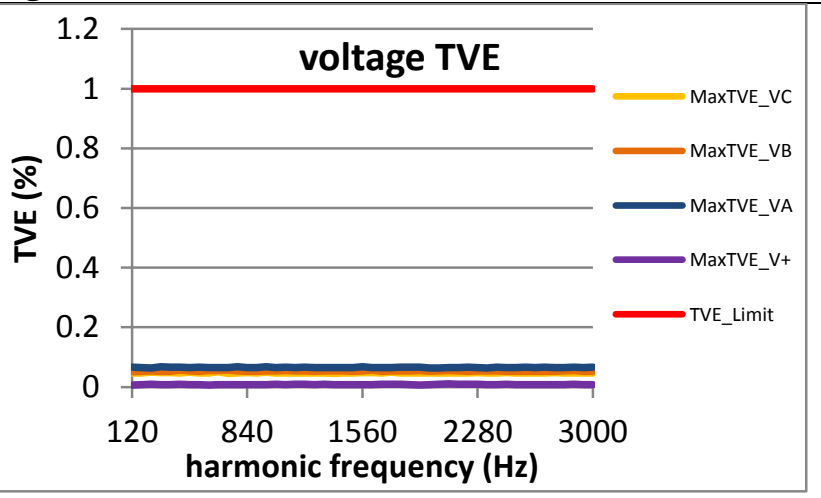

Figure 308: Fs = 20 FPS

Figure 309: Fs = 15 FPS

Figure 310: Fs = 12 FPS

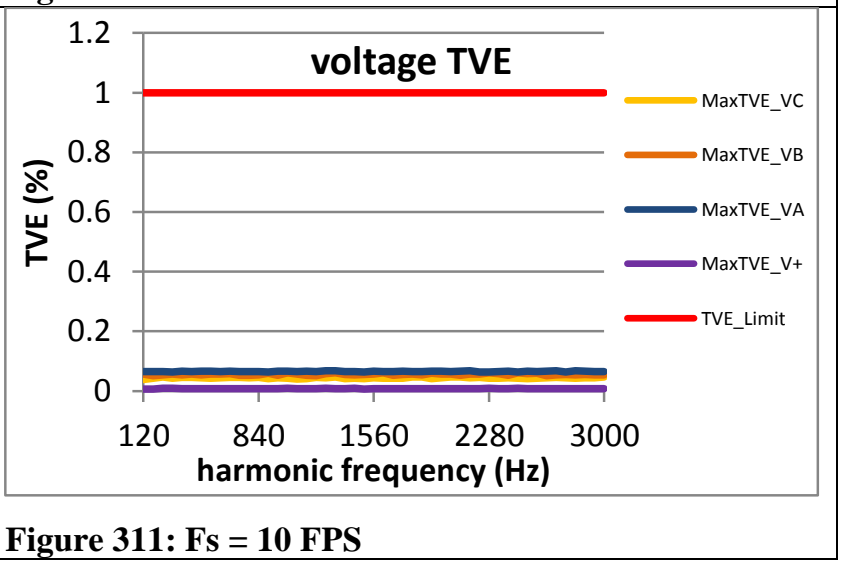




\subsection{Steady state harmonic distortion current TVE: P class}

4.6.1 C37.118.1 Annex C steady state harmonic distortion current TVE: P class
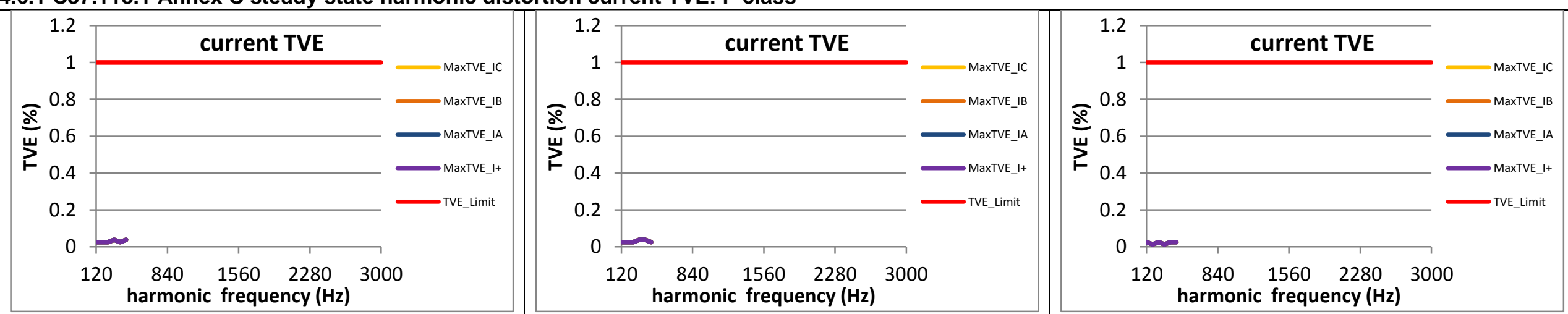

Figure 312: Fs = 60 FPS

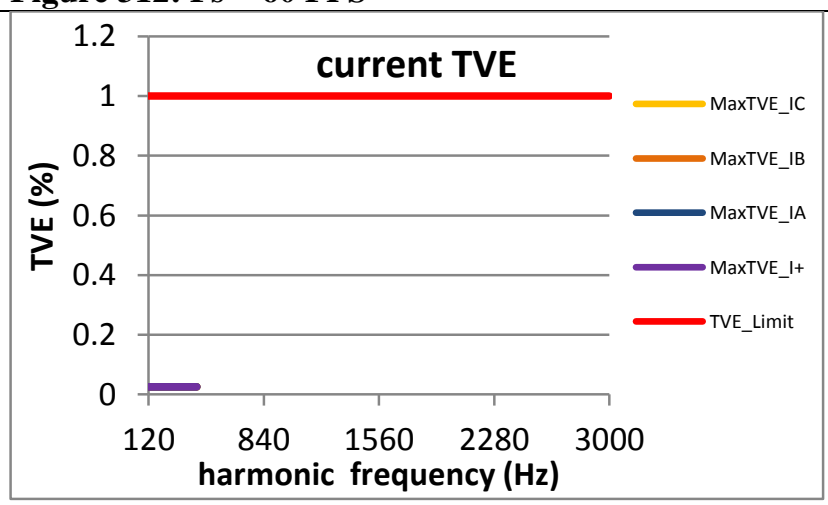

Figure 313: Fs = 30 FPS

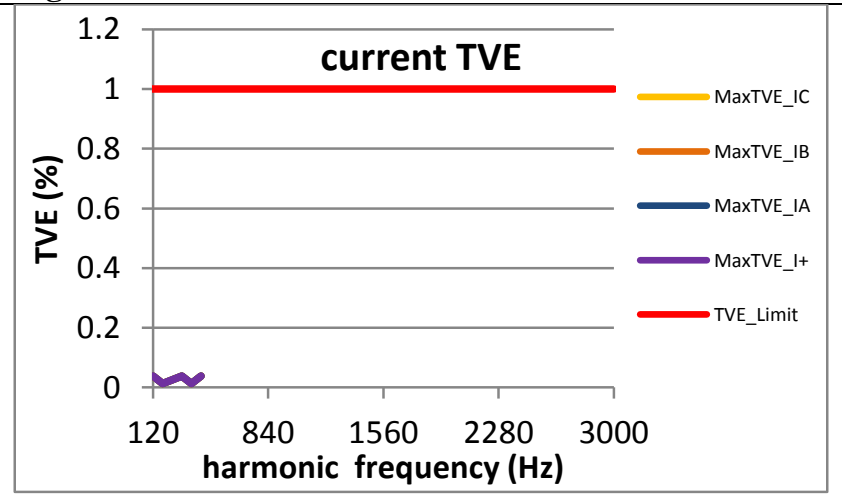

Figure 314: Fs = 20 FPS

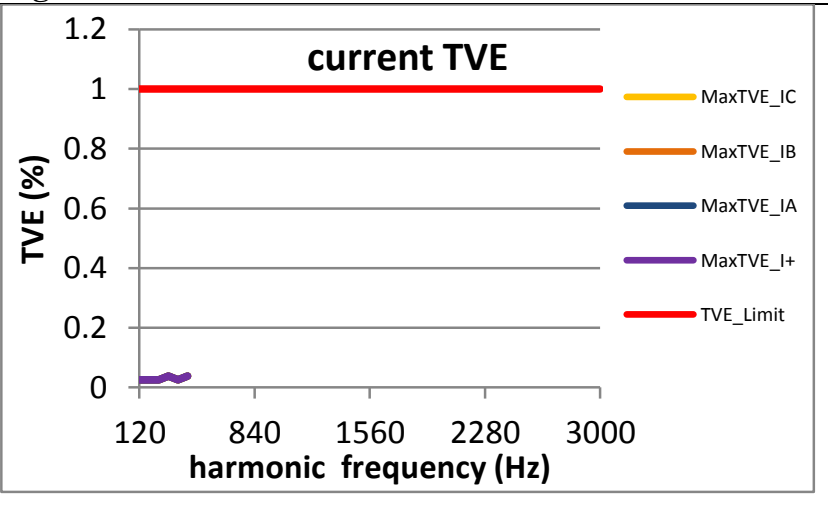

Figure 316: Fs = 12 FPS

Figure 317: Fs = 10 FPS 
4.6.2 PMU A steady state harmonic distortion current TVE: P class
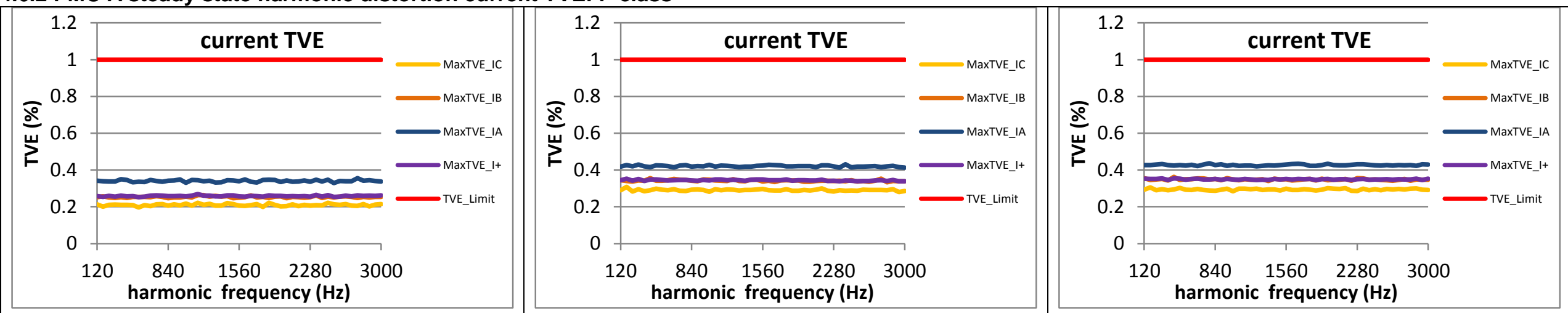

Figure 318: Fs = 60 FPS

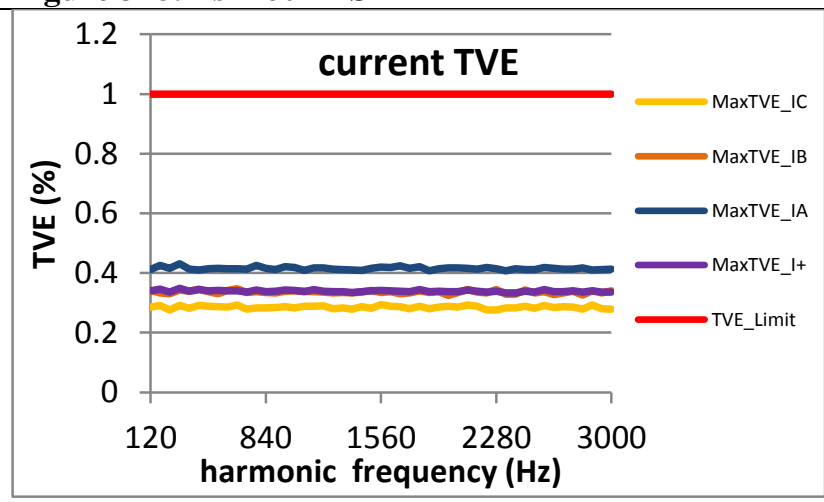

Figure 319: Fs = 30 FPS

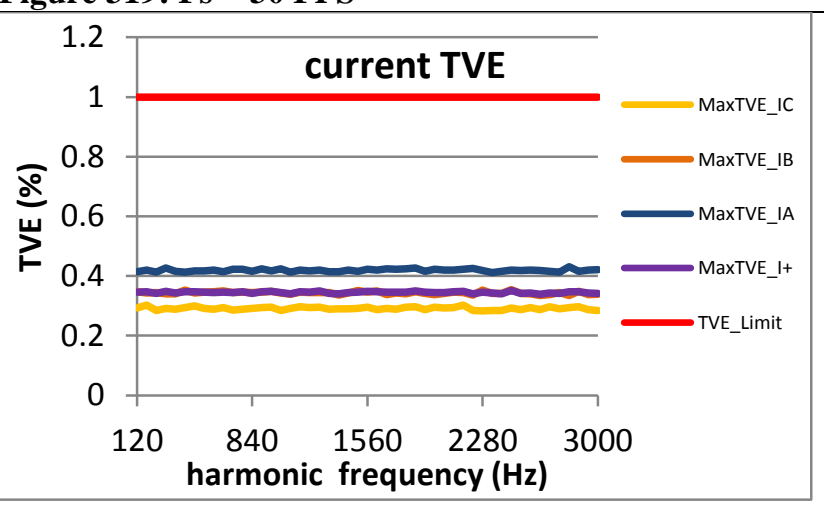

Figure 320: Fs = 20 FPS

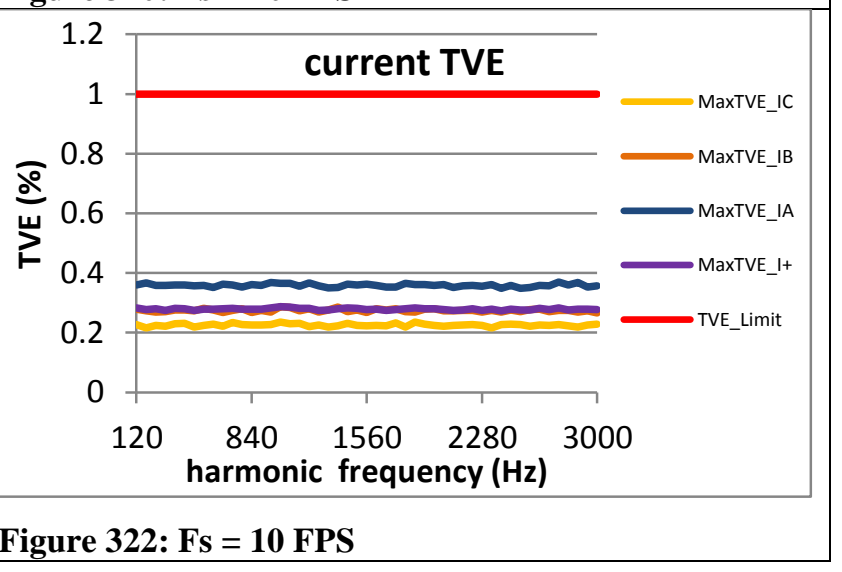

Figure 321: Fs = 15 FPS 
4.6.3 PMU B steady state harmonic distortion current TVE: P class
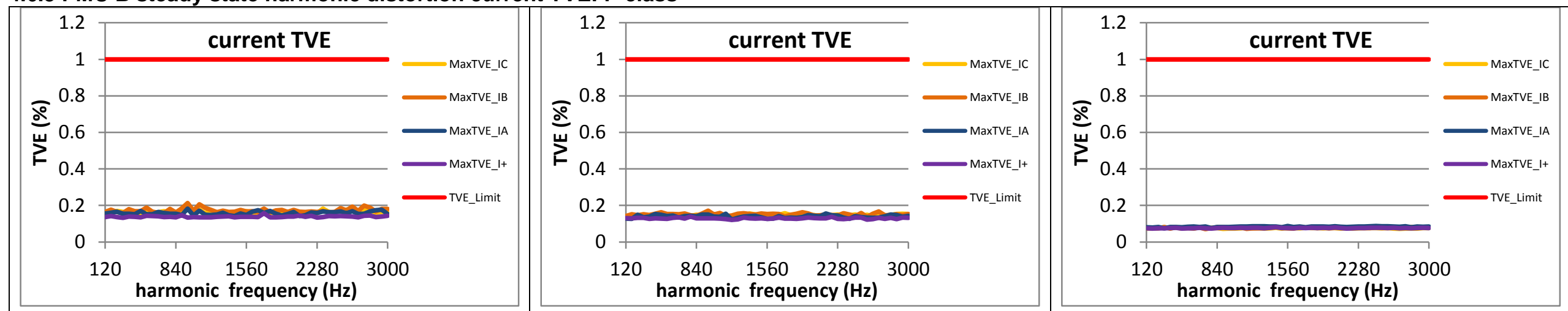

Figure 323: Fs = 60 FPS

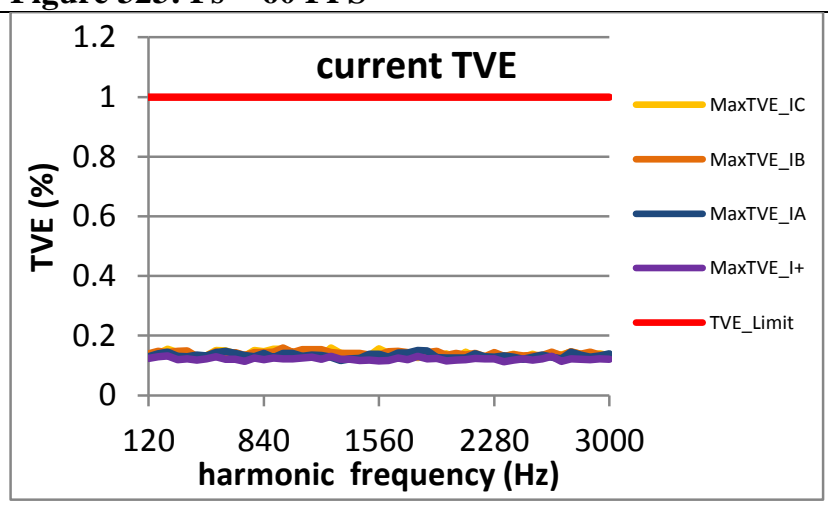

Figure 324: Fs = 30 FPS

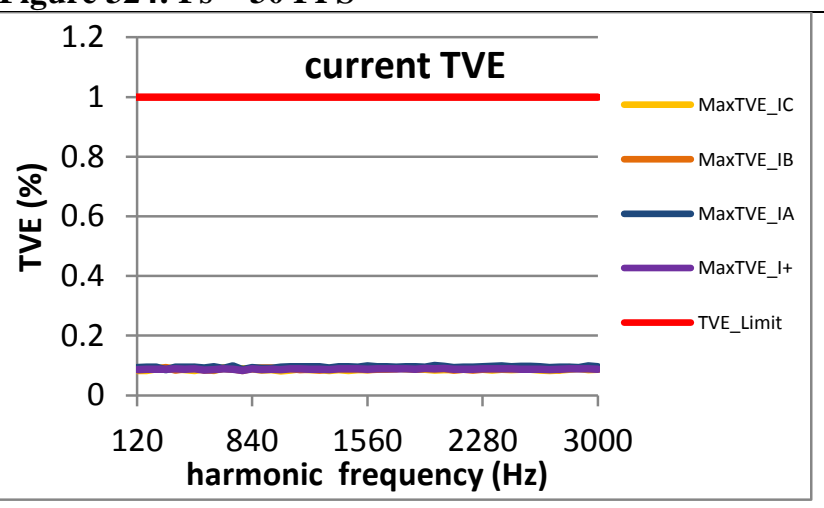

Figure 325: Fs = 20 FPS

Figure 326: Fs = 15 FPS

Figure 327: Fs = 12 FPS

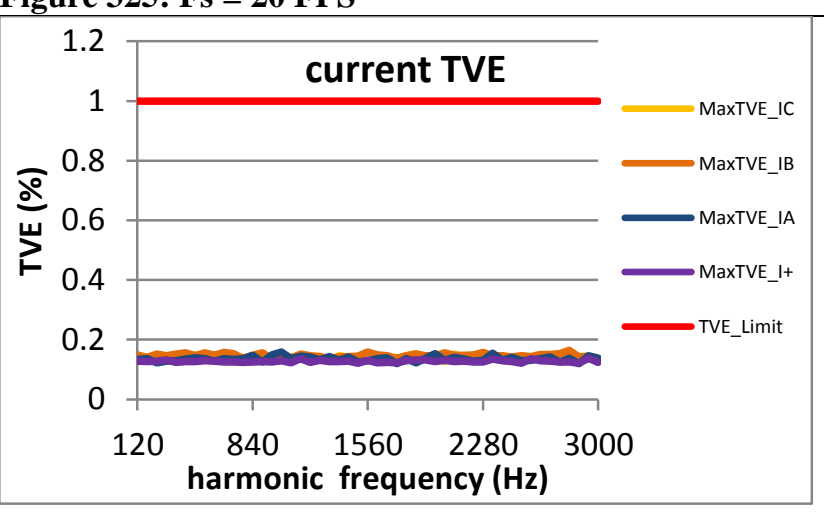

Figure 328: Fs = 10 FPS 
4.6.4 PMU C steady state harmonic distortion current TVE: P class
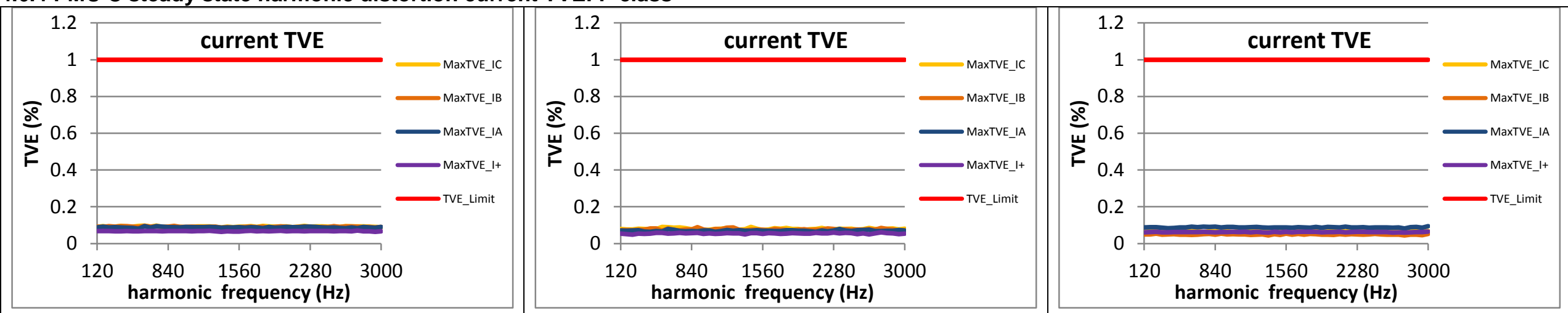

Figure 329: Fs = 60 FPS

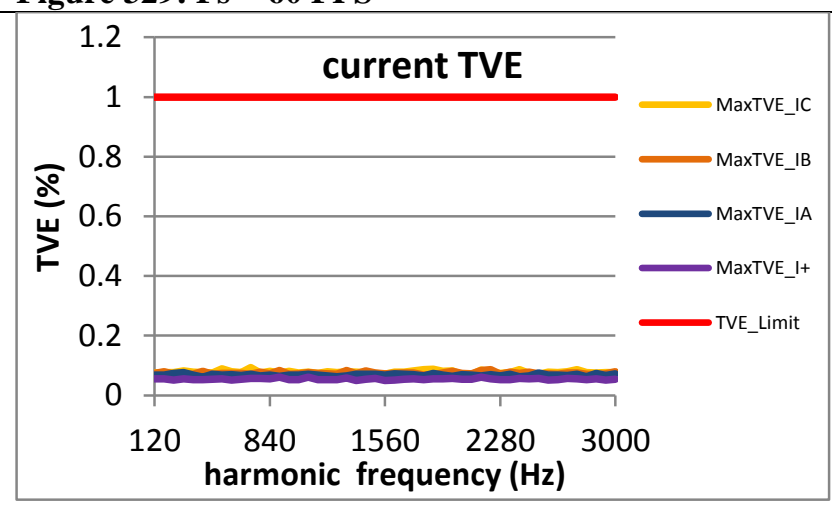

Figure 330: Fs = 30 FPS

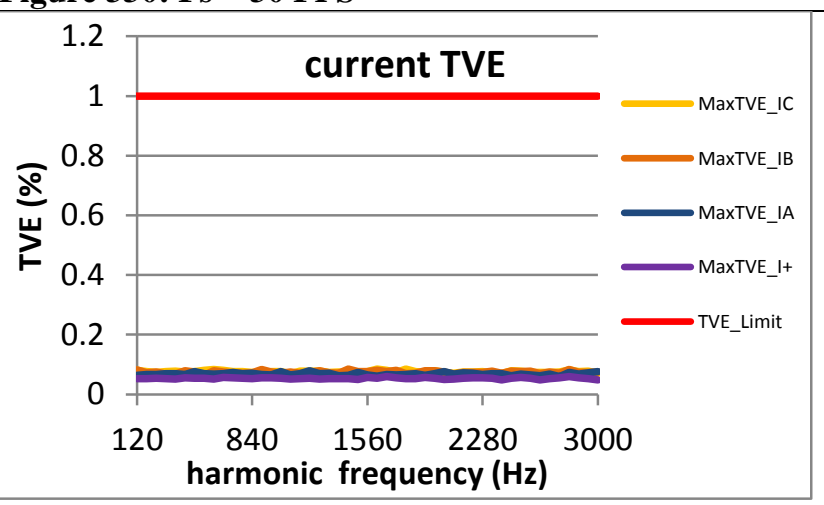

Figure 331: Fs = 20 FPS

Figure 332: Fs = 15 FPS

Figure 333: Fs = 12 FPS

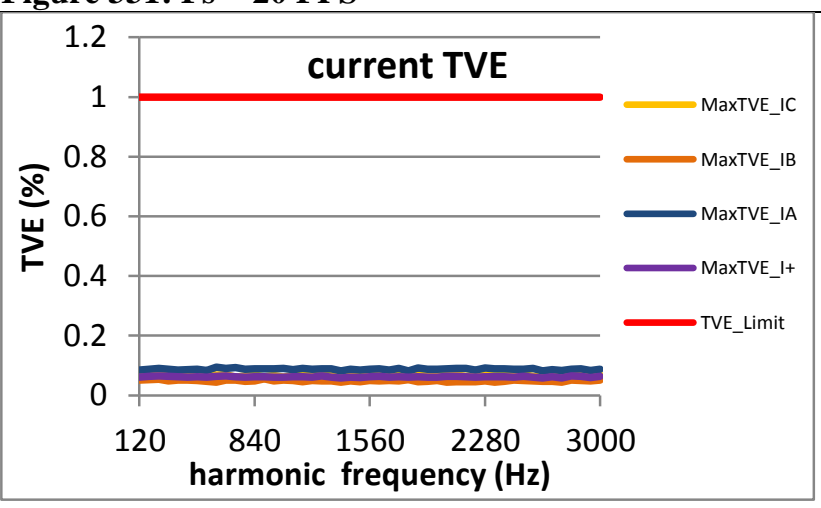

Figure 334: Fs = 10 FPS 
4.6.5 PMU D steady state harmonic distortion current TVE: P class
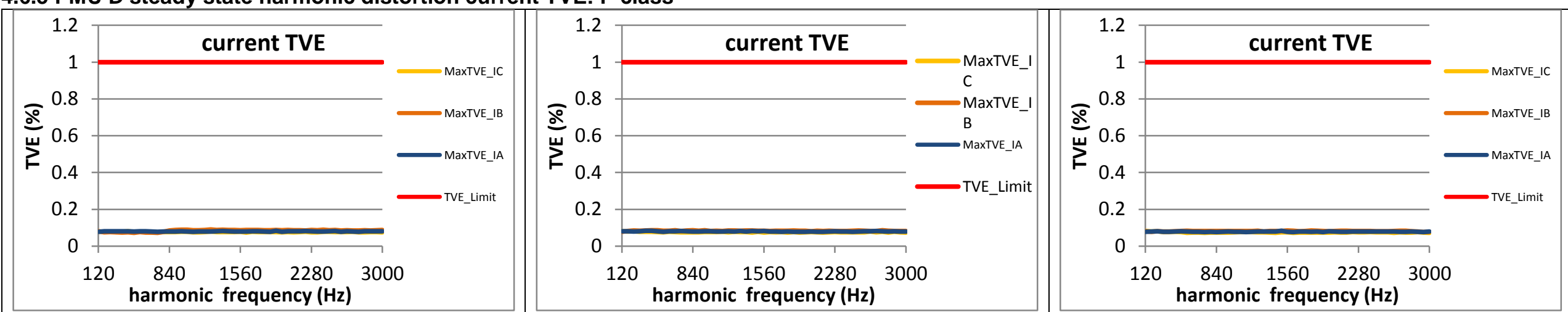

Figure 335: Fs = 60 FPS

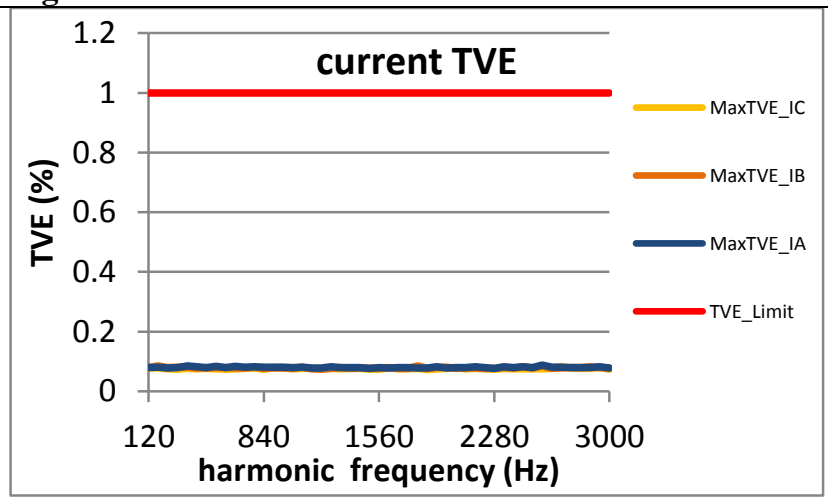

\section{Figure 336: Fs = 30 FPS}

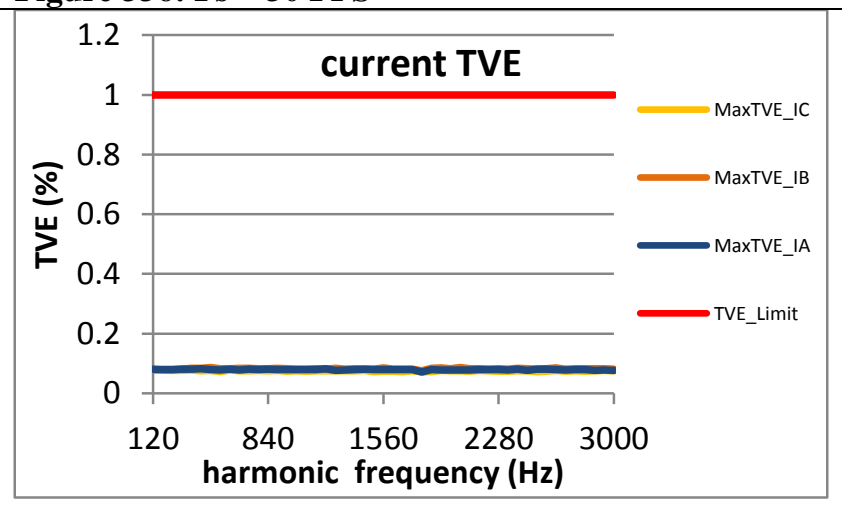

Figure 337: Fs = 20 FPS

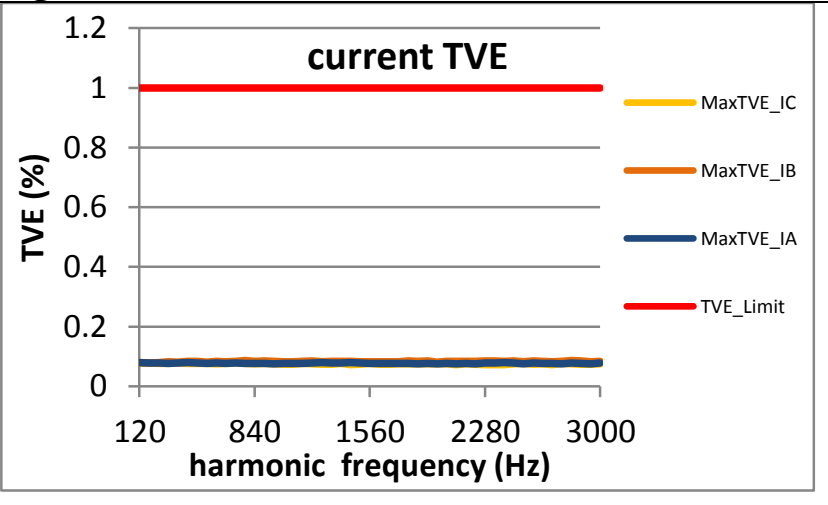

Figure 339: Fs = 12 FPS

Figure 340: Fs = 10 FPS

4.6.6 PMU E steady state harmonic distortion current TVE: P class

PMU E does not support $P$ class 
4.6.7 PMU F steady state harmonic distortion current TVE: P class
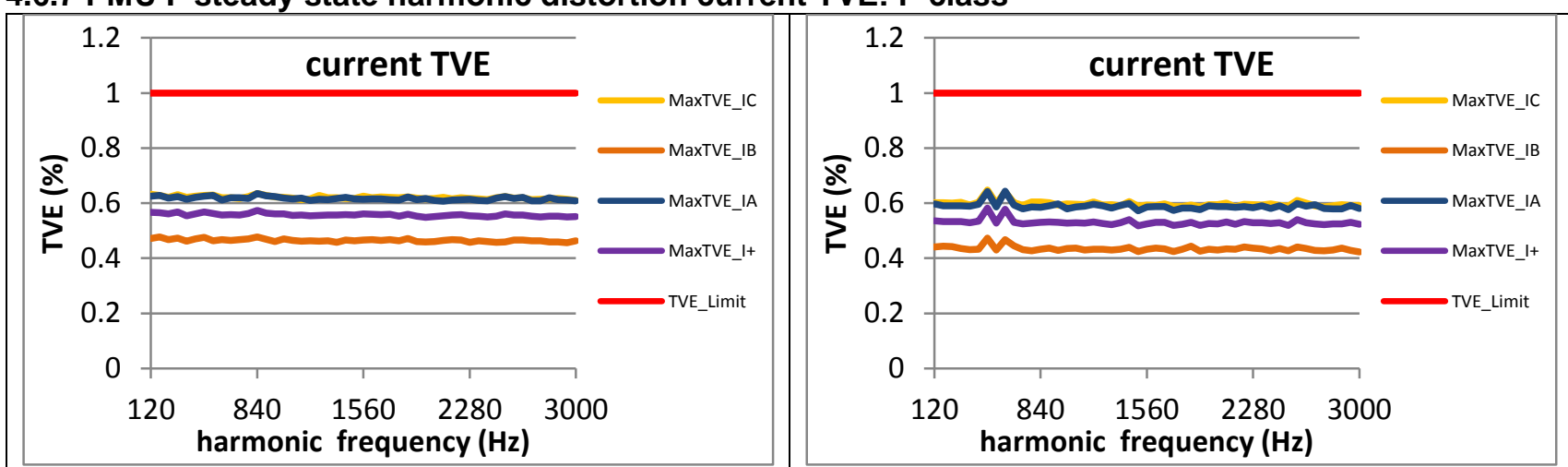

Figure 341: Fs = 60 FPS

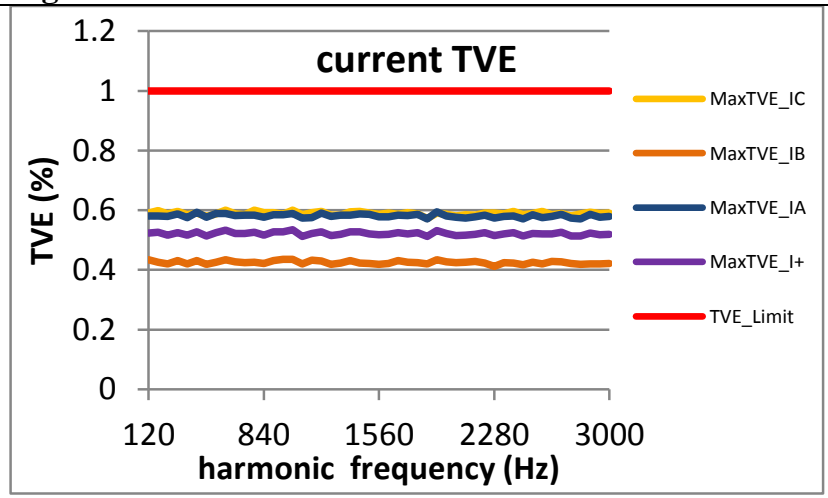

Figure 344: Fs = 15 FPS

\section{Figure 342: Fs = 30 FPS}

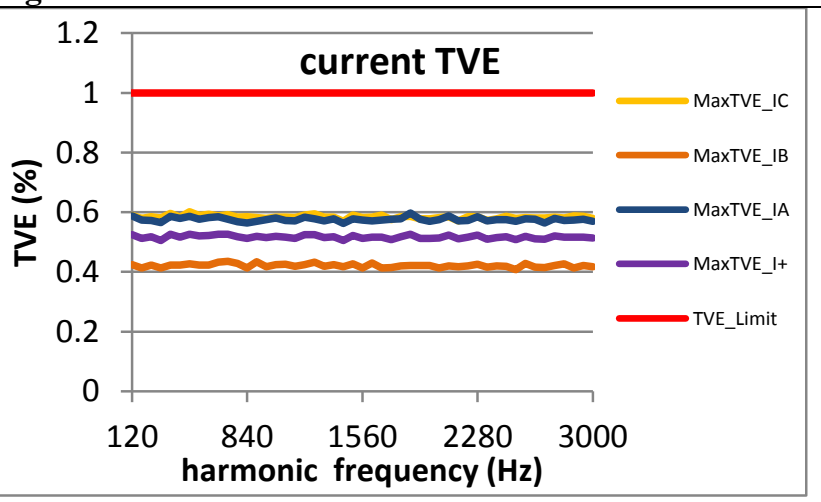

Figure 345: Fs $=12$ FPS

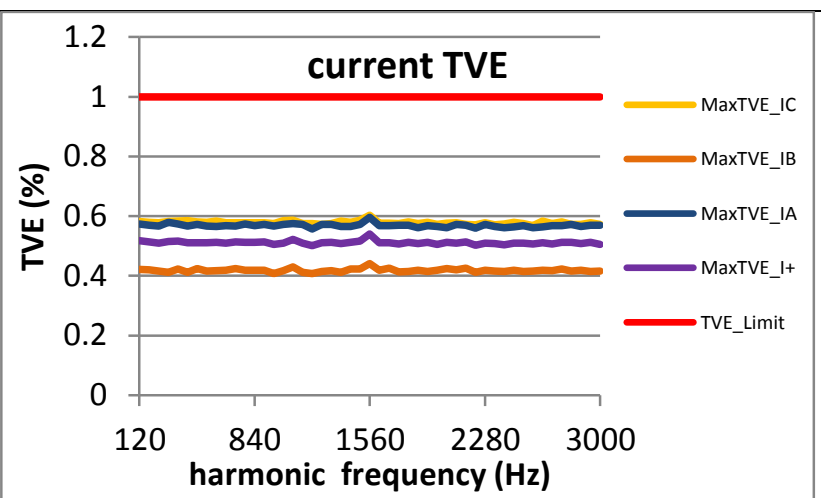

Figure 343: Fs = 20 FPS

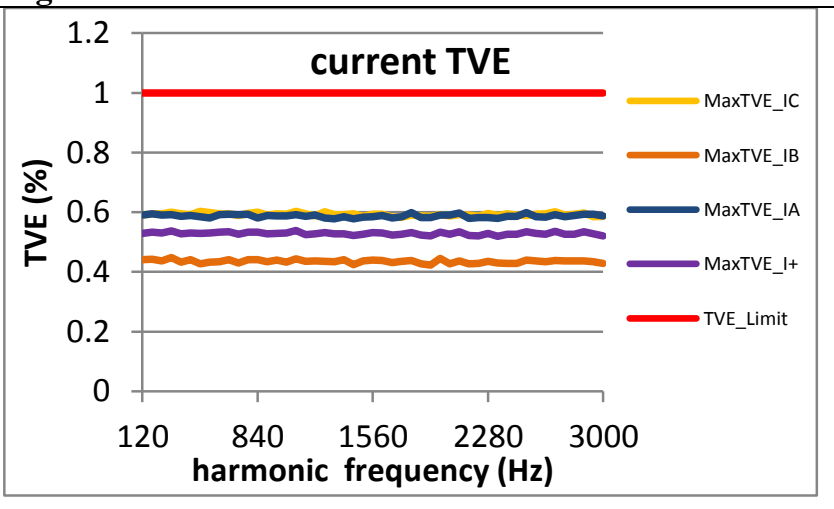

Figure 346: Fs $=10$ FPS

4.6.8 PMU G steady state harmonic distortion current TVE: P class

PMU G does not support P class 
4.6.9 PMU H steady state harmonic distortion current TVE: P class
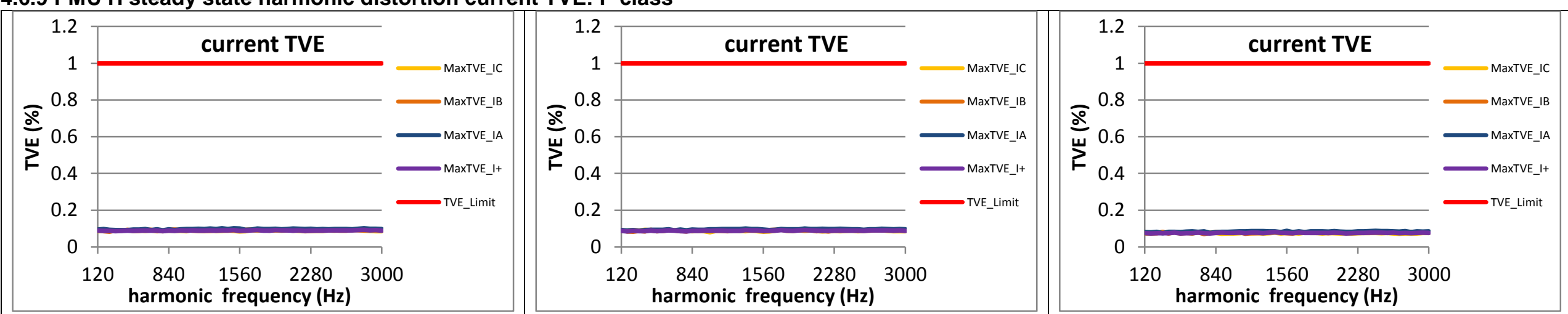

Figure 347: Fs = 60 FPS

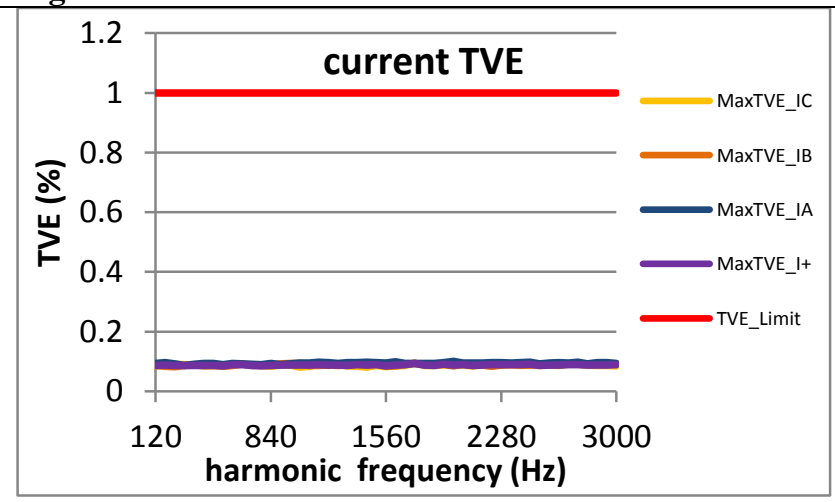

\section{Figure 348: Fs = 30 FPS}

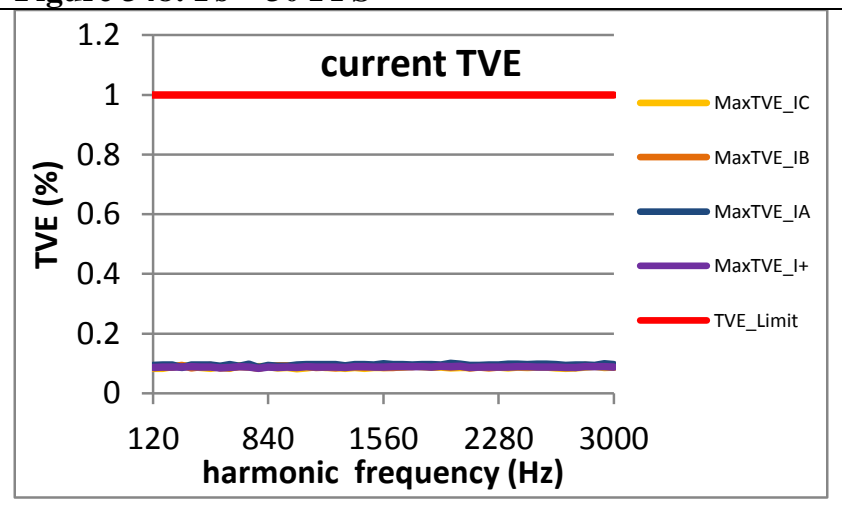

Figure 349: Fs = 20 FPS

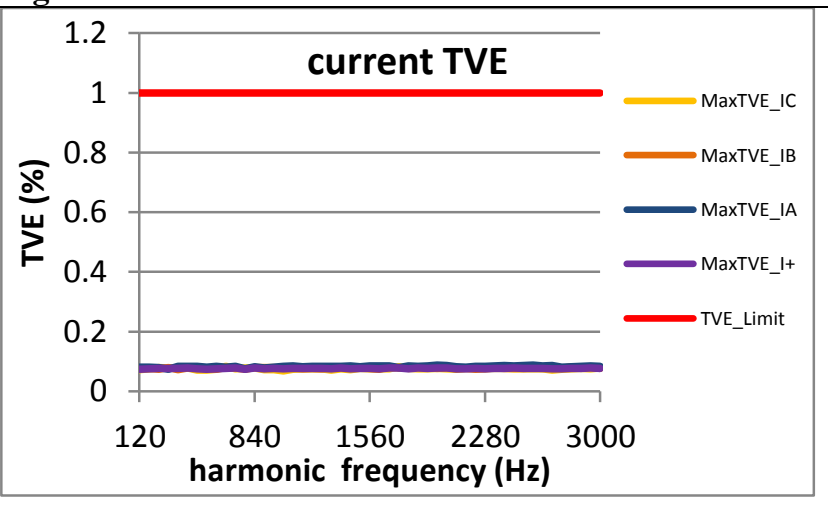

Figure 351: Fs = 12 FPS

Figure 352: Fs = 10 FPS

4.6.10 PMU I steady state harmonic distortion current TVE: P class

PMU I does not support P class 
4.6.11 PMU J steady state harmonic distortion current TVE: P class
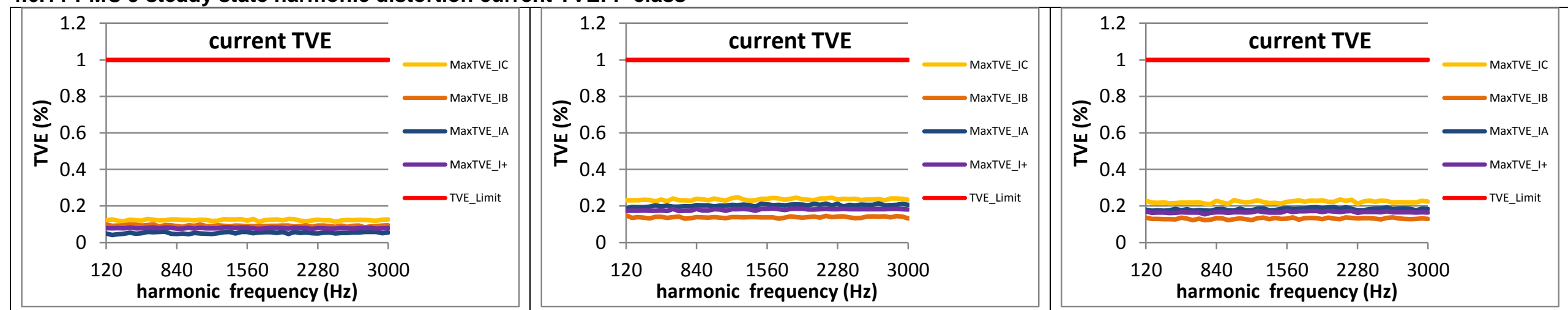

Figure 353: Fs = 60 FPS

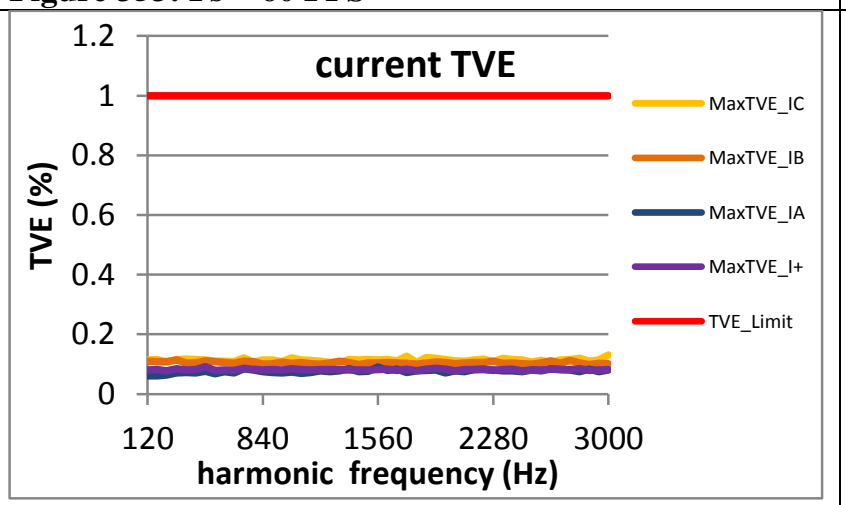

\section{Figure 354: Fs = 30 FPS}

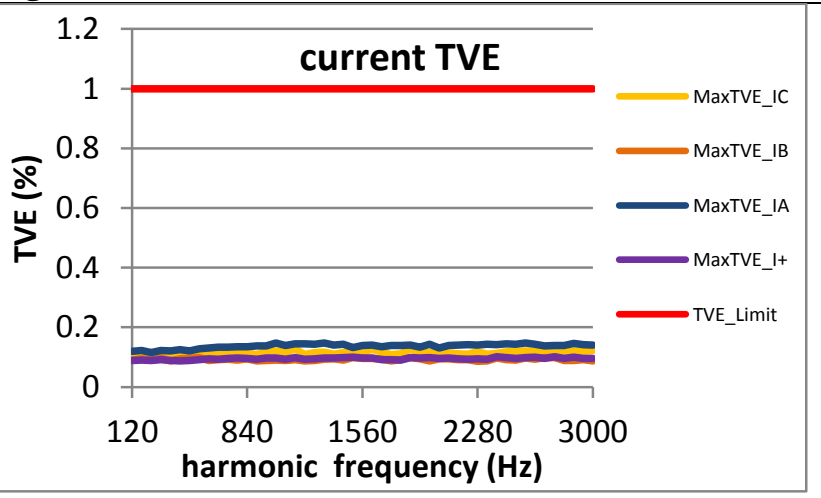

Figure 355: Fs = 20 FPS

Figure 356: Fs = 15 FPS

Figure 357: Fs = 12 FPS 


\subsection{Steady state frequency harmonic distortion frequency error: P class}

4.7.1 C37.118.1 Annex C steady harmonic distortion frequency error: $P$ class
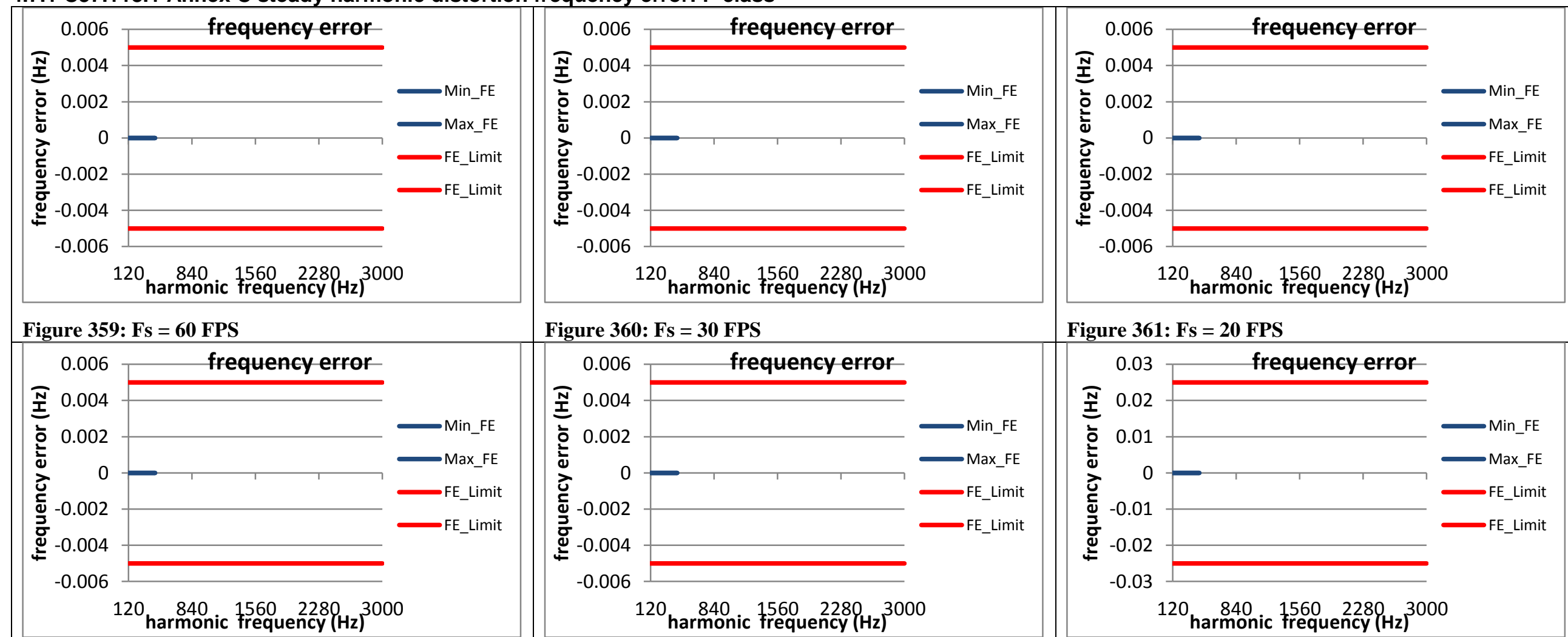

Figure 360: Fs = 30 FPS
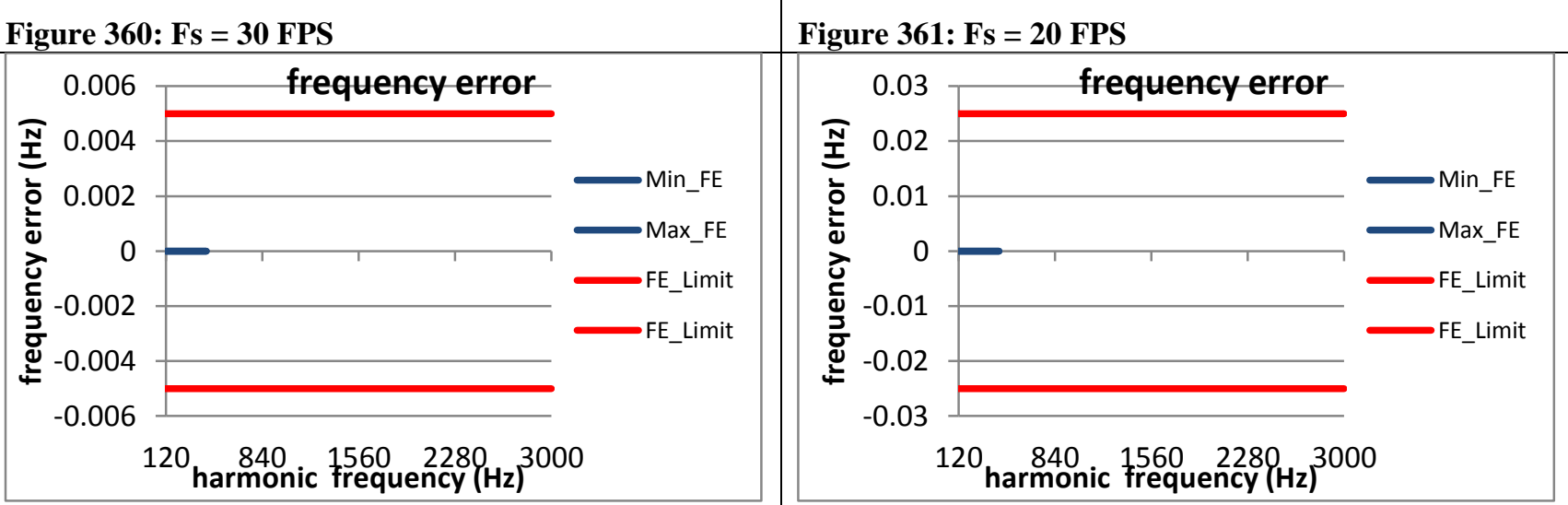

Figure 362; Fs = 15 FPS

Figure 363: Fs = 12 FPS

\section{Figure 364: Fs = 10 FPS}

The C37.118.1 Annex C model is a digital simulation with an internal sampling rate of 960 samples per second and so can only be tested with a harmonic frequency up to $420 \mathrm{~Hz}$. 
4.7.2 PMU A steady state harmonic distortion frequency error: P class

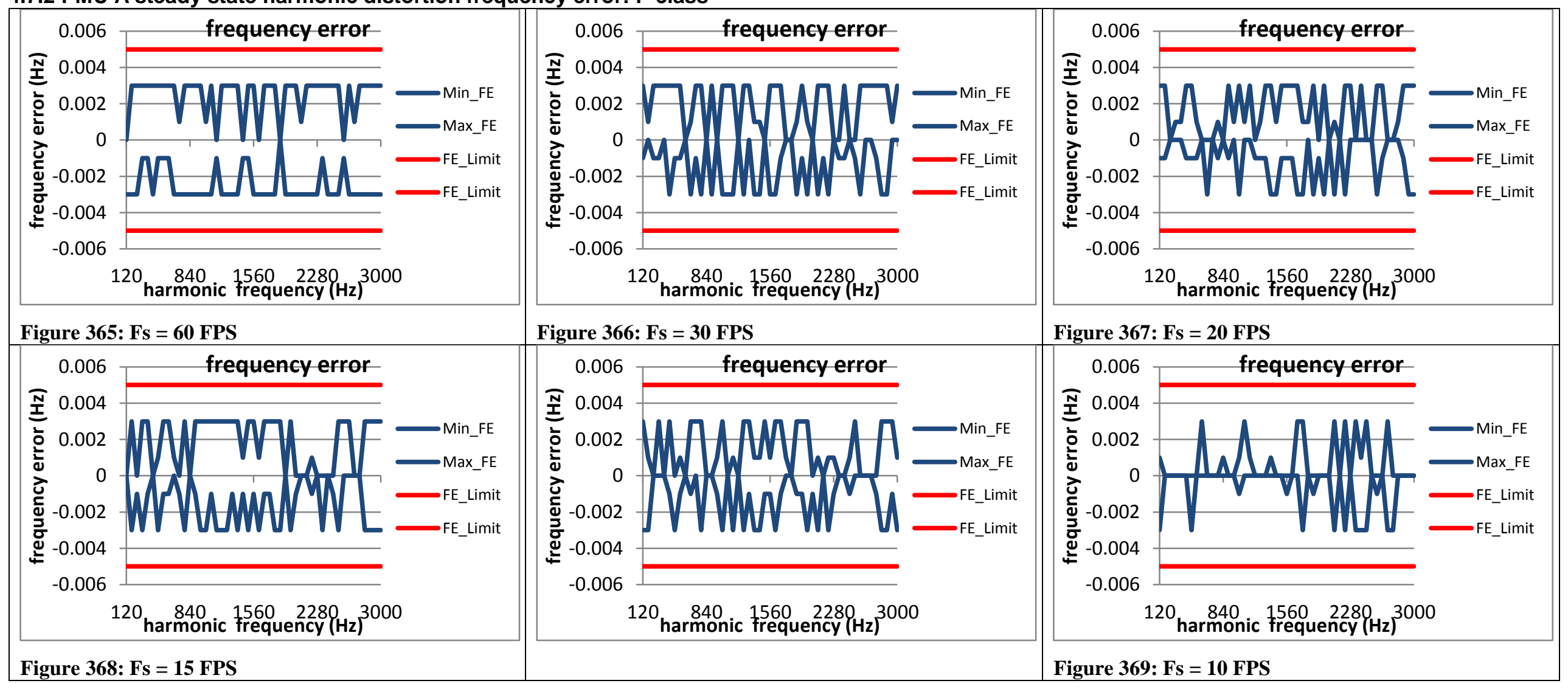


4.7.3 PMU B steady state harmonic distortion frequency error: $P$ class
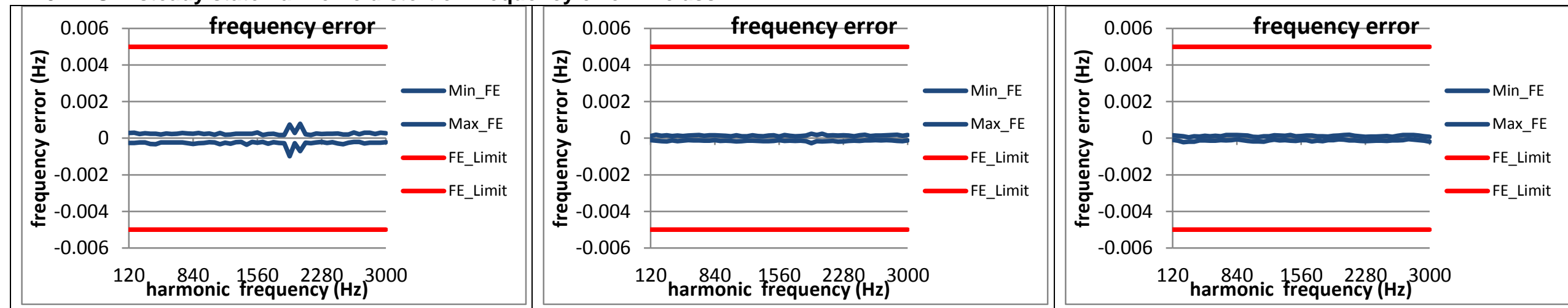

Figure 370: Fs = 60 FPS

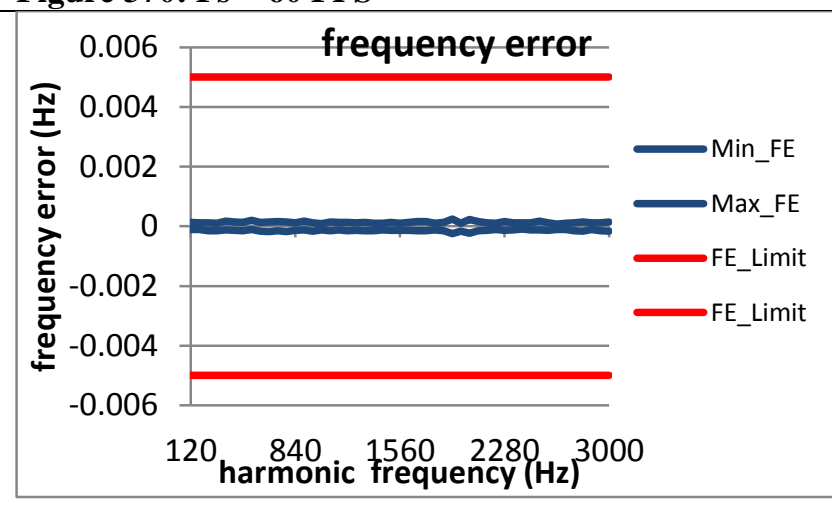

Figure 371: Fs = 30 FPS

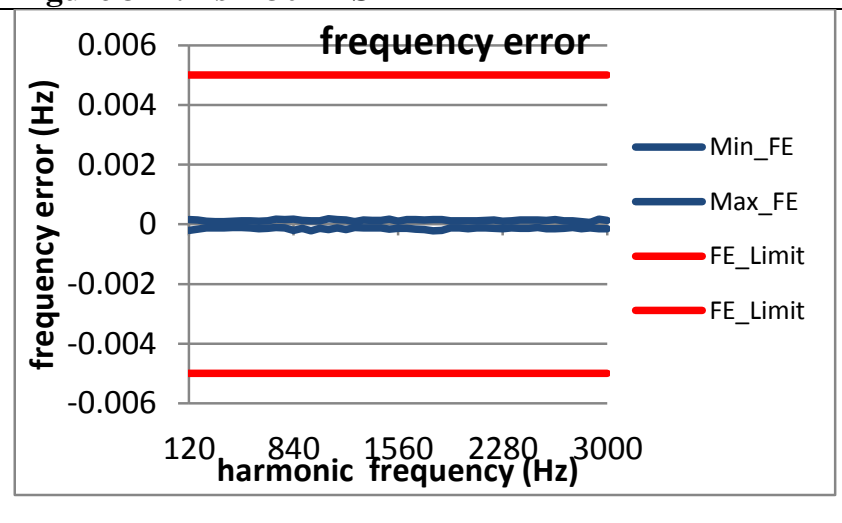

Figure 372: Fs = 20 FPS

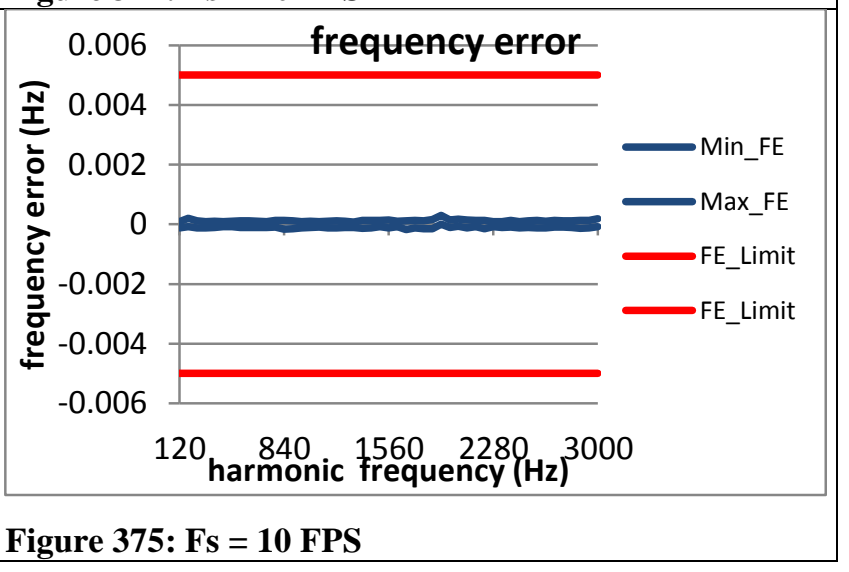


4.7.4 PMU C steady state harmonic distortion frequency error: $P$ class

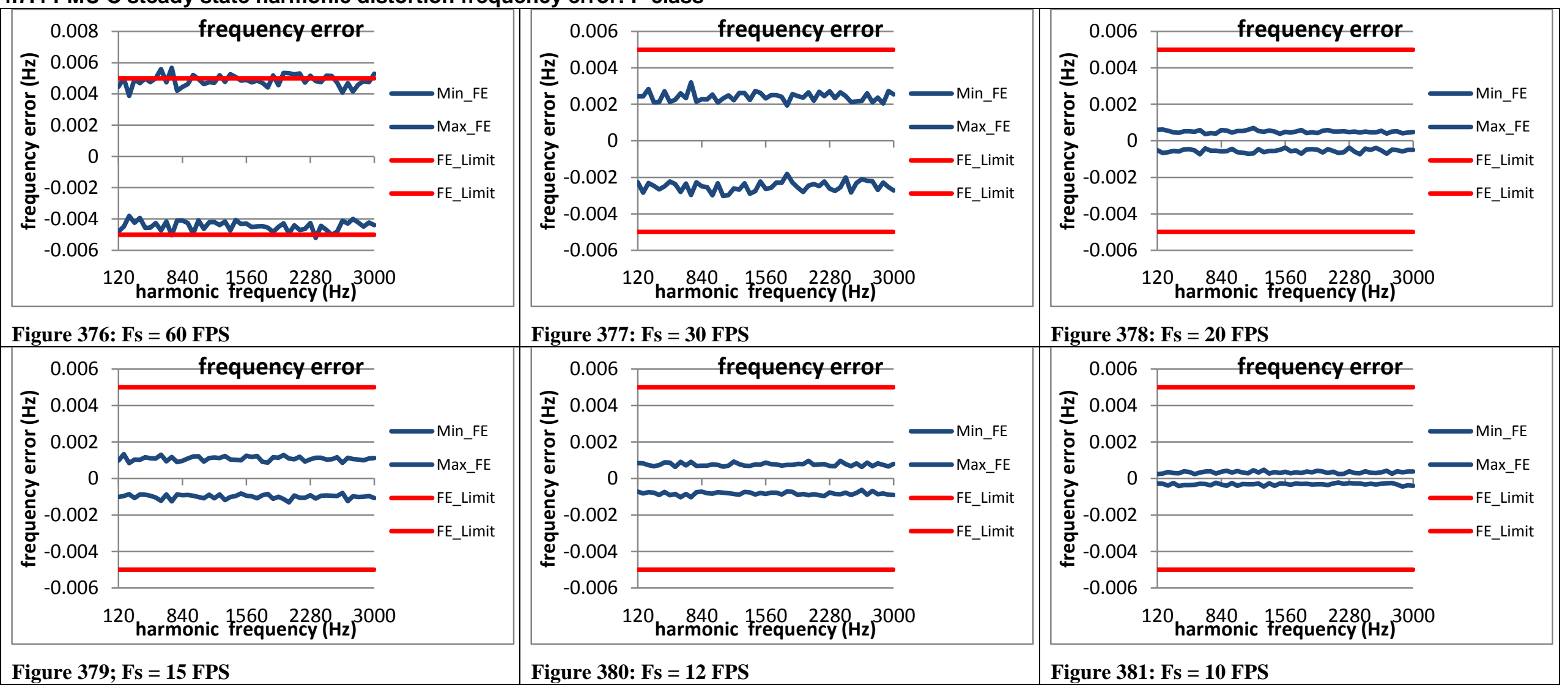


4.7.5 PMU D steady state harmonic distortion frequency error: $P$ class
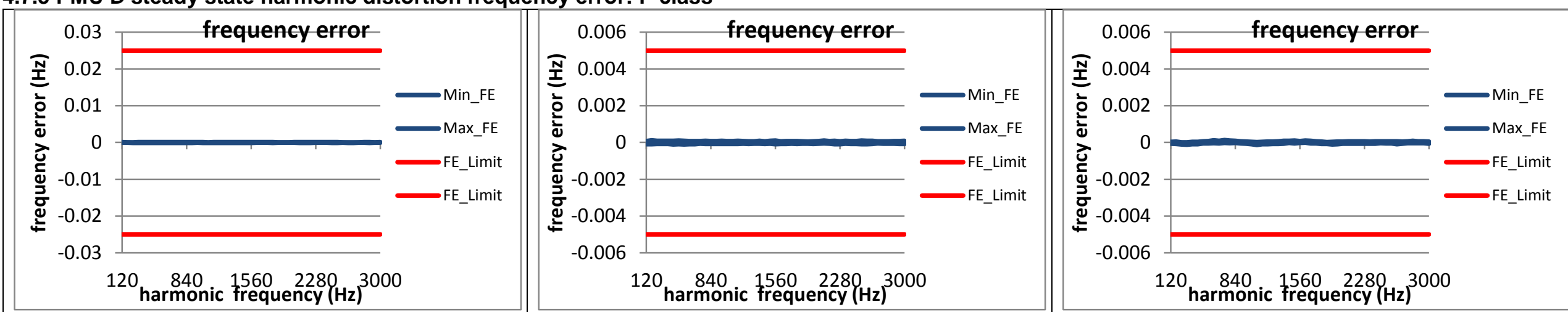

\section{Figure 382: Fs = 60 FPS}

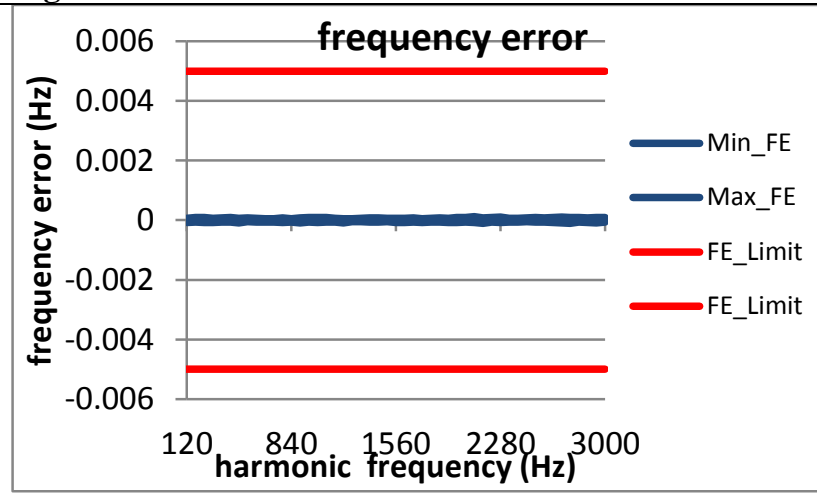

Figure 385; Fs $=15$ FPS

\section{Figure 383: Fs = 30 FPS}

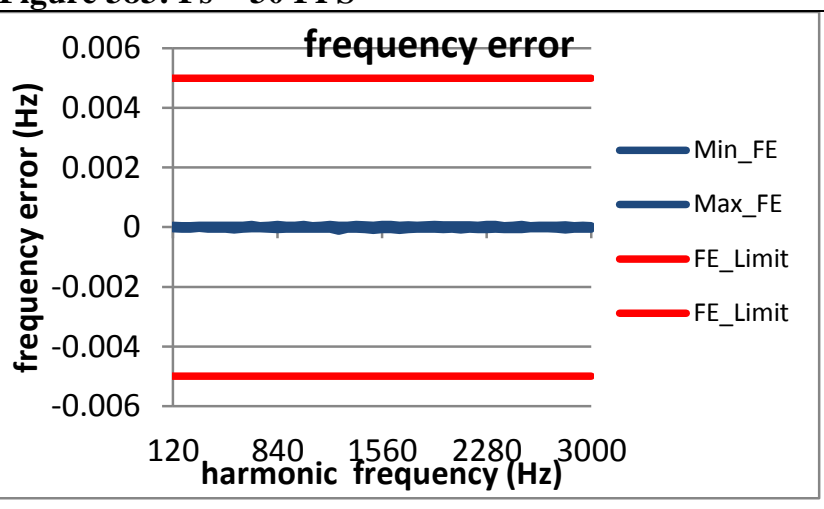

Figure 386: Fs $=12$ FPS

Figure 384: Fs = 20 FPS

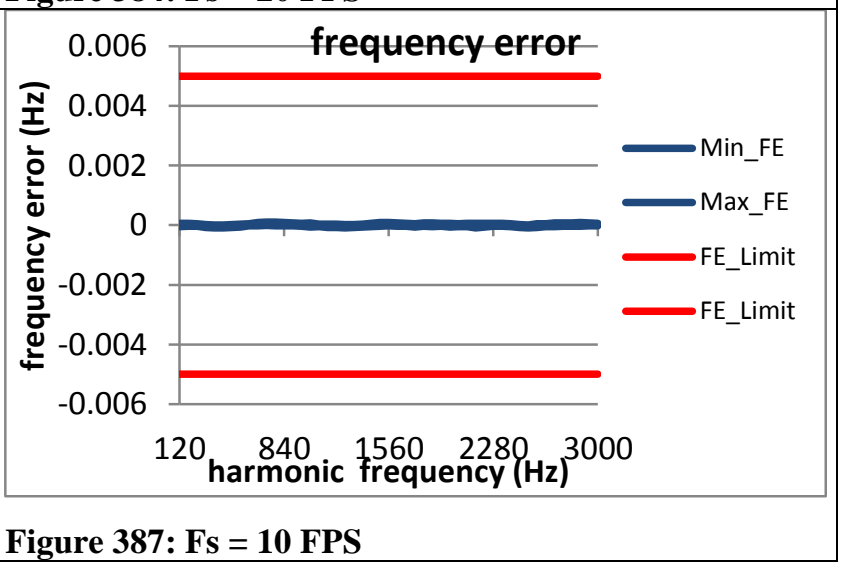

4.7.6 PMU E steady state harmonic distortion frequency error: $P$ class

PMU E does not support $P$ class 
4.7.7 PMU F steady state harmonic distortion frequency error: $P$ class
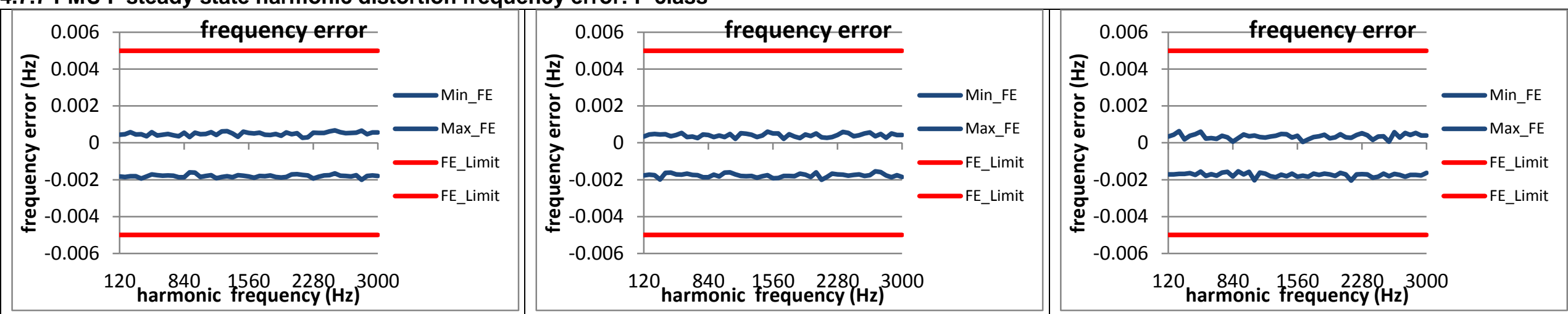

Figure 388: Fs = 60 FPS

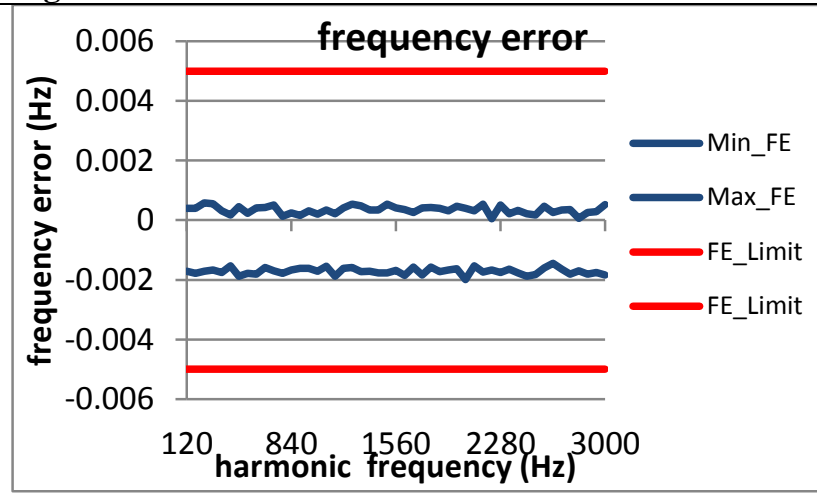

\section{Figure 389: Fs = 30 FPS}

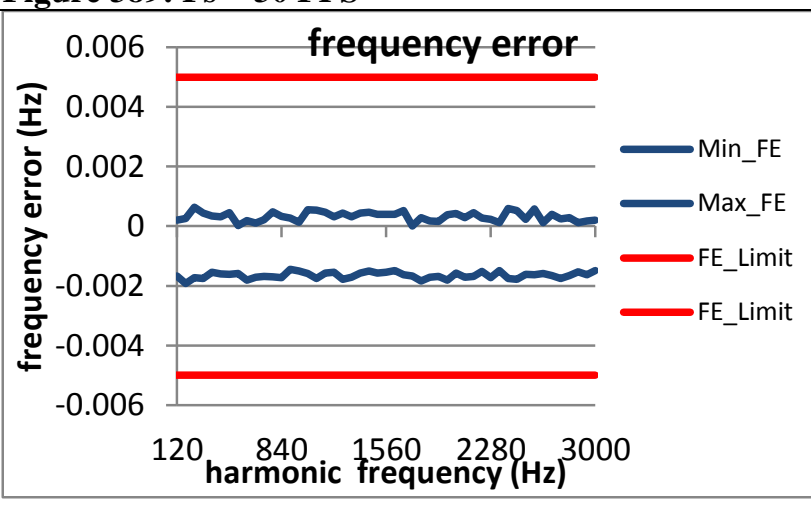

Figure 390: Fs = 20 FPS

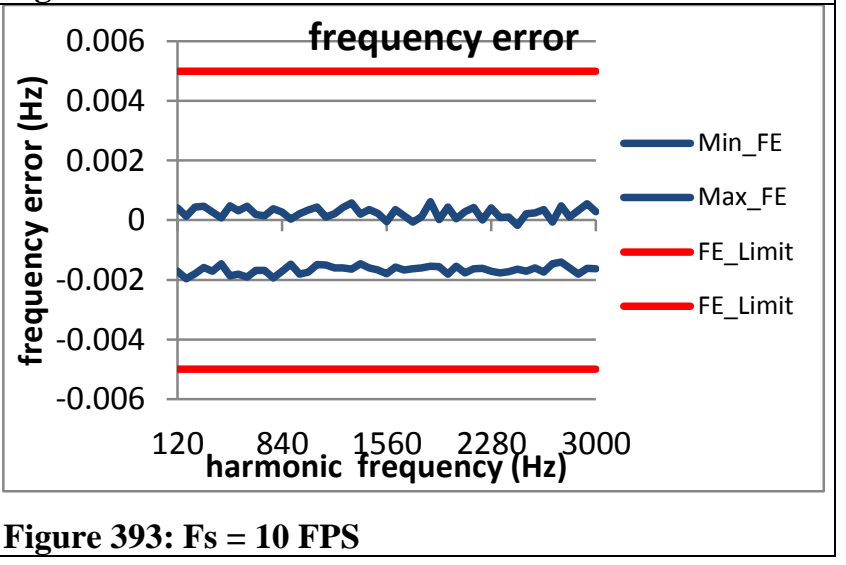

4.7.8 PMU G steady state harmonic distortion frequency error: P class

PMU G does not support P class 
4.7.9 PMU H steady state harmonic distortion frequency error: $P$ class
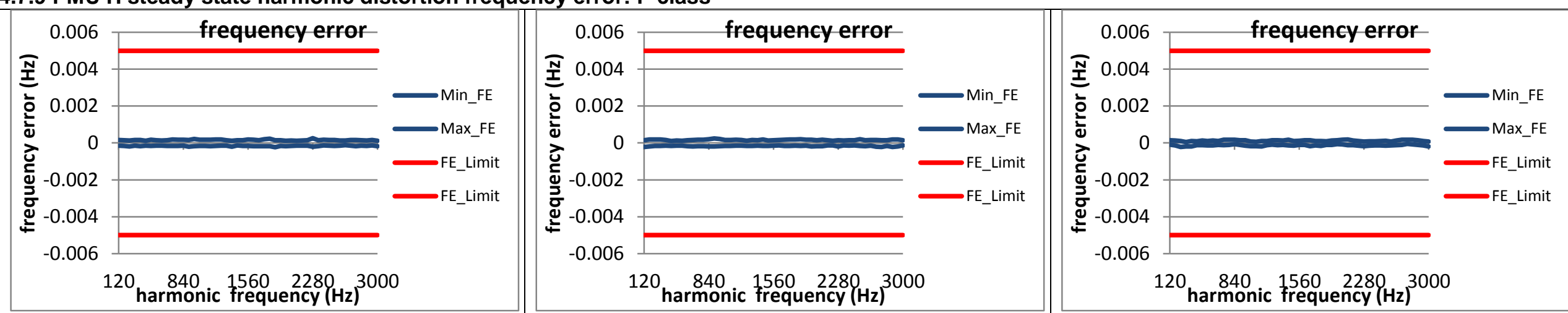

\section{Figure 394: Fs = 60 FPS}

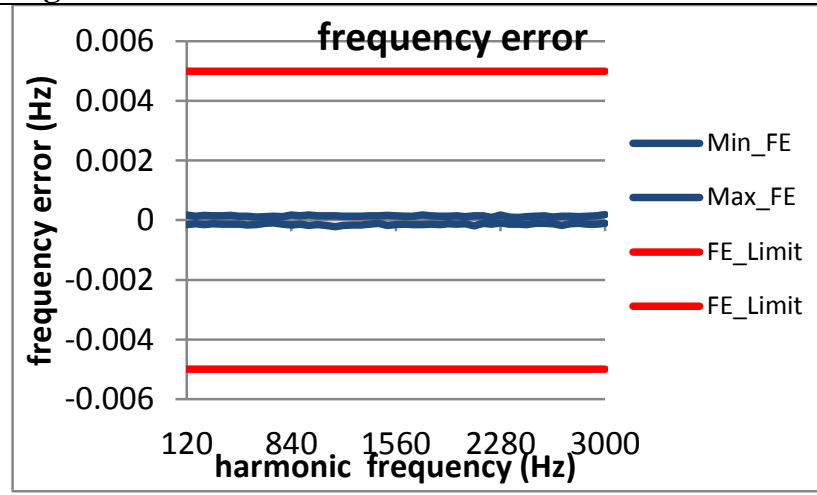

Figure 397; Fs = 15 FPS

\section{Figure 395: Fs = 30 FPS}

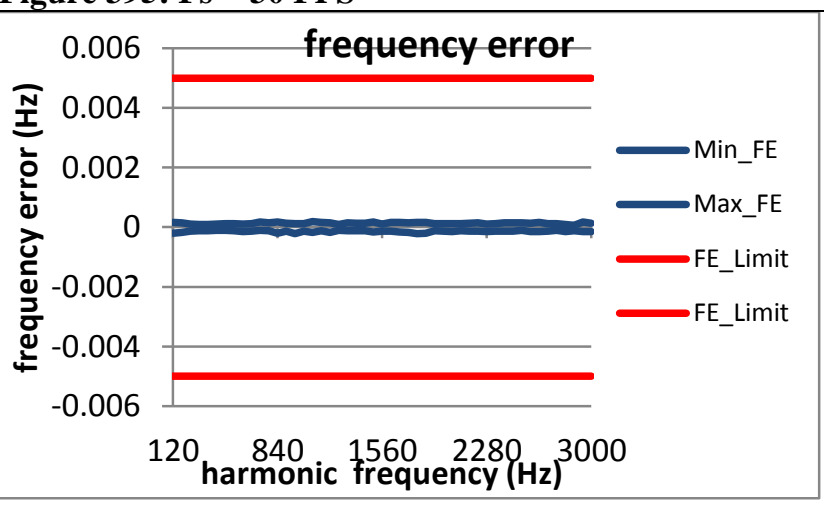

Figure 396: Fs = 20 FPS

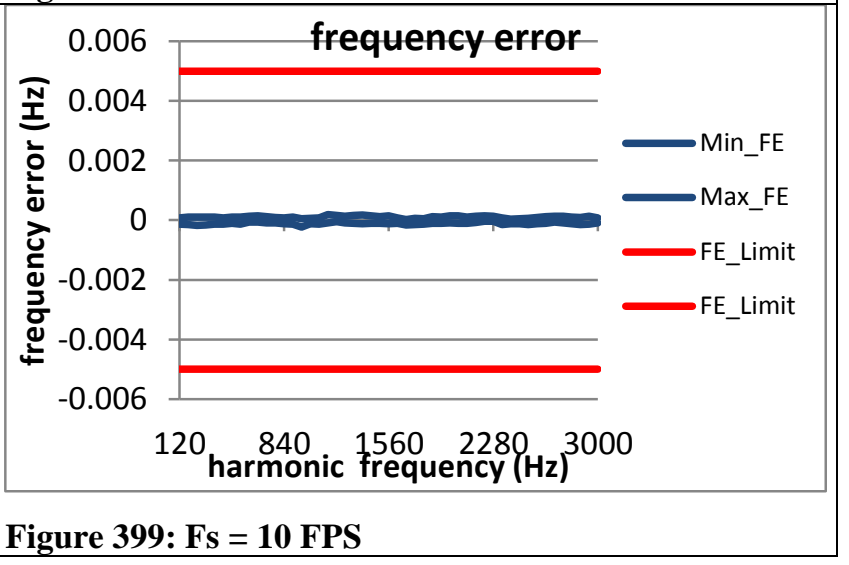

4.7.10 PMU I steady state harmonic distortion frequency error: $P$ class

PMU I does not support P class 
4.7.11 PMU J steady state harmonic distortion frequency error: $P$ class
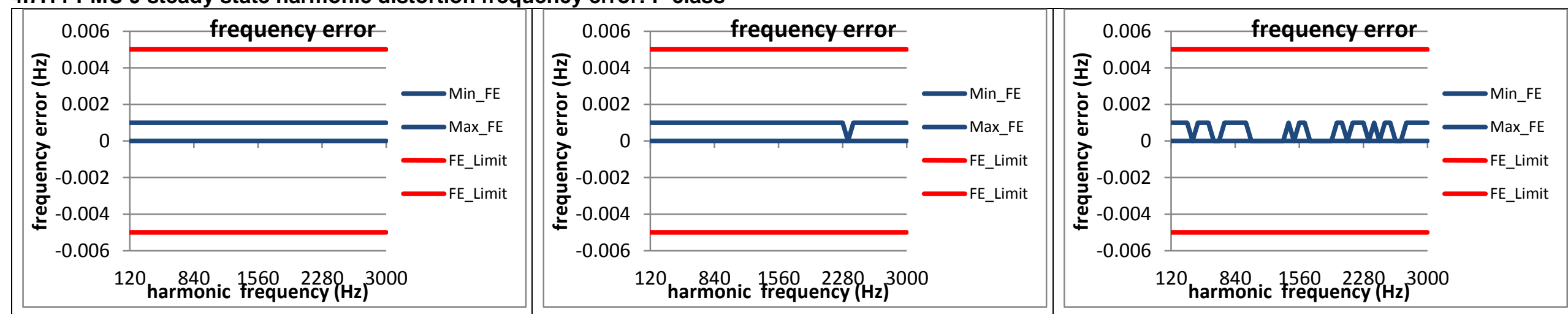

\section{Figure 400: Fs = 60 FPS}

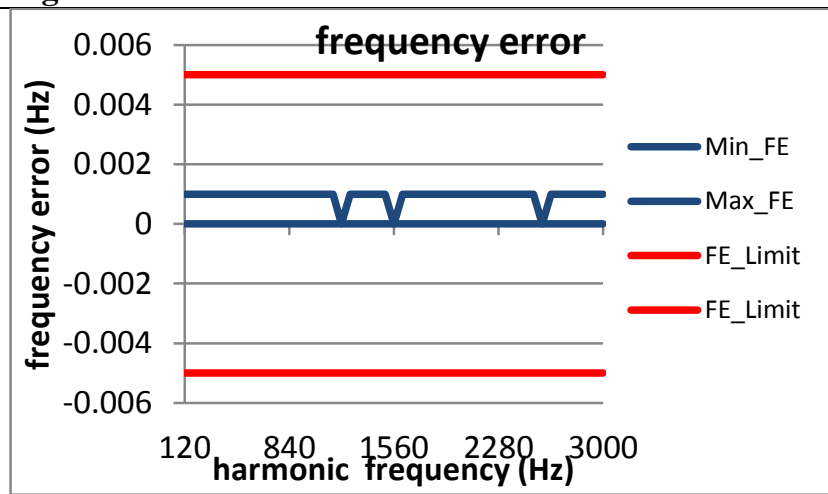

\section{Figure 401: Fs = 30 FPS}

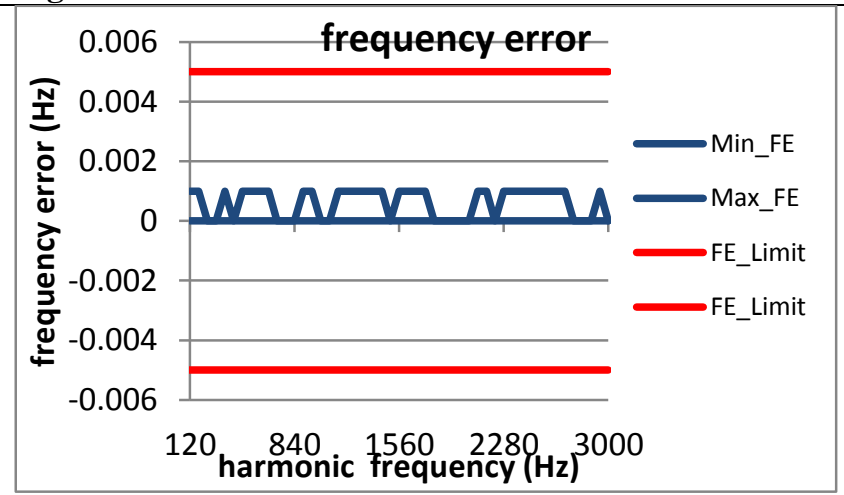

Figure 402: Fs = 20 FPS

Figure 403; Fs = 15 FPS

Figure 404: Fs = 12 FPS 


\subsection{Steady state harmonic distortion ROCOF error: P class}

\subsubsection{C37.118.1 Annex C steady harmonic distortion ROCOF error: P class}

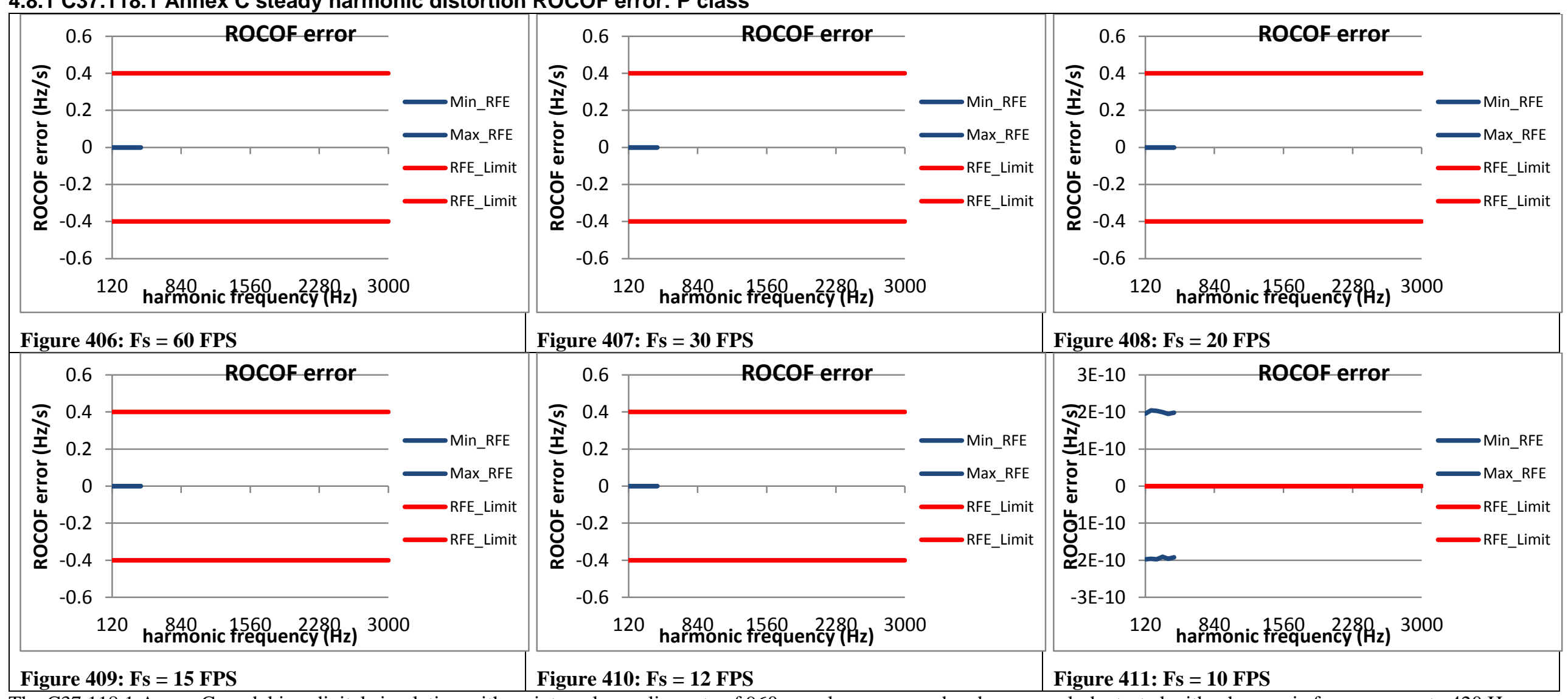

The C37.118.1 Annex C model is a digital simulation with an internal sampling rate of 960 samples per second and so can only be tested with a harmonic frequency up to $420 \mathrm{~Hz}$. 
4.8.2 PMU A steady state harmonic distortion ROCOF error: P class

\begin{tabular}{|c|c|c|}
\hline 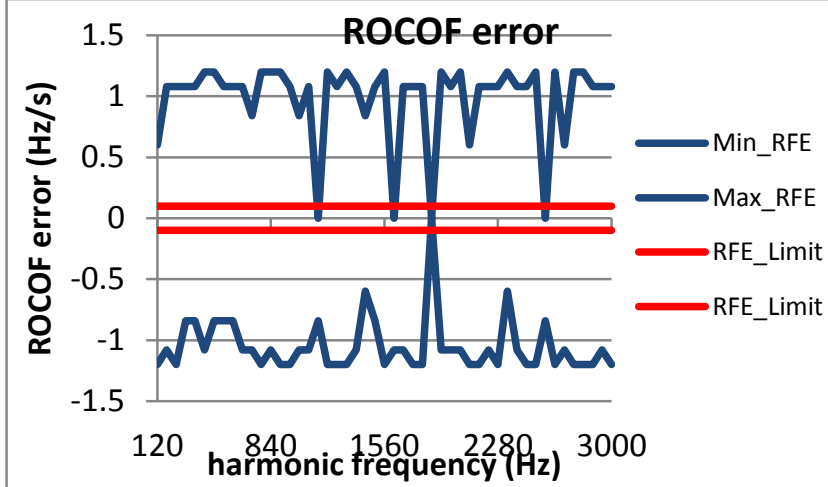 & 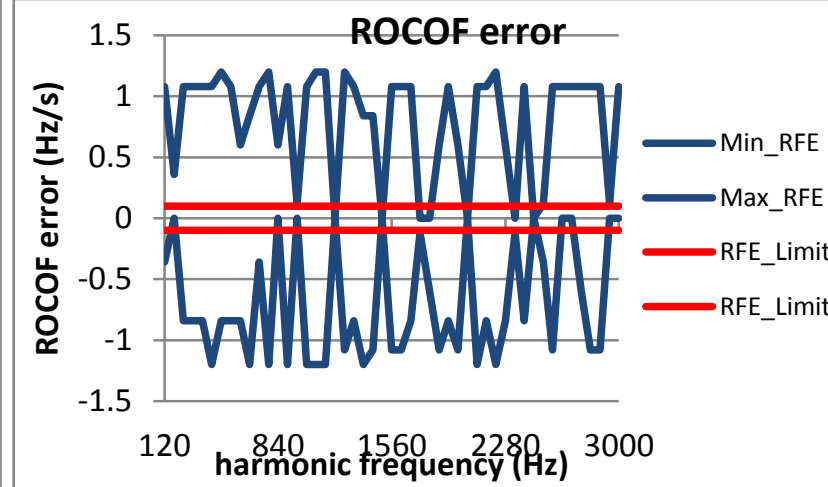 & 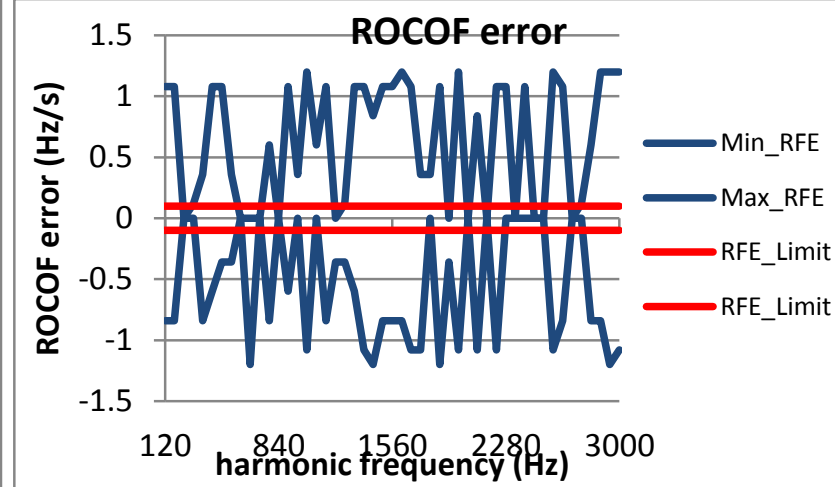 \\
\hline Figure 412: Fs = 60 FPS & Figure 413: Fs = 30 FPS & Figure 414: Fs = 20 FPS \\
\hline 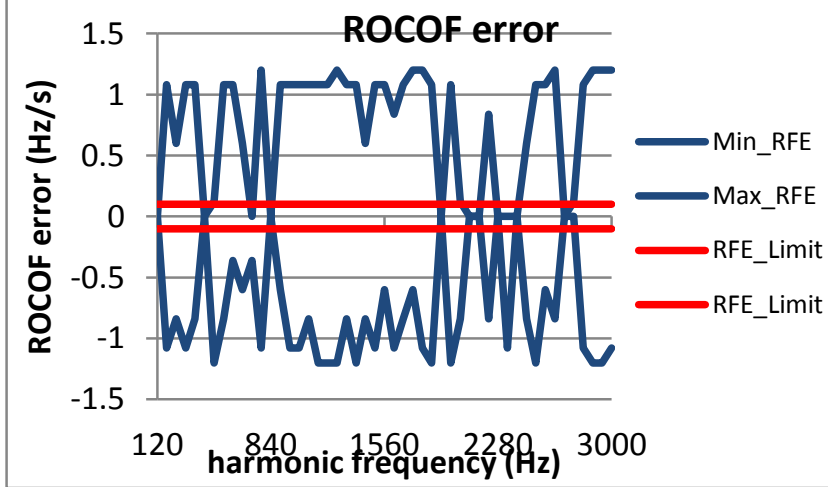 & & 120 \\
\hline Figure 415: Fs = 15 FPS & & \\
\hline
\end{tabular}


4.8.3 PMU B steady state harmonic distortion ROCOF error: P class

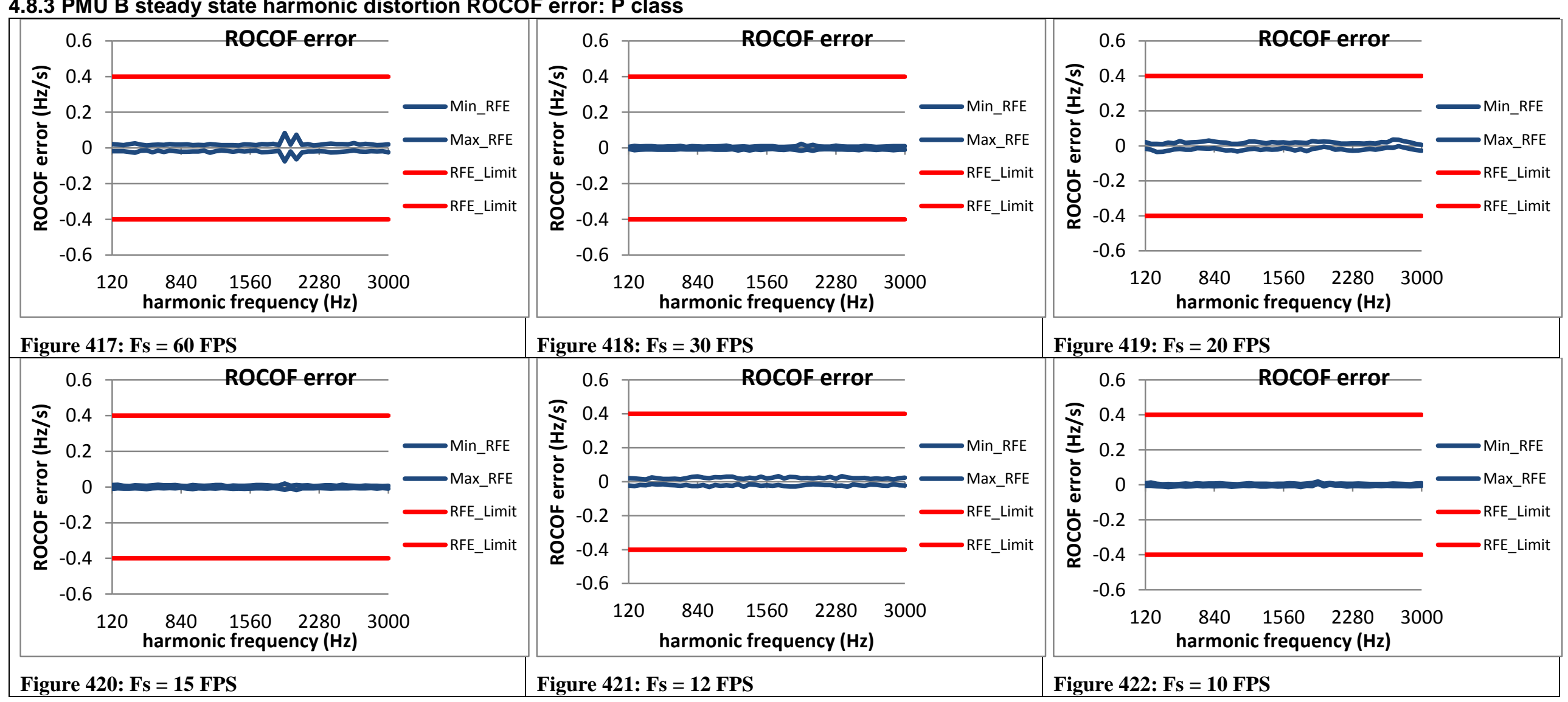


4.8.4 PMU C steady state harmonic distortion ROCOF error: P class

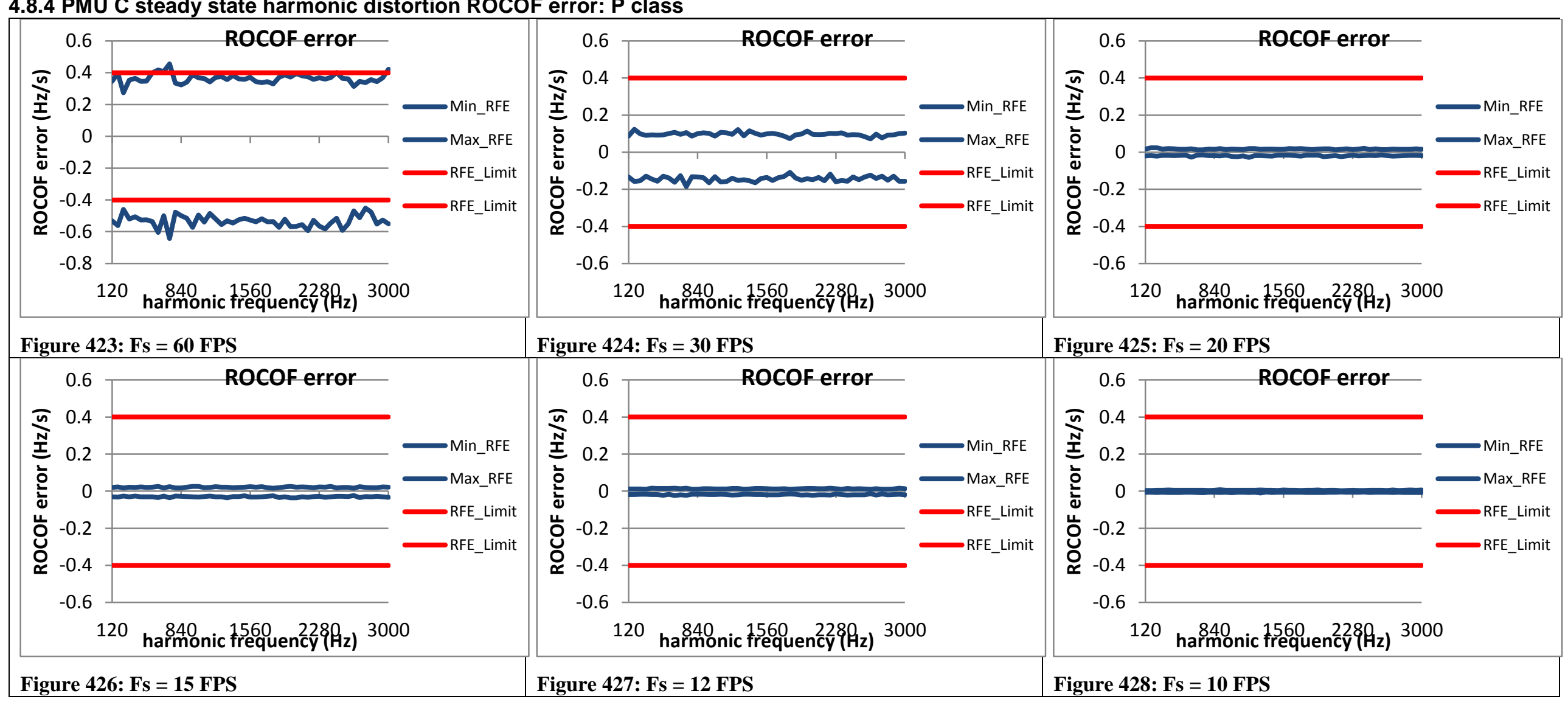


4.8.5 PMU D steady state harmonic distortion ROCOF error: P class

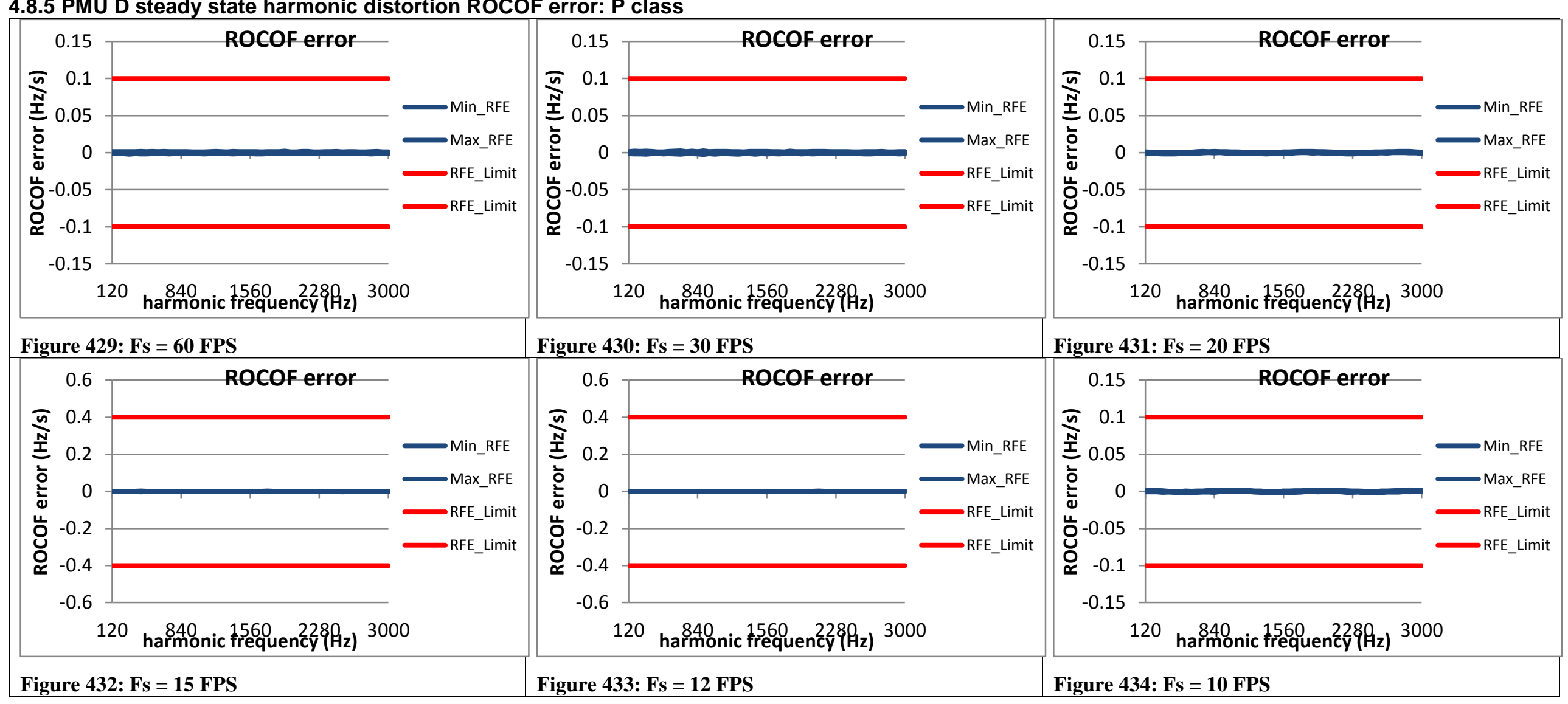

4.8.6 PMU E steady state harmonic distortion ROCOF error: $P$ class

PMU E does not support P class 
4.8.7 PMU F steady state harmonic distortion ROCOF error: $P$ class

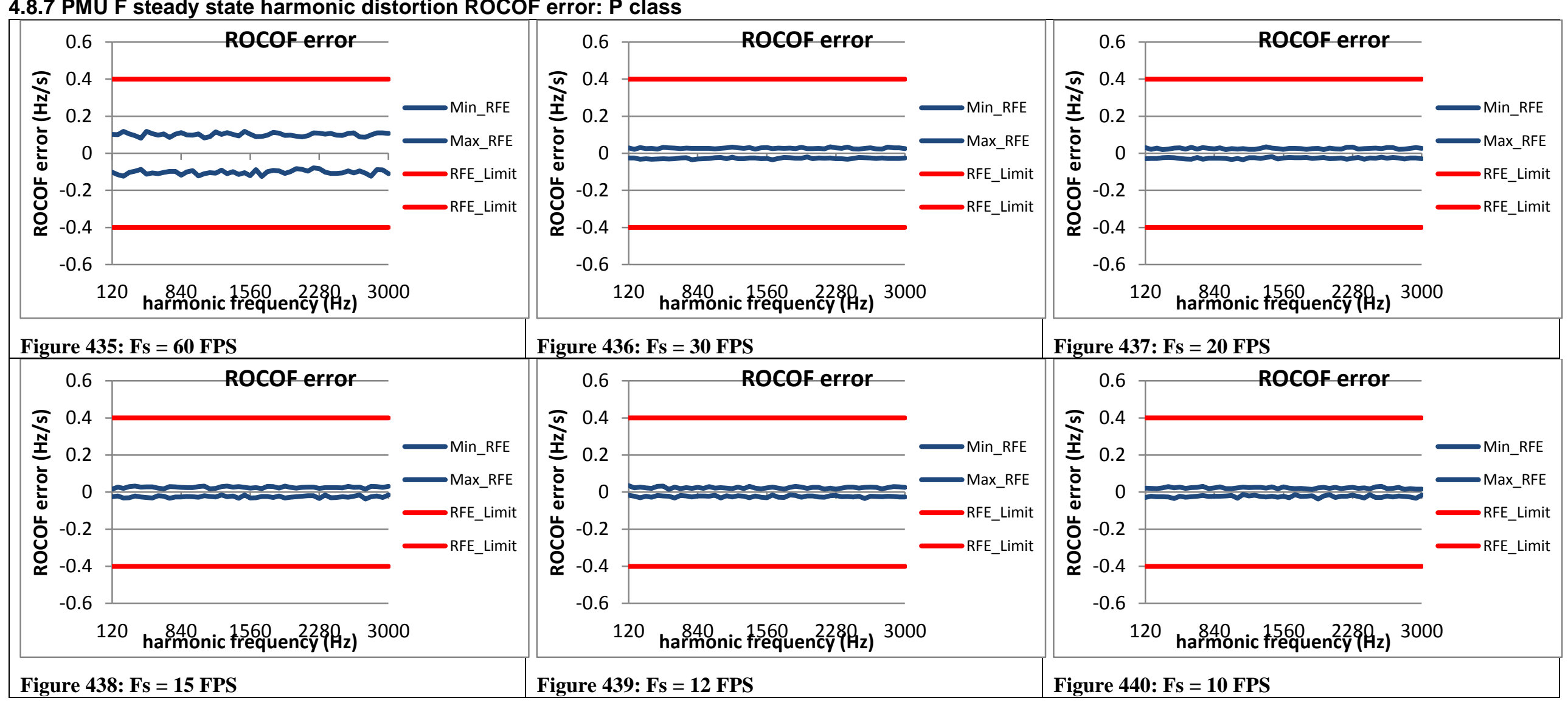

4.8.8 PMU G* steady state harmonic distortion ROCOF error: $P$ class

PMU G does not support P class 
4.8.9 PMU H steady state harmonic distortion ROCOF error: P class

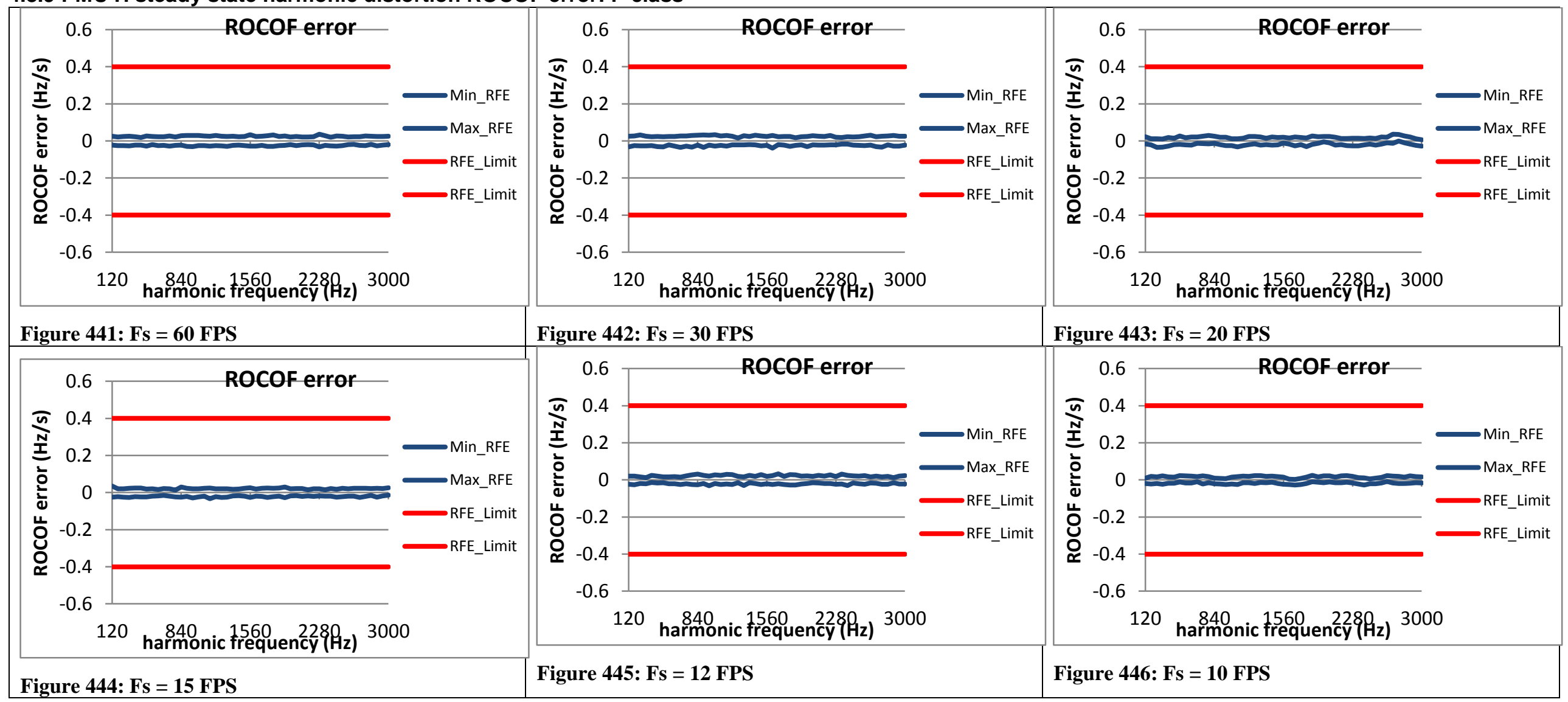

4.8.10 PMU I steady state harmonic distortion ROCOF error: P class

PMU I does not support P class 
4.8.11 PMU J steady state harmonic distortion ROCOF error: P class

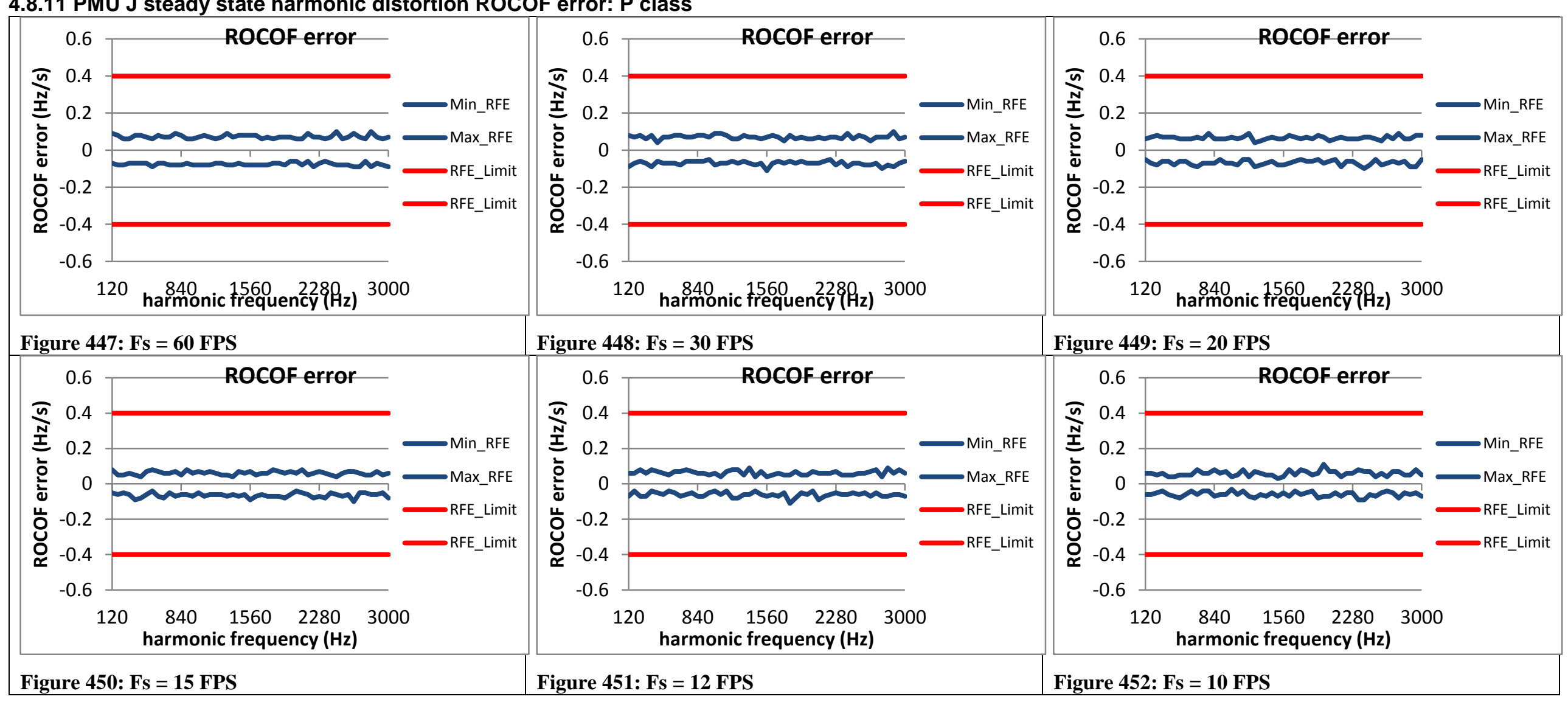




\section{Steady state out of band interfering signals test}

5. Steady state out of band interfering signals test.

5.1 Steady state out of band interfering signals test voltage TVE: M class.........

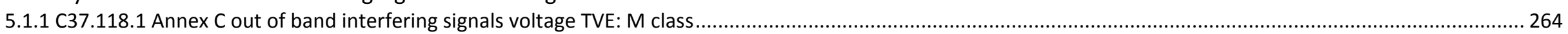

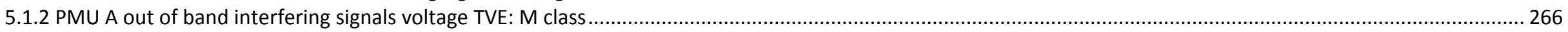

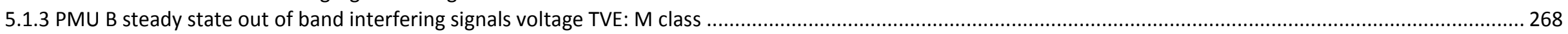

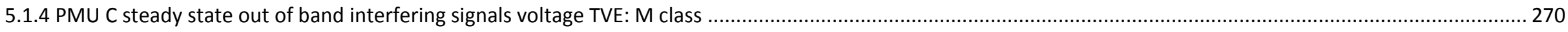

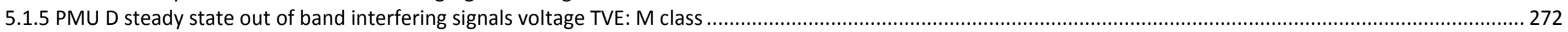

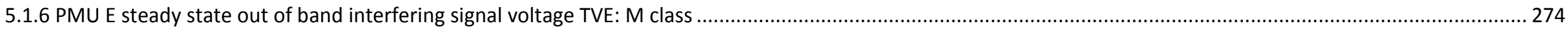

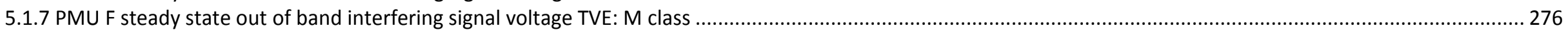

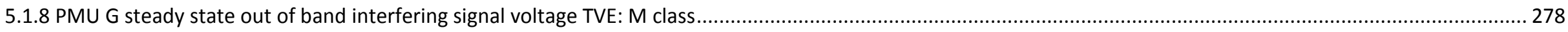

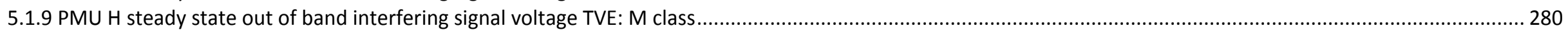

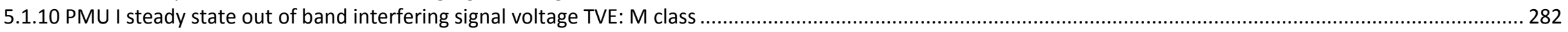

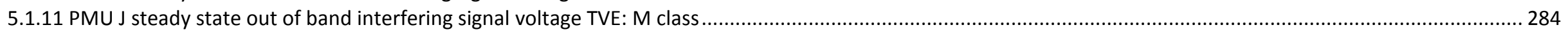

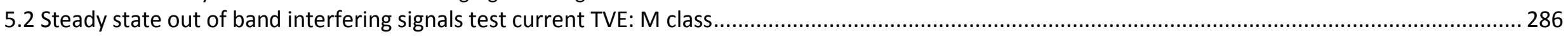

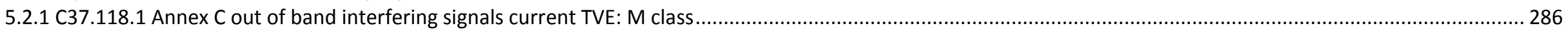

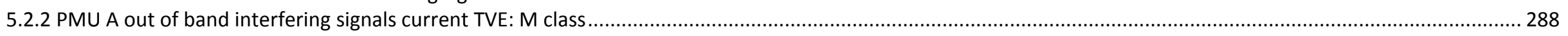

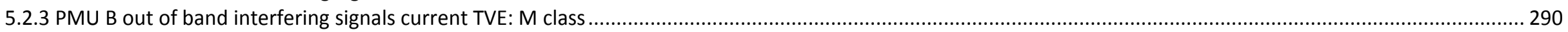

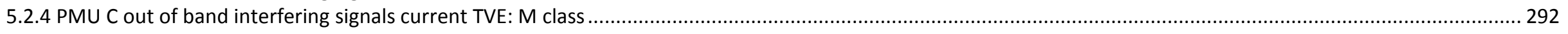

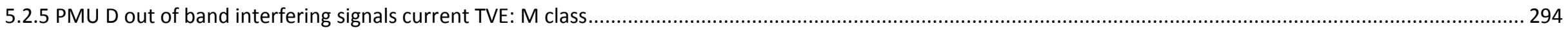

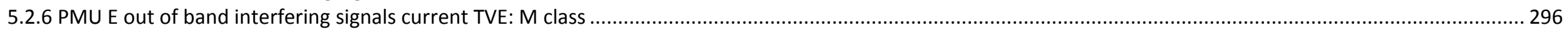

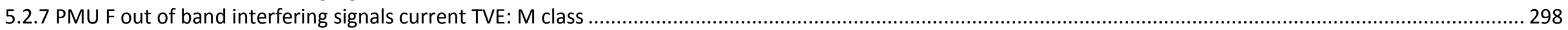

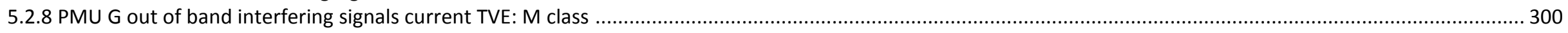

5.2.9 PMU H out of band interfering signals current TVE: M class

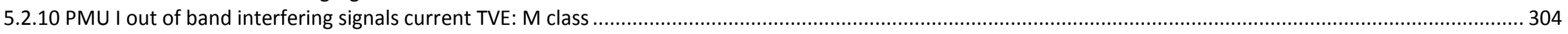

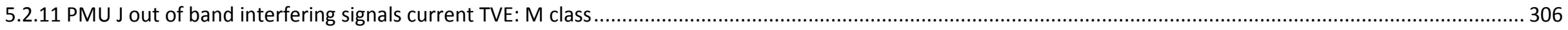

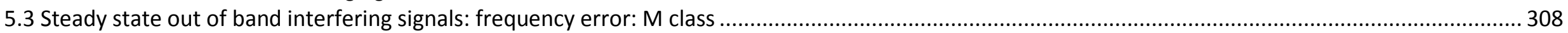

5.3.1 C37.118.1 Annex C steady state out of band interfering signals frequency error: M class ..................................................................................................... 308

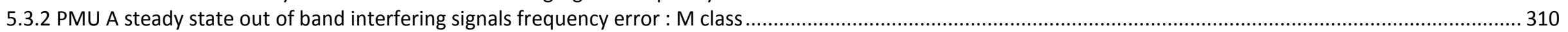

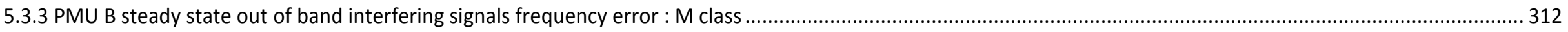

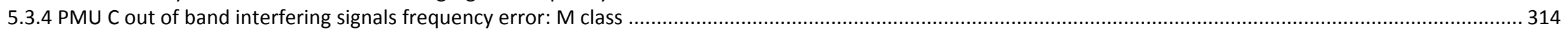

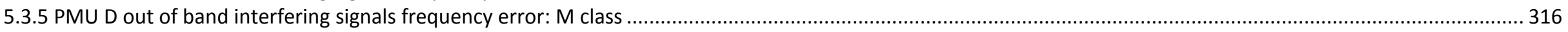

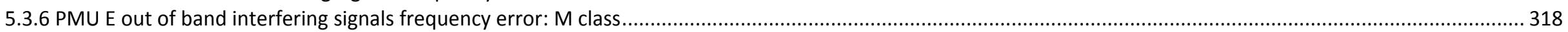

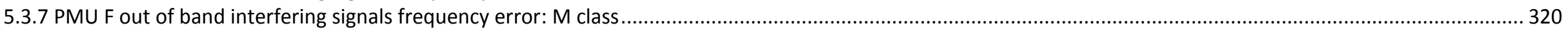

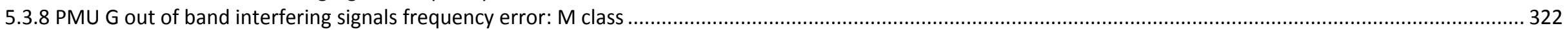

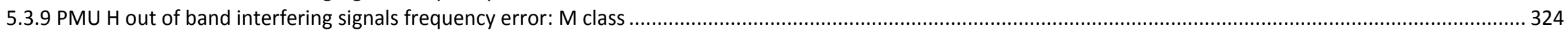

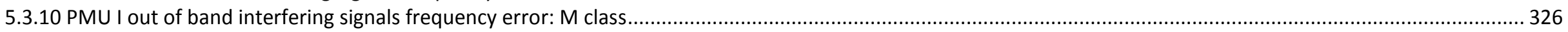

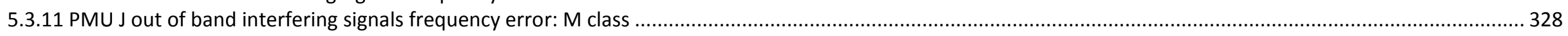

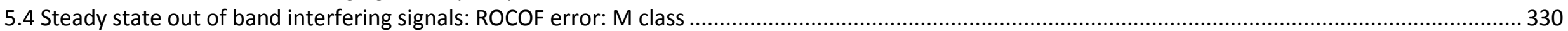


5.4.1 C37.118.1 Annex C steady state out of band interfering signals ROCOF error: M class.

5.4.2 PMU A steady state out of band interfering signals ROCOF error: M class.

5.4.3 PMU B steady state out of band interfering signals ROCOF error: $M$ class.

5.4.4 PMU C steady state out of band interfering signals ROCOF error: $M$ class.....

5.4.5 PMU D steady state out of band interfering signals ROCOF error: $M$ class

5.4.6 PMU E steady state out of band interfering signals ROCOF error: M class ...

5.4.7 PMU F steady state out of band interfering signals ROCOF error: $M$ class ...

5.4.8 PMU G* steady state out of band interfering signals ROCOF error: M class

5.4.9 PMU H steady state out of band interfering signals ROCOF error: $M$ class 
The steady state out of band interfering signals test is a series of tests where the PMU input signal is a combination of a fundamental frequency at nominal magnitude and an "out of band interfering signal" at $10 \%$ of nominal magnitude. Individual tests evaluate the PMU performance for steady state (unchanging in magnitude or frequency) for 5 seconds per test. In each subsequent test, the frequency of the interfering signal is incremented between $10 \mathrm{~Hz}$ and the nominal frequency minus the Nyquist frequency of the reporting rate $\left(\mathrm{F}_{0}-\mathrm{Fs} / 2\right)$ then from the nominal frequency plus the Nyquist frequency of the reporting rate $\left(\mathrm{F}_{0}+\right.$ $\mathrm{Fs} / 2$ ) up to the second harmonic of the nominal frequency. This set of interfering signals is repeated for three fundamental frequencies: nominal frequency minus $10 \%$ of the Nyquist of the reporting rate $\left(\mathrm{F}_{0}-0.1 \mathrm{Fs} / 2\right)$, the nominal frequency $\left(\mathrm{F}_{0}\right)$ and nominal frequency plus $10 \%$ of the Nyquist frequency $\left(\mathrm{F}_{0}+0.1 \mathrm{Fs} / 2\right)$.

\section{Test Plan:}

Apply a steady-state, balanced, three-phase signal to both current and voltage inputs, at nominal steady-state magnitude and nominal system frequency ( $50 \mathrm{~Hz}$ or $60 \mathrm{~Hz}$ ). Inject into the voltage and current inputs a positive-sequence interharmonic at

\section{Frequency $f_{\mathrm{i}}$ where $\left|f_{\mathrm{i}}-f_{0}\right| \geq \mathrm{F}_{\mathrm{s}} / 2$}

where

$F_{s}$ is the reporting rate

$f_{0}$ is the nominal frequency

$f_{i}$ is the frequency of the interfering signal

a) Add an interharmonic signal at $f_{\mathrm{i}}=f_{0}-\mathrm{F}_{\mathrm{s}} / 2$ at $10 \%$ nominal magnitude.

b) Wait for the system to settle.

c) Capture the PMU output for 5 seconds.

d) Calculate the errors: TVE, FE, RFE for each report.

e) Calculate the Max TVE, and FE.

f) Run step b) through step e) repeatedly, decreasing the interharmonic frequency exponentially until it reaches $10 \mathrm{~Hz}$ :

- For each test run, the interharmonic frequency decrements exponentially, thus providing many tests near $f_{i}=f_{0}-F_{s} / 2$ and fewer tests further away from $f_{i}=f_{0}-F_{s} / 2$. For example, the first decrease should be $0.1 \mathrm{~Hz}\left(f_{0}-F_{s} / 2-0.1\right)$, the next decrease $0.2 \mathrm{~Hz}\left(f_{0}-\mathrm{F}_{s} / 2-0.2\right)$, the third decrease $0.4 \mathrm{~Hz}$ $\left(f_{0}-\mathrm{F}_{\mathrm{s}} / 2-0.4\right)$, then $0.8 \mathrm{~Hz}\left(f_{0}-\mathrm{F}_{\mathrm{s}} / 2-0.8\right)$ until an interharmonic frequency below $10 \mathrm{~Hz}$ is reached. For the last frequency, use $10 \mathrm{~Hz}$ rather than the frequency below $10 \mathrm{~Hz}$.

g) Add an interharmonic signal at $f_{\mathrm{i}}=f_{0}+\mathrm{F}_{\mathrm{s}} / 2$ at $10 \%$ nominal magnitude.

h) Run step b) through step e) repeatedly, increasing the interharmonic frequency exponentially until it reaches $2 \times f_{0}$ :

- For each test run, the interharmonic frequency increments exponentially, thus providing many tests near $f_{i}=f_{0}+\mathrm{F}_{s} / 2$ and fewer tests further away from $f_{i}=f_{0}+\mathrm{F}_{\mathrm{s}} / 2$. For example, the first increase should be $0.1 \mathrm{~Hz}\left(f_{0}+\mathrm{F}_{\mathrm{s}} / 2+0.1\right)$, the next increase $0.2 \mathrm{~Hz}\left(f_{0}+\mathrm{F}_{\mathrm{s}} / 2+0.2\right)$, the third increase $0.4 \mathrm{~Hz}\left(f_{0}\right.$ 
$\left.+\mathrm{F}_{\mathrm{s}} / 2+0.4\right)$, then $0.8 \mathrm{~Hz}\left(f_{0}+\mathrm{F}_{\mathrm{s}} / 2+0.8\right)$, until an interharmonic frequency above $2 \times f_{0}$ is reached. For the last frequency use $2 \times f_{0}$ rather than the frequency above $2 \times f_{0}$..

i) Run all steps a) through $h$ ) two more times with the input signal frequency at nominal frequency plus and minus $10 \%$ of $F_{s} / 2$ (the Nyquist frequency of the reporting rate, $\left.F_{s}\right)$.

An example of TVE, FE and RFE from a single OOB test run taken at $F_{0}=60 \mathrm{~Hz}, \mathrm{Fs}=20 \mathrm{FPS}, \mathrm{M}$ class with a fundamental frequency at $59 \mathrm{~Hz}$ and out of band signal at $70 \mathrm{~Hz}$ and $10 \%$ of nominal magnitude is shown:

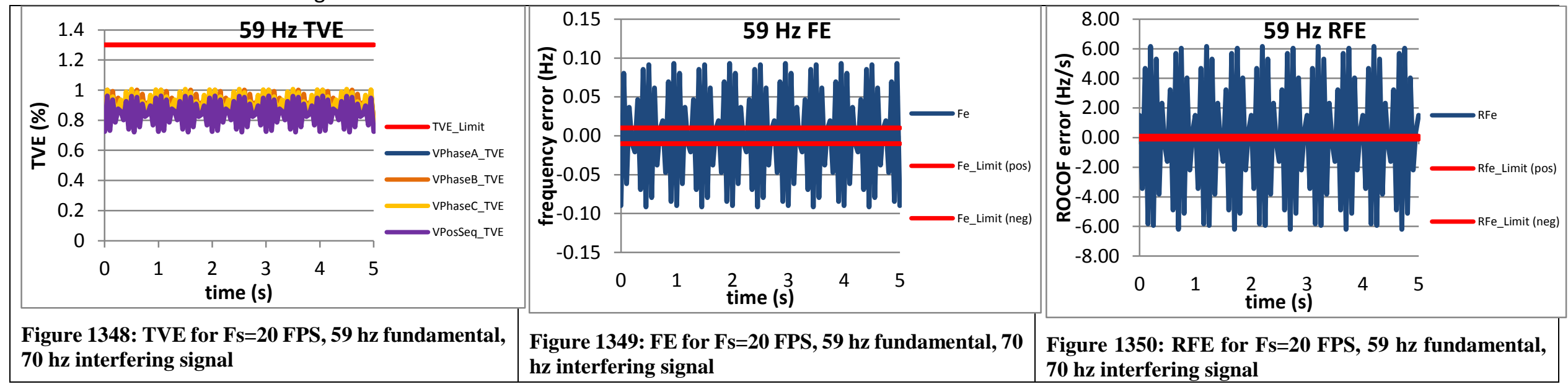

The maximum value from the above plots becomes a single data point from the below plots of TVE, FE and RFE for Fs $=20 \mathrm{FPS}$, at $59 \mathrm{~Hz}$ fundamental frequency. The data point is shown with a black arrow. Individual test runs insert a single out of band interfering frequency from $10 \mathrm{~Hz}$ to $120 \mathrm{~Hz}$ excluding the in-band frequencies between $50 \mathrm{~Hz}$ and $70 \mathrm{~Hz}$. 


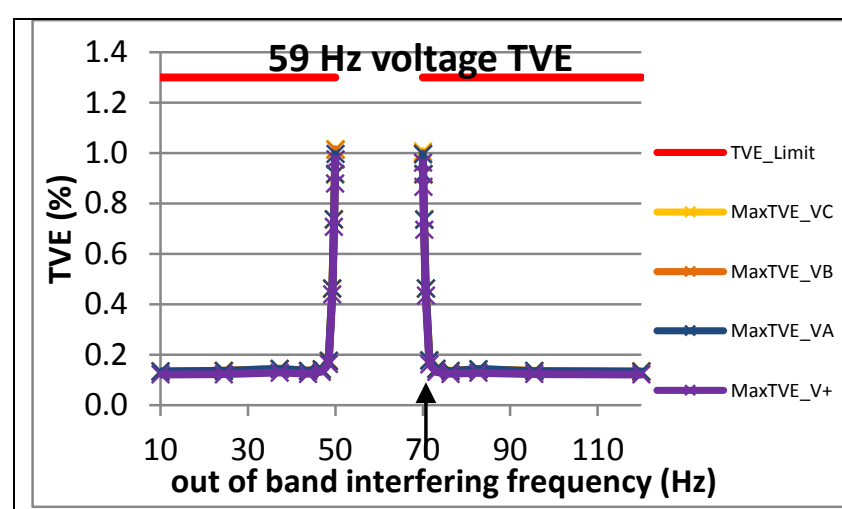

Figure 1351: Maximum TVE for Fs = $20 \mathrm{FPS}, 59 \mathrm{~Hz}$ fundamental frequency across all frequencies of out of band signals

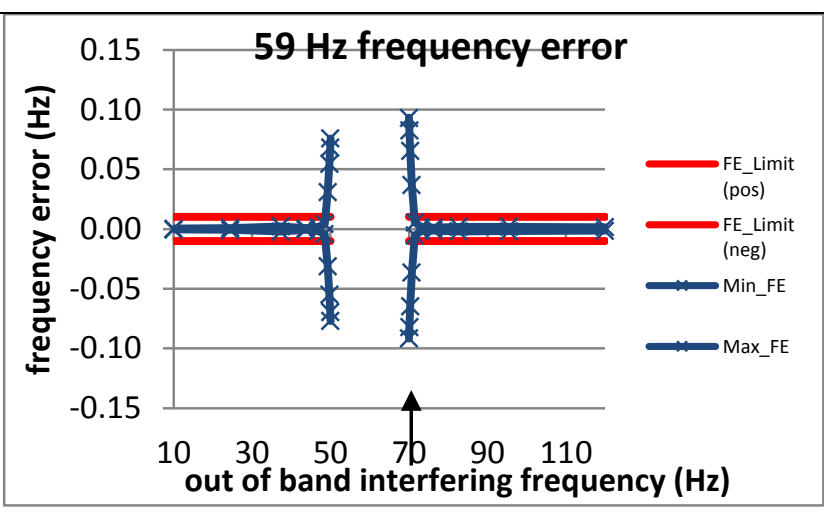

Figure 1352: Maximum and minimum FE for Fs $=20$ FPS, $59 \mathrm{~Hz}$ fundamental frequency across all frequencies of out of band signals

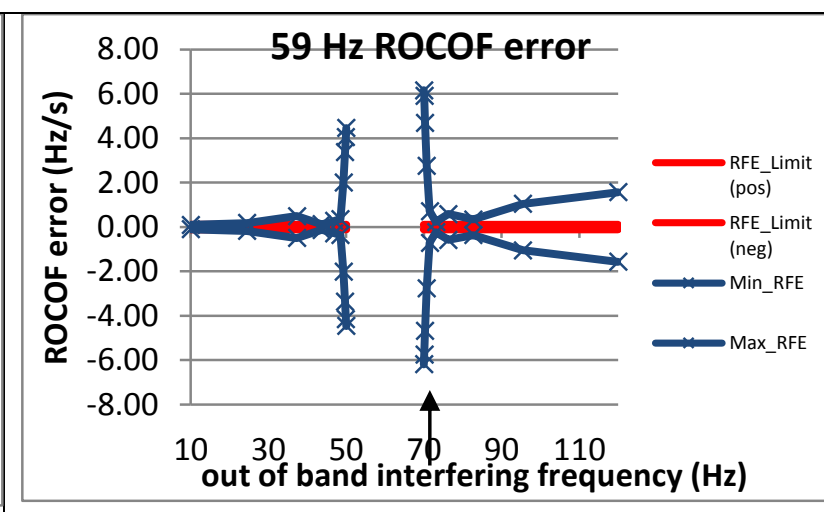

Figure 1353: Maximum and minimum RFE for Fs = $20 \mathrm{FPS}, 59 \mathrm{~Hz}$ fundamental frequency across all frequencies of out of band signals

Out of band interfering signals tests are run at three separate fundamental frequencies. For each measured result (TVE, FE, RFE), a table of plots is shown. Each row of three plots is from a single reporting rate $(F s)$, one for each of the input fundamental frequencies $\left(F_{0}-0.1 F s / 2\right),\left(F_{0}\right)$, $\left(F_{0}+0.1 F s / 2\right)$. The $x$-axis of all plots shows the frequency of the interfering signal (one interfering signal per test run). The $y$-axis of the TVE plots show the maximum TVE and the TVE limit in red. The $\mathrm{Y}$-axis of both the frequency error and ROCOF error plots show the maximum and minimum errors and the positive and negative error limits. When determining if the performance passes or fails a test, the maximum absolute value of the error can be compared against a positive error limit, showing both the maximum and minimum error allows us to see asymmetries and offsets in the error. 
Results from steady state out of band interfering signals test

\begin{tabular}{|c|c|c|c|c|c|c|c|c|c|c|c|c|c|c|c|c|c|c|c|c|c|c|c|c|c|c|c|c|c|c|c|c|c|c|c|c|}
\hline Fs (FPS) & & $10 n$ & & & $10 \mathrm{P}$ & & & $2 \mathrm{~N}$ & & & $12 \mathrm{P}$ & & & $5 \mathrm{~N}$ & & & $15 \mathrm{P}$ & & & $\mathrm{OM}$ & & & $20 P$ & & & $30 \mathrm{M}$ & & & $30 P$ & & & $60 \mathrm{M}$ & & & 60P & \\
\hline Test & $\begin{array}{l}\mathrm{T} \\
\mathrm{V} \\
\mathrm{E}\end{array}$ & $\begin{array}{l}F \\
E\end{array}$ & $\begin{array}{l}\mathrm{R} \\
\mathrm{F} \\
\mathrm{E}\end{array}$ & $\begin{array}{l}T \\
V \\
E\end{array}$ & $\begin{array}{l}F \\
E\end{array}$ & $\begin{array}{l}\mathrm{R} \\
\mathrm{F} \\
\mathrm{E}\end{array}$ & $\begin{array}{l}\mathrm{T} \\
\mathrm{V} \\
\mathrm{E}\end{array}$ & $\begin{array}{l}F \\
E\end{array}$ & $\begin{array}{l}\mathrm{R} \\
\mathrm{F} \\
\mathrm{E}\end{array}$ & $\begin{array}{l}\mathrm{T} \\
\mathrm{V} \\
\mathrm{E}\end{array}$ & $\begin{array}{l}F \\
E\end{array}$ & $\begin{array}{l}R \\
F \\
E\end{array}$ & $\begin{array}{l}\mathrm{T} \\
\mathrm{V} \\
\mathrm{E}\end{array}$ & $\begin{array}{l}F \\
E\end{array}$ & $\begin{array}{l}R \\
F \\
E\end{array}$ & $\begin{array}{l}\mathrm{T} \\
\mathrm{V} \\
\mathrm{E}\end{array}$ & $\begin{array}{l}F \\
E\end{array}$ & $\begin{array}{l}\mathrm{R} \\
\mathrm{F} \\
\mathrm{E}\end{array}$ & $\begin{array}{l}\mathrm{T} \\
\mathrm{V} \\
\mathrm{E}\end{array}$ & $\begin{array}{l}F \\
E\end{array}$ & $\begin{array}{l}\mathrm{R} \\
\mathrm{F} \\
\mathrm{E}\end{array}$ & $\begin{array}{l}\mathrm{T} \\
\mathrm{V} \\
\mathrm{E}\end{array}$ & $\begin{array}{l}\mathrm{F} \\
\mathrm{E}\end{array}$ & $\begin{array}{l}\mathrm{R} \\
\mathrm{F} \\
\mathrm{E}\end{array}$ & $\begin{array}{l}\mathrm{T} \\
\mathrm{V} \\
\mathrm{E}\end{array}$ & $\begin{array}{l}F \\
E\end{array}$ & $\begin{array}{l}\mathrm{R} \\
\mathrm{F} \\
\mathrm{E}\end{array}$ & $\begin{array}{l}\mathrm{T} \\
\mathrm{V} \\
\mathrm{E}\end{array}$ & $\begin{array}{l}F \\
E\end{array}$ & $\begin{array}{l}\mathrm{R} \\
\mathrm{F} \\
\mathrm{E}\end{array}$ & $\begin{array}{l}\mathrm{T} \\
\mathrm{V} \\
\mathrm{E}\end{array}$ & $\begin{array}{l}\mathrm{F} \\
\mathrm{E}\end{array}$ & $\begin{array}{l}\mathrm{R} \\
\mathrm{F} \\
\mathrm{E}\end{array}$ & $\begin{array}{l}\mathrm{T} \\
\mathrm{V} \\
\mathrm{E}\end{array}$ & $\begin{array}{l}F \\
E\end{array}$ & $\begin{array}{l}R \\
F \\
E\end{array}$ \\
\hline C37.118.1 Annex C & $\mathrm{P}$ & $\mathrm{P}$ & - & - & - & - & $\mathrm{P}$ & $\mathrm{P}$ & - & - & - & - & $\mathrm{P}$ & $\mathrm{P}$ & - & - & - & - & $\mathrm{P}$ & $\mathrm{P}$ & - & - & - & - & $\mathrm{P}$ & $\mathrm{P}$ & - & - & - & - & $\mathrm{P}$ & $\mathrm{P}$ & - & - & - & - \\
\hline PMU A & $\mathrm{P}$ & $\mathrm{P}$ & - & - & - & - & $\mathrm{P}$ & $\mathrm{P}$ & - & - & - & - & $\mathrm{P}$ & $\mathrm{P}$ & - & - & - & - & $\mathrm{P}$ & $\mathrm{F}$ & - & - & - & - & $\mathrm{P}$ & $\mathrm{F}$ & - & - & - & - & $\mathrm{P}$ & $\mathrm{F}$ & - & - & - & - \\
\hline PMU B & $\mathrm{F}$ & $\mathrm{F}$ & - & - & - & - & $\mathrm{F}$ & $\mathrm{F}$ & - & - & - & - & $\mathrm{F}$ & $\mathrm{F}$ & - & - & - & - & $\mathrm{F}$ & $\mathrm{F}$ & - & - & - & - & $\mathrm{P}$ & $\mathrm{F}$ & - & - & - & - & $P$ & $\mathrm{~F}$ & - & - & - & - \\
\hline PMU C & $\mathrm{F}$ & $\mathrm{F}$ & - & - & - & - & $\mathrm{F}$ & $\mathrm{F}$ & - & - & - & - & $\mathrm{F}$ & $\mathrm{F}$ & - & - & - & - & $\mathrm{F}$ & $\mathrm{F}$ & - & - & - & - & $\mathrm{F}$ & $\mathrm{F}$ & - & - & - & - & $P$ & $\mathrm{~F}$ & - & - & - & - \\
\hline PMU D & $\mathrm{P}$ & $P$ & - & - & - & - & $\mathrm{P}$ & $\mathrm{P}$ & - & - & - & - & $\mathrm{P}$ & $\mathrm{P}$ & - & - & - & - & $P$ & $\mathrm{P}$ & - & - & - & - & $P$ & $\mathrm{P}$ & - & - & - & - & $\mathrm{P}$ & $\mathrm{P}$ & - & - & - & - \\
\hline PMU E & $\mathrm{F}$ & $\mathrm{F}$ & - & - & - & - & & & & - & - & - & $\mathrm{F}$ & $\mathrm{F}$ & - & - & - & - & $\mathrm{F}$ & $\mathrm{F}$ & - & - & - & - & $\mathrm{F}$ & $\mathrm{F}$ & - & - & - & - & $\mathrm{P}$ & $\mathrm{F}$ & - & - & - & - \\
\hline PMU F & $\mathrm{P}$ & I & - & - & - & - & $\mathrm{P}$ & I & - & - & - & - & $\mathrm{P}$ & I & - & - & - & - & $\mathrm{P}$ & $\mathrm{F}$ & - & - & - & - & $\mathrm{P}$ & $\mathrm{F}$ & - & - & - & - & $P$ & $\mathrm{~F}$ & - & - & - & - \\
\hline PMU G* & $\mathrm{F}$ & $\mathrm{F}$ & - & - & - & - & $\mathrm{F}$ & $\mathrm{F}$ & - & - & - & - & $\mathrm{F}$ & $\mathrm{F}$ & - & - & - & - & $\mathrm{F}$ & $\mathrm{F}$ & - & - & - & - & $\mathrm{F}$ & $\mathrm{F}$ & - & - & - & - & - & - & - & - & - & - \\
\hline PMU H & $P$ & $P$ & - & - & - & - & $P$ & $P$ & - & - & - & - & $P$ & $P$ & - & - & - & - & $P$ & $P$ & - & - & - & - & $P$ & $P$ & - & - & - & - & $P$ & $P$ & - & - & - & - \\
\hline PMU I & $\mathrm{P}$ & $\mathrm{F}$ & - & - & - & - & $P$ & $F$ & - & - & - & - & $P$ & $F$ & - & - & - & - & $P$ & $\mathrm{~F}$ & - & - & - & - & $P$ & $\mathrm{~F}$ & - & - & - & - & $P$ & $\mathrm{~F}$ & - & - & - & - \\
\hline PMU J & $P$ & $P$ & - & - & - & - & $P$ & $P$ & - & - & - & - & $P$ & $P$ & - & - & - & - & $P$ & $P$ & - & - & - & - & $P$ & $P$ & - & - & - & - & $P$ & $P$ & - & - & - & - \\
\hline
\end{tabular}

$\mathrm{P}=$ Pass, $\mathrm{F}=$ Fail, $\mathrm{I}=$ Indeterminate 


\subsection{Steady state out of band interfering signals test voltage TVE: M class}

\subsubsection{C37.118.1 Annex $C$ out of band interfering signals voltage TVE: $M$ class}
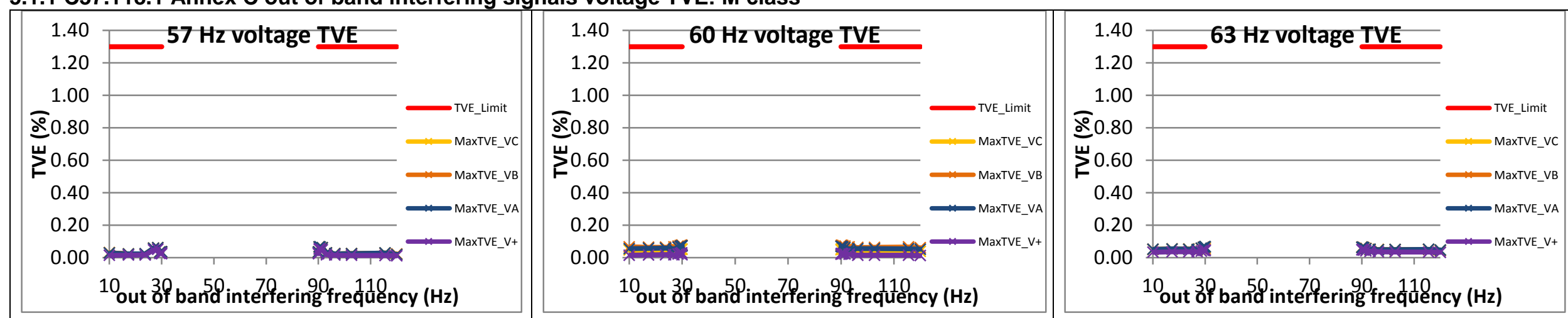

Figure 1354: Fs = 60 FPS, $57 \mathrm{~Hz}$ fundamental

Figure 1355: Fs = 60 FPS, $60 \mathrm{~Hz}$ fundamental

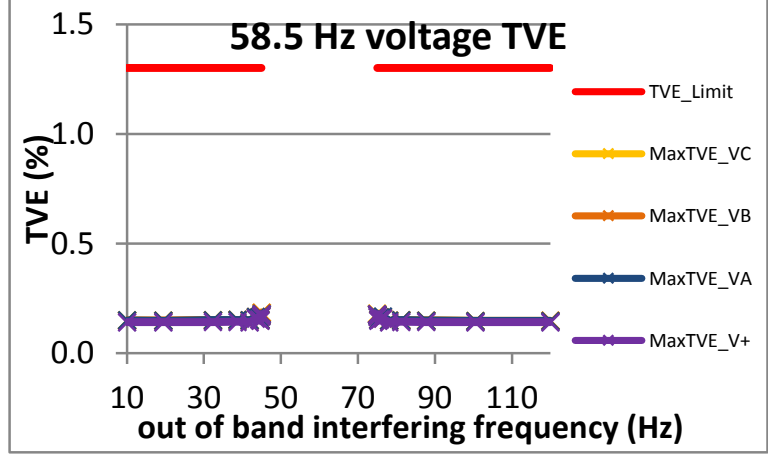

Figure 1357: Fs = 30 FPS, $58.5 \mathrm{~Hz}$ fundamental

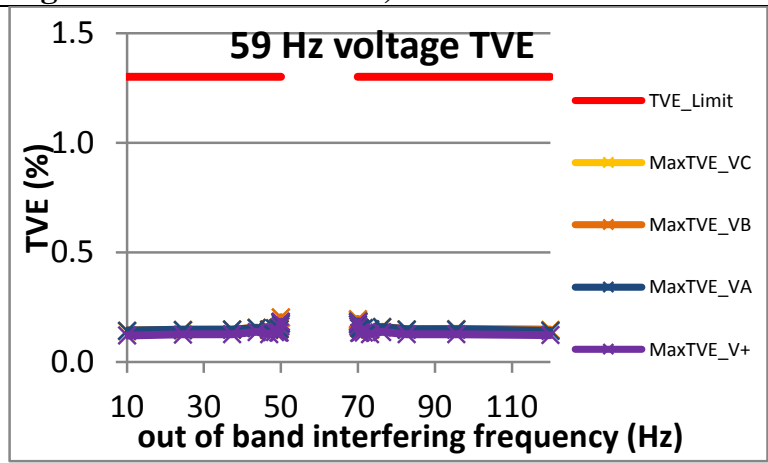

Figure 1360: Fs = 20 FPS, $59 \mathrm{~Hz}$ fundamental

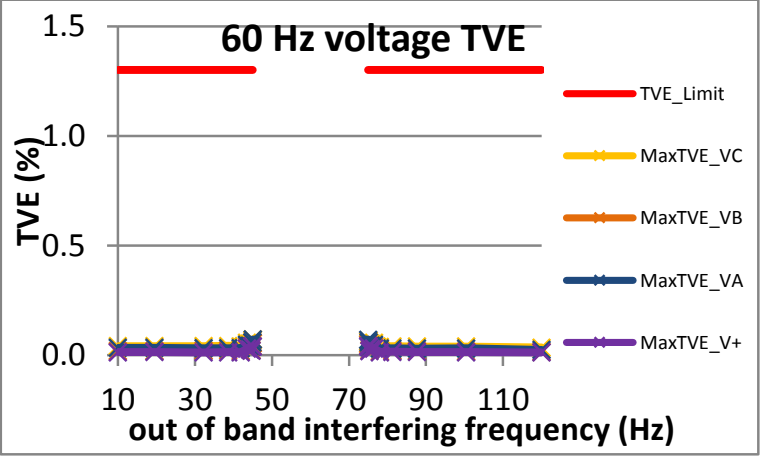

Figure 1358: Fs = 30 FPS, $60 \mathrm{~Hz}$ fundamental

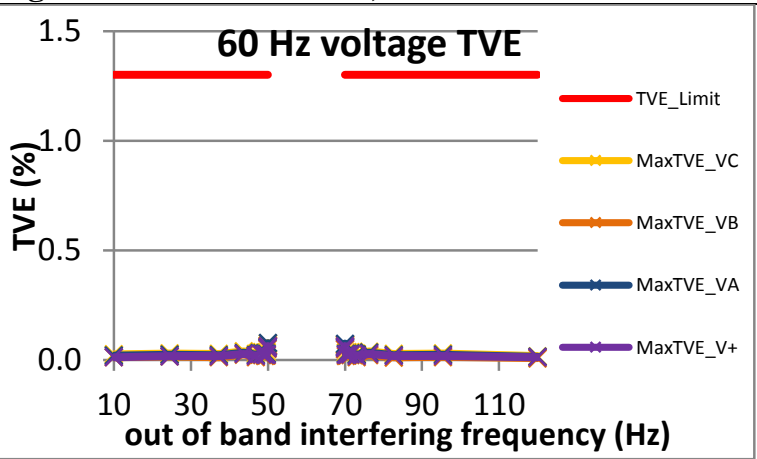

Figure 1361: Fs = 20 FPS, $60 \mathrm{~Hz}$ fundamental
Figure 1356: Fs = $60 \mathrm{FPS}, 63 \mathrm{~Hz}$ fundamental

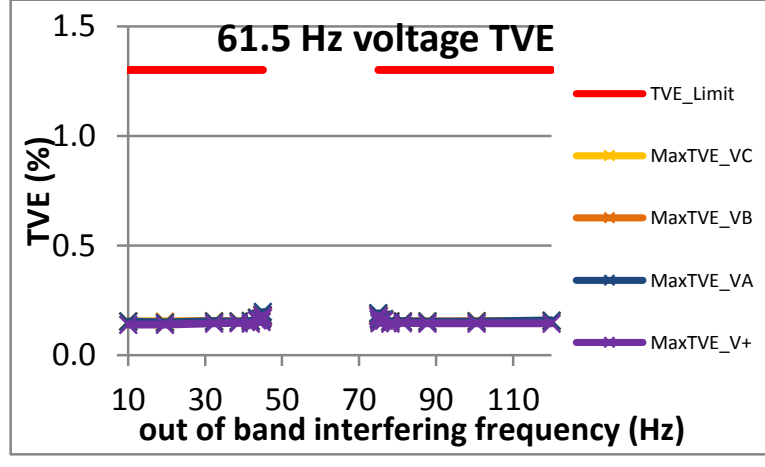

Figure 1359: Fs = 30 FPS, $61.5 \mathrm{~Hz}$ fundamental

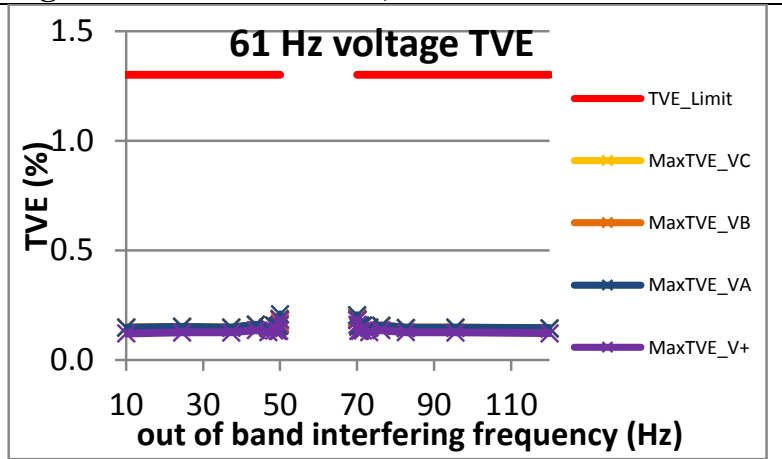

Figure 1362: Fs = 20 FPS, $61 \mathrm{~Hz}$ fundamental 


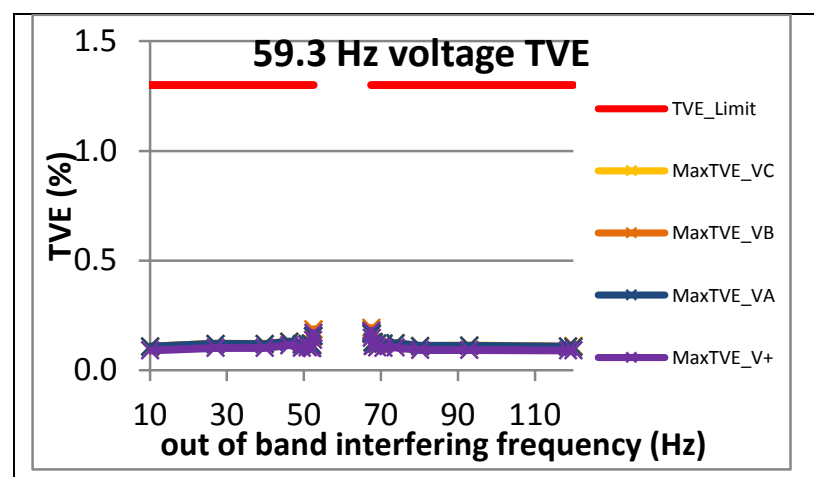

Figure 1363: Fs = 15 FPS, 59.3 Hz fundamental

Figure 1366: Fs = 12 FPS, $59.4 \mathrm{~Hz}$ fundamental

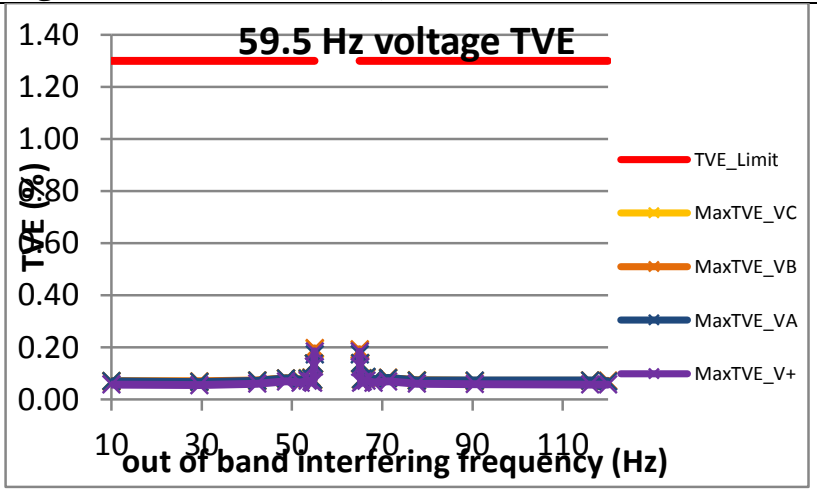

Figure 1369: Fs = 10 FPS, $59.5 \mathrm{~Hz}$ fundamental

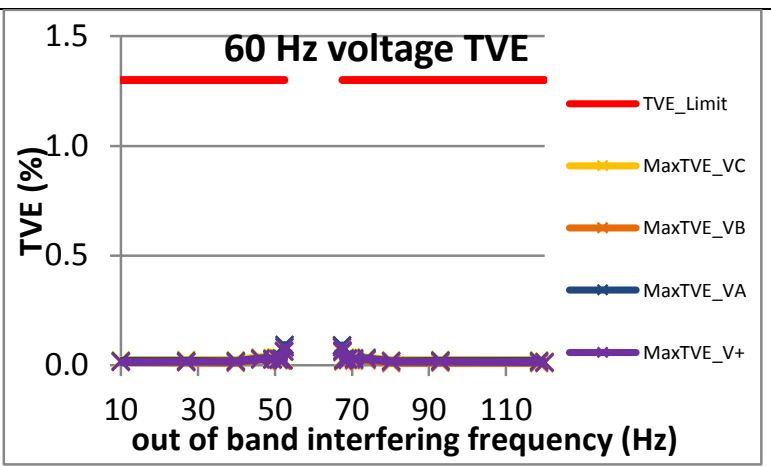

Figure 1364: Fs = 15 FPS, $60 \mathrm{~Hz}$ fundamental

Figure 1367: Fs = 12 FPS, $60 \mathrm{~Hz}$ fundamental

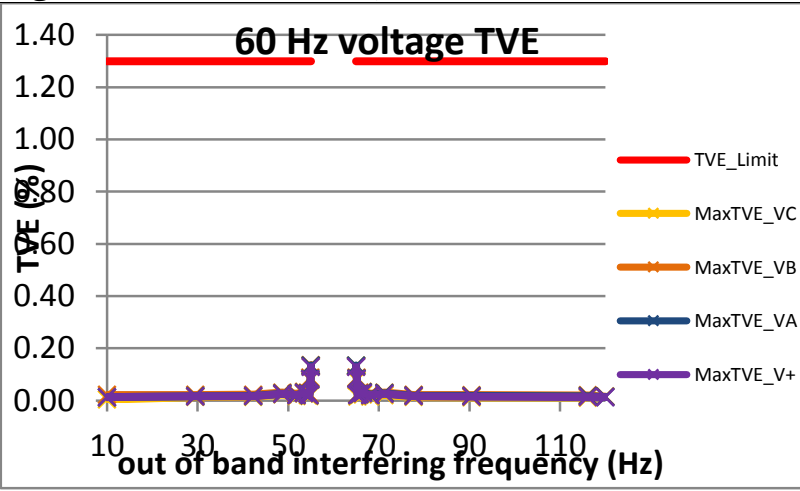

Figure 1370: Fs = $60 \mathrm{FPS}, 60 \mathrm{~Hz}$ fundamental

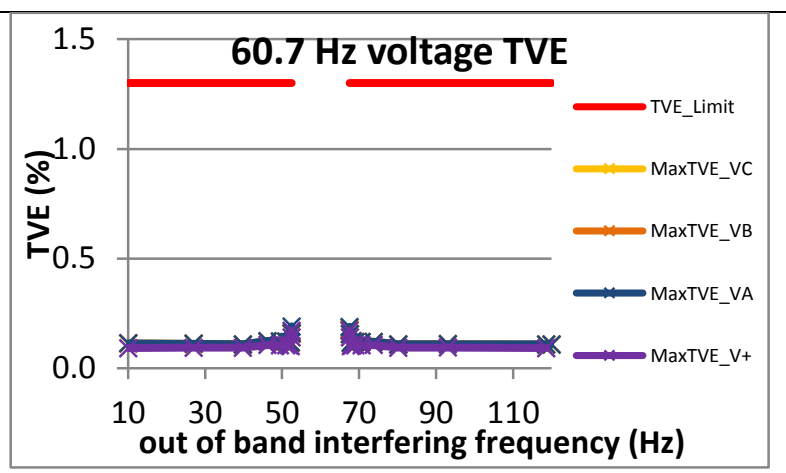

Figure 1365: Fs = 15 FPS, $60.7 \mathrm{~Hz}$ fundamental

Figure 1368: Fs = 12 FPS, $60.6 \mathrm{~Hz}$ fundamental

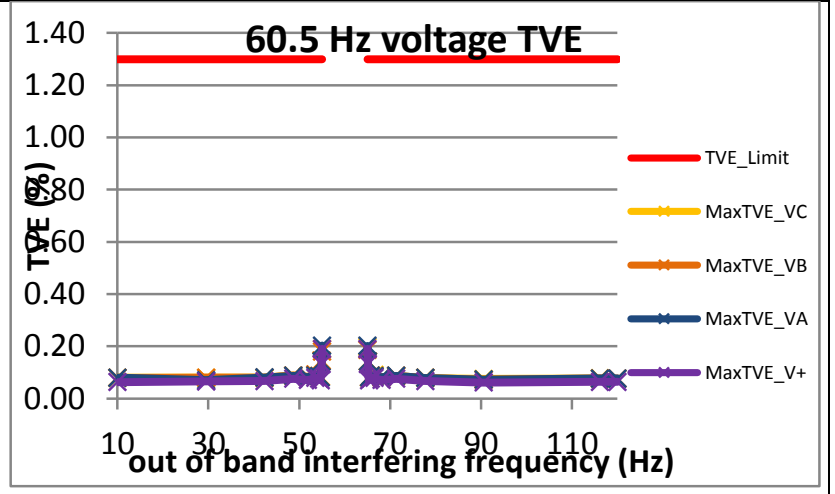

Figure 1371: Fs = 10 FPS, $60.5 \mathrm{~Hz}$ fundamental 
5.1.2 PMU A out of band interfering signals voltage TVE: M class

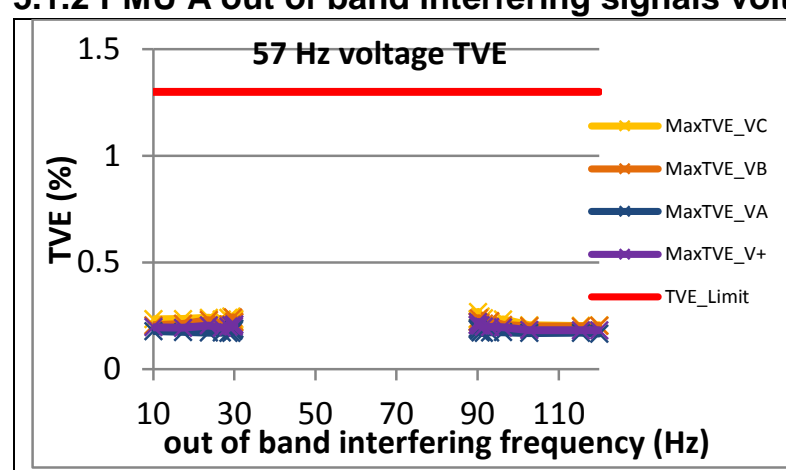

Figure 1372: Fs = 60 FPS, $57 \mathrm{~Hz}$ fundamental

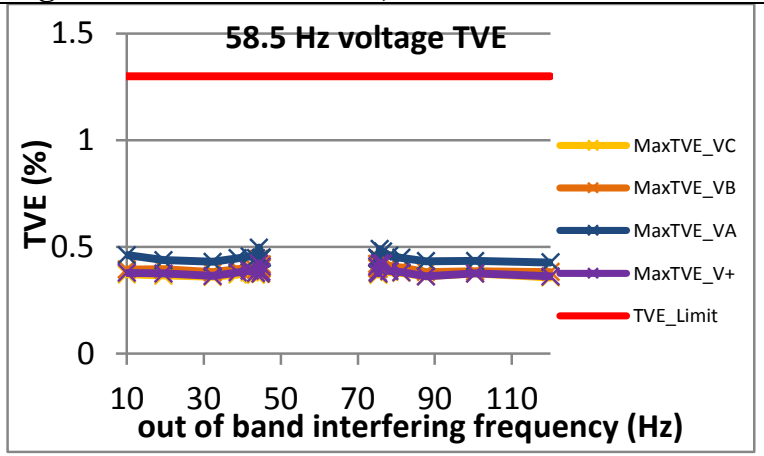

Figure 1375: Fs = 30 FPS, 58.5 Hz fundamental

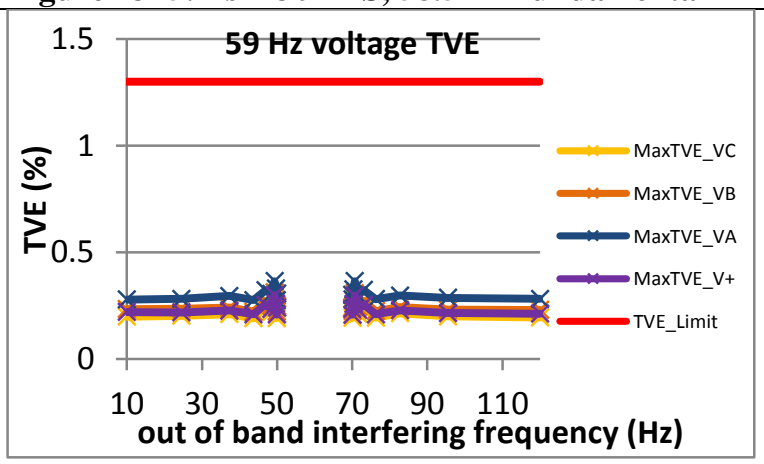

Figure 1378: Fs = 20 FPS, $59 \mathrm{~Hz}$ fundamental

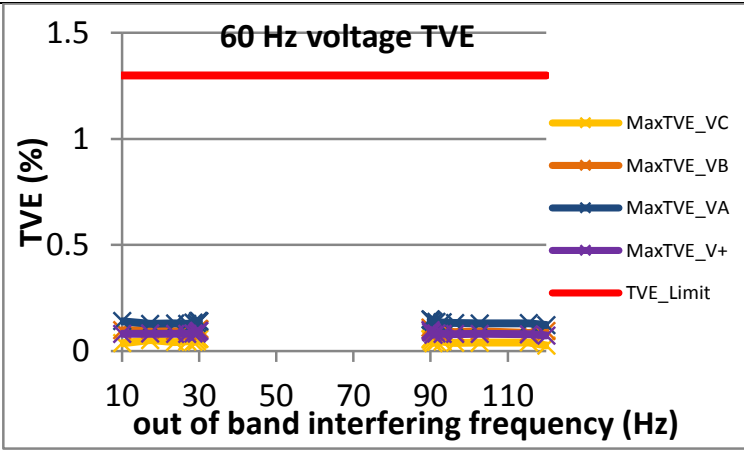

Figure 1373: Fs = $60 \mathrm{FPS}, 60 \mathrm{~Hz}$ fundamental

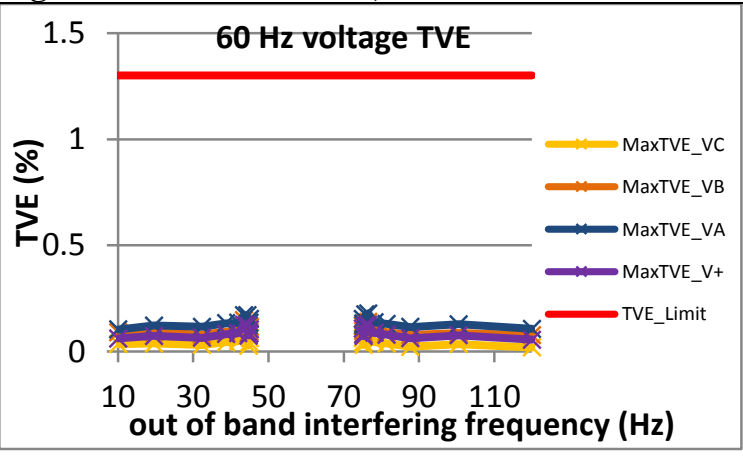

Figure 1376: Fs = $30 \mathrm{FPS}, 60 \mathrm{~Hz}$ fundamental

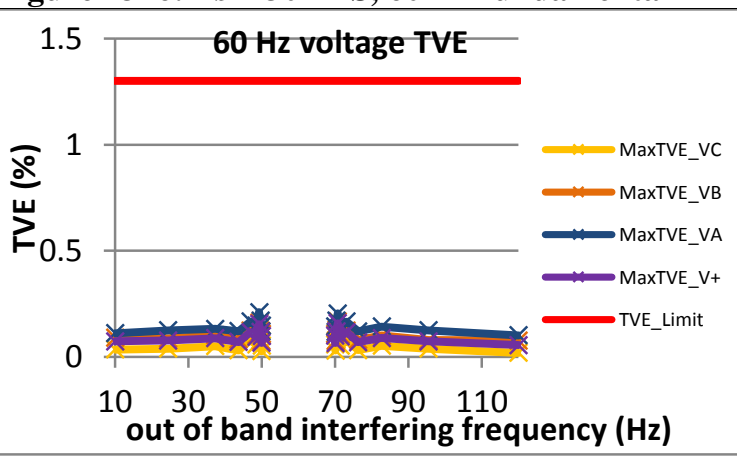

Figure 1379: Fs = 20 FPS, $60 \mathrm{~Hz}$ fundamental

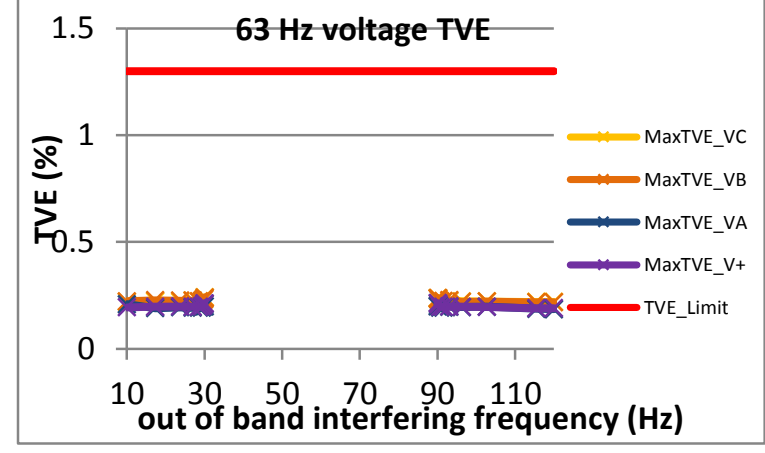

Figure 1374: Fs = $60 \mathrm{FPS}, 63 \mathrm{~Hz}$ fundamental

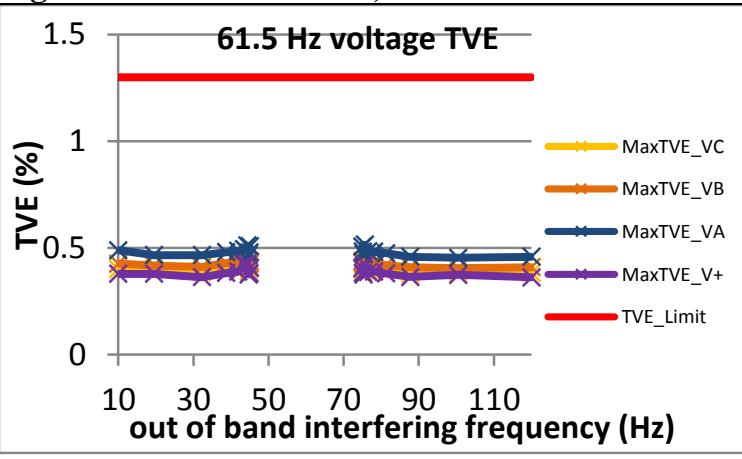

Figure 1377: Fs = 30 FPS, $61.5 \mathrm{~Hz}$ fundamental

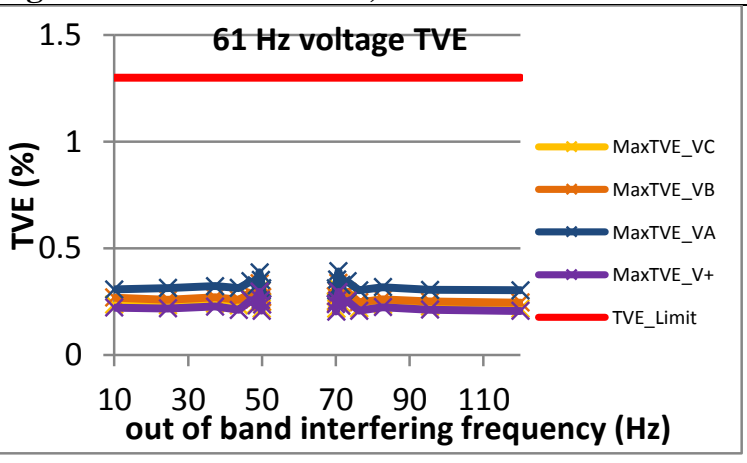

Figure 1380: Fs = 20 FPS, $61 \mathrm{~Hz}$ fundamental 


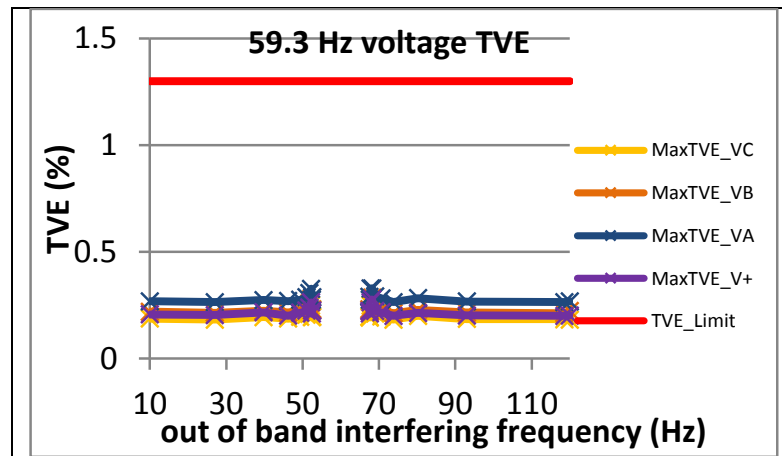

Figure 1381: Fs = 15 FPS, 59.3 Hz fundamental

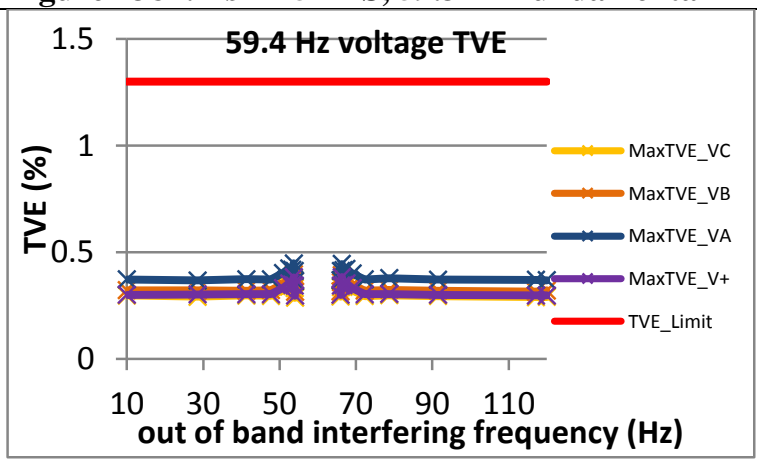

Figure 1384: Fs = 12 FPS, $59.4 \mathrm{~Hz}$ fundamental

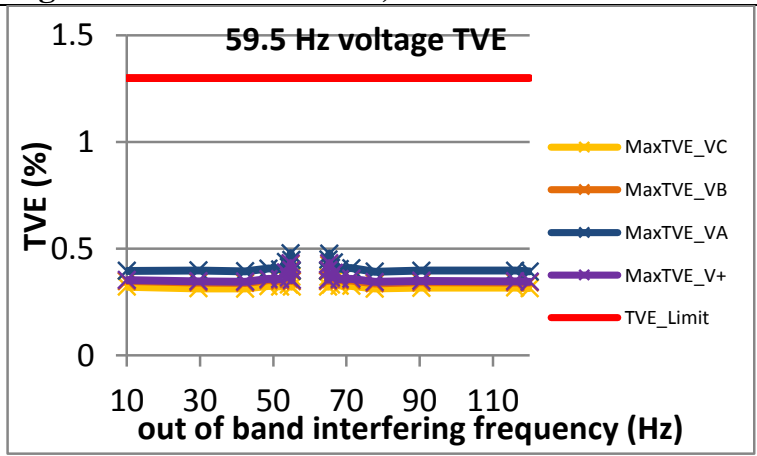

Figure 1387: Fs = 10 FPS, 59.5 Hz fundamental

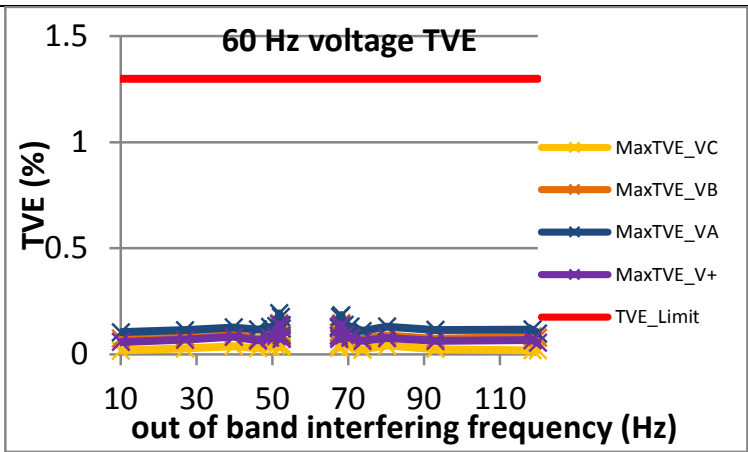

Figure 1382: Fs = $15 \mathrm{FPS}, 60 \mathrm{~Hz}$ fundamental

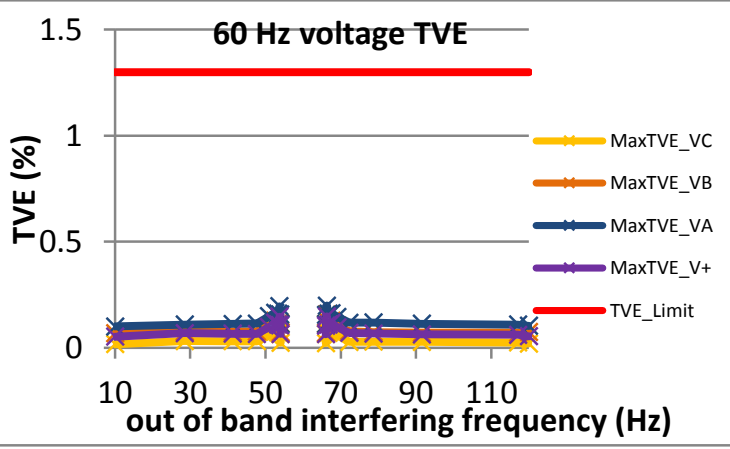

Figure 1385: Fs = $12 \mathrm{FPS}, 60 \mathrm{~Hz}$ fundamental

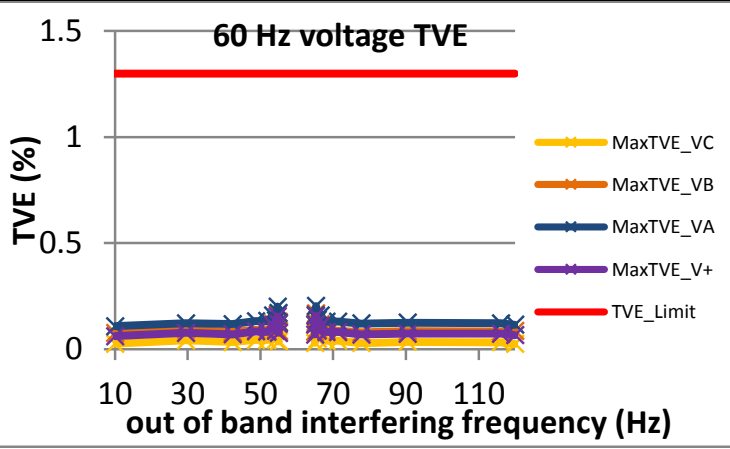

Figure 1388: Fs = $60 \mathrm{FPS}, 60 \mathrm{~Hz}$ fundamental

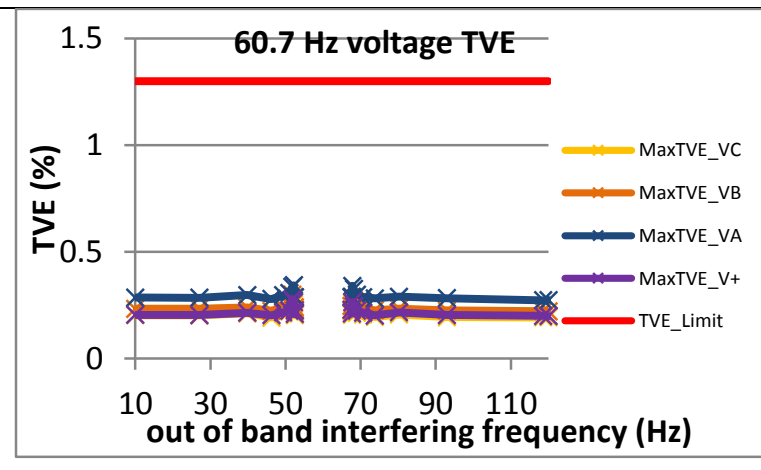

Figure 1383: Fs = 15 FPS, $60.7 \mathrm{~Hz}$ fundamental

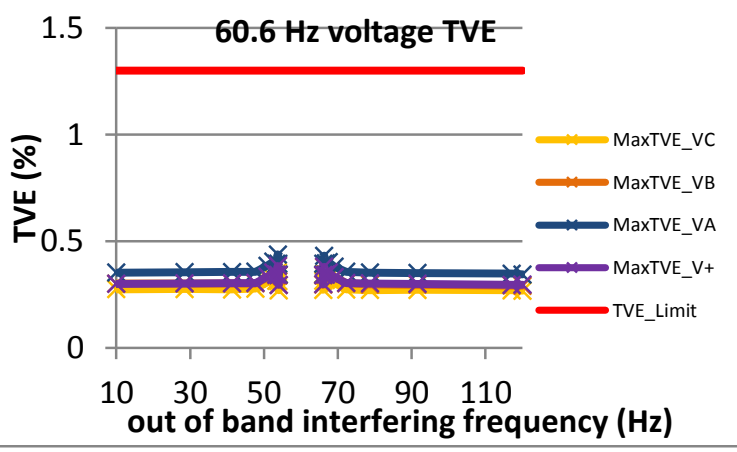

Figure 1386: Fs = 12 FPS, $60.6 \mathrm{~Hz}$ fundamental

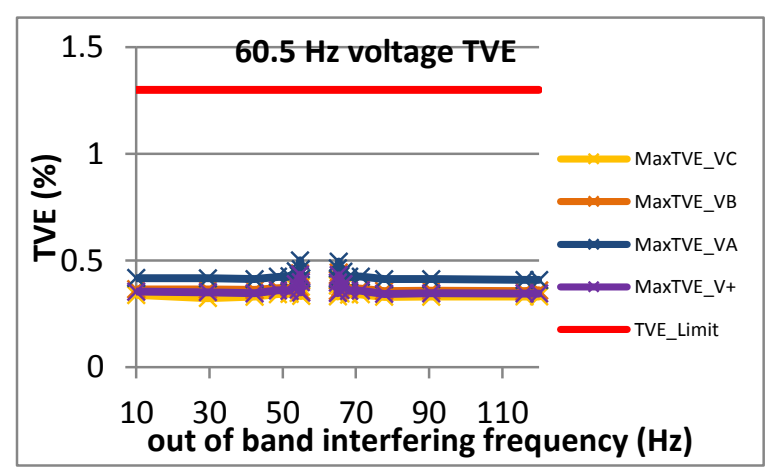

Figure 1389: Fs = 10 FPS, $60.5 \mathrm{~Hz}$ fundamental 
5.1.3 PMU B steady state out of band interfering signals voltage TVE: M class

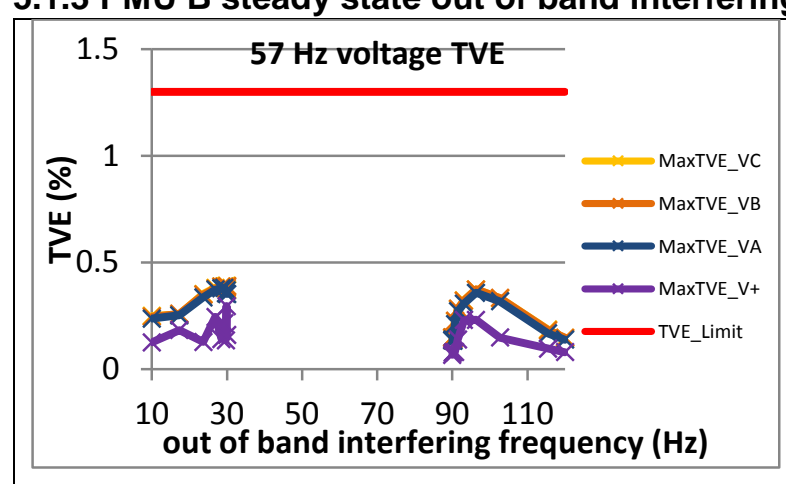

Figure 1390: Fs = 60 FPS, $57 \mathrm{~Hz}$ fundamental

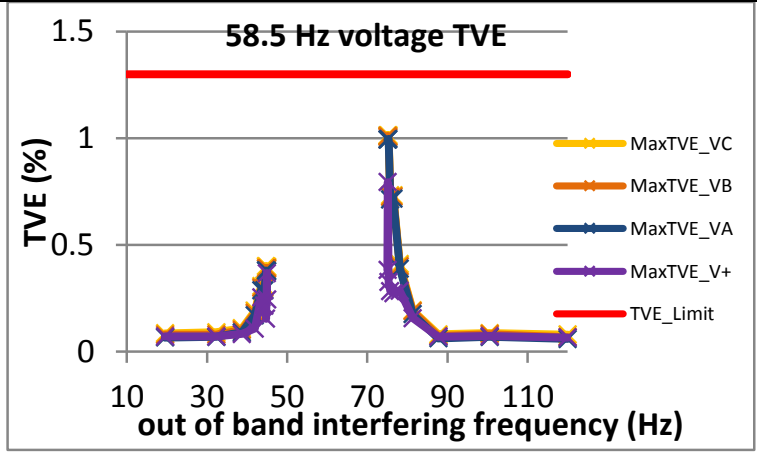

Figure 1393: Fs = 30 FPS, $58.5 \mathrm{~Hz}$ fundamental

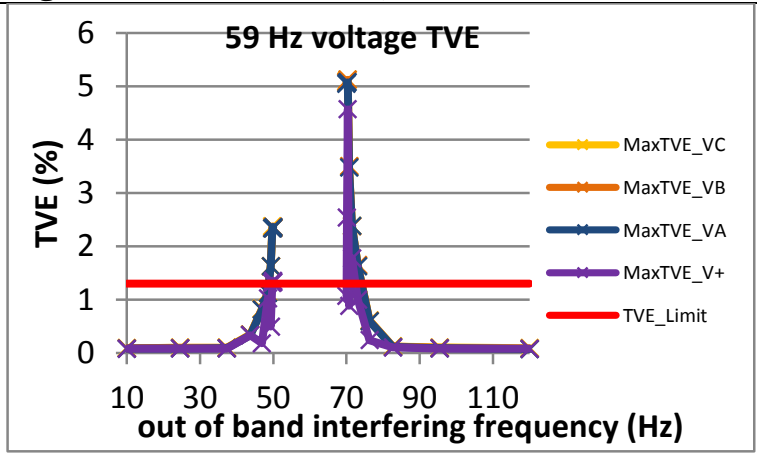

Figure 1396: Fs = 20 FPS, $59 \mathrm{~Hz}$ fundamental

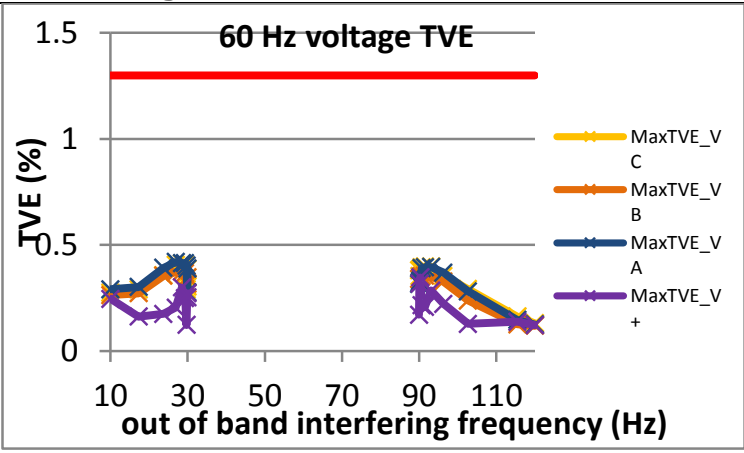

Figure 1391: Fs = $60 \mathrm{FPS}, 60 \mathrm{~Hz}$ fundamental

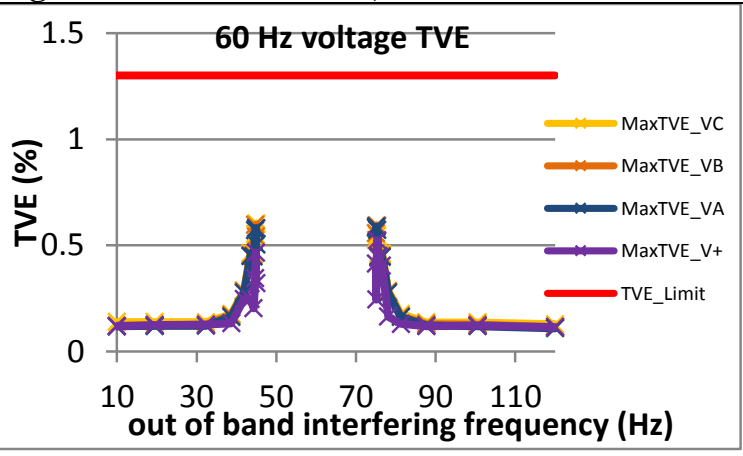

Figure 1394: Fs = $30 \mathrm{FPS}, 60 \mathrm{~Hz}$ fundamental

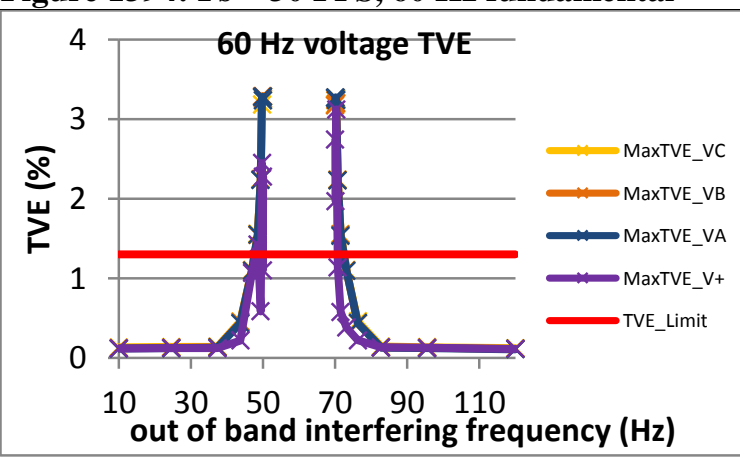

Figure 1397: Fs = 20 FPS, $60 \mathrm{~Hz}$ fundamental

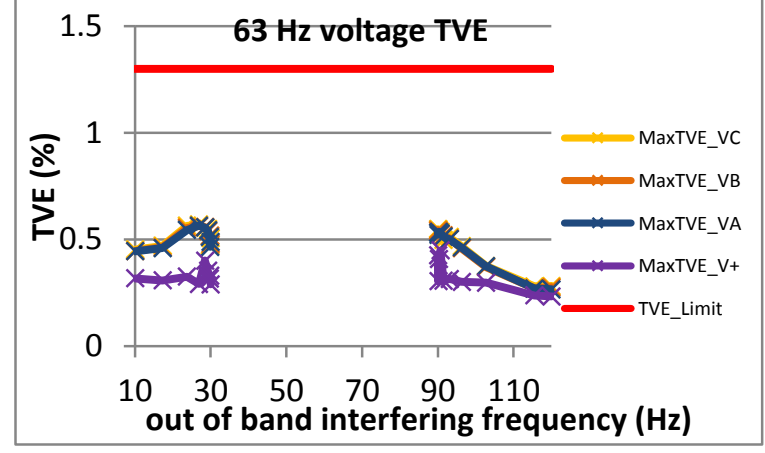

Figure 1392: Fs = $60 \mathrm{FPS}, 63 \mathrm{~Hz}$ fundamental

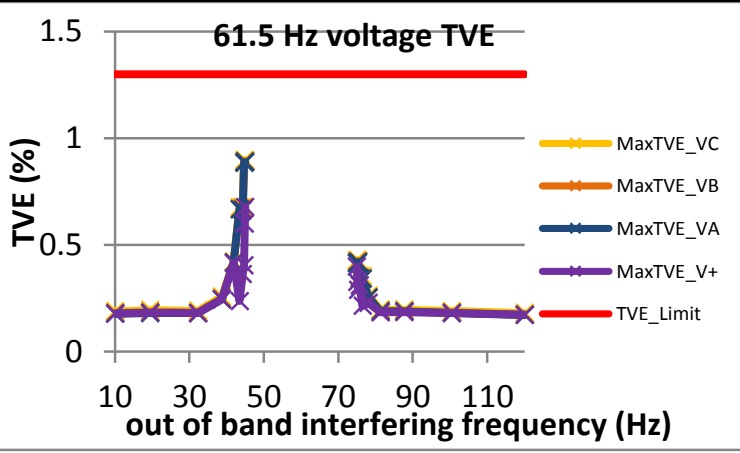

Figure 1395: Fs = 30 FPS, $61.5 \mathrm{~Hz}$ fundamental

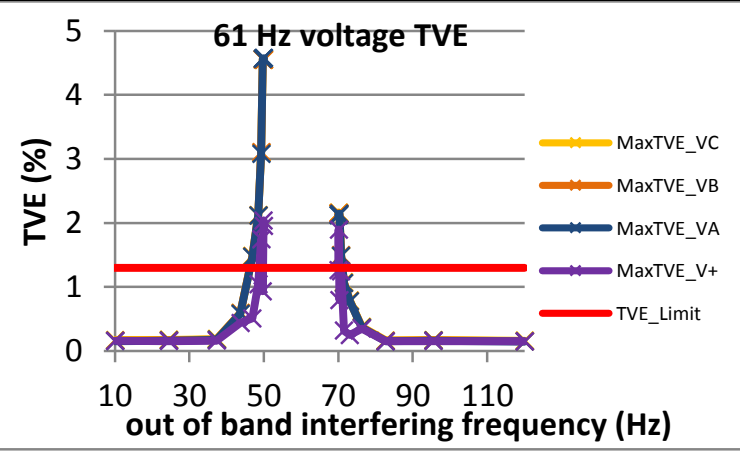

Figure 1398: Fs = 20 FPS, $61 \mathrm{~Hz}$ fundamental 


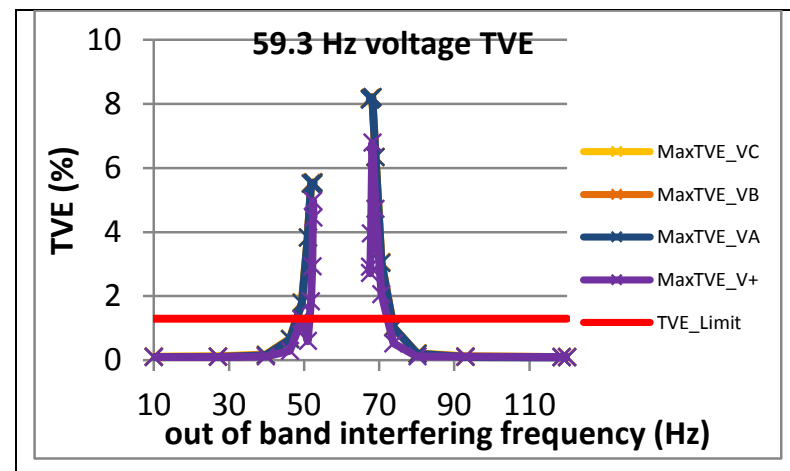

Figure 1399: Fs = 15 FPS, $59.3 \mathrm{~Hz}$ fundamental

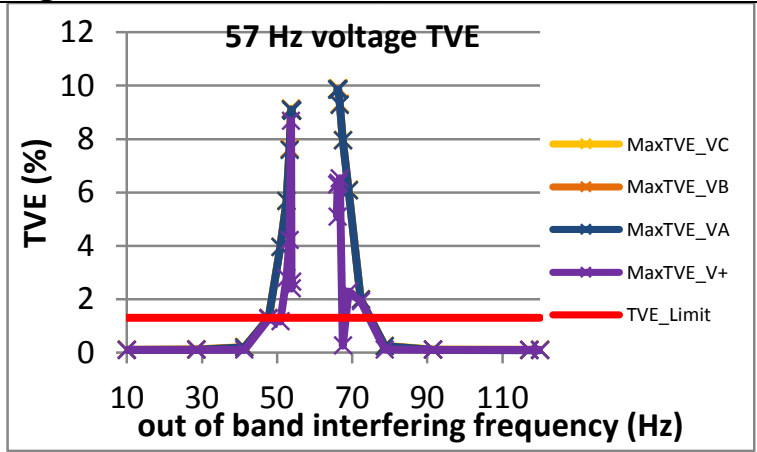

Figure 1402: Fs = 12 FPS, $59.4 \mathrm{~Hz}$ fundamental

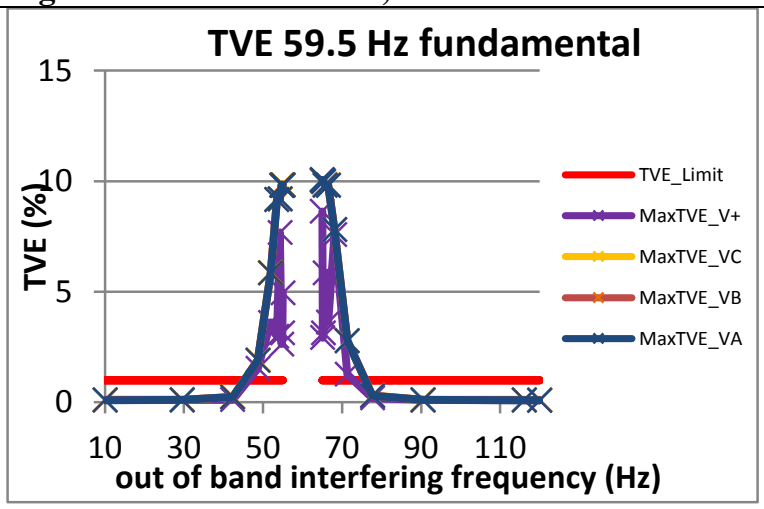

Figure 1405: Fs = 10 FPS, $59.5 \mathrm{~Hz}$ fundamental

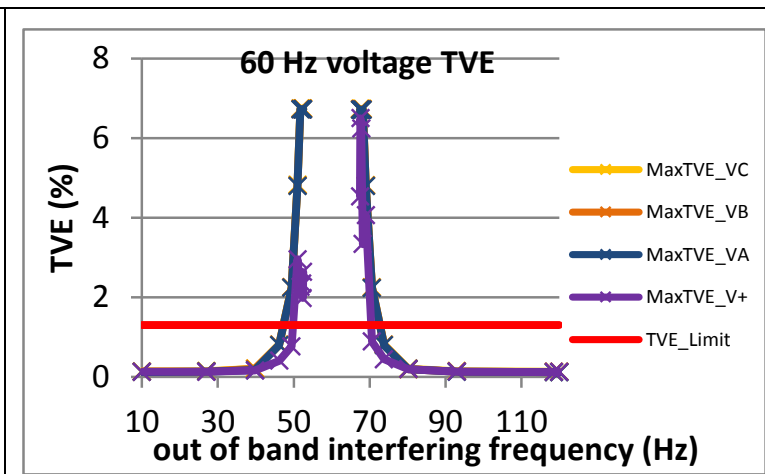

Figure 1400: Fs = 15 FPS, $60 \mathrm{~Hz}$ fundamental

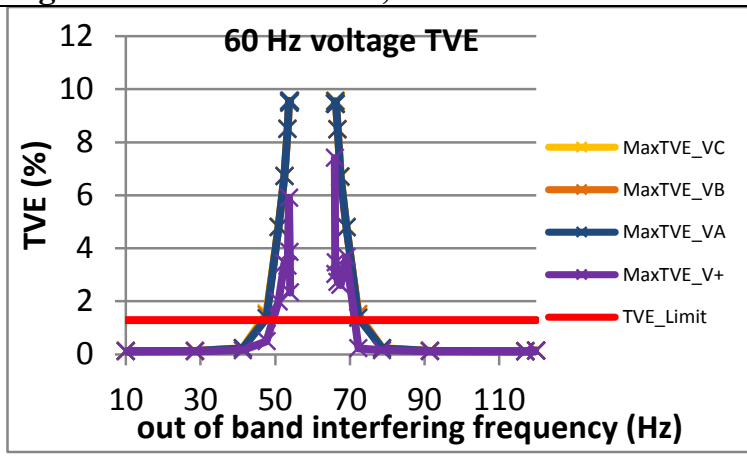

Figure 1403: Fs = $12 \mathrm{FPS}, 60 \mathrm{~Hz}$ fundamental

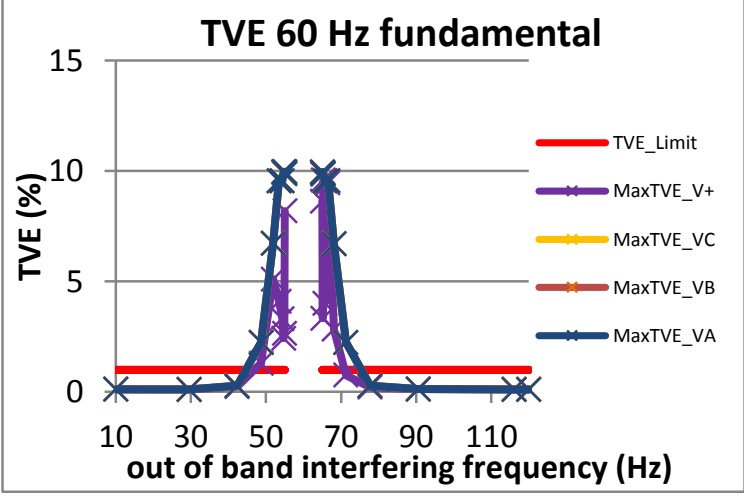

Figure 1406: Fs = $60 \mathrm{FPS}, 60 \mathrm{~Hz}$ fundamental

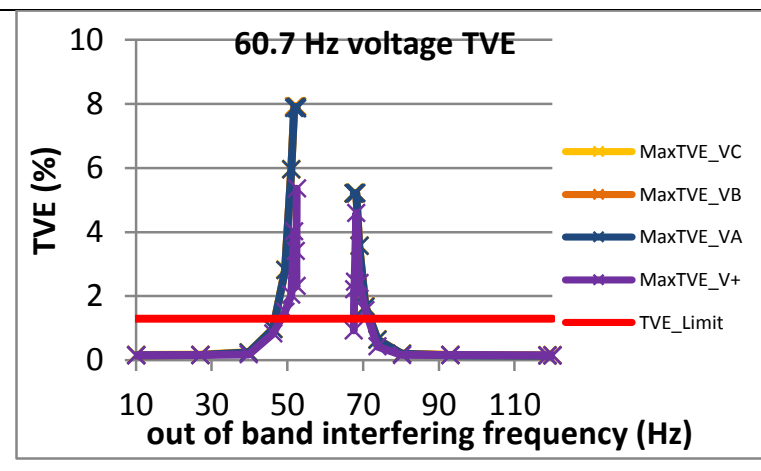

Figure 1401: Fs = 15 FPS, $60.7 \mathrm{~Hz}$ fundamental

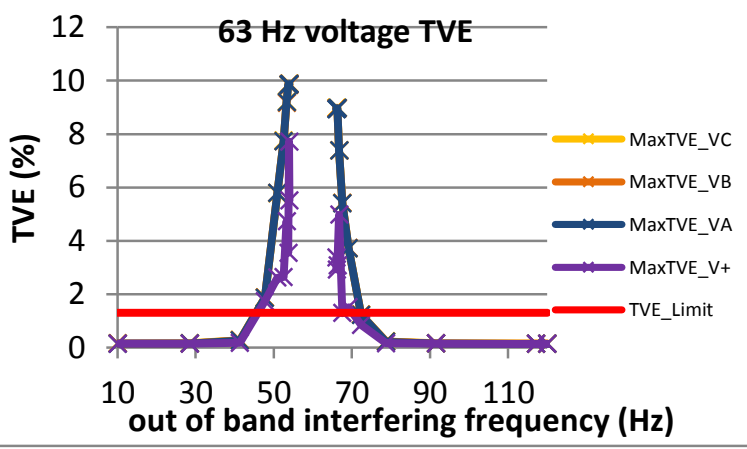

Figure 1404: Fs = 12 FPS, $60.6 \mathrm{~Hz}$ fundamental

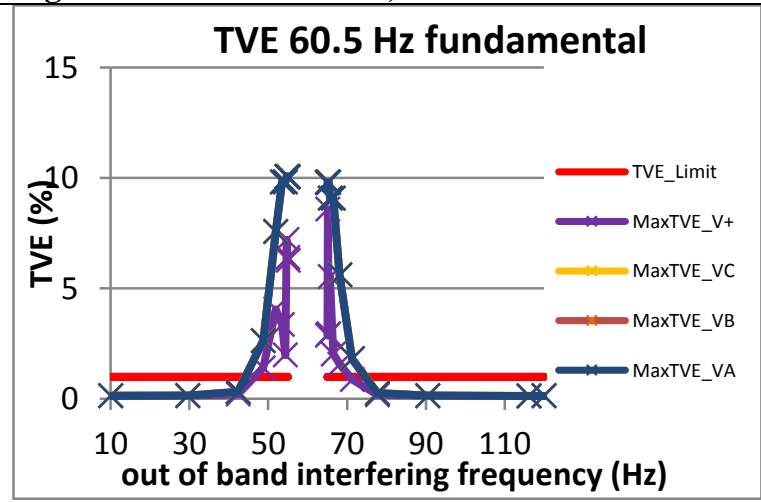

Figure 1407: Fs = 10 FPS, $60.5 \mathrm{~Hz}$ fundamental 
5.1.4 PMU C steady state out of band interfering signals voltage TVE: M class

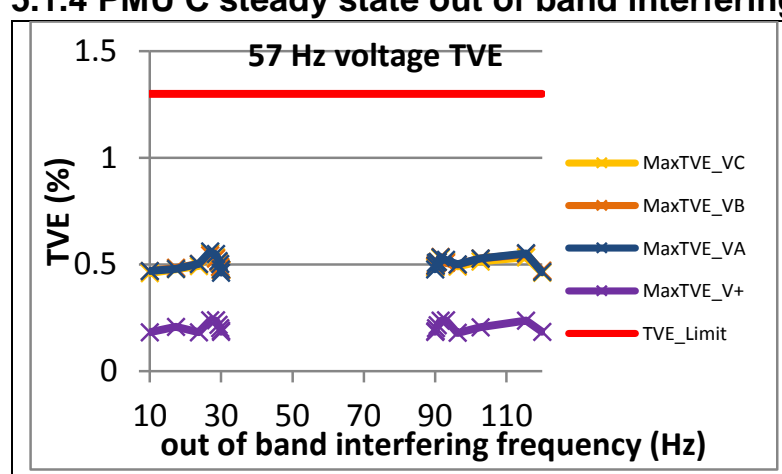

Figure 1408: Fs = 60 FPS, $57 \mathrm{~Hz}$ fundamental

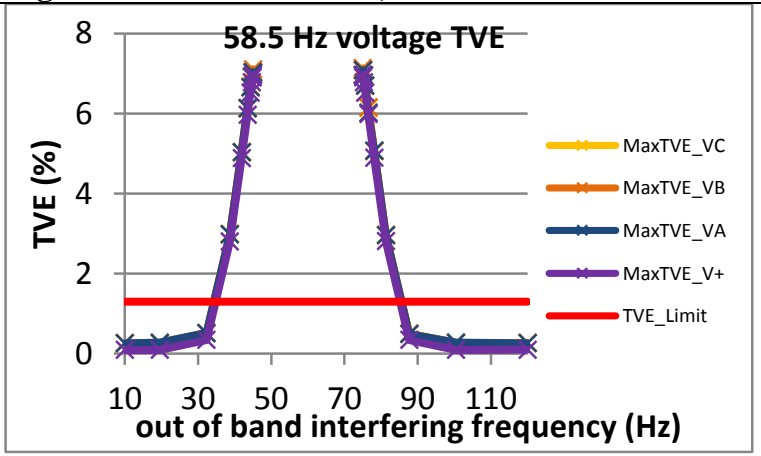

Figure 1411: Fs = 30 FPS, $58.5 \mathrm{~Hz}$ fundamental

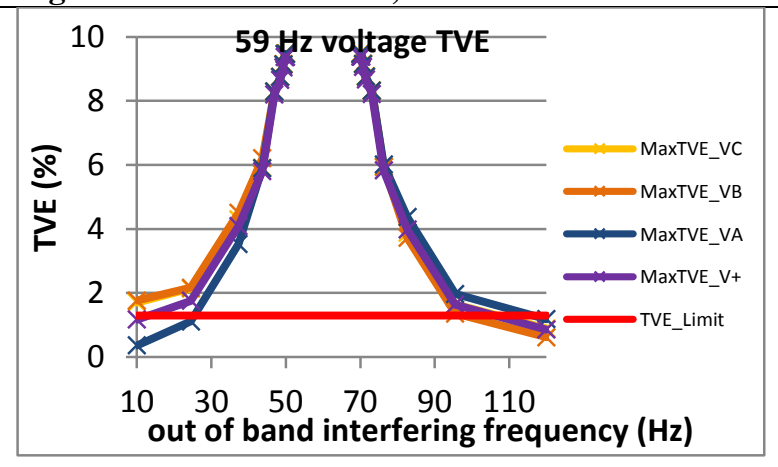

Figure 1414: Fs = 20 FPS, $59 \mathrm{~Hz}$ fundamental

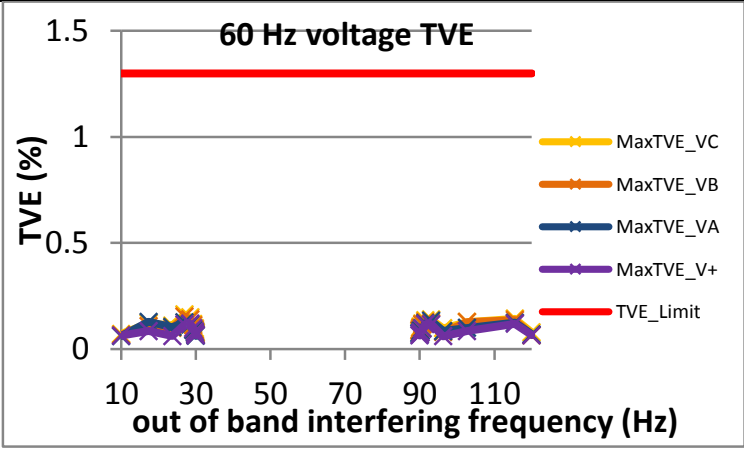

Figure 1409: Fs = 60 FPS, $60 \mathrm{~Hz}$ fundamental

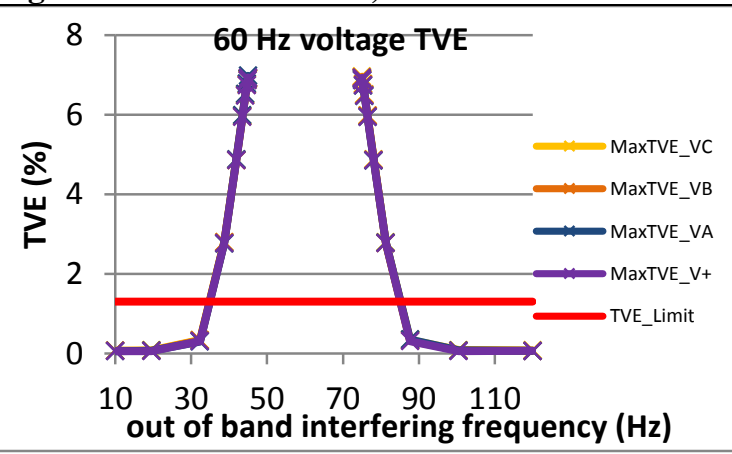

Figure 1412: Fs = $30 \mathrm{FPS}, 60 \mathrm{~Hz}$ fundamental

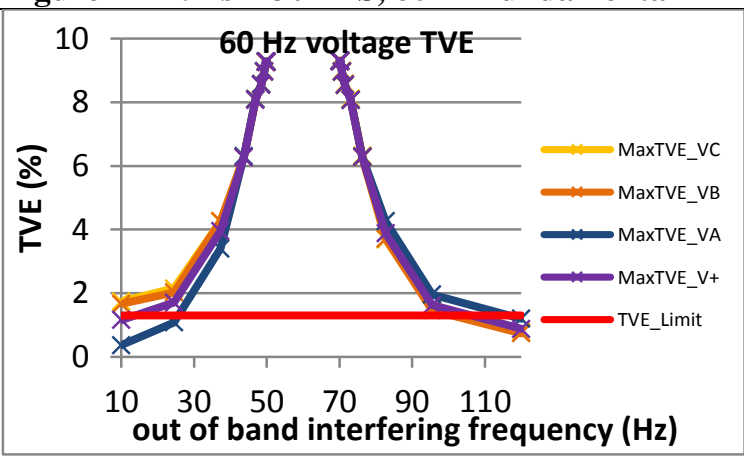

Figure 1415: Fs = 20 FPS, $60 \mathrm{~Hz}$ fundamental

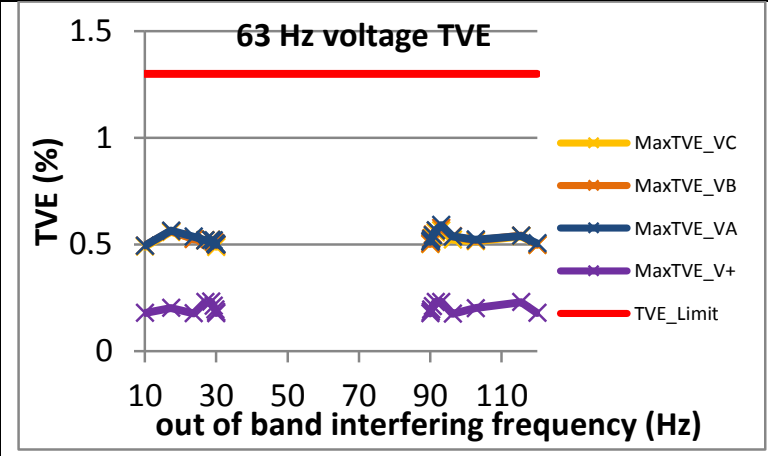

Figure 1410: Fs = $60 \mathrm{FPS}, 63 \mathrm{~Hz}$ fundamental

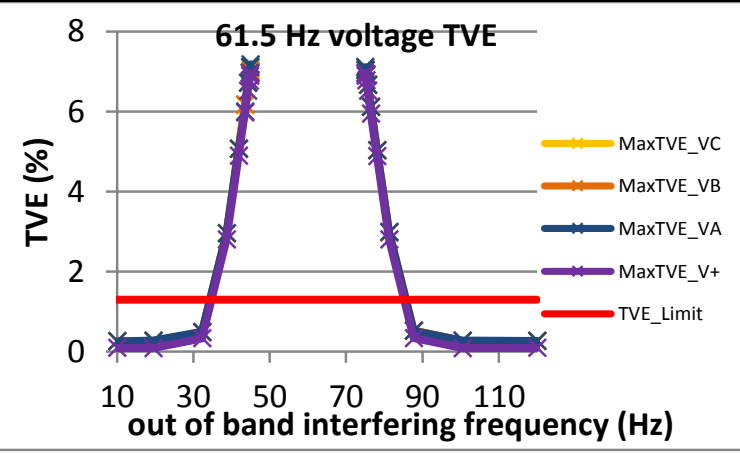

Figure 1413: Fs = $30 \mathrm{FPS}, 61.5 \mathrm{~Hz}$ fundamental

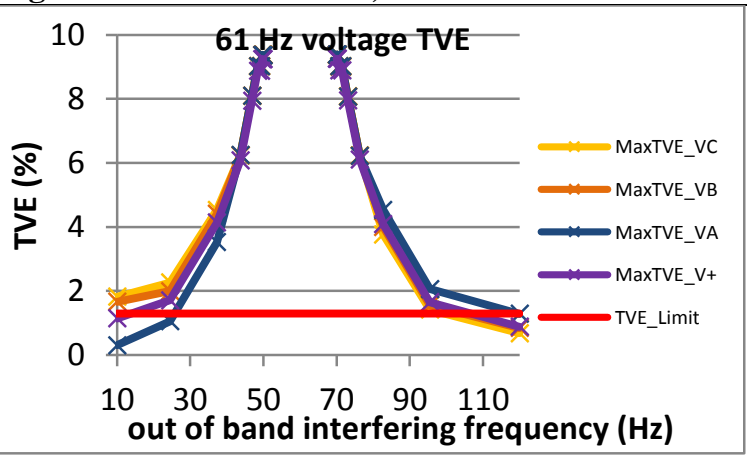

Figure 1416: Fs = 20 FPS, $61 \mathrm{~Hz}$ fundamental 


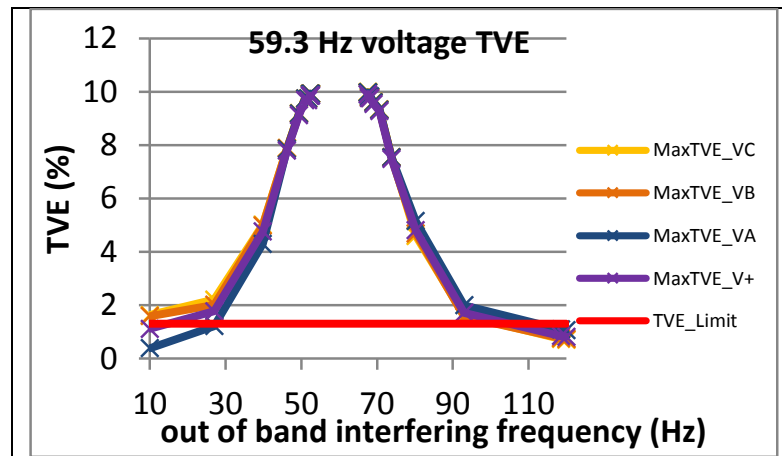

Figure 1417: Fs = 15 FPS, 59.3 Hz fundamental

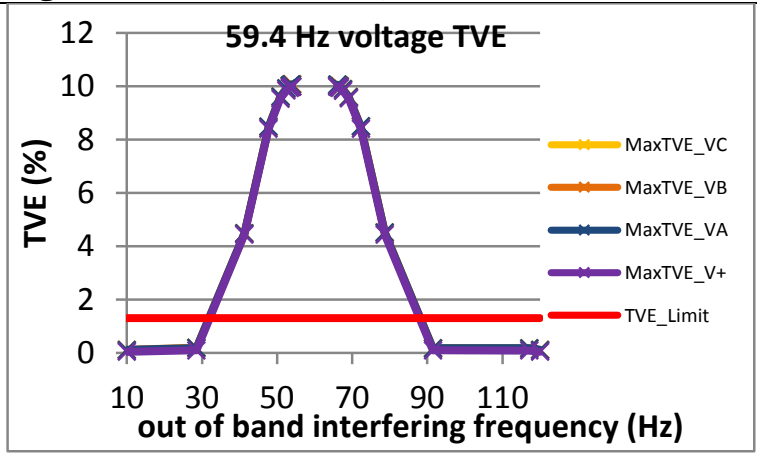

Figure 1420: Fs = 12 FPS, $59.4 \mathrm{~Hz}$ fundamental

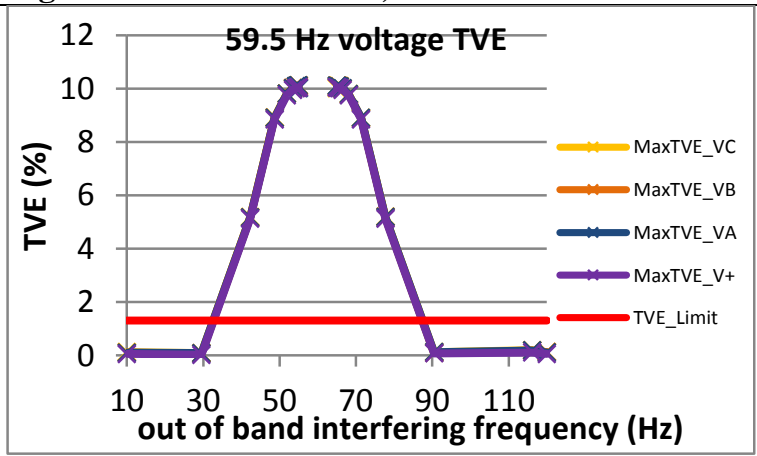

Figure 1423: Fs = 10 FPS, $59.5 \mathrm{~Hz}$ fundamental

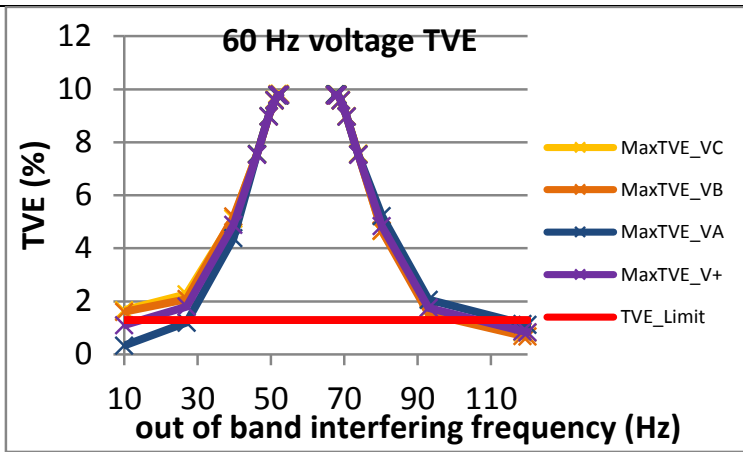

Figure 1418: Fs = 15 FPS, $60 \mathrm{~Hz}$ fundamental

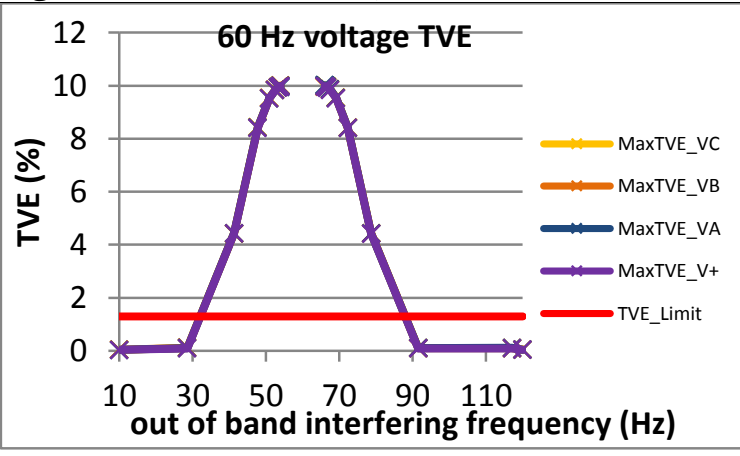

Figure 1421: Fs = $12 \mathrm{FPS}, 60 \mathrm{~Hz}$ fundamental

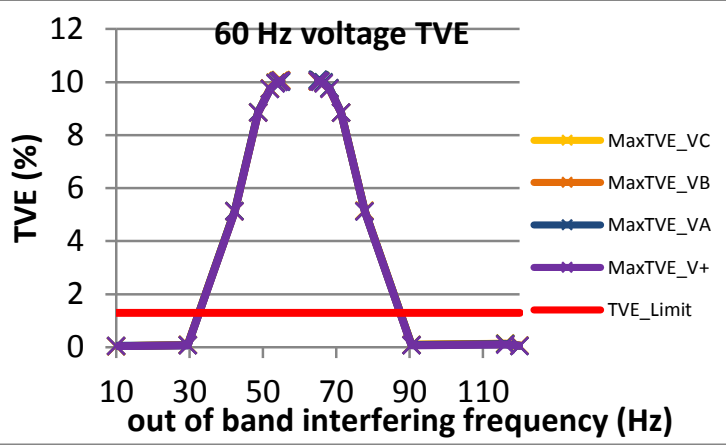

Figure 1424: Fs = $60 \mathrm{FPS}, 60 \mathrm{~Hz}$ fundamental

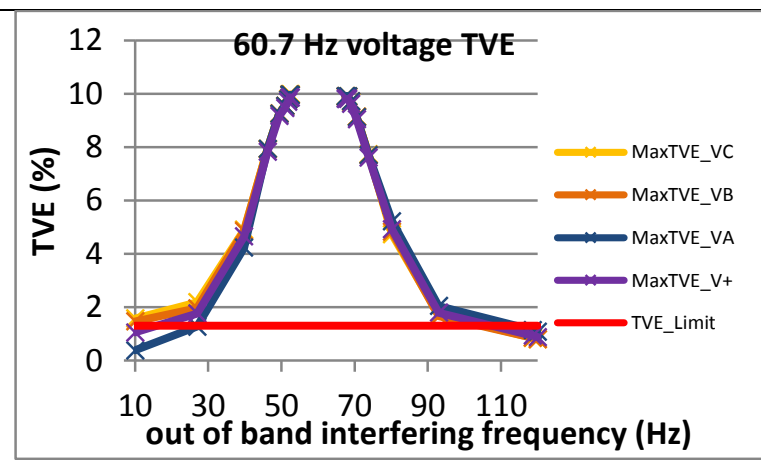

Figure 1419: Fs = 15 FPS, $60.7 \mathrm{~Hz}$ fundamental

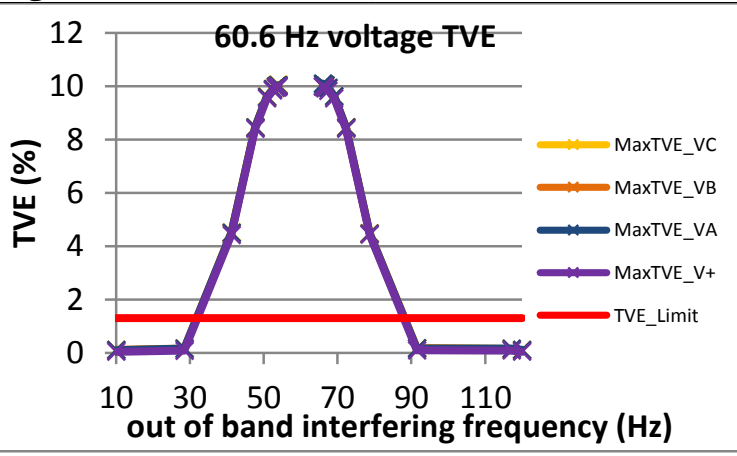

Figure 1422: Fs = 12 FPS, $60.6 \mathrm{~Hz}$ fundamental

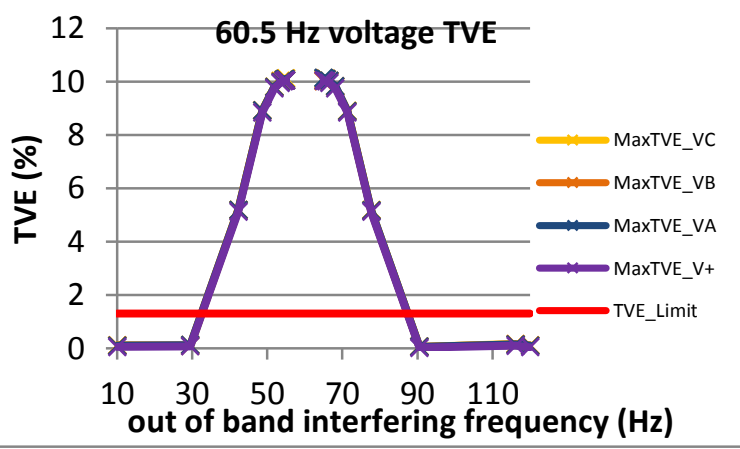

Figure 1425: Fs = 10 FPS, $60.5 \mathrm{~Hz}$ fundamental 
5.1.5 PMU D steady state out of band interfering signals voltage TVE: M class

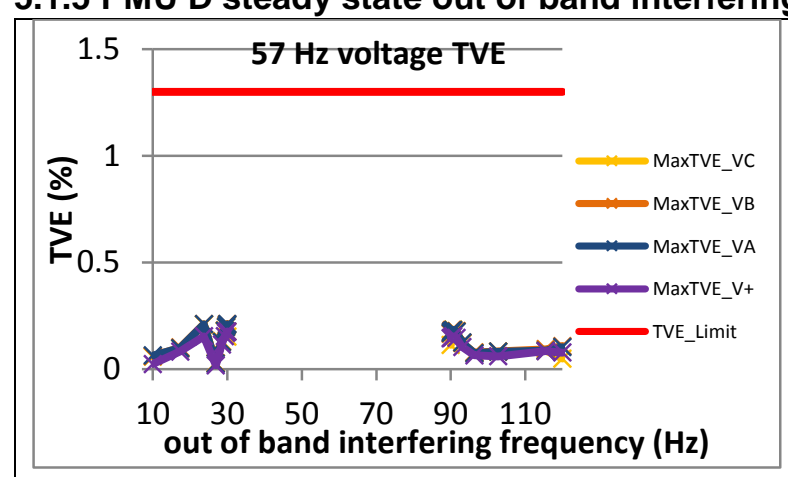

Figure 1426: Fs = 60 FPS, $57 \mathrm{~Hz}$ fundamental

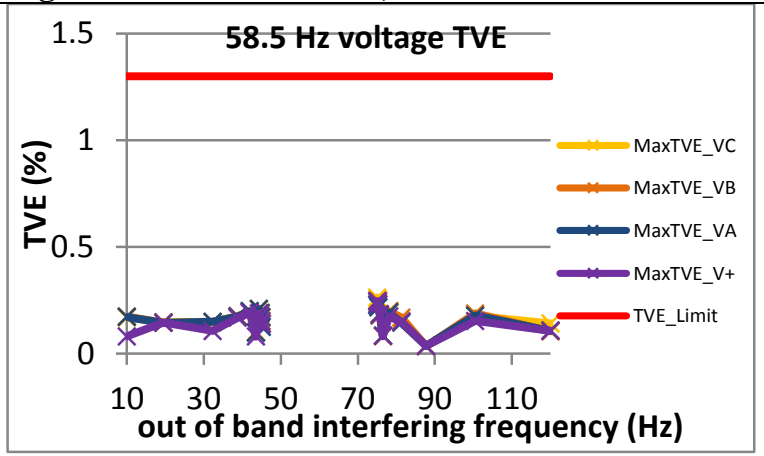

Figure 1429: Fs = 30 FPS, $58.5 \mathrm{~Hz}$ fundamental

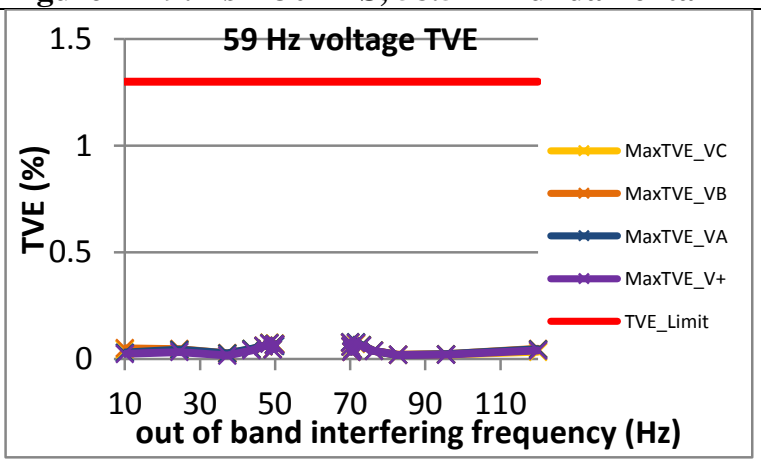

Figure 1432: Fs = 20 FPS, $59 \mathrm{~Hz}$ fundamental

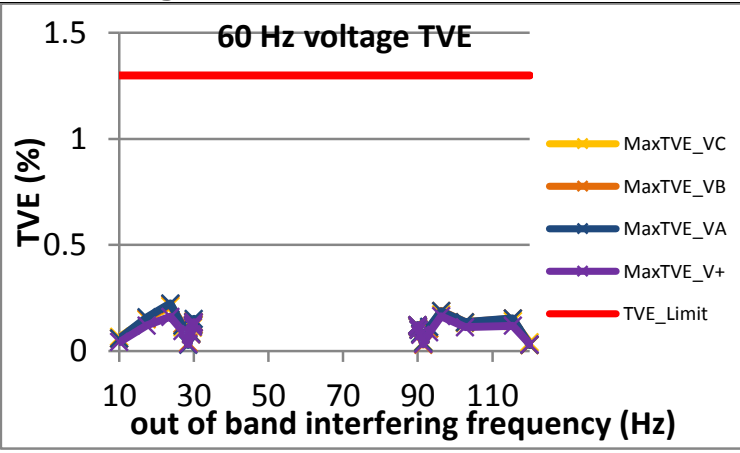

Figure 1427: Fs = $60 \mathrm{FPS}, 60 \mathrm{~Hz}$ fundamental

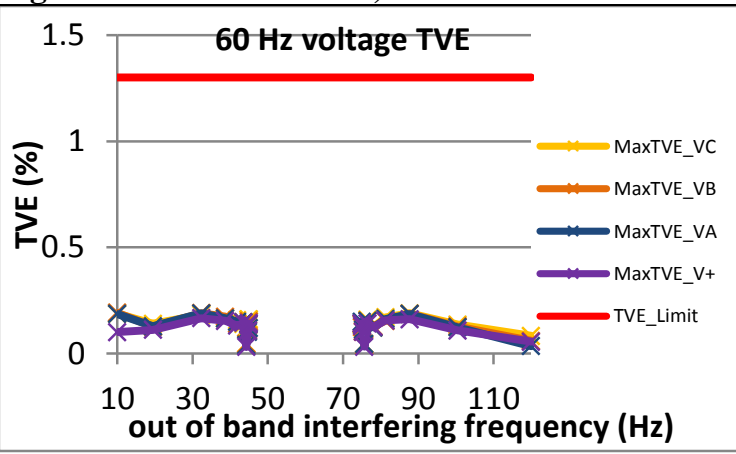

Figure 1430: Fs = $30 \mathrm{FPS}, 60 \mathrm{~Hz}$ fundamental

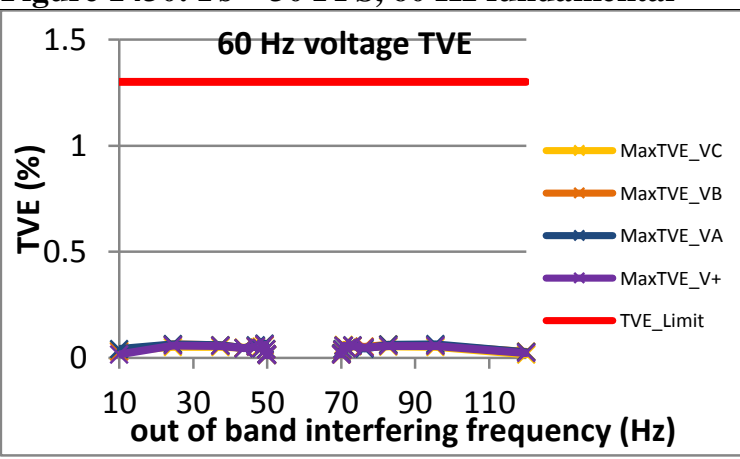

Figure 1433: Fs = 20 FPS, $60 \mathrm{~Hz}$ fundamental

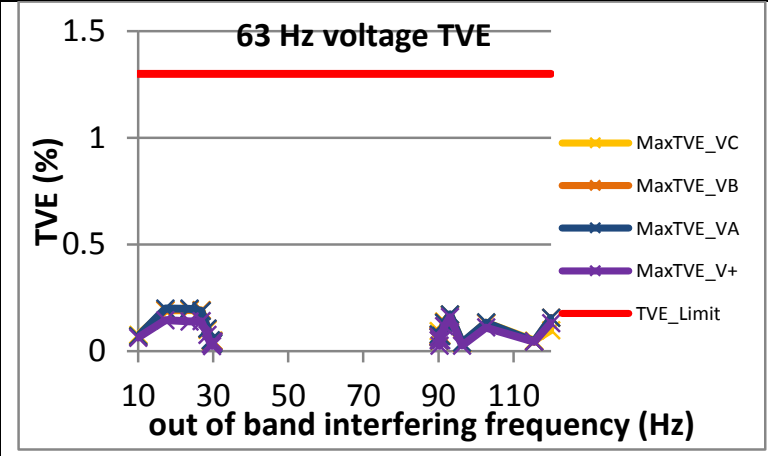

Figure 1428: Fs = $60 \mathrm{FPS}, 63 \mathrm{~Hz}$ fundamental

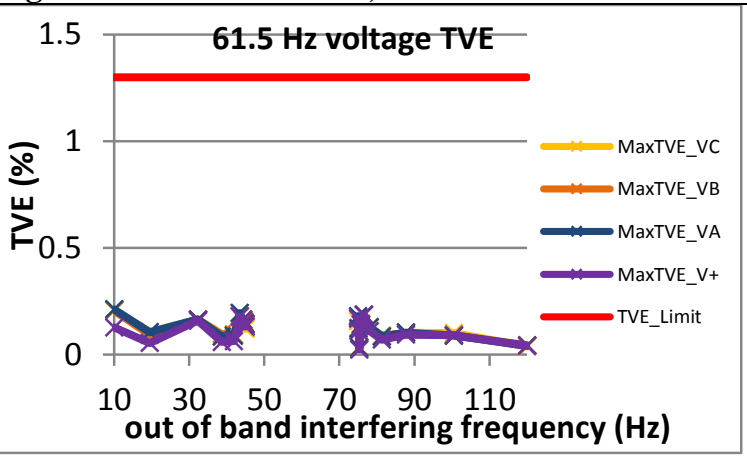

Figure 1431: Fs = $30 \mathrm{FPS}, 61.5 \mathrm{~Hz}$ fundamental

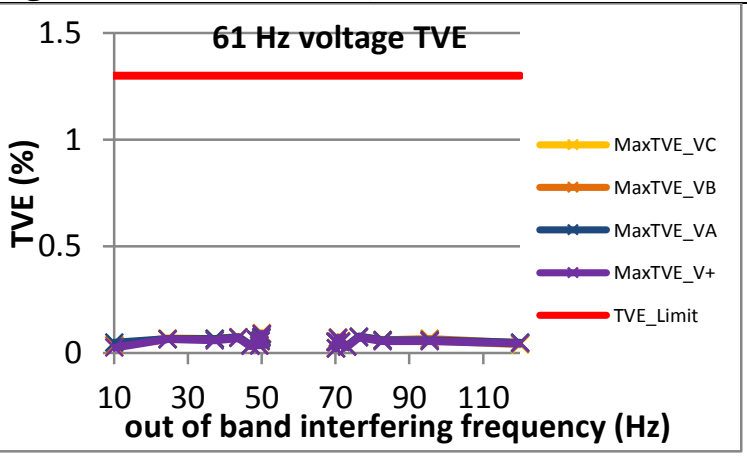

Figure 1434: Fs = 20 FPS, $61 \mathrm{~Hz}$ fundamental 


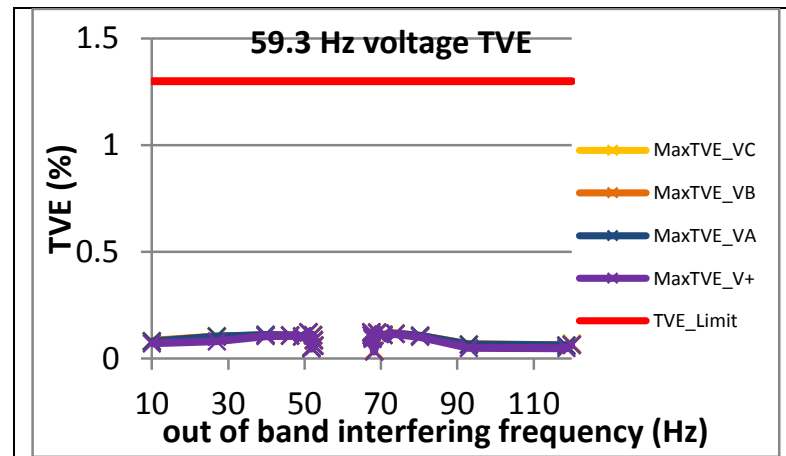

Figure 1435: Fs = 15 FPS, 59.3 Hz fundamental

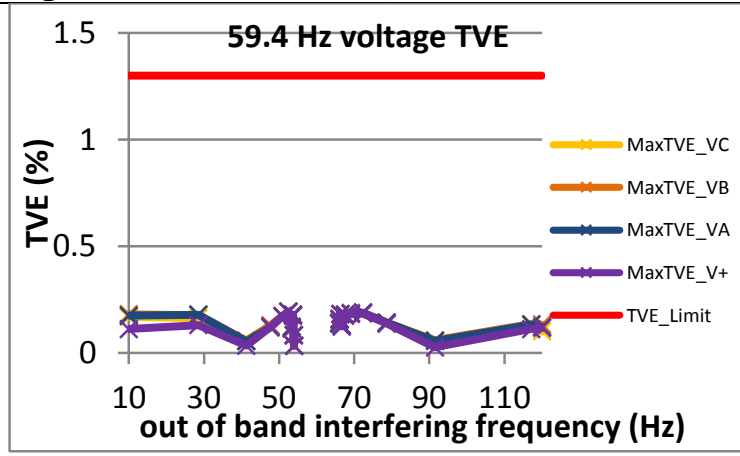

Figure 1438: Fs = 12 FPS, $59.4 \mathrm{~Hz}$ fundamental

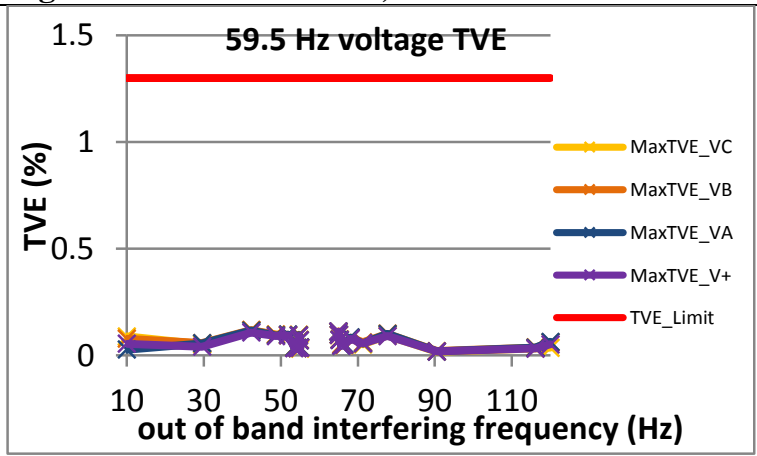

Figure 1441: Fs = 10 FPS, $59.5 \mathrm{~Hz}$ fundamental

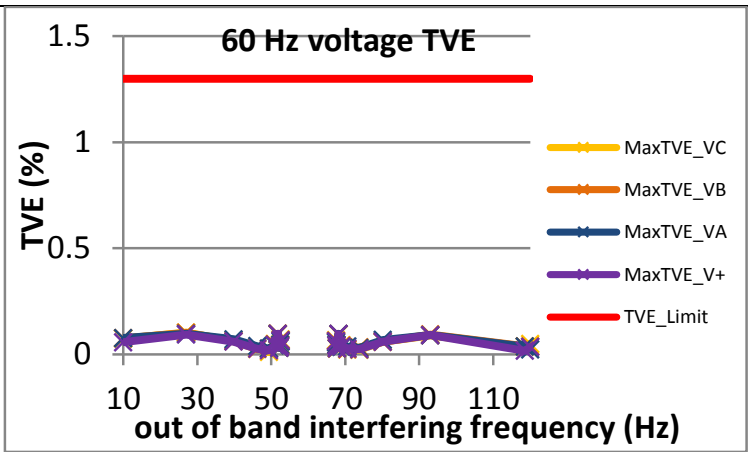

Figure 1436: Fs = 15 FPS, $60 \mathrm{~Hz}$ fundamental

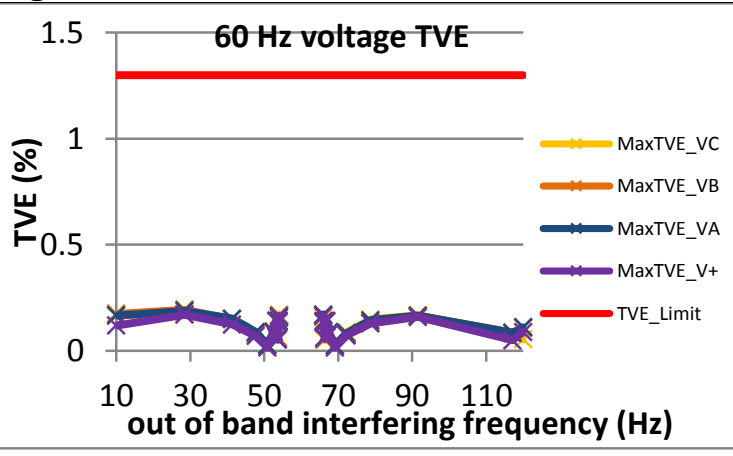

Figure 1439: Fs = $12 \mathrm{FPS}, 60 \mathrm{~Hz}$ fundamental

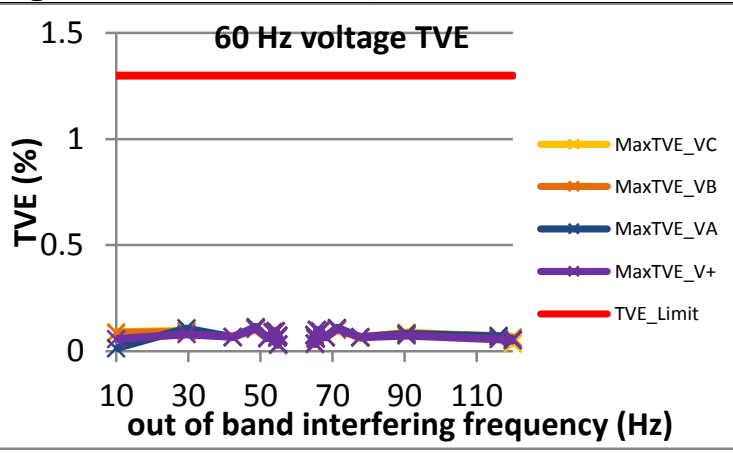

Figure 1442: Fs = $60 \mathrm{FPS}, 60 \mathrm{~Hz}$ fundamental

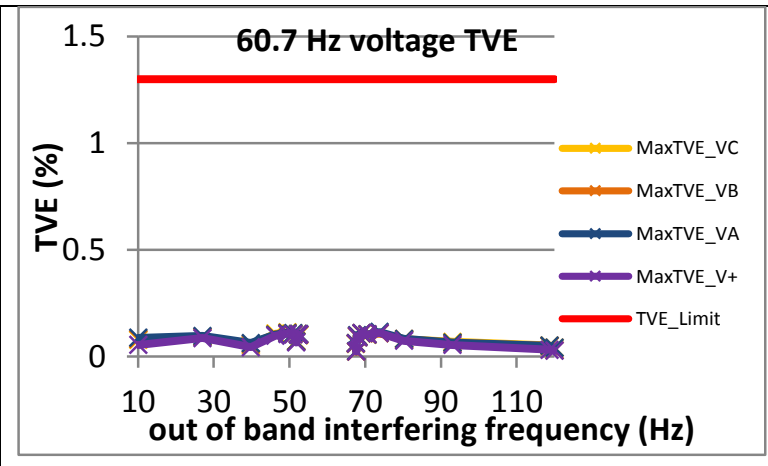

Figure 1437: Fs = 15 FPS, $60.7 \mathrm{~Hz}$ fundamental

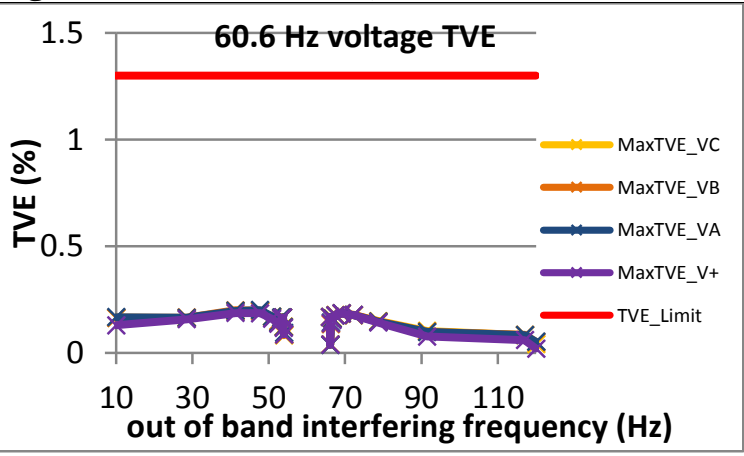

Figure 1440: Fs = 12 FPS, $60.6 \mathrm{~Hz}$ fundamental

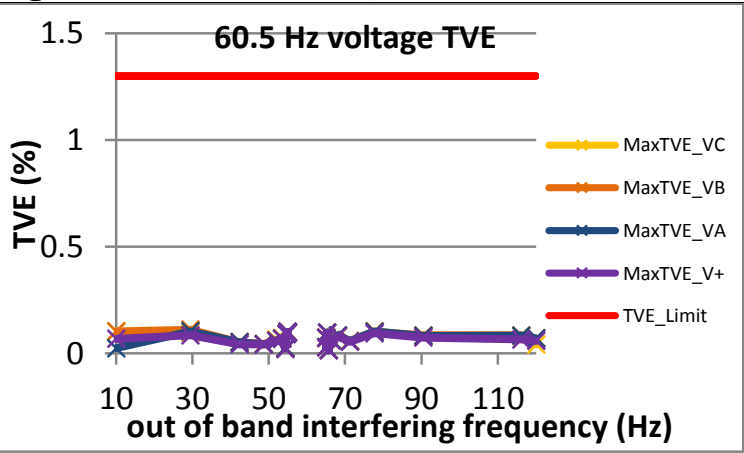

Figure 1443: Fs = 10 FPS, $60.5 \mathrm{~Hz}$ fundamental 
5.1.6 PMU E steady state out of band interfering signal voltage TVE: M class

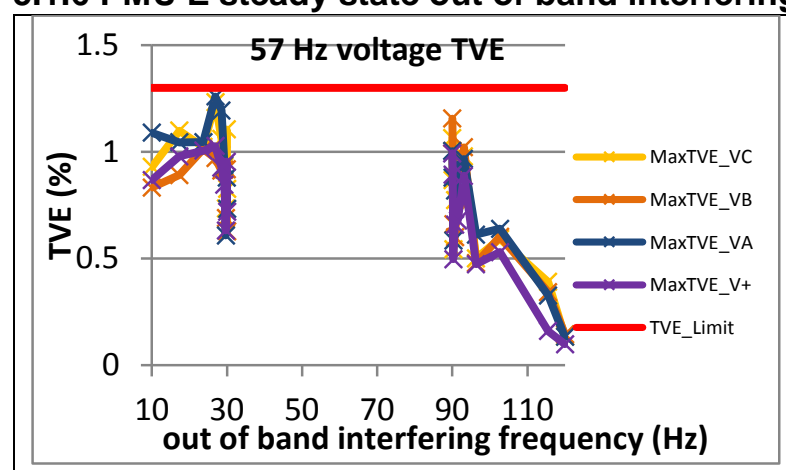

Figure 1444: Fs = 60 FPS, $57 \mathrm{~Hz}$ fundamental

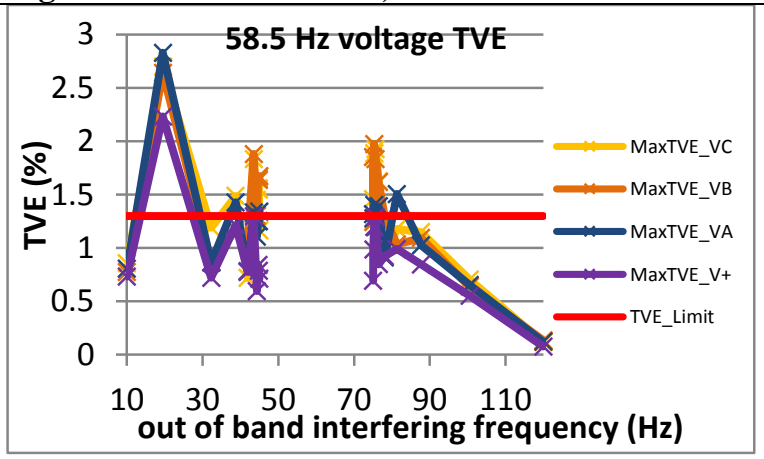

Figure 1447: Fs = 30 FPS, $58.5 \mathrm{~Hz}$ fundamental

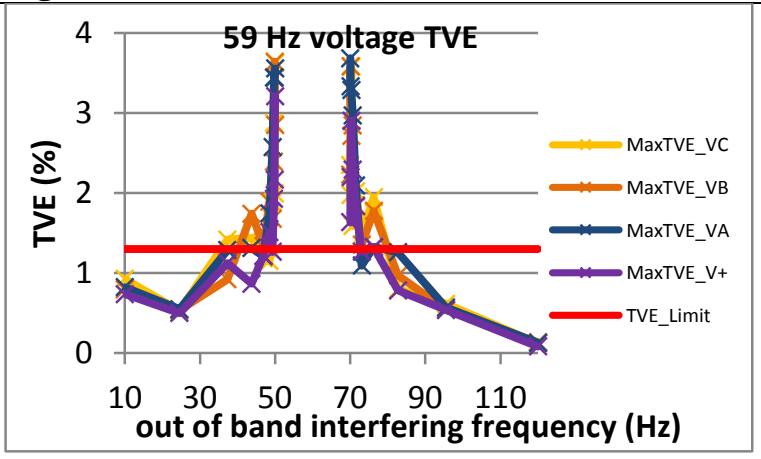

Figure 1450: Fs = 20 FPS, $59 \mathrm{~Hz}$ fundamental

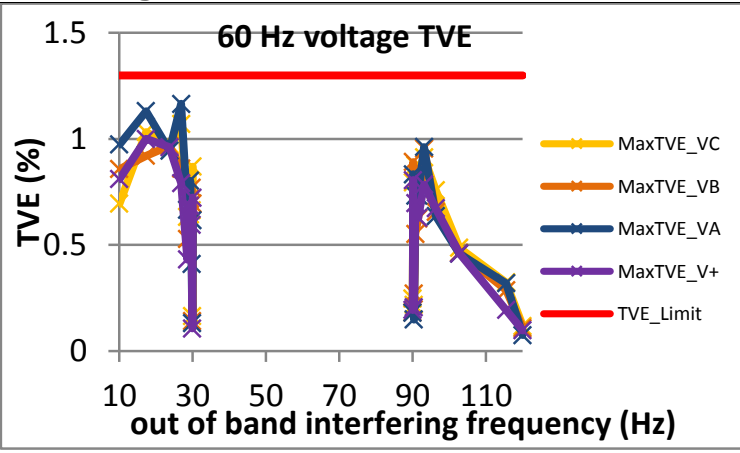

Figure 1445: Fs = $60 \mathrm{FPS}, 60 \mathrm{~Hz}$ fundamental

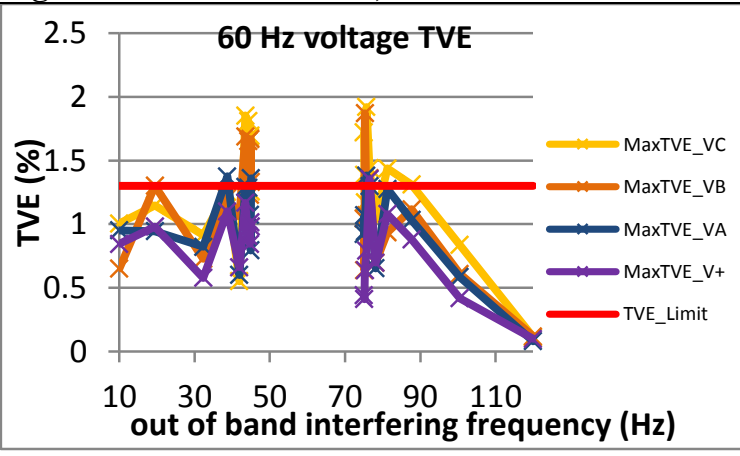

Figure 1448: Fs = $30 \mathrm{FPS}, 60 \mathrm{~Hz}$ fundamental

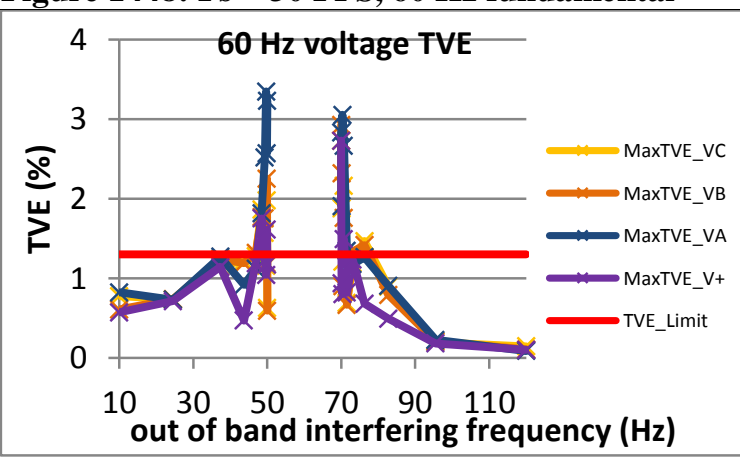

Figure 1451: Fs = 20 FPS, $60 \mathrm{~Hz}$ fundamental

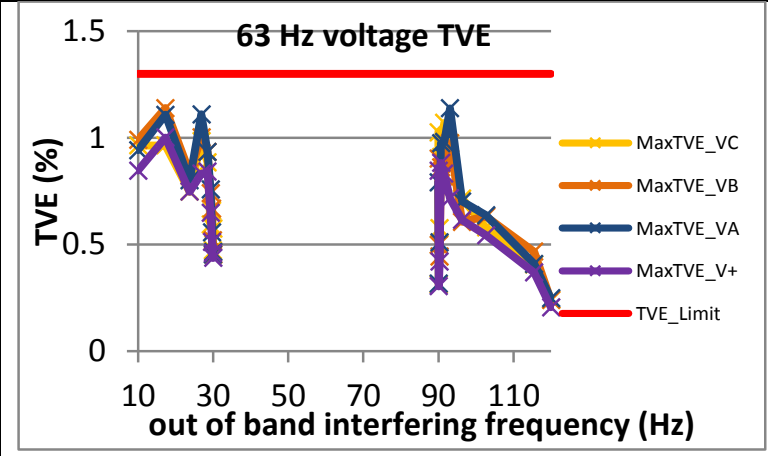

Figure 1446: Fs = $60 \mathrm{FPS}, 63 \mathrm{~Hz}$ fundamental

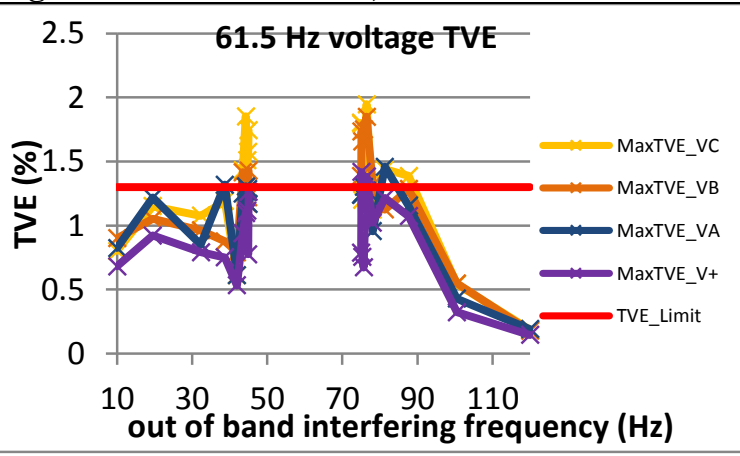

Figure 1449: Fs = 30 FPS, $61.5 \mathrm{~Hz}$ fundamental

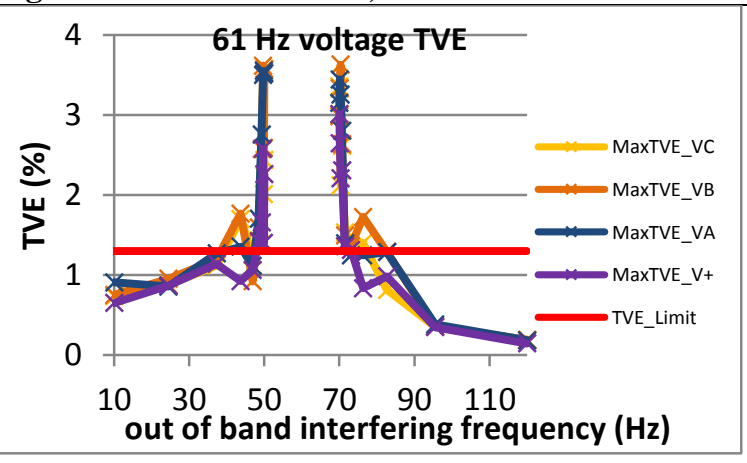

Figure 1452: Fs = 20 FPS, $61 \mathrm{~Hz}$ fundamental 


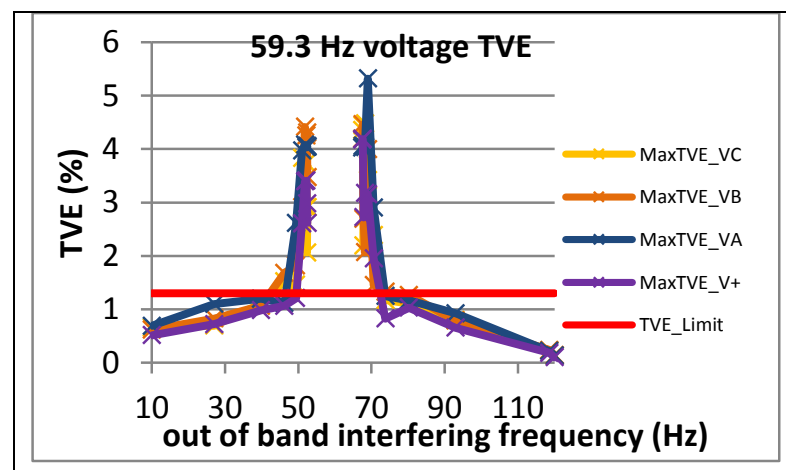

Figure 1453: Fs = 15 FPS, $59.3 \mathrm{~Hz}$ fundamental

Figure 1456: Fs = 12 FPS, $59.4 \mathrm{~Hz}$ fundamental

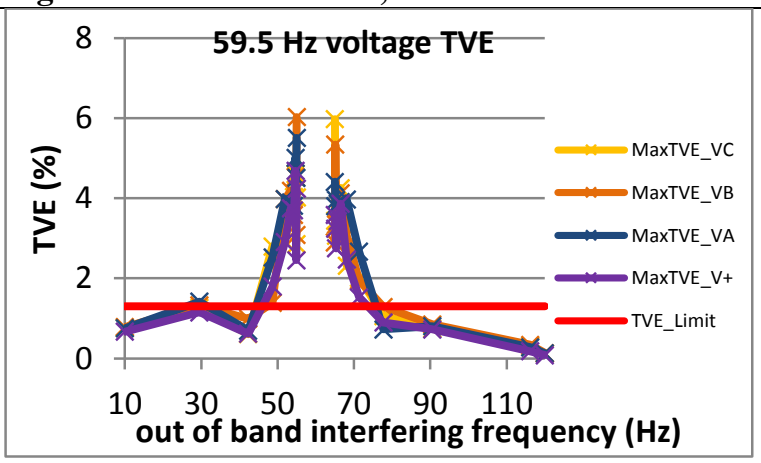

Figure 1459: Fs = 10 FPS, $59.5 \mathrm{~Hz}$ fundamental

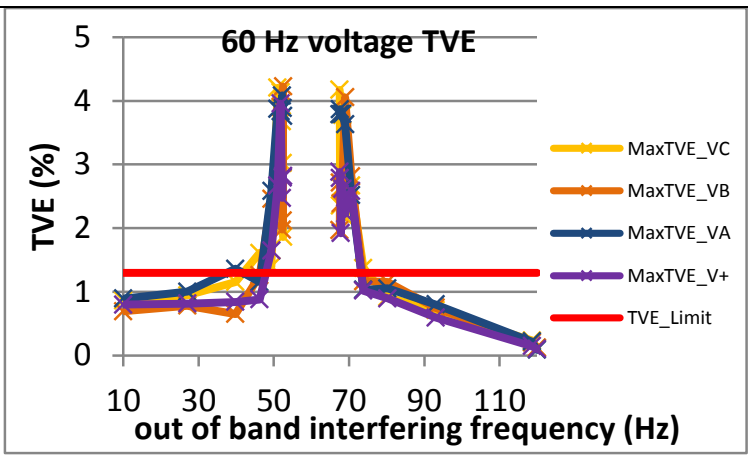

Figure 1454: Fs = 15 FPS, $60 \mathrm{~Hz}$ fundamental

Figure 1457: Fs = $12 \mathrm{FPS}, 60 \mathrm{~Hz}$ fundamental

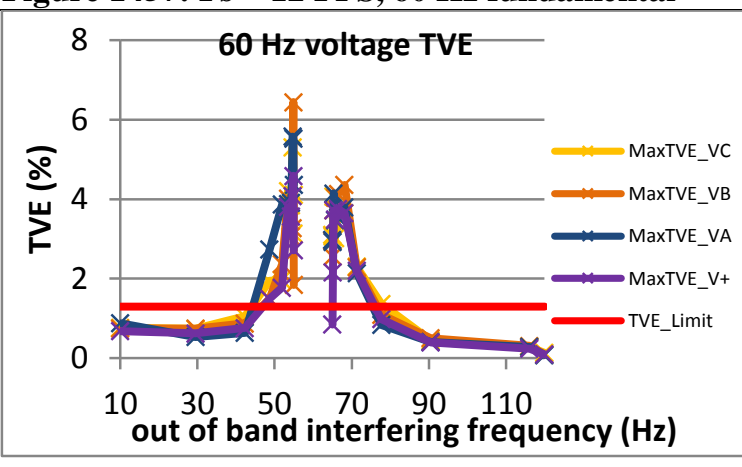

Figure 1460: Fs = $60 \mathrm{FPS}, 60 \mathrm{~Hz}$ fundamental

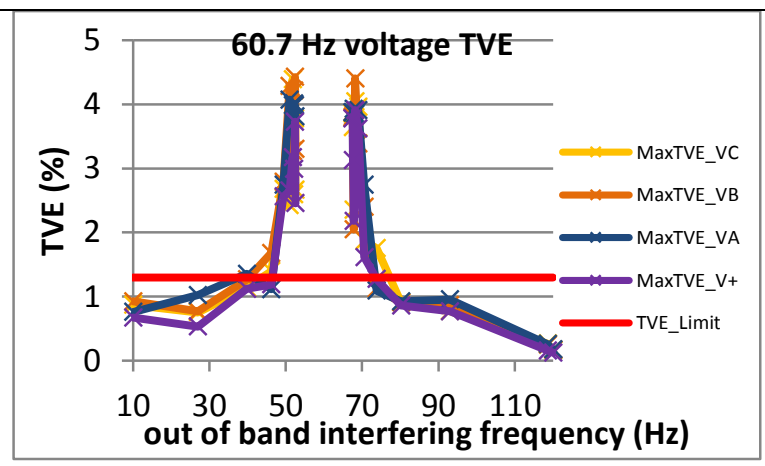

Figure 1455: Fs = 15 FPS, $60.7 \mathrm{~Hz}$ fundamental

Figure 1458: Fs = 12 FPS, $60.6 \mathrm{~Hz}$ fundamental

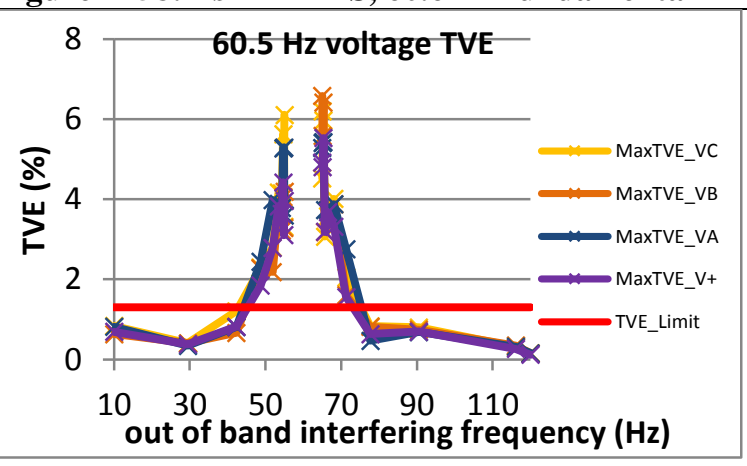

Figure 1461: Fs = 10 FPS, $60.5 \mathrm{~Hz}$ fundamental 
5.1.7 PMU F steady state out of band interfering signal voltage TVE: M class

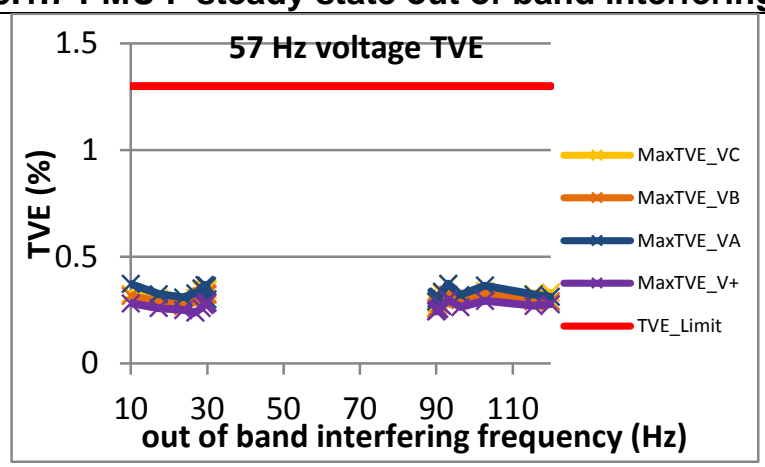

Figure 1462: $\mathrm{Fs}=60 \mathrm{FPS}, 57 \mathrm{~Hz}$ fundamental

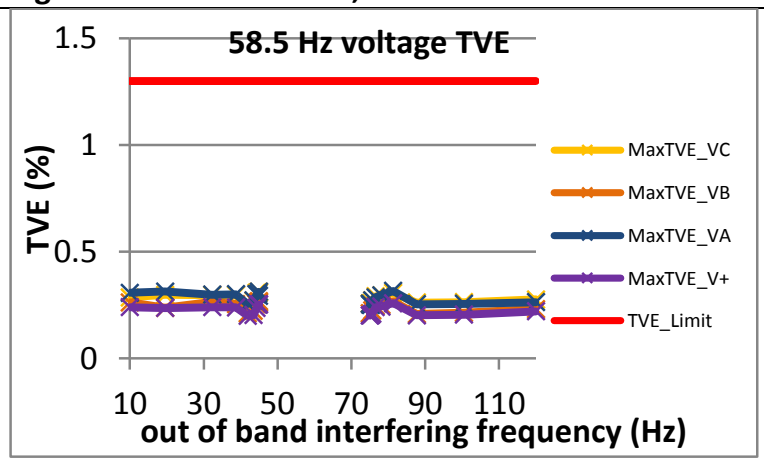

Figure 1465: Fs = $30 \mathrm{FPS}, 58.5 \mathrm{~Hz}$ fundamental

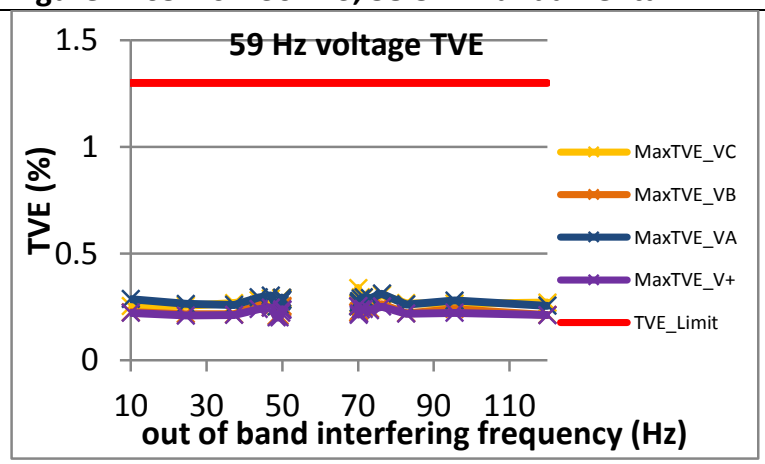

Figure 1468: Fs = $20 \mathrm{FPS}, 59 \mathrm{~Hz}$ fundamental

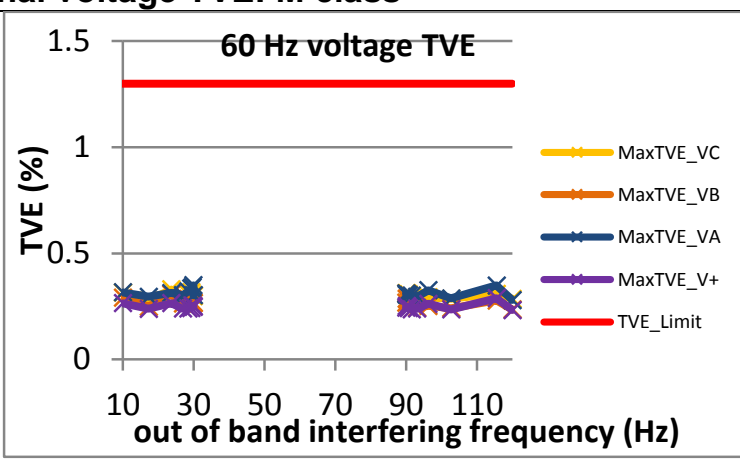

Figure 1463: Fs $=60 \mathrm{FPS}, 60 \mathrm{~Hz}$ fundamental

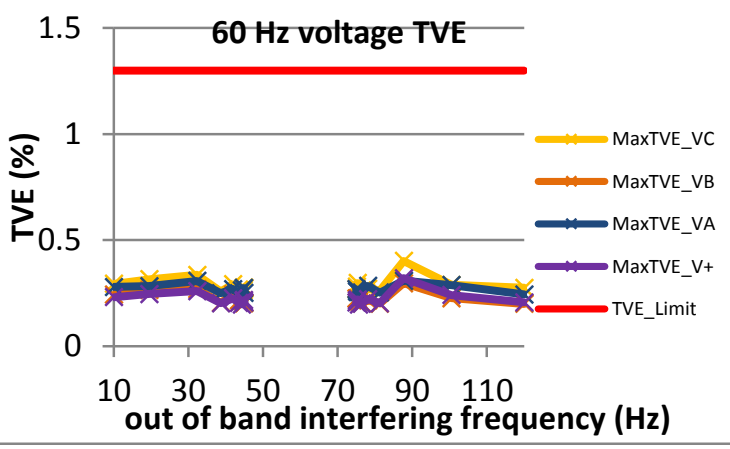

Figure 1466: $\mathrm{Fs}=30 \mathrm{FPS}, 60 \mathrm{~Hz}$ fundamental

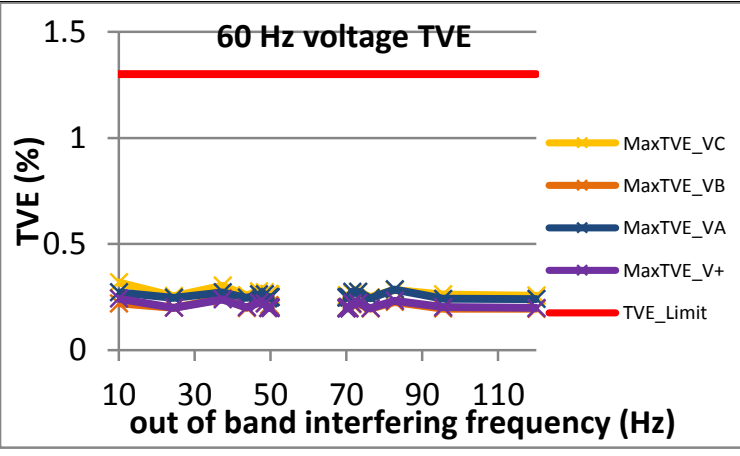

Figure 1469: Fs = $20 \mathrm{FPS}, 60 \mathrm{~Hz}$ fundamental

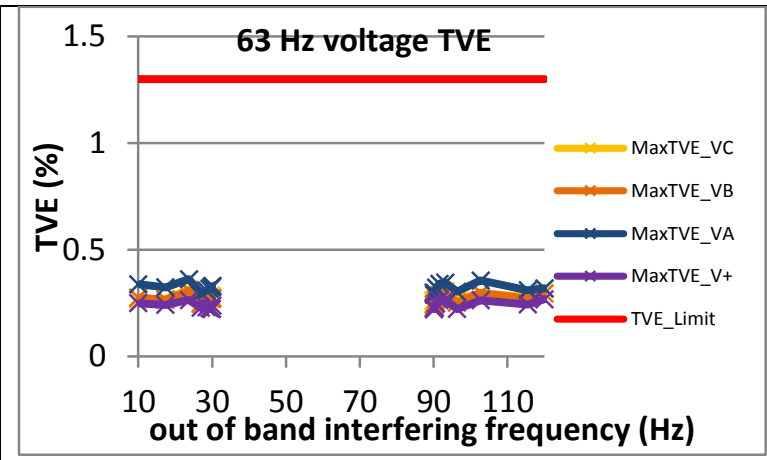

Figure 1464: Fs = $60 \mathrm{FPS}, 63 \mathrm{~Hz}$ fundamental

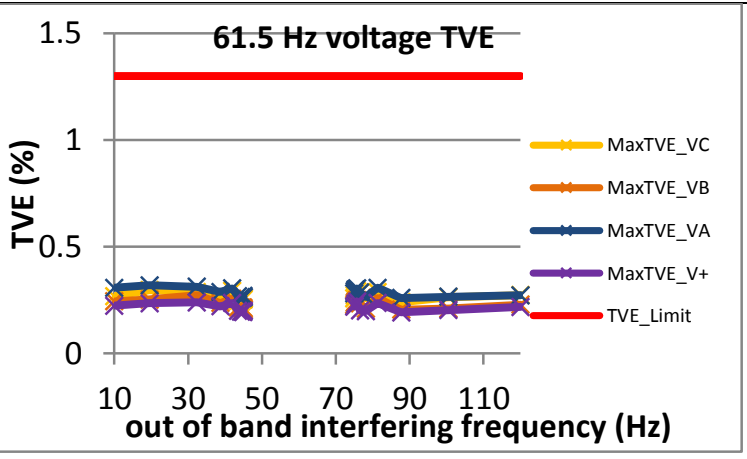

Figure 1467: Fs = $30 \mathrm{FPS}, 61.5 \mathrm{~Hz}$ fundamental

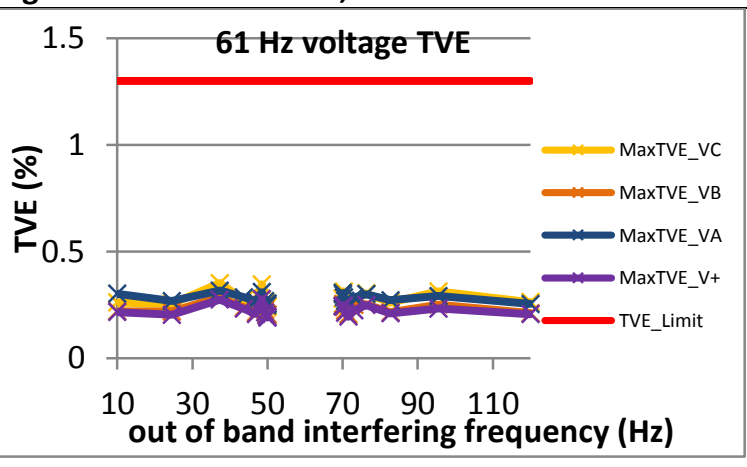

Figure 1470: Fs = $20 \mathrm{FPS}, 61 \mathrm{~Hz}$ fundamental 


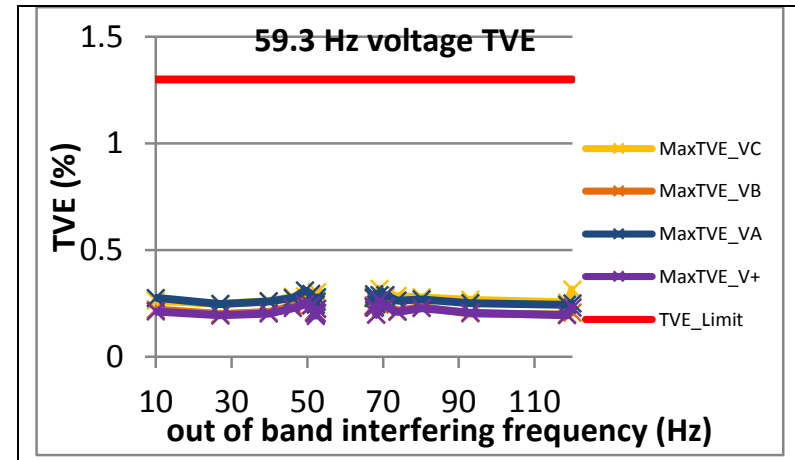

Figure 1471: Fs = $15 \mathrm{FPS}, 59.3 \mathrm{~Hz}$ fundamental

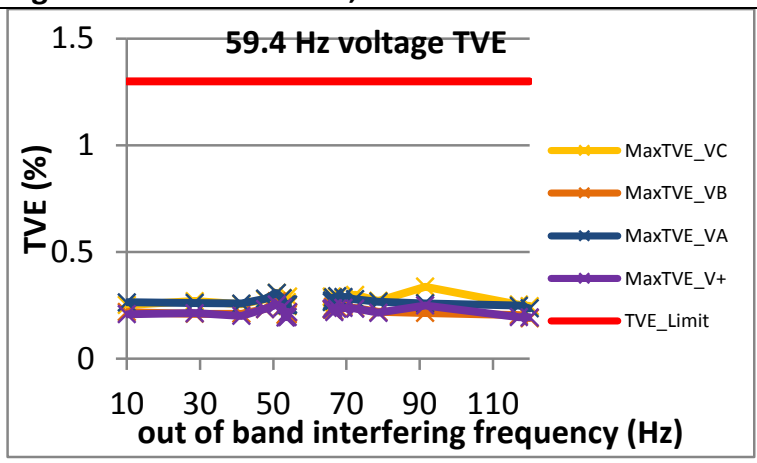

Figure 1474: Fs = $12 \mathrm{FPS}, 59.4 \mathrm{~Hz}$ fundamental

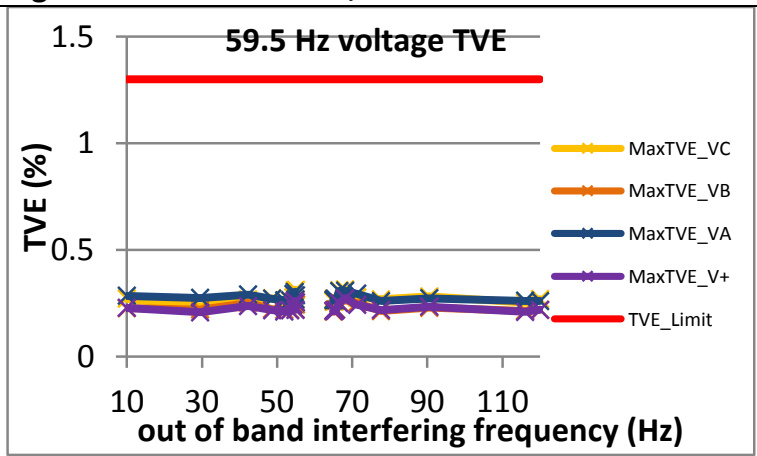

Figure 1477: Fs = $10 \mathrm{FPS}, 59.5 \mathrm{~Hz}$ fundamental

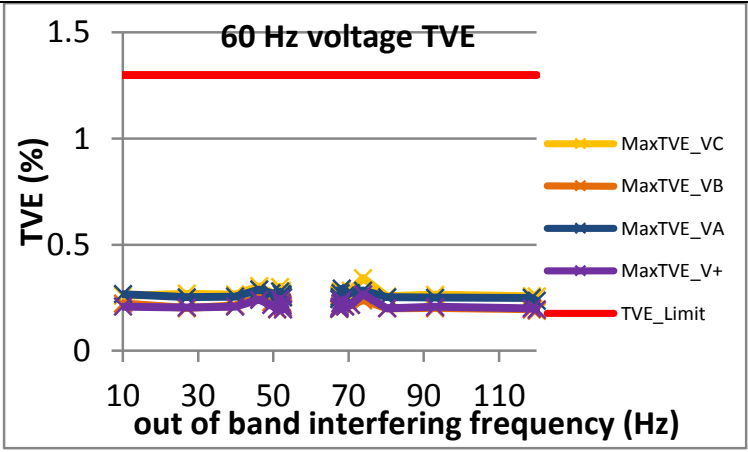

Figure 1472: Fs = $15 \mathrm{FPS}, 60 \mathrm{~Hz}$ fundamental

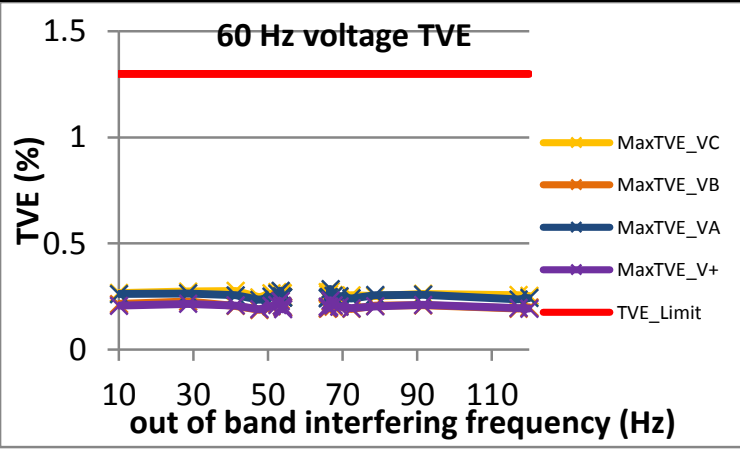

Figure 1475: Fs = $12 \mathrm{FPS}, 60 \mathrm{~Hz}$ fundamental

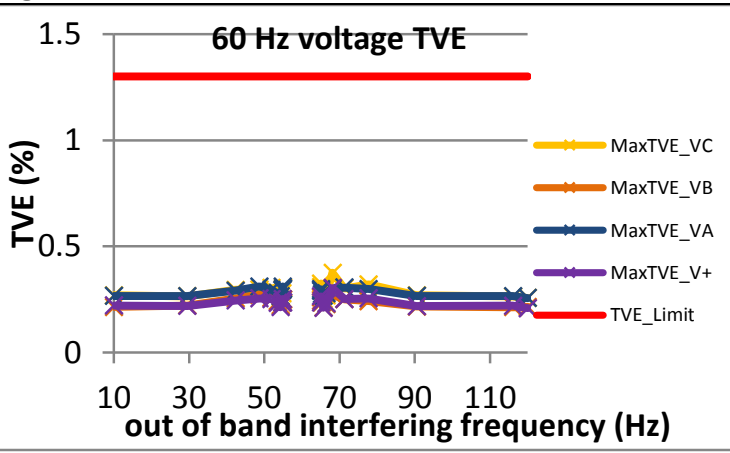

Figure 1478: Fs = $60 \mathrm{FPS}, 60 \mathrm{~Hz}$ fundamental

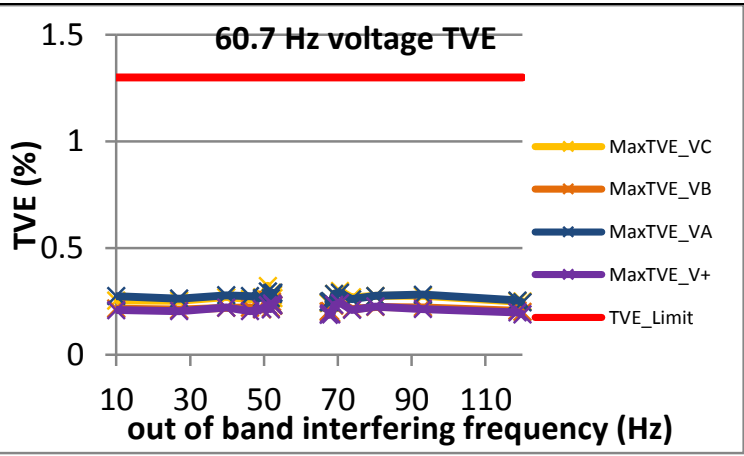

Figure 1473: Fs = $15 \mathrm{FPS}, 60.7 \mathrm{~Hz}$ fundamental

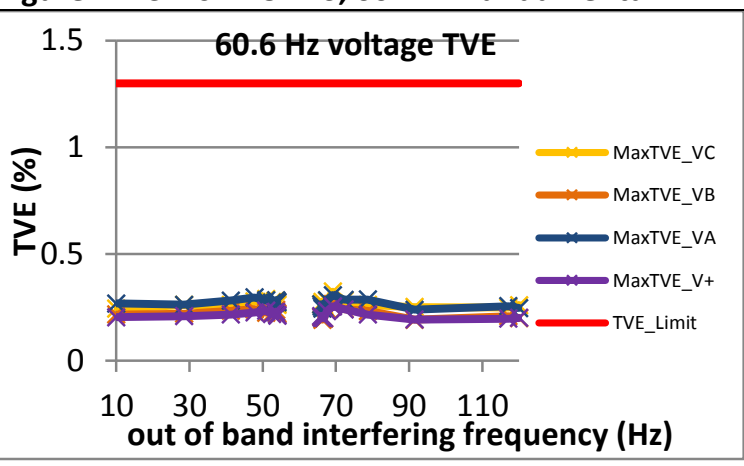

Figure 1476: Fs = $12 \mathrm{FPS}, 60.6 \mathrm{~Hz}$ fundamental

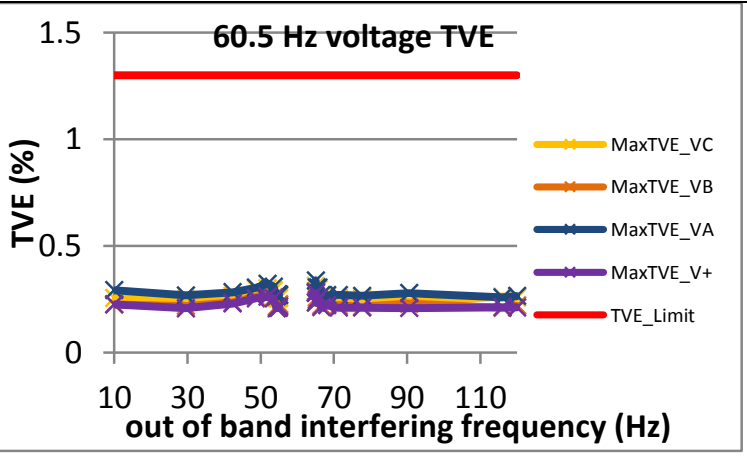

Figure 1479: Fs = $10 \mathrm{FPS}, 60.5 \mathrm{~Hz}$ fundamental 


\subsubsection{PMU G steady state out of band interfering signal voltage TVE: M class}

Figure 1480: Fs = 60 FPS, is not supported by this PMU

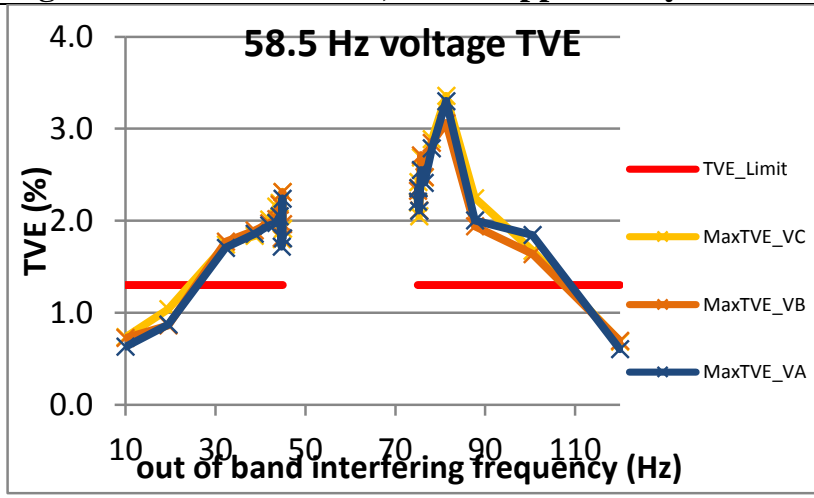

Figure 1481: Fs = 30 FPS, $58.5 \mathrm{~Hz}$ fundamental

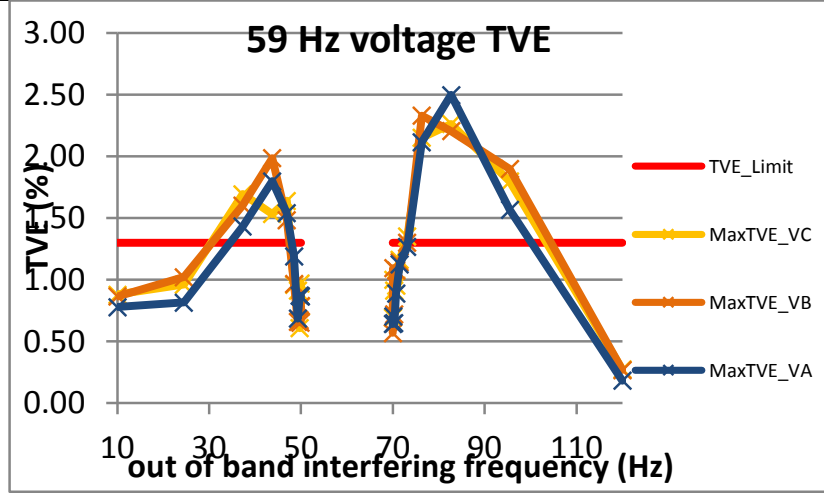

Figure 1484: Fs = 20 FPS, $59 \mathrm{~Hz}$ fundamental

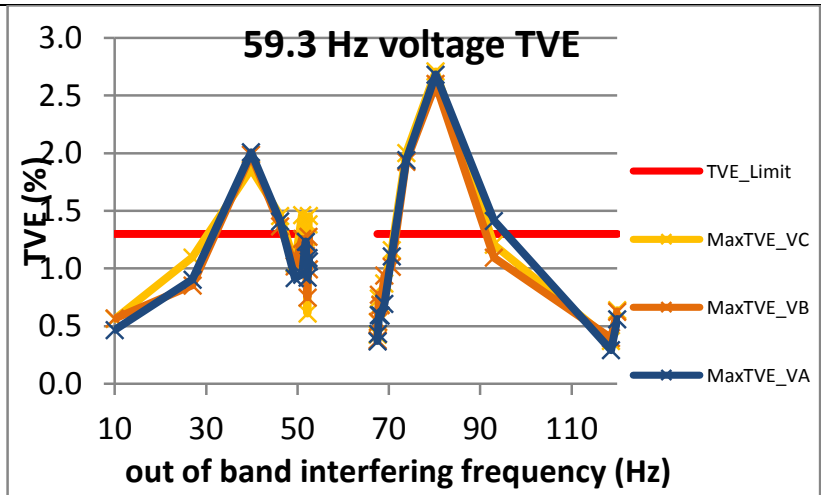

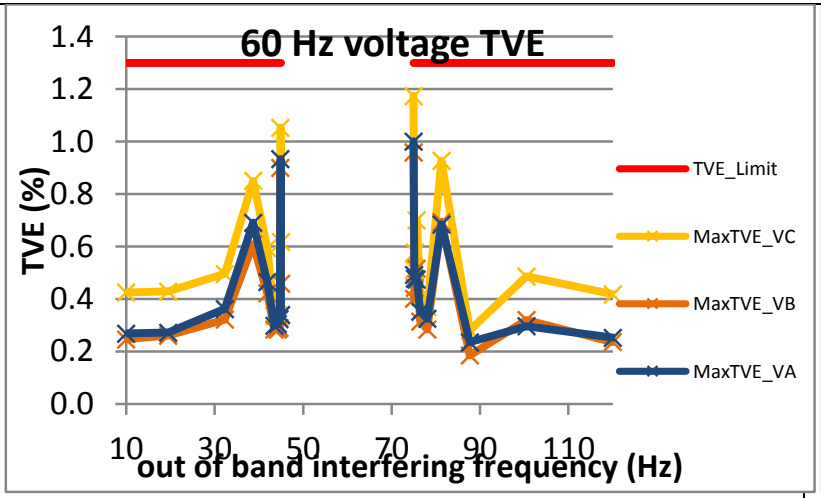

Figure 1482: Fs = $30 \mathrm{FPS}, 60 \mathrm{~Hz}$ fundamental

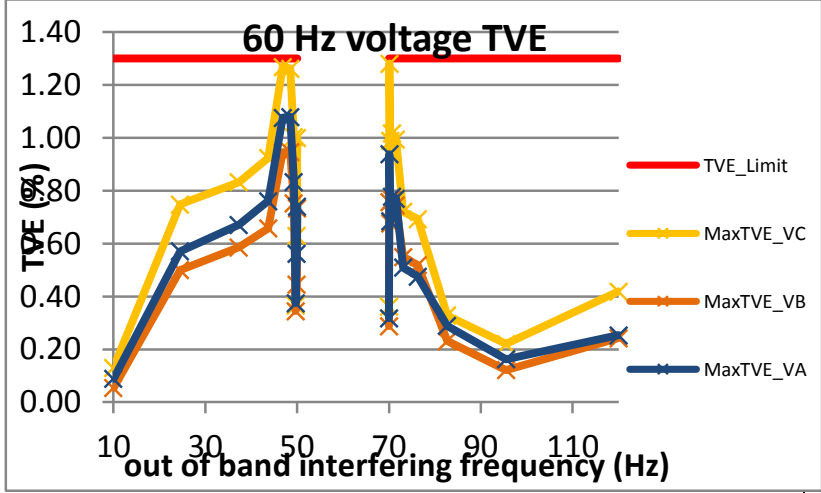

Figure 1485: Fs = 20 FPS, $60 \mathrm{~Hz}$ fundamental

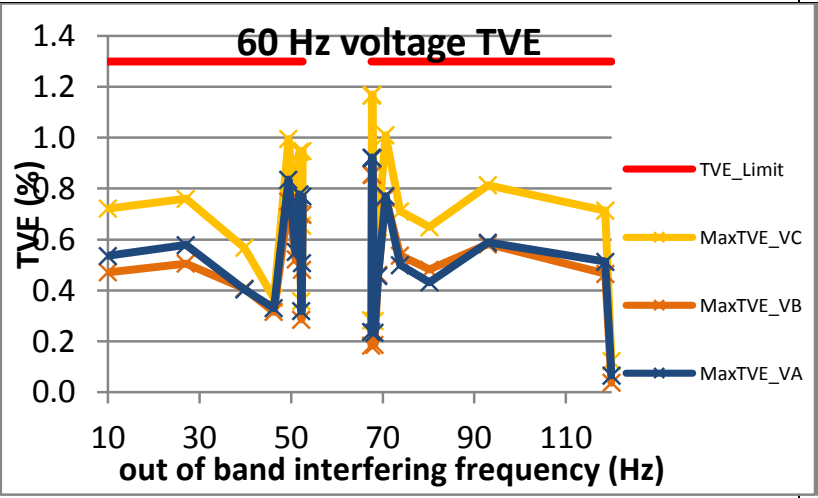

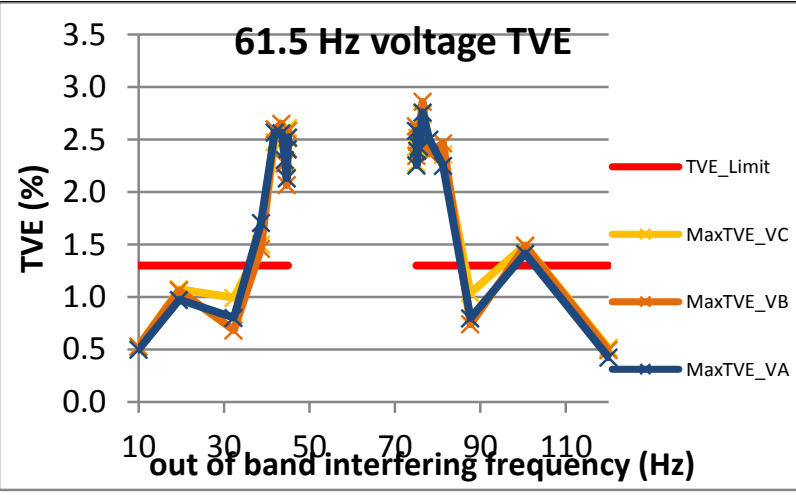

Figure 1483: Fs = 30 FPS, $61.5 \mathrm{~Hz}$ fundamental

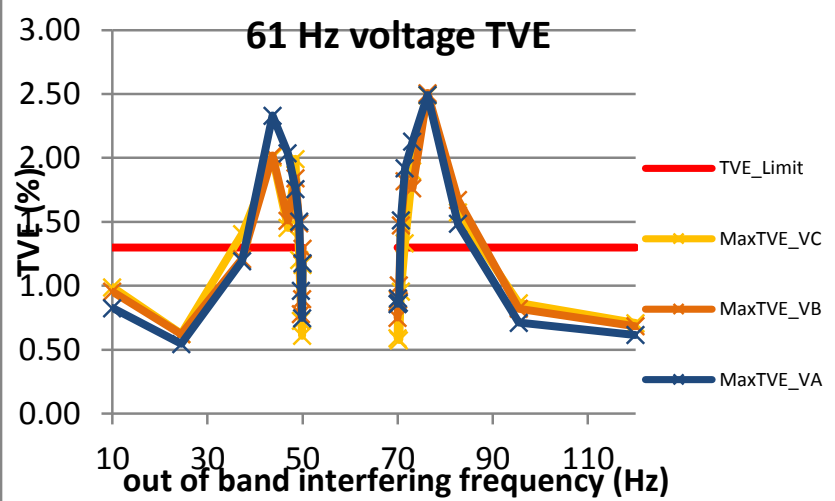

Figure 1486: Fs = 20 FPS, $61 \mathrm{~Hz}$ fundamental

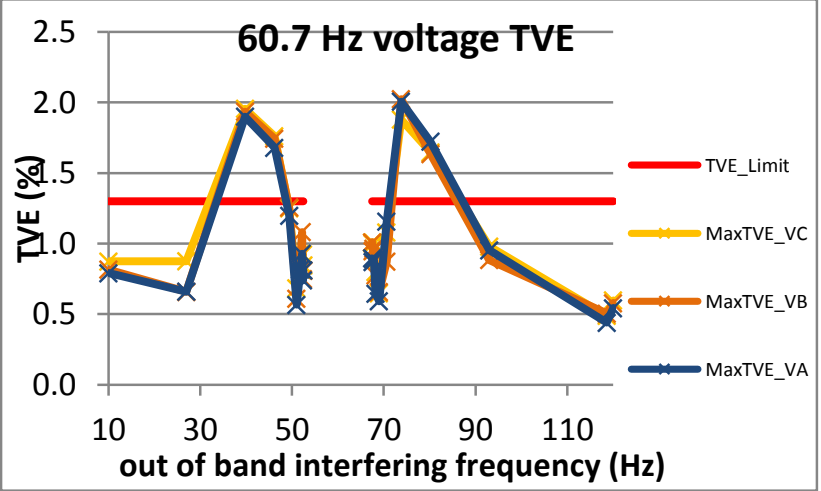




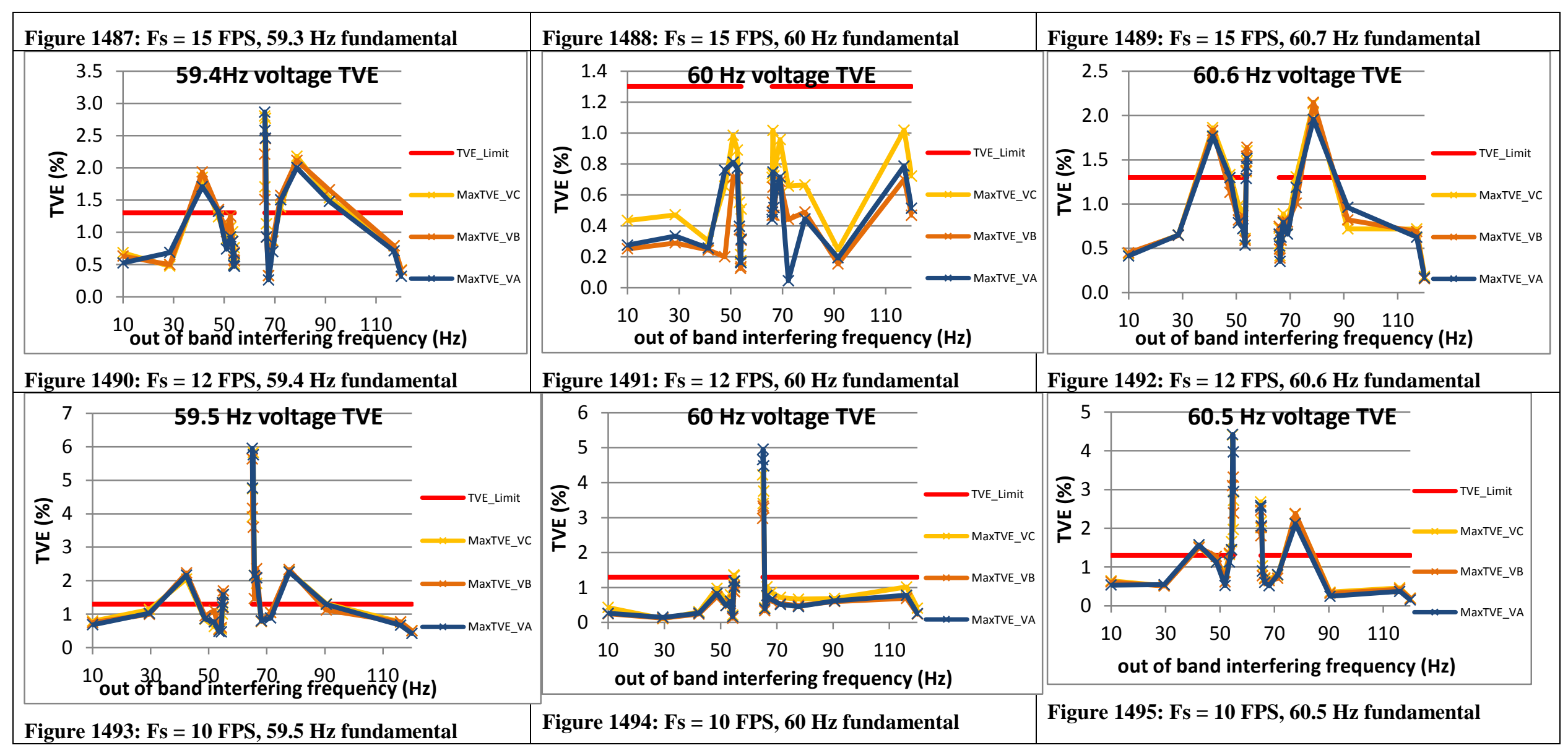


5.1.9 PMU H steady state out of band interfering signal voltage TVE: M class

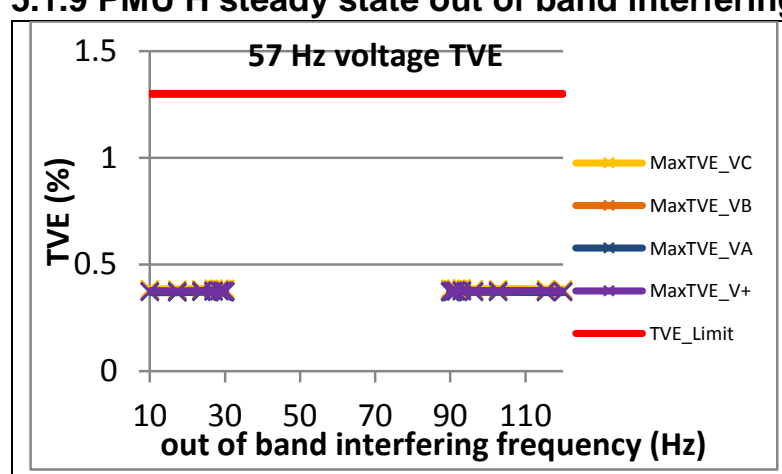

Figure 1496: Fs = 60 FPS, $57 \mathrm{~Hz}$ fundamental

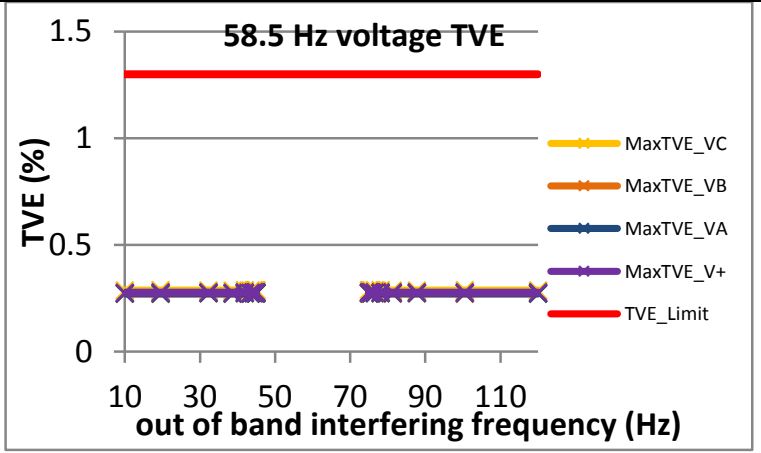

Figure 1499: Fs = 30 FPS, 58.5 Hz fundamental

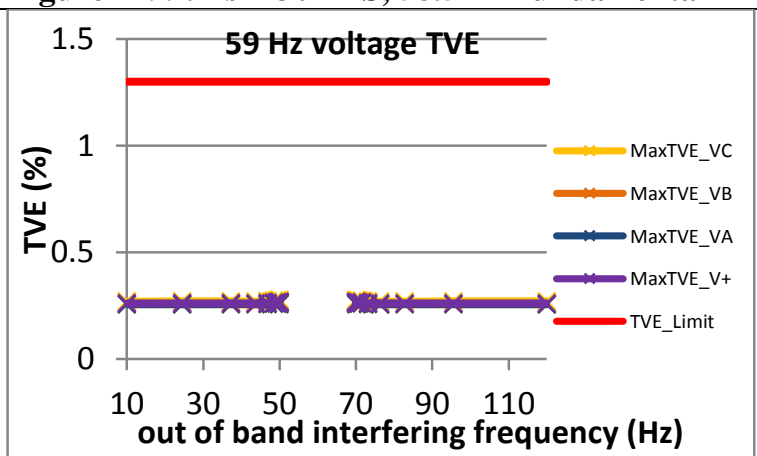

Figure 1502: Fs = 20 FPS, $59 \mathrm{~Hz}$ fundamental

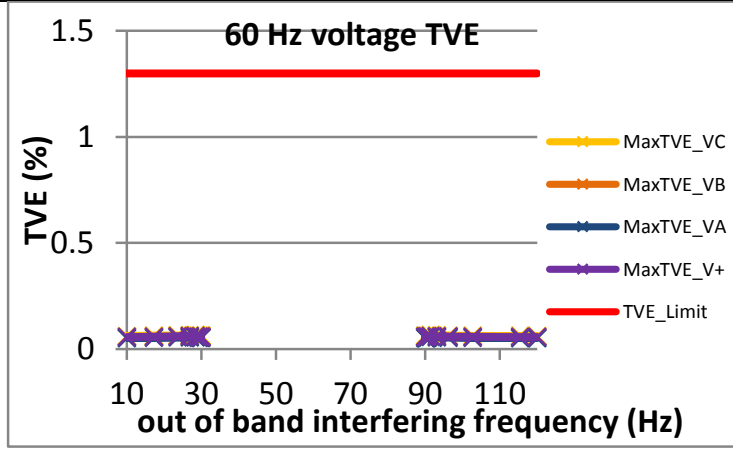

Figure 1497: Fs = $60 \mathrm{FPS}, 60 \mathrm{~Hz}$ fundamental

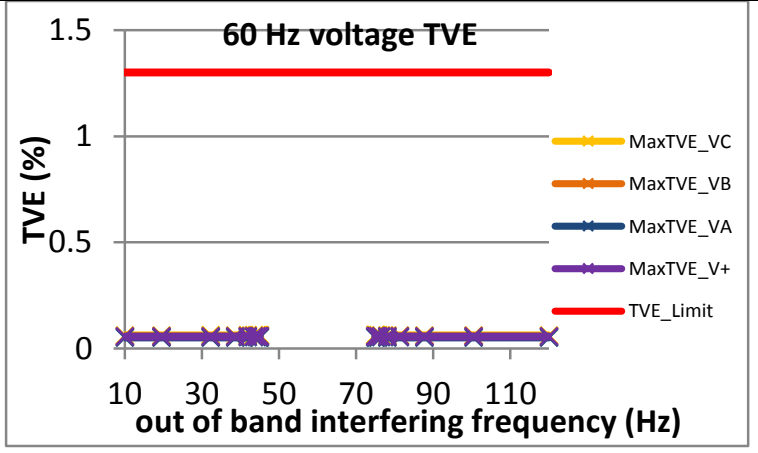

Figure 1500: Fs = $30 \mathrm{FPS}, 60 \mathrm{~Hz}$ fundamental

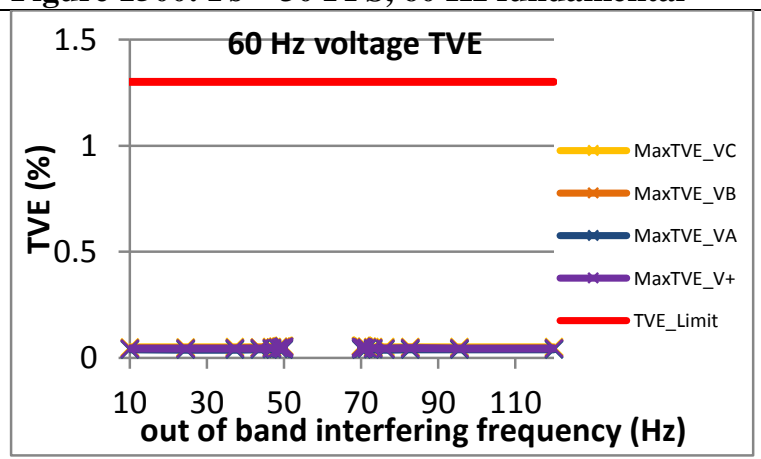

Figure 1503: Fs = 20 FPS, $60 \mathrm{~Hz}$ fundamental

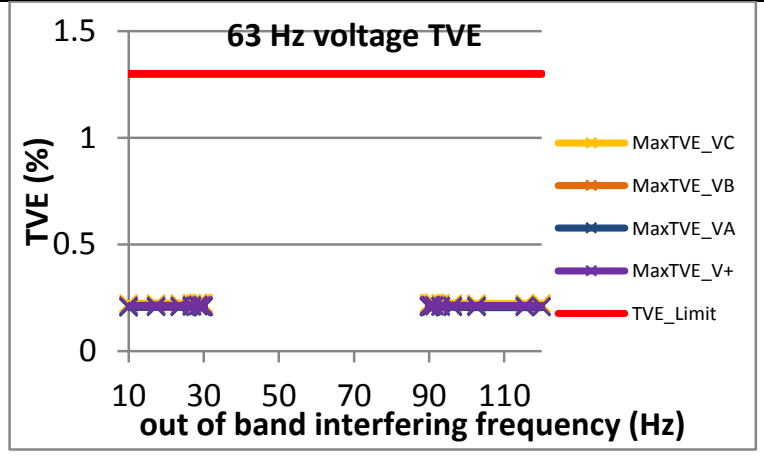

Figure 1498: Fs = 60 FPS, $63 \mathrm{~Hz}$ fundamental

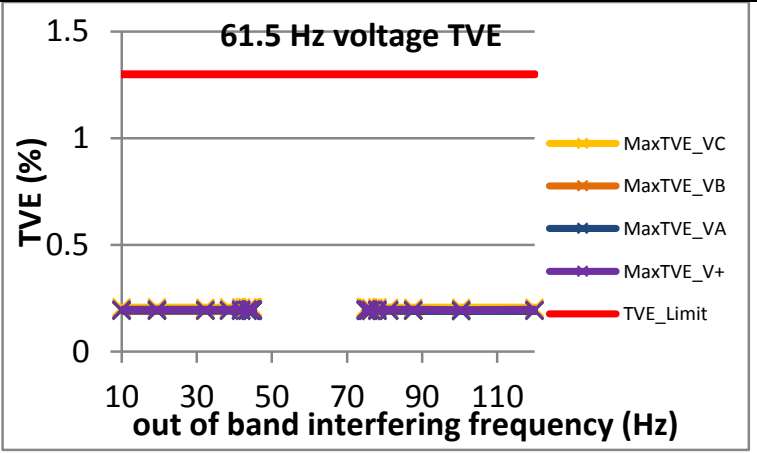

Figure 1501: Fs = $30 \mathrm{FPS}, 61.5 \mathrm{~Hz}$ fundamental

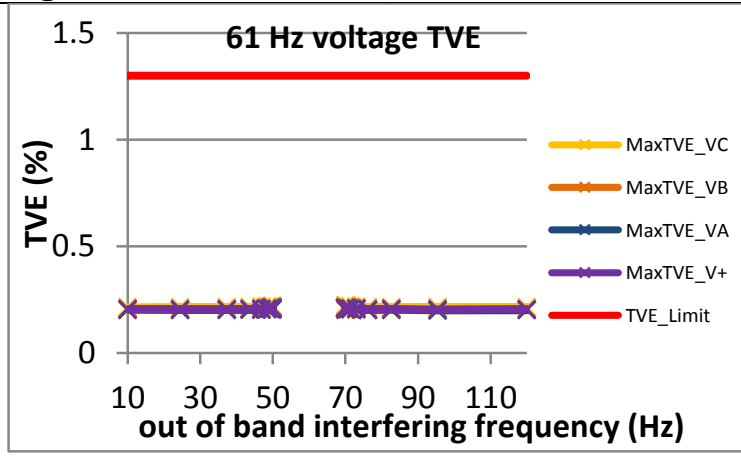

Figure 1504: Fs = 20 FPS, $61 \mathrm{~Hz}$ fundamental 

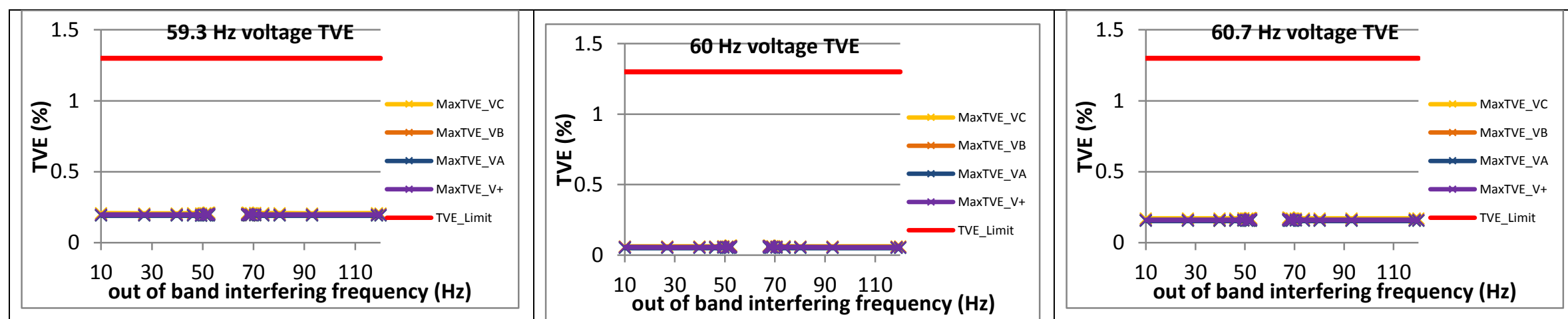

Figure 1505: Fs = 15 FPS, $59.3 \mathrm{~Hz}$ fundamental Figure 1506: Fs = $15 \mathrm{FPS}, 60 \mathrm{~Hz}$ fundamental
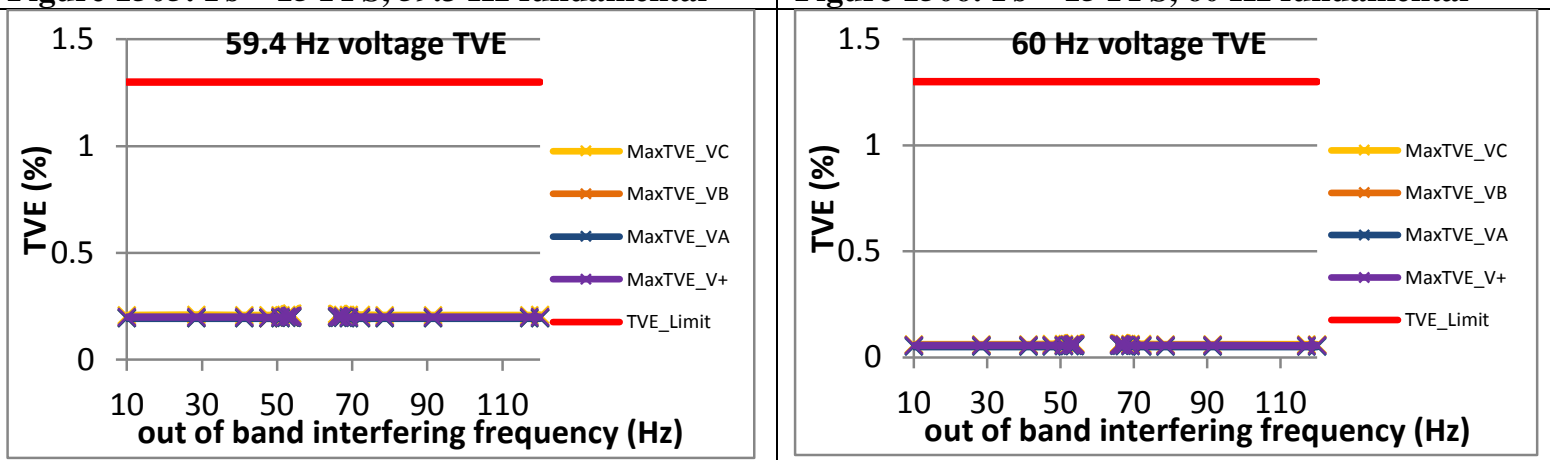

Figure 1507: Fs = 15 FPS, $60.7 \mathrm{~Hz}$ fundamental

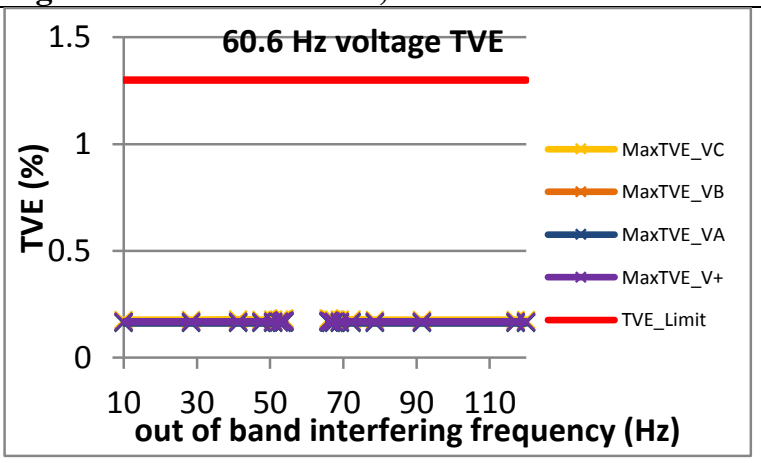

Figure 1508: Fs = 12 FPS, $59.4 \mathrm{~Hz}$ fundamental

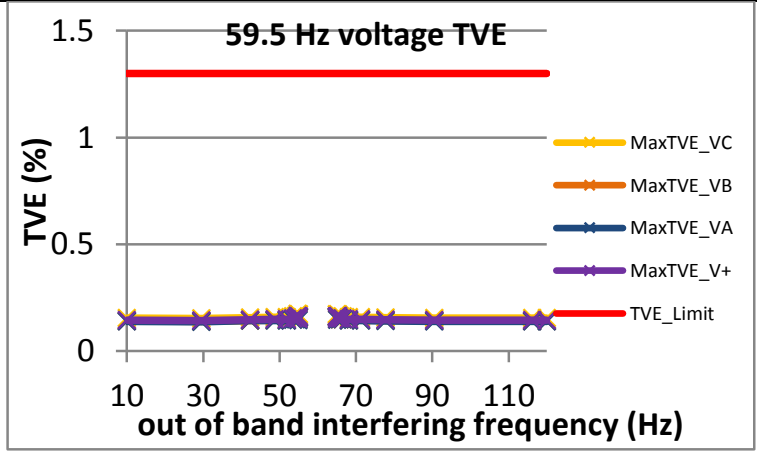

Figure 1509: Fs = 12 FPS, $60 \mathrm{~Hz}$ fundamental

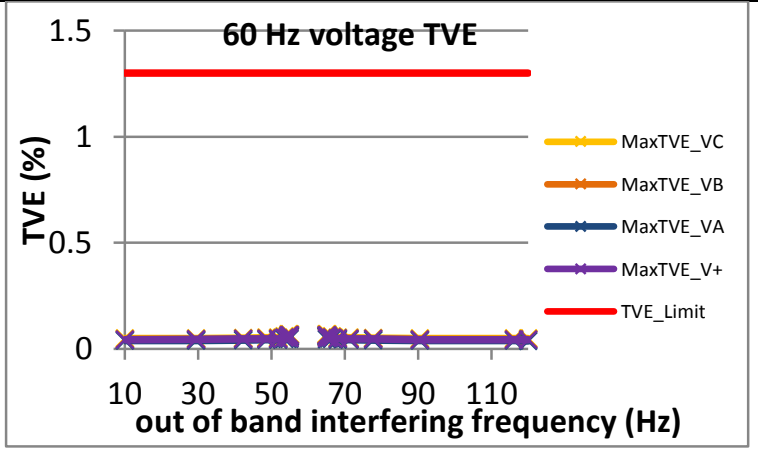

Figure 1510: Fs = 12 FPS, $60.6 \mathrm{~Hz}$ fundamental

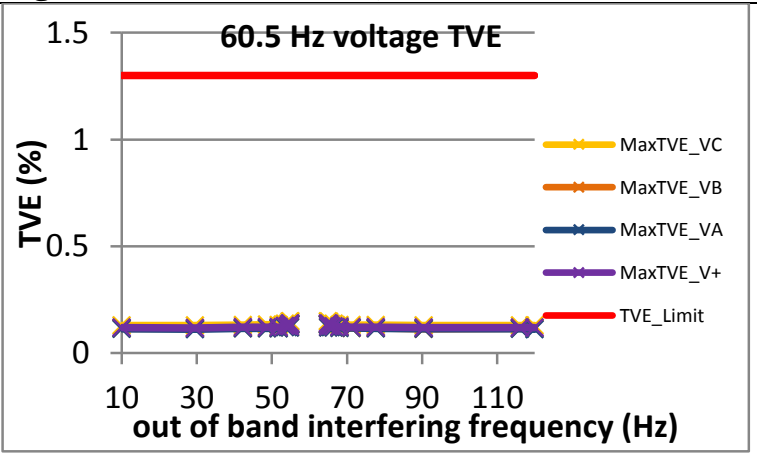

Figure 1511: Fs = 10 FPS, $59.5 \mathrm{~Hz}$ fundamental

Figure 1512: Fs = 60 FPS, $60 \mathrm{~Hz}$ fundamental 


\subsubsection{PMU I steady state out of band interfering signal voltage TVE: M class}

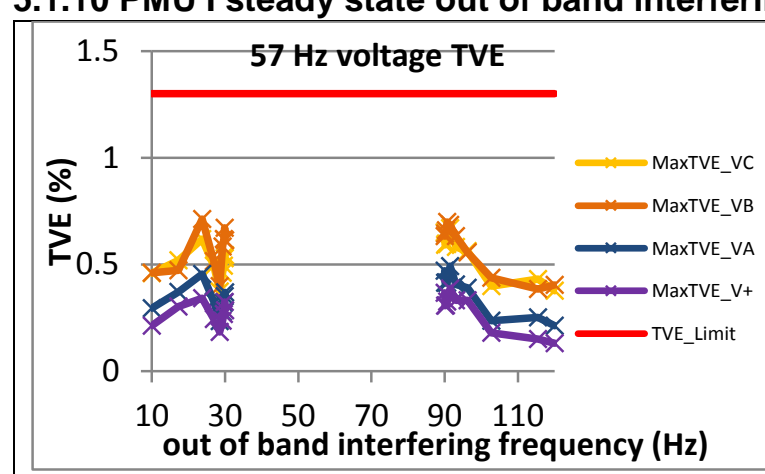

Figure 1514: Fs = 60 FPS, $57 \mathrm{~Hz}$ fundamental

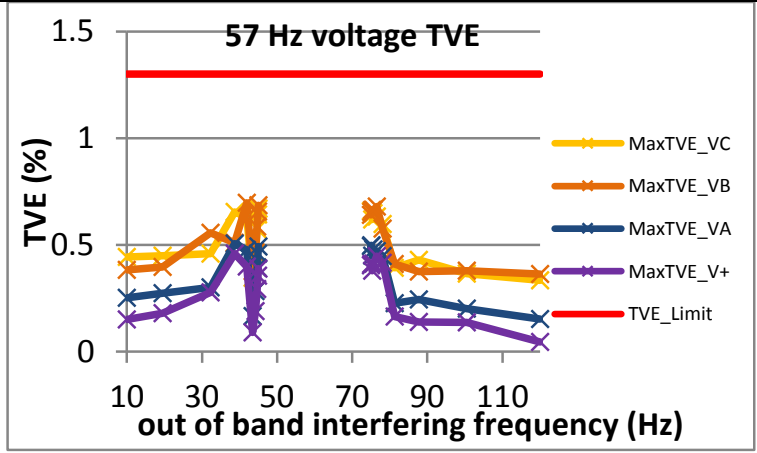

Figure 1517: Fs = 30 FPS, $58.5 \mathrm{~Hz}$ fundamental

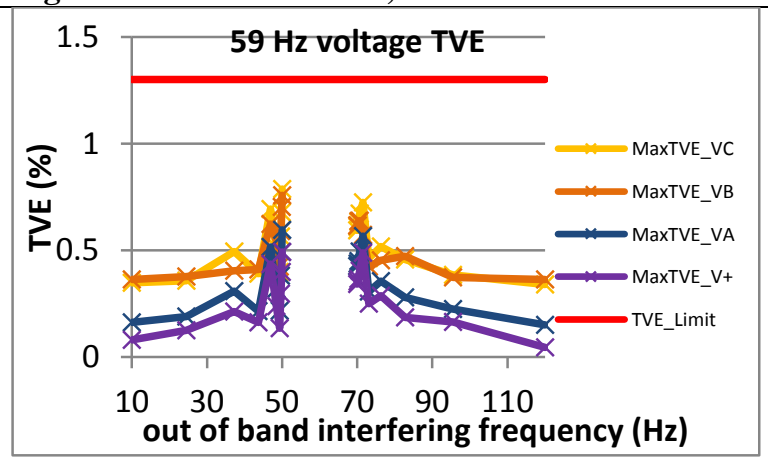

Figure 1520: Fs = 20 FPS, $59 \mathrm{~Hz}$ fundamental

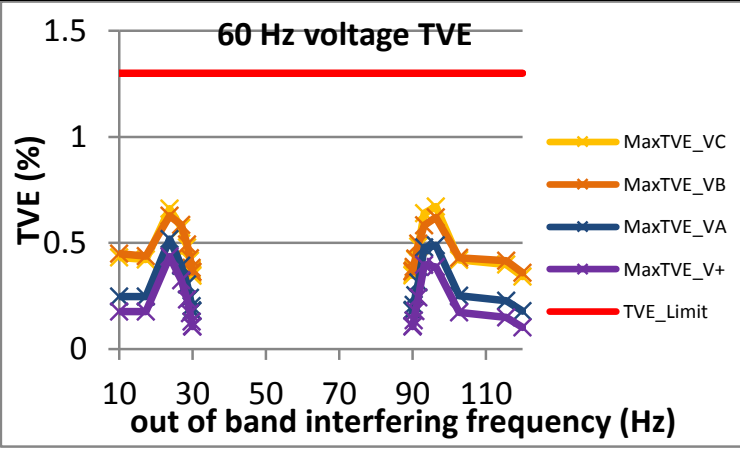

Figure 1515: Fs = $60 \mathrm{FPS}, 60 \mathrm{~Hz}$ fundamental

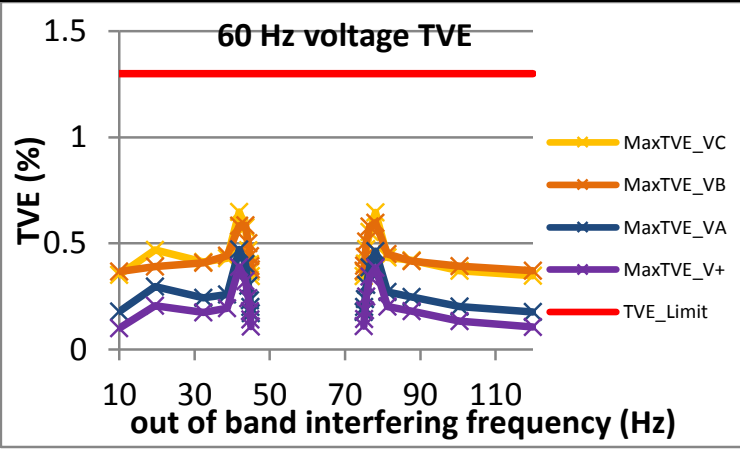

Figure 1518: Fs = $30 \mathrm{FPS}, 60 \mathrm{~Hz}$ fundamental

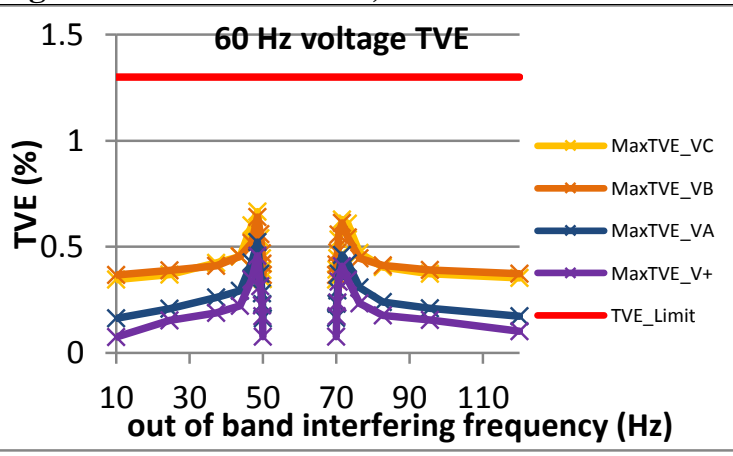

Figure 1521: Fs = 20 FPS, $60 \mathrm{~Hz}$ fundamental

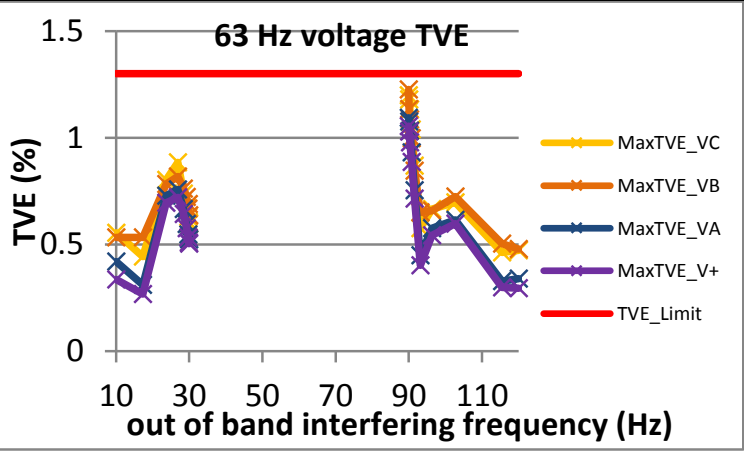

Figure 1516: Fs = $60 \mathrm{FPS}, 63 \mathrm{~Hz}$ fundamental

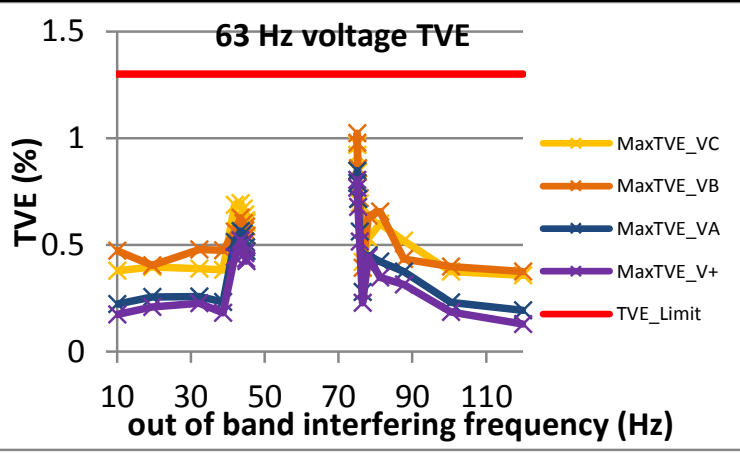

Figure 1519: Fs = $30 \mathrm{FPS}, 61.5 \mathrm{~Hz}$ fundamental

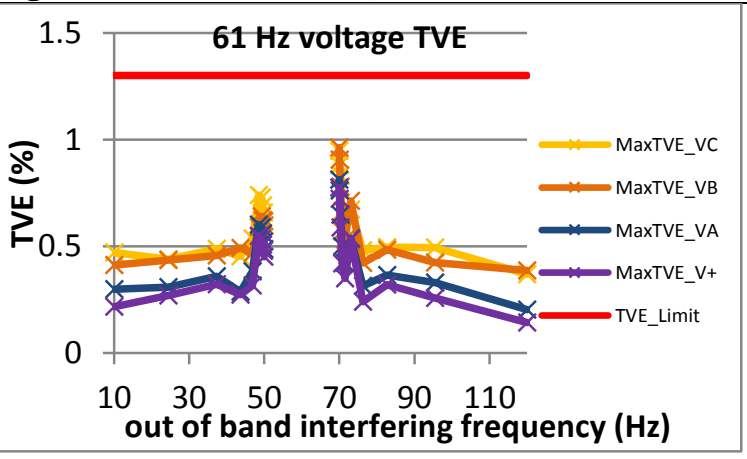

Figure 1522: Fs = $20 \mathrm{FPS}, 61 \mathrm{~Hz}$ fundamental 


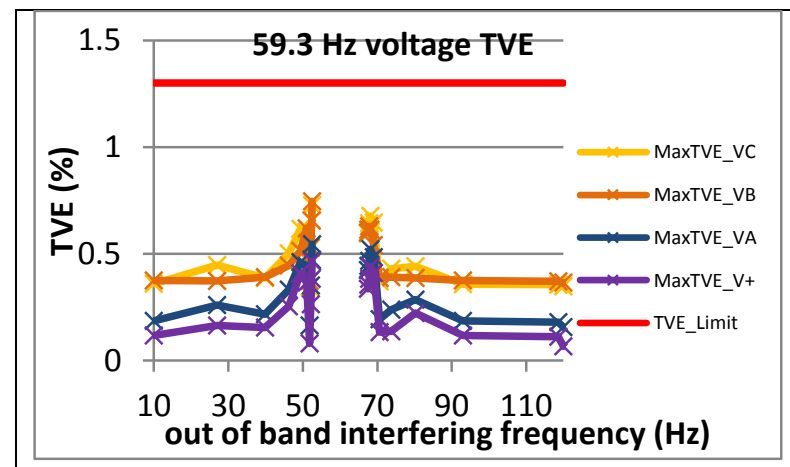

Figure 1523: Fs = 15 FPS, $59.3 \mathrm{~Hz}$ fundamental

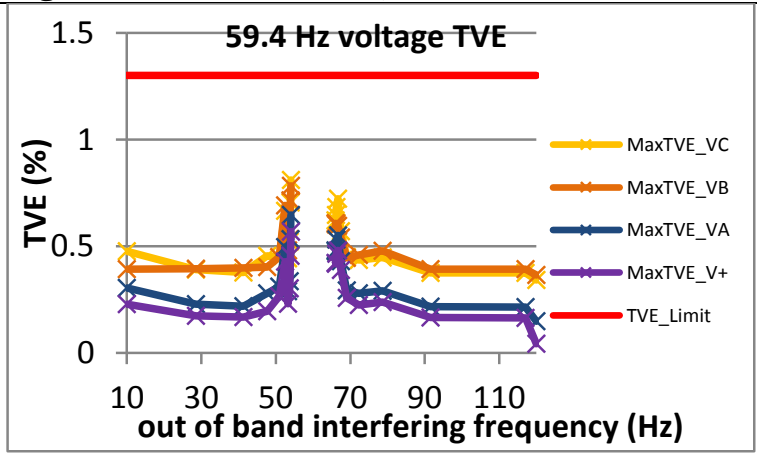

Figure 1526: Fs = 12 FPS, $59.4 \mathrm{~Hz}$ fundamental

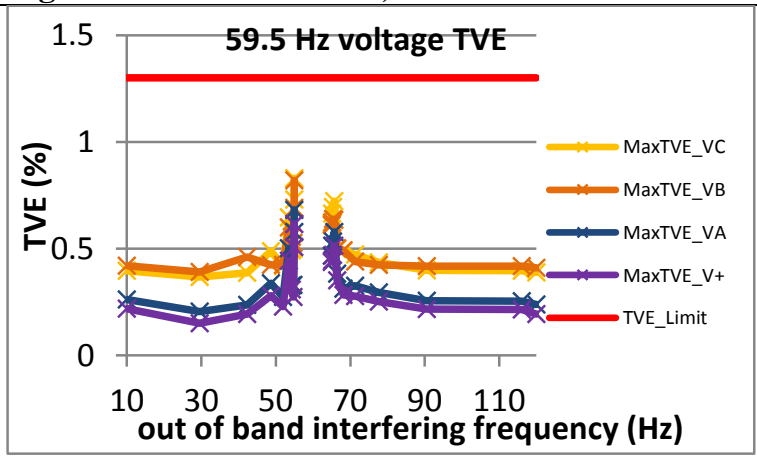

Figure 1529: Fs = 10 FPS, $59.5 \mathrm{~Hz}$ fundamental

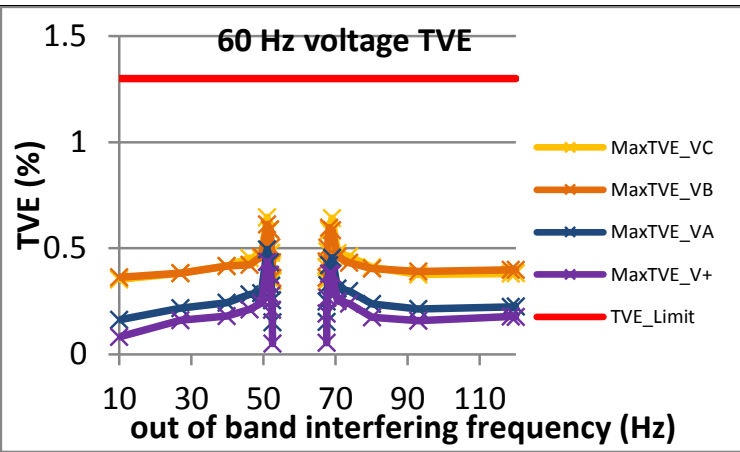

Figure 1524: Fs = 15 FPS, $60 \mathrm{~Hz}$ fundamental

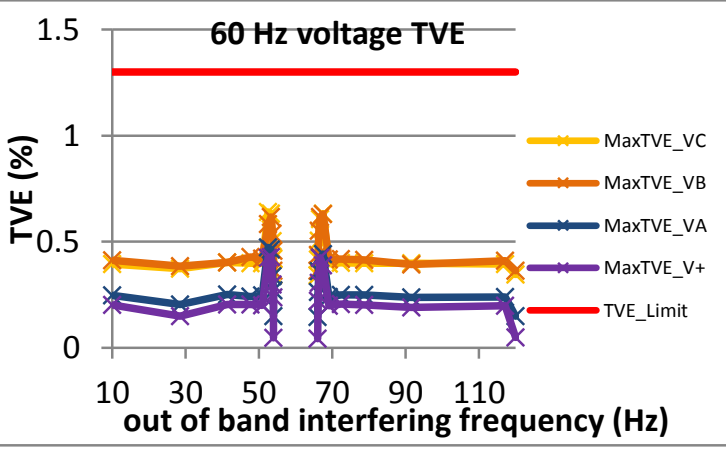

Figure 1527: Fs = $12 \mathrm{FPS}, 60 \mathrm{~Hz}$ fundamental

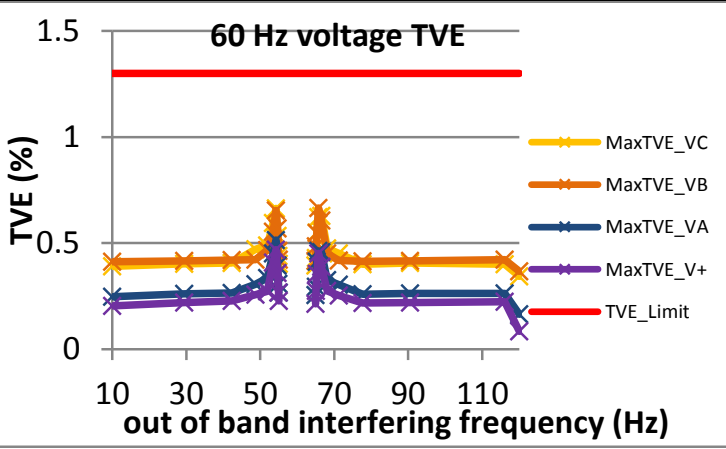

Figure 1530: Fs = $60 \mathrm{FPS}, 60 \mathrm{~Hz}$ fundamental

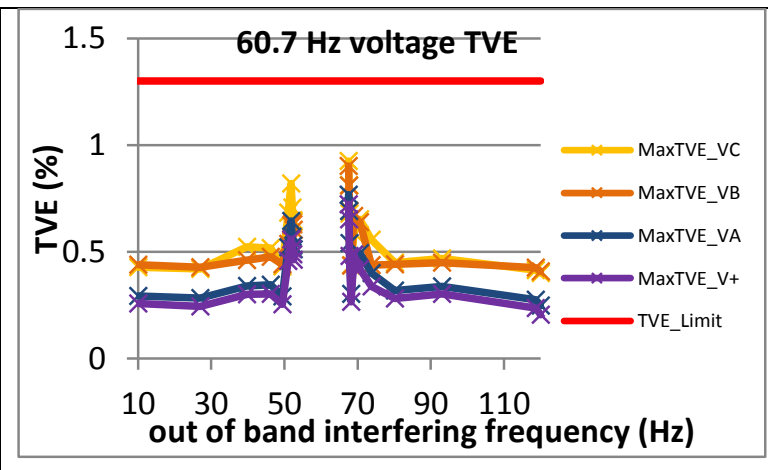

Figure 1525: Fs = 15 FPS, $60.7 \mathrm{~Hz}$ fundamental

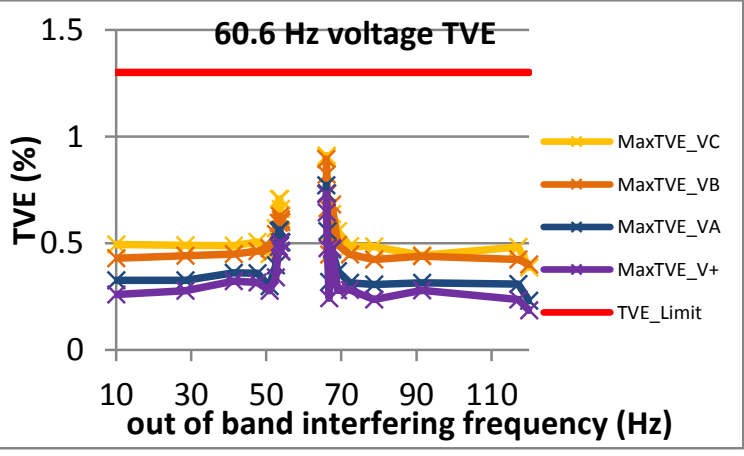

Figure 1528: Fs = 12 FPS, $60.6 \mathrm{~Hz}$ fundamental

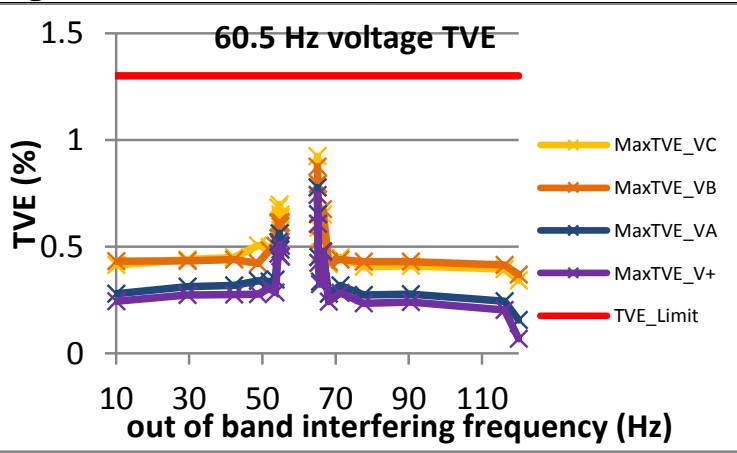

Figure 1531: Fs = 10 FPS, $60.5 \mathrm{~Hz}$ fundamental 
5.1.11 PMU J steady state out of band interfering signal voltage TVE: M class

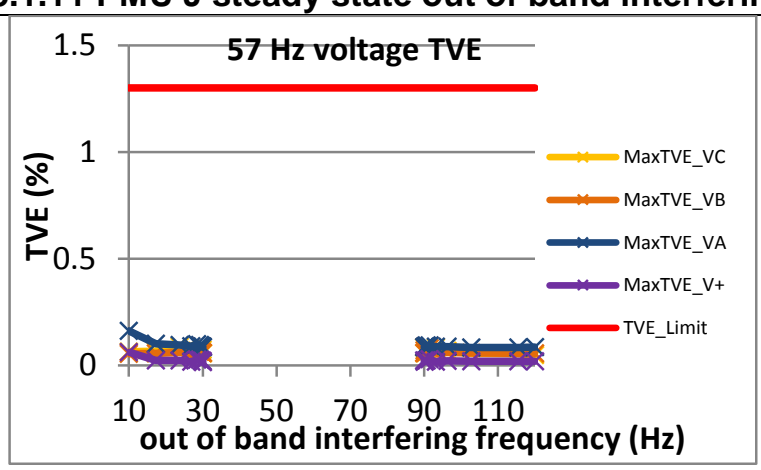

Figure 1532: $\mathrm{Fs}=60 \mathrm{FPS}, 57 \mathrm{~Hz}$ fundamental

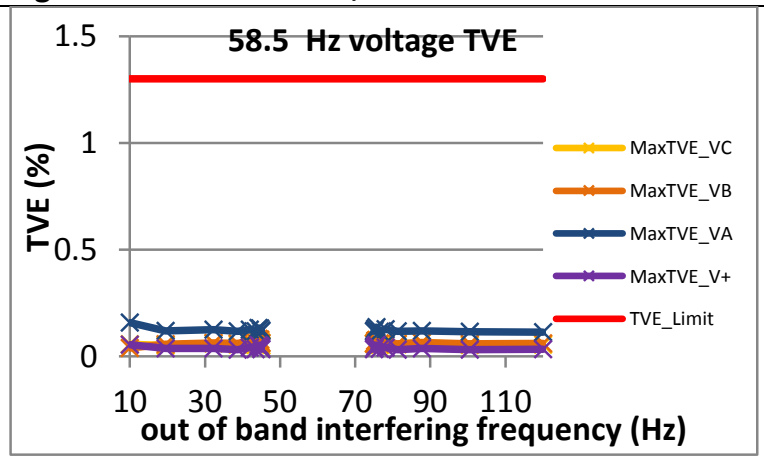

Figure 1535: Fs = $30 \mathrm{FPS}, 58.5 \mathrm{~Hz}$ fundamental

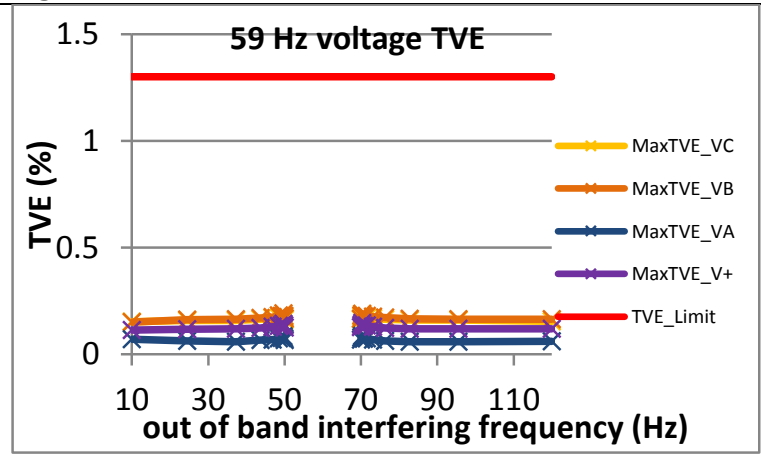

Figure 1538: Fs = $20 \mathrm{FPS}, 59 \mathrm{~Hz}$ fundamental

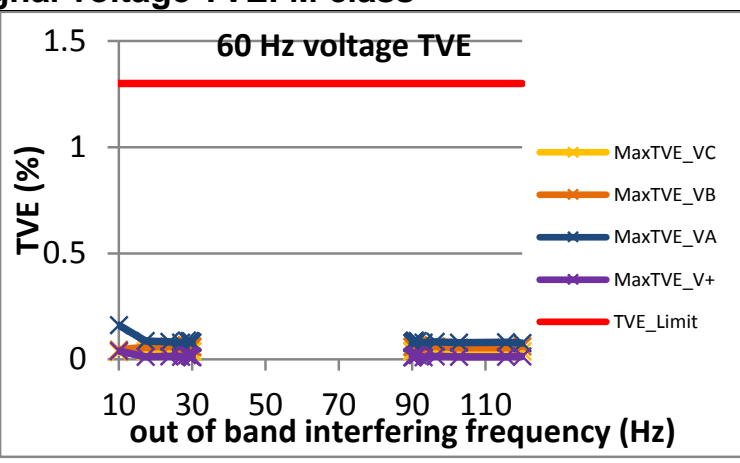

Figure 1533: $\mathrm{Fs}=60 \mathrm{FPS}, 60 \mathrm{~Hz}$ fundamental

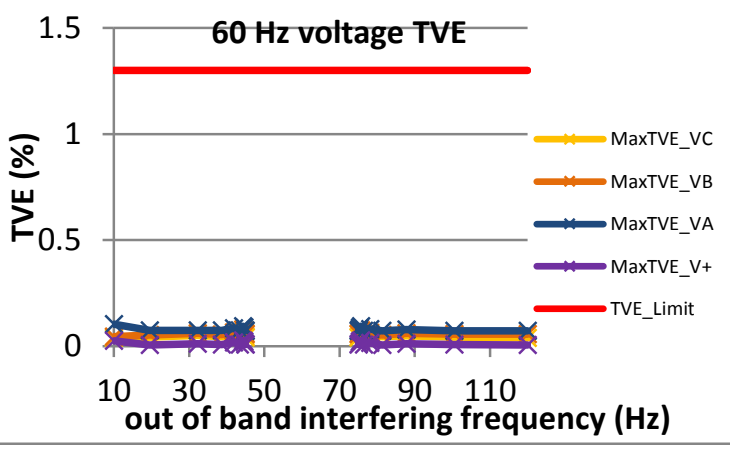

Figure 1536: $\mathrm{Fs}=30 \mathrm{FPS}, 60 \mathrm{~Hz}$ fundamental

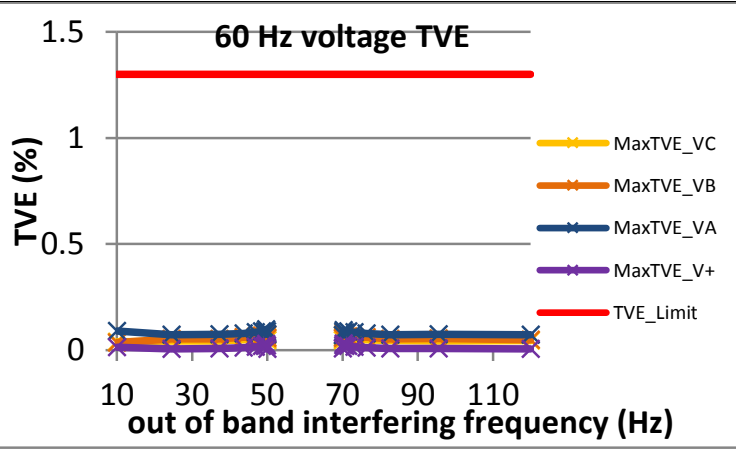

Figure 1539: Fs = $20 \mathrm{FPS}, 60 \mathrm{~Hz}$ fundamental

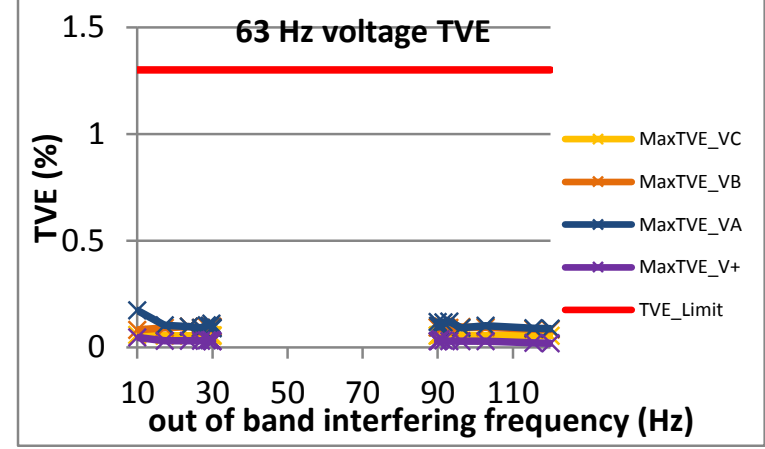

Figure 1534: $\mathrm{Fs}=60 \mathrm{FPS}, 63 \mathrm{~Hz}$ fundamental

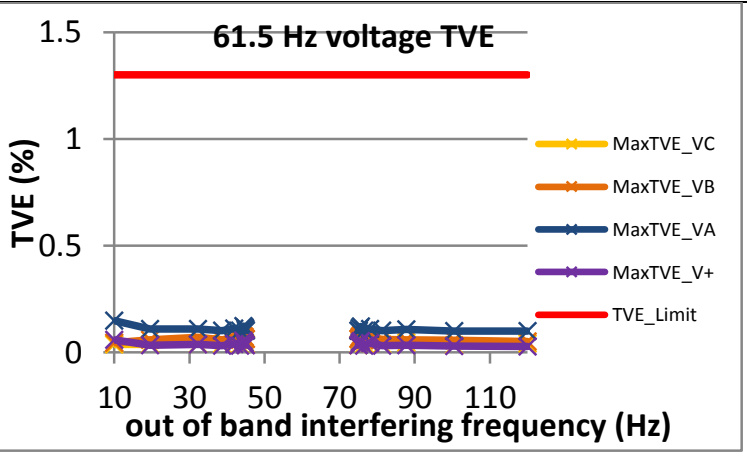

Figure 1537: Fs = $30 \mathrm{FPS}, 61.5 \mathrm{~Hz}$ fundamental

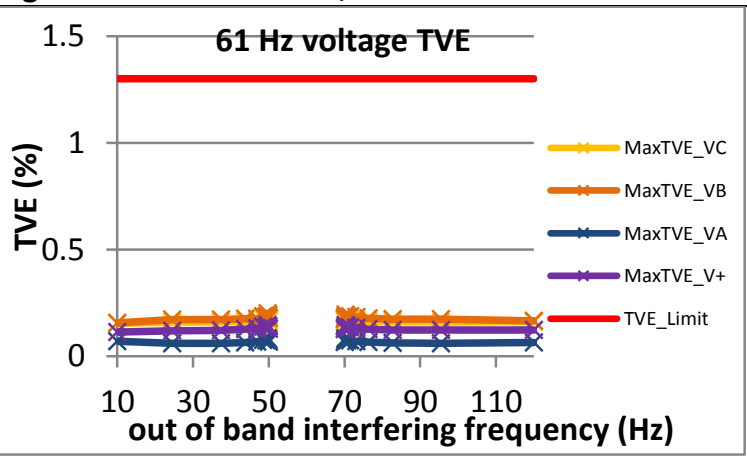

Figure 1540: Fs = $20 \mathrm{FPS}, 61 \mathrm{~Hz}$ fundamental 


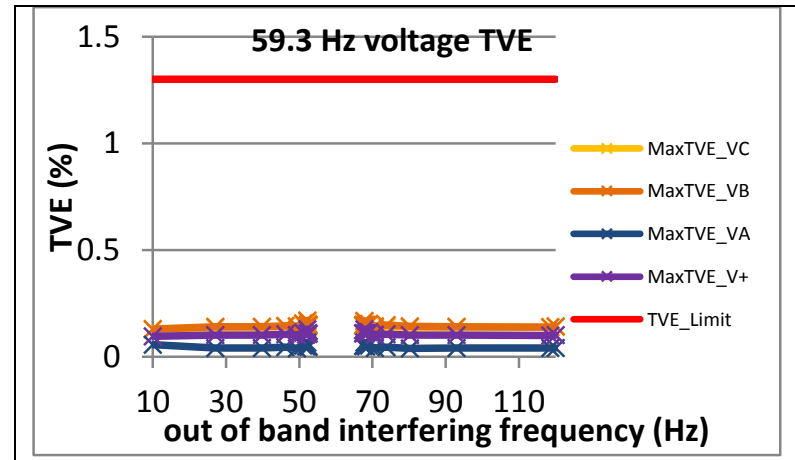

Figure 1541: Fs = $15 \mathrm{FPS}, 59.3 \mathrm{~Hz}$ fundamental

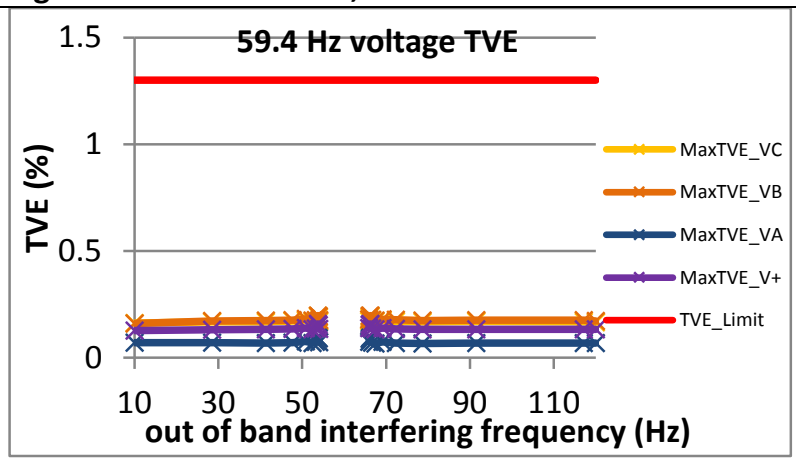

Figure 1544: Fs = $12 \mathrm{FPS}, 59.4 \mathrm{~Hz}$ fundamental

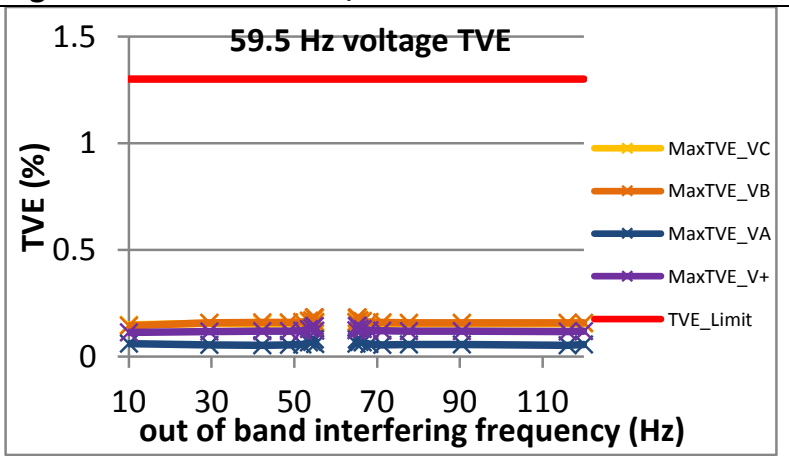

Figure 1547: Fs = $10 \mathrm{FPS}, 59.5 \mathrm{~Hz}$ fundamental

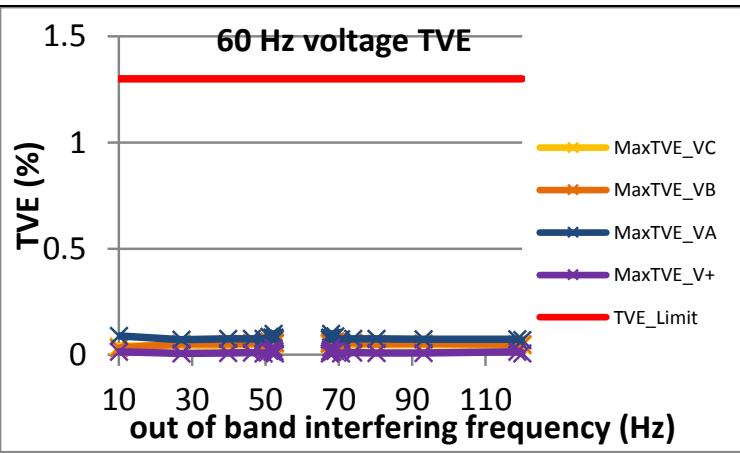

Figure 1542: Fs = $15 \mathrm{FPS}, 60 \mathrm{~Hz}$ fundamental

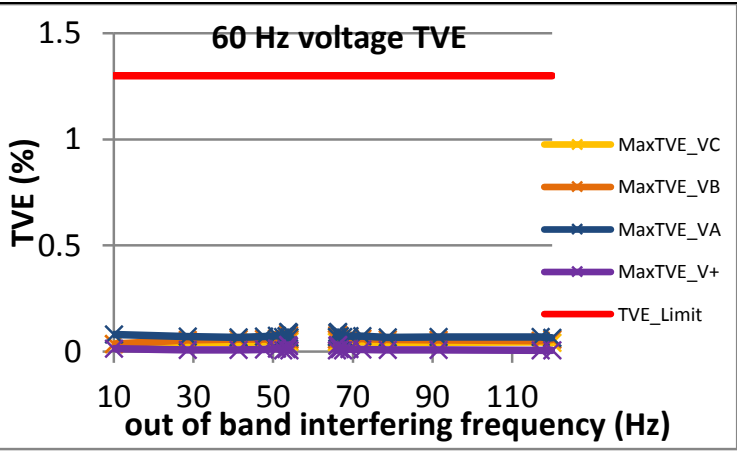

Figure 1545: Fs = $12 \mathrm{FPS}, 60 \mathrm{~Hz}$ fundamental

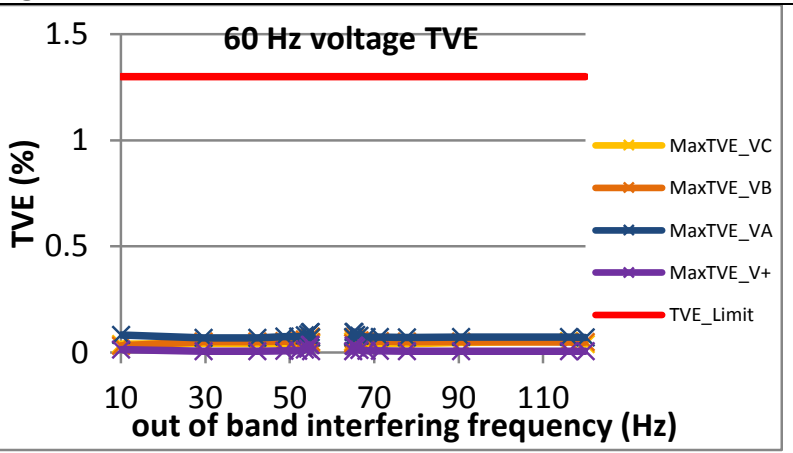

Figure 1548: Fs = $60 \mathrm{FPS}, 60 \mathrm{~Hz}$ fundamental

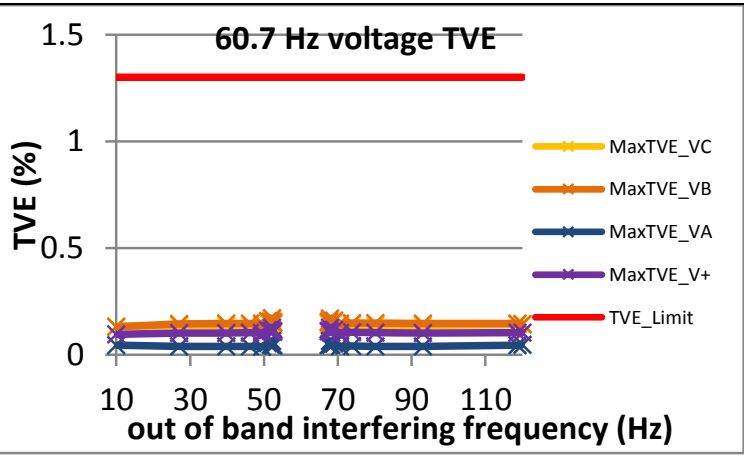

Figure 1543: Fs = $15 \mathrm{FPS}, 60.7 \mathrm{~Hz}$ fundamental

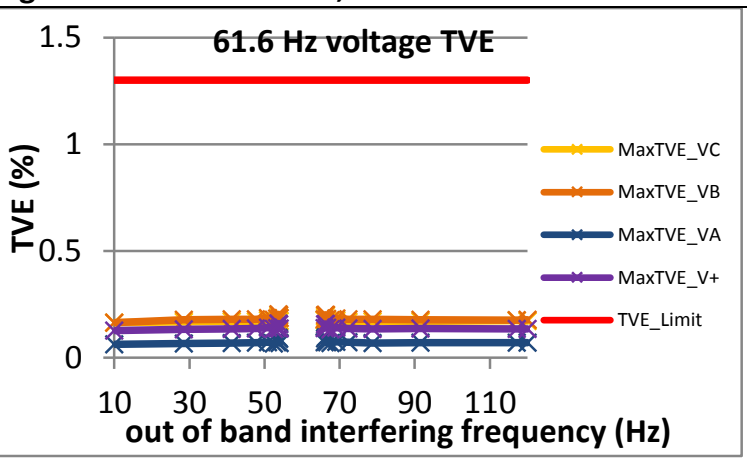

Figure 1546: Fs = $12 \mathrm{FPS}, 60.6 \mathrm{~Hz}$ fundamental

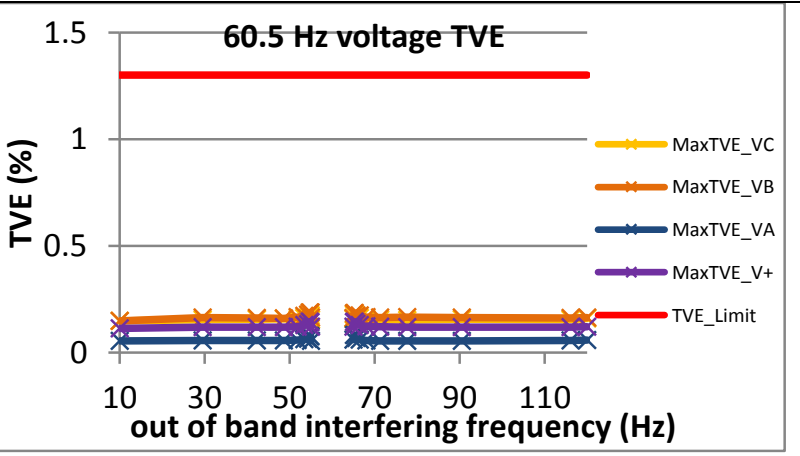

Figure 1549: Fs = $10 \mathrm{FPS}, 60.5 \mathrm{~Hz}$ fundamental 


\subsection{Steady state out of band interfering signals test current TVE: M class}

\subsubsection{C37.118.1 Annex $C$ out of band interfering signals current TVE: M class}
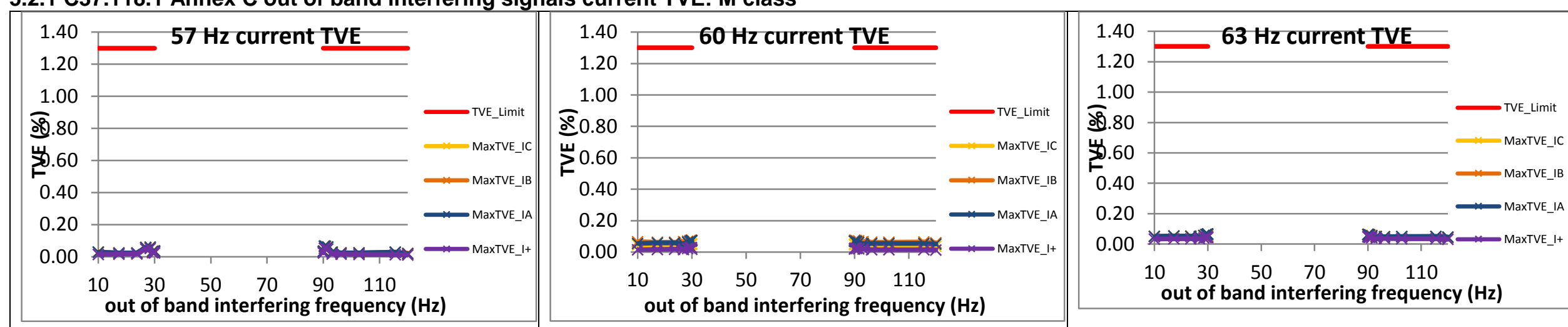

Figure 1550: Fs = 60 FPS, $57 \mathrm{~Hz}$ fundamental

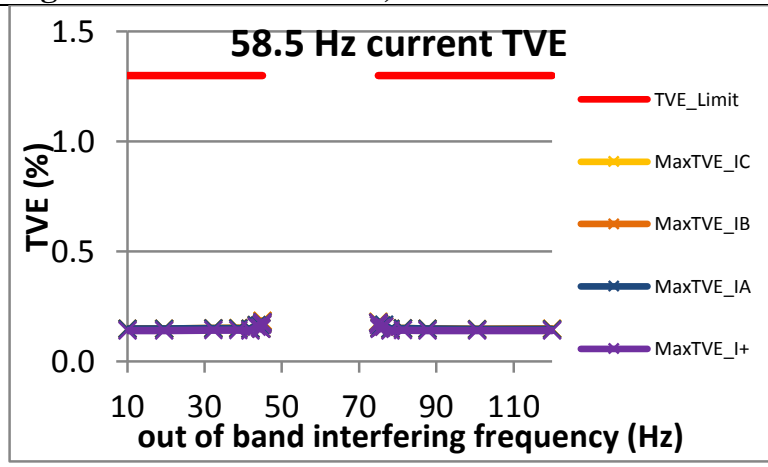

Figure 1551: Fs = $60 \mathrm{FPS}, 60 \mathrm{~Hz}$ fundamental

Figure 1552: Fs = 60 FPS, $63 \mathrm{~Hz}$ fundamental

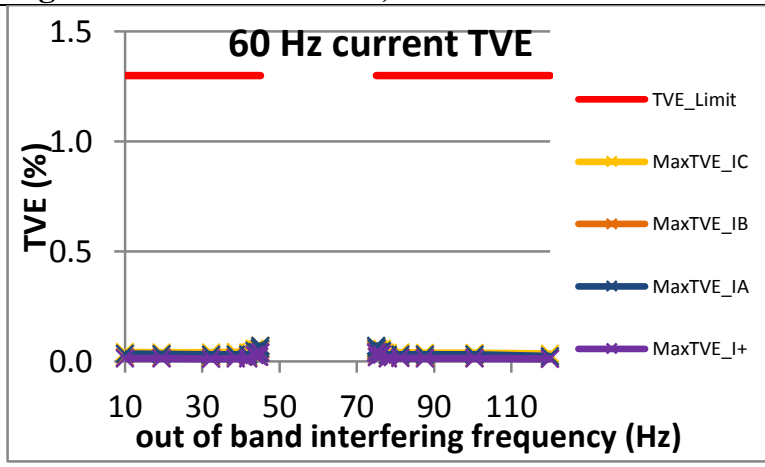

Figure 1553: Fs = $30 \mathrm{FPS}, 58.5 \mathrm{~Hz}$ fundamental

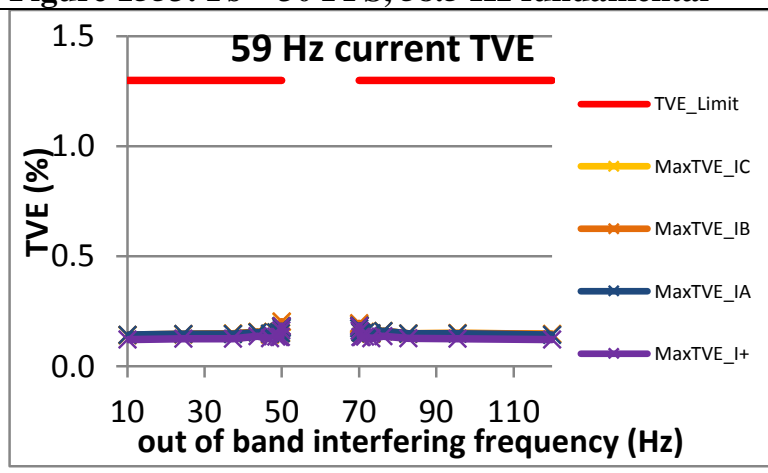

Figure 1556Fs = $20 \mathrm{FPS}, 59 \mathrm{~Hz}$ fundamental

Figure 1554: Fs $=30 \mathrm{FPS}, 60 \mathrm{~Hz}$ fundamental

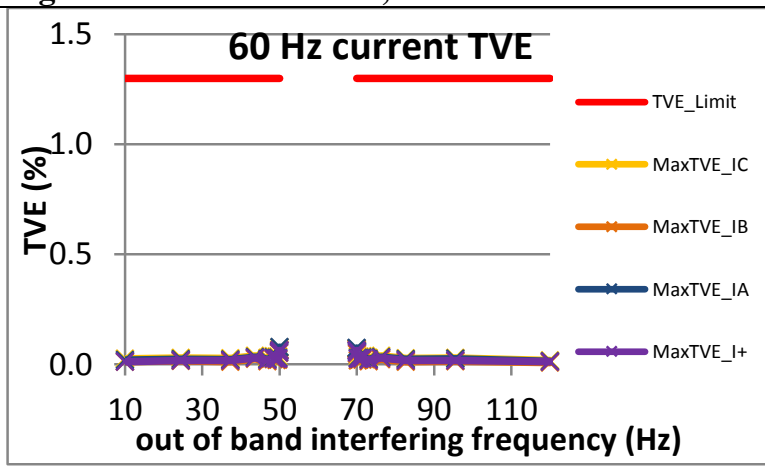

Figure 1557: Fs = 20 FPS, $60 \mathrm{~Hz}$ fundamental

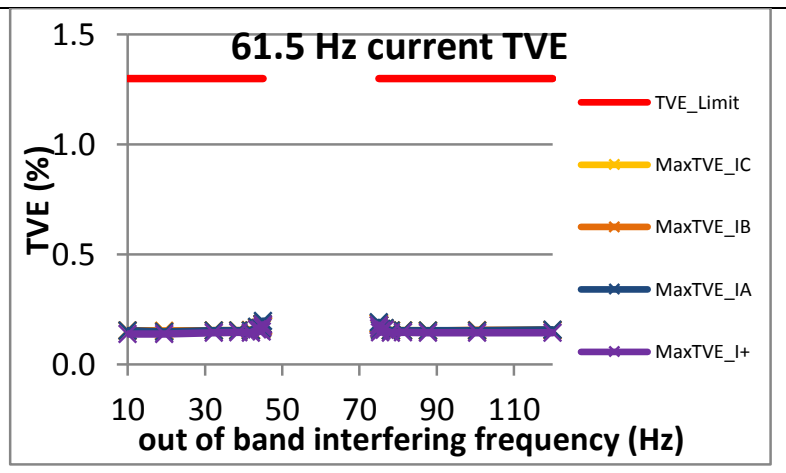

Figure 1555: Fs = 30 FPS, $61.5 \mathrm{~Hz}$ fundamental

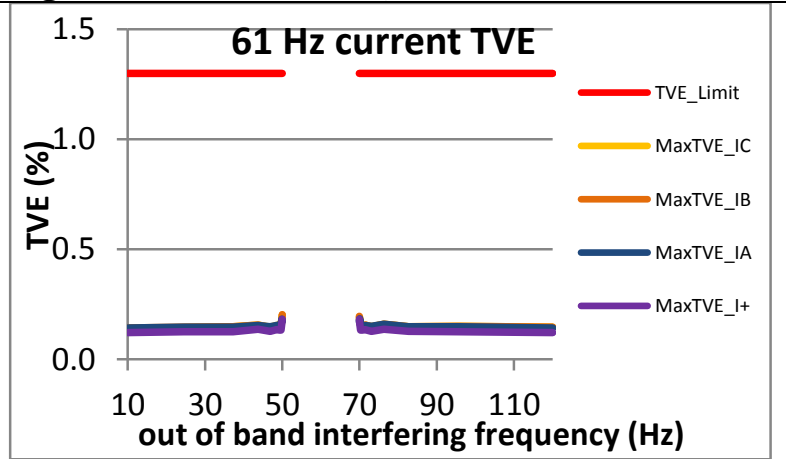

Figure 1558: Fs = 20 FPS, $61 \mathrm{~Hz}$ fundamental 


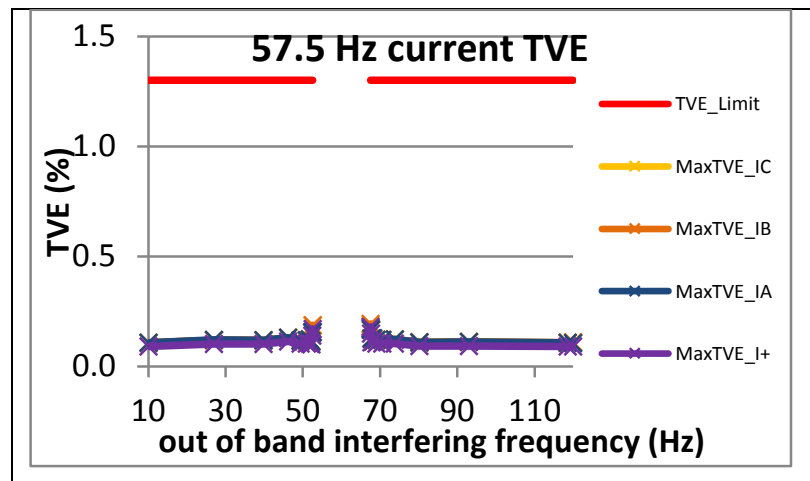

Figure 1559: Fs = 15 FPS, $59.3 \mathrm{~Hz}$ fundamental

Figure 1562: Fs = 12 FPS, $59.4 \mathrm{~Hz}$ fundamental

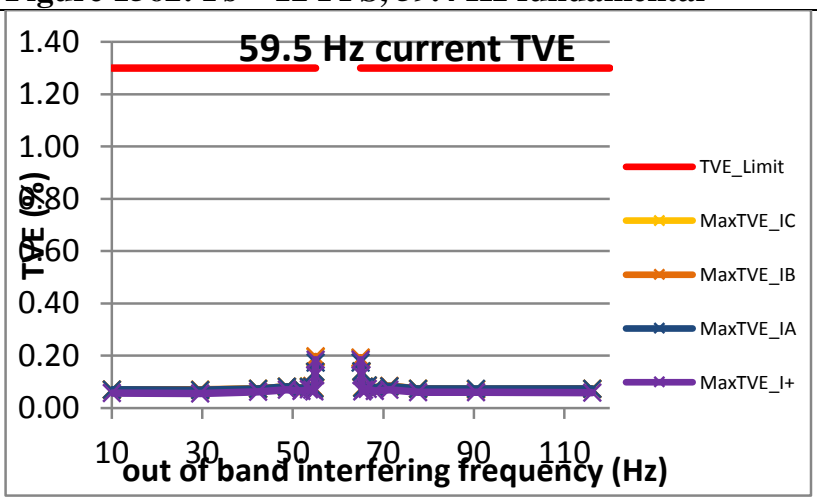

Figure 1565: Fs = $10 \mathrm{FPS}, 59.5 \mathrm{~Hz}$ fundamental

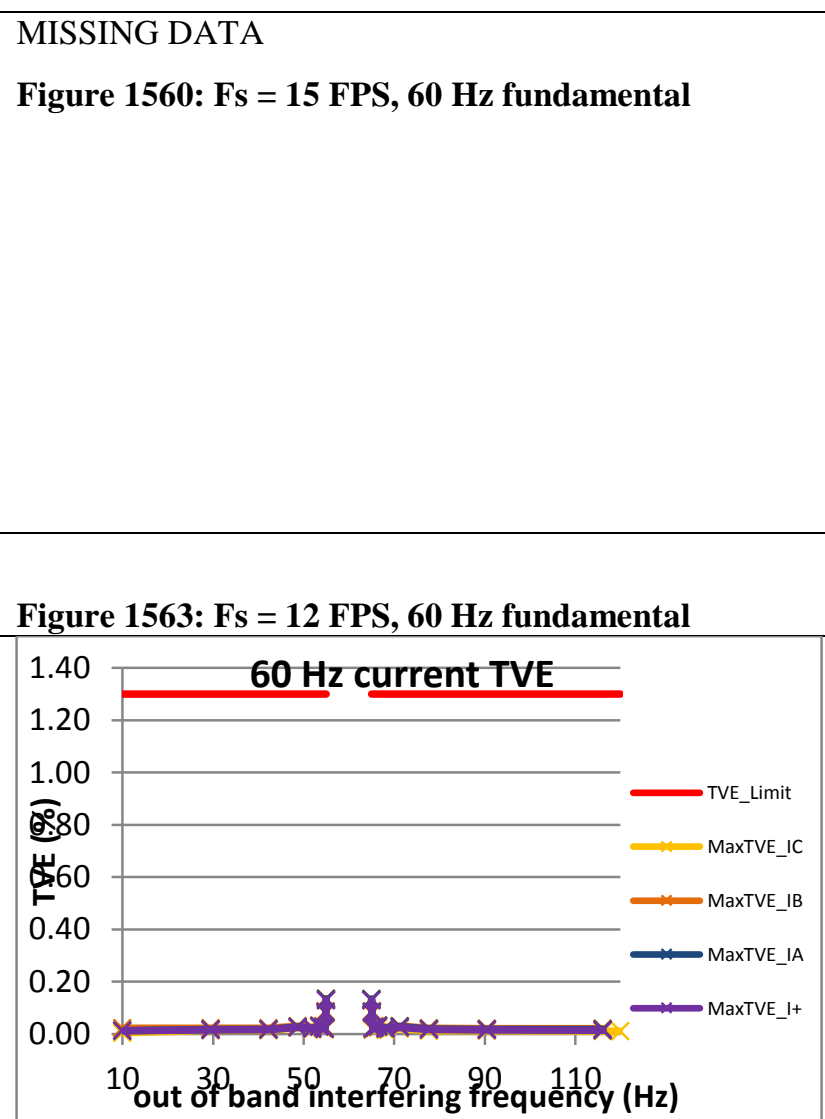

Figure 1566: Fs $=10 \mathrm{FPS}, 60 \mathrm{~Hz}$ fundamental
MISSING DATA

Figure 1561: Fs = 15 FPS, $60.7 \mathrm{~Hz}$ fundamental

Figure 1564: Fs = 12 FPS, $60.6 \mathrm{~Hz}$ fundamental

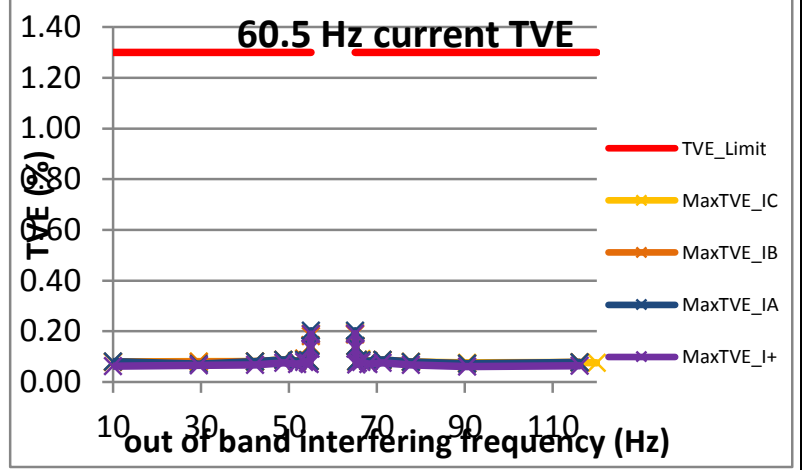

Figure 1567: Fs = $10 \mathrm{FPS}, 60.5 \mathrm{~Hz}$ fundamental 
5.2.2 PMU A out of band interfering signals current TVE: M class

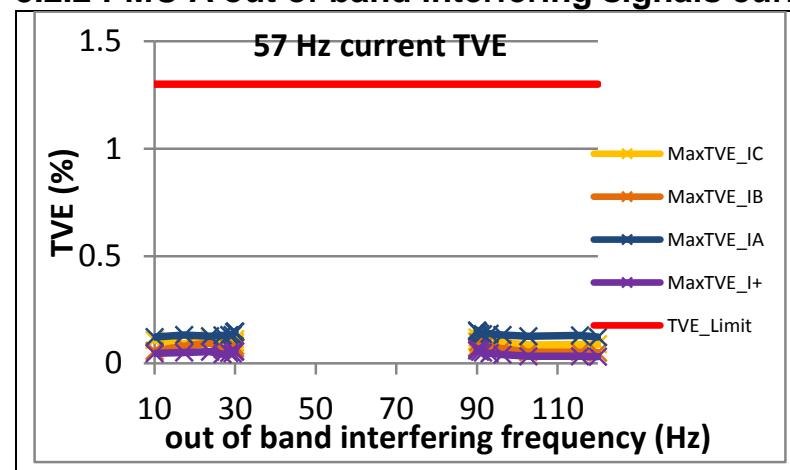

Figure 1568: Fs = 60 FPS, $57 \mathrm{~Hz}$ fundamental

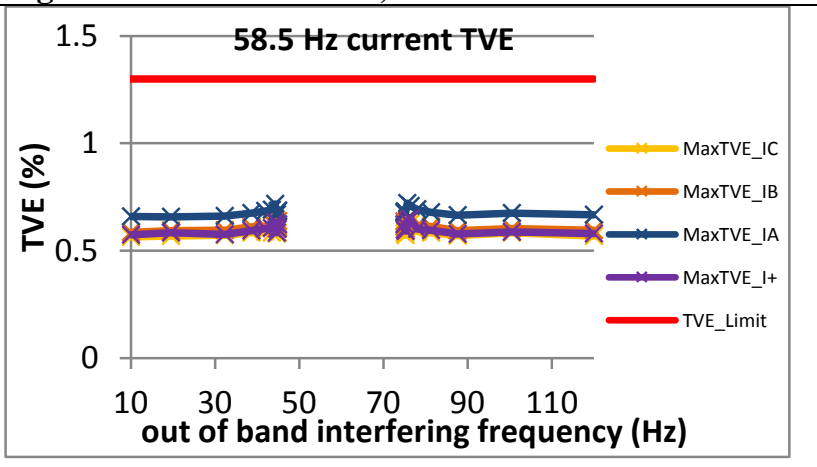

Figure 1571: Fs = 30 FPS, 58.5 Hz fundamental

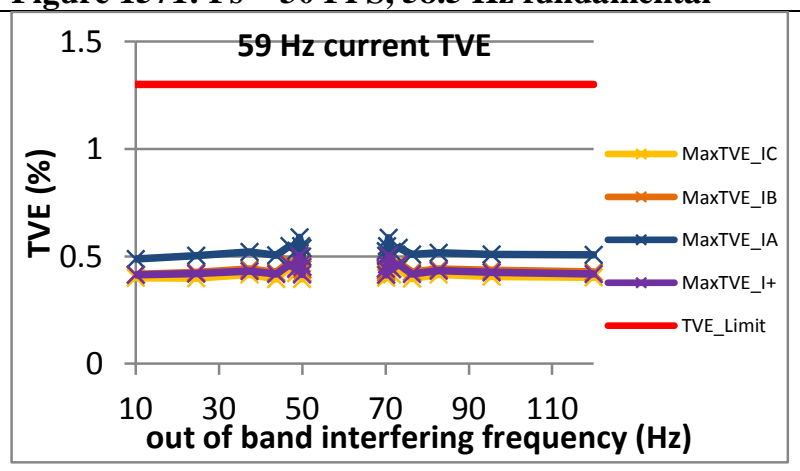

Figure 1574Fs = $20 \mathrm{FPS}, 59 \mathrm{~Hz}$ fundamental

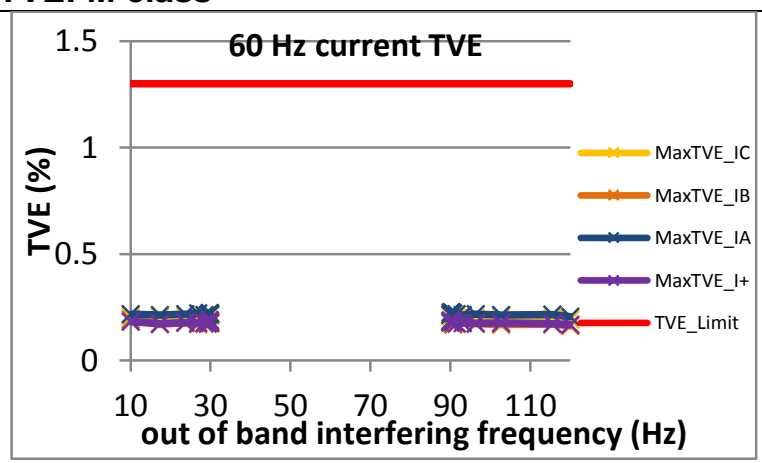

Figure 1569: Fs = $60 \mathrm{FPS}, 60 \mathrm{~Hz}$ fundamental

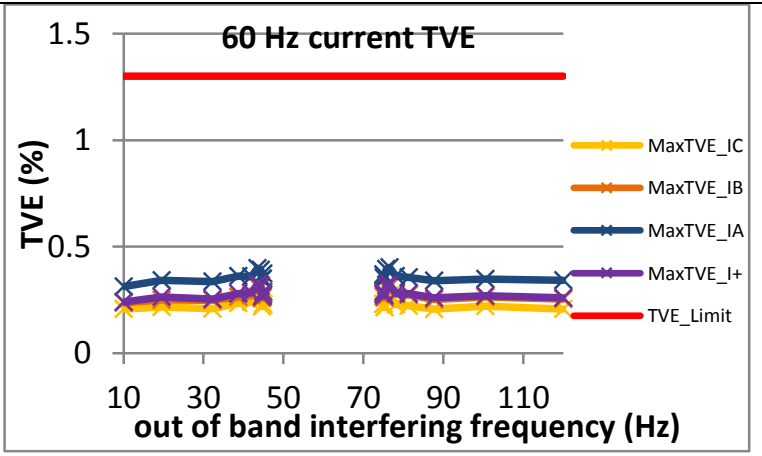

Figure 1572: Fs = $30 \mathrm{FPS}, 60 \mathrm{~Hz}$ fundamental

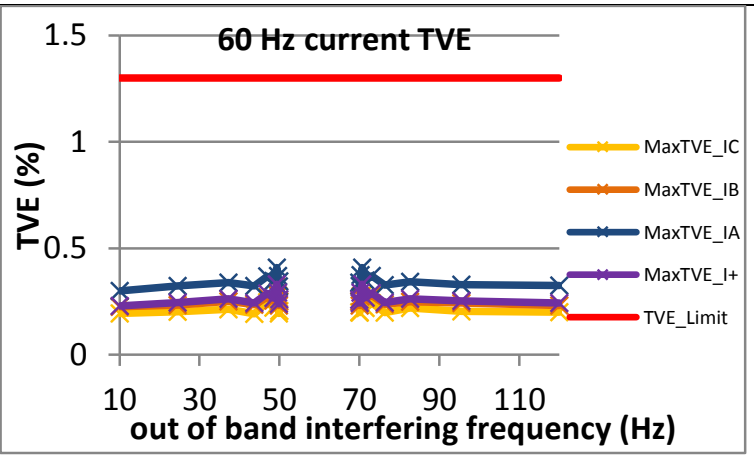

Figure 1575: Fs = 20 FPS, $60 \mathrm{~Hz}$ fundamental

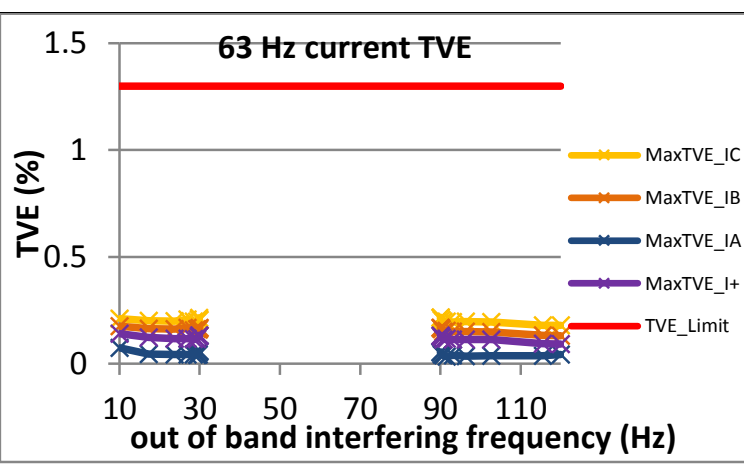

Figure 1570: Fs = 60 FPS, $63 \mathrm{~Hz}$ fundamental

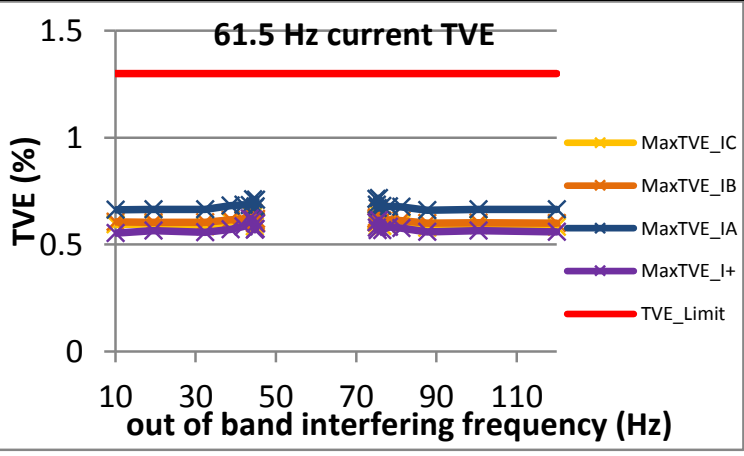

Figure 1573: Fs = 30 FPS, $61.5 \mathrm{~Hz}$ fundamental

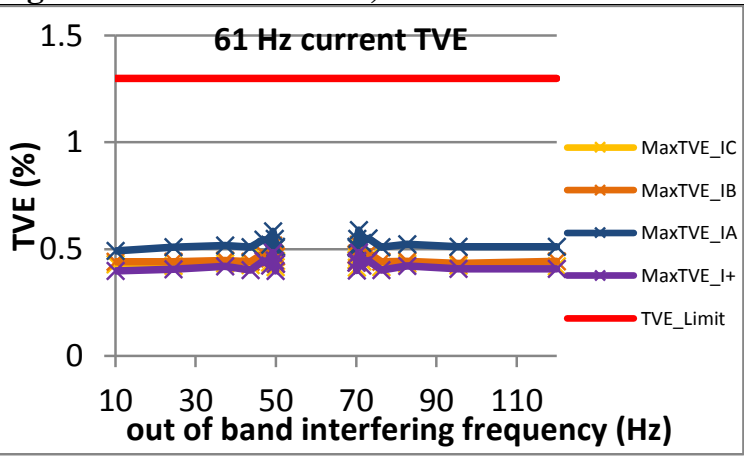

Figure 1576: Fs = 20 FPS, $61 \mathrm{~Hz}$ fundamental 


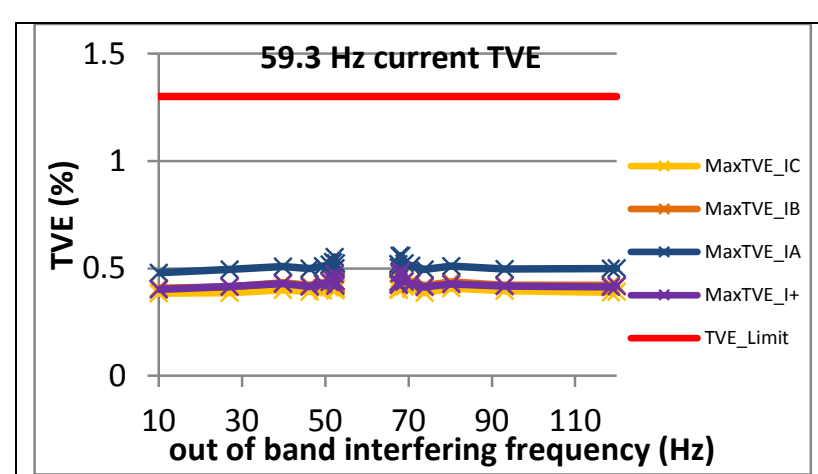

Figure 1577: Fs = 15 FPS, $59.3 \mathrm{~Hz}$ fundamental

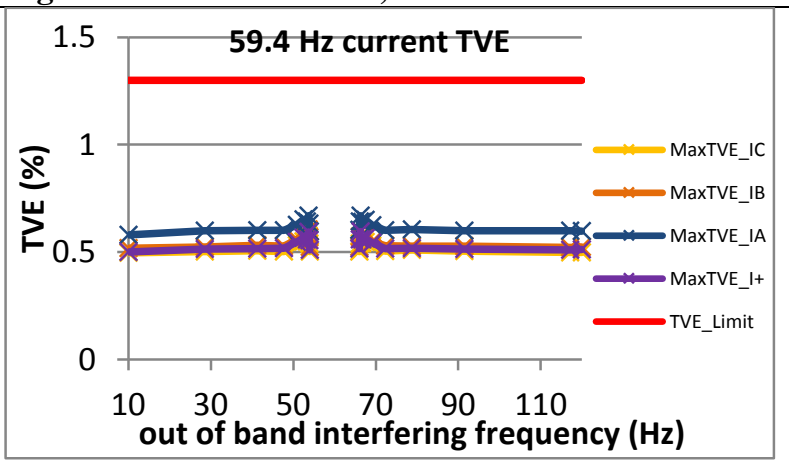

Figure 1580: Fs = 12 FPS, $59.4 \mathrm{~Hz}$ fundamental

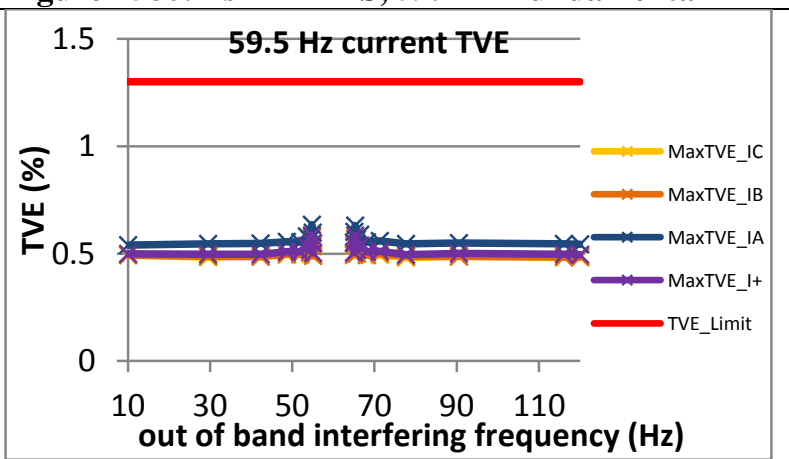

Figure 1583: Fs = 10 FPS, $59.5 \mathrm{~Hz}$ fundamental

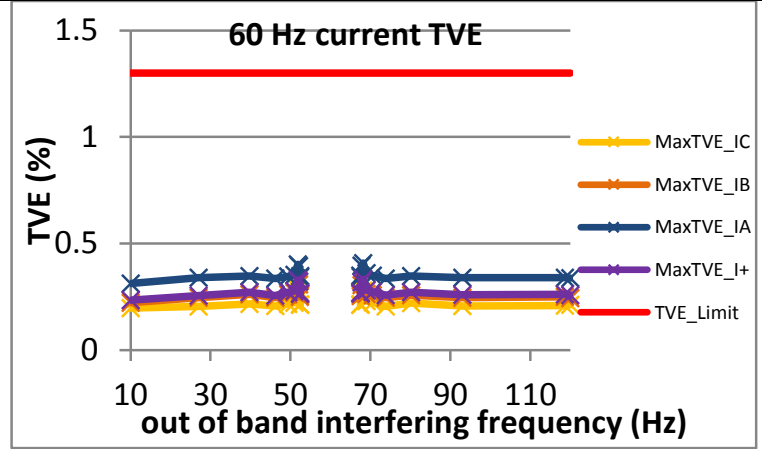

Figure 1578: Fs = 15 FPS, $60 \mathrm{~Hz}$ fundamental

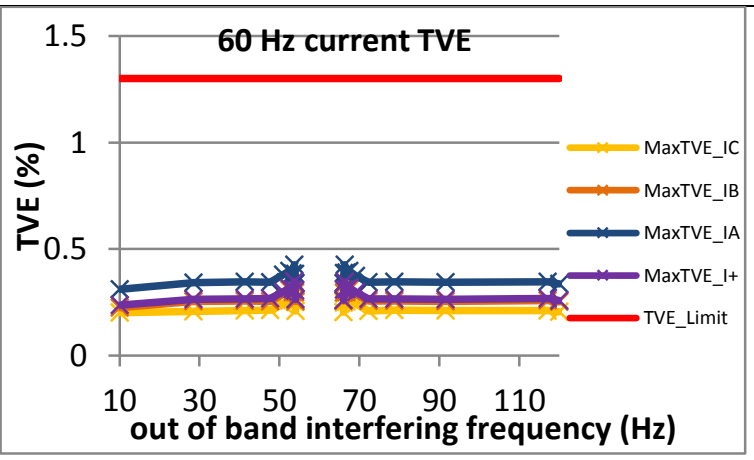

Figure 1581: Fs = 12 FPS, $60 \mathrm{~Hz}$ fundamental

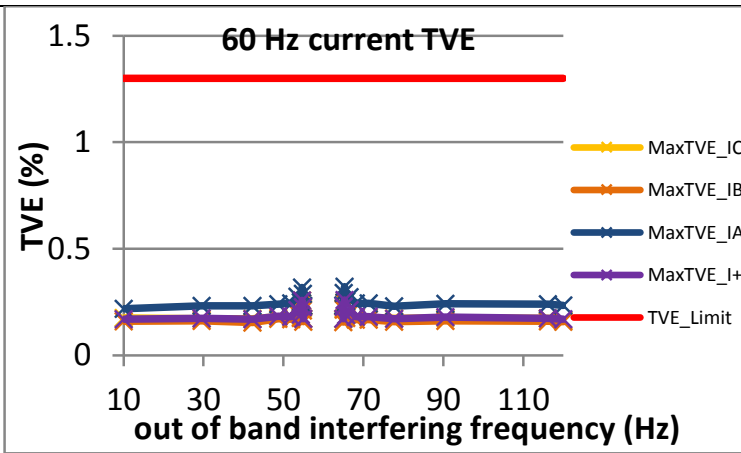

Figure 1584: Fs = 10 FPS, $60 \mathrm{~Hz}$ fundamental

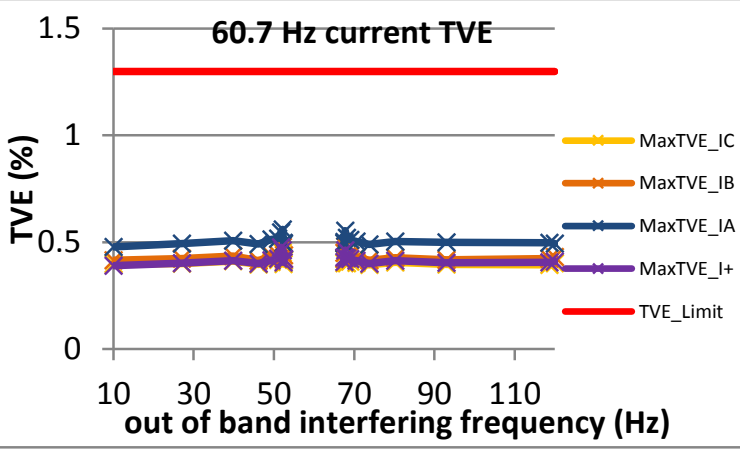

Figure 1579: Fs = 15 FPS, $60.7 \mathrm{~Hz}$ fundamental

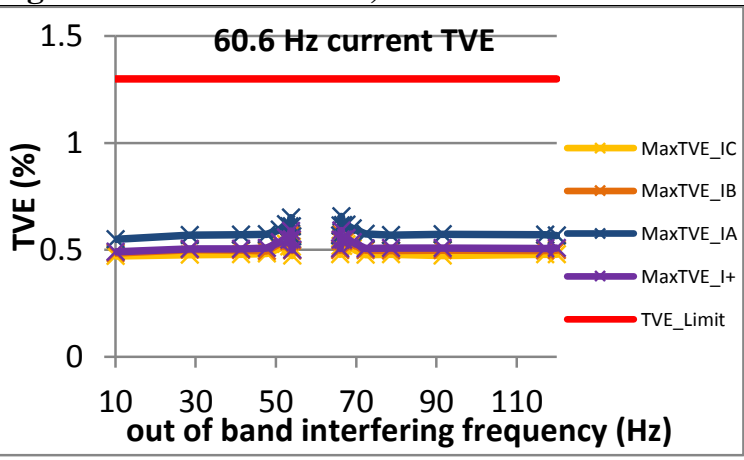

Figure 1582: Fs = $12 \mathrm{FPS}, 60.6 \mathrm{~Hz}$ fundamental

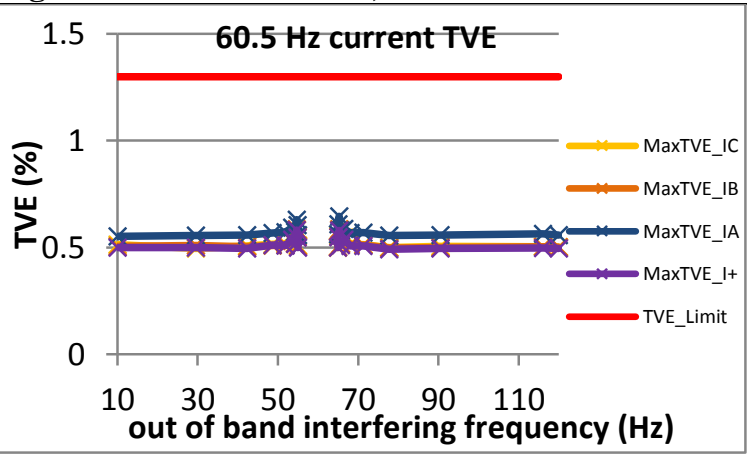

Figure 1585: Fs = $10 \mathrm{FPS}, 60.5 \mathrm{~Hz}$ fundamental 
5.2.3 PMU B out of band interfering signals current TVE: M class

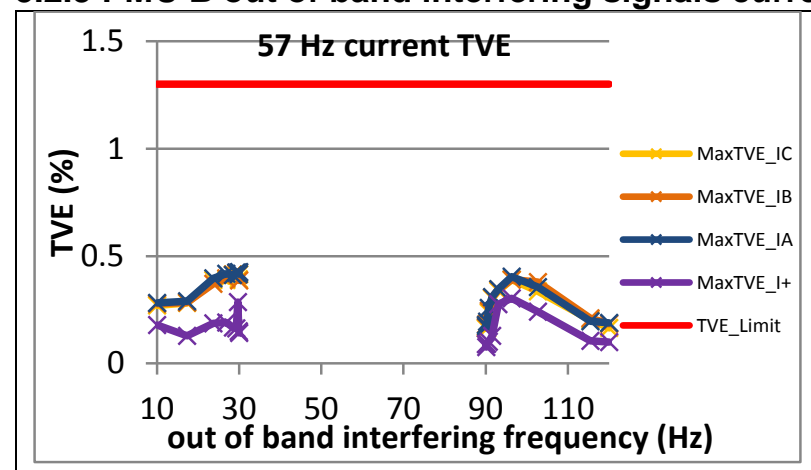

Figure 1586: Fs = 60 FPS, $57 \mathrm{~Hz}$ fundamental

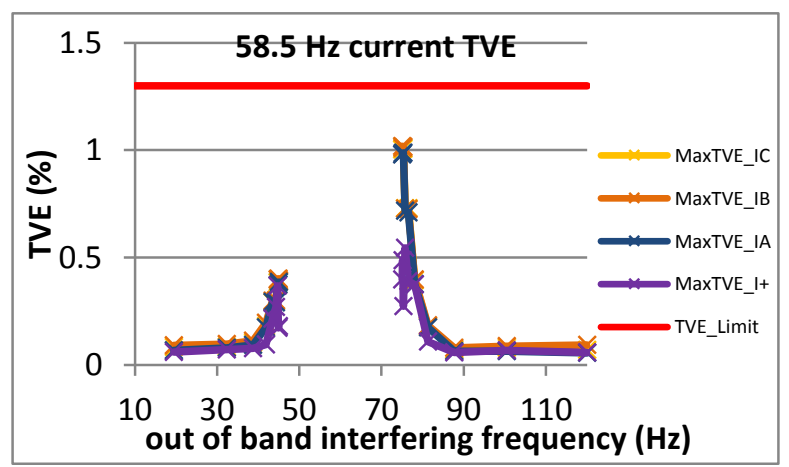

Figure 1589: Fs = 30 FPS, $58.5 \mathrm{~Hz}$ fundamental

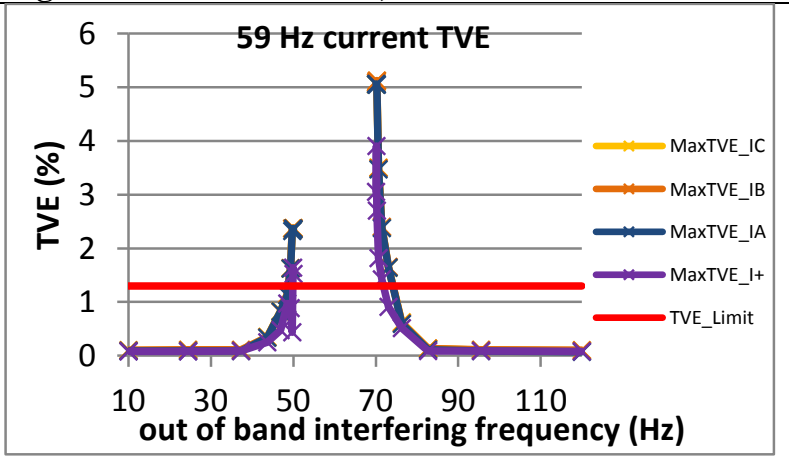

Figure 1592Fs = 20 FPS, $59 \mathrm{~Hz}$ fundamental

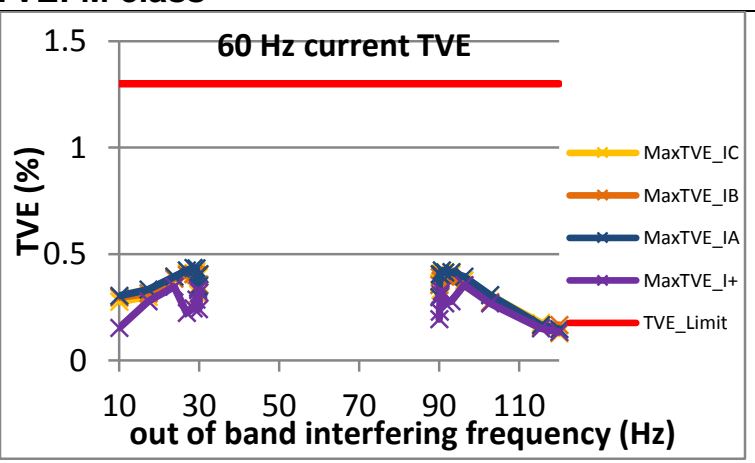

Figure 1587: Fs = $60 \mathrm{FPS}, 60 \mathrm{~Hz}$ fundamental

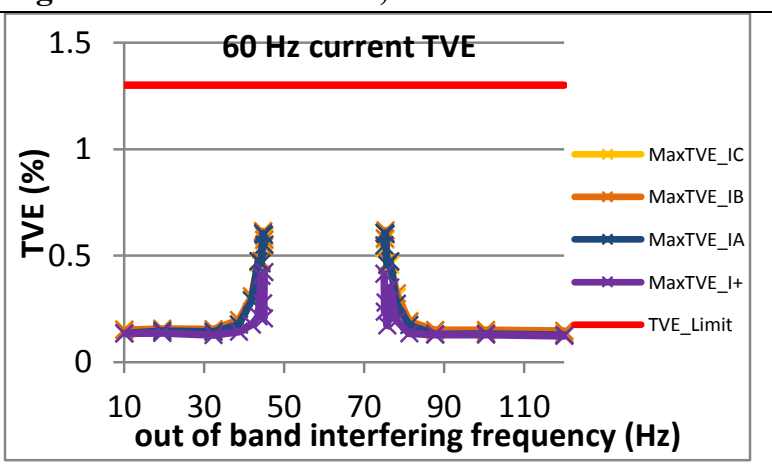

Figure 1590: Fs = $30 \mathrm{FPS}, 60 \mathrm{~Hz}$ fundamental

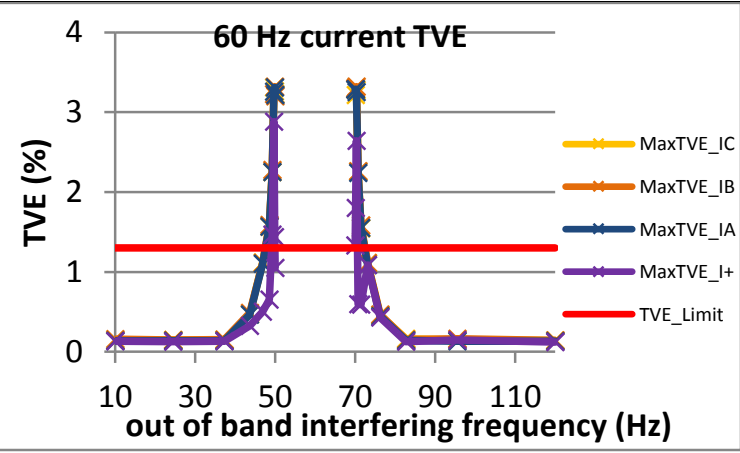

Figure 1593: Fs = $20 \mathrm{FPS}, 60 \mathrm{~Hz}$ fundamental

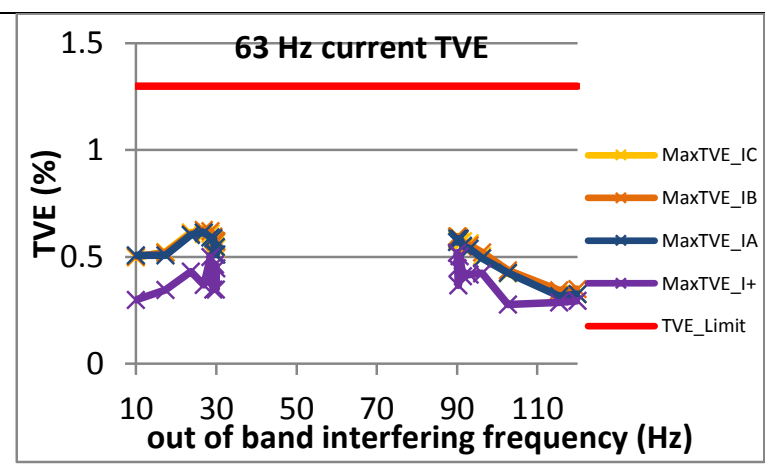

Figure 1588: Fs = 60 FPS, $63 \mathrm{~Hz}$ fundamental

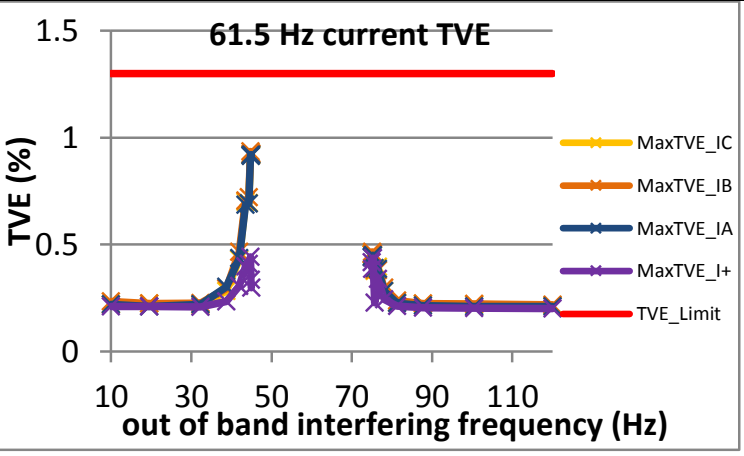

Figure 1591: Fs = 30 FPS, 61.5 Hz fundamental

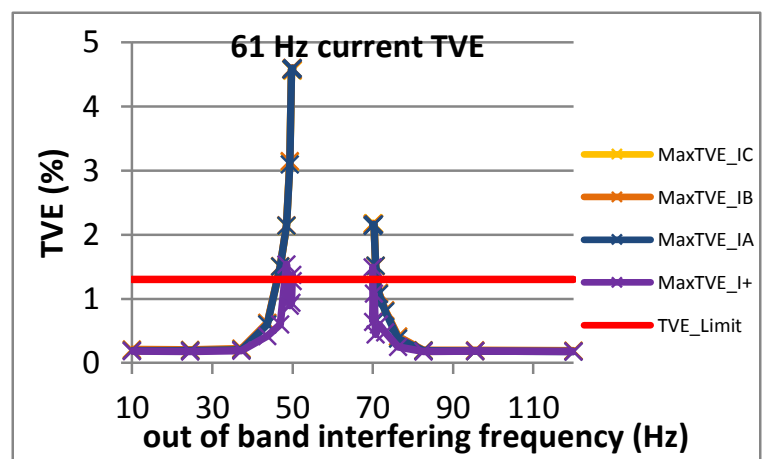

Figure 1594: Fs = 20 FPS, $61 \mathrm{~Hz}$ fundamental 


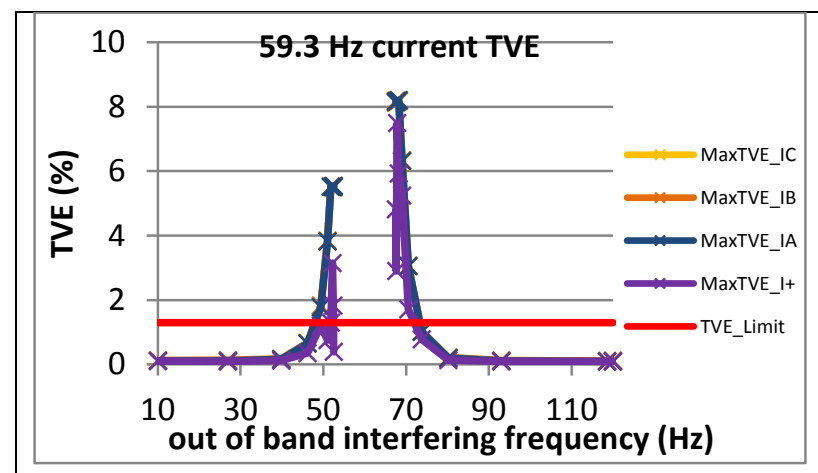

Figure 1595: Fs = 15 FPS, $59.3 \mathrm{~Hz}$ fundamental

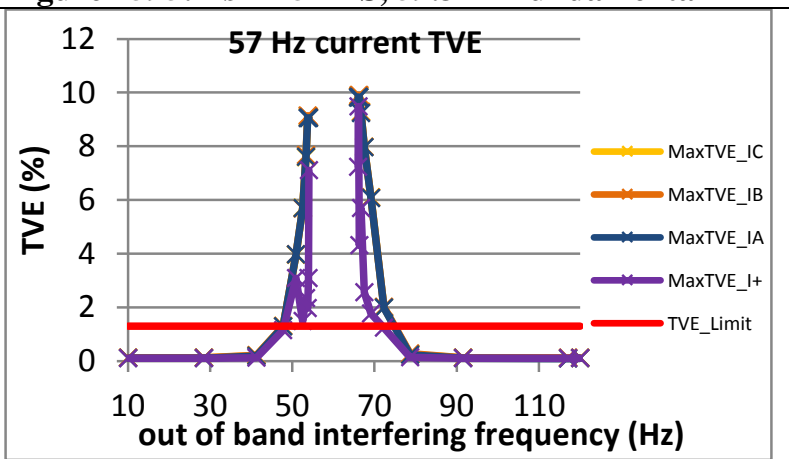

Figure 1598: Fs = 12 FPS, $59.4 \mathrm{~Hz}$ fundamental

Figure 1601: Fs = 10 FPS, $59.5 \mathrm{~Hz}$ fundamental

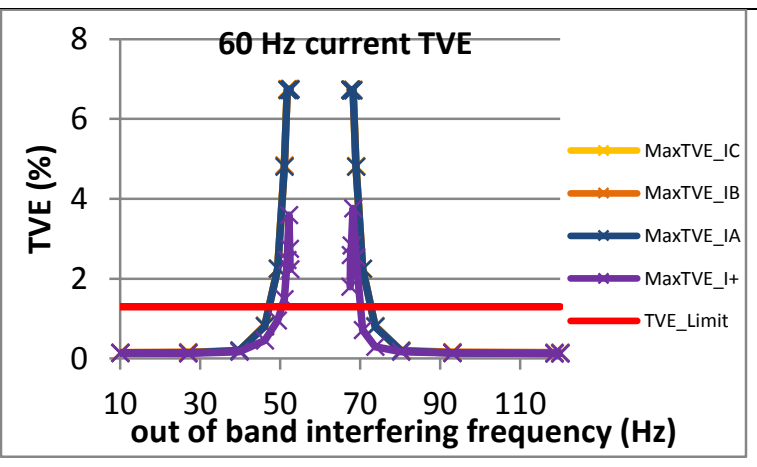

Figure 1596: Fs = 15 FPS, $60 \mathrm{~Hz}$ fundamental

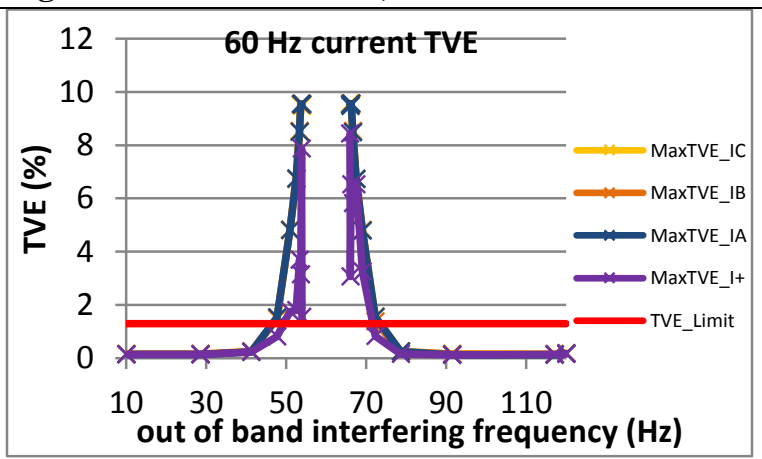

Figure 1599: Fs = $12 \mathrm{FPS}, 60 \mathrm{~Hz}$ fundamental

Figure 1602: Fs = $10 \mathrm{FPS}, 60 \mathrm{~Hz}$ fundamental

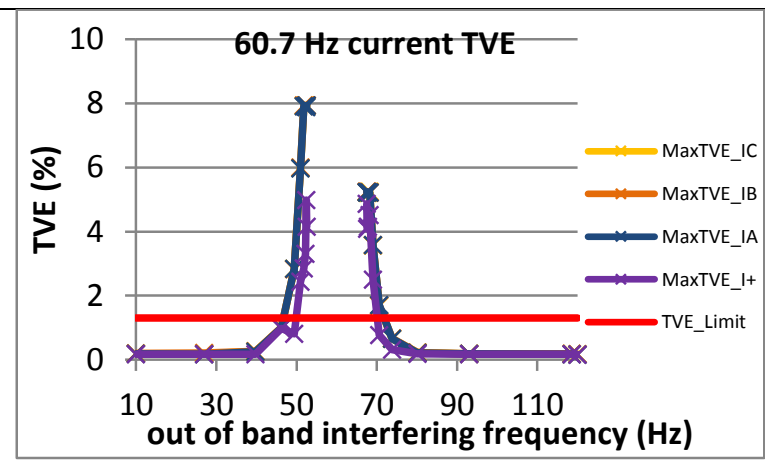

Figure 1597: Fs = $15 \mathrm{FPS}, 60.7 \mathrm{~Hz}$ fundamental

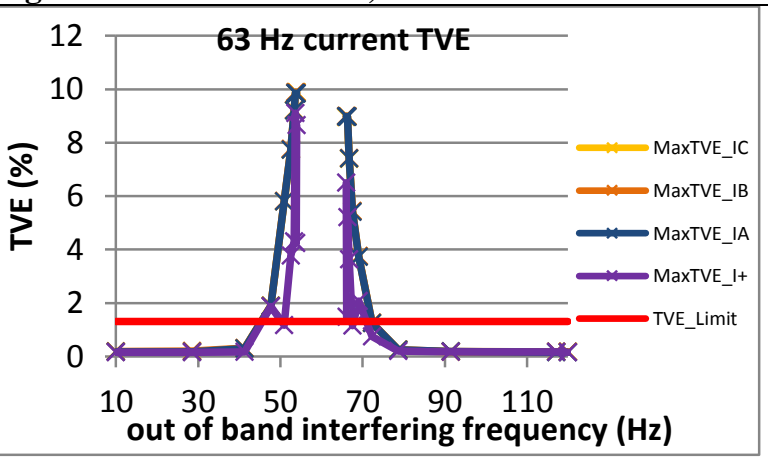

Figure 1600: Fs = 12 FPS, $60.6 \mathrm{~Hz}$ fundamental

Figure 1603: Fs = 10 FPS, $60.5 \mathrm{~Hz}$ fundamental 
5.2.4 PMU C out of band interfering signals current TVE: M class
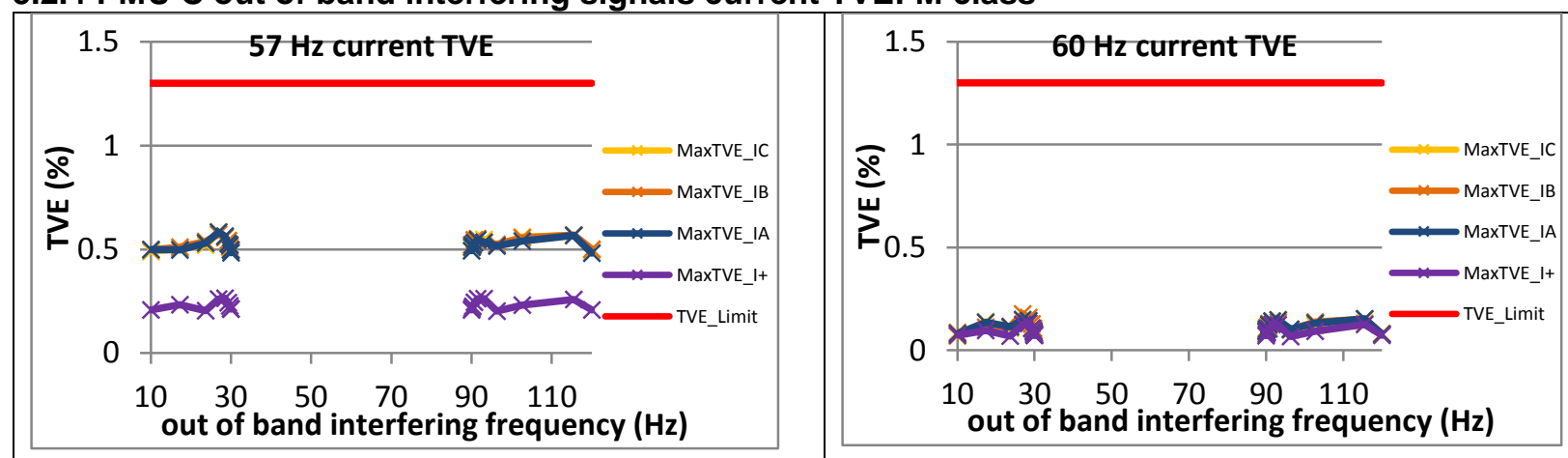

Figure 1605: Fs = $60 \mathrm{FPS}, 60 \mathrm{~Hz}$ fundamental

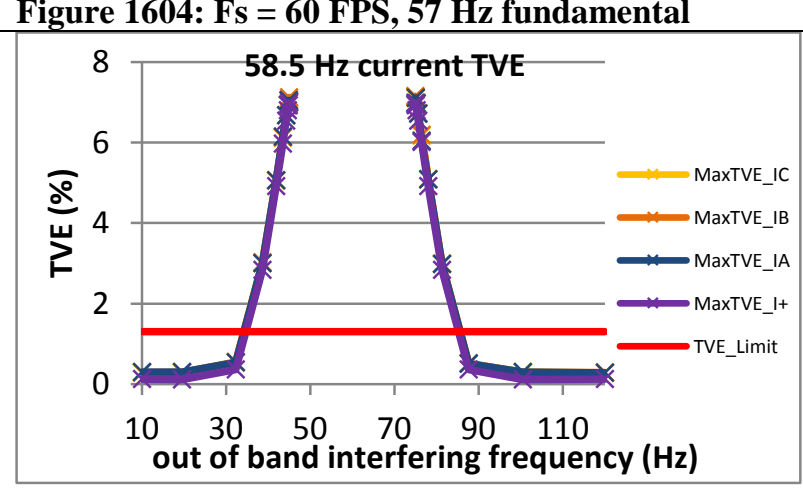

Figure 1607: Fs = 30 FPS, 58.5 Hz fundamental

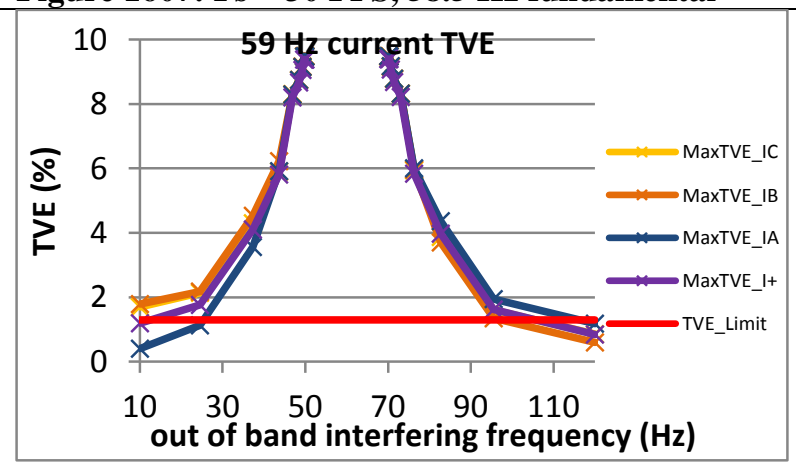

Figure 1610Fs = $20 \mathrm{FPS}, 59 \mathrm{~Hz}$ fundamental

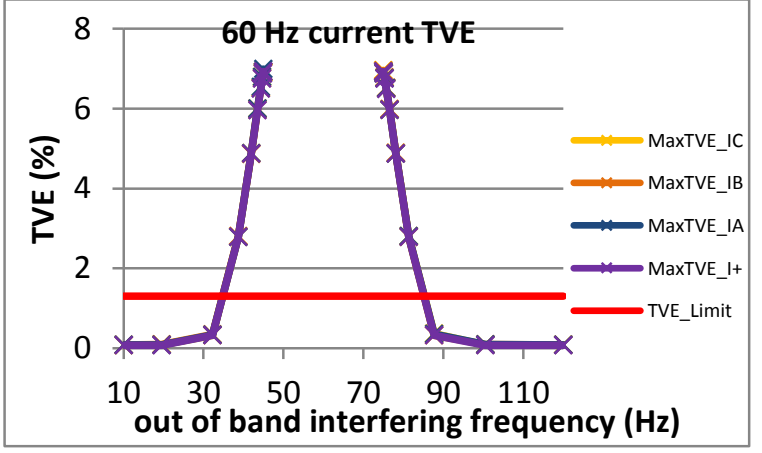

Figure 1608: Fs = $30 \mathrm{FPS}, 60 \mathrm{~Hz}$ fundamental

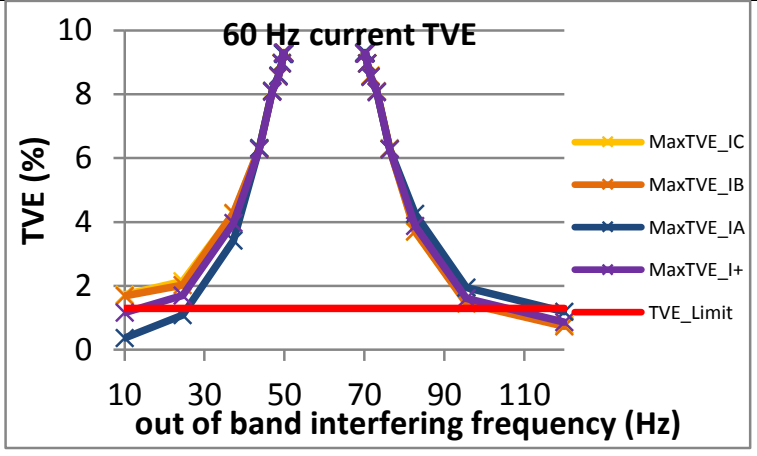

Figure 1611: Fs $=20 \mathrm{FPS}, 60 \mathrm{~Hz}$ fundamental

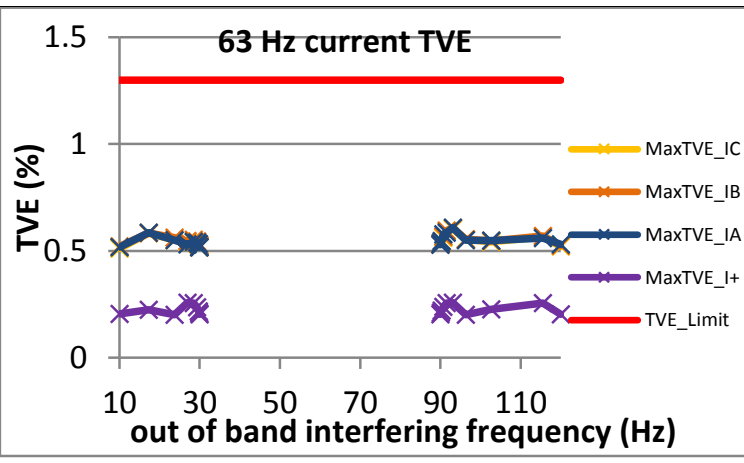

Figure 1606: Fs = 60 FPS, $63 \mathrm{~Hz}$ fundamental

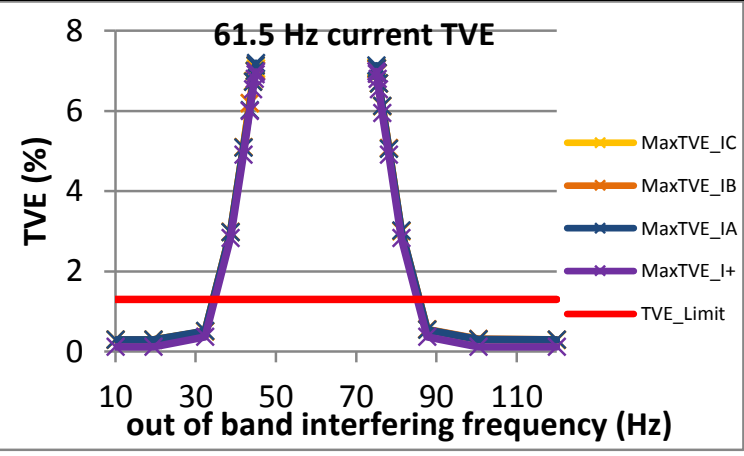

Figure 1609: Fs = 30 FPS, $61.5 \mathrm{~Hz}$ fundamental

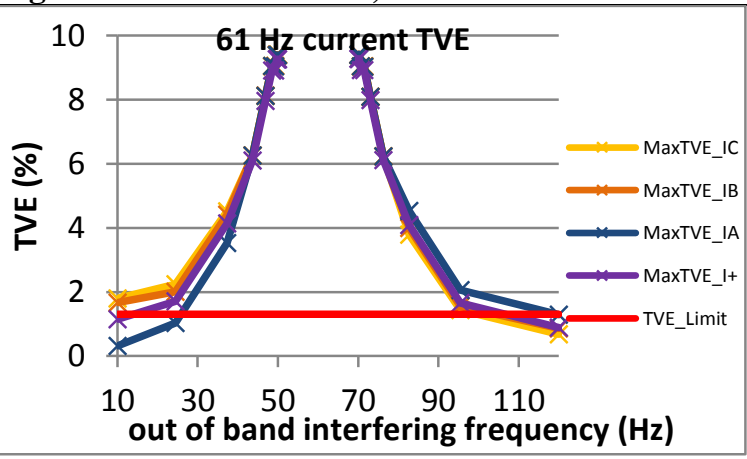

Figure 1612: Fs = 20 FPS, $61 \mathrm{~Hz}$ fundamental 


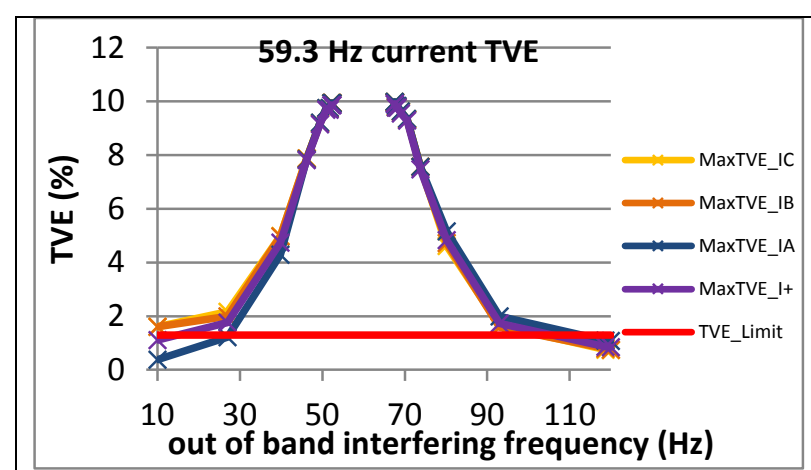

Figure 1613: Fs = 15 FPS, $59.3 \mathrm{~Hz}$ fundamental

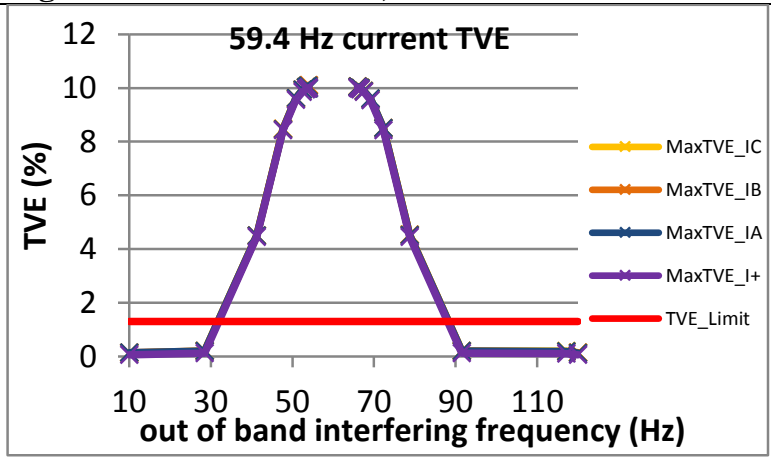

Figure 1616: Fs = 12 FPS, $59.4 \mathrm{~Hz}$ fundamental

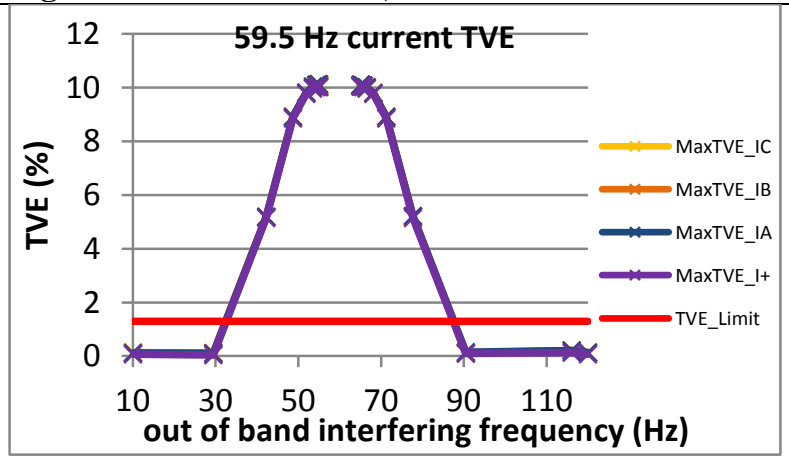

Figure 1619: Fs = 10 FPS, $59.5 \mathrm{~Hz}$ fundamental

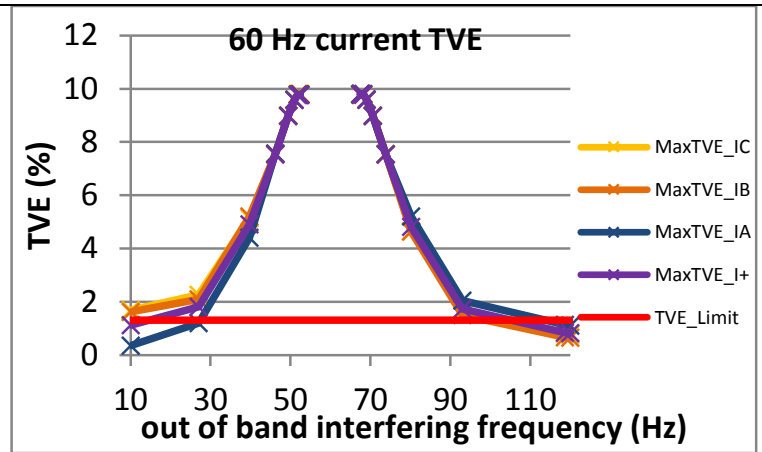

Figure 1614: Fs = $15 \mathrm{FPS}, 60 \mathrm{~Hz}$ fundamental

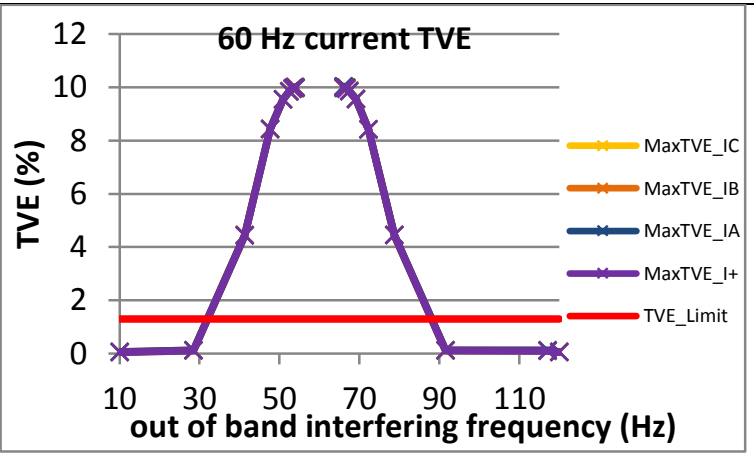

Figure 1617: Fs = 12 FPS, $60 \mathrm{~Hz}$ fundamental

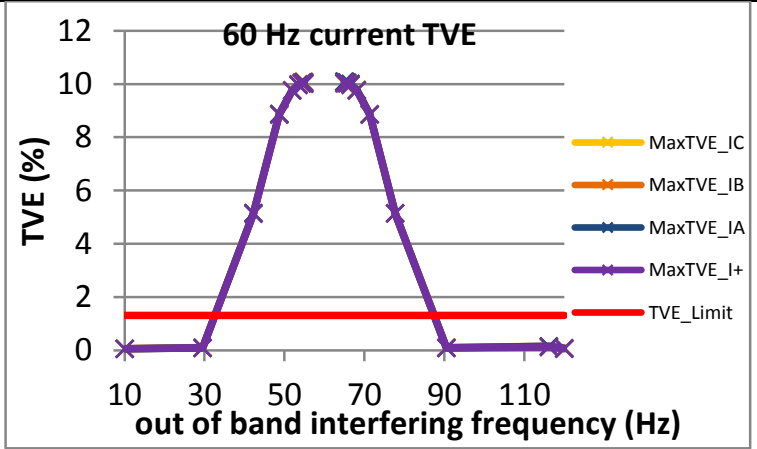

Figure 1620: Fs = 10 FPS, $60 \mathrm{~Hz}$ fundamental

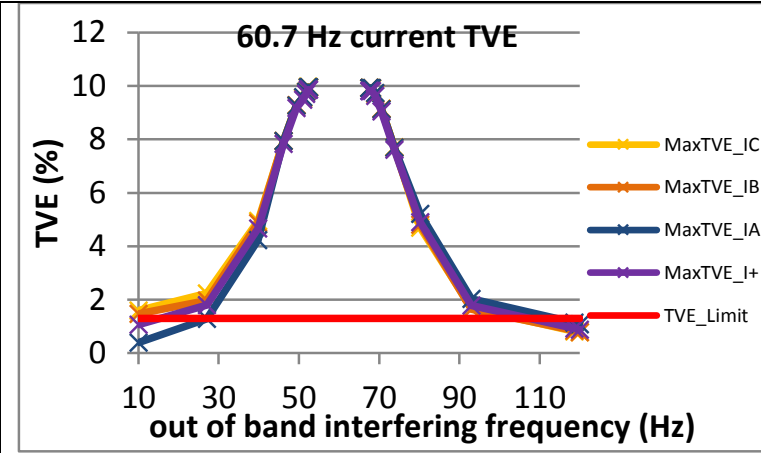

Figure 1615: Fs = 15 FPS, $60.7 \mathrm{~Hz}$ fundamental

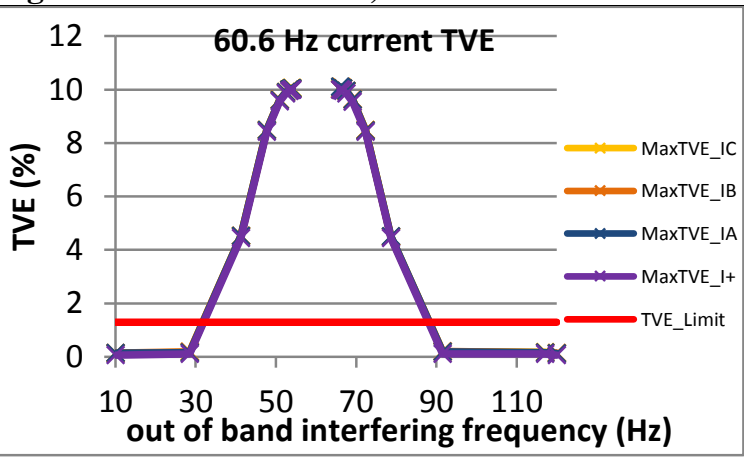

Figure 1618: Fs = 12 FPS, $60.6 \mathrm{~Hz}$ fundamental

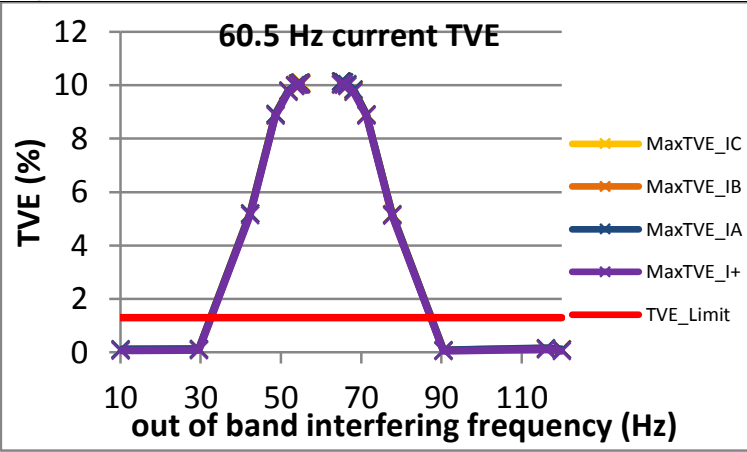

Figure 1621: Fs = $10 \mathrm{FPS}, 60.5 \mathrm{~Hz}$ fundamental 
5.2.5 PMU D out of band interfering signals current TVE: M class

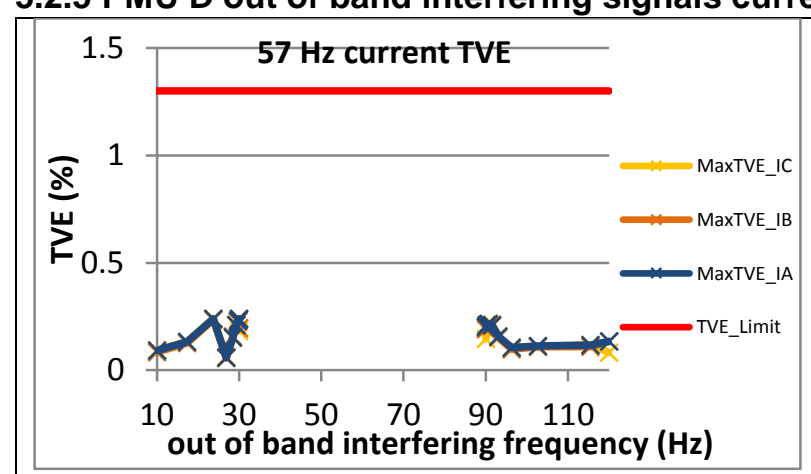

Figure 1622: Fs = 60 FPS, $57 \mathrm{~Hz}$ fundamental

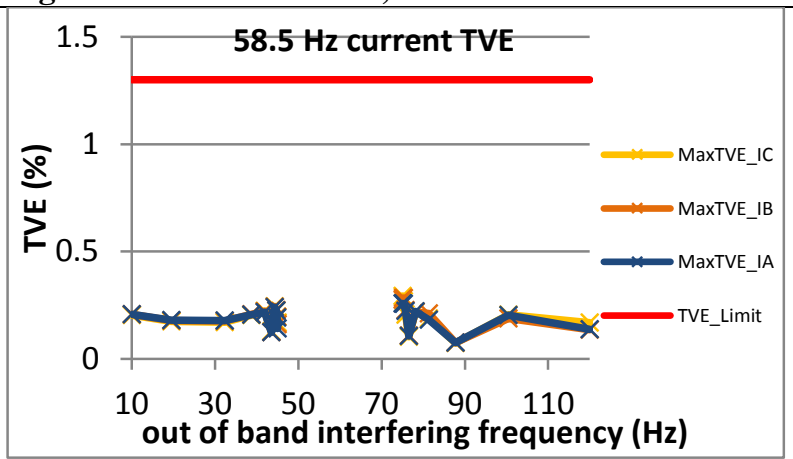

Figure 1625: Fs = 30 FPS, 58.5 Hz fundamental

Figure 1628Fs = 20 FPS, $59 \mathrm{~Hz}$ fundamental

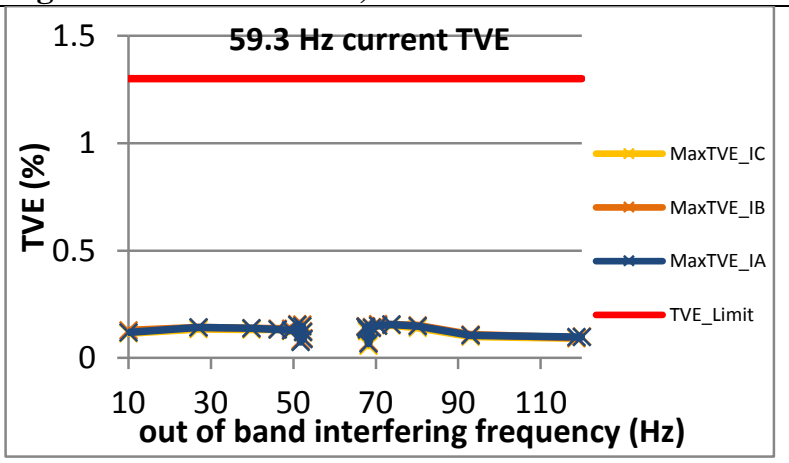

Figure 1631: Fs = 15 FPS, $59.3 \mathrm{~Hz}$ fundamental

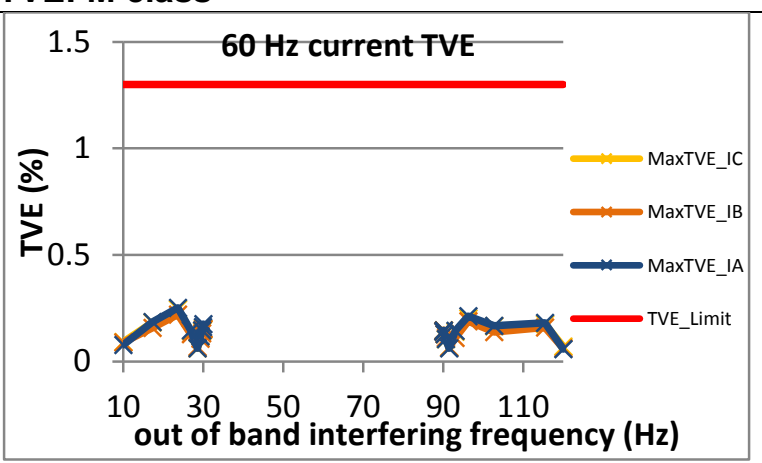

Figure 1623: Fs = $60 \mathrm{FPS}, 60 \mathrm{~Hz}$ fundamental

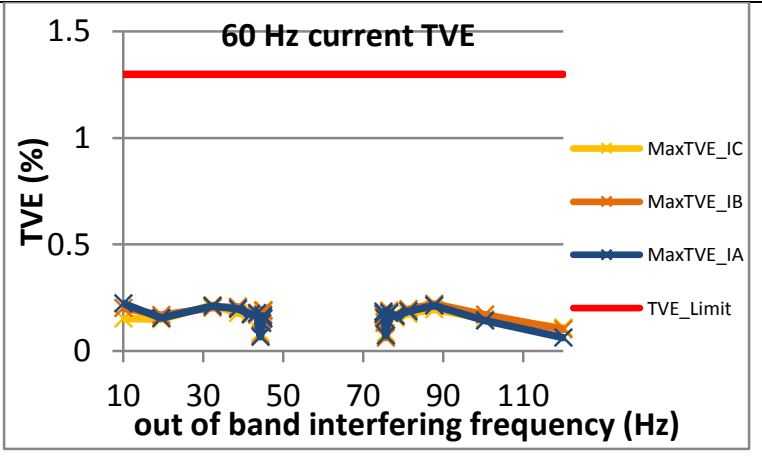

Figure 1626: Fs = 30 FPS, $60 \mathrm{~Hz}$ fundamental

Figure 1629: Fs $=20 \mathrm{FPS}, 60 \mathrm{~Hz}$ fundamental

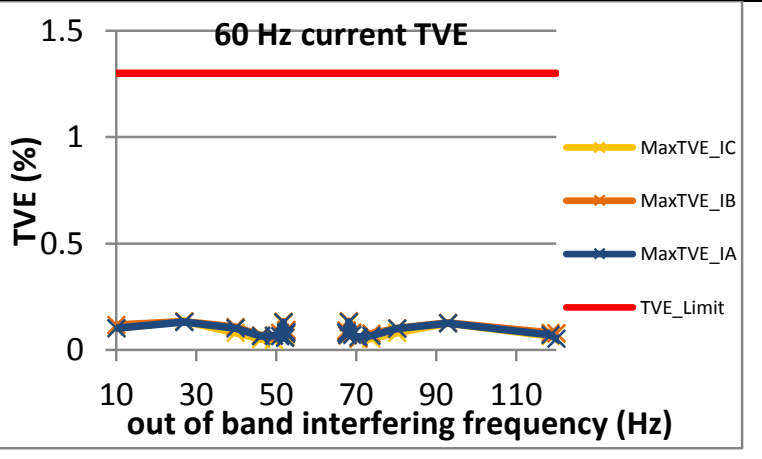

Figure 1632: Fs = $15 \mathrm{FPS}, 60 \mathrm{~Hz}$ fundamental

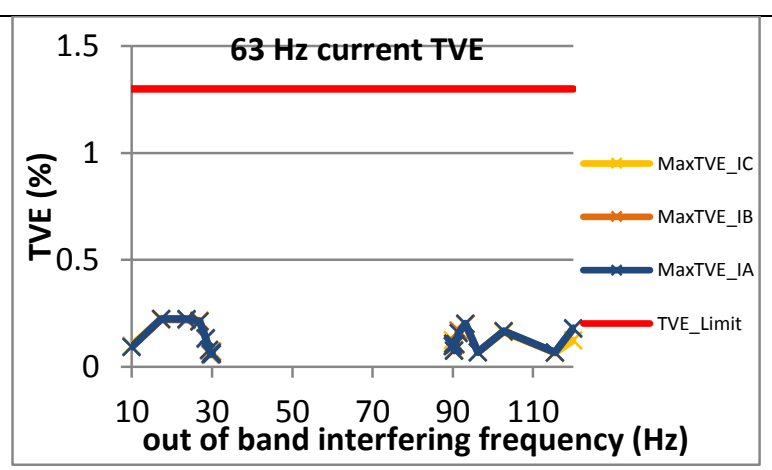

Figure 1624: Fs = $60 \mathrm{FPS}, 63 \mathrm{~Hz}$ fundamental

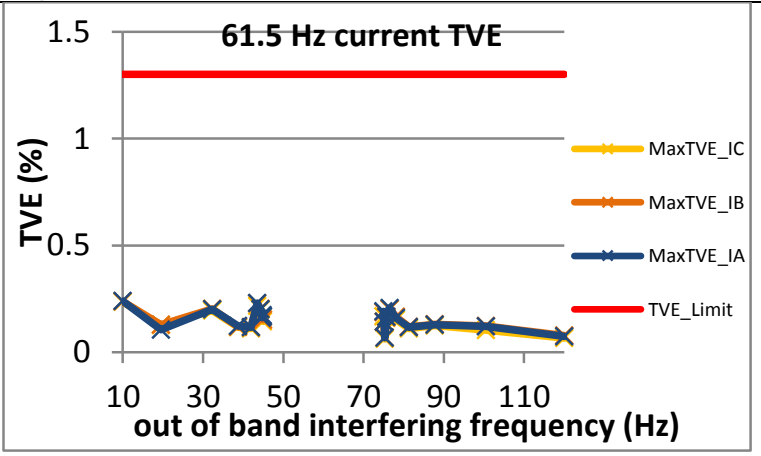

Figure 1627: Fs = 30 FPS, $61.5 \mathrm{~Hz}$ fundamental

Figure 1630: Fs = $20 \mathrm{FPS}, 61 \mathrm{~Hz}$ fundamental

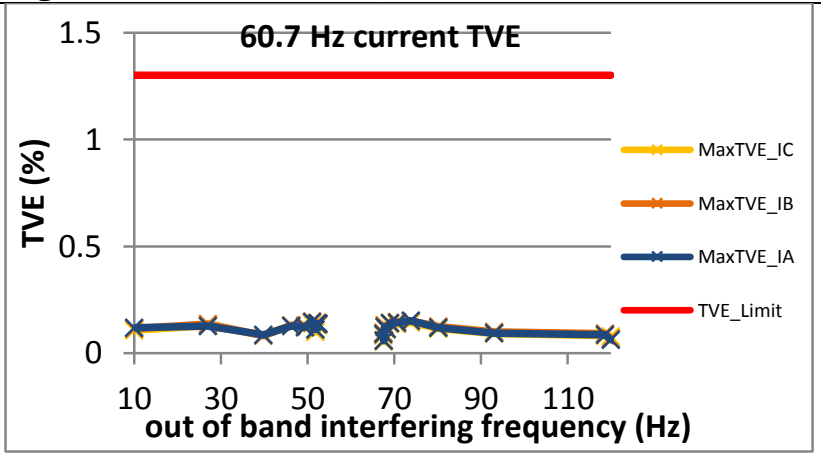

Figure 1633: Fs = 15 FPS, $60.7 \mathrm{~Hz}$ fundamental 

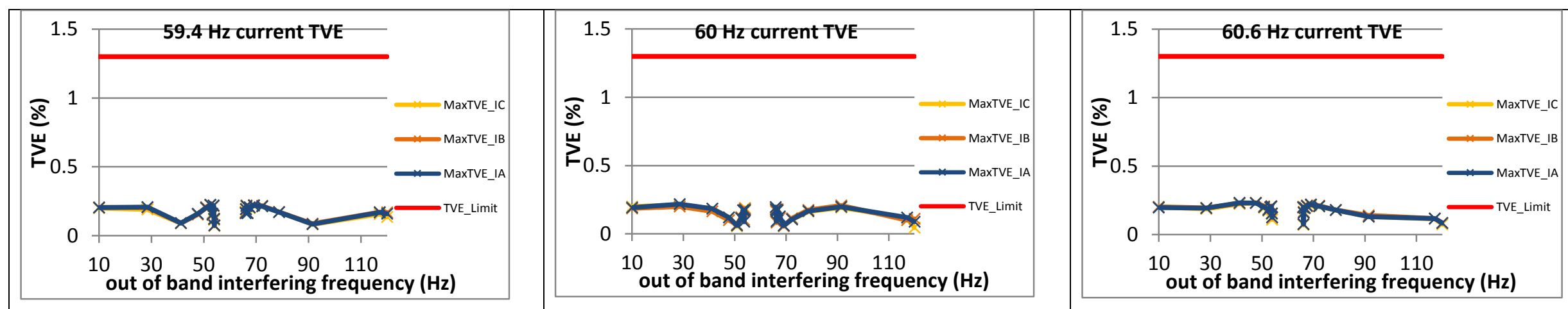

Figure 1634: Fs = 12 FPS, $59.4 \mathrm{~Hz}$ fundamental

Figure 1635: Fs = 12 FPS, $60 \mathrm{~Hz}$ fundamental
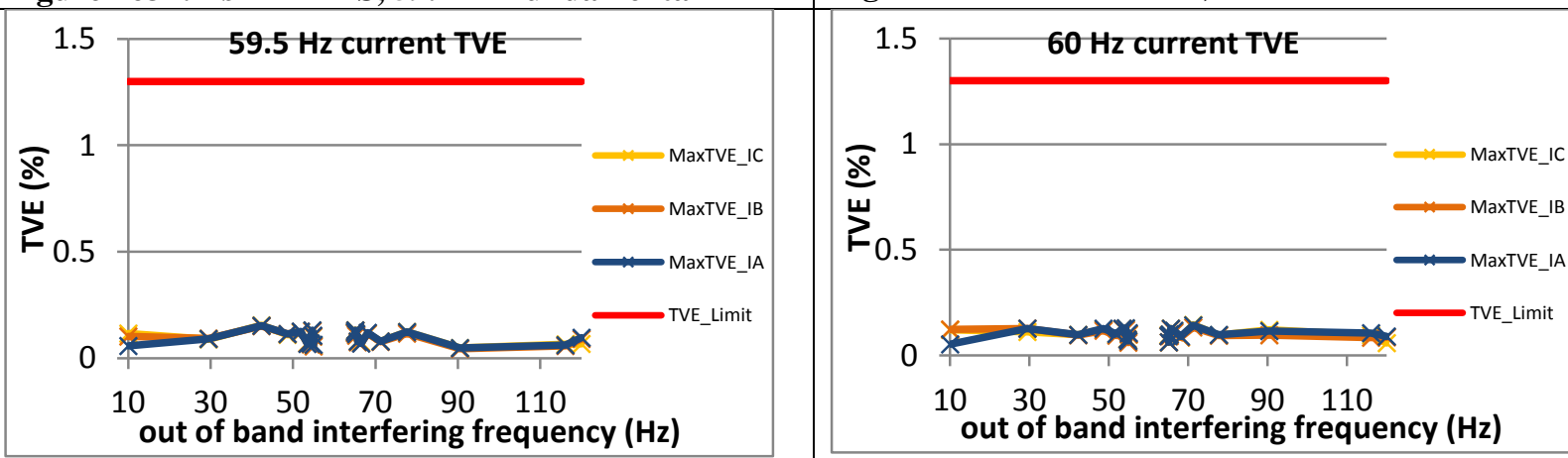

Figure 1638: Fs = $10 \mathrm{FPS}, 60 \mathrm{~Hz}$ fundamental

Figure 1636: Fs = 12 FPS, $60.6 \mathrm{~Hz}$ fundamental

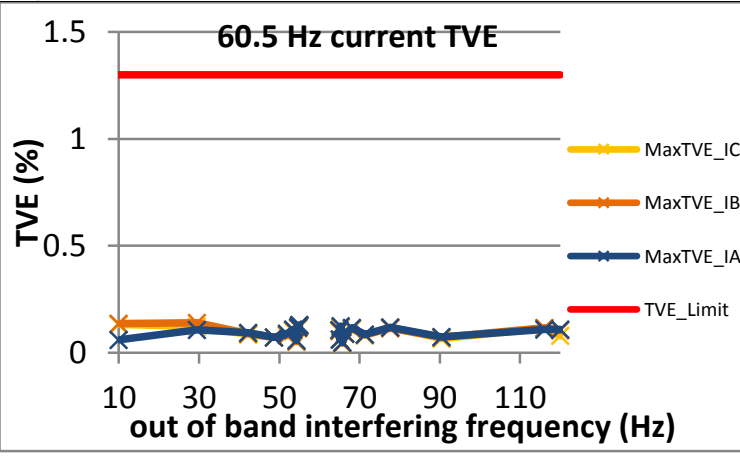

Figure 1639: Fs = $10 \mathrm{FPS}, 60.5 \mathrm{~Hz}$ fundamental 
5.2.6 PMU E out of band interfering signals current TVE: M class
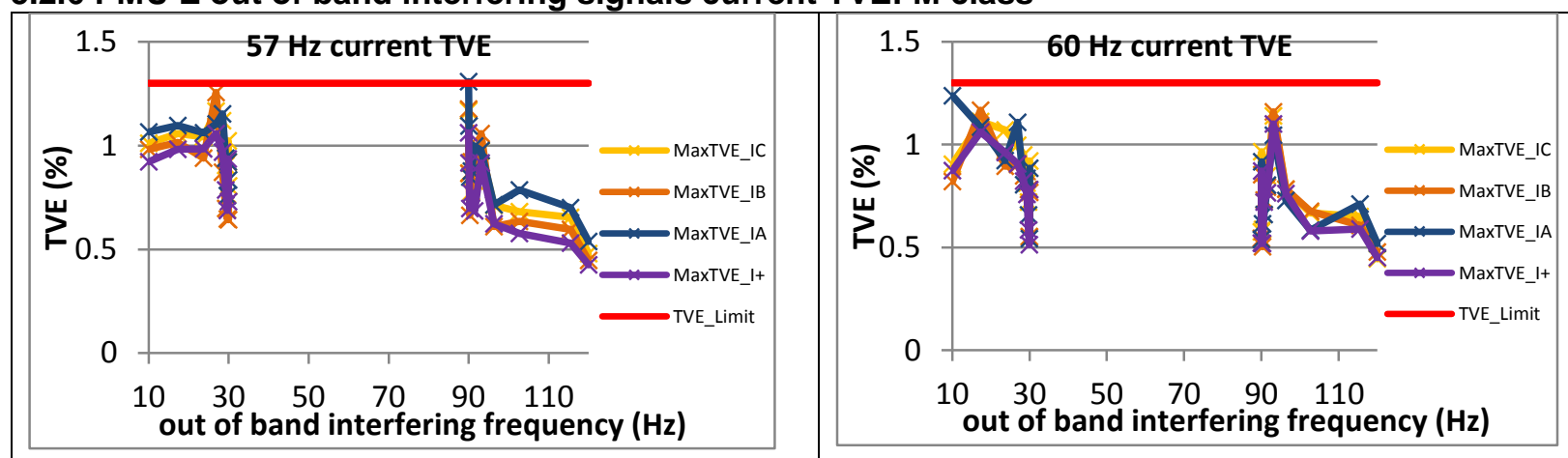

Figure 1641: Fs = $60 \mathrm{FPS}, 60 \mathrm{~Hz}$ fundamental

Figure 1640: Fs = 60 FPS, $57 \mathrm{~Hz}$ fundamental

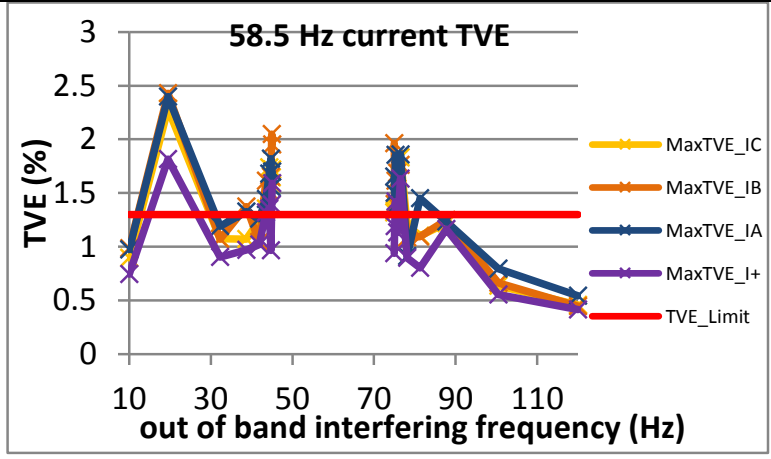

Figure 1643: Fs = 30 FPS, $58.5 \mathrm{~Hz}$ fundamental

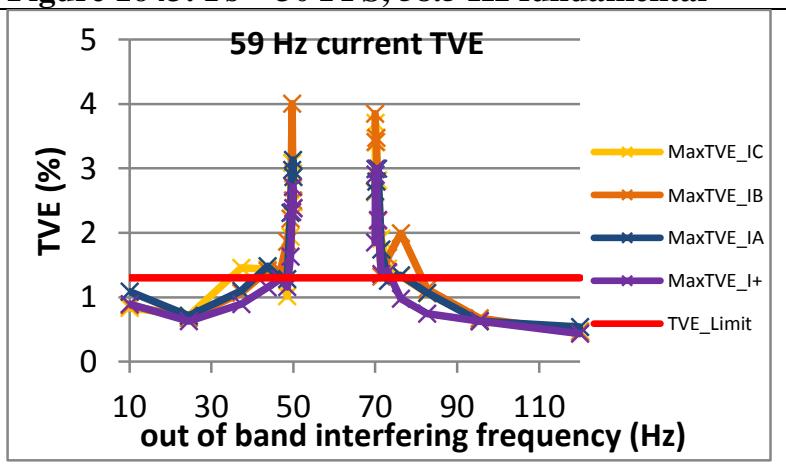

Figure 1646Fs = $20 \mathrm{FPS}, 59 \mathrm{~Hz}$ fundamental

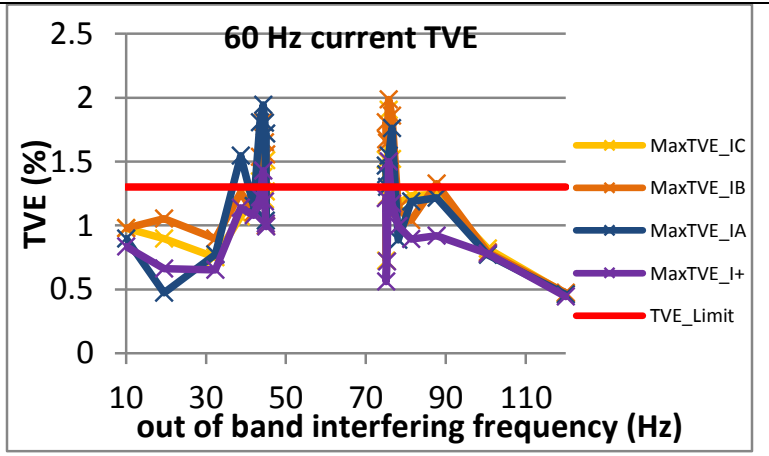

Figure 1644: Fs = $30 \mathrm{FPS}, 60 \mathrm{~Hz}$ fundamental

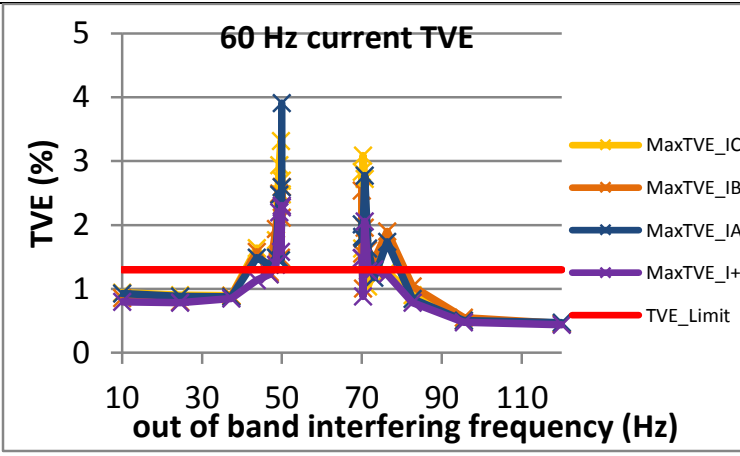

Figure 1647: Fs = $20 \mathrm{FPS}, 60 \mathrm{~Hz}$ fundamental

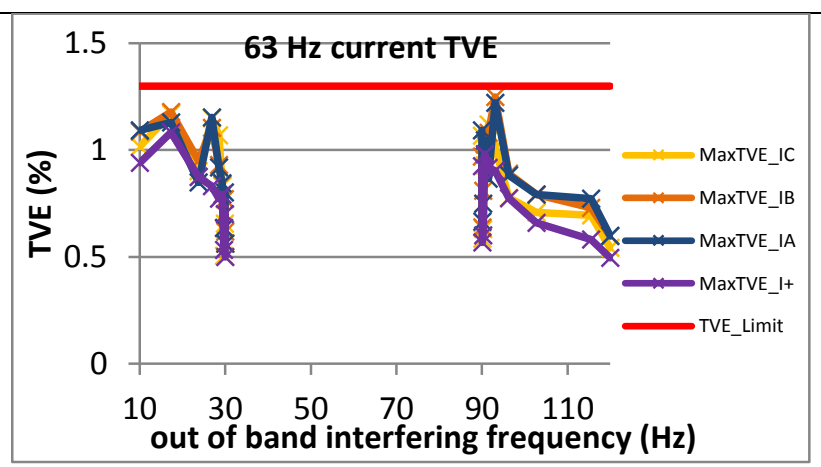

Figure 1642: Fs = 60 FPS, $63 \mathrm{~Hz}$ fundamental

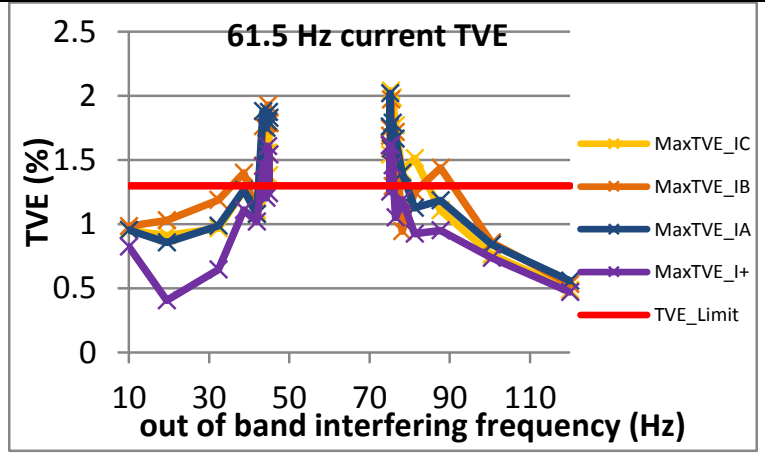

Figure 1645: Fs = 30 FPS, $61.5 \mathrm{~Hz}$ fundamental

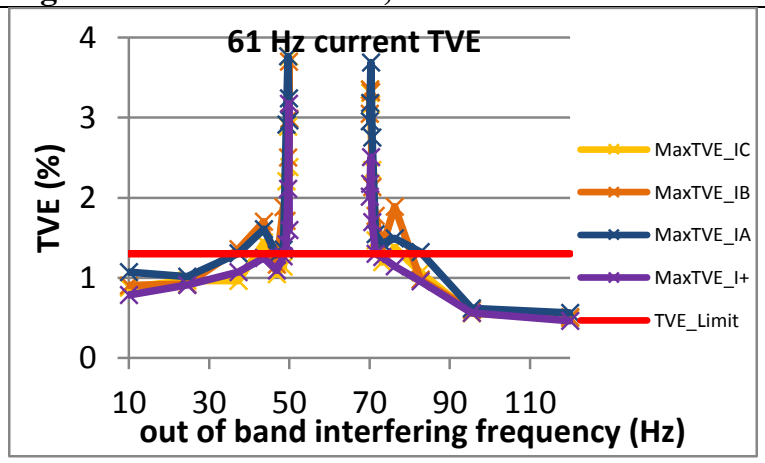

Figure 1648: Fs = $20 \mathrm{FPS}, 61 \mathrm{~Hz}$ fundamental 


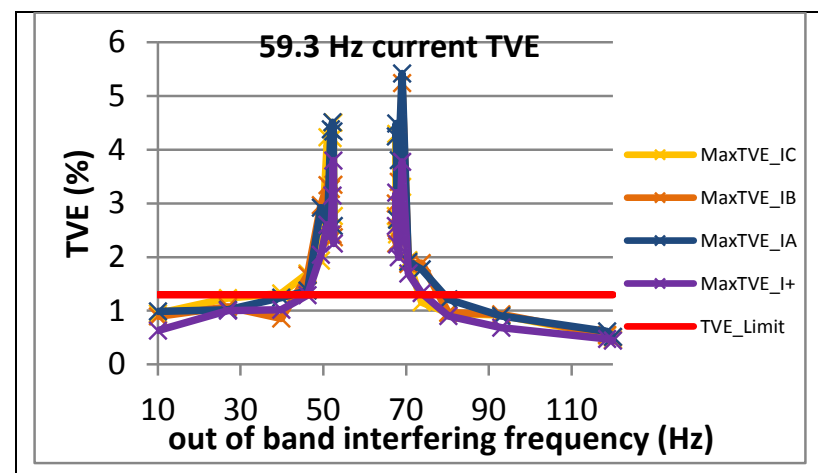

Figure 1649: Fs = 15 FPS, $59.3 \mathrm{~Hz}$ fundamental

Figure 1652: Fs = 12 FPS, $59.4 \mathrm{~Hz}$ fundamental

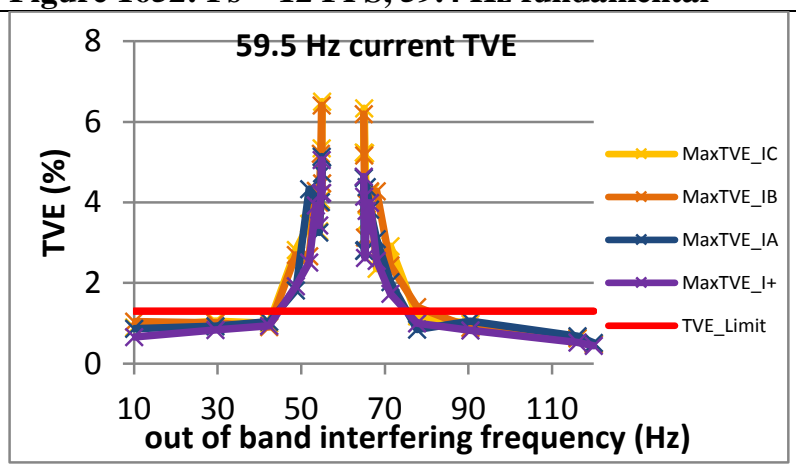

Figure 1655: Fs = 10 FPS, $59.5 \mathrm{~Hz}$ fundamental

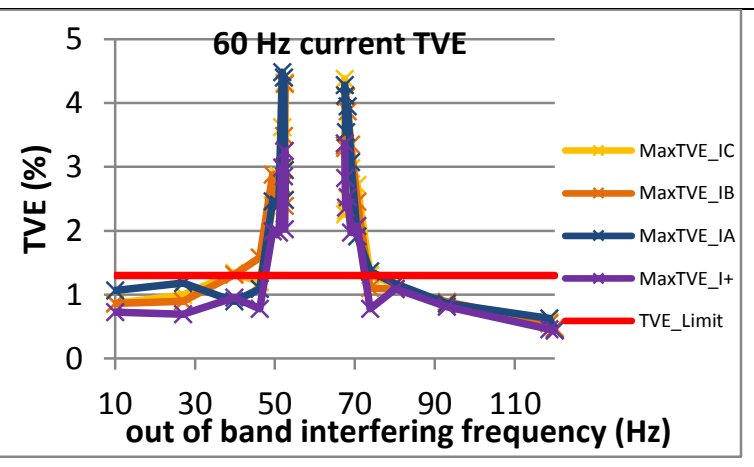

Figure 1650: Fs = $15 \mathrm{FPS}, 60 \mathrm{~Hz}$ fundamental

Figure 1653: Fs = $12 \mathrm{FPS}, 60 \mathrm{~Hz}$ fundamental

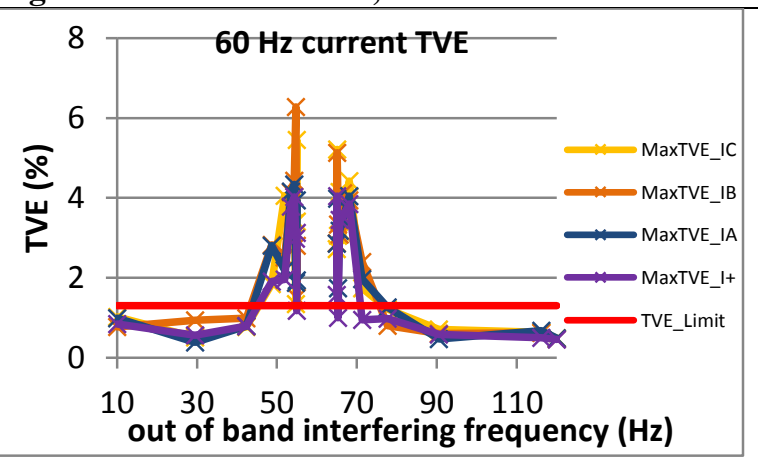

Figure 1656: Fs = $10 \mathrm{FPS}, 60 \mathrm{~Hz}$ fundamental

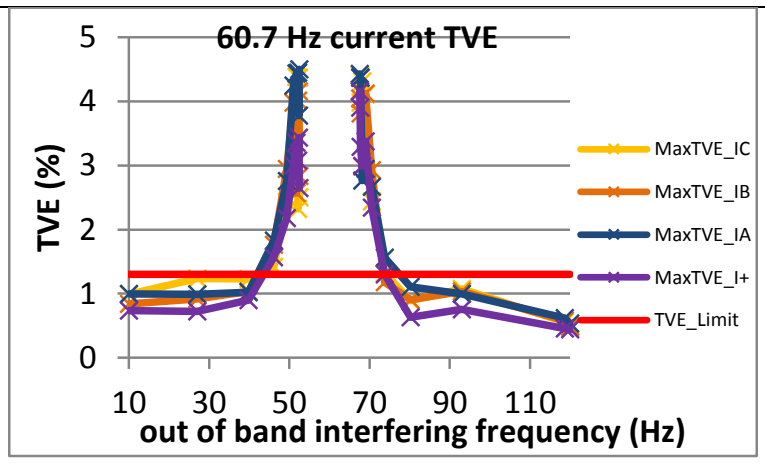

Figure 1651: Fs = 15 FPS, $60.7 \mathrm{~Hz}$ fundamental

Figure 1654: Fs = 12 FPS, $60.6 \mathrm{~Hz}$ fundamental

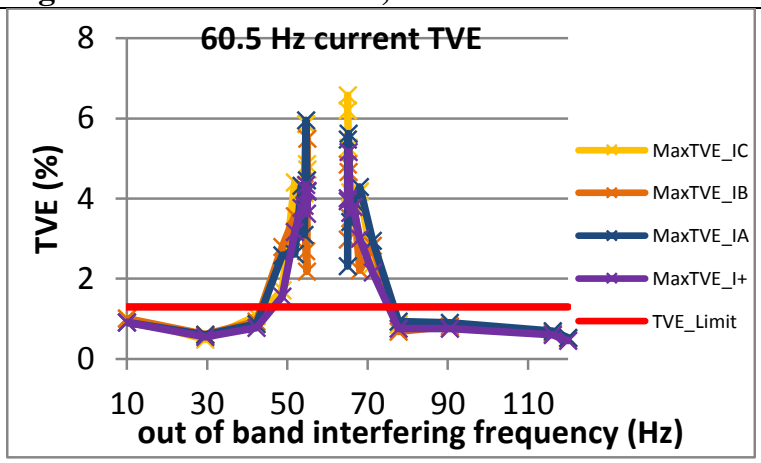

Figure 1657: Fs = $10 \mathrm{FPS}, 60.5 \mathrm{~Hz}$ fundamental 
5.2.7 PMU F out of band interfering signals current TVE: M class

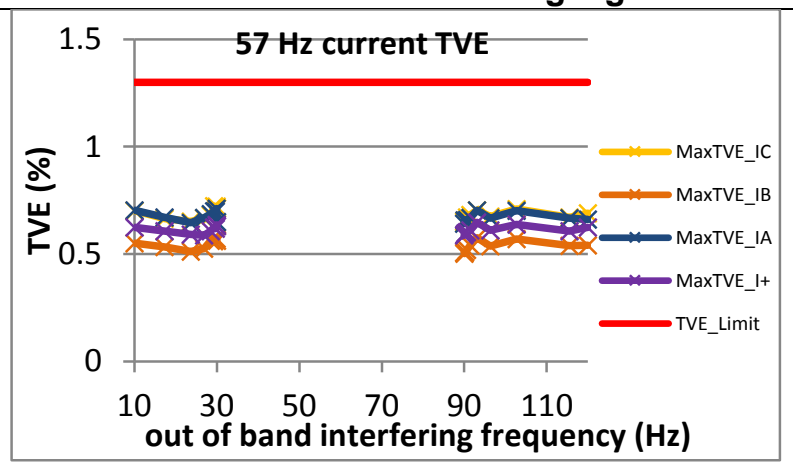

Figure 1658: Fs = $60 \mathrm{FPS}, 57 \mathrm{~Hz}$ fundamental

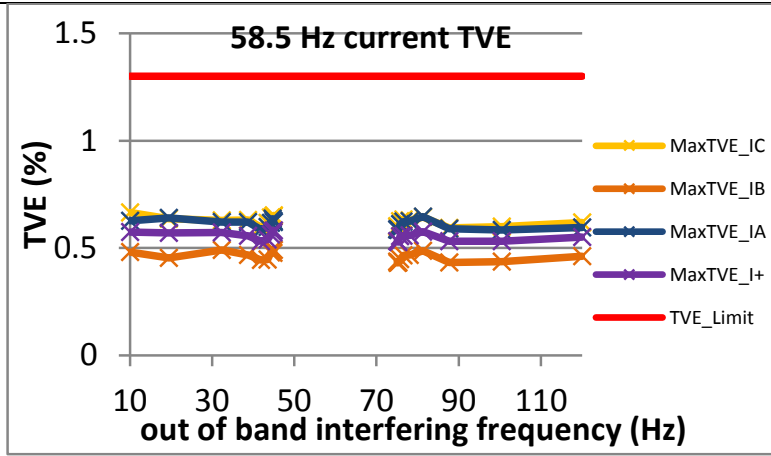

Figure 1661: Fs = $30 \mathrm{FPS}, 58.5 \mathrm{~Hz}$ fundamental

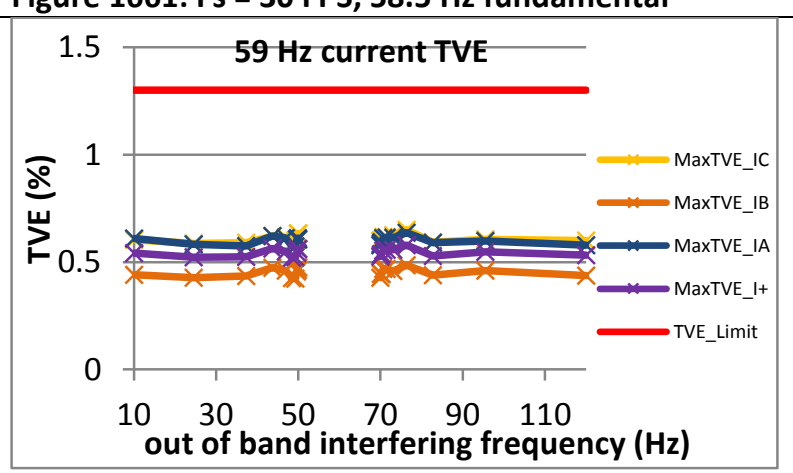

Figure 1664Fs $=20 \mathrm{FPS}, 59 \mathrm{~Hz}$ fundamental

Figure 1667: Fs = $15 \mathrm{FPS}, 59.3 \mathrm{~Hz}$ fundamental

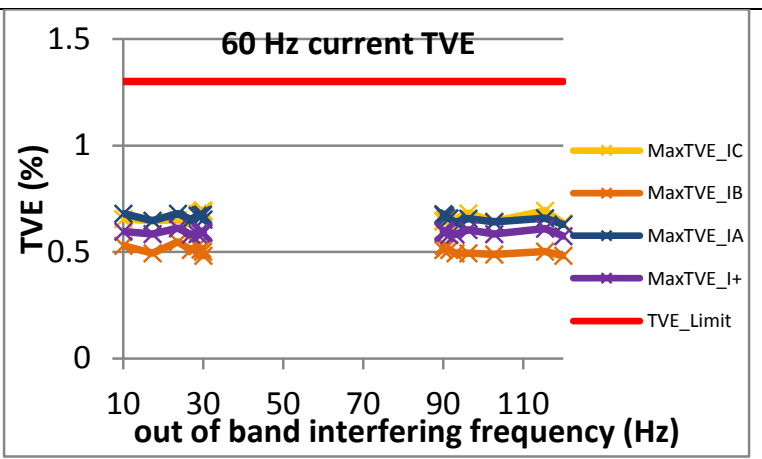

Figure 1659: Fs = $60 \mathrm{FPS}, 60 \mathrm{~Hz}$ fundamental

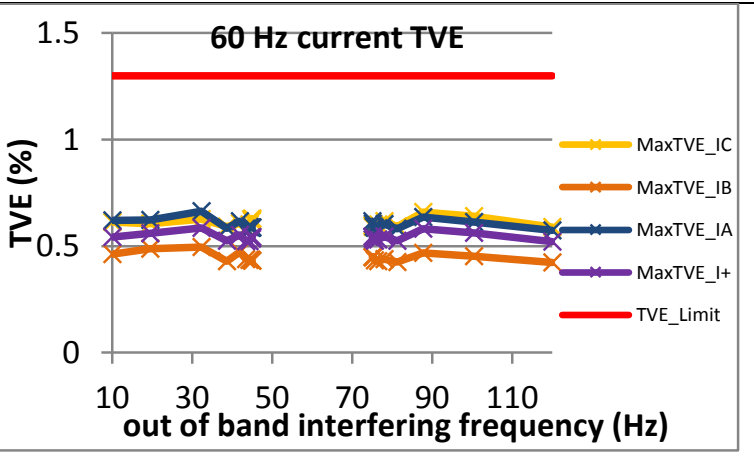

Figure 1662: Fs = $30 \mathrm{FPS}, 60 \mathrm{~Hz}$ fundamental

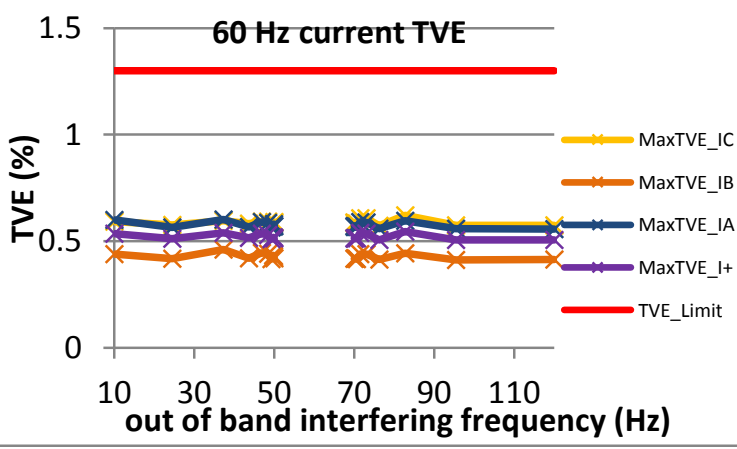

Figure 1665: Fs = 20 FPS, $60 \mathrm{~Hz}$ fundamental

Figure 1668: Fs = $15 \mathrm{FPS}, 60 \mathrm{~Hz}$ fundamental

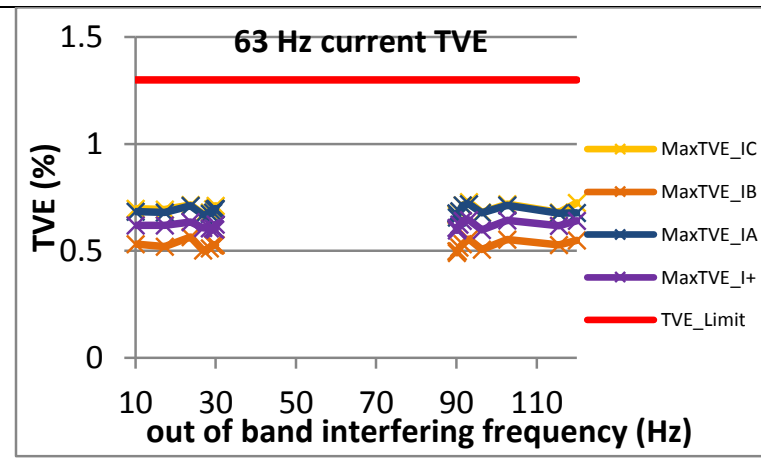

Figure 1660: Fs = $60 \mathrm{FPS}, 63 \mathrm{~Hz}$ fundamental

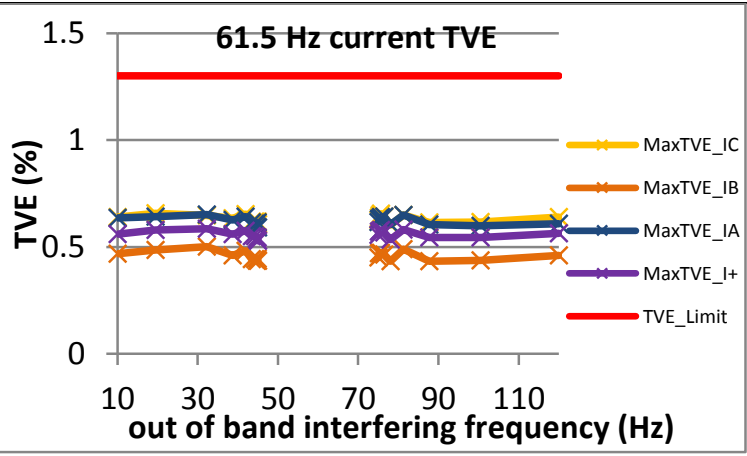

Figure 1663: Fs = 30 FPS, $61.5 \mathrm{~Hz}$ fundamental

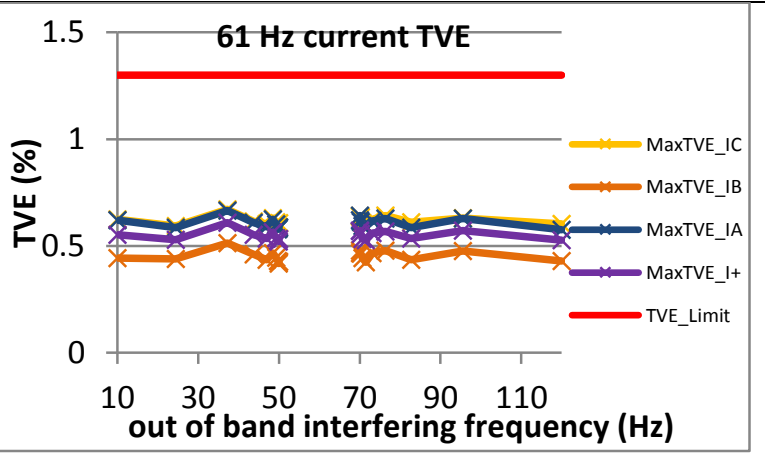

Figure 1666: Fs = $20 \mathrm{FPS}, 61 \mathrm{~Hz}$ fundamental

Figure 1669: Fs = $15 \mathrm{FPS}, 60.7 \mathrm{~Hz}$ fundamental 


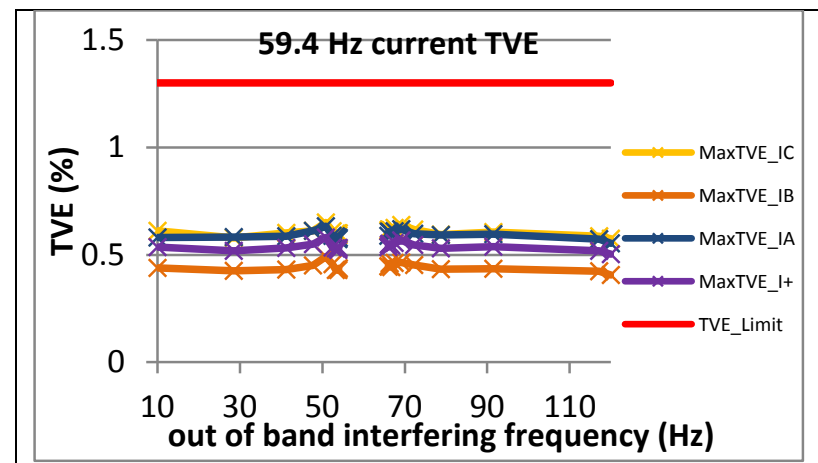

Figure 1670: Fs = $12 \mathrm{FPS}, 59.4 \mathrm{~Hz}$ fundamental

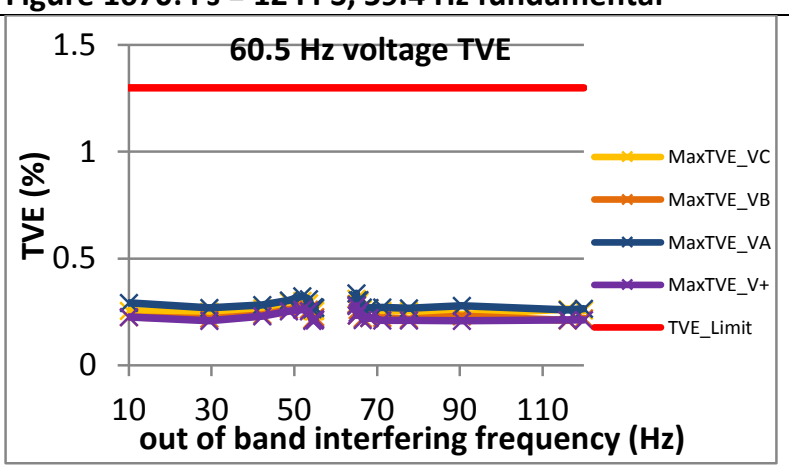

Figure 1673: Fs = $10 \mathrm{FPS}, 59.5 \mathrm{~Hz}$ fundamental

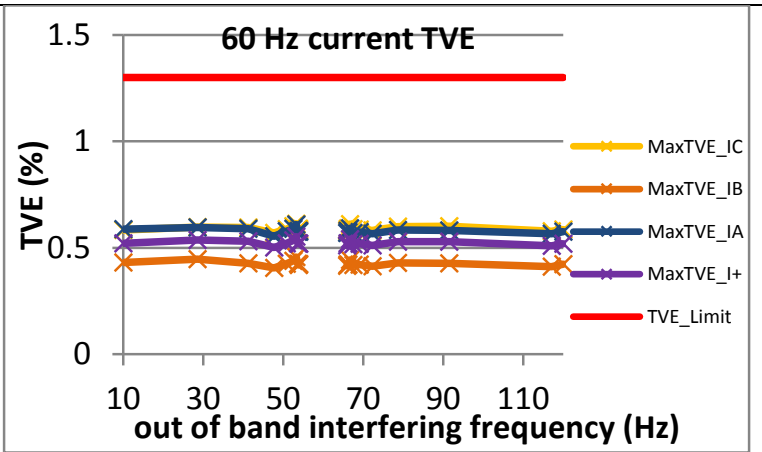

Figure 1671: Fs = $12 \mathrm{FPS}, 60 \mathrm{~Hz}$ fundamental

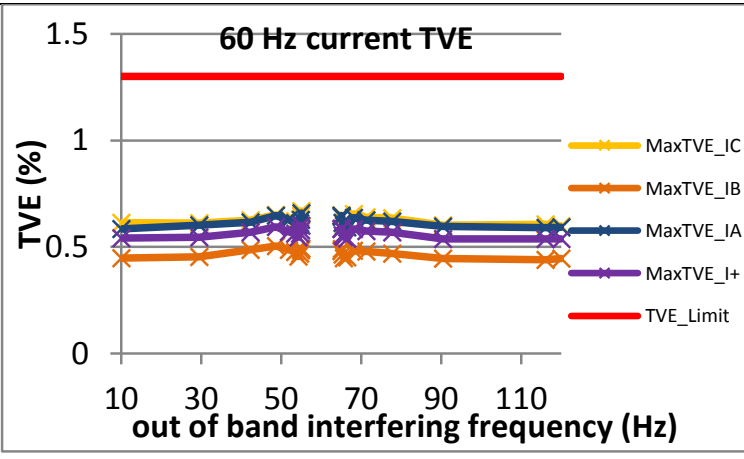

Figure 1674: Fs = $10 \mathrm{FPS}, 60 \mathrm{~Hz}$ fundamental

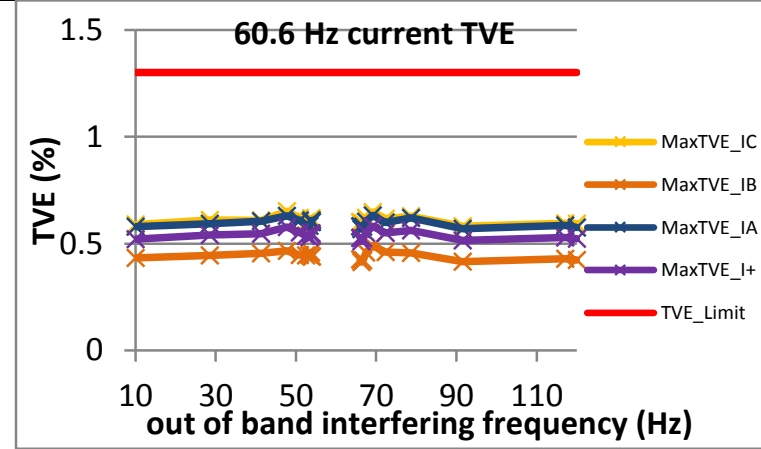

Figure 1672: Fs = $12 \mathrm{FPS}, 60.6 \mathrm{~Hz}$ fundamental

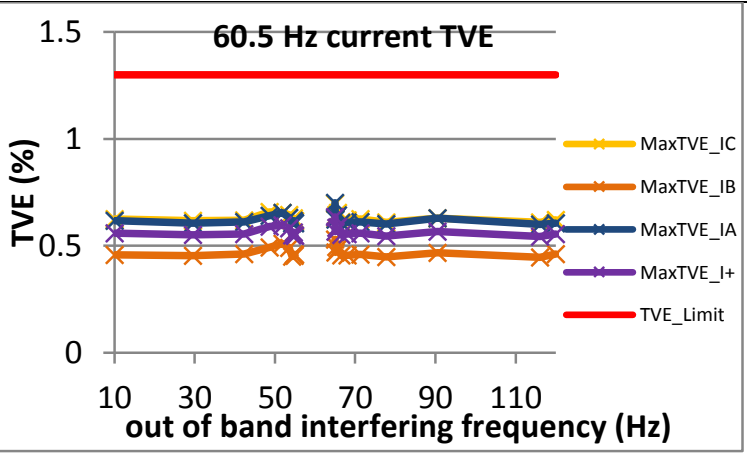

Figure 1675: Fs = $10 \mathrm{FPS}, 60.5 \mathrm{~Hz}$ fundamental 


\subsubsection{PMU G out of band interfering signals current TVE: M class}

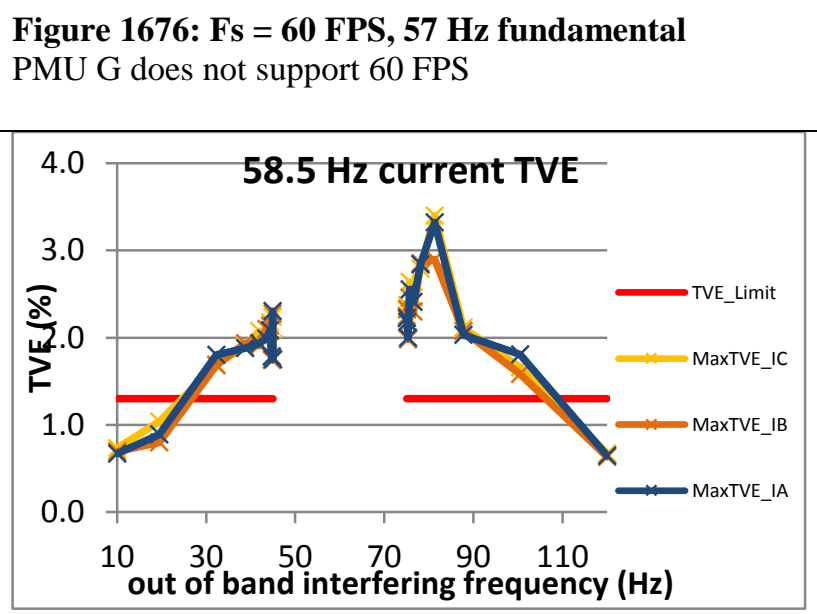

Figure 1679: Fs = 30 FPS, $58.5 \mathrm{~Hz}$ fundamental

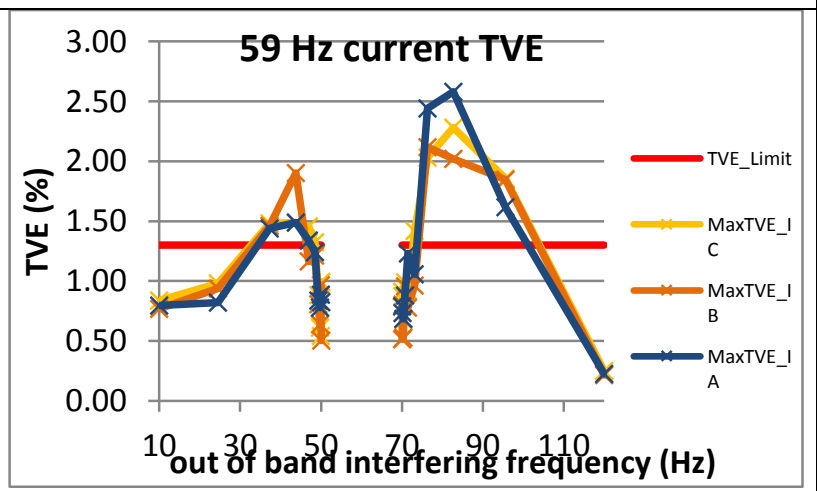

Figure 1682Fs = 20 FPS, $59 \mathrm{~Hz}$ fundamental
Figure 1677: Fs = 60 FPS, $60 \mathrm{~Hz}$ fundamental PMU G does not support 60 FPS

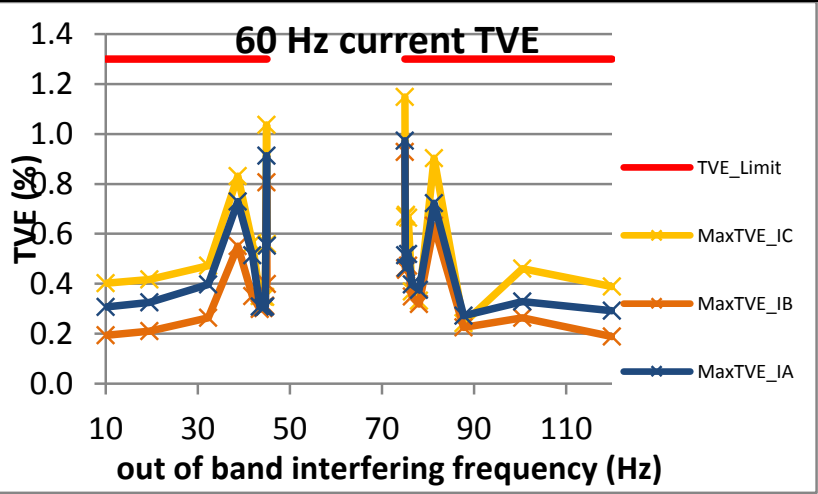

Figure 1680: Fs = 30 FPS, $60 \mathrm{~Hz}$ fundamental

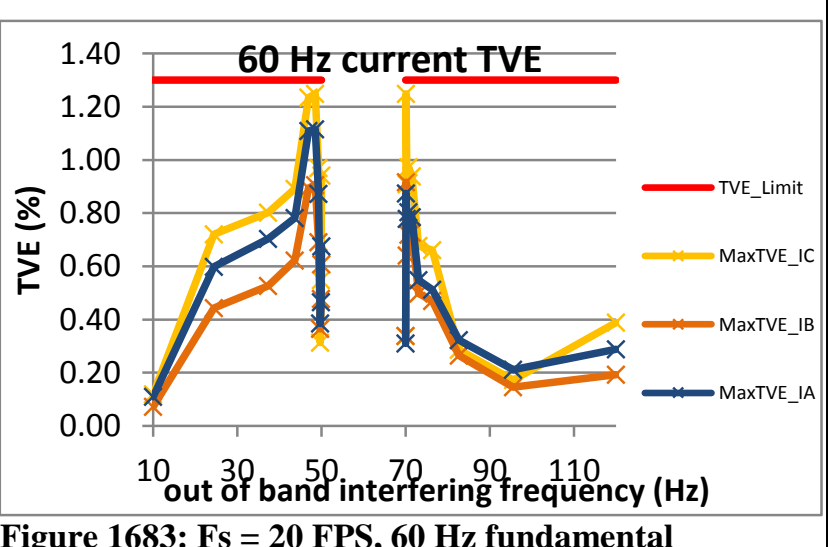

Figure 1683: Fs = 20 FPS, $60 \mathrm{~Hz}$ fundamental
Figure 1678: Fs = $60 \mathrm{FPS}, 63 \mathrm{~Hz}$ fundamental PMU G does not support 60 FPS

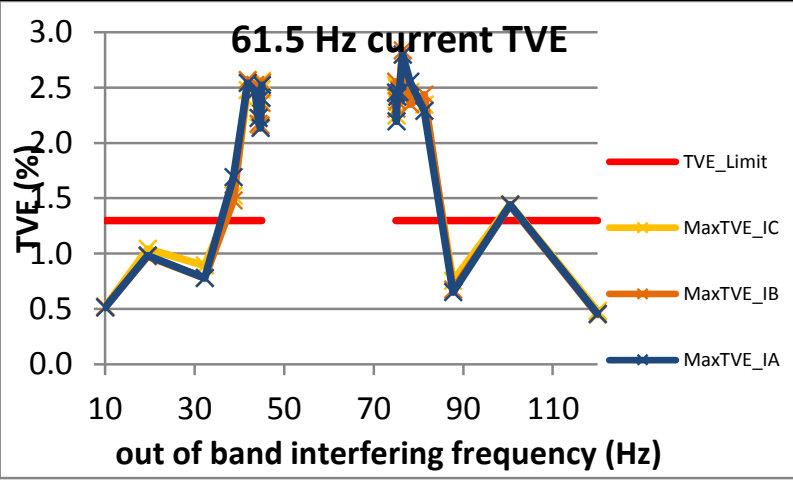

Figure 1681: Fs = 30 FPS, $61.5 \mathrm{~Hz}$ fundamental

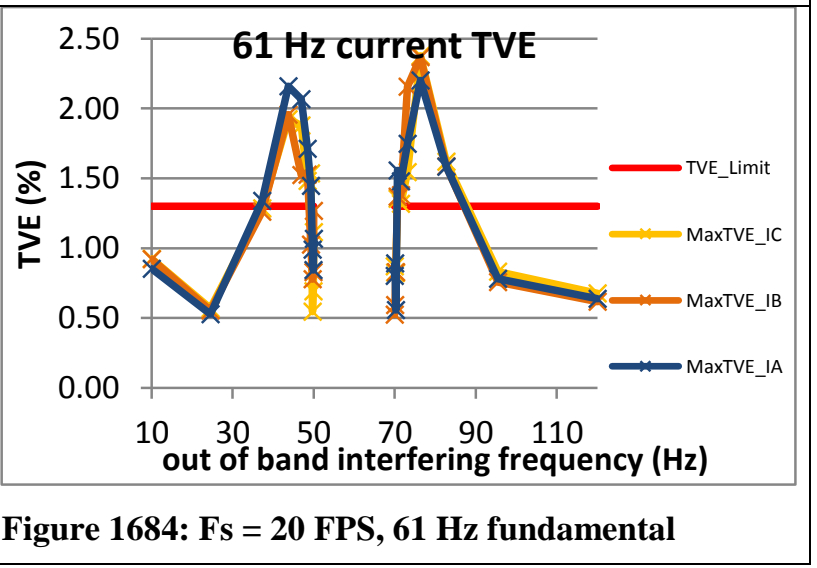



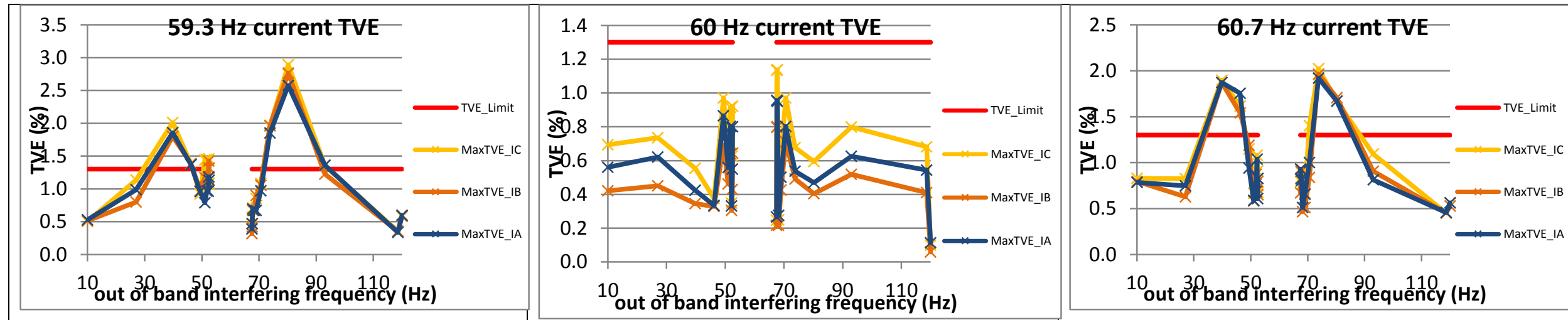

Figure 1685: Fs = 15 FPS, $59.3 \mathrm{~Hz}$ fundamental

Figure 1686: Fs = 15 FPS, $60 \mathrm{~Hz}$ fundamental
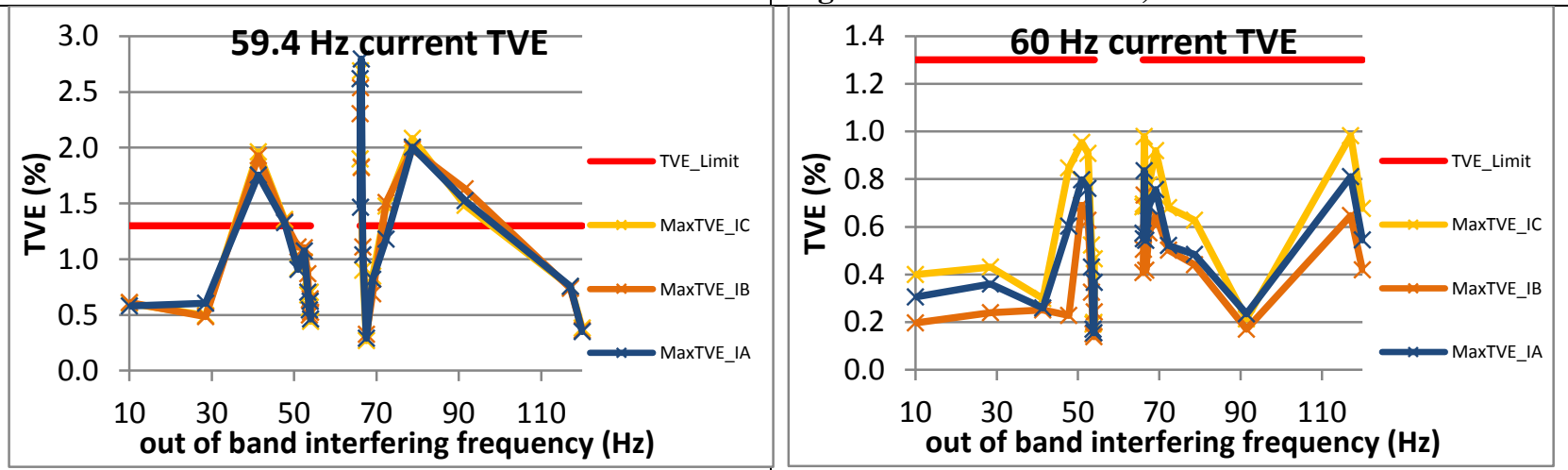

Figure 1687: Fs = 15 FPS, $60.7 \mathrm{~Hz}$ fundamental

Figure 1688: Fs = 12 FPS, $59.4 \mathrm{~Hz}$ fundamental Figure 1689: Fs = 12 FPS, $60 \mathrm{~Hz}$ fundamental
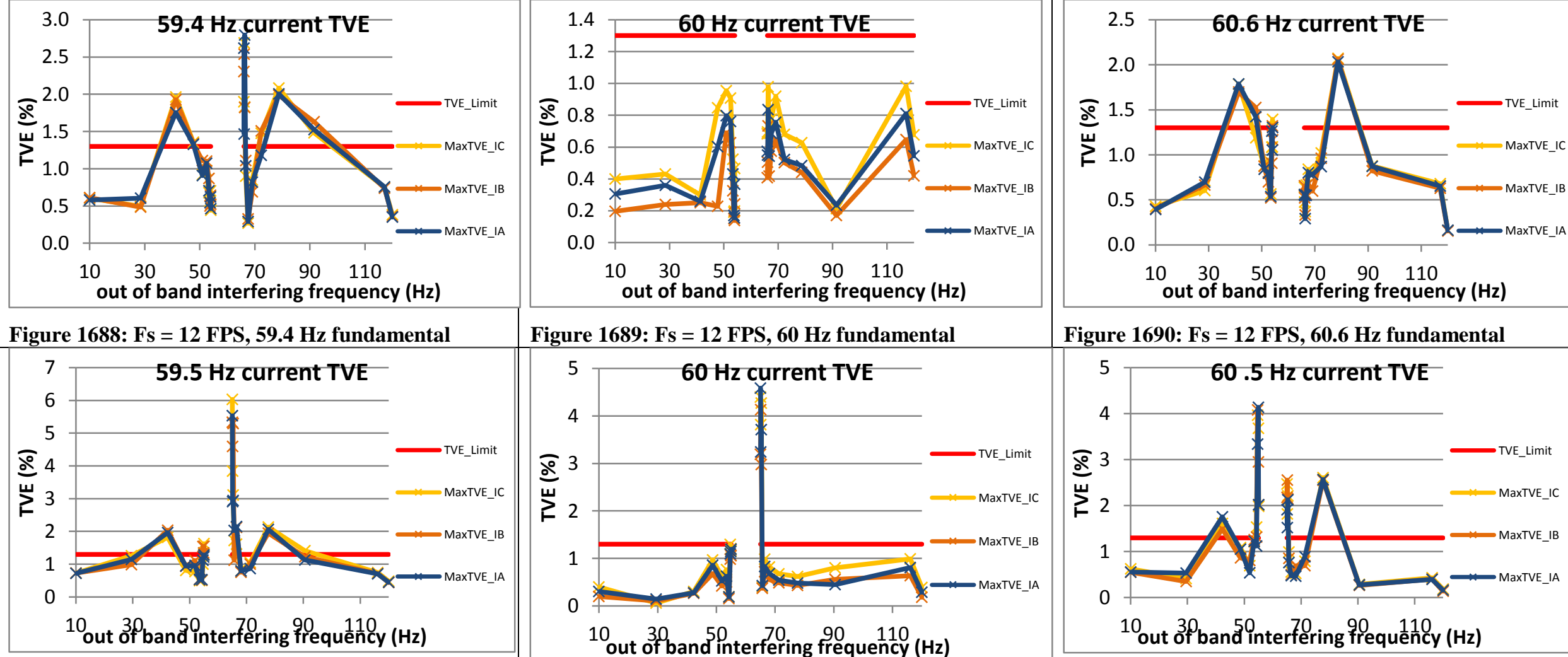

Figure 1691: Fs = 10 FPS, $59.5 \mathrm{~Hz}$ fundamental

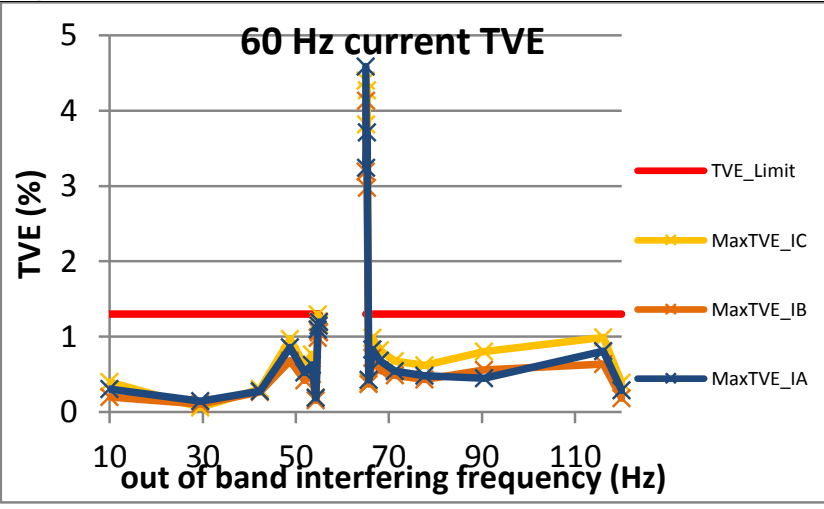

Figure 1690: Fs = 12 FPS, $60.6 \mathrm{~Hz}$ fundamental

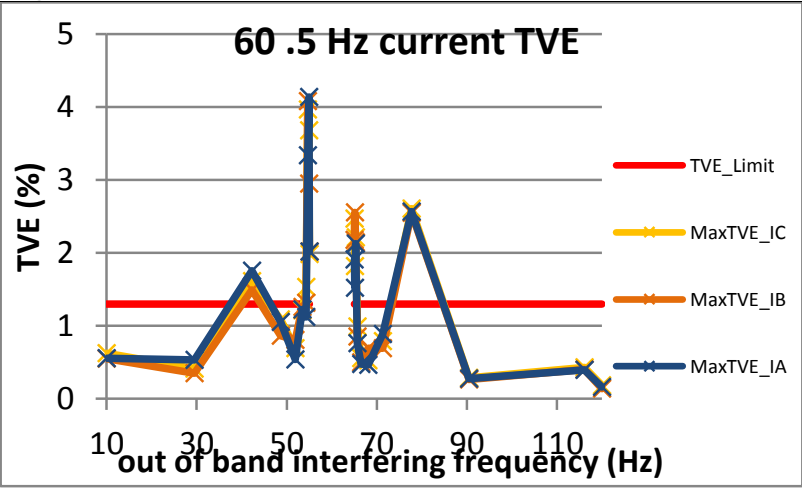

Figure 1692: Fs = $10 \mathrm{FPS}, 60 \mathrm{~Hz}$ fundamental

Figure 1693: Fs = 10 FPS, $60.5 \mathrm{~Hz}$ fundamental 
5.2.9 PMU H out of band interfering signals current TVE: M class
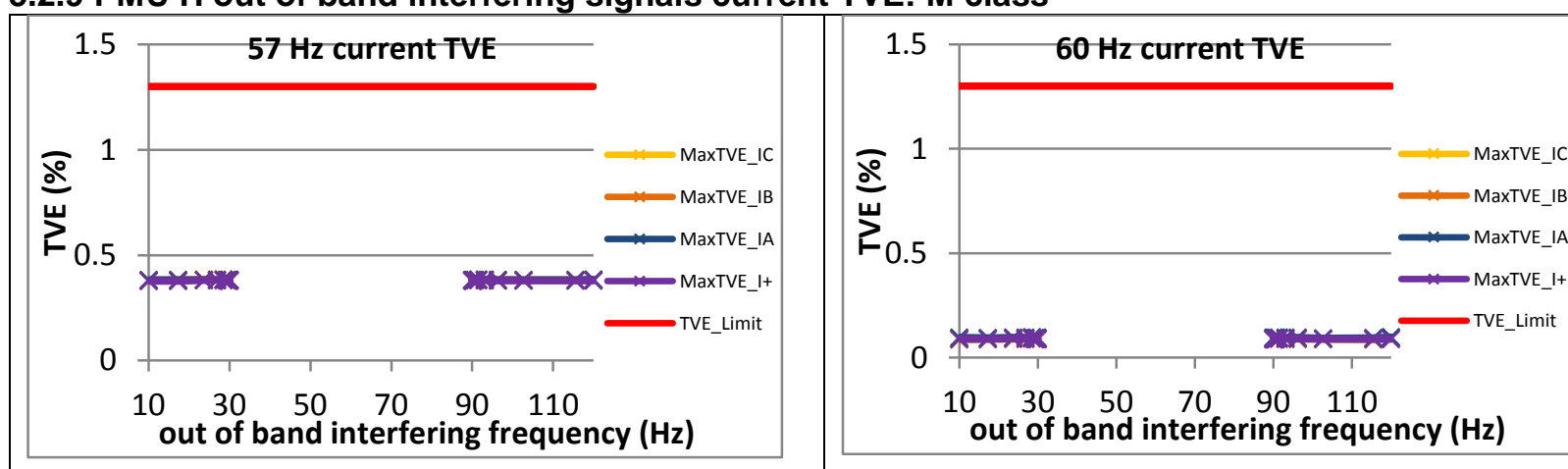

Figure 1695: Fs = $60 \mathrm{FPS}, 60 \mathrm{~Hz}$ fundamental

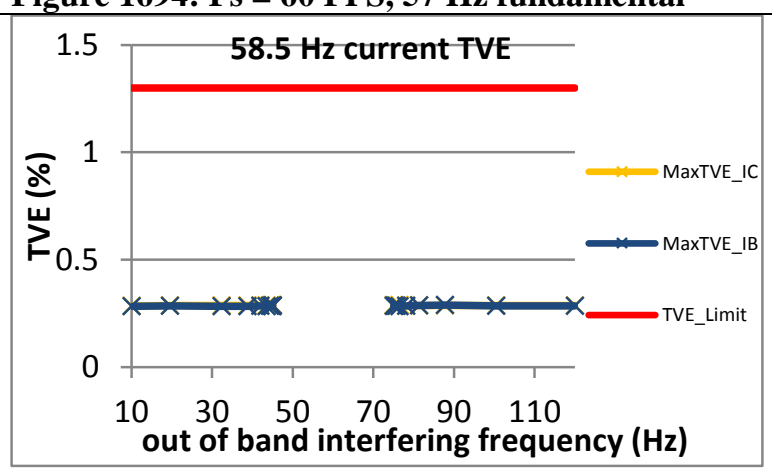

Figure 1697: Fs = 30 FPS, $58.5 \mathrm{~Hz}$ fundamental

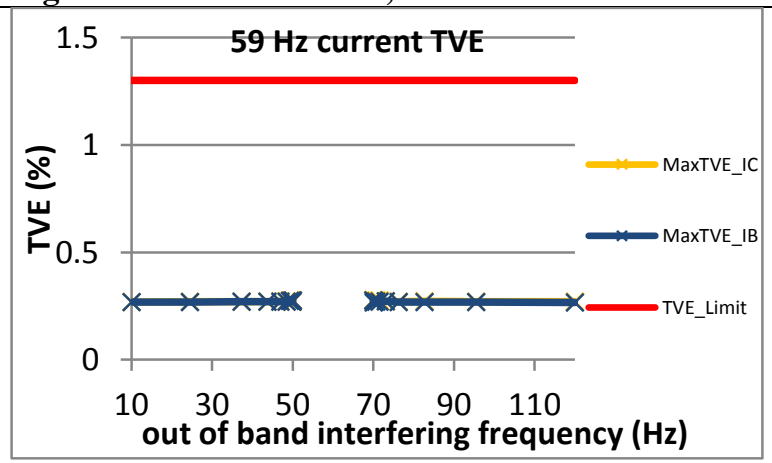

Figure 1700Fs = $20 \mathrm{FPS}, 59 \mathrm{~Hz}$ fundamental

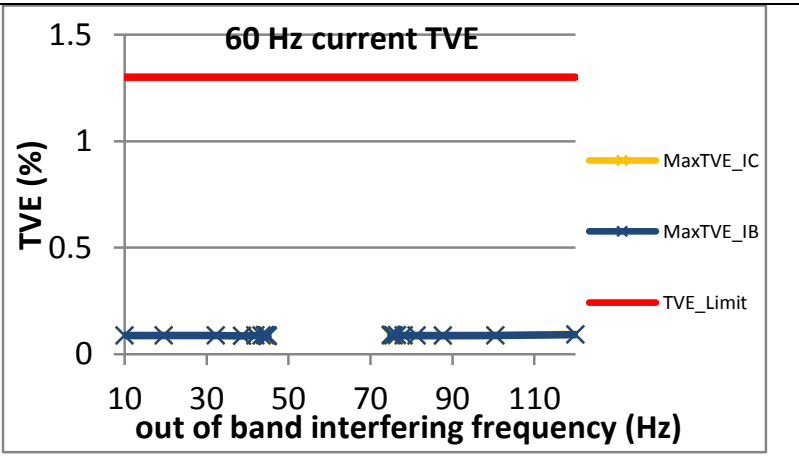

Figure 1698: Fs = $30 \mathrm{FPS}, 60 \mathrm{~Hz}$ fundamental

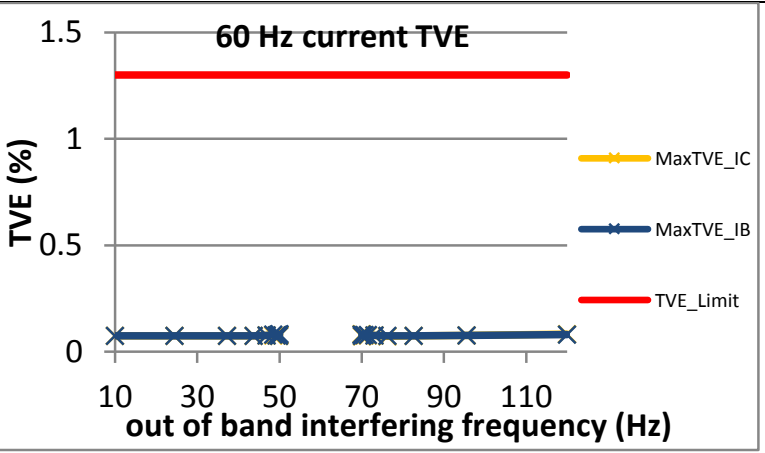

Figure 1701: Fs $=20$ FPS, $60 \mathrm{~Hz}$ fundamental

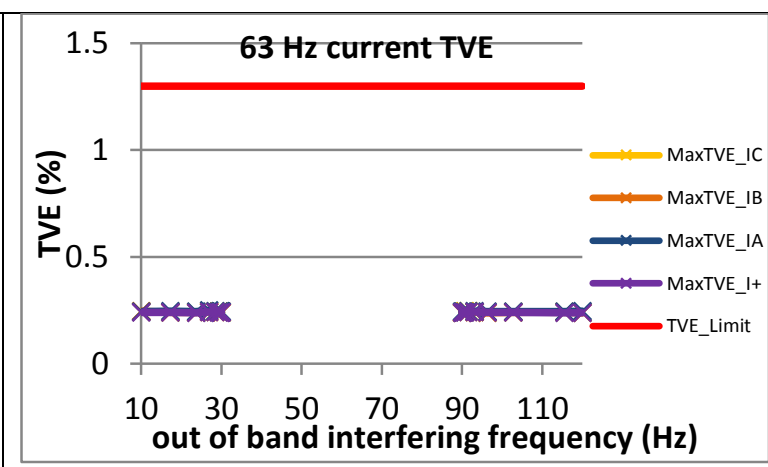

Figure 1696: Fs = 60 FPS, $63 \mathrm{~Hz}$ fundamental

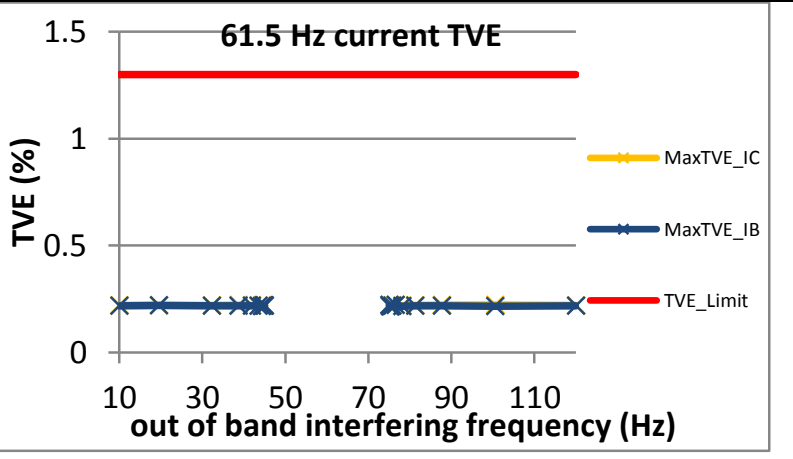

Figure 1699: Fs = 30 FPS, 61.5 Hz fundamental

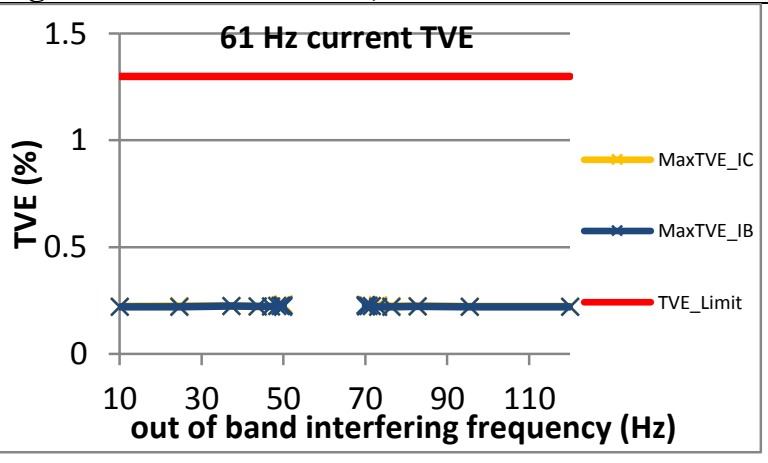

Figure 1702: Fs = 20 FPS, $61 \mathrm{~Hz}$ fundamental 


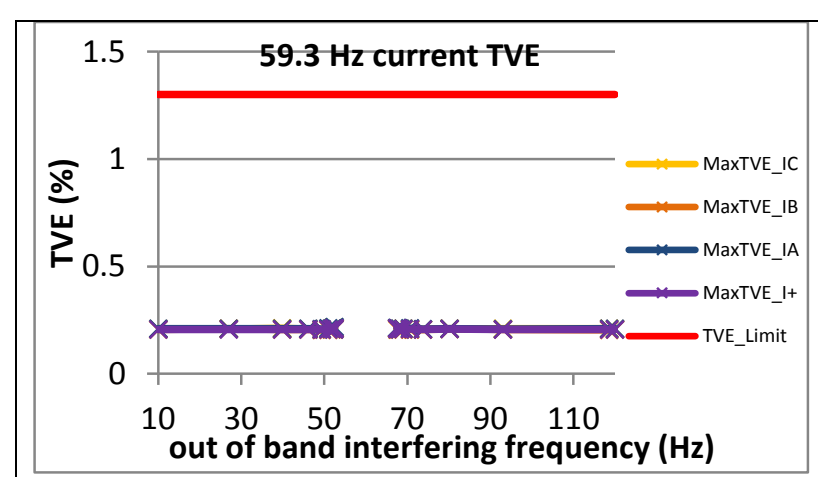

Figure 1703: Fs = 15 FPS, $59.3 \mathrm{~Hz}$ fundamental

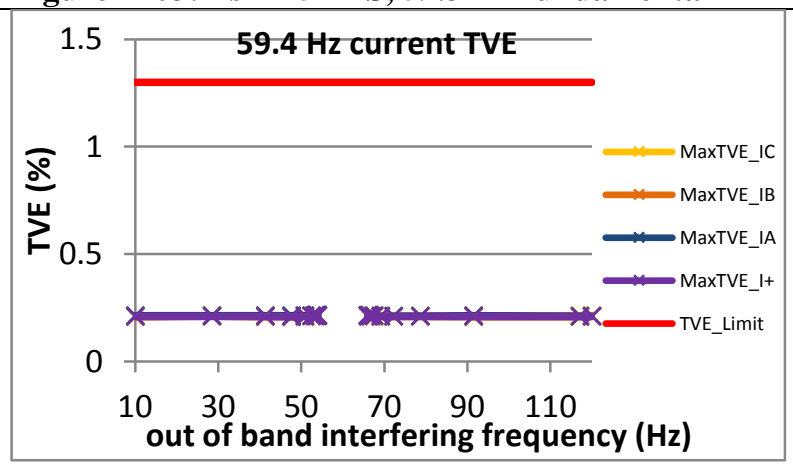

Figure 1706: Fs = 12 FPS, $59.4 \mathrm{~Hz}$ fundamental

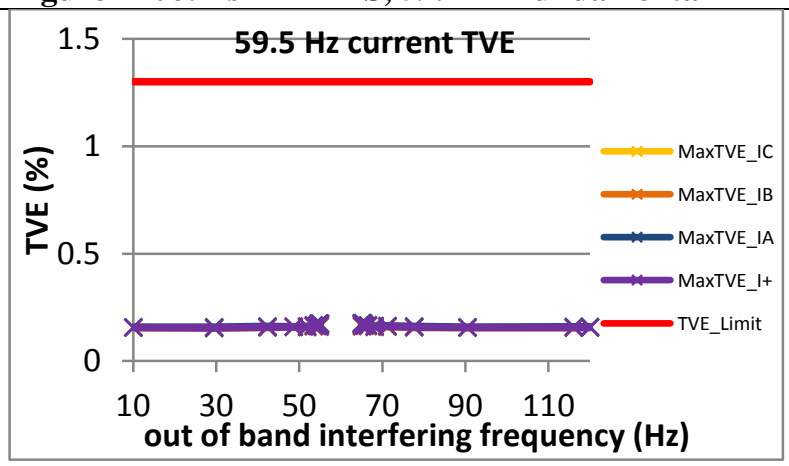

Figure 1709: Fs = 10 FPS, $59.5 \mathrm{~Hz}$ fundamental

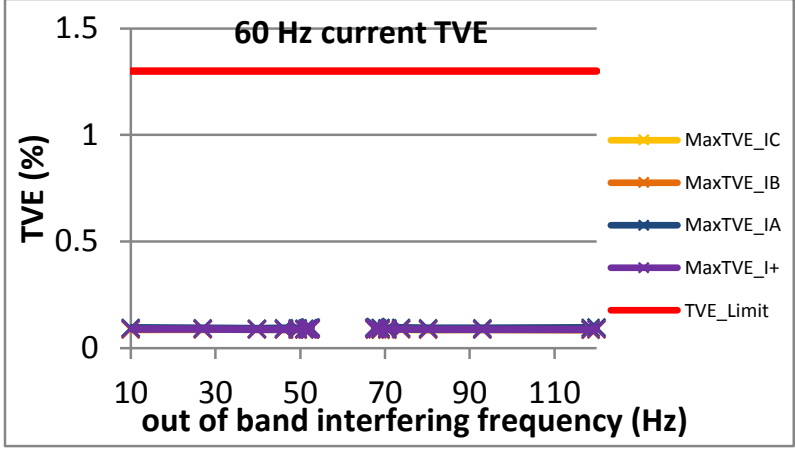

Figure 1704: Fs = 15 FPS, $60 \mathrm{~Hz}$ fundamental

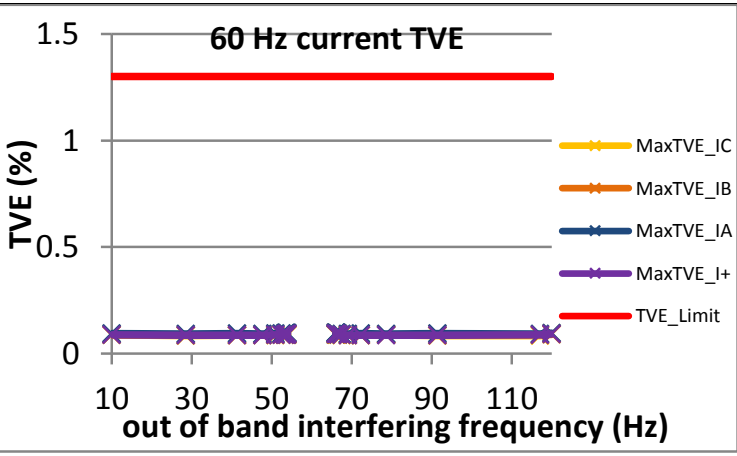

Figure 1707: Fs = 12 FPS, $60 \mathrm{~Hz}$ fundamental

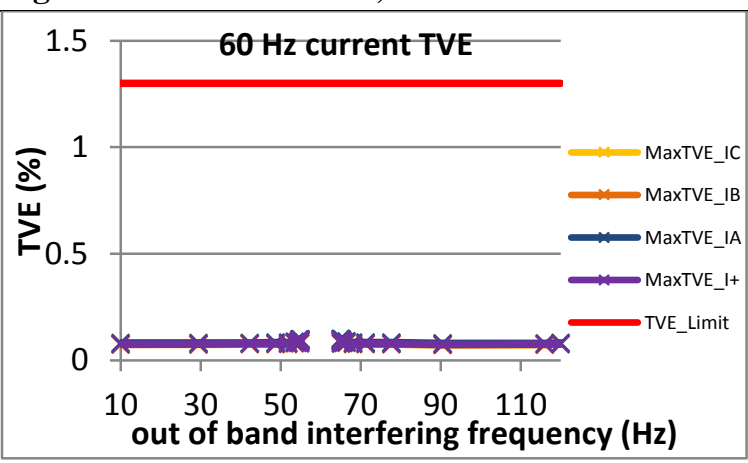

Figure 1710: Fs = $10 \mathrm{FPS}, 60 \mathrm{~Hz}$ fundamental

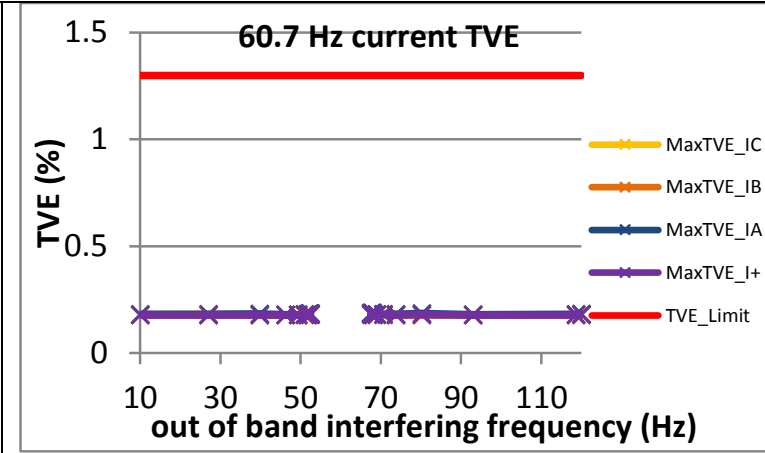

Figure 1705: Fs = 15 FPS, $60.7 \mathrm{~Hz}$ fundamental

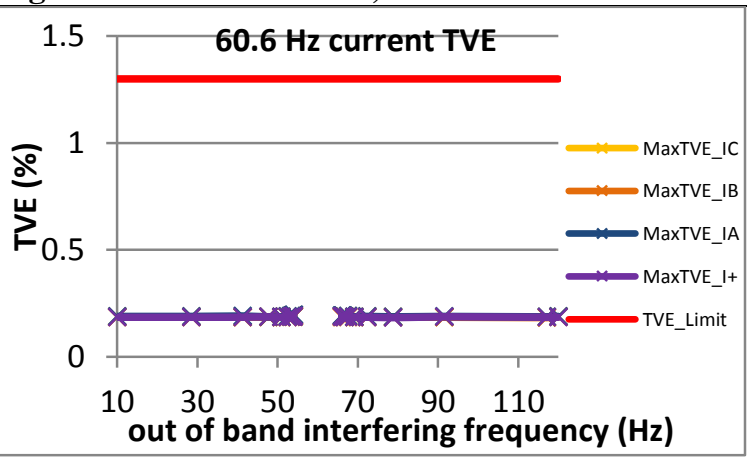

Figure 1708: Fs = 12 FPS, $60.6 \mathrm{~Hz}$ fundamental

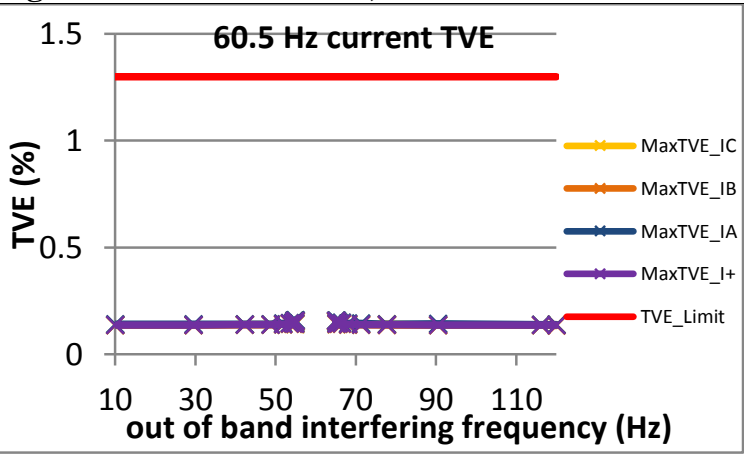

Figure 1711: Fs = 10 FPS, $60.5 \mathrm{~Hz}$ fundamental 
5.2.10 PMU I out of band interfering signals current TVE: M class

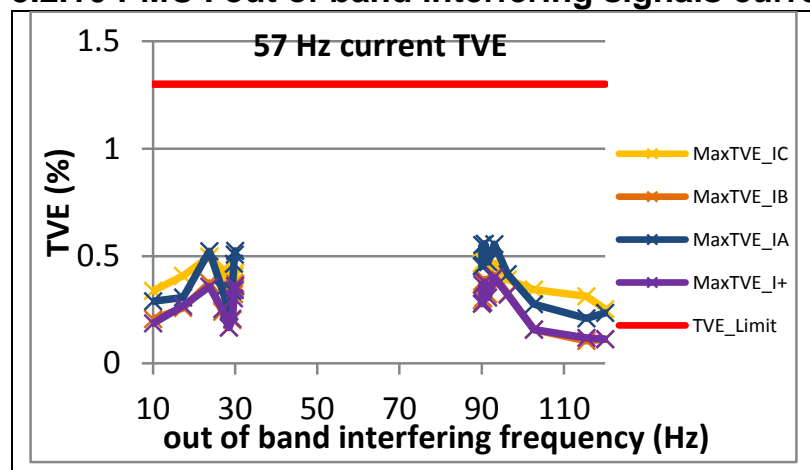

Figure 1712: Fs = 60 FPS, $57 \mathrm{~Hz}$ fundamental

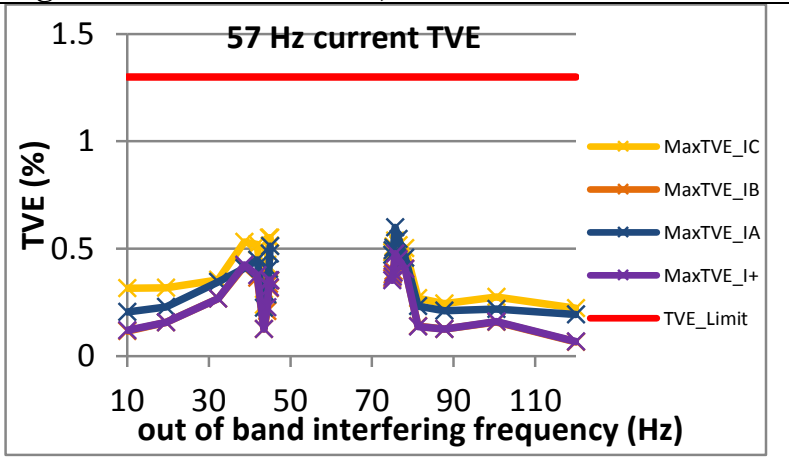

Figure 1715: Fs = 30 FPS, 58.5 Hz fundamental

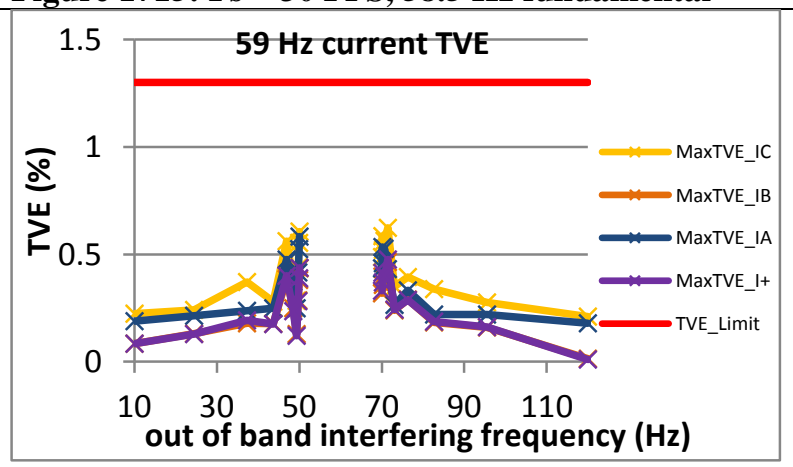

Figure 1718Fs = $20 \mathrm{FPS}, 59 \mathrm{~Hz}$ fundamental

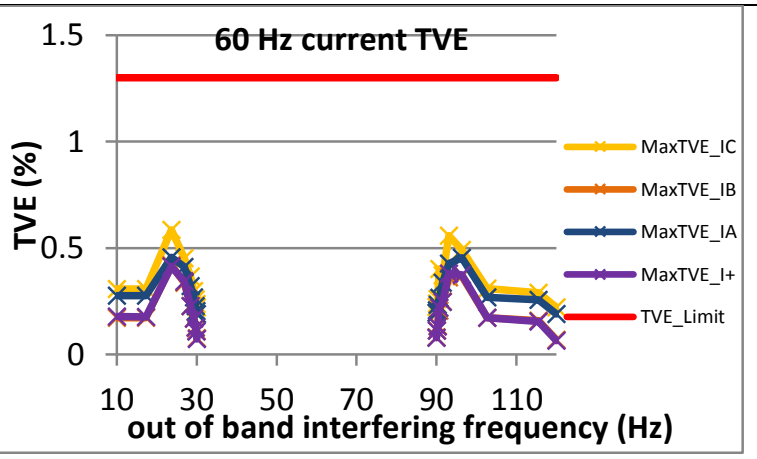

Figure 1713: Fs = $60 \mathrm{FPS}, 60 \mathrm{~Hz}$ fundamental

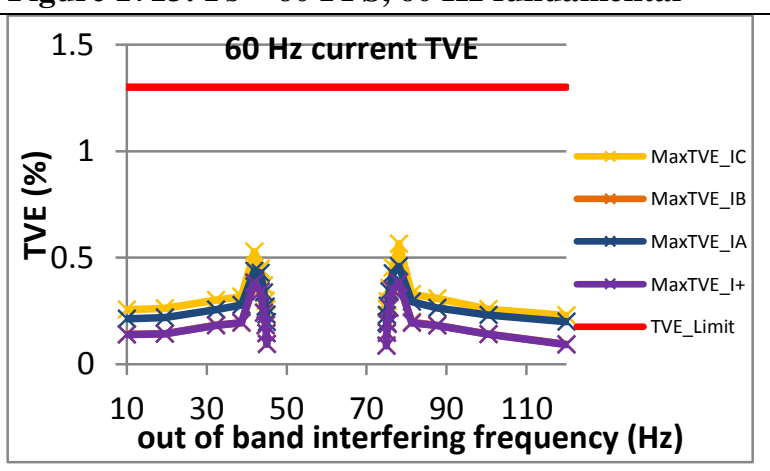

Figure 1716: Fs = $30 \mathrm{FPS}, 60 \mathrm{~Hz}$ fundamental

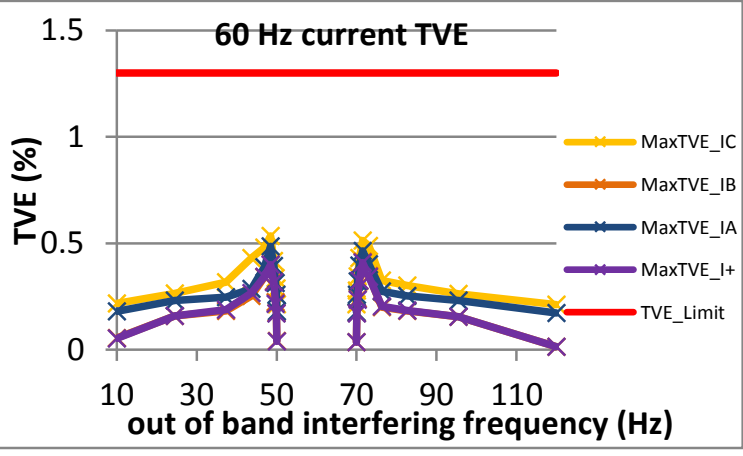

Figure 1719: Fs = 20 FPS, $60 \mathrm{~Hz}$ fundamental

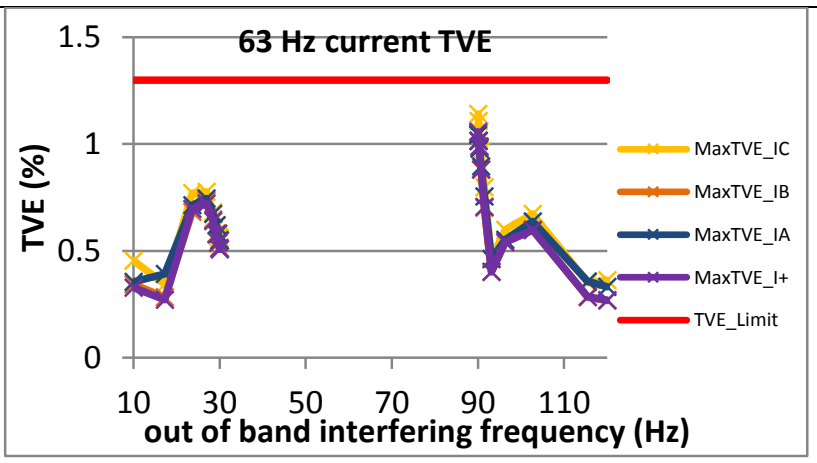

Figure 1714: Fs = 60 FPS, $63 \mathrm{~Hz}$ fundamental

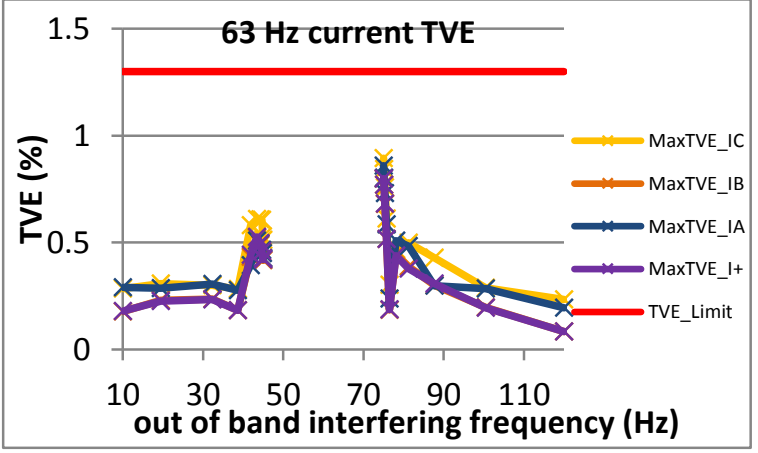

Figure 1717: Fs = 30 FPS, $61.5 \mathrm{~Hz}$ fundamental

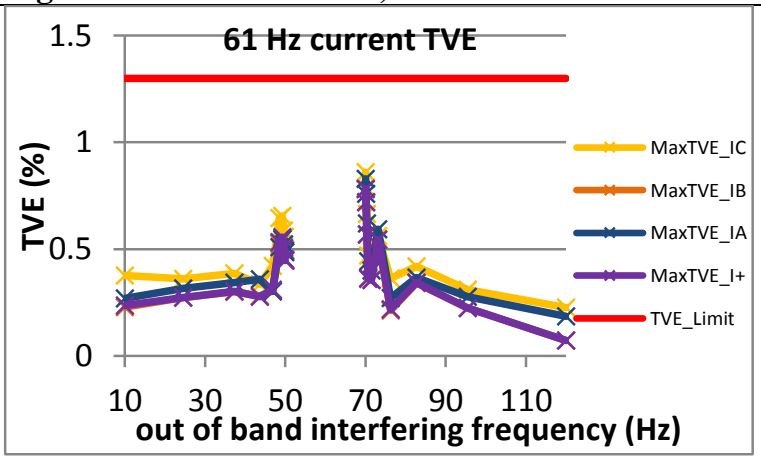

Figure 1720: Fs = 20 FPS, $61 \mathrm{~Hz}$ fundamental 


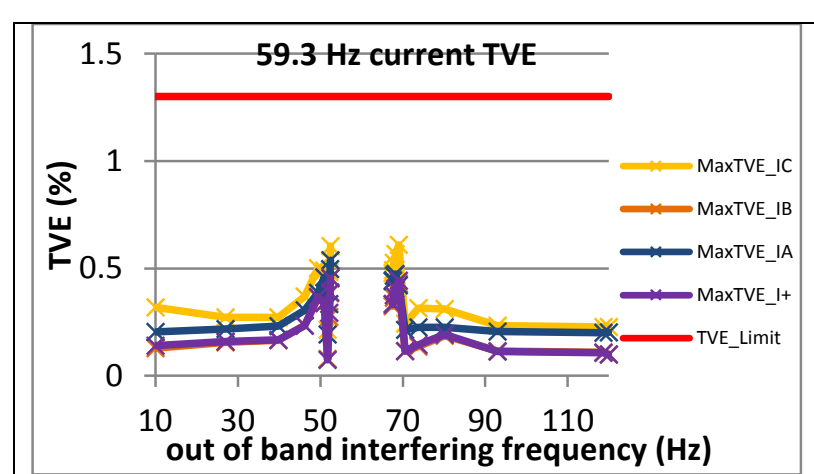

Figure 1721: Fs = 15 FPS, $59.3 \mathrm{~Hz}$ fundamental

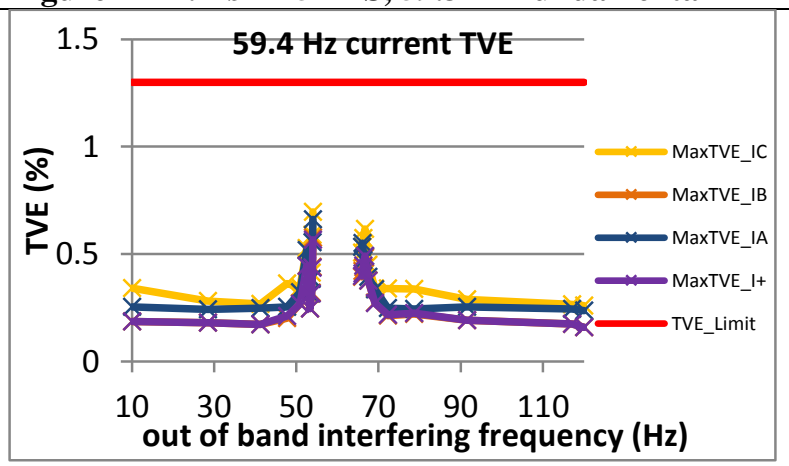

Figure 1724: Fs = 12 FPS, $59.4 \mathrm{~Hz}$ fundamental

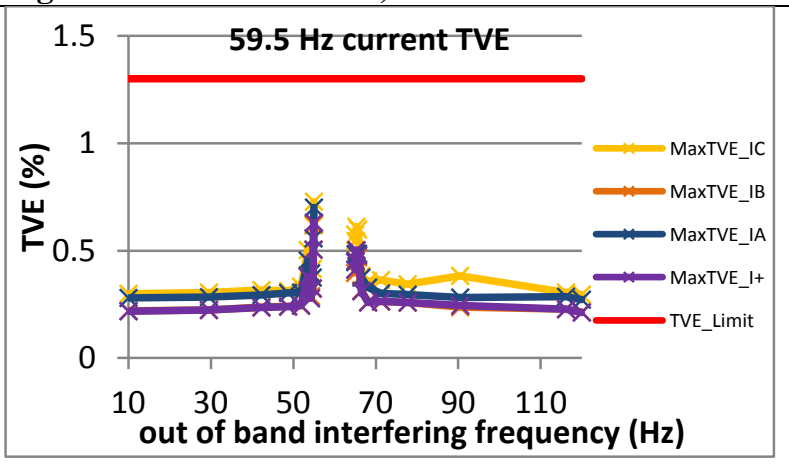

Figure 1727: Fs = 10 FPS, $59.5 \mathrm{~Hz}$ fundamental

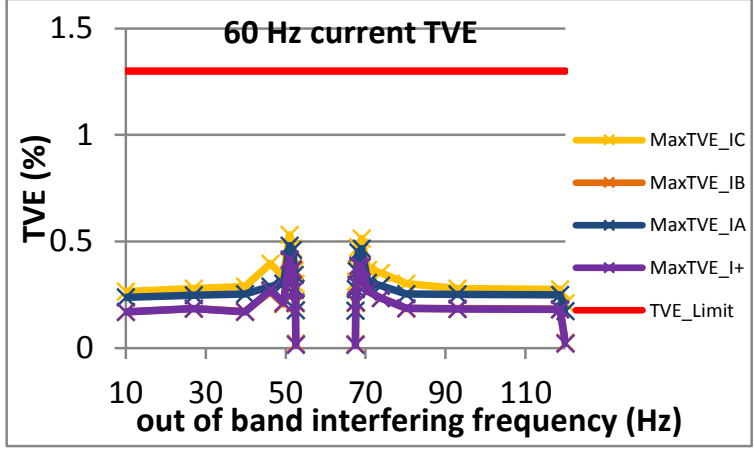

Figure 1722: Fs = $15 \mathrm{FPS}, 60 \mathrm{~Hz}$ fundamental

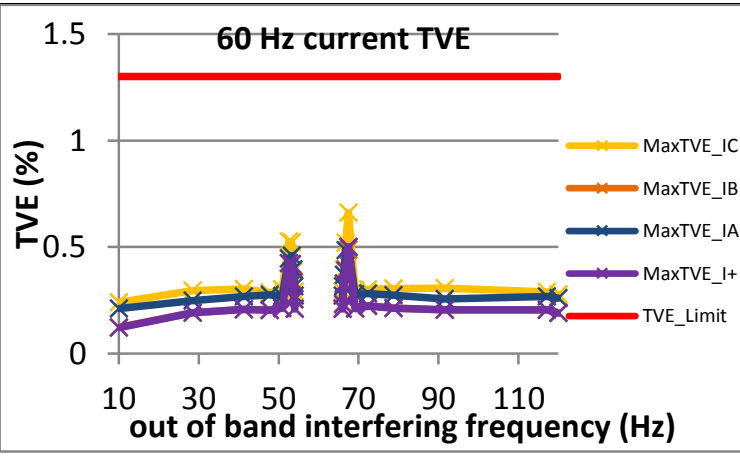

Figure 1725: Fs = 12 FPS, $60 \mathrm{~Hz}$ fundamental

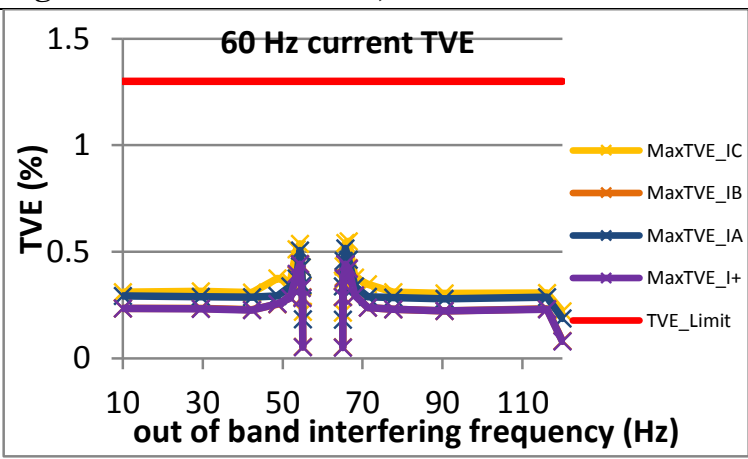

Figure 1728: Fs = 10 FPS, $60 \mathrm{~Hz}$ fundamental

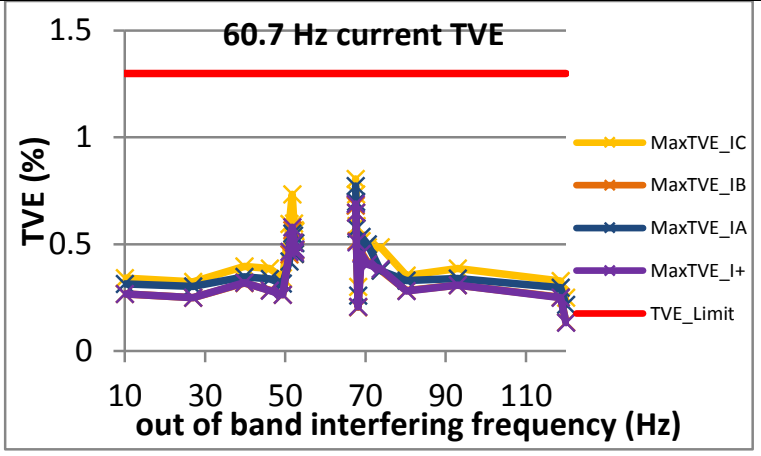

Figure 1723: Fs = 15 FPS, $60.7 \mathrm{~Hz}$ fundamental

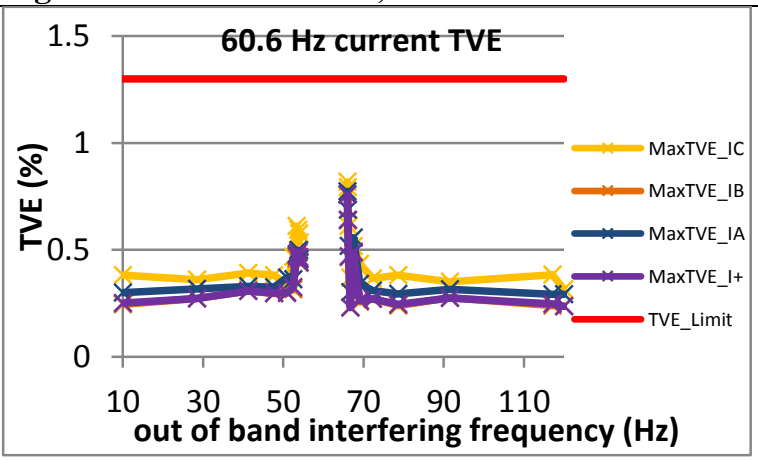

Figure 1726: Fs = $12 \mathrm{FPS}, 60.6 \mathrm{~Hz}$ fundamental

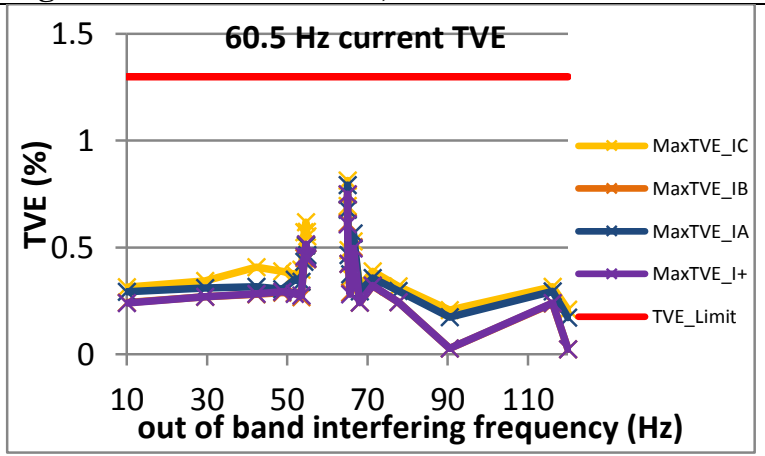

Figure 1729: Fs = 10 FPS, $60.5 \mathrm{~Hz}$ fundamental 
5.2.11 PMU J out of band interfering signals current TVE: M class
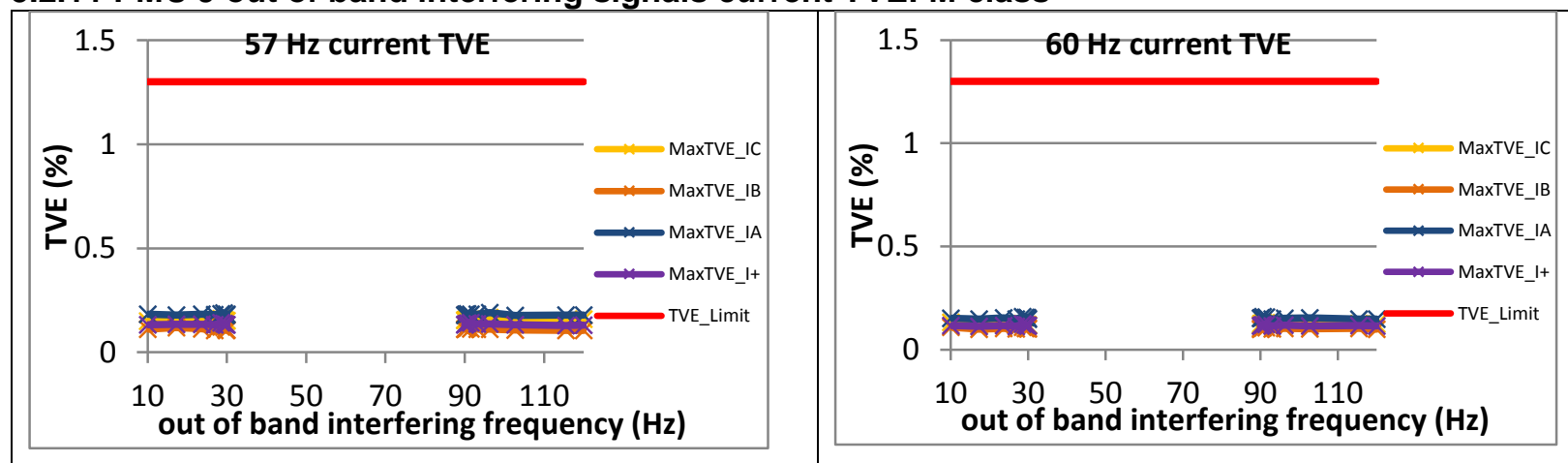

Figure 1731: Fs = $60 \mathrm{FPS}, 60 \mathrm{~Hz}$ fundamental

Figure 1730: Fs = $60 \mathrm{FPS}, 57 \mathrm{~Hz}$ fundamental

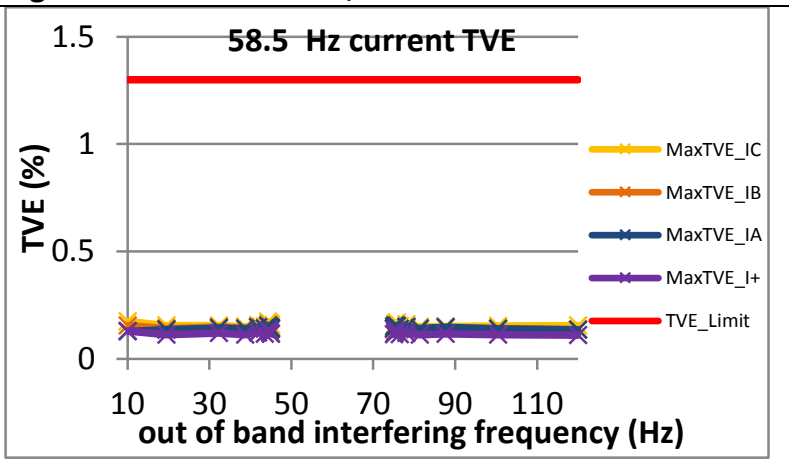

Figure 1733: Fs = $30 \mathrm{FPS}, 58.5 \mathrm{~Hz}$ fundamental

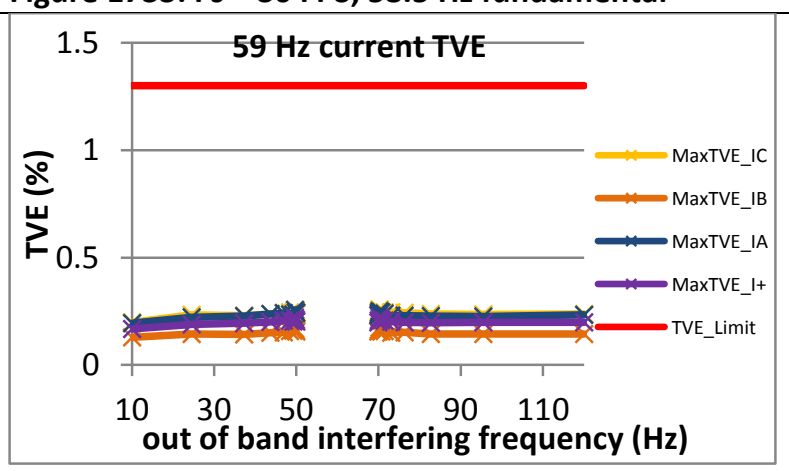

Figure 1736Fs = $20 \mathrm{FPS}, 59 \mathrm{~Hz}$ fundamental

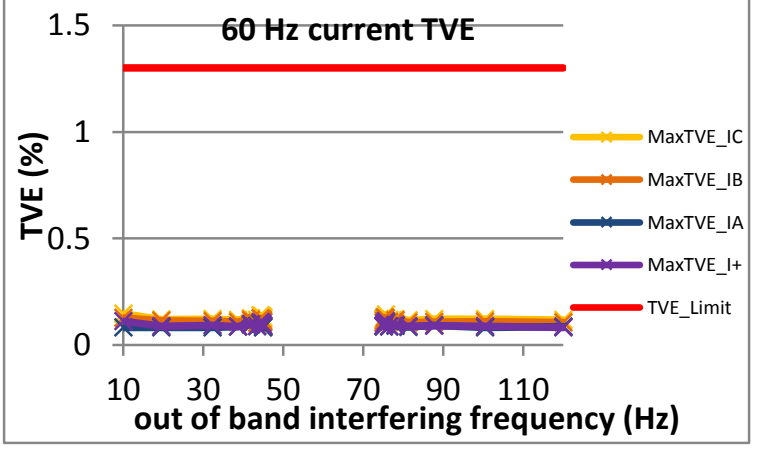

Figure 1734: Fs = $30 \mathrm{FPS}, 60 \mathrm{~Hz}$ fundamental

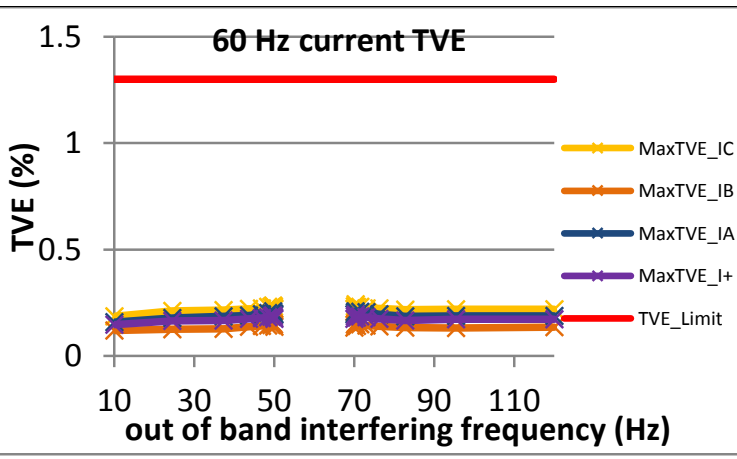

Figure 1737: Fs = 20 FPS, $60 \mathrm{~Hz}$ fundamental

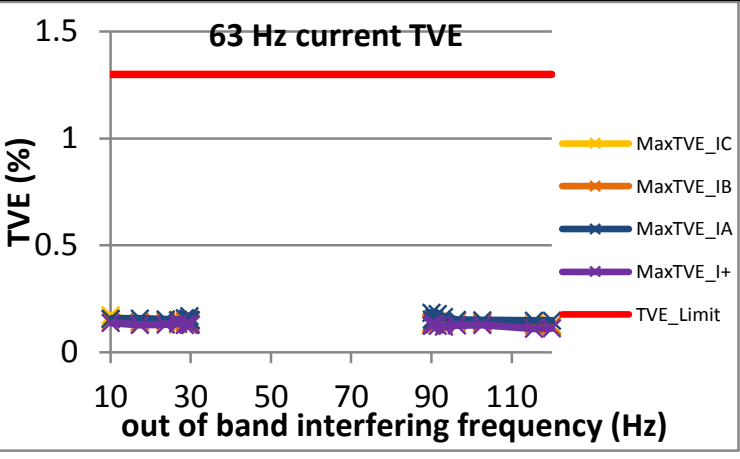

Figure 1732: Fs = $60 \mathrm{FPS}, 63 \mathrm{~Hz}$ fundamental

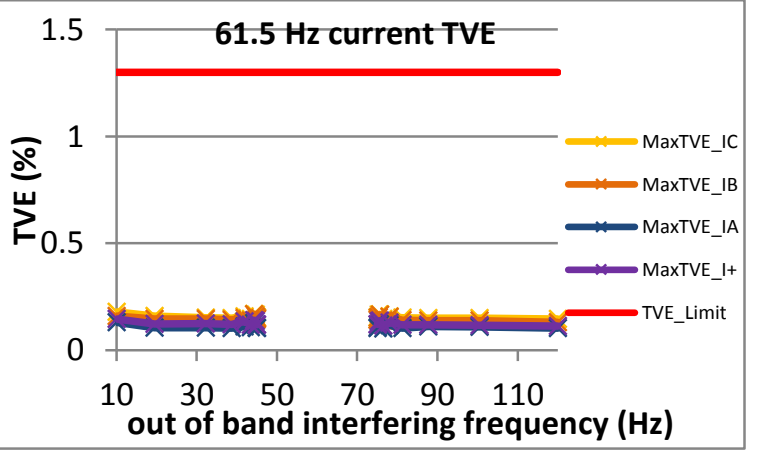

Figure 1735: Fs = $30 \mathrm{FPS}, 61.5 \mathrm{~Hz}$ fundamental

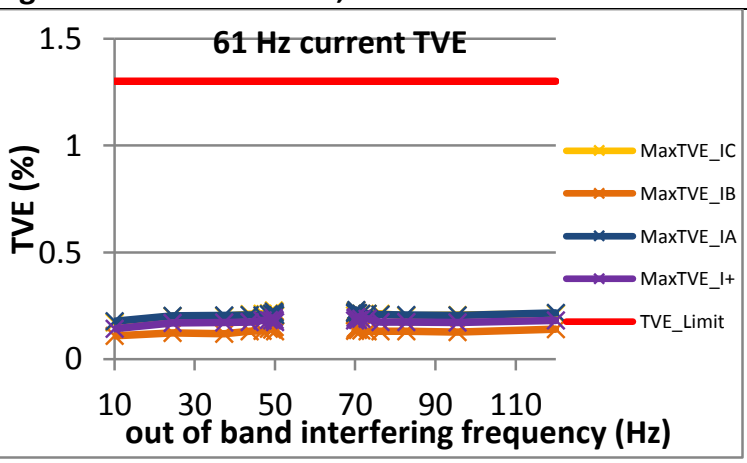

Figure 1738: Fs = 20 FPS, $61 \mathrm{~Hz}$ fundamental 

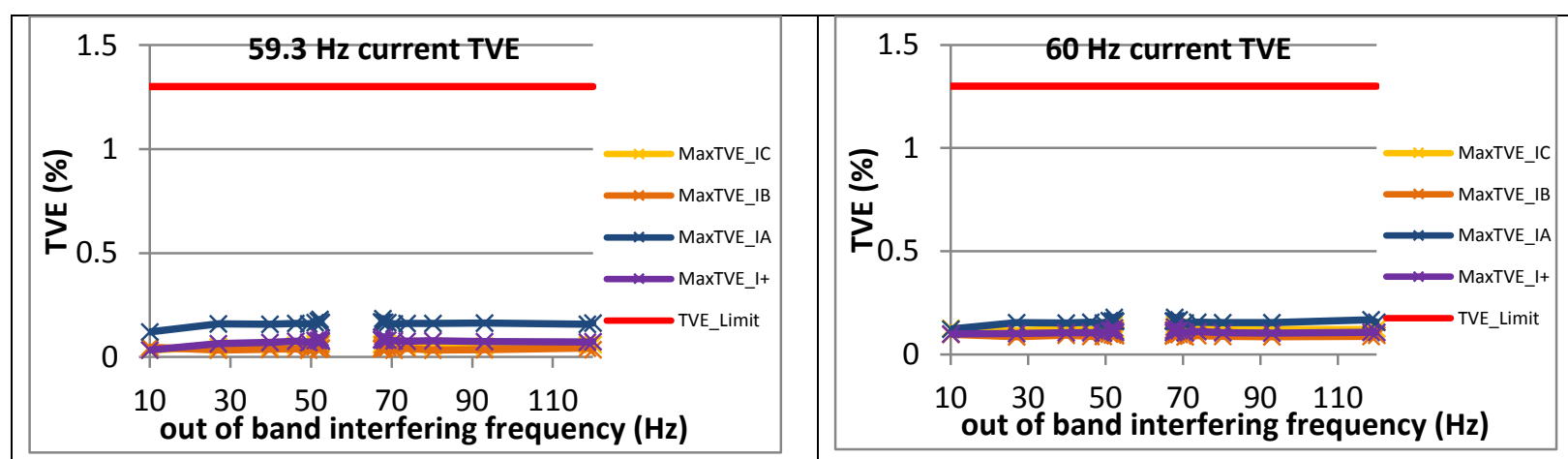

Figure 1740: Fs = 15 FPS, $60 \mathrm{~Hz}$ fundamental

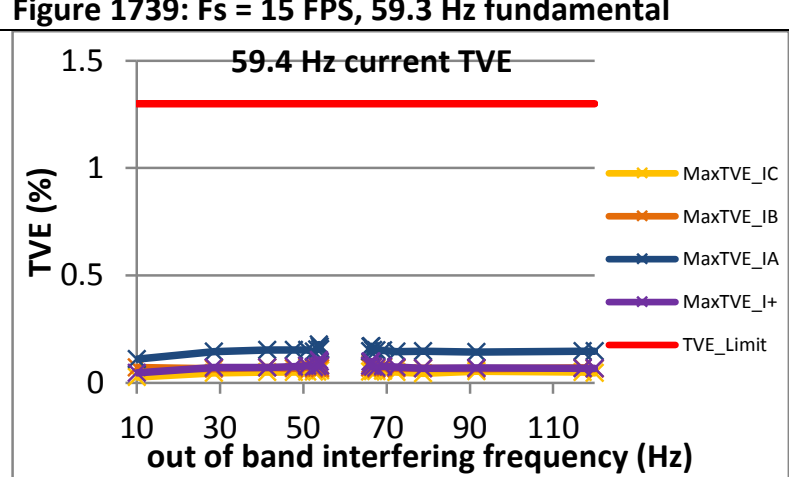

Figure 1742: Fs = $12 \mathrm{FPS}, 59.4 \mathrm{~Hz}$ fundamental

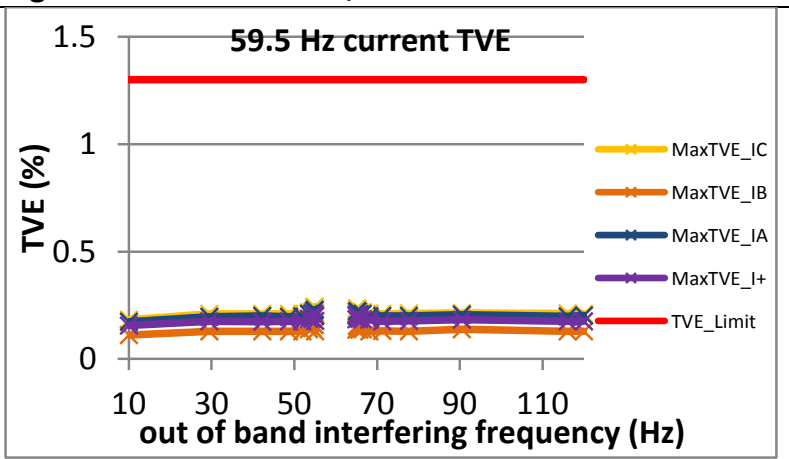

Figure 1745: Fs = 10 FPS, $59.5 \mathrm{~Hz}$ fundamental
Figure 1743: Fs = 12 FPS, $60 \mathrm{~Hz}$ fundamental

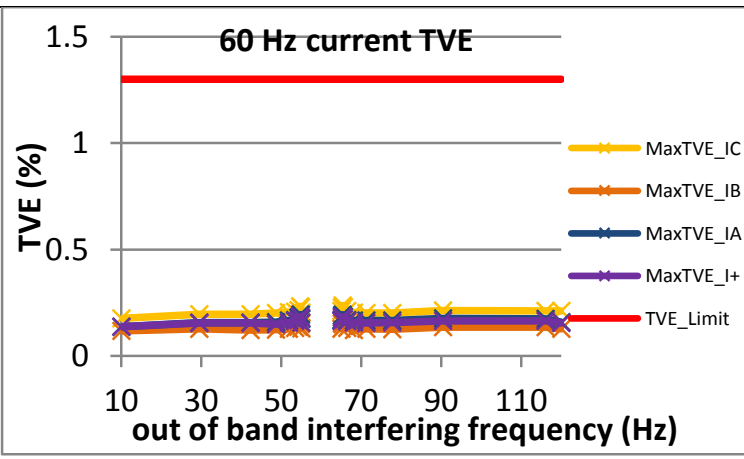

Figure 1746: Fs = $10 \mathrm{FPS}, 60 \mathrm{~Hz}$ fundamental

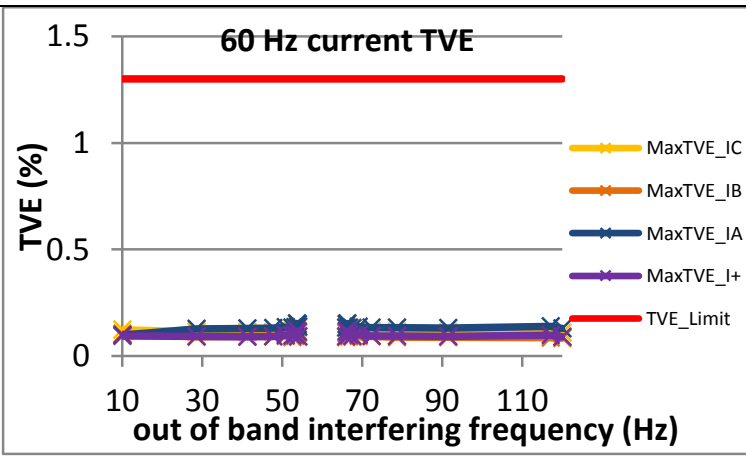

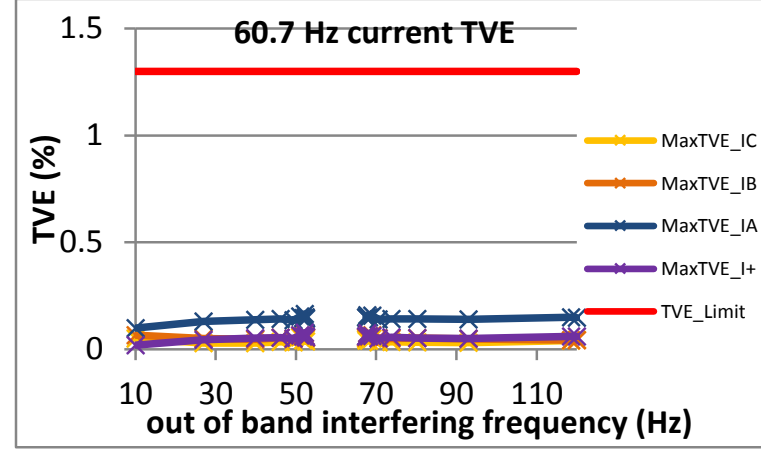

Figure 1741: Fs = 15 FPS, $60.7 \mathrm{~Hz}$ fundamental

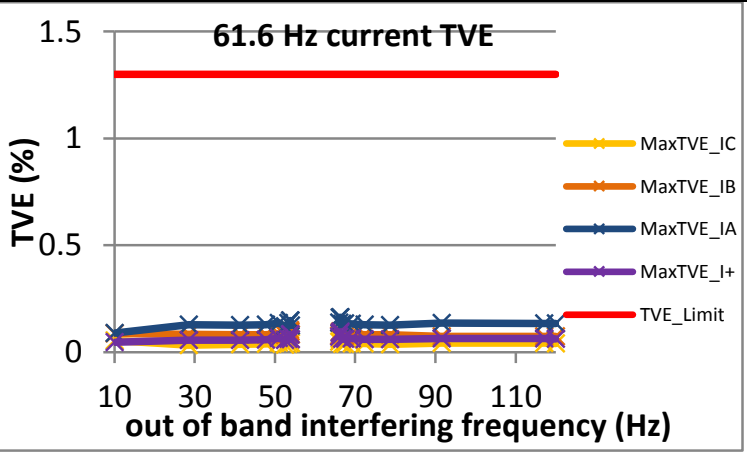

Figure 1744: Fs = $12 \mathrm{FPS}, 60.6 \mathrm{~Hz}$ fundamental

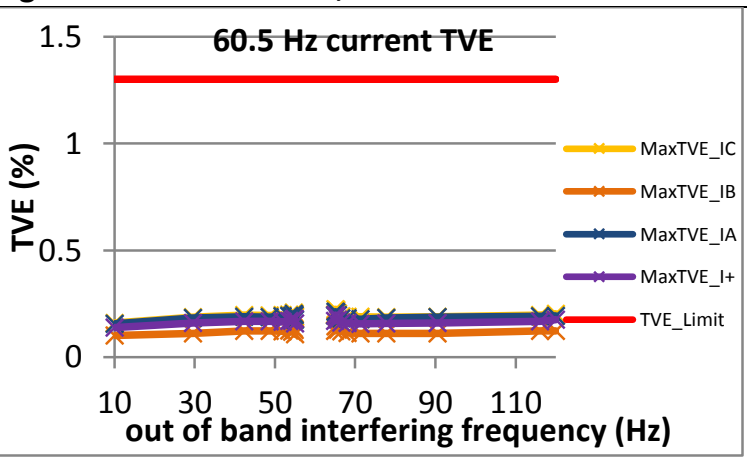

Figure 1747: Fs = 10 FPS, $60.5 \mathrm{~Hz}$ fundamental 
5.3 Steady state out of band interfering signals: frequency error: M class

5.3.1 C37.118.1 Annex $C$ steady state out of band interfering signals frequency error: $M$ class

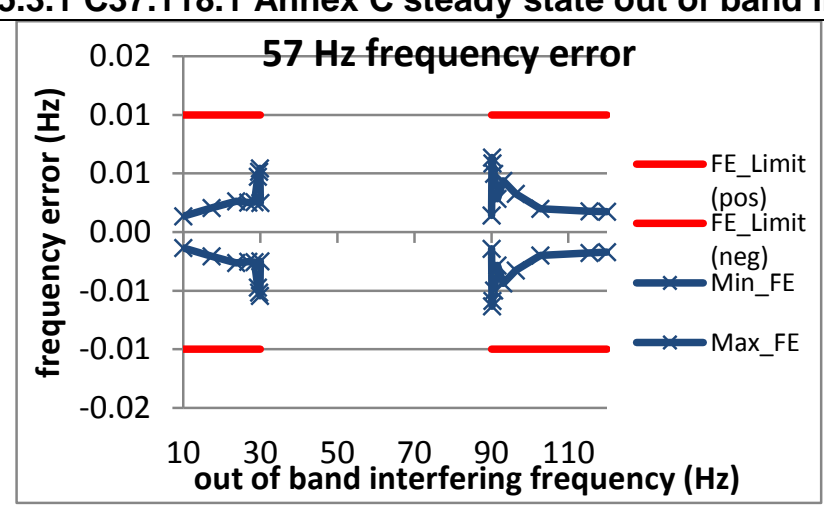

Figure 1748: Fs = 60 FPS, $57 \mathrm{~Hz}$ fundamental

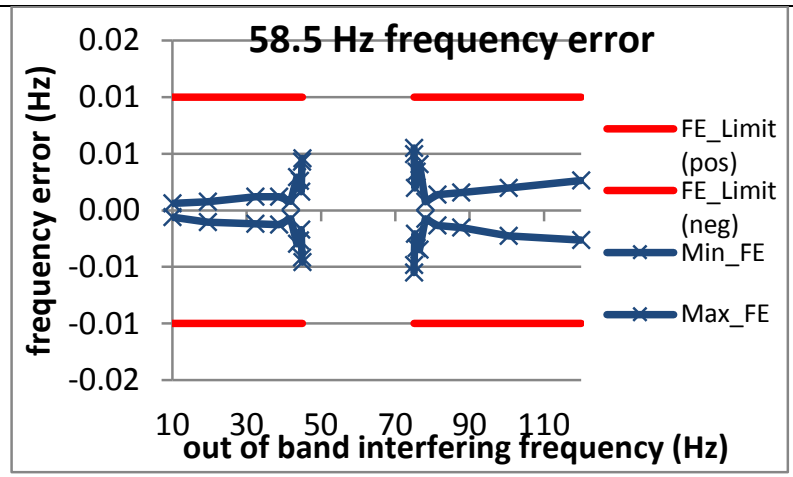

Figure 1751: Fs = $30 \mathrm{FPS}, 58.5 \mathrm{~Hz}$ fundamental

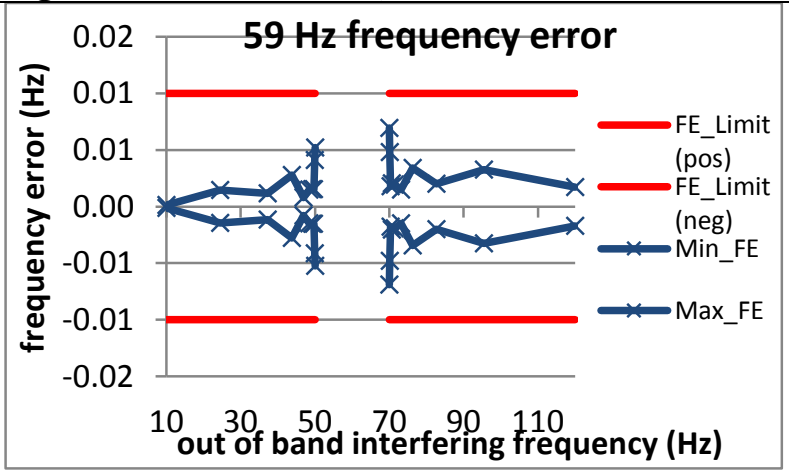

Figure 1754: Fs = 20 FPS, $59 \mathrm{~Hz}$ fundamental

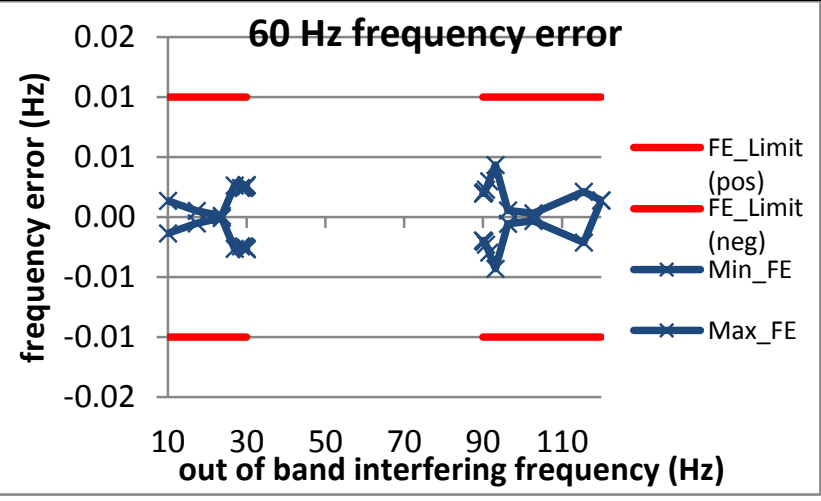

Figure 1749: Fs = $60 \mathrm{FPS}, 60 \mathrm{~Hz}$ fundamental

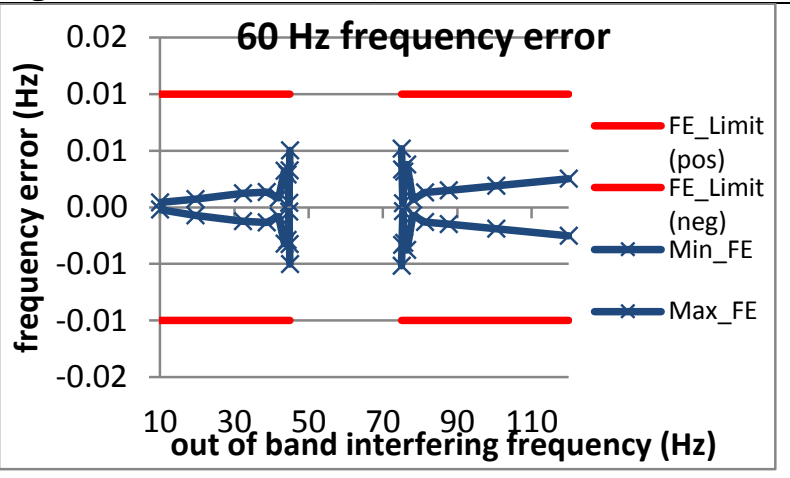

Figure 1752: Fs = 30 FPS, $60 \mathrm{~Hz}$ fundamental

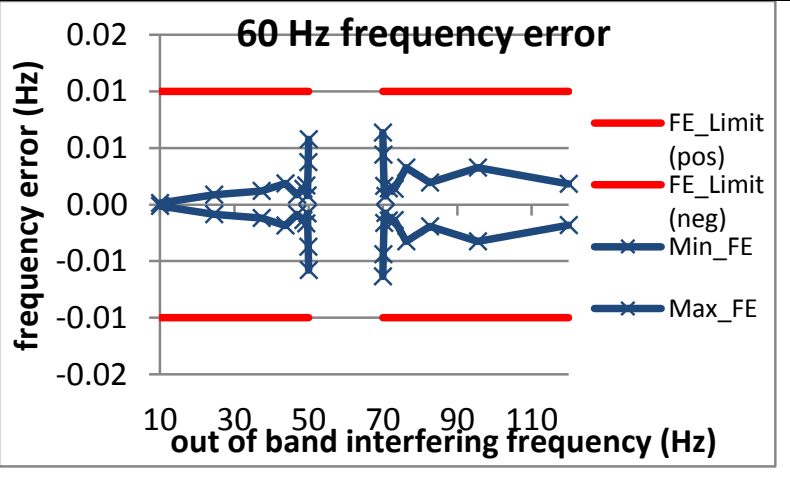

Figure 1755: Fs = 20 FPS, $60 \mathrm{~Hz}$ fundamental

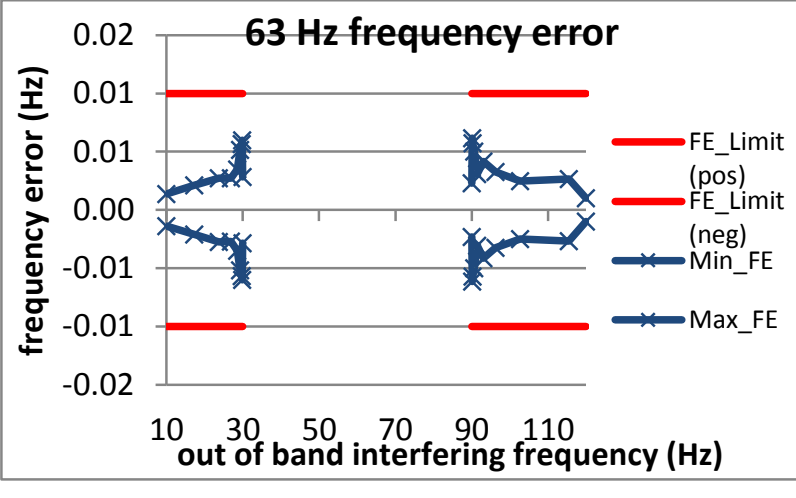

Figure 1750: Fs = $60 \mathrm{FPS}, 63 \mathrm{~Hz}$ fundamental

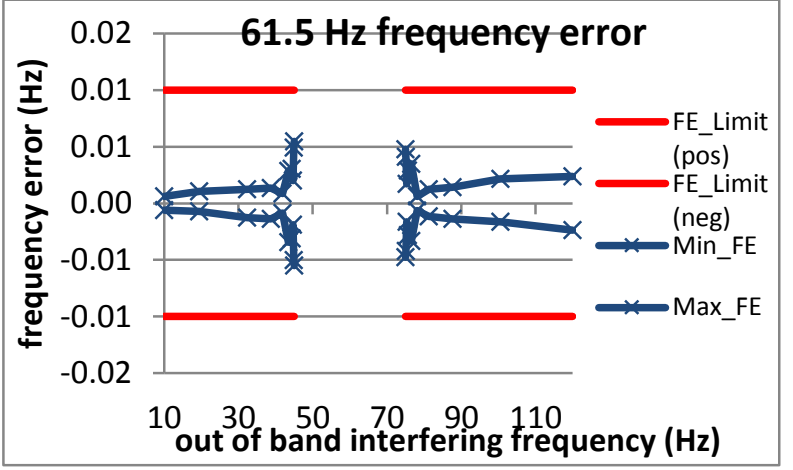

Figure 1753: Fs = 30 FPS, $61.5 \mathrm{~Hz}$ fundamental

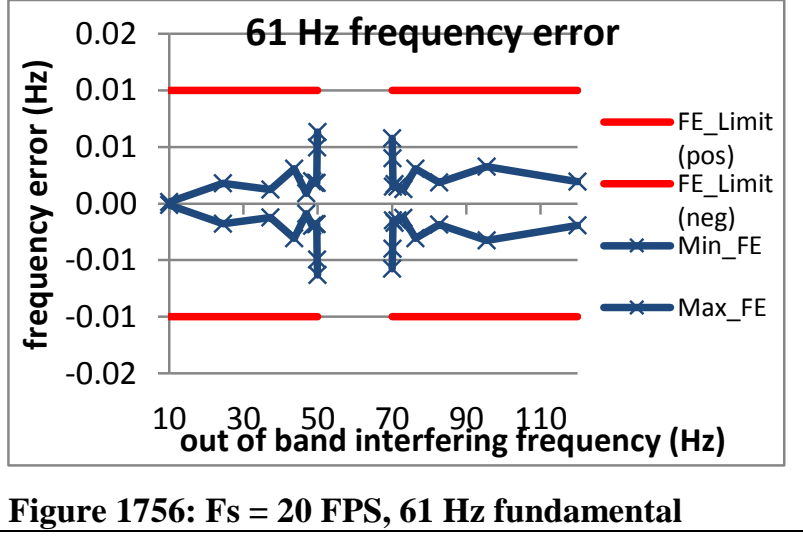




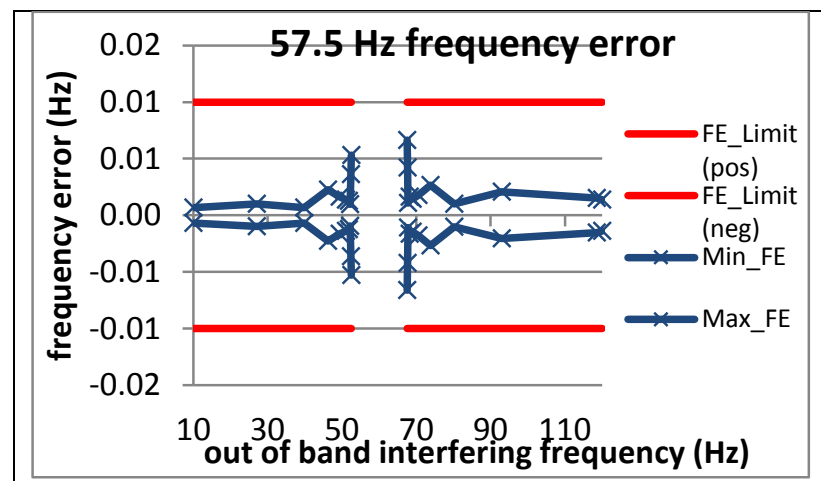

Figure 1757: Fs = 15 FPS, $59.3 \mathrm{~Hz}$ fundamental

Figure 1760: Fs = 12 FPS, $59.4 \mathrm{~Hz}$ fundamental

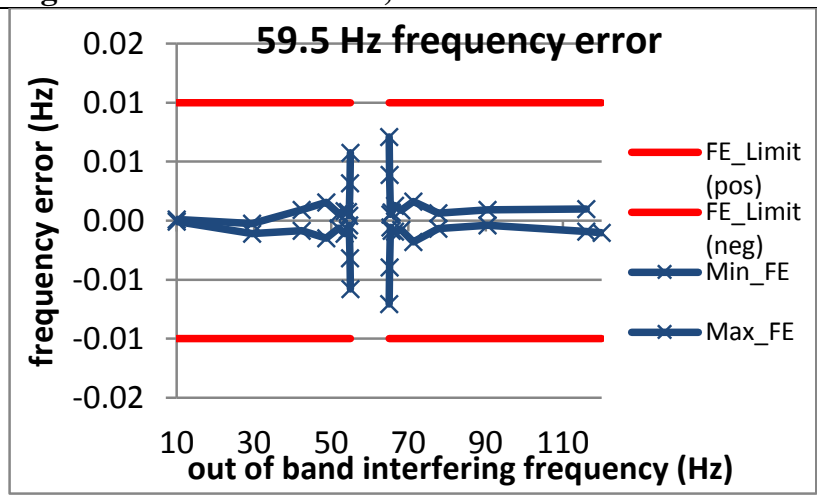

Figure 1763: Fs = 10 FPS, $59.5 \mathrm{~Hz}$ fundamental

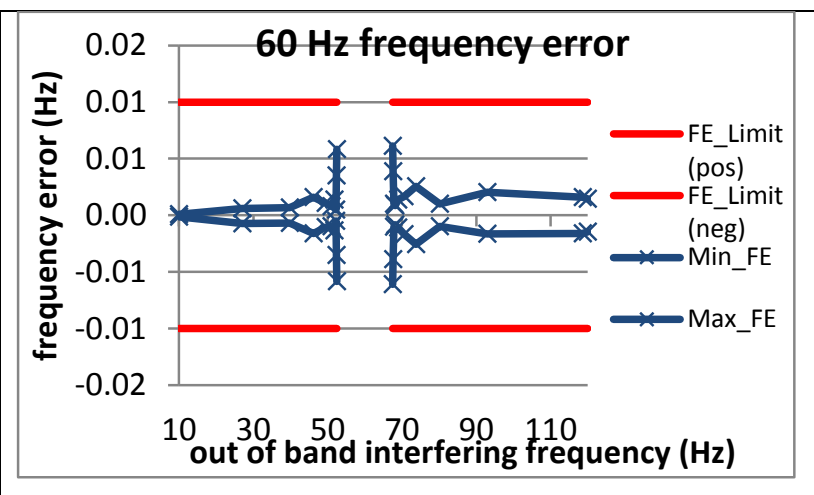

Figure 1758: Fs = 15 FPS, $60 \mathrm{~Hz}$ fundamental Figure 1761: Fs = 12 FPS, $60 \mathrm{~Hz}$ fundamental

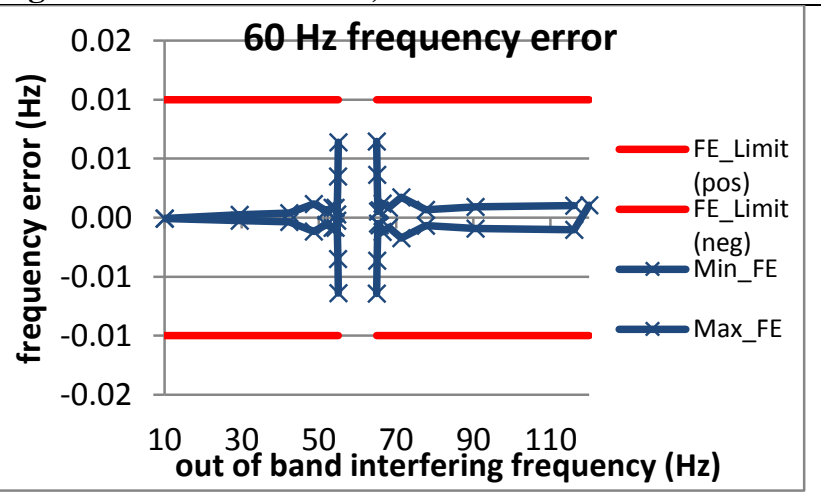

Figure 1764: Fs = $10 \mathrm{FPS}, 60 \mathrm{~Hz}$ fundamental

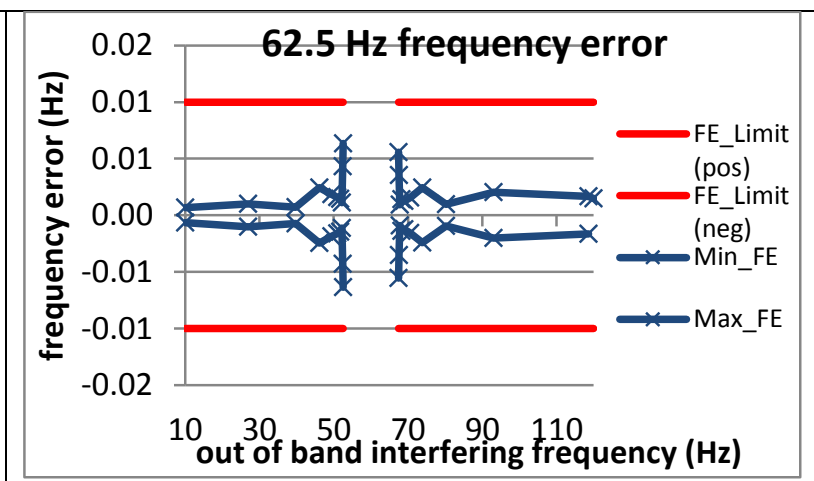

Figure 1759: Fs = 15 FPS, $60.7 \mathrm{~Hz}$ fundamental

Figure 1762: Fs = $12 \mathrm{FPS}, 60.6 \mathrm{~Hz}$ fundamental

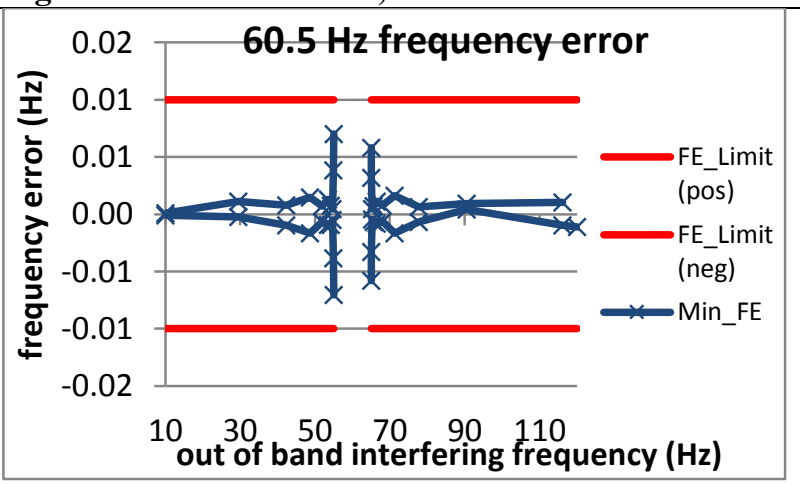

Figure 1765: Fs = 10 FPS, $60.5 \mathrm{~Hz}$ fundamental 
5.3.2 PMU A steady state out of band interfering signals frequency error : $M$ class

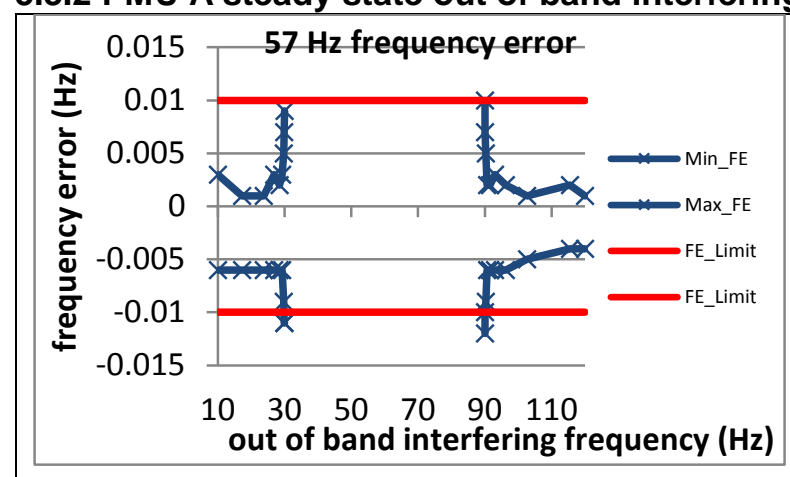

Figure 1766: Fs = 60 FPS, $57 \mathrm{~Hz}$ fundamental

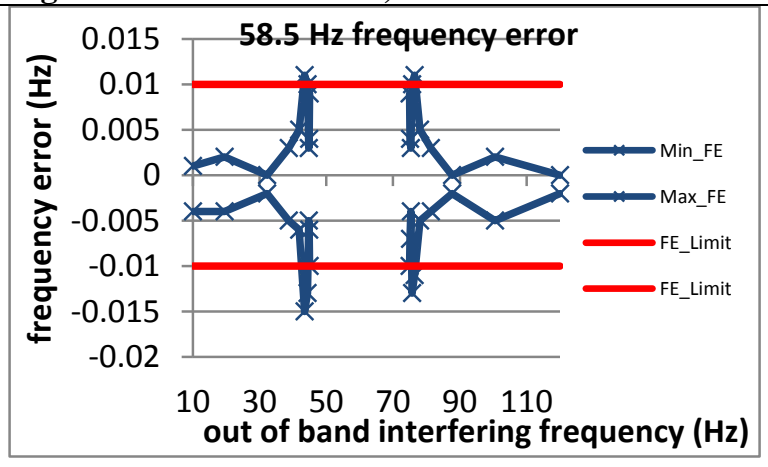

Figure 1769: Fs = 30 FPS, $58.5 \mathrm{~Hz}$ fundamental

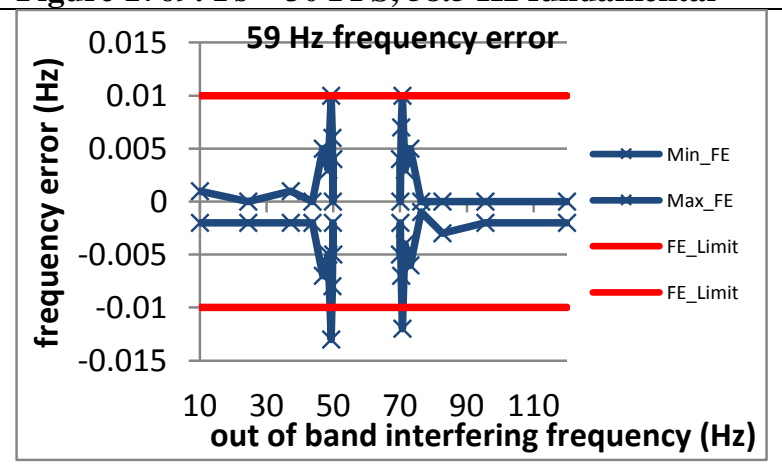

Figure 1772: Fs = 20 FPS, $59 \mathrm{~Hz}$ fundamental

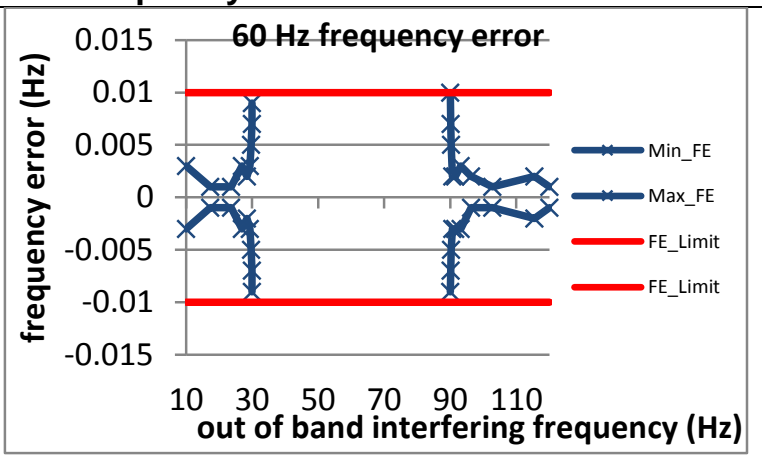

Figure 1767: Fs = $60 \mathrm{FPS}, 60 \mathrm{~Hz}$ fundamental

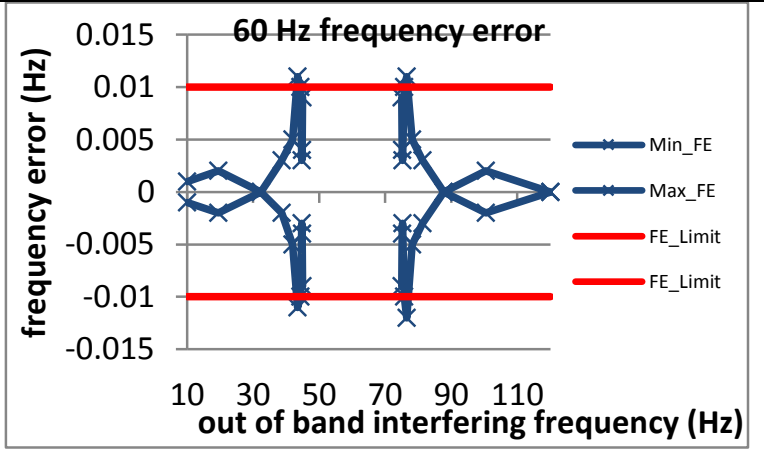

Figure 1770: Fs = $30 \mathrm{FPS}, 60 \mathrm{~Hz}$ fundamental

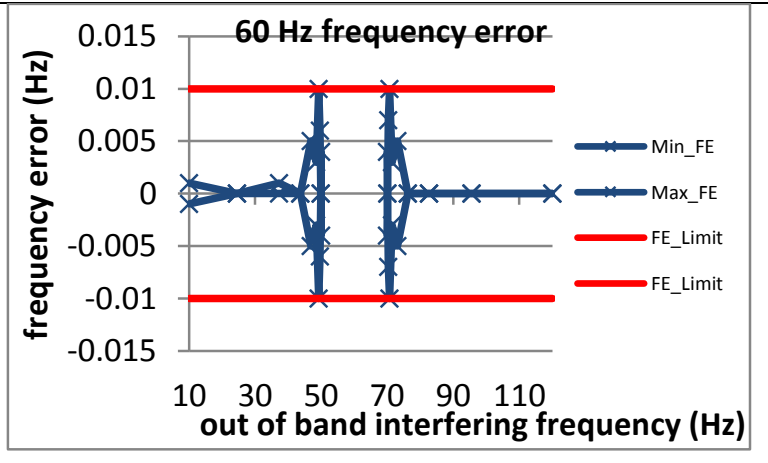

Figure 1773: Fs = $20 \mathrm{FPS}, 60 \mathrm{~Hz}$ fundamental

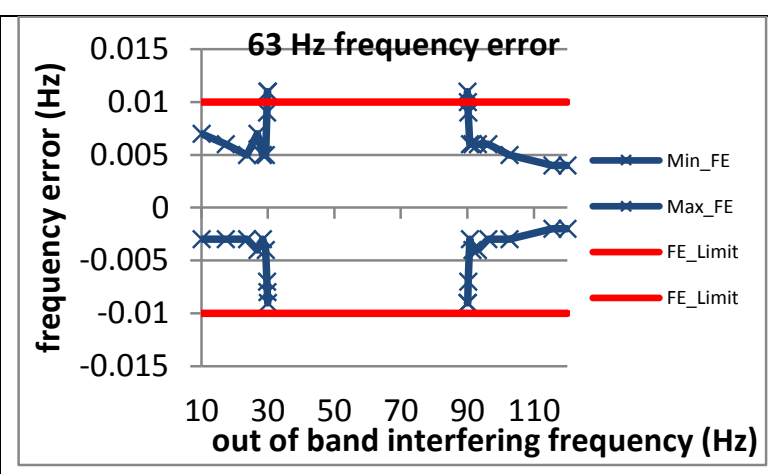

Figure 1768: Fs = 60 FPS, $63 \mathrm{~Hz}$ fundamental

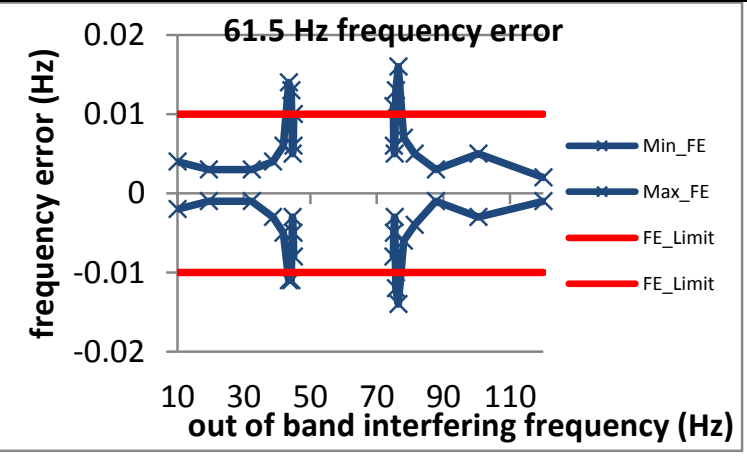

Figure 1771: Fs = 30 FPS, $61.5 \mathrm{~Hz}$ fundamental

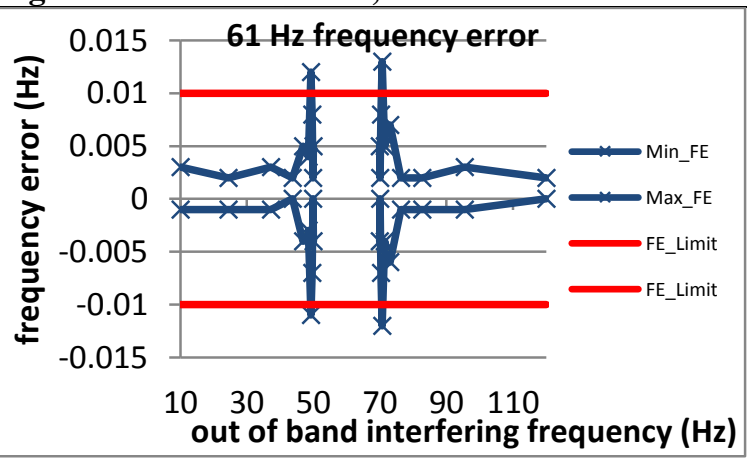

Figure 1774: Fs = $20 \mathrm{FPS}, 61 \mathrm{~Hz}$ fundamental 


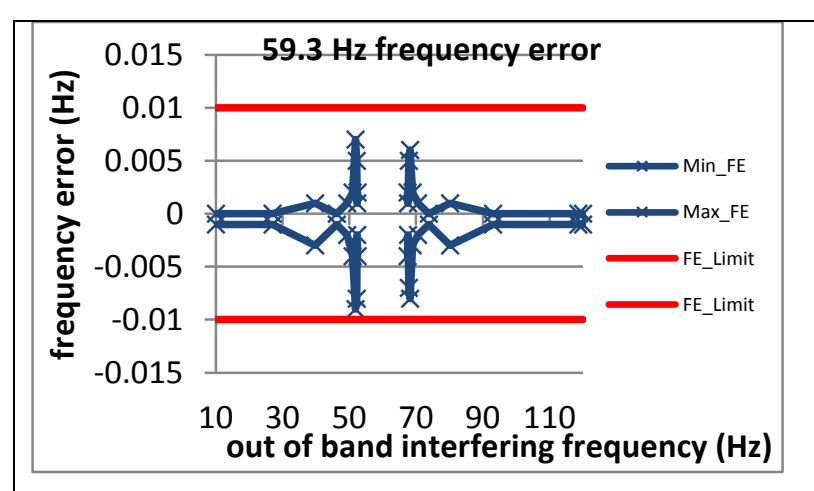

Figure 1775: Fs = 15 FPS, $59.3 \mathrm{~Hz}$ fundamental

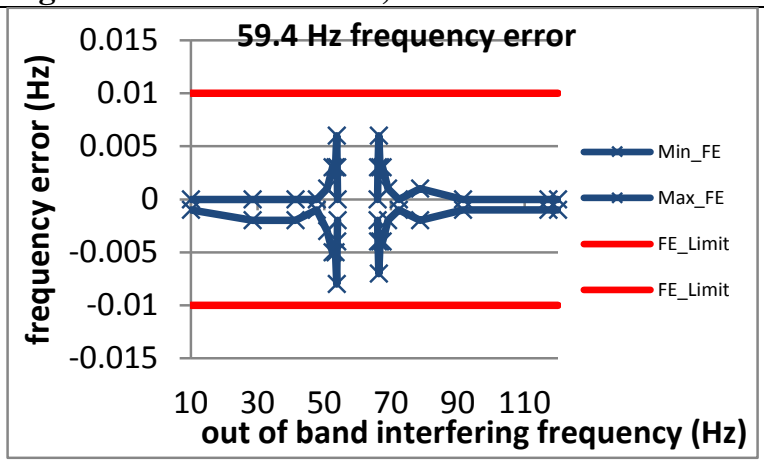

Figure 1778: Fs = 12 FPS, $59.4 \mathrm{~Hz}$ fundamental

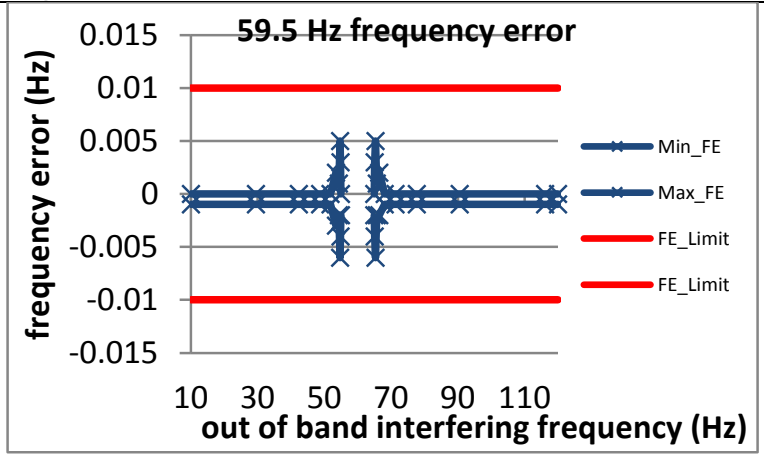

Figure 1781: Fs = 10 FPS, $59.5 \mathrm{~Hz}$ fundamental

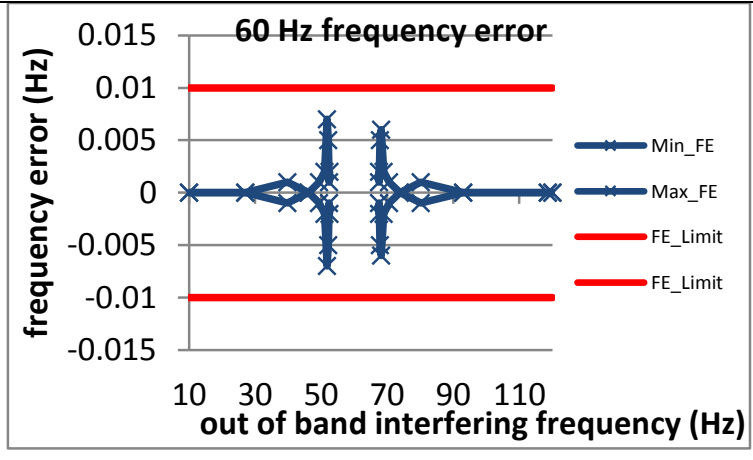

Figure 1776: Fs = $15 \mathrm{FPS}, 60 \mathrm{~Hz}$ fundamental

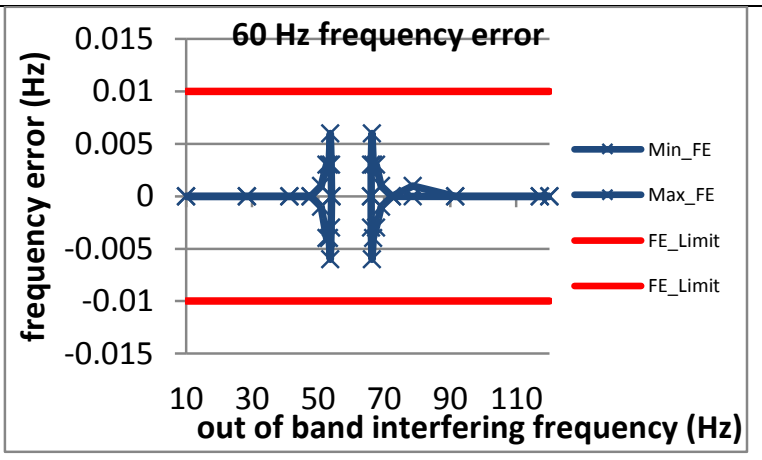

Figure 1779: Fs = 12 FPS, $60 \mathrm{~Hz}$ fundamental

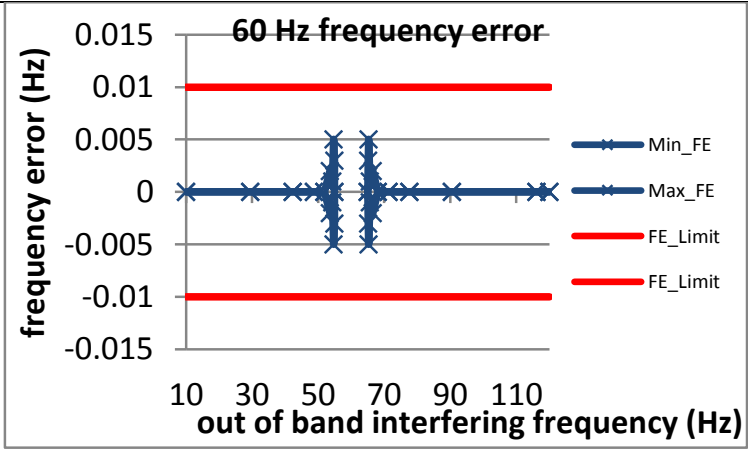

Figure 1782: Fs = $10 \mathrm{FPS}, 60 \mathrm{~Hz}$ fundamental

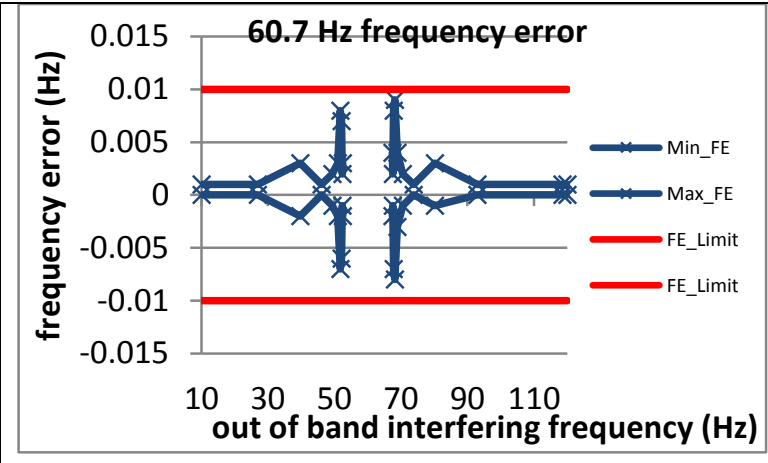

Figure 1777: Fs = 15 FPS, $60.7 \mathrm{~Hz}$ fundamental

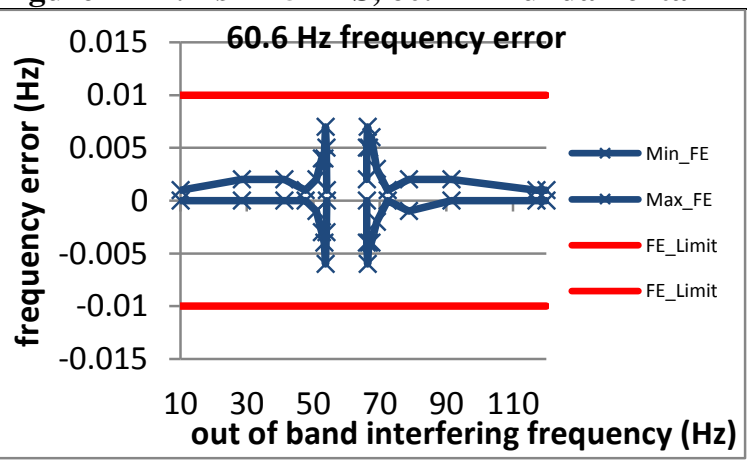

Figure 1780: Fs = 12 FPS, $60.6 \mathrm{~Hz}$ fundamental

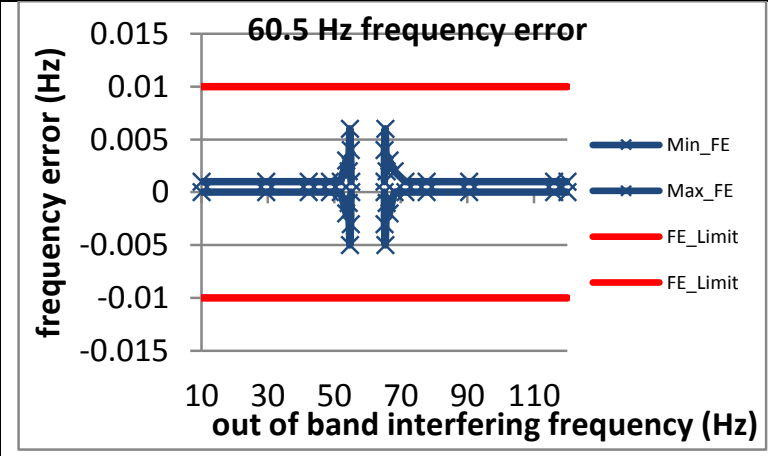

Figure 1783: Fs = 10 FPS, $60.5 \mathrm{~Hz}$ fundamental 
5.3.3 PMU B steady state out of band interfering signals frequency error : $M$ class

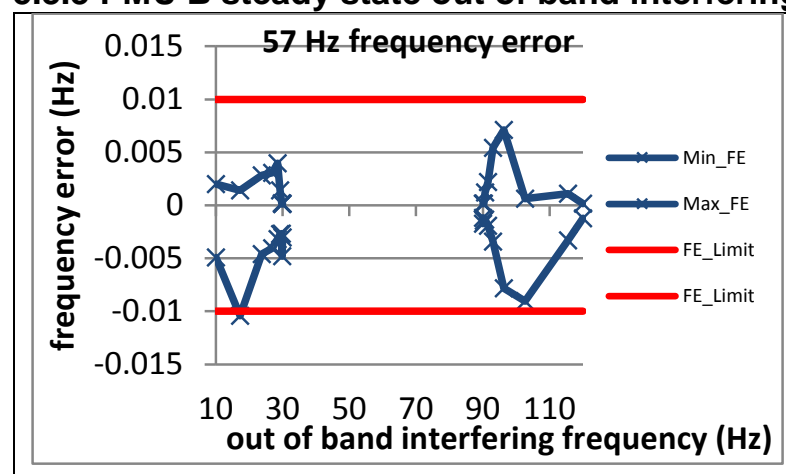

Figure 1784: Fs = 60 FPS, $57 \mathrm{~Hz}$ fundamental

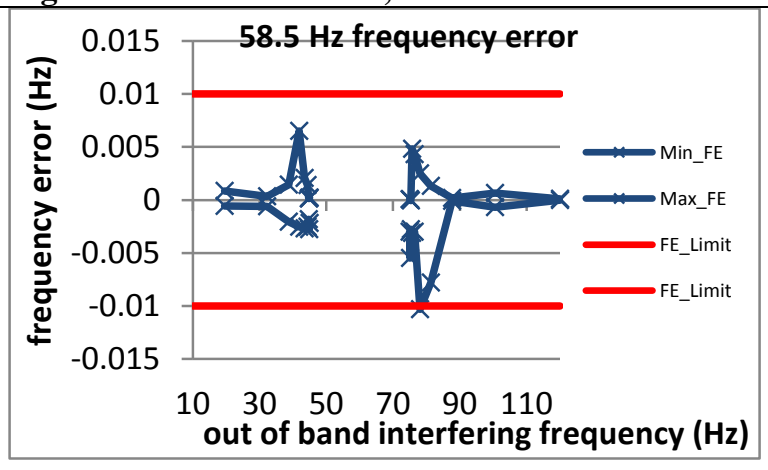

Figure 1787: Fs = 30 FPS, $58.5 \mathrm{~Hz}$ fundamental

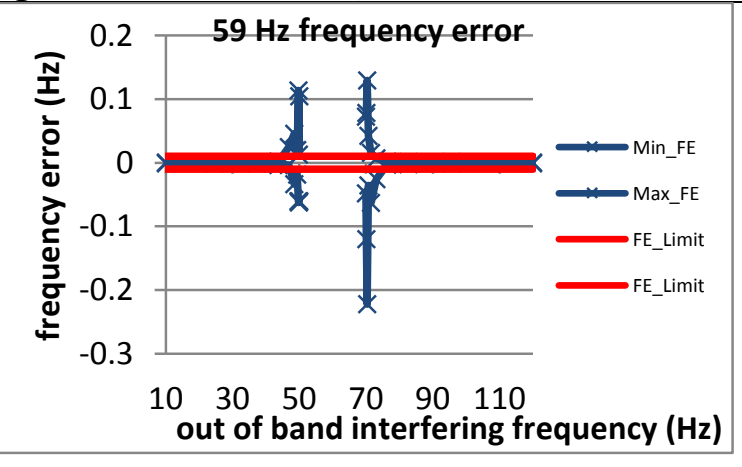

Figure 1790: Fs = 20 FPS, $59 \mathrm{~Hz}$ fundamental

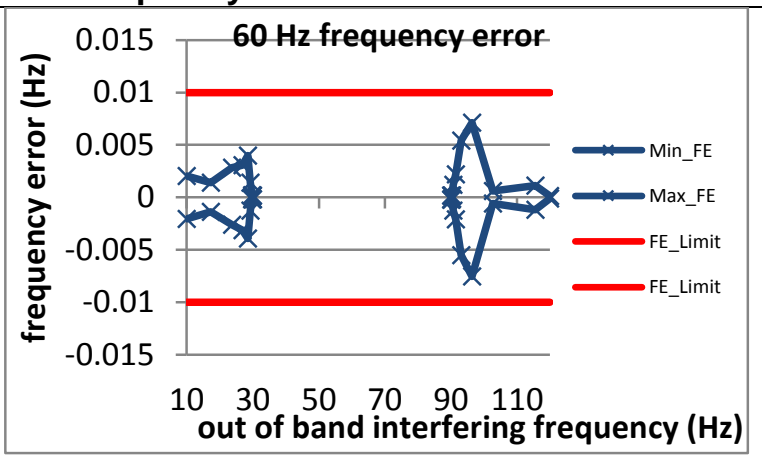

Figure 1785: Fs = $60 \mathrm{FPS}, 60 \mathrm{~Hz}$ fundamental

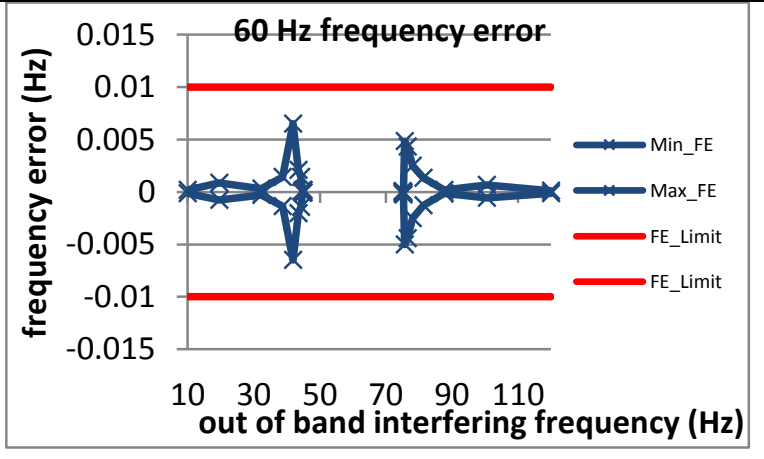

Figure 1788: Fs = $30 \mathrm{FPS}, 60 \mathrm{~Hz}$ fundamental

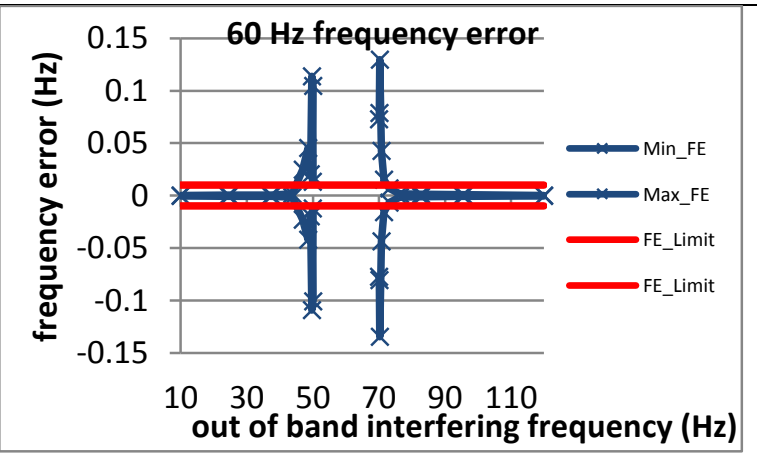

Figure 1791: Fs = $20 \mathrm{FPS}, 60 \mathrm{~Hz}$ fundamental

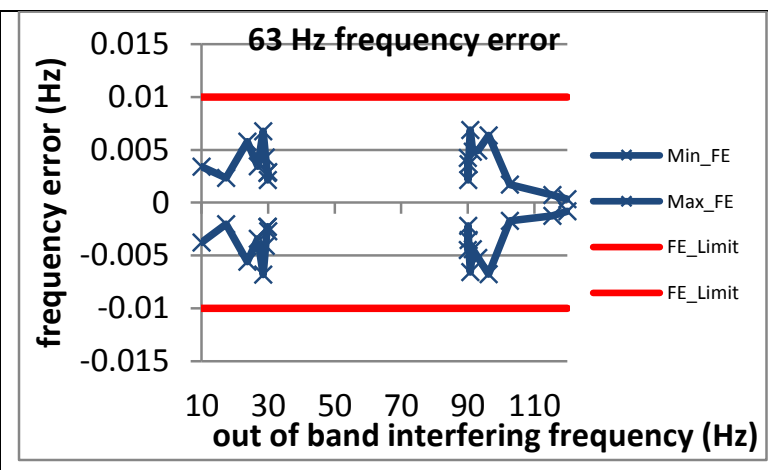

Figure 1786: Fs = 60 FPS, $63 \mathrm{~Hz}$ fundamental

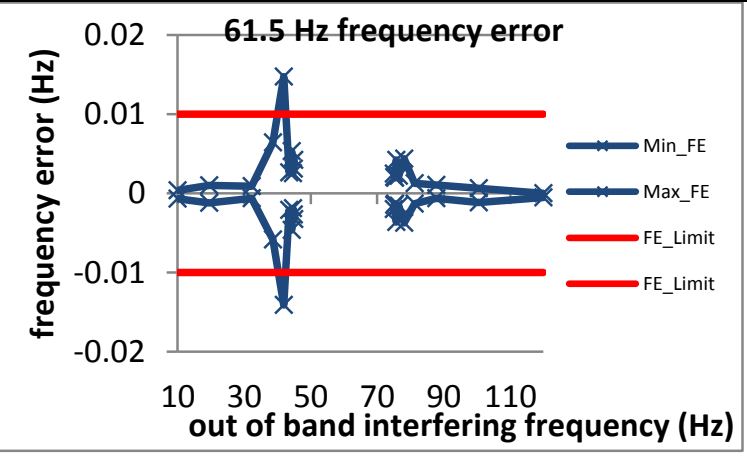

Figure 1789: Fs = 30 FPS, $61.5 \mathrm{~Hz}$ fundamental

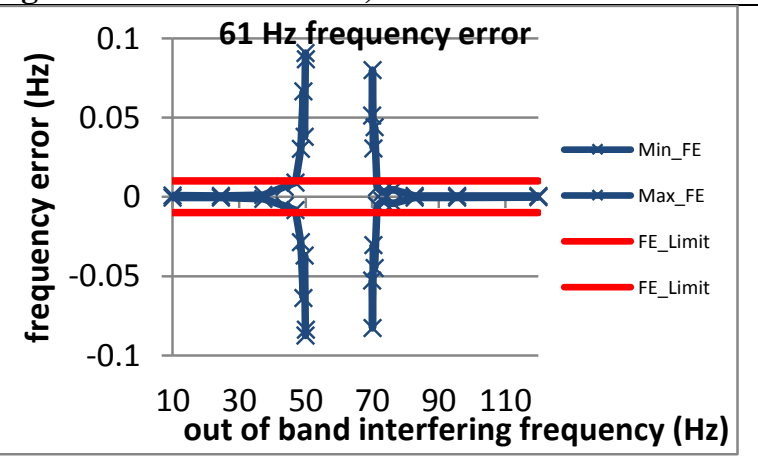

Figure 1792: Fs $=20 \mathrm{FPS}, 61 \mathrm{~Hz}$ fundamental 


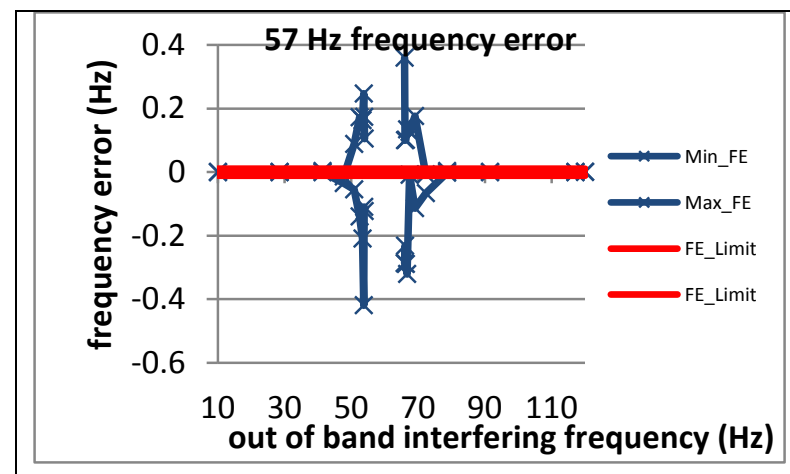

Figure 1793: Fs = 15 FPS, $59.3 \mathrm{~Hz}$ fundamental

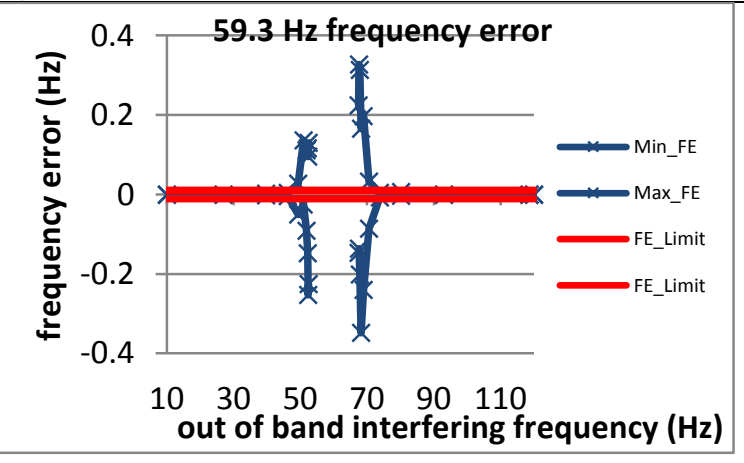

Figure 1796: Fs = 12 FPS, $59.4 \mathrm{~Hz}$ fundamental

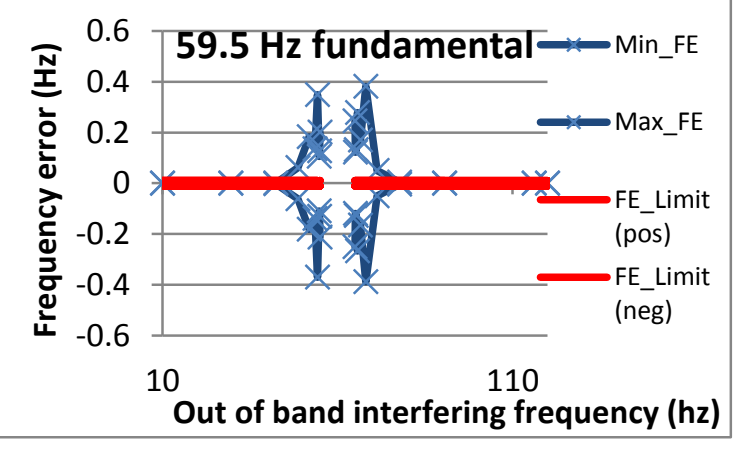

Figure 1799: Fs = 10 FPS, $59.5 \mathrm{~Hz}$ fundamental

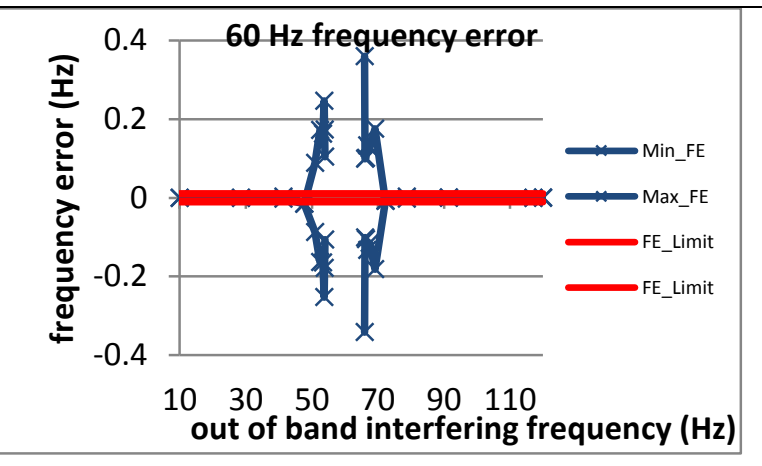

Figure 1794: Fs = 15 FPS, $60 \mathrm{~Hz}$ fundamental

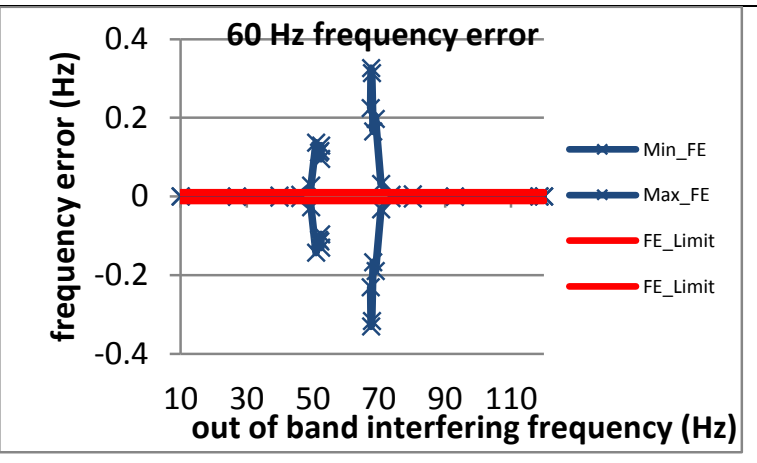

Figure 1797: Fs = 12 FPS, $60 \mathrm{~Hz}$ fundamental

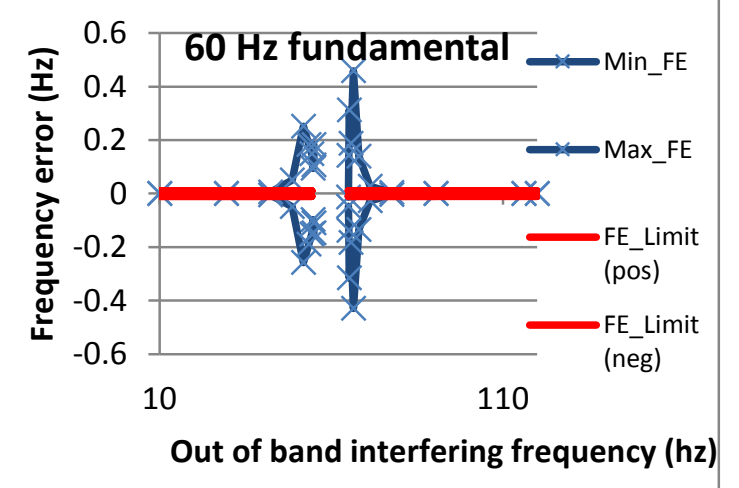

Figure 1800: Fs = $10 \mathrm{FPS}, 60 \mathrm{~Hz}$ fundamental

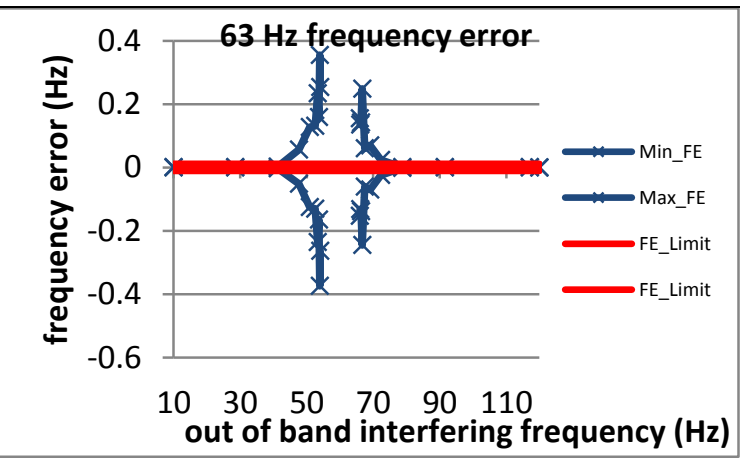

Figure 1795: Fs = 15 FPS, $60.7 \mathrm{~Hz}$ fundamental

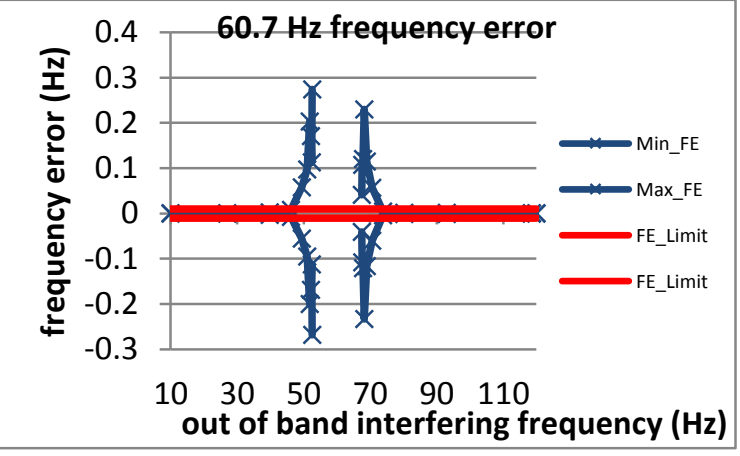

Figure 1798: Fs = 12 FPS, $60.6 \mathrm{~Hz}$ fundamental

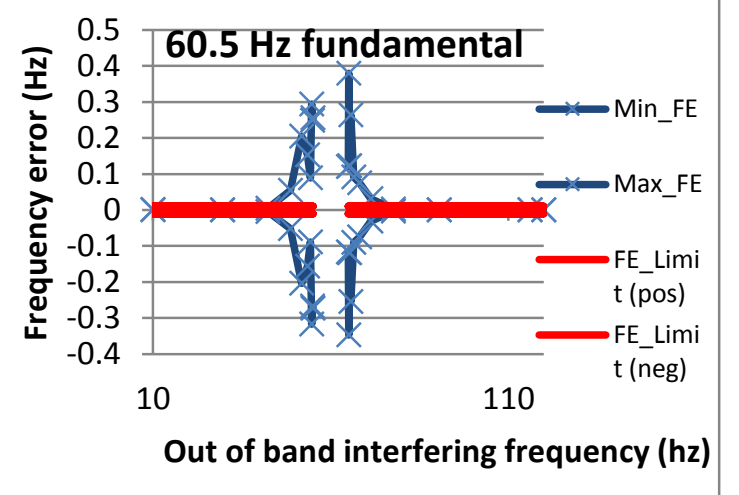

Figure 1801: Fs = 10 FPS, $60.5 \mathrm{~Hz}$ fundamental 
5.3.4 PMU $C$ out of band interfering signals frequency error: $M$ class

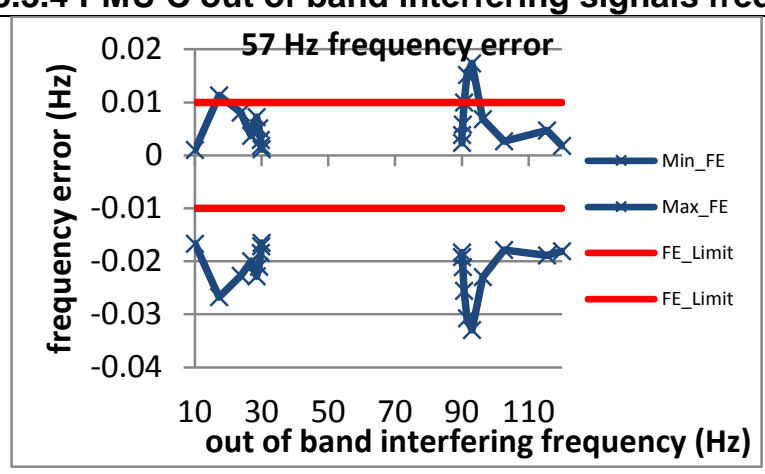

Figure 1802: Fs = 60 FPS, $57 \mathrm{~Hz}$ fundamental

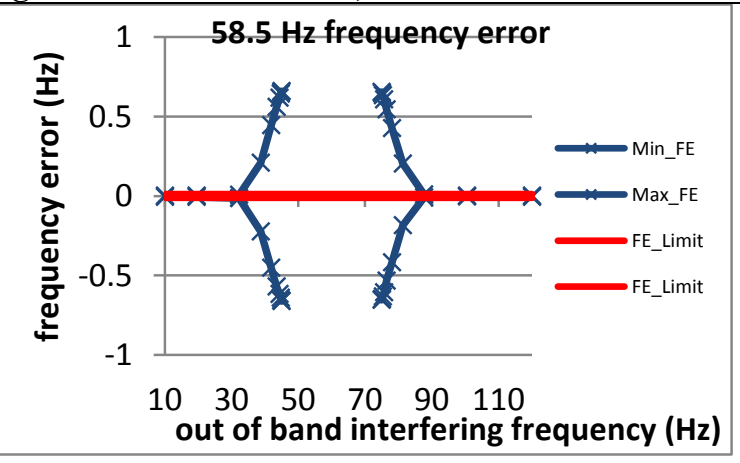

Figure 1805: Fs = 30 FPS, 58.5 Hz fundamental

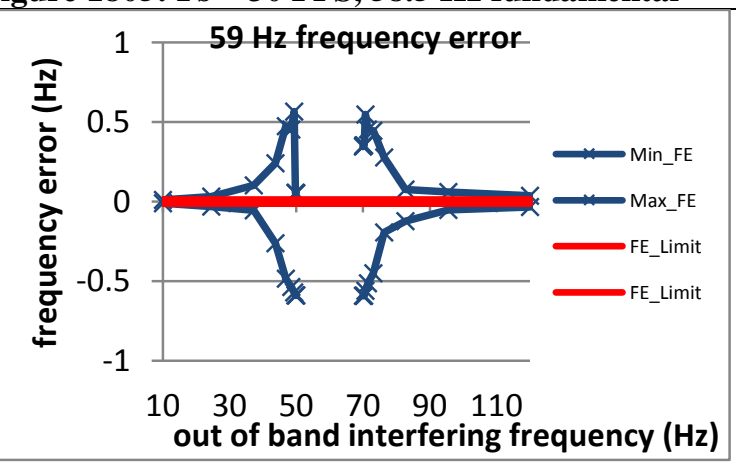

Figure 1808: Fs = 20 FPS, $59 \mathrm{~Hz}$ fundamental

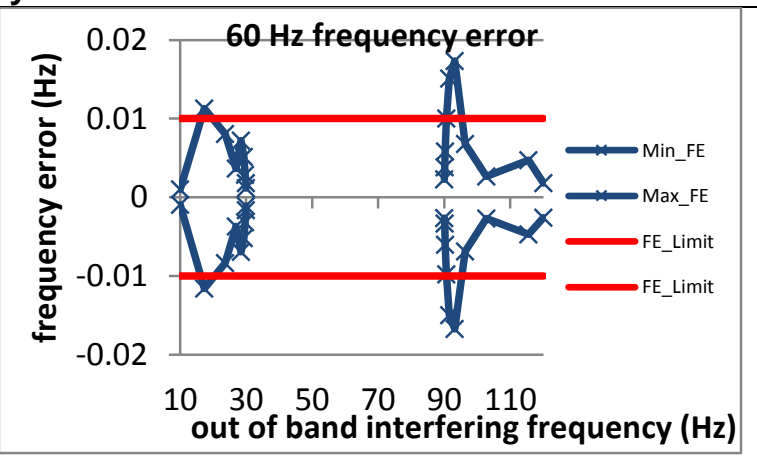

Figure 1803: Fs = $60 \mathrm{FPS}, 60 \mathrm{~Hz}$ fundamental

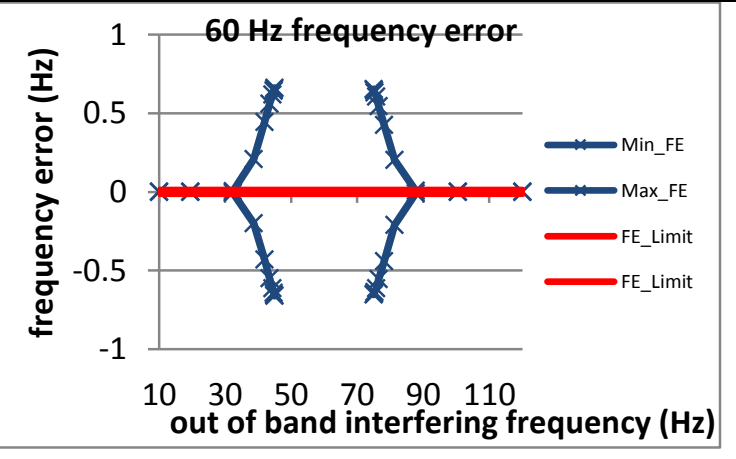

Figure 1806: Fs = $30 \mathrm{FPS}, 60 \mathrm{~Hz}$ fundamental

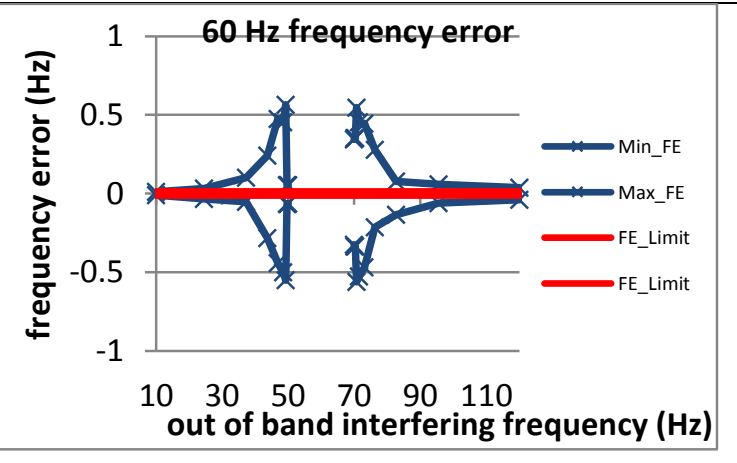

Figure 1809: Fs = 20 FPS, $60 \mathrm{~Hz}$ fundamental

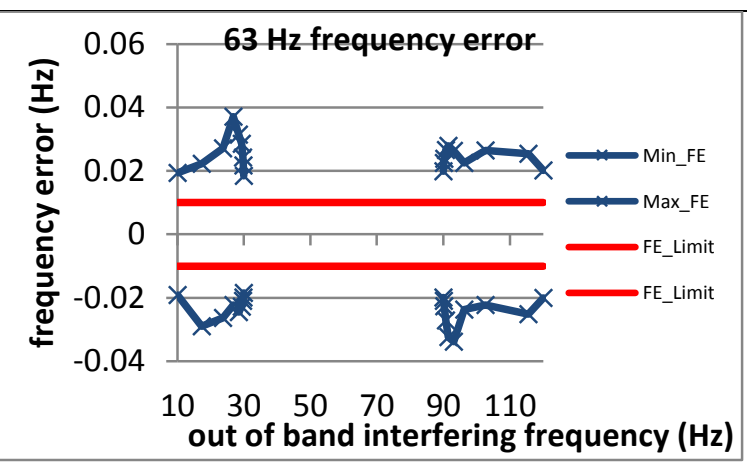

Figure 1804: Fs = $60 \mathrm{FPS}, 63 \mathrm{~Hz}$ fundamental

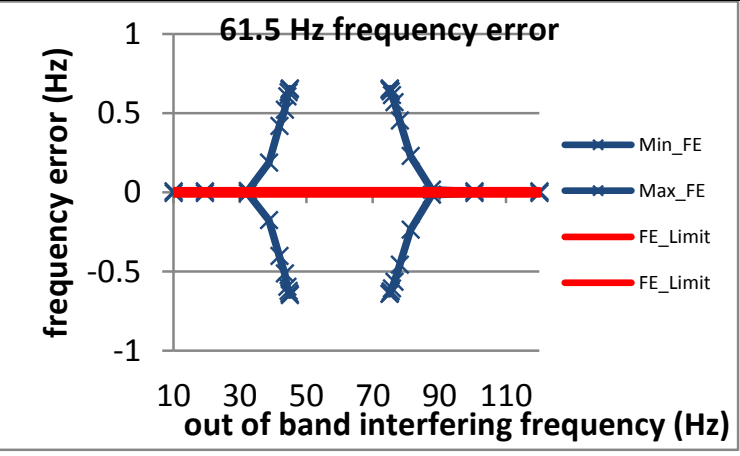

Figure 1807: Fs = 30 FPS, $61.5 \mathrm{~Hz}$ fundamental

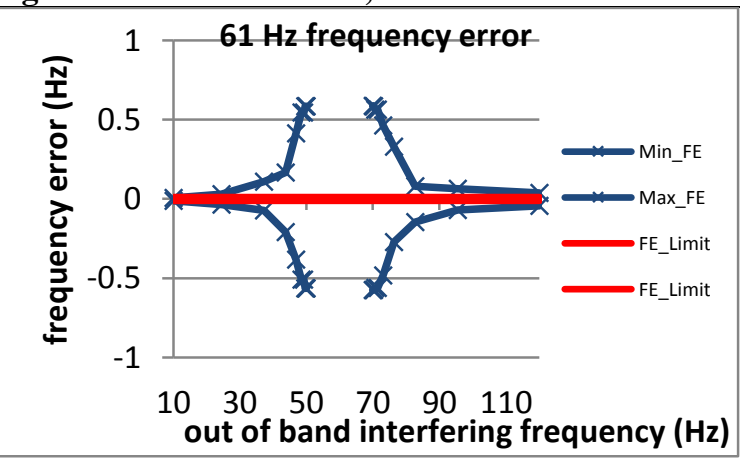

Figure 1810: Fs = $20 \mathrm{FPS}, 61 \mathrm{~Hz}$ fundamental 


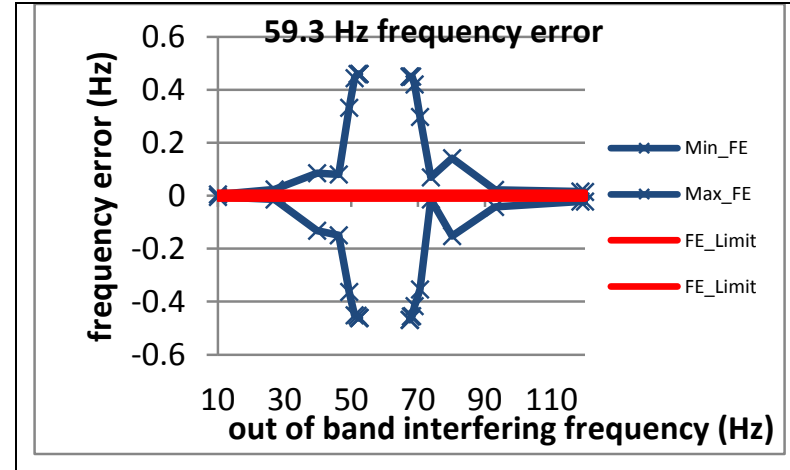

Figure 1811: Fs = 15 FPS, $59.3 \mathrm{~Hz}$ fundamental

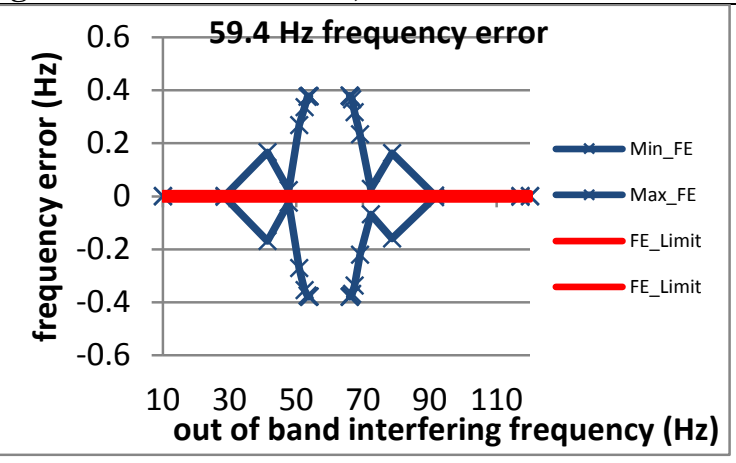

Figure 1814: Fs = 12 FPS, $59.4 \mathrm{~Hz}$ fundamental

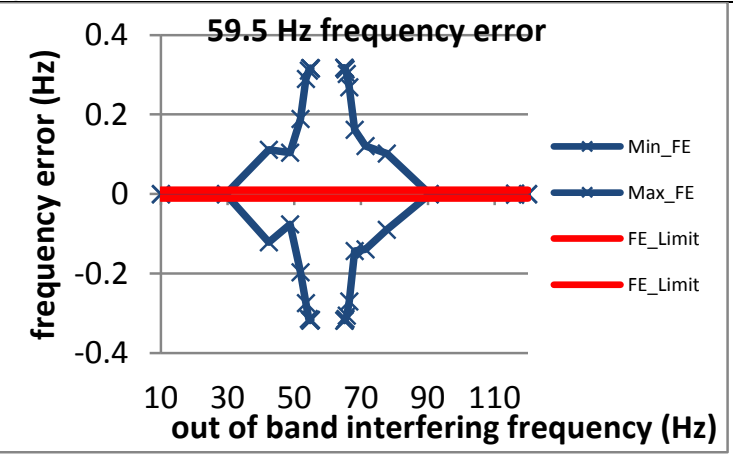

Figure 1817: Fs = $10 \mathrm{FPS}, 59.5 \mathrm{~Hz}$ fundamental

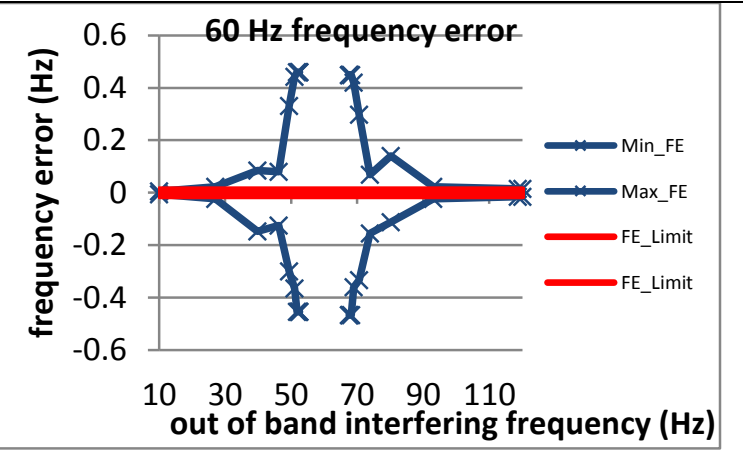

Figure 1812: Fs = $15 \mathrm{FPS}, 60 \mathrm{~Hz}$ fundamental

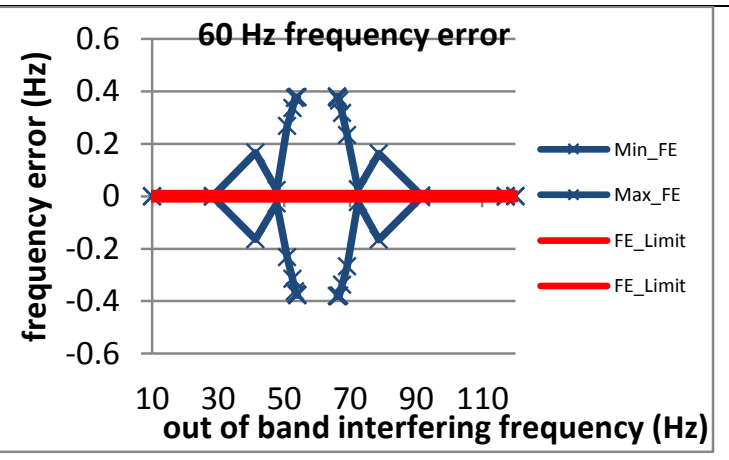

Figure 1815: Fs = 12 FPS, $60 \mathrm{~Hz}$ fundamental

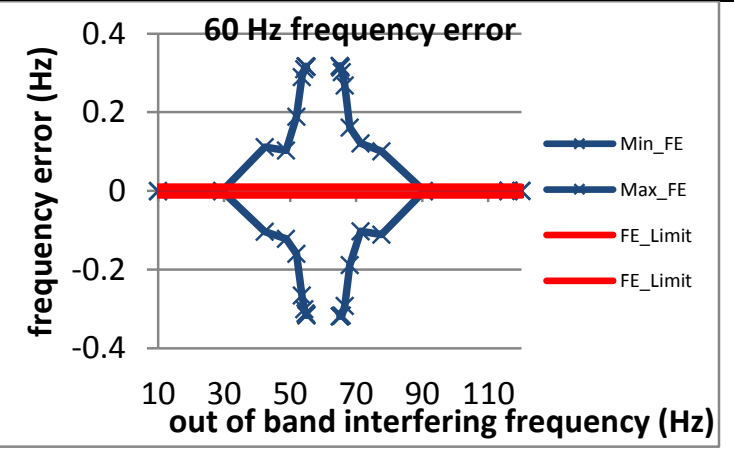

Figure 1818: Fs = $10 \mathrm{FPS}, 60 \mathrm{~Hz}$ fundamental

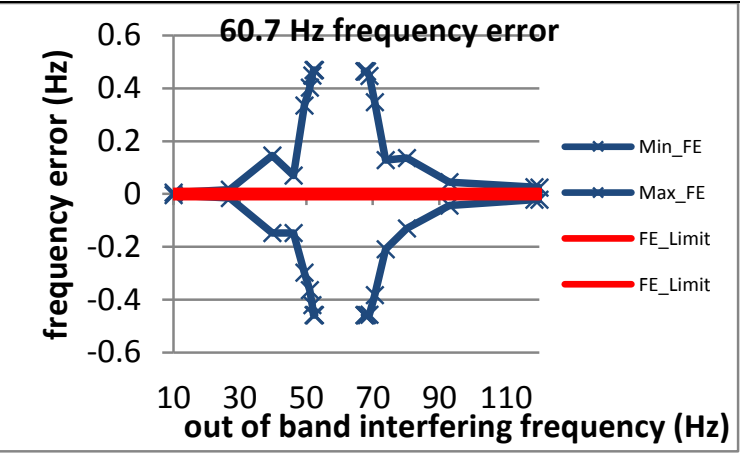

Figure 1813: Fs = 15 FPS, $60.7 \mathrm{~Hz}$ fundamental

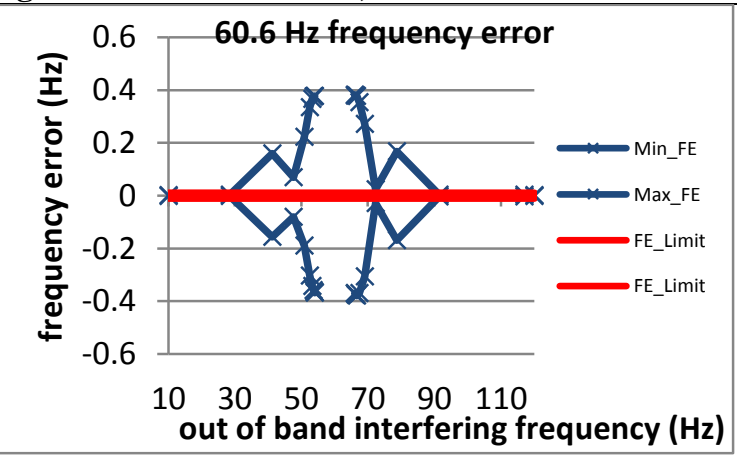

Figure 1816: Fs = 12 FPS, $60.6 \mathrm{~Hz}$ fundamental

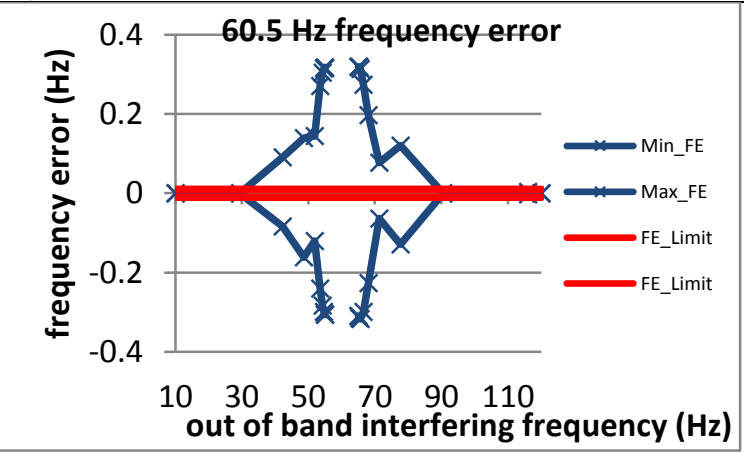

Figure 1819: Fs = $10 \mathrm{FPS}, 60.5 \mathrm{~Hz}$ fundamental 
5.3.5 PMU D out of band interfering signals frequency error: $M$ class

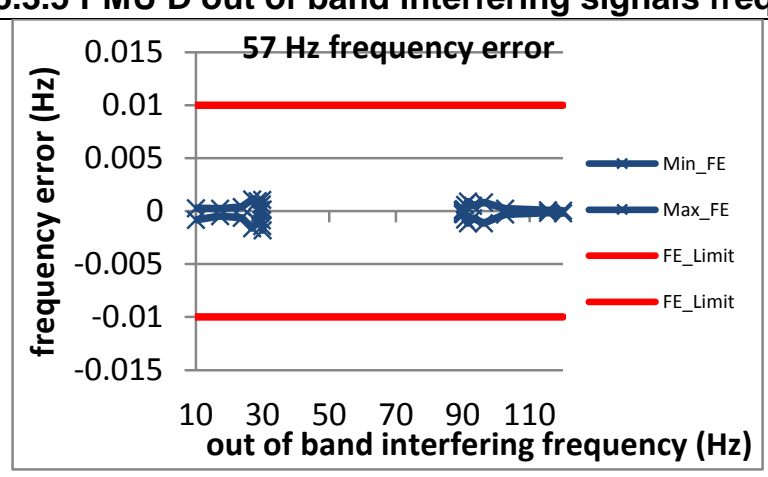

Figure 1820: Fs = 60 FPS, $57 \mathrm{~Hz}$ fundamental

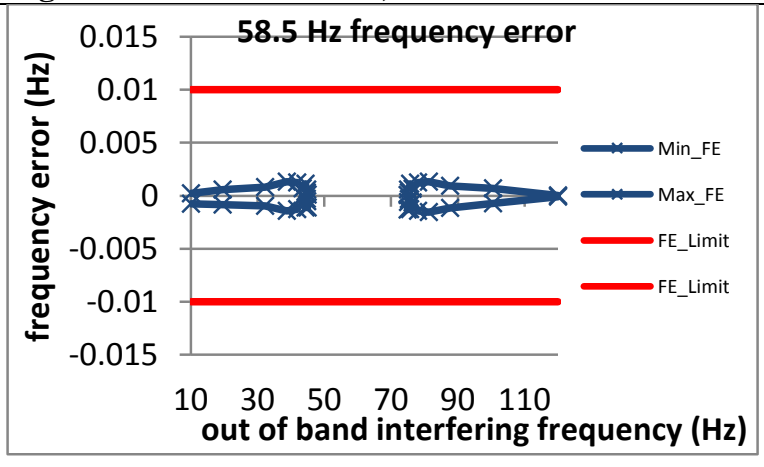

Figure 1823: Fs = 30 FPS, 58.5 Hz fundamental

Figure 1826: Fs = 20 FPS, $59 \mathrm{~Hz}$ fundamental

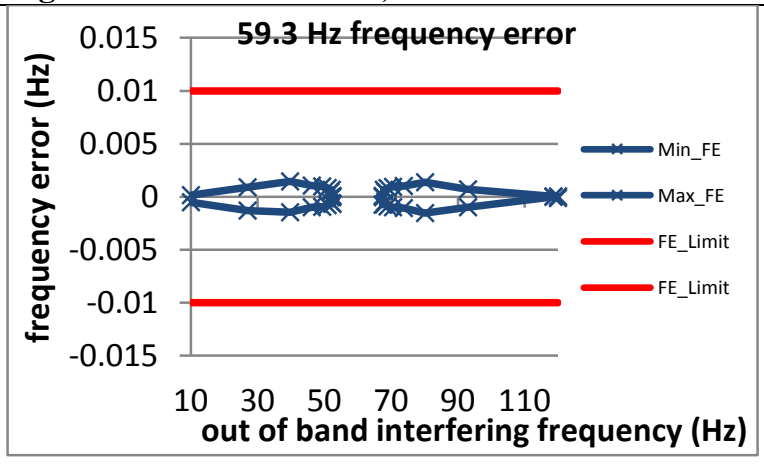

Figure 1829: Fs = 15 FPS, $59.3 \mathrm{~Hz}$ fundamental

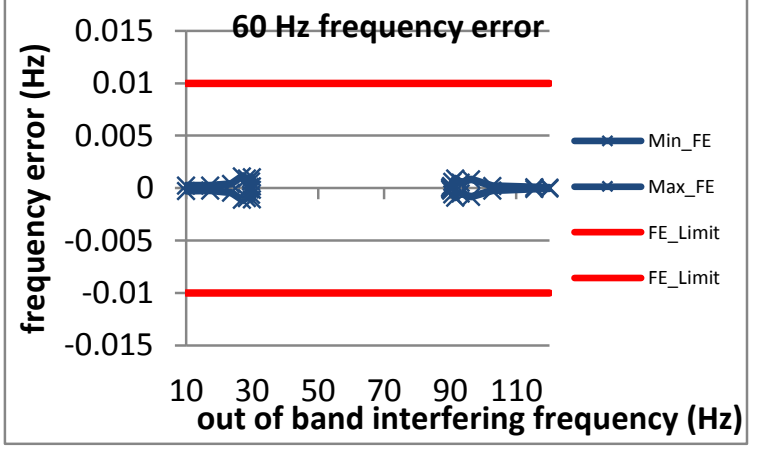

Figure 1821: Fs = $60 \mathrm{FPS}, 60 \mathrm{~Hz}$ fundamental

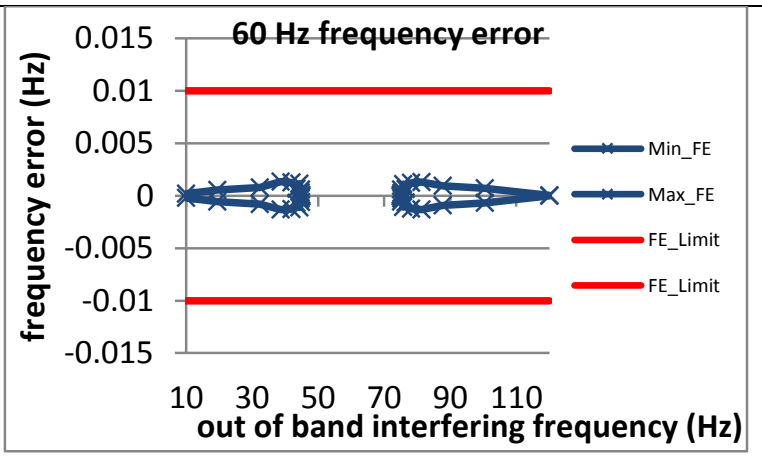

Figure 1824: Fs = 30 FPS, $60 \mathrm{~Hz}$ fundamental

Figure 1827: Fs = $20 \mathrm{FPS}, 60 \mathrm{~Hz}$ fundamental

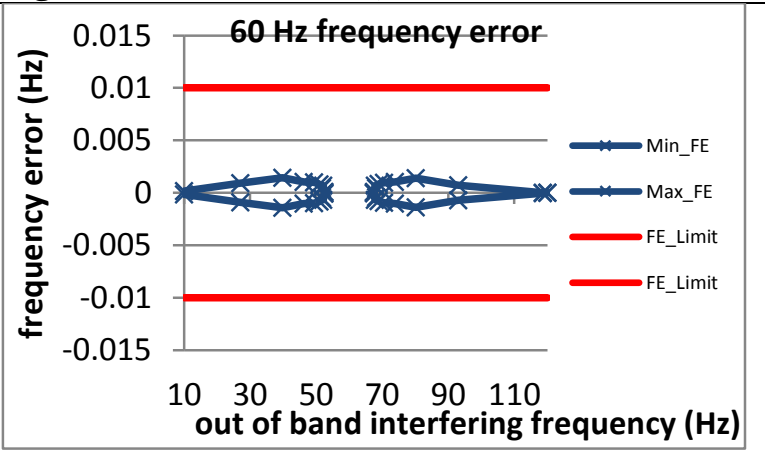

Figure 1830: Fs = $15 \mathrm{FPS}, 60 \mathrm{~Hz}$ fundamental

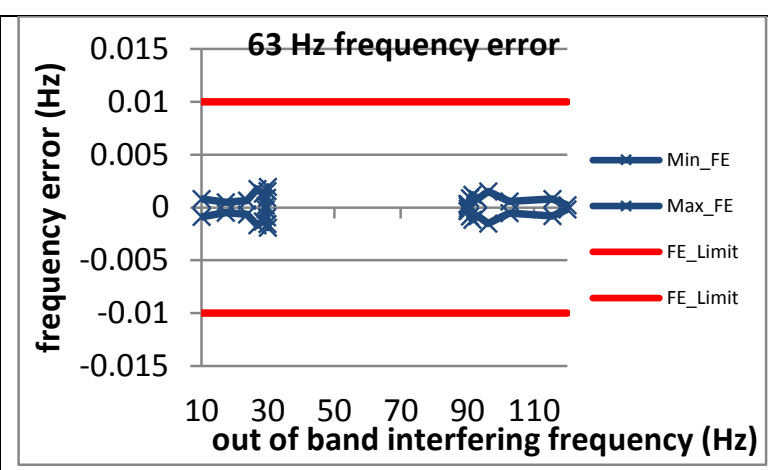

Figure 1822: Fs = $60 \mathrm{FPS}, 63 \mathrm{~Hz}$ fundamental

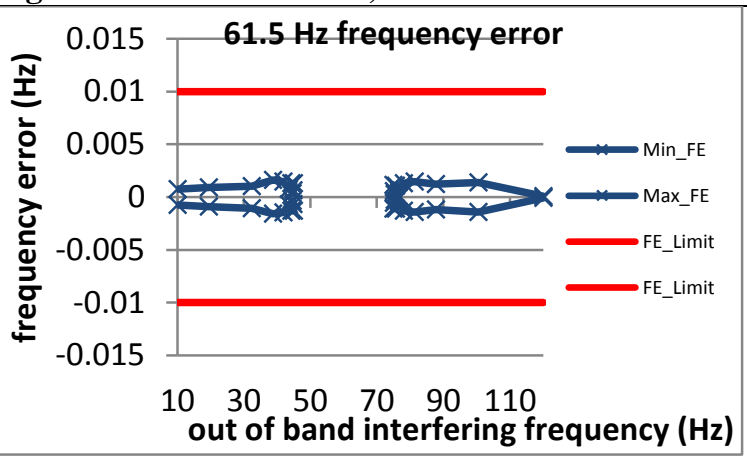

Figure 1825: Fs = 30 FPS, $61.5 \mathrm{~Hz}$ fundamental

Figure 1828: Fs = $20 \mathrm{FPS}, 61 \mathrm{~Hz}$ fundamental

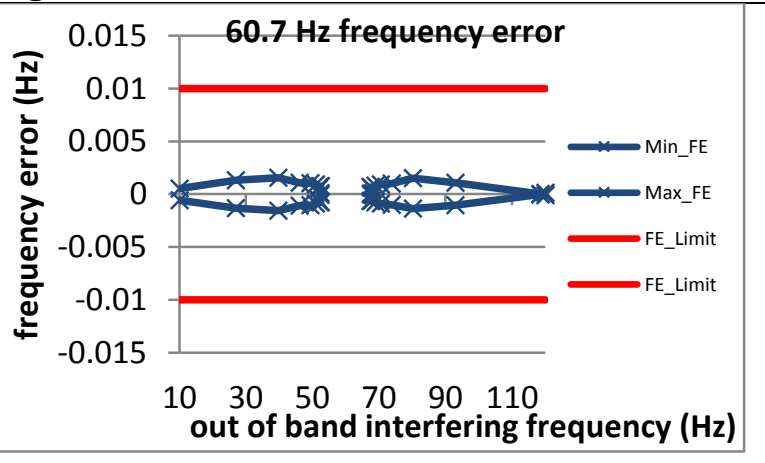

Figure 1831: Fs = 15 FPS, $60.7 \mathrm{~Hz}$ fundamental 

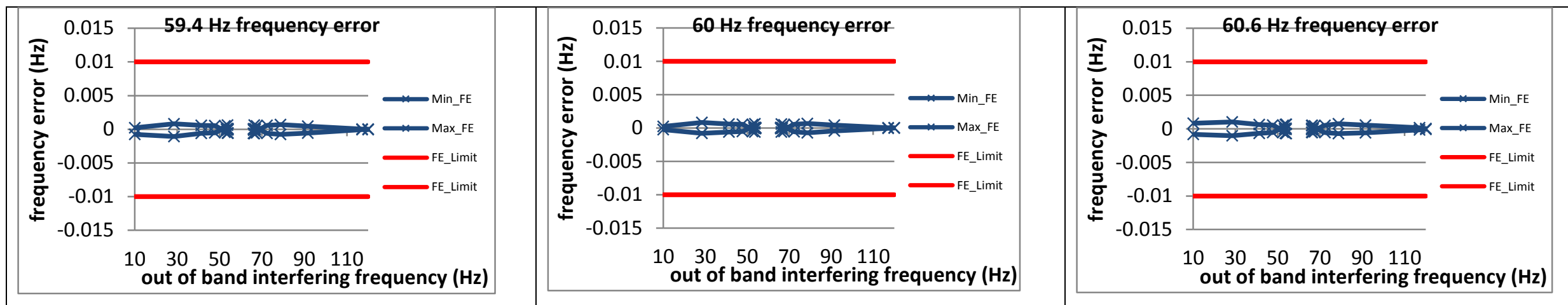

Figure 1832: Fs = 12 FPS, $59.4 \mathrm{~Hz}$ fundamental

Figure 1833: Fs = 12 FPS, $60 \mathrm{~Hz}$ fundamental
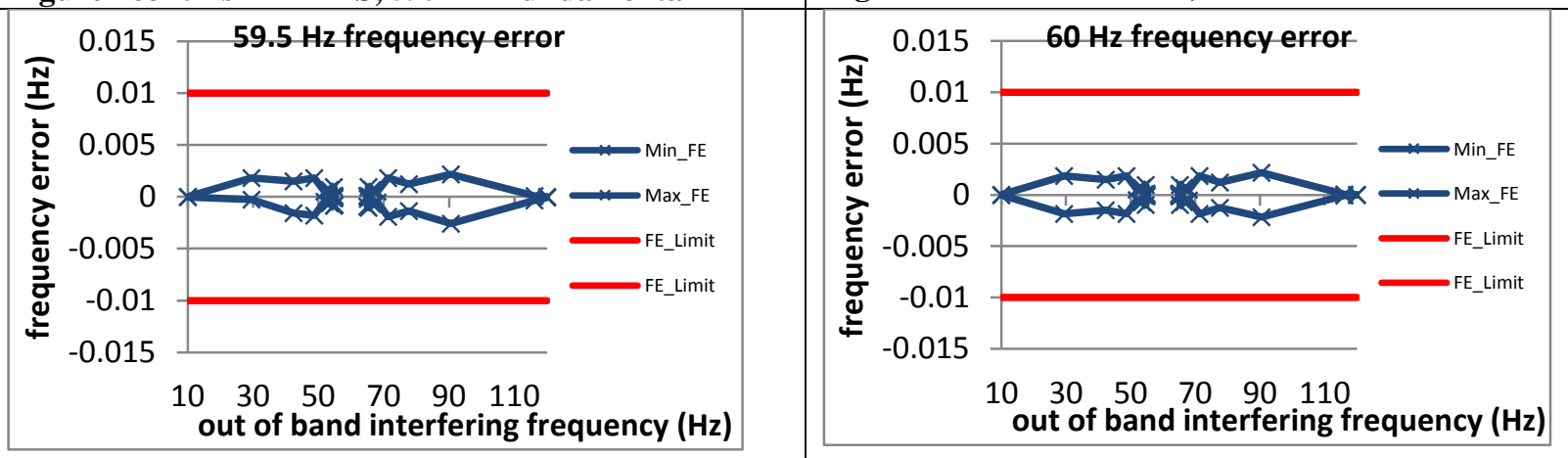

Figure 1834: Fs = 12 FPS, $60.6 \mathrm{~Hz}$ fundamental

Figure 1835: Fs = 10 FPS, 59.5 Hz fundamental

Figure 1836: Fs = 10 FPS, $60 \mathrm{~Hz}$ fundamental

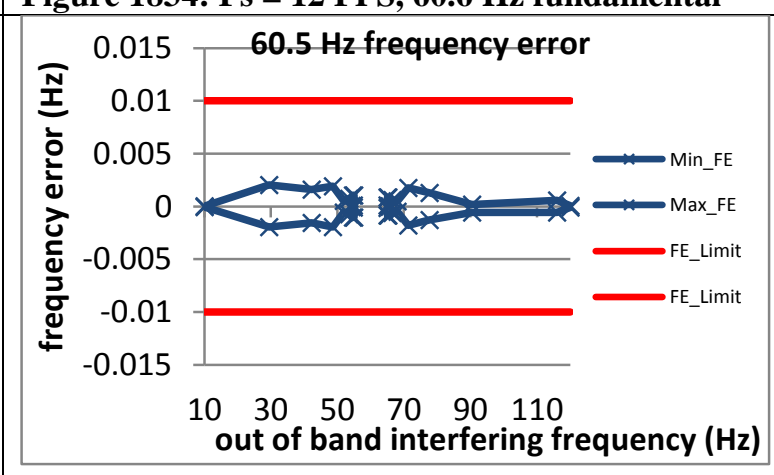

Figure 1837: Fs = 10 FPS, $60.5 \mathrm{~Hz}$ fundamental 
5.3.6 PMU E out of band interfering signals frequency error: $M$ class

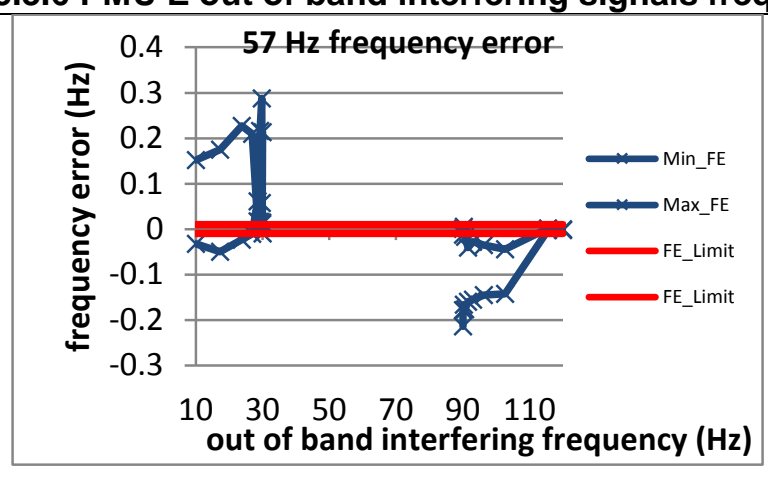

Figure 1838: Fs = $60 \mathrm{FPS}, 57 \mathrm{~Hz}$ fundamental

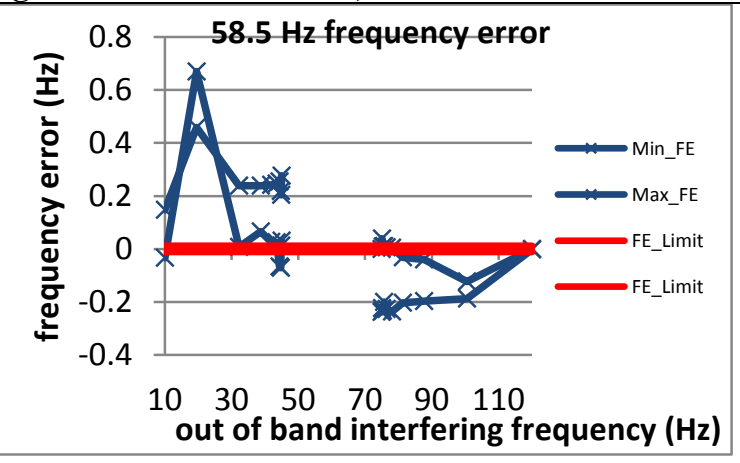

Figure 1841: Fs = 30 FPS, $58.5 \mathrm{~Hz}$ fundamental

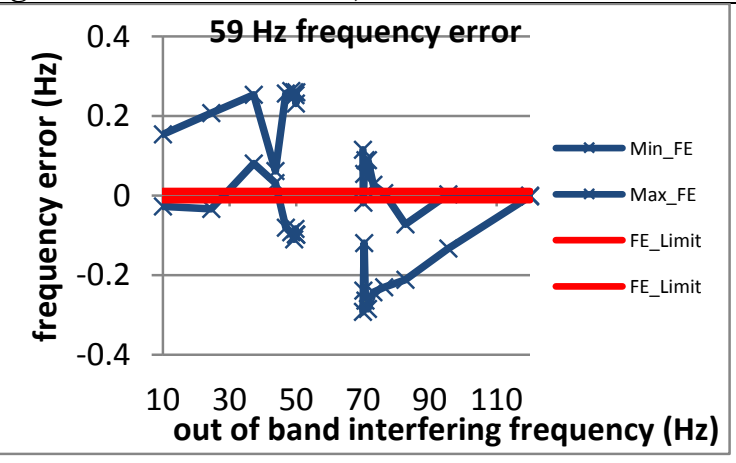

Figure 1844: Fs = 20 FPS, 59 Hz fundamental

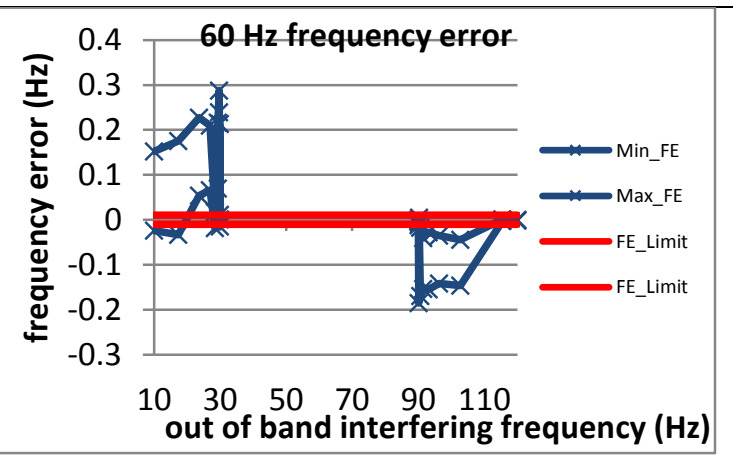

Figure 1839: Fs = 60 FPS, $60 \mathrm{~Hz}$ fundamental

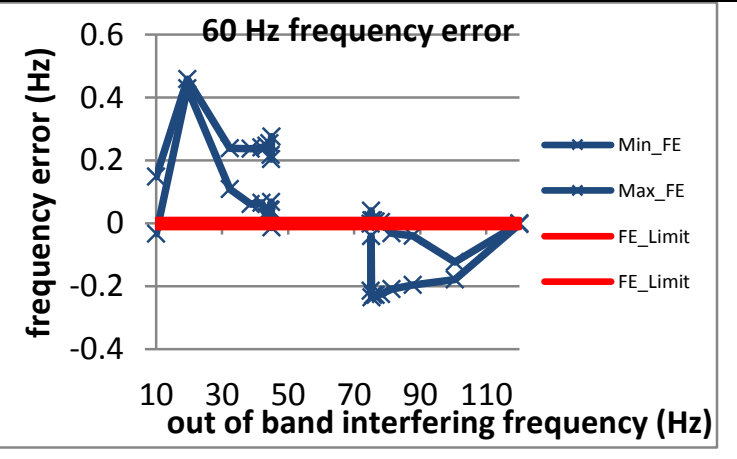

Figure 1842: Fs = $30 \mathrm{FPS}, 60 \mathrm{~Hz}$ fundamental

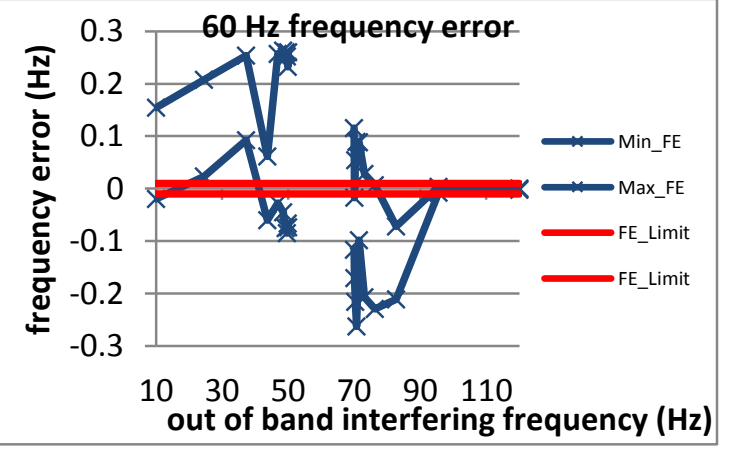

Figure 1845: Fs = 20 FPS, $60 \mathrm{~Hz}$ fundamental

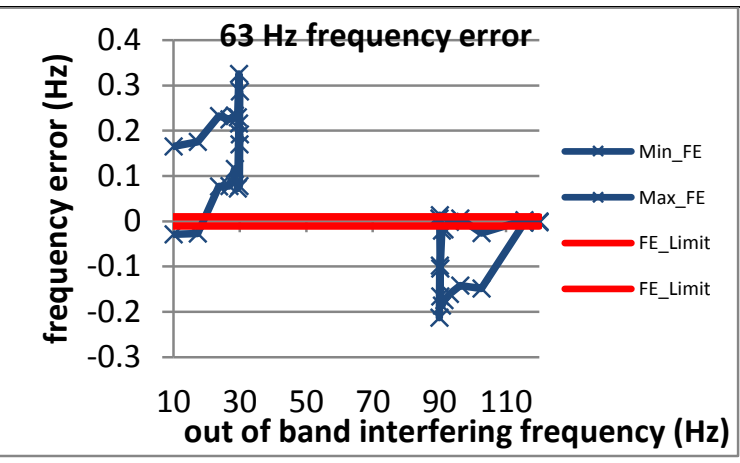

Figure 1840: Fs = 60 FPS, $63 \mathrm{~Hz}$ fundamental

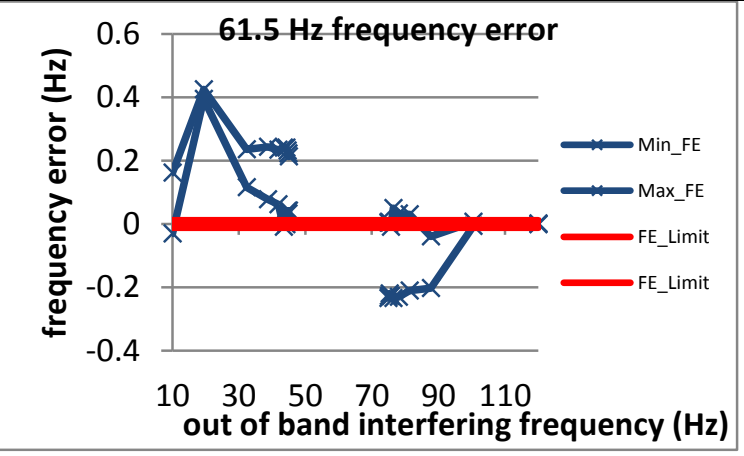

Figure 1843: Fs = 30 FPS, 61.5 Hz fundamental

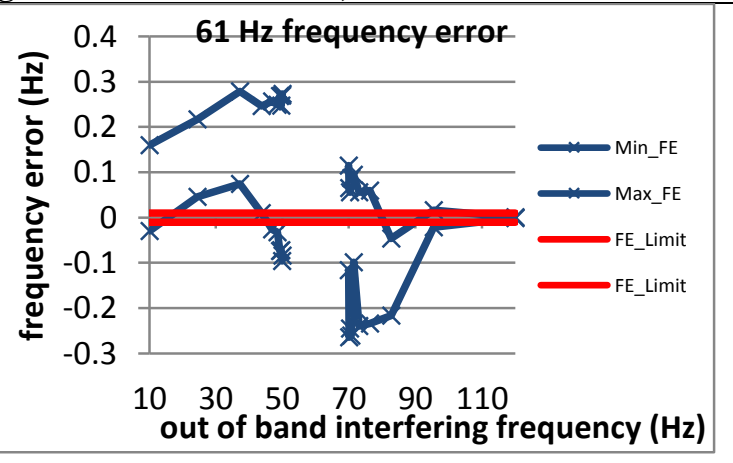

Figure 1846: Fs = 20 FPS, $61 \mathrm{~Hz}$ fundamental 


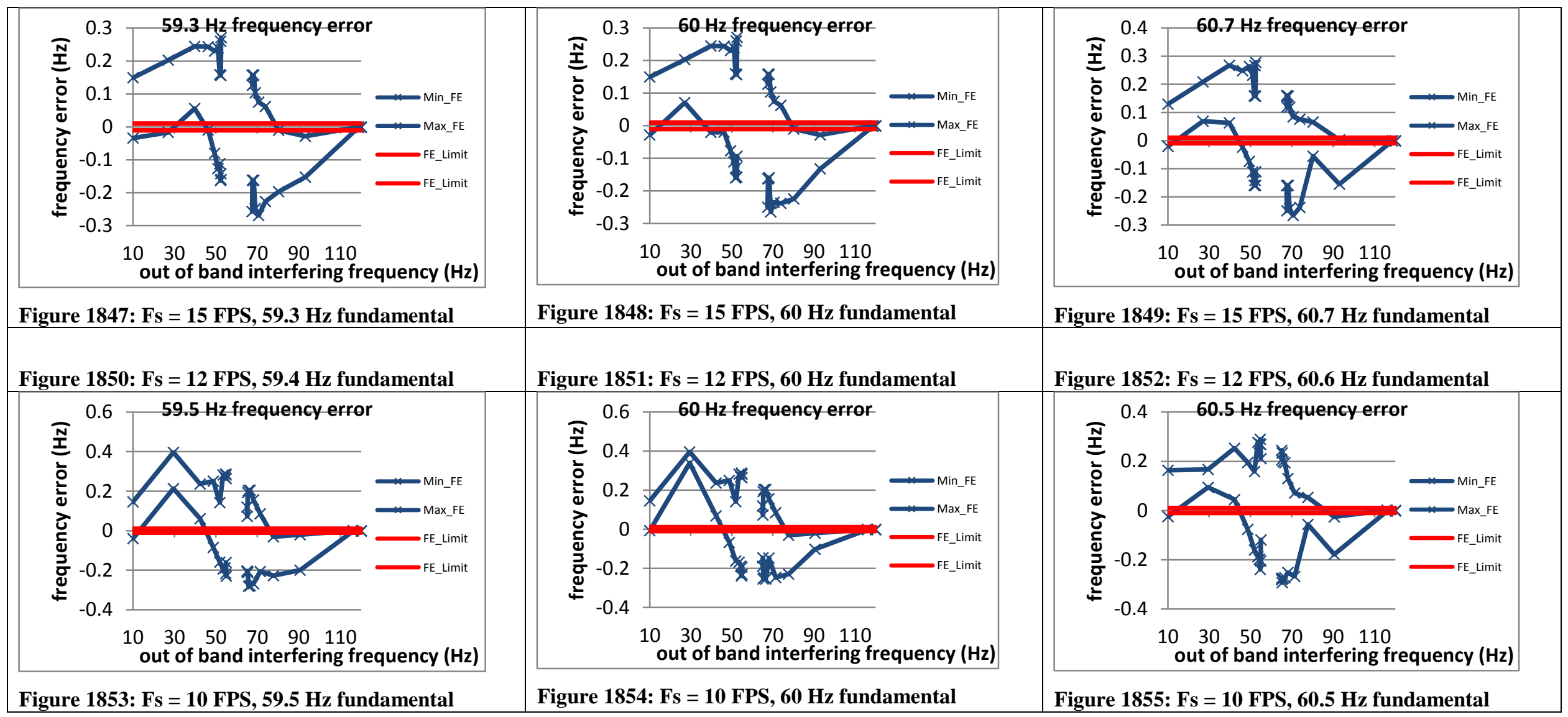


5.3.7 PMU F out of band interfering signals frequency error: $M$ class

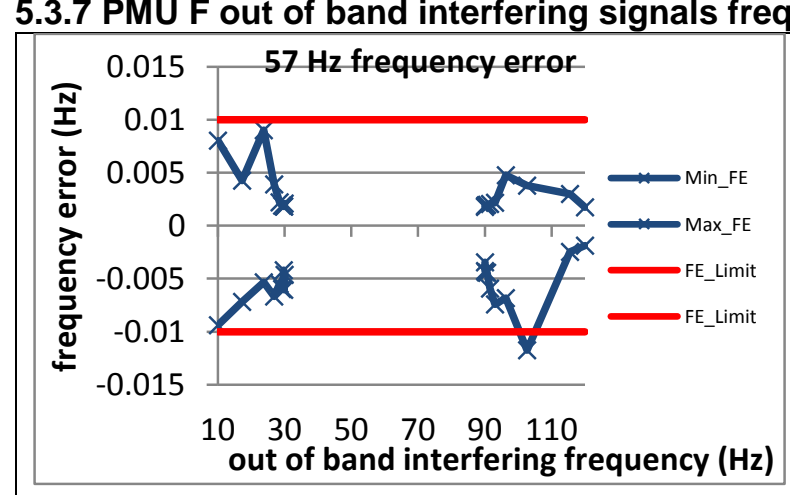

Figure 1856: Fs = 60 FPS, $57 \mathrm{~Hz}$ fundamental

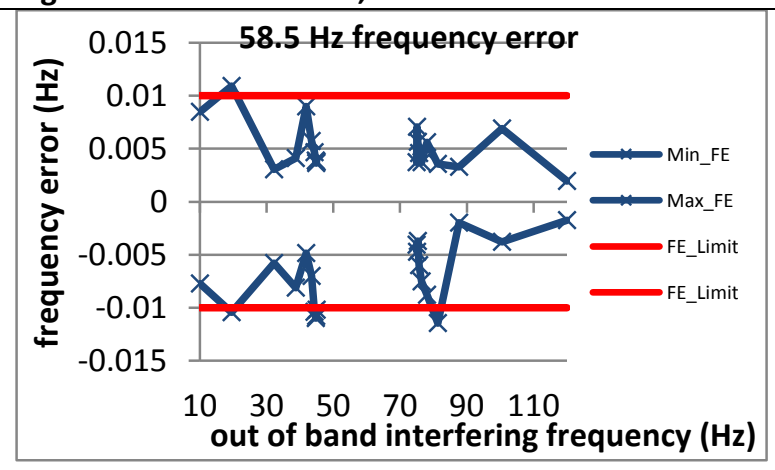

Figure 1859: Fs = $30 \mathrm{FPS}, 58.5 \mathrm{~Hz}$ fundamental

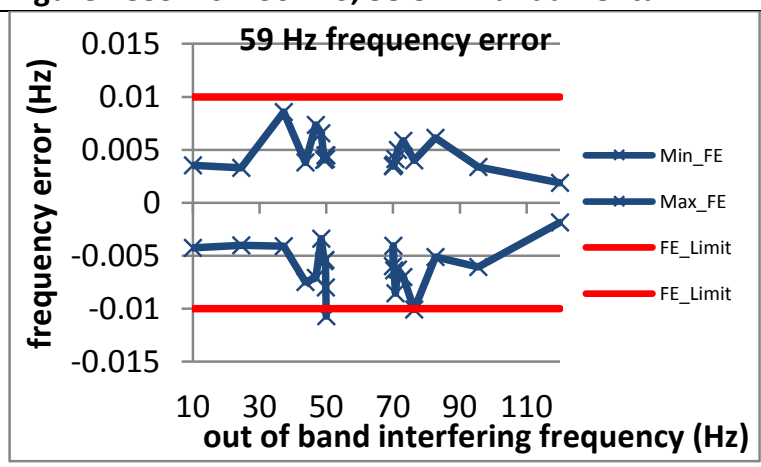

Figure 1862: Fs = $20 \mathrm{FPS}, 59 \mathrm{~Hz}$ fundamental

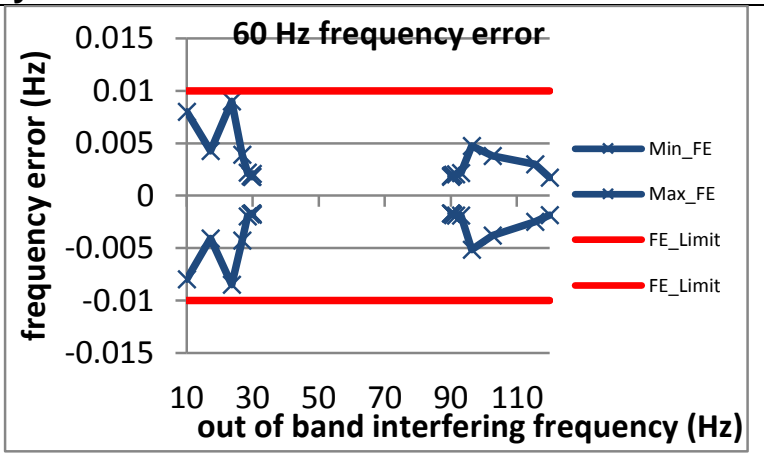

Figure 1857: Fs = $60 \mathrm{FPS}, 60 \mathrm{~Hz}$ fundamental

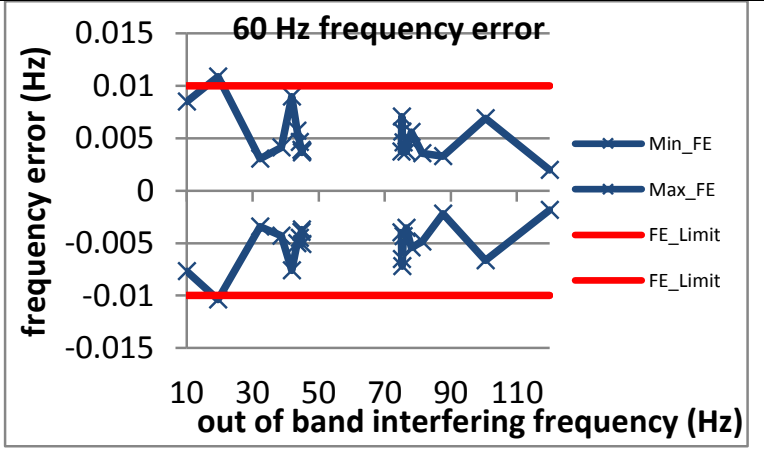

Figure 1860: Fs = $30 \mathrm{FPS}, 60 \mathrm{~Hz}$ fundamental

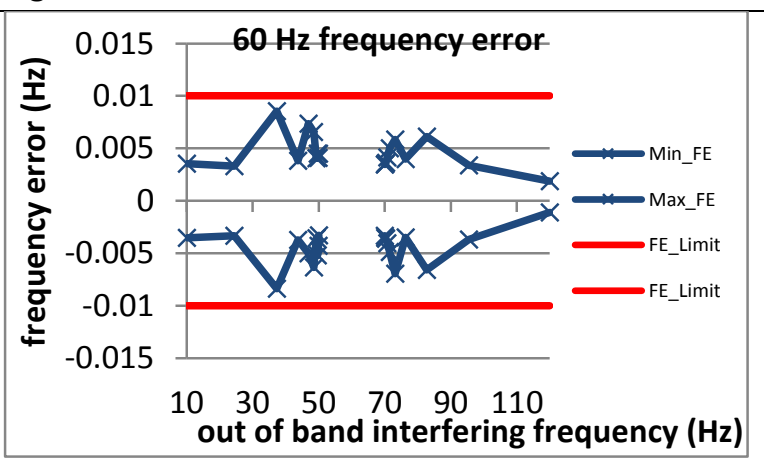

Figure 1863: Fs = $20 \mathrm{FPS}, 60 \mathrm{~Hz}$ fundamental

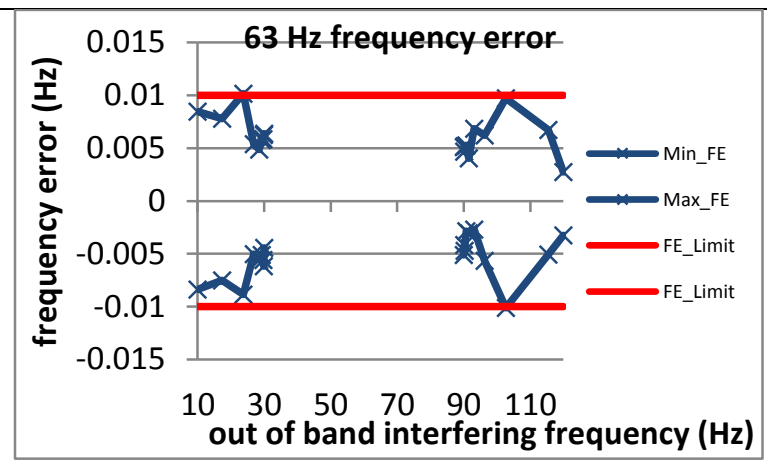

Figure 1858: Fs = $60 \mathrm{FPS}, 63 \mathrm{~Hz}$ fundamental

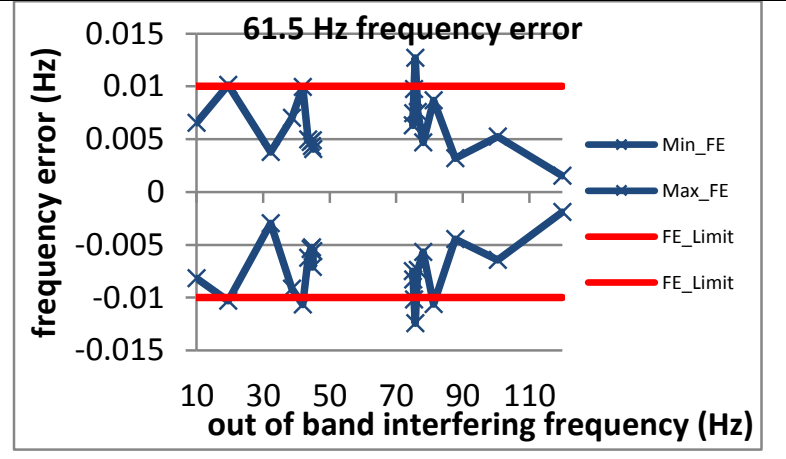

Figure 1861: Fs = $30 \mathrm{FPS}, 61.5 \mathrm{~Hz}$ fundamental

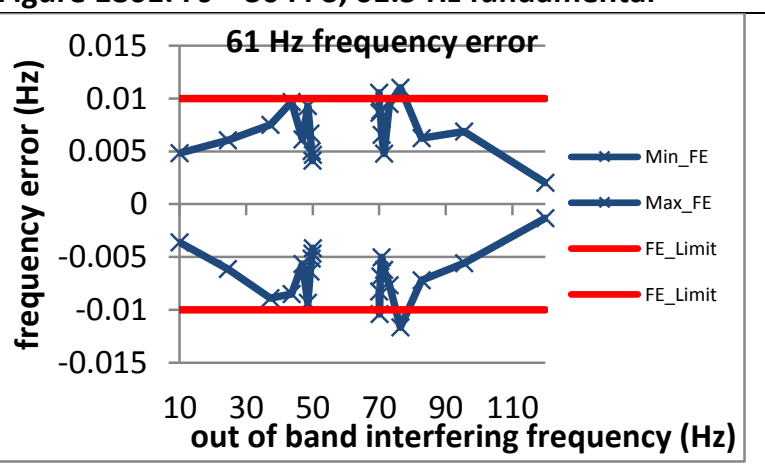

Figure 1864: Fs = 20 FPS, $61 \mathrm{~Hz}$ fundamental 


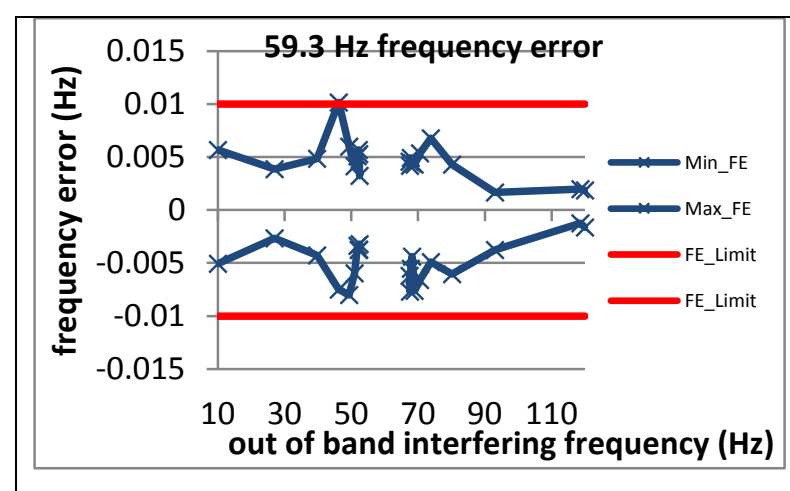

Figure 1865: Fs = $15 \mathrm{FPS}, 59.3 \mathrm{~Hz}$ fundamental

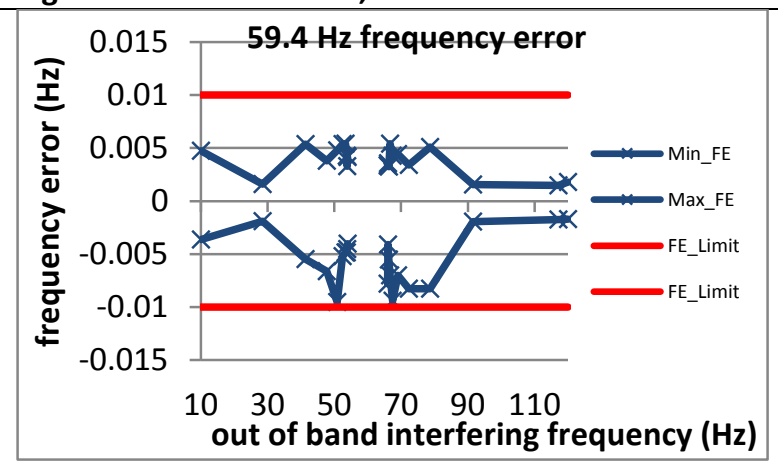

Figure 1868: $\mathrm{Fs}=12 \mathrm{FPS}, 59.4 \mathrm{~Hz}$ fundamental

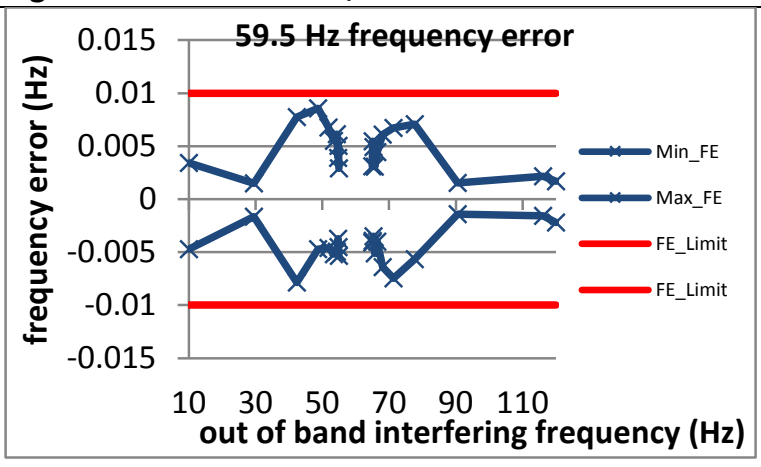

Figure 1871: Fs = $10 \mathrm{FPS}, 59.5 \mathrm{~Hz}$ fundamental

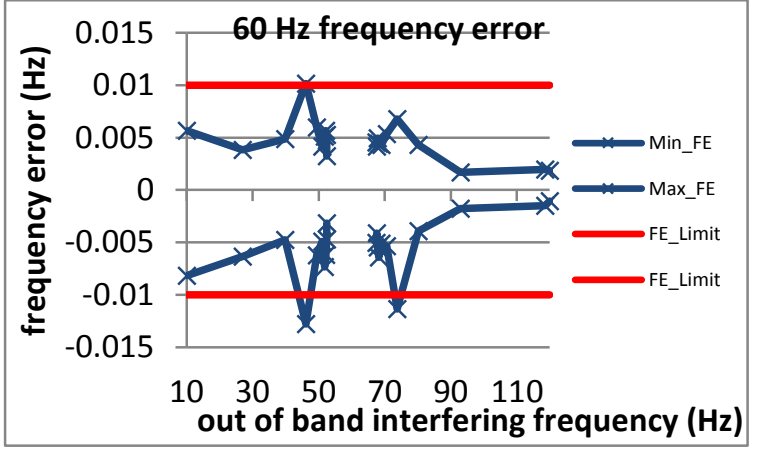

Figure 1866: Fs = $15 \mathrm{FPS}, 60 \mathrm{~Hz}$ fundamental

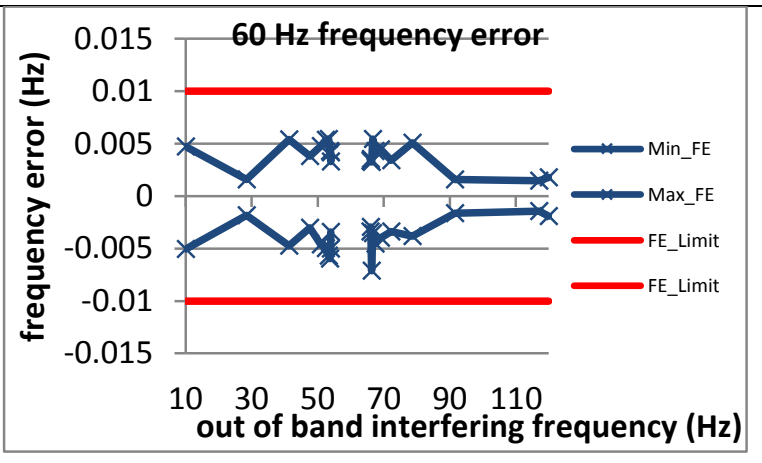

Figure 1869: $\mathrm{Fs}=12 \mathrm{FPS}, 60 \mathrm{~Hz}$ fundamental

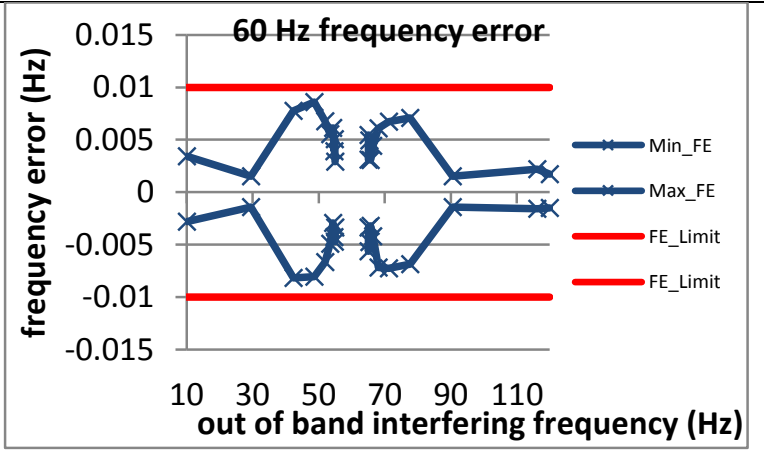

Figure 1872: Fs = $10 \mathrm{FPS}, 60 \mathrm{~Hz}$ fundamental

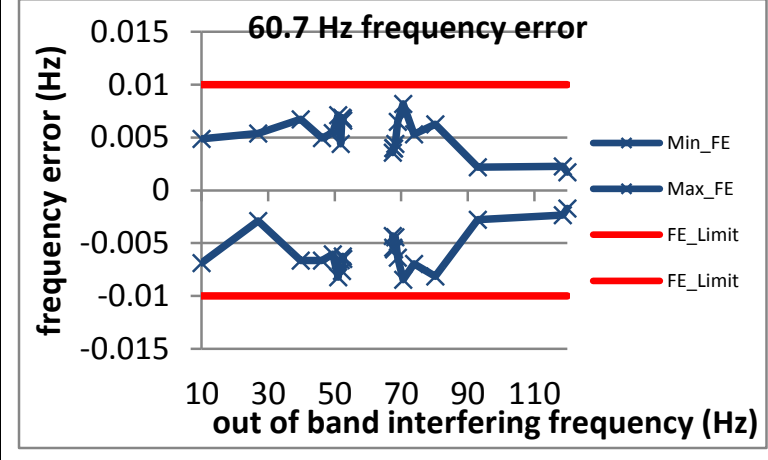

Figure 1867: Fs = $15 \mathrm{FPS}, 60.7 \mathrm{~Hz}$ fundamental

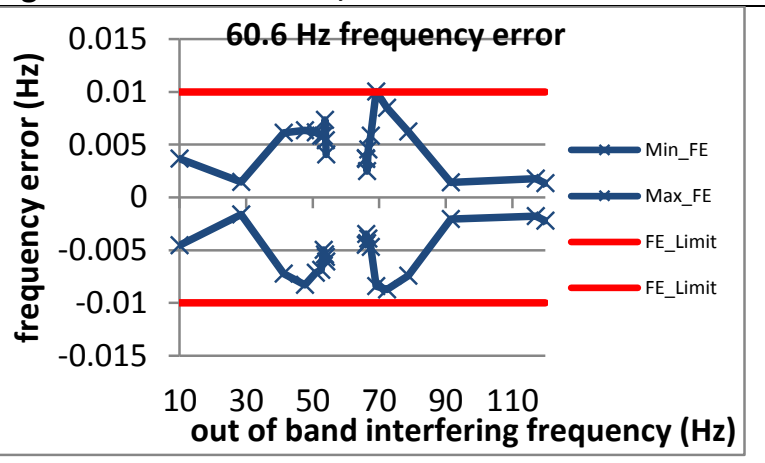

Figure 1870: Fs = $12 \mathrm{FPS}, 60.6 \mathrm{~Hz}$ fundamental

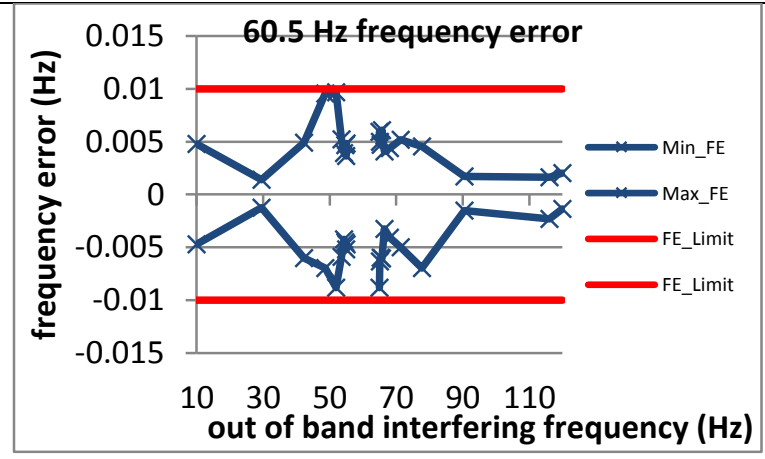

Figure 1873: Fs = $10 \mathrm{FPS}, 60.5 \mathrm{~Hz}$ fundamental 
5.3.8 PMU G out of band interfering signals frequency error: $M$ class

\begin{tabular}{|c|c|c|}
\hline $\begin{array}{l}60 \text { FPS is not supported by this PMU } \\
\text { Figure 1874: Fs = } 60 \text { FPS, } \mathbf{5 7} \mathbf{~ H z} \text { fundamental }\end{array}$ & $\begin{array}{l}60 \text { FPS is not supported by this PMU } \\
\text { Figure 1875: Fs = } \mathbf{6 0} \mathbf{F P S}, \mathbf{6 0} \mathbf{~ H z} \text { fundamental }\end{array}$ & $\begin{array}{l}60 \text { FPS is not supported by this PMU } \\
\text { Figure 1876: Fs = 60 FPS, } 63 \mathrm{~Hz} \text { fundamental }\end{array}$ \\
\hline 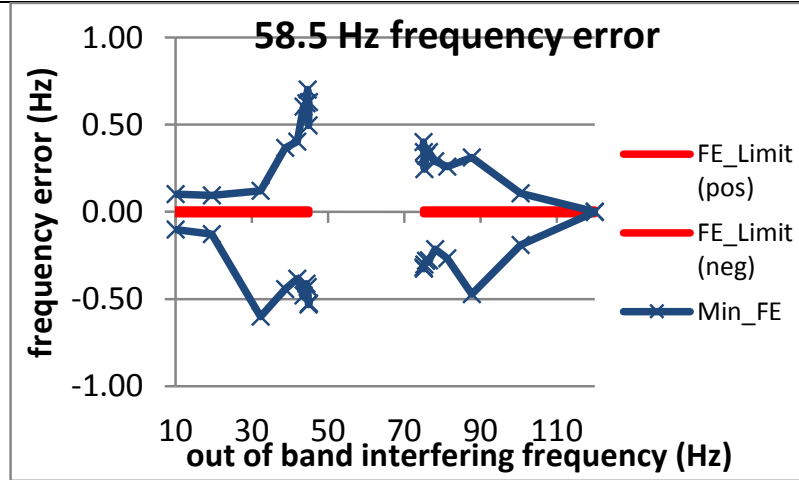 & Hz frequency error & $5 \mathrm{~Hz}$ frequency error \\
\hline Figure 1877: F & Figure 1878: Fs = $30 \mathrm{FPS}, 60 \mathrm{~Hz}$ fundamental & $30 \mathrm{FPS}, 61.5 \mathrm{~Hz}$ fundamental \\
\hline 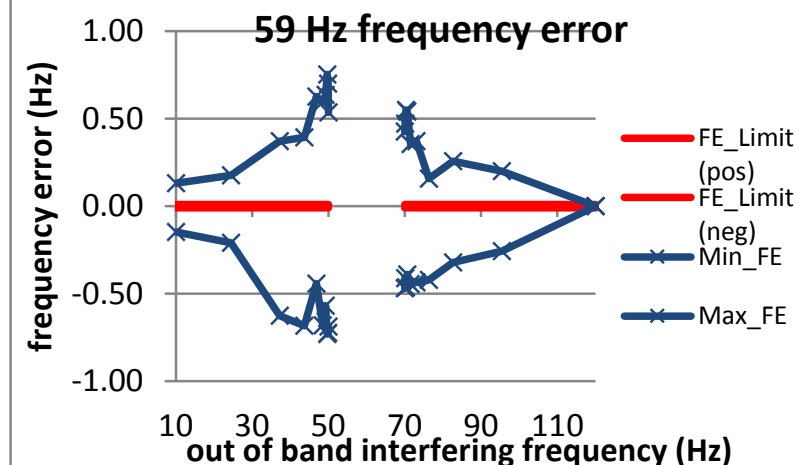 & 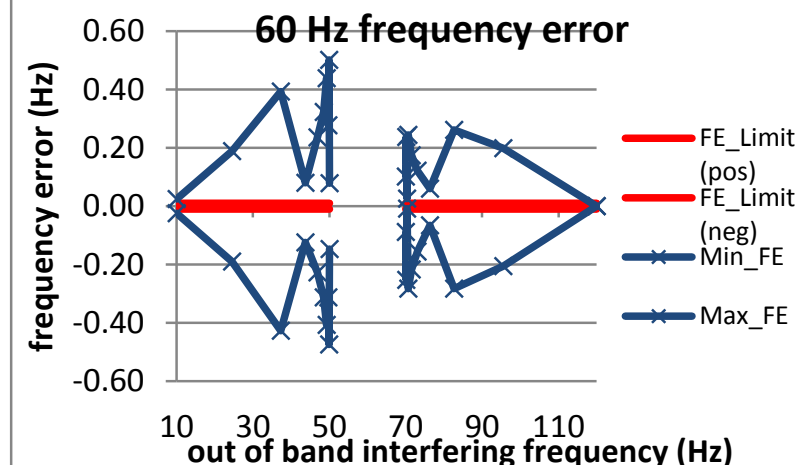 & 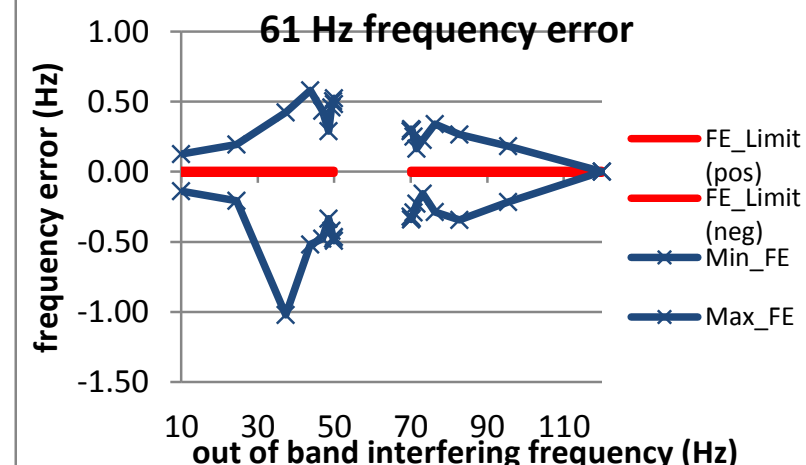 \\
\hline Figure 1880: Fs = 20 FPS, $59 \mathrm{~Hz}$ fundamental & Figure 1881: Fs = $20 \mathrm{FPS}, 60 \mathrm{~Hz}$ fundamental & Figure 1882: Fs = 20 FPS, $61 \mathrm{~Hz}$ fundamental \\
\hline
\end{tabular}



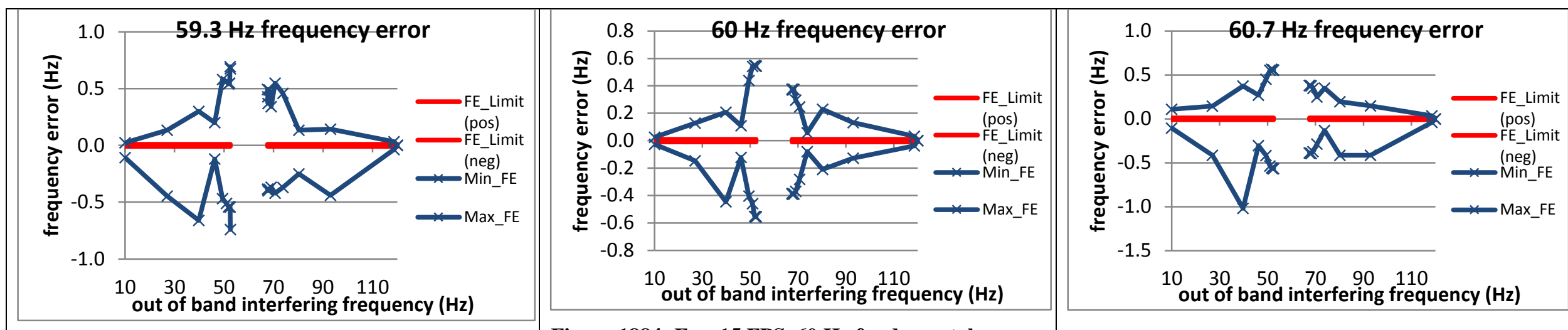

Figure 1883: Fs = 15 FPS, $59.3 \mathrm{~Hz}$ fundamental

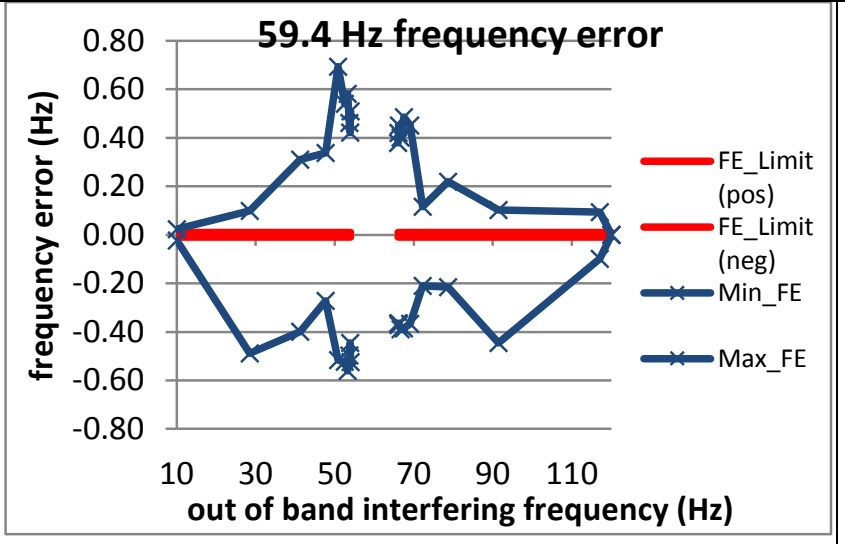

Figure 1884: Fs = $15 \mathrm{FPS}, 60 \mathrm{~Hz}$ fundamental

Figure 1885: Fs = 15 FPS, $60.7 \mathrm{~Hz}$ fundamental
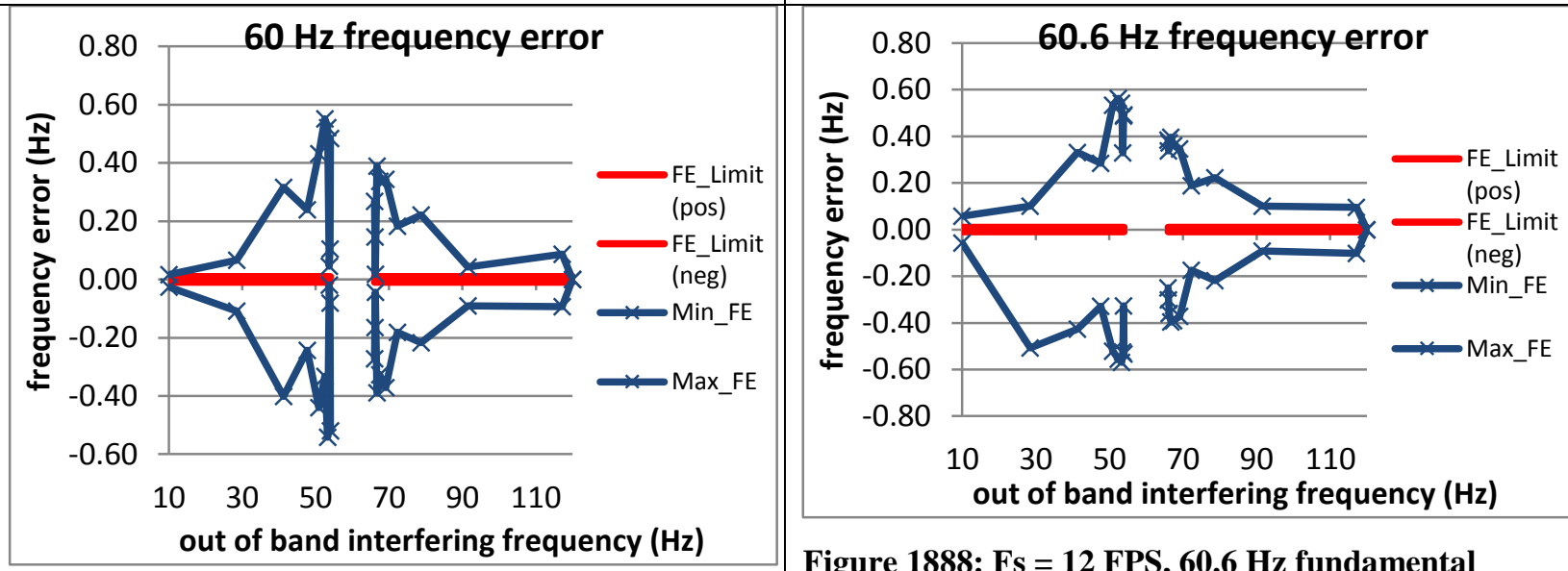

Figure 1886: Fs = 12 FPS, $59.4 \mathrm{~Hz}$ fundamental

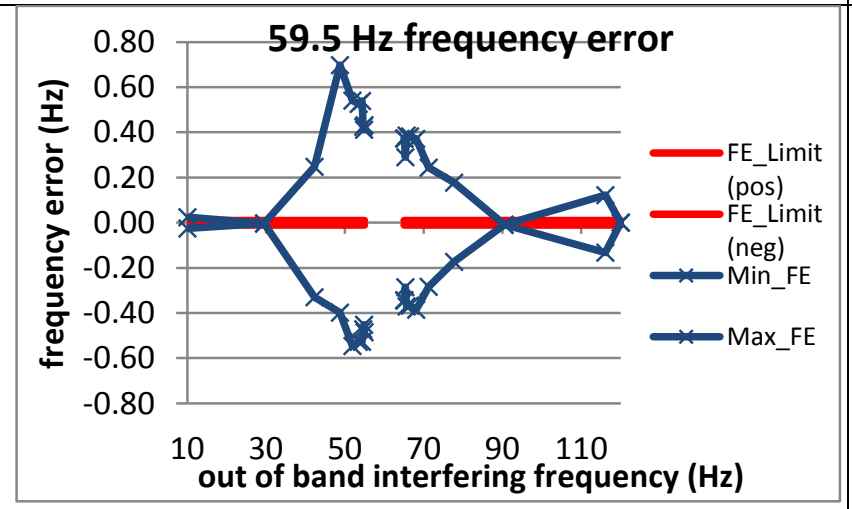

Figure 1887: Fs = $12 \mathrm{FPS}, 60 \mathrm{~Hz}$ fundamental

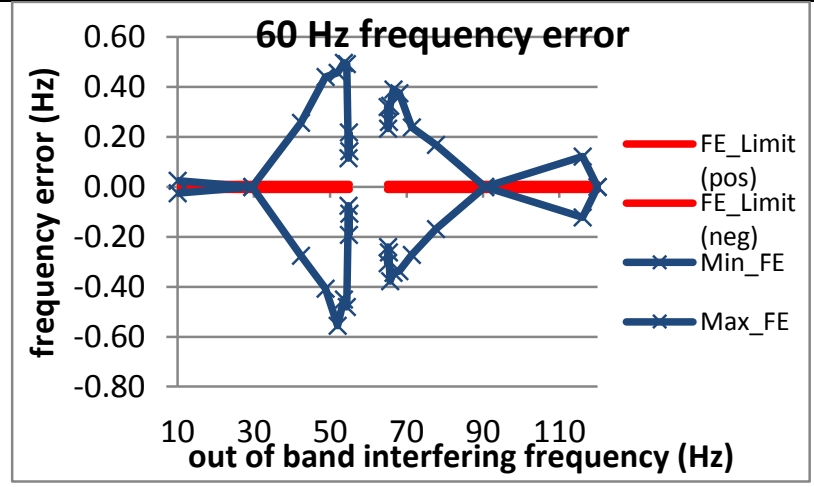

Figure 1888: Fs = 12 FPS, $60.6 \mathrm{~Hz}$ fundamental

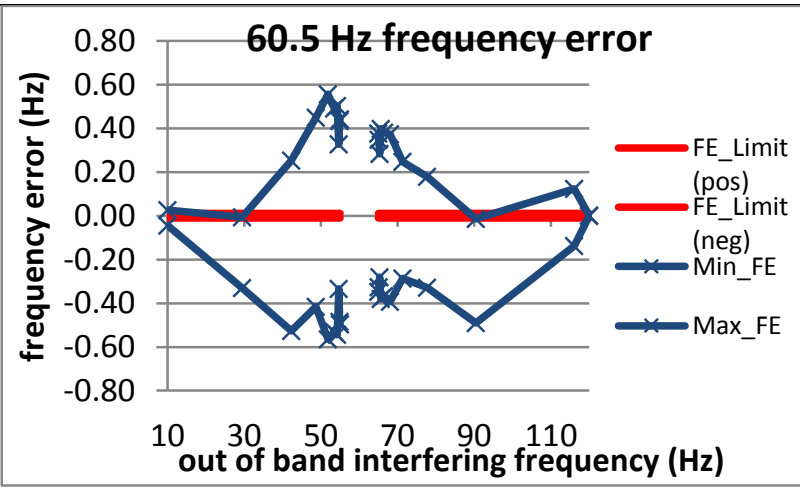

Figure 1890: Fs = $10 \mathrm{FPS}, 60 \mathrm{~Hz}$ fundamental

Figure 1891: Fs = 10 FPS, $60.5 \mathrm{~Hz}$ fundamental 
5.3.9 PMU H out of band interfering signals frequency error: $M$ class
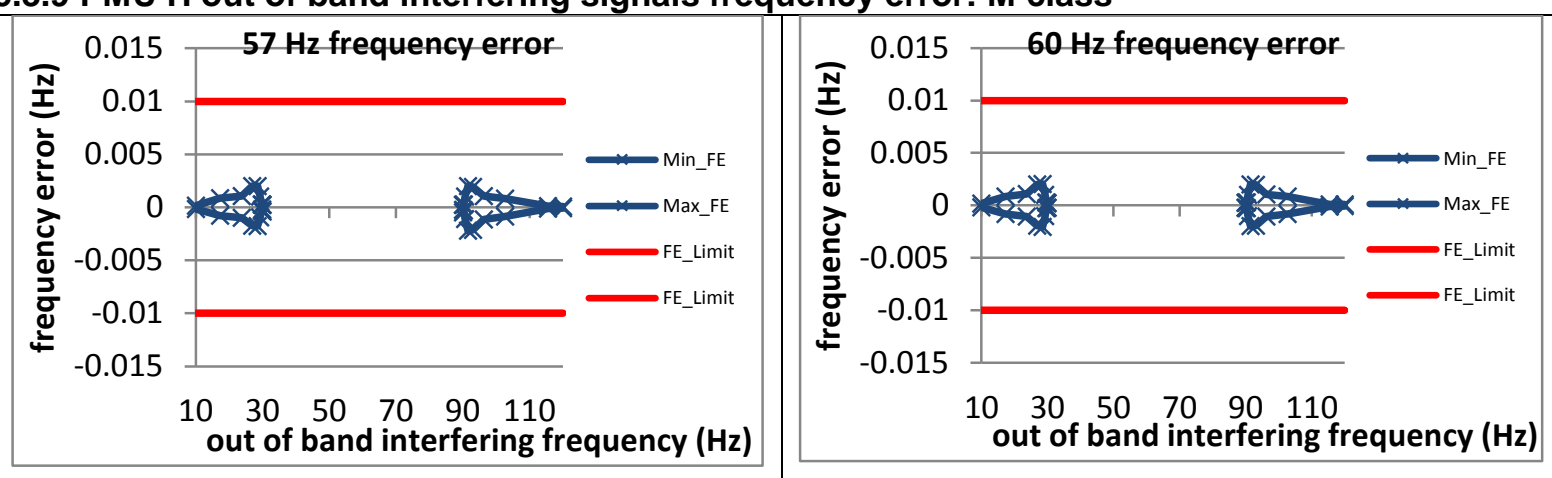

Figure 1892: Fs = 60 FPS, $57 \mathrm{~Hz}$ fundamental

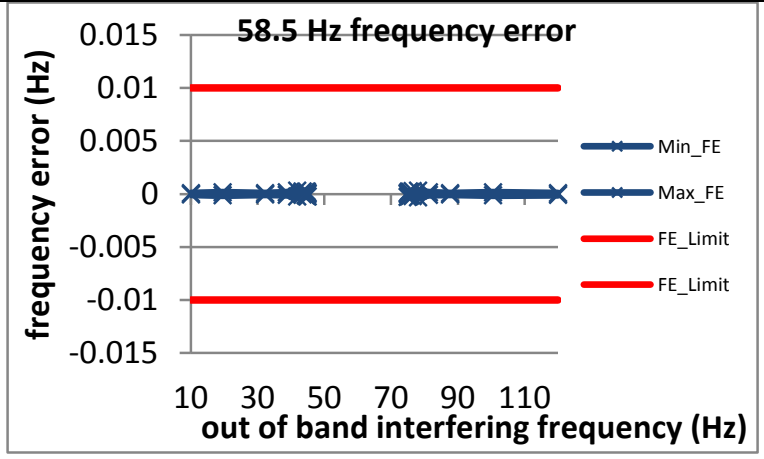

Figure 1895: Fs = 30 FPS, $58.5 \mathrm{~Hz}$ fundamental

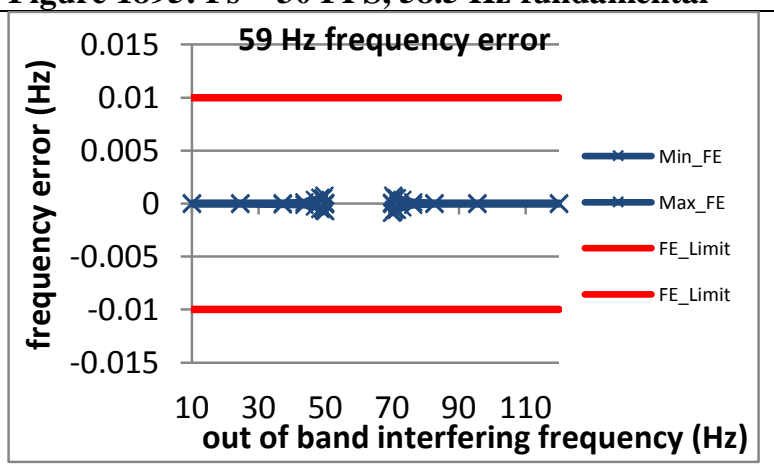

Figure 1898: Fs = 20 FPS, 59 Hz fundamental

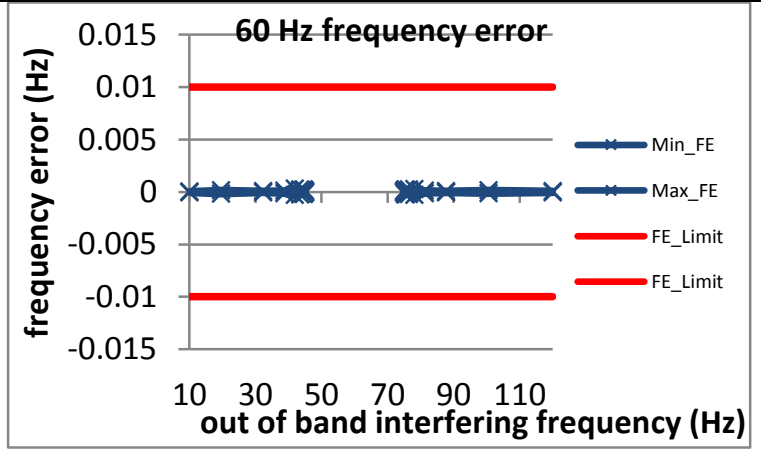

Figure 1896: Fs = 30 FPS, $60 \mathrm{~Hz}$ fundamental

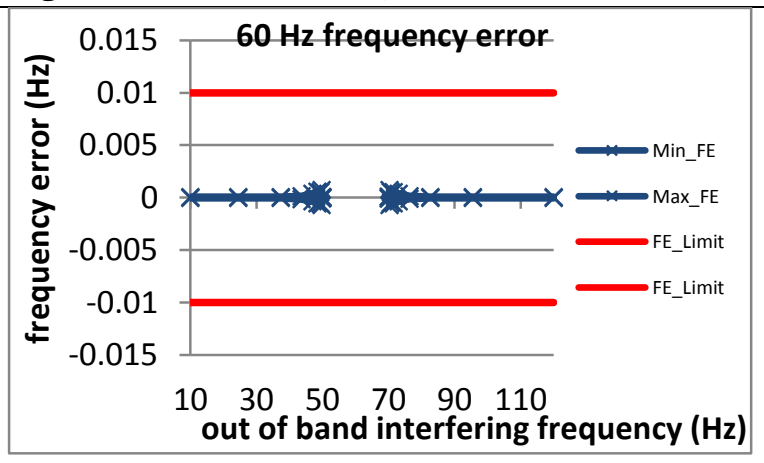

Figure 1899: Fs $=20$ FPS, $60 \mathrm{~Hz}$ fundamental
Figure 1893: Fs = 60 FPS, $60 \mathrm{~Hz}$ fundamental

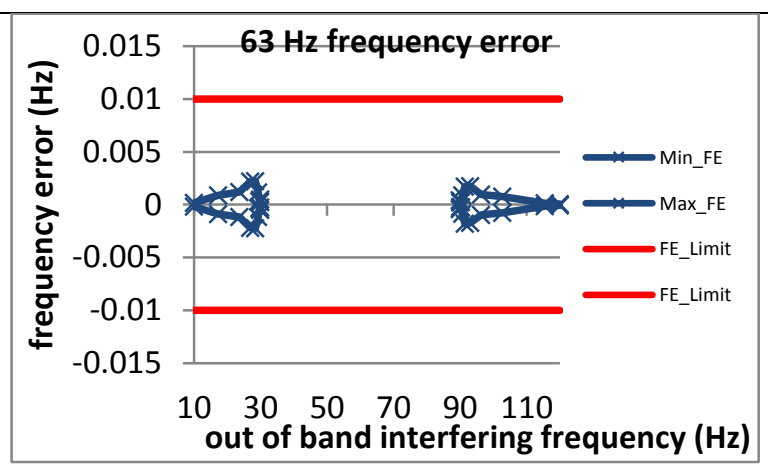

Figure 1894: Fs = $60 \mathrm{FPS}, 63 \mathrm{~Hz}$ fundamental

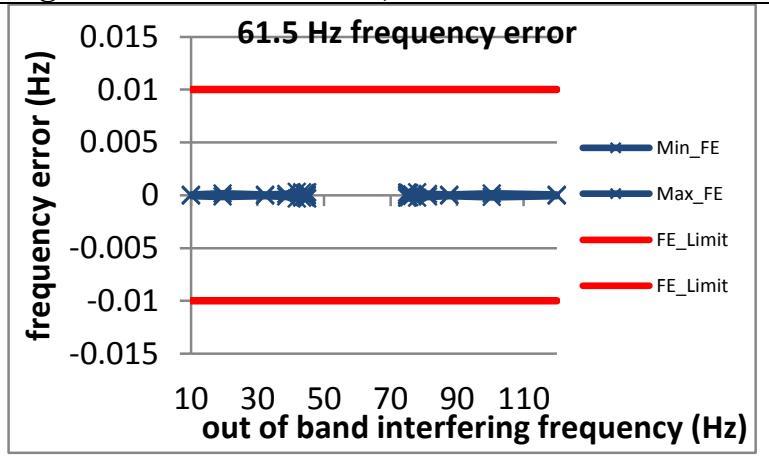

Figure 1897: Fs = 30 FPS, 61.5 Hz fundamental

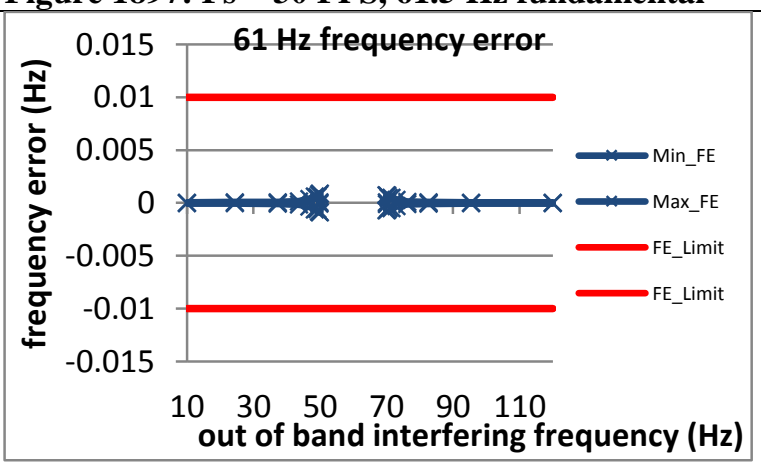

Figure 1900: Fs = 20 FPS, $61 \mathrm{~Hz}$ fundamental 

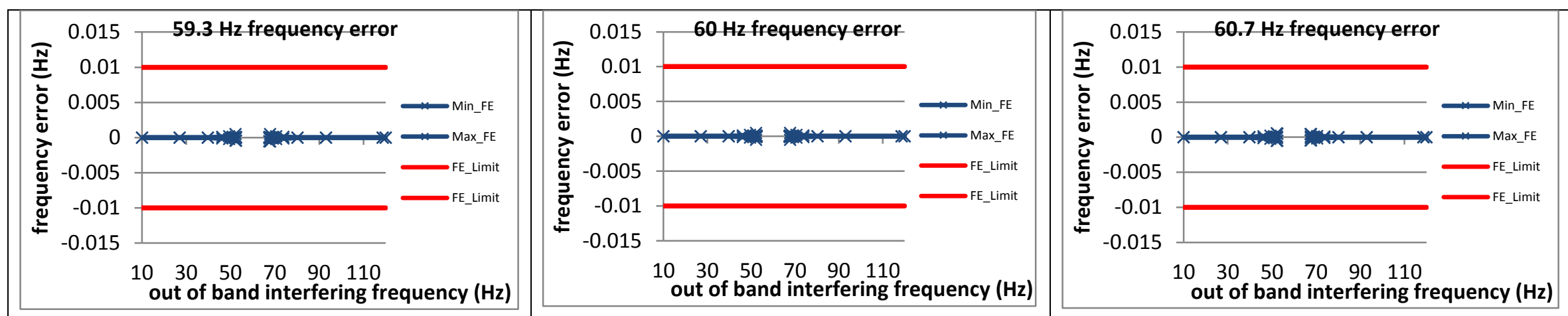

Figure 1901: Fs = 15 FPS, $59.3 \mathrm{~Hz}$ fundamental

Figure 1902: Fs = 15 FPS, $60 \mathrm{~Hz}$ fundamental
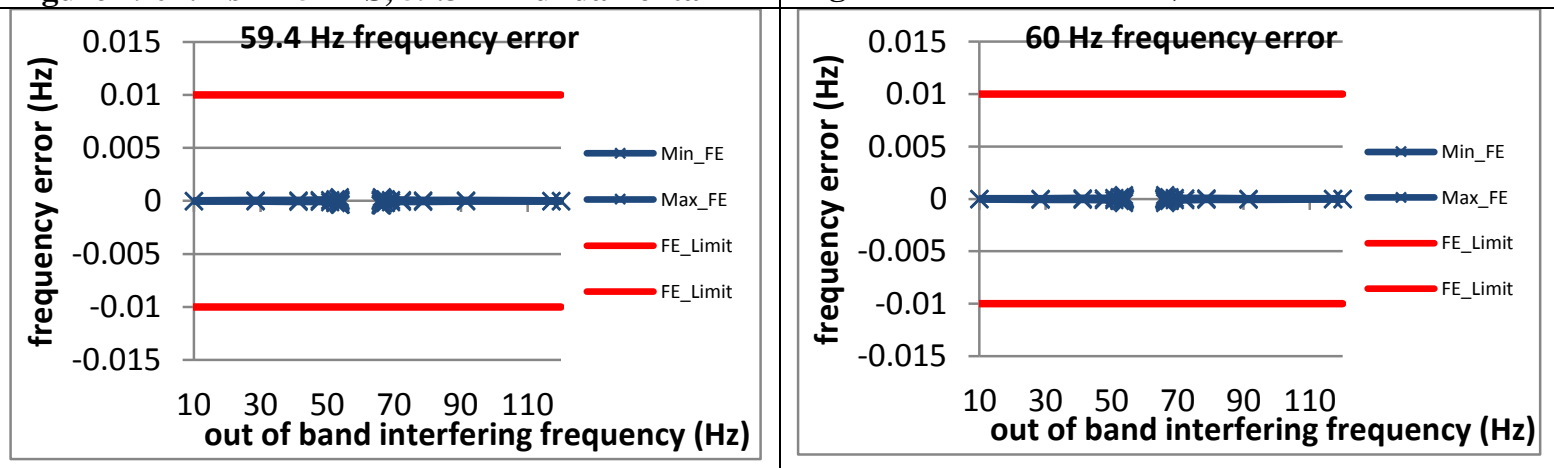

Figure 1903: Fs = 15 FPS, $60.7 \mathrm{~Hz}$ fundamental

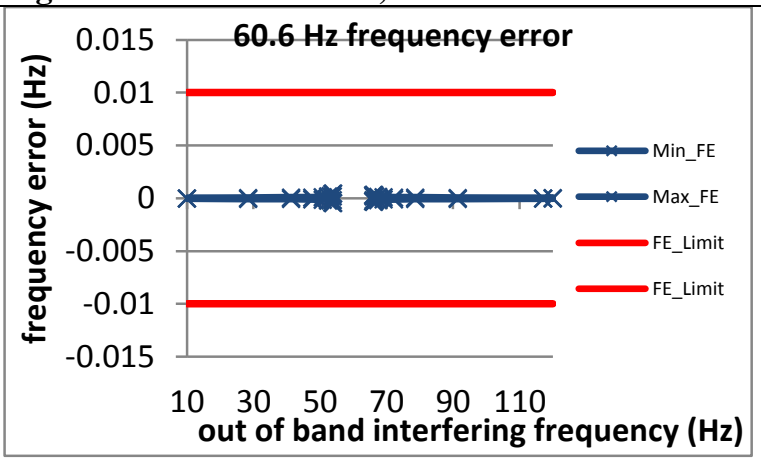

Figure 1904: Fs = 12 FPS, $59.4 \mathrm{~Hz}$ fundamental

Figure 1905: Fs = 12 FPS, $60 \mathrm{~Hz}$ fundamental
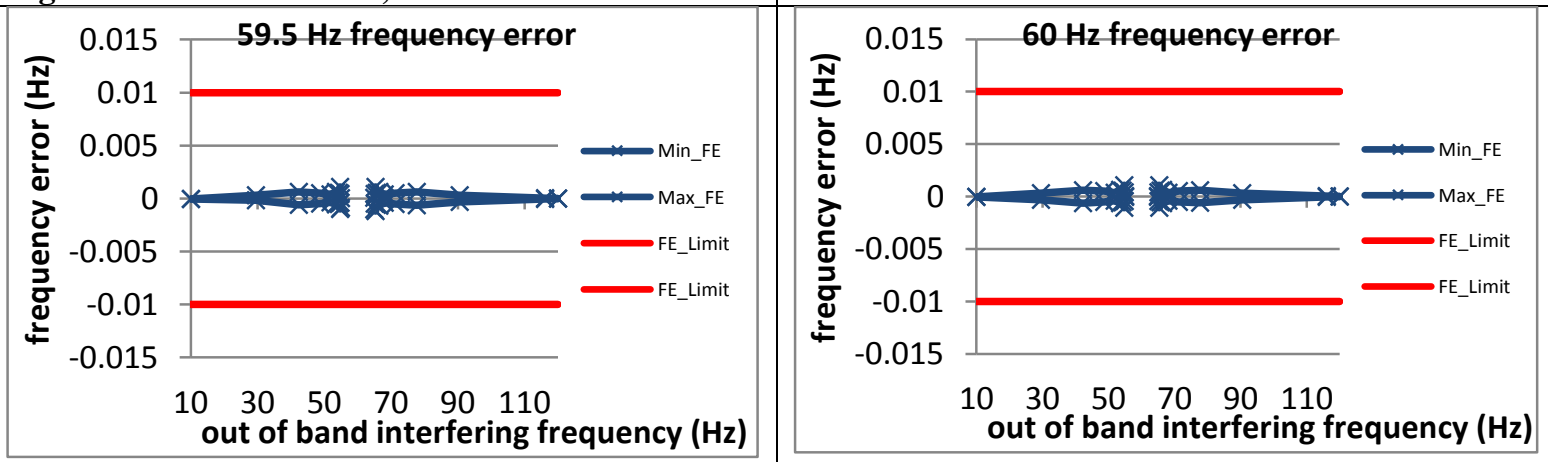

Figure 1906: Fs = $12 \mathrm{FPS}, 60.6 \mathrm{~Hz}$ fundamental

Figure 1907: Fs = 10 FPS, 59.5 Hz fundamental

Figure 1908: Fs = $10 \mathrm{FPS}, 60 \mathrm{~Hz}$ fundamental

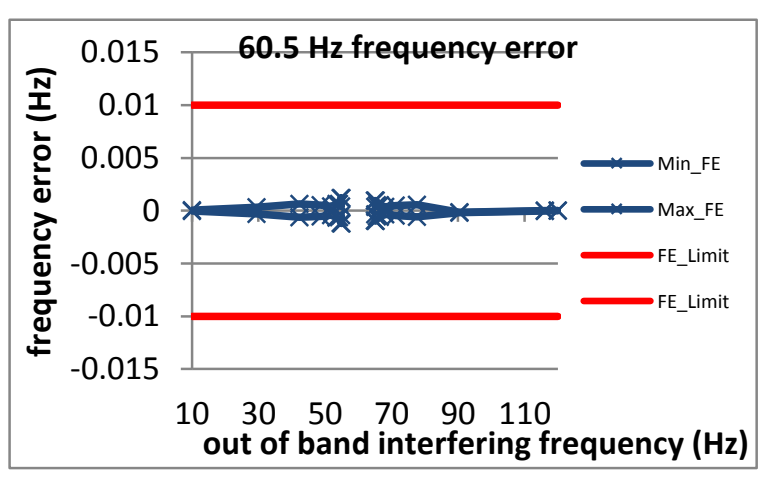

Figure 1909: Fs = 10 FPS, $60.5 \mathrm{~Hz}$ fundamental 
5.3.10 PMU I out of band interfering signals frequency error: $M$ class

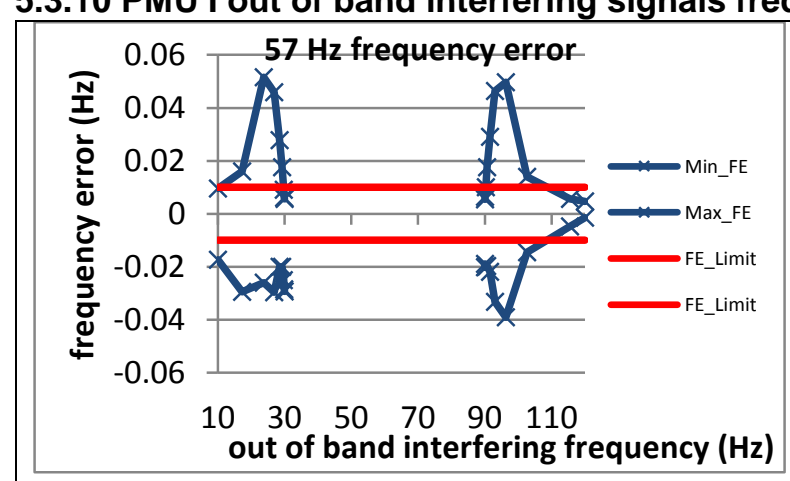

Figure 1910: Fs = 60 FPS, $57 \mathrm{~Hz}$ fundamental

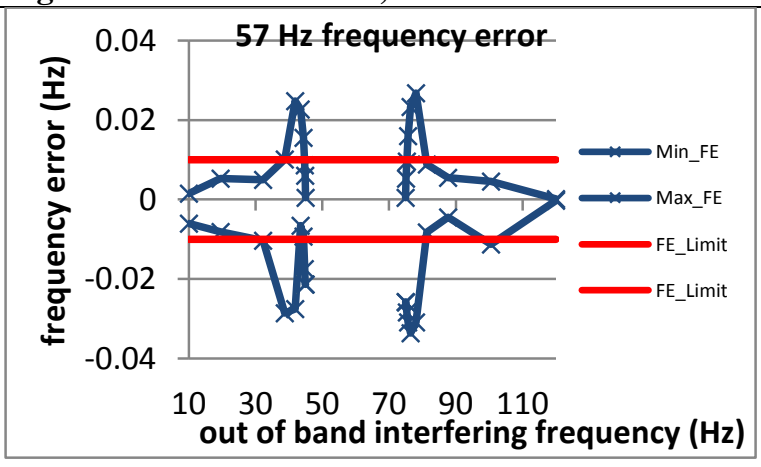

Figure 1913: Fs = 30 FPS, $58.5 \mathrm{~Hz}$ fundamental

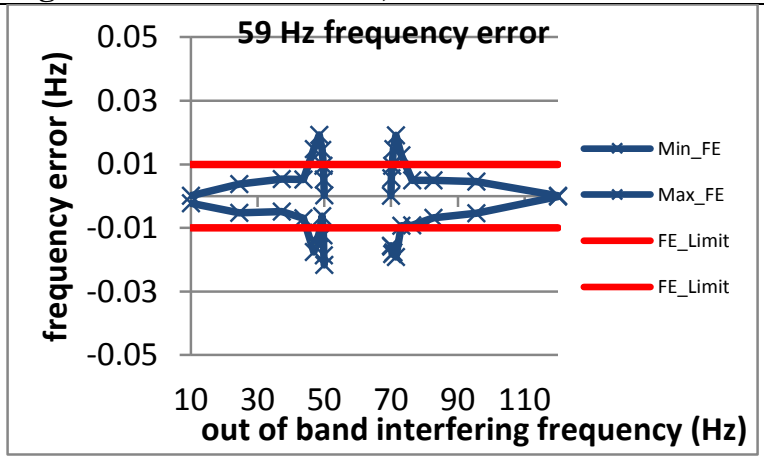

Figure 1916: Fs = 20 FPS, $59 \mathrm{~Hz}$ fundamental

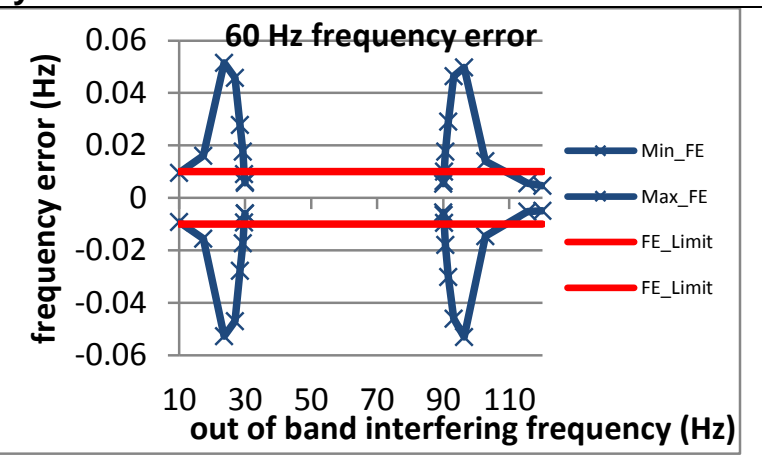

Figure 1911: Fs = $60 \mathrm{FPS}, 60 \mathrm{~Hz}$ fundamental

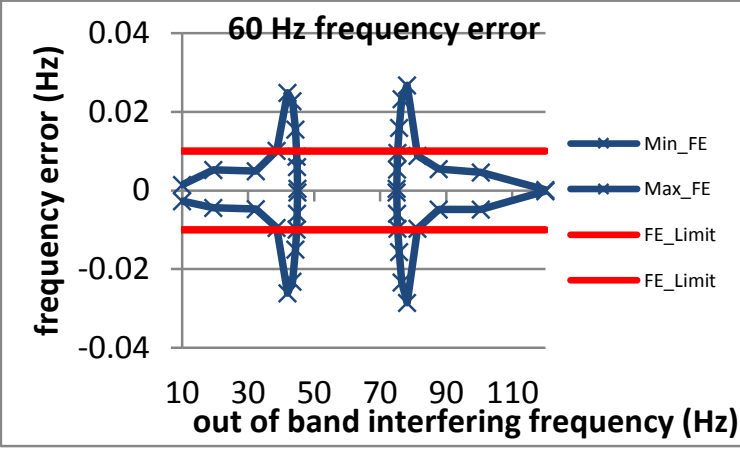

Figure 1914: Fs = $30 \mathrm{FPS}, 60 \mathrm{~Hz}$ fundamental

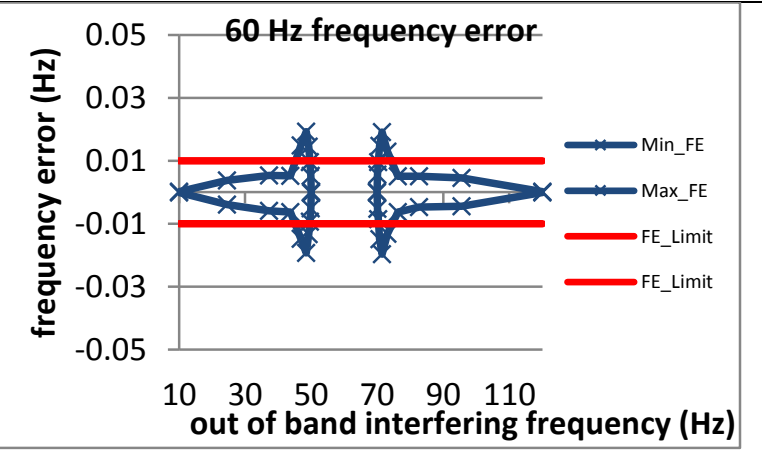

Figure 1917: Fs = $20 \mathrm{FPS}, 60 \mathrm{~Hz}$ fundamental

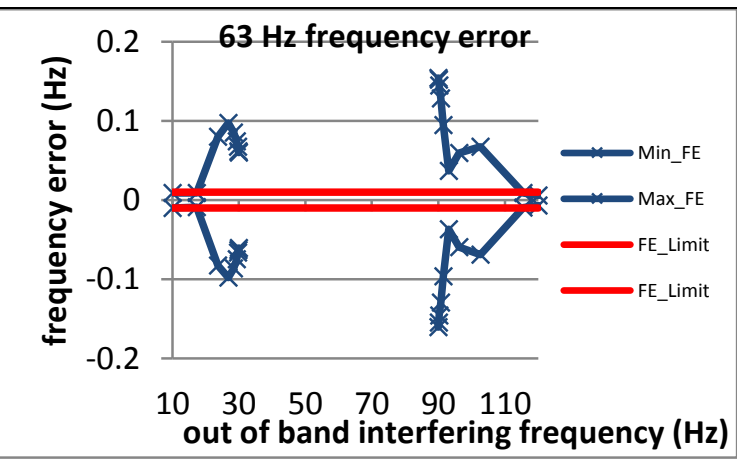

Figure 1912: Fs = $60 \mathrm{FPS}, 63 \mathrm{~Hz}$ fundamental

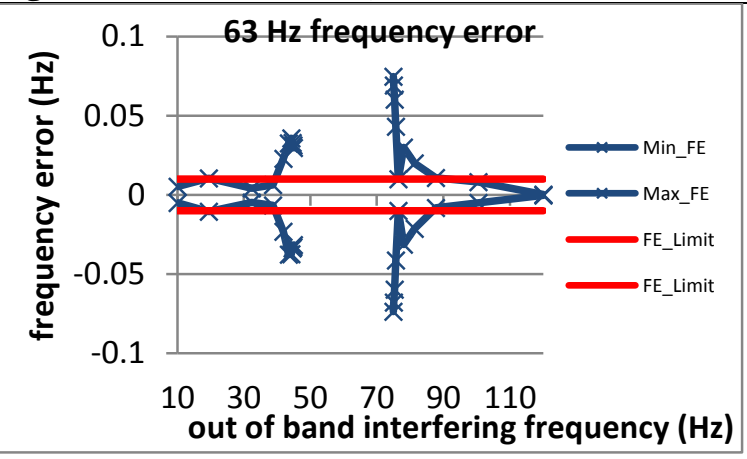

Figure 1915: Fs = 30 FPS, $61.5 \mathrm{~Hz}$ fundamental

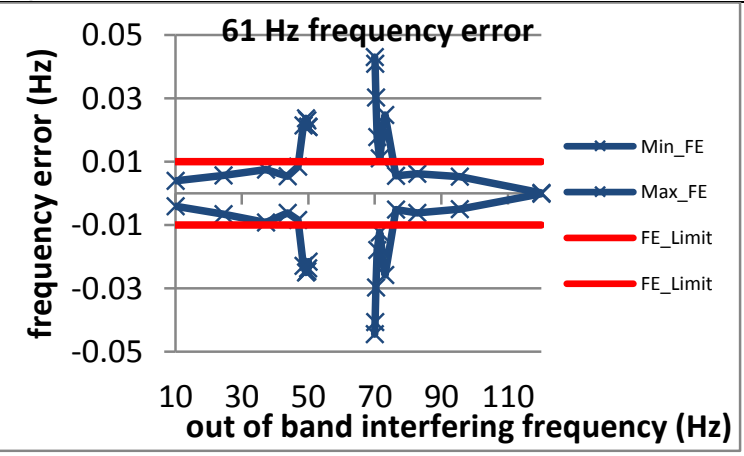

Figure 1918: Fs = 20 FPS, $61 \mathrm{~Hz}$ fundamental 


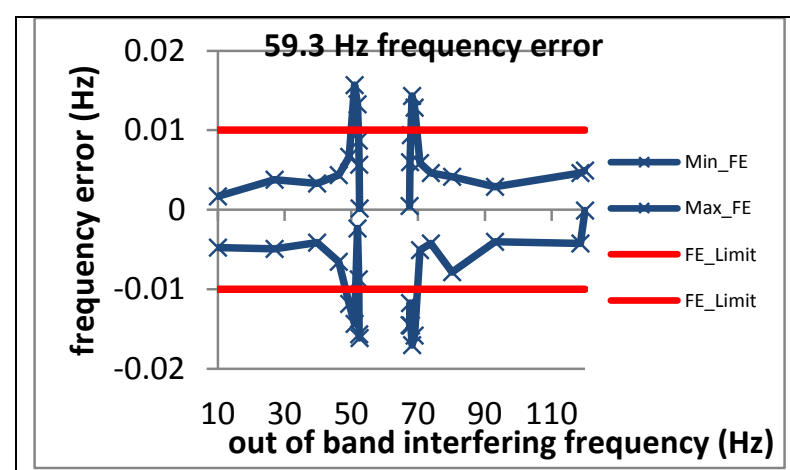

Figure 1919: Fs = 15 FPS, $59.3 \mathrm{~Hz}$ fundamental

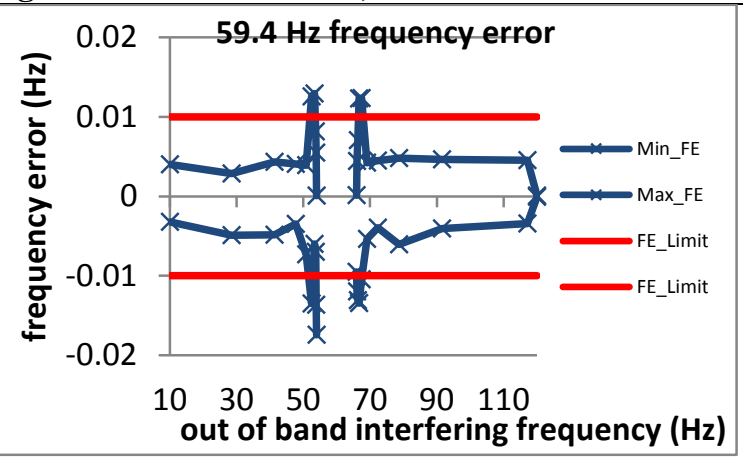

Figure 1922: Fs = 12 FPS, $59.4 \mathrm{~Hz}$ fundamental

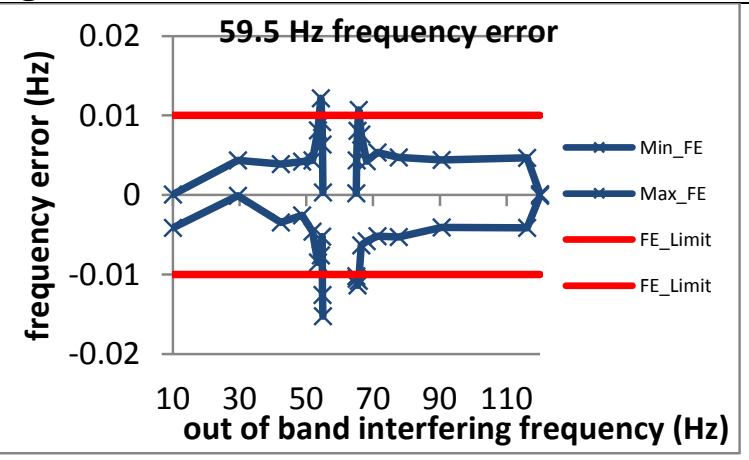

Figure 1925: Fs = 10 FPS, $59.5 \mathrm{~Hz}$ fundamental

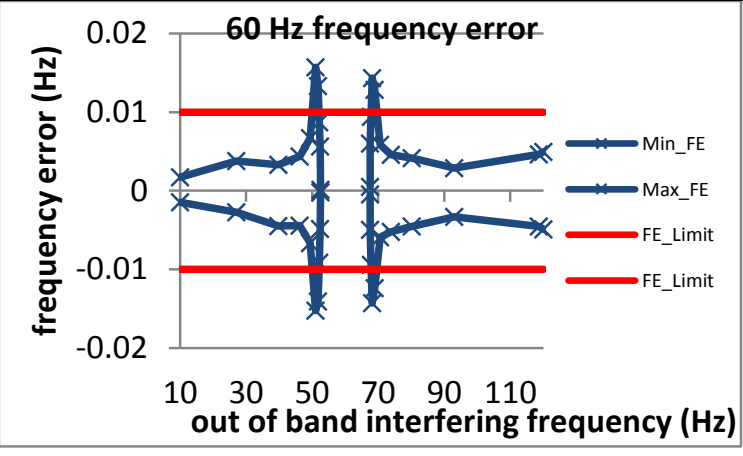

Figure 1920: Fs = $15 \mathrm{FPS}, 60 \mathrm{~Hz}$ fundamental

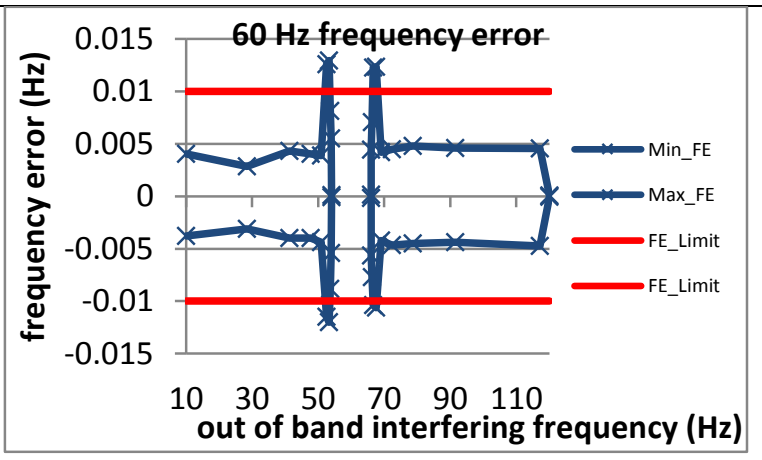

Figure 1923: Fs = 12 FPS, $60 \mathrm{~Hz}$ fundamental

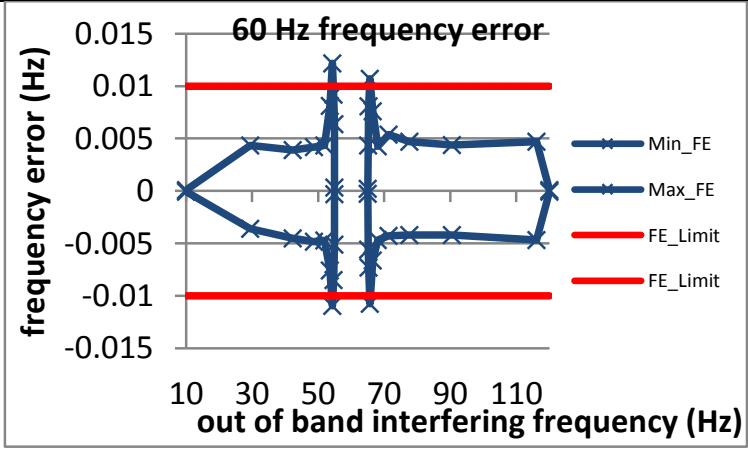

Figure 1926: Fs = $10 \mathrm{FPS}, 60 \mathrm{~Hz}$ fundamental

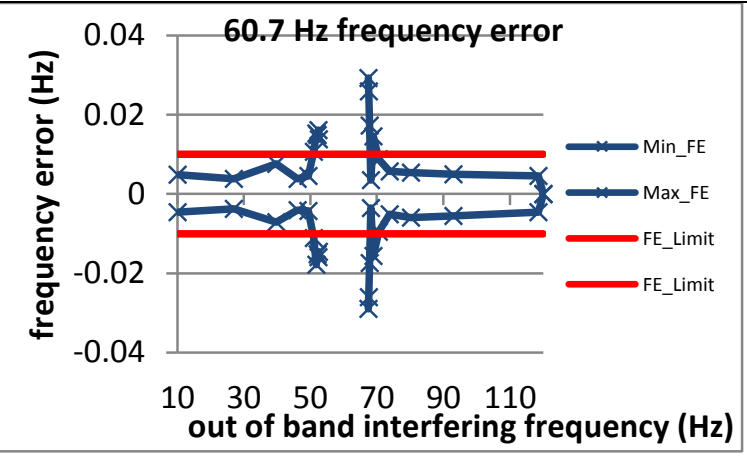

Figure 1921: Fs = 15 FPS, $60.7 \mathrm{~Hz}$ fundamental

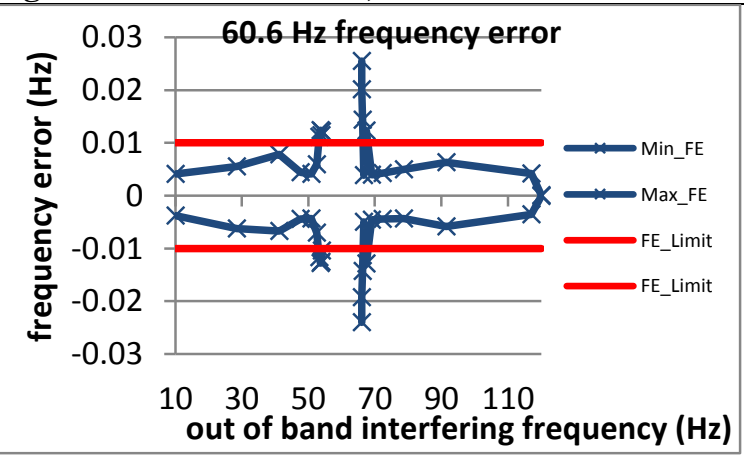

Figure 1924: Fs = 12 FPS, $60.6 \mathrm{~Hz}$ fundamental

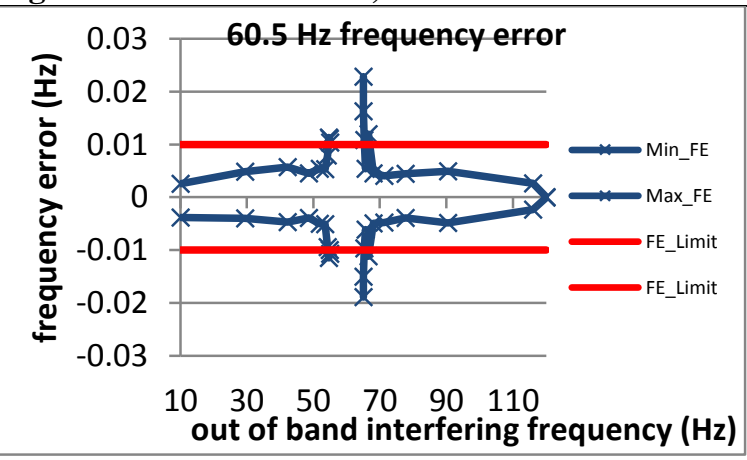

Figure 1927: Fs = 10 FPS, $60.5 \mathrm{~Hz}$ fundamental 
5.3.11 PMU J out of band interfering signals frequency error: $M$ class

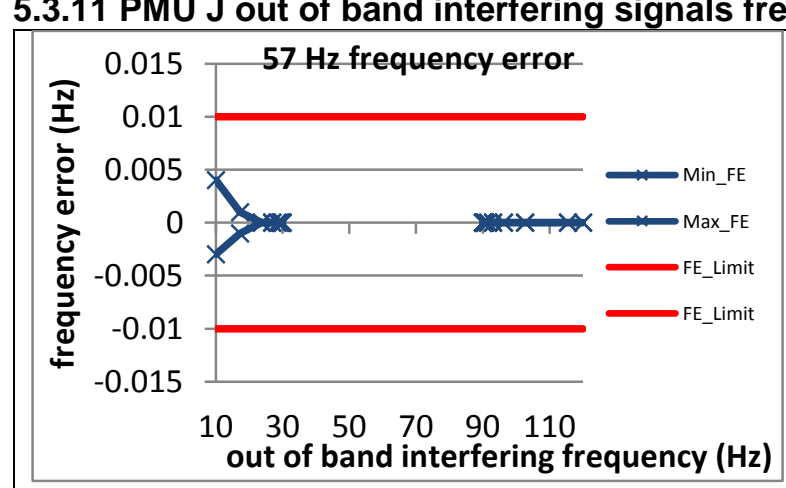

Figure 1928: Fs = $60 \mathrm{FPS}, 57 \mathrm{~Hz}$ fundamental

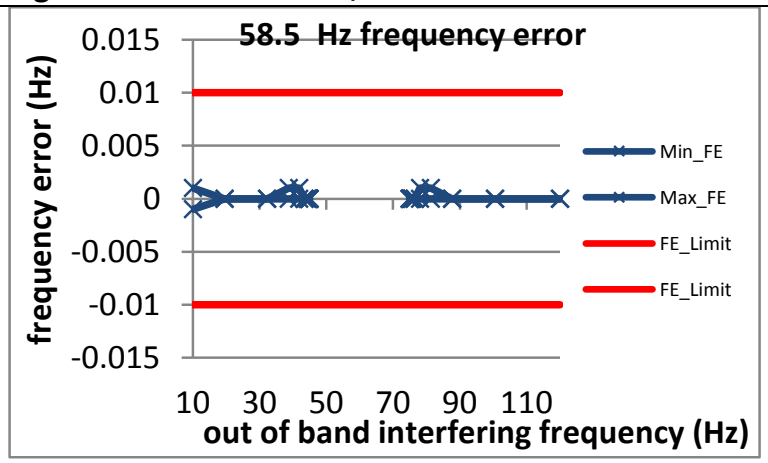

Figure 1931: Fs = $30 \mathrm{FPS}, 58.5 \mathrm{~Hz}$ fundamental

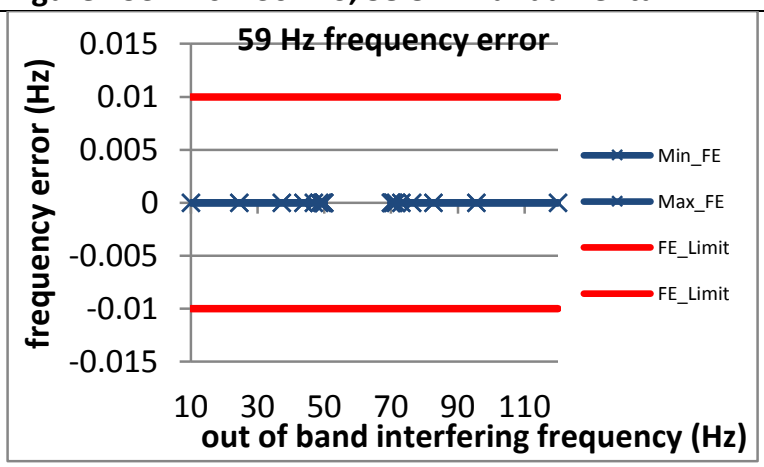

Figure 1934: Fs = 20 FPS, $59 \mathrm{~Hz}$ fundamental

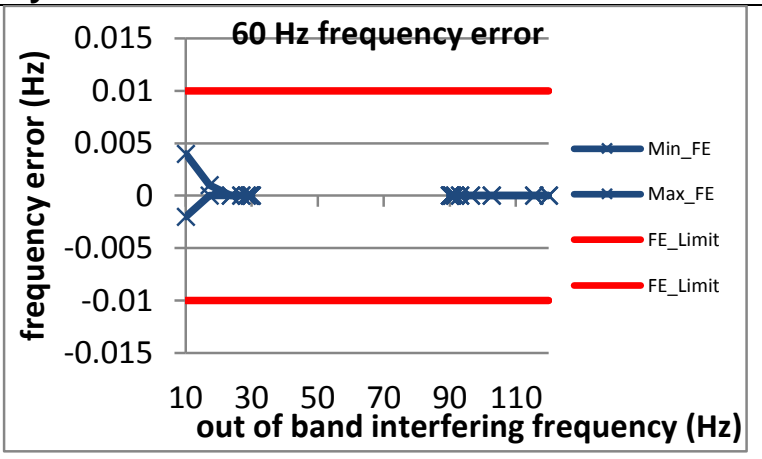

Figure 1929: Fs = $60 \mathrm{FPS}, 60 \mathrm{~Hz}$ fundamental

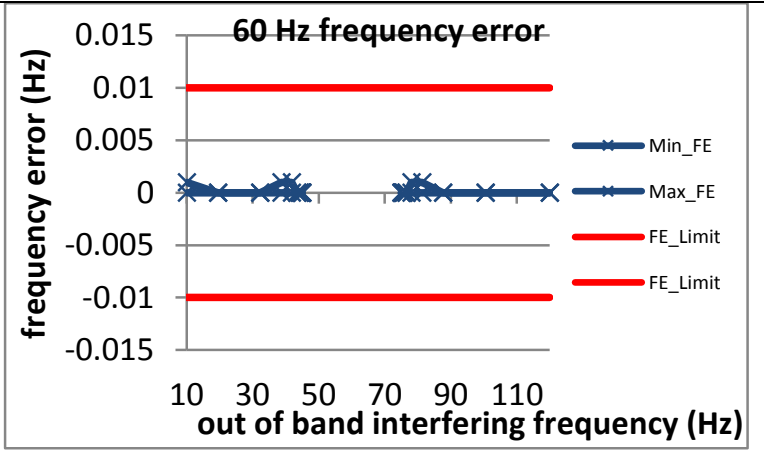

Figure 1932: Fs = $30 \mathrm{FPS}, 60 \mathrm{~Hz}$ fundamental

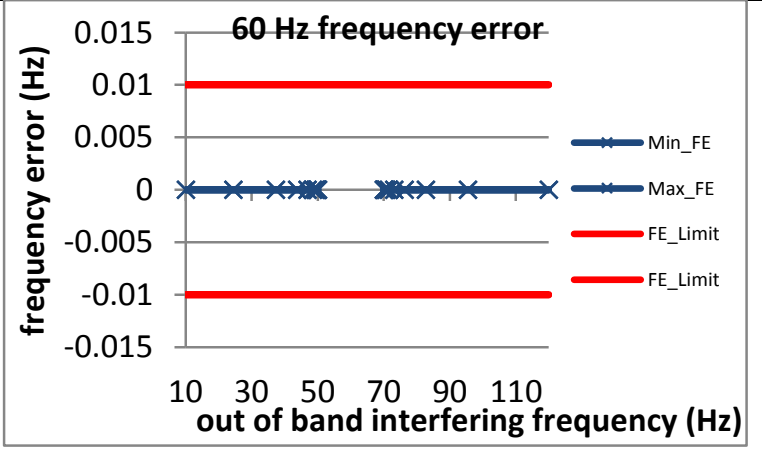

Figure 1935: Fs = 20 FPS, $60 \mathrm{~Hz}$ fundamental

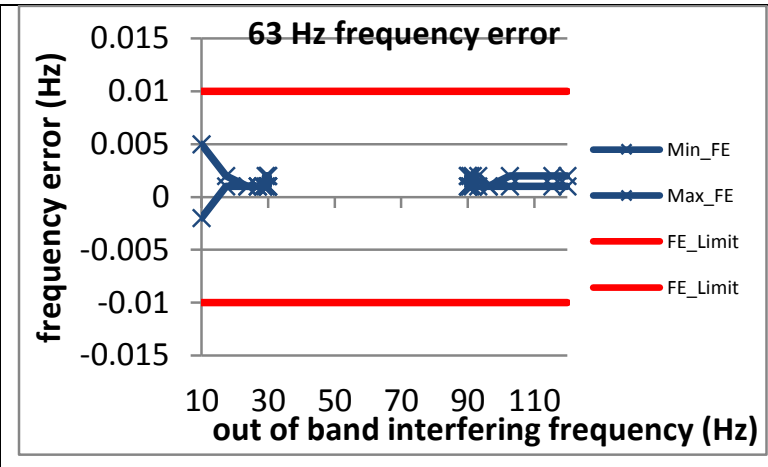

Figure 1930: Fs = 60 FPS, $63 \mathrm{~Hz}$ fundamental

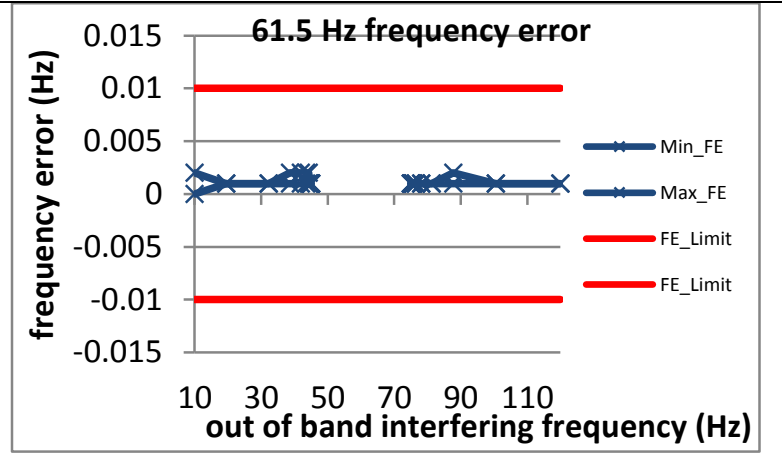

Figure 1933: Fs = $30 \mathrm{FPS}, 61.5 \mathrm{~Hz}$ fundamental

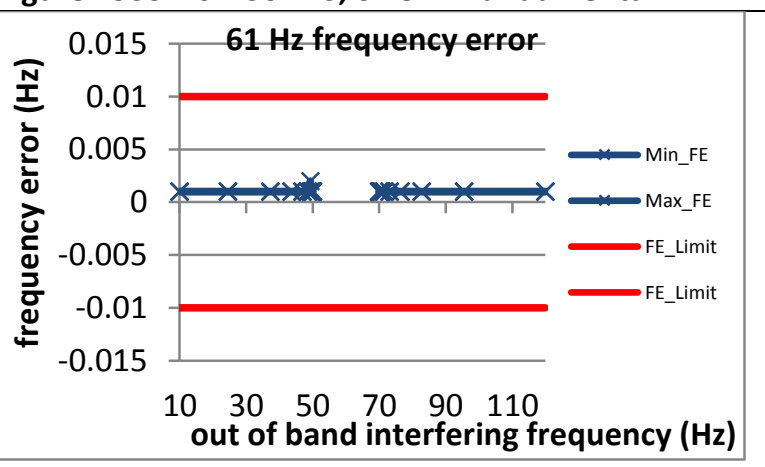

Figure 1936: Fs = $20 \mathrm{FPS}, 61 \mathrm{~Hz}$ fundamental 


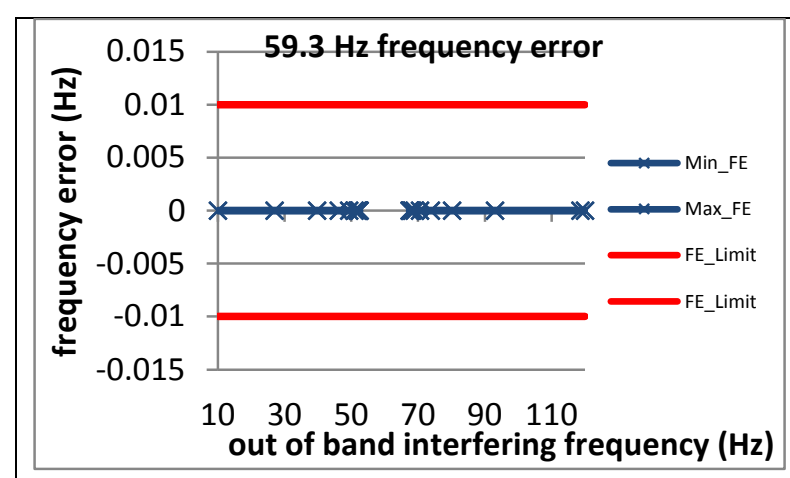

Figure 1937: Fs = $15 \mathrm{FPS}, 59.3 \mathrm{~Hz}$ fundamental

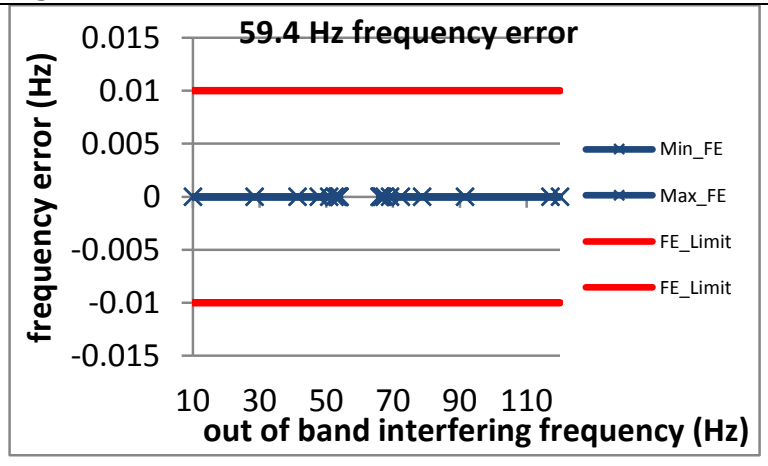

Figure 1940: $\mathrm{Fs}=12 \mathrm{FPS}, 59.4 \mathrm{~Hz}$ fundamental

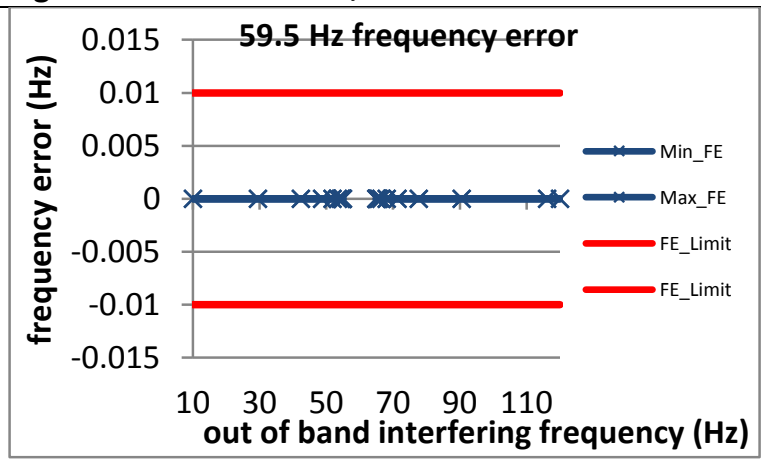

Figure 1943: Fs = $10 \mathrm{FPS}, 59.5 \mathrm{~Hz}$ fundamental

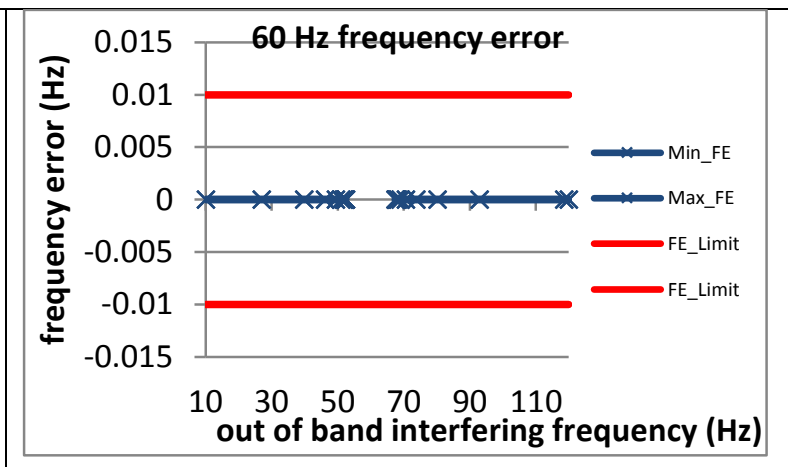

Figure 1938: Fs = $15 \mathrm{FPS}, 60 \mathrm{~Hz}$ fundamental

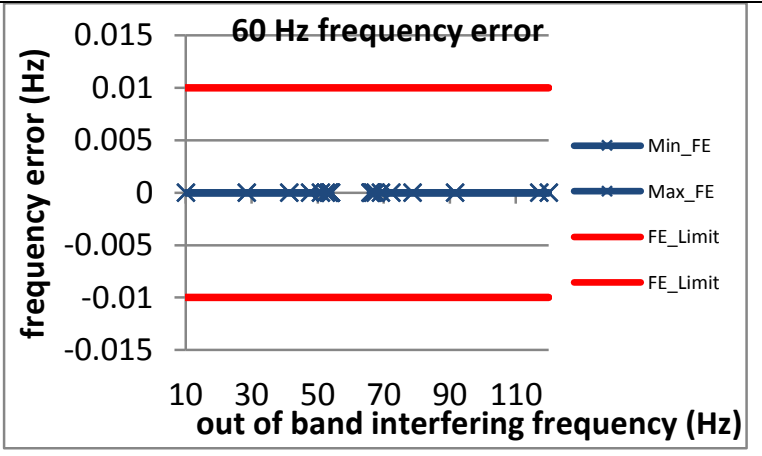

Figure 1941: Fs = $12 \mathrm{FPS}, 60 \mathrm{~Hz}$ fundamental

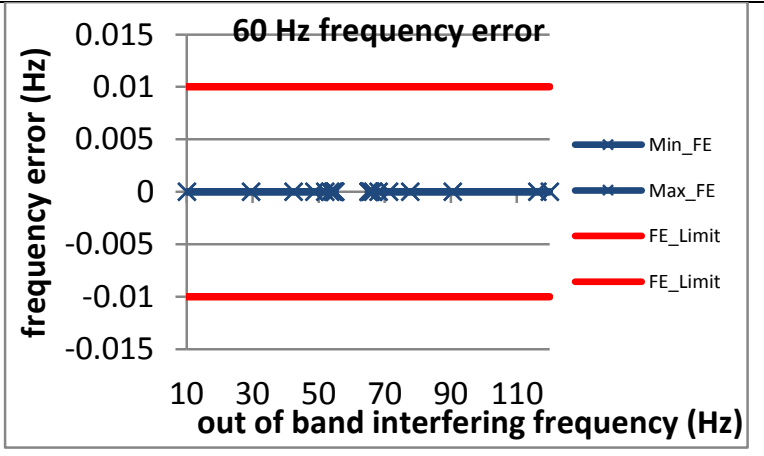

Figure 1944: Fs = $10 \mathrm{FPS}, 60 \mathrm{~Hz}$ fundamental

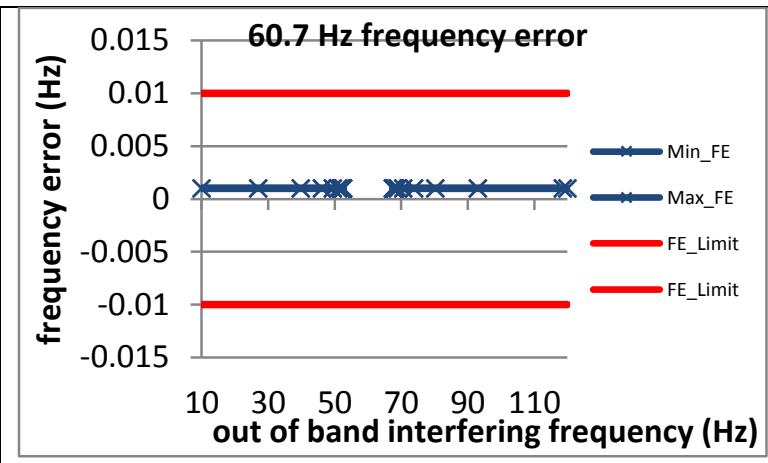

Figure 1939: $\mathrm{Fs}=15 \mathrm{FPS}, 60.7 \mathrm{~Hz}$ fundamental

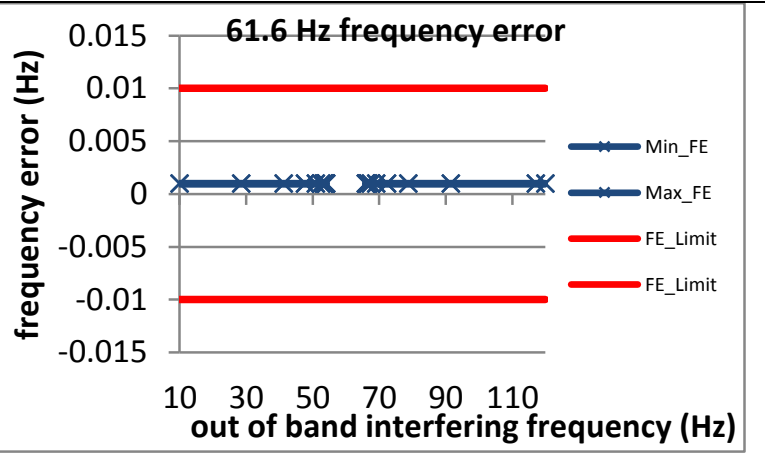

Figure 1942: Fs = $12 \mathrm{FPS}, 60.6 \mathrm{~Hz}$ fundamental

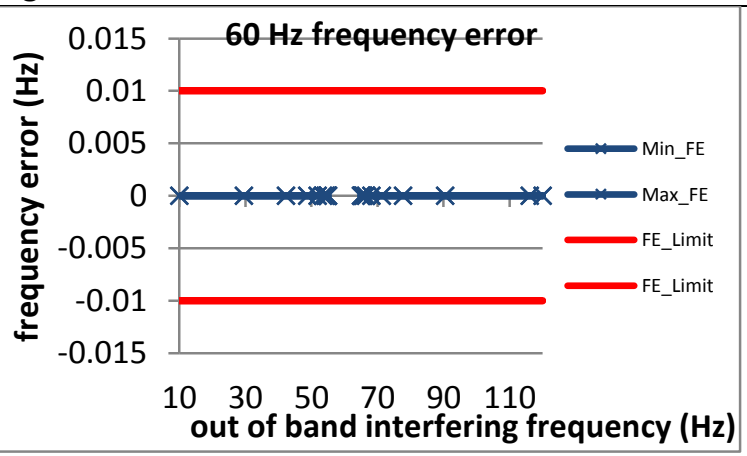

Figure 1945: Fs = $10 \mathrm{FPS}, 60.5 \mathrm{~Hz}$ fundamental 
5.4 Steady state out of band interfering signals: ROCOF error: M class

5.4.1 C37.118.1 Annex C steady state out of band interfering signals ROCOF error: M class

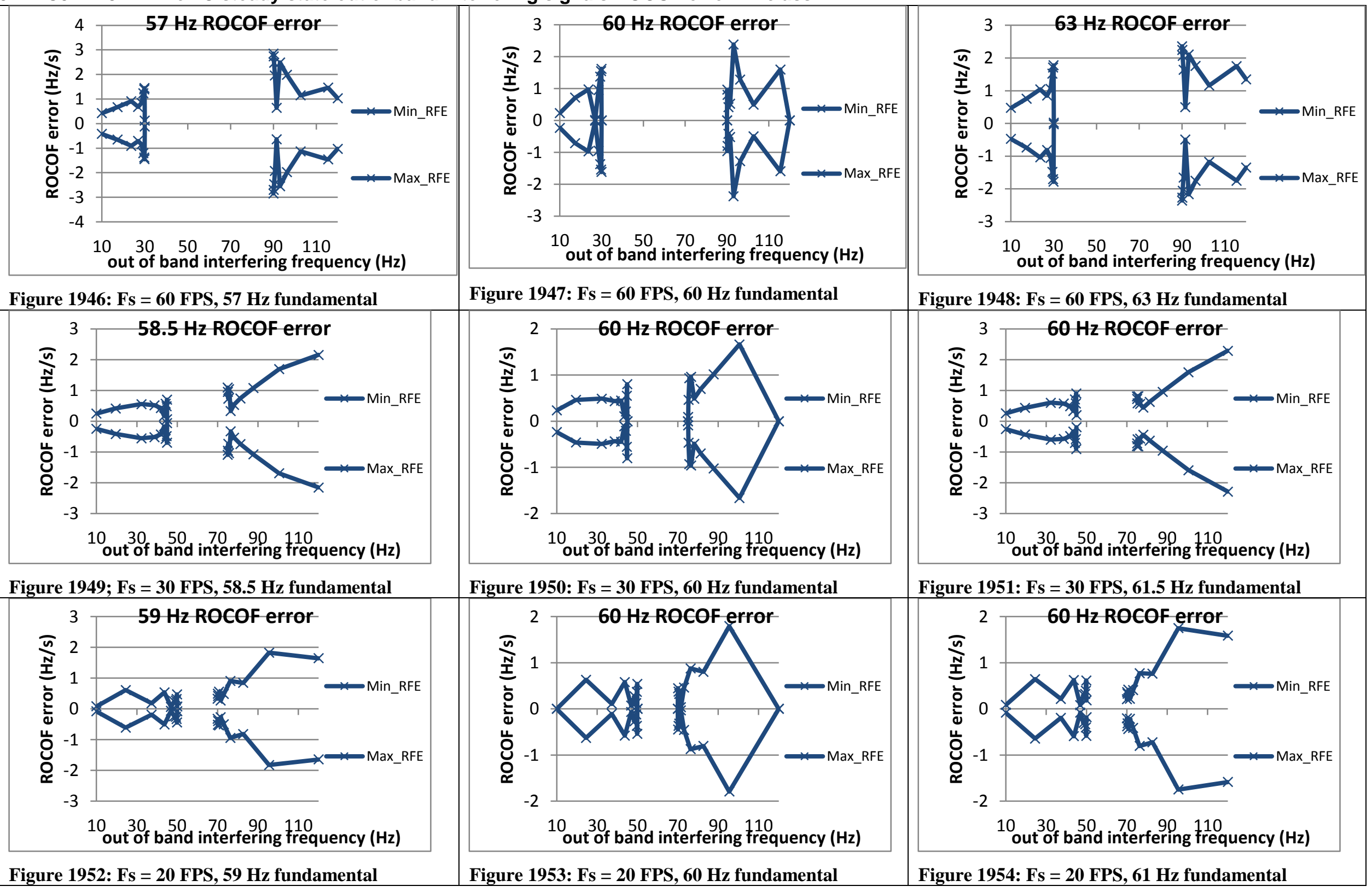




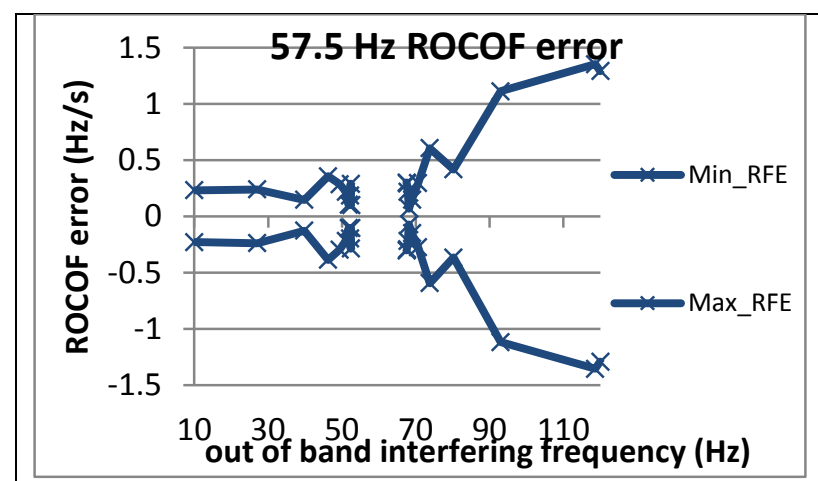

Figure 1955: Fs = 15 FPS, $59.3 \mathrm{~Hz}$ fundamental

Figure 1958: Fs = 12 FPS, 59.4 Hz fundamental

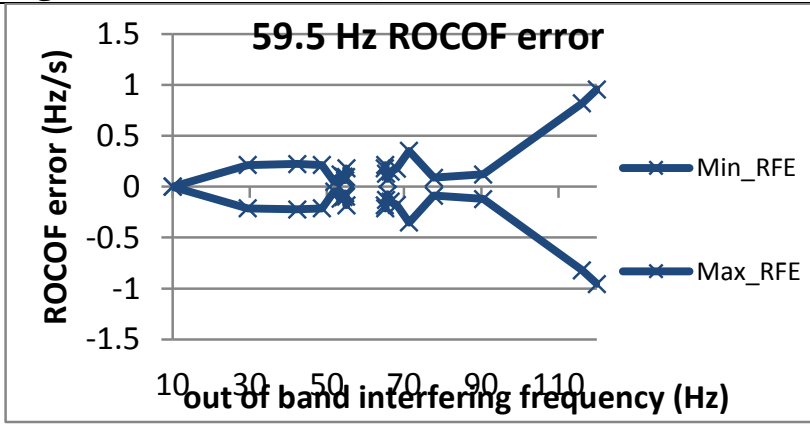

Figure 1961: Fs = 10 FPS, $59.5 \mathrm{~Hz}$ fundamental

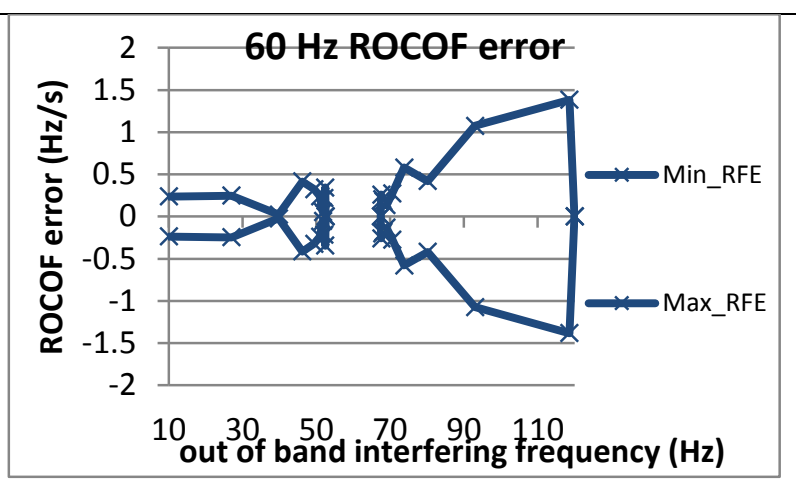

Figure 1956: Fs = 15 FPS, $60 \mathrm{~Hz}$ fundamental

Figure 1959: Fs = $12 \mathrm{FPS}, 60 \mathrm{~Hz}$ fundamental

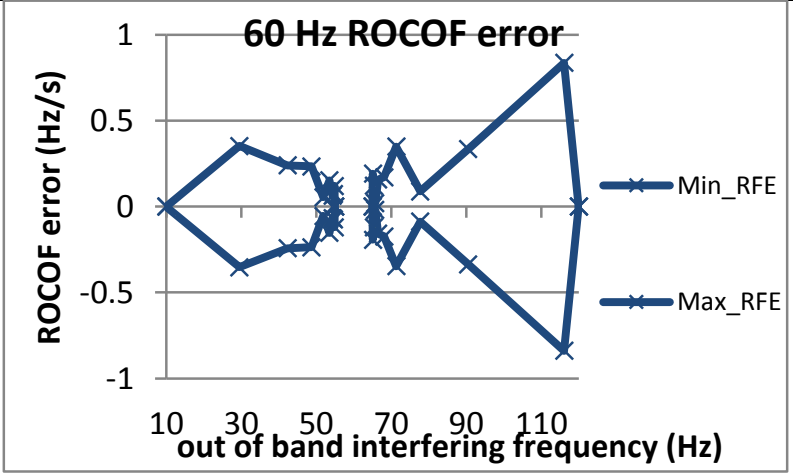

Figure 1962: Fs = 10 FPS, $60 \mathrm{~Hz}$ fundamental

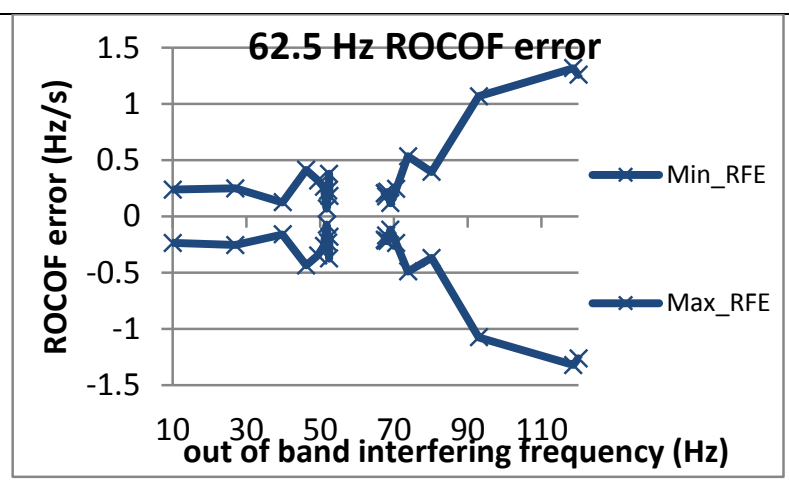

Figure 1957: Fs = 15 FPS, $60.7 \mathrm{~Hz}$ fundamental

Figure 1960: Fs = 12 FPS, $60.6 \mathrm{~Hz}$ fundamental

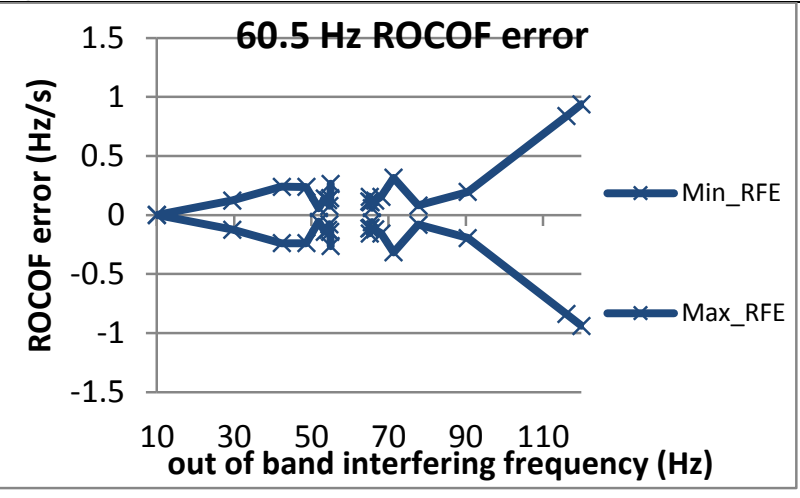

Figure 1963: Fs = 10 FPS, $60.5 \mathrm{~Hz}$ fundamental 
5.4.2 PMU A steady state out of band interfering signals ROCOF error: $M$ class

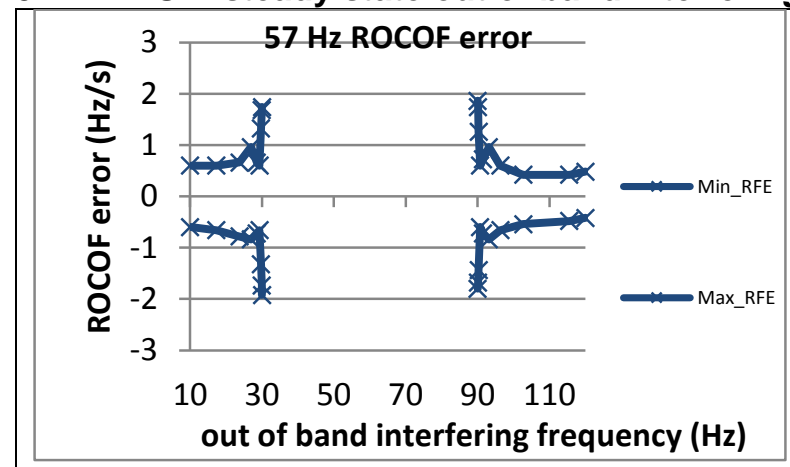

Figure 1964: Fs = 60 FPS, $57 \mathrm{~Hz}$ fundamental

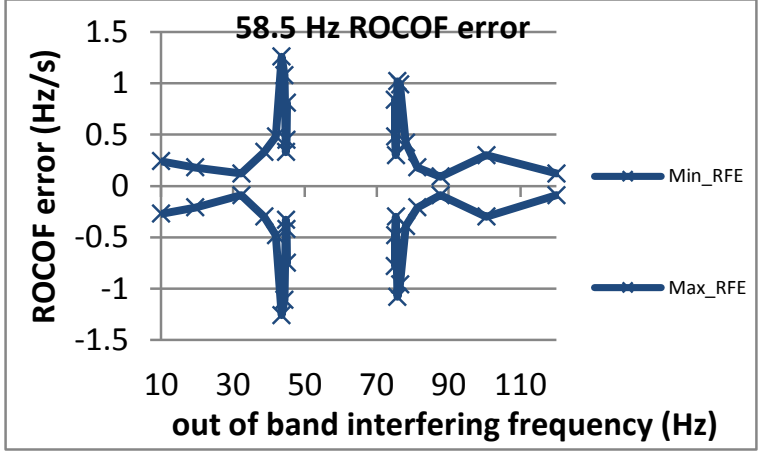

Figure 1967; Fs = 30 FPS, 58.5 Hz fundamental

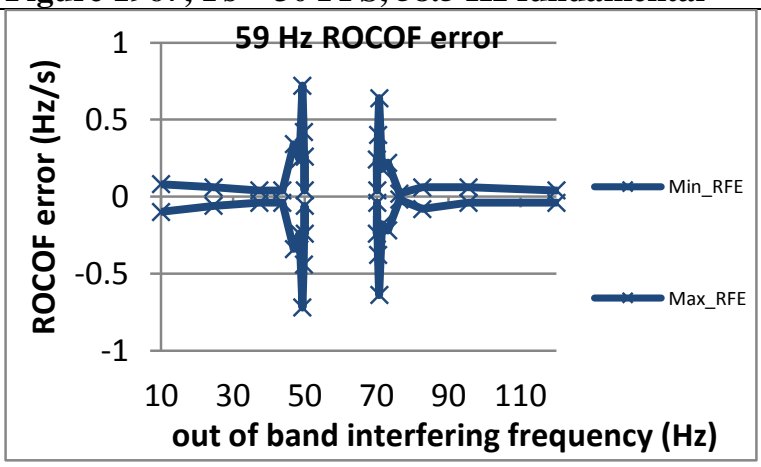

Figure 1970: Fs = 20 FPS, $59 \mathrm{~Hz}$ fundamental

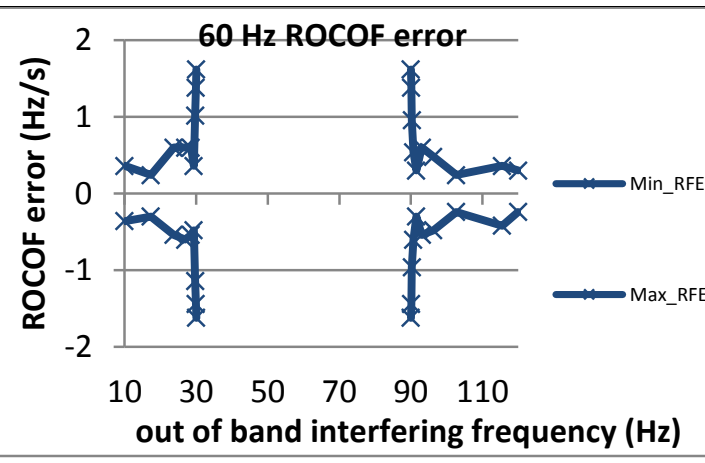

Figure 1965: Fs = 60 FPS, $60 \mathrm{~Hz}$ fundamental

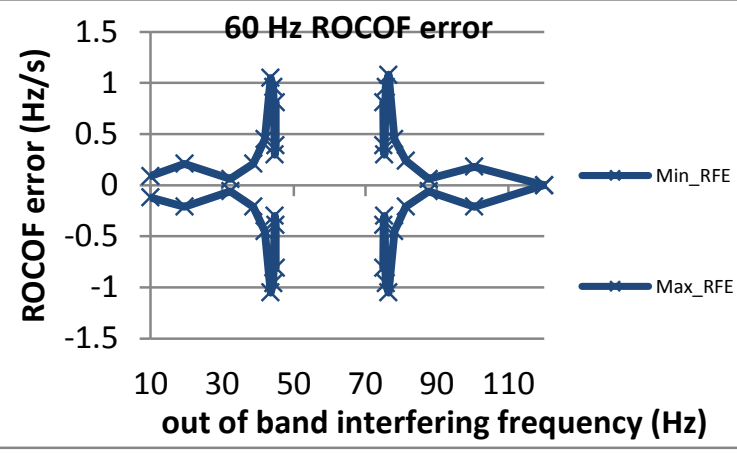

Figure 1968: Fs = $30 \mathrm{FPS}, 60 \mathrm{~Hz}$ fundamental

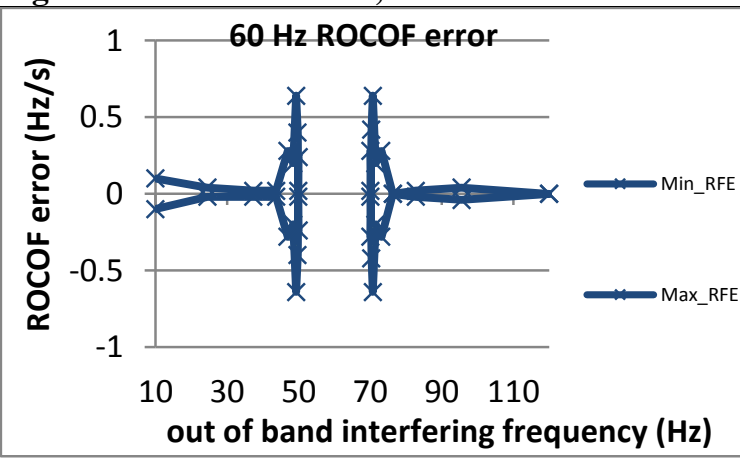

Figure 1971: Fs = $20 \mathrm{FPS}, 60 \mathrm{~Hz}$ fundamental

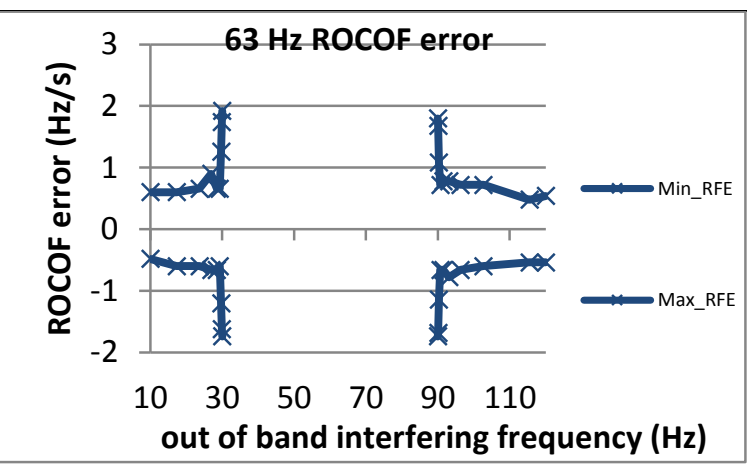

Figure 1966: Fs = 60 FPS, $63 \mathrm{~Hz}$ fundamental

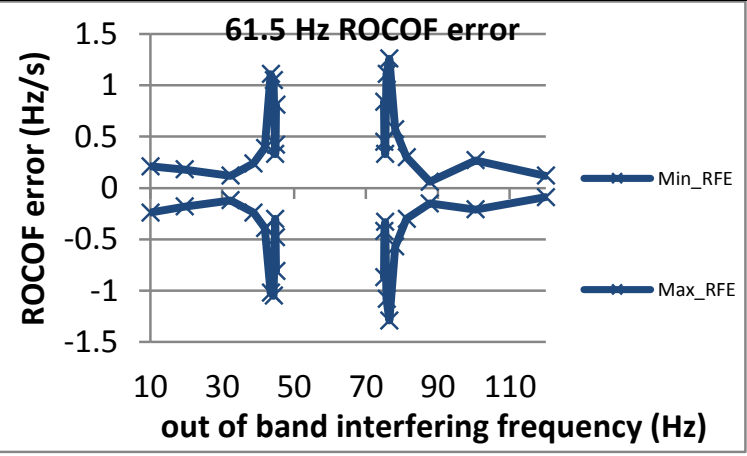

Figure 1969: Fs = 30 FPS, $61.5 \mathrm{~Hz}$ fundamental

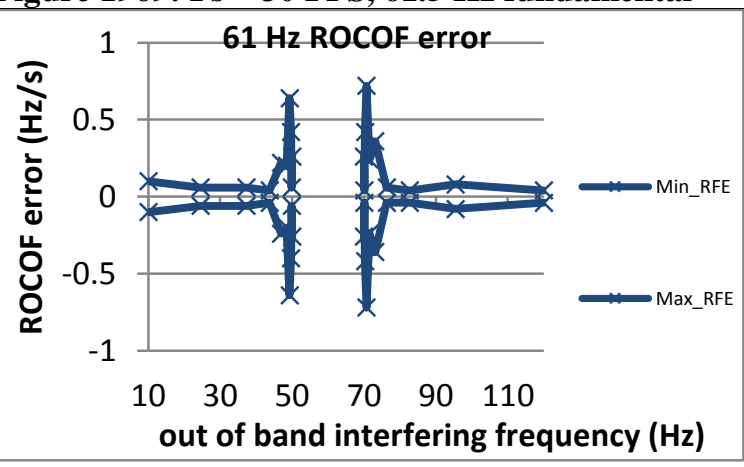

Figure 1972: Fs = 20 FPS, $61 \mathrm{~Hz}$ fundamental 


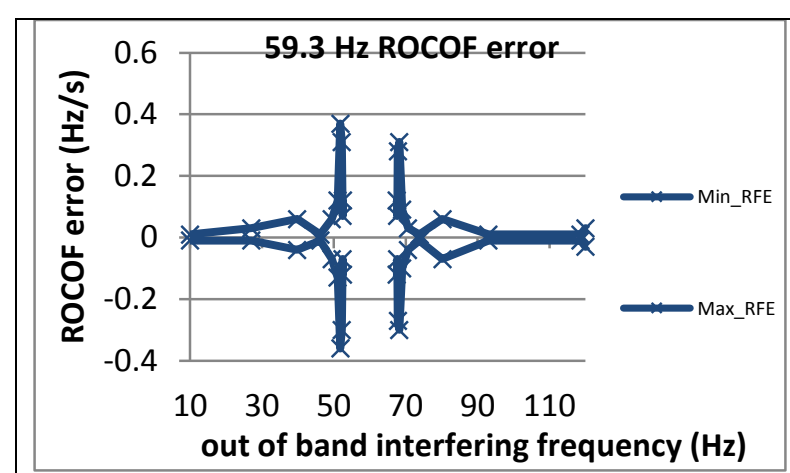

Figure 1973: Fs = 15 FPS, $59.3 \mathrm{~Hz}$ fundamental

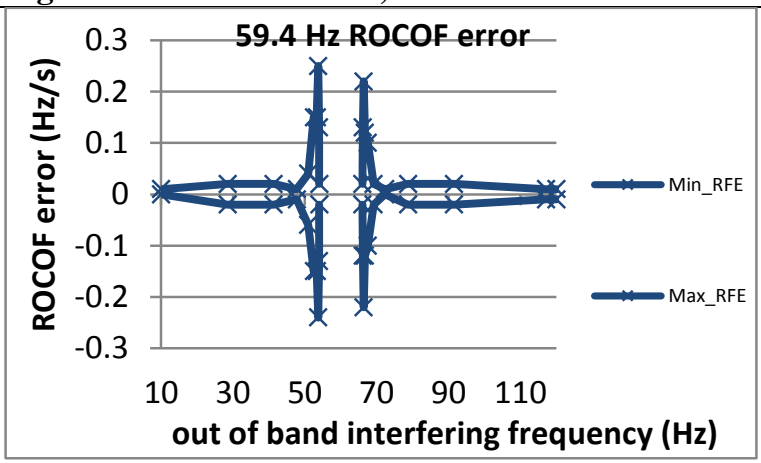

Figure 1976: Fs = 12 FPS, $59.4 \mathrm{~Hz}$ fundamental

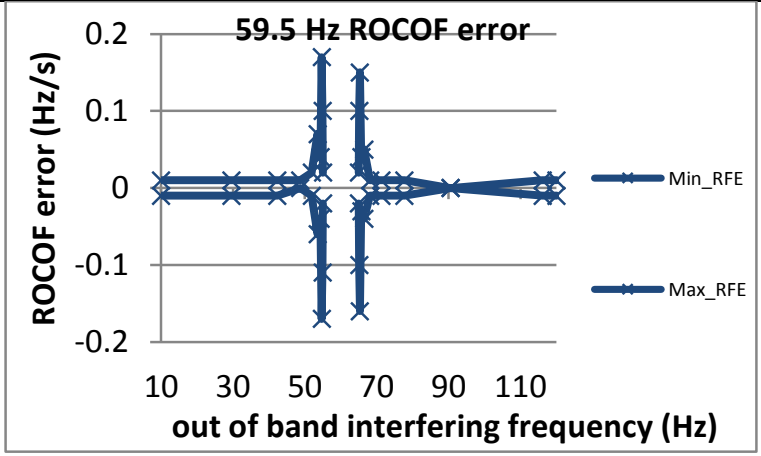

Figure 1979: Fs = 10 FPS, $59.5 \mathrm{~Hz}$ fundamental

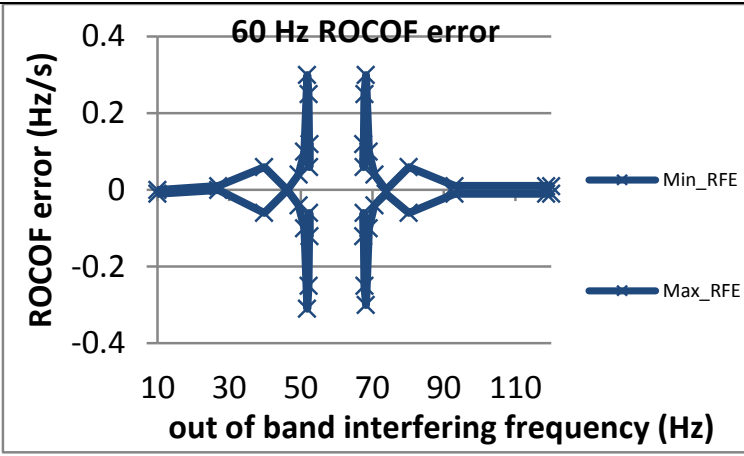

Figure 1974: Fs = 15 FPS, $60 \mathrm{~Hz}$ fundamental

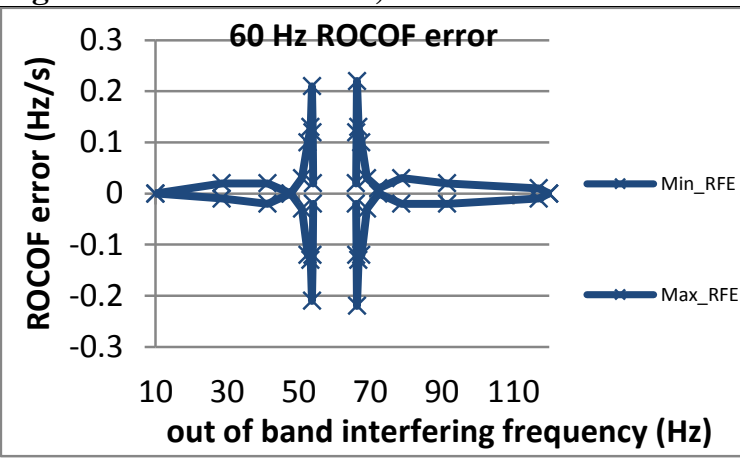

Figure 1977: Fs = 12 FPS, $60 \mathrm{~Hz}$ fundamental

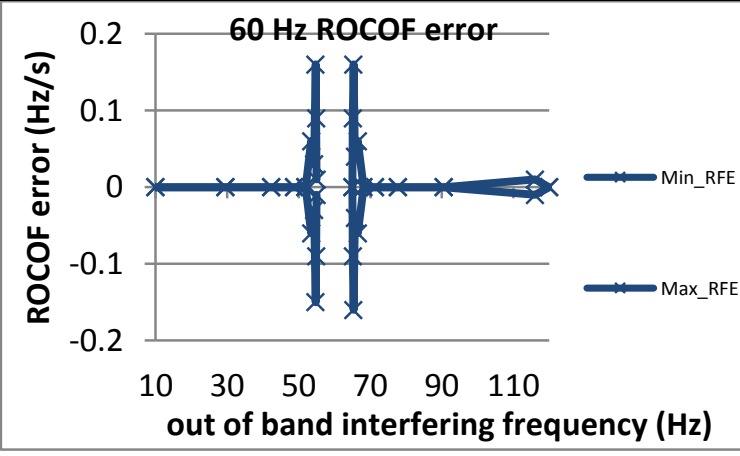

Figure 1980: Fs = $10 \mathrm{FPS}, 60 \mathrm{~Hz}$ fundamental

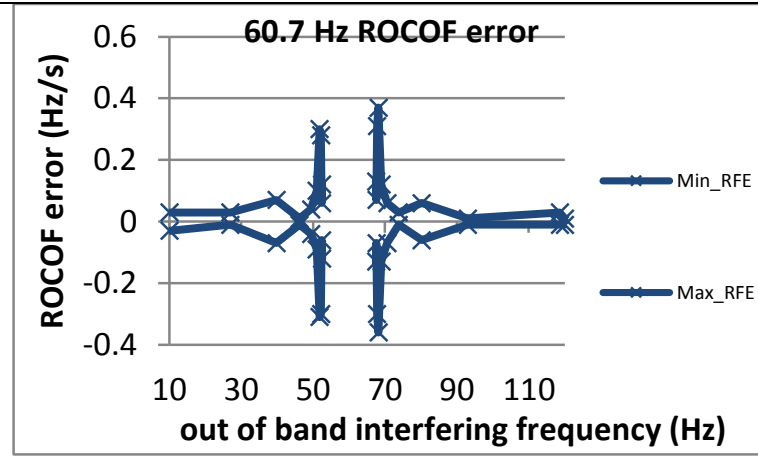

Figure 1975: Fs = 15 FPS, $60.7 \mathrm{~Hz}$ fundamental

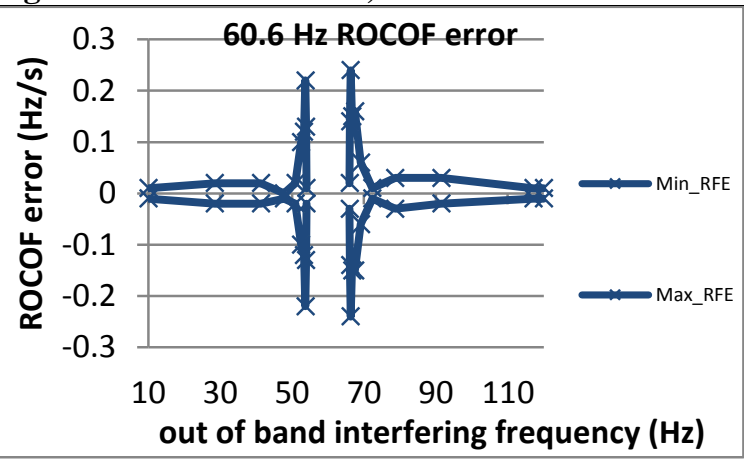

Figure 1978: Fs = 12 FPS, $60.6 \mathrm{~Hz}$ fundamental

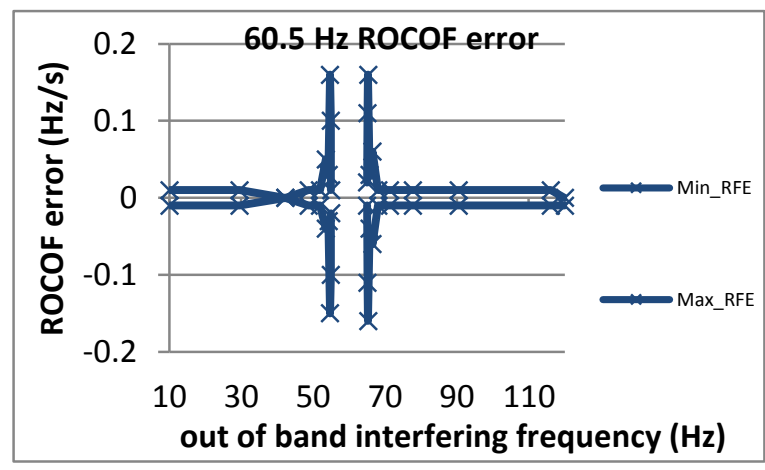

Figure 1981: Fs = 10 FPS, $60.5 \mathrm{~Hz}$ fundamental 
5.4.3 PMU B steady state out of band interfering signals ROCOF error: $M$ class

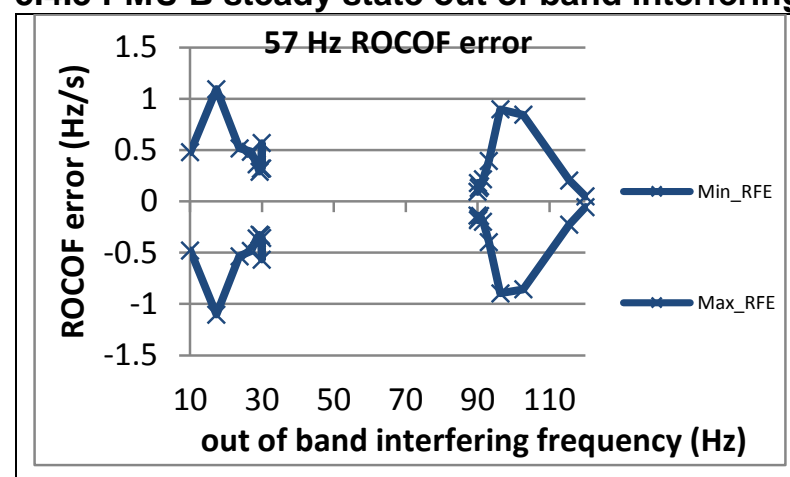

Figure 1982: Fs = 60 FPS, $57 \mathrm{~Hz}$ fundamental

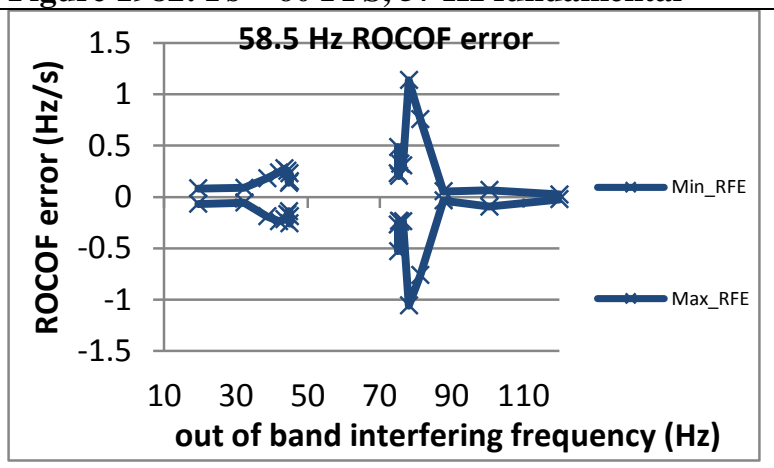

Figure 1985; Fs = 30 FPS, 58.5 Hz fundamental

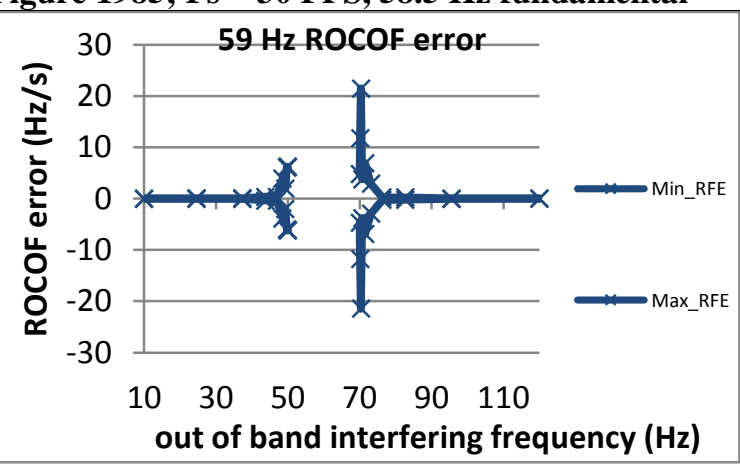

Figure 1988: Fs = 20 FPS, $59 \mathrm{~Hz}$ fundamental

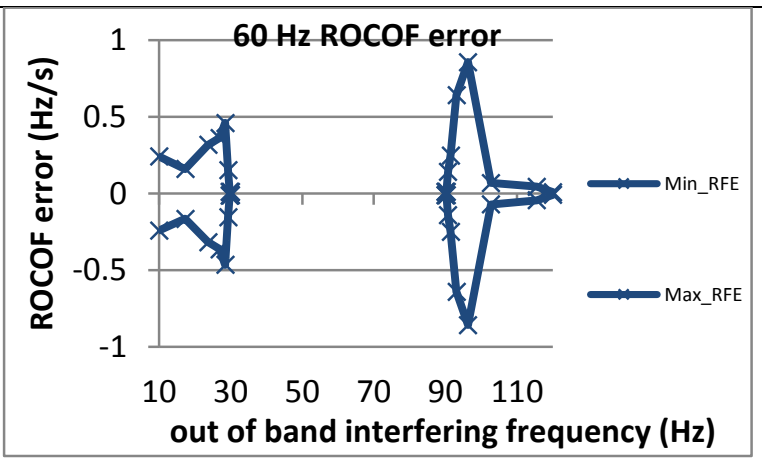

Figure 1983: Fs = 60 FPS, $60 \mathrm{~Hz}$ fundamental

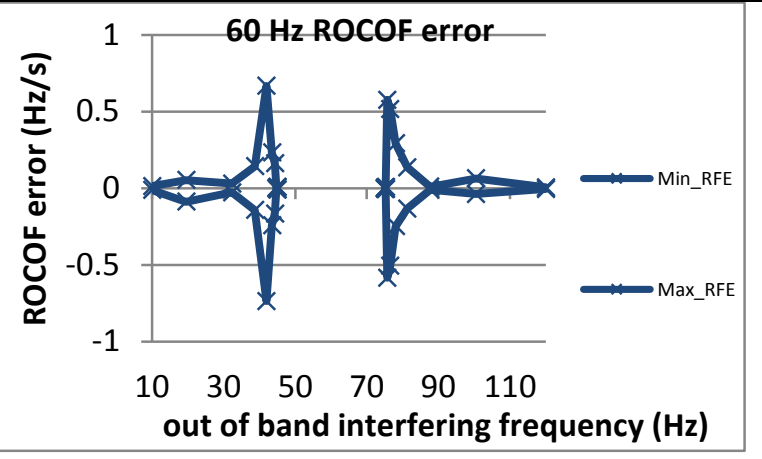

Figure 1986: Fs = $30 \mathrm{FPS}, 60 \mathrm{~Hz}$ fundamental

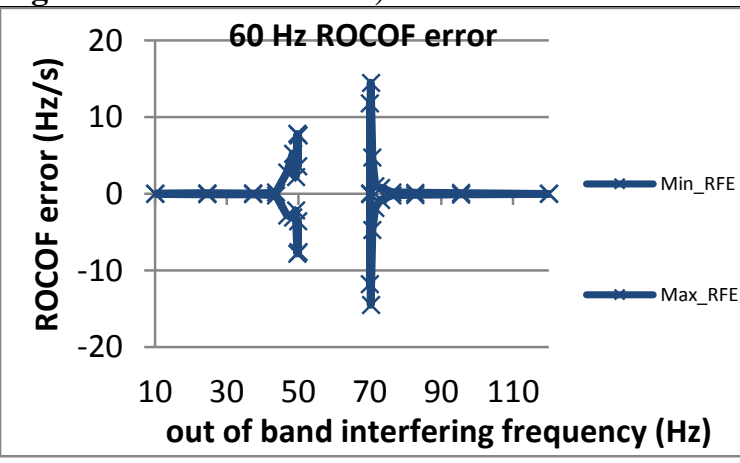

Figure 1989: Fs = $20 \mathrm{FPS}, 60 \mathrm{~Hz}$ fundamental

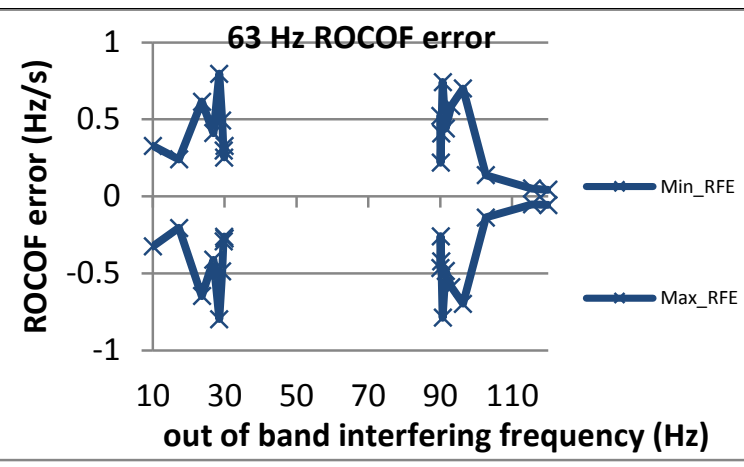

Figure 1984: Fs = $60 \mathrm{FPS}, 63 \mathrm{~Hz}$ fundamental

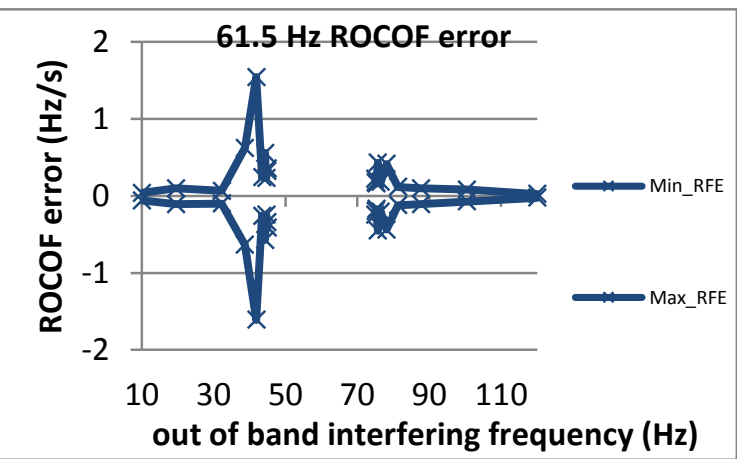

Figure 1987: Fs = 30 FPS, $61.5 \mathrm{~Hz}$ fundamental

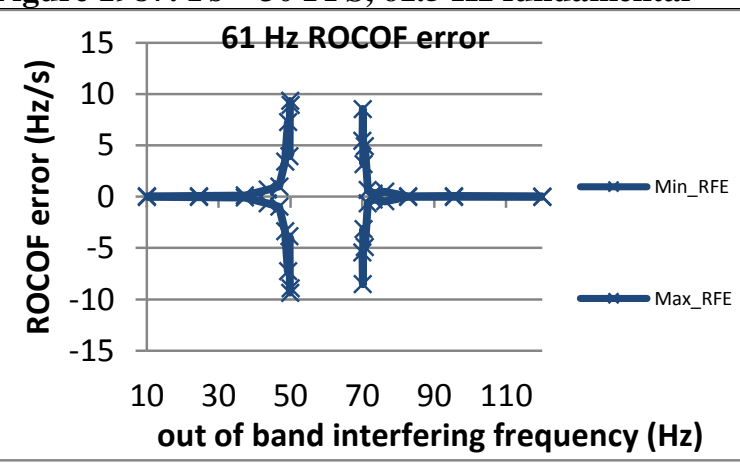

Figure 1990: Fs = 20 FPS, $61 \mathrm{~Hz}$ fundamental 


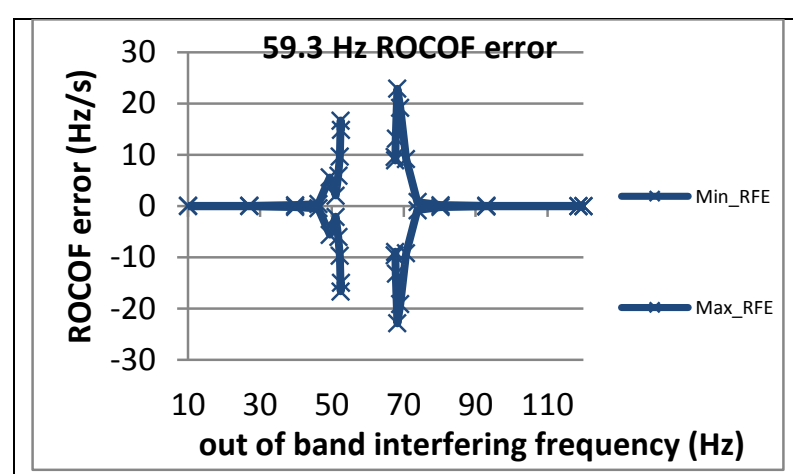

Figure 1991: Fs = 15 FPS, $59.3 \mathrm{~Hz}$ fundamental

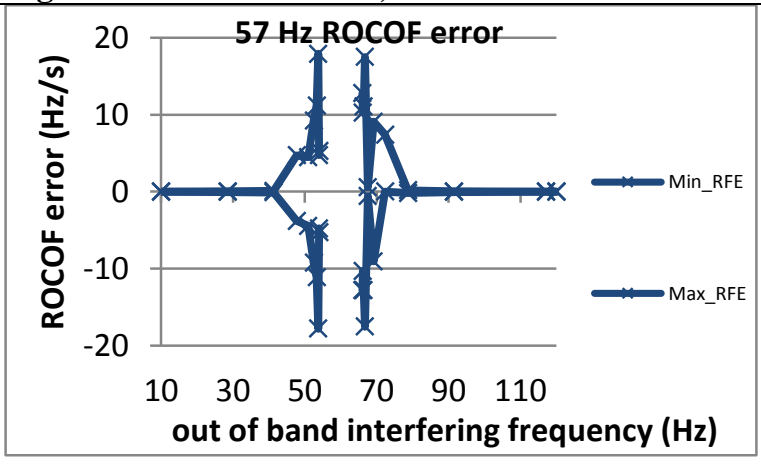

Figure 1994: Fs = 12 FPS, $59.4 \mathrm{~Hz}$ fundamental

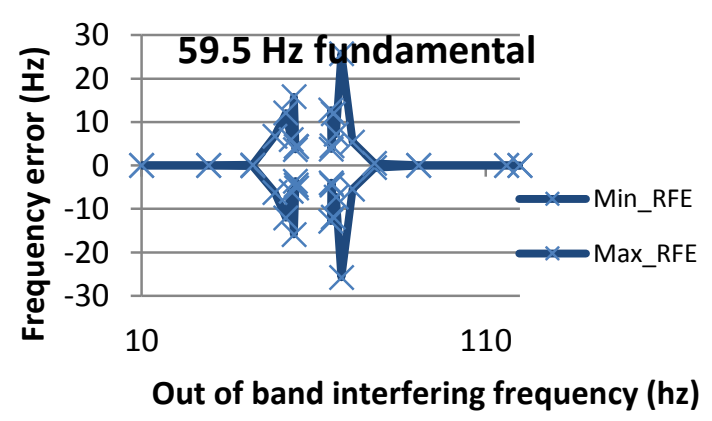

Figure 1997: Fs = 10 FPS, $59.5 \mathrm{~Hz}$ fundamental

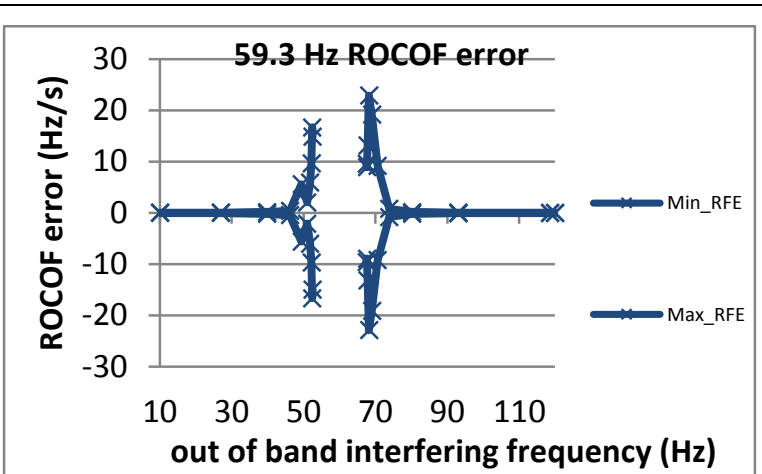

Figure 1992: $\mathrm{Fs}=15 \mathrm{FPS}, 60 \mathrm{~Hz}$ fundamental

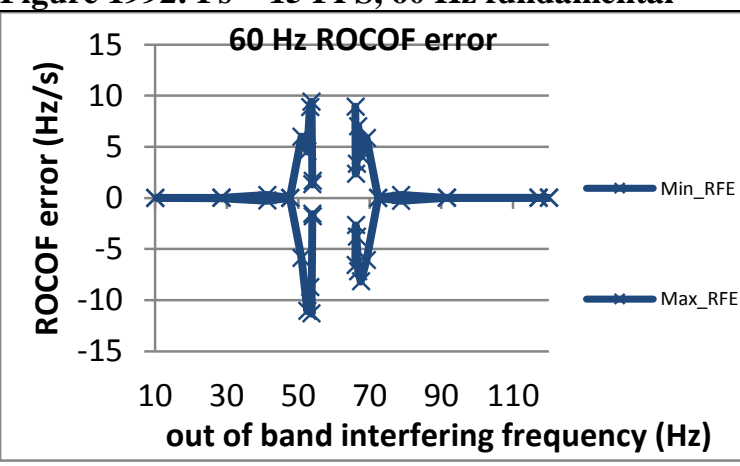

Figure 1995: Fs = 12 FPS, $60 \mathrm{~Hz}$ fundamental

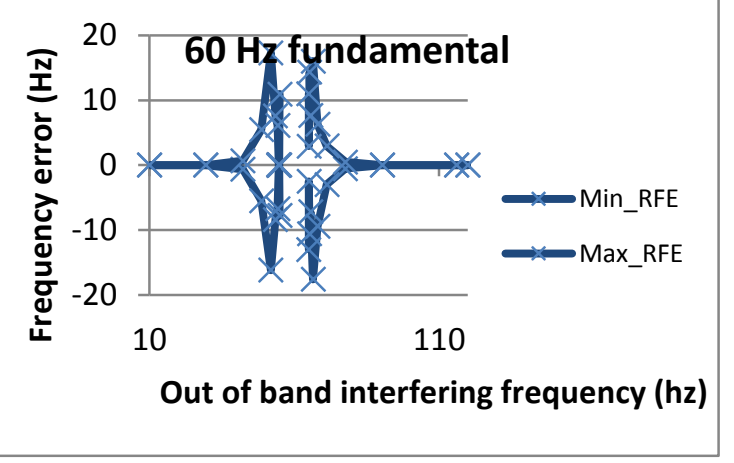

Figure 1998: Fs = $10 \mathrm{FPS}, 60 \mathrm{~Hz}$ fundamental

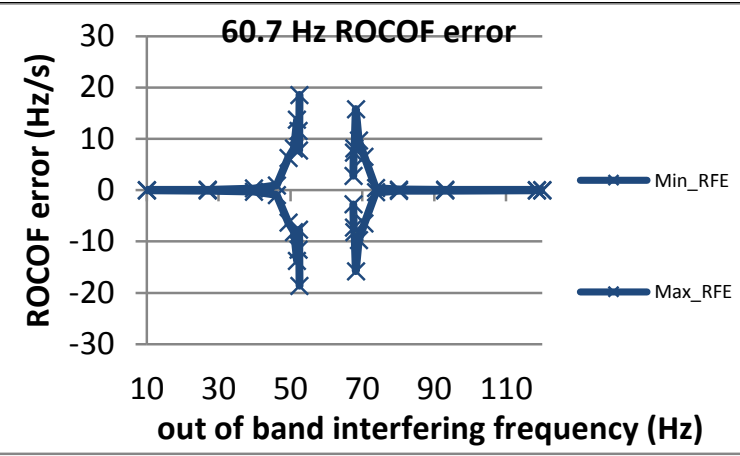

Figure 1993: Fs = 15 FPS, $60.7 \mathrm{~Hz}$ fundamental

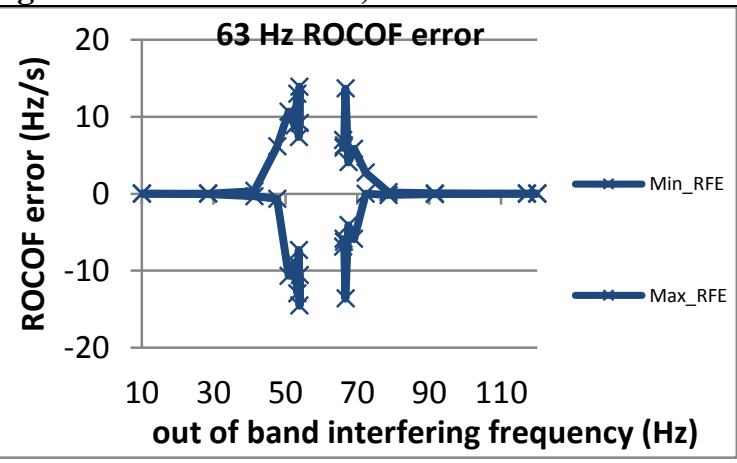

Figure 1996: Fs = 12 FPS, 60.6 Hz fundamental

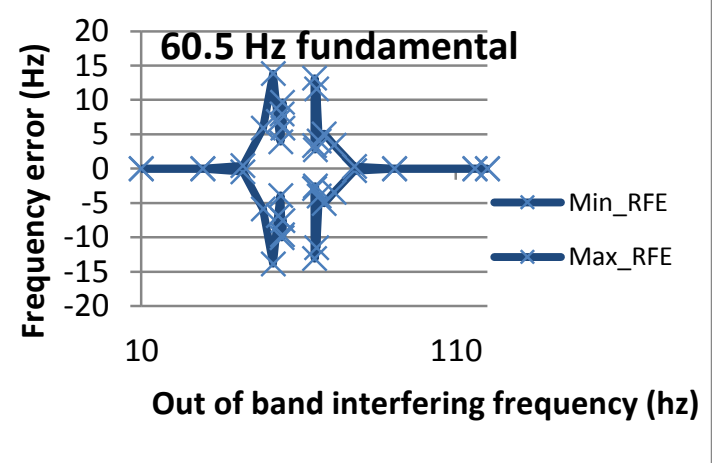

Figure 1999: Fs = 10 FPS, $60.5 \mathrm{~Hz}$ fundamental 
5.4.4 PMU C steady state out of band interfering signals ROCOF error: M class

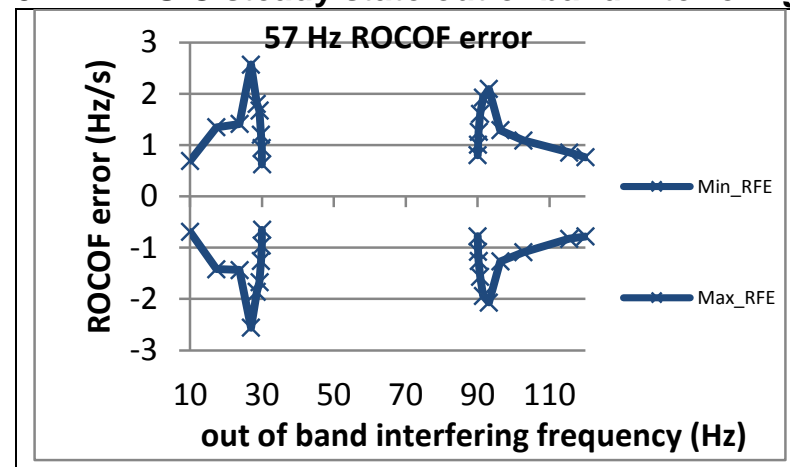

Figure 2000: Fs = 60 FPS, $57 \mathrm{~Hz}$ fundamental

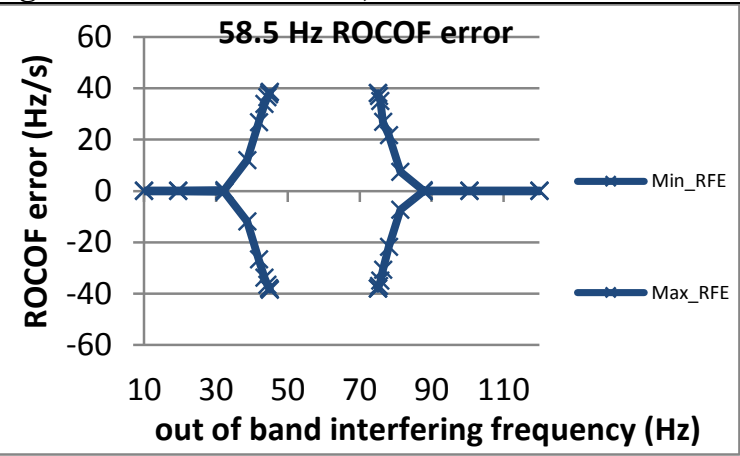

Figure 2003; Fs = 30 FPS, 58.5 Hz fundamental

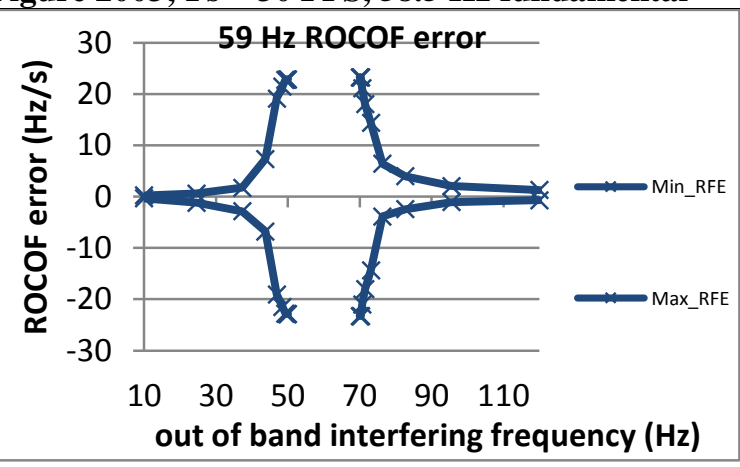

Figure 2006: Fs = 20 FPS, $59 \mathrm{~Hz}$ fundamental

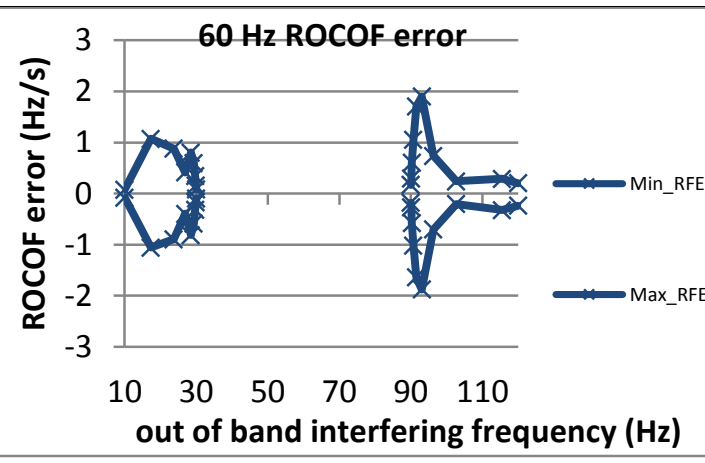

Figure 2001: Fs = $60 \mathrm{FPS}, 60 \mathrm{~Hz}$ fundamental

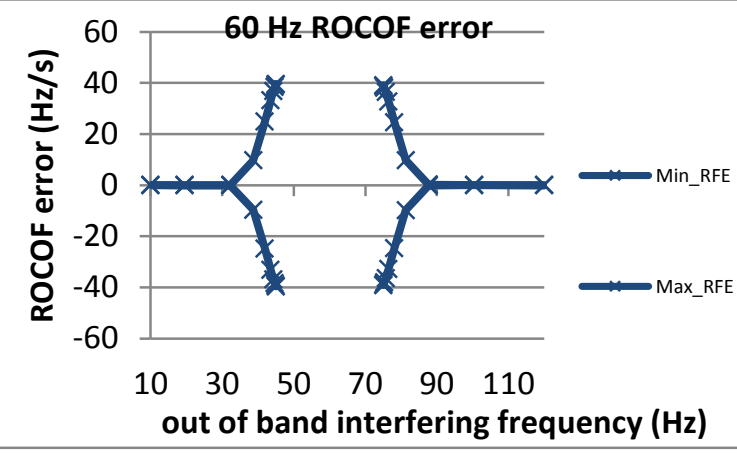

Figure 2004: Fs = $30 \mathrm{FPS}, 60 \mathrm{~Hz}$ fundamental

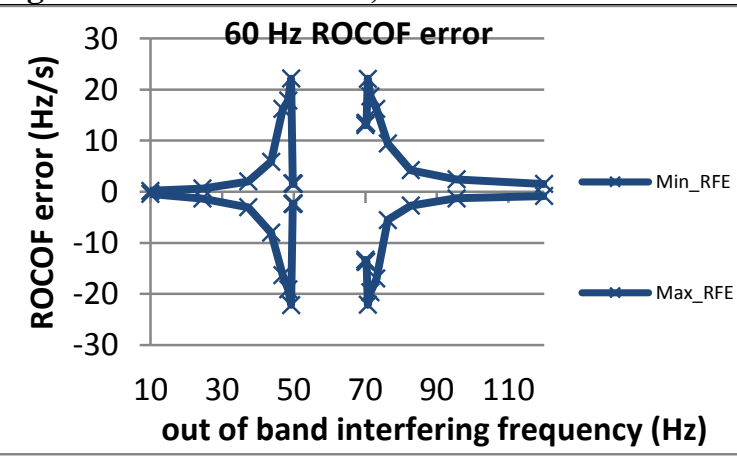

Figure 2007: Fs = $20 \mathrm{FPS}, 60 \mathrm{~Hz}$ fundamental

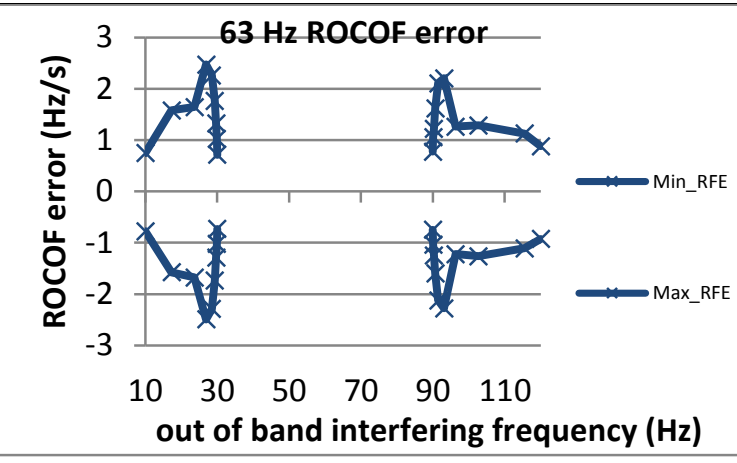

Figure 2002: Fs = $60 \mathrm{FPS}, 63 \mathrm{~Hz}$ fundamental

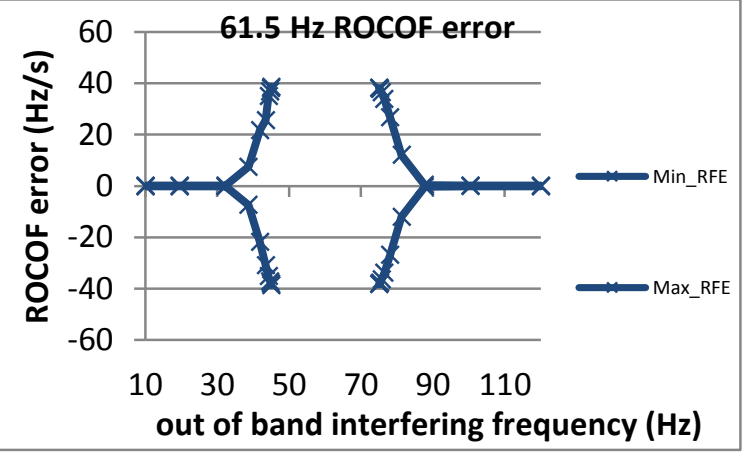

Figure 2005: Fs = 30 FPS, $61.5 \mathrm{~Hz}$ fundamental

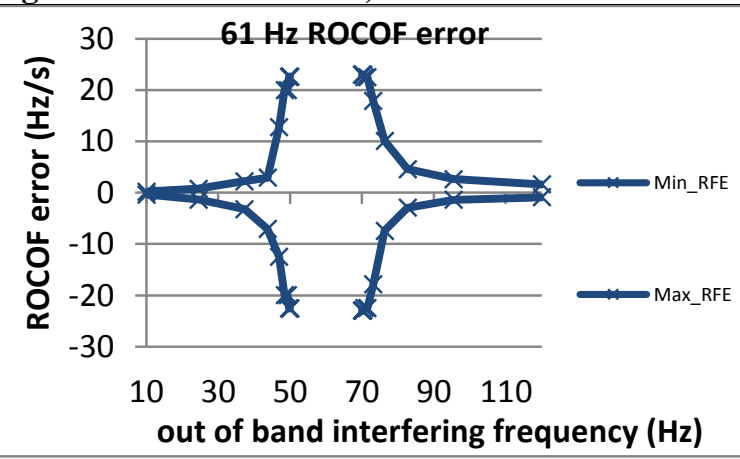

Figure 2008: Fs = $20 \mathrm{FPS}, 61 \mathrm{~Hz}$ fundamental 


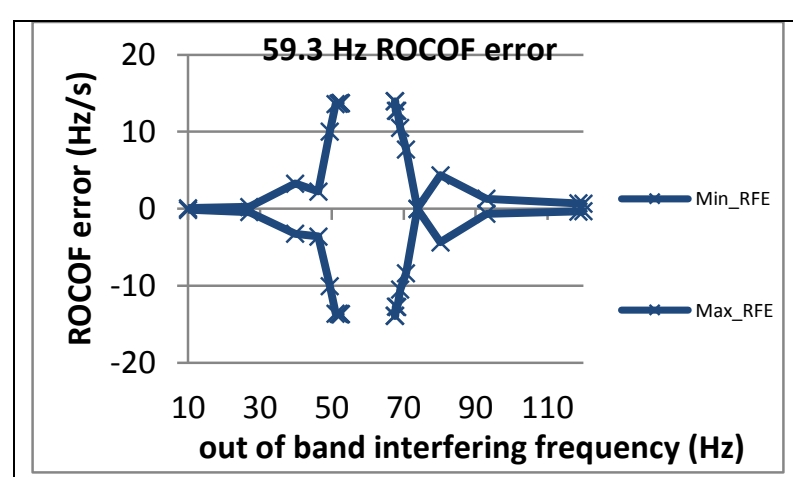

Figure 2009: Fs = 15 FPS, $59.3 \mathrm{~Hz}$ fundamental

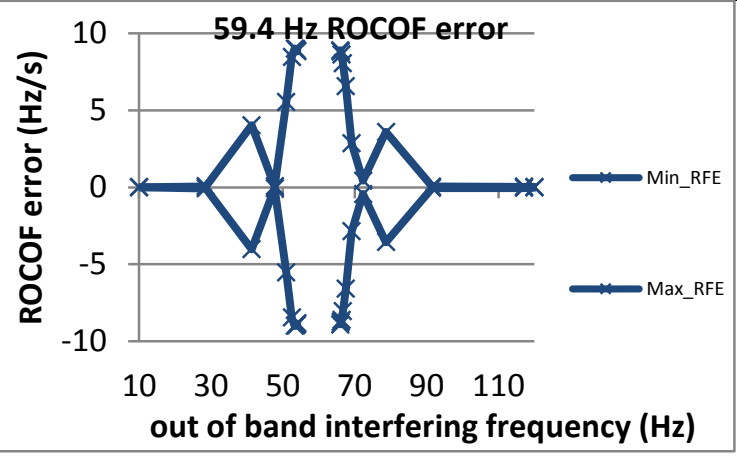

Figure 2012: Fs = 12 FPS, $59.4 \mathrm{~Hz}$ fundamental

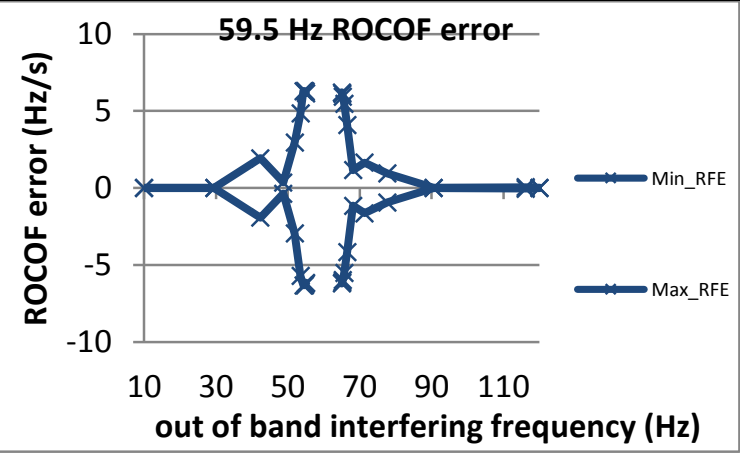

Figure 2015: Fs = 10 FPS, $59.5 \mathrm{~Hz}$ fundamental

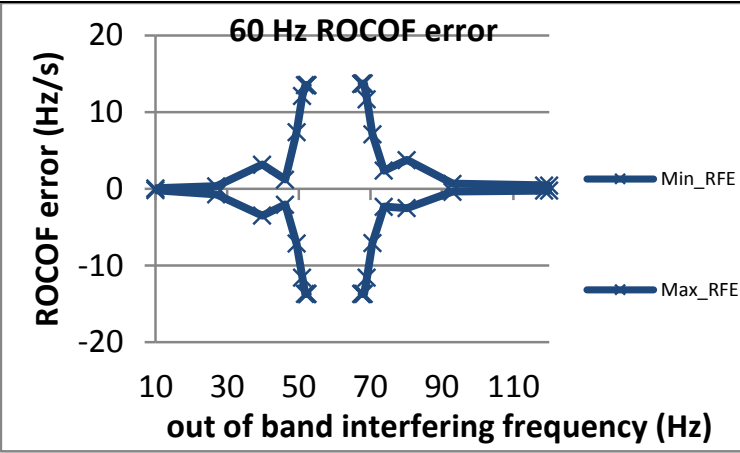

Figure 2010: Fs = 15 FPS, $60 \mathrm{~Hz}$ fundamental

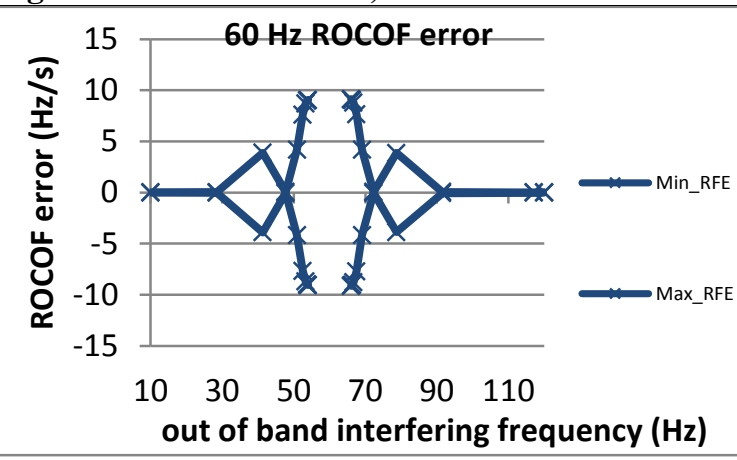

Figure 2013: Fs = $12 \mathrm{FPS}, 60 \mathrm{~Hz}$ fundamental

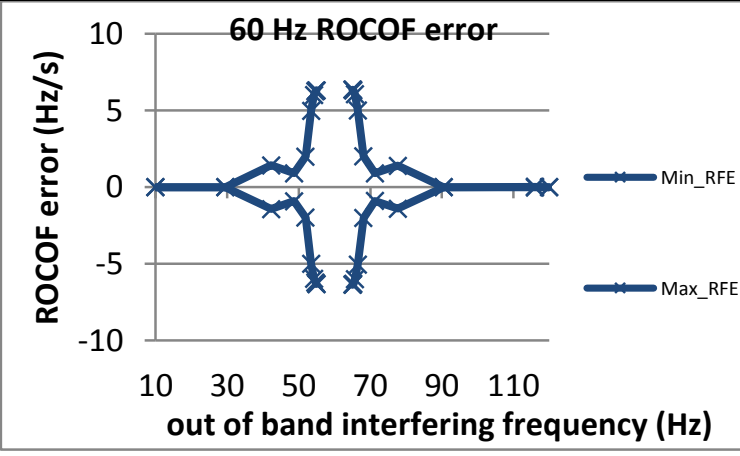

Figure 2016: Fs = 10 FPS, $60 \mathrm{~Hz}$ fundamental

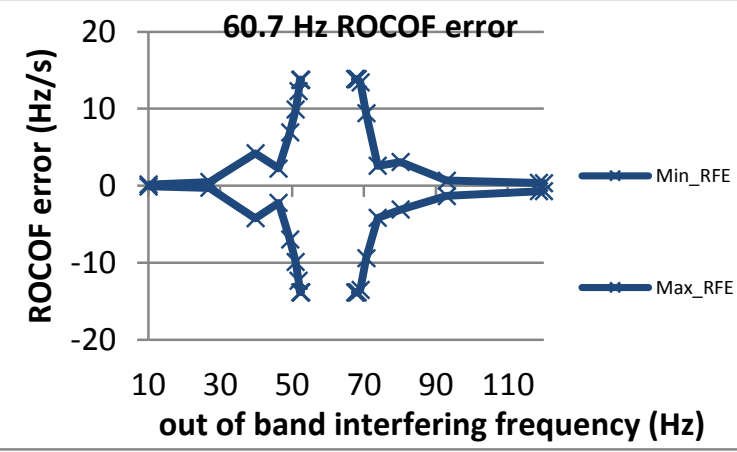

Figure 2011: Fs = 15 FPS, $60.7 \mathrm{~Hz}$ fundamental

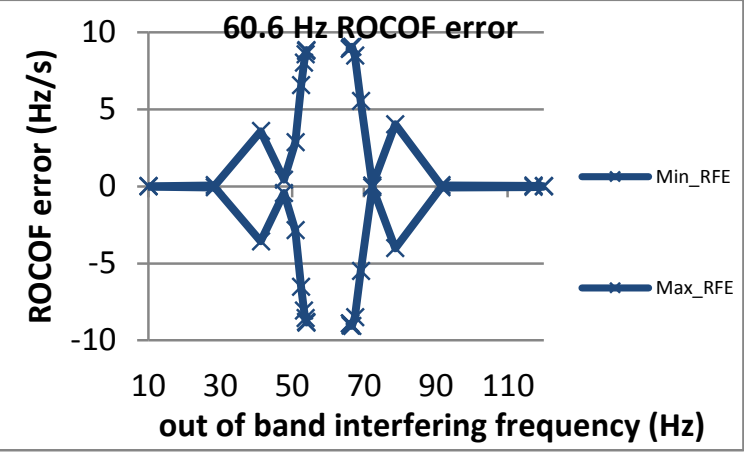

Figure 2014: Fs = 12 FPS, $60.6 \mathrm{~Hz}$ fundamental

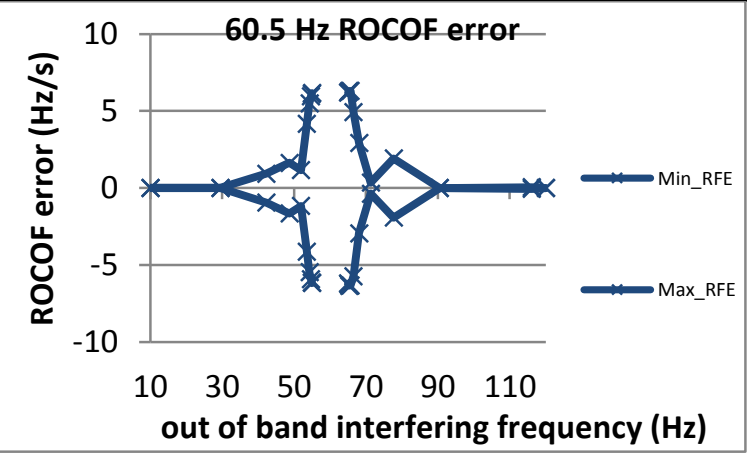

Figure 2017: Fs = 10 FPS, $60.5 \mathrm{~Hz}$ fundamental 
5.4.5 PMU D steady state out of band interfering signals ROCOF error: M class

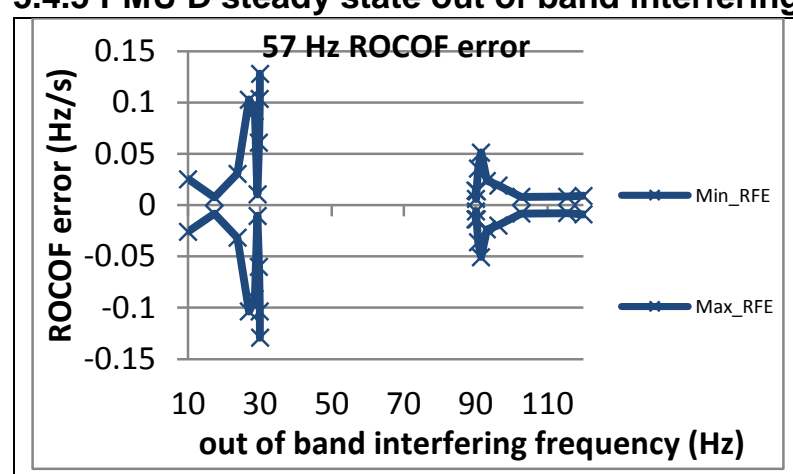

Figure 2018: Fs = 60 FPS, $57 \mathrm{~Hz}$ fundamental

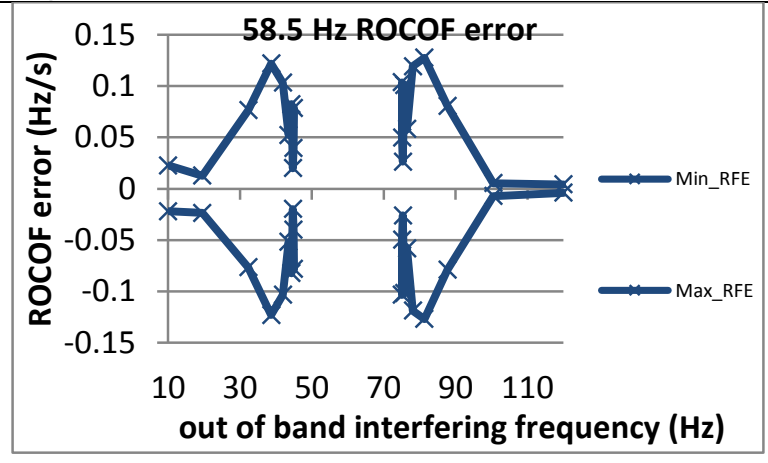

Figure 2021; Fs = 30 FPS, $58.5 \mathrm{~Hz}$ fundamental

Figure 2024: Fs = 20 FPS, $59 \mathrm{~Hz}$ fundamental

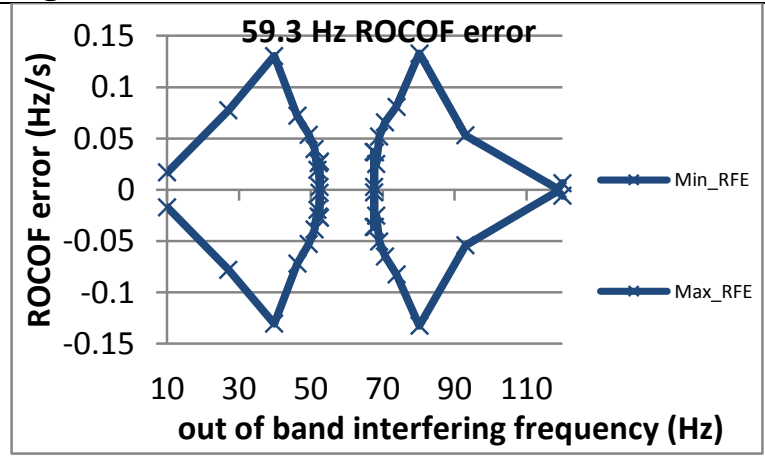

Figure 2027: Fs = 15 FPS, $59.3 \mathrm{~Hz}$ fundamental

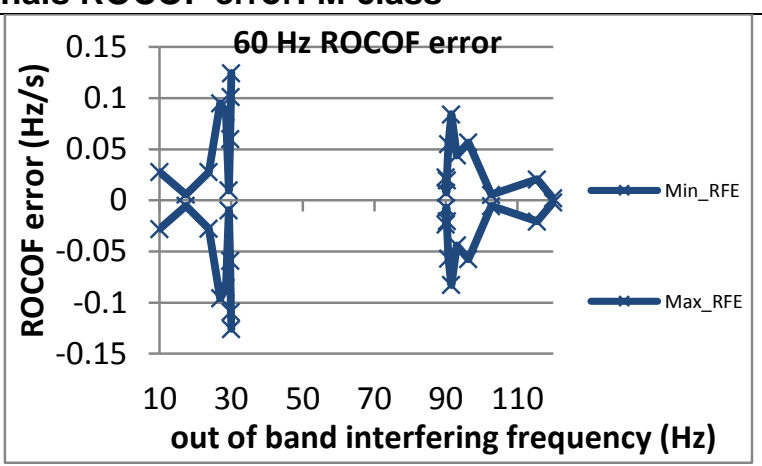

Figure 2019: Fs = 60 FPS, $60 \mathrm{~Hz}$ fundamental

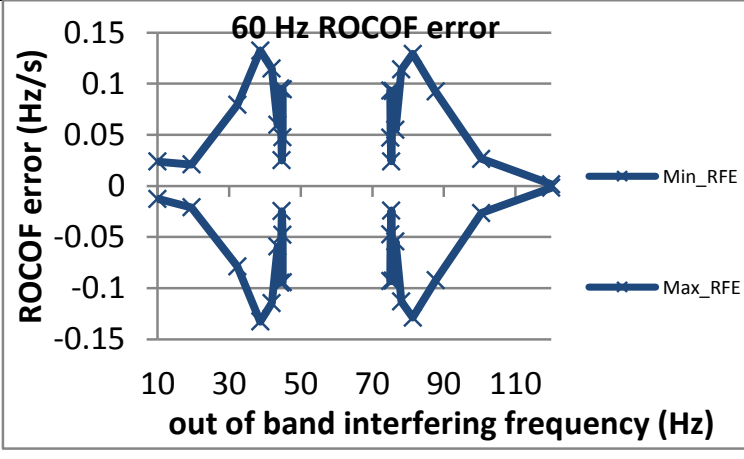

Figure 2022: Fs = $30 \mathrm{FPS}, 60 \mathrm{~Hz}$ fundamental

Figure 2025: Fs = $20 \mathrm{FPS}, 60 \mathrm{~Hz}$ fundamental

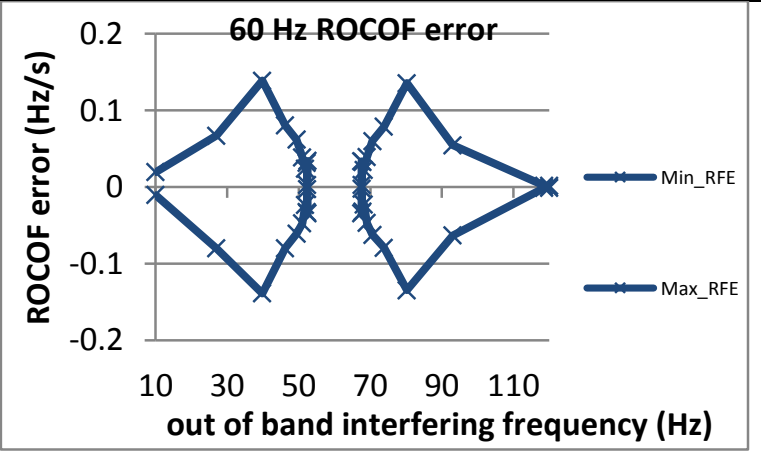

Figure 2028: Fs = 15 FPS, $60 \mathrm{~Hz}$ fundamental

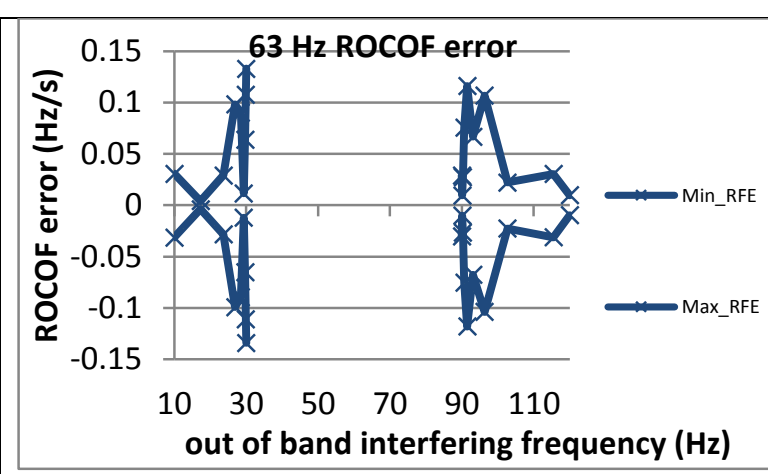

Figure 2020: Fs = 60 FPS, $63 \mathrm{~Hz}$ fundamental

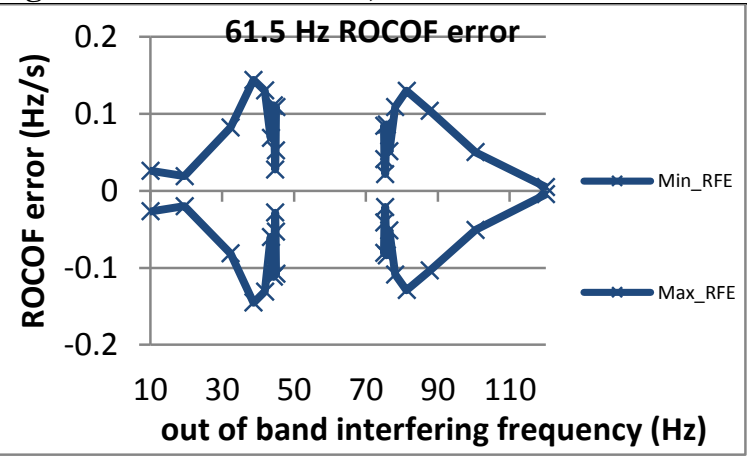

Figure 2023: Fs = 30 FPS, $61.5 \mathrm{~Hz}$ fundamental

Figure 2026: Fs = $20 \mathrm{FPS}, 61 \mathrm{~Hz}$ fundamental

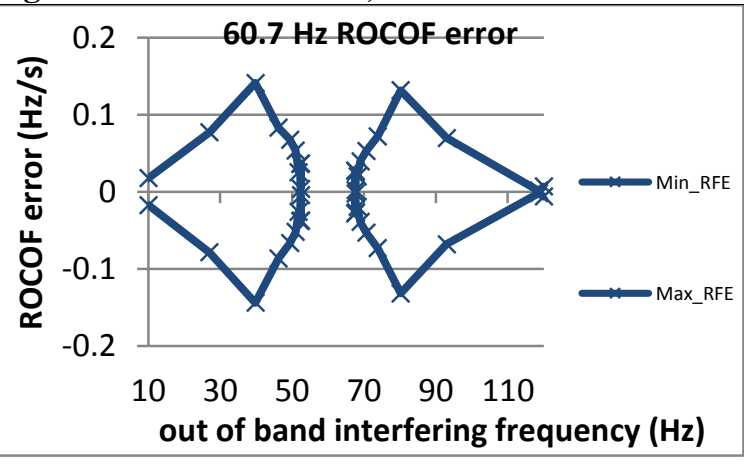

Figure 2029: Fs = 15 FPS, $60.7 \mathrm{~Hz}$ fundamental 


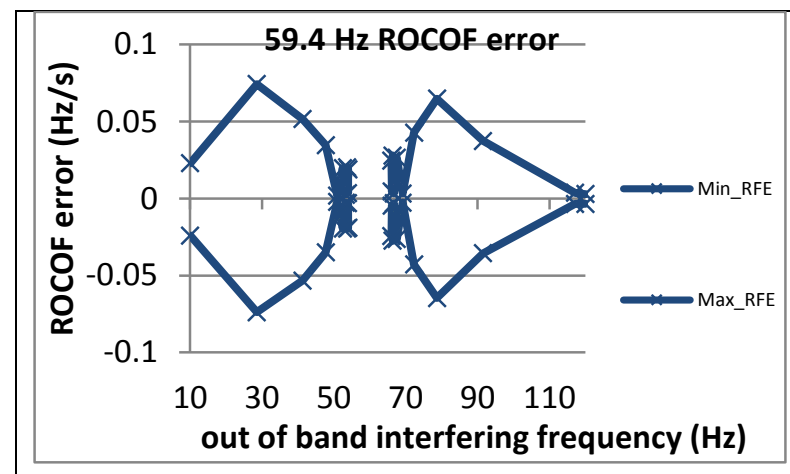

Figure 2030: Fs = 12 FPS, $59.4 \mathrm{~Hz}$ fundamental

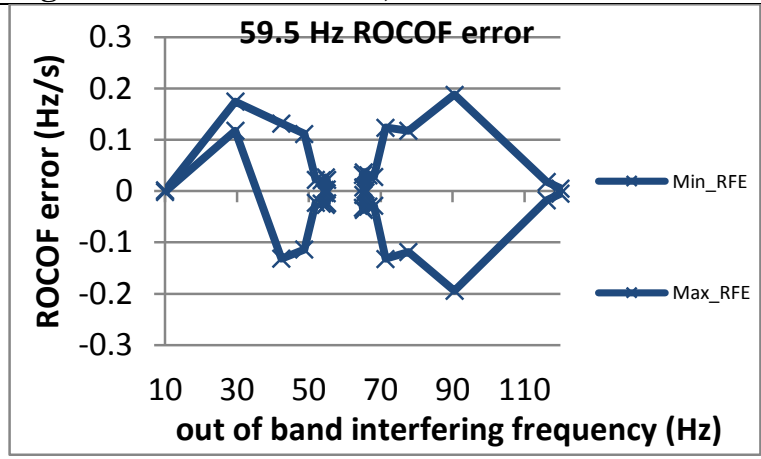

Figure 2033: Fs = 10 FPS, $59.5 \mathrm{~Hz}$ fundamental

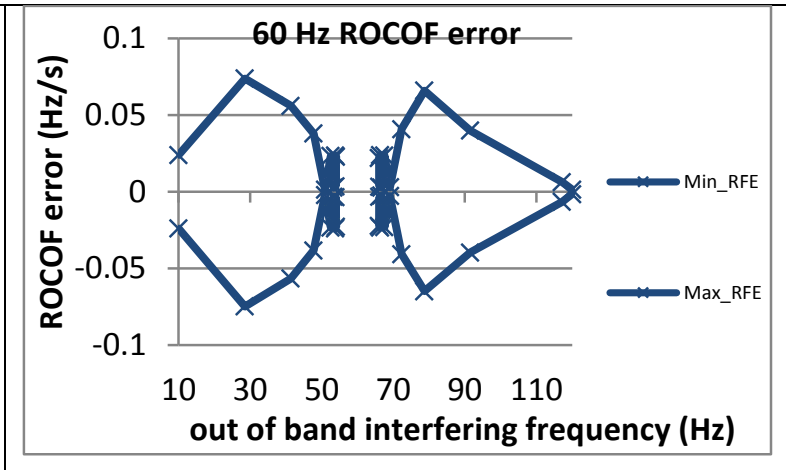

Figure 2031: Fs = $12 \mathrm{FPS}, 60 \mathrm{~Hz}$ fundamental

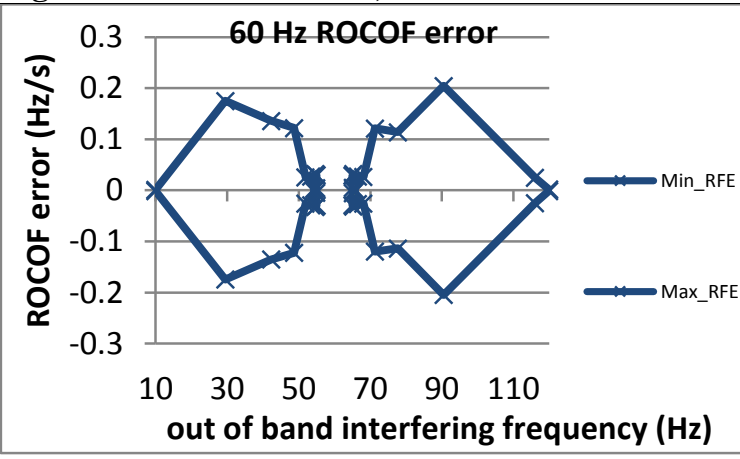

Figure 2034: Fs = 10 FPS, $60 \mathrm{~Hz}$ fundamental

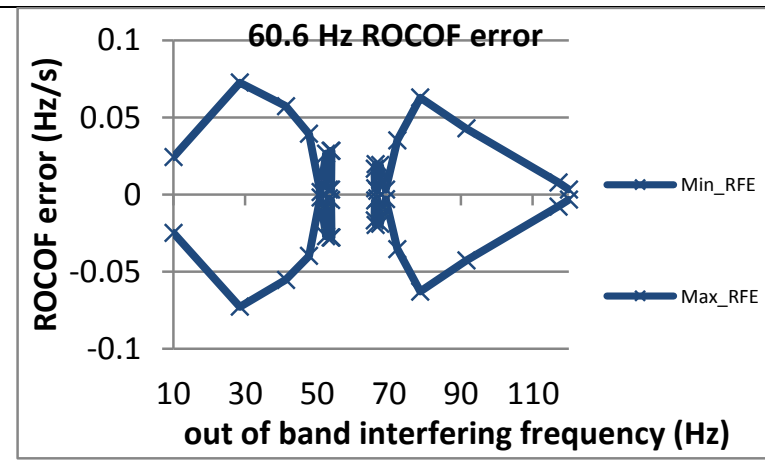

Figure 2032: Fs = 12 FPS, $60.6 \mathrm{~Hz}$ fundamental

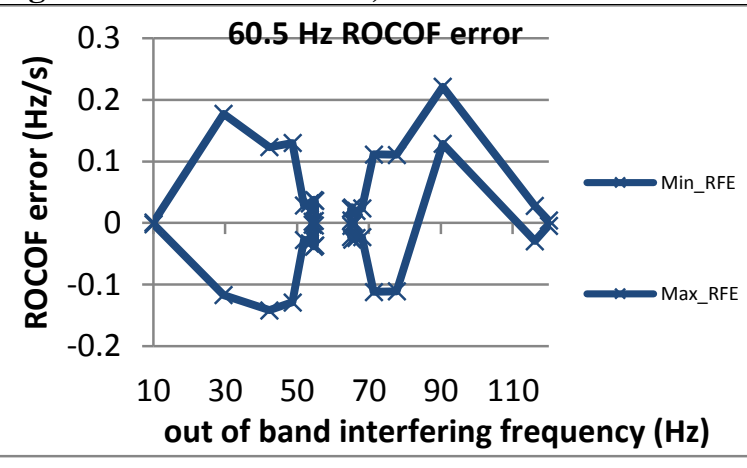

Figure 2035: Fs = 10 FPS, $60.5 \mathrm{~Hz}$ fundamental 
5.4.6 PMU E steady state out of band interfering signals ROCOF error: M class

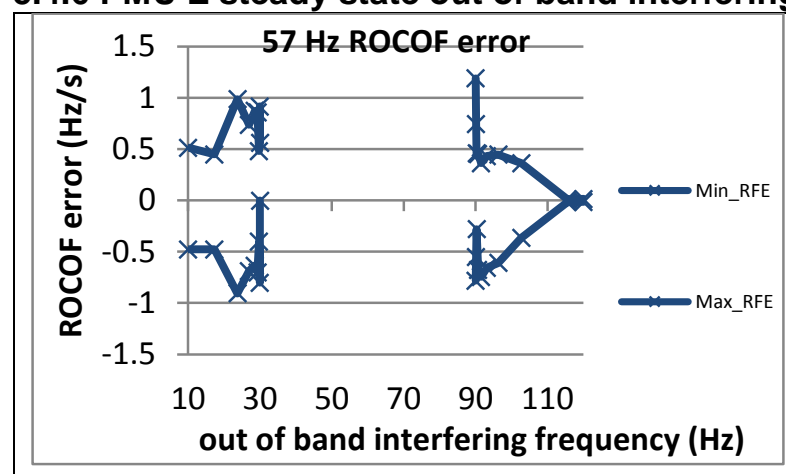

Figure 2036: Fs = 60 FPS, $57 \mathrm{~Hz}$ fundamental

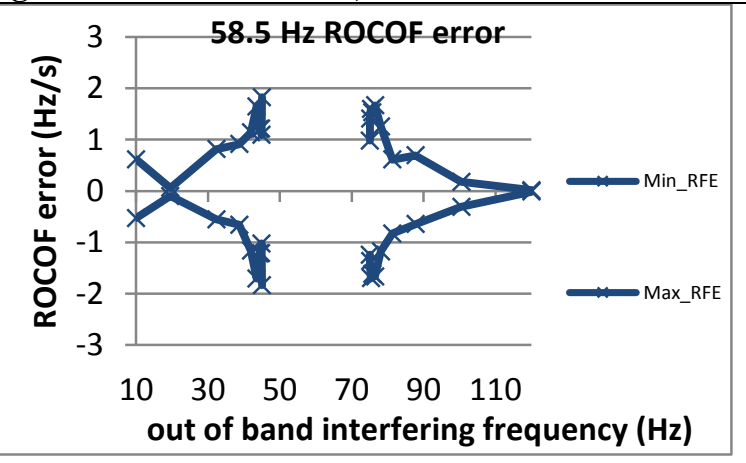

Figure 2039; Fs = 30 FPS, 58.5 Hz fundamental

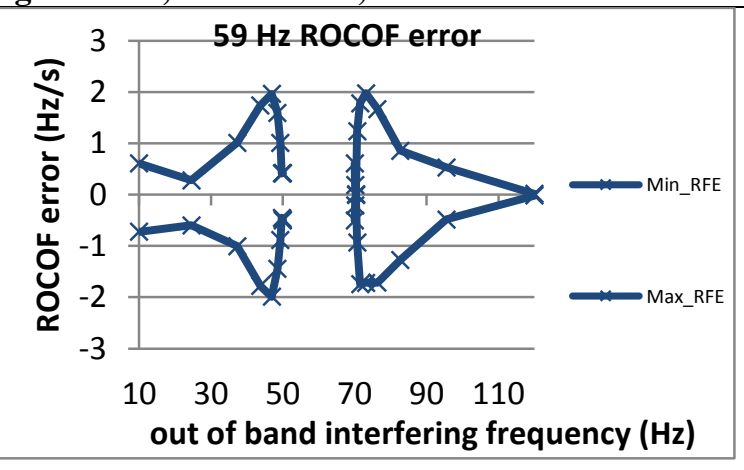

Figure 2042: Fs = 20 FPS, $59 \mathrm{~Hz}$ fundamental

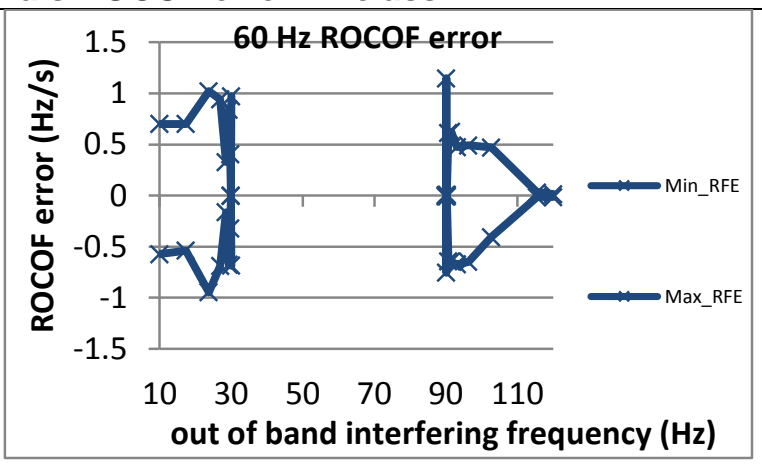

Figure 2037: Fs = $60 \mathrm{FPS}, 60 \mathrm{~Hz}$ fundamental

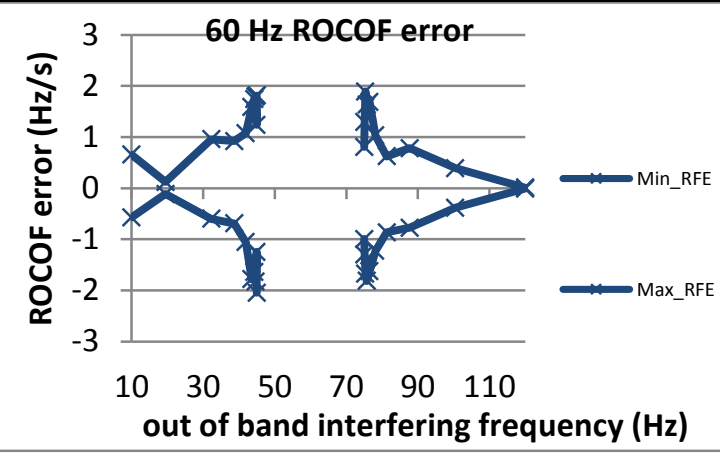

Figure 2040: Fs = $30 \mathrm{FPS}, 60 \mathrm{~Hz}$ fundamental

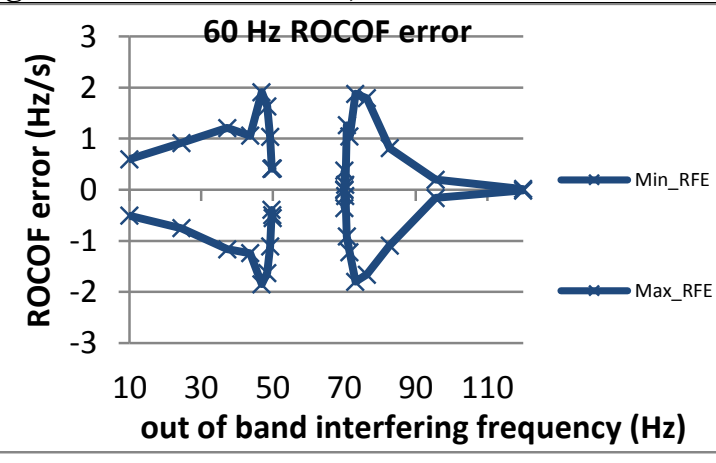

Figure 2043: Fs = 20 FPS, $60 \mathrm{~Hz}$ fundamental

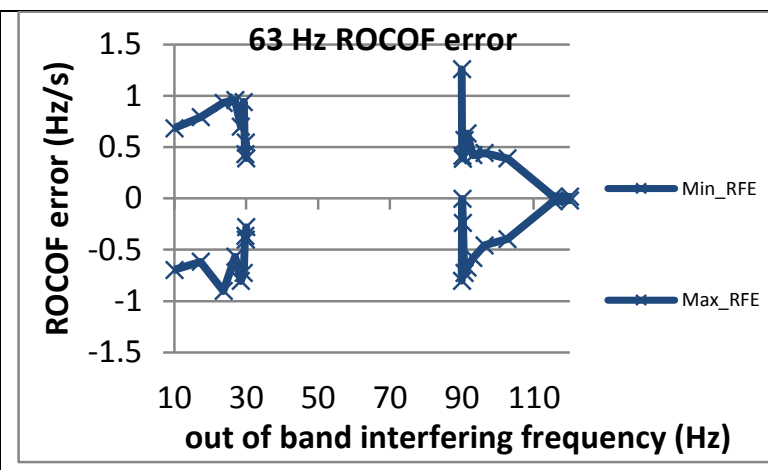

Figure 2038: Fs = 60 FPS, $63 \mathrm{~Hz}$ fundamental

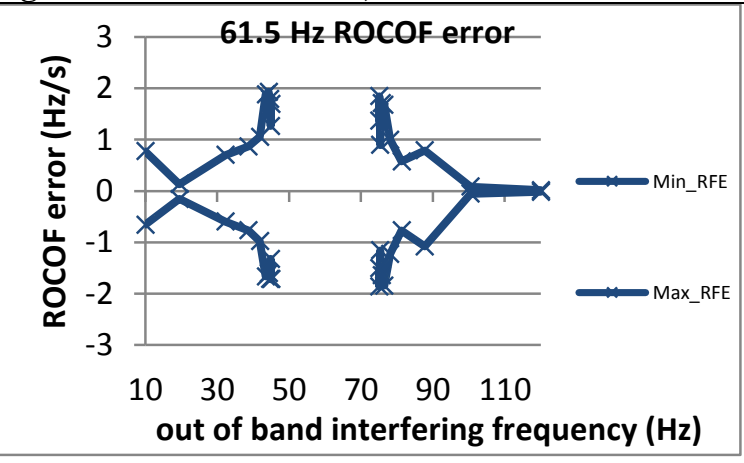

Figure 2041: Fs = 30 FPS, $61.5 \mathrm{~Hz}$ fundamental

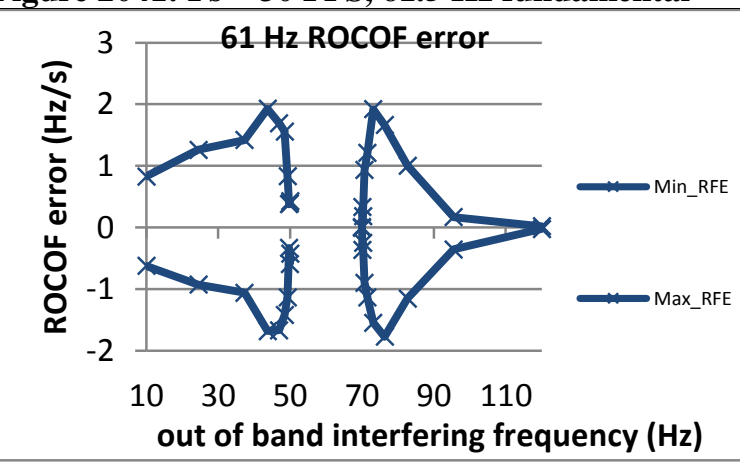

Figure 2044: Fs = 20 FPS, $61 \mathrm{~Hz}$ fundamental 


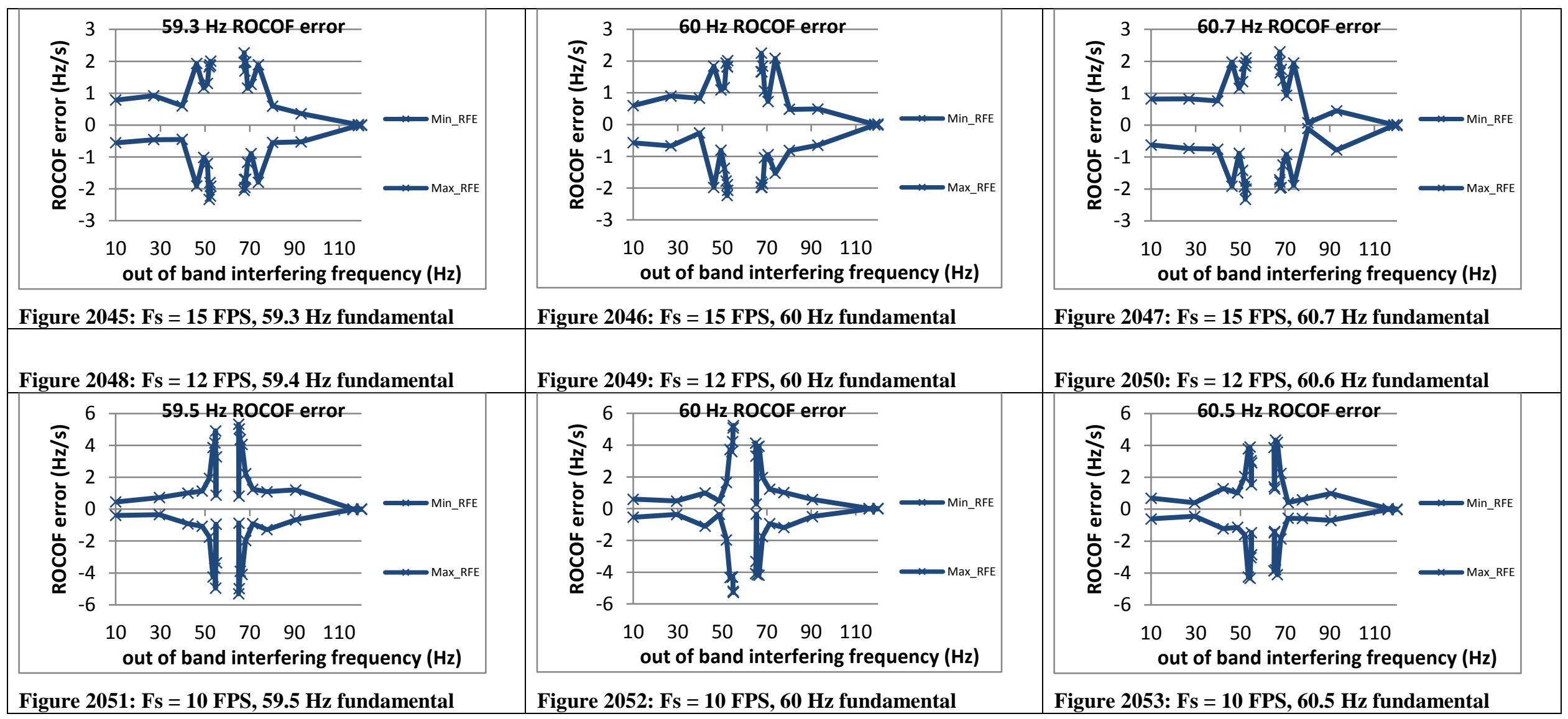


5.4.7 PMU F steady state out of band interfering signals ROCOF error: M class

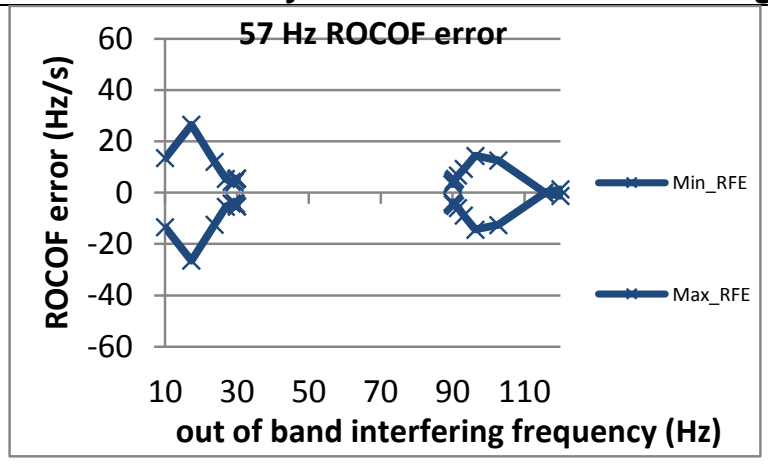

Figure 2054: $\mathrm{Fs}=60 \mathrm{FPS}, 57 \mathrm{~Hz}$ fundamental

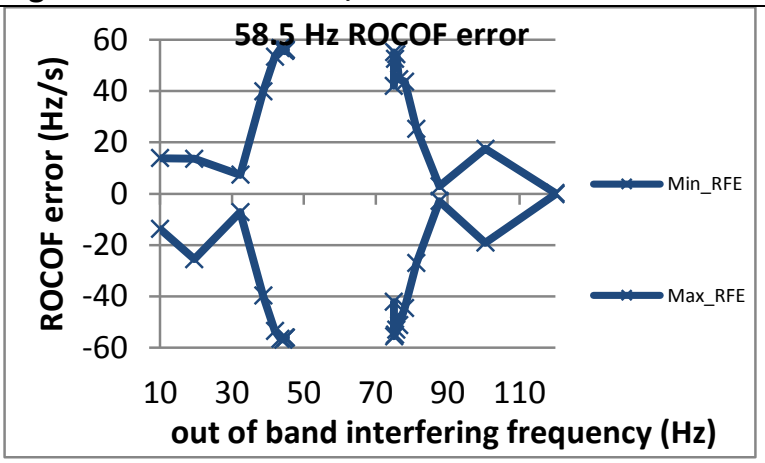

Figure 2057; Fs = $30 \mathrm{FPS}, 58.5 \mathrm{~Hz}$ fundamental

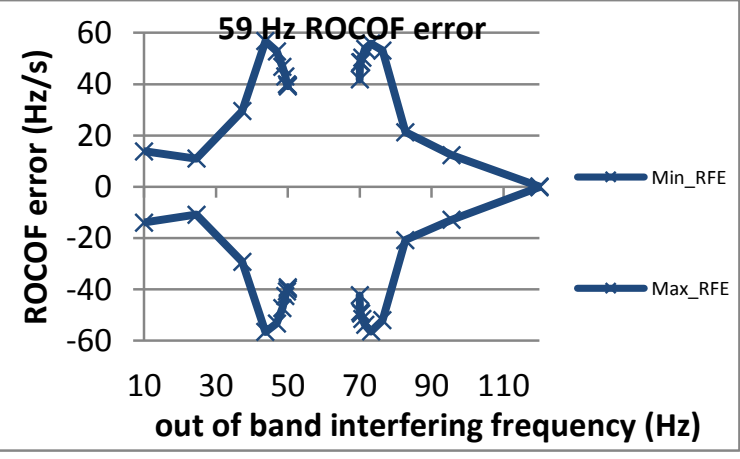

Figure 2060: Fs $=20 \mathrm{FPS}, 59 \mathrm{~Hz}$ fundamental

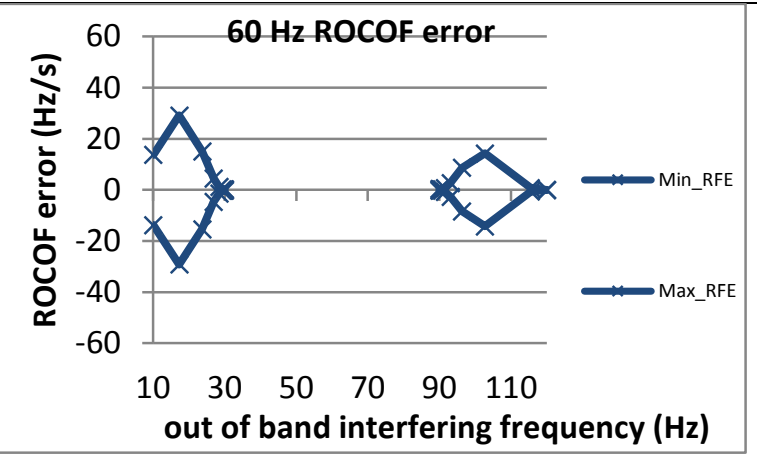

Figure 2055: $\mathrm{Fs}=60 \mathrm{FPS}, 60 \mathrm{~Hz}$ fundamental

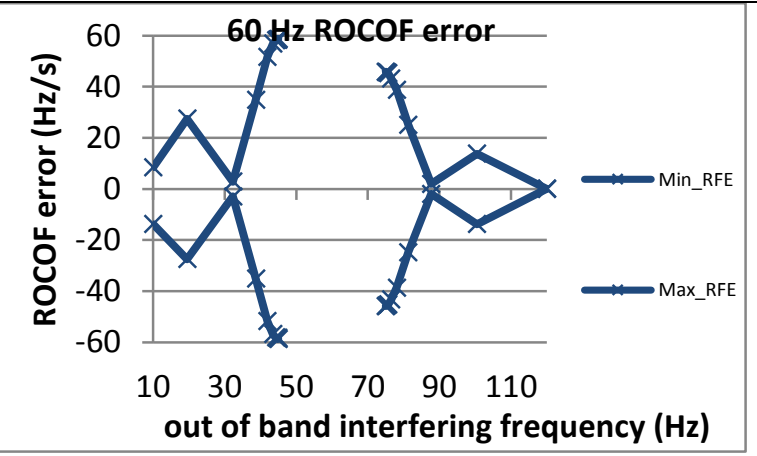

Figure 2058: $\mathrm{Fs}=30 \mathrm{FPS}, 60 \mathrm{~Hz}$ fundamental

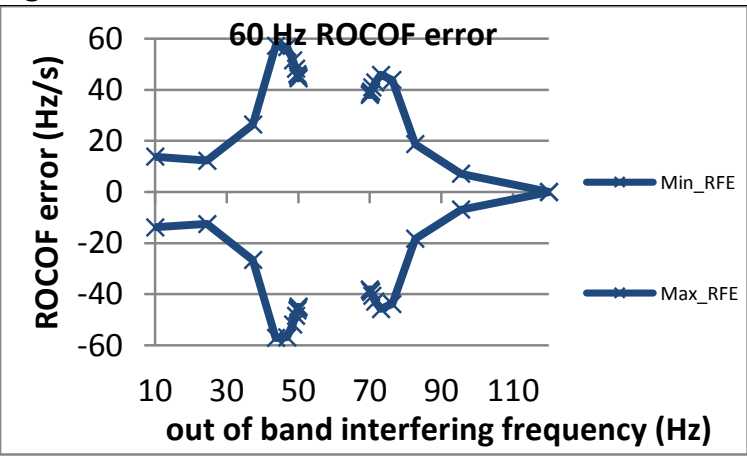

Figure 2061: Fs = $20 \mathrm{FPS}, 60 \mathrm{~Hz}$ fundamental

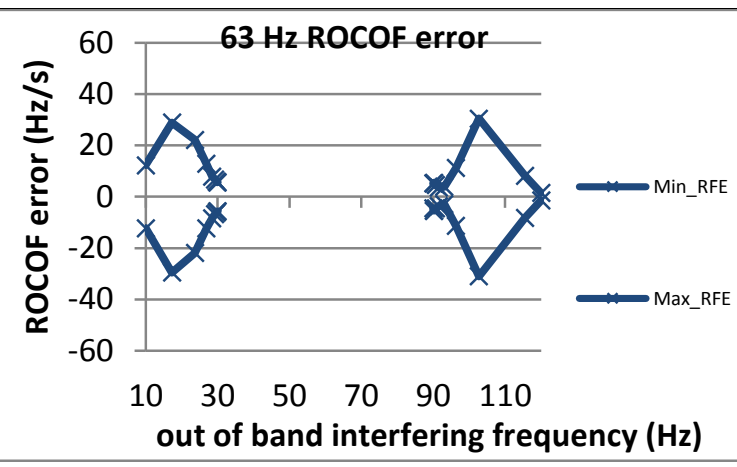

Figure 2056: $\mathrm{Fs}=60 \mathrm{FPS}, 63 \mathrm{~Hz}$ fundamental

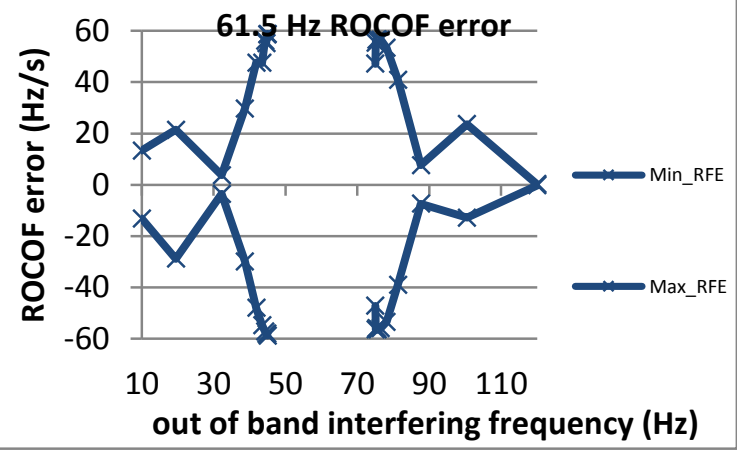

Figure 2059: $\mathrm{Fs}=30 \mathrm{FPS}, 61.5 \mathrm{~Hz}$ fundamental

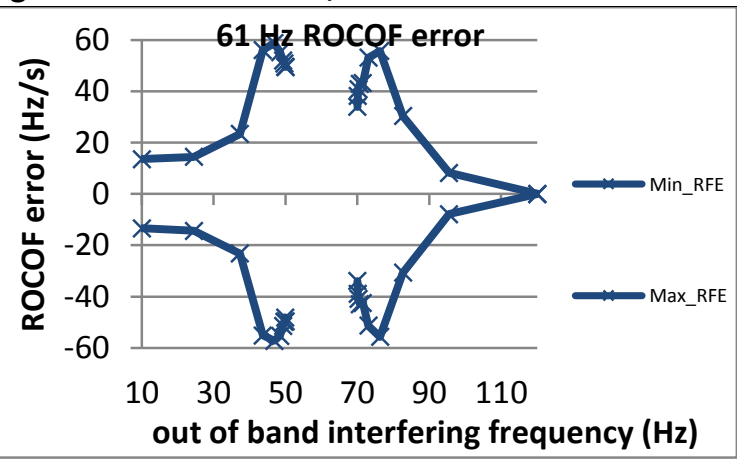

Figure 2062: Fs = $20 \mathrm{FPS}, 61 \mathrm{~Hz}$ fundamental 


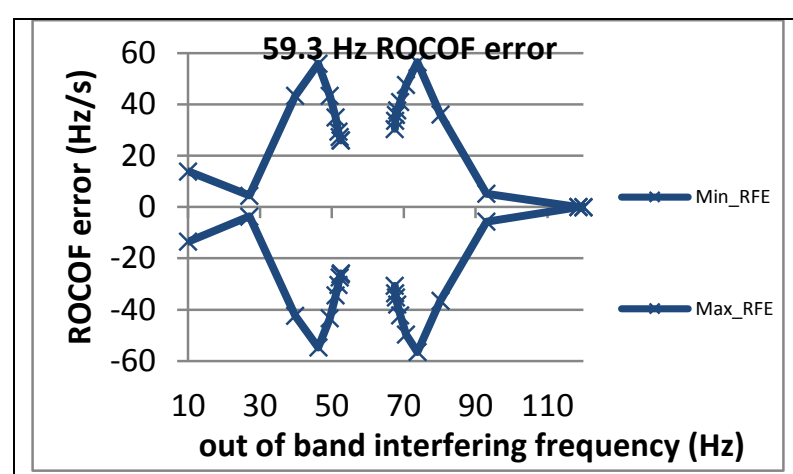

Figure 2063: Fs = $15 \mathrm{FPS}, 59.3 \mathrm{~Hz}$ fundamental

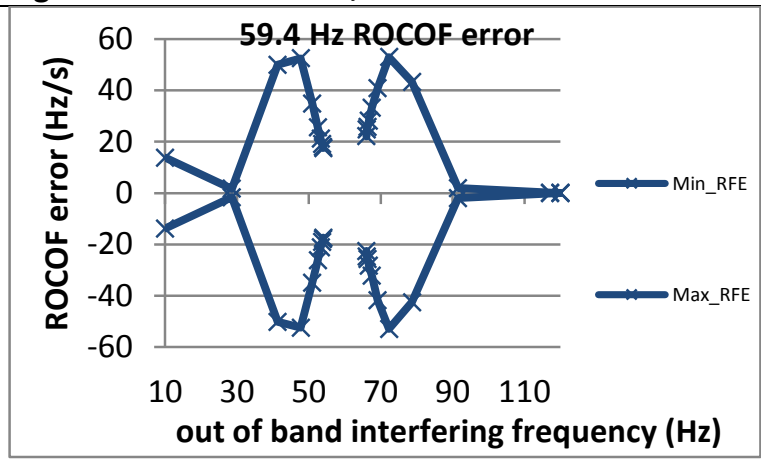

Figure 2066: Fs = $12 \mathrm{FPS}, 59.4 \mathrm{~Hz}$ fundamental

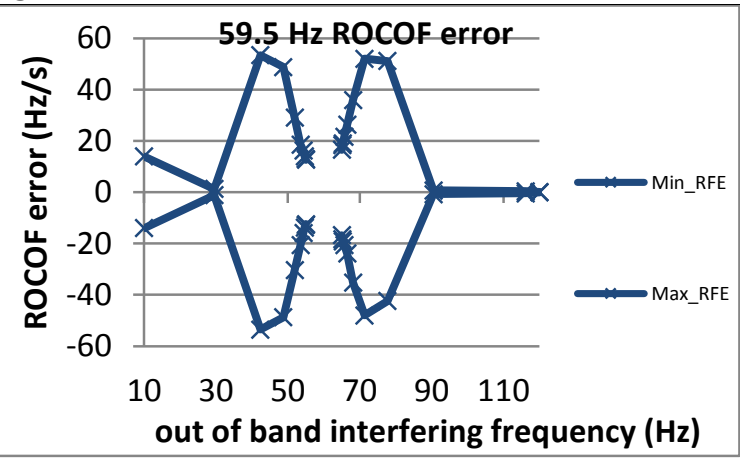

Figure 2069: Fs = 10 FPS, $59.5 \mathrm{~Hz}$ fundamental

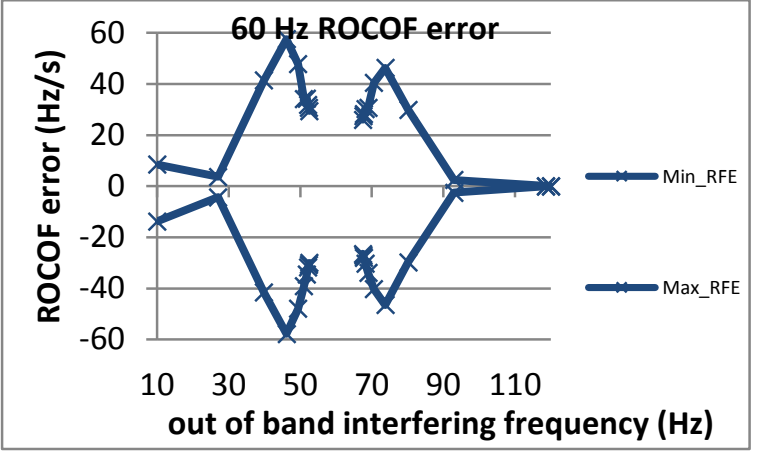

Figure 2064: Fs = $15 \mathrm{FPS}, 60 \mathrm{~Hz}$ fundamental

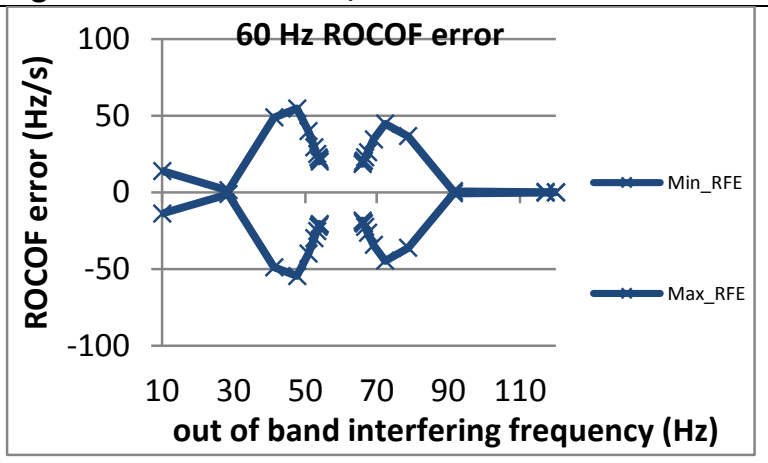

Figure 2067: Fs = 12 FPS, $60 \mathrm{~Hz}$ fundamental

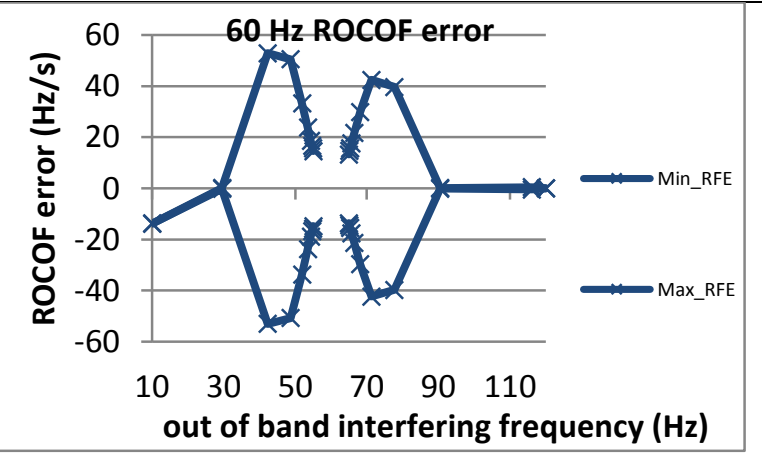

Figure 2070: Fs = $10 \mathrm{FPS}, 60 \mathrm{~Hz}$ fundamental

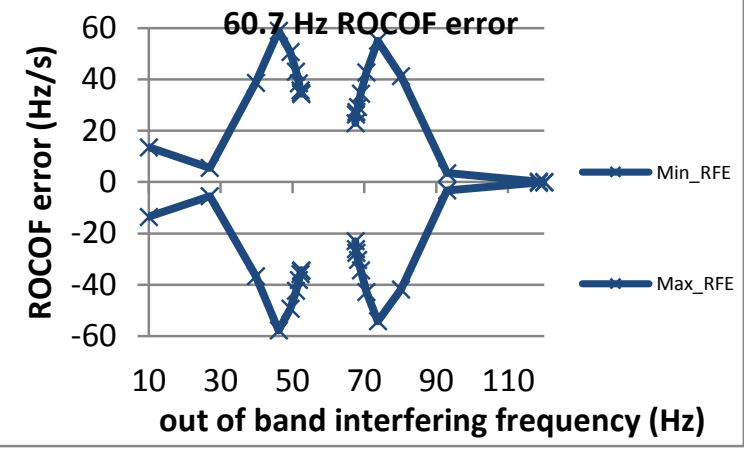

Figure 2065: Fs = $15 \mathrm{FPS}, 60.7 \mathrm{~Hz}$ fundamental

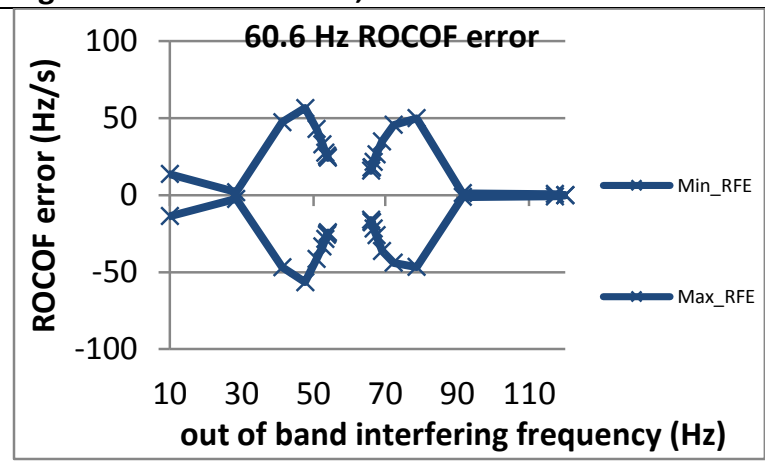

Figure 2068: Fs = $12 \mathrm{FPS}, 60.6 \mathrm{~Hz}$ fundamental

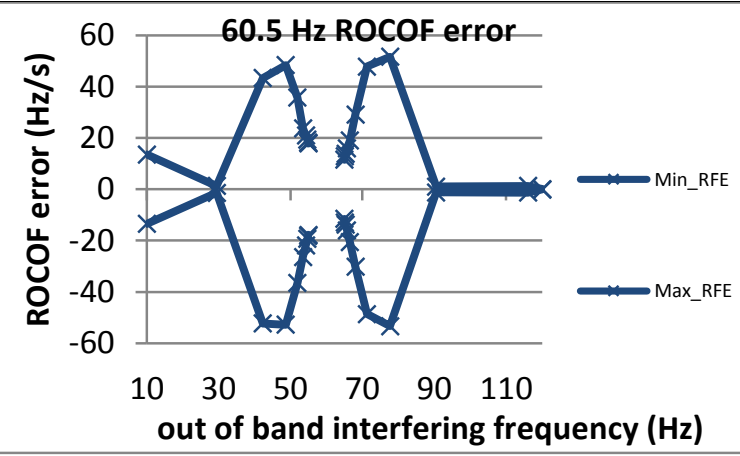

Figure 2071: Fs = 10 FPS, $60.5 \mathrm{~Hz}$ fundamental 
5.4.8 PMU G* steady state out of band interfering signals ROCOF error: M class

Figure 2072: Fs $=60$ FPS is not supported by this PMU
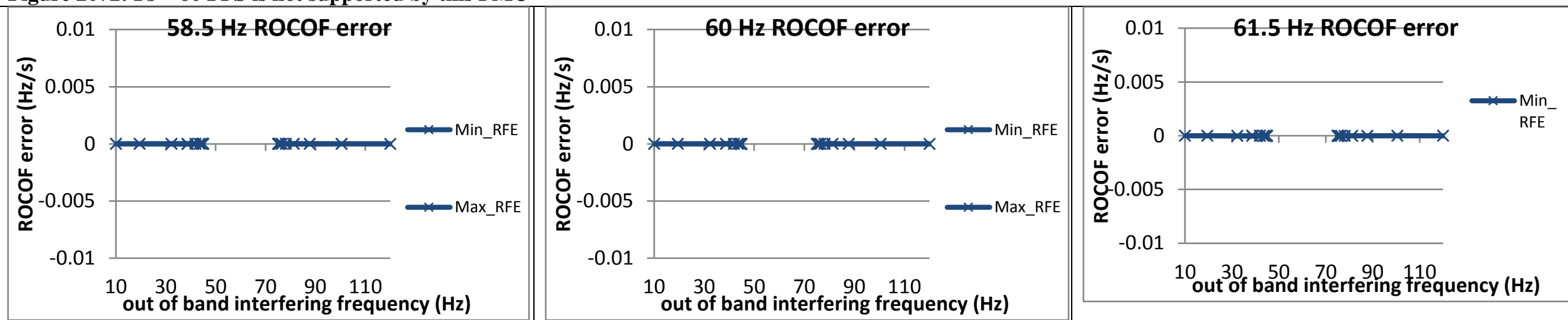

Figure 2073; Fs = 30 FPS, 58.5 Hz fundamental

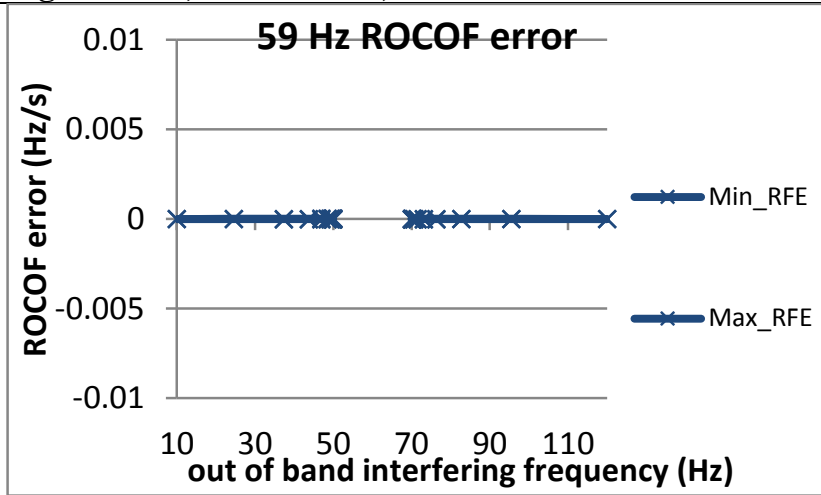

Figure 2074: Fs = $30 \mathrm{FPS}, 60 \mathrm{~Hz}$ fundamental

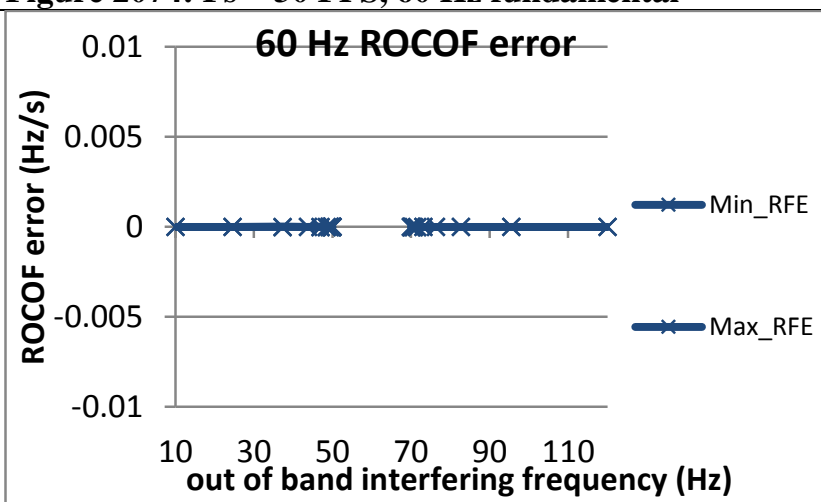

Figure 2075: Fs = 30 FPS, $61.5 \mathrm{~Hz}$ fundamental

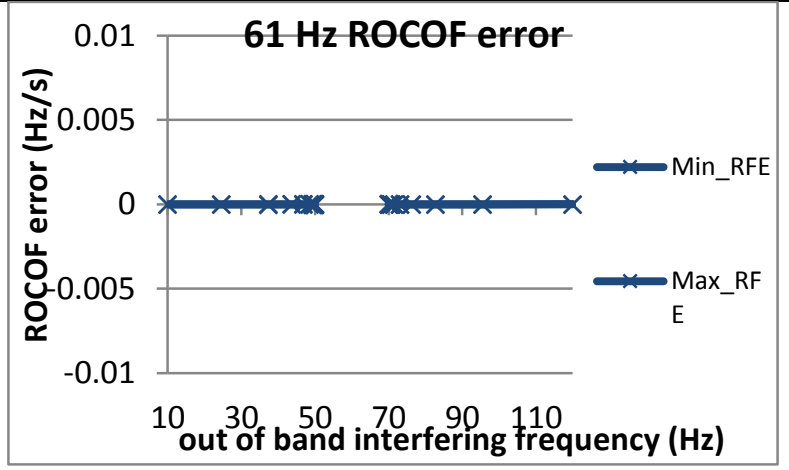

Figure 2076: Fs = 20 FPS, $59 \mathrm{~Hz}$ fundamental

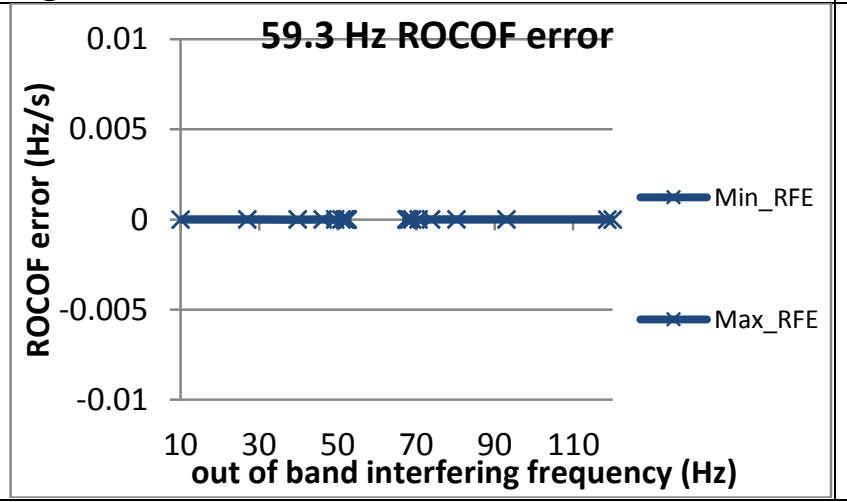

Figure 2077: Fs = 20 FPS, $60 \mathrm{~Hz}$ fundamental

Figure 2078: Fs = 20 FPS, $61 \mathrm{~Hz}$ fundamental
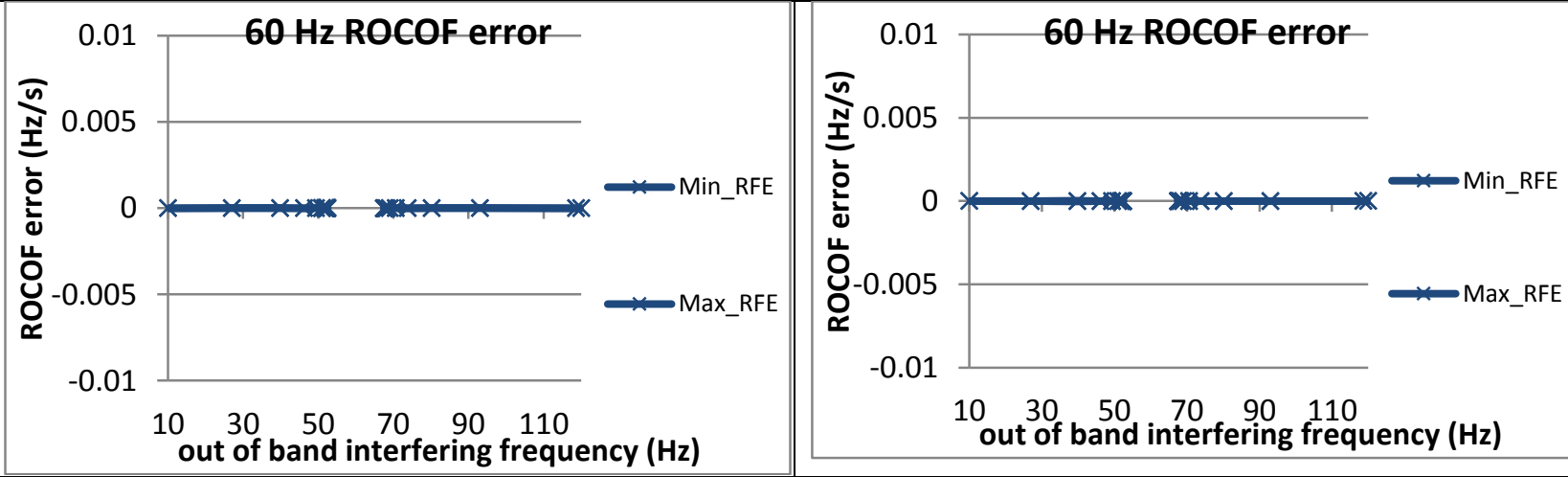


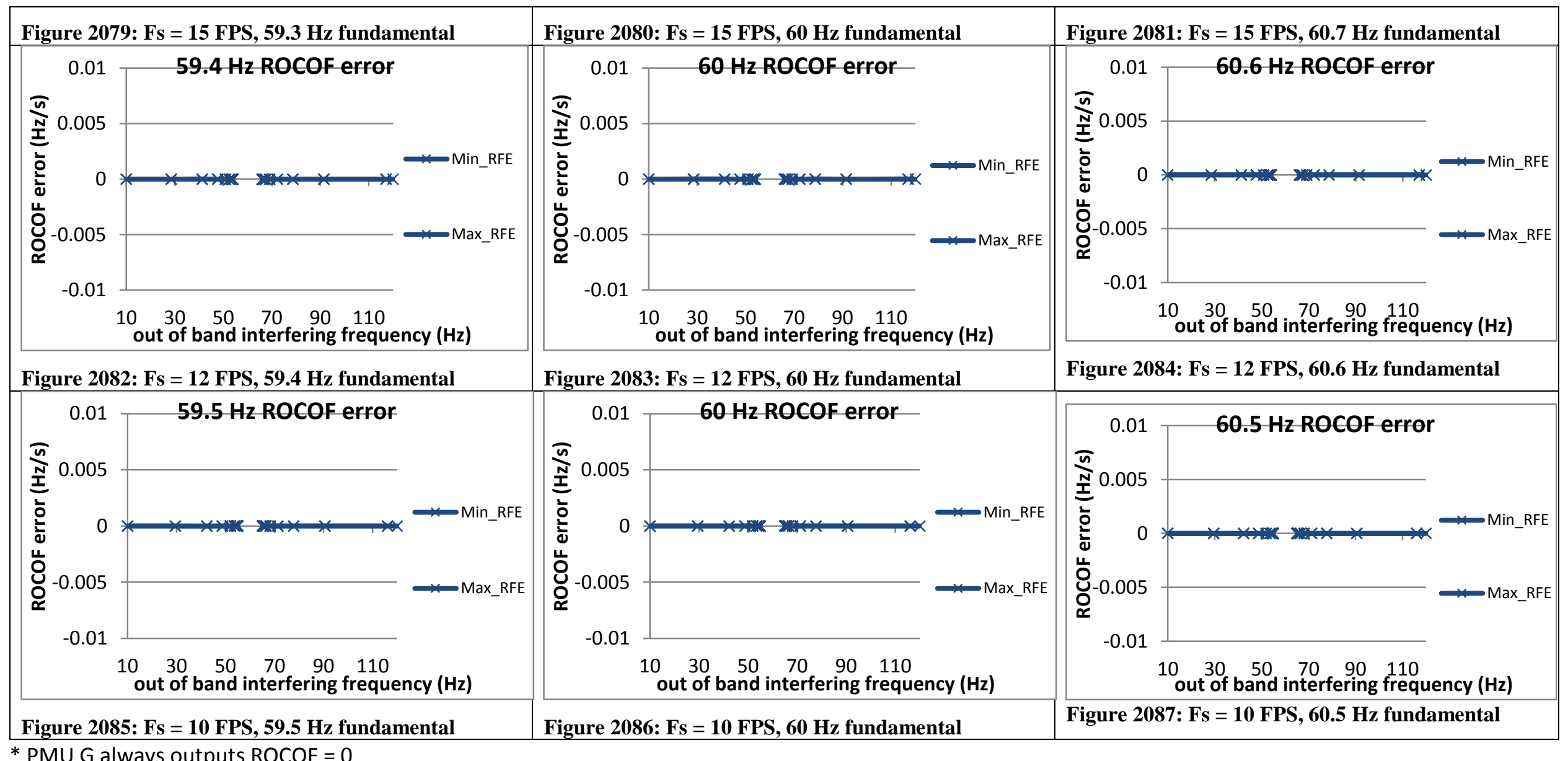


5.4.9 PMU H steady state out of band interfering signals ROCOF error: M class

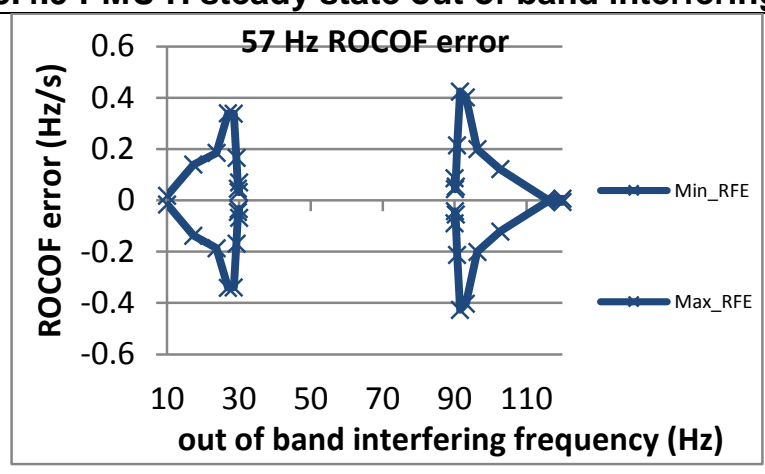

Figure 2088: Fs = 60 FPS, $57 \mathrm{~Hz}$ fundamental

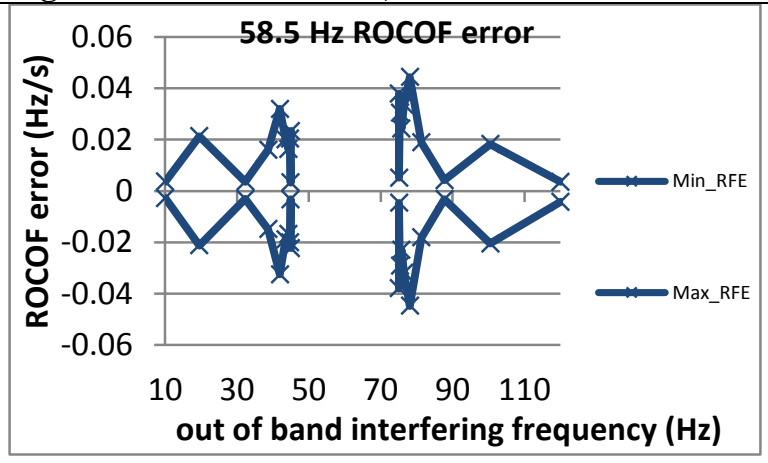

Figure 2091; Fs = 30 FPS, 58.5 Hz fundamental

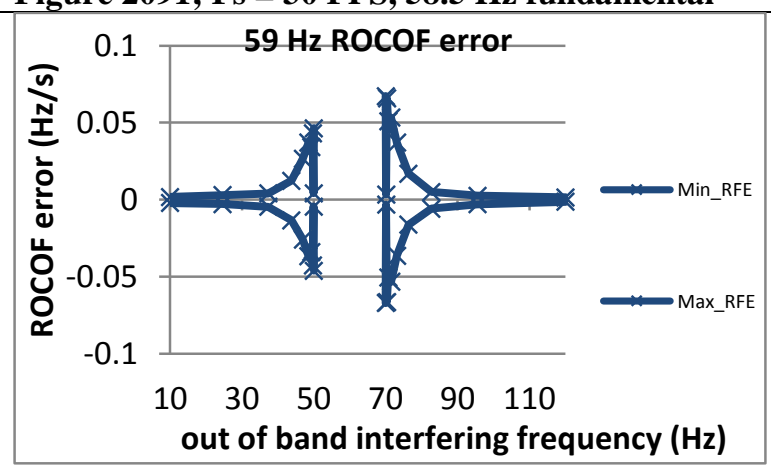

Figure 2094: Fs = 20 FPS, $59 \mathrm{~Hz}$ fundamental

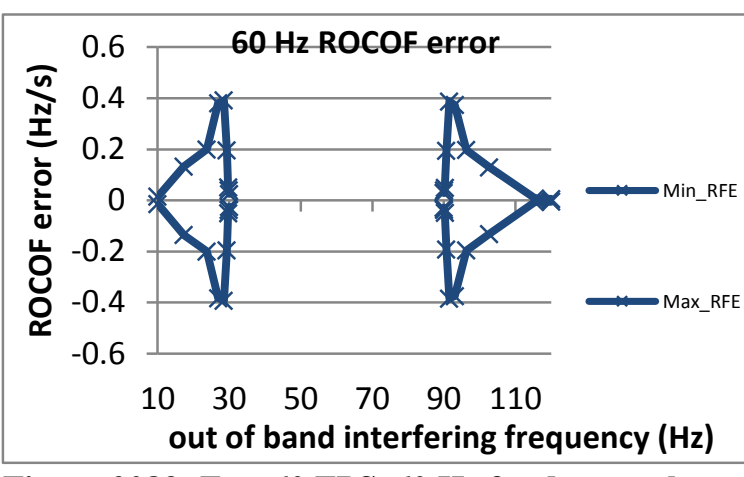

Figure 2089: Fs = 60 FPS, $60 \mathrm{~Hz}$ fundamental

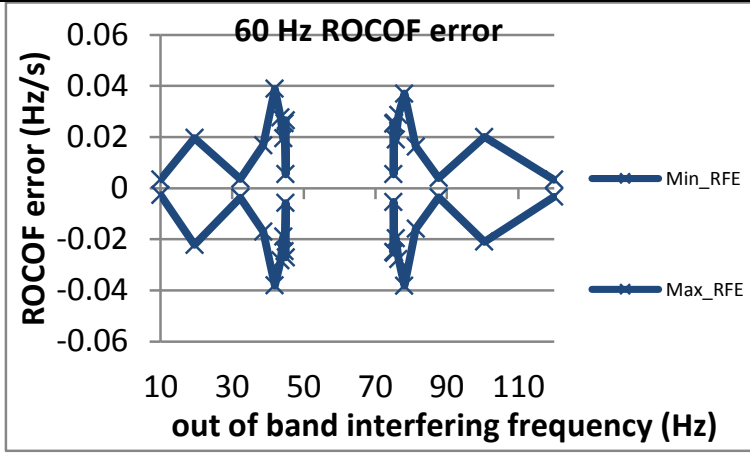

Figure 2092: Fs = $30 \mathrm{FPS}, 60 \mathrm{~Hz}$ fundamental

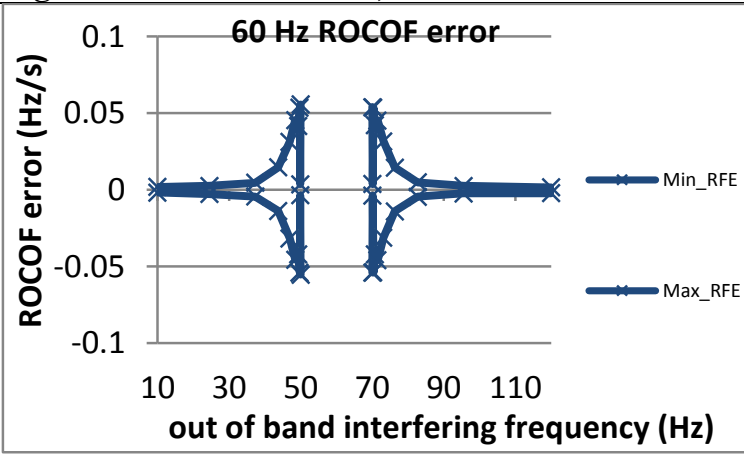

Figure 2095: Fs = $20 \mathrm{FPS}, 60 \mathrm{~Hz}$ fundamental

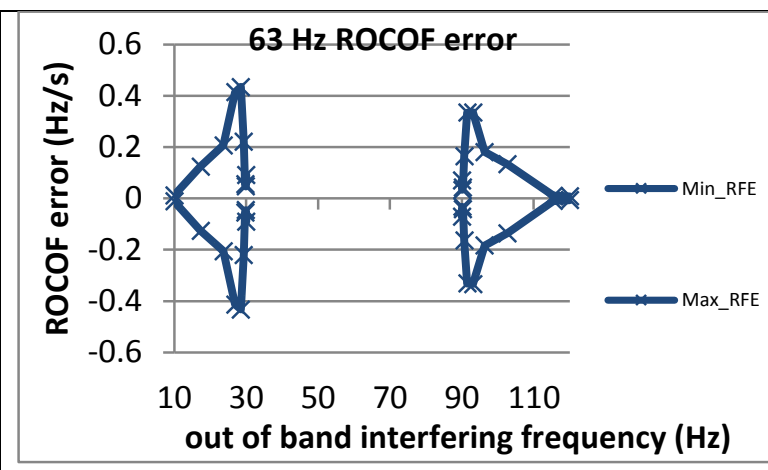

Figure 2090: Fs = 60 FPS, $63 \mathrm{~Hz}$ fundamental

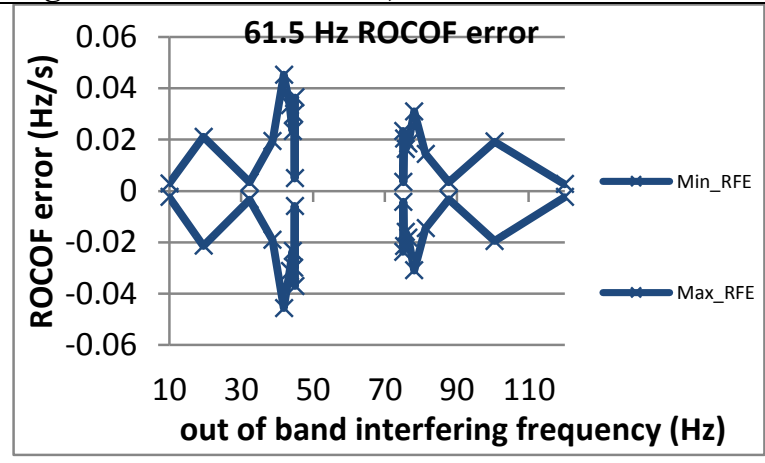

Figure 2093: Fs = 30 FPS, $61.5 \mathrm{~Hz}$ fundamental

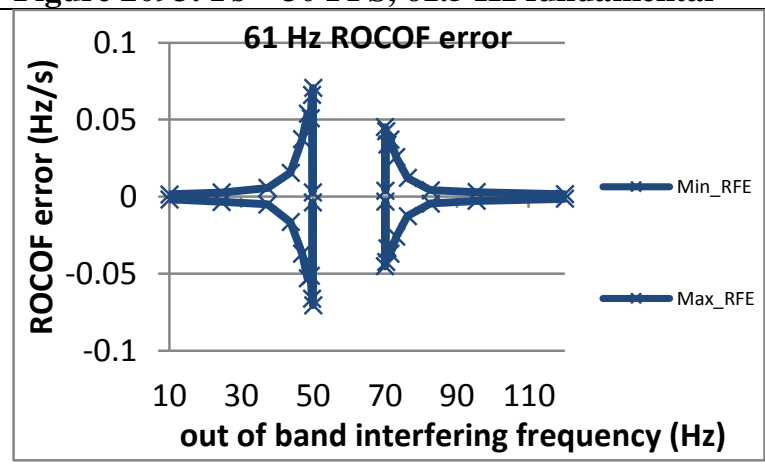

Figure 2096: Fs = 20 FPS, $61 \mathrm{~Hz}$ fundamental 


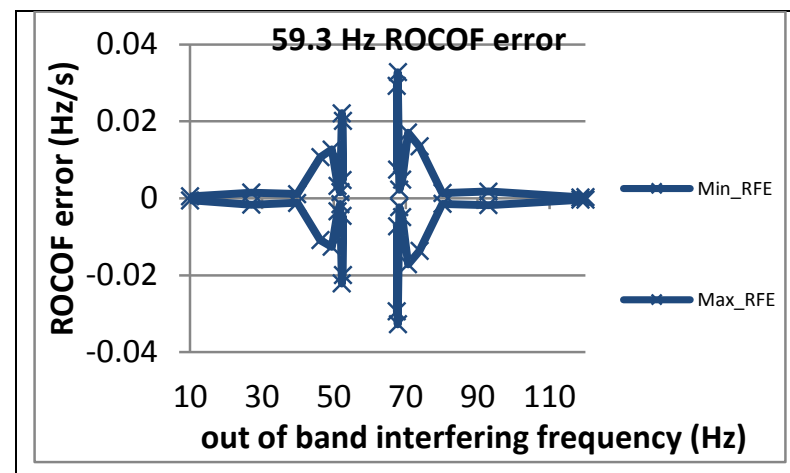

Figure 2097: Fs = 15 FPS, $59.3 \mathrm{~Hz}$ fundamental

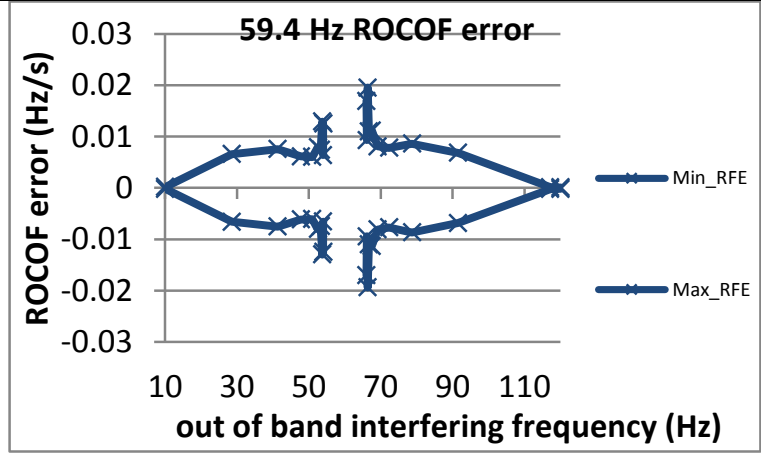

Figure 2100: Fs = 12 FPS, $59.4 \mathrm{~Hz}$ fundamental

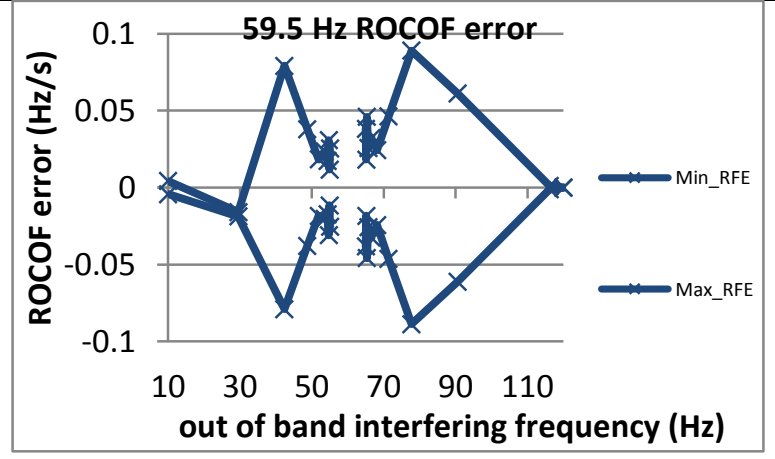

Figure 2103: Fs = 10 FPS, $59.5 \mathrm{~Hz}$ fundamental

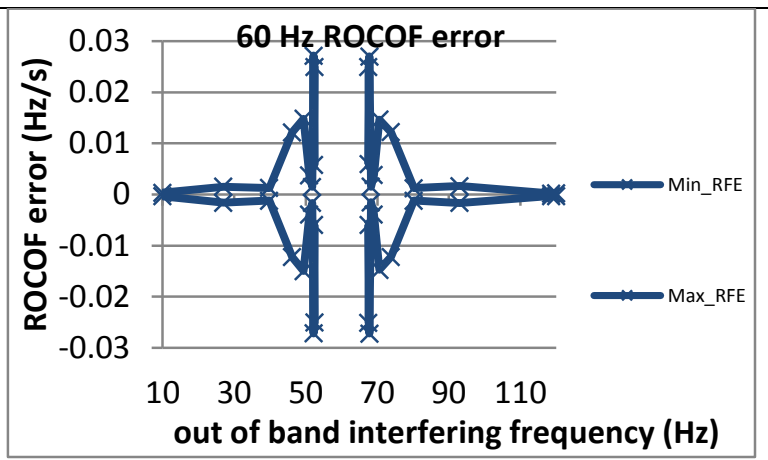

Figure 2098: Fs = 15 FPS, $60 \mathrm{~Hz}$ fundamental

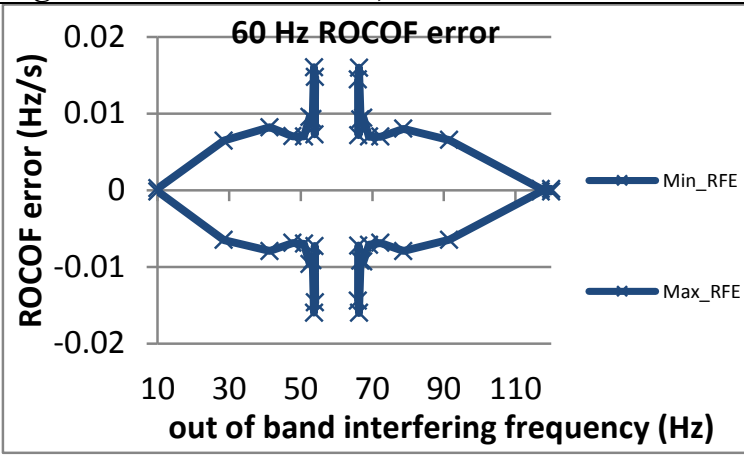

Figure 2101: Fs = $12 \mathrm{FPS}, 60 \mathrm{~Hz}$ fundamental

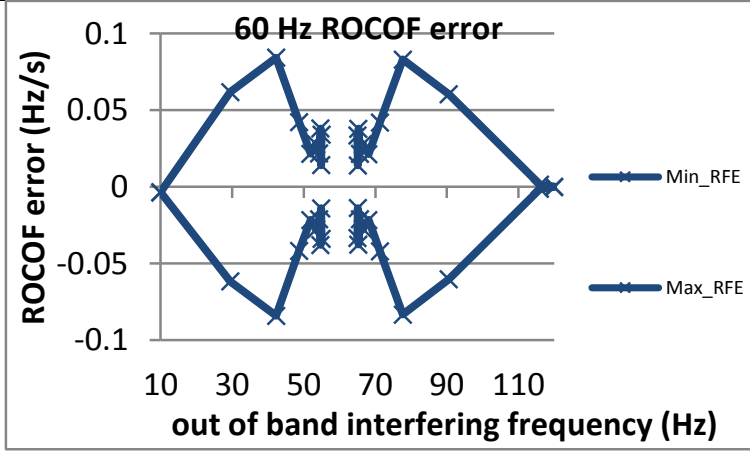

Figure 2104: Fs = 10 FPS, $60 \mathrm{~Hz}$ fundamental

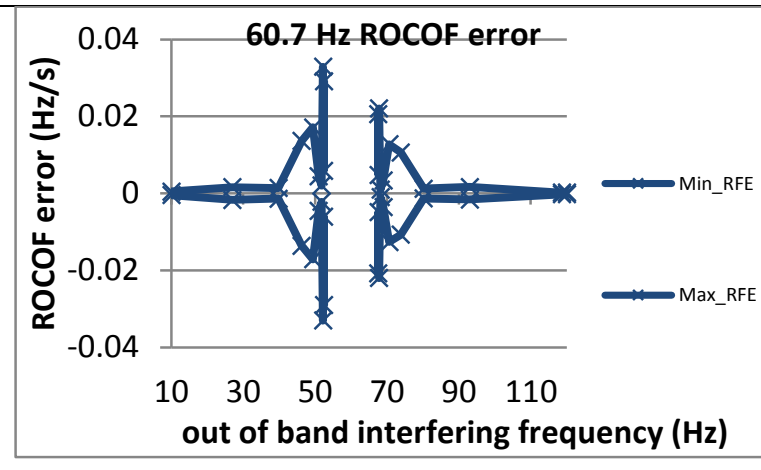

Figure 2099: Fs = 15 FPS, $60.7 \mathrm{~Hz}$ fundamental

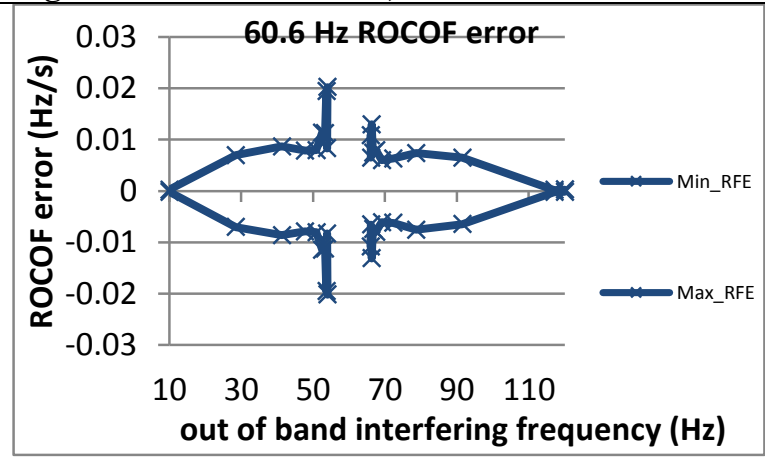

Figure 2102: Fs = 12 FPS, $60.6 \mathrm{~Hz}$ fundamental

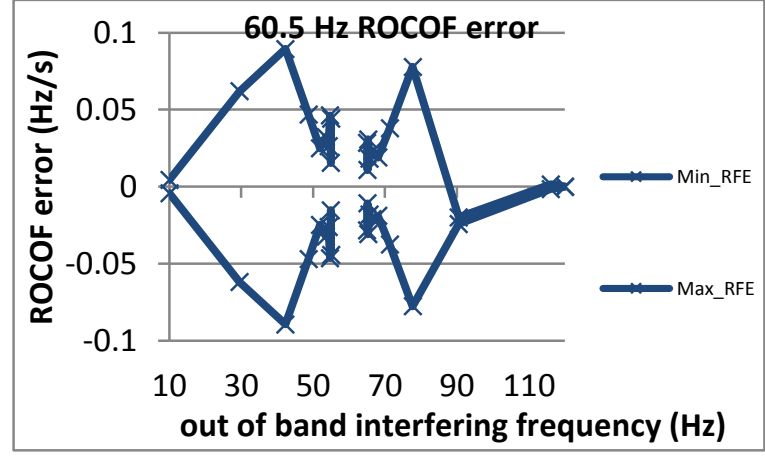

Figure 2105: Fs = $10 \mathrm{FPS}, 60.5 \mathrm{~Hz}$ fundamental 
5.4.10 PMU I steady state out of band interfering signals ROCOF error: M class

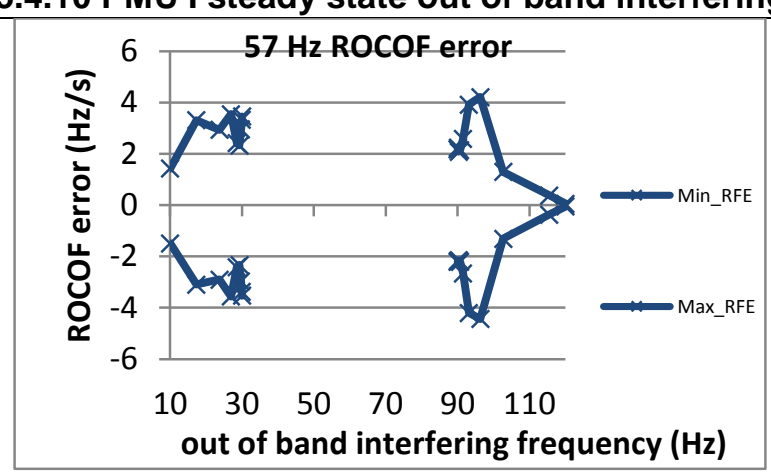

Figure 2106: Fs = 60 FPS, $57 \mathrm{~Hz}$ fundamental

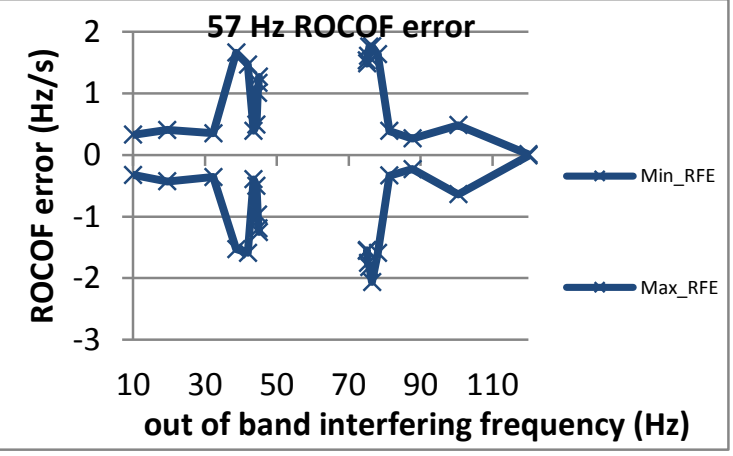

Figure 2109; Fs = 30 FPS, $58.5 \mathrm{~Hz}$ fundamental

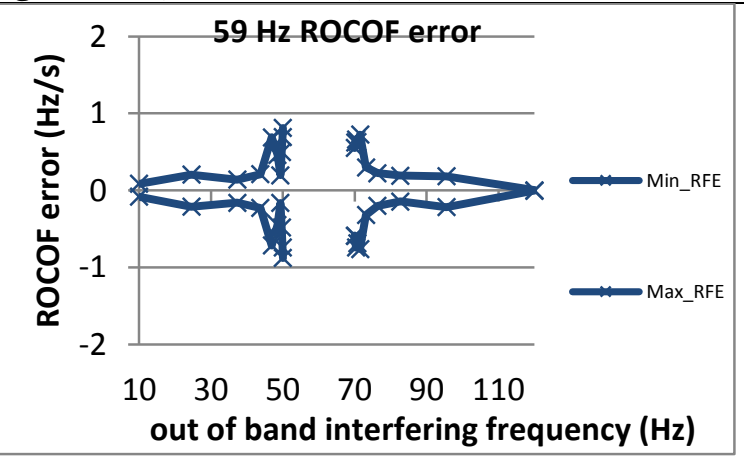

Figure 2112: Fs = 20 FPS, $59 \mathrm{~Hz}$ fundamental

Figure 2115: Fs = 15 FPS, $59.3 \mathrm{~Hz}$ fundamental

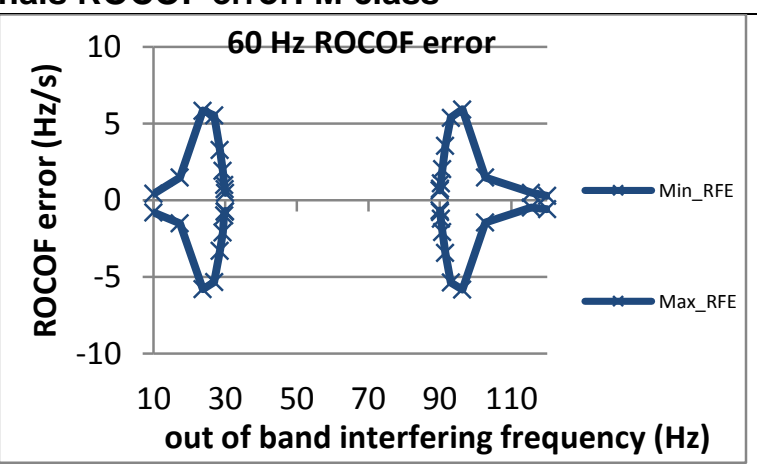

Figure 2107: Fs = $60 \mathrm{FPS}, 60 \mathrm{~Hz}$ fundamental

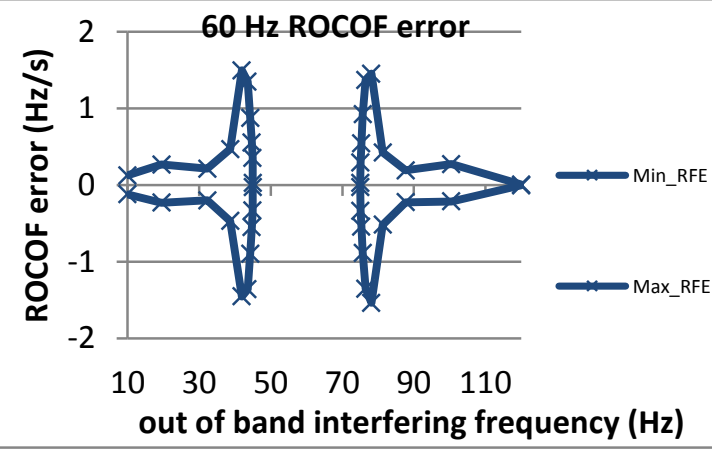

Figure 2110: Fs = $30 \mathrm{FPS}, 60 \mathrm{~Hz}$ fundamental

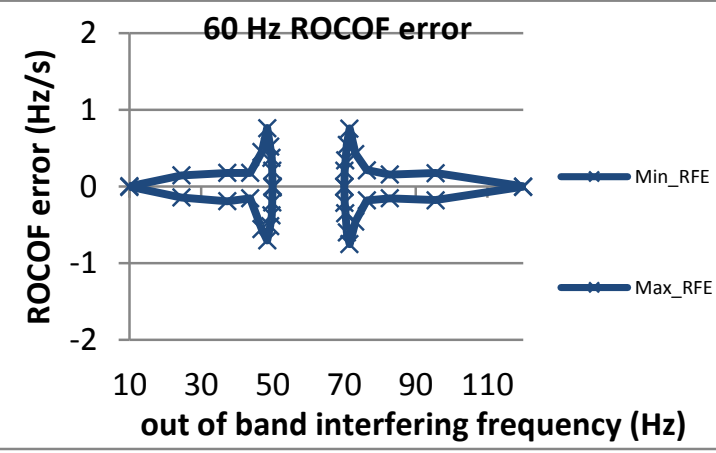

Figure 2113: Fs = $20 \mathrm{FPS}, 60 \mathrm{~Hz}$ fundamental

Figure 2116: Fs = 15 FPS, $60 \mathrm{~Hz}$ fundamental

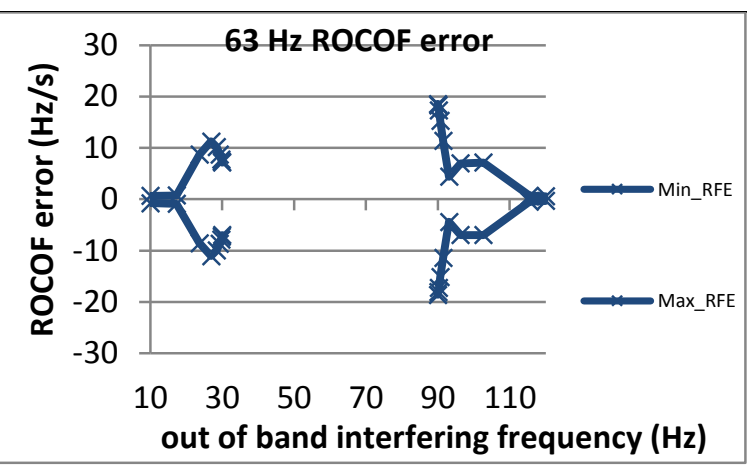

Figure 2108: Fs = $60 \mathrm{FPS}, 63 \mathrm{~Hz}$ fundamental

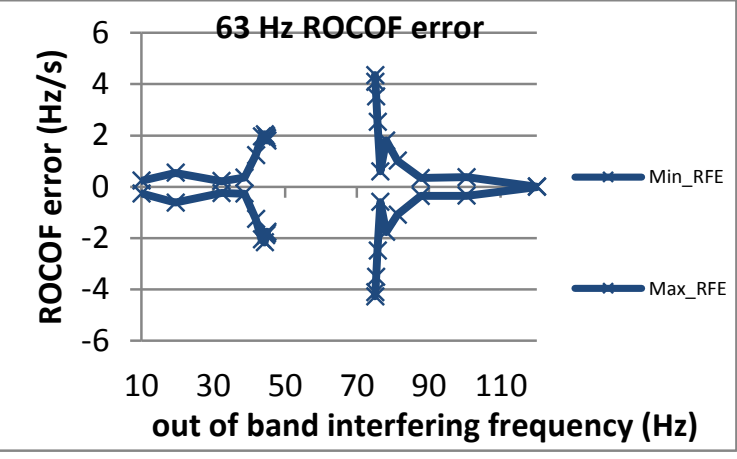

Figure 2111: Fs = 30 FPS, $61.5 \mathrm{~Hz}$ fundamental

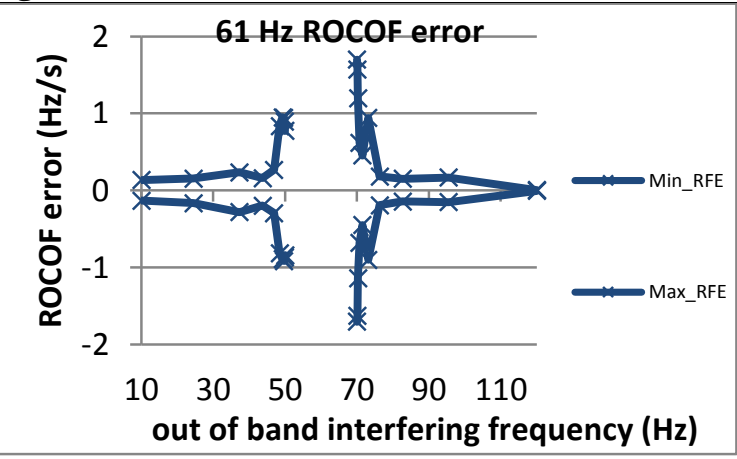

Figure 2114: Fs = 20 FPS, $61 \mathrm{~Hz}$ fundamental

Figure 2117: Fs = 15 FPS, $60.7 \mathrm{~Hz}$ fundamental 


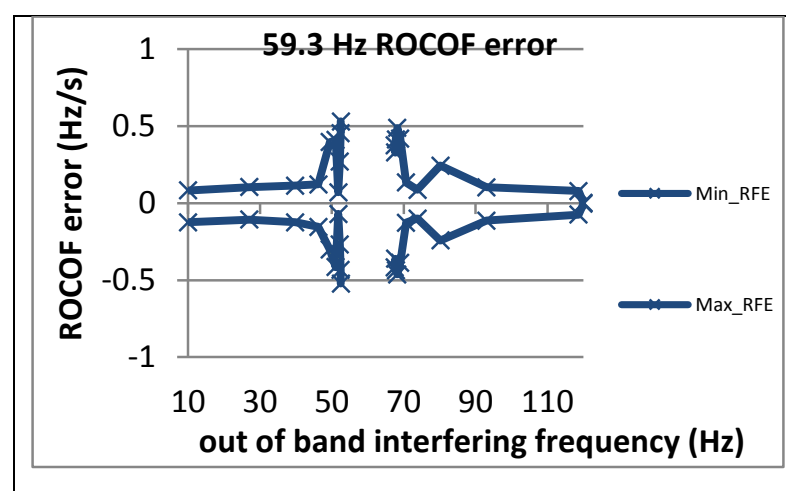

Figure 2118: Fs = 12 FPS, $59.4 \mathrm{~Hz}$ fundamental

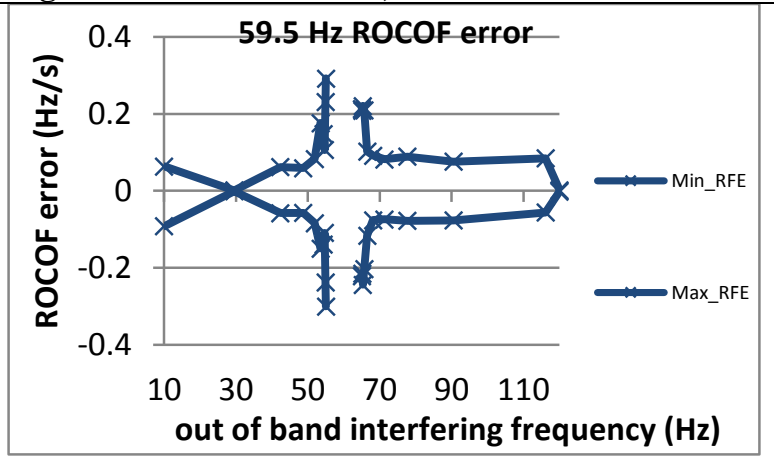

Figure 2121: Fs = 10 FPS, $59.5 \mathrm{~Hz}$ fundamental

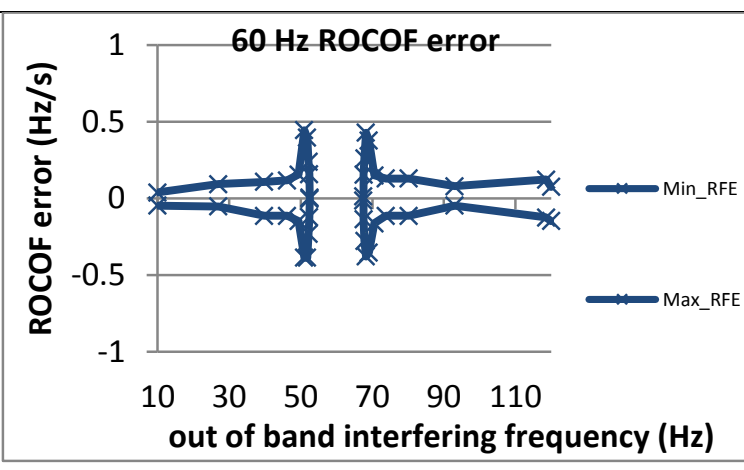

Figure 2119: Fs = 12 FPS, $60 \mathrm{~Hz}$ fundamental

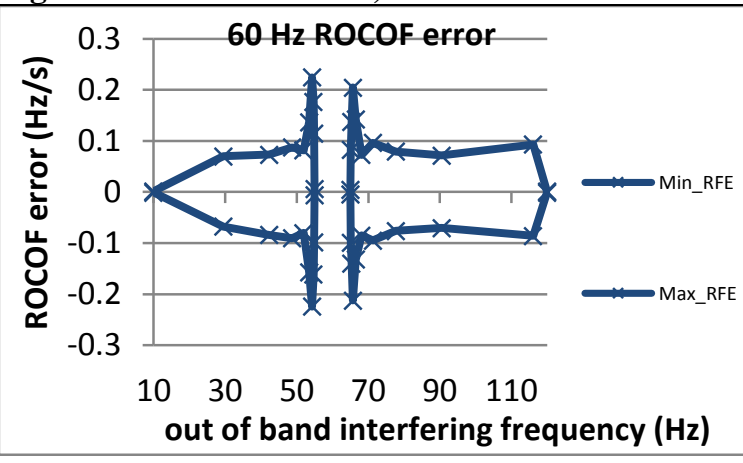

Figure 2122: Fs = $10 \mathrm{FPS}, 60 \mathrm{~Hz}$ fundamental

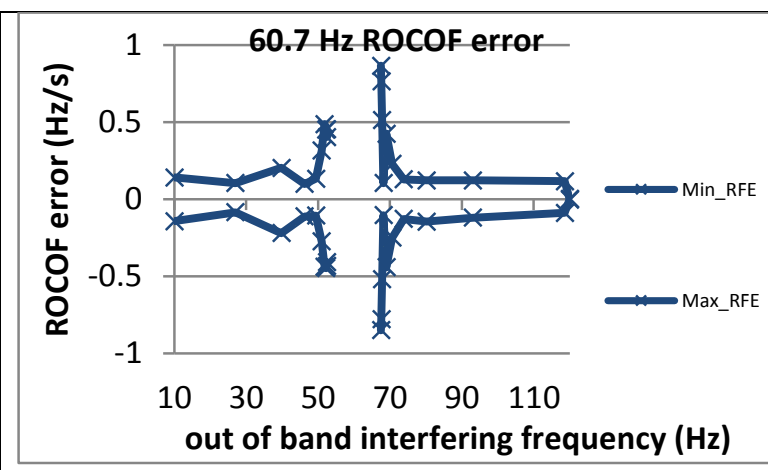

Figure 2120: Fs = 12 FPS, $60.6 \mathrm{~Hz}$ fundamental

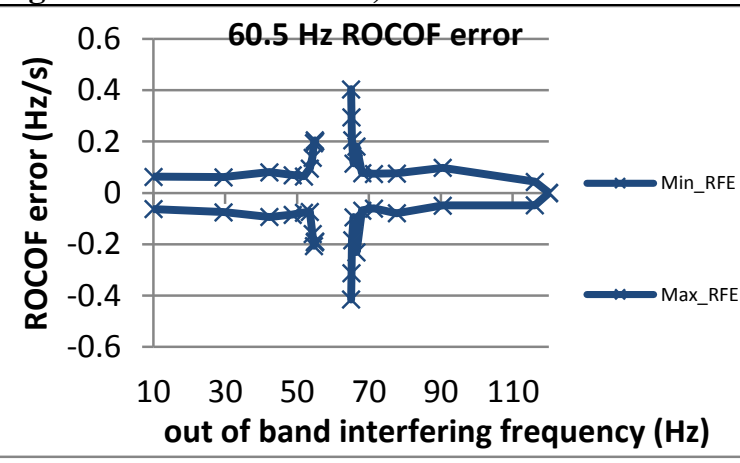

Figure 2123: Fs = 10 FPS, $60.5 \mathrm{~Hz}$ fundamental 
5.4.11 PMU J steady state out of band interfering signals ROCOF error: M class

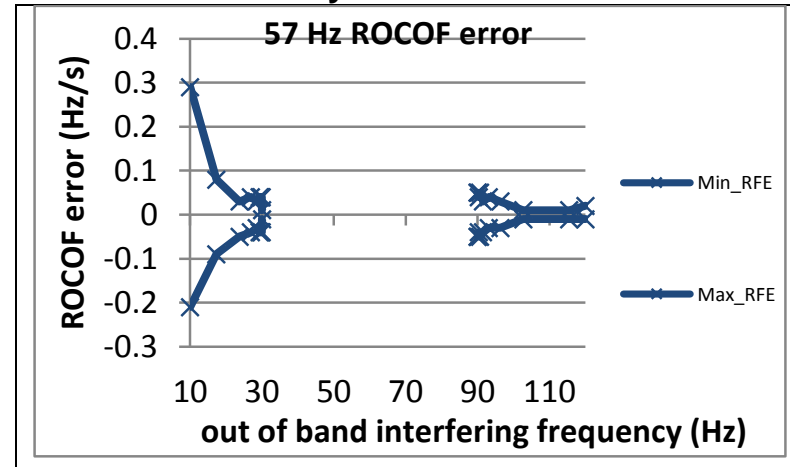

Figure 2124: $\mathrm{Fs}=60 \mathrm{FPS}, 57 \mathrm{~Hz}$ fundamental

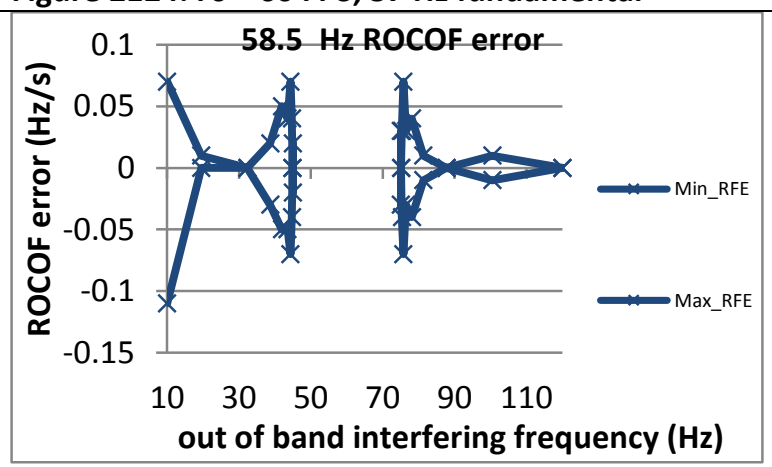

Figure 2127; Fs = $30 \mathrm{FPS}, 58.5 \mathrm{~Hz}$ fundamental

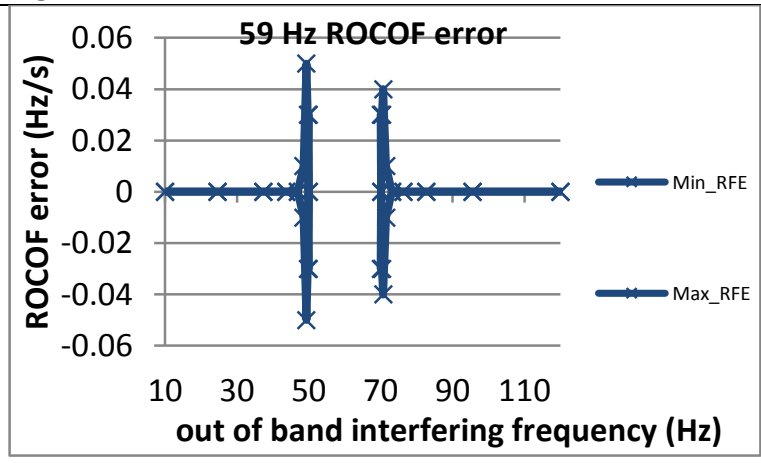

Figure 2130: Fs $=20 \mathrm{FPS}, 59 \mathrm{~Hz}$ fundamental

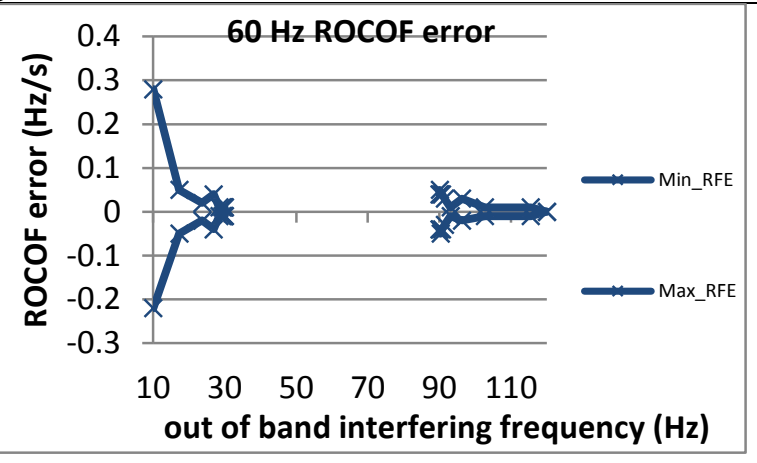

Figure 2125: $\mathrm{Fs}=60 \mathrm{FPS}, 60 \mathrm{~Hz}$ fundamental

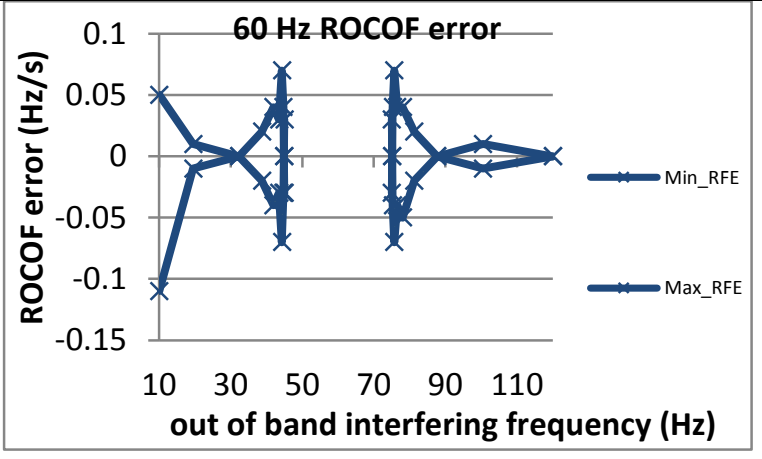

Figure 2128: $\mathrm{Fs}=30 \mathrm{FPS}, 60 \mathrm{~Hz}$ fundamental

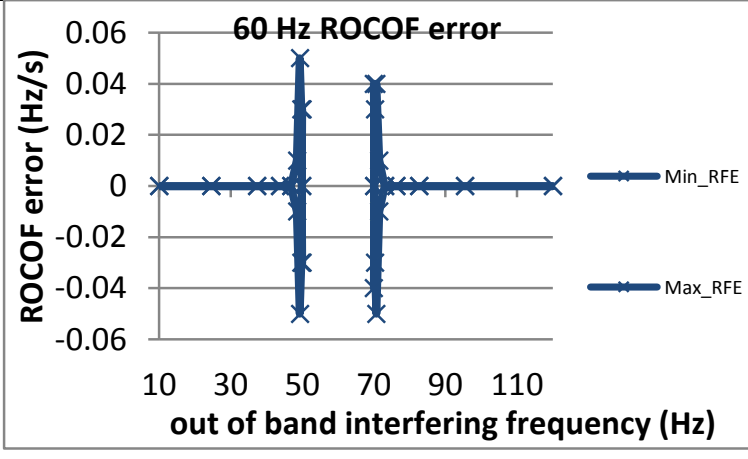

Figure 2131: Fs = $20 \mathrm{FPS}, 60 \mathrm{~Hz}$ fundamental

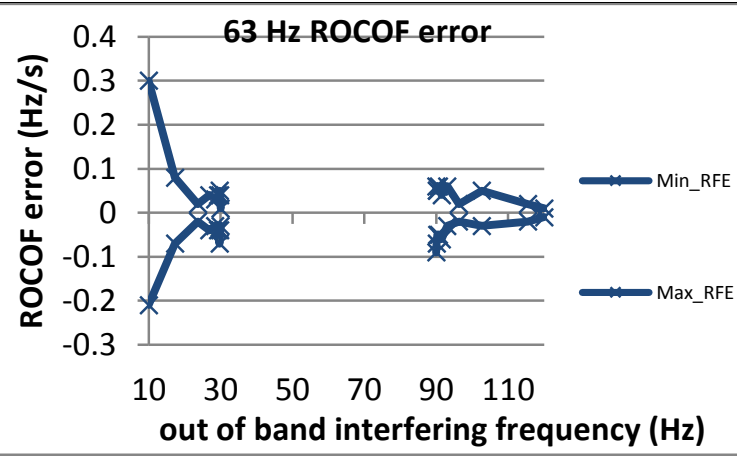

Figure 2126: Fs = $60 \mathrm{FPS}, 63 \mathrm{~Hz}$ fundamental

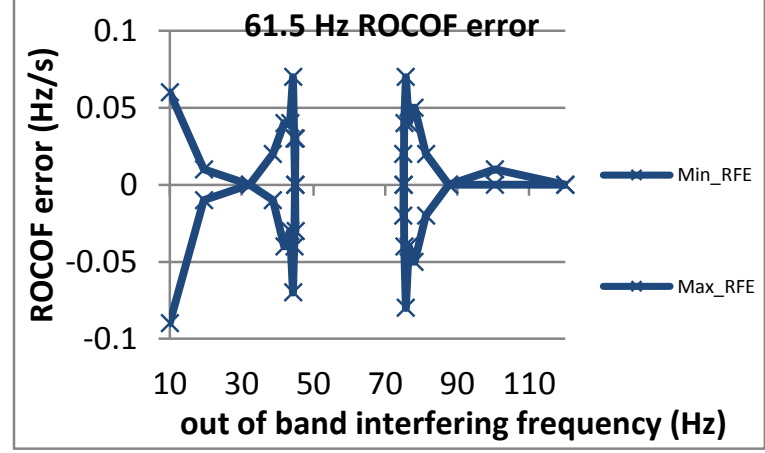

Figure 2129: $\mathrm{Fs}=30 \mathrm{FPS}, 61.5 \mathrm{~Hz}$ fundamental

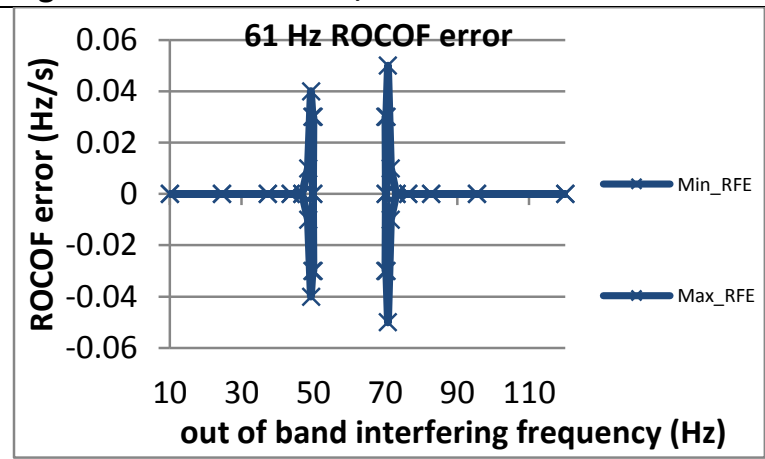

Figure 2132: Fs = $20 \mathrm{FPS}, 61 \mathrm{~Hz}$ fundamental 


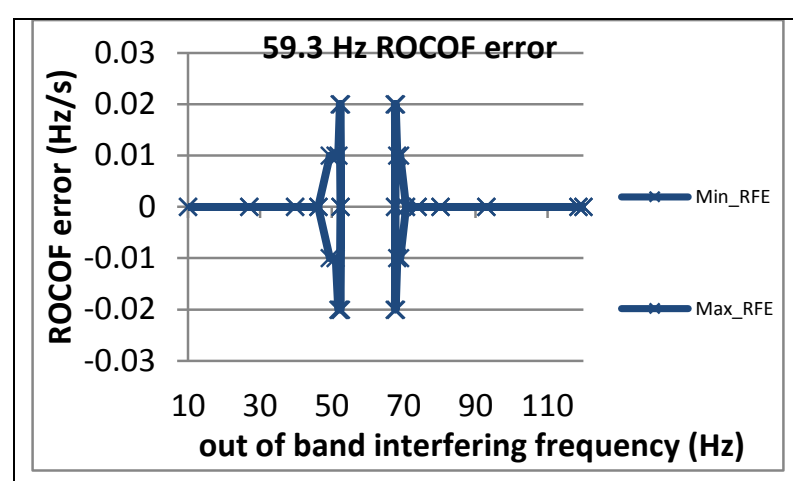

Figure 2133: $\mathrm{Fs}=15 \mathrm{FPS}, 59.3 \mathrm{~Hz}$ fundamental

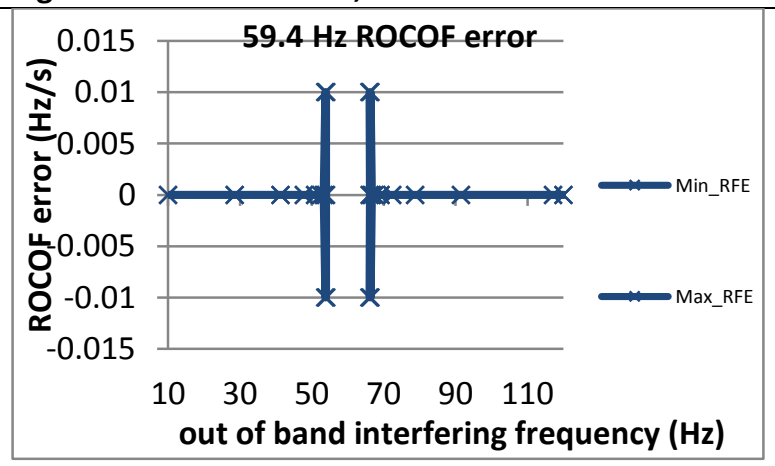

Figure 2136: Fs = $12 \mathrm{FPS}, 59.4 \mathrm{~Hz}$ fundamental

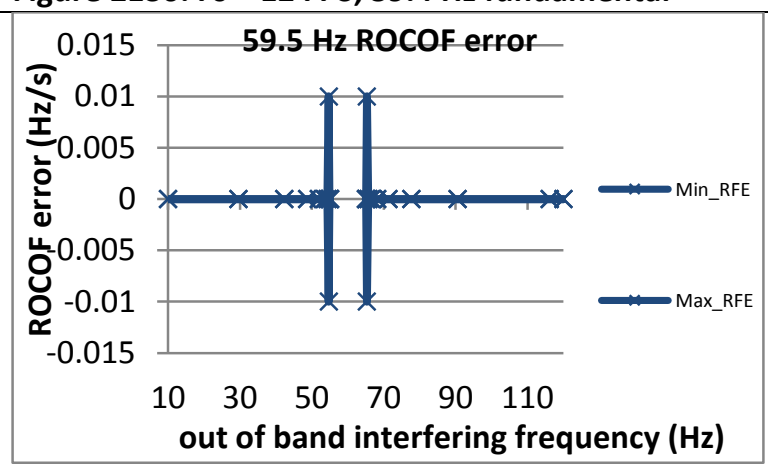

Figure 2139: Fs = $10 \mathrm{FPS}, 59.5 \mathrm{~Hz}$ fundamental

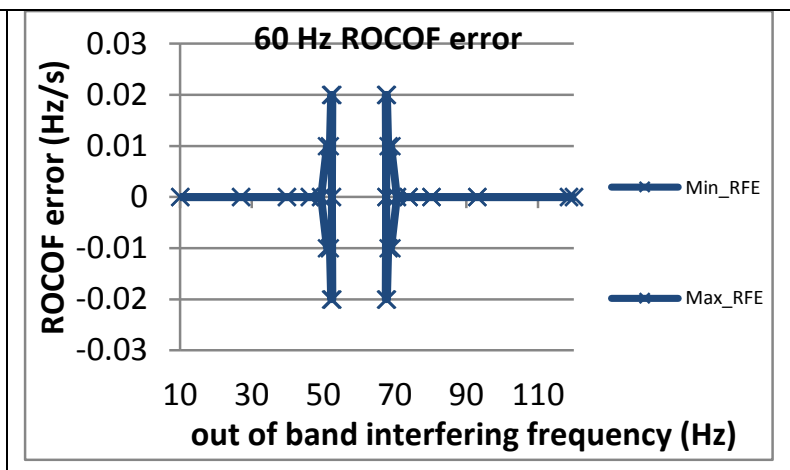

Figure 2134: Fs = $15 \mathrm{FPS}, 60 \mathrm{~Hz}$ fundamental

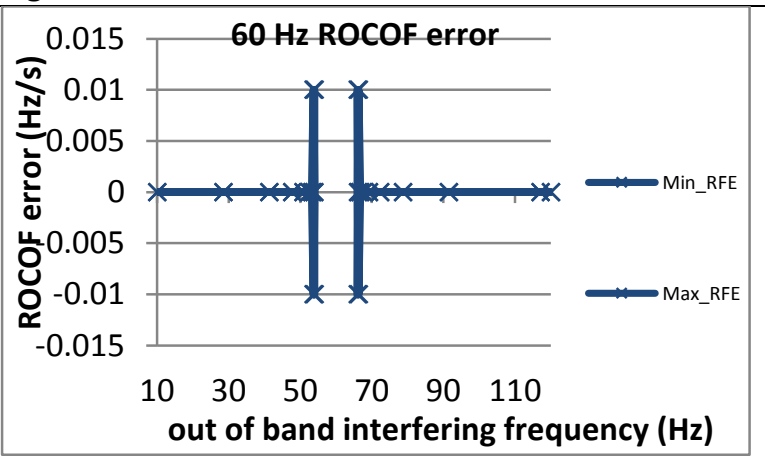

Figure 2137: Fs $=12 \mathrm{FPS}, 60 \mathrm{~Hz}$ fundamental

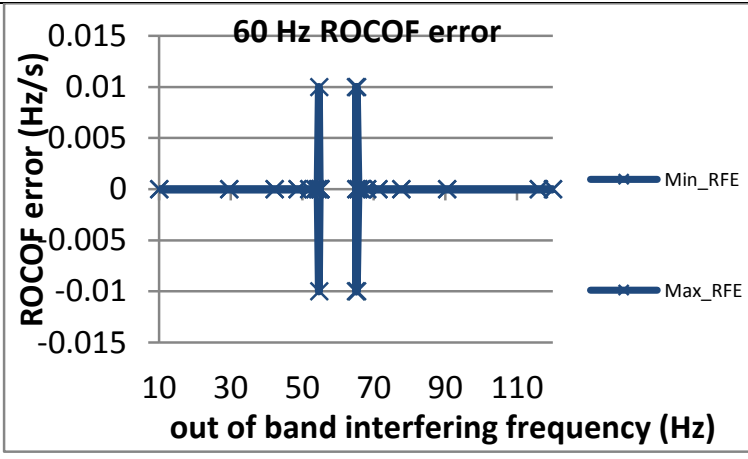

Figure 2140: Fs = $10 \mathrm{FPS}, 60 \mathrm{~Hz}$ fundamental

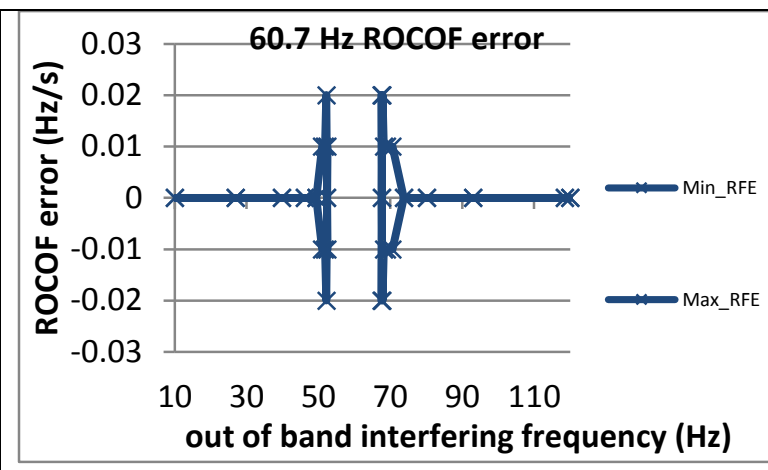

Figure 2135: $\mathrm{Fs}=15 \mathrm{FPS}, 60.7 \mathrm{~Hz}$ fundamental

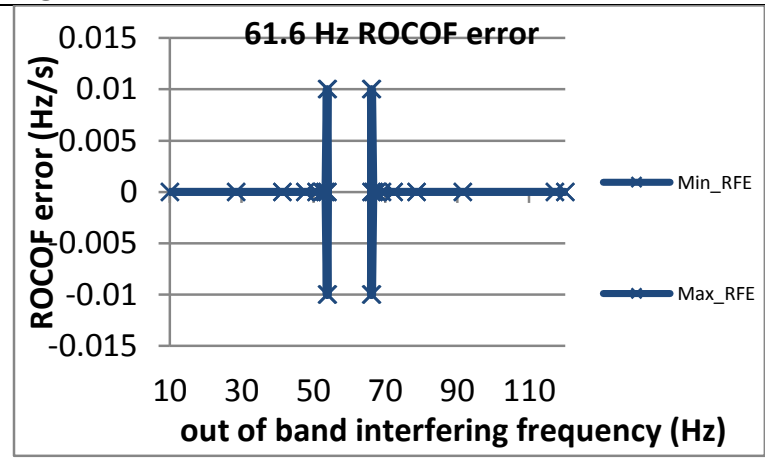

Figure 2138: $\mathrm{Fs}=12 \mathrm{FPS}, 60.6 \mathrm{~Hz}$ fundamental

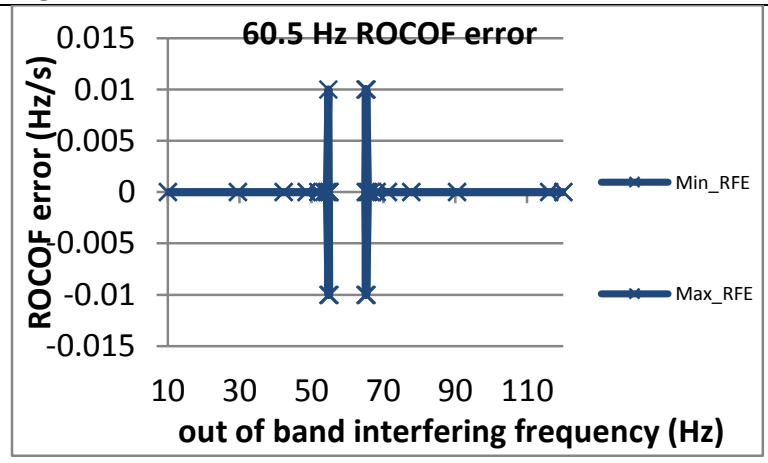

Figure 2141: Fs = $10 \mathrm{FPS}, 60.5 \mathrm{~Hz}$ fundamental 


\section{Dynamic ramp of system frequency}

\section{Table of Contents}

6. Dynamic ramp of system frequency

6.1 Dynamic ramp of system frequency voltage TVE, M class......

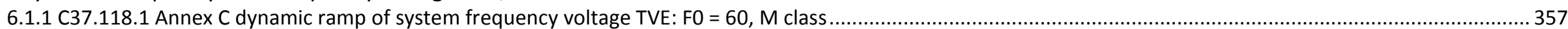

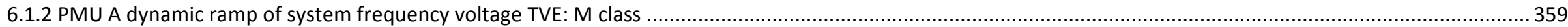

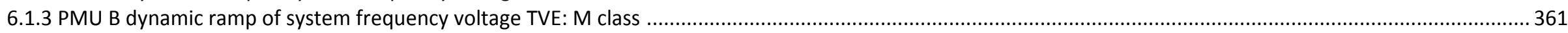

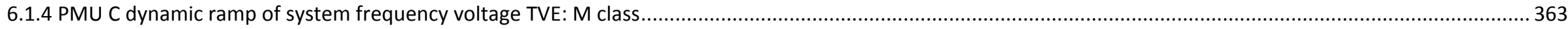

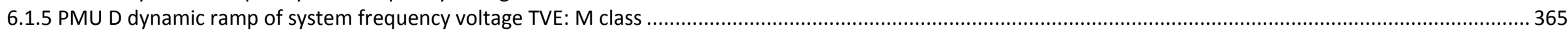

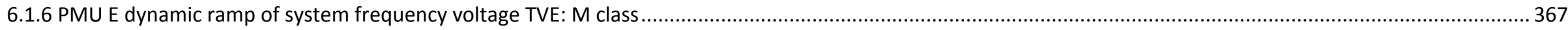

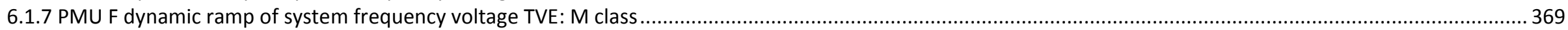

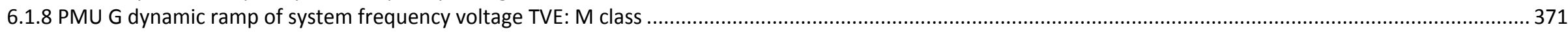

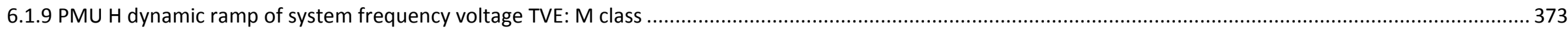

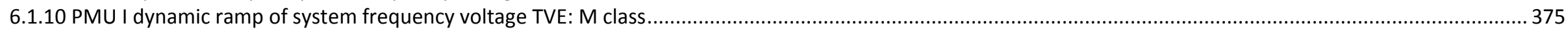

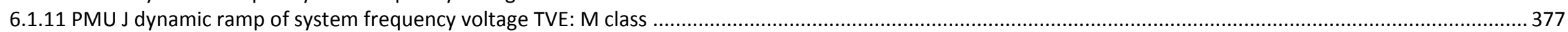

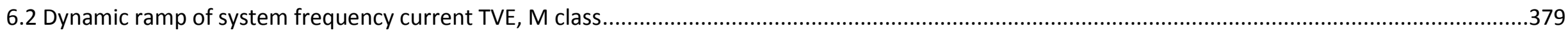

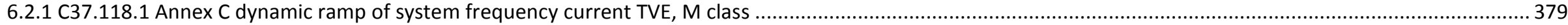

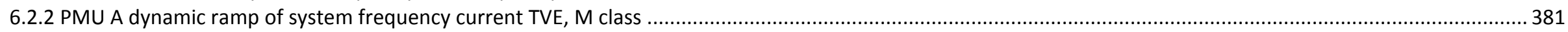

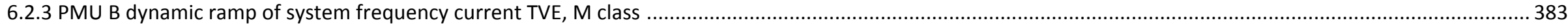

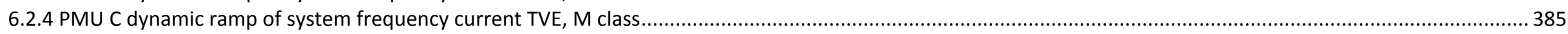

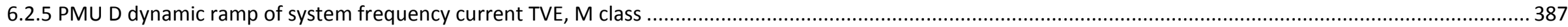

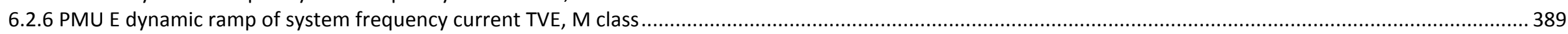

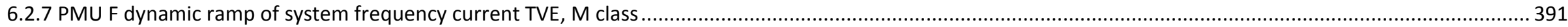

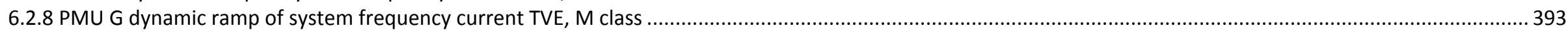

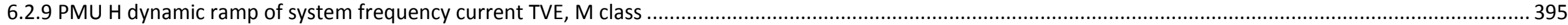

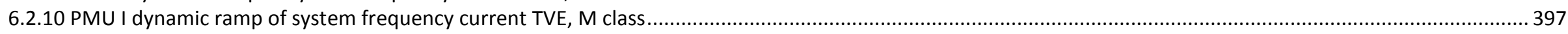

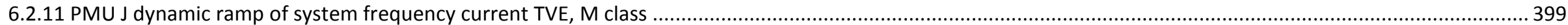

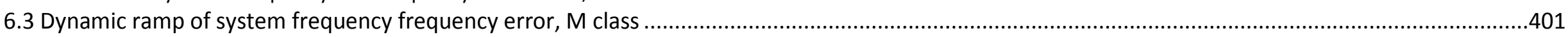

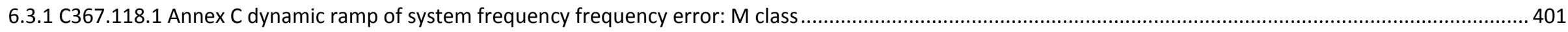

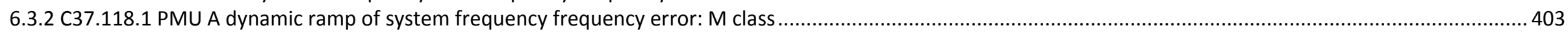

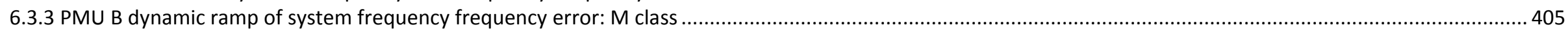

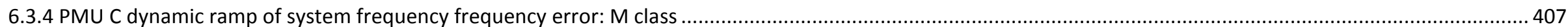

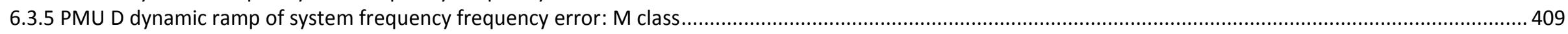

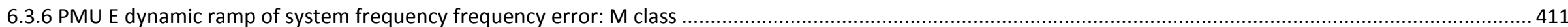

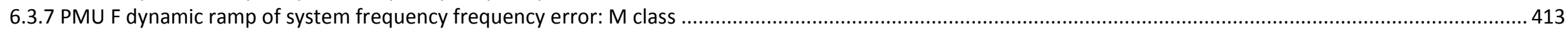


6.3.8 PMU G dynamic ramp of system frequency frequency error: $\mathrm{M}$ class.

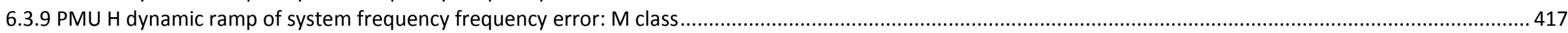

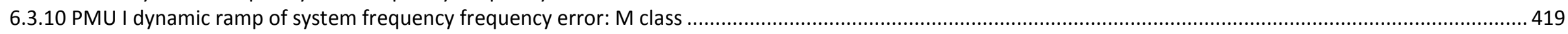

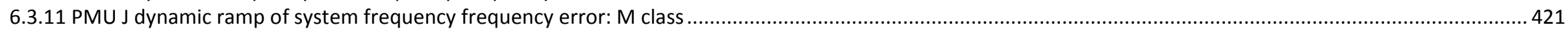

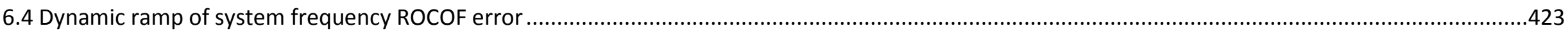

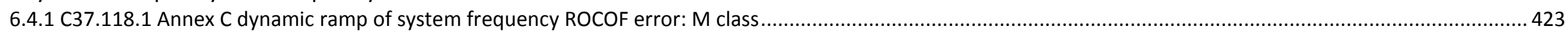

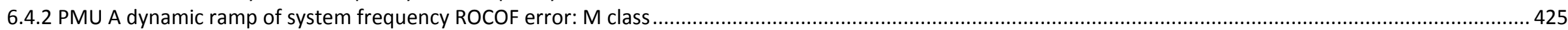

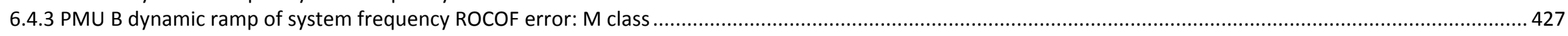

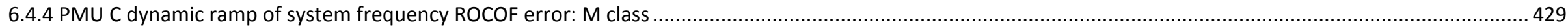

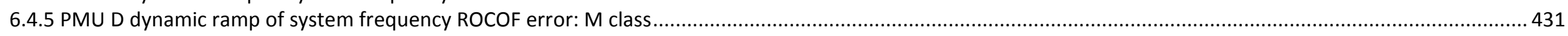

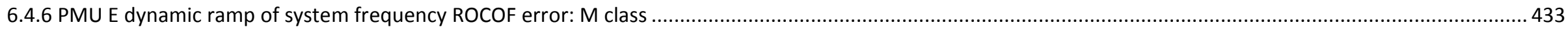

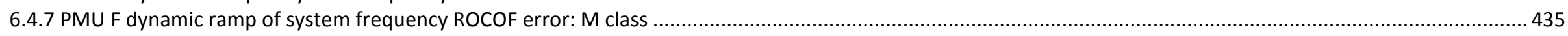

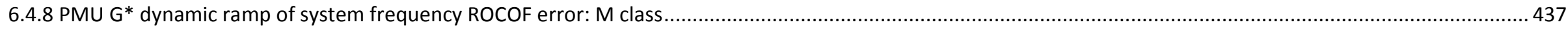

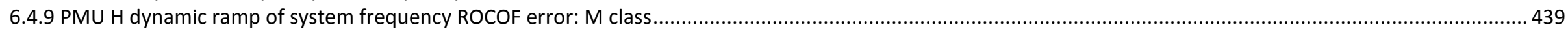

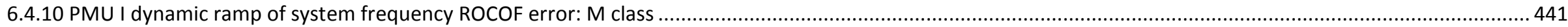

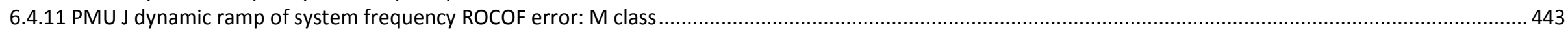

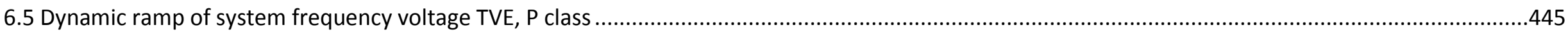

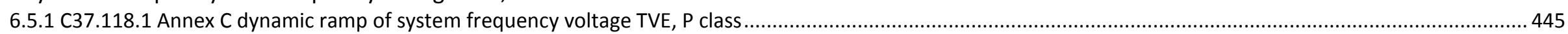

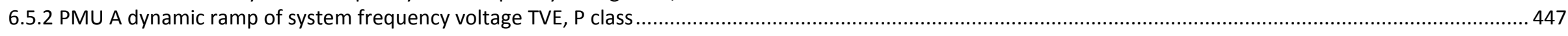

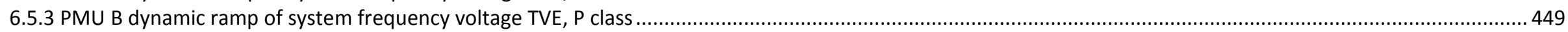

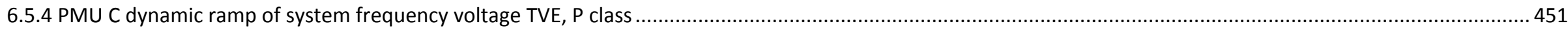

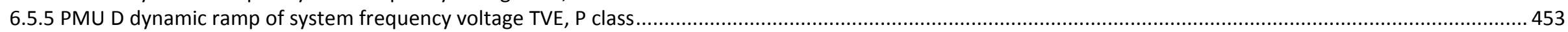

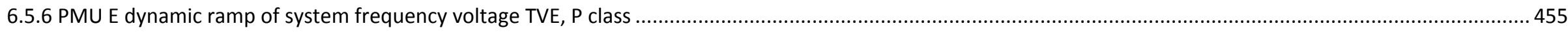

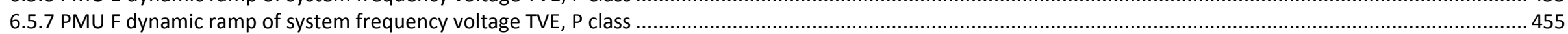

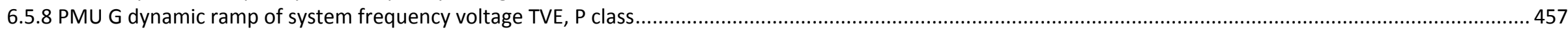

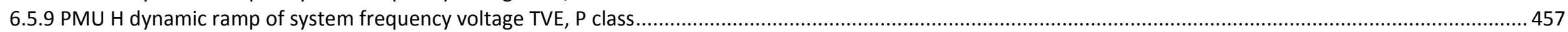

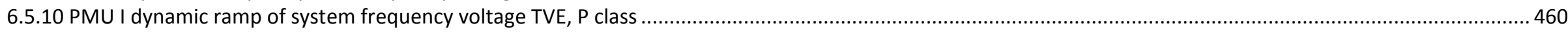

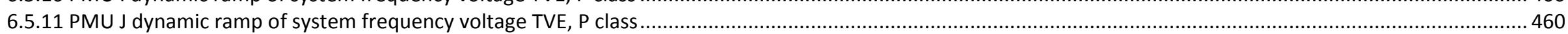

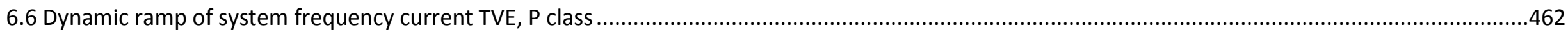

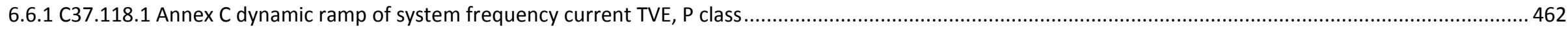

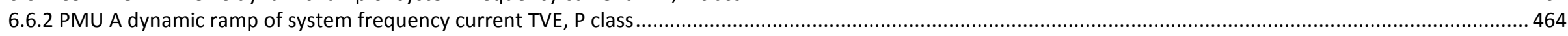

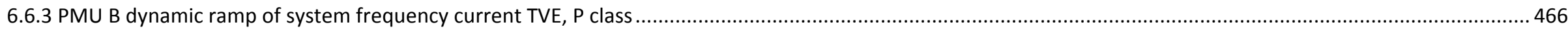

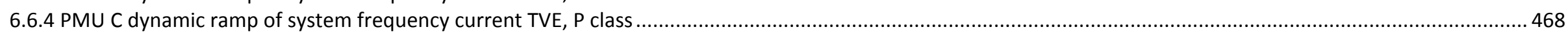

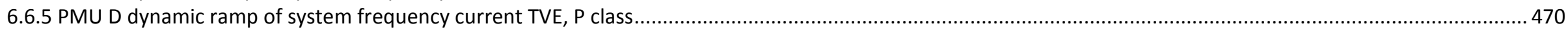

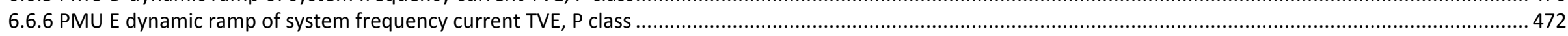

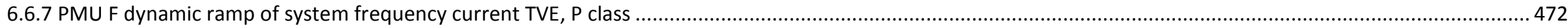

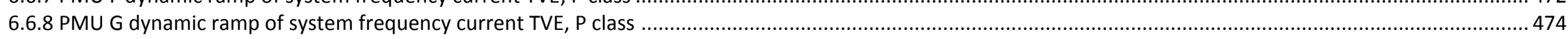

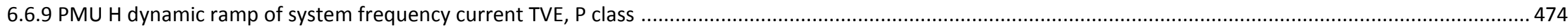

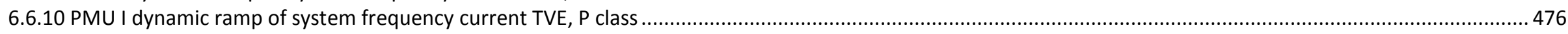

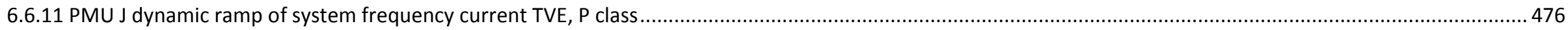


6.7 Dynamic ramp of system frequency frequency, $P$ class.....

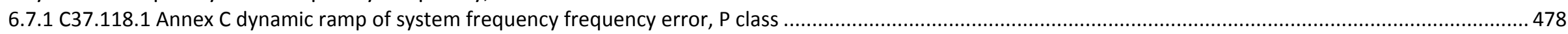

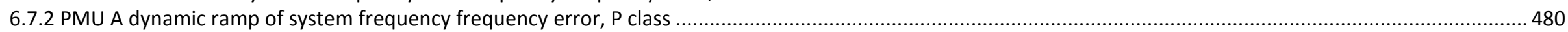

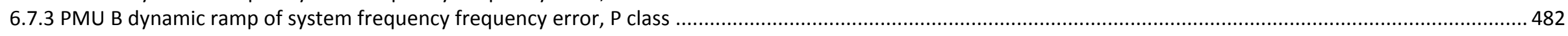

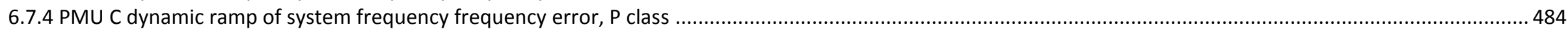

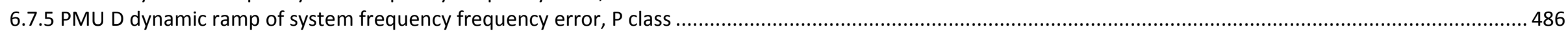

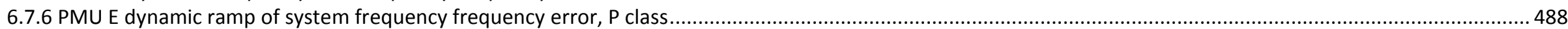

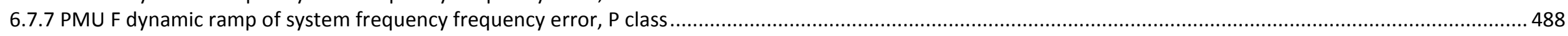

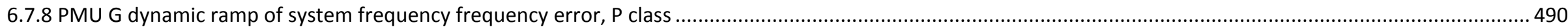

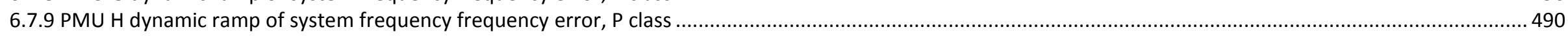

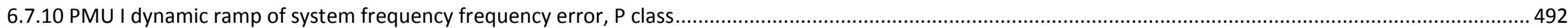

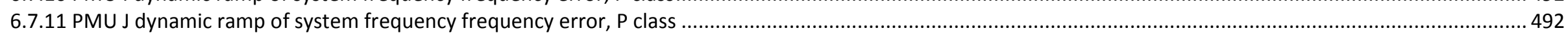

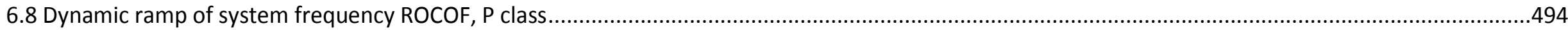

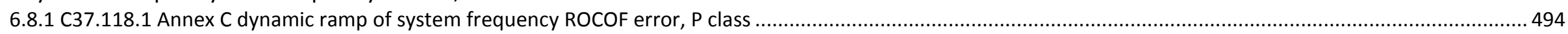

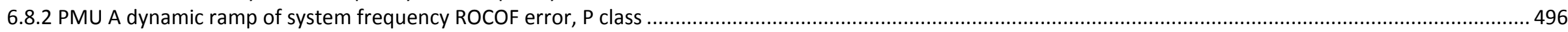

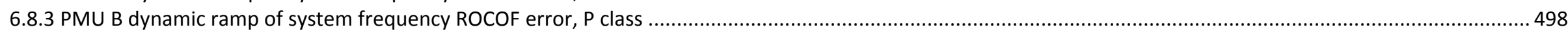

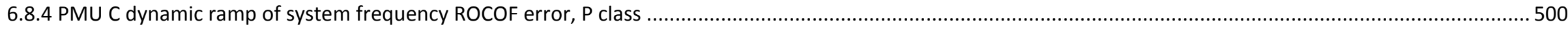

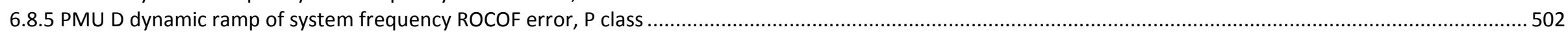

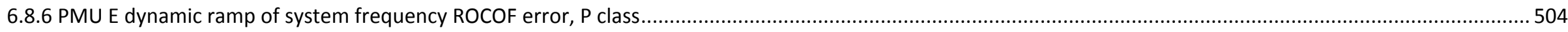

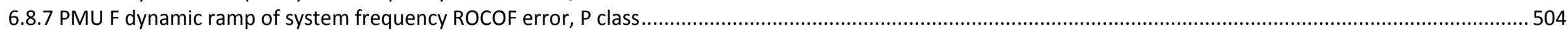

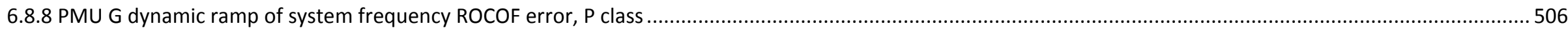

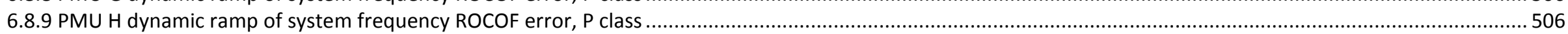

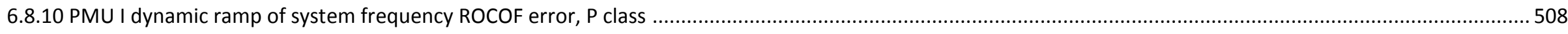

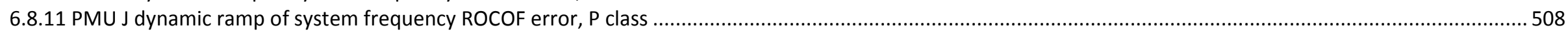


IEEE Std. C37.118.1-2011 table 7 specifies the minimum range of influence quantity over which PMU shall be within given TVE limit:

For $\mathrm{M}$ class the range is the lesser of:
○ $\mathrm{FO} \pm \mathrm{Fs} / 5 \mathrm{~Hz}$
○ $\mathrm{FO} \pm 5 \mathrm{~Hz}$

For $\mathrm{P}$ class the range is $\mathrm{FO} \pm 2 \mathrm{~Hz}$.

IEEE Std. C37.118.1a states that the error calculation shall exclude measurements during the first seven sample periods for $M$ class and two sample periods for $P$ class before and after a change in the test ROCOF. In the tests below; the ramp of system frequency test is performed by first applying a beginning steady state frequency at the required range. The standard requires two transition time samples $(\mathrm{Nt})$ and a ramp rate $(\mathrm{R})$ of $\pm 1 \mathrm{~Hz} /$ second. The beginning frequency is:

\section{Test plan:}

a) Begin with input at nominal magnitude and lower frequency range.

b) Wait for the system to settle.

c) Begin ramping the frequency with a positive ramp rate of $1 \mathrm{~Hz} / \mathrm{s}$, ramp until the upper frequency range is reached and hold at that frequency for at least the settling period.

d) Calculate the errors: TVE, FE, and RFE for each report.

e) Calculate the max TVE, FE, and RFE excluding data during the exclusion intervals as required by the standard.

f) Hold the frequency constant for at least the settling period, and then begin ramping the frequency at the negative ramp rate.

g) Calculate the max TVE, FE, and RFE excluding data during the exclusion intervals as required by the standard.

The ramp test is performed twice, once with ramp rates $=+1 \mathrm{~Hz} / \mathrm{s}$ and again with $-1 \mathrm{~Hz} / \mathrm{s}$. The plots below show the TVE, Fe and RFe for individual test runs in columns showing the positive and negative ramp, and rows showing the PMU response at various reporting rates. The $\mathrm{X}$ axis of the plots are the ramp time and the $\mathrm{Y}$ axis are TVE, Fe and RFe respectively. The excluded measurements are not shown in the plots.
M class limits:
- TVE: $1 \%$
P class limits:
- Fe: $0.01 \mathrm{~Hz}$
- TVE: $1 \%$
- Fe: $0.01 \mathrm{~Hz}$
- RFe: $0.2 \mathrm{~Hz} / \mathrm{s}$
- $\mathrm{RFe}: 0.4 \mathrm{~Hz} / \mathrm{s}$ 


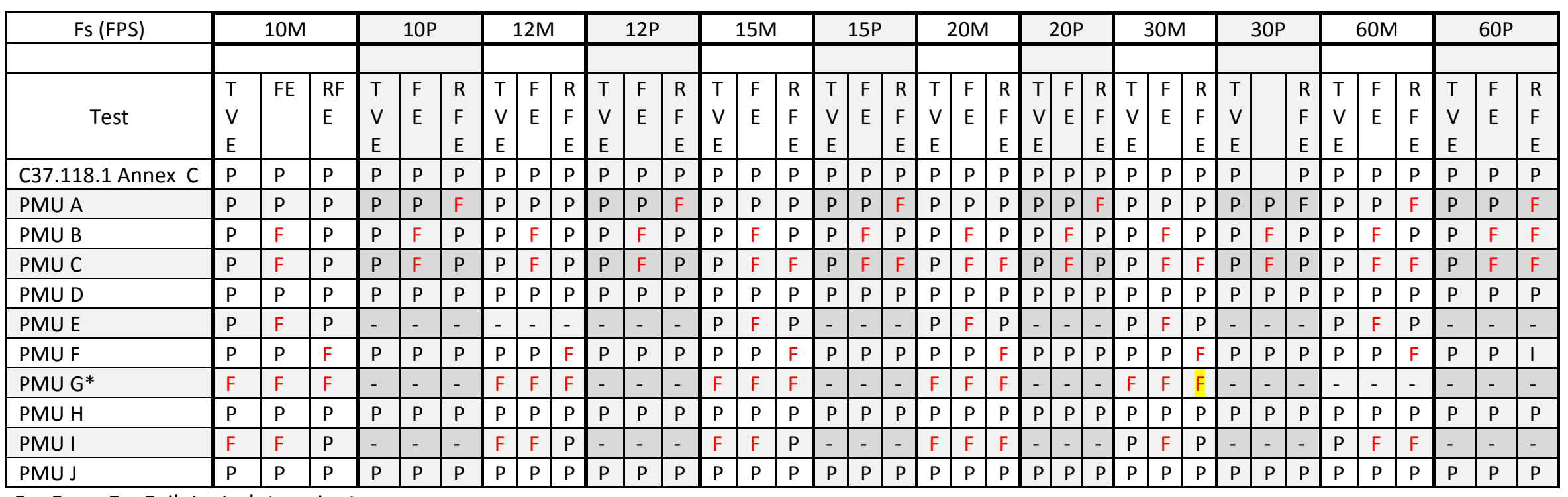

$\mathrm{P}=$ Pass, $\mathrm{F}=$ Fail, $\mathrm{I}=$ Indeterminate

* PMU G always outputs ROCOF $=0$ 


\subsection{Dynamic ramp of system frequency voltage TVE, M class}

\subsubsection{C37.118.1 Annex C dynamic ramp of system frequency voltage TVE: $F 0=60, M$ class}
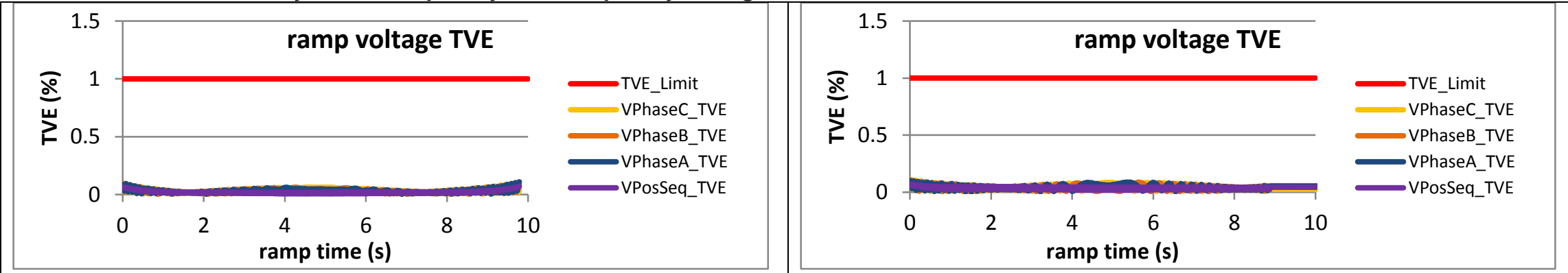

Figure 2142: Fs = $60 \mathrm{FPS}$, ramp from $55 \mathrm{~Hz}$ to $65 \mathrm{~Hz}$ at $+1 \mathrm{~Hz} / \mathrm{s}$
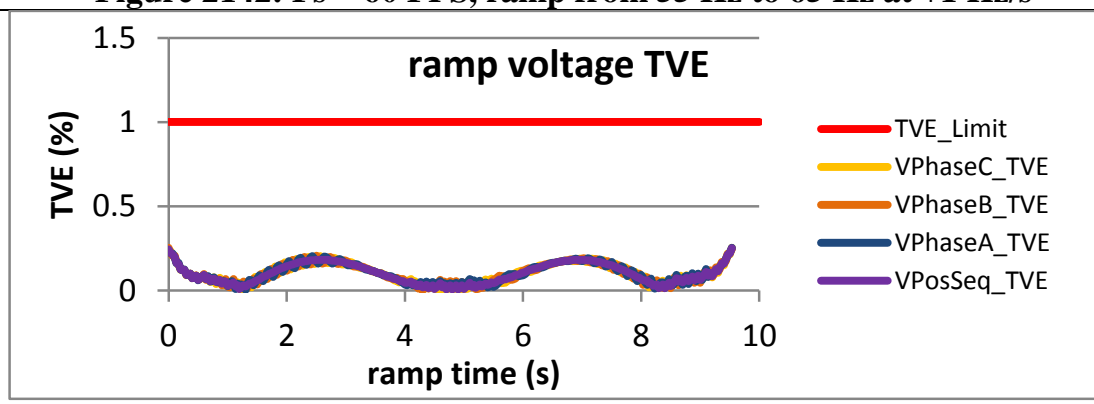

Figure 2144: Fs = $30 \mathrm{FPS}$, ramp from $55 \mathrm{~Hz}$ to $65 \mathrm{~Hz}$ at $+1 \mathrm{~Hz} / \mathrm{s}$

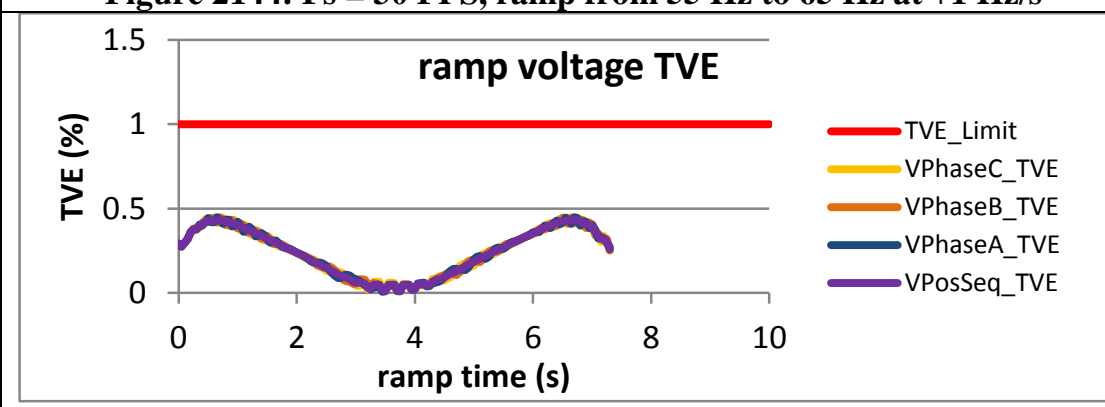

Figure 2146: Fs = 20 FPS, ramp from $56 \mathrm{~Hz}$ to $64 \mathrm{~Hz}$ at $+1 \mathrm{~Hz} / \mathrm{s}$

Figure 2143: Fs = $60 \mathrm{FPS}$, ramp from $65 \mathrm{~Hz}$ to $55 \mathrm{~Hz}$ at $-1 \mathrm{~Hz} / \mathrm{s}$

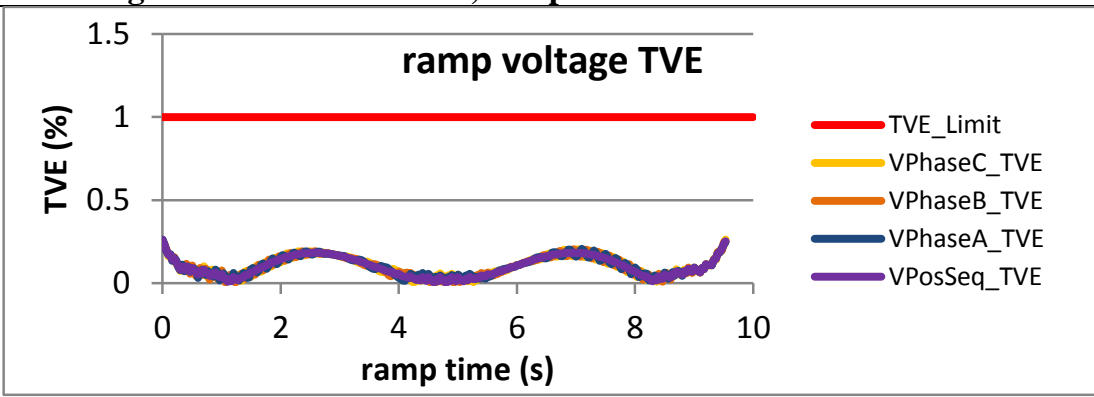

Figure 2145: Fs = $30 \mathrm{FPS}$, ramp from $65 \mathrm{~Hz}$ to $55 \mathrm{~Hz}$ at $-1 \mathrm{~Hz} / \mathrm{s}$

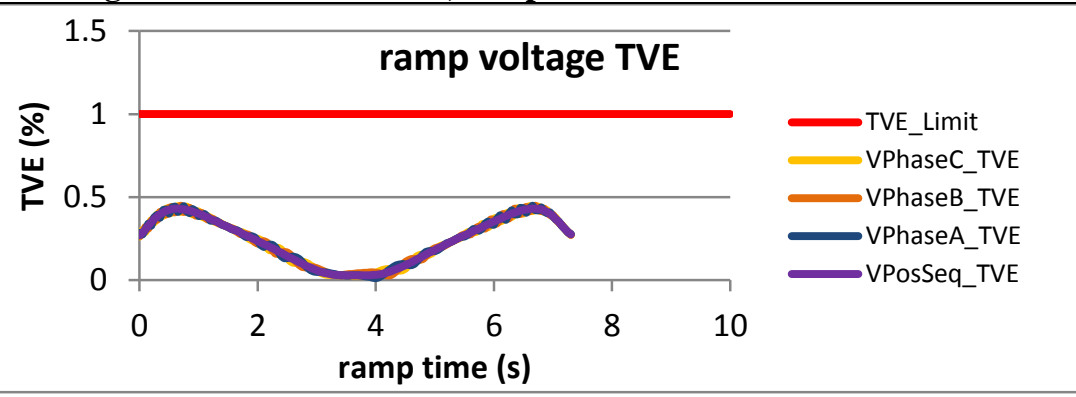

Figure 2147: Fs = $20 \mathrm{FPS}$, ramp from $64 \mathrm{~Hz}$ to $56 \mathrm{~Hz}$ at $-1 \mathrm{~Hz} / \mathrm{s}$ 

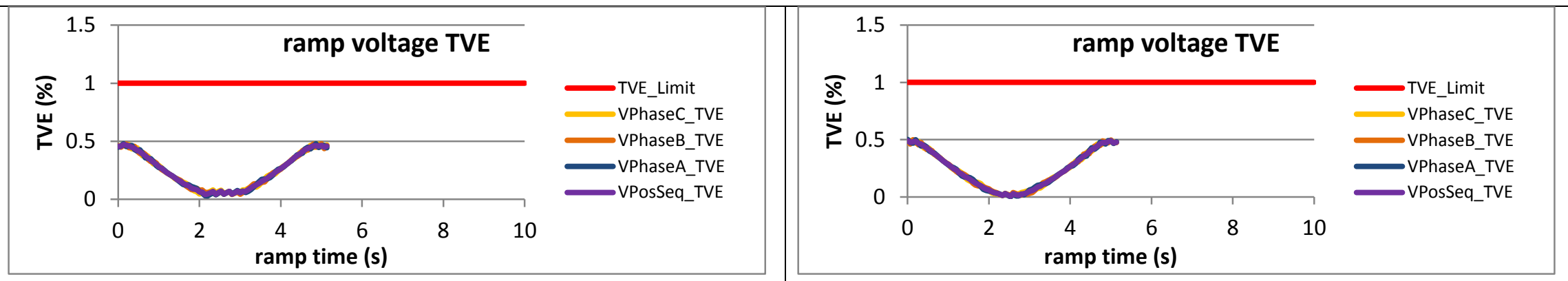

Figure 2148: Fs = $15 \mathrm{FPS}$, ramp from $57 \mathrm{~Hz}$ to $63 \mathrm{~Hz}$ at $+1 \mathrm{~Hz} / \mathrm{s}$

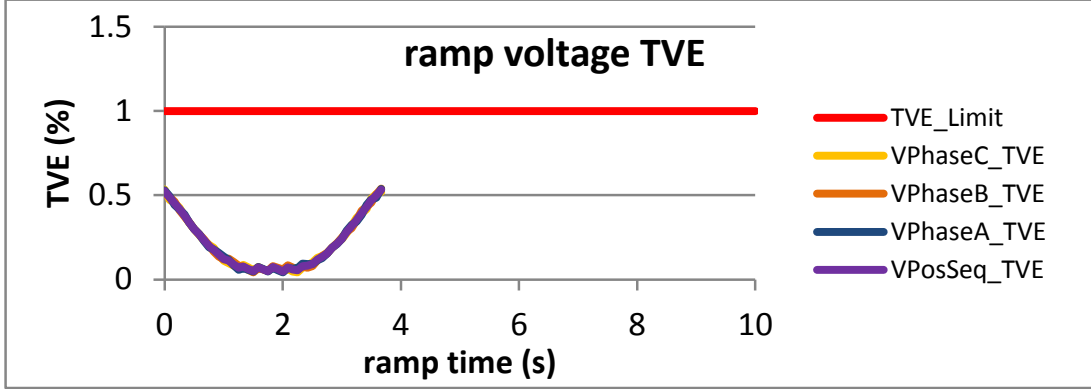

Figure 2150: Fs = 12 FPS, ramp from $58.6 \mathrm{~Hz}$ to $62.4 \mathrm{~Hz}$ at $+1 \mathrm{~Hz} / \mathrm{s}$
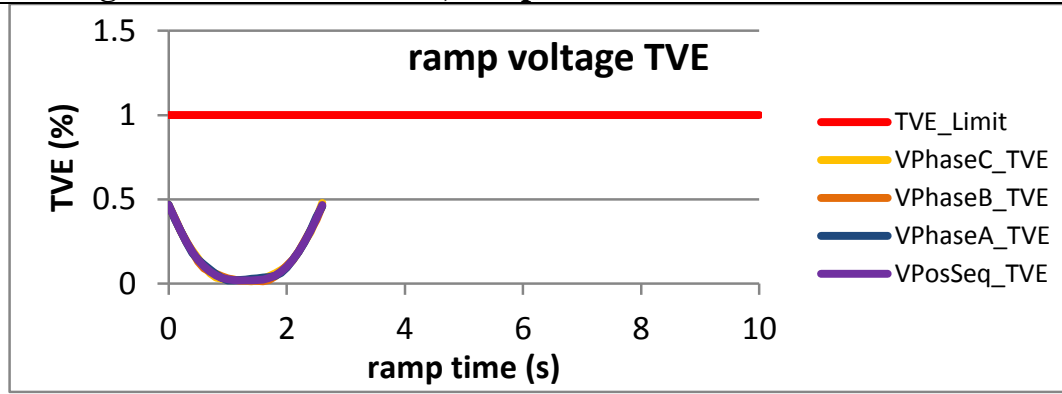

Figure 2152: Fs = $10 \mathrm{FPS}$, ramp from $58 \mathrm{~Hz}$ to $62 \mathrm{~Hz}$ at $+1 \mathrm{~Hz} / \mathrm{s}$

Figure 2149: Fs = $15 \mathrm{FPS}$, ramp from $63 \mathrm{~Hz}$ to $57 \mathrm{~Hz}$ at $-1 \mathrm{~Hz} / \mathrm{s}$

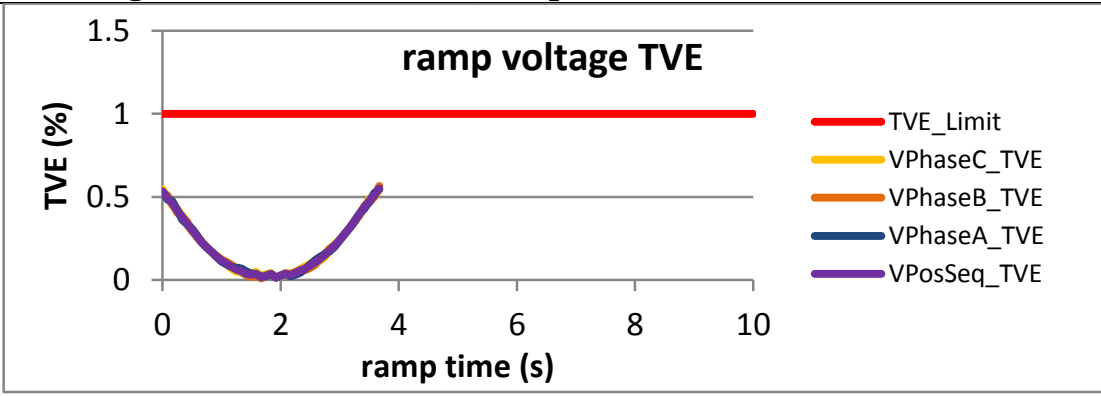

Figure 2151: Fs = 12 FPS, ramp from $62.4 \mathrm{~Hz}$ to $58.6 \mathrm{~Hz}$ at $-1 \mathrm{~Hz} / \mathrm{s}$

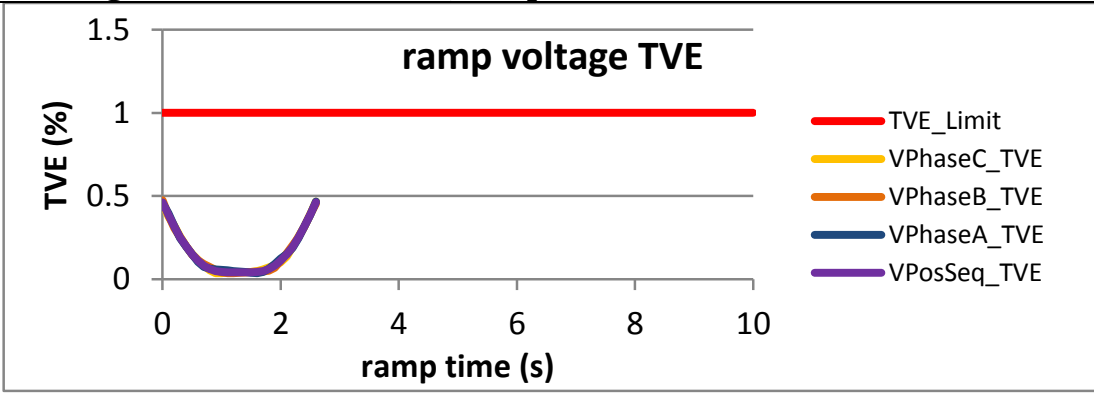

Figure 2153: Fs = $10 \mathrm{FPS}$, ramp from $62 \mathrm{~Hz}$ to $58 \mathrm{~Hz}$ at $-1 \mathrm{~Hz} / \mathrm{s}$ 
6.1.2 PMU A dynamic ramp of system frequency voltage TVE: M class

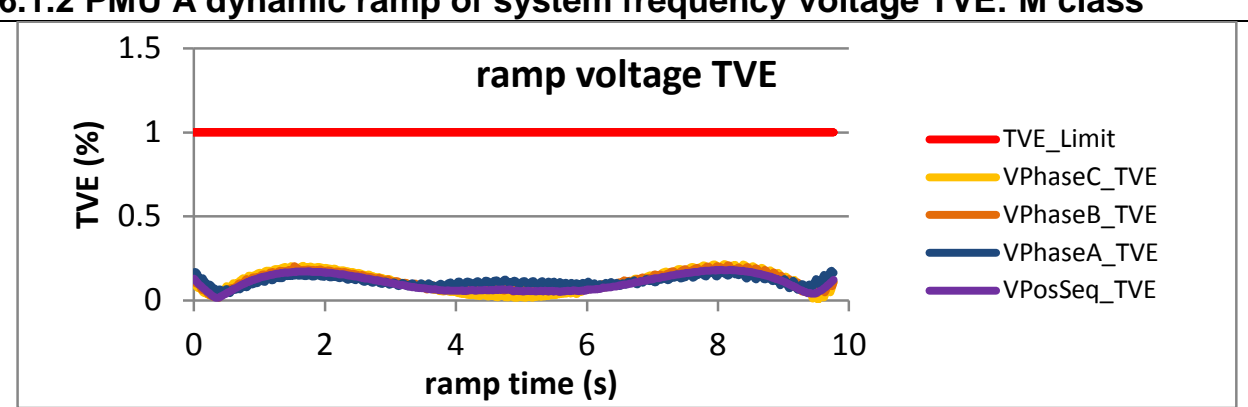

Figure 2154: Fs = $60 \mathrm{FPS}$, ramp from $55 \mathrm{~Hz}$ to $65 \mathrm{~Hz}$ at $+1 \mathrm{~Hz} / \mathrm{s}$

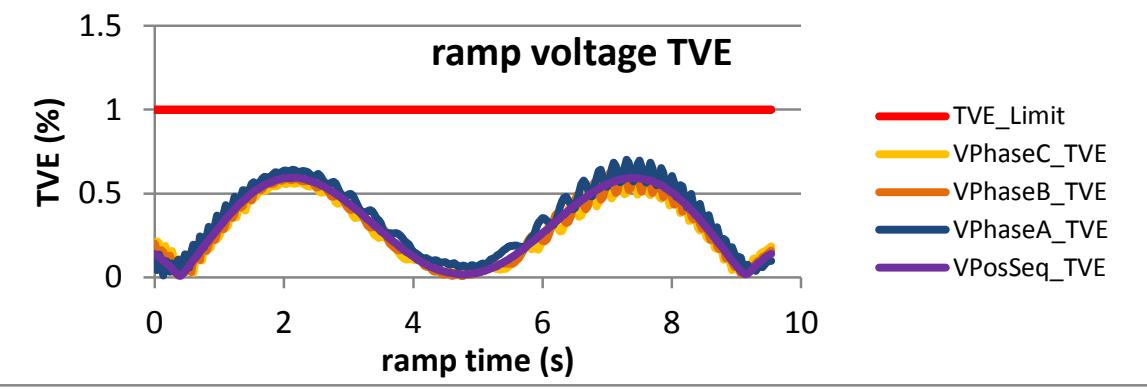

Figure 2156: Fs = $30 \mathrm{FPS}$, ramp from $55 \mathrm{~Hz}$ to $65 \mathrm{~Hz}$ at $+1 \mathrm{~Hz} / \mathrm{s}$

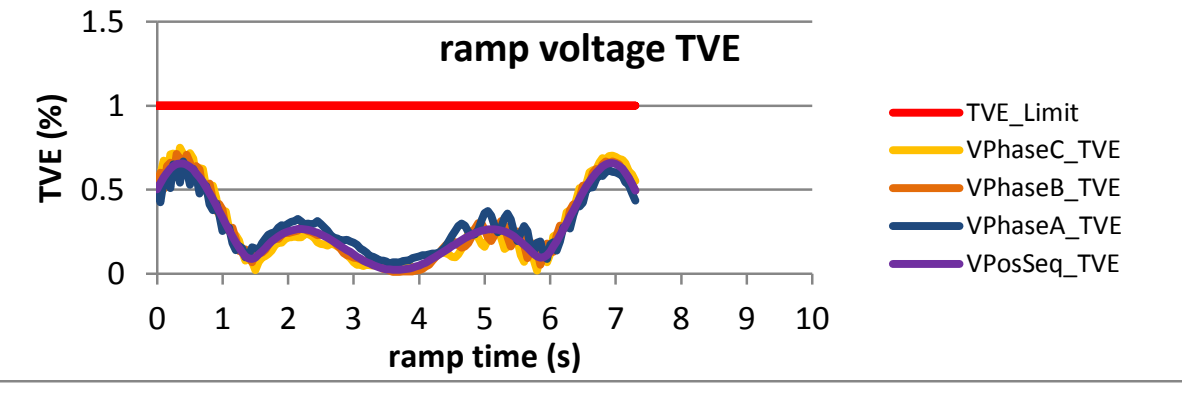

Figure 2158: Fs = 20 FPS, ramp from $56 \mathrm{~Hz}$ to $64 \mathrm{~Hz}$ at $+1 \mathrm{~Hz} / \mathrm{s}$

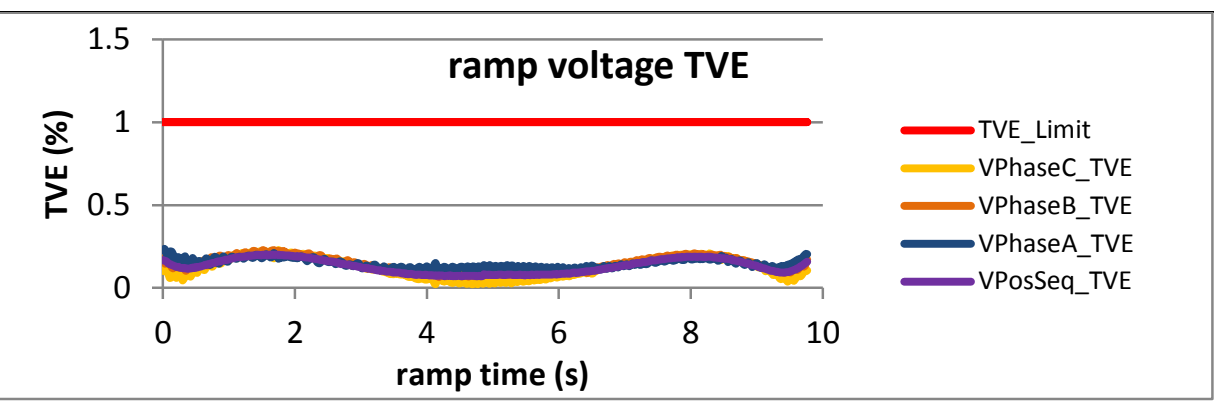

Figure 2155: Fs = $60 \mathrm{FPS}$, ramp from $65 \mathrm{~Hz}$ to $55 \mathrm{~Hz}$ at $-1 \mathrm{~Hz} / \mathrm{s}$

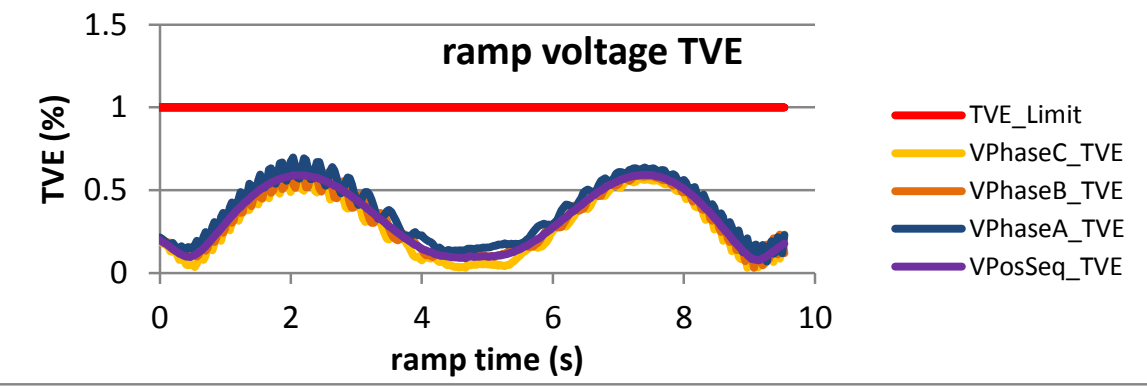

Figure 2157: Fs = $30 \mathrm{FPS}$, ramp from $65 \mathrm{~Hz}$ to $55 \mathrm{~Hz}$ at $-1 \mathrm{~Hz} / \mathrm{s}$

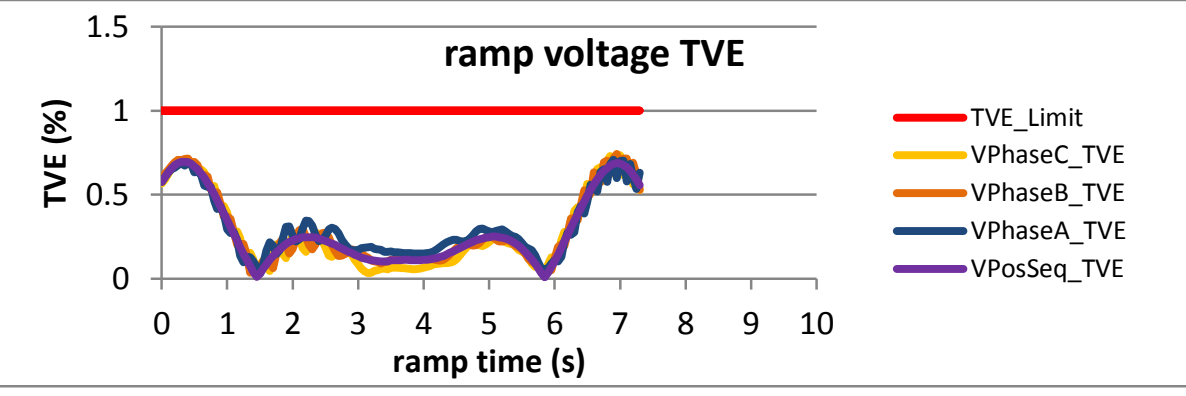

Figure 2159: Fs = $20 \mathrm{FPS}$, ramp from $64 \mathrm{~Hz}$ to $56 \mathrm{~Hz}$ at $-1 \mathrm{~Hz} / \mathrm{s}$ 


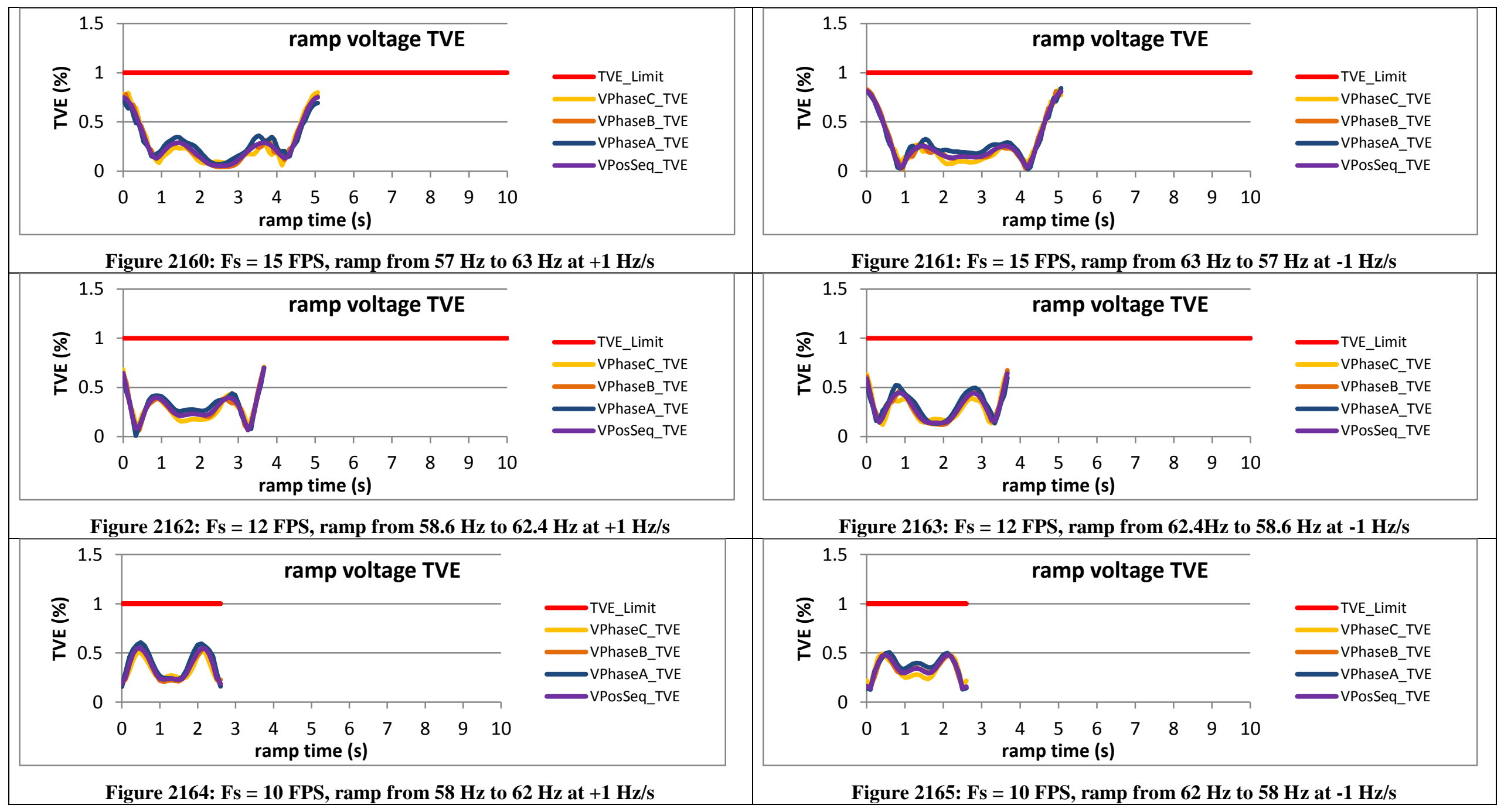


6.1.3 PMU B dynamic ramp of system frequency voltage TVE: M class

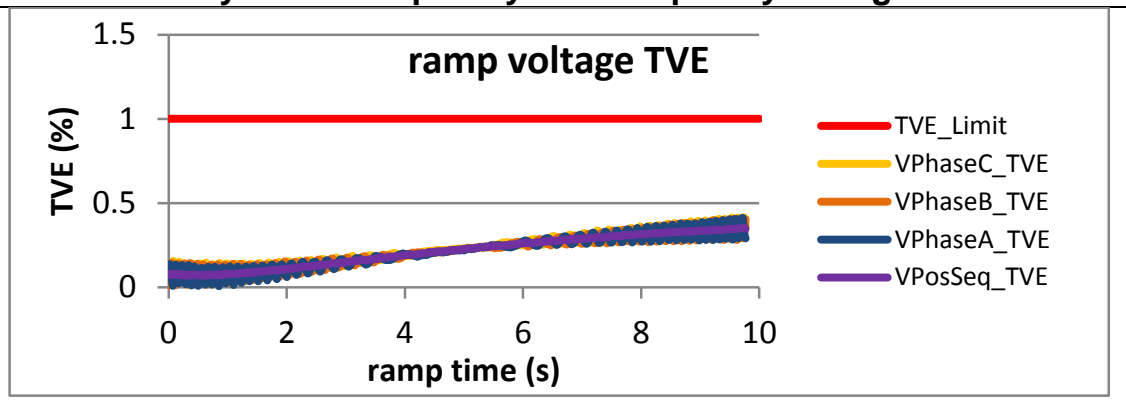

Figure 2166: Fs = $60 \mathrm{FPS}$, ramp from $55 \mathrm{~Hz}$ to $65 \mathrm{~Hz}$ at $+1 \mathrm{~Hz} / \mathrm{s}$

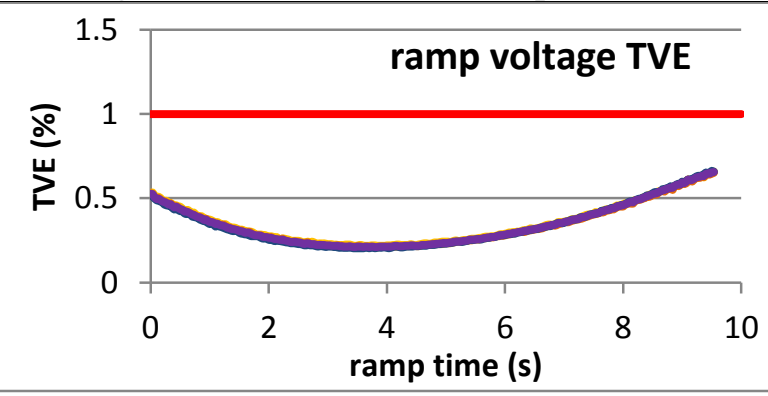

Figure 2168: Fs = $30 \mathrm{FPS}$, ramp from $55 \mathrm{~Hz}$ to $65 \mathrm{~Hz}$ at $+1 \mathrm{~Hz} / \mathrm{s}$

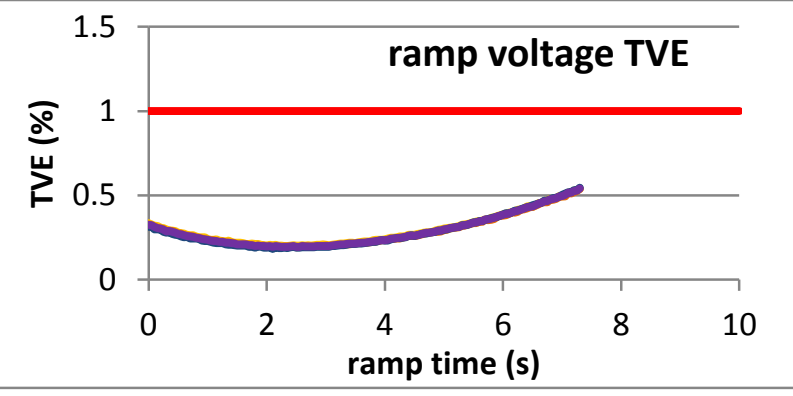

Figure 2170: Fs = $20 \mathrm{FPS}$, ramp from $56 \mathrm{~Hz}$ to $64 \mathrm{~Hz}$ at $+1 \mathrm{~Hz} / \mathrm{s}$

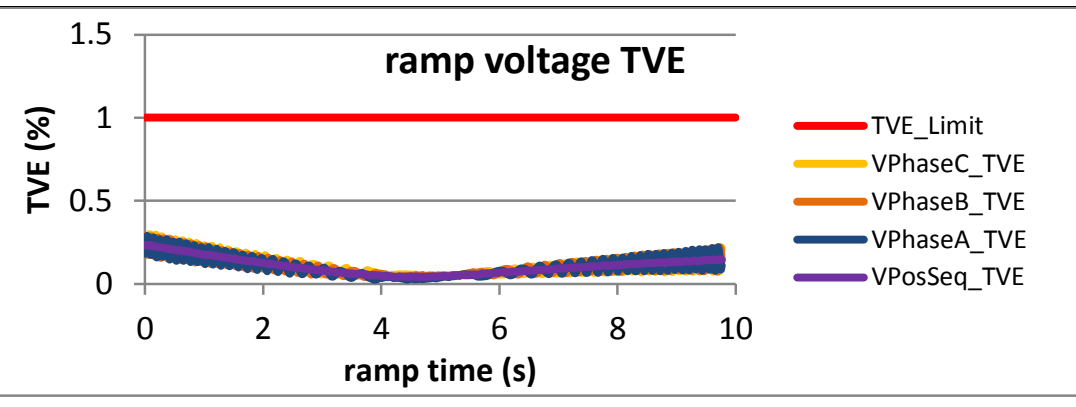

Figure 2167: Fs = 60 FPS, ramp from $65 \mathrm{~Hz}$ to $55 \mathrm{~Hz}$ at $-1 \mathrm{~Hz} / \mathrm{s}$

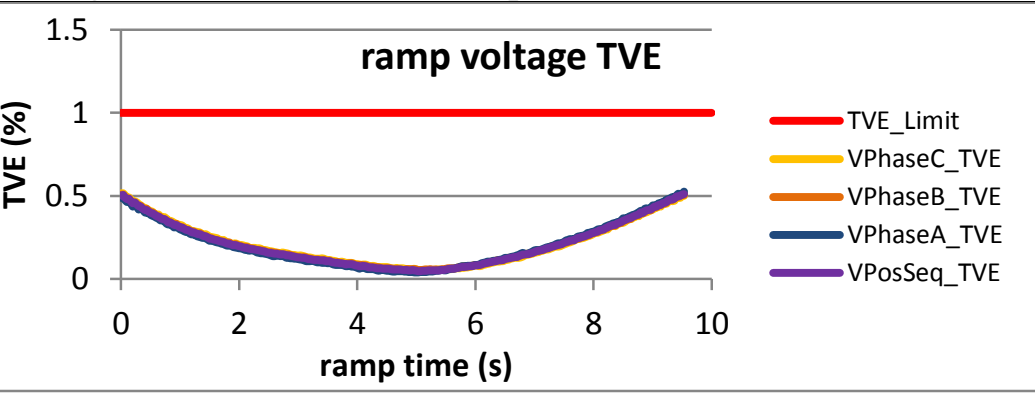

Figure 2169: Fs = $30 \mathrm{FPS}$, ramp from $65 \mathrm{~Hz}$ to $55 \mathrm{~Hz}$ at $-1 \mathrm{~Hz} / \mathrm{s}$

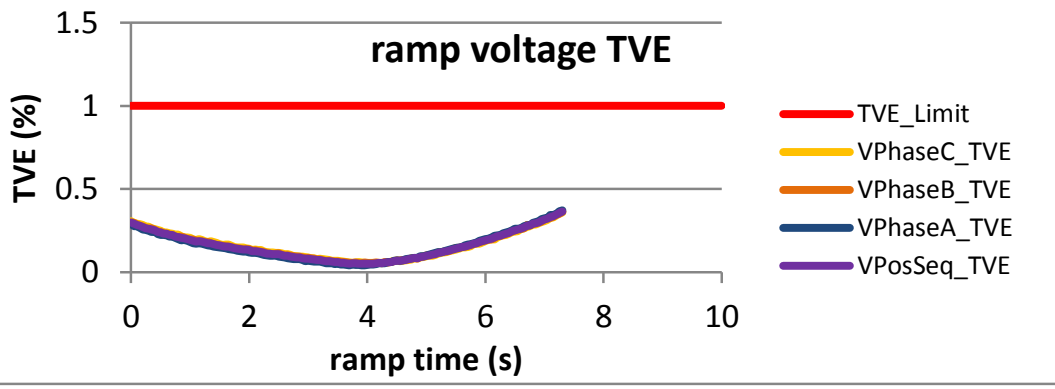

Figure 2171: Fs = $20 \mathrm{FPS}$, ramp from $64 \mathrm{~Hz}$ to $56 \mathrm{~Hz}$ at $-1 \mathrm{~Hz} / \mathrm{s}$ 

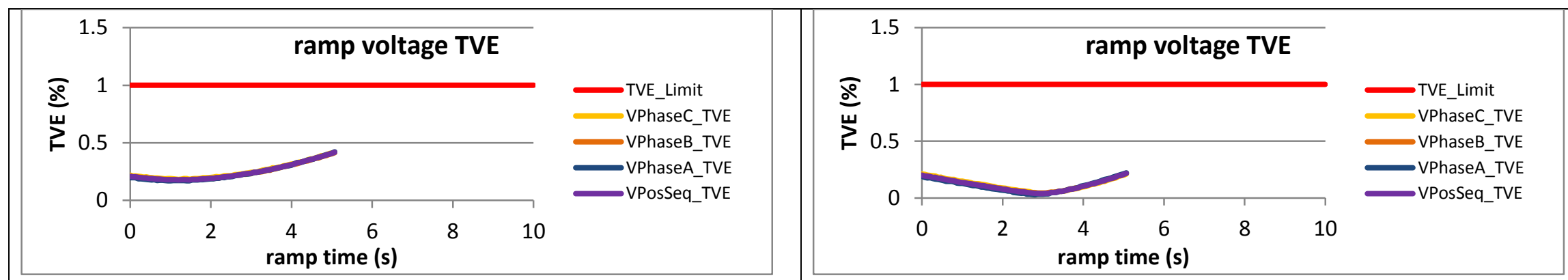

Figure 2172: Fs = $15 \mathrm{FPS}$, ramp from $57 \mathrm{~Hz}$ to $63 \mathrm{~Hz}$ at $+1 \mathrm{~Hz} / \mathrm{s}$

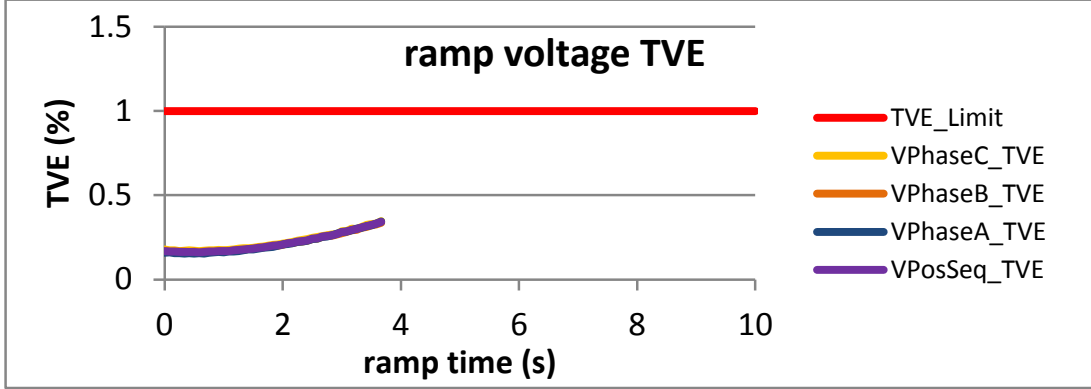

Figure 2174: Fs = 12 FPS, ramp from $58.6 \mathrm{~Hz}$ to $62.4 \mathrm{~Hz}$ at $+1 \mathrm{~Hz} / \mathrm{s}$
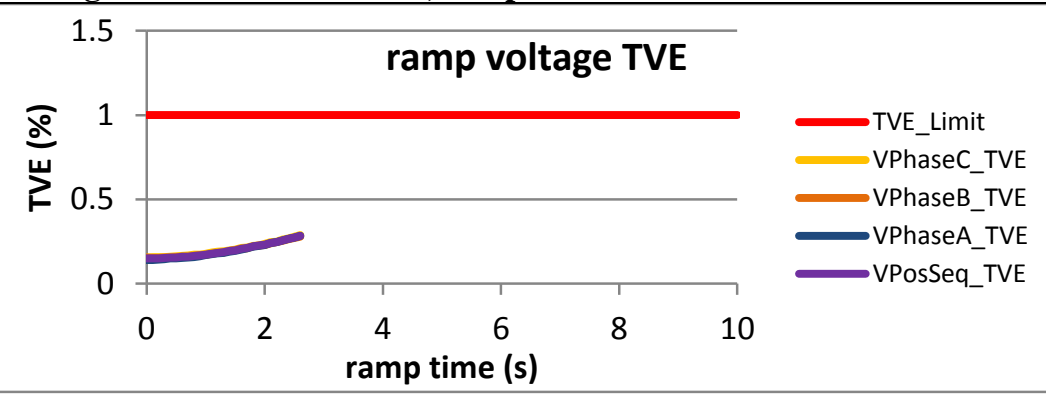

ramp time $(s)$

Figure 2176: Fs = $10 \mathrm{FPS}$, ramp from $58 \mathrm{~Hz}$ to $62 \mathrm{~Hz}$ at $+1 \mathrm{~Hz} / \mathrm{s}$

Figure 2173: Fs = $15 \mathrm{FPS}$, ramp from $63 \mathrm{~Hz}$ to $57 \mathrm{~Hz}$ at $-1 \mathrm{~Hz} / \mathrm{s}$

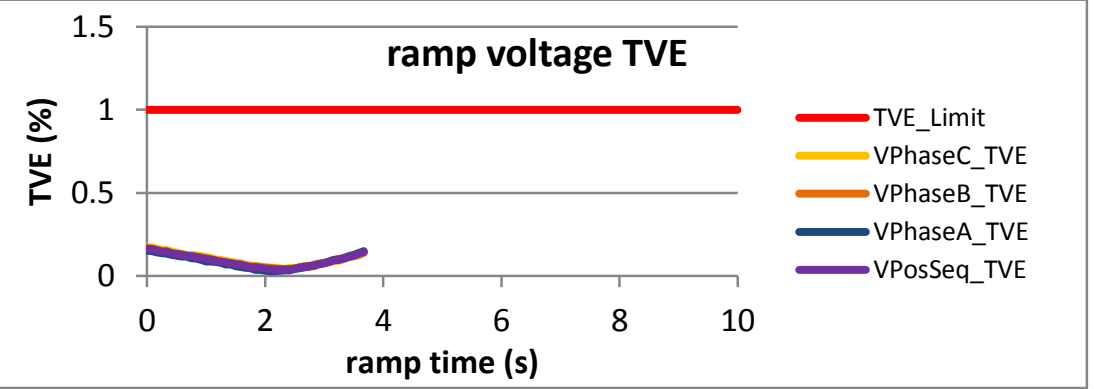

Figure 2175: Fs = 12 FPS, ramp from $62.4 \mathrm{~Hz}$ to $58.6 \mathrm{~Hz}$ at $-1 \mathrm{~Hz} / \mathrm{s}$

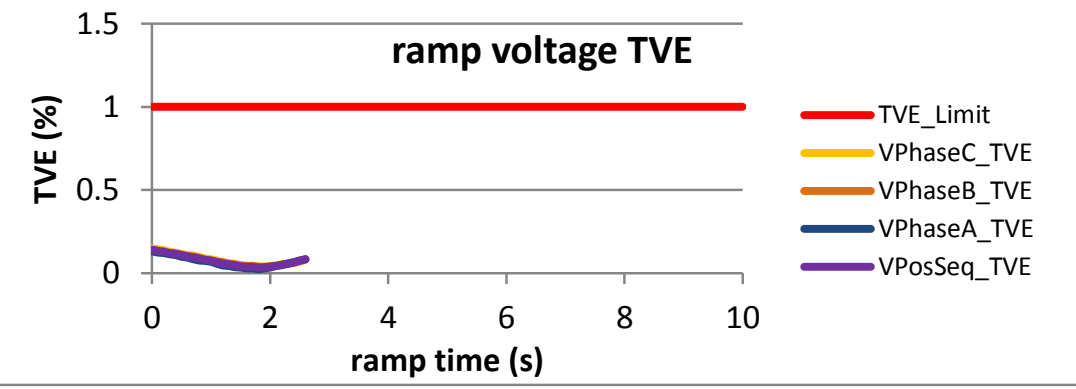

Figure 2177: Fs = $10 \mathrm{FPS}$, ramp from $62 \mathrm{~Hz}$ to $58 \mathrm{~Hz}$ at $-1 \mathrm{~Hz} / \mathrm{s}$ 
6.1.4 PMU C dynamic ramp of system frequency voltage TVE: M class

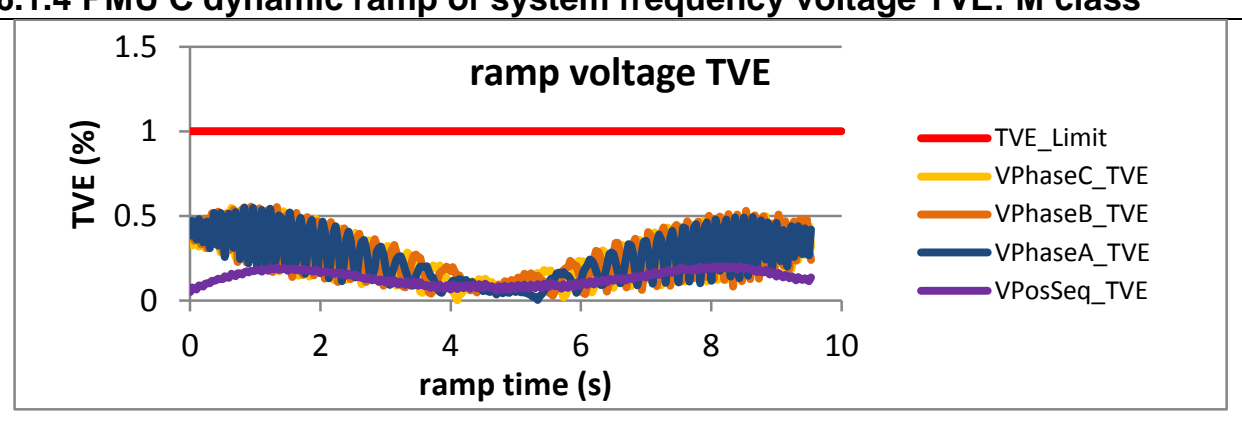

Figure 2178: Fs = 60 FPS, ramp from $55 \mathrm{~Hz}$ to $65 \mathrm{~Hz}$ at $+1 \mathrm{~Hz} / \mathrm{s}$

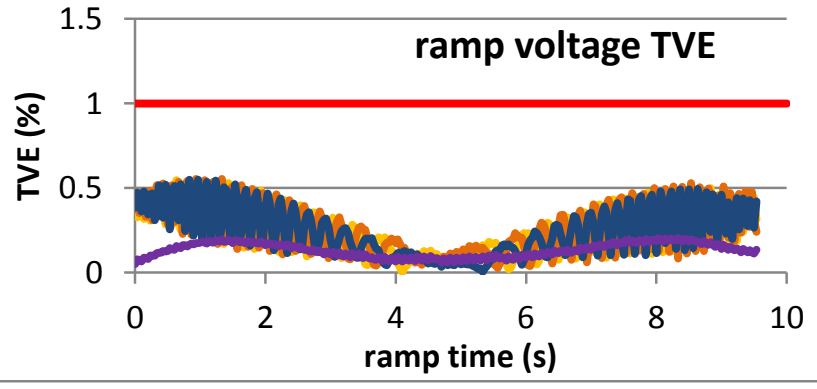

Figure 2180: Fs = $30 \mathrm{FPS}$, ramp from $55 \mathrm{~Hz}$ to $65 \mathrm{~Hz}$ at $+1 \mathrm{~Hz} / \mathrm{s}$

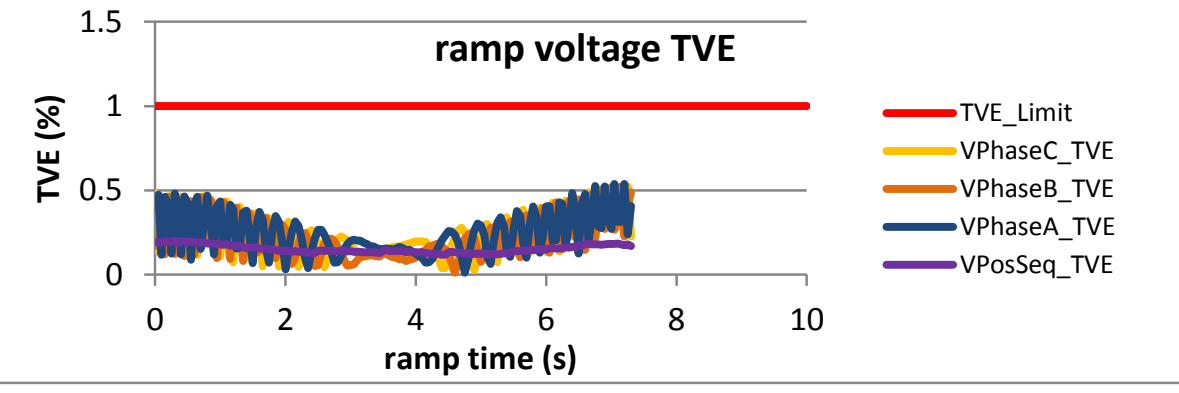

Figure 2182: Fs = $20 \mathrm{FPS}$, ramp from $56 \mathrm{~Hz}$ to $64 \mathrm{~Hz}$ at $+1 \mathrm{~Hz} / \mathrm{s}$

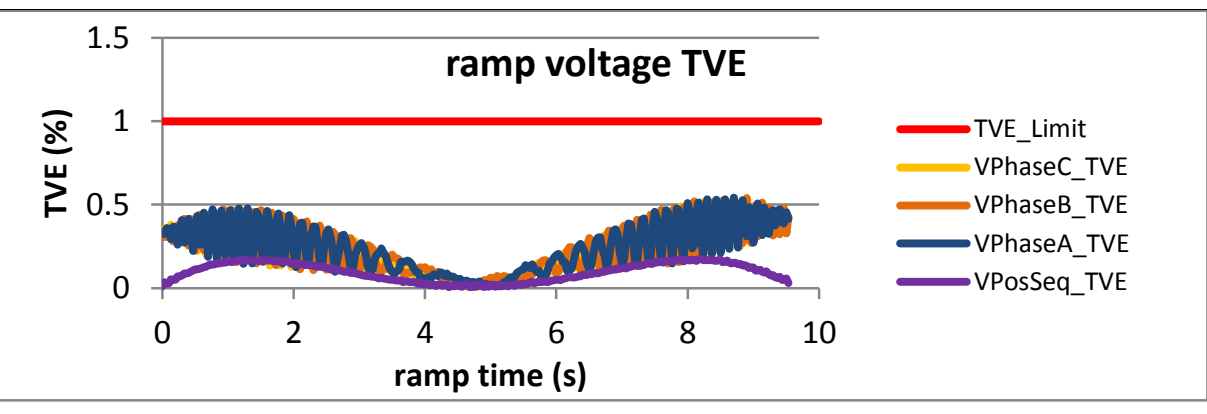

Figure 2179: Fs = $60 \mathrm{FPS}$, ramp from $65 \mathrm{~Hz}$ to $55 \mathrm{~Hz}$ at $-1 \mathrm{~Hz} / \mathrm{s}$

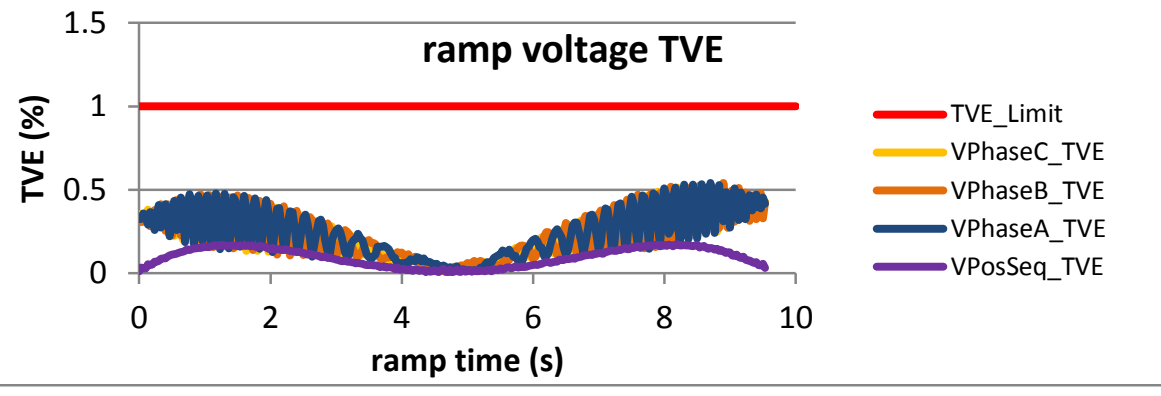

Figure 2181: Fs = $30 \mathrm{FPS}$, ramp from $65 \mathrm{~Hz}$ to $55 \mathrm{~Hz}$ at $-1 \mathrm{~Hz} / \mathrm{s}$

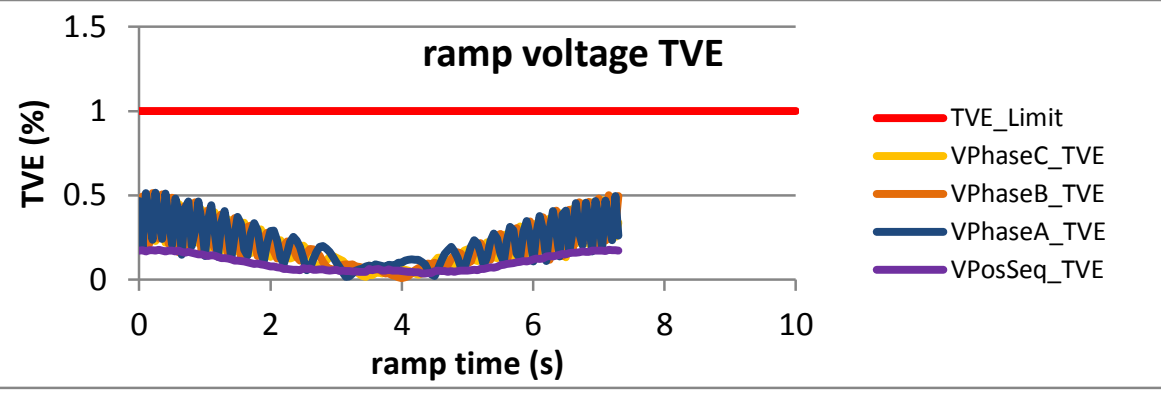

Figure 2183: Fs = $20 \mathrm{FPS}$, ramp from $64 \mathrm{~Hz}$ to $56 \mathrm{~Hz}$ at $-1 \mathrm{~Hz} / \mathrm{s}$ 


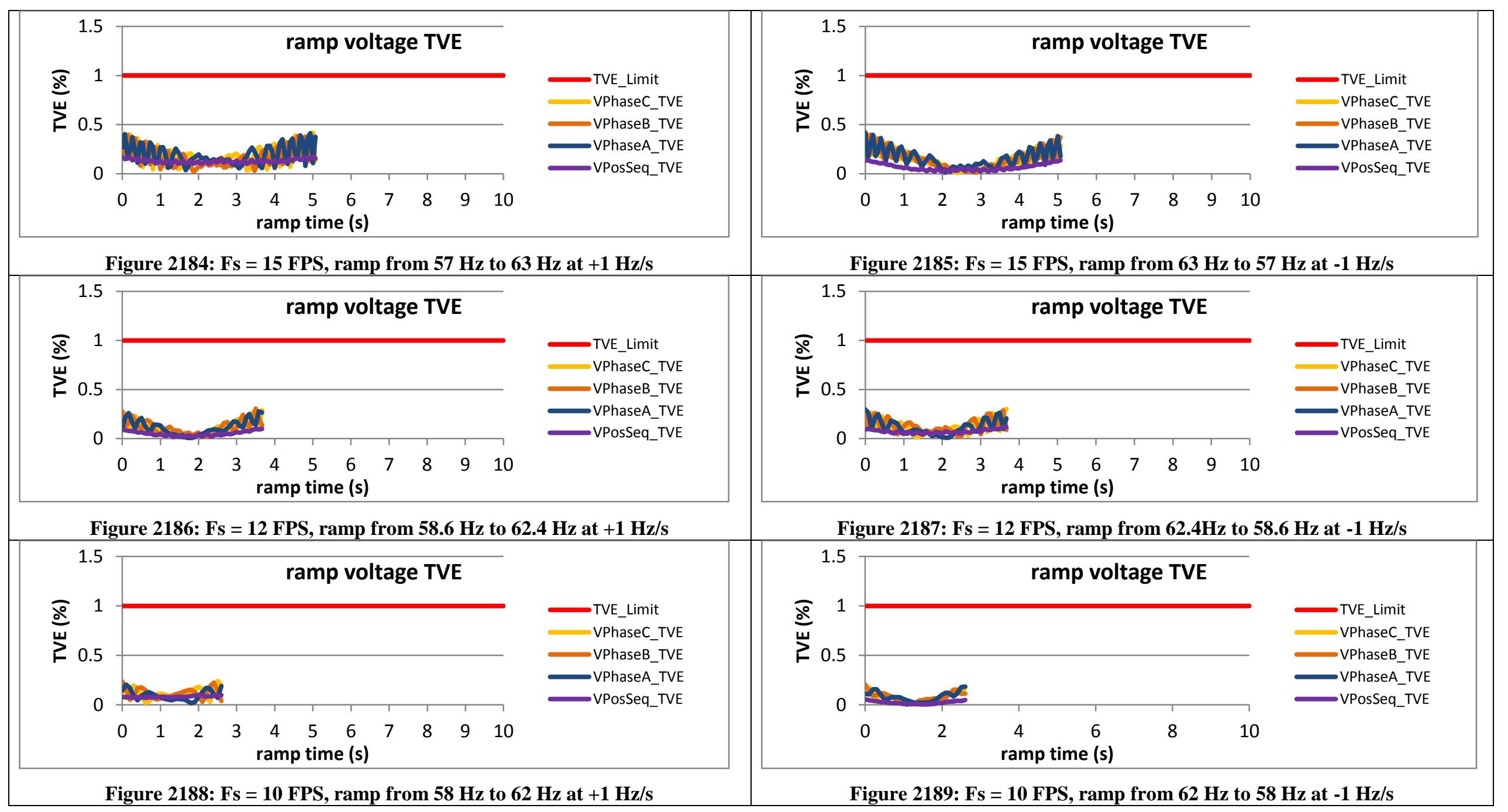


6.1.5 PMU D dynamic ramp of system frequency voltage TVE: M class

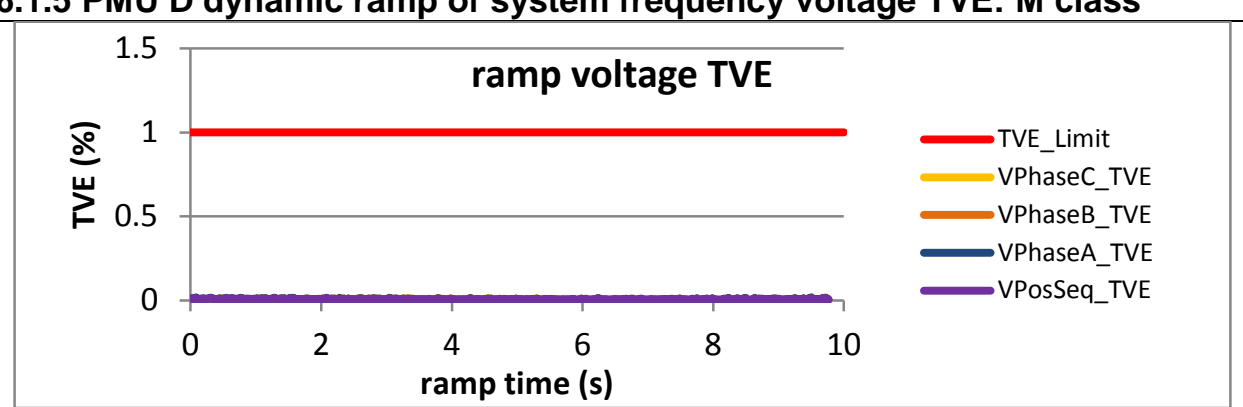

Figure 2190: Fs = $60 \mathrm{FPS}$, ramp from $55 \mathrm{~Hz}$ to $65 \mathrm{~Hz}$ at $+1 \mathrm{~Hz} / \mathrm{s}$

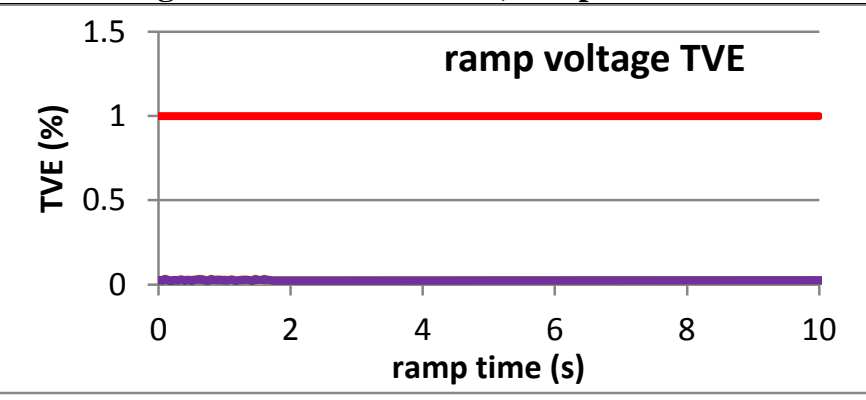

Figure 2192: Fs = $30 \mathrm{FPS}$, ramp from $55 \mathrm{~Hz}$ to $65 \mathrm{~Hz}$ at $+1 \mathrm{~Hz} / \mathrm{s}$

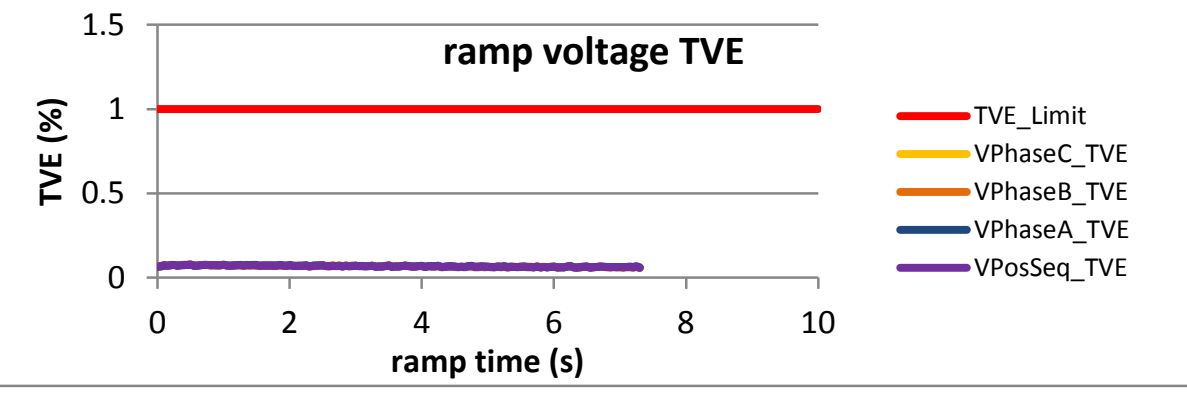

Figure 2194: Fs = $20 \mathrm{FPS}$, ramp from $56 \mathrm{~Hz}$ to $64 \mathrm{~Hz}$ at $+1 \mathrm{~Hz} / \mathrm{s}$

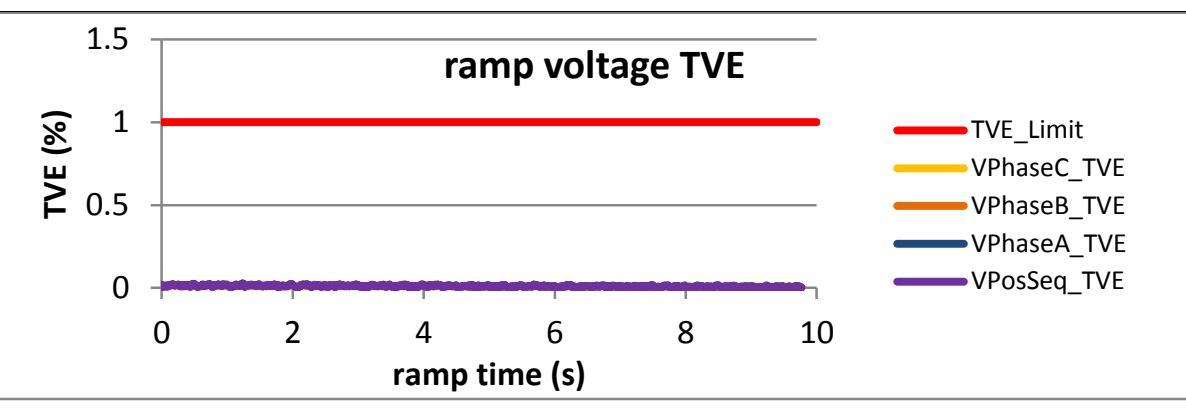

Figure 2191: Fs = 60 FPS, ramp from $65 \mathrm{~Hz}$ to $55 \mathrm{~Hz}$ at $-1 \mathrm{~Hz} / \mathrm{s}$

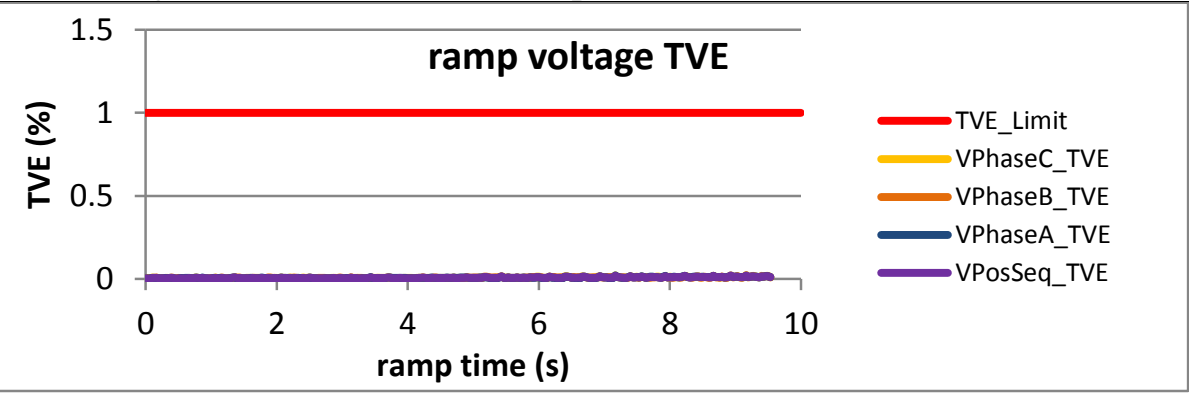

Figure 2193: Fs = $30 \mathrm{FPS}$, ramp from $65 \mathrm{~Hz}$ to $55 \mathrm{~Hz}$ at $-1 \mathrm{~Hz} / \mathrm{s}$

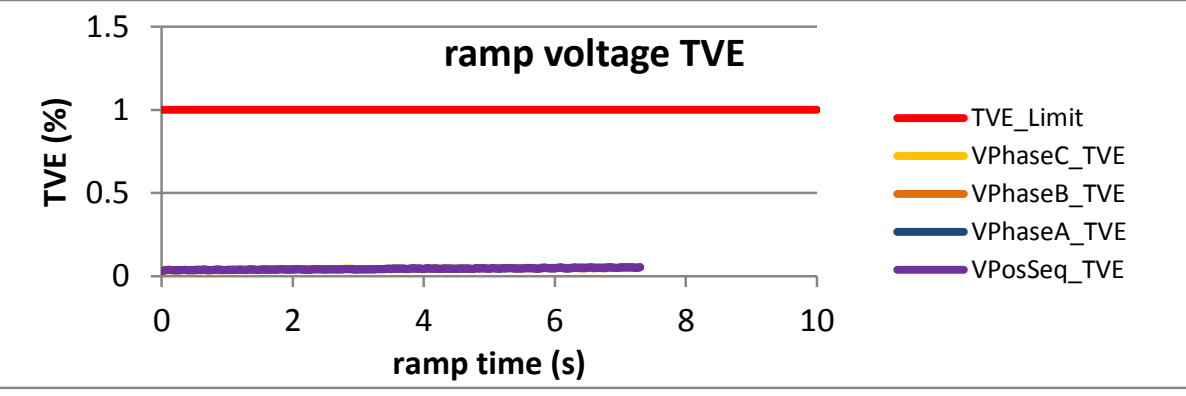

Figure 2195: Fs = $20 \mathrm{FPS}$, ramp from $64 \mathrm{~Hz}$ to $56 \mathrm{~Hz}$ at $-1 \mathrm{~Hz} / \mathrm{s}$ 

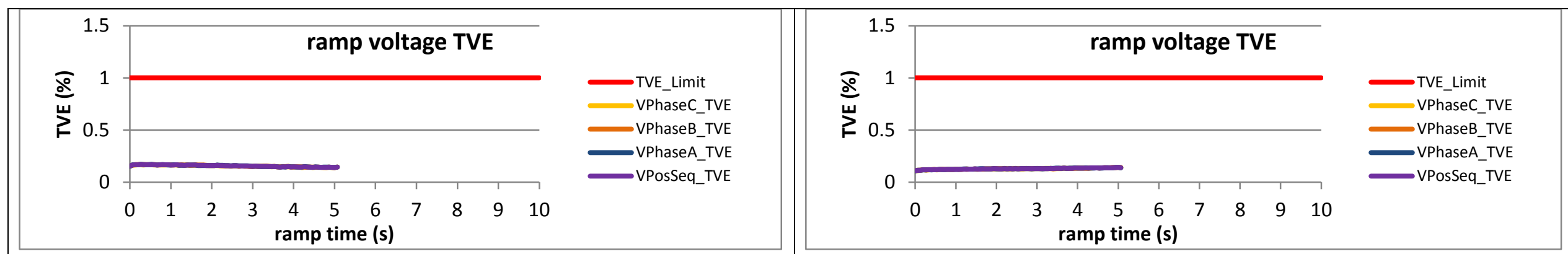

Figure 2196: Fs = $15 \mathrm{FPS}$, ramp from $57 \mathrm{~Hz}$ to $63 \mathrm{~Hz}$ at $+1 \mathrm{~Hz} / \mathrm{s}$

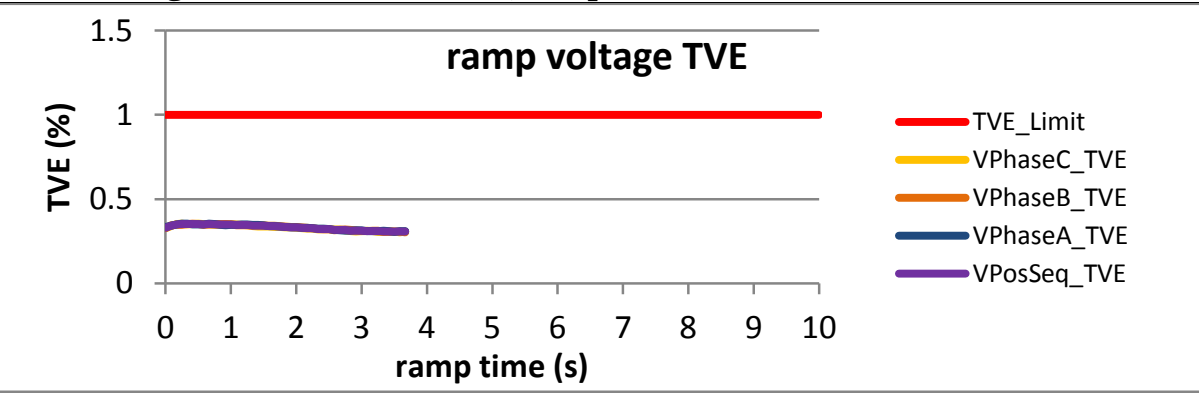

Figure 2197: Fs = $15 \mathrm{FPS}$, ramp from $63 \mathrm{~Hz}$ to $57 \mathrm{~Hz}$ at $-1 \mathrm{~Hz} / \mathrm{s}$

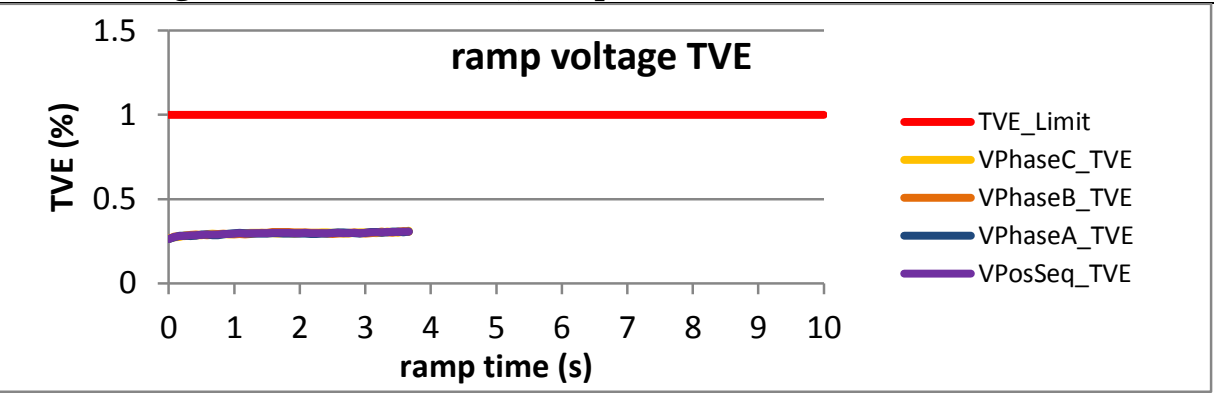

Figure 2198: Fs = 12 FPS, ramp from $58.6 \mathrm{~Hz}$ to $62.4 \mathrm{~Hz}$ at $+1 \mathrm{~Hz} / \mathrm{s}$
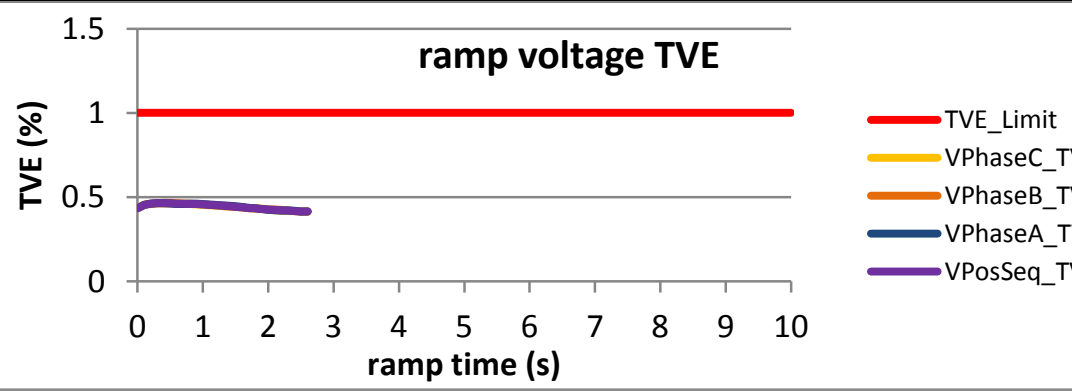

VPhaseC_TVE

VPhaseB_TVE

VPhaseA_TVE

-VPosSeq_TVE ramp time (s)

Figure 2200: Fs = $10 \mathrm{FPS}$, ramp from $58 \mathrm{~Hz}$ to $62 \mathrm{~Hz}$ at $+1 \mathrm{~Hz} / \mathrm{s}$

Figure 2199: Fs = 12 FPS, ramp from $62.4 \mathrm{~Hz}$ to $58.6 \mathrm{~Hz}$ at $-1 \mathrm{~Hz} / \mathrm{s}$
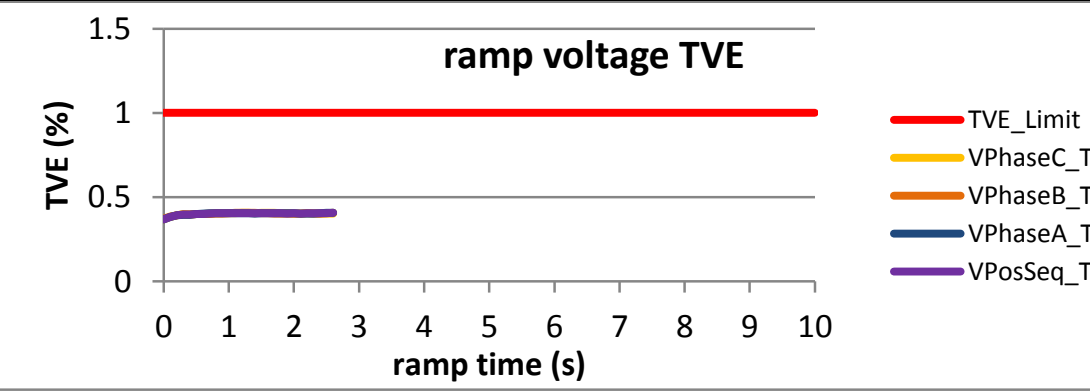

VPhaseC TVE

VPhaseB_TVE

-VPhaseA_TVE

-VPosSeq_TVE

ramp time (s)

Figure 2201: Fs = $10 \mathrm{FPS}$, ramp from $62 \mathrm{~Hz}$ to $58 \mathrm{~Hz}$ at $-1 \mathrm{~Hz} / \mathrm{s}$ 
6.1.6 PMU E dynamic ramp of system frequency voltage TVE: M class

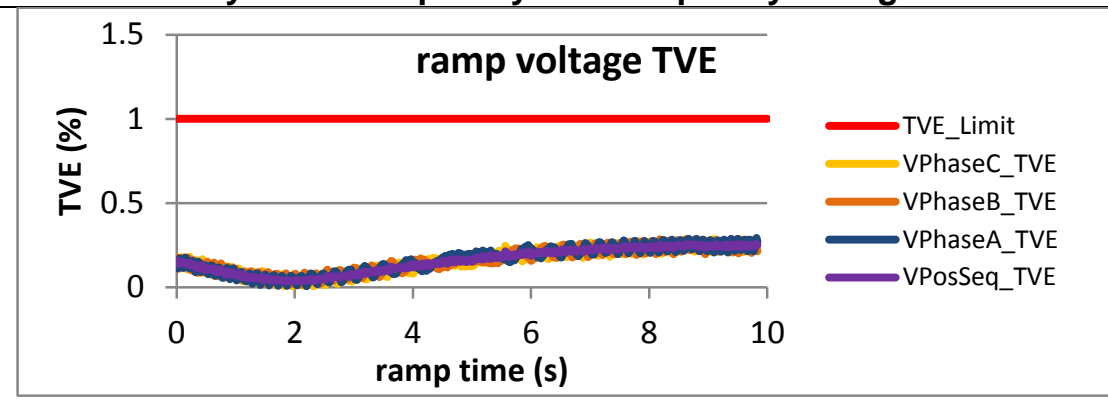

Figure 2202: Fs = $60 \mathrm{FPS}$, ramp from $55 \mathrm{~Hz}$ to $65 \mathrm{~Hz}$ at $+1 \mathrm{~Hz} / \mathrm{s}$

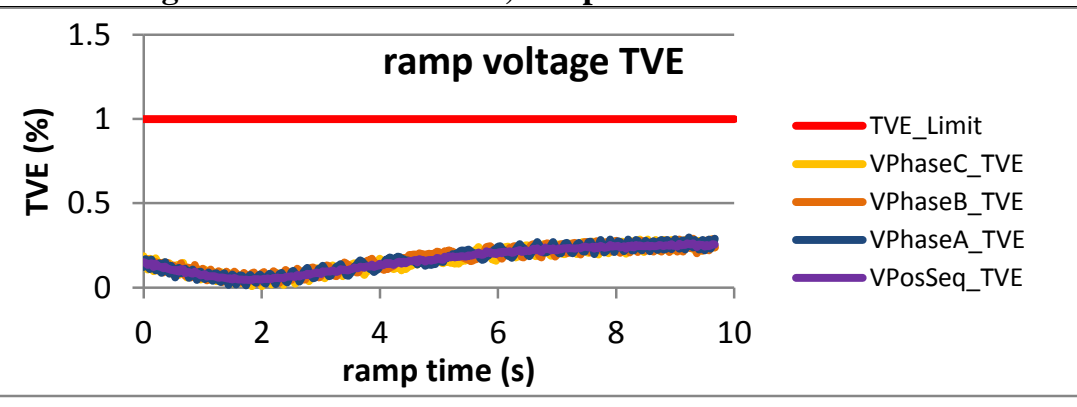

Figure 2204: Fs = $30 \mathrm{FPS}$, ramp from $55 \mathrm{~Hz}$ to $65 \mathrm{~Hz}$ at $+1 \mathrm{~Hz} / \mathrm{s}$

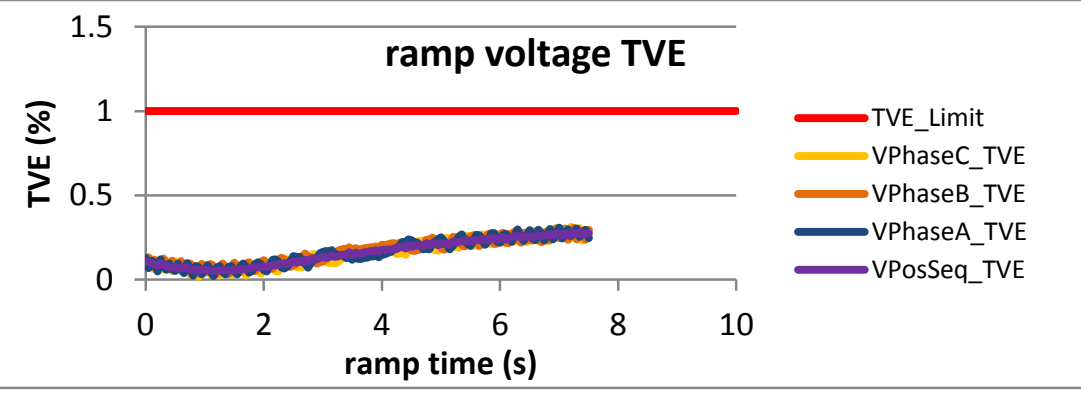

Figure 2206: Fs $=20 \mathrm{FPS}$, ramp from $56 \mathrm{~Hz}$ to $64 \mathrm{~Hz}$ at $+1 \mathrm{~Hz} / \mathrm{s}$

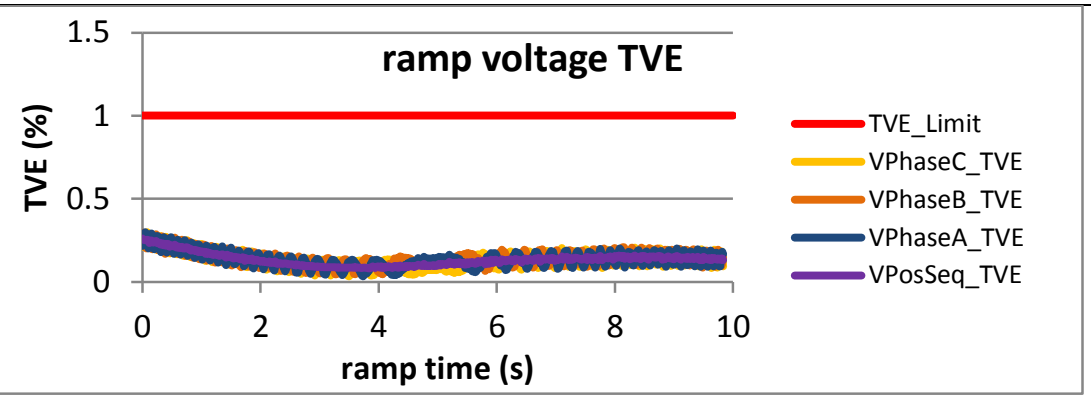

Figure 2203: Fs = $60 \mathrm{FPS}$, ramp from $65 \mathrm{~Hz}$ to $55 \mathrm{~Hz}$ at $-1 \mathrm{~Hz} / \mathrm{s}$

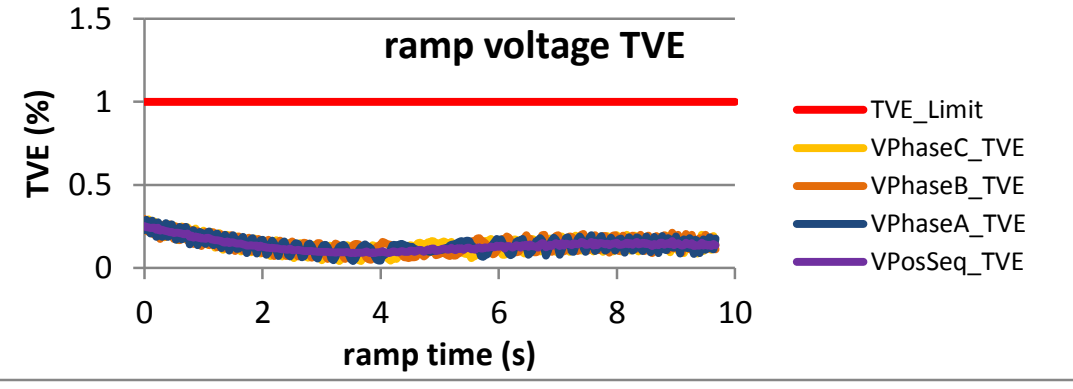

Figure 2205: Fs = $30 \mathrm{FPS}$, ramp from $65 \mathrm{~Hz}$ to $55 \mathrm{~Hz}$ at $-1 \mathrm{~Hz} / \mathrm{s}$

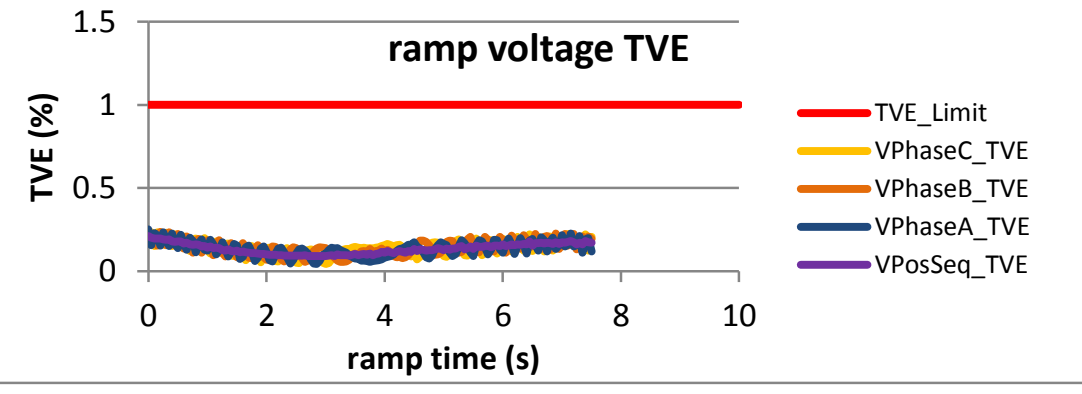

Figure 2207: Fs = $20 \mathrm{FPS}$, ramp from $64 \mathrm{~Hz}$ to $56 \mathrm{~Hz}$ at $-1 \mathrm{~Hz} / \mathrm{s}$ 


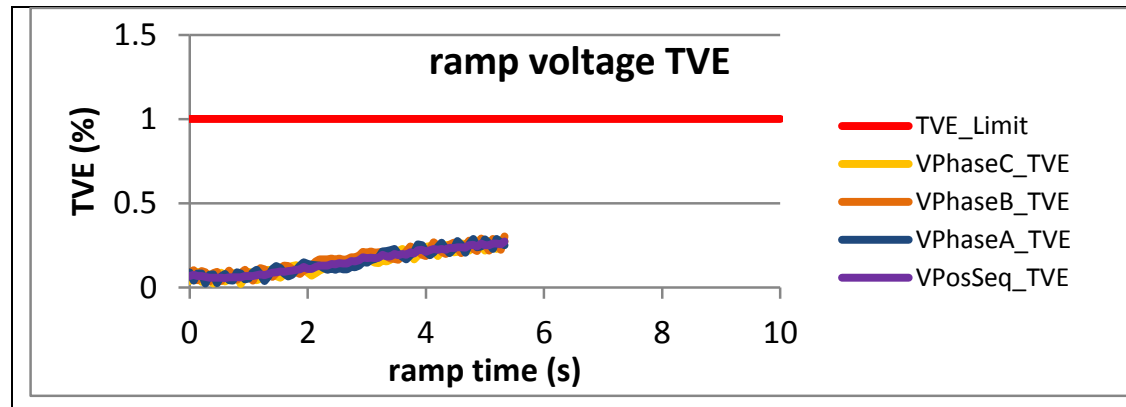

Figure 2208: Fs = $15 \mathrm{FPS}$, ramp from $57 \mathrm{~Hz}$ to $63 \mathrm{~Hz}$ at $+1 \mathrm{~Hz} / \mathrm{s}$

$\|$

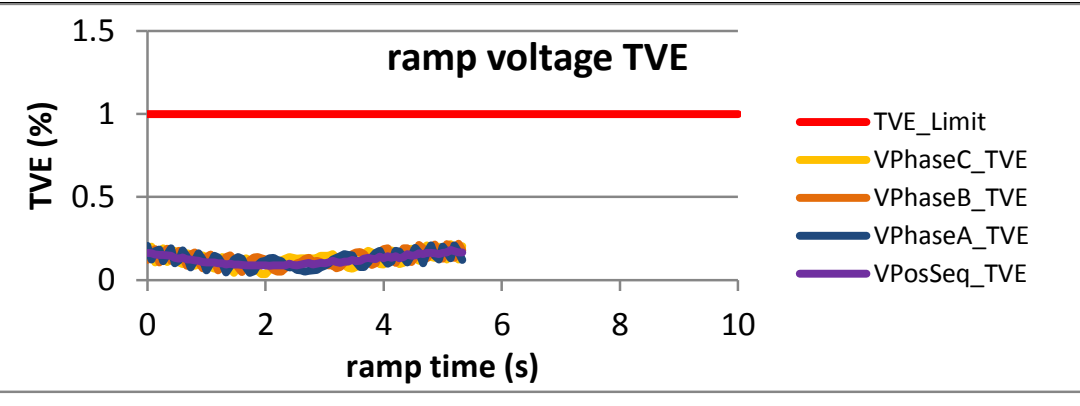

Figure 2209: Fs = $15 \mathrm{FPS}$, ramp from $63 \mathrm{~Hz}$ to $57 \mathrm{~Hz}$ at $-1 \mathrm{~Hz} / \mathrm{s}$

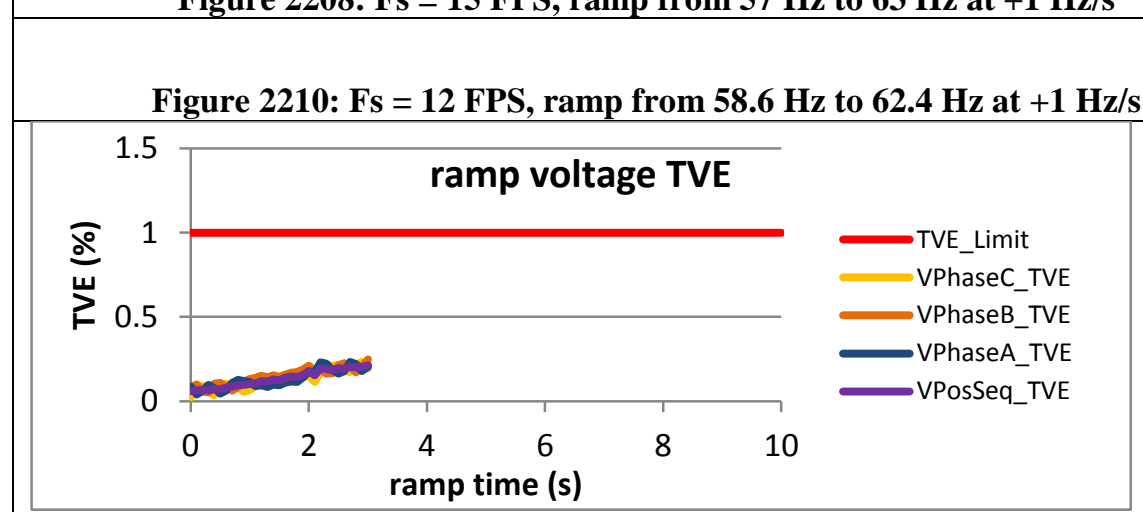

Figure 2211: Fs = 12 FPS, ramp from $62.4 \mathrm{~Hz}$ to $58.6 \mathrm{~Hz}$ at $-1 \mathrm{~Hz} / \mathrm{s}$

Figure 2212: Fs = $10 \mathrm{FPS}$, ramp from $58 \mathrm{~Hz}$ to $62 \mathrm{~Hz}$ at $+1 \mathrm{~Hz} / \mathrm{s}$

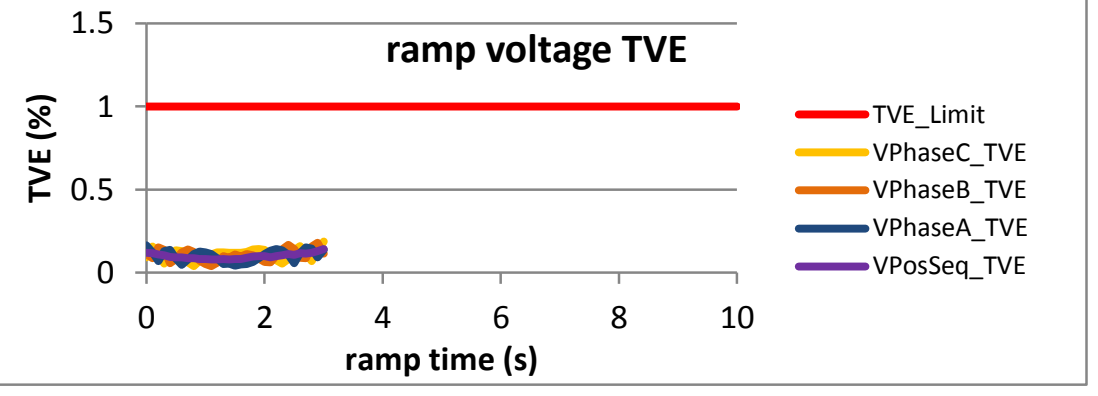

Figure 2213: Fs = $10 \mathrm{FPS}$, ramp from $62 \mathrm{~Hz}$ to $58 \mathrm{~Hz}$ at $-1 \mathrm{~Hz} / \mathrm{s}$ 
6.1.7 PMU F dynamic ramp of system frequency voltage TVE: M class

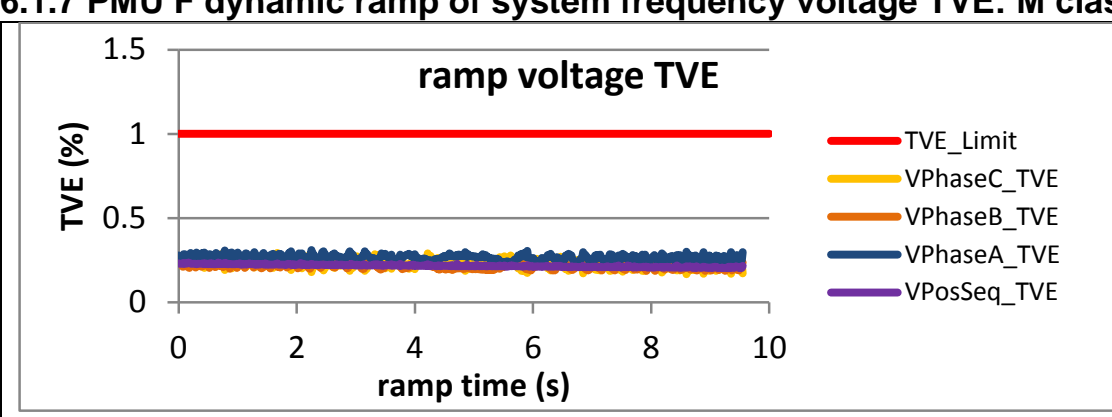

Figure 2214: Fs = $60 \mathrm{FPS}$, ramp from $55 \mathrm{~Hz}$ to $65 \mathrm{~Hz}$ at $+1 \mathrm{~Hz} / \mathrm{s}$

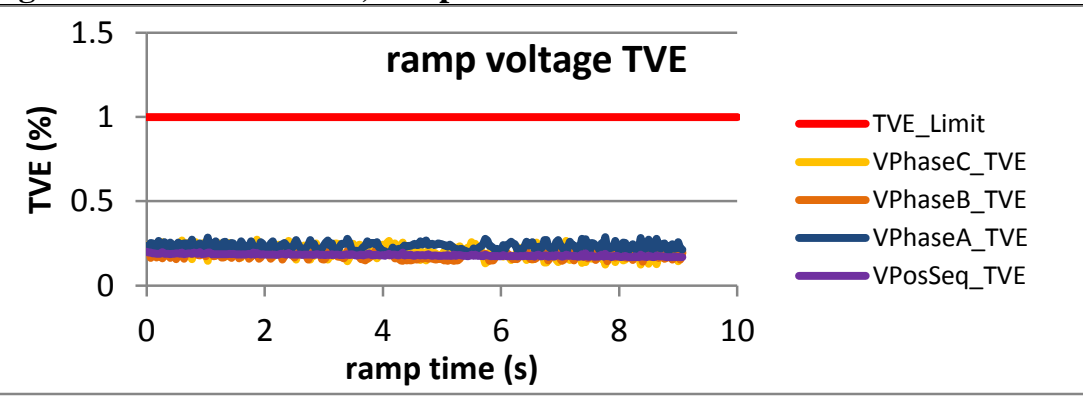

Figure 2216: Fs = $30 \mathrm{FPS}$, ramp from $55 \mathrm{~Hz}$ to $65 \mathrm{~Hz}$ at $+1 \mathrm{~Hz} / \mathrm{s}$

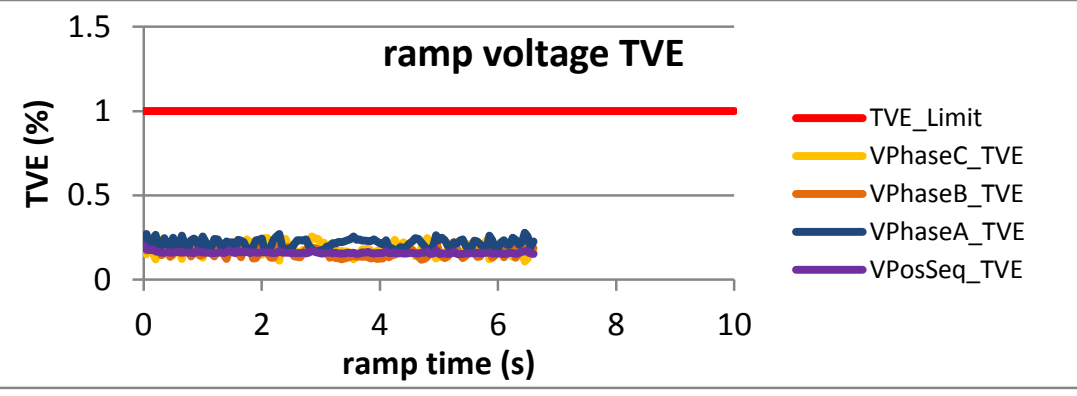

Figure 2218: Fs = $20 \mathrm{FPS}$, ramp from $56 \mathrm{~Hz}$ to $64 \mathrm{~Hz}$ at $+1 \mathrm{~Hz} / \mathrm{s}$

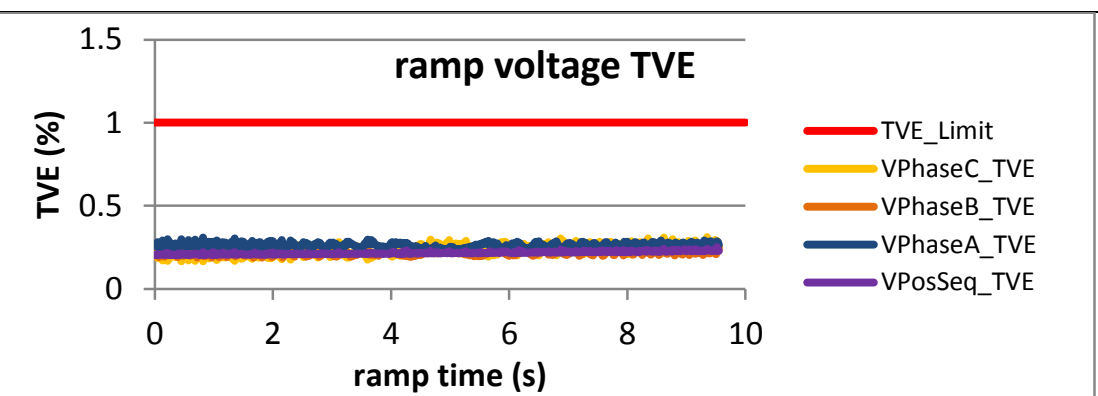

Figure 2215: Fs = $60 \mathrm{FPS}$, ramp from $65 \mathrm{~Hz}$ to $55 \mathrm{~Hz}$ at $-1 \mathrm{~Hz} / \mathrm{s}$

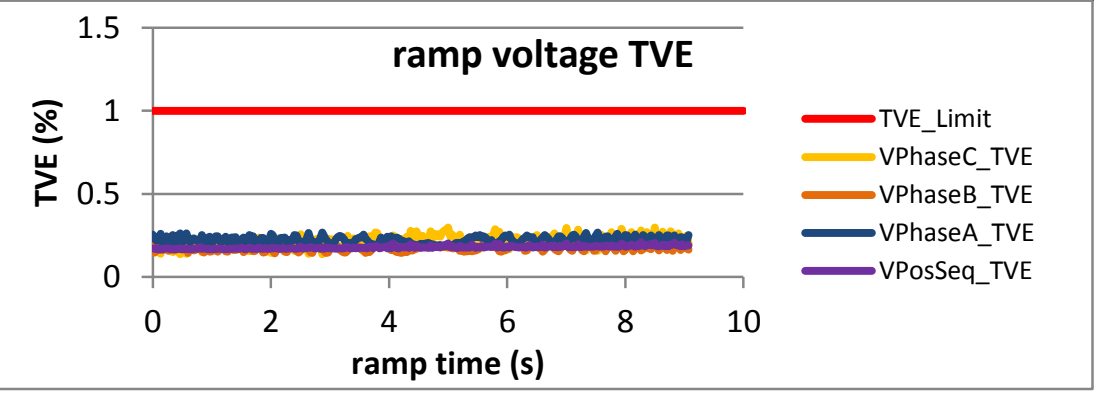

Figure 2217: Fs = $30 \mathrm{FPS}$, ramp from $65 \mathrm{~Hz}$ to $55 \mathrm{~Hz}$ at $-1 \mathrm{~Hz} / \mathrm{s}$

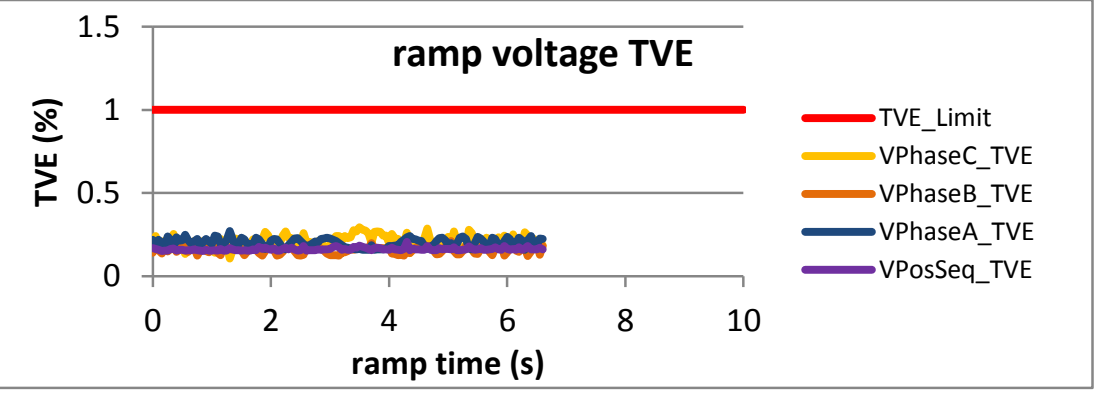

Figure 2219: Fs = 20 FPS, ramp from $64 \mathrm{~Hz}$ to $56 \mathrm{~Hz}$ at $-1 \mathrm{~Hz} / \mathrm{s}$ 


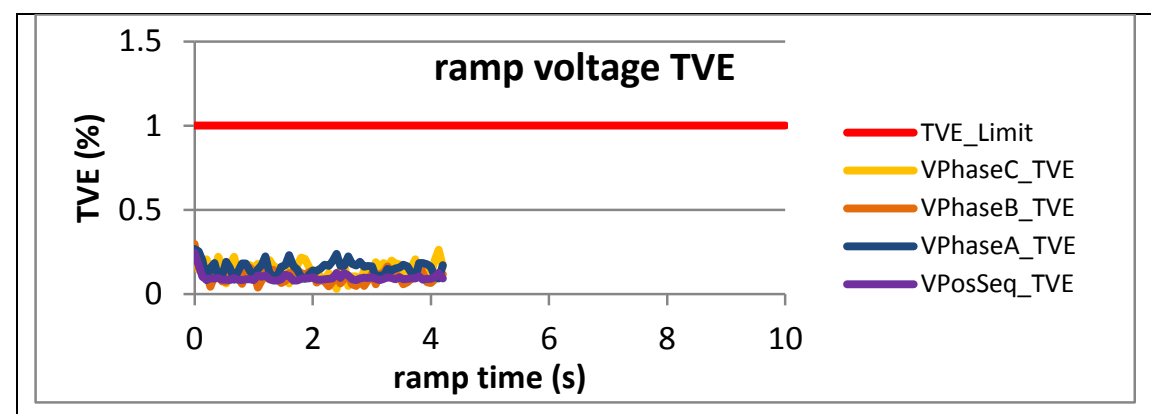

Figure 2220: Fs = $15 \mathrm{FPS}$, ramp from $57 \mathrm{~Hz}$ to $63 \mathrm{~Hz}$ at $+1 \mathrm{~Hz} / \mathrm{s}$

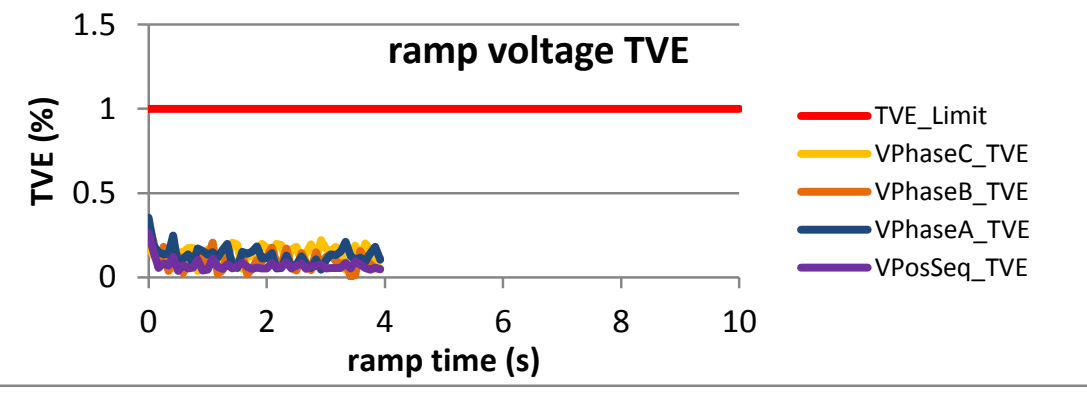

Figure 2221: Fs = $15 \mathrm{FPS}$, ramp from $63 \mathrm{~Hz}$ to $57 \mathrm{~Hz}$ at $-1 \mathrm{~Hz} / \mathrm{s}$
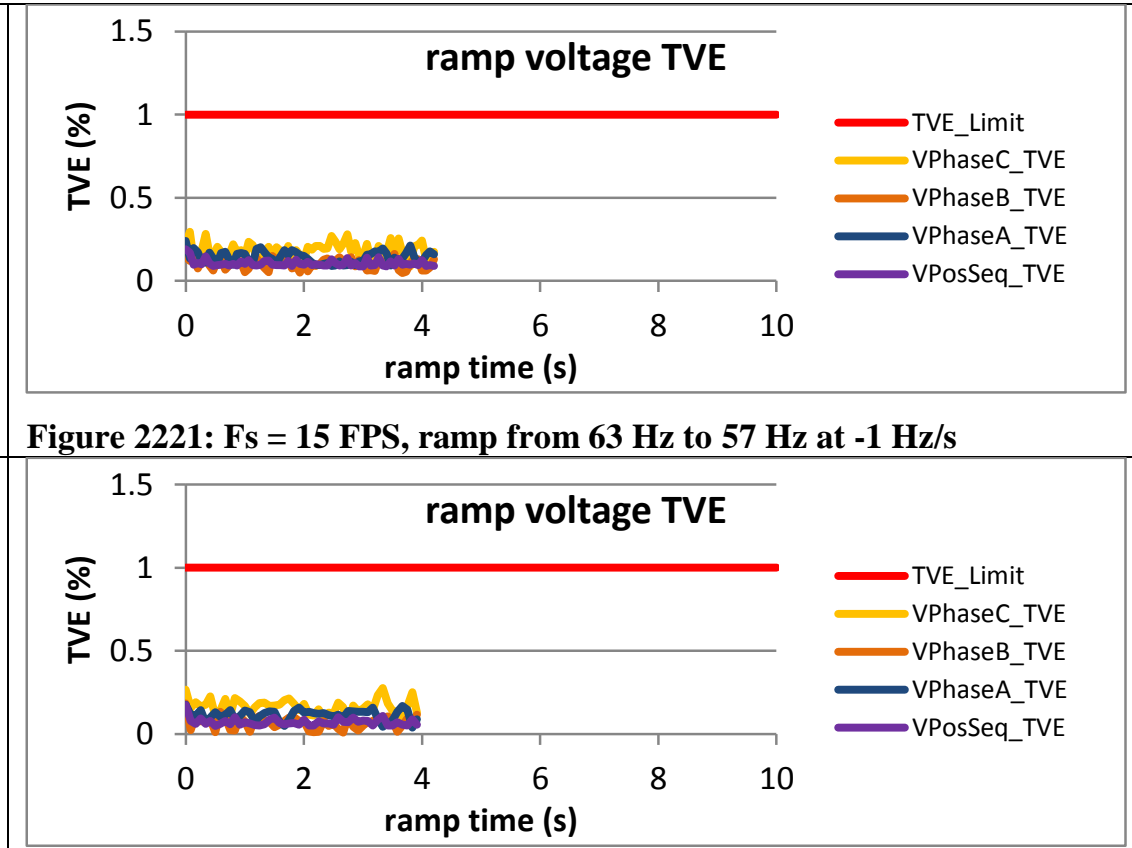

Figure 2222: Fs = 12 FPS, ramp from $58.6 \mathrm{~Hz}$ to $62.4 \mathrm{~Hz}$ at $+1 \mathrm{~Hz} / \mathrm{s}$

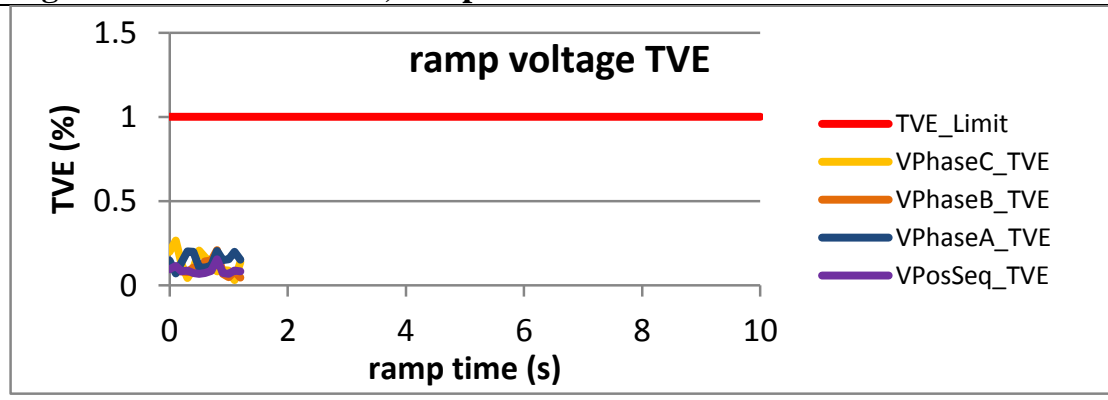

Figure 2224: Fs = $10 \mathrm{FPS}$, ramp from $58 \mathrm{~Hz}$ to $62 \mathrm{~Hz}$ at $+1 \mathrm{~Hz} / \mathrm{s}$

Figure 2223: Fs = 12 FPS, ramp from $62.4 \mathrm{~Hz}$ to $58.6 \mathrm{~Hz}$ at $-1 \mathrm{~Hz} / \mathrm{s}$

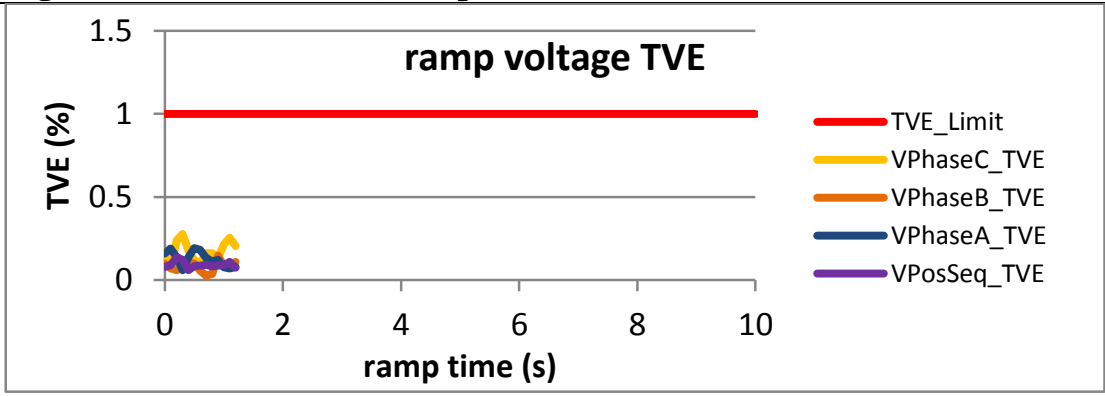

Figure 2225: Fs = $10 \mathrm{FPS}$, ramp from $62 \mathrm{~Hz}$ to $58 \mathrm{~Hz}$ at $-1 \mathrm{~Hz} / \mathrm{s}$ 
6.1.8 PMU G dynamic ramp of system frequency voltage TVE: M class

Figure 2226: Fs = 60 FPS is not supported by this PMU

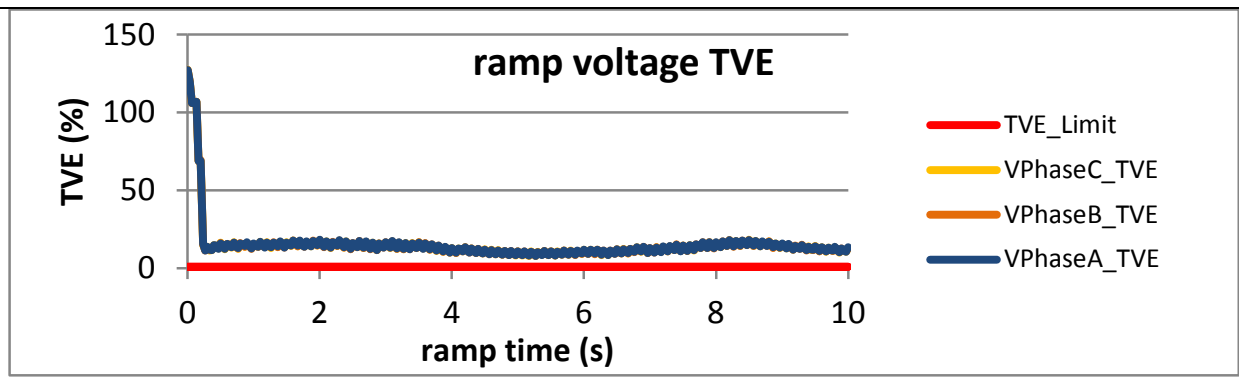

Figure 2227: Fs = $30 \mathrm{FPS}$, ramp from $55 \mathrm{~Hz}$ to $65 \mathrm{~Hz}$ at $+1 \mathrm{~Hz} / \mathrm{s}$

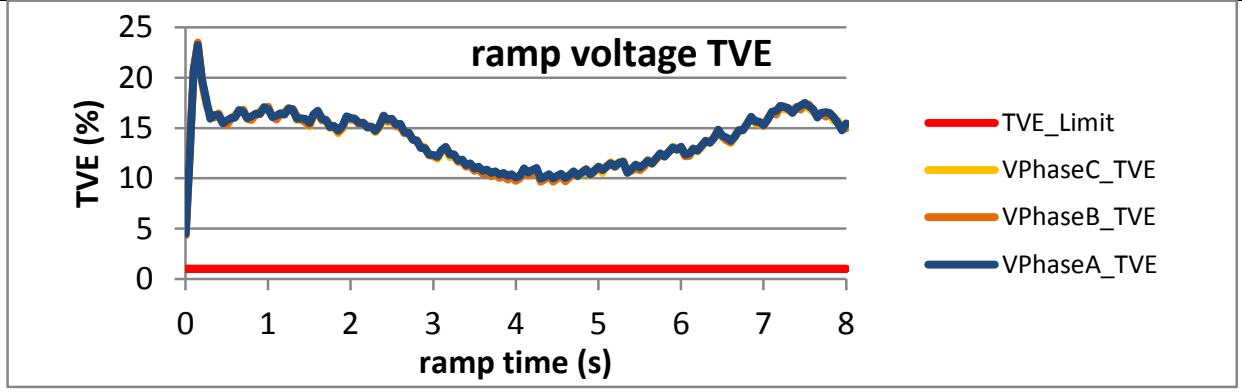

Figure 2229: Fs = $20 \mathrm{FPS}$, ramp from $56 \mathrm{~Hz}$ to $64 \mathrm{~Hz}$ at $+1 \mathrm{~Hz} / \mathrm{s}$

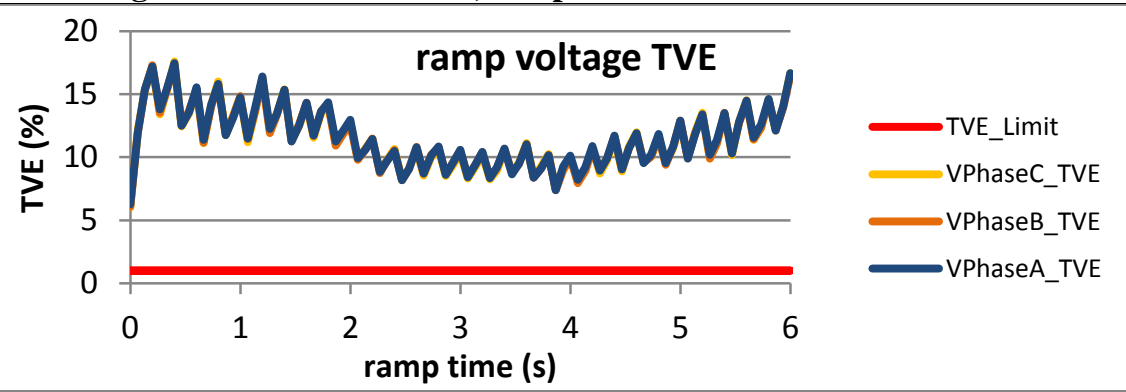

Figure 2231: Fs = $15 \mathrm{FPS}$, ramp from $57 \mathrm{~Hz}$ to $63 \mathrm{~Hz}$ at $+1 \mathrm{~Hz} / \mathrm{s}$

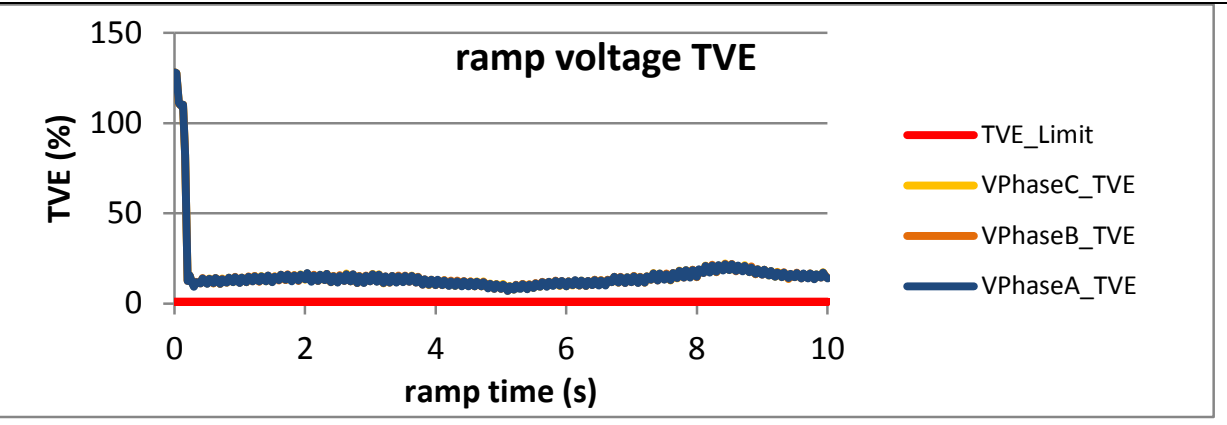

Figure 2228: Fs = $30 \mathrm{FPS}$, ramp from $65 \mathrm{~Hz}$ to $55 \mathrm{~Hz}$ at $-1 \mathrm{~Hz} / \mathrm{s}$

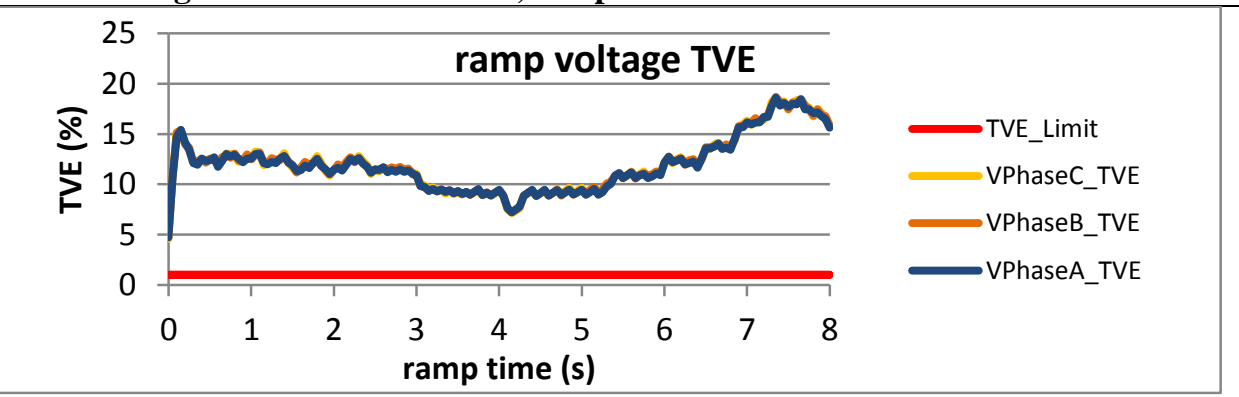

Figure 2230: Fs = $20 \mathrm{FPS}$, ramp from $64 \mathrm{~Hz}$ to $56 \mathrm{~Hz}$ at $-1 \mathrm{~Hz} / \mathrm{s}$

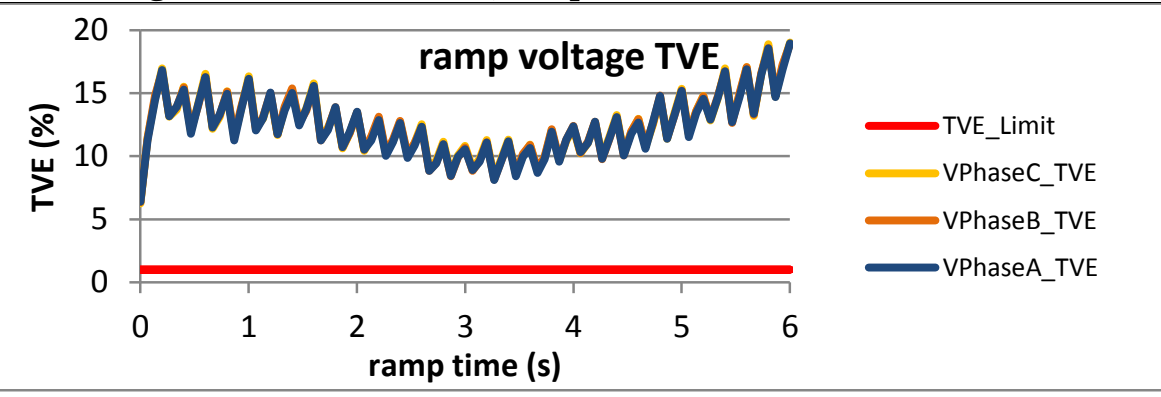

Figure 2232: Fs = $15 \mathrm{FPS}$, ramp from $63 \mathrm{~Hz}$ to $57 \mathrm{~Hz}$ at $-1 \mathrm{~Hz} / \mathrm{s}$ 


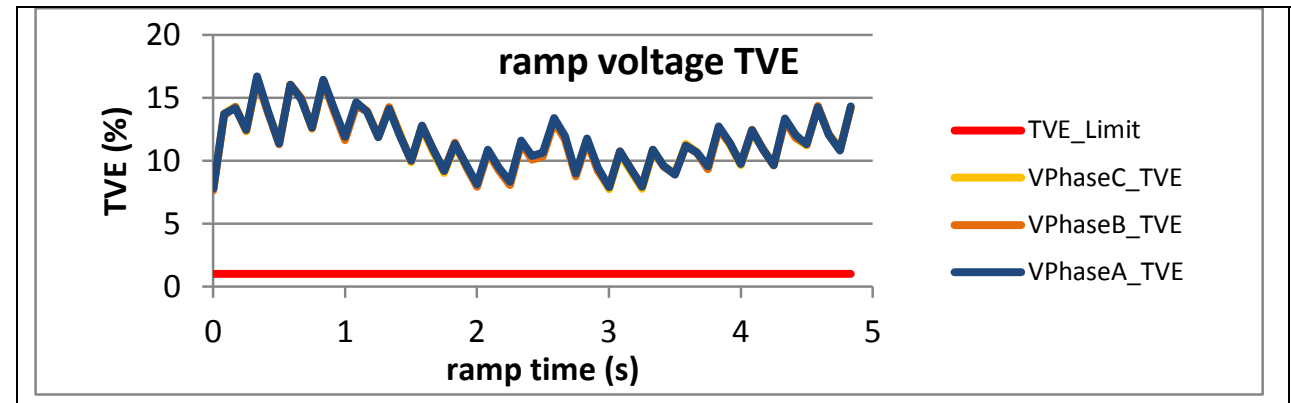

Figure 2233: Fs = 12 FPS, ramp from $57.6 \mathrm{~Hz}$ to $62.4 \mathrm{~Hz}$ at $+1 \mathrm{~Hz} / \mathrm{s}$

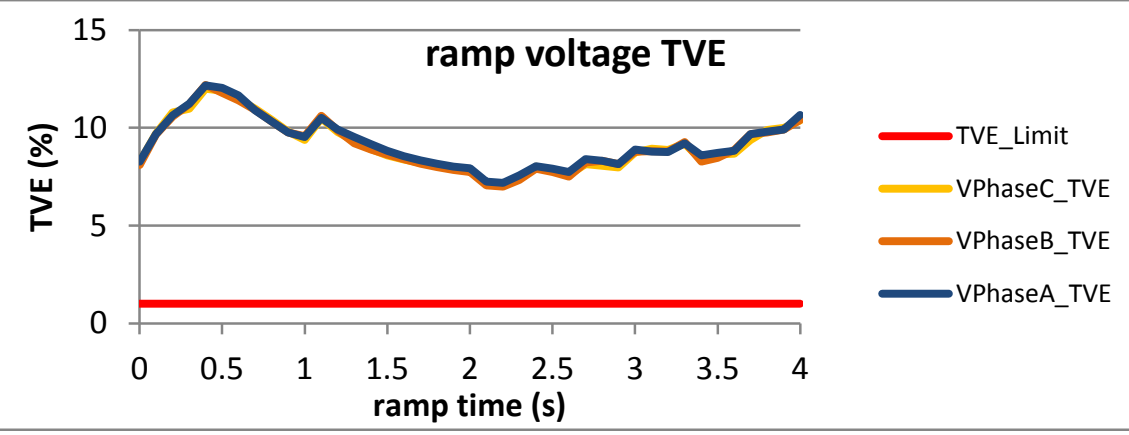

Figure 2235: Fs = $10 \mathrm{FPS}$, ramp from $58 \mathrm{~Hz}$ to $62 \mathrm{~Hz}$ at $+1 \mathrm{~Hz} / \mathrm{s}$

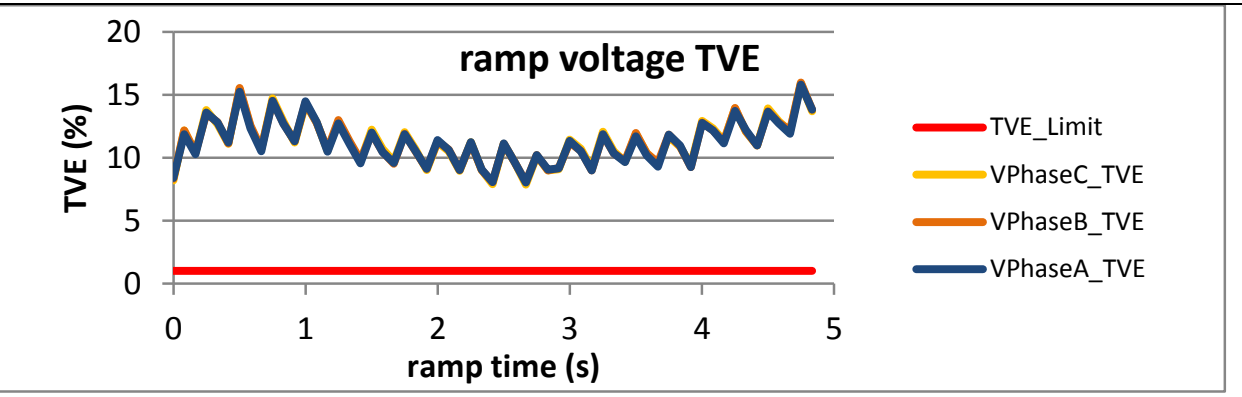

Figure 2234: Fs = 12 FPS, ramp from $62.4 \mathrm{~Hz}$ to $57.6 \mathrm{~Hz}$ at $-1 \mathrm{~Hz} / \mathrm{s}$

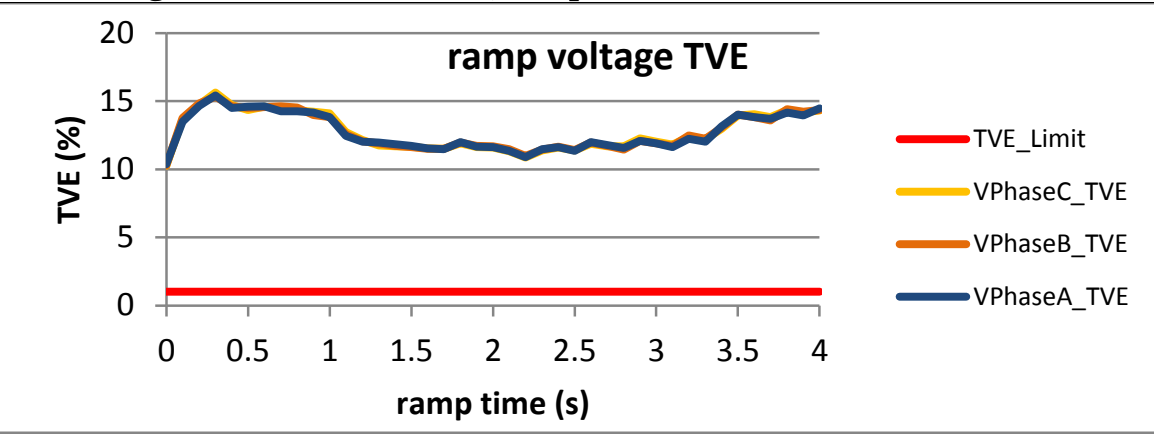

Figure 2236: Fs = $10 \mathrm{FPS}$, ramp from $62 \mathrm{~Hz}$ to $58 \mathrm{~Hz}$ at $-1 \mathrm{~Hz} / \mathrm{s}$ 
6.1.9 PMU H dynamic ramp of system frequency voltage TVE: M class
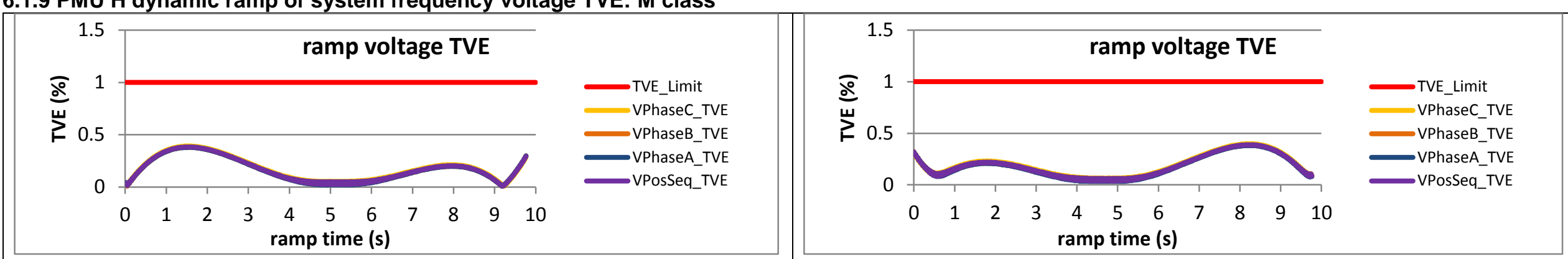

Figure 2237: Fs = $60 \mathrm{FPS}$, ramp from $55 \mathrm{~Hz}$ to $65 \mathrm{~Hz}$ at $+1 \mathrm{~Hz} / \mathrm{s}$

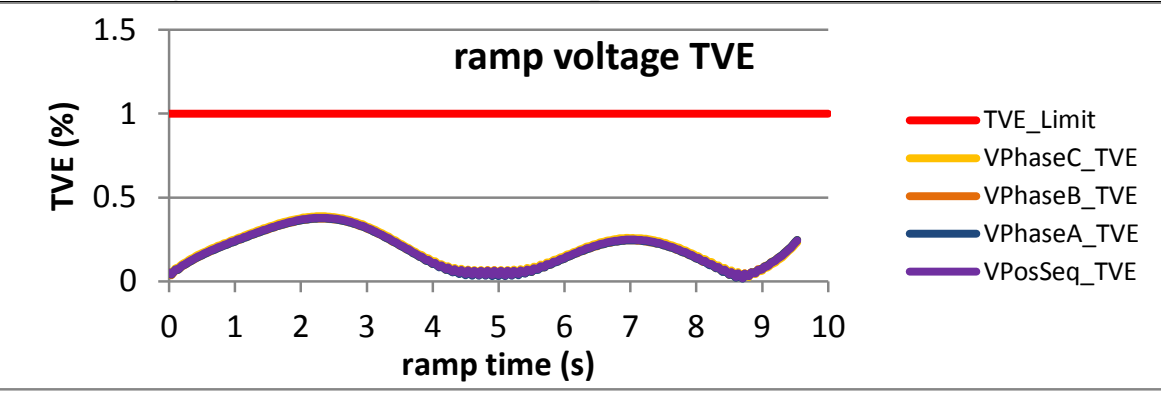

Figure 2239: Fs = $30 \mathrm{FPS}$, ramp from $55 \mathrm{~Hz}$ to $65 \mathrm{~Hz}$ at $+1 \mathrm{~Hz} / \mathrm{s}$

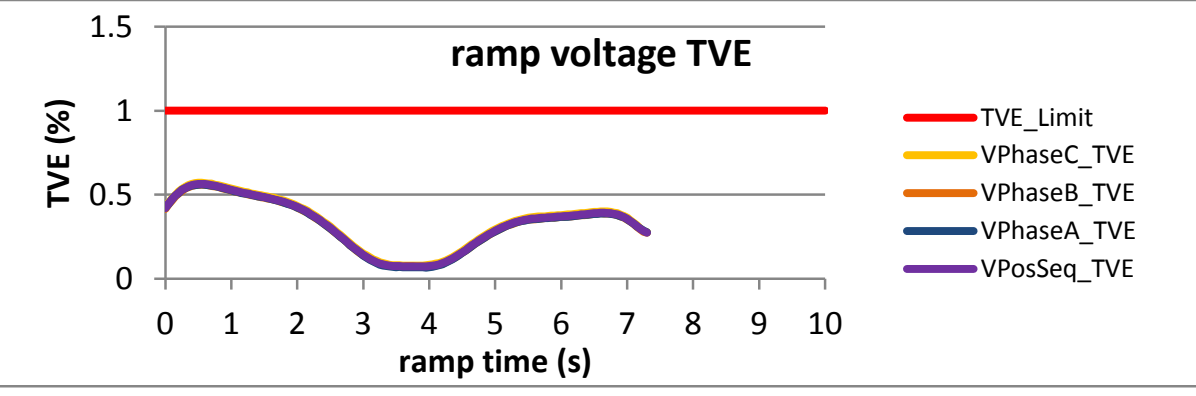

Figure 2241: Fs = $20 \mathrm{FPS}$, ramp from $56 \mathrm{~Hz}$ to $64 \mathrm{~Hz}$ at $+1 \mathrm{~Hz} / \mathrm{s}$

Figure 2238: Fs = $60 \mathrm{FPS}$, ramp from $65 \mathrm{~Hz}$ to $55 \mathrm{~Hz}$ at $-1 \mathrm{~Hz} / \mathrm{s}$

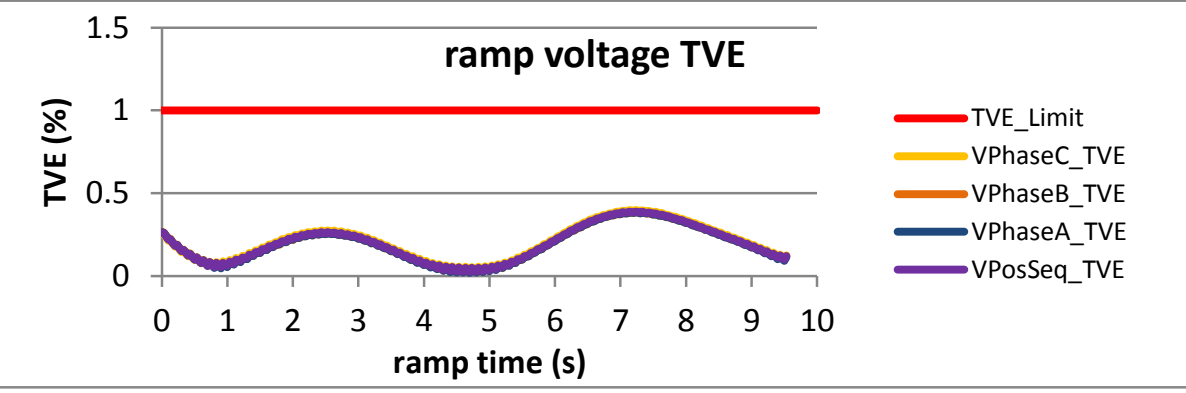

Figure 2240: Fs = $30 \mathrm{FPS}$, ramp from $65 \mathrm{~Hz}$ to $55 \mathrm{~Hz}$ at $-1 \mathrm{~Hz} / \mathrm{s}$

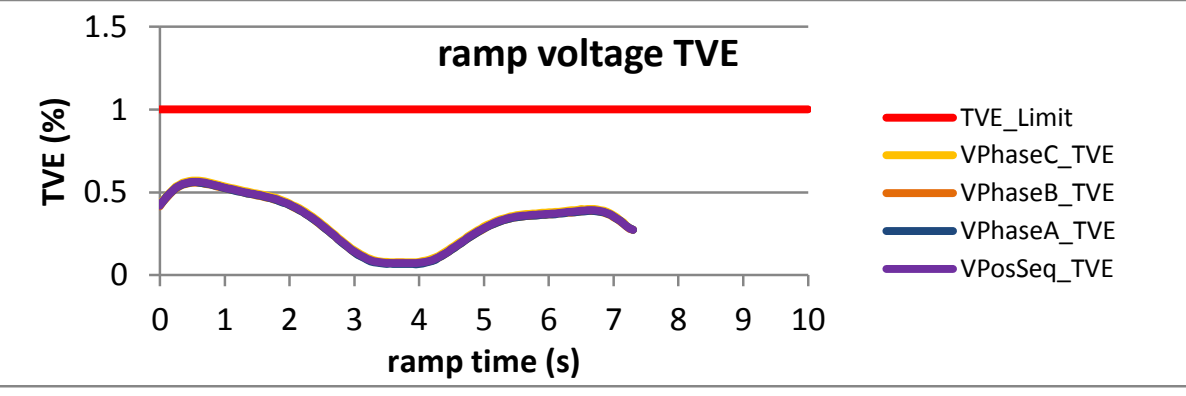

Figure 2242: Fs = $20 \mathrm{FPS}$, ramp from $64 \mathrm{~Hz}$ to $56 \mathrm{~Hz}$ at $-1 \mathrm{~Hz} / \mathrm{s}$ 


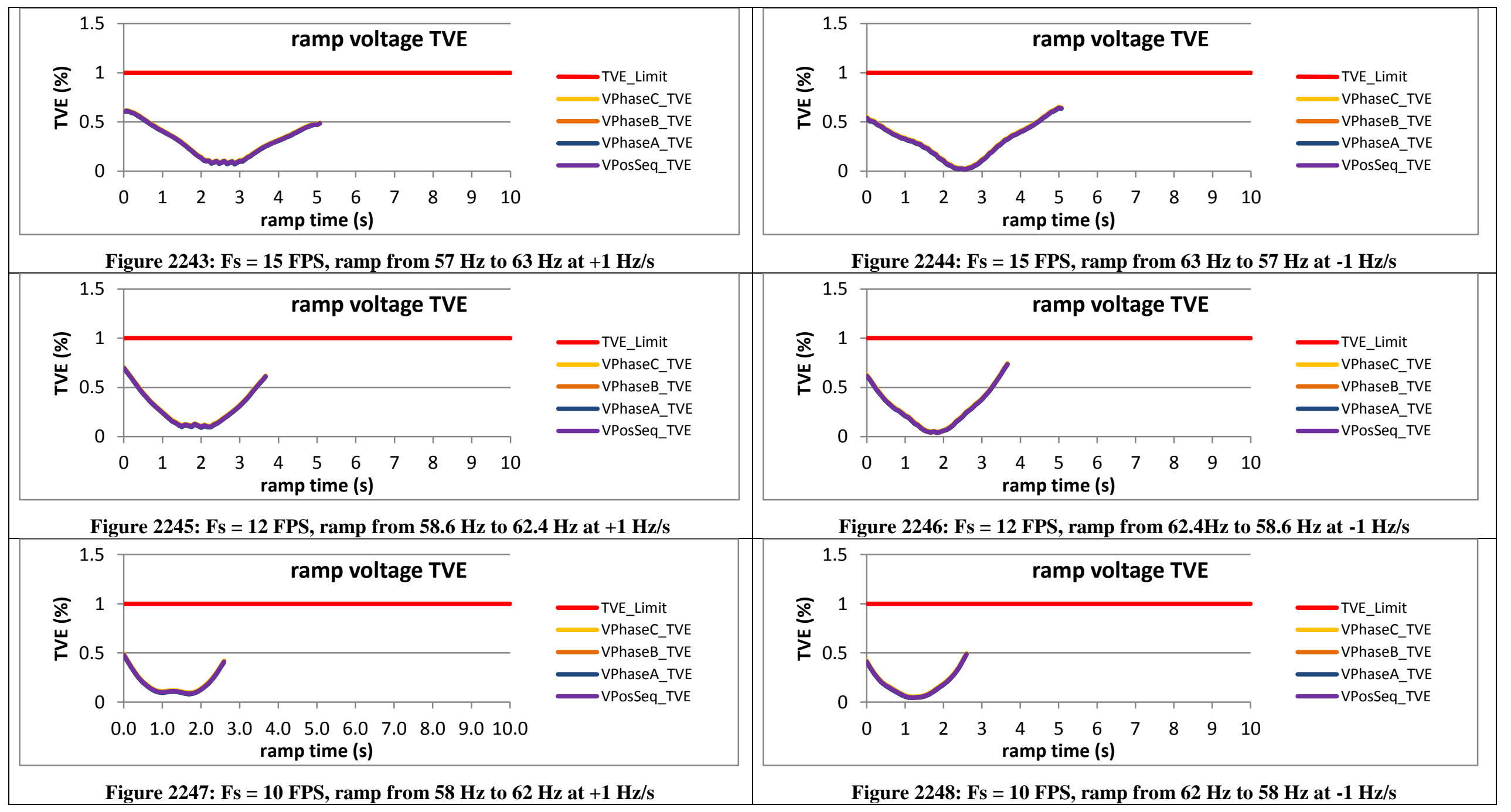


6.1.10 PMU I dynamic ramp of system frequency voltage TVE: M class

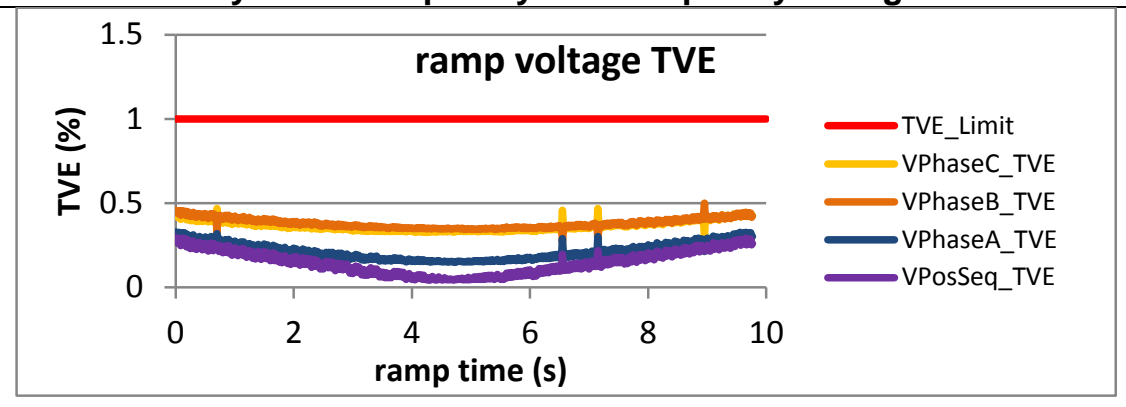

Figure 2249: Fs = 60 FPS, ramp from $55 \mathrm{~Hz}$ to $65 \mathrm{~Hz}$ at $+1 \mathrm{~Hz} / \mathrm{s}$

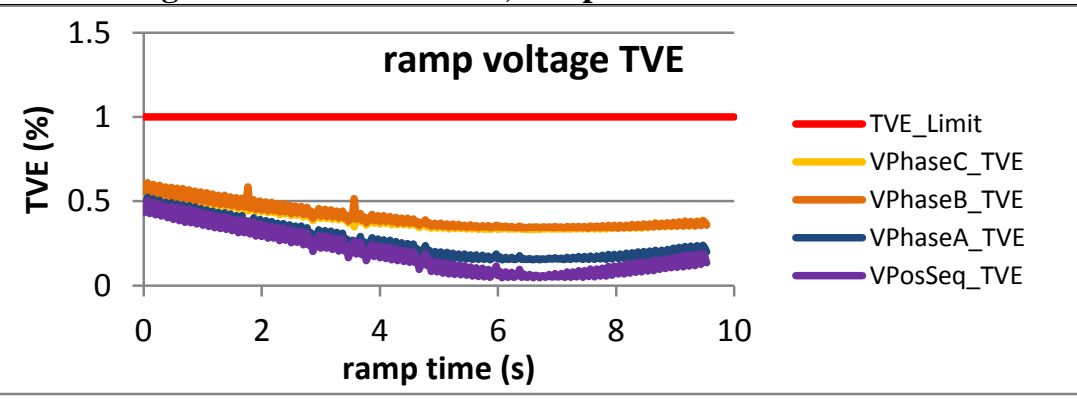

Figure 2251: Fs = $30 \mathrm{FPS}$, ramp from $55 \mathrm{~Hz}$ to $65 \mathrm{~Hz}$ at $+1 \mathrm{~Hz} / \mathrm{s}$

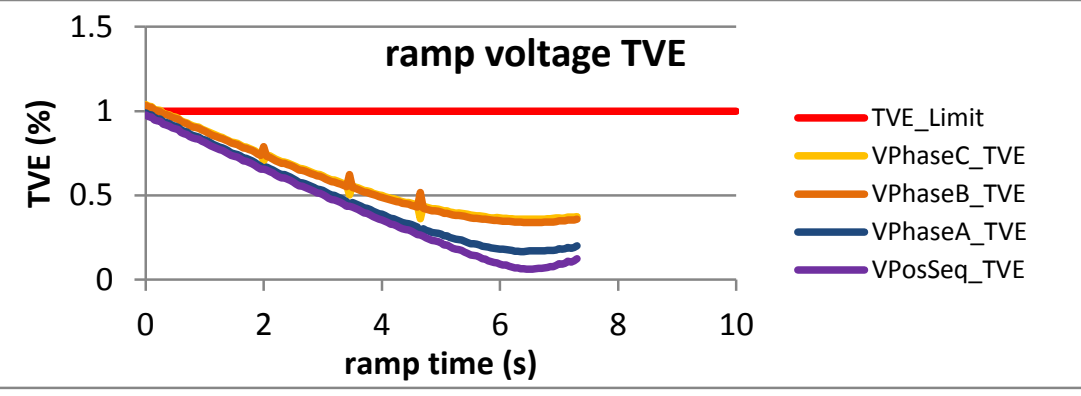

Figure 2253: Fs $=20 \mathrm{FPS}$, ramp from $56 \mathrm{~Hz}$ to $64 \mathrm{~Hz}$ at $+1 \mathrm{~Hz} / \mathrm{s}$

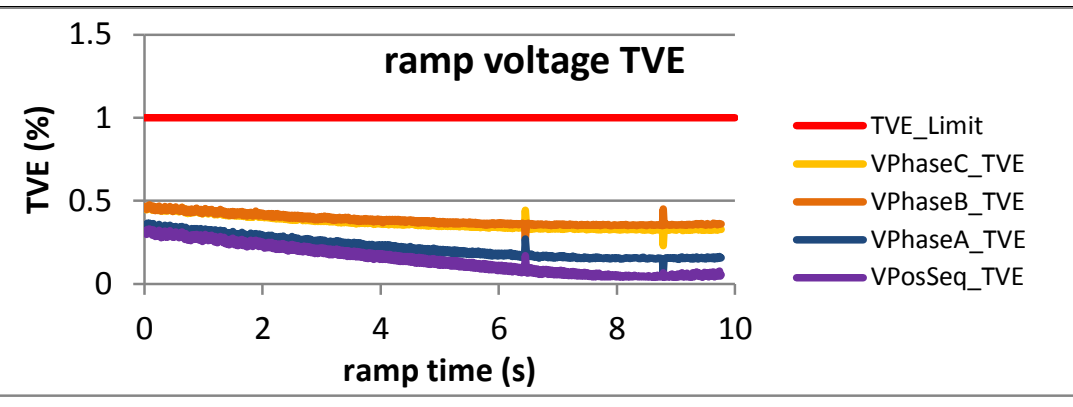

Figure 2250: Fs = $60 \mathrm{FPS}$, ramp from $65 \mathrm{~Hz}$ to $55 \mathrm{~Hz}$ at $-1 \mathrm{~Hz} / \mathrm{s}$

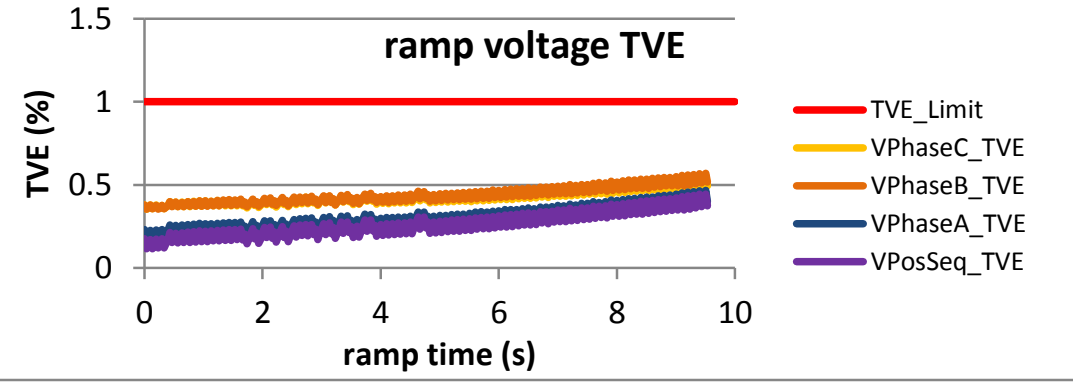

Figure 2252: Fs = $30 \mathrm{FPS}$, ramp from $65 \mathrm{~Hz}$ to $55 \mathrm{~Hz}$ at $-1 \mathrm{~Hz} / \mathrm{s}$

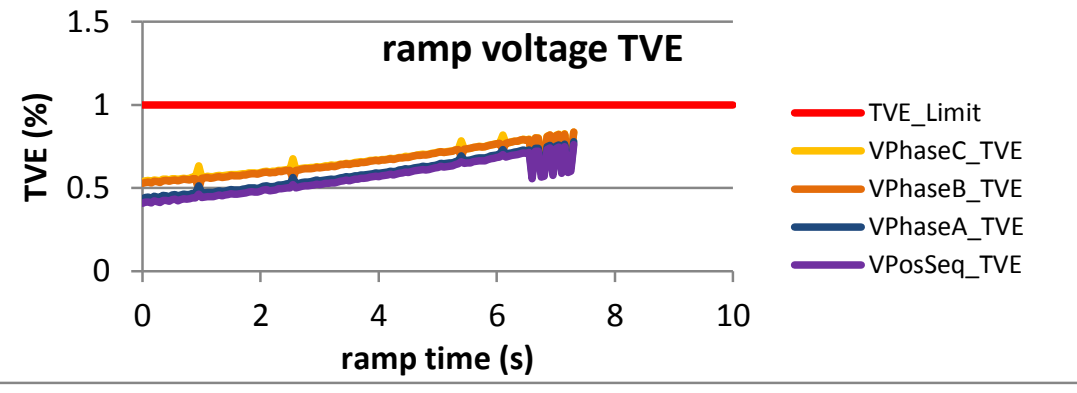

Figure 2254: Fs = $20 \mathrm{FPS}$, ramp from $64 \mathrm{~Hz}$ to $56 \mathrm{~Hz}$ at $-1 \mathrm{~Hz} / \mathrm{s}$ 


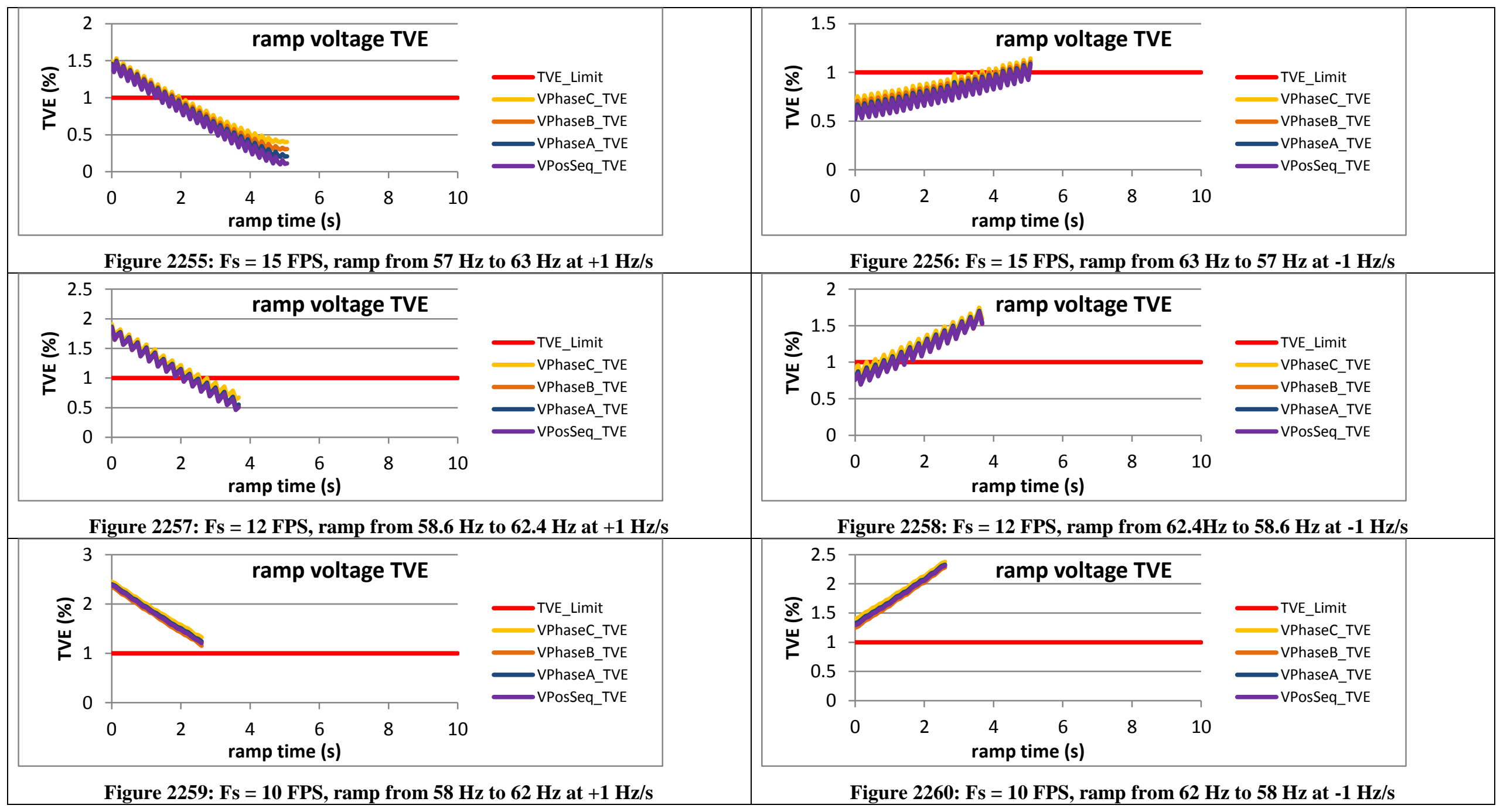


6.1.11 PMU J dynamic ramp of system frequency voltage TVE: M class

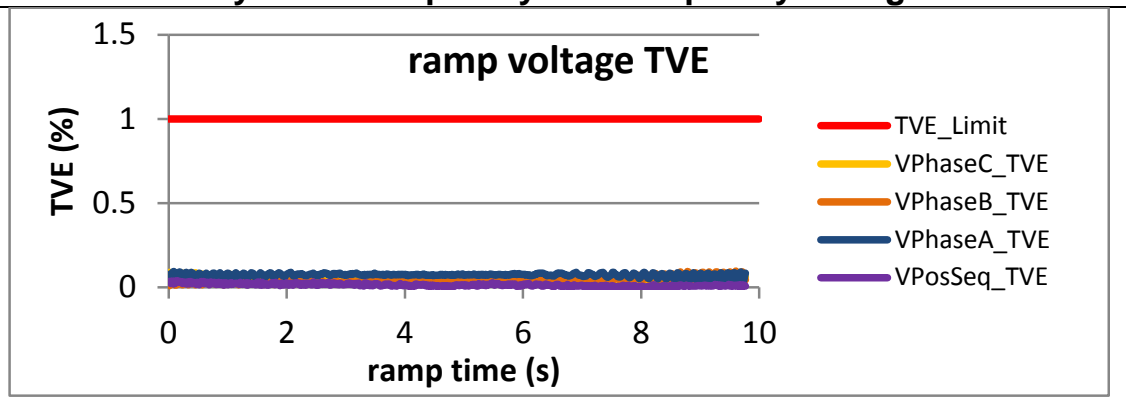

Figure 2261: Fs = $60 \mathrm{FPS}$, ramp from $55 \mathrm{~Hz}$ to $65 \mathrm{~Hz}$ at $+1 \mathrm{~Hz} / \mathrm{s}$

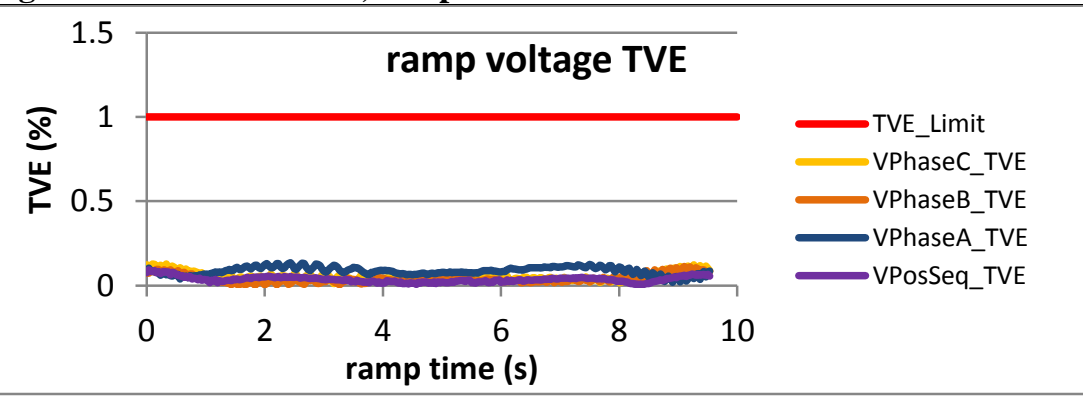

Figure 2263: Fs = $30 \mathrm{FPS}$, ramp from $55 \mathrm{~Hz}$ to $65 \mathrm{~Hz}$ at $+1 \mathrm{~Hz} / \mathrm{s}$

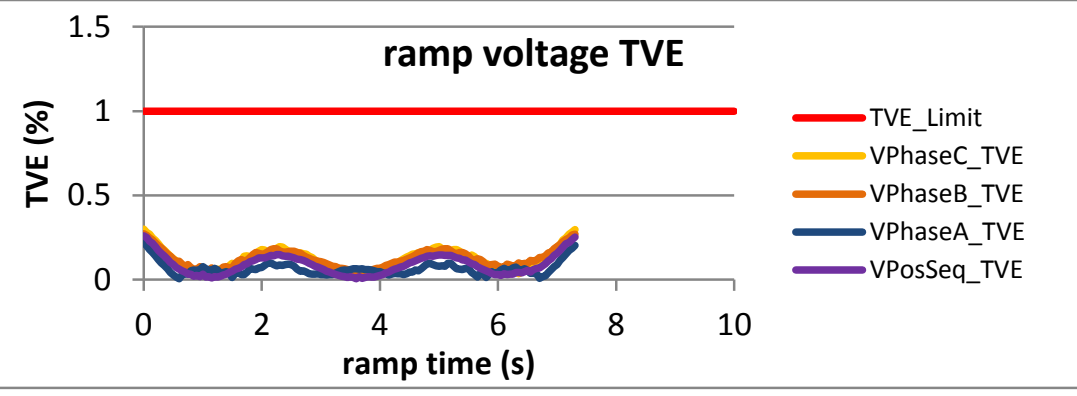

Figure 2265: Fs = 20 FPS, ramp from $56 \mathrm{~Hz}$ to $64 \mathrm{~Hz}$ at $+1 \mathrm{~Hz} / \mathrm{s}$

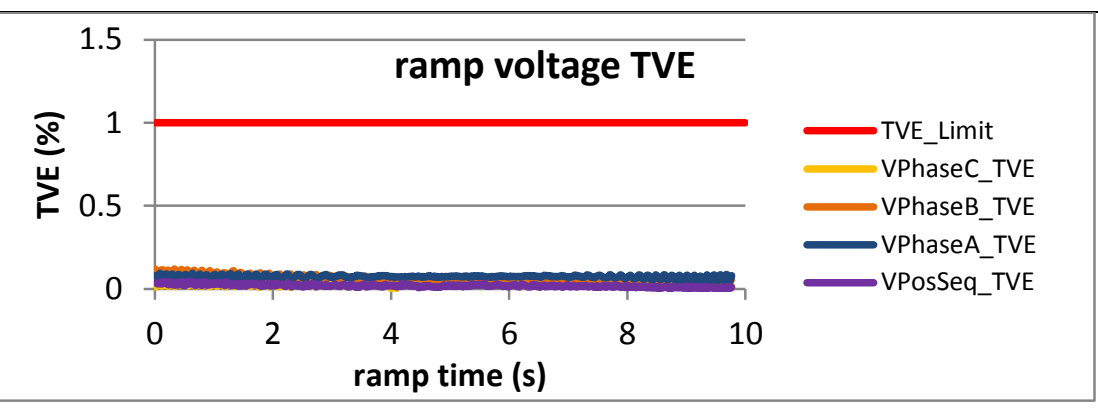

Figure 2262: Fs = $60 \mathrm{FPS}$, ramp from $65 \mathrm{~Hz}$ to $55 \mathrm{~Hz}$ at $-1 \mathrm{~Hz} / \mathrm{s}$

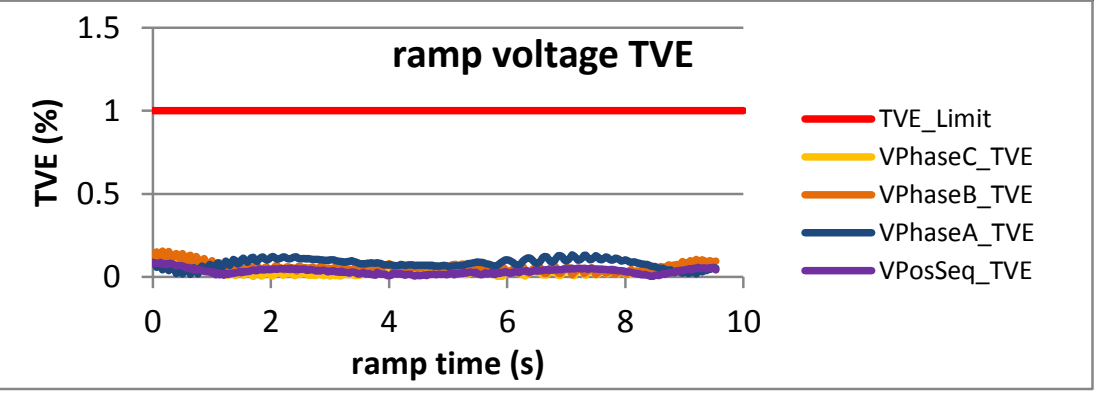

Figure 2264: Fs = $30 \mathrm{FPS}$, ramp from $65 \mathrm{~Hz}$ to $55 \mathrm{~Hz}$ at $-1 \mathrm{~Hz} / \mathrm{s}$

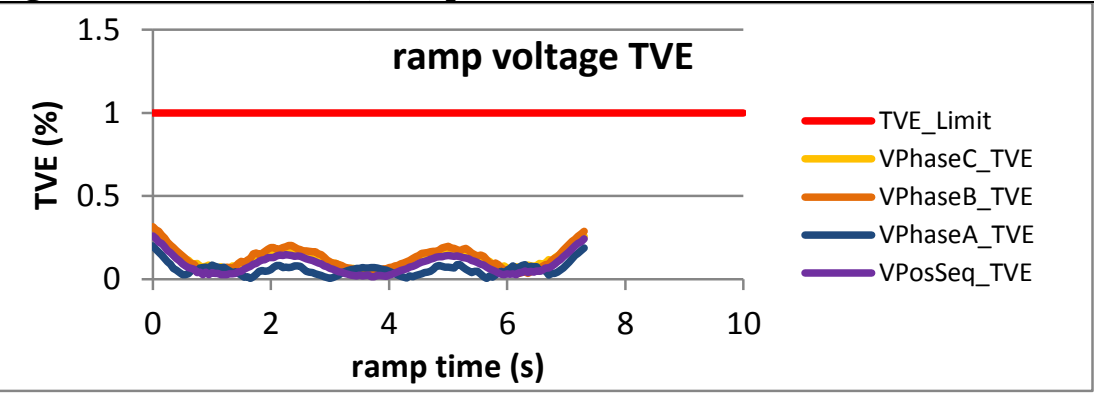

Figure 2266: Fs = $20 \mathrm{FPS}$, ramp from $64 \mathrm{~Hz}$ to $56 \mathrm{~Hz}$ at $-1 \mathrm{~Hz} / \mathrm{s}$ 


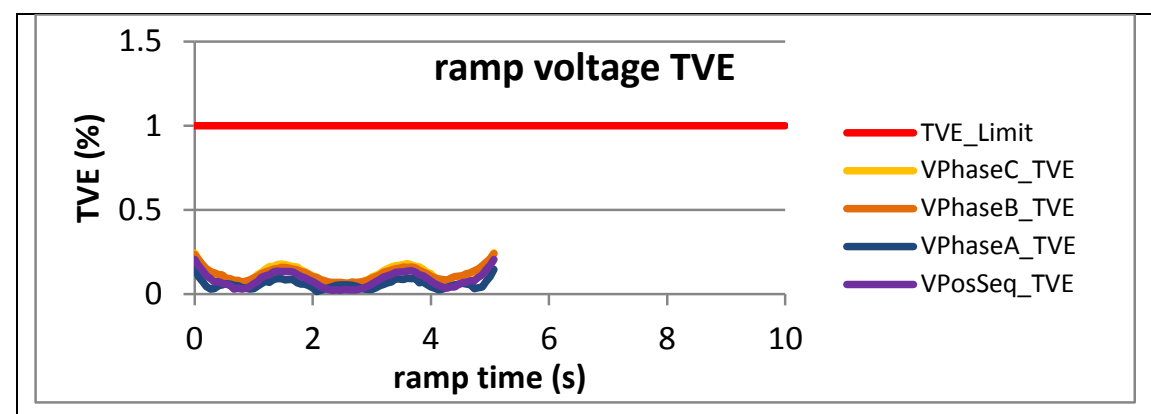

Figure 2267: Fs = $15 \mathrm{FPS}$, ramp from $57 \mathrm{~Hz}$ to $63 \mathrm{~Hz}$ at $+1 \mathrm{~Hz} / \mathrm{s}$

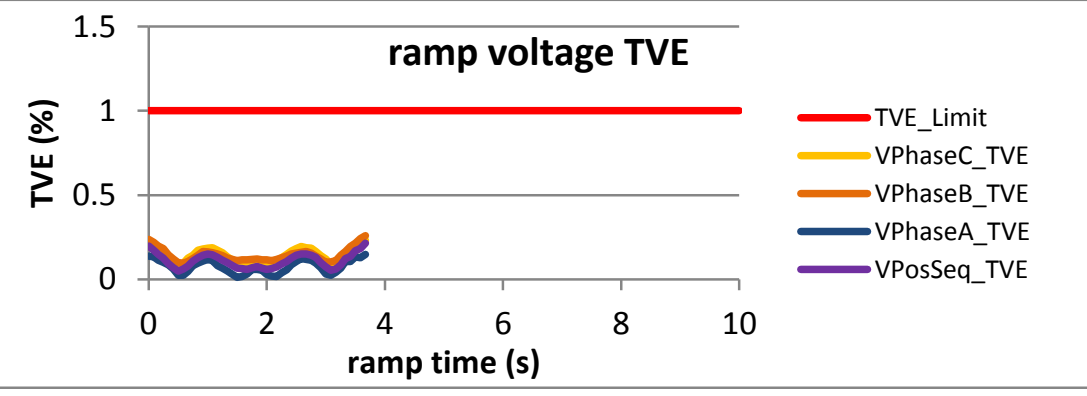

Figure 2268: Fs = $15 \mathrm{FPS}$, ramp from $63 \mathrm{~Hz}$ to $57 \mathrm{~Hz}$ at $-1 \mathrm{~Hz} / \mathrm{s}$
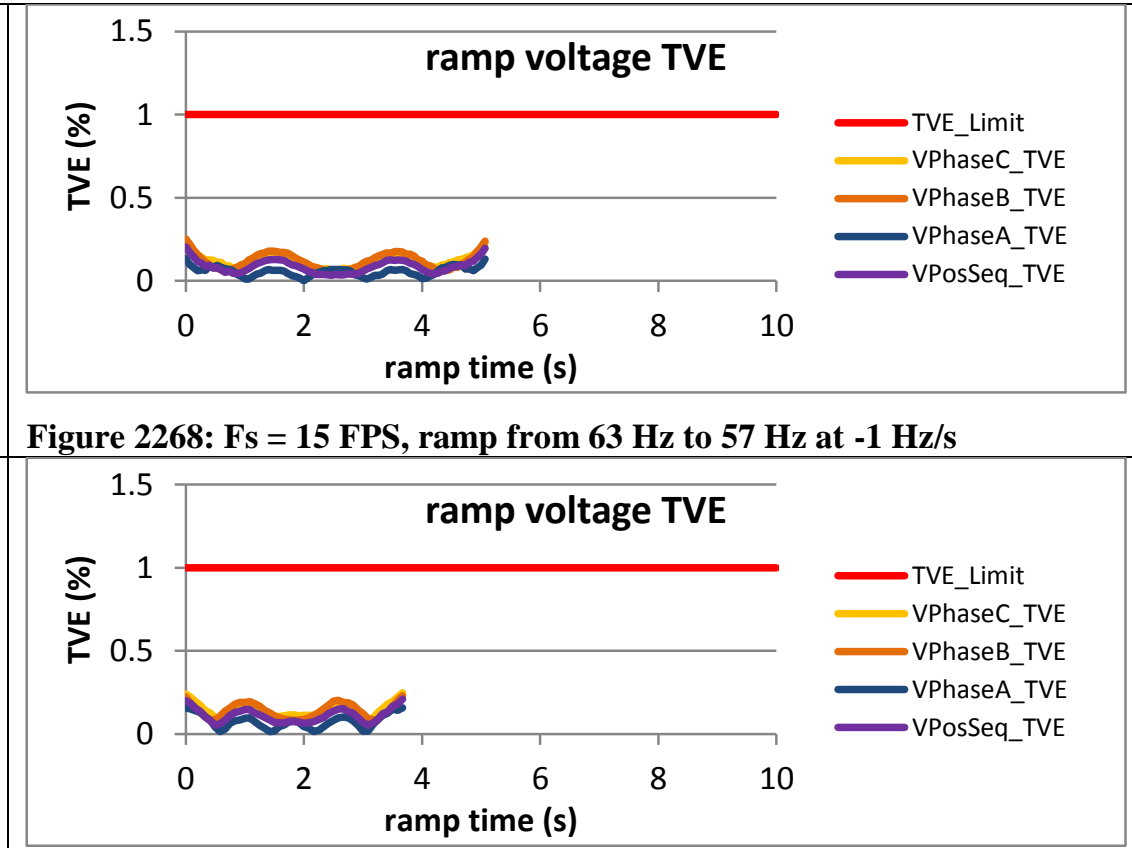

Figure 2269: Fs = 12 FPS, ramp from $58.6 \mathrm{~Hz}$ to $62.4 \mathrm{~Hz}$ at $+1 \mathrm{~Hz} / \mathrm{s}$

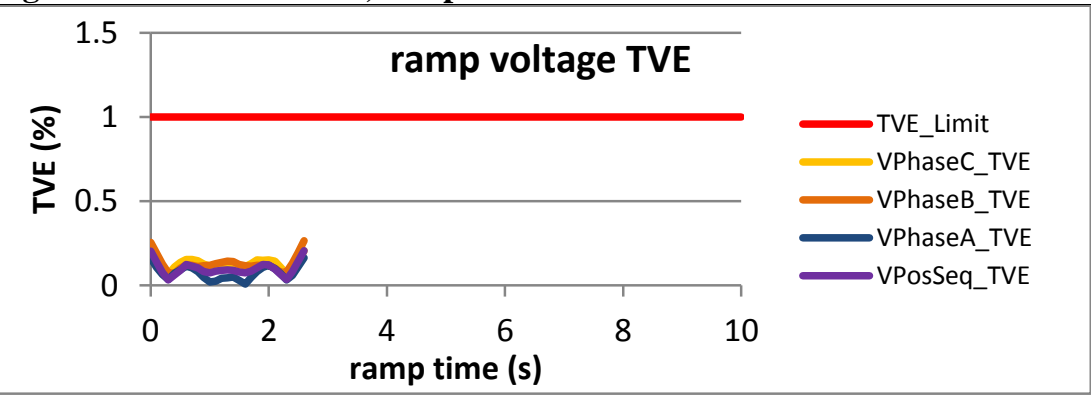

Figure 2271: Fs = $10 \mathrm{FPS}$, ramp from $58 \mathrm{~Hz}$ to $62 \mathrm{~Hz}$ at $+1 \mathrm{~Hz} / \mathrm{s}$

Figure 2270: Fs = 12 FPS, ramp from $62.4 \mathrm{~Hz}$ to $58.6 \mathrm{~Hz}$ at $-1 \mathrm{~Hz} / \mathrm{s}$

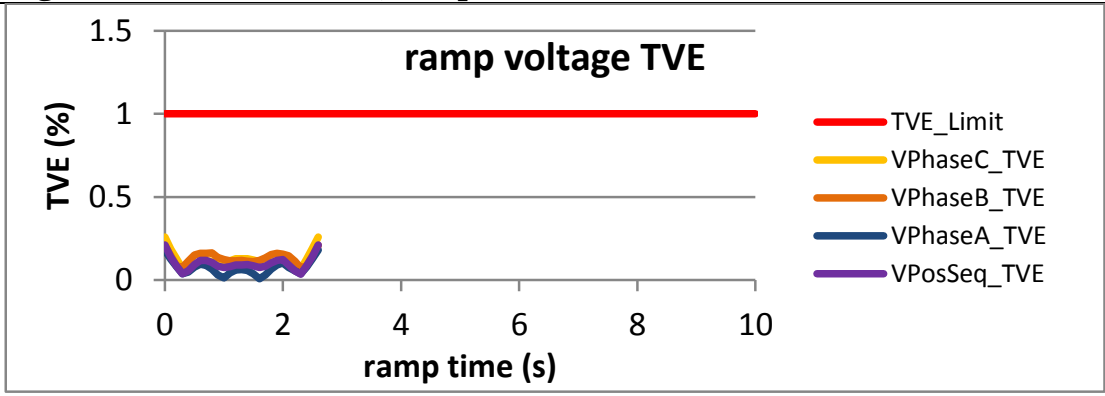

Figure 2272: Fs = $10 \mathrm{FPS}$, ramp from $62 \mathrm{~Hz}$ to $58 \mathrm{~Hz}$ at $-1 \mathrm{~Hz} / \mathrm{s}$ 


\subsection{Dynamic ramp of system frequency current TVE, M class}

\subsubsection{C37.118.1 Annex C dynamic ramp of system frequency current TVE, M class}

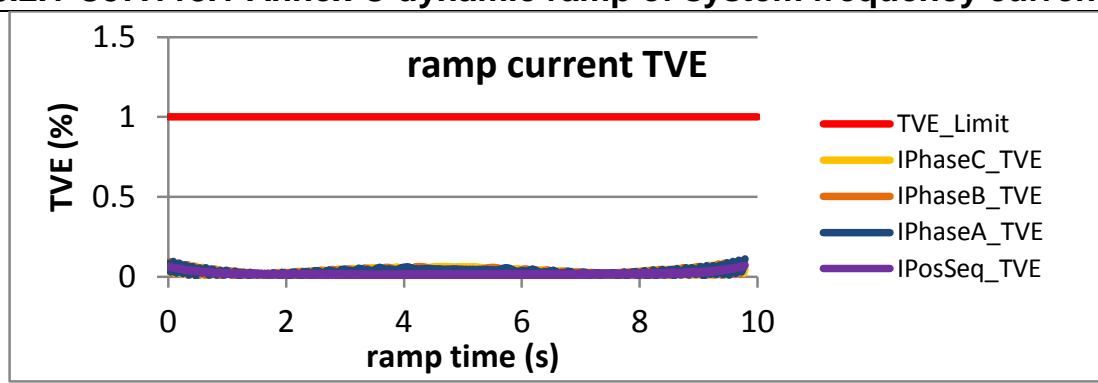

Figure 2273: Fs = $60 \mathrm{FPS}$, ramp from $55 \mathrm{~Hz}$ to $65 \mathrm{~Hz}$ at $+1 \mathrm{~Hz} / \mathrm{s}$

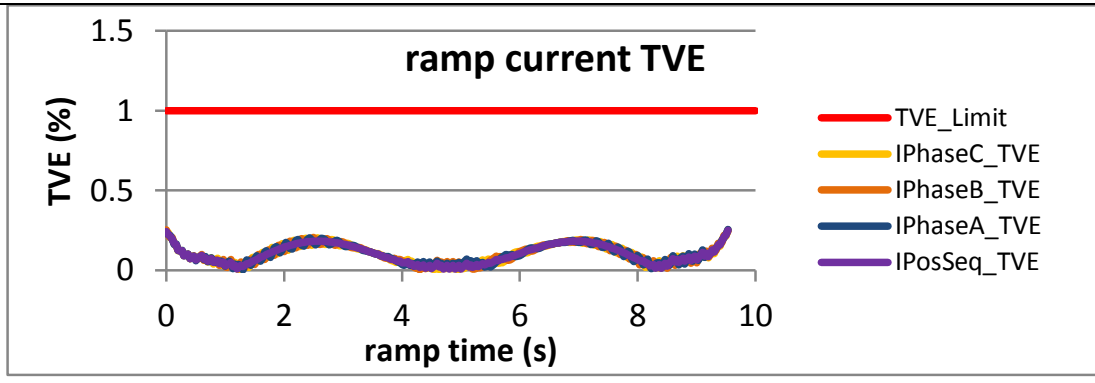

Figure 2275: Fs = $30 \mathrm{FPS}$, ramp from $55 \mathrm{~Hz}$ to $65 \mathrm{~Hz}$ at $+1 \mathrm{~Hz} / \mathrm{s}$

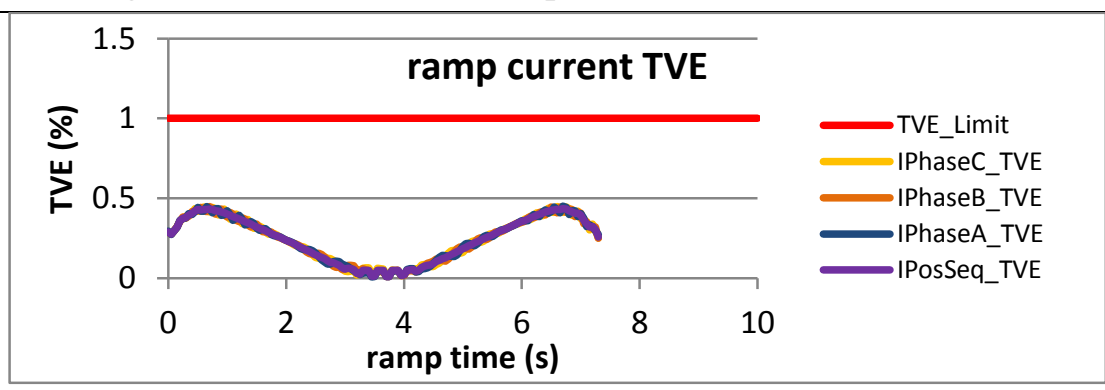

Figure 2277: Fs = $20 \mathrm{FPS}$, ramp from $56 \mathrm{~Hz}$ to $64 \mathrm{~Hz}$ at $+1 \mathrm{~Hz} / \mathrm{s}$

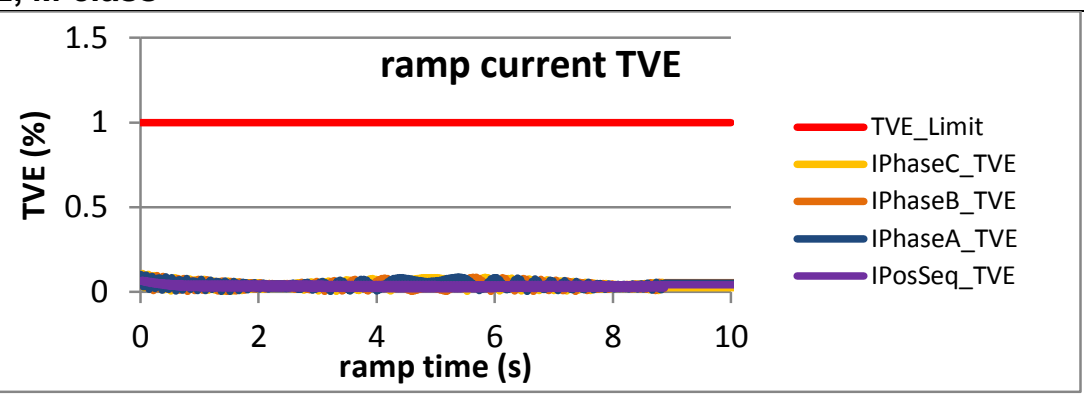

Figure 2274: Fs = $60 \mathrm{FPS}$, ramp from $65 \mathrm{~Hz}$ to $55 \mathrm{~Hz}$ at $-1 \mathrm{~Hz} / \mathrm{s}$

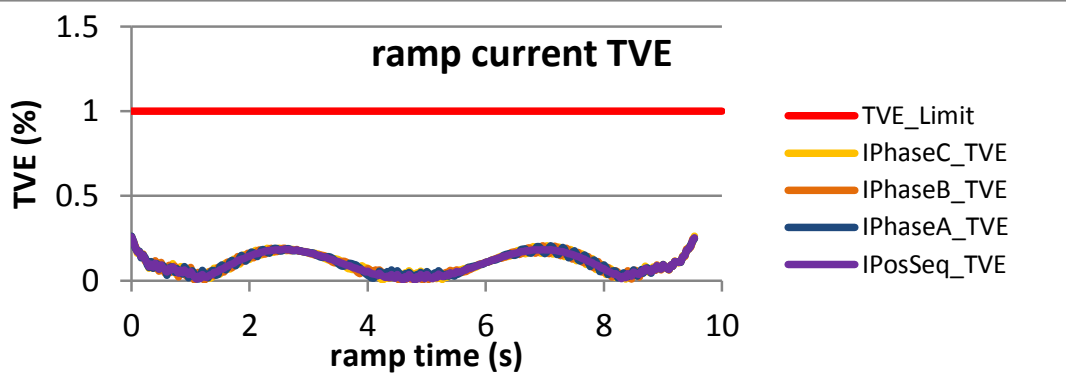

Figure 2276: Fs = $30 \mathrm{FPS}$, ramp from $65 \mathrm{~Hz}$ to $55 \mathrm{~Hz}$ at $-1 \mathrm{~Hz} / \mathrm{s}$
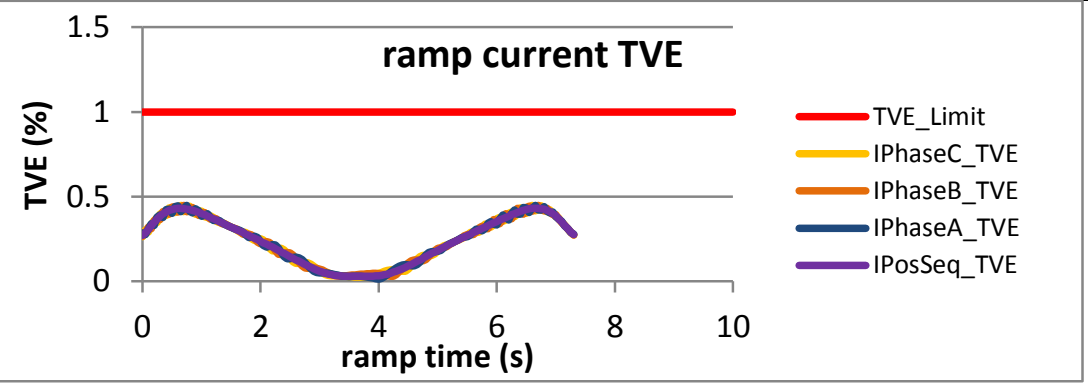

Figure 2278: Fs = $20 \mathrm{FPS}$, ramp from $64 \mathrm{~Hz}$ to $56 \mathrm{~Hz}$ at $-1 \mathrm{~Hz} / \mathrm{s}$ 


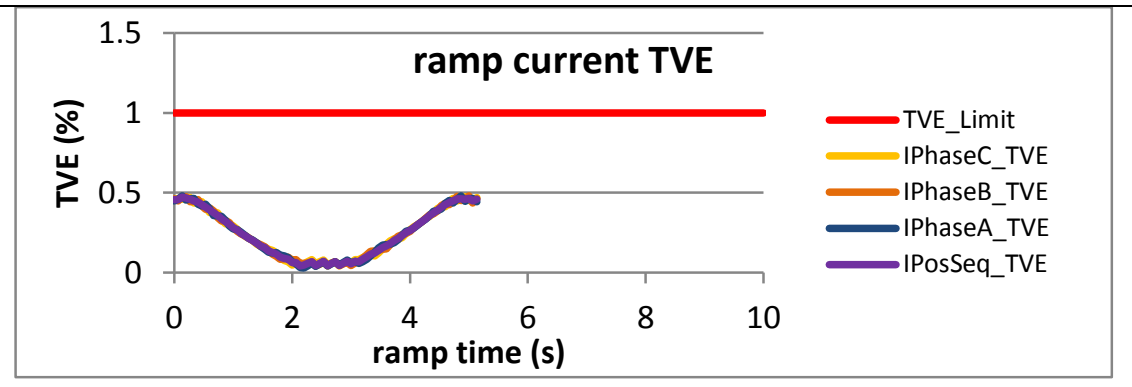

Figure 2279: Fs = 15 FPS, ramp from $57 \mathrm{~Hz}$ to $63 \mathrm{~Hz}$ at $+1 \mathrm{~Hz} / \mathrm{s}$

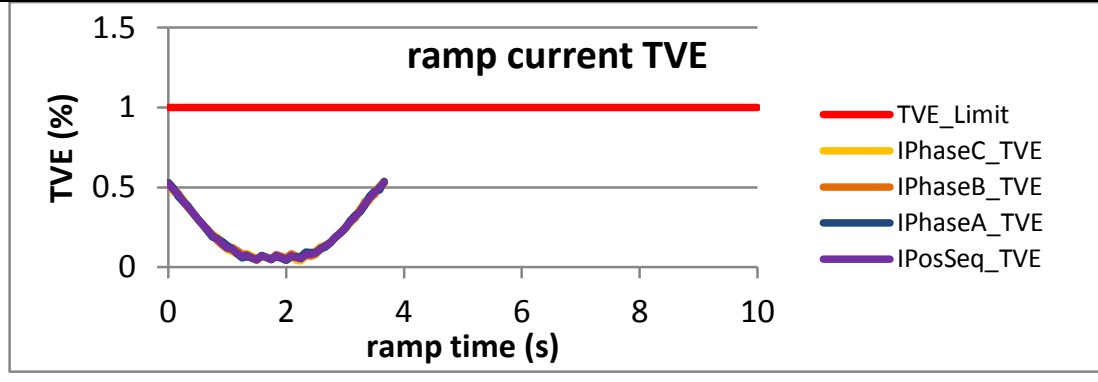

Figure 2281: Fs = 12 FPS, ramp from $58.6 \mathrm{~Hz}$ to $62.4 \mathrm{~Hz}$ at $+1 \mathrm{~Hz} / \mathrm{s}$
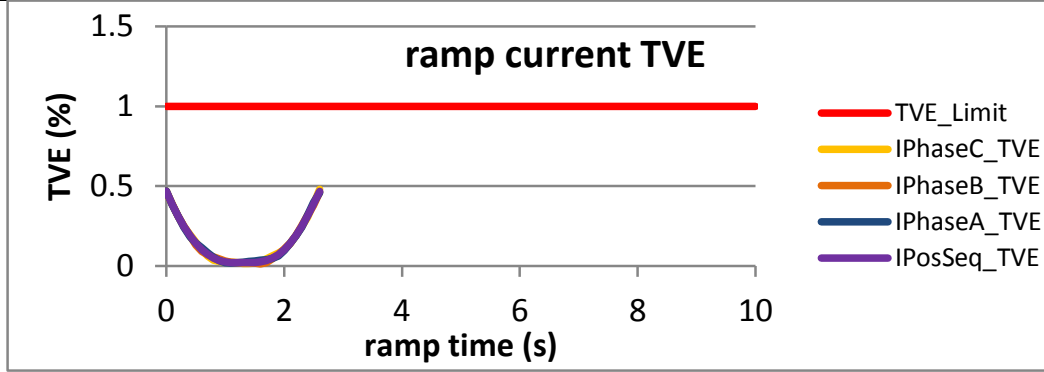

Figure 2283: Fs = $10 \mathrm{FPS}$, ramp from $58 \mathrm{~Hz}$ to $62 \mathrm{~Hz}$ at $+1 \mathrm{~Hz} / \mathrm{s}$

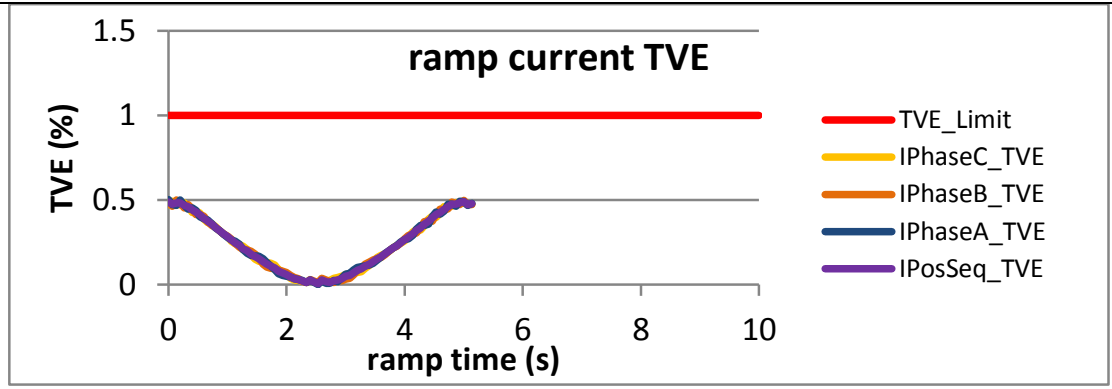

Figure 2280: Fs = $15 \mathrm{FPS}$, ramp from $63 \mathrm{~Hz}$ to $57 \mathrm{~Hz}$ at $-1 \mathrm{~Hz} / \mathrm{s}$

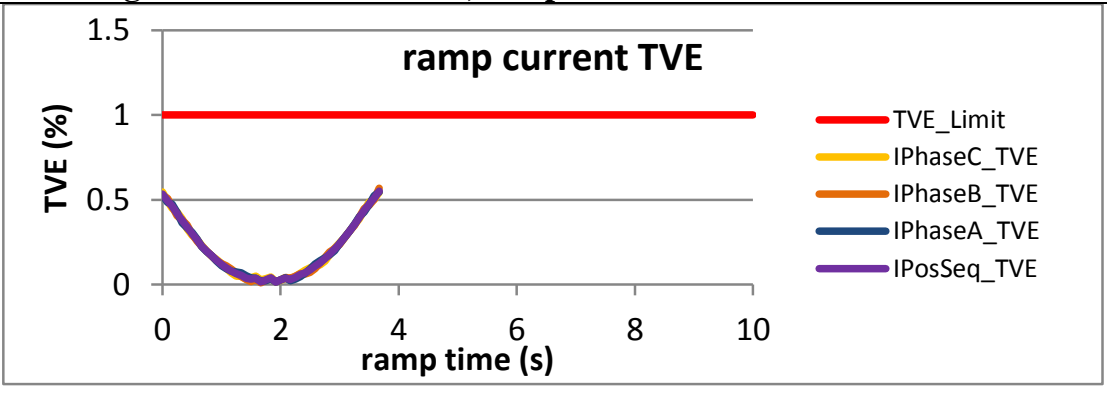

Figure 2282: Fs = 12 FPS, ramp from $62.4 \mathrm{~Hz}$ to $58.6 \mathrm{~Hz}$ at $-1 \mathrm{~Hz} / \mathrm{s}$

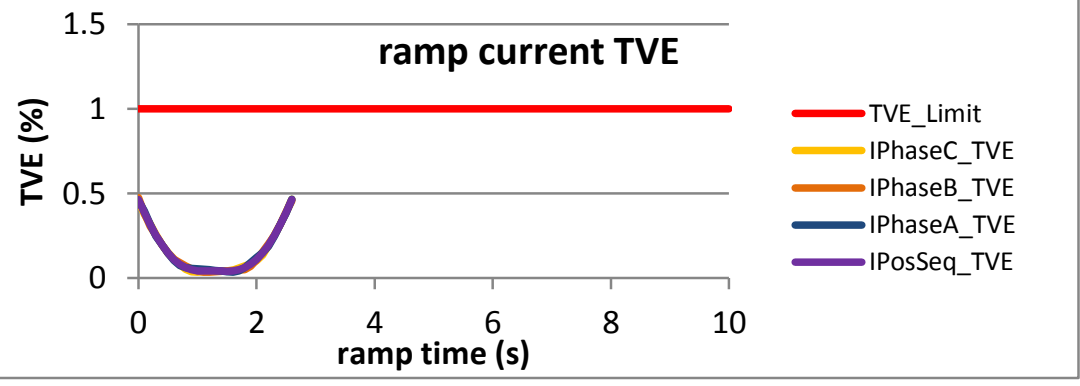

Figure 2284: Fs = $10 \mathrm{FPS}$, ramp from $62 \mathrm{~Hz}$ to $58 \mathrm{~Hz}$ at $-1 \mathrm{~Hz} / \mathrm{s}$ 
6.2.2 PMU A dynamic ramp of system frequency current TVE, M class
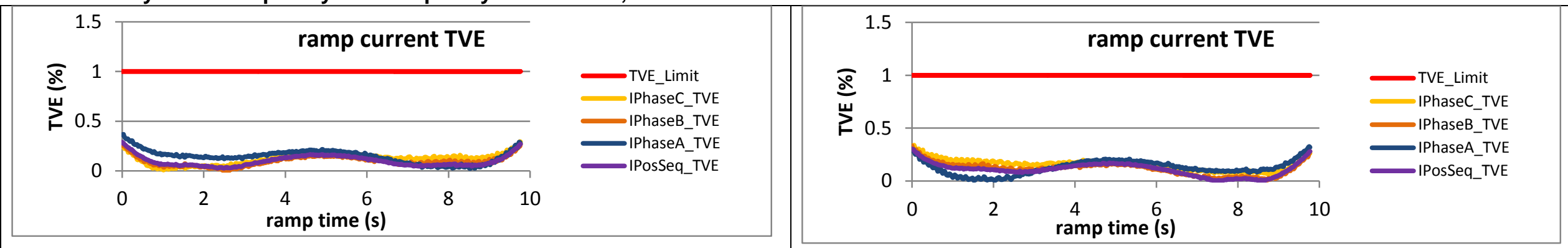

Figure 2285: Fs = $60 \mathrm{FPS}$, ramp from $55 \mathrm{~Hz}$ to $65 \mathrm{~Hz}$ at $+1 \mathrm{~Hz} / \mathrm{s}$
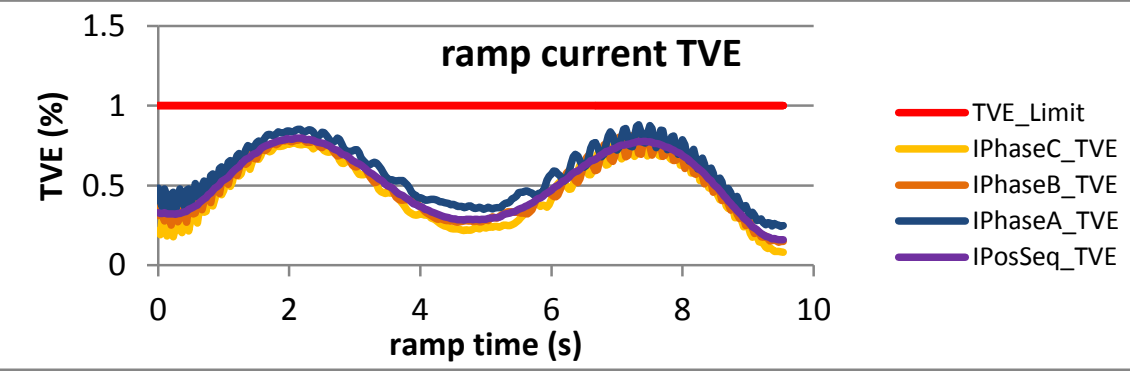

Figure 2287: Fs = $30 \mathrm{FPS}$, ramp from $55 \mathrm{~Hz}$ to $65 \mathrm{~Hz}$ at $+1 \mathrm{~Hz} / \mathrm{s}$
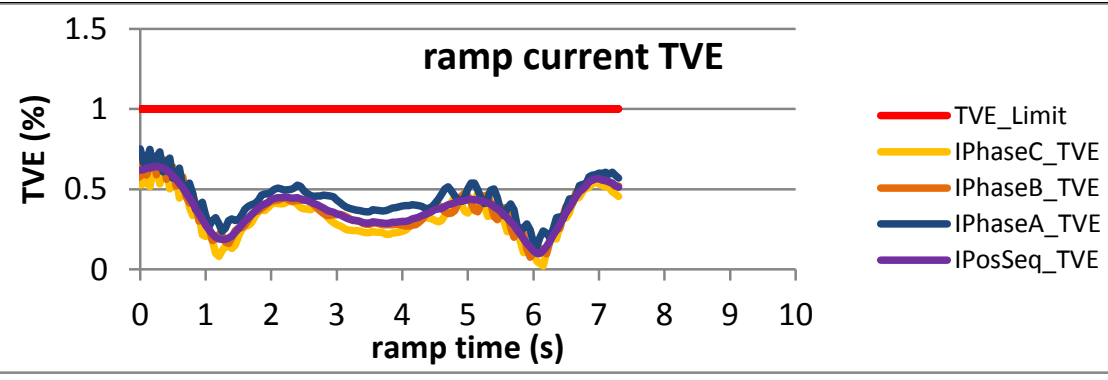

Figure 2289: Fs $=20 \mathrm{FPS}$, ramp from $56 \mathrm{~Hz}$ to $64 \mathrm{~Hz}$ at $+1 \mathrm{~Hz} / \mathrm{s}$

Figure 2286: Fs = 60 FPS, ramp from $65 \mathrm{~Hz}$ to $55 \mathrm{~Hz}$ at $-1 \mathrm{~Hz} / \mathrm{s}$

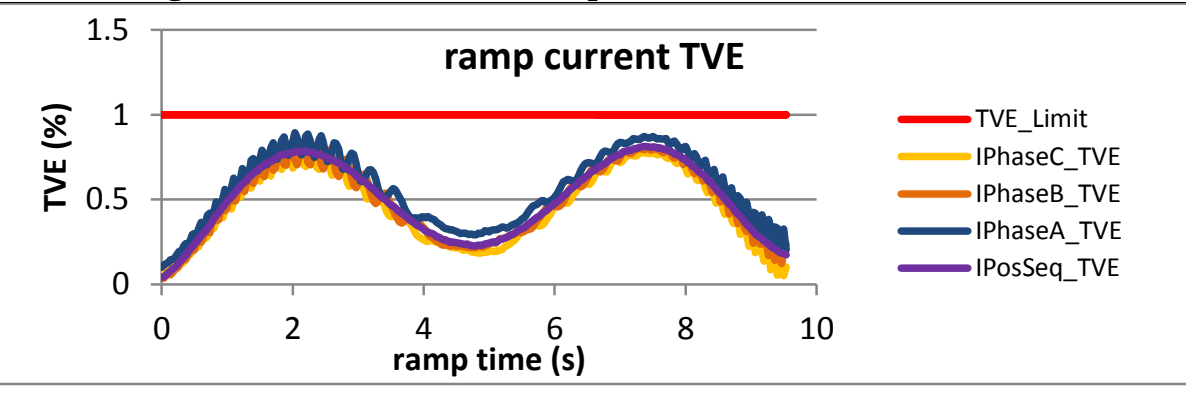

Figure 2288: Fs = $30 \mathrm{FPS}$, ramp from $65 \mathrm{~Hz}$ to $55 \mathrm{~Hz}$ at $-1 \mathrm{~Hz} / \mathrm{s}$
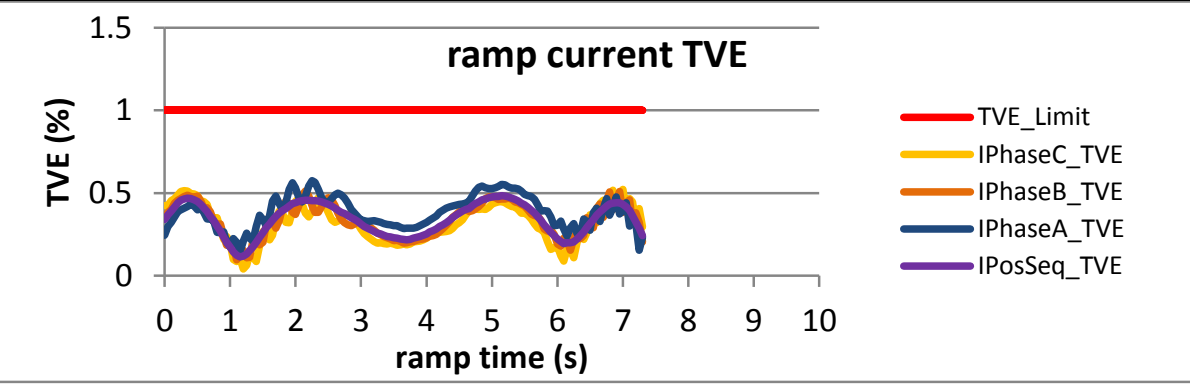

Figure 2290: Fs = $20 \mathrm{FPS}$, ramp from $64 \mathrm{~Hz}$ to $56 \mathrm{~Hz}$ at $-1 \mathrm{~Hz} / \mathrm{s}$ 


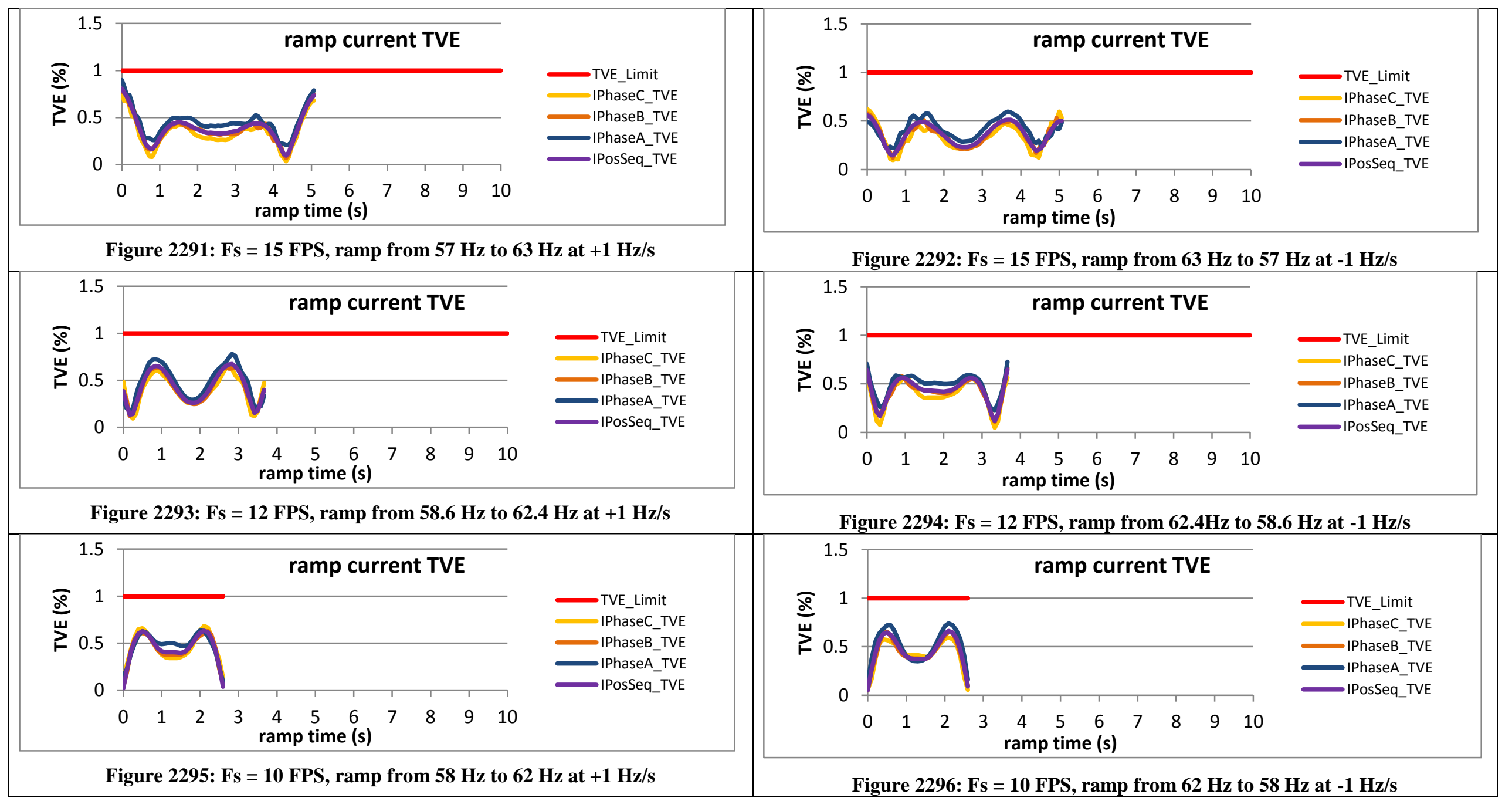


6.2.3 PMU B dynamic ramp of system frequency current TVE, M class
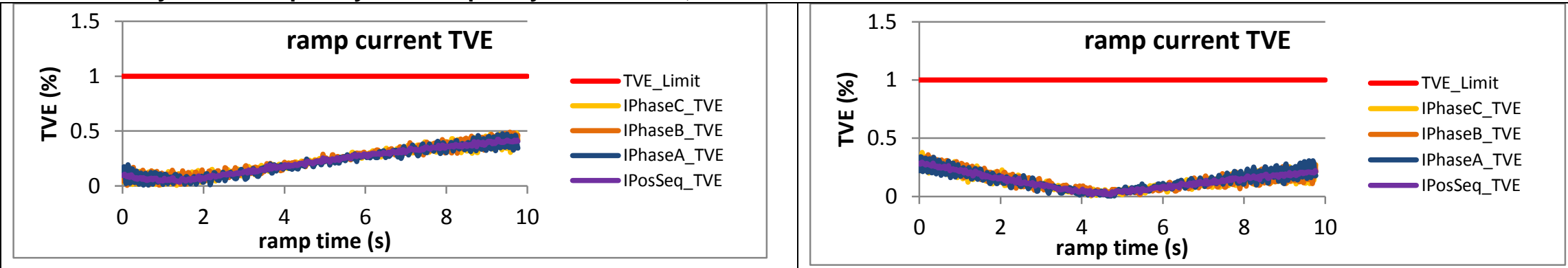

Figure 2297: Fs = $60 \mathrm{FPS}$, ramp from $55 \mathrm{~Hz}$ to $65 \mathrm{~Hz}$ at $+1 \mathrm{~Hz} / \mathrm{s}$
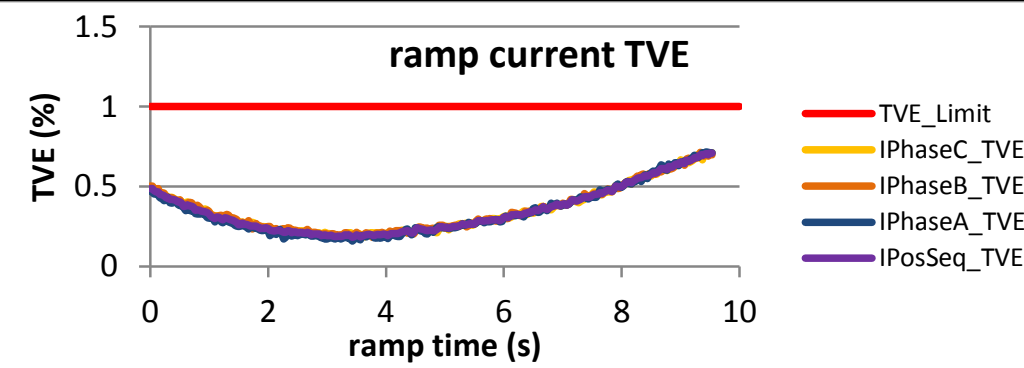

Figure 2299: Fs = $30 \mathrm{FPS}$, ramp from $55 \mathrm{~Hz}$ to $65 \mathrm{~Hz}$ at $+1 \mathrm{~Hz} / \mathrm{s}$

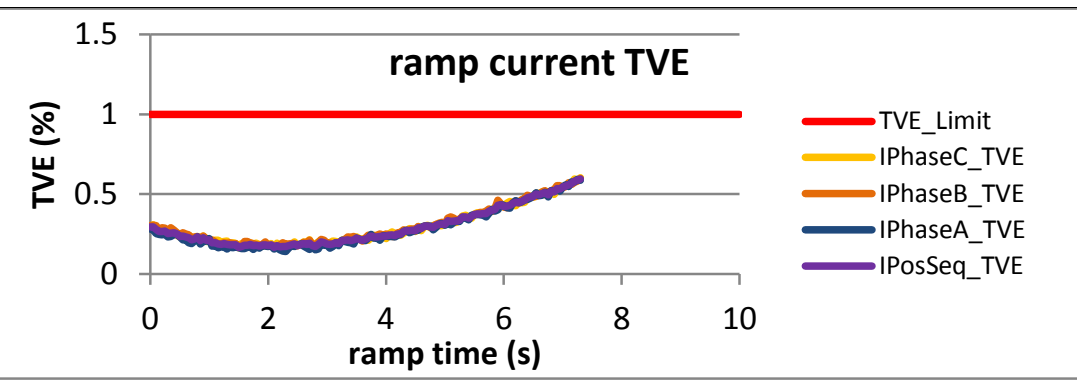

Figure 2301: Fs = $20 \mathrm{FPS}$, ramp from $56 \mathrm{~Hz}$ to $64 \mathrm{~Hz}$ at $+1 \mathrm{~Hz} / \mathrm{s}$

Figure 2298: Fs = $60 \mathrm{FPS}$, ramp from $65 \mathrm{~Hz}$ to $55 \mathrm{~Hz}$ at $-1 \mathrm{~Hz} / \mathrm{s}$

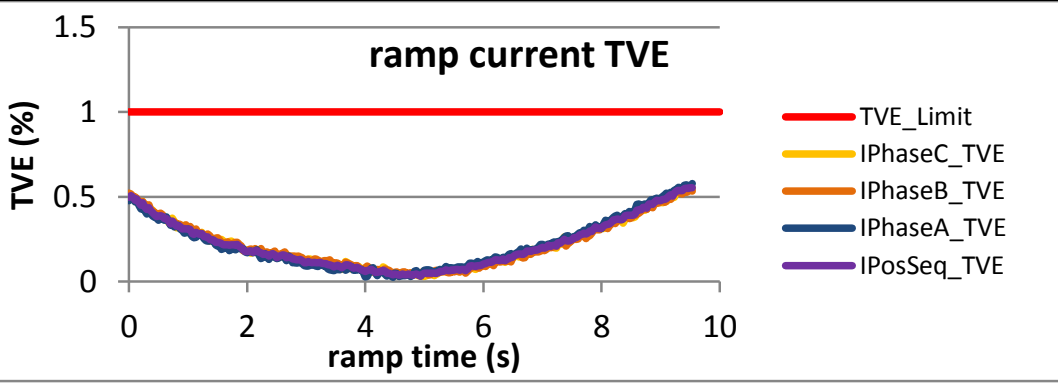

Figure 2300: Fs = $30 \mathrm{FPS}$, ramp from $65 \mathrm{~Hz}$ to $55 \mathrm{~Hz}$ at $-1 \mathrm{~Hz} / \mathrm{s}$

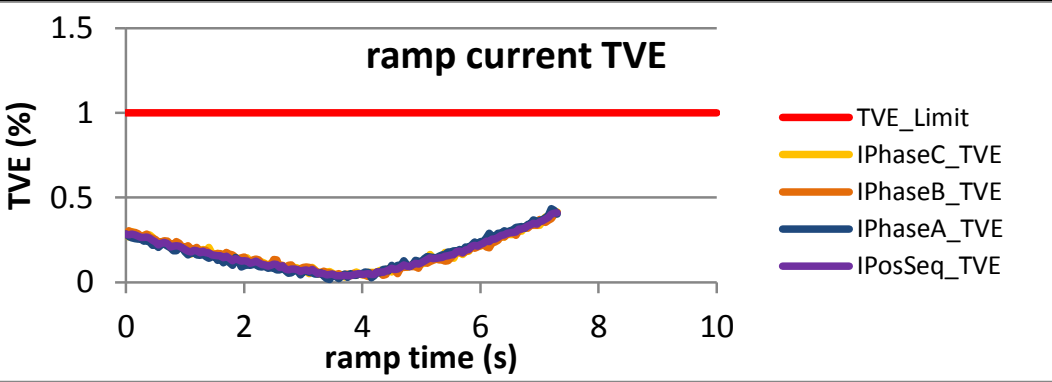

Figure 2302: Fs = $20 \mathrm{FPS}$, ramp from $64 \mathrm{~Hz}$ to $56 \mathrm{~Hz}$ at $-1 \mathrm{~Hz} / \mathrm{s}$ 

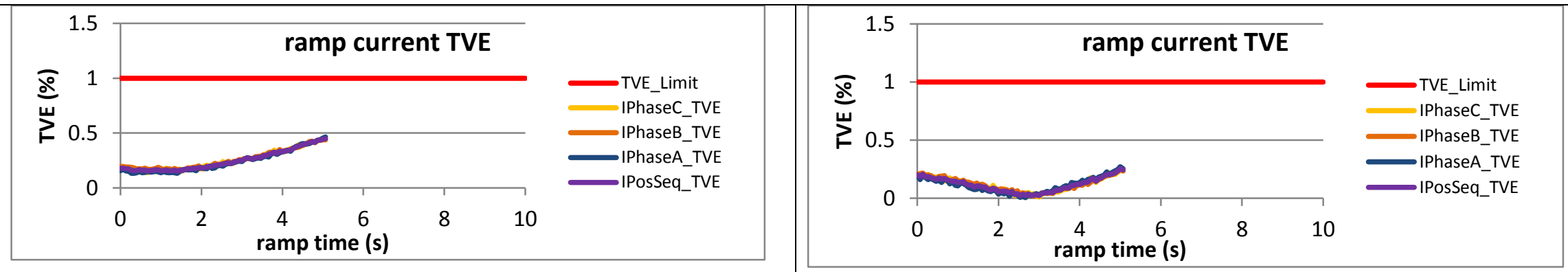

Figure 2303: Fs = $15 \mathrm{FPS}$, ramp from $57 \mathrm{~Hz}$ to $63 \mathrm{~Hz}$ at $+1 \mathrm{~Hz} / \mathrm{s}$

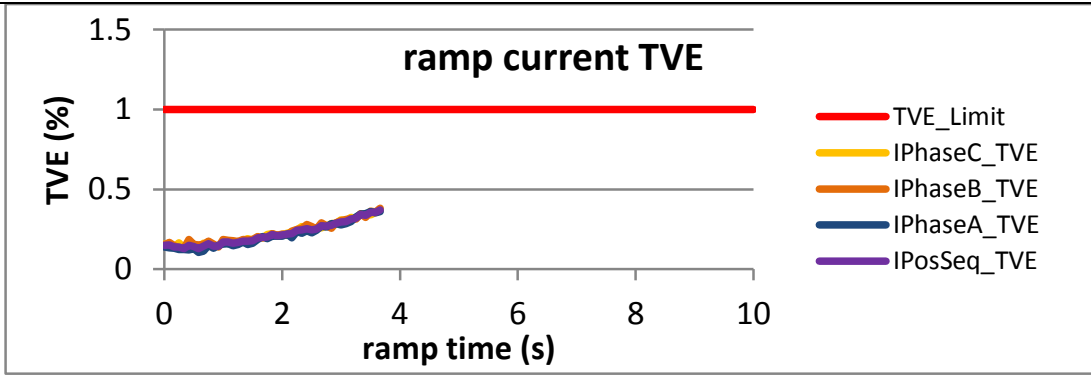

Figure 2305: Fs = 12 FPS, ramp from $58.6 \mathrm{~Hz}$ to $62.4 \mathrm{~Hz}$ at $+1 \mathrm{~Hz} / \mathrm{s}$

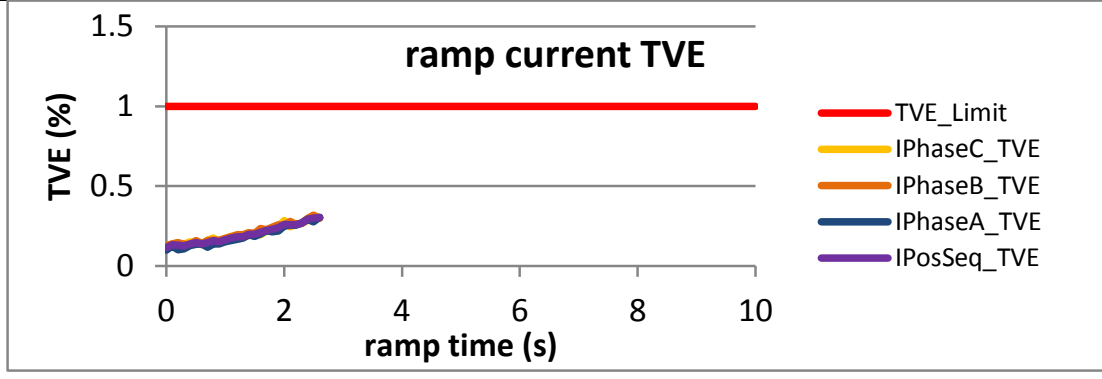

Figure 2307: Fs = $10 \mathrm{FPS}$, ramp from $58 \mathrm{~Hz}$ to $62 \mathrm{~Hz}$ at $+1 \mathrm{~Hz} / \mathrm{s}$

Figure 2304: Fs = $15 \mathrm{FPS}$, ramp from $63 \mathrm{~Hz}$ to $57 \mathrm{~Hz}$ at $-1 \mathrm{~Hz} / \mathrm{s}$

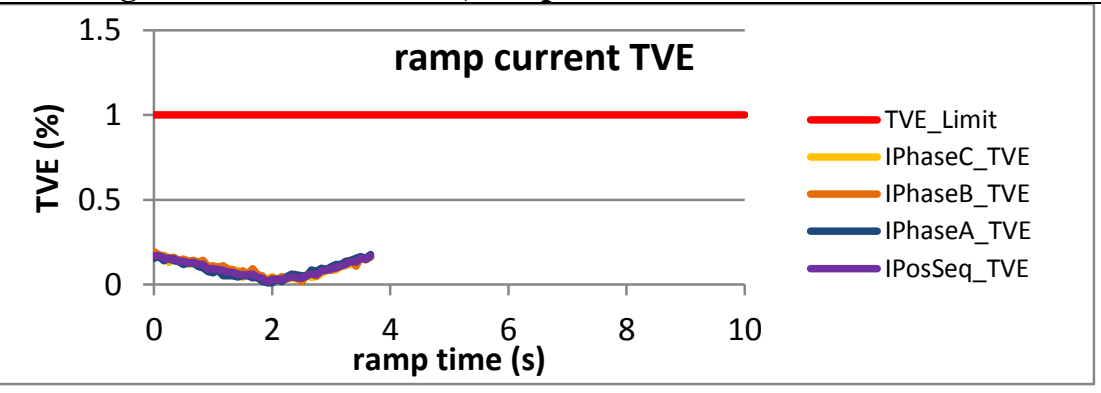

Figure 2306: Fs = 12 FPS, ramp from $62.4 \mathrm{~Hz}$ to $58.6 \mathrm{~Hz}$ at $-1 \mathrm{~Hz} / \mathrm{s}$

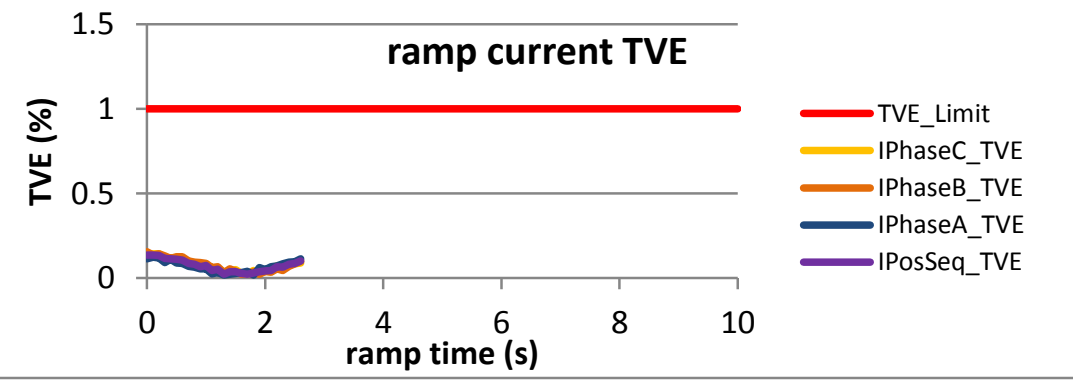

Figure 2308: Fs = $10 \mathrm{FPS}$, ramp from $62 \mathrm{~Hz}$ to $58 \mathrm{~Hz}$ at $-1 \mathrm{~Hz} / \mathrm{s}$ 
6.2.4 PMU C dynamic ramp of system frequency current TVE, M class
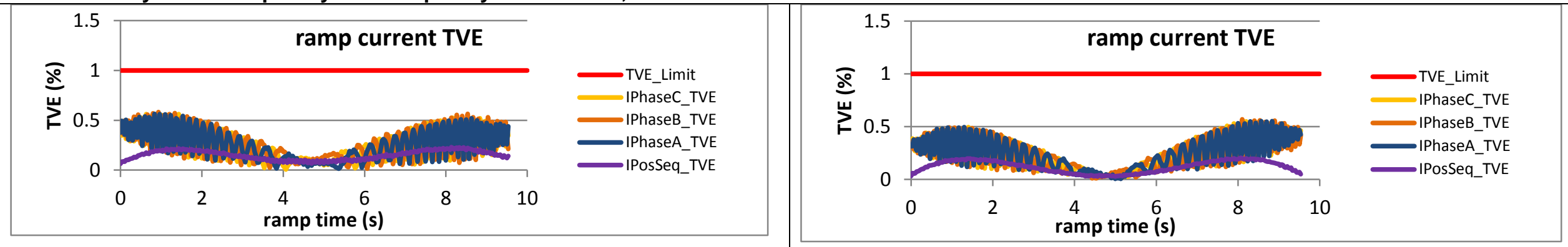

Figure 2309: Fs = $60 \mathrm{FPS}$, ramp from $55 \mathrm{~Hz}$ to $65 \mathrm{~Hz}$ at $+1 \mathrm{~Hz} / \mathrm{s}$
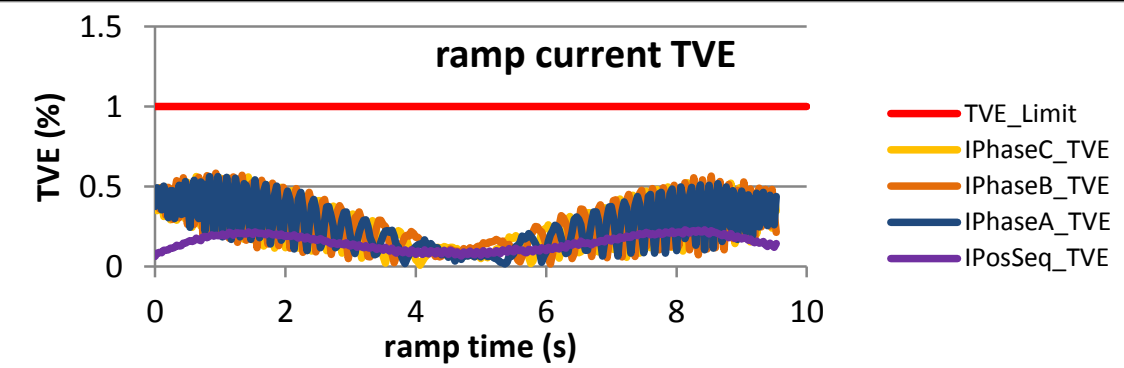

Figure 2311: Fs = $30 \mathrm{FPS}$, ramp from $55 \mathrm{~Hz}$ to $65 \mathrm{~Hz}$ at $+1 \mathrm{~Hz} / \mathrm{s}$

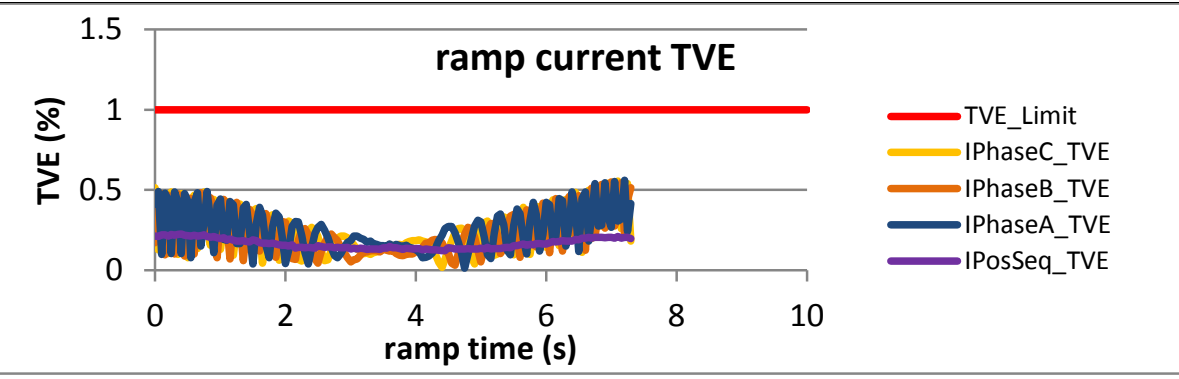

Figure 2313: Fs = 20 FPS, ramp from $56 \mathrm{~Hz}$ to $64 \mathrm{~Hz}$ at $+1 \mathrm{~Hz} / \mathrm{s}$

Figure 2310: Fs = $60 \mathrm{FPS}$, ramp from $65 \mathrm{~Hz}$ to $55 \mathrm{~Hz}$ at $-1 \mathrm{~Hz} / \mathrm{s}$

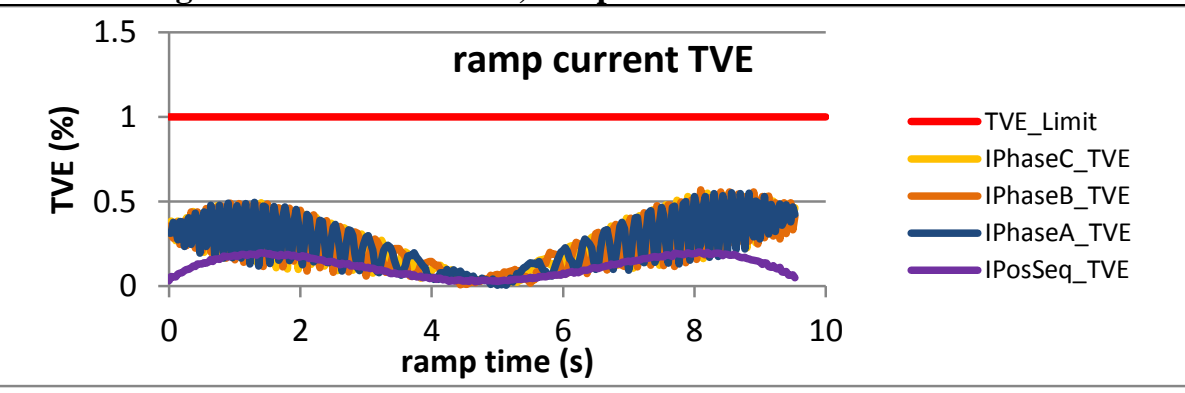

Figure 2312: Fs = $30 \mathrm{FPS}$, ramp from $65 \mathrm{~Hz}$ to $55 \mathrm{~Hz}$ at $-1 \mathrm{~Hz} / \mathrm{s}$
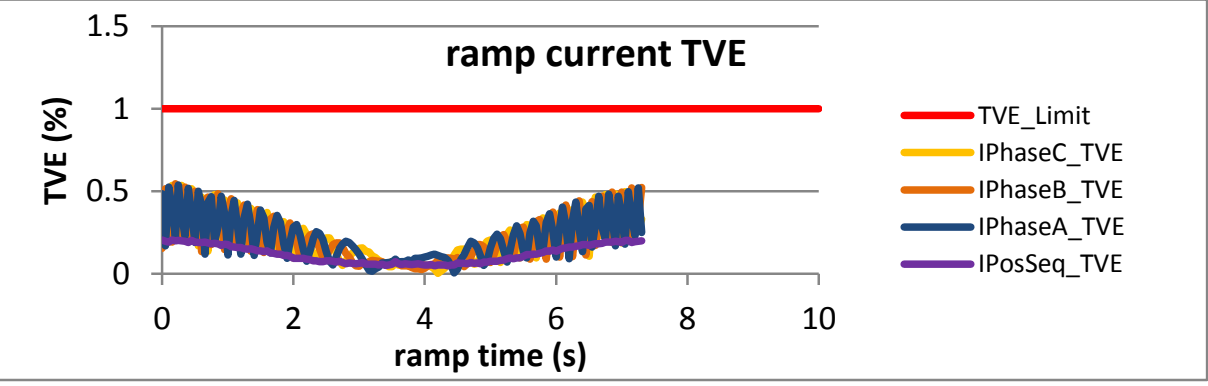

Figure 2314: Fs = $20 \mathrm{FPS}$, ramp from $64 \mathrm{~Hz}$ to $56 \mathrm{~Hz}$ at $-1 \mathrm{~Hz} / \mathrm{s}$ 


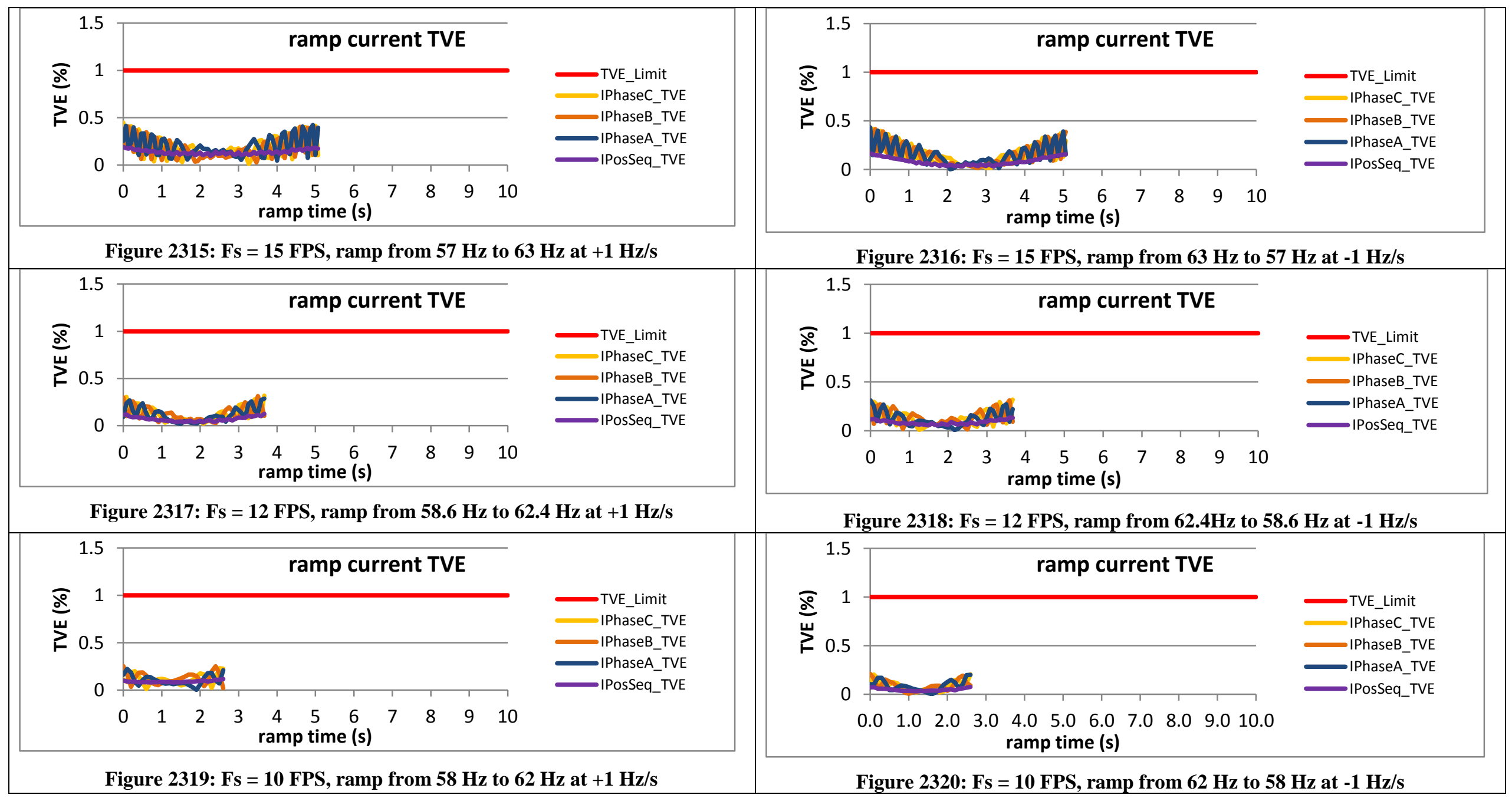


6.2.5 PMU D dynamic ramp of system frequency current TVE, M class
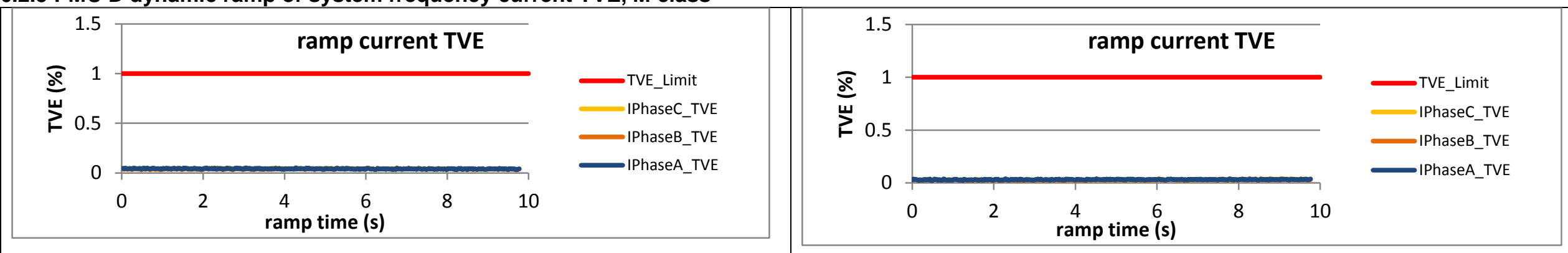

Figure 2321: Fs = $60 \mathrm{FPS}$, ramp from $55 \mathrm{~Hz}$ to $65 \mathrm{~Hz}$ at $+1 \mathrm{~Hz} / \mathrm{s}$

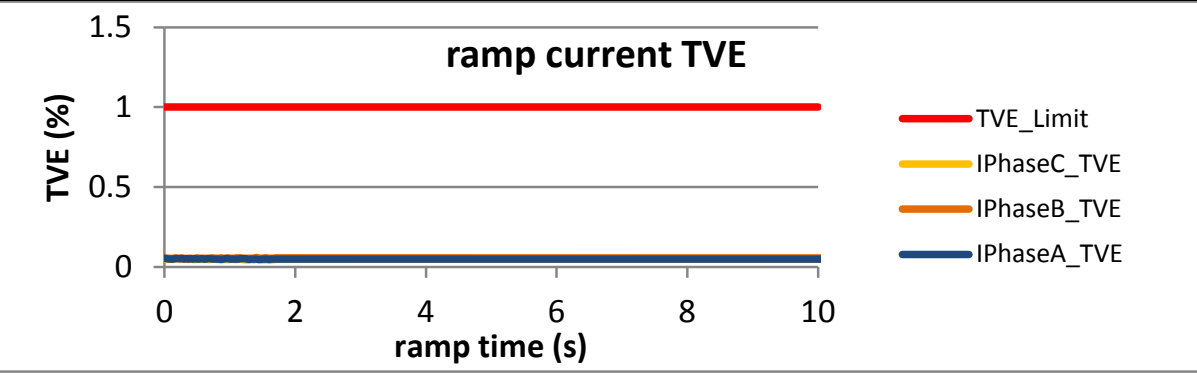

Figure 2323: Fs = $30 \mathrm{FPS}$, ramp from $55 \mathrm{~Hz}$ to $65 \mathrm{~Hz}$ at $+1 \mathrm{~Hz} / \mathrm{s}$

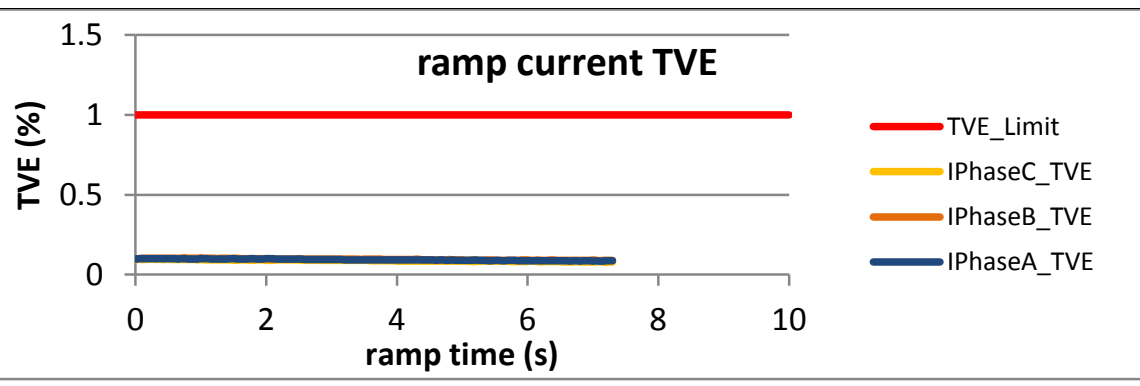

Figure 2325: Fs = $20 \mathrm{FPS}$, ramp from $56 \mathrm{~Hz}$ to $64 \mathrm{~Hz}$ at $+1 \mathrm{~Hz} / \mathrm{s}$

Figure 2322: Fs = $60 \mathrm{FPS}$, ramp from $65 \mathrm{~Hz}$ to $55 \mathrm{~Hz}$ at $-1 \mathrm{~Hz} / \mathrm{s}$

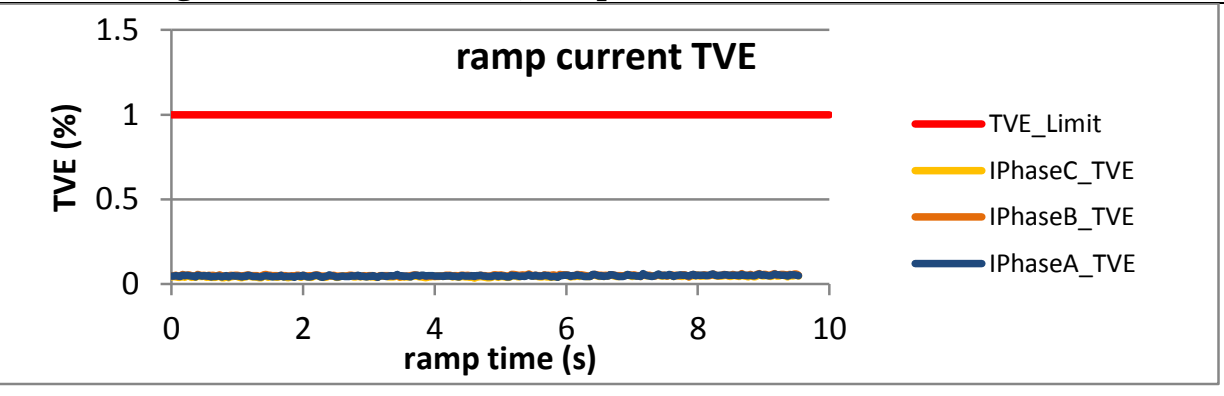

Figure 2324: Fs = $30 \mathrm{FPS}$, ramp from $65 \mathrm{~Hz}$ to $55 \mathrm{~Hz}$ at $-1 \mathrm{~Hz} / \mathrm{s}$

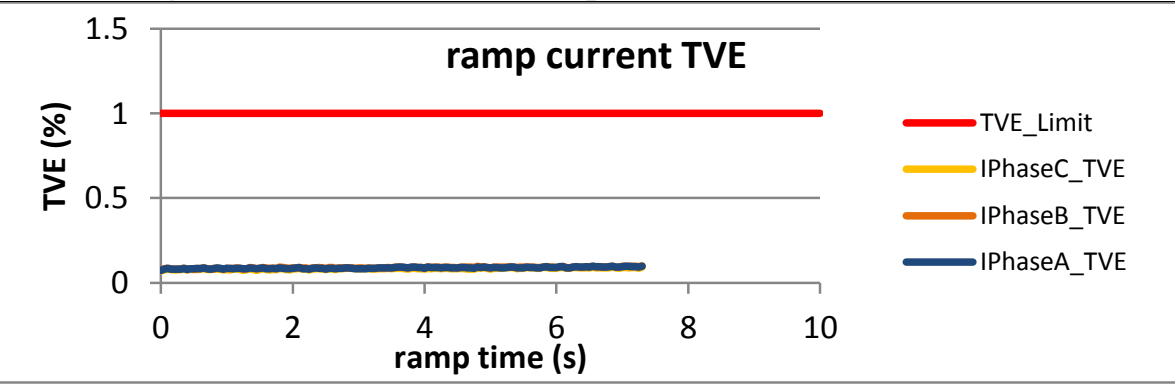

Figure 2326: Fs = $20 \mathrm{FPS}$, ramp from $64 \mathrm{~Hz}$ to $56 \mathrm{~Hz}$ at $-1 \mathrm{~Hz} / \mathrm{s}$ 

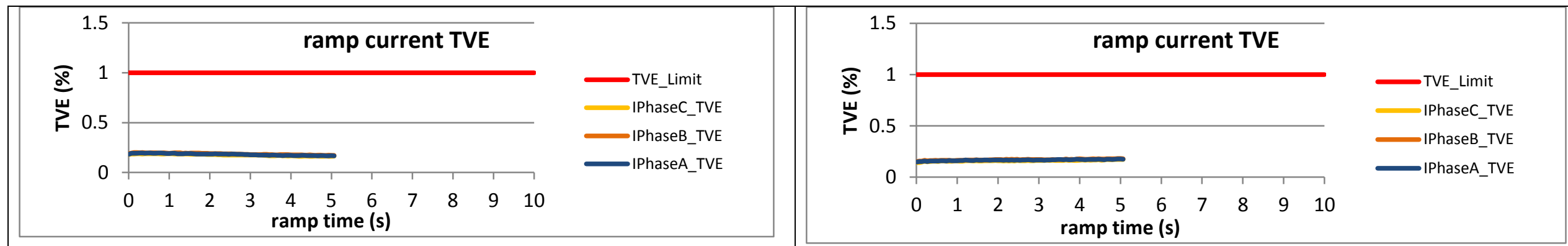

Figure 2327: Fs = $15 \mathrm{FPS}$, ramp from $57 \mathrm{~Hz}$ to $63 \mathrm{~Hz}$ at $+1 \mathrm{~Hz} / \mathrm{s}$

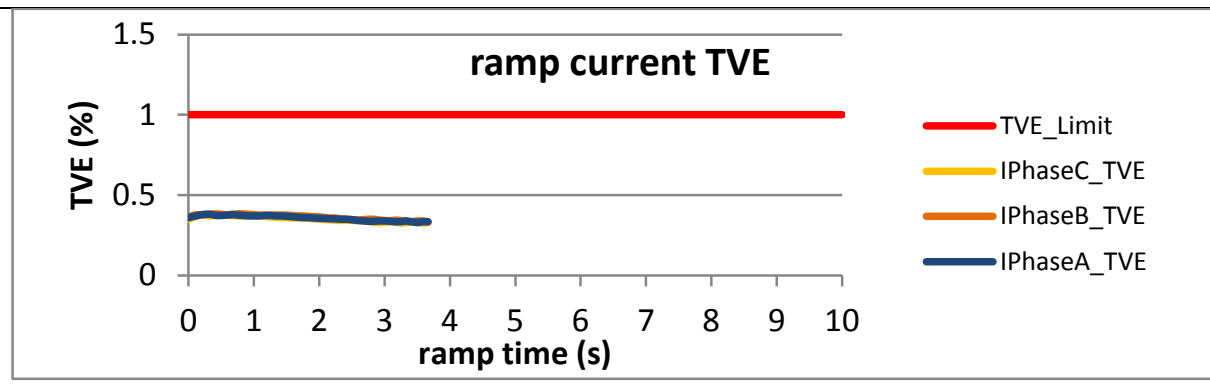

Figure 2329: Fs = 12 FPS, ramp from $58.6 \mathrm{~Hz}$ to $62.4 \mathrm{~Hz}$ at $+1 \mathrm{~Hz} / \mathrm{s}$

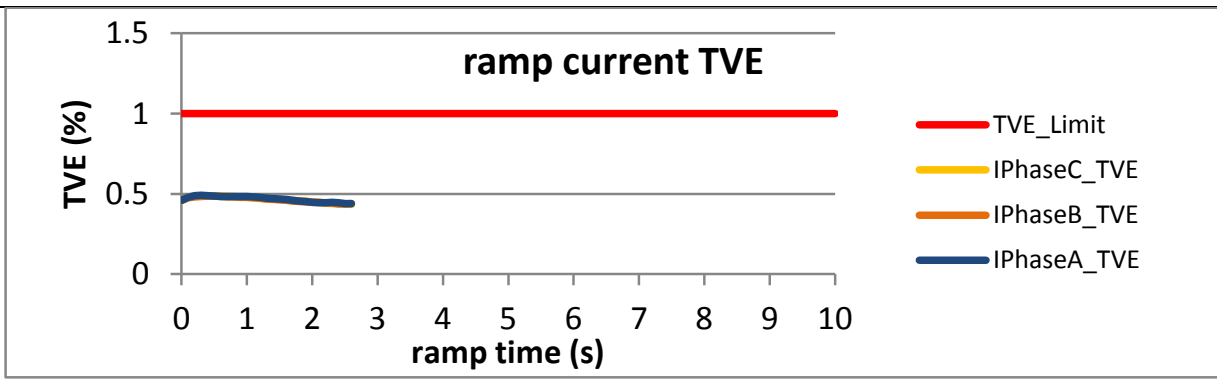

Figure 2331: Fs = $10 \mathrm{FPS}$, ramp from $58 \mathrm{~Hz}$ to $62 \mathrm{~Hz}$ at $+1 \mathrm{~Hz} / \mathrm{s}$

Figure 2328: Fs = $15 \mathrm{FPS}$, ramp from $63 \mathrm{~Hz}$ to $57 \mathrm{~Hz}$ at $-1 \mathrm{~Hz} / \mathrm{s}$

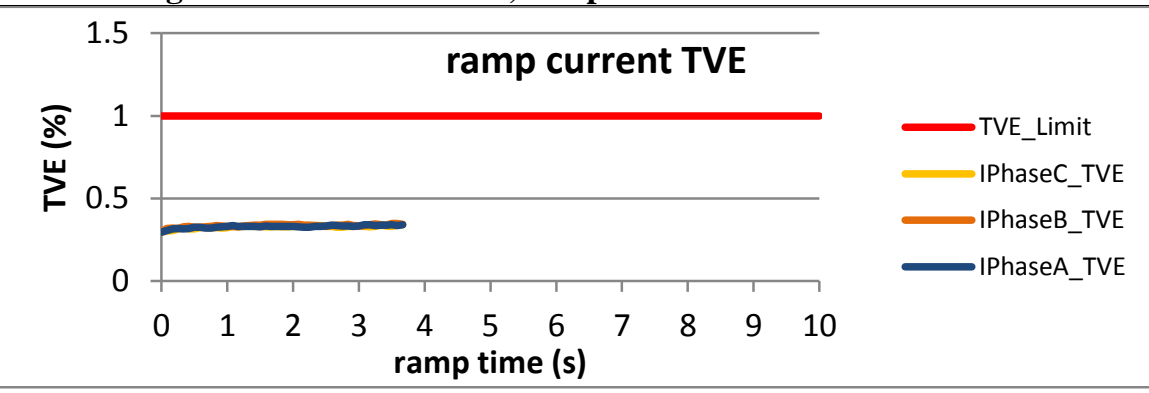

Figure 2330: Fs = 12 FPS, ramp from $62.4 \mathrm{~Hz}$ to $58.6 \mathrm{~Hz}$ at $-1 \mathrm{~Hz} / \mathrm{s}$

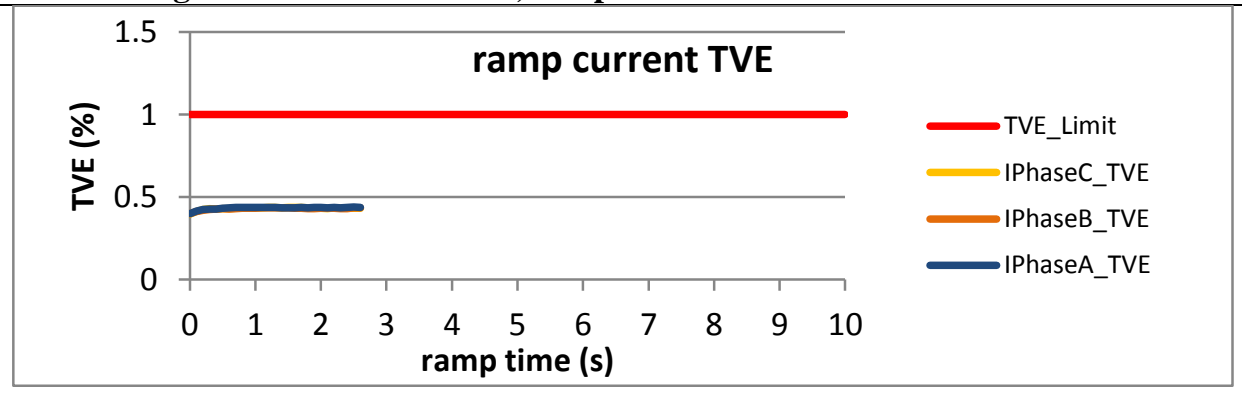

Figure 2332: Fs = $10 \mathrm{FPS}$, ramp from $62 \mathrm{~Hz}$ to $58 \mathrm{~Hz}$ at $-1 \mathrm{~Hz} / \mathrm{s}$ 
6.2.6 PMU E dynamic ramp of system frequency current TVE, M class

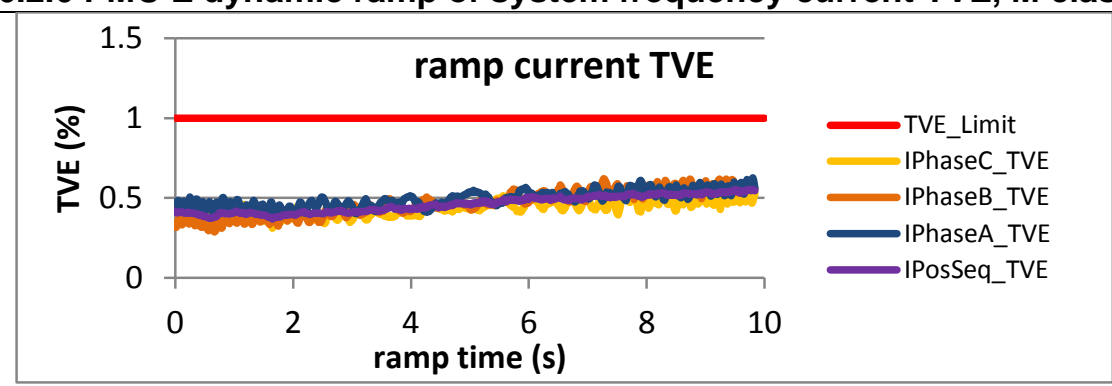

Figure 2333: Fs = $60 \mathrm{FPS}$, ramp from $55 \mathrm{~Hz}$ to $65 \mathrm{~Hz}$ at $+1 \mathrm{~Hz} / \mathrm{s}$

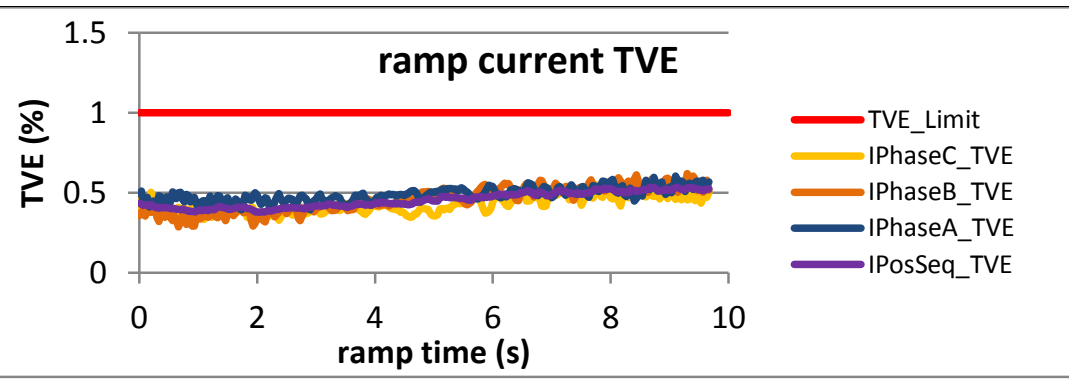

Figure 2335: Fs = $30 \mathrm{FPS}$, ramp from $55 \mathrm{~Hz}$ to $65 \mathrm{~Hz}$ at $+1 \mathrm{~Hz} / \mathrm{s}$

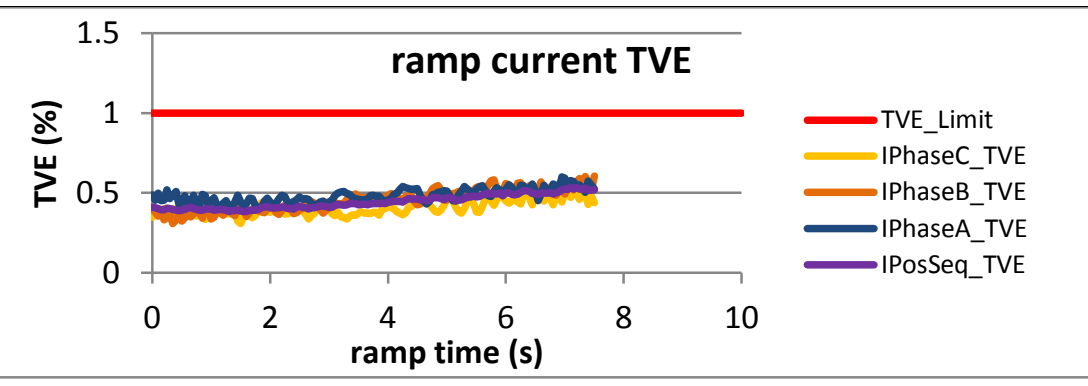

Figure 2337: Fs = $20 \mathrm{FPS}$, ramp from $56 \mathrm{~Hz}$ to $64 \mathrm{~Hz}$ at $+1 \mathrm{~Hz} / \mathrm{s}$

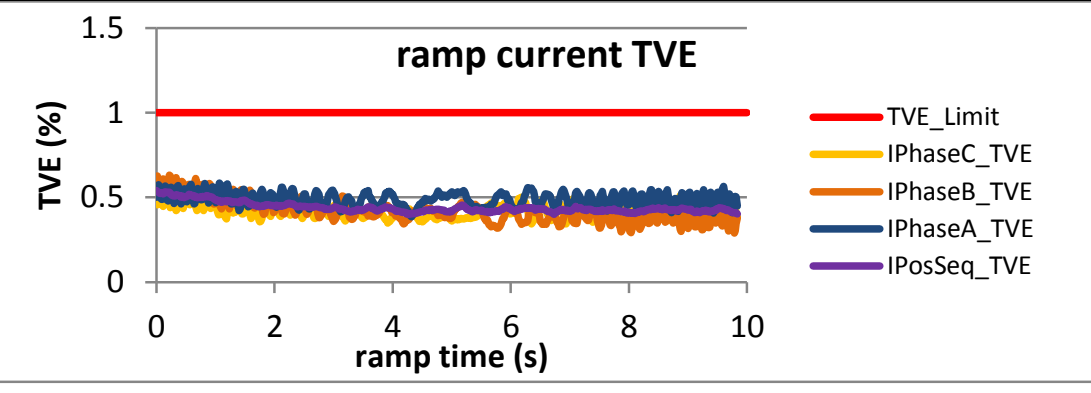

Figure 2334: Fs = $60 \mathrm{FPS}$, ramp from $65 \mathrm{~Hz}$ to $55 \mathrm{~Hz}$ at $-1 \mathrm{~Hz} / \mathrm{s}$
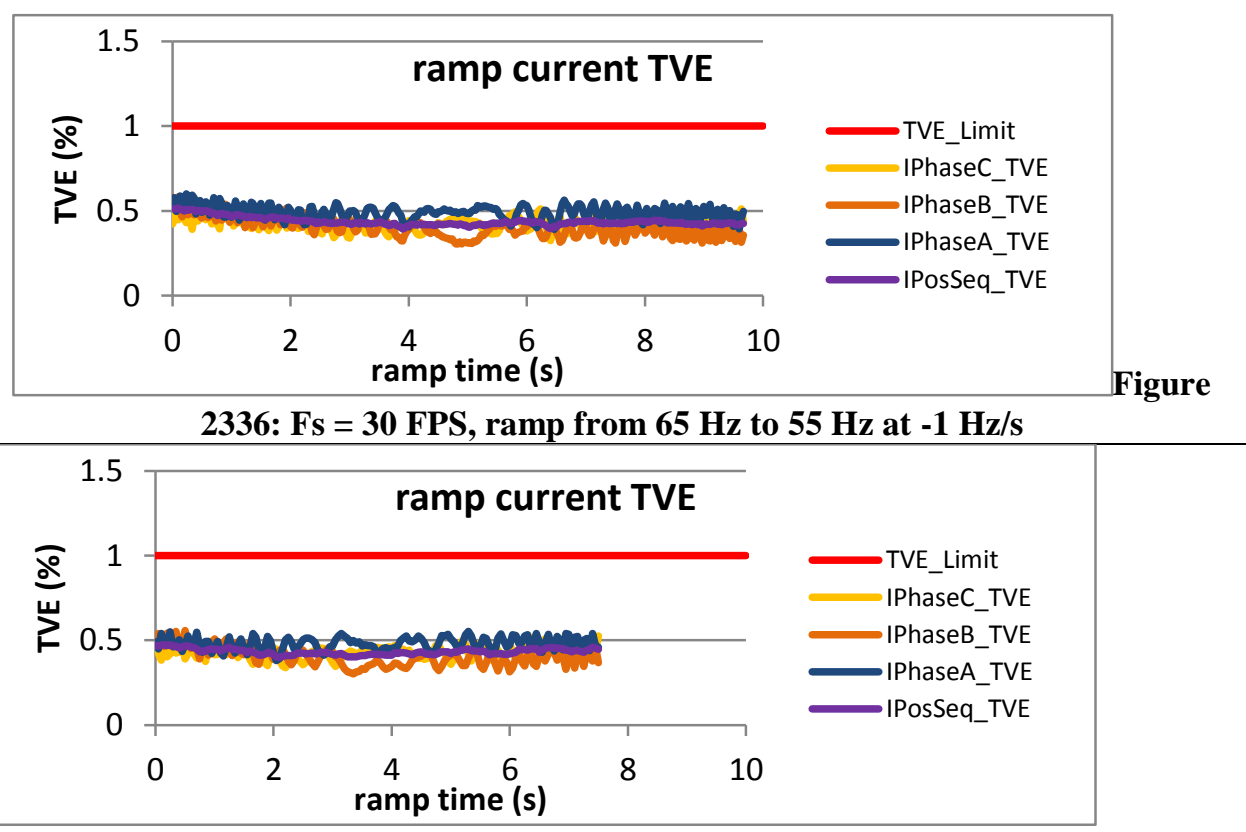

Figure 2338: Fs = $20 \mathrm{FPS}$, ramp from $64 \mathrm{~Hz}$ to $56 \mathrm{~Hz}$ at $-1 \mathrm{~Hz} / \mathrm{s}$ 

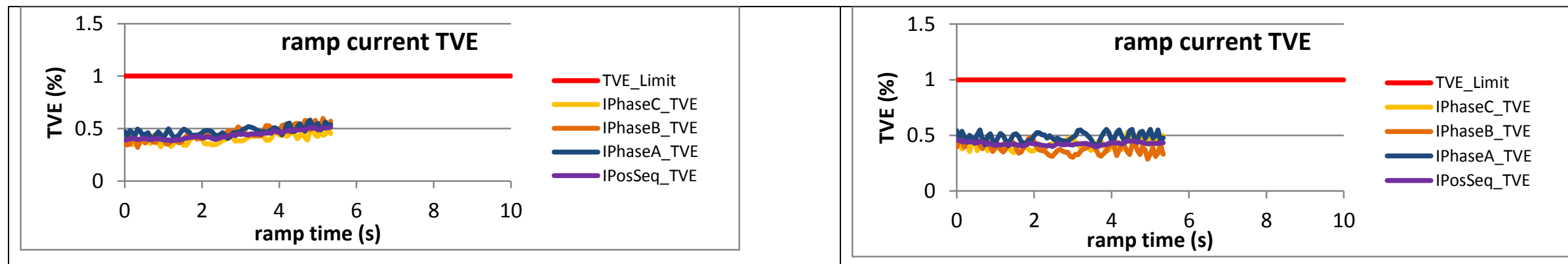

Figure 2339: Fs = $15 \mathrm{FPS}$, ramp from $57 \mathrm{~Hz}$ to $63 \mathrm{~Hz}$ at $+1 \mathrm{~Hz} / \mathrm{s}$

Figure 2340: Fs = $15 \mathrm{FPS}$, ramp from $63 \mathrm{~Hz}$ to $57 \mathrm{~Hz}$ at $-1 \mathrm{~Hz} / \mathrm{s}$

Figure 2341: Fs = 12 FPS, ramp from $58.6 \mathrm{~Hz}$ to $62.4 \mathrm{~Hz}$ at $+1 \mathrm{~Hz} / \mathrm{s}$

Figure 2342: Fs = 12 FPS, ramp from $62.4 \mathrm{~Hz}$ to $58.6 \mathrm{~Hz}$ at $-1 \mathrm{~Hz} / \mathrm{s}$

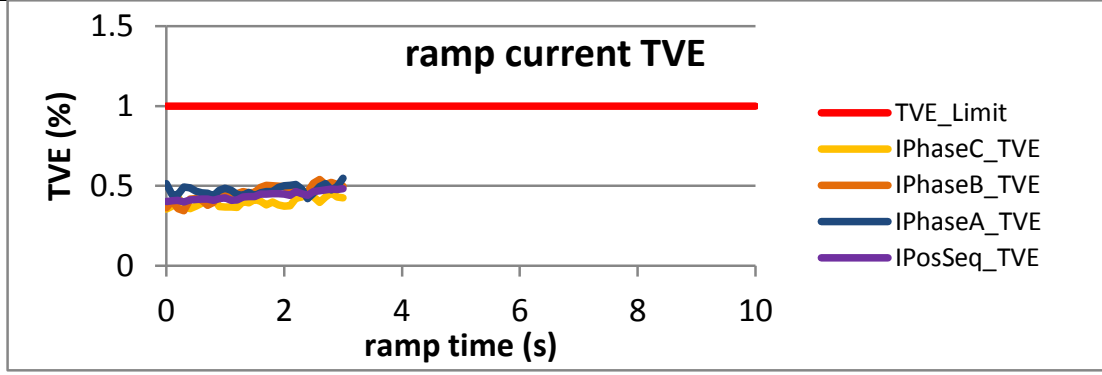

Figure 2343: Fs = $10 \mathrm{FPS}$, ramp from $58 \mathrm{~Hz}$ to $62 \mathrm{~Hz}$ at $+1 \mathrm{~Hz} / \mathrm{s}$

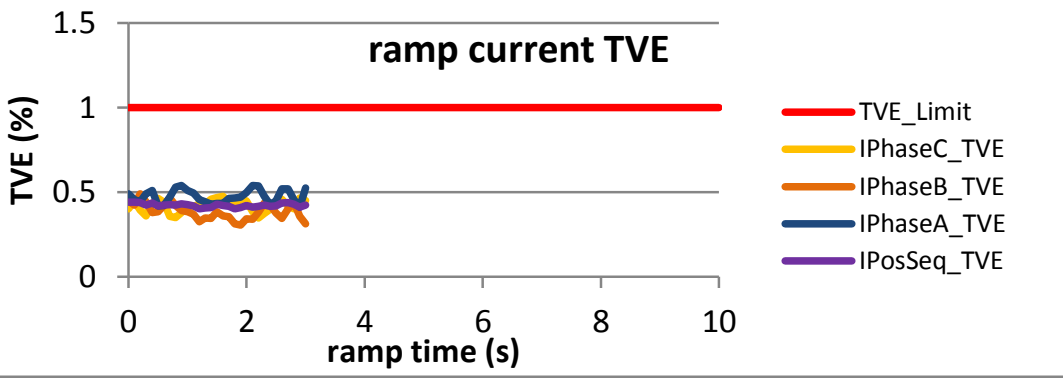

Figure 2344: Fs = $10 \mathrm{FPS}$, ramp from $62 \mathrm{~Hz}$ to $58 \mathrm{~Hz}$ at $-1 \mathrm{~Hz} / \mathrm{s}$ 
6.2.7 PMU F dynamic ramp of system frequency current TVE, M class

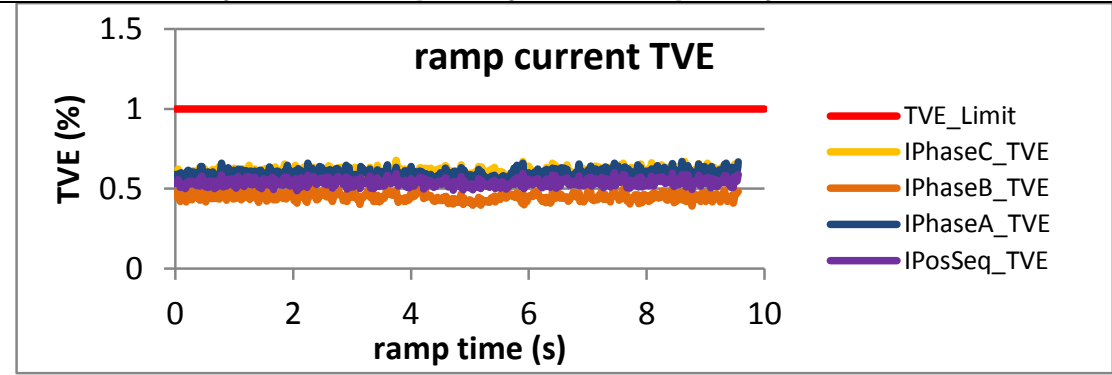

Figure 2345: Fs = $60 \mathrm{FPS}$, ramp from $55 \mathrm{~Hz}$ to $65 \mathrm{~Hz}$ at $+1 \mathrm{~Hz} / \mathrm{s}$

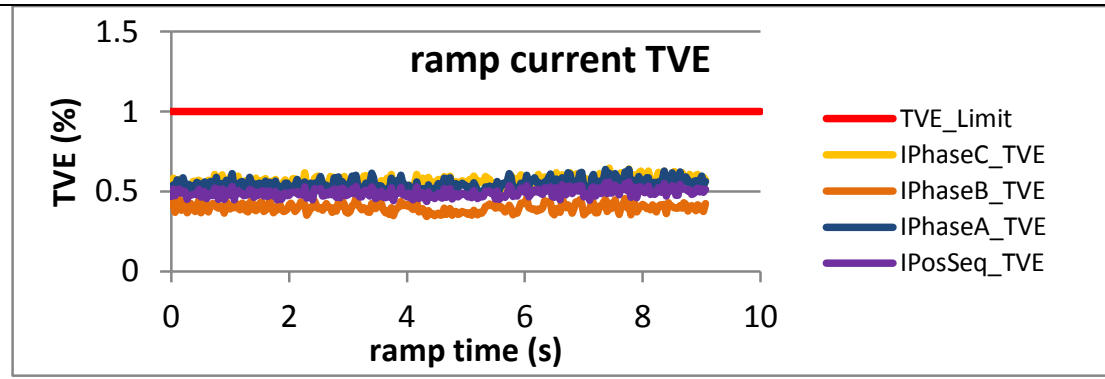

Figure 2347: Fs = $30 \mathrm{FPS}$, ramp from $55 \mathrm{~Hz}$ to $65 \mathrm{~Hz}$ at $+1 \mathrm{~Hz} / \mathrm{s}$

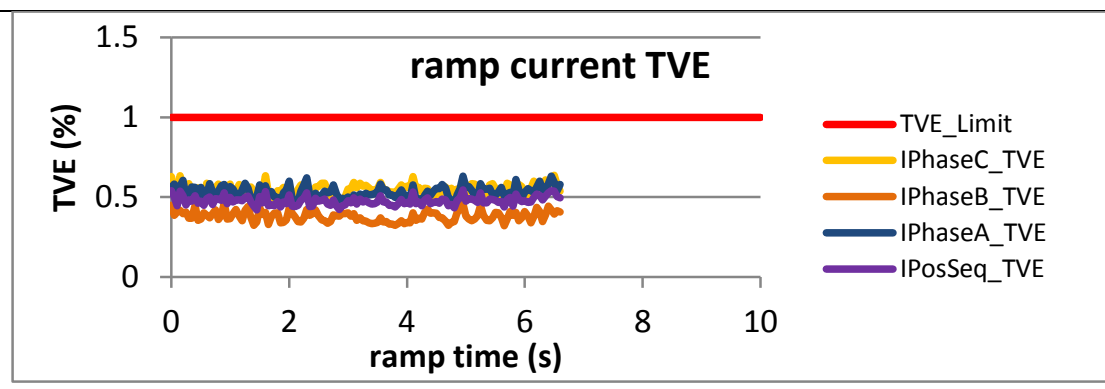

Figure 2349: Fs = $20 \mathrm{FPS}$, ramp from $56 \mathrm{~Hz}$ to $64 \mathrm{~Hz}$ at $+1 \mathrm{~Hz} / \mathrm{s}$

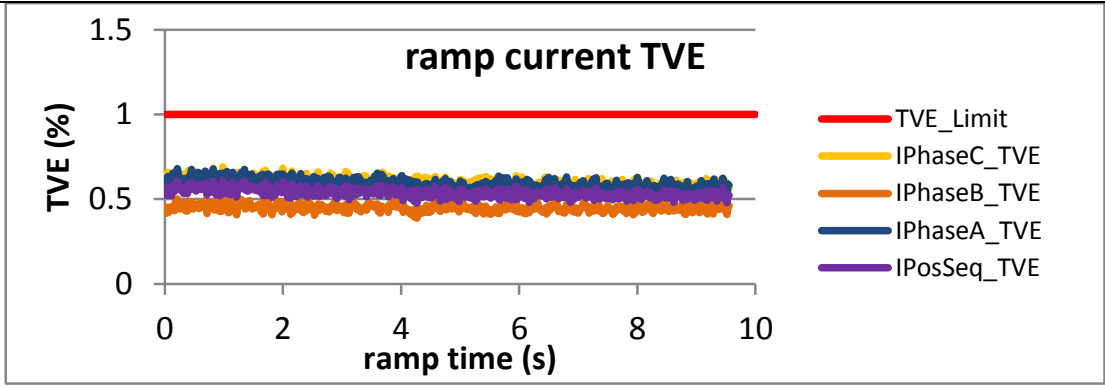

Figure 2346: Fs = $60 \mathrm{FPS}$, ramp from $65 \mathrm{~Hz}$ to $55 \mathrm{~Hz}$ at $-1 \mathrm{~Hz} / \mathrm{s}$

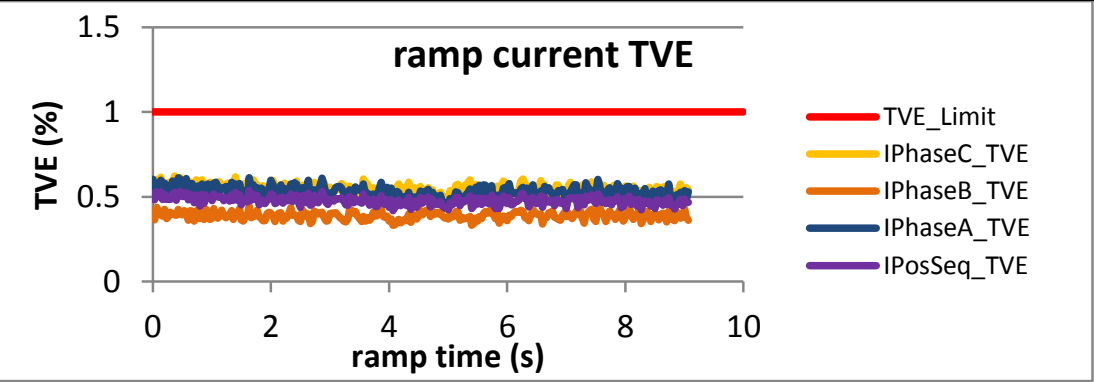

Figure 2348: Fs = $30 \mathrm{FPS}$, ramp from $65 \mathrm{~Hz}$ to $55 \mathrm{~Hz}$ at $-1 \mathrm{~Hz} / \mathrm{s}$

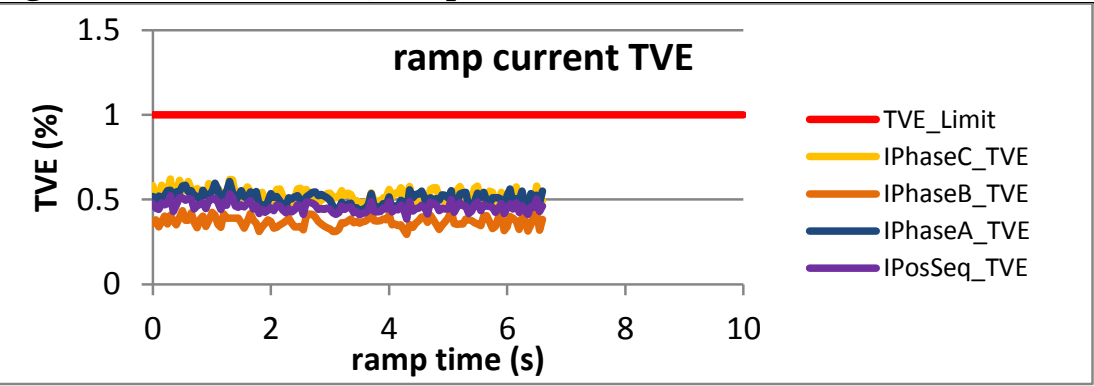

Figure 2350: Fs = $20 \mathrm{FPS}$, ramp from $64 \mathrm{~Hz}$ to $56 \mathrm{~Hz}$ at $-1 \mathrm{~Hz} / \mathrm{s}$ 


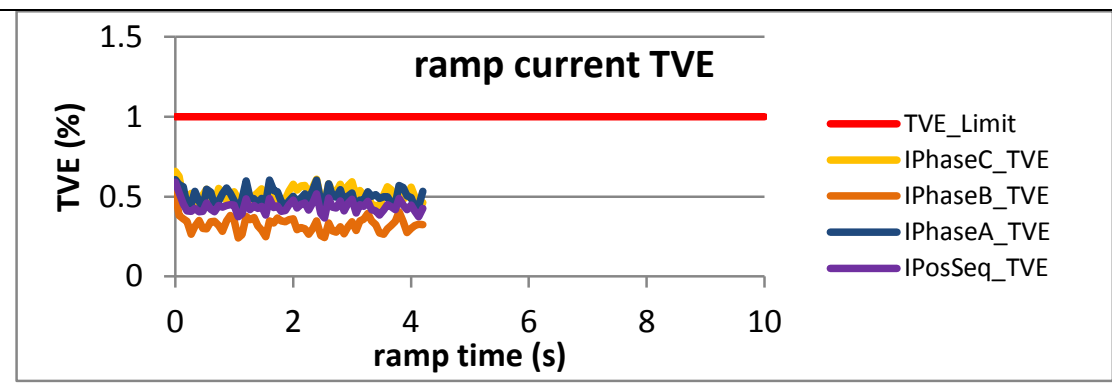

Figure 2351: Fs = $15 \mathrm{FPS}$, ramp from $57 \mathrm{~Hz}$ to $63 \mathrm{~Hz}$ at $+1 \mathrm{~Hz} / \mathrm{s}$

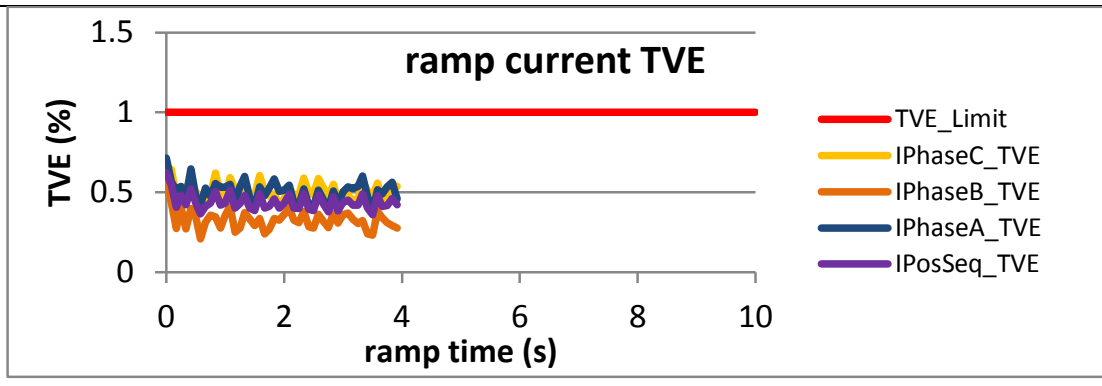

Figure 2353: Fs = 12 FPS, ramp from $58.6 \mathrm{~Hz}$ to $62.4 \mathrm{~Hz}$ at $+1 \mathrm{~Hz} / \mathrm{s}$

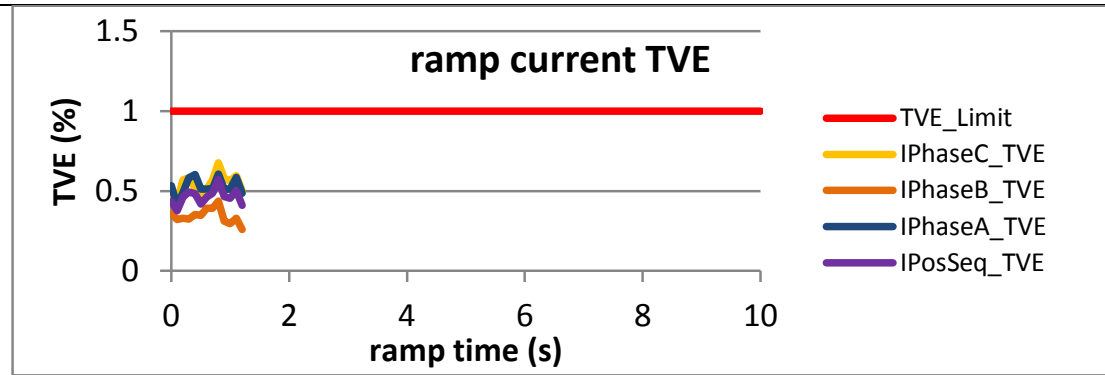

Figure 2355: Fs = $10 \mathrm{FPS}$, ramp from $58 \mathrm{~Hz}$ to $62 \mathrm{~Hz}$ at $+1 \mathrm{~Hz} / \mathrm{s}$

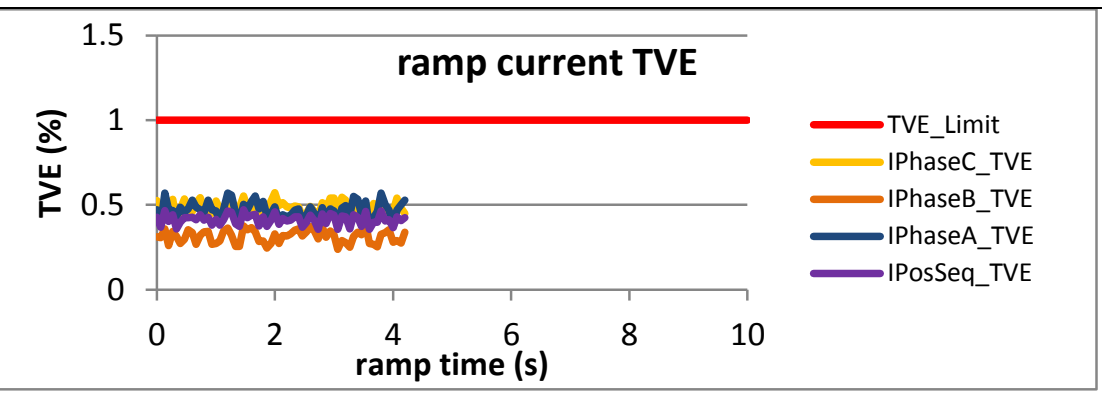

Figure 2352: Fs = $15 \mathrm{FPS}$, ramp from $63 \mathrm{~Hz}$ to $57 \mathrm{~Hz}$ at $-1 \mathrm{~Hz} / \mathrm{s}$

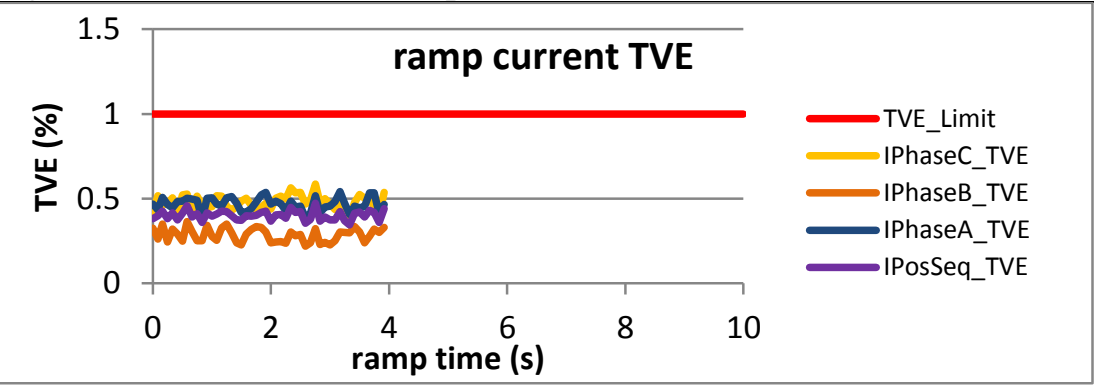

Figure 2354: Fs = 12 FPS, ramp from $62.4 \mathrm{~Hz}$ to $58.6 \mathrm{~Hz}$ at $-1 \mathrm{~Hz} / \mathrm{s}$

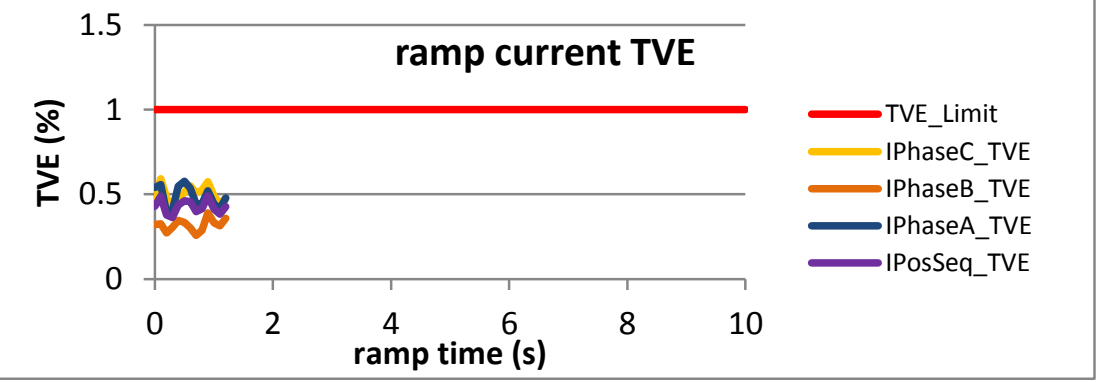

Figure 2356: Fs = $10 \mathrm{FPS}$, ramp from $62 \mathrm{~Hz}$ to $58 \mathrm{~Hz}$ at $-1 \mathrm{~Hz} / \mathrm{s}$ 


\subsubsection{PMU G dynamic ramp of system frequency current TVE, M class}

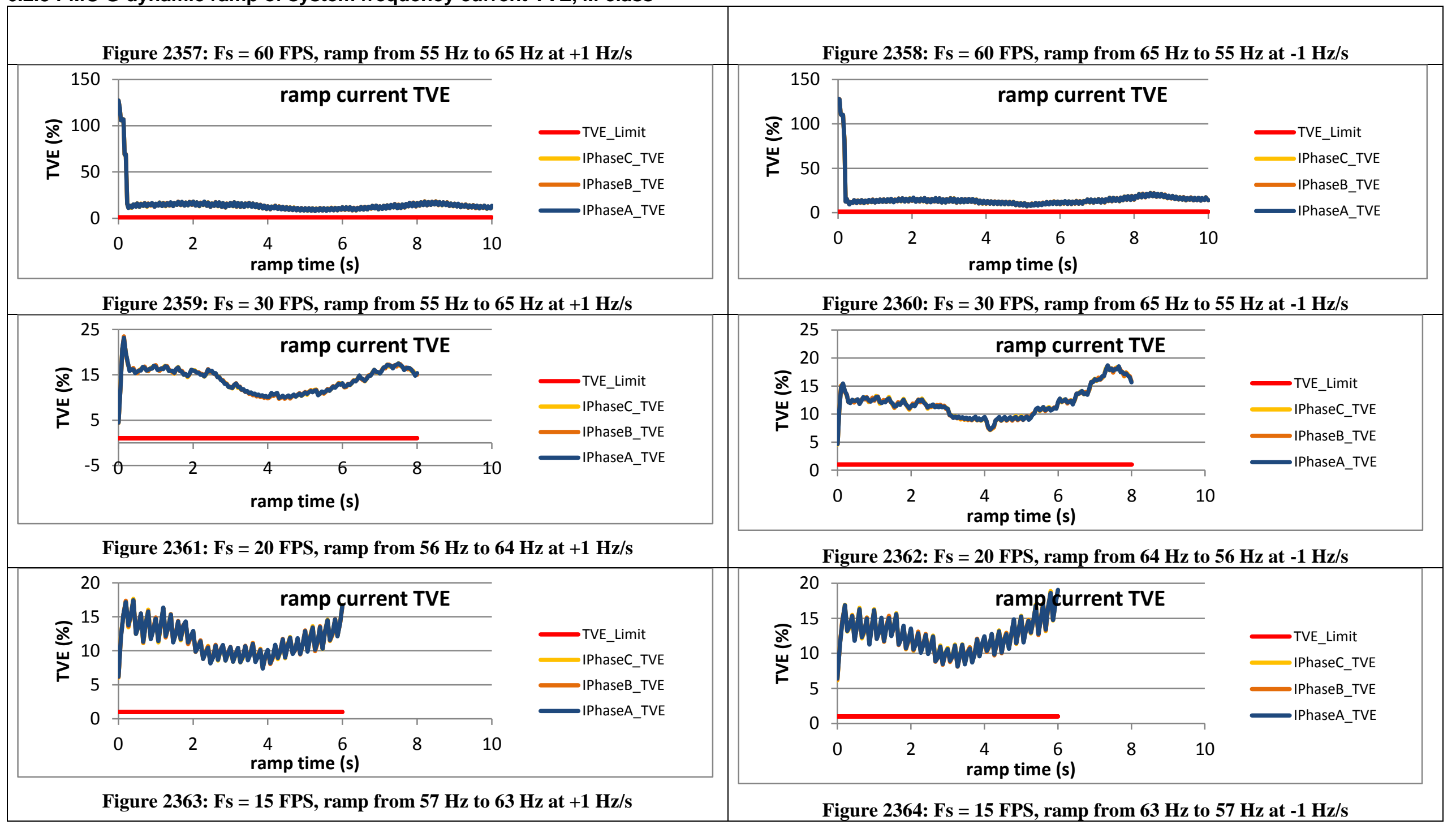



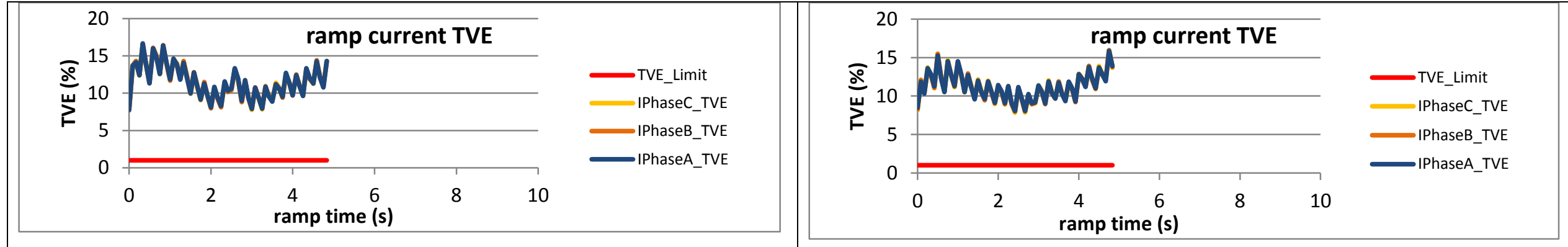

Figure 2365: Fs = 12 FPS, ramp from $57.6 \mathrm{~Hz}$ to $62.4 \mathrm{~Hz}$ at $+1 \mathrm{~Hz} / \mathrm{s}$

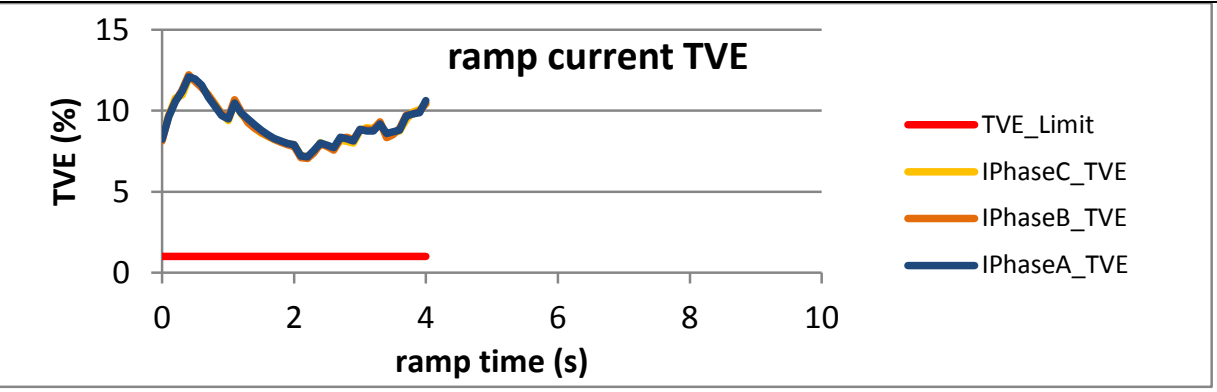

Figure 2367: Fs = $10 \mathrm{FPS}$, ramp from $58 \mathrm{~Hz}$ to $62 \mathrm{~Hz}$ at $+1 \mathrm{~Hz} / \mathrm{s}$

Figure 2366: Fs = $12 \mathrm{FPS}$, ramp from $62.4 \mathrm{~Hz}$ to $57.6 \mathrm{~Hz}$ at $-1 \mathrm{~Hz} / \mathrm{s}$

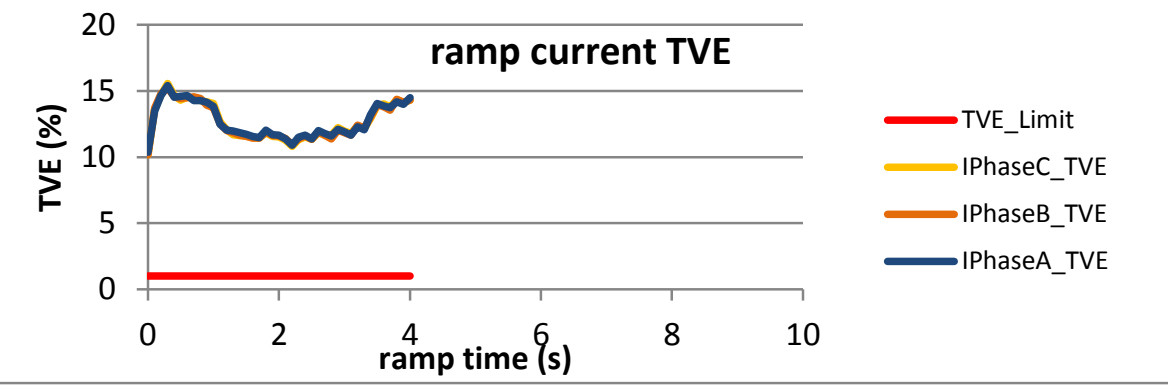

Figure 2368: Fs = $10 \mathrm{FPS}$, ramp from $62 \mathrm{~Hz}$ to $58 \mathrm{~Hz}$ at $-1 \mathrm{~Hz} / \mathrm{s}$ 
6.2.9 PMU H dynamic ramp of system frequency current TVE, M class

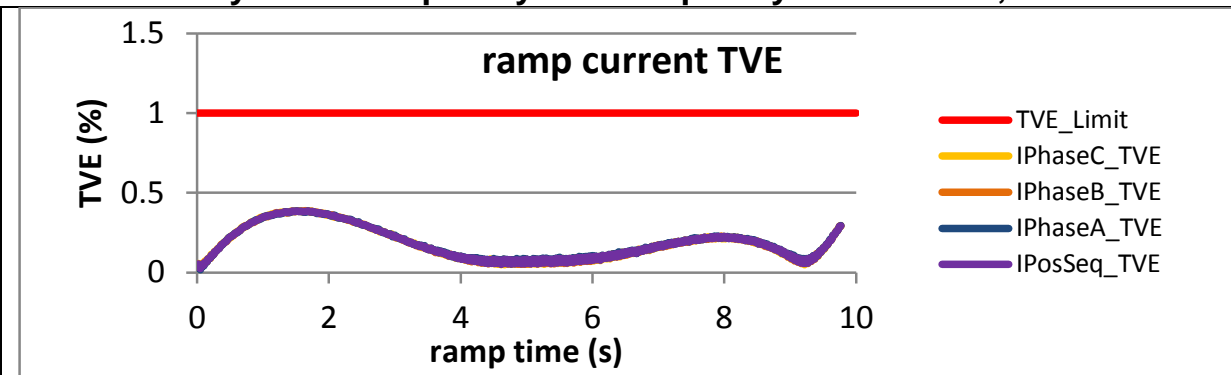

Figure 2369: Fs = $60 \mathrm{FPS}$, ramp from $55 \mathrm{~Hz}$ to $65 \mathrm{~Hz}$ at $+1 \mathrm{~Hz} / \mathrm{s}$

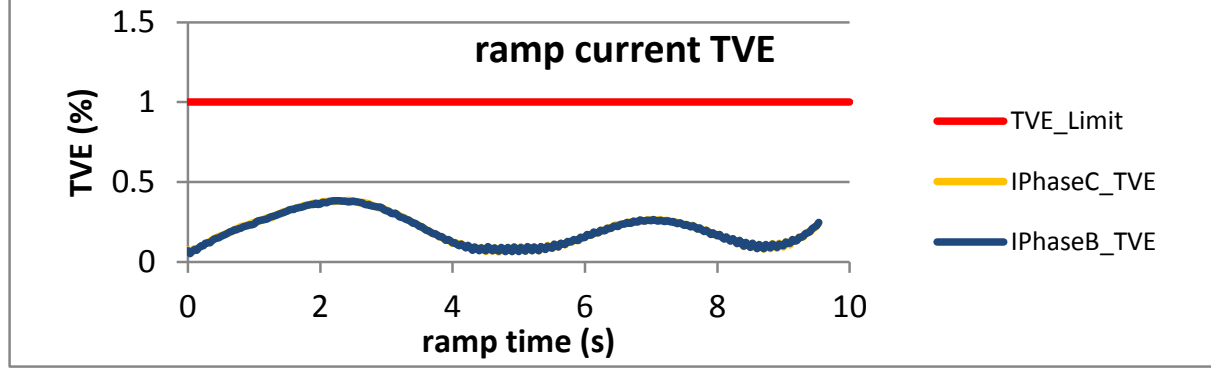

Figure 2371: Fs = $30 \mathrm{FPS}$, ramp from $55 \mathrm{~Hz}$ to $65 \mathrm{~Hz}$ at $+1 \mathrm{~Hz} / \mathrm{s}$

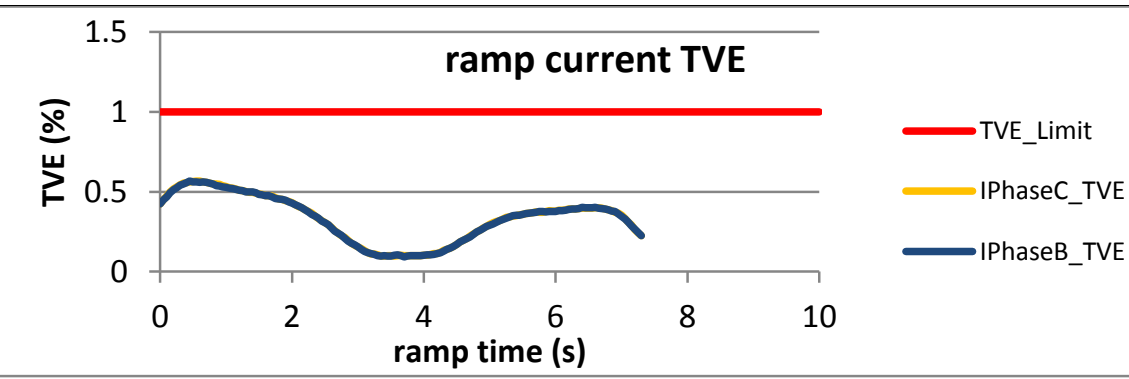

Figure 2373: Fs = 20 FPS, ramp from $56 \mathrm{~Hz}$ to $64 \mathrm{~Hz}$ at $+1 \mathrm{~Hz} / \mathrm{s}$

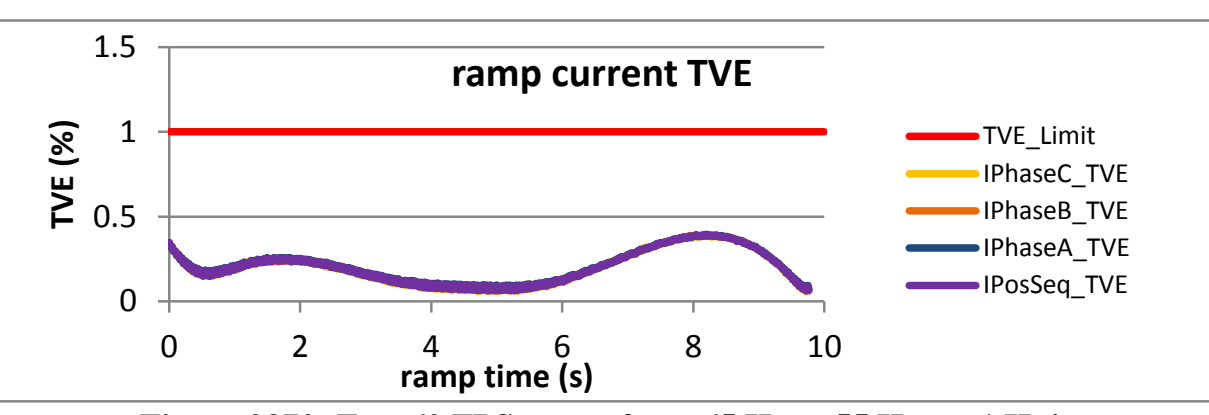

Figure 2370: Fs = 60 FPS, ramp from $65 \mathrm{~Hz}$ to $55 \mathrm{~Hz}$ at $-1 \mathrm{~Hz} / \mathrm{s}$

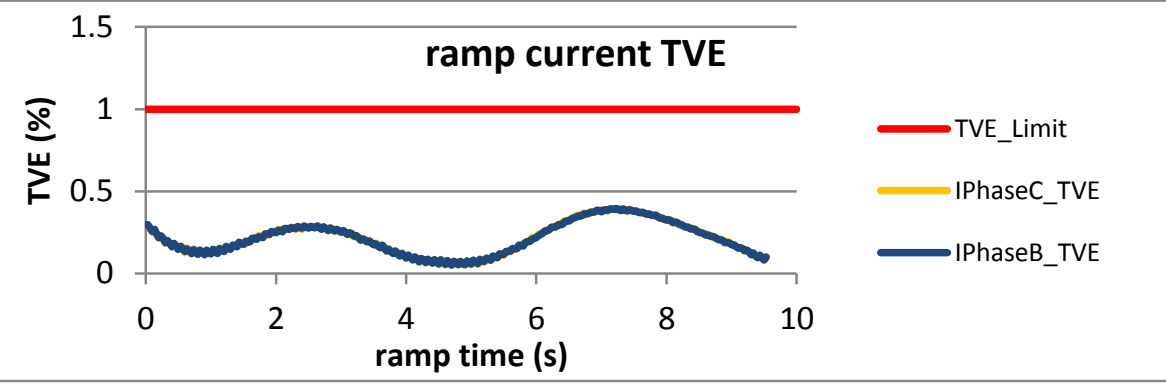

Figure 2372: Fs = $30 \mathrm{FPS}$, ramp from $65 \mathrm{~Hz}$ to $55 \mathrm{~Hz}$ at $-1 \mathrm{~Hz} / \mathrm{s}$

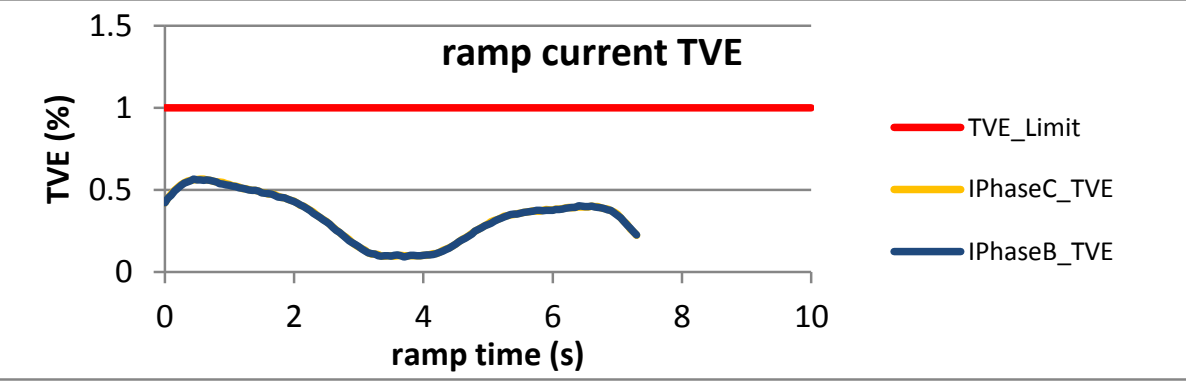

Figure 2374: Fs = $20 \mathrm{FPS}$, ramp from $64 \mathrm{~Hz}$ to $56 \mathrm{~Hz}$ at $-1 \mathrm{~Hz} / \mathrm{s}$ 


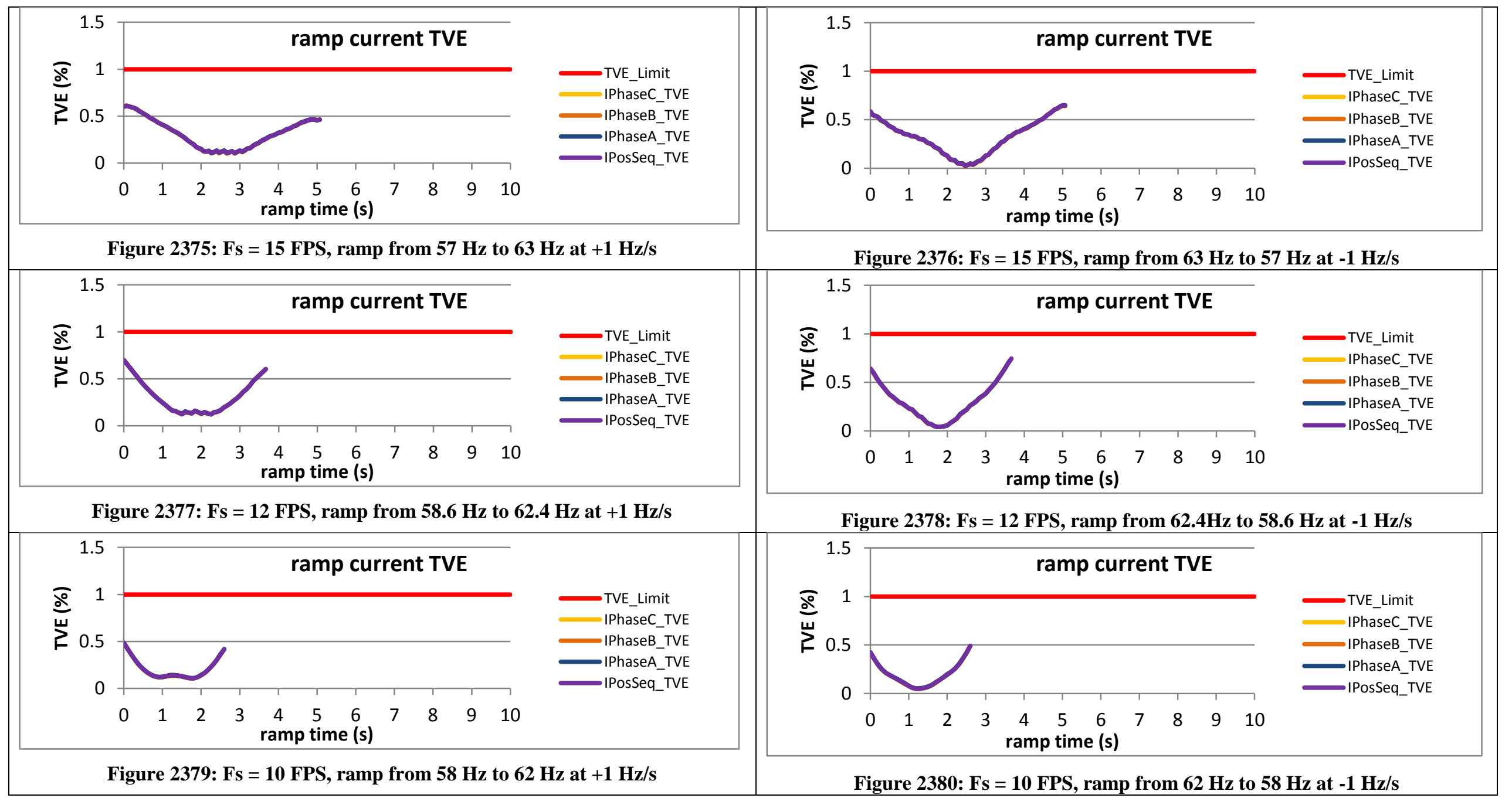


6.2.10 PMU I dynamic ramp of system frequency current TVE, M class

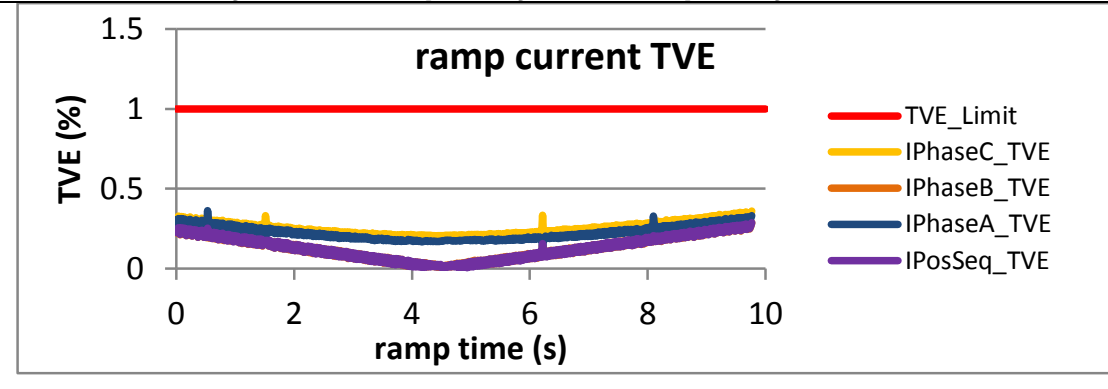

Figure 2381: Fs = $60 \mathrm{FPS}$, ramp from $55 \mathrm{~Hz}$ to $65 \mathrm{~Hz}$ at $+1 \mathrm{~Hz} / \mathrm{s}$

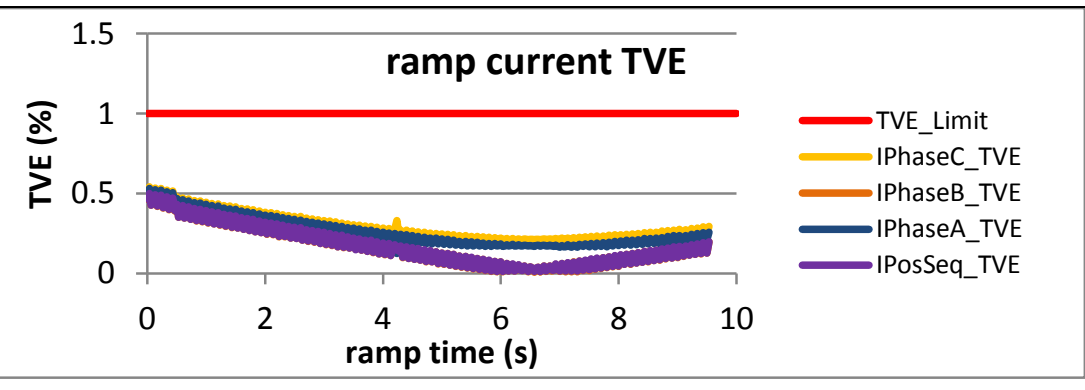

Figure 2383: Fs = $30 \mathrm{FPS}$, ramp from $55 \mathrm{~Hz}$ to $65 \mathrm{~Hz}$ at $+1 \mathrm{~Hz} / \mathrm{s}$

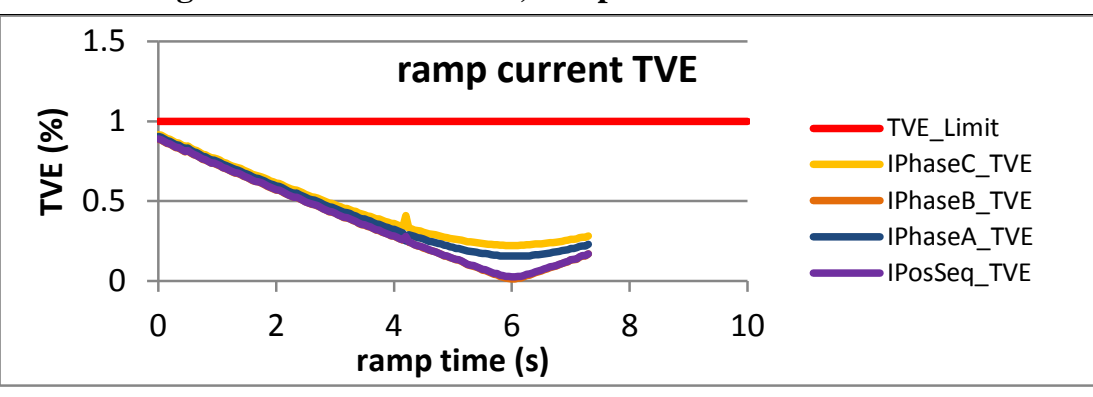

Figure 2385: Fs = $20 \mathrm{FPS}$, ramp from $56 \mathrm{~Hz}$ to $64 \mathrm{~Hz}$ at $+1 \mathrm{~Hz} / \mathrm{s}$

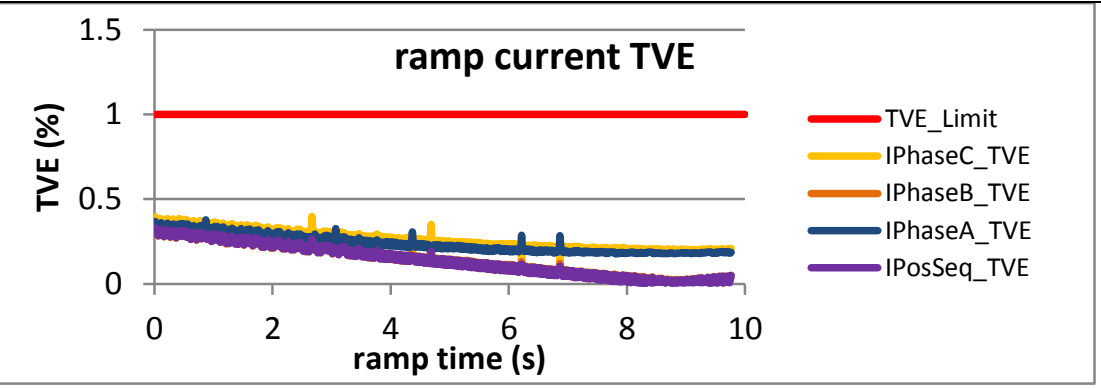

Figure 2382: Fs = $60 \mathrm{FPS}$, ramp from $65 \mathrm{~Hz}$ to $55 \mathrm{~Hz}$ at $-1 \mathrm{~Hz} / \mathrm{s}$

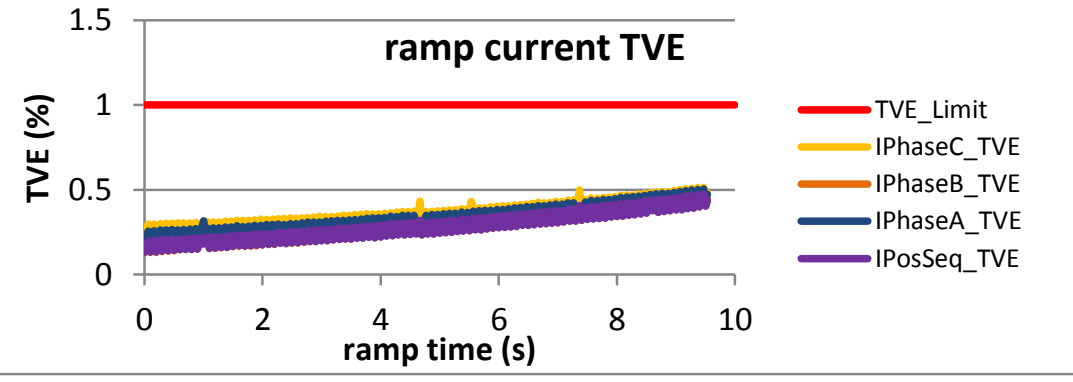

Figure 2384: Fs = $30 \mathrm{FPS}$, ramp from $65 \mathrm{~Hz}$ to $55 \mathrm{~Hz}$ at $-1 \mathrm{~Hz} / \mathrm{s}$

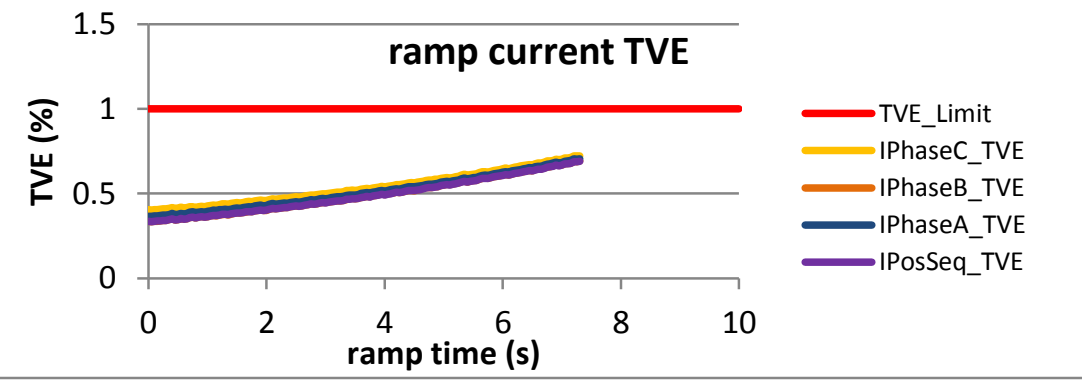

Figure 2386: Fs = $20 \mathrm{FPS}$, ramp from $64 \mathrm{~Hz}$ to $56 \mathrm{~Hz}$ at $-1 \mathrm{~Hz} / \mathrm{s}$ 


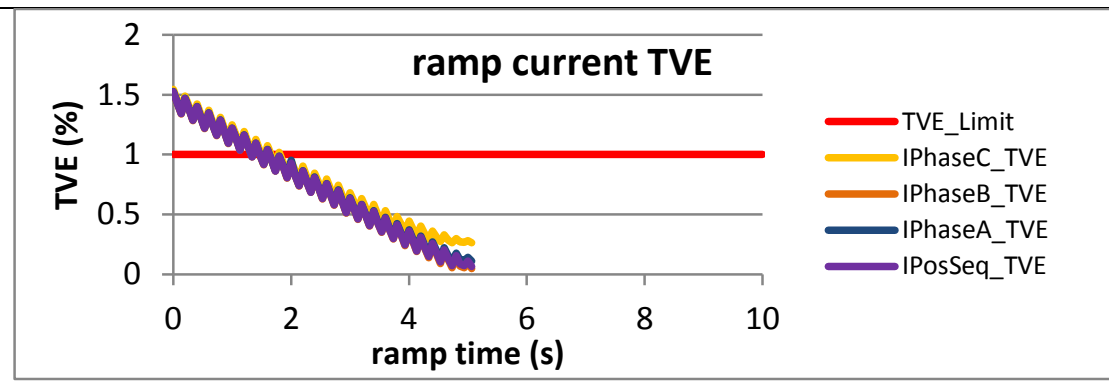

Figure 2387: Fs = $15 \mathrm{FPS}$, ramp from $57 \mathrm{~Hz}$ to $63 \mathrm{~Hz}$ at $+1 \mathrm{~Hz} / \mathrm{s}$

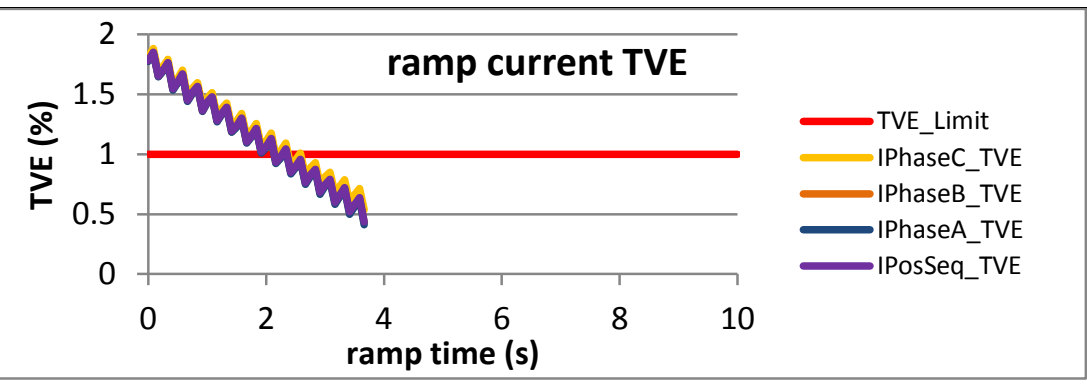

Figure 2389: Fs = 12 FPS, ramp from $58.6 \mathrm{~Hz}$ to $62.4 \mathrm{~Hz}$ at $+1 \mathrm{~Hz} / \mathrm{s}$

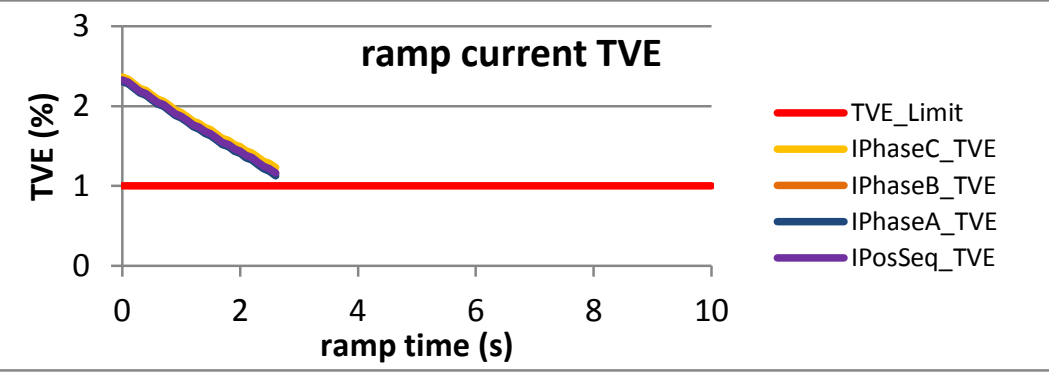

Figure 2391: Fs = $10 \mathrm{FPS}$, ramp from $58 \mathrm{~Hz}$ to $62 \mathrm{~Hz}$ at $+1 \mathrm{~Hz} / \mathrm{s}$

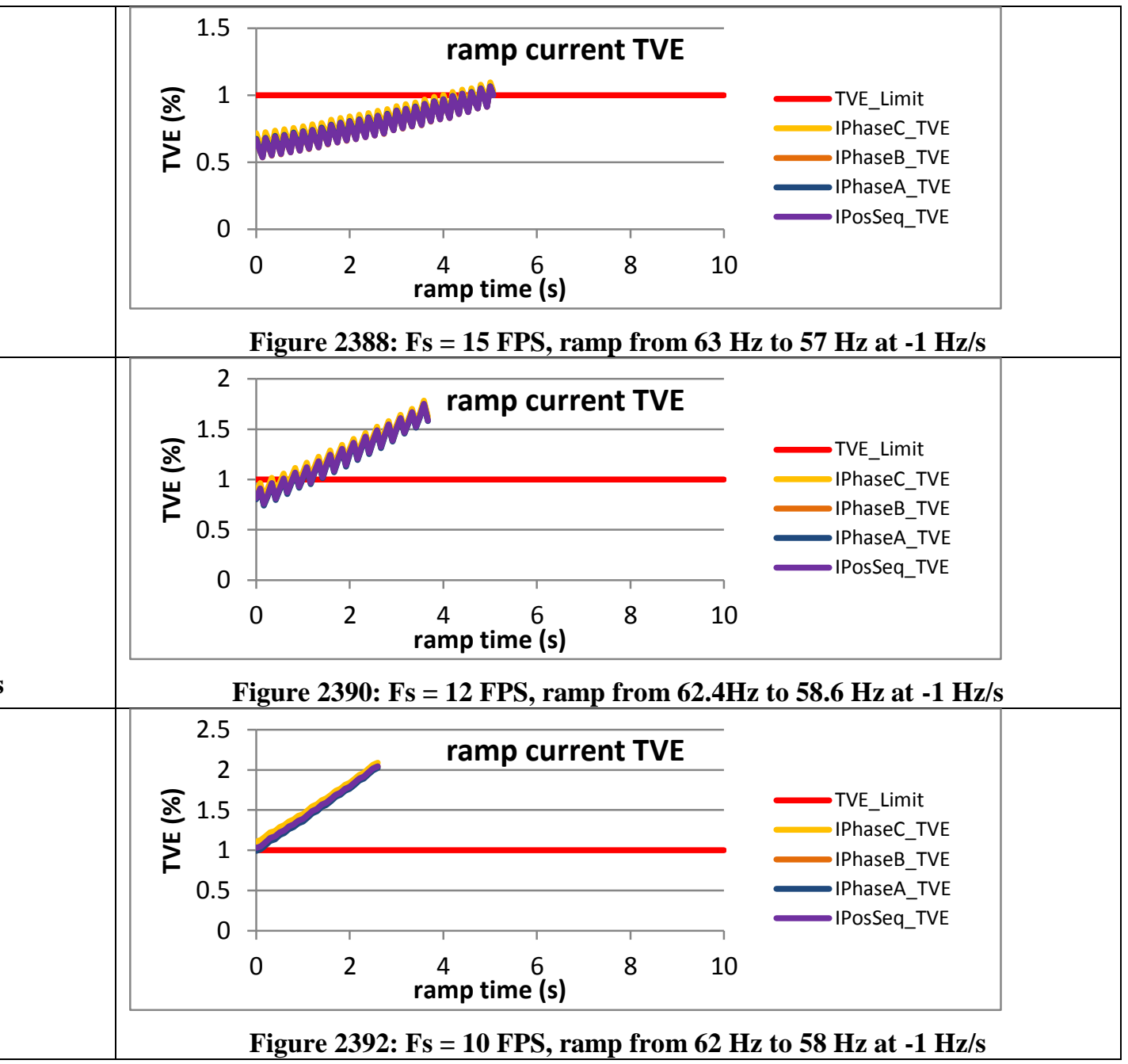


6.2.11 PMU J dynamic ramp of system frequency current TVE, M class

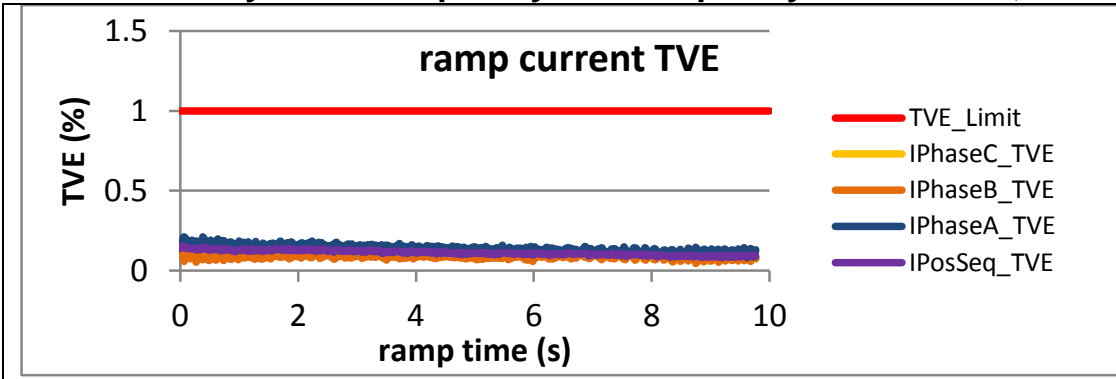

Figure 2393: Fs = $60 \mathrm{FPS}$, ramp from $55 \mathrm{~Hz}$ to $65 \mathrm{~Hz}$ at $+1 \mathrm{~Hz} / \mathrm{s}$

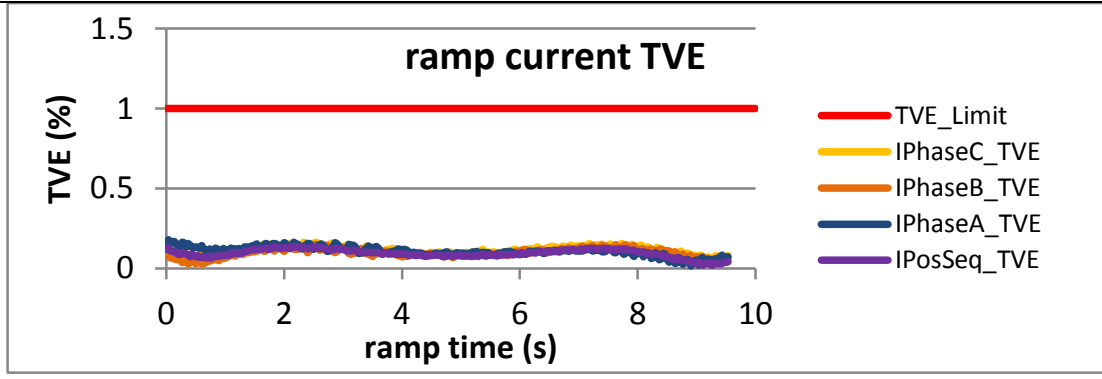

Figure 2395: Fs = $30 \mathrm{FPS}$, ramp from $55 \mathrm{~Hz}$ to $65 \mathrm{~Hz}$ at $+1 \mathrm{~Hz} / \mathrm{s}$

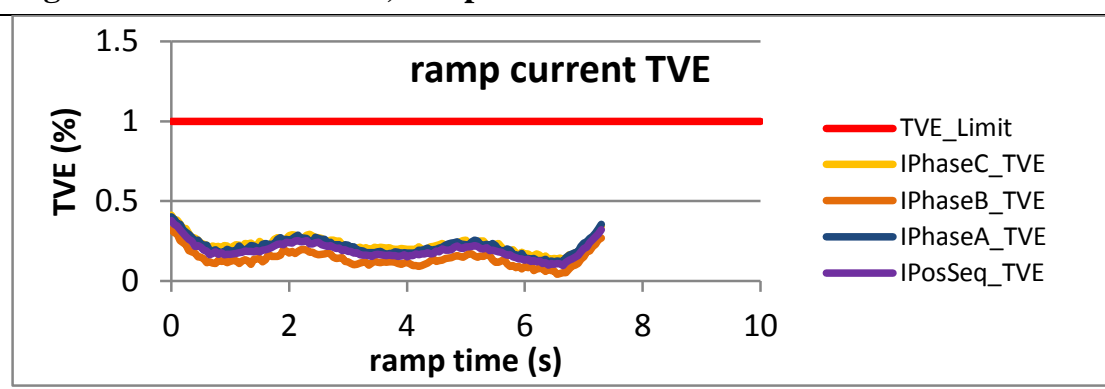

Figure 2397: Fs = $20 \mathrm{FPS}$, ramp from $56 \mathrm{~Hz}$ to $64 \mathrm{~Hz}$ at $+1 \mathrm{~Hz} / \mathrm{s}$

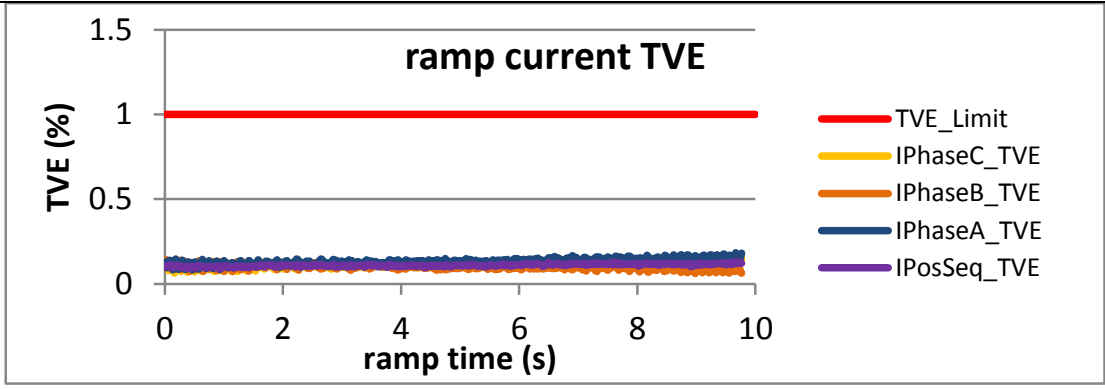

Figure 2394: Fs = $60 \mathrm{FPS}$, ramp from $65 \mathrm{~Hz}$ to $55 \mathrm{~Hz}$ at $-1 \mathrm{~Hz} / \mathrm{s}$

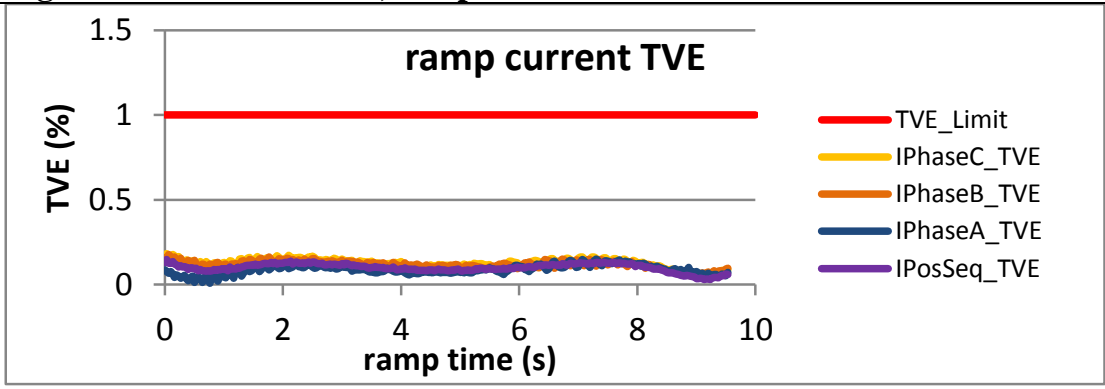

Figure 2396: Fs = $30 \mathrm{FPS}$, ramp from $65 \mathrm{~Hz}$ to $55 \mathrm{~Hz}$ at $-1 \mathrm{~Hz} / \mathrm{s}$

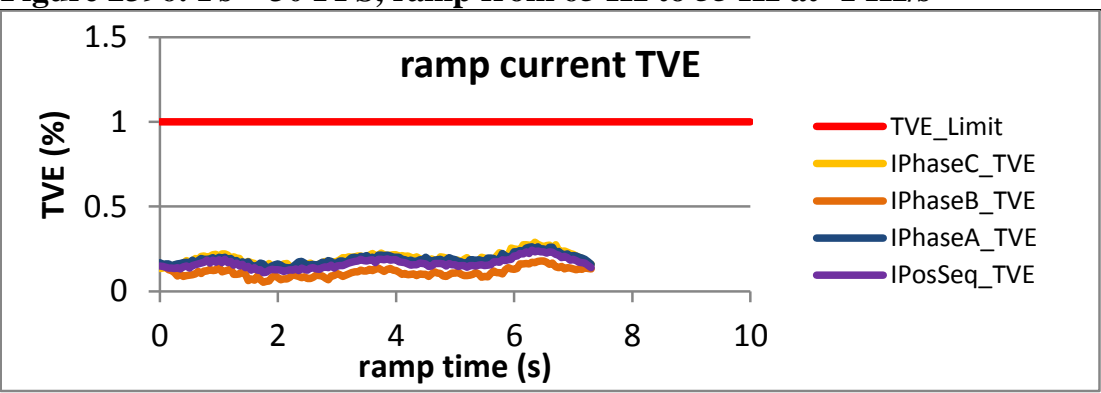

Figure 2398: Fs = 20 FPS, ramp from $64 \mathrm{~Hz}$ to $56 \mathrm{~Hz}$ at $-1 \mathrm{~Hz} / \mathrm{s}$ 


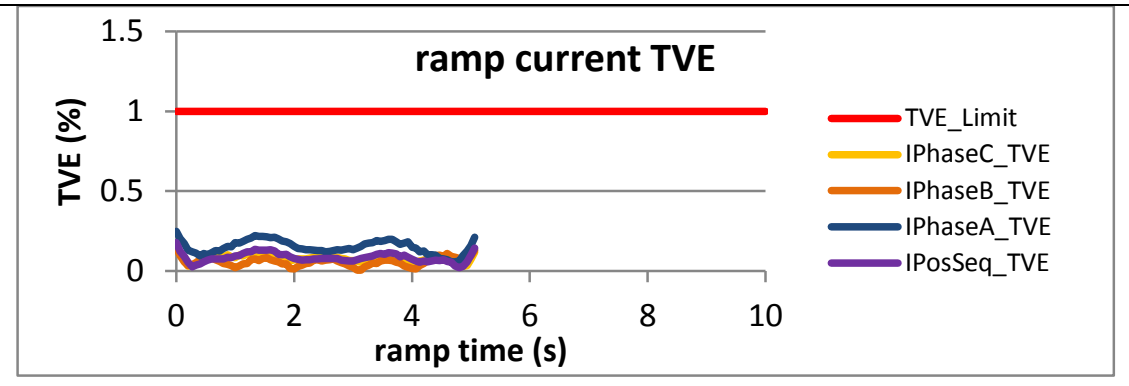

Figure 2399: Fs = $15 \mathrm{FPS}$, ramp from $57 \mathrm{~Hz}$ to $63 \mathrm{~Hz}$ at $+1 \mathrm{~Hz} / \mathrm{s}$

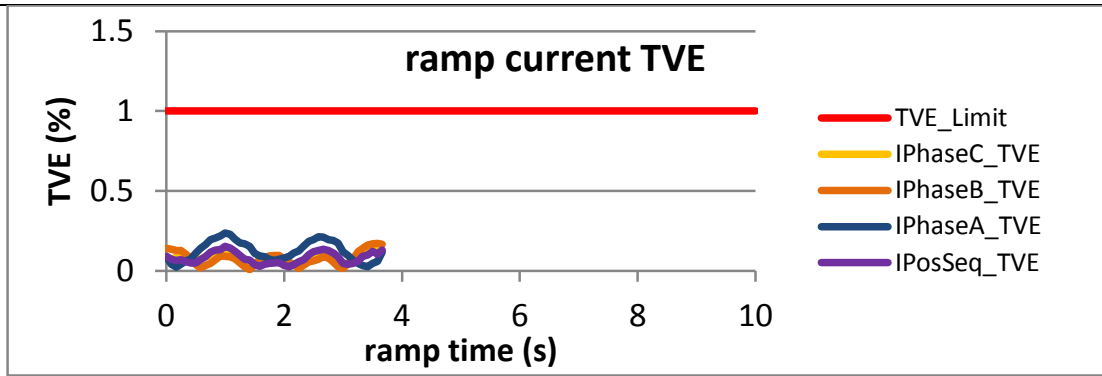

Figure 2401: Fs = 12 FPS, ramp from $58.6 \mathrm{~Hz}$ to $62.4 \mathrm{~Hz}$ at $+1 \mathrm{~Hz} / \mathrm{s}$

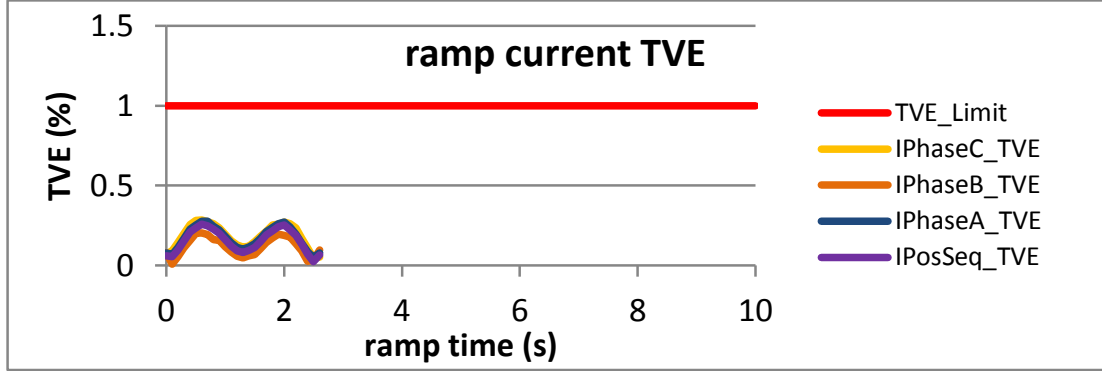

Figure 2403: Fs = $10 \mathrm{FPS}$, ramp from $58 \mathrm{~Hz}$ to $62 \mathrm{~Hz}$ at $+1 \mathrm{~Hz} / \mathrm{s}$

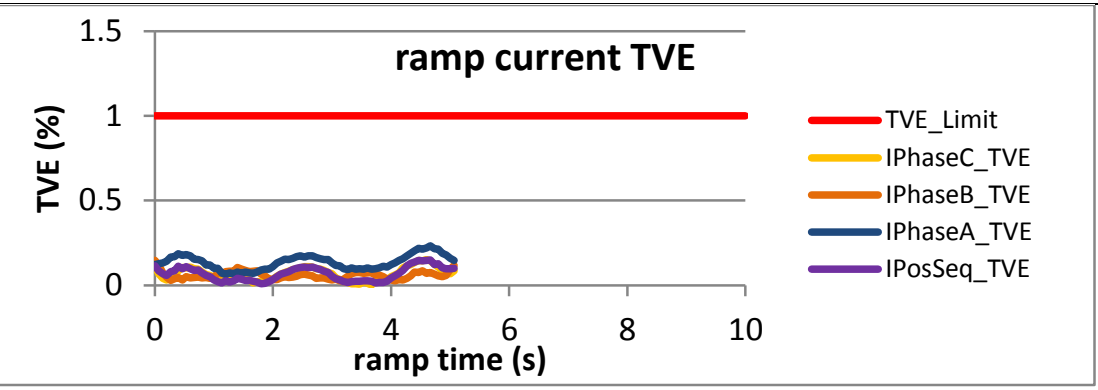

Figure 2400: Fs = $15 \mathrm{FPS}$, ramp from $63 \mathrm{~Hz}$ to $57 \mathrm{~Hz}$ at $-1 \mathrm{~Hz} / \mathrm{s}$

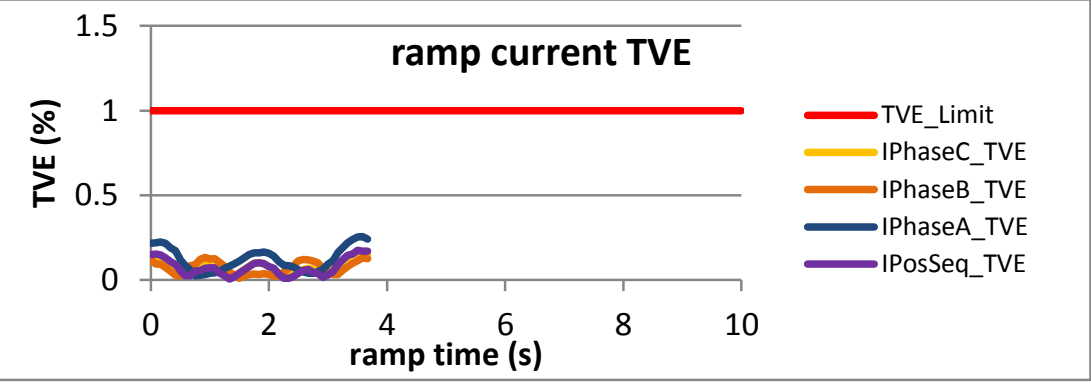

Figure 2402: Fs = 12 FPS, ramp from $62.4 \mathrm{~Hz}$ to $58.6 \mathrm{~Hz}$ at $-1 \mathrm{~Hz} / \mathrm{s}$

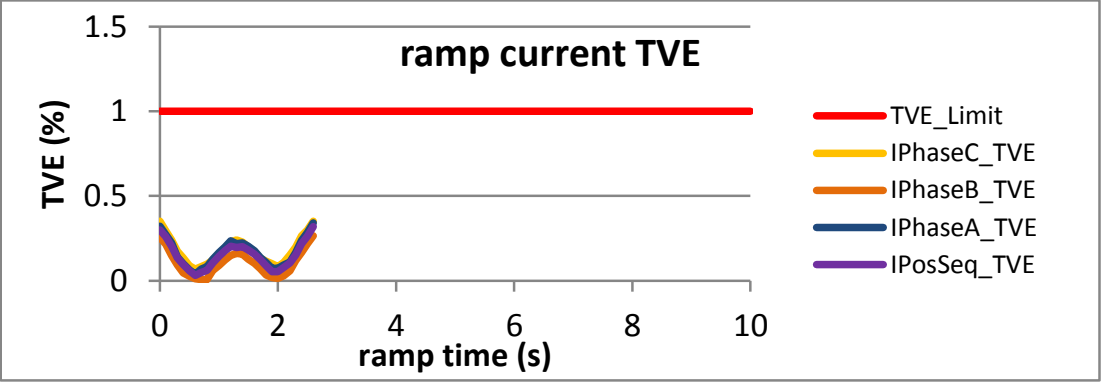

Figure 2404: Fs = $10 \mathrm{FPS}$, ramp from $62 \mathrm{~Hz}$ to $58 \mathrm{~Hz}$ at $-1 \mathrm{~Hz} / \mathrm{s}$ 


\subsection{Dynamic ramp of system frequency frequency error, M class}

\subsubsection{C367.118.1 Annex $C$ dynamic ramp of system frequency frequency error: $M$ class}
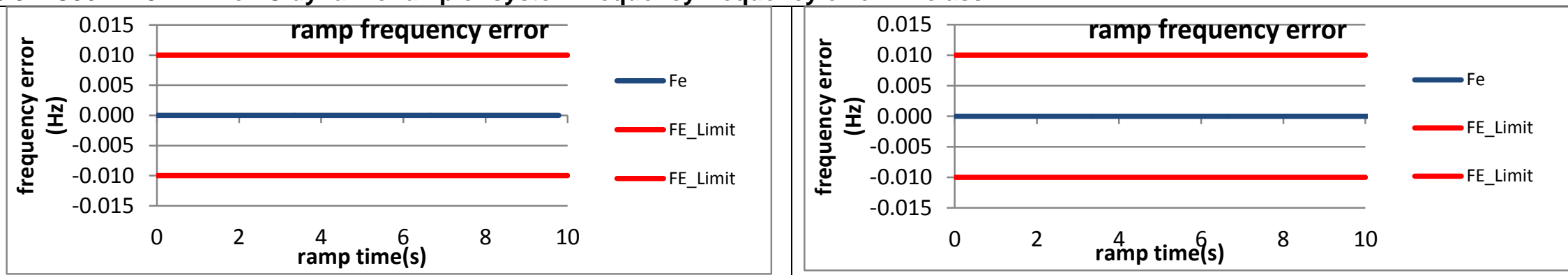

Figure 2405: Fs = 60 FPS, ramp from $55 \mathrm{~Hz}$ to $65 \mathrm{~Hz}$ at $+1 \mathrm{~Hz} / \mathrm{s}$

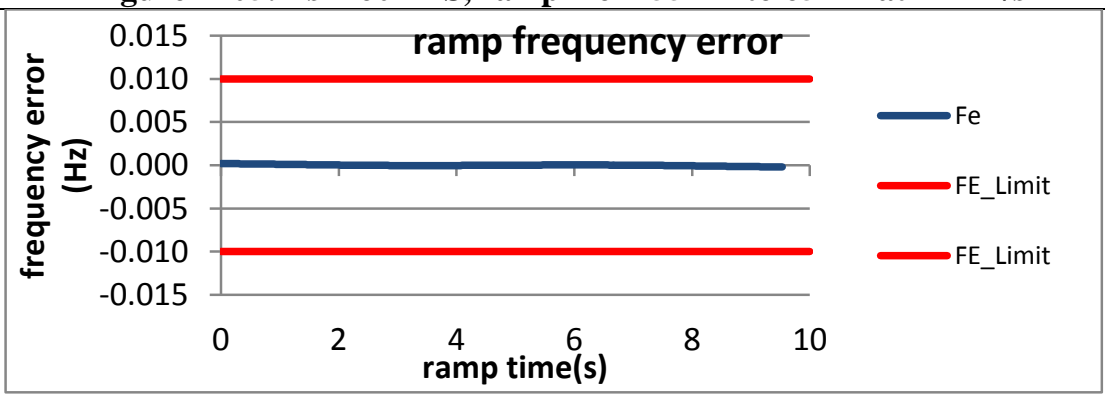

Figure 2407: Fs = $30 \mathrm{FPS}$, ramp from $55 \mathrm{~Hz}$ to $65 \mathrm{~Hz}$ at $+1 \mathrm{~Hz} / \mathrm{s}$

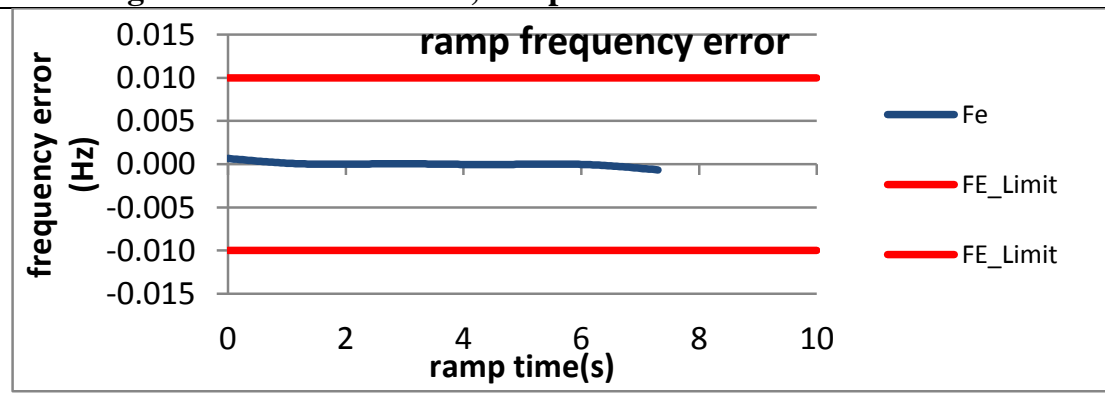

Figure 2409: Fs = 20 FPS, ramp from $56 \mathrm{~Hz}$ to $64 \mathrm{~Hz}$ at $+1 \mathrm{~Hz} / \mathrm{s}$

Figure 2406: Fs = $60 \mathrm{FPS}$, ramp from $65 \mathrm{~Hz}$ to $55 \mathrm{~Hz}$ at $-1 \mathrm{~Hz} / \mathrm{s}$

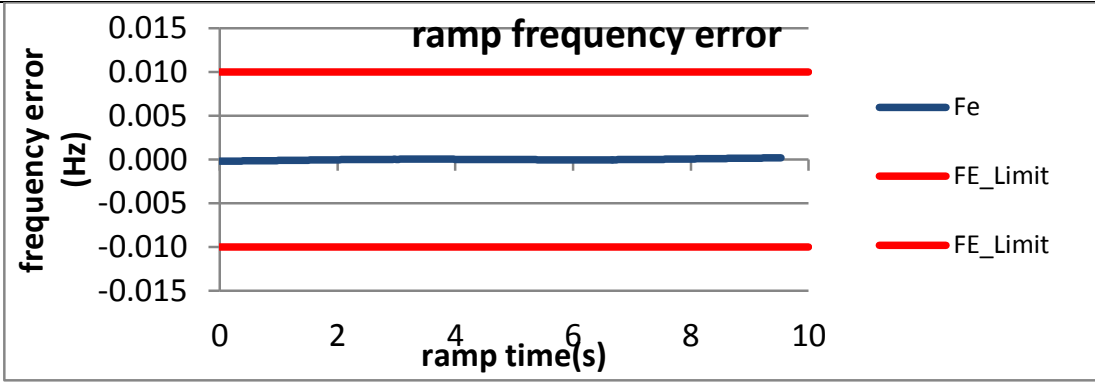

Figure 2408: Fs = $30 \mathrm{FPS}$, ramp from $65 \mathrm{~Hz}$ to $55 \mathrm{~Hz}$ at $-1 \mathrm{~Hz} / \mathrm{s}$

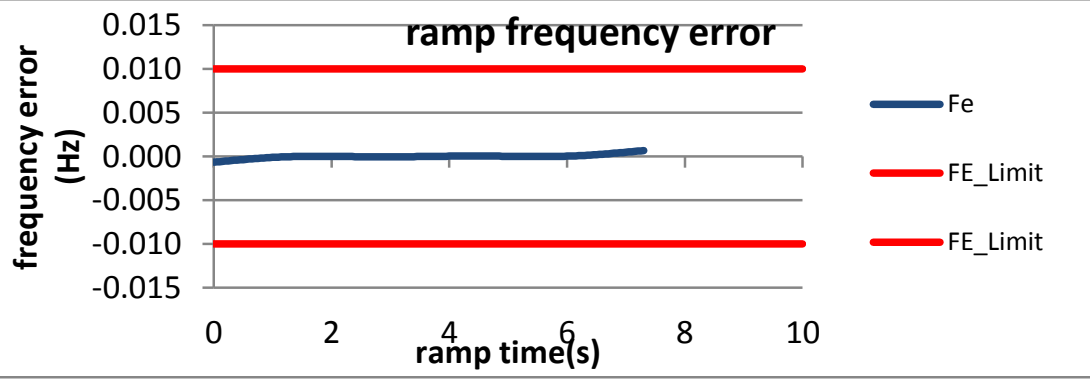

Figure 2410: Fs = $20 \mathrm{FPS}$, ramp from $64 \mathrm{~Hz}$ to $56 \mathrm{~Hz}$ at $-1 \mathrm{~Hz} / \mathrm{s}$ 

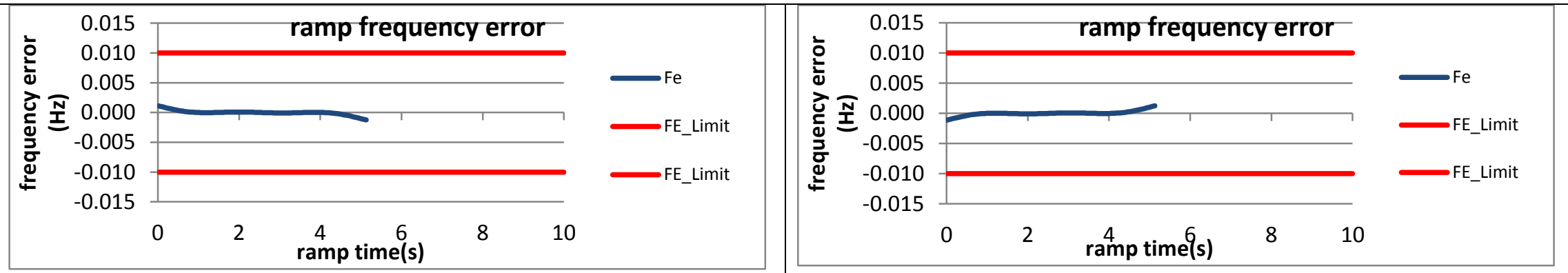

Figure 2411: Fs = $15 \mathrm{FPS}$, ramp from $57 \mathrm{~Hz}$ to $63 \mathrm{~Hz}$ at $+1 \mathrm{~Hz} / \mathrm{s}$

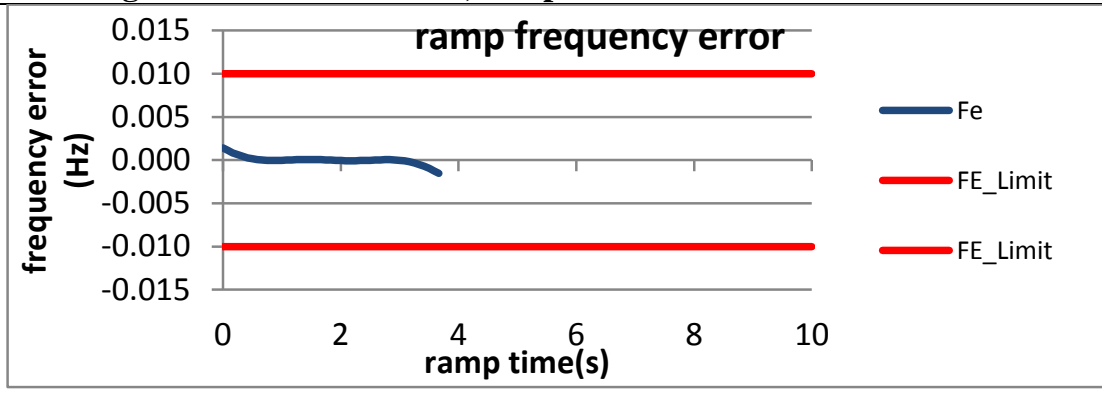

Figure 2413: Fs = 12 FPS, ramp from $58.6 \mathrm{~Hz}$ to $62.4 \mathrm{~Hz}$ at $+1 \mathrm{~Hz} / \mathrm{s}$

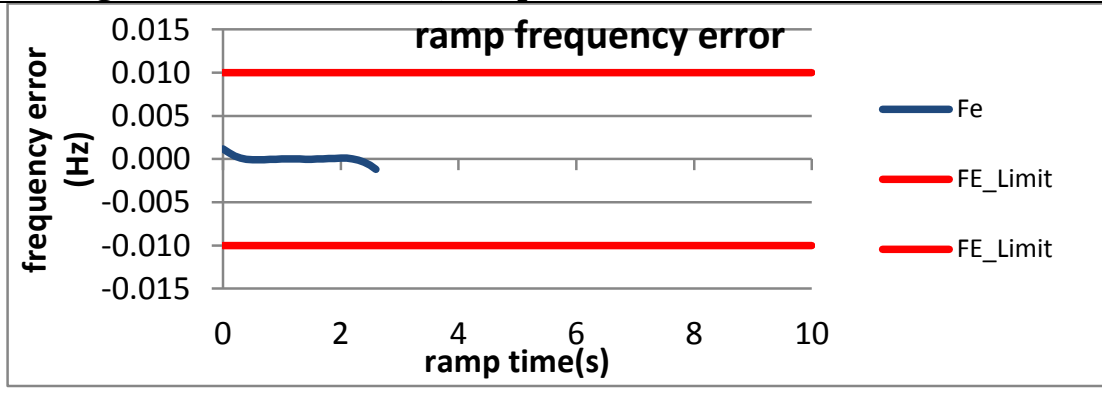

Figure 2415: Fs = $10 \mathrm{FPS}$, ramp from $58 \mathrm{~Hz}$ to $62 \mathrm{~Hz}$ at $+1 \mathrm{~Hz} / \mathrm{s}$

Figure 2412: Fs = $15 \mathrm{FPS}$, ramp from $63 \mathrm{~Hz}$ to $57 \mathrm{~Hz}$ at $-1 \mathrm{~Hz} / \mathrm{s}$

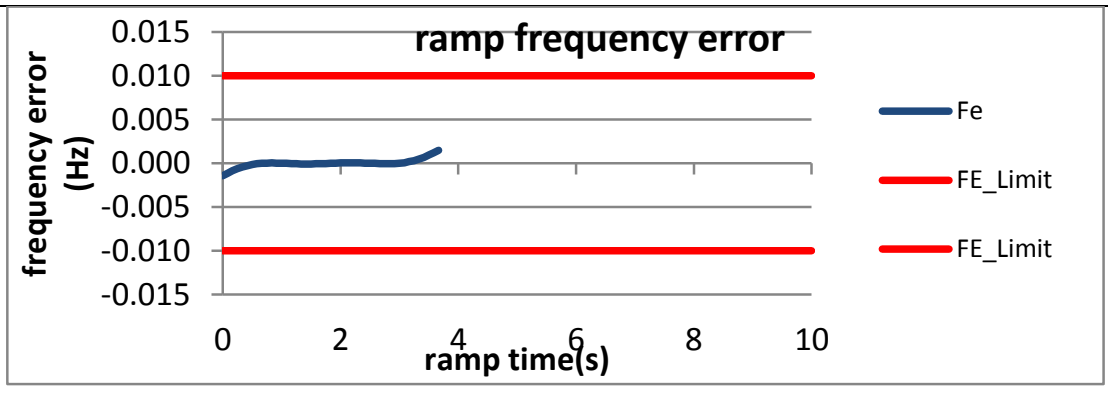

Figure 2414: Fs = 12 FPS, ramp from 62.4Hz to $58.6 \mathrm{~Hz}$ at $-1 \mathrm{~Hz} / \mathrm{s}$

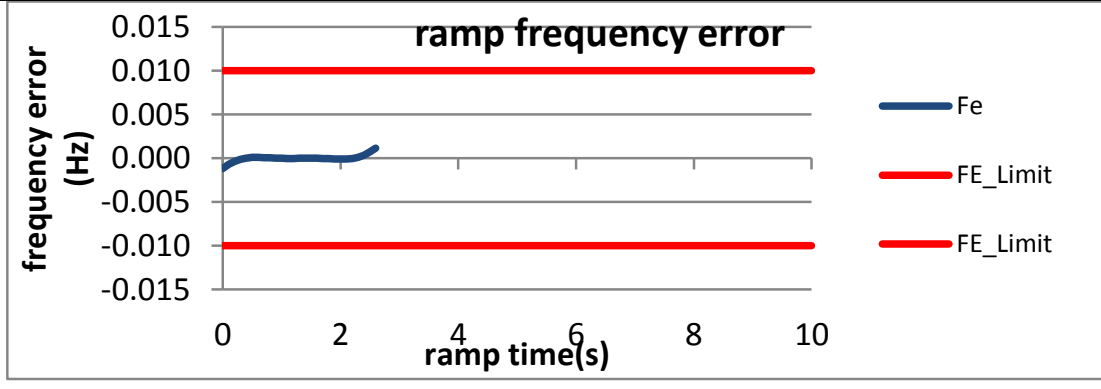

Figure 2416: Fs = $10 \mathrm{FPS}$, ramp from $62 \mathrm{~Hz}$ to $58 \mathrm{~Hz}$ at $-1 \mathrm{~Hz} / \mathrm{s}$ 
6.3.2 C37.118.1 PMU A dynamic ramp of system frequency frequency error: $M$ class

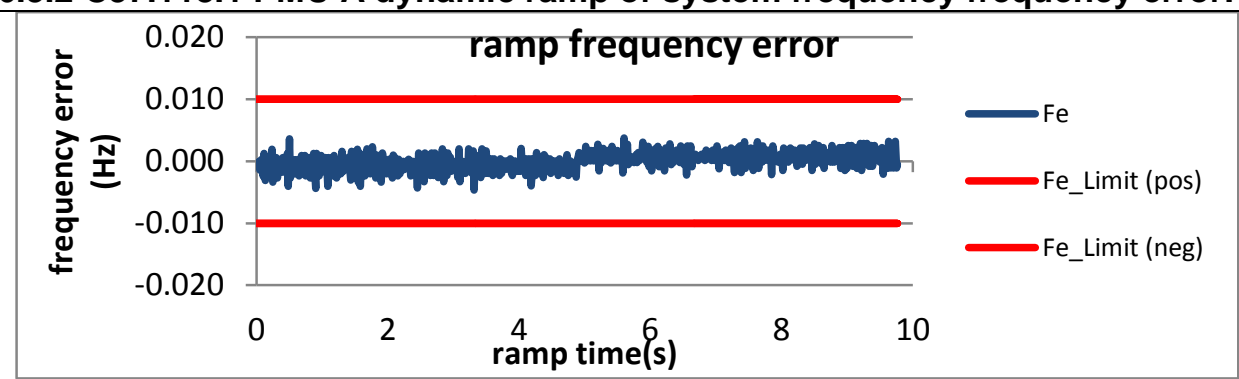

Figure 2417: Fs = $60 \mathrm{FPS}$, ramp from $55 \mathrm{~Hz}$ to $65 \mathrm{~Hz}$ at $+1 \mathrm{~Hz} / \mathrm{s}$

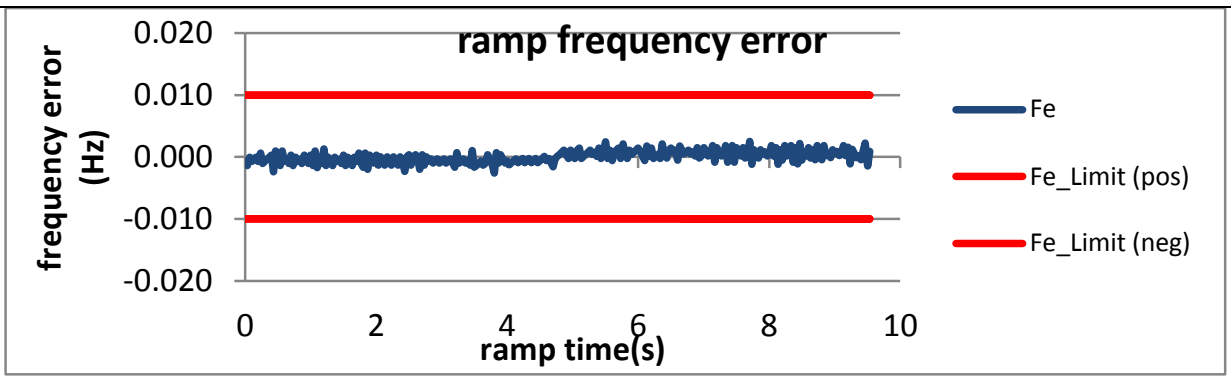

Figure 2419: Fs = $30 \mathrm{FPS}$, ramp from $55 \mathrm{~Hz}$ to $65 \mathrm{~Hz}$ at $+1 \mathrm{~Hz} / \mathrm{s}$

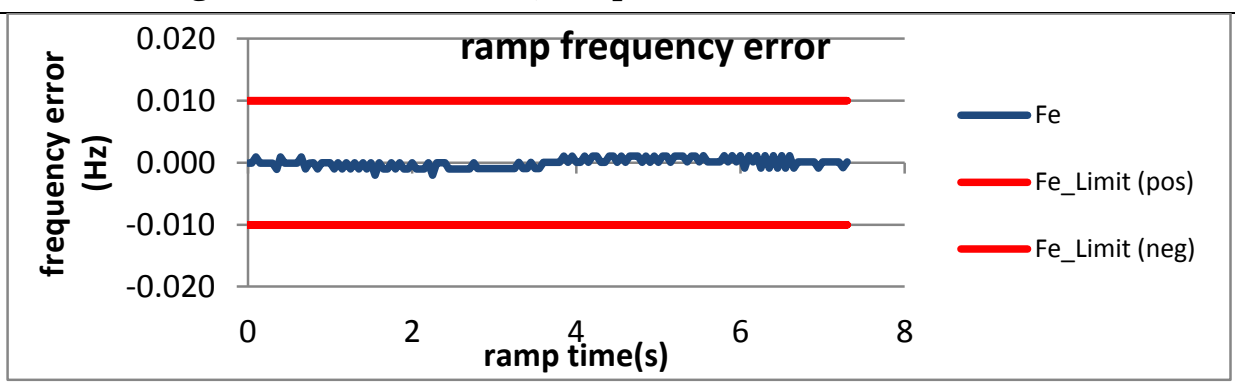

Figure 2421: Fs = $20 \mathrm{FPS}$, ramp from $56 \mathrm{~Hz}$ to $64 \mathrm{~Hz}$ at $+1 \mathrm{~Hz} / \mathrm{s}$

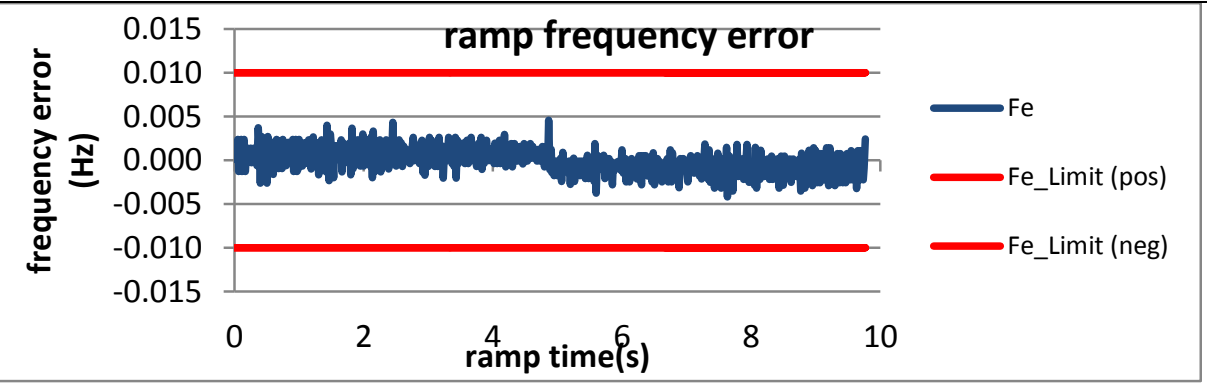

Figure 2418: Fs = $60 \mathrm{FPS}$, ramp from $65 \mathrm{~Hz}$ to $55 \mathrm{~Hz}$ at $-1 \mathrm{~Hz} / \mathrm{s}$

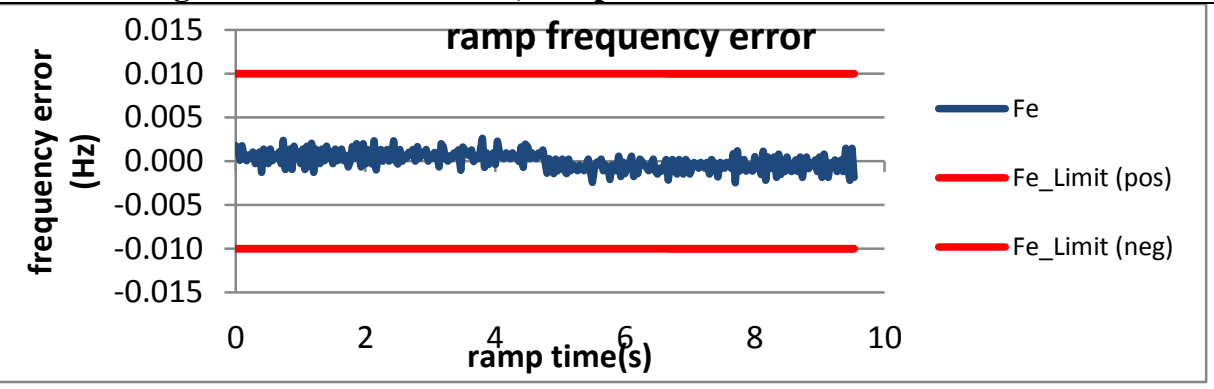

Figure 2420: Fs = $30 \mathrm{FPS}$, ramp from $65 \mathrm{~Hz}$ to $55 \mathrm{~Hz}$ at $-1 \mathrm{~Hz} / \mathrm{s}$

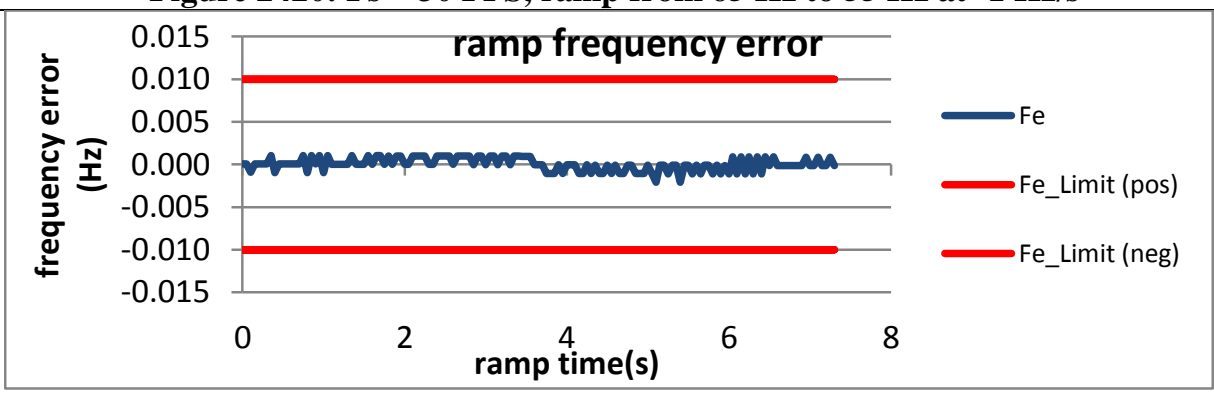

Figure 2422: Fs = $20 \mathrm{FPS}$, ramp from $64 \mathrm{~Hz}$ to $56 \mathrm{~Hz}$ at $-1 \mathrm{~Hz} / \mathrm{s}$ 


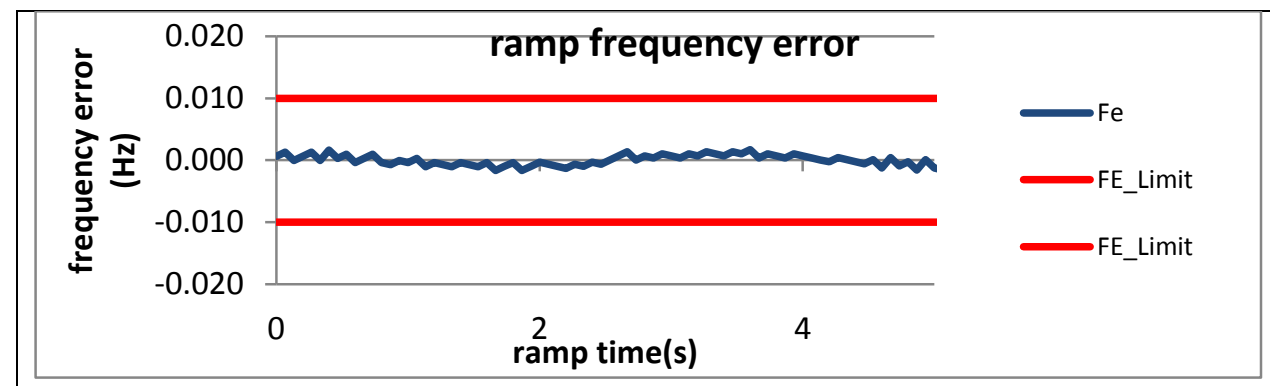

Figure 2423: Fs = $15 \mathrm{FPS}$, ramp from $57 \mathrm{~Hz}$ to $63 \mathrm{~Hz} \mathrm{at}+1 \mathrm{~Hz} / \mathrm{s}$

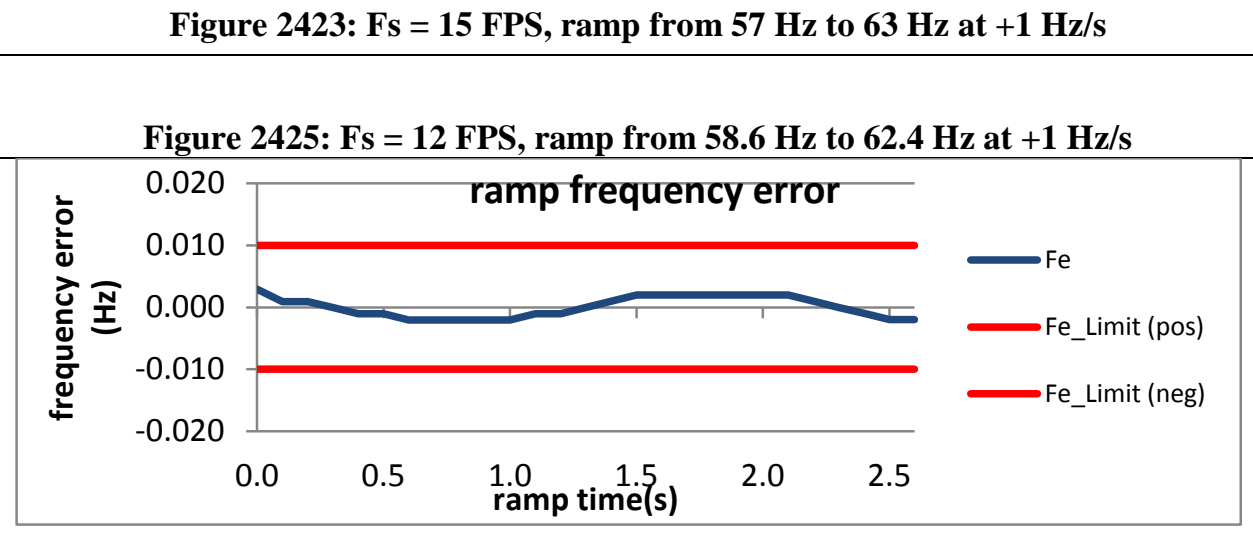

Figure 2427: Fs = $10 \mathrm{FPS}$, ramp from $58 \mathrm{~Hz}$ to $62 \mathrm{~Hz}$ at $+1 \mathrm{~Hz} / \mathrm{s}$

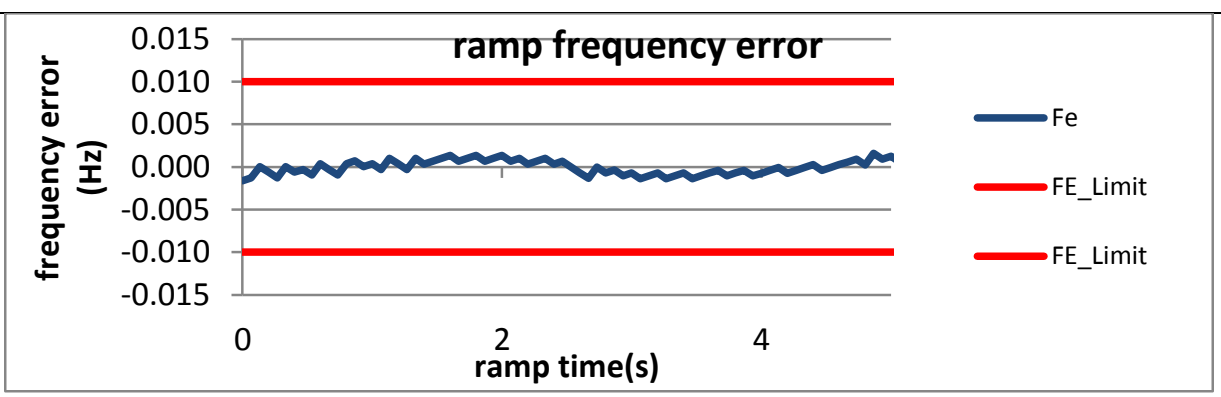

Figure 2424: Fs = $15 \mathrm{FPS}$, ramp from $63 \mathrm{~Hz}$ to $57 \mathrm{~Hz}$ at $-1 \mathrm{~Hz} / \mathrm{s}$

Figure 2426: Fs = 12 FPS, ramp from $62.4 \mathrm{~Hz}$ to $58.6 \mathrm{~Hz}$ at $-1 \mathrm{~Hz} / \mathrm{s}$

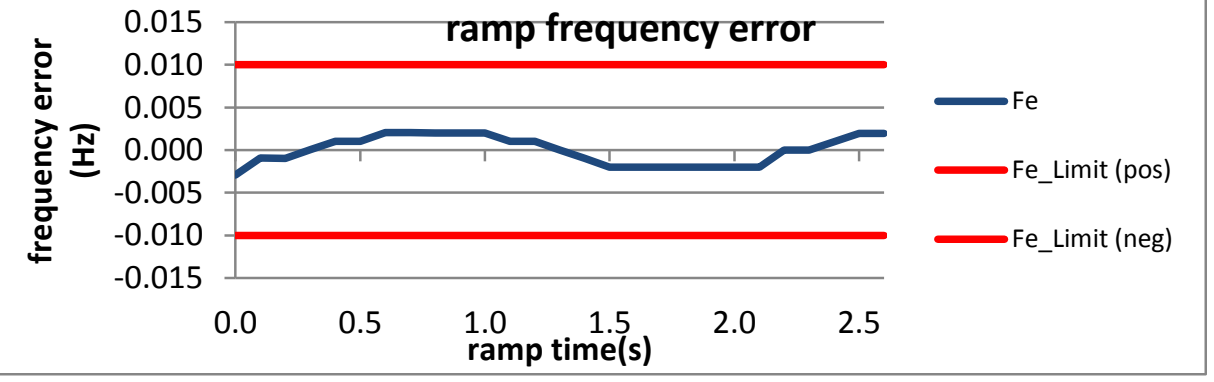

Figure 2428: Fs = $10 \mathrm{FPS}$, ramp from $62 \mathrm{~Hz}$ to $58 \mathrm{~Hz}$ at $-1 \mathrm{~Hz} / \mathrm{s}$ 
6.3.3 PMU B dynamic ramp of system frequency frequency error: M class

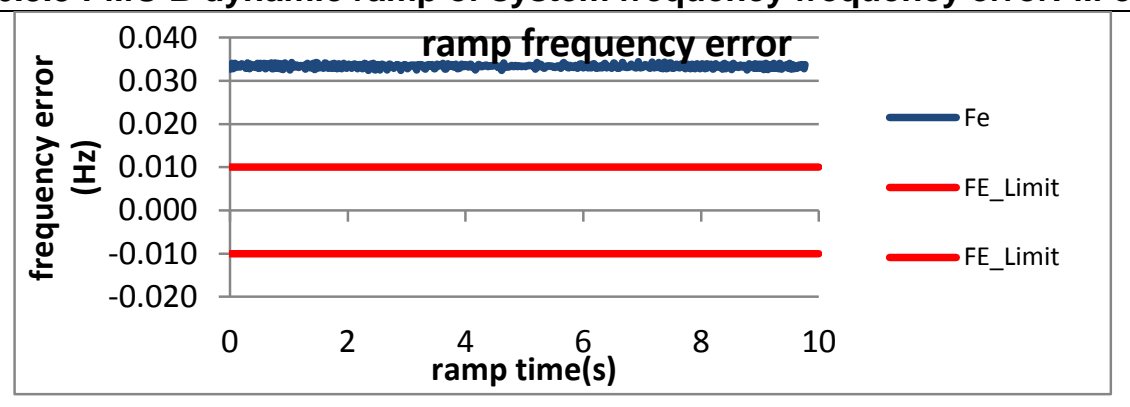

Figure 2429: Fs = $60 \mathrm{FPS}$, ramp from $55 \mathrm{~Hz}$ to $65 \mathrm{~Hz}$ at $+1 \mathrm{~Hz} / \mathrm{s}$

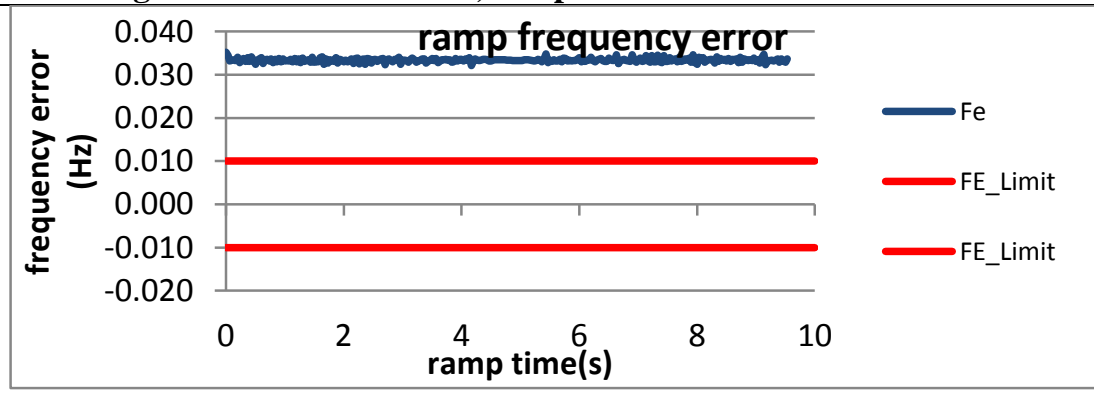

Figure 2431: Fs = $30 \mathrm{FPS}$, ramp from $55 \mathrm{~Hz}$ to $65 \mathrm{~Hz}$ at $+1 \mathrm{~Hz} / \mathrm{s}$

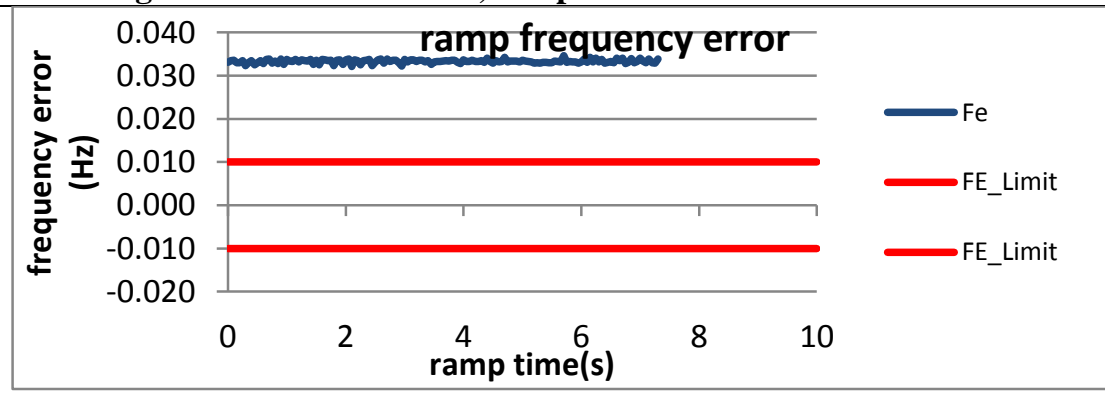

Figure 2433: Fs = 20 FPS, ramp from $56 \mathrm{~Hz}$ to $64 \mathrm{~Hz}$ at $+1 \mathrm{~Hz} / \mathrm{s}$

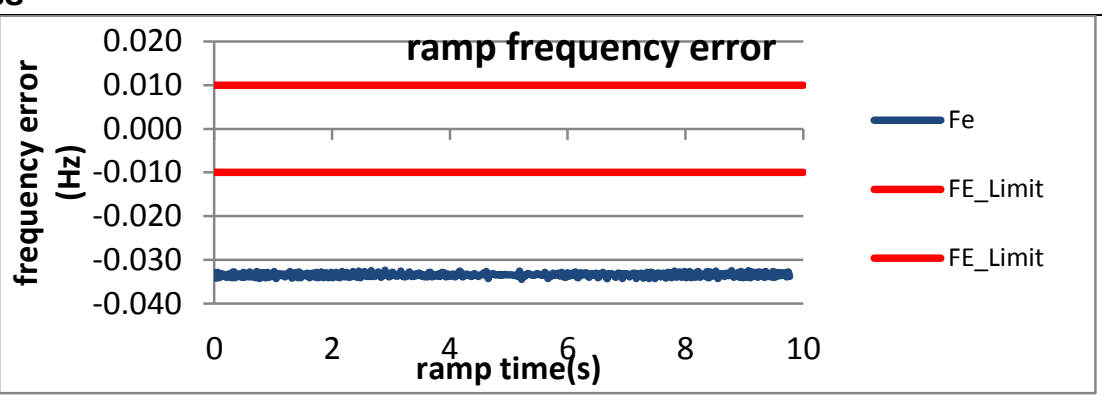

Figure 2430: Fs = $60 \mathrm{FPS}$, ramp from $65 \mathrm{~Hz}$ to $55 \mathrm{~Hz}$ at $-1 \mathrm{~Hz} / \mathrm{s}$

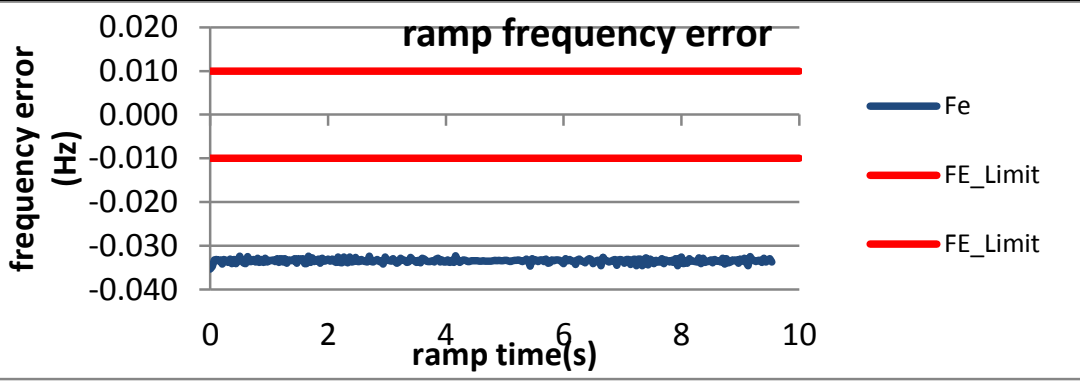

Figure 2432: Fs = $30 \mathrm{FPS}$, ramp from $65 \mathrm{~Hz}$ to $55 \mathrm{~Hz}$ at $-1 \mathrm{~Hz} / \mathrm{s}$

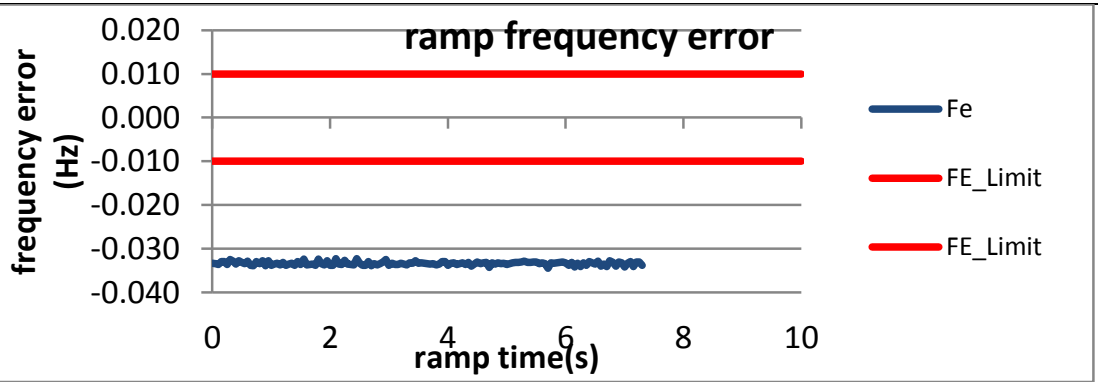

Figure 2434: Fs = $20 \mathrm{FPS}$, ramp from $64 \mathrm{~Hz}$ to $56 \mathrm{~Hz}$ at $-1 \mathrm{~Hz} / \mathrm{s}$ 

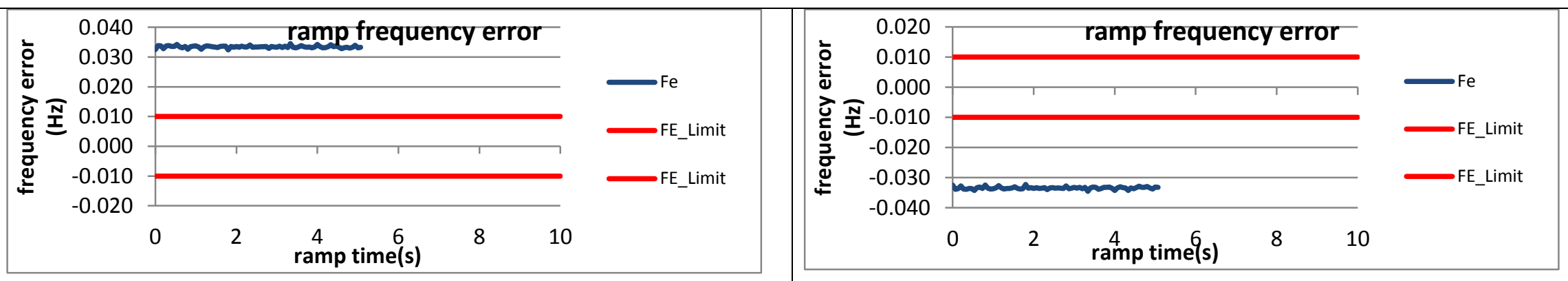

Figure 2435: Fs = $15 \mathrm{FPS}$, ramp from $57 \mathrm{~Hz}$ to $63 \mathrm{~Hz}$ at $+1 \mathrm{~Hz} / \mathrm{s}$

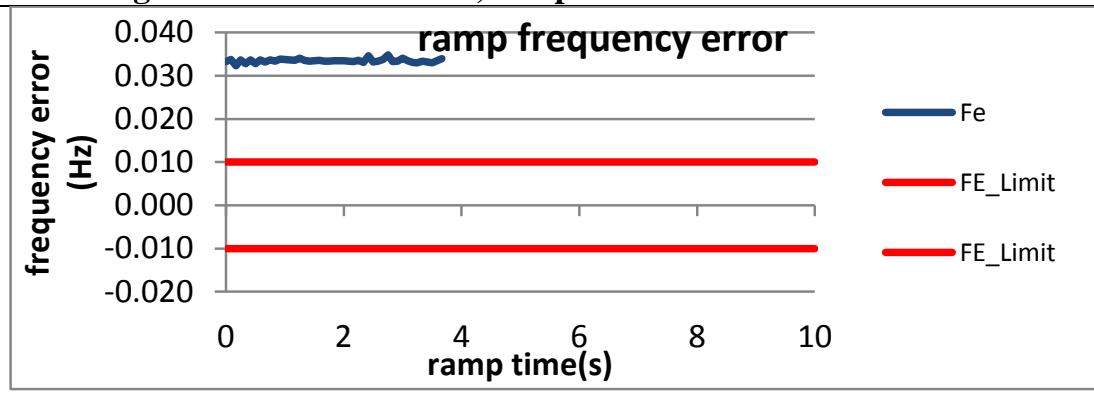

Figure 2437: Fs = 12 FPS, ramp from $58.6 \mathrm{~Hz}$ to $62.4 \mathrm{~Hz}$ at $+1 \mathrm{~Hz} / \mathrm{s}$

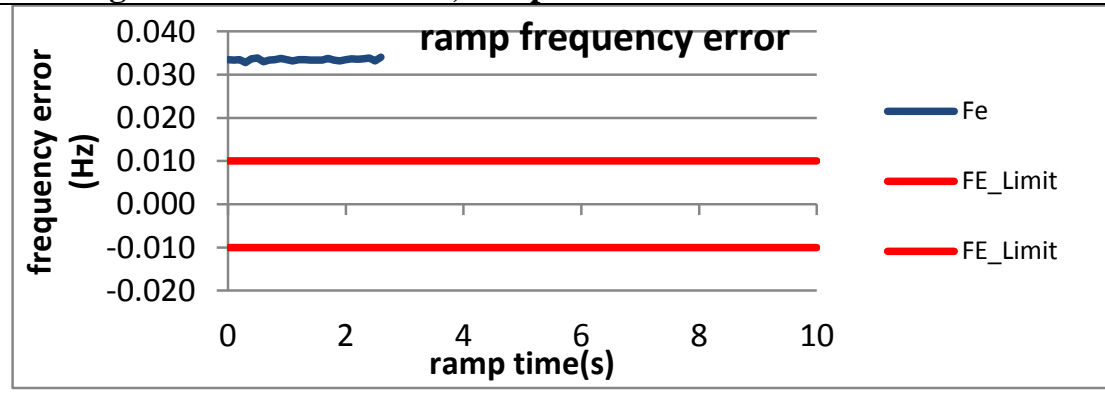

Figure 2439: Fs = $10 \mathrm{FPS}$, ramp from $58 \mathrm{~Hz}$ to $62 \mathrm{~Hz}$ at $+1 \mathrm{~Hz} / \mathrm{s}$

Figure 2436: Fs = $15 \mathrm{FPS}$, ramp from $63 \mathrm{~Hz}$ to $57 \mathrm{~Hz}$ at $-1 \mathrm{~Hz} / \mathrm{s}$

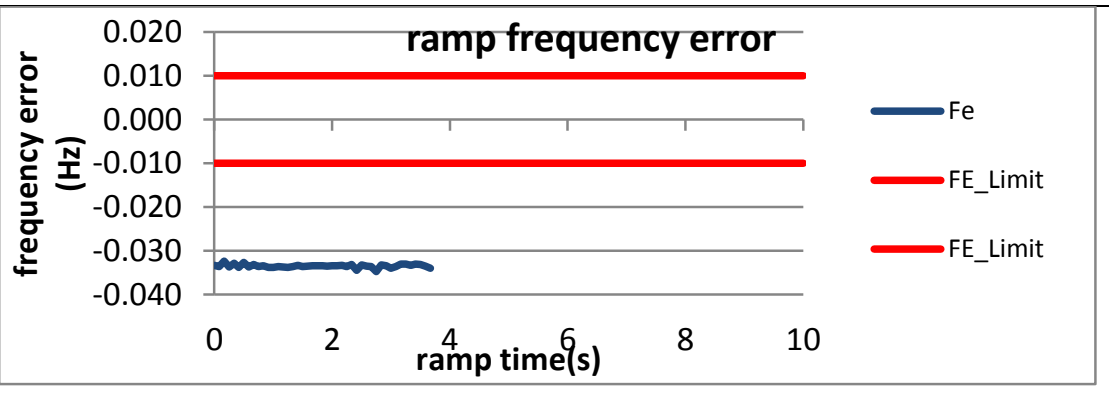

Figure 2438: Fs = 12 FPS, ramp from $62.4 \mathrm{~Hz}$ to $58.6 \mathrm{~Hz}$ at $-1 \mathrm{~Hz} / \mathrm{s}$

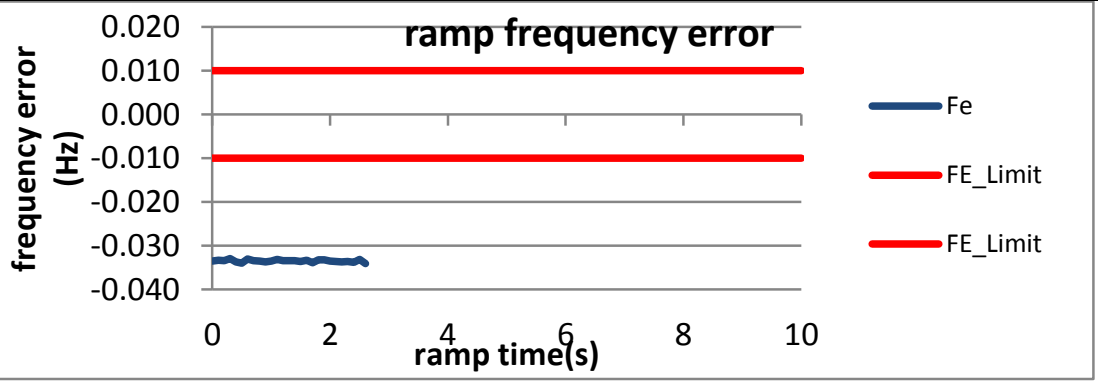

Figure 2440: Fs = $10 \mathrm{FPS}$, ramp from $62 \mathrm{~Hz}$ to $58 \mathrm{~Hz}$ at $-1 \mathrm{~Hz} / \mathrm{s}$ 
6.3.4 PMU C dynamic ramp of system frequency frequency error: $M$ class

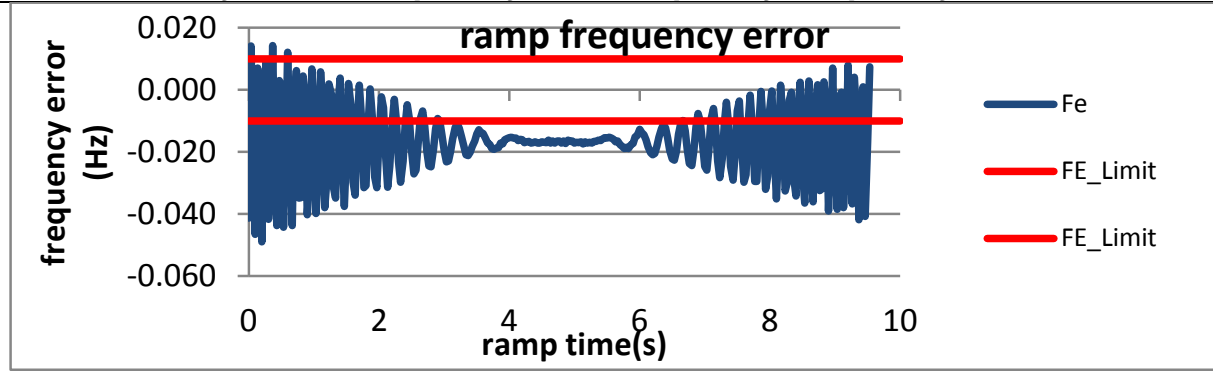

Figure 2441: Fs = $60 \mathrm{FPS}$, ramp from $55 \mathrm{~Hz}$ to $65 \mathrm{~Hz}$ at $+1 \mathrm{~Hz} / \mathrm{s}$

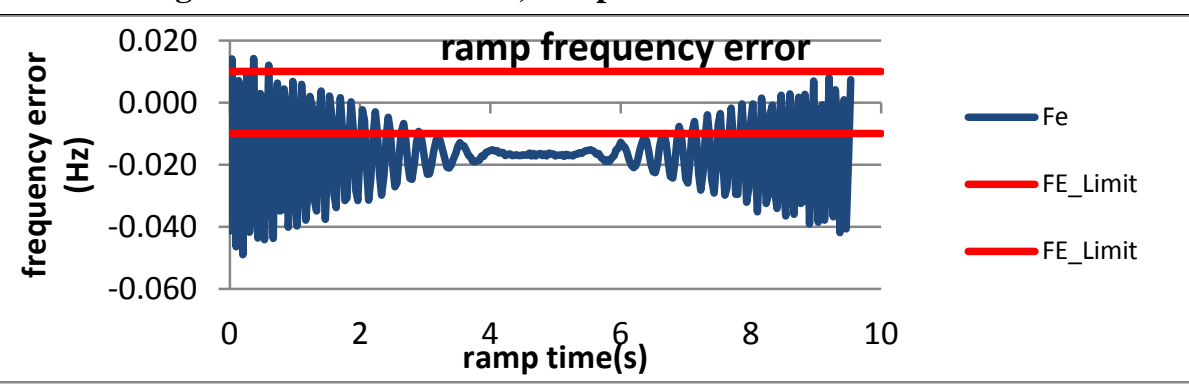

Figure 2443: Fs = $30 \mathrm{FPS}$, ramp from $55 \mathrm{~Hz}$ to $65 \mathrm{~Hz}$ at $+1 \mathrm{~Hz} / \mathrm{s}$

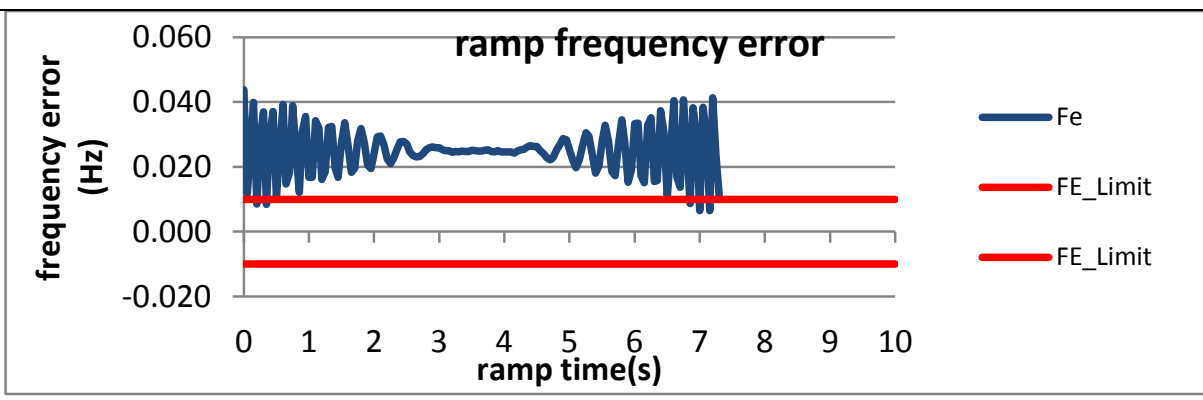

Figure 2445: Fs = 20 FPS, ramp from $56 \mathrm{~Hz}$ to $64 \mathrm{~Hz}$ at $+1 \mathrm{~Hz} / \mathrm{s}$

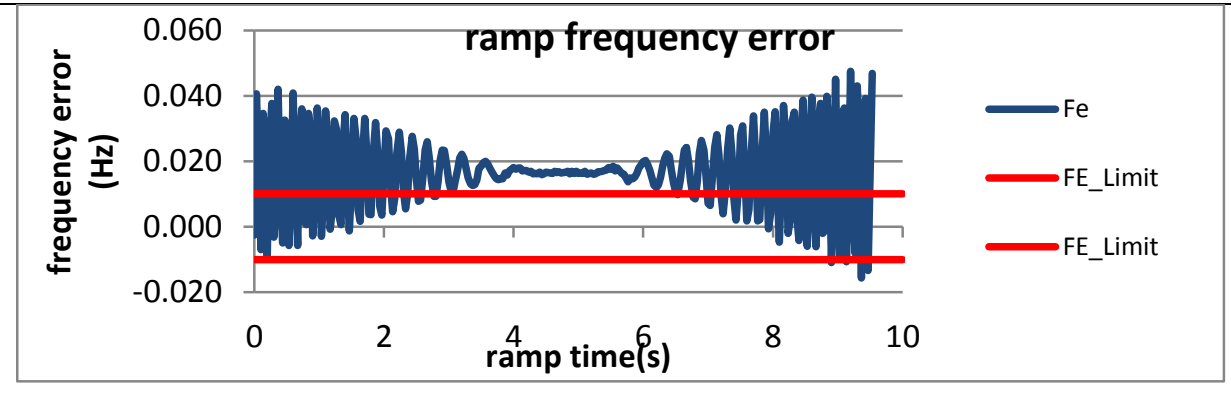

Figure 2442: Fs = $60 \mathrm{FPS}$, ramp from $65 \mathrm{~Hz}$ to $55 \mathrm{~Hz}$ at $-1 \mathrm{~Hz} / \mathrm{s}$

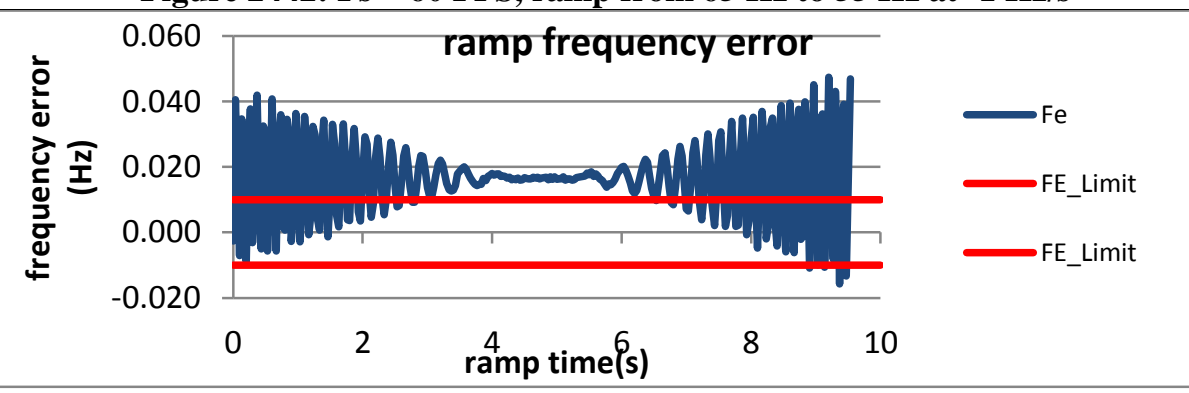

Figure 2444: Fs = $30 \mathrm{FPS}$, ramp from $65 \mathrm{~Hz}$ to $55 \mathrm{~Hz}$ at $-1 \mathrm{~Hz} / \mathrm{s}$

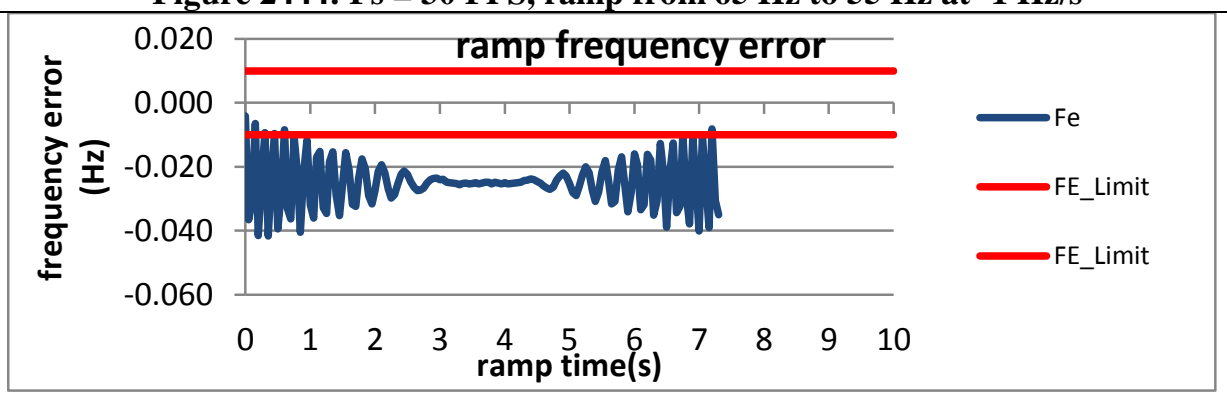

Figure 2446: Fs = $20 \mathrm{FPS}$, ramp from $64 \mathrm{~Hz}$ to $56 \mathrm{~Hz}$ at $-1 \mathrm{~Hz} / \mathrm{s}$ 


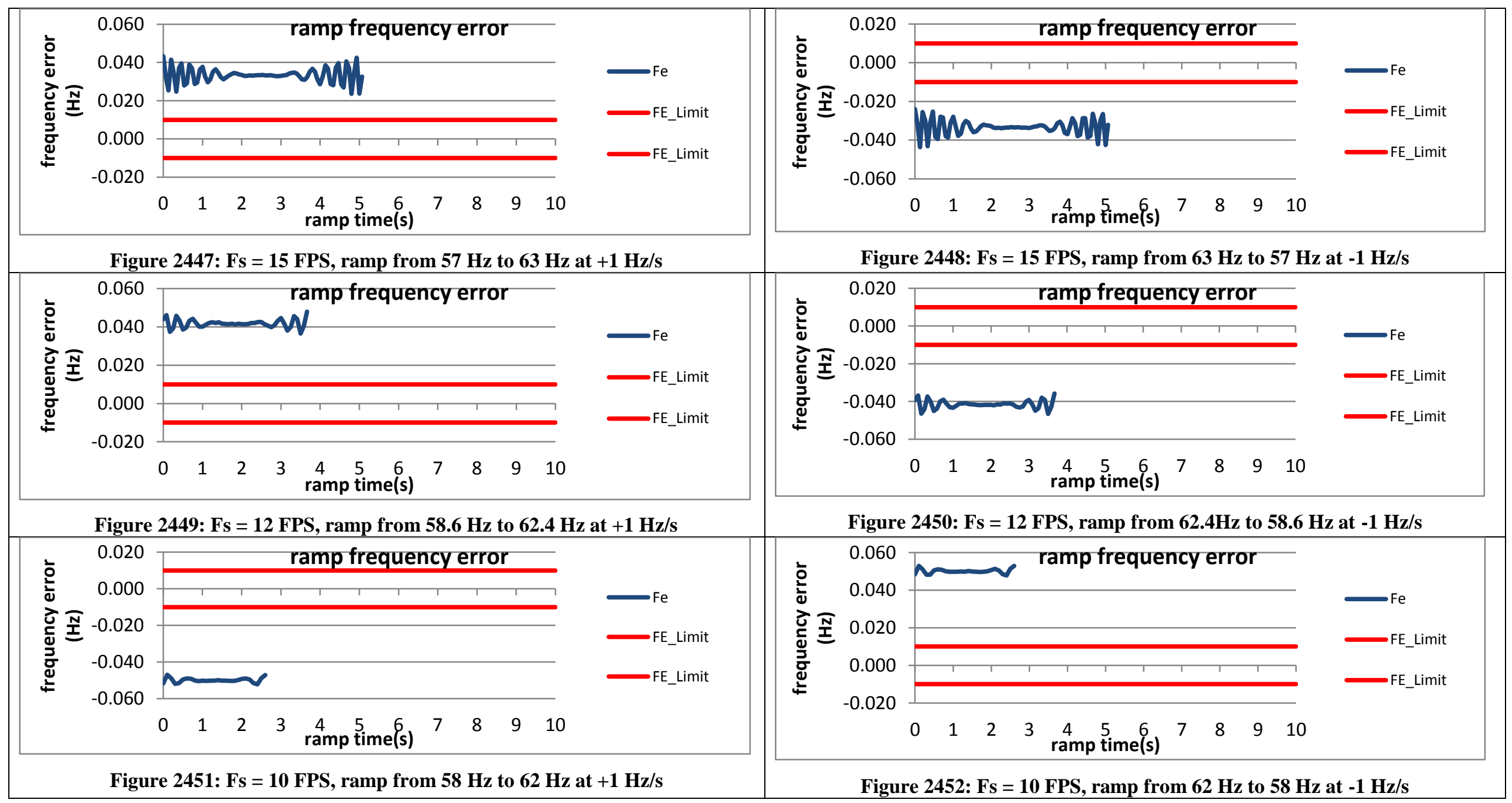


6.3.5 PMU D dynamic ramp of system frequency frequency error: $M$ class

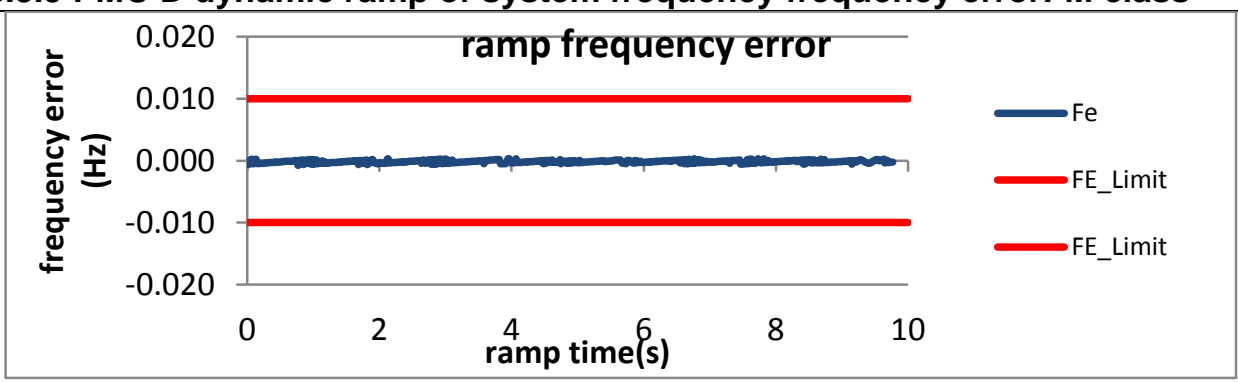

Figure 2453: Fs = $60 \mathrm{FPS}$, ramp from $55 \mathrm{~Hz}$ to $65 \mathrm{~Hz}$ at $+1 \mathrm{~Hz} / \mathrm{s}$

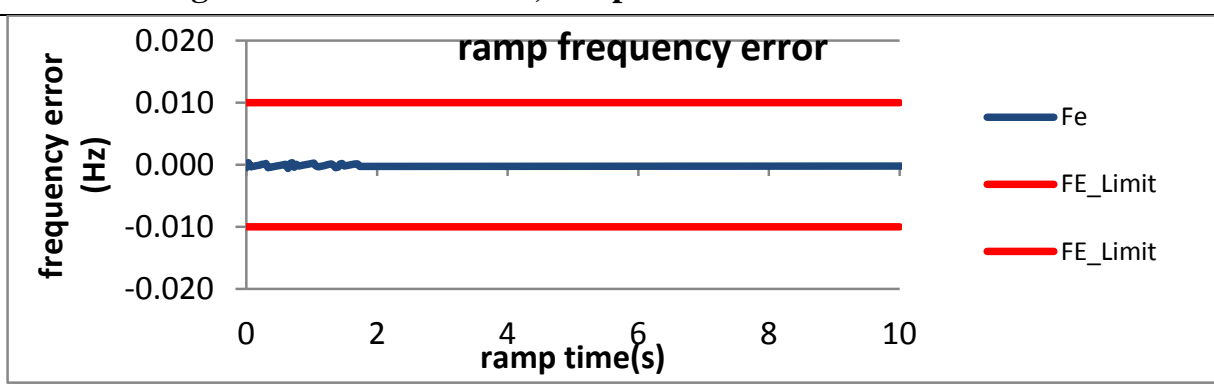

Figure 2455: Fs = $30 \mathrm{FPS}$, ramp from $55 \mathrm{~Hz}$ to $65 \mathrm{~Hz}$ at $+1 \mathrm{~Hz} / \mathrm{s}$

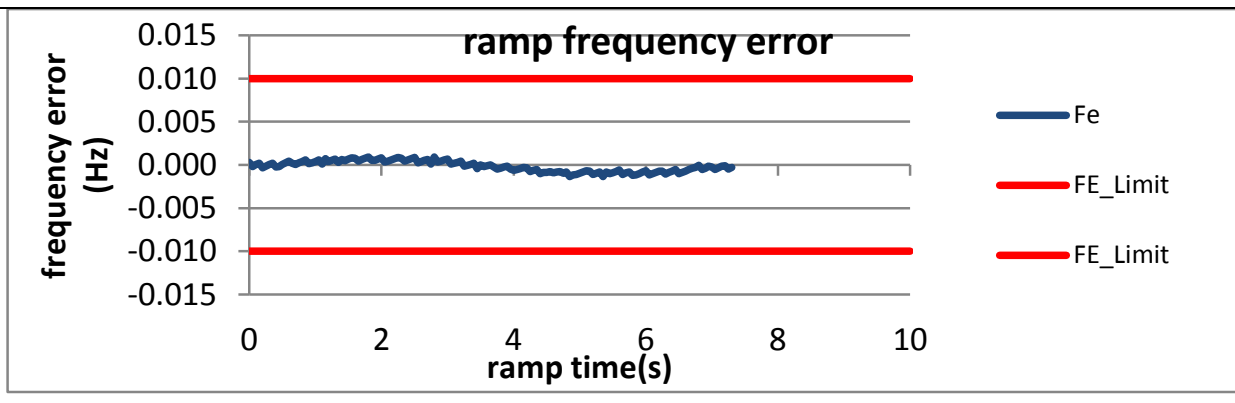

Figure 2457: Fs = $20 \mathrm{FPS}$, ramp from $56 \mathrm{~Hz}$ to $64 \mathrm{~Hz}$ at $+1 \mathrm{~Hz} / \mathrm{s}$

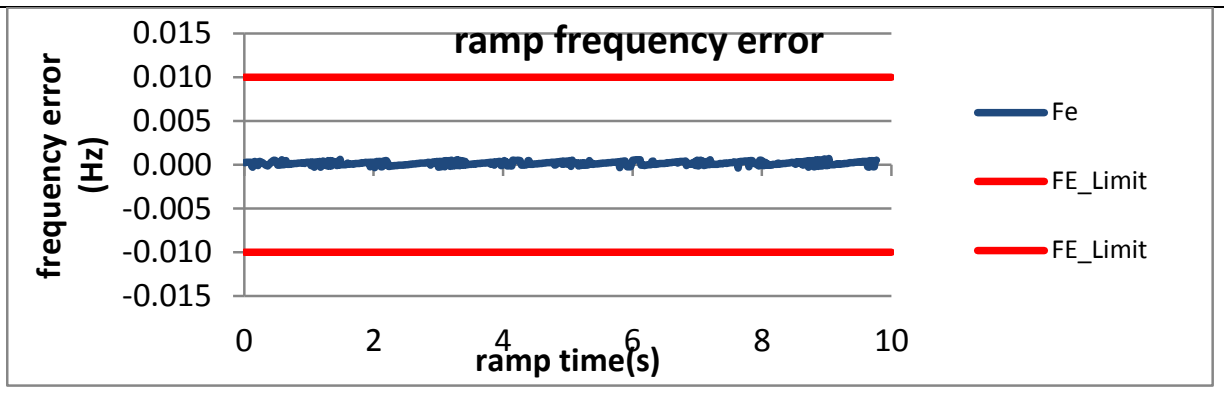

Figure 2454: Fs = $60 \mathrm{FPS}$, ramp from $65 \mathrm{~Hz}$ to $55 \mathrm{~Hz}$ at $-1 \mathrm{~Hz} / \mathrm{s}$

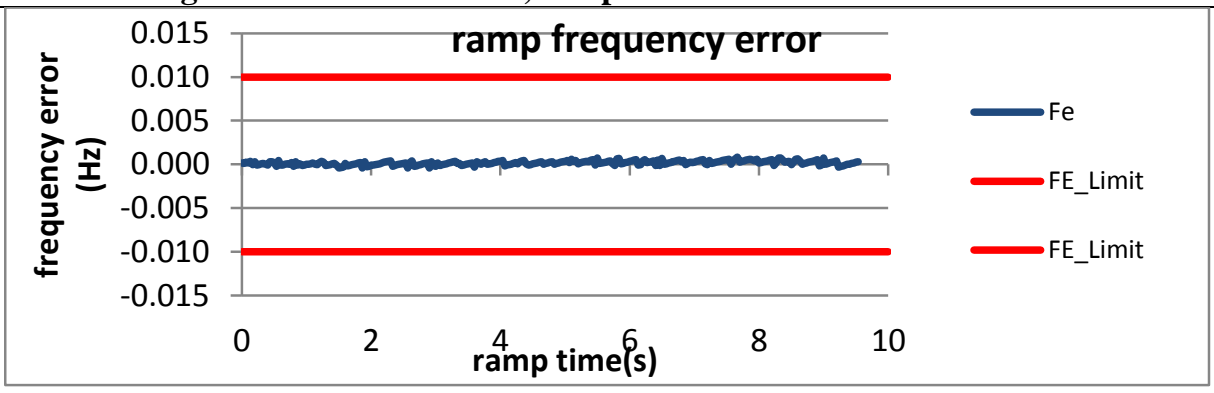

Figure 2456: Fs = $30 \mathrm{FPS}$, ramp from $65 \mathrm{~Hz}$ to $55 \mathrm{~Hz}$ at $-1 \mathrm{~Hz} / \mathrm{s}$

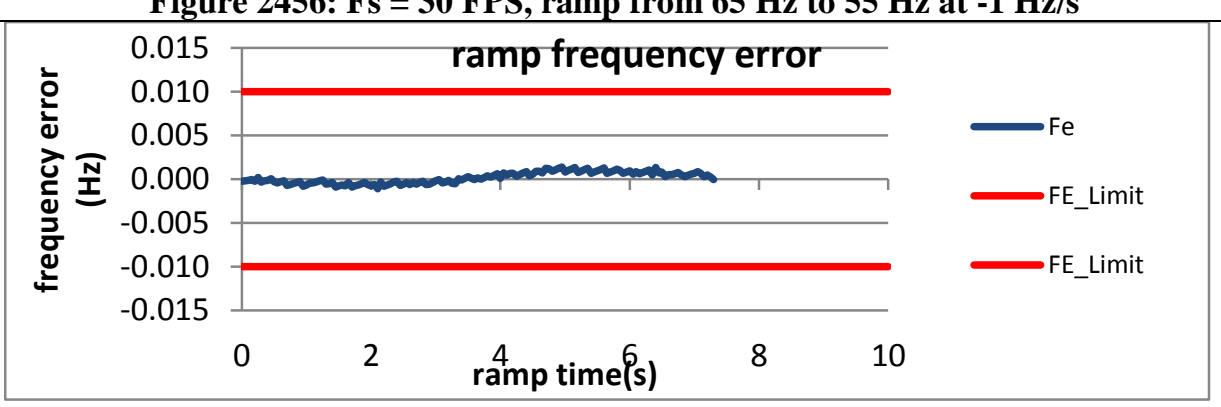

Figure 2458: Fs = $20 \mathrm{FPS}$, ramp from $64 \mathrm{~Hz}$ to $56 \mathrm{~Hz}$ at $-1 \mathrm{~Hz} / \mathrm{s}$ 

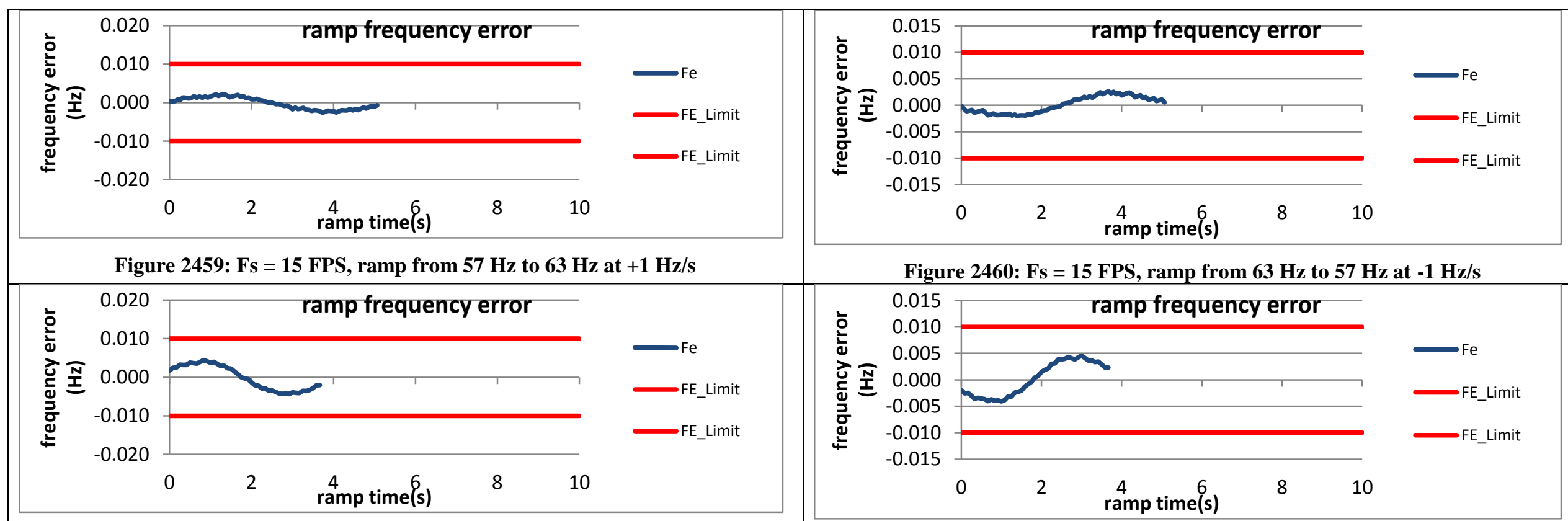

Figure 2460: Fs = $15 \mathrm{FPS}$, ramp from $63 \mathrm{~Hz}$ to $57 \mathrm{~Hz}$ at $-1 \mathrm{~Hz} / \mathrm{s}$

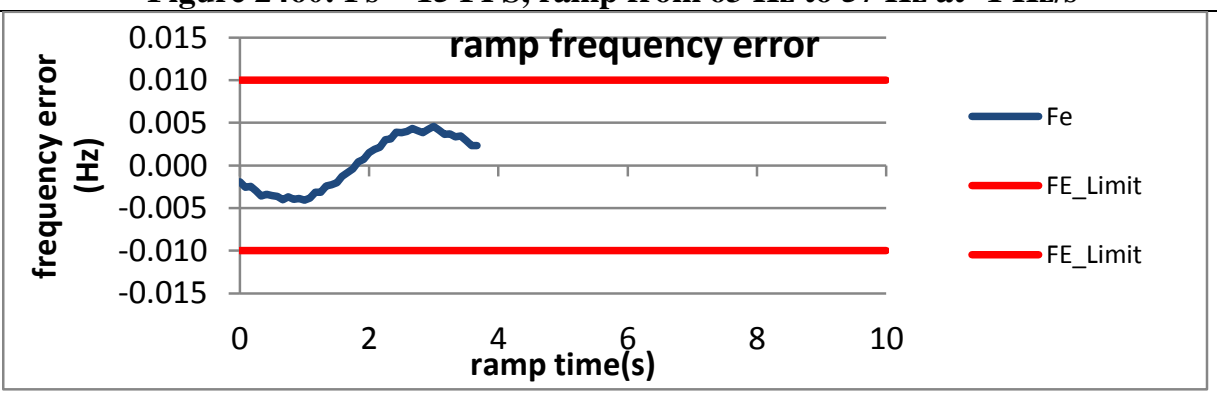

Figure 2461: Fs = 12 FPS, ramp from $58.6 \mathrm{~Hz}$ to $62.4 \mathrm{~Hz}$ at $+1 \mathrm{~Hz} / \mathrm{s}$

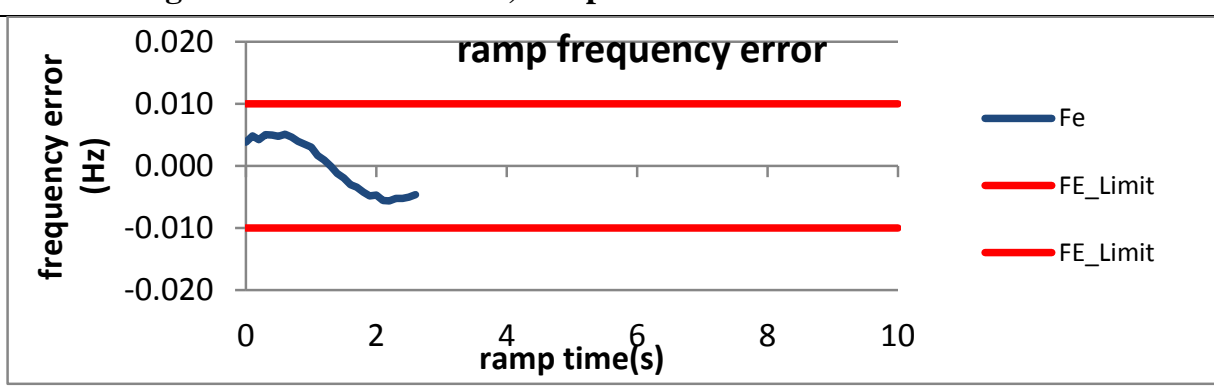

Figure 2463: Fs = $10 \mathrm{FPS}$, ramp from $58 \mathrm{~Hz}$ to $62 \mathrm{~Hz}$ at $+1 \mathrm{~Hz} / \mathrm{s}$

Figure 2462: Fs = 12 FPS, ramp from $62.4 \mathrm{~Hz}$ to $58.6 \mathrm{~Hz}$ at $-1 \mathrm{~Hz} / \mathrm{s}$

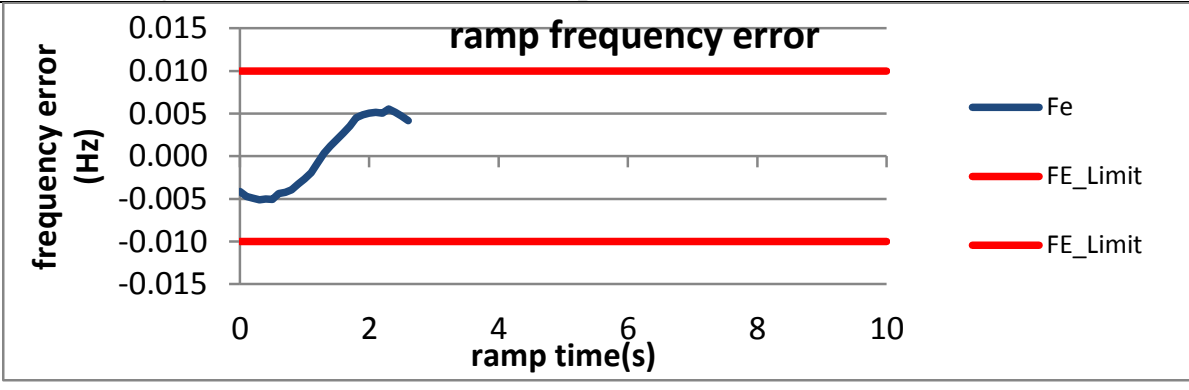

Figure 2464: Fs = $10 \mathrm{FPS}$, ramp from $62 \mathrm{~Hz}$ to $58 \mathrm{~Hz}$ at $-1 \mathrm{~Hz} / \mathrm{s}$ 
6.3.6 PMU E dynamic ramp of system frequency frequency error: M class
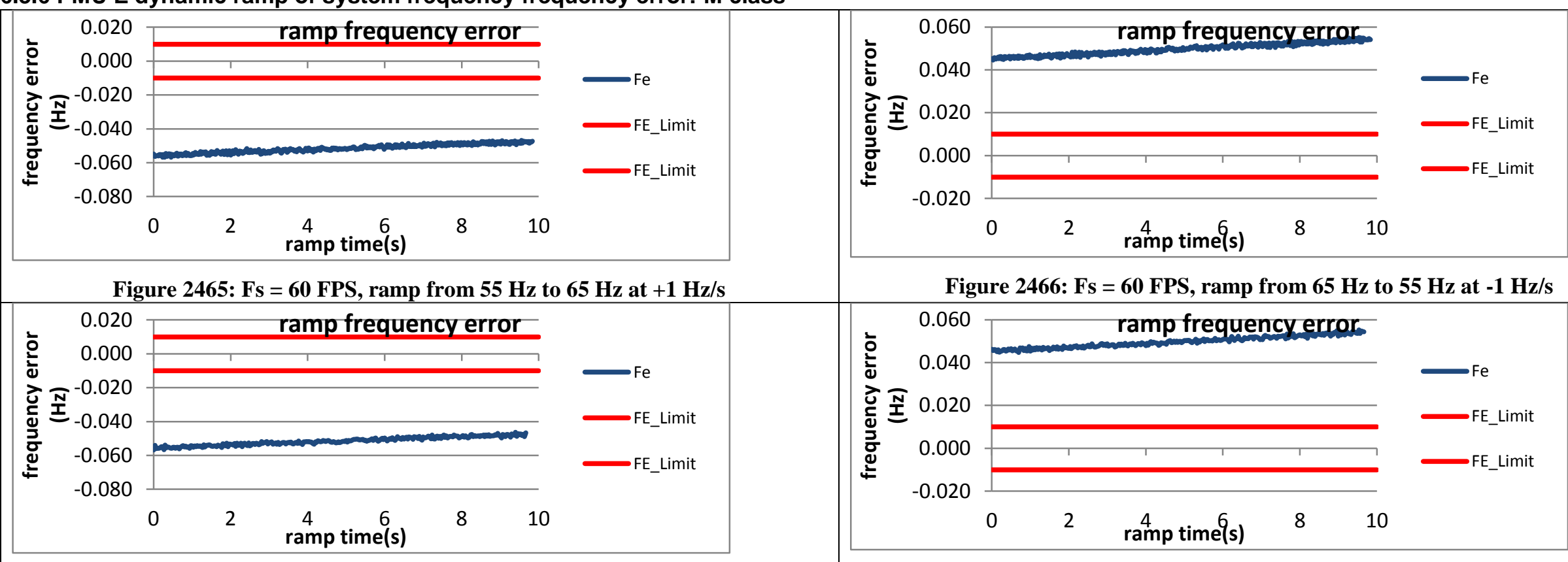

Figure 2466: Fs = $60 \mathrm{FPS}$, ramp from $65 \mathrm{~Hz}$ to $55 \mathrm{~Hz}$ at $-1 \mathrm{~Hz} / \mathrm{s}$

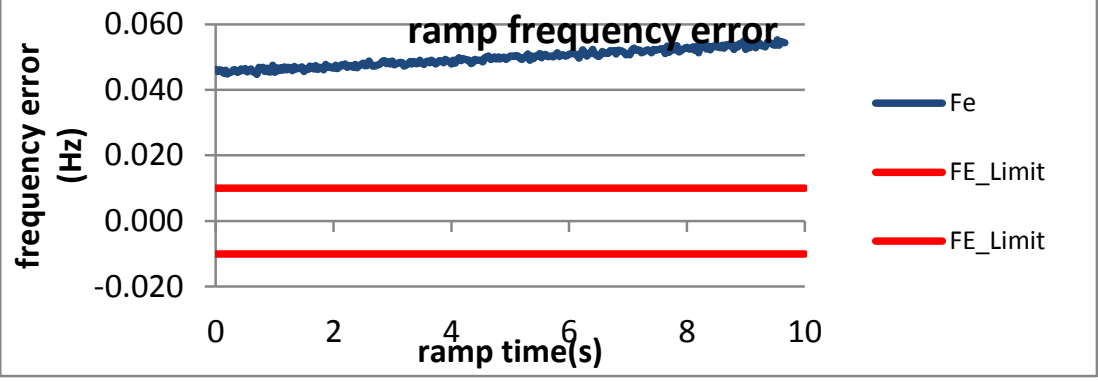

Figure 2467: Fs = $30 \mathrm{FPS}$, ramp from $55 \mathrm{~Hz}$ to $65 \mathrm{~Hz}$ at $+1 \mathrm{~Hz} / \mathrm{s}$

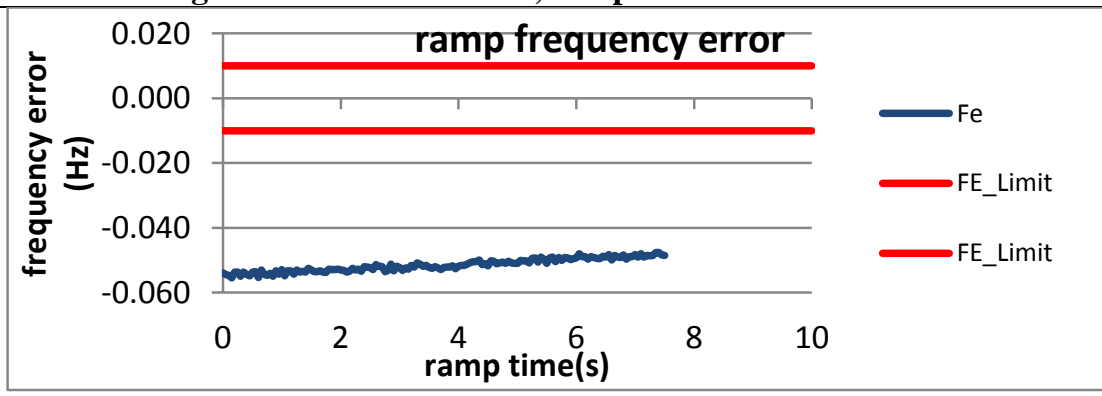

Figure 2468: Fs = $30 \mathrm{FPS}$, ramp from $65 \mathrm{~Hz}$ to $55 \mathrm{~Hz}$ at $-1 \mathrm{~Hz} / \mathrm{s}$

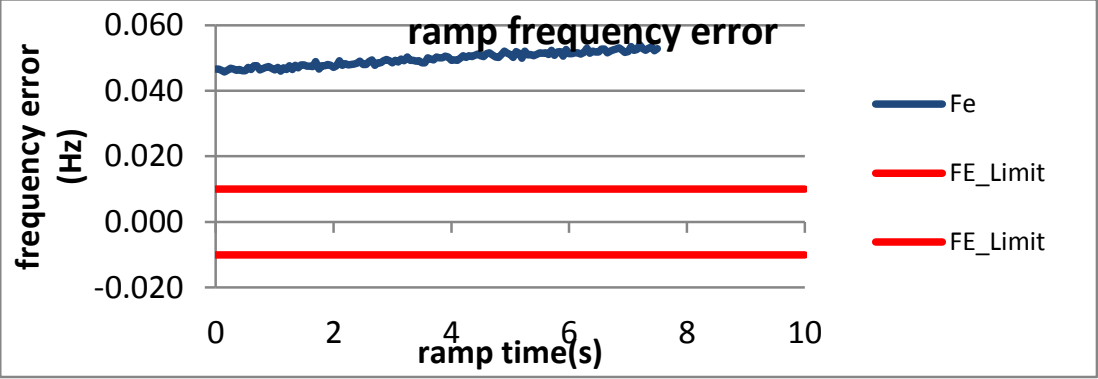

Figure 2469: Fs = $20 \mathrm{FPS}$, ramp from $56 \mathrm{~Hz}$ to $64 \mathrm{~Hz}$ at $+1 \mathrm{~Hz} / \mathrm{s}$

Figure 2470: Fs = $20 \mathrm{FPS}$, ramp from $64 \mathrm{~Hz}$ to $56 \mathrm{~Hz}$ at $-1 \mathrm{~Hz} / \mathrm{s}$ 

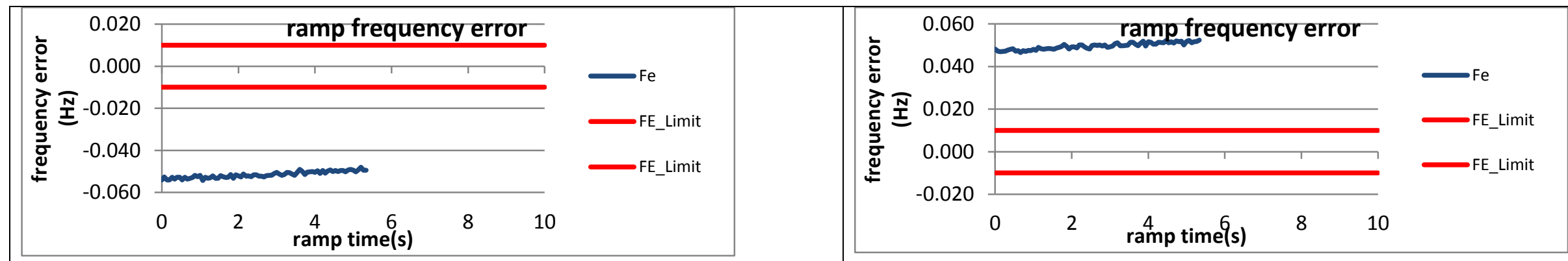

Figure 2471: Fs = $15 \mathrm{FPS}$, ramp from $57 \mathrm{~Hz}$ to $63 \mathrm{~Hz}$ at $+1 \mathrm{~Hz} / \mathrm{s}$

Figure 2472: Fs = $15 \mathrm{FPS}$, ramp from $63 \mathrm{~Hz}$ to $57 \mathrm{~Hz}$ at $-1 \mathrm{~Hz} / \mathrm{s}$

Figure 2473: Fs = 12 FPS, ramp from $58.6 \mathrm{~Hz}$ to $62.4 \mathrm{~Hz}$ at $+1 \mathrm{~Hz} / \mathrm{s}$

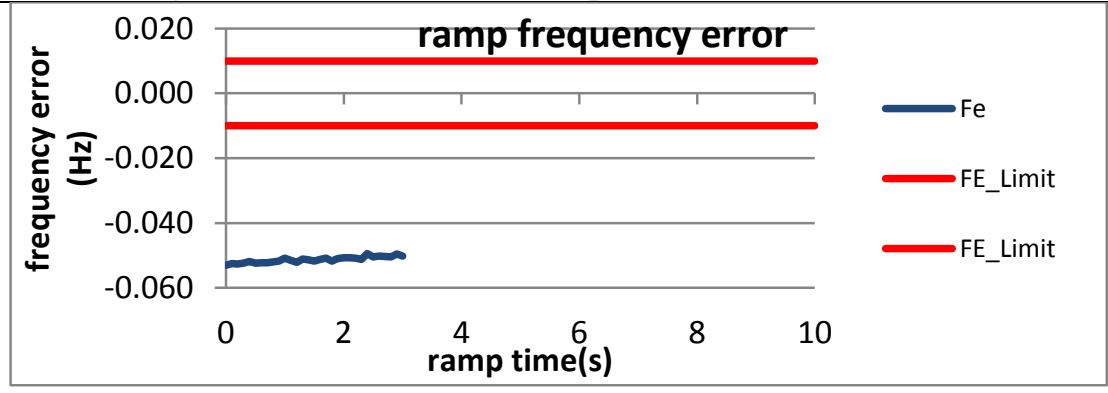

Figure 2474: Fs = 12 FPS, ramp from $62.4 \mathrm{~Hz}$ to $58.6 \mathrm{~Hz}$ at $-1 \mathrm{~Hz} / \mathrm{s}$

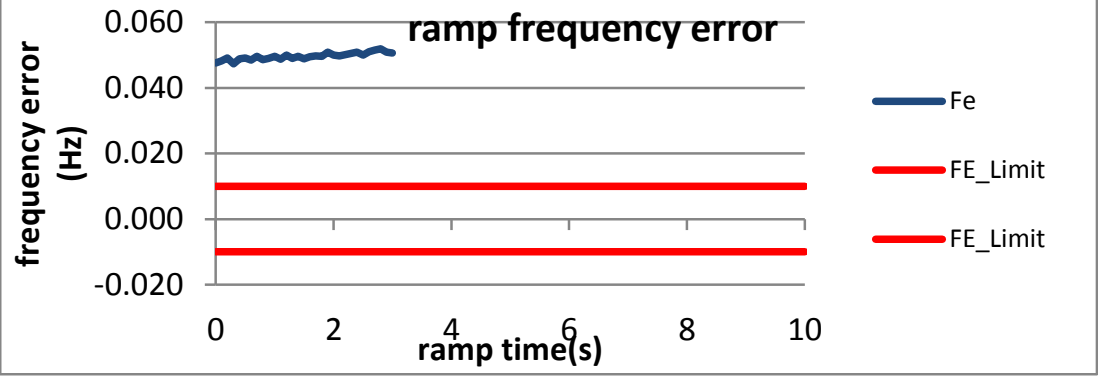

Figure 2475: Fs = $10 \mathrm{FPS}$, ramp from $58 \mathrm{~Hz}$ to $62 \mathrm{~Hz}$ at $+1 \mathrm{~Hz} / \mathrm{s}$

Figure 2476: Fs = $10 \mathrm{FPS}$, ramp from $62 \mathrm{~Hz}$ to $58 \mathrm{~Hz}$ at $-1 \mathrm{~Hz} / \mathrm{s}$ 
6.3.7 PMU F dynamic ramp of system frequency frequency error: M class
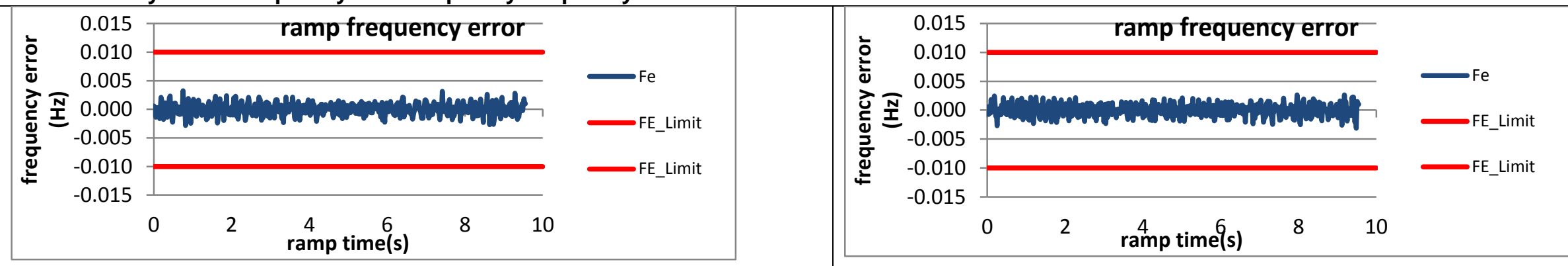

Figure 2477: Fs = $60 \mathrm{FPS}$, ramp from $55 \mathrm{~Hz}$ to $65 \mathrm{~Hz}$ at $+1 \mathrm{~Hz} / \mathrm{s}$

Figure 2478: Fs = $60 \mathrm{FPS}$, ramp from $65 \mathrm{~Hz}$ to $55 \mathrm{~Hz}$ at $-1 \mathrm{~Hz} / \mathrm{s}$
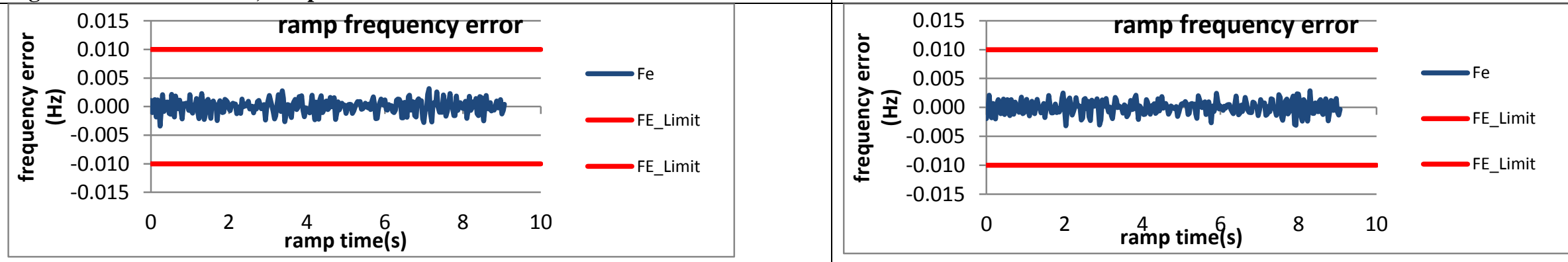

Figure 2479: Fs = $30 \mathrm{FPS}$, ramp from $55 \mathrm{~Hz}$ to $65 \mathrm{~Hz}$ at $+1 \mathrm{~Hz} / \mathrm{s}$

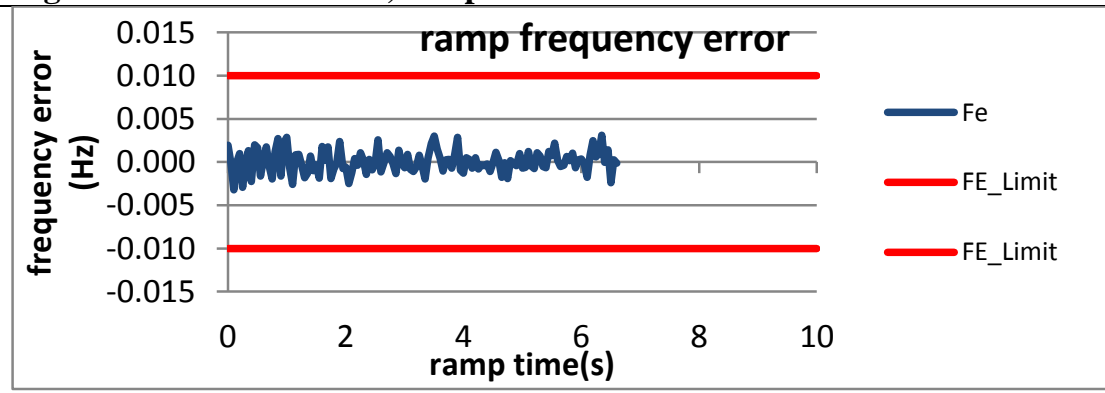

Figure 2480: Fs = $30 \mathrm{FPS}$, ramp from $65 \mathrm{~Hz}$ to $55 \mathrm{~Hz}$ at $-1 \mathrm{~Hz} / \mathrm{s}$

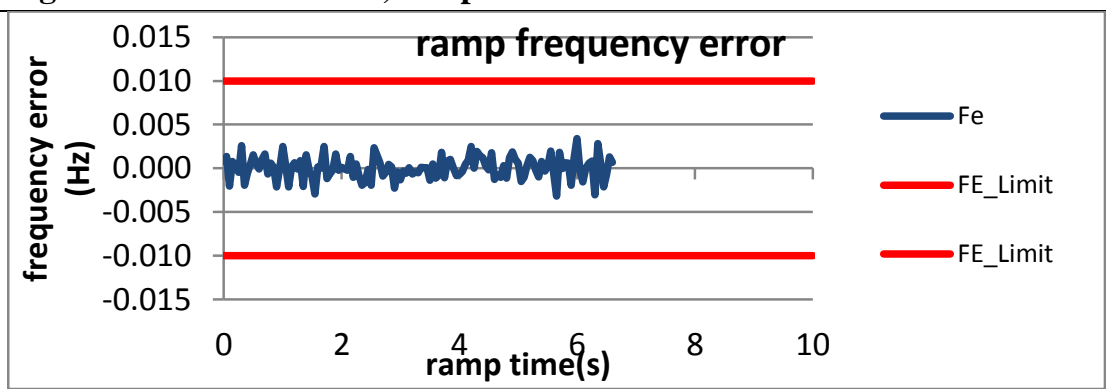

Figure 2481: Fs = $20 \mathrm{FPS}$, ramp from $56 \mathrm{~Hz}$ to $64 \mathrm{~Hz}$ at $+1 \mathrm{~Hz} / \mathrm{s}$

Figure 2482: Fs = 20 FPS, ramp from $64 \mathrm{~Hz}$ to $56 \mathrm{~Hz}$ at $-1 \mathrm{~Hz} / \mathrm{s}$ 

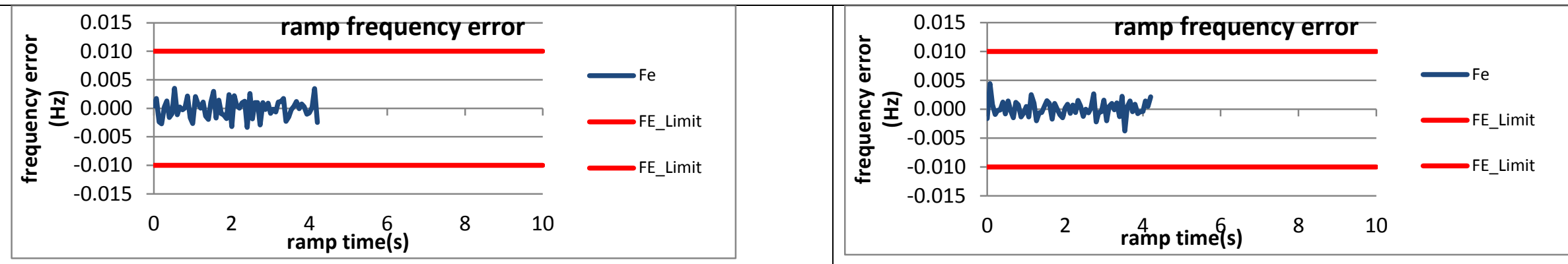

Figure 2483: Fs = $15 \mathrm{FPS}$, ramp from $57 \mathrm{~Hz}$ to $63 \mathrm{~Hz}$ at $+1 \mathrm{~Hz} / \mathrm{s}$

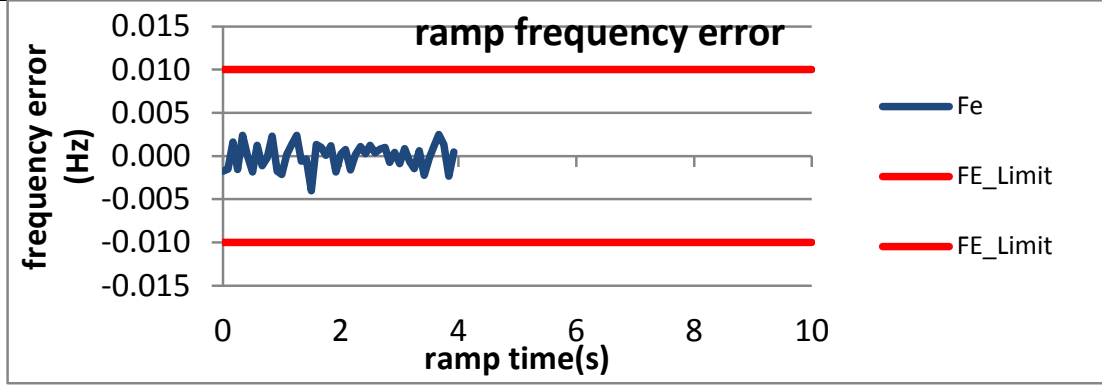

Figure 2484: Fs = $15 \mathrm{FPS}$, ramp from $63 \mathrm{~Hz}$ to $57 \mathrm{~Hz}$ at $-1 \mathrm{~Hz} / \mathrm{s}$

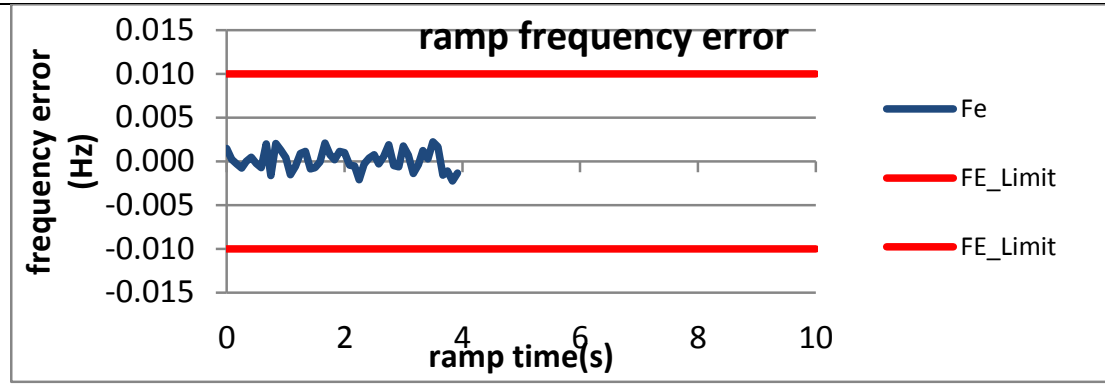

Figure 2485: Fs = 12 FPS, ramp from $58.6 \mathrm{~Hz}$ to $62.4 \mathrm{~Hz}$ at $+1 \mathrm{~Hz} / \mathrm{s}$

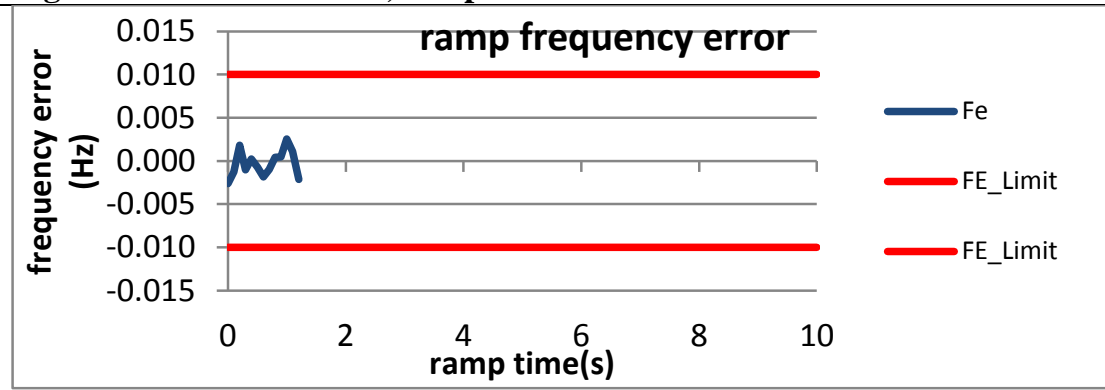

Figure 2486: Fs = 12 FPS, ramp from $62.4 \mathrm{~Hz}$ to $58.6 \mathrm{~Hz}$ at $-1 \mathrm{~Hz} / \mathrm{s}$

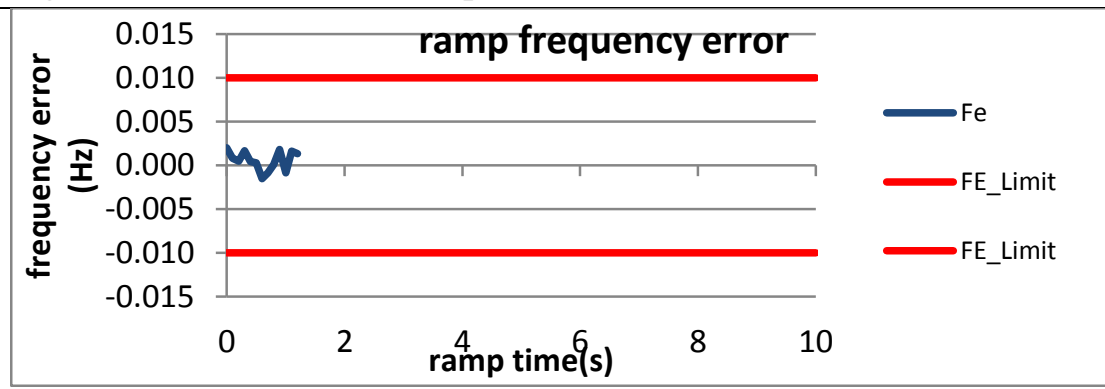

Figure 2487: Fs = $10 \mathrm{FPS}$, ramp from $58 \mathrm{~Hz}$ to $62 \mathrm{~Hz}$ at $+1 \mathrm{~Hz} / \mathrm{s}$

Figure 2488: Fs = $10 \mathrm{FPS}$, ramp from $62 \mathrm{~Hz}$ to $58 \mathrm{~Hz}$ at $-1 \mathrm{~Hz} / \mathrm{s}$ 
6.3.8 PMU G dynamic ramp of system frequency frequency error: M class

Figure 2489: Fs = 60 FPS is not supported by this PMU
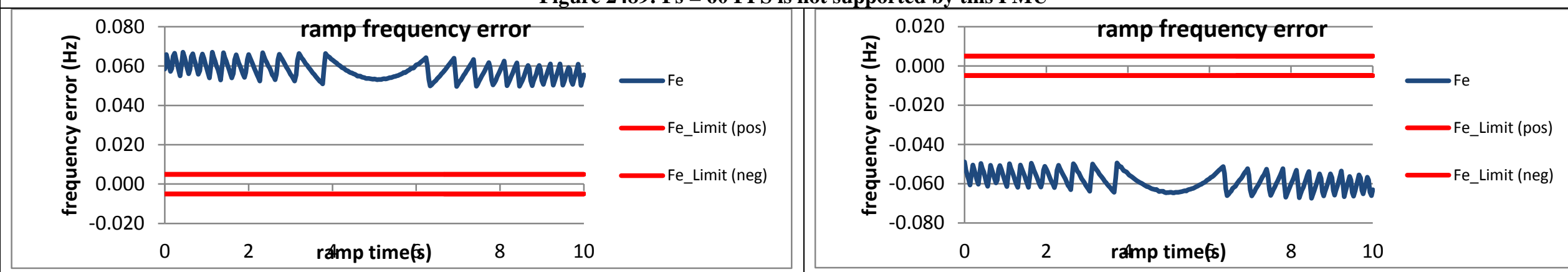

Figure 2490: Fs = $30 \mathrm{FPS}$, ramp from $55 \mathrm{~Hz}$ to $65 \mathrm{~Hz}$ at $+1 \mathrm{~Hz} / \mathrm{s}$
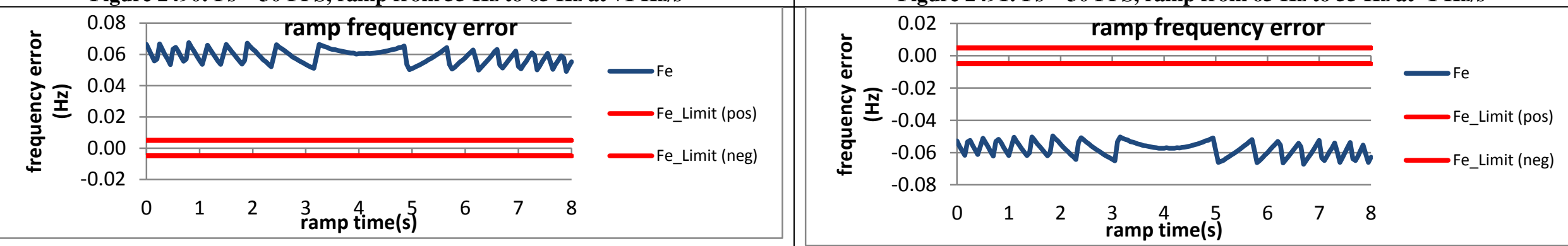

Figure 2493: Fs = $20 \mathrm{FPS}$, ramp from $64 \mathrm{~Hz}$ to $56 \mathrm{~Hz}$ at $-1 \mathrm{~Hz} / \mathrm{s}$

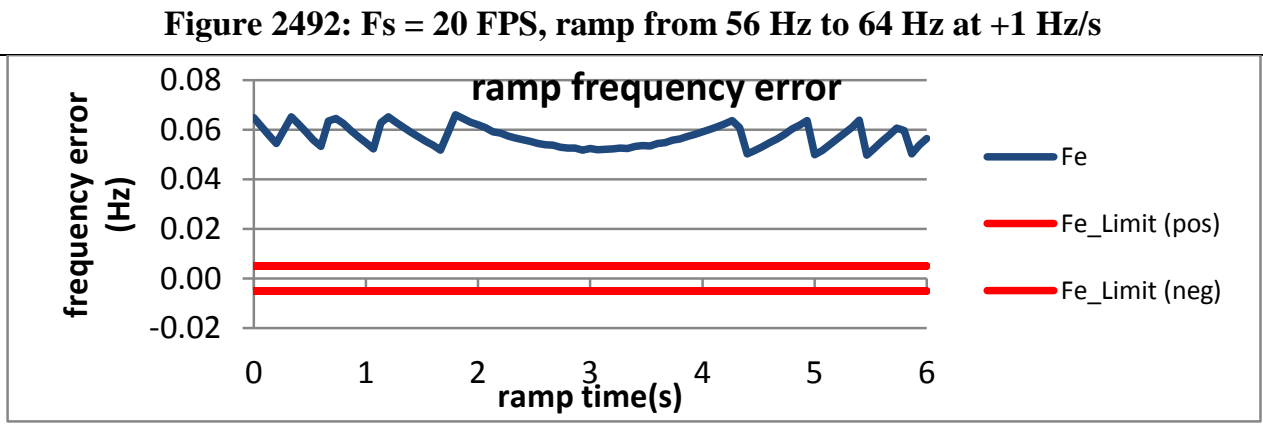

Figure 2494: Fs = $15 \mathrm{FPS}$, ramp from $57 \mathrm{~Hz}$ to $63 \mathrm{~Hz}$ at $+1 \mathrm{~Hz} / \mathrm{s}$

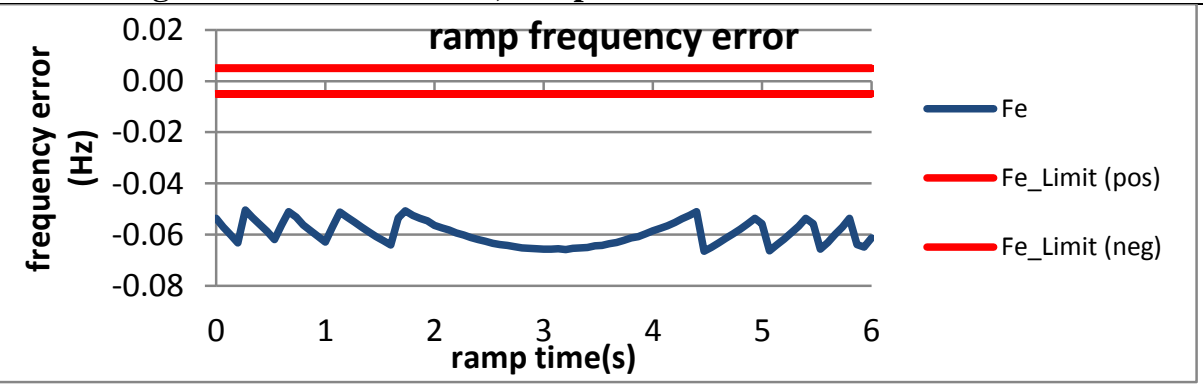

Figure 2495: Fs = $15 \mathrm{FPS}$, ramp from $63 \mathrm{~Hz}$ to $57 \mathrm{~Hz}$ at $-1 \mathrm{~Hz} / \mathrm{s}$ 

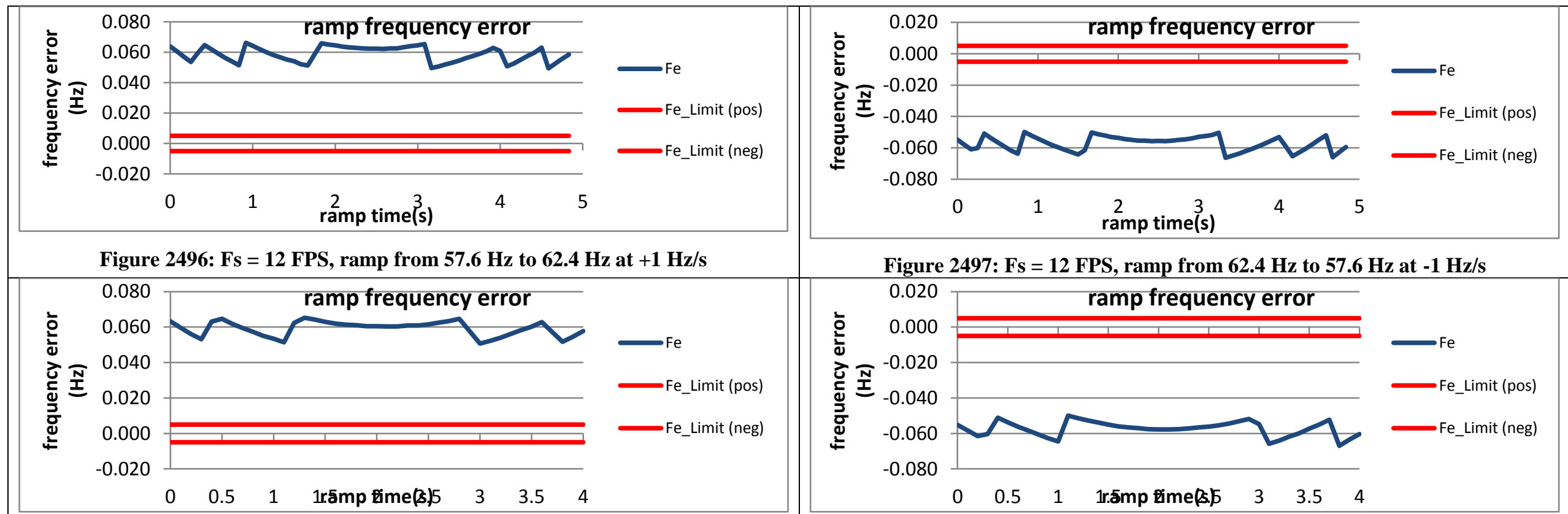

Figure 2497: Fs = 12 FPS, ramp from $62.4 \mathrm{~Hz}$ to $57.6 \mathrm{~Hz}$ at $-1 \mathrm{~Hz} / \mathrm{s}$

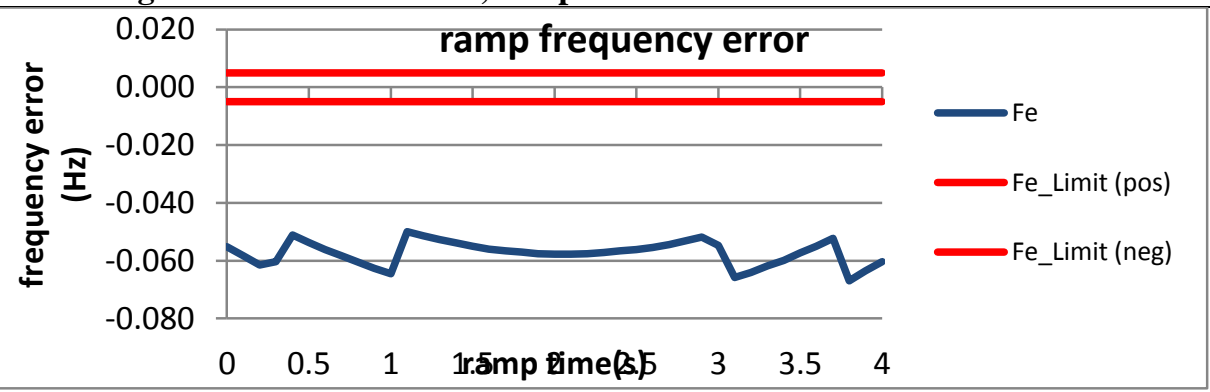

Figure 2498: Fs = $10 \mathrm{FPS}$, ramp from $58 \mathrm{~Hz}$ to $62 \mathrm{~Hz}$ at $+1 \mathrm{~Hz} / \mathrm{s}$

Figure 2499: Fs = $10 \mathrm{FPS}$, ramp from $62 \mathrm{~Hz}$ to $58 \mathrm{~Hz}$ at $-1 \mathrm{~Hz} / \mathrm{s}$ 
6.3.9 PMU H dynamic ramp of system frequency frequency error: $M$ class

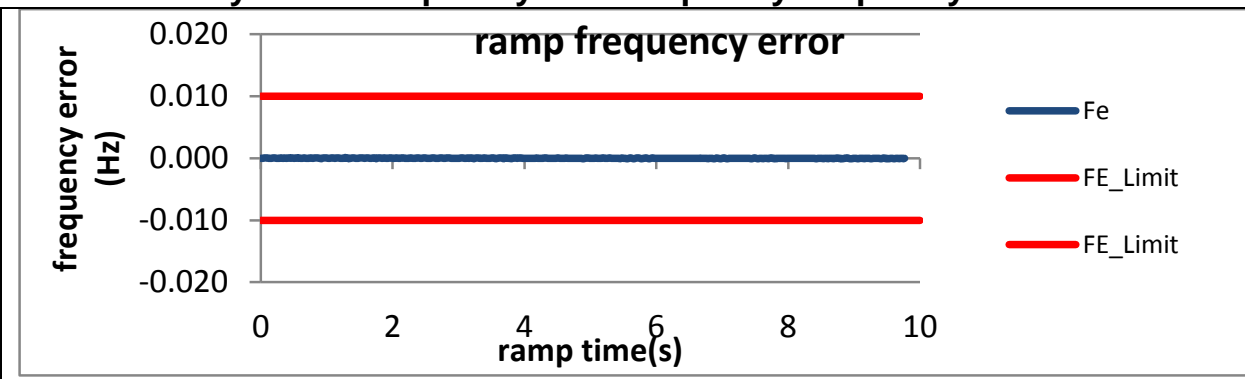

Figure 2500: Fs = $60 \mathrm{FPS}$, ramp from $55 \mathrm{~Hz}$ to $65 \mathrm{~Hz}$ at $+1 \mathrm{~Hz} / \mathrm{s}$

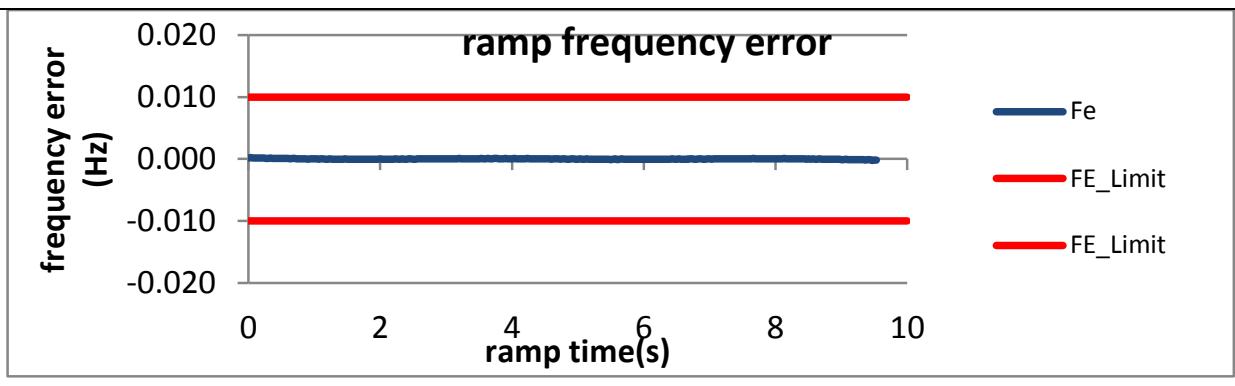

Figure 2502: Fs = $30 \mathrm{FPS}$, ramp from $55 \mathrm{~Hz}$ to $65 \mathrm{~Hz}$ at $+1 \mathrm{~Hz} / \mathrm{s}$

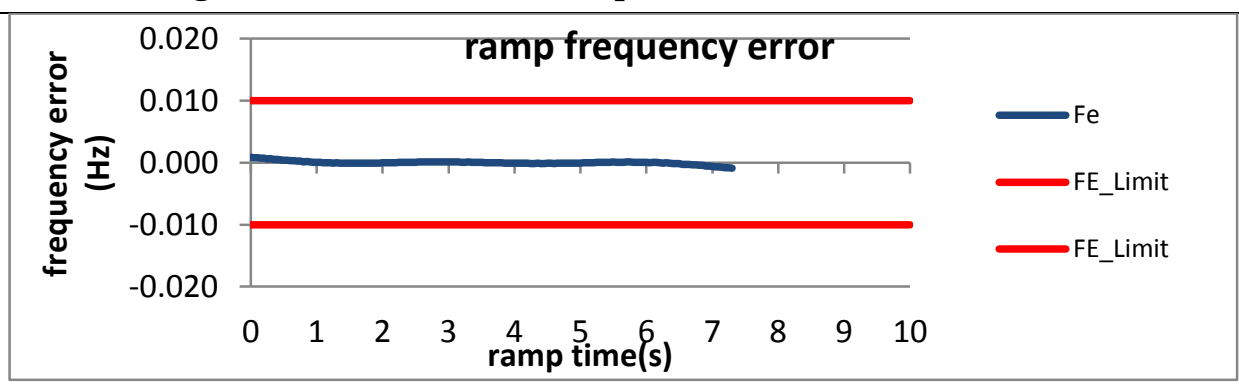

Figure 2504: Fs = $20 \mathrm{FPS}$, ramp from $56 \mathrm{~Hz}$ to $64 \mathrm{~Hz}$ at $+1 \mathrm{~Hz} / \mathrm{s}$

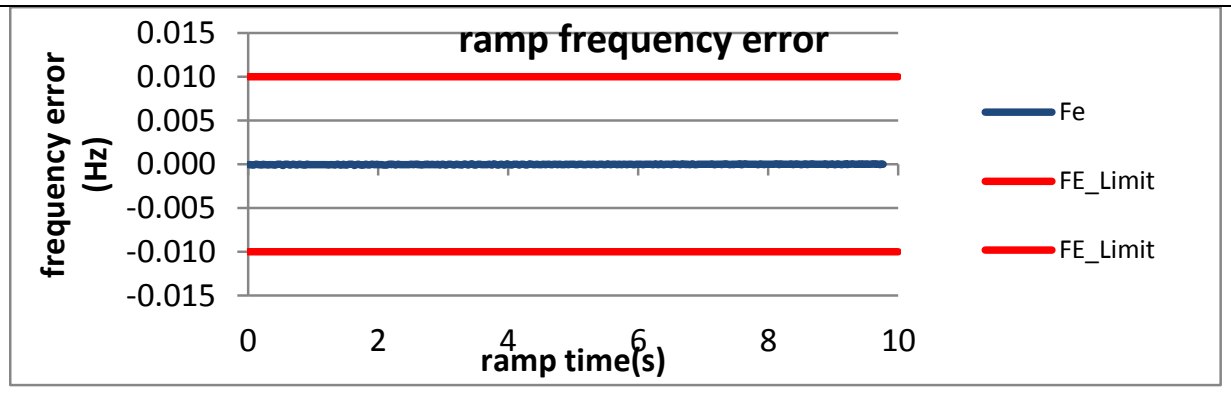

Figure 2501: Fs = $60 \mathrm{FPS}$, ramp from $65 \mathrm{~Hz}$ to $55 \mathrm{~Hz}$ at $-1 \mathrm{~Hz} / \mathrm{s}$

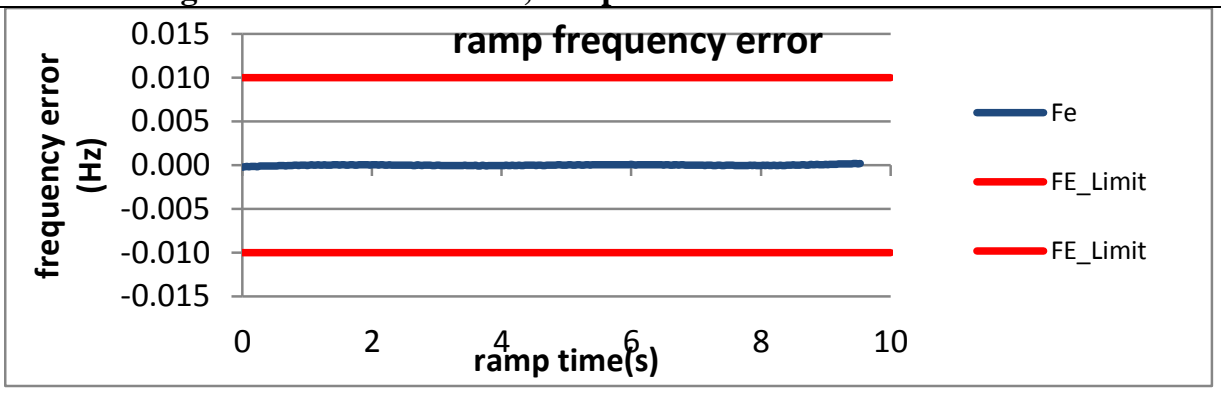

Figure 2503: Fs = $30 \mathrm{FPS}$, ramp from $65 \mathrm{~Hz}$ to $55 \mathrm{~Hz}$ at $-1 \mathrm{~Hz} / \mathrm{s}$

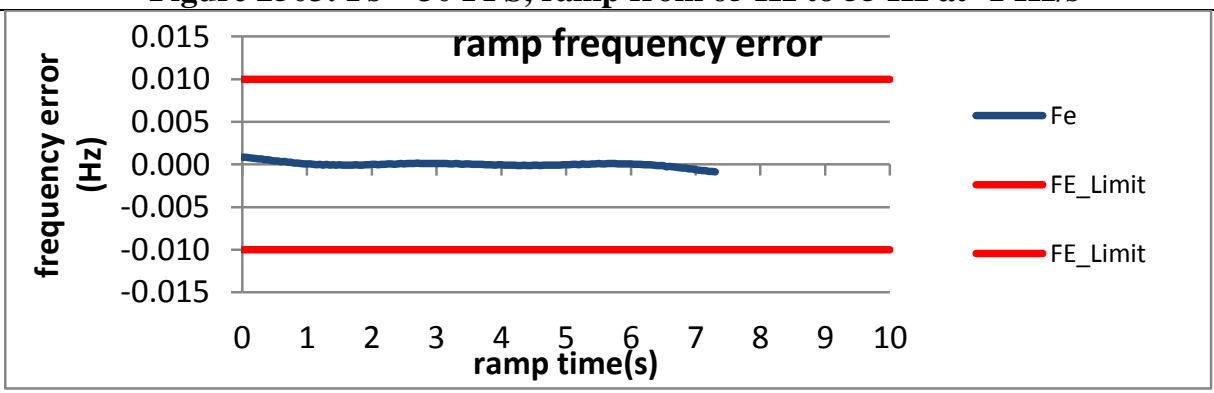

Figure 2505: Fs = $20 \mathrm{FPS}$, ramp from $64 \mathrm{~Hz}$ to $56 \mathrm{~Hz}$ at $-1 \mathrm{~Hz} / \mathrm{s}$ 

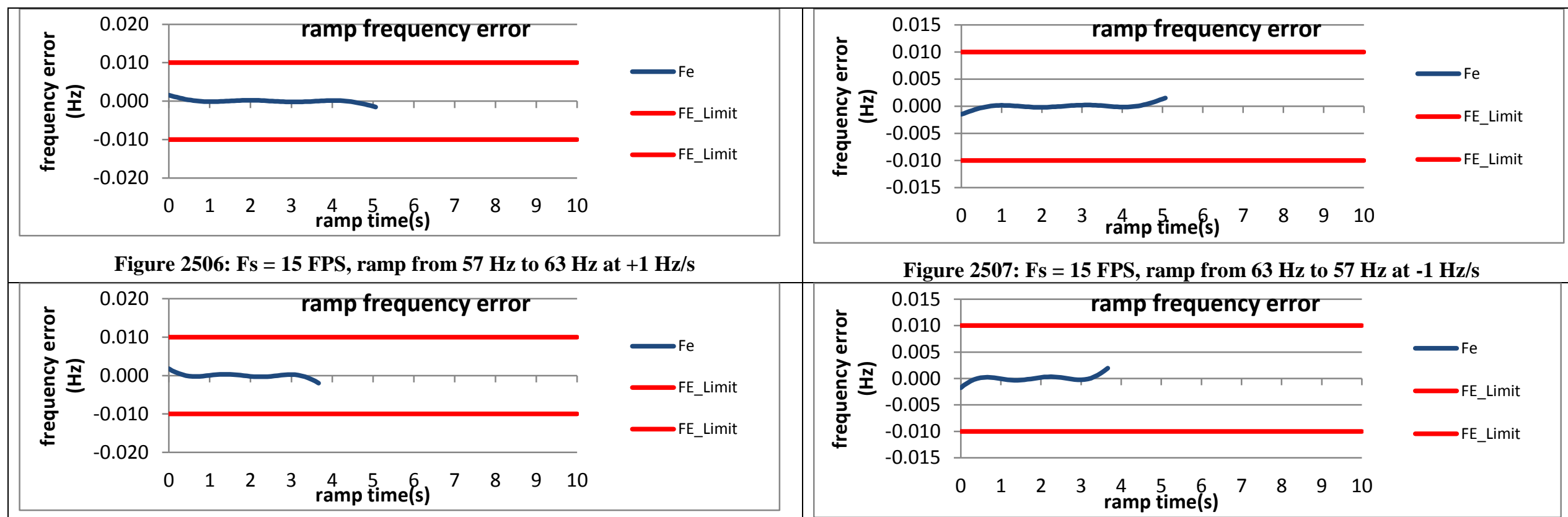

Figure 2507: Fs = $15 \mathrm{FPS}$, ramp from $63 \mathrm{~Hz}$ to $57 \mathrm{~Hz}$ at $-1 \mathrm{~Hz} / \mathrm{s}$

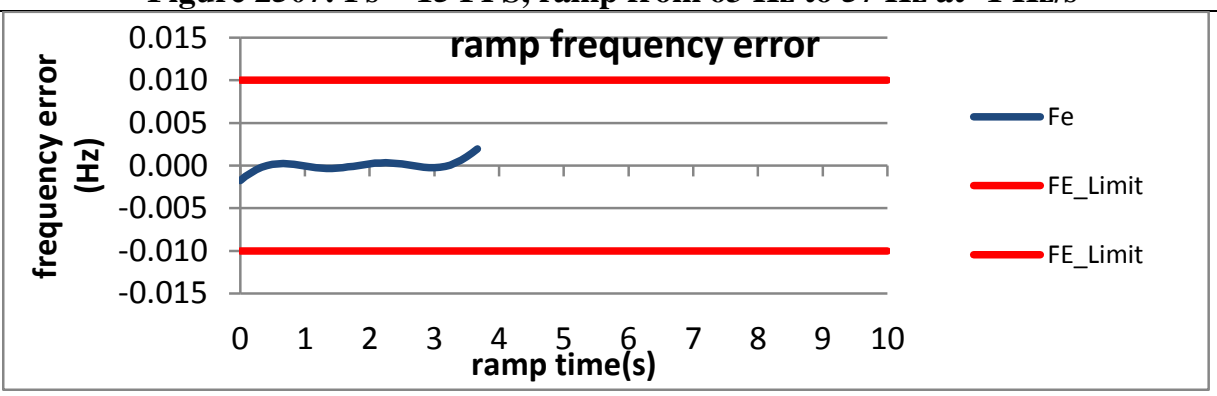

Figure 2508: Fs = 12 FPS, ramp from $58.6 \mathrm{~Hz}$ to $62.4 \mathrm{~Hz}$ at $+1 \mathrm{~Hz} / \mathrm{s}$

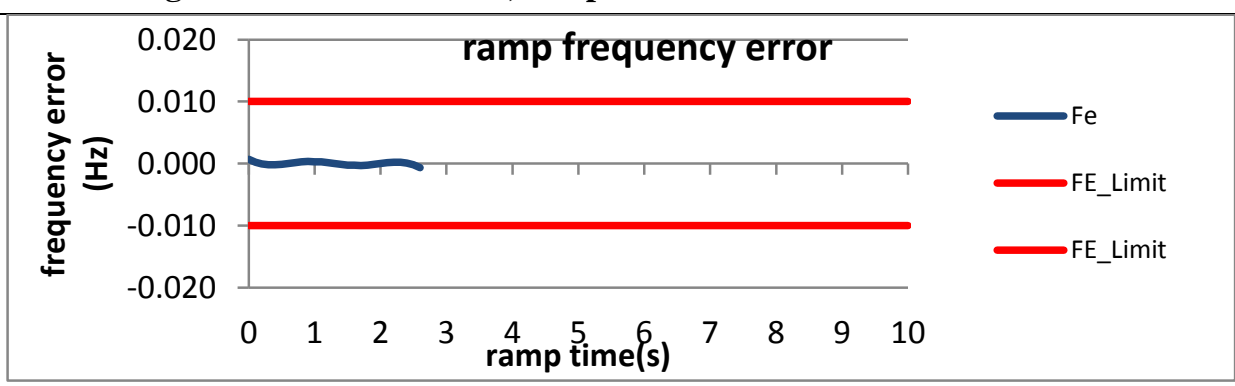

Figure 2510: Fs = $10 \mathrm{FPS}$, ramp from $58 \mathrm{~Hz}$ to $62 \mathrm{~Hz}$ at $+1 \mathrm{~Hz} / \mathrm{s}$

Figure 2509: Fs = 12 FPS, ramp from $62.4 \mathrm{~Hz}$ to $58.6 \mathrm{~Hz}$ at $-1 \mathrm{~Hz} / \mathrm{s}$

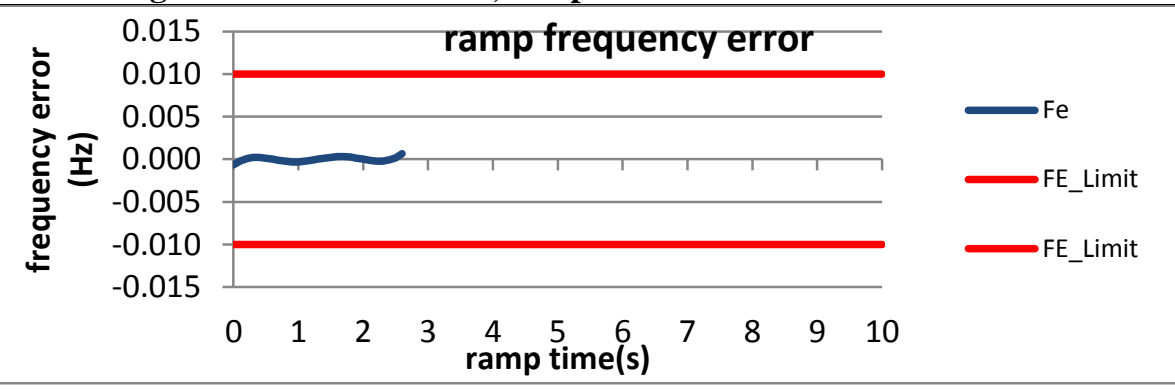

Figure 2511: Fs = $10 \mathrm{FPS}$, ramp from $62 \mathrm{~Hz}$ to $58 \mathrm{~Hz}$ at $-1 \mathrm{~Hz} / \mathrm{s}$ 
6.3.10 PMU I dynamic ramp of system frequency frequency error: M class
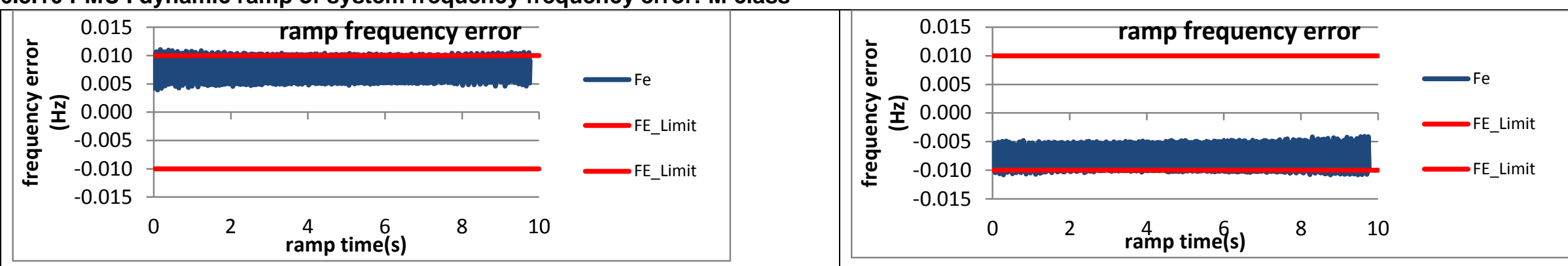

Figure 2512: Fs = $60 \mathrm{FPS}$, ramp from $55 \mathrm{~Hz}$ to $65 \mathrm{~Hz}$ at $+1 \mathrm{~Hz} / \mathrm{s}$

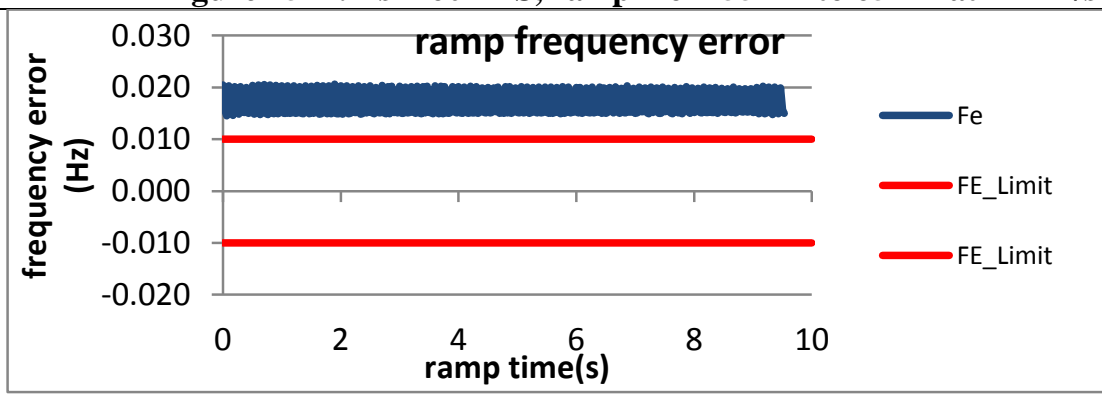

Figure 2513: Fs = $60 \mathrm{FPS}$, ramp from $65 \mathrm{~Hz}$ to $55 \mathrm{~Hz}$ at $-1 \mathrm{~Hz} / \mathrm{s}$

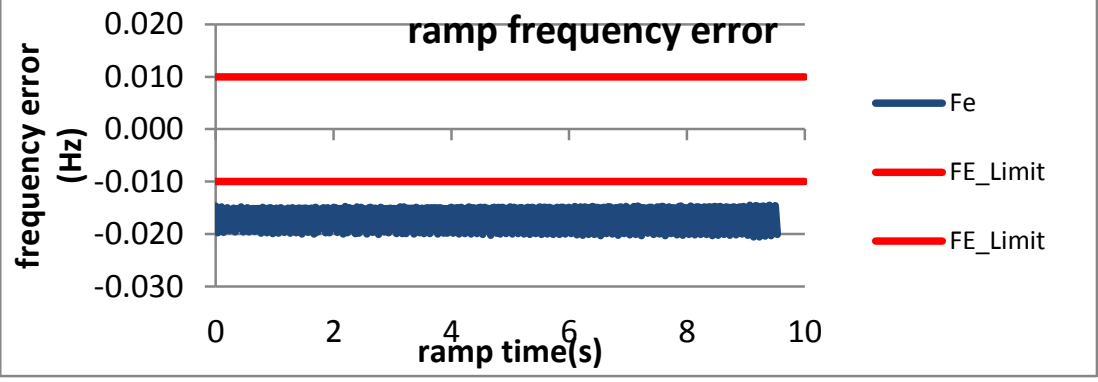

Figure 2514: Fs = $30 \mathrm{FPS}$, ramp from $55 \mathrm{~Hz}$ to $65 \mathrm{~Hz}$ at $+1 \mathrm{~Hz} / \mathrm{s}$

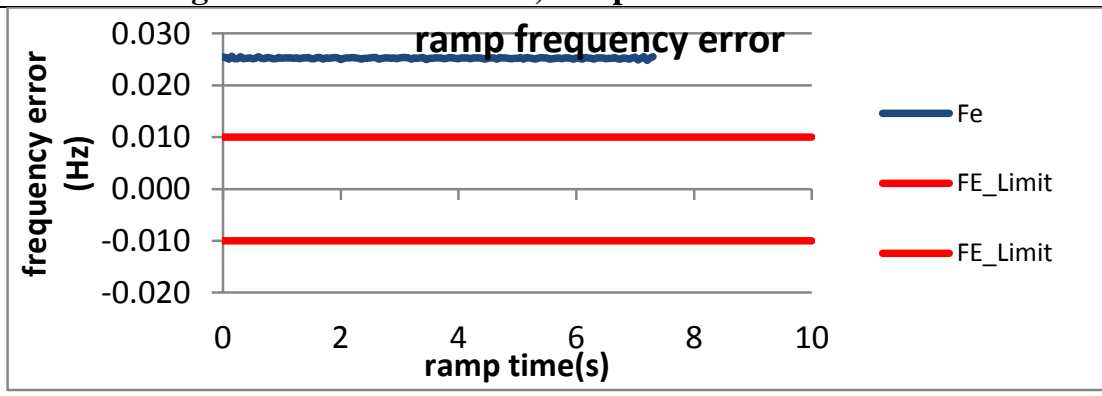

Figure 2515: Fs = $30 \mathrm{FPS}$, ramp from $65 \mathrm{~Hz}$ to $55 \mathrm{~Hz}$ at $-1 \mathrm{~Hz} / \mathrm{s}$

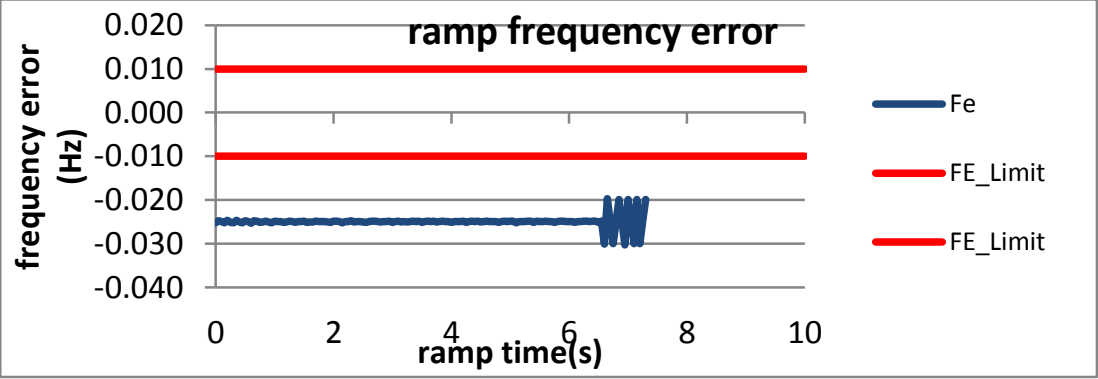

Figure 2516: Fs = 20 FPS, ramp from $56 \mathrm{~Hz}$ to $64 \mathrm{~Hz}$ at $+1 \mathrm{~Hz} / \mathrm{s}$

Figure 2517: Fs = $20 \mathrm{FPS}$, ramp from $64 \mathrm{~Hz}$ to $56 \mathrm{~Hz}$ at $-1 \mathrm{~Hz} / \mathrm{s}$ 

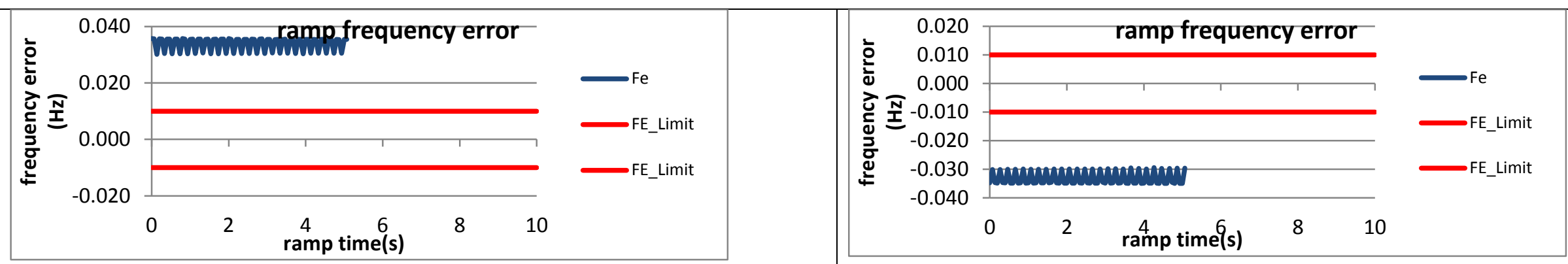

Figure 2518: Fs = $15 \mathrm{FPS}$, ramp from $57 \mathrm{~Hz}$ to $63 \mathrm{~Hz}$ at $+1 \mathrm{~Hz} / \mathrm{s}$

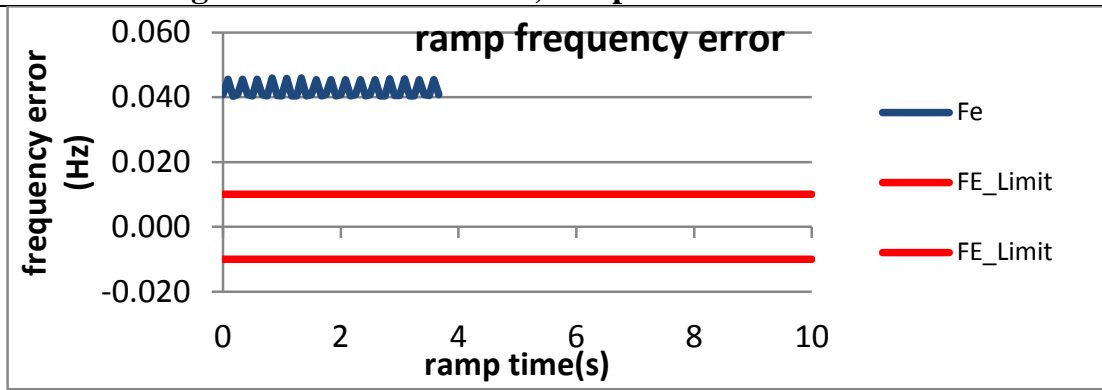

Figure 2519: Fs = $15 \mathrm{FPS}$, ramp from $63 \mathrm{~Hz}$ to $57 \mathrm{~Hz}$ at $-1 \mathrm{~Hz} / \mathrm{s}$

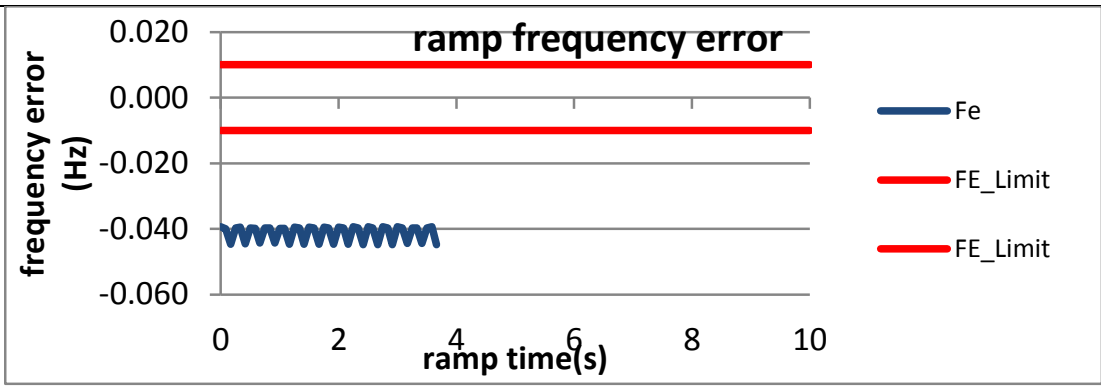

Figure 2520: Fs = $12 \mathrm{FPS}$, ramp from $58.6 \mathrm{~Hz}$ to $62.4 \mathrm{~Hz}$ at $+1 \mathrm{~Hz} / \mathrm{s}$

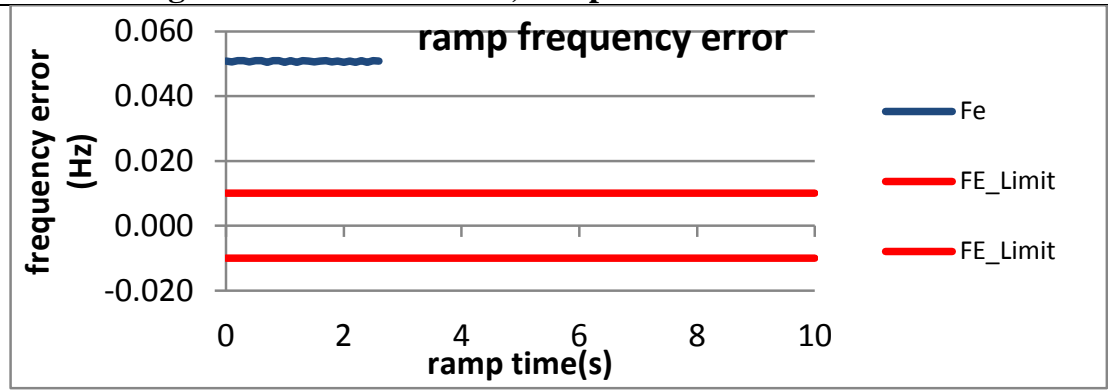

Figure 2521: Fs = 12 FPS, ramp from $62.4 \mathrm{~Hz}$ to $58.6 \mathrm{~Hz}$ at $-1 \mathrm{~Hz} / \mathrm{s}$

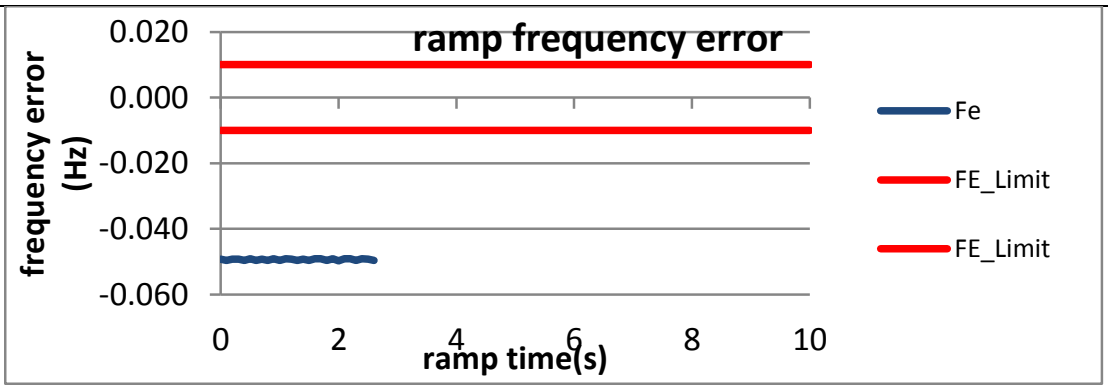

Figure 2522: Fs = $10 \mathrm{FPS}$, ramp from $58 \mathrm{~Hz}$ to $62 \mathrm{~Hz}$ at $+1 \mathrm{~Hz} / \mathrm{s}$

Figure 2523: Fs = $10 \mathrm{FPS}$, ramp from $62 \mathrm{~Hz}$ to $58 \mathrm{~Hz}$ at $-1 \mathrm{~Hz} / \mathrm{s}$ 
6.3.11 PMU J dynamic ramp of system frequency frequency error: $M$ class
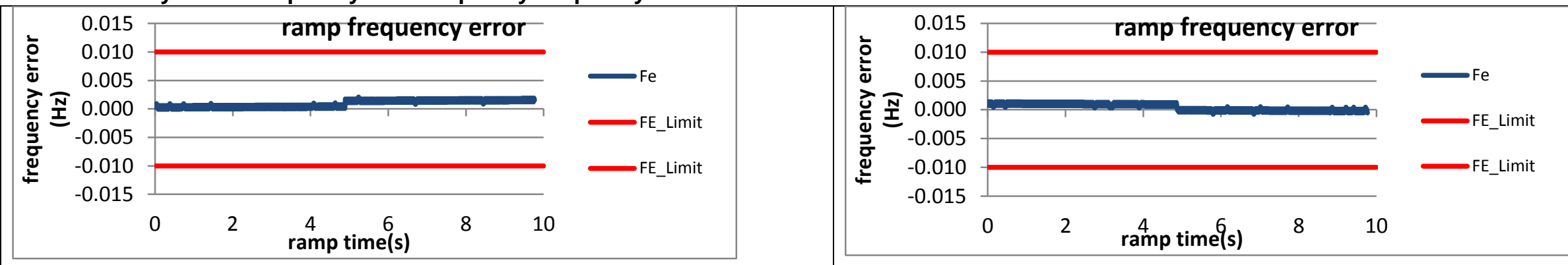

Figure 2524: Fs = $60 \mathrm{FPS}$, ramp from $55 \mathrm{~Hz}$ to $65 \mathrm{~Hz}$ at $+1 \mathrm{~Hz} / \mathrm{s}$

Figure 2525: Fs = $60 \mathrm{FPS}$, ramp from $65 \mathrm{~Hz}$ to $55 \mathrm{~Hz}$ at $-1 \mathrm{~Hz} / \mathrm{s}$
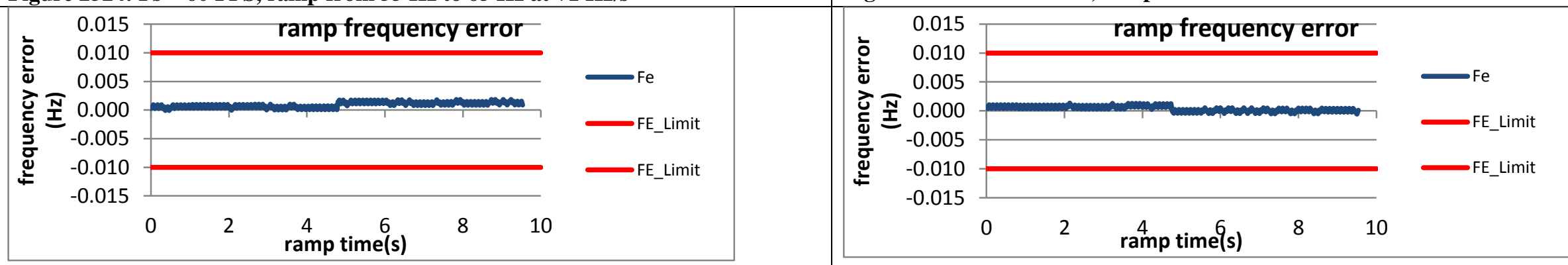

Figure 2526: Fs = $30 \mathrm{FPS}$, ramp from $55 \mathrm{~Hz}$ to $65 \mathrm{~Hz}$ at $+1 \mathrm{~Hz} / \mathrm{s}$

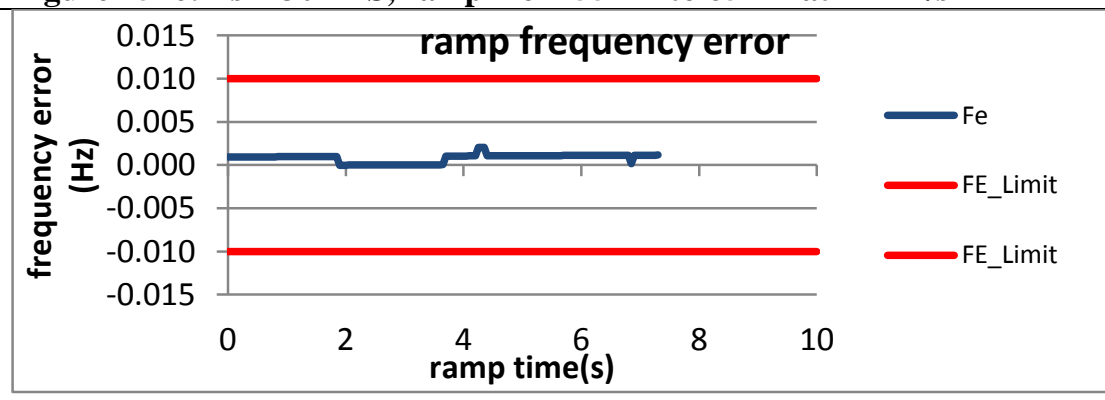

Figure 2527: Fs = $30 \mathrm{FPS}$, ramp from $65 \mathrm{~Hz}$ to $55 \mathrm{~Hz}$ at $-1 \mathrm{~Hz} / \mathrm{s}$

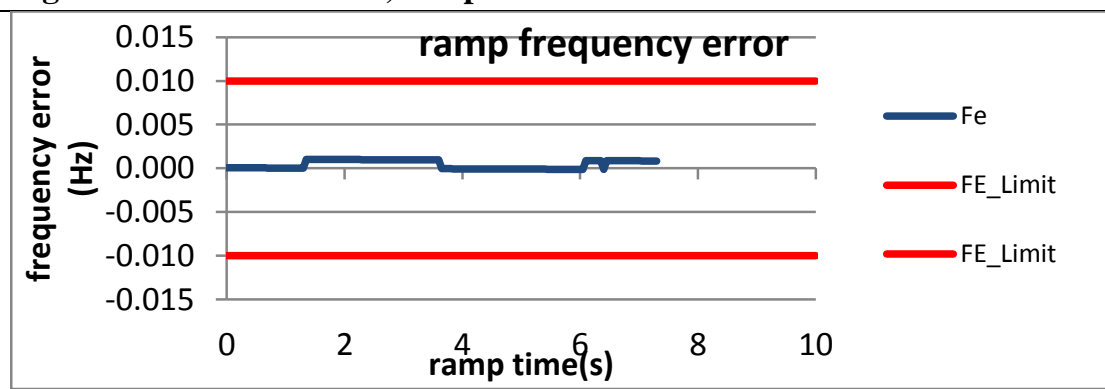

Figure 2528: Fs = $20 \mathrm{FPS}$, ramp from $56 \mathrm{~Hz}$ to $64 \mathrm{~Hz}$ at $+1 \mathrm{~Hz} / \mathrm{s}$

Figure 2529: Fs = $20 \mathrm{FPS}$, ramp from $64 \mathrm{~Hz}$ to $56 \mathrm{~Hz}$ at $-1 \mathrm{~Hz} / \mathrm{s}$ 


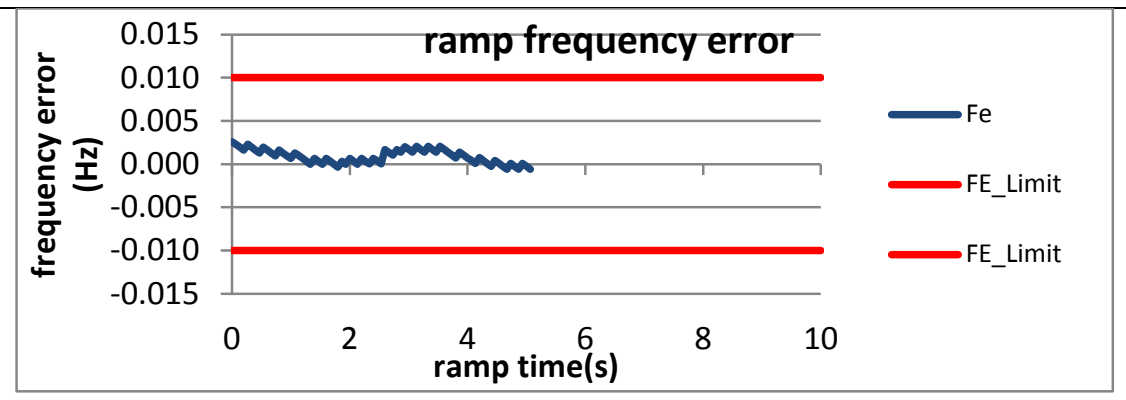

Figure 2530: Fs = $15 \mathrm{FPS}$, ramp from $57 \mathrm{~Hz}$ to $63 \mathrm{~Hz}$ at $+1 \mathrm{~Hz} / \mathrm{s}$

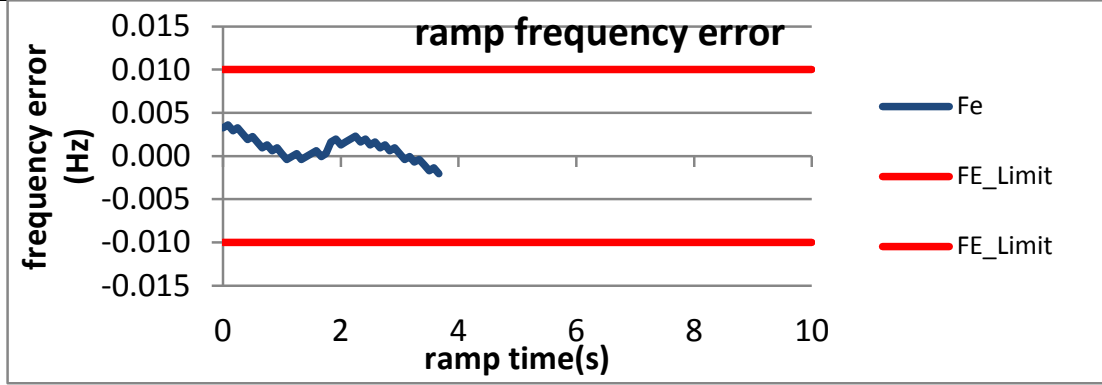

Figure 2532: Fs = 12 FPS, ramp from $58.6 \mathrm{~Hz}$ to $62.4 \mathrm{~Hz}$ at $+1 \mathrm{~Hz} / \mathrm{s}$

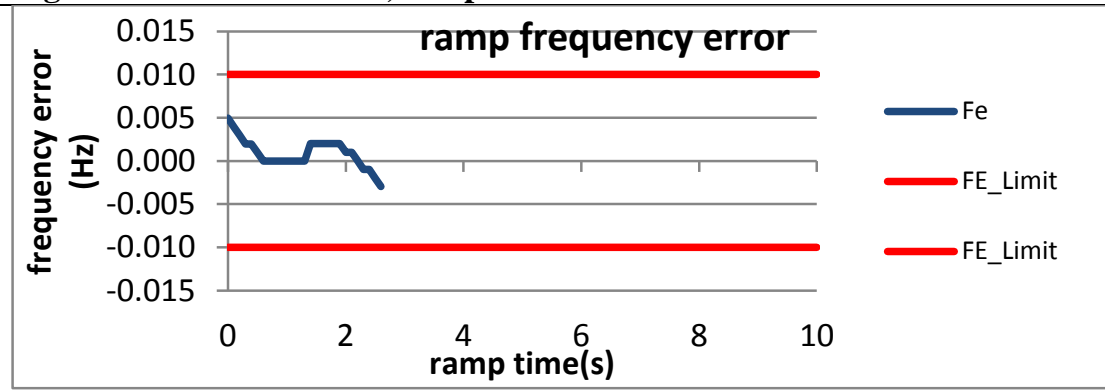

Figure 2534: Fs = 10 FPS, ramp from $58 \mathrm{~Hz}$ to $62 \mathrm{~Hz}$ at $+1 \mathrm{~Hz} / \mathrm{s}$

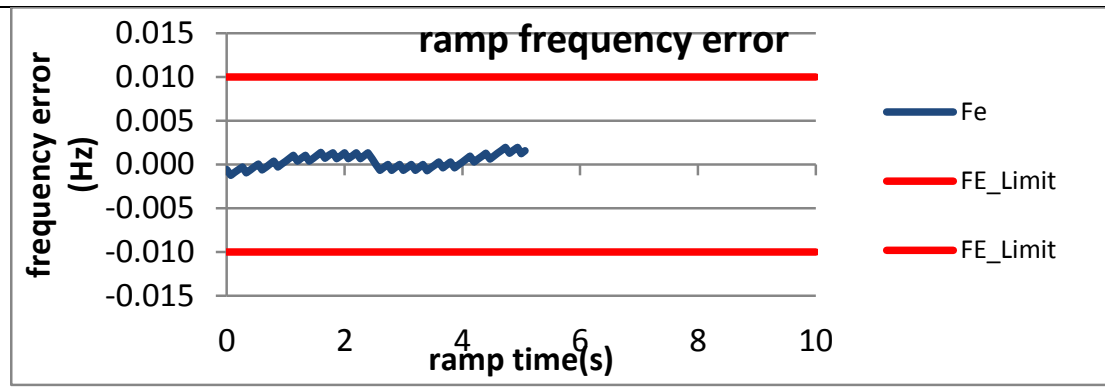

Figure 2531: Fs = $15 \mathrm{FPS}$, ramp from $63 \mathrm{~Hz}$ to $57 \mathrm{~Hz}$ at $-1 \mathrm{~Hz} / \mathrm{s}$

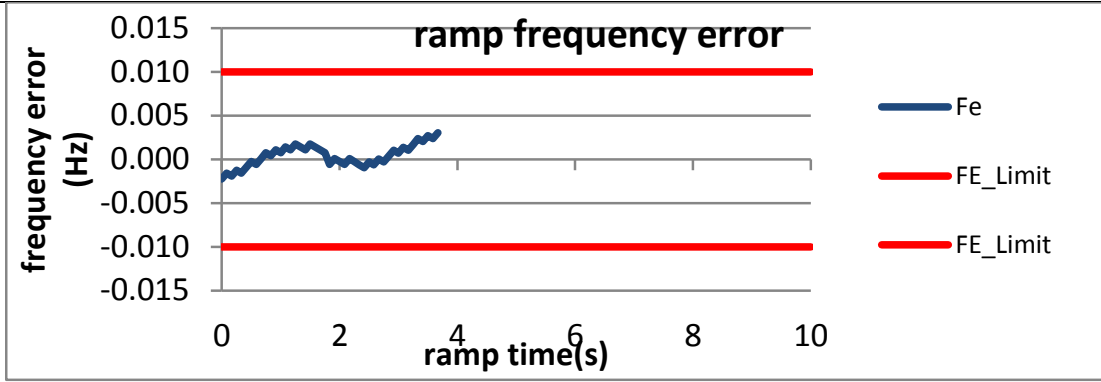

Figure 2533: Fs = 12 FPS, ramp from $62.4 \mathrm{~Hz}$ to $58.6 \mathrm{~Hz}$ at $-1 \mathrm{~Hz} / \mathrm{s}$

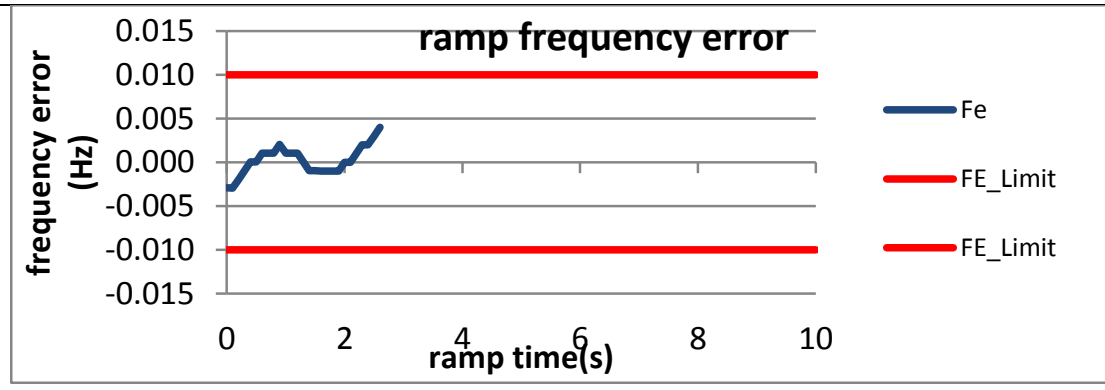

Figure 2535: Fs = $10 \mathrm{FPS}$, ramp from $62 \mathrm{~Hz}$ to $58 \mathrm{~Hz}$ at $-1 \mathrm{~Hz} / \mathrm{s}$ 


\subsection{Dynamic ramp of system frequency ROCOF error}

\subsubsection{C37.118.1 Annex C dynamic ramp of system frequency ROCOF error: M class}

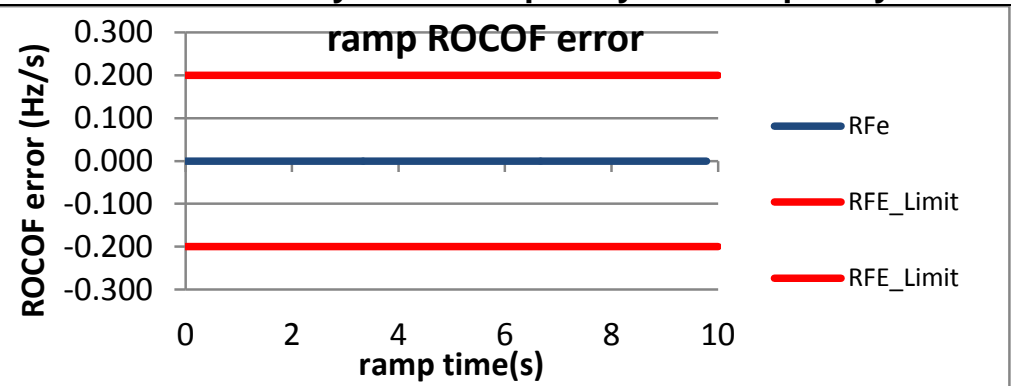

Figure 2536: Fs = $60 \mathrm{FPS}$, ram from $55 \mathrm{~Hz}$ to $65 \mathrm{~Hz}$ at $+1 \mathrm{~Hz} / \mathrm{s}$

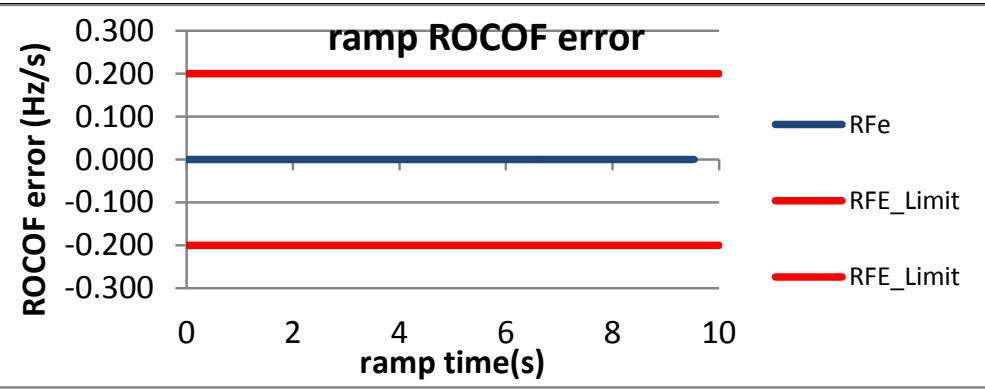

Figure 2538: Fs = $30 \mathrm{FPS}$, ram from $55 \mathrm{~Hz}$ to $65 \mathrm{~Hz}$ at $+1 \mathrm{~Hz} / \mathrm{s}$

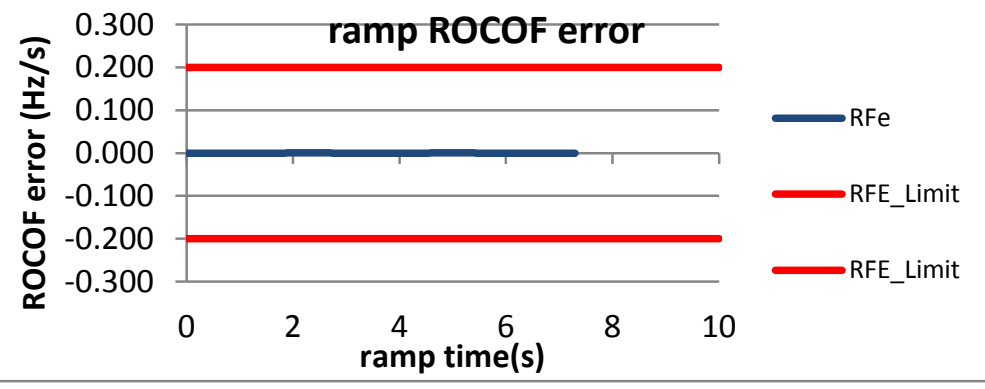

Figure 2540: Fs = $20 \mathrm{FPS}$, ramp from $56 \mathrm{~Hz}$ to $64 \mathrm{~Hz}$ at $+1 \mathrm{~Hz} / \mathrm{s}$

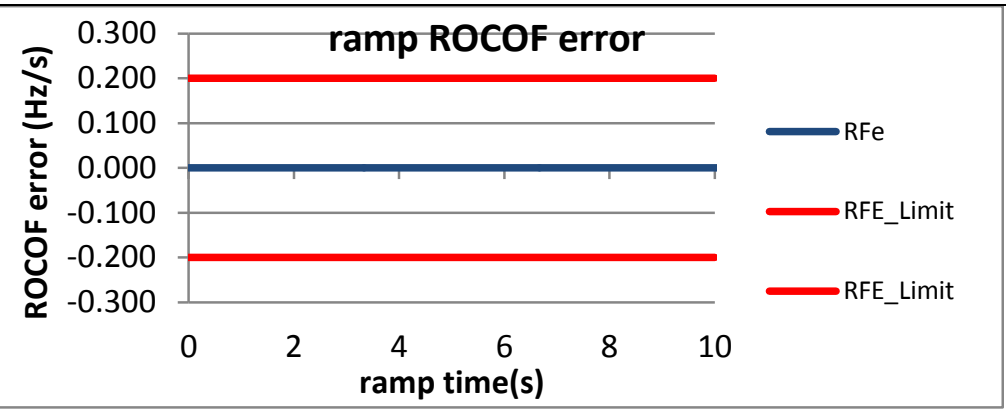

Figure 2537: Fs = $60 \mathrm{FPS}$, ram from $65 \mathrm{~Hz}$ to $55 \mathrm{~Hz}$ at $-1 \mathrm{~Hz} / \mathrm{s}$

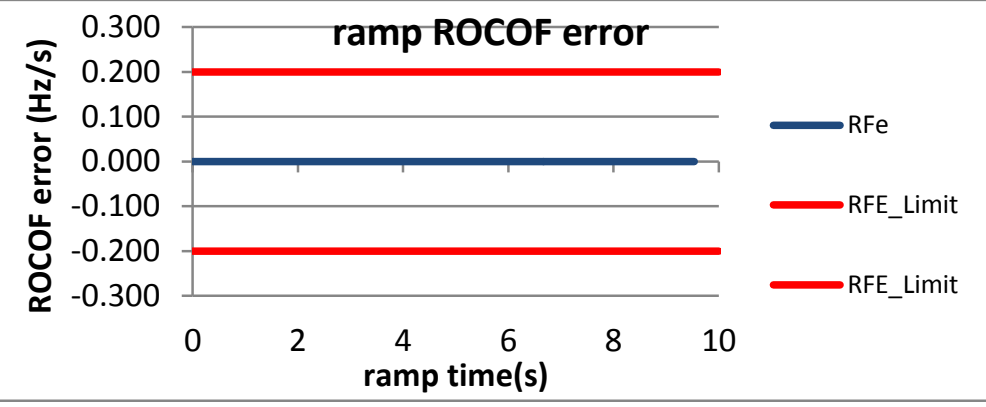

Figure 2539: Fs = $30 \mathrm{FPS}$, ram from $65 \mathrm{~Hz}$ to $55 \mathrm{~Hz}$ at $-1 \mathrm{~Hz} / \mathrm{s}$

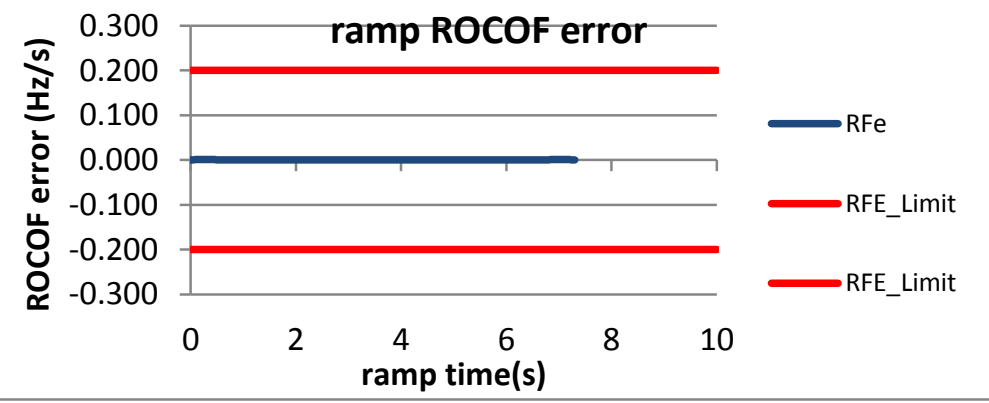

Figure 2541: Fs = $20 \mathrm{FPS}$, ramp from $64 \mathrm{~Hz}$ to $56 \mathrm{~Hz}$ at $=1 \mathrm{~Hz} / \mathrm{s}$ 


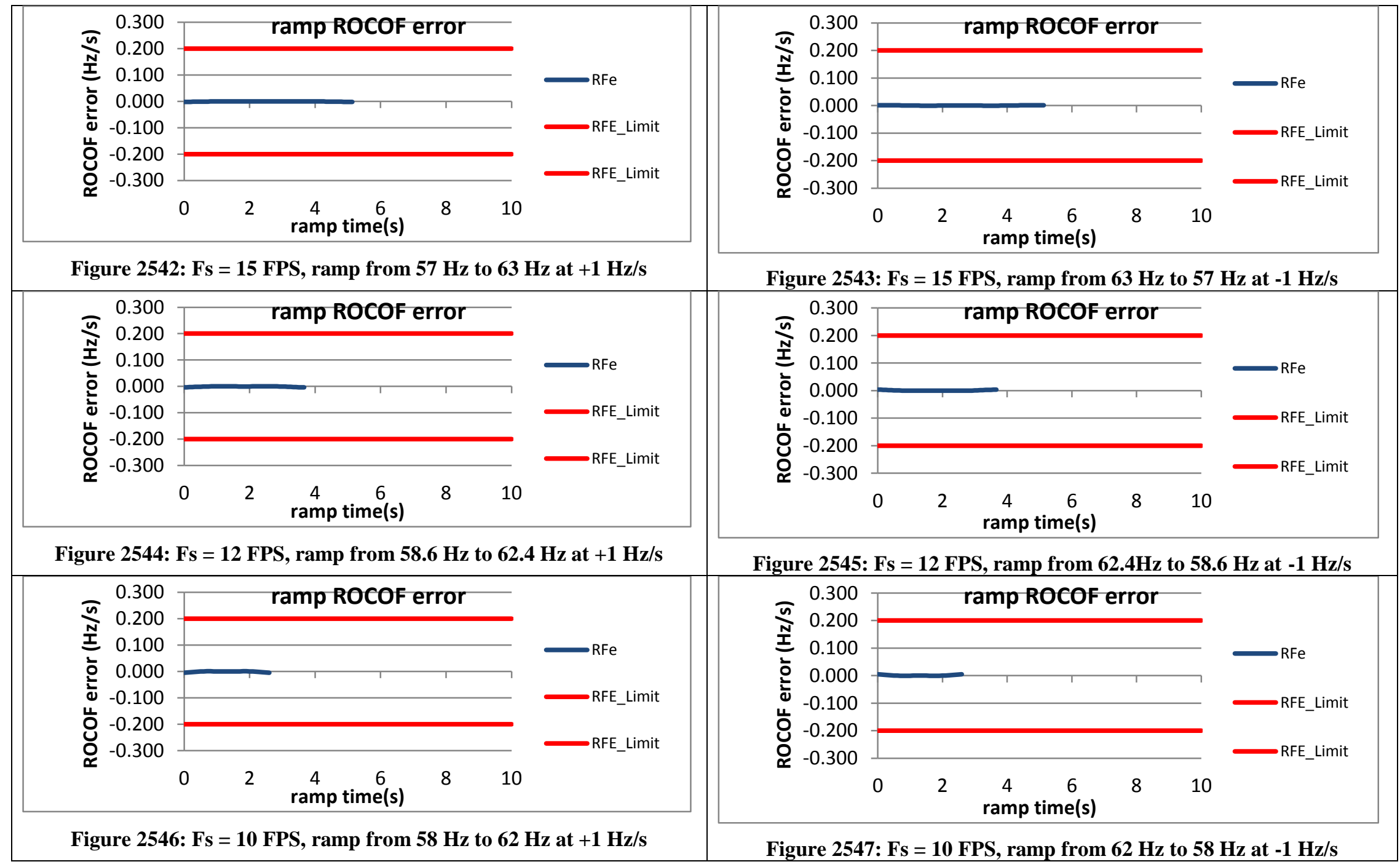


6.4.2 PMU A dynamic ramp of system frequency ROCOF error: M class

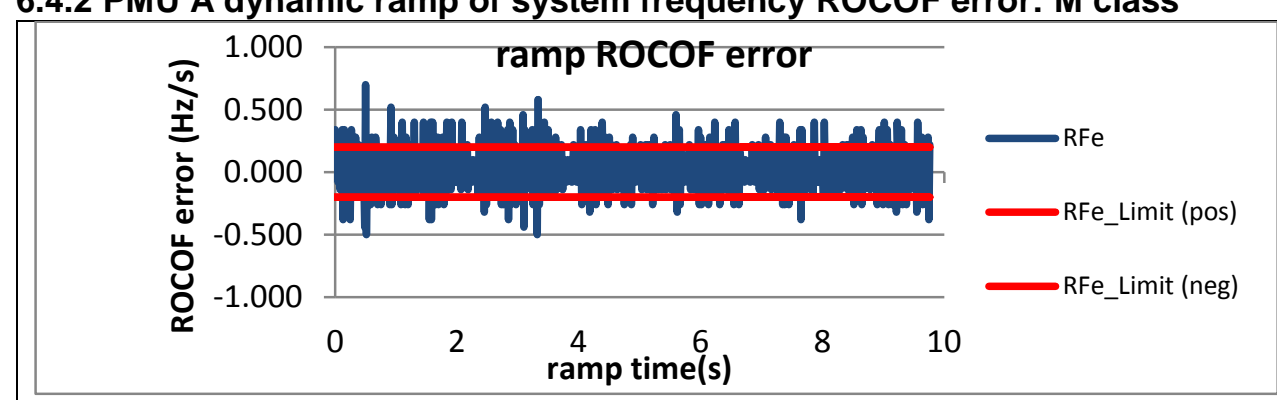

Figure 2548: Fs = $60 \mathrm{FPS}$, ram from $55 \mathrm{~Hz}$ to $65 \mathrm{~Hz}$ at $+1 \mathrm{~Hz} / \mathrm{s}$

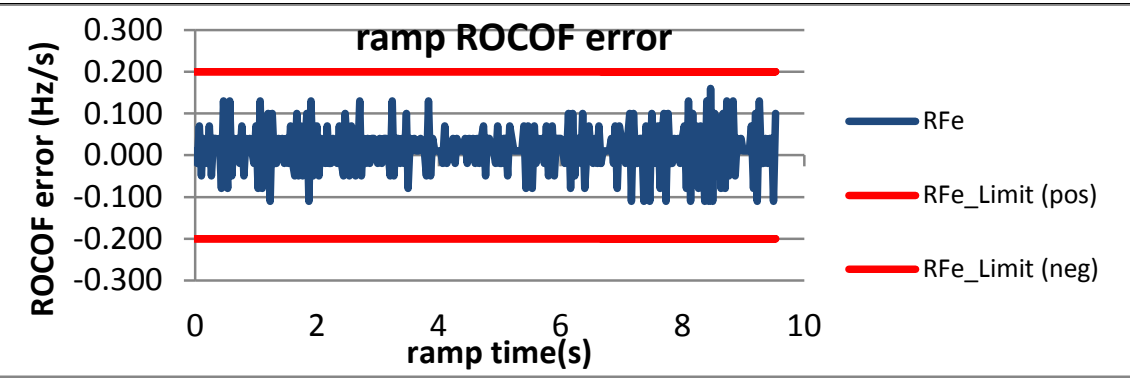

Figure 2550: Fs = $30 \mathrm{FPS}$, ram from $55 \mathrm{~Hz}$ to $65 \mathrm{~Hz}$ at $+1 \mathrm{~Hz} / \mathrm{s}$

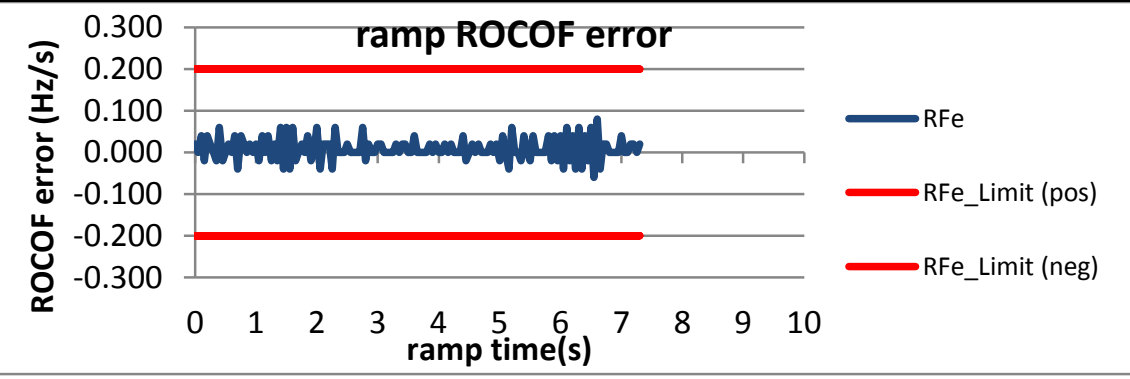

Figure 2552: Fs $=20 \mathrm{FPS}$, ramp from $56 \mathrm{~Hz}$ to $64 \mathrm{~Hz}$ at $+1 \mathrm{~Hz} / \mathrm{s}$

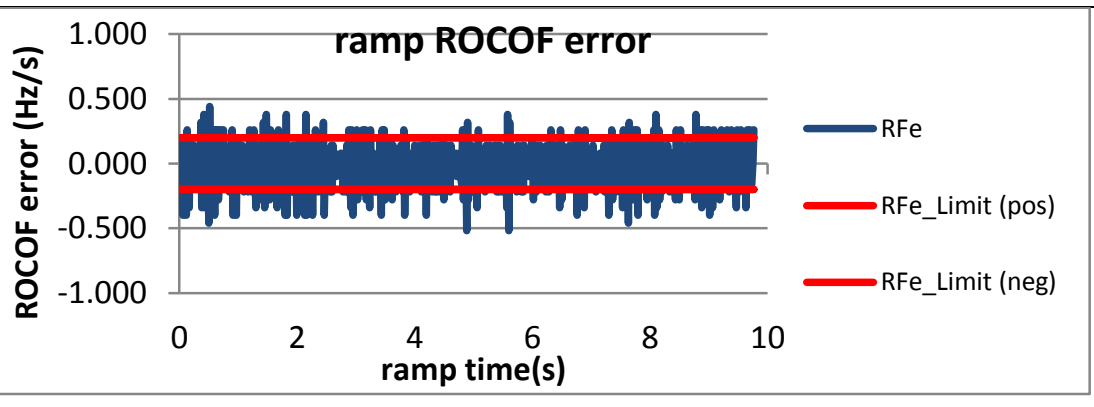

Figure 2549: Fs = $60 \mathrm{FPS}$, ram from $65 \mathrm{~Hz}$ to $55 \mathrm{~Hz}$ at $-1 \mathrm{~Hz} / \mathrm{s}$

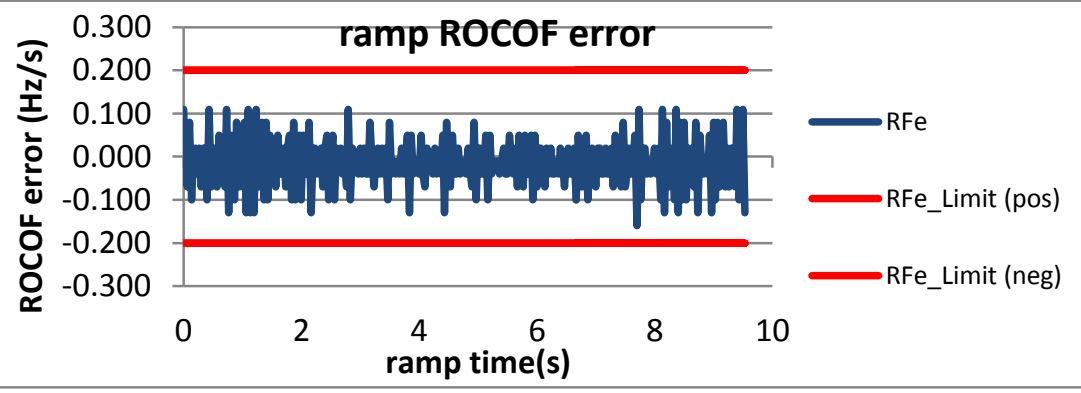

Figure 2551: Fs = $30 \mathrm{FPS}$, ram from $65 \mathrm{~Hz}$ to $55 \mathrm{~Hz}$ at $-1 \mathrm{~Hz} / \mathrm{s}$

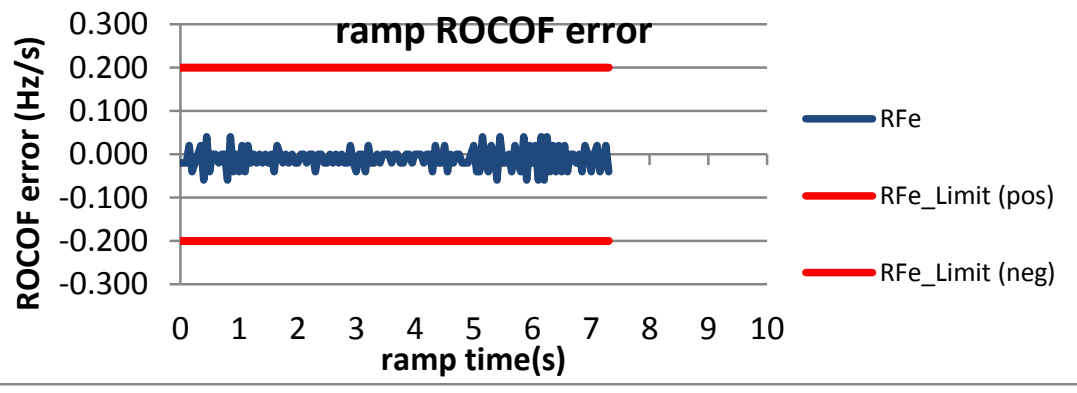

Figure 2553: Fs = 20 FPS, ramp from $64 \mathrm{~Hz}$ to $56 \mathrm{~Hz}$ at $=1 \mathrm{~Hz} / \mathrm{s}$ 


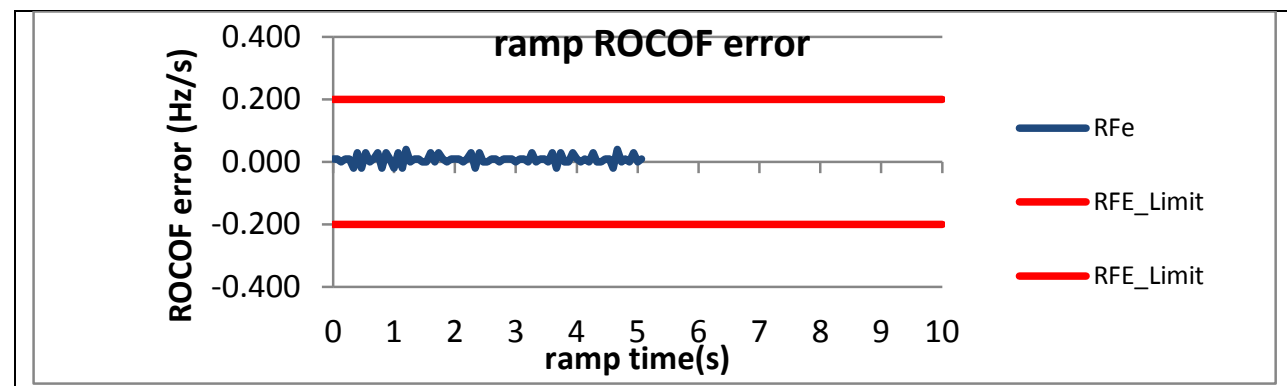

Figure 2554: Fs = $15 \mathrm{FPS}$, ramp from $57 \mathrm{~Hz}$ to $63 \mathrm{~Hz}$ at $+1 \mathrm{~Hz} / \mathrm{s}$

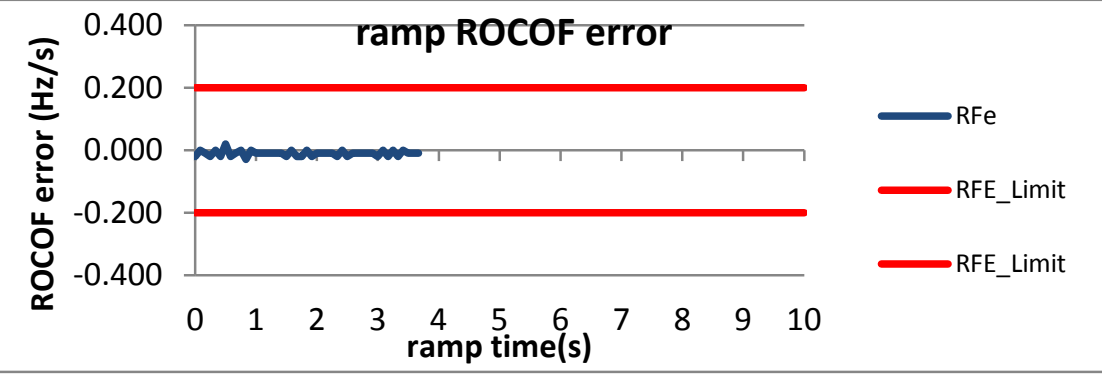

Figure 2556: Fs = 12 FPS, ramp from $58.6 \mathrm{~Hz}$ to $62.4 \mathrm{~Hz}$ at $+1 \mathrm{~Hz} / \mathrm{s}$

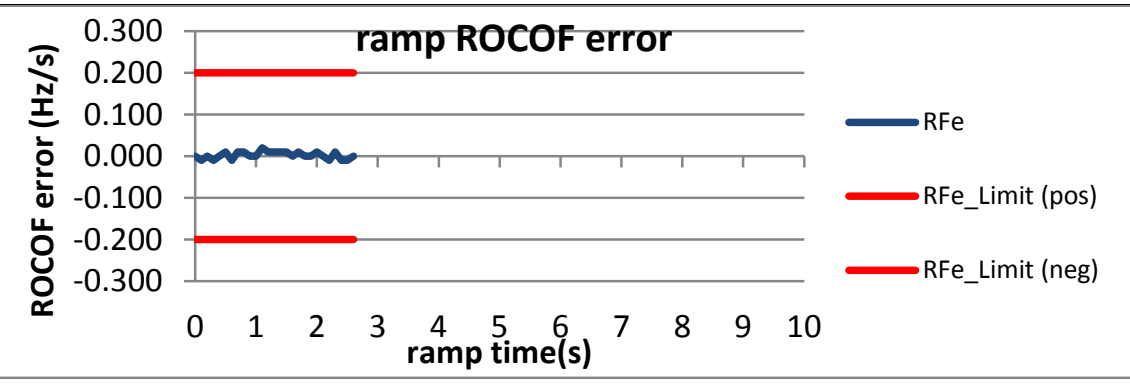

Figure 2558: Fs = $10 \mathrm{FPS}$, ramp from $58 \mathrm{~Hz}$ to $62 \mathrm{~Hz}$ at $+1 \mathrm{~Hz} / \mathrm{s}$

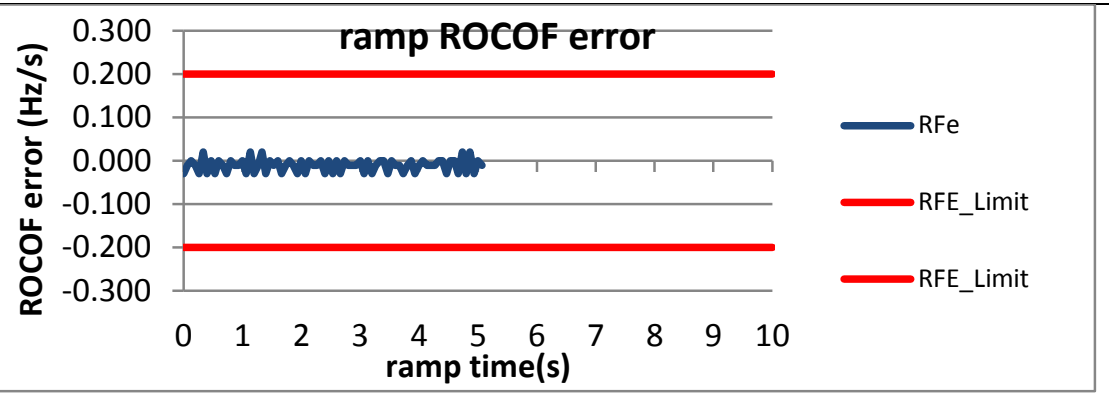

Figure 2555: Fs = $15 \mathrm{FPS}$, ramp from $63 \mathrm{~Hz}$ to $57 \mathrm{~Hz}$ at $-1 \mathrm{~Hz} / \mathrm{s}$

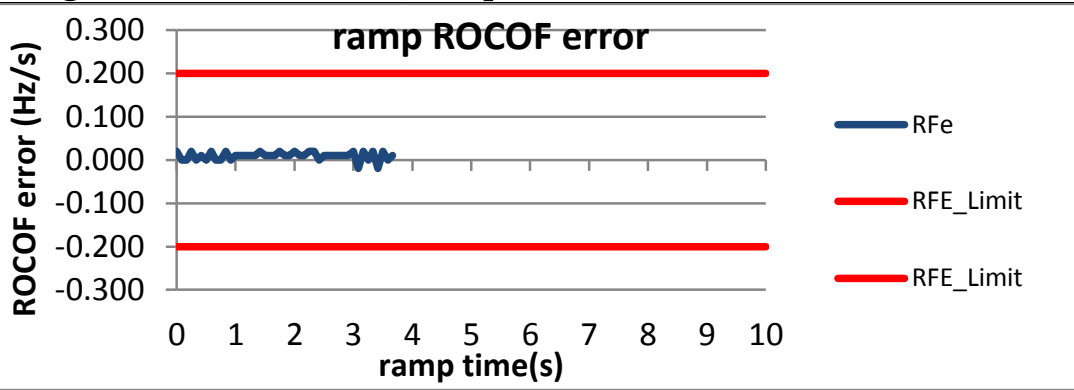

Figure 2557: Fs = $12 \mathrm{FPS}$, ramp from $62.4 \mathrm{~Hz}$ to $58.6 \mathrm{~Hz}$ at $-1 \mathrm{~Hz} / \mathrm{s}$

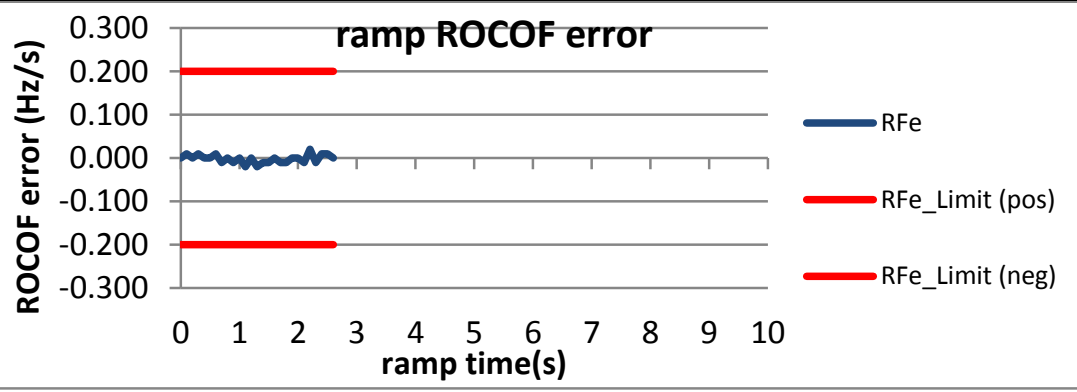

Figure 2559: Fs = $10 \mathrm{FPS}$, ramp from $62 \mathrm{~Hz}$ to $58 \mathrm{~Hz}$ at $-1 \mathrm{~Hz} / \mathrm{s}$ 
6.4.3 PMU B dynamic ramp of system frequency ROCOF error: M class

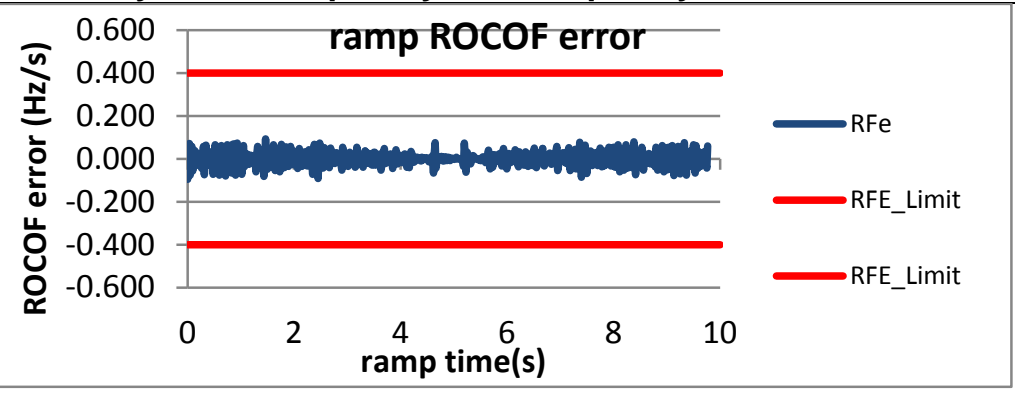

Figure 2560: Fs = $60 \mathrm{FPS}$, ram from $55 \mathrm{~Hz}$ to $65 \mathrm{~Hz}$ at $+1 \mathrm{~Hz} / \mathrm{s}$

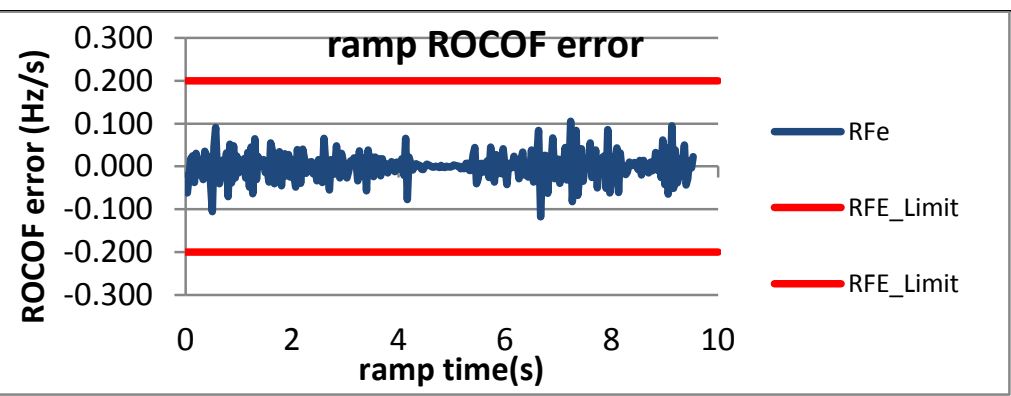

Figure 2562: Fs = $30 \mathrm{FPS}$, ram from $55 \mathrm{~Hz}$ to $65 \mathrm{~Hz}$ at $+1 \mathrm{~Hz} / \mathrm{s}$

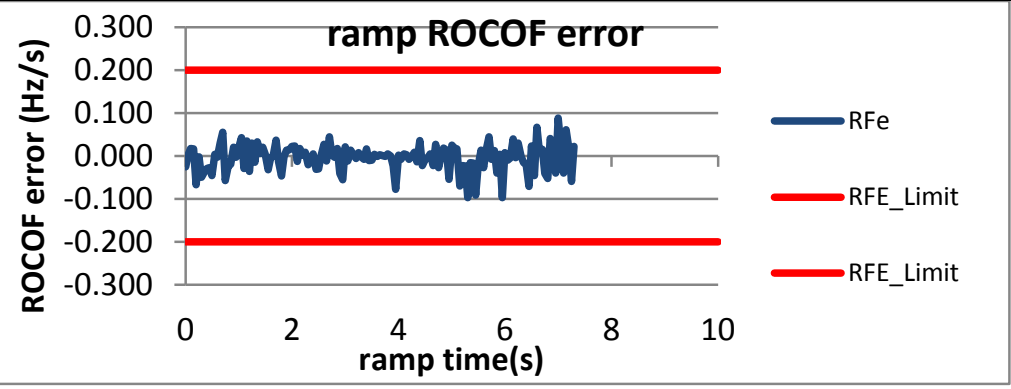

Figure 2564: Fs = $20 \mathrm{FPS}$, ramp from $56 \mathrm{~Hz}$ to $64 \mathrm{~Hz}$ at $+1 \mathrm{~Hz} / \mathrm{s}$

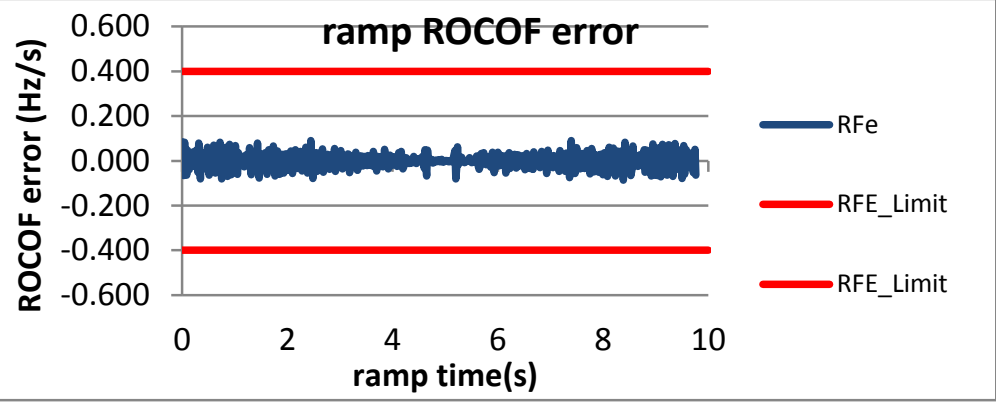

Figure 2561: Fs = $60 \mathrm{FPS}$, ram from $65 \mathrm{~Hz}$ to $55 \mathrm{~Hz}$ at $-1 \mathrm{~Hz} / \mathrm{s}$

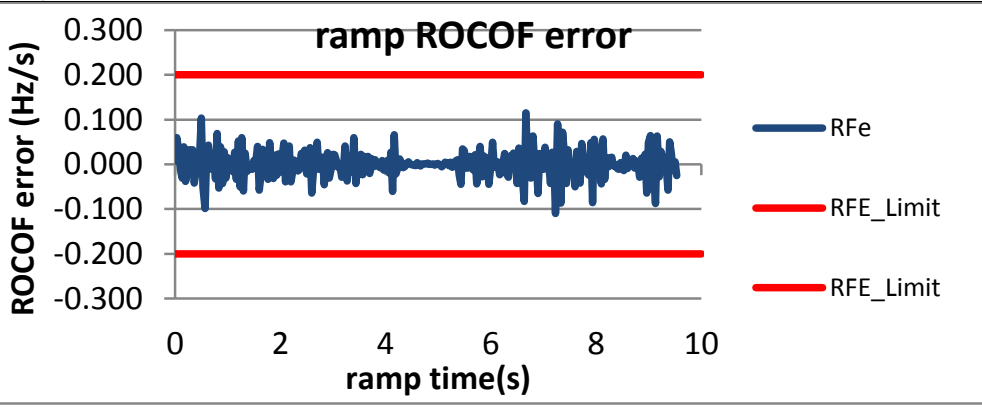

Figure 2563: Fs = $30 \mathrm{FPS}$, ram from $65 \mathrm{~Hz}$ to $55 \mathrm{~Hz}$ at $-1 \mathrm{~Hz} / \mathrm{s}$

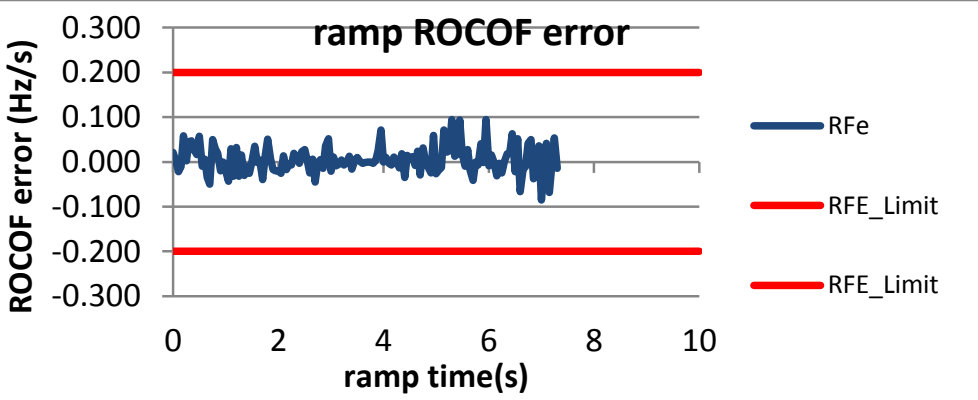

Figure 2565: Fs = $20 \mathrm{FPS}$, ramp from $64 \mathrm{~Hz}$ to $56 \mathrm{~Hz}$ at =1 Hz/s 


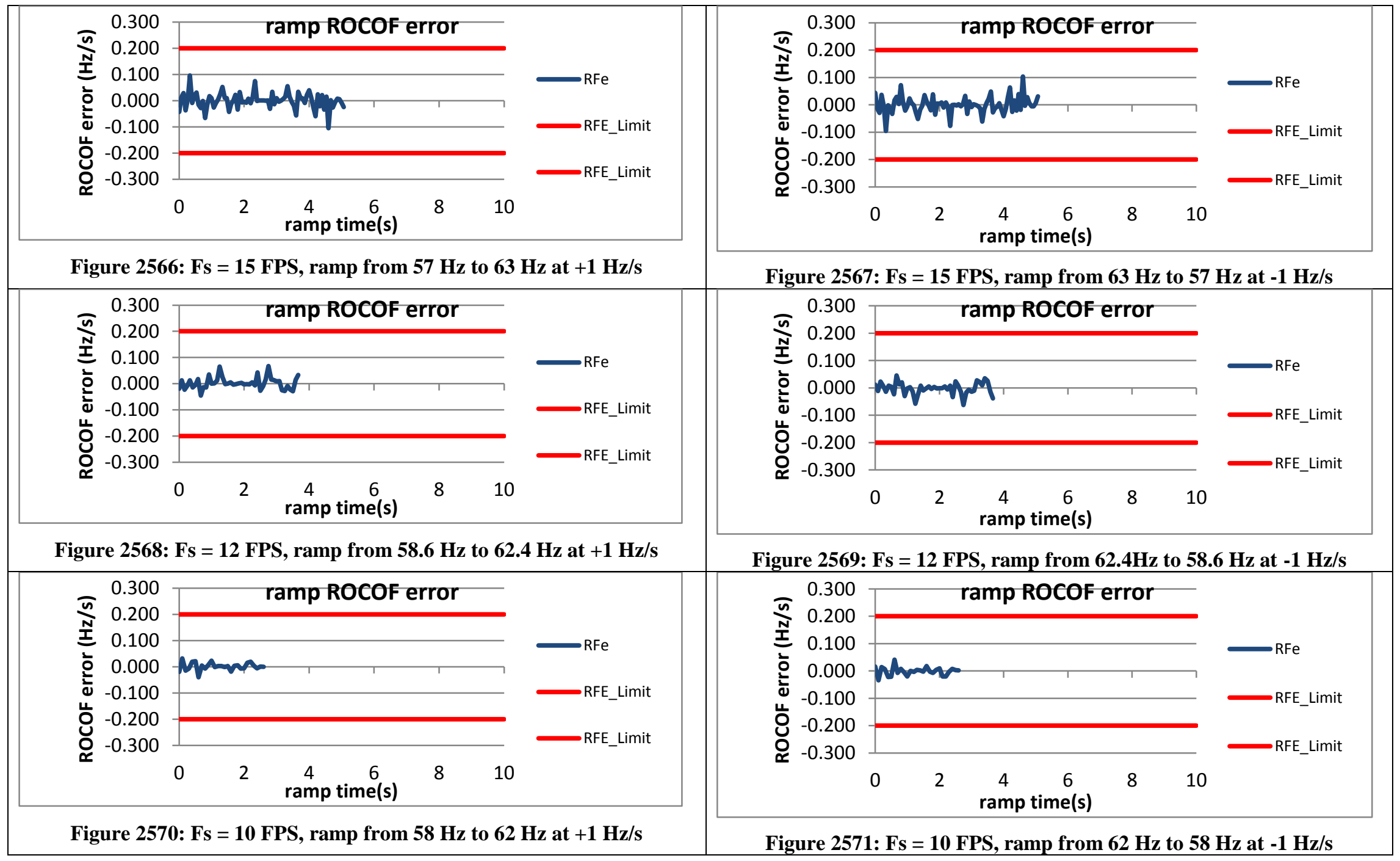


6.4.4 PMU C dynamic ramp of system frequency ROCOF error: M class

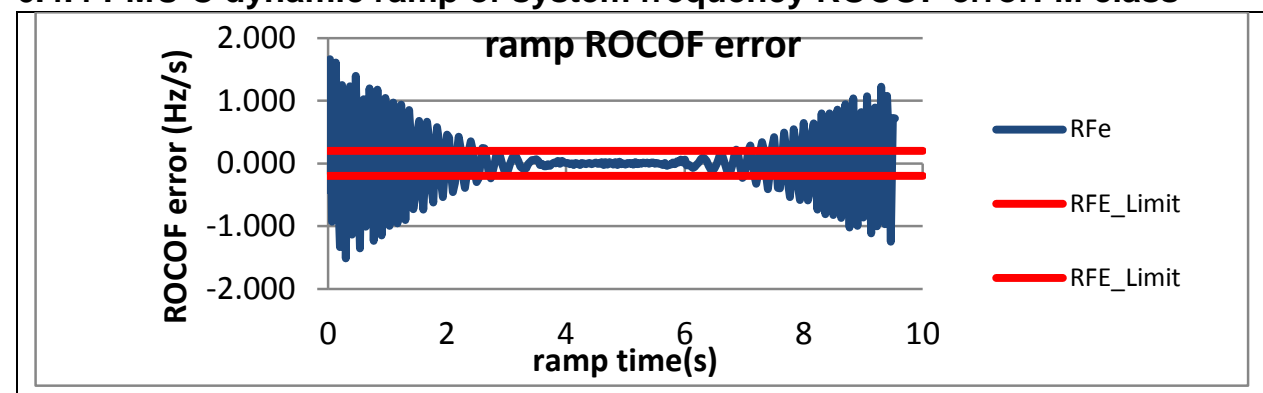

Figure 2572: Fs = $60 \mathrm{FPS}$, ram from $55 \mathrm{~Hz}$ to $65 \mathrm{~Hz}$ at $+1 \mathrm{~Hz} / \mathrm{s}$

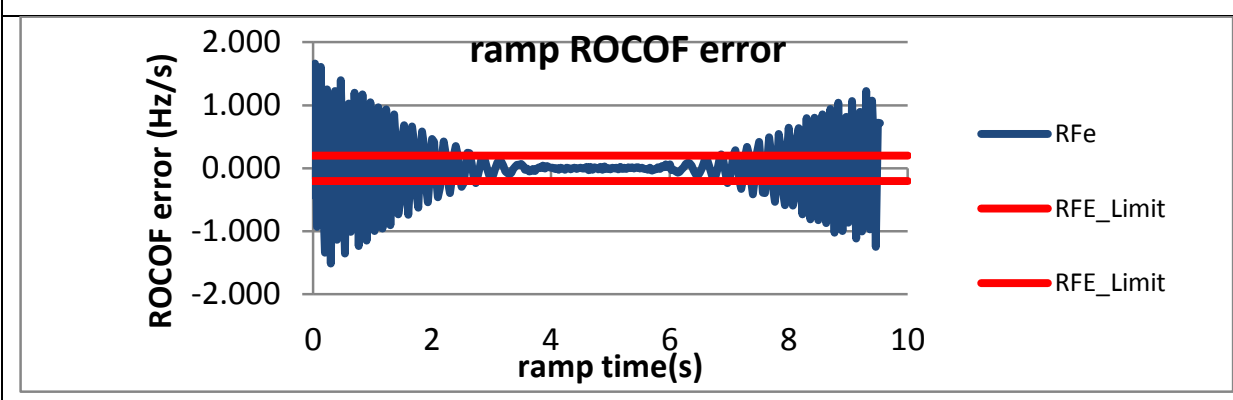

Figure 2574: Fs = $30 \mathrm{FPS}$, ram from $55 \mathrm{~Hz}$ to $65 \mathrm{~Hz}$ at $+1 \mathrm{~Hz} / \mathrm{s}$

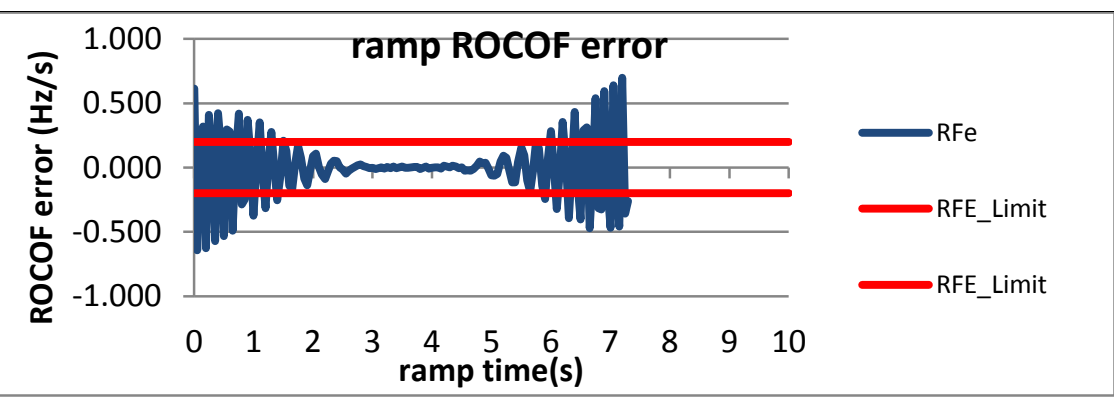

Figure 2576: Fs = $20 \mathrm{FPS}$, ramp from $56 \mathrm{~Hz}$ to $64 \mathrm{~Hz}$ at $+1 \mathrm{~Hz} / \mathrm{s}$

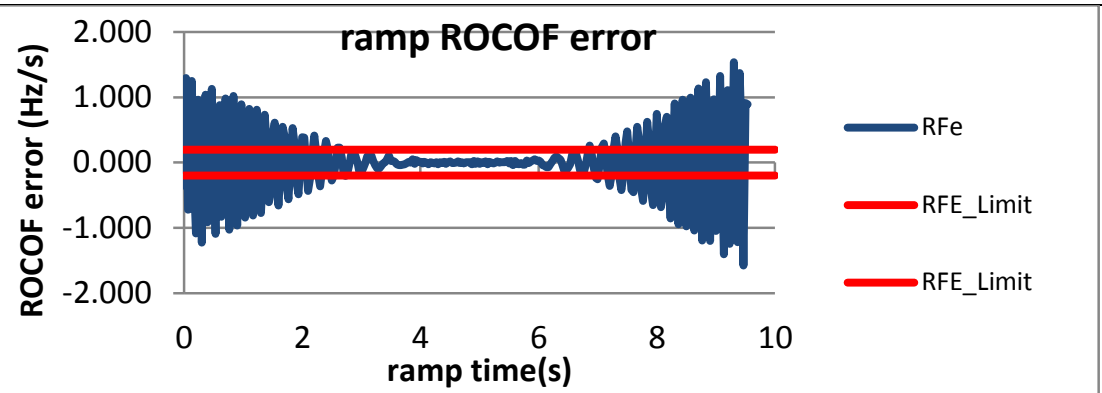

Figure 2573: Fs = $60 \mathrm{FPS}$, ram from $65 \mathrm{~Hz}$ to $55 \mathrm{~Hz}$ at $-1 \mathrm{~Hz} / \mathrm{s}$

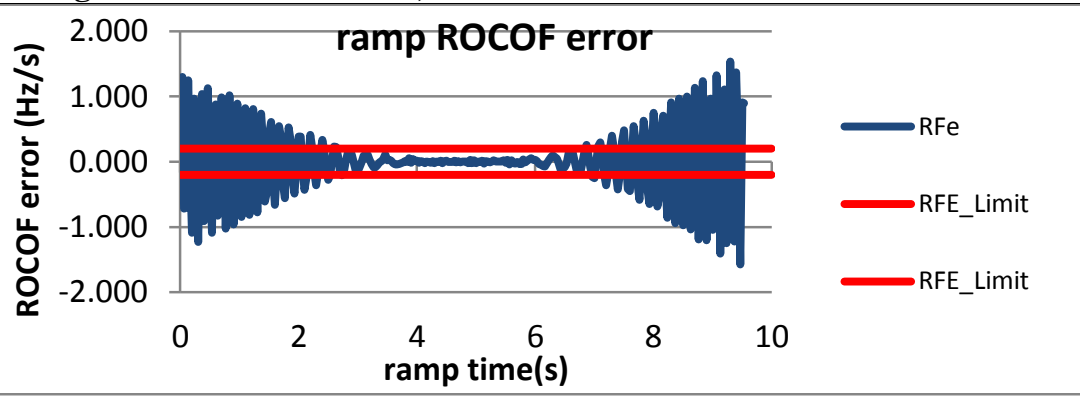

Figure 2575: Fs = $30 \mathrm{FPS}$, ram from $65 \mathrm{~Hz}$ to $55 \mathrm{~Hz}$ at $-1 \mathrm{~Hz} / \mathrm{s}$

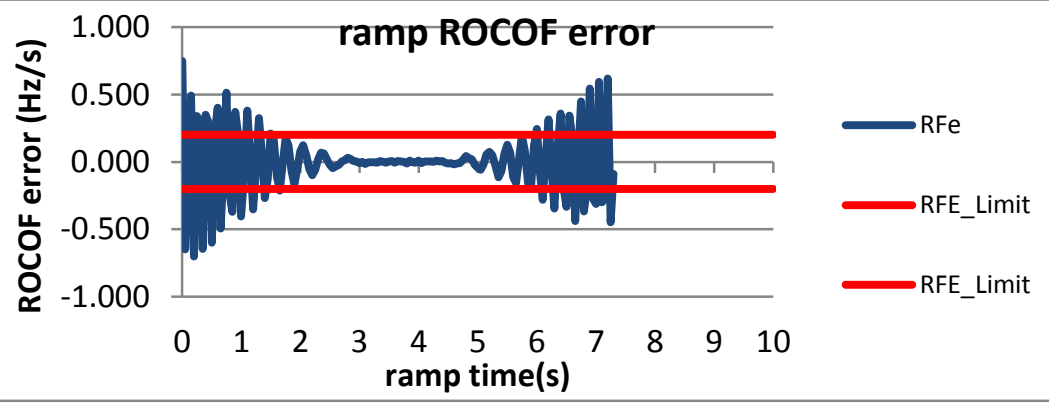

Figure 2577: Fs = $20 \mathrm{FPS}$, ramp from $64 \mathrm{~Hz}$ to $56 \mathrm{~Hz}$ at $=1 \mathrm{~Hz} / \mathrm{s}$ 


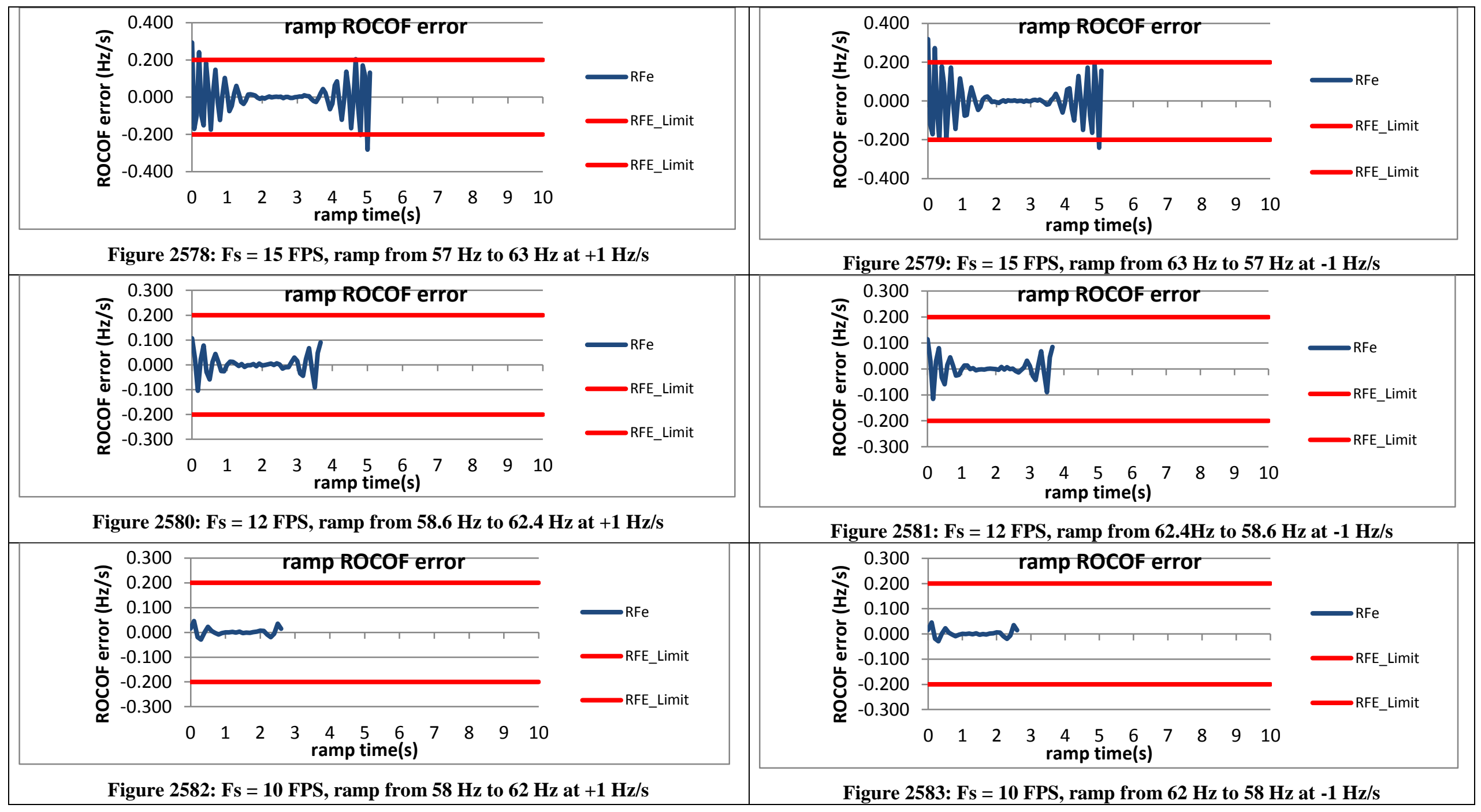


6.4.5 PMU D dynamic ramp of system frequency ROCOF error: M class

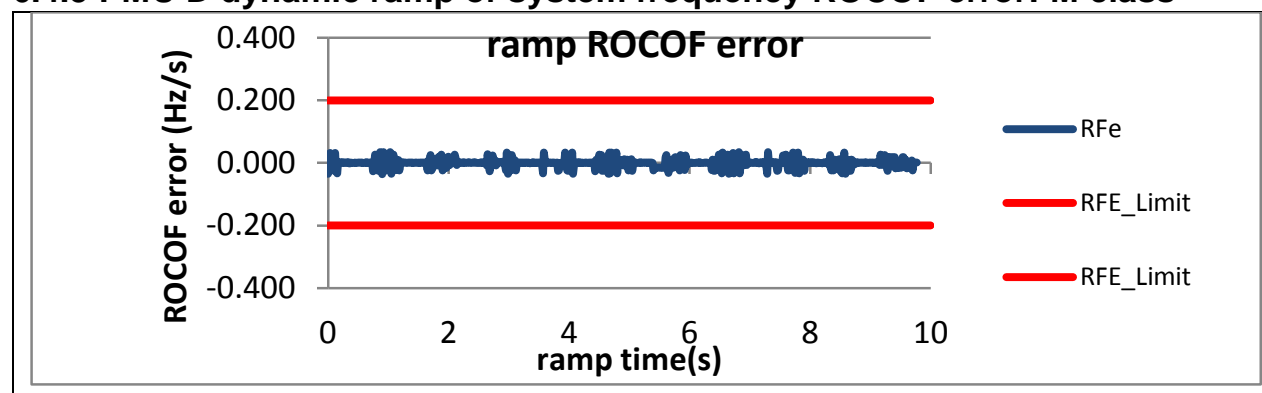

Figure 2584: Fs = $60 \mathrm{FPS}$, ram from $55 \mathrm{~Hz}$ to $65 \mathrm{~Hz}$ at $+1 \mathrm{~Hz} / \mathrm{s}$

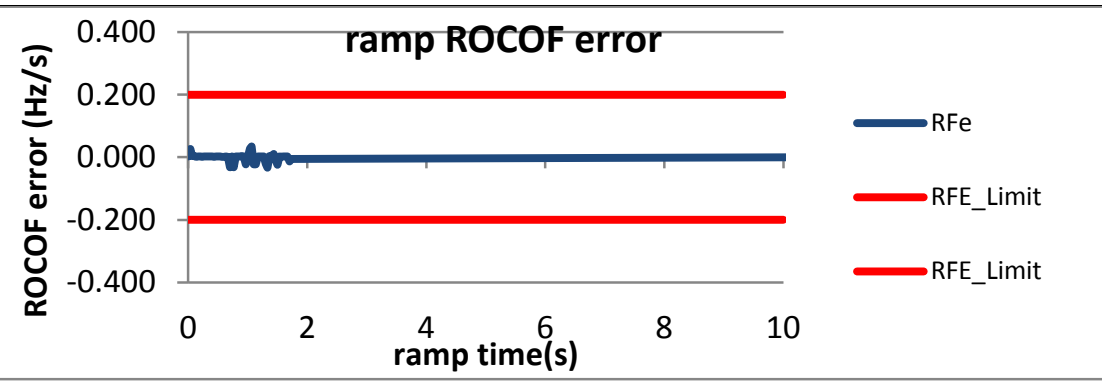

Figure 2586: Fs = $30 \mathrm{FPS}$, ram from $55 \mathrm{~Hz}$ to $65 \mathrm{~Hz}$ at $+1 \mathrm{~Hz} / \mathrm{s}$

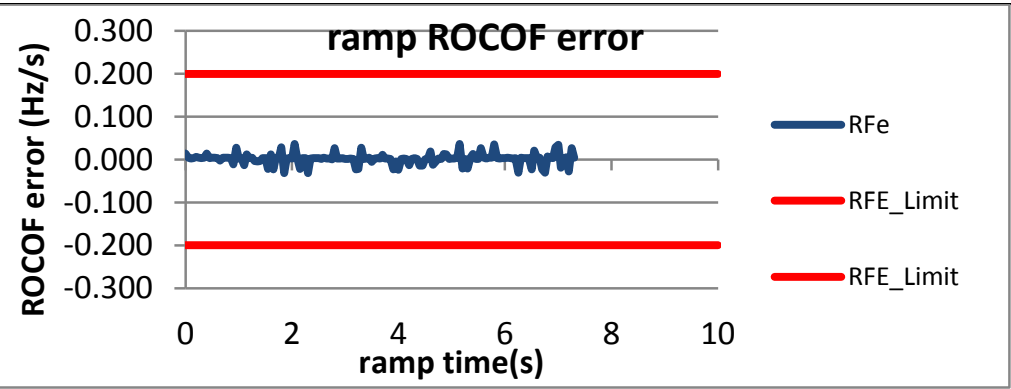

Figure 2588: Fs = $20 \mathrm{FPS}$, ramp from $56 \mathrm{~Hz}$ to $64 \mathrm{~Hz}$ at $+1 \mathrm{~Hz} / \mathrm{s}$

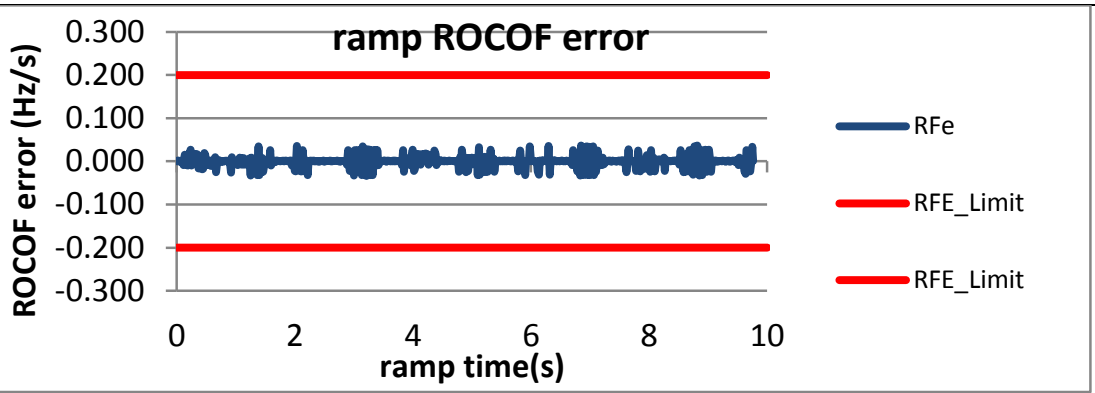

Figure 2585: Fs = $60 \mathrm{FPS}$, ram from $65 \mathrm{~Hz}$ to $55 \mathrm{~Hz}$ at $-1 \mathrm{~Hz} / \mathrm{s}$

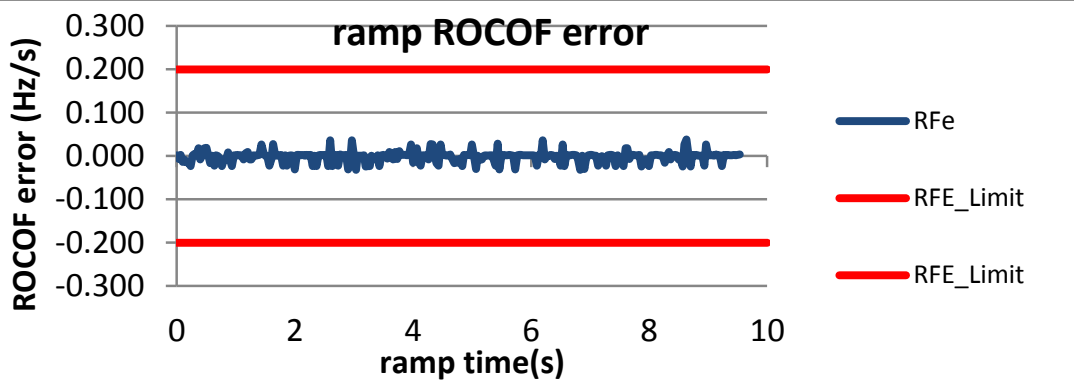

Figure 2587: Fs = $30 \mathrm{FPS}$, ram from $65 \mathrm{~Hz}$ to $55 \mathrm{~Hz}$ at $-1 \mathrm{~Hz} / \mathrm{s}$

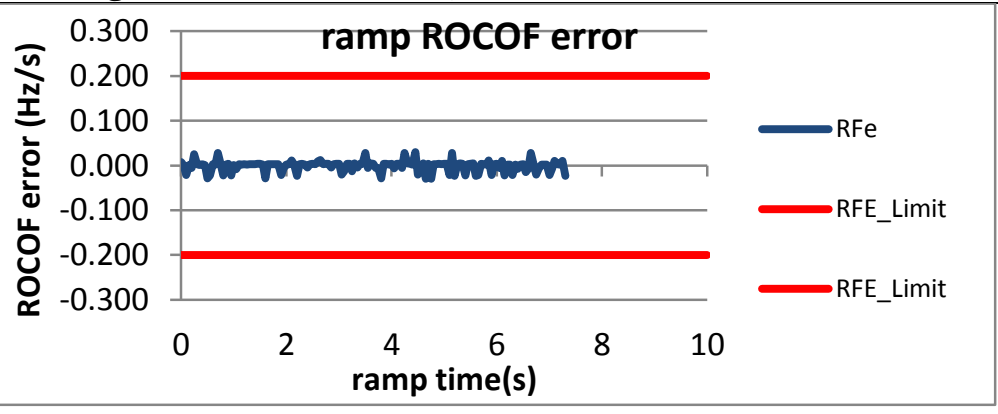

Figure 2589: Fs = 20 FPS, ramp from $64 \mathrm{~Hz}$ to $56 \mathrm{~Hz}$ at $=1 \mathrm{~Hz} / \mathrm{s}$ 


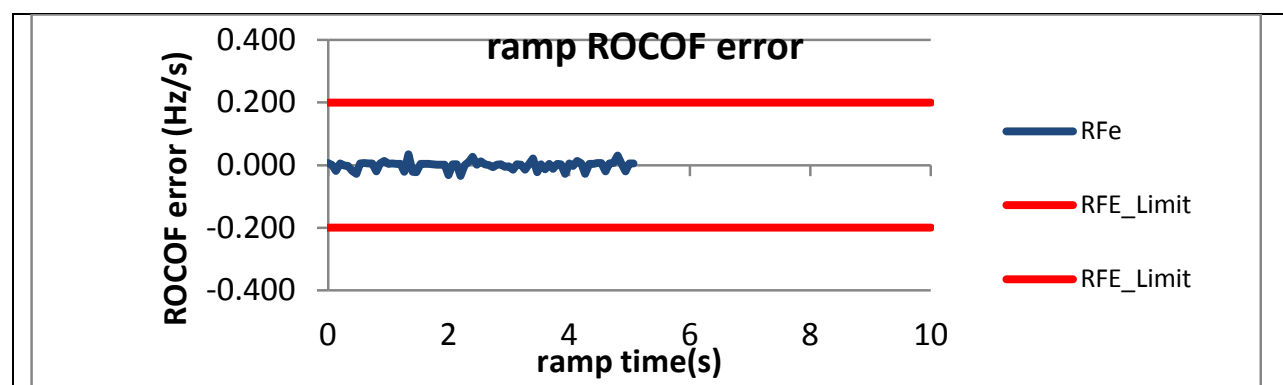

Figure 2590: Fs = $15 \mathrm{FPS}$, ramp from $57 \mathrm{~Hz}$ to $63 \mathrm{~Hz}$ at $+1 \mathrm{~Hz} / \mathrm{s}$

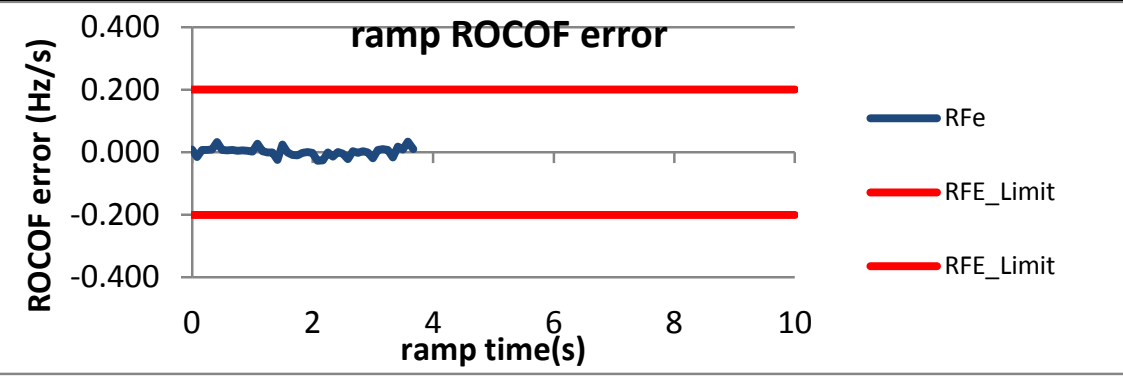

Figure 2592: Fs = 12 FPS, ramp from $58.6 \mathrm{~Hz}$ to $62.4 \mathrm{~Hz}$ at $+1 \mathrm{~Hz} / \mathrm{s}$

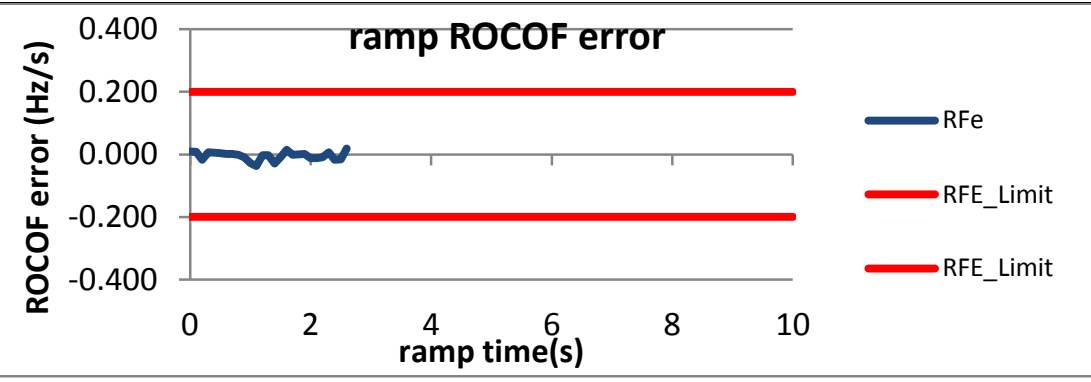

Figure 2594: Fs = $10 \mathrm{FPS}$, ramp from $58 \mathrm{~Hz}$ to $62 \mathrm{~Hz}$ at $+1 \mathrm{~Hz} / \mathrm{s}$

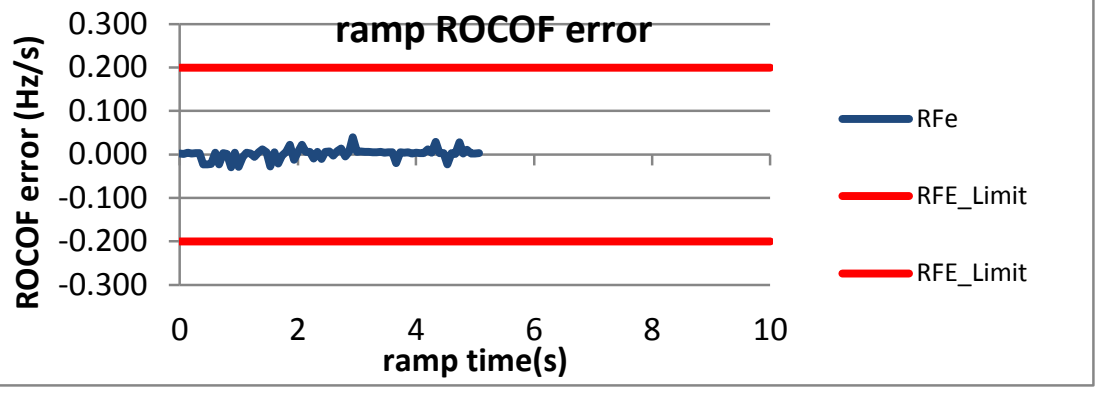

Figure 2591: Fs = $15 \mathrm{FPS}$, ramp from $63 \mathrm{~Hz}$ to $57 \mathrm{~Hz}$ at $-1 \mathrm{~Hz} / \mathrm{s}$

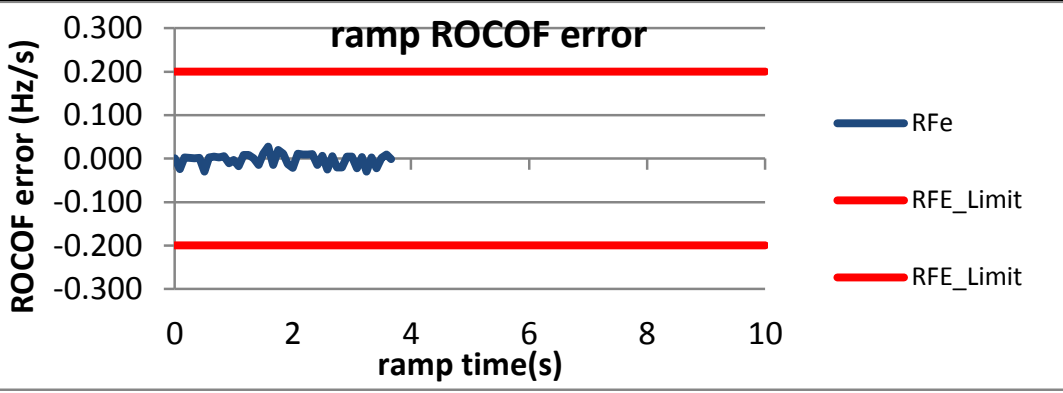

Figure 2593: Fs = $12 \mathrm{FPS}$, ramp from $62.4 \mathrm{~Hz}$ to $58.6 \mathrm{~Hz}$ at $-1 \mathrm{~Hz} / \mathrm{s}$

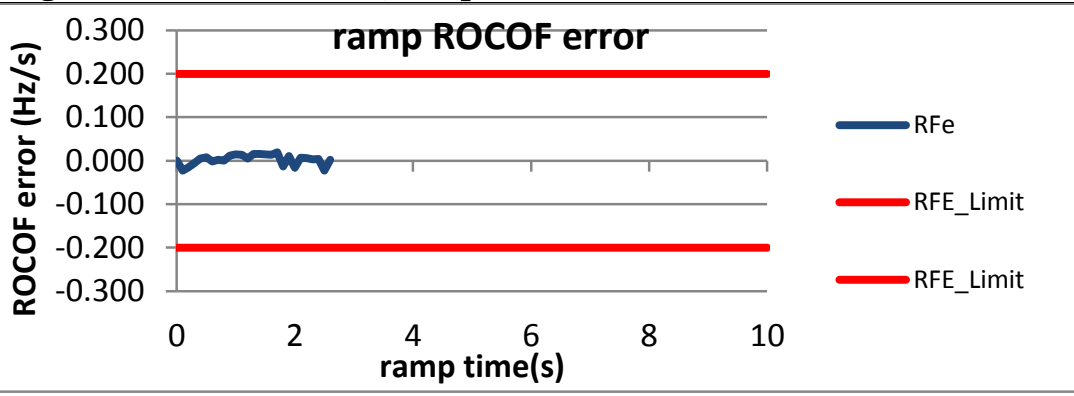

Figure 2595: Fs = $10 \mathrm{FPS}$, ramp from $62 \mathrm{~Hz}$ to $58 \mathrm{~Hz}$ at $-1 \mathrm{~Hz} / \mathrm{s}$ 
6.4.6 PMU E dynamic ramp of system frequency ROCOF error: M class
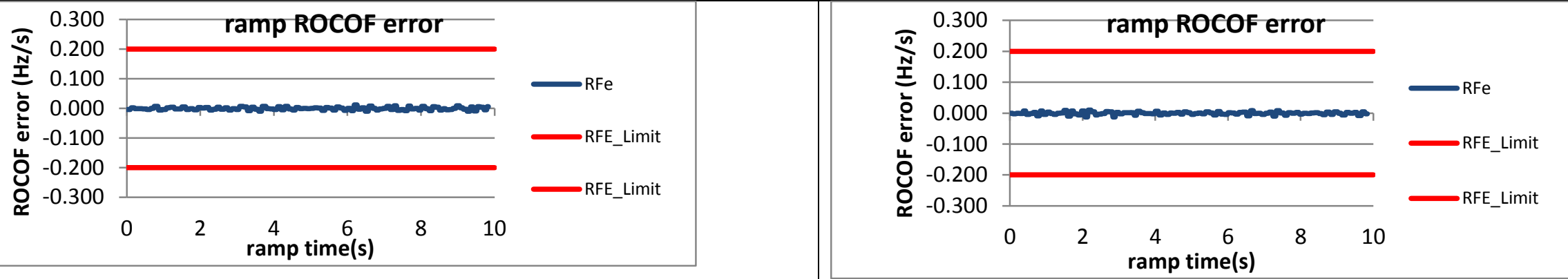

Figure 2596: Fs = $60 \mathrm{FPS}$, ram from $55 \mathrm{~Hz}$ to $65 \mathrm{~Hz}$ at $+1 \mathrm{~Hz} / \mathrm{s}$

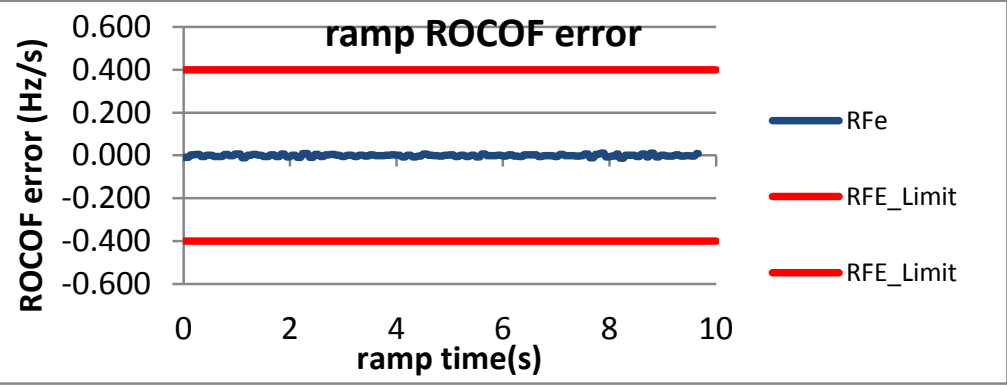

Figure 2597: Fs = $60 \mathrm{FPS}$, ram from $65 \mathrm{~Hz}$ to $55 \mathrm{~Hz}$ at $-1 \mathrm{~Hz} / \mathrm{s}$

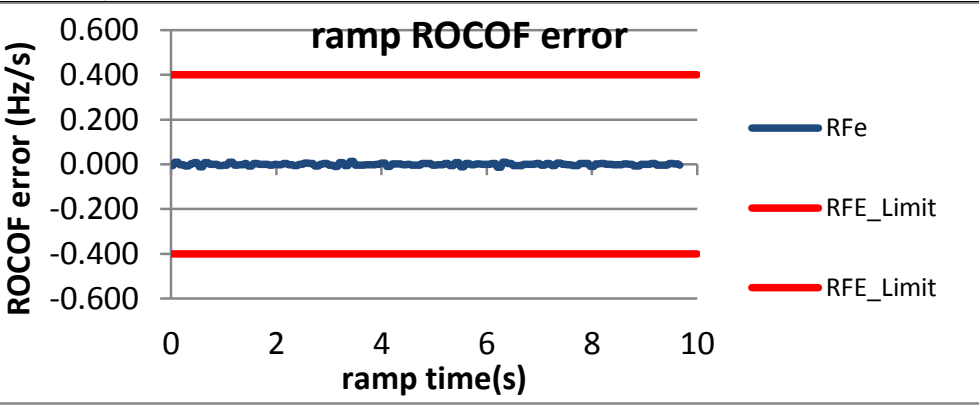

Figure 2598: Fs = $30 \mathrm{FPS}$, ram from $55 \mathrm{~Hz}$ to $65 \mathrm{~Hz}$ at $+1 \mathrm{~Hz} / \mathrm{s}$

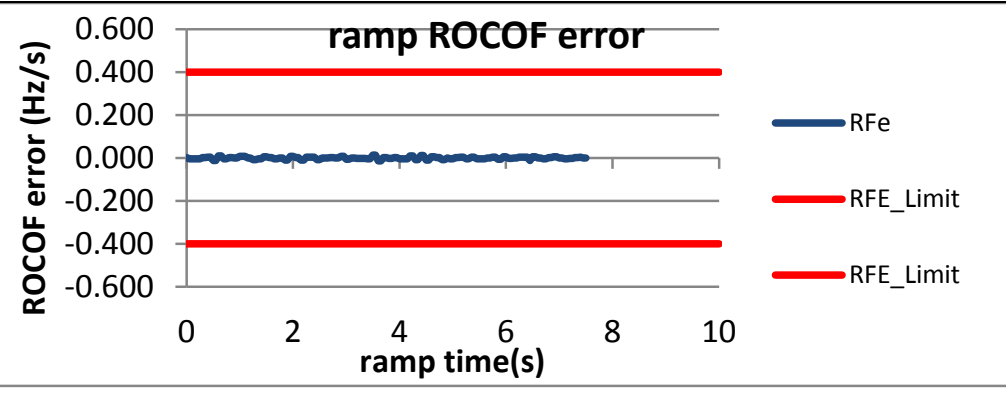

Figure 2599: Fs = $30 \mathrm{FPS}$, ram from $65 \mathrm{~Hz}$ to $55 \mathrm{~Hz}$ at $-1 \mathrm{~Hz} / \mathrm{s}$

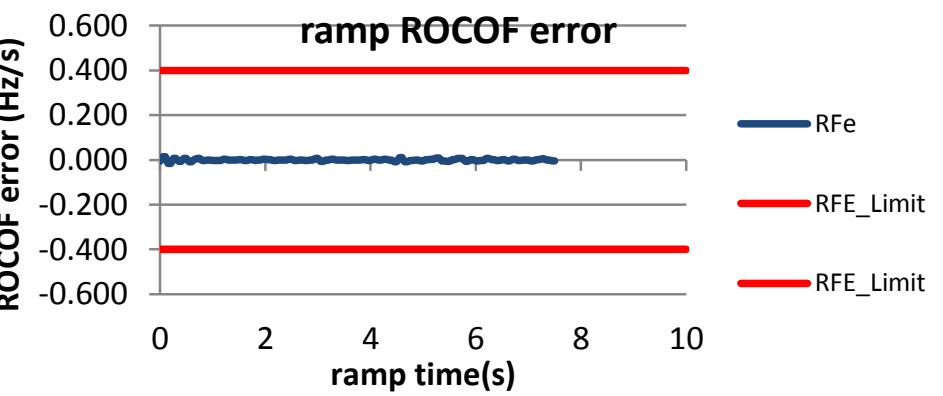

Figure 2600: Fs = $20 \mathrm{FPS}$, ramp from $56 \mathrm{~Hz}$ to $64 \mathrm{~Hz}$ at $+1 \mathrm{~Hz} / \mathrm{s}$

Figure 2601: Fs = $20 \mathrm{FPS}$, ramp from $64 \mathrm{~Hz}$ to $56 \mathrm{~Hz}$ at $=1 \mathrm{~Hz} / \mathrm{s}$ 

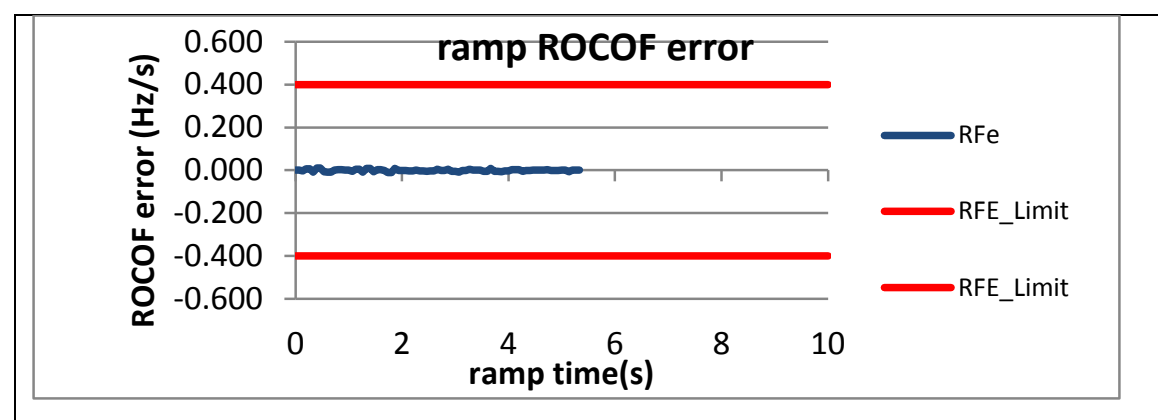

Figure 2602: Fs = $15 \mathrm{FPS}$, ramp from $57 \mathrm{~Hz}$ to $63 \mathrm{~Hz}$ at $+1 \mathrm{~Hz} / \mathrm{s}$

Figure 2604: Fs = 12 FPS, ramp from $58.6 \mathrm{~Hz}$ to $62.4 \mathrm{~Hz}$ at $+1 \mathrm{~Hz} / \mathrm{s}$

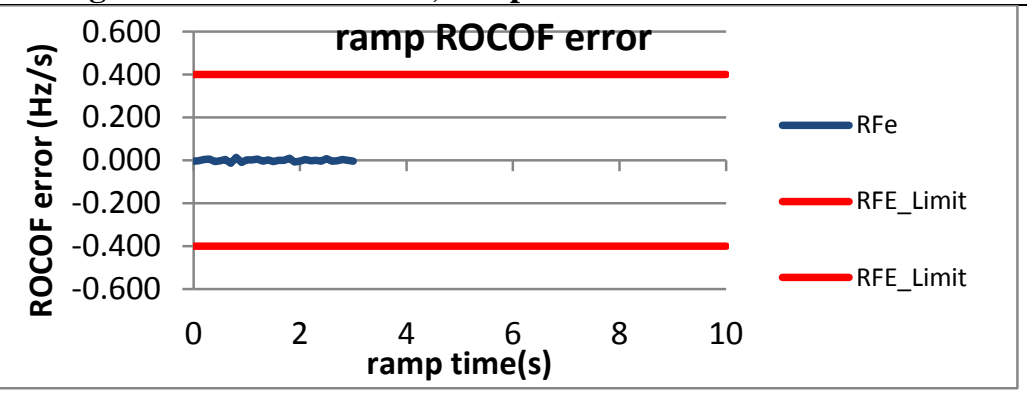

Figure 2606: Fs = $10 \mathrm{FPS}$, ramp from $58 \mathrm{~Hz}$ to $62 \mathrm{~Hz}$ at $+1 \mathrm{~Hz} / \mathrm{s}$

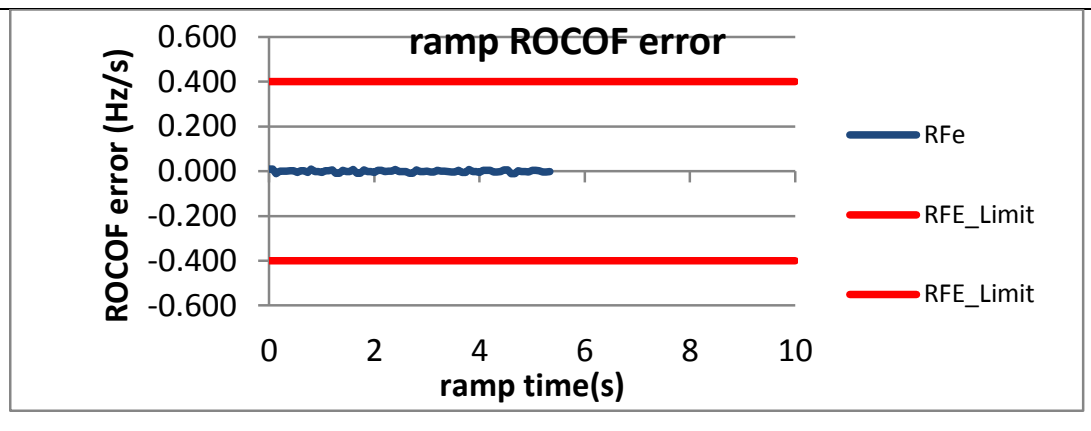

Figure 2603: Fs = $15 \mathrm{FPS}$, ramp from $63 \mathrm{~Hz}$ to $57 \mathrm{~Hz}$ at $-1 \mathrm{~Hz} / \mathrm{s}$

Figure 2605: Fs = 12 FPS, ramp from $62.4 \mathrm{~Hz}$ to $58.6 \mathrm{~Hz}$ at $-1 \mathrm{~Hz} / \mathrm{s}$

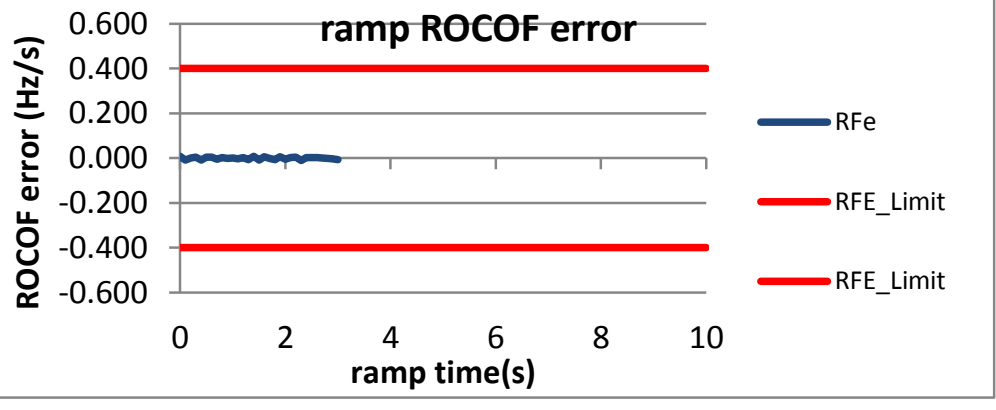

Figure 2607: Fs = $10 \mathrm{FPS}$, ramp from $62 \mathrm{~Hz}$ to $58 \mathrm{~Hz}$ at $-1 \mathrm{~Hz} / \mathrm{s}$ 
6.4.7 PMU F dynamic ramp of system frequency ROCOF error: M class

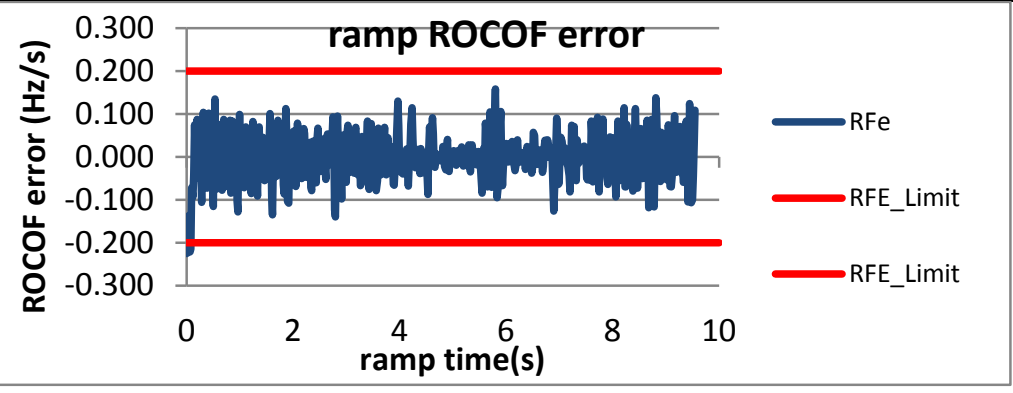

Figure 2608: Fs = $60 \mathrm{FPS}$, ram from $55 \mathrm{~Hz}$ to $65 \mathrm{~Hz}$ at $+1 \mathrm{~Hz} / \mathrm{s}$

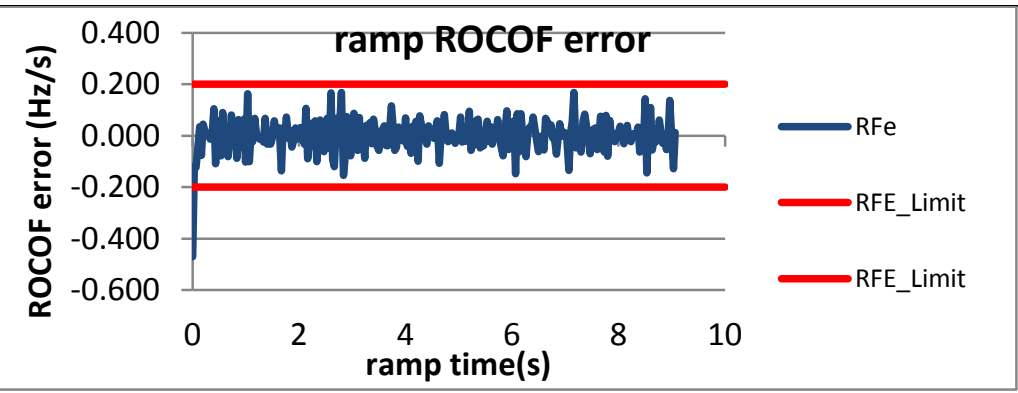

Figure 2610: Fs = $30 \mathrm{FPS}$, ram from $55 \mathrm{~Hz}$ to $65 \mathrm{~Hz}$ at $+1 \mathrm{~Hz} / \mathrm{s}$

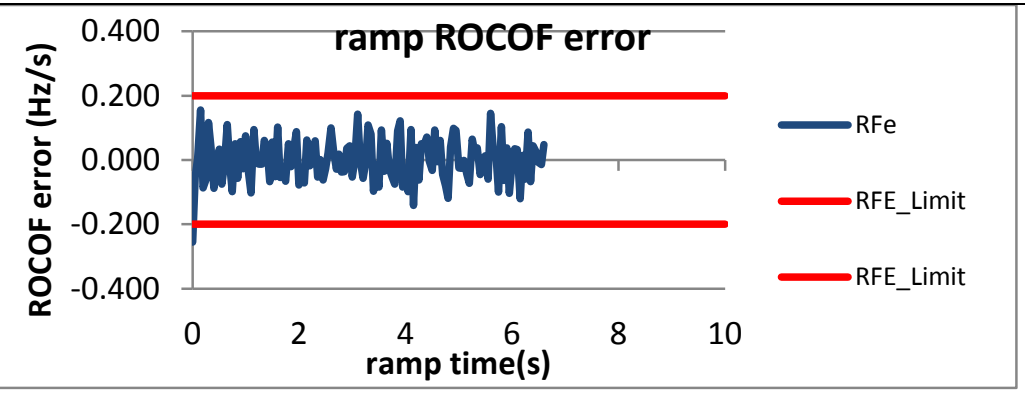

Figure 2612: Fs = $20 \mathrm{FPS}$, ramp from $56 \mathrm{~Hz}$ to $64 \mathrm{~Hz}$ at $+1 \mathrm{~Hz} / \mathrm{s}$

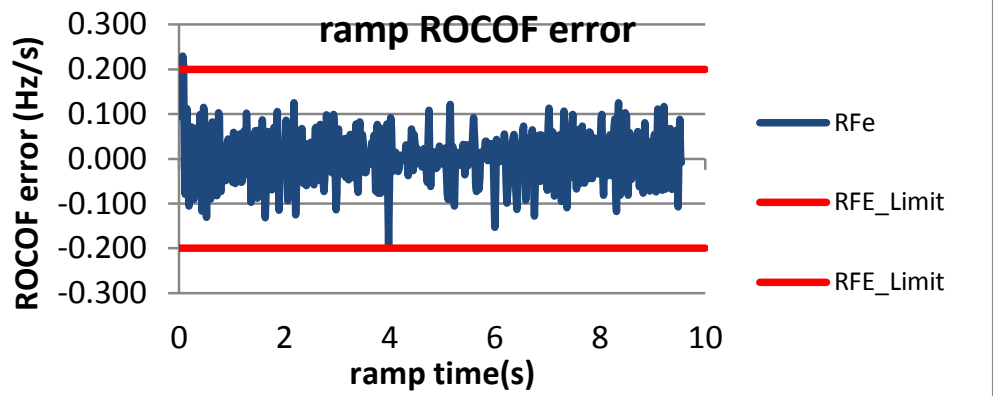

Figure 2609: Fs = $60 \mathrm{FPS}$, ram from $65 \mathrm{~Hz}$ to $55 \mathrm{~Hz}$ at $-1 \mathrm{~Hz} / \mathrm{s}$

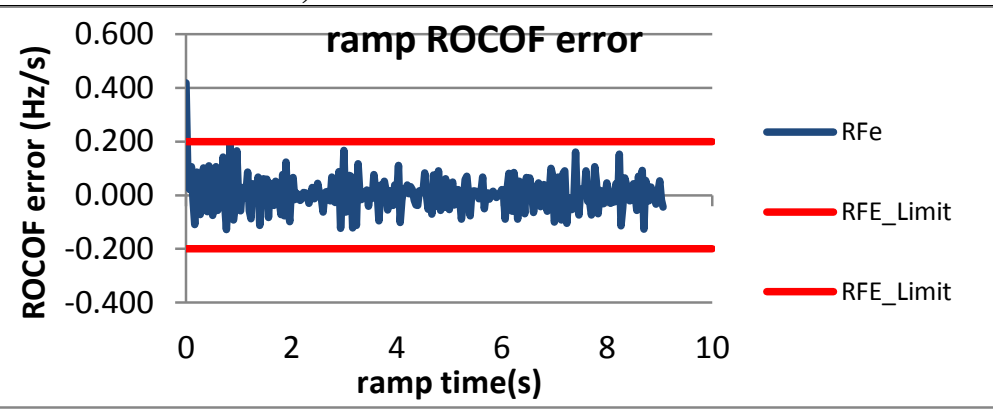

Figure 2611: Fs = $30 \mathrm{FPS}$, ram from $65 \mathrm{~Hz}$ to $55 \mathrm{~Hz}$ at $-1 \mathrm{~Hz} / \mathrm{s}$

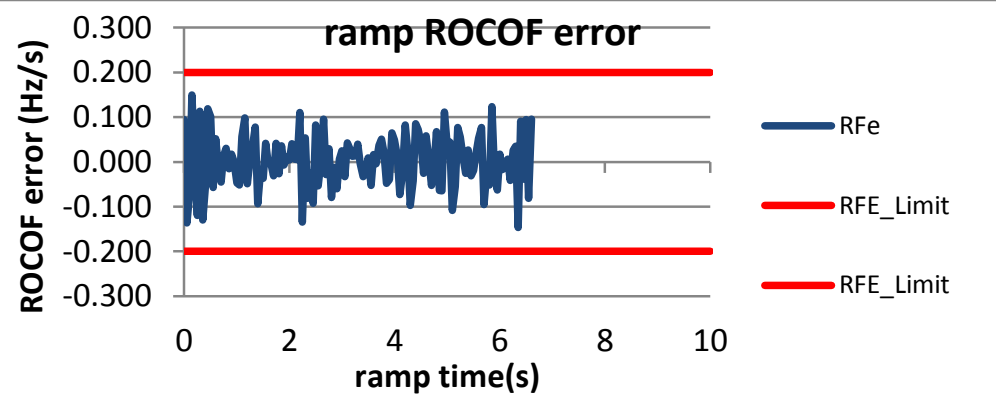

Figure 2613: Fs = 20 FPS, ramp from $64 \mathrm{~Hz}$ to $56 \mathrm{~Hz}$ at $=1 \mathrm{~Hz} / \mathrm{s}$ 


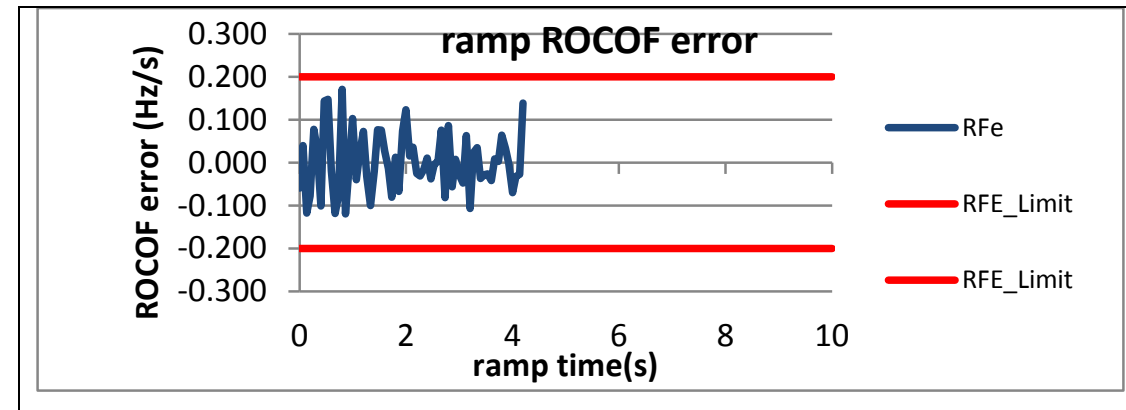

Figure 2614: Fs = $15 \mathrm{FPS}$, ramp from $57 \mathrm{~Hz}$ to $63 \mathrm{~Hz}$ at $+1 \mathrm{~Hz} / \mathrm{s}$

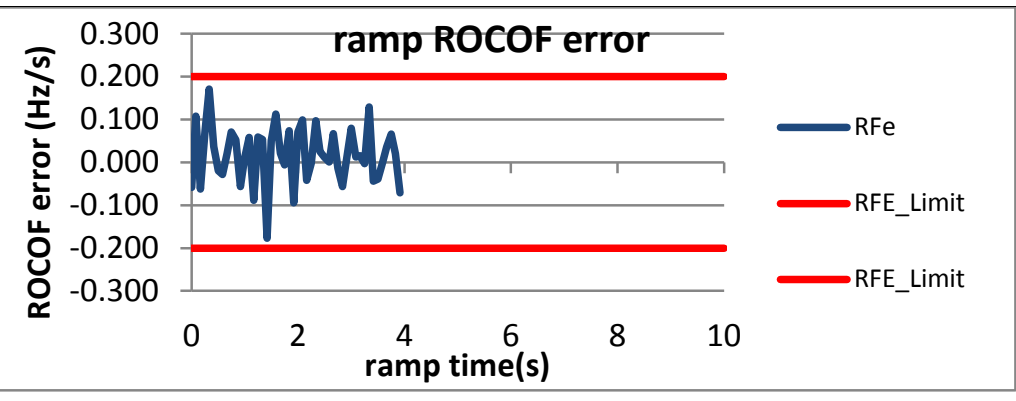

Figure 2616: Fs = 12 FPS, ramp from $58.6 \mathrm{~Hz}$ to $62.4 \mathrm{~Hz}$ at $+1 \mathrm{~Hz} / \mathrm{s}$

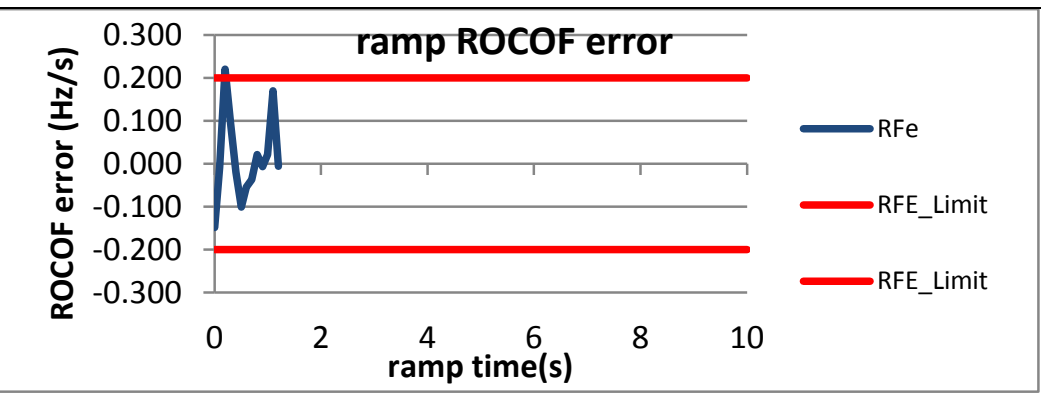

Figure 2618: Fs = $10 \mathrm{FPS}$, ramp from $58 \mathrm{~Hz}$ to $62 \mathrm{~Hz}$ at $+1 \mathrm{~Hz} / \mathrm{s}$

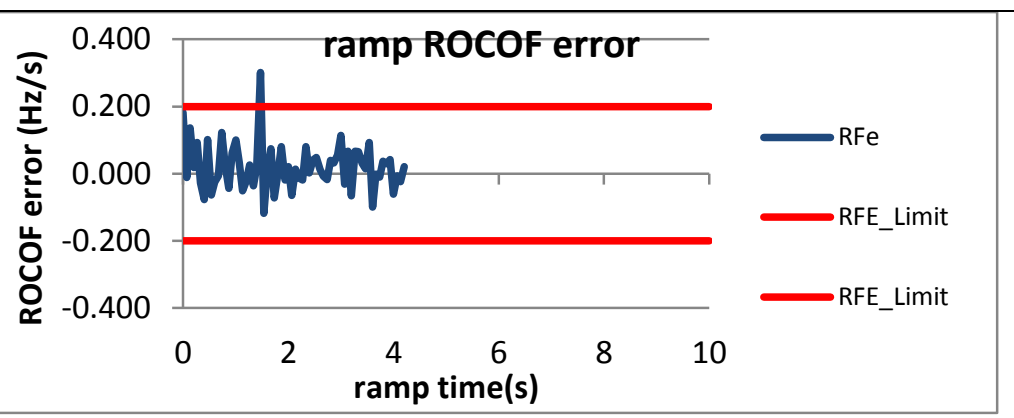

Figure 2615: Fs = $15 \mathrm{FPS}$, ramp from $63 \mathrm{~Hz}$ to $57 \mathrm{~Hz}$ at $-1 \mathrm{~Hz} / \mathrm{s}$

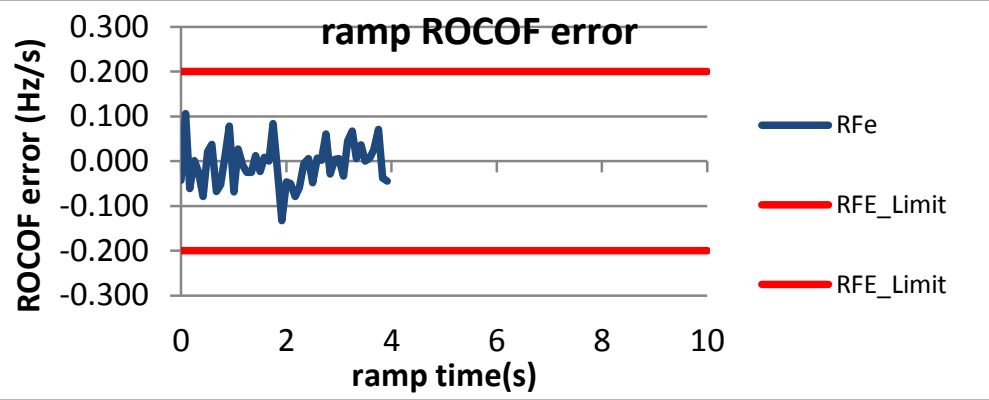

Figure 2617: Fs = 12 FPS, ramp from $62.4 \mathrm{~Hz}$ to $58.6 \mathrm{~Hz}$ at $-1 \mathrm{~Hz} / \mathrm{s}$

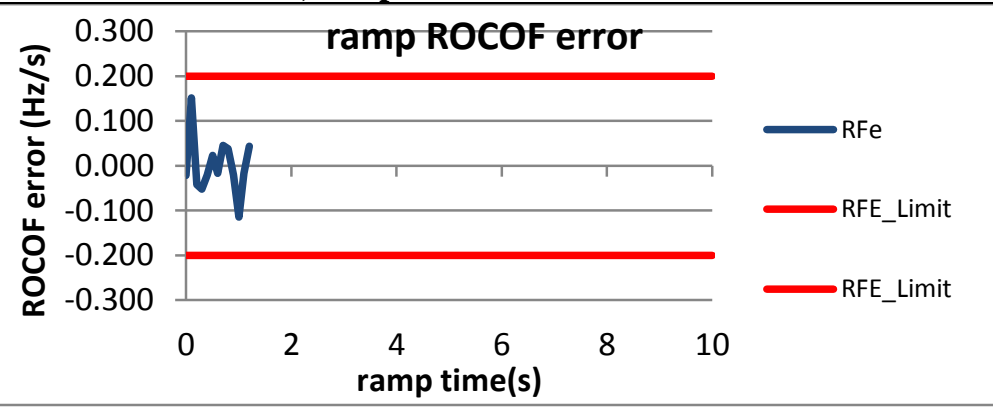

Figure 2619: Fs = $10 \mathrm{FPS}$, ramp from $62 \mathrm{~Hz}$ to $58 \mathrm{~Hz}$ at $-1 \mathrm{~Hz} / \mathrm{s}$ 


\subsubsection{PMU G* dynamic ramp of system frequency ROCOF error: M class}

Figure 2620: Fs = 60 FPS is not supported by this PMU
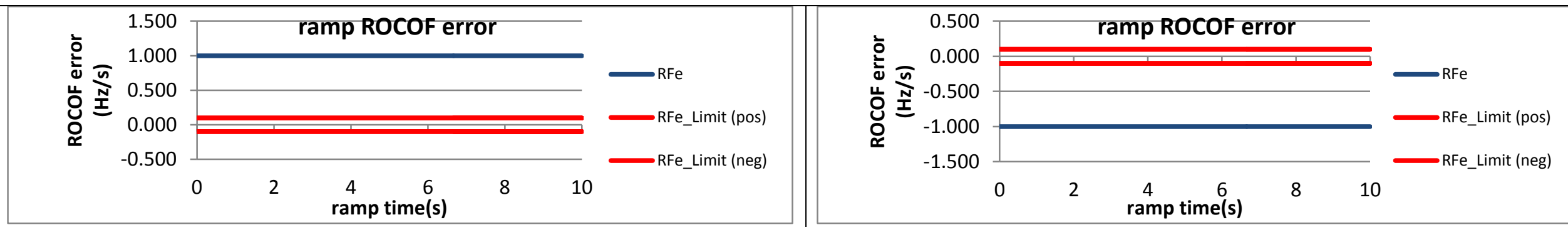

Figure 2621: Fs = $30 \mathrm{FPS}$, ram from $55 \mathrm{~Hz}$ to $65 \mathrm{~Hz}$ at $+1 \mathrm{~Hz} / \mathrm{s}$

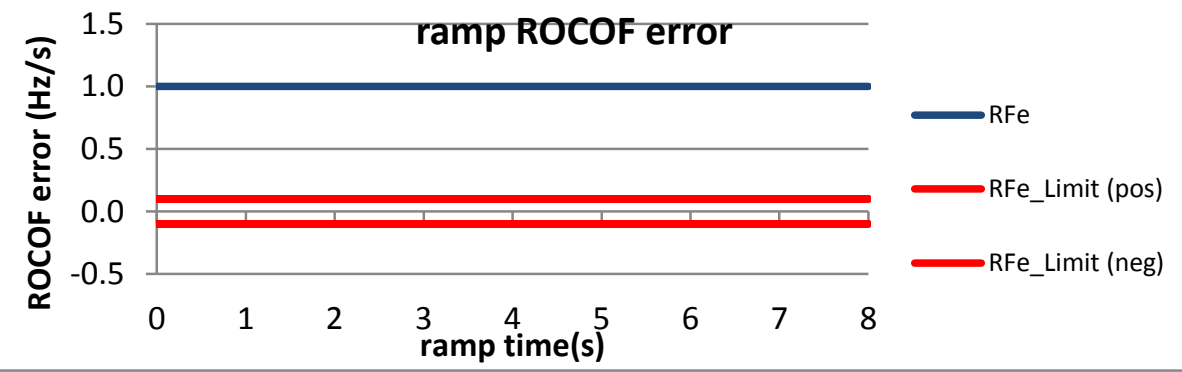

Figure 2622: Fs = $30 \mathrm{FPS}$, ram from $65 \mathrm{~Hz}$ to $55 \mathrm{~Hz}$ at $-1 \mathrm{~Hz} / \mathrm{s}$

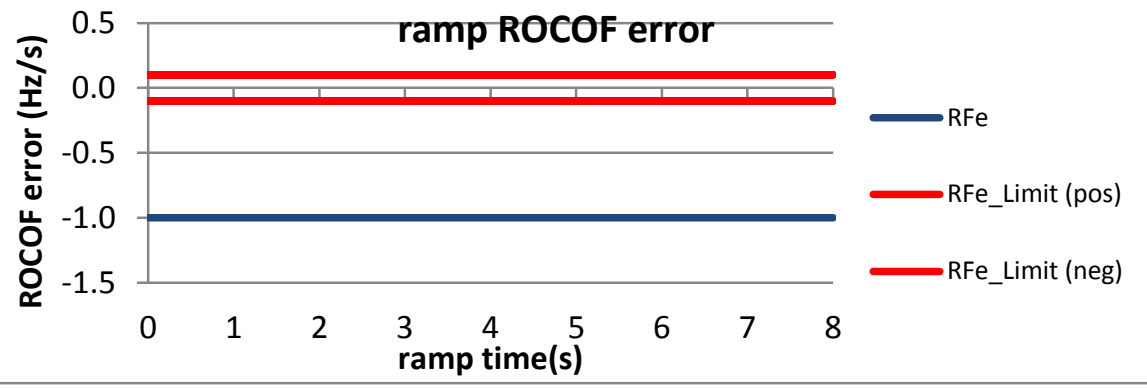

Figure 2623: Fs = $20 \mathrm{FPS}$, ramp from $56 \mathrm{~Hz}$ to $64 \mathrm{~Hz}$ at $+1 \mathrm{~Hz} / \mathrm{s}$

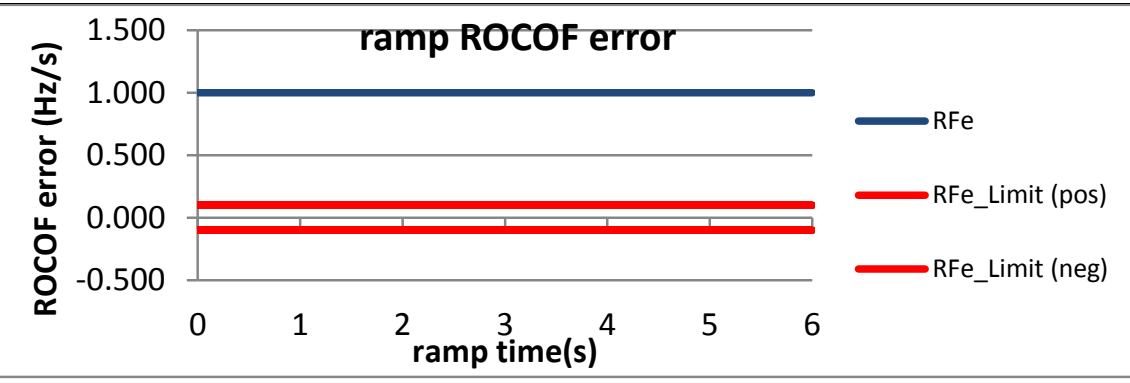

Figure 2624: Fs = $20 \mathrm{FPS}$, ramp from $64 \mathrm{~Hz}$ to $56 \mathrm{~Hz}$ at =1 Hz/s

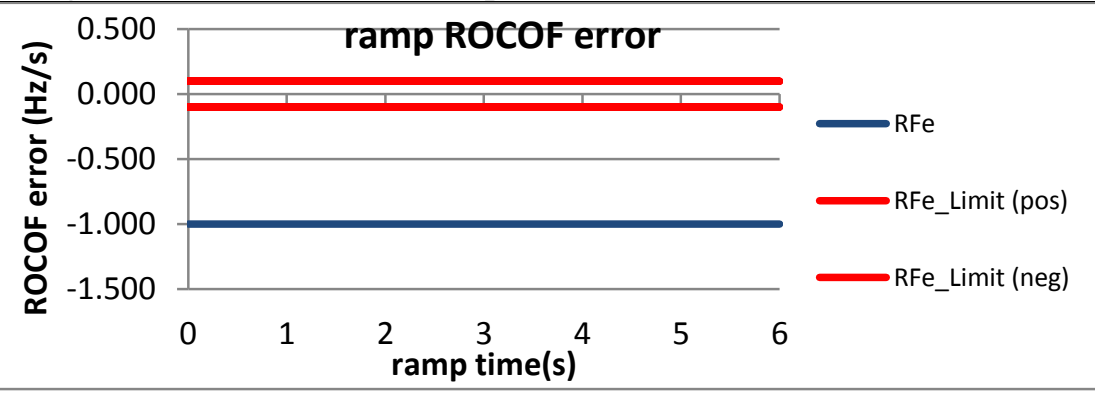

Figure 2625: Fs = 15 FPS, ramp from $57 \mathrm{~Hz}$ to $63 \mathrm{~Hz}$ at $+1 \mathrm{~Hz} / \mathrm{s}$

Figure 2626: Fs = $15 \mathrm{FPS}$, ramp from $63 \mathrm{~Hz}$ to $57 \mathrm{~Hz}$ at $-1 \mathrm{~Hz} / \mathrm{s}$ 


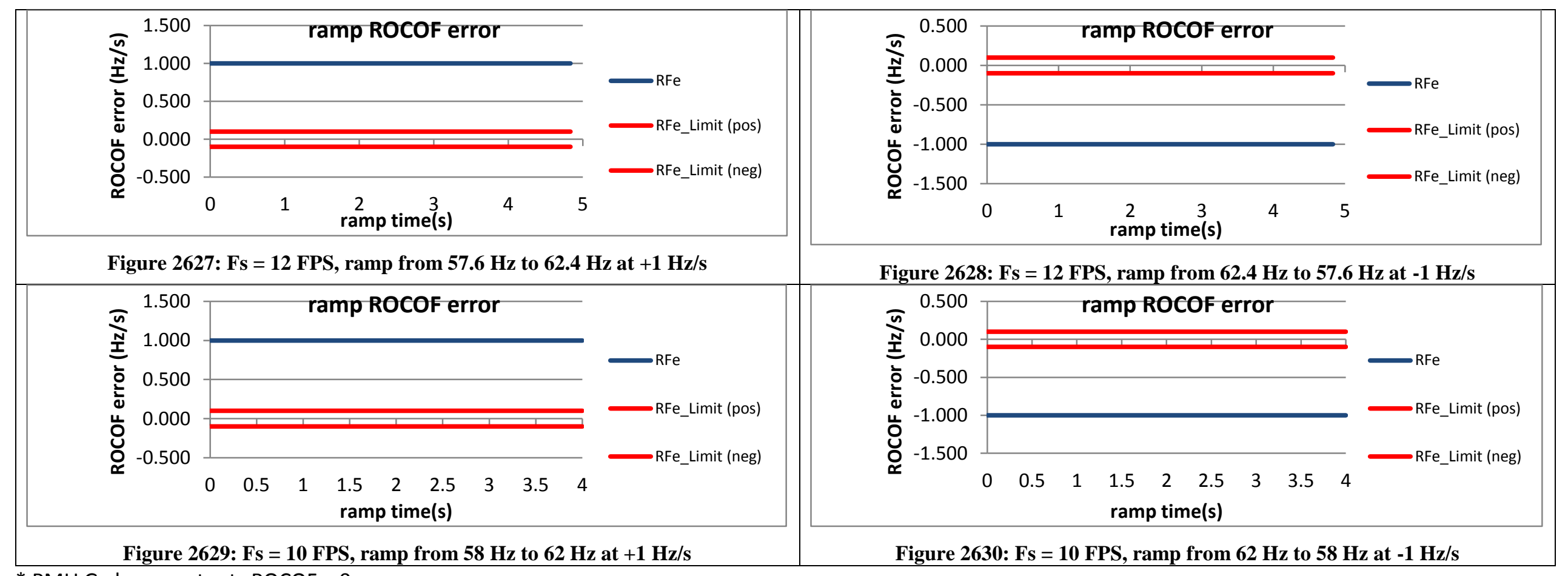

* PMU G always outputs ROCOF $=0$. 
6.4.9 PMU H dynamic ramp of system frequency ROCOF error: M class

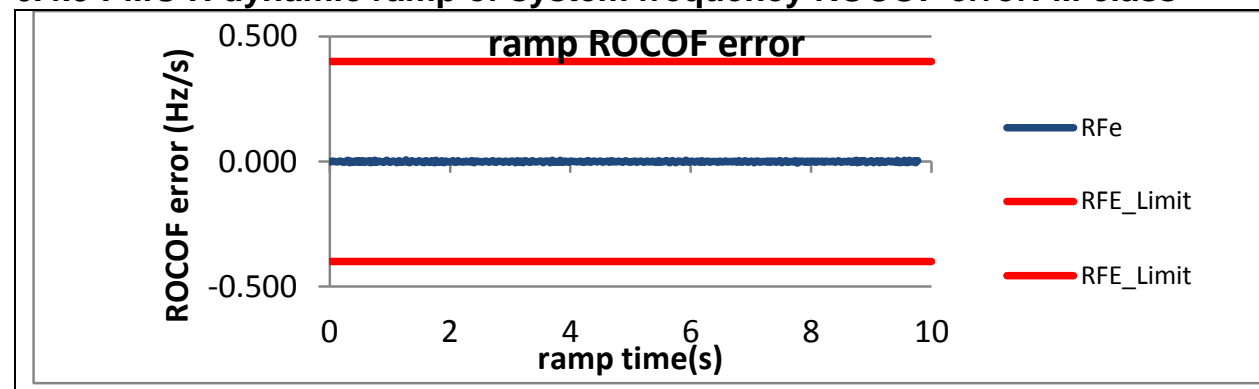

Figure 2631: Fs = $60 \mathrm{FPS}$, ramp from $55 \mathrm{~Hz}$ to $65 \mathrm{~Hz}$ at $+1 \mathrm{~Hz} / \mathrm{s}$

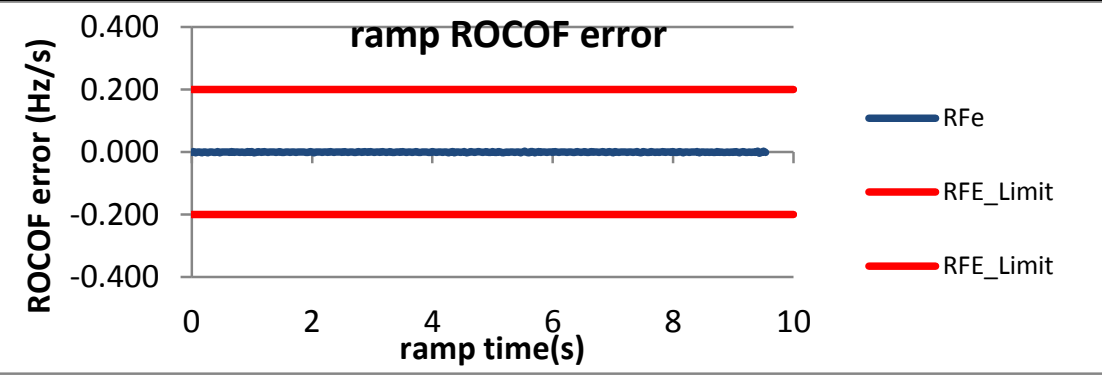

Figure 2633: Fs = $30 \mathrm{FPS}$, ramp from $55 \mathrm{~Hz}$ to $65 \mathrm{~Hz}$ at $+1 \mathrm{~Hz} / \mathrm{s}$

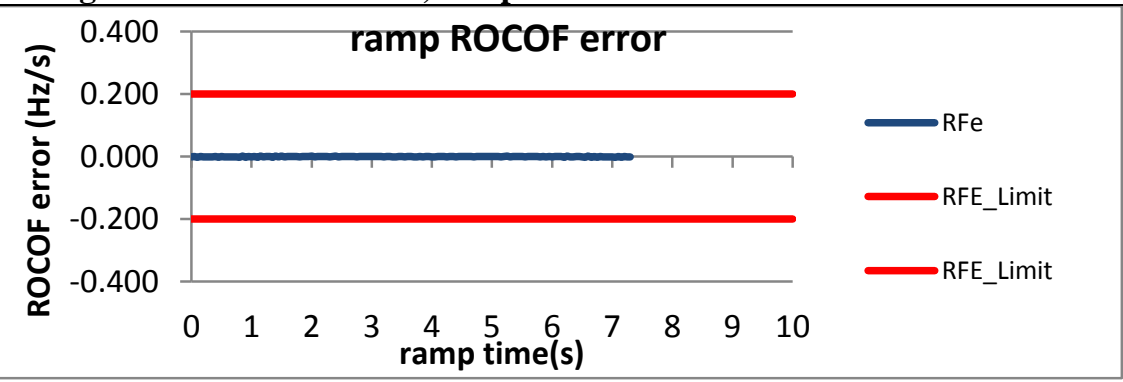

Figure 2635: Fs = $20 \mathrm{FPS}$, ramp from $56 \mathrm{~Hz}$ to $64 \mathrm{~Hz}$ at $+1 \mathrm{~Hz} / \mathrm{s}$

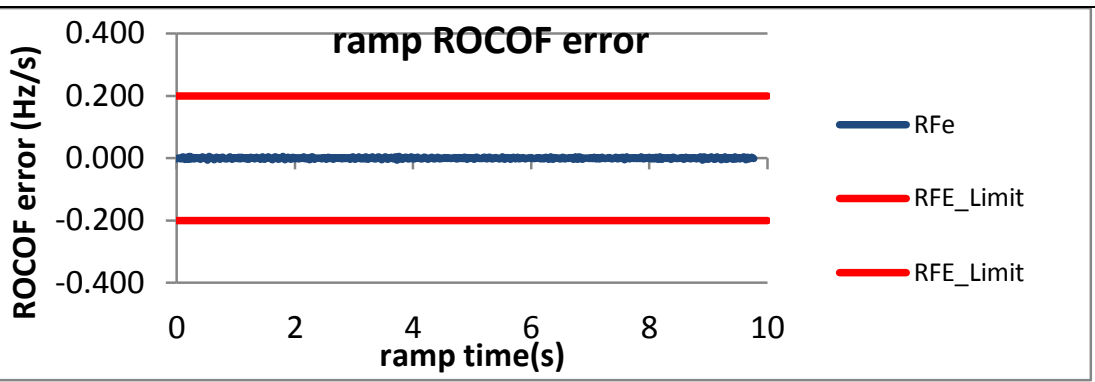

Figure 2632: Fs = $60 \mathrm{FPS}$, ramp from $65 \mathrm{~Hz}$ to $55 \mathrm{~Hz}$ at $-1 \mathrm{~Hz} / \mathrm{s}$

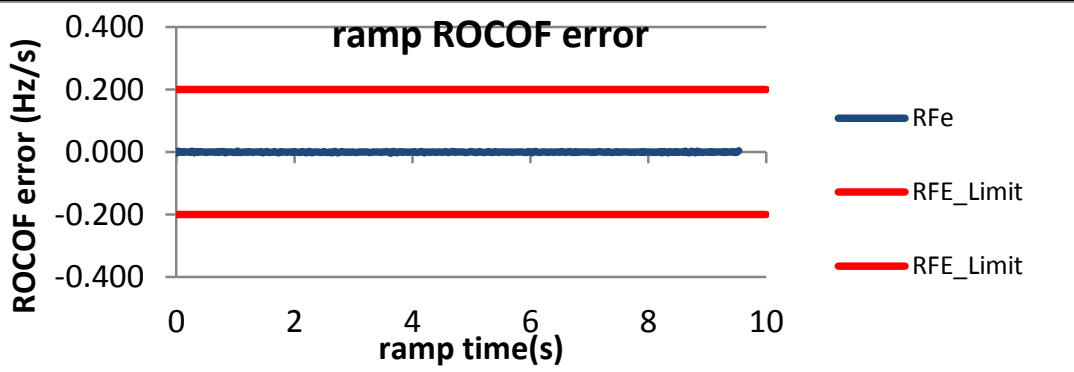

Figure 2634: Fs = $30 \mathrm{FPS}$, ramp from $65 \mathrm{~Hz}$ to $55 \mathrm{~Hz}$ at $-1 \mathrm{~Hz} / \mathrm{s}$

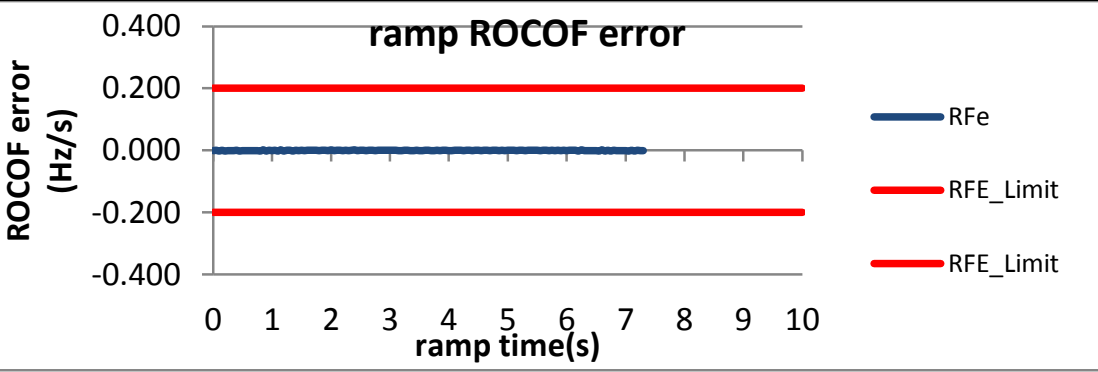

Figure 2636: Fs = $20 \mathrm{FPS}$, ramp from $64 \mathrm{~Hz}$ to $56 \mathrm{~Hz}$ at $=1 \mathrm{~Hz} / \mathrm{s}$ 


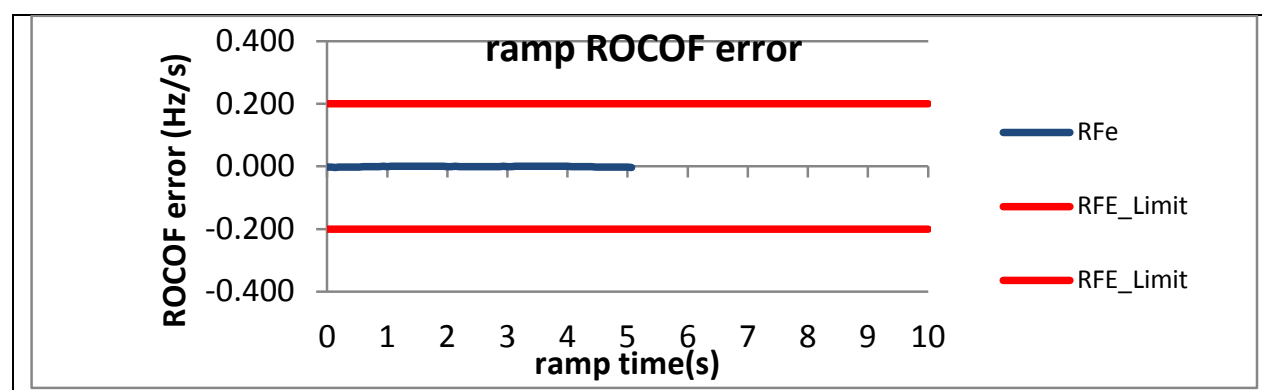

Figure 2637: Fs = $15 \mathrm{FPS}$, ramp from $57 \mathrm{~Hz}$ to $63 \mathrm{~Hz}$ at $+1 \mathrm{~Hz} / \mathrm{s}$

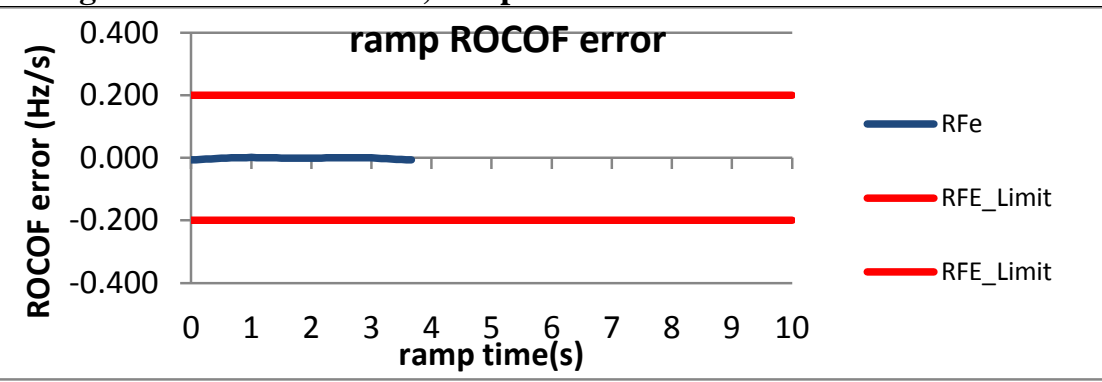

Figure 2639: Fs = 12 FPS, ramp from $58.6 \mathrm{~Hz}$ to $62.4 \mathrm{~Hz}$ at $+1 \mathrm{~Hz} / \mathrm{s}$

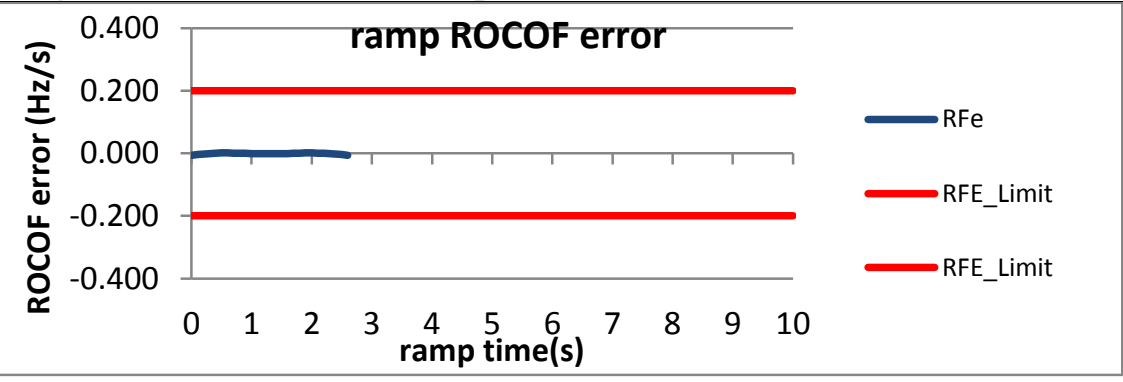

Figure 2641: Fs = $10 \mathrm{FPS}$, ramp from $58 \mathrm{~Hz}$ to $62 \mathrm{~Hz}$ at $+1 \mathrm{~Hz} / \mathrm{s}$

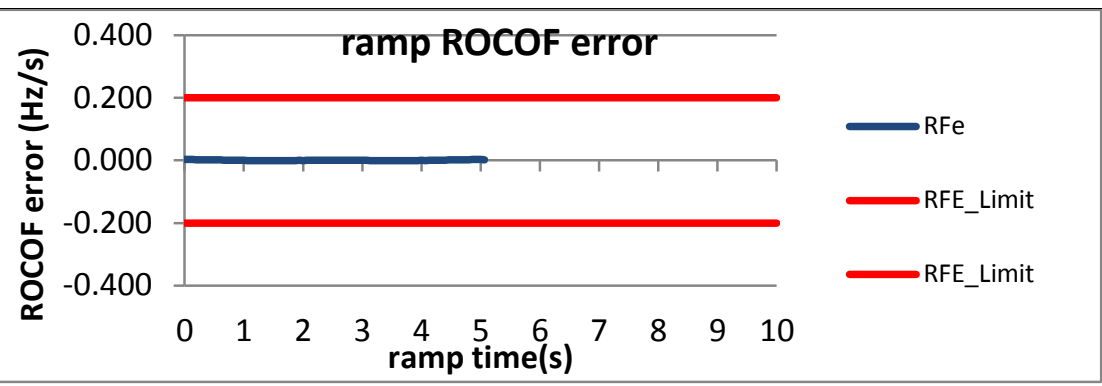

Figure 2638: Fs = $15 \mathrm{FPS}$, ramp from $63 \mathrm{~Hz}$ to $57 \mathrm{~Hz}$ at $-1 \mathrm{~Hz} / \mathrm{s}$

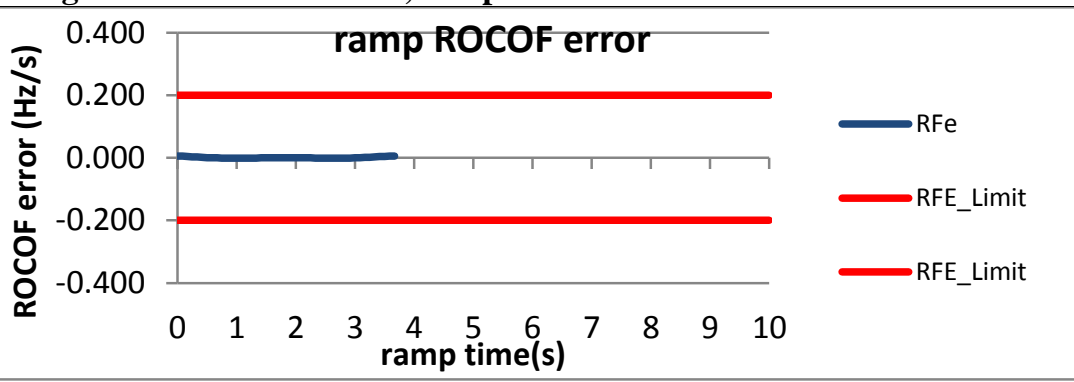

Figure 2640: Fs = 12 FPS, ramp from $62.4 \mathrm{~Hz}$ to $58.6 \mathrm{~Hz}$ at $-1 \mathrm{~Hz} / \mathrm{s}$

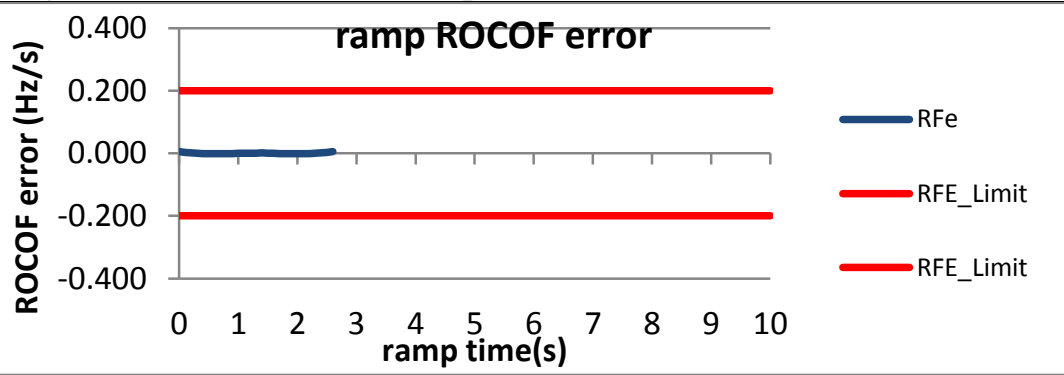

Figure 2642: Fs = $10 \mathrm{FPS}$, ramp from $62 \mathrm{~Hz}$ to $58 \mathrm{~Hz}$ at $-1 \mathrm{~Hz} / \mathrm{s}$ 
6.4.10 PMU I dynamic ramp of system frequency ROCOF error: M class

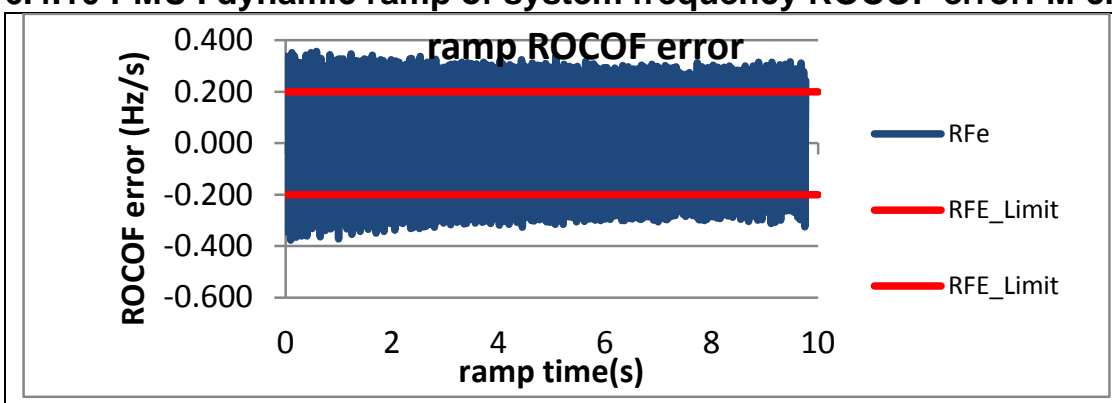

Figure 2643: Fs = $60 \mathrm{FPS}$, ram from $55 \mathrm{~Hz}$ to $65 \mathrm{~Hz}$ at $+1 \mathrm{~Hz} / \mathrm{s}$

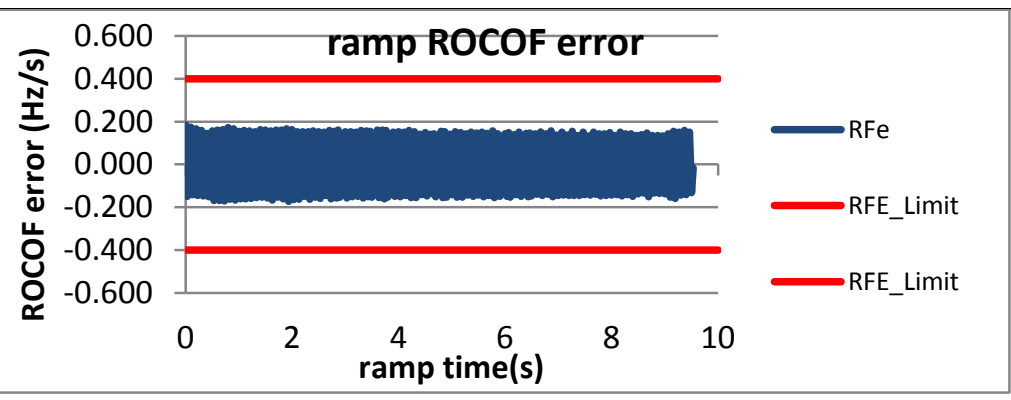

Figure 2645: Fs = $30 \mathrm{FPS}$, ram from $55 \mathrm{~Hz}$ to $65 \mathrm{~Hz}$ at $+1 \mathrm{~Hz} / \mathrm{s}$

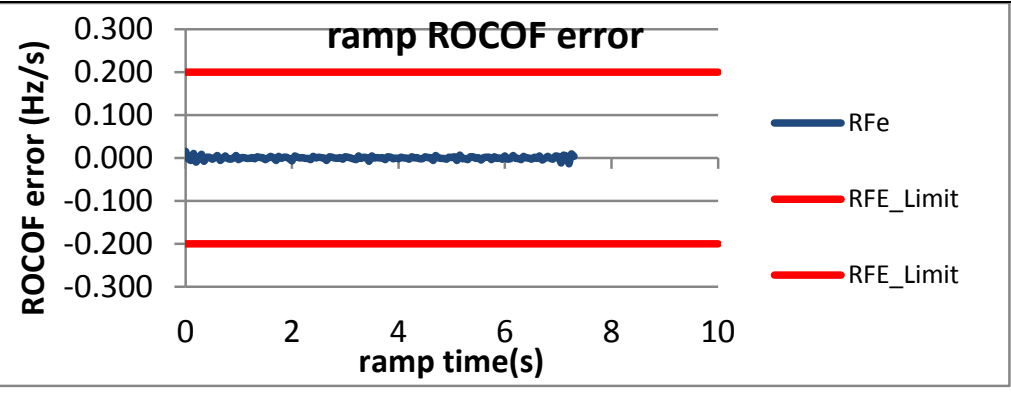

Figure 2647: Fs = $20 \mathrm{FPS}$, ramp from $56 \mathrm{~Hz}$ to $64 \mathrm{~Hz}$ at $+1 \mathrm{~Hz} / \mathrm{s}$

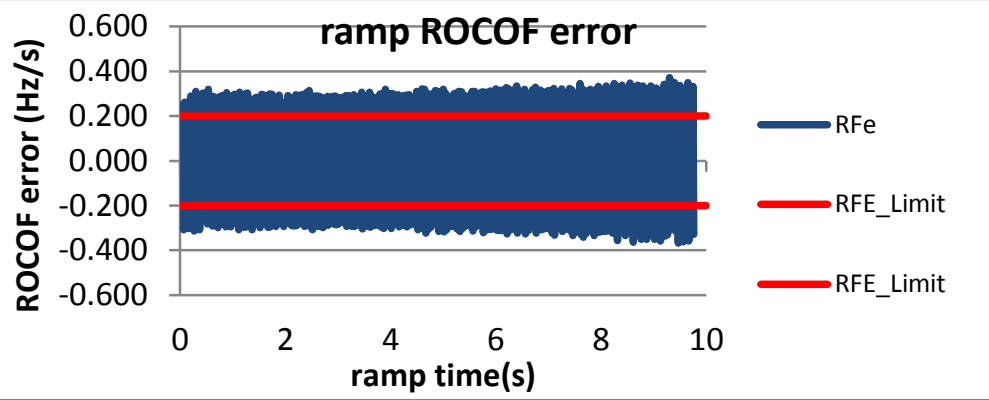

Figure 2644: Fs = $60 \mathrm{FPS}$, ram from $65 \mathrm{~Hz}$ to $55 \mathrm{~Hz}$ at $-1 \mathrm{~Hz} / \mathrm{s}$

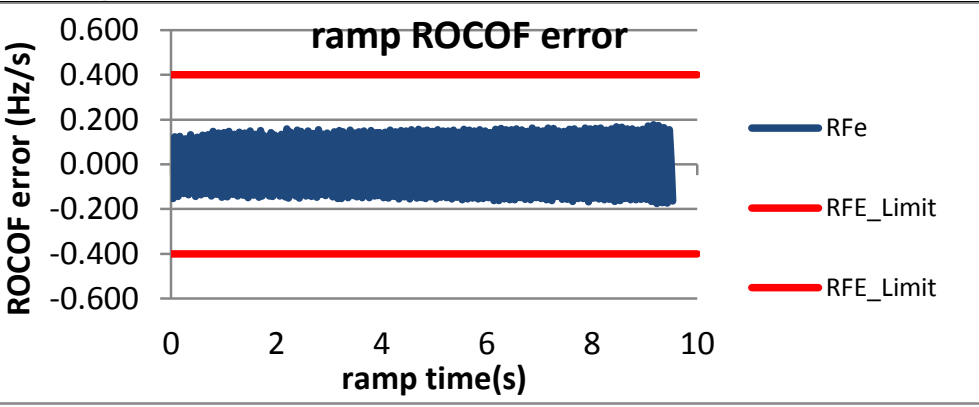

Figure 2646: Fs = $30 \mathrm{FPS}$, ram from $65 \mathrm{~Hz}$ to $55 \mathrm{~Hz}$ at $-1 \mathrm{~Hz} / \mathrm{s}$

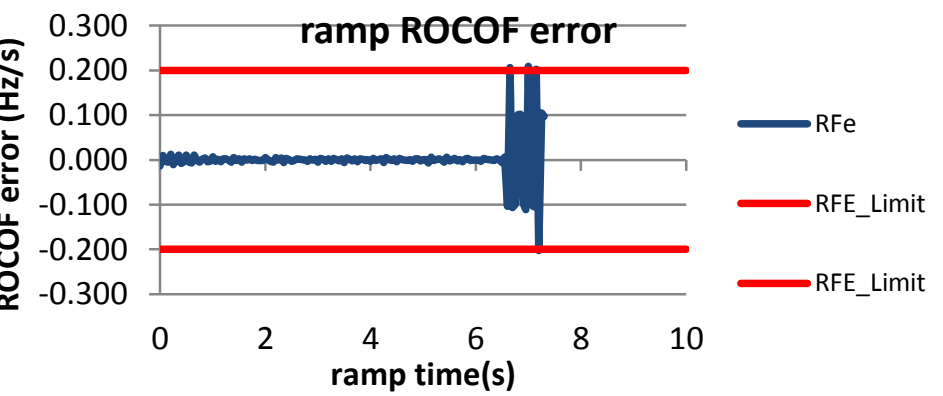

Figure 2648: Fs = $20 \mathrm{FPS}$, ramp from $64 \mathrm{~Hz}$ to $56 \mathrm{~Hz}$ at $=1 \mathrm{~Hz} / \mathrm{s}$ 


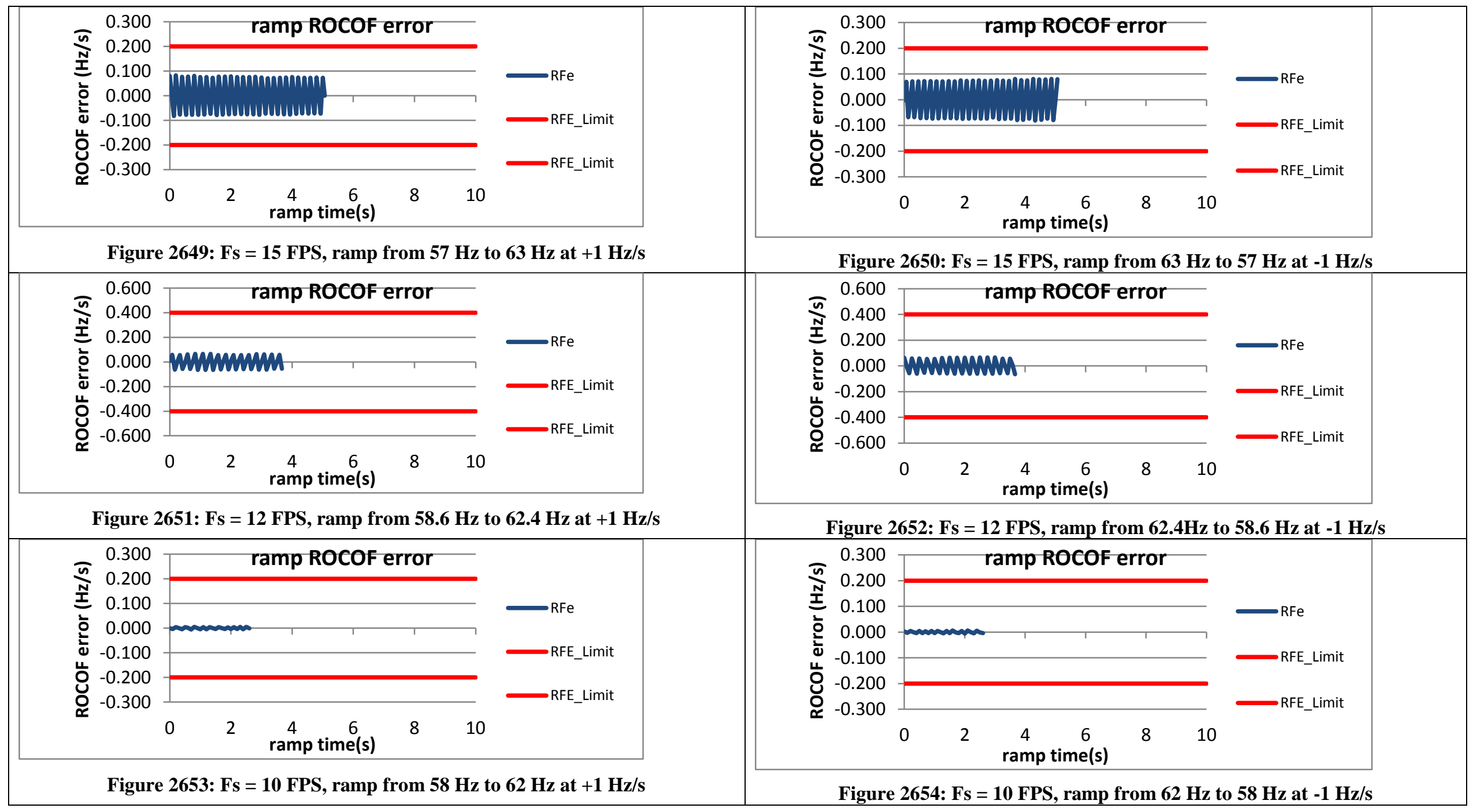


6.4.11 PMU J dynamic ramp of system frequency ROCOF error: M class
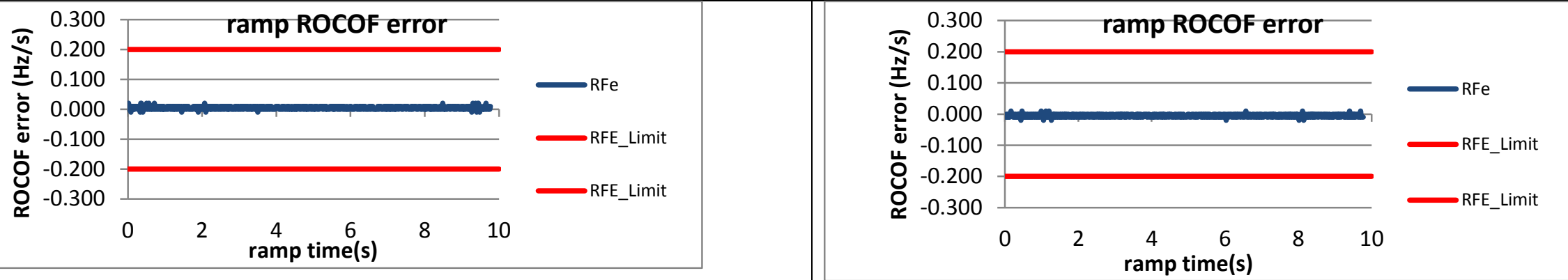

Figure 2655: Fs = $60 \mathrm{FPS}$, ram from $55 \mathrm{~Hz}$ to $65 \mathrm{~Hz}$ at $+1 \mathrm{~Hz} / \mathrm{s}$

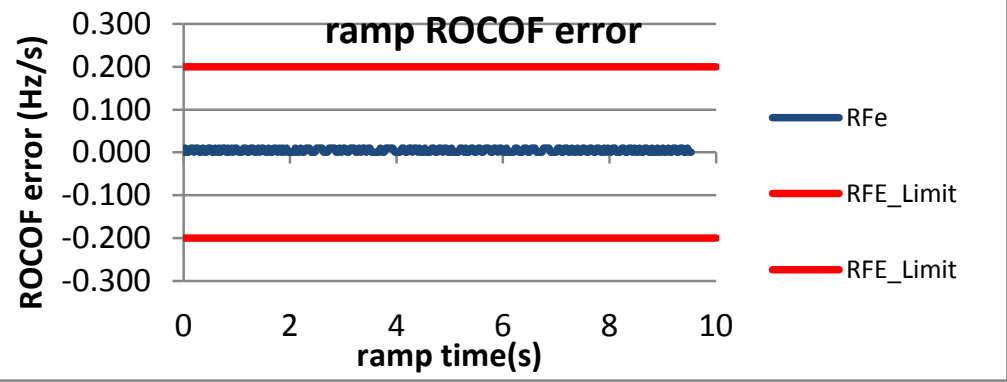

Figure 2657: Fs = $30 \mathrm{FPS}$, ram from $55 \mathrm{~Hz}$ to $65 \mathrm{~Hz}$ at $+1 \mathrm{~Hz} / \mathrm{s}$

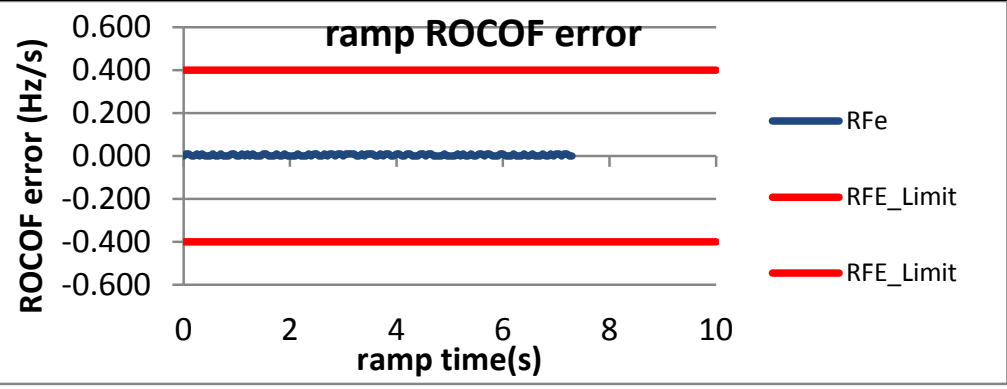

Figure 2659: Fs = $20 \mathrm{FPS}$, ramp from $56 \mathrm{~Hz}$ to $64 \mathrm{~Hz}$ at $+1 \mathrm{~Hz} / \mathrm{s}$

Figure 2656: Fs = $60 \mathrm{FPS}$, ram from $65 \mathrm{~Hz}$ to $55 \mathrm{~Hz}$ at $-1 \mathrm{~Hz} / \mathrm{s}$

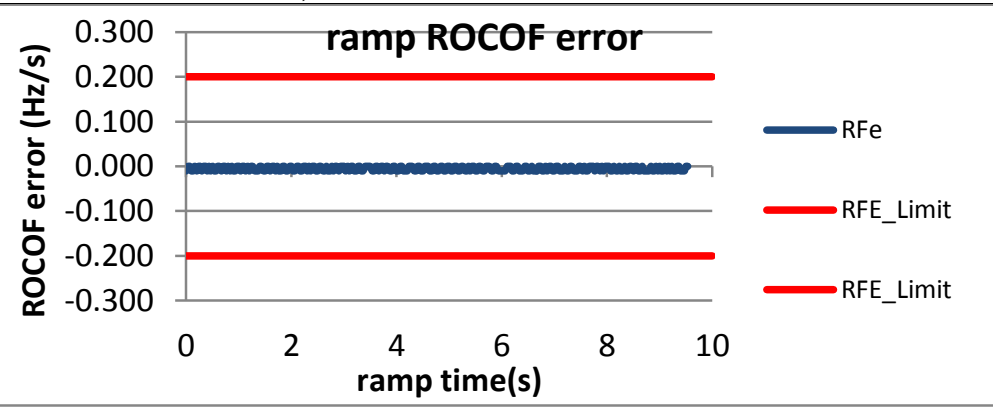

Figure 2658: Fs = $30 \mathrm{FPS}$, ram from $65 \mathrm{~Hz}$ to $55 \mathrm{~Hz}$ at $-1 \mathrm{~Hz} / \mathrm{s}$

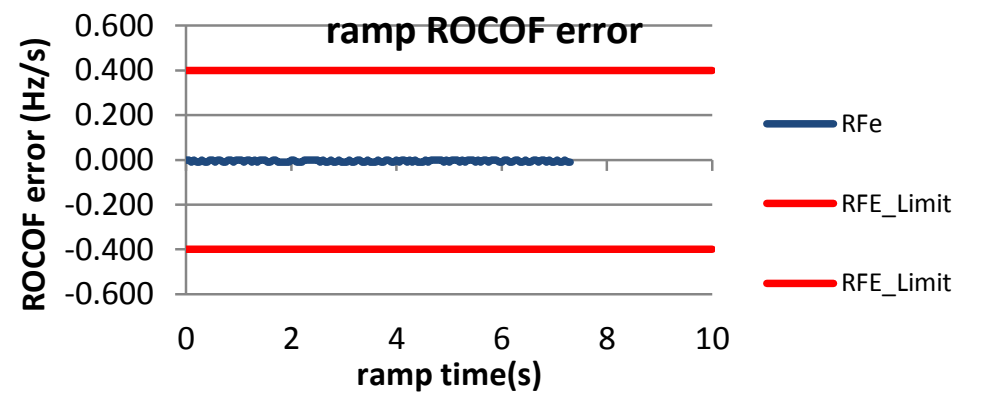

Figure 2660: Fs = 20 FPS, ramp from $64 \mathrm{~Hz}$ to $56 \mathrm{~Hz}$ at $=1 \mathrm{~Hz} / \mathrm{s}$ 


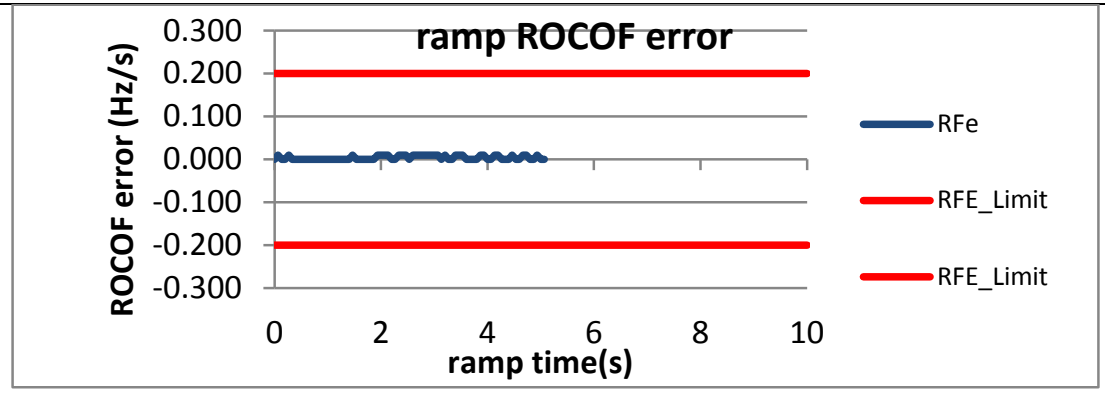

Figure 2661: Fs = $15 \mathrm{FPS}$, ramp from $57 \mathrm{~Hz}$ to $63 \mathrm{~Hz}$ at $+1 \mathrm{~Hz} / \mathrm{s}$

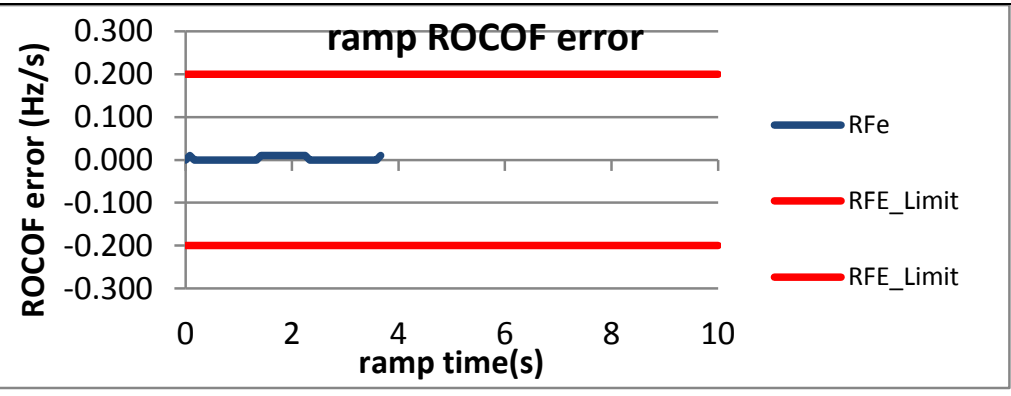

Figure 2663: Fs = 12 FPS, ramp from $58.6 \mathrm{~Hz}$ to $62.4 \mathrm{~Hz}$ at $+1 \mathrm{~Hz} / \mathrm{s}$

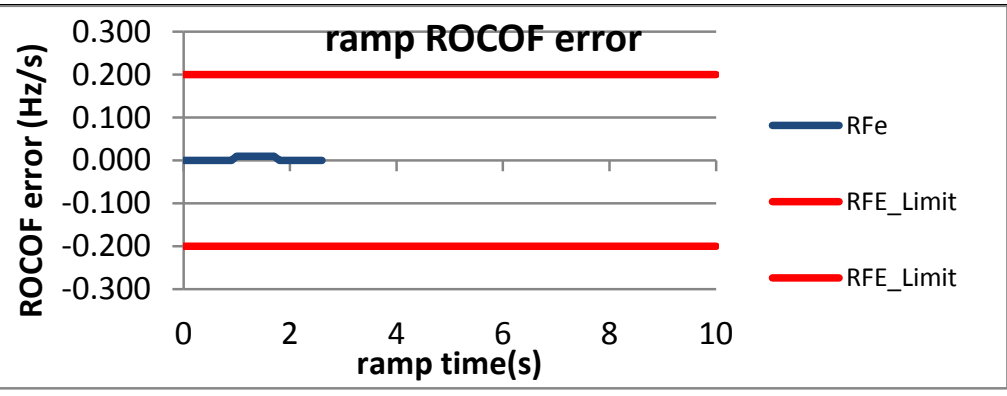

Figure 2665: Fs = $10 \mathrm{FPS}$, ramp from $58 \mathrm{~Hz}$ to $62 \mathrm{~Hz}$ at $+1 \mathrm{~Hz} / \mathrm{s}$

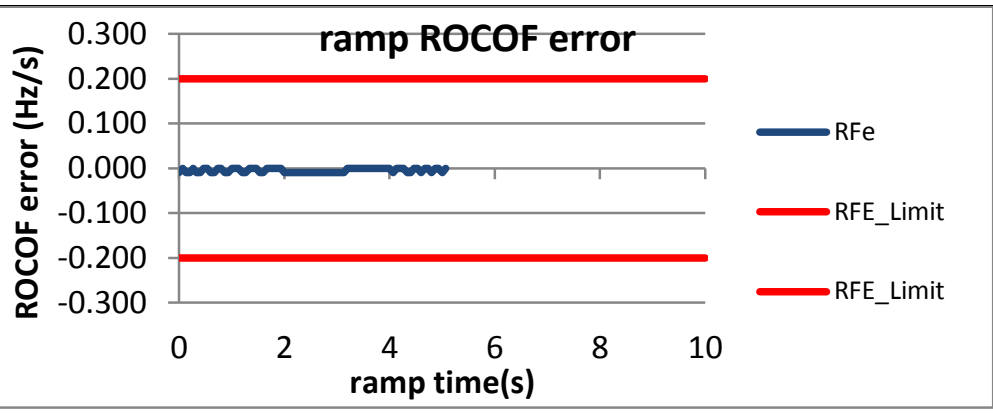

Figure 2662: Fs = $15 \mathrm{FPS}$, ramp from $63 \mathrm{~Hz}$ to $57 \mathrm{~Hz}$ at $-1 \mathrm{~Hz} / \mathrm{s}$

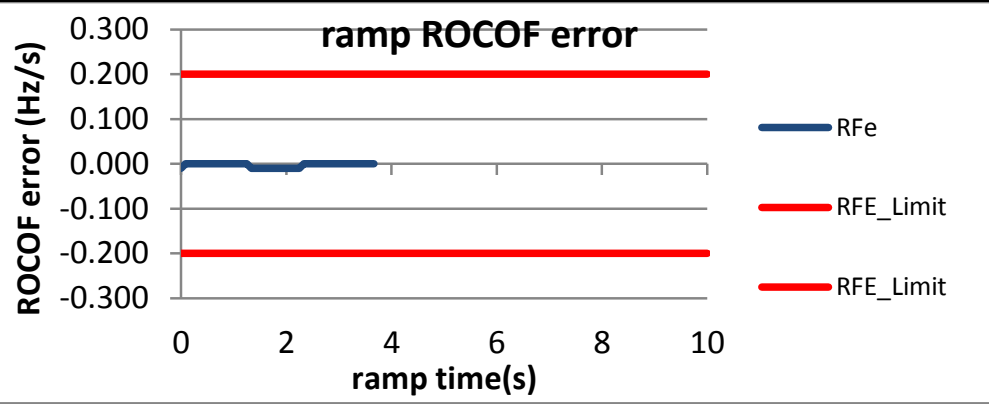

Figure 2664: Fs = 12 FPS, ramp from $62.4 \mathrm{~Hz}$ to $58.6 \mathrm{~Hz}$ at $-1 \mathrm{~Hz} / \mathrm{s}$

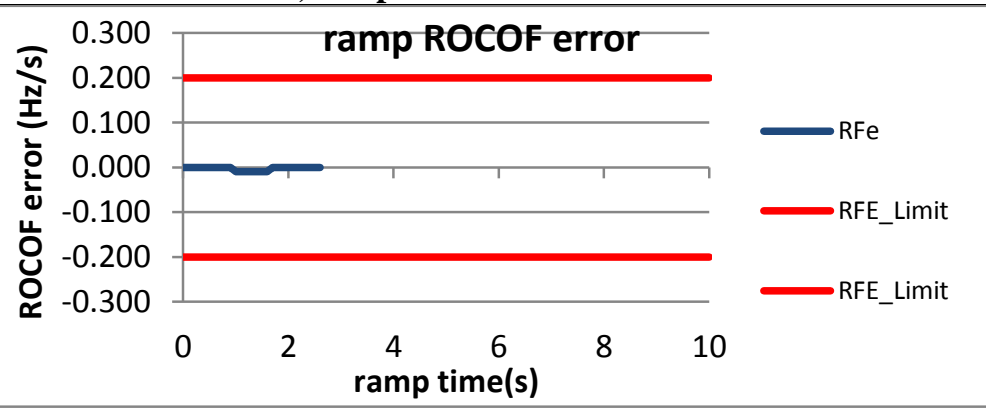

Figure 2666: Fs = $10 \mathrm{FPS}$, ramp from $62 \mathrm{~Hz}$ to $58 \mathrm{~Hz}$ at $-1 \mathrm{~Hz} / \mathrm{s}$ 


\subsection{Dynamic ramp of system frequency voltage TVE, $P$ class}

\subsubsection{C37.118.1 Annex C dynamic ramp of system frequency voltage TVE, P class}
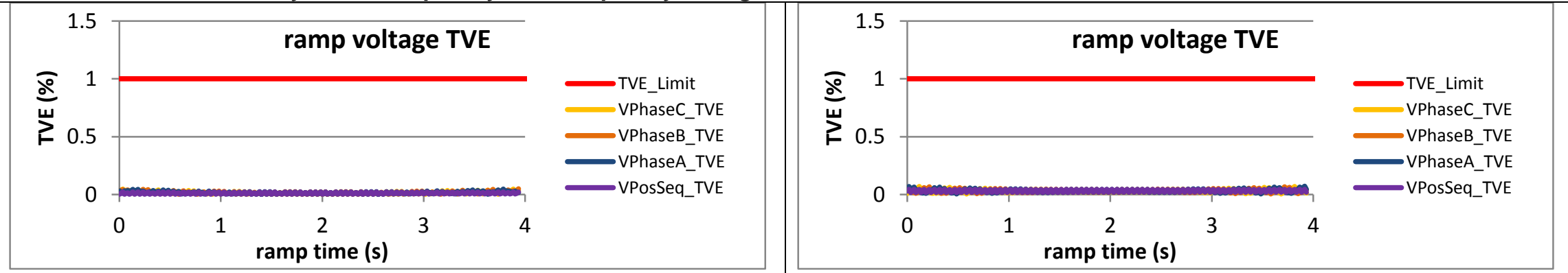

Figure 2667: Fs = 60 FPS, ramp from $58 \mathrm{~Hz}$ to $62 \mathrm{~Hz}$ at $+1 \mathrm{~Hz} / \mathrm{s}$

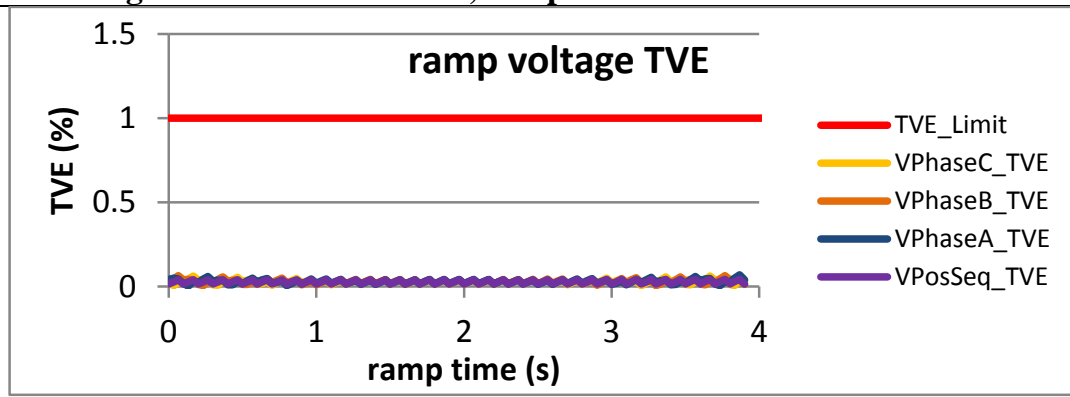

Figure 2669: Fs = $30 \mathrm{FPS}$, ramp from $58 \mathrm{~Hz}$ to $62 \mathrm{~Hz}$ at $+1 \mathrm{~Hz} / \mathrm{s}$

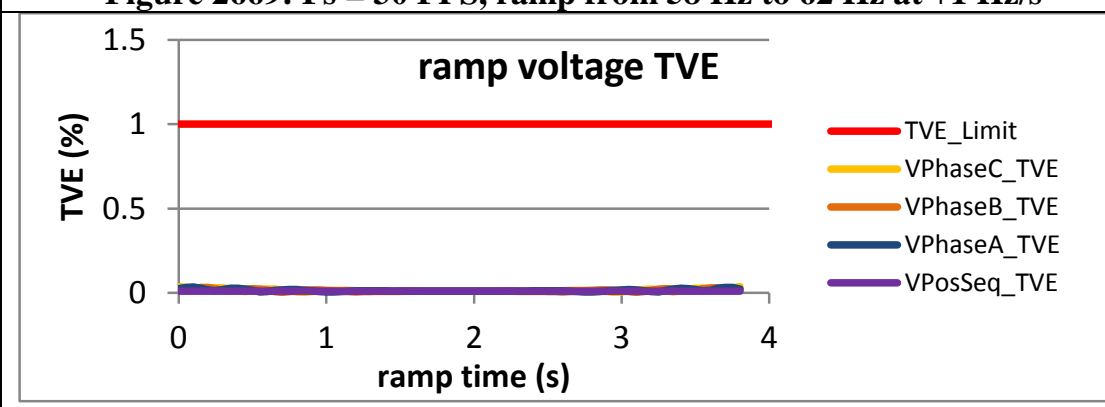

Figure 2671: Fs = 20 FPS, ramp from $58 \mathrm{~Hz}$ to $62 \mathrm{~Hz}$ at $+1 \mathrm{~Hz} / \mathrm{s}$

Figure 2668: Fs = $60 \mathrm{FPS}$, ramp from $62 \mathrm{~Hz}$ to $55 \mathrm{~Hz}$ at $-1 \mathrm{~Hz} / \mathrm{s}$

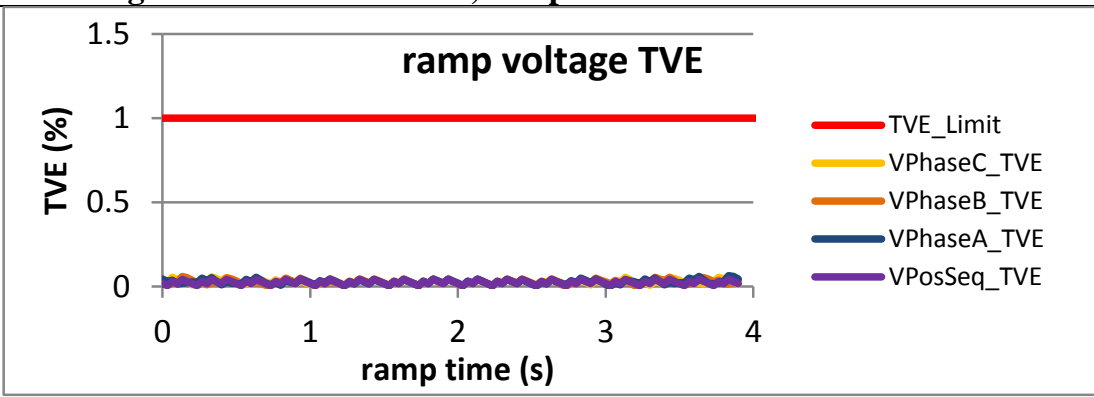

Figure 2670: Fs = $30 \mathrm{FPS}$, ramp from $62 \mathrm{~Hz}$ to $55 \mathrm{~Hz}$ at $-1 \mathrm{~Hz} / \mathrm{s}$

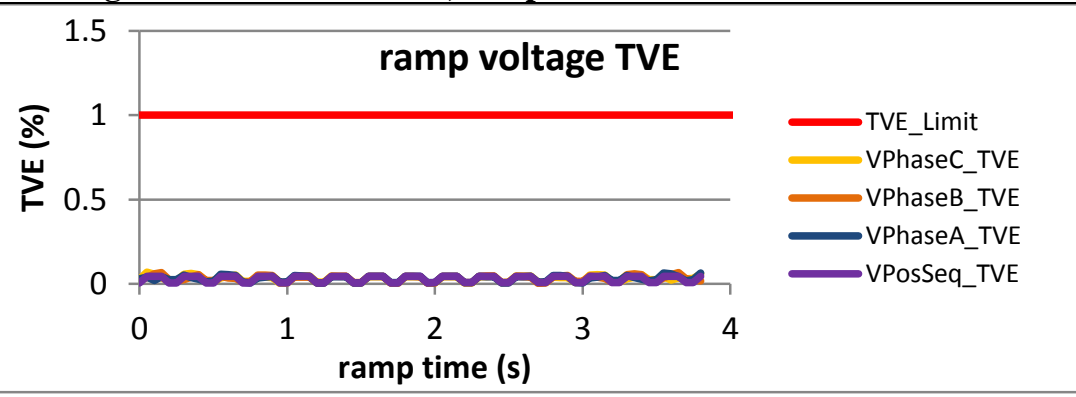

Figure 2672: Fs = $20 \mathrm{FPS}$, ramp from $64 \mathrm{~Hz}$ to $56 \mathrm{~Hz}$ at $-1 \mathrm{~Hz} / \mathrm{s}$ 

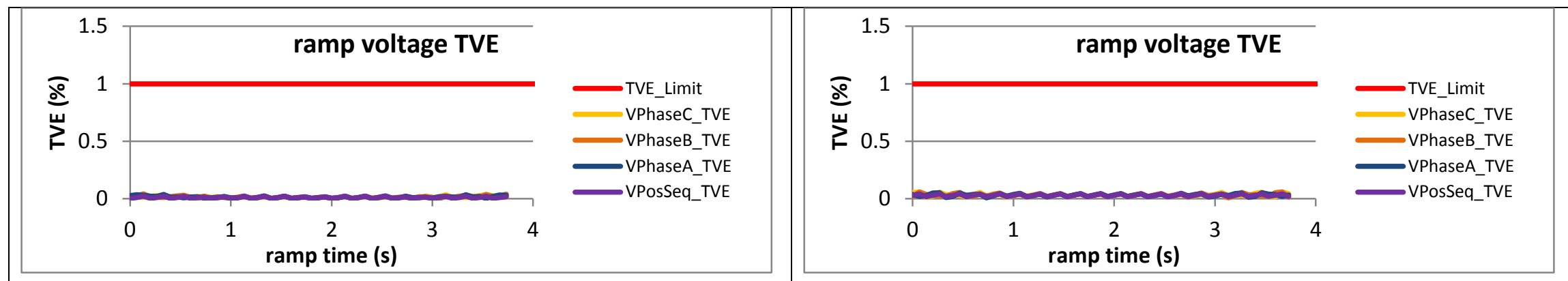

Figure 2673: Fs = $15 \mathrm{FPS}$, ramp from $58 \mathrm{~Hz}$ to $62 \mathrm{~Hz}$ at $+1 \mathrm{~Hz} / \mathrm{s}$

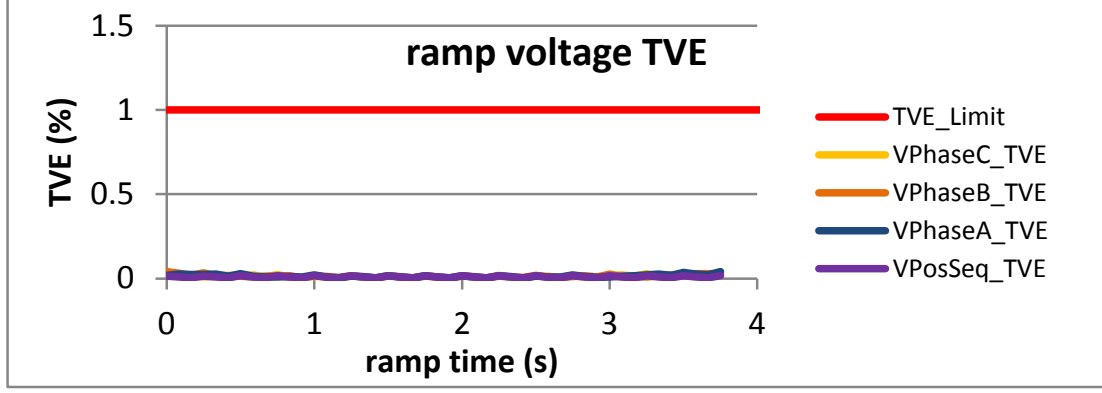

Figure 2675: Fs = $12 \mathrm{FPS}$, ramp from $58 \mathrm{~Hz}$ to $62 \mathrm{~Hz}$ at $+1 \mathrm{~Hz} / \mathrm{s}$

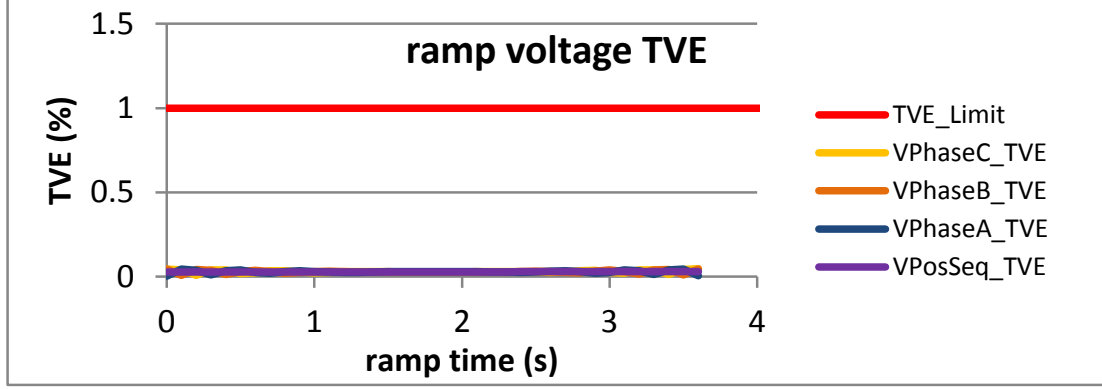

Figure 2677: Fs = $10 \mathrm{FPS}$, ramp from $58 \mathrm{~Hz}$ to $62 \mathrm{~Hz}$ at $+1 \mathrm{~Hz} / \mathrm{s}$

Figure 2674: Fs = $15 \mathrm{FPS}$, ramp from $62 \mathrm{~Hz}$ to $57 \mathrm{~Hz}$ at $-1 \mathrm{~Hz} / \mathrm{s}$

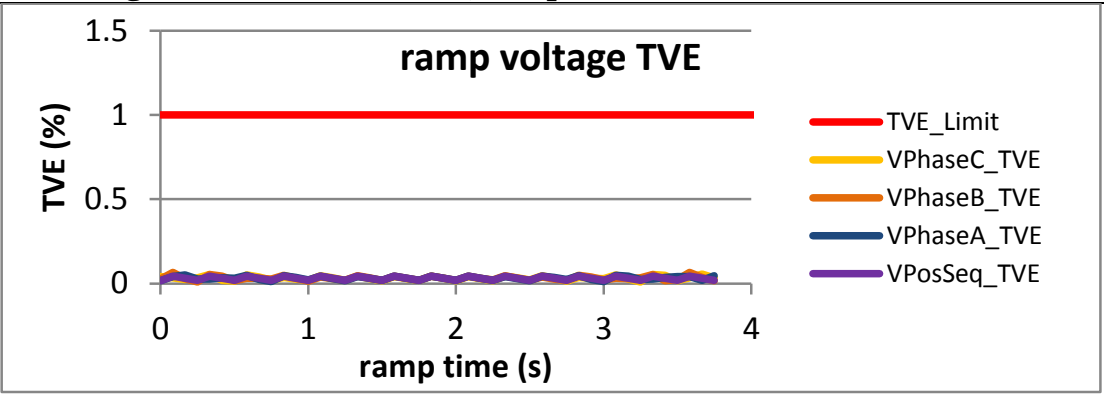

Figure 2676: Fs = $12 \mathrm{FPS}$, ramp from $62 \mathrm{~Hz}$ to $58.6 \mathrm{~Hz}$ at $-1 \mathrm{~Hz} / \mathrm{s}$

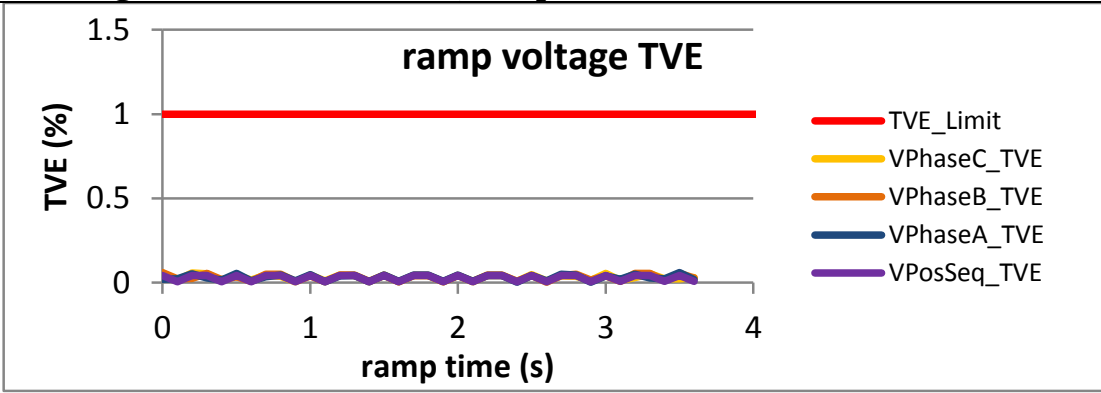

Figure 2678: Fs = $10 \mathrm{FPS}$, ramp from $62 \mathrm{~Hz}$ to $58 \mathrm{~Hz}$ at $-1 \mathrm{~Hz} / \mathrm{s}$ 
6.5.2 PMU A dynamic ramp of system frequency voltage TVE, P class

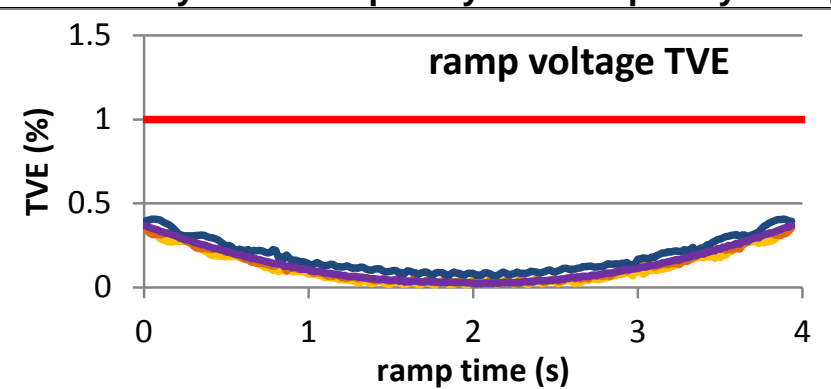

Figure 2679: Fs = $60 \mathrm{FPS}$, ramp from $58 \mathrm{~Hz}$ to $62 \mathrm{~Hz}$ at $+1 \mathrm{~Hz} / \mathrm{s}$

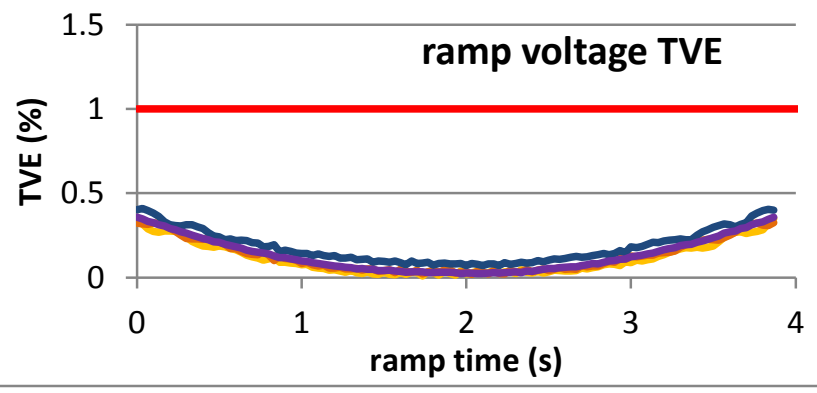

Figure 2681: Fs $=30 \mathrm{FPS}$, ramp from $58 \mathrm{~Hz}$ to $62 \mathrm{~Hz}$ at $+1 \mathrm{~Hz} / \mathrm{s}$

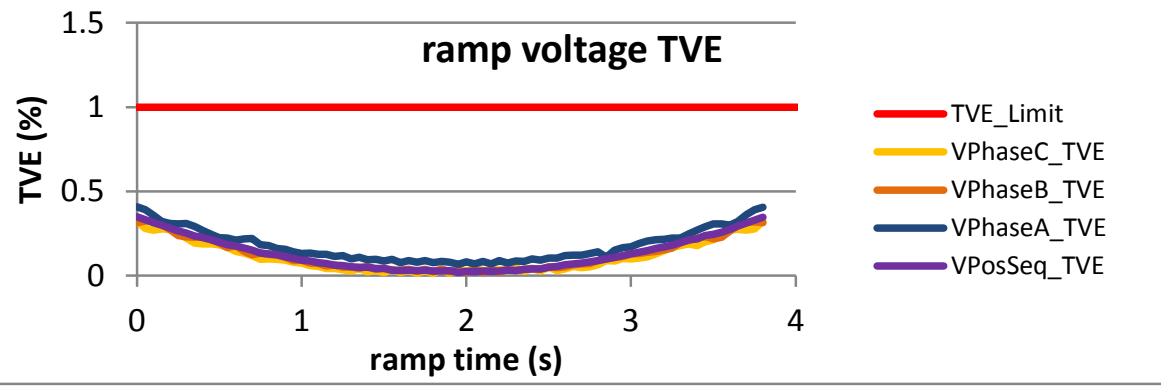

Figure 2683: Fs = 20 FPS, ramp from $58 \mathrm{~Hz}$ to $62 \mathrm{~Hz}$ at $+1 \mathrm{~Hz} / \mathrm{s}$

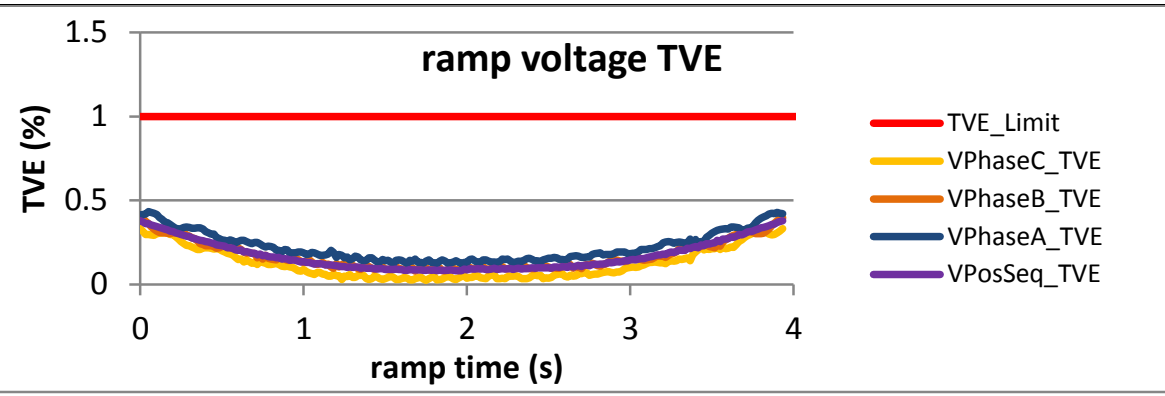

Figure 2680: Fs = $60 \mathrm{FPS}$, ramp from $62 \mathrm{~Hz}$ to $58 \mathrm{~Hz}$ at $-1 \mathrm{~Hz} / \mathrm{s}$

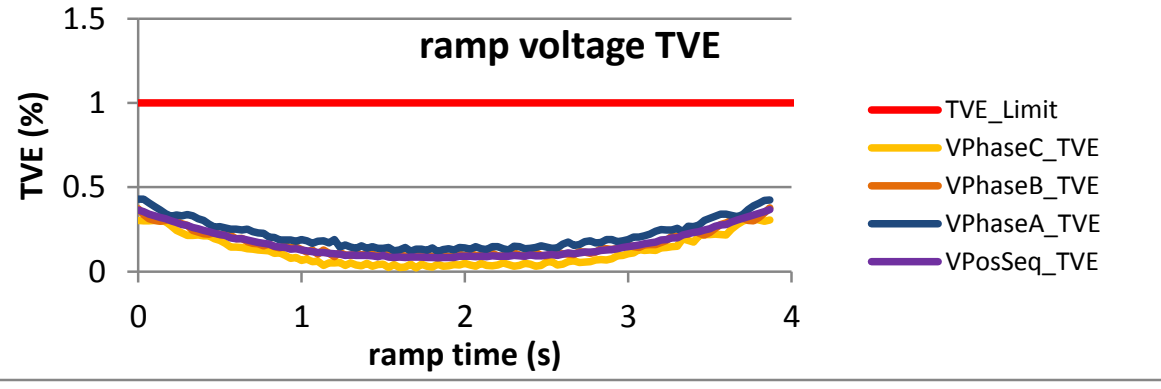

Figure 2682: Fs = $30 \mathrm{FPS}$, ramp from $62 \mathrm{~Hz}$ to $58 \mathrm{~Hz}$ at $-1 \mathrm{~Hz} / \mathrm{s}$

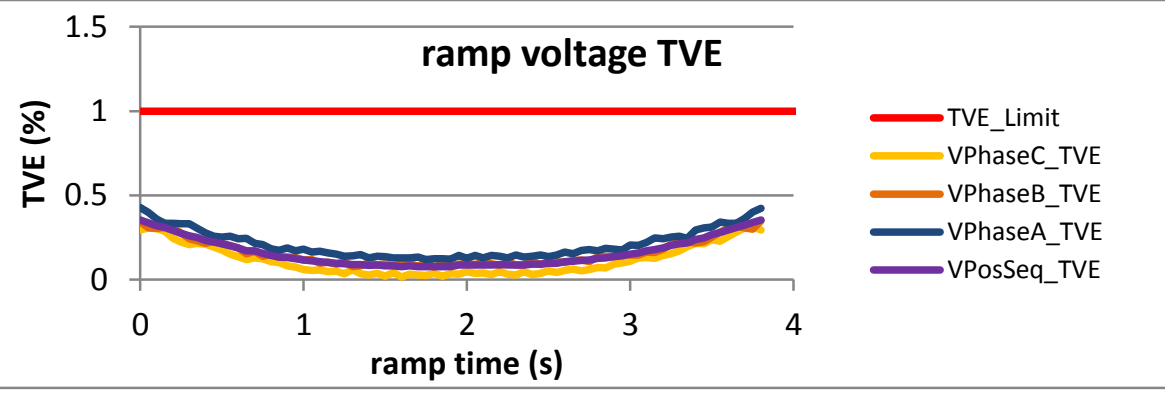

Figure 2684: Fs = $20 \mathrm{FPS}$, ramp from $62 \mathrm{~Hz}$ to $58 \mathrm{~Hz}$ at $-1 \mathrm{~Hz} / \mathrm{s}$ 

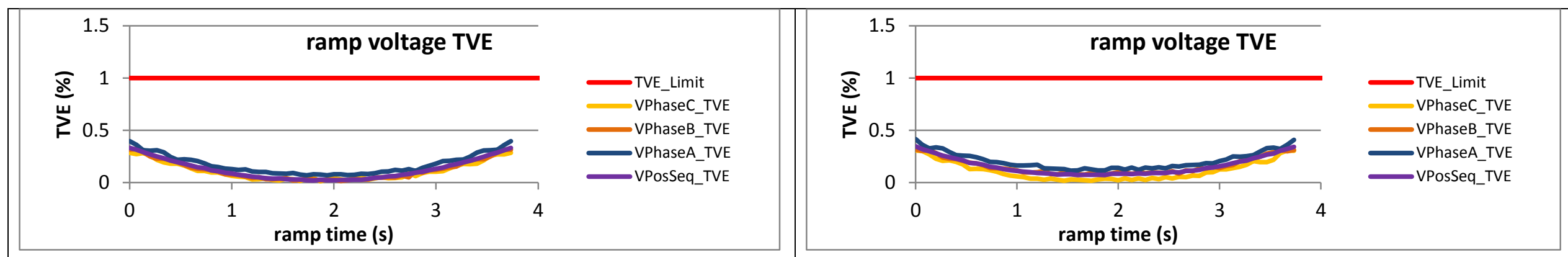

Figure 2685: Fs = $15 \mathrm{FPS}$, ramp from $58 \mathrm{~Hz}$ to $62 \mathrm{~Hz}$ at $+1 \mathrm{~Hz} / \mathrm{s}$

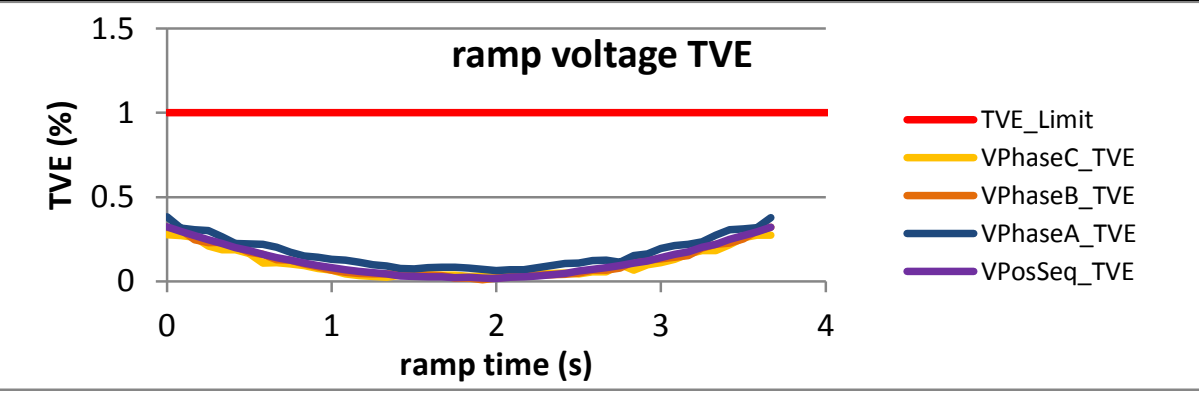

Figure 2686: Fs = $15 \mathrm{FPS}$, ramp from $62 \mathrm{~Hz}$ to $58 \mathrm{~Hz}$ at $-1 \mathrm{~Hz} / \mathrm{s}$

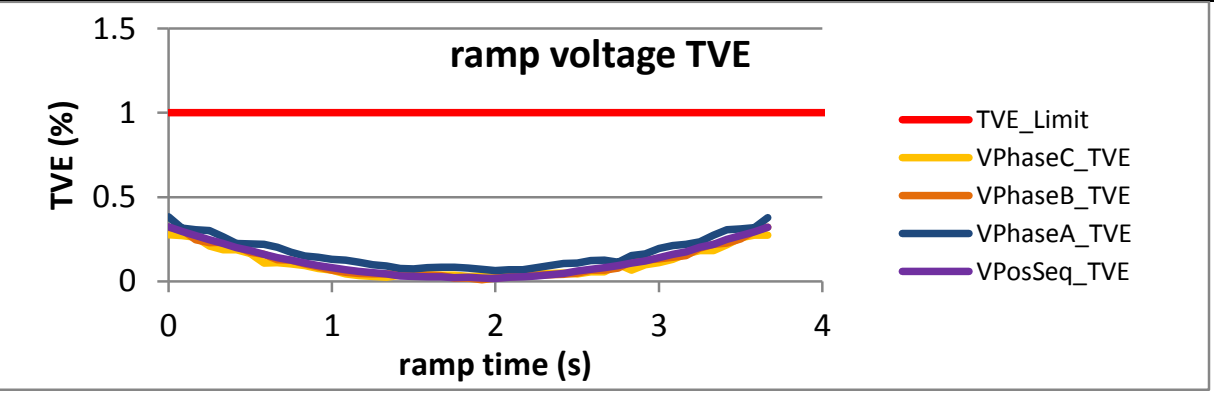

Figure 2687: Fs = $12 \mathrm{FPS}$, ramp $58 \mathrm{~Hz}$ to $62 \mathrm{~Hz}$ at $+1 \mathrm{~Hz} / \mathrm{s}$

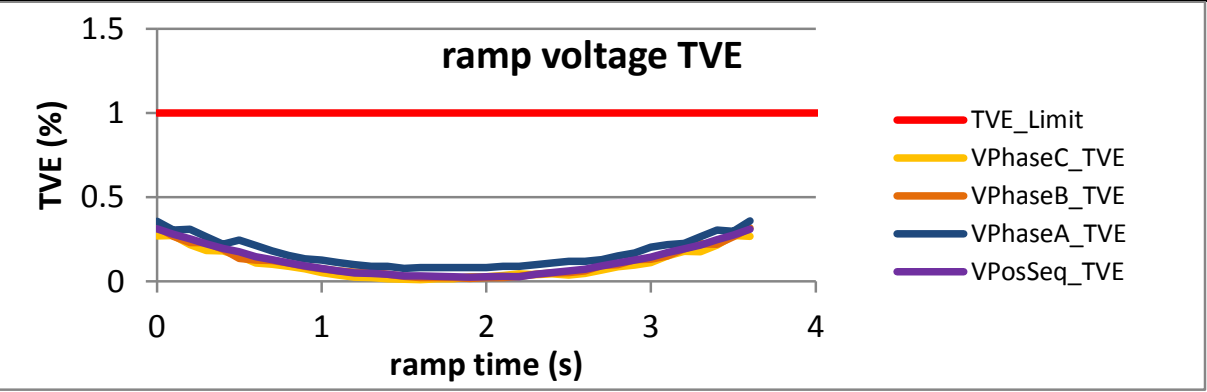

Figure 2688: Fs = $12 \mathrm{FPS}$, ramp from $62 \mathrm{~Hz}$ to $58 \mathrm{~Hz}$ at $-1 \mathrm{~Hz} / \mathrm{s}$

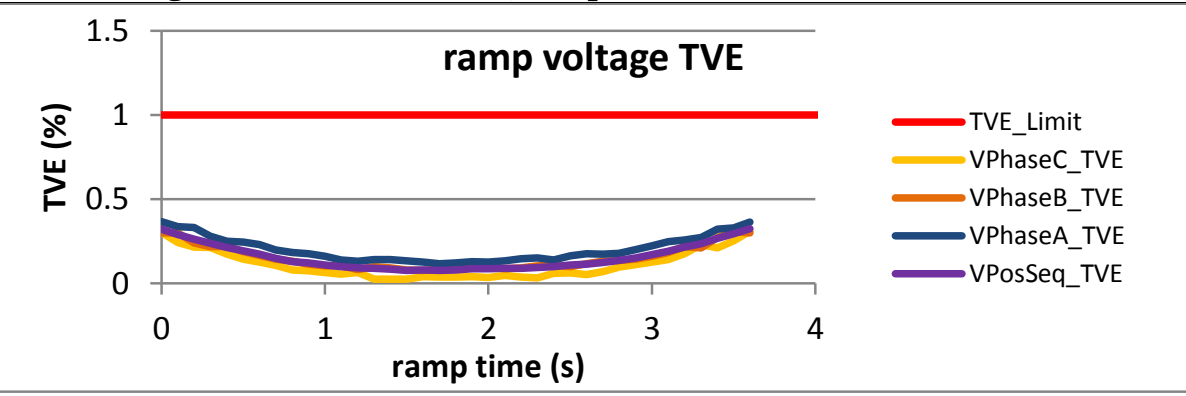

Figure 2689: Fs = $10 \mathrm{FPS}$, ramp from $58 \mathrm{~Hz}$ to $62 \mathrm{~Hz}$ at $+1 \mathrm{~Hz} / \mathrm{s}$

Figure 2690: Fs = $10 \mathrm{FPS}$, ramp from $62 \mathrm{~Hz}$ to $58 \mathrm{~Hz}$ at $-1 \mathrm{~Hz} / \mathrm{s}$ 
6.5.3 PMU B dynamic ramp of system frequency voltage TVE, P class

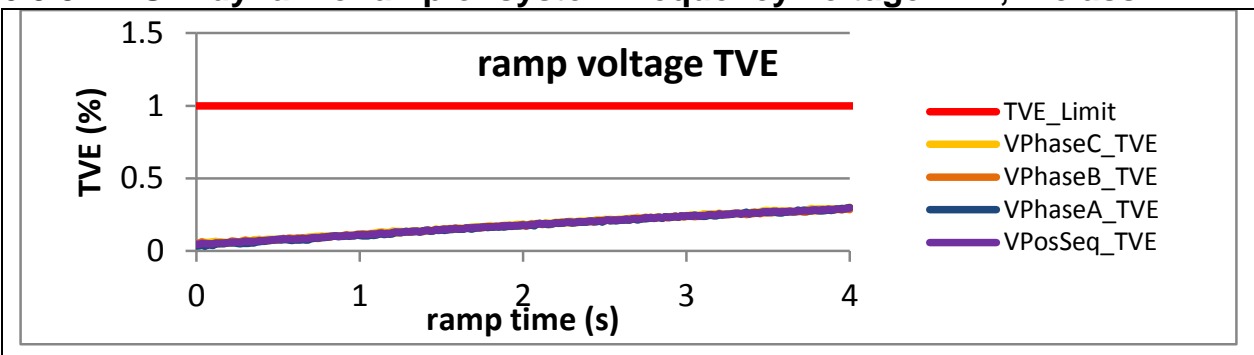

Figure 2691: Fs = $60 \mathrm{FPS}$, ramp from $55 \mathrm{~Hz}$ to $65 \mathrm{~Hz}$ at $+1 \mathrm{~Hz} / \mathrm{s}$

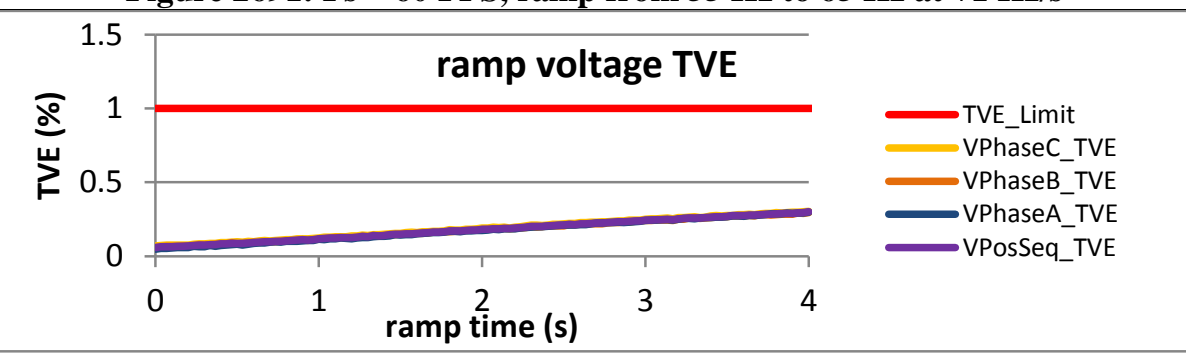

Figure 2693: Fs = $30 \mathrm{FPS}$, ramp from $55 \mathrm{~Hz}$ to $65 \mathrm{~Hz}$ at $+1 \mathrm{~Hz} / \mathrm{s}$

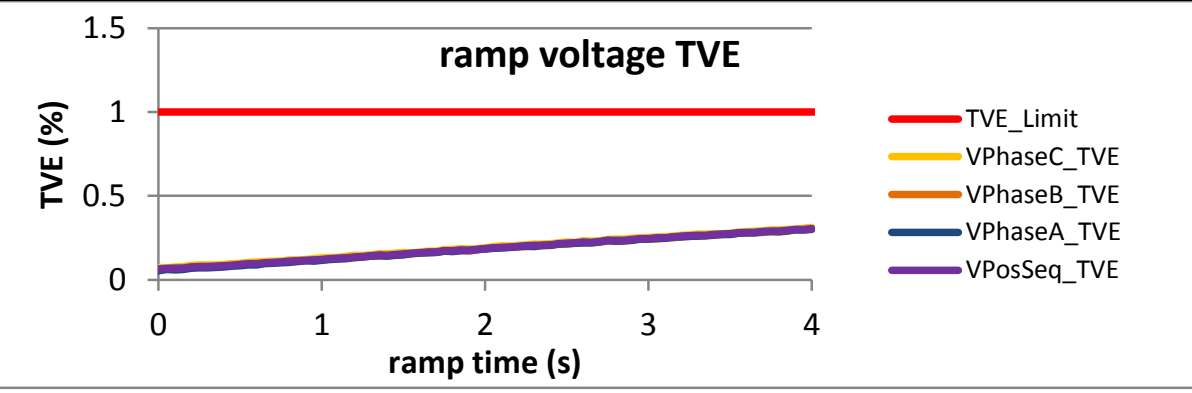

Figure 2695: Fs = $20 \mathrm{FPS}$, ramp from $56 \mathrm{~Hz}$ to $64 \mathrm{~Hz}$ at $+1 \mathrm{~Hz} / \mathrm{s}$

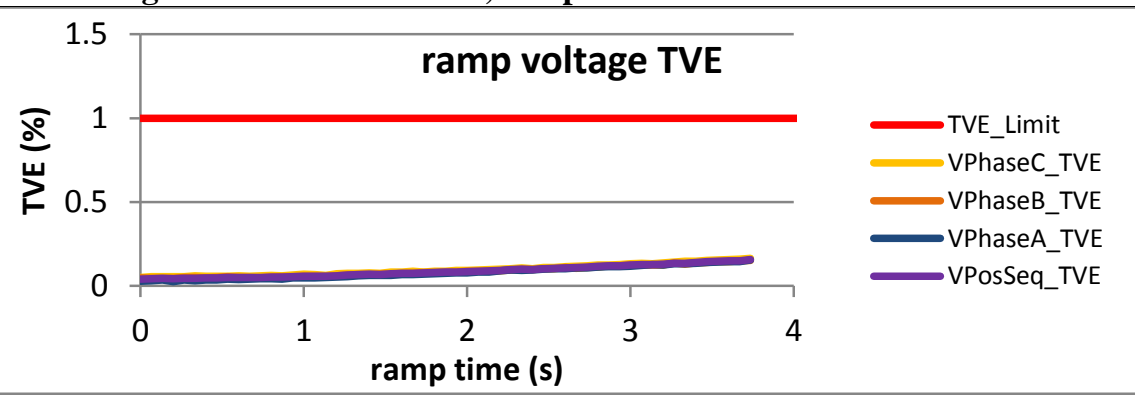

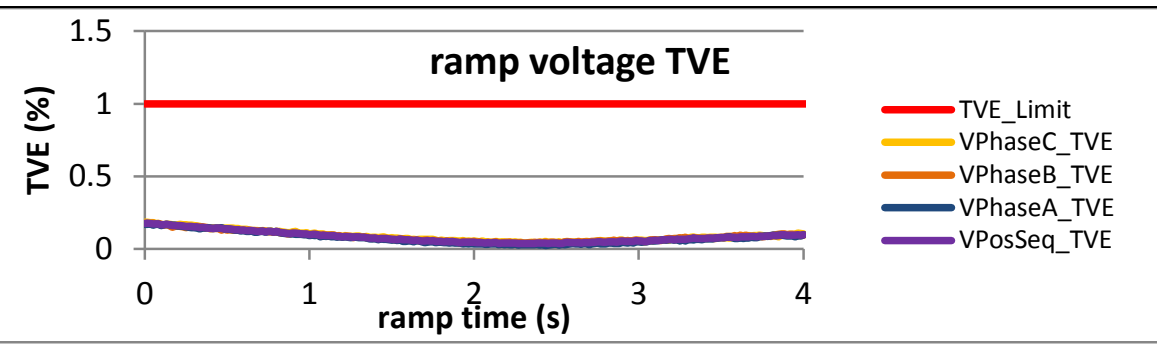

Figure 2692: Fs = $60 \mathrm{FPS}$, ramp from $65 \mathrm{~Hz}$ to $55 \mathrm{~Hz}$ at $-1 \mathrm{~Hz} / \mathrm{s}$

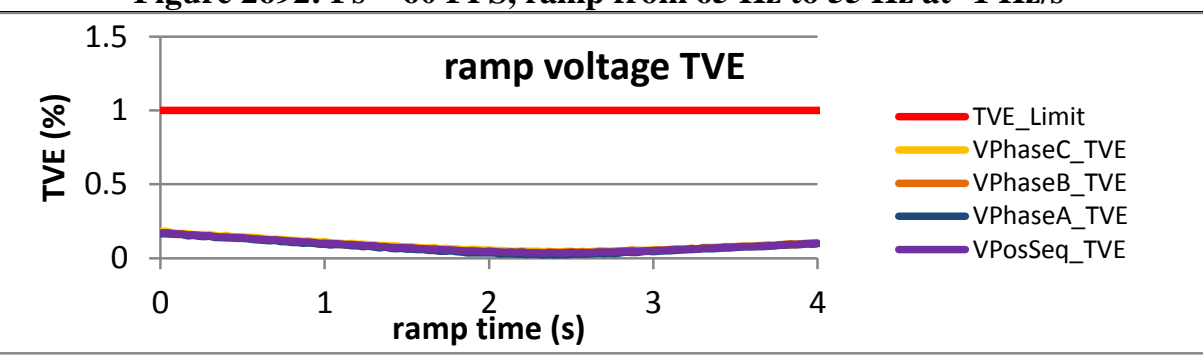

Figure 2694: Fs = $30 \mathrm{FPS}$, ramp from $65 \mathrm{~Hz}$ to $55 \mathrm{~Hz}$ at $-1 \mathrm{~Hz} / \mathrm{s}$

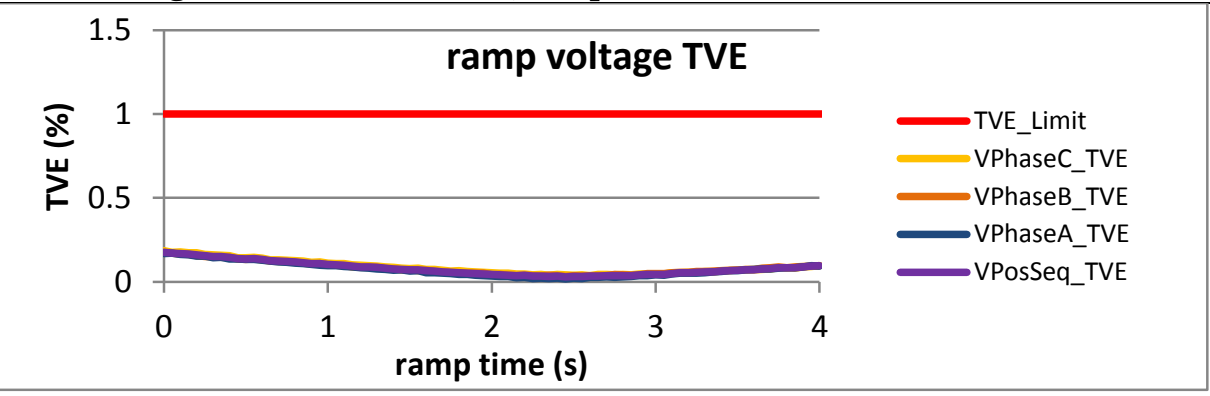

Figure 2696: Fs = 20 FPS, ramp from $64 \mathrm{~Hz}$ to $56 \mathrm{~Hz}$ at $-1 \mathrm{~Hz} / \mathrm{s}$

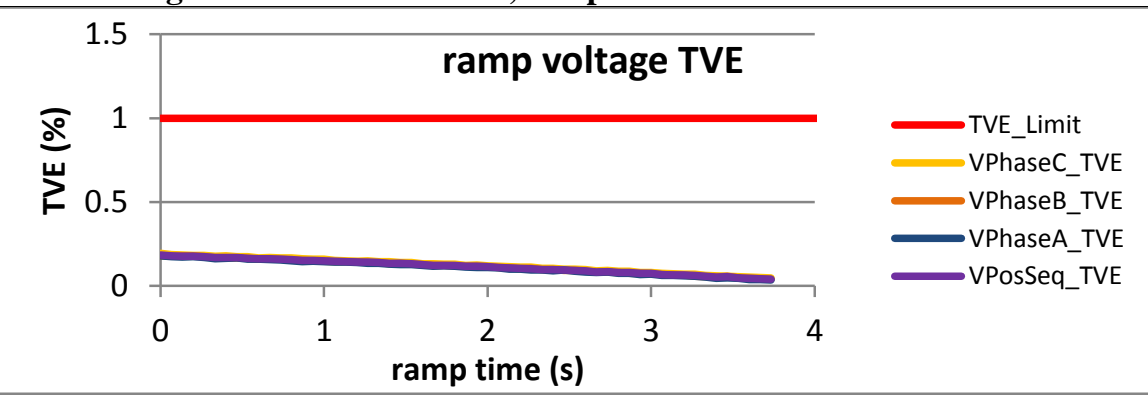




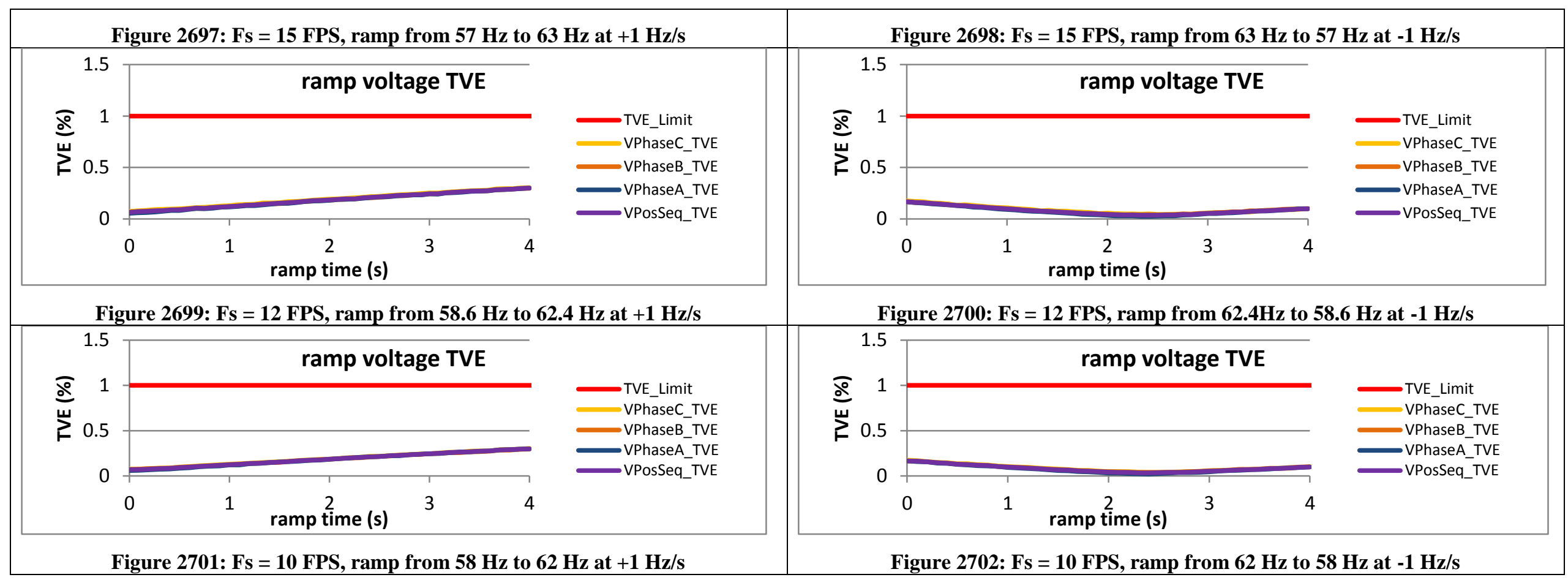


6.5.4 PMU C dynamic ramp of system frequency voltage TVE, $P$ class

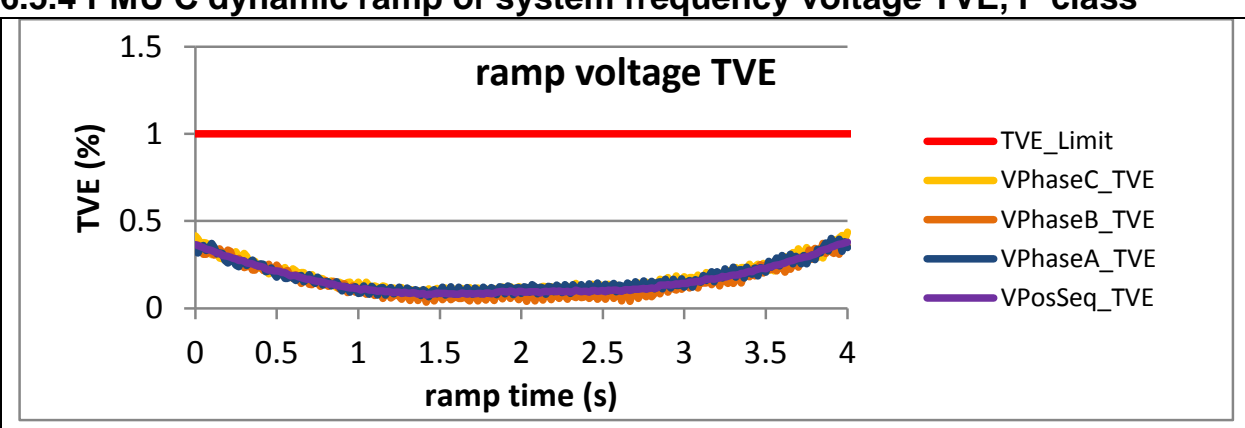

Figure 2703: Fs = 60 FPS, ramp from $55 \mathrm{~Hz}$ to $65 \mathrm{~Hz}$ at $+1 \mathrm{~Hz} / \mathrm{s}$

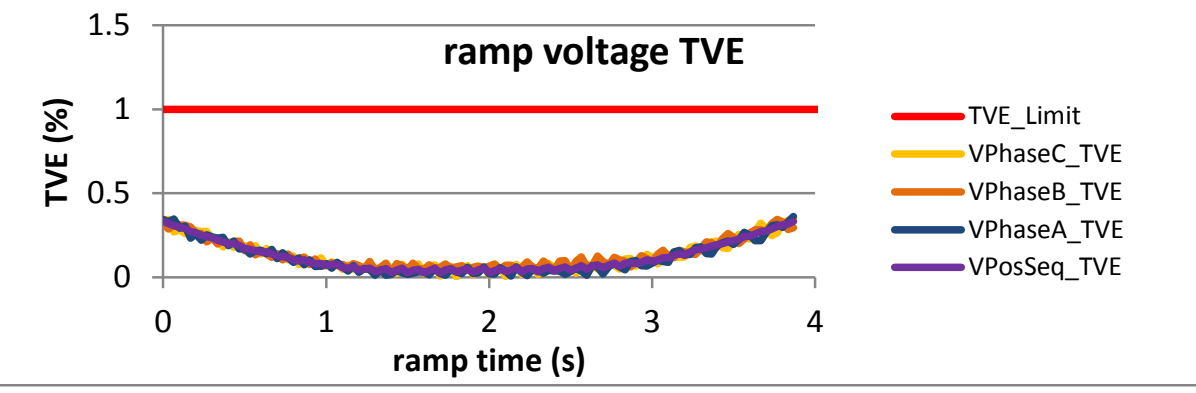

Figure 2705: Fs $=30 \mathrm{FPS}$, ramp from $55 \mathrm{~Hz}$ to $65 \mathrm{~Hz}$ at $+1 \mathrm{~Hz} / \mathrm{s}$

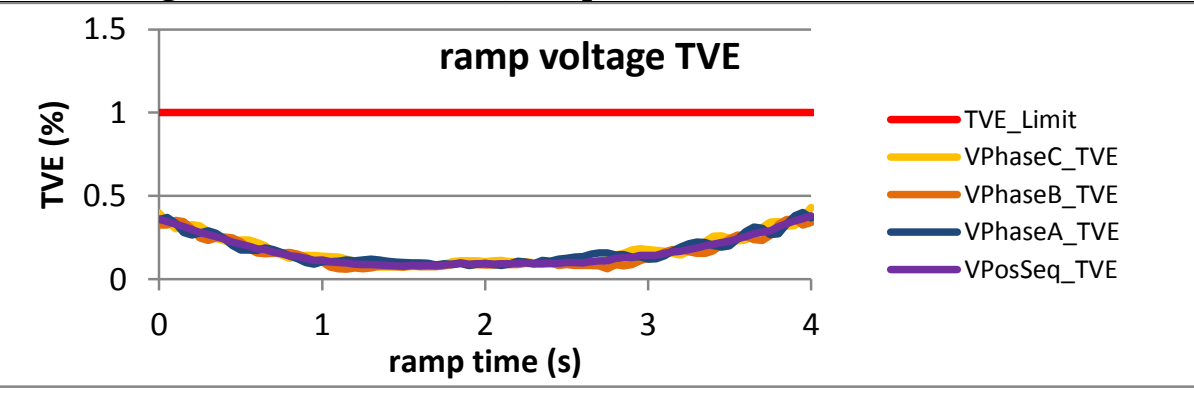

Figure 2707: Fs $=20 \mathrm{FPS}$, ramp from $56 \mathrm{~Hz}$ to $64 \mathrm{~Hz}$ at $+1 \mathrm{~Hz} / \mathrm{s}$

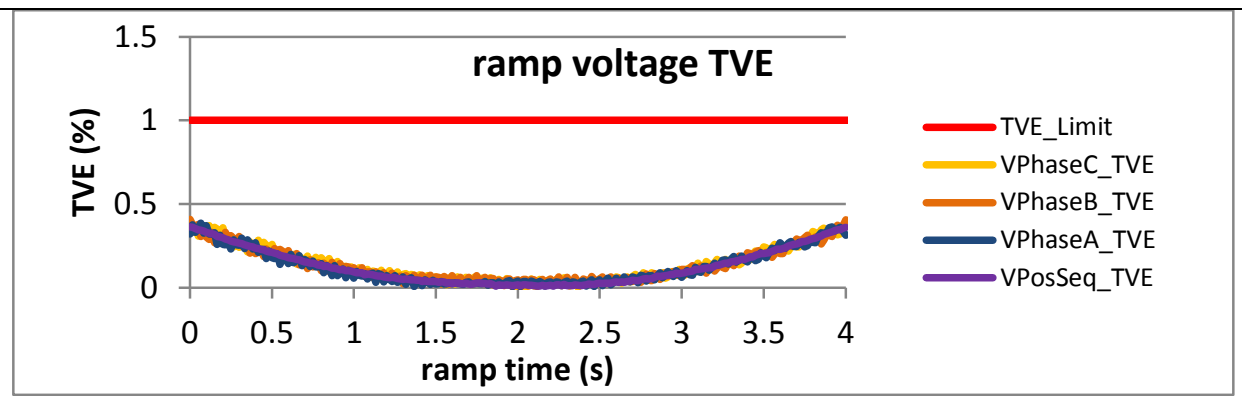

Figure 2704: Fs = $60 \mathrm{FPS}$, ramp from $65 \mathrm{~Hz}$ to $55 \mathrm{~Hz}$ at $-1 \mathrm{~Hz} / \mathrm{s}$

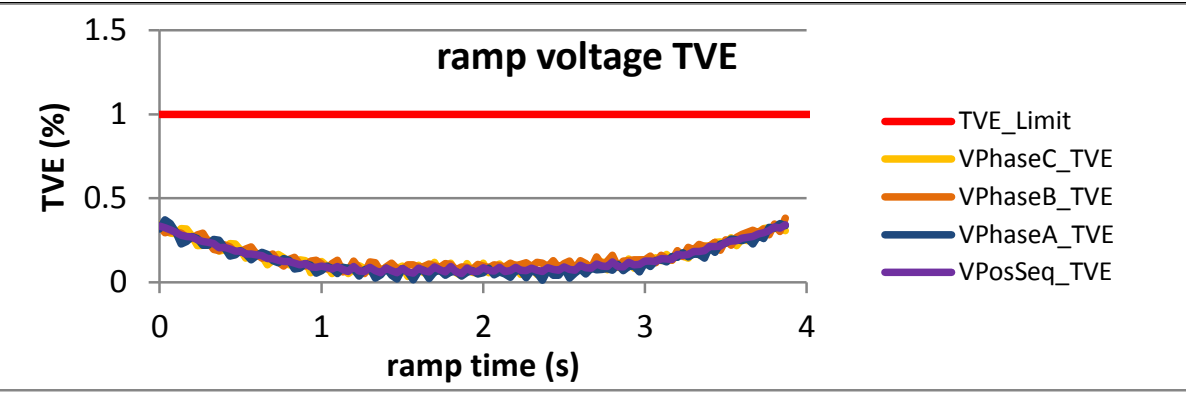

Figure 2706: Fs = $30 \mathrm{FPS}$, ramp from $65 \mathrm{~Hz}$ to $55 \mathrm{~Hz}$ at $-1 \mathrm{~Hz} / \mathrm{s}$

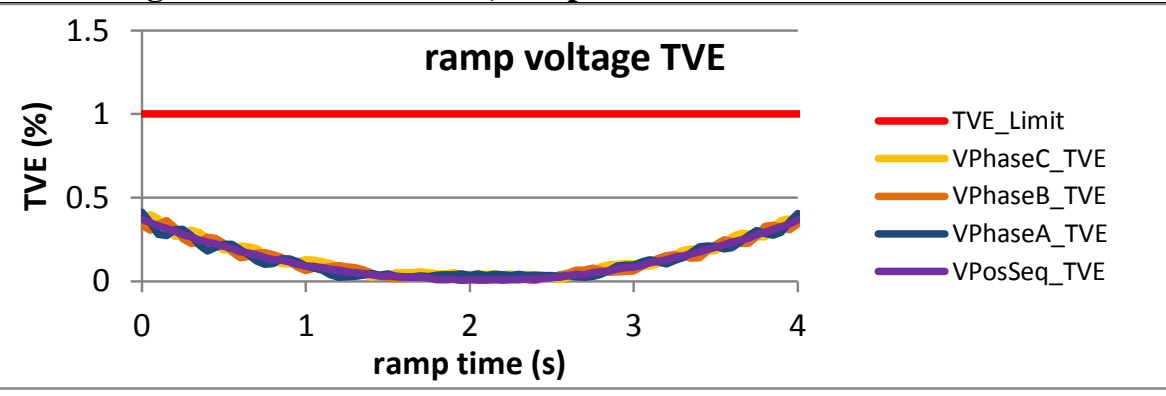

Figure 2708: Fs = $20 \mathrm{FPS}$, ramp from $64 \mathrm{~Hz}$ to $56 \mathrm{~Hz}$ at $-1 \mathrm{~Hz} / \mathrm{s}$ 


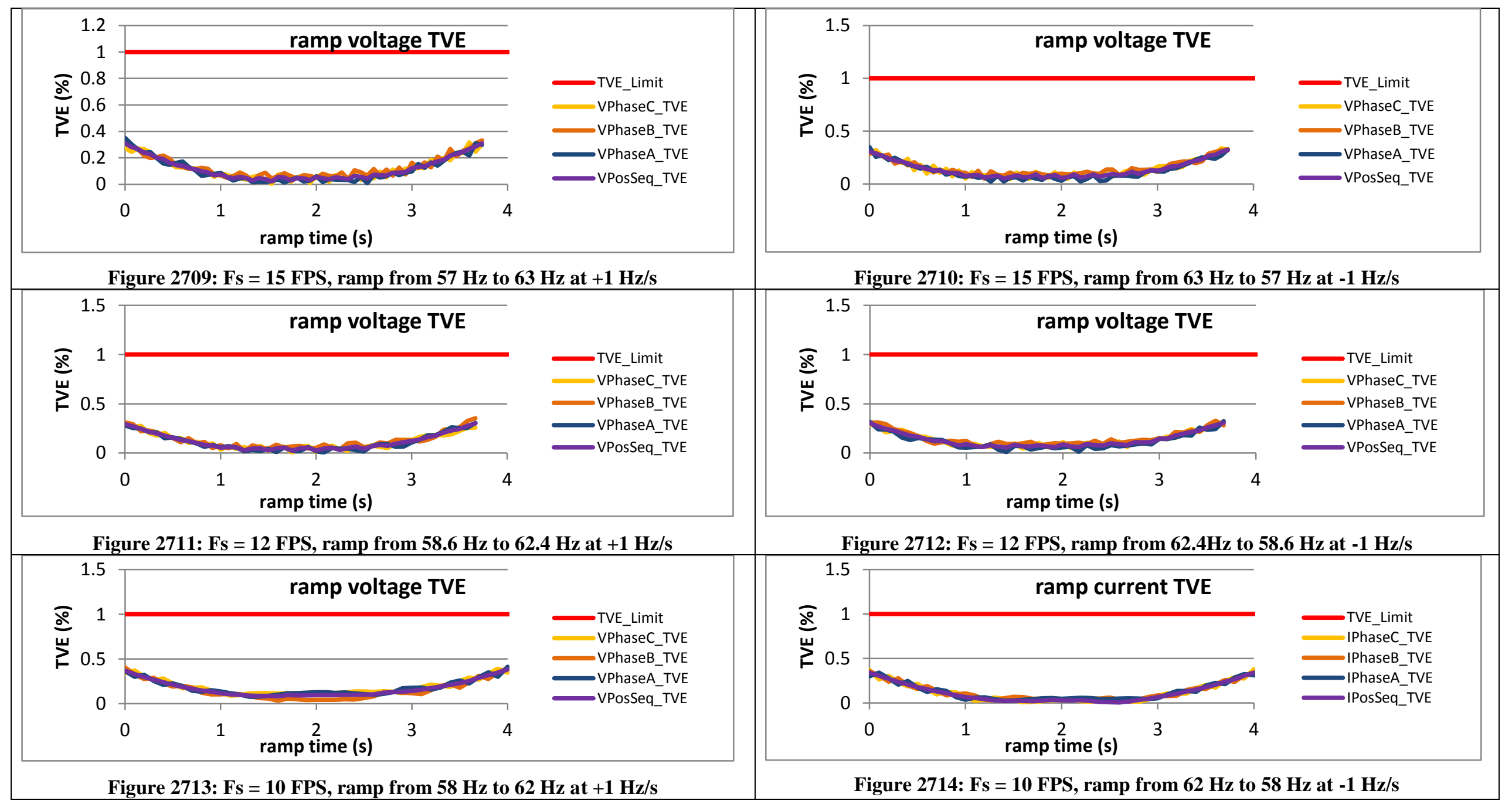


6.5.5 PMU D dynamic ramp of system frequency voltage TVE, P class

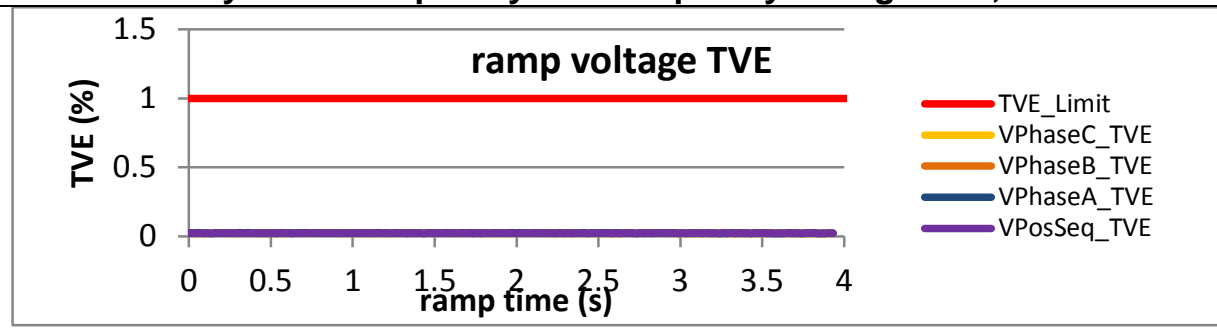

Figure 2715: Fs = $60 \mathrm{FPS}$, ramp from $58 \mathrm{~Hz}$ to $62 \mathrm{~Hz}$ at $+1 \mathrm{~Hz} / \mathrm{s}$

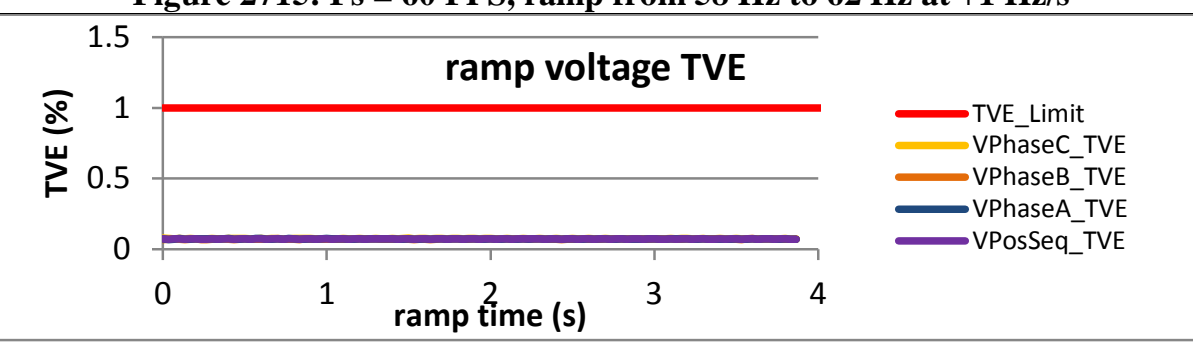

Figure 2717: Fs = $30 \mathrm{FPS}$, ramp from $58 \mathrm{~Hz}$ to $62 \mathrm{~Hz}$ at $+1 \mathrm{~Hz} / \mathrm{s}$

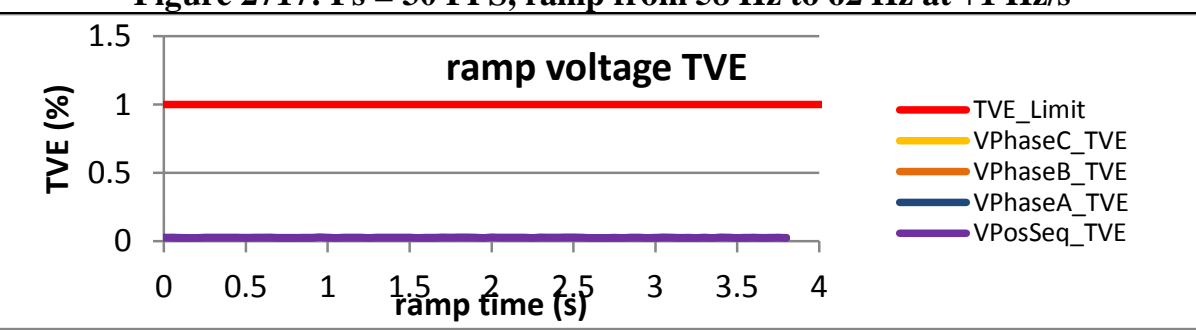

Figure 2719: Fs = $20 \mathrm{FPS}$, ramp from $58 \mathrm{~Hz}$ to $62 \mathrm{~Hz}$ at $+1 \mathrm{~Hz} / \mathrm{s}$

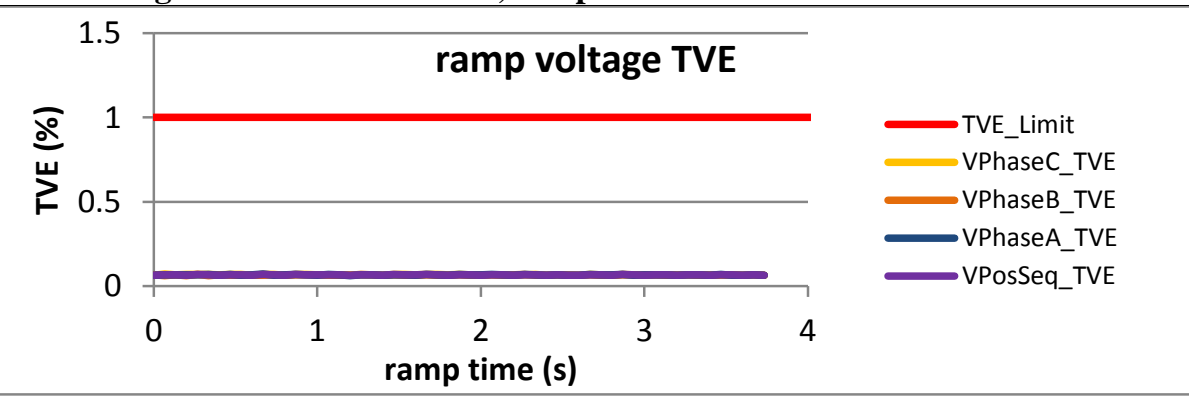

Figure 2721: Fs = $15 \mathrm{FPS}$, ramp from $58 \mathrm{~Hz}$ to $62 \mathrm{~Hz}$ at $+1 \mathrm{~Hz} / \mathrm{s}$
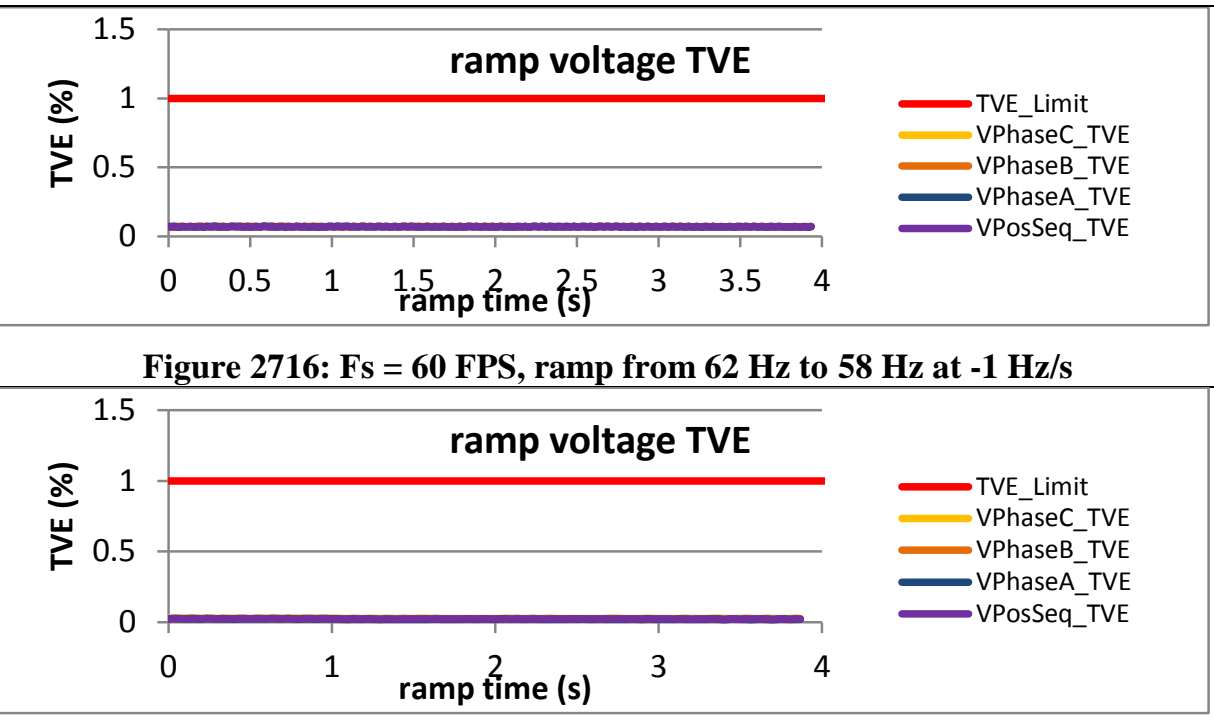

Figure 2718: Fs = $30 \mathrm{FPS}$, ramp from $62 \mathrm{~Hz}$ to $58 \mathrm{~Hz}$ at $-1 \mathrm{~Hz} / \mathrm{s}$

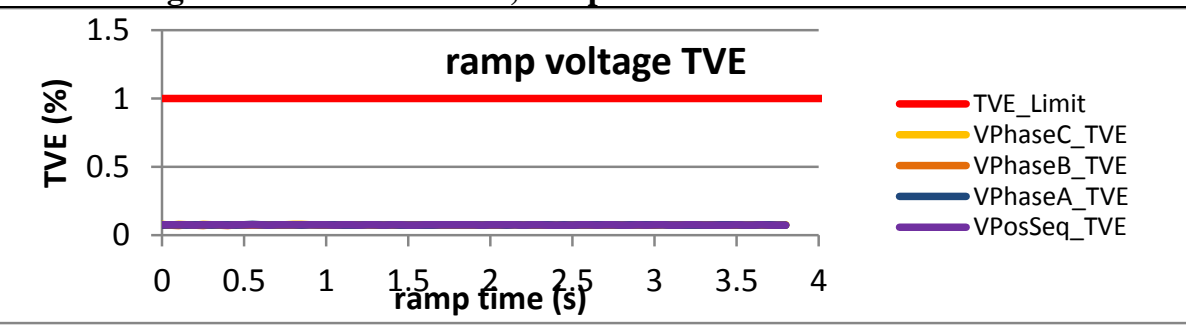

Figure 2720: Fs = $20 \mathrm{FPS}$, ramp from $62 \mathrm{~Hz}$ to $58 \mathrm{~Hz}$ at $-1 \mathrm{~Hz} / \mathrm{s}$

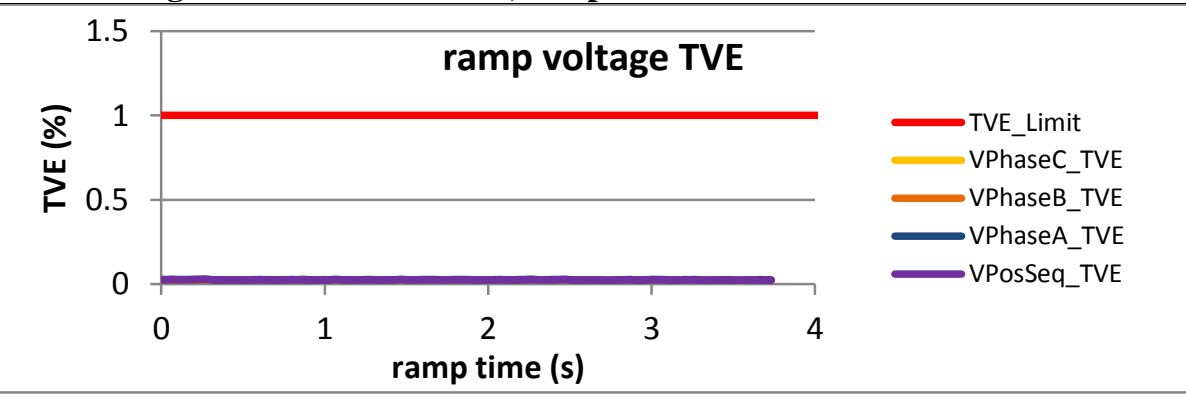

Figure 2722: Fs = $15 \mathrm{FPS}$, ramp from $62 \mathrm{~Hz}$ to $58 \mathrm{~Hz}$ at $-1 \mathrm{~Hz} / \mathrm{s}$ 

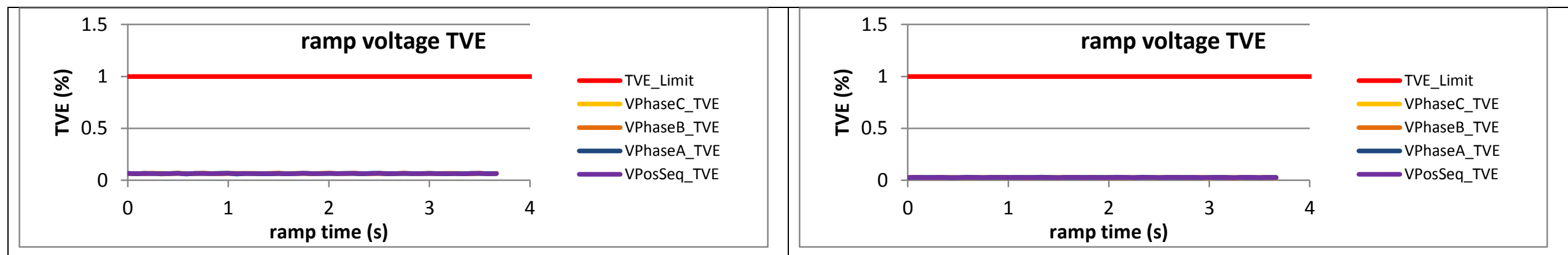

Figure 2723: Fs = $12 \mathrm{FPS}$, ramp from $58 \mathrm{~Hz}$ to $62 \mathrm{~Hz}$ at $+1 \mathrm{~Hz} / \mathrm{s}$

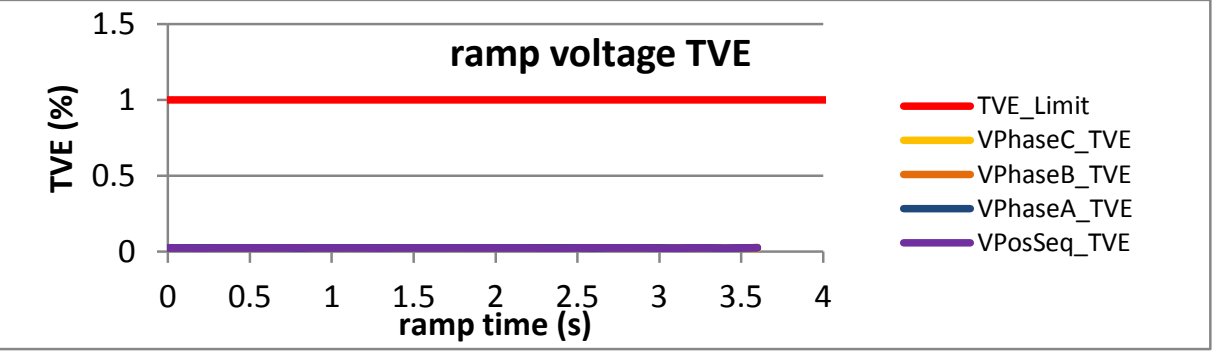

Figure 2725: Fs = $10 \mathrm{FPS}$, ramp from $58 \mathrm{~Hz}$ to $62 \mathrm{~Hz}$ at $+1 \mathrm{~Hz} / \mathrm{s}$

Figure 2724: Fs = $12 \mathrm{FPS}$, ramp from $62 \mathrm{~Hz}$ to $58 \mathrm{~Hz}$ at $-1 \mathrm{~Hz} / \mathrm{s}$

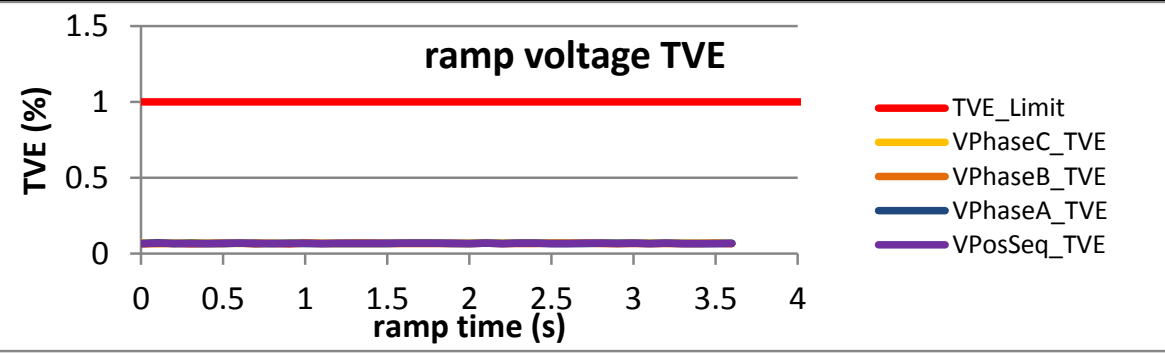

Figure 2726: Fs = $10 \mathrm{FPS}$, ramp from $62 \mathrm{~Hz}$ to $58 \mathrm{~Hz}$ at $-1 \mathrm{~Hz} / \mathrm{s}$ 
6.5.6 PMU E dynamic ramp of system frequency voltage TVE, $P$ class

PMU E does not support P class.

6.5.7 PMU F dynamic ramp of system frequency voltage TVE, $P$ class

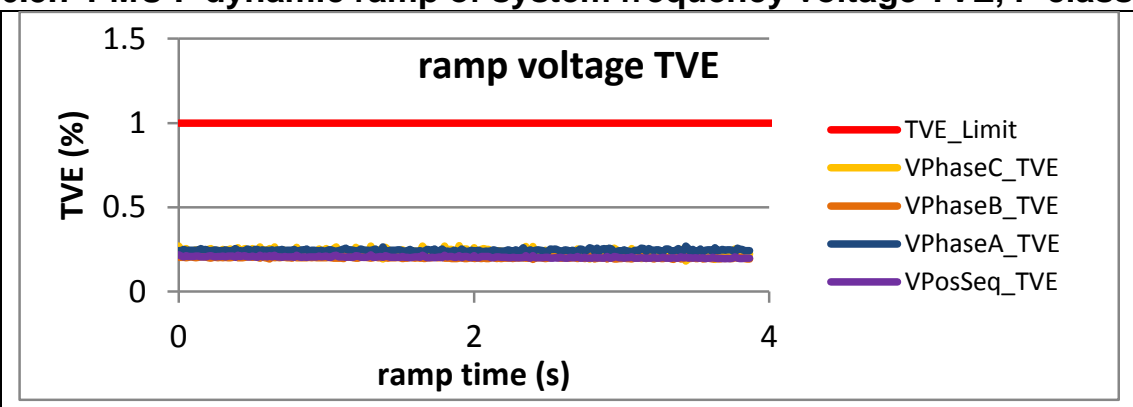

Figure 2727: Fs = $60 \mathrm{FPS}$, ramp from $55 \mathrm{~Hz}$ to $65 \mathrm{~Hz}$ at $+1 \mathrm{~Hz} / \mathrm{s}$

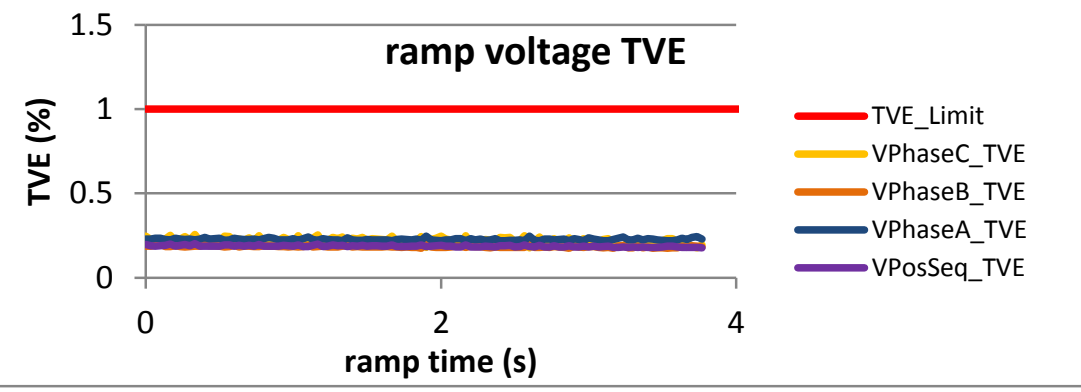

Figure 2729: Fs = $30 \mathrm{FPS}$, ramp from $55 \mathrm{~Hz}$ to $65 \mathrm{~Hz}$ at $+1 \mathrm{~Hz} / \mathrm{s}$

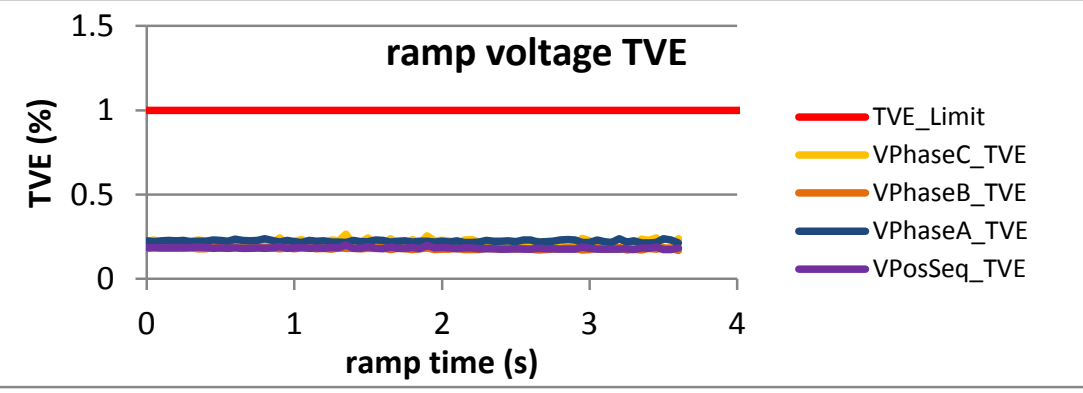

Figure 2731: Fs = $20 \mathrm{FPS}$, ramp from $56 \mathrm{~Hz}$ to $64 \mathrm{~Hz}$ at $+1 \mathrm{~Hz} / \mathrm{s}$

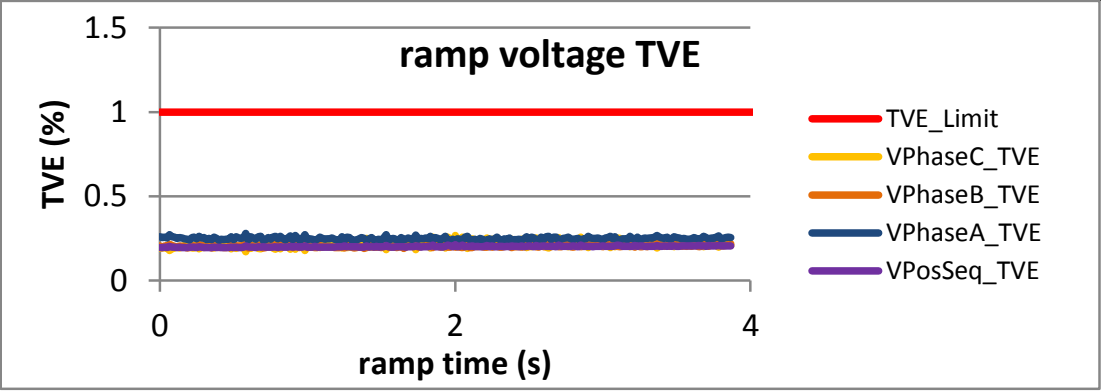

Figure 2728: Fs = $60 \mathrm{FPS}$, ramp from $65 \mathrm{~Hz}$ to $55 \mathrm{~Hz}$ at $-1 \mathrm{~Hz} / \mathrm{s}$

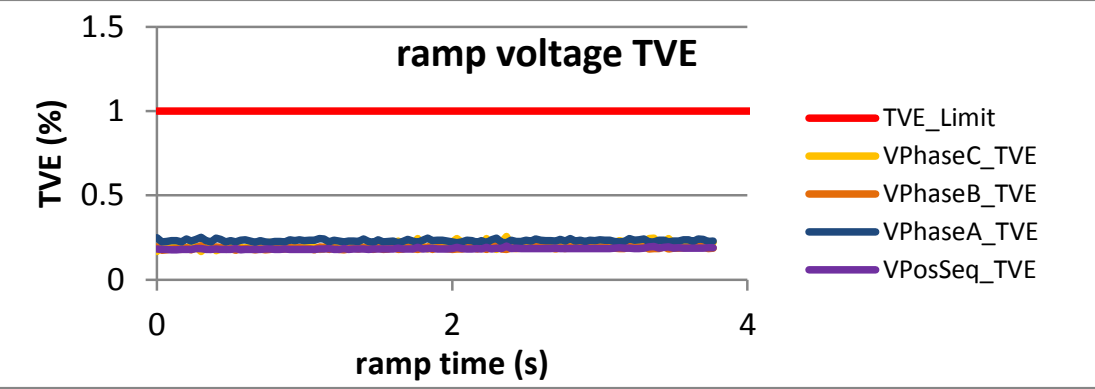

Figure 2730: Fs = $30 \mathrm{FPS}$, ramp from $65 \mathrm{~Hz}$ to $55 \mathrm{~Hz}$ at $-1 \mathrm{~Hz} / \mathrm{s}$

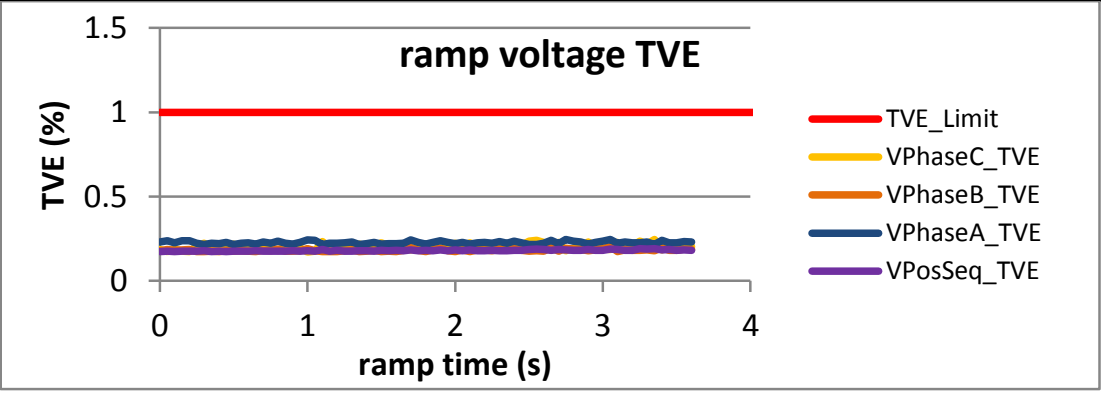

Figure 2732: Fs = $20 \mathrm{FPS}$, ramp from $64 \mathrm{~Hz}$ to $56 \mathrm{~Hz}$ at $-1 \mathrm{~Hz} / \mathrm{s}$ 


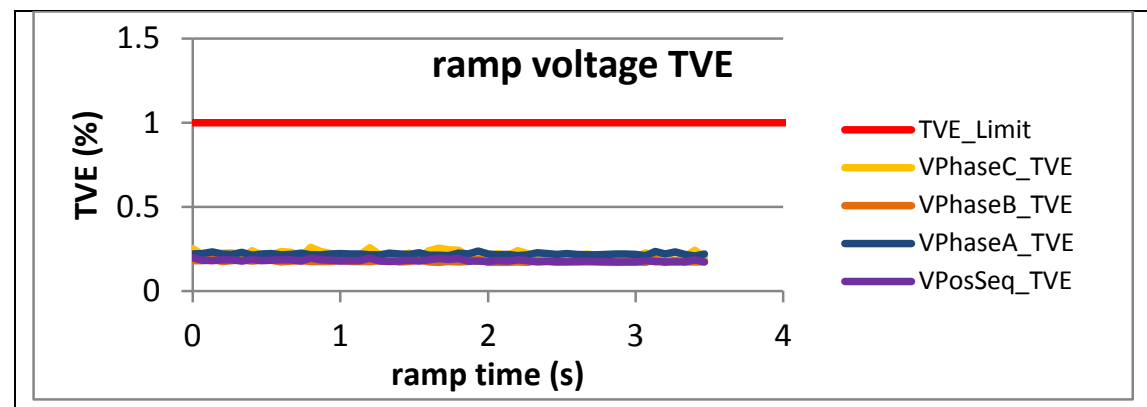

Figure 2733: Fs = $15 \mathrm{FPS}$, ramp from $57 \mathrm{~Hz}$ to $63 \mathrm{~Hz}$ at $+1 \mathrm{~Hz} / \mathrm{s}$

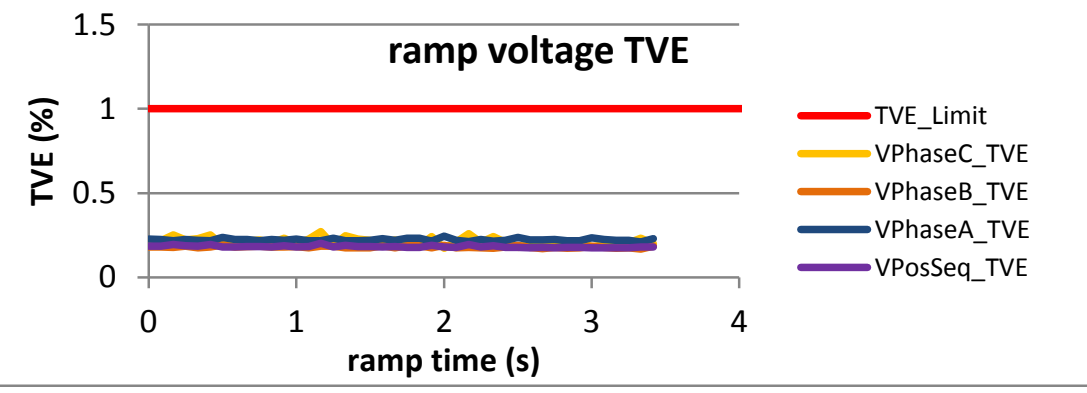

Figure 2735: Fs = 12 FPS, ramp from $58.6 \mathrm{~Hz}$ to $62.4 \mathrm{~Hz}$ at $+1 \mathrm{~Hz} / \mathrm{s}$

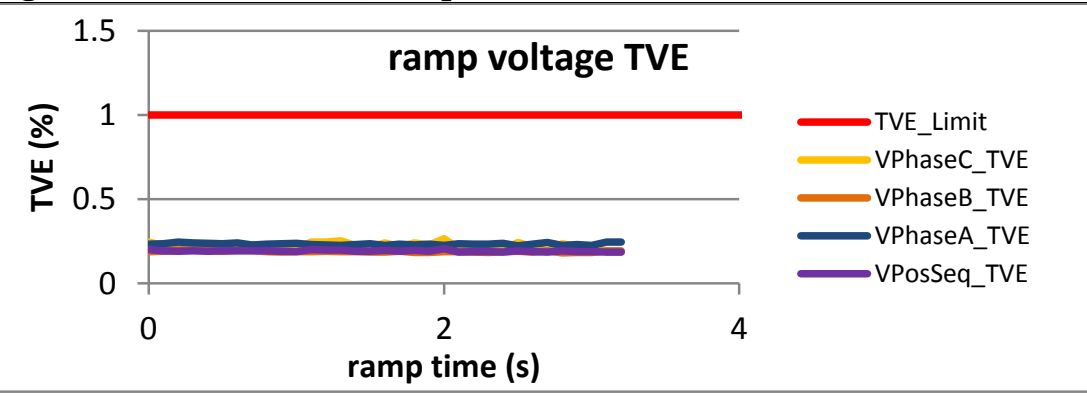

Figure 2737: Fs = $10 \mathrm{FPS}$, ramp from $58 \mathrm{~Hz}$ to $62 \mathrm{~Hz}$ at $+1 \mathrm{~Hz} / \mathrm{s}$

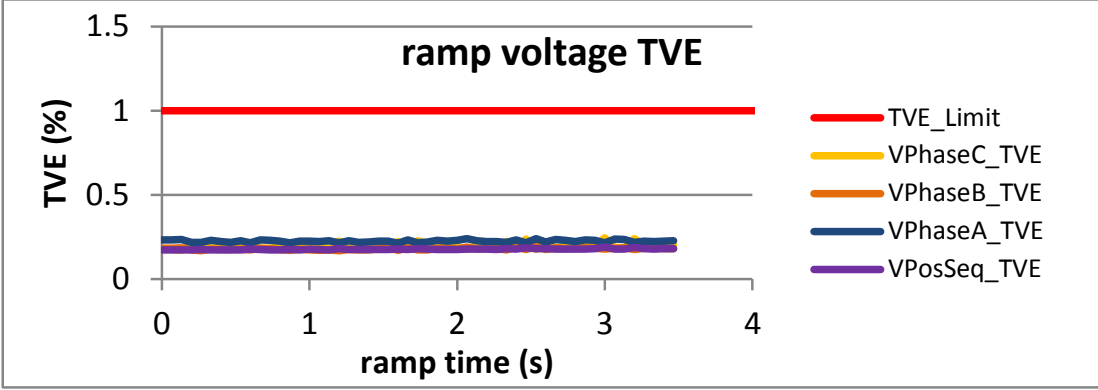

Figure 2734: Fs = $15 \mathrm{FPS}$, ramp from $63 \mathrm{~Hz}$ to $57 \mathrm{~Hz}$ at $-1 \mathrm{~Hz} / \mathrm{s}$

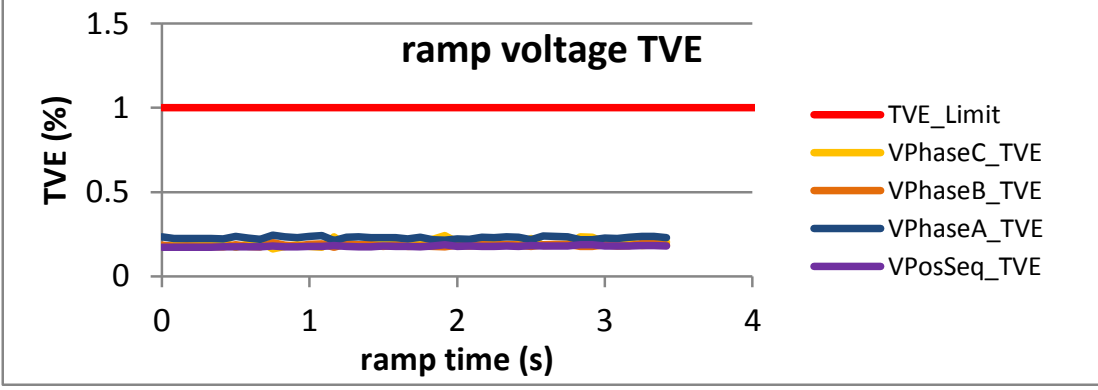

Figure 2736: Fs = 12 FPS, ramp from $62.4 \mathrm{~Hz}$ to $58.6 \mathrm{~Hz}$ at $-1 \mathrm{~Hz} / \mathrm{s}$

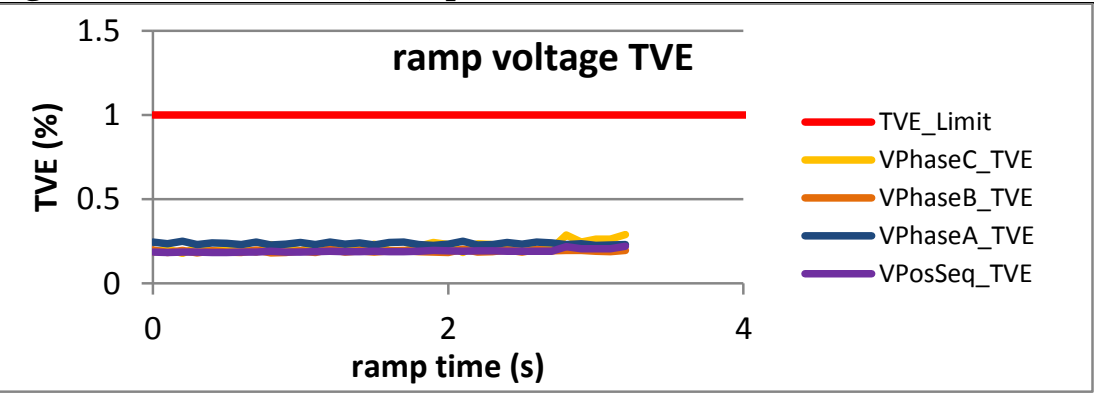

Figure 2738: Fs = $10 \mathrm{FPS}$, ramp from $62 \mathrm{~Hz}$ to $58 \mathrm{~Hz}$ at $-1 \mathrm{~Hz} / \mathrm{s}$ 
6.5.8 PMU G dynamic ramp of system frequency voltage TVE, $P$ class PMU G does not support P class.

6.5.9 PMU H dynamic ramp of system frequency voltage TVE, P class

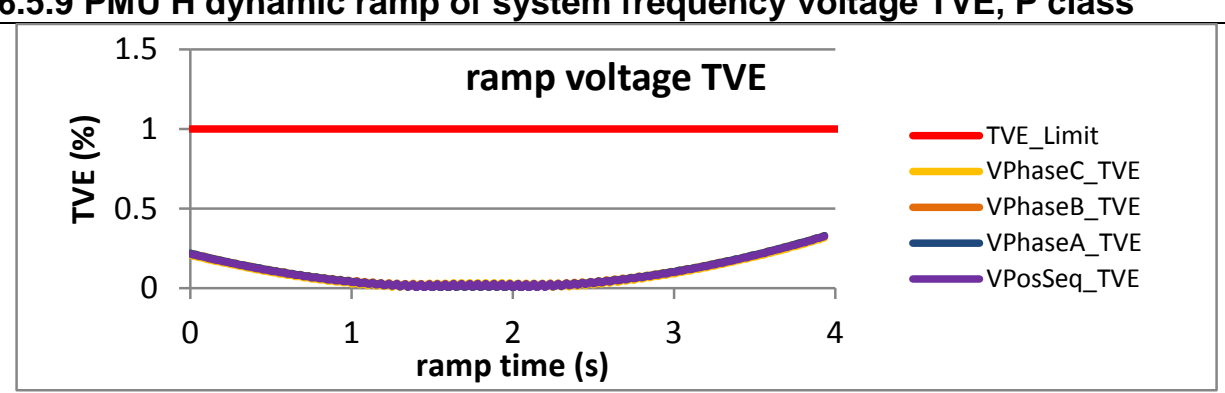

Figure 2739: Fs = $60 \mathrm{FPS}$, ramp from $58 \mathrm{~Hz}$ to $62 \mathrm{~Hz}$ at $+1 \mathrm{~Hz} / \mathrm{s}$

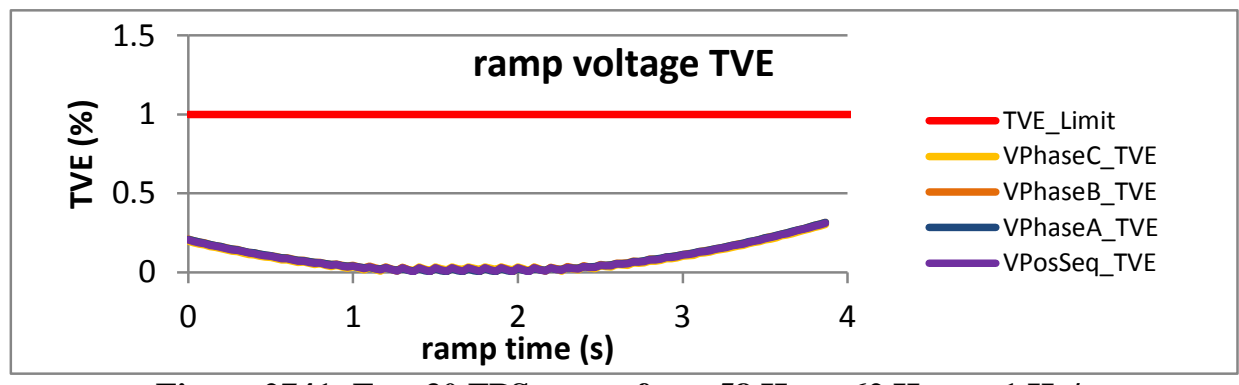

Figure 2741: Fs = $30 \mathrm{FPS}$, ramp from $58 \mathrm{~Hz}$ to $62 \mathrm{~Hz}$ at $+1 \mathrm{~Hz} / \mathrm{s}$

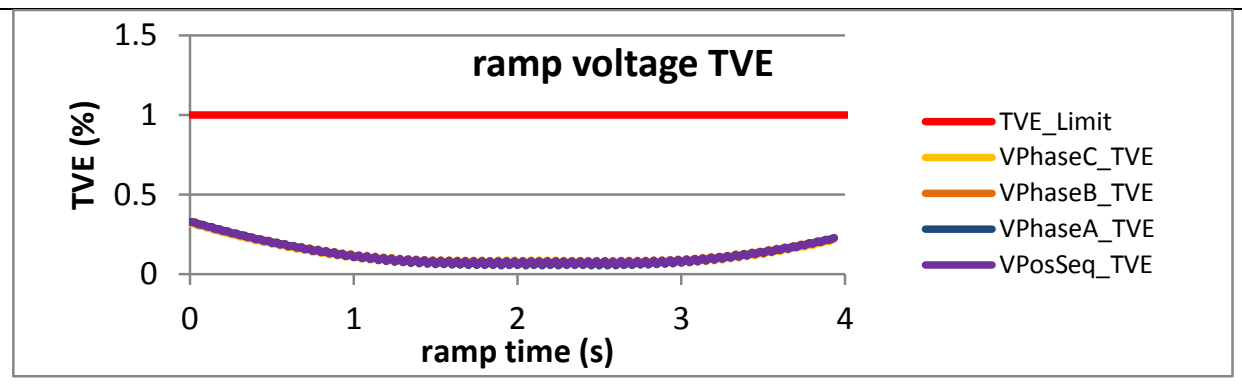

Figure 2740: Fs = $60 \mathrm{FPS}$, ramp from $62 \mathrm{~Hz}$ to $58 \mathrm{~Hz}$ at $-1 \mathrm{~Hz} / \mathrm{s}$

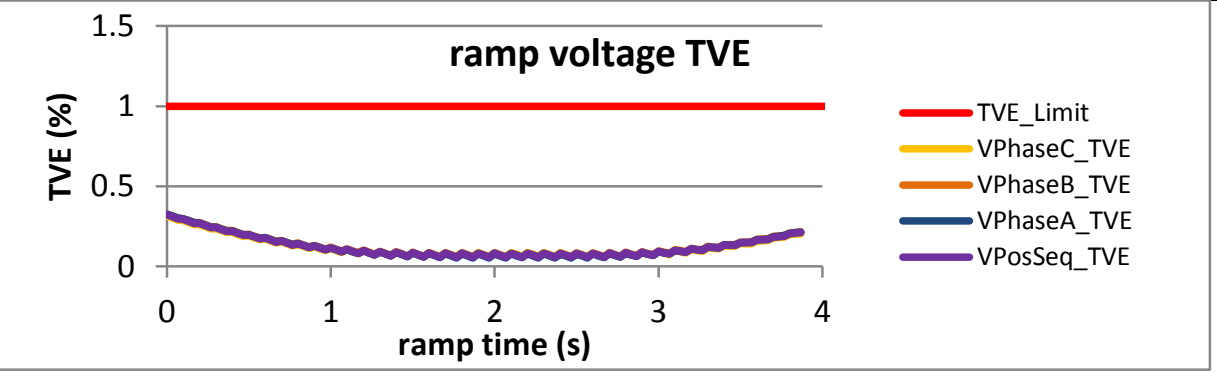

Figure 2742: Fs = $30 \mathrm{FPS}$, ramp from $62 \mathrm{~Hz}$ to $58 \mathrm{~Hz}$ at $-1 \mathrm{~Hz} / \mathrm{s}$ 

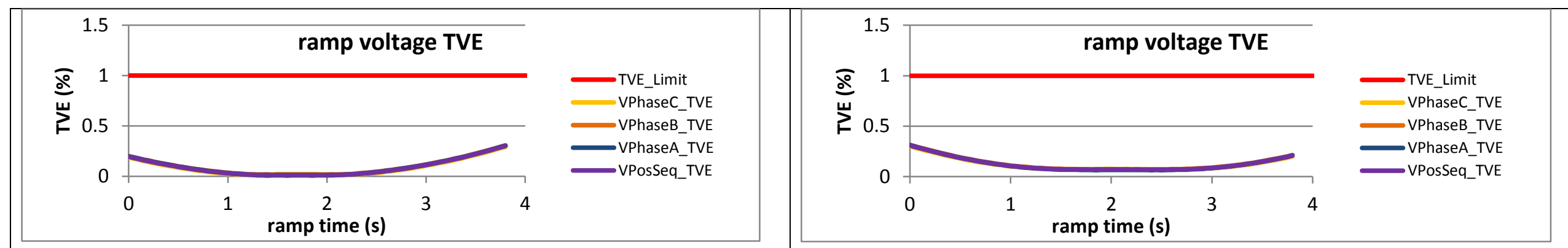

Figure 2743: Fs = 20 FPS, ramp from $58 \mathrm{~Hz}$ to $62 \mathrm{~Hz}$ at $+1 \mathrm{~Hz} / \mathrm{s}$

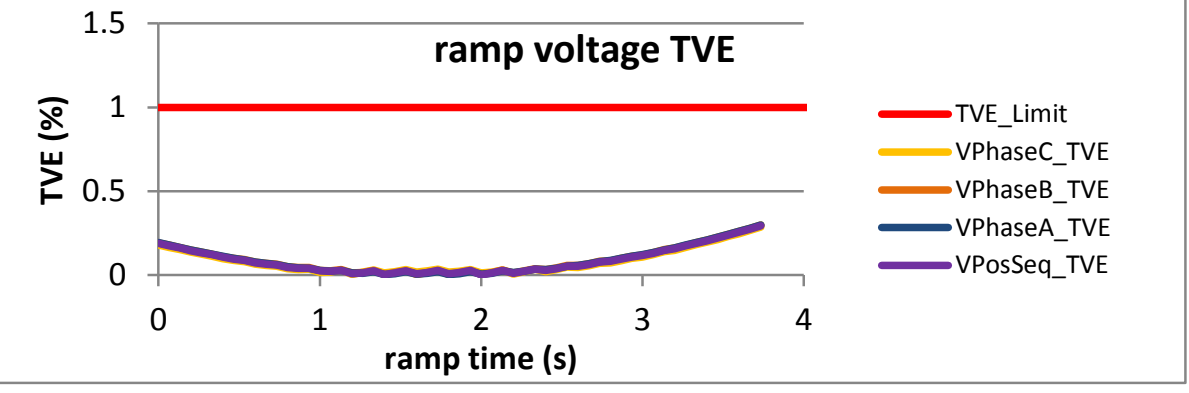

Figure 2744: Fs = $20 \mathrm{FPS}$, ramp from $62 \mathrm{~Hz}$ to $58 \mathrm{~Hz}$ at $-1 \mathrm{~Hz} / \mathrm{s}$

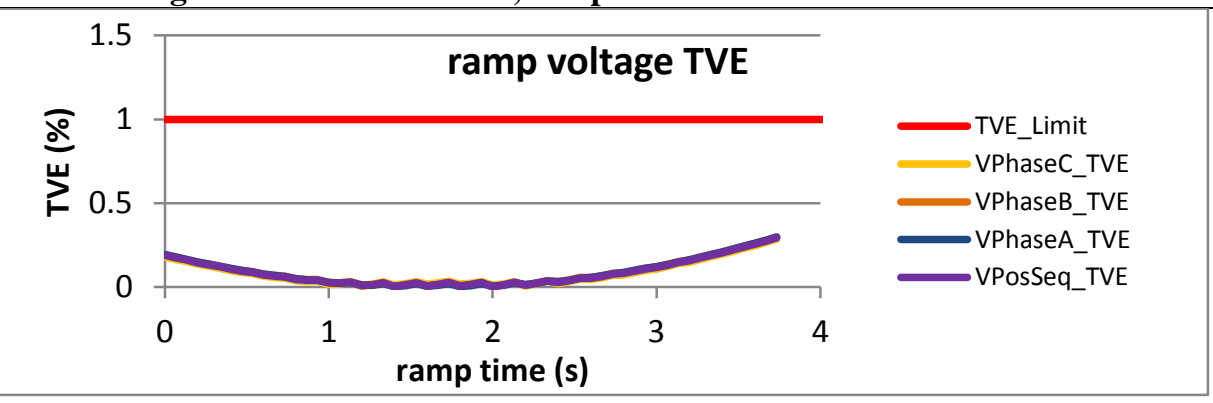

Figure 2745: Fs = $15 \mathrm{FPS}$, ramp from $58 \mathrm{~Hz}$ to $62 \mathrm{~Hz}$ at $+1 \mathrm{~Hz} / \mathrm{s}$

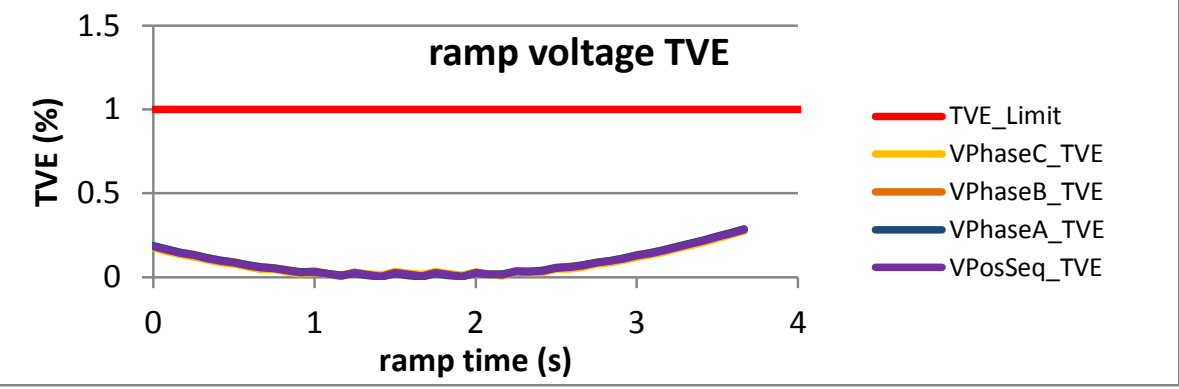

Figure 2746: Fs = $15 \mathrm{FPS}$, ramp from $63 \mathrm{~Hz}$ to $58 \mathrm{~Hz}$ at $-1 \mathrm{~Hz} / \mathrm{s}$

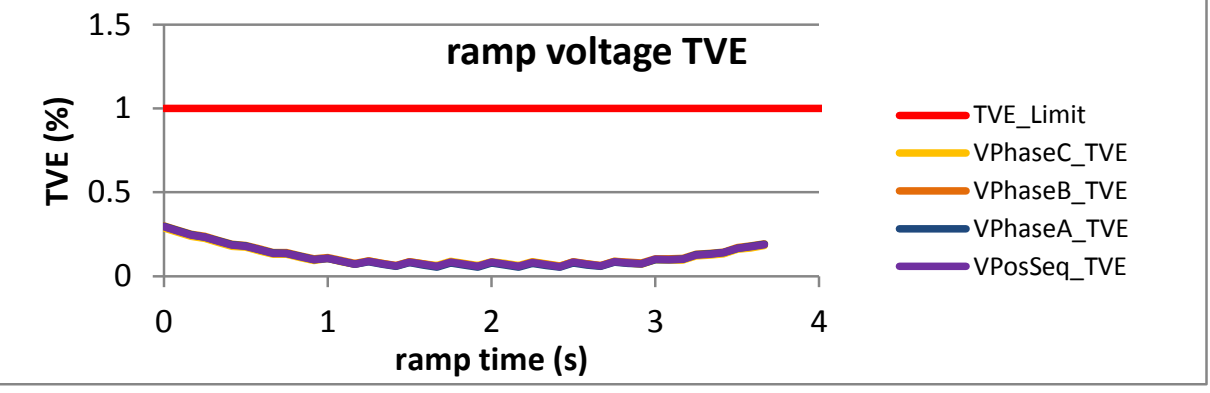

Figure 2747: Fs = 12 FPS, ramp from $58.6 \mathrm{~Hz}$ to $62 \mathrm{~Hz}$ at $+1 \mathrm{~Hz} / \mathrm{s}$

Figure 2748: Fs = $12 \mathrm{FPS}$, ramp from $62 \mathrm{~Hz}$ to $58 \mathrm{~Hz}$ at $-1 \mathrm{~Hz} / \mathrm{s}$ 


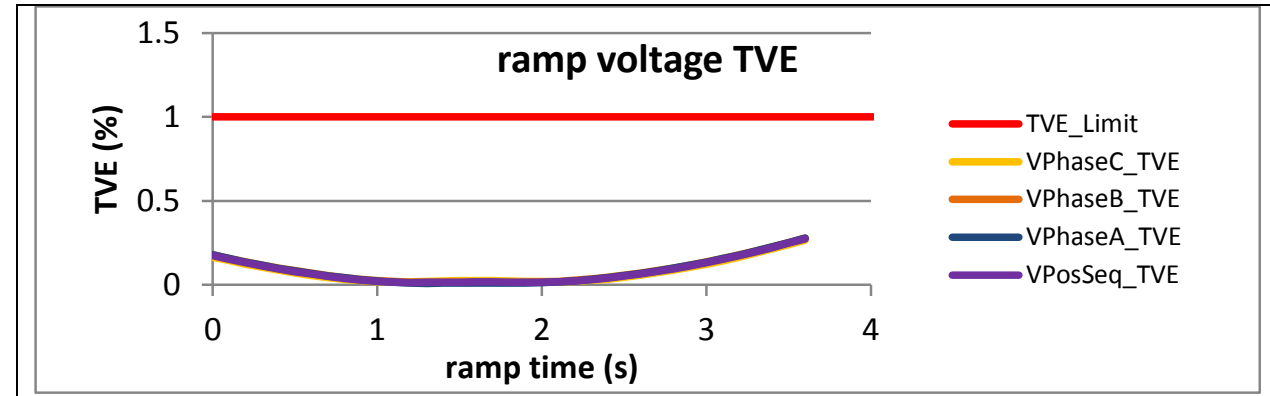

Figure 2749: Fs = $10 \mathrm{FPS}$, ramp from $58 \mathrm{~Hz}$ to $62 \mathrm{~Hz}$ at $+1 \mathrm{~Hz} / \mathrm{s}$

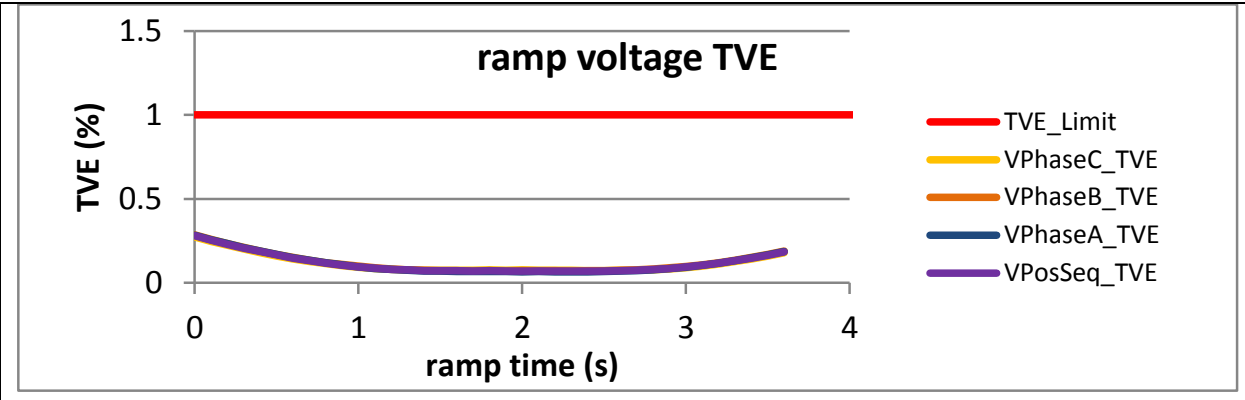

Figure 2750: Fs = $10 \mathrm{FPS}$, ramp from $62 \mathrm{~Hz}$ to $58 \mathrm{~Hz}$ at $-1 \mathrm{~Hz} / \mathrm{s}$ 
6.5.10 PMU I dynamic ramp of system frequency voltage TVE, $P$ class

PMU I does not support P class

\subsubsection{PMU J dynamic ramp of system frequency voltage TVE, P class}

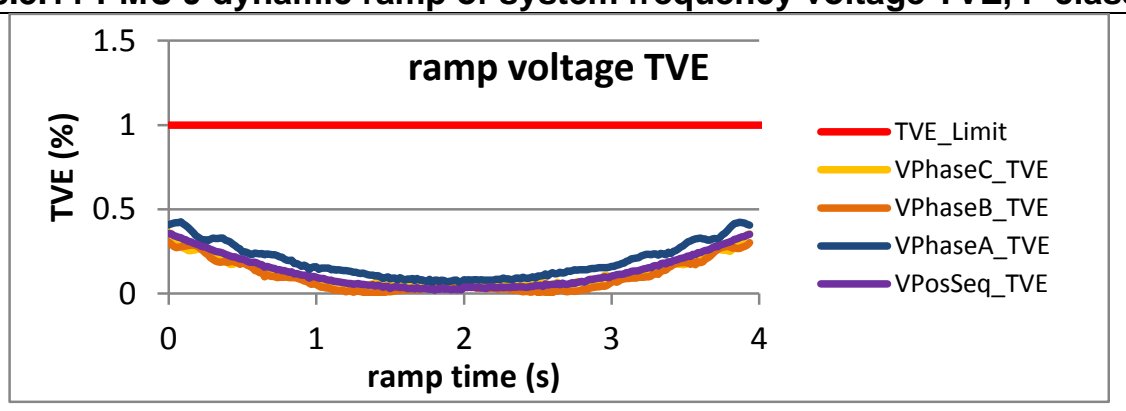

Figure 2751: Fs = $60 \mathrm{FPS}$, ramp from $55 \mathrm{~Hz}$ to $65 \mathrm{~Hz}$ at $+1 \mathrm{~Hz} / \mathrm{s}$

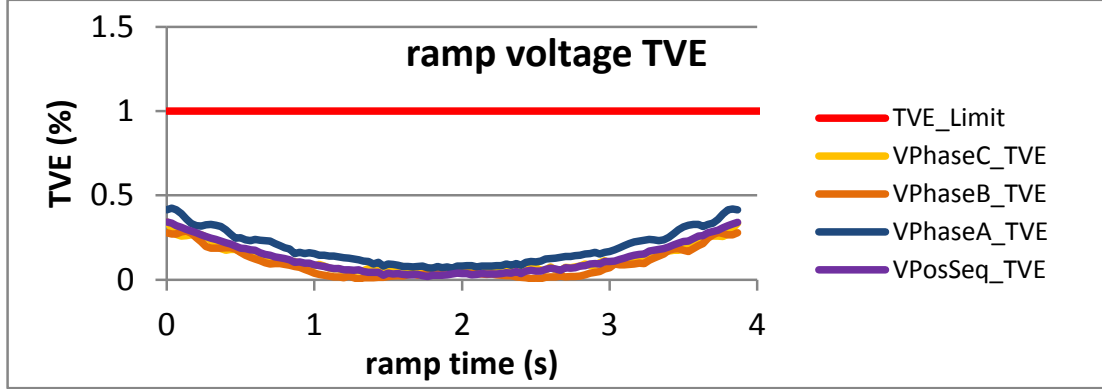

Figure 2753: Fs = $30 \mathrm{FPS}$, ramp from $55 \mathrm{~Hz}$ to $65 \mathrm{~Hz}$ at $+1 \mathrm{~Hz} / \mathrm{s}$

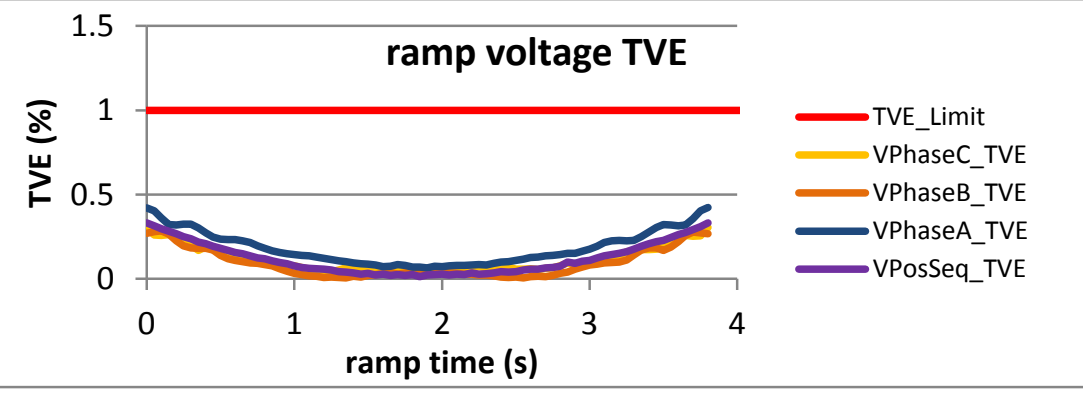

Figure 2755: Fs $=20 \mathrm{FPS}$, ramp from $56 \mathrm{~Hz}$ to $64 \mathrm{~Hz}$ at $+1 \mathrm{~Hz} / \mathrm{s}$

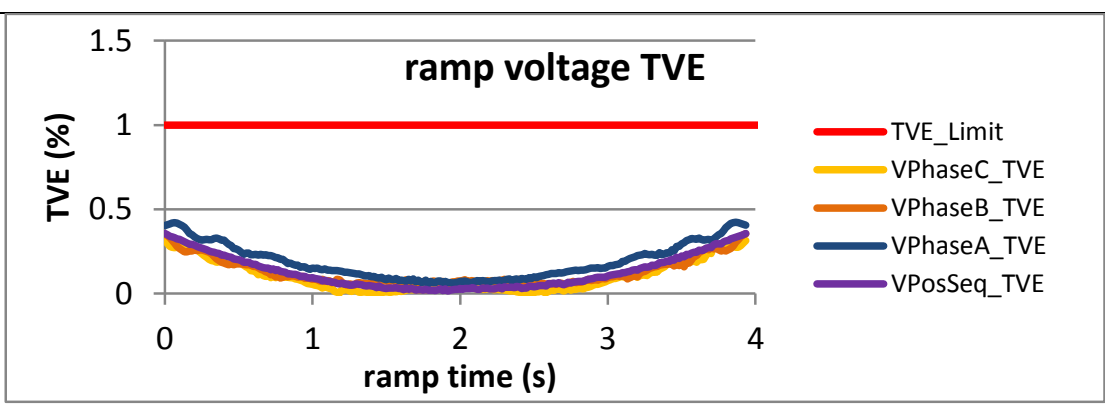

Figure 2752: Fs = $60 \mathrm{FPS}$, ramp from $65 \mathrm{~Hz}$ to $55 \mathrm{~Hz}$ at $-1 \mathrm{~Hz} / \mathrm{s}$

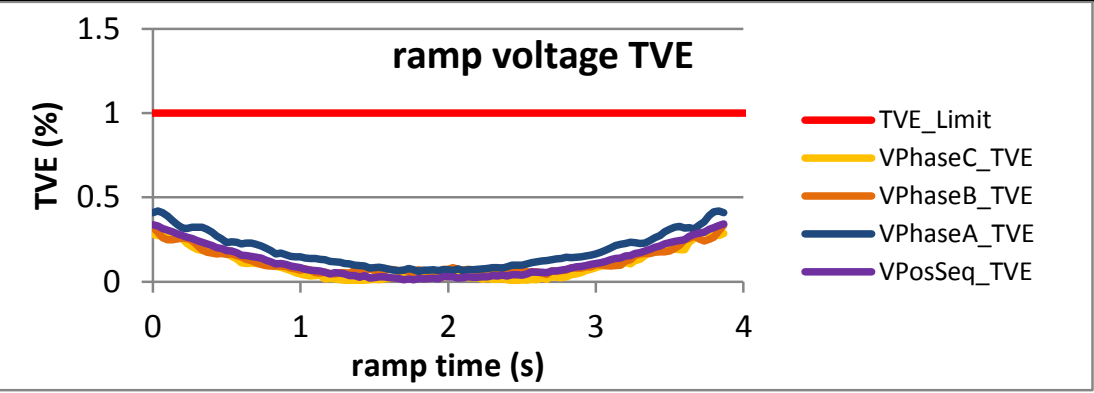

Figure 2754: Fs = $30 \mathrm{FPS}$, ramp from $65 \mathrm{~Hz}$ to $55 \mathrm{~Hz}$ at $-1 \mathrm{~Hz} / \mathrm{s}$

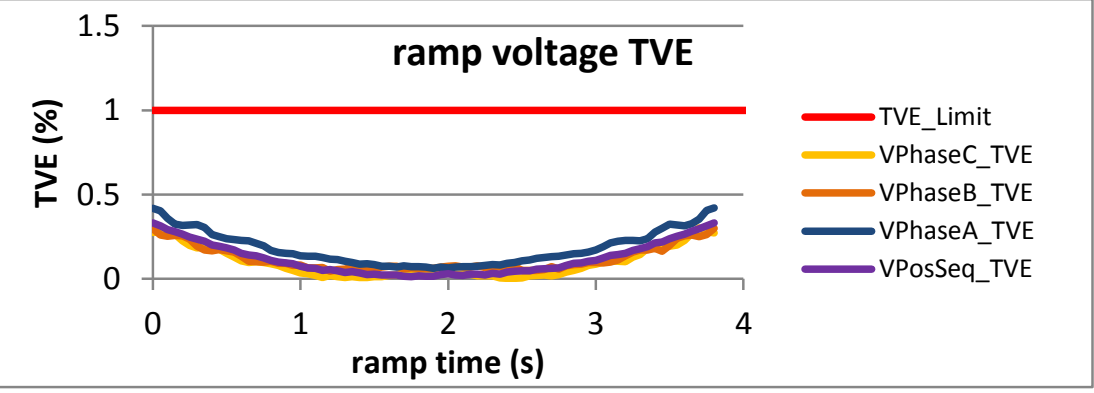

Figure 2756: Fs = $20 \mathrm{FPS}$, ramp from $64 \mathrm{~Hz}$ to $56 \mathrm{~Hz}$ at $-1 \mathrm{~Hz} / \mathrm{s}$ 


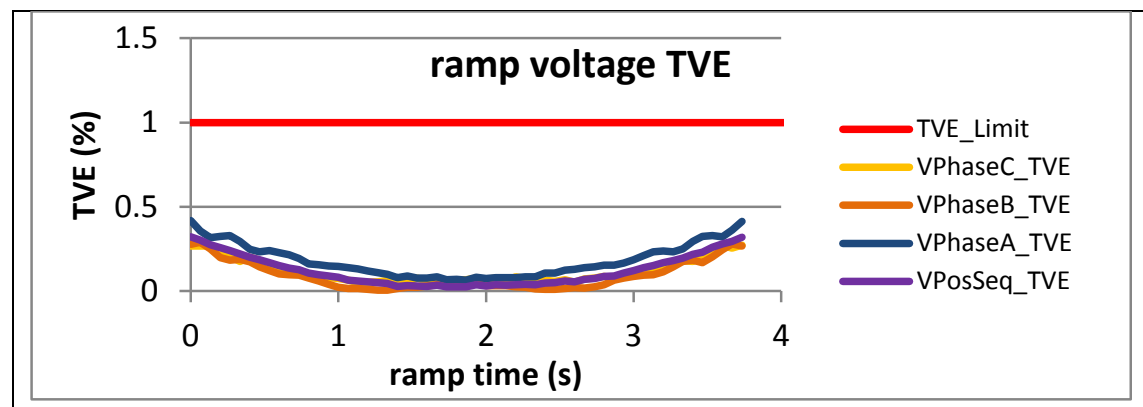

Figure 2757: Fs = $15 \mathrm{FPS}$, ramp from $57 \mathrm{~Hz}$ to $63 \mathrm{~Hz}$ at $+1 \mathrm{~Hz} / \mathrm{s}$

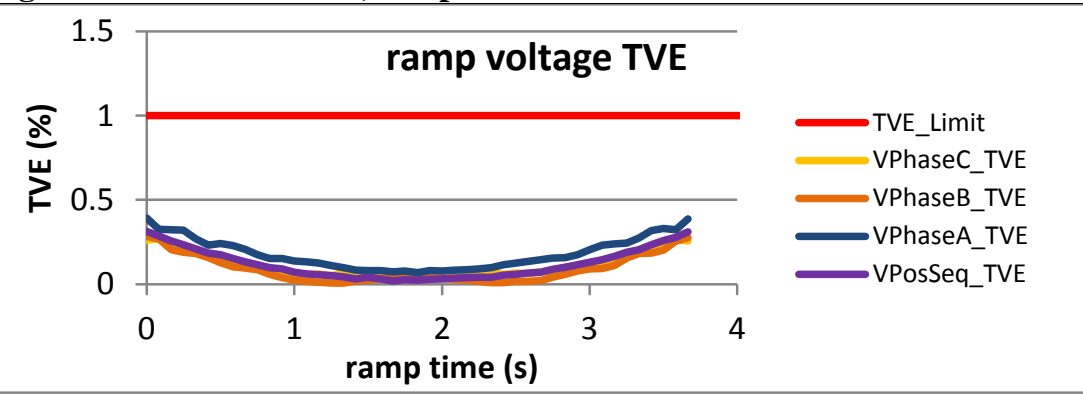

Figure 2759: Fs = 12 FPS, ramp from $58.6 \mathrm{~Hz}$ to $62.4 \mathrm{~Hz}$ at $+1 \mathrm{~Hz} / \mathrm{s}$

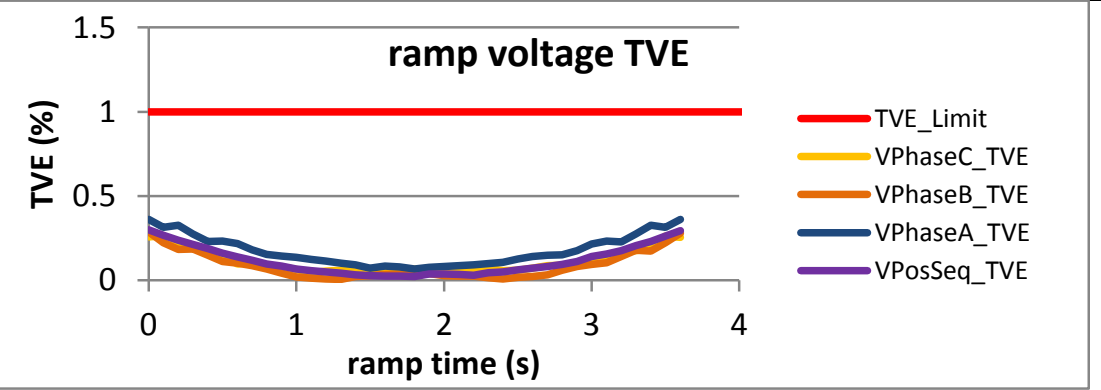

Figure 2761: Fs = $10 \mathrm{FPS}$, ramp from $58 \mathrm{~Hz}$ to $62 \mathrm{~Hz}$ at $+1 \mathrm{~Hz} / \mathrm{s}$

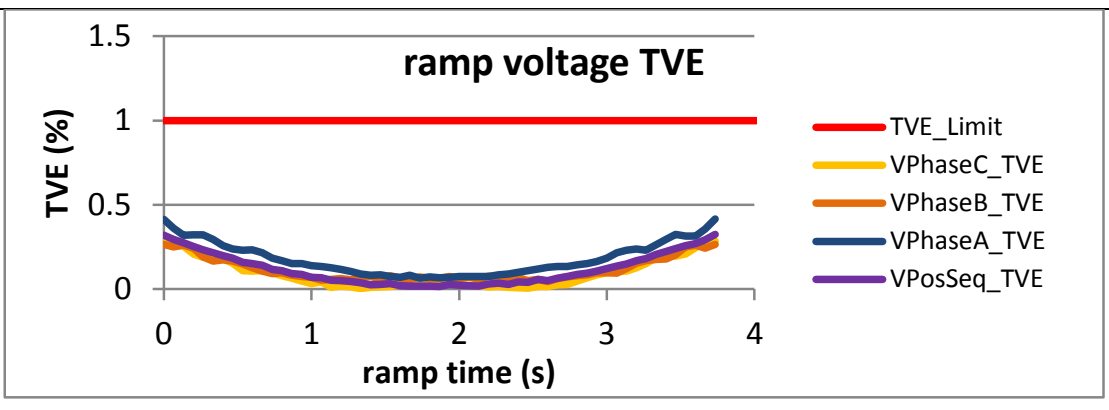

Figure 2758: Fs = $15 \mathrm{FPS}$, ramp from $63 \mathrm{~Hz}$ to $57 \mathrm{~Hz}$ at $-1 \mathrm{~Hz} / \mathrm{s}$

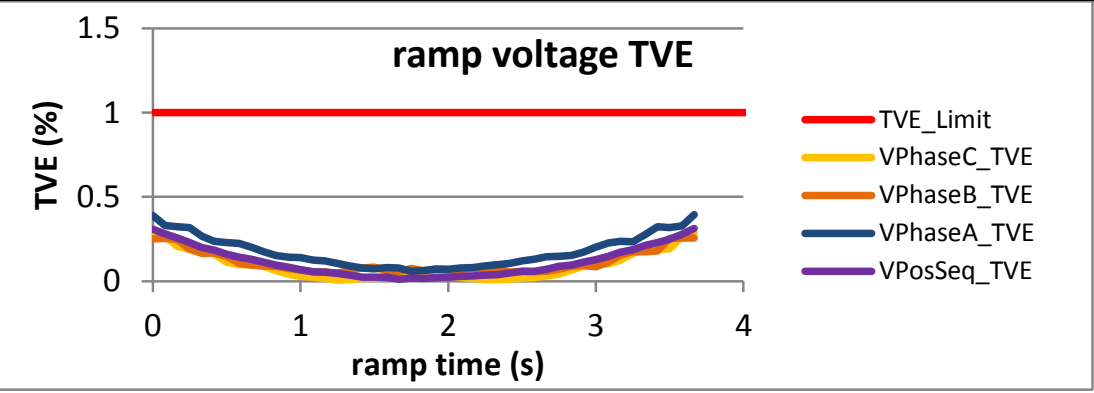

Figure 2760: Fs = $12 \mathrm{FPS}$, ramp from $62.4 \mathrm{~Hz}$ to $58.6 \mathrm{~Hz}$ at $-1 \mathrm{~Hz} / \mathrm{s}$

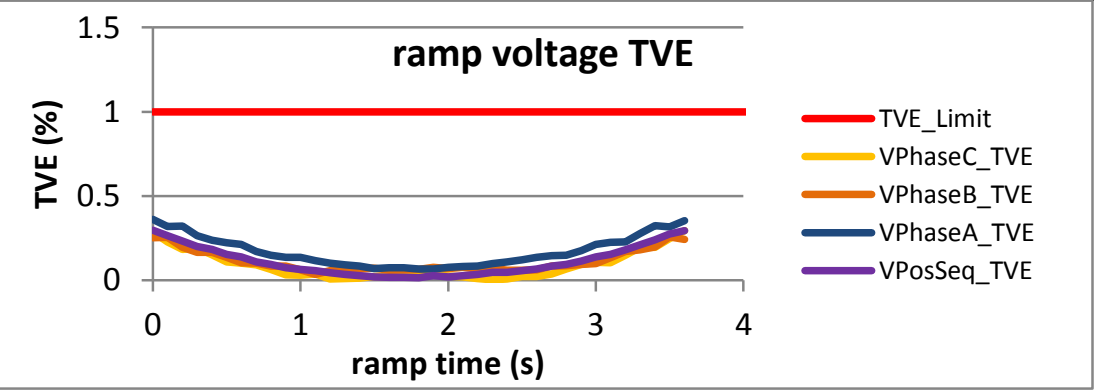

Figure 2762: Fs = $10 \mathrm{FPS}$, ramp from $62 \mathrm{~Hz}$ to $58 \mathrm{~Hz}$ at $-1 \mathrm{~Hz} / \mathrm{s}$ 


\subsection{Dynamic ramp of system frequency current TVE, P class}

\subsubsection{C37.118.1 Annex C dynamic ramp of system frequency current TVE, P class}

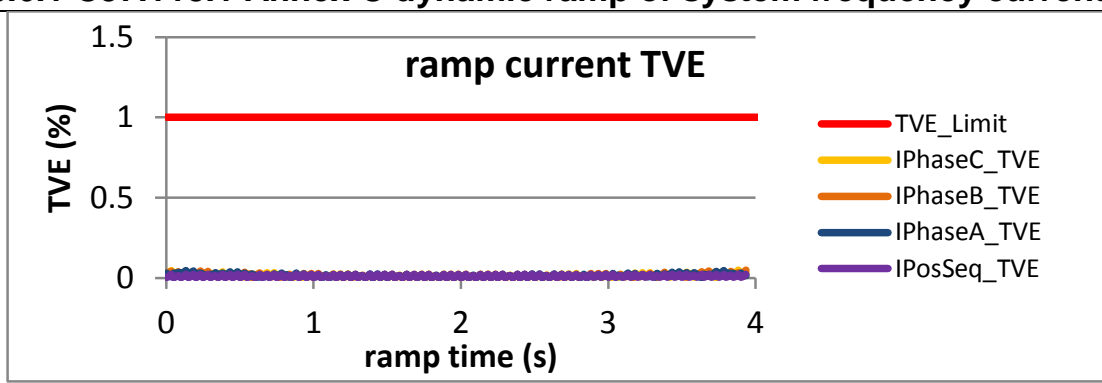

Figure 2763: Fs = $60 \mathrm{FPS}$, ramp from $58 \mathrm{~Hz}$ to $62 \mathrm{~Hz}$ at $+1 \mathrm{~Hz} / \mathrm{s}$

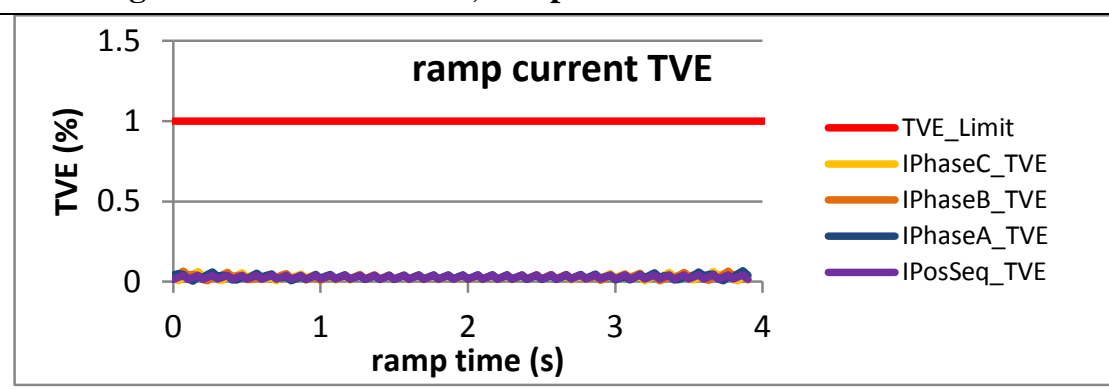

Figure 2765: Fs = $30 \mathrm{FPS}$, ramp from $58 \mathrm{~Hz}$ to $62 \mathrm{~Hz}$ at $+1 \mathrm{~Hz} / \mathrm{s}$

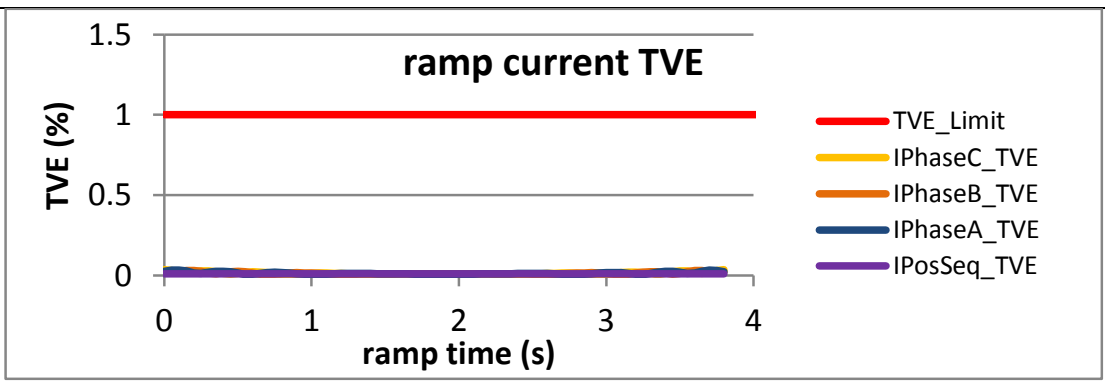

Figure 2767: Fs = $20 \mathrm{FPS}$, ramp from $58 \mathrm{~Hz}$ to $62 \mathrm{~Hz}$ at $+1 \mathrm{~Hz} / \mathrm{s}$

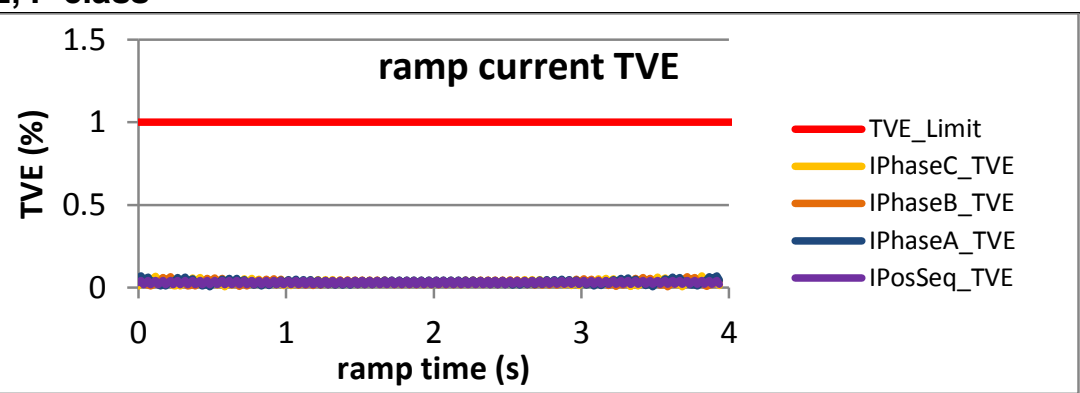

Figure 2764: Fs = $60 \mathrm{FPS}$, ramp from $62 \mathrm{~Hz}$ to $58 \mathrm{~Hz}$ at $-1 \mathrm{~Hz} / \mathrm{s}$

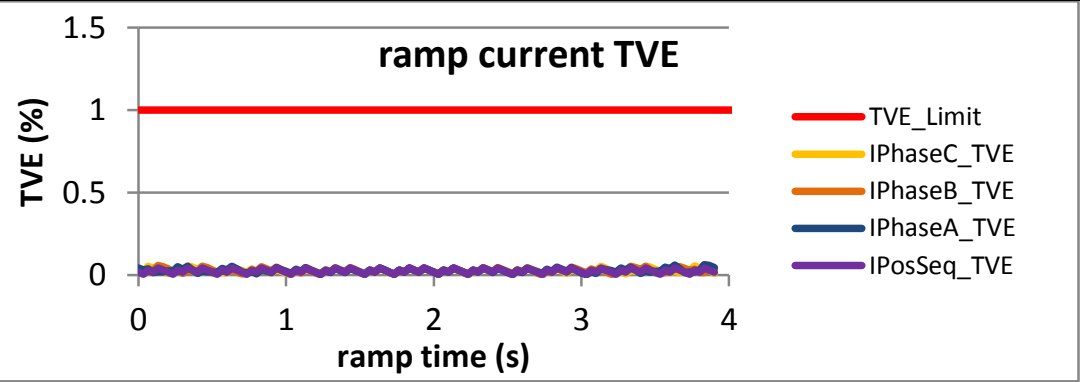

Figure 2766: Fs = $30 \mathrm{FPS}$, ramp from $62 \mathrm{~Hz}$ to $58 \mathrm{~Hz}$ at $-1 \mathrm{~Hz} / \mathrm{s}$

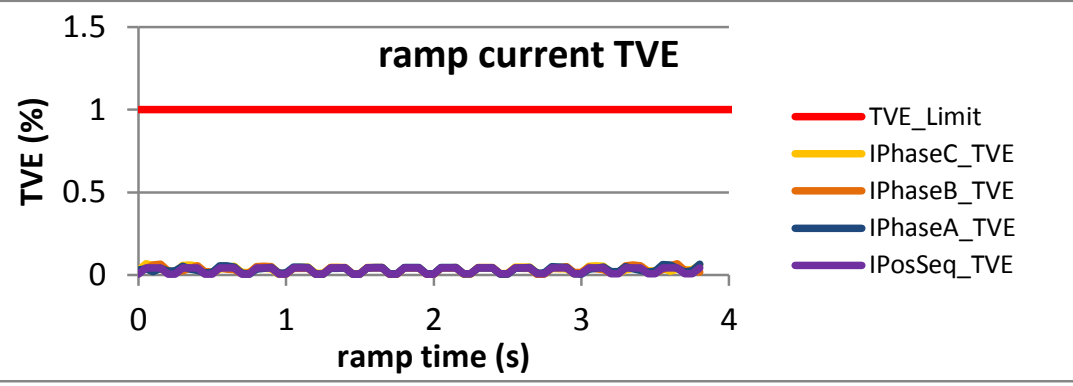

Figure 2768: Fs = $20 \mathrm{FPS}$, ramp from $62 \mathrm{~Hz}$ to $58 \mathrm{~Hz}$ at $-1 \mathrm{~Hz} / \mathrm{s}$ 


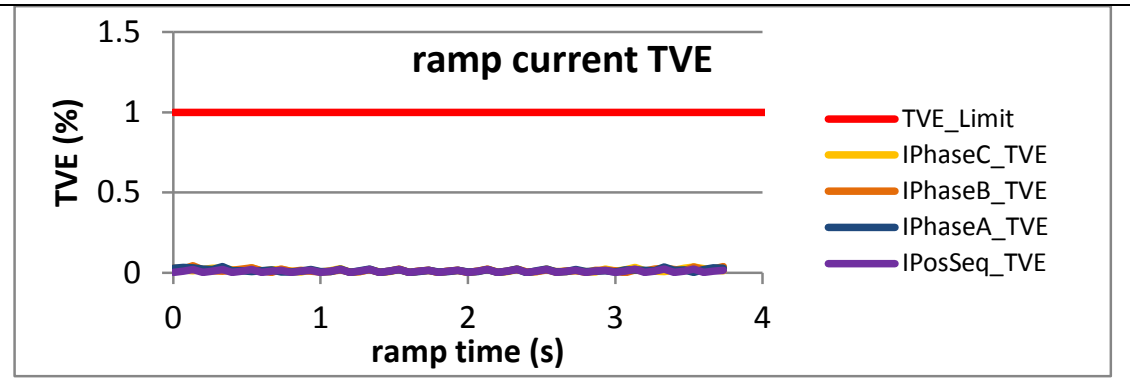

Figure 2769: Fs = $15 \mathrm{FPS}$, ramp from $58 \mathrm{~Hz}$ to $62 \mathrm{~Hz}$ at $+1 \mathrm{~Hz} / \mathrm{s}$

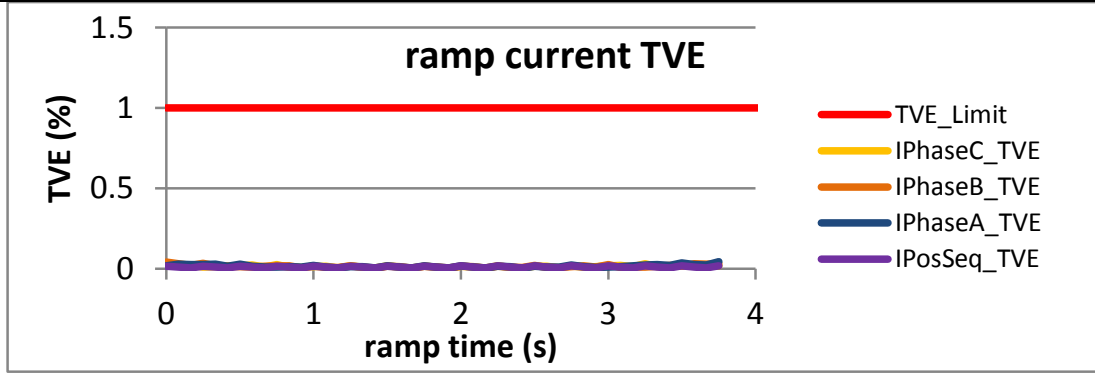

Figure 2771: Fs = $12 \mathrm{FPS}$, ramp from $58 \mathrm{~Hz}$ to $62 \mathrm{~Hz}$ at $+1 \mathrm{~Hz} / \mathrm{s}$

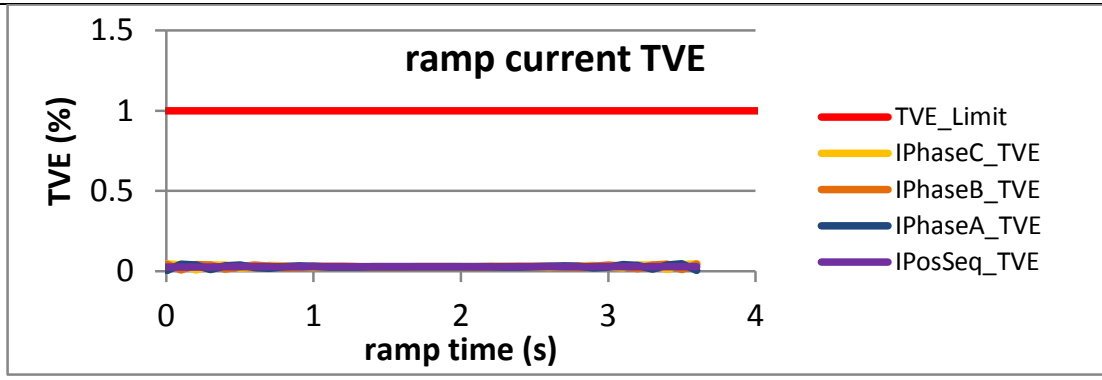

Figure 2773: Fs = $10 \mathrm{FPS}$, ramp from $58 \mathrm{~Hz}$ to $62 \mathrm{~Hz}$ at $+1 \mathrm{~Hz} / \mathrm{s}$

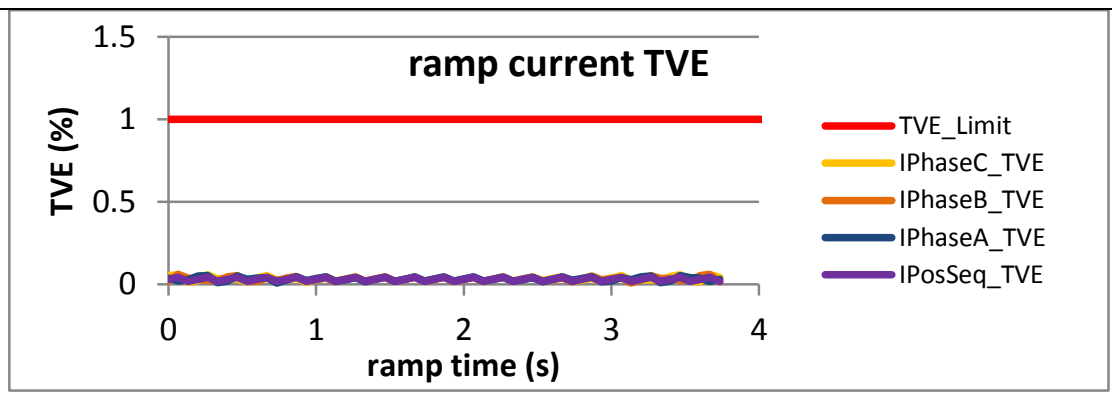

Figure 2770: Fs = $15 \mathrm{FPS}$, ramp from $62 \mathrm{~Hz}$ to $58 \mathrm{~Hz}$ at $-1 \mathrm{~Hz} / \mathrm{s}$

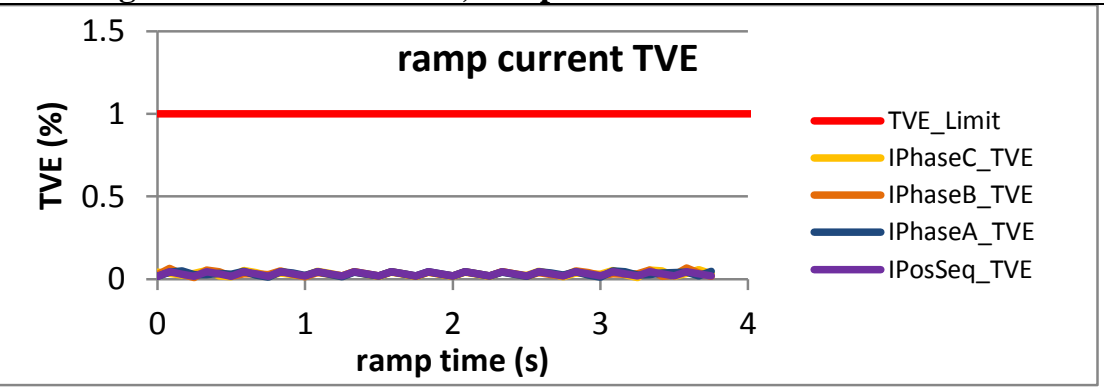

Figure 2772: Fs = $12 \mathrm{FPS}$, ramp from $62 \mathrm{~Hz}$ to $58 \mathrm{~Hz}$ at $-1 \mathrm{~Hz} / \mathrm{s}$

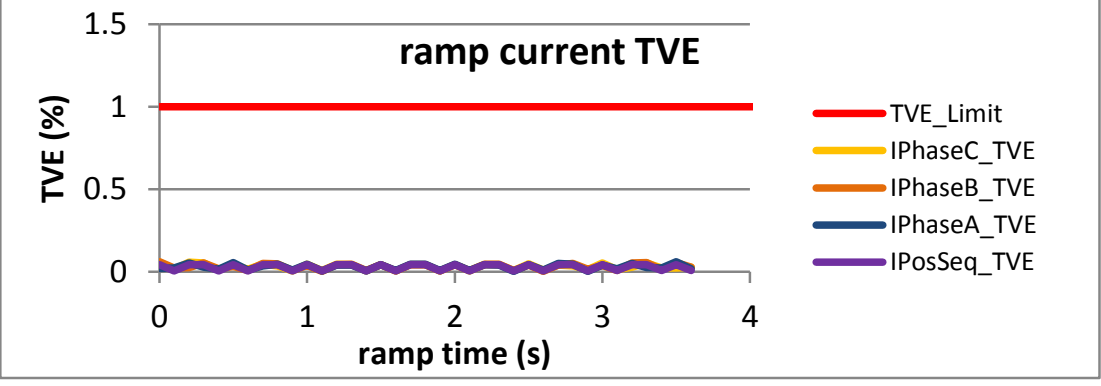

Figure 2774: Fs = $10 \mathrm{FPS}$, ramp from $62 \mathrm{~Hz}$ to $58 \mathrm{~Hz}$ at $-1 \mathrm{~Hz} / \mathrm{s}$ 
6.6.2 PMU A dynamic ramp of system frequency current TVE, $P$ class

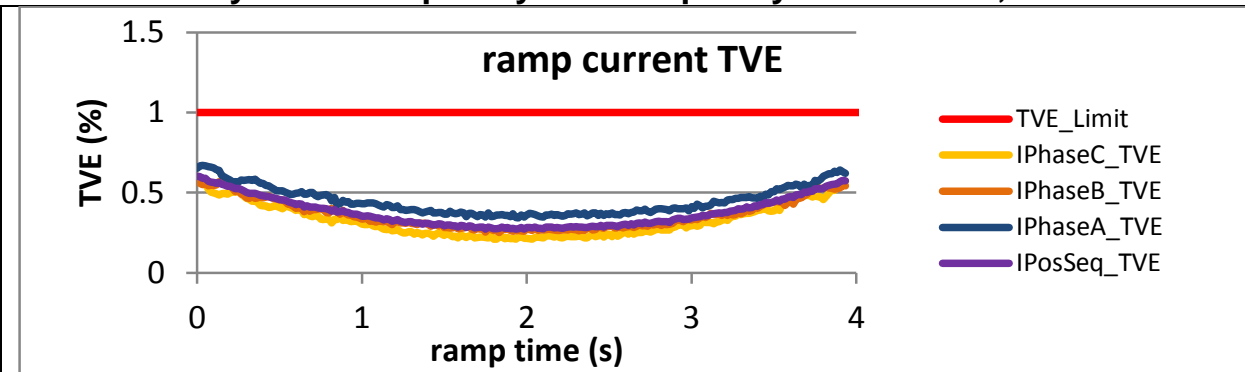

Figure 2775: Fs = $60 \mathrm{FPS}$, ramp from $58 \mathrm{~Hz}$ to $62 \mathrm{~Hz}$ at $+1 \mathrm{~Hz} / \mathrm{s}$

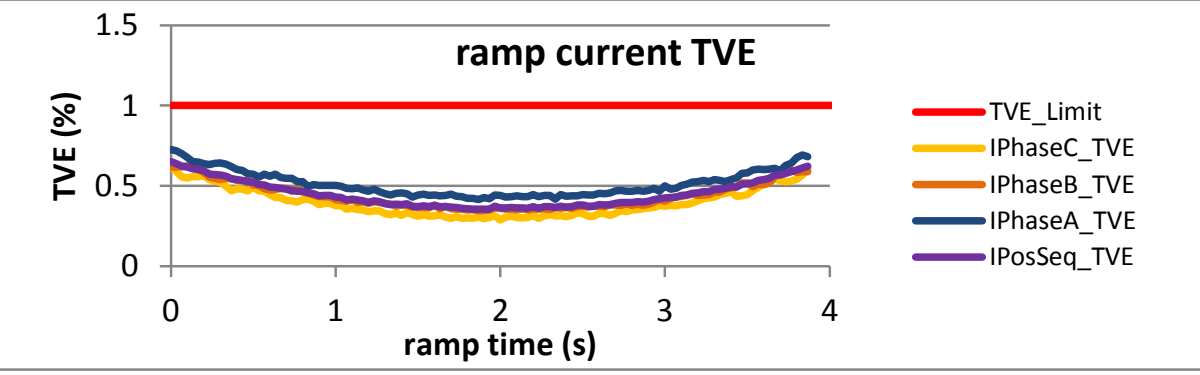

Figure 2777: Fs = $30 \mathrm{FPS}$, ramp from $58 \mathrm{~Hz}$ to $62 \mathrm{~Hz}$ at $+1 \mathrm{~Hz} / \mathrm{s}$

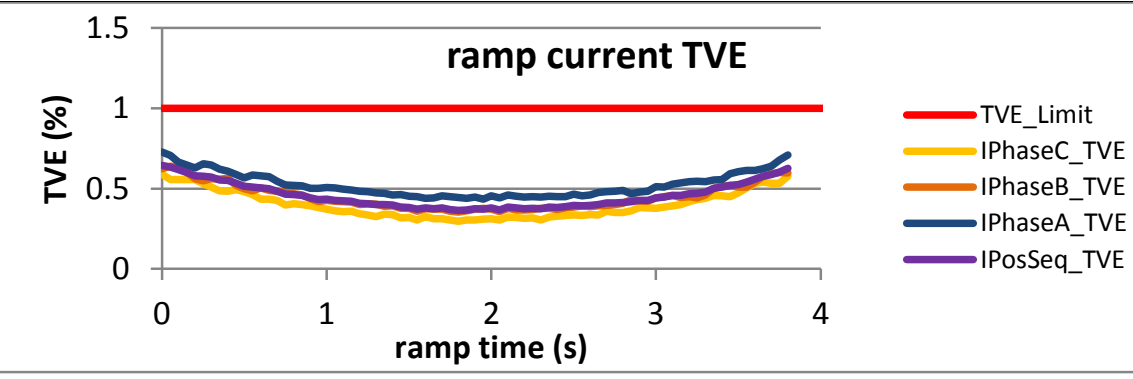

Figure 2779: Fs = $20 \mathrm{FPS}$, ramp from $58 \mathrm{~Hz}$ to $62 \mathrm{~Hz}$ at $+1 \mathrm{~Hz} / \mathrm{s}$

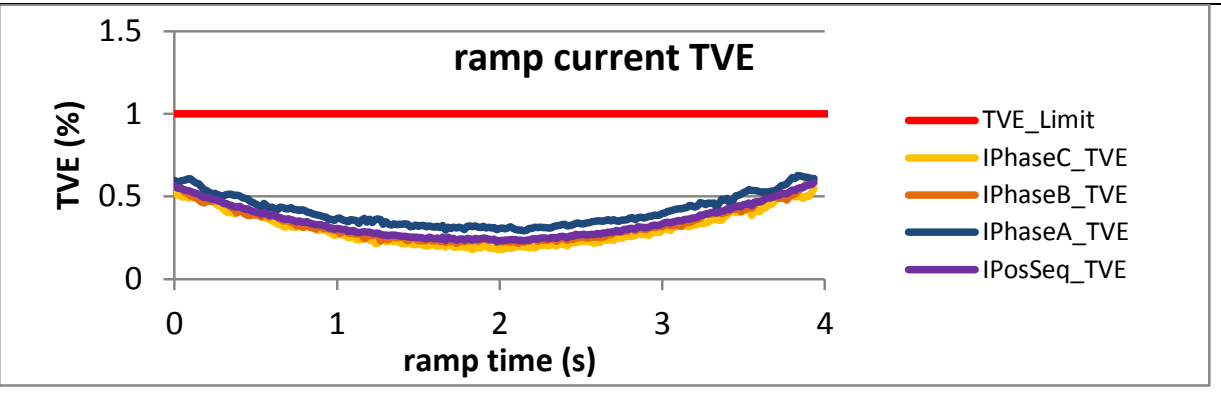

Figure 2776: Fs = $60 \mathrm{FPS}$, ramp from $62 \mathrm{~Hz}$ to $58 \mathrm{~Hz}$ at $-1 \mathrm{~Hz} / \mathrm{s}$

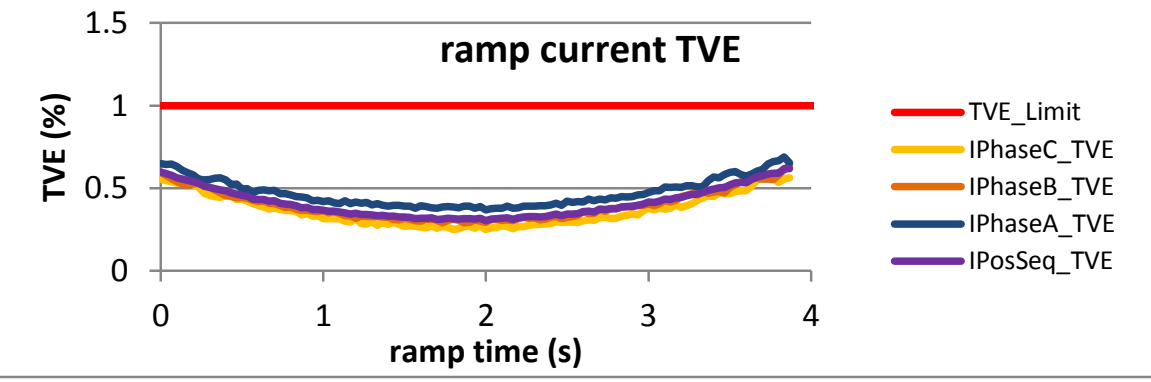

Figure 2778: Fs = $30 \mathrm{FPS}$, ramp from $62 \mathrm{~Hz}$ to $58 \mathrm{~Hz}$ at $-1 \mathrm{~Hz} / \mathrm{s}$

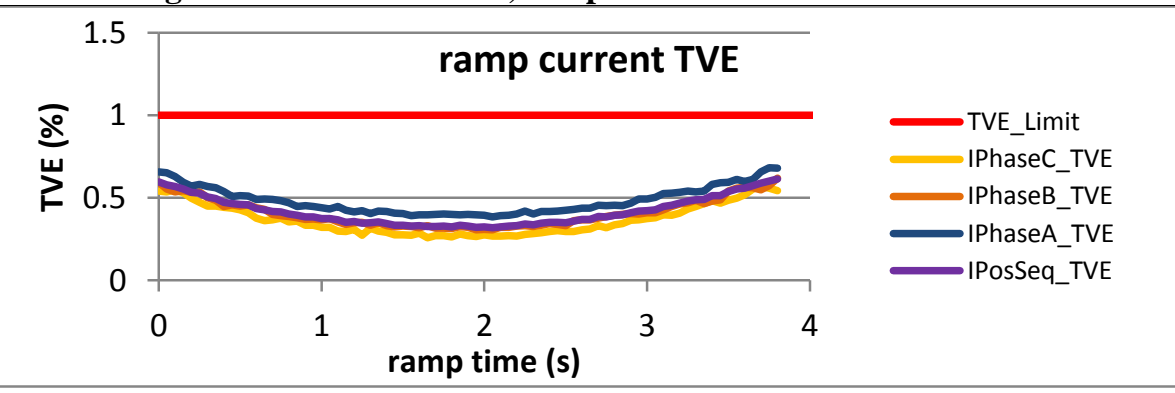

Figure 2780: Fs = $20 \mathrm{FPS}$, ramp from $62 \mathrm{~Hz}$ to $58 \mathrm{~Hz}$ at $-1 \mathrm{~Hz} / \mathrm{s}$ 

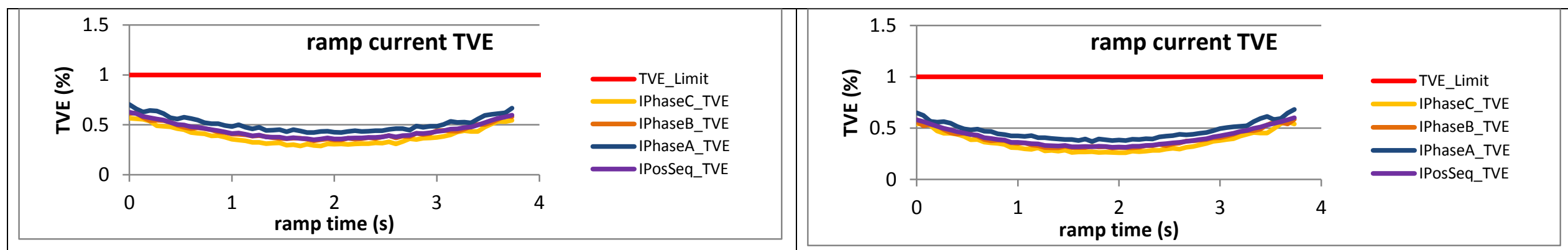

Figure 2781: Fs = $15 \mathrm{FPS}$, ramp from $58 \mathrm{~Hz}$ to $62 \mathrm{~Hz}$ at $+1 \mathrm{~Hz} / \mathrm{s}$

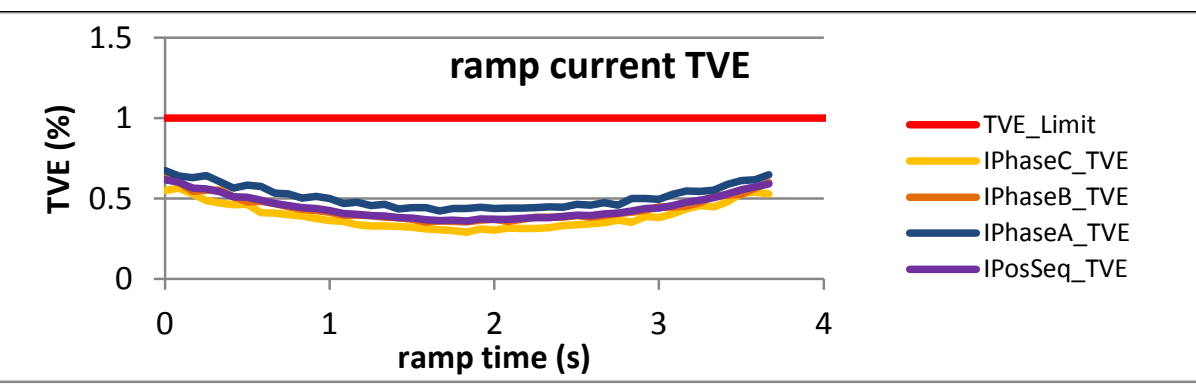

Figure 2783: Fs = 12 FPS, ramp from $58 \mathrm{~Hz}$ to $62 \mathrm{~Hz}$ at $+1 \mathrm{~Hz} / \mathrm{s}$

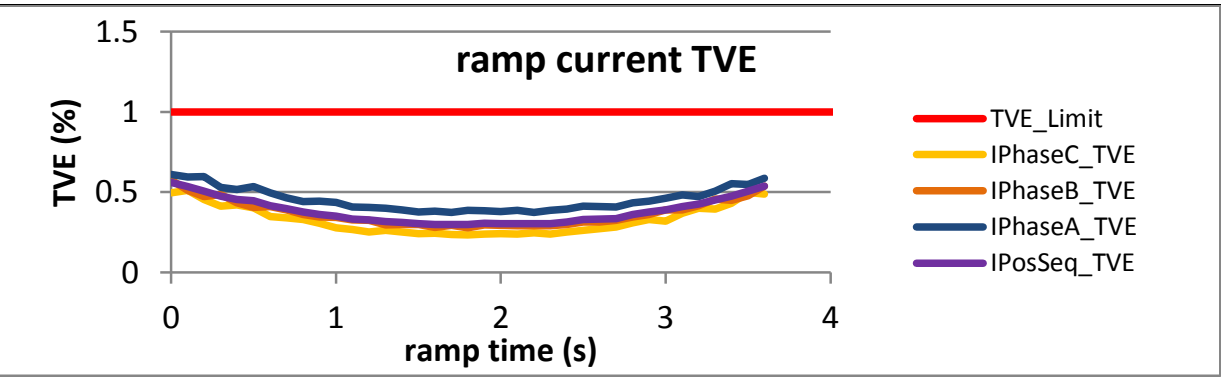

Figure 2785: Fs = $10 \mathrm{FPS}$, ramp from $58 \mathrm{~Hz}$ to $62 \mathrm{~Hz}$ at $+1 \mathrm{~Hz} / \mathrm{s}$

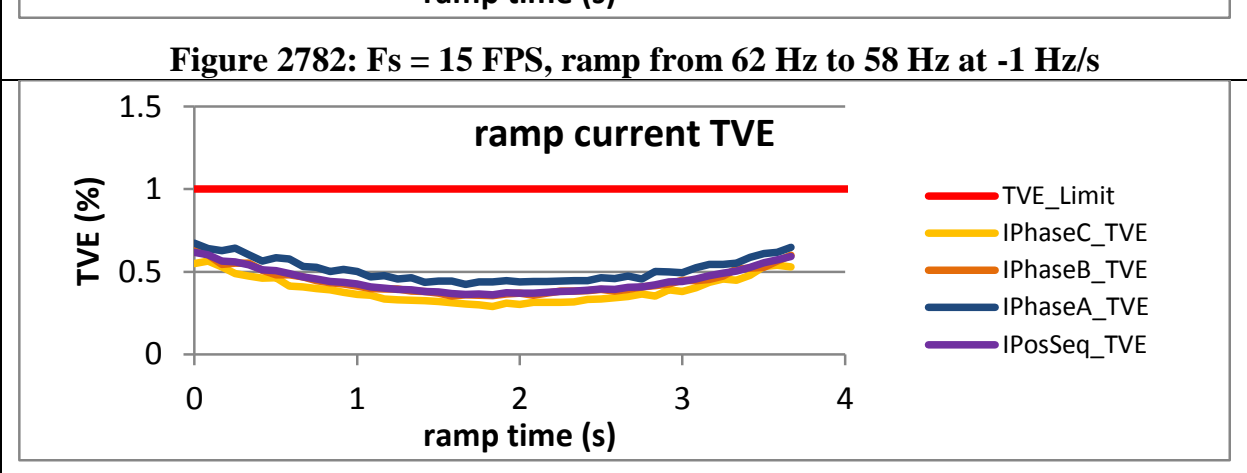

Figure 2784: Fs = $12 \mathrm{FPS}$, ramp from $62 \mathrm{~Hz}$ to $58 \mathrm{~Hz}$ at $-1 \mathrm{~Hz} / \mathrm{s}$

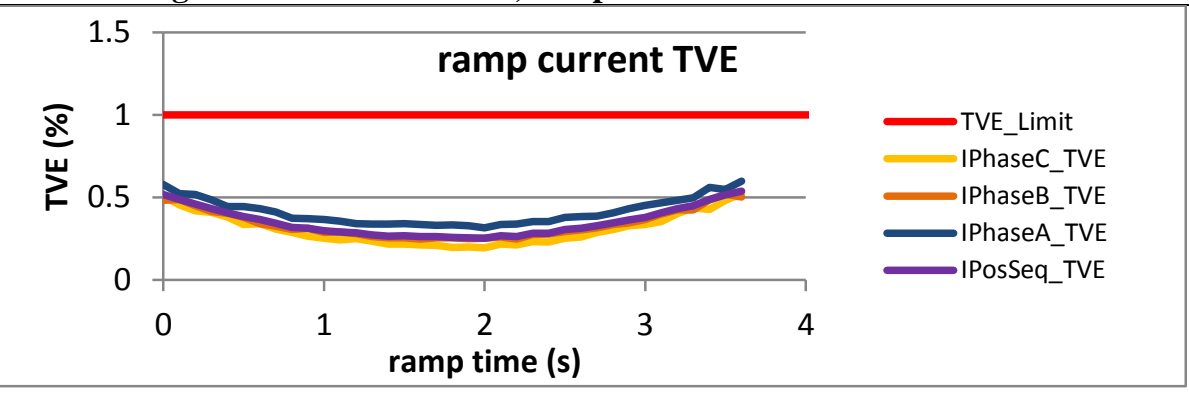

Figure 2786: Fs = $10 \mathrm{FPS}$, ramp from $62 \mathrm{~Hz}$ to $58 \mathrm{~Hz}$ at $-1 \mathrm{~Hz} / \mathrm{s}$ 
6.6.3 PMU B dynamic ramp of system frequency current TVE, $P$ class
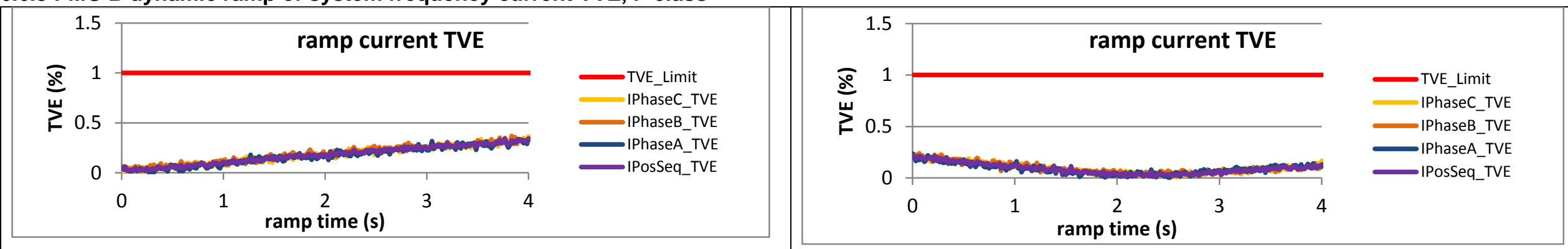

Figure 2787: Fs = $60 \mathrm{FPS}$, ramp from $55 \mathrm{~Hz}$ to $65 \mathrm{~Hz}$ at $+1 \mathrm{~Hz} / \mathrm{s}$

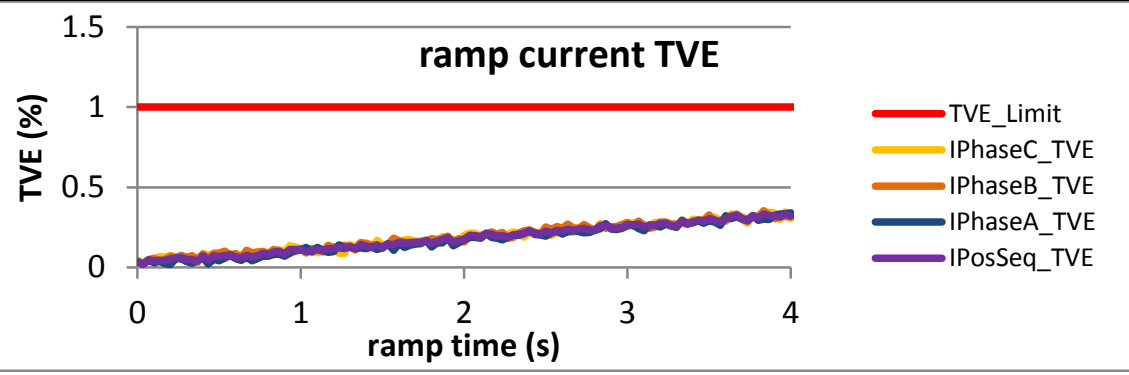

Figure 2789: Fs = $30 \mathrm{FPS}$, ramp from $55 \mathrm{~Hz}$ to $65 \mathrm{~Hz}$ at $+1 \mathrm{~Hz} / \mathrm{s}$

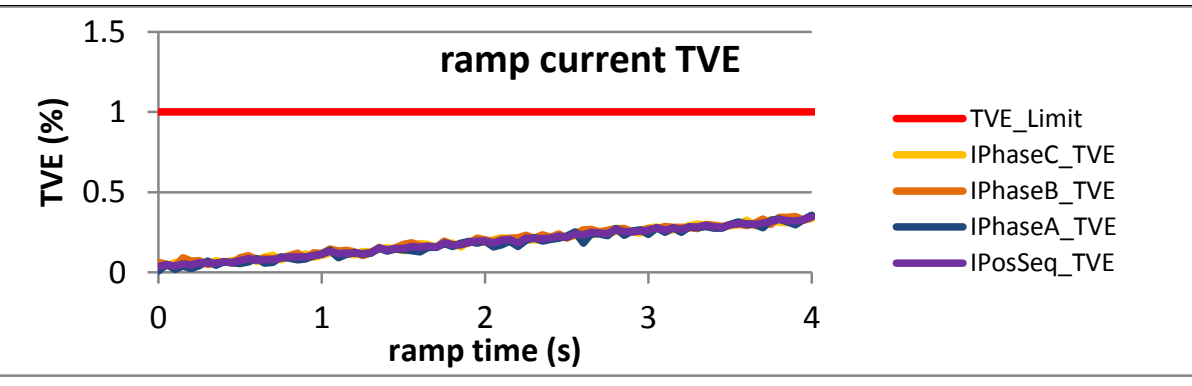

Figure 2791: Fs = $20 \mathrm{FPS}$, ramp from $56 \mathrm{~Hz}$ to $64 \mathrm{~Hz}$ at $+1 \mathrm{~Hz} / \mathrm{s}$

Figure 2788: Fs = $60 \mathrm{FPS}$, ramp from $65 \mathrm{~Hz}$ to $55 \mathrm{~Hz}$ at $-1 \mathrm{~Hz} / \mathrm{s}$

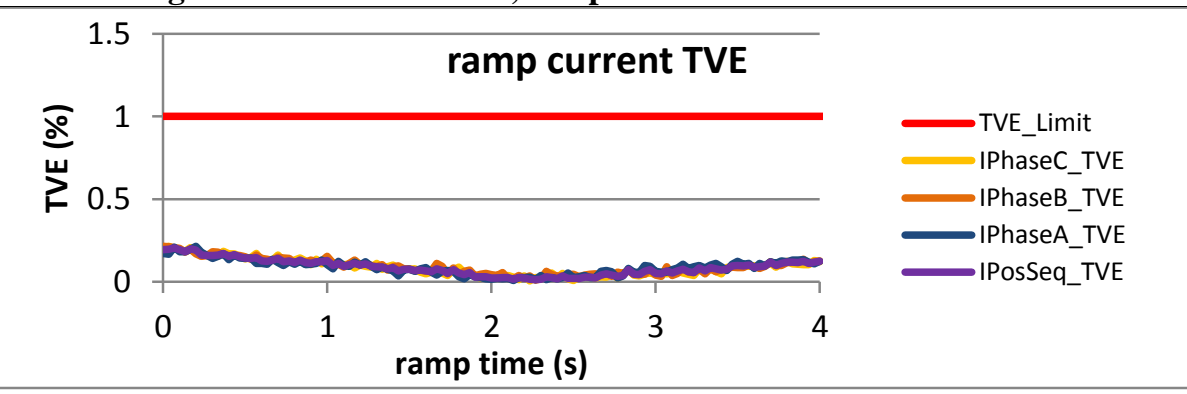

Figure 2790: Fs = $30 \mathrm{FPS}$, ramp from $65 \mathrm{~Hz}$ to $55 \mathrm{~Hz}$ at $-1 \mathrm{~Hz} / \mathrm{s}$

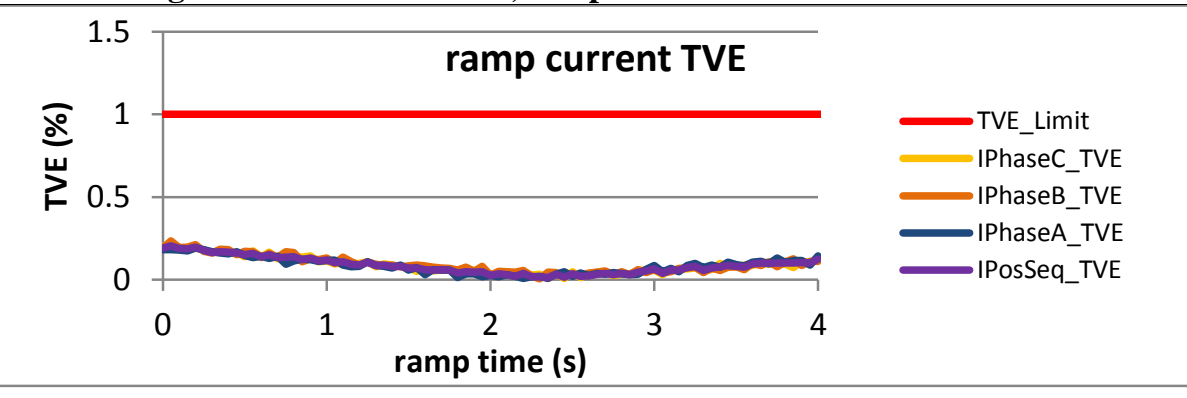

Figure 2792: Fs = $20 \mathrm{FPS}$, ramp from $64 \mathrm{~Hz}$ to $56 \mathrm{~Hz}$ at $-1 \mathrm{~Hz} / \mathrm{s}$ 

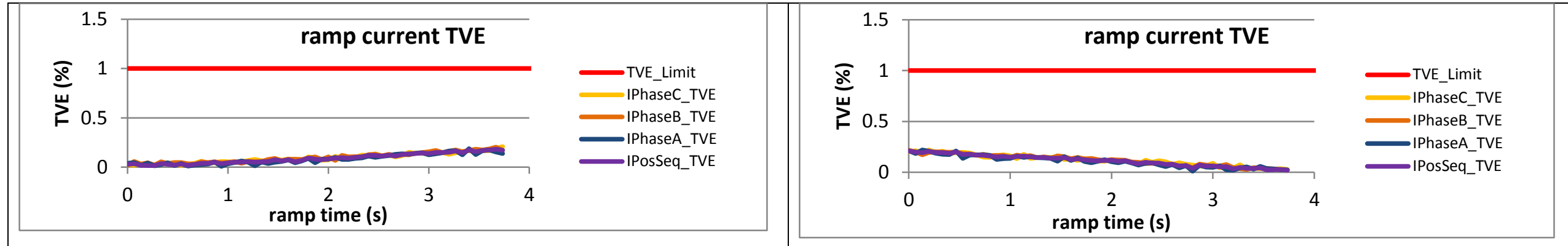

Figure 2793: Fs = $15 \mathrm{FPS}$, ramp from $57 \mathrm{~Hz}$ to $63 \mathrm{~Hz}$ at $+1 \mathrm{~Hz} / \mathrm{s}$

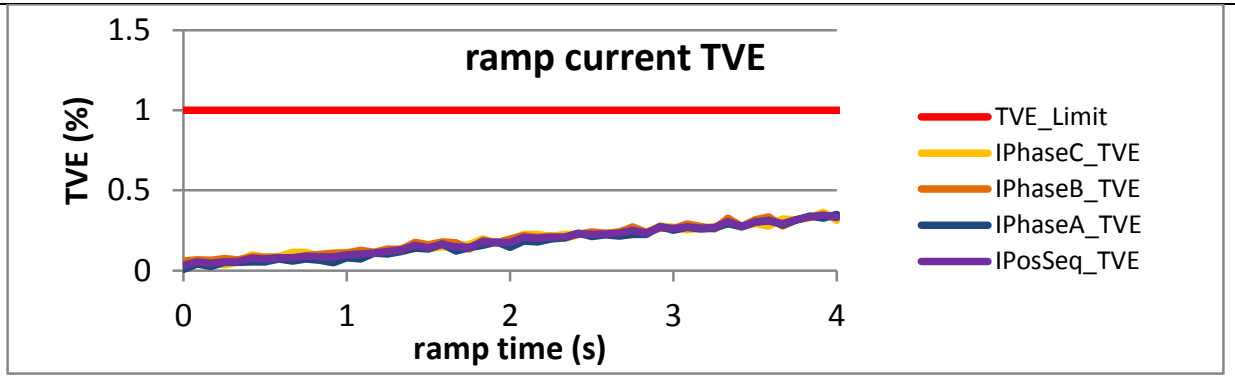

Figure 2794: Fs = $15 \mathrm{FPS}$, ramp from $63 \mathrm{~Hz}$ to $57 \mathrm{~Hz}$ at $-1 \mathrm{~Hz} / \mathrm{s}$

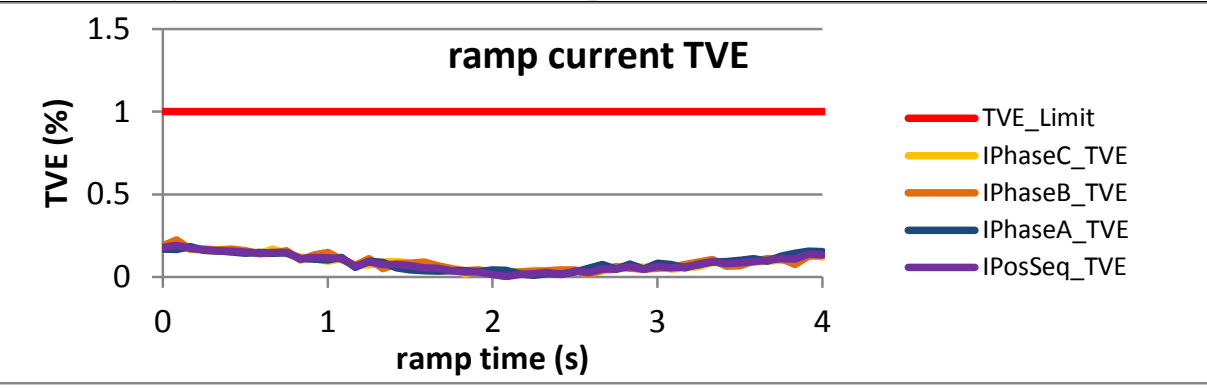

Figure 2795: Fs = 12 FPS, ramp from $58.6 \mathrm{~Hz}$ to $62.4 \mathrm{~Hz}$ at $+1 \mathrm{~Hz} / \mathrm{s}$ 
6.6.4 PMU C dynamic ramp of system frequency current TVE, P class
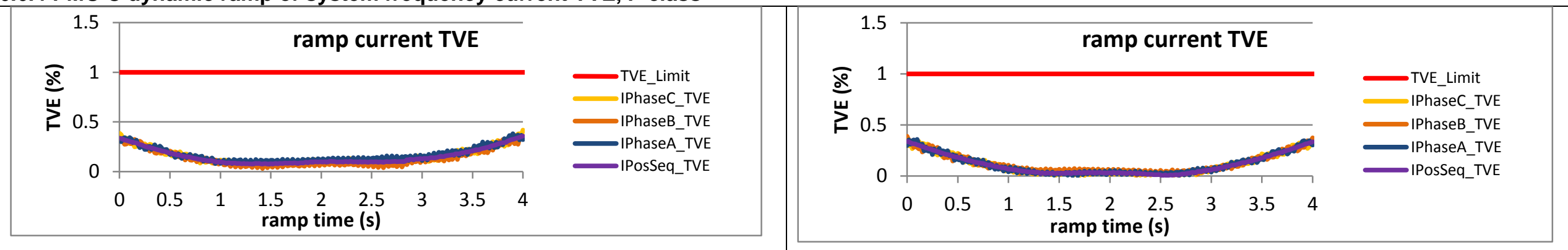

Figure 2799: Fs = $60 \mathrm{FPS}$, ramp from $55 \mathrm{~Hz}$ to $65 \mathrm{~Hz}$ at $+1 \mathrm{~Hz} / \mathrm{s}$

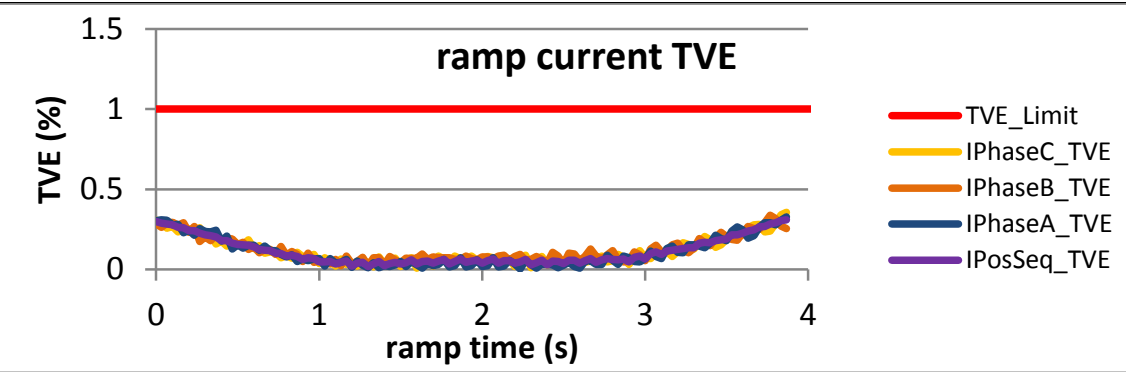

Figure 2801: Fs = $30 \mathrm{FPS}$, ramp from $55 \mathrm{~Hz}$ to $65 \mathrm{~Hz}$ at $+1 \mathrm{~Hz} / \mathrm{s}$

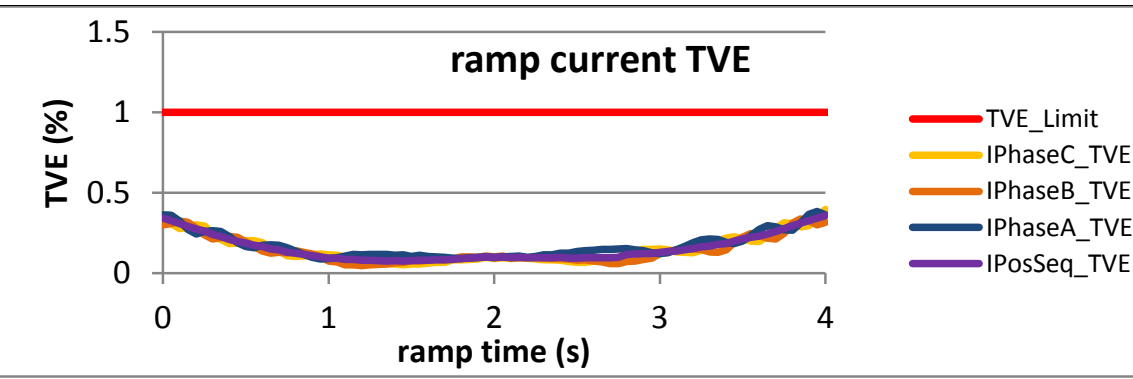

Figure 2803: Fs = 20 FPS, ramp from $56 \mathrm{~Hz}$ to $64 \mathrm{~Hz}$ at $+1 \mathrm{~Hz} / \mathrm{s}$

Figure 2800: Fs = $60 \mathrm{FPS}$, ramp from $65 \mathrm{~Hz}$ to $55 \mathrm{~Hz}$ at $-1 \mathrm{~Hz} / \mathrm{s}$

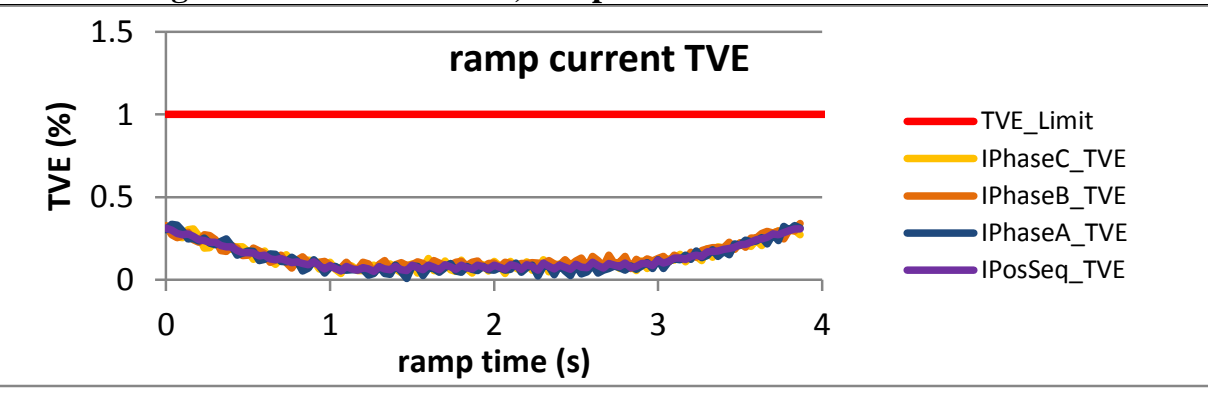

Figure 2802: Fs = $30 \mathrm{FPS}$, ramp from $65 \mathrm{~Hz}$ to $55 \mathrm{~Hz}$ at $-1 \mathrm{~Hz} / \mathrm{s}$

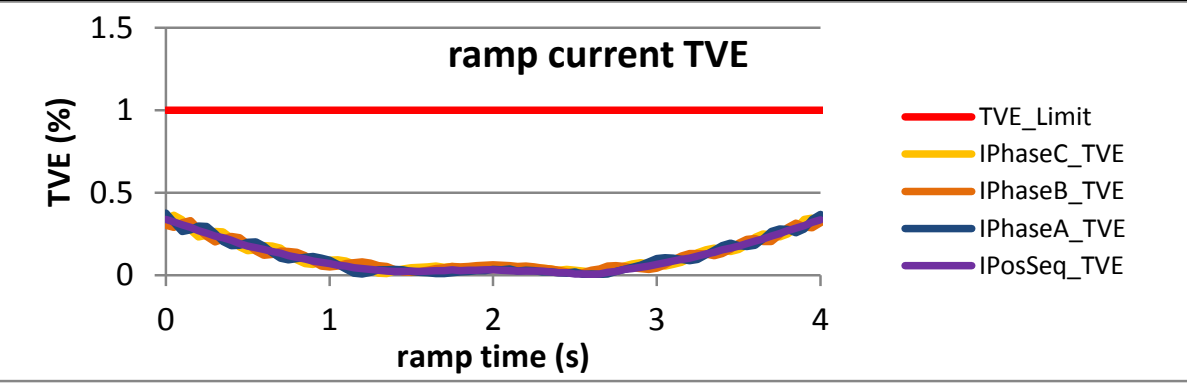

Figure 2804: Fs = $20 \mathrm{FPS}$, ramp from $64 \mathrm{~Hz}$ to $56 \mathrm{~Hz}$ at $-1 \mathrm{~Hz} / \mathrm{s}$ 


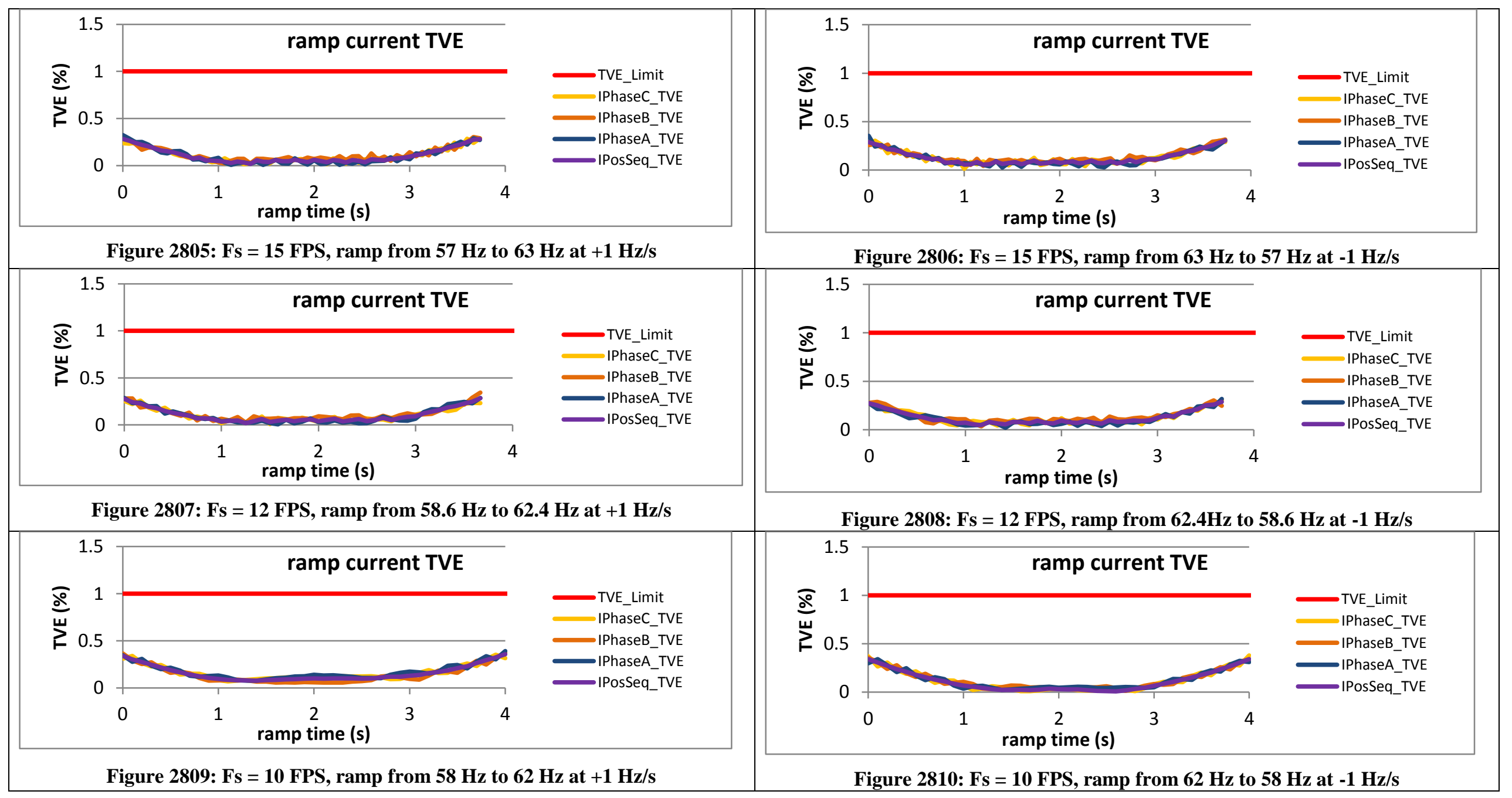


6.6.5 PMU D dynamic ramp of system frequency current TVE, $P$ class

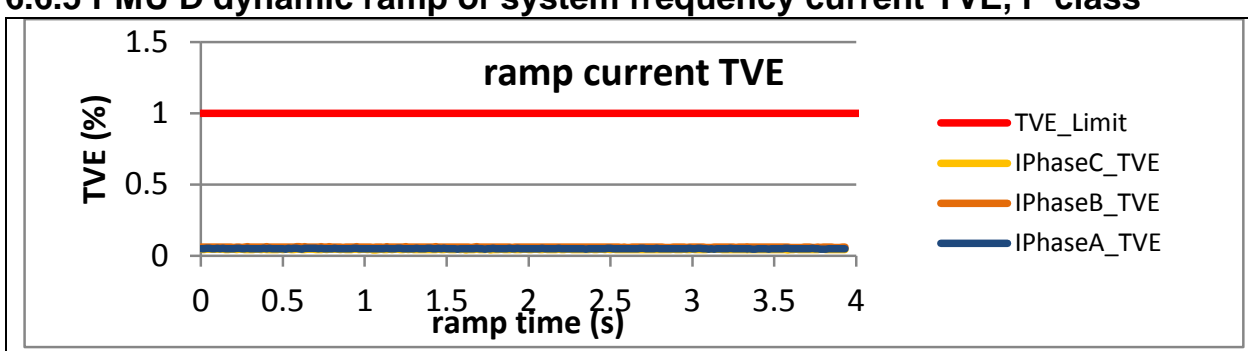

Figure 2811: Fs = $60 \mathrm{FPS}$, ramp from $58 \mathrm{~Hz}$ to $62 \mathrm{~Hz}$ at $+1 \mathrm{~Hz} / \mathrm{s}$

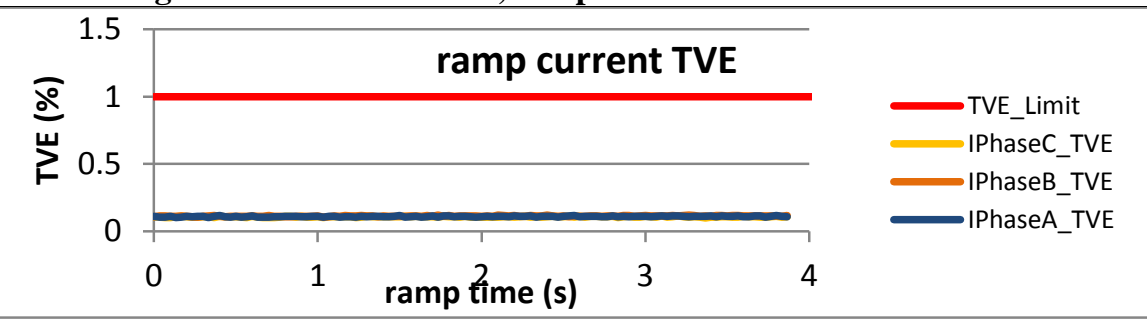

Figure 2813: Fs = $30 \mathrm{FPS}$, ramp from $58 \mathrm{~Hz}$ to $62 \mathrm{~Hz}$ at $+1 \mathrm{~Hz} / \mathrm{s}$

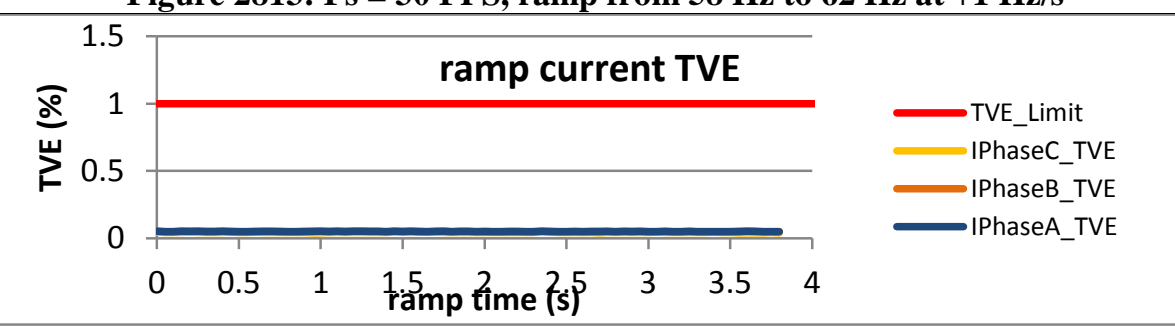

Figure 2815: Fs = $20 \mathrm{FPS}$, ramp from $58 \mathrm{~Hz}$ to $62 \mathrm{~Hz}$ at $+1 \mathrm{~Hz} / \mathrm{s}$

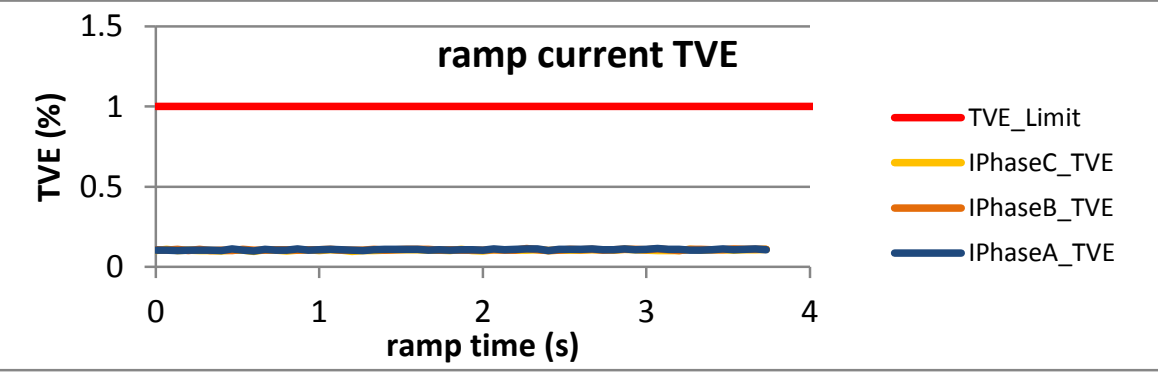

Figure 2817: Fs = $15 \mathrm{FPS}$, ramp from $58 \mathrm{~Hz}$ to $62 \mathrm{~Hz}$ at $+1 \mathrm{~Hz} / \mathrm{s}$

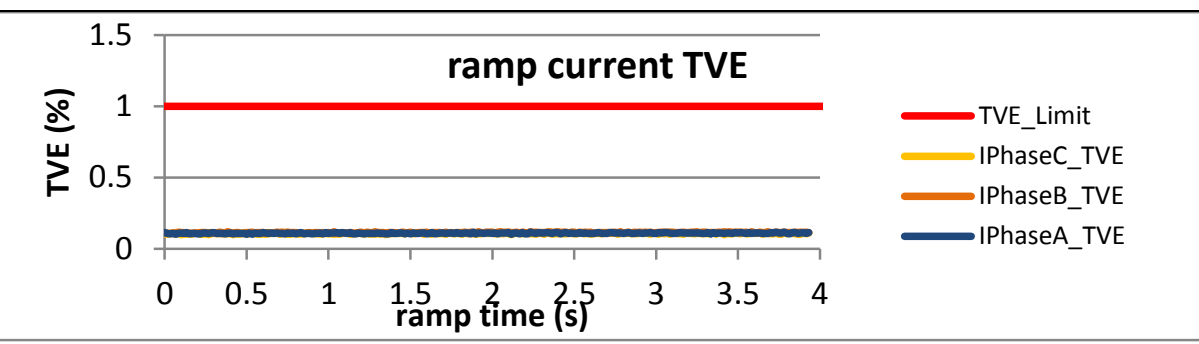

Figure 2812: Fs = $60 \mathrm{FPS}$, ramp from $62 \mathrm{~Hz}$ to $58 \mathrm{~Hz}$ at $-1 \mathrm{~Hz} / \mathrm{s}$

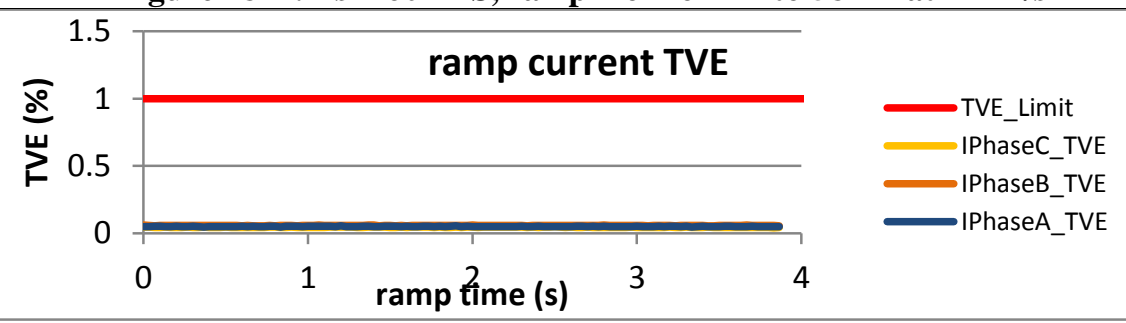

Figure 2814: Fs = $30 \mathrm{FPS}$, ramp from $62 \mathrm{~Hz}$ to $58 \mathrm{~Hz}$ at $-1 \mathrm{~Hz} / \mathrm{s}$

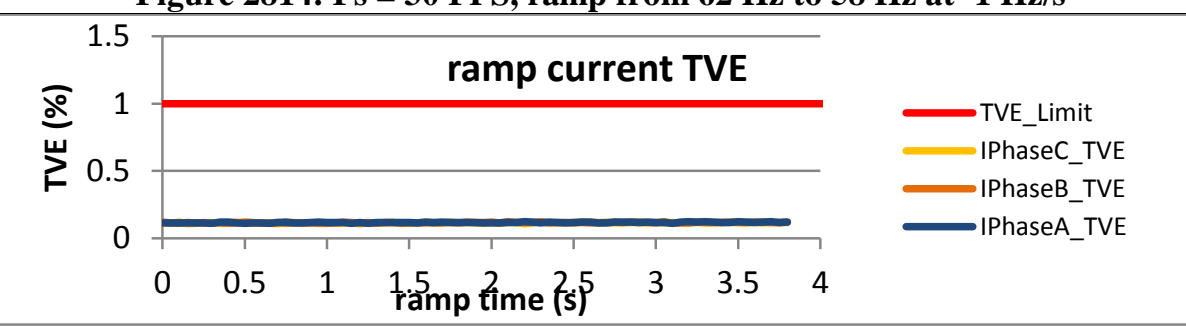

Figure 2816: Fs = $20 \mathrm{FPS}$, ramp from $62 \mathrm{~Hz}$ to $58 \mathrm{~Hz}$ at $-1 \mathrm{~Hz} / \mathrm{s}$

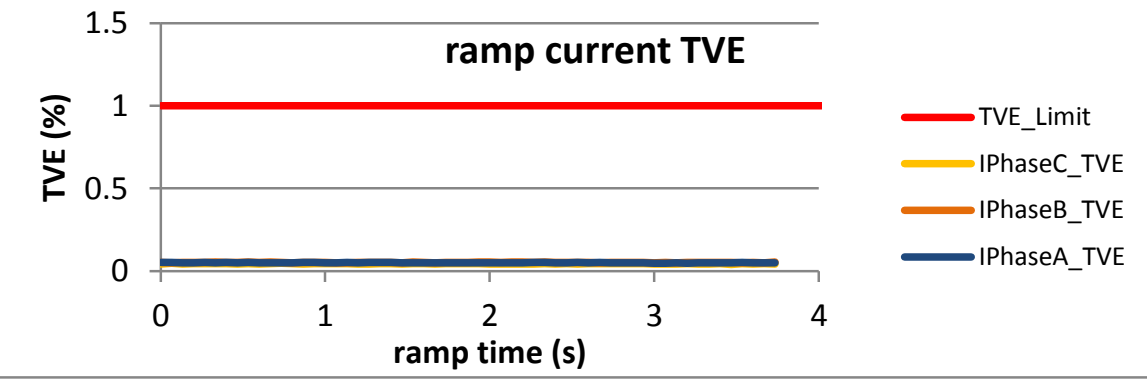

Figure 2818: Fs = $15 \mathrm{FPS}$, ramp from $62 \mathrm{~Hz}$ to $58 \mathrm{~Hz}$ at $-1 \mathrm{~Hz} / \mathrm{s}$ 


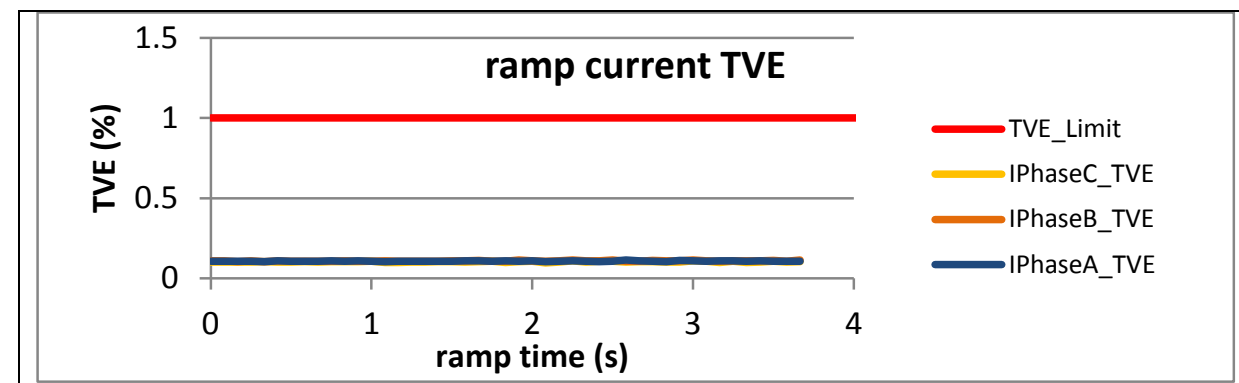

Figure 2819: Fs = 12 FPS, ramp from $58 \mathrm{~Hz}$ to $62 \mathrm{~Hz}$ at $+1 \mathrm{~Hz} / \mathrm{s}$

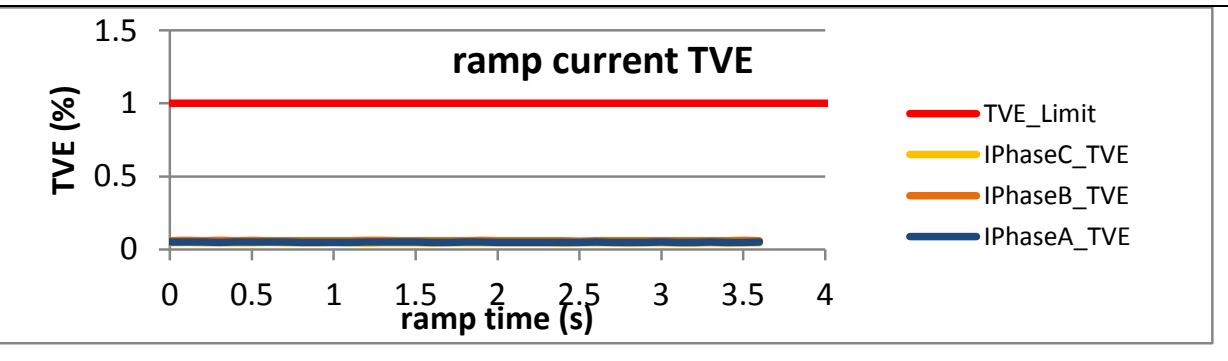

Figure 2821: Fs = $10 \mathrm{FPS}$, ramp from $58 \mathrm{~Hz}$ to $62 \mathrm{~Hz}$ at $+1 \mathrm{~Hz} / \mathrm{s}$

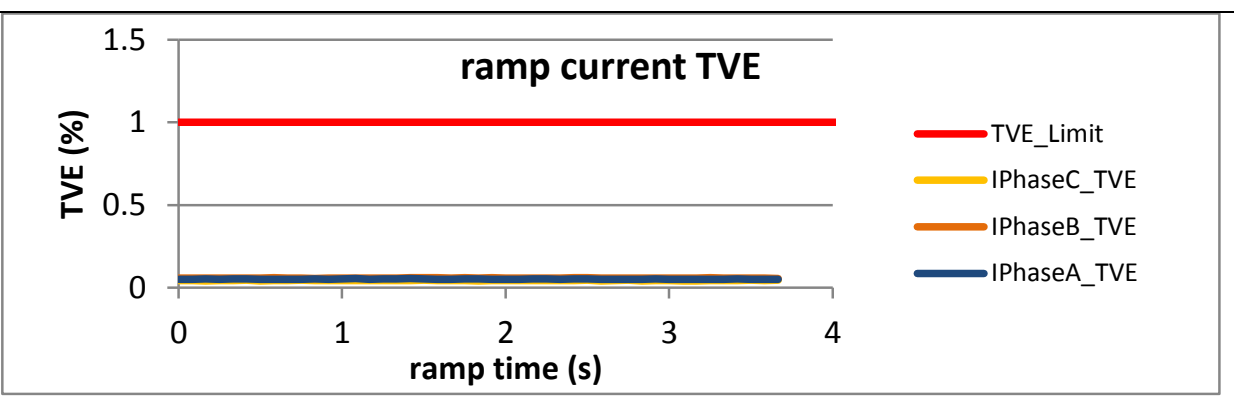

Figure 2820: Fs = $12 \mathrm{FPS}$, ramp from $62 \mathrm{~Hz}$ to $58 \mathrm{~Hz}$ at $-1 \mathrm{~Hz} / \mathrm{s}$

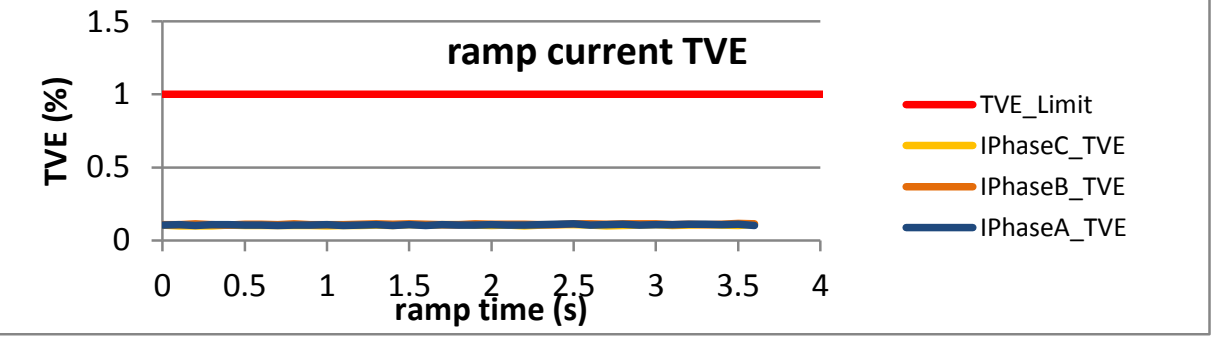

Figure 2822: Fs = $10 \mathrm{FPS}$, ramp from $62 \mathrm{~Hz}$ to $58 \mathrm{~Hz}$ at $-1 \mathrm{~Hz} / \mathrm{s}$ 
6.6.6 PMU E dynamic ramp of system frequency current TVE, P class PMU E does not support P class

\subsubsection{PMU F dynamic ramp of system frequency current TVE, $P$ class}

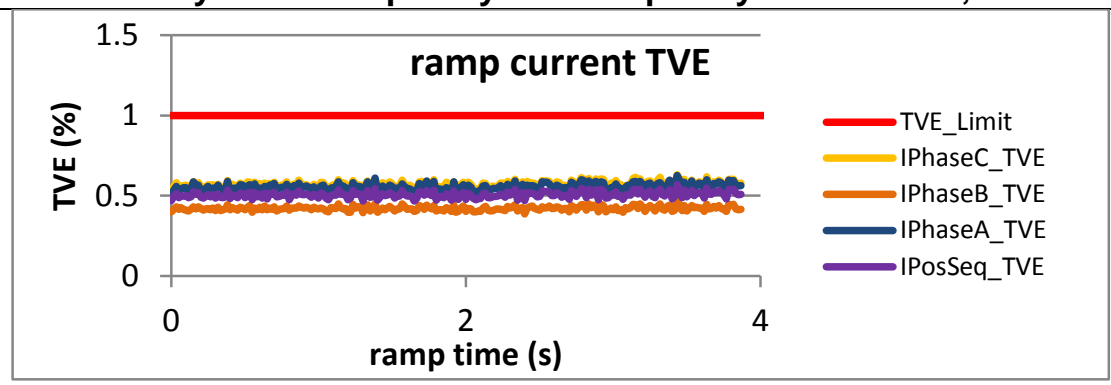

Figure 2823: Fs = $60 \mathrm{FPS}$, ramp from $55 \mathrm{~Hz}$ to $65 \mathrm{~Hz}$ at $+1 \mathrm{~Hz} / \mathrm{s}$

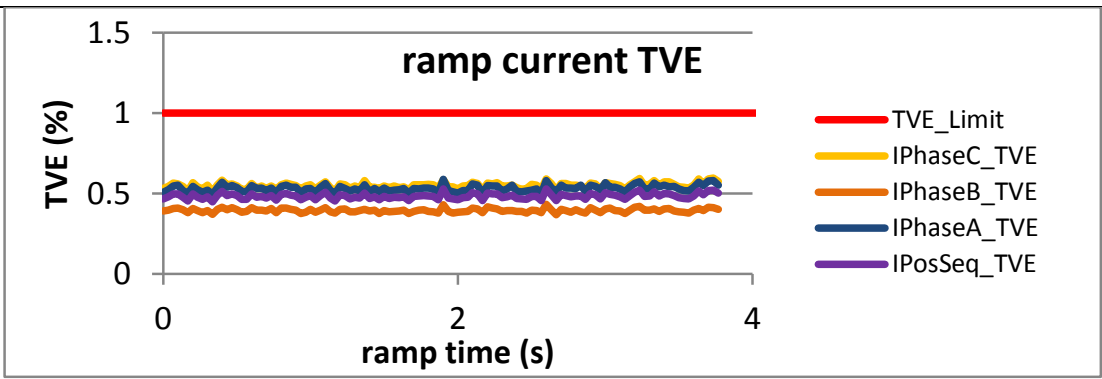

Figure 2825: Fs = $30 \mathrm{FPS}$, ramp from $55 \mathrm{~Hz}$ to $65 \mathrm{~Hz}$ at $+1 \mathrm{~Hz} / \mathrm{s}$

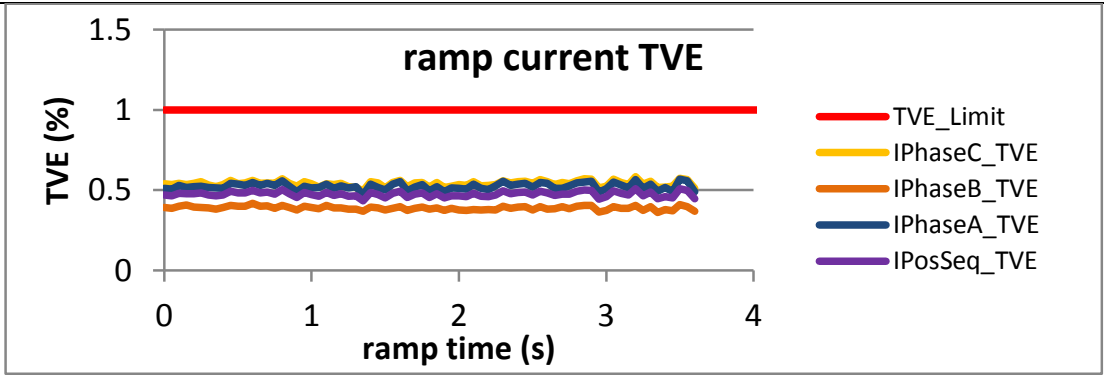

Figure 2827: Fs = $20 \mathrm{FPS}$, ramp from $56 \mathrm{~Hz}$ to $64 \mathrm{~Hz}$ at $+1 \mathrm{~Hz} / \mathrm{s}$

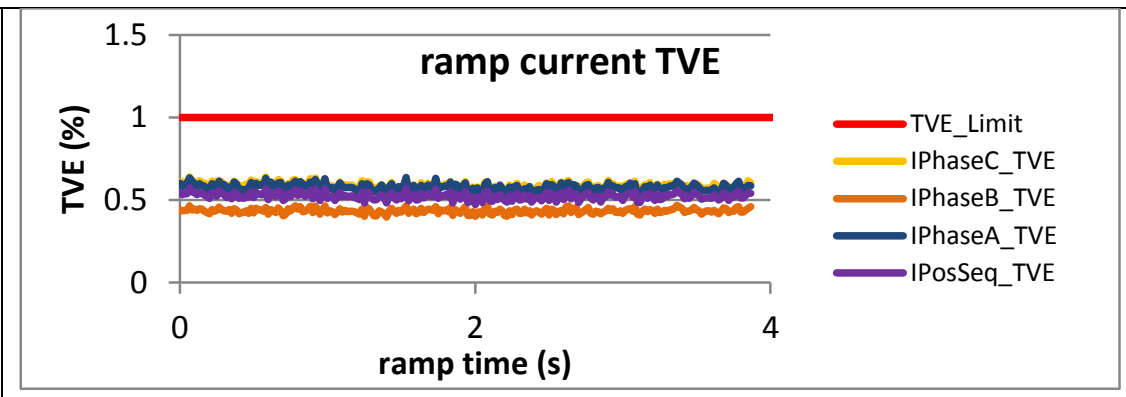

Figure 2824: Fs = $60 \mathrm{FPS}$, ramp from $65 \mathrm{~Hz}$ to $55 \mathrm{~Hz}$ at $-1 \mathrm{~Hz} / \mathrm{s}$

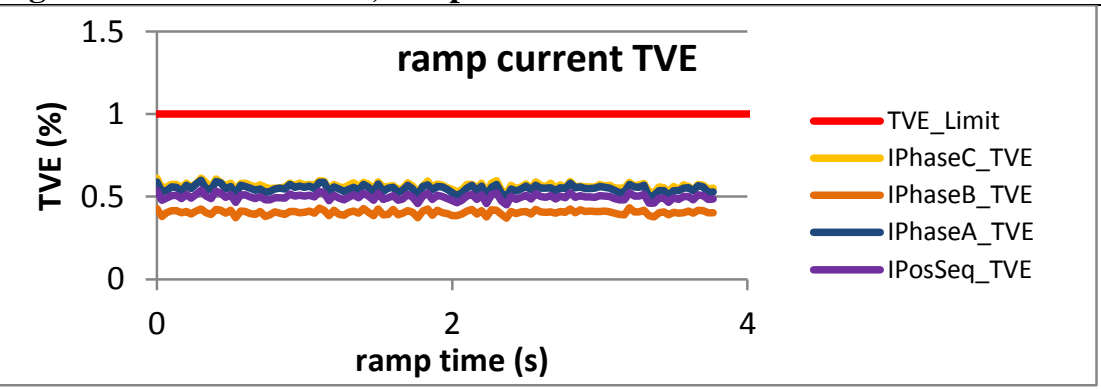

Figure 2826: Fs = $30 \mathrm{FPS}$, ramp from $65 \mathrm{~Hz}$ to $55 \mathrm{~Hz}$ at $-1 \mathrm{~Hz} / \mathrm{s}$

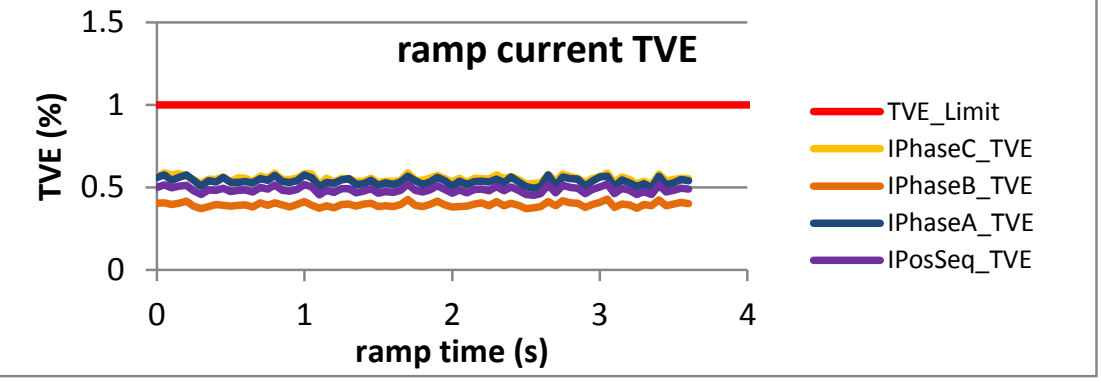

Figure 2828: Fs = $20 \mathrm{FPS}$, ramp from $64 \mathrm{~Hz}$ to $56 \mathrm{~Hz}$ at $-1 \mathrm{~Hz} / \mathrm{s}$ 


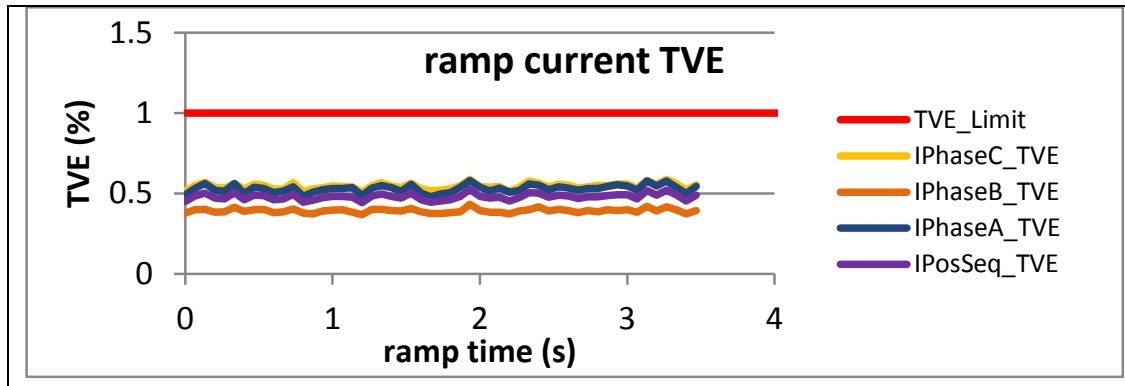

Figure 2829: Fs = $15 \mathrm{FPS}$, ramp from $57 \mathrm{~Hz}$ to $63 \mathrm{~Hz}$ at $+1 \mathrm{~Hz} / \mathrm{s}$

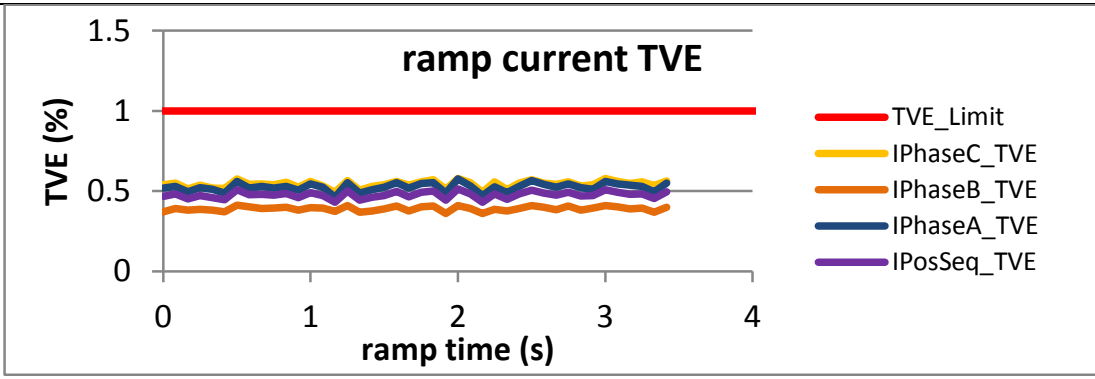

Figure 2831: Fs = 12 FPS, ramp from $58.6 \mathrm{~Hz}$ to $62.4 \mathrm{~Hz}$ at $+1 \mathrm{~Hz} / \mathrm{s}$

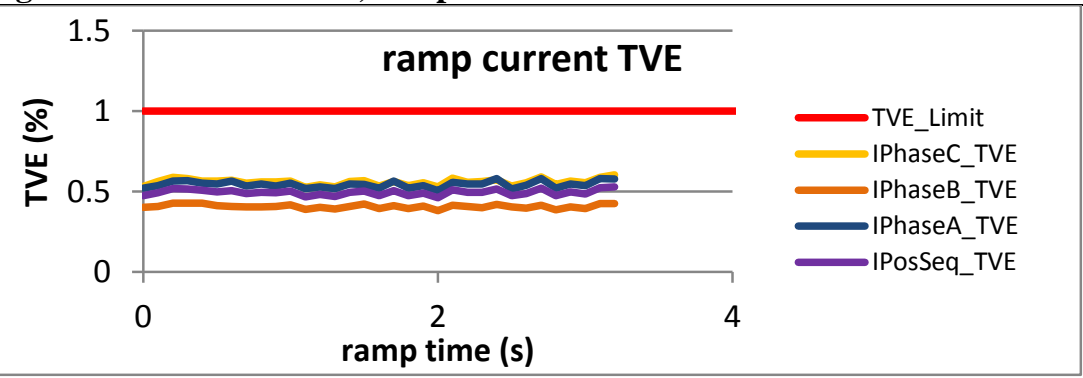

Figure 2833: Fs = $10 \mathrm{FPS}$, ramp from $58 \mathrm{~Hz}$ to $62 \mathrm{~Hz}$ at $+1 \mathrm{~Hz} / \mathrm{s}$

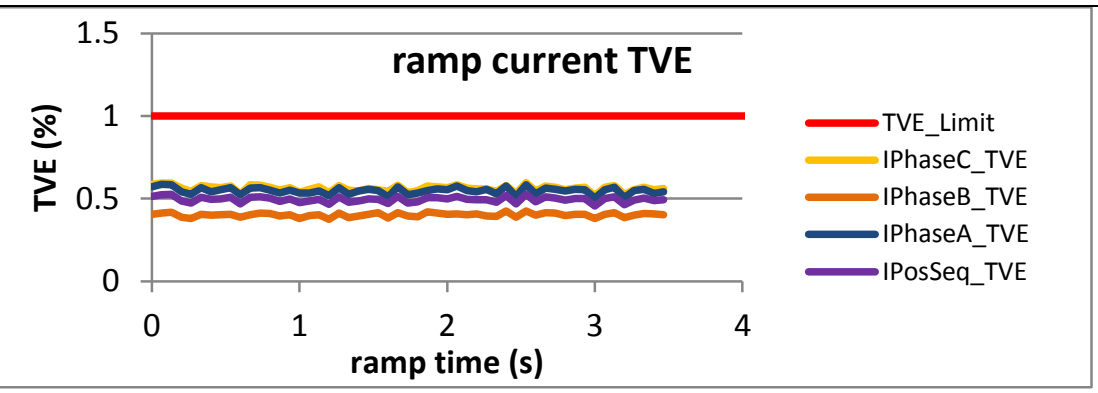

Figure 2830: Fs = $15 \mathrm{FPS}$, ramp from $63 \mathrm{~Hz}$ to $57 \mathrm{~Hz}$ at $-1 \mathrm{~Hz} / \mathrm{s}$

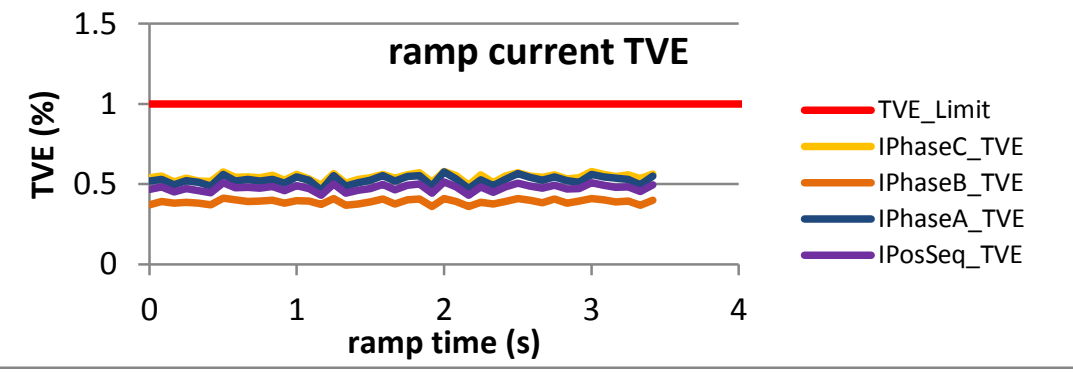

Figure 2832: Fs = 12 FPS, ramp from $62.4 \mathrm{~Hz}$ to $58.6 \mathrm{~Hz}$ at $-1 \mathrm{~Hz} / \mathrm{s}$

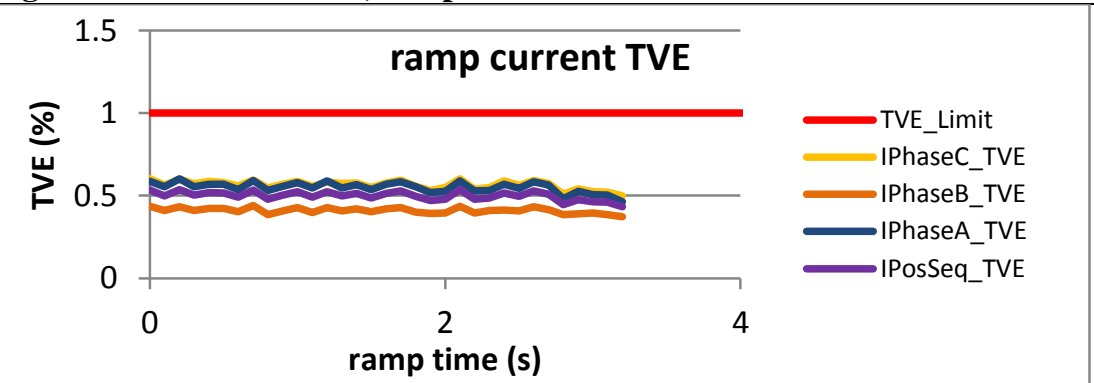

Figure 2834: Fs = $10 \mathrm{FPS}$, ramp from $62 \mathrm{~Hz}$ to $58 \mathrm{~Hz}$ at $-1 \mathrm{~Hz} / \mathrm{s}$ 
6.6.8 PMU G dynamic ramp of system frequency current TVE, P class PMU G does not support P class.

6.6.9 PMU H dynamic ramp of system frequency current TVE, P class

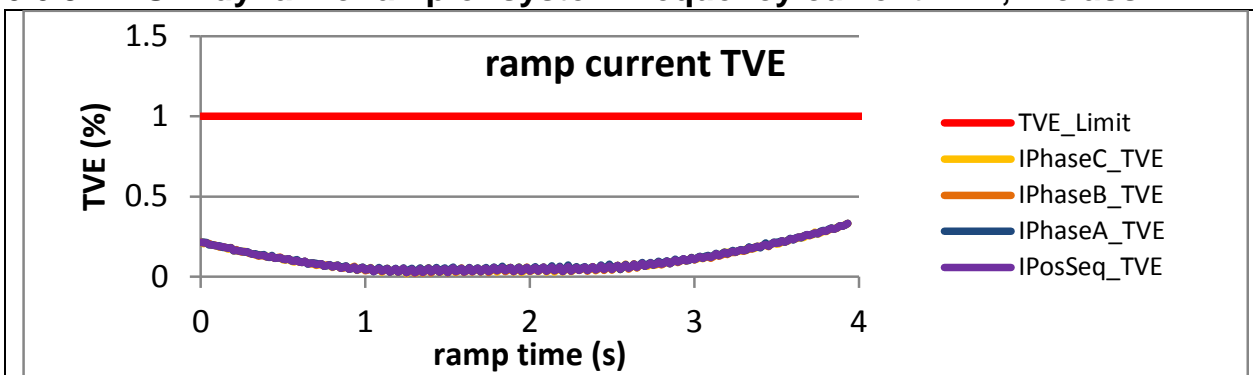

Figure 2835: Fs = $60 \mathrm{FPS}$, ramp from $55 \mathrm{~Hz}$ to $65 \mathrm{~Hz}$ at $+1 \mathrm{~Hz} / \mathrm{s}$

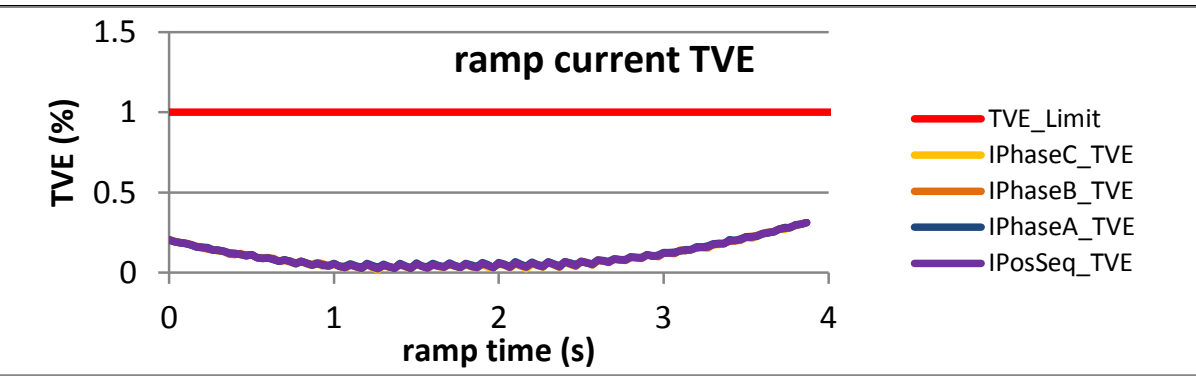

Figure 2837: Fs = $30 \mathrm{FPS}$, ramp from $55 \mathrm{~Hz}$ to $65 \mathrm{~Hz}$ at $+1 \mathrm{~Hz} / \mathrm{s}$

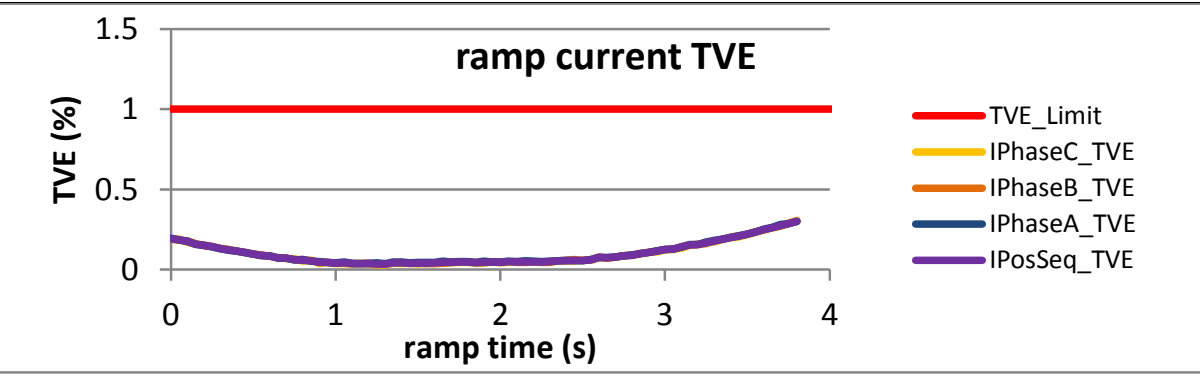

Figure 2839: Fs = $20 \mathrm{FPS}$, ramp from $56 \mathrm{~Hz}$ to $64 \mathrm{~Hz}$ at $+1 \mathrm{~Hz} / \mathrm{s}$

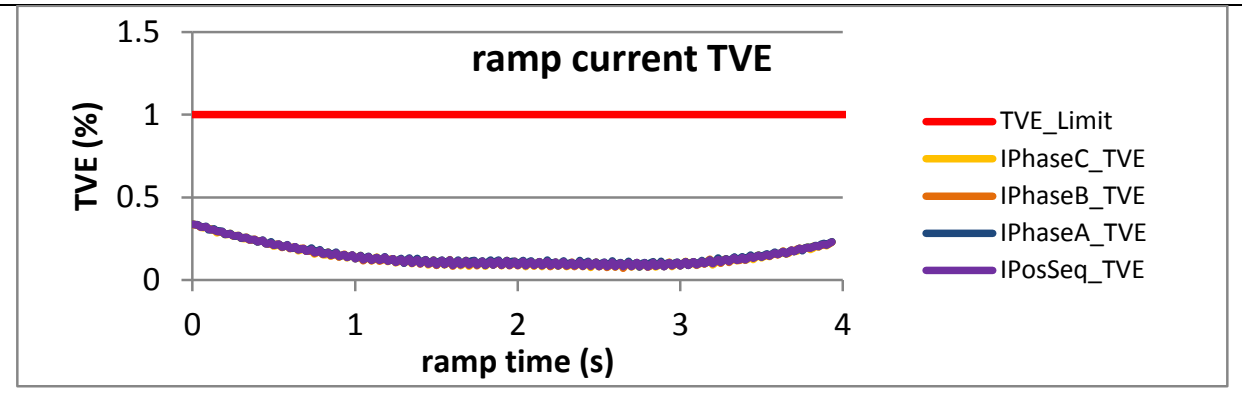

Figure 2836: Fs = 60 FPS, ramp from $65 \mathrm{~Hz}$ to $55 \mathrm{~Hz}$ at $-1 \mathrm{~Hz} / \mathrm{s}$

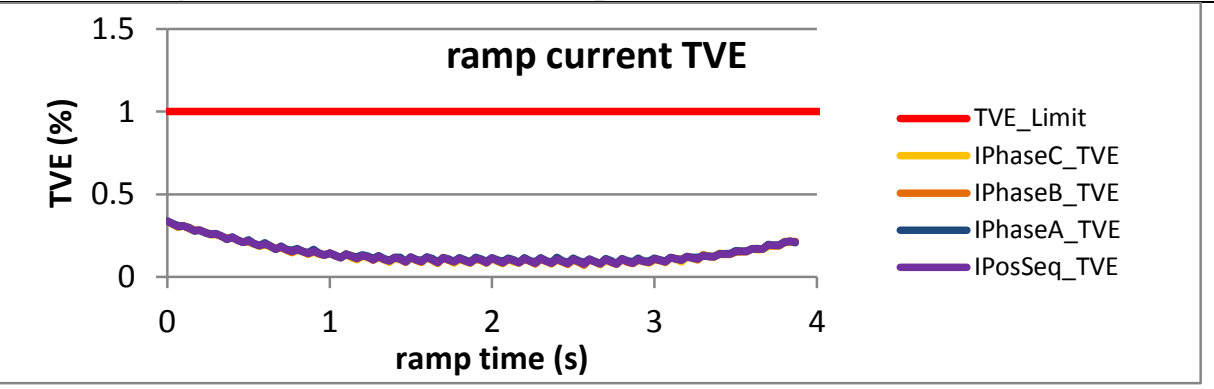

Figure 2838: Fs = $30 \mathrm{FPS}$, ramp from $65 \mathrm{~Hz}$ to $55 \mathrm{~Hz}$ at $-1 \mathrm{~Hz} / \mathrm{s}$

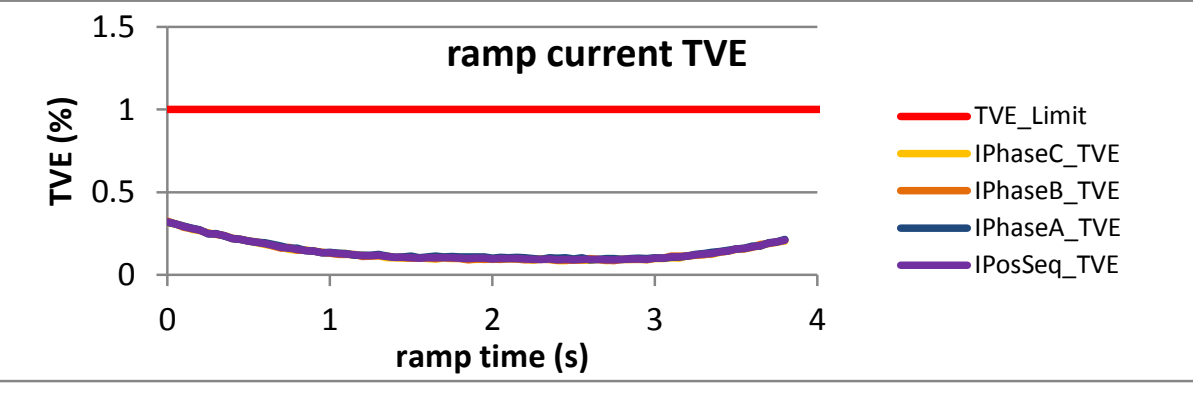

Figure 2840: Fs = $20 \mathrm{FPS}$, ramp from $64 \mathrm{~Hz}$ to $56 \mathrm{~Hz}$ at $-1 \mathrm{~Hz} / \mathrm{s}$ 

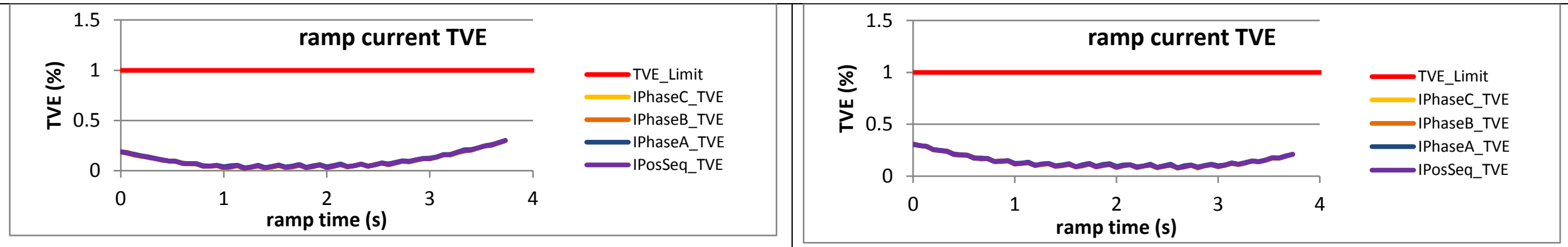

Figure 2841: Fs = $15 \mathrm{FPS}$, ramp from $57 \mathrm{~Hz}$ to $63 \mathrm{~Hz}$ at $+1 \mathrm{~Hz} / \mathrm{s}$
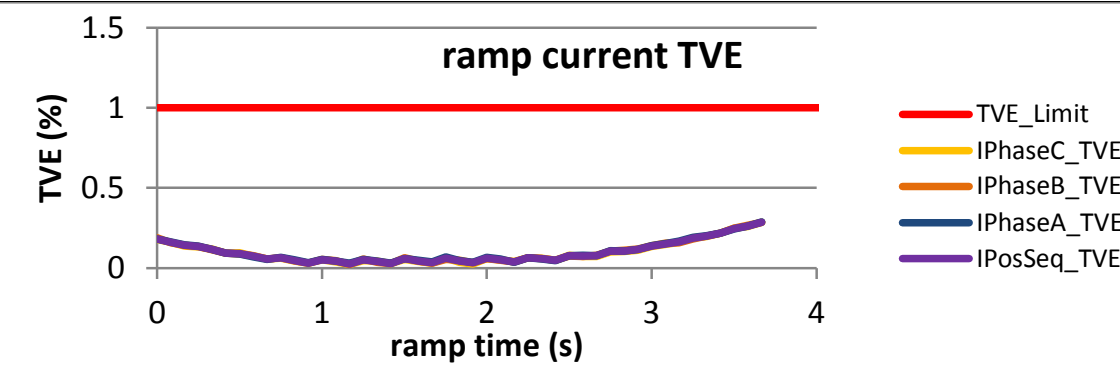

Figure 2842: Fs = $15 \mathrm{FPS}$, ramp from $63 \mathrm{~Hz}$ to $57 \mathrm{~Hz}$ at $-1 \mathrm{~Hz} / \mathrm{s}$

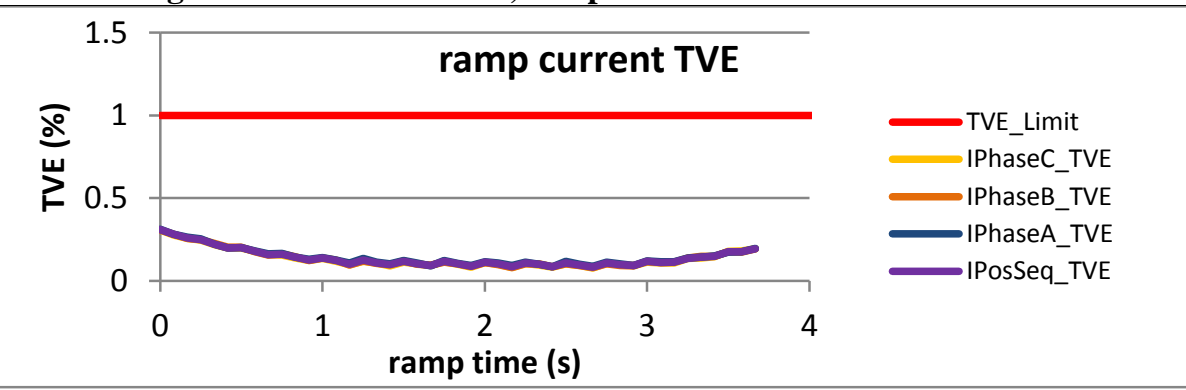

Figure 2843: Fs = 12 FPS, ramp from $58.6 \mathrm{~Hz}$ to $62.4 \mathrm{~Hz}$ at $+1 \mathrm{~Hz} / \mathrm{s}$
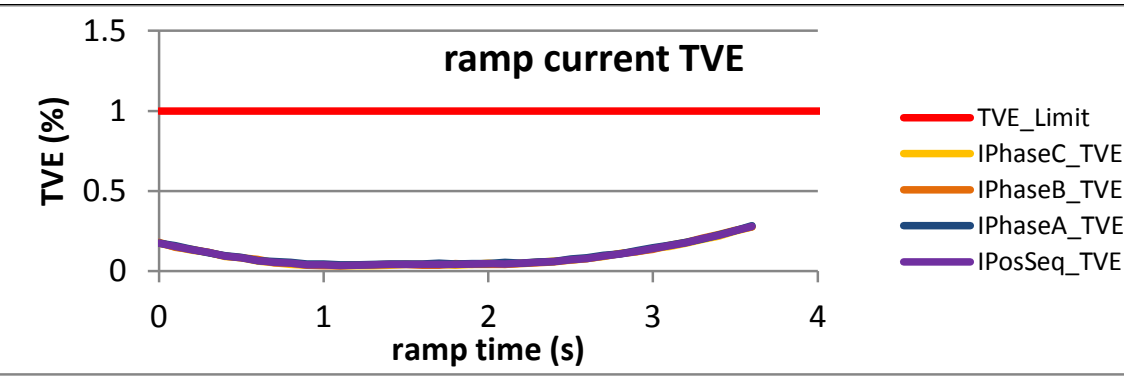

$\longrightarrow$ IPhaseB TVE

IPhaseA_TVE

$\longrightarrow$ IPosSeq_TVE

ramp time (s)

Figure 2845: Fs = $10 \mathrm{FPS}$, ramp from $58 \mathrm{~Hz}$ to $62 \mathrm{~Hz}$ at $+1 \mathrm{~Hz} / \mathrm{s}$

Figure 2844: Fs = $12 \mathrm{FPS}$, ramp from $62.4 \mathrm{~Hz}$ to $58.6 \mathrm{~Hz}$ at $-1 \mathrm{~Hz} / \mathrm{s}$

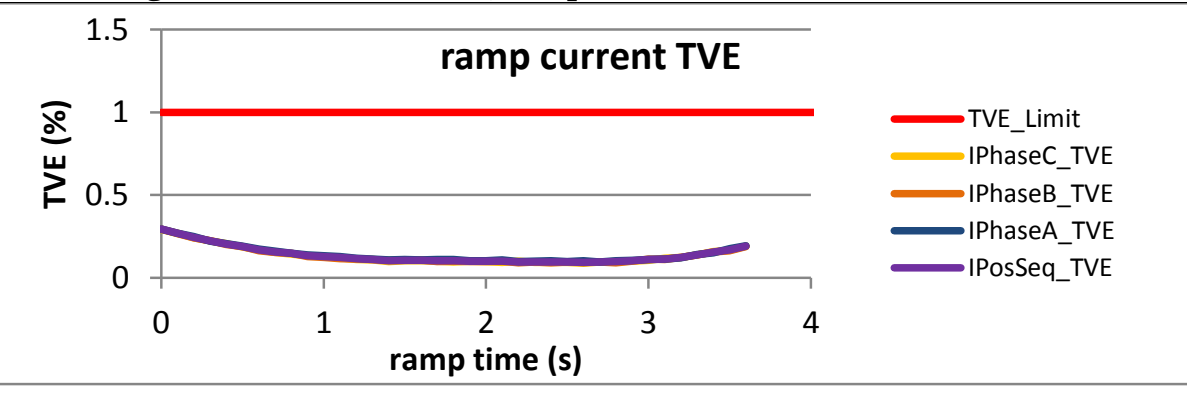

Figure 2846: Fs = $10 \mathrm{FPS}$, ramp from $62 \mathrm{~Hz}$ to $58 \mathrm{~Hz}$ at $-1 \mathrm{~Hz} / \mathrm{s}$ 
6.6.10 PMU I dynamic ramp of system frequency current TVE, P class PMU I does not support P class

\subsubsection{PMU J dynamic ramp of system frequency current TVE, P class}

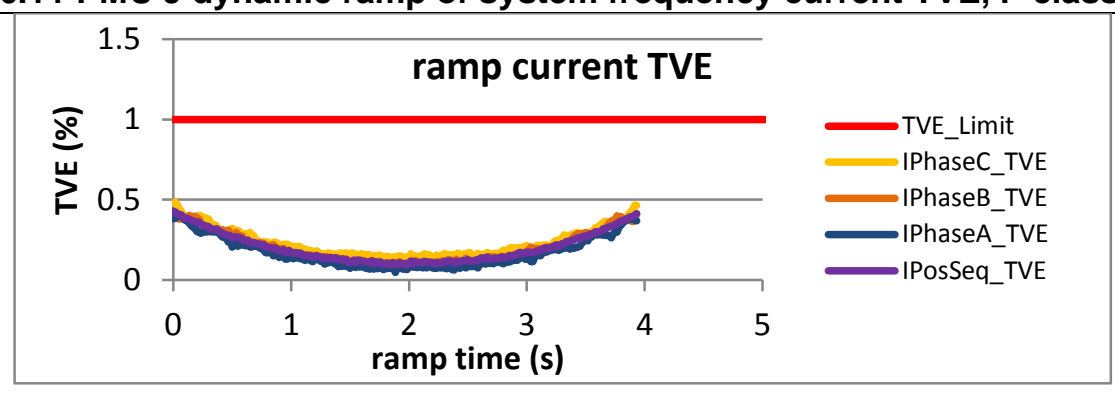

Figure 2847: Fs = $60 \mathrm{FPS}$, ramp from $55 \mathrm{~Hz}$ to $65 \mathrm{~Hz}$ at $+1 \mathrm{~Hz} / \mathrm{s}$

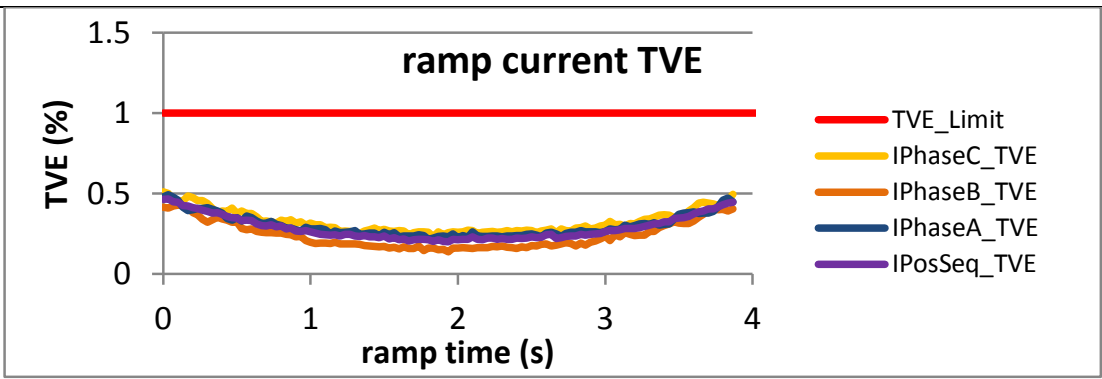

Figure 2849: Fs = $30 \mathrm{FPS}$, ramp from $55 \mathrm{~Hz}$ to $65 \mathrm{~Hz}$ at $+1 \mathrm{~Hz} / \mathrm{s}$

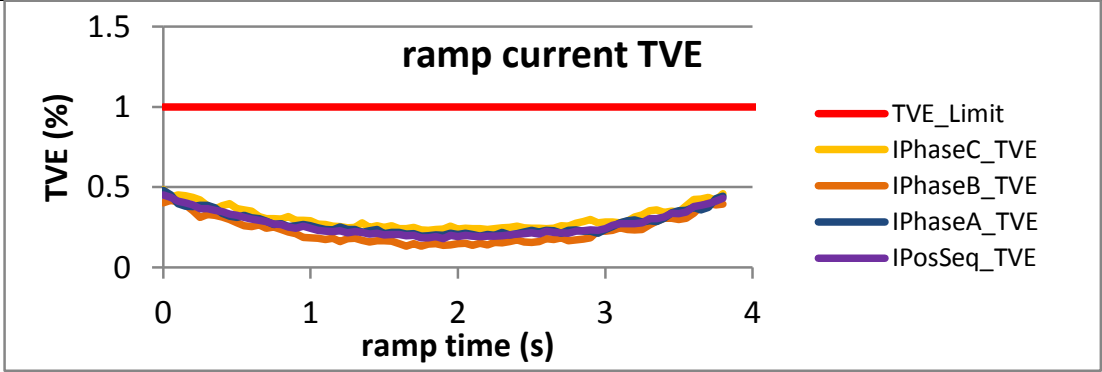

Figure 2851: Fs = 20 FPS, ramp from $56 \mathrm{~Hz}$ to $64 \mathrm{~Hz}$ at $+1 \mathrm{~Hz} / \mathrm{s}$

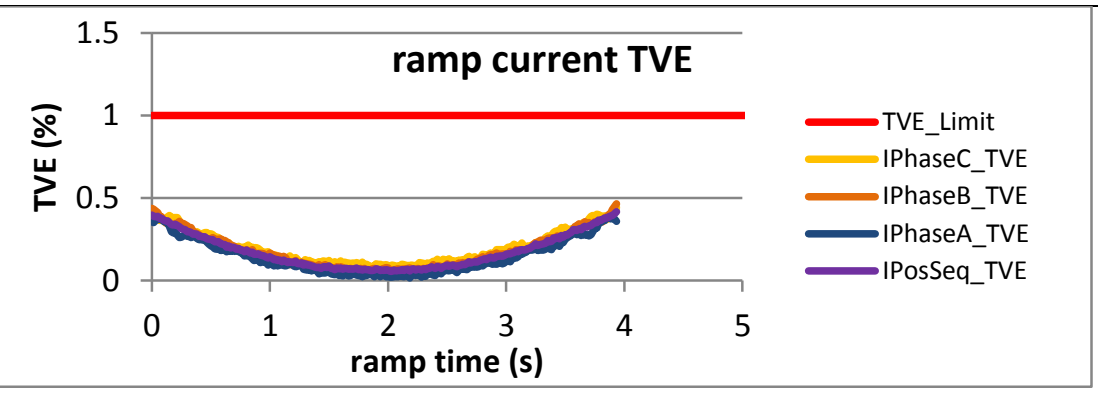

Figure 2848: Fs = $60 \mathrm{FPS}$, ramp from $65 \mathrm{~Hz}$ to $55 \mathrm{~Hz}$ at $-1 \mathrm{~Hz} / \mathrm{s}$

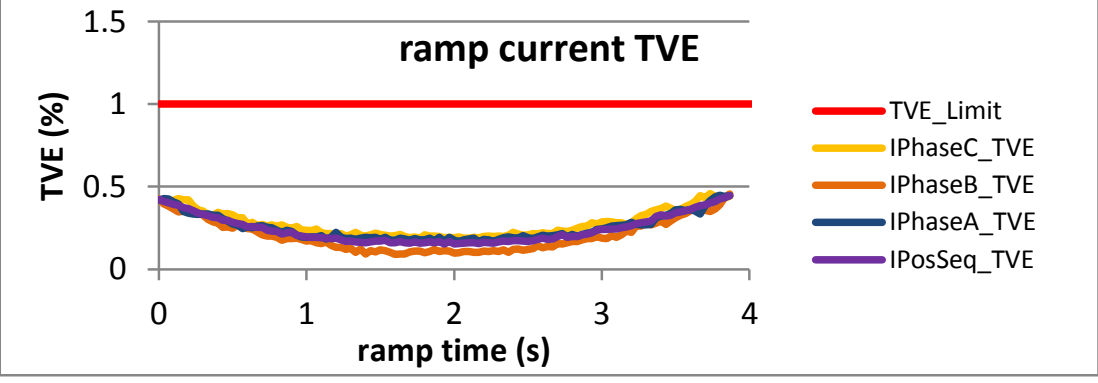

Figure 2850: Fs = $30 \mathrm{FPS}$, ramp from $65 \mathrm{~Hz}$ to $55 \mathrm{~Hz}$ at $-1 \mathrm{~Hz} / \mathrm{s}$

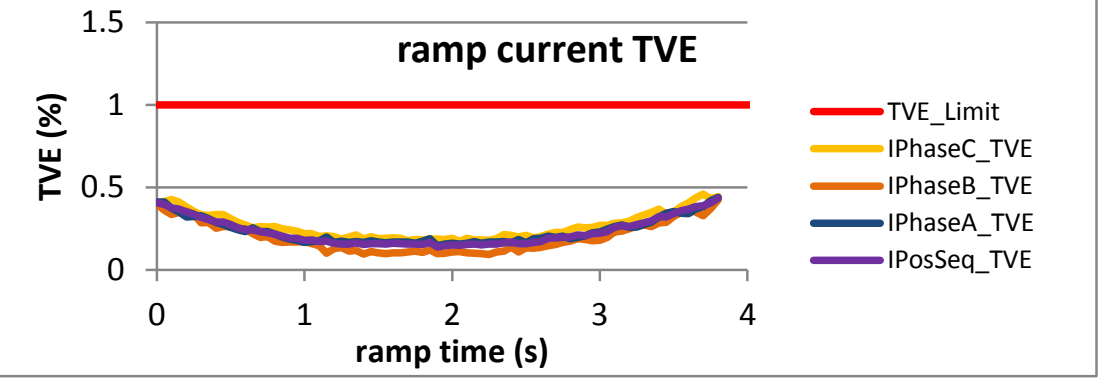

Figure 2852: Fs = $20 \mathrm{FPS}$, ramp from $64 \mathrm{~Hz}$ to $56 \mathrm{~Hz}$ at $-1 \mathrm{~Hz} / \mathrm{s}$ 


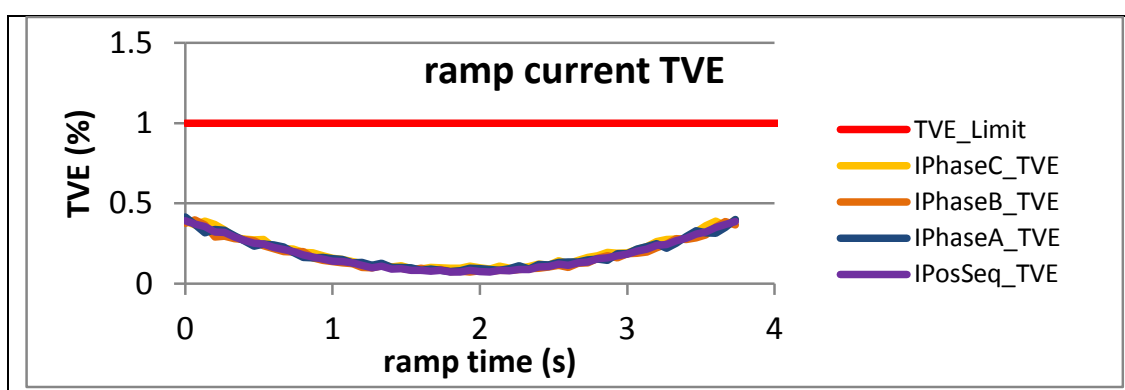

Figure 2853: Fs = $15 \mathrm{FPS}$, ramp from $57 \mathrm{~Hz}$ to $63 \mathrm{~Hz}$ at $+1 \mathrm{~Hz} / \mathrm{s}$

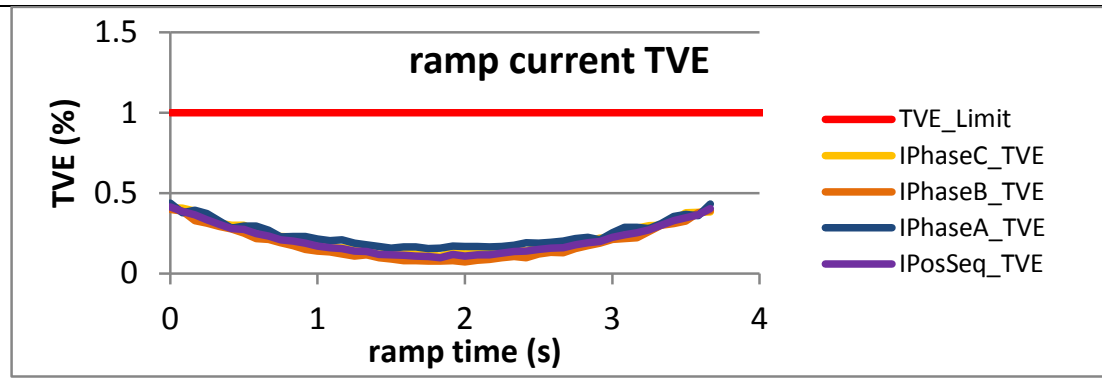

Figure 2855: Fs = 12 FPS, ramp from $58.6 \mathrm{~Hz}$ to $62.4 \mathrm{~Hz}$ at $+1 \mathrm{~Hz} / \mathrm{s}$

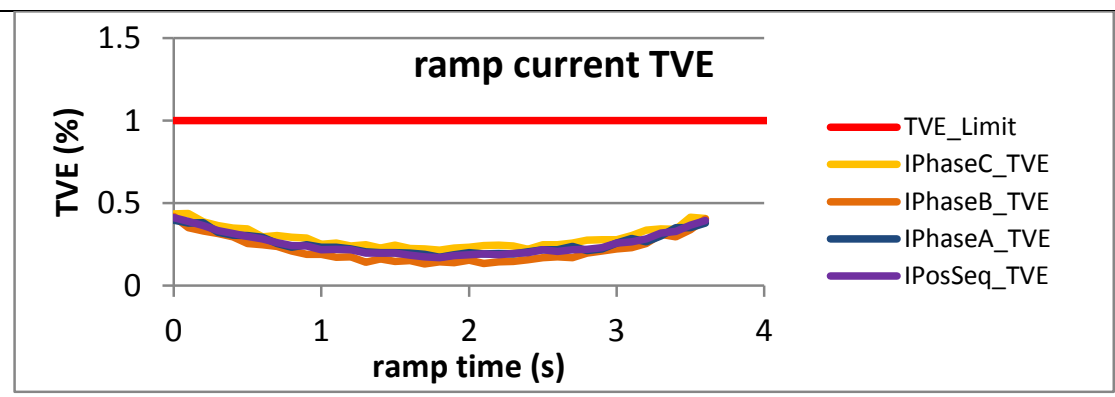

Figure 2857: Fs = $10 \mathrm{FPS}$, ramp from $58 \mathrm{~Hz}$ to $62 \mathrm{~Hz}$ at $+1 \mathrm{~Hz} / \mathrm{s}$

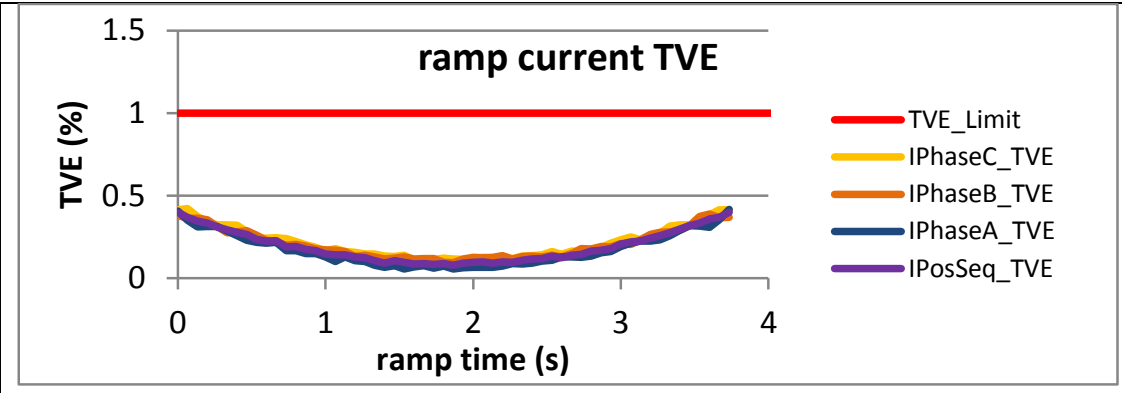

Figure 2854: Fs = $15 \mathrm{FPS}$, ramp from $63 \mathrm{~Hz}$ to $57 \mathrm{~Hz}$ at $-1 \mathrm{~Hz} / \mathrm{s}$

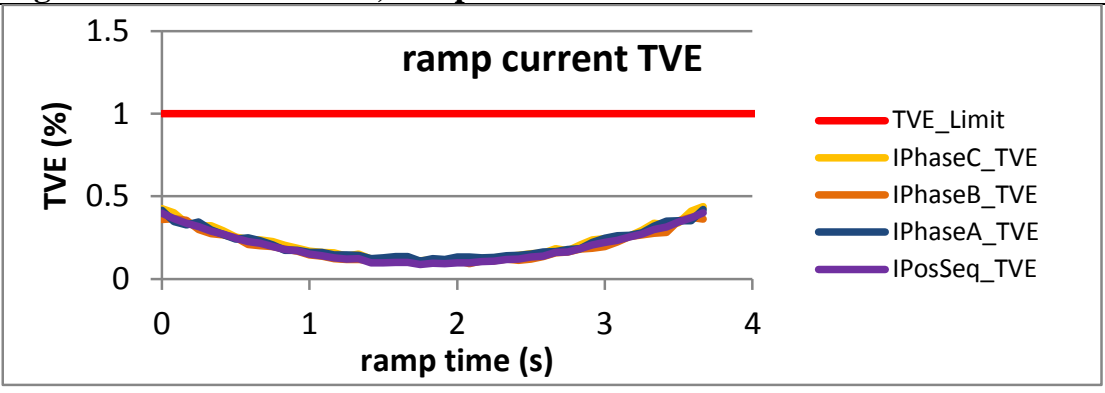

Figure 2856: Fs = 12 FPS, ramp from $62.4 \mathrm{~Hz}$ to $58.6 \mathrm{~Hz}$ at $-1 \mathrm{~Hz} / \mathrm{s}$

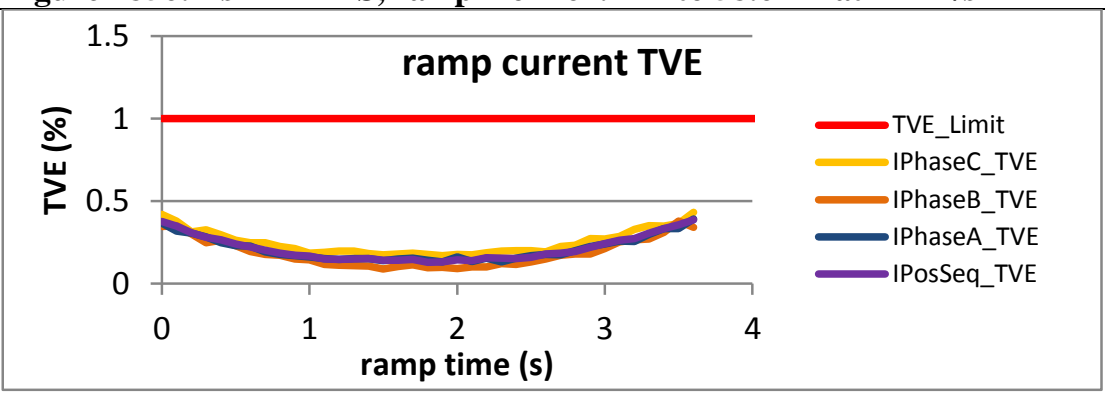

Figure 2858: Fs = $10 \mathrm{FPS}$, ramp from $62 \mathrm{~Hz}$ to $58 \mathrm{~Hz}$ at $-1 \mathrm{~Hz} / \mathrm{s}$ 


\subsection{Dynamic ramp of system frequency frequency, $P$ class}

\subsubsection{C37.118.1 Annex $\mathrm{C}$ dynamic ramp of system frequency frequency error, $\mathrm{P}$ class}
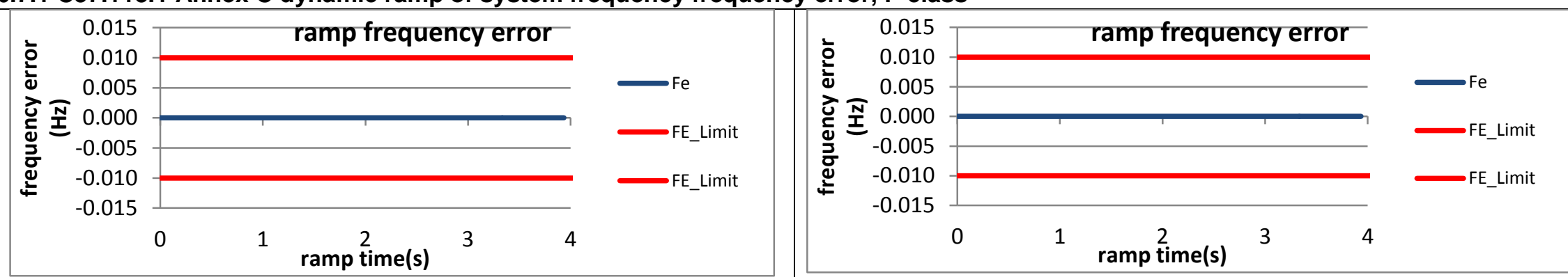

Figure 2859: Fs = $60 \mathrm{FPS}$, ramp from $58 \mathrm{~Hz}$ to $62 \mathrm{~Hz}$ at $+1 \mathrm{~Hz} / \mathrm{s}$

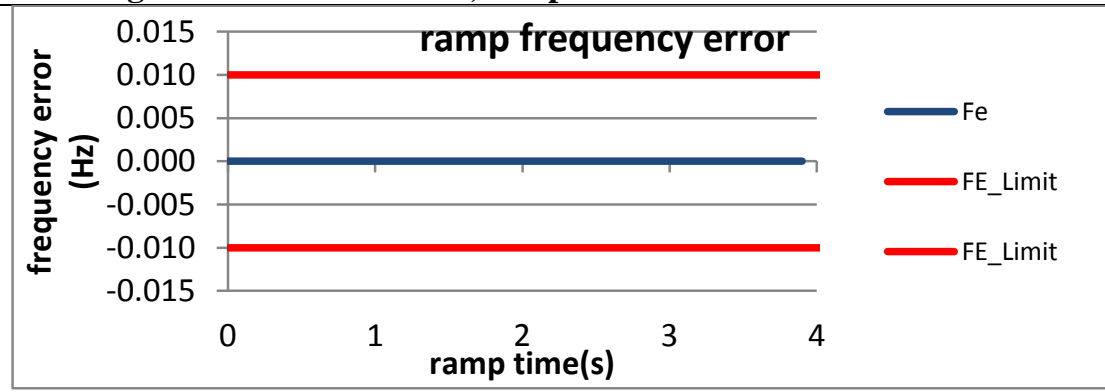

Figure 2861: Fs = $30 \mathrm{FPS}$, ramp from $58 \mathrm{~Hz}$ to $62 \mathrm{~Hz}$ at $+1 \mathrm{~Hz} / \mathrm{s}$

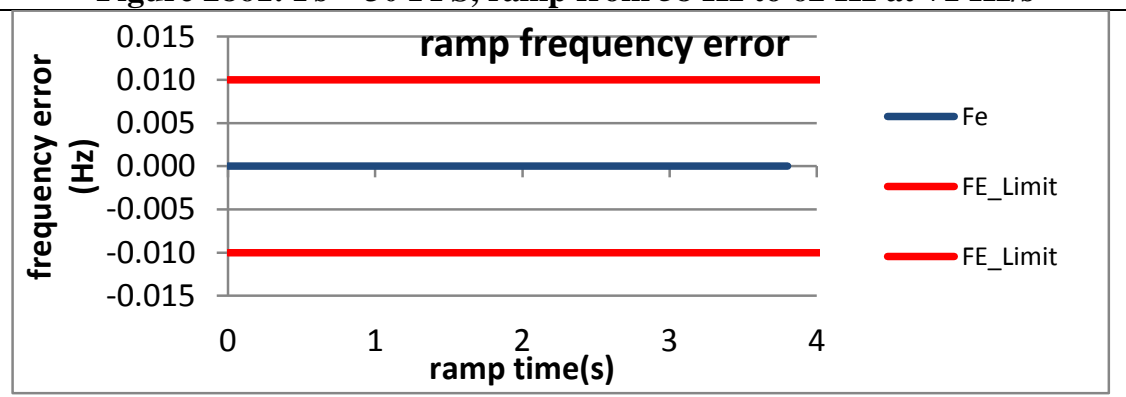

Figure 2863: Fs = $20 \mathrm{FPS}$, ramp from $58 \mathrm{~Hz}$ to $62 \mathrm{~Hz}$ at $+1 \mathrm{~Hz} / \mathrm{s}$

Figure 2860: Fs = $60 \mathrm{FPS}$, ramp from $62 \mathrm{~Hz}$ to $58 \mathrm{~Hz}$ at $-1 \mathrm{~Hz} / \mathrm{s}$

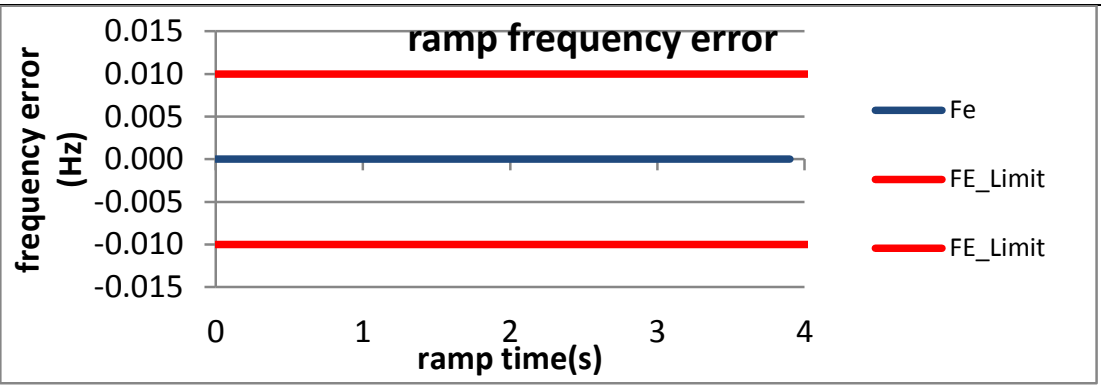

Figure 2862: Fs = $30 \mathrm{FPS}$, ramp from $62 \mathrm{~Hz}$ to $58 \mathrm{~Hz}$ at $-1 \mathrm{~Hz} / \mathrm{s}$

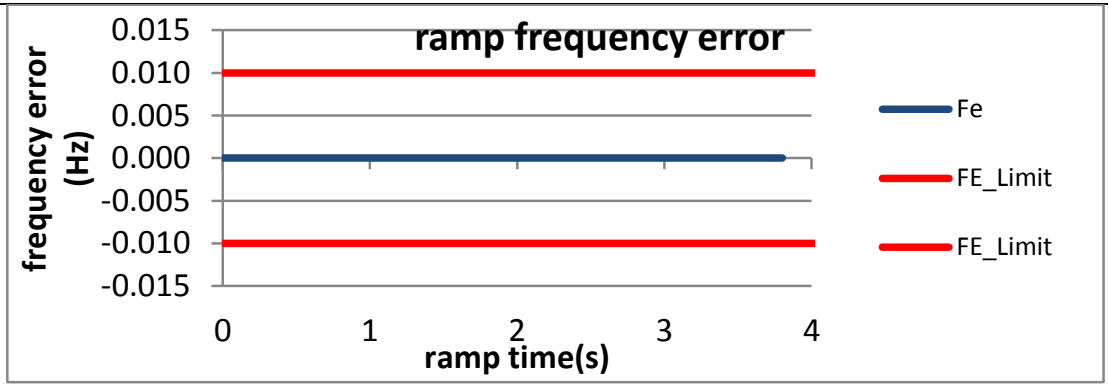

Figure 2864: Fs = $20 \mathrm{FPS}$, ramp from $62 \mathrm{~Hz}$ to $58 \mathrm{~Hz}$ at $-1 \mathrm{~Hz} / \mathrm{s}$ 

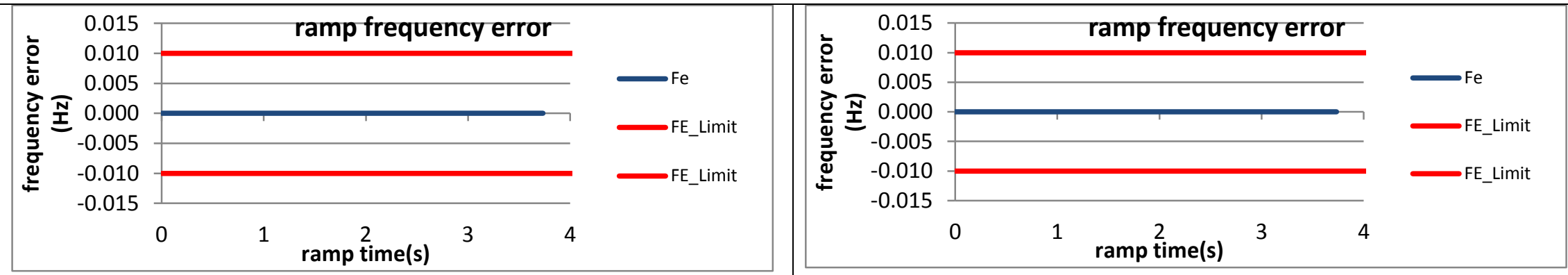

Figure 2865: Fs = $15 \mathrm{FPS}$, ramp from $58 \mathrm{~Hz}$ to $62 \mathrm{~Hz}$ at $+1 \mathrm{~Hz} / \mathrm{s}$

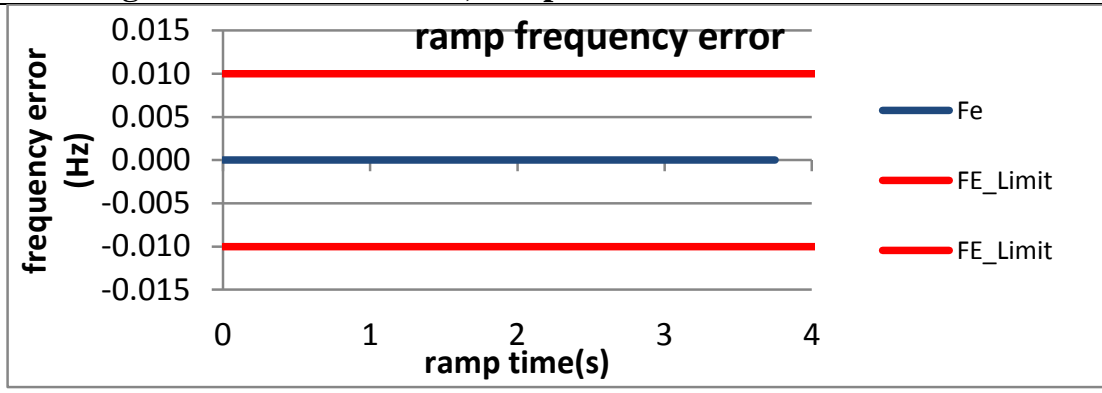

Figure 2866: Fs = $15 \mathrm{FPS}$, ramp from $62 \mathrm{~Hz}$ to $58 \mathrm{~Hz}$ at $-1 \mathrm{~Hz} / \mathrm{s}$

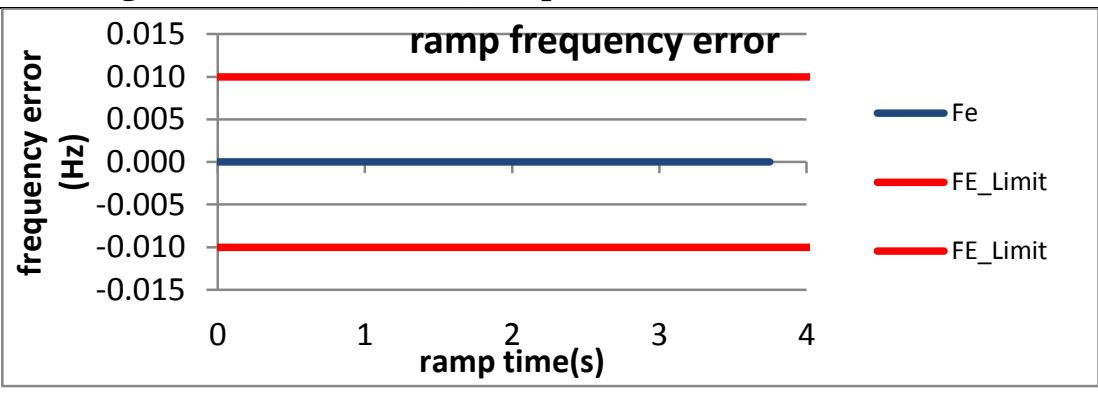

Figure 2867: Fs = $12 \mathrm{FPS}$, ramp from $58 \mathrm{~Hz}$ to $62 \mathrm{~Hz}$ at $+1 \mathrm{~Hz} / \mathrm{s}$

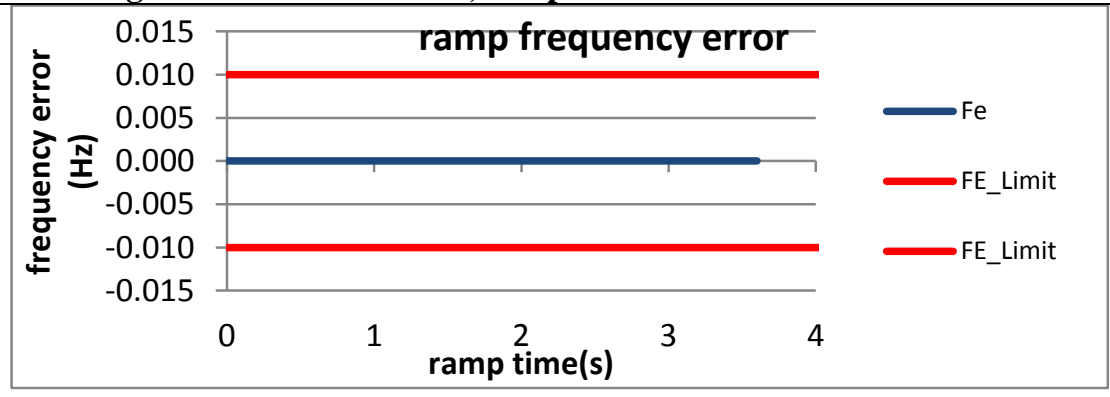

Figure 2869: Fs = $10 \mathrm{FPS}$, ramp from $58 \mathrm{~Hz}$ to $62 \mathrm{~Hz}$ at $+1 \mathrm{~Hz} / \mathrm{s}$

Figure 2868: Fs = $12 \mathrm{FPS}$, ramp from $62 \mathrm{~Hz}$ to $58 \mathrm{~Hz}$ at $-1 \mathrm{~Hz} / \mathrm{s}$

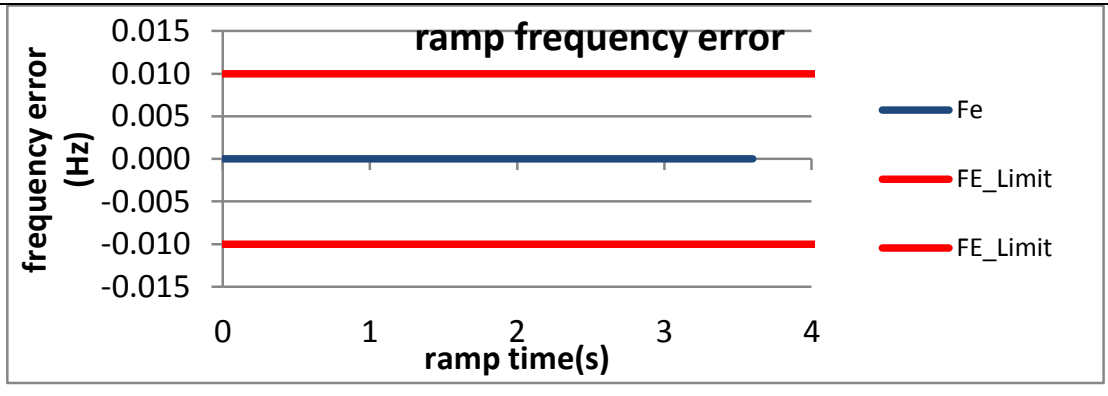

Figure 2870: Fs = $10 \mathrm{FPS}$, ramp from $62 \mathrm{~Hz}$ to $58 \mathrm{~Hz}$ at $-1 \mathrm{~Hz} / \mathrm{s}$ 
6.7.2 PMU A dynamic ramp of system frequency frequency error, $P$ class
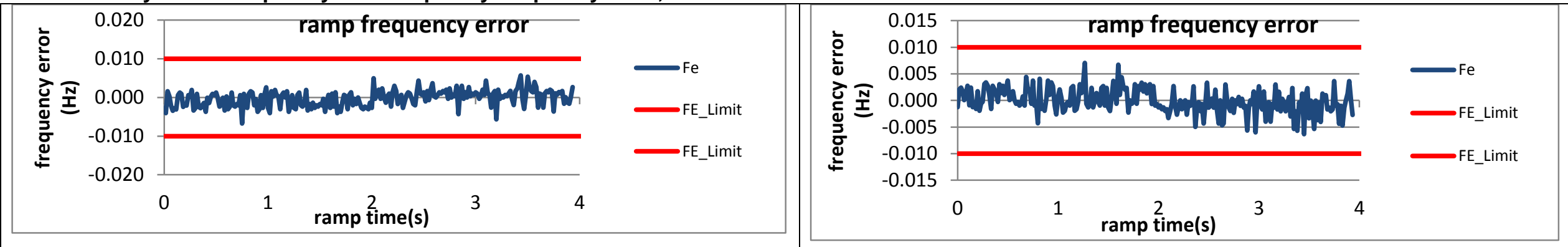

Figure 2871: Fs = $60 \mathrm{FPS}$, ramp from $58 \mathrm{~Hz}$ to $62 \mathrm{~Hz}$ at $+1 \mathrm{~Hz} / \mathrm{s}$

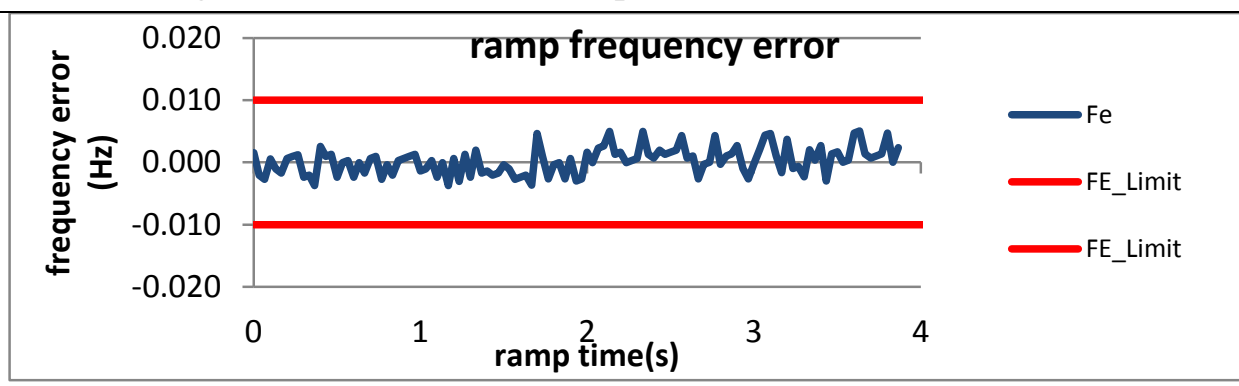

Figure 2872: Fs = $60 \mathrm{FPS}$, ramp from $62 \mathrm{~Hz}$ to $58 \mathrm{~Hz}$ at $-1 \mathrm{~Hz} / \mathrm{s}$

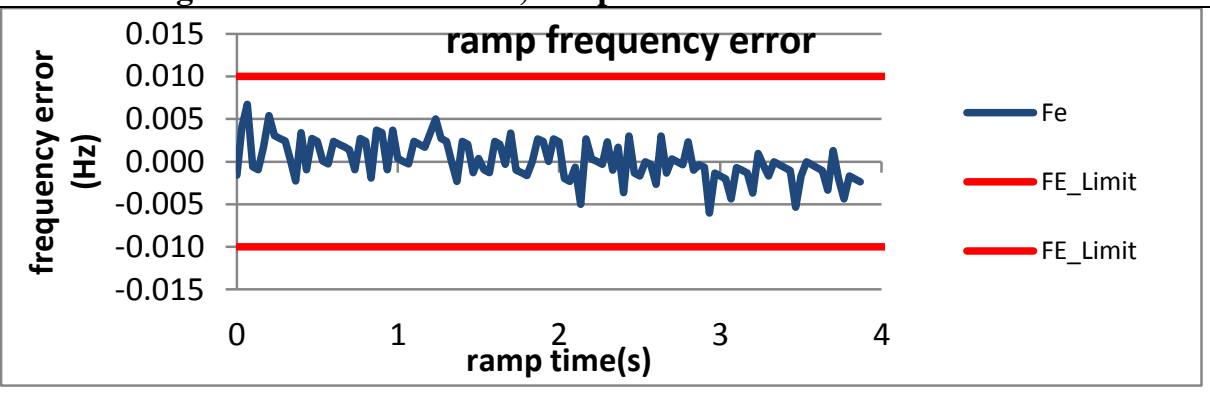

Figure 2873: Fs = $30 \mathrm{FPS}$, ramp from $58 \mathrm{~Hz}$ to $62 \mathrm{~Hz}$ at $+1 \mathrm{~Hz} / \mathrm{s}$

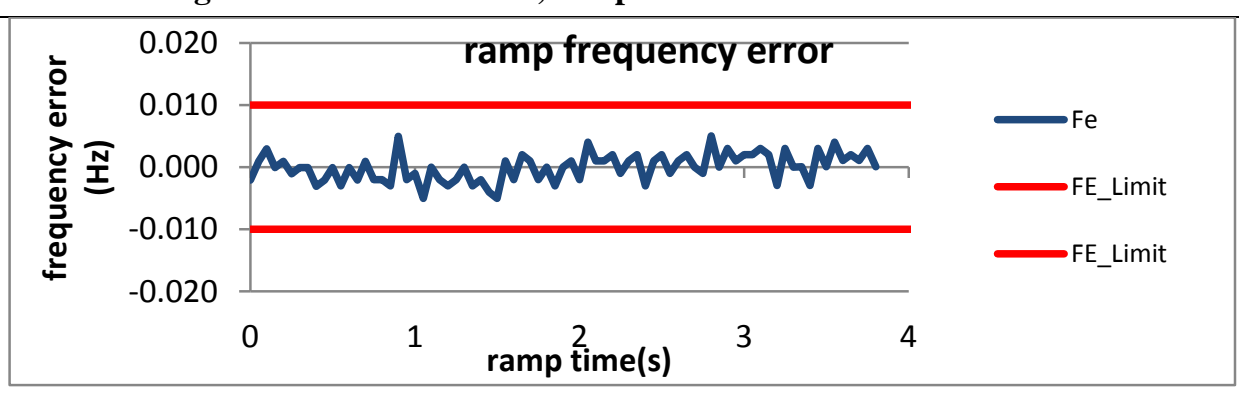

Figure 2875: Fs = $20 \mathrm{FPS}$, ramp from $58 \mathrm{~Hz}$ to $62 \mathrm{~Hz}$ at $+1 \mathrm{~Hz} / \mathrm{s}$

Figure 2874: Fs = $30 \mathrm{FPS}$, ramp from $62 \mathrm{~Hz}$ to $58 \mathrm{~Hz}$ at $-1 \mathrm{~Hz} / \mathrm{s}$

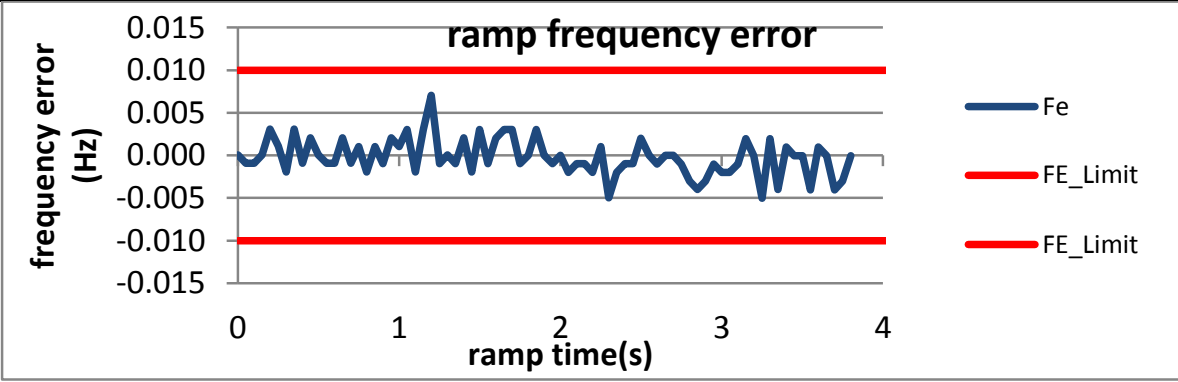

Figure 2876: Fs = $20 \mathrm{FPS}$, ramp from $62 \mathrm{~Hz}$ to $58 \mathrm{~Hz}$ at $-1 \mathrm{~Hz} / \mathrm{s}$ 

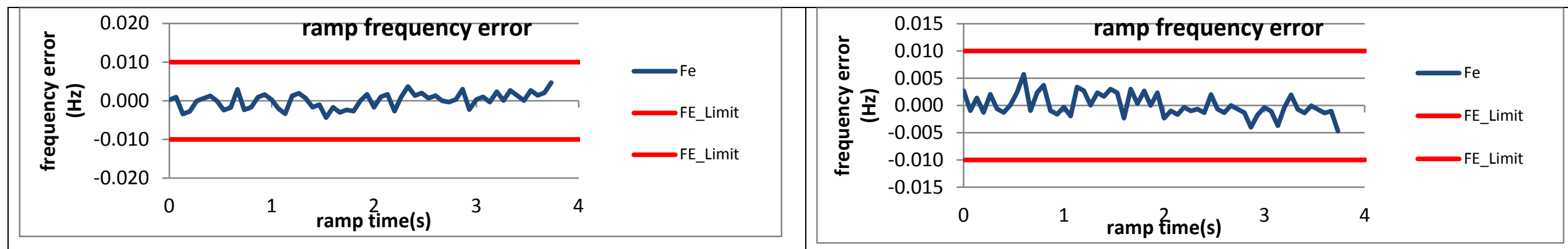

Figure 2877: Fs = $15 \mathrm{FPS}$, ramp from $58 \mathrm{~Hz}$ to $62 \mathrm{~Hz}$ at $+1 \mathrm{~Hz} / \mathrm{s}$

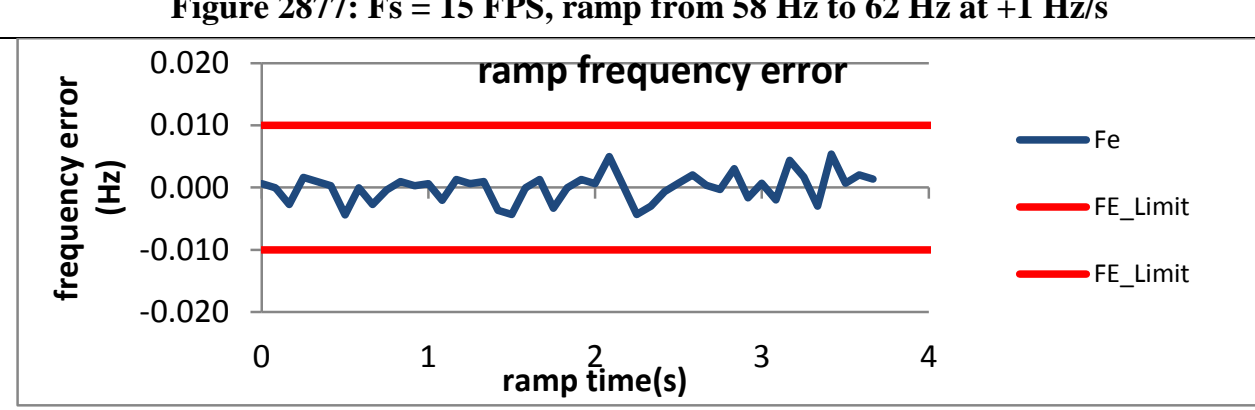

Figure 2878: Fs = $15 \mathrm{FPS}$, ramp from $62 \mathrm{~Hz}$ to $58 \mathrm{~Hz}$ at $-1 \mathrm{~Hz} / \mathrm{s}$

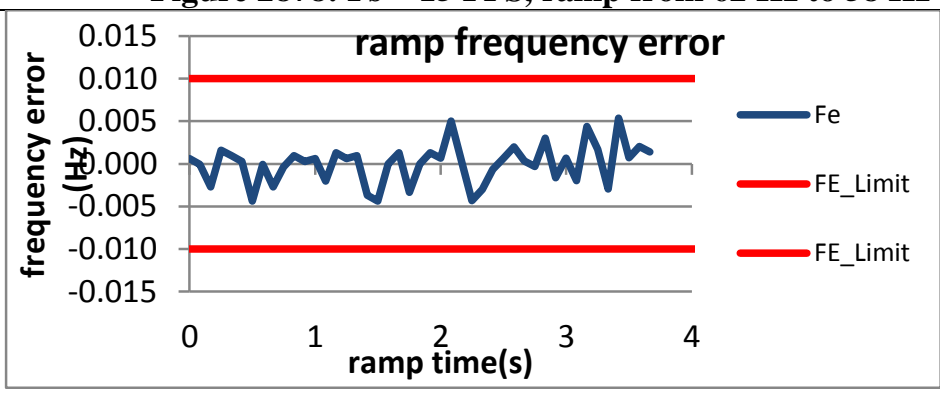

Figure 2879: Fs = $12 \mathrm{FPS}$, ramp from $58 \mathrm{~Hz}$ to $62 \mathrm{~Hz}$ at $+1 \mathrm{~Hz} / \mathrm{s}$

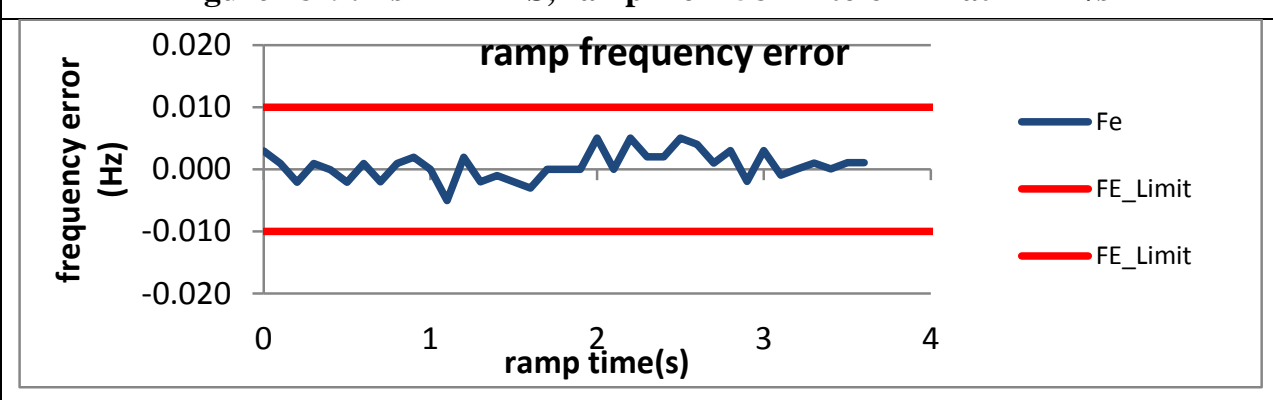

Figure 2881: Fs = $10 \mathrm{FPS}$, ramp from $58 \mathrm{~Hz}$ to $62 \mathrm{~Hz}$ at $+1 \mathrm{~Hz} / \mathrm{s}$

Figure 2880: Fs = $12 \mathrm{FPS}$, ramp from $62 \mathrm{~Hz}$ to $58 \mathrm{~Hz}$ at $-1 \mathrm{~Hz} / \mathrm{s}$

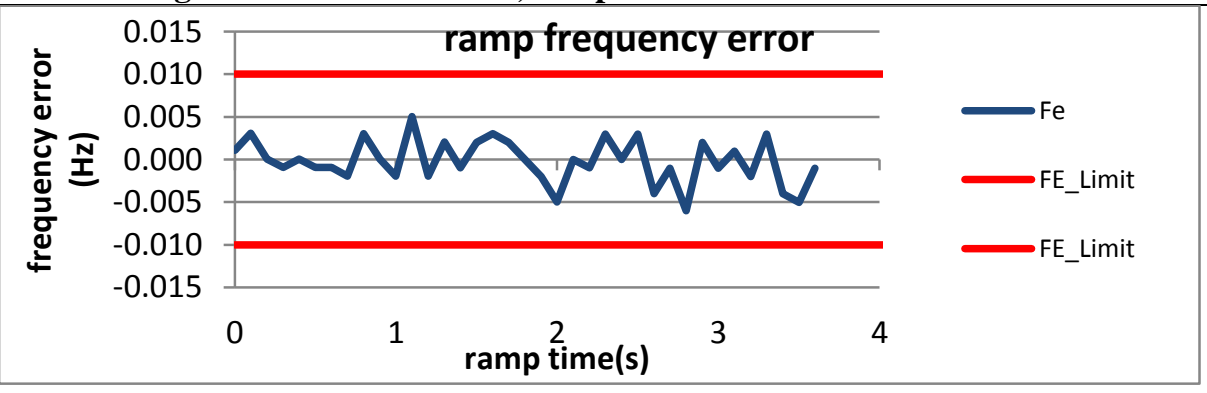

Figure 2882: Fs = $10 \mathrm{FPS}$, ramp from $62 \mathrm{~Hz}$ to $58 \mathrm{~Hz}$ at $-1 \mathrm{~Hz} / \mathrm{s}$ 
6.7.3 PMU B dynamic ramp of system frequency frequency error, $P$ class

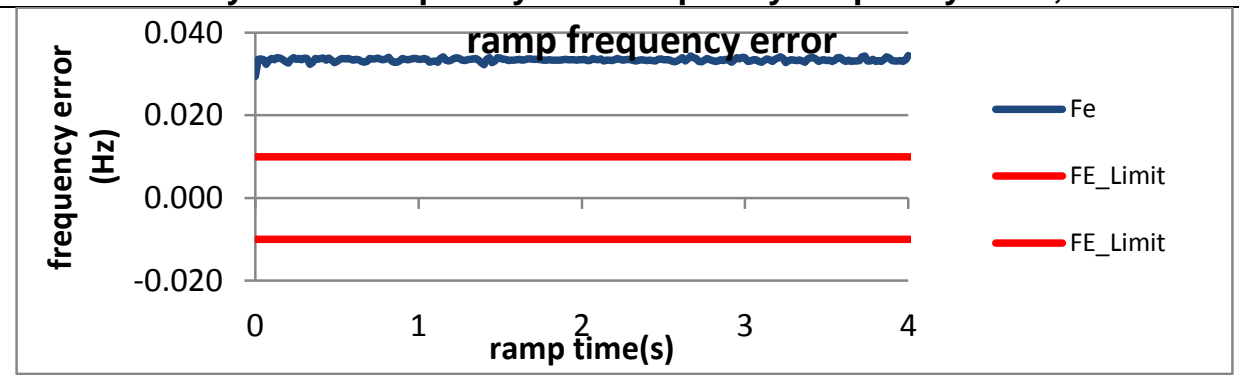

Figure 2883: Fs = $60 \mathrm{FPS}$, ramp from $55 \mathrm{~Hz}$ to $65 \mathrm{~Hz}$ at $+1 \mathrm{~Hz} / \mathrm{s}$

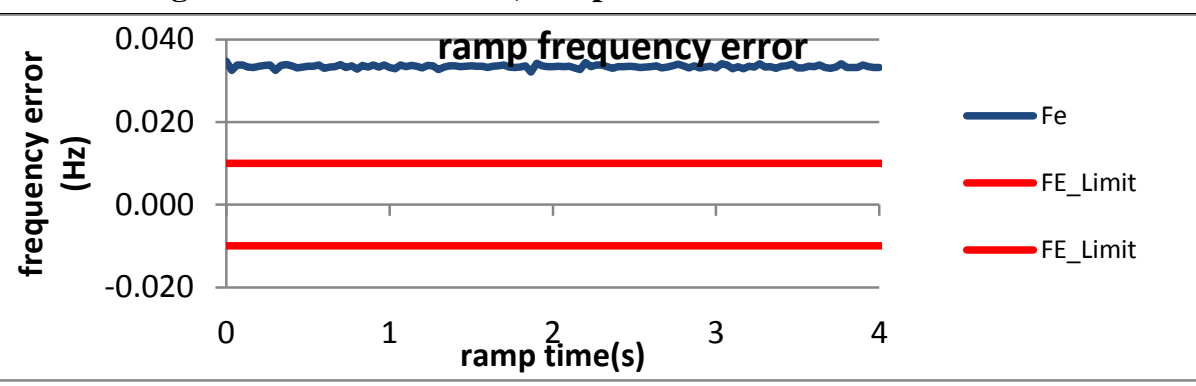

Figure 2885: Fs = $30 \mathrm{FPS}$, ramp from $55 \mathrm{~Hz}$ to $65 \mathrm{~Hz}$ at $+1 \mathrm{~Hz} / \mathrm{s}$

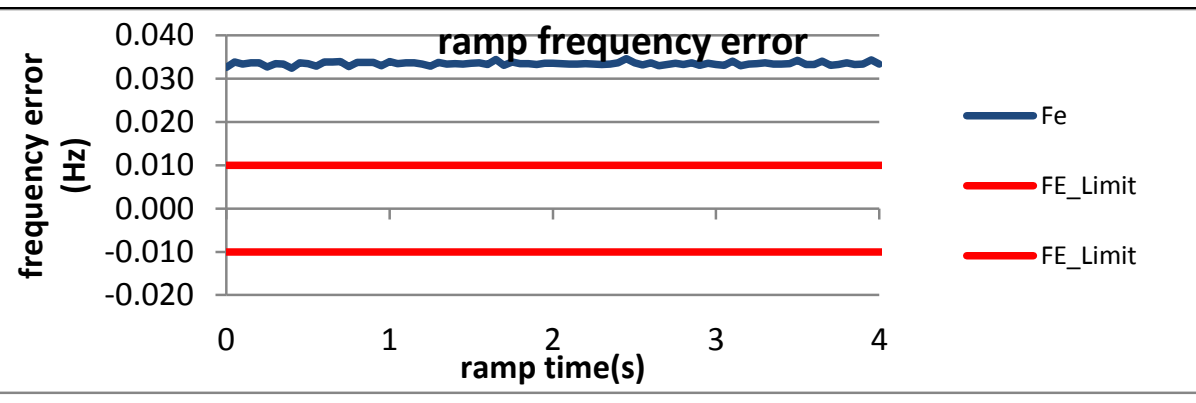

Figure 2887: Fs = $20 \mathrm{FPS}$, ramp from $56 \mathrm{~Hz}$ to $64 \mathrm{~Hz}$ at $+1 \mathrm{~Hz} / \mathrm{s}$

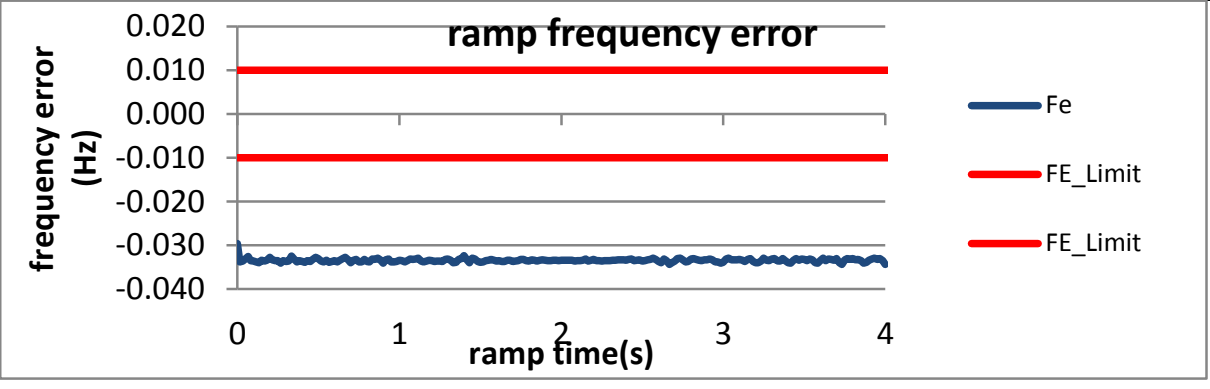

Figure 2884: Fs = $60 \mathrm{FPS}$, ramp from $65 \mathrm{~Hz}$ to $55 \mathrm{~Hz}$ at $-1 \mathrm{~Hz} / \mathrm{s}$

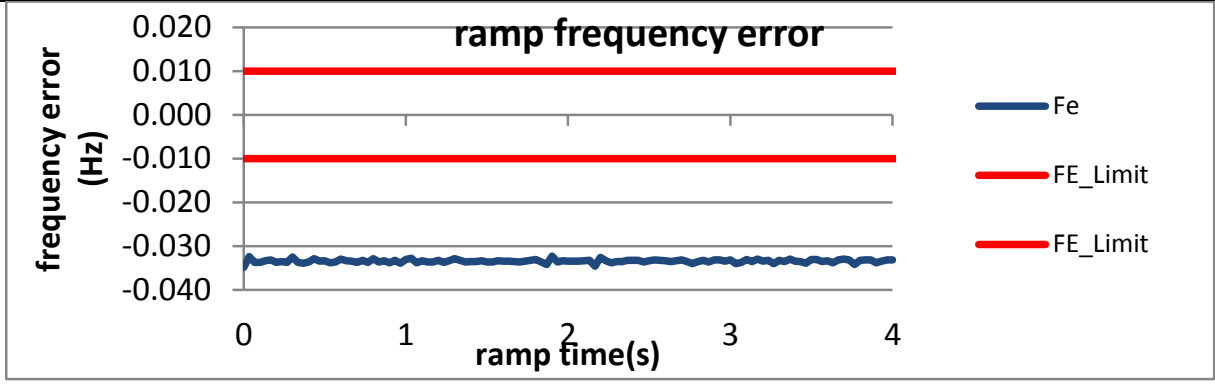

Figure 2886: Fs = $30 \mathrm{FPS}$, ramp from $65 \mathrm{~Hz}$ to $55 \mathrm{~Hz}$ at $-1 \mathrm{~Hz} / \mathrm{s}$

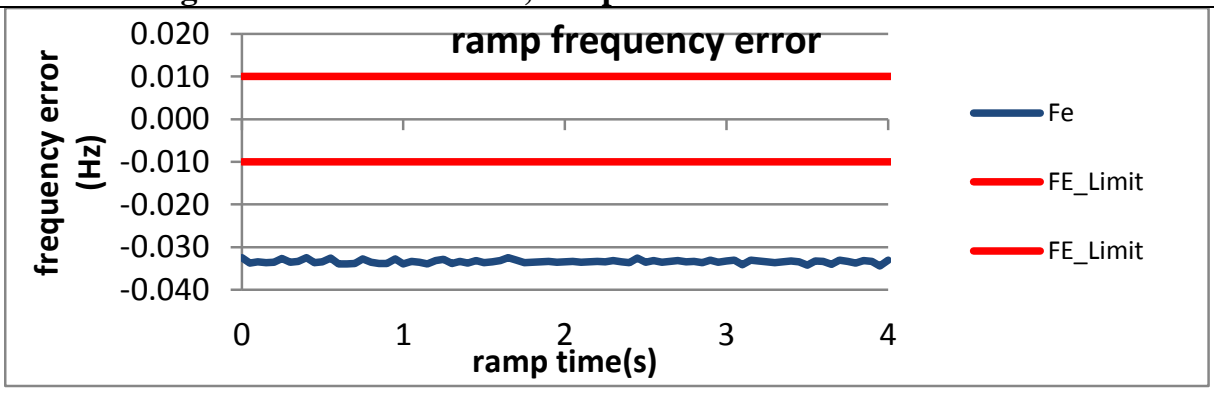

Figure 2888: Fs = $20 \mathrm{FPS}$, ramp from $64 \mathrm{~Hz}$ to $56 \mathrm{~Hz}$ at $-1 \mathrm{~Hz} / \mathrm{s}$ 


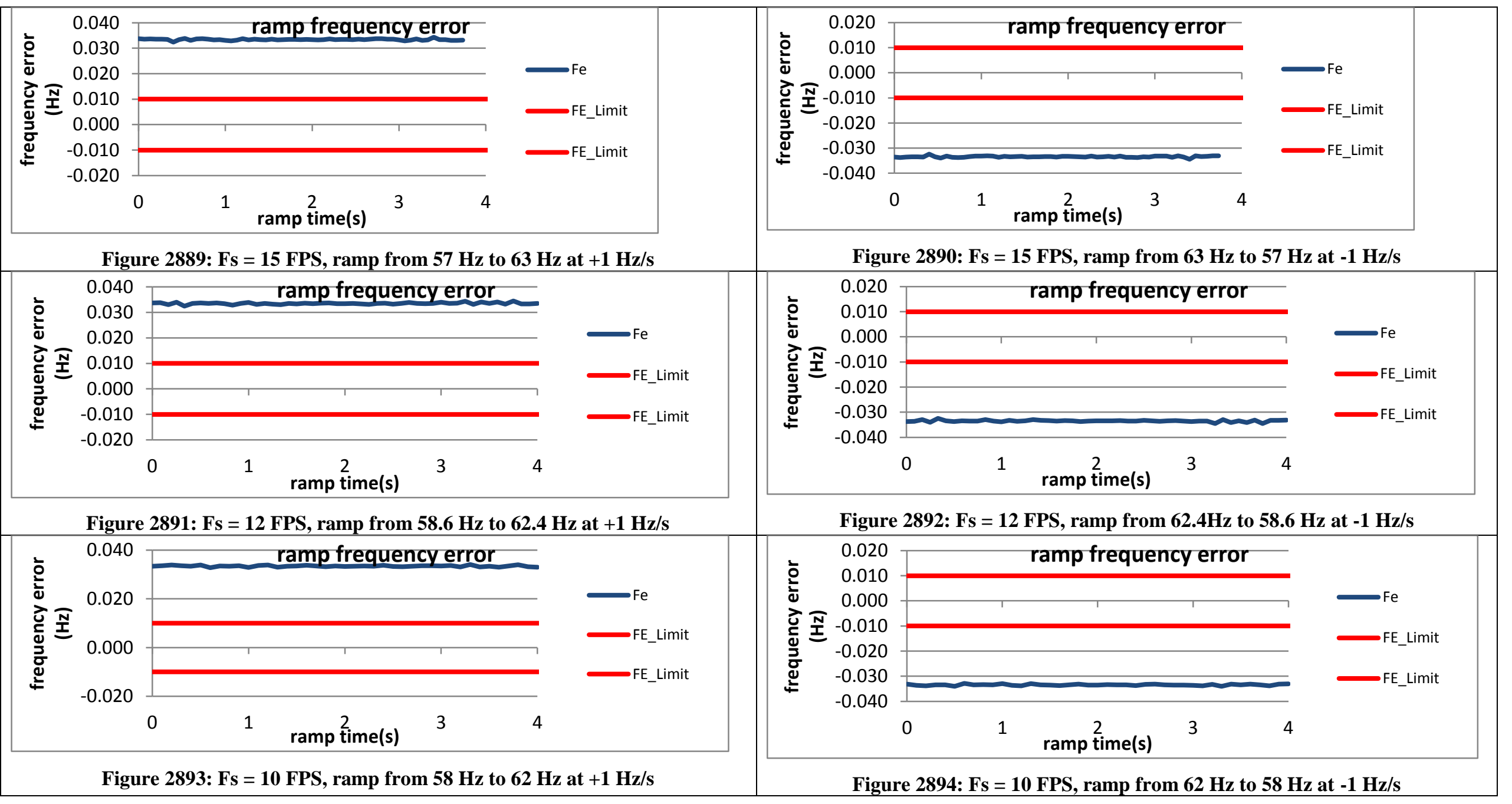


6.7.4 PMU C dynamic ramp of system frequency frequency error, $P$ class
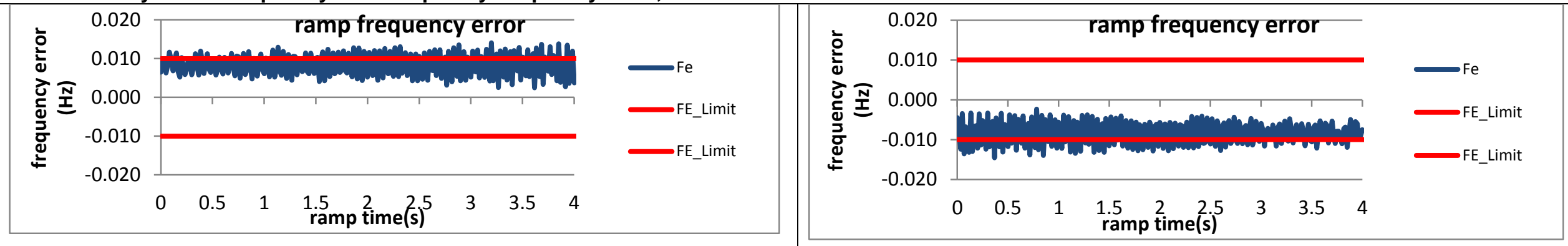

Figure 2895: Fs = $60 \mathrm{FPS}$, ramp from $55 \mathrm{~Hz}$ to $65 \mathrm{~Hz}$ at $+1 \mathrm{~Hz} / \mathrm{s}$

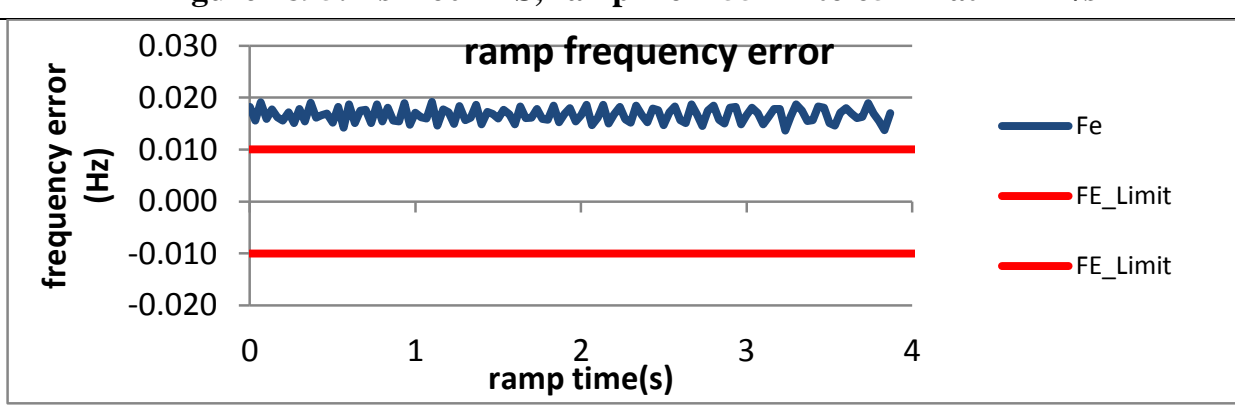

Figure 2896: Fs = $60 \mathrm{FPS}$, ramp from $65 \mathrm{~Hz}$ to $55 \mathrm{~Hz}$ at $-1 \mathrm{~Hz} / \mathrm{s}$

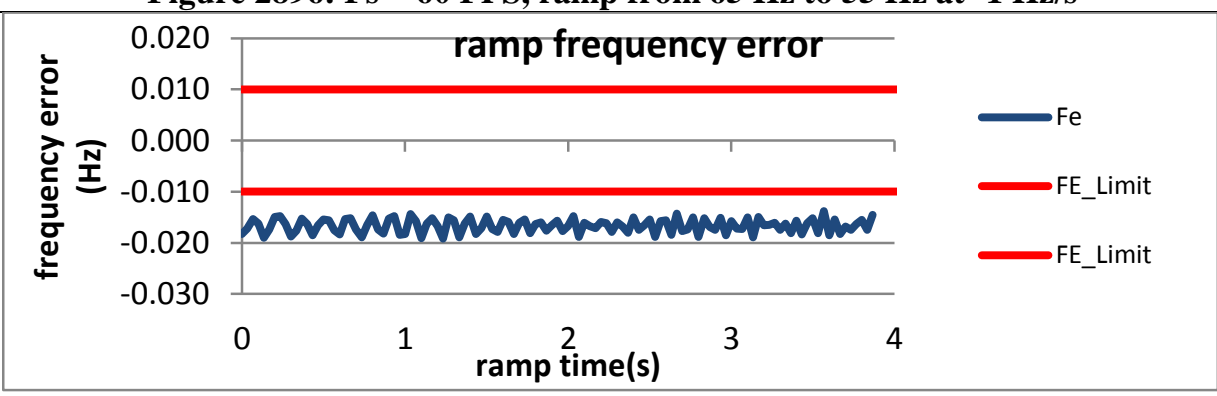

Figure 2897: Fs = $30 \mathrm{FPS}$, ramp from $55 \mathrm{~Hz}$ to $65 \mathrm{~Hz}$ at $+1 \mathrm{~Hz} / \mathrm{s}$

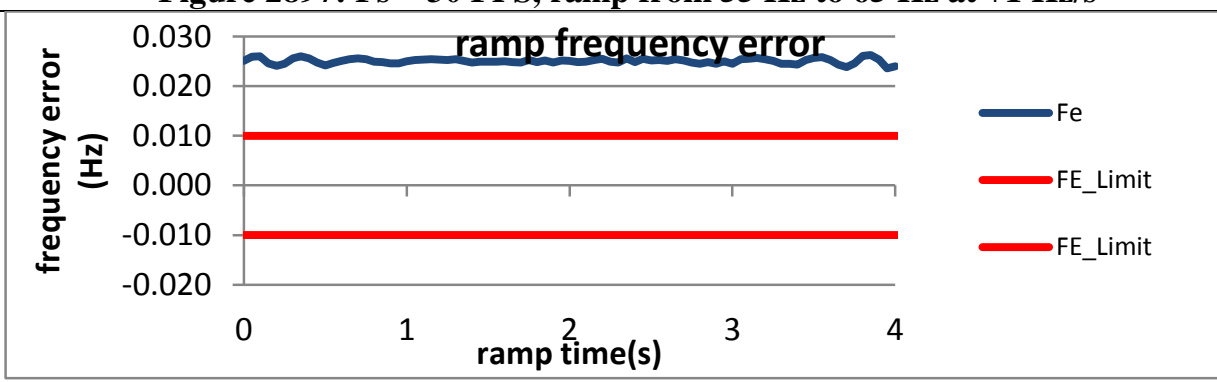

Figure 2898: Fs = $30 \mathrm{FPS}$, ramp from $65 \mathrm{~Hz}$ to $55 \mathrm{~Hz}$ at $-1 \mathrm{~Hz} / \mathrm{s}$

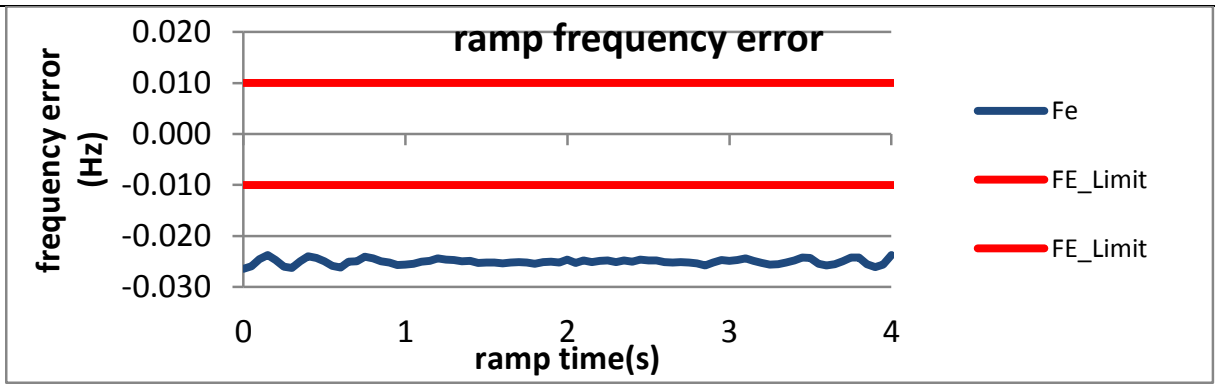

Figure 2899: Fs = $20 \mathrm{FPS}$, ramp from $56 \mathrm{~Hz}$ to $64 \mathrm{~Hz}$ at $+1 \mathrm{~Hz} / \mathrm{s}$

Figure 2900: Fs = $20 \mathrm{FPS}$, ramp from $64 \mathrm{~Hz}$ to $56 \mathrm{~Hz}$ at $-1 \mathrm{~Hz} / \mathrm{s}$ 


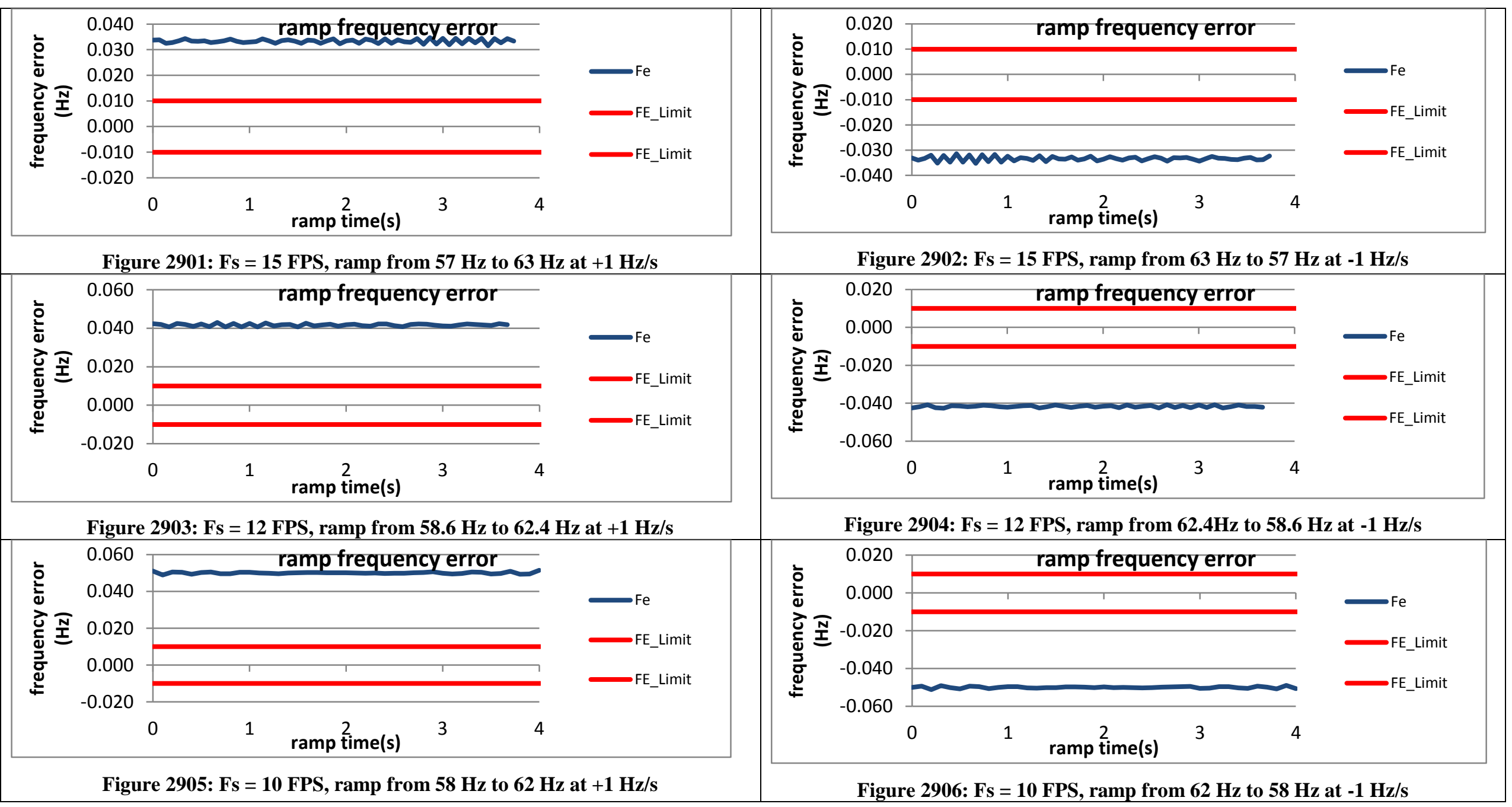


6.7.5 PMU D dynamic ramp of system frequency frequency error, $P$ class

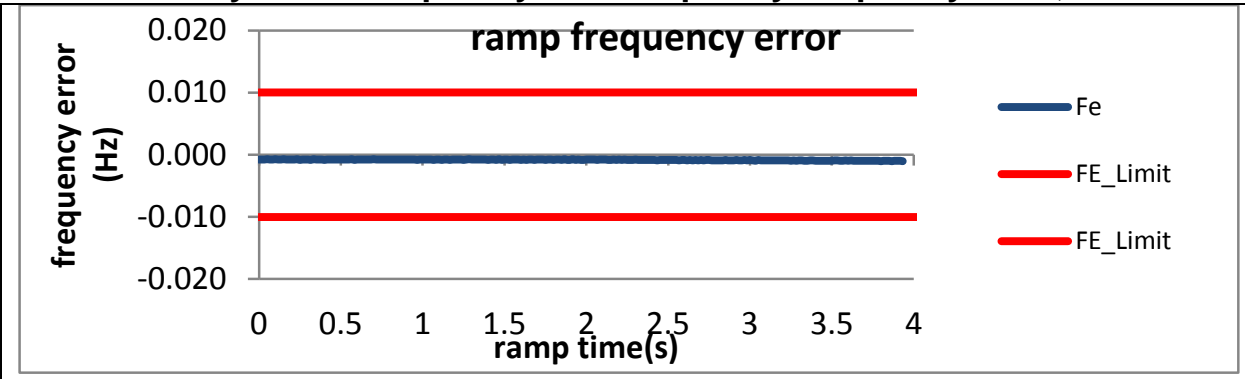

Figure 2907: Fs = $60 \mathrm{FPS}$, ramp from $58 \mathrm{~Hz}$ to $62 \mathrm{~Hz}$ at $+1 \mathrm{~Hz} / \mathrm{s}$

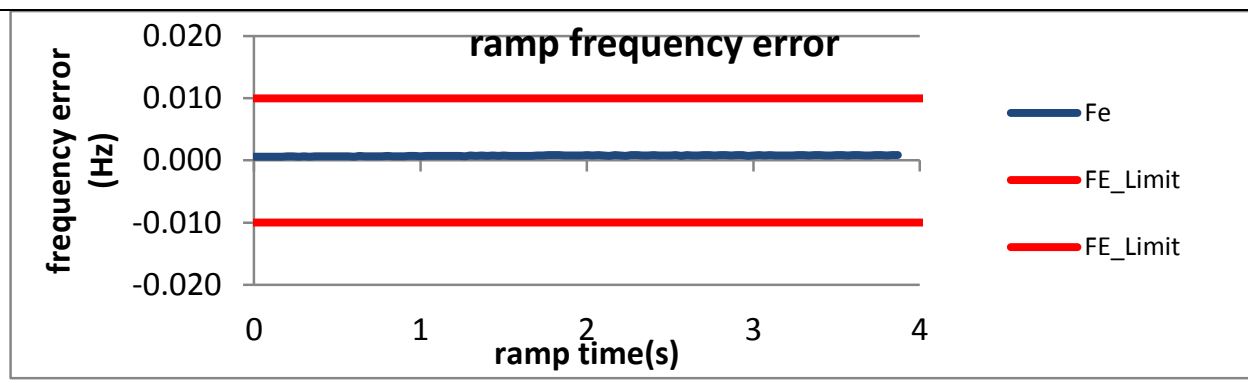

Figure 2909: Fs = $30 \mathrm{FPS}$, ramp from $58 \mathrm{~Hz}$ to $62 \mathrm{~Hz}$ at $+1 \mathrm{~Hz} / \mathrm{s}$

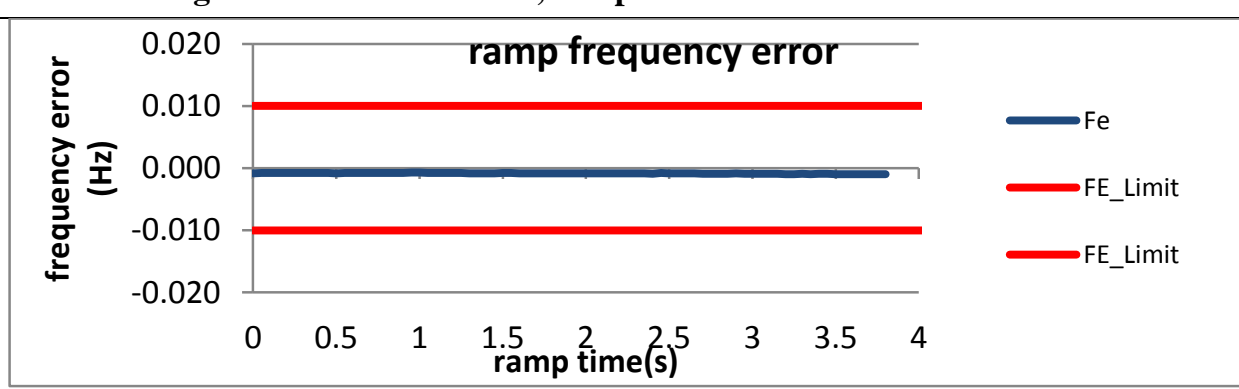

Figure 2911: Fs = $20 \mathrm{FPS}$, ramp from $58 \mathrm{~Hz}$ to $62 \mathrm{~Hz}$ at $+1 \mathrm{~Hz} / \mathrm{s}$

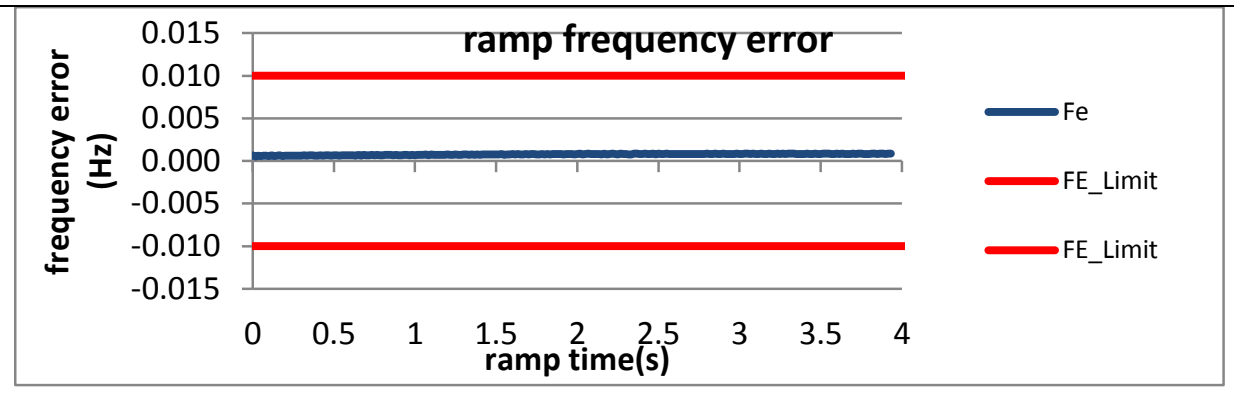

Figure 2908: Fs = $60 \mathrm{FPS}$, ramp from $62 \mathrm{~Hz}$ to $58 \mathrm{~Hz}$ at $-1 \mathrm{~Hz} / \mathrm{s}$

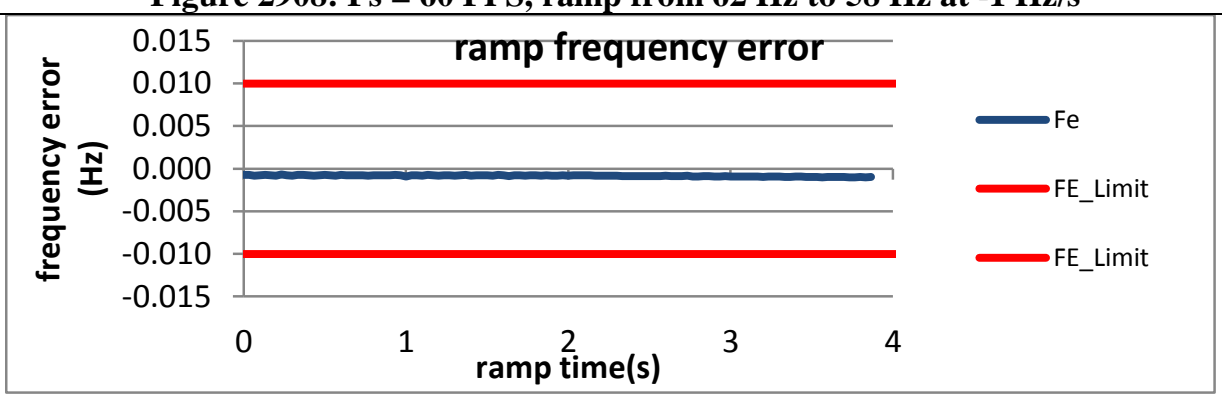

Figure 2910: Fs = $30 \mathrm{FPS}$, ramp from $62 \mathrm{~Hz}$ to $58 \mathrm{~Hz}$ at $-1 \mathrm{~Hz} / \mathrm{s}$

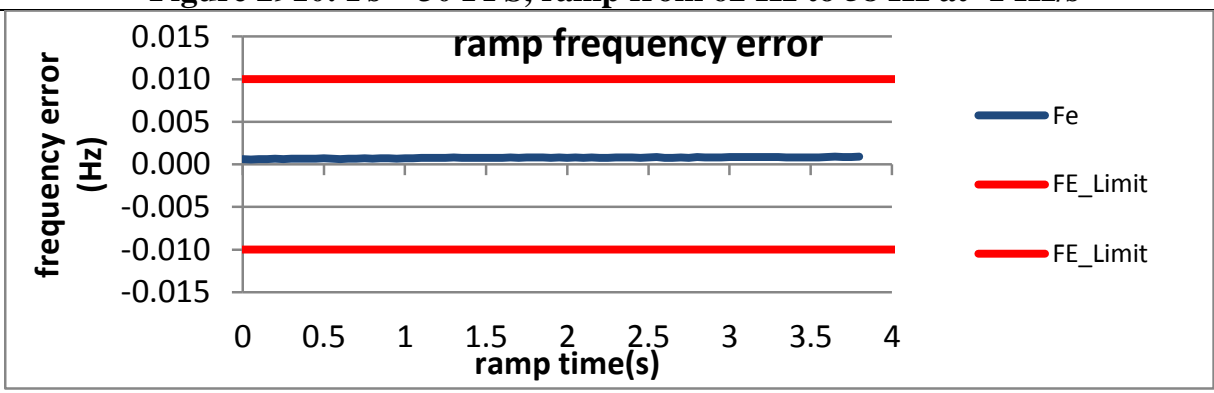

Figure 2912: Fs = $20 \mathrm{FPS}$, ramp from $62 \mathrm{~Hz}$ to $58 \mathrm{~Hz}$ at $-1 \mathrm{~Hz} / \mathrm{s}$ 


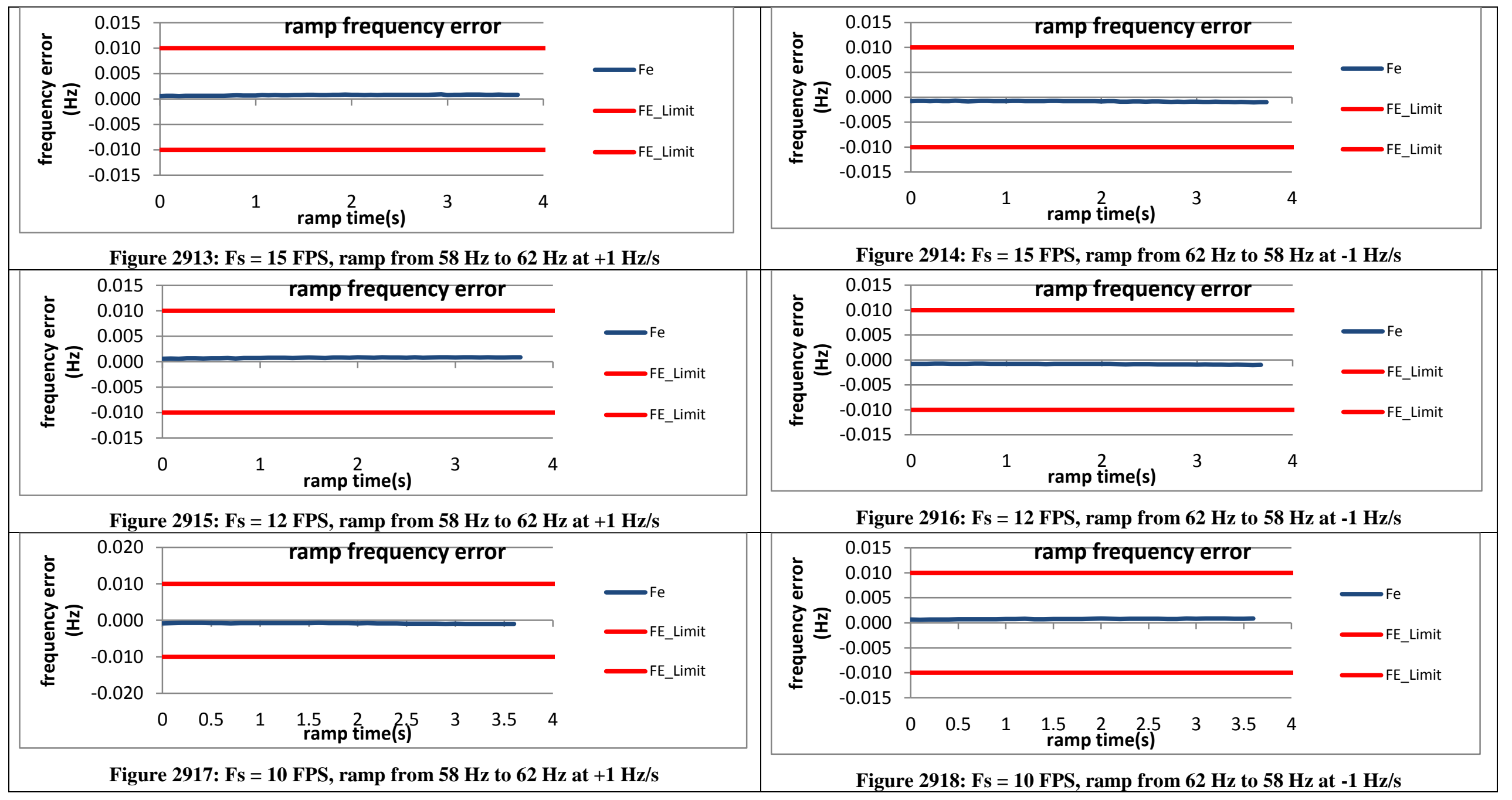


6.7.6 PMU E dynamic ramp of system frequency frequency error, $P$ class PMU E does not support P class.

6.7.7 PMU F dynamic ramp of system frequency frequency error, $P$ class
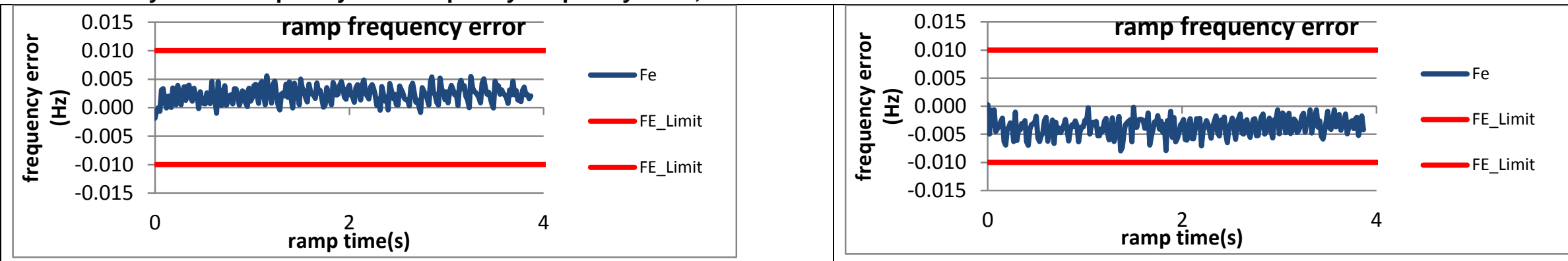

Figure 2919: Fs = $60 \mathrm{FPS}$, ramp from $55 \mathrm{~Hz}$ to $65 \mathrm{~Hz}$ at $+1 \mathrm{~Hz} / \mathrm{s}$

Figure 2920: Fs = $60 \mathrm{FPS}$, ramp from $65 \mathrm{~Hz}$ to $55 \mathrm{~Hz}$ at $-1 \mathrm{~Hz} / \mathrm{s}$
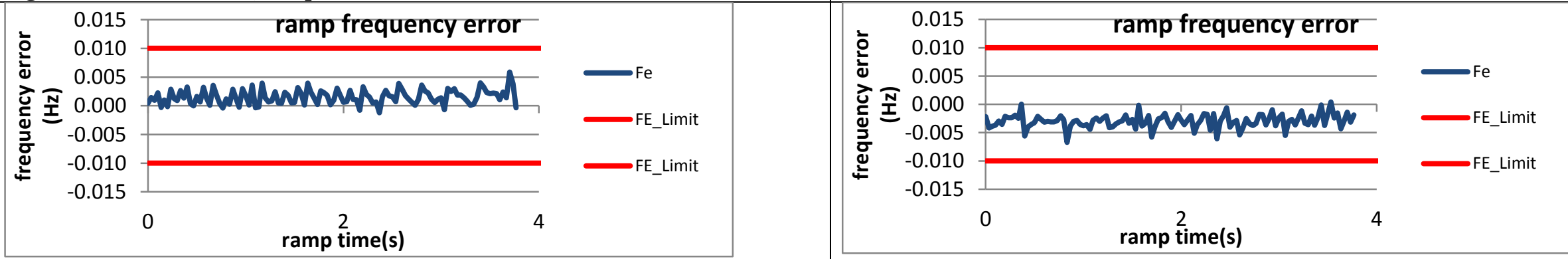

Figure 2921: Fs = $30 \mathrm{FPS}$, ramp from $55 \mathrm{~Hz}$ to $65 \mathrm{~Hz}$ at $+1 \mathrm{~Hz} / \mathrm{s}$

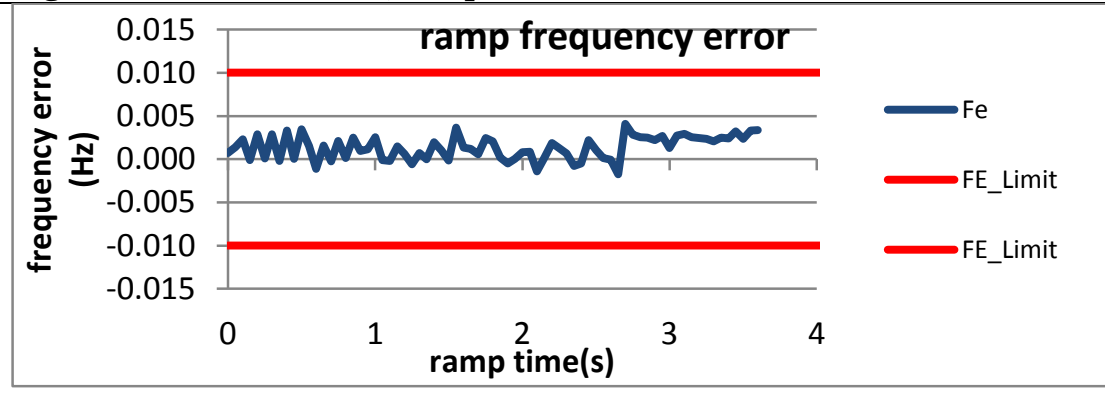

Figure 2923: Fs = $20 \mathrm{FPS}$, ramp from $56 \mathrm{~Hz}$ to $64 \mathrm{~Hz}$ at $+1 \mathrm{~Hz} / \mathrm{s}$

Figure 2922: Fs = $30 \mathrm{FPS}$, ramp from $65 \mathrm{~Hz}$ to $55 \mathrm{~Hz}$ at $-1 \mathrm{~Hz} / \mathrm{s}$

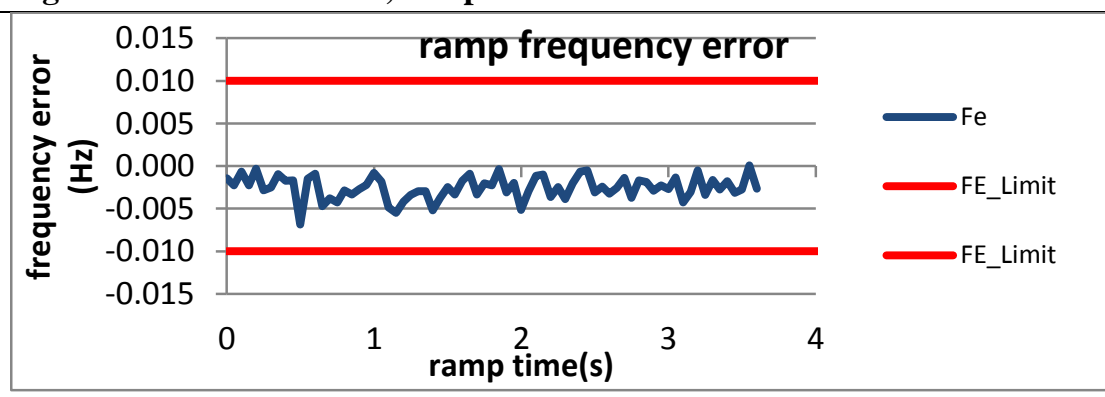

Figure 2924: Fs = $20 \mathrm{FPS}$, ramp from $64 \mathrm{~Hz}$ to $56 \mathrm{~Hz}$ at $-1 \mathrm{~Hz} / \mathrm{s}$ 

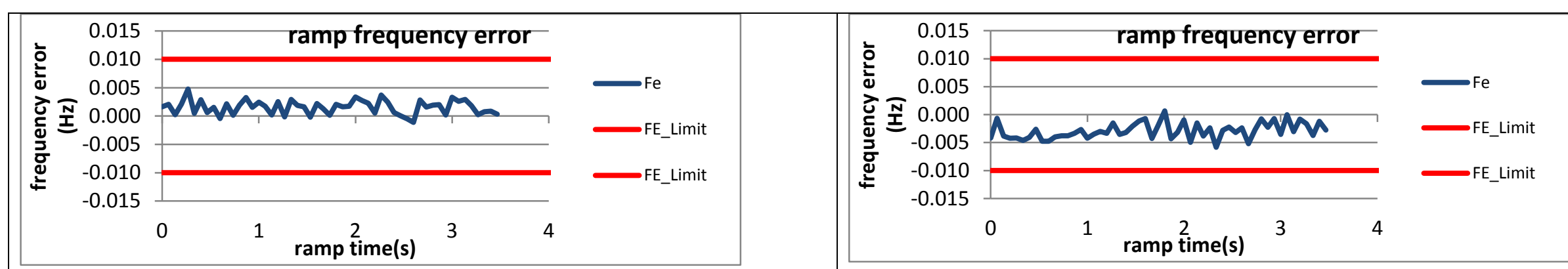

Figure 2925: Fs = $15 \mathrm{FPS}$, ramp from $57 \mathrm{~Hz}$ to $63 \mathrm{~Hz}$ at $+1 \mathrm{~Hz} / \mathrm{s}$

Figure 2926: Fs = $15 \mathrm{FPS}$, ramp from $63 \mathrm{~Hz}$ to $57 \mathrm{~Hz}$ at $-1 \mathrm{~Hz} / \mathrm{s}$
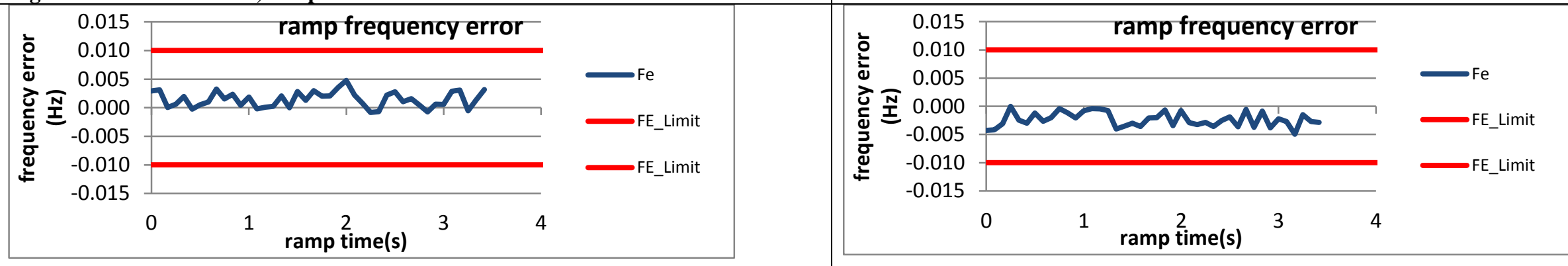

Figure 2927: Fs = 12 FPS, ramp from $58.6 \mathrm{~Hz}$ to $62.4 \mathrm{~Hz}$ at $+1 \mathrm{~Hz} / \mathrm{s}$

Figure 2928: Fs = 12 FPS, ramp from $62.4 \mathrm{~Hz}$ to $58.6 \mathrm{~Hz}$ at $-1 \mathrm{~Hz} / \mathrm{s}$

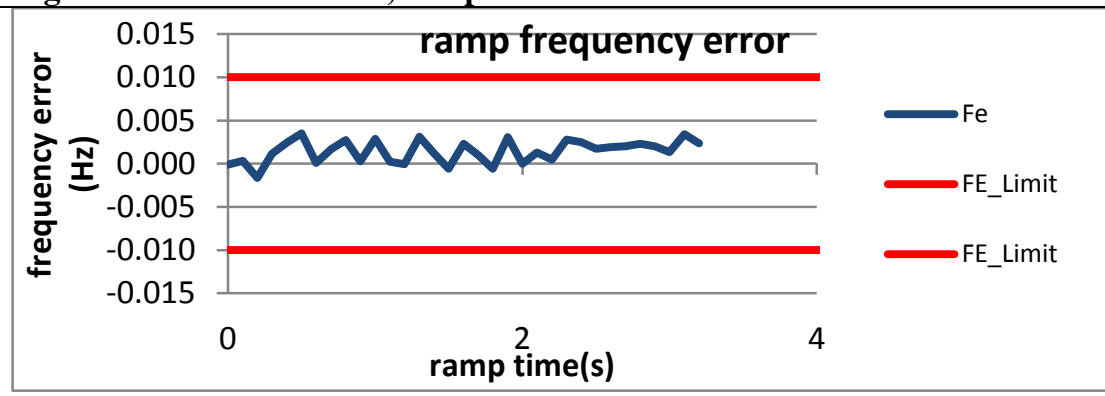

Figure 2929: Fs = $10 \mathrm{FPS}$, ramp from $58 \mathrm{~Hz}$ to $62 \mathrm{~Hz}$ at $+1 \mathrm{~Hz} / \mathrm{s}$

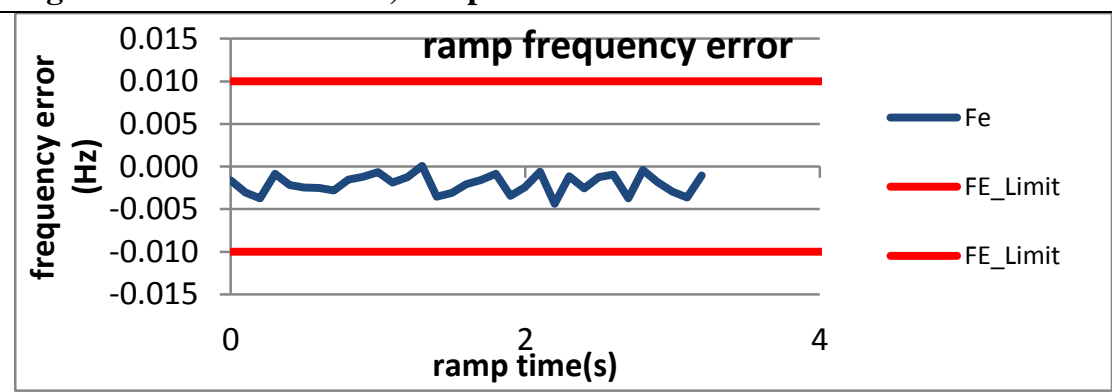

Figure 2930: Fs = $10 \mathrm{FPS}$, ramp from $62 \mathrm{~Hz}$ to $58 \mathrm{~Hz}$ at $-1 \mathrm{~Hz} / \mathrm{s}$ 
6.7.8 PMU G dynamic ramp of system frequency frequency error, $P$ class PMU G does not support P class.

\subsubsection{PMU H dynamic ramp of system frequency frequency error, $\mathrm{P}$ class}
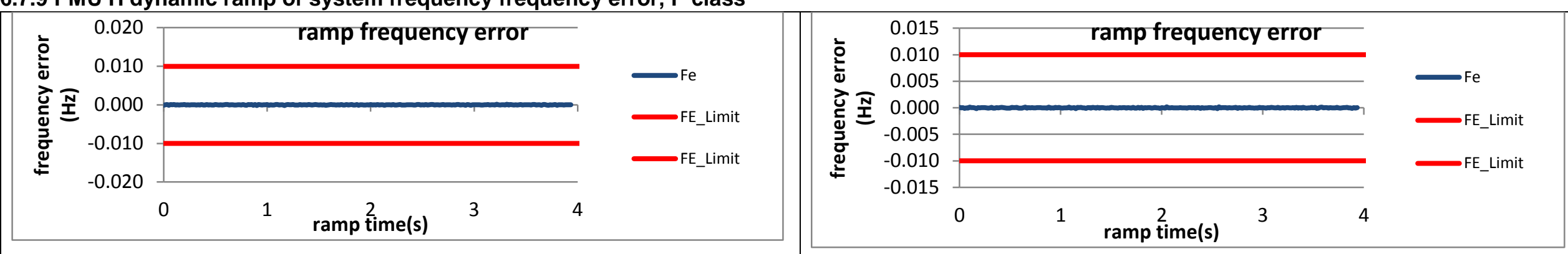

Figure 2931: Fs = $60 \mathrm{FPS}$, ramp from $55 \mathrm{~Hz}$ to $65 \mathrm{~Hz}$ at $+1 \mathrm{~Hz} / \mathrm{s}$

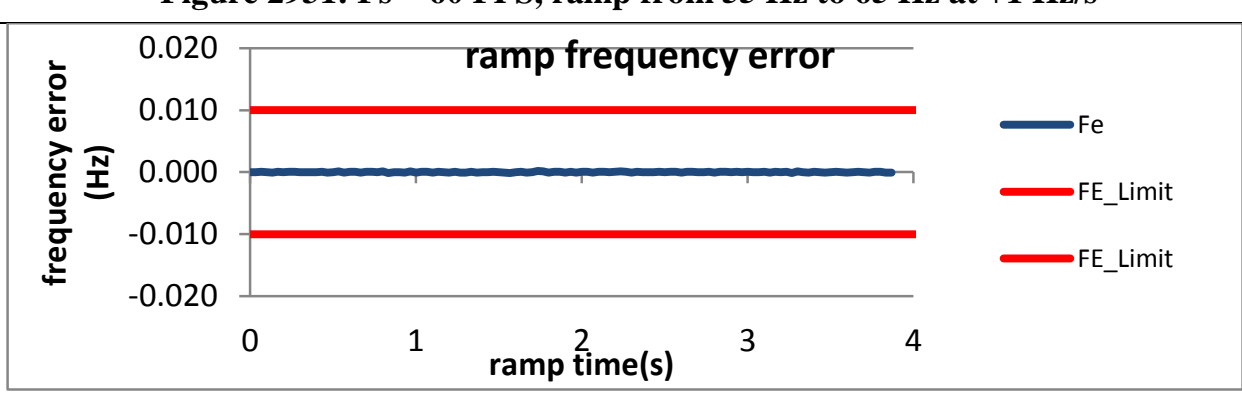

Figure 2932: Fs = $60 \mathrm{FPS}$, ramp from $65 \mathrm{~Hz}$ to $55 \mathrm{~Hz}$ at $-1 \mathrm{~Hz} / \mathrm{s}$

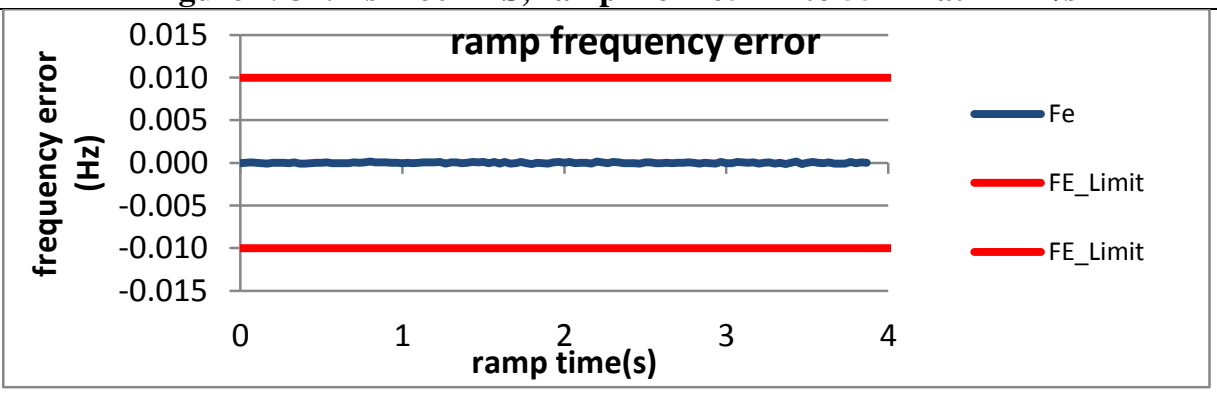

Figure 2933: Fs = $30 \mathrm{FPS}$, ramp from $55 \mathrm{~Hz}$ to $65 \mathrm{~Hz}$ at $+1 \mathrm{~Hz} / \mathrm{s}$

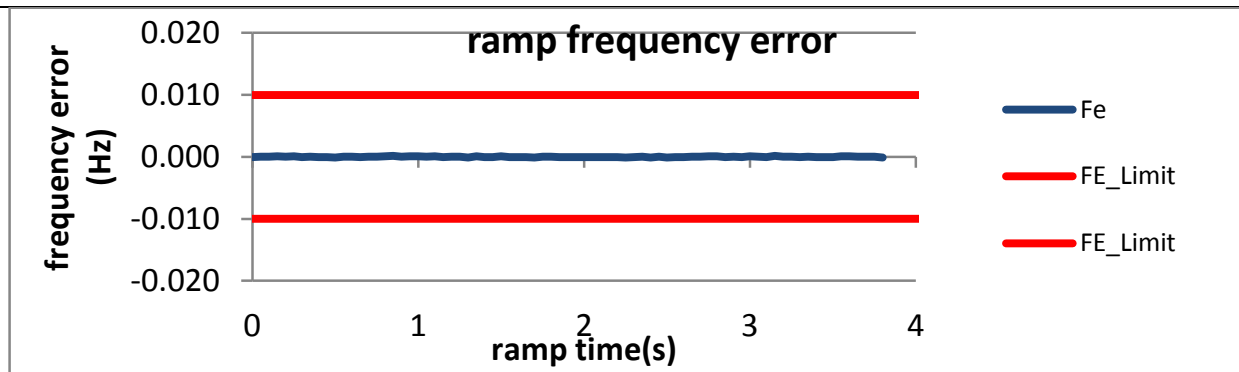

Figure 2935: Fs = $20 \mathrm{FPS}$, ramp from $56 \mathrm{~Hz}$ to $64 \mathrm{~Hz}$ at $+1 \mathrm{~Hz} / \mathrm{s}$

Figure 2934: Fs = $30 \mathrm{FPS}$, ramp from $65 \mathrm{~Hz}$ to $55 \mathrm{~Hz}$ at $-1 \mathrm{~Hz} / \mathrm{s}$

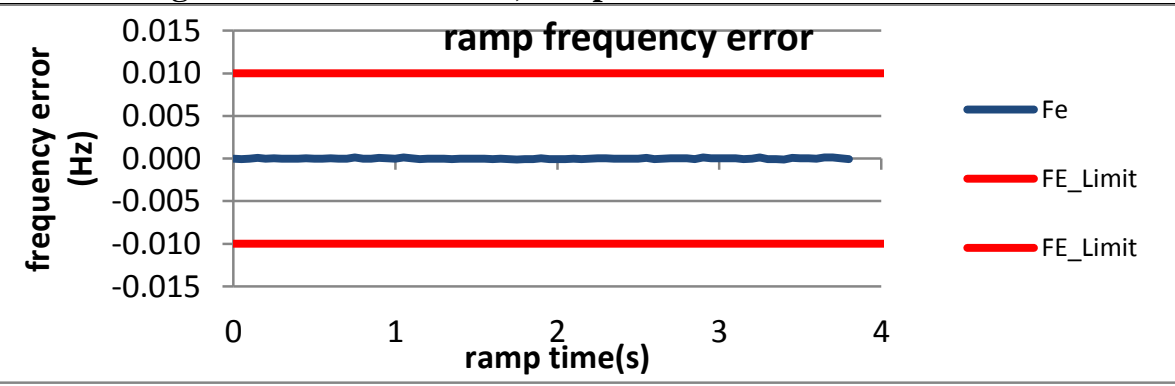

Figure 2936: Fs = $20 \mathrm{FPS}$, ramp from $64 \mathrm{~Hz}$ to $56 \mathrm{~Hz}$ at $-1 \mathrm{~Hz} / \mathrm{s}$ 


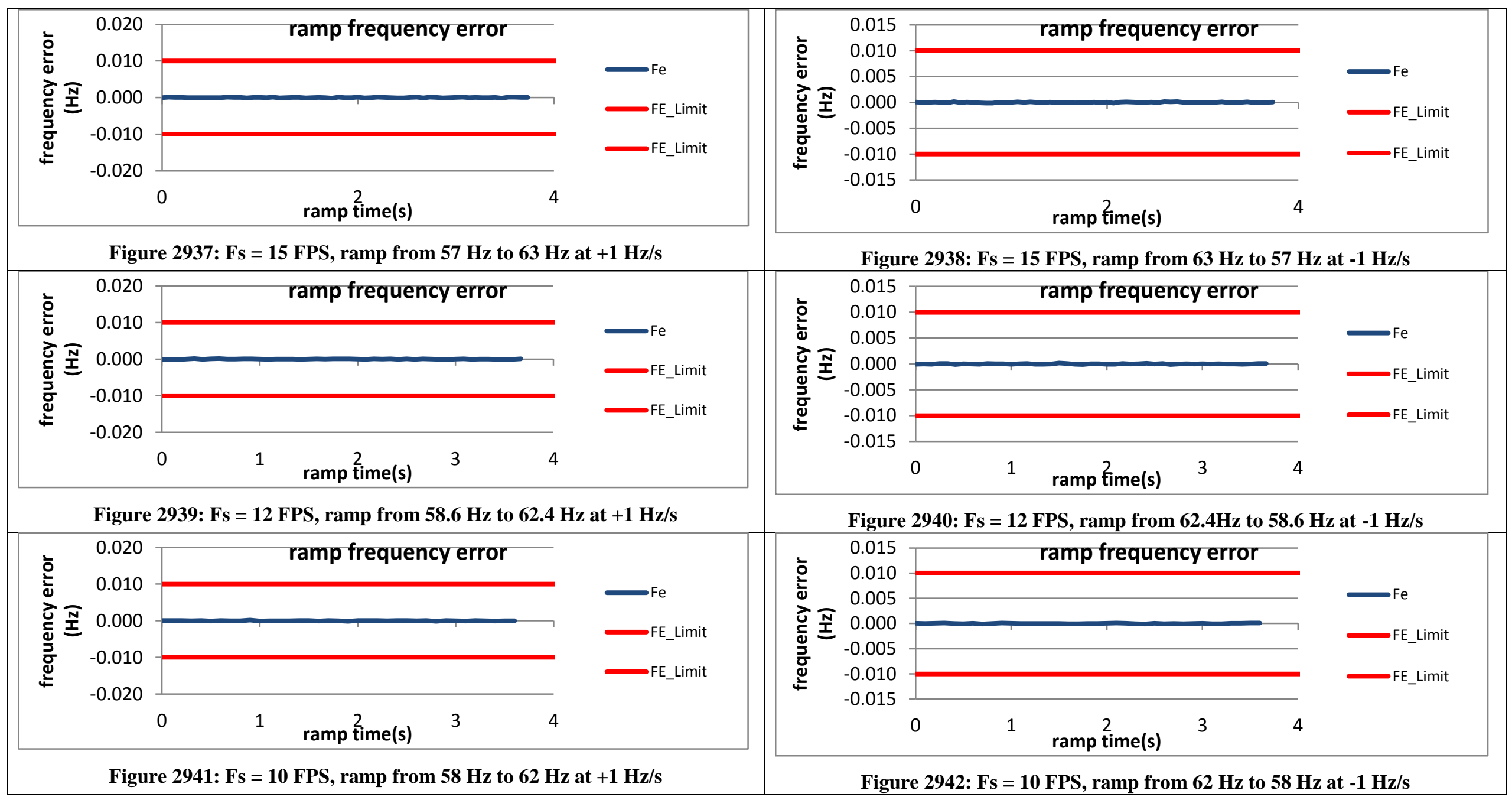


6.7.10 PMU I dynamic ramp of system frequency frequency error, $P$ class PMU I does not support P class

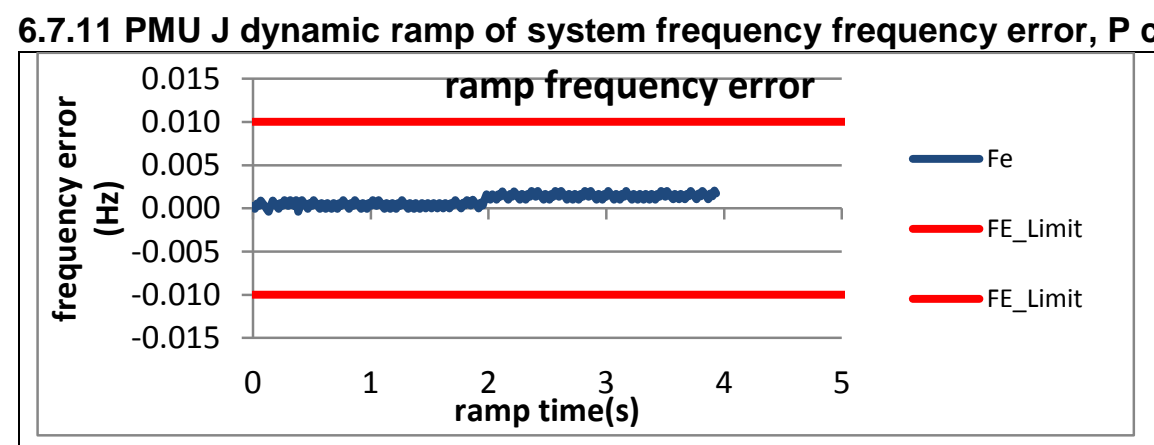

Figure 2943: Fs = $60 \mathrm{FPS}$, ramp from $55 \mathrm{~Hz}$ to $65 \mathrm{~Hz}$ at $+1 \mathrm{~Hz} / \mathrm{s}$

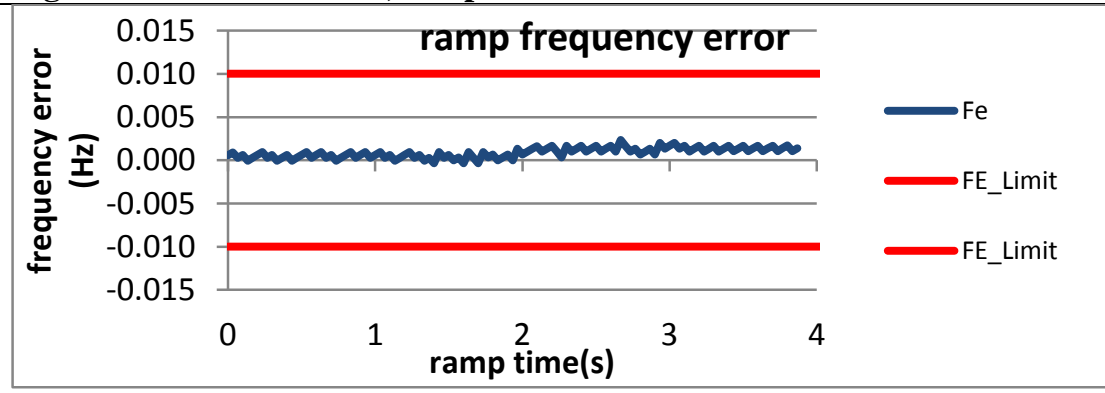

Figure 2945: Fs = $30 \mathrm{FPS}$, ramp from $55 \mathrm{~Hz}$ to $65 \mathrm{~Hz}$ at $+1 \mathrm{~Hz} / \mathrm{s}$

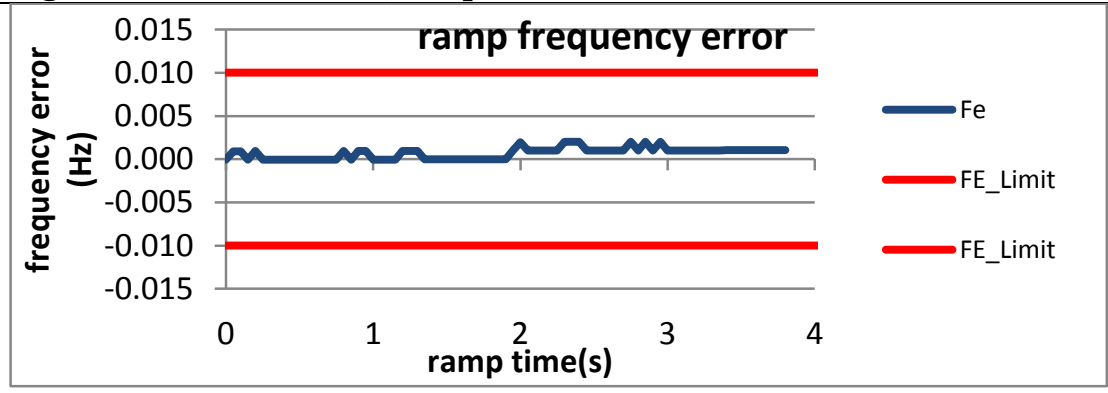

Figure 2947: Fs = $20 \mathrm{FPS}$, ramp from $56 \mathrm{~Hz}$ to $64 \mathrm{~Hz}$ at $+1 \mathrm{~Hz} / \mathrm{s}$

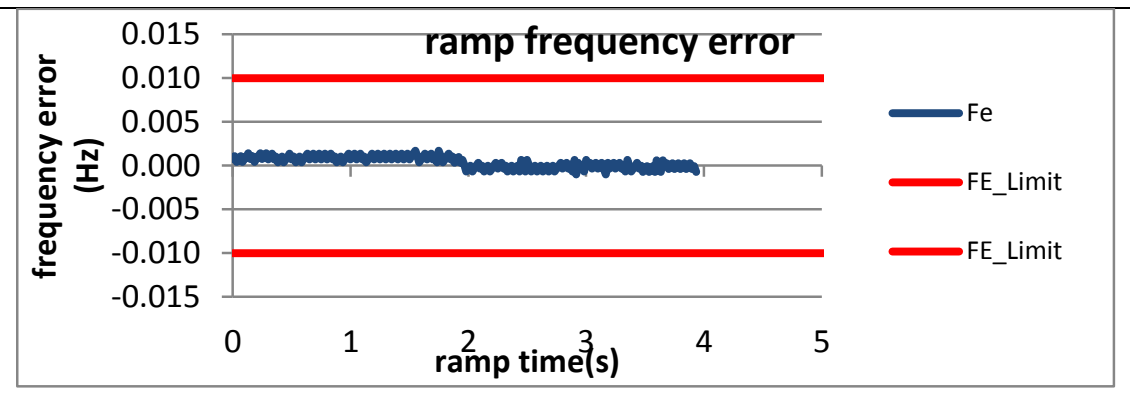

Figure 2944: Fs = $60 \mathrm{FPS}$, ramp from $65 \mathrm{~Hz}$ to $55 \mathrm{~Hz}$ at $-1 \mathrm{~Hz} / \mathrm{s}$

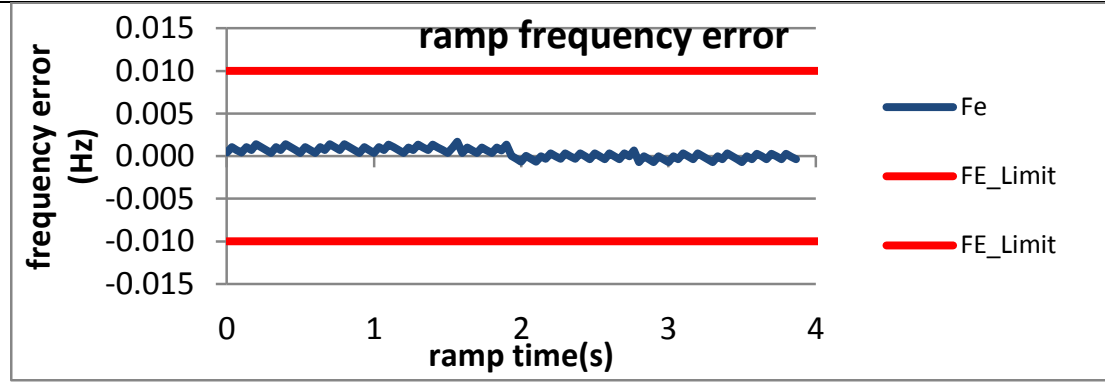

Figure 2946: Fs = $30 \mathrm{FPS}$, ramp from $65 \mathrm{~Hz}$ to $55 \mathrm{~Hz}$ at $-1 \mathrm{~Hz} / \mathrm{s}$

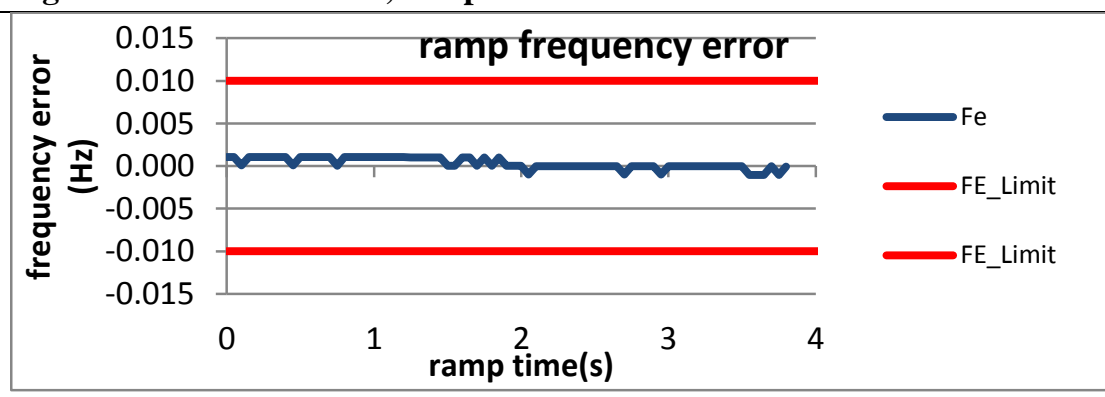

Figure 2948: Fs = $20 \mathrm{FPS}$, ramp from $64 \mathrm{~Hz}$ to $56 \mathrm{~Hz}$ at $-1 \mathrm{~Hz} / \mathrm{s}$ 

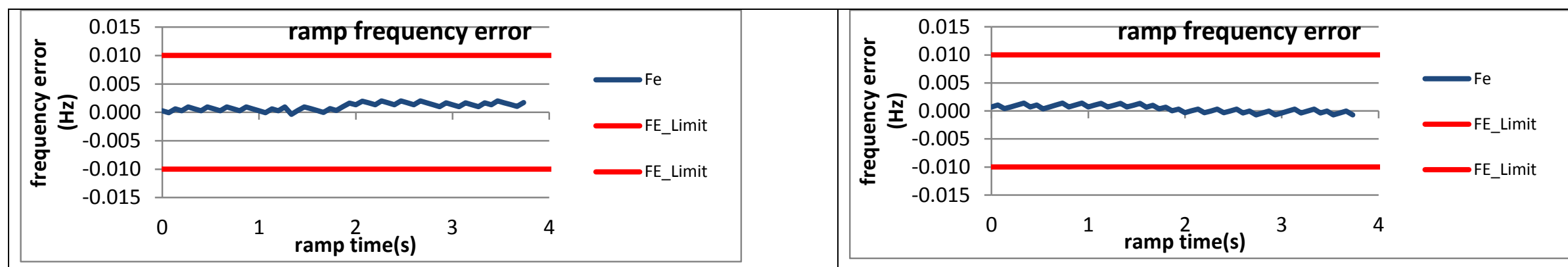

Figure 2949: Fs = $15 \mathrm{FPS}$, ramp from $57 \mathrm{~Hz}$ to $63 \mathrm{~Hz}$ at $+1 \mathrm{~Hz} / \mathrm{s}$

Figure 2950: Fs = $15 \mathrm{FPS}$, ramp from $63 \mathrm{~Hz}$ to $57 \mathrm{~Hz}$ at $-1 \mathrm{~Hz} / \mathrm{s}$
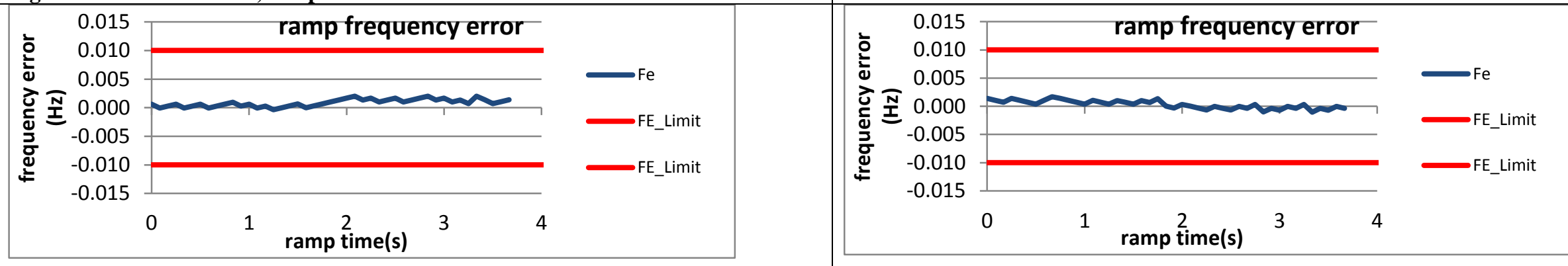

Figure 2951: Fs = 12 FPS, ramp from $58.6 \mathrm{~Hz}$ to $62.4 \mathrm{~Hz}$ at $+1 \mathrm{~Hz} / \mathrm{s}$

Figure 2952: Fs = 12 FPS, ramp from $62.4 \mathrm{~Hz}$ to $58.6 \mathrm{~Hz}$ at $-1 \mathrm{~Hz} / \mathrm{s}$

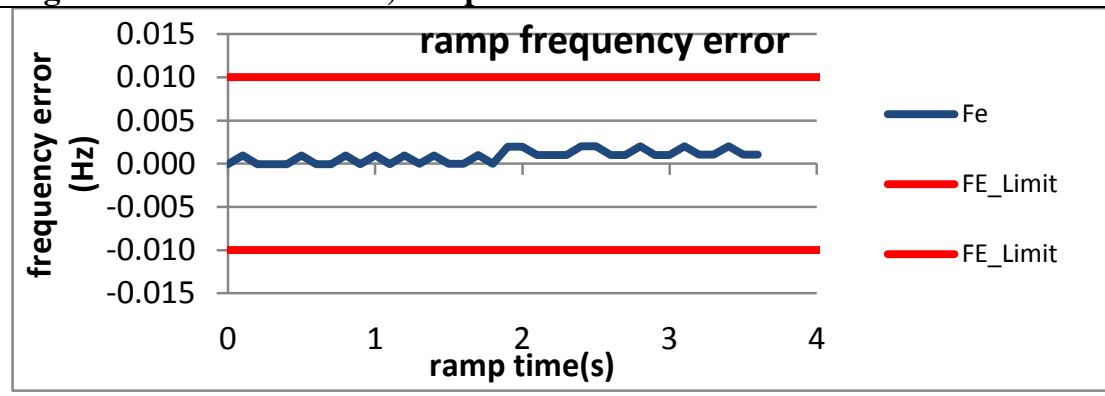

Figure 2953: Fs = $10 \mathrm{FPS}$, ramp from $58 \mathrm{~Hz}$ to $62 \mathrm{~Hz}$ at $+1 \mathrm{~Hz} / \mathrm{s}$

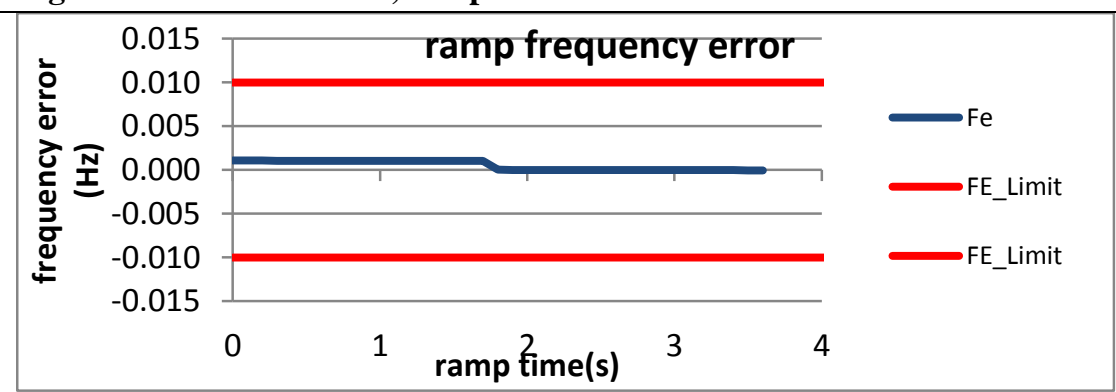

Figure 2954: Fs = $10 \mathrm{FPS}$, ramp from $62 \mathrm{~Hz}$ to $58 \mathrm{~Hz}$ at $-1 \mathrm{~Hz} / \mathrm{s}$ 


\subsection{Dynamic ramp of system frequency ROCOF, P class}

\subsubsection{C37.118.1 Annex $C$ dynamic ramp of system frequency ROCOF error, $P$ class}

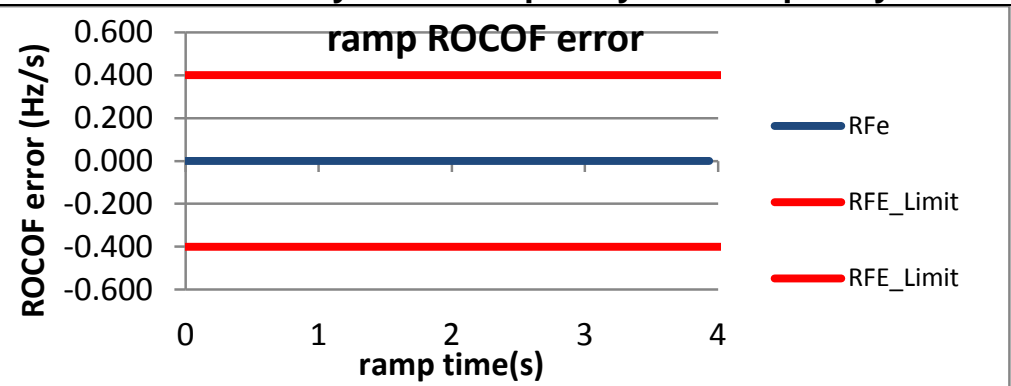

Figure 2955: Fs = $60 \mathrm{FPS}$, ram from $58 \mathrm{~Hz}$ to $62 \mathrm{~Hz}$ at $+1 \mathrm{~Hz} / \mathrm{s}$

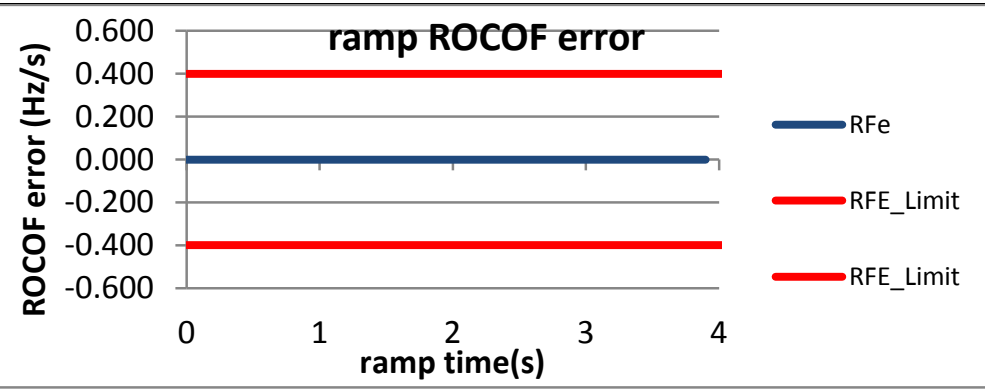

Figure 2957: Fs = $30 \mathrm{FPS}$, ram from $58 \mathrm{~Hz}$ to $62 \mathrm{~Hz}$ at $+1 \mathrm{~Hz} / \mathrm{s}$

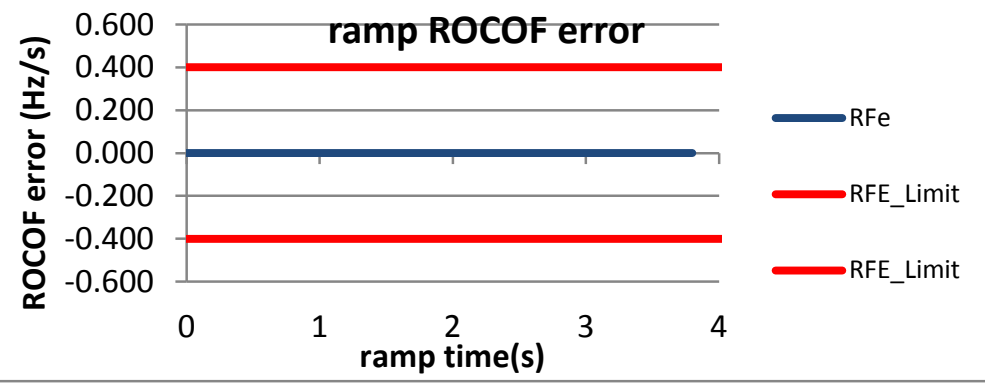

Figure 2959: Fs = $20 \mathrm{FPS}$, ramp from $58 \mathrm{~Hz}$ to $62 \mathrm{~Hz}$ at $+1 \mathrm{~Hz} / \mathrm{s}$

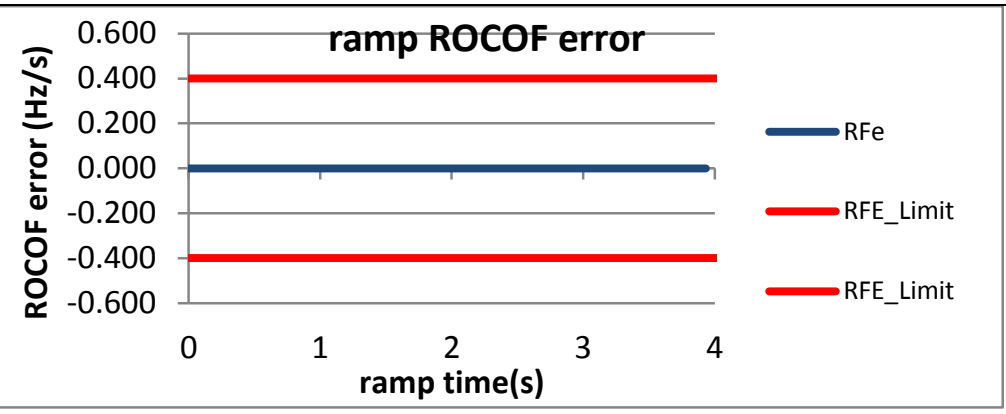

Figure 2956: Fs = $60 \mathrm{FPS}$, ram from $62 \mathrm{~Hz}$ to $58 \mathrm{~Hz}$ at $-1 \mathrm{~Hz} / \mathrm{s}$

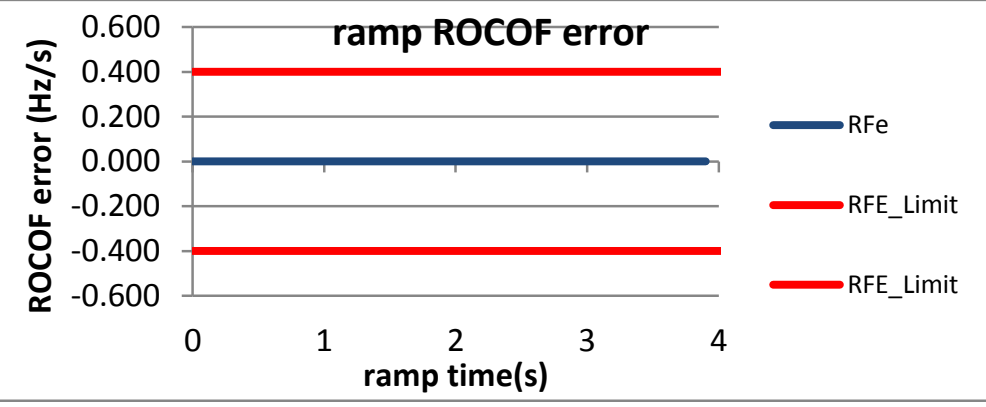

Figure 2958: Fs = $30 \mathrm{FPS}$, ram from $62 \mathrm{~Hz}$ to $58 \mathrm{~Hz}$ at $-1 \mathrm{~Hz} / \mathrm{s}$

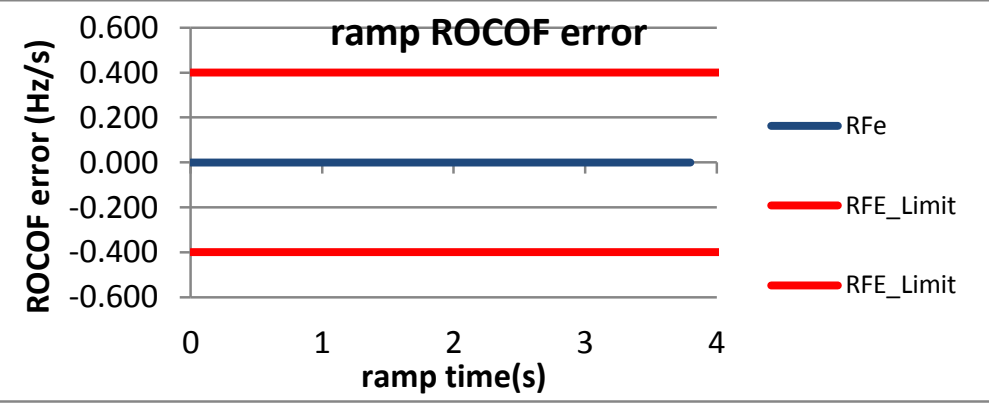

Figure 2960: Fs = $20 \mathrm{FPS}$, ramp from $62 \mathrm{~Hz}$ to $58 \mathrm{~Hz}$ at $=1 \mathrm{~Hz} / \mathrm{s}$ 


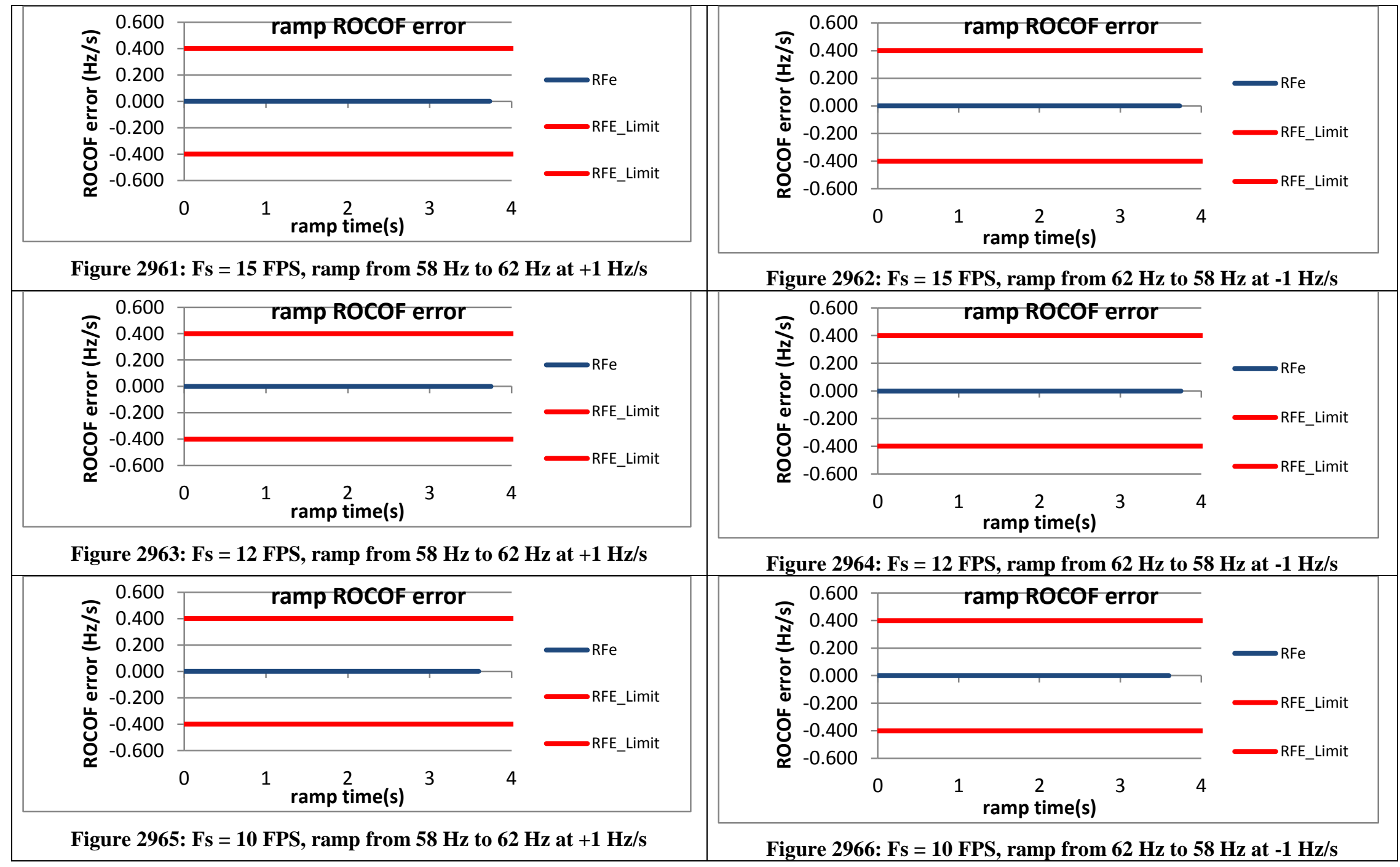


6.8.2 PMU A dynamic ramp of system frequency ROCOF error, $P$ class

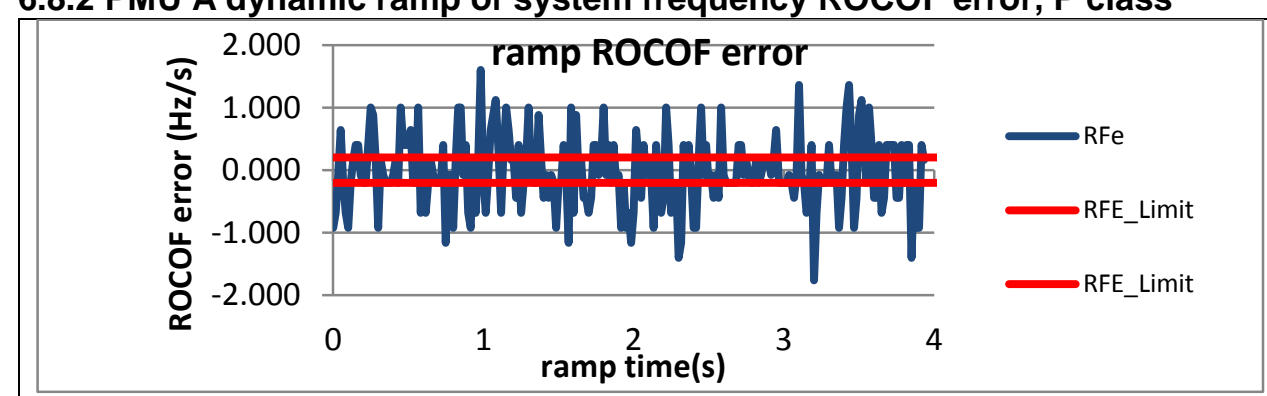

Figure 2967: Fs = $60 \mathrm{FPS}$, ram from $58 \mathrm{~Hz}$ to $62 \mathrm{~Hz}$ at $+1 \mathrm{~Hz} / \mathrm{s}$

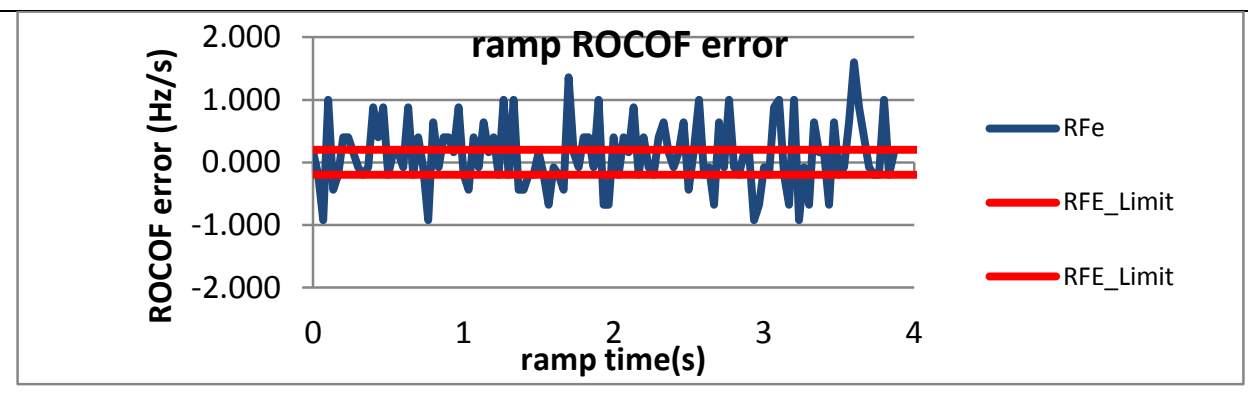

Figure 2969: Fs = $30 \mathrm{FPS}$, ram from $58 \mathrm{~Hz}$ to $62 \mathrm{~Hz}$ at $+1 \mathrm{~Hz} / \mathrm{s}$

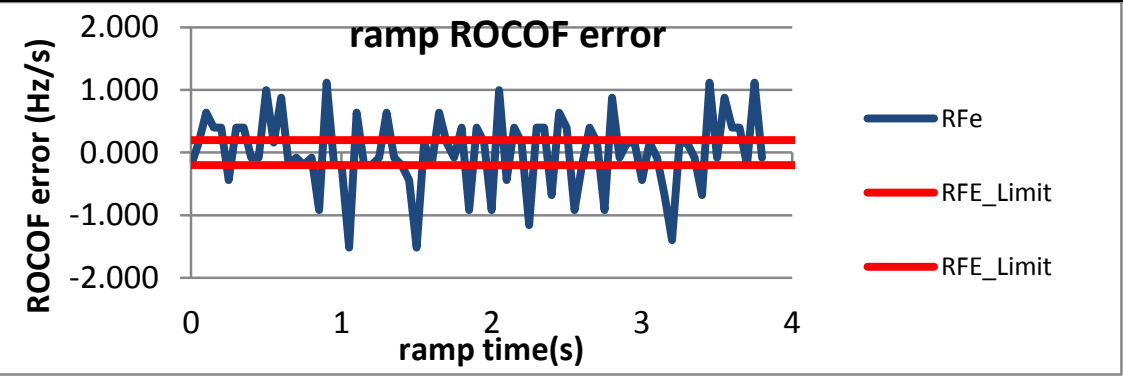

Figure 2971: Fs = $20 \mathrm{FPS}$, ramp from $58 \mathrm{~Hz}$ to $62 \mathrm{~Hz}$ at $+1 \mathrm{~Hz} / \mathrm{s}$

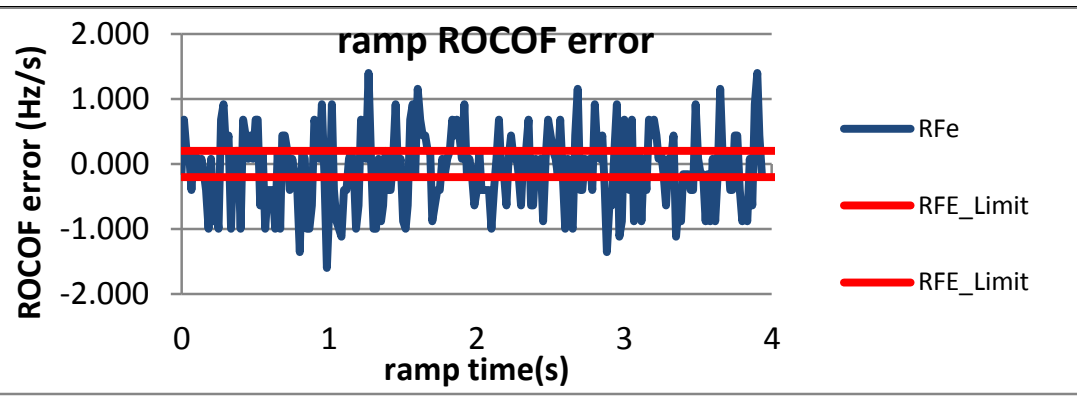

Figure 2968: Fs = 60 FPS, ram from $62 \mathrm{~Hz}$ to $58 \mathrm{~Hz}$ at $-1 \mathrm{~Hz} / \mathrm{s}$

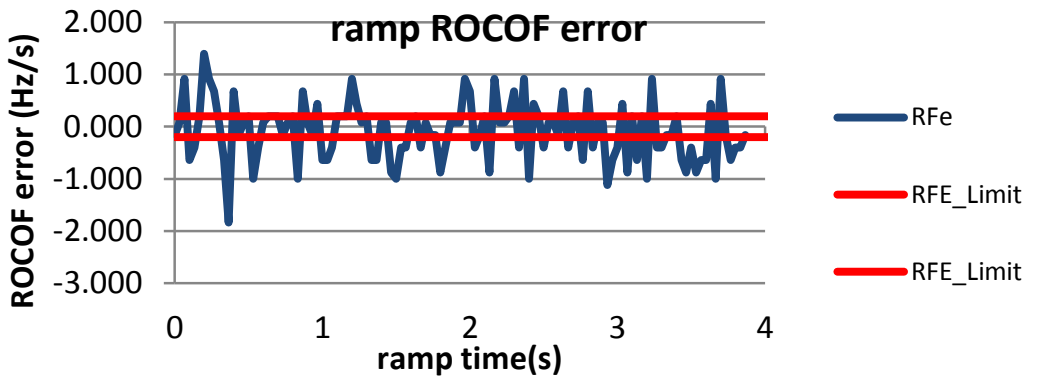

Figure 2970: Fs = $30 \mathrm{FPS}$, ram from $62 \mathrm{~Hz}$ to $58 \mathrm{~Hz}$ at $-1 \mathrm{~Hz} / \mathrm{s}$

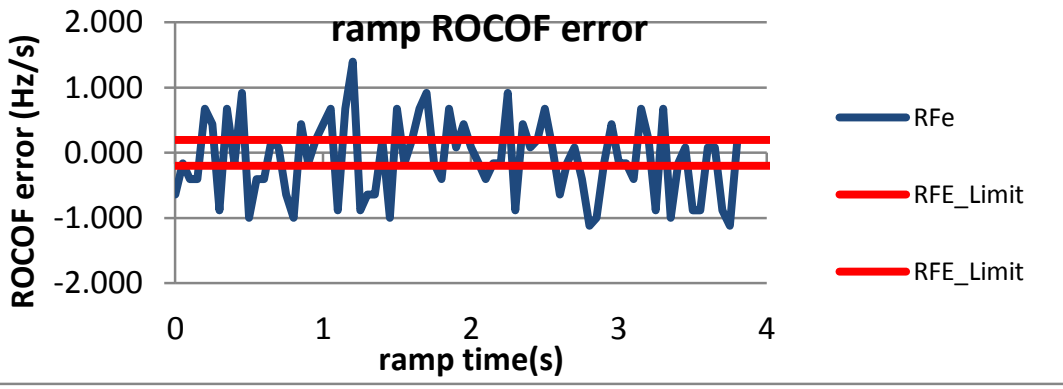

Figure 2972: Fs = $20 \mathrm{FPS}$, ramp from $62 \mathrm{~Hz}$ to $58 \mathrm{~Hz}$ at $=1 \mathrm{~Hz} / \mathrm{s}$ 


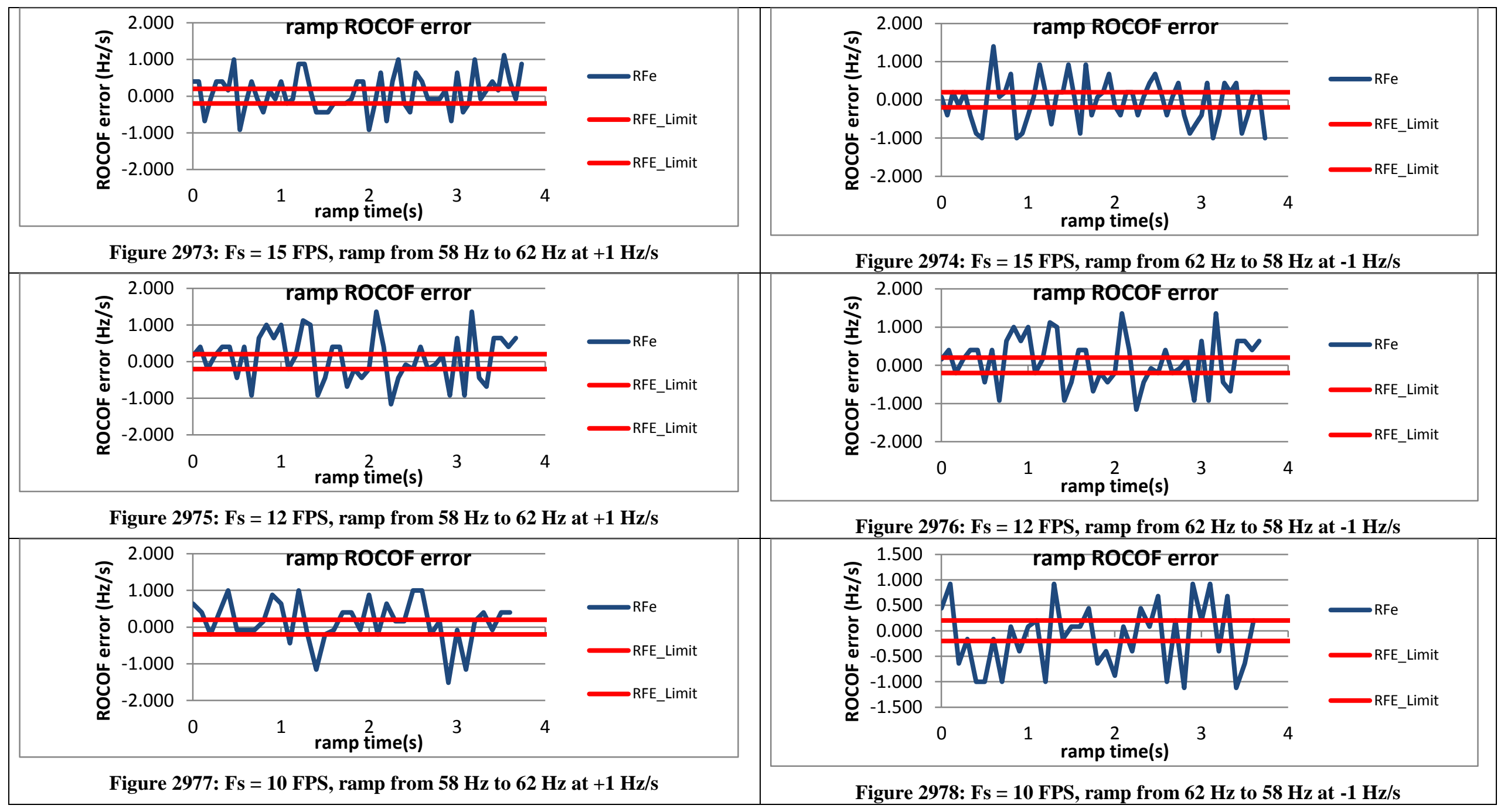


6.8.3 PMU B dynamic ramp of system frequency ROCOF error, $P$ class

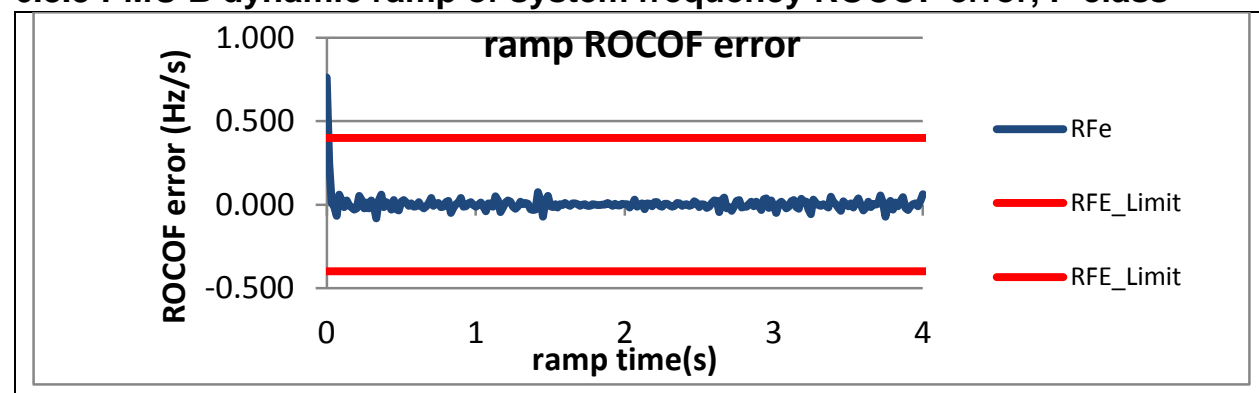

Figure 2979: Fs = $60 \mathrm{FPS}$, ram from $55 \mathrm{~Hz}$ to $65 \mathrm{~Hz}$ at $+1 \mathrm{~Hz} / \mathrm{s}$

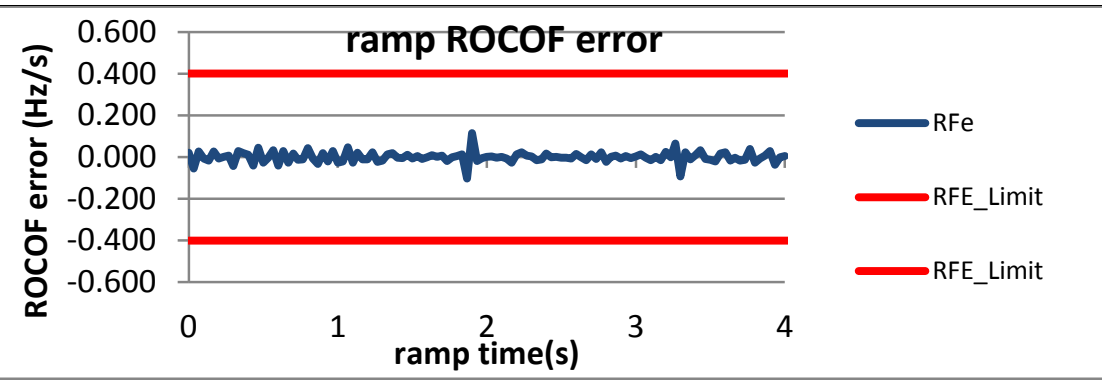

Figure 2981: Fs = $30 \mathrm{FPS}$, ram from $55 \mathrm{~Hz}$ to $65 \mathrm{~Hz}$ at $+1 \mathrm{~Hz} / \mathrm{s}$

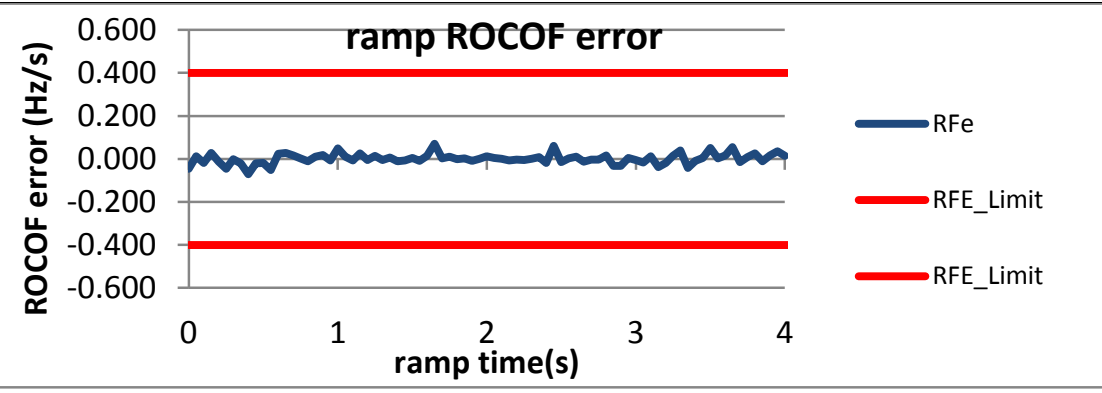

Figure 2983: Fs = $20 \mathrm{FPS}$, ramp from $56 \mathrm{~Hz}$ to $64 \mathrm{~Hz}$ at $+1 \mathrm{~Hz} / \mathrm{s}$

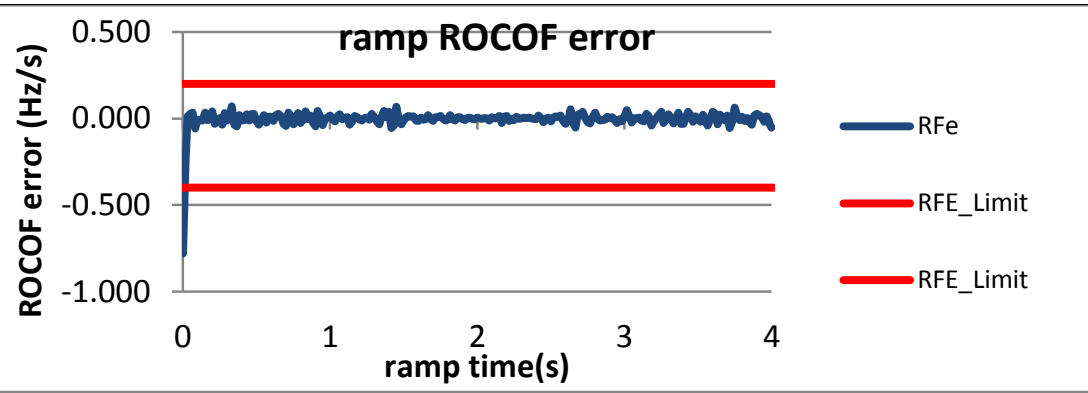

Figure 2980: Fs = $60 \mathrm{FPS}$, ram from $65 \mathrm{~Hz}$ to $55 \mathrm{~Hz}$ at $-1 \mathrm{~Hz} / \mathrm{s}$

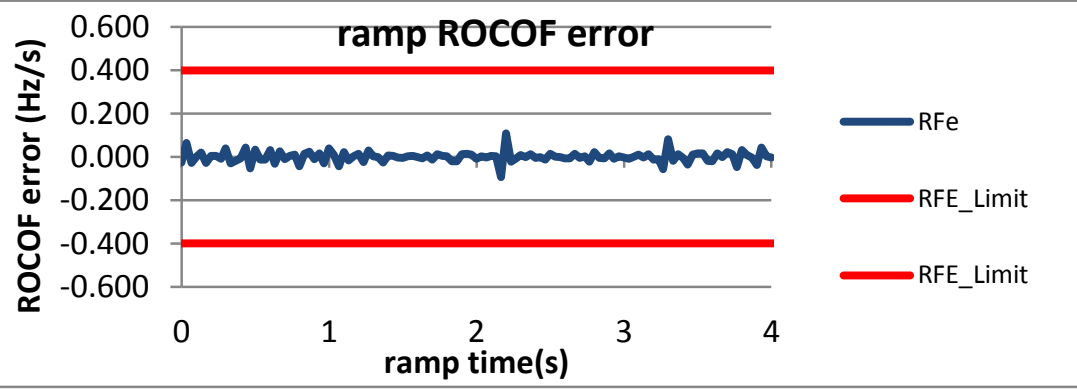

Figure 2982: Fs = $30 \mathrm{FPS}$, ram from $65 \mathrm{~Hz}$ to $55 \mathrm{~Hz}$ at $-1 \mathrm{~Hz} / \mathrm{s}$

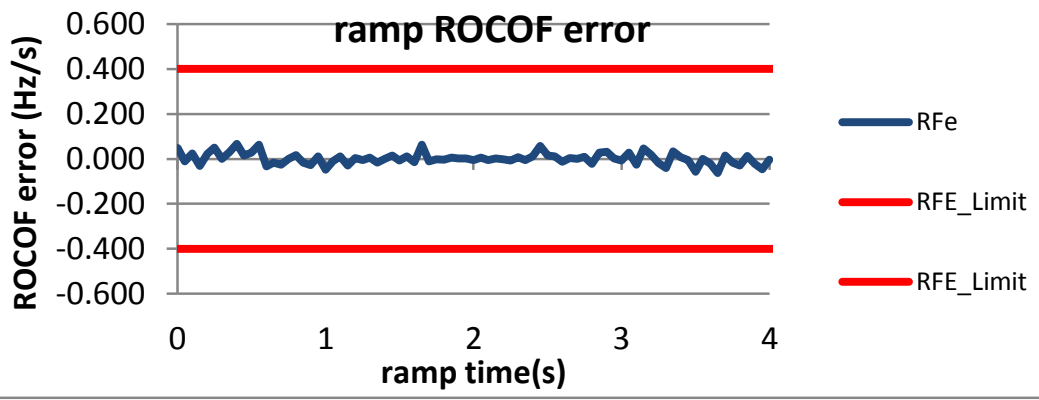

Figure 2984: Fs = $20 \mathrm{FPS}$, ramp from $64 \mathrm{~Hz}$ to $56 \mathrm{~Hz}$ at =1 Hz/s 


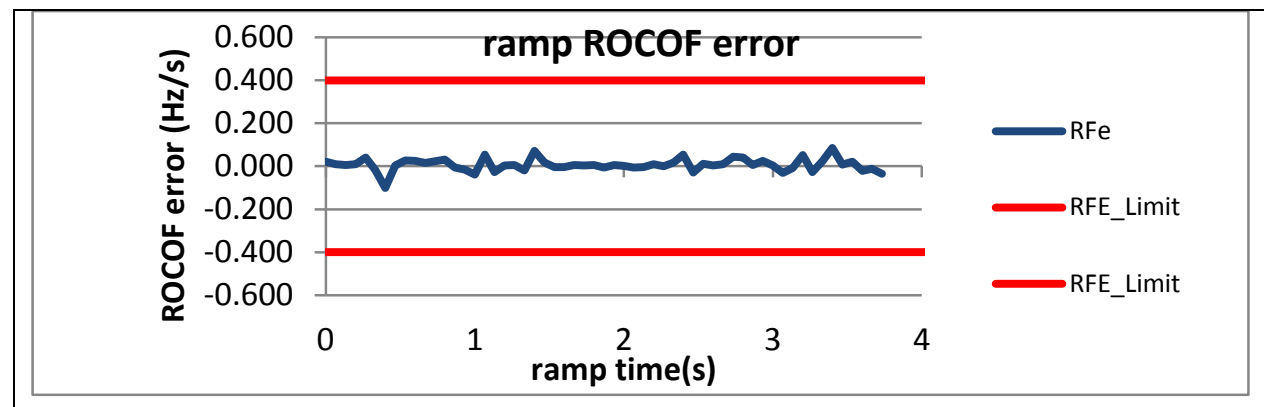

Figure 2985: Fs = $15 \mathrm{FPS}$, ramp from $57 \mathrm{~Hz}$ to $63 \mathrm{~Hz}$ at $+1 \mathrm{~Hz} / \mathrm{s}$

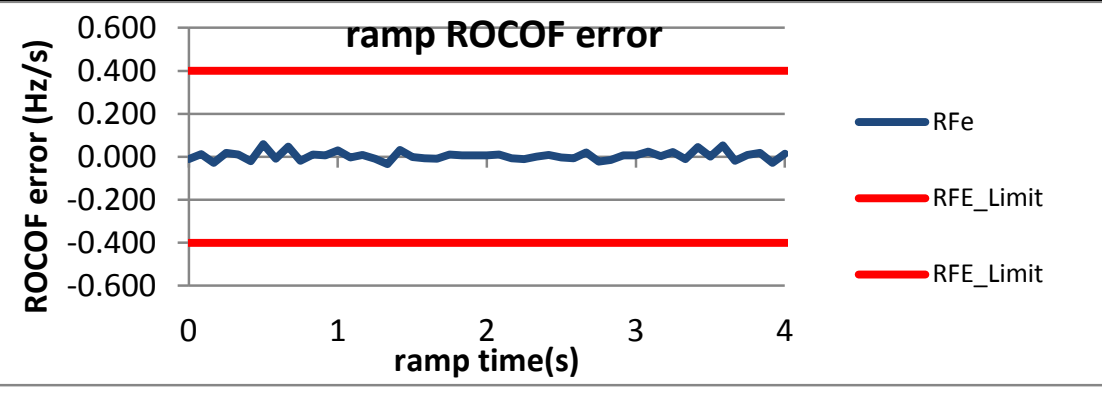

Figure 2987: Fs = 12 FPS, ramp from $58.6 \mathrm{~Hz}$ to $62.4 \mathrm{~Hz}$ at $+1 \mathrm{~Hz} / \mathrm{s}$

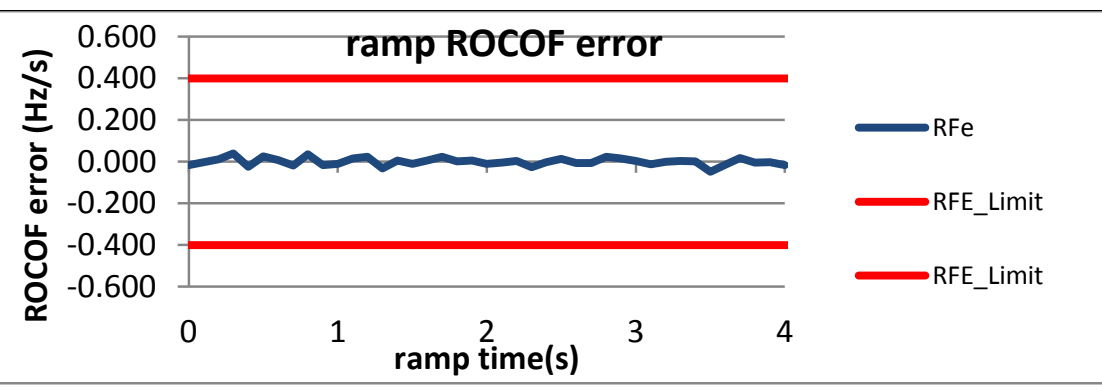

Figure 2989: Fs = $10 \mathrm{FPS}$, ramp from $58 \mathrm{~Hz}$ to $62 \mathrm{~Hz}$ at $+1 \mathrm{~Hz} / \mathrm{s}$

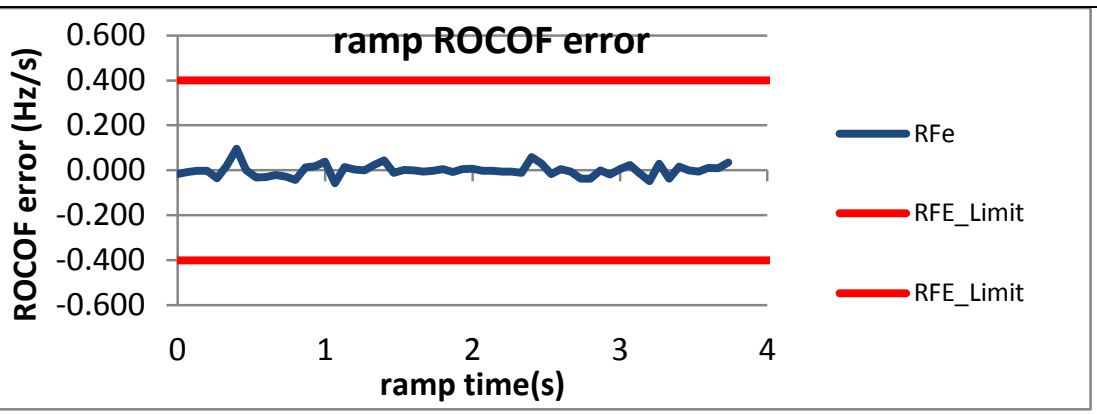

Figure 2986: Fs = $15 \mathrm{FPS}$, ramp from $63 \mathrm{~Hz}$ to $57 \mathrm{~Hz}$ at $-1 \mathrm{~Hz} / \mathrm{s}$

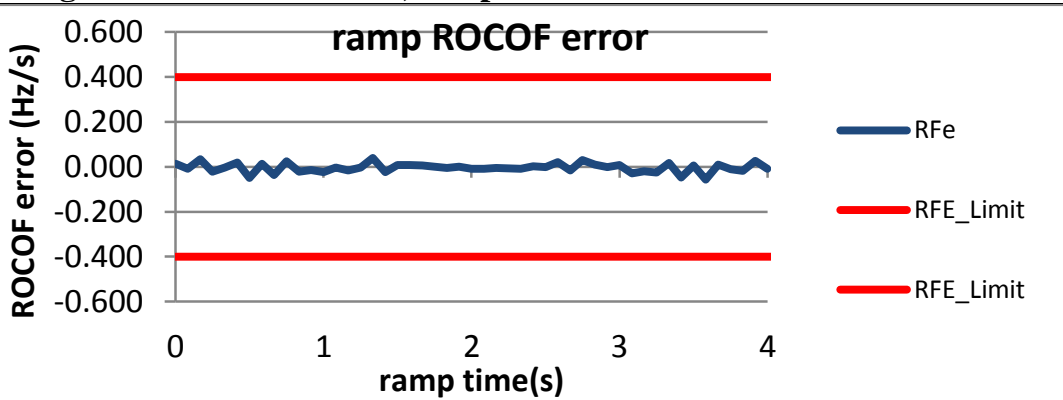

Figure 2988: Fs = 12 FPS, ramp from $62.4 \mathrm{~Hz}$ to $58.6 \mathrm{~Hz}$ at $-1 \mathrm{~Hz} / \mathrm{s}$

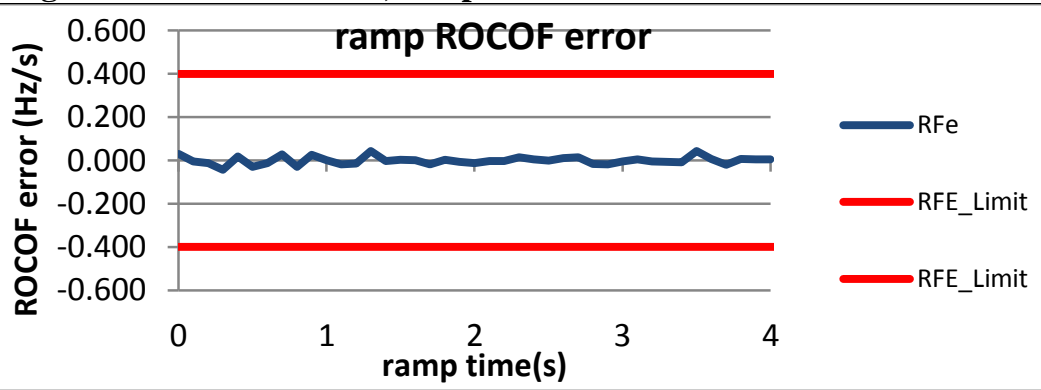

Figure 2990: Fs = $10 \mathrm{FPS}$, ramp from $62 \mathrm{~Hz}$ to $58 \mathrm{~Hz}$ at $-1 \mathrm{~Hz} / \mathrm{s}$ 
6.8.4 PMU C dynamic ramp of system frequency ROCOF error, $\mathrm{P}$ class

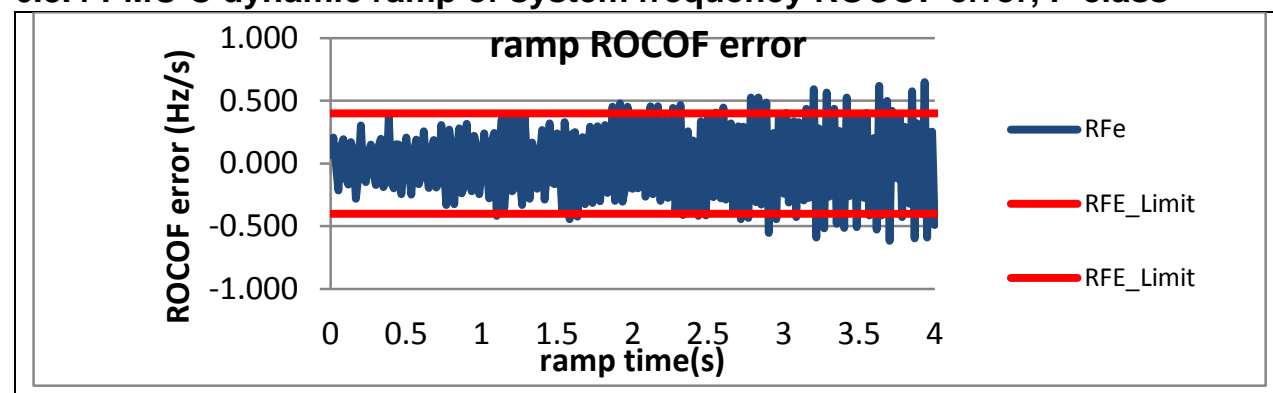

Figure 2991: Fs = $60 \mathrm{FPS}$, ram from $55 \mathrm{~Hz}$ to $65 \mathrm{~Hz}$ at $+1 \mathrm{~Hz} / \mathrm{s}$

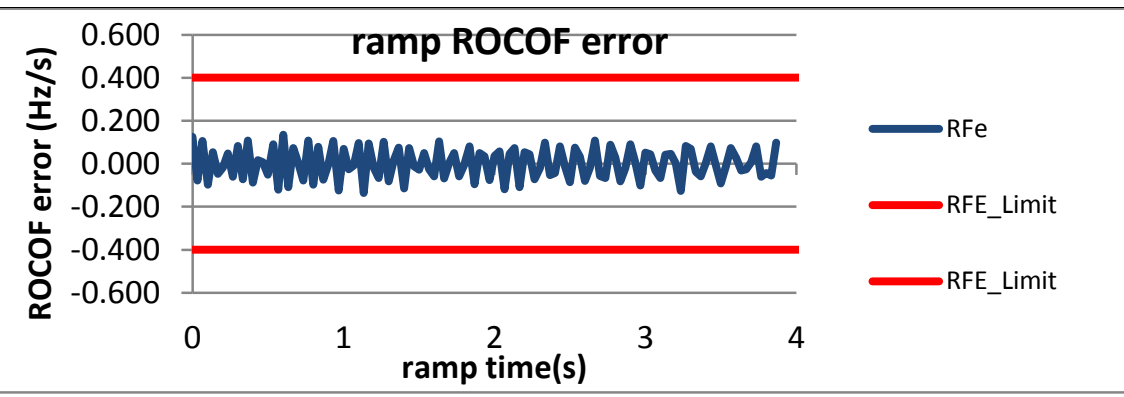

Figure 2993: Fs = $30 \mathrm{FPS}$, ram from $55 \mathrm{~Hz}$ to $65 \mathrm{~Hz}$ at $+1 \mathrm{~Hz} / \mathrm{s}$

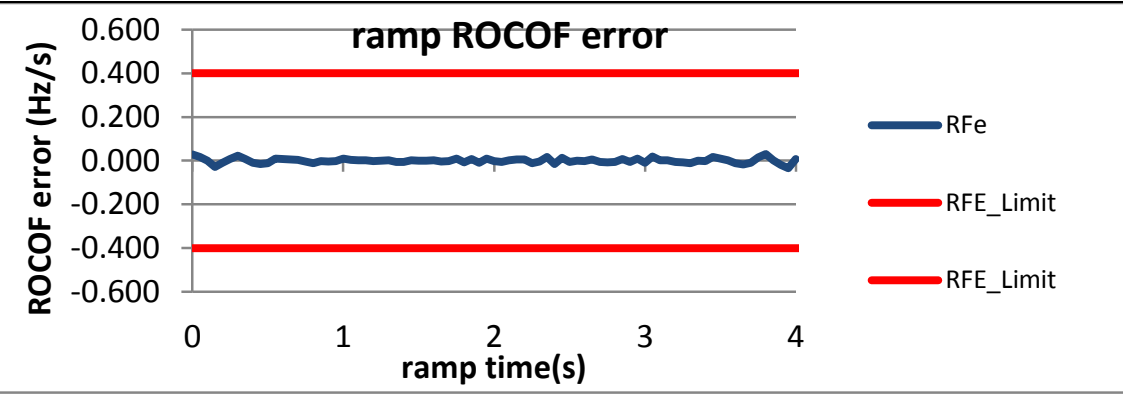

Figure 2995: Fs = 20 FPS, ramp from $56 \mathrm{~Hz}$ to $64 \mathrm{~Hz}$ at $+1 \mathrm{~Hz} / \mathrm{s}$

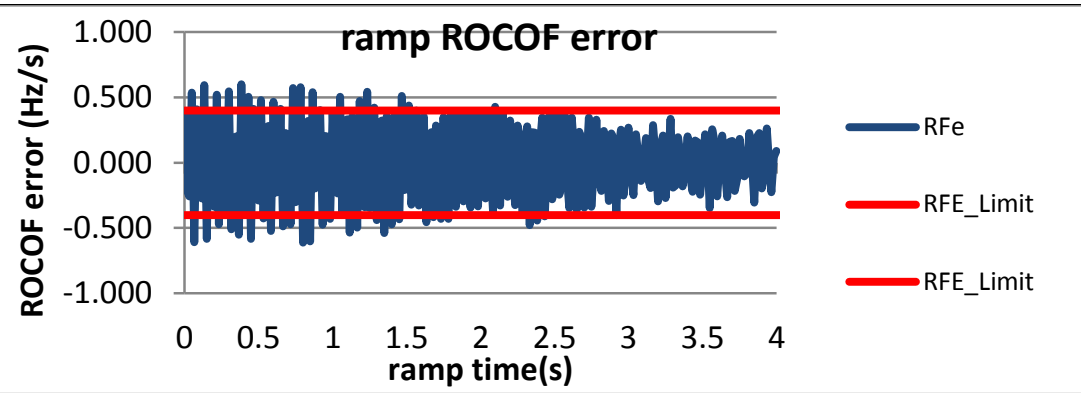

Figure 2992: Fs = $60 \mathrm{FPS}$, ram from $65 \mathrm{~Hz}$ to $55 \mathrm{~Hz}$ at $-1 \mathrm{~Hz} / \mathrm{s}$

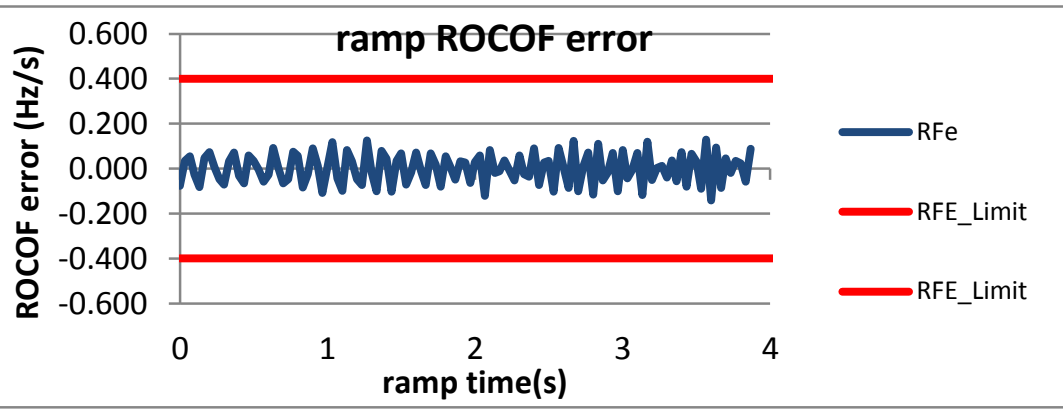

Figure 2994: Fs = $30 \mathrm{FPS}$, ram from $65 \mathrm{~Hz}$ to $55 \mathrm{~Hz}$ at $-1 \mathrm{~Hz} / \mathrm{s}$

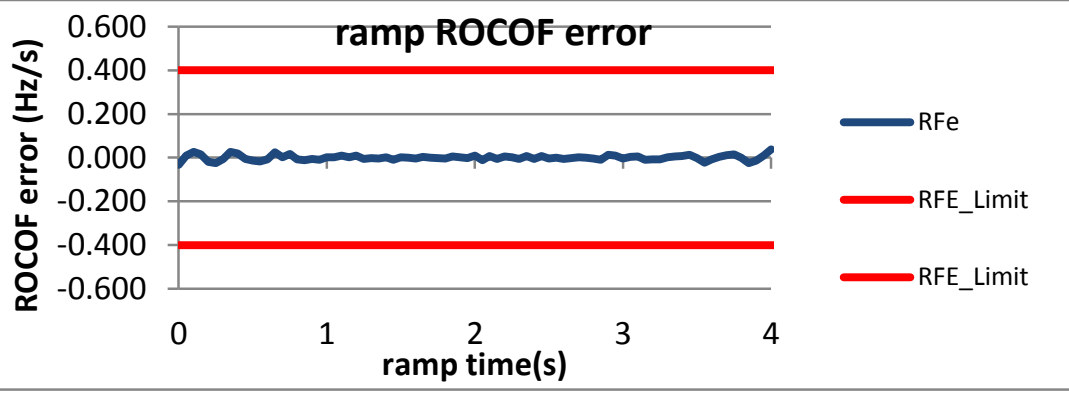

Figure 2996: Fs = $20 \mathrm{FPS}$, ramp from $64 \mathrm{~Hz}$ to $56 \mathrm{~Hz}$ at $=1 \mathrm{~Hz} / \mathrm{s}$ 


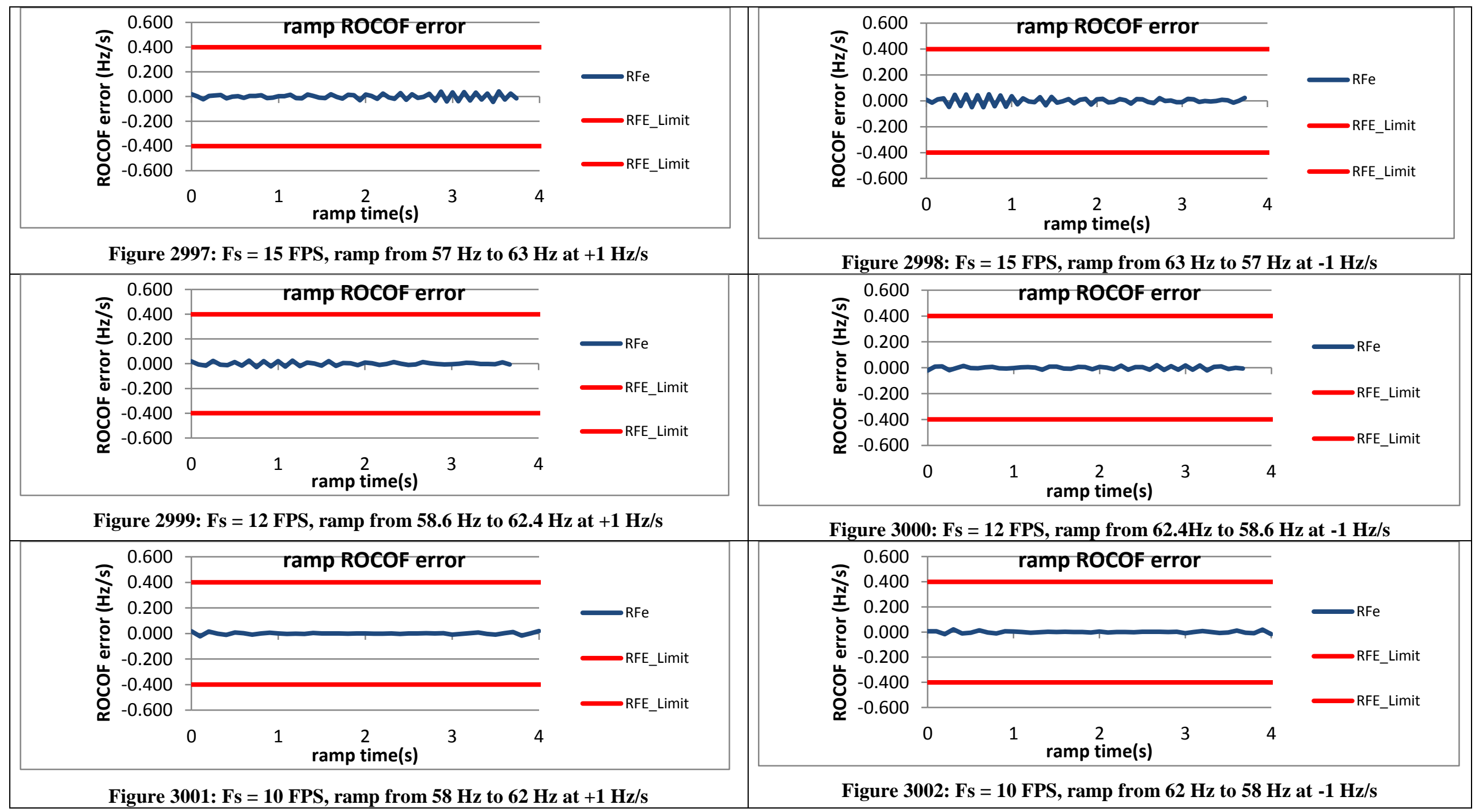


6.8.5 PMU D dynamic ramp of system frequency ROCOF error, $P$ class
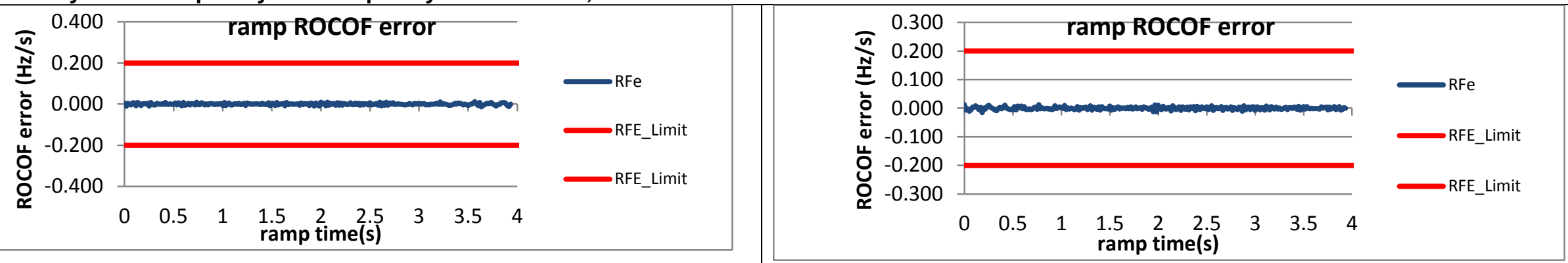

Figure 3003: Fs = $60 \mathrm{FPS}$, ram from $55 \mathrm{~Hz}$ to $65 \mathrm{~Hz}$ at $+1 \mathrm{~Hz} / \mathrm{s}$

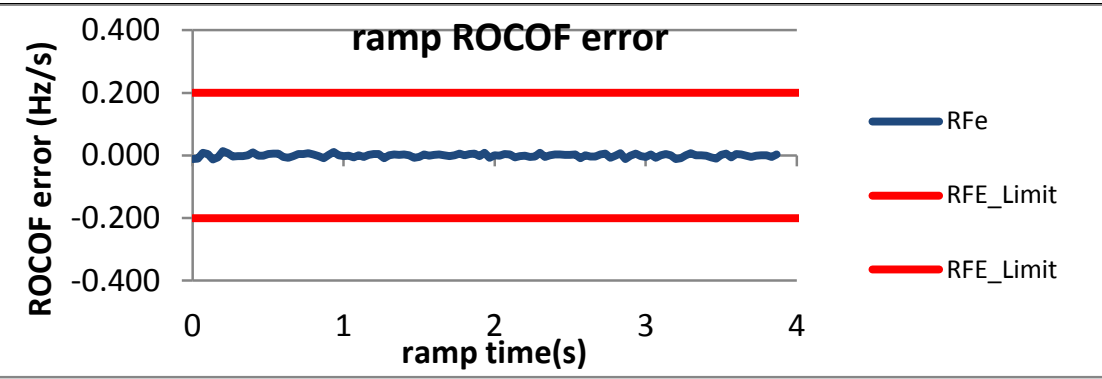

Figure 3004: Fs = $60 \mathrm{FPS}$, ram from $62 \mathrm{~Hz}$ to $58 \mathrm{~Hz}$ at $-1 \mathrm{~Hz} / \mathrm{s}$

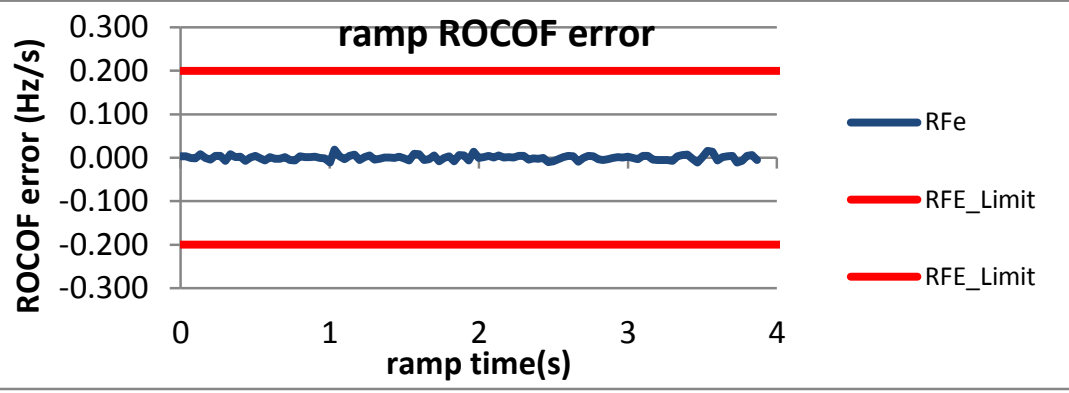

Figure 3005: Fs = $30 \mathrm{FPS}$, ram from $55 \mathrm{~Hz}$ to $65 \mathrm{~Hz}$ at $+1 \mathrm{~Hz} / \mathrm{s}$

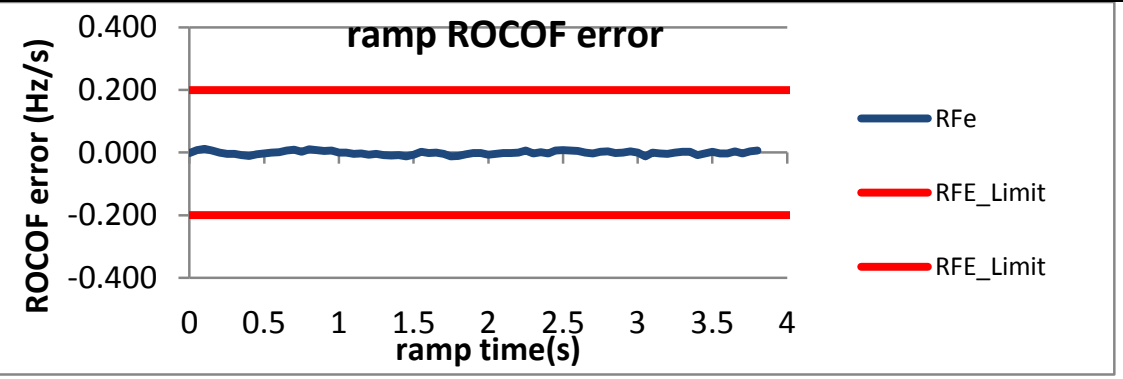

Figure 3006: Fs = 30 FPS, ram from $62 \mathrm{~Hz}$ to $58 \mathrm{~Hz}$ at $-1 \mathrm{~Hz} / \mathrm{s}$

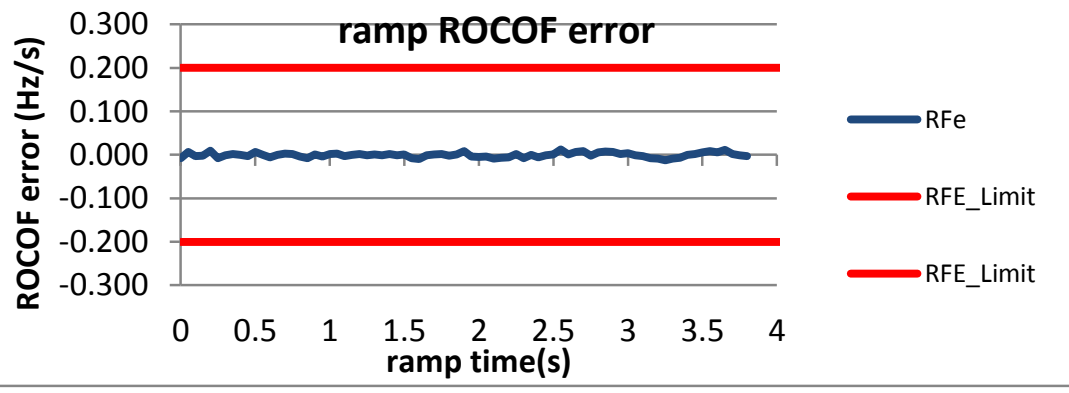

Figure 3007: Fs = $20 \mathrm{FPS}$, ramp from $56 \mathrm{~Hz}$ to $64 \mathrm{~Hz}$ at $+1 \mathrm{~Hz} / \mathrm{s}$

Figure 3008: Fs = $20 \mathrm{FPS}$, ramp from $62 \mathrm{~Hz}$ to $58 \mathrm{~Hz}$ at $=1 \mathrm{~Hz} / \mathrm{s}$ 


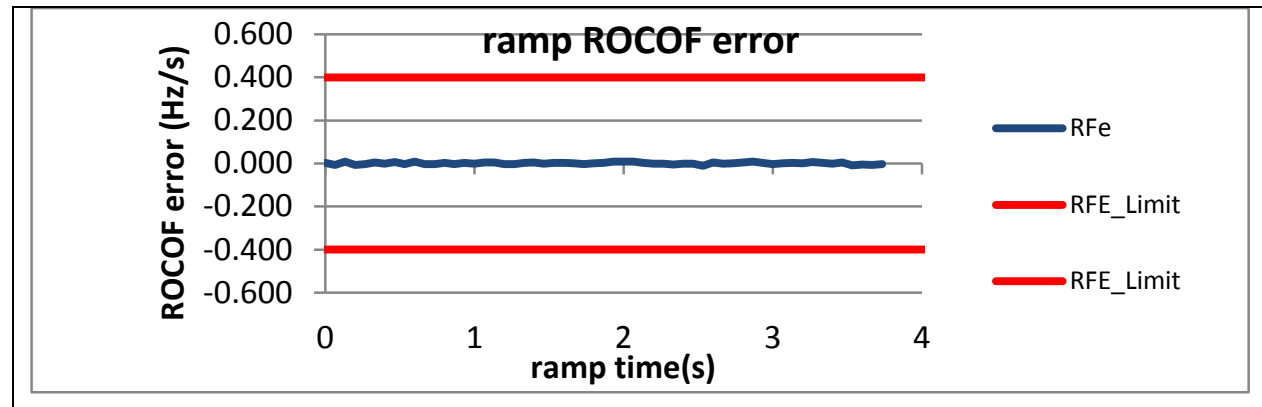

Figure 3009: Fs = $15 \mathrm{FPS}$, ramp from $57 \mathrm{~Hz}$ to $63 \mathrm{~Hz}$ at $+1 \mathrm{~Hz} / \mathrm{s}$

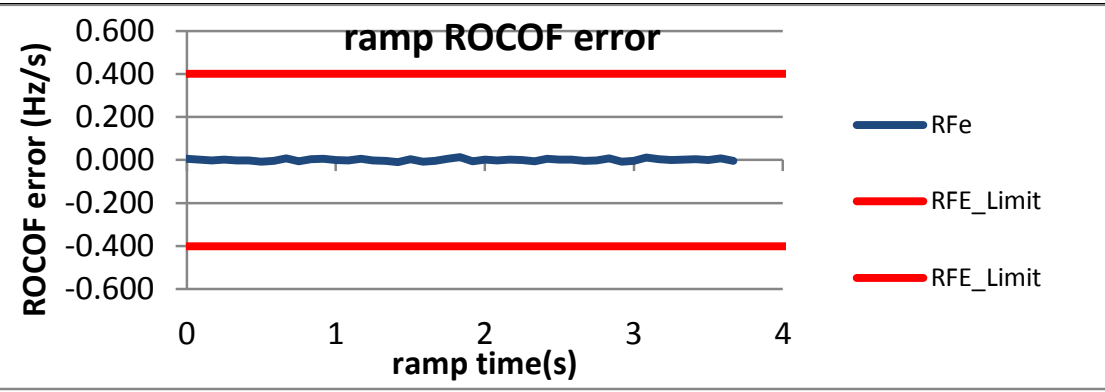

Figure 3011: Fs = 12 FPS, ramp from $58.6 \mathrm{~Hz}$ to $62.4 \mathrm{~Hz}$ at $+1 \mathrm{~Hz} / \mathrm{s}$

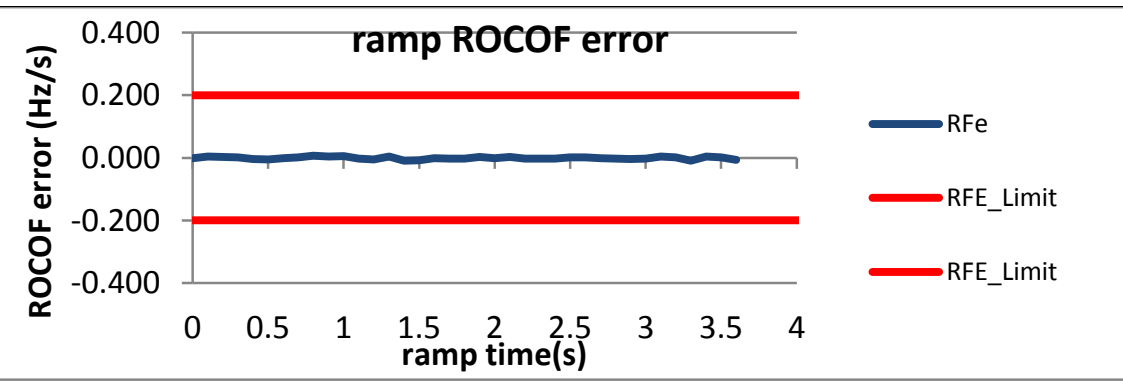

Figure 3013: Fs = $10 \mathrm{FPS}$, ramp from $58 \mathrm{~Hz}$ to $62 \mathrm{~Hz}$ at $+1 \mathrm{~Hz} / \mathrm{s}$

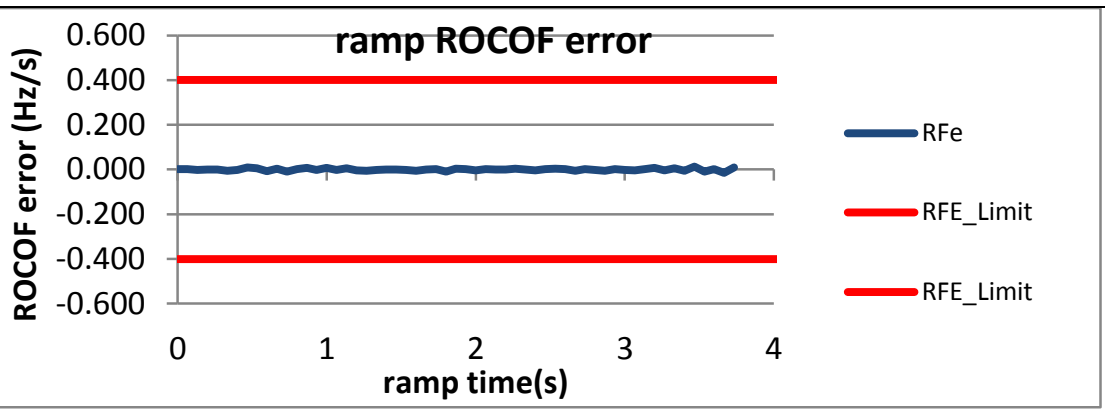

Figure 3010: Fs = $15 \mathrm{FPS}$, ramp from $62 \mathrm{~Hz}$ to $58 \mathrm{~Hz}$ at $-1 \mathrm{~Hz} / \mathrm{s}$

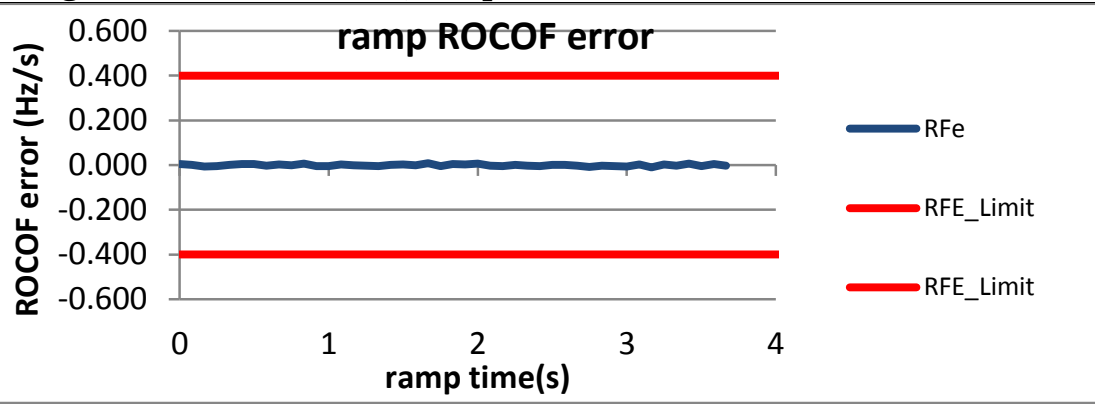

Figure 3012: Fs = $12 \mathrm{FPS}$, ramp from $62.4 \mathrm{~Hz}$ to $58 \mathrm{~Hz}$ at $-1 \mathrm{~Hz} / \mathrm{s}$

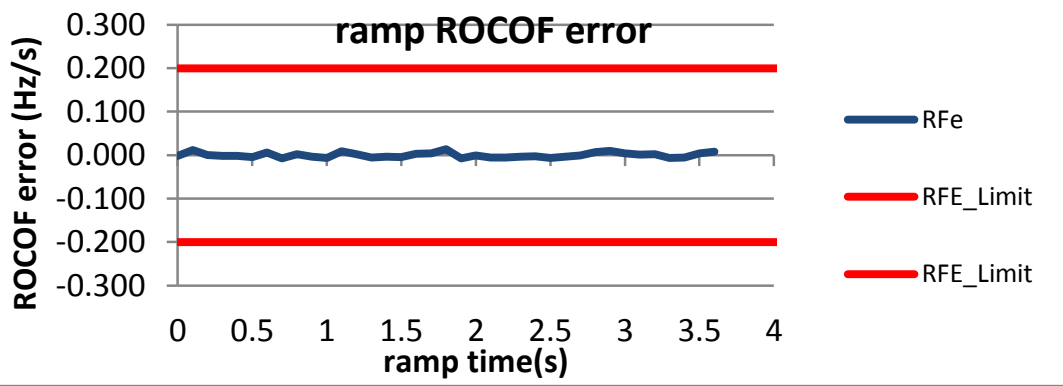

Figure 3014: Fs = $10 \mathrm{FPS}$, ramp from $62 \mathrm{~Hz}$ to $58 \mathrm{~Hz}$ at $-1 \mathrm{~Hz} / \mathrm{s}$ 
6.8.6 PMU E dynamic ramp of system frequency ROCOF error, $P$ class PMU E does not support P class.

6.8.7 PMU F dynamic ramp of system frequency ROCOF error, $P$ class

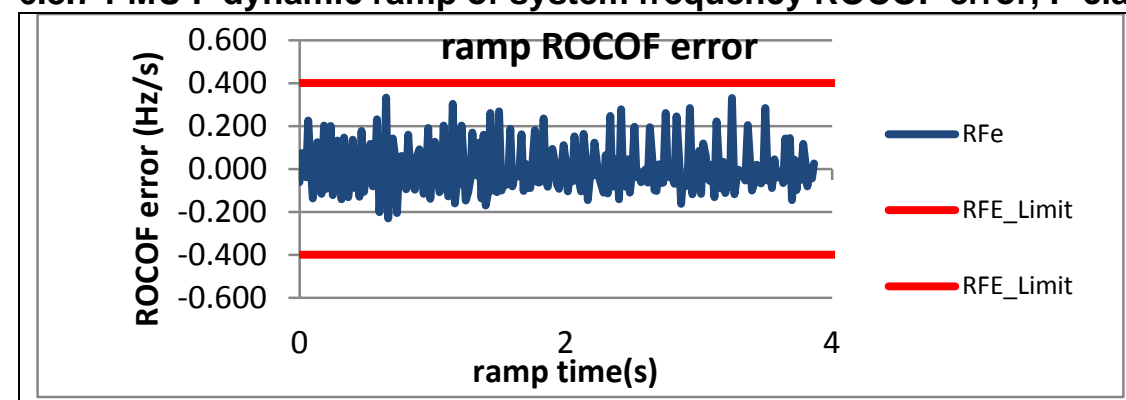

Figure 3015: Fs = $60 \mathrm{FPS}$, ram from $55 \mathrm{~Hz}$ to $65 \mathrm{~Hz}$ at $+1 \mathrm{~Hz} / \mathrm{s}$

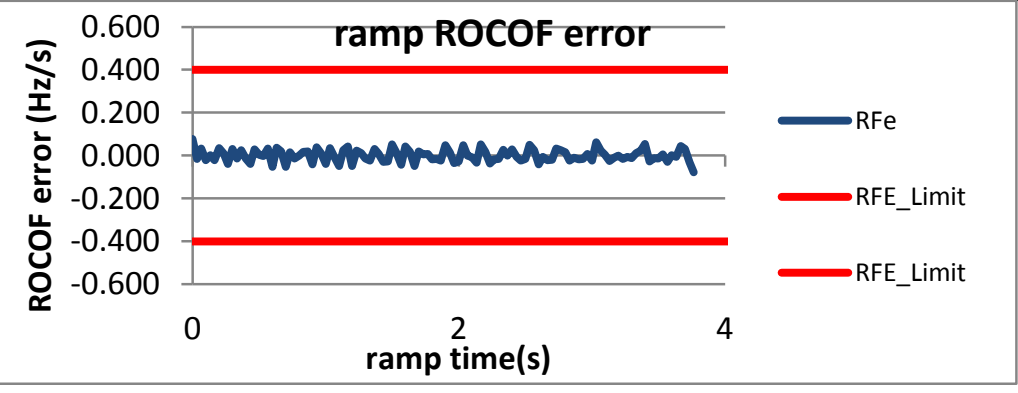

Figure 3017: Fs = $30 \mathrm{FPS}$, ramp from $55 \mathrm{~Hz}$ to $65 \mathrm{~Hz}$ at $+1 \mathrm{~Hz} / \mathrm{s}$

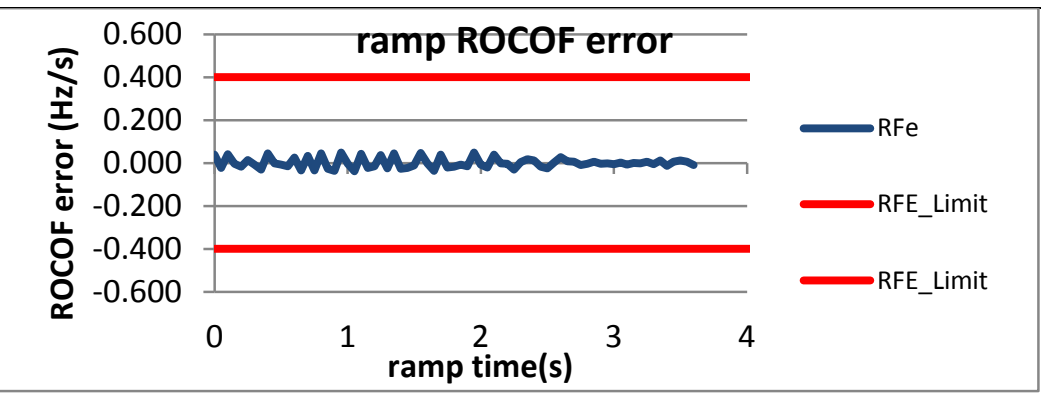

Figure 3019: Fs = $20 \mathrm{FPS}$, ramp from $56 \mathrm{~Hz}$ to $64 \mathrm{~Hz}$ at $+1 \mathrm{~Hz} / \mathrm{s}$

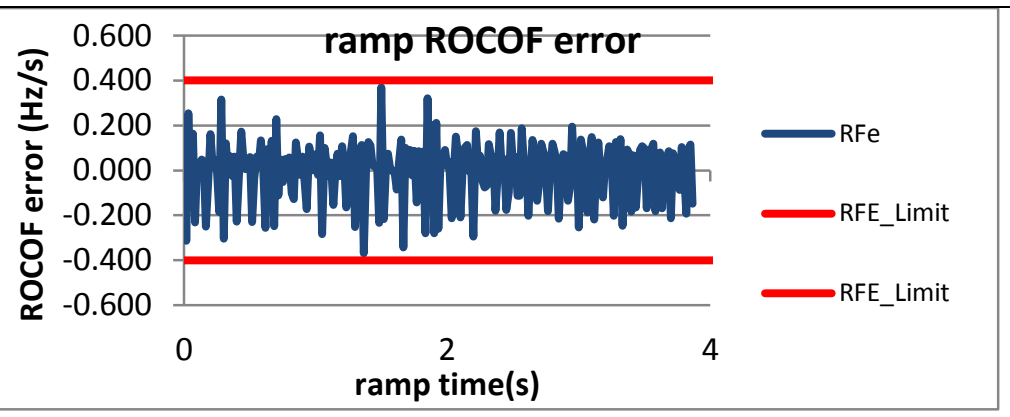

Figure 3016: Fs = $60 \mathrm{FPS}$, ram from $65 \mathrm{~Hz}$ to $55 \mathrm{~Hz}$ at $-1 \mathrm{~Hz} / \mathrm{s}$

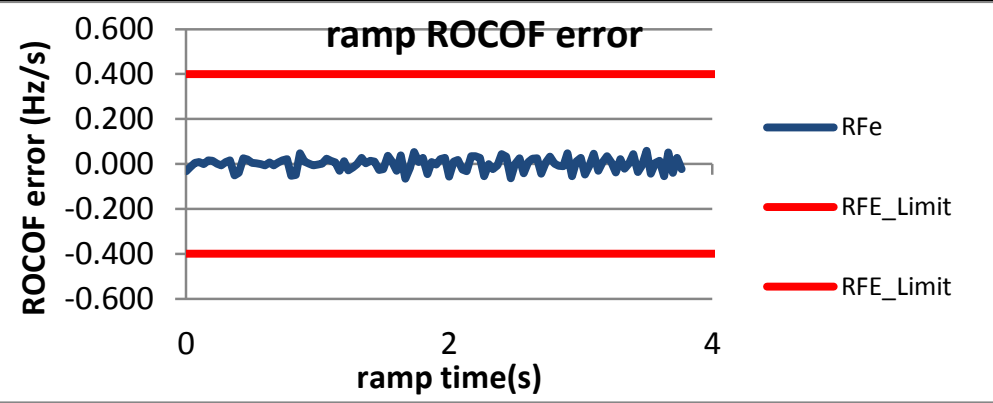

Figure 3018: Fs = $30 \mathrm{FPS}$, ram from $65 \mathrm{~Hz}$ to $55 \mathrm{~Hz}$ at $-1 \mathrm{~Hz} / \mathrm{s}$

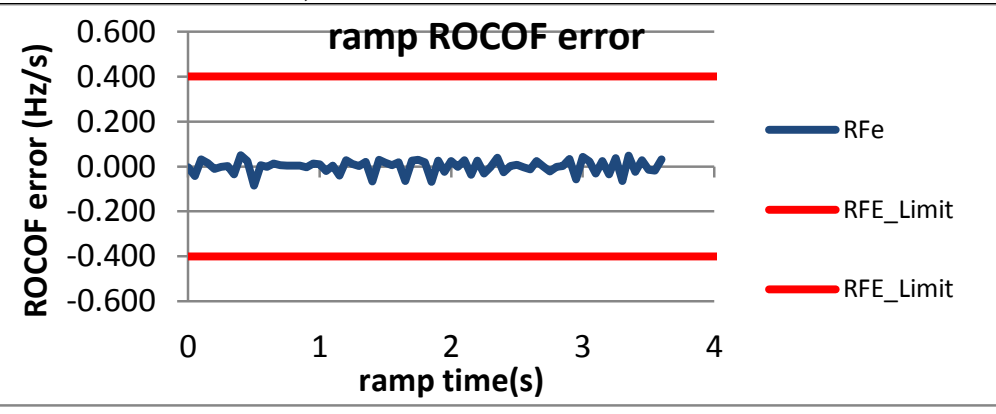

Figure 3020: Fs = $20 \mathrm{FPS}$, ramp from $64 \mathrm{~Hz}$ to $56 \mathrm{~Hz}$ at $=1 \mathrm{~Hz} / \mathrm{s}$ 


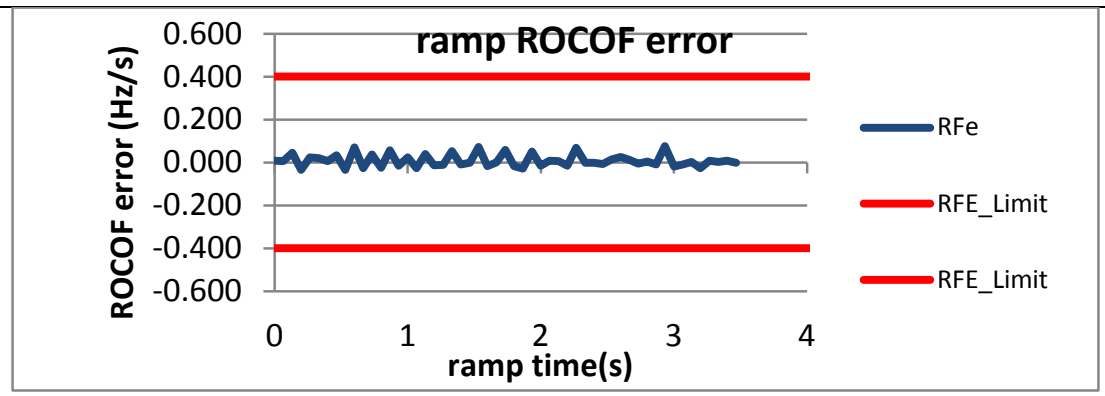

Figure 3021: Fs = 15 FPS, ramp from $57 \mathrm{~Hz}$ to $63 \mathrm{~Hz}$ at $+1 \mathrm{~Hz} / \mathrm{s}$

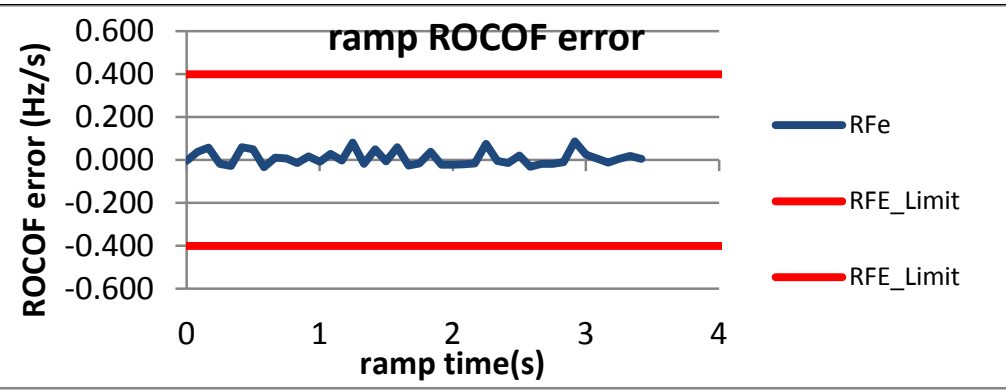

Figure 3023: Fs = 12 FPS, ramp from $58.6 \mathrm{~Hz}$ to $62.4 \mathrm{~Hz}$ at $+1 \mathrm{~Hz} / \mathrm{s}$

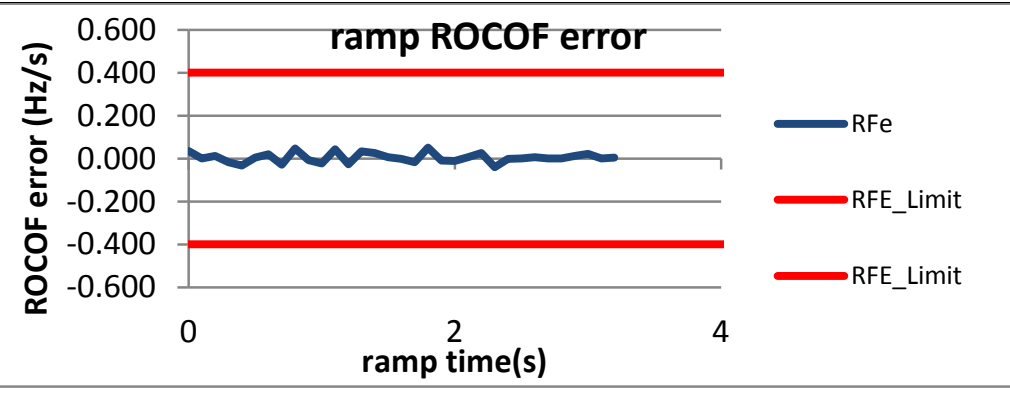

Figure 3025: Fs = $10 \mathrm{FPS}$, ramp from $58 \mathrm{~Hz}$ to $62 \mathrm{~Hz}$ at $+1 \mathrm{~Hz} / \mathrm{s}$

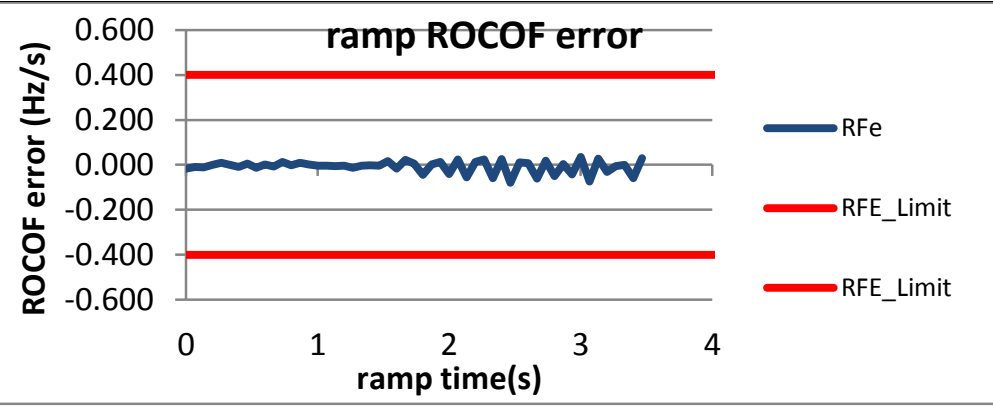

Figure 3022: Fs = $15 \mathrm{FPS}$, ramp from $63 \mathrm{~Hz}$ to $57 \mathrm{~Hz}$ at $-1 \mathrm{~Hz} / \mathrm{s}$

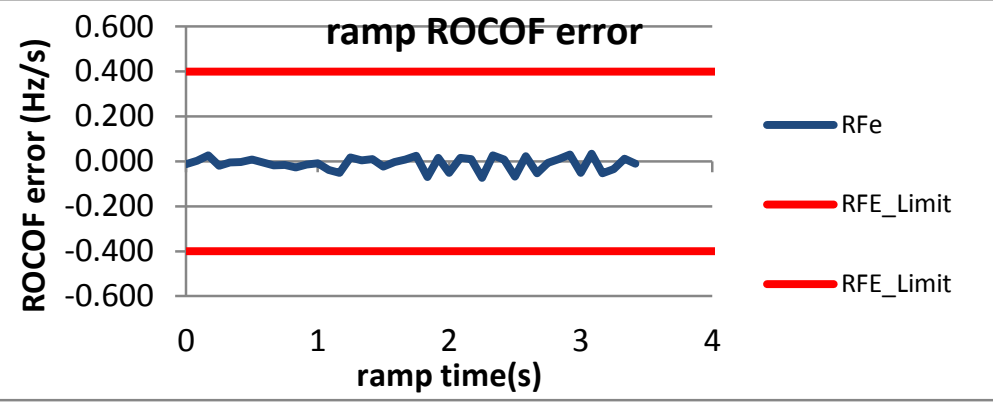

Figure 3024: Fs = 12 FPS, ramp from $62.4 \mathrm{~Hz}$ to $58.6 \mathrm{~Hz}$ at $-1 \mathrm{~Hz} / \mathrm{s}$

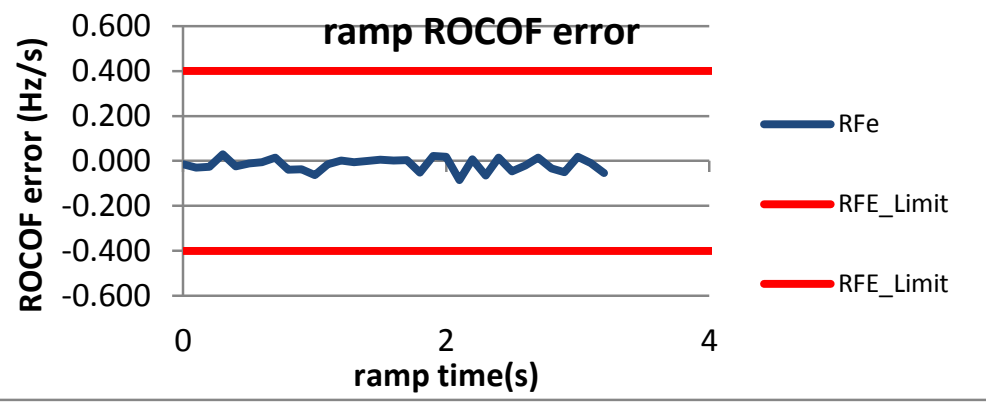

Figure 3026: Fs = $10 \mathrm{FPS}$, ramp from $62 \mathrm{~Hz}$ to $58 \mathrm{~Hz}$ at $-1 \mathrm{~Hz} / \mathrm{s}$ 
6.8.8 PMU G dynamic ramp of system frequency ROCOF error, $P$ class PMU G does not support P class.

\subsubsection{PMU H dynamic ramp of system frequency ROCOF error, $\mathrm{P}$ class}

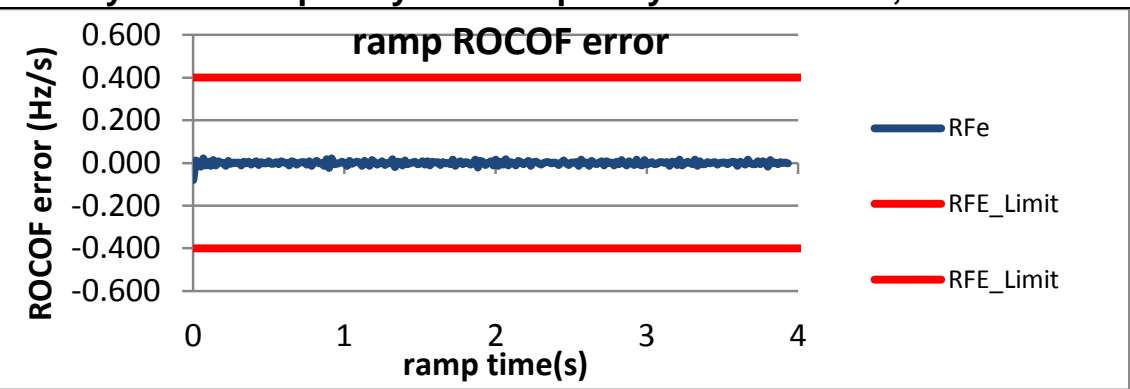

Figure 3027: Fs = $60 \mathrm{FPS}$, ramp from $58 \mathrm{~Hz}$ to $62 \mathrm{~Hz}$ at $+1 \mathrm{~Hz} / \mathrm{s}$

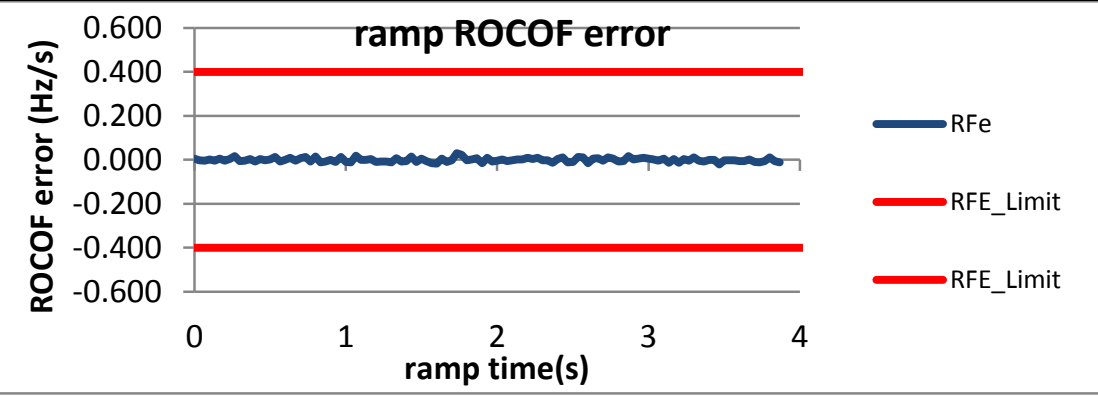

Figure 3029: Fs = $30 \mathrm{FPS}$, ramp from $58 \mathrm{~Hz}$ to $62 \mathrm{~Hz}$ at $+1 \mathrm{~Hz} / \mathrm{s}$

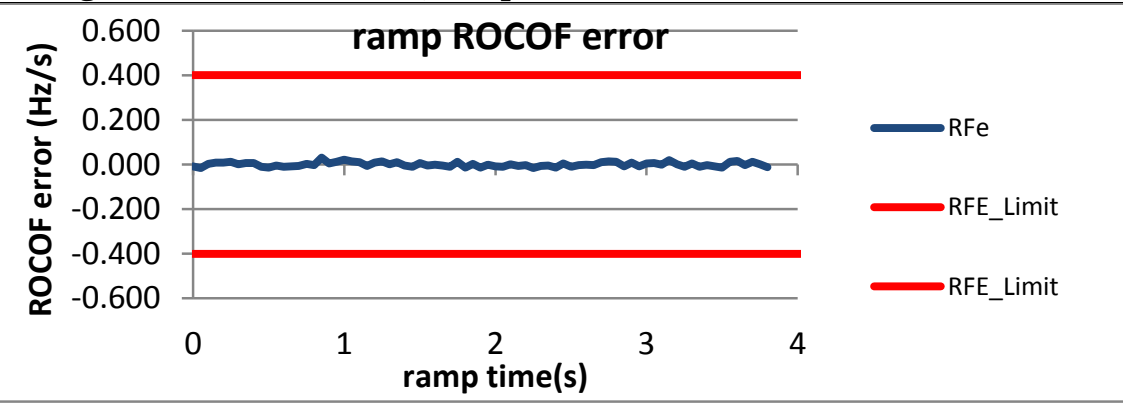

Figure 3031: Fs $=20 \mathrm{FPS}$, ramp from $58 \mathrm{~Hz}$ to $62 \mathrm{~Hz}$ at $+1 \mathrm{~Hz} / \mathrm{s}$

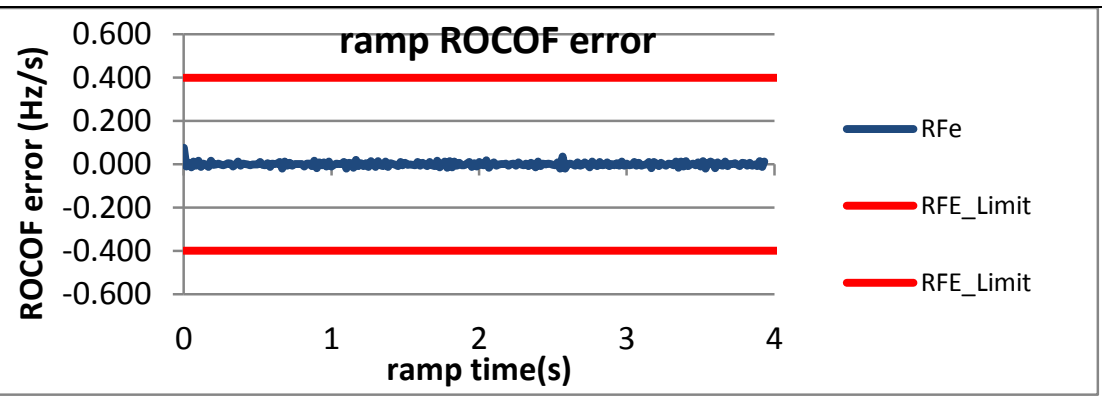

Figure 3028: Fs = $60 \mathrm{FPS}$, ramp from $62 \mathrm{~Hz}$ to $58 \mathrm{~Hz}$ at $-1 \mathrm{~Hz} / \mathrm{s}$

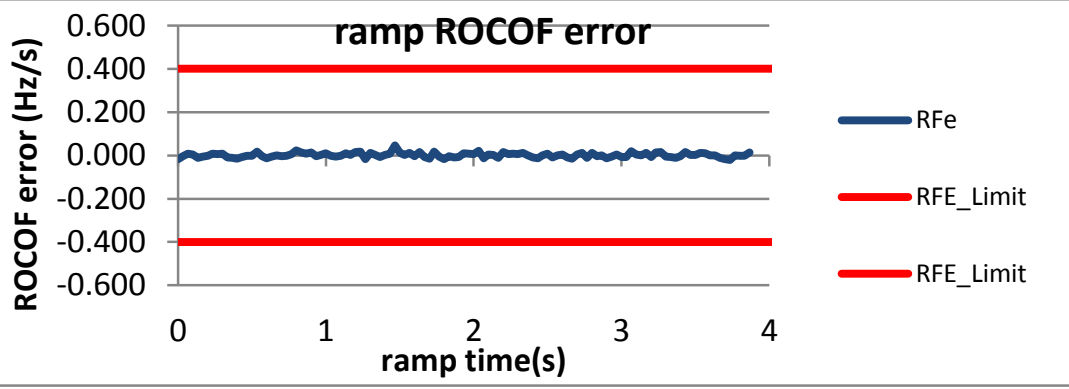

Figure 3030: Fs = $30 \mathrm{FPS}$, ram from $62 \mathrm{~Hz}$ to $58 \mathrm{~Hz}$ at $-1 \mathrm{~Hz} / \mathrm{s}$

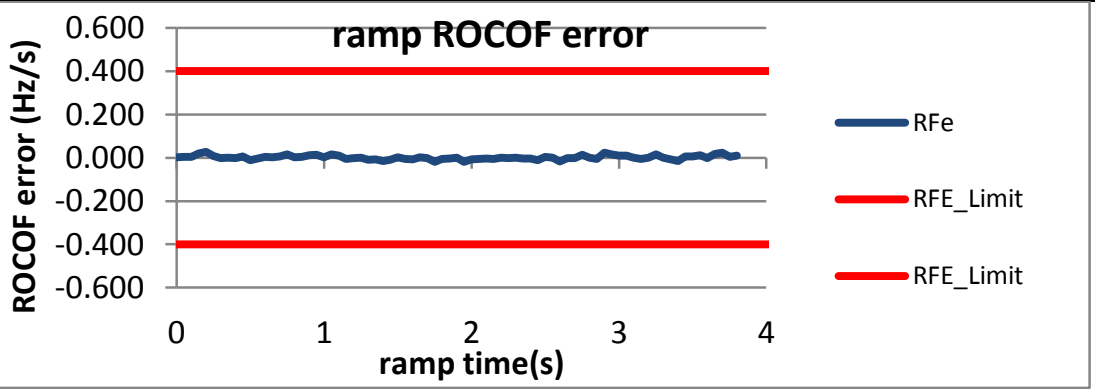

Figure 3032: Fs = $20 \mathrm{FPS}$, ramp from $62 \mathrm{~Hz}$ to $58 \mathrm{~Hz}$ at $=1 \mathrm{~Hz} / \mathrm{s}$ 

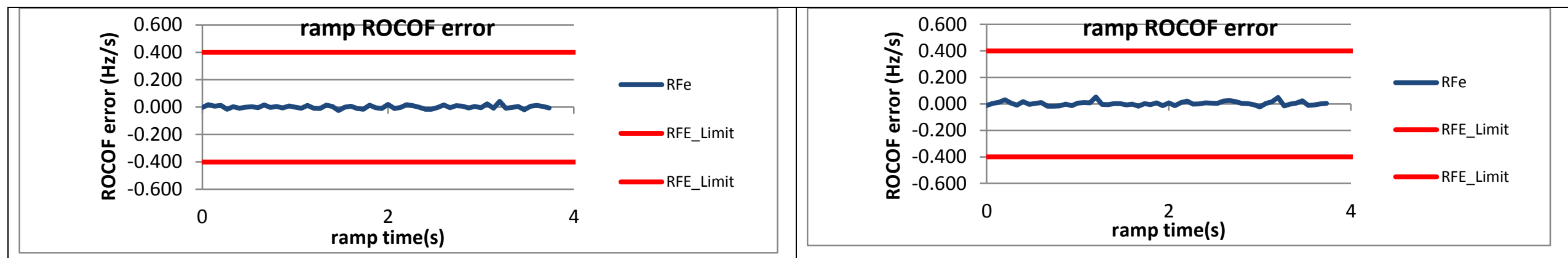

Figure 3033: Fs = $15 \mathrm{FPS}$, ramp from $58 \mathrm{~Hz}$ to $62 \mathrm{~Hz}$ at $+1 \mathrm{~Hz} / \mathrm{s}$

Figure 3034: Fs = $15 \mathrm{FPS}$, ramp from $62 \mathrm{~Hz}$ to $58 \mathrm{~Hz}$ at $-1 \mathrm{~Hz} / \mathrm{s}$
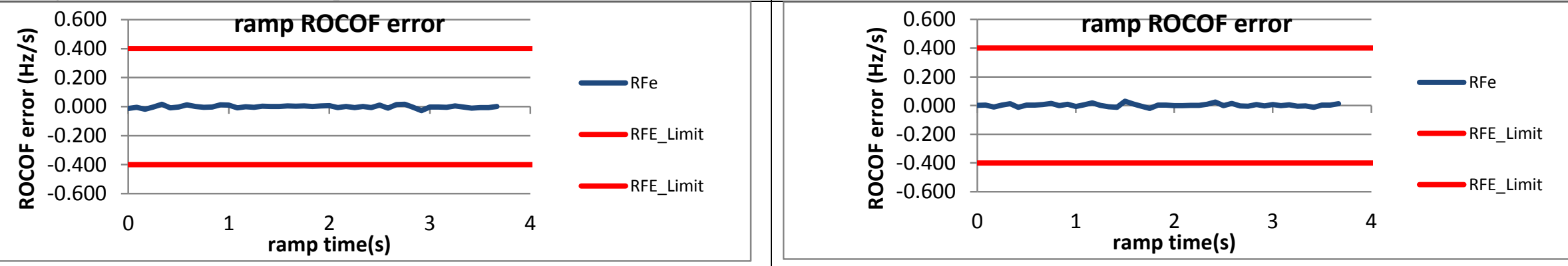

Figure 3035: Fs = $12 \mathrm{FPS}$, ramp from $58 \mathrm{~Hz}$ to $62 \mathrm{~Hz}$ at $+1 \mathrm{~Hz} / \mathrm{s}$

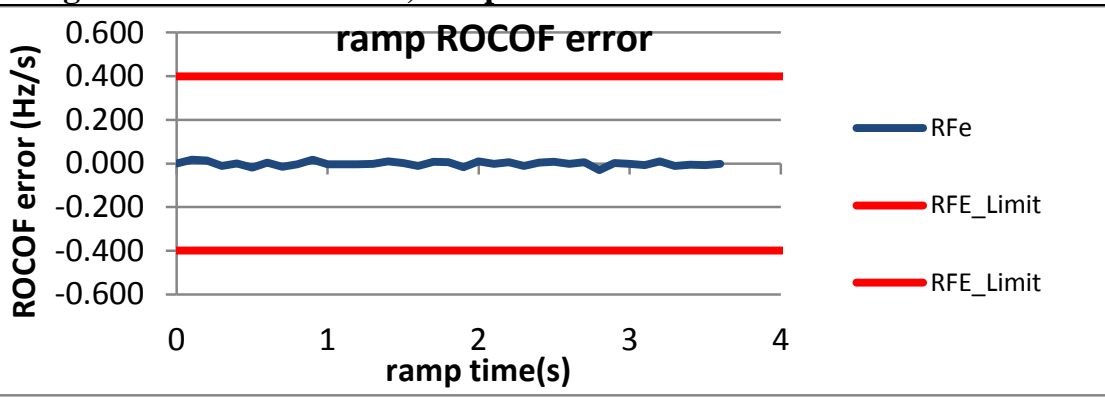

Figure 3036: Fs = $12 \mathrm{FPS}$, ramp from $62 \mathrm{~Hz}$ to $58 \mathrm{~Hz}$ at $-1 \mathrm{~Hz} / \mathrm{s}$

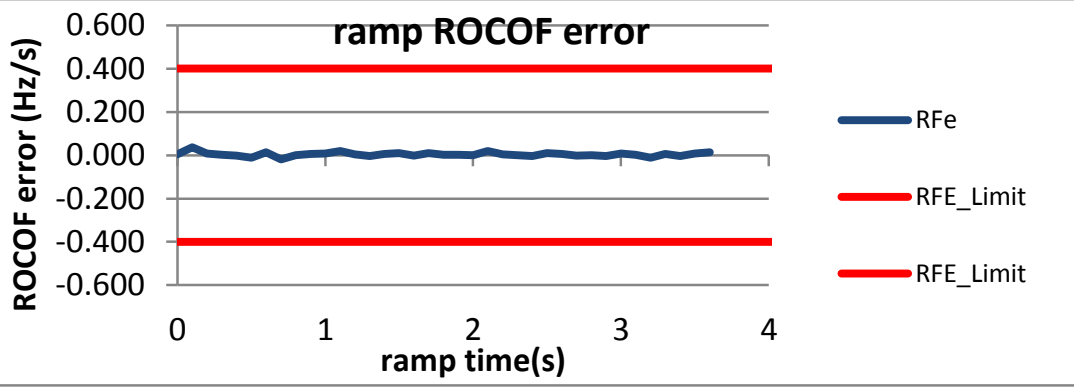

Figure 3037: Fs = $10 \mathrm{FPS}$, ramp from $58 \mathrm{~Hz}$ to $62 \mathrm{~Hz}$ at $+1 \mathrm{~Hz} / \mathrm{s}$

Figure 3038: Fs = $10 \mathrm{FPS}$, ramp from $62 \mathrm{~Hz}$ to $58 \mathrm{~Hz}$ at $-1 \mathrm{~Hz} / \mathrm{s}$ 
6.8.10 PMU I dynamic ramp of system frequency ROCOF error, $P$ class PMU I does not support $P$ class

6.8.11 PMU J dynamic ramp of system frequency ROCOF error, $P$ class

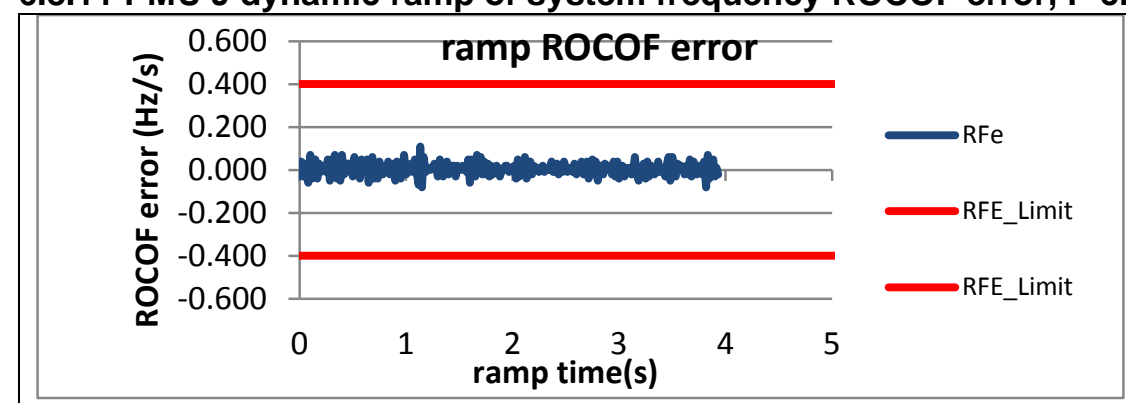

Figure 3039: Fs = $60 \mathrm{FPS}$, ram from $55 \mathrm{~Hz}$ to $65 \mathrm{~Hz}$ at $+1 \mathrm{~Hz} / \mathrm{s}$

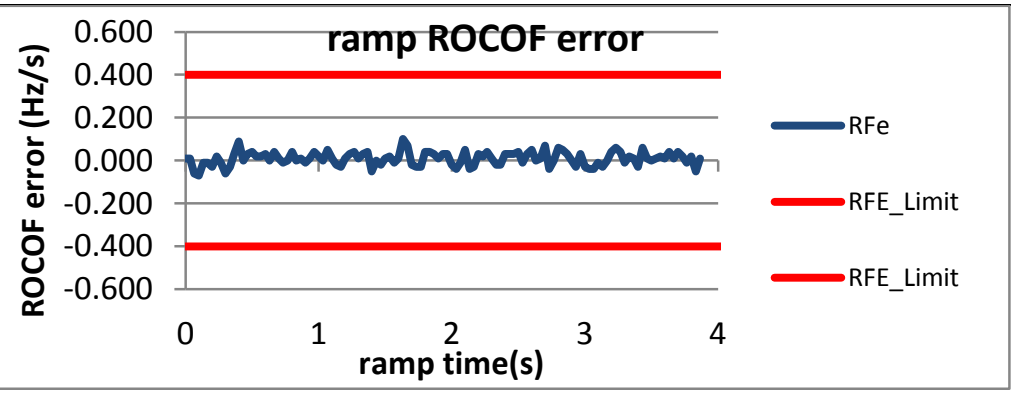

Figure 3041: Fs = $30 \mathrm{FPS}$, ramp from $55 \mathrm{~Hz}$ to $65 \mathrm{~Hz}$ at $+1 \mathrm{~Hz} / \mathrm{s}$

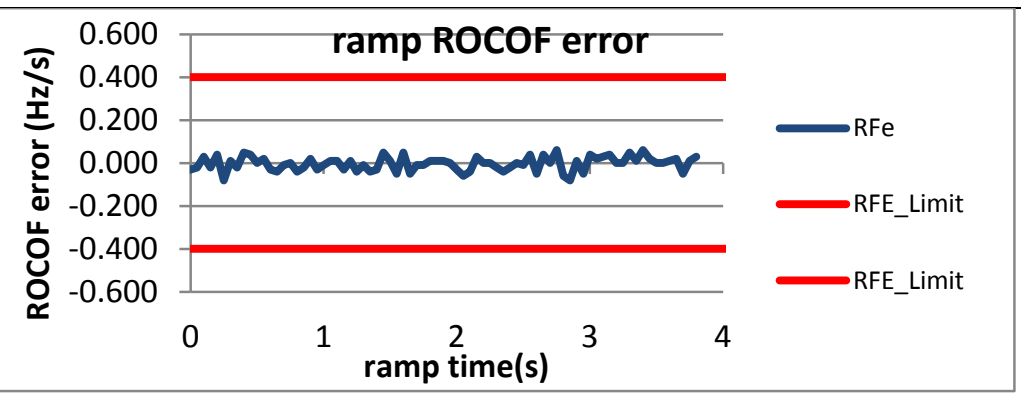

Figure 3043: Fs = $20 \mathrm{FPS}$, ramp from $56 \mathrm{~Hz}$ to $64 \mathrm{~Hz}$ at $+1 \mathrm{~Hz} / \mathrm{s}$

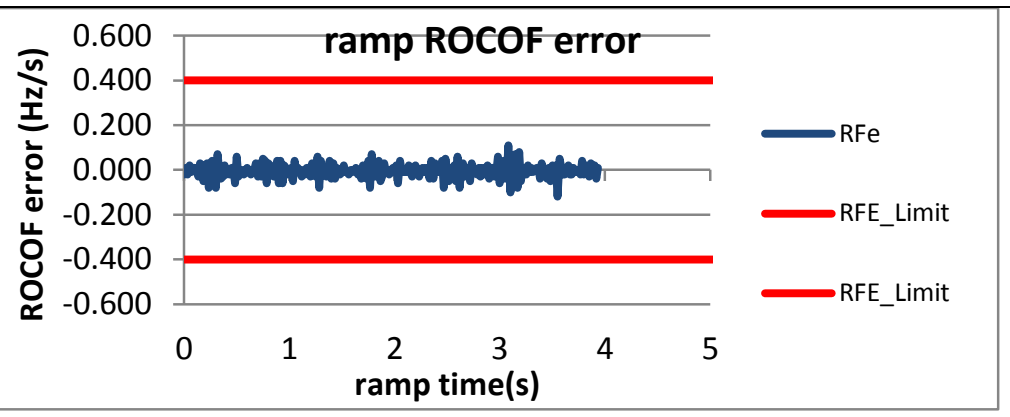

Figure 3040: Fs = $60 \mathrm{FPS}$, ram from $65 \mathrm{~Hz}$ to $55 \mathrm{~Hz}$ at $-1 \mathrm{~Hz} / \mathrm{s}$

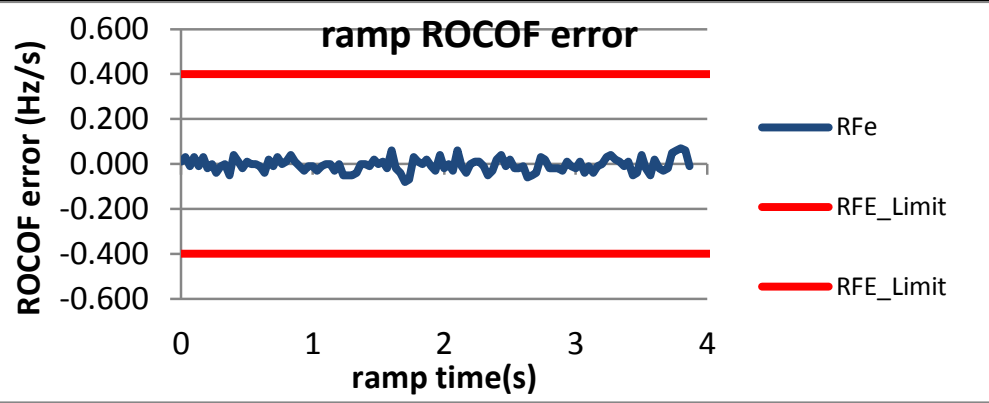

Figure 3042: Fs = $30 \mathrm{FPS}$, ram from $65 \mathrm{~Hz}$ to $55 \mathrm{~Hz}$ at $-1 \mathrm{~Hz} / \mathrm{s}$

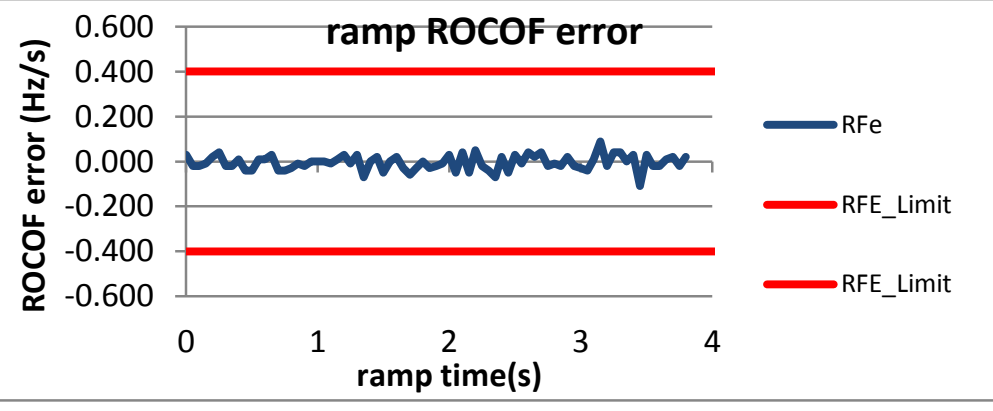

Figure 3044: Fs = $20 \mathrm{FPS}$, ramp from $64 \mathrm{~Hz}$ to $56 \mathrm{~Hz}$ at $=1 \mathrm{~Hz} / \mathrm{s}$ 


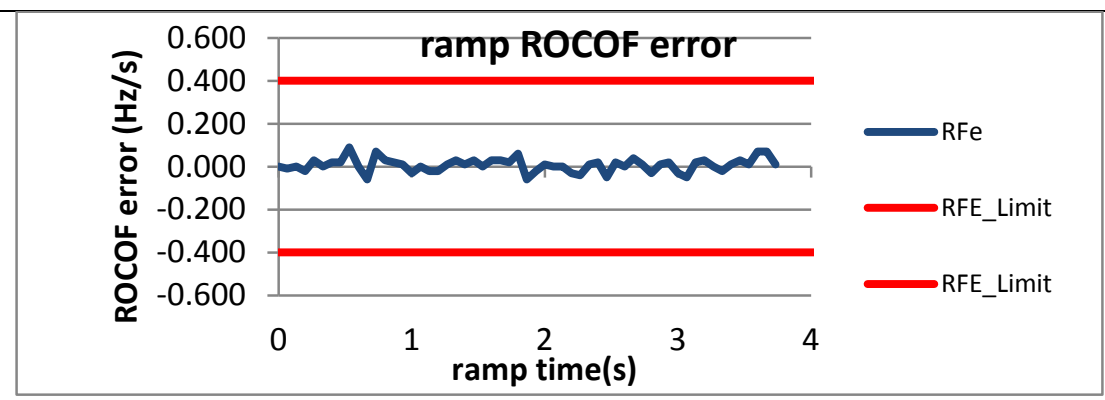

Figure 3045: Fs = $15 \mathrm{FPS}$, ramp from $57 \mathrm{~Hz}$ to $63 \mathrm{~Hz}$ at $+1 \mathrm{~Hz} / \mathrm{s}$

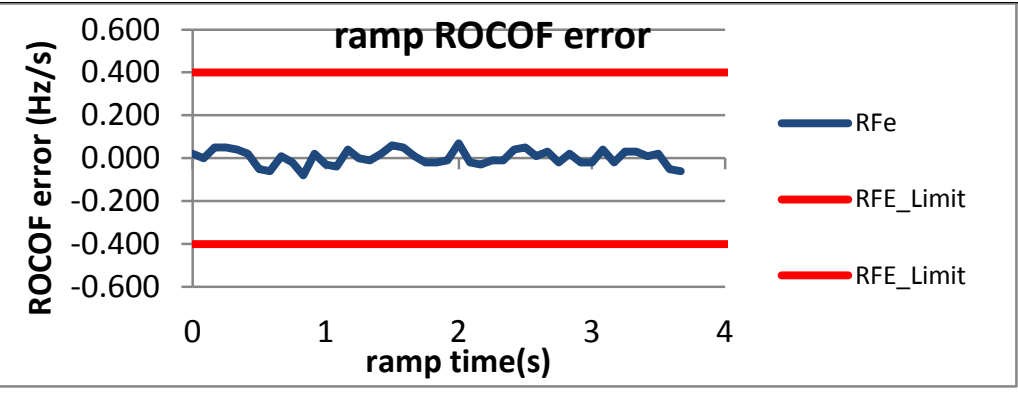

Figure 3047: Fs = 12 FPS, ramp from $58.6 \mathrm{~Hz}$ to $62.4 \mathrm{~Hz}$ at $+1 \mathrm{~Hz} / \mathrm{s}$

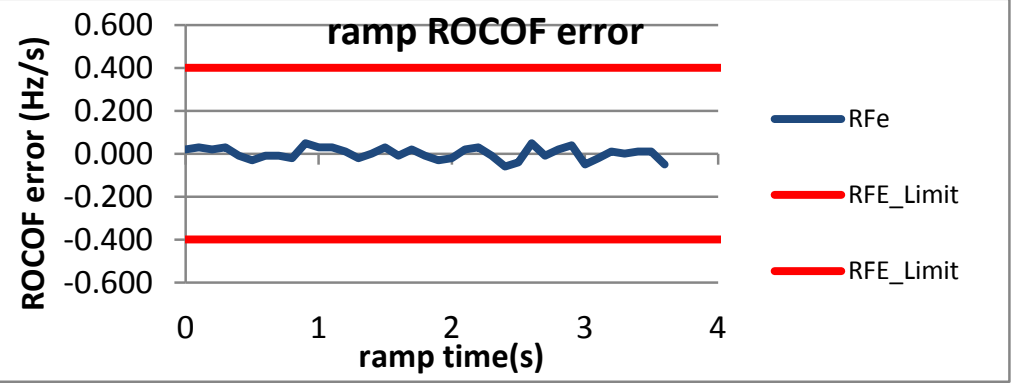

Figure 3049: Fs = $10 \mathrm{FPS}$, ramp from $58 \mathrm{~Hz}$ to $62 \mathrm{~Hz}$ at $+1 \mathrm{~Hz} / \mathrm{s}$

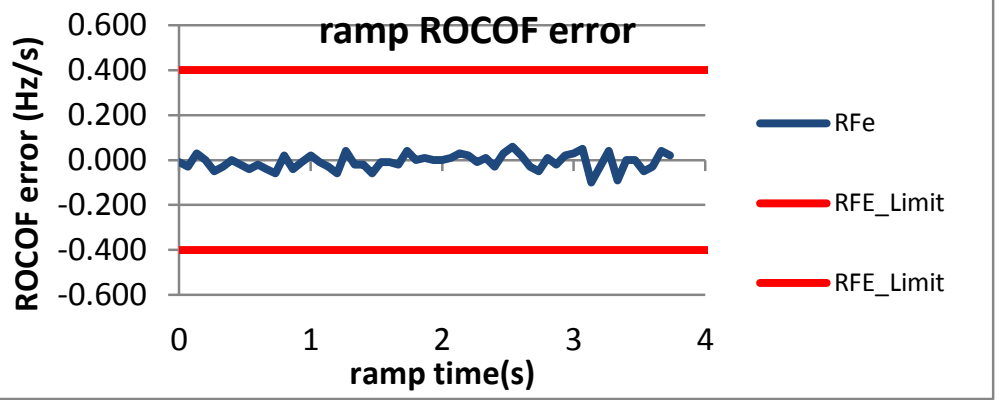

Figure 3046: Fs = $15 \mathrm{FPS}$, ramp from $63 \mathrm{~Hz}$ to $57 \mathrm{~Hz}$ at $-1 \mathrm{~Hz} / \mathrm{s}$

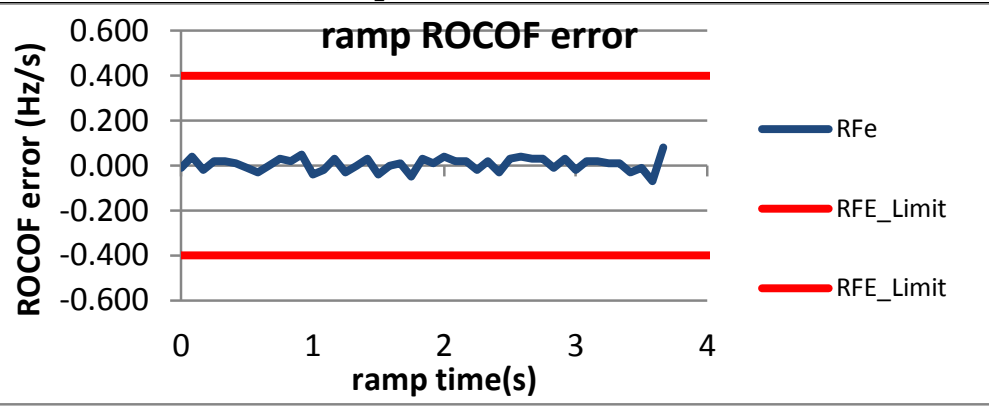

Figure 3048: Fs = 12 FPS, ramp from $62.4 \mathrm{~Hz}$ to $58.6 \mathrm{~Hz}$ at $-1 \mathrm{~Hz} / \mathrm{s}$

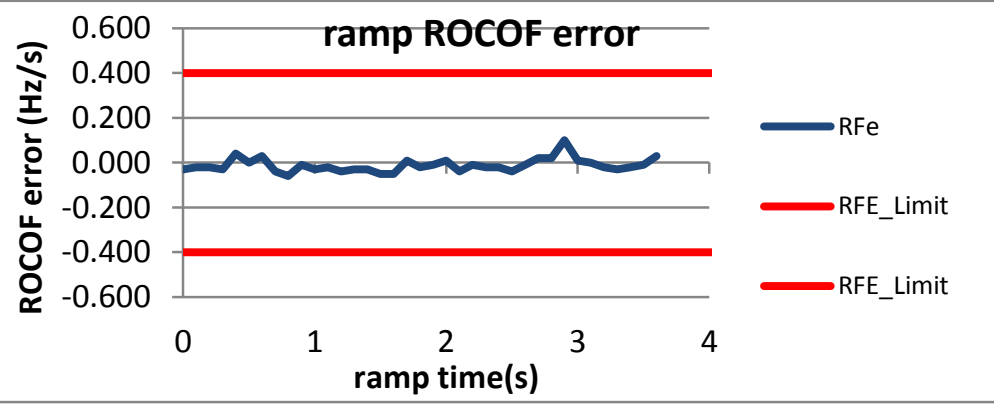

Figure 3050: Fs = $10 \mathrm{FPS}$, ramp from $62 \mathrm{~Hz}$ to $58 \mathrm{~Hz}$ at $-1 \mathrm{~Hz} / \mathrm{s}$ 


\section{Dynamic bandwidth measurement: phase modulation}

\section{Table of Contents}

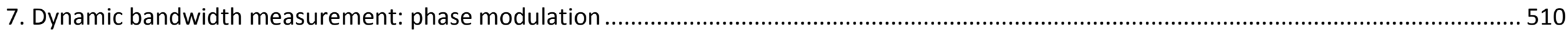

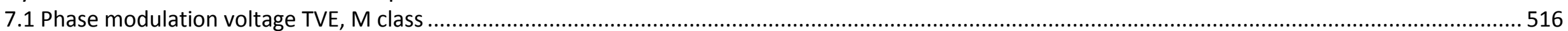

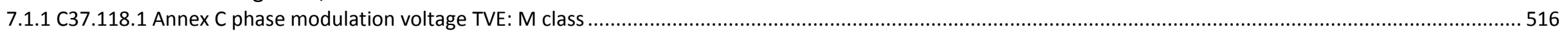

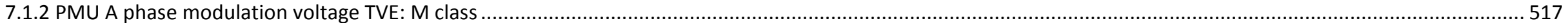

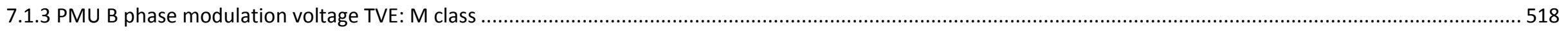

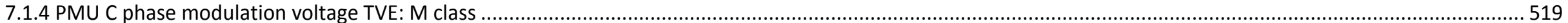

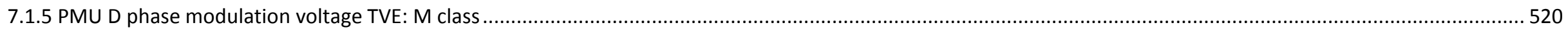

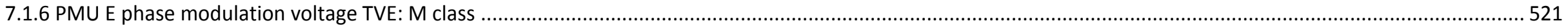

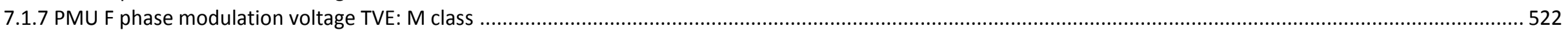

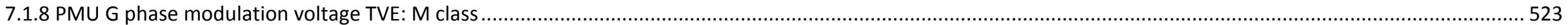

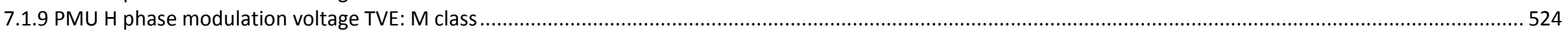

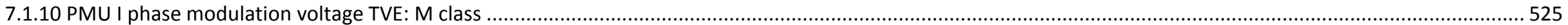

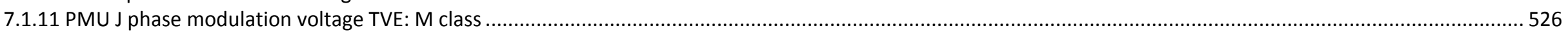

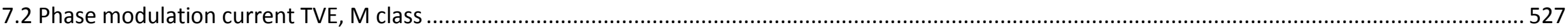

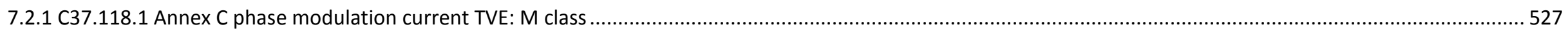

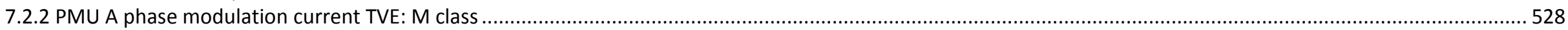

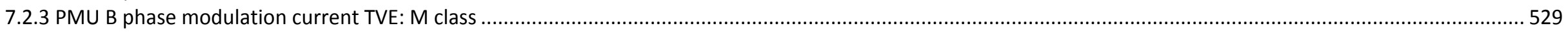

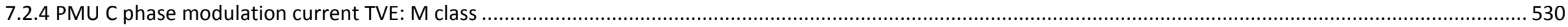

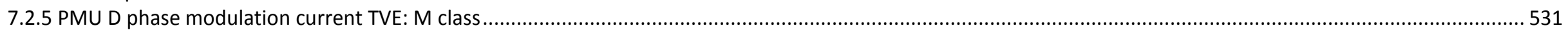

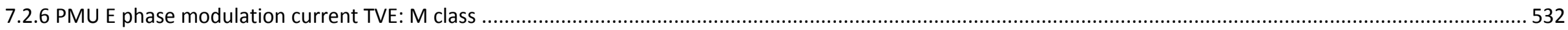

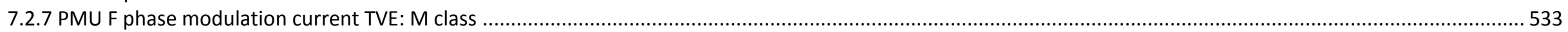

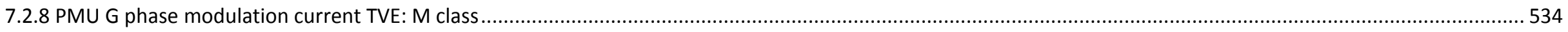

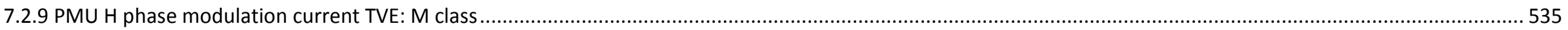

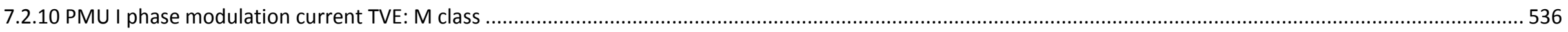

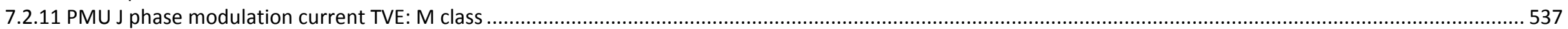

7.3 Dynamic bandwidth measurement: phase modulation frequency error, M class .................................................................................................... 538

7.3.1 C37.118.1 Annex C dynamic bandwidth measurement: phase modulation frequency error: M class ......................................................................................... 538

7.3.2 PMU A dynamic bandwidth measurement: phase modulation frequency error: M class .................................................................................................... 539

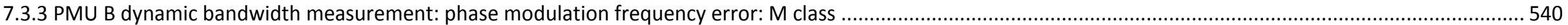

7.3.4 PMU C dynamic bandwidth measurement: phase modulation frequency error: M class .................................................................................................... 541

7.3.5 PMU D dynamic bandwidth measurement: phase modulation frequency error: M class ...................................................................................................... 542

7.3.6 PMU E dynamic bandwidth measurement: phase modulation frequency error: M class ..................................................................................................... 543

7.3.7 PMU F dynamic bandwidth measurement: phase modulation frequency error: M class .................................................................................................... 544

7.3.8 PMU G dynamic bandwidth measurement: phase modulation frequency error: M class …………....................................................................................... 545 
7.3.9 PMU H dynamic bandwidth measurement: phase modulation frequency error: M class ……............................................................................................... 546

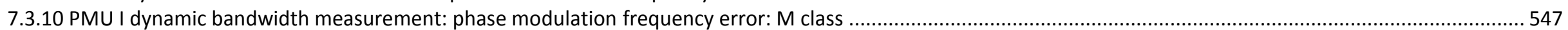

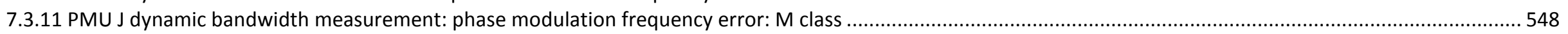

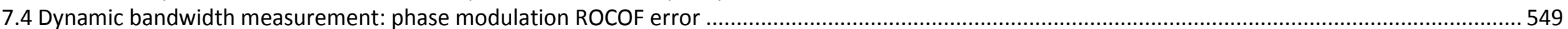

7.4.1 C37.118.1 Annex C dynamic bandwidth measurement: phase modulation ROCOF error: M class ....................................................................................... 549

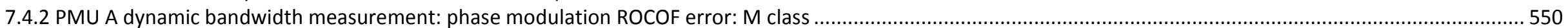

7.4.3 PMU B dynamic bandwidth measurement: phase modulation ROCOF error: M class …................................................................................................. 551

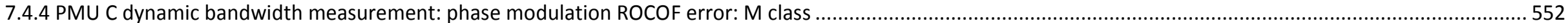

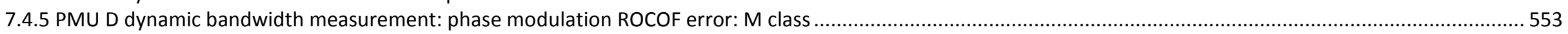

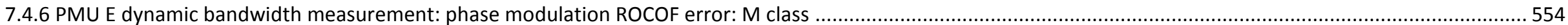

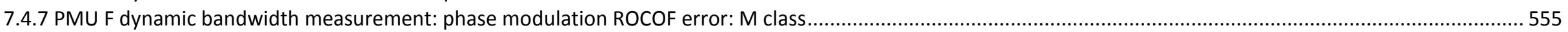

7.4.8 PMU G * dynamic bandwidth measurement: phase modulation ROCOF error: M class ............................................................................................................... 556

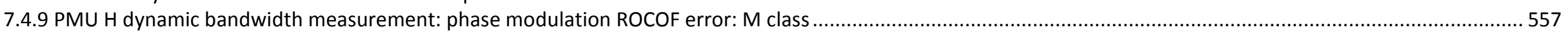

7.4.10 PMU I dynamic bandwidth measurement: phase modulation ROCOF error: M class ..................................................................................................... 558

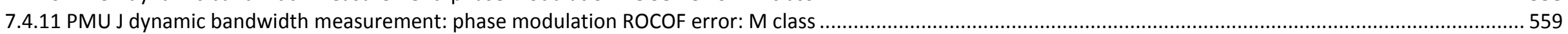

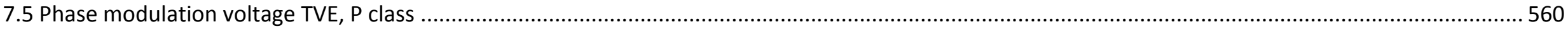

7.5.1 C37.118.1 Annex C dynamic bandwidth measurement: phase modulation voltage TVE: P class ................................................................................................ 560

7.5.2 PMU A dynamic bandwidth measurement: phase modulation voltage TVE: P class ....................................................................................................... 561

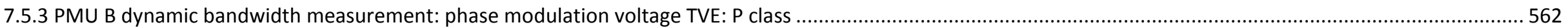

7.5.4 PMU C dynamic bandwidth measurement: phase modulation voltage TVE: P class .................................................................................................................. 563

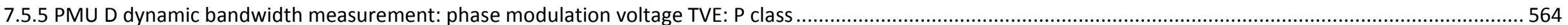

7.5.6 PMU E dynamic bandwidth measurement: phase modulation voltage TVE: P class ........................................................................................................ 564

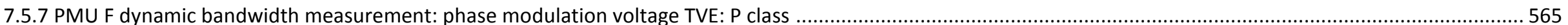

7.5.8 PMU G dynamic bandwidth measurement: phase modulation voltage TVE: P class ............................................................................................................. 565

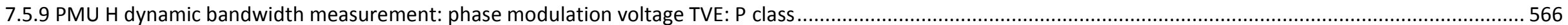

7.5.10 PMU I dynamic bandwidth measurement: phase modulation voltage TVE: P class ................................................................................................................ 566

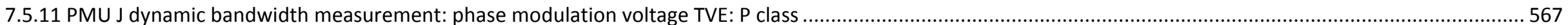

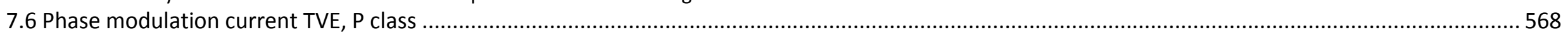

7.6.1 C37.118.1 Annex C dynamic bandwidth measurement: phase modulation current TVE: P class .............................................................................................. 568

7.6.2 PMU A dynamic bandwidth measurement: phase modulation current TVE: P class ........................................................................................................... 569

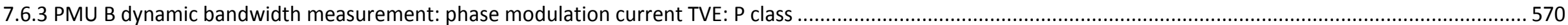

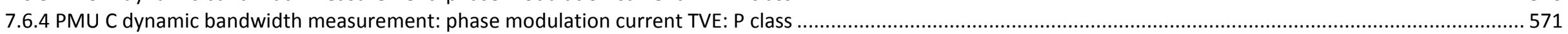

7.6.5 PMU D dynamic bandwidth measurement: phase modulation current TVE: P class ......................................................................................................... 572

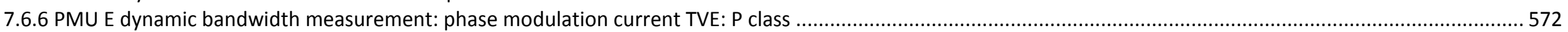

7.6.7 PMU F dynamic bandwidth measurement: phase modulation current TVE: P class ............................................................................................................. 573

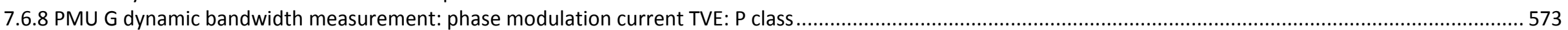

7.6.9 PMU H dynamic bandwidth measurement: phase modulation current TVE: P class ..............................................................................................................5 574

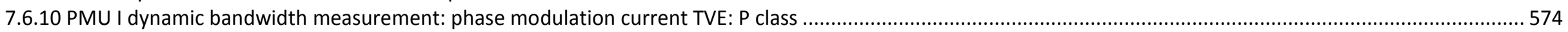

7.6.11 PMU J dynamic bandwidth measurement: phase modulation current TVE: P class .................................................................................................................. 575

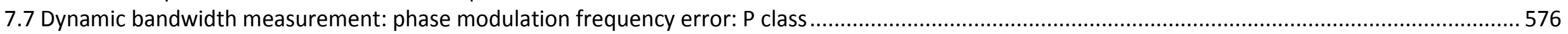

7.7.1 C37.118.1 Annex C dynamic bandwidth measurement: phase modulation frequency error: P class ......................................................................................... 576 
7.7.2 PMU A dynamic bandwidth measurement: phase modulation frequency error: $\mathrm{P}$ class

7.7.3 PMU B dynamic bandwidth measurement: phase modulation frequency error: $P$ class

7.7.4 PMU $C$ dynamic bandwidth measurement: phase modulation frequency error: $P$ class

579

7.7.5 PMU D dynamic bandwidth measurement: phase modulation frequency error: $P$ class

580

7.7.6 PMU E dynamic bandwidth measurement: phase modulation frequency error: $P$ class

580

7.7.7 PMU F dynamic bandwidth measurement: phase modulation frequency error: $P$ class

581

7.7.8 PMU G dynamic bandwidth measurement: phase modulation frequency error: $P$ class ............

581

7.7.9 PMU H dynamic bandwidth measurement: phase modulation frequency error: $P$ class

582

7.7.10 PMU I dynamic bandwidth measurement: phase modulation frequency error: $P$ class

582

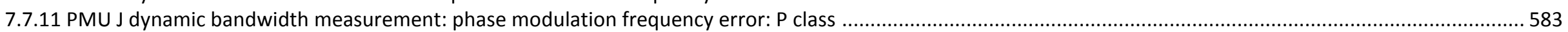

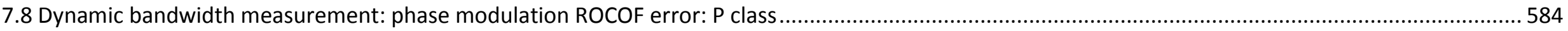

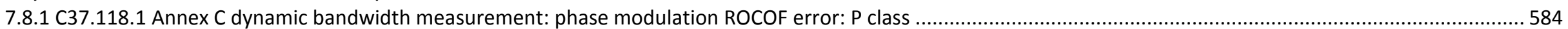

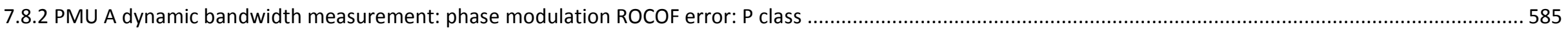

7.8.3 PMU B dynamic bandwidth measurement: phase modulation ROCOF error: P class ........................................................................................................... 586

7.8.4 PMU C dynamic bandwidth measurement: phase modulation ROCOF error: P class............................................................................................................... 587

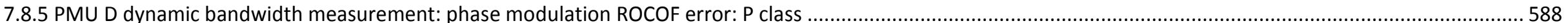

7.8.6 PMU E dynamic bandwidth measurement: phase modulation ROCOF error: P class .............................................................................................................. 588

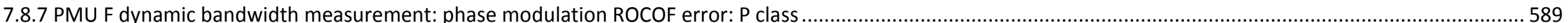

7.8.8 PMU G dynamic bandwidth measurement: phase modulation ROCOF error: P class ....................................................................................................... 589

7.8.9 PMU H dynamic bandwidth measurement: phase modulation ROCOF error: P class ......................................................................................................... 590

7.8.10 PMU I dynamic bandwidth measurement: phase modulation ROCOF error: P class .............................................................................................................. 590

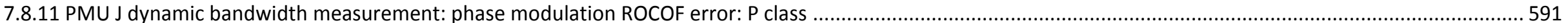


The measurement bandwidth tests apply modulated input signals to the PMU to ensure that the in-band performance is within limits. The phase modulation tests are a series of individual dynamic tests where the input signal phase is modulated at a $10 \%$ index of modulation. Individual tests are run with $0.2 \mathrm{~Hz}$ modulation frequency increments beginning at $0.1 \mathrm{~Hz}$ until the modulation frequency has reached the lesser of Fs/5 Hz or $5 \mathrm{~Hz}$ for $\mathrm{M}$ class and $2 \mathrm{~Hz}$ for $\mathrm{P}$ class PMUs.

The maximum TVE, Fe and RFe of all tests are compared against the limits for TVE, Fe, and RFe to determine if the unit passes or fails the test.

C37.118.1-2011 amended by C37.118.1a-2014 specifies the limits as:

- maxTVE: $3 \%$

- $\max \mathrm{Fe}:$

○ M class: $0.06 \times \min (\mathrm{Fs} / 5,5)$

○ class: $0.03 \times \min (\mathrm{Fs} / 10,2)$

- $\max R F e$ :

○ M class: $0.18 \times \pi \times(\min (\mathrm{Fs} / 5,5))^{\wedge} 2$

$\circ$ P class: $0.18 \times \pi \times(\min (\mathrm{Fs} / 10,2))^{\wedge} 2$

The test plan for measurement bandwidth is as follows:

$X_{m}$ is the peak amplitude of the input signal

$\omega_{0}$ is the nominal power system frequency in radians/second $\left(2 \pi F_{0}\right)$

$\omega$ is the modulation frequency in radians/second

$\mathrm{k}_{\mathrm{x}}$ is the amplitude modulation index

$k_{a}$ is the phase angle modulation index

$t$ is time

a) Begin with phase modulated input at $\omega / 2 \pi=0.1 \mathrm{~Hz}, \mathrm{k}_{\mathrm{x}}=0.1, \mathrm{k}_{\mathrm{a}}=0$.

b) Wait for the system to settle.

c) Capture the PMU output for at least 2 full cycles of modulation or 5 seconds, whichever is greater.

d) Calculate the errors: TVE, FE, RFE for each report.

e) Calculate the Max TVE, FE and RFE.

f) Increase the modulation frequency $\omega / 2 \pi$ by $0.2 \mathrm{~Hz}$.

g) Repeat steps $b$ through $f$ until the upper frequency range limit is reached. 
To illustrate an example of one PMU's response to one test, the below plots of TVE, Fe and RFe are shown from the test run on PMU B at Fs $=30$ FPS and a phase modulation frequency of $4.1 \mathrm{~Hz}$ :

Example data from one run of dynamic phase modulation test: PMU B at Fs = 30 FPS, $4.1 \mathrm{~Hz}$ modulation frequency

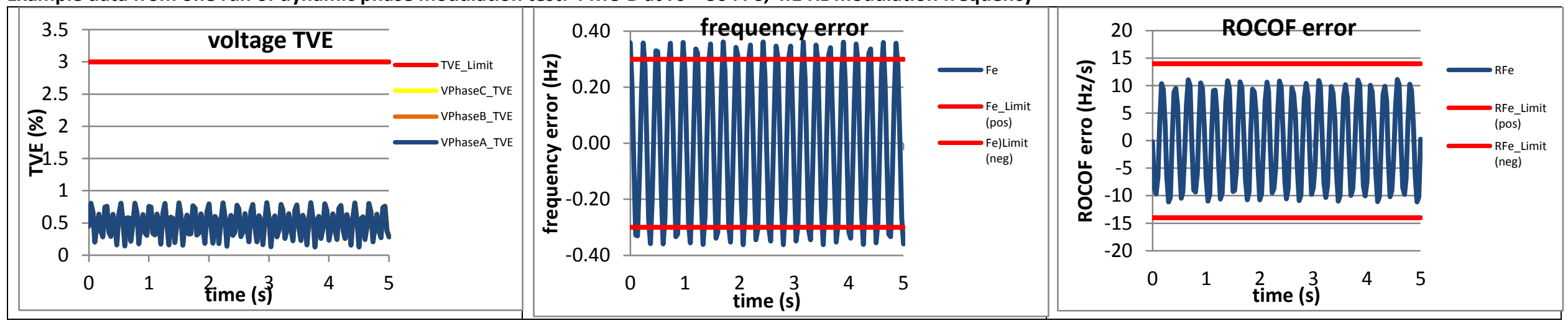

The plots in the body of this section are a compilation of data for all tests made at $0.2 \mathrm{~Hz}$ modulation frequency intervals from each reporting rate. The $\mathrm{X}$ axis is the modulation frequency and the $\mathrm{Y}$ axis is TVE, Fe or RFe respectively. In the example below, an arrow indicates the maximum value from the example above.

Example compiled data from all runs of dynamic phase modulation tests from a single reporting rate: PMU B at Fs = 30 FPS
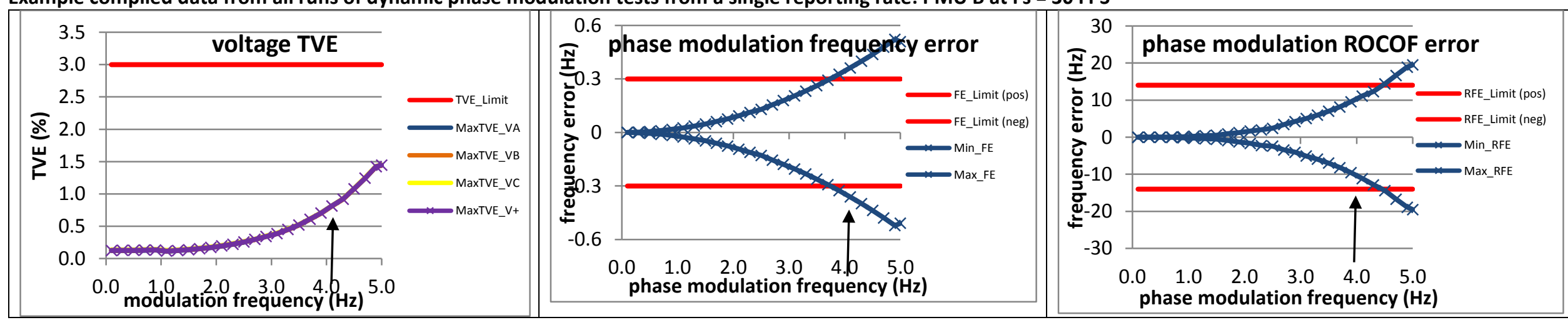
Results from the dynamic phase modulation tests

\begin{tabular}{|c|c|c|c|c|c|c|c|c|c|c|c|c|c|c|c|c|c|c|c|c|c|c|c|c|c|c|c|c|c|c|c|c|c|c|c|c|}
\hline \multirow{4}{*}{\begin{tabular}{|l} 
Fs (FPS) \\
Test
\end{tabular}} & \multicolumn{3}{|c|}{$10 \mathrm{M}$} & \multicolumn{3}{|c|}{$10 P$} & \multicolumn{3}{|c|}{$12 \mathrm{M}$} & \multicolumn{3}{|c|}{$12 \mathrm{P}$} & \multicolumn{3}{|c|}{$15 \mathrm{M}$} & \multicolumn{3}{|c|}{$15 \mathrm{P}$} & \multicolumn{3}{|c|}{$20 \mathrm{M}$} & \multicolumn{3}{|c|}{$20 P$} & \multicolumn{3}{|c|}{$30 \mathrm{M}$} & \multicolumn{3}{|c|}{$30 P$} & \multicolumn{3}{|c|}{$60 \mathrm{M}$} & \multicolumn{3}{|c|}{$60 P$} \\
\hline & $\mathrm{T}$ & $\mathrm{F}$ & $R$ & $\mathrm{~T}$ & $F$ & $R$ & $\mathrm{~T}$ & $\mathrm{~F}$ & $\mathrm{R}$ & $\mathrm{T}$ & $F$ & $\mathrm{R}$ & $\mathrm{T}$ & $F$ & $\mathrm{R}$ & $\mathrm{T}$ & $\mathrm{F}$ & $R$ & $\mathrm{~T}$ & $\mathrm{~F}$ & $\mathrm{R}$ & $\mathrm{T}$ & $\mathrm{F}$ & $\mathrm{R}$ & $\mathrm{T}$ & $\mathrm{F}$ & $\mathrm{R}$ & $\mathrm{T}$ & $F$ & $R$ & $\mathrm{~T}$ & $F$ & $R$ & $\mathrm{~T}$ & $F$ & $\mathrm{R}$ \\
\hline & V & $E$ & $\mathrm{~F}$ & V & $E$ & $F$ & V & $E$ & $\mathrm{~F}$ & V & $E$ & $F$ & V & $E$ & $\mathrm{~F}$ & V & $E$ & $\mathrm{~F}$ & V & $E$ & $\mathrm{~F}$ & V & $E$ & $\mathrm{~F}$ & $\mathrm{~V}$ & $E$ & $\mathrm{~F}$ & V & $E$ & $F$ & V & $E$ & $\mathrm{~F}$ & V & $E$ & $F$ \\
\hline & $\mathrm{E}$ & & $E$ & $\mathrm{E}$ & & $E$ & $\mathrm{E}$ & & $\mathrm{E}$ & $\mathrm{E}$ & & $\mathrm{E}$ & $\mathrm{E}$ & & $\mathrm{E}$ & $\mathrm{E}$ & & $\mathrm{E}$ & $\mathrm{E}$ & & $\mathrm{E}$ & $E$ & & $\mathrm{E}$ & $E$ & & $\mathrm{E}$ & $E$ & & $E$ & $E$ & & $E$ & $\mathrm{E}$ & & $\mathrm{E}$ \\
\hline C37.118.1 Annex C & $\mathrm{P}$ & $\mathrm{P}$ & $\mathrm{P}$ & $\mathrm{P}$ & $\mathrm{P}$ & $P$ & $\mathrm{P}$ & $P$ & $\mathrm{P}$ & $\mathrm{P}$ & $\mathrm{P}$ & $\mathrm{P}$ & $\mathrm{P}$ & $\mathrm{P}$ & $\mathrm{P}$ & $\mathrm{P}$ & $\mathrm{P}$ & $\mathrm{P}$ & $\mathrm{P}$ & $\mathrm{P}$ & $\mathrm{P}$ & $\mathrm{P}$ & $\mathrm{P}$ & $\mathrm{P}$ & $\mathrm{P}$ & $\mathrm{P}$ & $P$ & $\mathrm{P}$ & $\mathrm{P}$ & $\mathrm{P}$ & $\mathrm{P}$ & $\mathrm{P}$ & $\mathrm{P}$ & $P$ & $\mathrm{P}$ & $\mathrm{P}$ \\
\hline PMU A & $\mathrm{P}$ & $\mathrm{P}$ & $\mathrm{F}$ & $\mathrm{P}$ & $\mathrm{P}$ & $\mathrm{F}$ & $\mathrm{P}$ & $\mathrm{P}$ & $\mathrm{F}$ & $\mathrm{P}$ & $\mathrm{P}$ & $\mathrm{F}$ & $\mathrm{P}$ & $\mathrm{P}$ & $\mathrm{F}$ & $\mathrm{P}$ & $\mathrm{P}$ & $\mathrm{F}$ & $\mathrm{P}$ & $\mathrm{P}$ & $\mathrm{F}$ & $\mathrm{P}$ & $\mathrm{P}$ & $\mathrm{P}$ & $\mathrm{P}$ & $\mathrm{P}$ & $\mathrm{F}$ & $\mathrm{P}$ & $\mathrm{P}$ & $\mathrm{P}$ & $\mathrm{P}$ & $\mathrm{P}$ & $\mathrm{P}$ & $\mathrm{P}$ & $\mathrm{P}$ & $\mathrm{P}$ \\
\hline PMU B & $\mathrm{P}$ & $\mathrm{P}$ & $\mathrm{P}$ & $\mathrm{P}$ & $\mathrm{P}$ & $\mathrm{P}$ & $\mathrm{P}$ & $P$ & $\mathrm{P}$ & $\mathrm{P}$ & $P$ & $\mathrm{P}$ & $P$ & $\mathrm{~F}$ & $P$ & $\mathrm{P}$ & $\mathrm{P}$ & $P$ & $\mathrm{P}$ & $\mathrm{F}$ & $\mathrm{F}$ & $\mathrm{P}$ & $\mathrm{P}$ & $\mathrm{P}$ & $\mathrm{P}$ & $\mathrm{F}$ & $\mathrm{F}$ & $P$ & $P$ & $P$ & $\mathrm{P}$ & $\mathrm{F}$ & $\mathrm{F}$ & $P$ & $\mathrm{P}$ & $\mathrm{P}$ \\
\hline PMU C & $\mathrm{P}$ & $\mathrm{P}$ & $\mathrm{F}$ & $P$ & $\mathrm{P}$ & $P$ & $\mathrm{P}$ & $P$ & $\mathrm{~F}$ & $\mathrm{P}$ & $P$ & $\mathrm{P}$ & $P$ & $\mathrm{P}$ & $\mathrm{F}$ & $\mathrm{P}$ & $\mathrm{P}$ & $P$ & $\mathrm{P}$ & $\mathrm{P}$ & $\mathrm{F}$ & $\mathrm{P}$ & $\mathrm{P}$ & $\mathrm{P}$ & $\mathrm{P}$ & $\mathrm{P}$ & $\mathrm{F}$ & $P$ & $\mathrm{P}$ & $\mathrm{P}$ & $P$ & $\mathrm{P}$ & $P$ & $P$ & $\mathrm{P}$ & $\mathrm{P}$ \\
\hline PMU D & $\mathrm{P}$ & $\mathrm{P}$ & $\mathrm{P}$ & $\mathrm{P}$ & $\mathrm{P}$ & $\mathrm{P}$ & $\mathrm{P}$ & $\mathrm{P}$ & $\mathrm{P}$ & $\mathrm{P}$ & $\mathrm{P}$ & $\mathrm{P}$ & $\mathrm{P}$ & $\mathrm{P}$ & $\mathrm{P}$ & $\mathrm{P}$ & $\mathrm{P}$ & $\mathrm{P}$ & $\mathrm{P}$ & $\mathrm{P}$ & $\mathrm{P}$ & $\mathrm{P}$ & $\mathrm{P}$ & $\mathrm{P}$ & $\mathrm{P}$ & $\mathrm{P}$ & $P$ & $\mathrm{P}$ & $\mathrm{P}$ & $P$ & $\mathrm{P}$ & $\mathrm{P}$ & $\mathrm{P}$ & $\mathrm{P}$ & $\mathrm{P}$ & $\mathrm{P}$ \\
\hline PMU E & $\mathrm{P}$ & $\mathrm{I}$ & $\mathrm{P}$ & - & - & - & - & - & - & - & - & - & $\mathrm{P}$ & $\mathrm{F}$ & $\mathrm{F}$ & - & - & - & $\mathrm{F}$ & $\mathrm{F}$ & $\mathrm{F}$ & - & - & - & $\mathrm{F}$ & $\mathrm{F}$ & $\mathrm{F}$ & - & - & - & $\mathrm{F}$ & $\mathrm{F}$ & $\mathrm{F}$ & - & - & - \\
\hline PMU F & $\mathrm{P}$ & $\mathrm{P}$ & $\mathrm{F}$ & $\mathrm{P}$ & $\mathrm{P}$ & $\mathrm{P}$ & $\mathrm{P}$ & $\mathrm{P}$ & $\mathrm{F}$ & $\mathrm{P}$ & $\mathrm{P}$ & $\mathrm{P}$ & $\mathrm{F}$ & $\mathrm{P}$ & $\mathrm{F}$ & $\mathrm{P}$ & $\mathrm{P}$ & $\mathrm{P}$ & $\mathrm{P}$ & $\mathrm{P}$ & $\mathrm{P}$ & $\mathrm{P}$ & $\mathrm{P}$ & $\mathrm{P}$ & $\mathrm{P}$ & $\mathrm{P}$ & $\mathrm{P}$ & $\mathrm{P}$ & $\mathrm{P}$ & $\mathrm{P}$ & $\mathrm{P}$ & $\mathrm{P}$ & $\mathrm{P}$ & $\mathrm{P}$ & $\mathrm{P}$ & $\mathrm{P}$ \\
\hline PMU G* & $\mathrm{F}$ & $\mathrm{F}$ & $\mathrm{F}$ & - & - & - & $\mathrm{F}$ & $\mathrm{F}$ & $\mathrm{F}$ & - & - & - & $\mathrm{F}$ & $\mathrm{F}$ & $\mathrm{F}$ & - & - & - & $\mathrm{F}$ & $\mathrm{F}$ & $\mathrm{F}$ & - & - & - & $\mathrm{F}$ & $\mathrm{F}$ & $P$ & - & - & - & - & - & - & - & - & - \\
\hline PMU H & $\mathrm{P}$ & $\mathrm{P}$ & $\mathrm{P}$ & $\mathrm{P}$ & $\mathrm{P}$ & $\mathrm{P}$ & $\mathrm{P}$ & $\mathrm{P}$ & $\mathrm{P}$ & $\mathrm{P}$ & $\mathrm{P}$ & $\mathrm{P}$ & $\mathrm{P}$ & $\mathrm{P}$ & $\mathrm{P}$ & $\mathrm{P}$ & $\mathrm{P}$ & $\mathrm{P}$ & $\mathrm{P}$ & $\mathrm{P}$ & $\mathrm{P}$ & $\mathrm{P}$ & $\mathrm{P}$ & $\mathrm{P}$ & $\mathrm{P}$ & $\mathrm{P}$ & $\mathrm{P}$ & $\mathrm{P}$ & $\mathrm{P}$ & $\mathrm{P}$ & $\mathrm{P}$ & $\mathrm{P}$ & $\mathrm{P}$ & $\mathrm{P}$ & $\mathrm{P}$ & $\mathrm{P}$ \\
\hline PMU I & $\mathrm{F}$ & $\mathrm{I}$ & 1 & - & - & - & $\mathrm{F}$ & 1 & 1 & - & - & - & $\mathrm{F}$ & I & I & - & - & - & $\mathrm{F}$ & I & I & - & - & - & $\mathrm{F}$ & $\mathrm{P}$ & 1 & - & - & - & $\mathrm{P}$ & $\mathrm{P}$ & $\mathrm{P}$ & - & - & - \\
\hline PMU J & $\mathrm{P}$ & $\mathrm{P}$ & $P$ & $\mathrm{P}$ & $\mathrm{P}$ & $\mathrm{P}$ & $\mathrm{P}$ & $\mathrm{P}$ & $\mathrm{P}$ & $\mathrm{P}$ & $\mathrm{P}$ & $\mathrm{P}$ & $\mathrm{P}$ & $\mathrm{P}$ & $\mathrm{P}$ & $\mathrm{P}$ & $\mathrm{P}$ & $\mathrm{P}$ & $\mathrm{P}$ & $\mathrm{P}$ & $\mathrm{P}$ & $\mathrm{P}$ & $\mathrm{P}$ & $\mathrm{P}$ & $\mathrm{P}$ & $\mathrm{P}$ & $\mathrm{P}$ & $\mathrm{P}$ & $\mathrm{P}$ & $P$ & $\mathrm{P}$ & $\mathrm{P}$ & $P$ & $\mathrm{P}$ & $\mathrm{P}$ & $\mathrm{P}$ \\
\hline
\end{tabular}

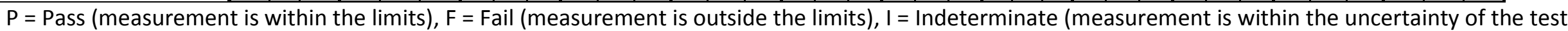
instrument of the limits)

* PMU G ROCOF output is always 0. 


\subsection{Phase modulation voltage TVE, M class}

\subsubsection{C37.118.1 Annex C phase modulation voltage TVE: M class}

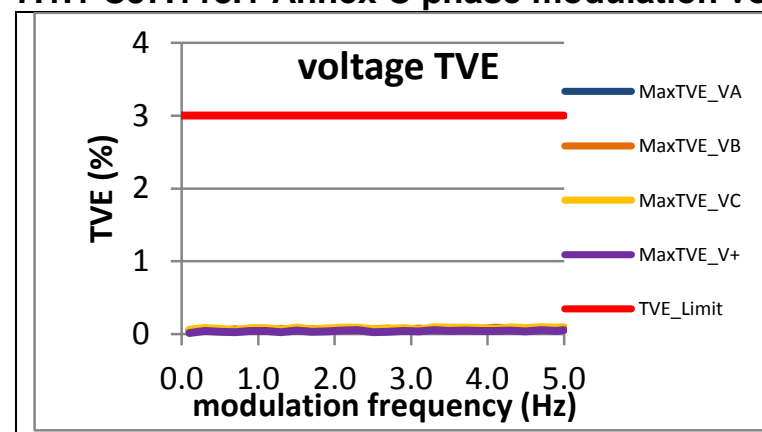

Figure 3051: Fs = 60 FPS

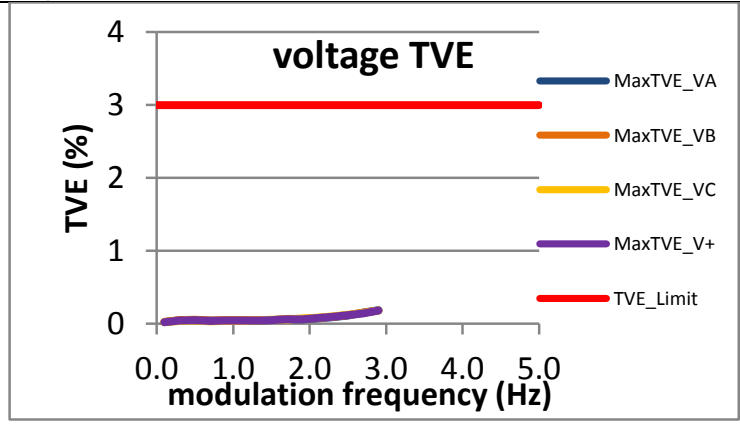

Figure 3054: Fs = 15 FPS

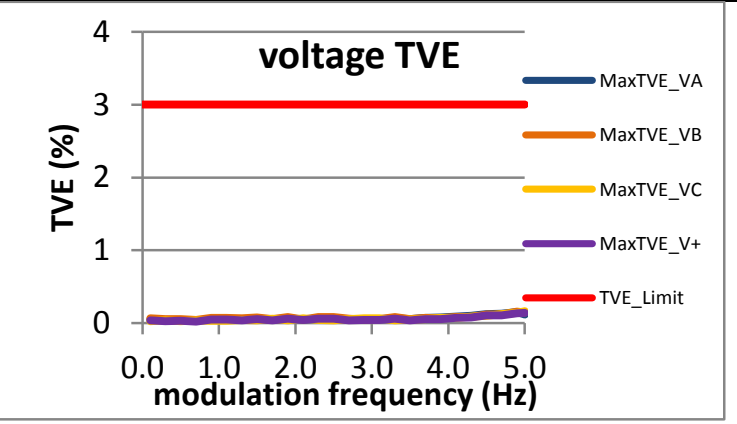

Figure 3052: Fs = 30 FPS

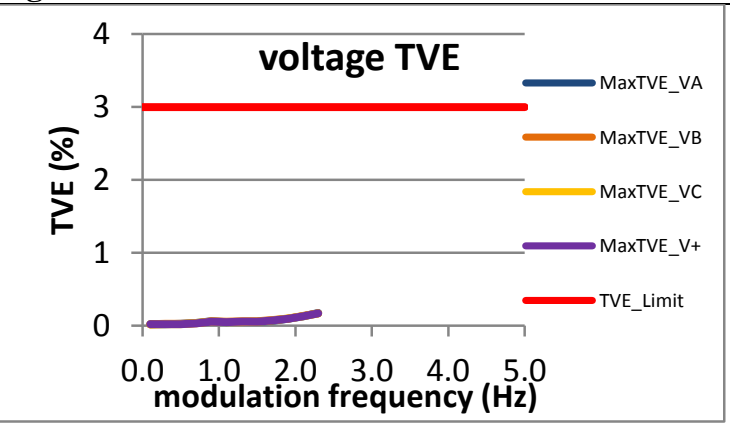

Figure 3055: Fs = 12 FPS

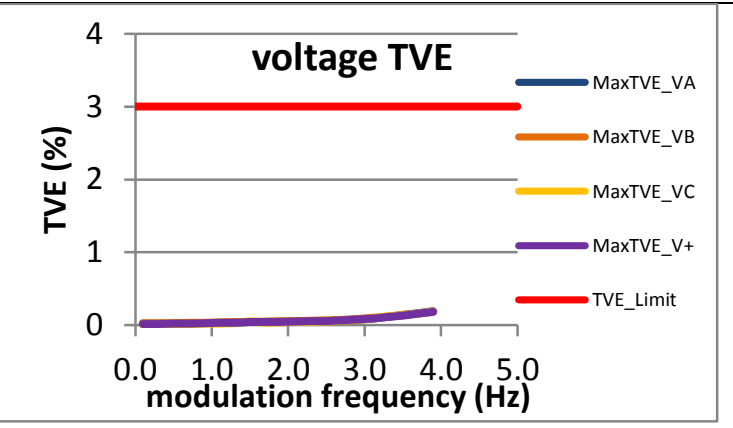

Figure 3053: Fs = 20 FPS

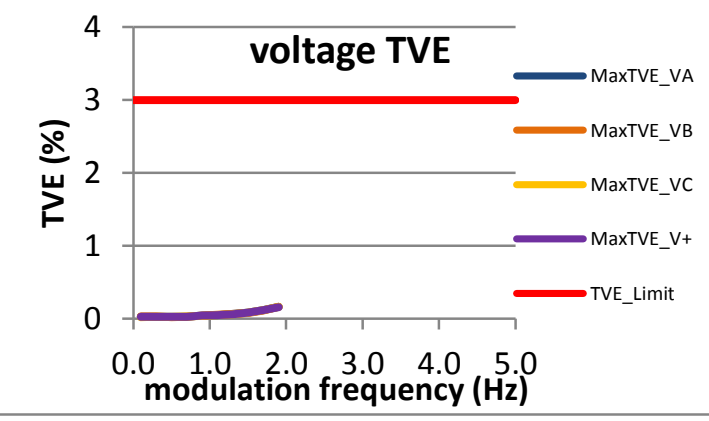

Figure 3056: Fs = 10 FPS 
7.1.2 PMU A phase modulation voltage TVE: M class
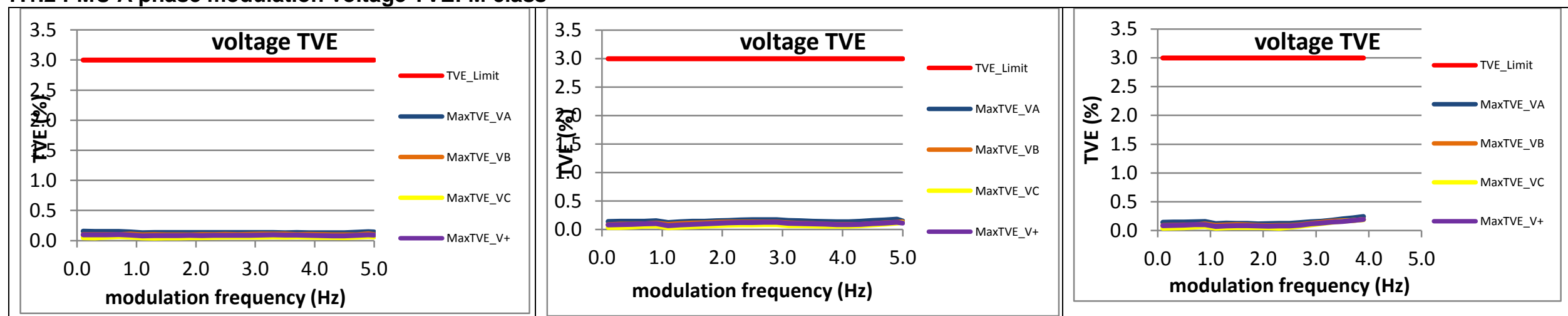

Figure 3057: Fs = 60 FPS

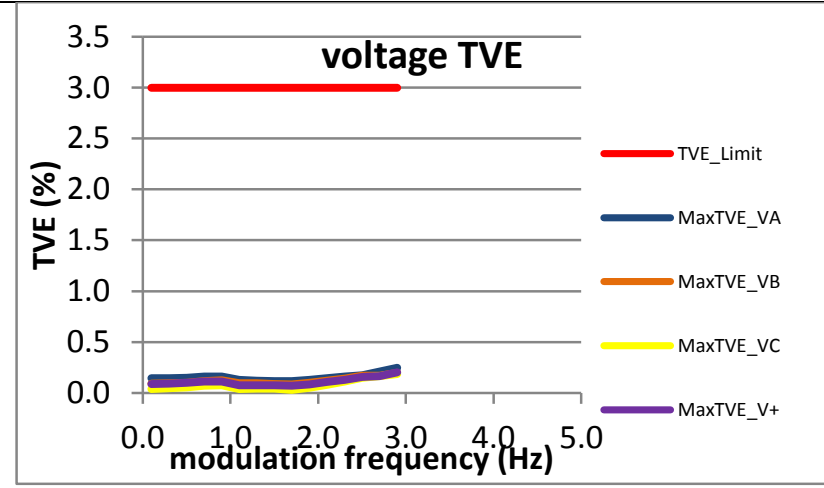

Figure 3058: Fs = 30 FPS

Figure 3059: Fs = 20 FPS

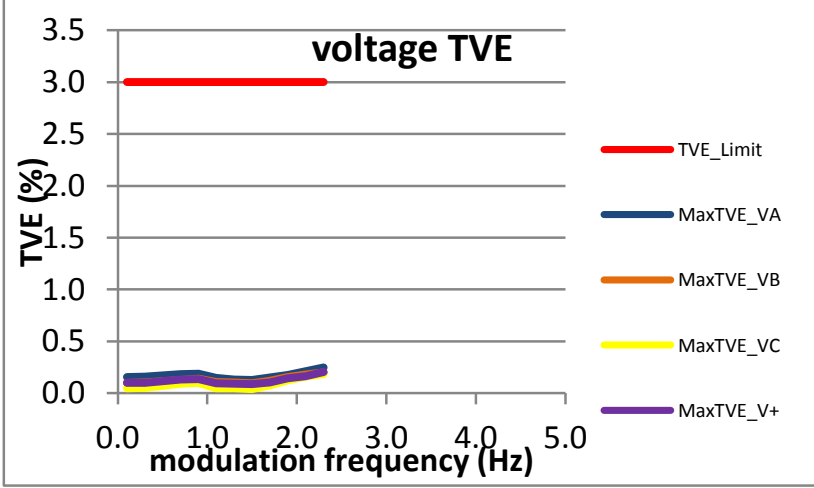

Figure 3061: Fs = 12 FPS

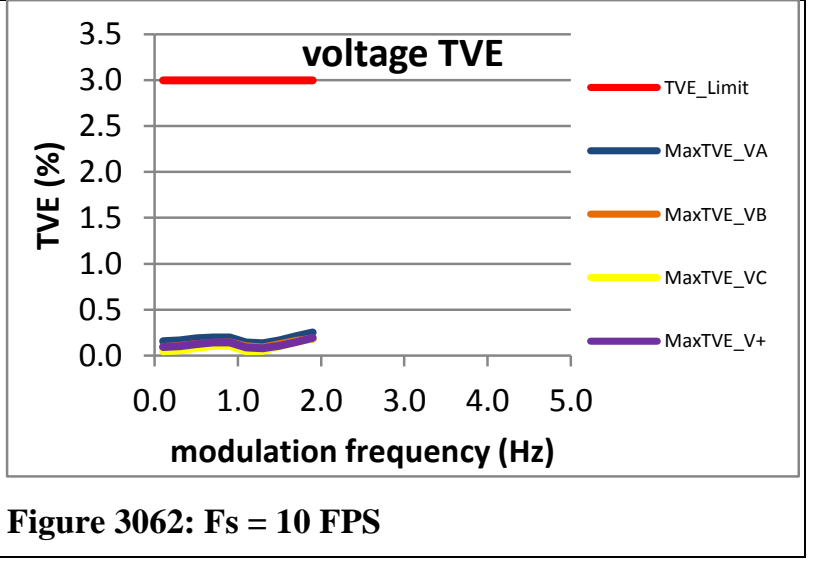


7.1.3 PMU B phase modulation voltage TVE: M class

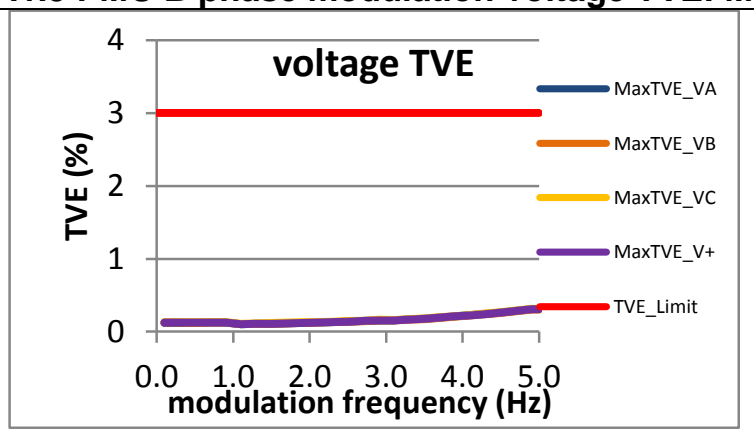

Figure 3063: Fs = 60 FPS

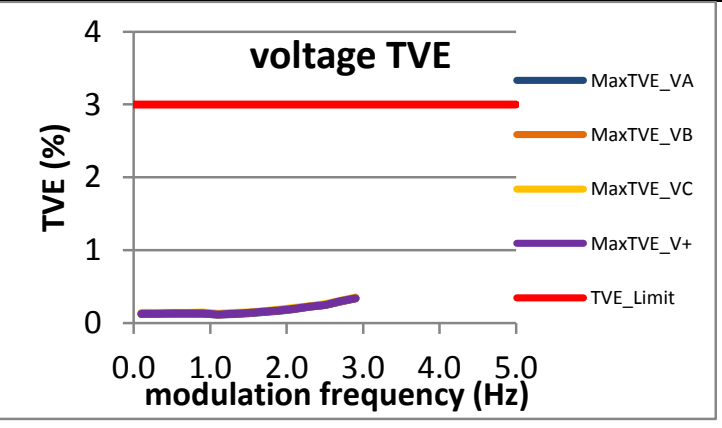

Figure 3066: Fs = 15 FPS

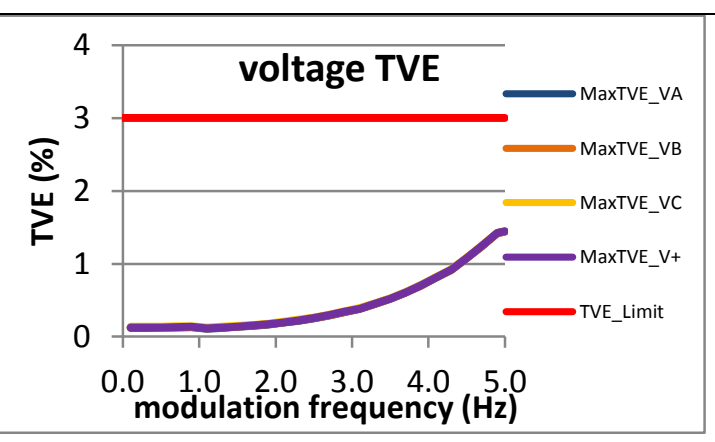

Figure 3064: Fs = 30 FPS

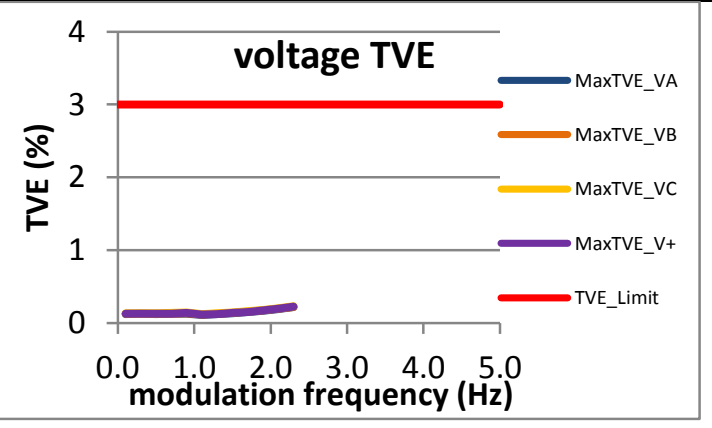

Figure 3067: Fs = 12 FPS

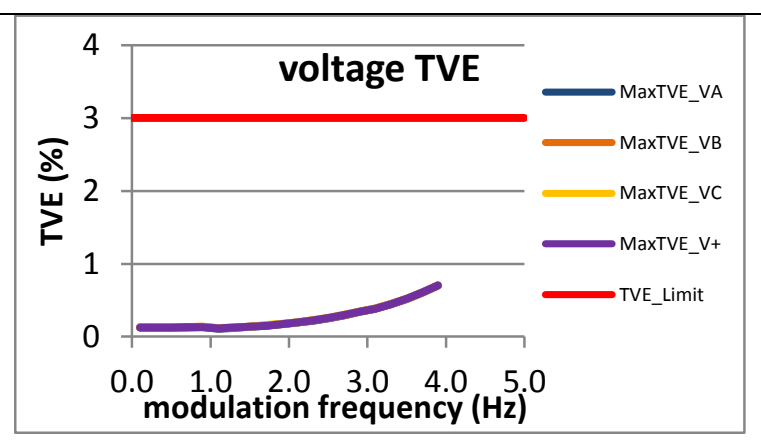

Figure 3065: Fs = 20 FPS

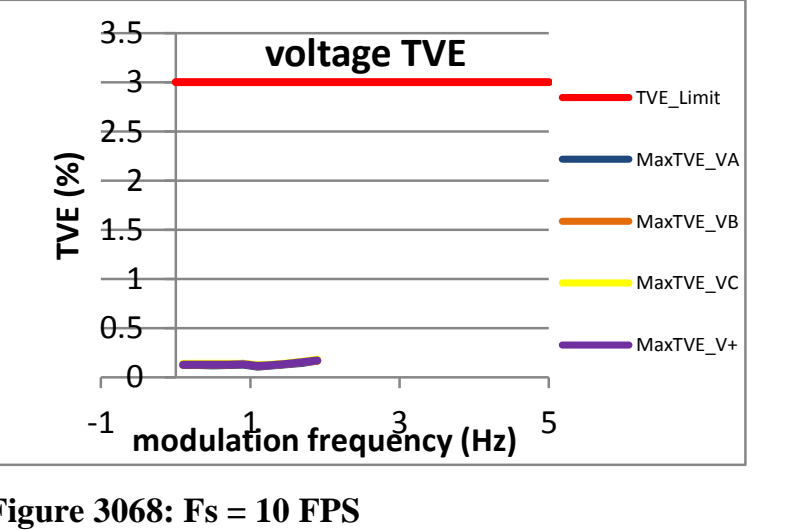




\subsubsection{PMU C phase modulation voltage TVE: M class}

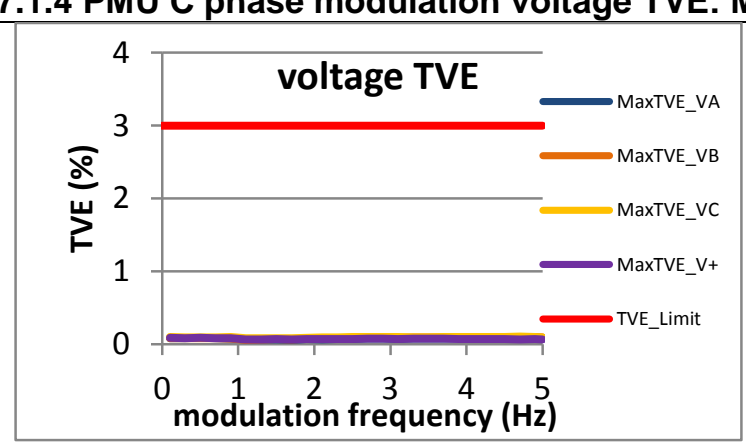

Figure 3069: Fs = 60 FPS

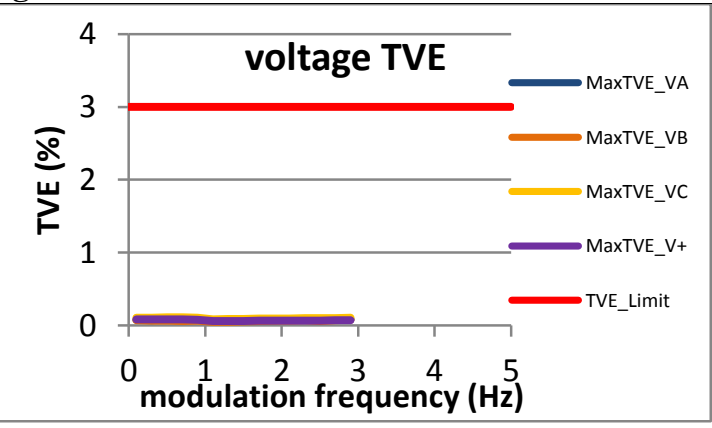

Figure 3072: Fs = 15 FPS

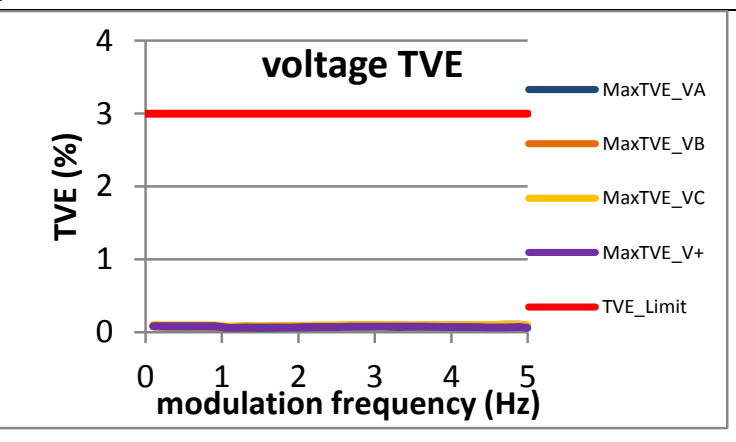

Figure 3070: Fs = 30 FPS

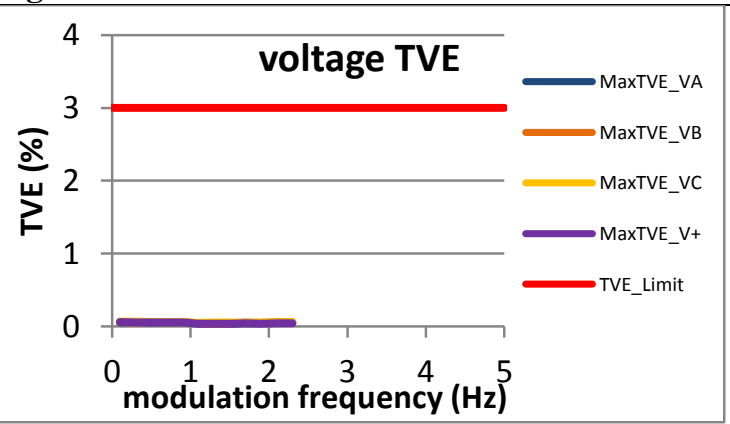

Figure 3073: Fs = 12 FPS

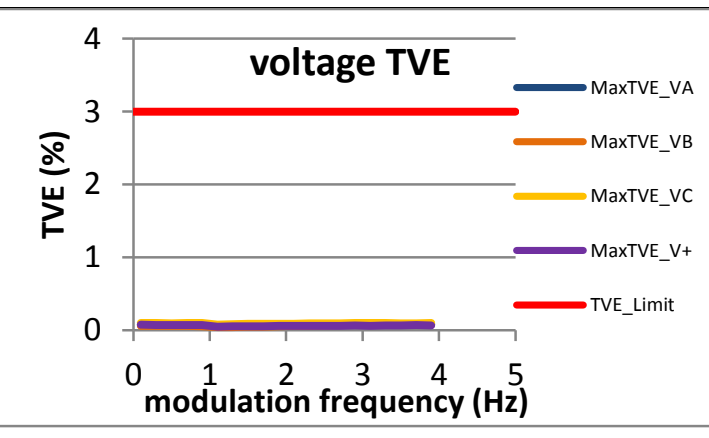

Figure 3071: Fs = 20 FPS

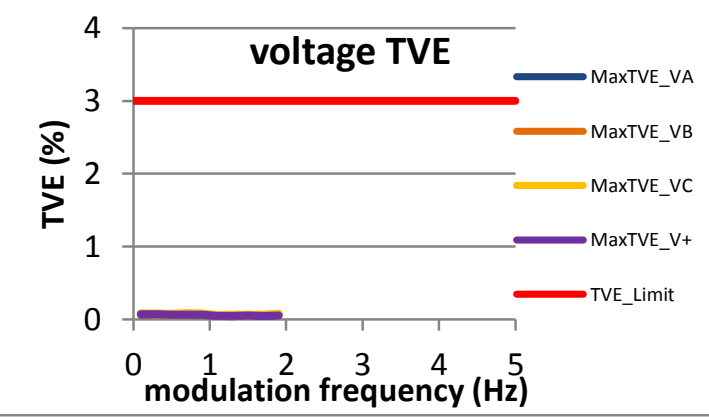

Figure 3074: Fs = 10 FPS 


\subsubsection{PMU D phase modulation voltage TVE: M class}

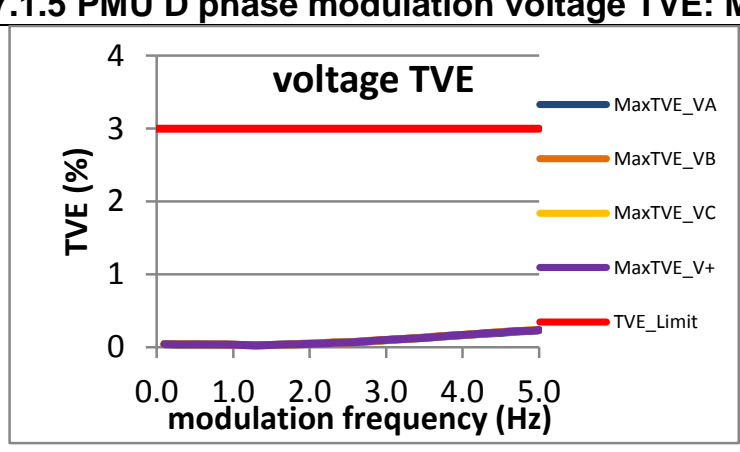

Figure 3075: Fs = 60 FPS

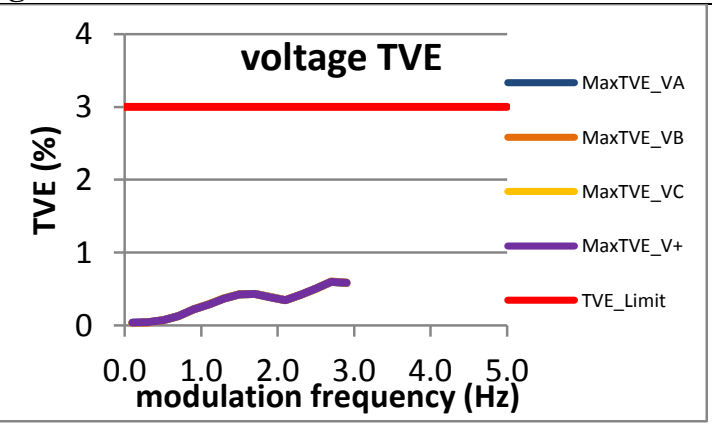

Figure 3078: Fs = 15 FPS

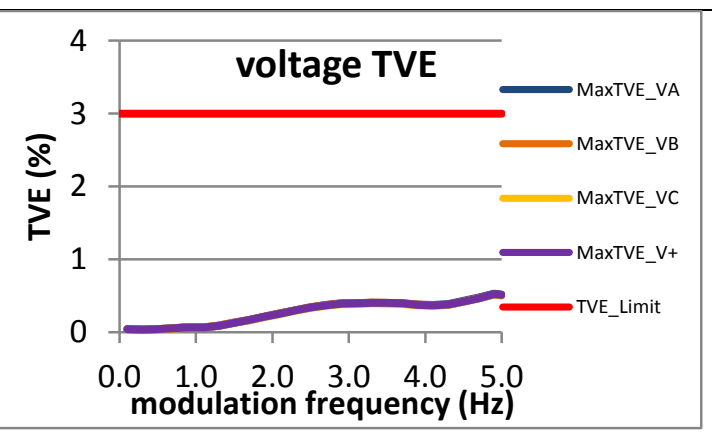

Figure 3076: Fs = 30 FPS

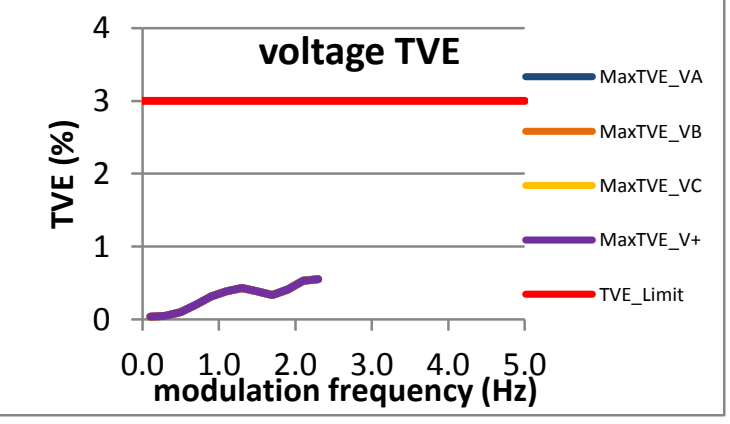

Figure 3079: Fs = 12FPS

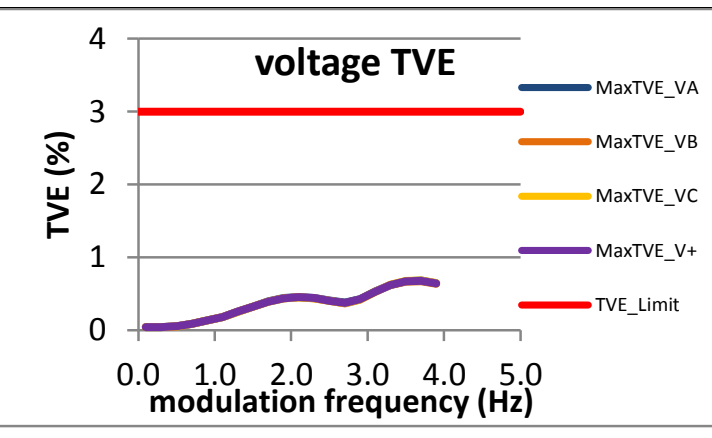

Figure 3077: Fs = 20 FPS

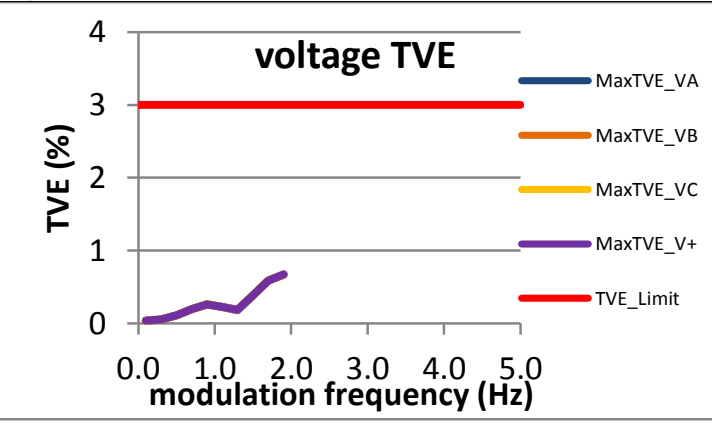

Figure 3080: Fs = 10 FPS 
7.1.6 PMU E phase modulation voltage TVE: M class

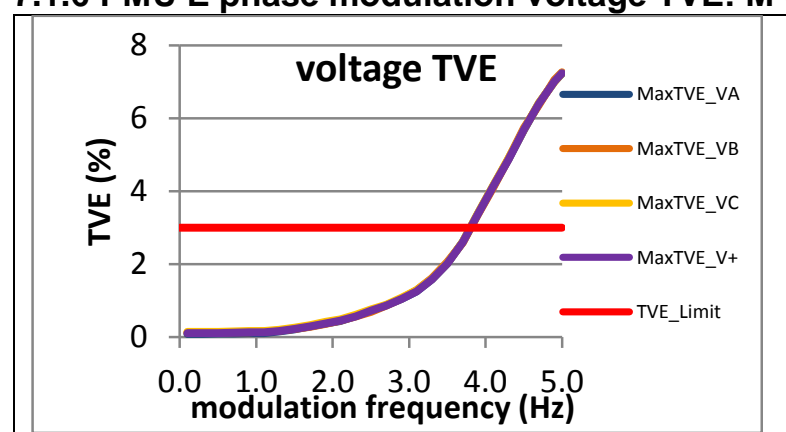

Figure 3081: Fs = 60 FPS

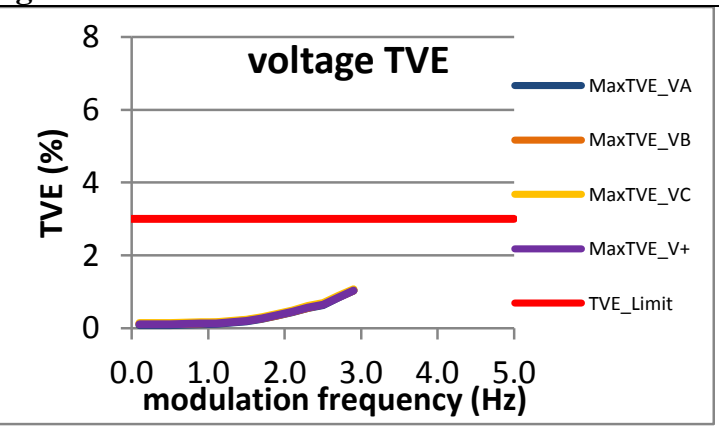

Figure 3084: Fs = 15 FPS

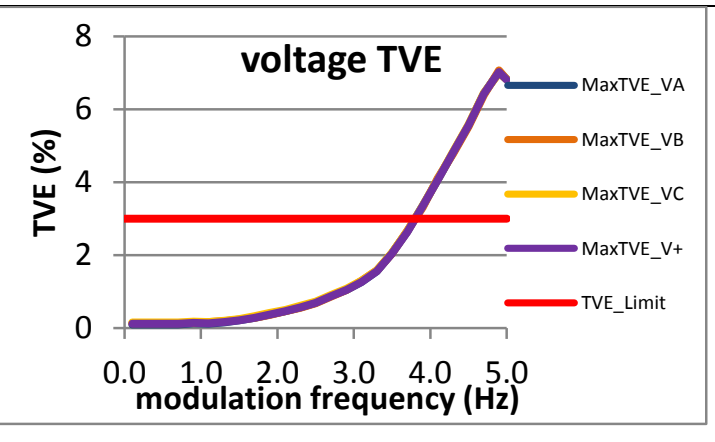

Figure 3082: Fs = 30 FPS MISSING DATA

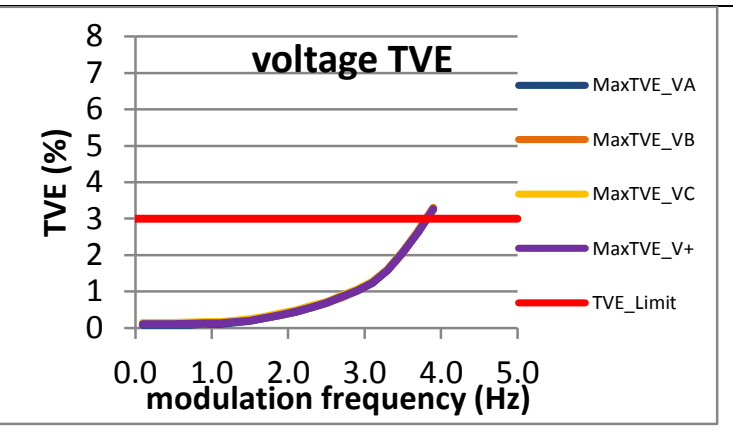

Figure 3083: Fs = 20 FPS

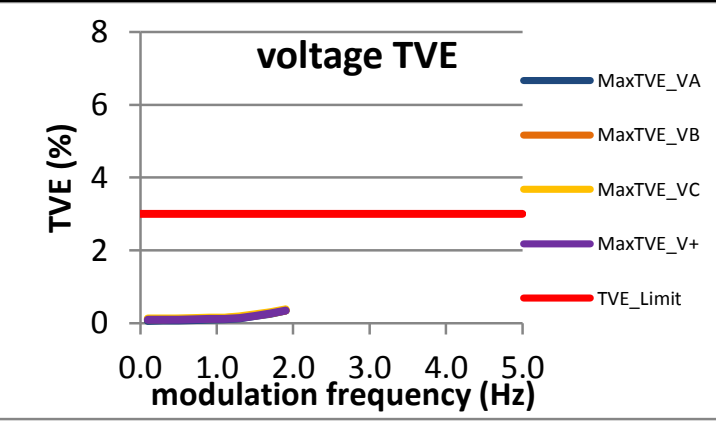

Figure 3085: Fs = 10 FPS 
7.1.7 PMU F phase modulation voltage TVE: M class

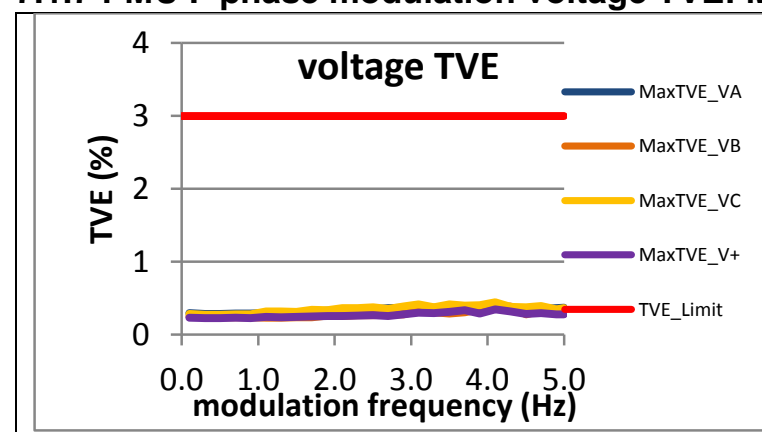

Figure 3086: Fs $=60$ FPS

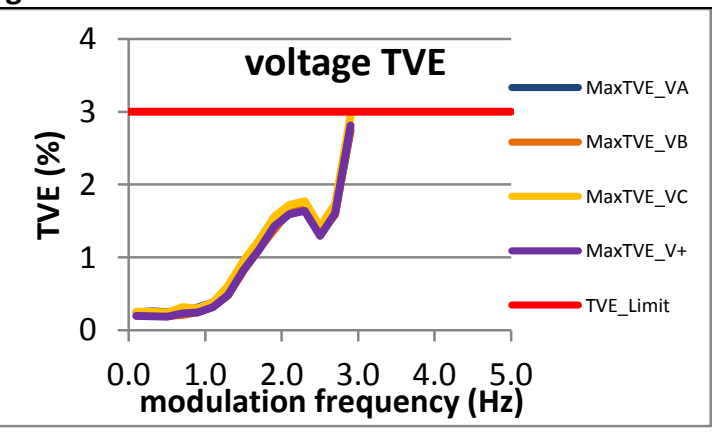

Figure 3089: Fs = 15 FPS

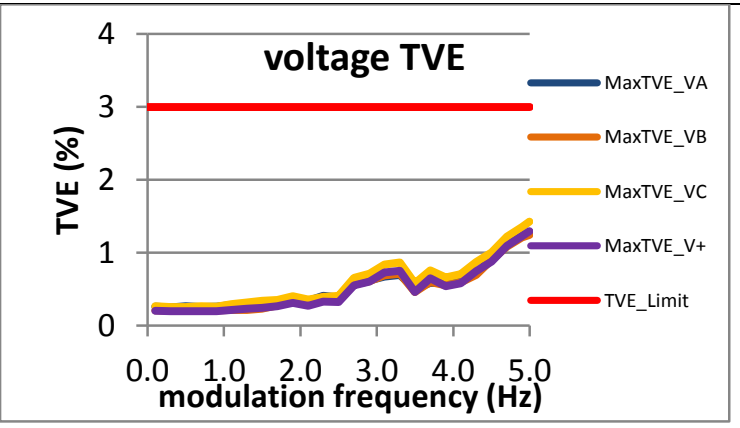

Figure 3087: Fs $=30 \mathrm{FPS}$

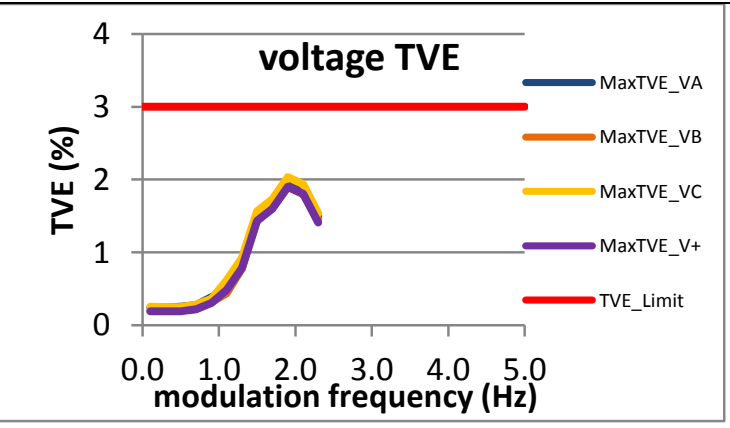

Figure 3090: Fs =12 FPS

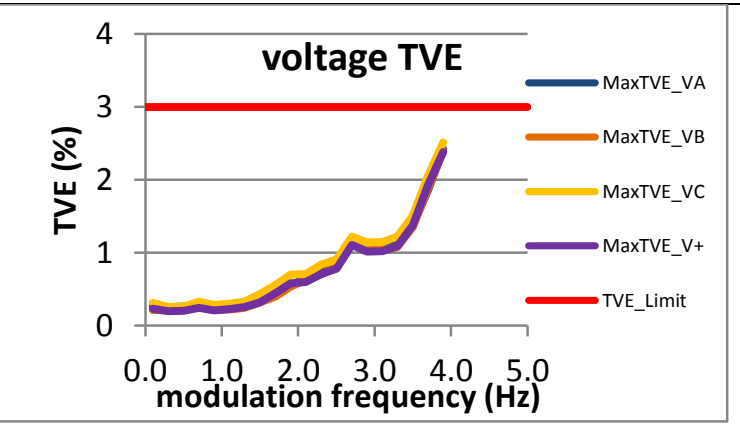

Figure 3088: $\mathrm{Fs}=20 \mathrm{FPS}$

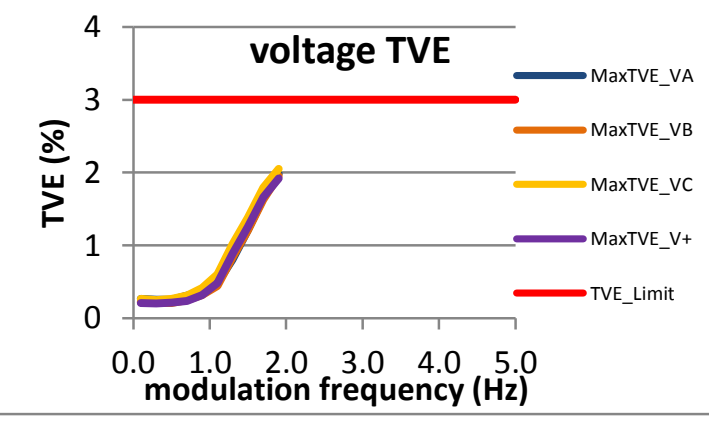

Figure 3091: Fs = 10 FPS 
7.1.8 PMU G phase modulation voltage TVE: M class

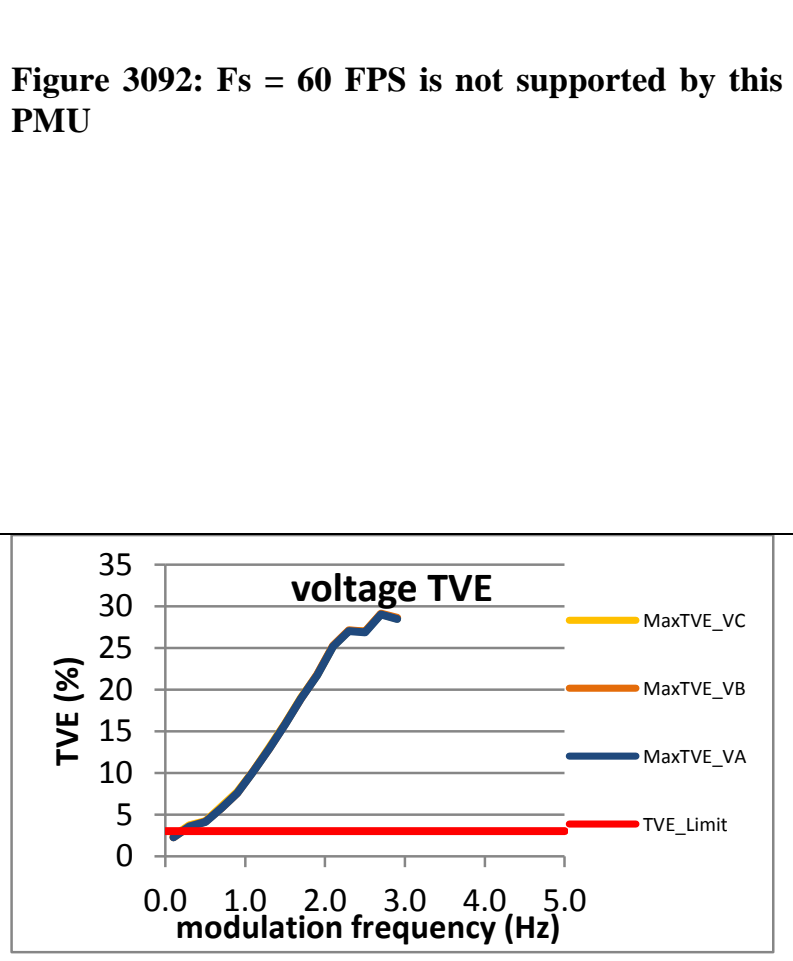

Figure 3095: Fs = 15 FPS

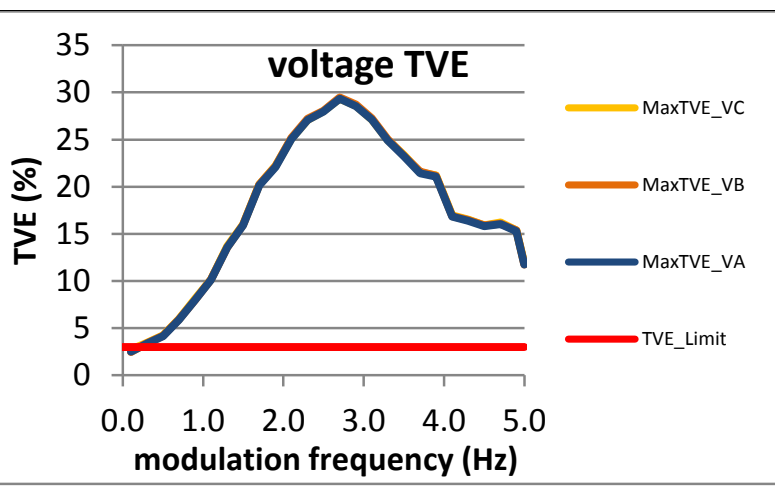

Figure 3093: Fs = 30 FPS

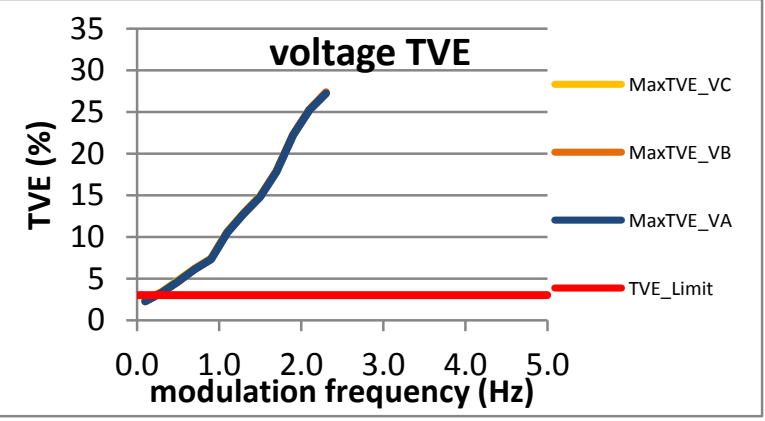

Figure 3096: Fs = 12 FPS

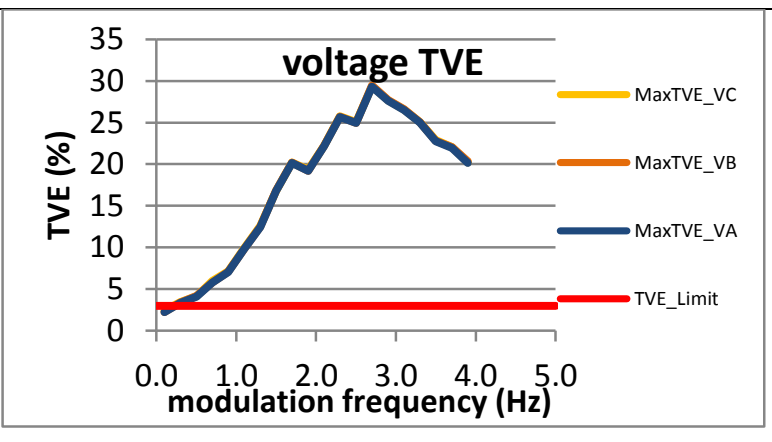

Figure 3094: Fs = 20 FPS

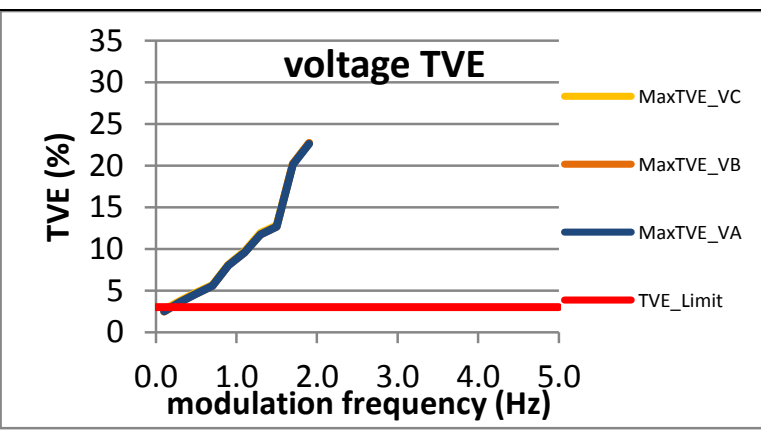

Figure 3097: Fs = 10 FPS 


\subsubsection{PMU H phase modulation voltage TVE: M class}

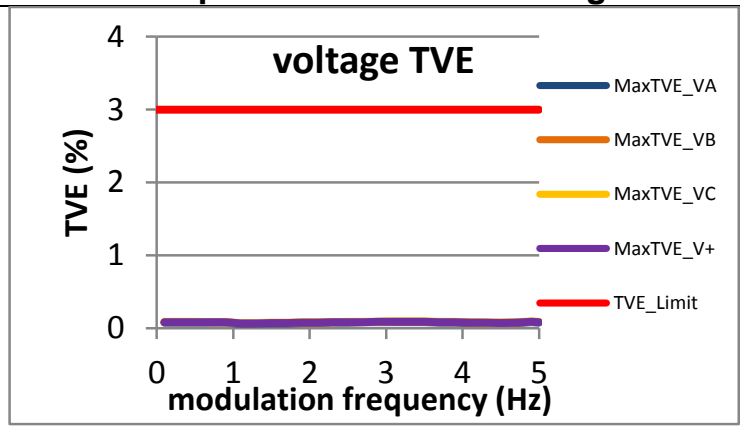

Figure 3098: Fs = 60 FPS

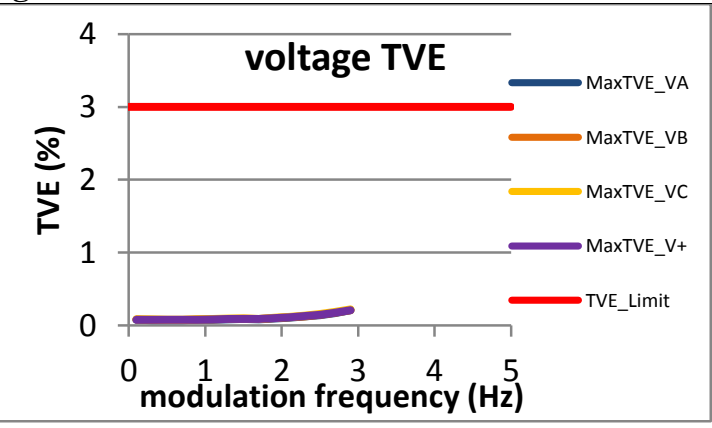

Figure 3101: Fs = 15 FPS

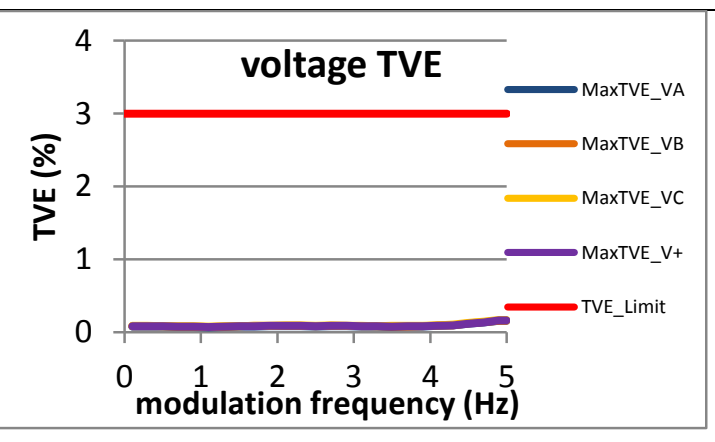

Figure 3099: Fs = 30 FPS

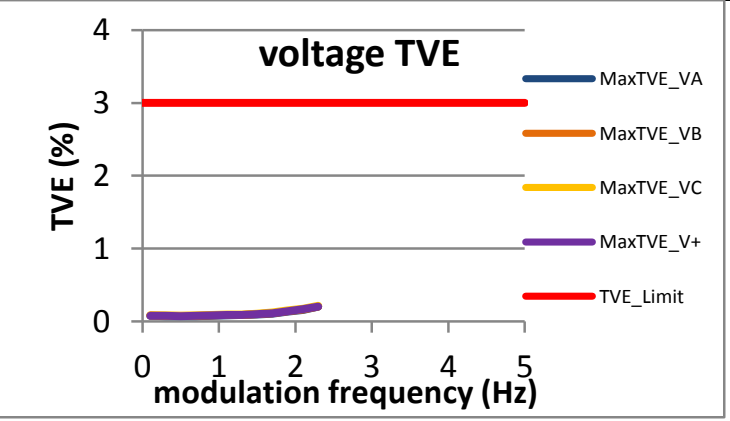

Figure 3102: Fs = 12 FPS

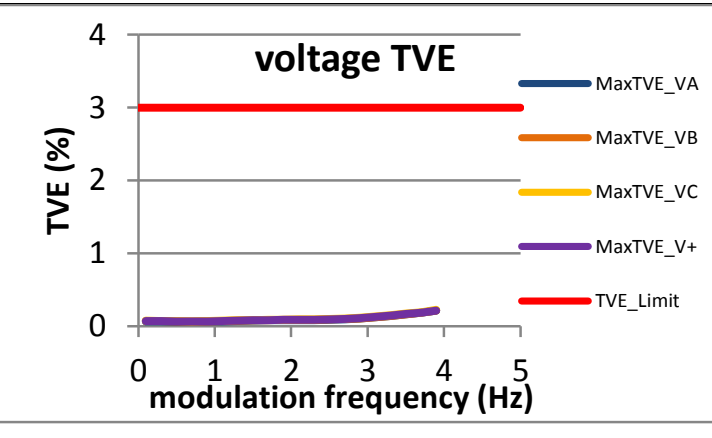

Figure 3100: Fs = 20 FPS

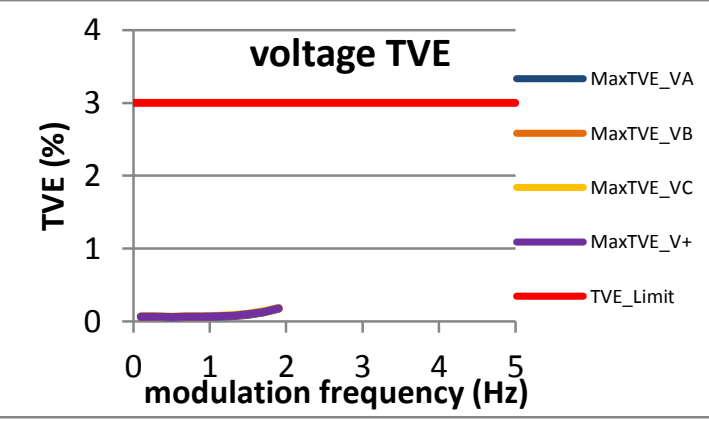

Figure 3103: Fs = 10 FPS 
7.1.10 PMU I phase modulation voltage TVE: M class

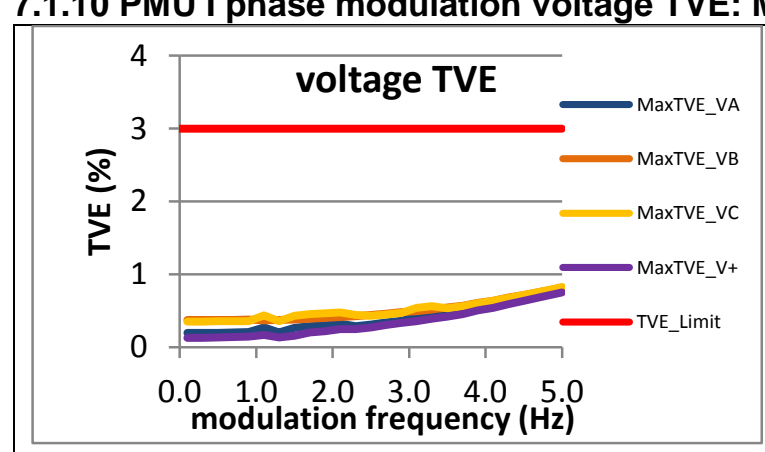

Figure 3104: Fs = 60 FPS

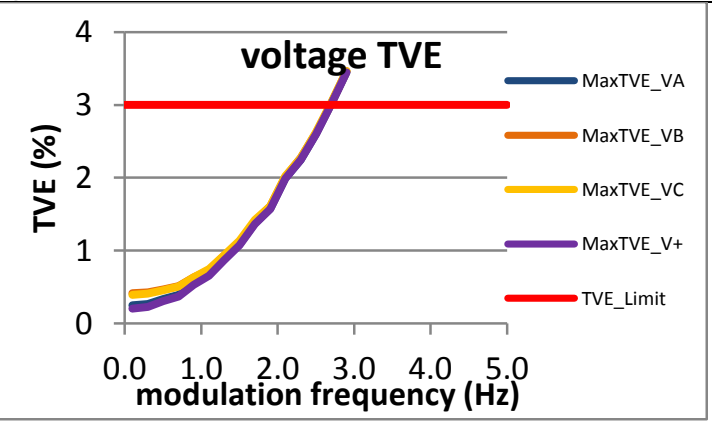

Figure 3107: Fs = 15 FPS

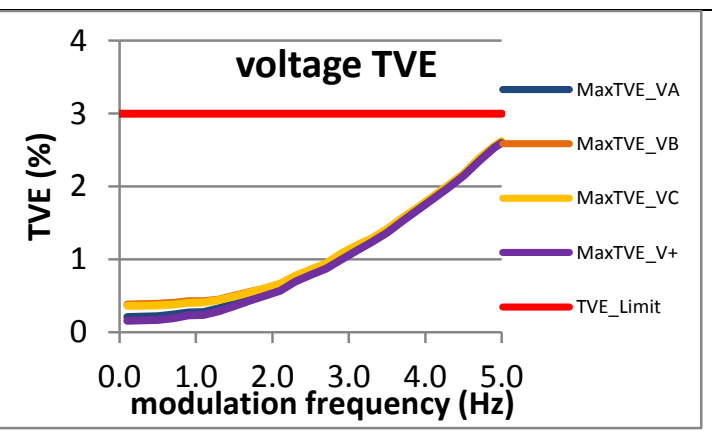

Figure 3105: Fs = 30 FPS

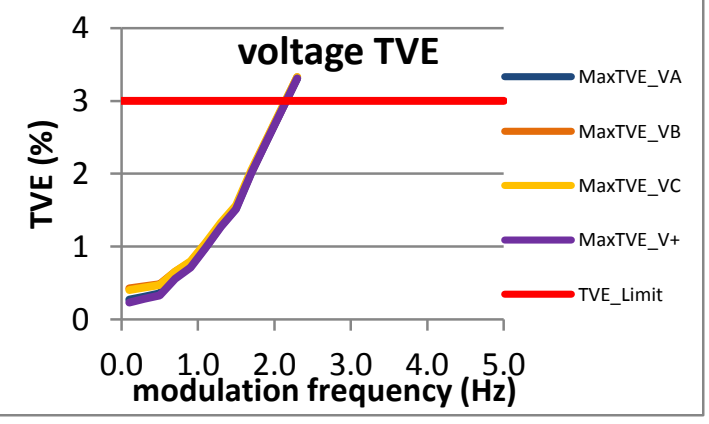

Figure 3108: Fs = 12 FPS

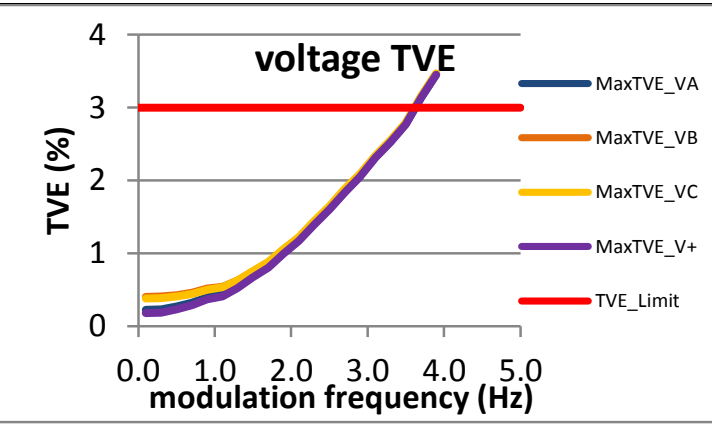

Figure 3106: Fs = 20 FPS

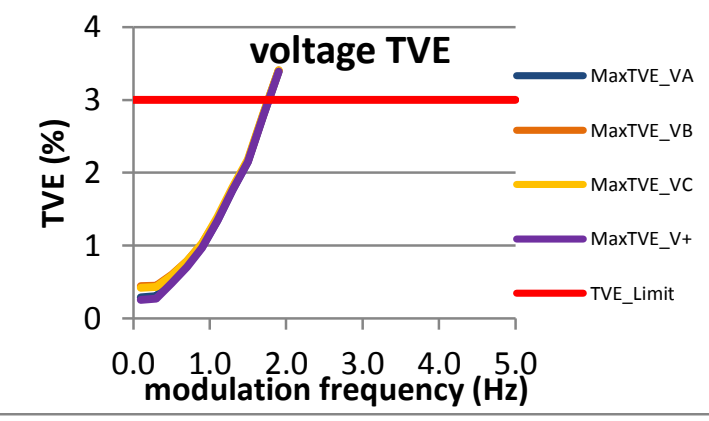

Figure 3109: Fs = 10 FPS 
7.1.11 PMU J phase modulation voltage TVE: M class

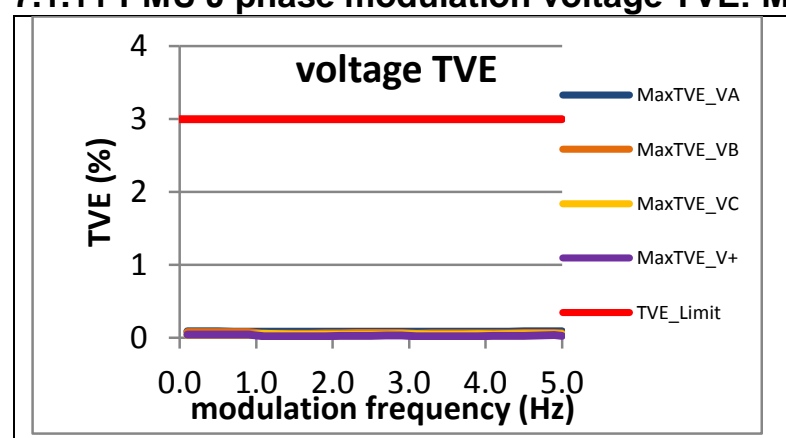

Figure 3110: Fs = 60 FPS

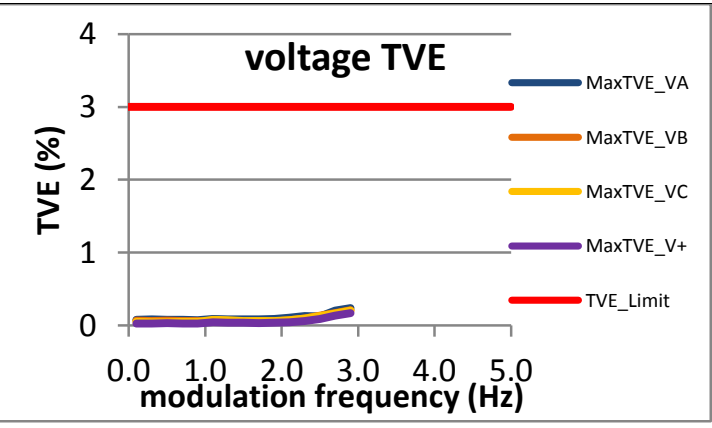

Figure 3113: Fs = 15 FPS

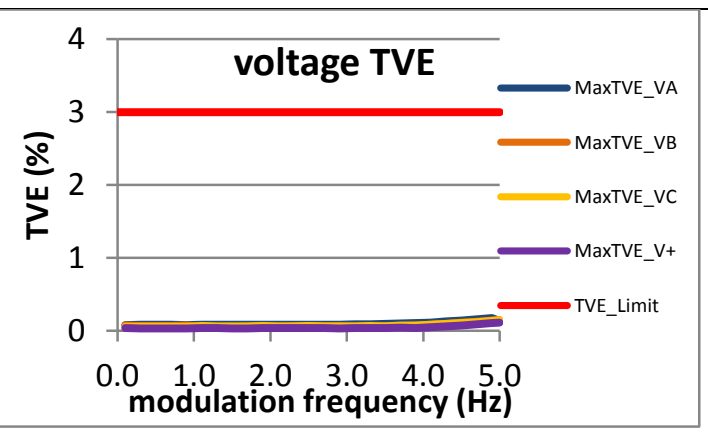

Figure 3111: Fs = 30 FPS

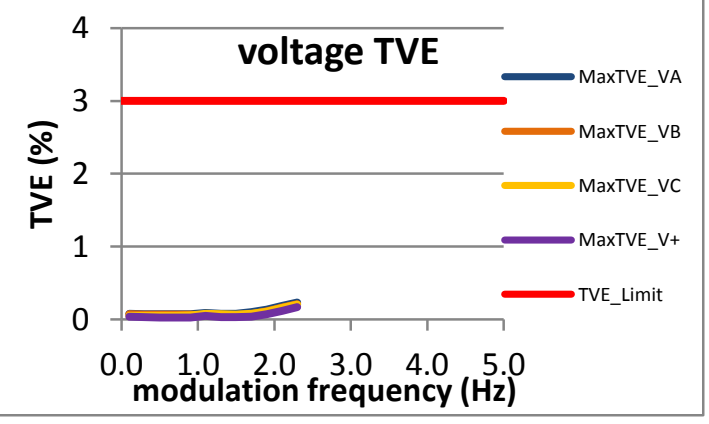

Figure 3114: Fs =12 FPS

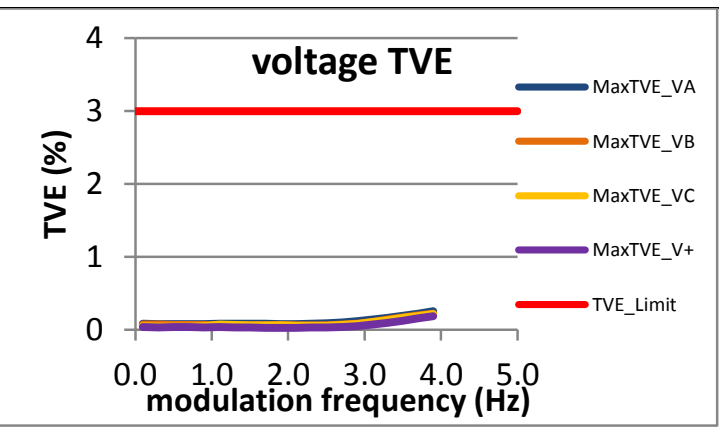

Figure 3112: Fs = 20 FPS

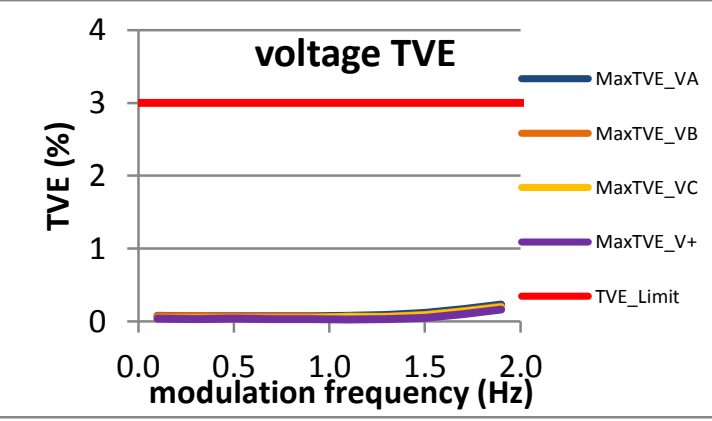

Figure 3115: Fs = $10 \mathrm{FPS}$ 


\subsection{Phase modulation current TVE, M class}

\subsubsection{C37.118.1 Annex C phase modulation current TVE: M class}

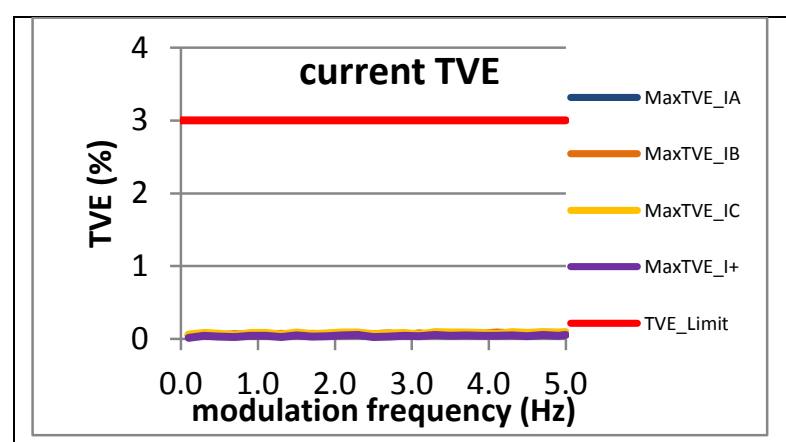

Figure 3116: Fs = 60 FPS

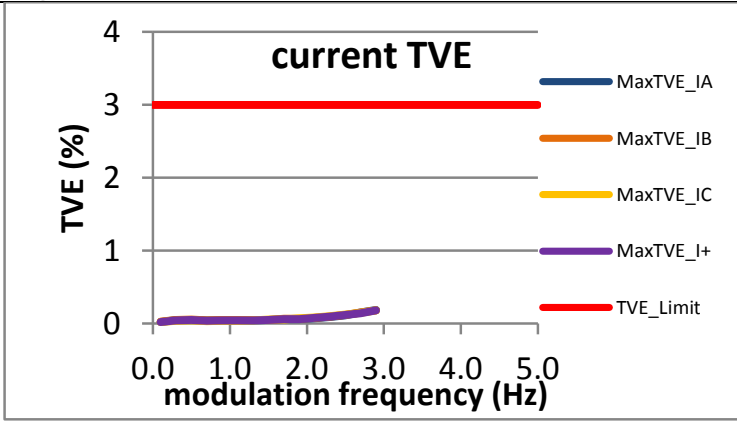

Figure 3119: Fs = 15 FPS

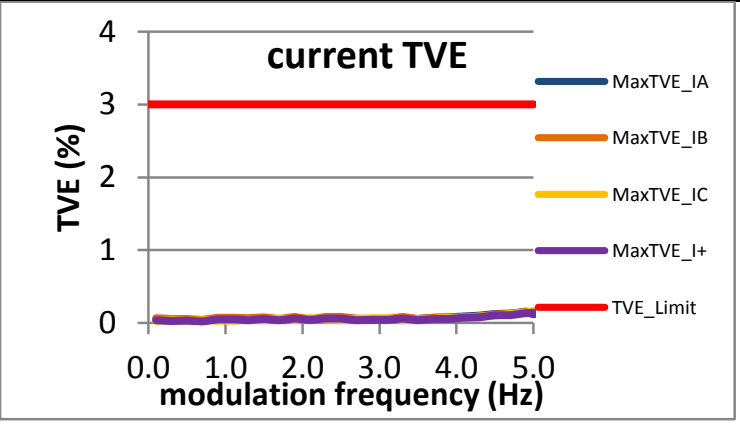

Figure 3117: Fs = 30 FPS

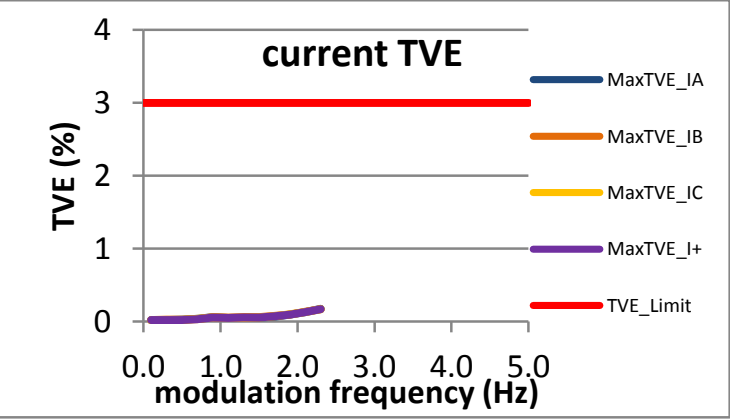

Figure 3120: Fs = 12 FPS

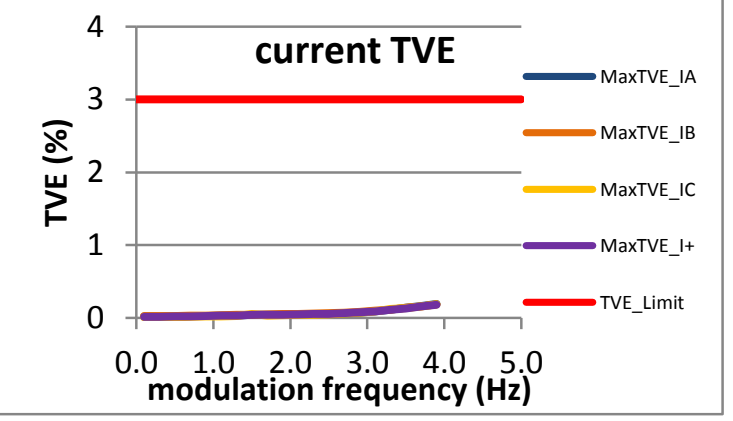

Figure 3118: Fs = 20 FPS

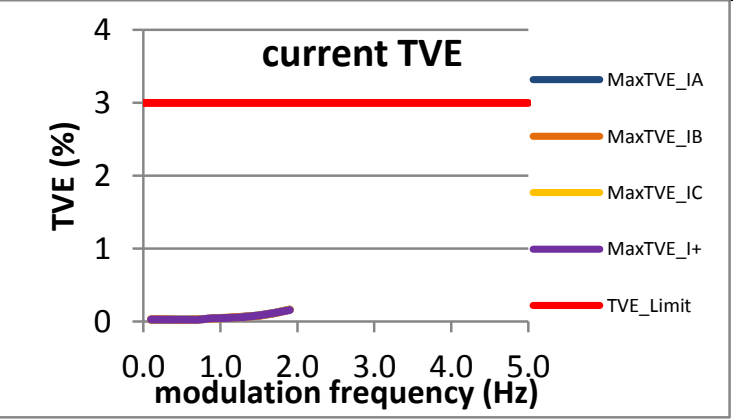

Figure 3121: Fs = 10 FPS 


\subsubsection{PMU A phase modulation current TVE: M class}

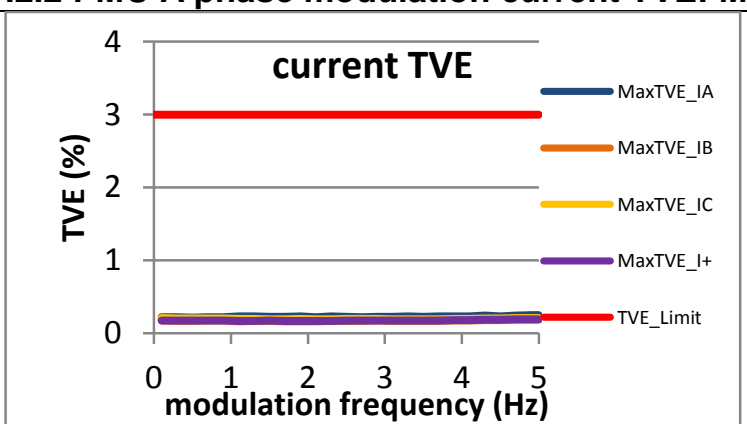

Figure 3122: Fs = 60 FPS

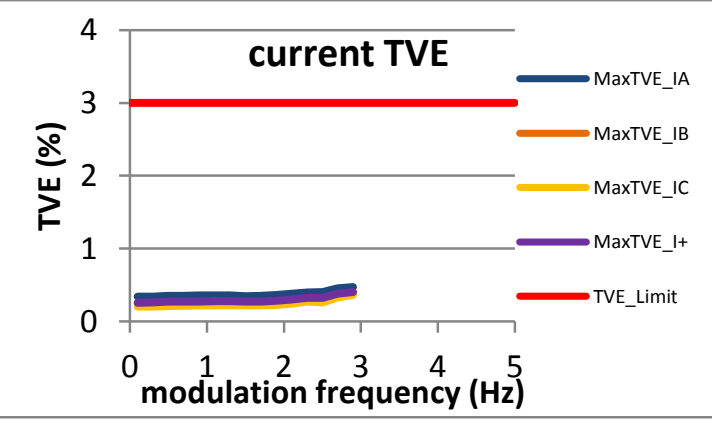

Figure 3125: Fs = 15 FPS

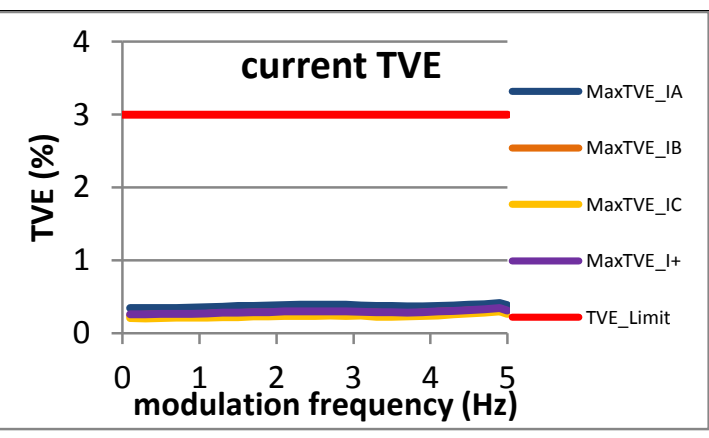

Figure 3123: Fs = 30 FPS

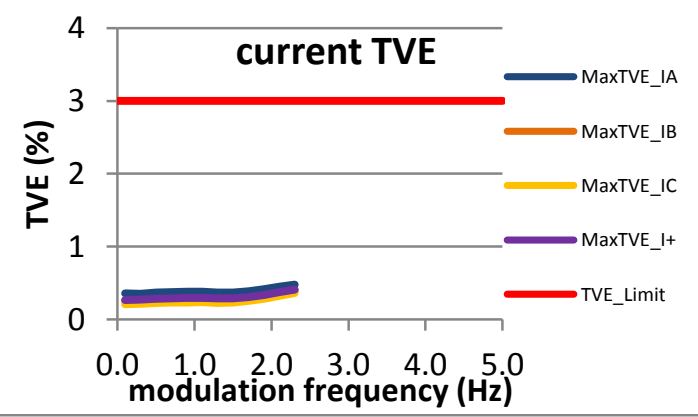

Figure 3126: Fs = 12FPS

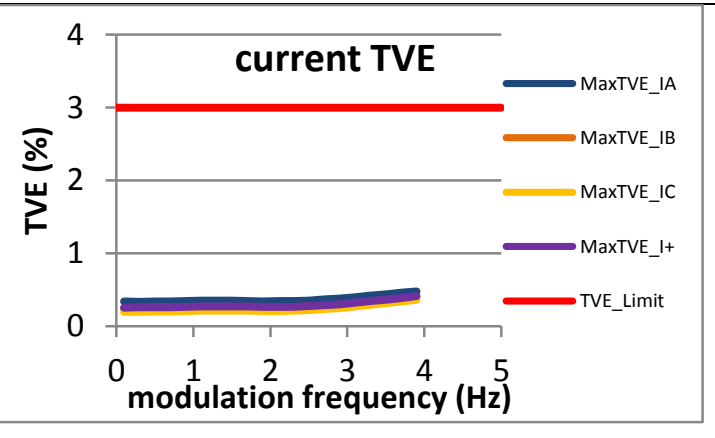

Figure 3124: Fs = 20 FPS

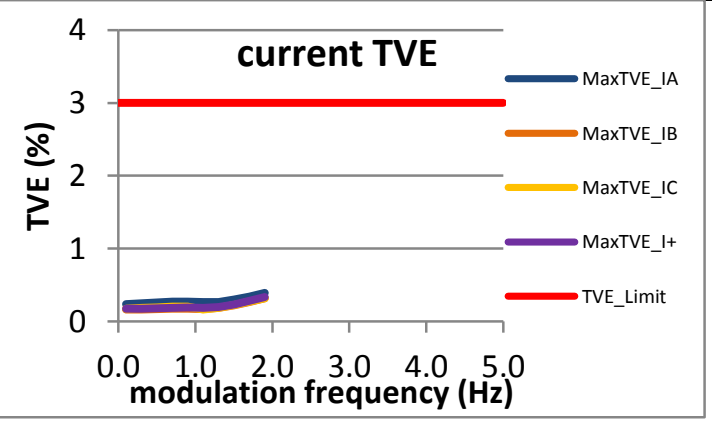

Figure 3127: Fs = 10 FPS 
7.2.3 PMU B phase modulation current TVE: M class

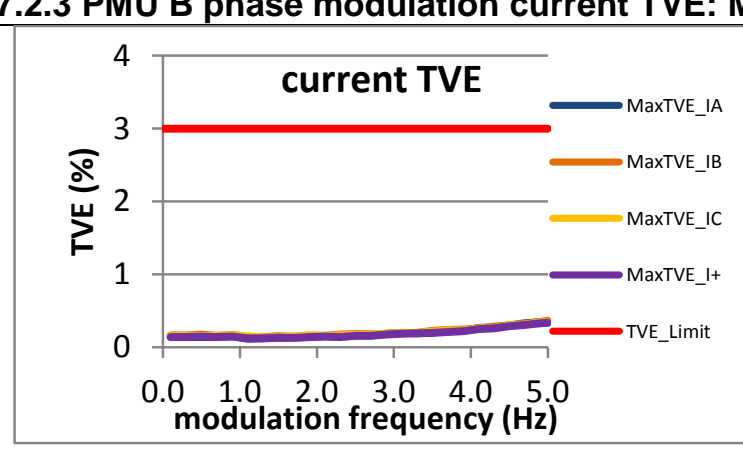

Figure 3128: Fs = 60 FPS

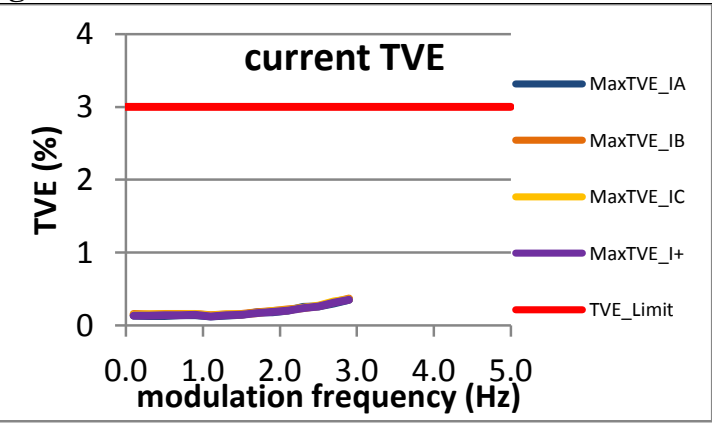

Figure 3131: Fs = 15 FPS

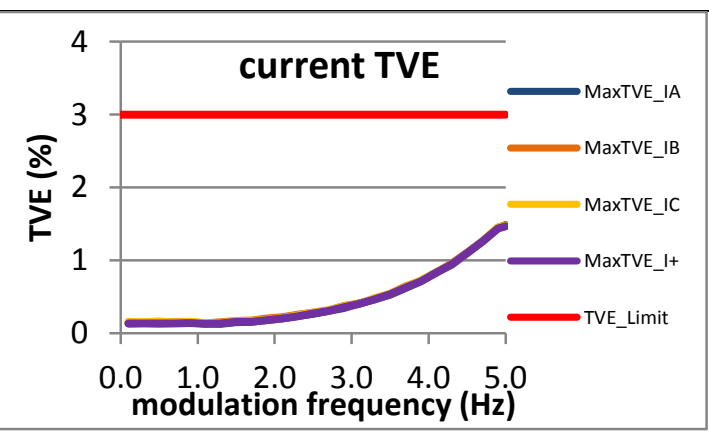

Figure 3129: Fs = 30 FPS

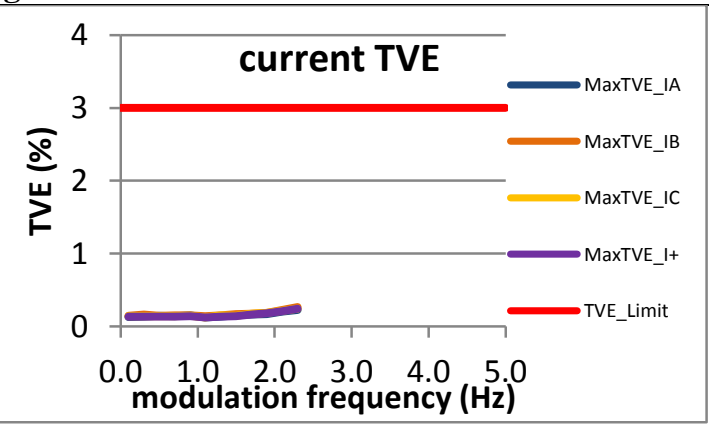

Figure 3132: Fs = 12 FPS

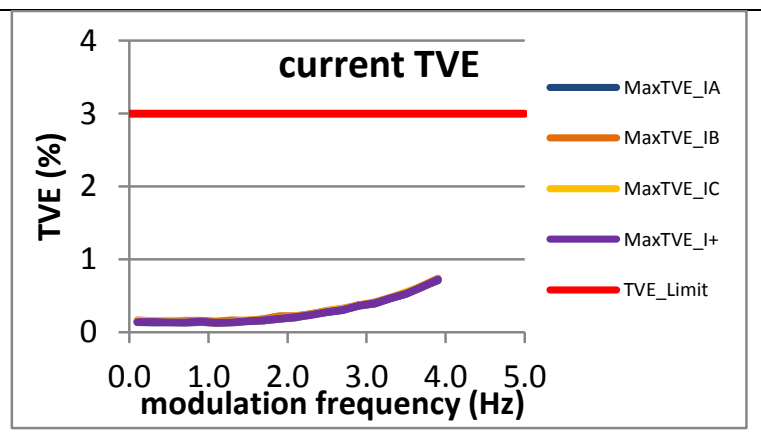

Figure 3130: Fs = 20 FPS

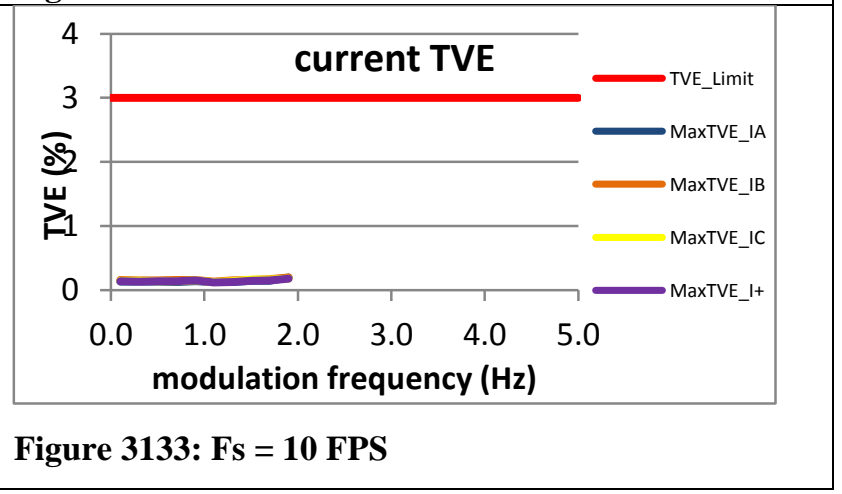


7.2.4 PMU C phase modulation current TVE: M class

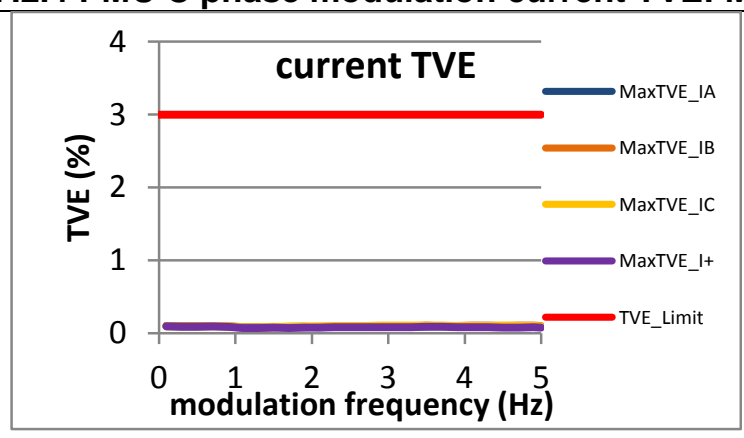

Figure 3134: Fs = 60 FPS

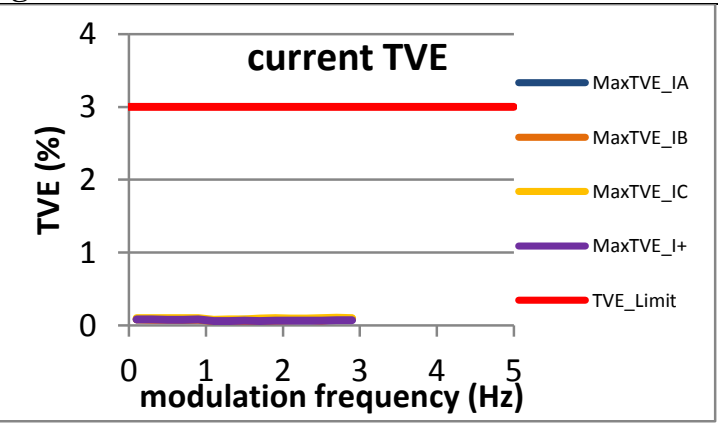

Figure 3137: Fs = 15 FPS

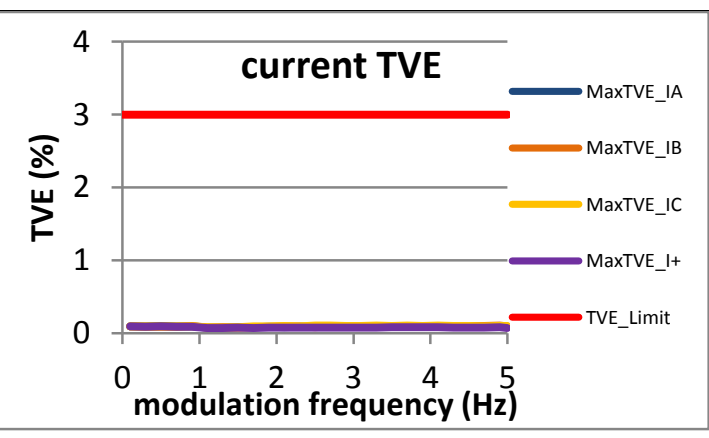

Figure 3135: Fs = 30 FPS

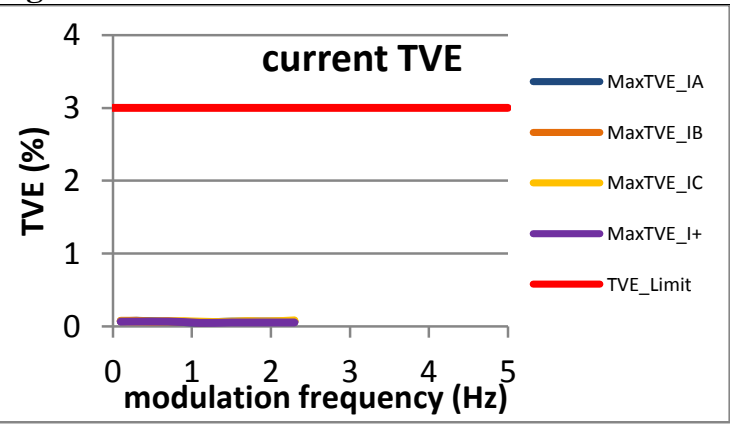

Figure 3138: Fs = 12 FPS

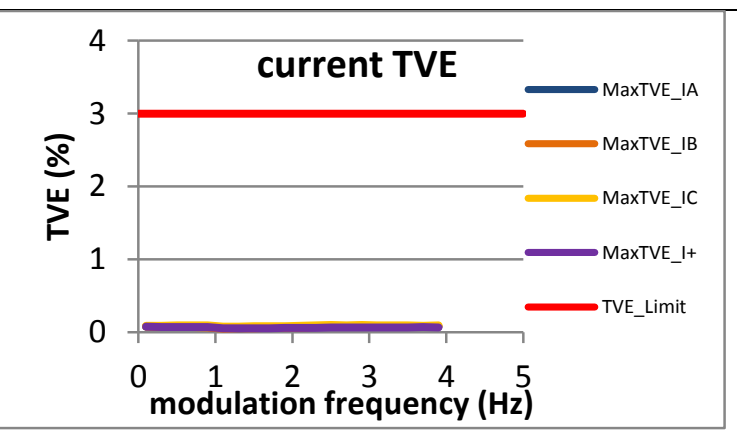

Figure 3136: Fs = 20 FPS

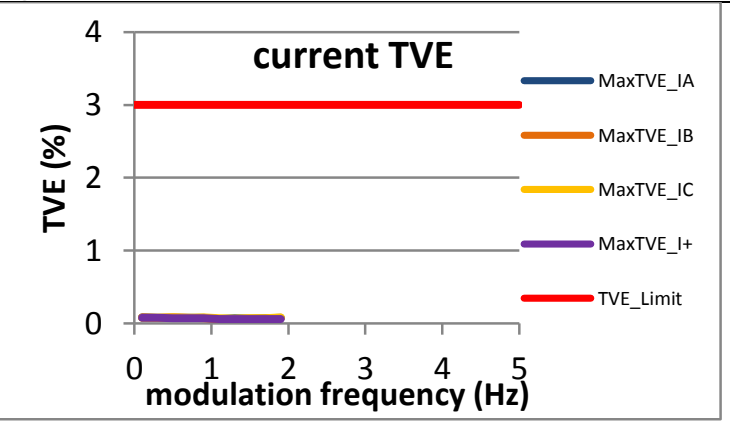

Figure 3139: Fs = 10 FPS 


\subsubsection{PMU D phase modulation current TVE: M class}

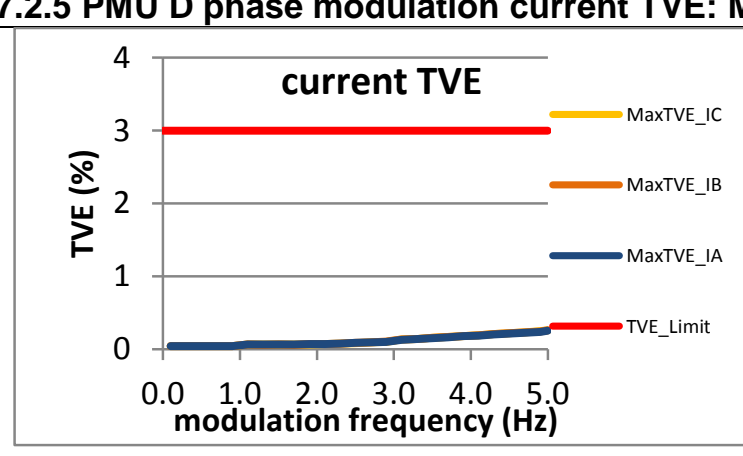

Figure 3140: Fs = 60 FPS

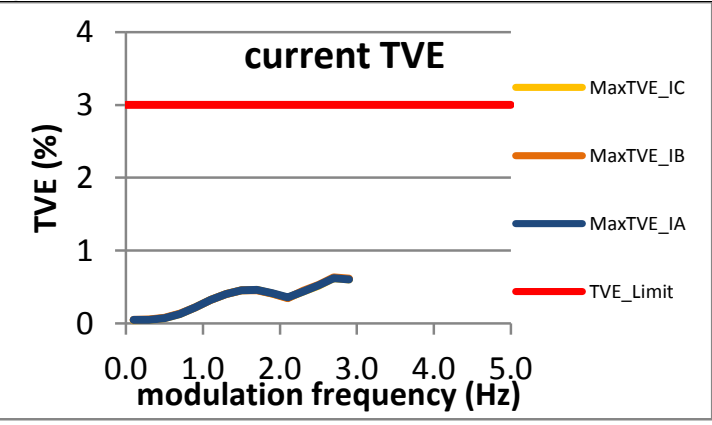

Figure 3143: Fs = 15 FPS

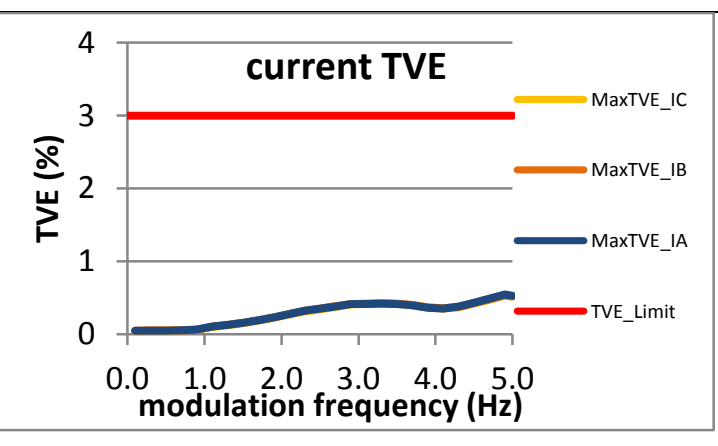

Figure 3141: Fs = 30 FPS

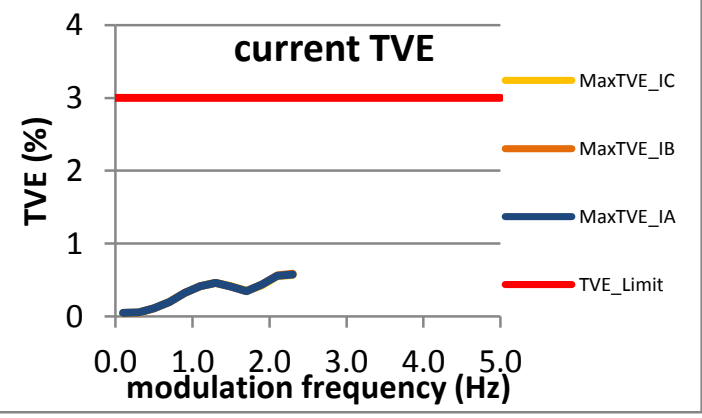

Figure 3144: Fs = 12 FPS

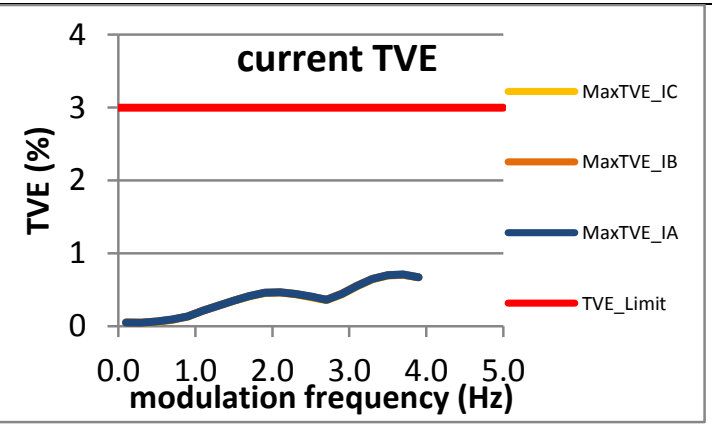

Figure 3142: Fs = 20 FPS

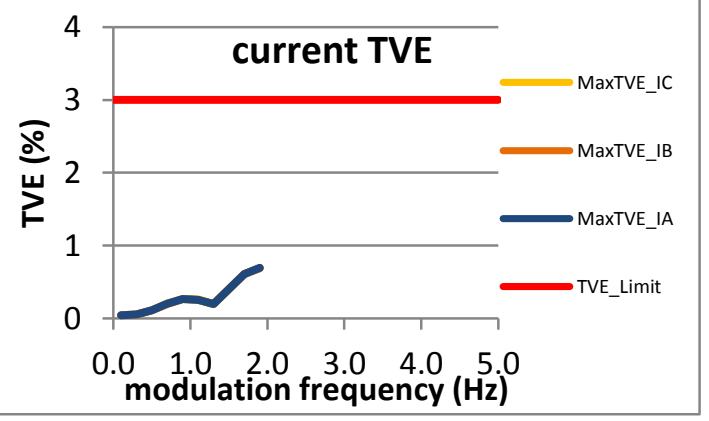

Figure 3145: Fs = 10 FPS 


\subsubsection{PMU E phase modulation current TVE: M class}

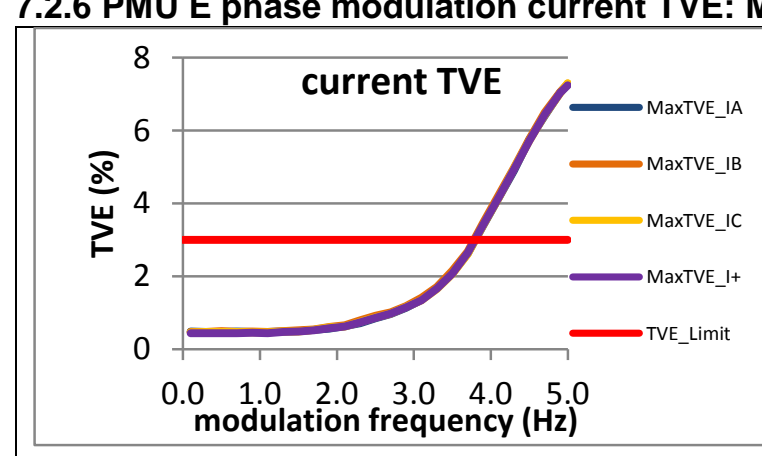

Figure 3146: Fs = 60 FPS

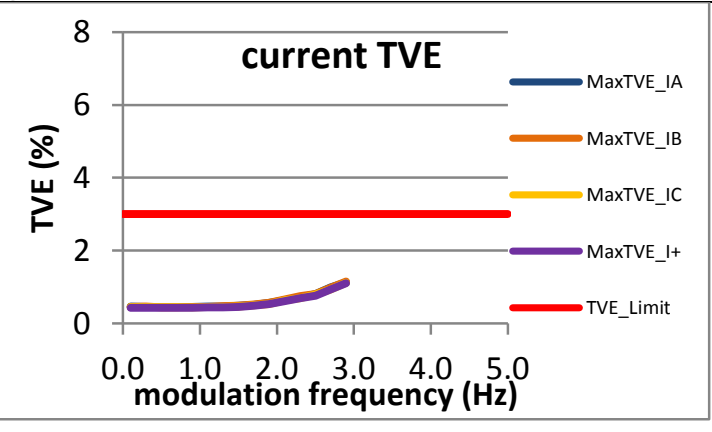

Figure 3149: Fs = 15 FPS

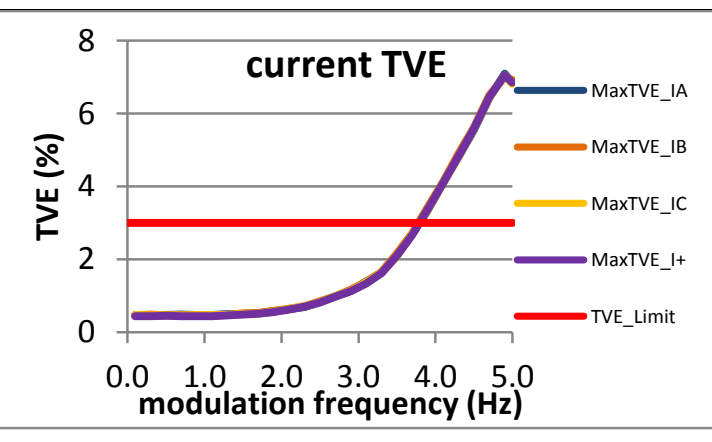

Figure 3147: Fs = 30 FPS MISSING DATA

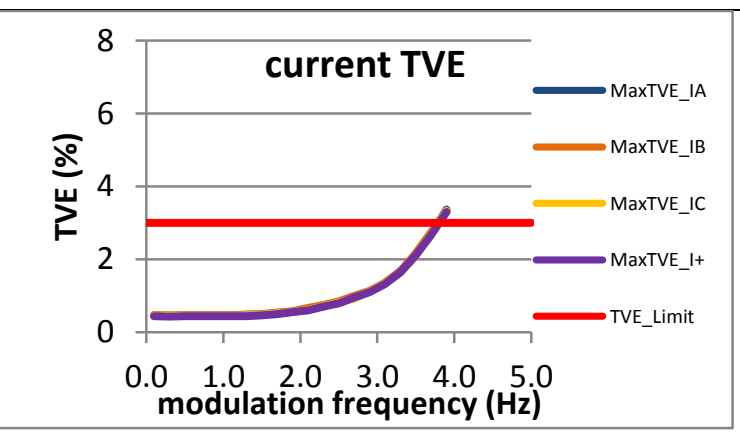

Figure 3148: Fs = 20 FPS

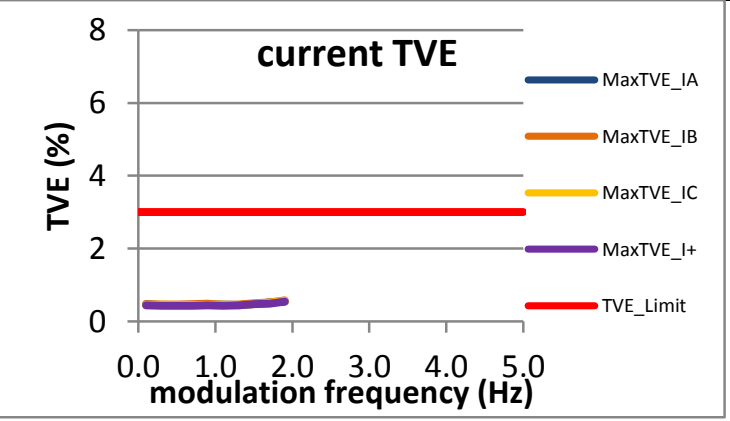

Figure 3150: Fs = 10 FPS 
7.2.7 PMU F phase modulation current TVE: M class

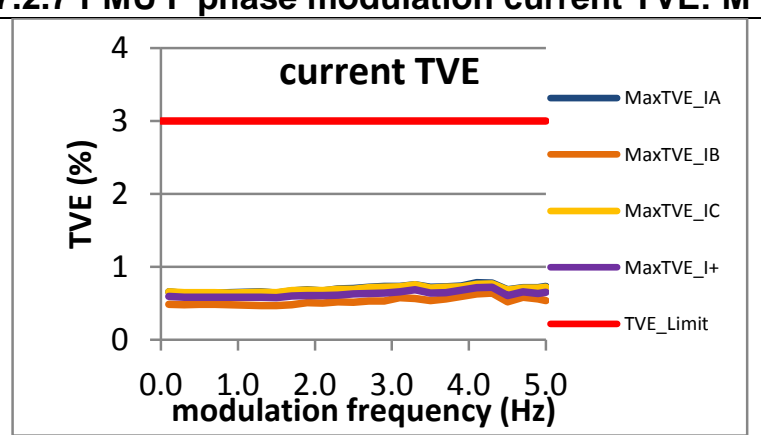

Figure 3151: Fs = 60 FPS

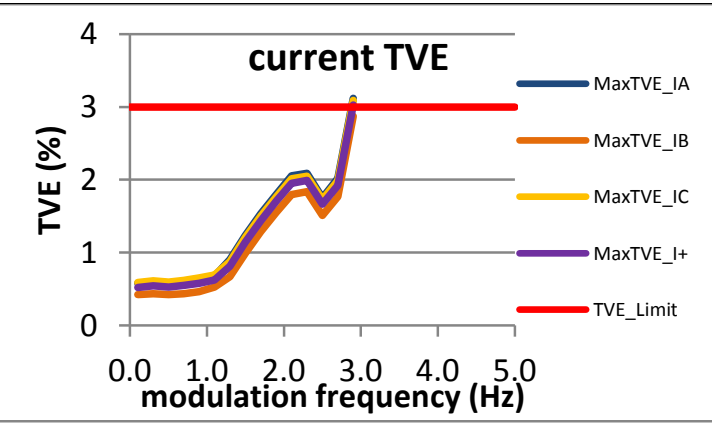

Figure 3154: Fs = 15 FPS

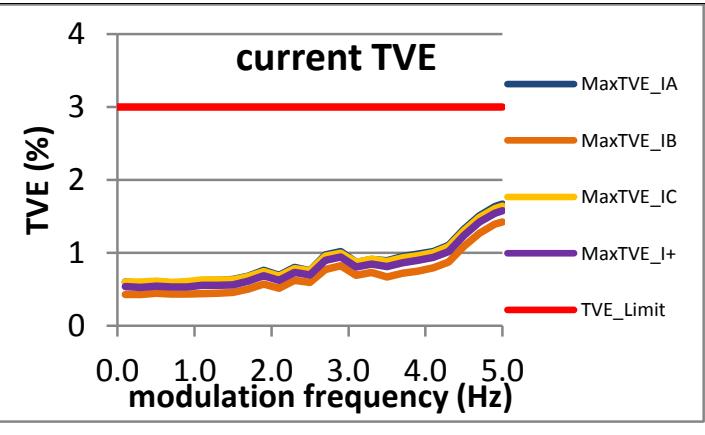

Figure 3152: Fs = 30 FPS

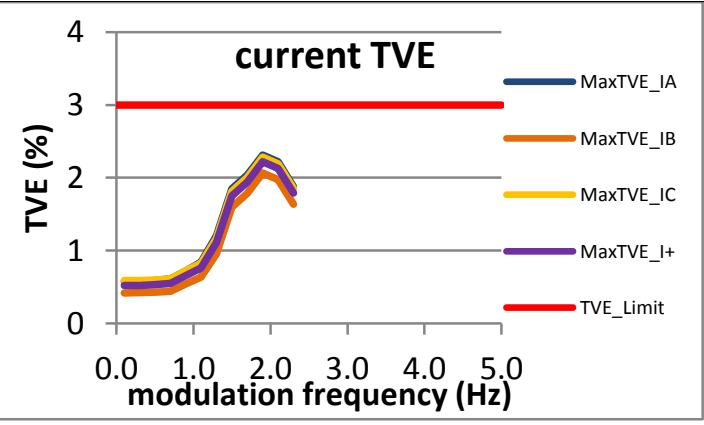

Figure 3155: Fs = 12 FPS

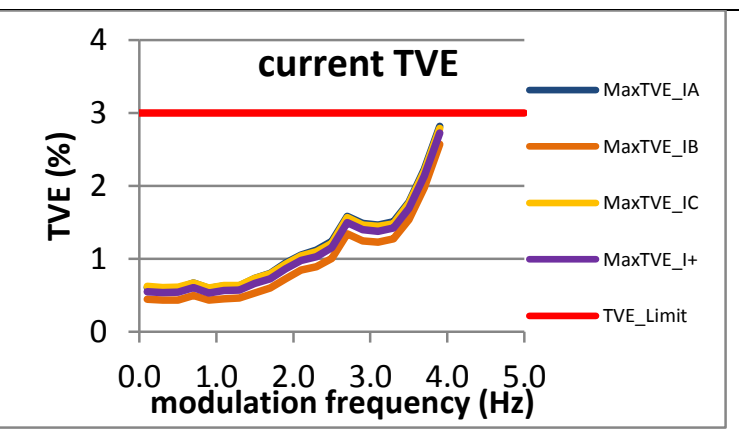

Figure 3153: Fs = 20 FPS

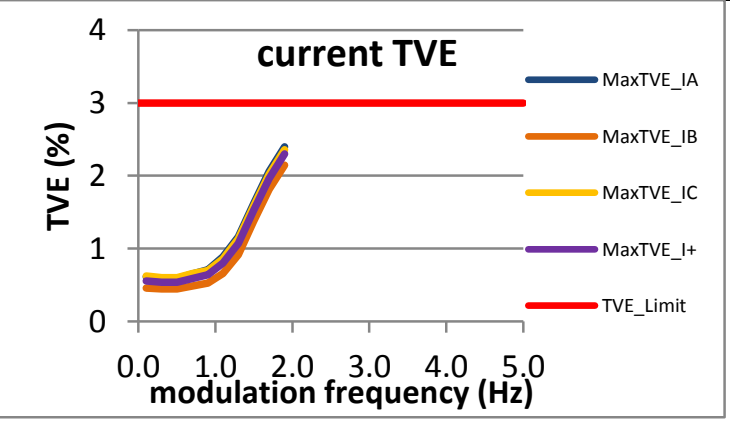

Figure 3156: Fs = 10 FPS 
7.2.8 PMU G phase modulation current TVE: M class

Figure 3157: Fs = 60 FPS is not supported by this PMU

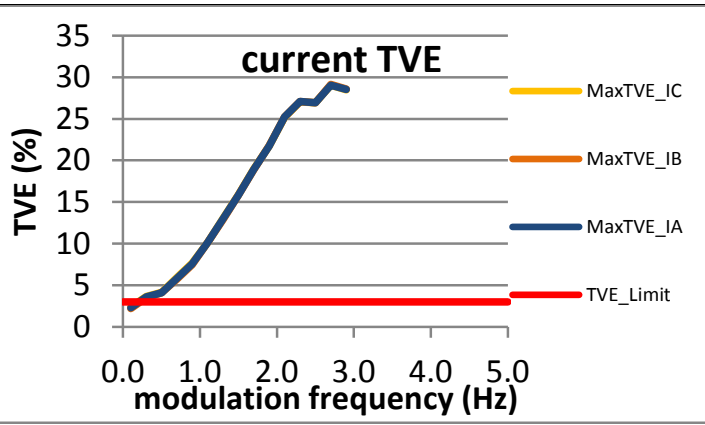

Figure 3160: Fs = 15 FPS

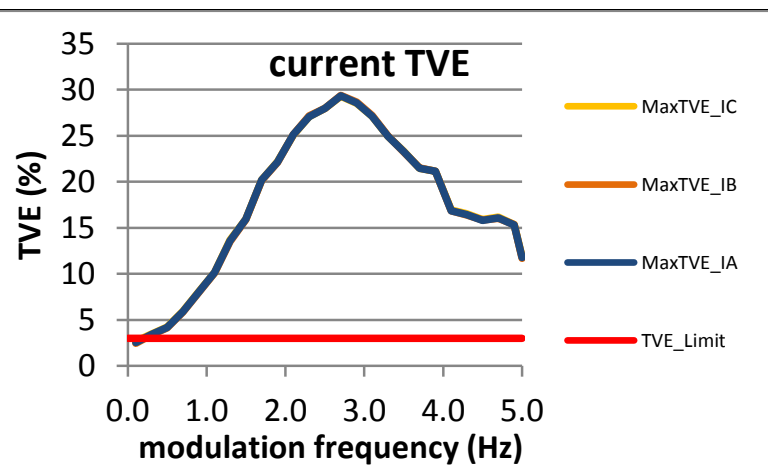

Figure 3158: Fs = 30 FPS

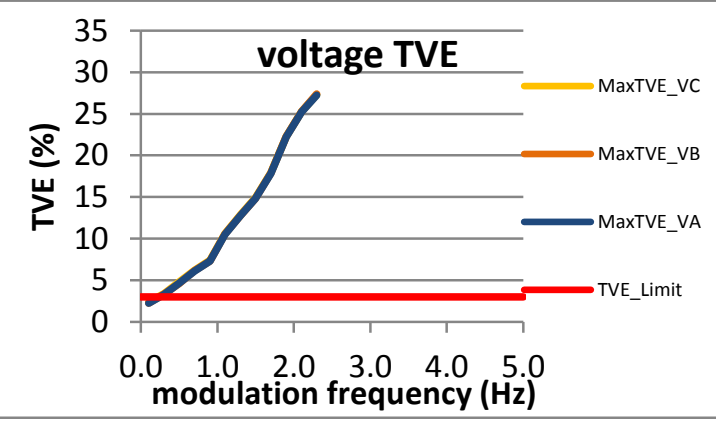

Figure 3161: Fs = 12 FPS

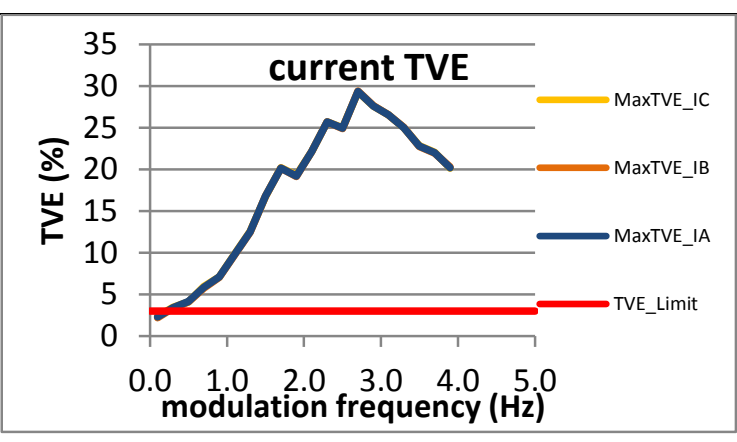

Figure 3159: Fs = 20 FPS

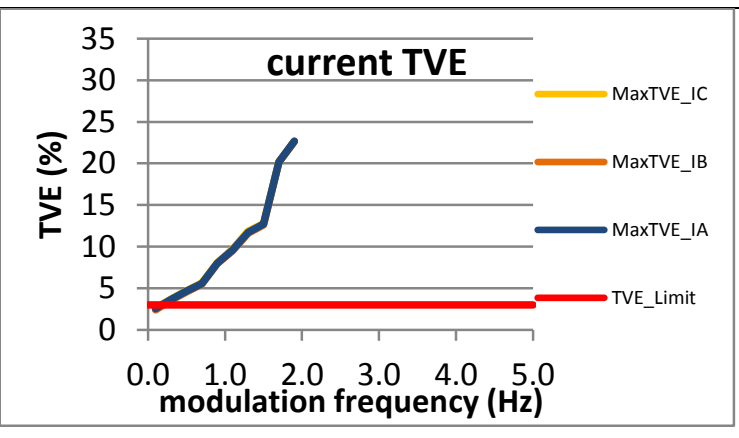

Figure 3162: Fs = 10 FPS 
7.2.9 PMU H phase modulation current TVE: M class

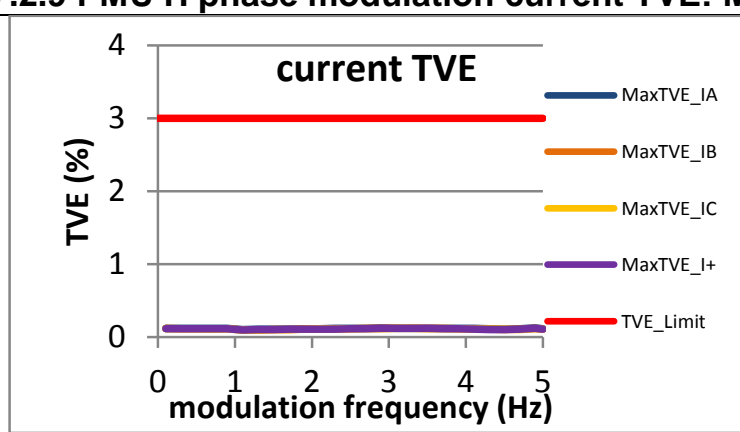

Figure 3163: Fs = 60 FPS

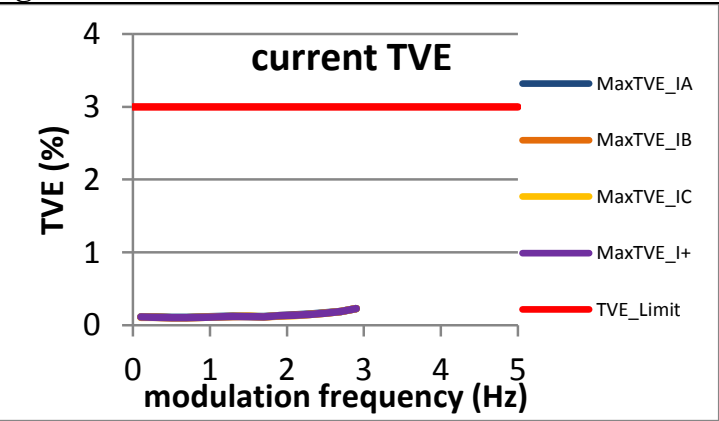

Figure 3166: Fs = 15 FPS

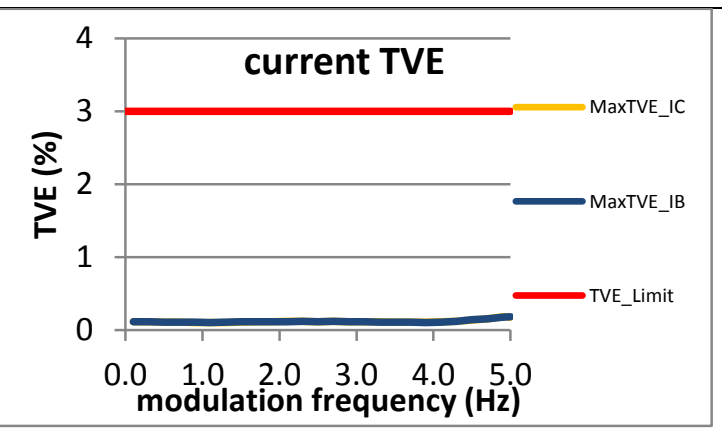

Figure 3164: Fs = 30 FPS

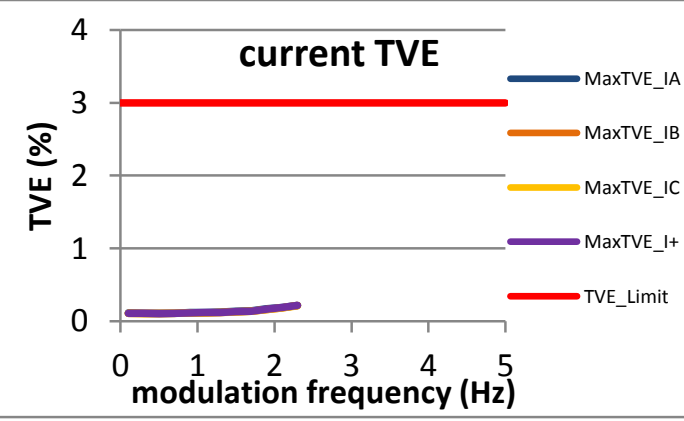

Figure 3167: Fs = 12 FPS

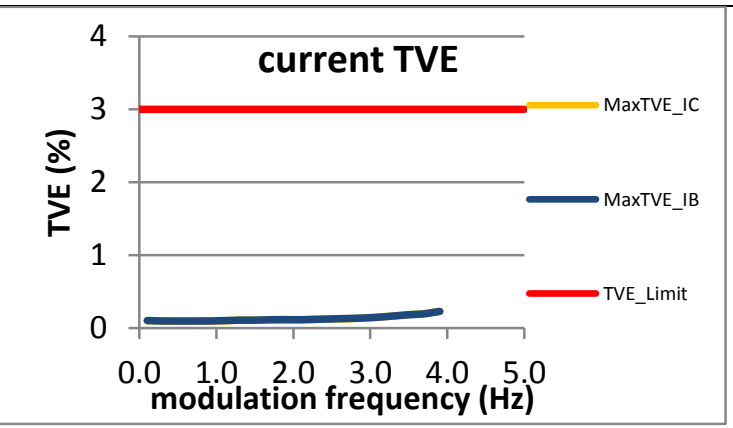

Figure 3165: Fs = 20 FPS

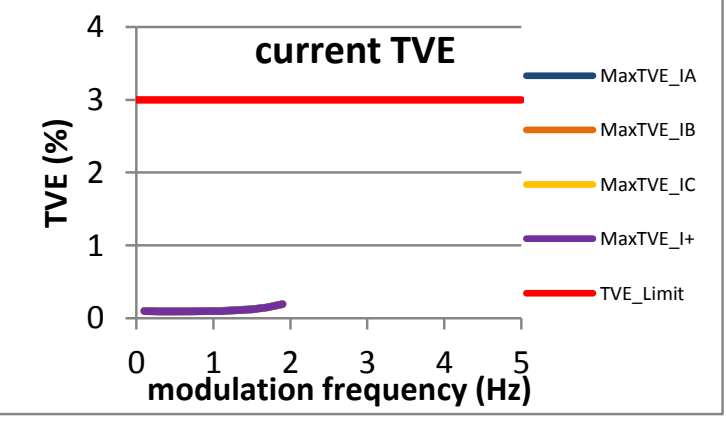

Figure 3168: Fs = 10 FPS 
7.2.10 PMU I phase modulation current TVE: M class

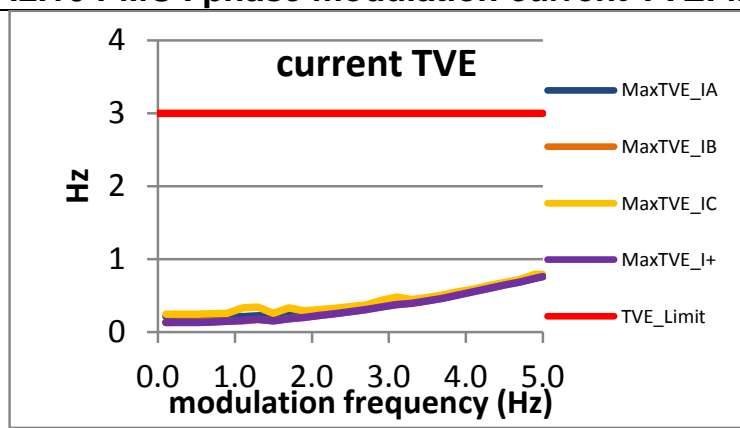

Figure 3169: Fs = 60 FPS

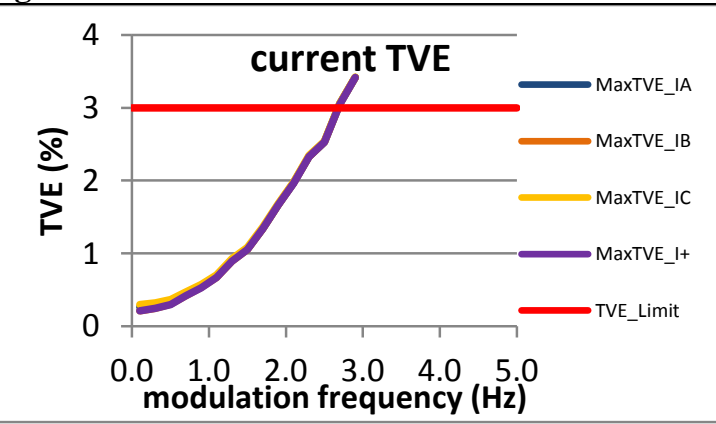

Figure 3172: Fs = 15 FPS

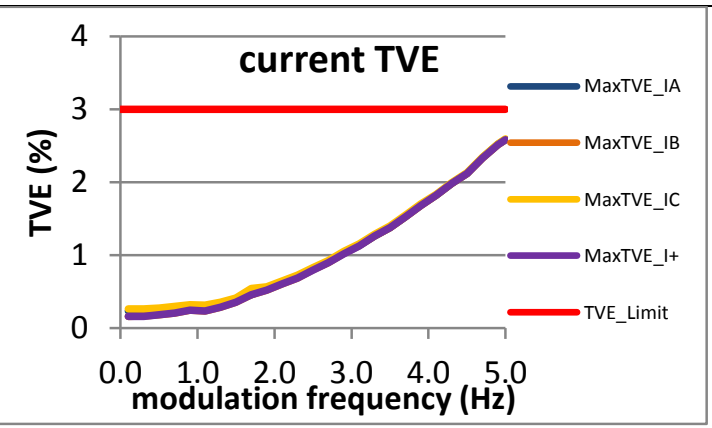

Figure 3170: Fs = 30 FPS

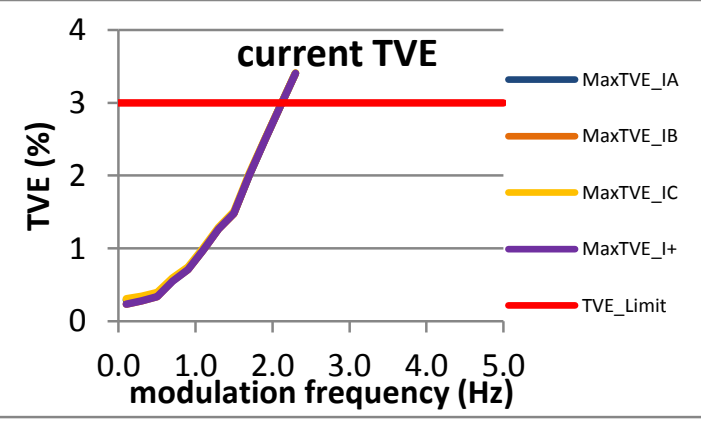

Figure 3173: Fs = 12 FPS

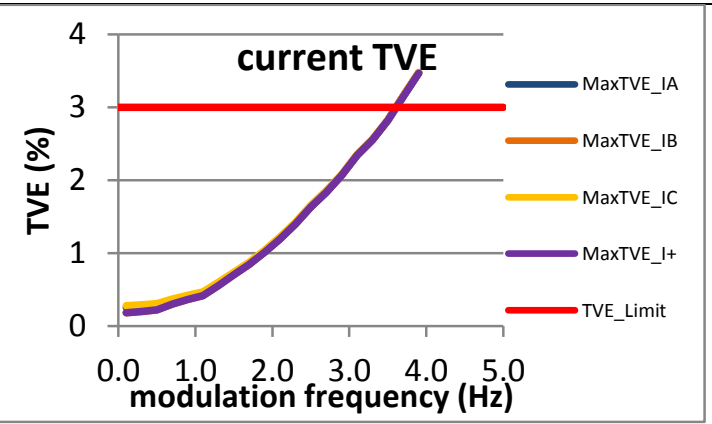

Figure 3171: Fs = 20 FPS

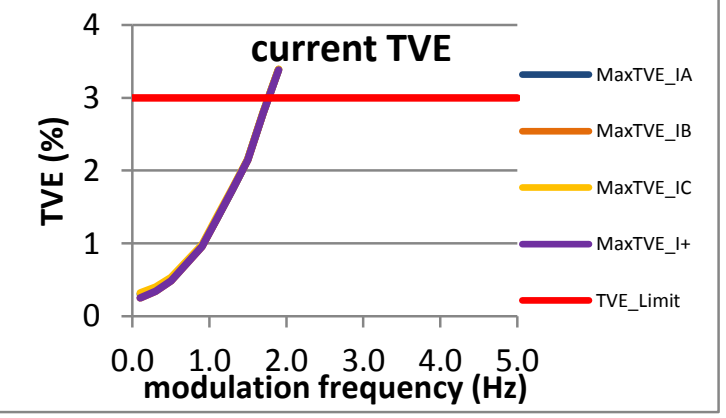

Figure 3174: Fs = 10 FPS 
7.2.11 PMU J phase modulation current TVE: M class

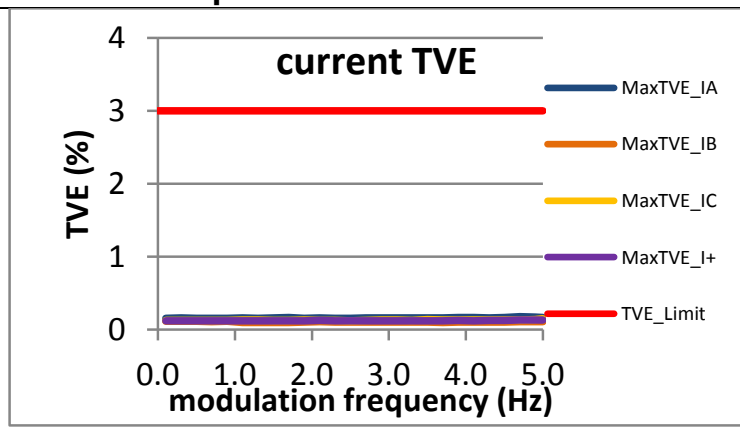

Figure 3175: Fs = 60 FPS

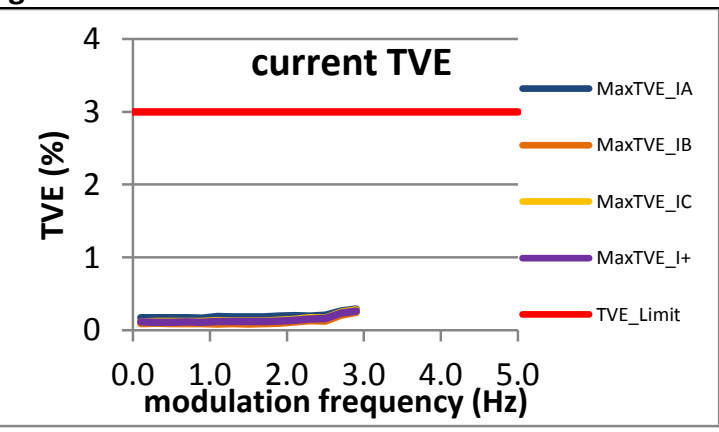

Figure 3178: Fs = 15 FPS

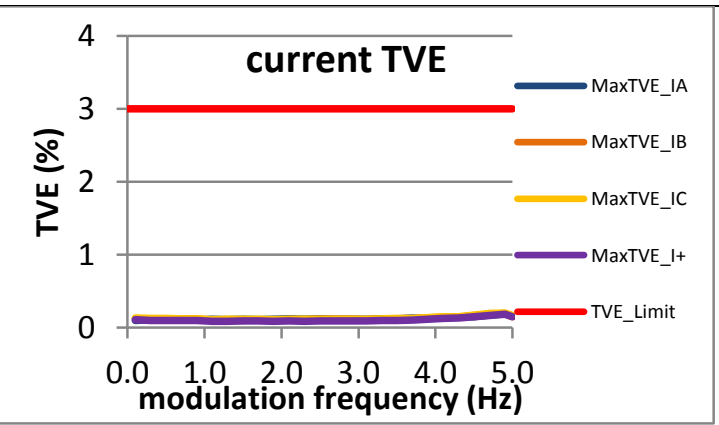

Figure 3176: Fs = 30 FPS

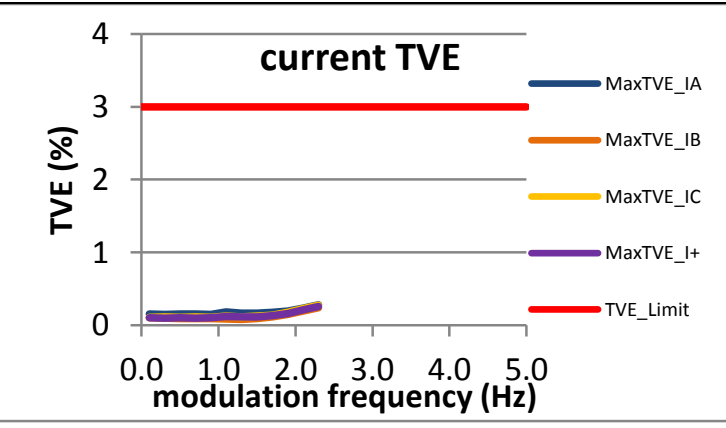

Figure 3179: Fs = 12 FPS

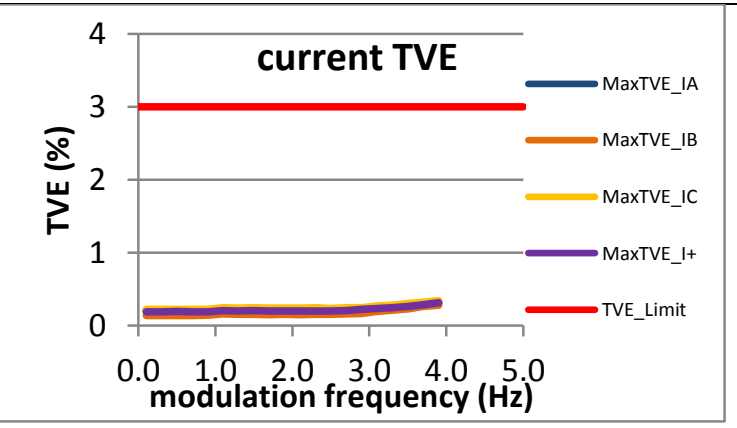

Figure 3177: Fs = 20 FPS

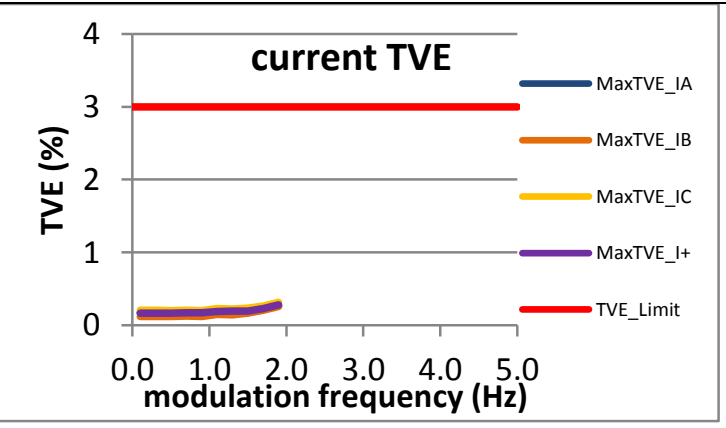

Figure 3180: Fs = 10 FPS 


\subsection{Dynamic bandwidth measurement: phase modulation frequency error, $M$ class}

7.3.1 C37.118.1 Annex C dynamic bandwidth measurement: phase modulation frequency error: M class

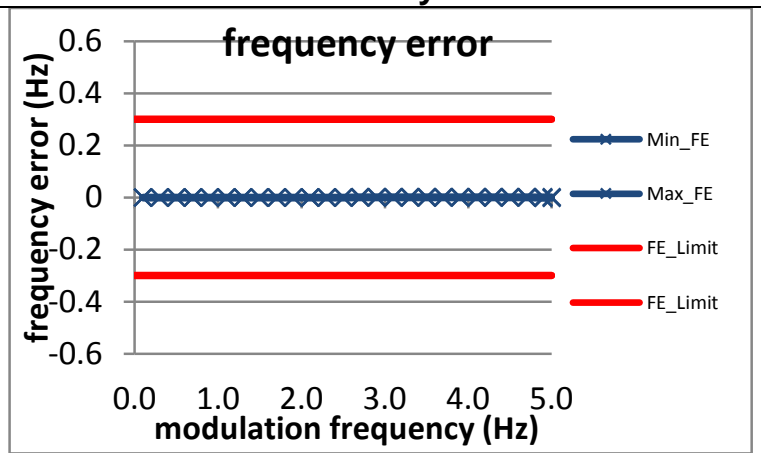

Figure 3181: Fs = 60 FPS

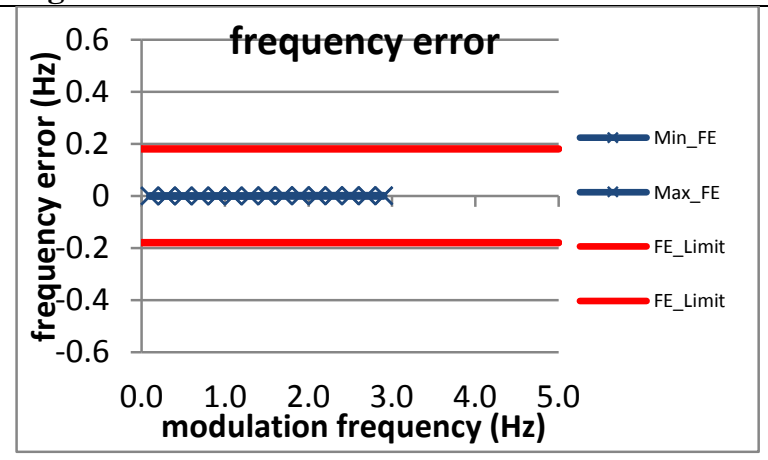

Figure 3184: Fs = 15 FPS

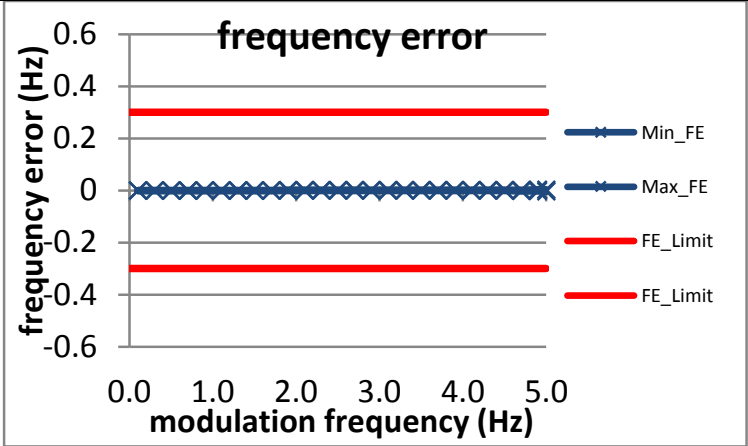

Figure 3182: Fs = 30 FPS

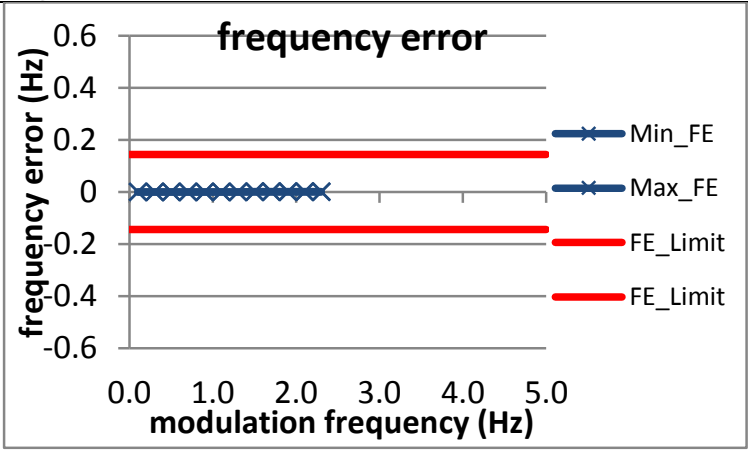

Figure 3185: Fs = 12 FPS

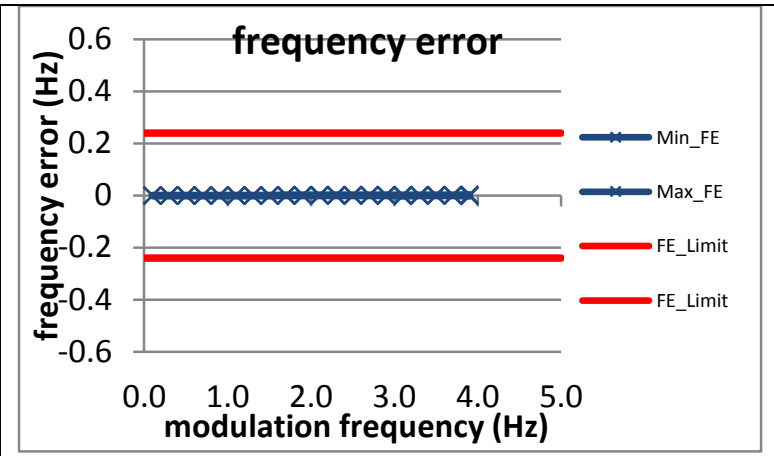

Figure 3183: Fs = 20 FPS

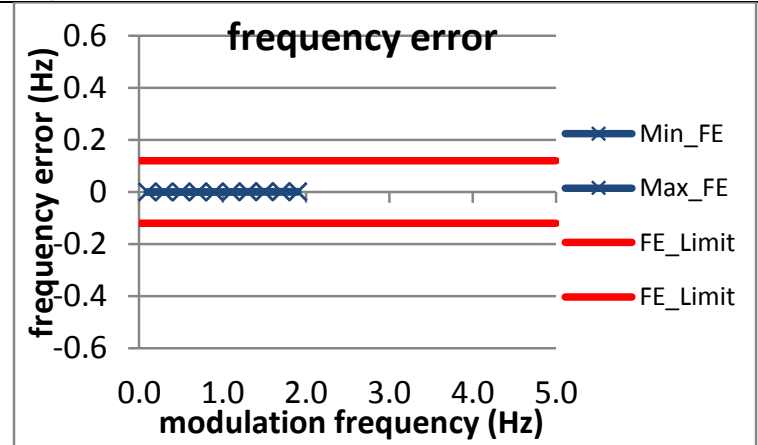

Figure 3186: Fs = 10 FPS 
7.3.2 PMU A dynamic bandwidth measurement: phase modulation frequency error: $M$ class

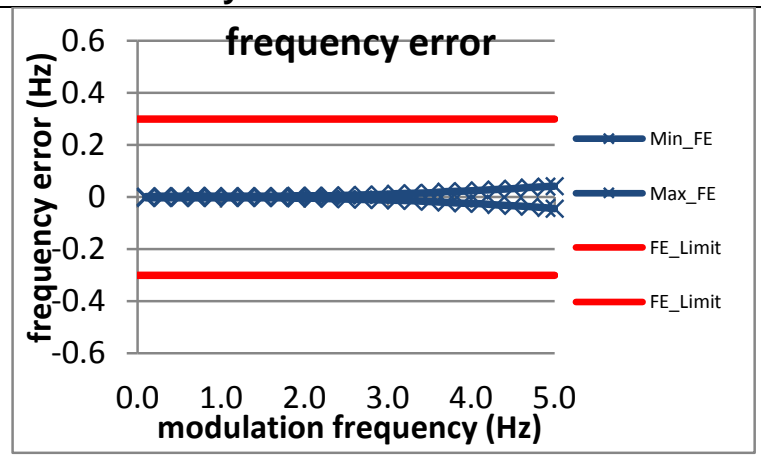

Figure 3187: Fs = 60 FPS

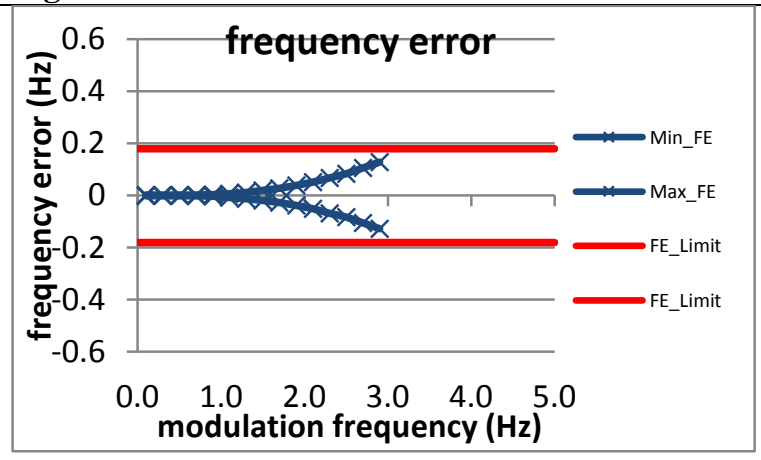

Figure 3190: Fs = 15 FPS

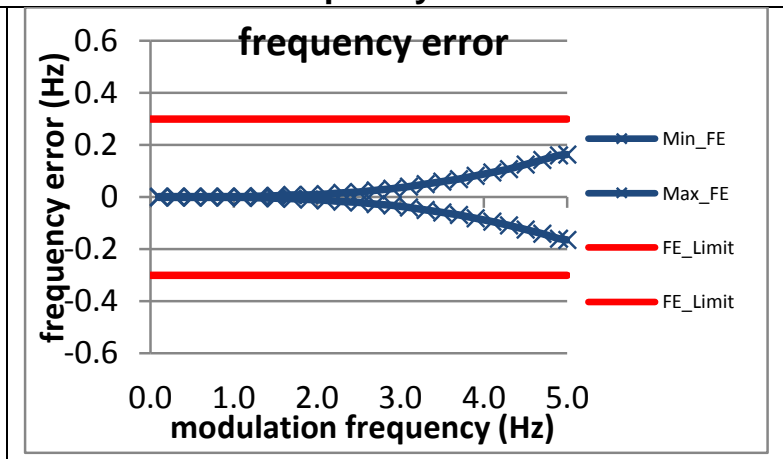

Figure 3188: Fs = 30 FPS

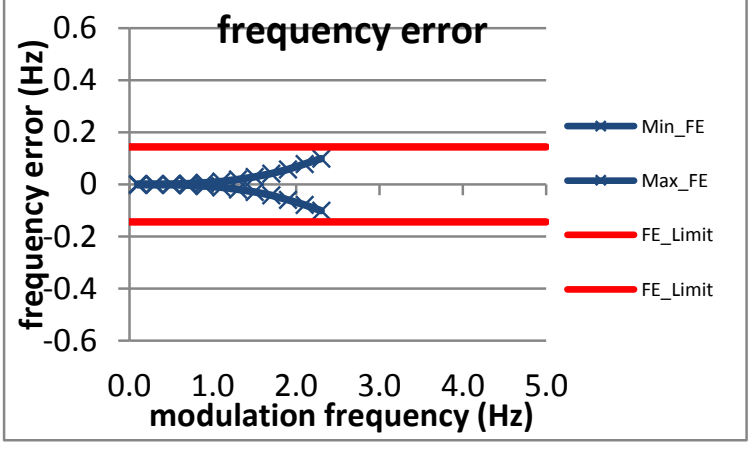

Figure 3191: Fs = 12FPS

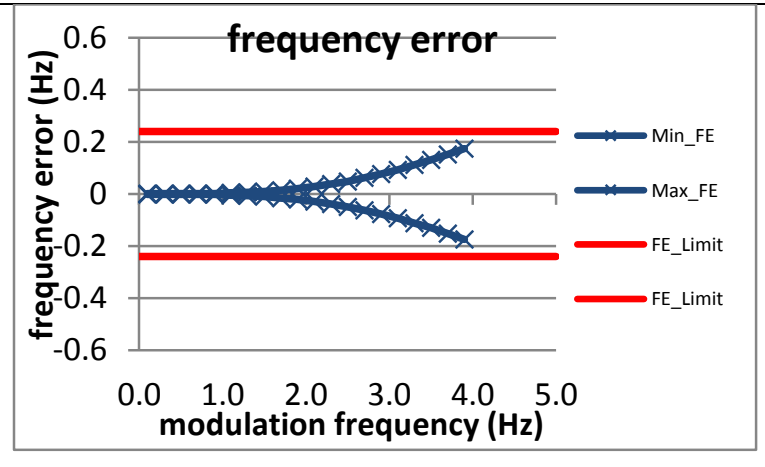

Figure 3189: Fs = 20 FPS

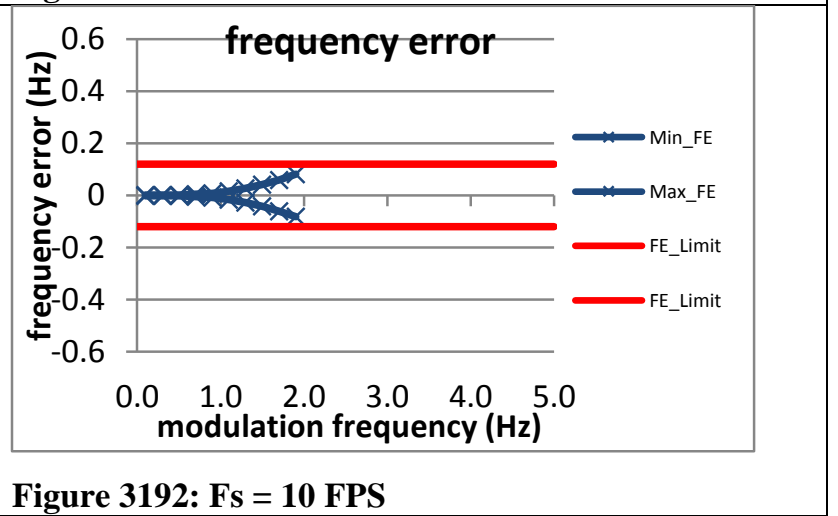


7.3.3 PMU B dynamic bandwidth measurement: phase modulation frequency error: $M$ class

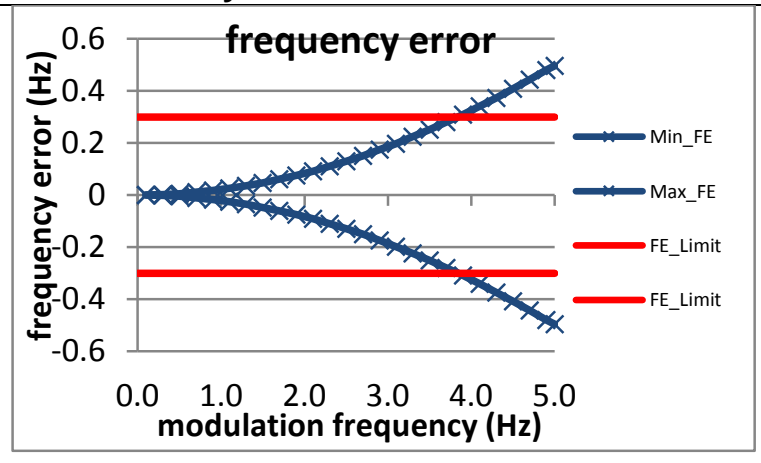

Figure 3193: Fs = 60 FPS

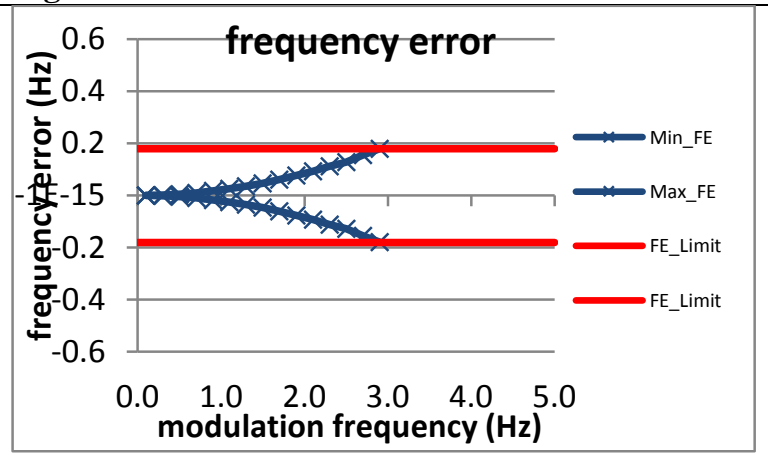

Figure 3196: Fs = 15 FPS

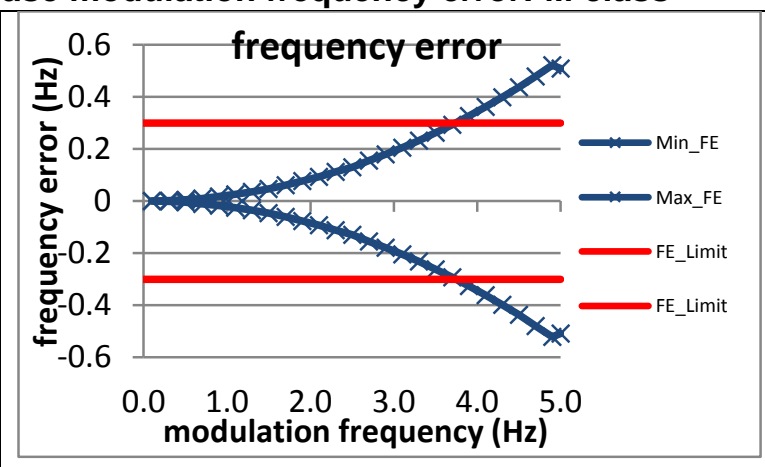

Figure 3194: Fs = 30 FPS

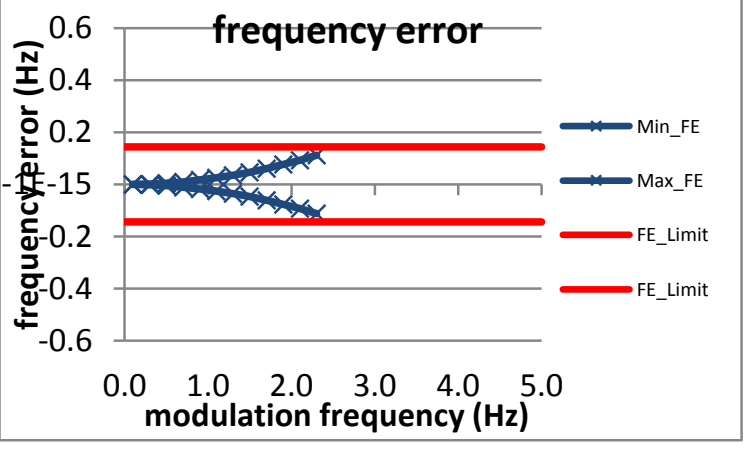

Figure 3197: Fs = 12 FPS

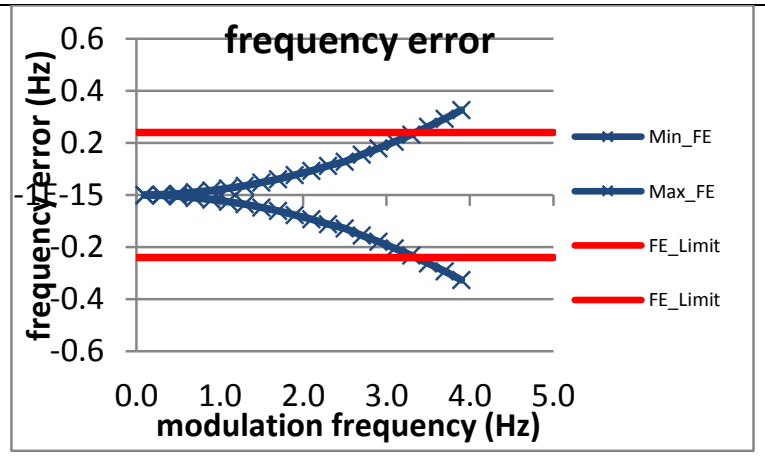

Figure 3195: Fs = 20 FPS

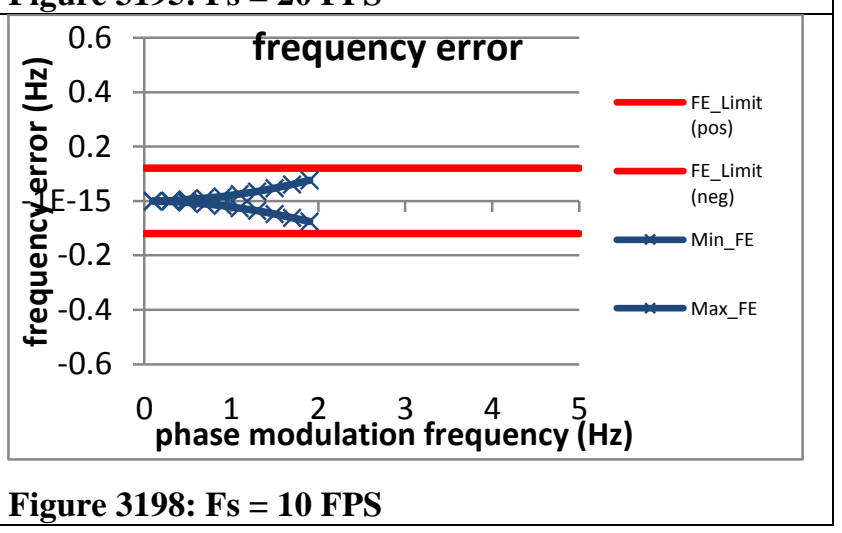


7.3.4 PMU C dynamic bandwidth measurement: phase modulation frequency error: $M$ class

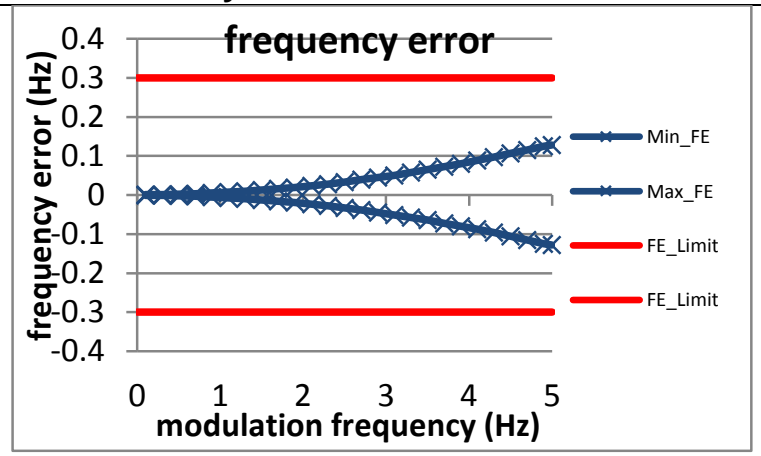

Figure 3199: Fs = 60 FPS

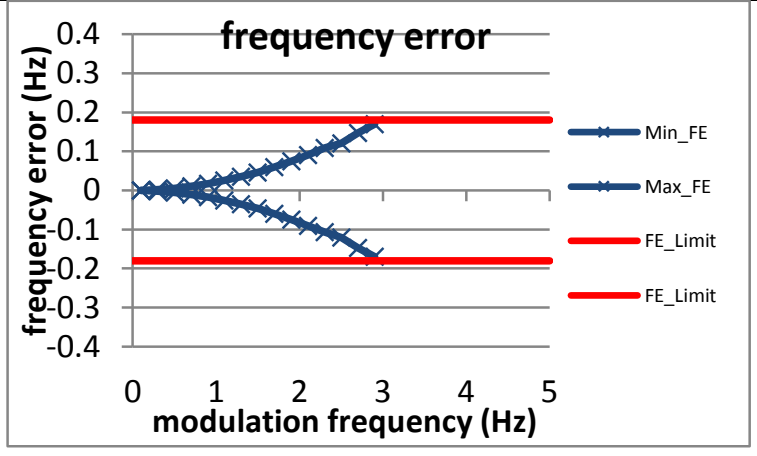

Figure 3202: Fs = 15 FPS

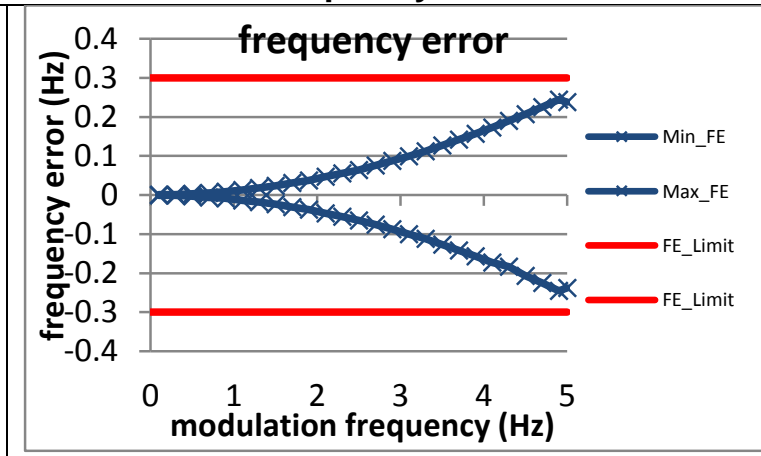

Figure 3200: Fs = 30 FPS

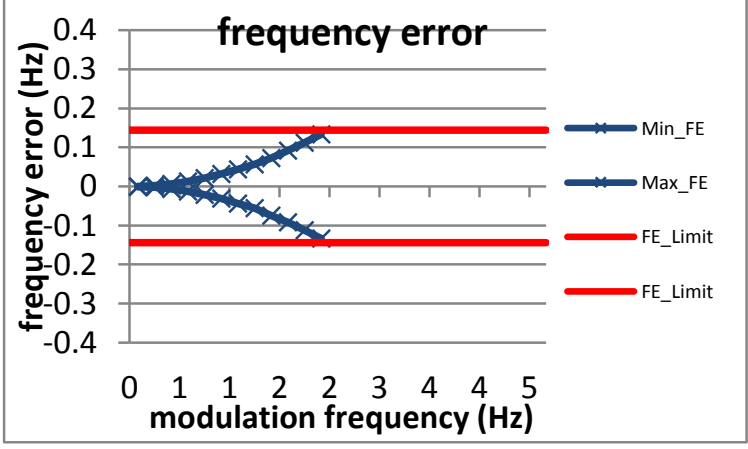

Figure 3203; Fs = 12 FPS

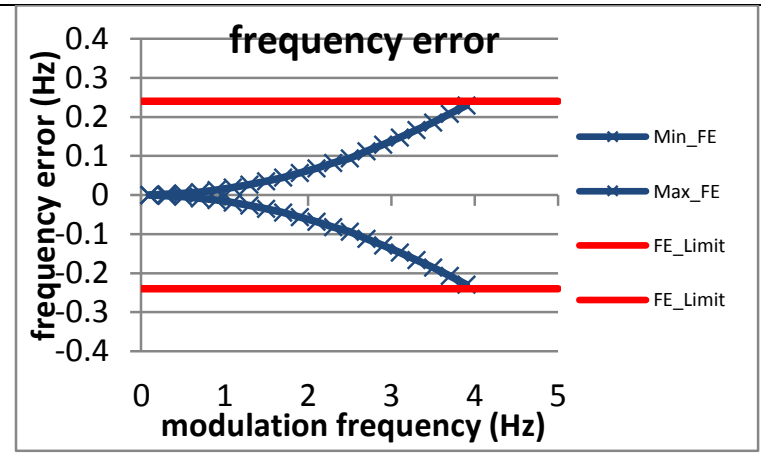

Figure 3201: Fs = 20 FPS

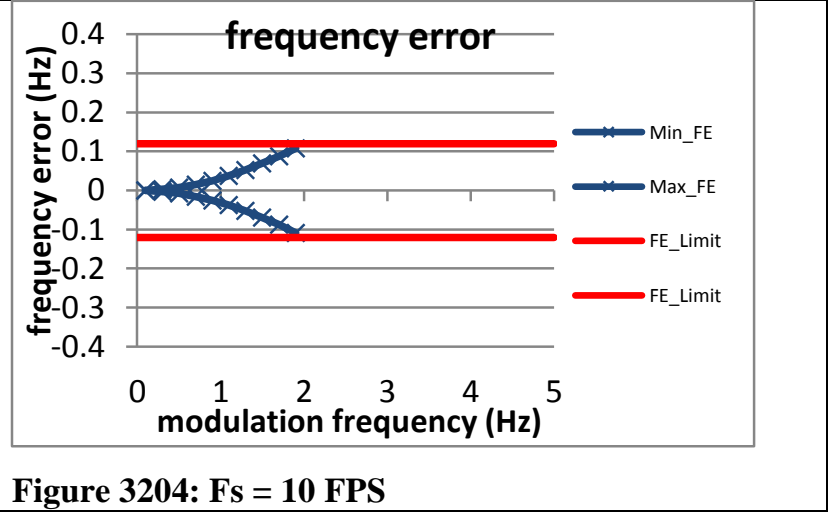


7.3.5 PMU D dynamic bandwidth measurement: phase modulation frequency error: $M$ class

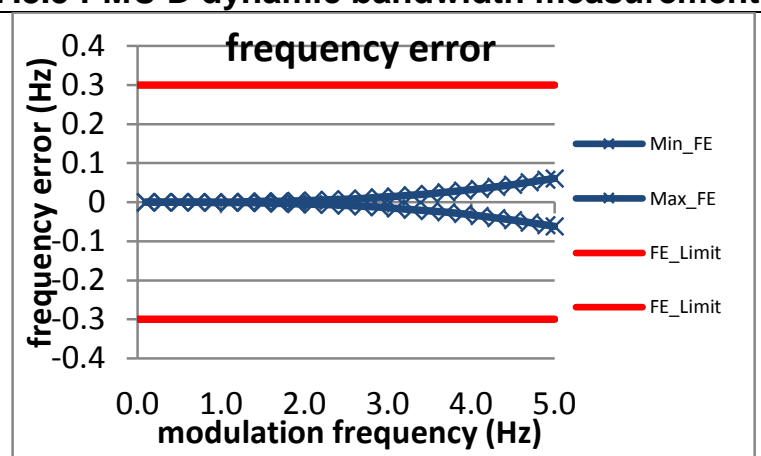

Figure 3205: Fs = 60 FPS

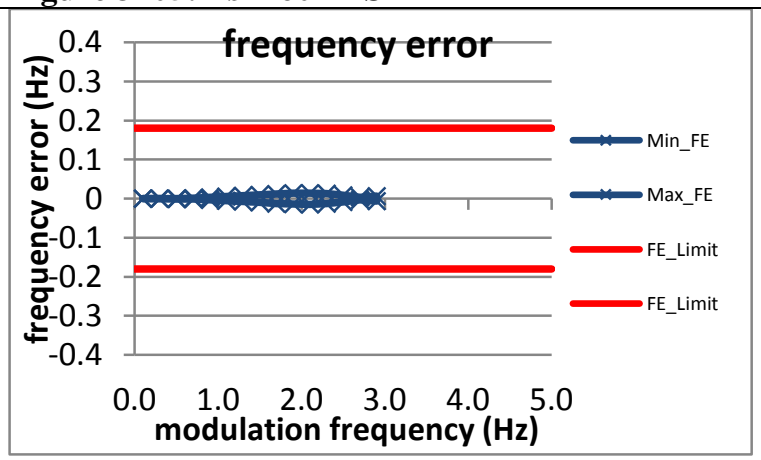

Figure 3208: Fs = 15 FPS

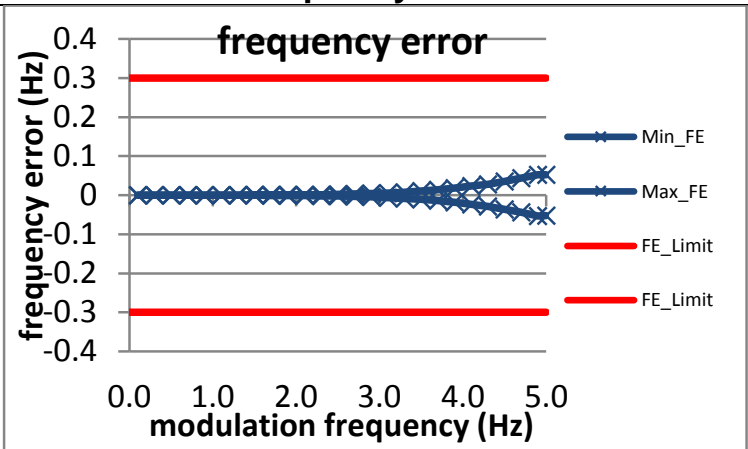

Figure 3206: Fs = 30 FPS

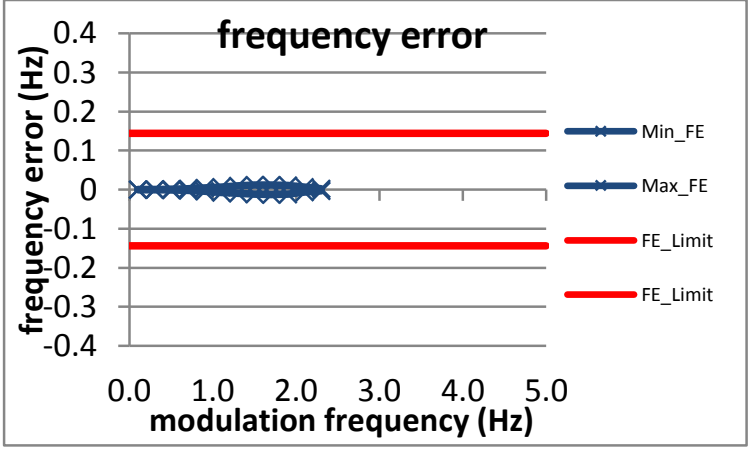

Figure 3209: Fs = 12 FPS

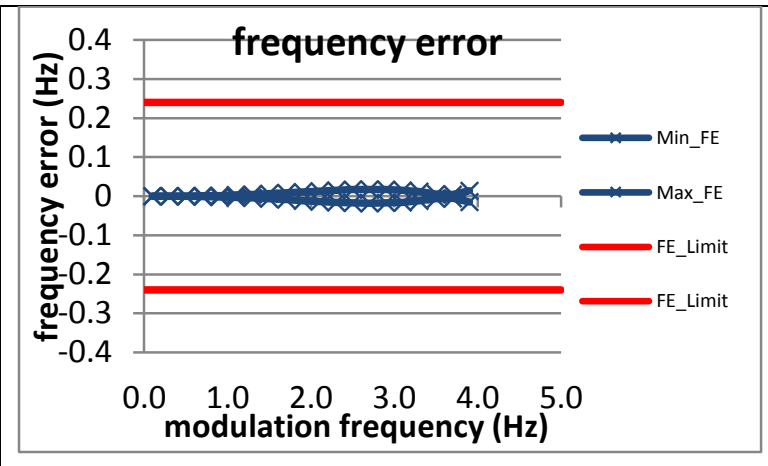

Figure 3207: Fs = 20 FPS

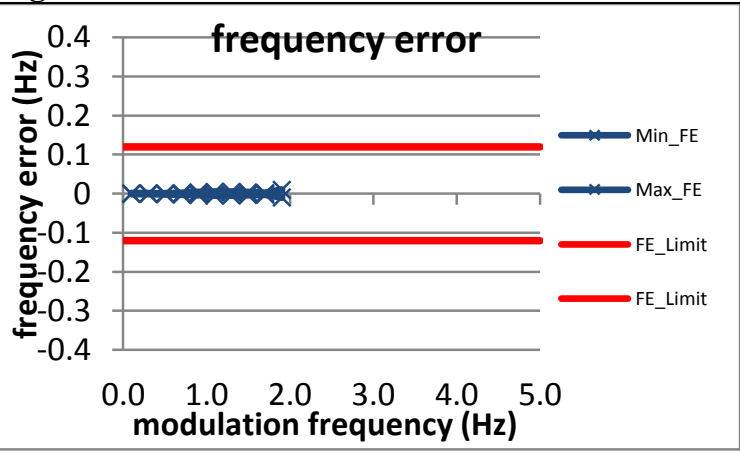

Figure 3210: Fs = 10 FPS 
7.3.6 PMU E dynamic bandwidth measurement: phase modulation frequency error: M class

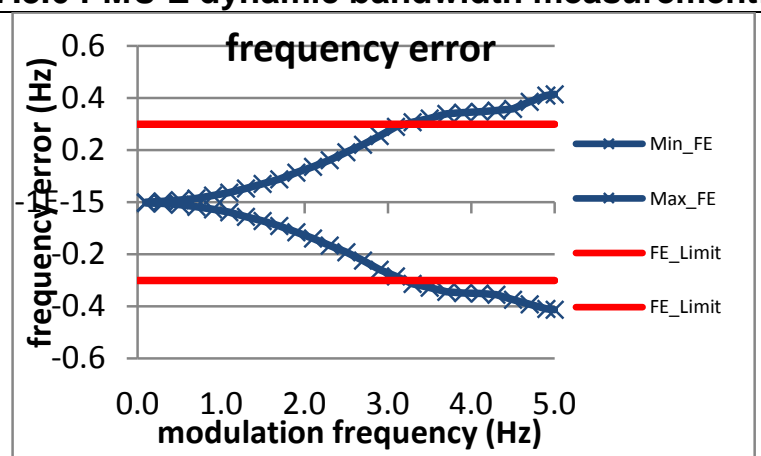

Figure 3211: Fs = 60 FPS

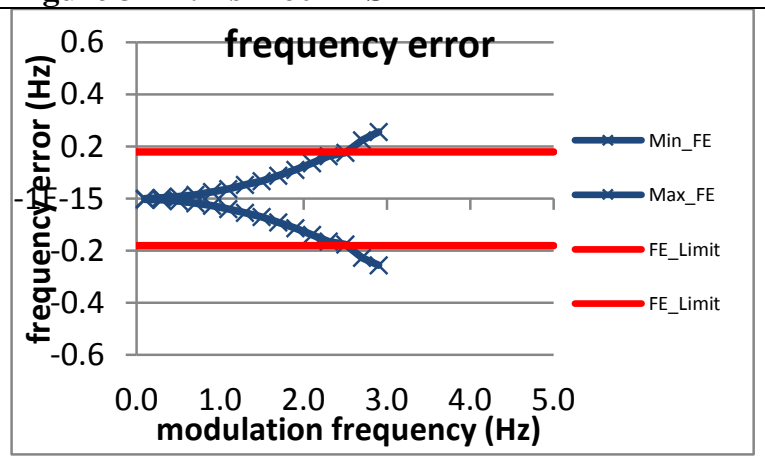

Figure 3214: Fs = 15 FPS

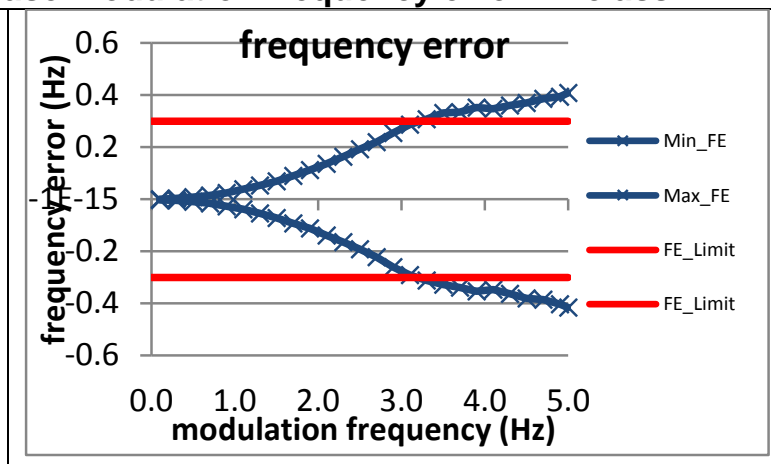

Figure 3212: Fs = 30 FPS

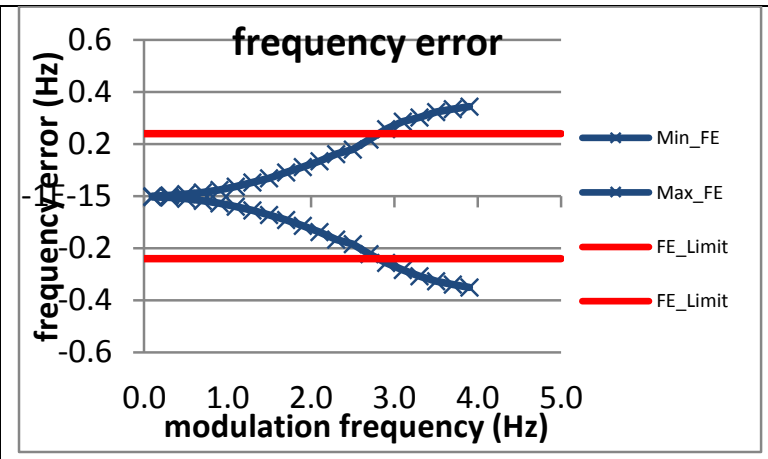

Figure 3213: Fs = 20 FPS

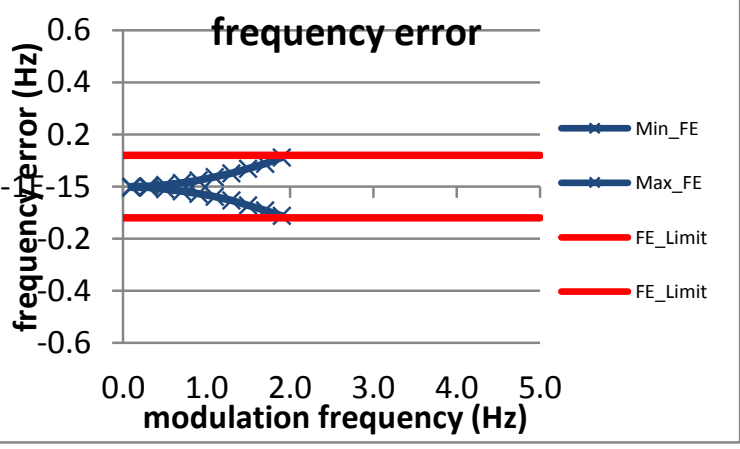

Figure 3215: Fs = 10 FPS 
7.3.7 PMU F dynamic bandwidth measurement: phase modulation frequency error: M class

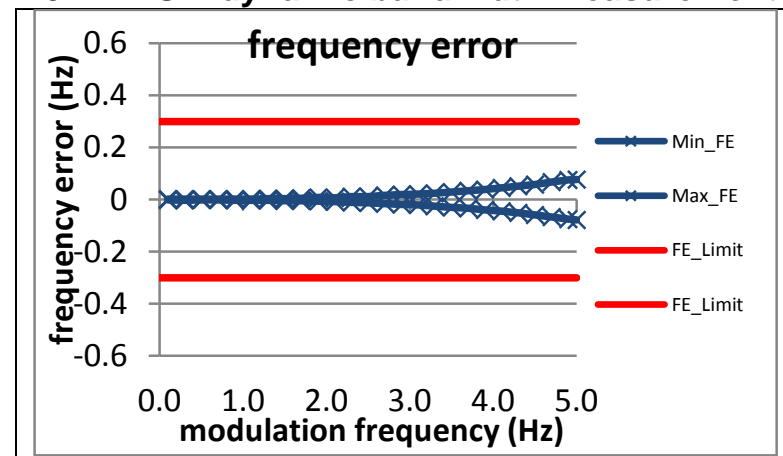

Figure 3216: Fs $=60 \mathrm{FPS}$

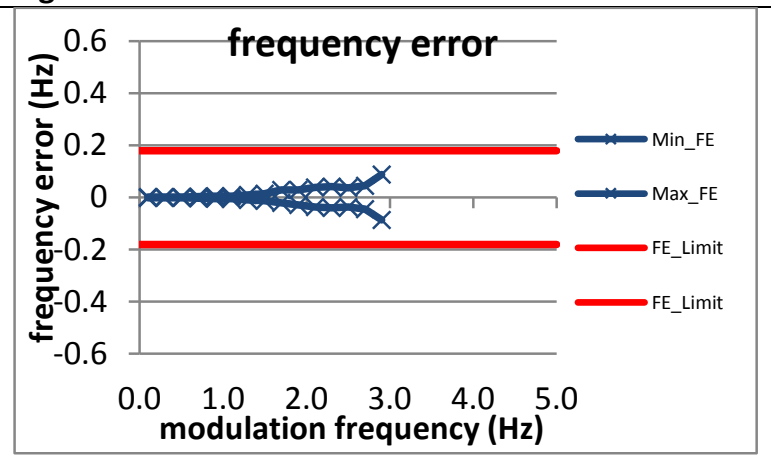

Figure 3219: Fs = 15 FPS

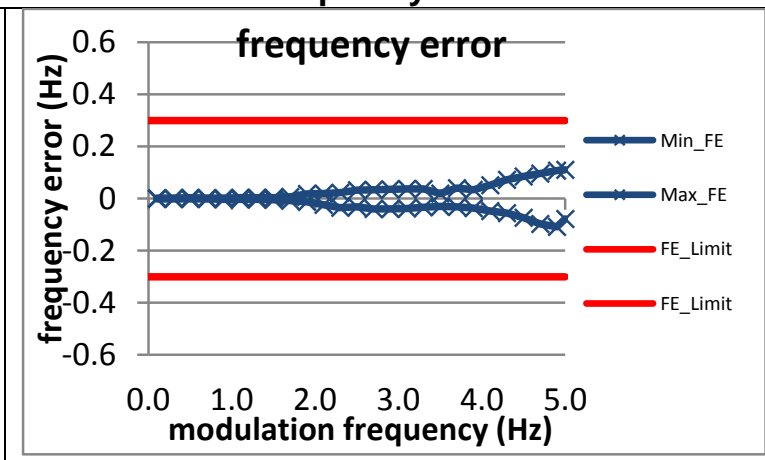

Figure 3217: $\mathrm{Fs}=\mathbf{3 0}$ FPS

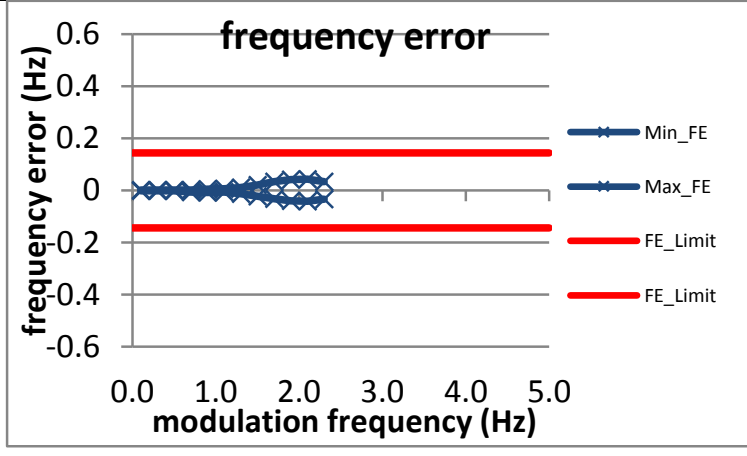

Figure 3220: Fs = 12 FPS

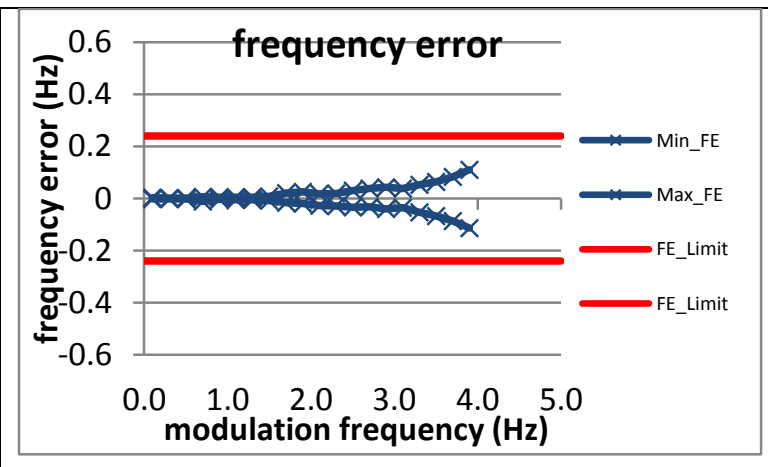

Figure 3218: $\mathrm{Fs}=20 \mathrm{FPS}$

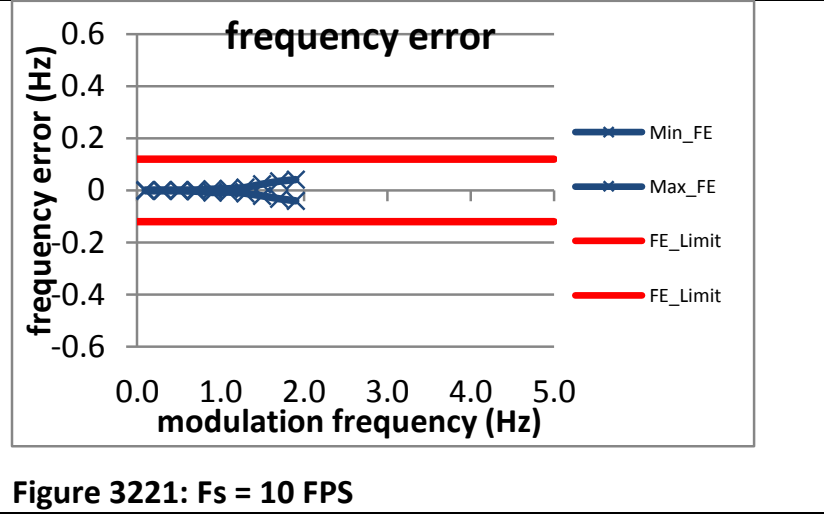


7.3.8 PMU G dynamic bandwidth measurement: phase modulation frequency error: $M$ class

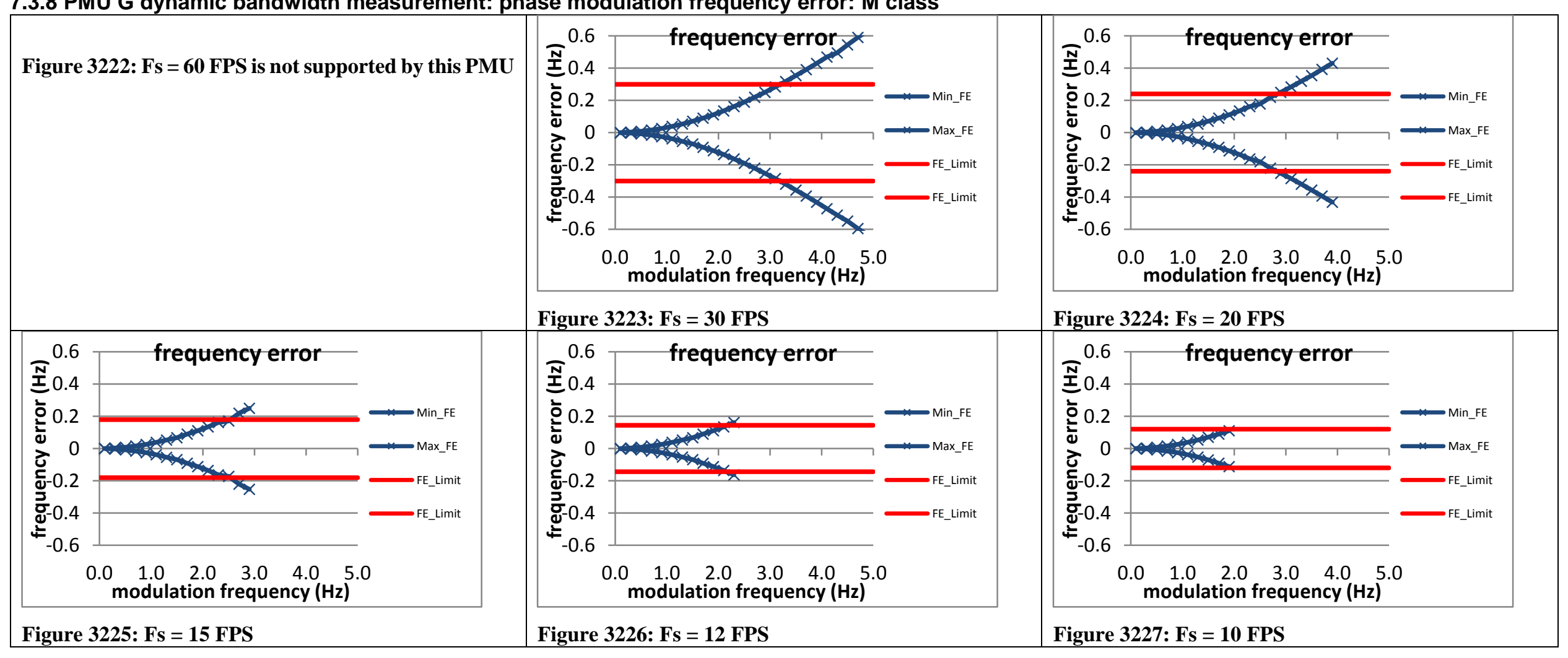


7.3.9 PMU H dynamic bandwidth measurement: phase modulation frequency error: $M$ class

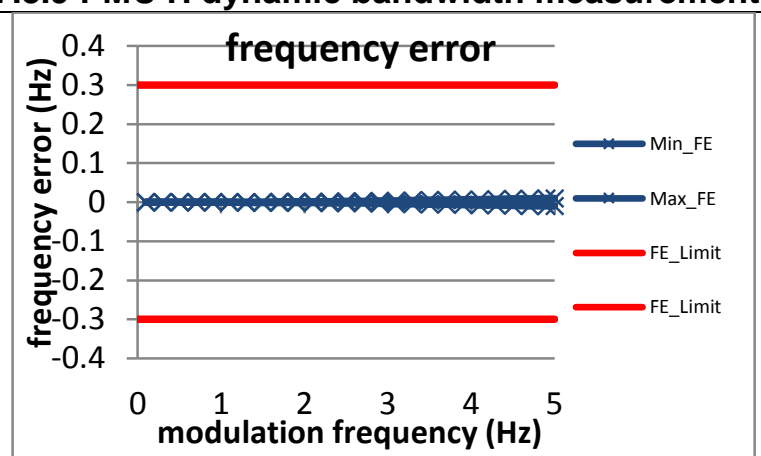

Figure 3228: Fs = 60 FPS

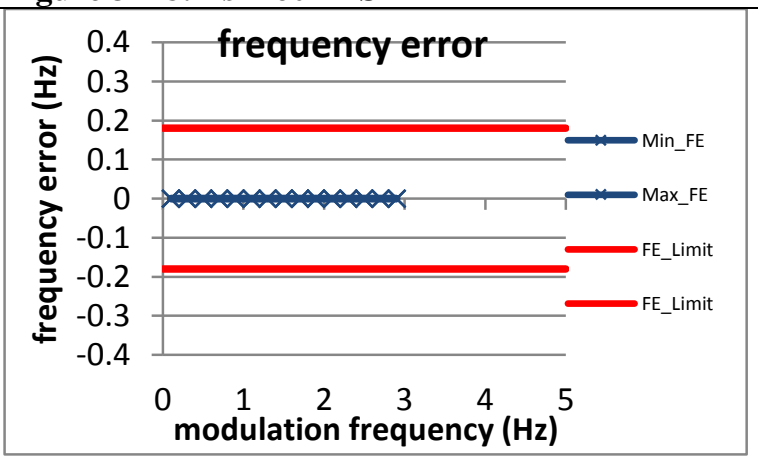

Figure 3231: Fs = 15 FPS

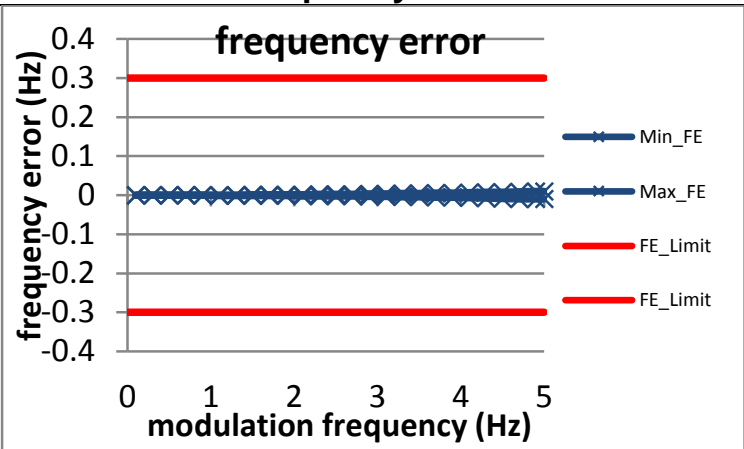

Figure 3229: Fs = 30 FPS

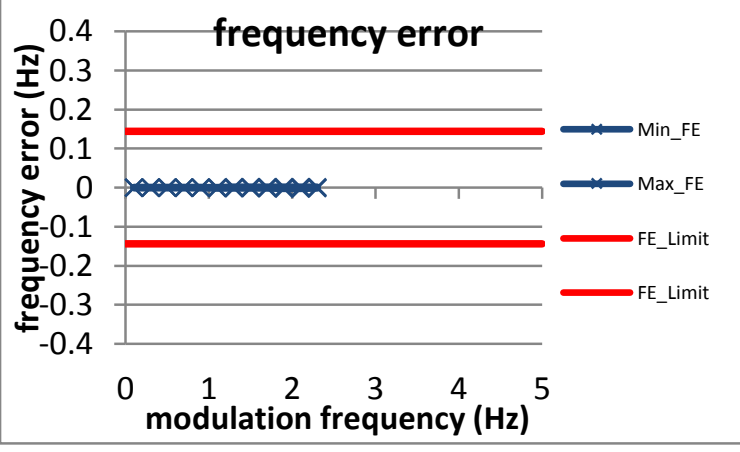

Figure 3232: Fs = 12 FPS

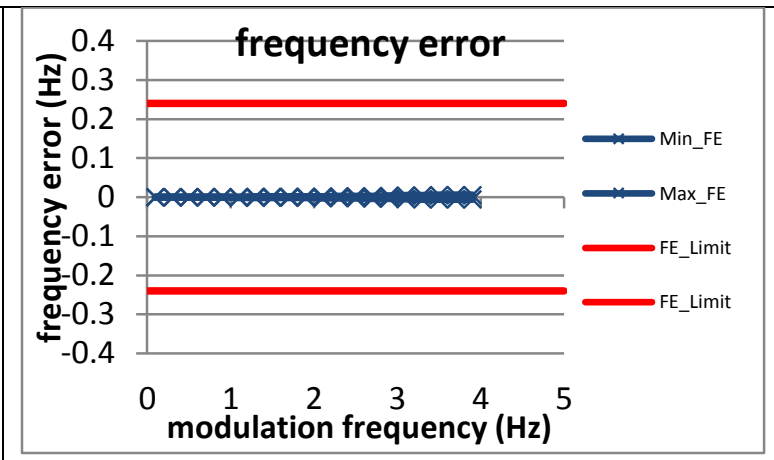

Figure 3230: Fs = 20 FPS

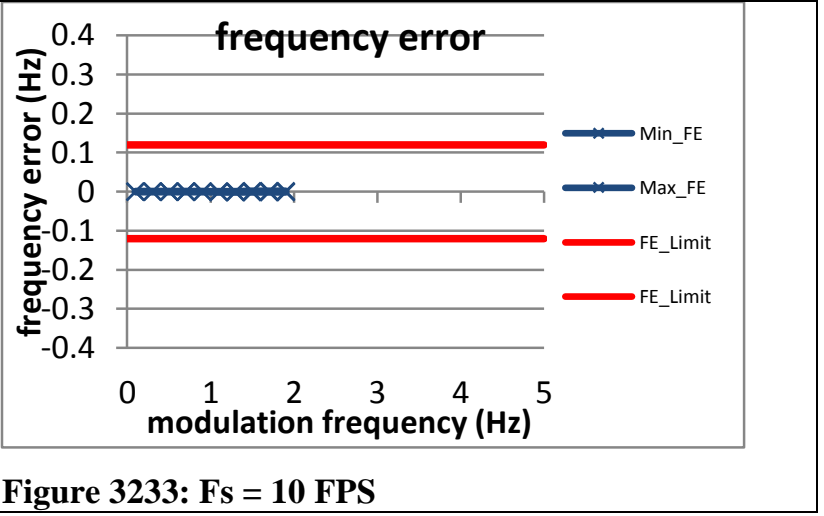


7.3.10 PMU I dynamic bandwidth measurement: phase modulation frequency error: M class

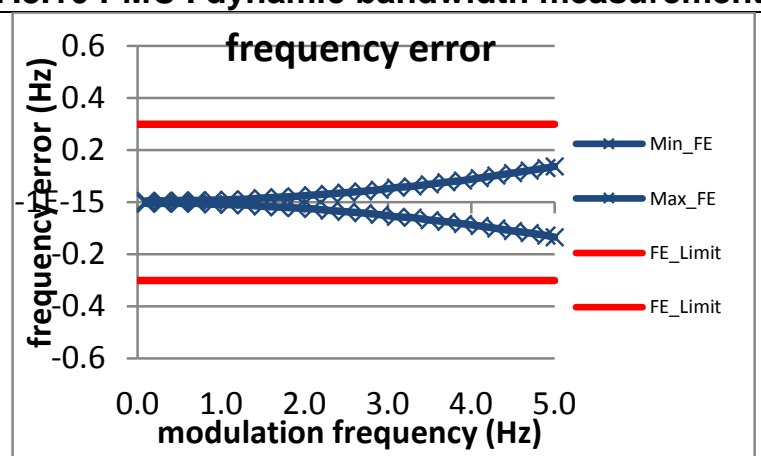

Figure 3234: Fs = 60 FPS

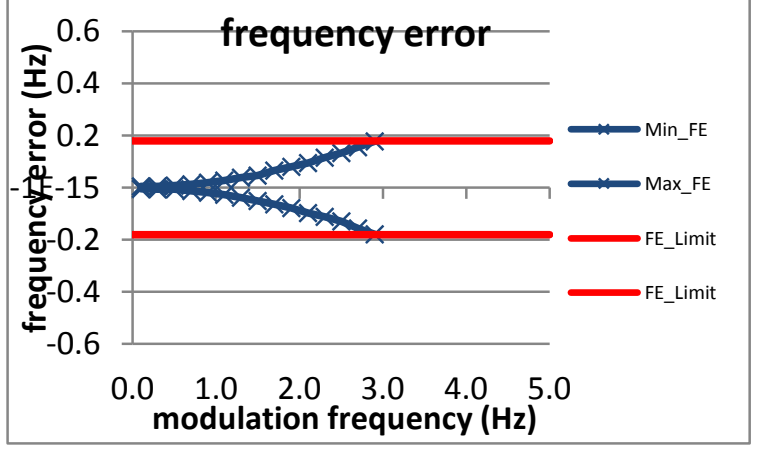

Figure 3237: Fs = 15 FPS

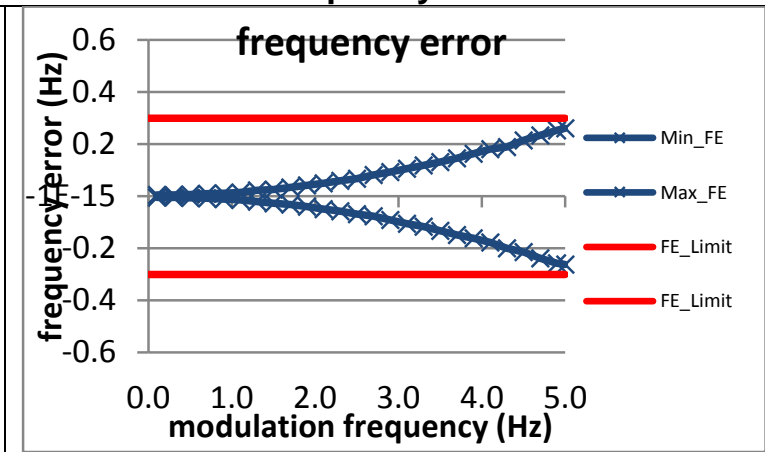

Figure 3235: Fs = 30 FPS

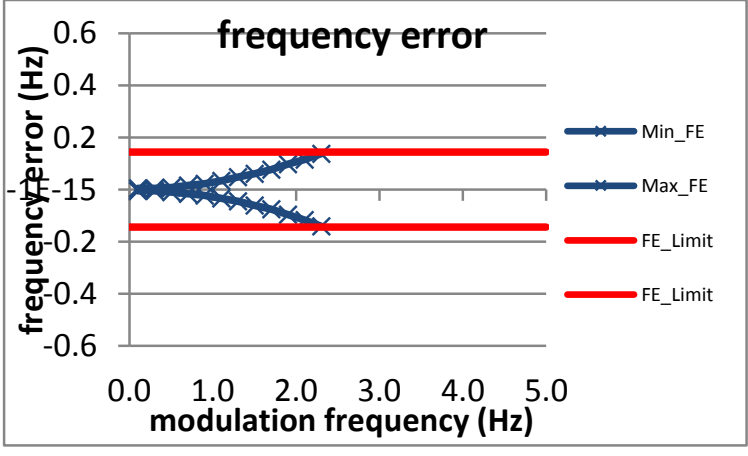

Figure 3238: Fs = 12 FPS

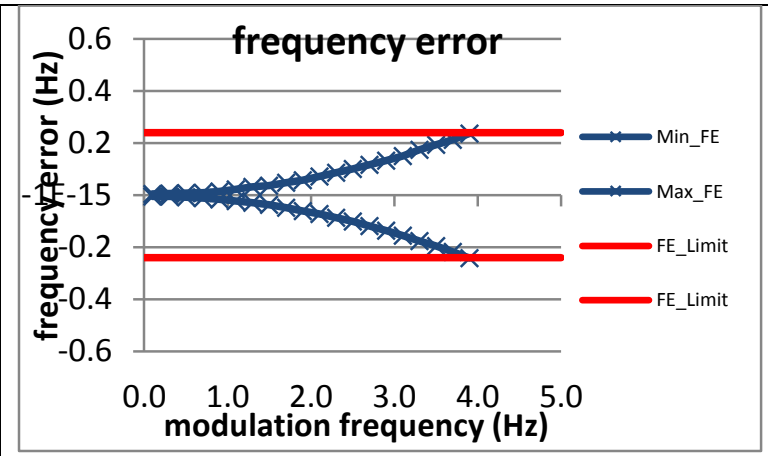

Figure 3236: Fs = 20 FPS

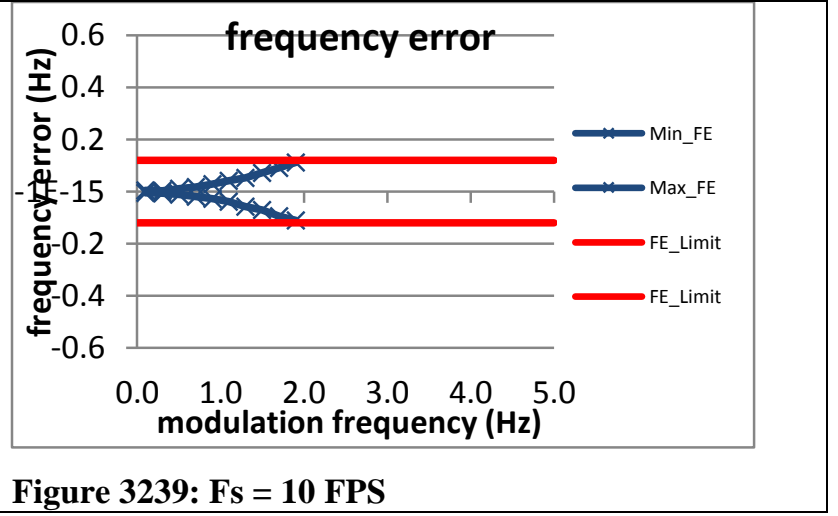


7.3.11 PMU J dynamic bandwidth measurement: phase modulation frequency error: $\mathrm{M}$ class

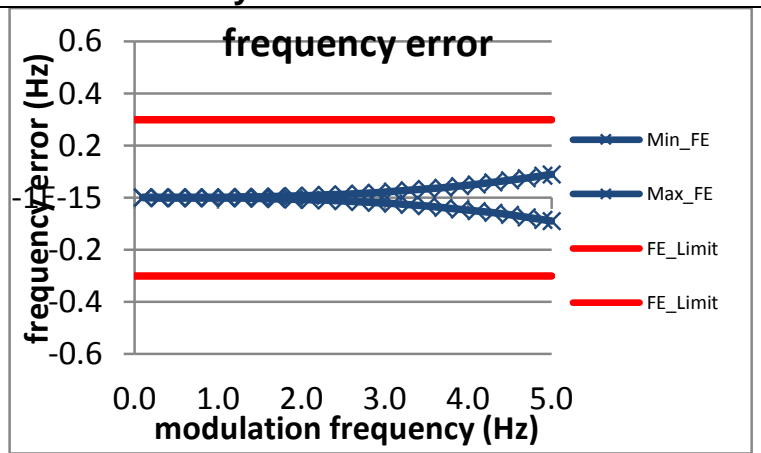

Figure 3240: Fs = 60 FPS

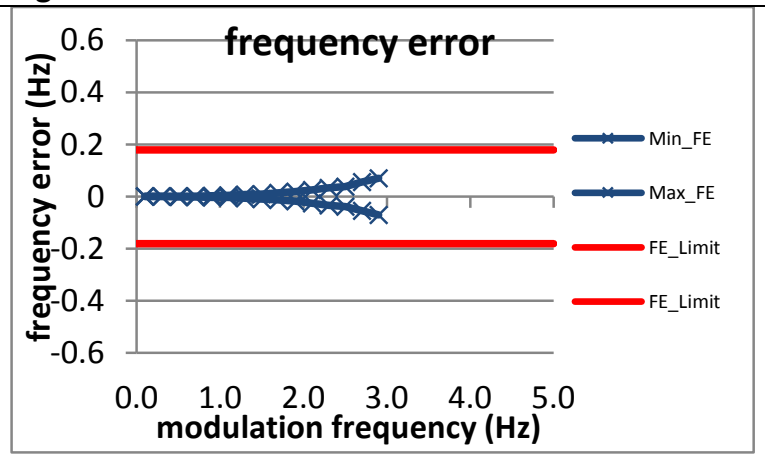

Figure 3243: Fs = 15 FPS

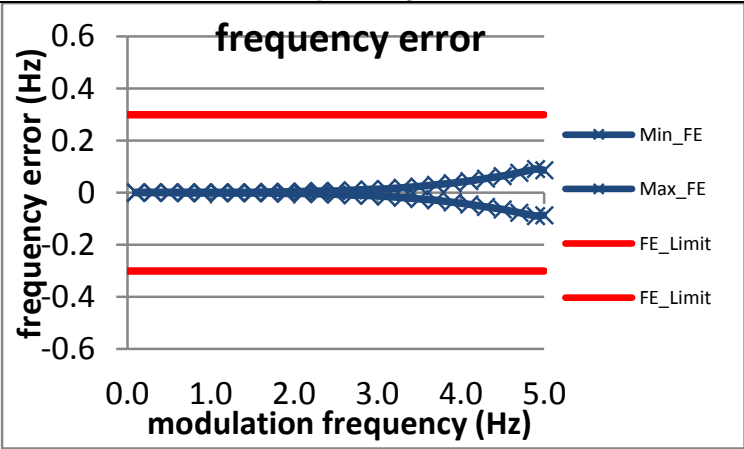

Figure 3241: Fs = 30 FPS

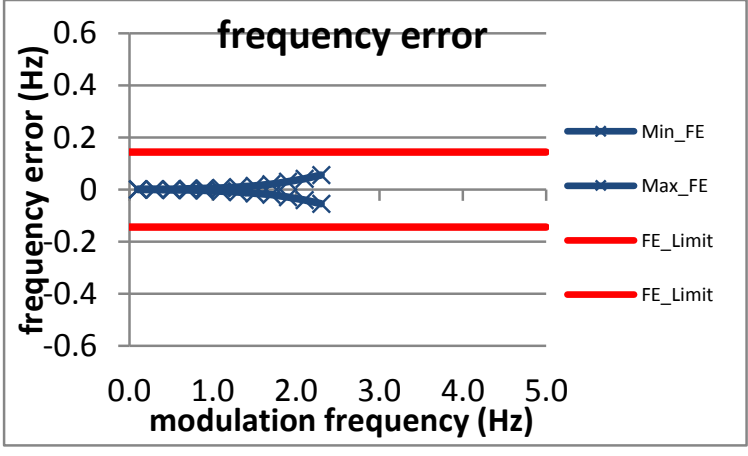

Figure 3244: Fs = 12 FPS

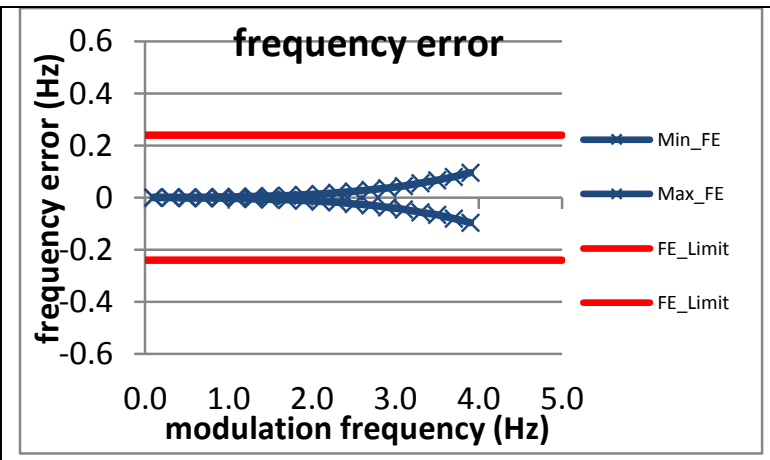

Figure 3242: Fs = 20 FPS

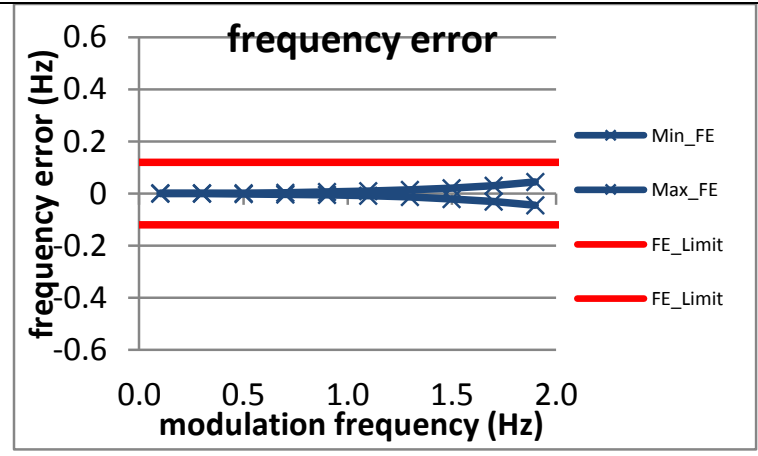

Figure 3245: Fs = 10 FPS 


\subsection{Dynamic bandwidth measurement: phase modulation ROCOF error}

7.4.1 C37.118.1 Annex C dynamic bandwidth measurement: phase modulation ROCOF error: $M$ class

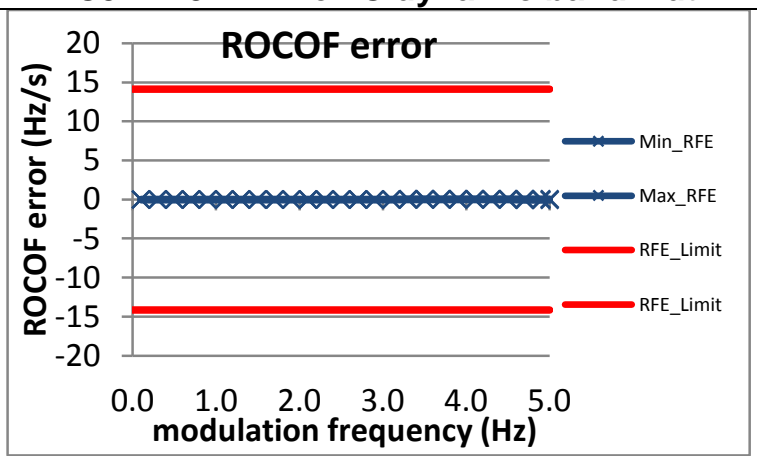

Figure 3246: Fs = 60 FPS

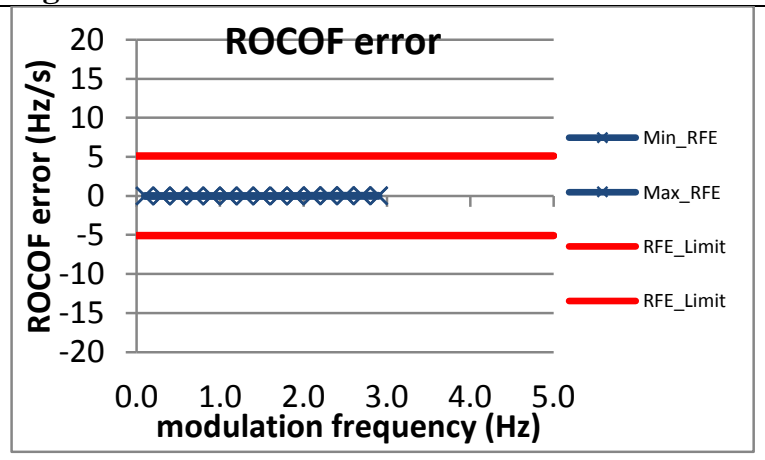

Figure 3249: Fs = 15 FPS

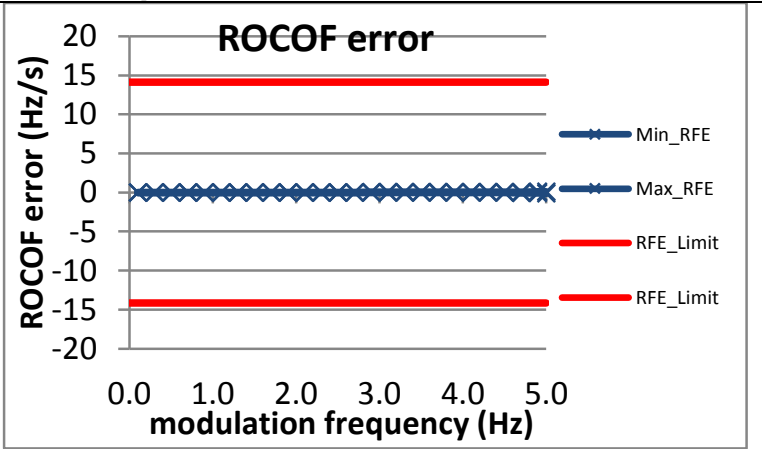

Figure 3247: Fs = 30 FPS

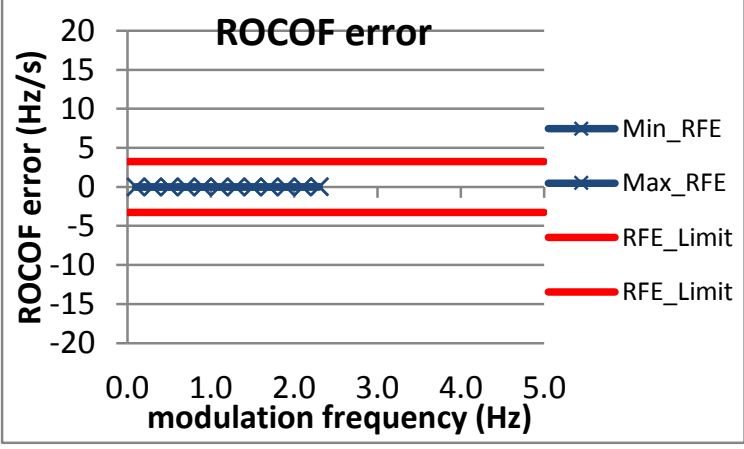

Figure 3250: Fs = 12 FPS

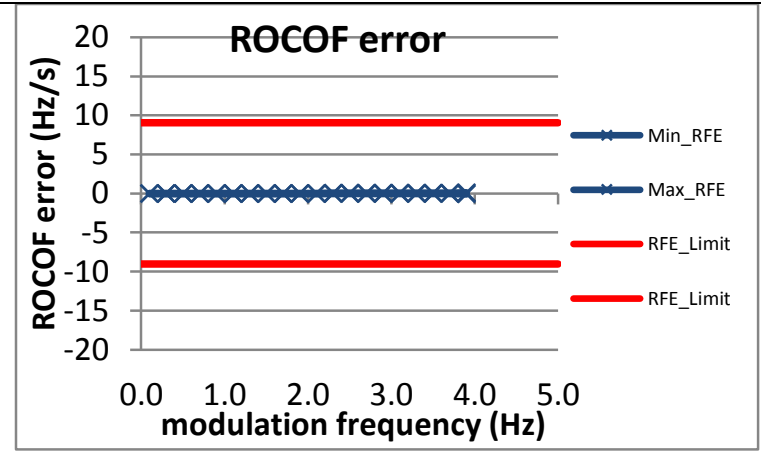

Figure 3248: Fs = 20 FPS

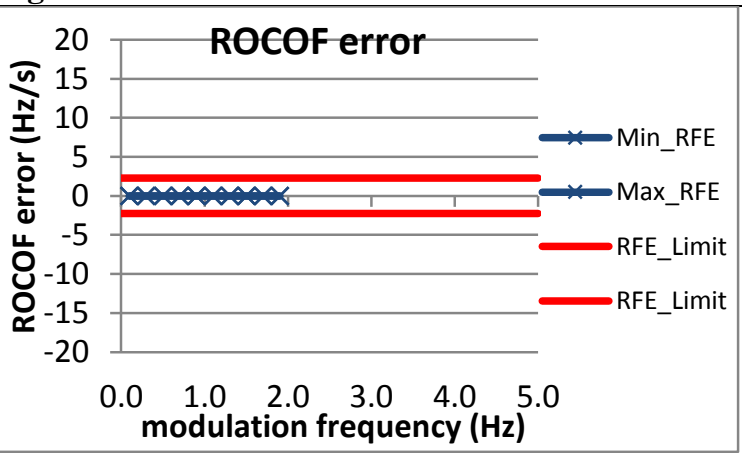

Figure 3251: Fs = 10 FPS 
7.4.2 PMU A dynamic bandwidth measurement: phase modulation ROCOF error: M class

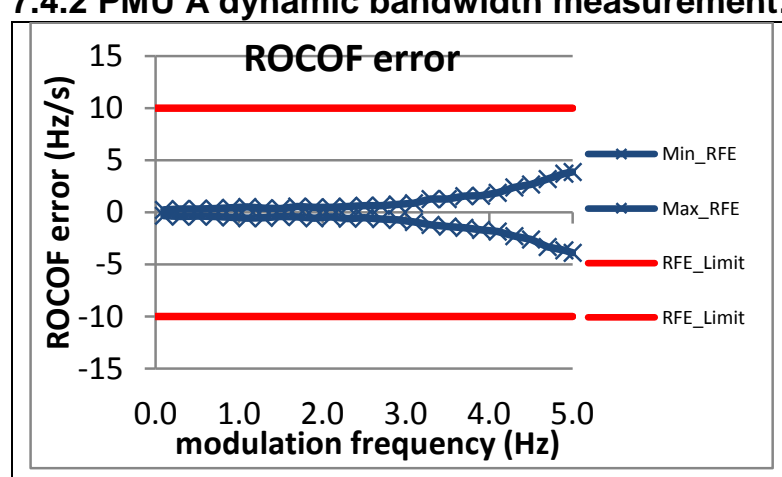

Figure 3252: Fs = 60 FPS

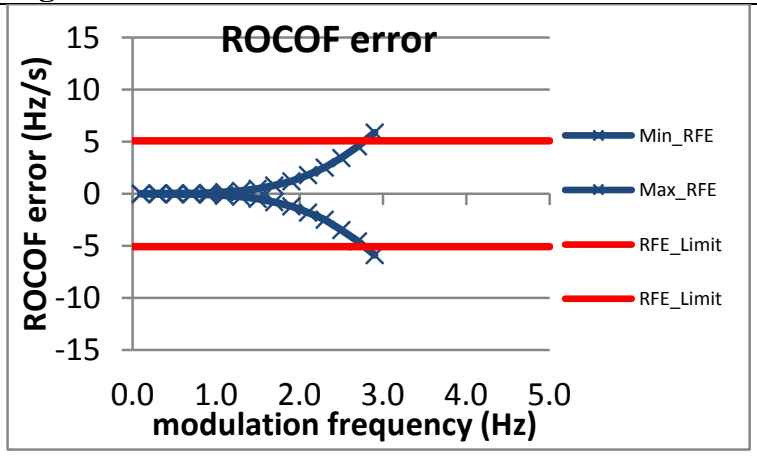

Figure 3255: Fs = 15 FPS

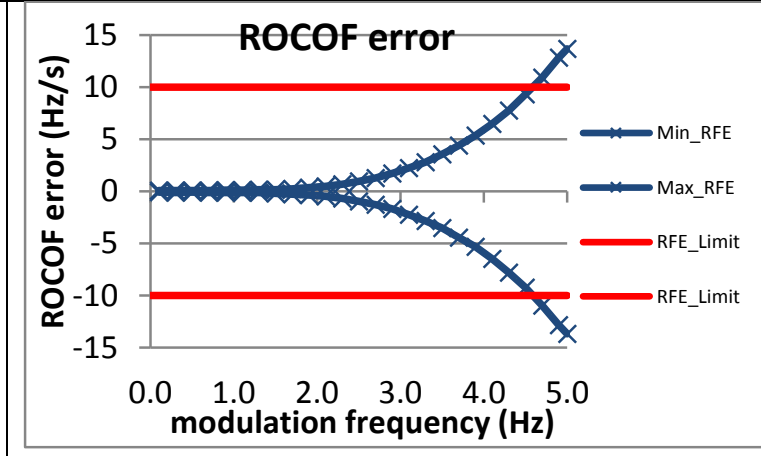

Figure 3253: Fs = 30 FPS

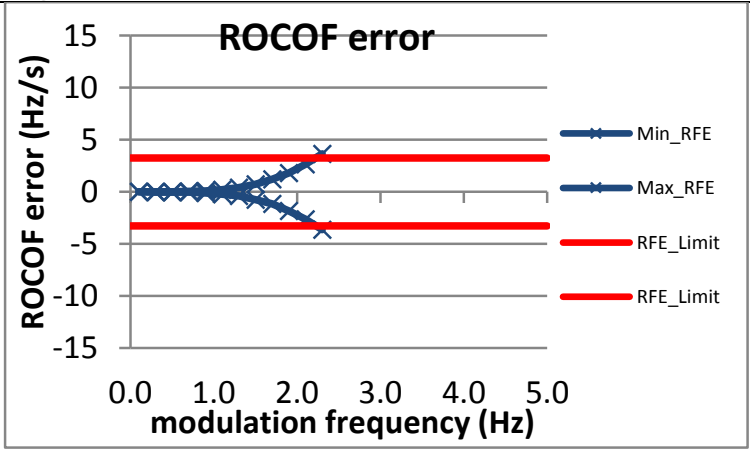

Figure 3256: Fs = 12FPS

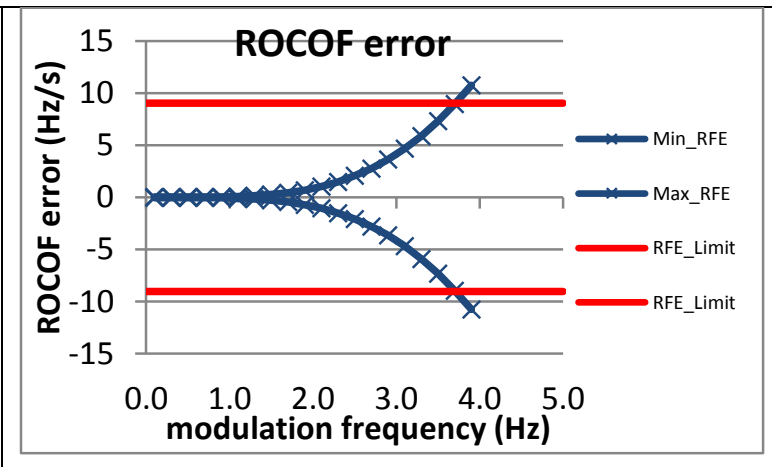

Figure 3254: Fs = 20 FPS

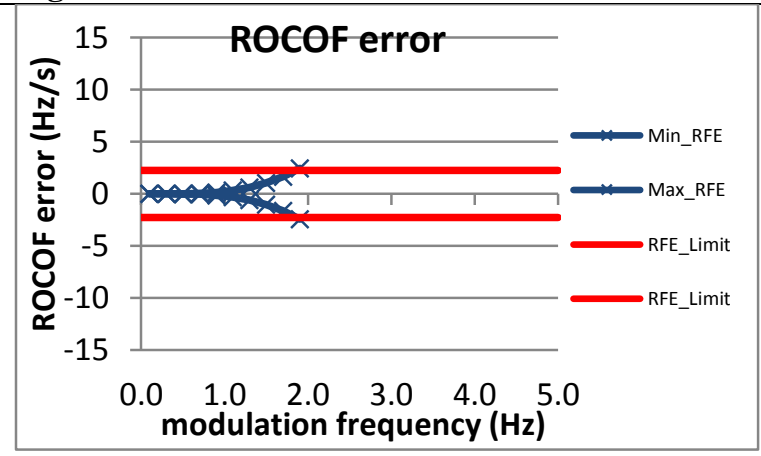

Figure 3257: Fs = 10 FPS 
7.4.3 PMU B dynamic bandwidth measurement: phase modulation ROCOF error: M class

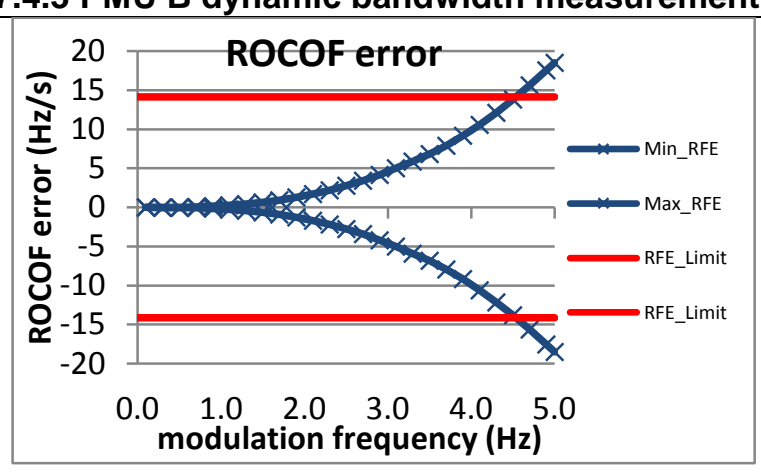

Figure 3258: Fs $=60 \mathrm{FPS}$

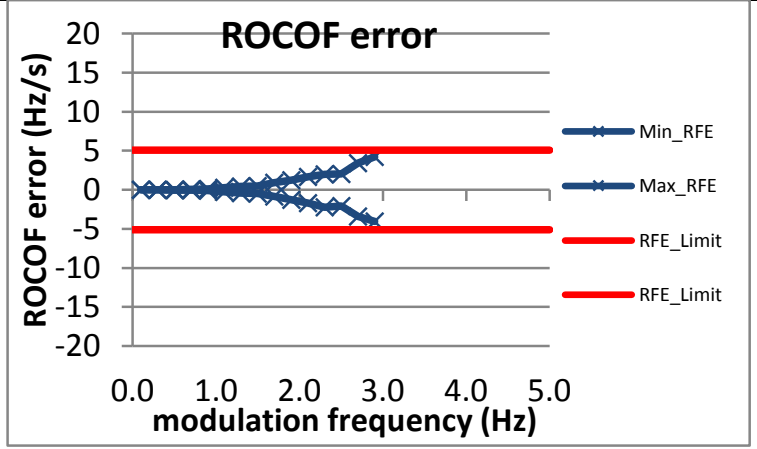

Figure 3261: Fs = 15 FPS

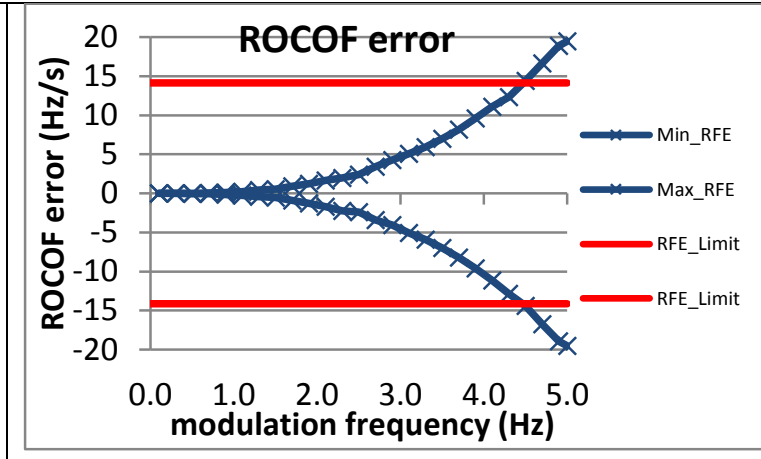

Figure 3259: Fs = 30 FPS

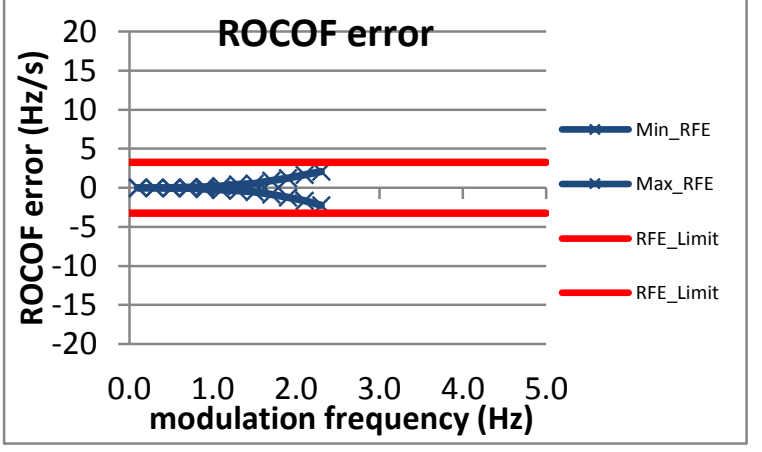

Figure 3262: Fs = 12 FPS

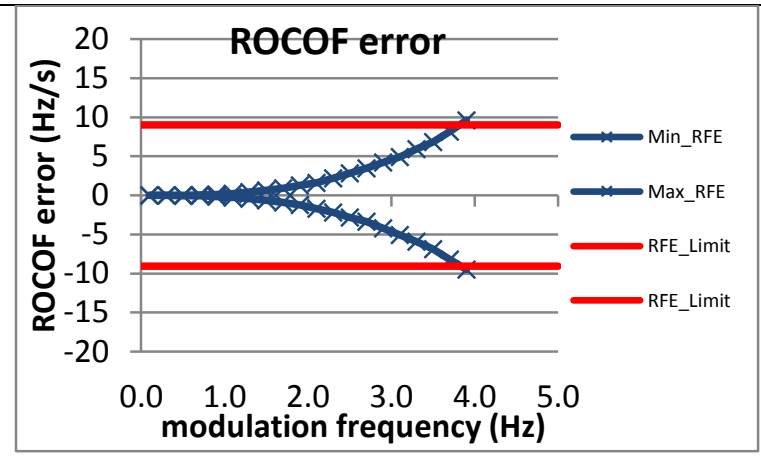

Figure 3260: Fs = 20 FPS

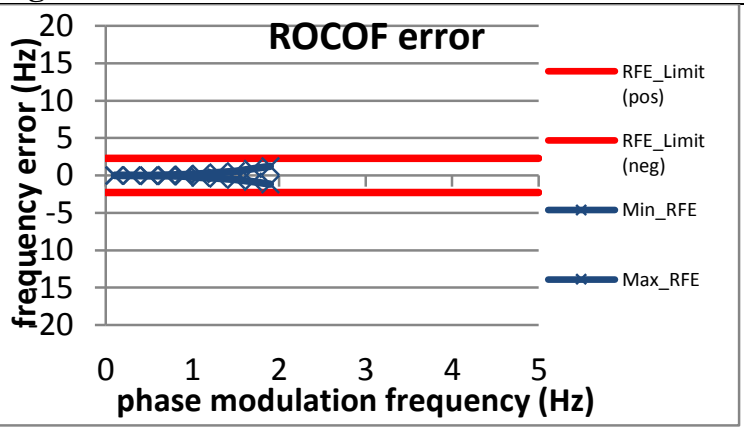

Figure 3263: Fs = 10 FPS 
7.4.4 PMU C dynamic bandwidth measurement: phase modulation ROCOF error: $M$ class

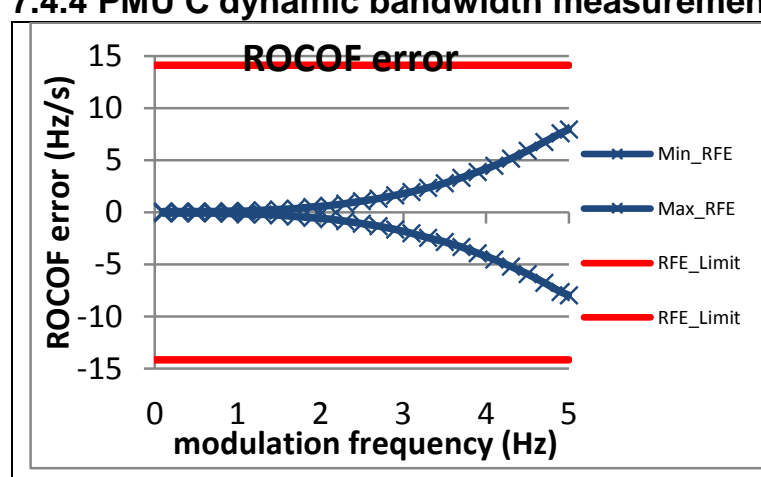

Figure 3264: Fs = 60 FPS

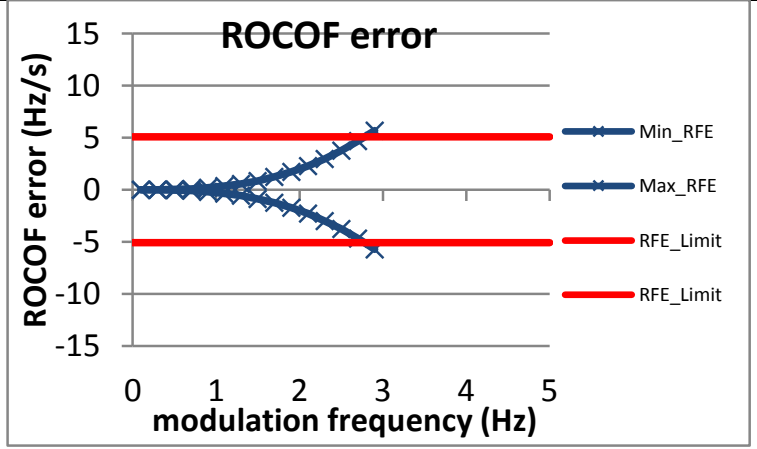

Figure 3267: Fs = 15 FPS

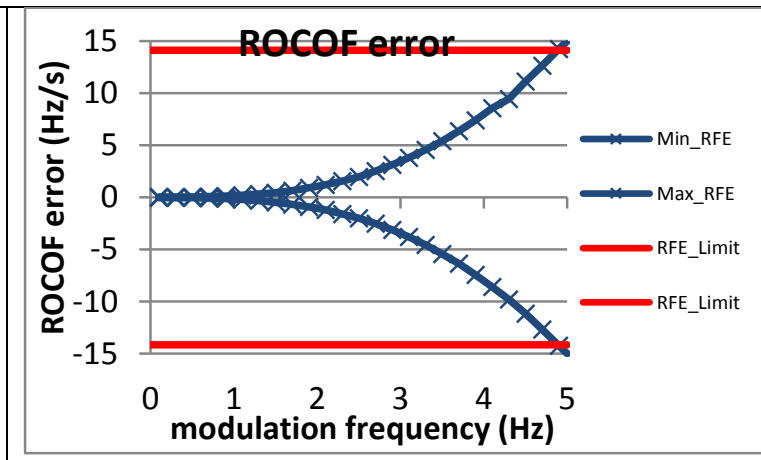

Figure 3265: Fs = 30 FPS

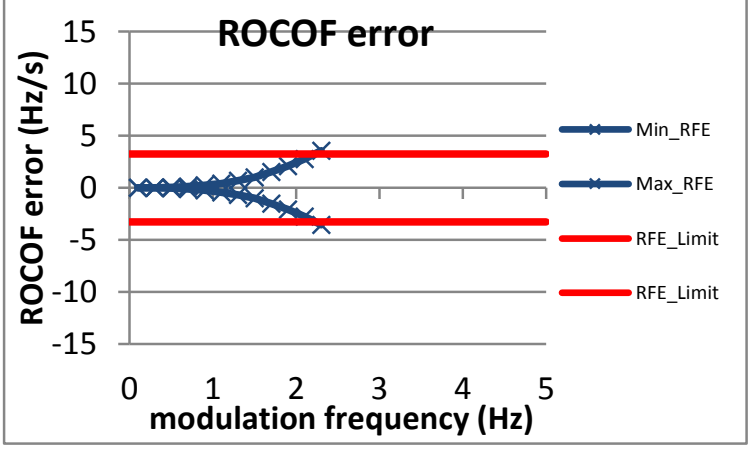

Figure 3268: Fs = 12 FPS

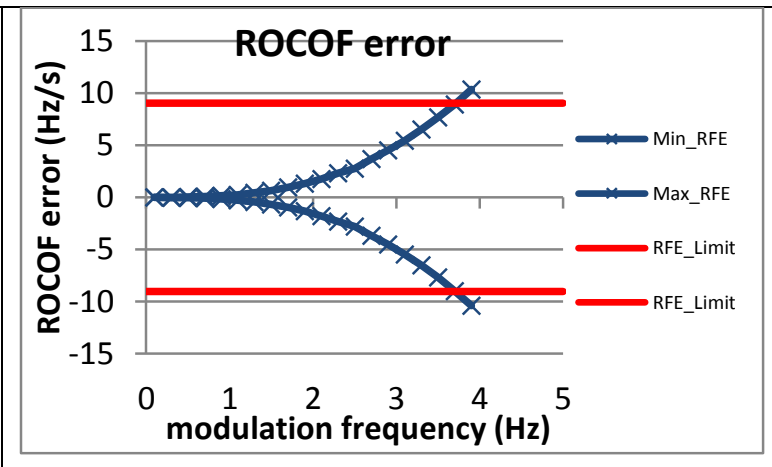

Figure 3266: Fs = 20 FPS

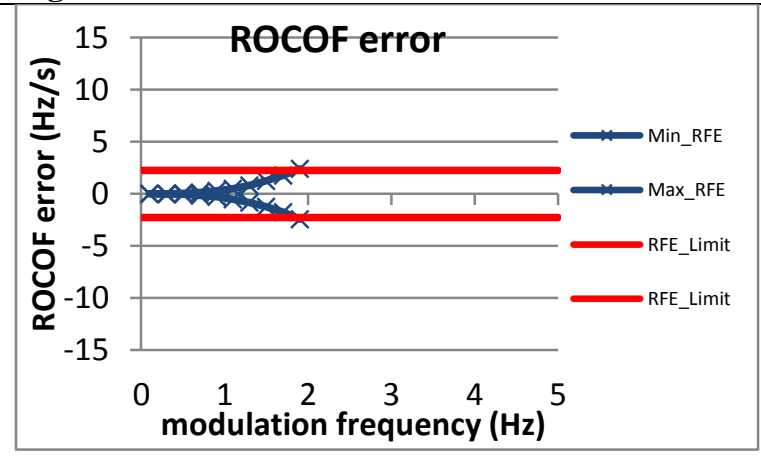

Figure 3269: Fs = 10 FPS 
7.4.5 PMU D dynamic bandwidth measurement: phase modulation ROCOF error: M class

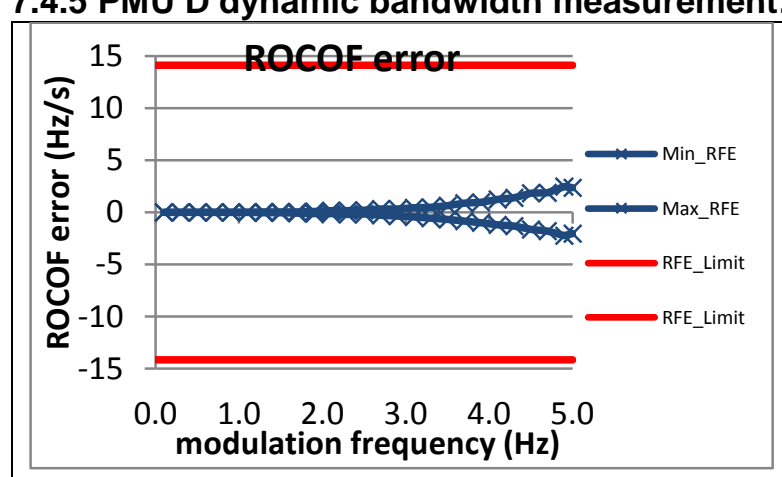

Figure 3270: Fs = 60 FPS

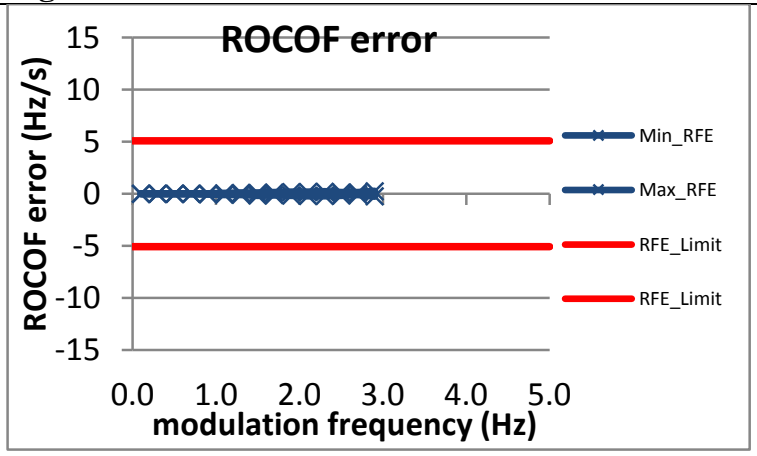

Figure 3273: Fs = 15 FPS

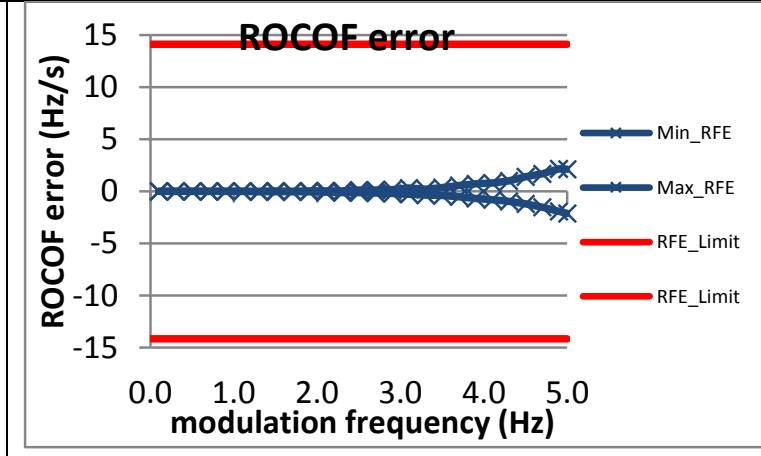

Figure 3271: Fs = 30 FPS

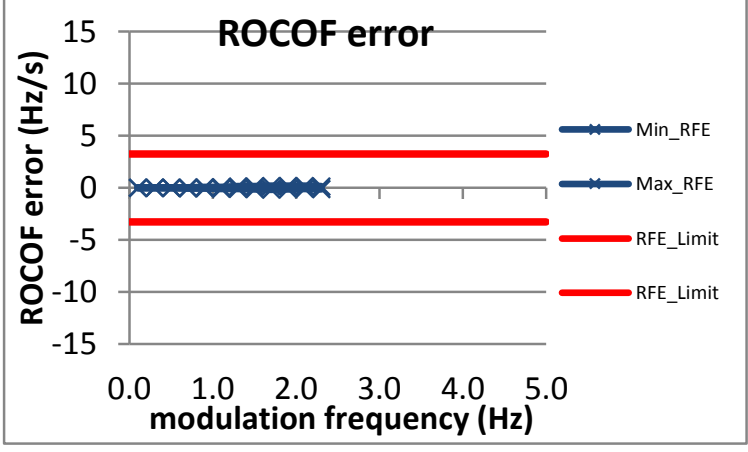

Figure 3274: Fs = 12 FPS

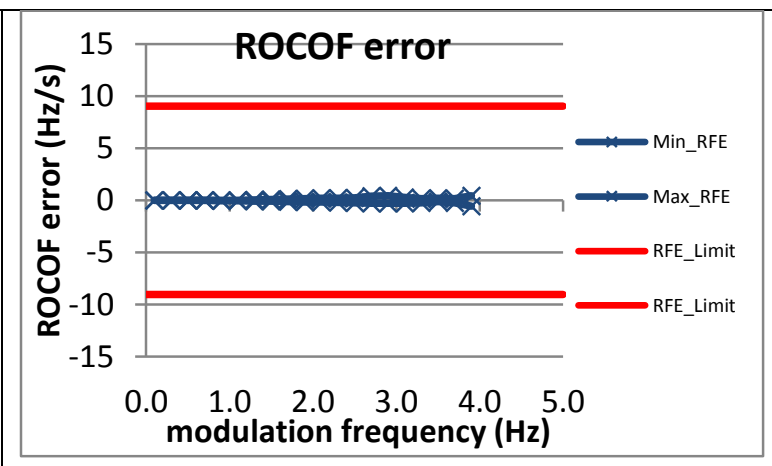

Figure 3272: Fs = 20 FPS

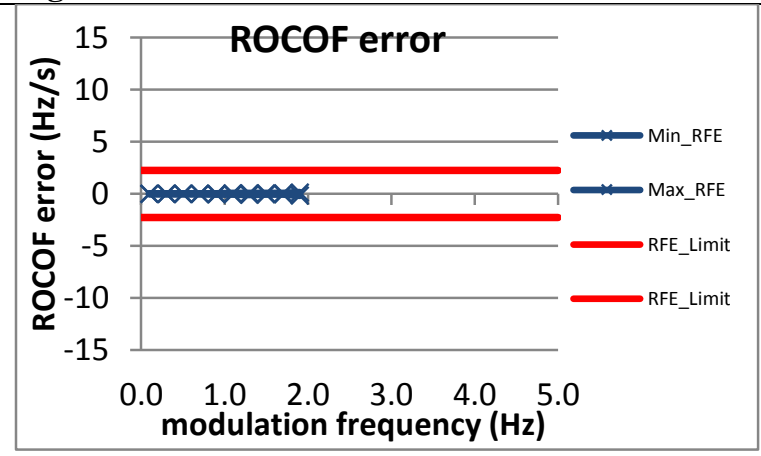

Figure 3275: Fs = 10 FPS 
7.4.6 PMU E dynamic bandwidth measurement: phase modulation ROCOF error: M class

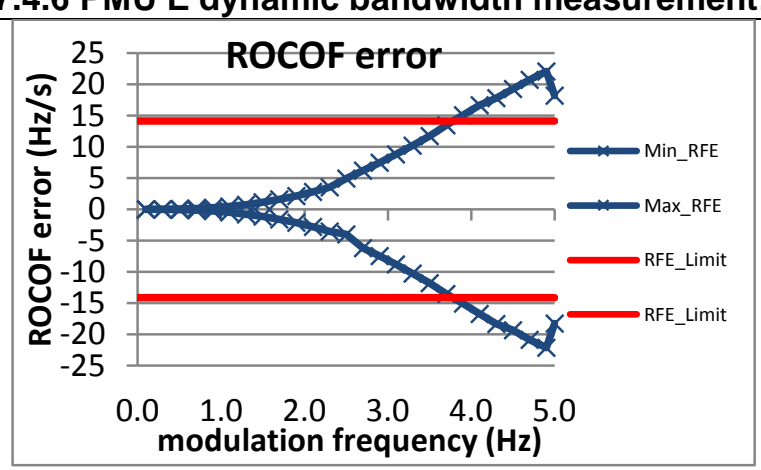

Figure 3276: Fs = 60 FPS

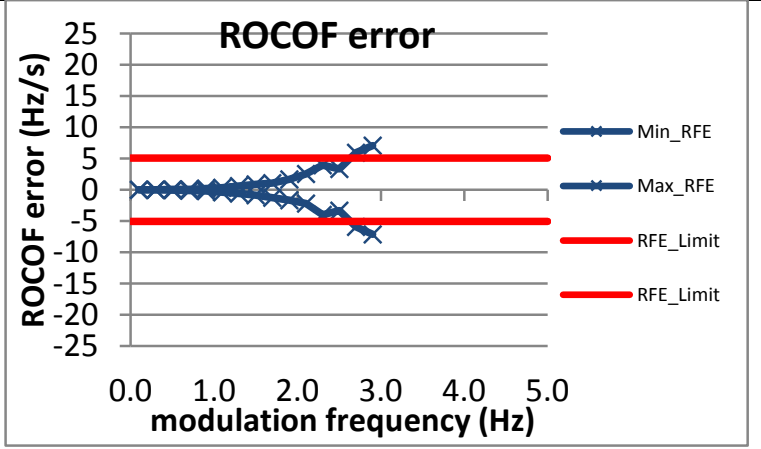

Figure 3279: Fs = 15 FPS

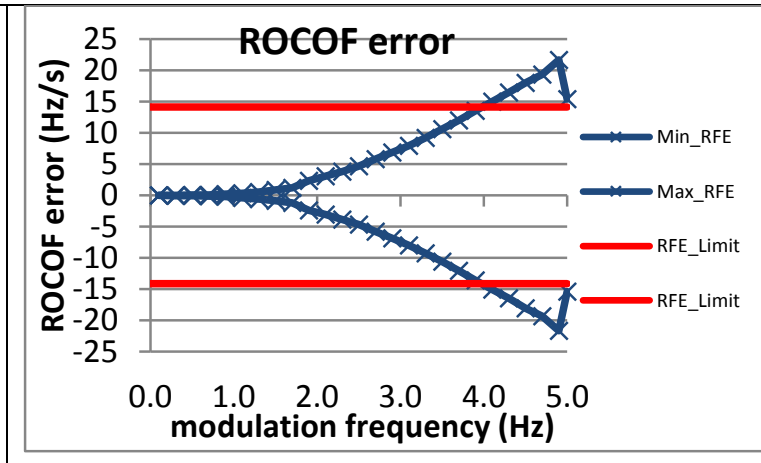

Figure 3277: Fs = 30 FPS

MISSING DATA

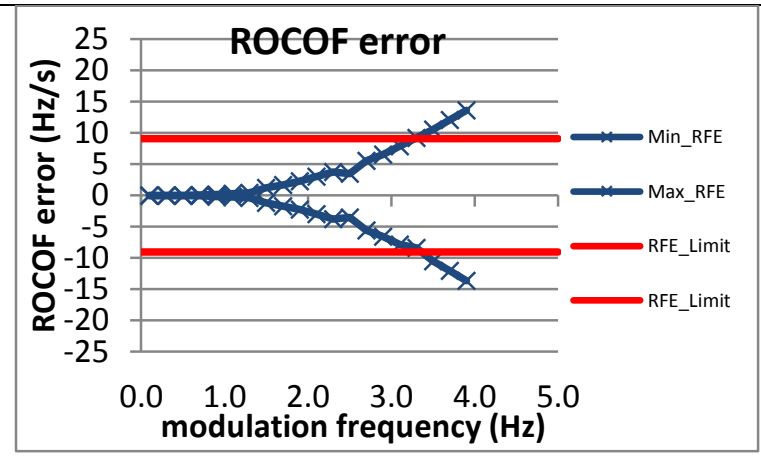

Figure 3278: Fs = 20 FPS

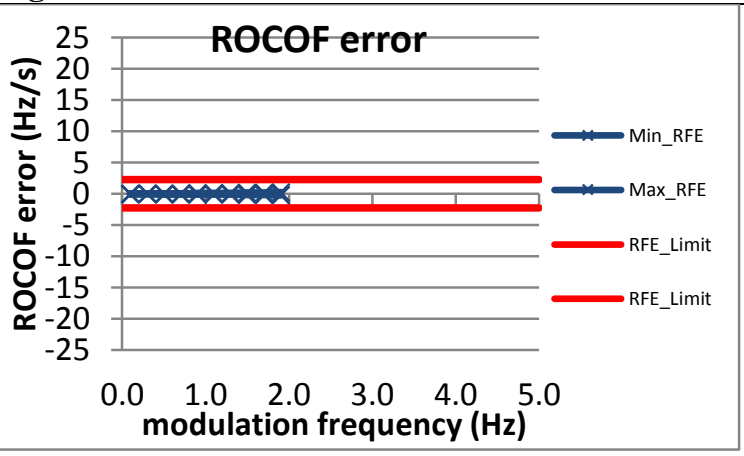

Figure 3280: Fs = 10 FPS 


\subsubsection{PMU F dynamic bandwidth measurement: phase modulation ROCOF error: M class}

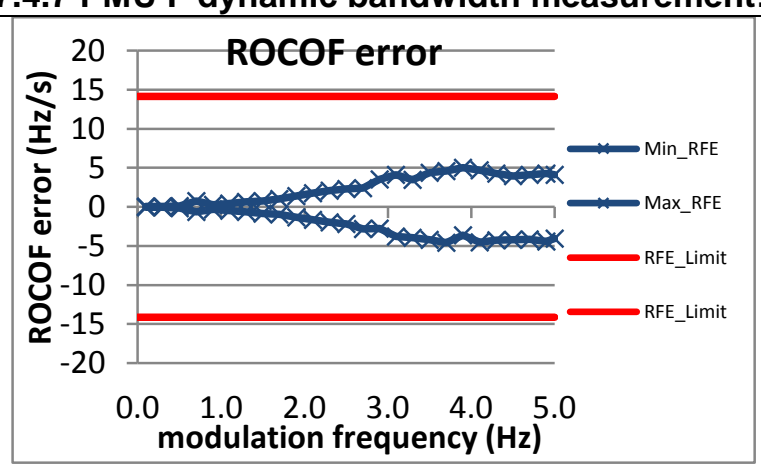

Figure 3281: Fs = $60 \mathrm{FPS}$

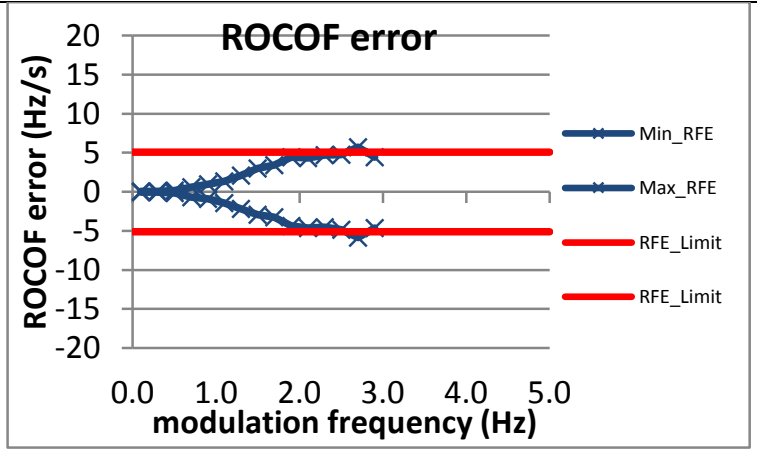

Figure 3284: Fs = 15 FPS

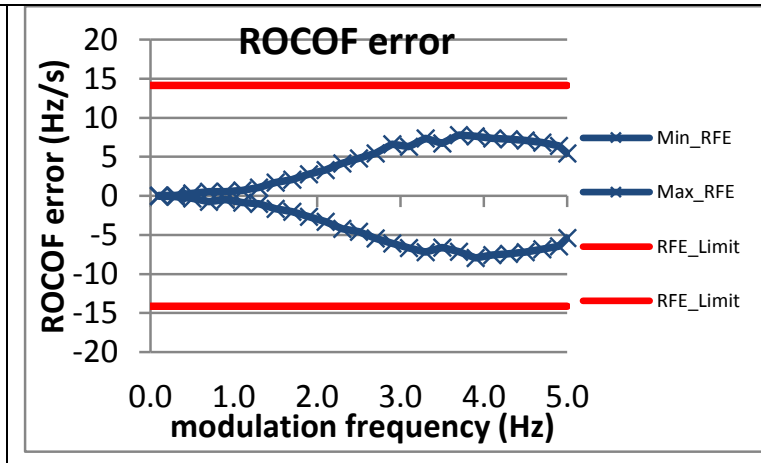

Figure 3282: $\mathrm{Fs}=30 \mathrm{FPS}$

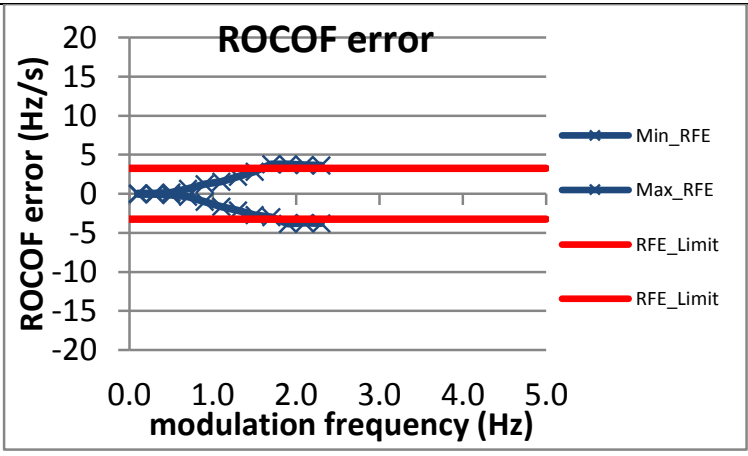

Figure 3285: Fs = 12 FPS

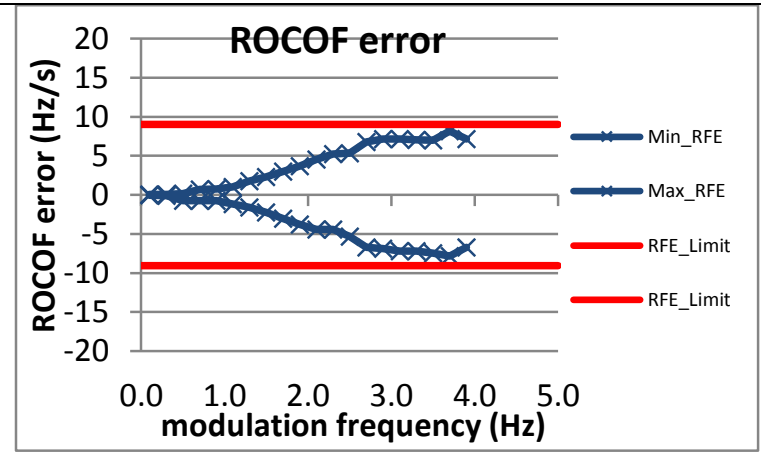

Figure 3283: Fs = 20 FPS

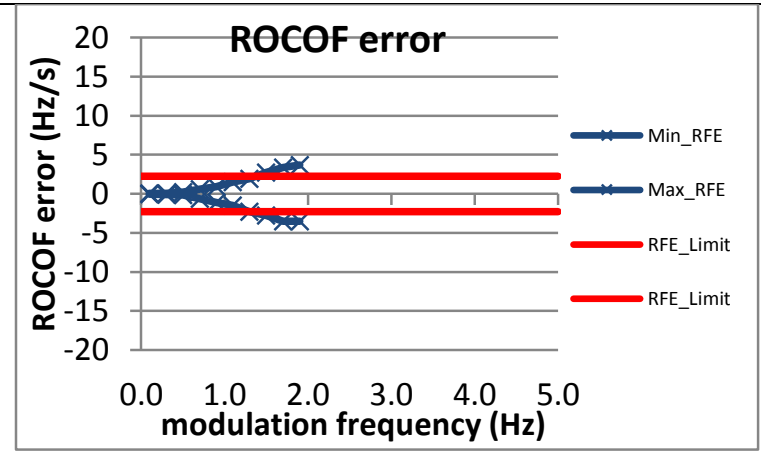

Figure 3286: Fs = 10 FPS 
7.4.8 PMU G * dynamic bandwidth measurement: phase modulation ROCOF error: M class

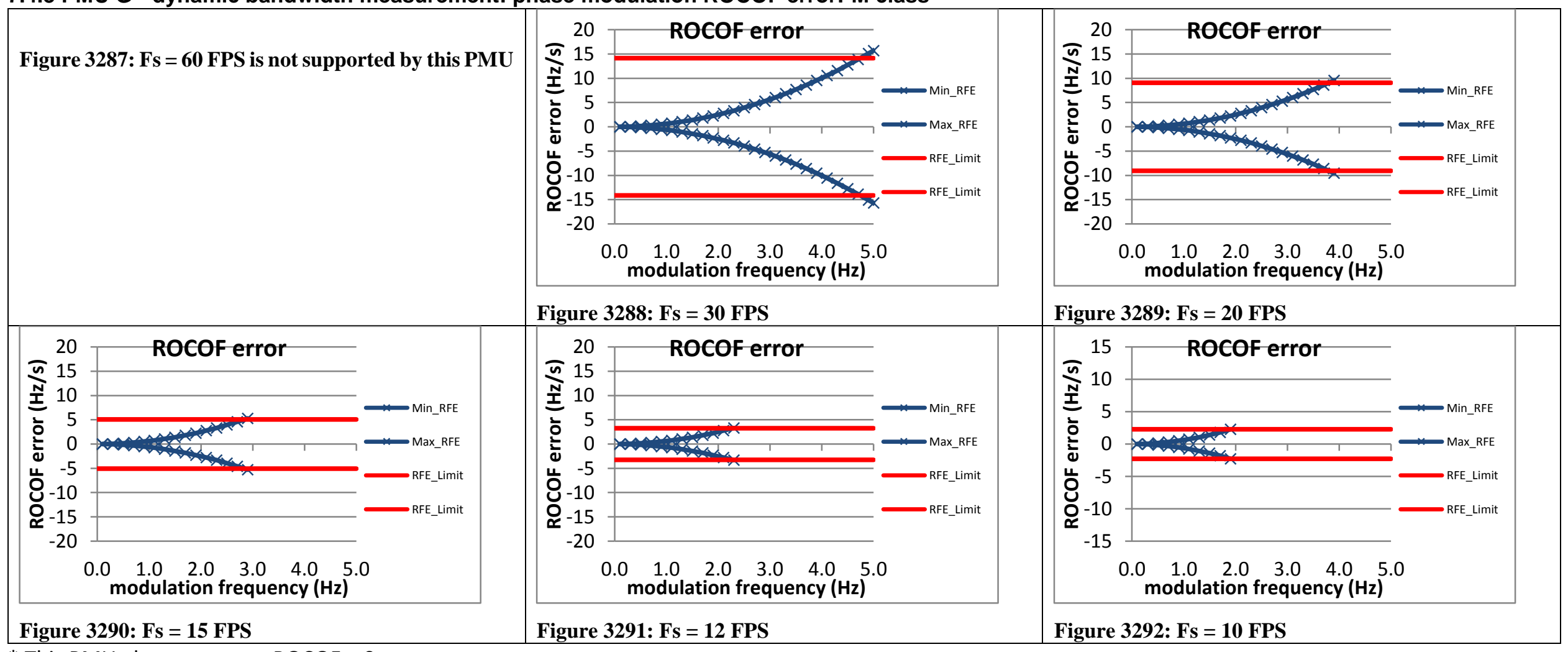

* This PMU always outputs ROCOF $=0$ 
7.4.9 PMU H dynamic bandwidth measurement: phase modulation ROCOF error: M class

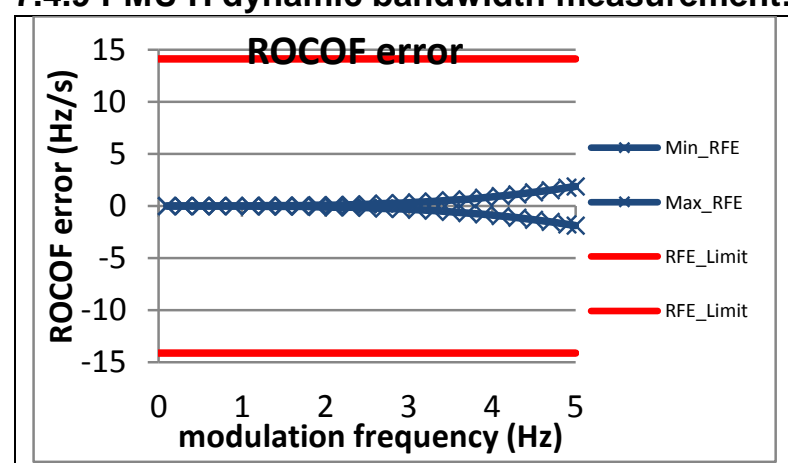

Figure 3293: Fs = 60 FPS

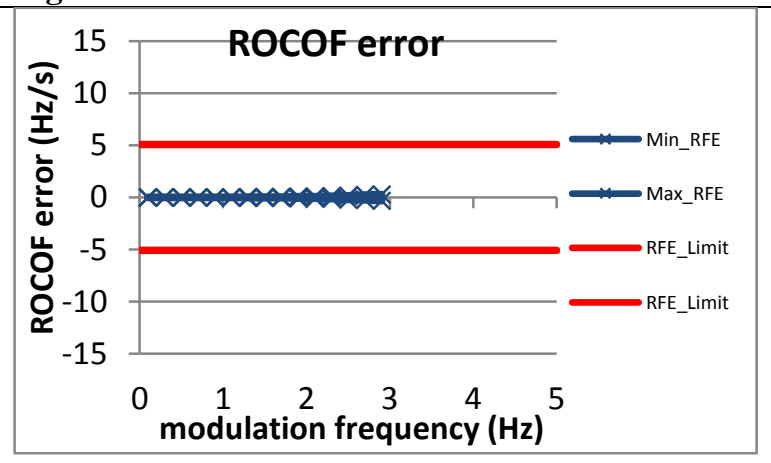

Figure 3296: Fs = 15 FPS

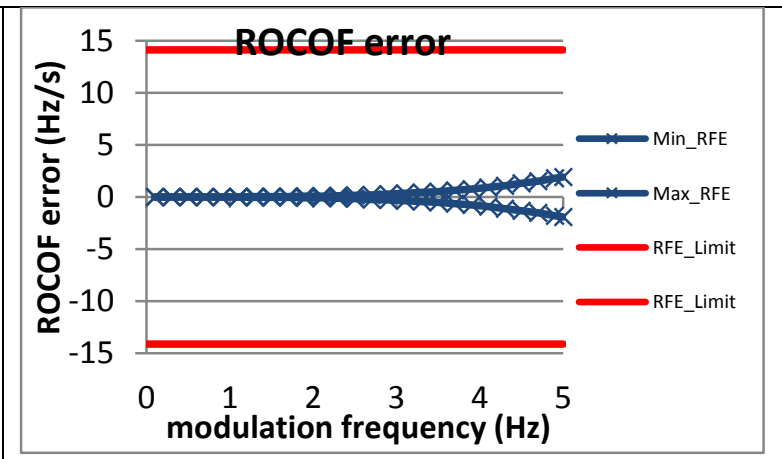

Figure 3294: Fs = 30 FPS

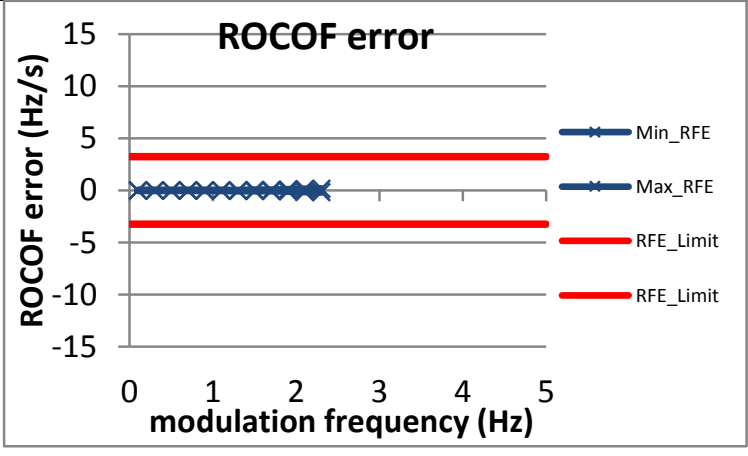

Figure 3297: Fs = 12 FPS

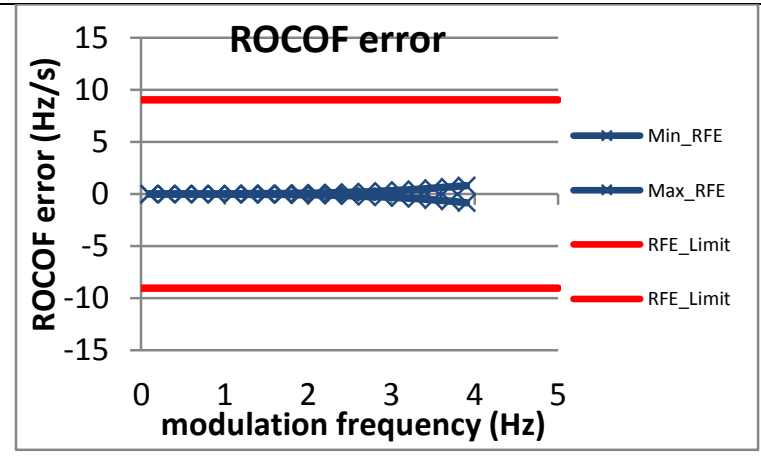

Figure 3295: Fs = 20 FPS

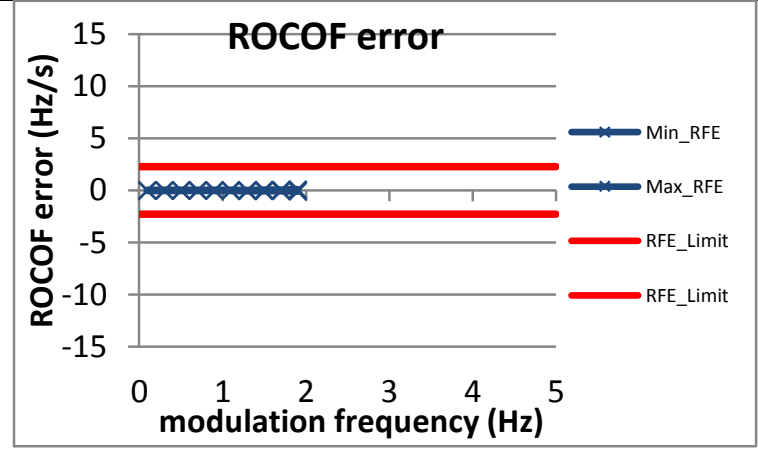

Figure 3298: Fs = 10 FPS 
7.4.10 PMU I dynamic bandwidth measurement: phase modulation ROCOF error: M class

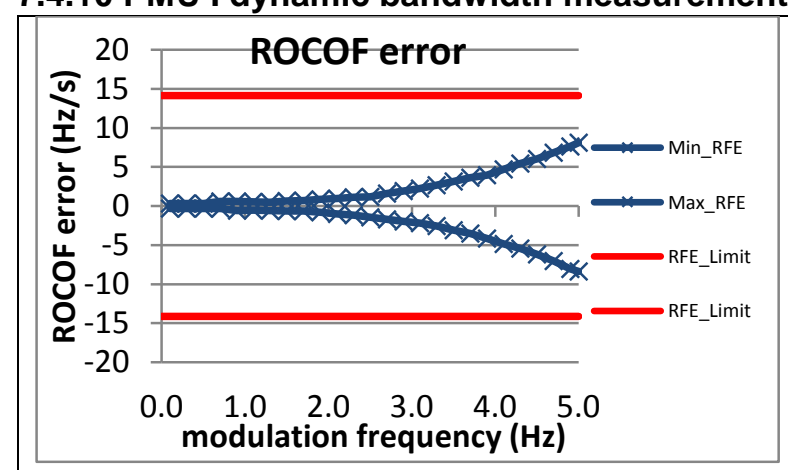

Figure 3299: Fs = 60 FPS

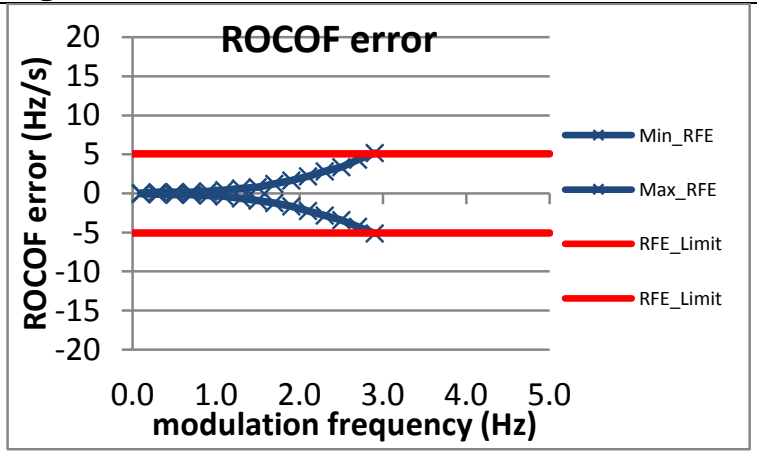

Figure 3302: Fs = 15 FPS

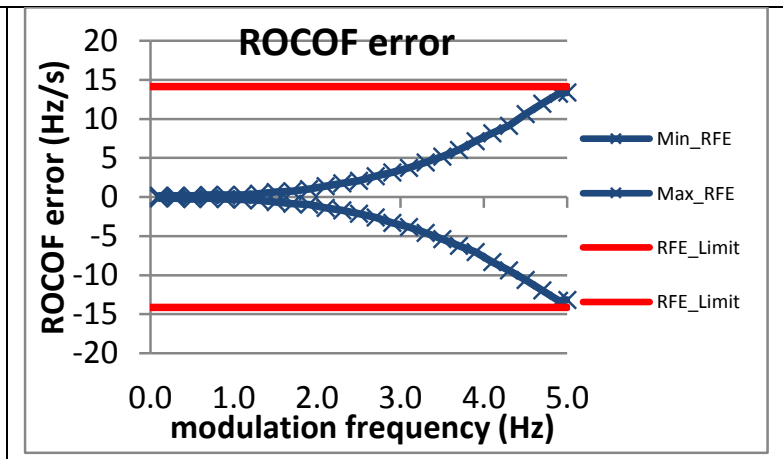

Figure 3300: Fs = 30 FPS

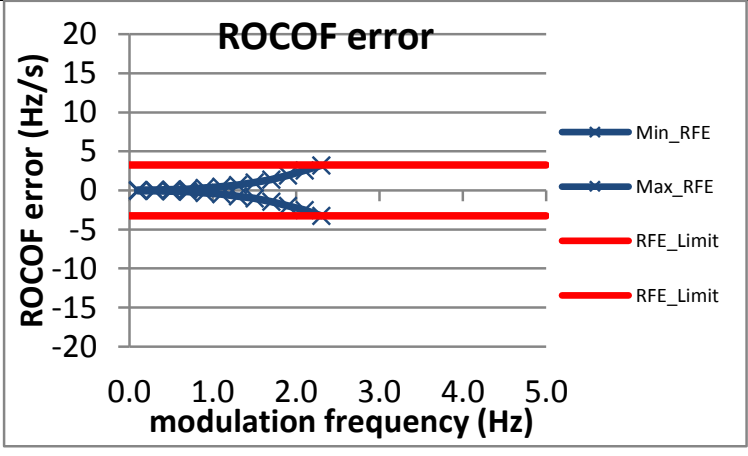

Figure 3303: Fs = 12 FPS

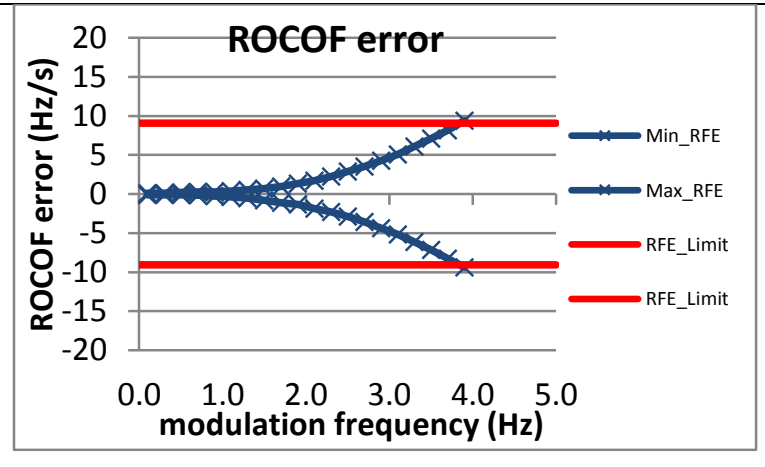

Figure 3301: Fs = 20 FPS

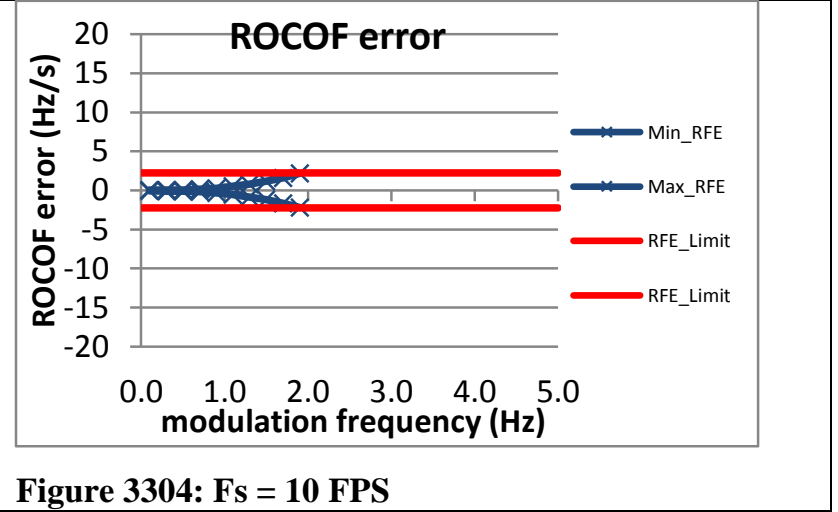


7.4.11 PMU J dynamic bandwidth measurement: phase modulation ROCOF error: M class

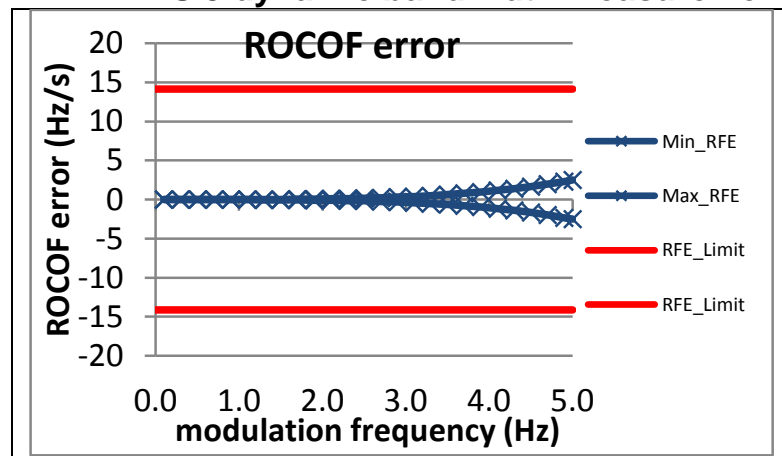

Figure 3305: Fs $=60$ FPS

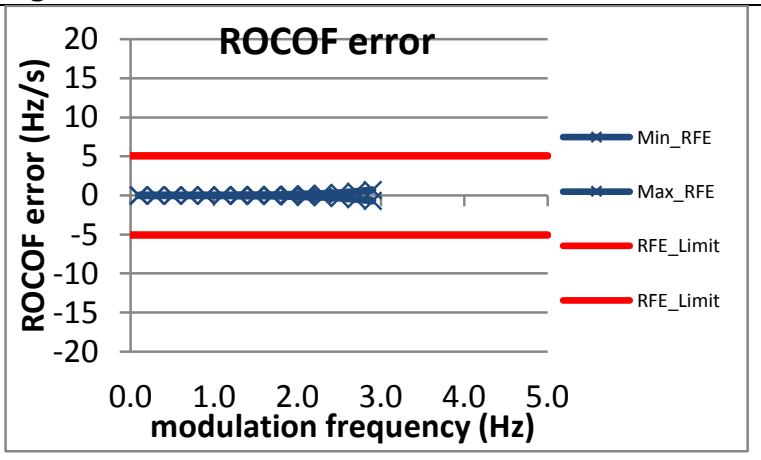

Figure 3308: Fs = 15 FPS

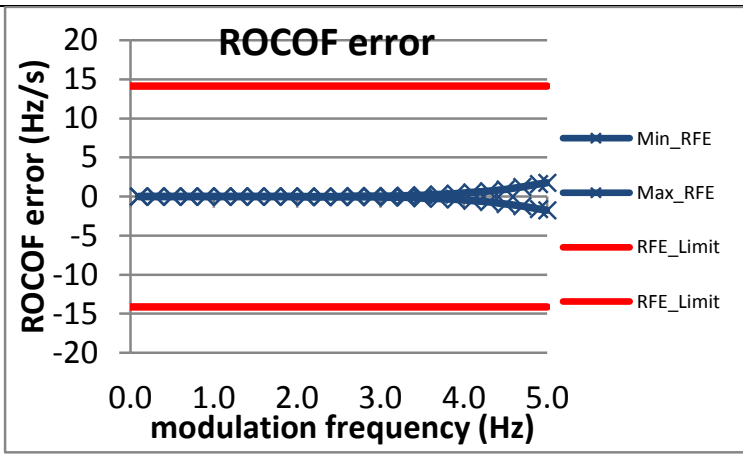

Figure 3306: $\mathrm{Fs}=30 \mathrm{FPS}$

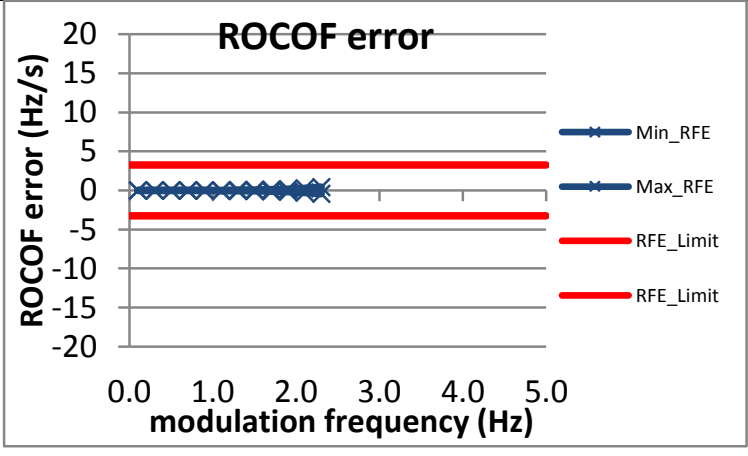

Figure 3309: Fs = 12 FPS

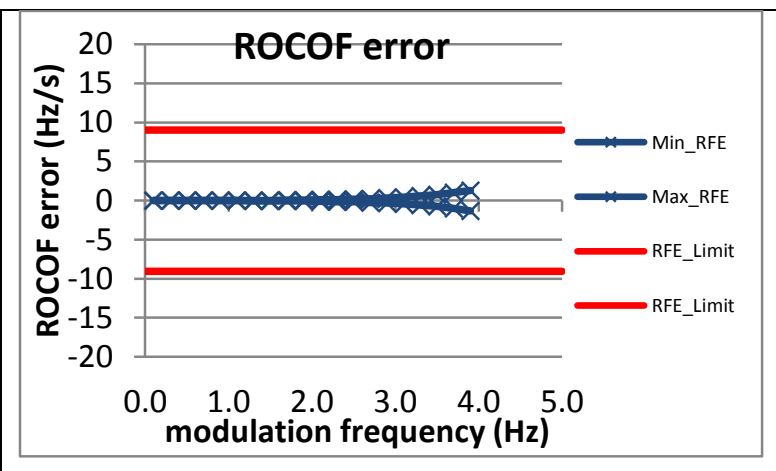

Figure 3307: Fs = 20 FPS

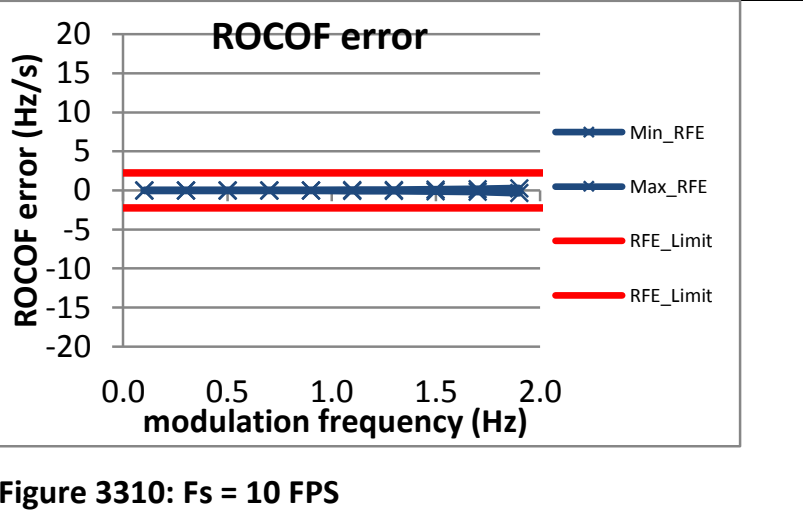




\subsection{Phase modulation voltage TVE, P class}

7.5.1 C37.118.1 Annex C dynamic bandwidth measurement: phase modulation voltage TVE: P class

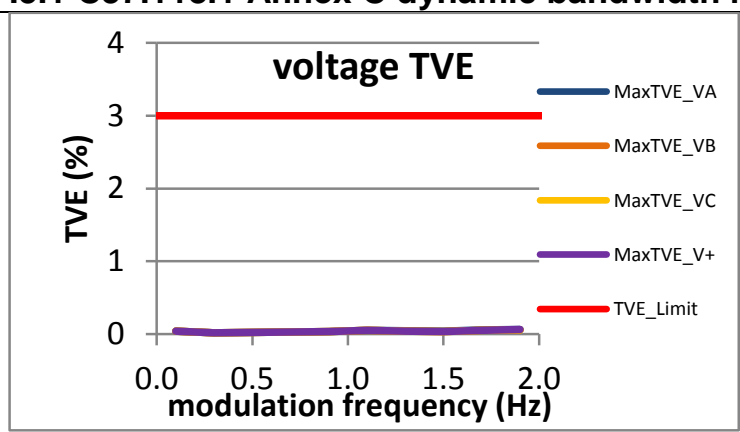

Figure 3311: Fs = 60 FPS

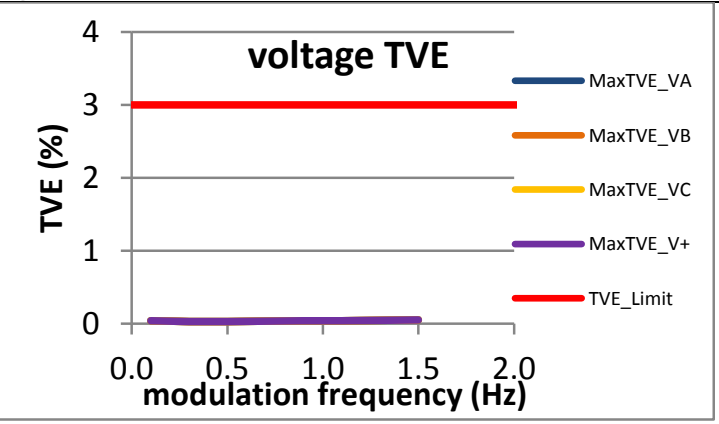

Figure 3314: Fs = 15 FPS

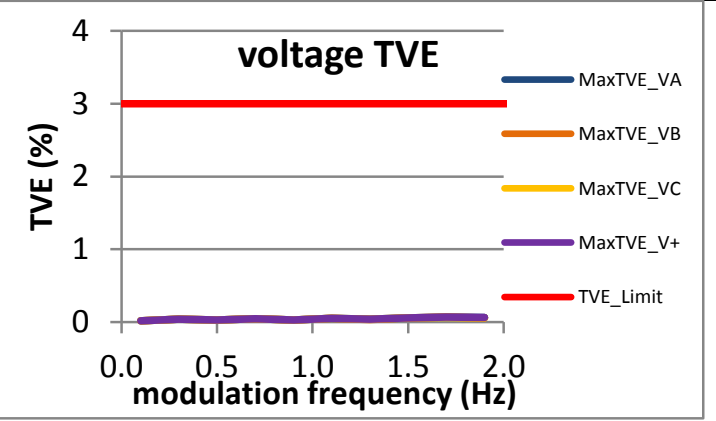

Figure 3312: Fs = 30 FPS

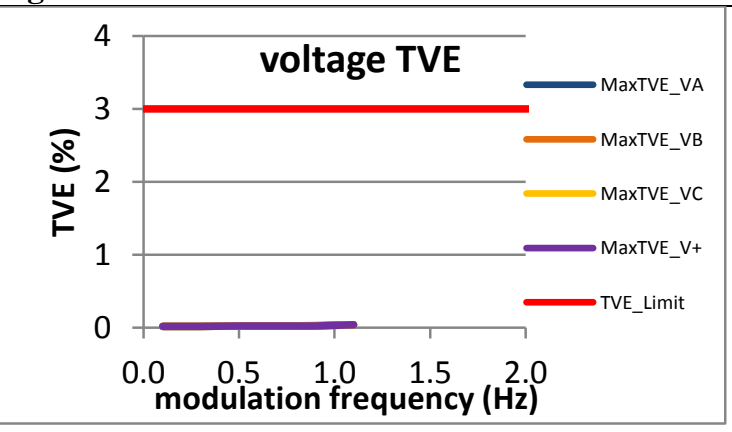

Figure 3315: Fs = 12 FPS

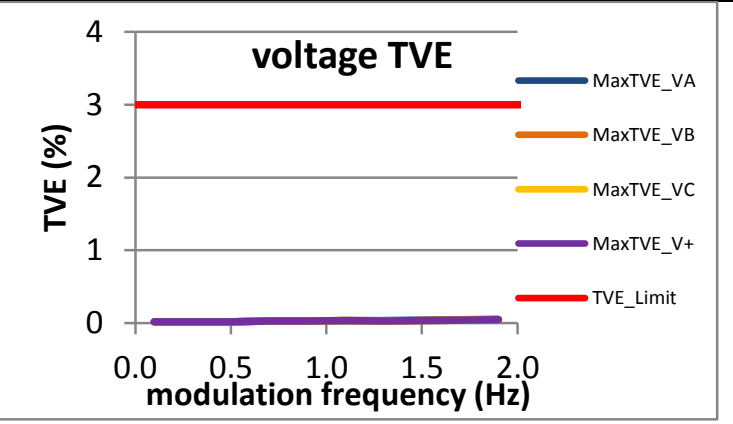

Figure 3313: Fs = 20 FPS

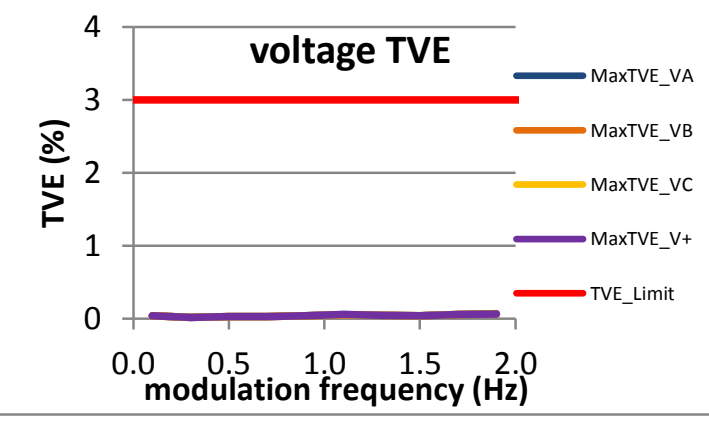

Figure 3316: Fs = 10 FPS 


\subsubsection{PMU A dynamic bandwidth measurement: phase modulation voltage TVE: P class}

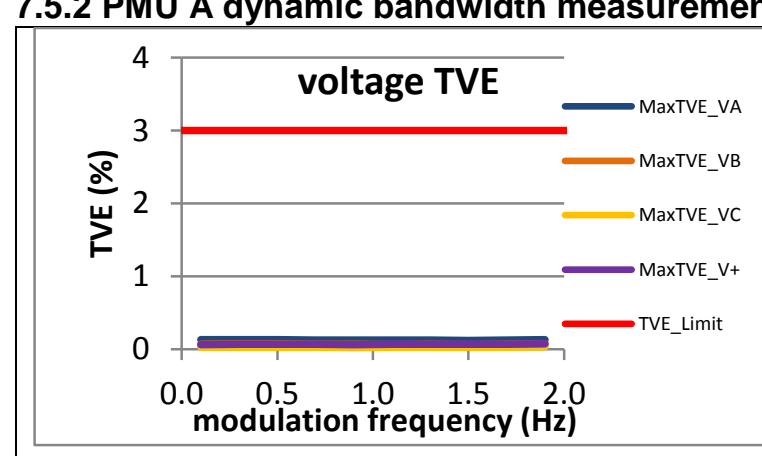

Figure 3317: Fs = 60 FPS

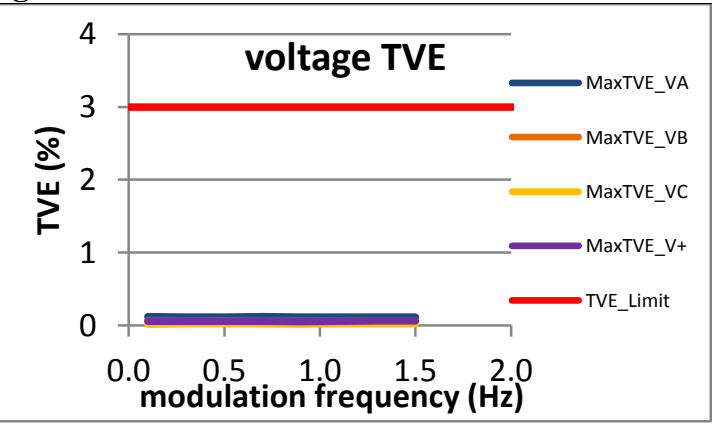

Figure 3320: Fs = 15 FPS

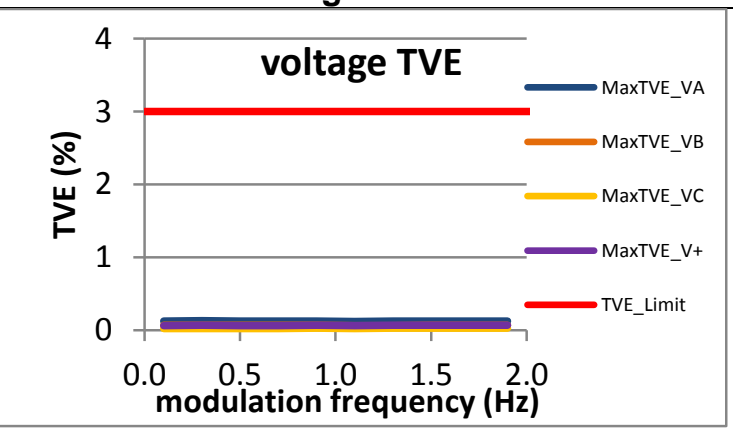

Figure 3318: Fs = 30 FPS

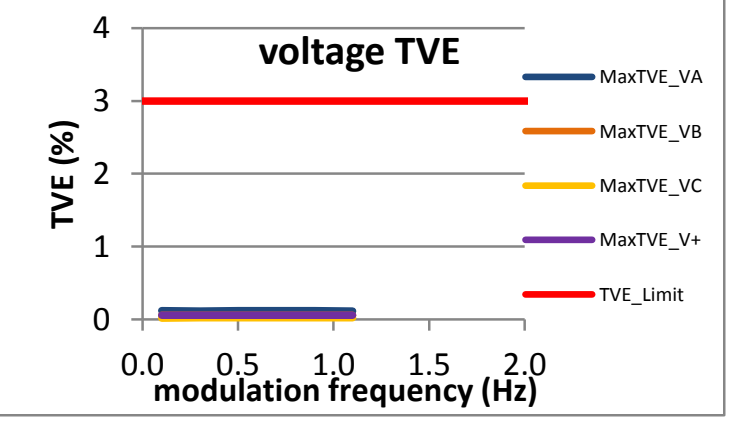

Figure 3321: Fs = 12 FPS

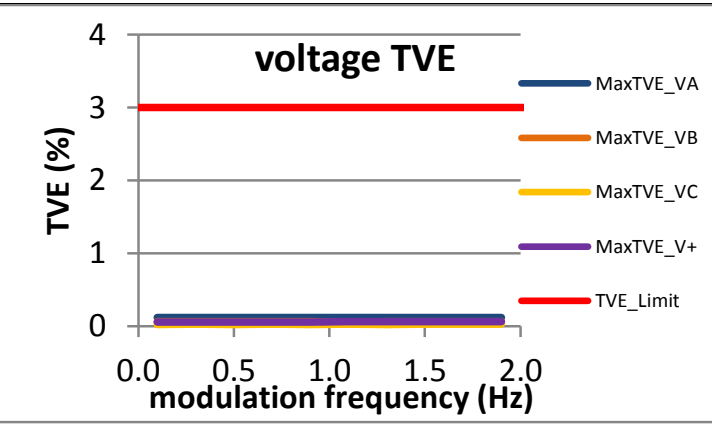

Figure 3319:Fs = 20 FPS

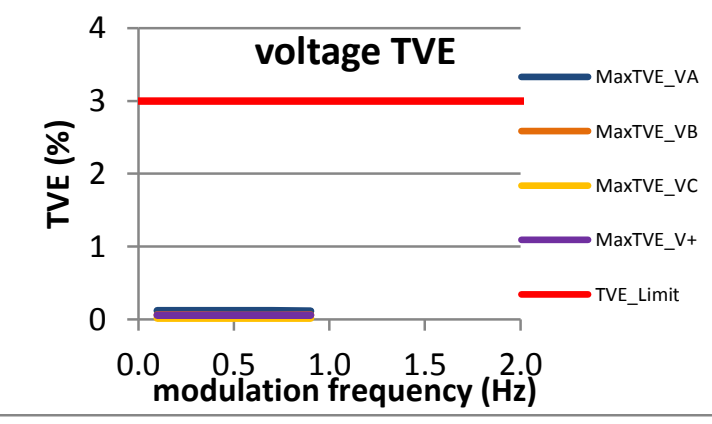

Figure 3322: Fs = 10 FPS 
7.5.3 PMU B dynamic bandwidth measurement: phase modulation voltage TVE: P class

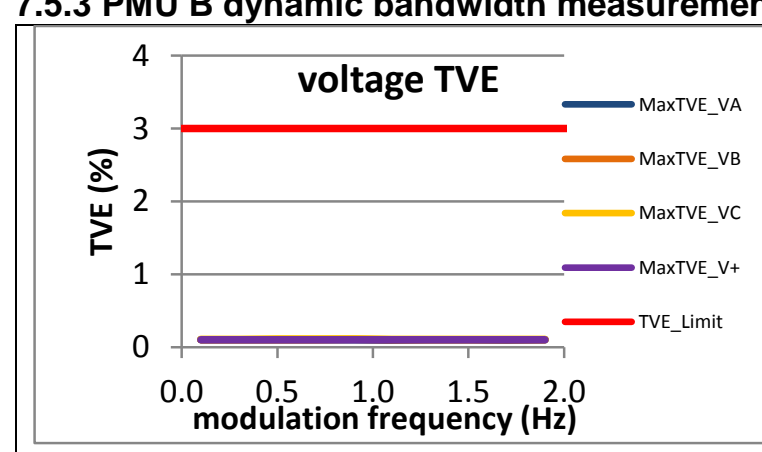

Figure 3323: Fs = 60 FPS

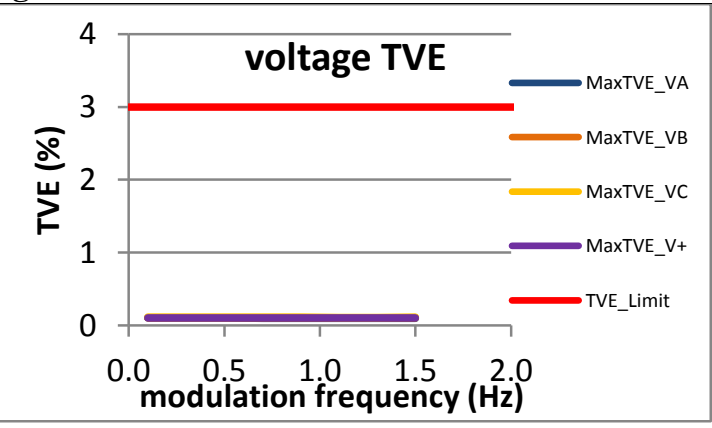

Figure 3326: Fs = 15 FPS

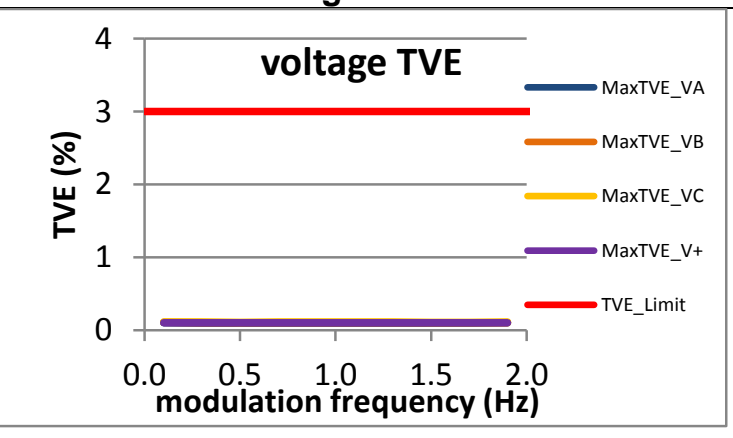

Figure 3324: Fs = 30 FPS

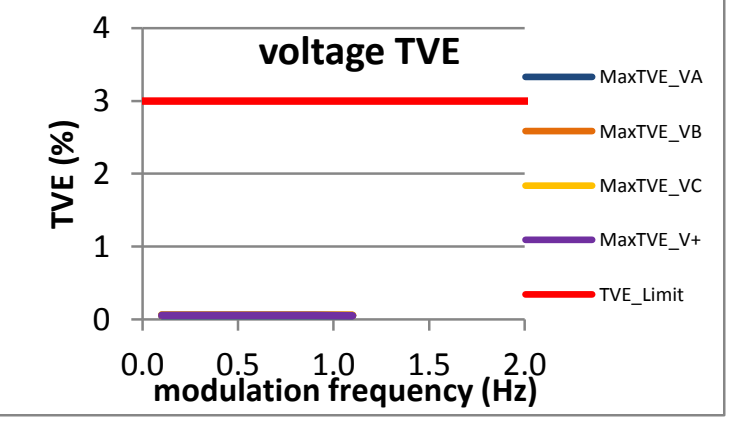

Figure 3327: Fs = 12 FPS

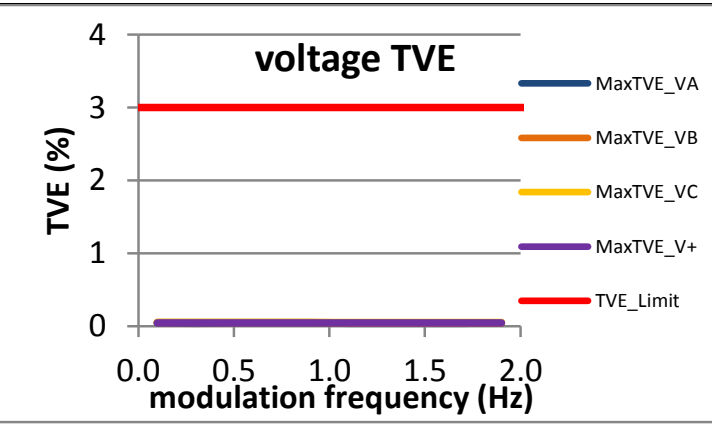

Figure 3325: Fs = 20 FPS

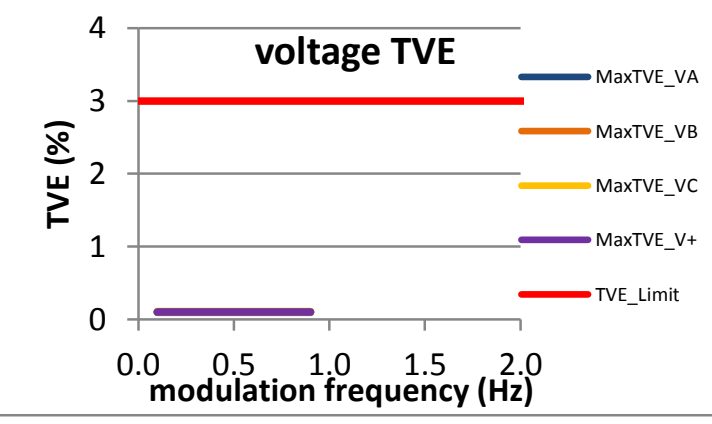

Figure 3328: Fs = 10 FPS 
7.5.4 PMU C dynamic bandwidth measurement: phase modulation voltage TVE: P class

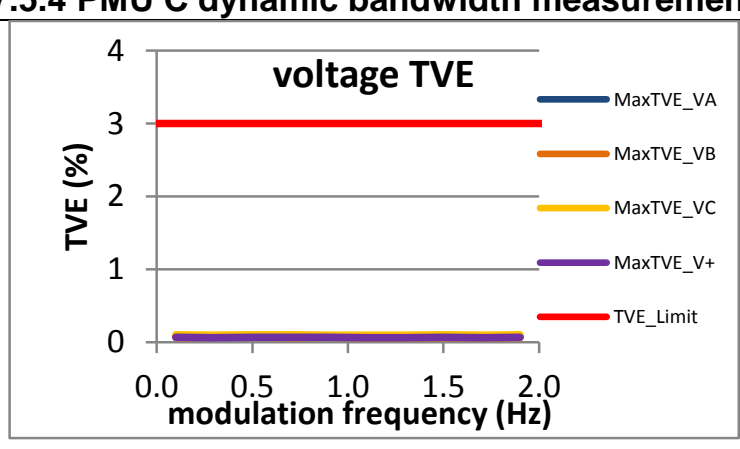

Figure 3329: Fs = 60 FPS

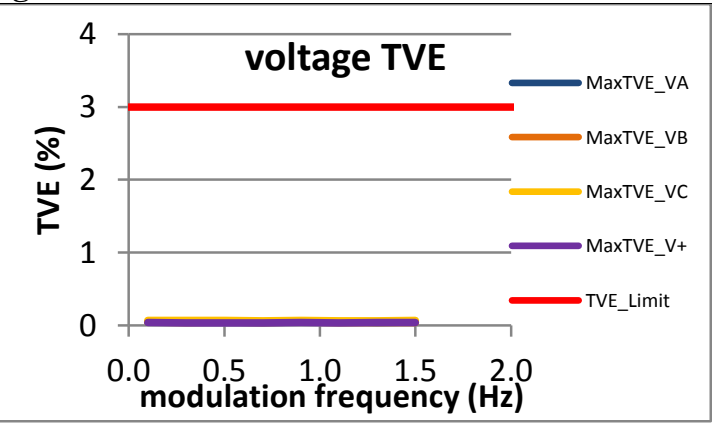

Figure 3332: Fs = 15 FPS

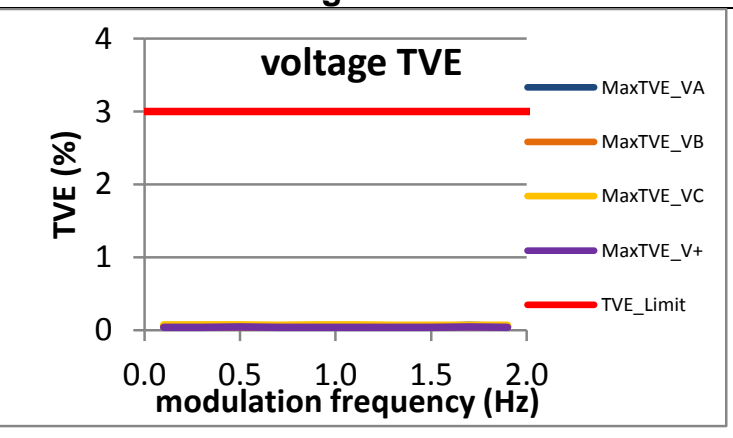

Figure 3330: Fs = 30 FPS

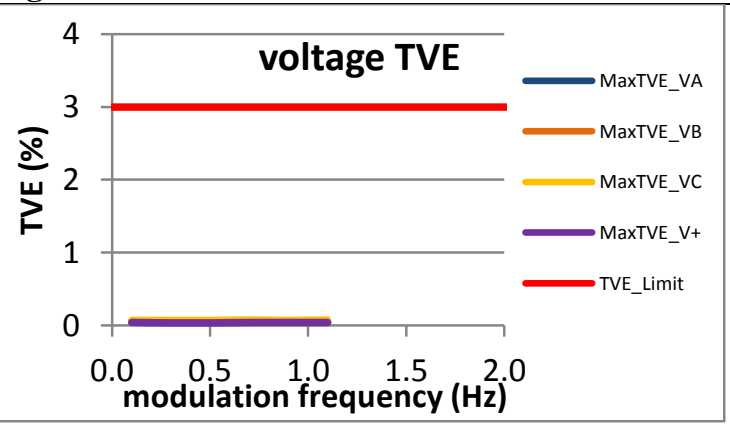

Figure 3333: Fs = 12 FPS

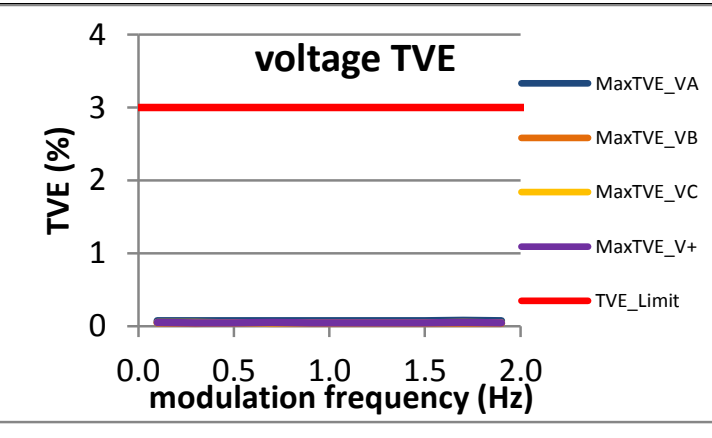

Figure 3331: Fs = 20 FPS

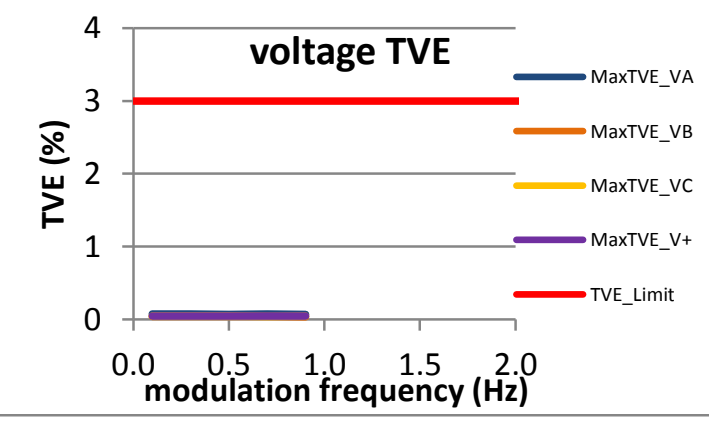

Figure 3334: Fs = 10 FPS 
7.5.5 PMU D dynamic bandwidth measurement: phase modulation voltage TVE: P class

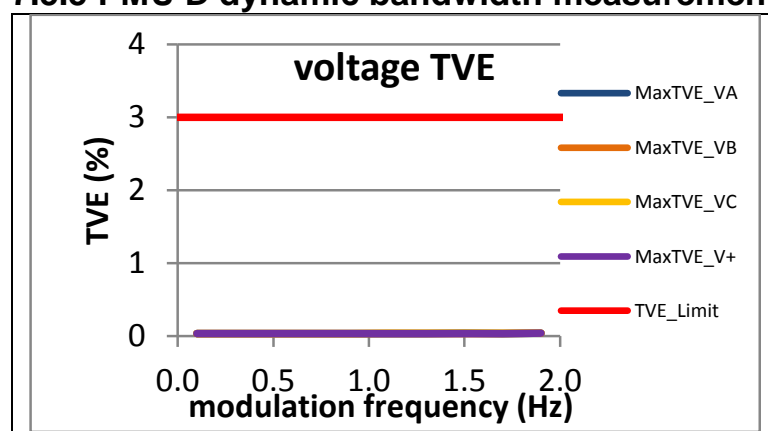

Figure 3335: Fs = 60 FPS

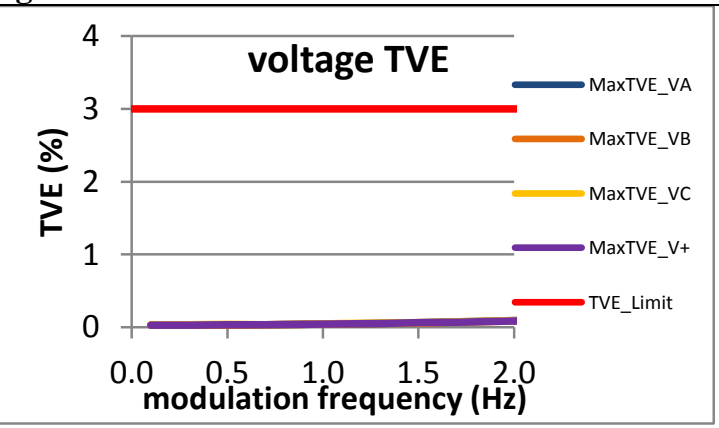

Figure 3338: Fs = 15 FPS

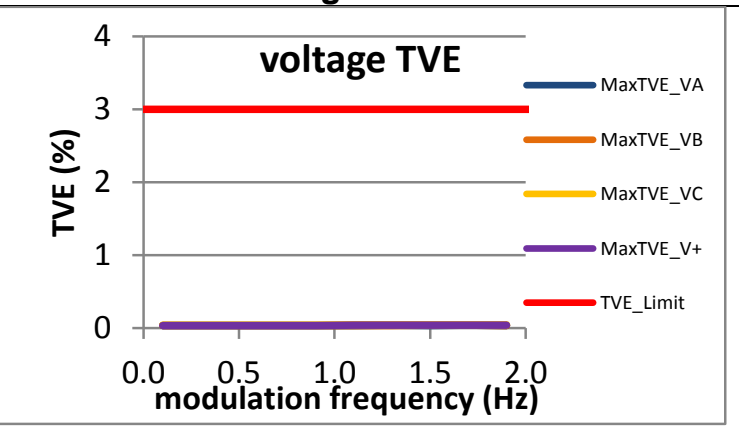

Figure 3336: Fs = 30 FPS

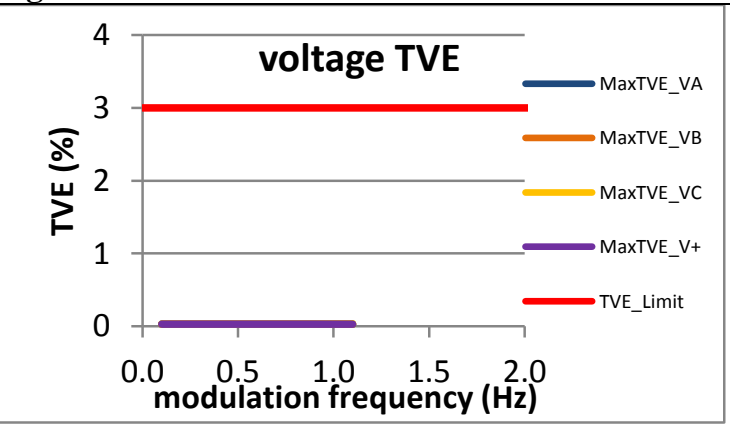

Figure 3339: Fs = 12 FPS

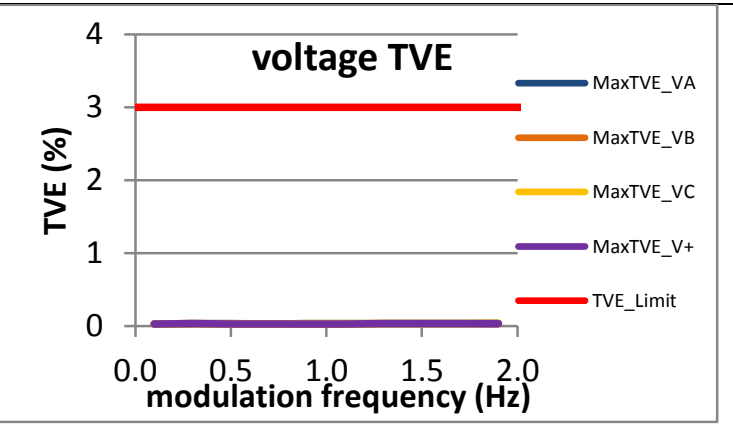

Figure 3337: Fs = 20 FPS

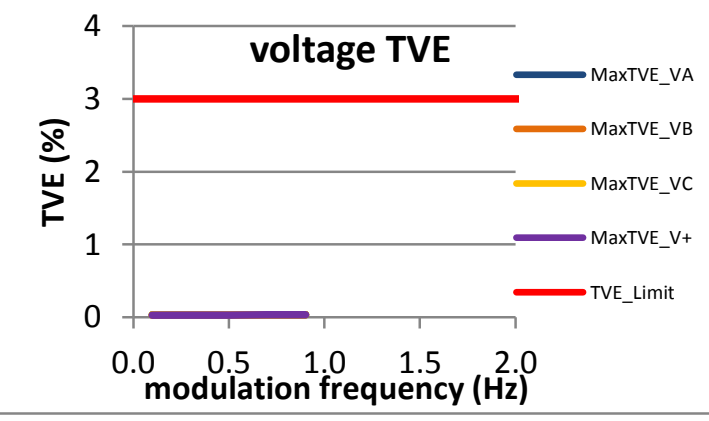

Figure 3340: Fs = 10 FPS

7.5.6 PMU E dynamic bandwidth measurement: phase modulation voltage TVE: P class

PMU E does not support P class 
7.5.7 PMU F dynamic bandwidth measurement: phase modulation voltage TVE: P class

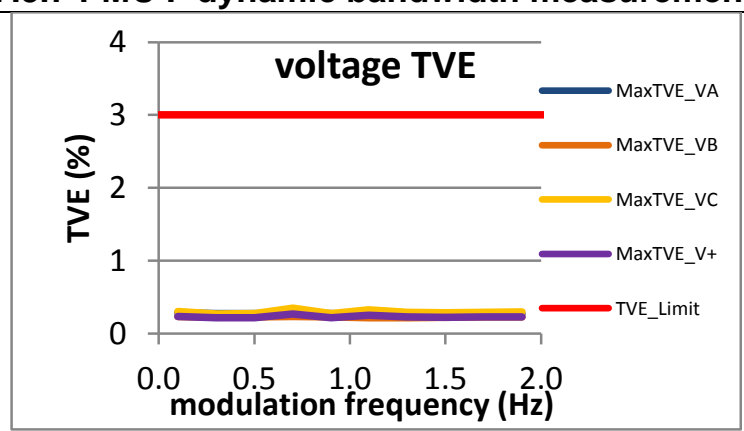

Figure 3341: Fs = 60 FPS

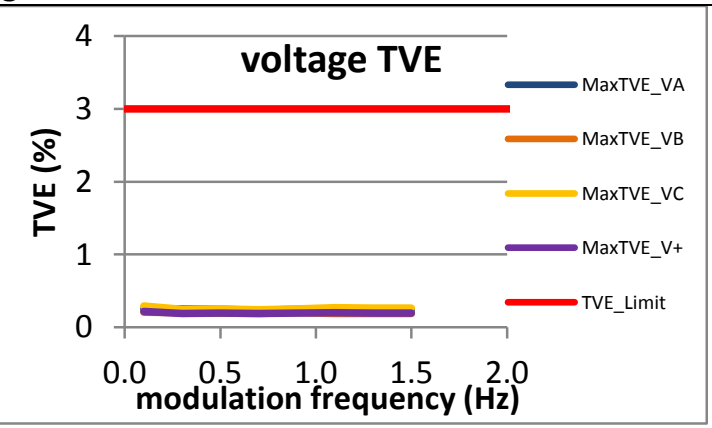

Figure 3344: Fs = 15 FPS

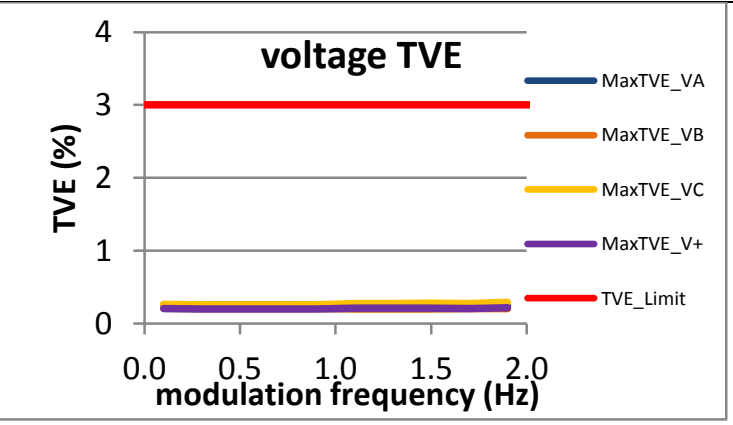

Figure 3342: Fs = 30 FPS

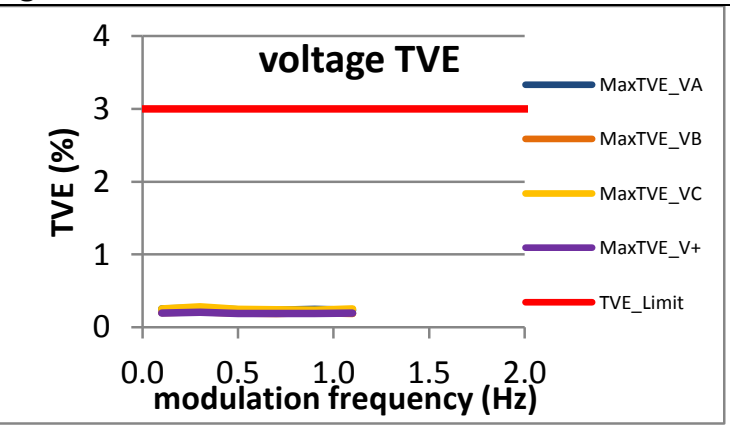

Figure 3345: Fs = 12 FPS

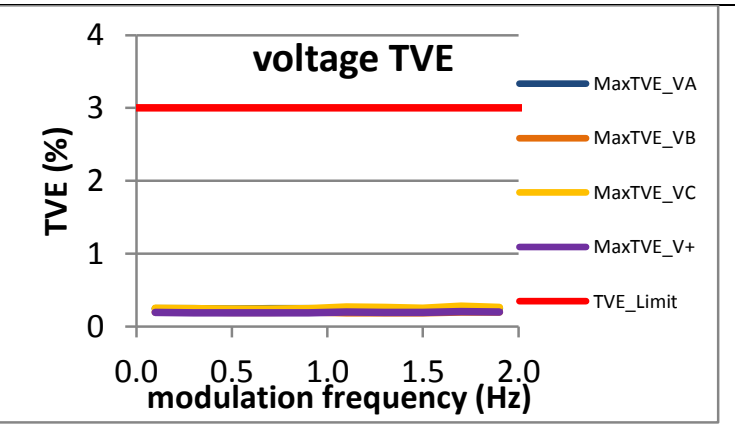

Figure 3343: Fs = 20 FPS

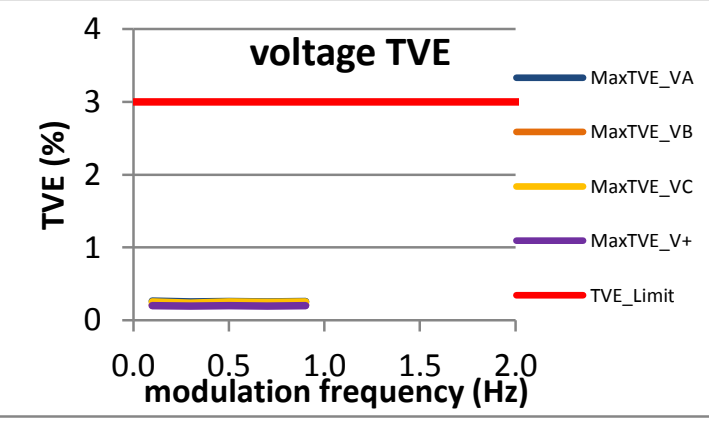

Figure 3346: Fs = 10 FPS

7.5.8 PMU G dynamic bandwidth measurement: phase modulation voltage TVE: P class

PMU G does not support P class. 
7.5.9 PMU H dynamic bandwidth measurement: phase modulation voltage TVE: P class

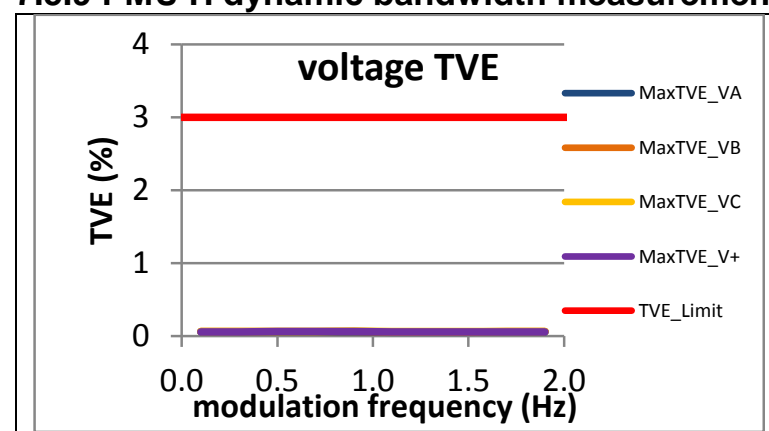

Figure 3347: Fs = 60 FPS

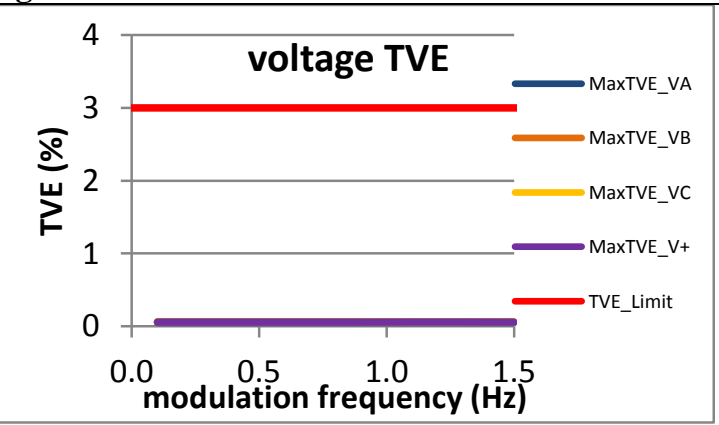

Figure 3350: Fs = 15 FPS

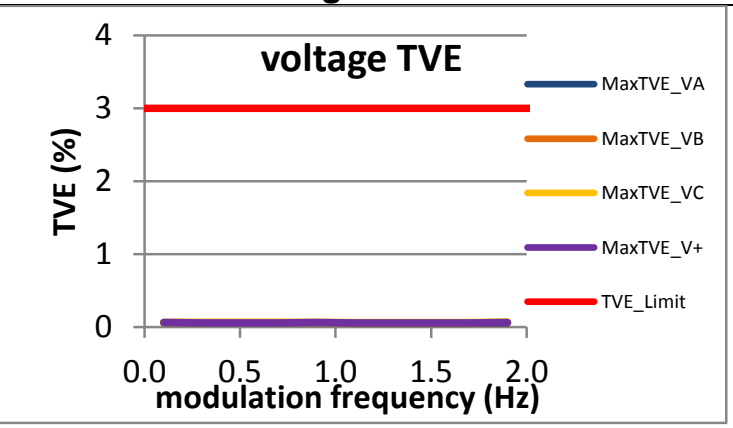

Figure 3348: Fs = 30 FPS

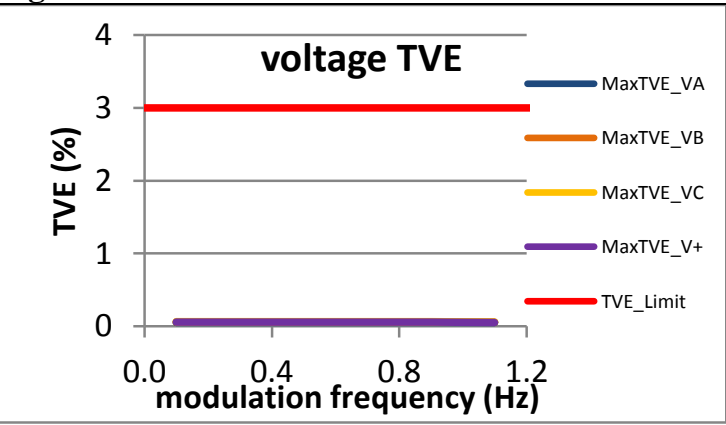

Figure 3351: Fs = 12 FPS

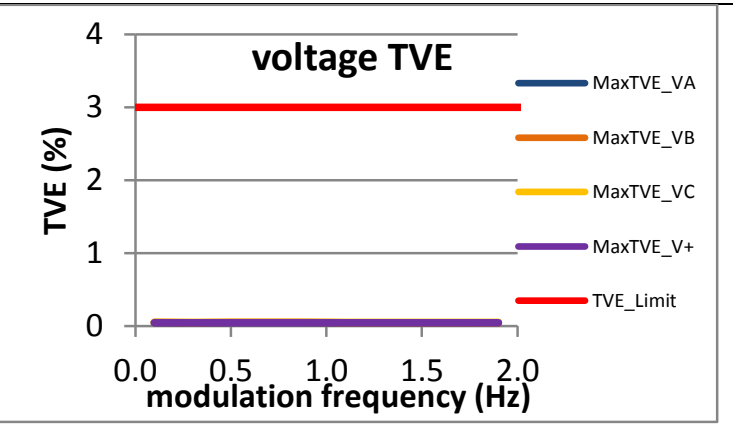

Figure 3349: Fs = 20 FPS

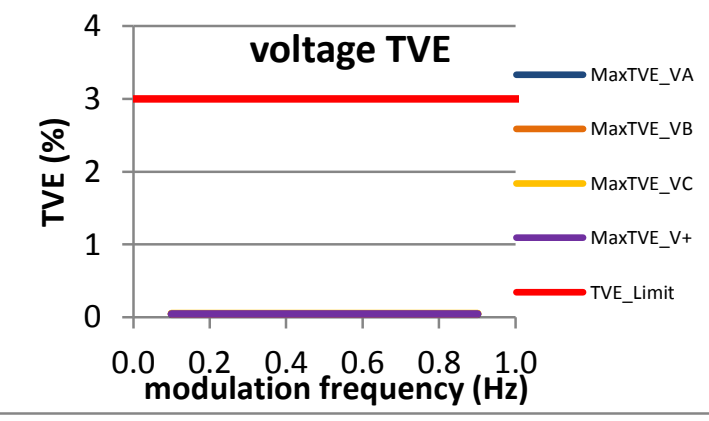

Figure 3352: Fs = 10 FPS

7.5.10 PMU I dynamic bandwidth measurement: phase modulation voltage TVE: P class

PMU I does not support P class 
7.5.11 PMU J dynamic bandwidth measurement: phase modulation voltage TVE: P class

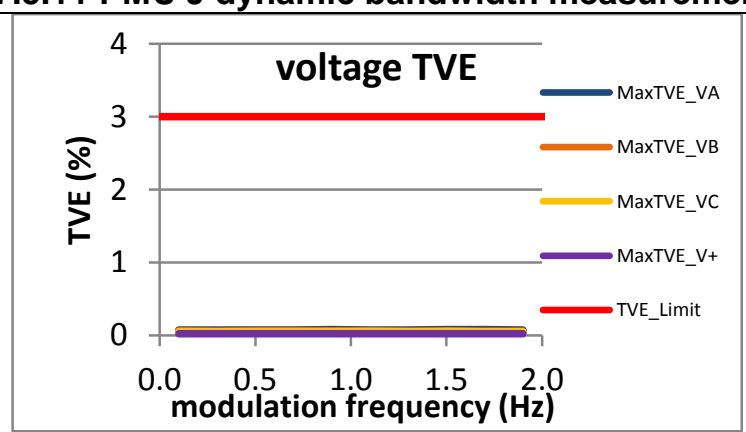

Figure 3353: Fs = 60 FPS

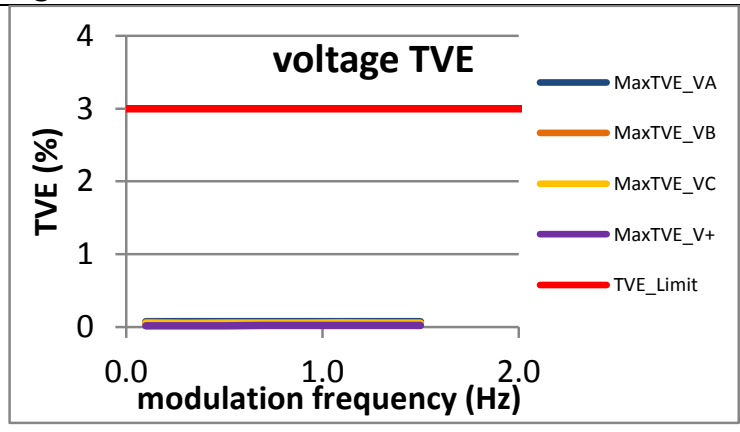

Figure 3356: Fs = 15 FPS

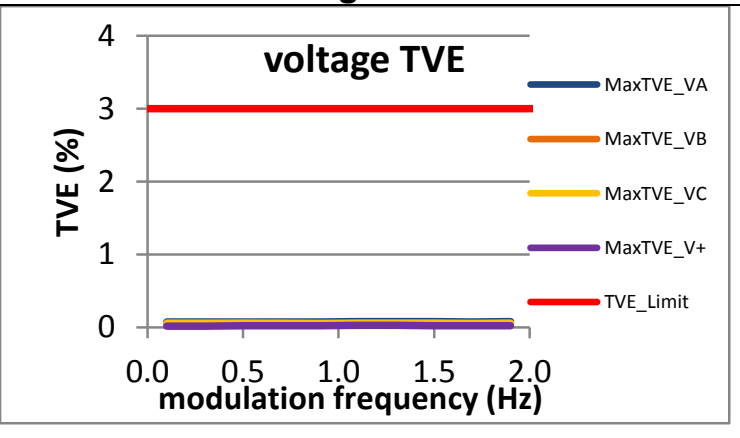

Figure 3354: Fs $=30$ FPS

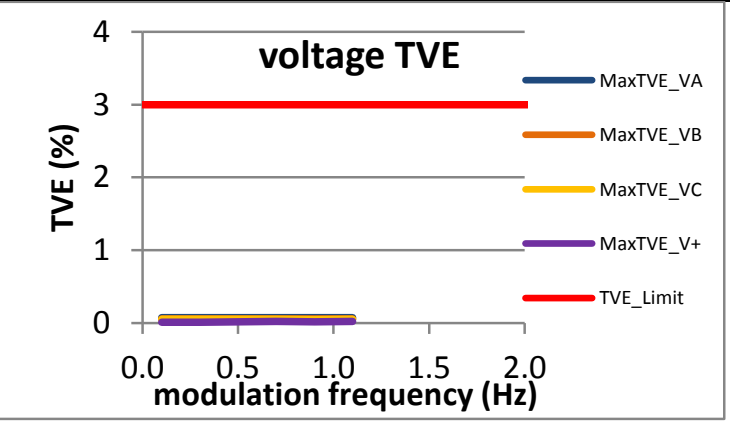

Figure 3357: Fs = 12 FPS

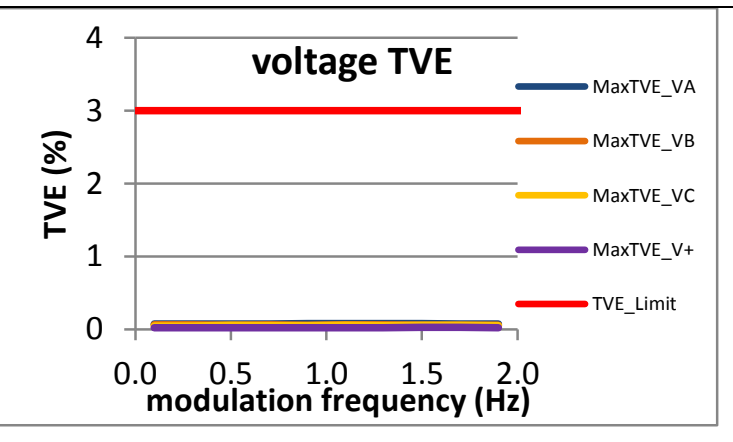

Figure 3355: Fs = 20 FPS

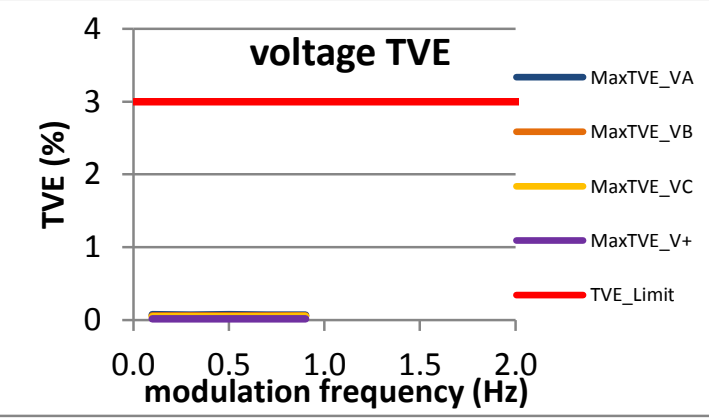

Figure 3358: Fs = 10 FPS 


\subsection{Phase modulation current TVE, P class}

7.6.1 C37.118.1 Annex C dynamic bandwidth measurement: phase modulation current TVE: P class

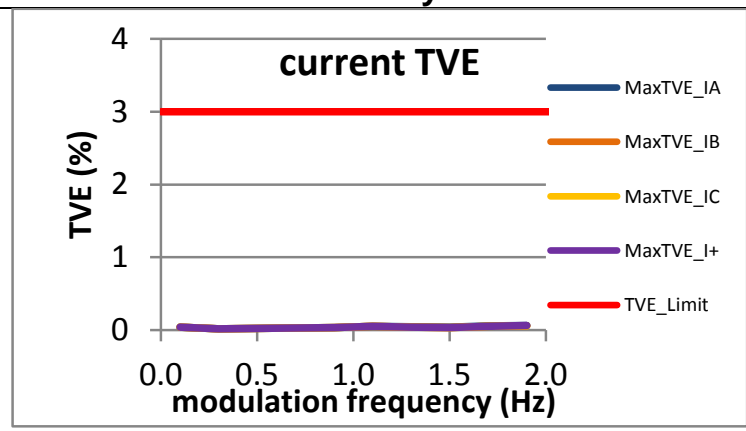

Figure 3359: Fs = 60 FPS

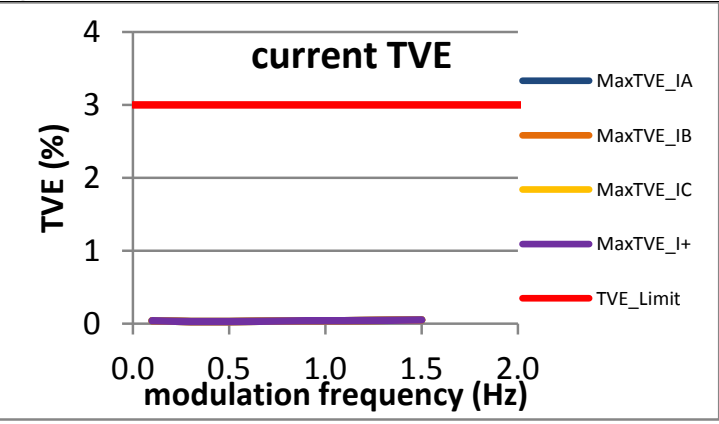

Figure 3362: Fs = 15 FPS

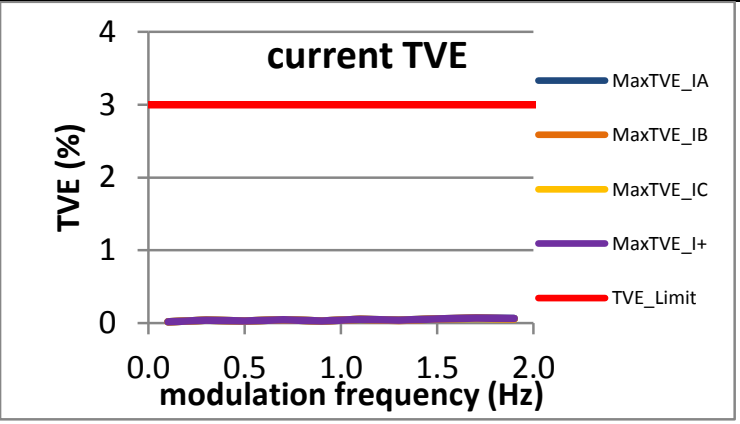

Figure 3360: Fs = 30 FPS

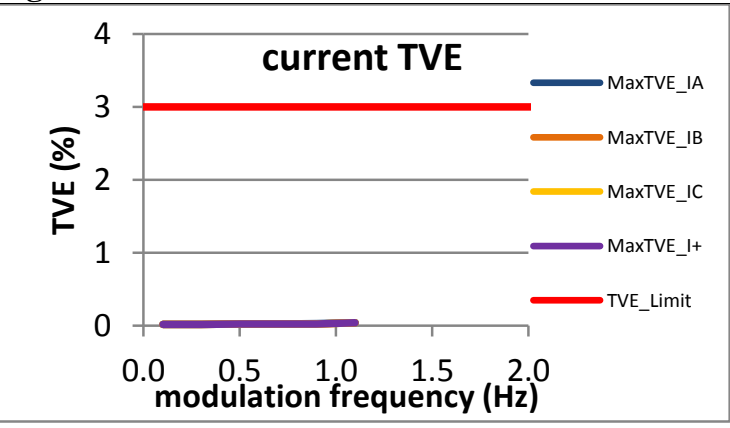

Figure 3363: Fs = 12 FPS

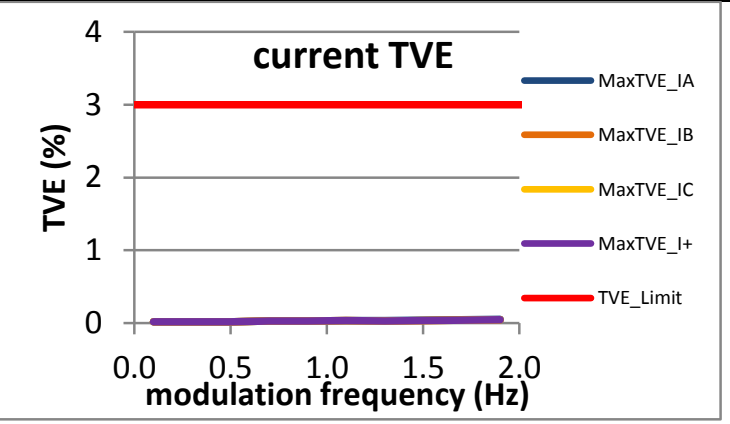

Figure 3361: Fs = 20 FPS

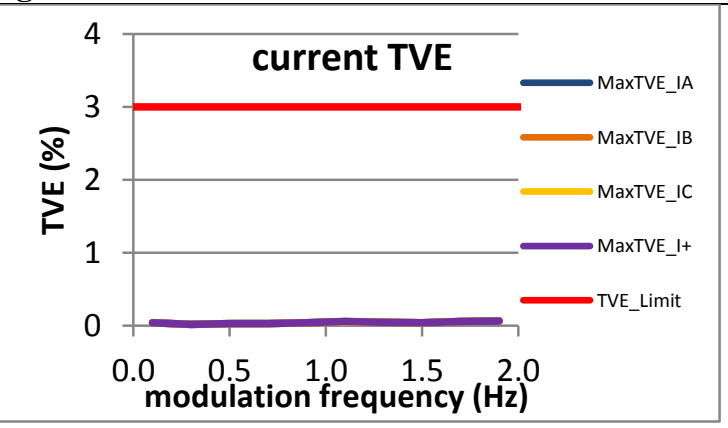

Figure 3364: Fs = 10 FPS 
7.6.2 PMU A dynamic bandwidth measurement: phase modulation current TVE: P class

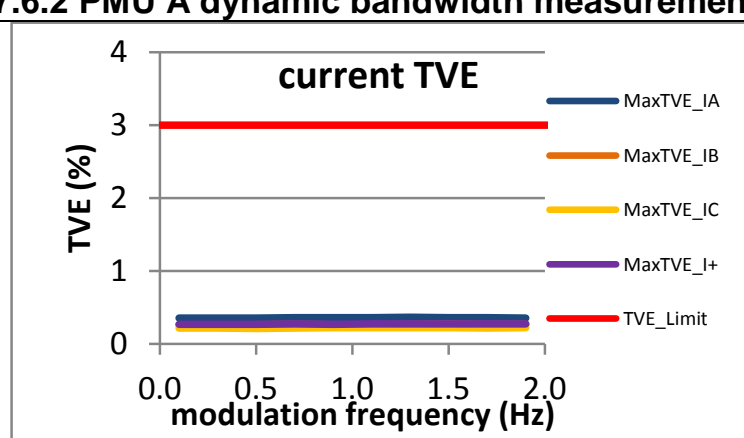

Figure 3365: Fs = 60 FPS

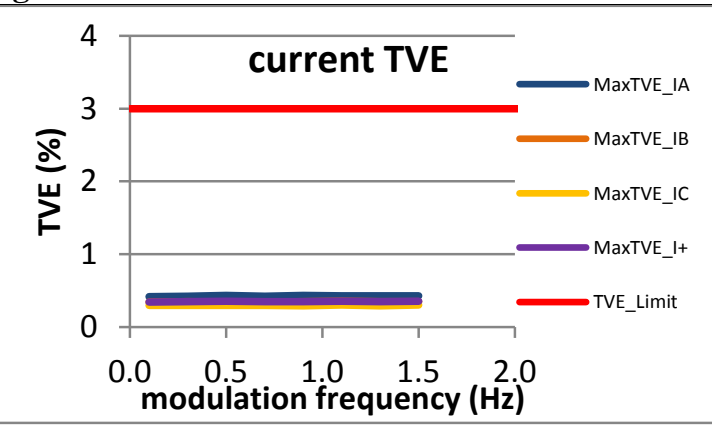

Figure 3368: Fs = 15 FPS

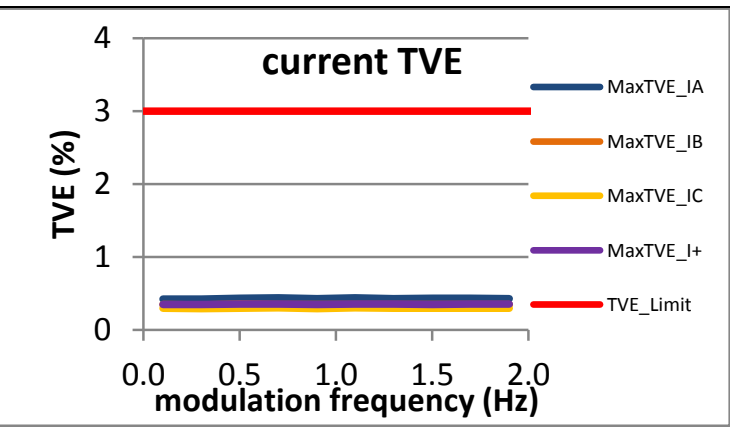

Figure 3366: Fs = 30 FPS

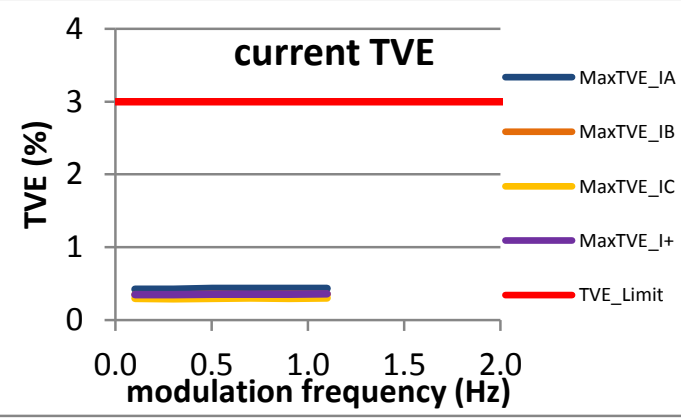

Figure 3369: Fs = 12 FPS

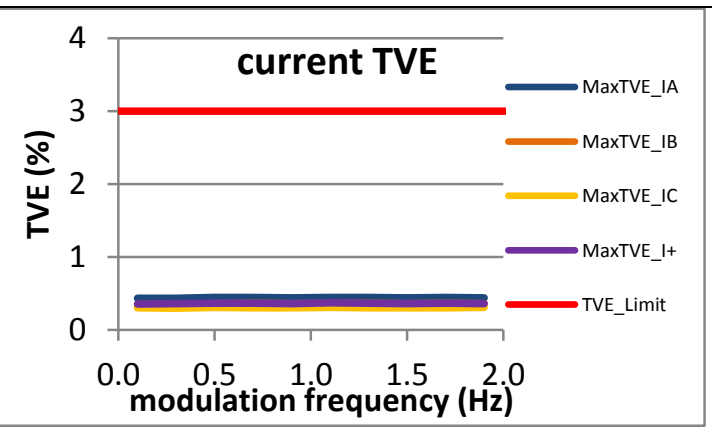

Figure 3367:Fs $=20$ FPS

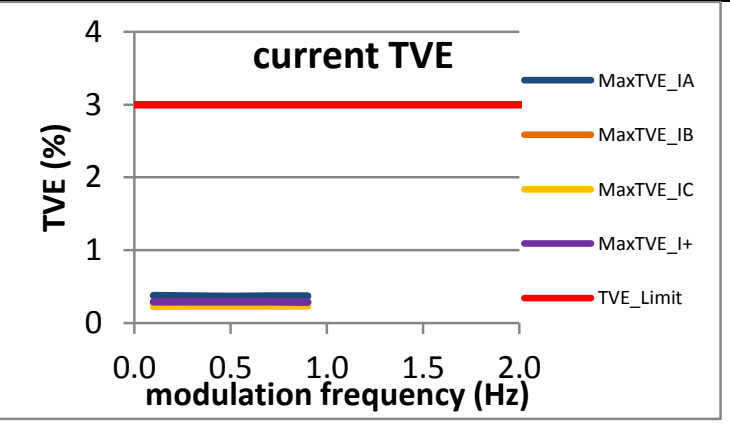

Figure 3370: Fs = 10 FPS 
7.6.3 PMU B dynamic bandwidth measurement: phase modulation current TVE: P class

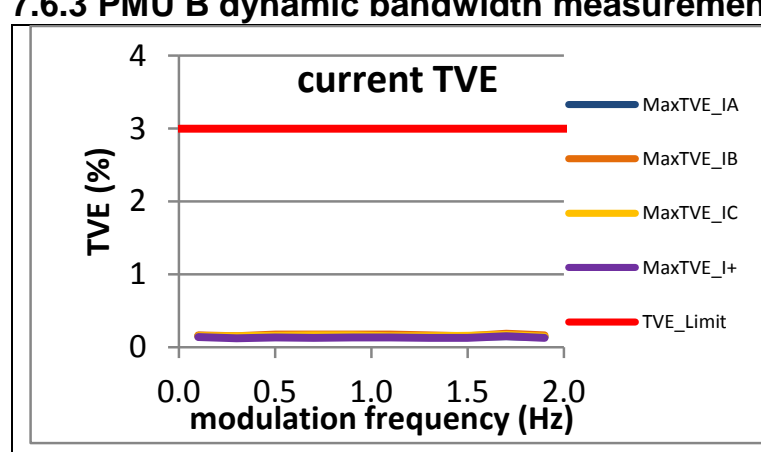

Figure 3371: Fs = 60 FPS

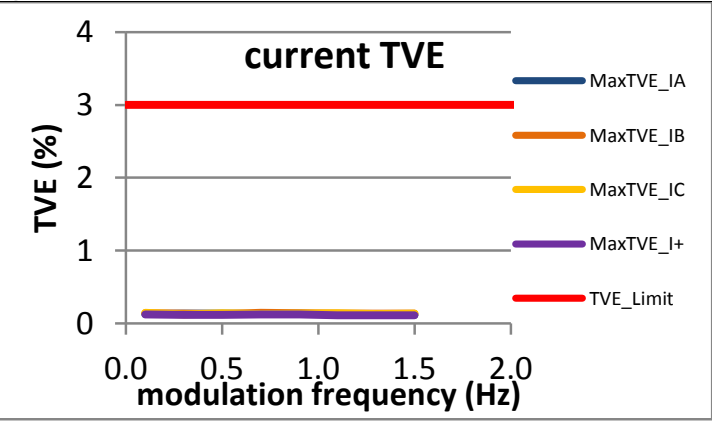

Figure 3374: Fs = 15 FPS

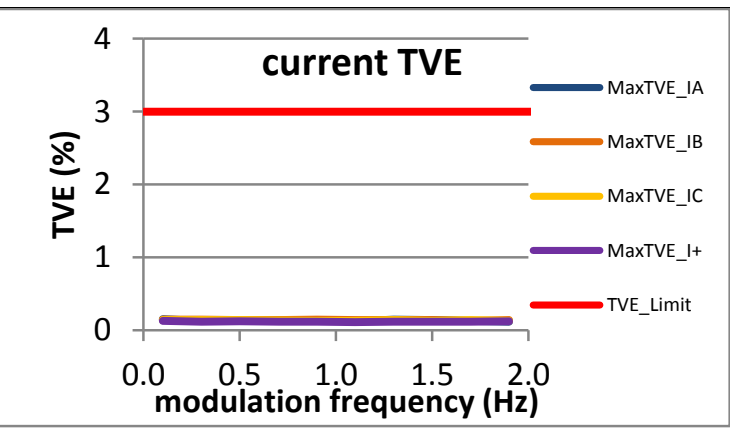

Figure 3372: Fs = 30 FPS

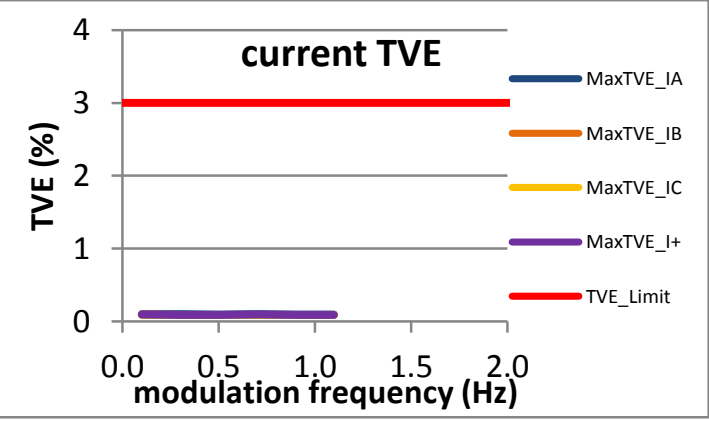

Figure 3375: Fs = 12 FPS

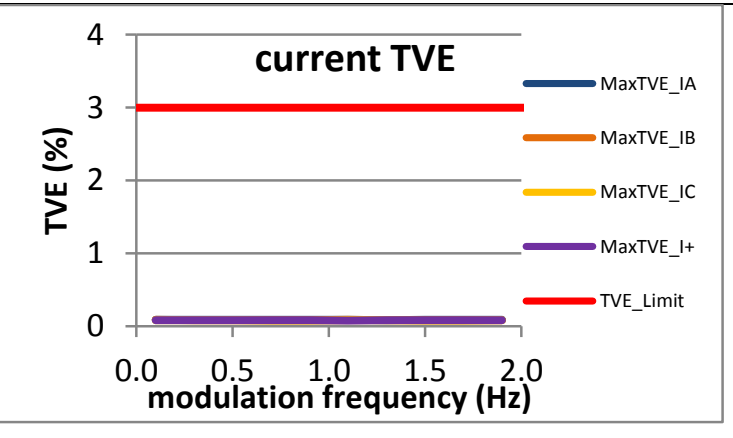

Figure 3373: Fs = 20 FPS

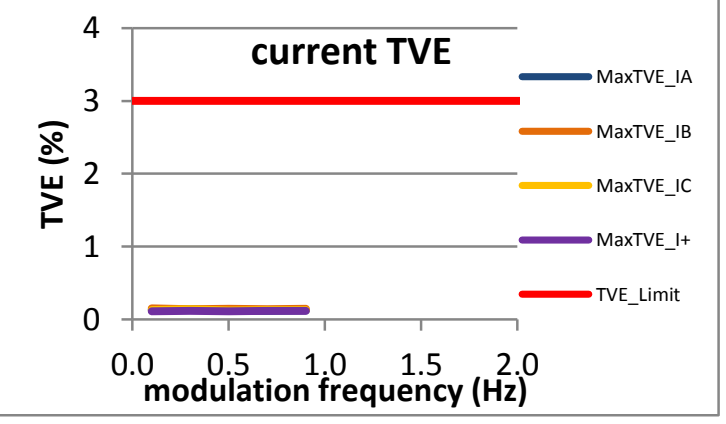

Figure 3376: Fs = 10 FPS 
7.6.4 PMU C dynamic bandwidth measurement: phase modulation current TVE: P class

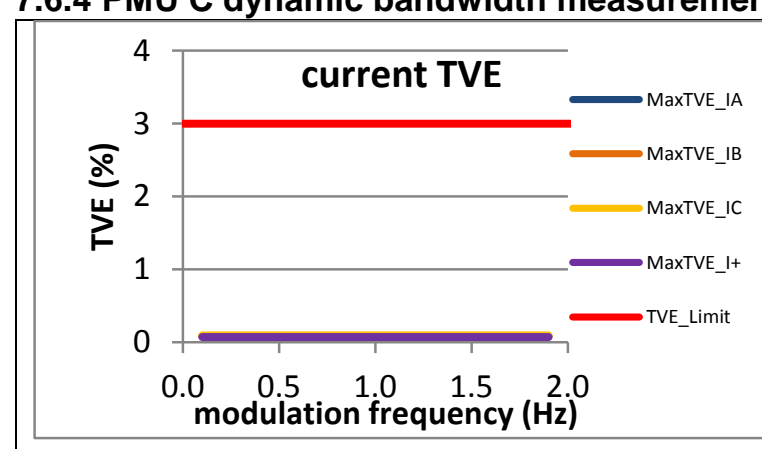

Figure 3377: Fs = 60 FPS

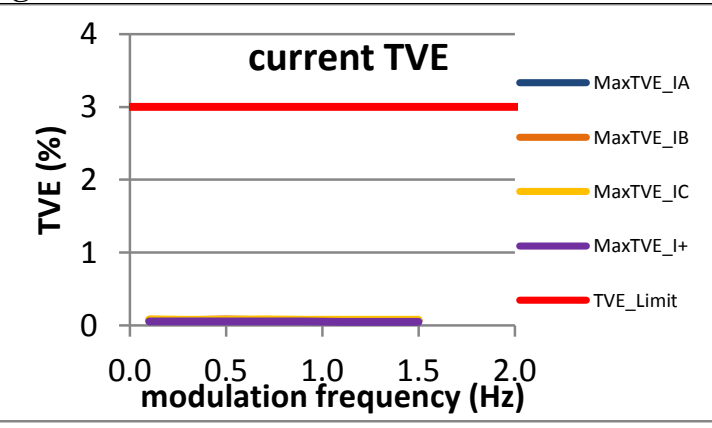

Figure 3380: Fs = 15 FPS

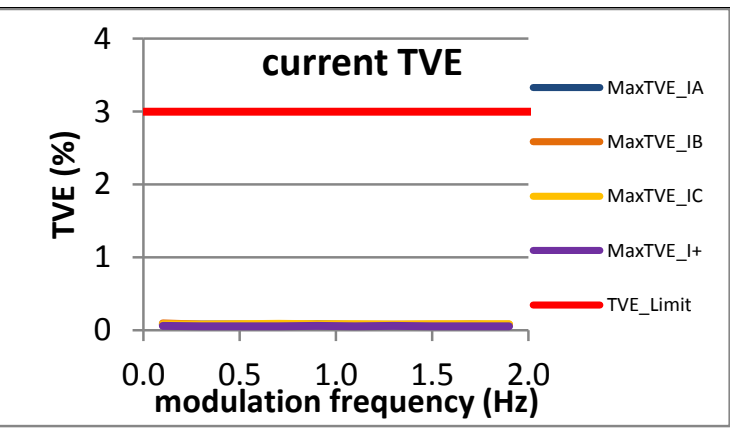

Figure 3378: Fs = 30 FPS

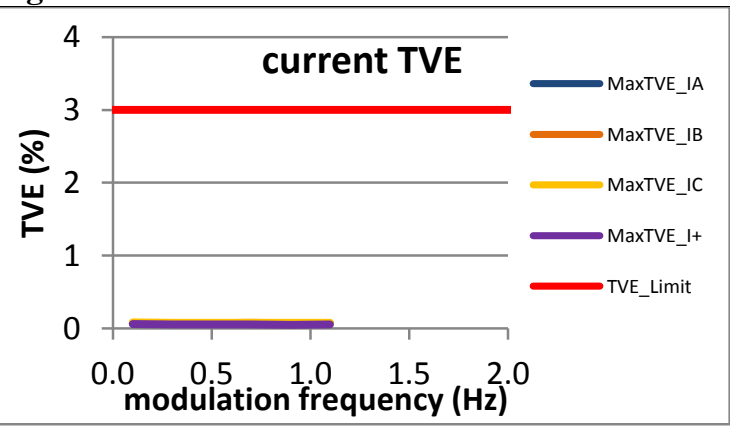

Figure 3381: Fs = 12 FPS

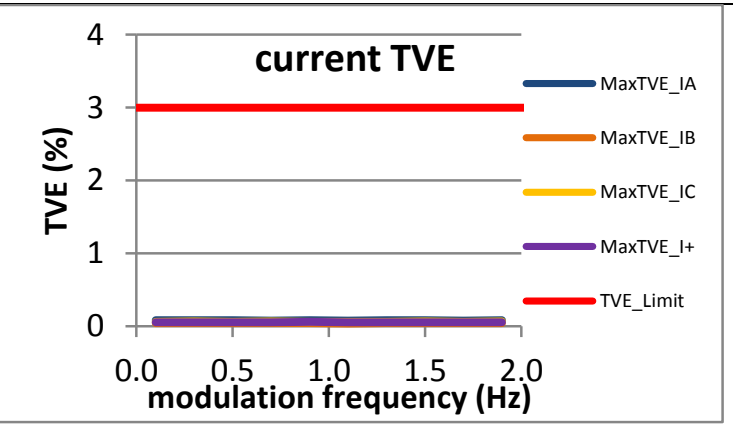

Figure 3379: Fs = 20 FPS

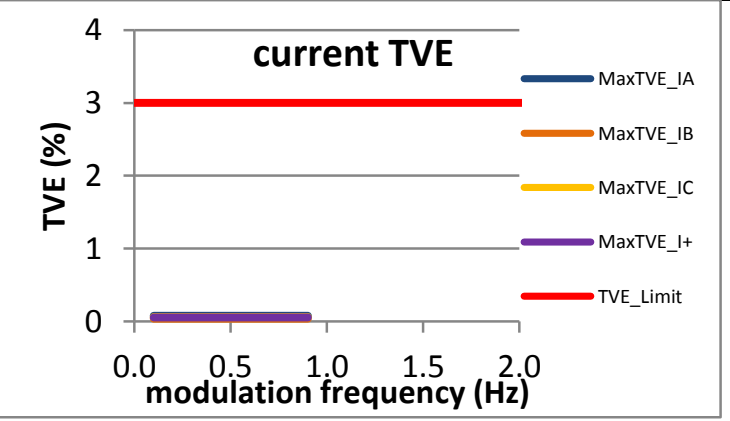

Figure 3382: Fs = 10 FPS 
7.6.5 PMU D dynamic bandwidth measurement: phase modulation current TVE: P class

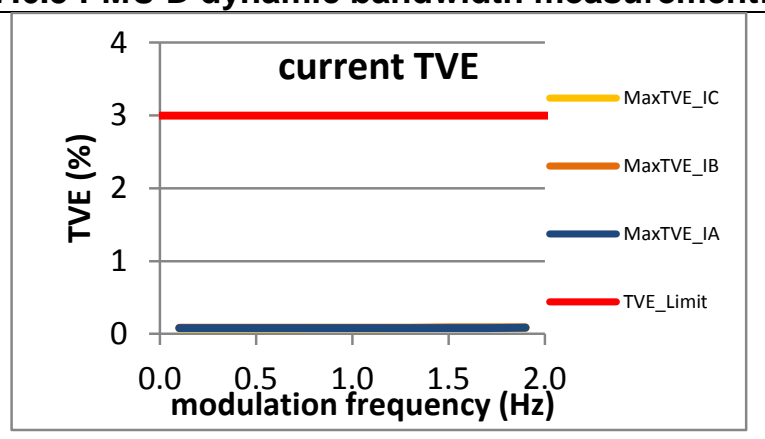

Figure 3383: Fs = 60 FPS

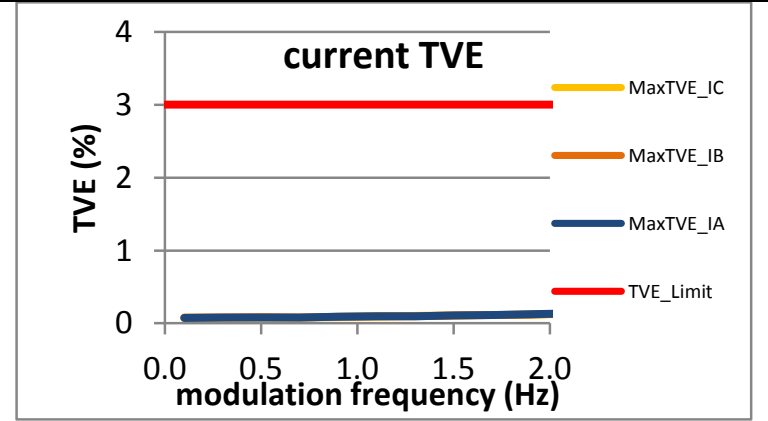

Figure 3386: Fs = 15 FPS

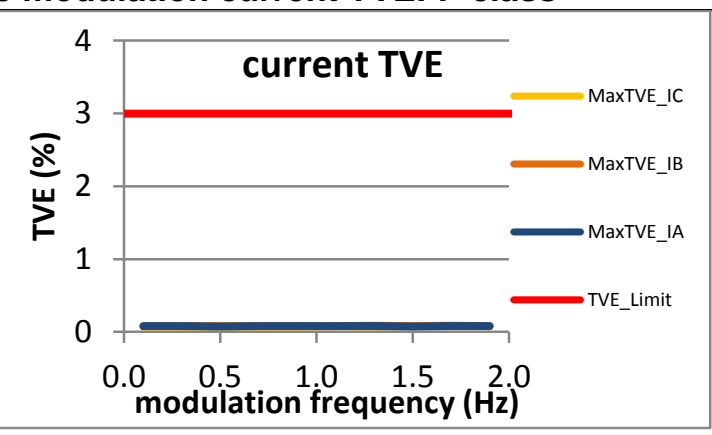

Figure 3384: Fs = 30 FPS

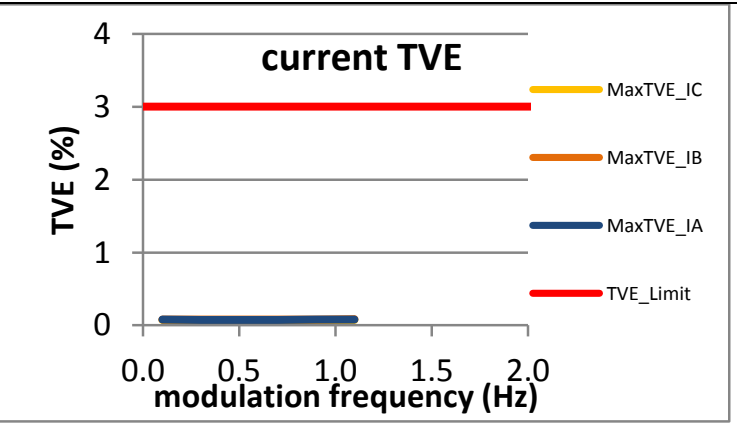

Figure 3387: Fs = 12 FPS

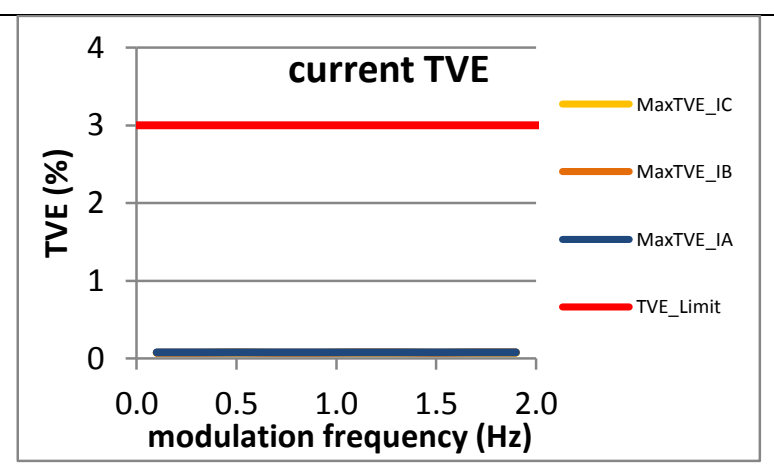

Figure 3385: Fs = 20 FPS

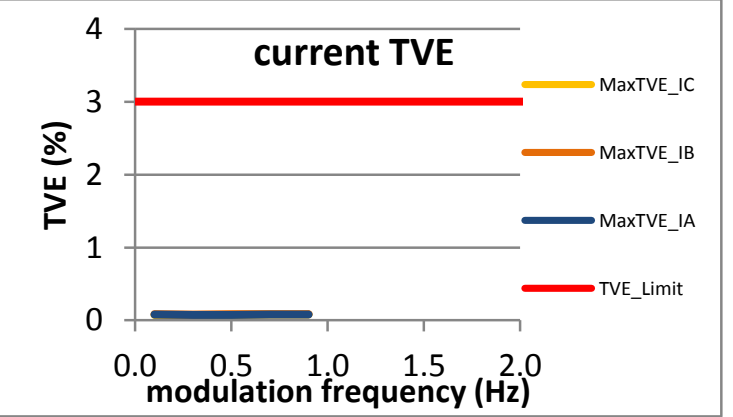

Figure 3388: Fs = 10 FPS

7.6.6 PMU E dynamic bandwidth measurement: phase modulation current TVE: P class

PMU E does not support P class 
7.6.7 PMU F dynamic bandwidth measurement: phase modulation current TVE: P class

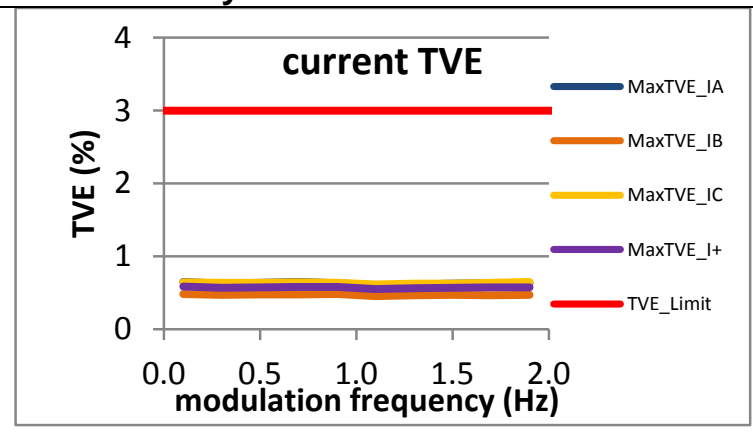

Figure 3389: Fs = 60 FPS

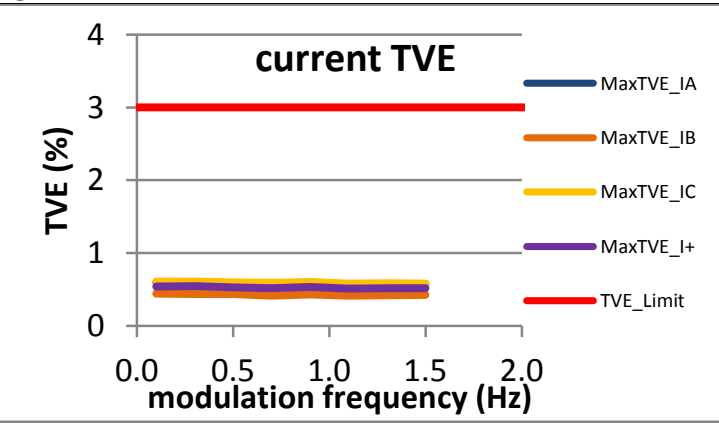

Figure 3392: Fs = 15 FPS

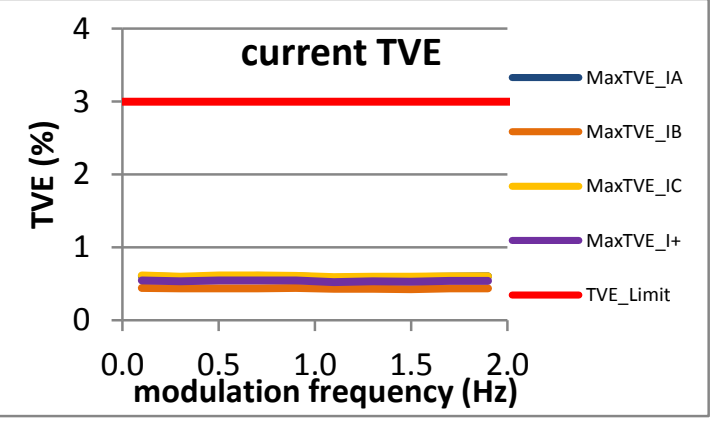

Figure 3390: Fs = 30 FPS

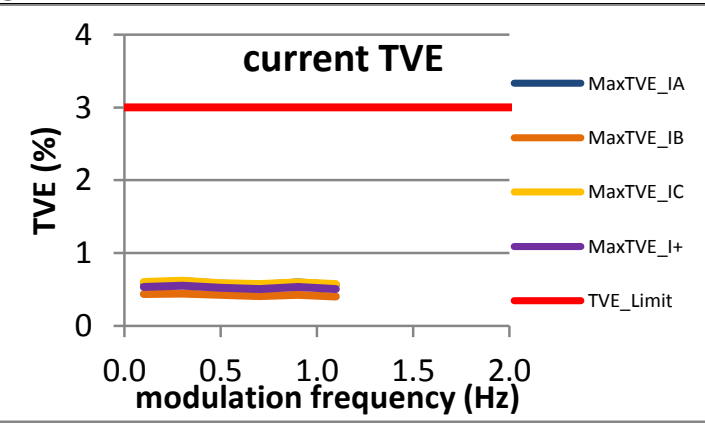

Figure 3393: Fs = 12 FPS

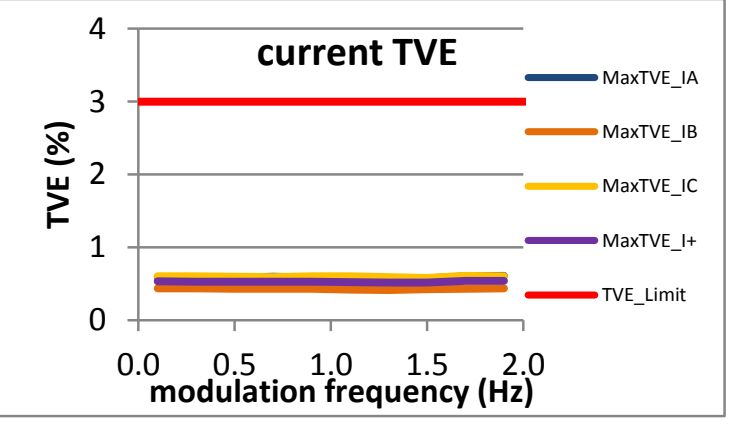

Figure 3391: Fs = 20 FPS

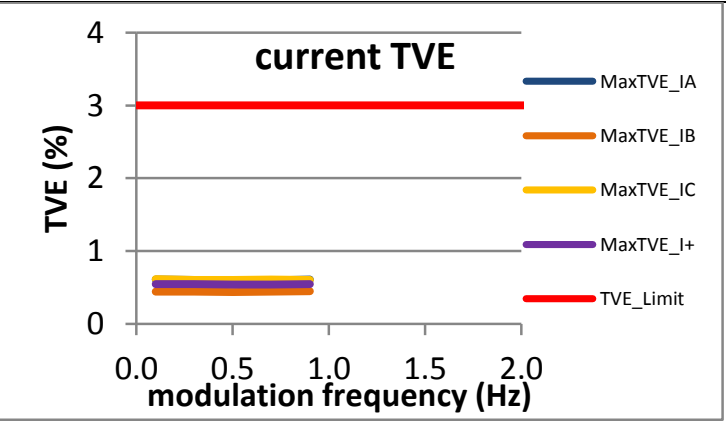

Figure 3394: Fs = 10 FPS

7.6.8 PMU G dynamic bandwidth measurement: phase modulation current TVE: P class

PMU G does not support P class 
7.6.9 PMU H dynamic bandwidth measurement: phase modulation current TVE: P class

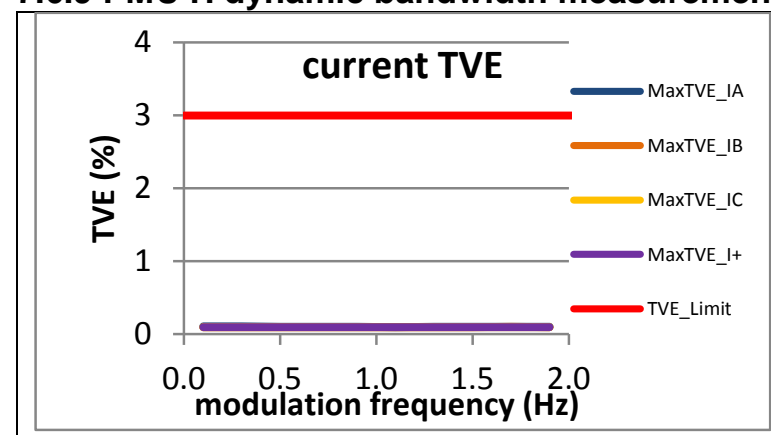

Figure 3395: Fs = 60 FPS

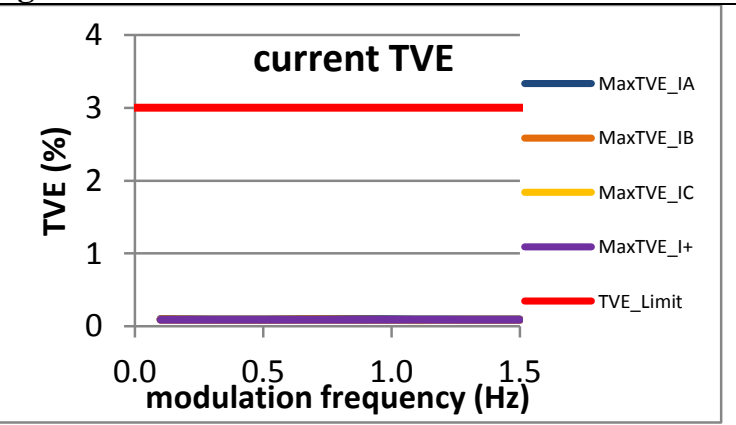

Figure 3398: Fs = 15 FPS

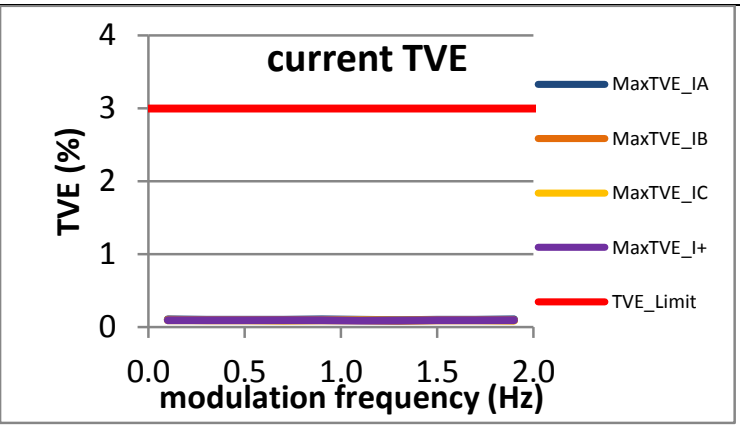

Figure 3396: Fs = 30 FPS

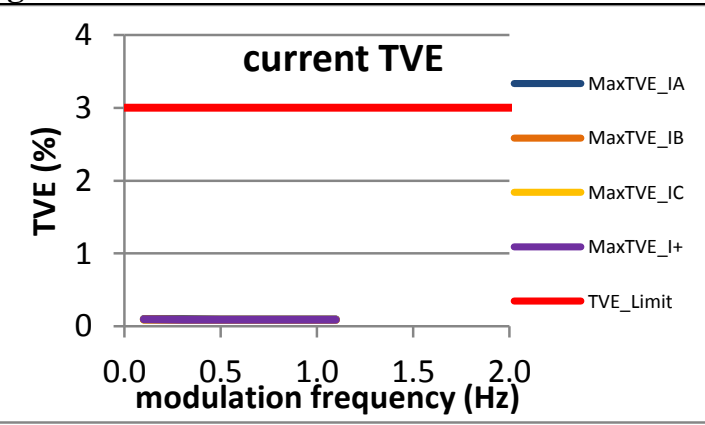

Figure 3399: Fs = 12 FPS

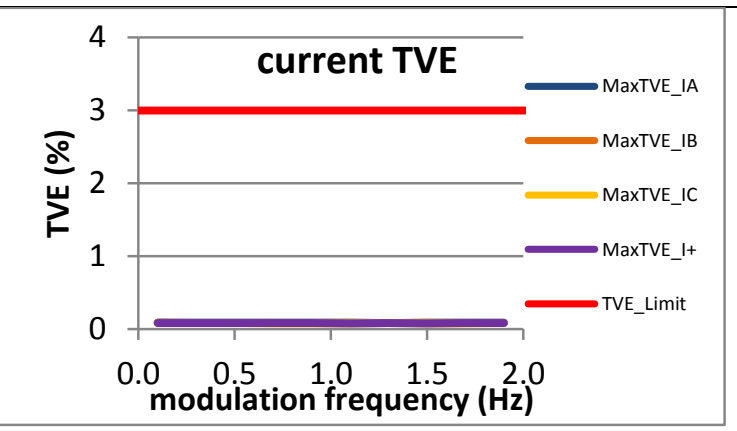

Figure 3397: Fs = 20 FPS

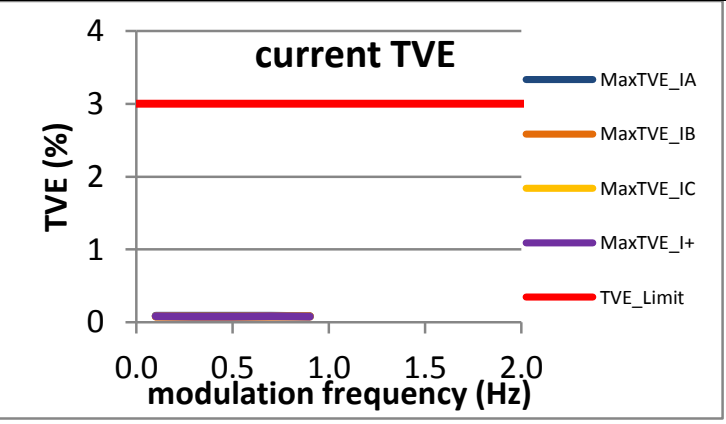

Figure 3400: Fs = 10 FPS

7.6.10 PMU I dynamic bandwidth measurement: phase modulation current TVE: P class

PMU I does not support P class 
7.6.11 PMU J dynamic bandwidth measurement: phase modulation current TVE: P class

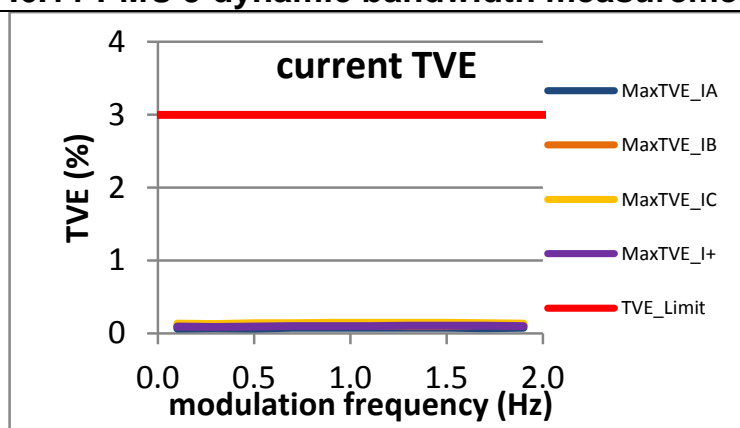

Figure 3401: Fs = 60 FPS

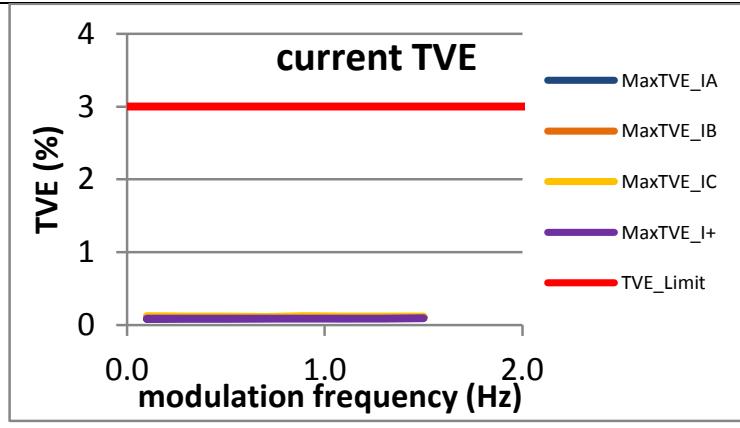

Figure 3404: Fs = 15 FPS

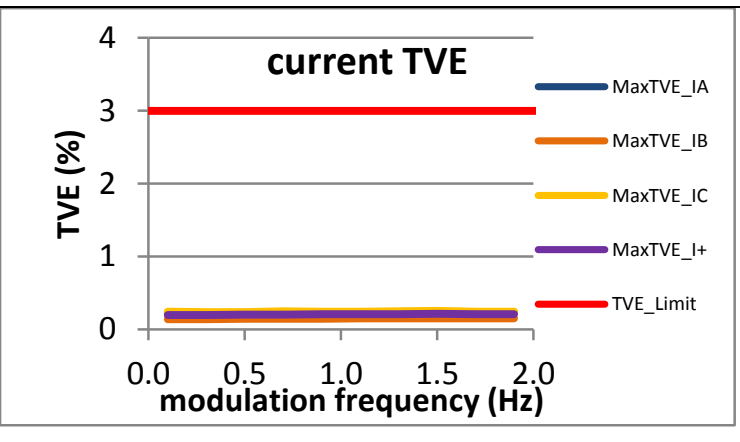

Figure 3402: Fs = 30 FPS

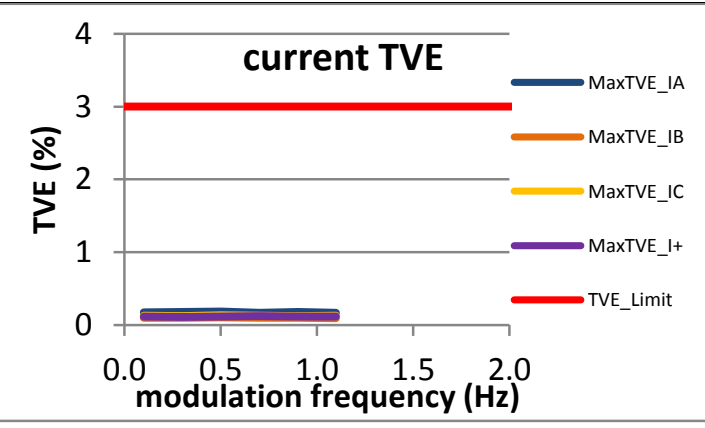

Figure 3405: Fs = 12 FPS

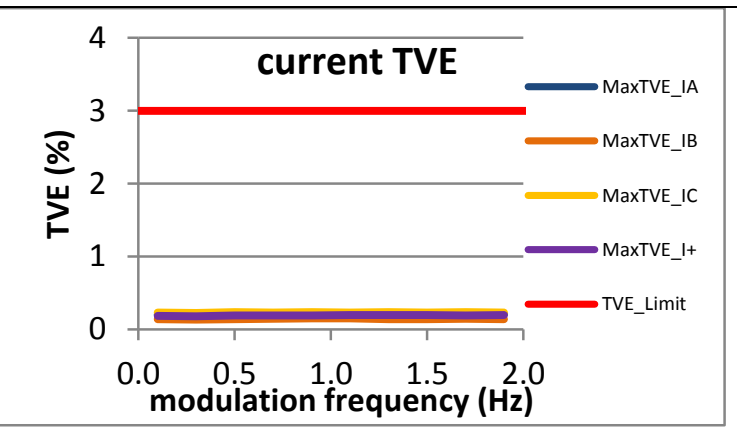

Figure 3403: Fs = 20 FPS

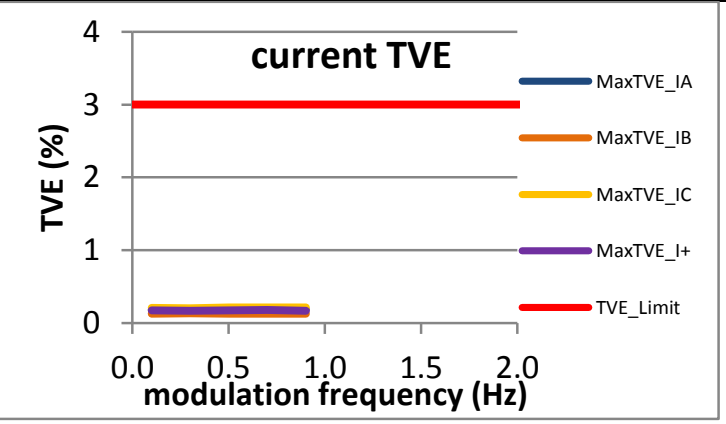

Figure 3406: Fs = 10 FPS 


\subsection{Dynamic bandwidth measurement: phase modulation frequency error: P class}

7.7.1 C37.118.1 Annex $C$ dynamic bandwidth measurement: phase modulation frequency error: $P$ class

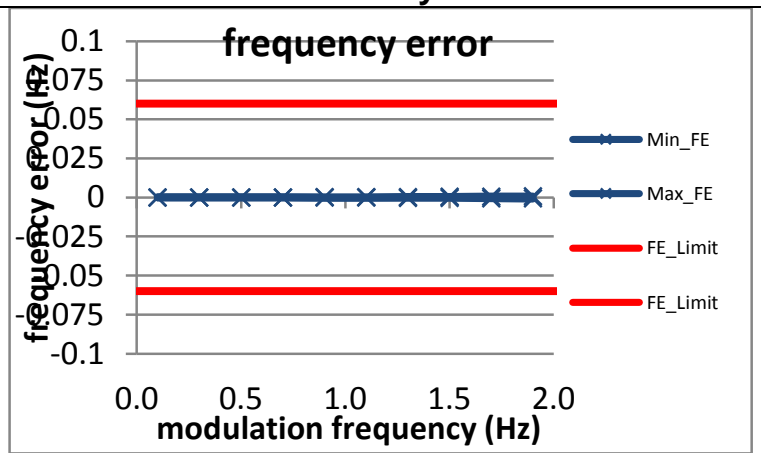

Figure 3407: Fs = 60 FPS

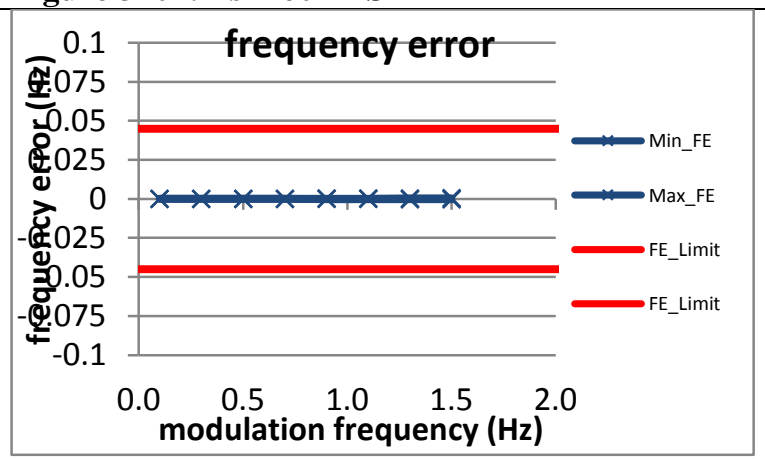

Figure 3410: Fs = 15 FPS

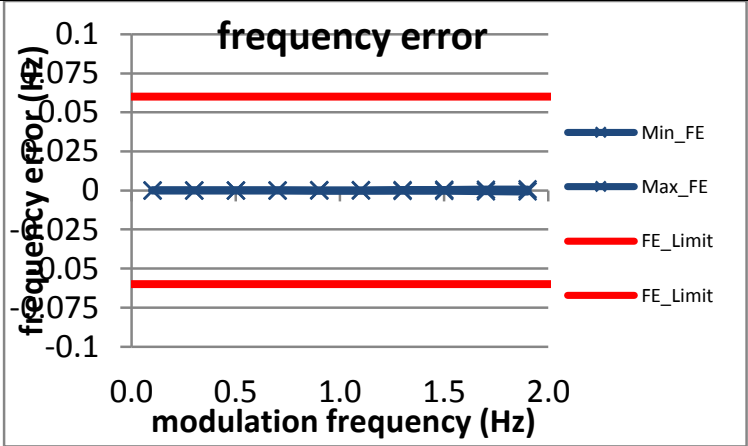

Figure 3408: Fs = 30 FPS

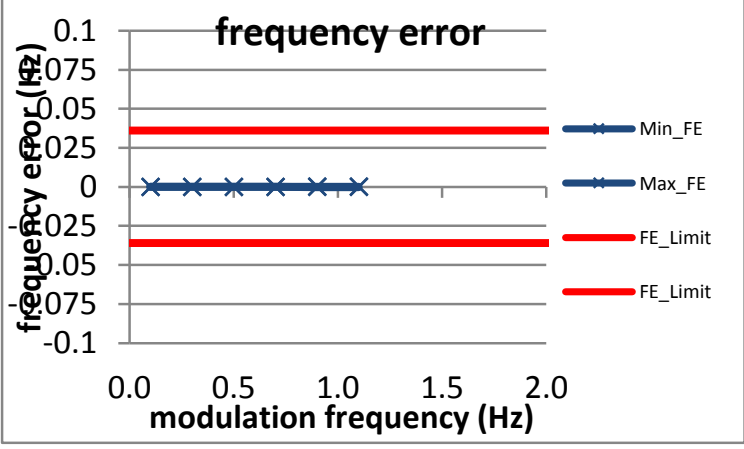

Figure 3411: Fs = 12 FPS

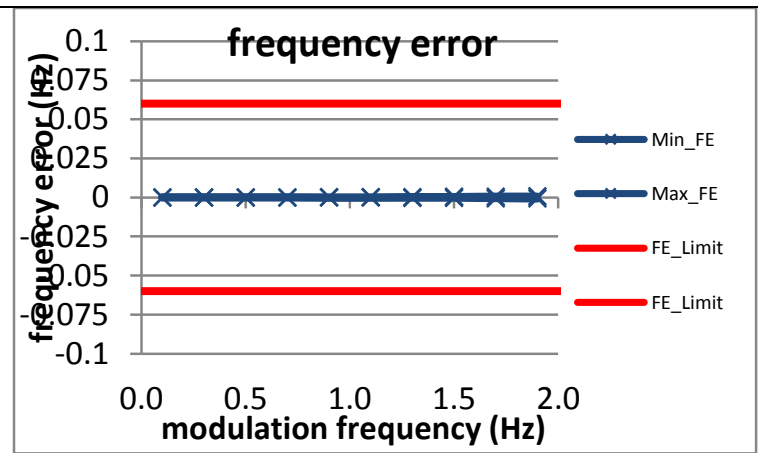

Figure 3409: Fs = 20 FPS

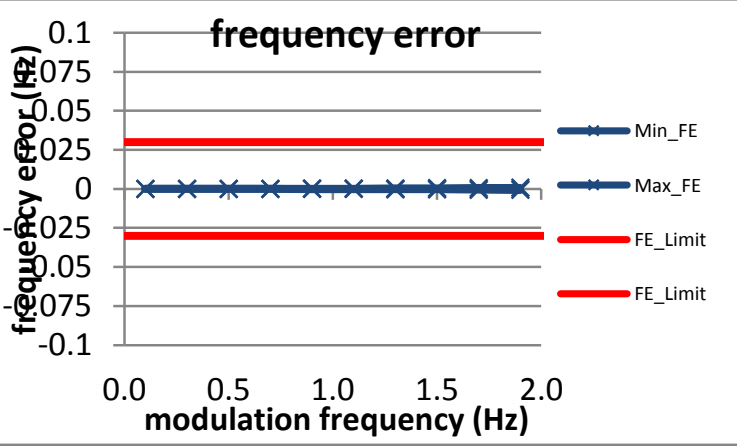

Figure 3412: Fs = 10 FPS 


\subsubsection{PMU A dynamic bandwidth measurement: phase modulation frequency error: $\mathbf{P}$ class}

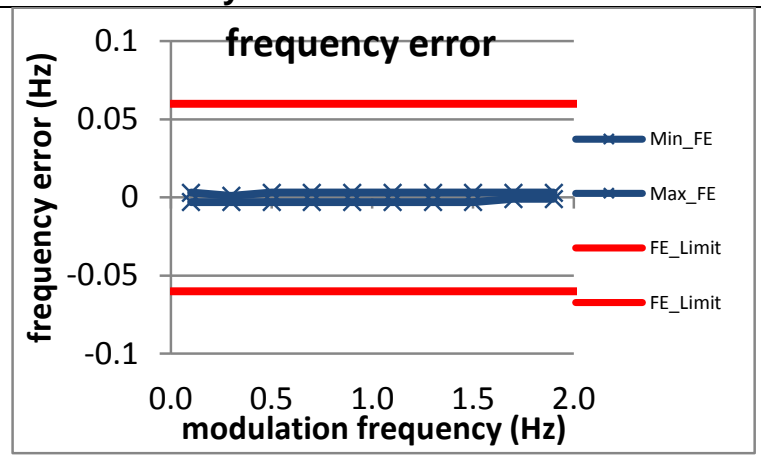

Figure 3413: Fs = 60 FPS

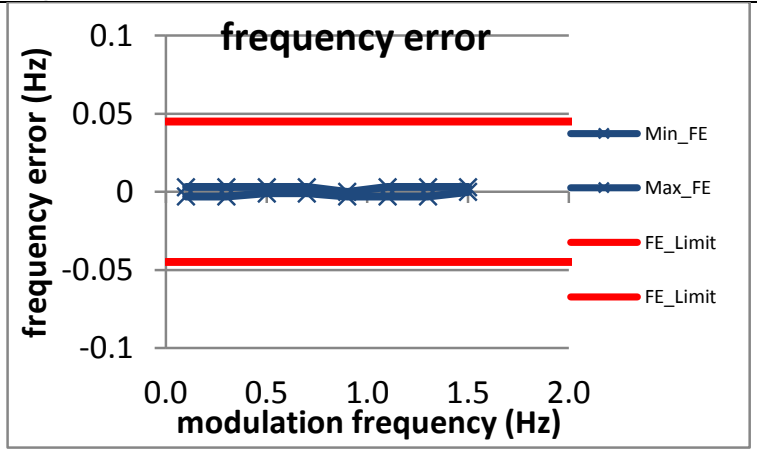

Figure 3416: Fs = 15 FPS

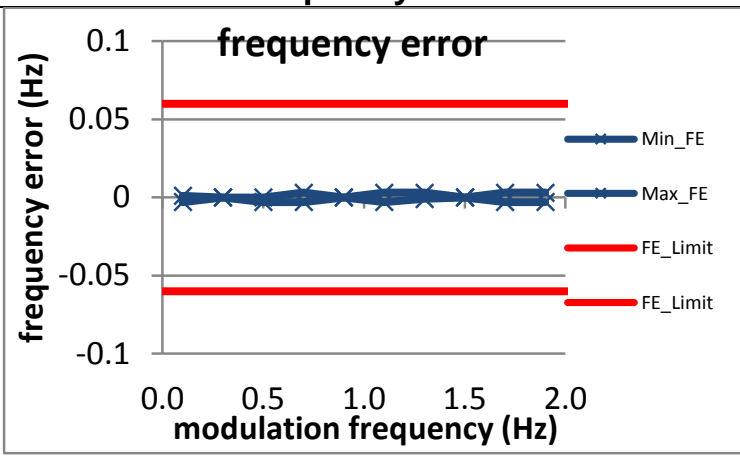

Figure 3414: Fs = 30 FPS

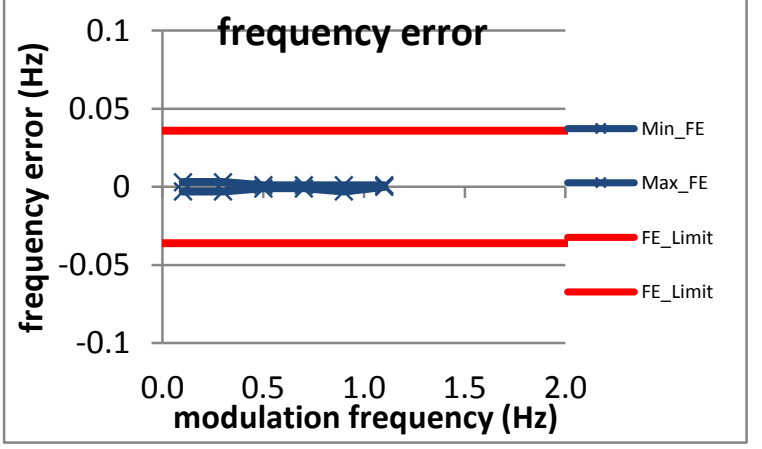

Figure 3417: Fs = 12 FPS

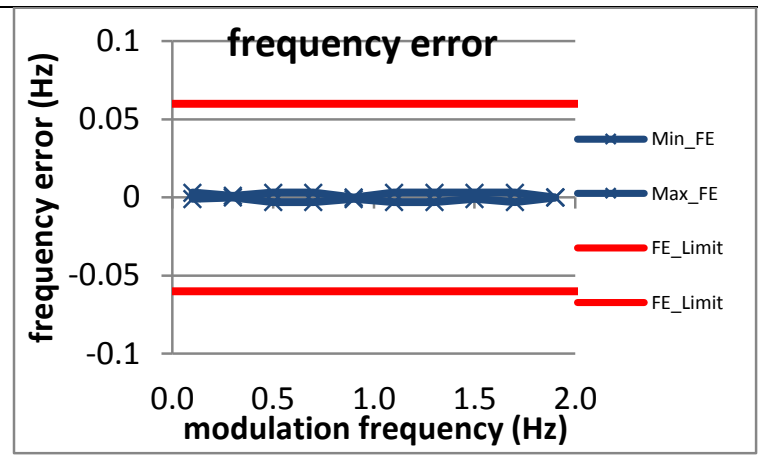

Figure 3415: Fs = 20 FPS

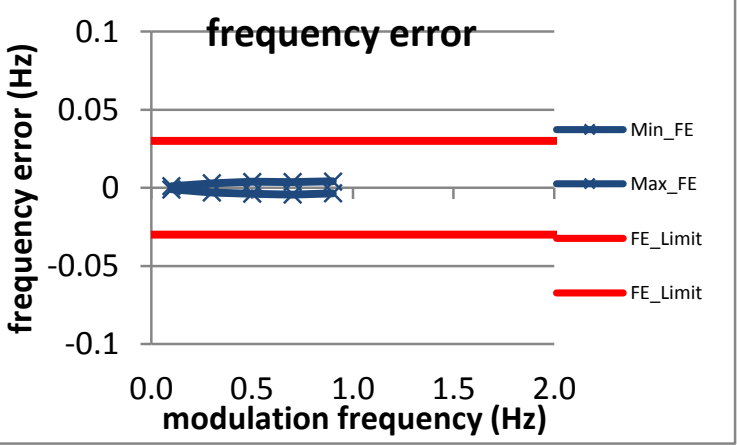

Figure 3418: Fs = 10 FPS 


\subsubsection{PMU B dynamic bandwidth measurement: phase modulation frequency error: $\mathbf{P}$ class}

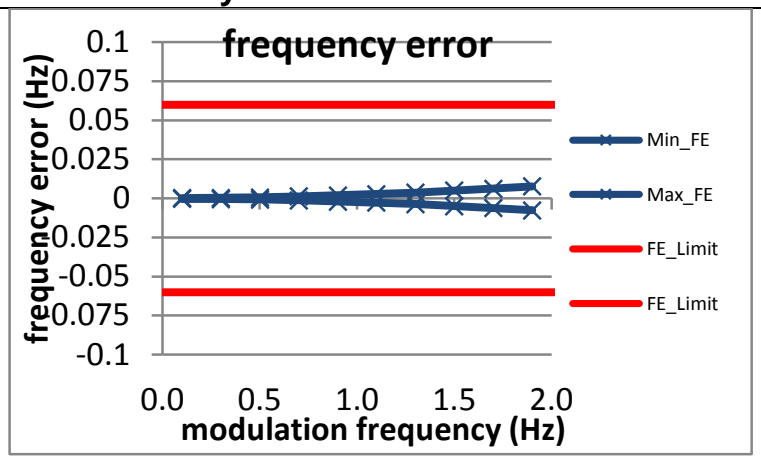

Figure 3419: Fs = 60 FPS

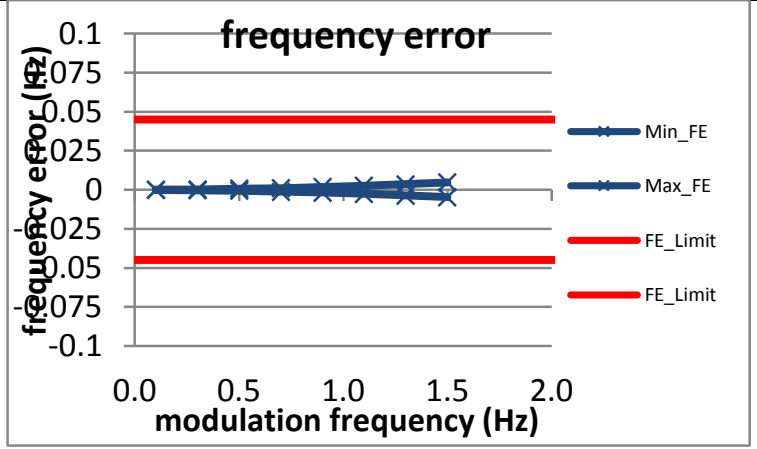

Figure 3422: Fs = 15 FPS

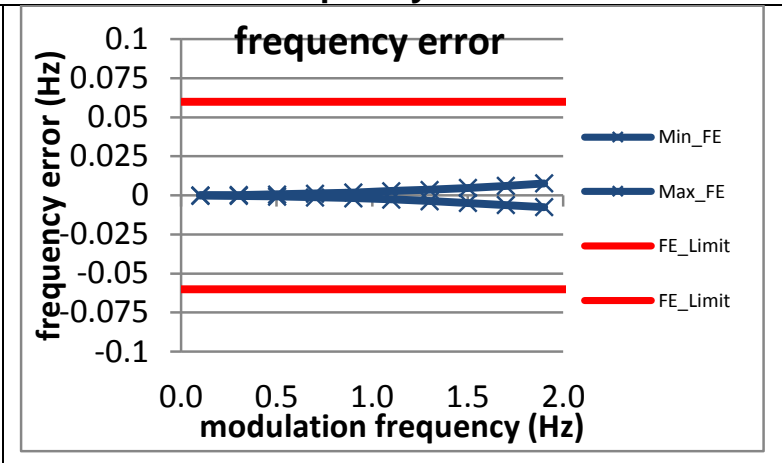

Figure 3420: Fs = 30 FPS

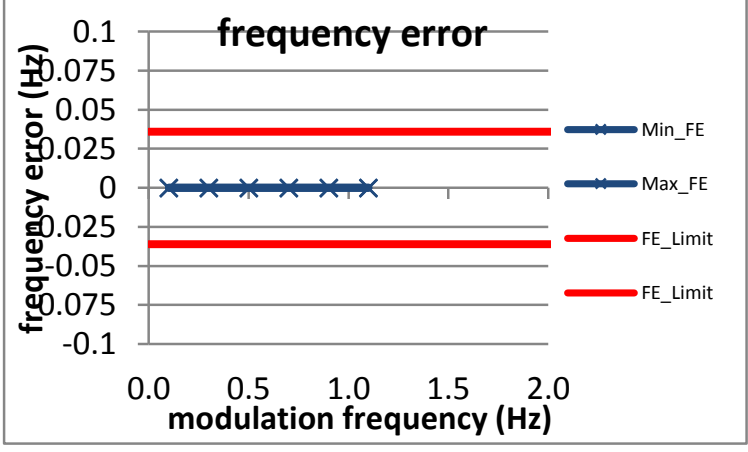

Figure 3423: Fs = 12 FPS

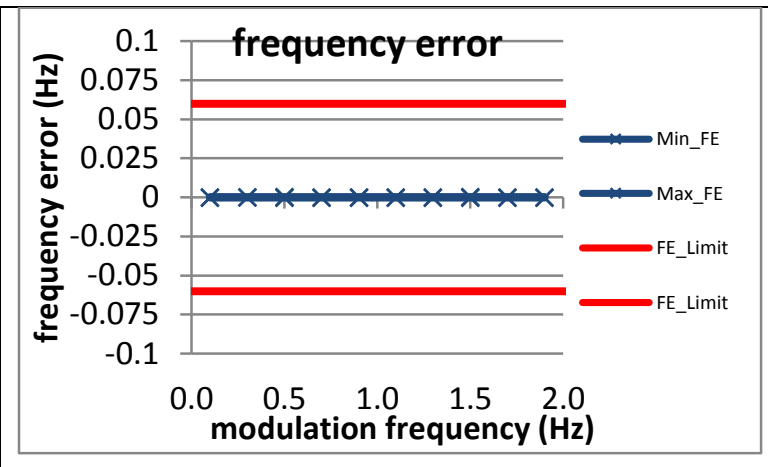

Figure 3421: Fs = 20 FPS

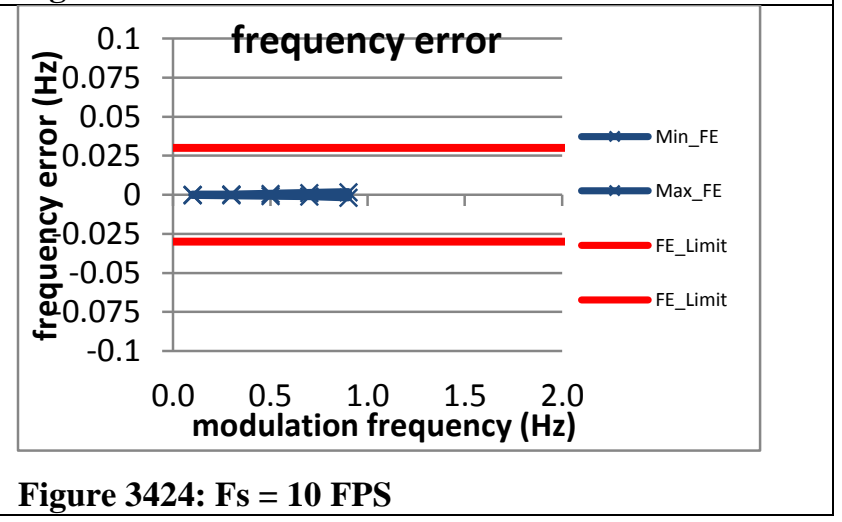




\subsubsection{PMU C dynamic bandwidth measurement: phase modulation frequency error: $P$ class}

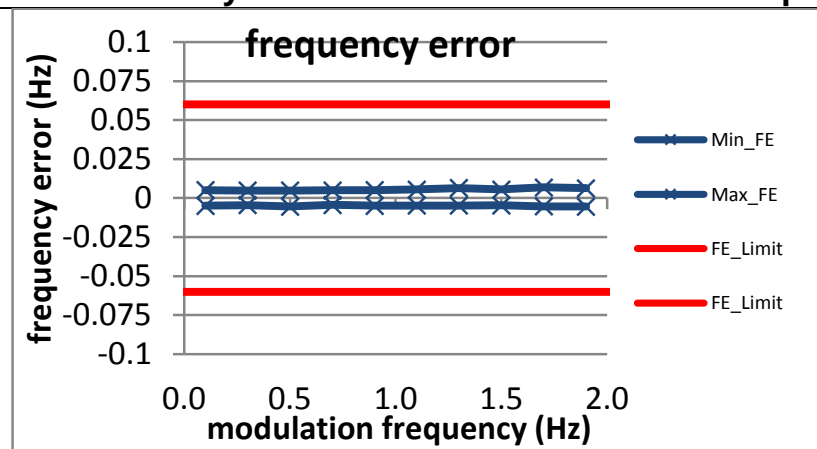

\section{Fi gure 3425: Fs = 60 FPS}

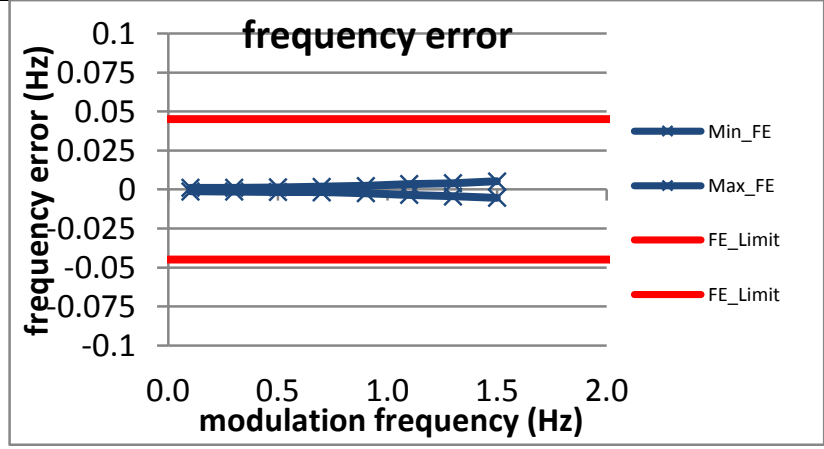

Figure 3428: Fs = 15 FPS

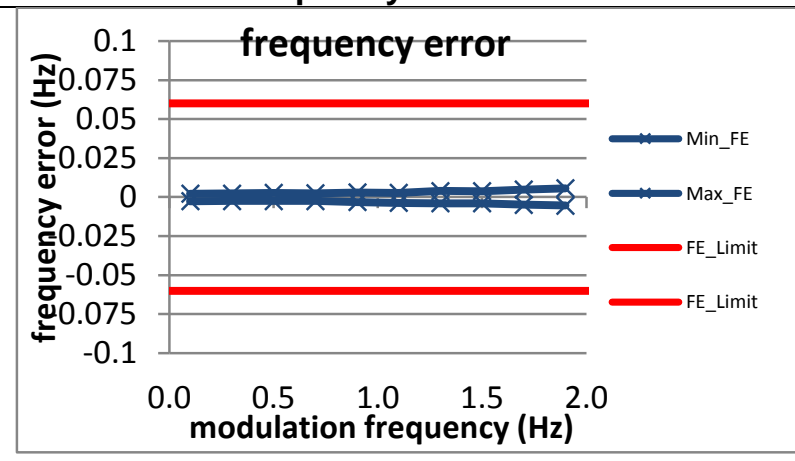

Figure 3426: Fs = 30 FPS

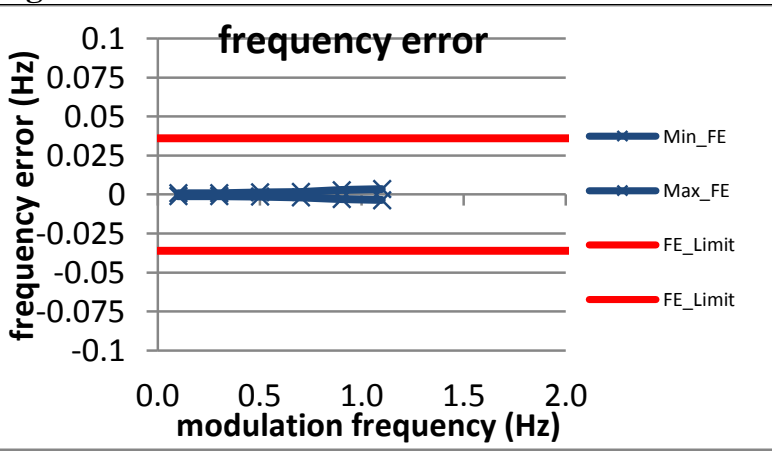

Figure 3429: Fs = 12 FPS

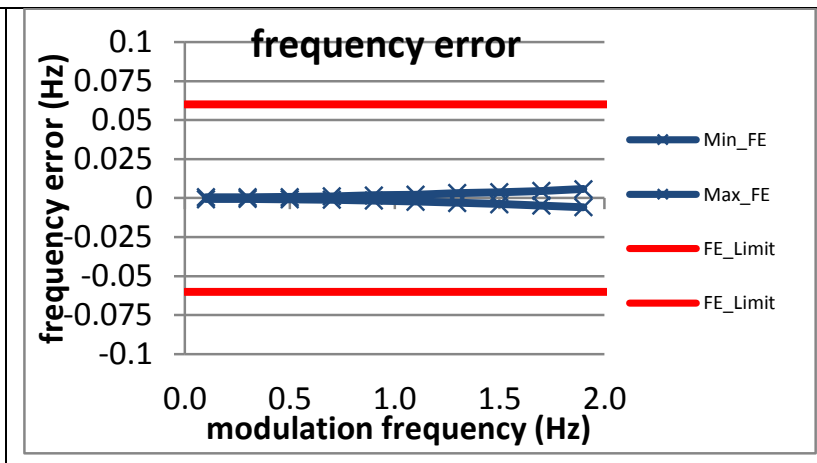

Figure 3427: Fs = 20 FPS

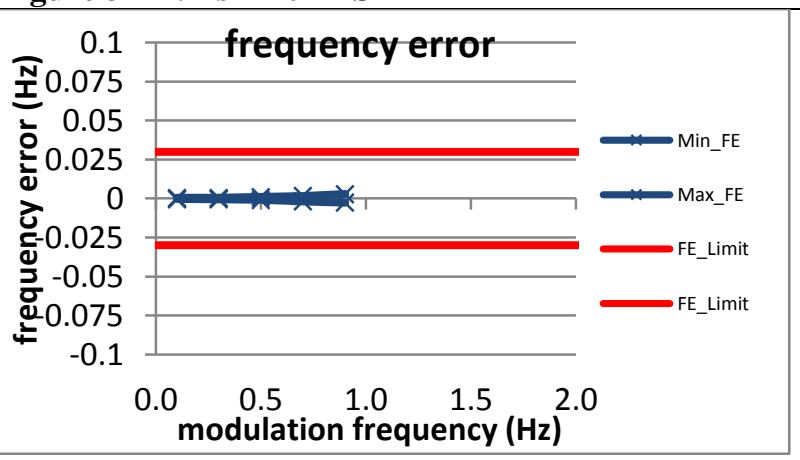

Figure 3430: Fs = 10 FPS 
7.7.5 PMU D dynamic bandwidth measurement: phase modulation frequency error: $P$ class

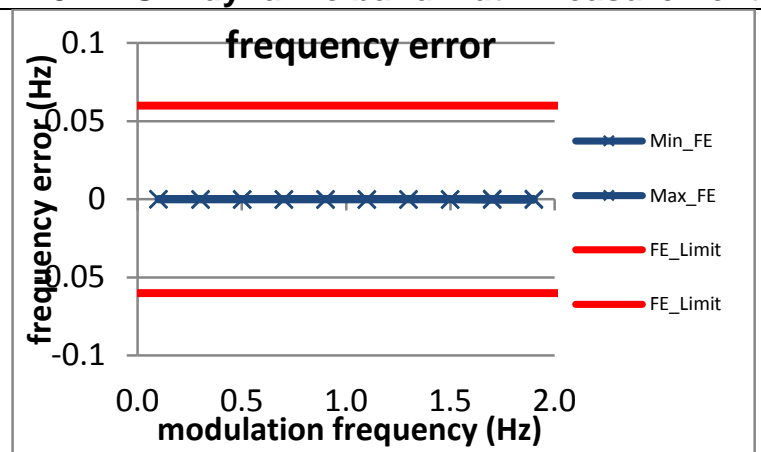

Figure 3431: Fs = 60 FPS

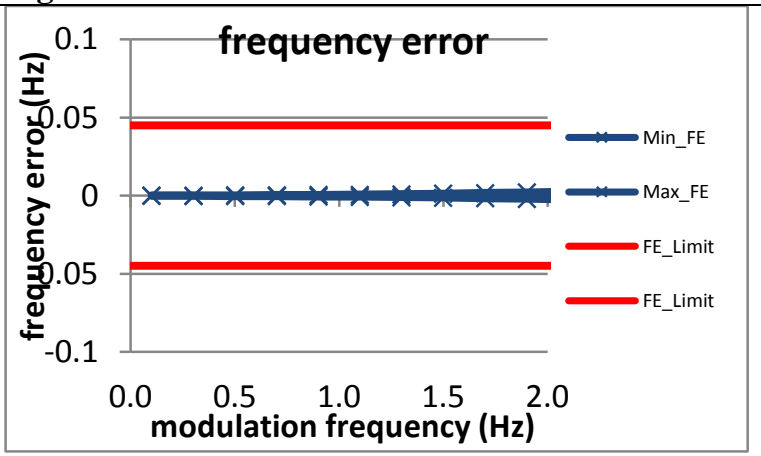

Figure 3434: Fs = 15 FPS

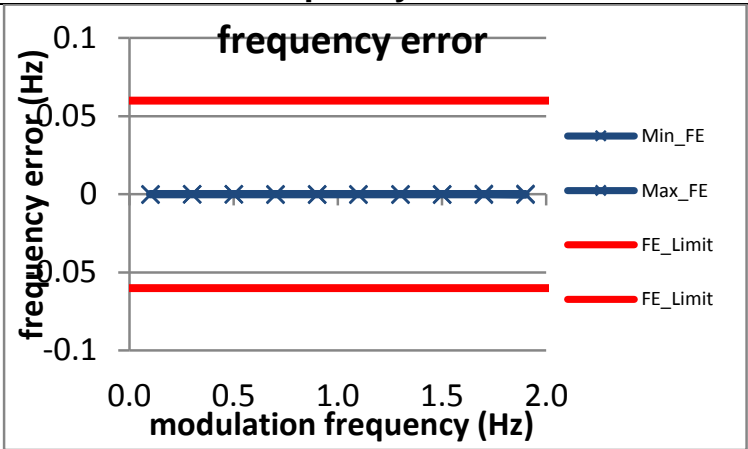

Figure 3432: Fs = 30 FPS

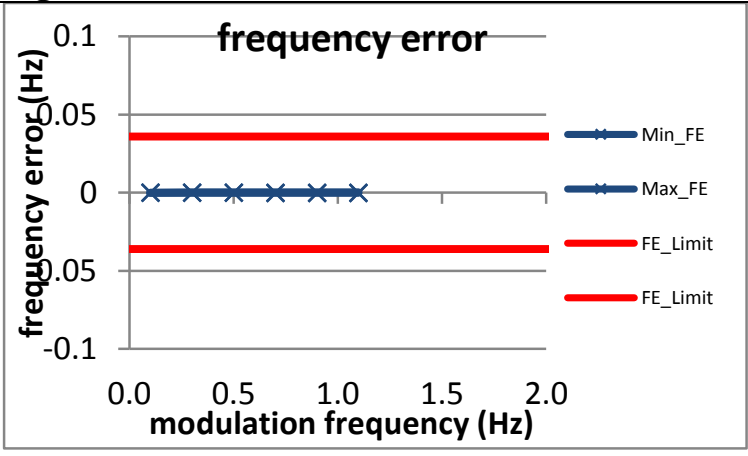

Figure 3435: Fs = 12 FPS

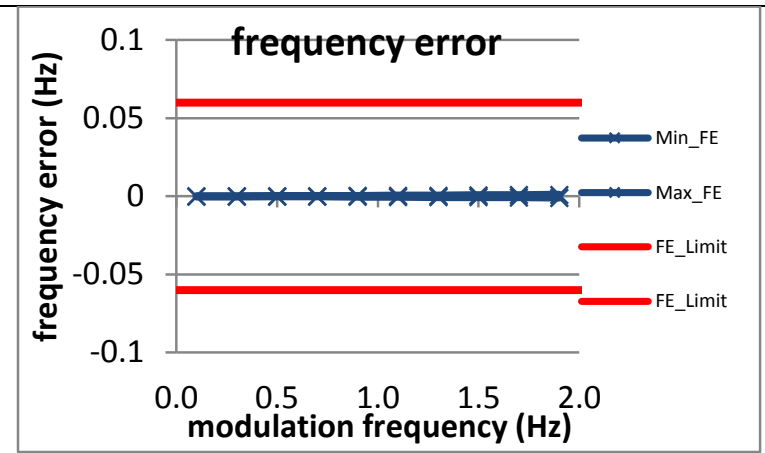

Figure 3433: Fs = 20 FPS

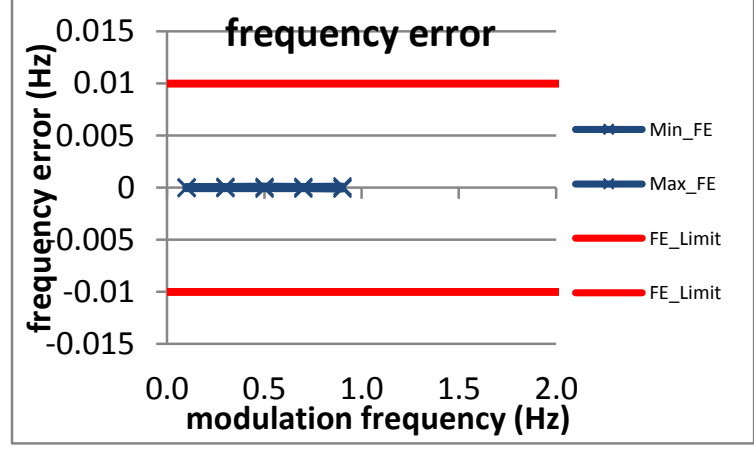

Figure 3436: Fs = 10 FPS

7.7.6 PMU E dynamic bandwidth measurement: phase modulation frequency error: $P$ class

PMU E does not support P class 
7.7.7 PMU F dynamic bandwidth measurement: phase modulation frequency error: $P$ class

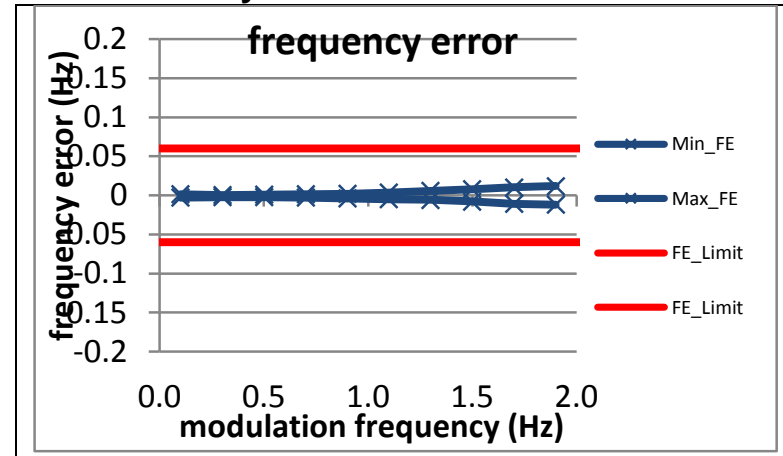

Figure 3437: Fs $=60$ FPS

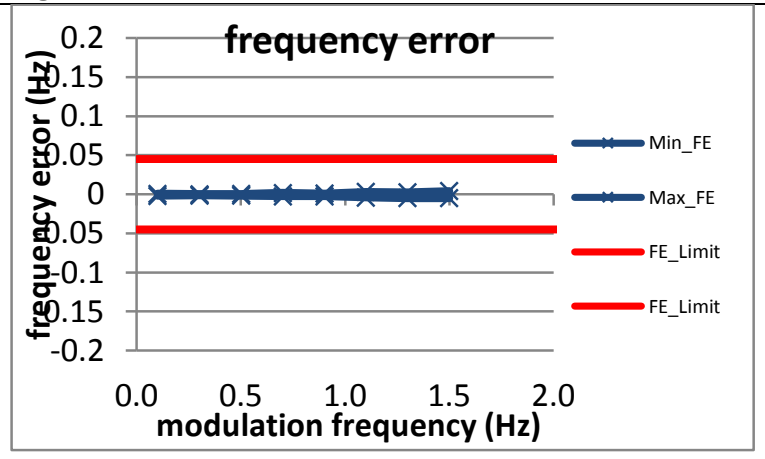

Figure 3440: Fs = 15 FPS

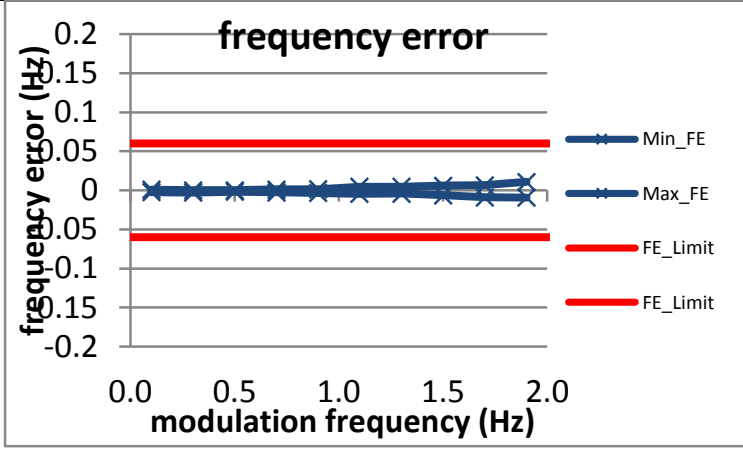

Figure 3438: Fs = 30 FPS

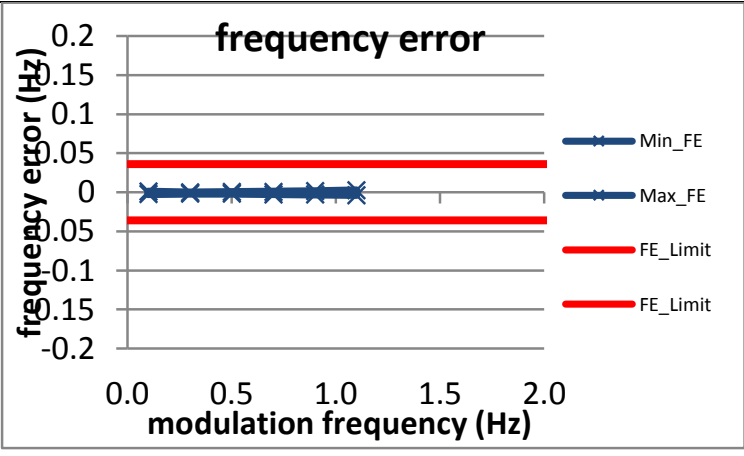

Figure 3441: Fs = 12 FPS

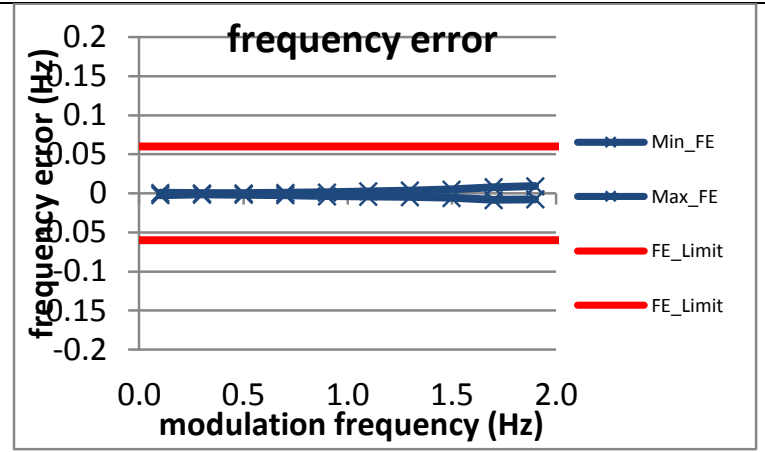

Figure 3439: Fs = 20 FPS

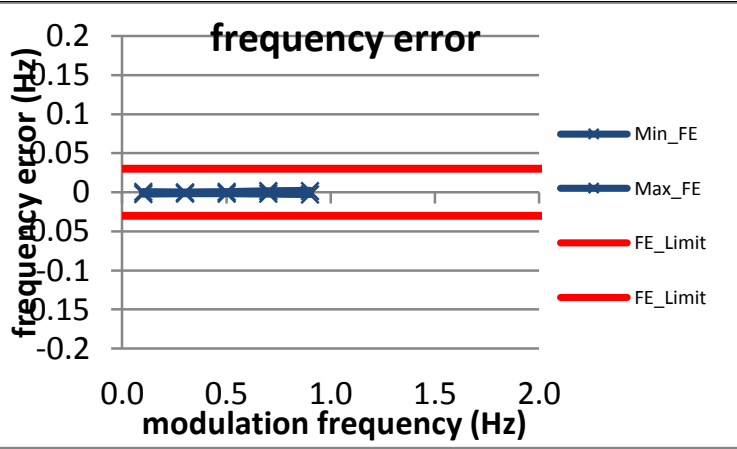

Figure 3442: Fs = 10 FPS

7.7.8 PMU G dynamic bandwidth measurement: phase modulation frequency error: $P$ class

PMU G does not support P class. 
7.7.9 PMU H dynamic bandwidth measurement: phase modulation frequency error: P class
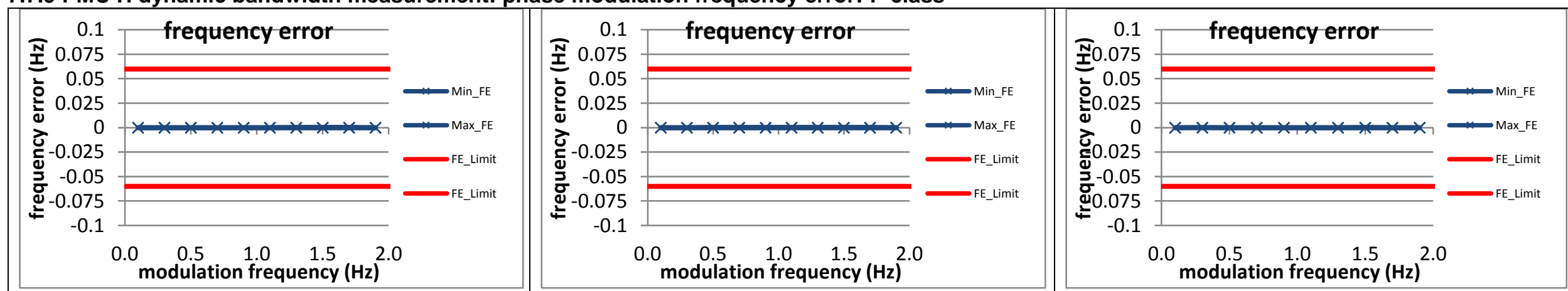

Figure 3443: Fs = 60 FPS

Figure 3444: Fs = 30 FPS

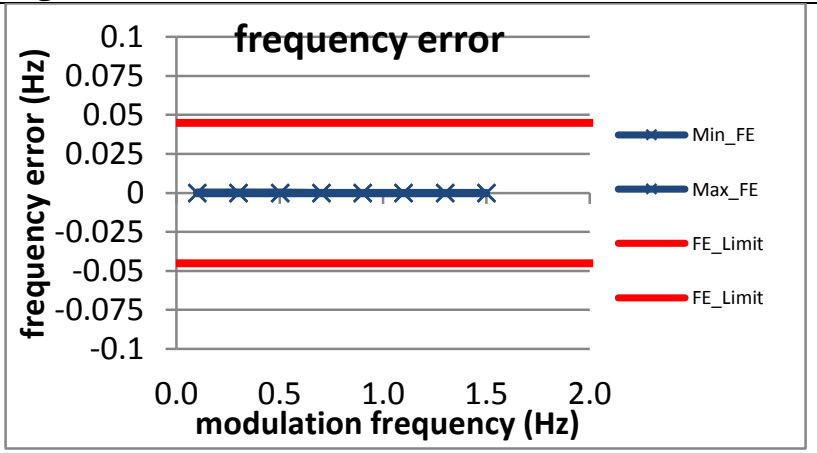

Figure 3446: Fs = 15 FPS

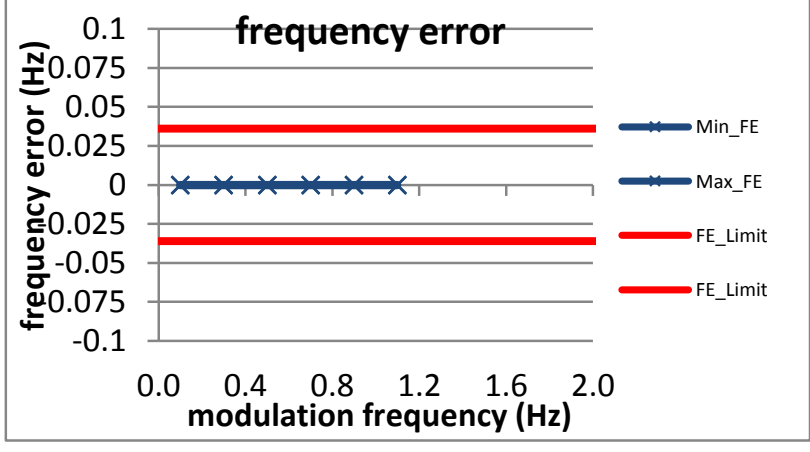

Figure 3447: Fs = 12 FPS
Figure 3445: Fs = 20 FPS

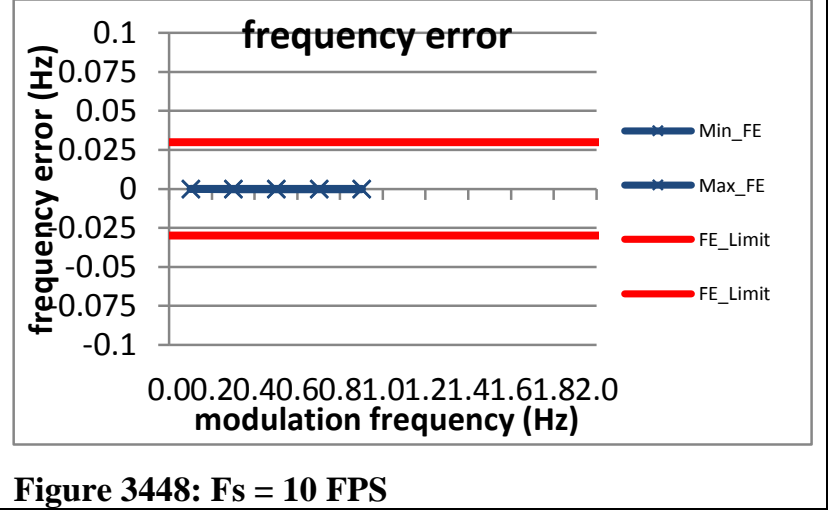

\subsubsection{PMU I dynamic bandwidth measurement: phase modulation frequency error: $P$ class}

PMU I does not support P class 
7.7.11 PMU J dynamic bandwidth measurement: phase modulation frequency error: P class

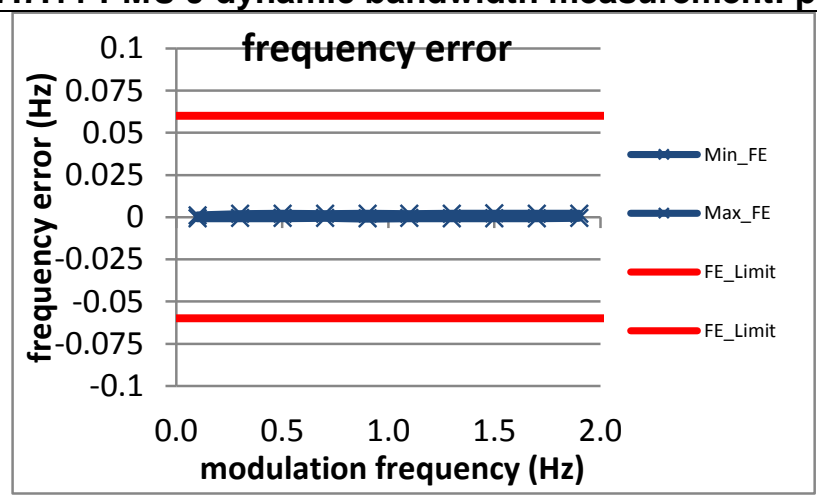

Figure 3449: Fs = 60 FPS

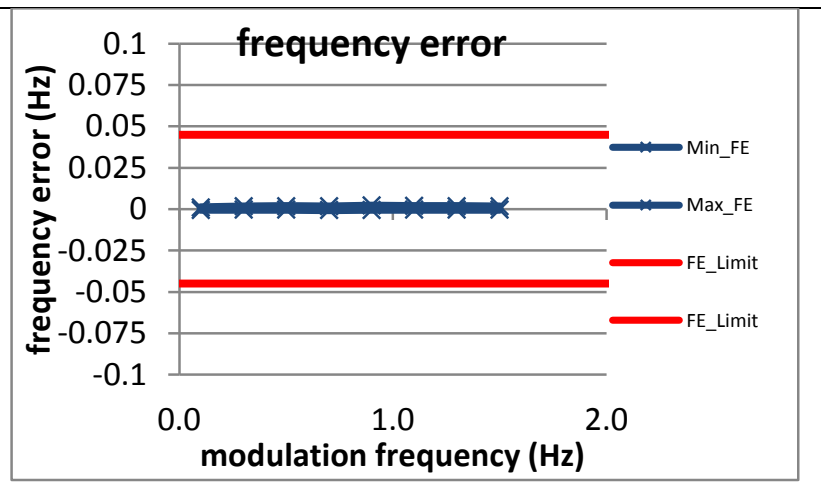

Figure 3452: Fs = 15 FPS

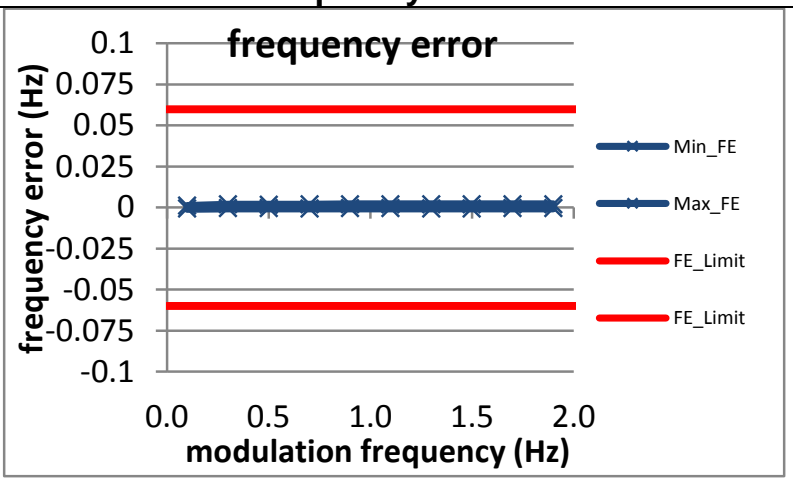

Figure 3450: Fs = 30 FPS

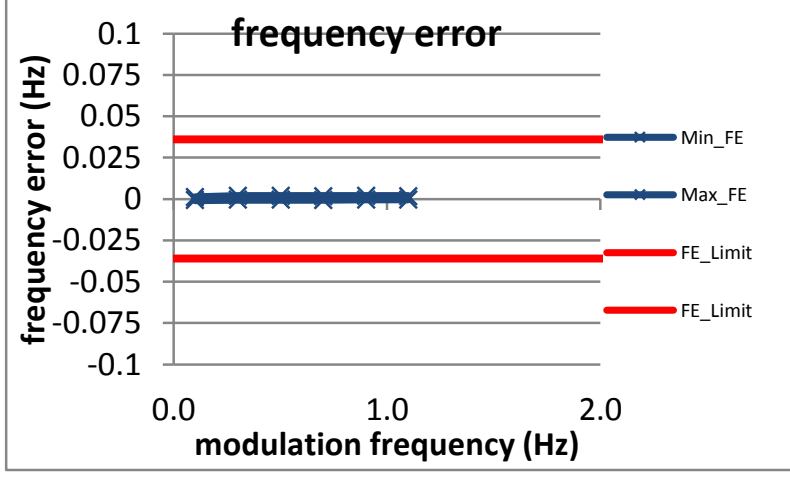

Figure 3453: Fs = 12 FPS

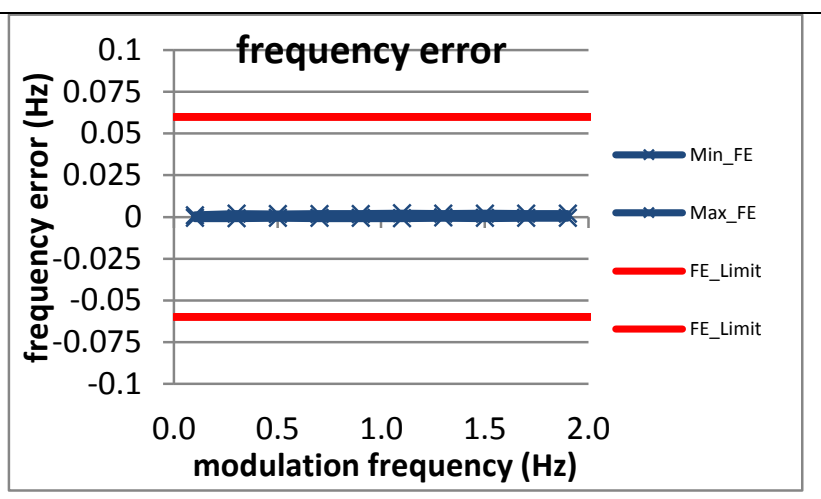

Figure 3451: Fs = 20 FPS

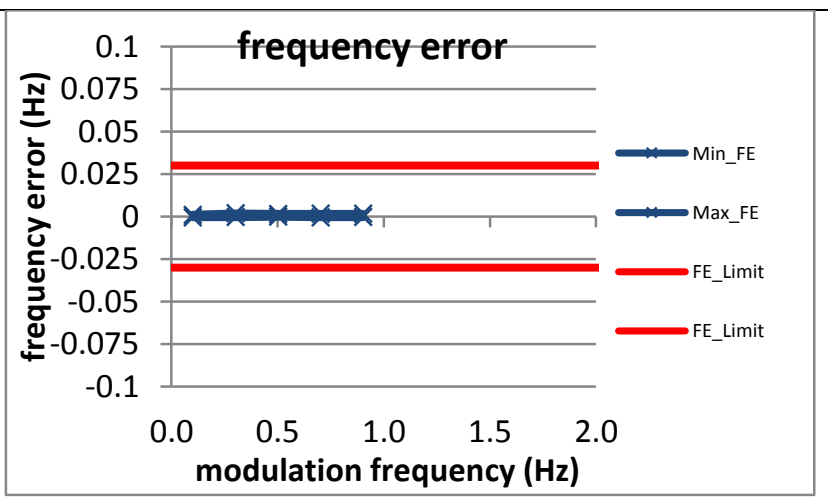

Figure 3454: Fs = 10 FPS 


\subsection{Dynamic bandwidth measurement: phase modulation ROCOF error: P class}

7.8.1 C37.118.1 Annex C dynamic bandwidth measurement: phase modulation ROCOF error: P class

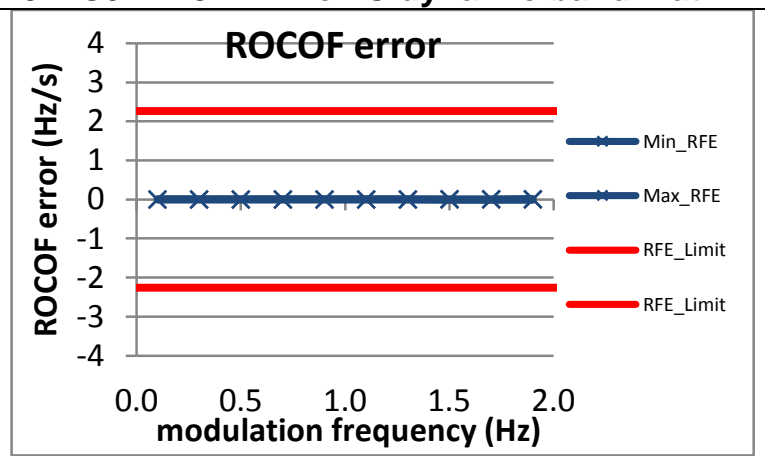

Figure 3455: Fs = 60 FPS

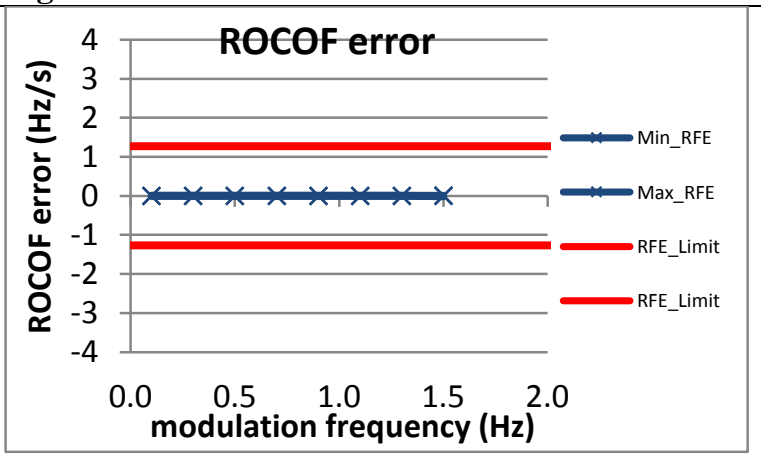

Figure 3458: Fs = 15 FPS

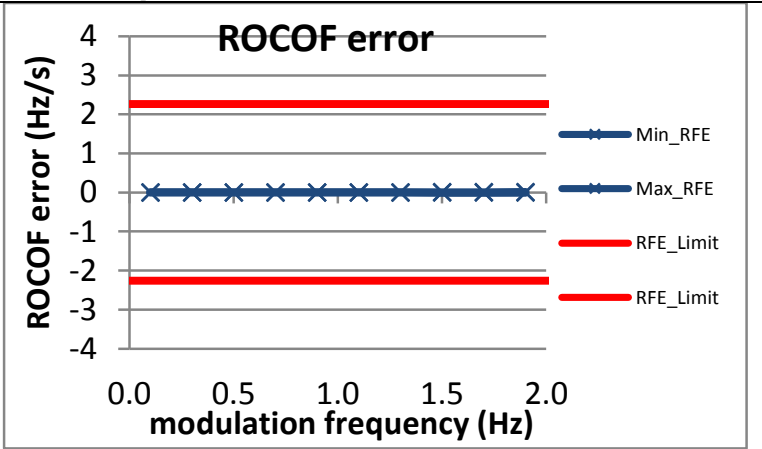

Figure 3456: Fs = 30 FPS

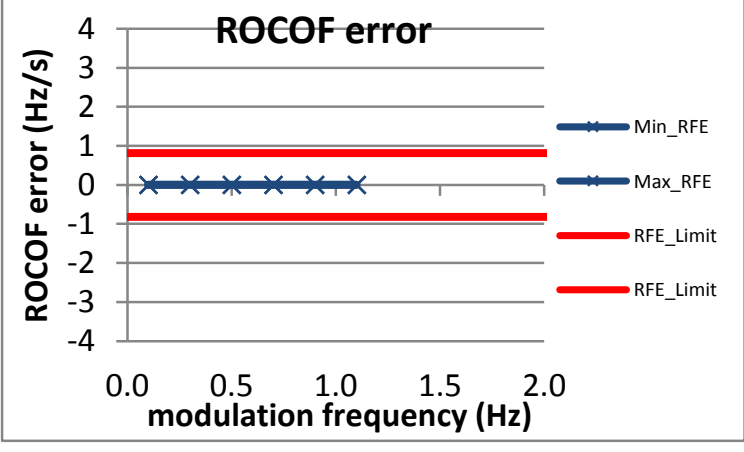

Figure 3459: Fs = 12 FPS

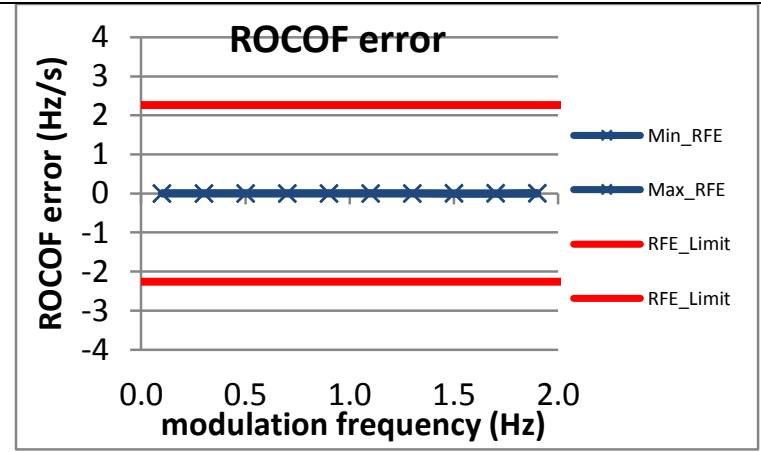

Figure 3457: Fs = 20 FPS

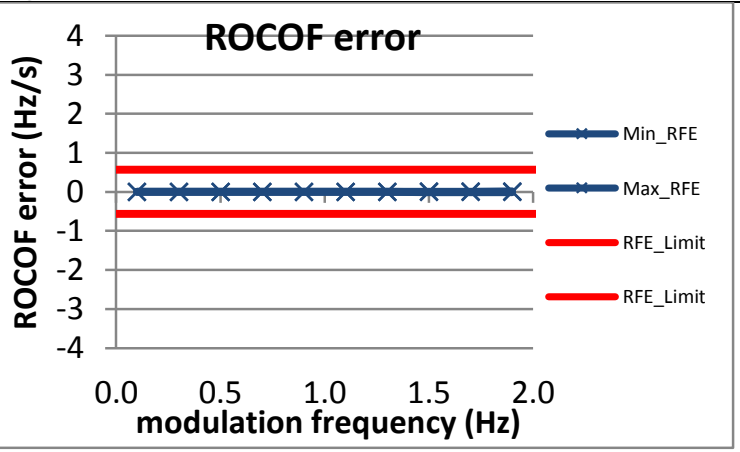

Figure 3460: Fs = 10 FPS 
7.8.2 PMU A dynamic bandwidth measurement: phase modulation ROCOF error: $P$ class

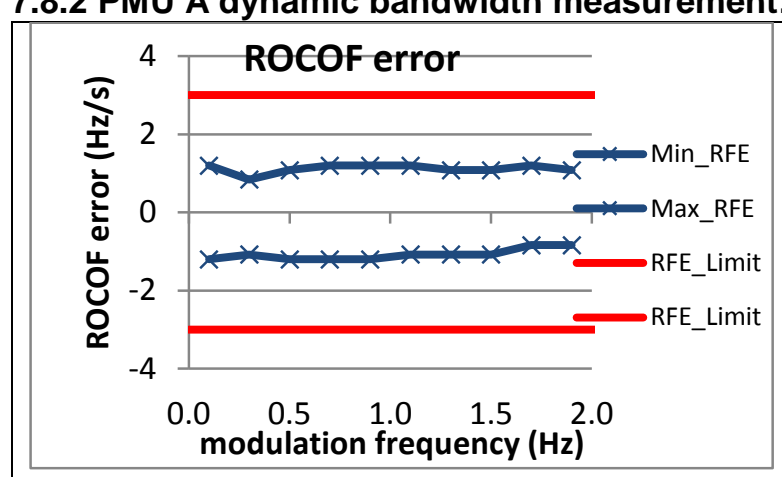

Figure 3461: Fs = 60 FPS

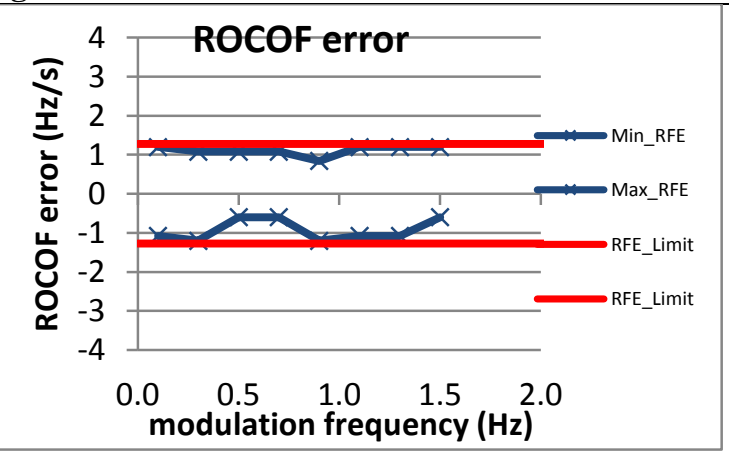

Figure 3464: Fs = 15 FPS

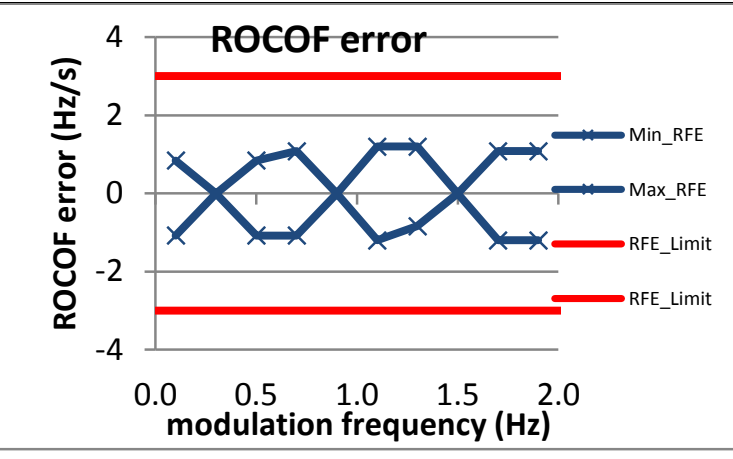

Figure 3462: Fs = 30 FPS

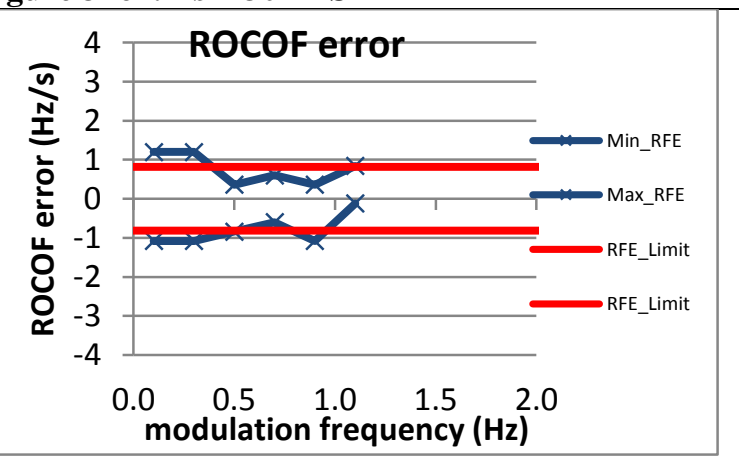

Figure 3465: Fs = 12 FPS

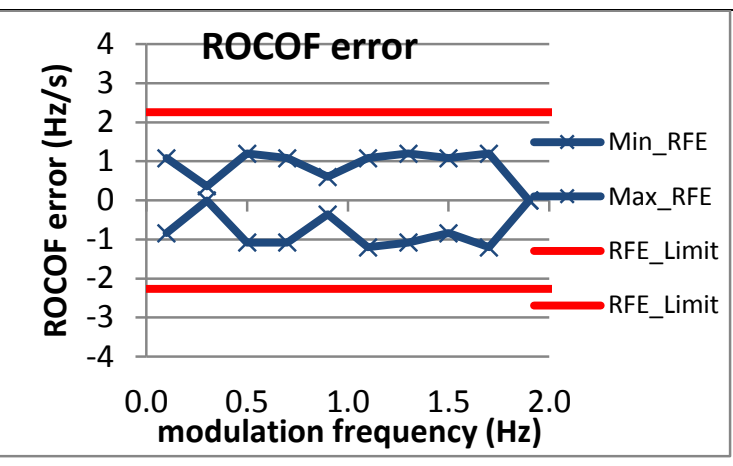

Figure 3463: Fs = 20 FPS

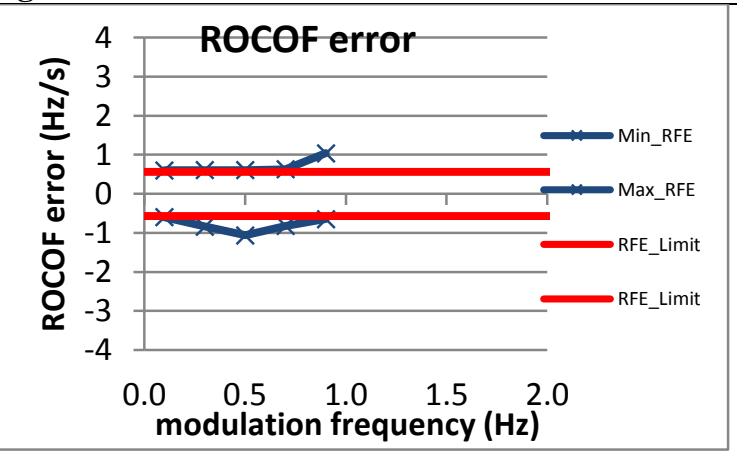

Figure 3466: Fs = 10 FPS 
7.8.3 PMU B dynamic bandwidth measurement: phase modulation ROCOF error: $P$ class

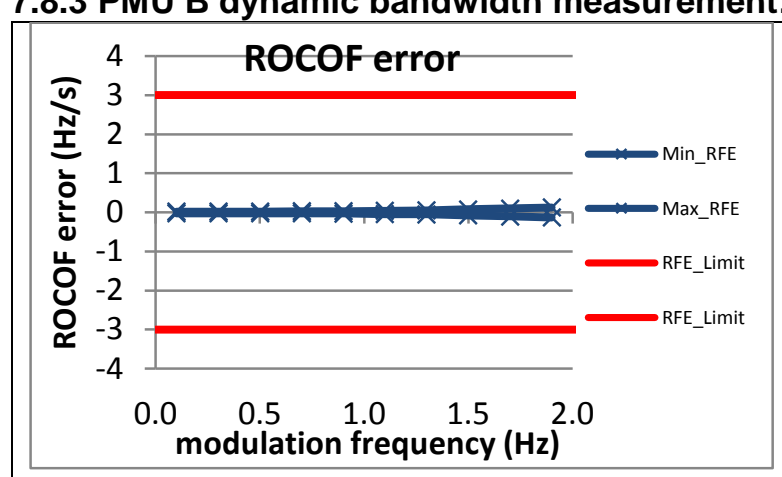

Figure 3467: Fs = 60 FPS

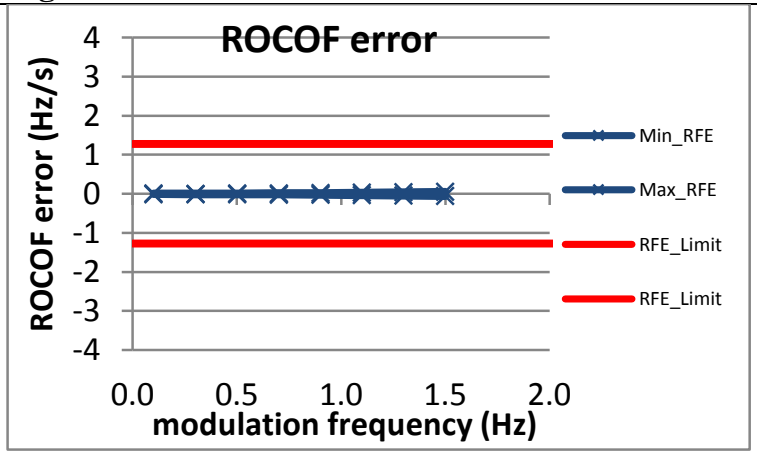

Figure 3470: Fs = 15 FPS

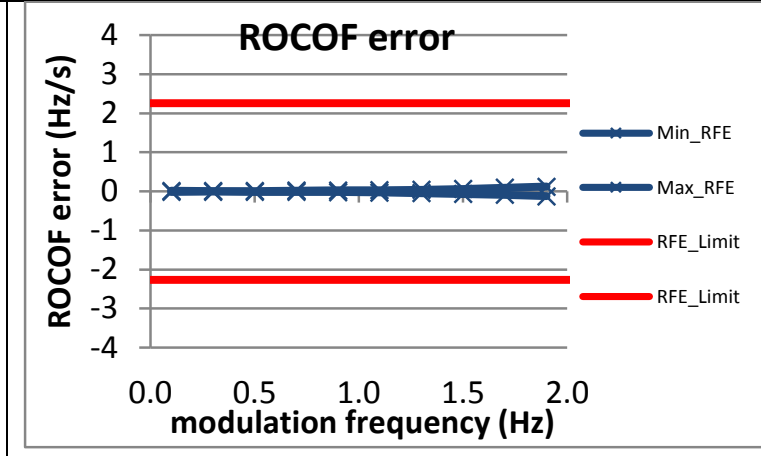

Figure 3468: Fs = 30 FPS

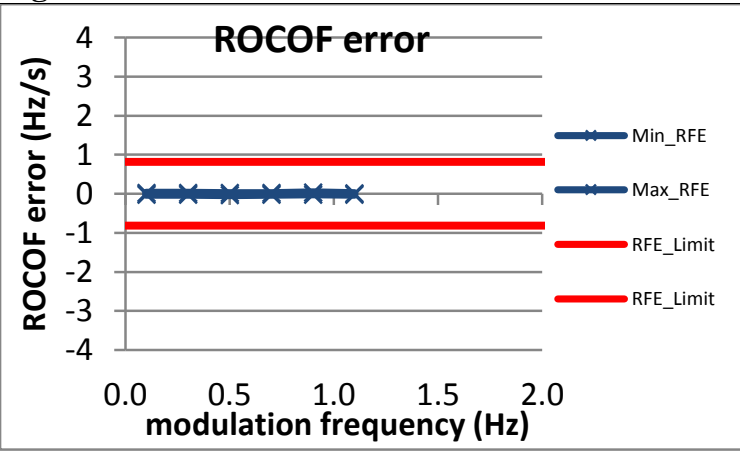

Figure 3471: Fs = 12 FPS

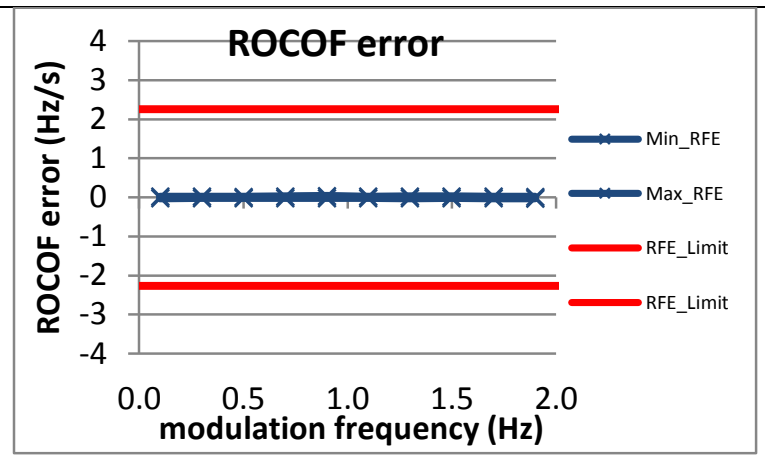

Figure 3469: Fs = 20 FPS

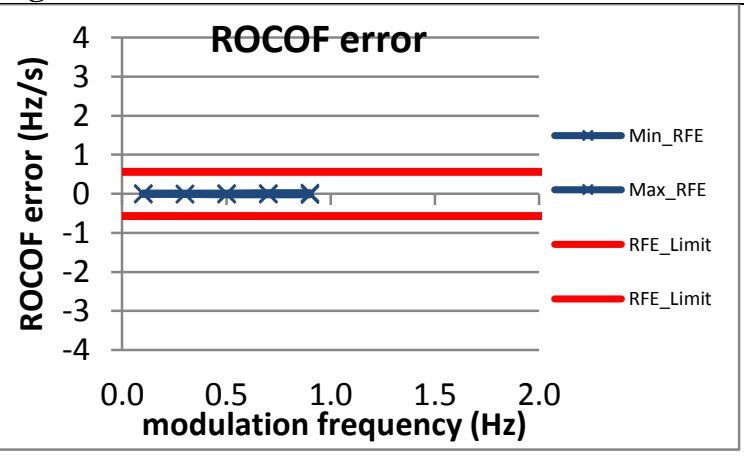

Figure 3472: Fs = 10 FPS 
7.8.4 PMU C dynamic bandwidth measurement: phase modulation ROCOF error: P class

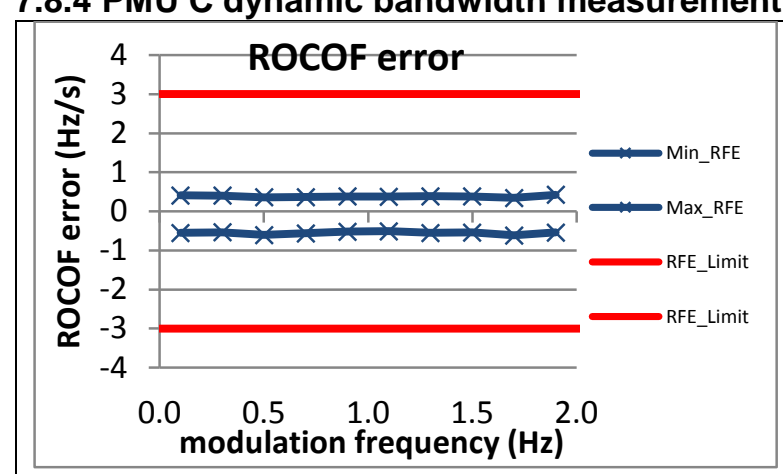

Figure 3473: Fs = 60 FPS

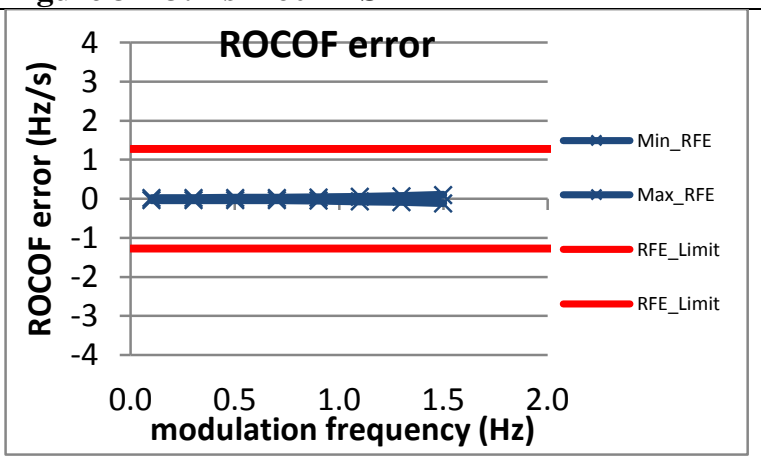

Figure 3476: Fs = 15 FPS

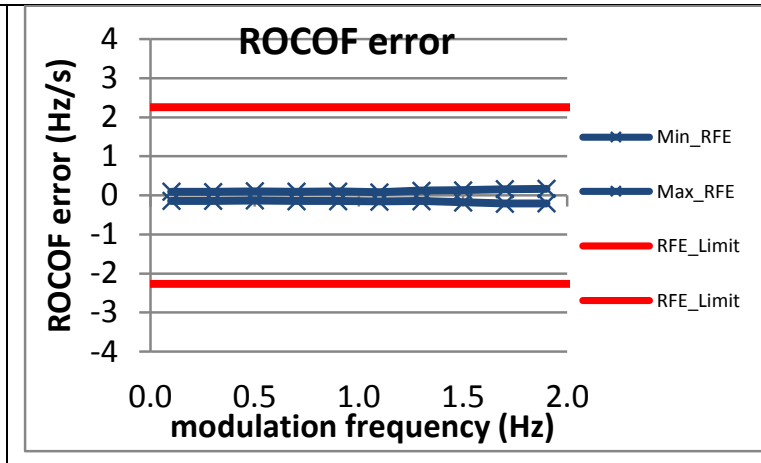

Figure 3474: Fs = 30 FPS

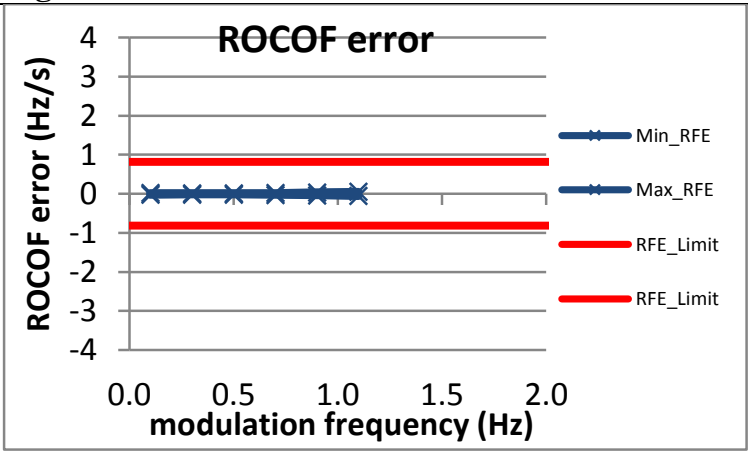

Figure 3477: Fs = 12 FPS

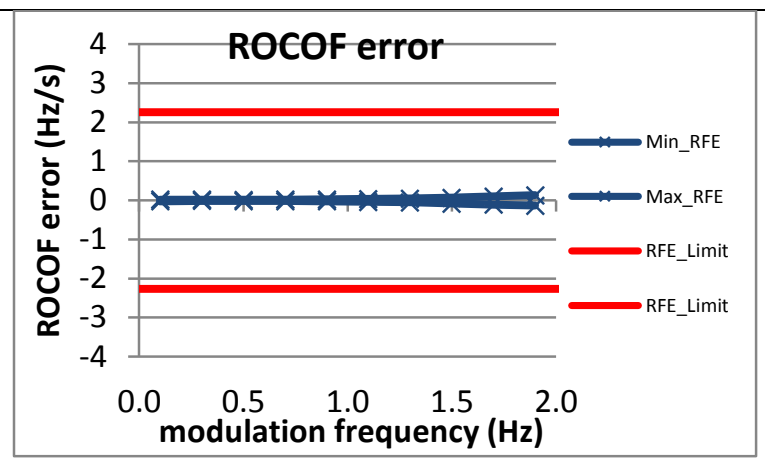

Figure 3475: Fs = 20 FPS

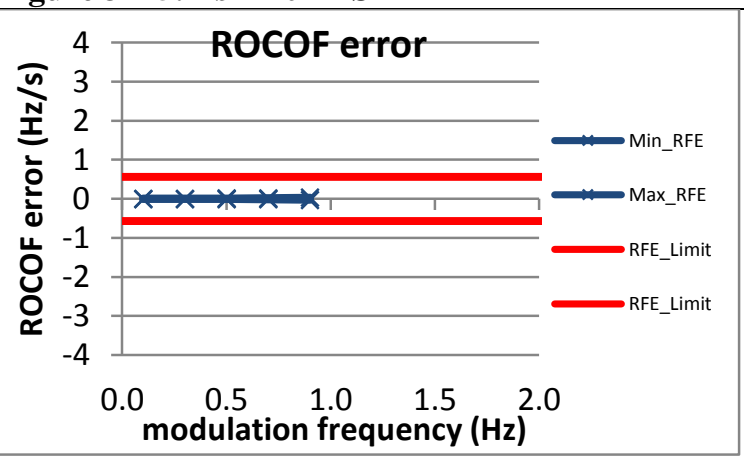

Figure 3478: Fs = 10 FPS 
7.8.5 PMU D dynamic bandwidth measurement: phase modulation ROCOF error: P class

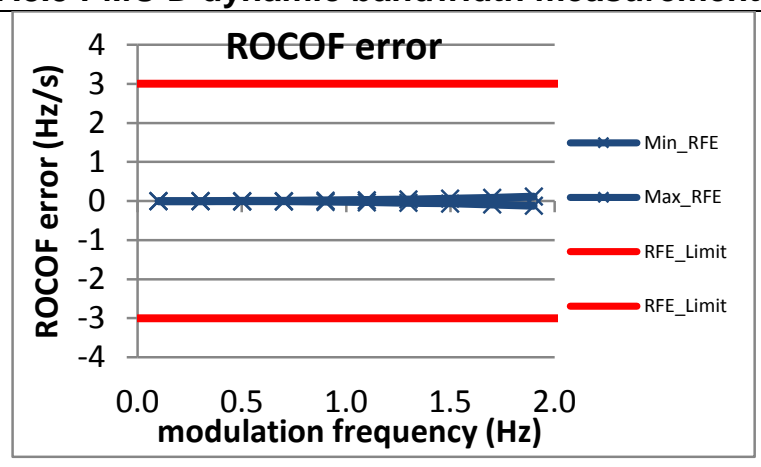

Figure 3479: Fs = 60 FPS

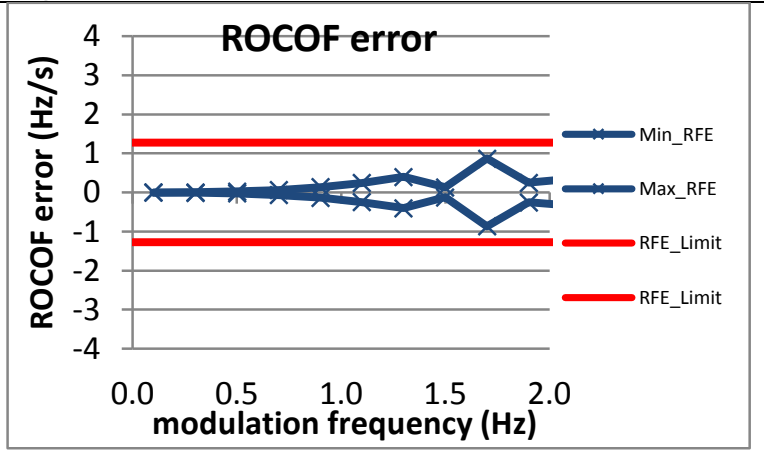

Figure 3482: Fs = 15 FPS

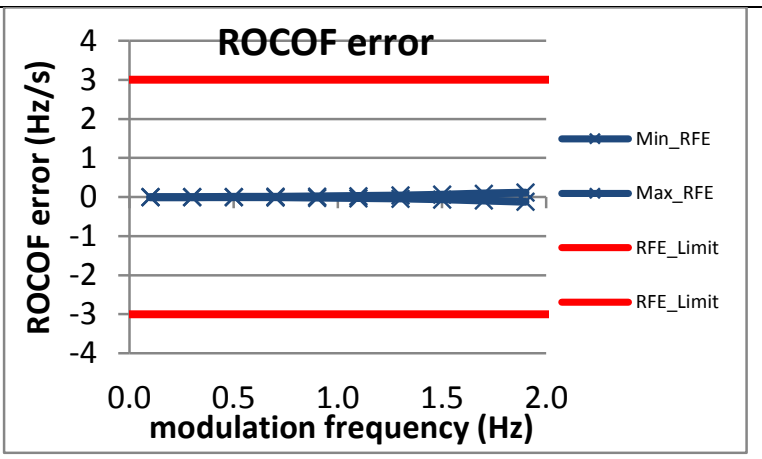

Figure 3480: Fs = 30 FPS

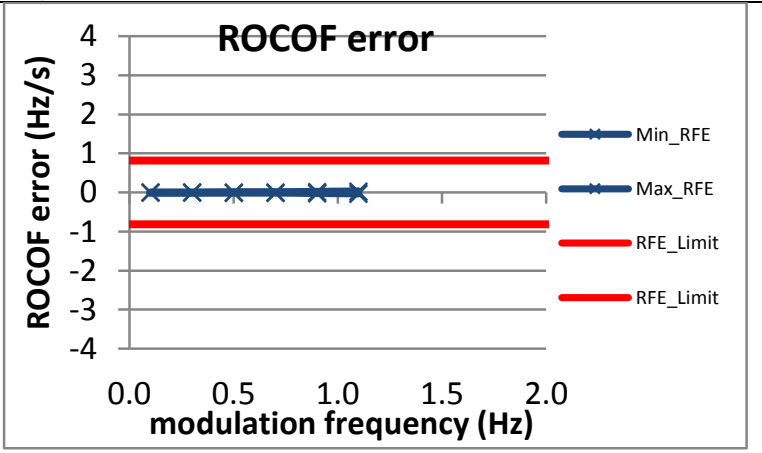

Figure 3483: Fs = 12 FPS

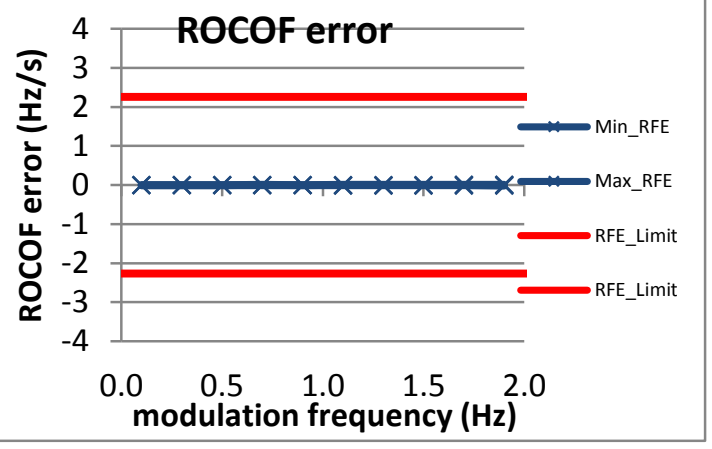

Figure 3481: Fs = 20 FPS

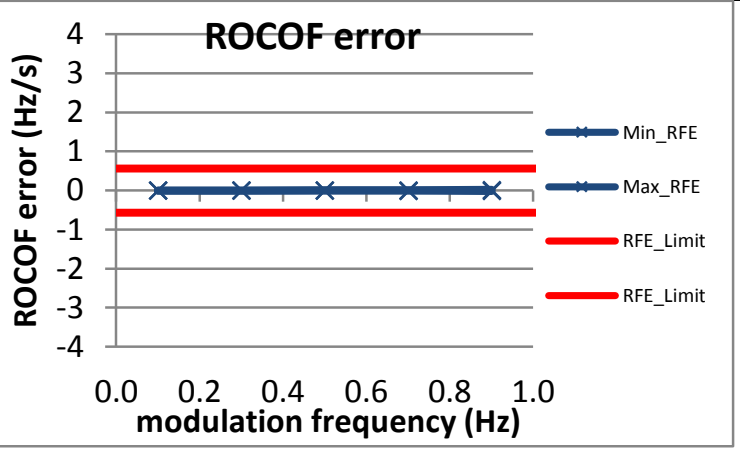

Figure 3484: Fs = 10 FPS

7.8.6 PMU E dynamic bandwidth measurement: phase modulation ROCOF error: P class

PMU E does not support P class 
7.8.7 PMU F dynamic bandwidth measurement: phase modulation ROCOF error: $P$ class

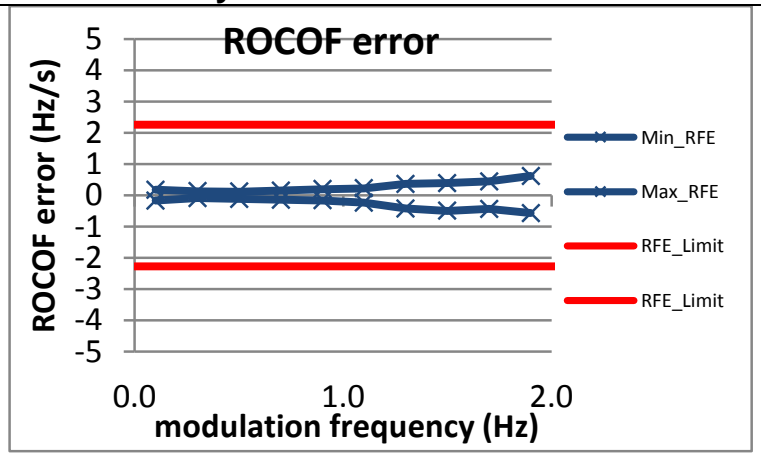

Figure 3485: Fs $=60$ FPS

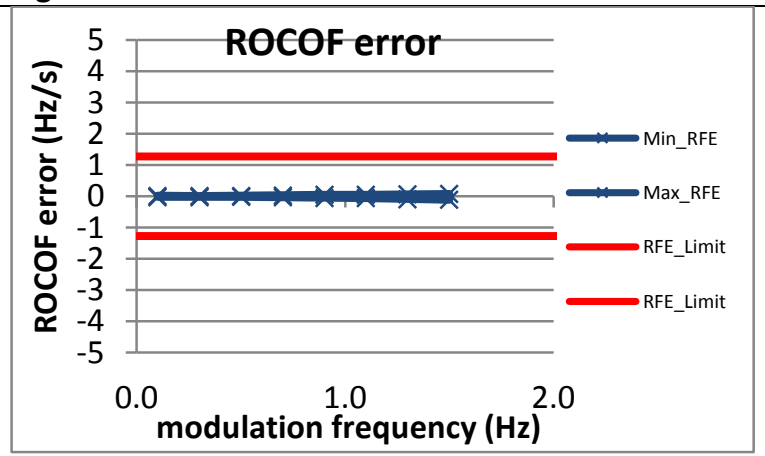

Figure 3488: Fs = 15 FPS

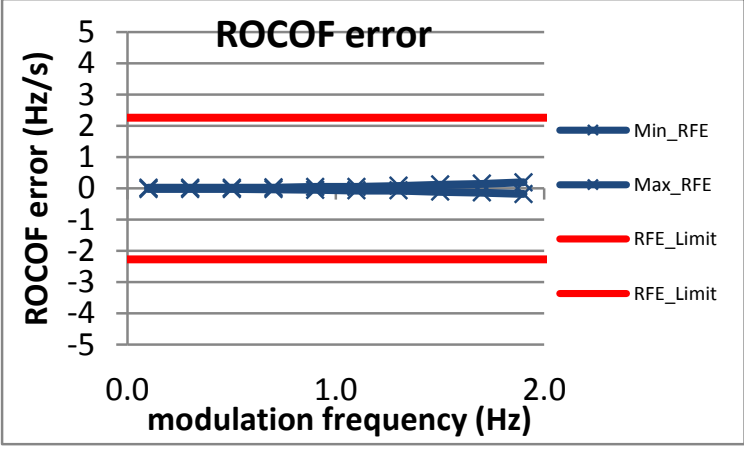

Figure 3486: $\mathrm{Fs}=30 \mathrm{FPS}$

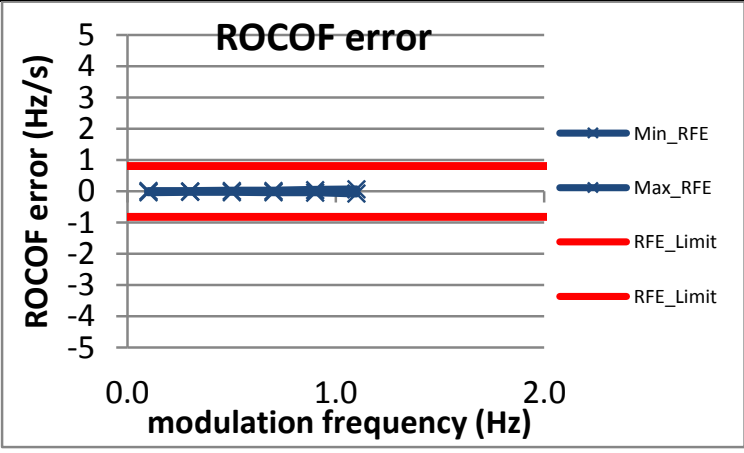

Figure 3489: Fs = 12 FPS

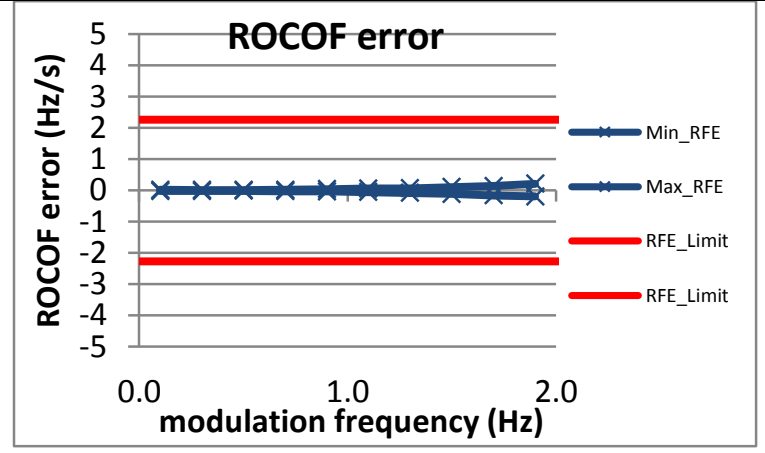

Figure 3487: $\mathrm{Fs}=20 \mathrm{FPS}$

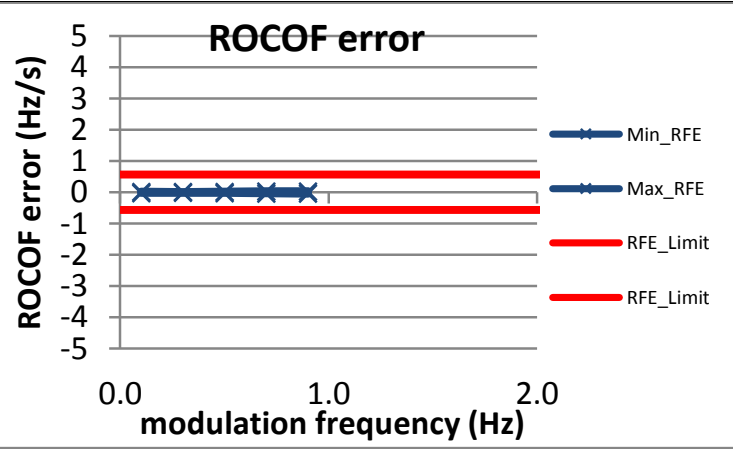

Figure 3490: Fs = 10 FPS

7.8.8 PMU G dynamic bandwidth measurement: phase modulation ROCOF error: P class

PMU G does not support P class 
7.8.9 PMU H dynamic bandwidth measurement: phase modulation ROCOF error: $P$ class

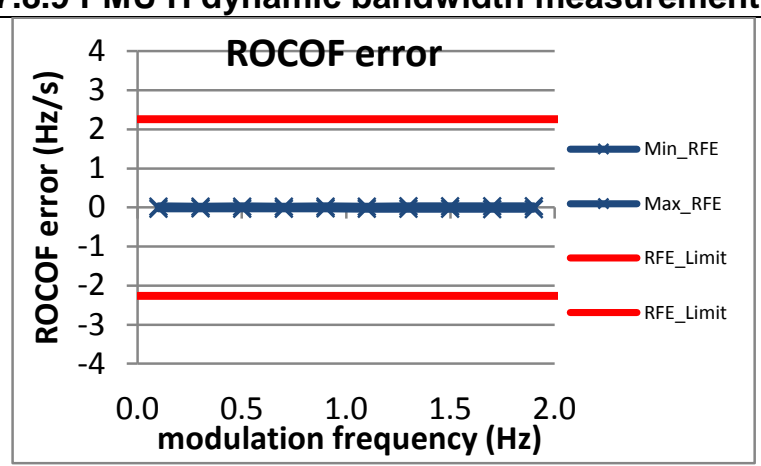

Figure 3491: Fs = 60 FPS

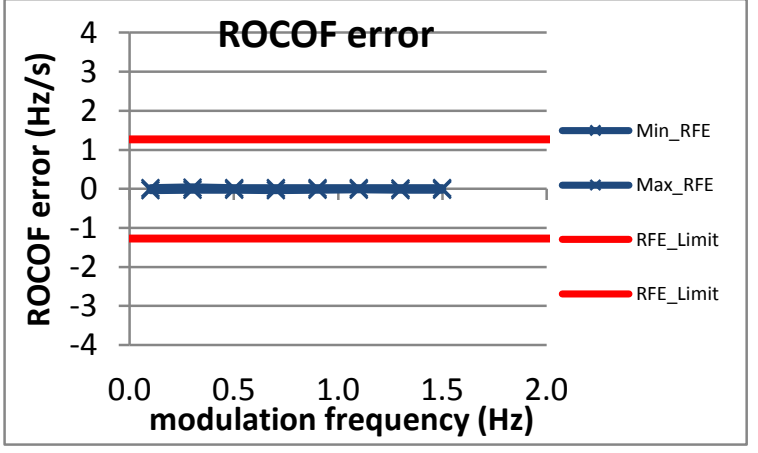

Figure 3494: Fs = 15 FPS

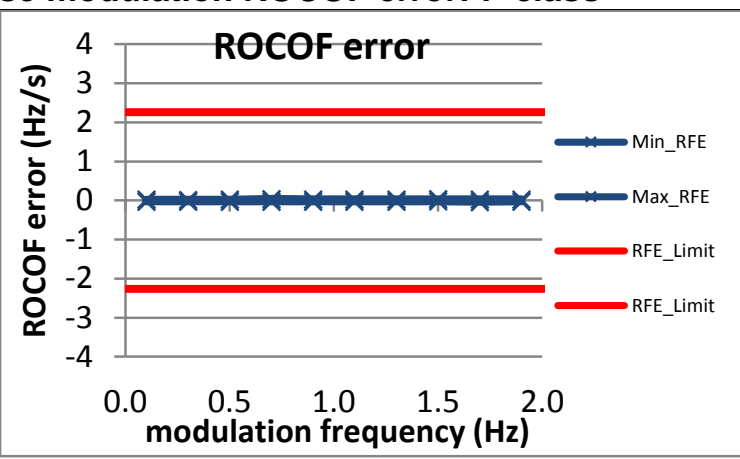

Figure 3492: Fs = 30 FPS

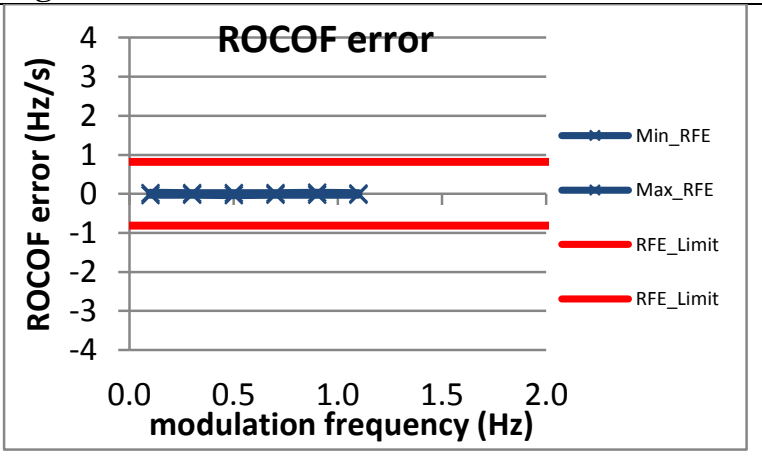

Figure 3495: Fs = 12 FPS

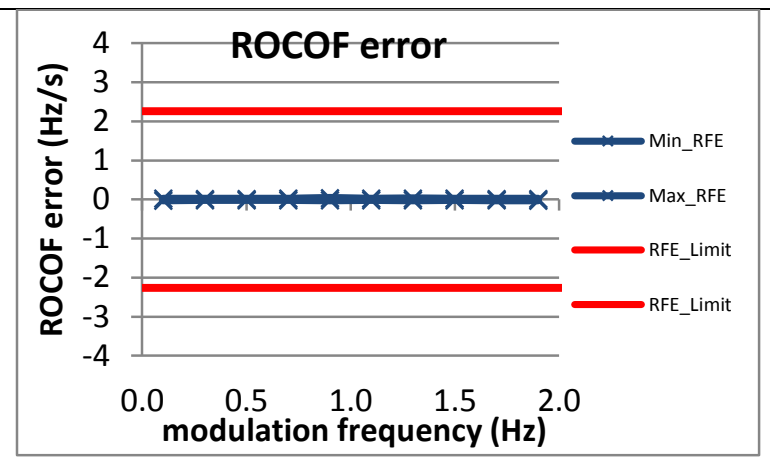

Figure 3493: Fs = 20 FPS

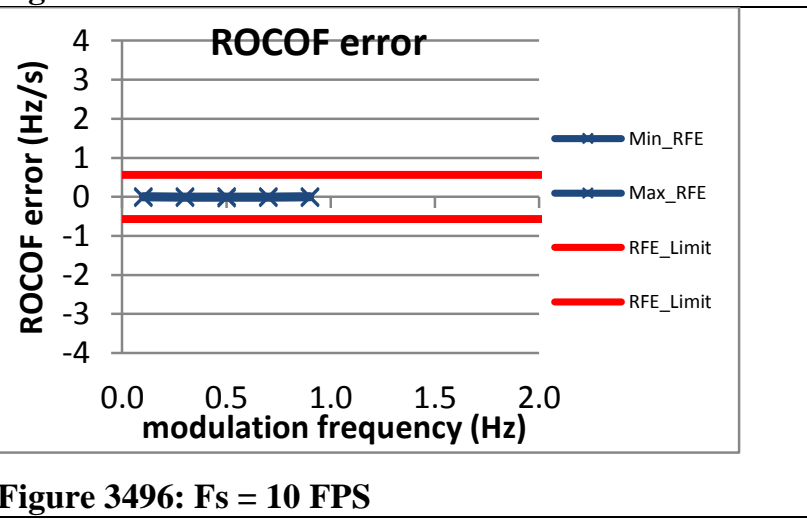

7.8.10 PMU I dynamic bandwidth measurement: phase modulation ROCOF error: P class

PMU I does not support P class 
7.8.11 PMU J dynamic bandwidth measurement: phase modulation ROCOF error: $\mathbf{P}$ class

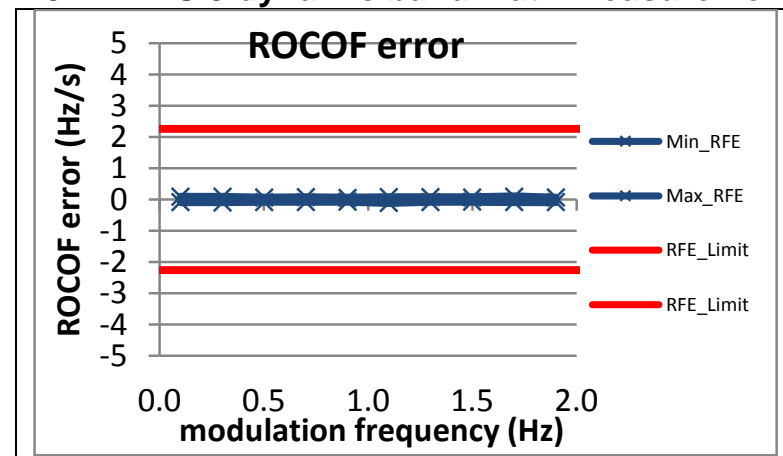

Figure 3497: Fs $=60$ FPS

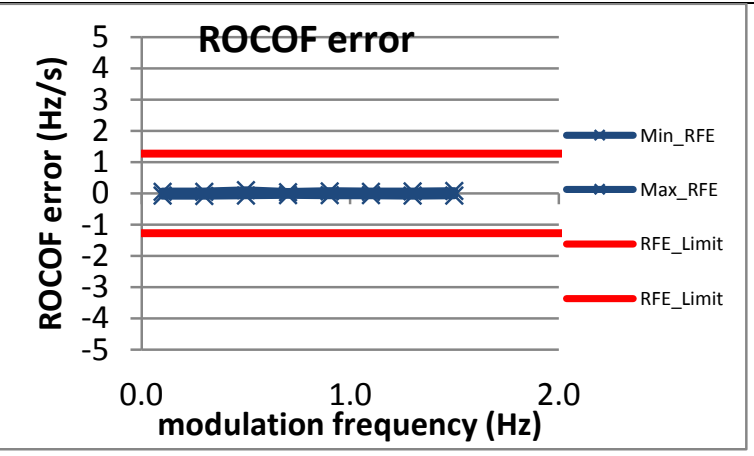

Figure 3500: Fs = 15 FPS

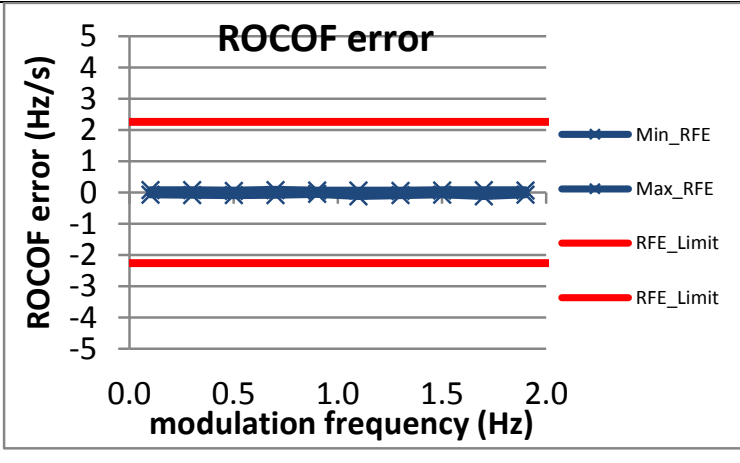

Figure 3498: $\mathrm{Fs}=30 \mathrm{FPS}$

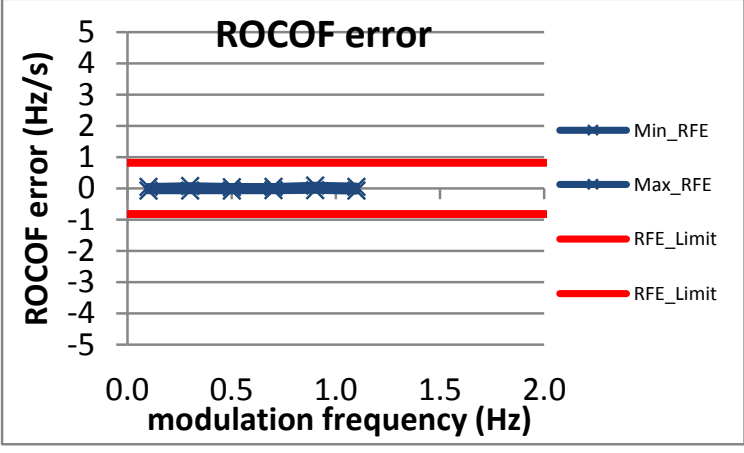

Figure 3501: Fs = 12 FPS

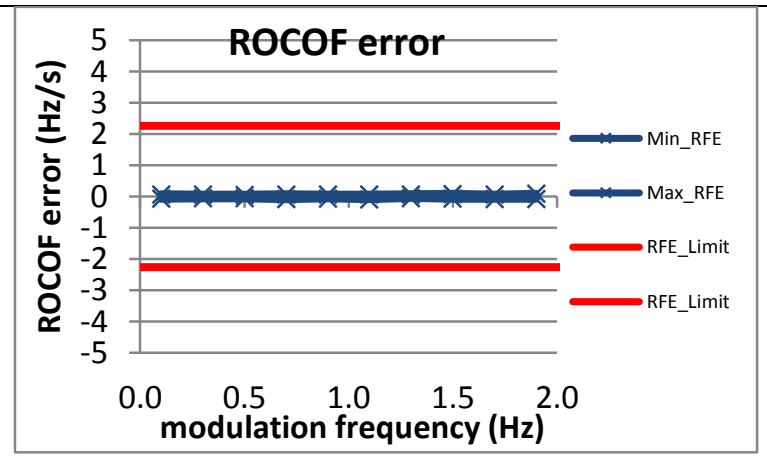

Figure 3499: $\mathrm{Fs}=20 \mathrm{FPS}$

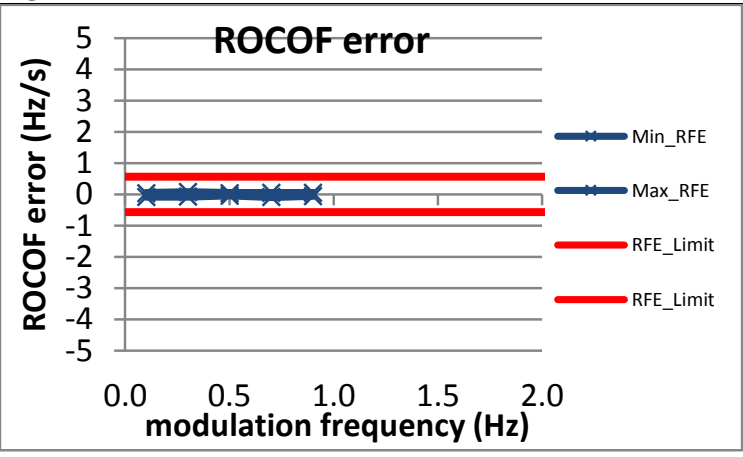

Figure 3502: Fs = 10 FPS 


\section{Dynamic bandwidth measurement: amplitude modulation}

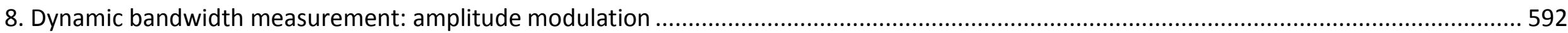

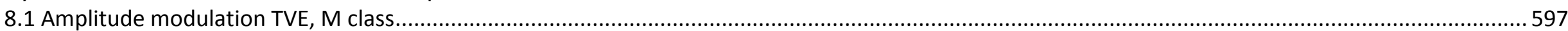

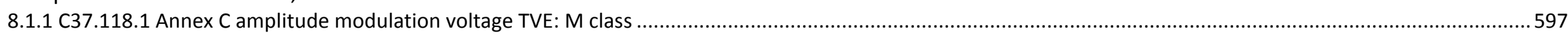

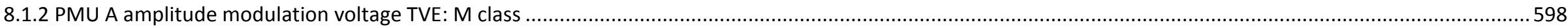

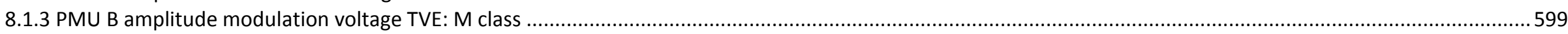

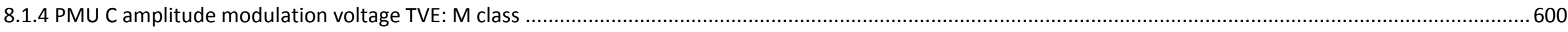

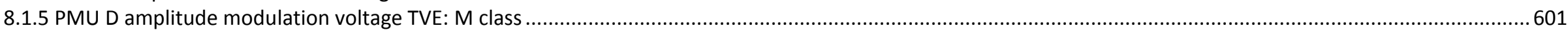

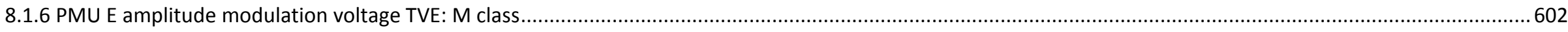

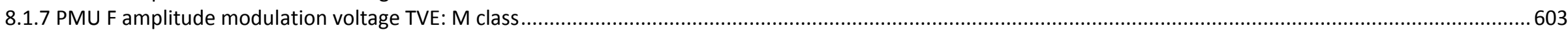

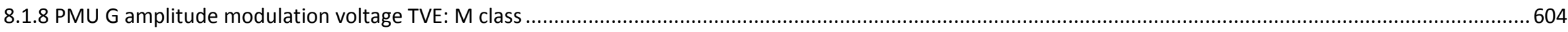

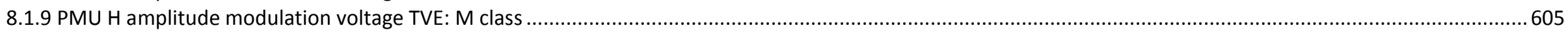

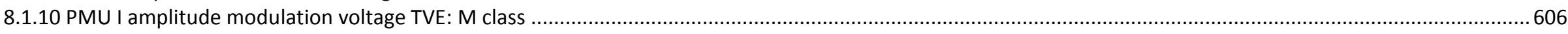

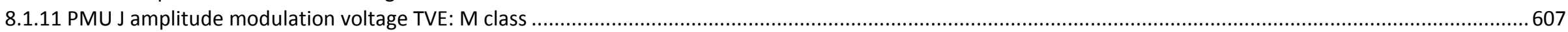

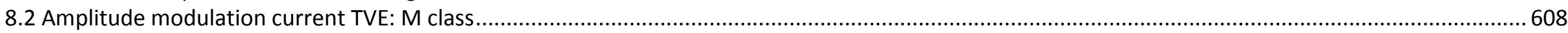

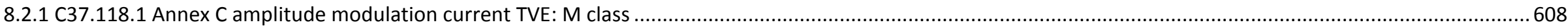

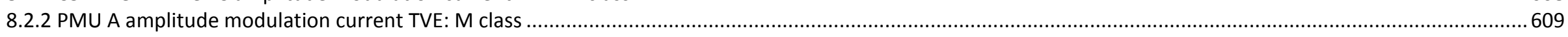

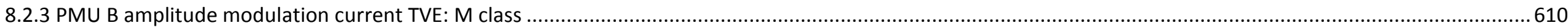

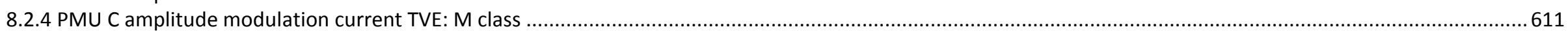

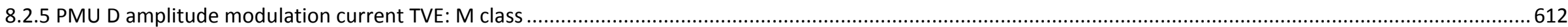

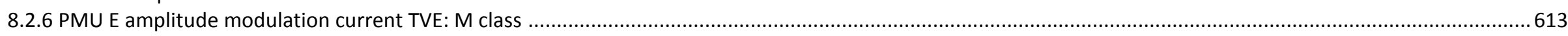

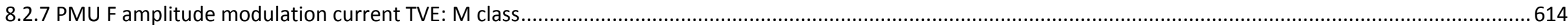

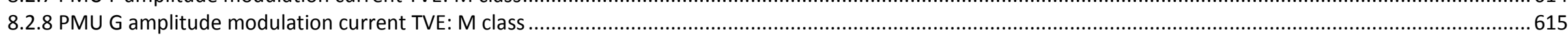

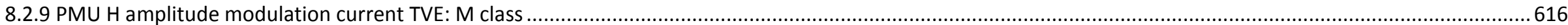

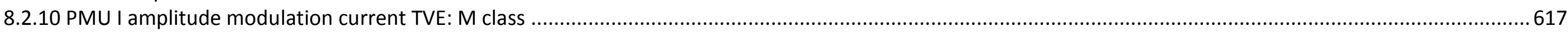

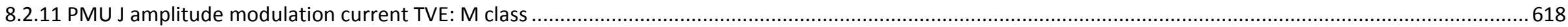

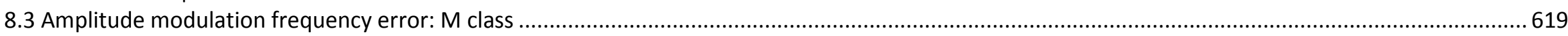

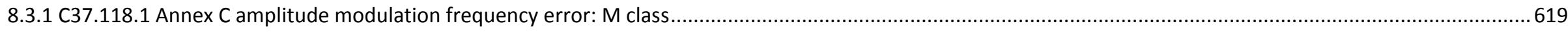

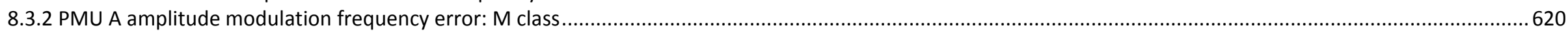

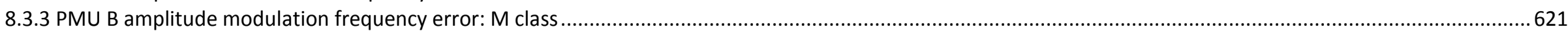

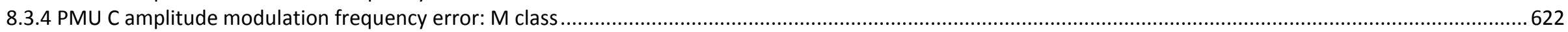

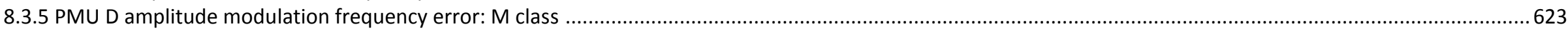

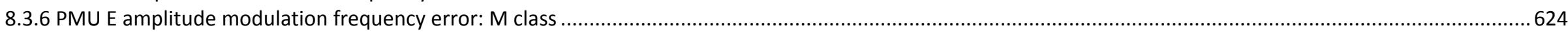

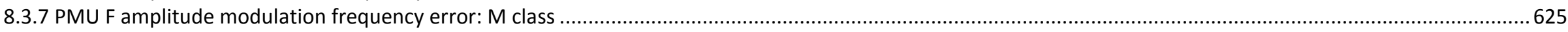

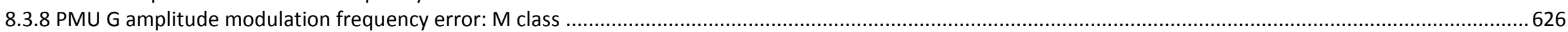

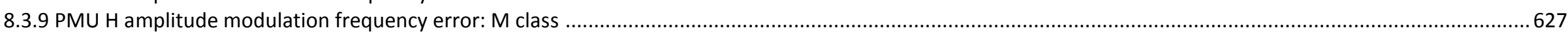

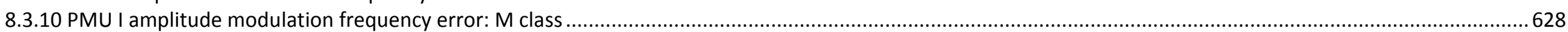

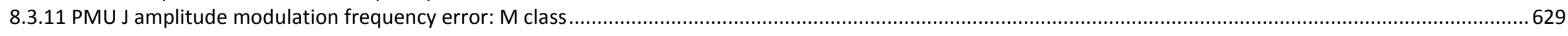

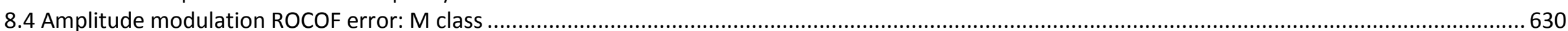


8.4.1 C37.118.1 Annex C amplitude modulation ROCOF error: M class...

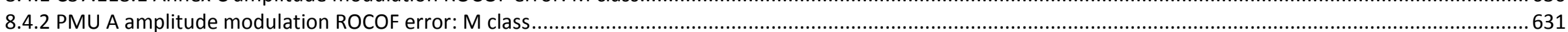

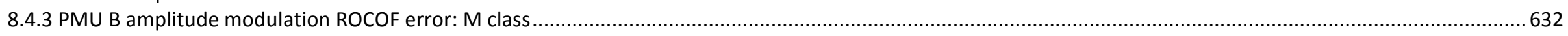

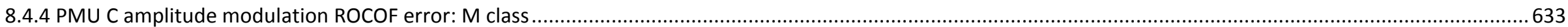

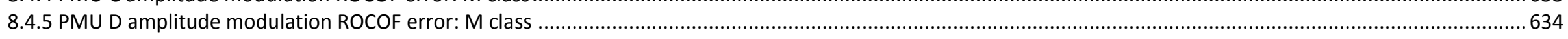

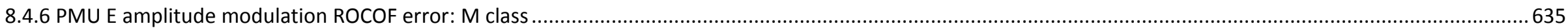

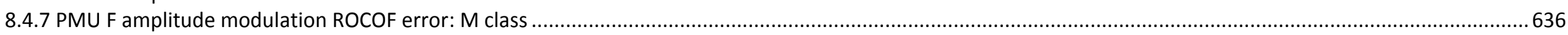

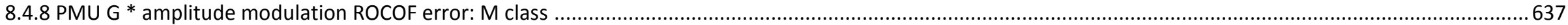

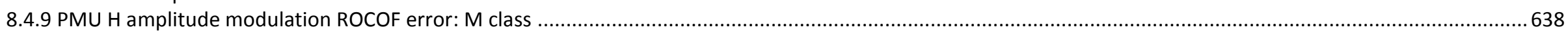

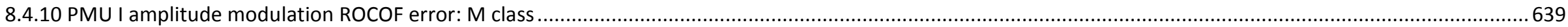

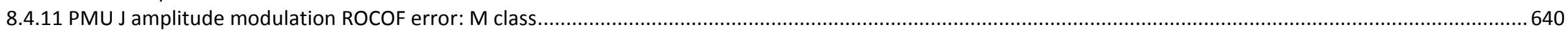

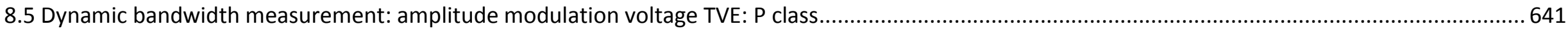

8.5.1 C37.118.1 Annex C dynamic bandwidth measurement: amplitude modulation voltage TVE: P class ......................................................................................641

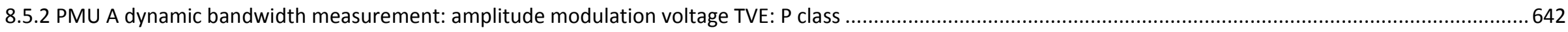

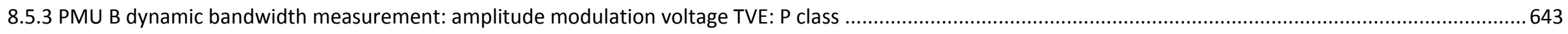

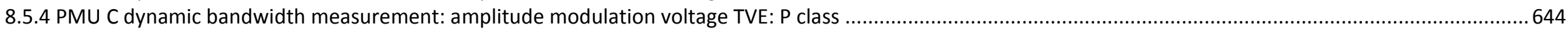

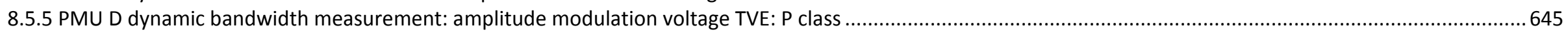

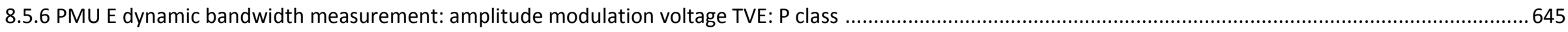

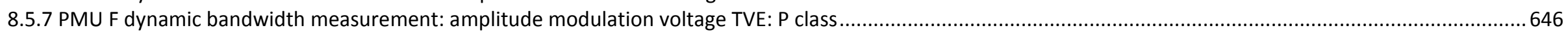

8.5.8 PMU G dynamic bandwidth measurement: amplitude modulation voltage TVE: P class ..................................................................................................646

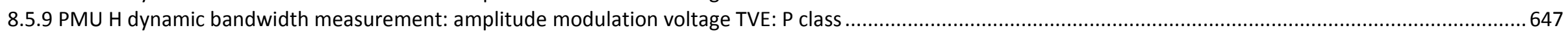

8.5.10 PMU I dynamic bandwidth measurement: amplitude modulation voltage TVE: P class .....................................................................................................647

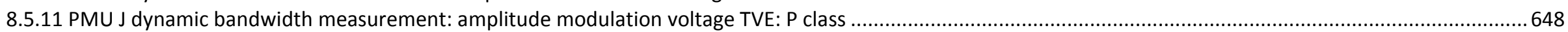

8.6 Dynamic bandwidth measurement: amplitude modulation current TVE: P class .............................................................................................. 649

8.6.1 C37.118.1 Annex C dynamic bandwidth measurement: amplitude modulation current TVE: P class .................................................................................... 649

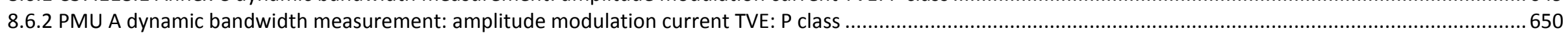

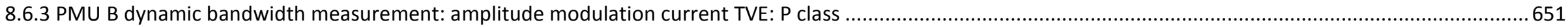

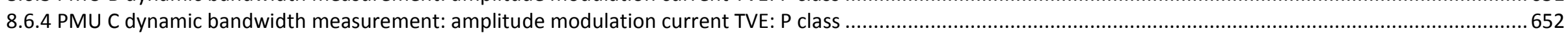

8.6.5 PMU D dynamic bandwidth measurement: amplitude modulation current TVE: P class .................................................................................................... 653

8.6.6 PMU E dynamic bandwidth measurement: amplitude modulation current TVE: P class ………...................................................................................... 653

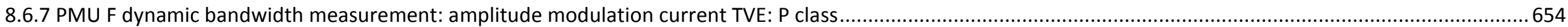

8.6.8 PMU G dynamic bandwidth measurement: amplitude modulation current TVE: P class ............................................................................................... 654

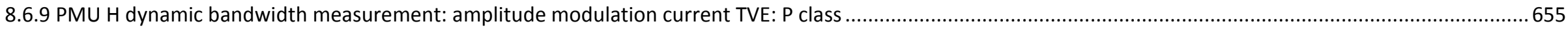

8.6.10 PMU I dynamic bandwidth measurement: amplitude modulation current TVE: P class ..................................................................................................65 655

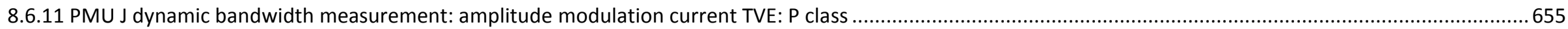

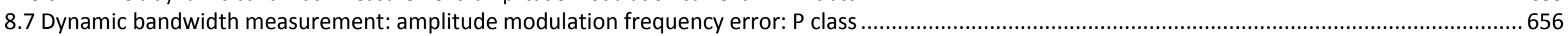

8.7.1 C37.118.1 Annex C dynamic bandwidth measurement: amplitude modulation frequency error: P class ................................................................................ 656

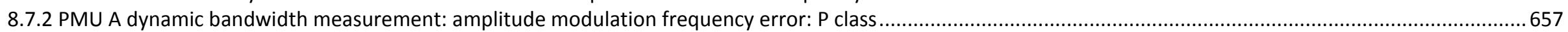

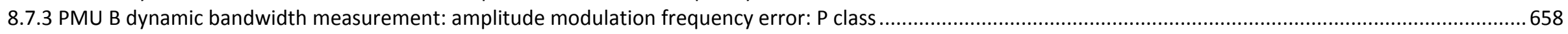

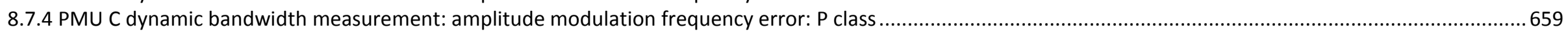

8.7.5 PMU D dynamic bandwidth measurement: amplitude modulation frequency error: P class ................................................................................................ 660 
8.7.6 PMU E dynamic bandwidth measurement: amplitude modulation frequency error: $\mathrm{P}$ class

8.7.7 PMU F dynamic bandwidth measurement: amplitude modulation frequency error: $P$ class 661

8.7.8 PMU G dynamic bandwidth measurement: amplitude modulation frequency error: $\mathrm{P}$ class 661

8.7.9 PMU H dynamic bandwidth measurement: amplitude modulation frequency error: $\mathrm{P}$ class 662

8.7.10 PMU I dynamic bandwidth measurement: amplitude modulation frequency error: $\mathrm{P}$ class

662

8.7.11 PMU J dynamic bandwidth measurement: amplitude modulation frequency error: $P$ class......... 663

8.8 dynamic bandwidth measurement: amplitude modulation ROCOF error: $P$ class

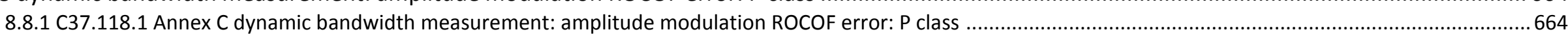

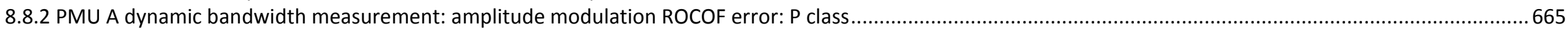

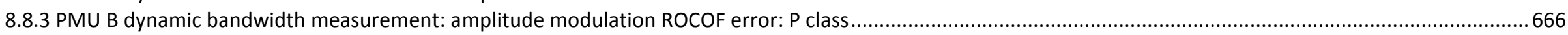

8.8.4 PMU C dynamic bandwidth measurement: amplitude modulation ROCOF error: P class .....................................................................................................67 667

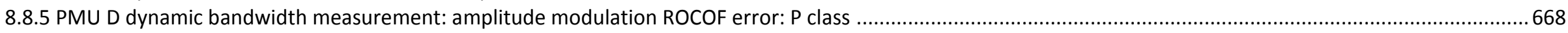

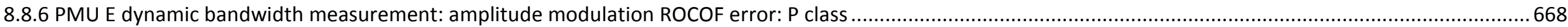

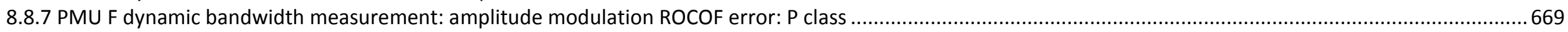

8.8.8 PMU G dynamic bandwidth measurement: amplitude modulation ROCOF error: P class ........................................................................................................69 669

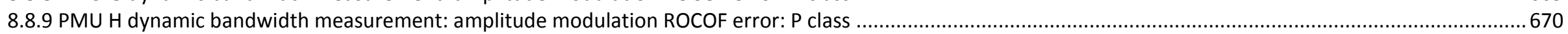

8.8.10 PMU I dynamic bandwidth measurement: amplitude modulation ROCOF error: P class .......................................................................................................670

8.8.11 PMU J dynamic bandwidth measurement: amplitude modulation ROCOF error: P class .................................................................................................... 671 
The measurement bandwidth tests apply modulated input signals to the PMU to ensure that the in-band performance is within limits. The amplitude modulation tests are a series of individual dynamic tests where the input signal amplitude is modulated at a $10 \%$ index of modulation. Individual tests are run with $0.2 \mathrm{~Hz}$ modulation frequency increments beginning at $0.1 \mathrm{~Hz}$ until the modulation frequency has reached the lesser of $\mathrm{Fs} / 5 \mathrm{~Hz}$ or $5 \mathrm{~Hz}$ for $\mathrm{M}$ class and $2 \mathrm{~Hz}$ for P class PMUs.

The maximum TVE, Fe and RFe of all tests are compared against the limits for TVE, Fe, and RFe to determine if the unit passes or fails the test.

C37.118.1-2011 amended by C37.118.1a-2014 specifies the limits as:

- maxTVE: $3 \%$

- max Fe:

$\circ$ M class: $0.06 \times \min (\mathrm{Fs} / 5,5)$

○ class: $0.03 \times \min (\mathrm{Fs} / 10,2)$

- $\max R F e$ :

○ M class: $0.18 \times \pi \times(\min (\mathrm{Fs} / 5,5))^{\wedge} 2$

$\circ$ P class: $0.18 \times \pi \times(\min (\mathrm{Fs} / 10,2))^{\wedge} 2$

The test plan for measurement bandwidth is as follows:

$X_{m}$ is the peak amplitude of the input signal

$\omega_{0}$ is the nominal power system frequency in radians/second $\left(2 \pi F_{0}\right)$

$\omega$ is the modulation frequency in radians/second

$\mathrm{k}_{\mathrm{x}}$ is the amplitude modulation index

$k_{a}$ is the phase angle modulation index

$t$ is time

a) Begin with phase modulated input at $\omega / 2 \pi=0.1 \mathrm{~Hz}, \mathrm{k}_{\mathrm{x}}=0.1, \mathrm{k}_{\mathrm{a}}=0$.

b) Wait for the system to settle.

c) Capture the PMU output for at least 2 full cycles of modulation or 5 seconds, whichever is greater.

d) Calculate the errors: TVE, FE, RFE for each report.

e) Calculate the Max TVE, FE and RFE.

f) Increase the modulation frequency $\omega / 2 \pi$ by $0.2 \mathrm{~Hz}$.

g) Repeat steps $b$ through $f$ until the upper frequency range limit is reached. 
Results from the dynamic phase modulation tests

\begin{tabular}{|c|c|c|c|c|c|c|c|c|c|c|c|c|c|c|c|c|c|c|c|c|c|c|c|c|c|c|c|c|c|c|c|c|c|c|c|c|}
\hline Fs (FPS) & & $10 \mathrm{~N}$ & & & $10 \mathrm{P}$ & & & $2 \mathrm{M}$ & & & $12 \mathrm{P}$ & & & $15 \mathrm{M}$ & & & $5 P$ & & & $20 \mathrm{M}$ & & & $O P$ & & & $30 \mathrm{M}$ & & & $30 \mathrm{P}$ & & & $60 \mathrm{M}$ & & & $60 \mathrm{P}$ & \\
\hline & $T$ & $\mathrm{FE}$ & $\mathrm{RF}$ & $\mathrm{T}$ & $\mathrm{F}$ & $R$ & $T$ & $\mathrm{~F}$ & $\mathrm{R}$ & $\mathrm{T}$ & $\mathrm{F}$ & $R$ & $T$ & $F$ & $\mathrm{R}$ & $\mathrm{T}$ & $\mathrm{F}$ & $\mathrm{R}$ & $T$ & $\mathrm{~F}$ & $R$ & $T$ & $F$ & \begin{tabular}{|l|l|} 
& \\
\end{tabular} & $\bar{T}$ & $\mathrm{~F}$ & $R$ & $T$ & & $\mathrm{R}$ & $\bar{T}$ & $\bar{F}$ & $R$ & $\mathrm{~T}$ & $F$ & $\bar{R}$ \\
\hline & E & & & $\mathrm{E}$ & & $\mathrm{E}$ & E & & $\mathrm{E}$ & E & & $\mathrm{E}$ & $E$ & & $\mathrm{E}$ & $E$ & & $\mathrm{E}$ & $E$ & & $\mathrm{E}$ & $\mathrm{E}$ & & $E$ & $\mathrm{E}$ & & $E$ & $E$ & & $\mathrm{E}$ & E & & $E$ & $E$ & & E \\
\hline PMU A & $P$ & $P$ & $P$ & $P$ & $P$ & $\mathrm{~F}$ & $\mathrm{P}$ & $\mathrm{P}$ & $P$ & $P$ & $P$ & $\mathrm{~F}$ & $\mathrm{P}$ & $P$ & $P$ & $P$ & $\mathrm{P}$ & $\mathrm{F}$ & $P$ & $P$ & $P$ & $\mathrm{P}$ & $\mathrm{P}$ & $\mathrm{F}$ & $P$ & $P$ & $P$ & $P$ & $P$ & $P$ & $P$ & $P$ & $P$ & $P$ & $P$ & $P$ \\
\hline PMU B & $\mathrm{P}$ & $\mathrm{P}$ & $\mathrm{P}$ & $\mathrm{P}$ & $\mathrm{P}$ & $\mathrm{P}$ & $\mathrm{P}$ & $\mathrm{P}$ & $\mathrm{P}$ & $\mathrm{P}$ & $\mathrm{P}$ & $\mathrm{P}$ & $\mathrm{P}$ & $\mathrm{P}$ & $\mathrm{P}$ & $P$ & $\mathrm{P}$ & $\mathrm{P}$ & $\mathrm{P}$ & $\mathrm{P}$ & $P$ & $\mathrm{P}$ & $\mathrm{P}$ & \begin{tabular}{|l|}
$\mathrm{P}$ \\
\end{tabular} & $\mathrm{P}$ & $\mathrm{P}$ & $\mathrm{P}$ & $\mathrm{P}$ & $\mathrm{P}$ & $\mathrm{P}$ & $\mathrm{P}$ & $P$ & $P$ & $P$ & $\mathrm{P}$ & $\mathrm{P}$ \\
\hline PMU C & $P$ & $P$ & $P$ & $P$ & $\mathrm{P}$ & $P$ & $\mathrm{P}$ & $\mathrm{P}$ & $P$ & $\mathrm{P}$ & $P$ & $P$ & $P$ & $P$ & $P$ & $P$ & $\mathrm{P}$ & $P$ & $P$ & $P$ & $P$ & $P$ & $\mathrm{P}$ & \begin{tabular}{|l|l|}
$P$ \\
\end{tabular} & $P$ & $P$ & $P$ & $P$ & $P$ & $P$ & $\mathrm{P}$ & $P$ & $P$ & $P$ & $P$ & $P$ \\
\hline PMU F & $P$ & $P$ & $P$ & $P$ & $P$ & $P$ & $\mathrm{~F}$ & $\mathrm{P}$ & $P$ & $P$ & $P$ & $P$ & $F$ & $P$ & $P$ & $P$ & $\mathrm{P}$ & $P$ & $P$ & $P$ & $P$ & $P$ & $\mathrm{P}$ & \begin{tabular}{|l|l|}
$P$ & \\
\end{tabular} & $P$ & $P$ & $\mathrm{P}$ & $P$ & $P$ & $P$ & $P$ & $P$ & $P$ & $P$ & $\mathrm{P}$ & $P$ \\
\hline PMU G* & $\mathrm{F}$ & $P$ & $P$ & - & - & - & $F$ & $P$ & $P$ & 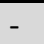 & - & - & $\mathrm{F}$ & $P$ & $P$ & - & - & - & $F$ & $P$ & $P$ & - & - & - & $\mathrm{F}$ & $P$ & $P$ & - & - & - & - & - & - & - & - & - \\
\hline PMU H & $P$ & $P$ & $P$ & $P$ & $P$ & $P$ & $P$ & P & $P$ & $P$ & $P$ & $P$ & $P$ & $P$ & $P$ & $P$ & $\begin{array}{ll}\mathrm{P} \\
\end{array}$ & $P$ & $P$ & $P$ & $P$ & $P$ & $P$ & \begin{tabular}{|l|l|}
$P$ \\
\end{tabular} & $P$ & $P$ & \begin{tabular}{|l|}
$P$ \\
\end{tabular} & $P$ & $P$ & $P$ & $P$ & $P$ & $P$ & $P$ & $P$ & $P$ \\
\hline PMU I & $\mathrm{F}$ & $P$ & $P$ & - & - & - & $\mathrm{F}$ & $\bar{P}$ & $P$ & - & 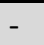 & - & $\mathrm{F}$ & $P$ & $P$ & - & - & - & $F$ & $P$ & $P$ & - & - & - & $\mathrm{F}$ & $P$ & 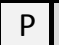 & - & 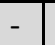 & - & $P$ & $P$ & $P$ & - & - & - \\
\hline PMU J & $P$ & $P$ & $P$ & $P$ & $P$ & $P$ & $P$ & $\bar{P}$ & $P$ & $P$ & $P$ & $P$ & $P$ & $P$ & $P$ & $P$ & $\bar{P}$ & $P$ & $P$ & $P$ & $P$ & $P$ & $\mathrm{P}$ & \begin{tabular}{|l|l|}
$P$ \\
\end{tabular} & $P$ & $P$ & $P$ & $P$ & $P$ & $\bar{P}$ & $P$ & $P$ & $P$ & $P$ & $P$ & $P$ \\
\hline
\end{tabular}

$\mathrm{P}=$ Pass (measurement is within the limits), $\mathrm{F}=$ Fail (measurement is outside the limits), I = Indeterminate (measurement is within the uncertainty of the test instrument of the limits)

* PMU G ROCOF output is always 0 


\subsection{Amplitude modulation TVE, M class}

\subsubsection{C37.118.1 Annex $C$ amplitude modulation voltage TVE: $M$ class}
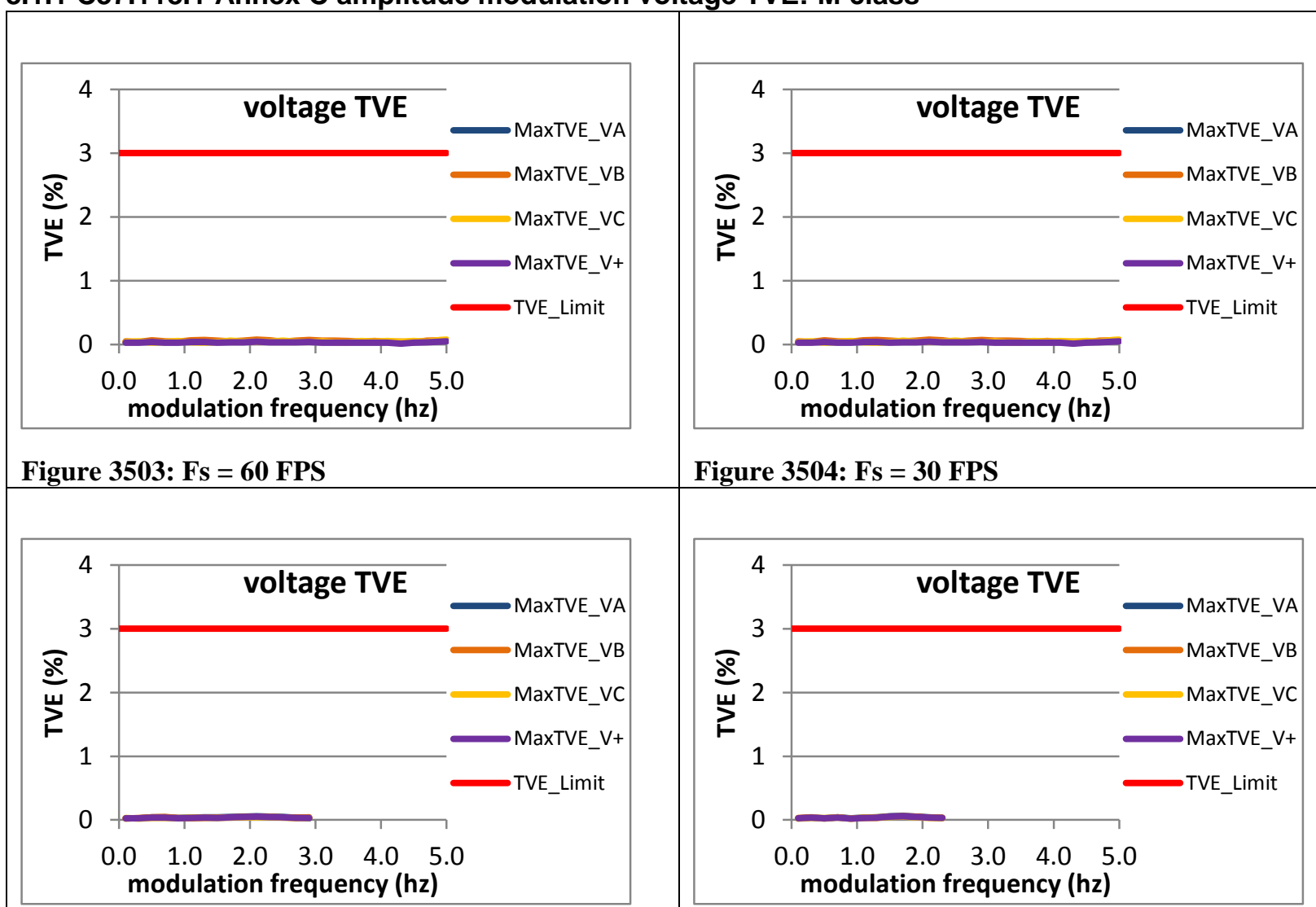

Figure 3504: Fs = 30 FPS

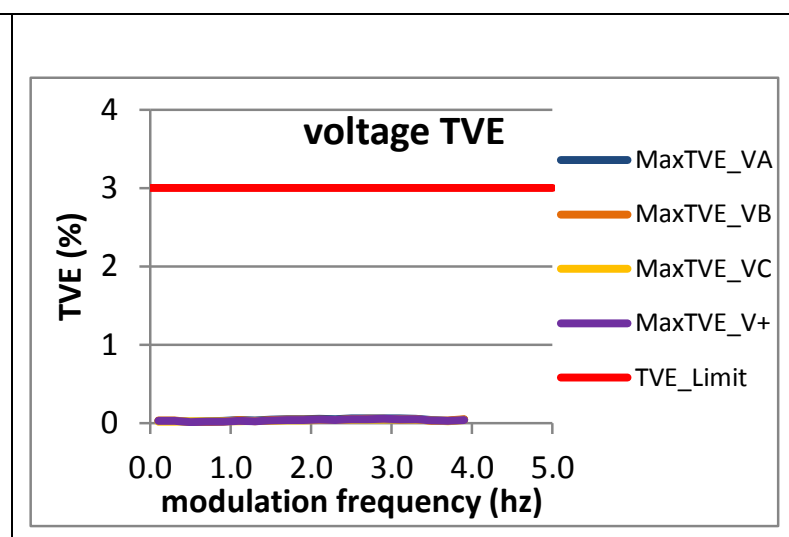

\section{Figure 3505: Fs = 20 FPS}

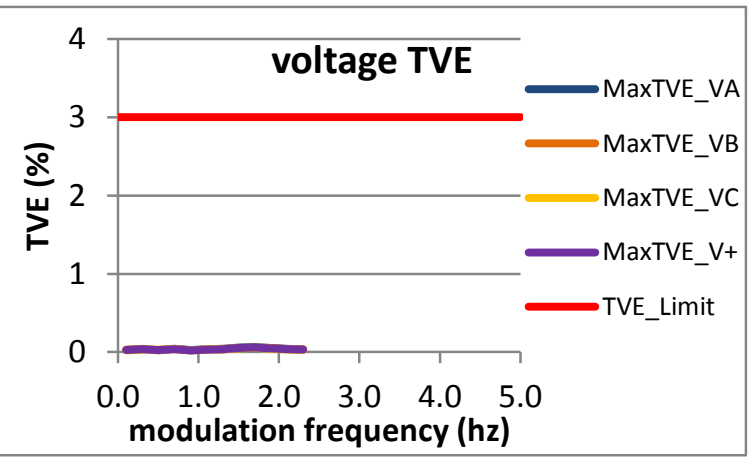

Figure 3507: Fs = 12 FPS

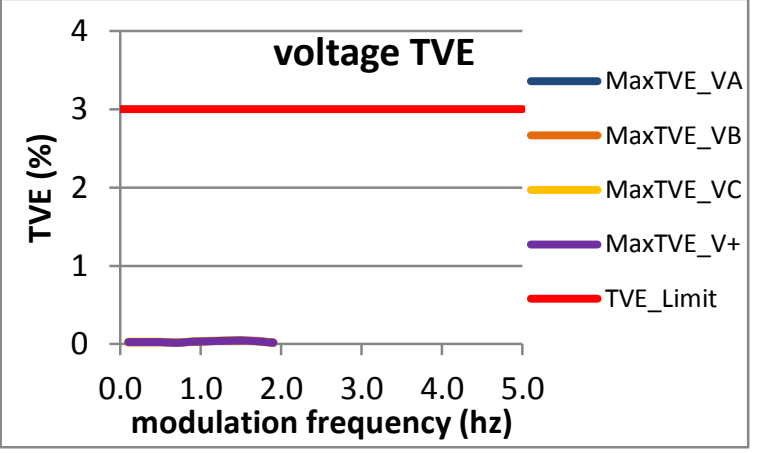

Figure 3506: Fs = 15 FPS

Figure 3508: Fs $=10$ FPS 


\subsubsection{PMU A amplitude modulation voltage TVE: M class}

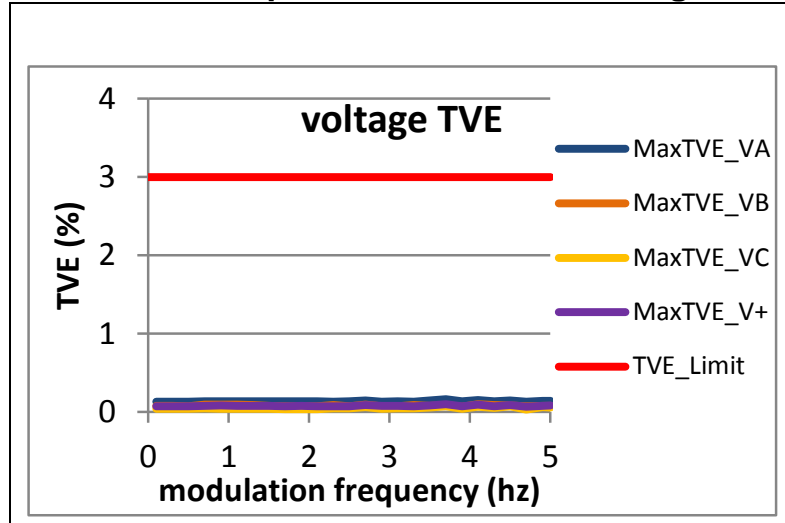

Figure 3509: Fs = 60 FPS

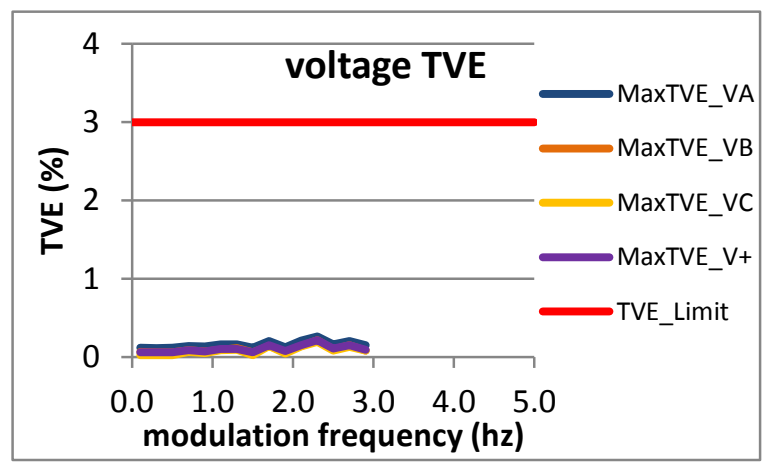

Figure 3512: Fs = 15 FPS

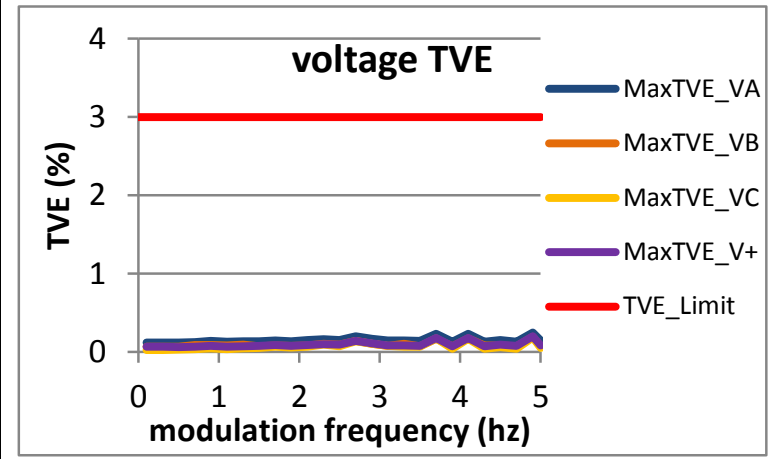

Figure 3510: Fs = 30 FPS

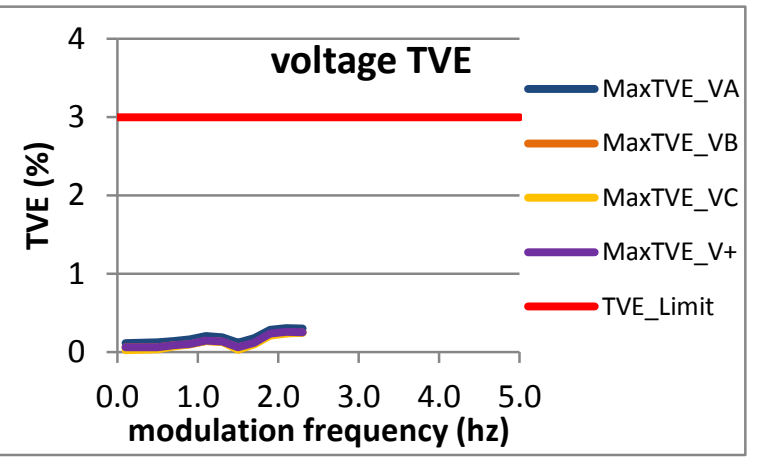

Figure 3513: Fs = 12 FPS

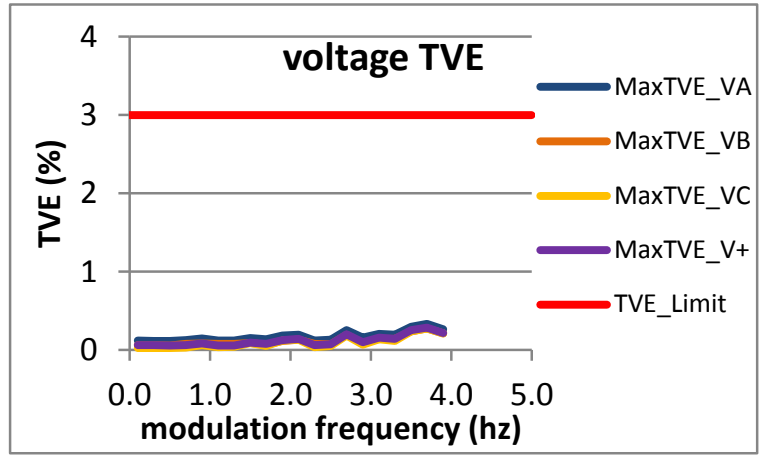

Figure 3511: Fs = 20 FPS

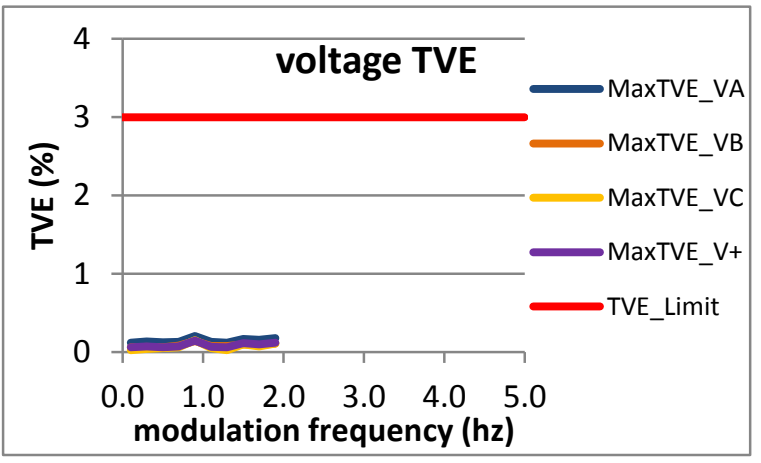

Figure 3514: Fs = 10 FPS 


\subsubsection{PMU B amplitude modulation voltage TVE: M class}
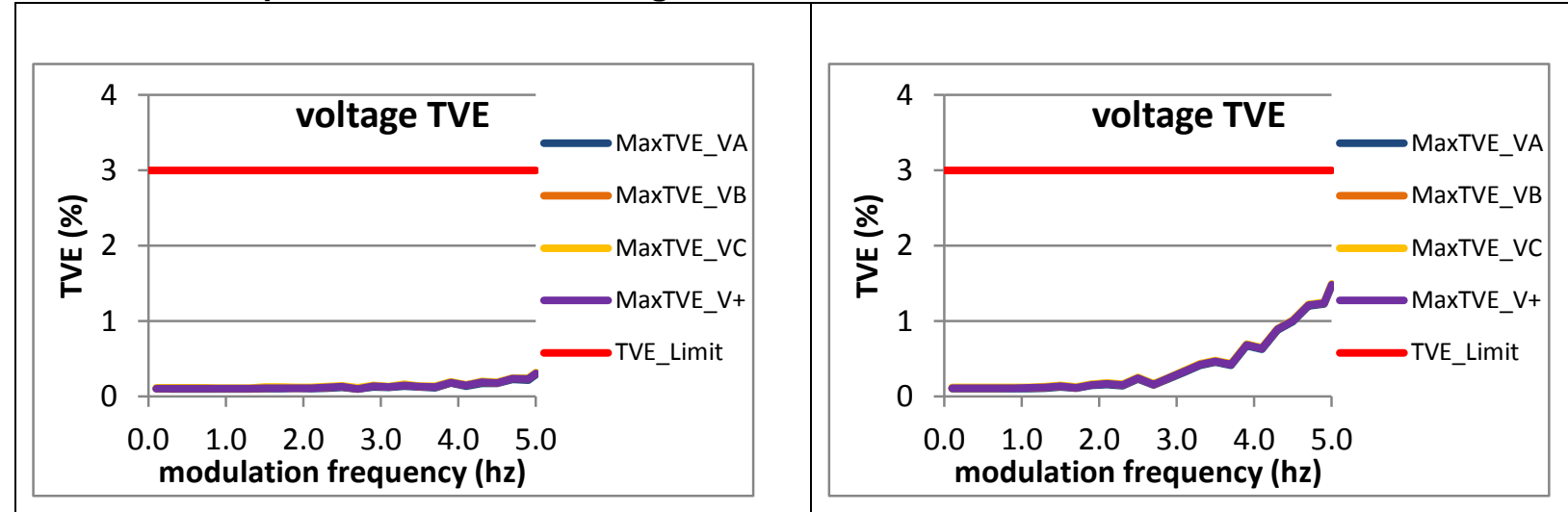

Figure 3516: Fs = 30 FPS

Figure 3515: Fs = 60 FPS

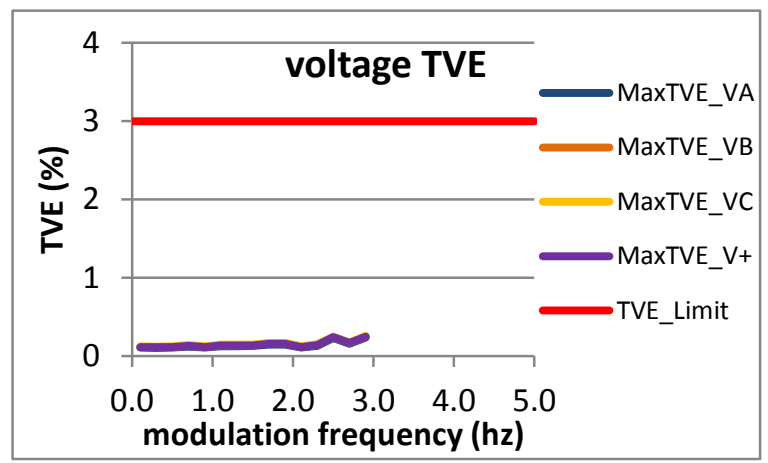

Figure 3518: Fs = 15 FPS

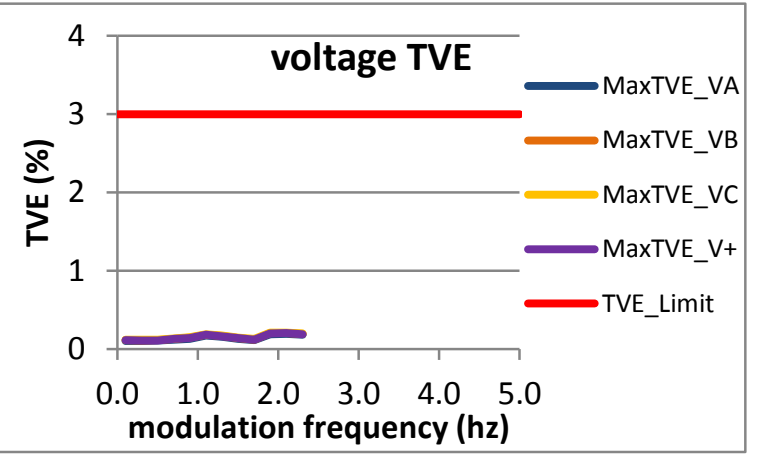

Figure 3519: Fs = 12 FPS

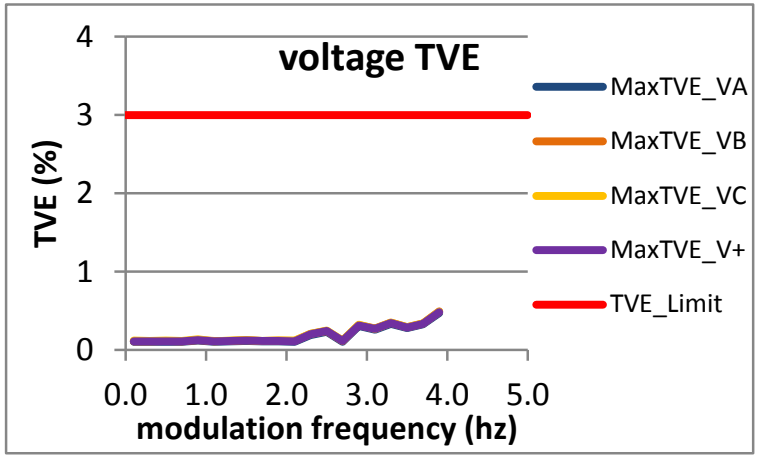

Figure 3517: Fs = 20 FPS

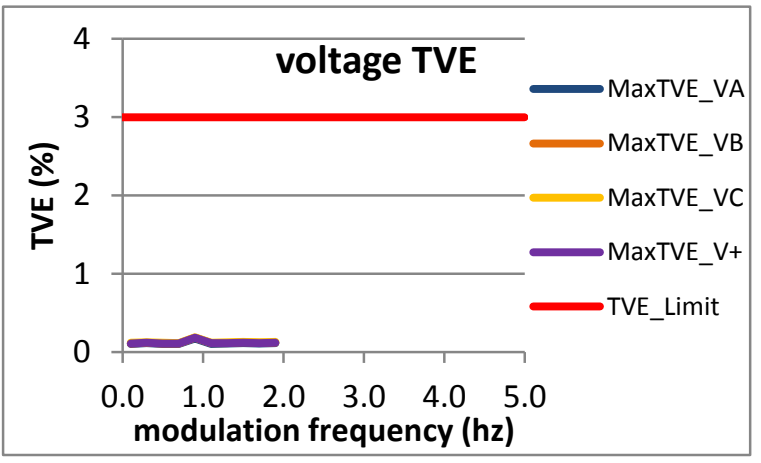

Figure 3520: Fs = 10 FPS 


\subsubsection{PMU C amplitude modulation voltage TVE: M class}

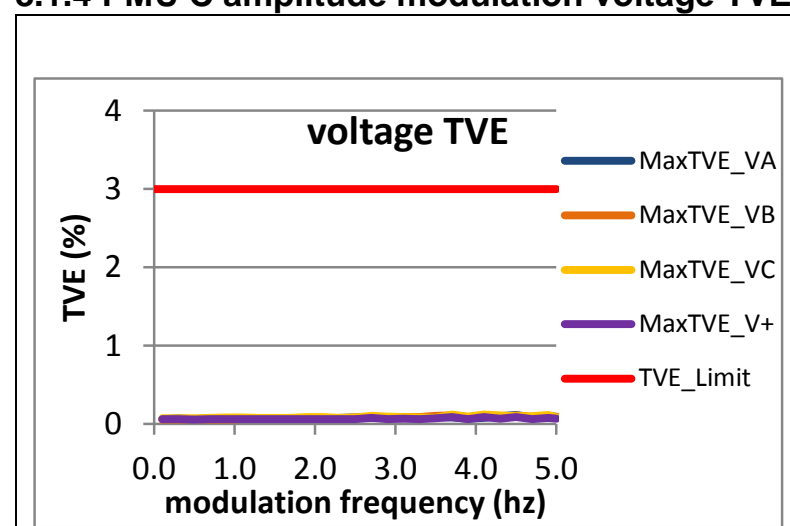

Figure 3521: Fs = 60 FPS

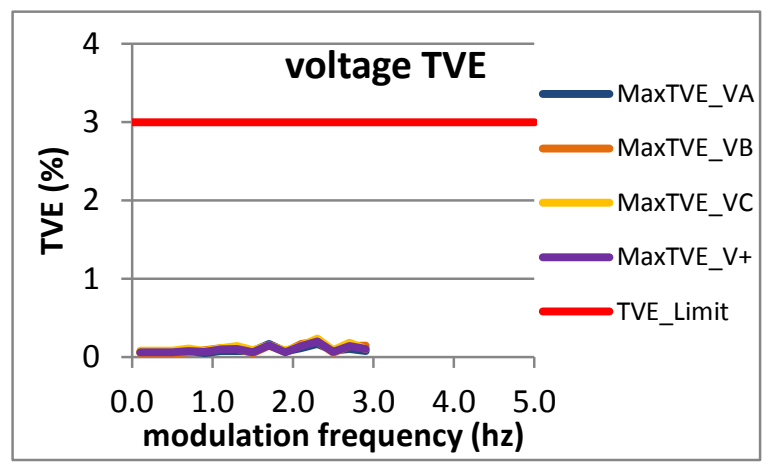

Figure 3524: Fs = 15 FPS

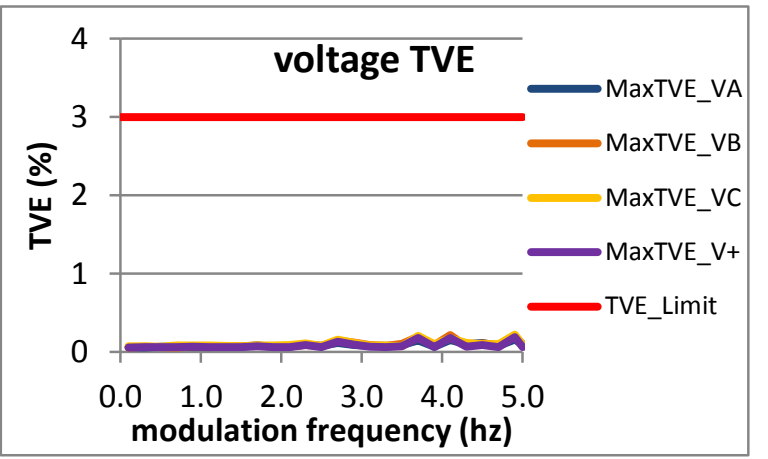

Figure 3522: Fs = 30 FPS

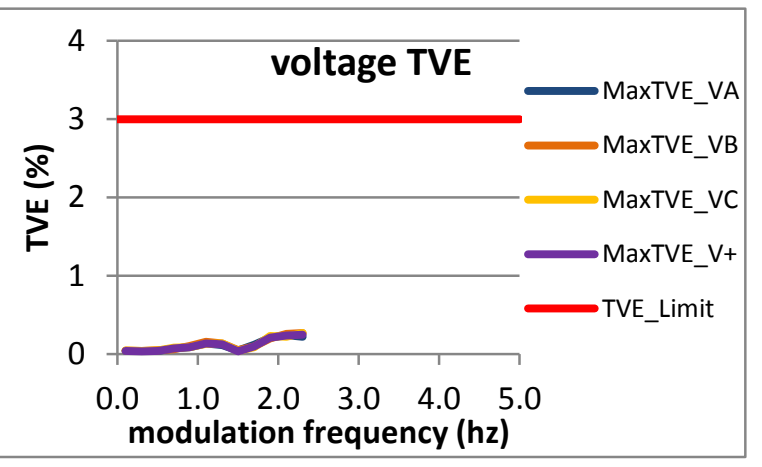

Figure 3525: Fs = 12 FPS

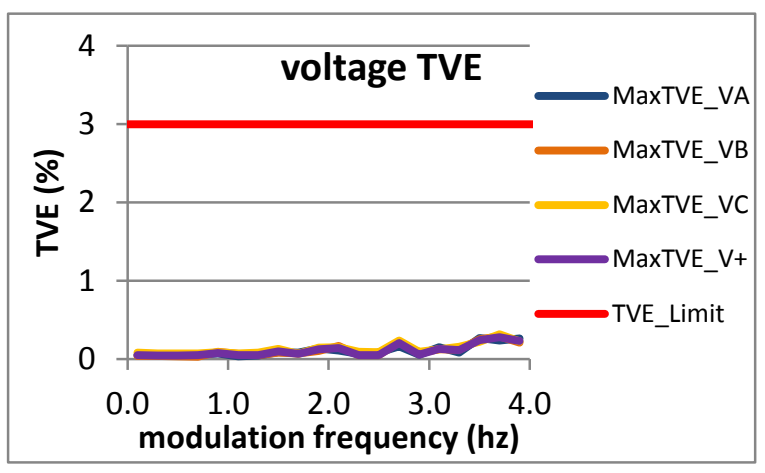

Figure 3523: Fs = 20 FPS

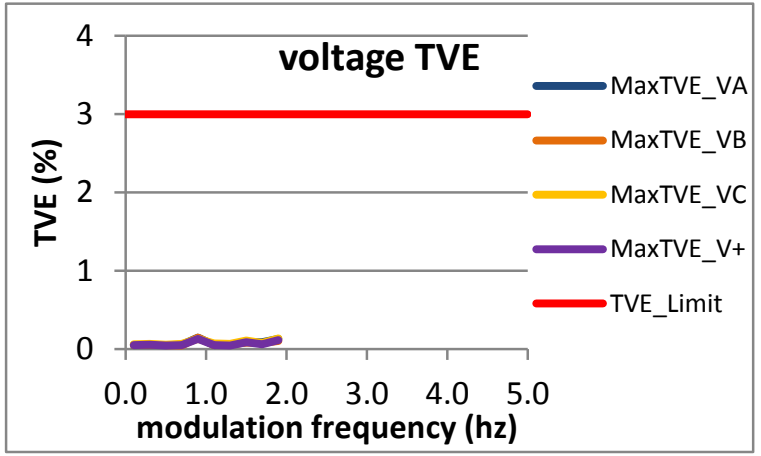

Figure 3526: Fs = 10 FPS 


\subsubsection{PMU D amplitude modulation voltage TVE: M class}
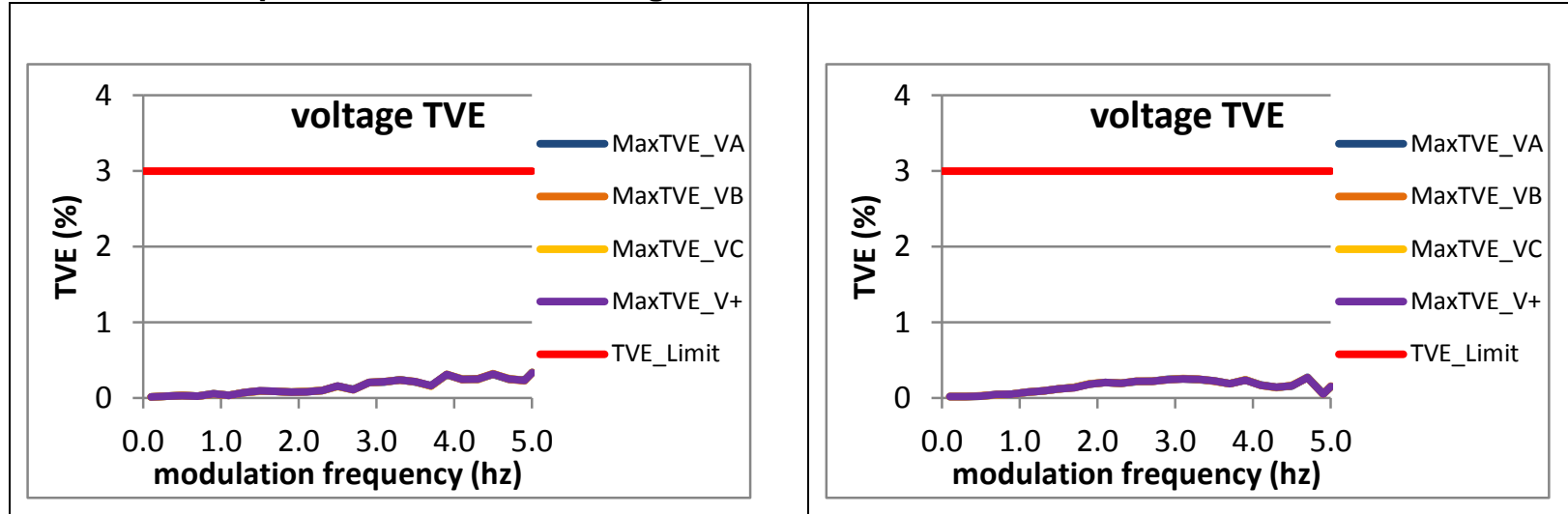

Figure 3528: Fs = 30 FPS

Figure 3527: Fs = 60 FPS

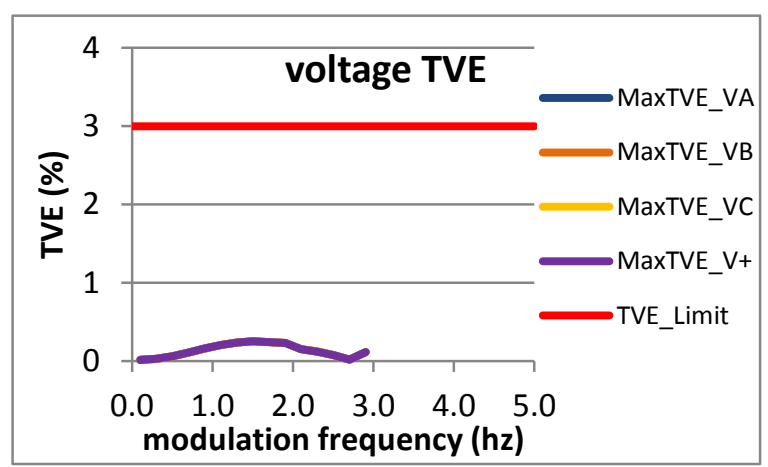

Figure 3530: Fs = 15 FPS

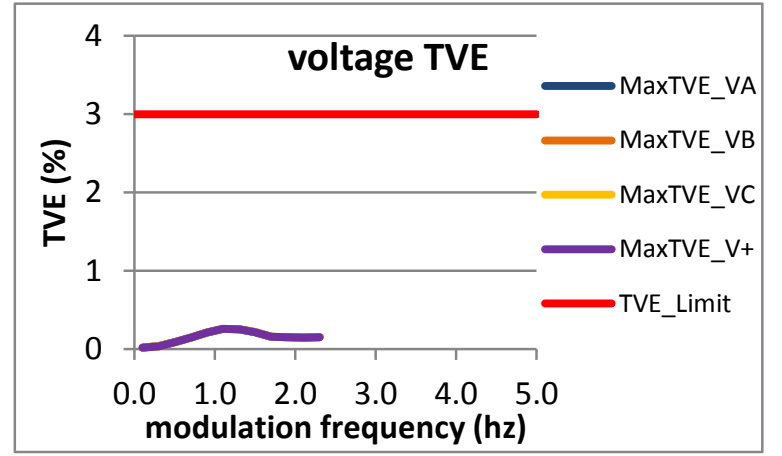

Figure 3531: Fs = 12 FPS

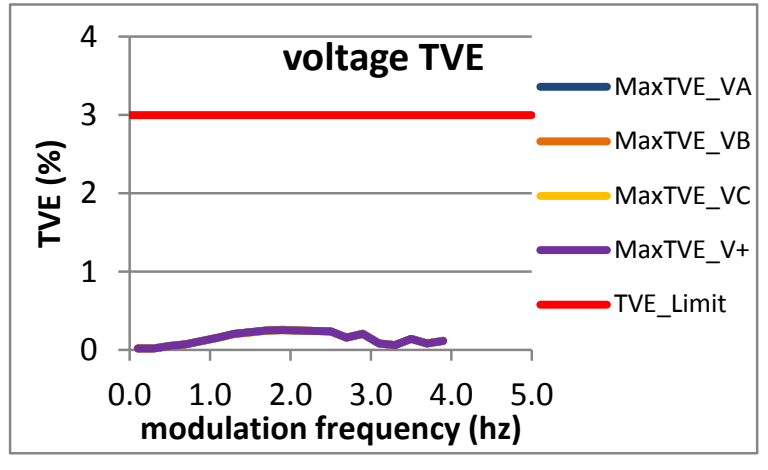

Figure 3529: Fs = 20 FPS

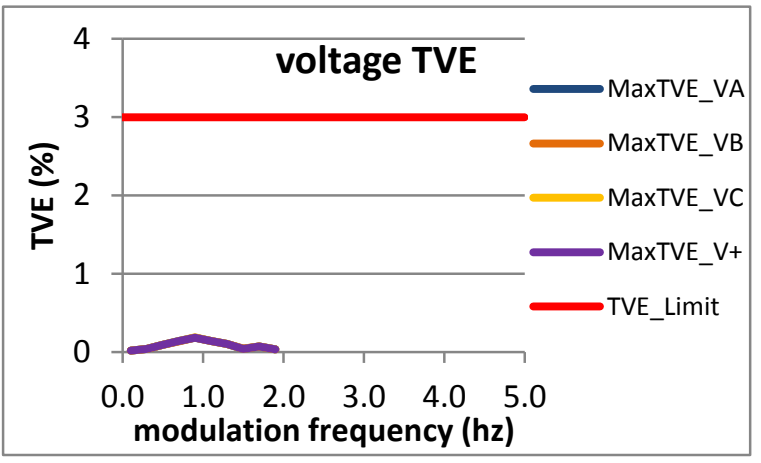

Figure 3532: Fs = 10 FPS 


\subsubsection{PMU E amplitude modulation voltage TVE: M class}

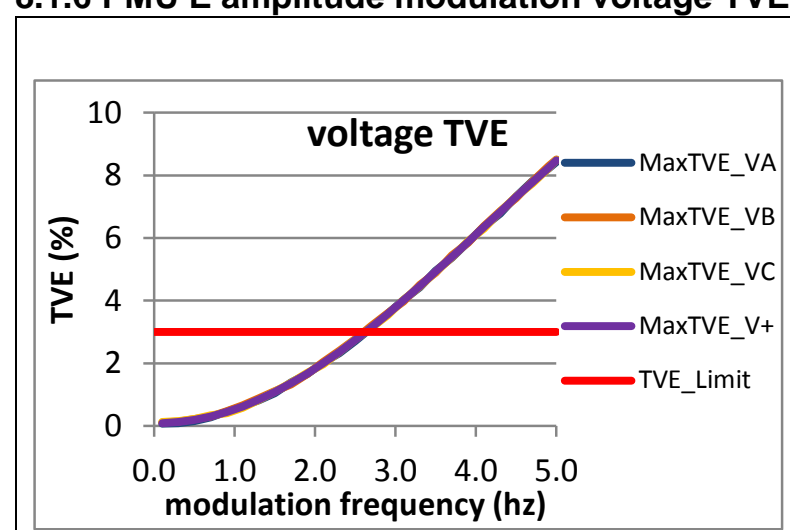

Figure 3533: Fs = 60 FPS

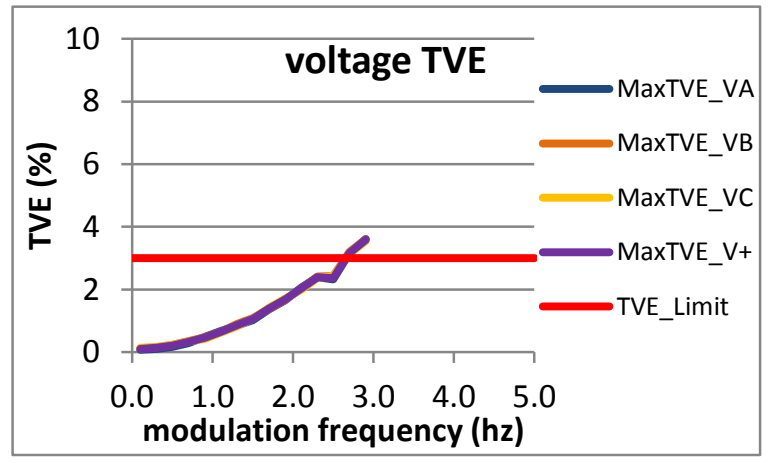

Figure 3536: Fs = 15 FPS

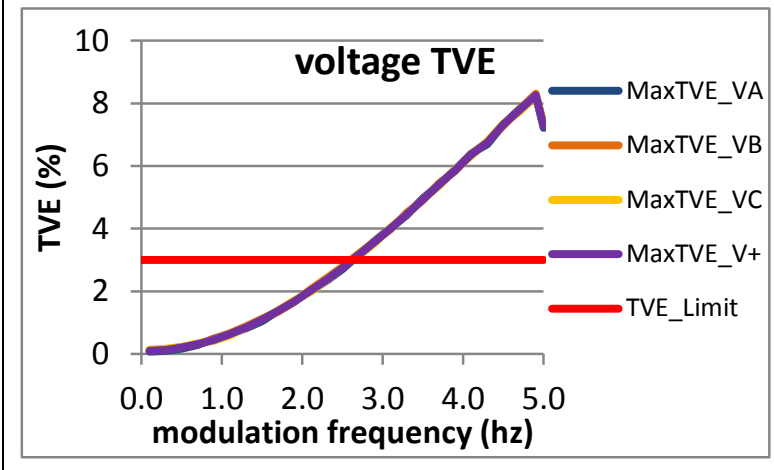

Figure 3534: Fs = 30 FPS

Figure 3537: Fs = 12 FPS data was lost.

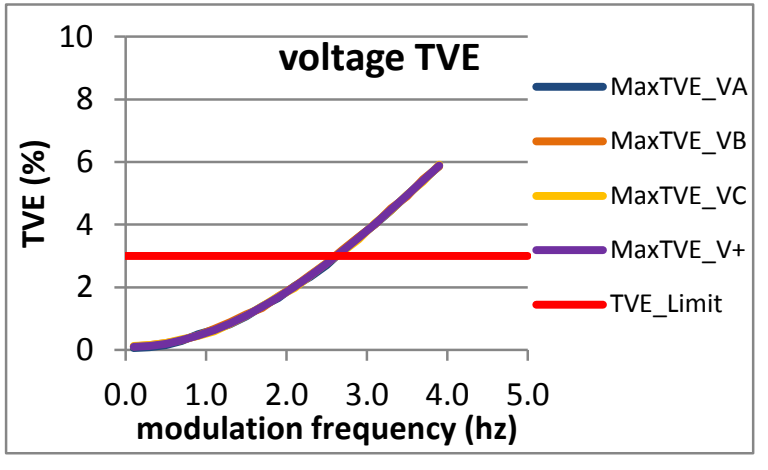

Figure 3535: Fs = 20 FPS

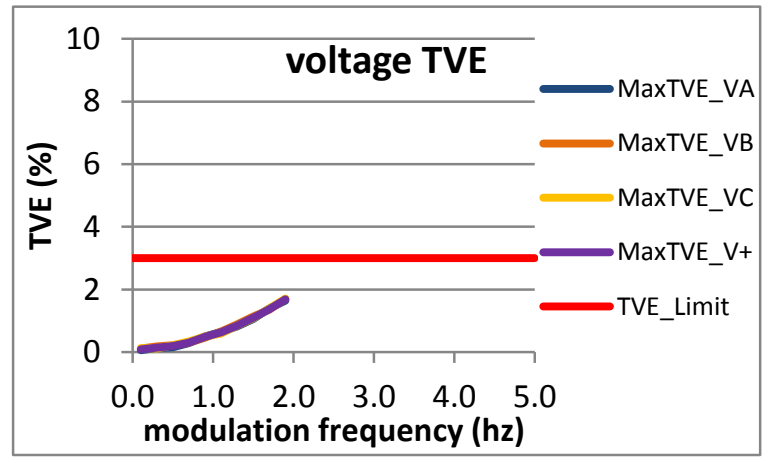

Figure 3538: Fs = 10 FPS 


\subsubsection{PMU F amplitude modulation voltage TVE: M class}

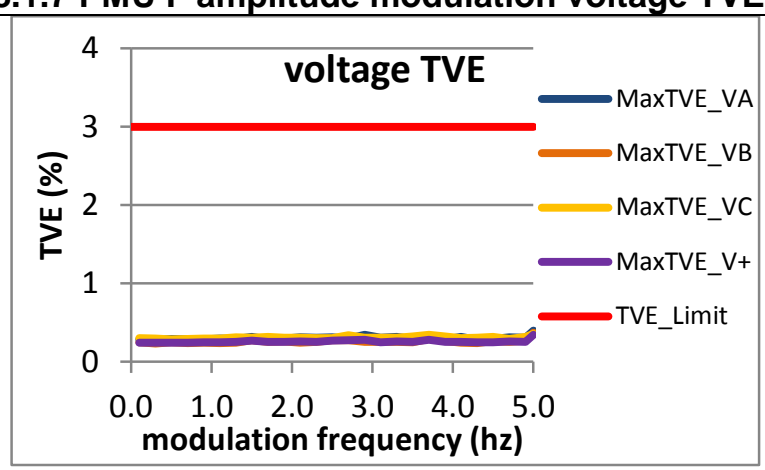

Figure 3539: Fs = 60 FPS

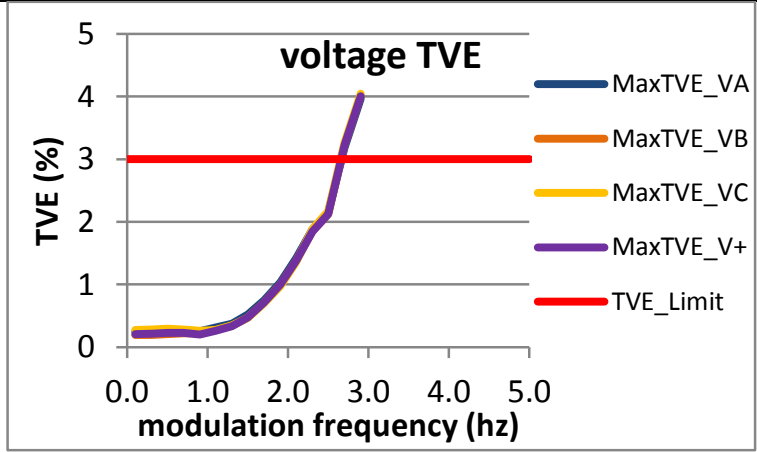

Figure 3542: Fs = 15 FPS

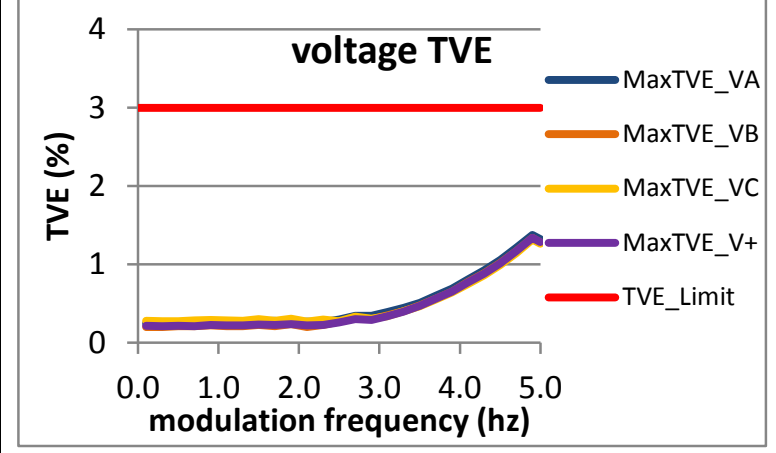

Figure 3540: Fs = 30 FPS

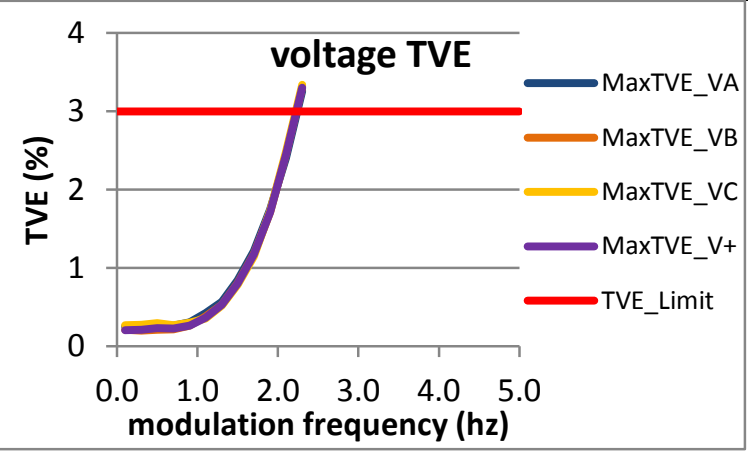

Figure 3543: Fs = 12 FPS

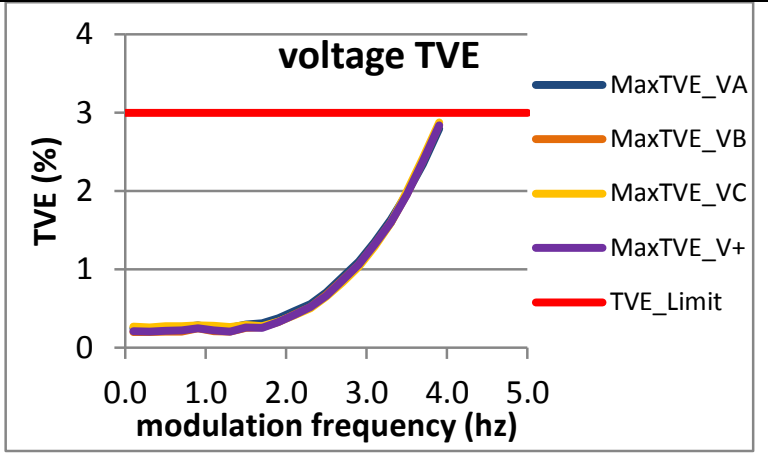

Figure 3541: Fs = 20 FPS

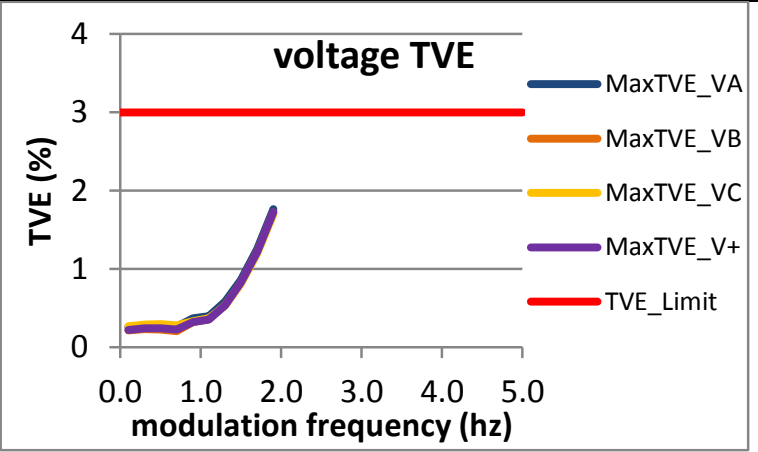

Figure 3544: Fs = 10 FPS 


\subsubsection{PMU G amplitude modulation voltage TVE: M class}

Figure 3545: Fs = 60 FPS is not supported by this PMU

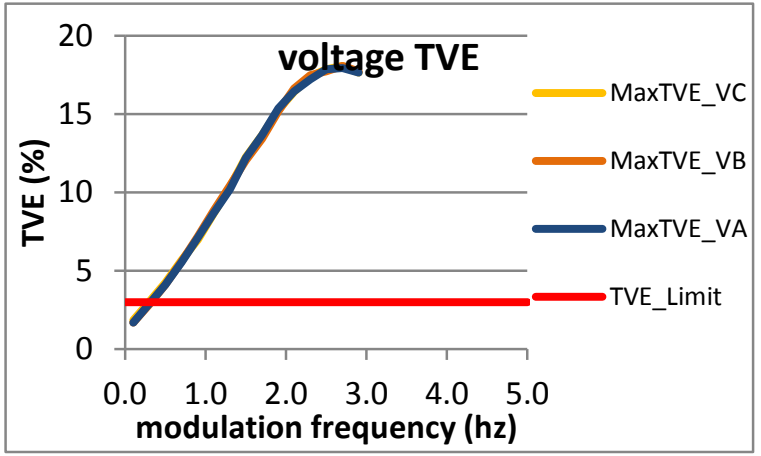

Figure 3548: Fs = 15 FPS

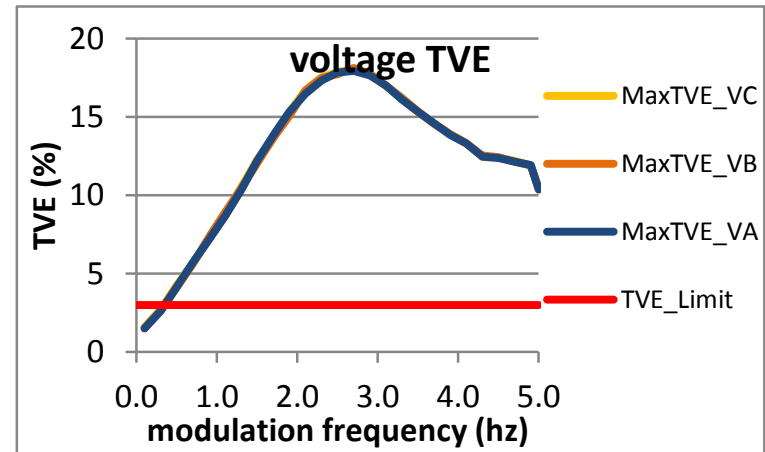

Figure 3546: Fs = 30 FPS

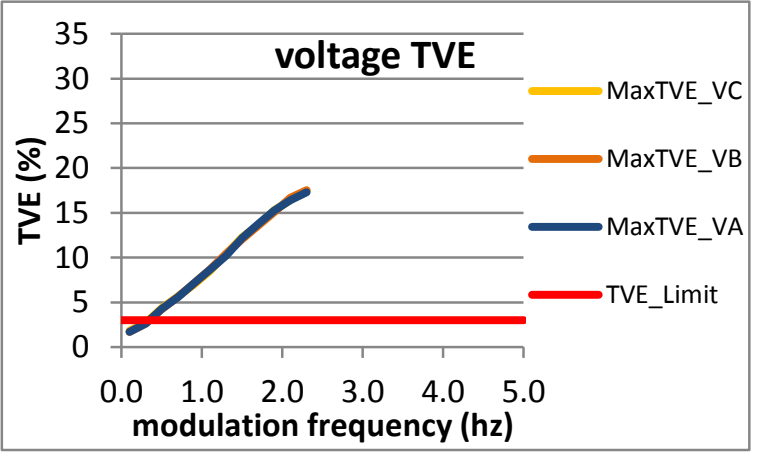

Figure 3549: Fs = 12 FPS

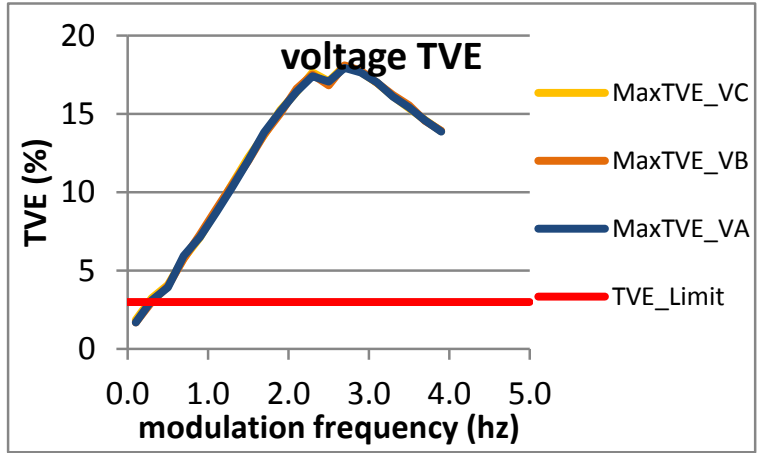

Figure 3547: Fs $=20$ FPS

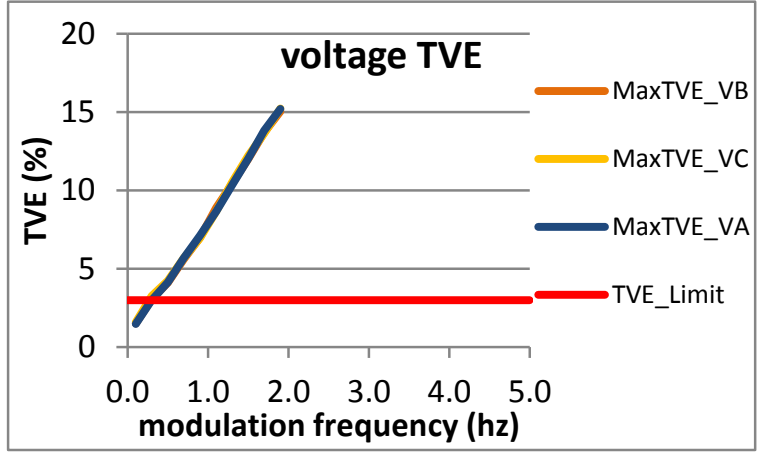

Figure 3550: Fs = 10 FPS 


\subsubsection{PMU H amplitude modulation voltage TVE: M class}
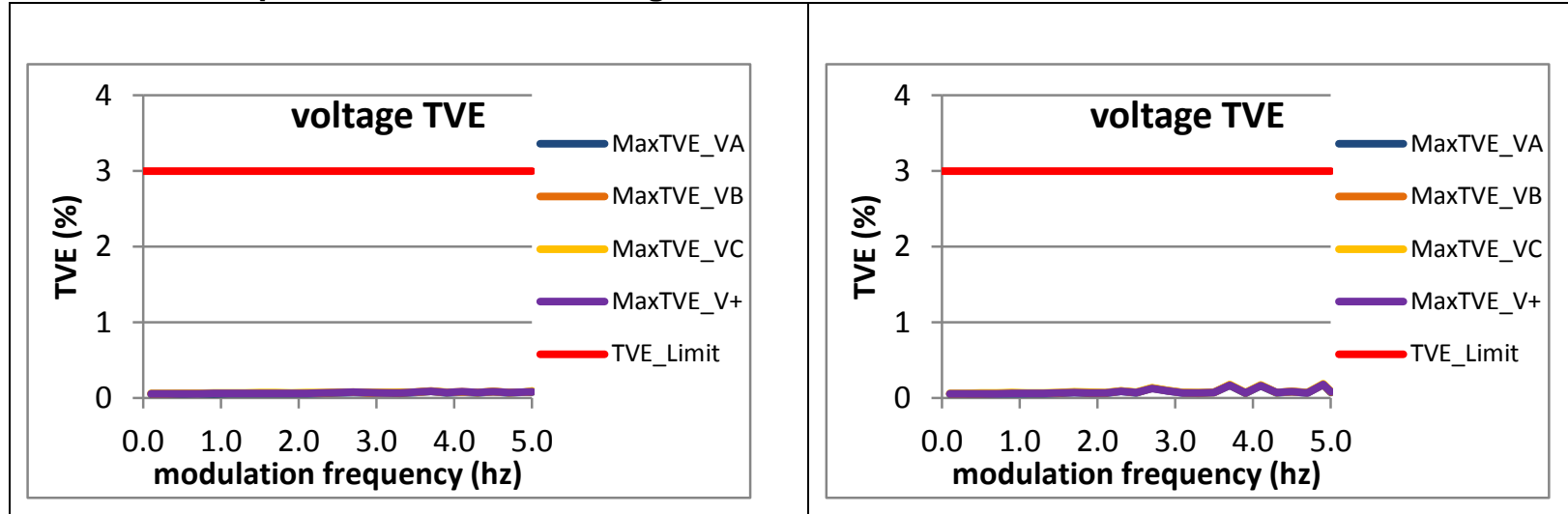

Figure 3552: Fs = 30 FPS

Figure 3551: Fs = 60 FPS

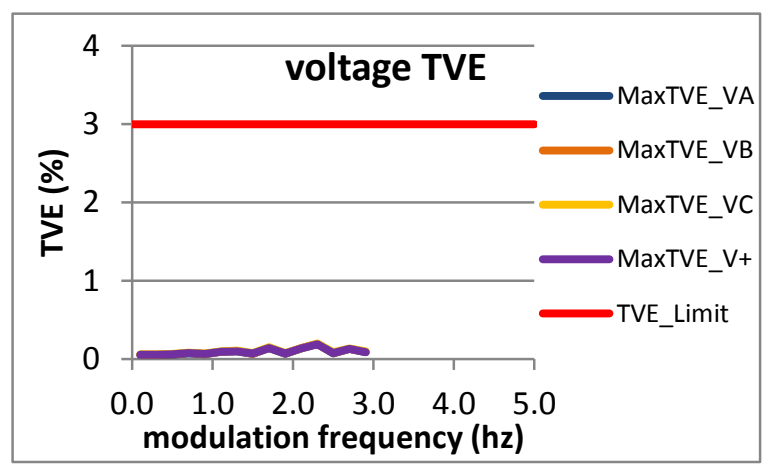

Figure 3554: Fs = 15 FPS

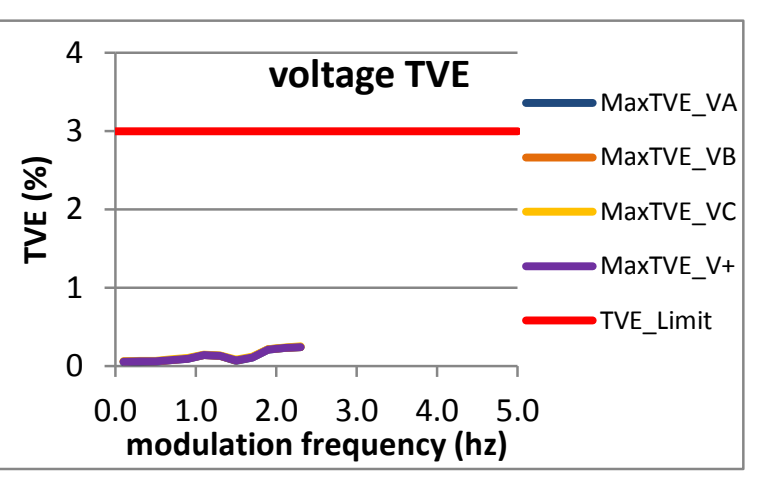

Figure 3555: Fs = 12 FPS

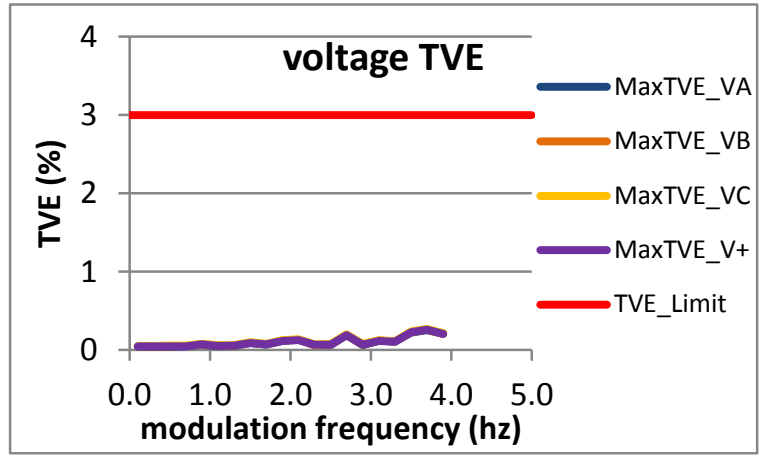

Figure 3553: Fs = 20 FPS

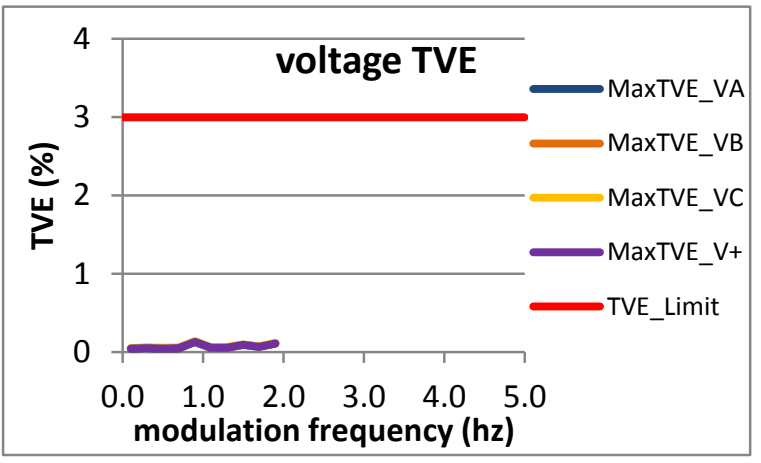

Figure 3556: Fs = 10 FPS 


\subsubsection{PMU I amplitude modulation voltage TVE: M class}

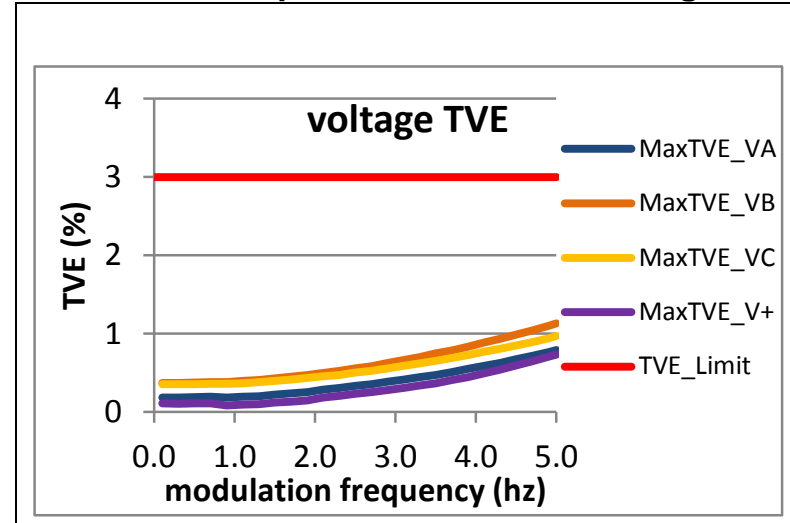

Figure 3557: Fs = 60 FPS

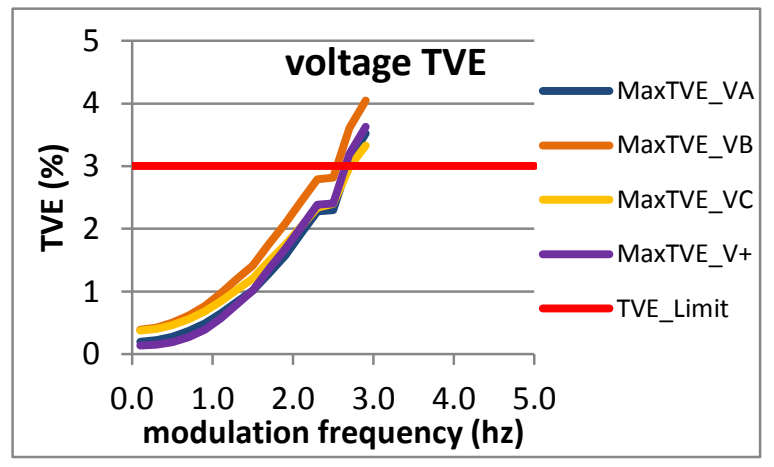

Figure 3560: Fs = 15 FPS

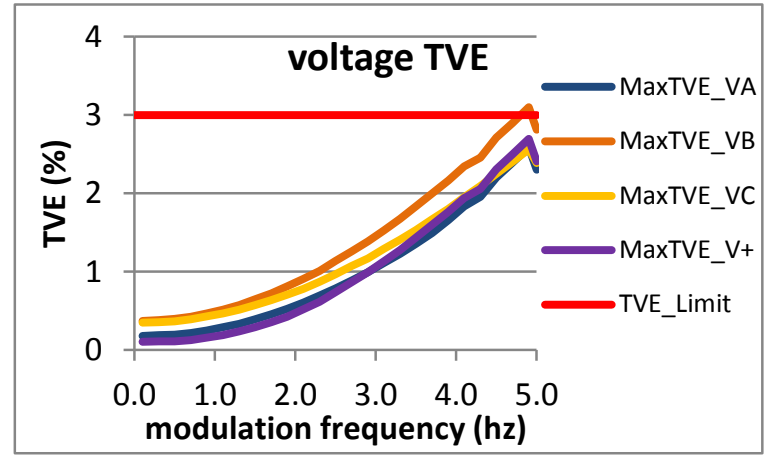

Figure 3558: Fs = 30 FPS

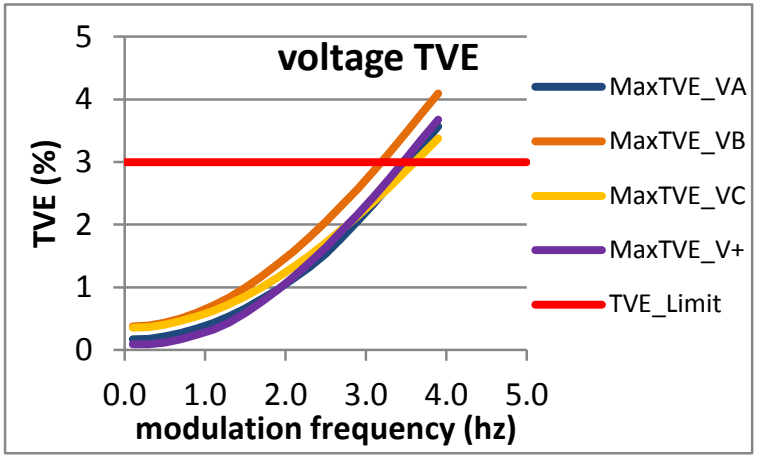

Figure 3559: Fs = 20 FPS

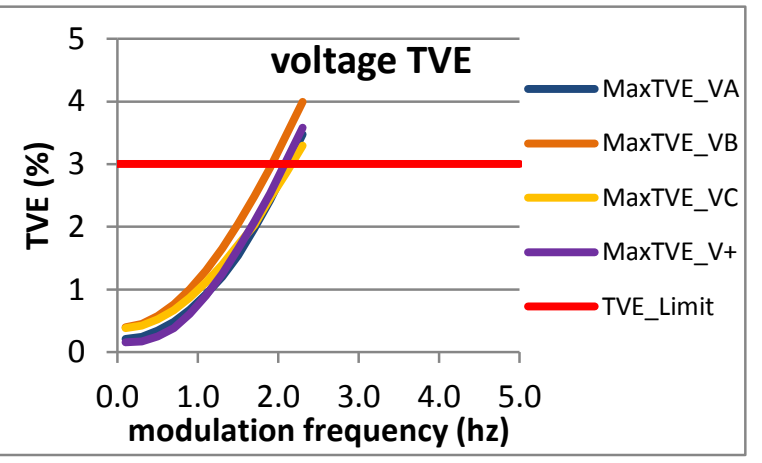

Figure 3561: Fs = 12 FPS

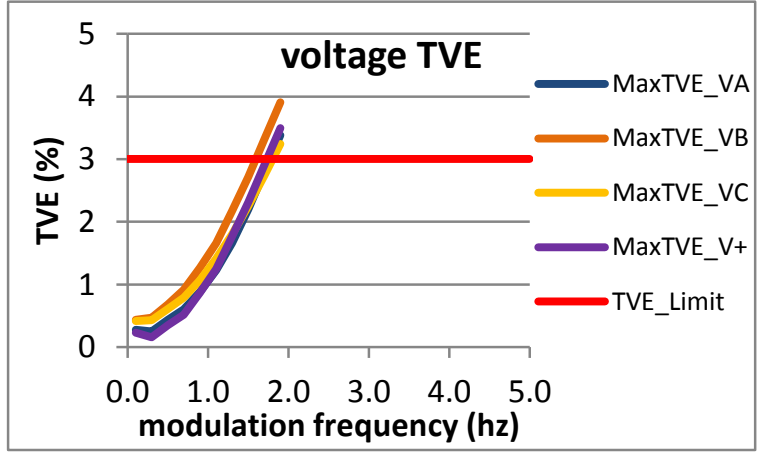

Figure 3562: Fs = 10 FPS 


\subsubsection{PMU J amplitude modulation voltage TVE: M class}

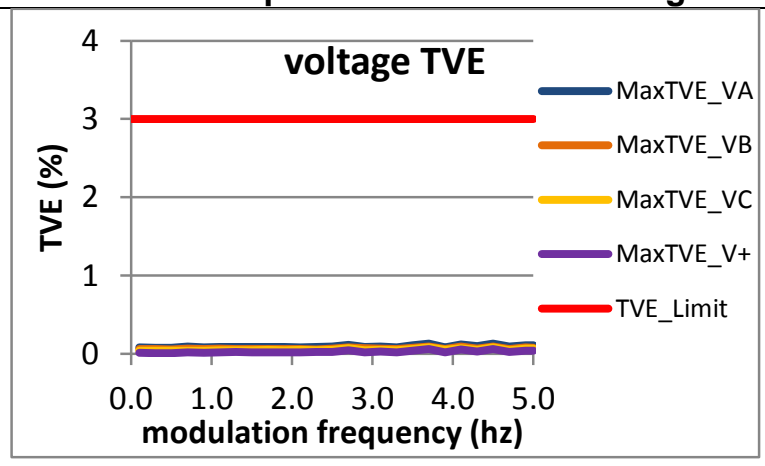

Figure 3563: Fs = 60 FPS

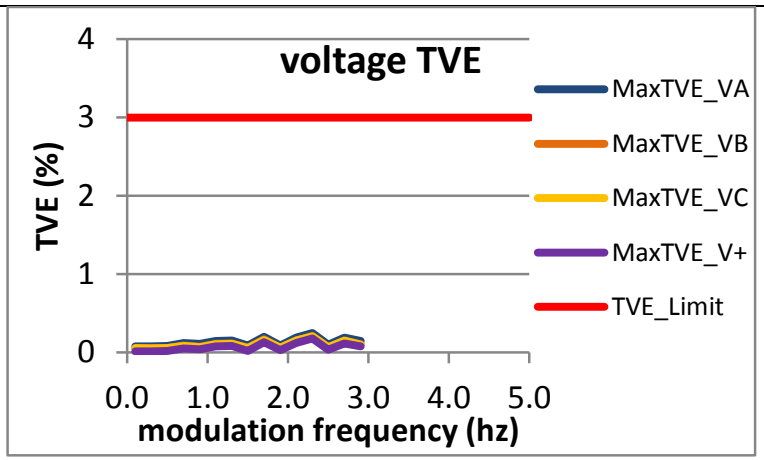

Figure 3566: Fs = 15 FPS

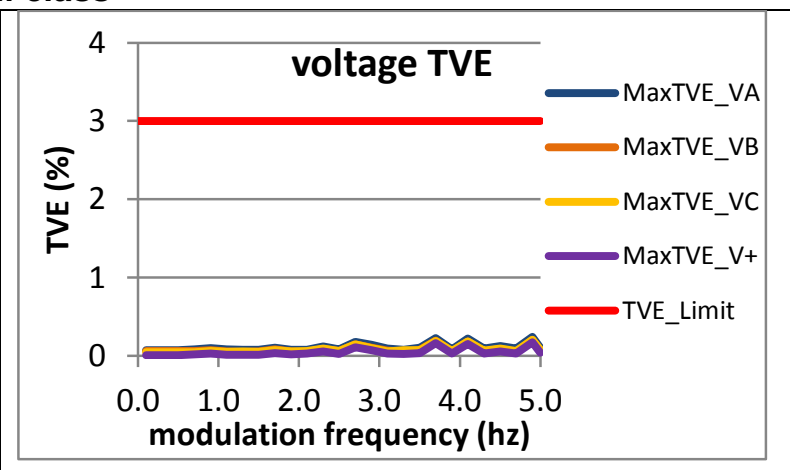

Figure 3564: Fs = 30 FPS

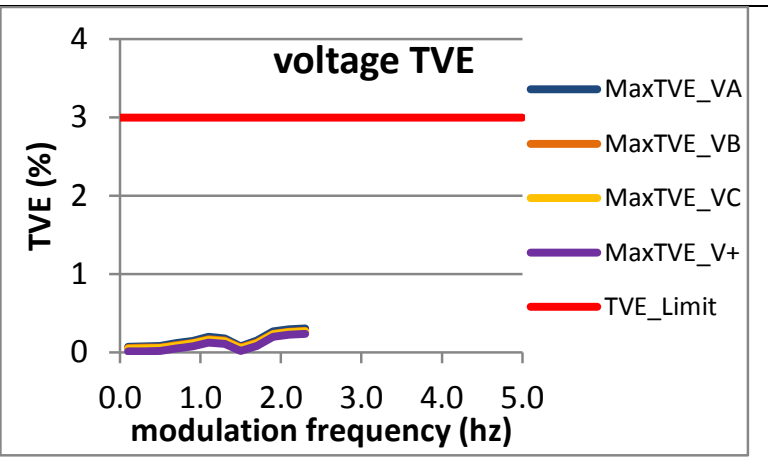

Figure 3567: Fs = 12 FPS

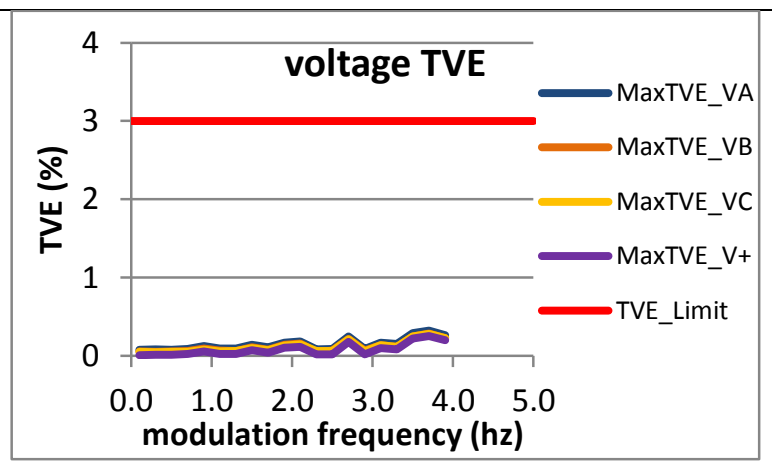

Figure 3565: Fs = 20 FPS

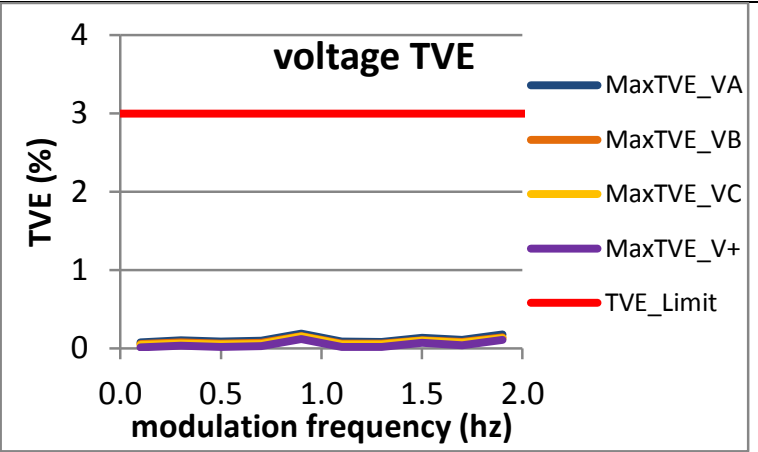

Figure 3568: Fs = 10 FPS 


\subsection{Amplitude modulation current TVE: M class}

\subsubsection{C37.118.1 Annex C amplitude modulation current TVE: M class}

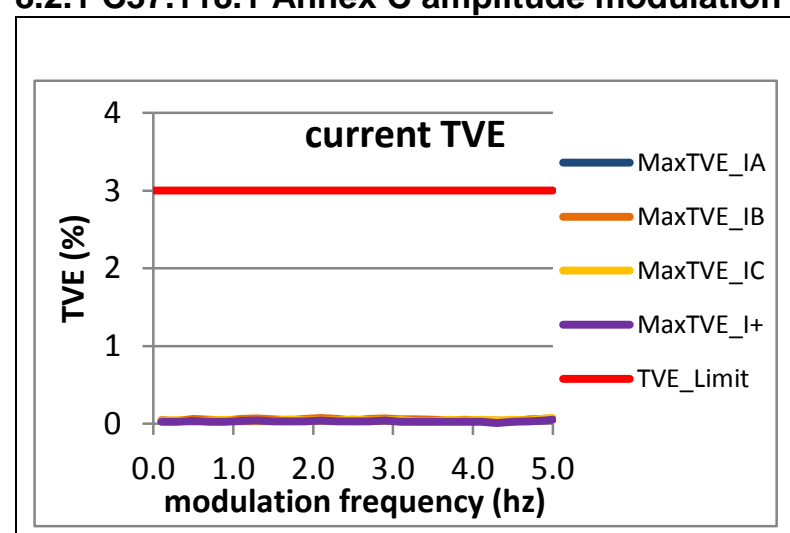

Figure 3569: Fs = 60 FPS

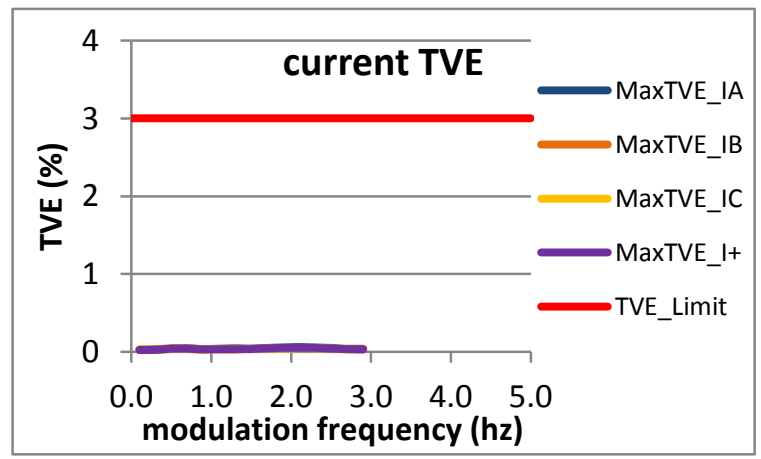

Figure 3572: Fs = 15 FPS

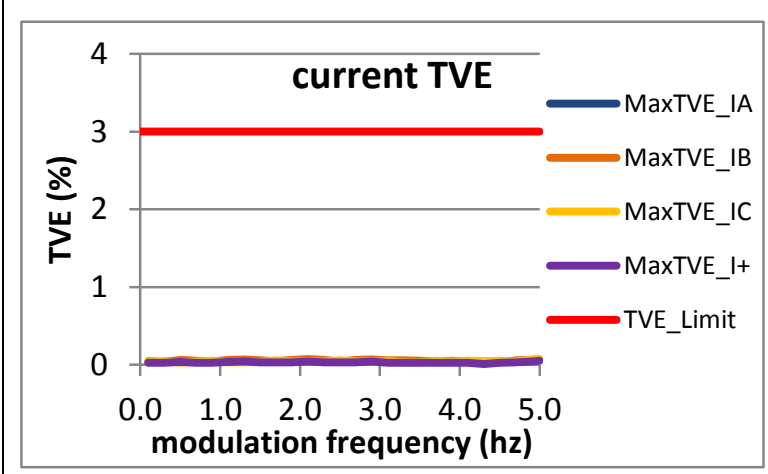

Figure 3570: Fs = 30 FPS

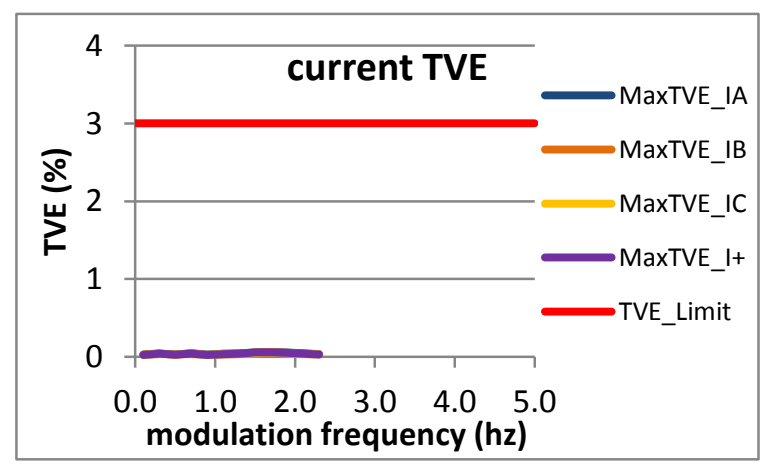

Figure 3573: Fs = 12 FPS

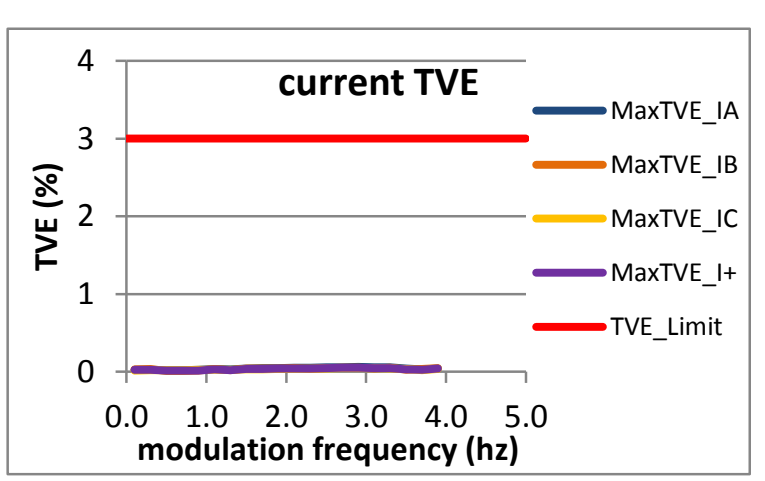

Figure 3571: Fs = 20 FPS

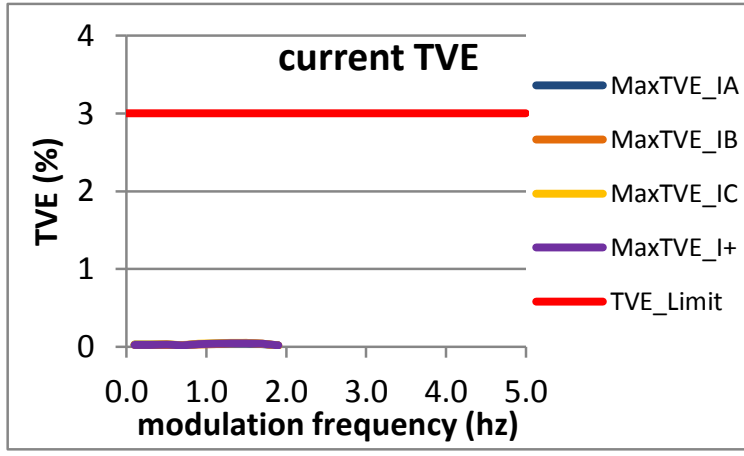

Figure 3574: Fs = 10 FPS 
8.2.2 PMU A amplitude modulation current TVE: M class

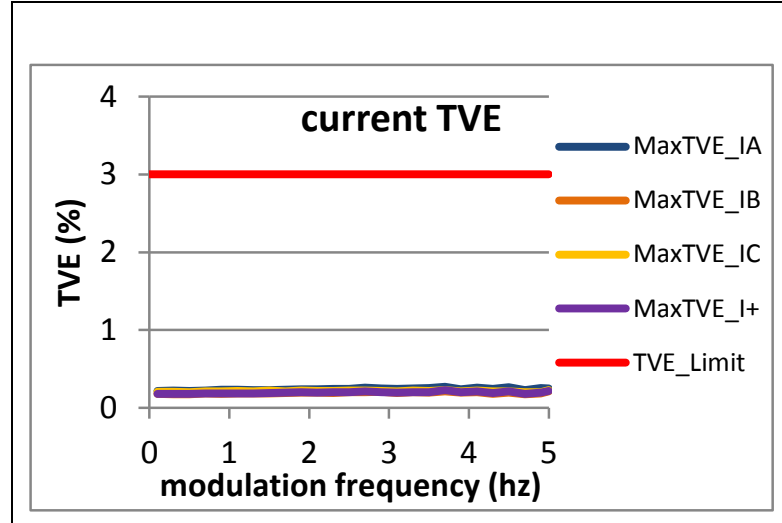

Figure 3575: Fs = 60 FPS

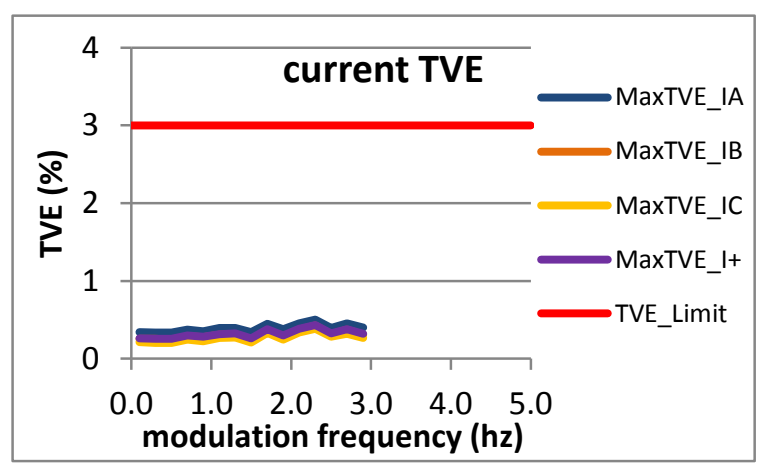

Figure 3578: Fs = 15 FPS

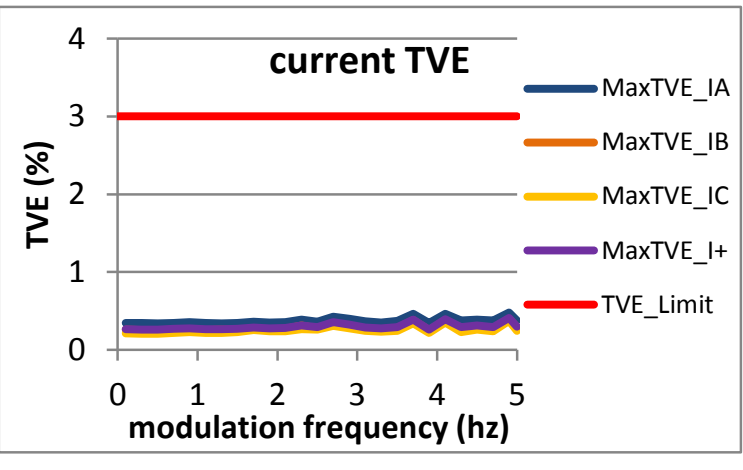

Figure 3576: Fs = 30 FPS

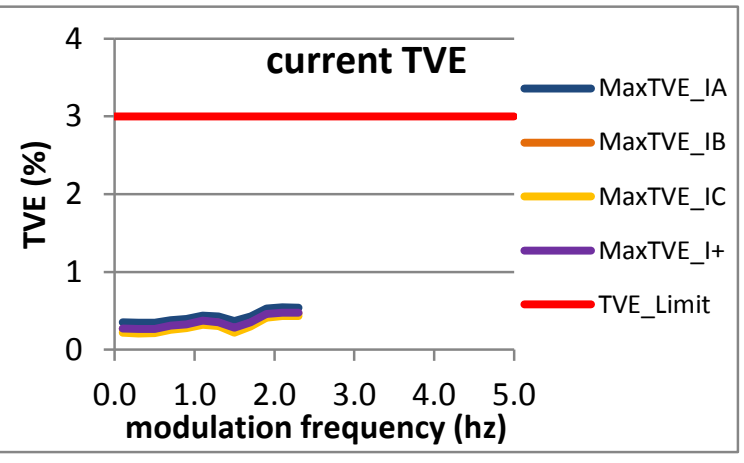

Figure 3579: Fs = 12 FPS

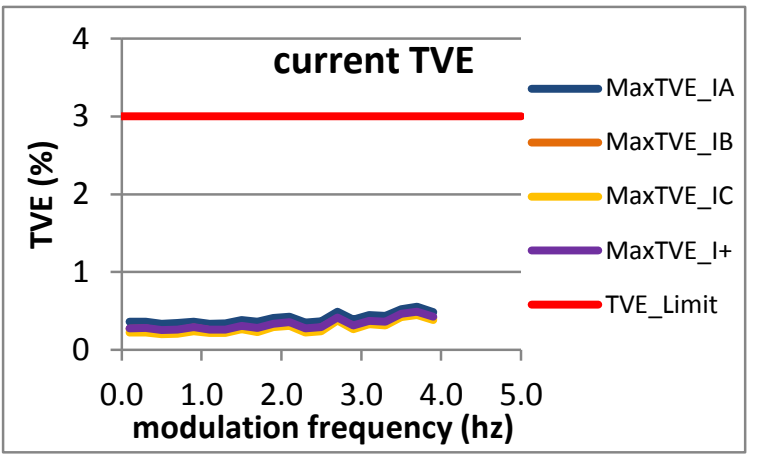

Figure 3577: Fs = 20 FPS

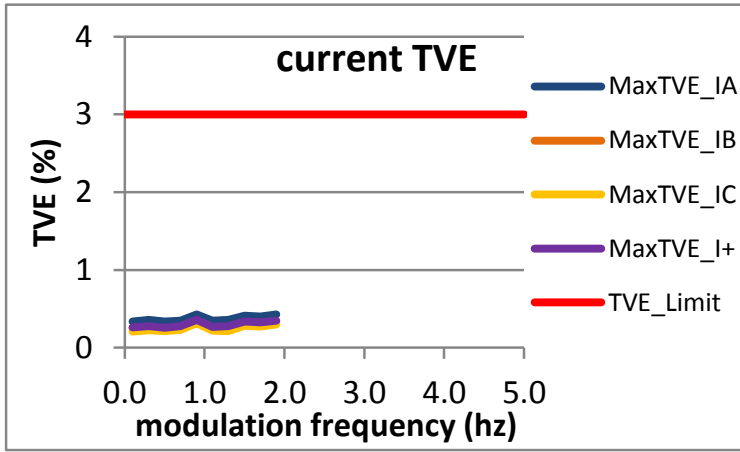

Figure 3580: Fs = 10 FPS 
8.2.3 PMU B amplitude modulation current TVE: M class

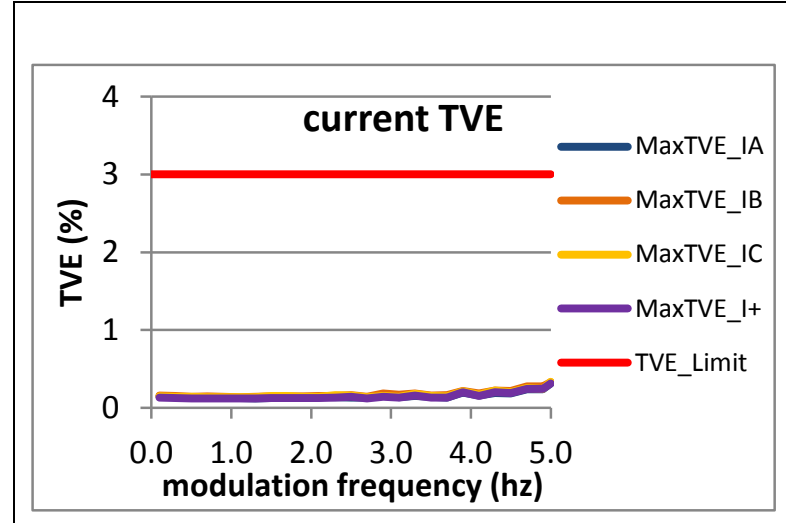

Figure 3581: Fs = 60 FPS

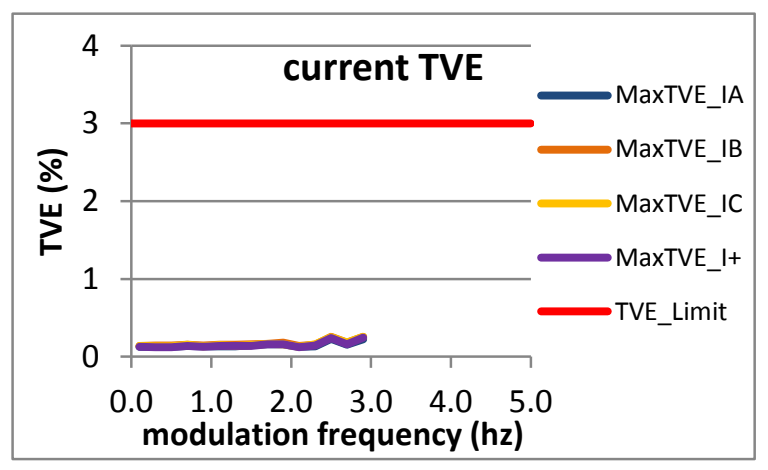

Figure 3584: Fs = 15 FPS

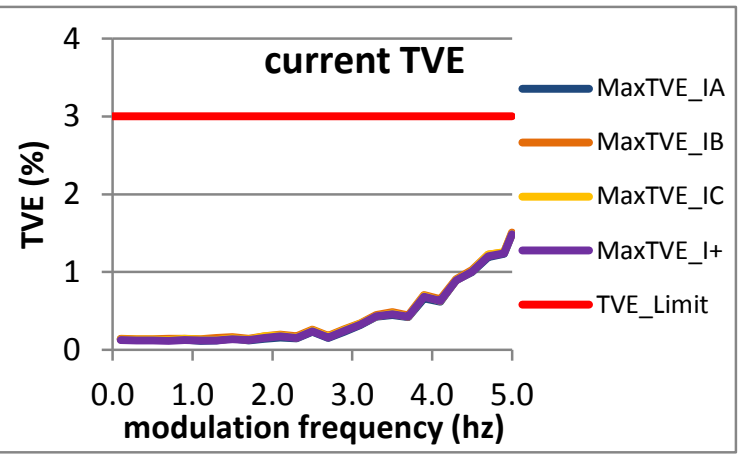

Figure 3582: Fs = 30 FPS

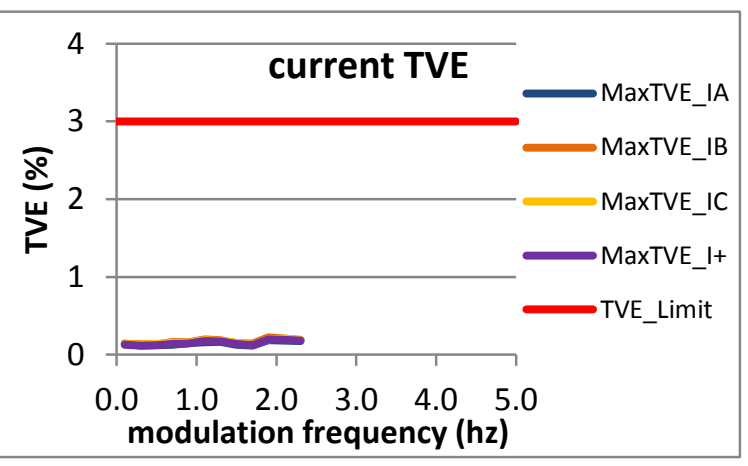

Figure 3585: Fs = 12 FPS

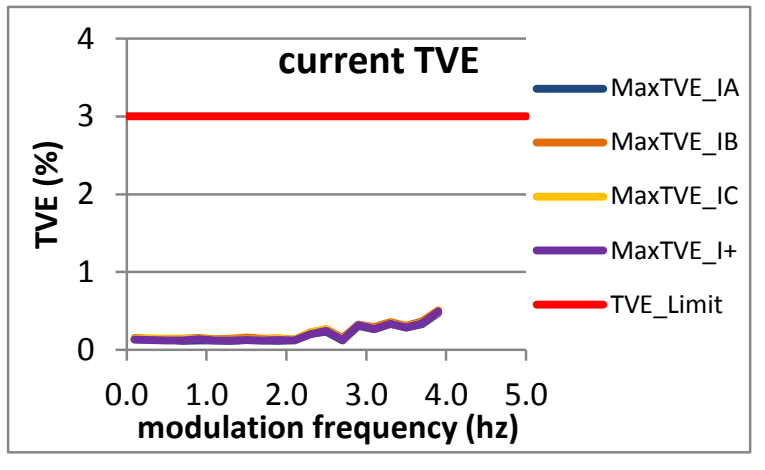

Figure 3583: Fs = 20 FPS

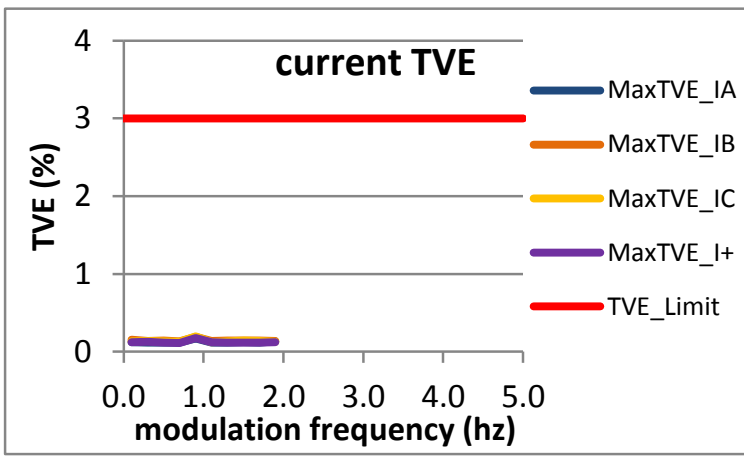

Figure 3586: Fs = 10 FPS 
8.2.4 PMU C amplitude modulation current TVE: M class

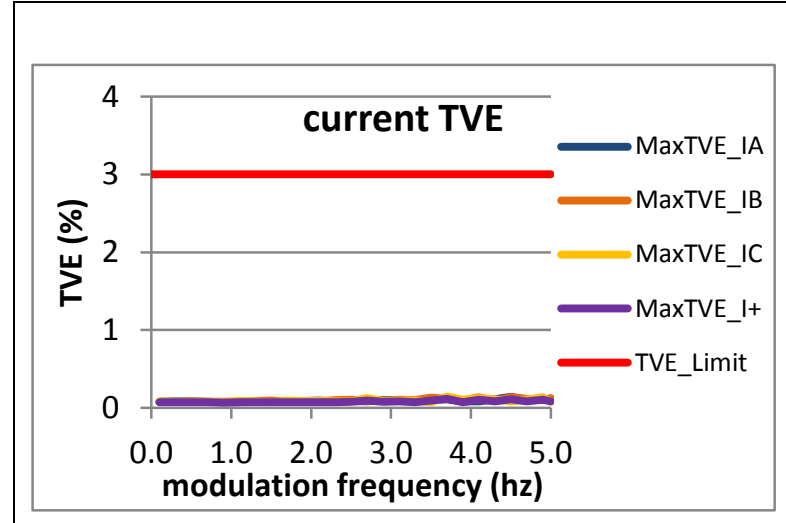

Figure 3587: Fs = 60 FPS

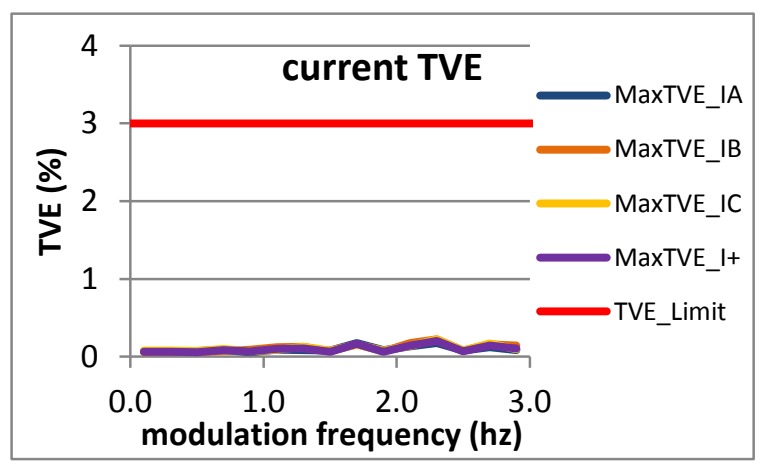

Figure 3590: Fs = 15 FPS

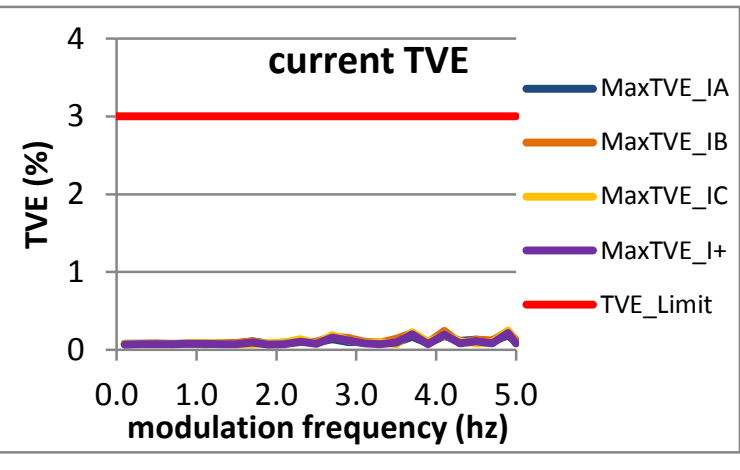

Figure 3588: Fs = 30 FPS

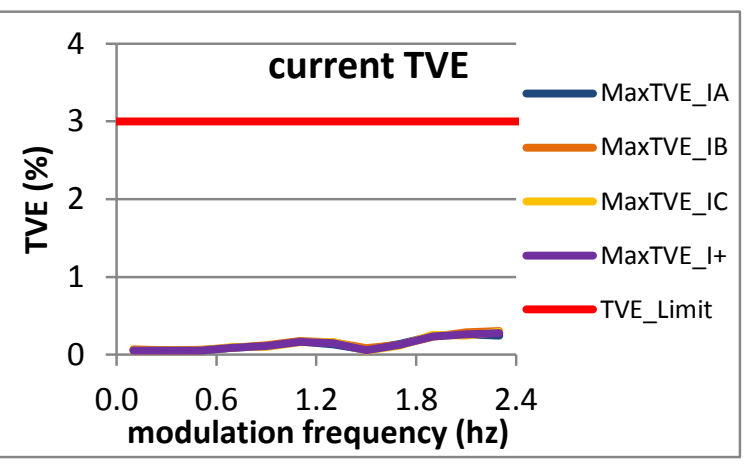

Figure 3591: Fs = 12 FPS

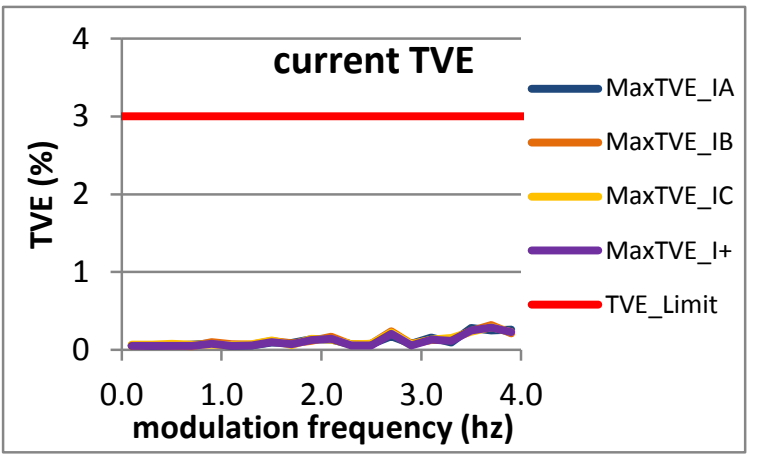

Figure 3589: Fs = 20 FPS

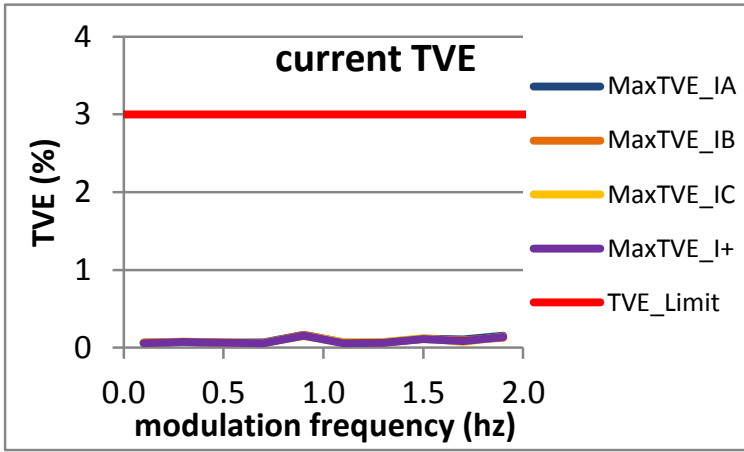

Figure 3592: Fs = 10 FPS 
8.2.5 PMU D amplitude modulation current TVE: M class

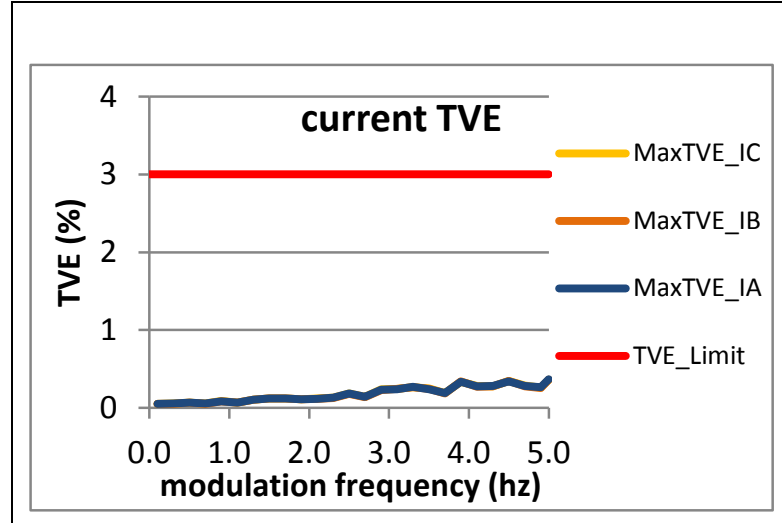

Figure 3593: Fs = 60 FPS

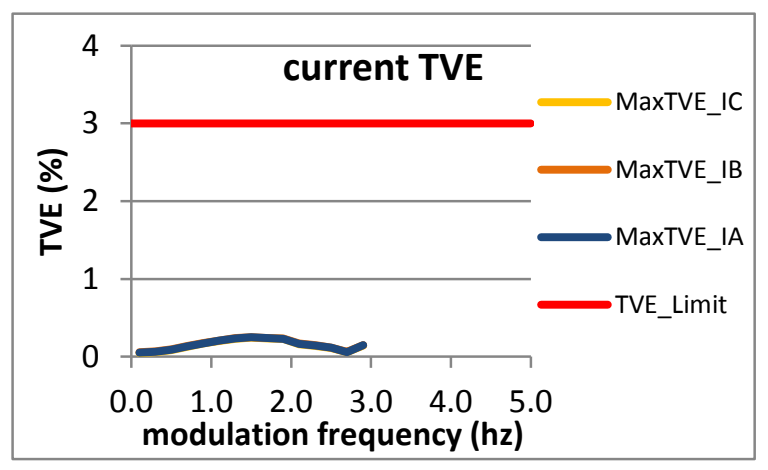

Figure 3596: Fs = 15 FPS

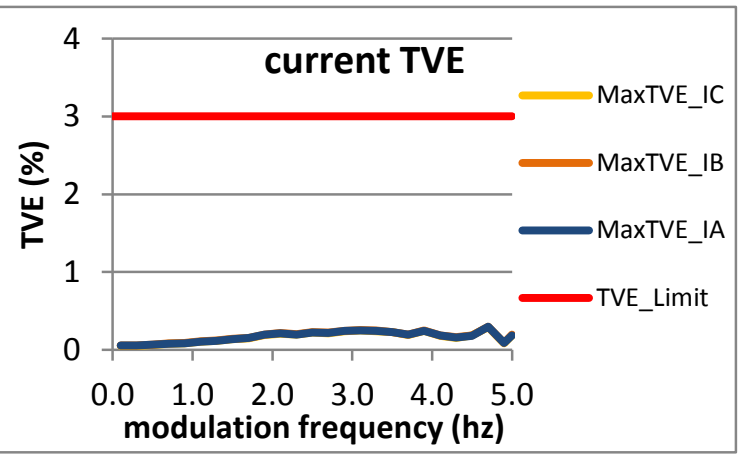

Figure 3594: Fs = 30 FPS

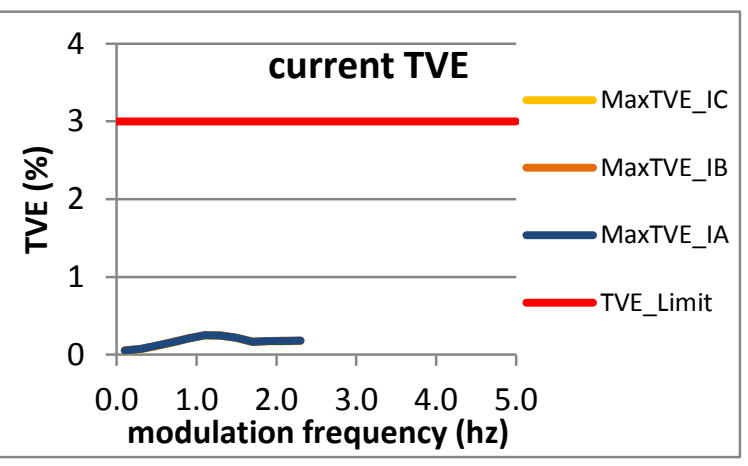

Figure 3597: Fs = 12 FPS

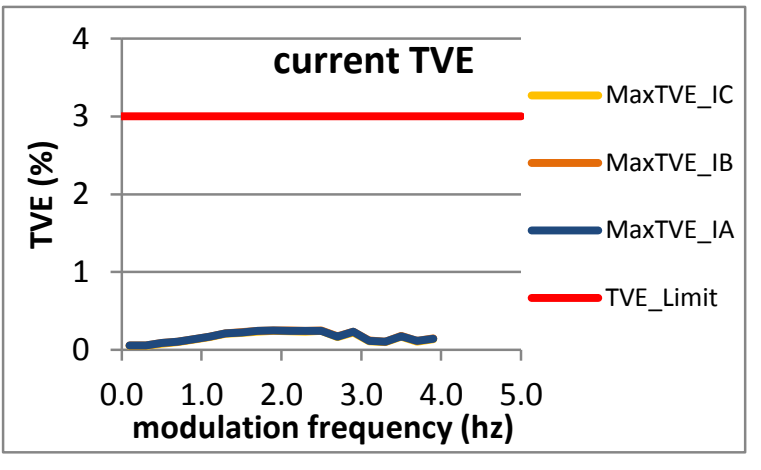

Figure 3595: Fs = 20 FPS

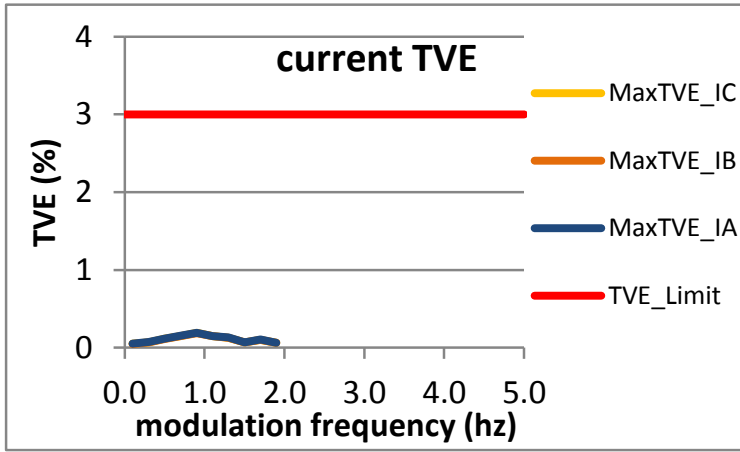

Figure 3598: Fs = 10 FPS 
8.2.6 PMU E amplitude modulation current TVE: M class

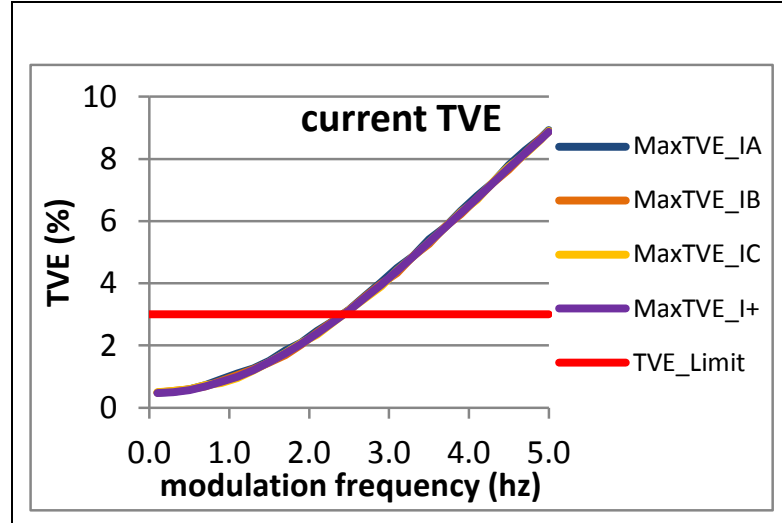

Figure 3599: Fs = 60 FPS

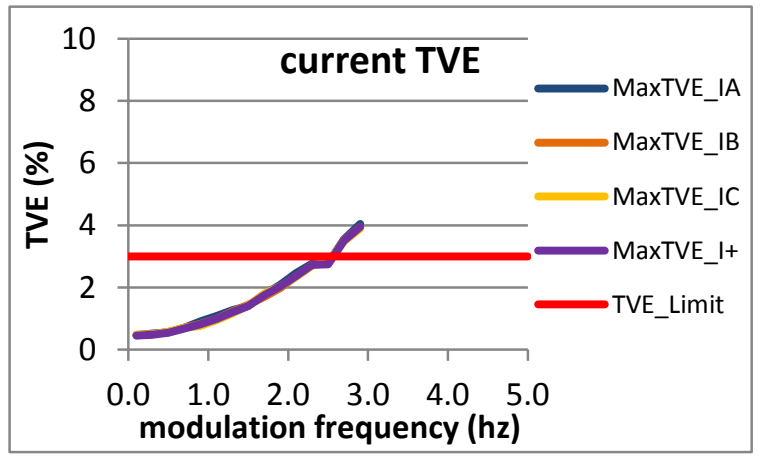

Figure 3602: Fs = 15 FPS

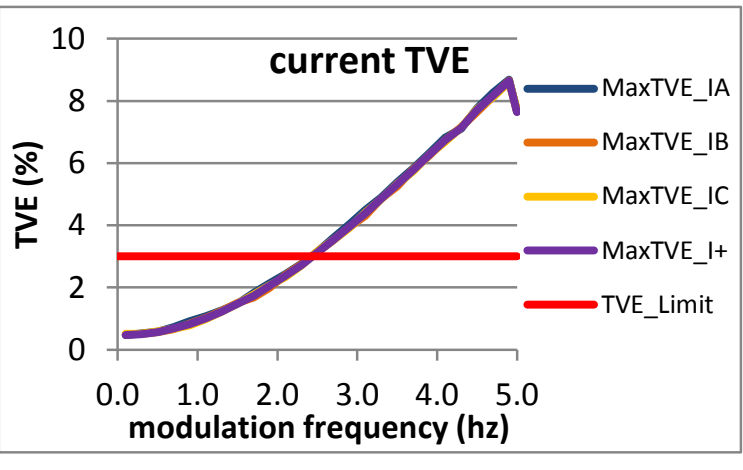

Figure 3600: Fs = 30 FPS

Figure 3603: Fs = 12 FPS data was lost.

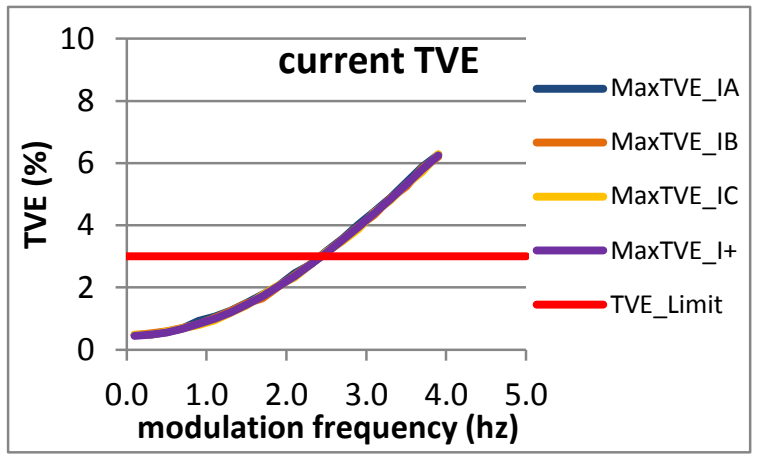

Figure 3601: Fs = 20 FPS

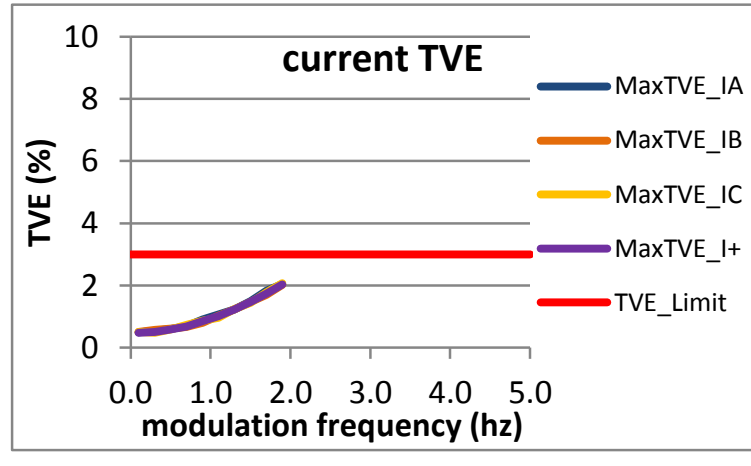

Figure 3604: Fs = 10 FPS 
8.2.7 PMU F amplitude modulation current TVE: M class

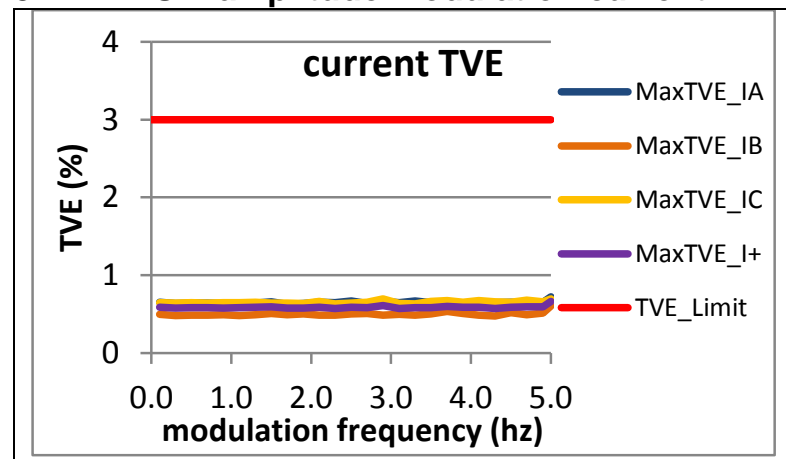

Figure 3605: Fs = 60 FPS

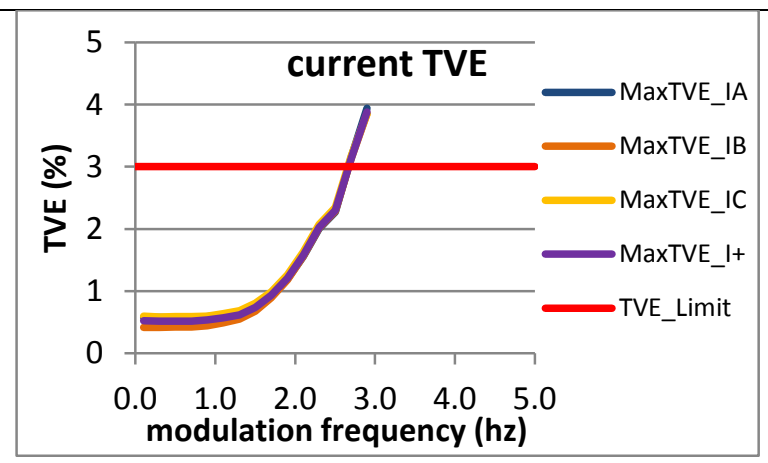

Figure 3608: Fs = 15 FPS

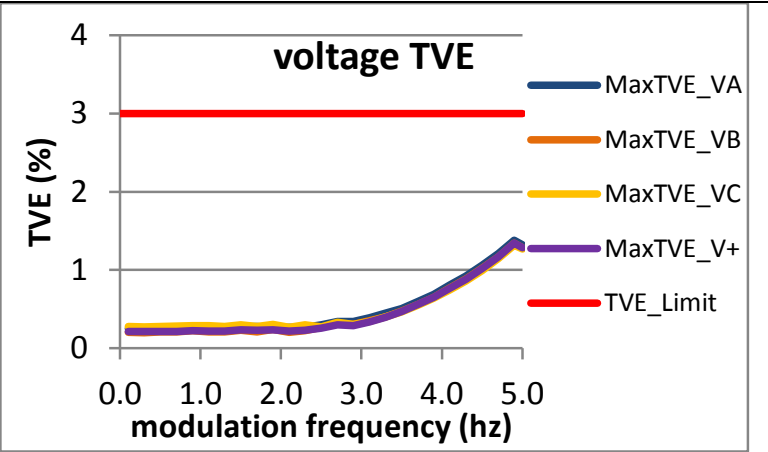

Figure 3606: Fs = 30 FPS

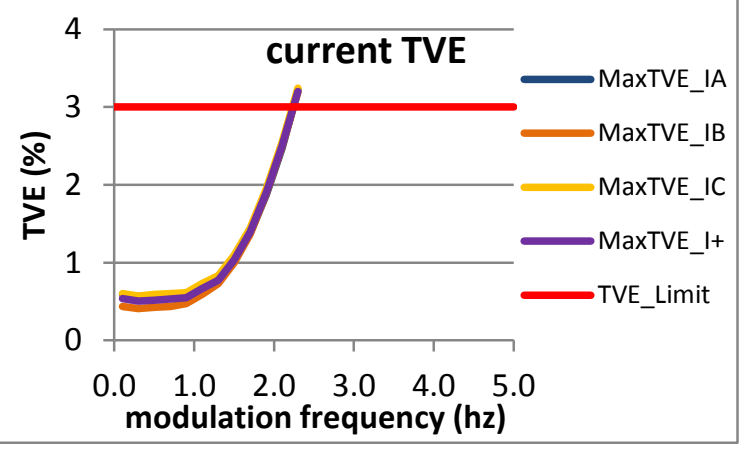

Figure 3609: Fs = 12 FPS

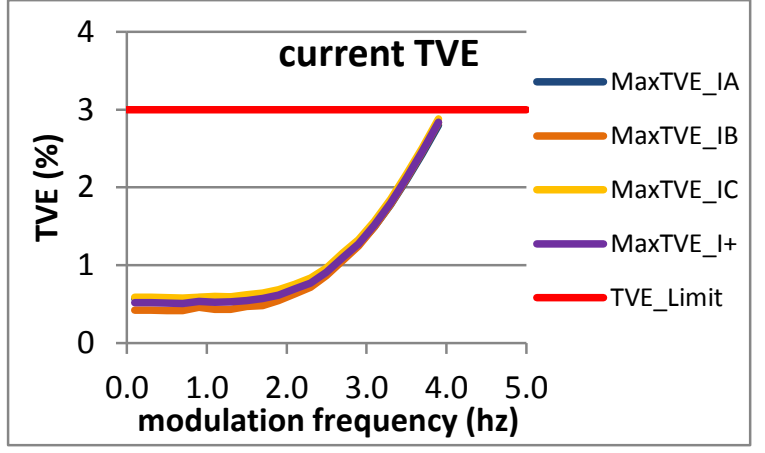

Figure 3607: Fs = 20 FPS

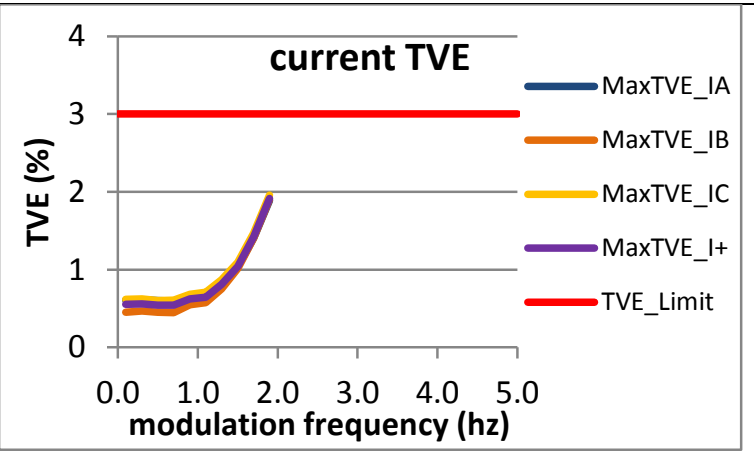

Figure 3610: Fs = 10 FPS 
8.2.8 PMU G amplitude modulation current TVE: M class

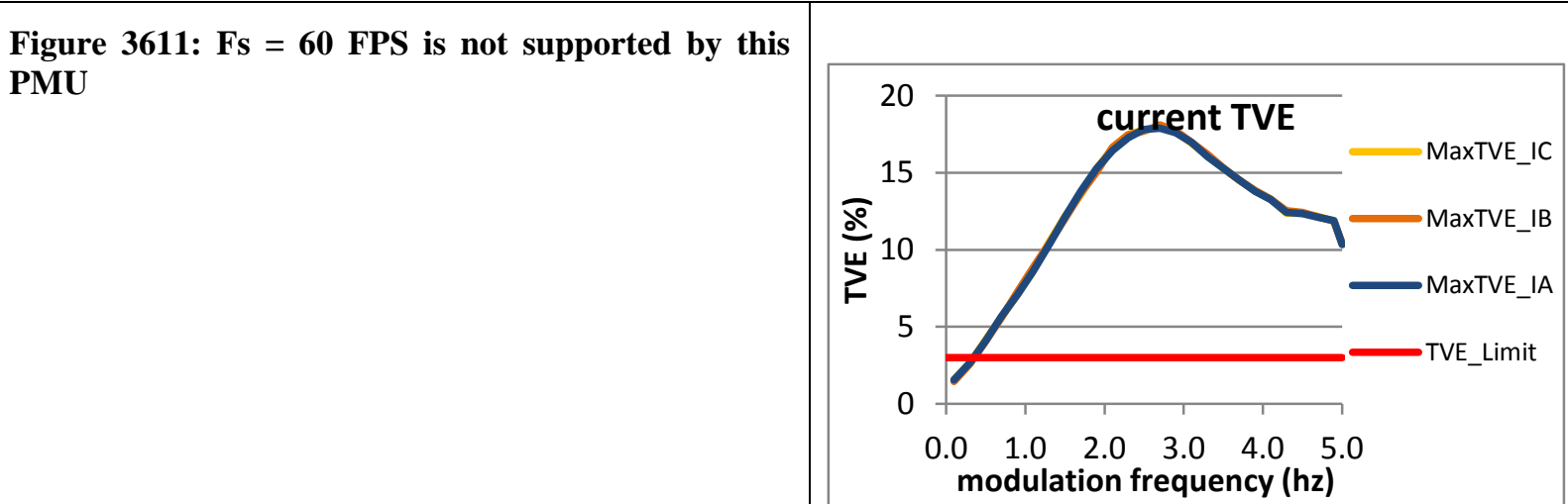

Figure 3612: Fs = 30 FPS

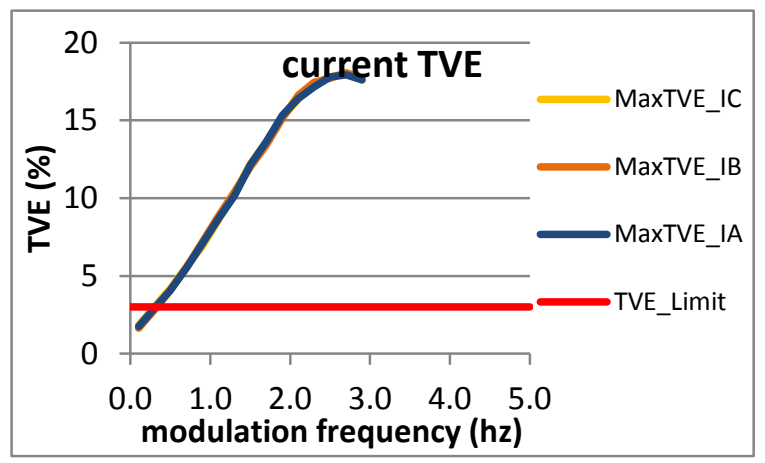

Figure 3614: Fs = 15 FPS

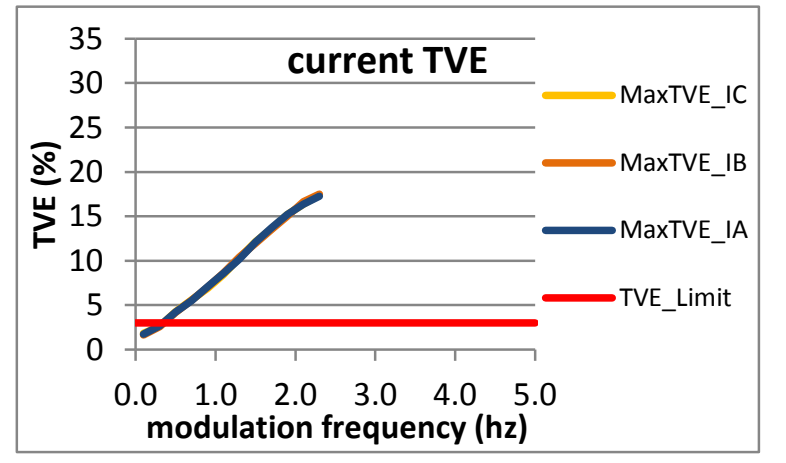

Figure 3615: Fs = 12 FPS

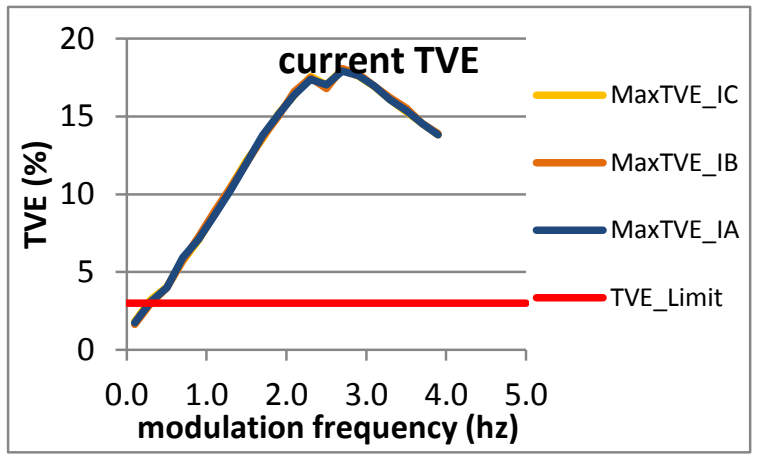

Figure 3613: Fs = 20 FPS

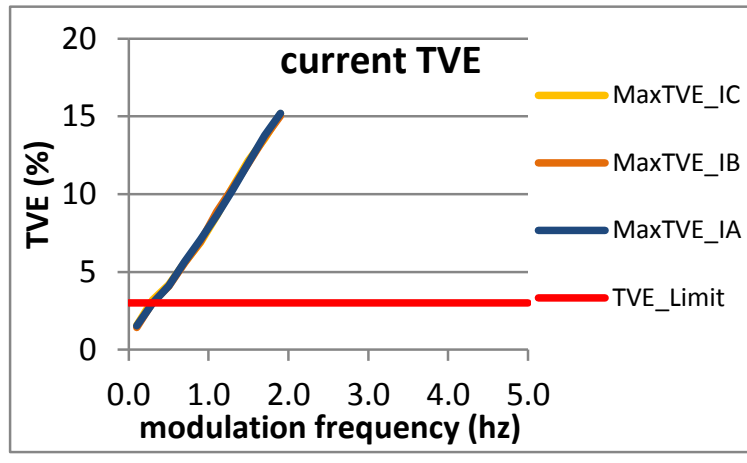

Figure 3616: Fs = 10 FPS 
8.2.9 PMU H amplitude modulation current TVE: M class

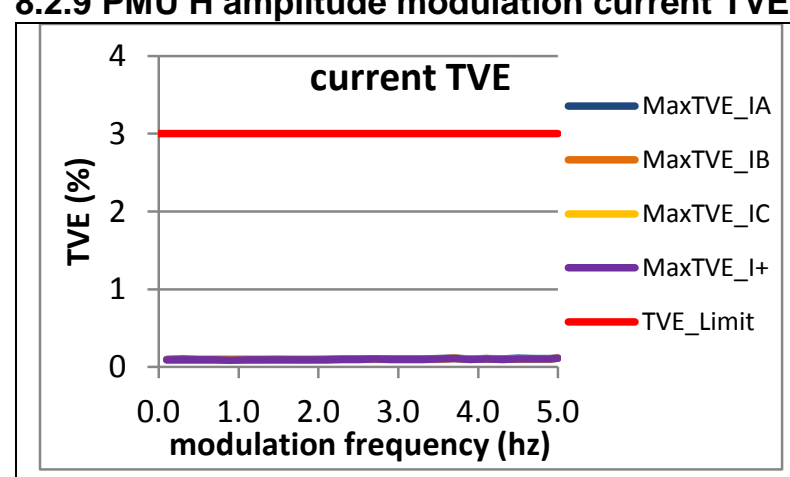

Figure 3617: Fs = 60 FPS

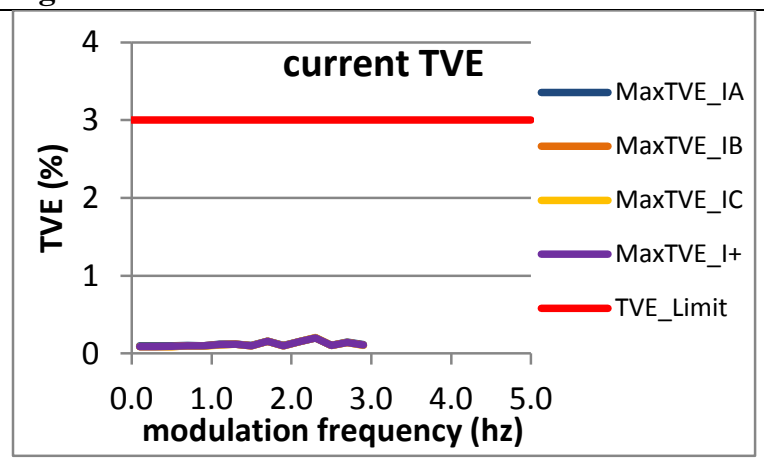

Figure 3620: Fs = 15 FPS

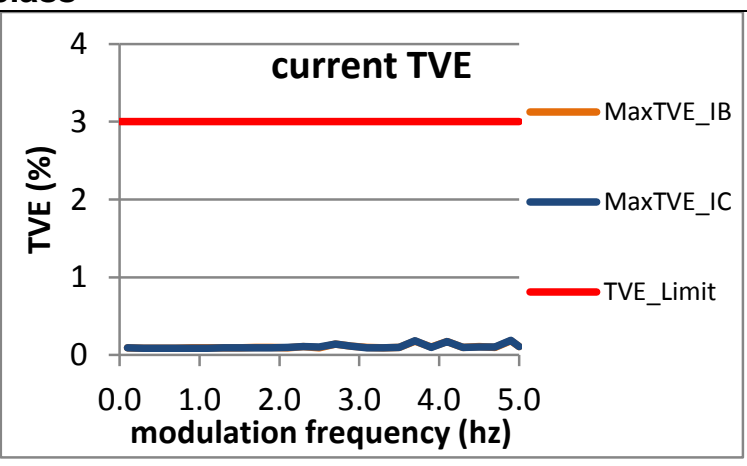

Figure 3618: Fs = 30 FPS

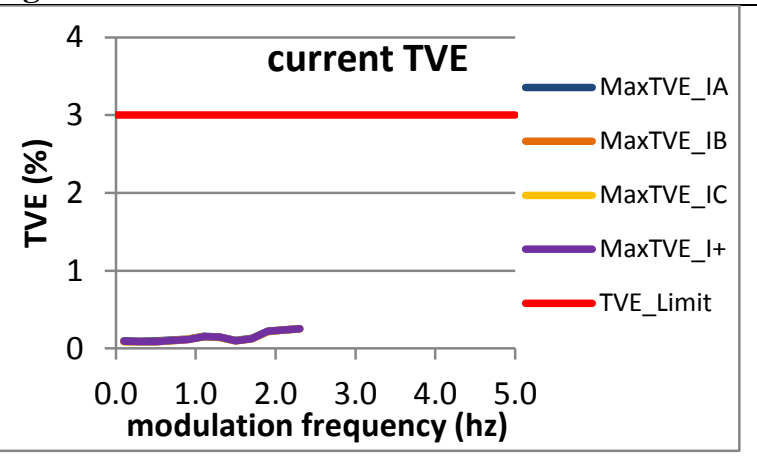

Figure 3621: Fs = 12 FPS

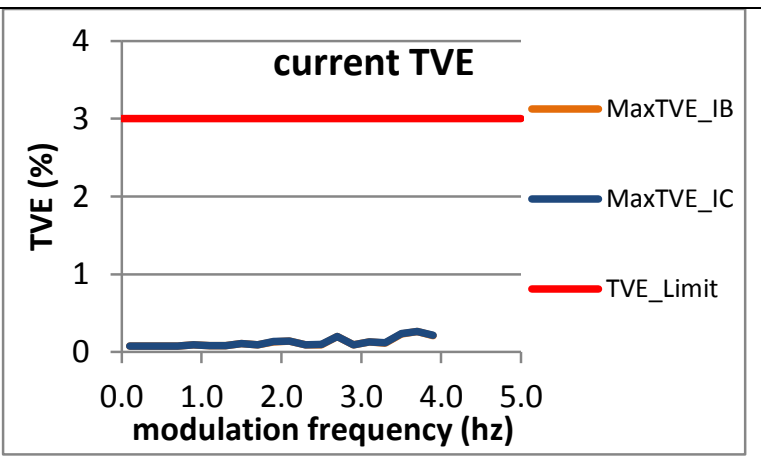

Figure 3619: Fs = 20 FPS

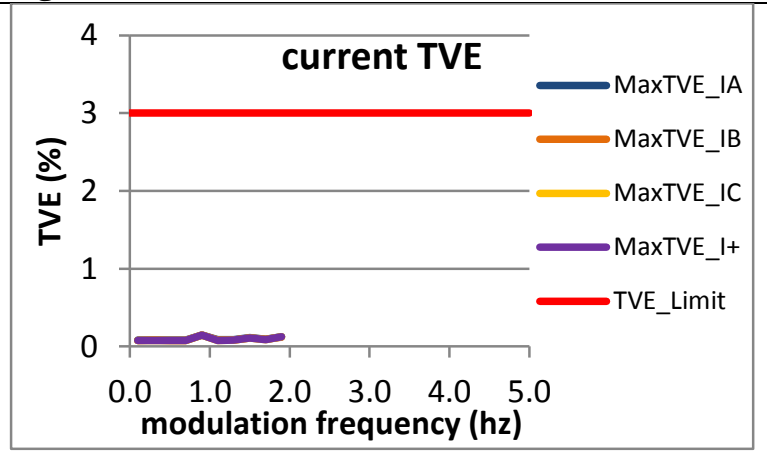

Figure 3622: Fs = 10 FPS 
8.2.10 PMU I amplitude modulation current TVE: M class

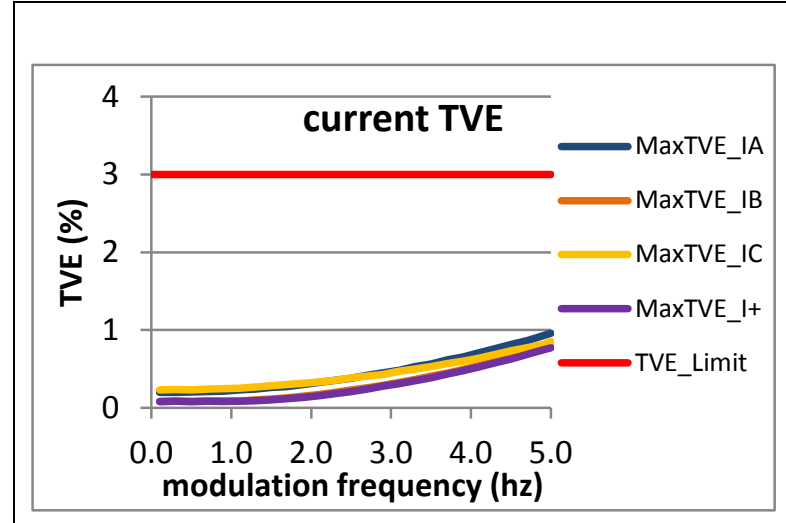

Figure 3623: Fs = 60 FPS

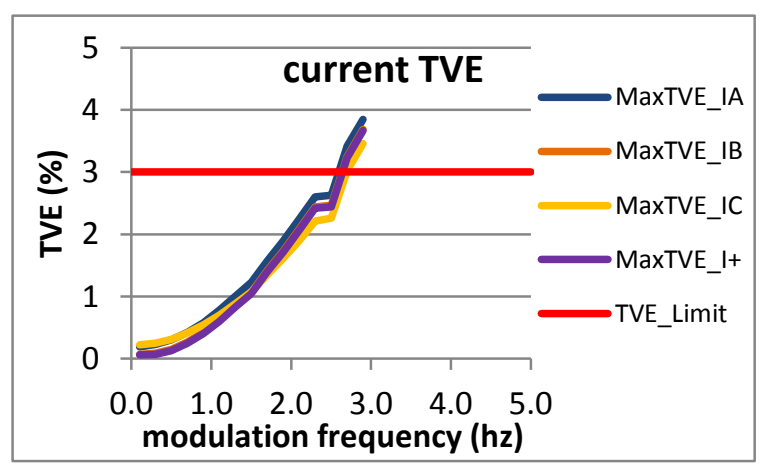

Figure 3626: Fs = 15 FPS

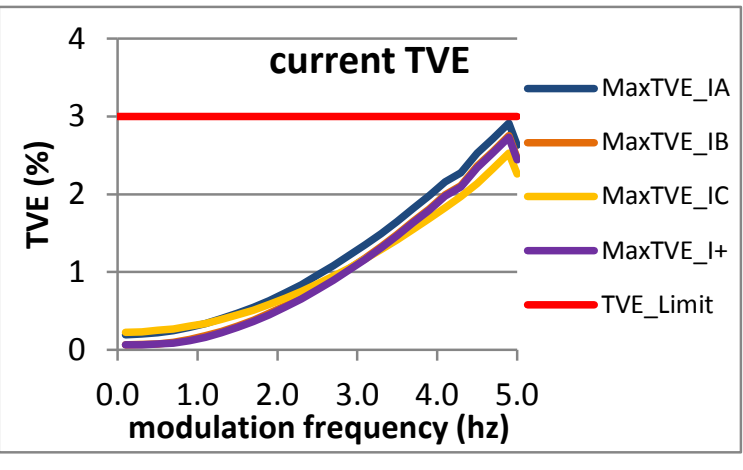

Figure 3624: Fs = 30 FPS

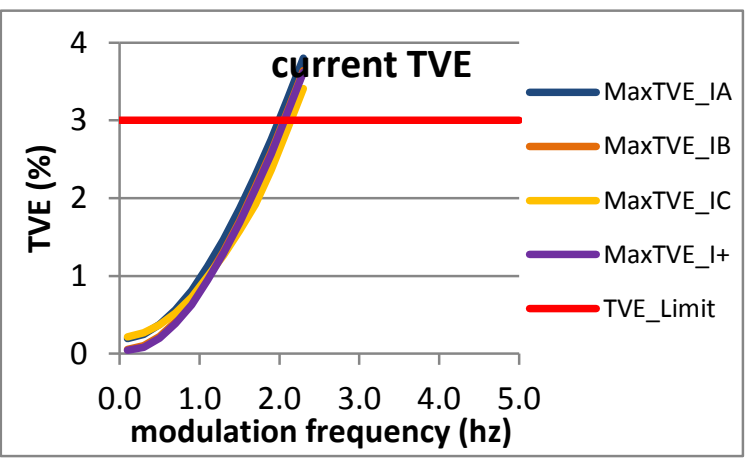

Figure 3627: Fs = 12 FPS

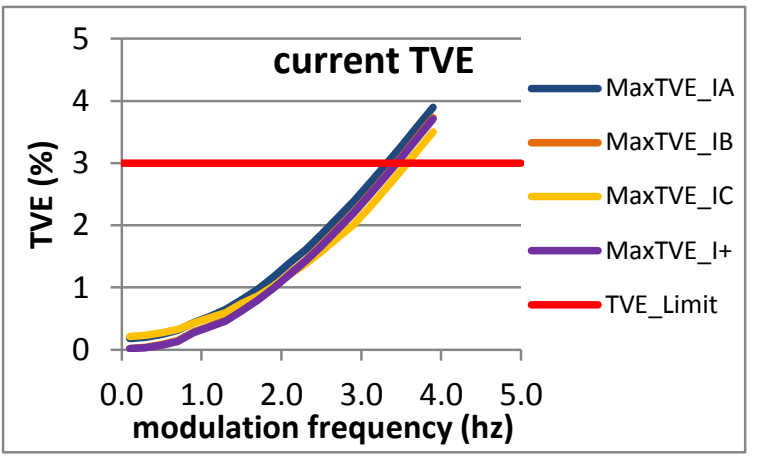

Figure 3625: Fs = 20 FPS

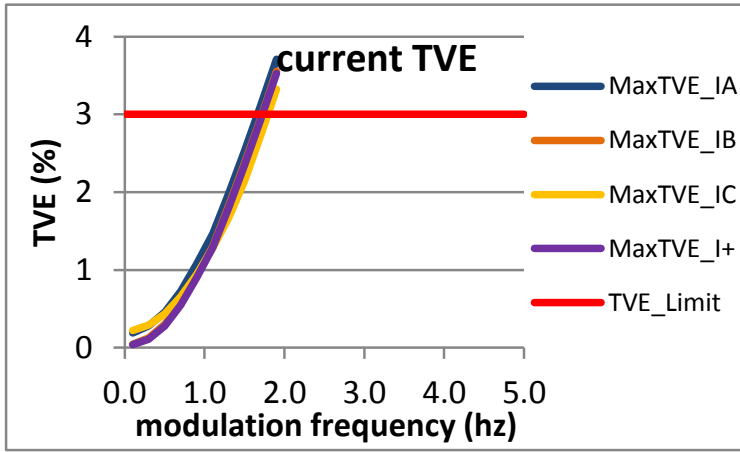

Figure 3628: Fs = 10 FPS 
8.2.11 PMU J amplitude modulation current TVE: M class

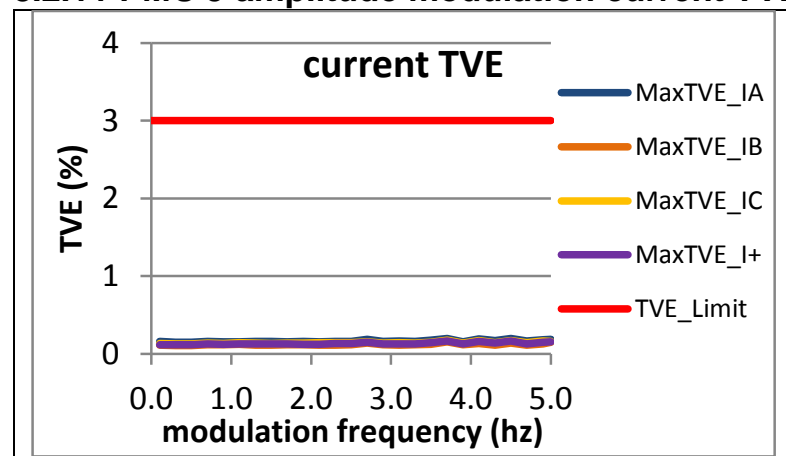

Figure 3629: Fs $=60$ FPS

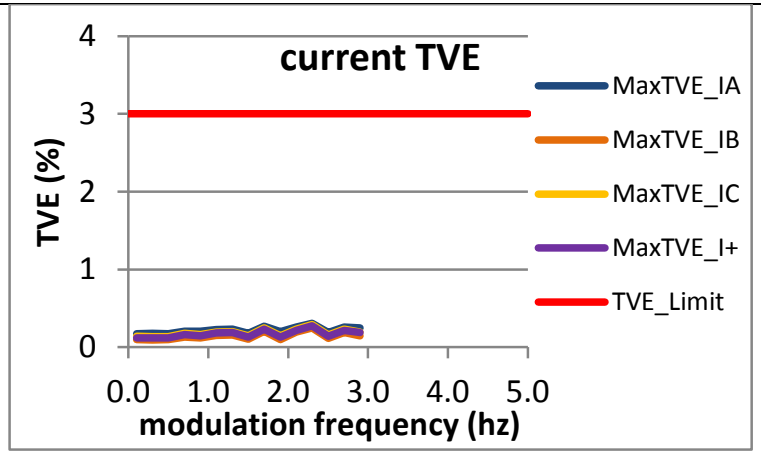

Figure 3632: Fs = 15 FPS

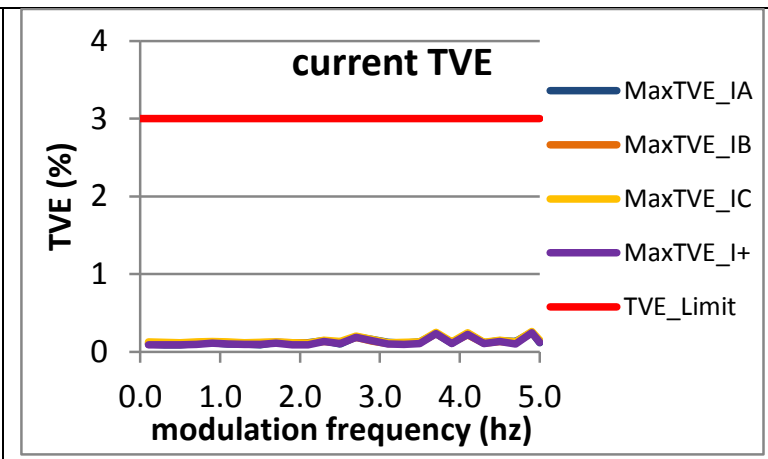

Figure 3630: Fs = 30 FPS

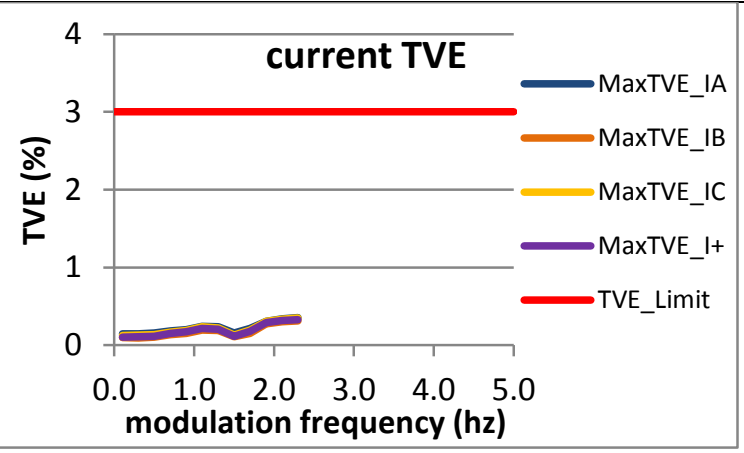

Figure 3633: Fs = 12 FPS

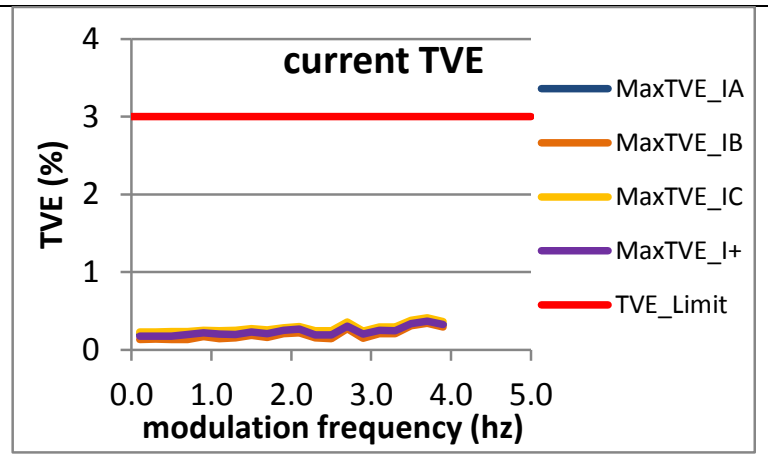

Figure 3631: Fs = 20 FPS

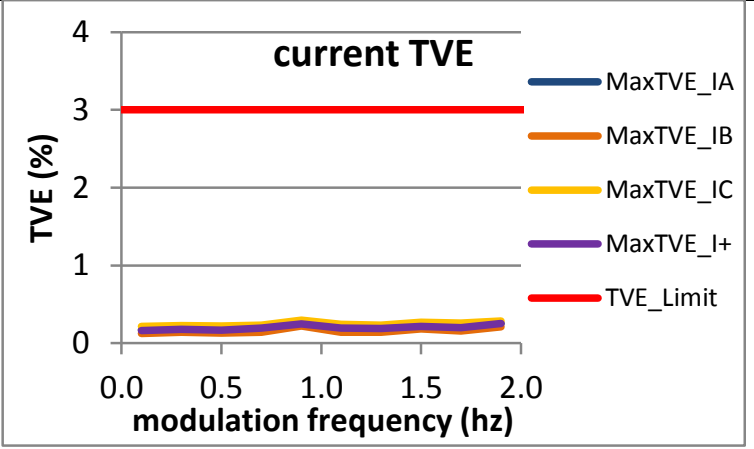

Figure 3634: Fs = 10 FPS 


\subsection{Amplitude modulation frequency error: M class}

\subsubsection{C37.118.1 Annex C amplitude modulation frequency error: M class}

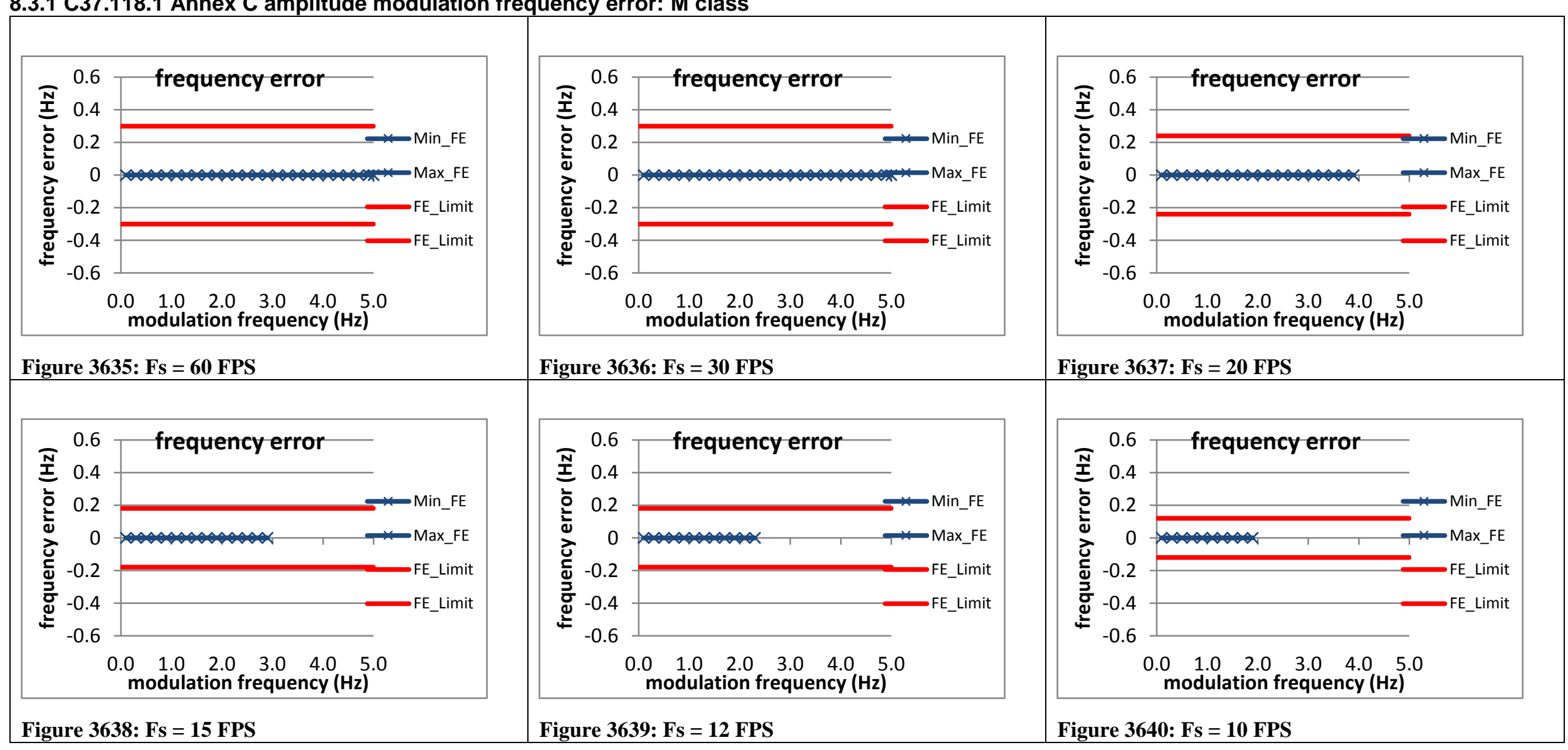


8.3.2 PMU A amplitude modulation frequency error: M class

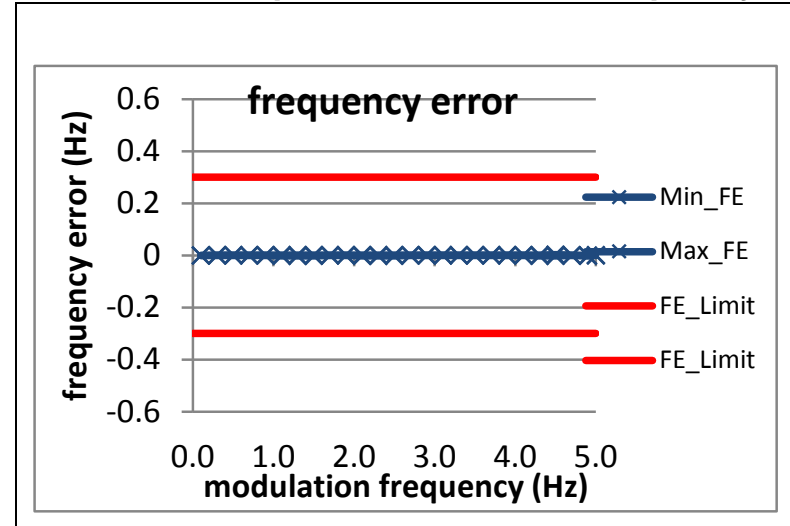

Figure 3641: Fs = 60 FPS

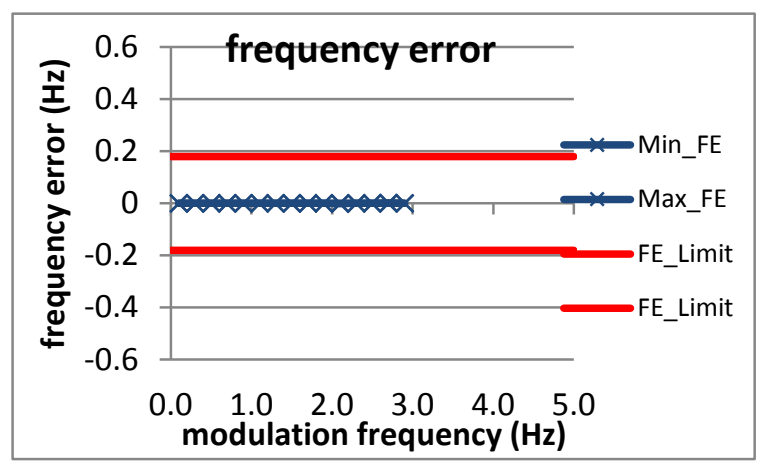

Figure 3644: Fs = 15 FPS

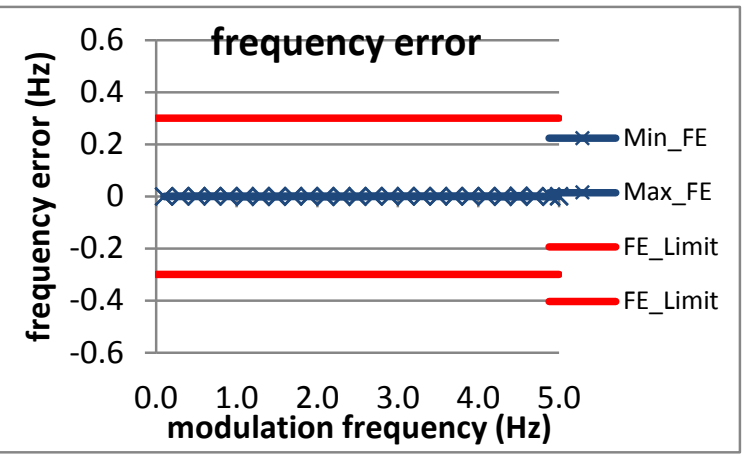

Figure 3642: Fs = 30 FPS

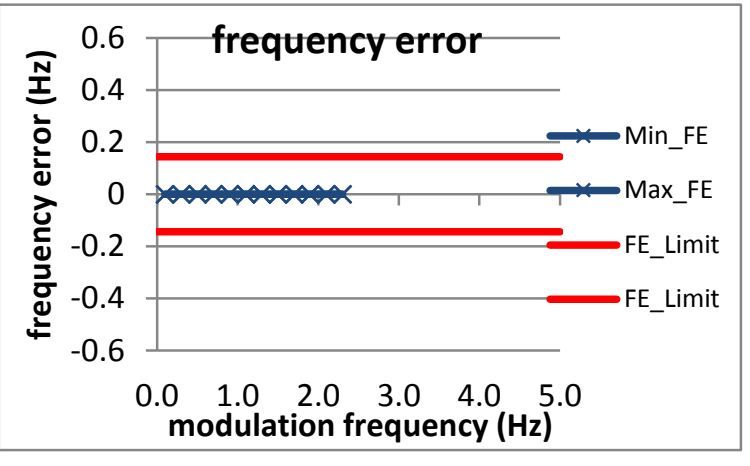

Figure 3645: Fs = 12 FPS

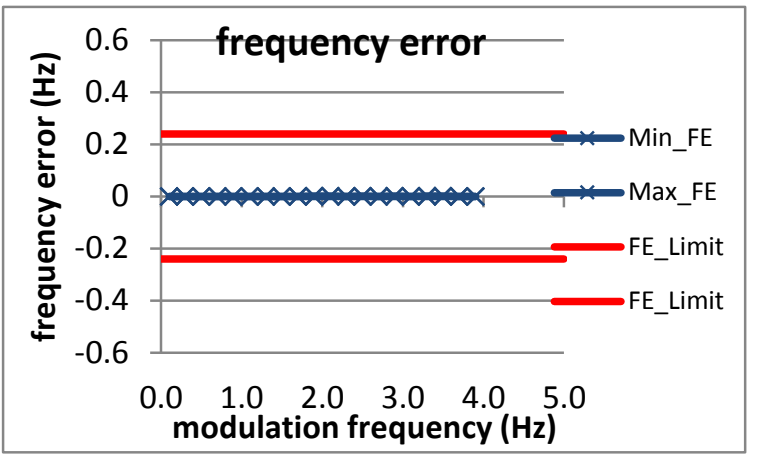

Figure 3643: Fs = 20 FPS

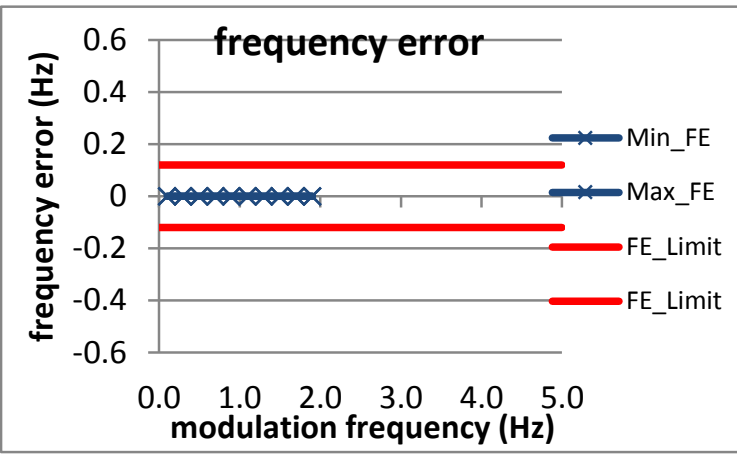

Figure 3646: Fs = 10 FPS 
8.3.3 PMU B amplitude modulation frequency error: M class

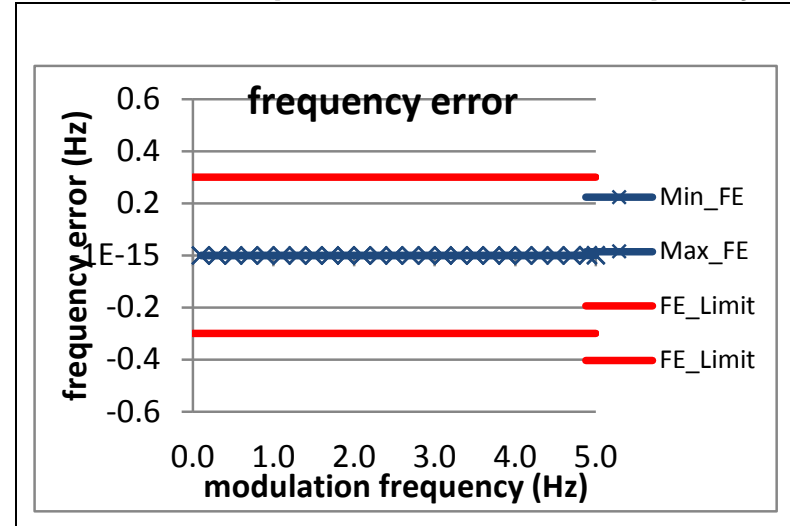

Figure 3647: Fs = 60 FPS

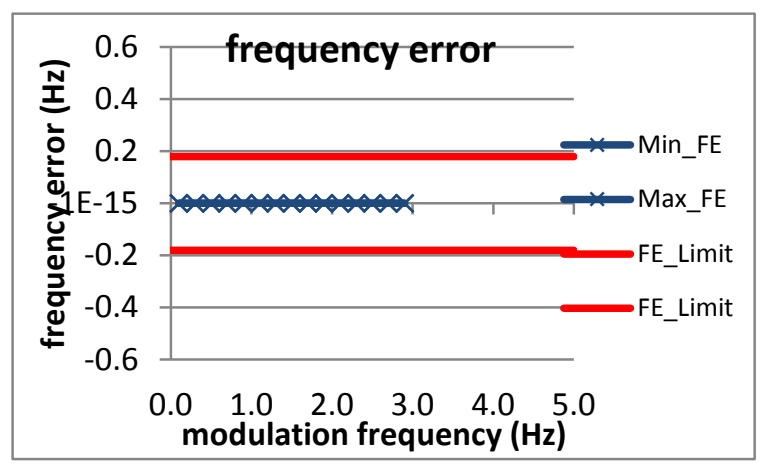

Figure 3650: Fs = 15 FPS

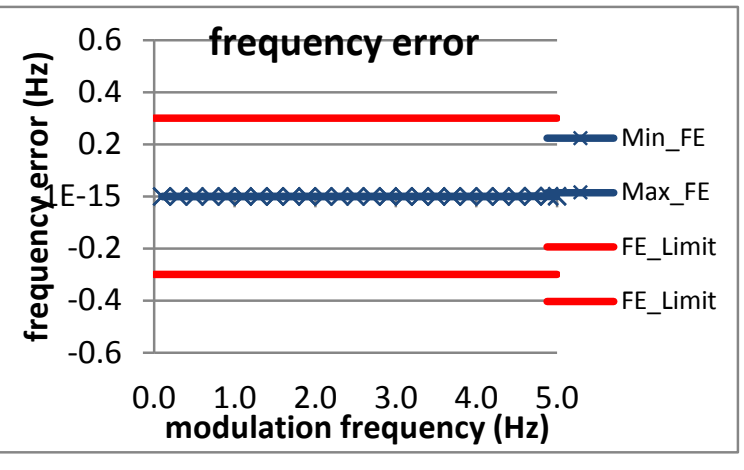

Figure 3648: Fs = 30 FPS

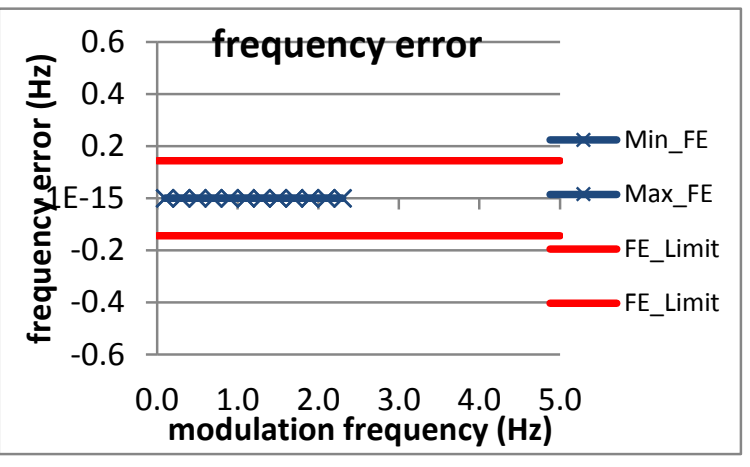

Figure 3651: Fs = 12 FPS

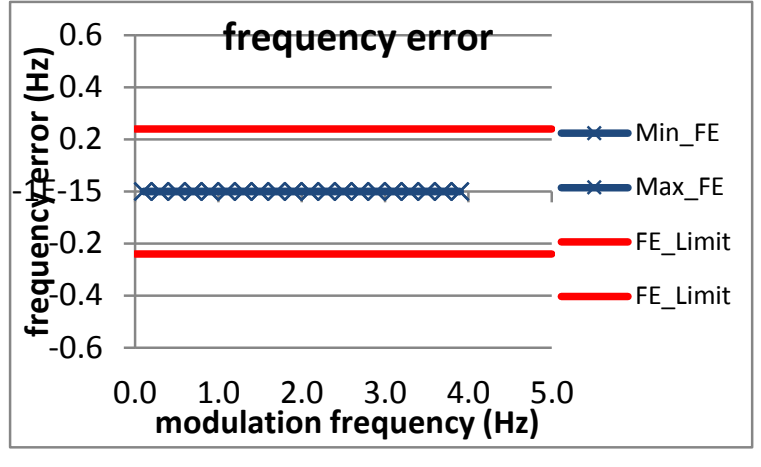

Figure 3649: Fs = 20 FPS

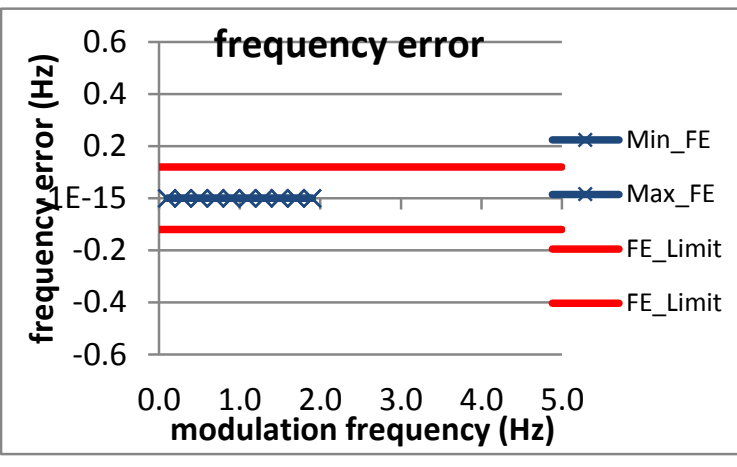

Figure 3652: Fs = 10 FPS 
8.3.4 PMU C amplitude modulation frequency error: M class

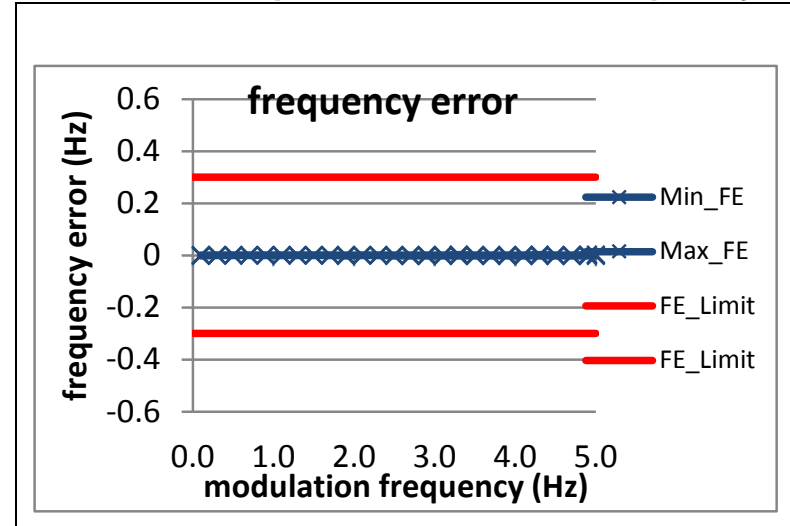

Figure 3653: Fs = 60 FPS

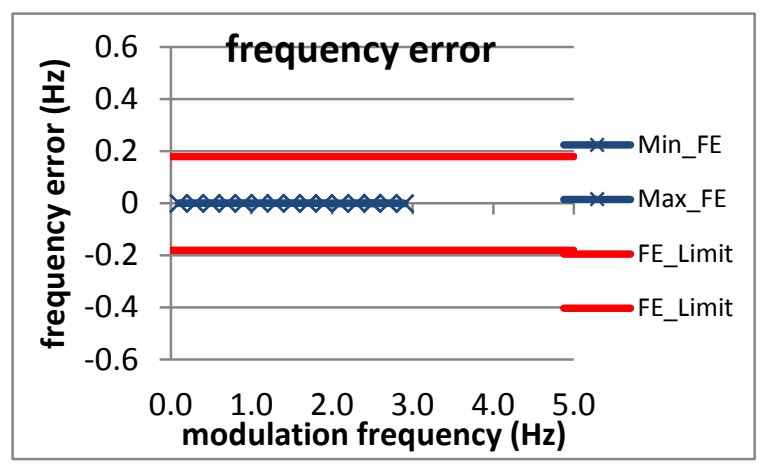

Figure 3656: Fs = 15 FPS

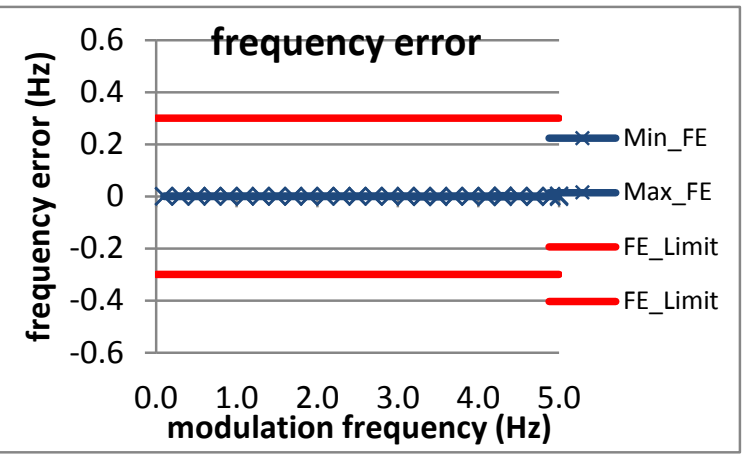

Figure 3654: Fs = 30 FPS

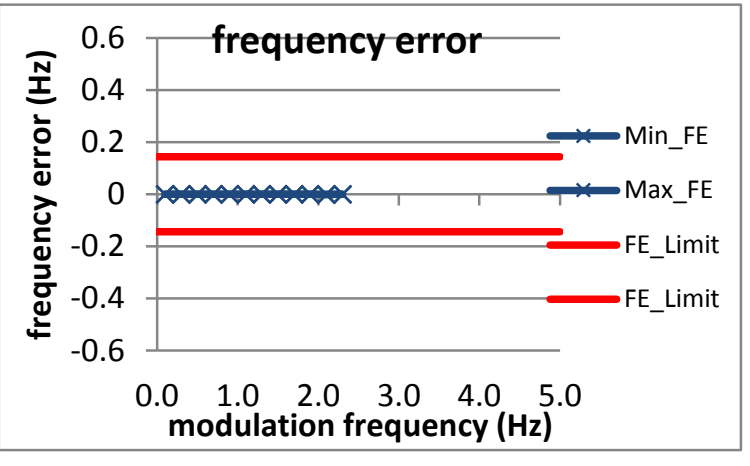

Figure 3657: Fs = 12 FPS

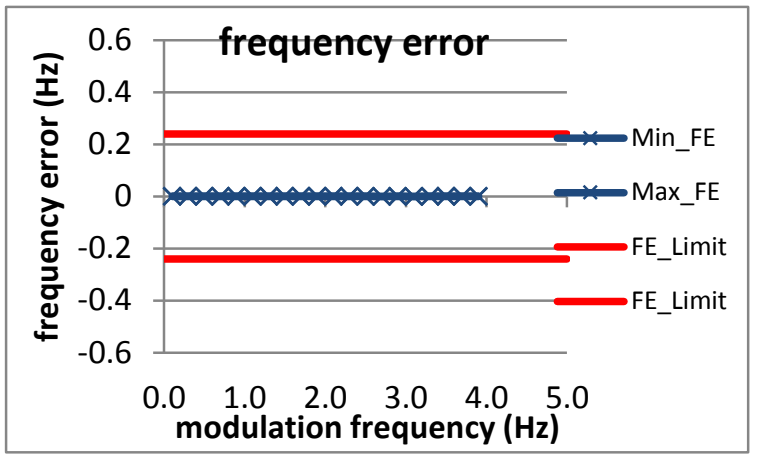

Figure 3655: Fs = 20 FPS

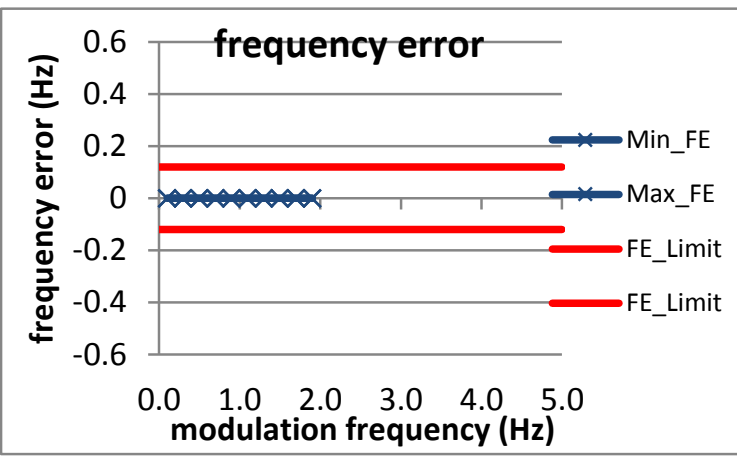

Figure 3658: Fs = 10 FPS 
8.3.5 PMU D amplitude modulation frequency error: $M$ class

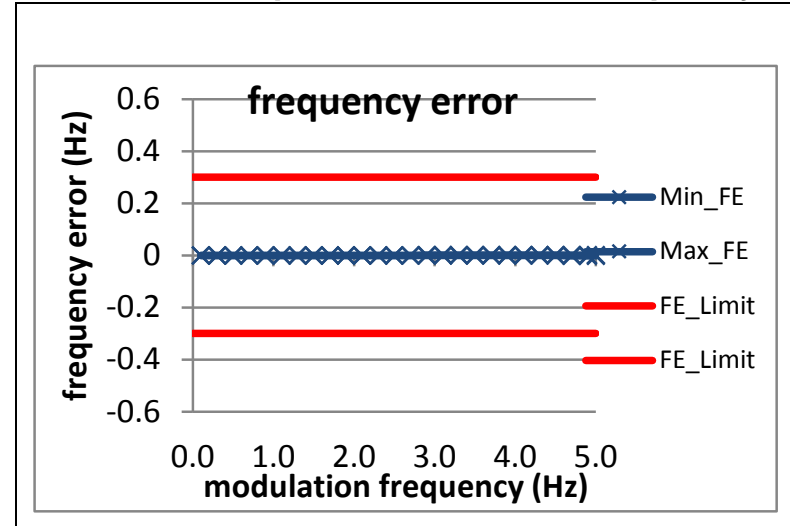

Figure 3659: Fs = 60 FPS

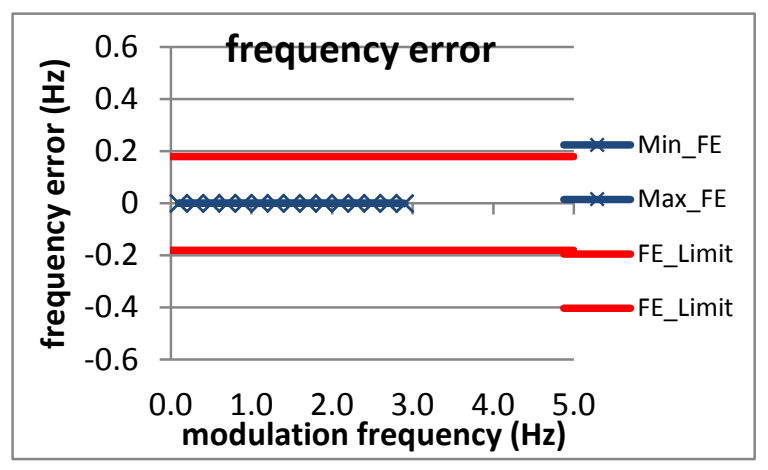

Figure 3662: Fs = 15 FPS

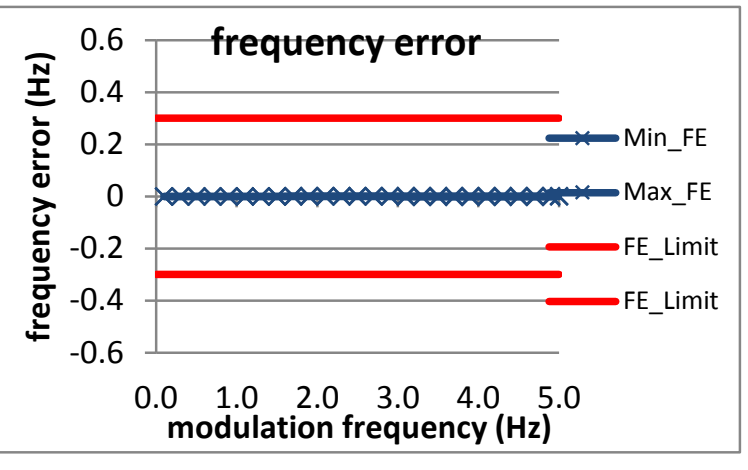

Figure 3660: Fs = 30 FPS

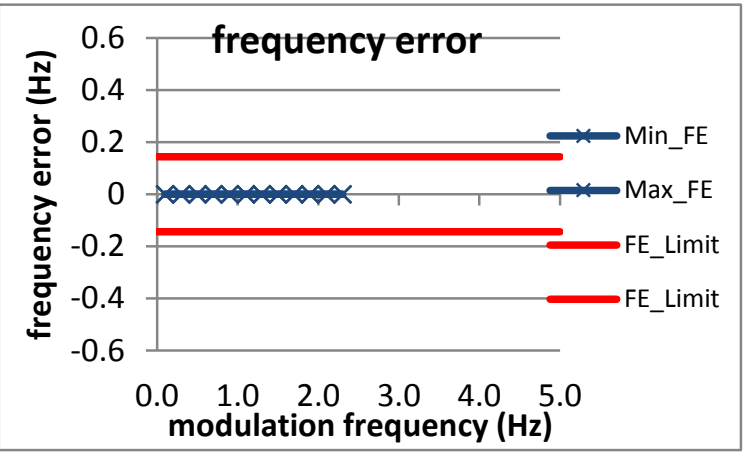

Figure 3663: Fs = 12 FPS

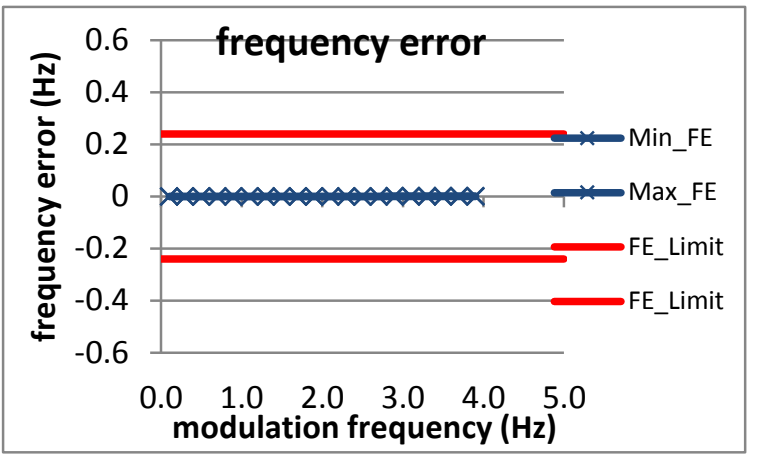

Figure 3661: Fs = 20 FPS

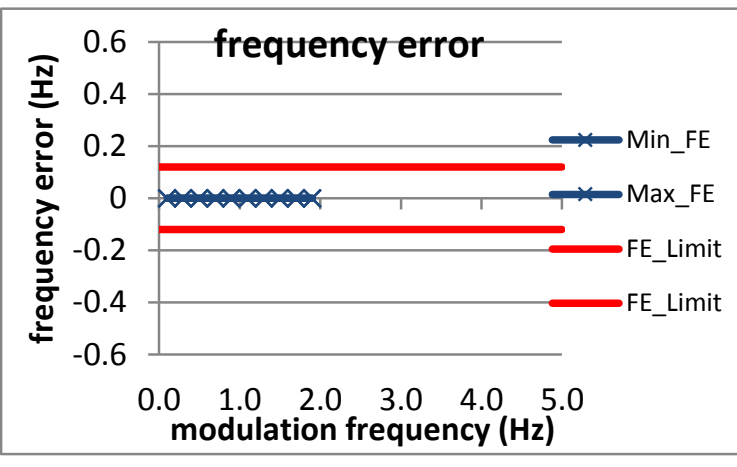

Figure 3664: Fs = 10 FPS 
8.3.6 PMU E amplitude modulation frequency error: M class

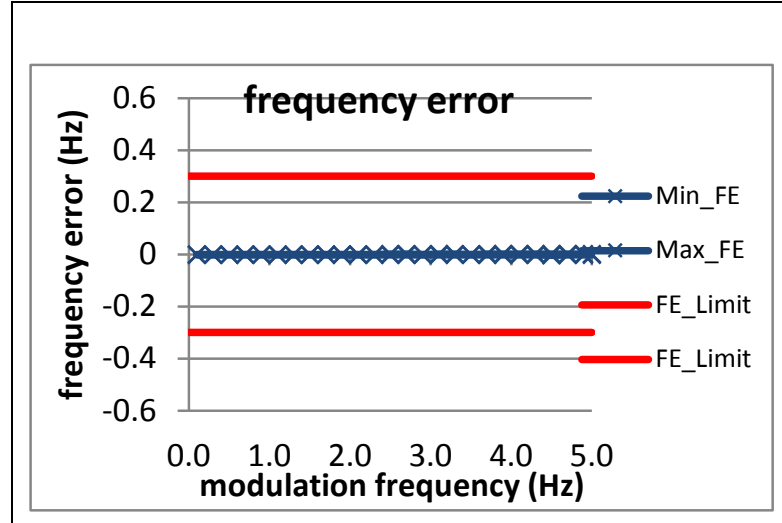

Figure 3665: Fs = 60 FPS

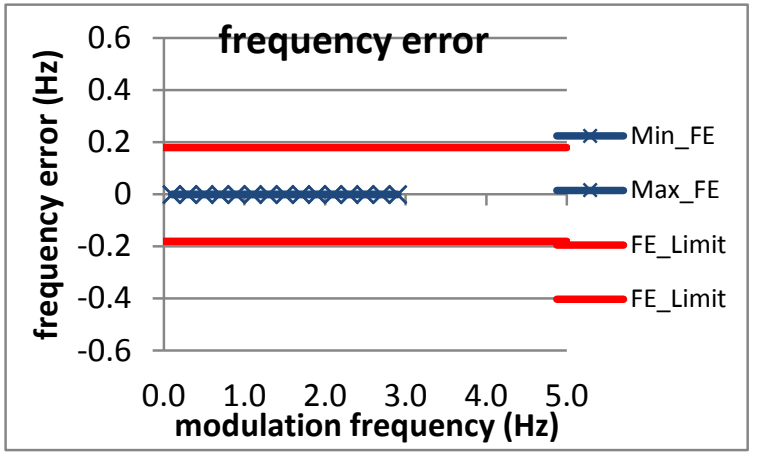

Figure 3668: Fs = 15 FPS

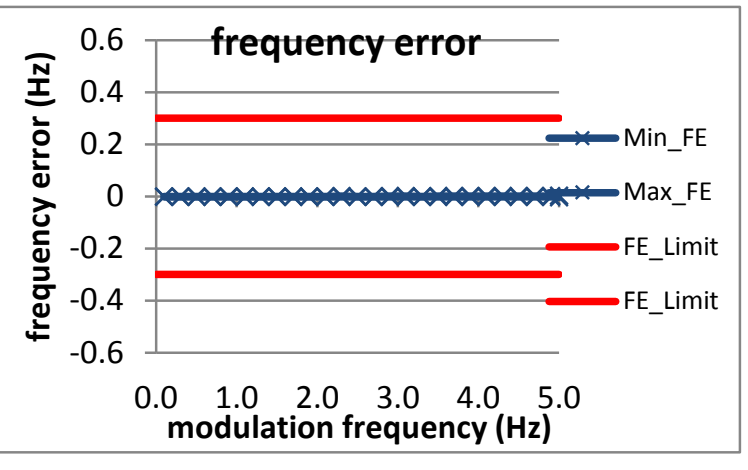

Figure 3666: Fs = 30 FPS

Figure 3669: Fs = 12 FPS data was lost

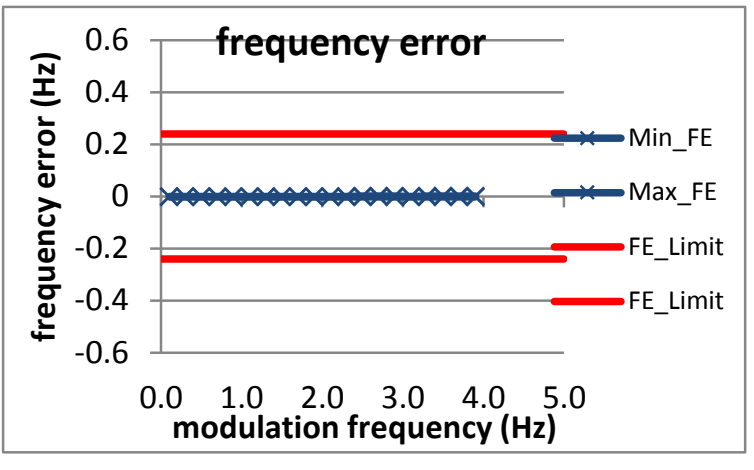

Figure 3667: Fs $=20$ FPS

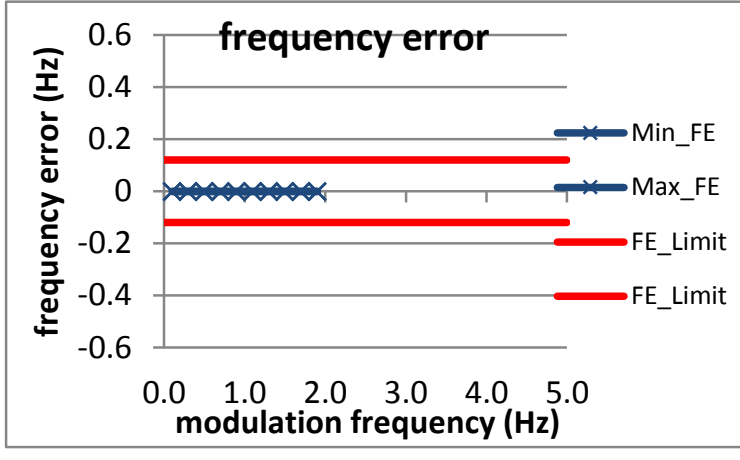

Figure 3670: Fs = 10 FPS 
8.3.7 PMU F amplitude modulation frequency error: M class

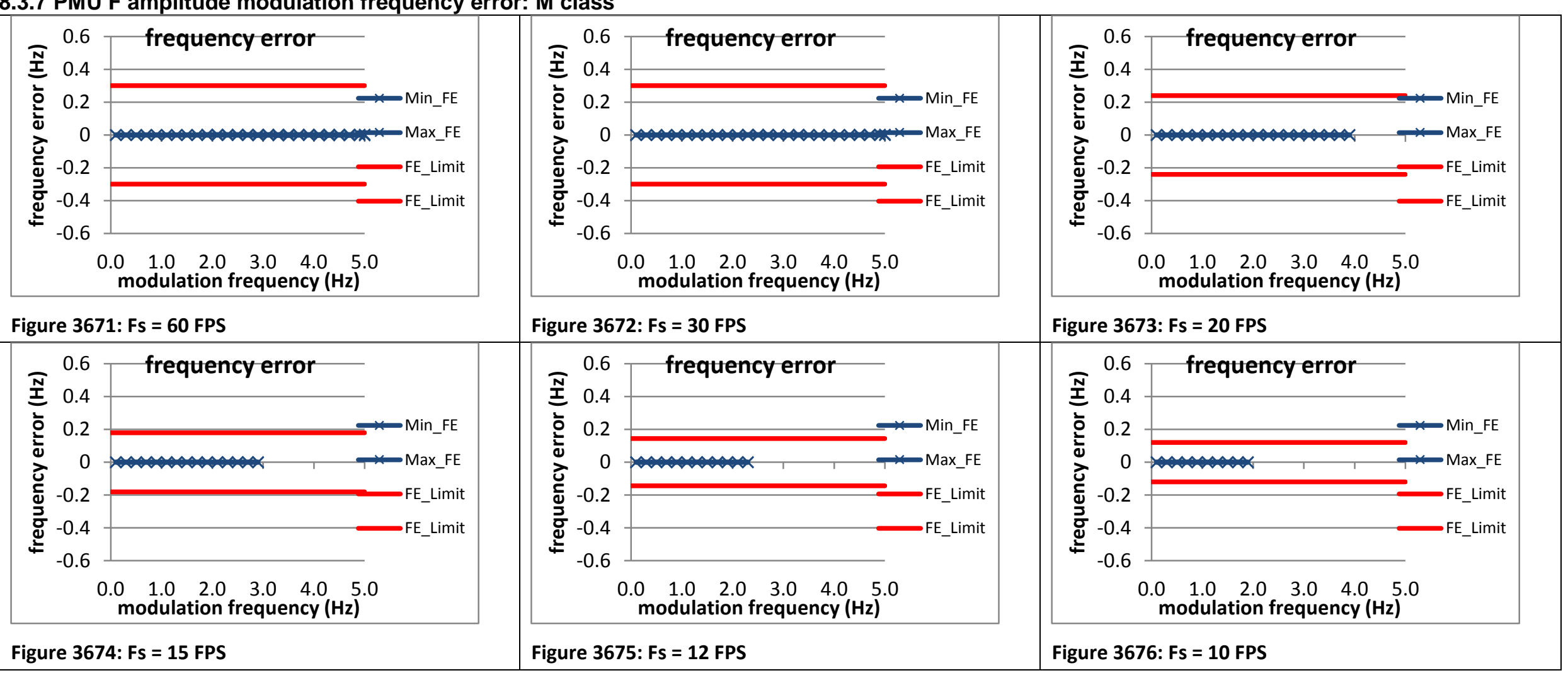


8.3.8 PMU G amplitude modulation frequency error: M class

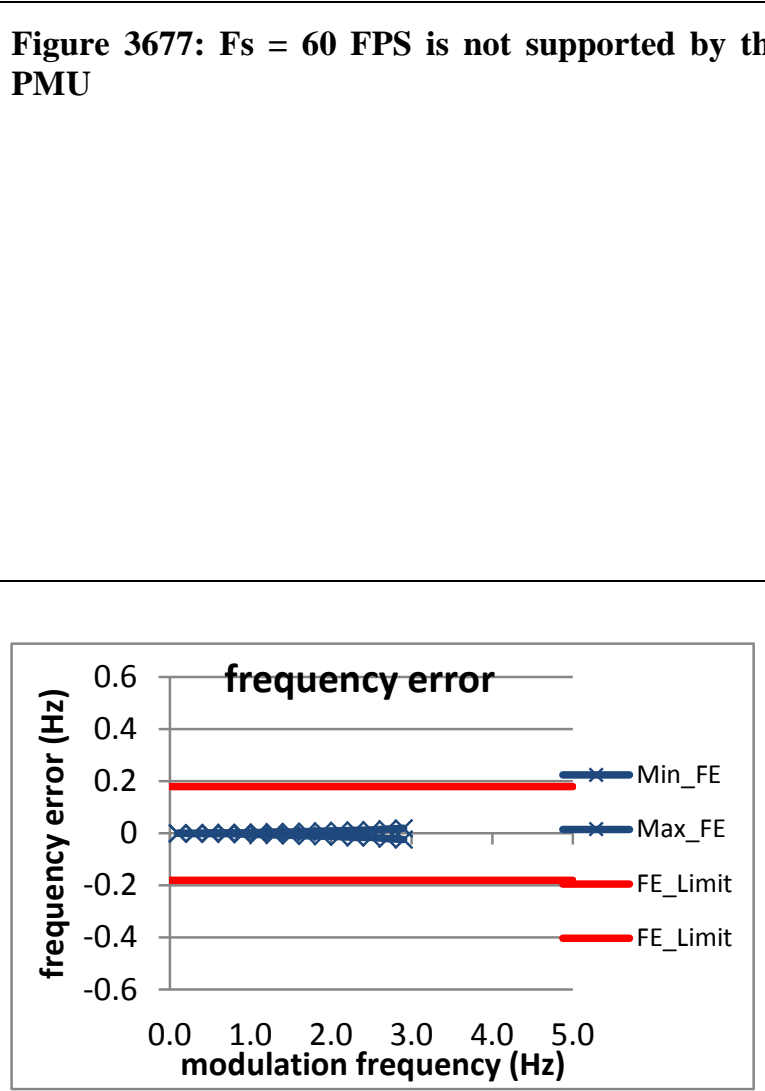

Figure 3680: Fs = 15 FPS

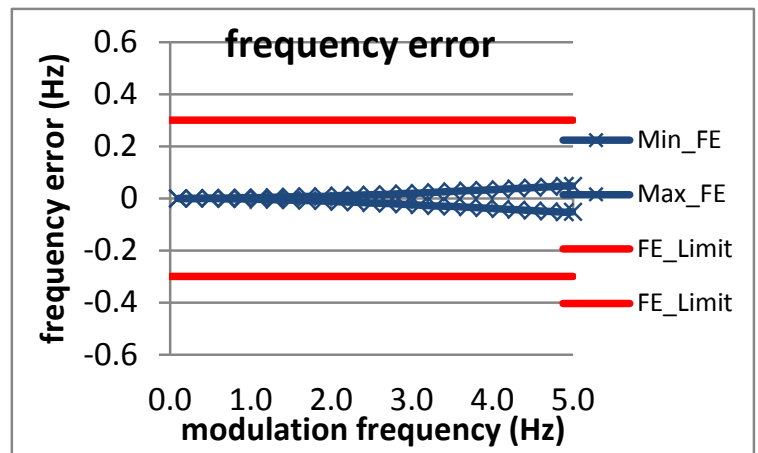

Figure 3678: Fs = 30 FPS

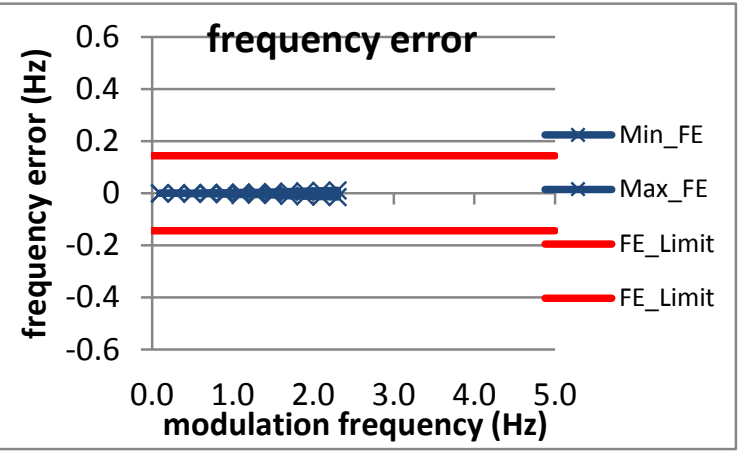

Figure 3681: Fs = 12 FPS

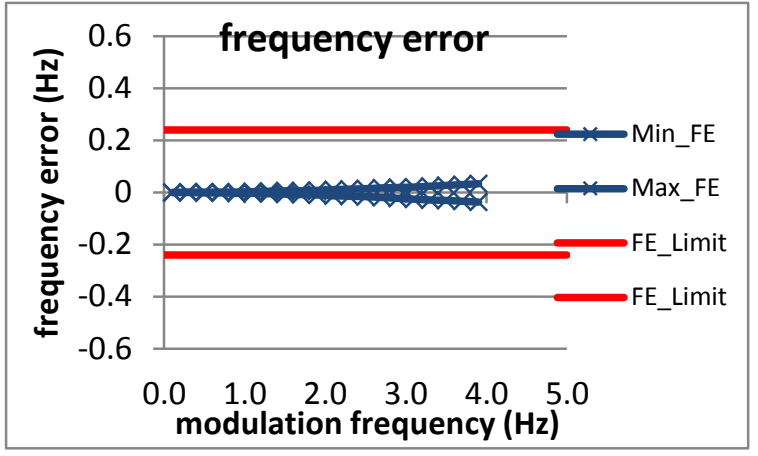

Figure 3679: Fs $=20$ FPS

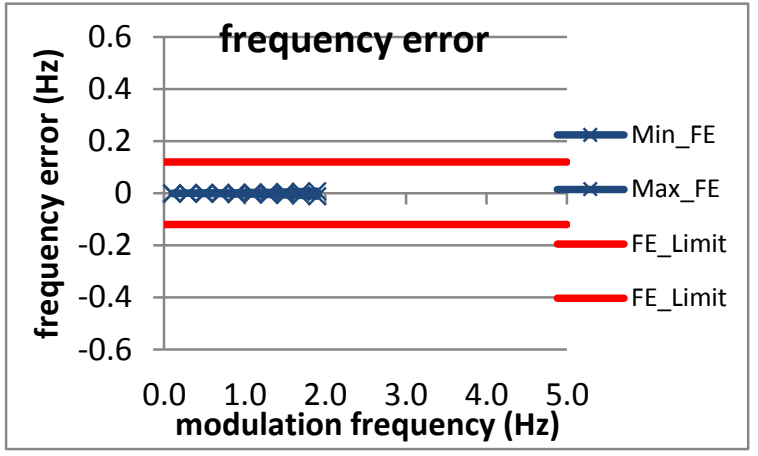

Figure 3682: Fs = 10 FPS 
8.3.9 PMU H amplitude modulation frequency error: $M$ class

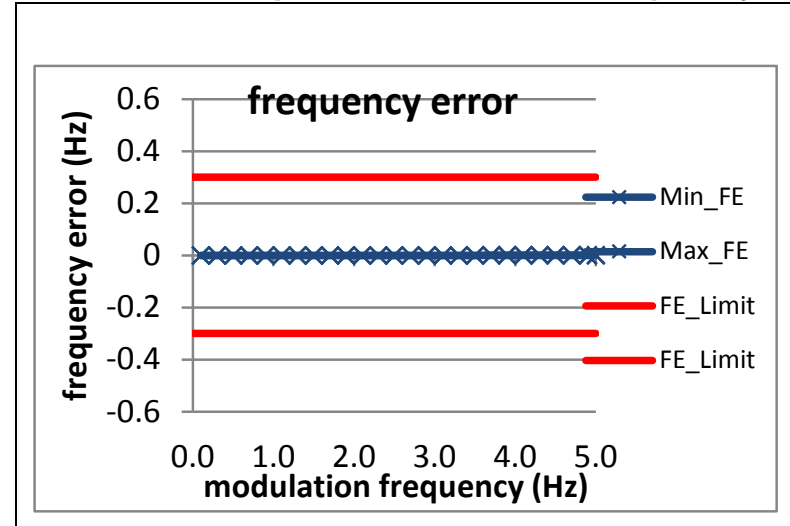

Figure 3683: Fs = 60 FPS

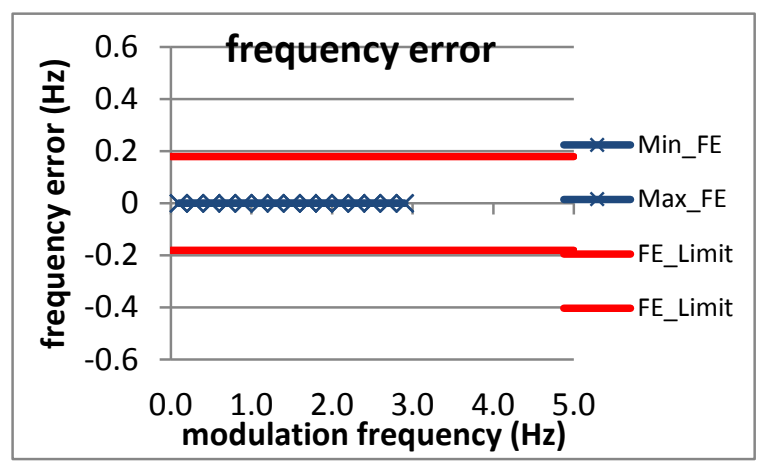

Figure 3686: Fs = 15 FPS

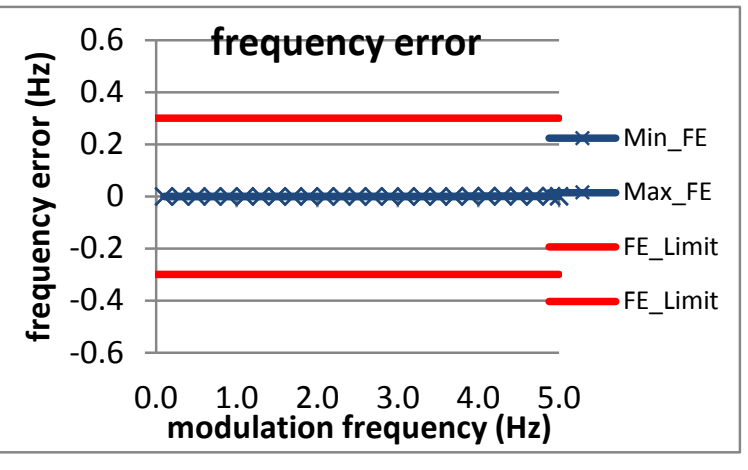

Figure 3684: Fs = 30 FPS

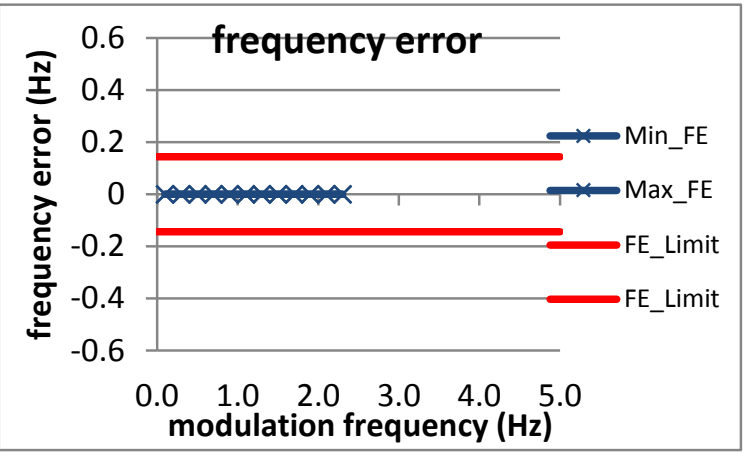

Figure 3687: Fs = 12 FPS

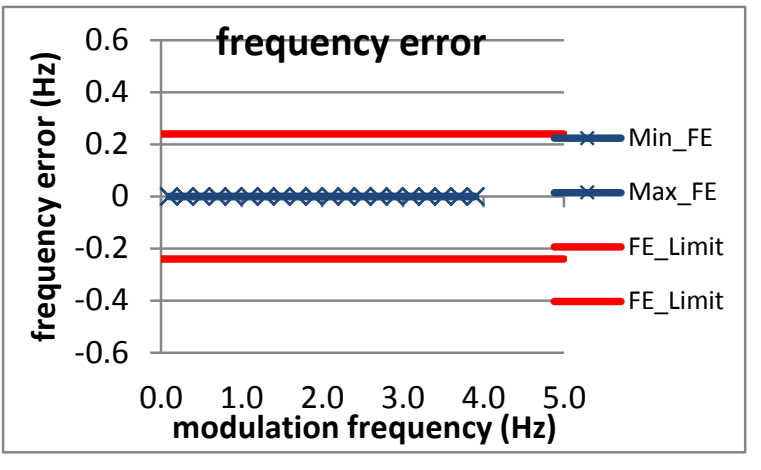

Figure 3685: Fs = 20 FPS

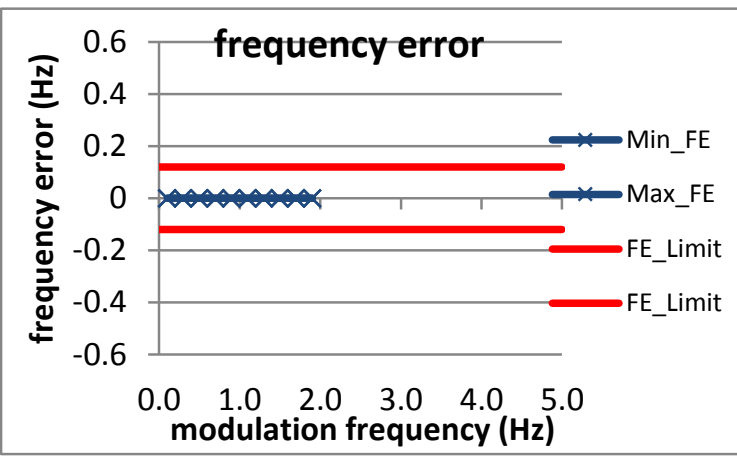

Figure 3688: Fs = 10 FPS 


\subsubsection{PMU I amplitude modulation frequency error: M class}

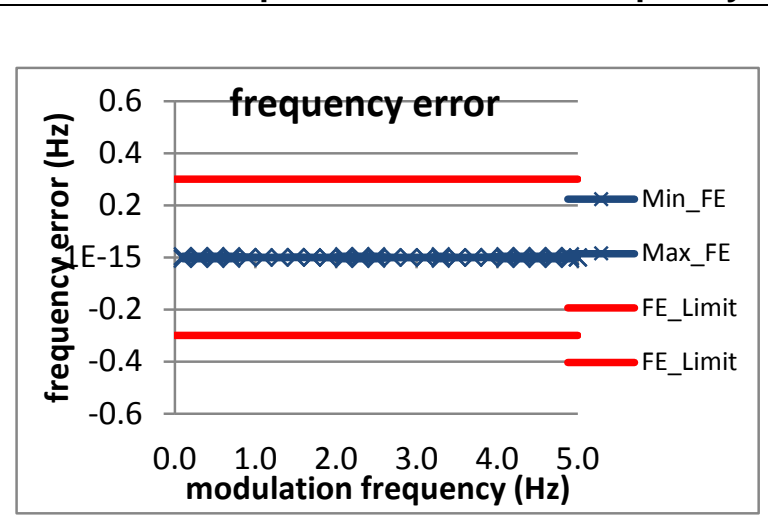

Figure 3689: Fs = 60 FPS

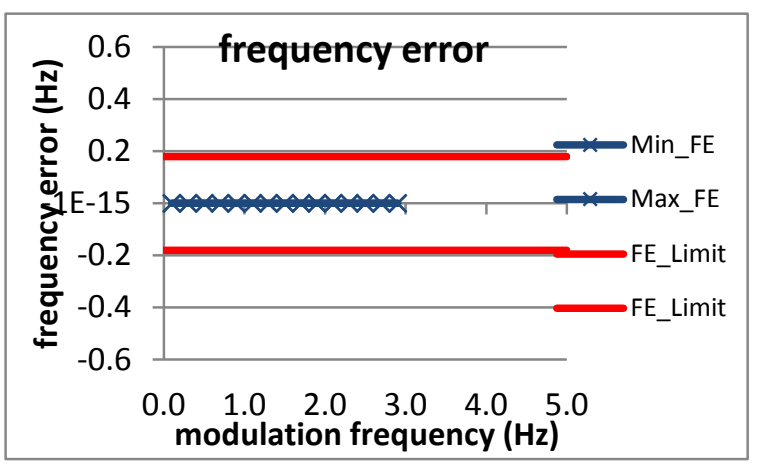

Figure 3692: Fs = 15 FPS

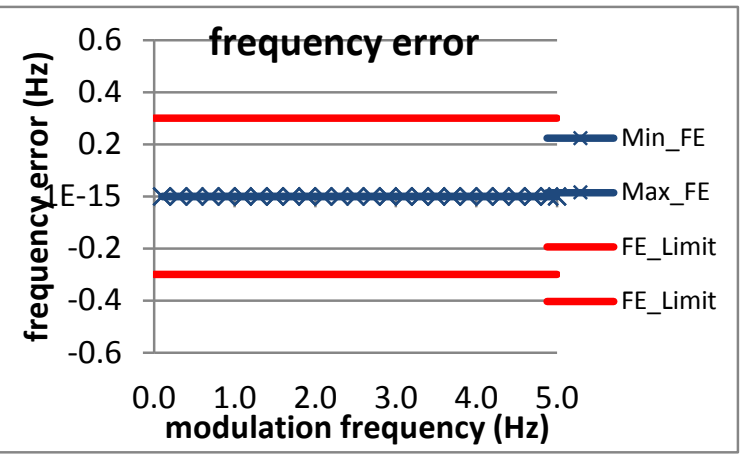

Figure 3690: Fs = 30 FPS

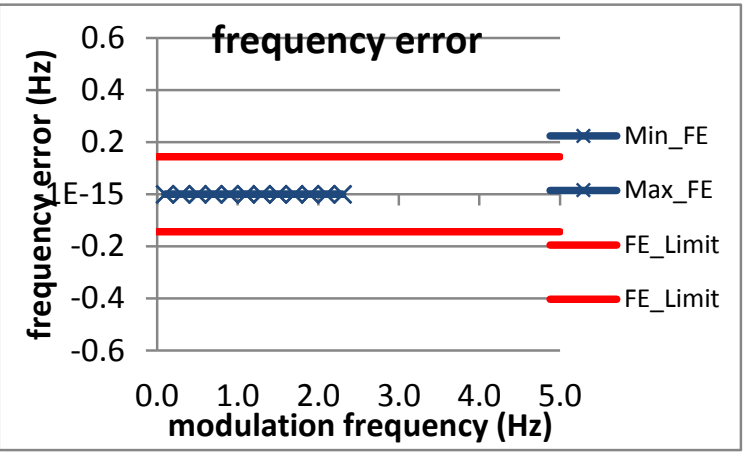

Figure 3693: Fs = 12 FPS

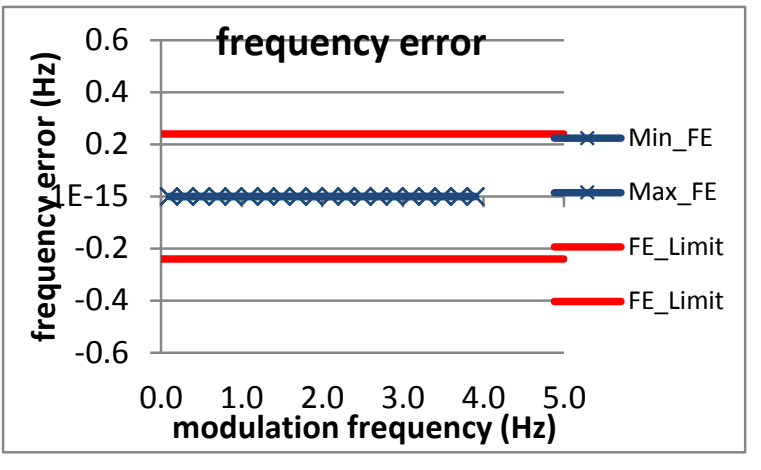

Figure 3691: Fs = 20 FPS

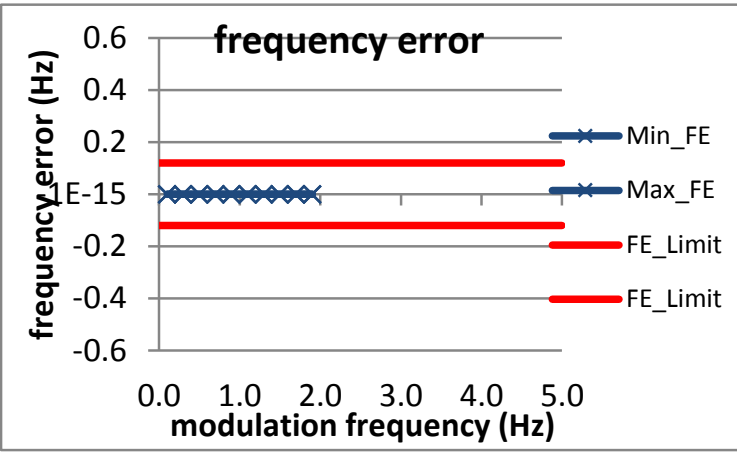

Figure 3694: Fs = 10 FPS 
8.3.11 PMU J amplitude modulation frequency error: $M$ class

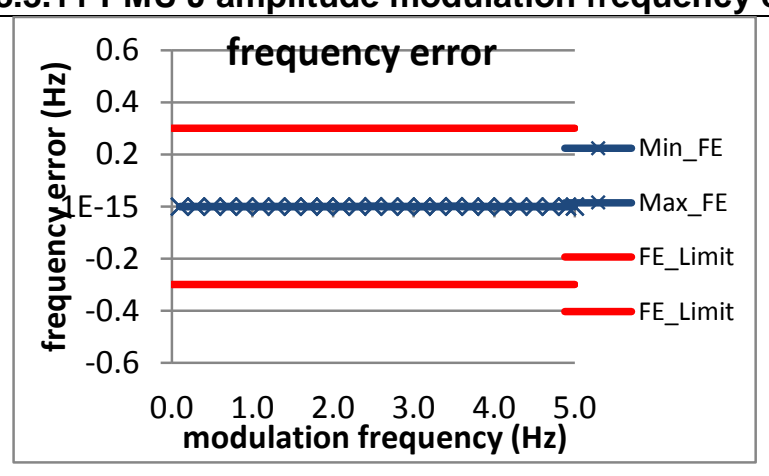

Figure 3695: Fs = 60 FPS

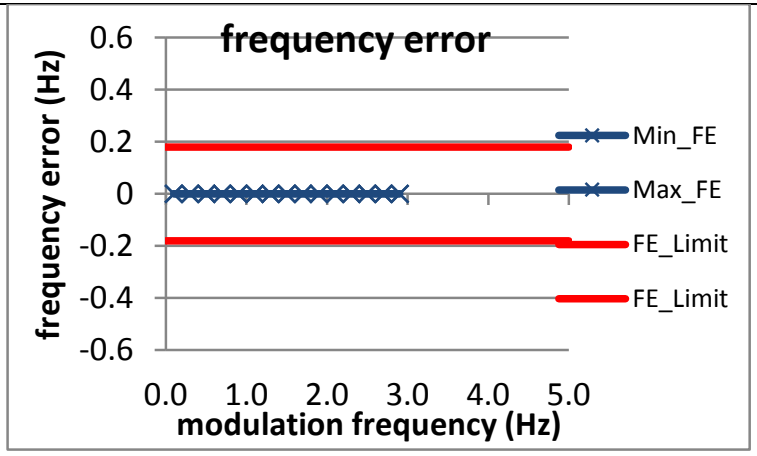

Figure 3698: Fs = 15 FPS

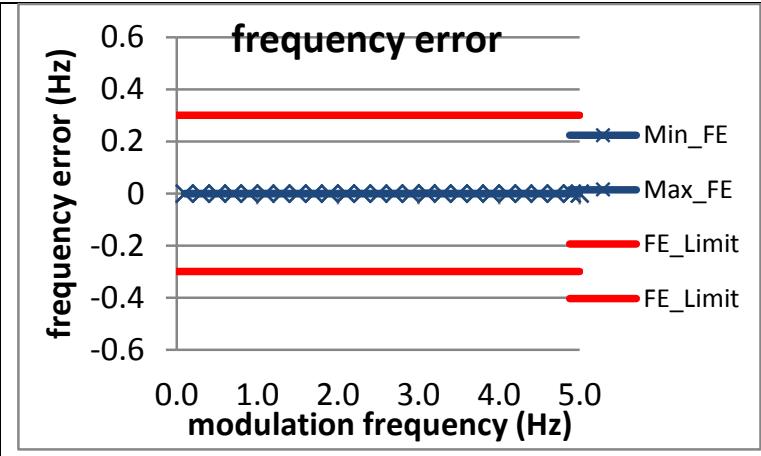

Figure 3696: Fs = 30 FPS

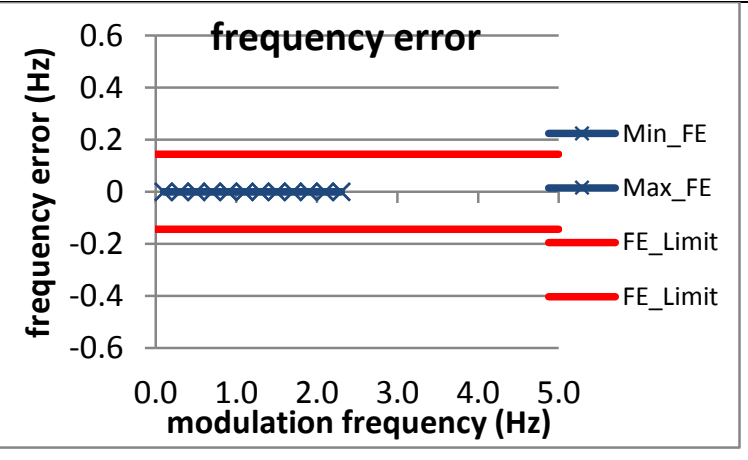

Figure 3699: Fs = 12 FPS

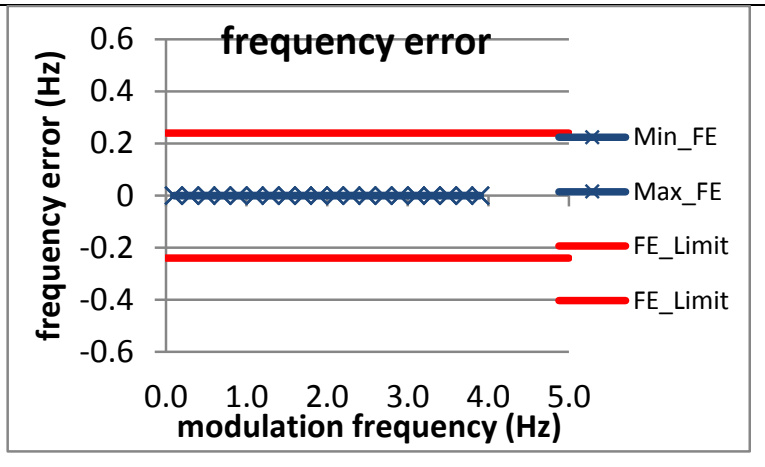

Figure 3697: Fs = 20 FPS

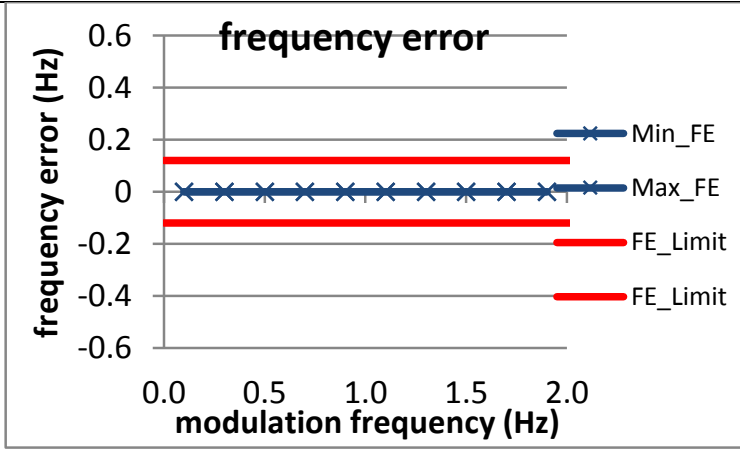

Figure 3700: Fs = 10 FPS 


\subsection{Amplitude modulation ROCOF error: M class}

\subsubsection{C37.118.1 Annex C amplitude modulation ROCOF error: M class}

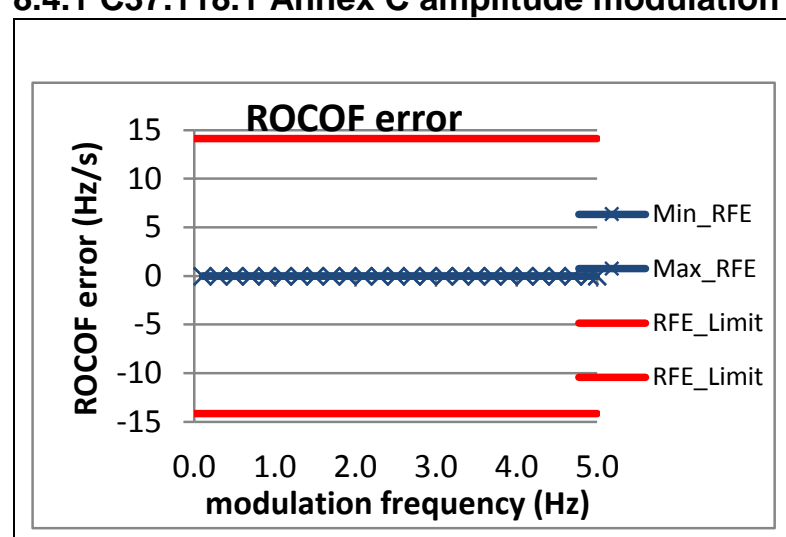

Figure 3701: Fs = 60 FPS

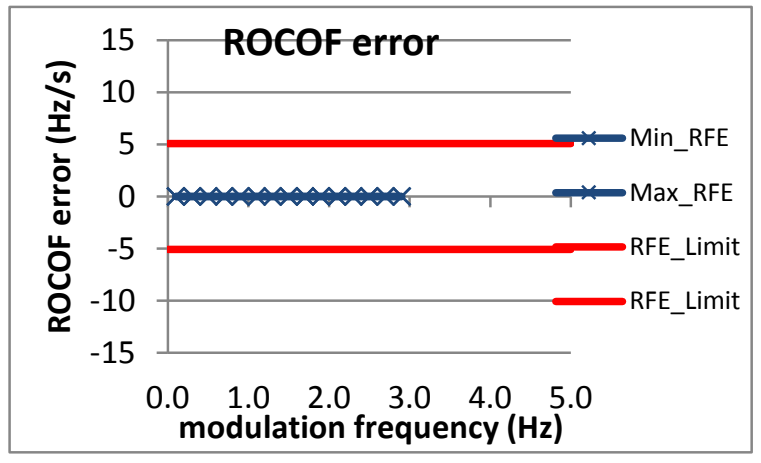

Figure 3704: Fs = 15 FPS

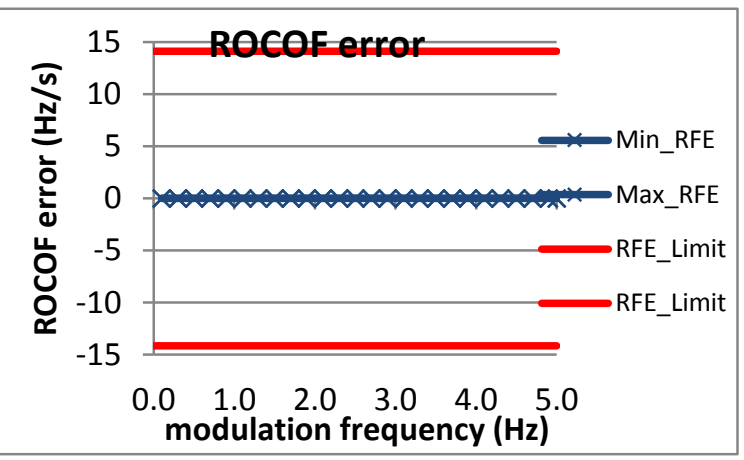

Figure 3702: Fs = 30 FPS

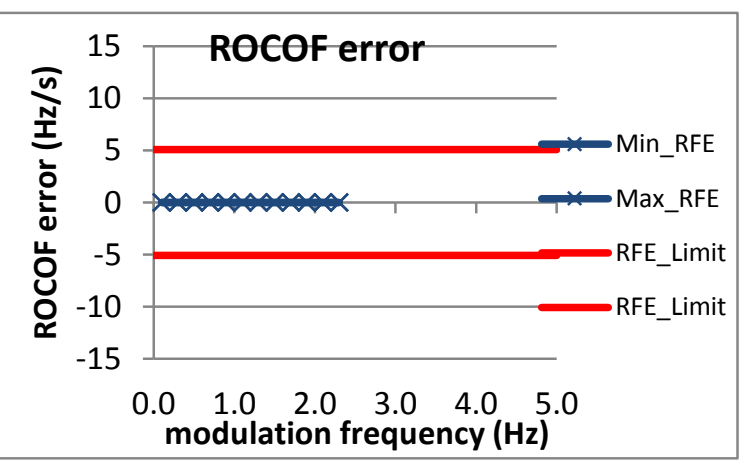

Figure 3705: Fs = 12 FPS

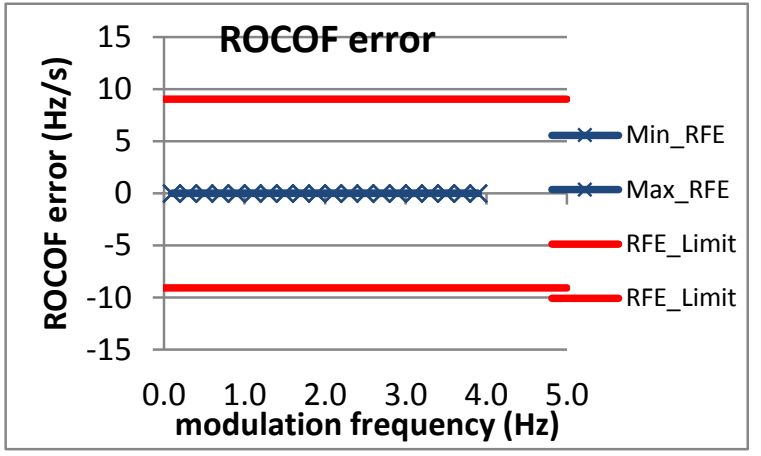

Figure 3703: Fs = 20 FPS

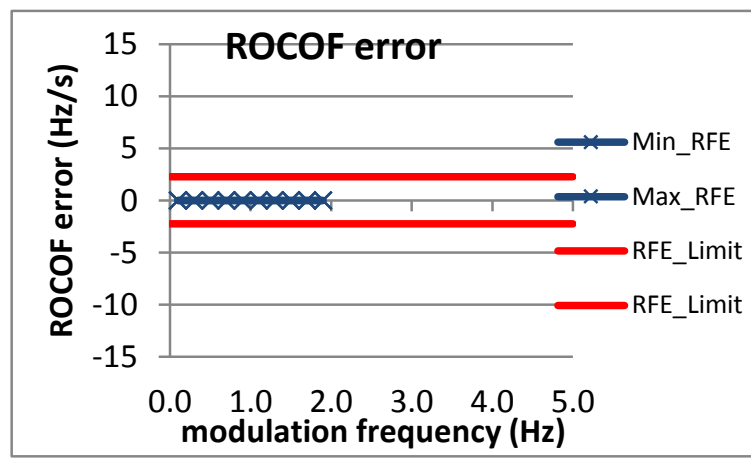

Figure 3706: Fs = 10 FPS 
8.4.2 PMU A amplitude modulation ROCOF error: M class

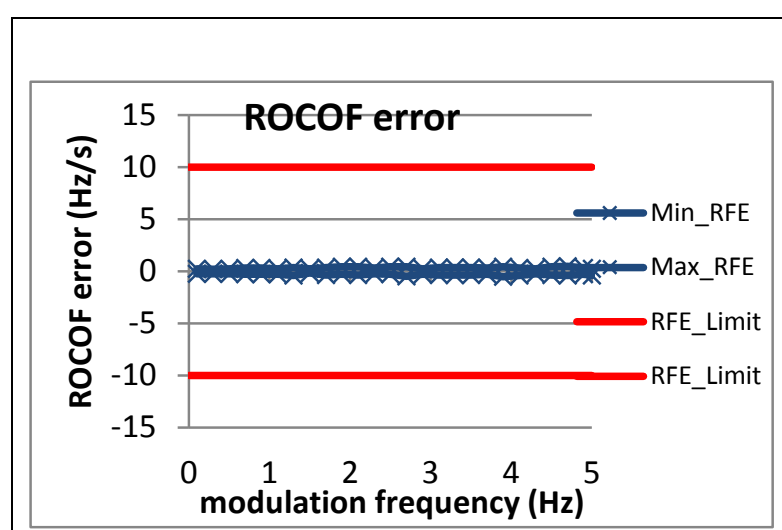

Figure 3707: Fs = 60 FPS

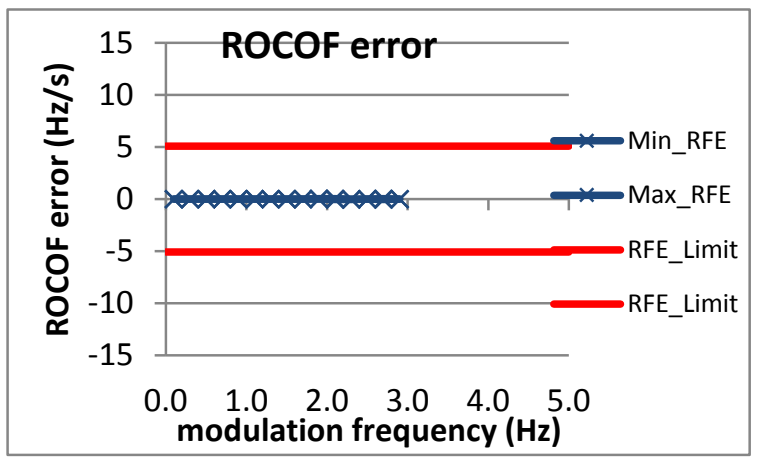

Figure 3710: Fs = 15 FPS

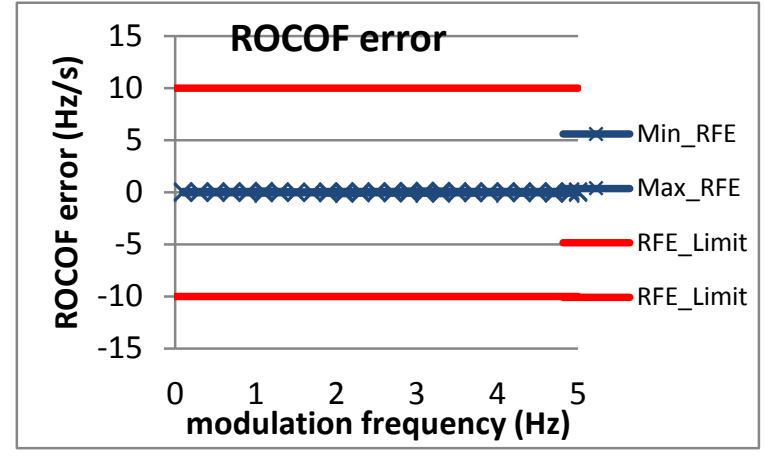

Figure 3708: Fs = 30 FPS

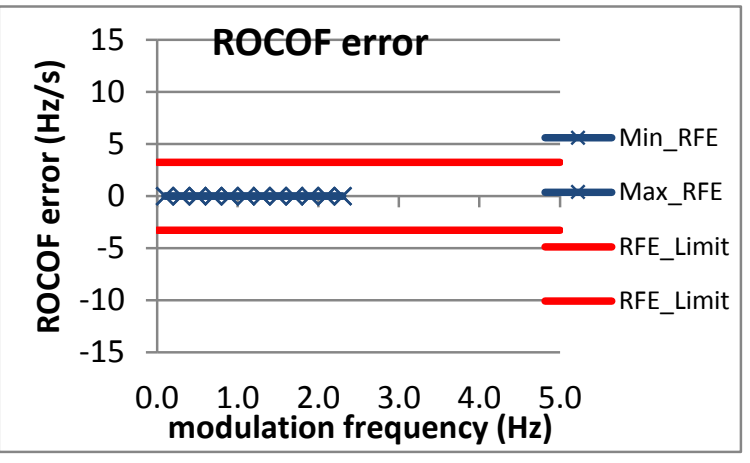

Figure 3711: Fs = 12 FPS

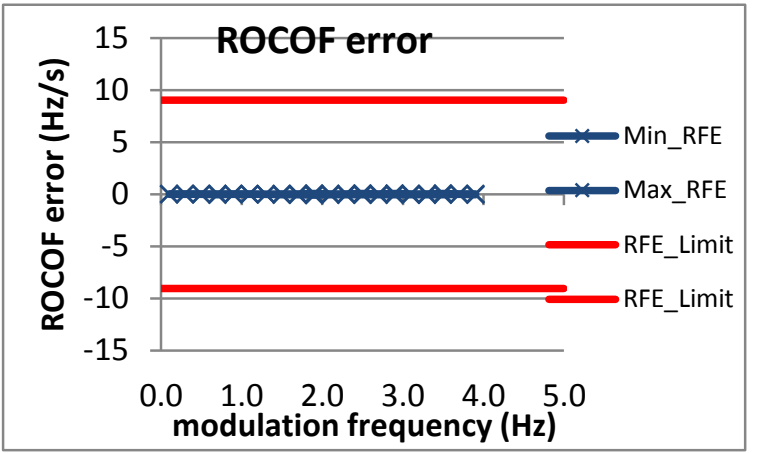

Figure 3709: Fs = 20 FPS

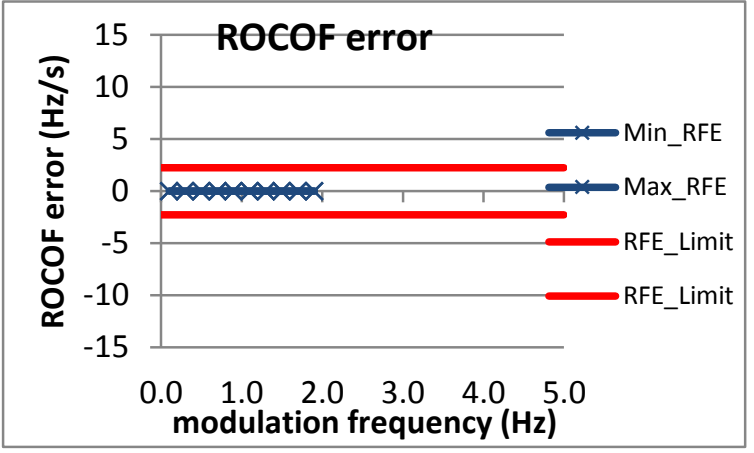

Figure 3712: Fs = 10 FPS 
8.4.3 PMU B amplitude modulation ROCOF error: M class

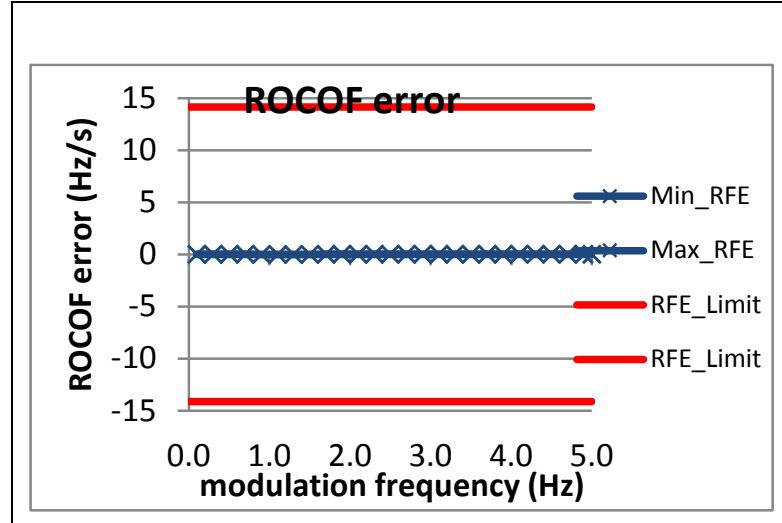

Figure 3713: Fs = 60 FPS

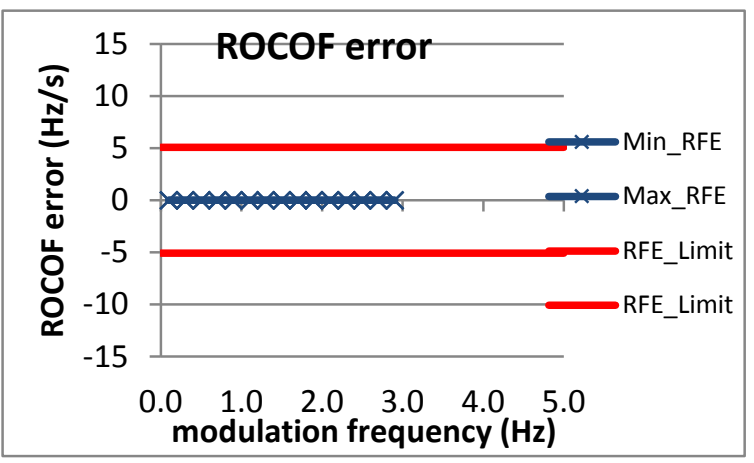

Figure 3716: Fs = 15 FPS

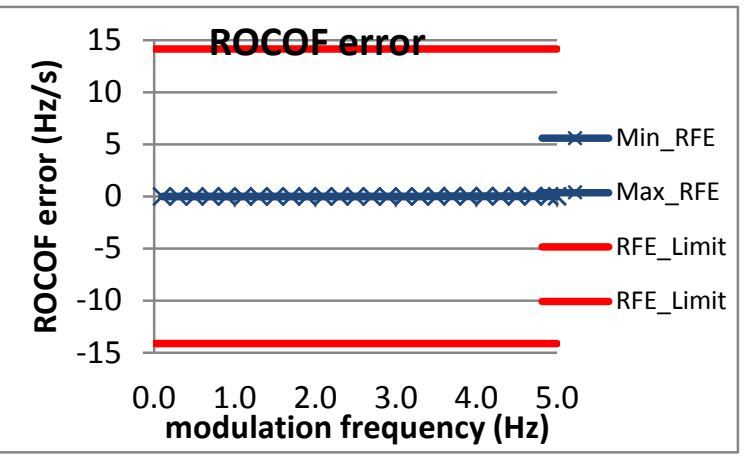

Figure 3714: Fs = 30 FPS

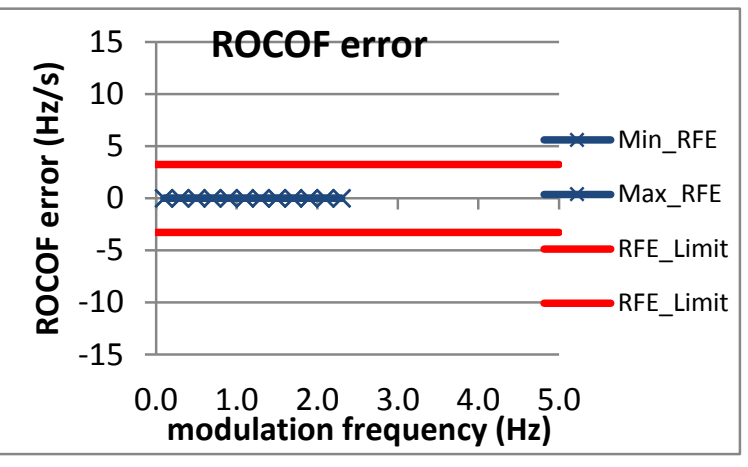

Figure 3717: Fs = 12 FPS

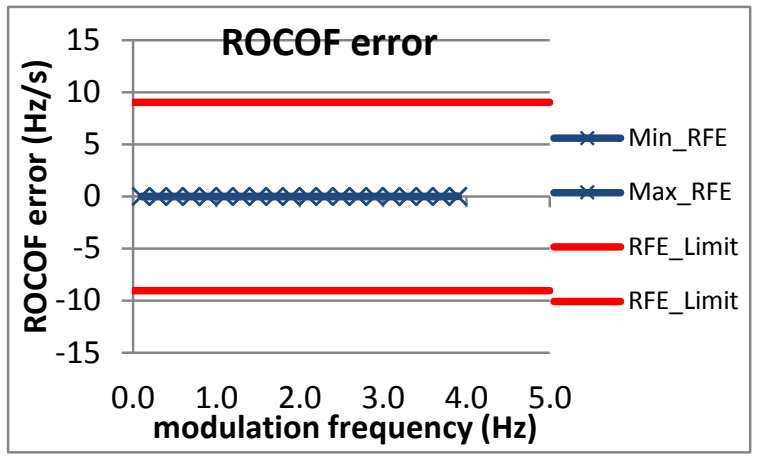

Figure 3715: Fs = 20 FPS

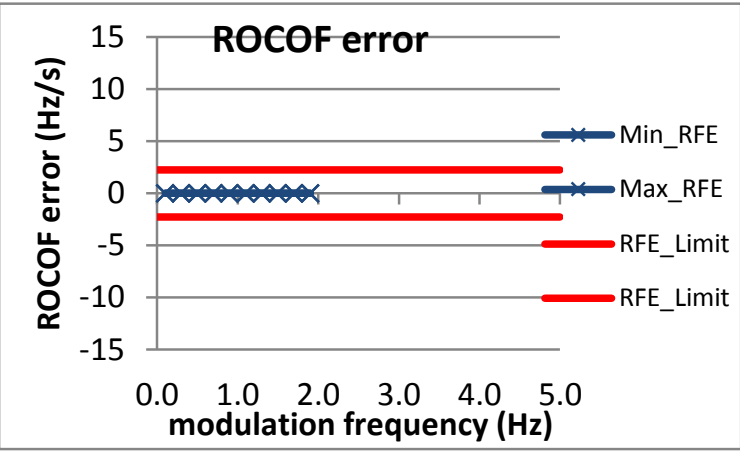

Figure 3718: Fs = 10 FPS 
8.4.4 PMU C amplitude modulation ROCOF error: M class

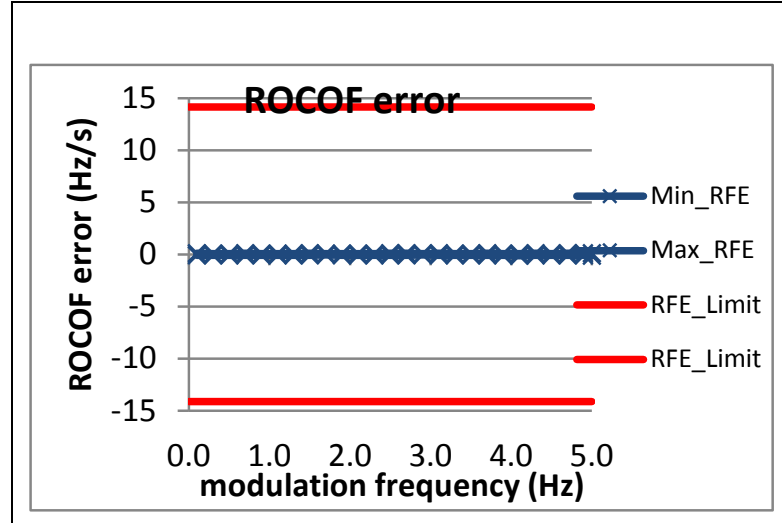

Figure 3719: Fs = 60 FPS

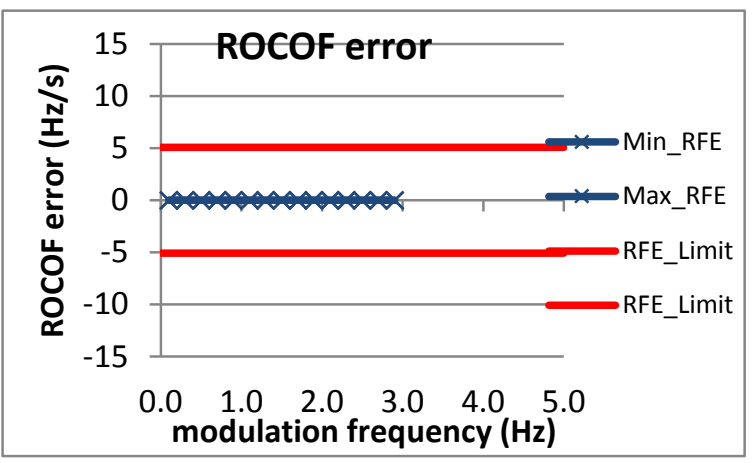

Figure 3722: Fs = 15 FPS

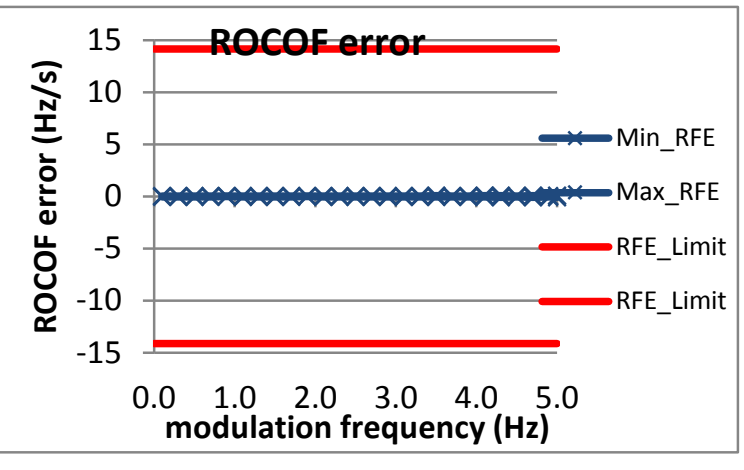

Figure 3720: Fs = 30 FPS

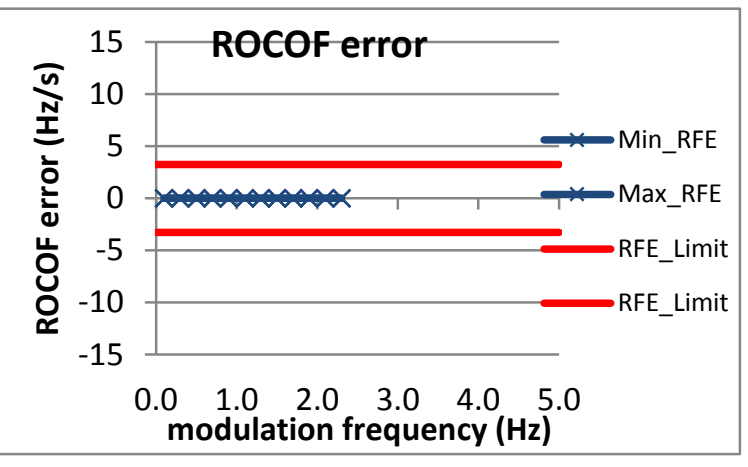

Figure 3723: Fs = 12 FPS

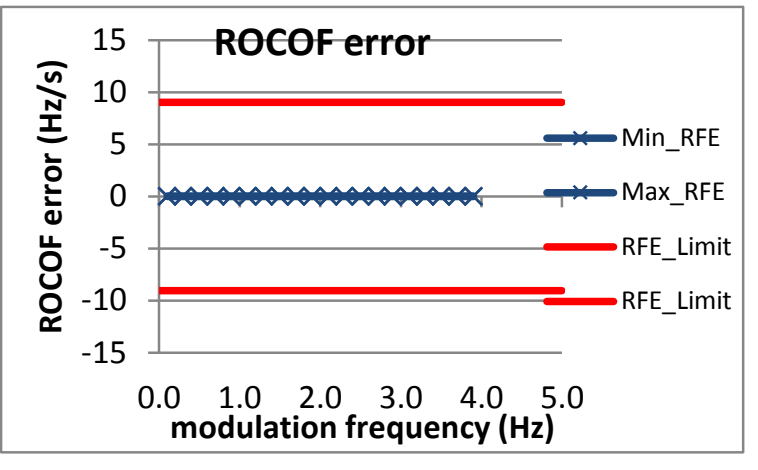

Figure 3721: Fs = 20 FPS

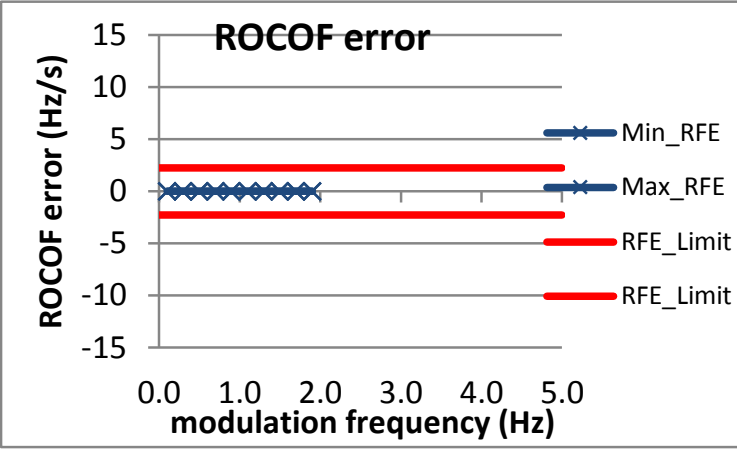

Figure 3724: Fs = 10 FPS 
8.4.5 PMU D amplitude modulation ROCOF error: M class

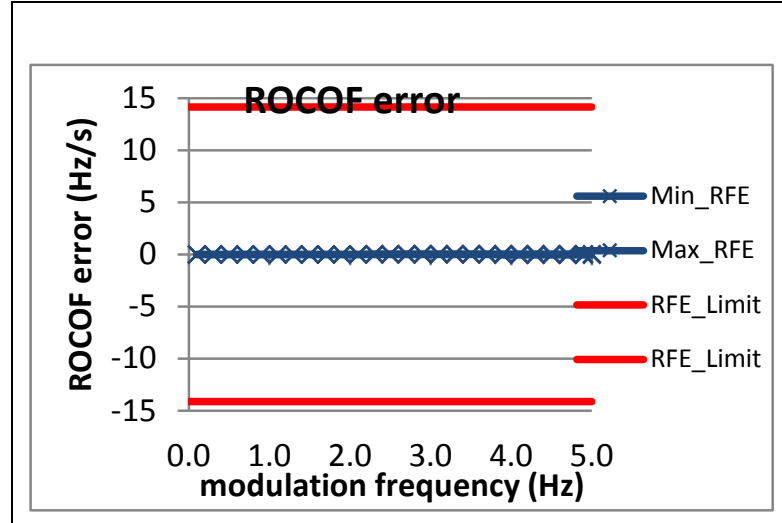

Figure 3725: Fs = 60 FPS

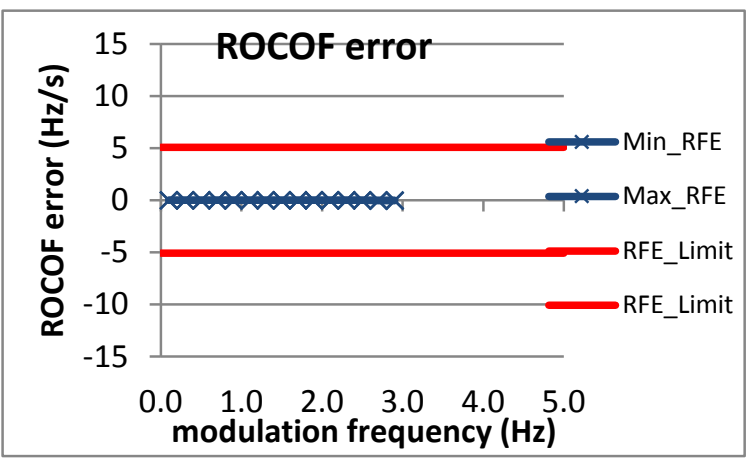

Figure 3728: Fs = 15 FPS

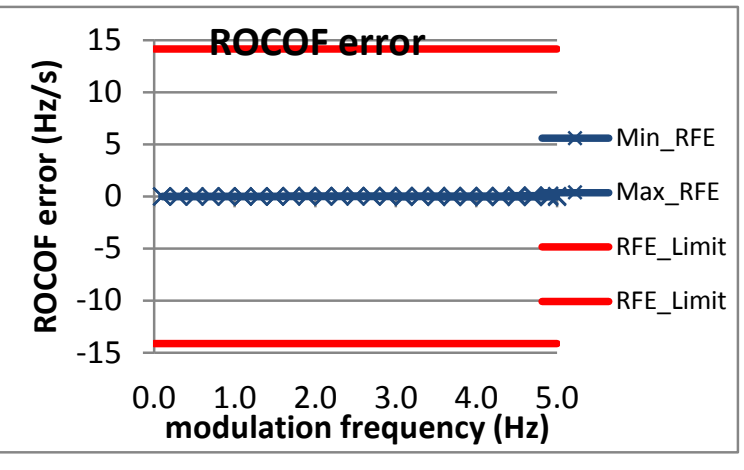

Figure 3726: Fs = 30 FPS

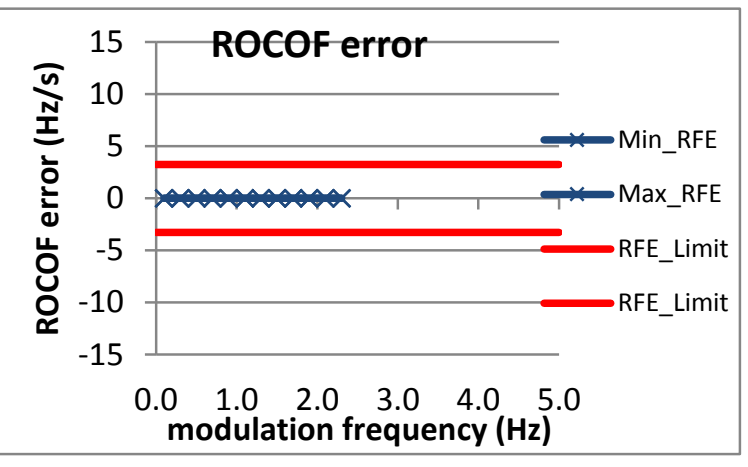

Figure 3729: Fs = 12 FPS

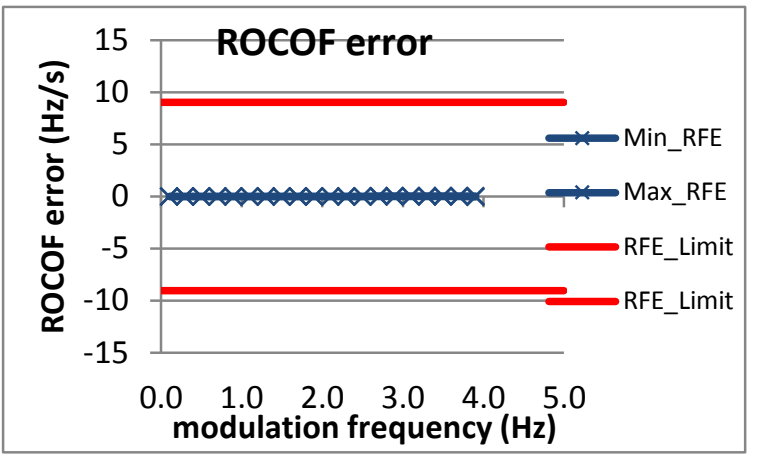

Figure 3727: Fs $=20$ FPS

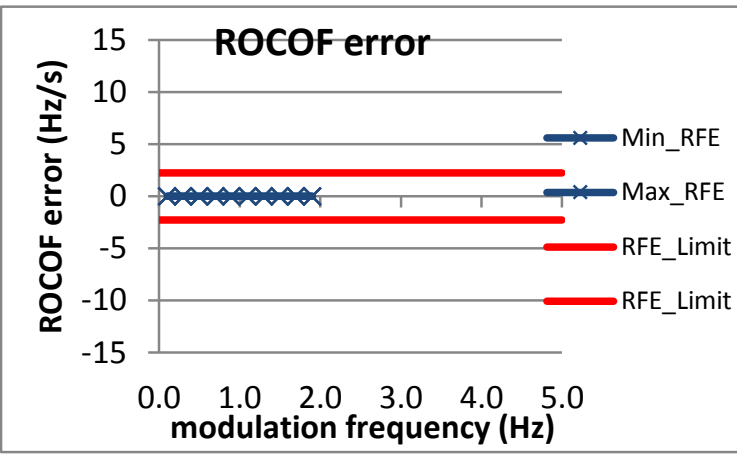

Figure 3730: Fs = 10 FPS 
8.4.6 PMU E amplitude modulation ROCOF error: M class

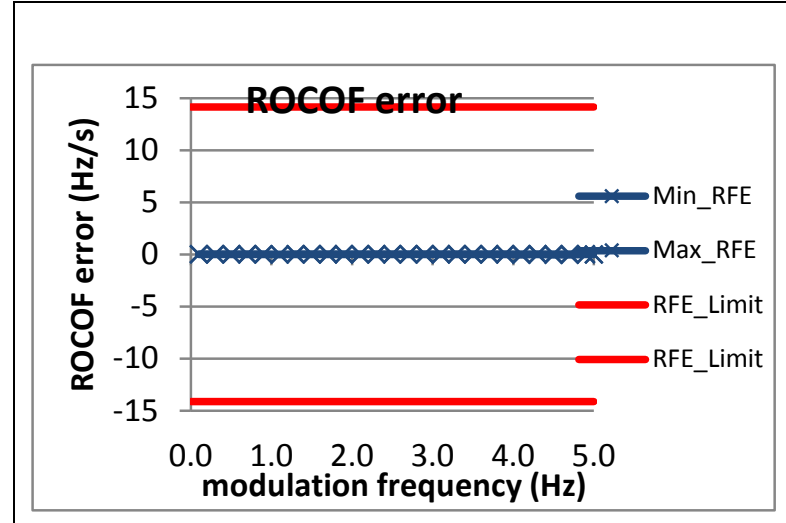

Figure 3731: Fs = 60 FPS

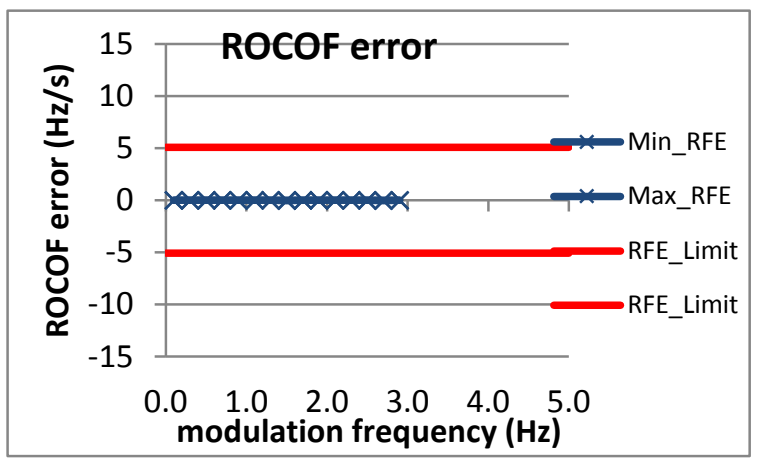

Figure 3734: Fs = 15 FPS

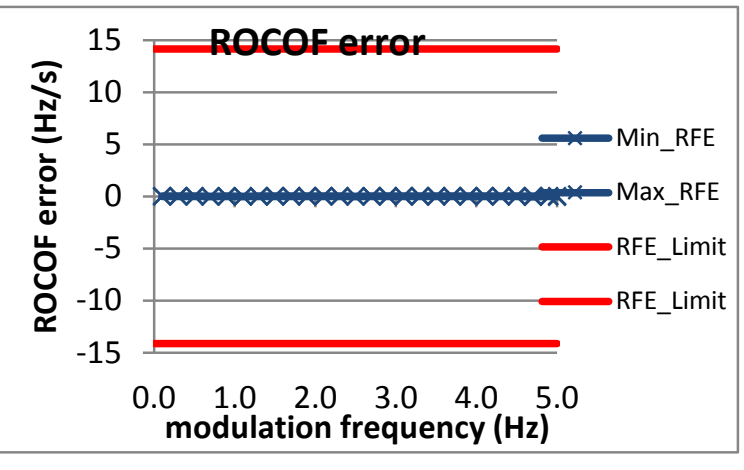

Figure 3732: Fs = 30 FPS

Figure 3735: Fs = 12 FPS data was lost

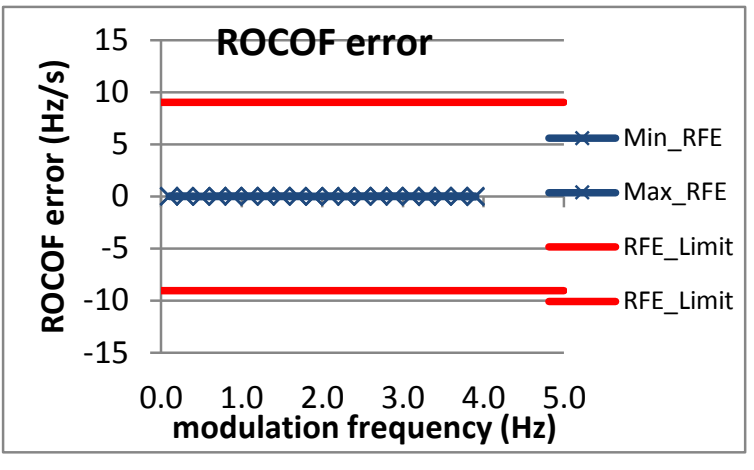

Figure 3733: Fs = 20 FPS

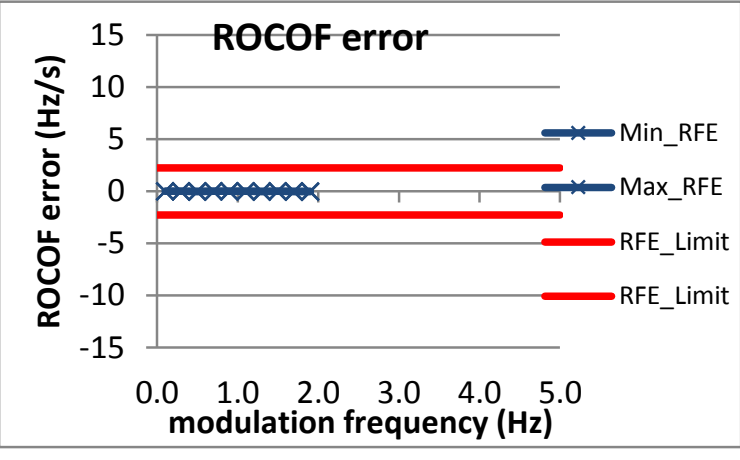

Figure 3736: Fs = 10 FPS 
8.4.7 PMU F amplitude modulation ROCOF error: M class

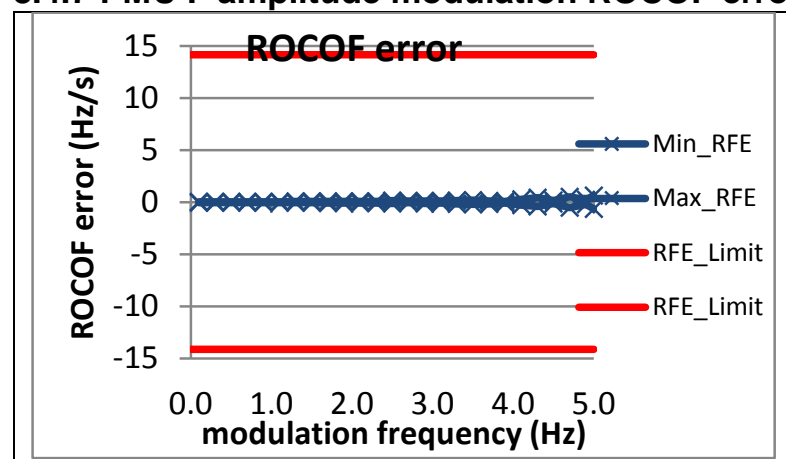

Figure 3737: Fs = 60 FPS

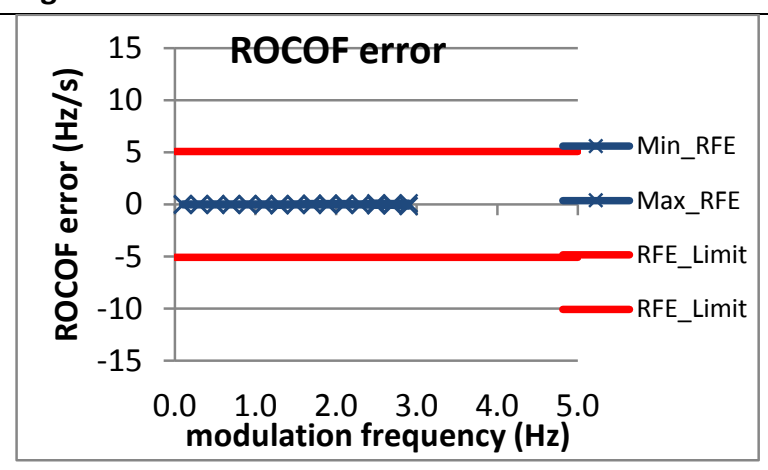

Figure 3740: Fs = 15 FPS

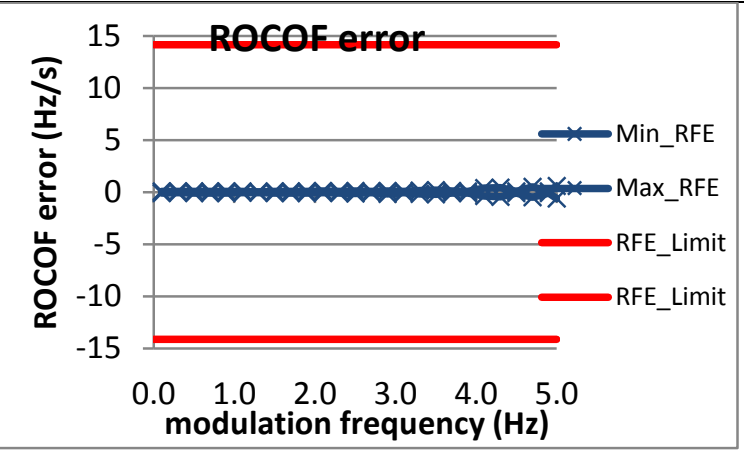

Figure 3738: Fs = 30 FPS

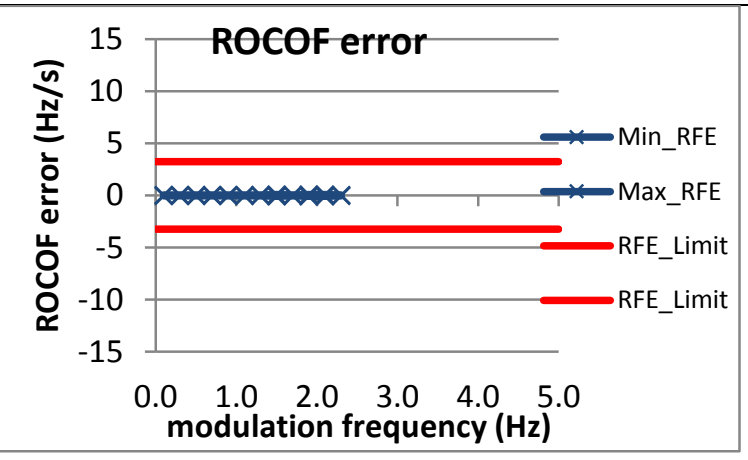

Figure 3741: Fs = 12 FPS

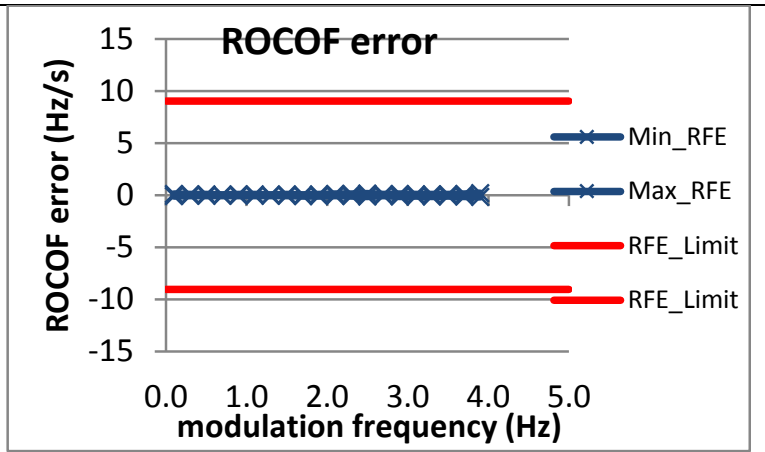

Figure 3739: Fs = 20 FPS

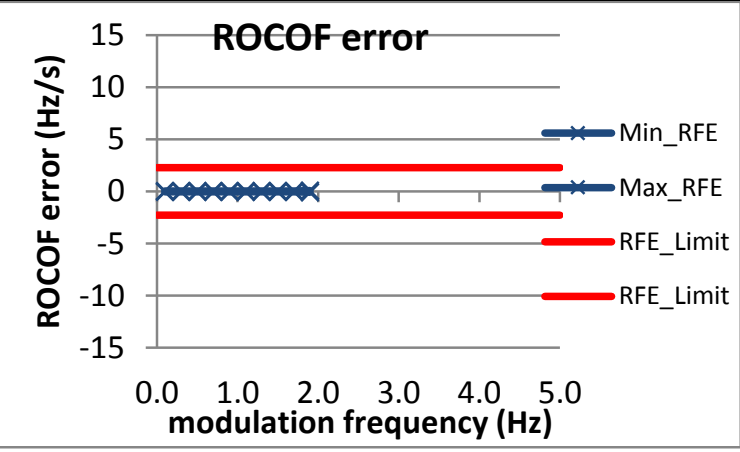

Figure 3742: Fs = 10 FPS 


\subsubsection{PMU G * amplitude modulation ROCOF error: M class}

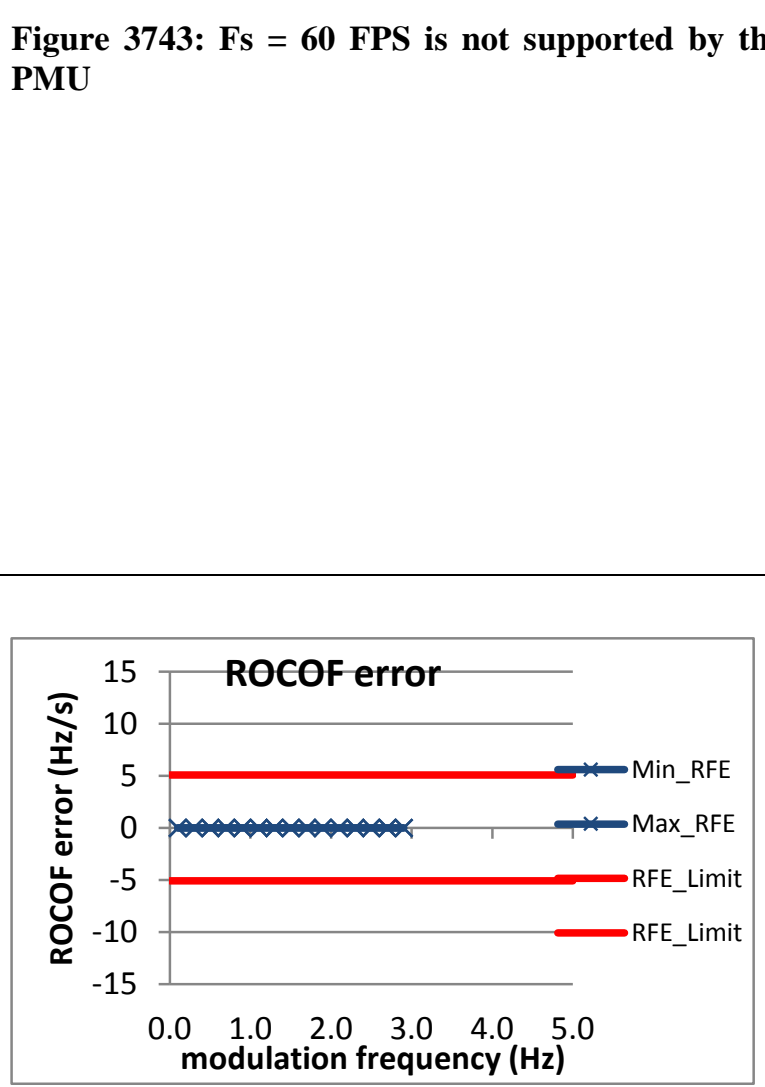

Figure 3746: Fs = 15 FPS

* PMU G always outputs ROCOF $=0$

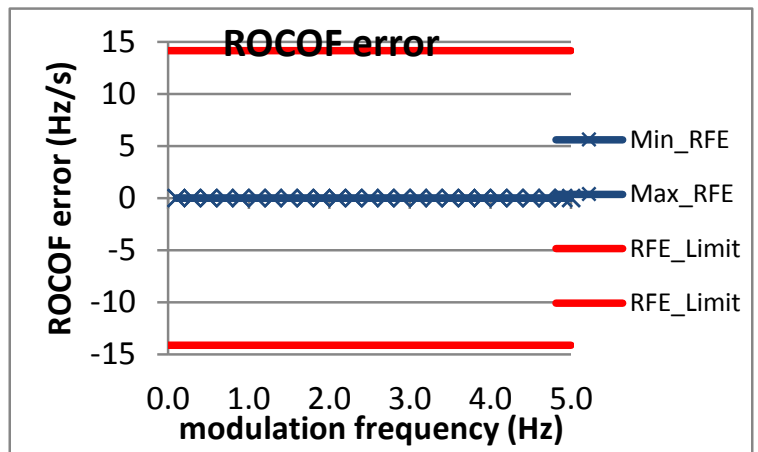

Figure 3744: Fs = 30 FPS

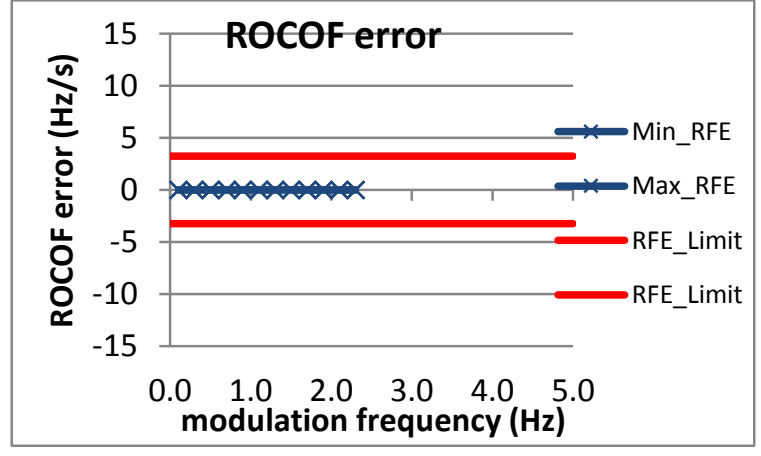

Figure 3747: Fs = 12 FPS

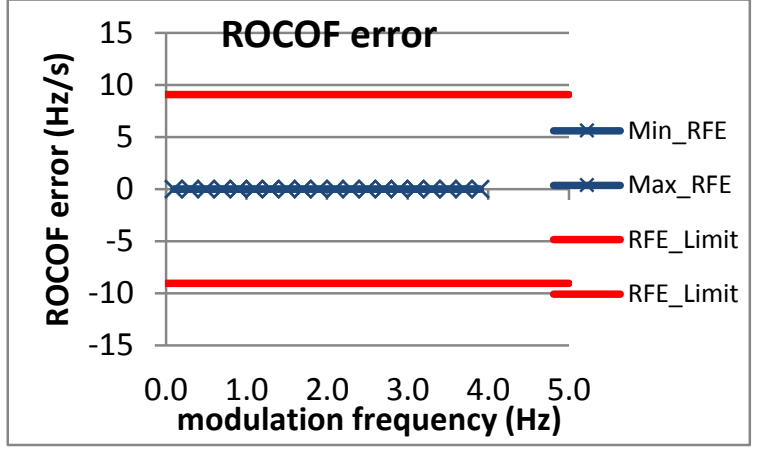

Figure 3745: Fs = 20 FPS

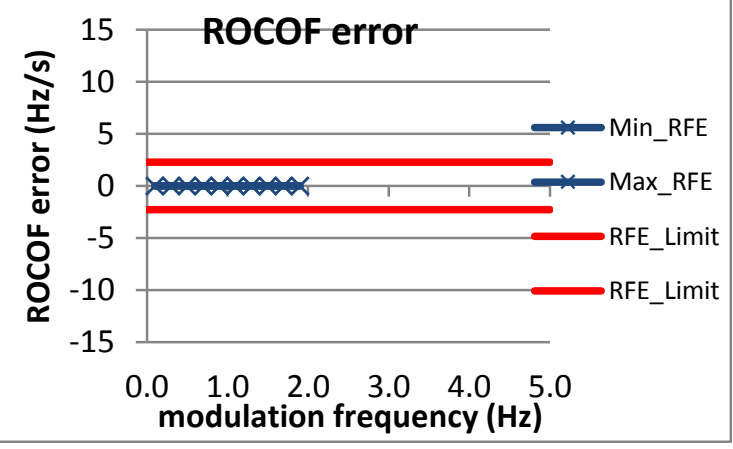

Figure 3748: Fs = 10 FPS 


\subsubsection{PMU H amplitude modulation ROCOF error: M class}

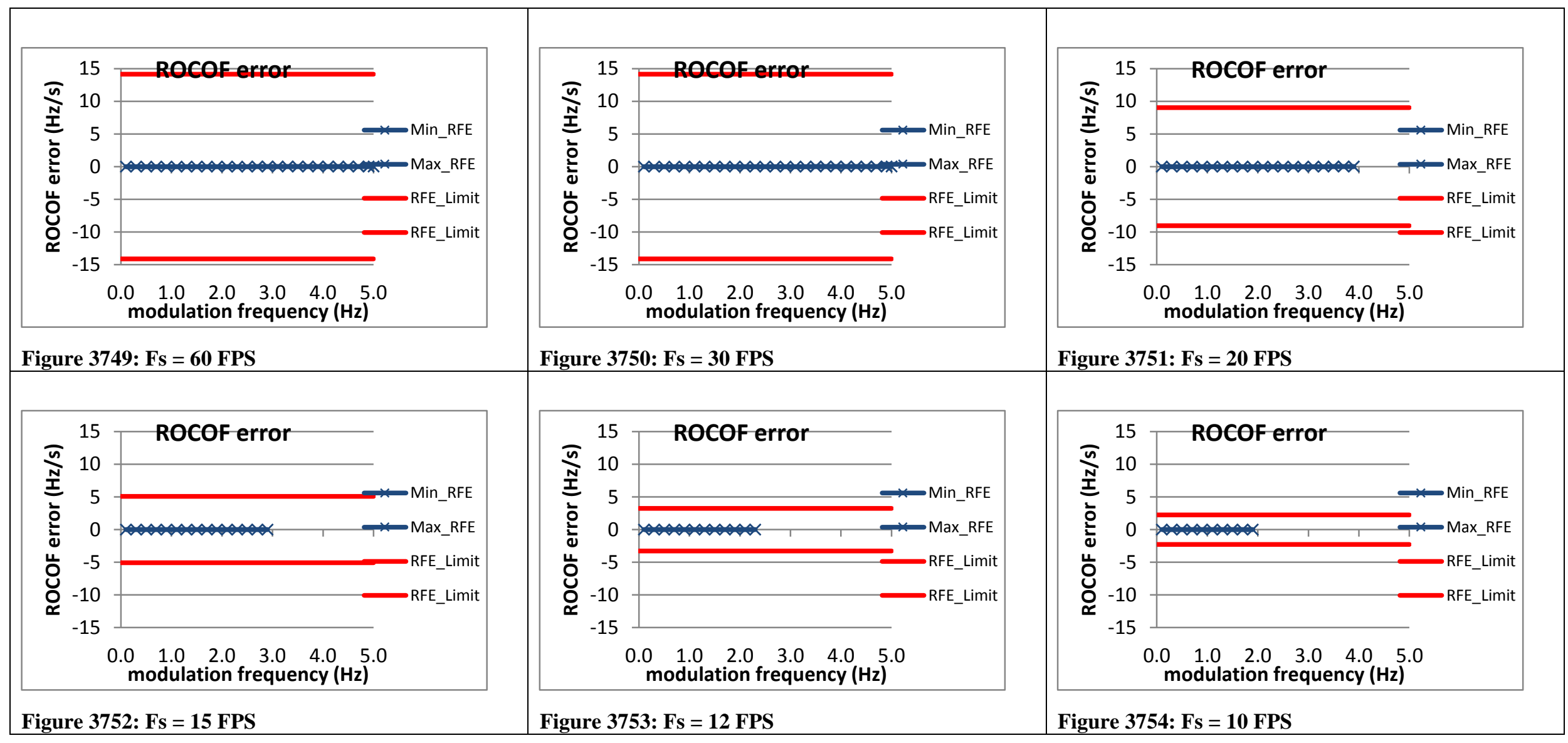




\subsubsection{PMU I amplitude modulation ROCOF error: M class}

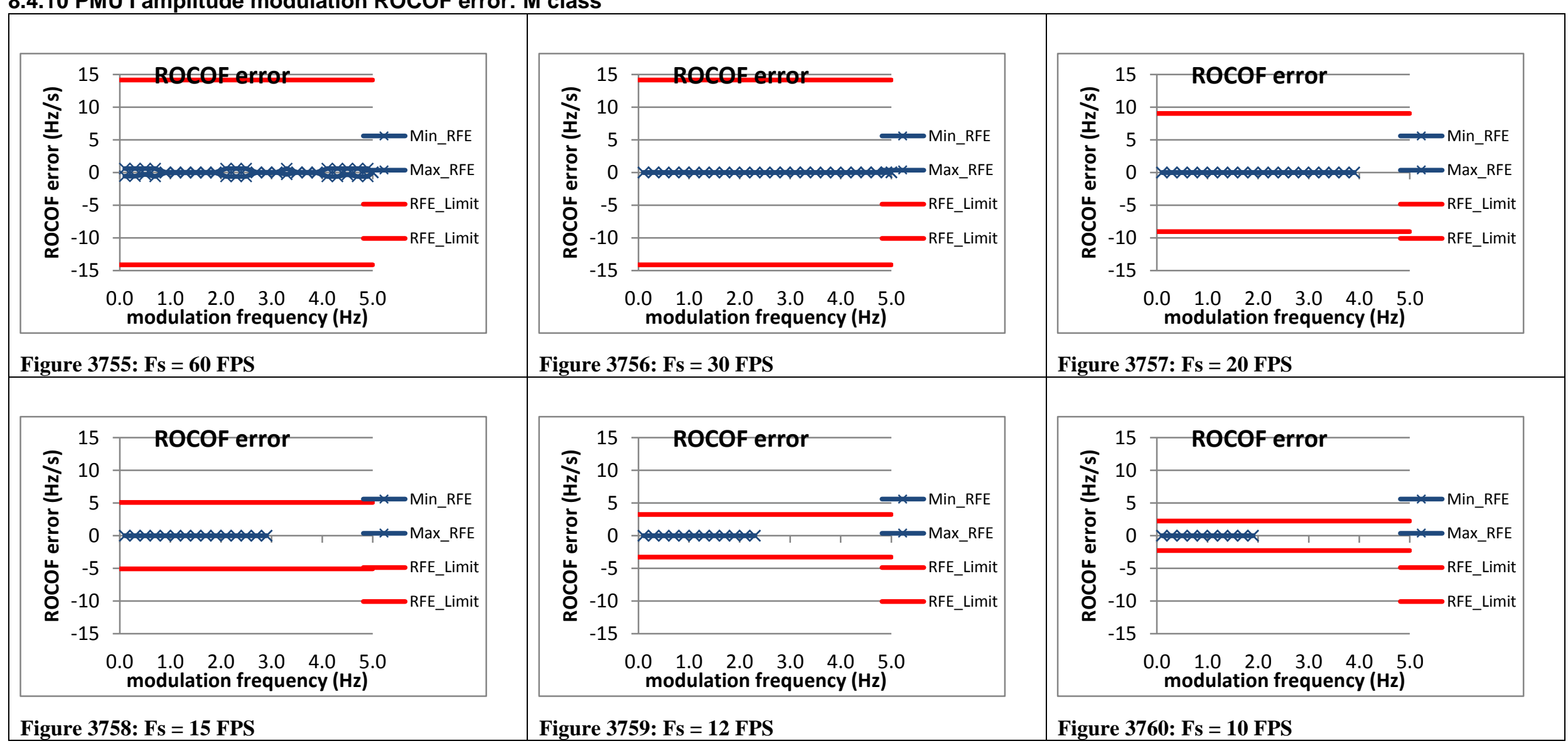




\subsubsection{PMU J amplitude modulation ROCOF error: M class}

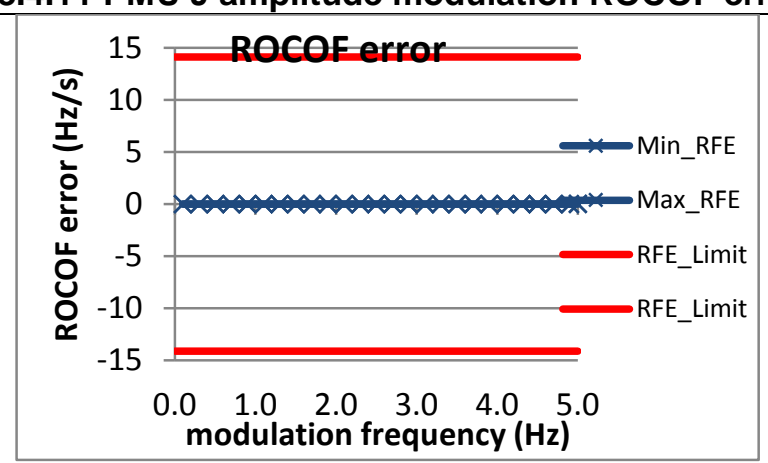

Figure 3761: Fs = 60 FPS
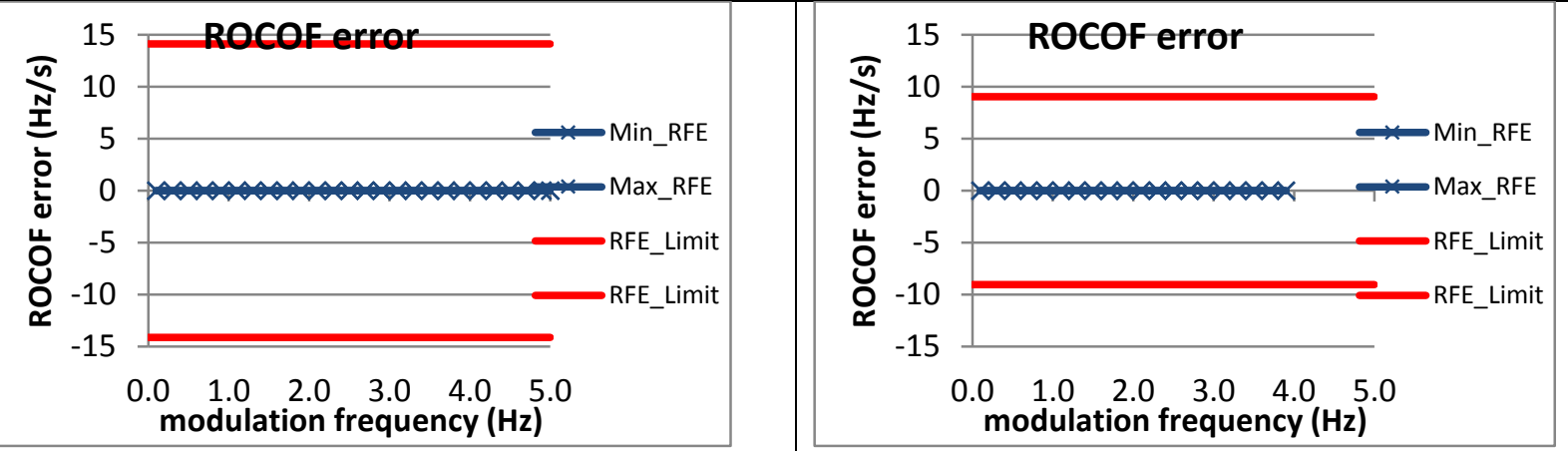

Figure 3762: Fs = 30 FPS

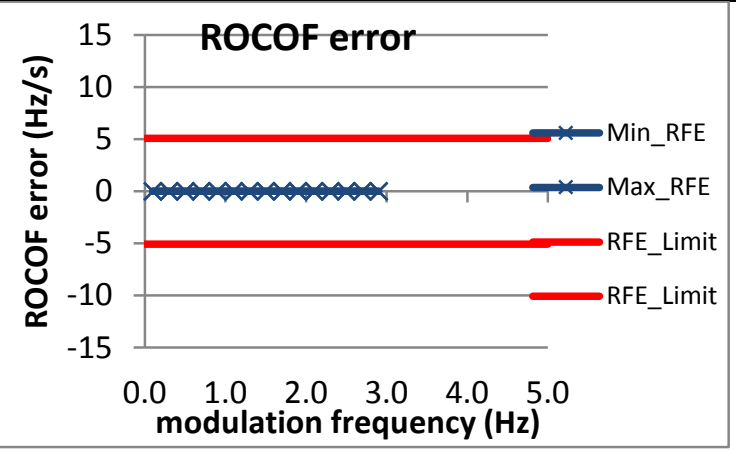

Figure 3764: Fs = 15 FPS

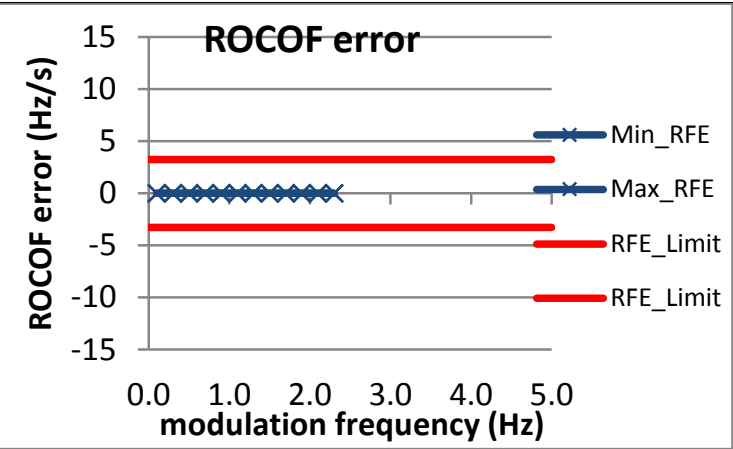

Figure 3765: Fs = 12 FPS
Figure 3763: Fs = 20 FPS

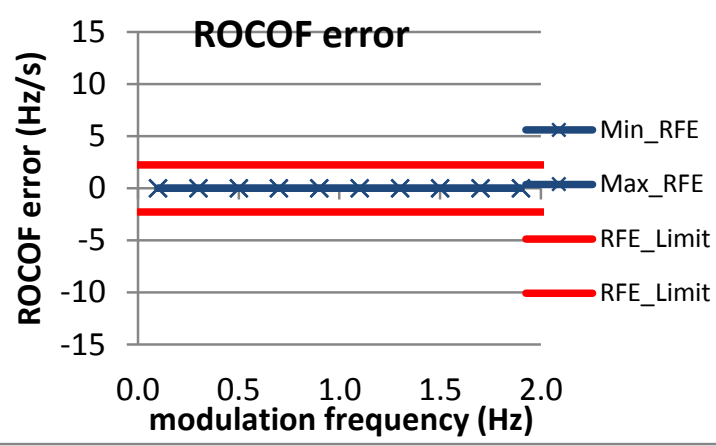

Figure 3766: Fs = 10 FPS 
8.5 Dynamic bandwidth measurement: amplitude modulation voltage TVE: P class

8.5.1 C37.118.1 Annex C dynamic bandwidth measurement: amplitude modulation voltage TVE: P class

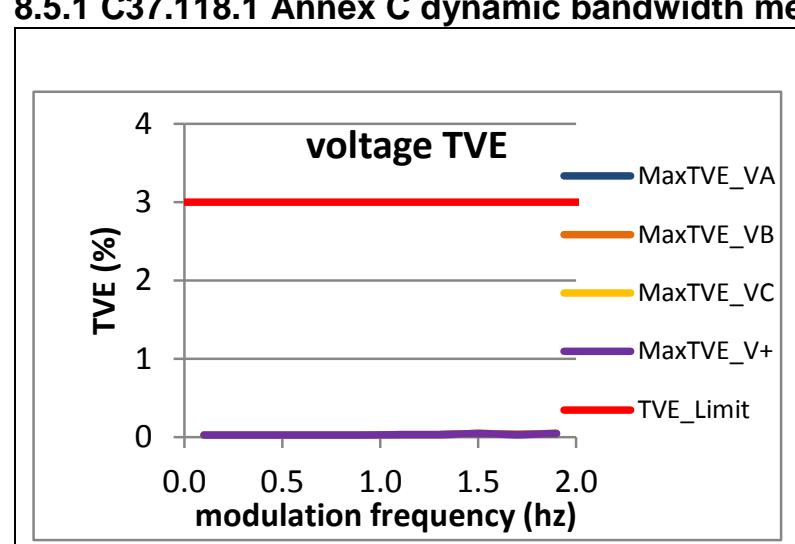

Figure 3767: Fs = 60 FPS
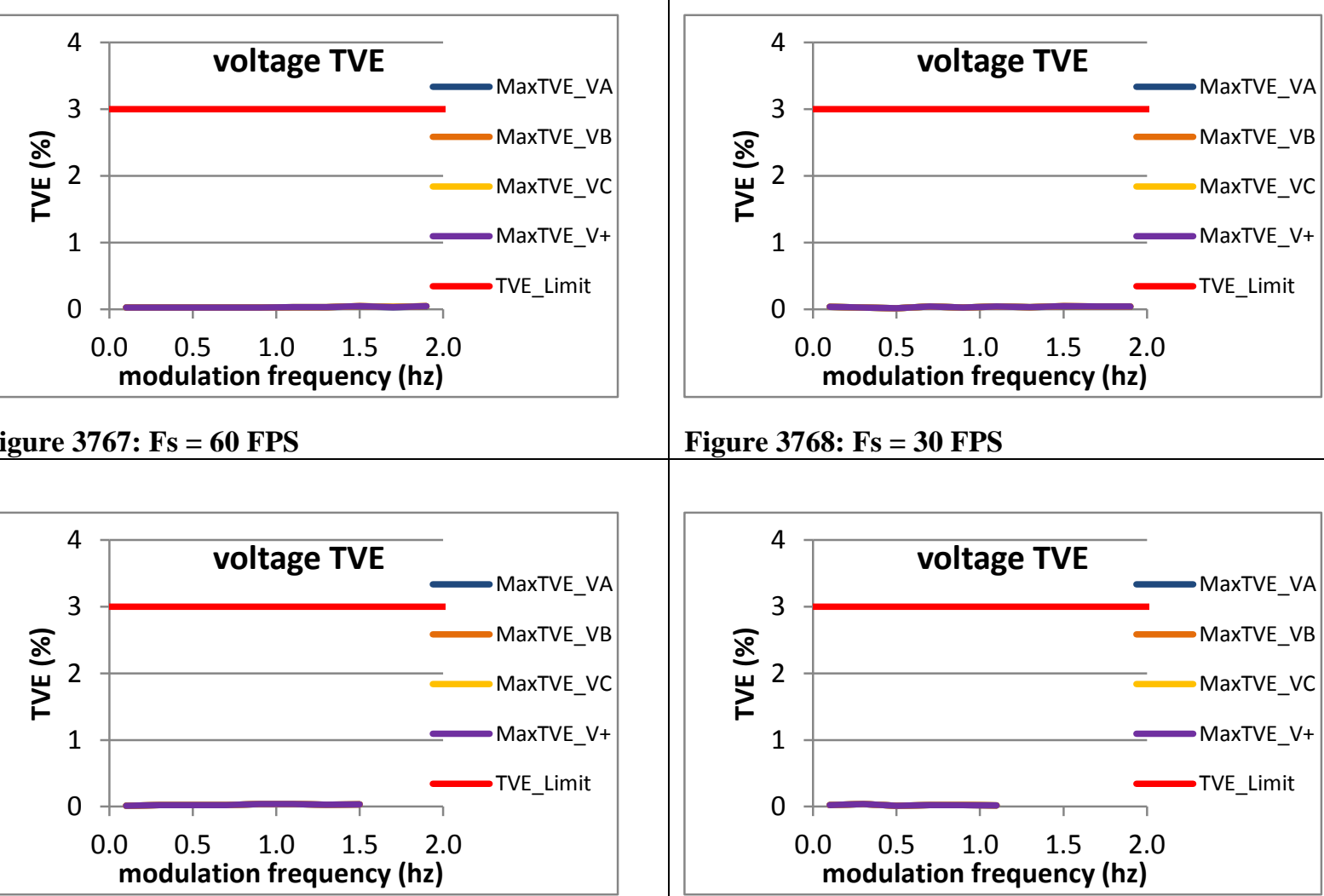

Figure 3770: Fs = 15 FPS

\section{Figure 3768: Fs = 30 FPS \\ Figure 3768: Fs = 30 FPS}

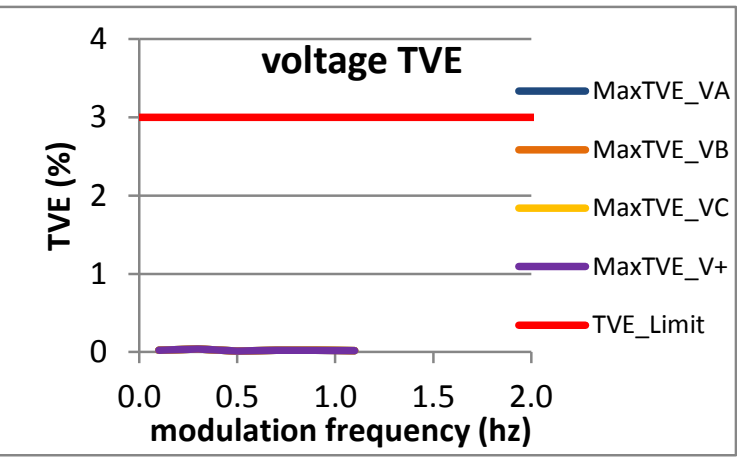

Figure 3771: Fs = 12 FPS

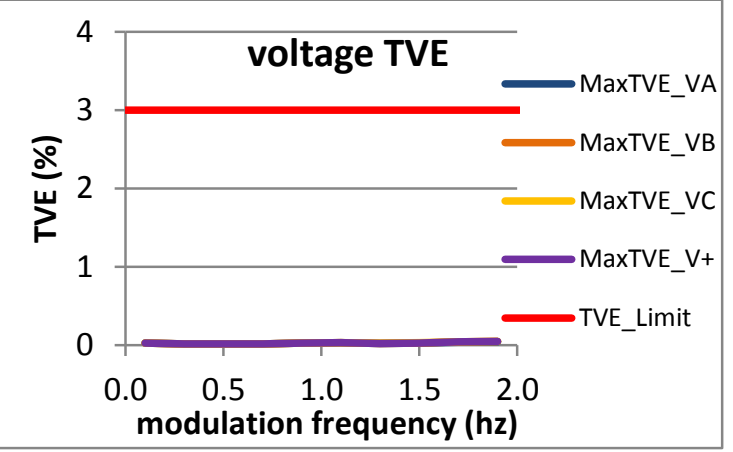

Figure 3769: Fs = 20 FPS

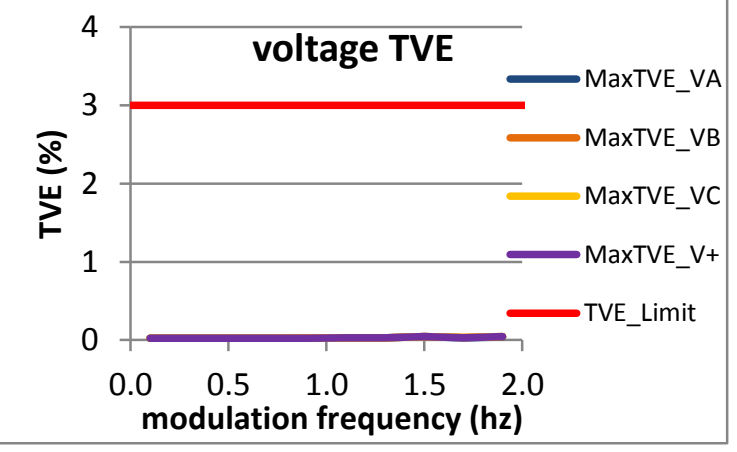

Figure 3772: Fs = 10 FPS 


\subsubsection{PMU A dynamic bandwidth measurement: amplitude modulation voltage TVE: P class}

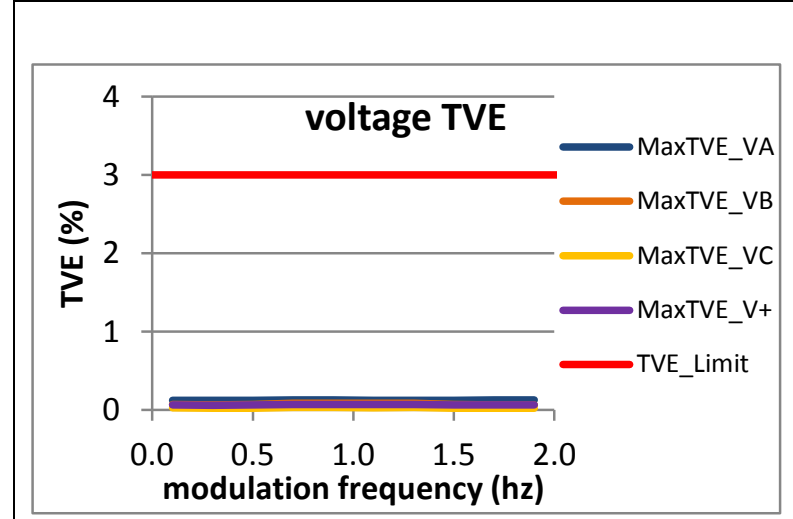

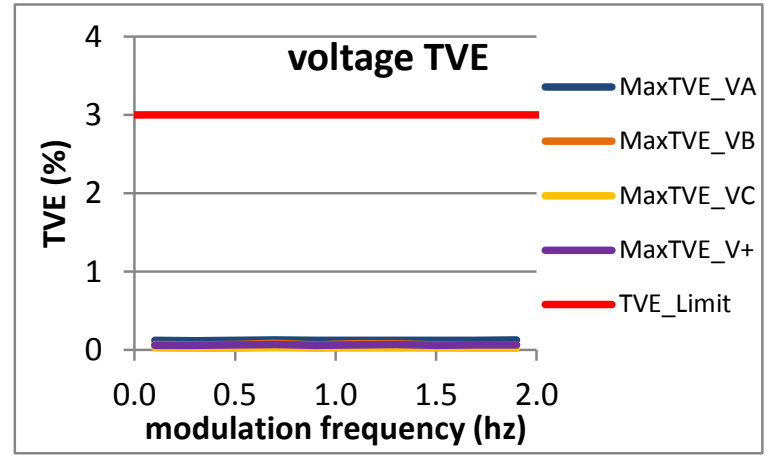

Figure 3774: Fs = 30 FPS

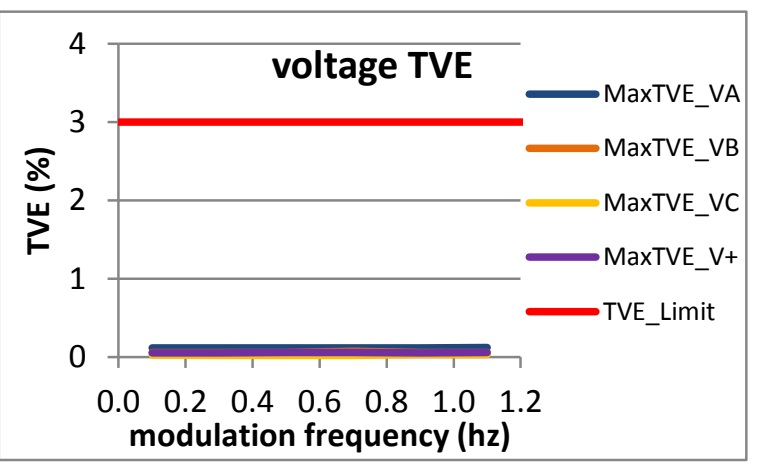

Figure 3777: Fs = 12 FPS

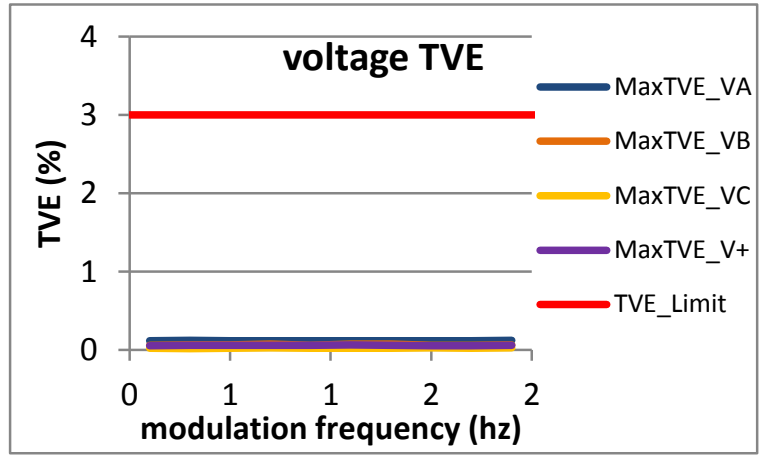

Figure 3775: Fs = 20 FPS

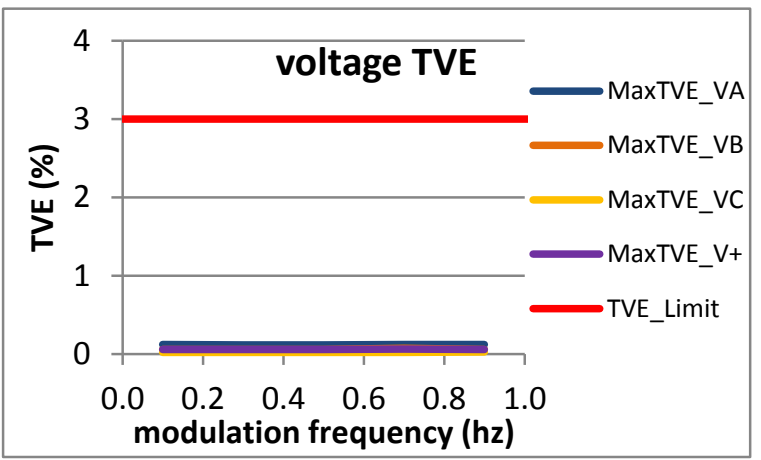

Figure 3778: Fs = 10 FPS 


\subsubsection{PMU B dynamic bandwidth measurement: amplitude modulation voltage TVE: P class}

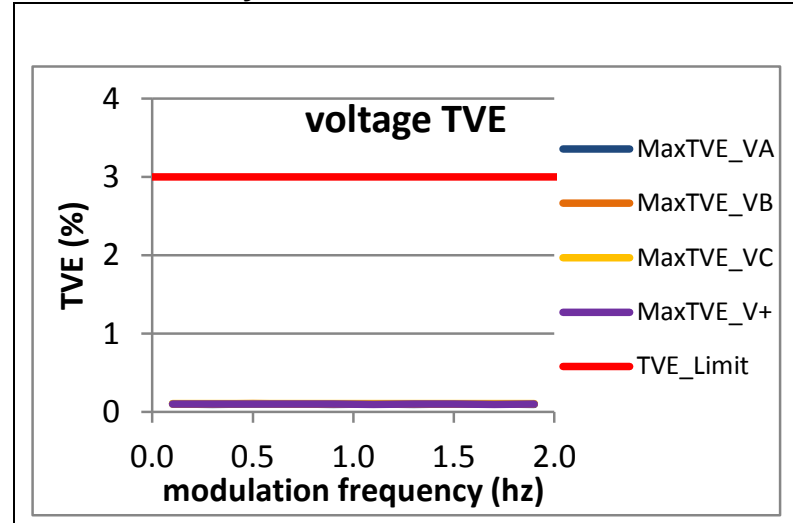

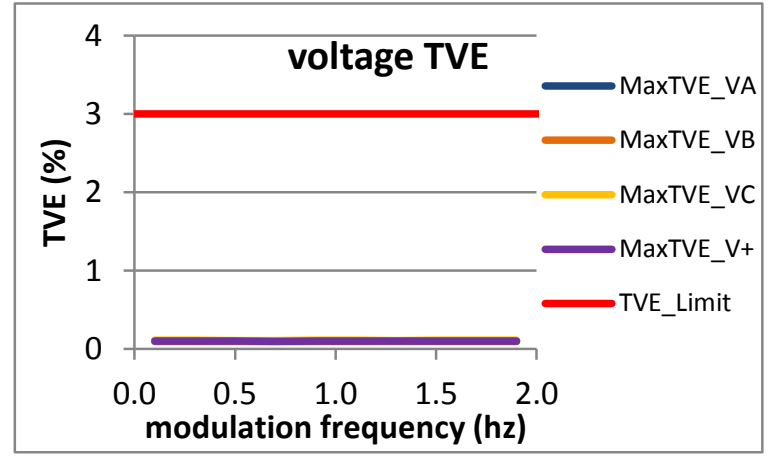

Figure 3780: Fs = 30 FPS

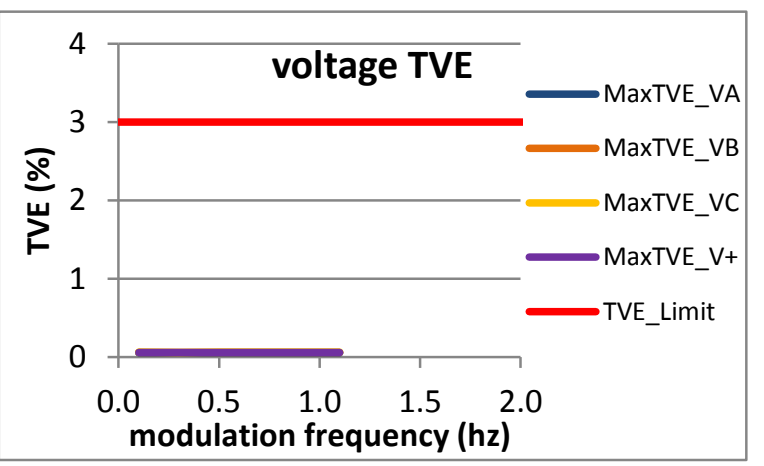

Figure 3783: Fs = 12 FPS

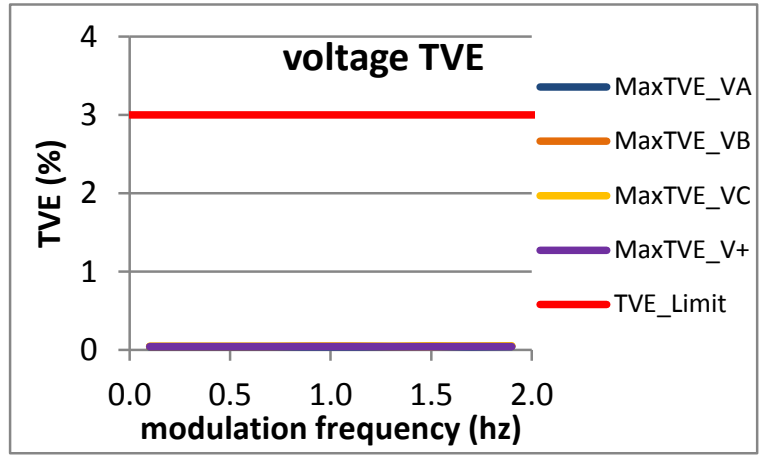

Figure 3781: Fs $=20$ FPS

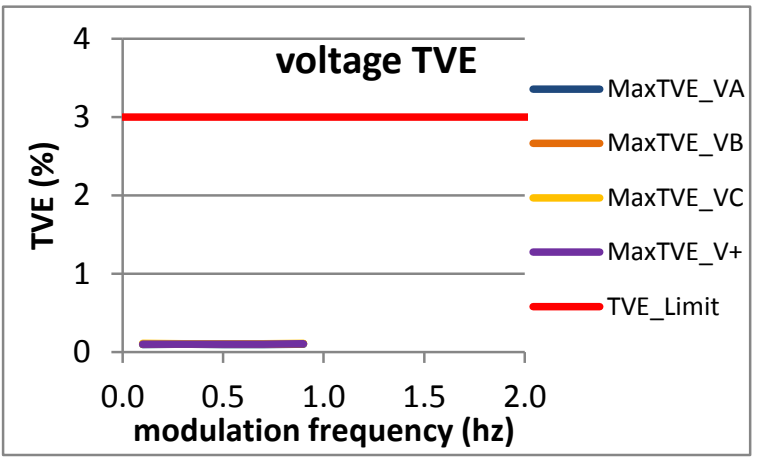

Figure 3784: Fs = 10 FPS 


\subsubsection{PMU C dynamic bandwidth measurement: amplitude modulation voltage TVE: P class}

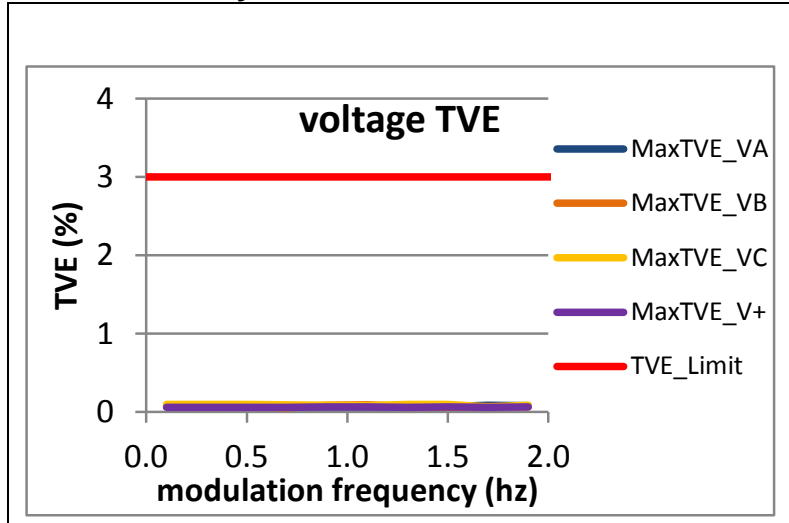

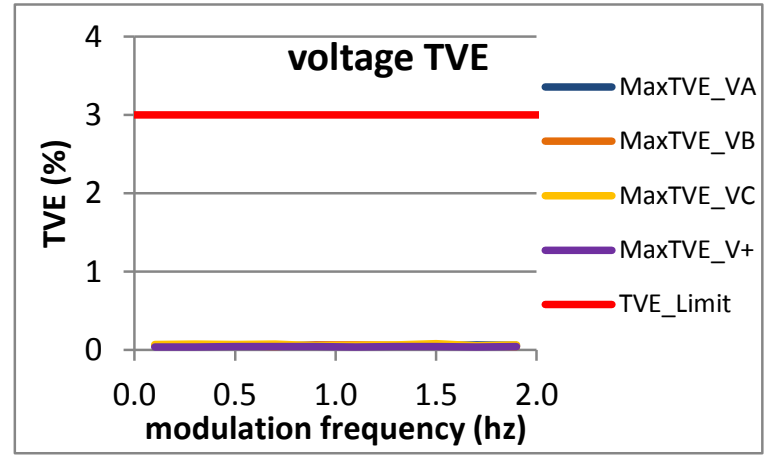

Figure 3786: Fs = 30 FPS

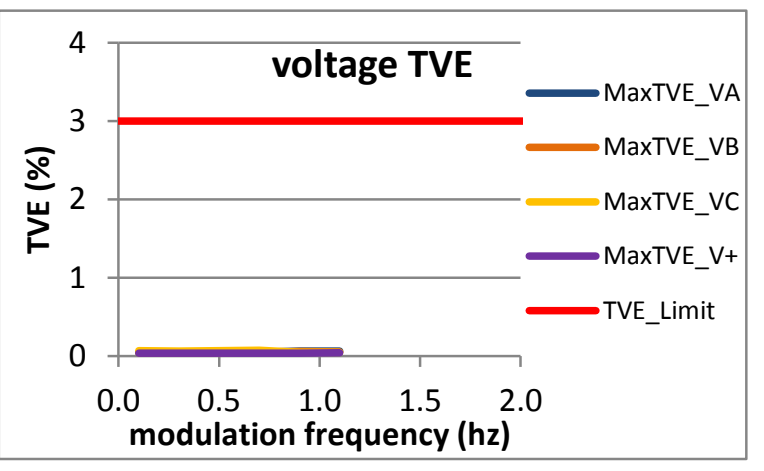

Figure 3789: Fs = 12 FPS

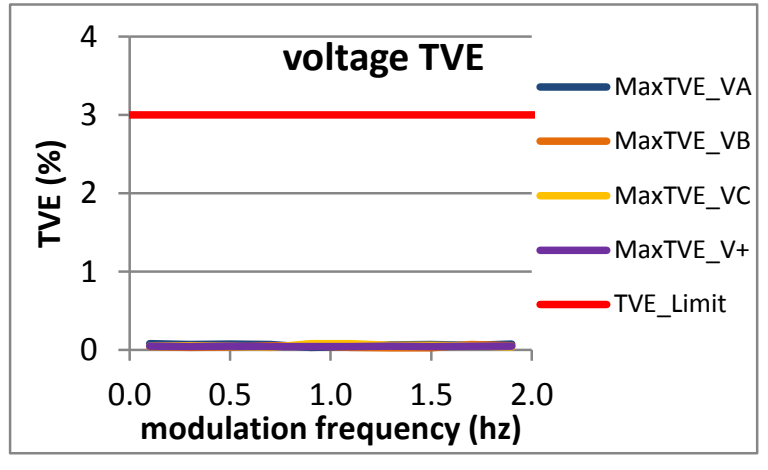

Figure 3787: Fs $=20$ FPS

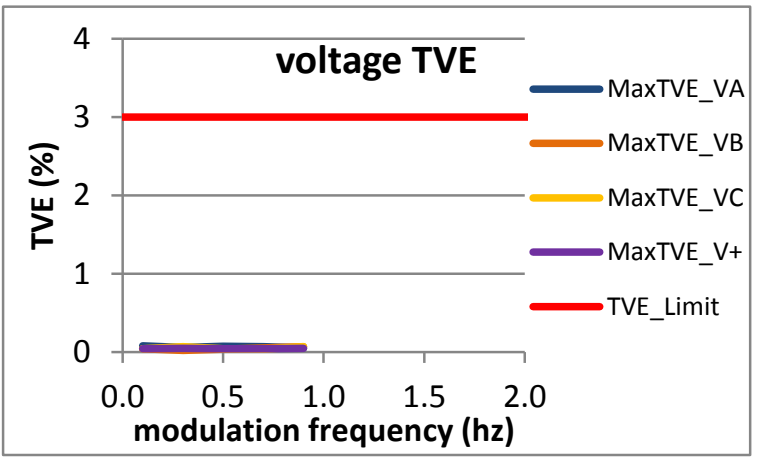

Figure 3790: Fs = 10 FPS 
8.5.5 PMU D dynamic bandwidth measurement: amplitude modulation voltage TVE: P class

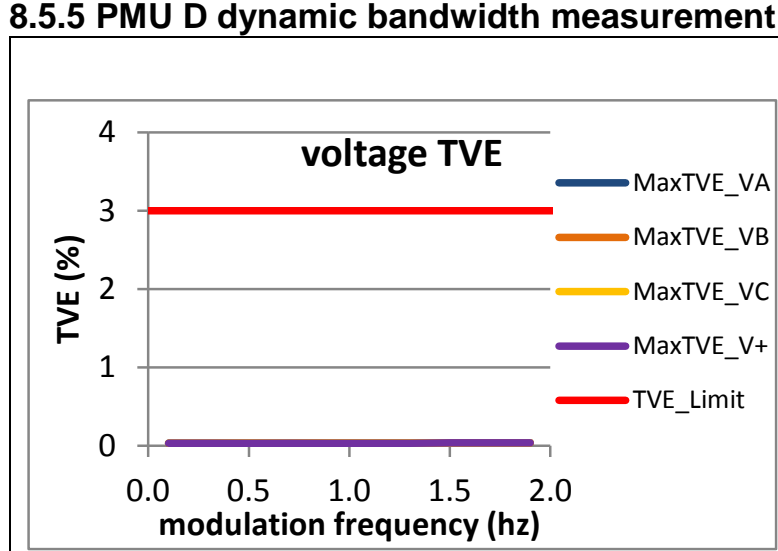

Figure 3791: Fs = 60 FPS

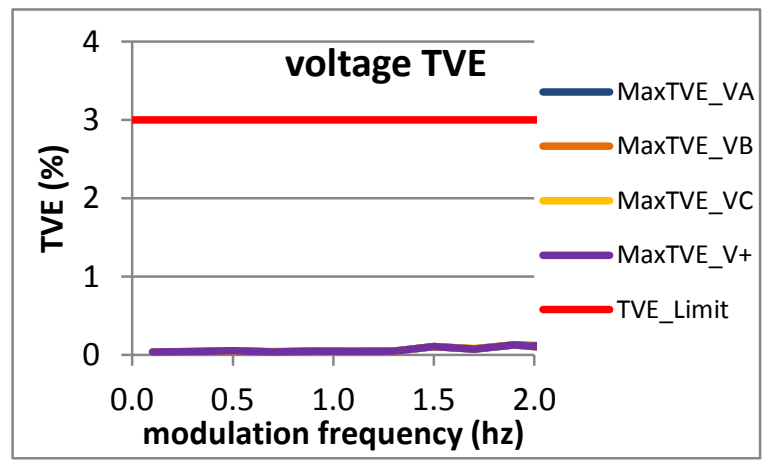

Figure 3794: Fs = 15 FPS

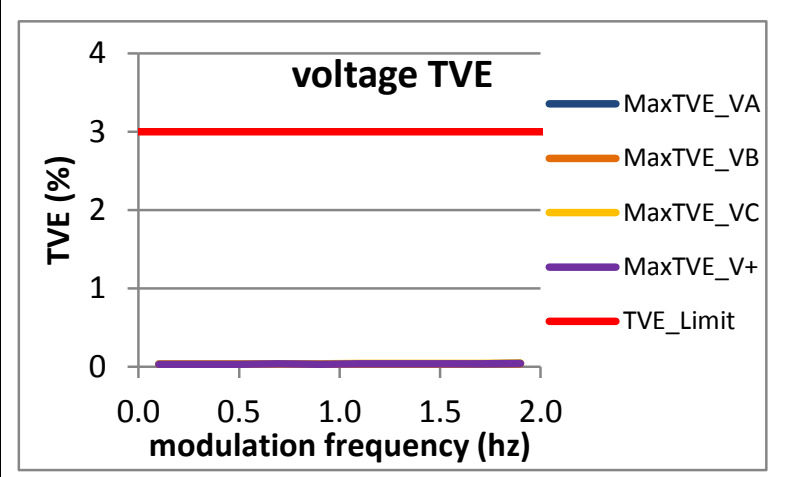

Figure 3792: Fs = 30 FPS

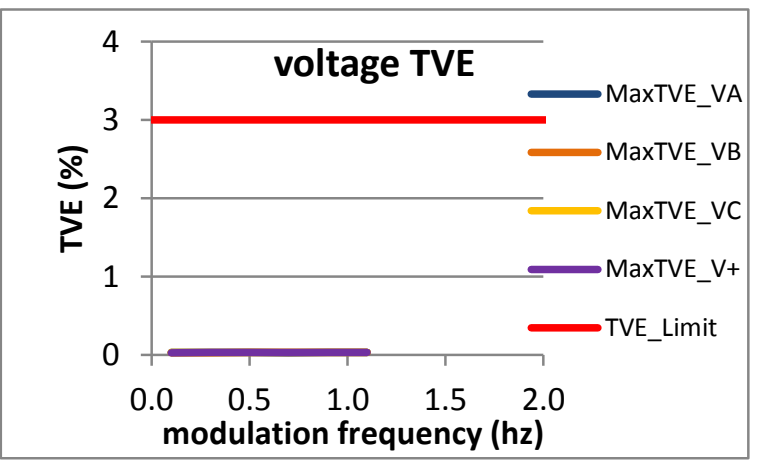

Figure 3795: Fs = 12 FPS

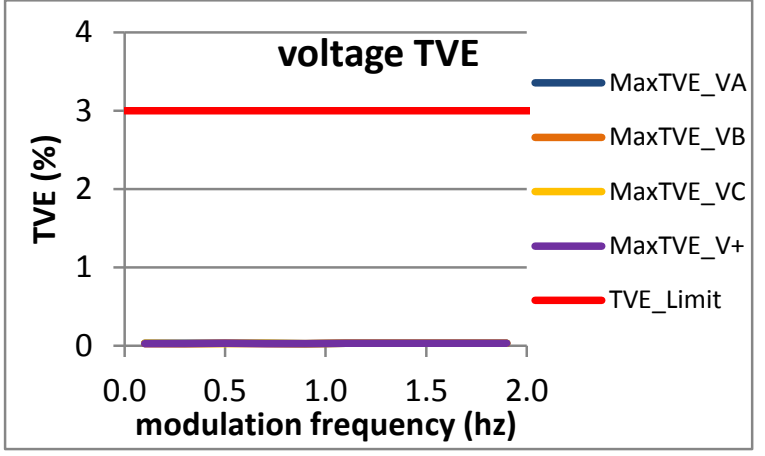

Figure 3793: Fs = 20 FPS

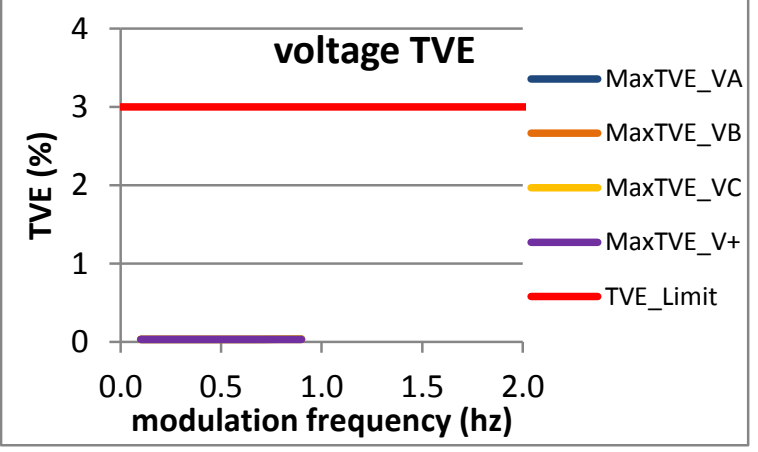

Figure 3796: Fs = 10 FPS

8.5.6 PMU E dynamic bandwidth measurement: amplitude modulation voltage TVE: P class

PMU E does not support P class 
8.5.7 PMU F dynamic bandwidth measurement: amplitude modulation voltage TVE: P class

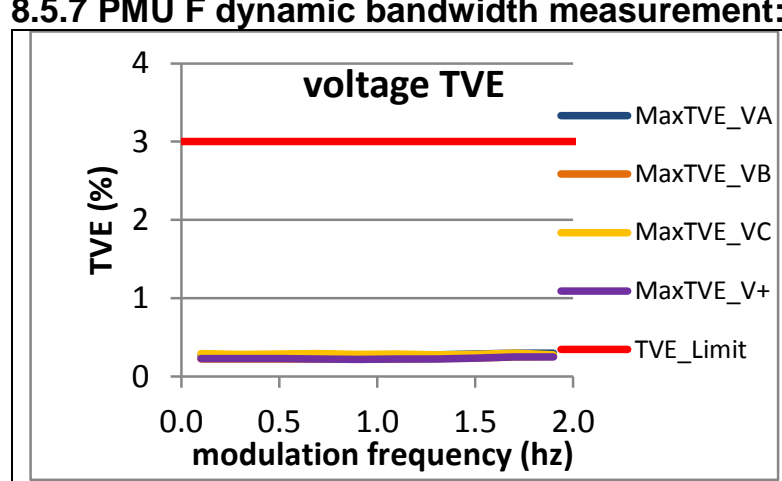

Figure 3797: Fs = 60 FPS

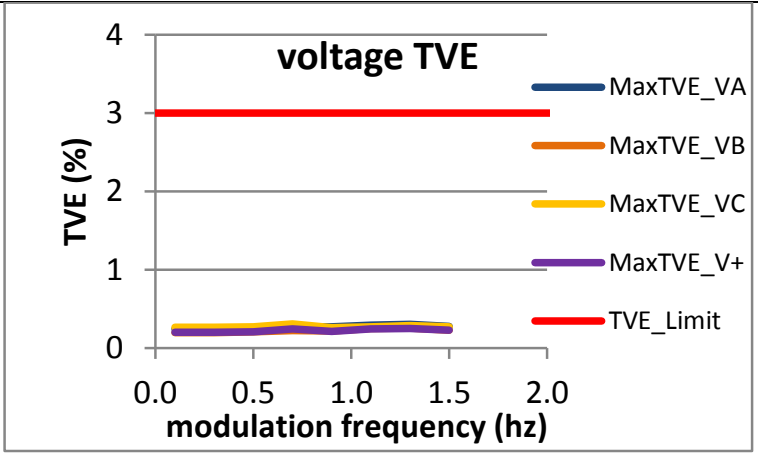

Figure 3800: Fs = 15 FPS

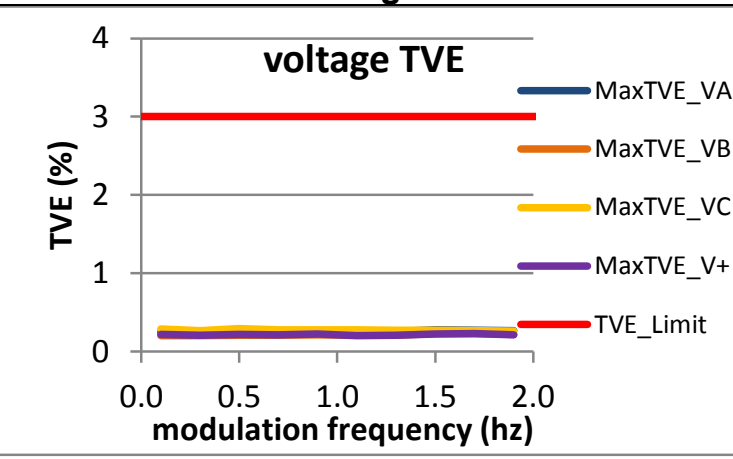

Figure 3798: Fs = 30 FPS

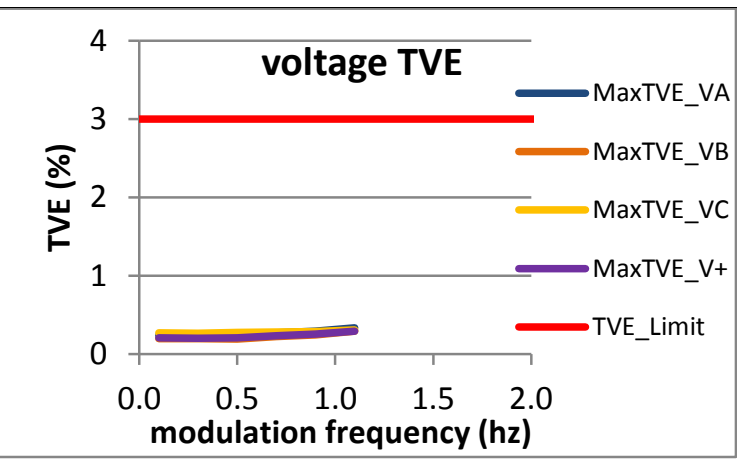

Figure 3801: Fs = 12 FPS

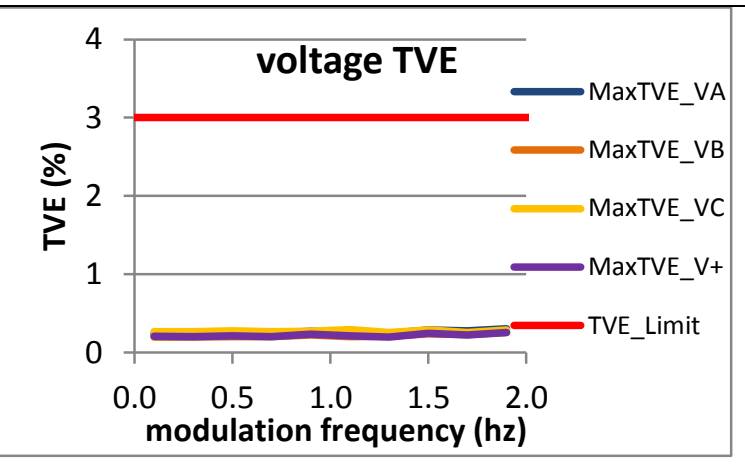

Figure 3799: Fs $=20$ FPS

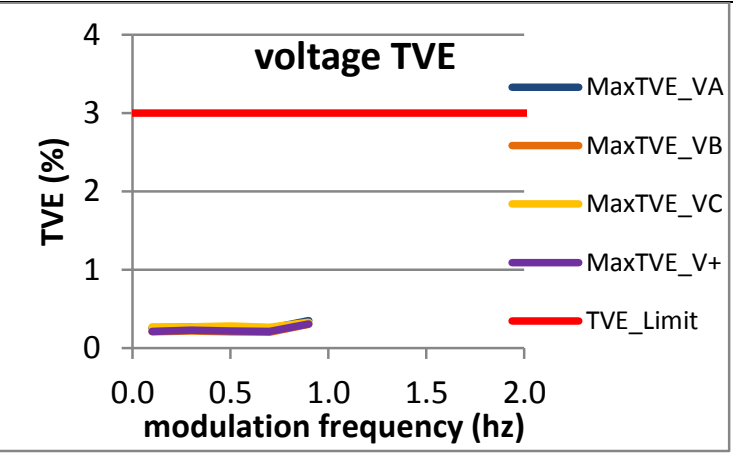

Figure 3802: Fs = 10 FPS

8.5.8 PMU G dynamic bandwidth measurement: amplitude modulation voltage TVE: P class PMU G does not support P class 
8.5.9 PMU H dynamic bandwidth measurement: amplitude modulation voltage TVE: P class

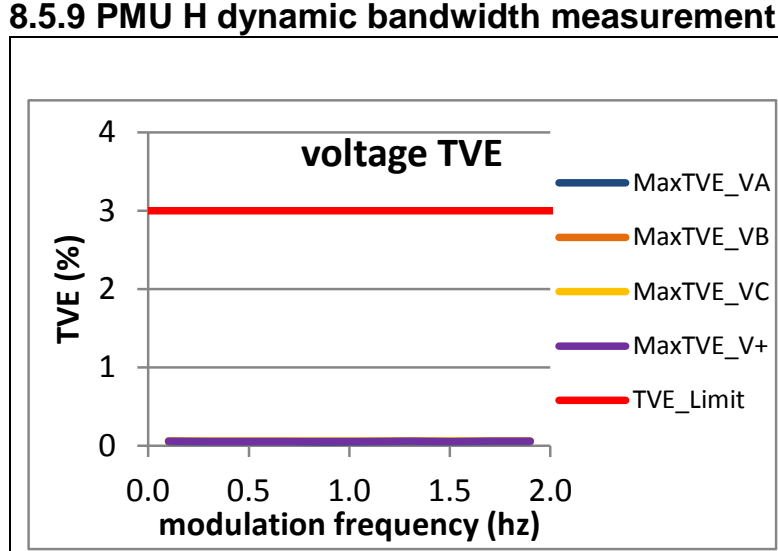

Figure 3803: Fs = 60 FPS

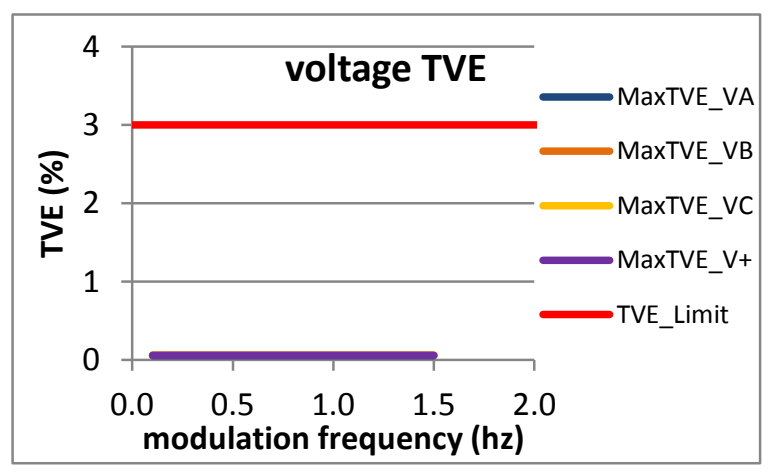

Figure 3806: Fs = 15 FPS

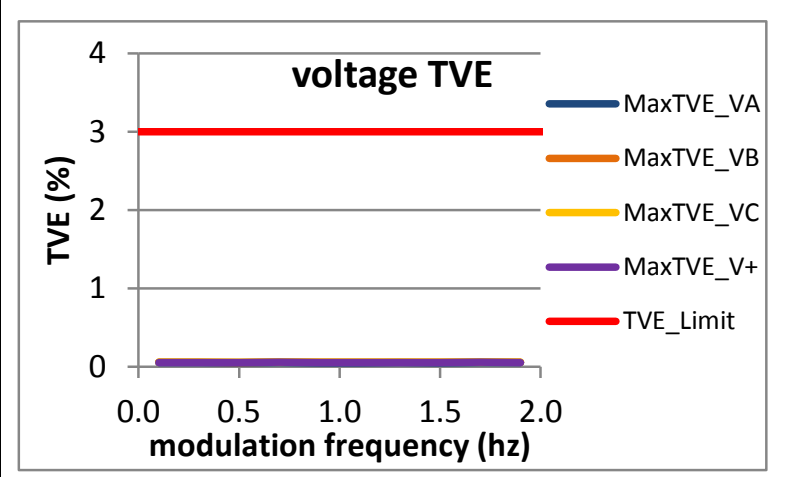

Figure 3804: Fs = 30 FPS

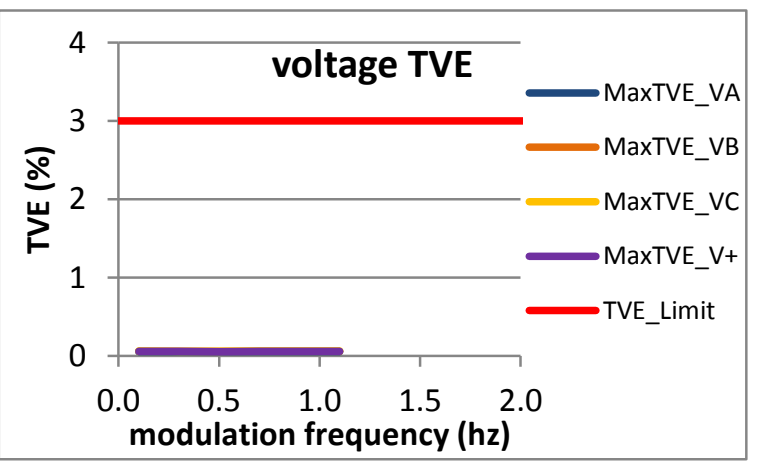

Figure 3807: Fs = 12 FPS

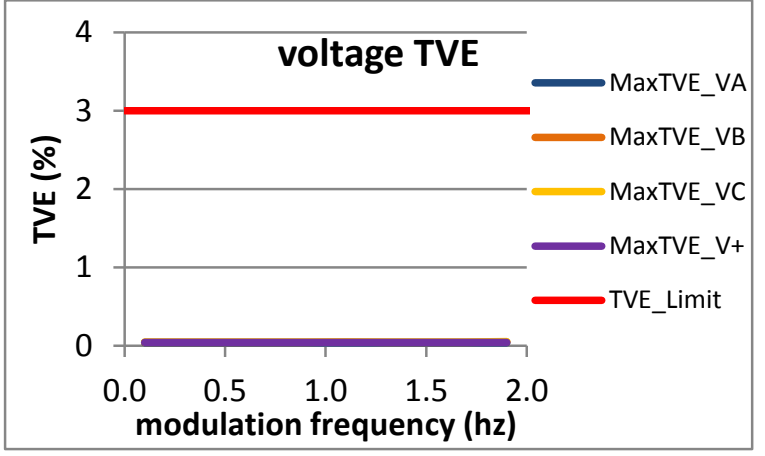

Figure 3805: Fs $=20$ FPS

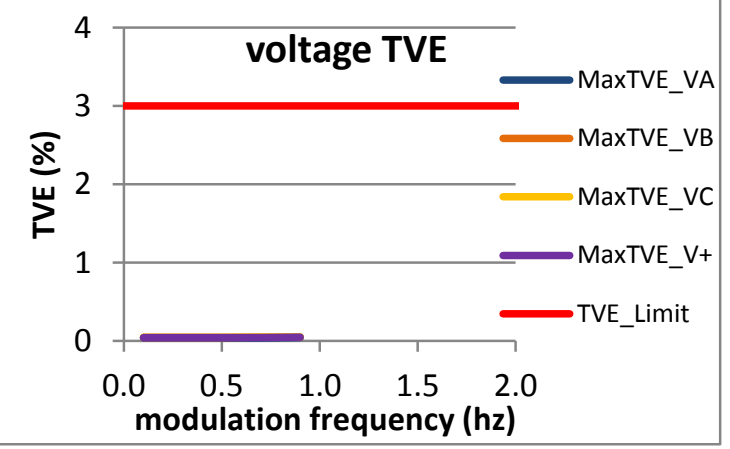

Figure 3808: Fs = 10 FPS

8.5.10 PMU I dynamic bandwidth measurement: amplitude modulation voltage TVE: P class

PMU I does not support P class 
8.5.11 PMU J dynamic bandwidth measurement: amplitude modulation voltage TVE: P class

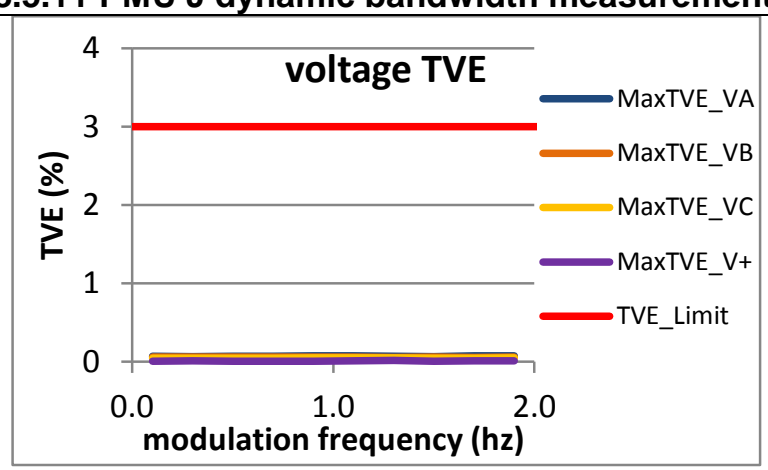

Figure 3809: Fs = 60 FPS

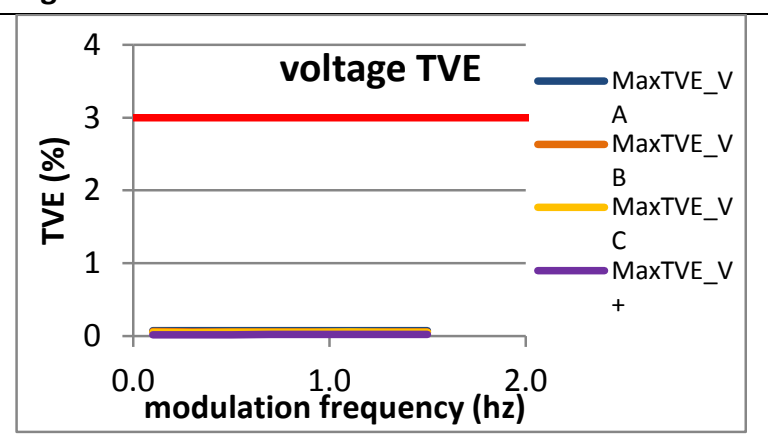

Figure 3812: Fs = 15 FPS

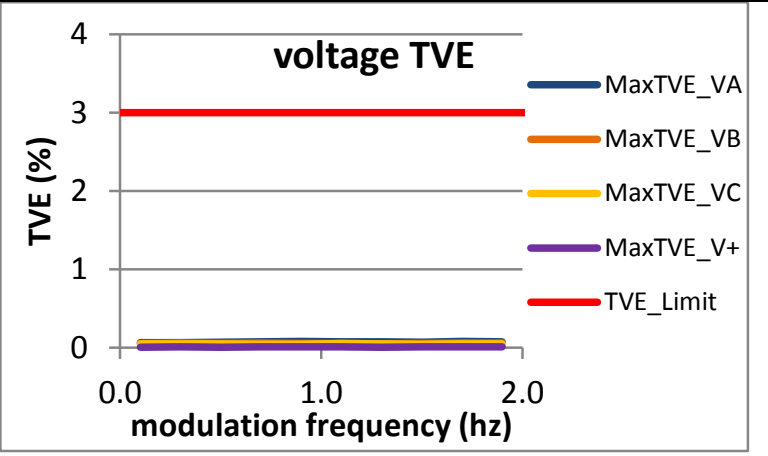

Figure 3810: Fs = 30 FPS

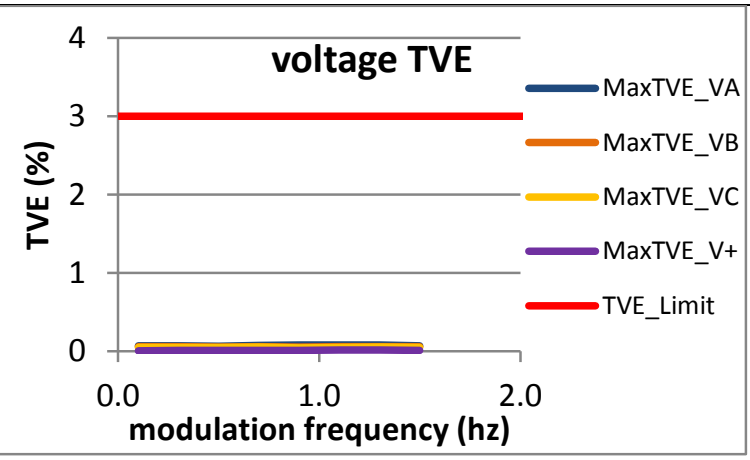

Figure 3813: Fs = 12 FPS

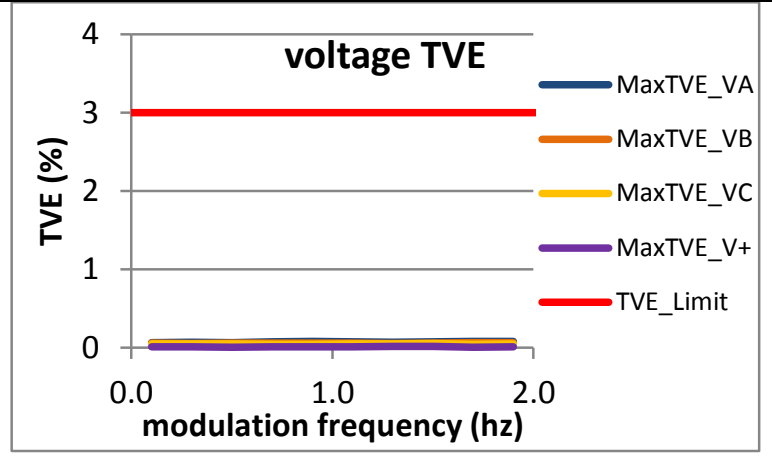

Figure 3811: Fs = 20 FPS

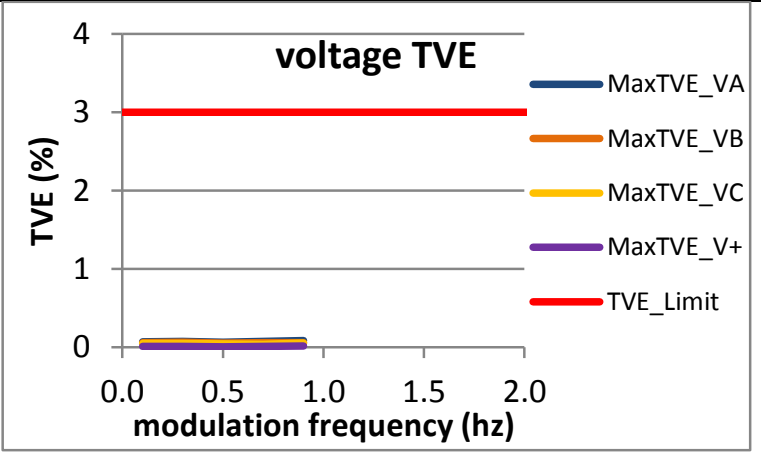

Figure 3814: Fs = 10 FPS 
8.6 Dynamic bandwidth measurement: amplitude modulation current TVE: P class

8.6.1 C37.118.1 Annex C dynamic bandwidth measurement: amplitude modulation current TVE: P class

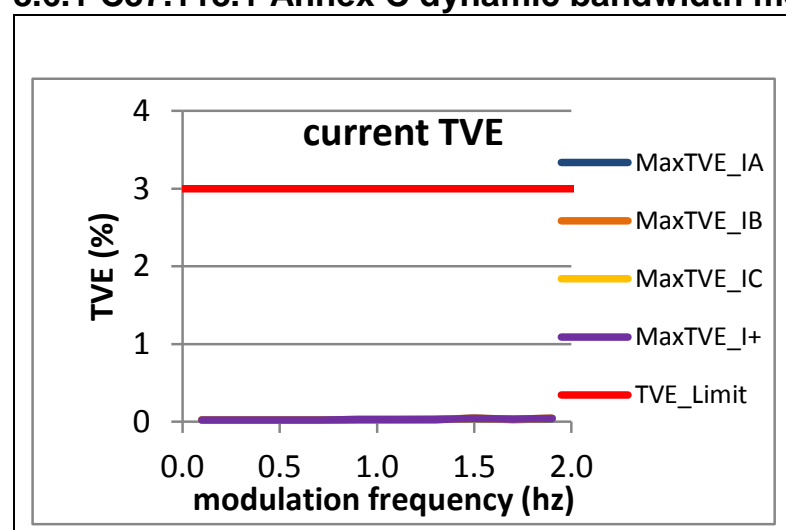

Figure 3815: Fs = 60 FPS

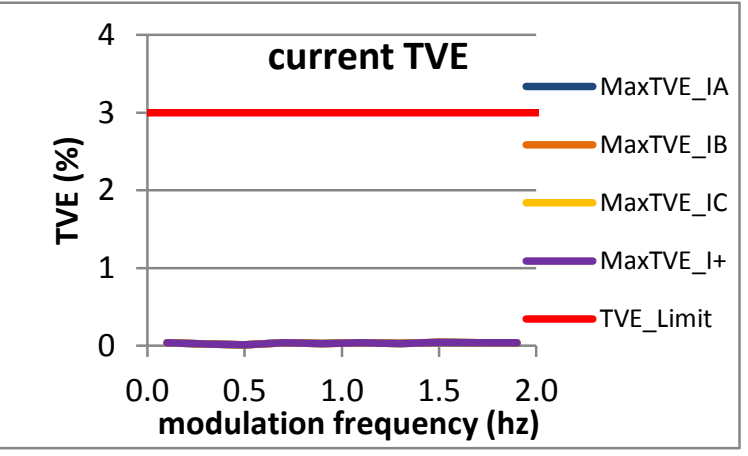

Figure 3816: Fs = 30 FPS

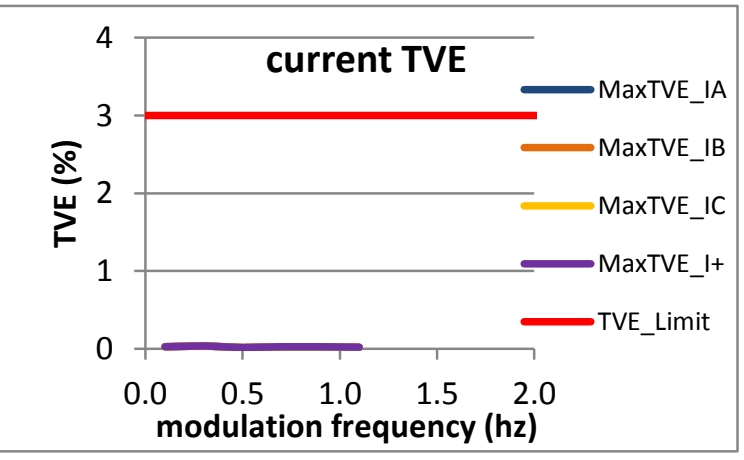

Figure 3819: Fs = 12 FPS

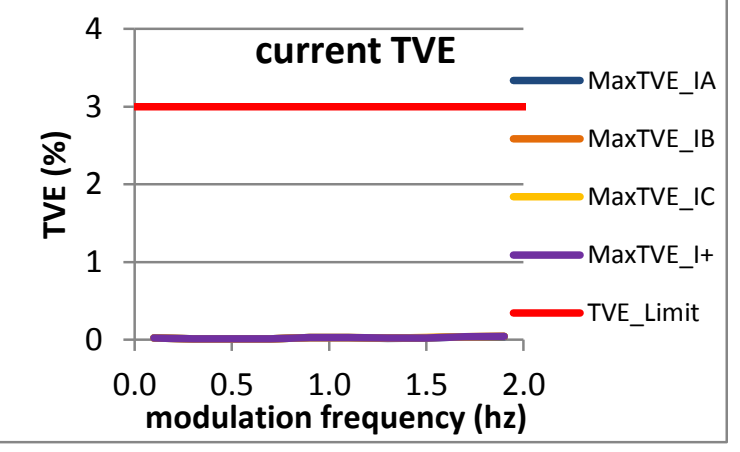

Figure 3817: Fs = 20 FPS

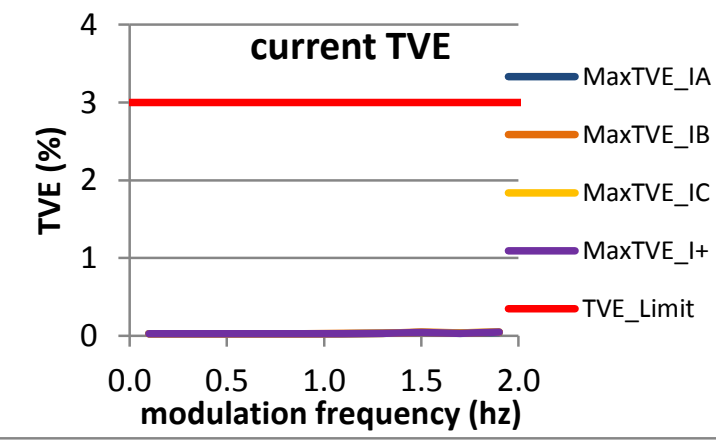

Figure 3820: Fs = 10 FPS 
8.6.2 PMU A dynamic bandwidth measurement: amplitude modulation current TVE: P class

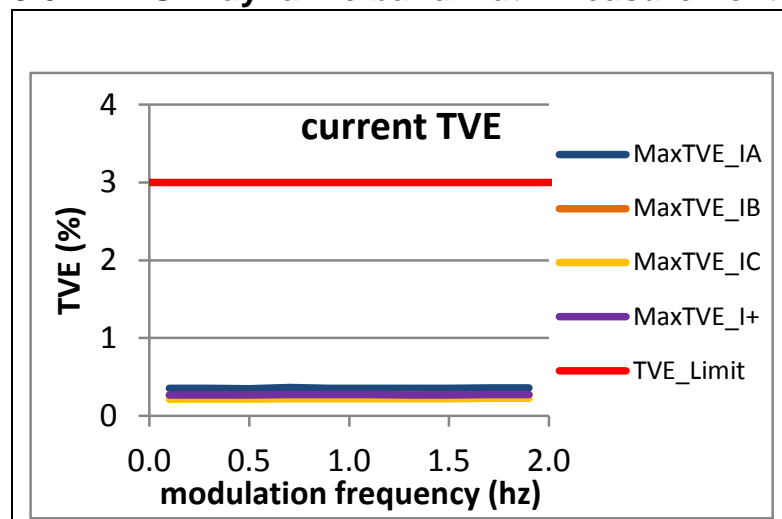

Figure 3821: Fs = 60 FPS

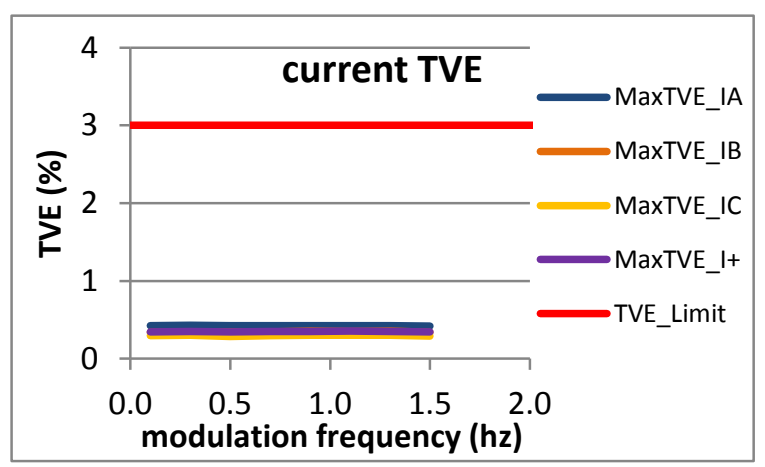

Figure 3824: Fs = 15 FPS

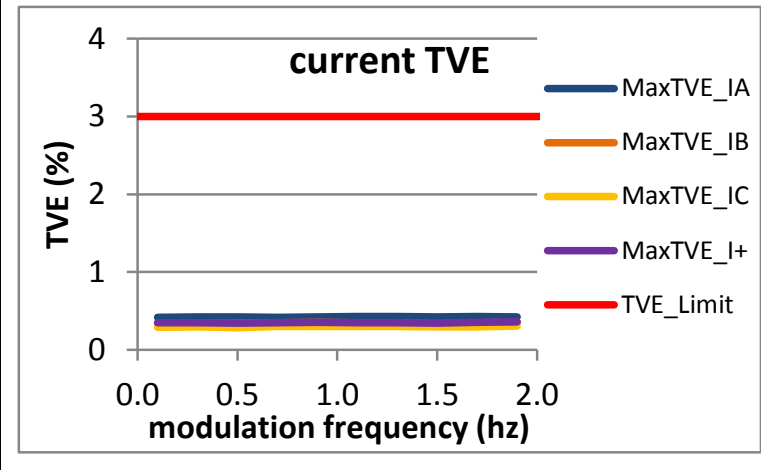

Figure 3822: Fs = 30 FPS

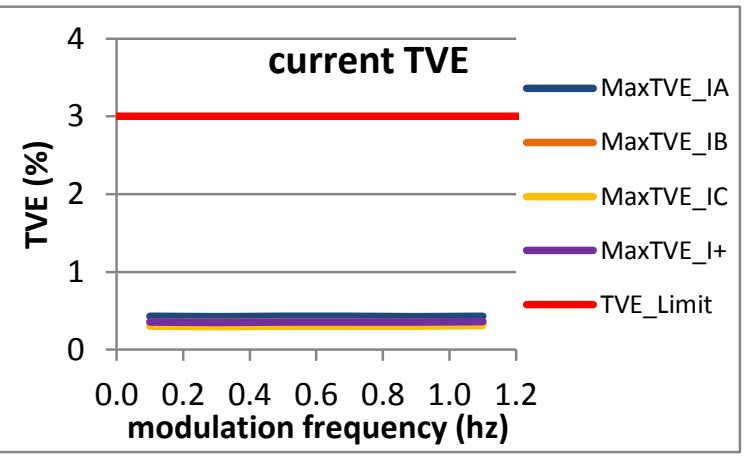

Figure 3825: Fs = 12 FPS

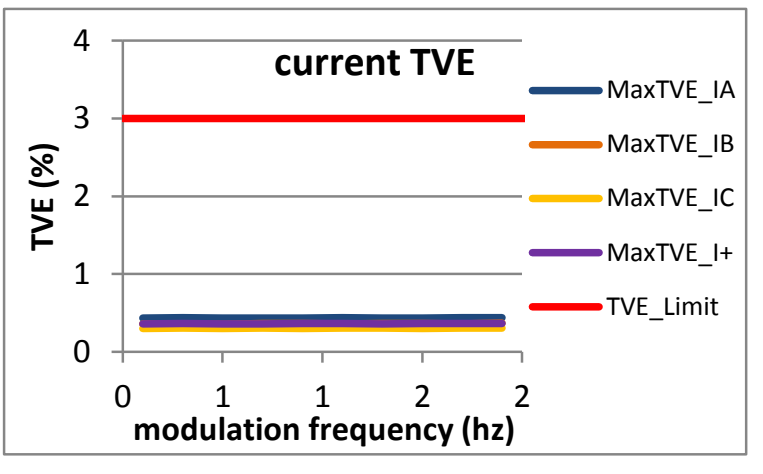

Figure 3823: Fs = 20 FPS

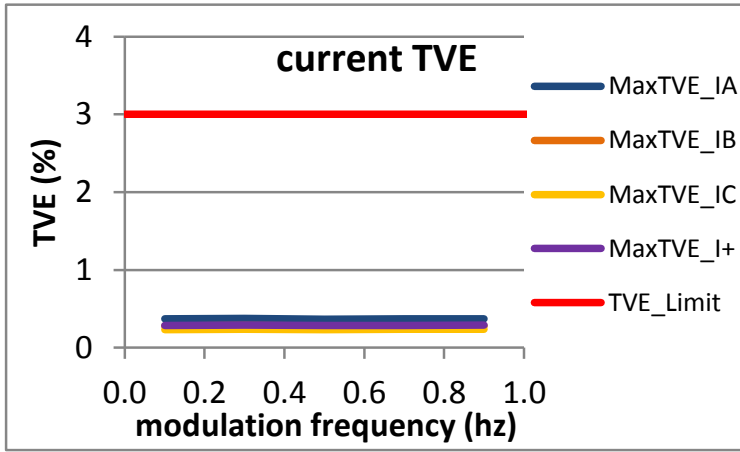

Figure 3826: $\mathrm{fs}=10 \mathrm{FPS}$ 
8.6.3 PMU B dynamic bandwidth measurement: amplitude modulation current TVE: P class

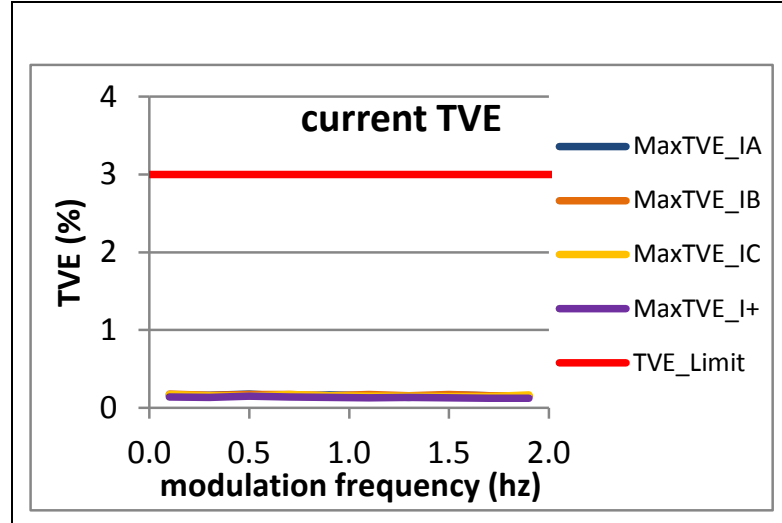

Figure 3827: Fs = 60 FPS

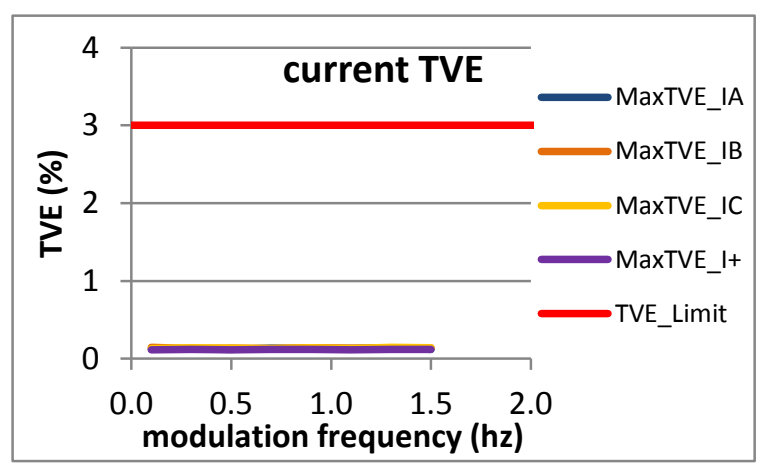

Figure 3830: Fs = 15 FPS

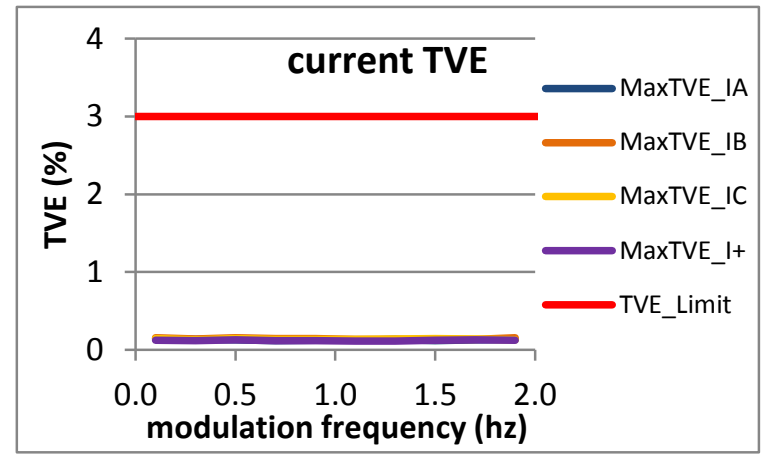

Figure 3828: Fs = 30 FPS

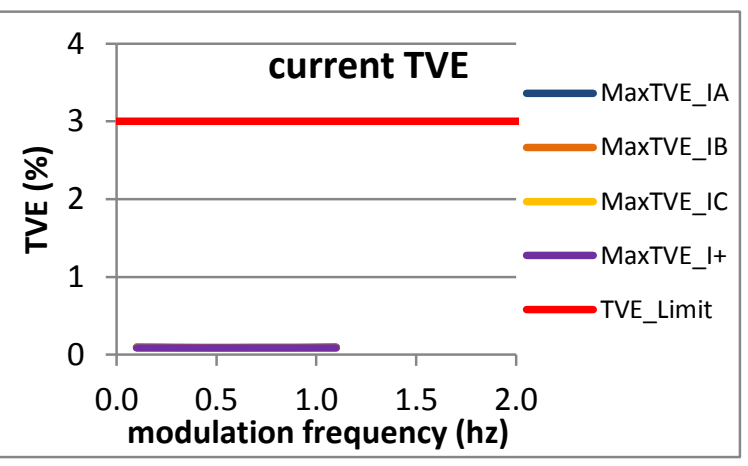

Figure 3831: Fs = 12 FPS

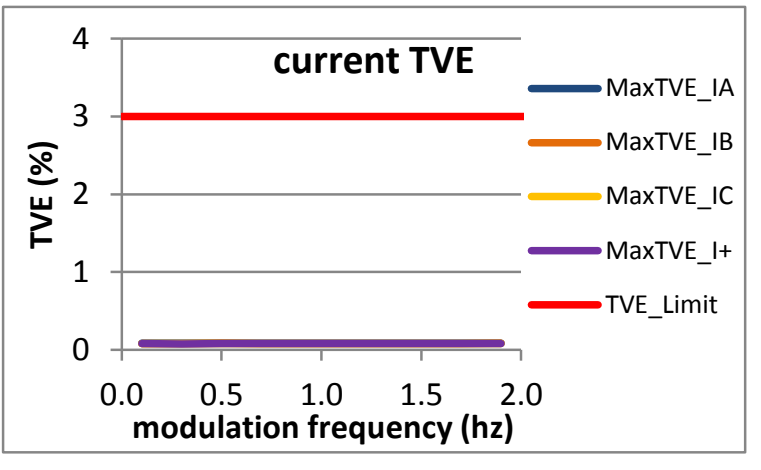

Figure 3829: Fs = 20 FPS

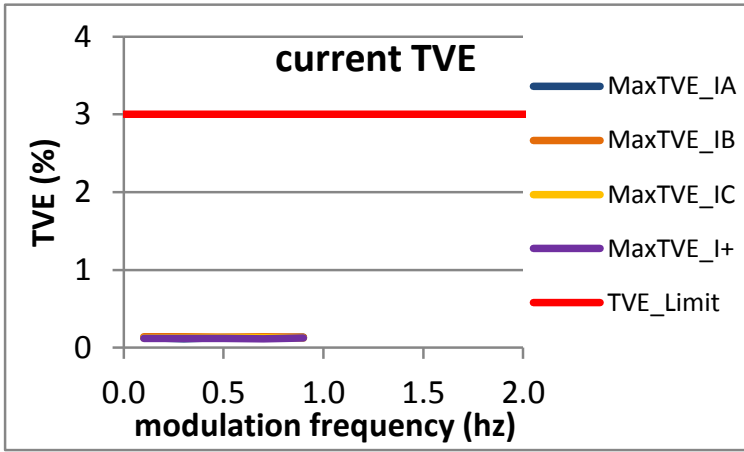

Figure 3832: Fs $=10$ FPS 
8.6.4 PMU C dynamic bandwidth measurement: amplitude modulation current TVE: P class

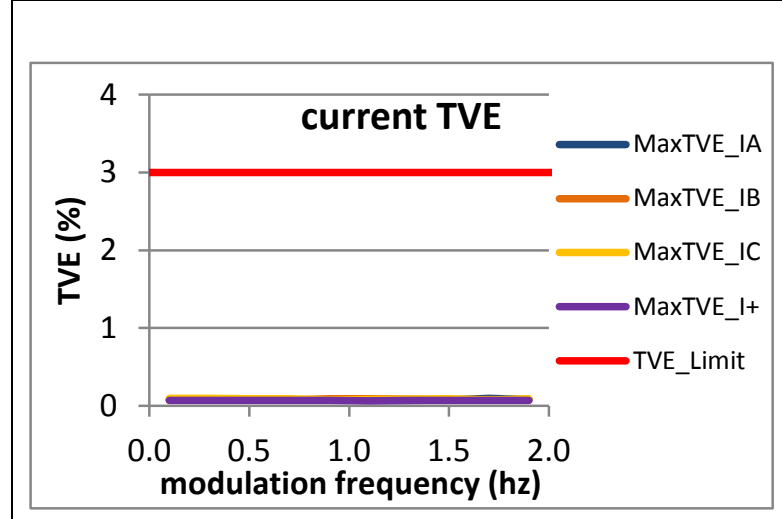

Figure 3833: Fs = 60 FPS

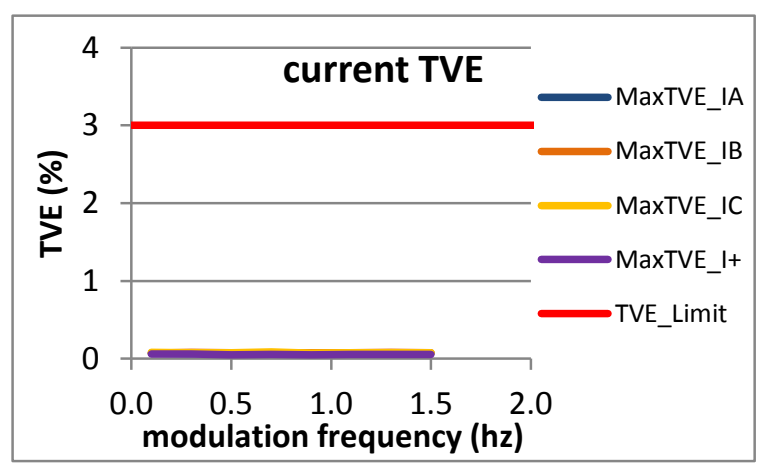

Figure 3836: Fs = 15 FPS

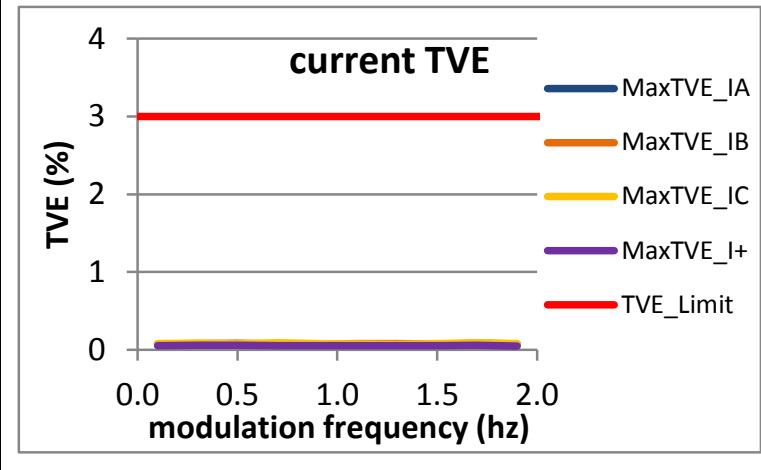

Figure 3834: Fs = 30 FPS

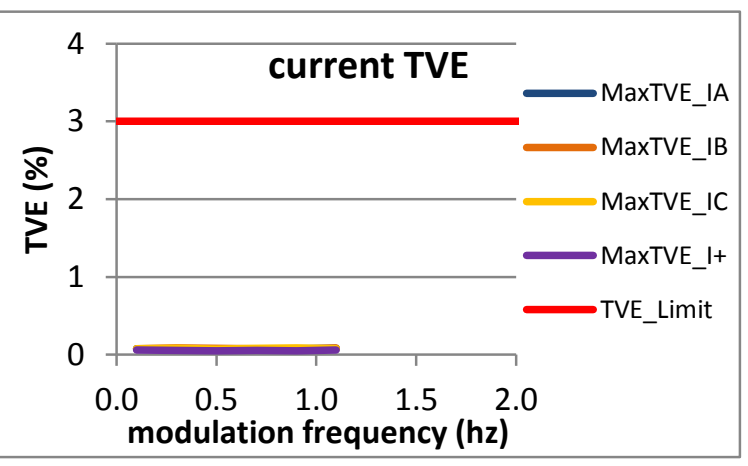

Figure 3837: Fs = 12 FPS

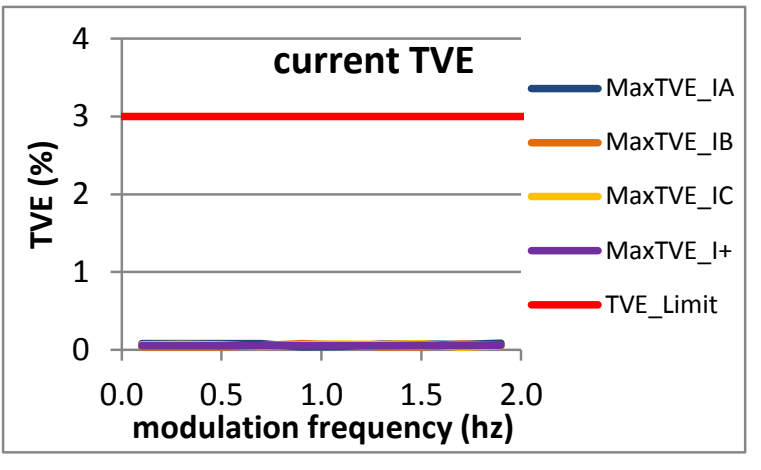

Figure 3835: Fs = 20 FPS

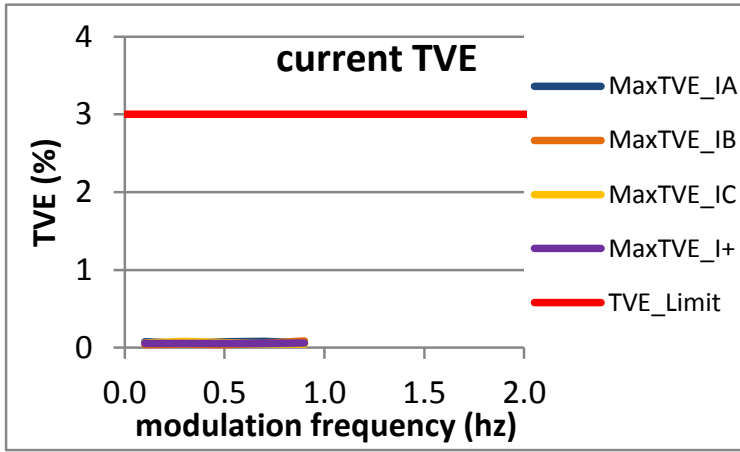

Figure 3838: Fs = 10 FPS 
8.6.5 PMU D dynamic bandwidth measurement: amplitude modulation current TVE: P class

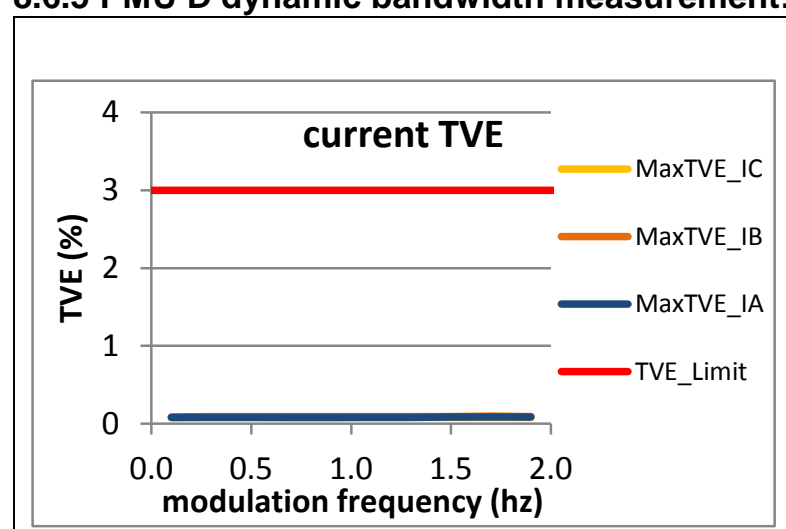

Figure 3839: Fs = 60 FPS

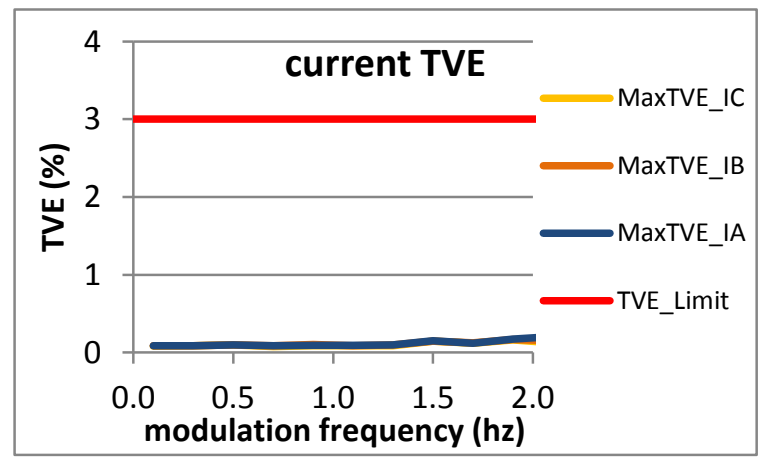

Figure 3842: Fs = 15 FPS

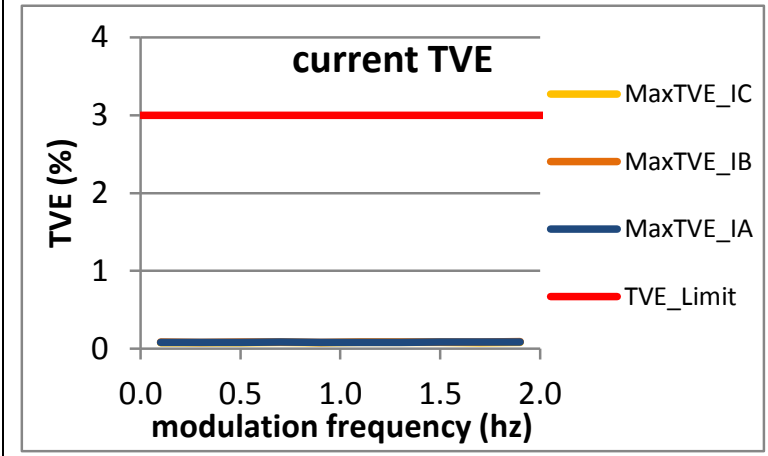

Figure 3840: Fs = 30 FPS

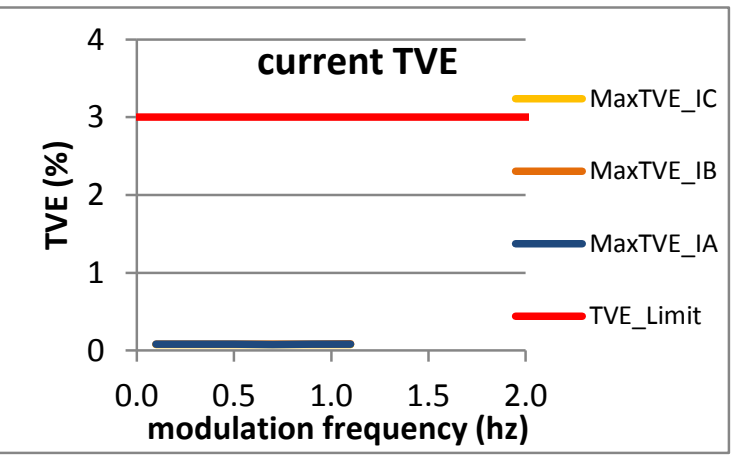

Figure 3843: Fs = 12 FPS

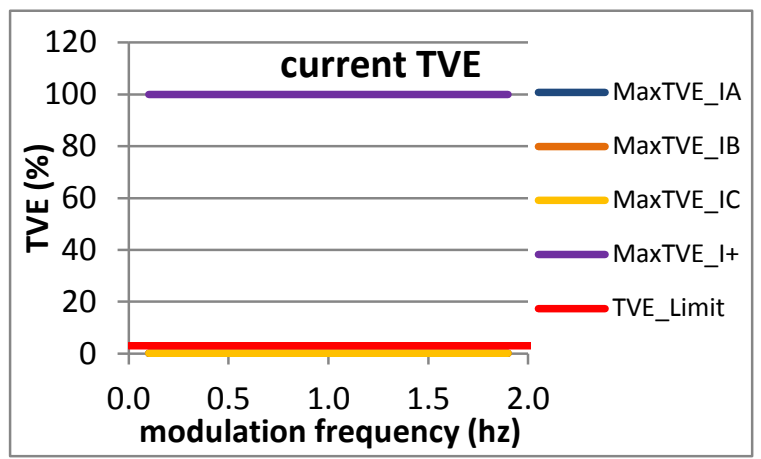

Figure 3841: Fs = 20 FPS

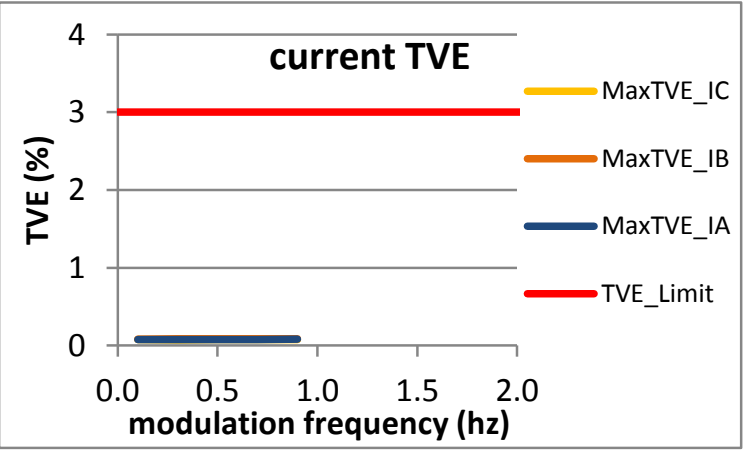

Figure 3844: Fs = 10 FPS

8.6.6 PMU E dynamic bandwidth measurement: amplitude modulation current TVE: P class

PMU E does not support P class 
8.6.7 PMU F dynamic bandwidth measurement: amplitude modulation current TVE: P class

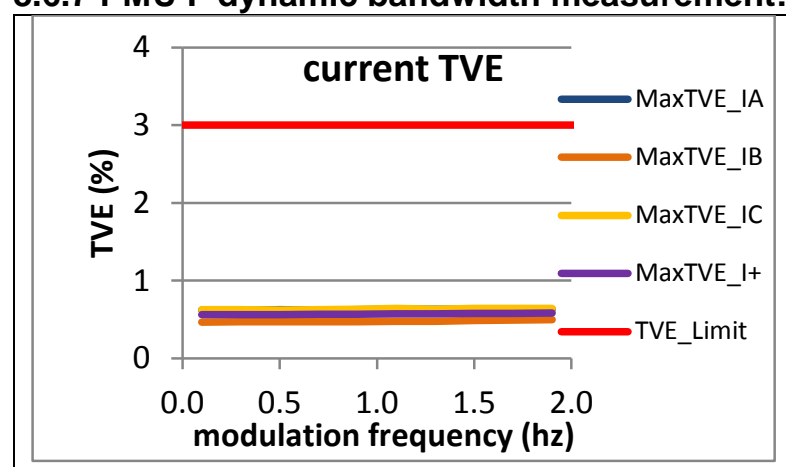

Figure 3845: Fs = 60 FPS

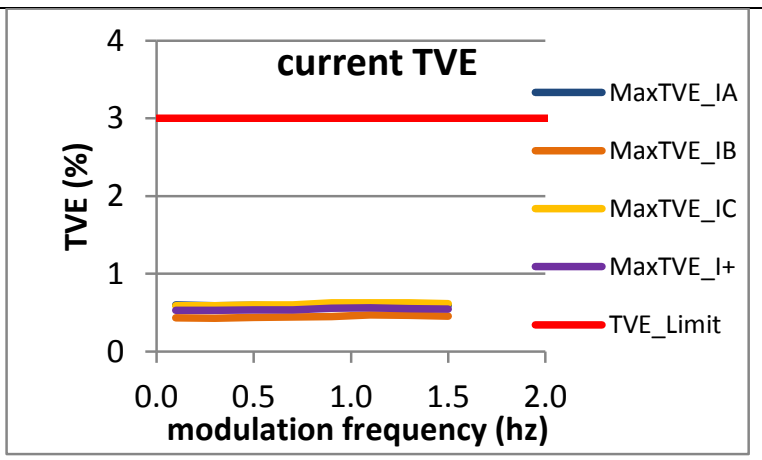

Figure 3848: Fs = 15 FPS

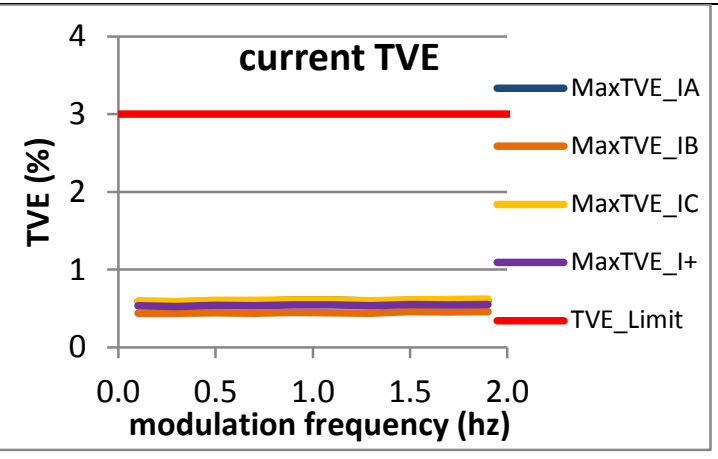

Figure 3846: Fs = 30 FPS

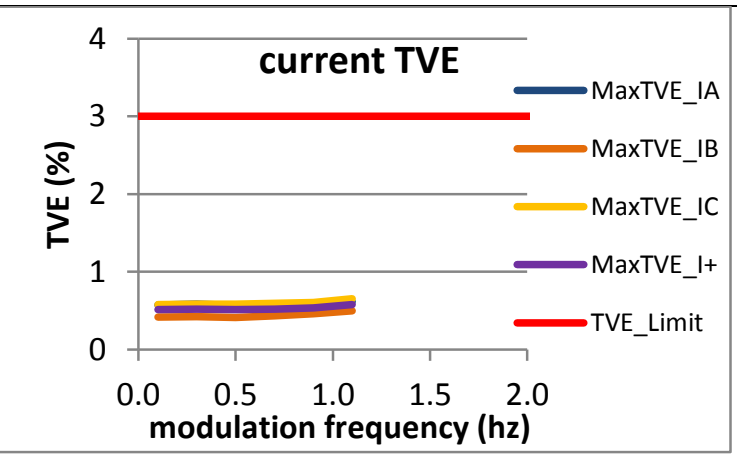

Figure 3849: Fs = 12 FPS

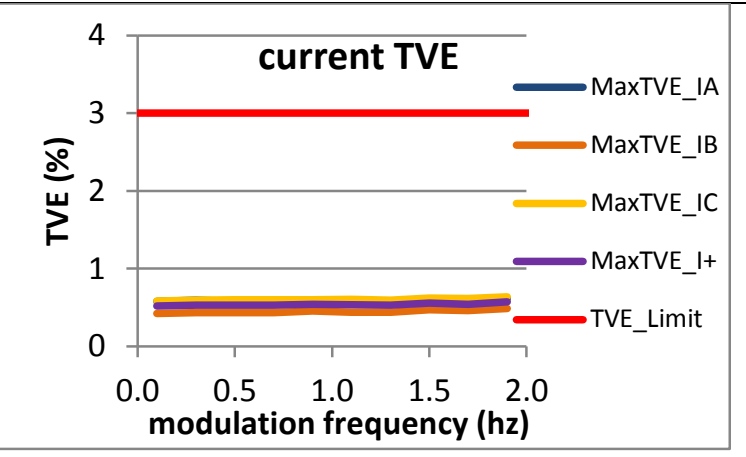

Figure 3847: Fs = 20 FPS

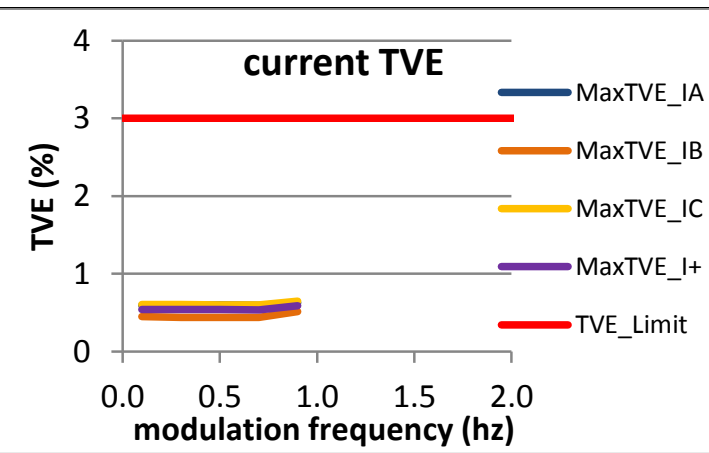

Figure 3850: Fs = 10 FPS

8.6.8 PMU G dynamic bandwidth measurement: amplitude modulation current TVE: P class

PMU G does not support P class 


\subsubsection{PMU H dynamic bandwidth measurement: amplitude modulation current TVE: P class}

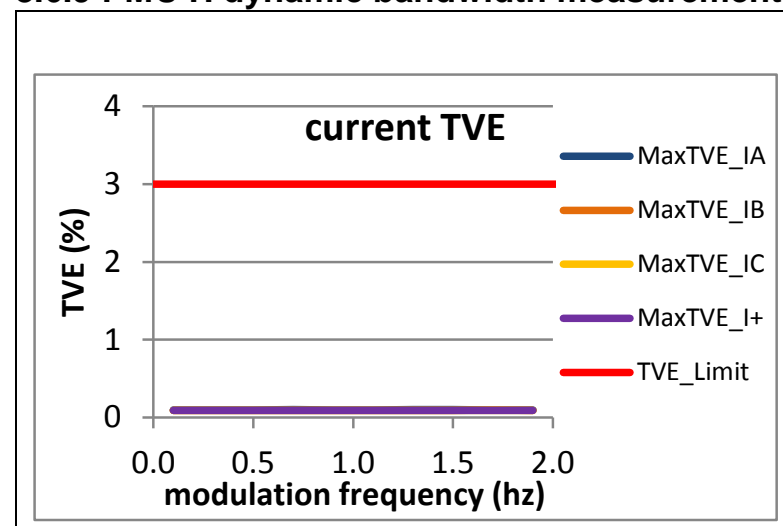

Figure 3851: Fs = 60 FPS

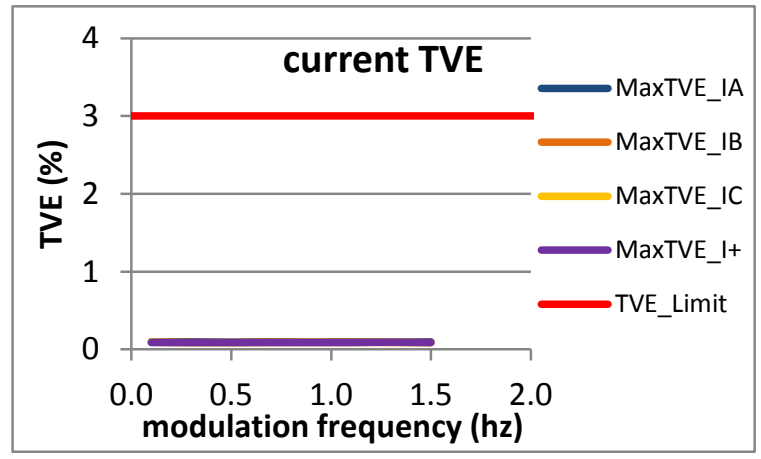

Figure 3854: Fs = 15 FPS

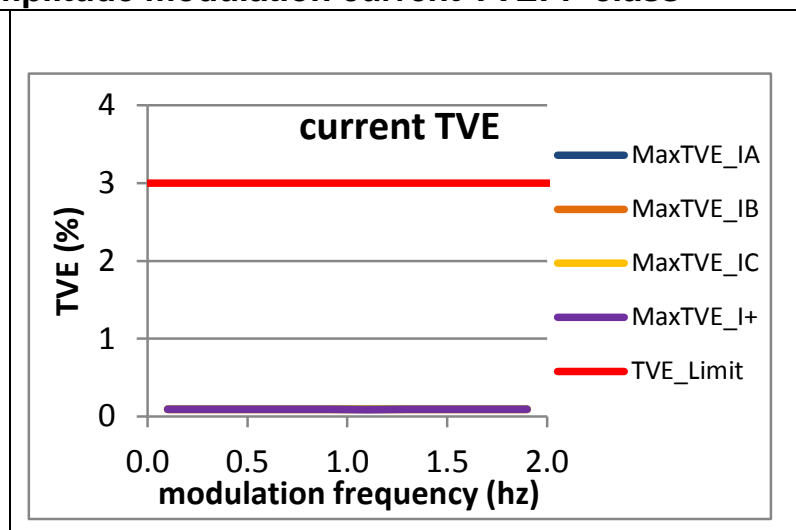

Figure 3852: Fs = 30 FPS

(n)

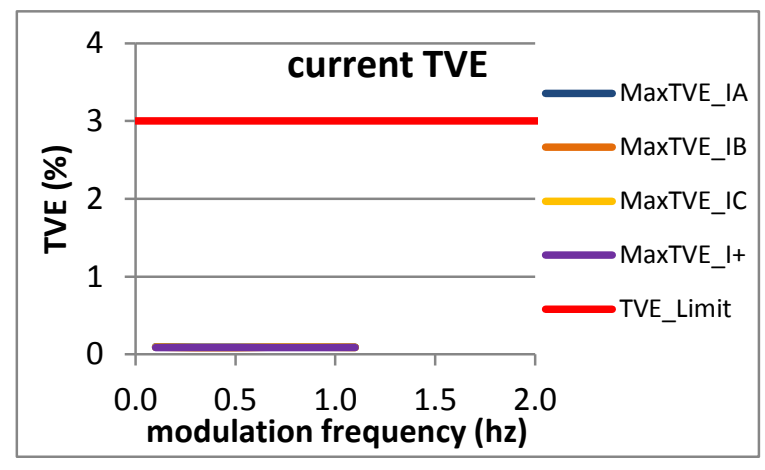

Figure 3855: Fs = 12 FPS

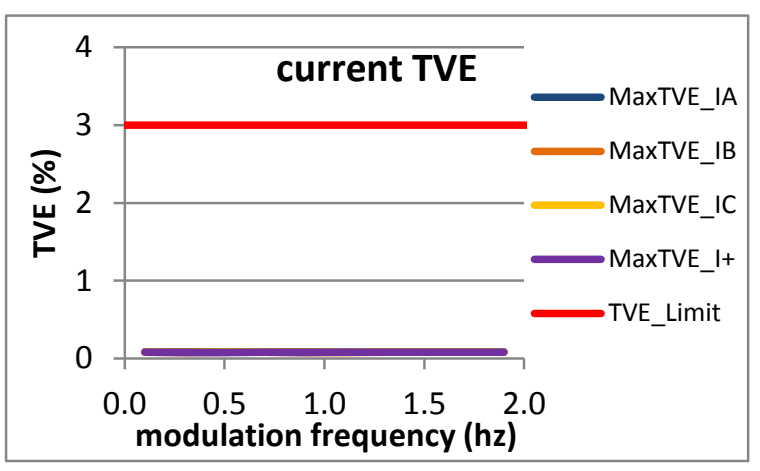

Figure 3853: Fs = 20 FPS

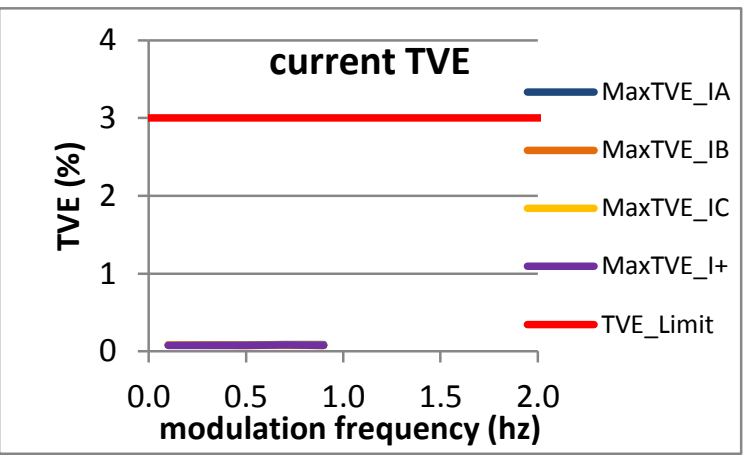

Figure 3856: Fs = 10 FPS

8.6.10 PMU I dynamic bandwidth measurement: amplitude modulation current TVE: P class

PMU I does not support P class

8.6.11 PMU J dynamic bandwidth measurement: amplitude modulation current TVE: P class 


\subsection{Dynamic bandwidth measurement: amplitude modulation frequency error: P class}

\subsubsection{C37.118.1 Annex C dynamic bandwidth measurement: amplitude modulation frequency error: $P$ class}

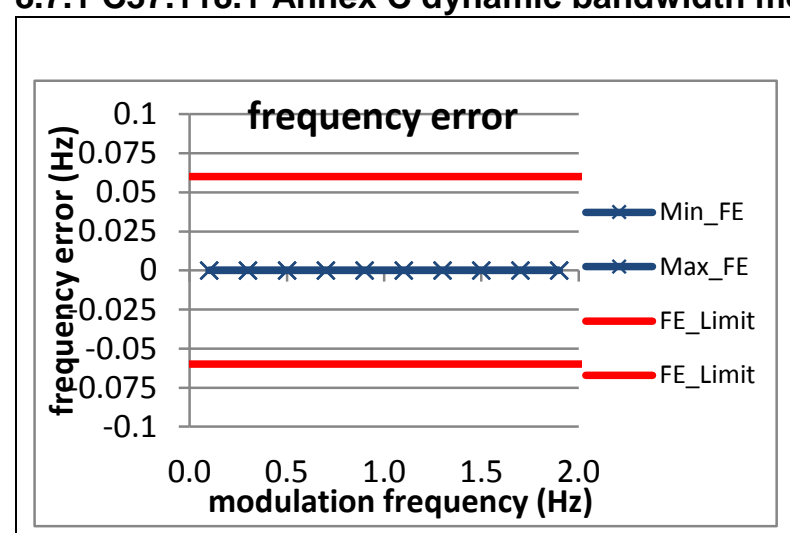

Figure 3857: Fs = 60 FPS

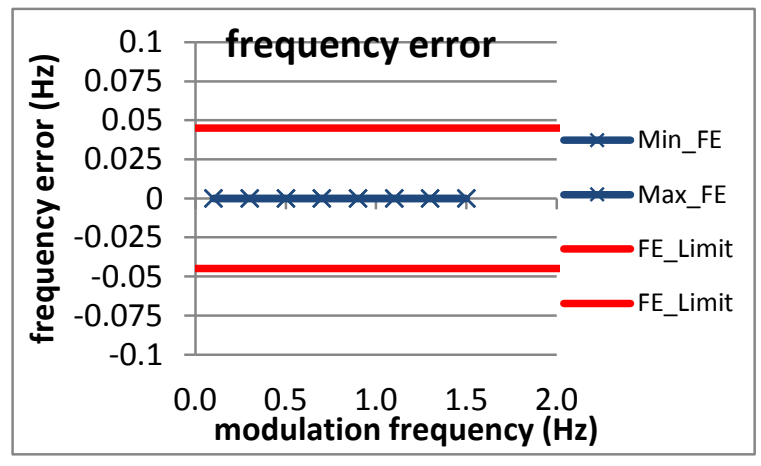

Figure 3860: Fs = 15 FPS

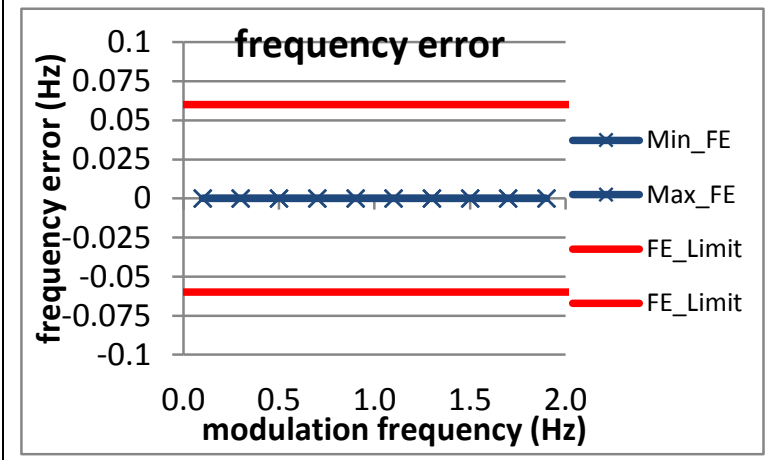

Figure 3858: Fs = 30 FPS

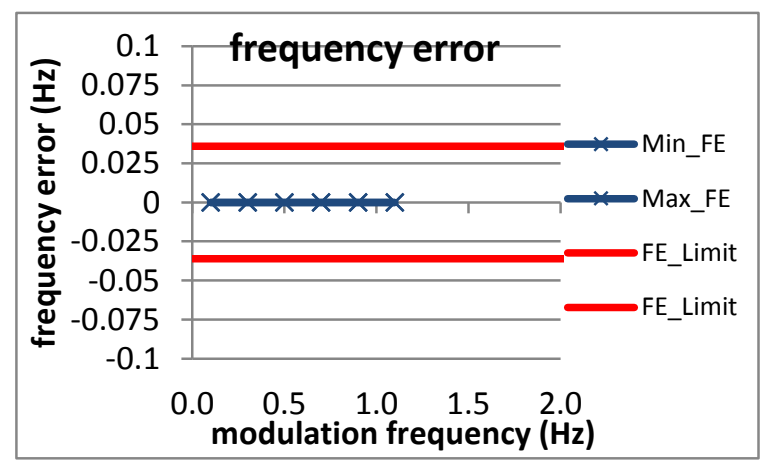

Figure 3861: Fs = 12 FPS

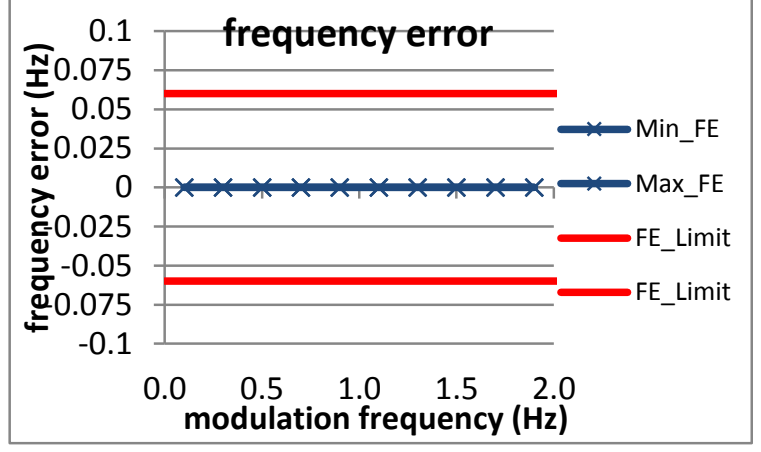

Figure 3859: Fs = 20 FPS

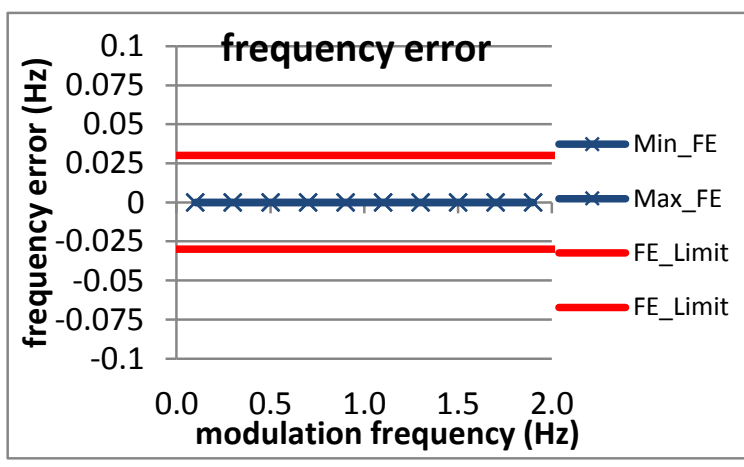

Figure 3862: Fs = 10 FPS 
8.7.2 PMU A dynamic bandwidth measurement: amplitude modulation frequency error: $P$ class

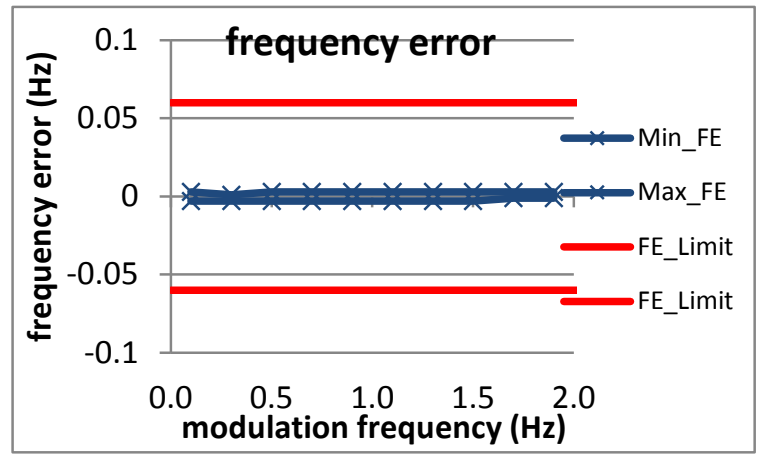

Figure 3863: Fs = 60 FPS

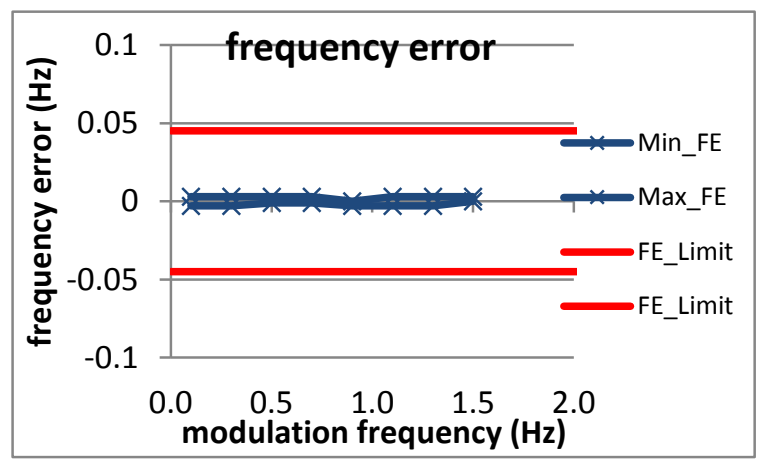

Figure 3866: Fs = 15 FPS

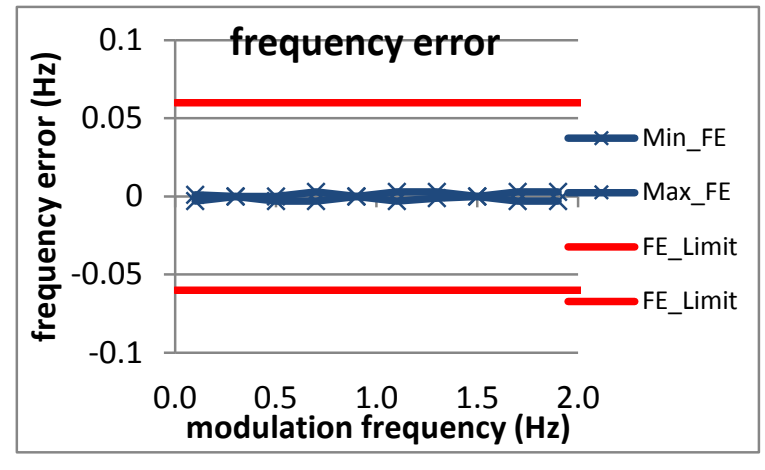

Figure 3864: Fs = 30 FPS

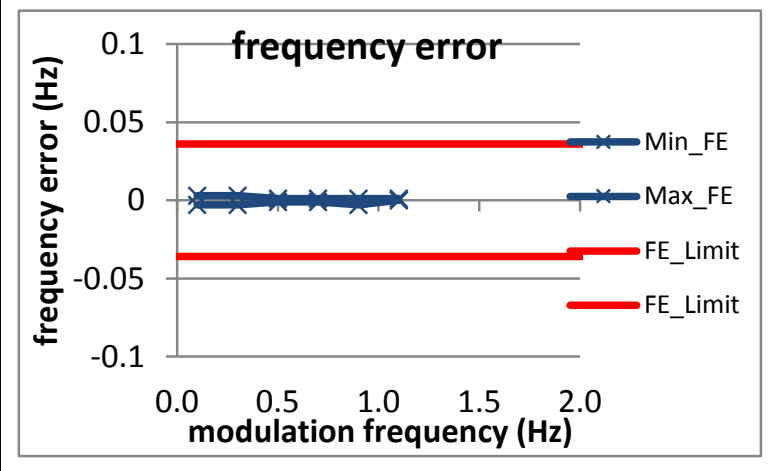

Figure 3867: Fs = 12 FPS

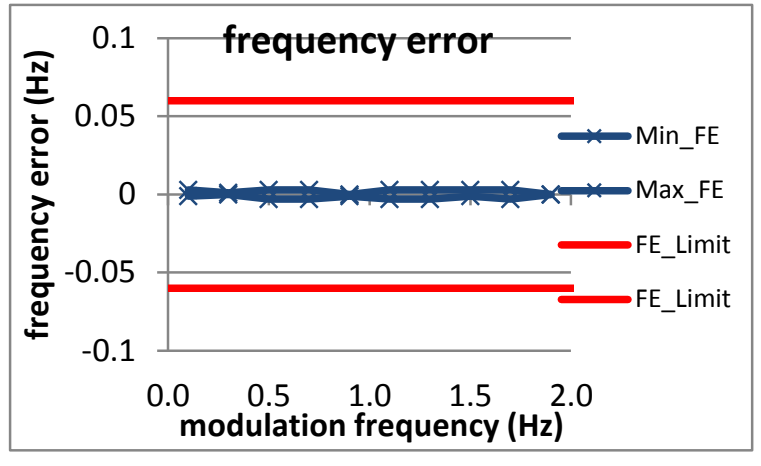

Figure 3865: Fs = 20 FPS

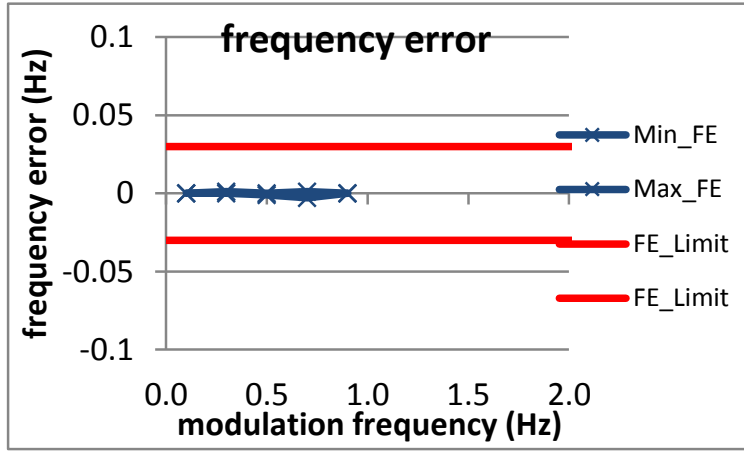

Figure 3868: Fs = 10 FPS 
8.7.3 PMU B dynamic bandwidth measurement: amplitude modulation frequency error: P class

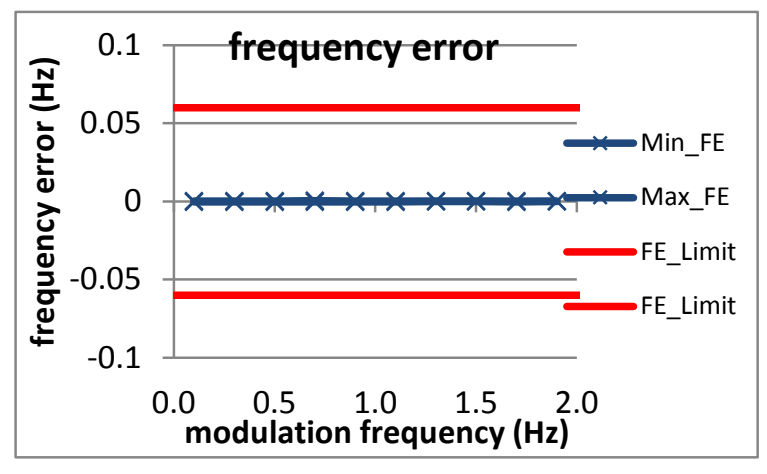

Figure 3869: Fs = 60 FPS

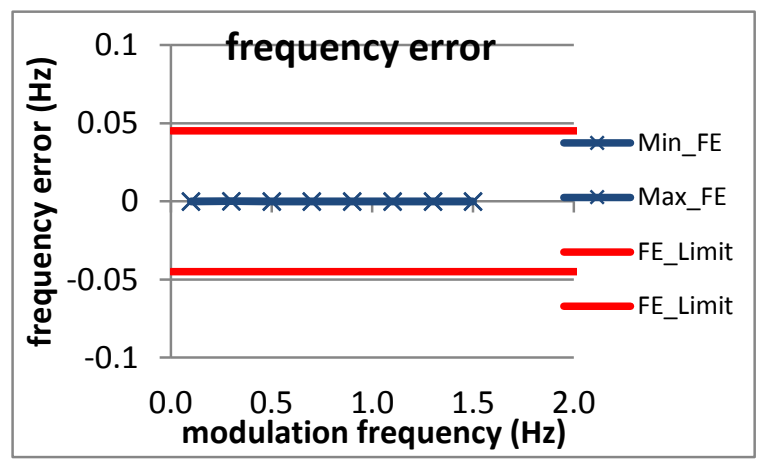

Figure 3872: Fs = 15 FPS

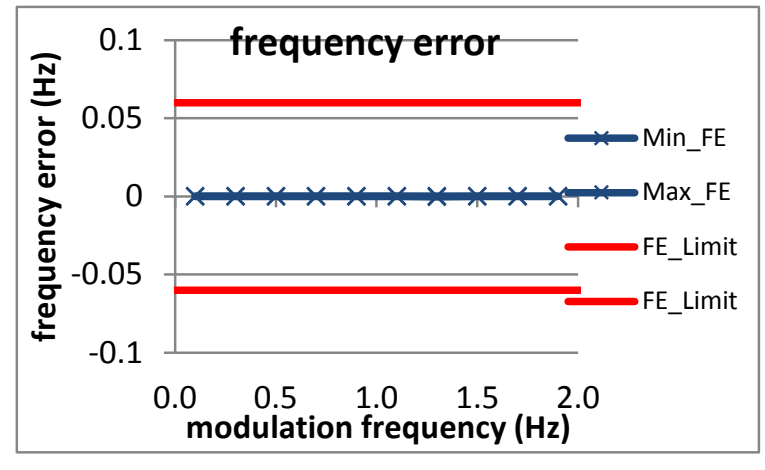

Figure 3870: Fs = 30 FPS

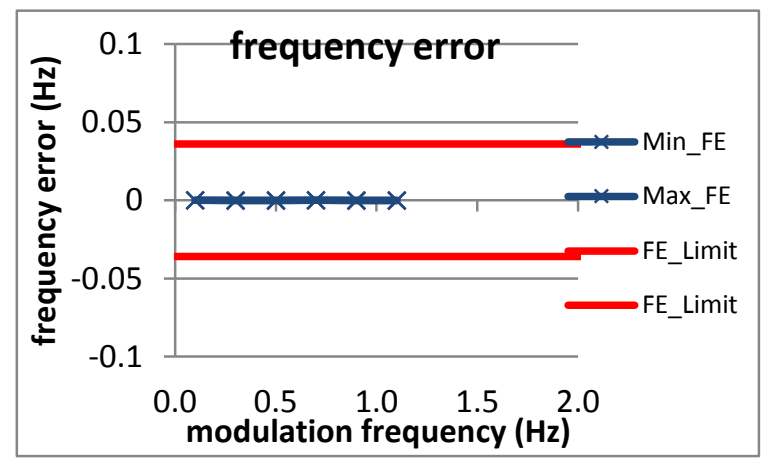

Figure 3873: Fs = 12 FPS

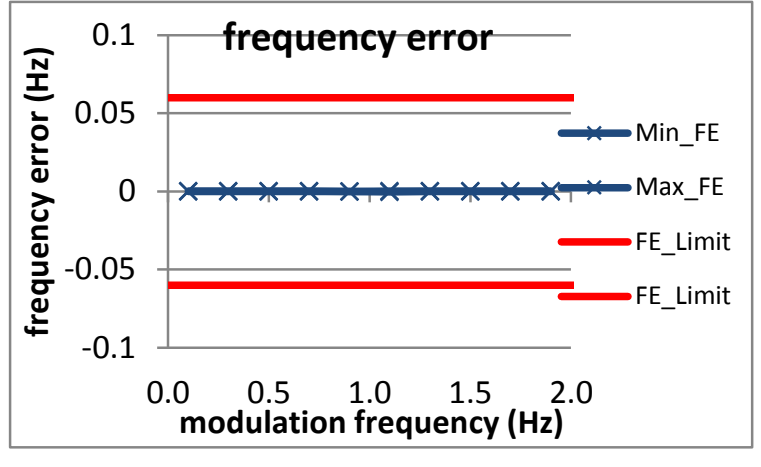

Figure 3871: Fs = 20 FPS

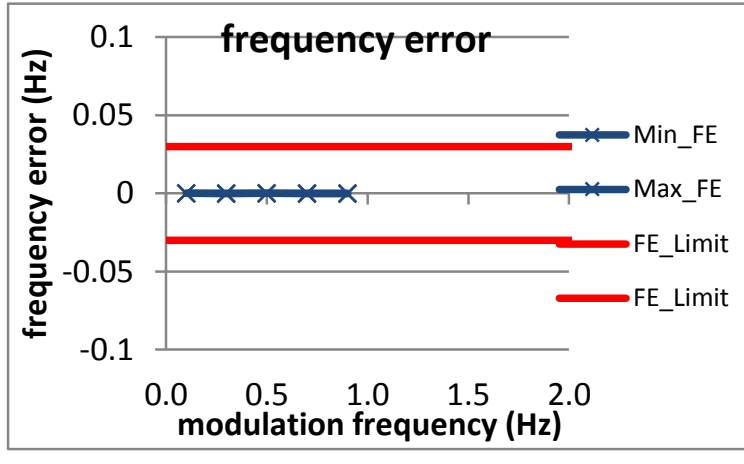

Figure 3874: Fs = 10 FPS 
8.7.4 PMU C dynamic bandwidth measurement: amplitude modulation frequency error: $P$ class

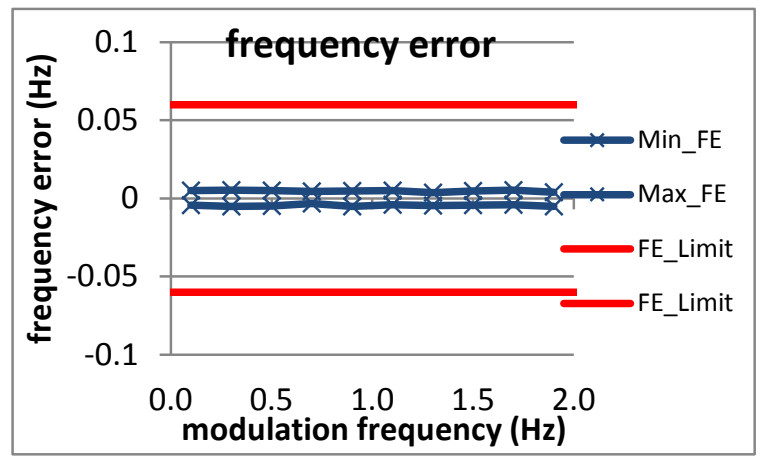

Figure 3875: Fs = 60 FPS

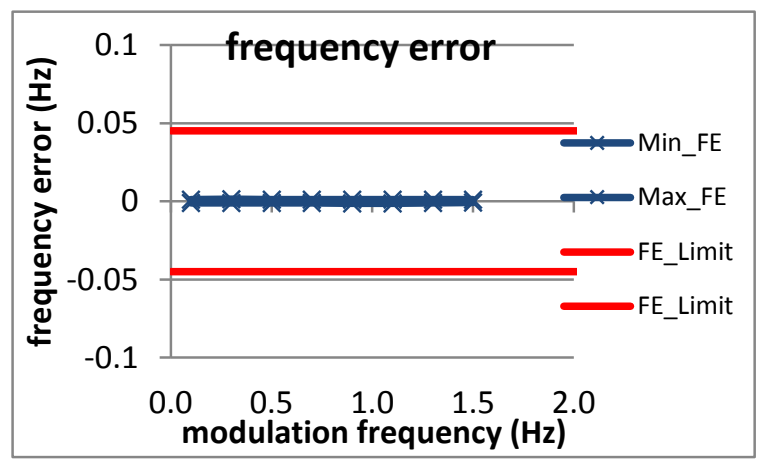

Figure 3878: Fs = 15 FPS

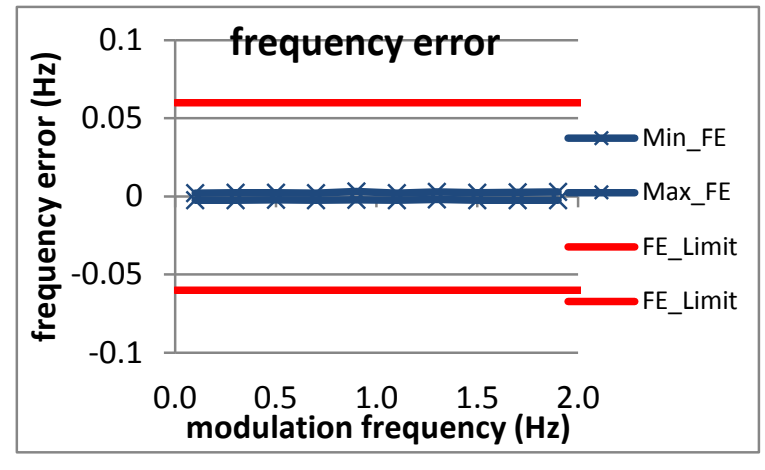

Figure 3876: Fs = 30 FPS

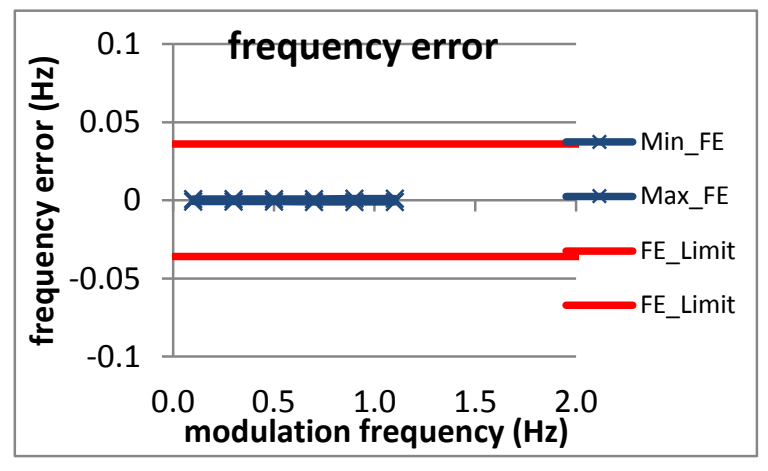

Figure 3879: Fs = 12 FPS

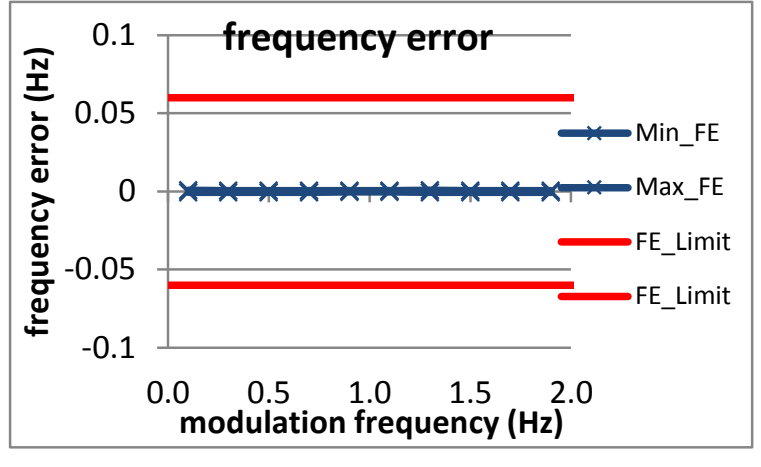

Figure 3877: Fs $=20$ FPS

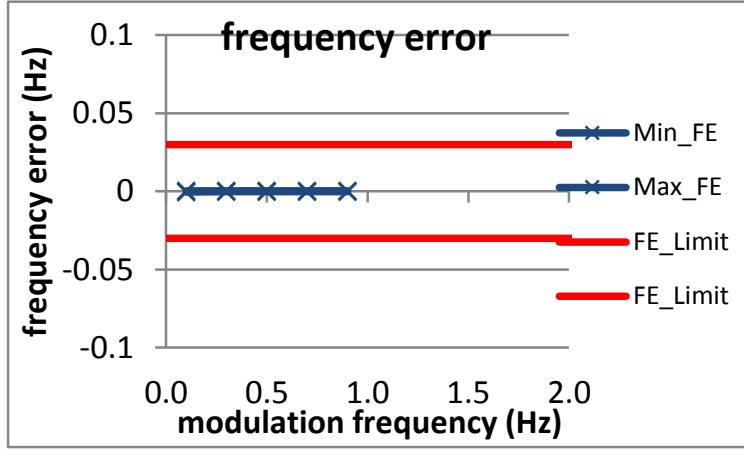

Figure 3880: Fs = 10 FPS 
8.7.5 PMU D dynamic bandwidth measurement: amplitude modulation frequency error: P class

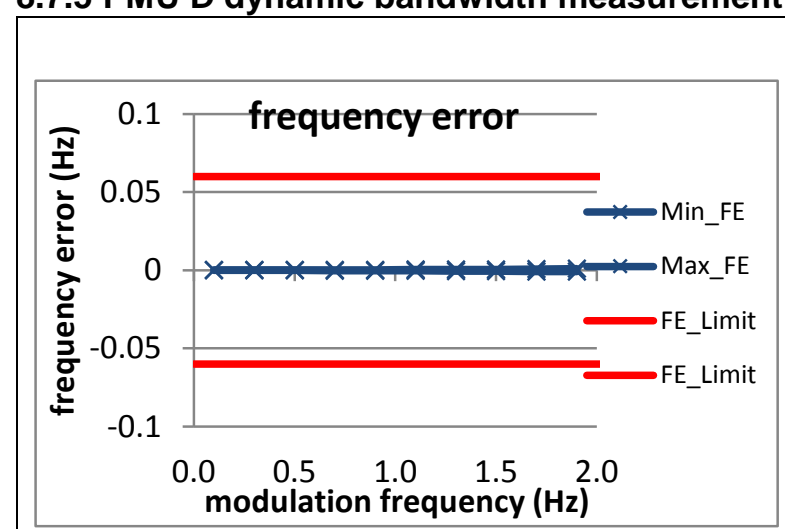

Figure 3881: Fs = 60 FPS

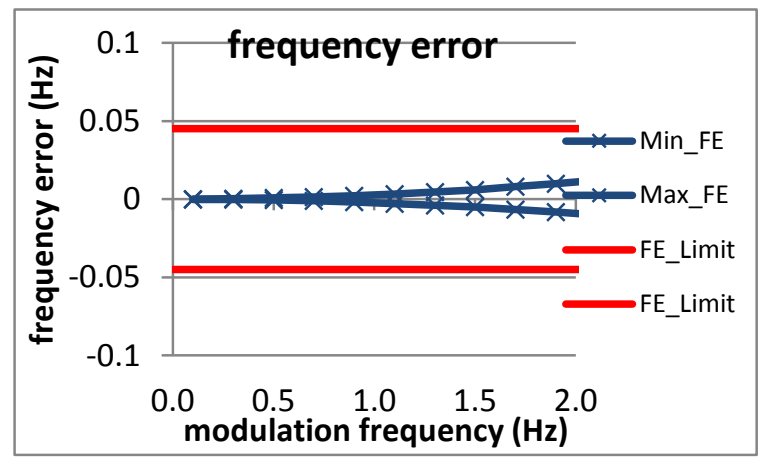

Figure 3884: Fs = 15 FPS

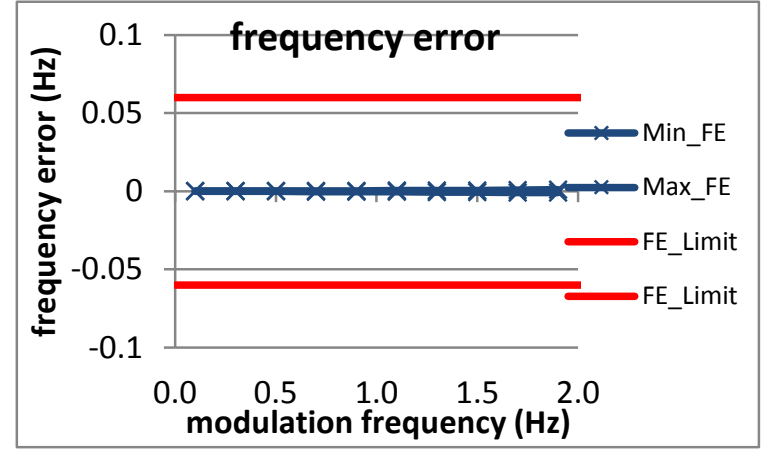

Figure 3882: Fs = 30 FPS

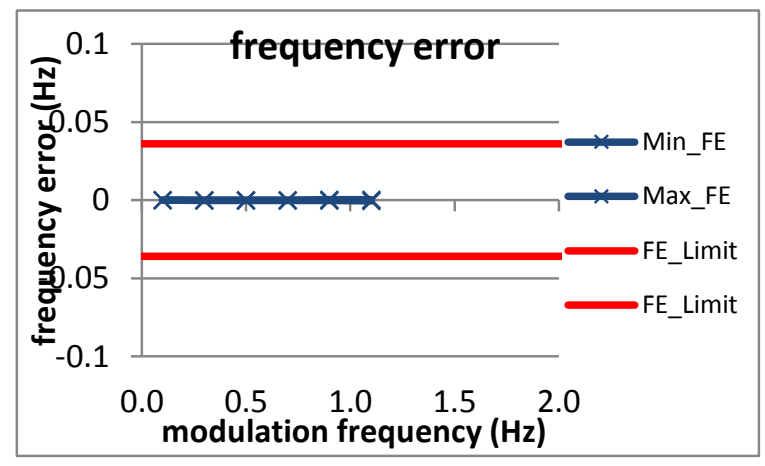

Figure 3885: Fs = 12 FPS

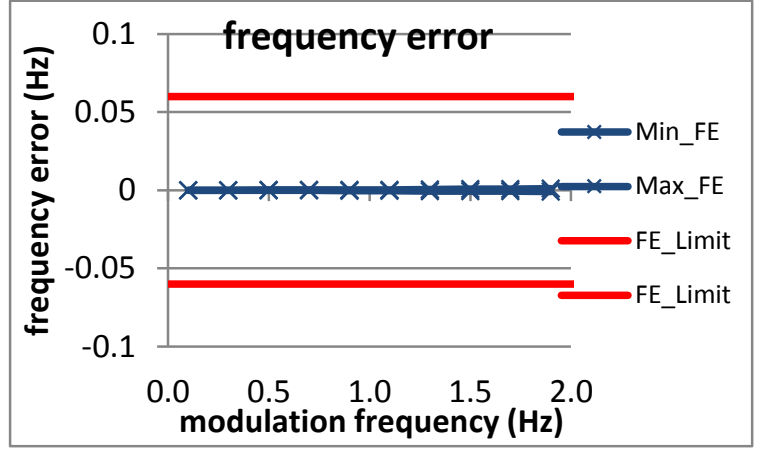

Figure 3883: Fs = 20 FPS

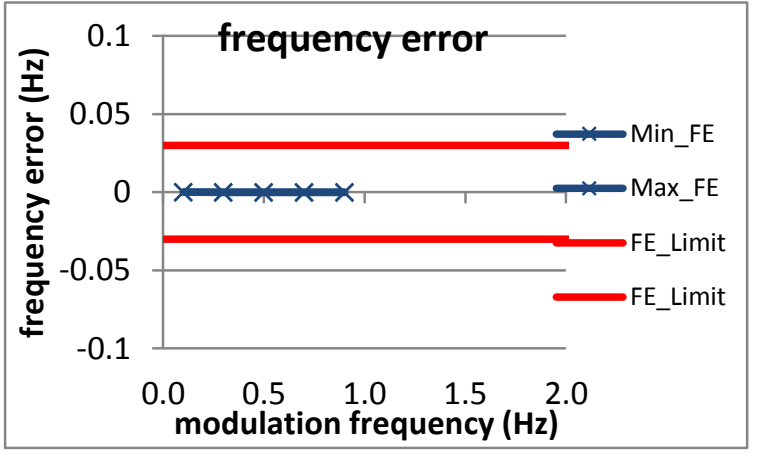

Figure 3886: Fs = 10 FPS

8.7.6 PMU E dynamic bandwidth measurement: amplitude modulation frequency error: $P$ class

PMU E does not support P class 
8.7.7 PMU F dynamic bandwidth measurement: amplitude modulation frequency error: $P$ class

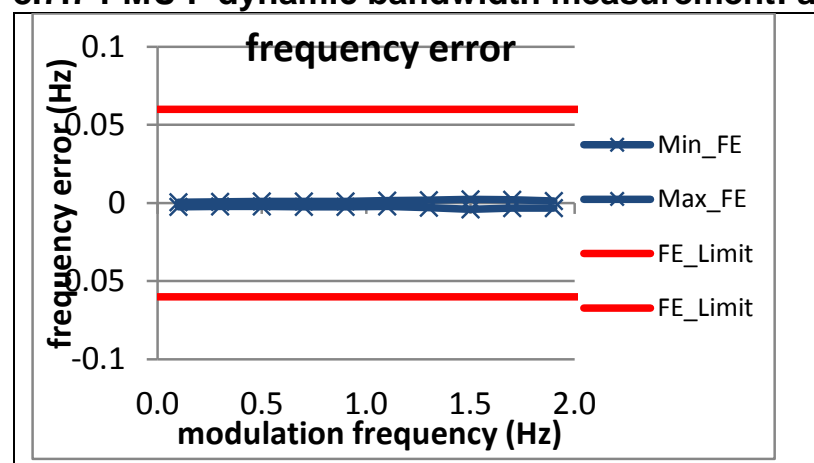

Figure 3887: Fs = 60 FPS

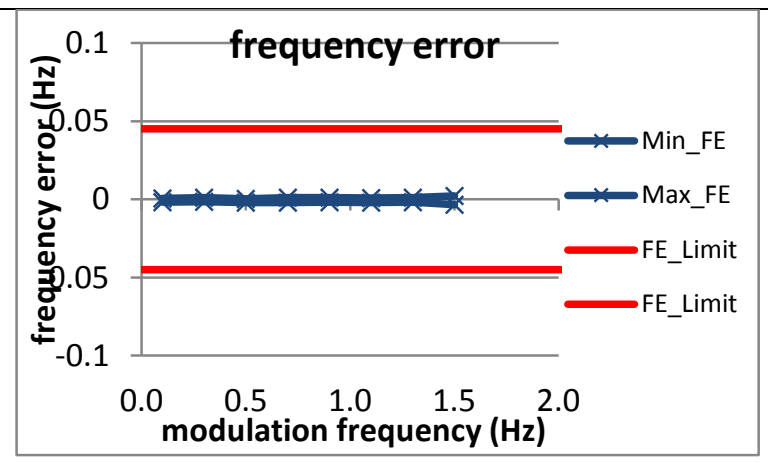

Figure 3890: Fs = 15 FPS

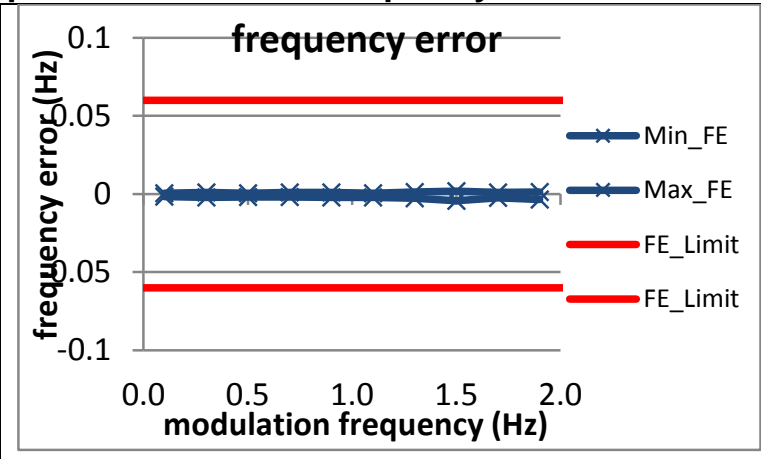

Figure 3888: Fs = 30 FPS

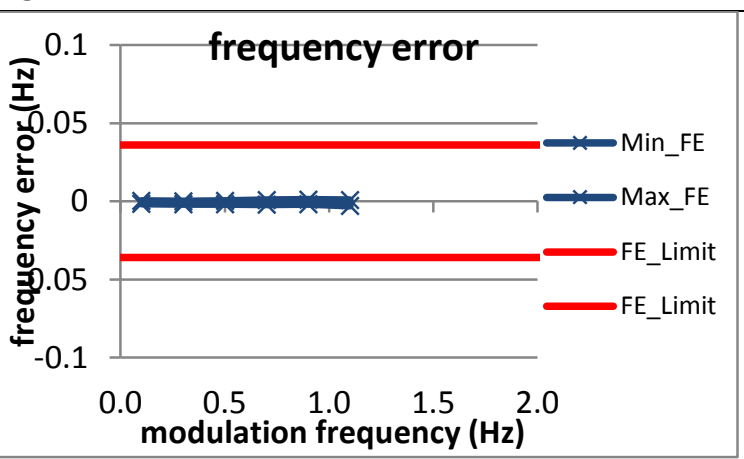

Figure 3891: Fs = 12 FPS

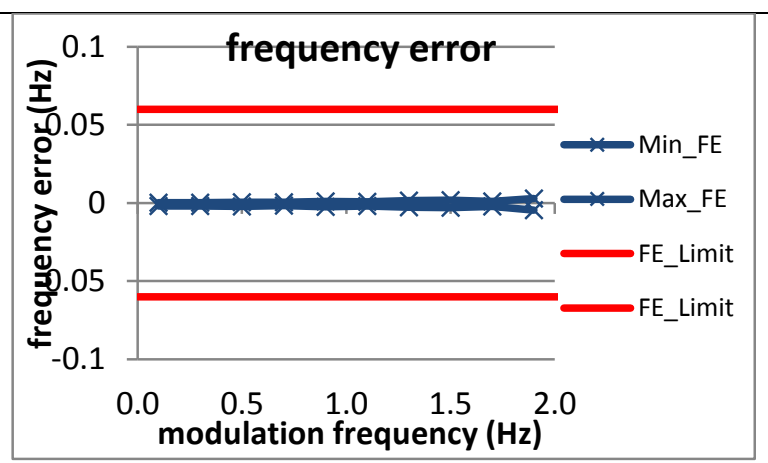

Figure 3889: Fs = 20 FPS

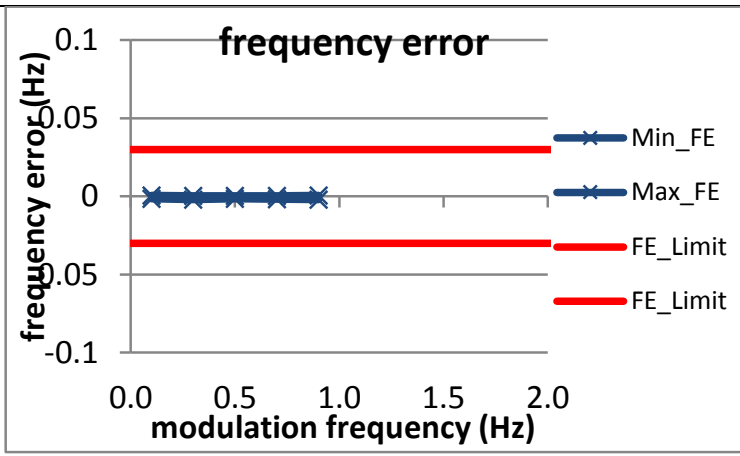

Figure 3892: Fs = 10 FPS

8.7.8 PMU G dynamic bandwidth measurement: amplitude modulation frequency error: P class PMU G does not support P class 
8.7.9 PMU H dynamic bandwidth measurement: amplitude modulation frequency error: P class

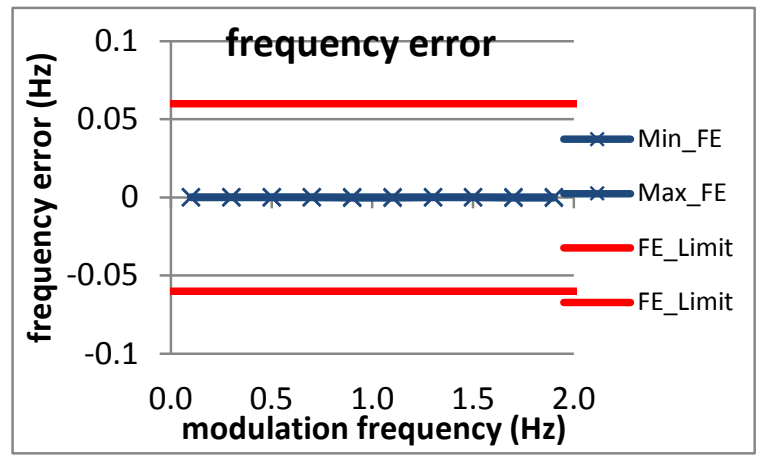

Figure 3893: Fs = 60 FPS

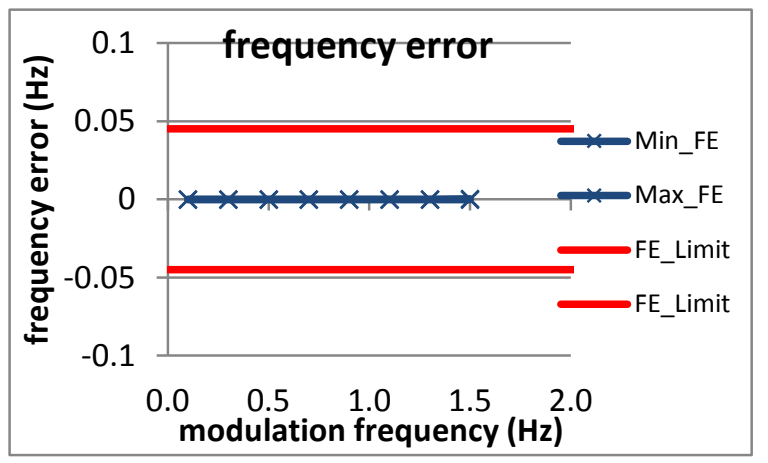

Figure 3896: Fs = 15 FPS

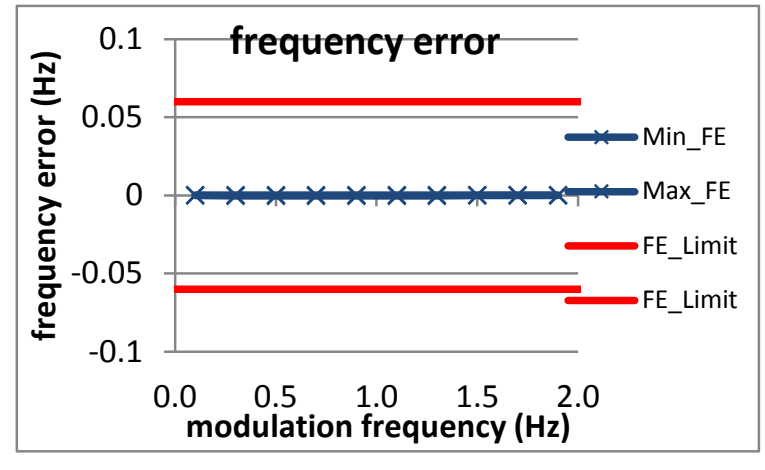

Figure 3894: Fs = 30 FPS

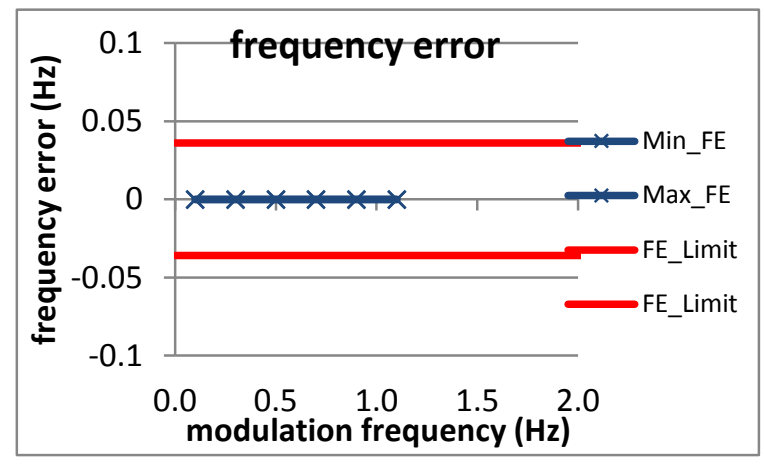

Figure 3897: Fs = 12 FPS

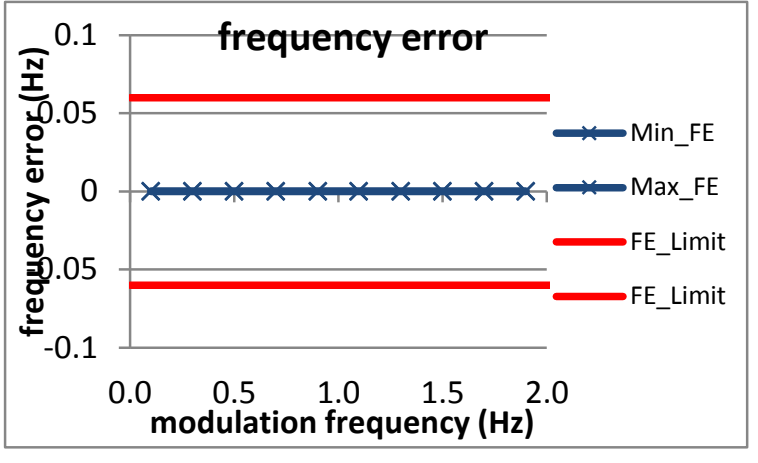

Figure 3895: Fs $=20$ FPS

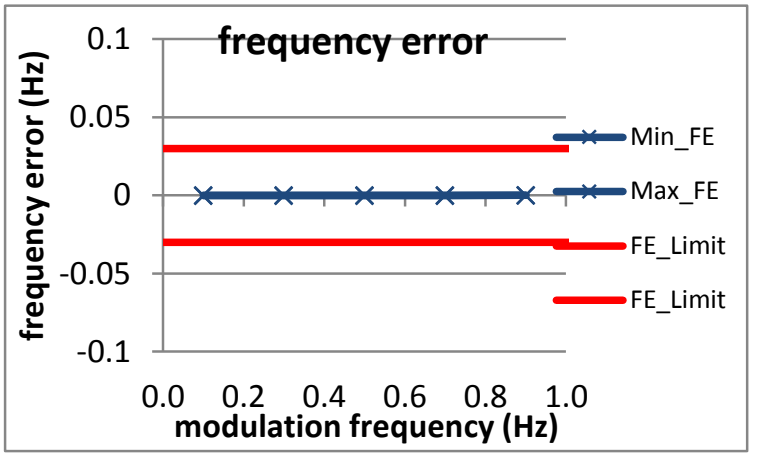

Figure 3898: Fs = 10 FPS

\subsubsection{PMU I dynamic bandwidth measurement: amplitude modulation frequency error: P class}

PMU I does not support P class 
8.7.11 PMU J dynamic bandwidth measurement: amplitude modulation frequency error: $P$ class

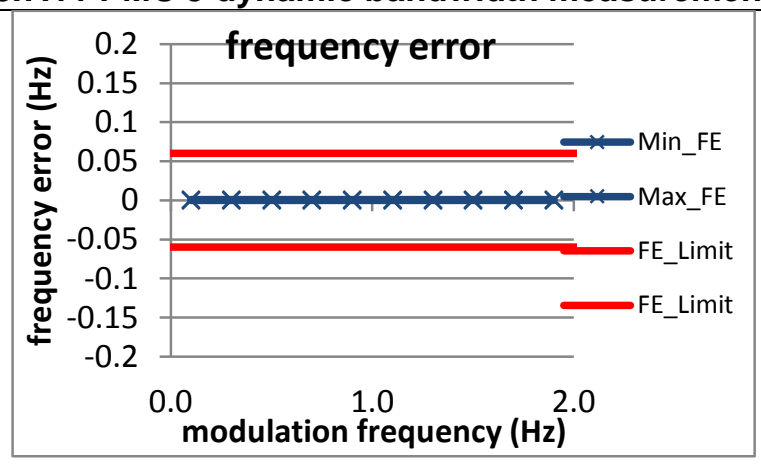

Figure 3899: Fs = 60 FPS

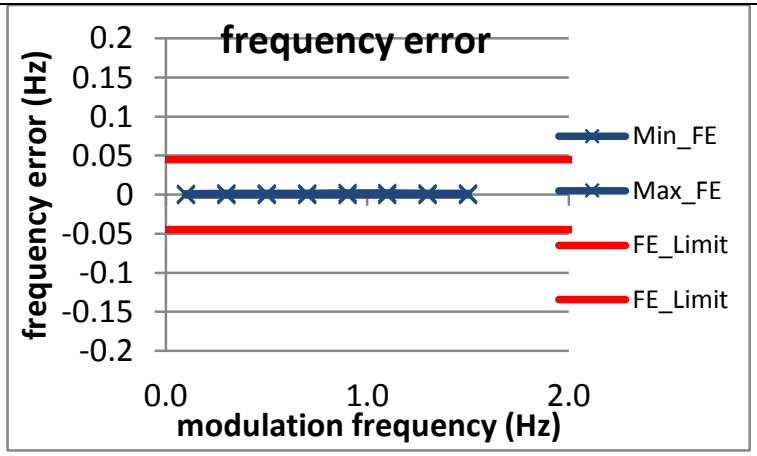

Figure 3902: Fs = 15 FPS

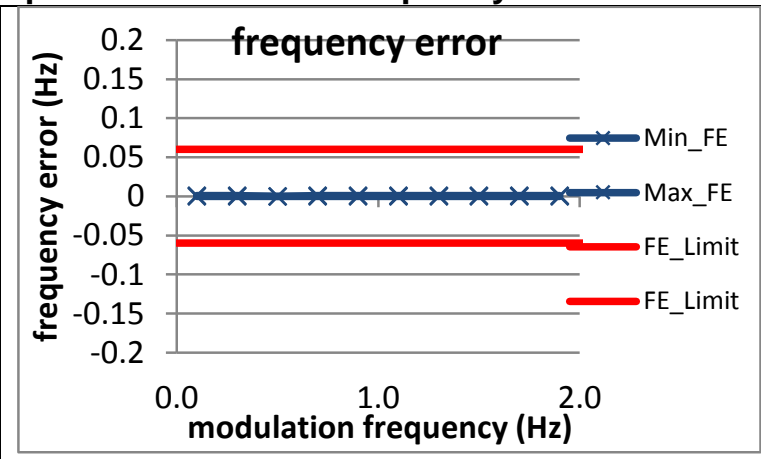

Figure 3900: Fs = 30 FPS

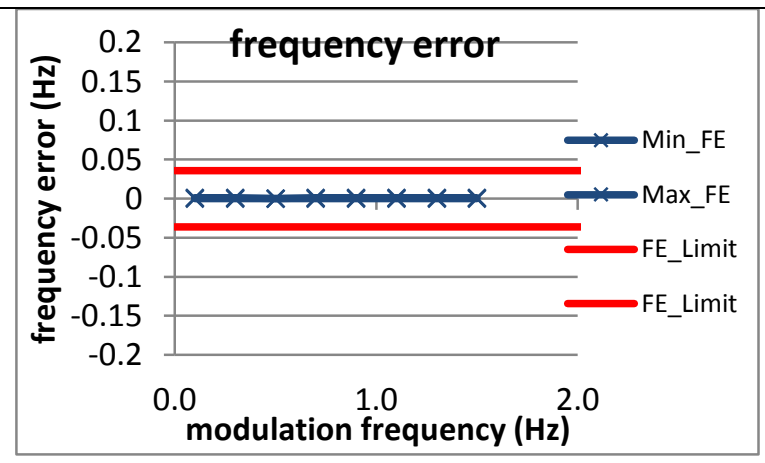

Figure 3903: Fs = 12 FPS

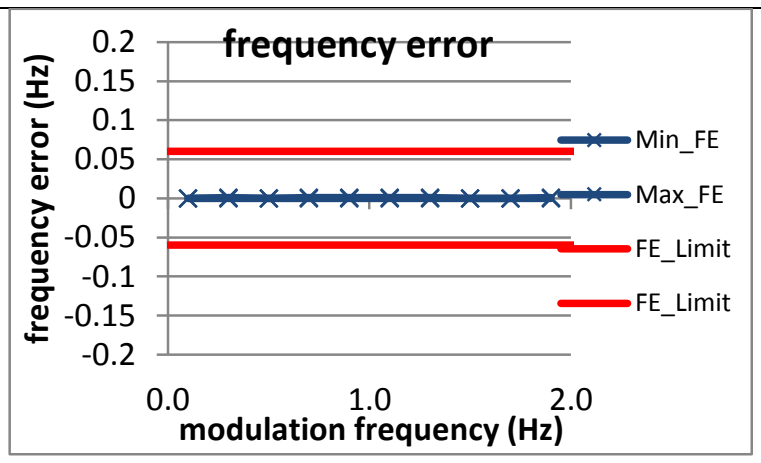

Figure 3901: Fs = 20 FPS

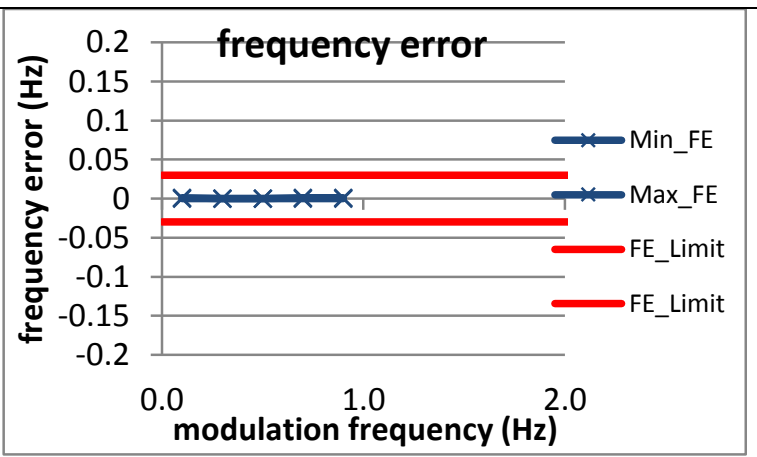

Figure 3904: Fs = 10 FPS 
8.8 dynamic bandwidth measurement: amplitude modulation ROCOF error: P class

8.8.1 C37.118.1 Annex C dynamic bandwidth measurement: amplitude modulation ROCOF error: P class

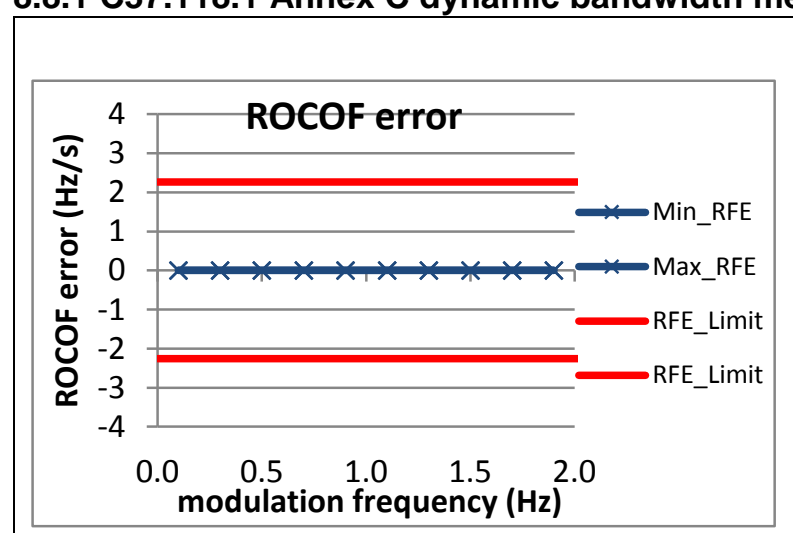

Figure 3905: Fs = 60 FPS

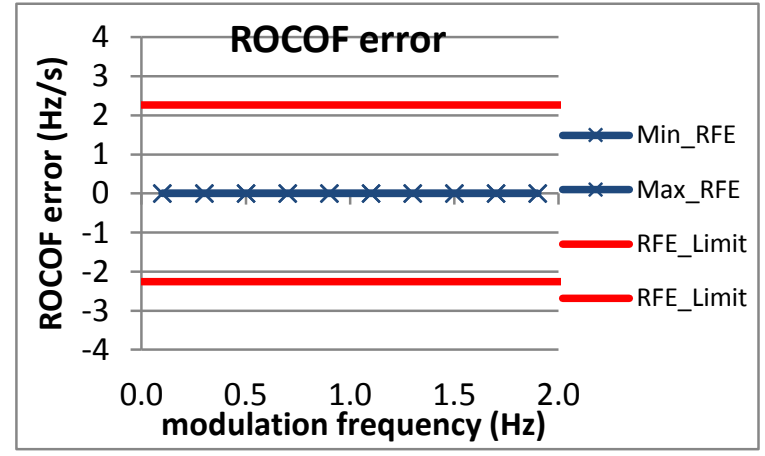

Figure 3906: Fs = 30 FPS

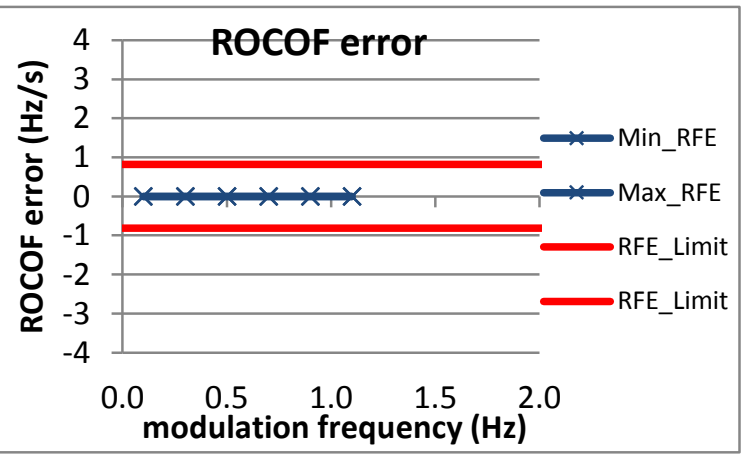

Figure 3909: Fs = 12 FPS

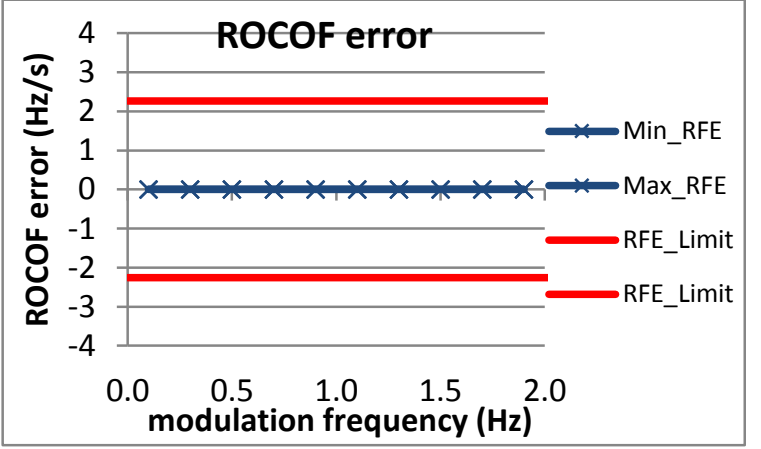

Figure 3907: Fs = 20 FPS

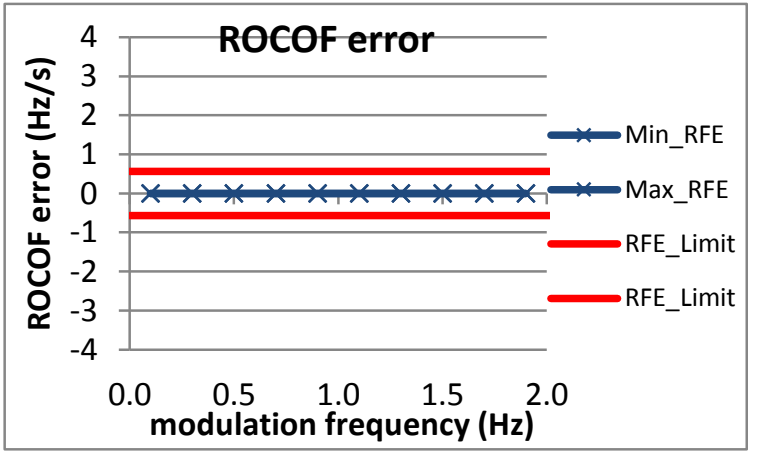

Figure 3910: Fs = 10 FPS

Figure 3908: Fs = 15 FPS 
8.8.2 PMU A dynamic bandwidth measurement: amplitude modulation ROCOF error: P class

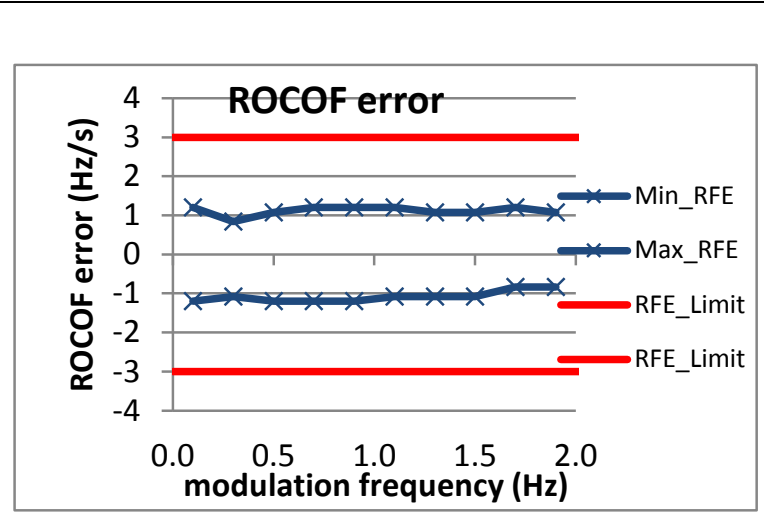

Figure 3911: Fs = 60 FPS

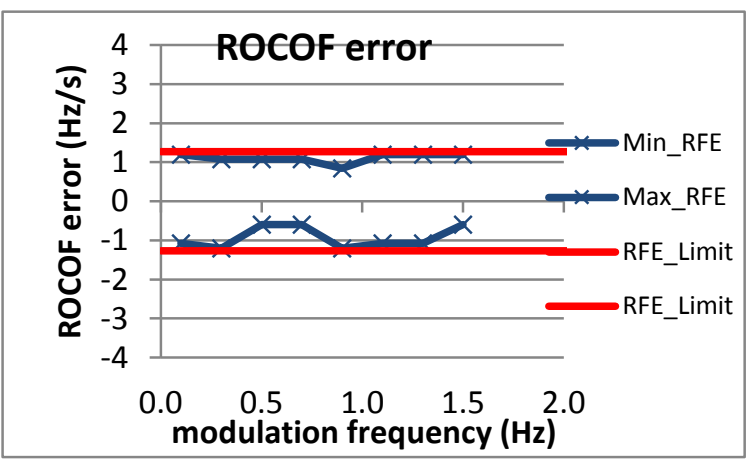

Figure 3914: Fs = 15 FPS

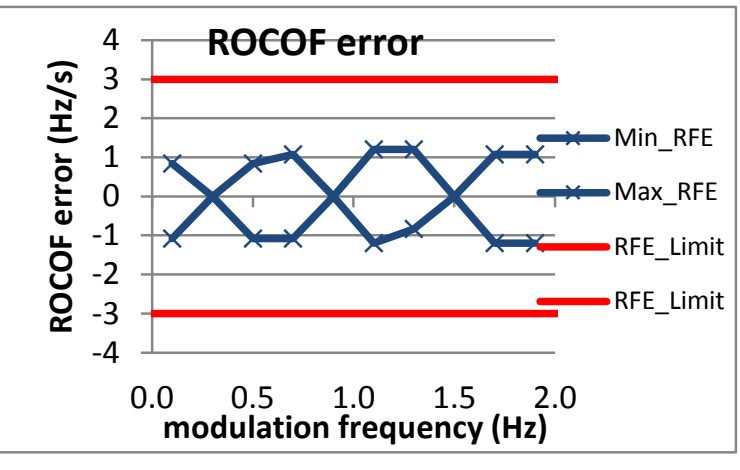

Figure 3912: Fs = 30 FPS

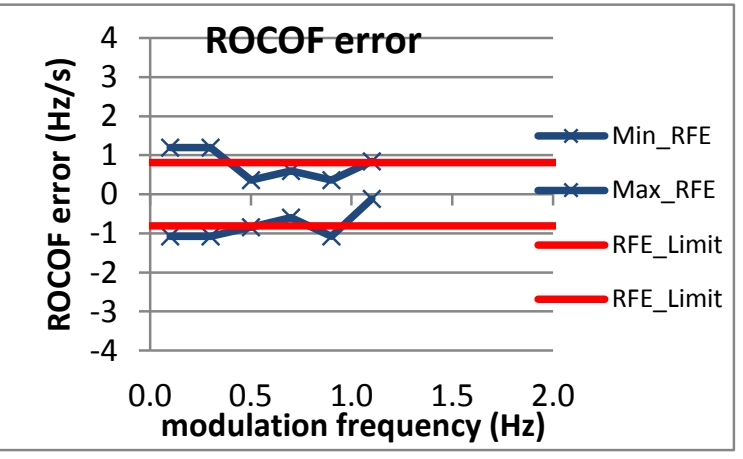

Figure 3915: Fs = 12 FPS

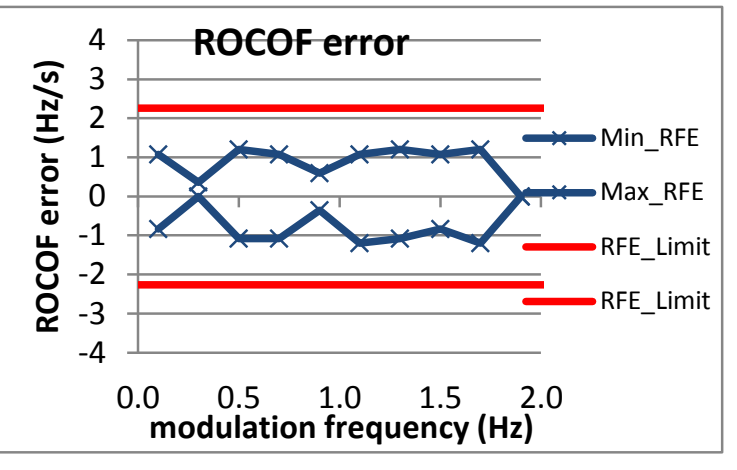

Figure 3913: Fs = 20 FPS

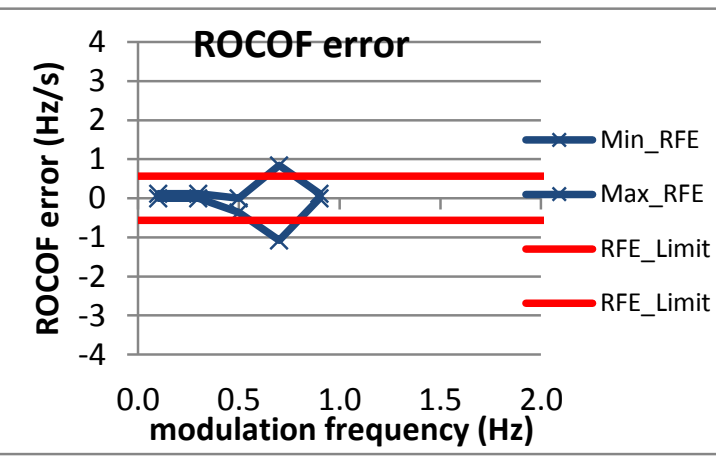

Figure 3916: Fs = 10 FPS 
8.8.3 PMU B dynamic bandwidth measurement: amplitude modulation ROCOF error: P class

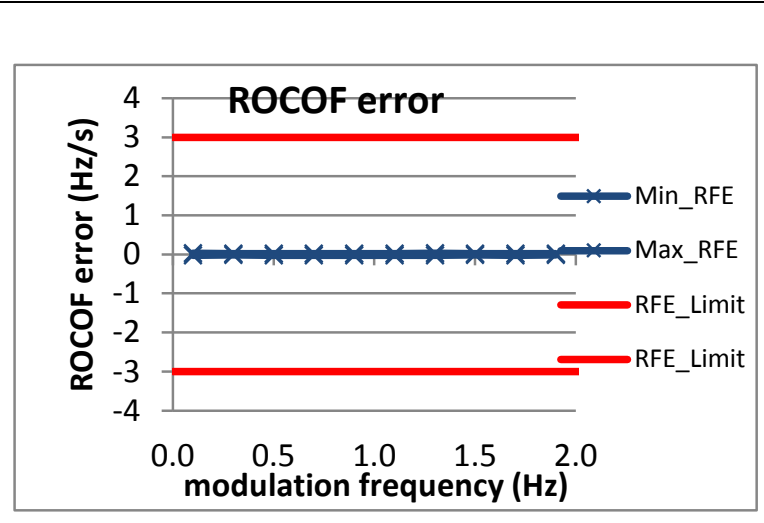

Figure 3917: Fs = 60 FPS

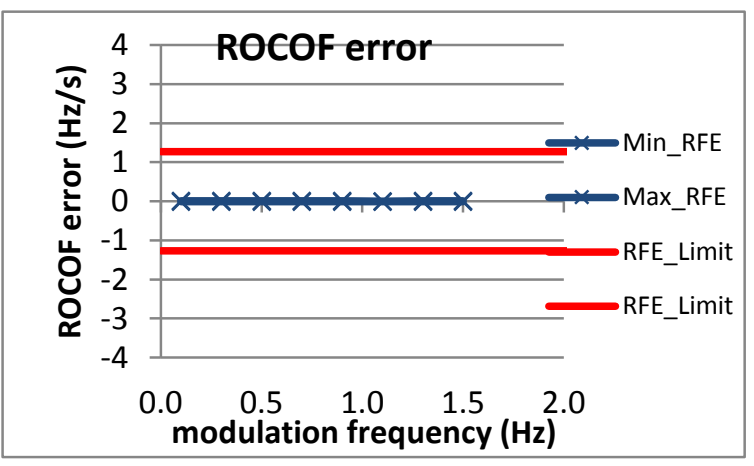

Figure 3920: Fs = 15 FPS

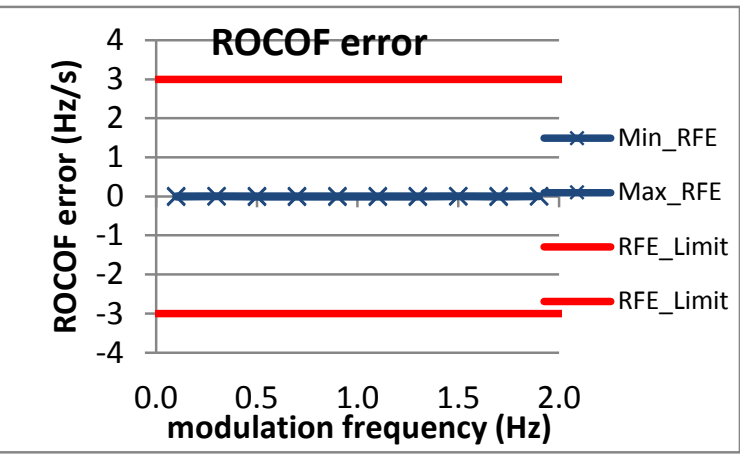

Figure 3918: Fs = 30 FPS

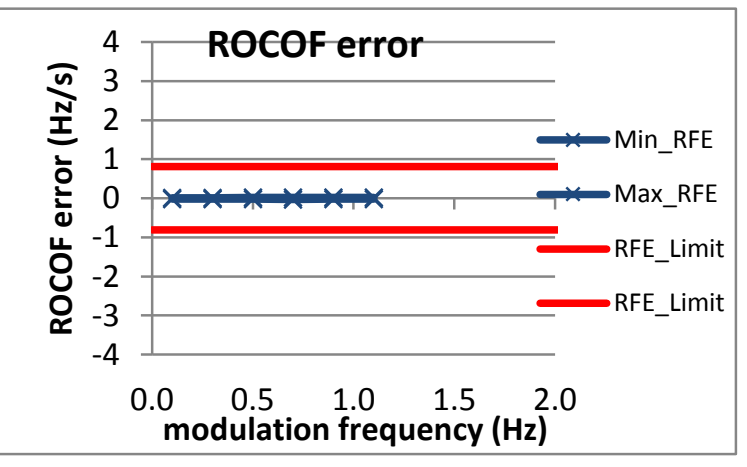

Figure 3921: Fs = 12 FPS

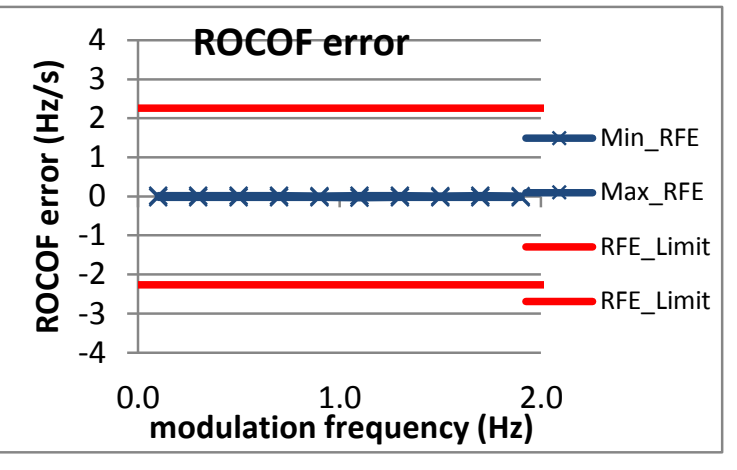

Figure 3919: Fs = 20 FPS

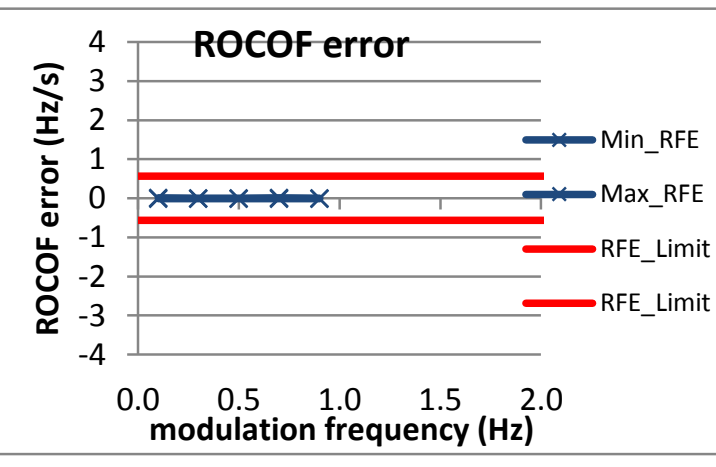

Figure 3922: Fs = 10 FPS 
8.8.4 PMU C dynamic bandwidth measurement: amplitude modulation ROCOF error: P class

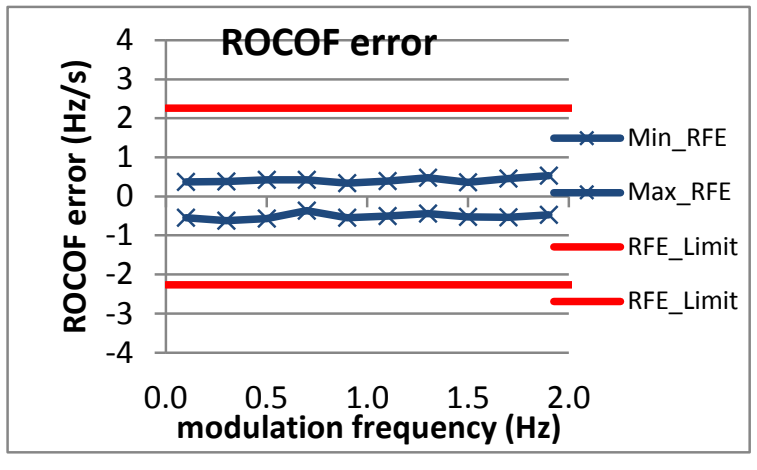

Figure 3923: Fs = 60 FPS

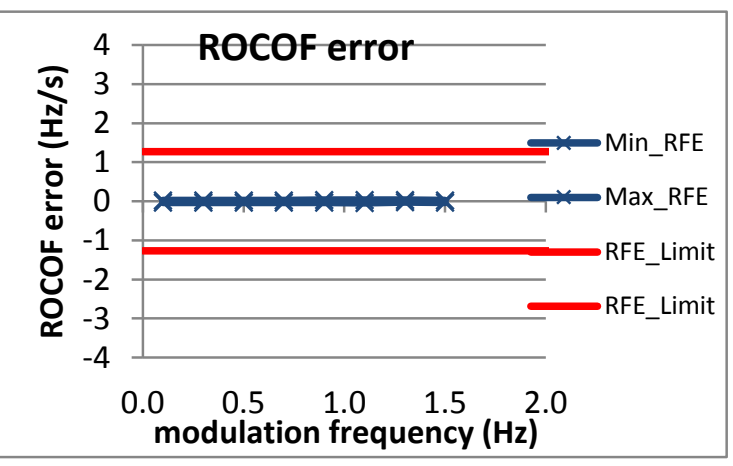

Figure 3926: Fs = 15 FPS

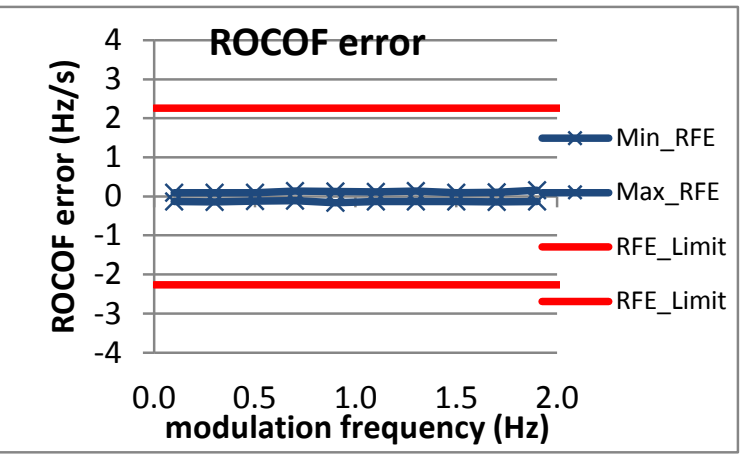

Figure 3924: Fs = 30 FPS

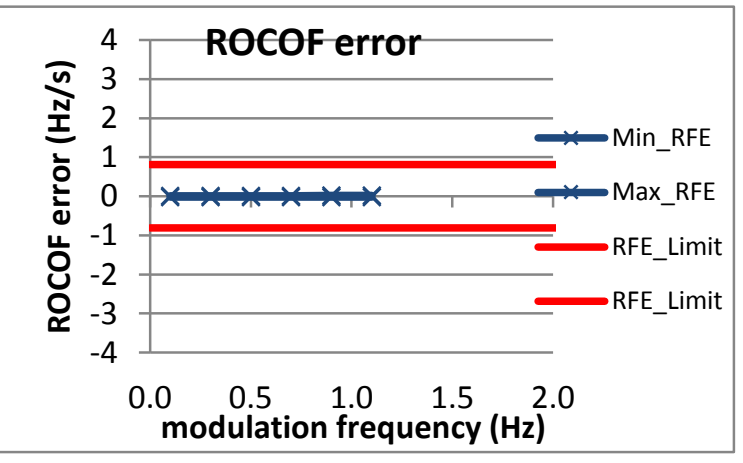

Figure 3927: Fs = 12 FPS

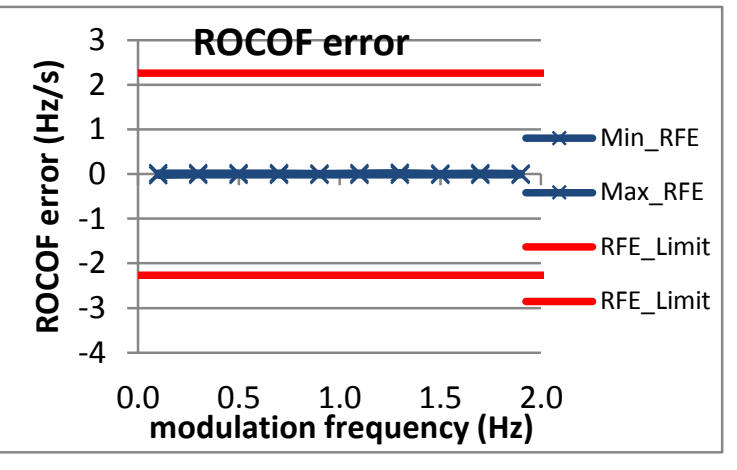

Figure 3925: Fs = 20 FPS

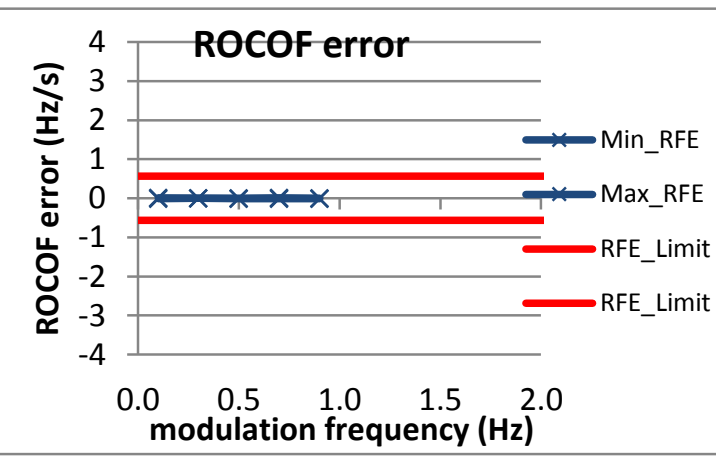

Figure 3928: Fs = 10 FPS 
8.8.5 PMU D dynamic bandwidth measurement: amplitude modulation ROCOF error: P class

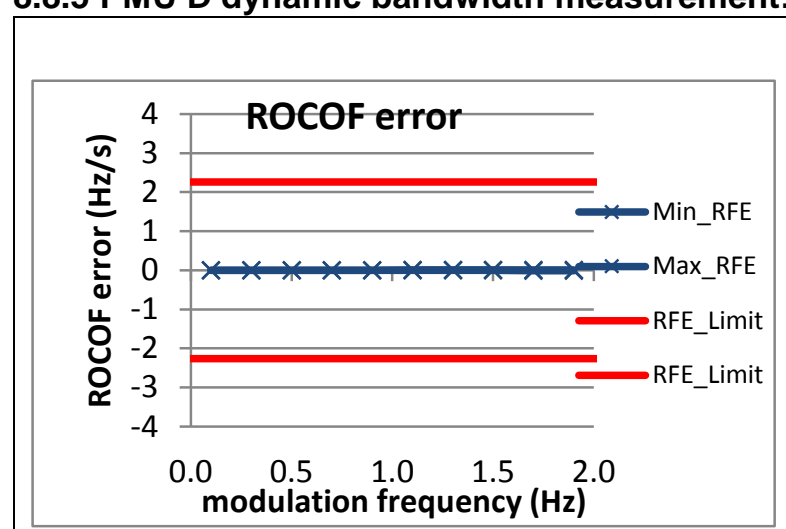

Figure 3929: Fs = 60 FPS

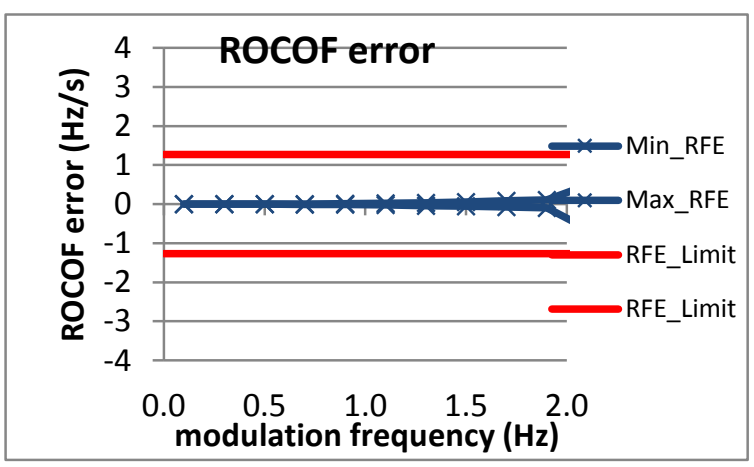

Figure 3932: Fs = 15 FPS

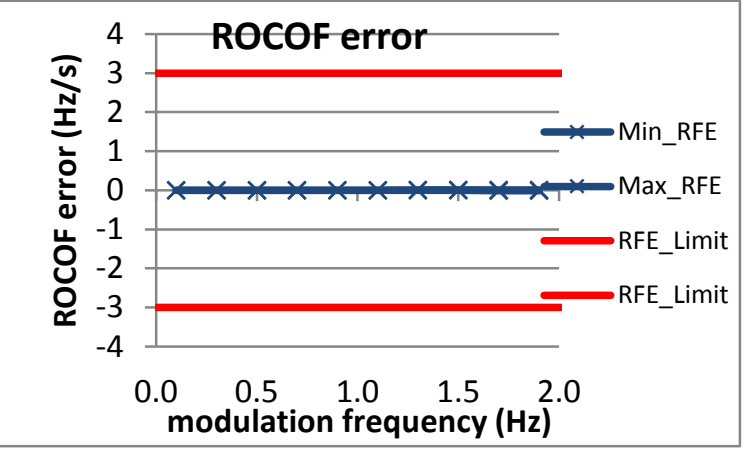

Figure 3930: Fs = 30 FPS

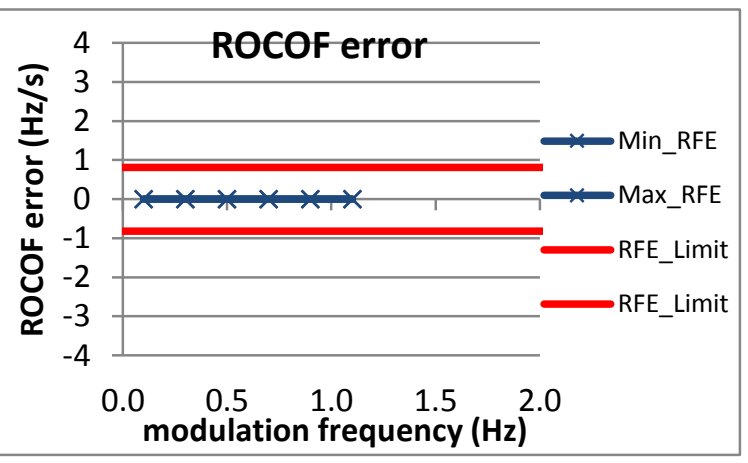

Figure 3933: Fs = 12 FPS

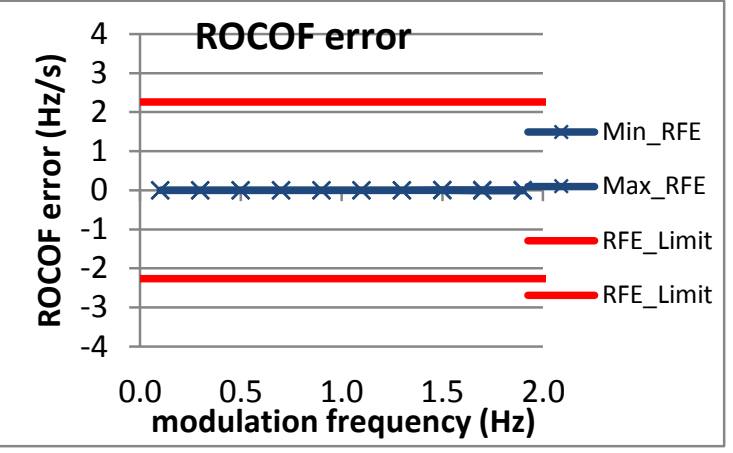

Figure 3931: Fs = 20 FPS

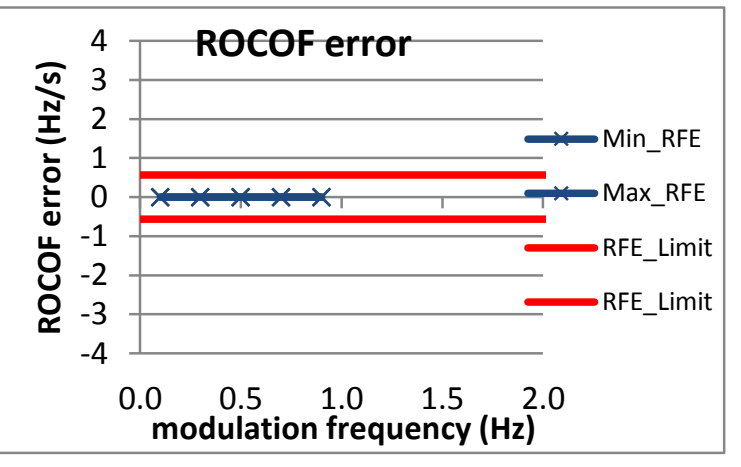

Figure 3934: Fs = 10 FPS

8.8.6 PMU E dynamic bandwidth measurement: amplitude modulation ROCOF error: P class

PMU E does not support P class 
8.8.7 PMU F dynamic bandwidth measurement: amplitude modulation ROCOF error: P class
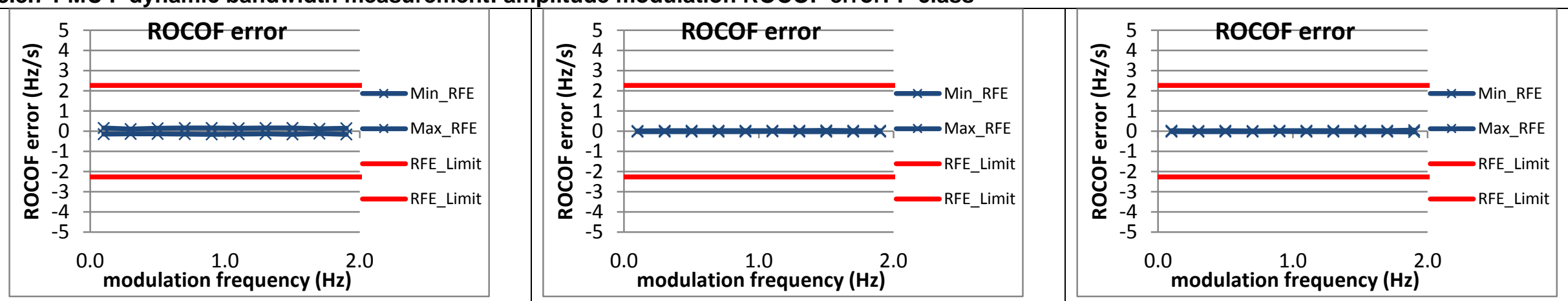

Figure 3935: Fs = 60 FPS

Figure 3936: Fs = 30 FPS

Figure 3937: Fs = 20 FPS
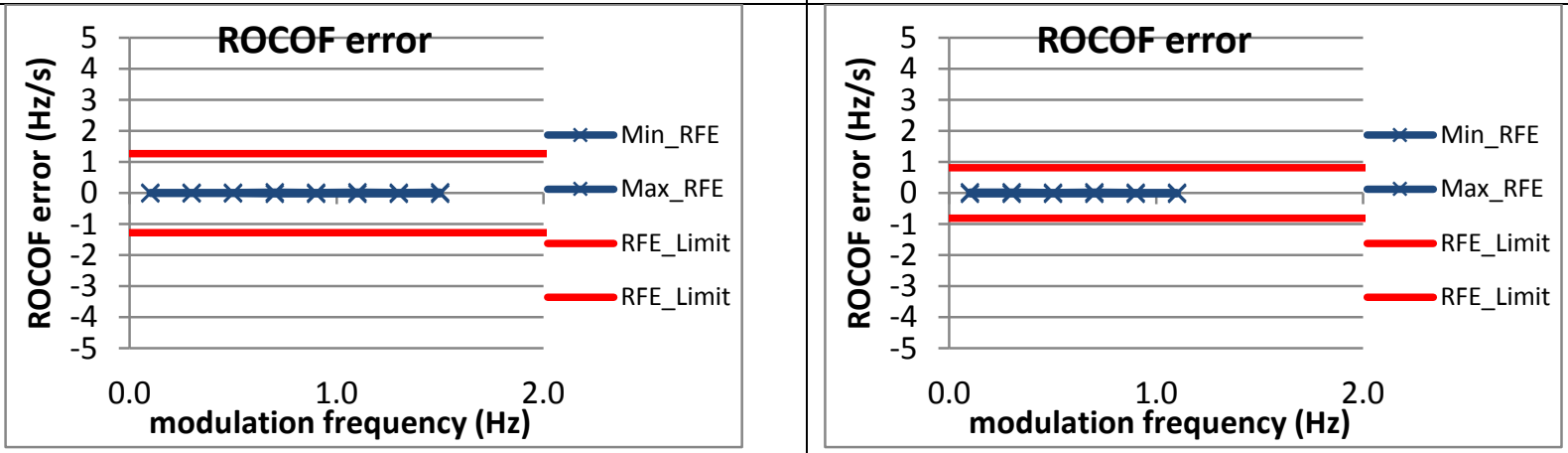

Figure 3939: Fs = 12 FPS

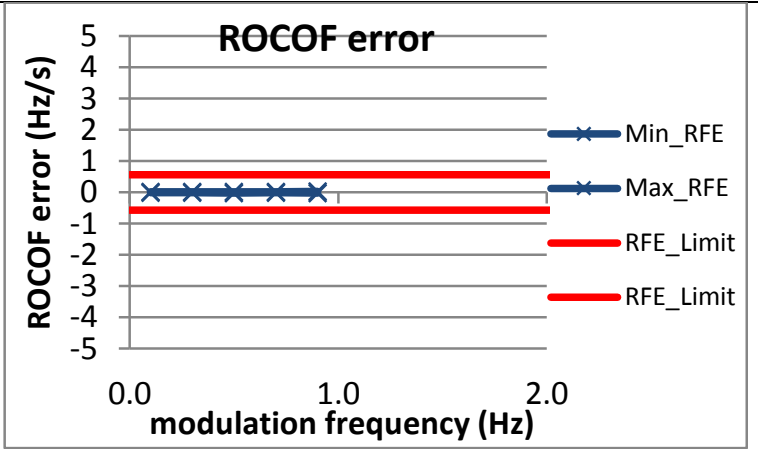

Figure 3938: Fs = 15 FPS

Figure 3940: Fs = 10 FPS

8.8.8 PMU G dynamic bandwidth measurement: amplitude modulation ROCOF error: P class

PMU G does not support P class 
8.8.9 PMU H dynamic bandwidth measurement: amplitude modulation ROCOF error: P class

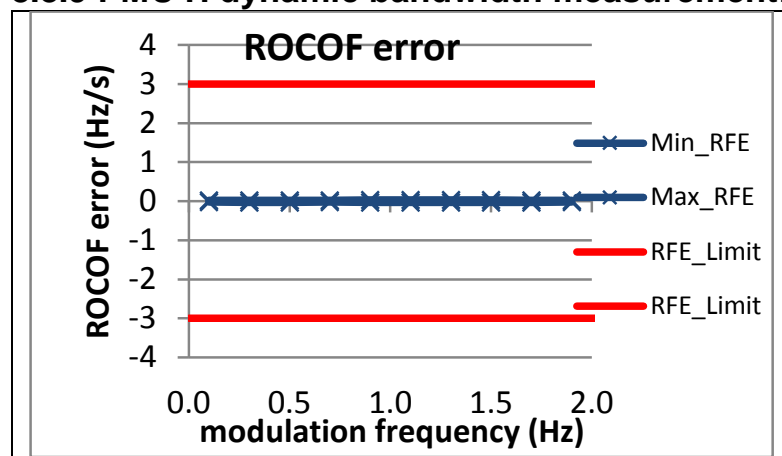

Figure 3941: Fs = 60 FPS

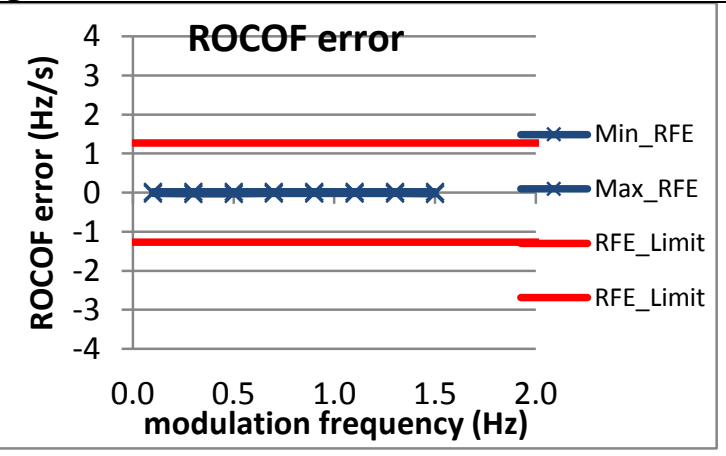

Figure 3944: Fs = 15 FPS

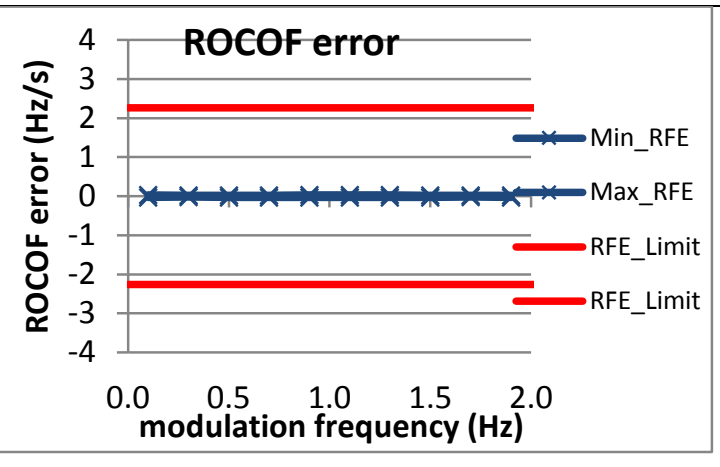

Figure 3942: Fs = 30 FPS

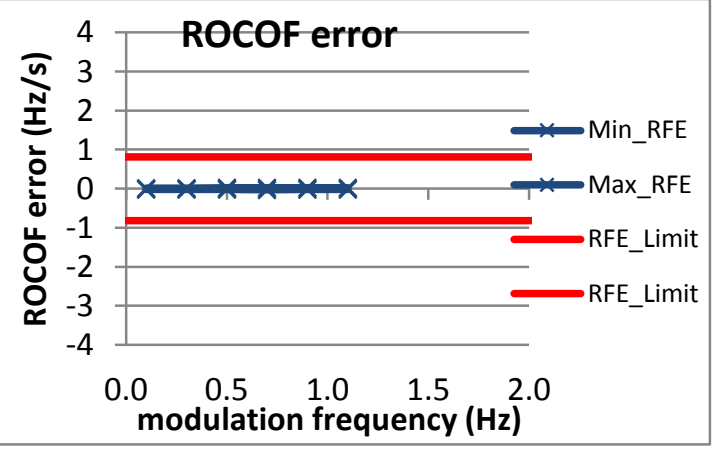

Figure 3945: Fs = 12 FPS

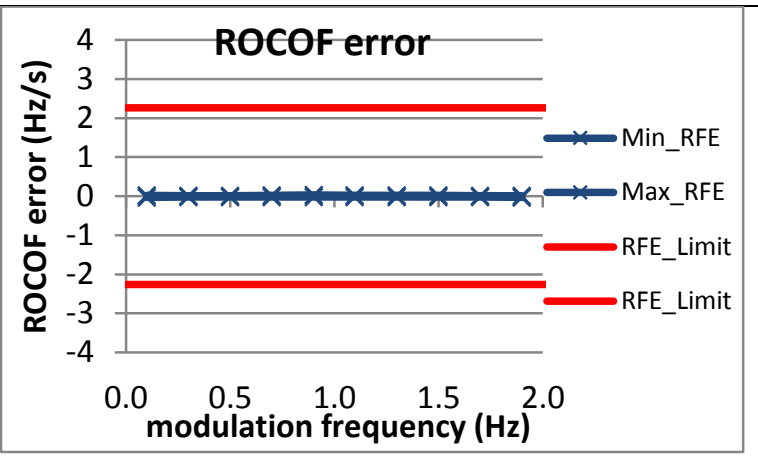

Figure 3943: Fs = 20 FPS

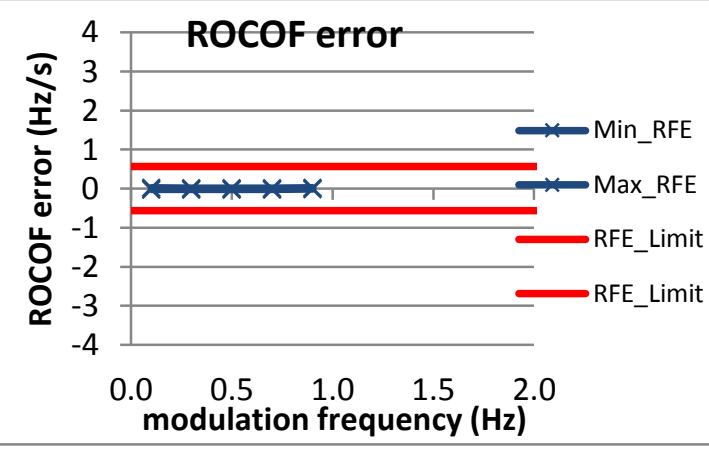

Figure 3946: Fs = 10 FPS

8.8.10 PMU I dynamic bandwidth measurement: amplitude modulation ROCOF error: P class

PMU I does not support P class 
8.8.11 PMU J dynamic bandwidth measurement: amplitude modulation ROCOF error: P class

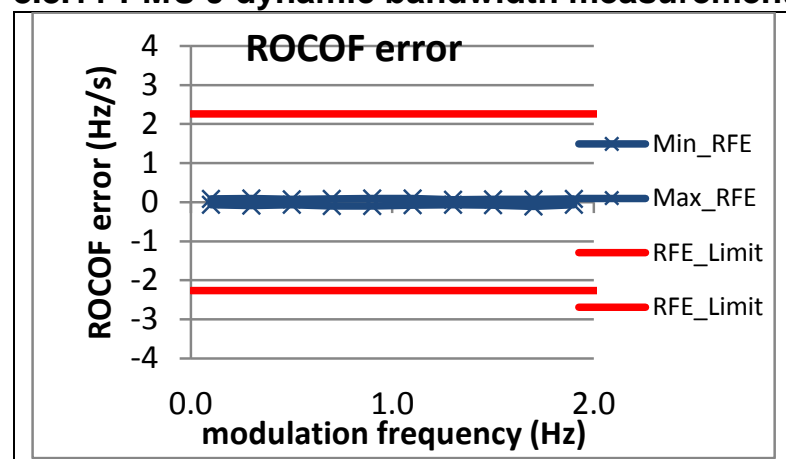

Figure 3947: Fs = 60 FPS

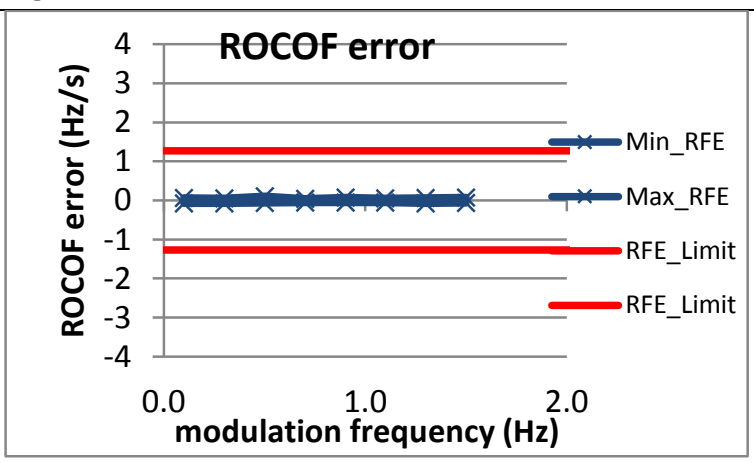

Figure 3950: Fs = 15 FPS

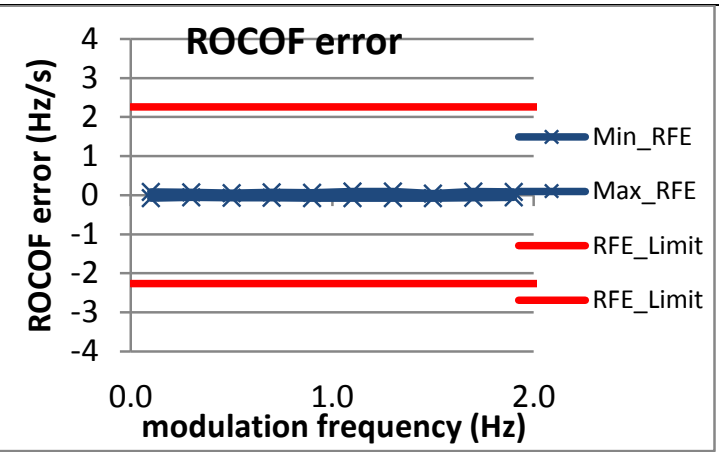

Figure 3948: Fs = 30 FPS

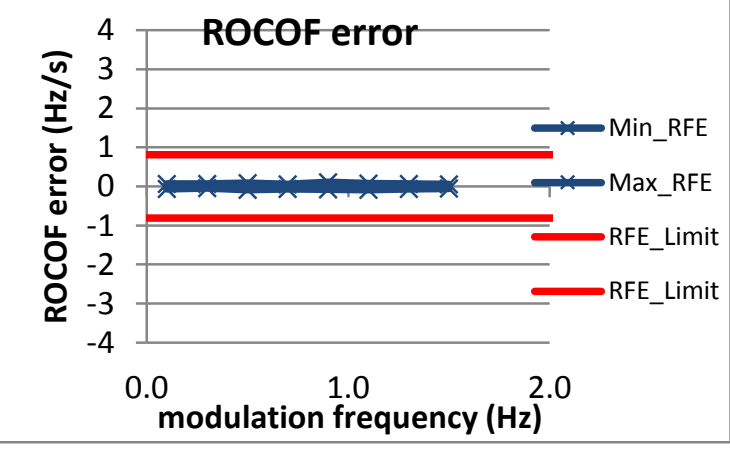

Figure 3951: Fs = 12 FPS

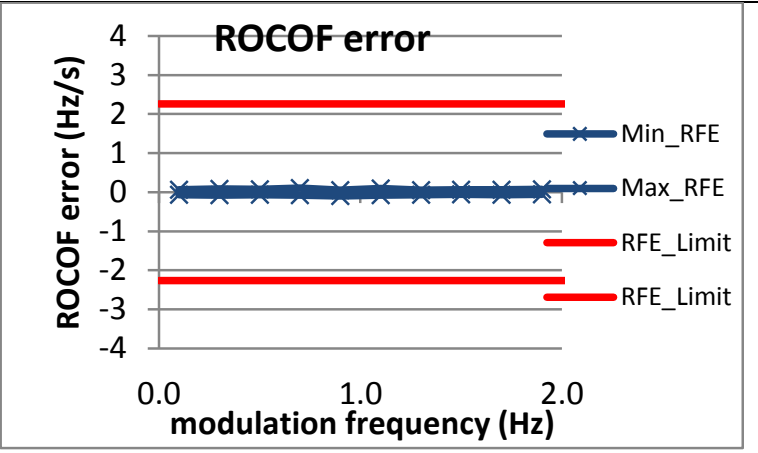

Figure 3949: Fs = 20 FPS

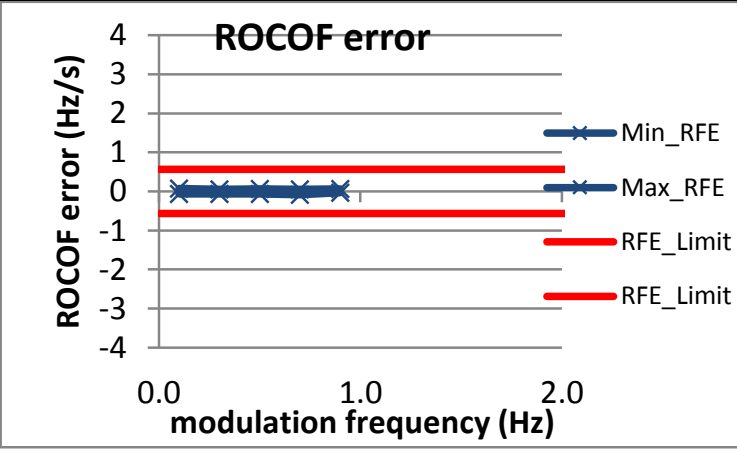

Figure 3952: Fs = 10 FPS 


\section{Dynamic step change in phase}

9. Dynamic step change in phase

9.1 Dynamic step change in phase: voltage response time: ...

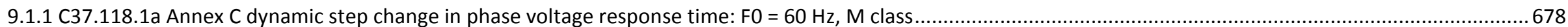

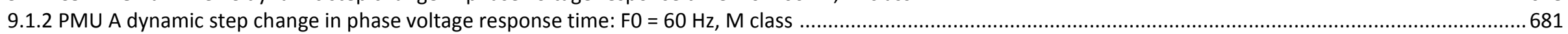

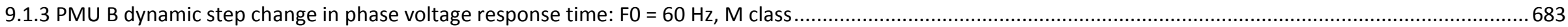

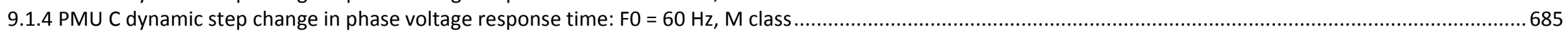

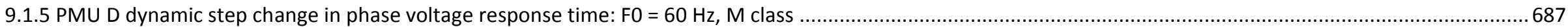

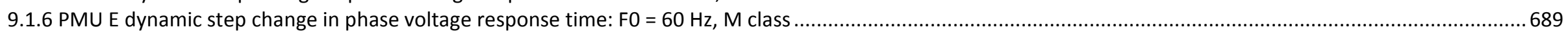

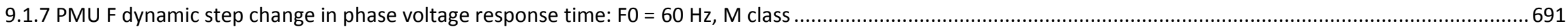

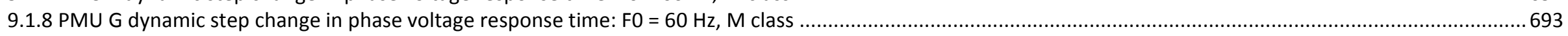

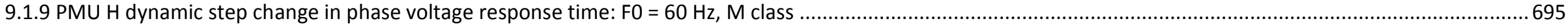

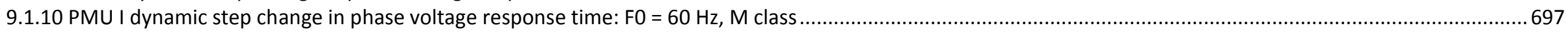

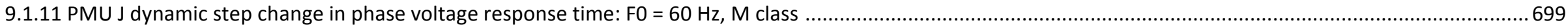

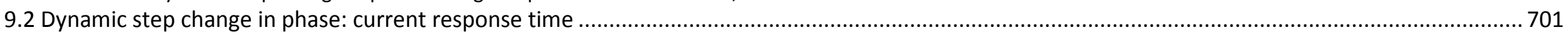

9.2.1 C37.118.1-2011 Annex C dynamic step change in phase current response time: $\mathrm{FO}=60 \mathrm{~Hz}$, M class .......................................................................... 701

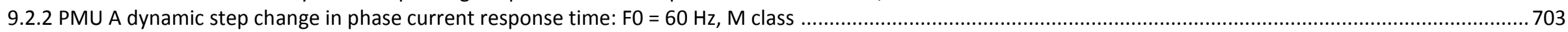

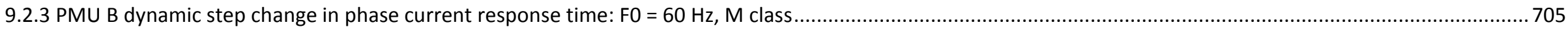

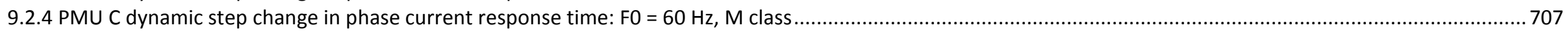

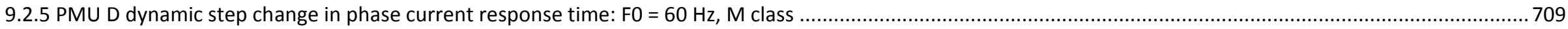

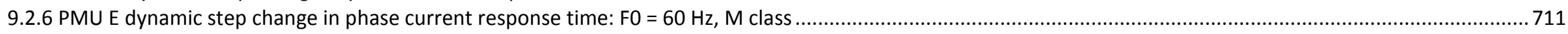

9.2.7 PMU F dynamic step change in phase current response time: $\mathrm{FO}=60 \mathrm{~Hz}$, M class ........................................................................................................... 713

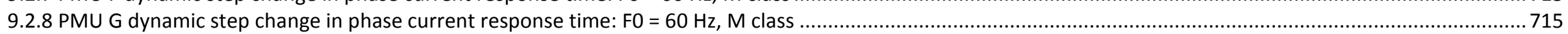

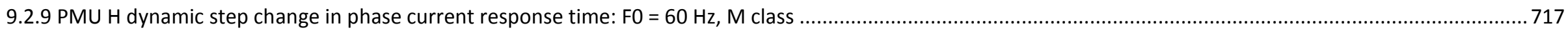

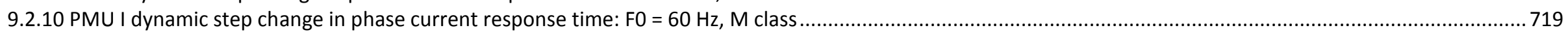

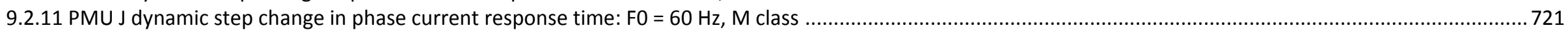

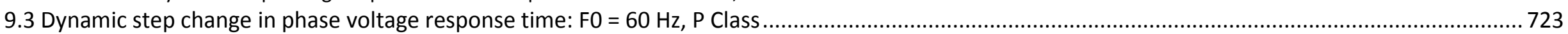

9.3.1 C37.118.1a Annex C dynamic step change in phase voltage response time: $\mathrm{FO}=60 \mathrm{~Hz}$, P class ................................................................................ 723

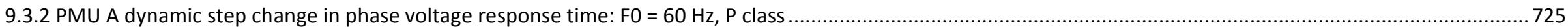

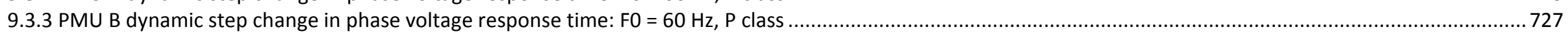

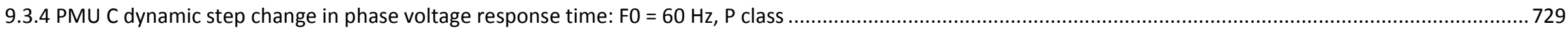

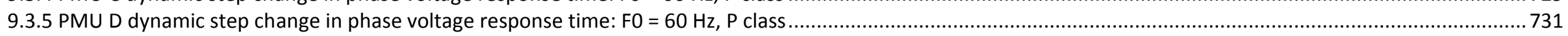

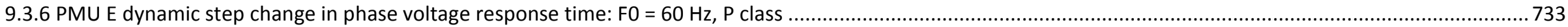

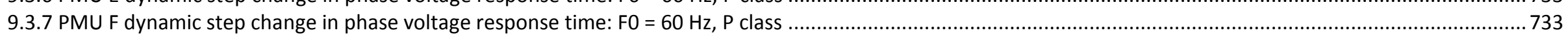

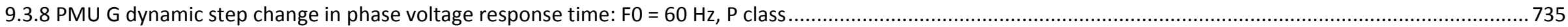

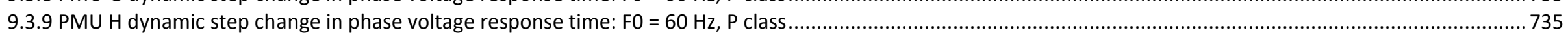

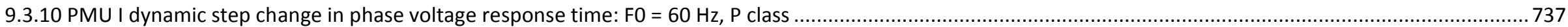


9.3.11 PMU J dynamic step change in phase voltage response time: $\mathrm{FO}=60 \mathrm{~Hz}$, P class.

9.4 Dynamic step change in phase current response time: $F 0=60 \mathrm{~Hz}$, P Class.....

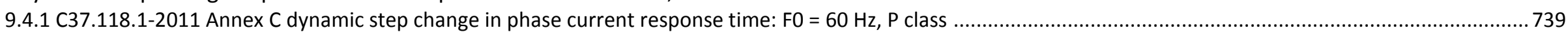

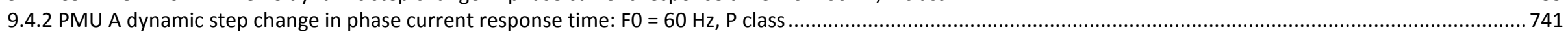

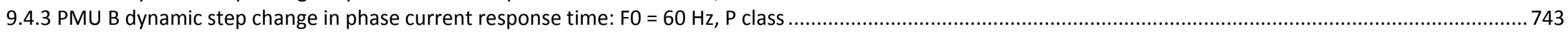

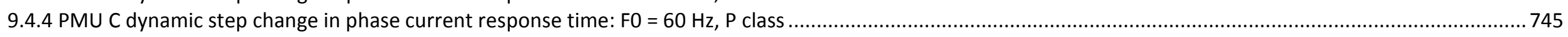

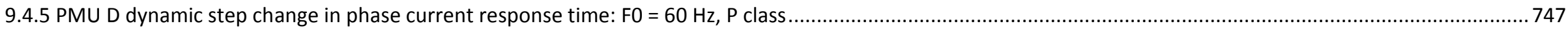

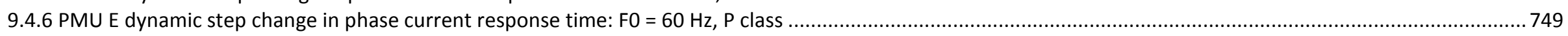

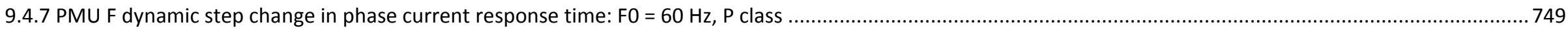

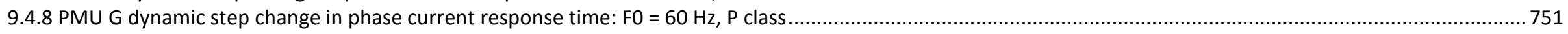

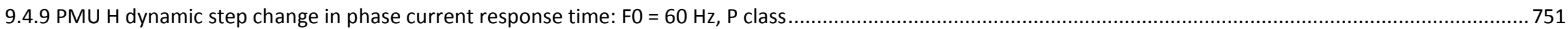

9.4.10 PMU I dynamic step change in phase current response time: $\mathrm{FO}=60 \mathrm{~Hz}$, P class .......................................................................................................... 752

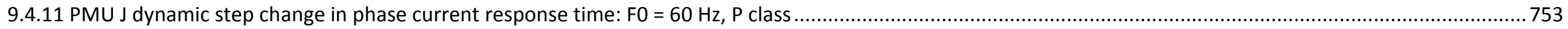

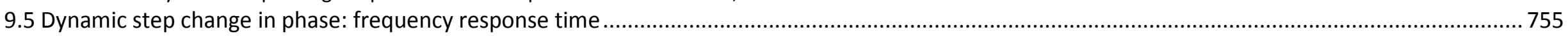

9.5.1 C37.118.1-2011 Annex C dynamic step change in phase frequency response time: $\mathrm{F0}=60 \mathrm{~Hz}, \mathrm{M}$ class ............................................................................. 755

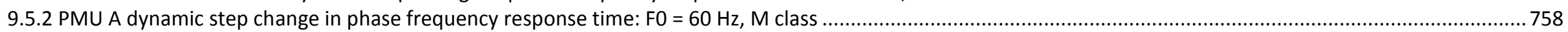

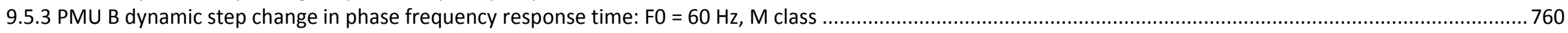

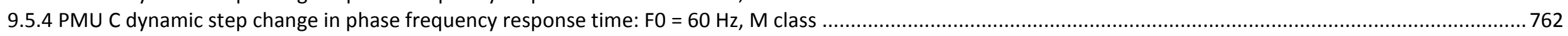

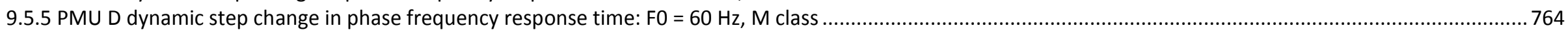

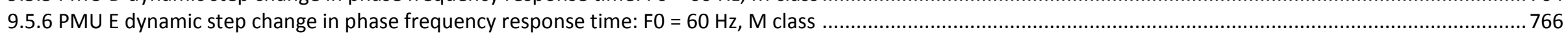

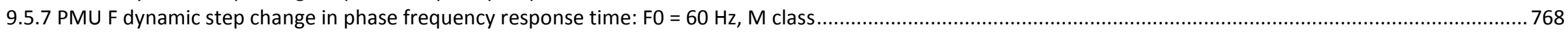

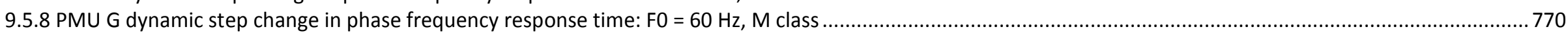

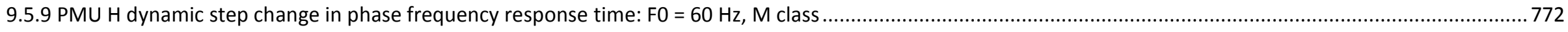

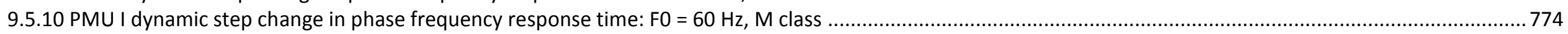

9.5.11 PMU J dynamic step change in phase frequency response time: $\mathrm{FO}=60 \mathrm{~Hz}$, M class ..................................................................................................... 776

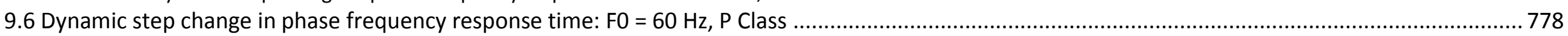

9.6.1 C37.118.1-2011 Annex C dynamic step change in phase frequency response time: $\mathrm{FO}=60 \mathrm{~Hz}$, P class ............................................................................778

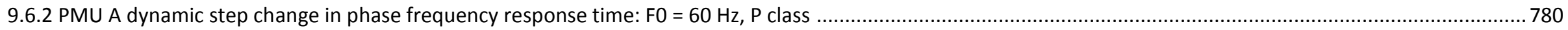

9.6.3 PMU B dynamic step change in phase frequency response time: $\mathrm{FO}=60 \mathrm{~Hz}$, P class................................................................................................... 782

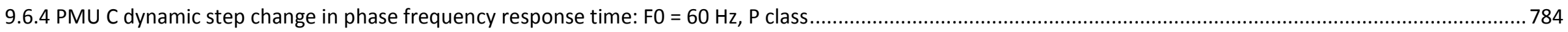

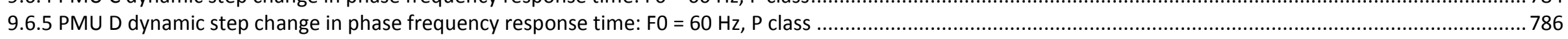

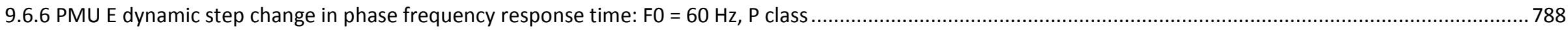

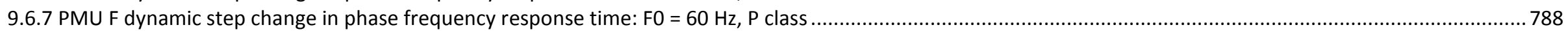

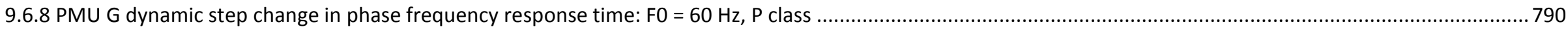

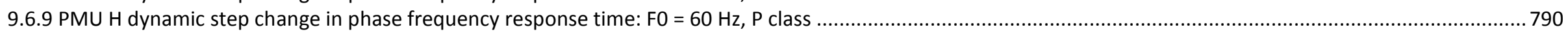

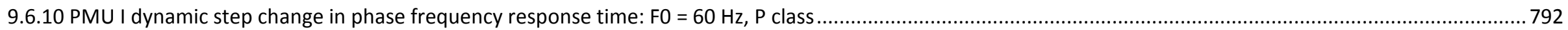

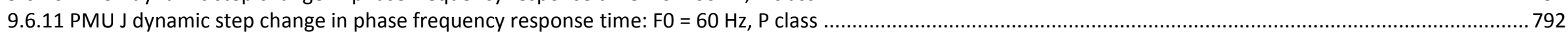

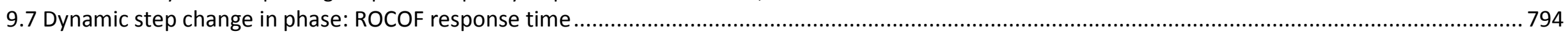

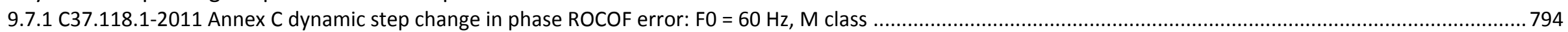

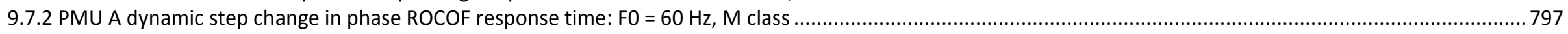


9.7.3 PMU B dynamic step change in phase ROCOF response time: $F 0=60 \mathrm{~Hz}$, M class 799 9.7.4 PMU C dynamic step change in phase ROCOF response time: $F 0=60 \mathrm{~Hz}$, M class

9.7.5 PMU D dynamic step change in phase ROCOF response time: $F 0=60 \mathrm{~Hz}, \mathrm{M}$ class....

9.7.6 PMU E dynamic step change in phase ROCOF response time: $\mathrm{FO}=60 \mathrm{~Hz}, \mathrm{M}$ class

9.7.7 PMU F dynamic step change in phase ROCOF response time: $F 0=60 \mathrm{~Hz}, \mathrm{M}$ class.....

9.7.8 PMU G* dynamic step change in phase ROCOF response time: $F 0=60 \mathrm{~Hz}$, M class

9.7.9 PMU H dynamic step change in phase ROCOF response time: $\mathrm{FO}=60 \mathrm{~Hz}$, M class.

9.7.10 PMU I dynamic step change in phase ROCOF response time: $\mathrm{FO}=60 \mathrm{~Hz}$, M class

9.7.11 PMU J dynamic step change in phase ROCOF response time: $F 0=60 \mathrm{~Hz}$, M class

9.8 Dynamic step change in phase: ROCOF response time: $P$ class

9.8.1 C37.118.1-2011 Annex C dynamic step change in phase ROCOF error: $\mathrm{FO}=60 \mathrm{~Hz}$, P class.

9.8.2 PMU A dynamic step change in phase ROCOF response time: $F 0=60 \mathrm{~Hz}$, P class

9.8.3 PMU B dynamic step change in phase ROCOF response time: $F 0=60 \mathrm{~Hz}$, P class

9.8.4 PMU C dynamic step change in phase ROCOF response time: $F O=60 \mathrm{~Hz}$, P class

9.8.5 PMU D dynamic step change in phase ROCOF response time: $F 0=60 \mathrm{~Hz}, \mathrm{P}$ class

9.8.6 PMU E dynamic step change in phase ROCOF response time: $F 0=60 \mathrm{~Hz}$, P class

9.8.7 PMU F dynamic step change in phase ROCOF response time: $F 0=60 \mathrm{~Hz}$, P class

9.8.8 PMU G dynamic step change in phase ROCOF response time: $F 0=60 \mathrm{~Hz}$, P class ...

9.8.9 PMU H dynamic step change in phase ROCOF response time: $F 0=60 \mathrm{~Hz}$, P class

9.8.10 PMU I dynamic step change in phase ROCOF response time: $\mathrm{FO}=60 \mathrm{~Hz}$, $\mathrm{P}$ class

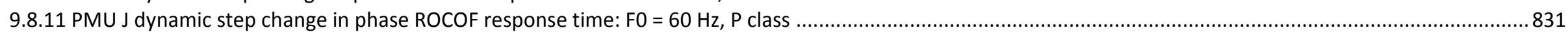

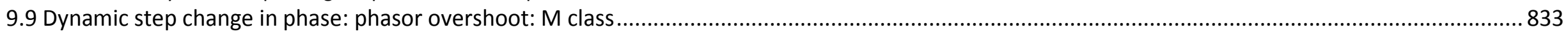

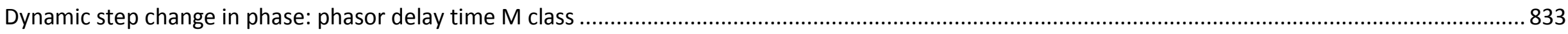

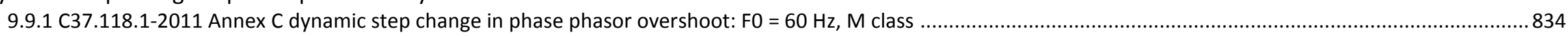

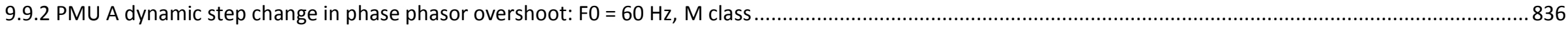

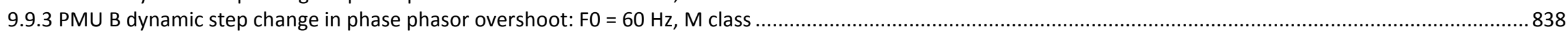

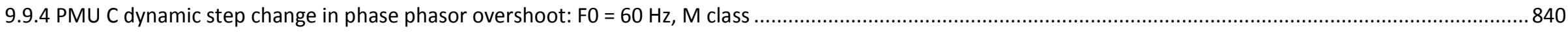

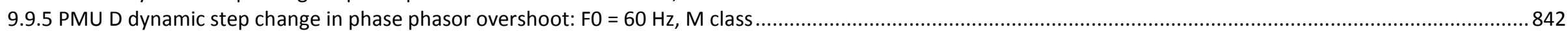

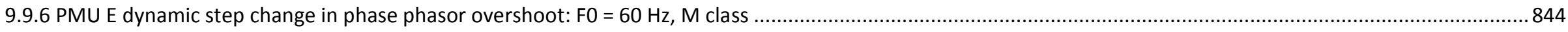

9.9.7 PMU F dynamic step change in phase phasor overshoot: $\mathrm{FO}=60 \mathrm{~Hz}$, M class

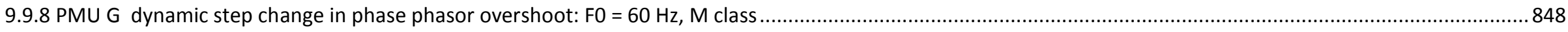

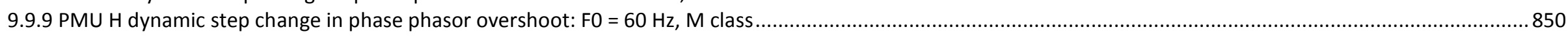

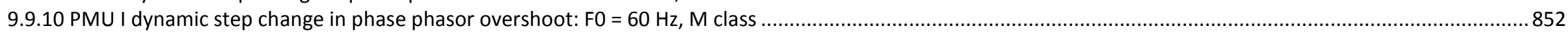

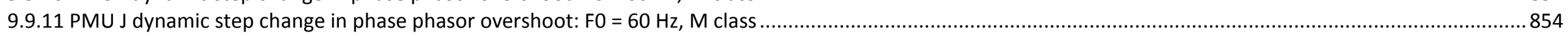

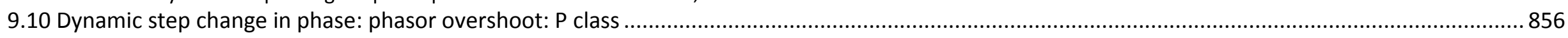

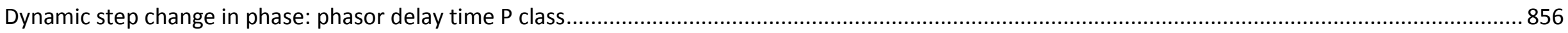

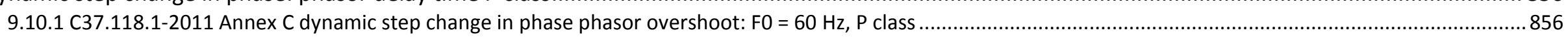

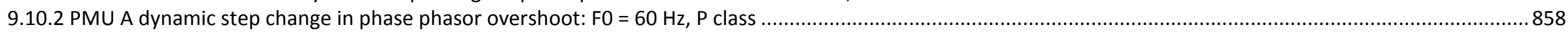

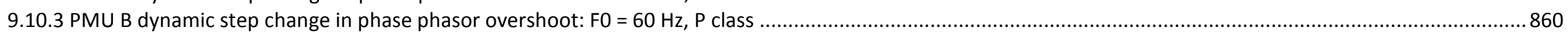


This publication is available free of charge from $\mathrm{http}: / / \mathrm{dx}$.doi.org/10.6028/NIST.IR.8106

9.10.4 PMU C dynamic step change in phase phasor overshoot: $\mathrm{F0}=60 \mathrm{~Hz}$, P class

9.10.5 PMU D dynamic step change in phase phasor overshoot: $F 0=60 \mathrm{~Hz}$, P class

9.10.6 PMU E dynamic step change in phase phasor overshoot: $\mathrm{FO}=60 \mathrm{~Hz}, \mathrm{P}$ class

9.10.7 PMU F dynamic step change in phase phasor overshoot: $\mathrm{FO}=60 \mathrm{~Hz}, \mathrm{P}$ class

9.10.8 PMU G* dynamic step change in phase phasor overshoot: $F 0=60 \mathrm{~Hz}$, P clas

9.10.9 PMU H dynamic step change in phase phasor overshoot: $F 0=60 \mathrm{~Hz}$, P class

9.10.10 PMU I dynamic step change in phase phasor overshoot: $F 0=60 \mathrm{~Hz}$, P class

9.10.11 PMU J dynamic step change in phase phasor overshoot: $F 0=60 \mathrm{~Hz}$, P class 
Performance during step changes in phase is determined by applying $\pm 10 \%$ step changes of phase. Response time and delay time of the phasors, frequency, and ROCOF measurements are compared to the limits. Overshoot of the phasors are compared to the limits.

A recommended practice for measuring a PMU's response to step changes in input is to run a series of $n$ step sub-tests where the occurrence of the step is displaced in time relative to the PMU report-time. During each $\left(n^{\text {th }}\right)$ iteration of 10 sub-tests, the step occurs $1 / 10$ th of a reporting period further from the time of the report than the previous iteration of the subtest. The results are combined to trace an equivalent time sampled response curve. During each sub-test, the phase is stepped, the response data are gathered, and the phase is returned to its previous value in preparation for the next sub-test in the series. At the end of the series of sub-tests, the TVE, FE, RFE results and the PMU reported phase data from all sub-tests are indexed and overlaid to create smooth curves approximating the step response of the PMU. From the curves, delay time, response time, and overshoot/undershoot shall be calculated

The test plan for step change in phase and magnitude is as follows:

a) For the first test, let $\mathrm{n}=0$.

b) Begin with three-phase balanced input at nominal amplitude and frequency. Hold steady state for at least 1 second plus one response time period.

c) At the beginning of a reporting cycle plus $n /\left(10 \times F_{s}\right)$ (i.e., $n \times$ reporting period/10) step the magnitude by $+10 \%$ of the nominal magnitude and hold steady state for at least 1 second plus one response time period.

d) Gather the PMU data, return the influence quantity to nominal and wait for the PMU to settle.

e) Increment $n$ by one $(n=n+1)$, then repeat step c) through step d) until $n=10-1$.

f) Index and overlay the PMU data to obtain a smooth response curve.

g) Repeat the tests for negative magnitude step.

IEEE Std. C37.118.1a-2014 sets the M class response time limit for $M$ class phasors at $7 / F_{s}$ and for frequency and ROCOF at the greater of $14 / F_{s}$ or $14 / F_{0}$. IEEE Std. C37.118.1a-2014 sets the $P$ class response time limit for phasors at $2 / F_{0}$ the delay time limit at $1 /\left(4 F_{s}\right)$ and the overshoot at $5 \%$ of the step magnitude. IEEE Std. C37.118.1a-2014 sets the $P$ class response time limit for frequency at $4.5 / F_{0}$ and for ROCOF at $6 / F_{0}$.

IEEE Std C37.118.1-2011 sets the M class phasor delay time at $1 /\left(4 \mathrm{~F}_{\mathrm{s}}\right)$ and the max overshoot at $10 \%$ of the step magnitude.

IEEE Std C37.118.1-2011 sets the P class phasor delay time at $1 /\left(4 \mathrm{~F}_{s}\right)$ and the max overshoot at $5 \%$ of the step magnitude.

The response time plots in this section show the limit of TVE, Fe, or RFe as a green horizontal line. A red vertical line appears at the response time limits. note that the limits are not interpolated, the first vertical red line appears at the first report which is above the TVE, FE or RFE limit and the second red line is at the response time limit away from the first. The PMU response must return and remain below the green line before the second vertical red line is crossed in order to comply with the response time limit.

The overshoot plots in this section show red horizontal lines at the overshoot/undershoot limits around the magnitude before and after the step. 
9.1 Dynamic step change in phase: voltage response time:

\begin{tabular}{|c|c|c|c|c|c|c|c|c|c|c|c|c|}
\hline Fs (FPS) & $10 \mathrm{M}$ & $10 \mathrm{P}$ & $12 \mathrm{M}$ & $12 \mathrm{P}$ & $15 \mathrm{M}$ & $15 \mathrm{P}$ & $20 \mathrm{M}$ & $20 \mathrm{P}$ & $30 \mathrm{M}$ & $30 \mathrm{P}$ & $60 \mathrm{M}$ & $60 \mathrm{P}$ \\
\hline C37.118.1 Annex C & $\mathrm{P}$ & $\mathrm{P}$ & $\mathrm{P}$ & $\mathrm{P}$ & $\mathrm{P}$ & $\mathrm{P}$ & $\mathrm{P}$ & $\mathrm{P}$ & $\mathrm{P}$ & $\mathrm{P}$ & $\mathrm{P}$ & $\mathrm{P}$ \\
\hline PMU A & $\mathrm{P}$ & $\mathrm{P}$ & $\mathrm{P}$ & $\mathrm{P}$ & $\mathrm{P}$ & $\mathrm{P}$ & $\mathrm{P}$ & $\mathrm{P}$ & $\mathrm{P}$ & $\mathrm{P}$ & $\mathrm{P}$ & $\mathrm{P}$ \\
\hline $\mathrm{PMU} \mathrm{B}$ & $\mathrm{P}$ & $\mathrm{F}$ & $\mathrm{P}$ & $\mathrm{F}$ & $\mathrm{P}$ & $\mathrm{F}$ & $\mathrm{P}$ & $\mathrm{F}$ & $\mathrm{P}$ & $\mathrm{F}$ & $\mathrm{P}$ & $\mathrm{F}$ \\
\hline $\mathrm{PMU} \mathrm{C}$ & $\mathrm{P}$ & $\mathrm{P}$ & $\mathrm{P}$ & $\mathrm{P}$ & $\mathrm{P}$ & $\mathrm{P}$ & $\mathrm{P}$ & $\mathrm{P}$ & $\mathrm{P}$ & $\mathrm{P}$ & $\mathrm{P}$ & $\mathrm{P}$ \\
\hline $\mathrm{PMU} \mathrm{D}$ & $\mathrm{P}$ & $\mathrm{I}$ & $\mathrm{P}$ & $\mathrm{I}$ & $\mathrm{P}$ & $\mathrm{I}$ & $\mathrm{P}$ & $\mathrm{I}$ & $\mathrm{P}$ & $\mathrm{I}$ & $\mathrm{P}$ & $\mathrm{I}$ \\
\hline PMU E & $\mathrm{P}$ & - & $\mathrm{P}$ & - & $\mathrm{P}$ & - & $\mathrm{P}$ & - & $\mathrm{P}$ & - & $\mathrm{F}$ & - \\
\hline PMU F & $\mathrm{P}$ & $\mathrm{P}$ & $\mathrm{P}$ & $\mathrm{P}$ & $\mathrm{P}$ & $\mathrm{P}$ & $\mathrm{P}$ & $\mathrm{P}$ & $\mathrm{P}$ & $\mathrm{P}$ & $\mathrm{P}$ & $\mathrm{P}$ \\
\hline PMU G & $\mathrm{P}$ & - & $\mathrm{P}$ & - & $\mathrm{P}$ & - & $\mathrm{P}$ & - & $\mathrm{F}$ & - & - & - \\
\hline PMU H & $\mathrm{P}$ & $\mathrm{P}$ & $\mathrm{P}$ & $\mathrm{P}$ & $\mathrm{P}$ & $\mathrm{P}$ & $\mathrm{P}$ & $\mathrm{P}$ & $\mathrm{P}$ & $\mathrm{P}$ & $\mathrm{P}$ & $\mathrm{P}$ \\
\hline $\mathrm{PMU} \mathrm{I}$ & $\mathrm{P}$ & - & $\mathrm{P}$ & - & $\mathrm{P}$ & - & $\mathrm{P}$ & - & $\mathrm{P}$ & - & $\mathrm{P}$ & - \\
\hline PMU J & $\mathrm{P}$ & $\mathrm{P}$ & $\mathrm{P}$ & $\mathrm{P}$ & $\mathrm{P}$ & $\mathrm{P}$ & $\mathrm{P}$ & $\mathrm{P}$ & $\mathrm{P}$ & $\mathrm{P}$ & $\mathrm{P}$ & $\mathrm{P}$ \\
\hline
\end{tabular}

$P=$ pass,$F=$ fail,$I=$ indeterminate 
9.1.1 C37.118.1a Annex C dynamic step change in phase voltage response time: $F 0=60 \mathrm{~Hz}, \mathrm{M}$ class

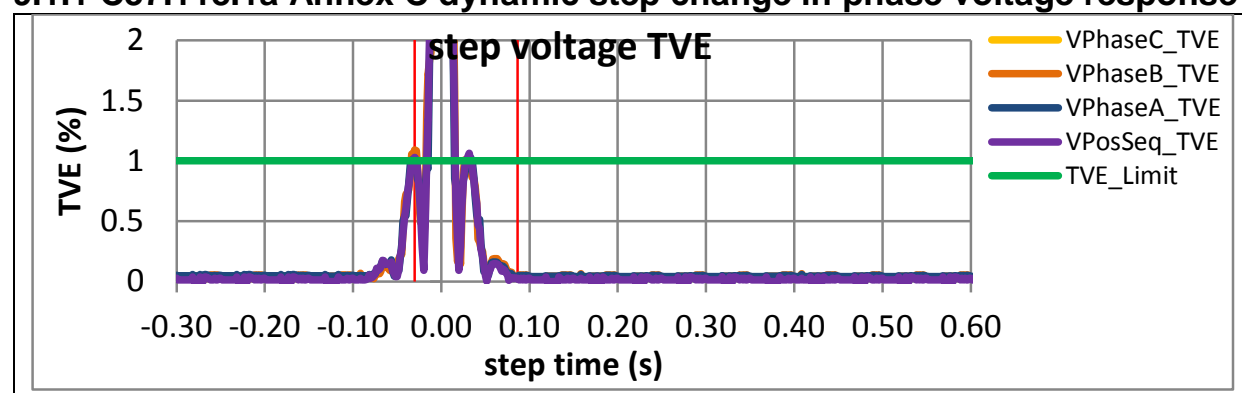

Figure 3953: Fs = 60 FPS, +10 degree phase step

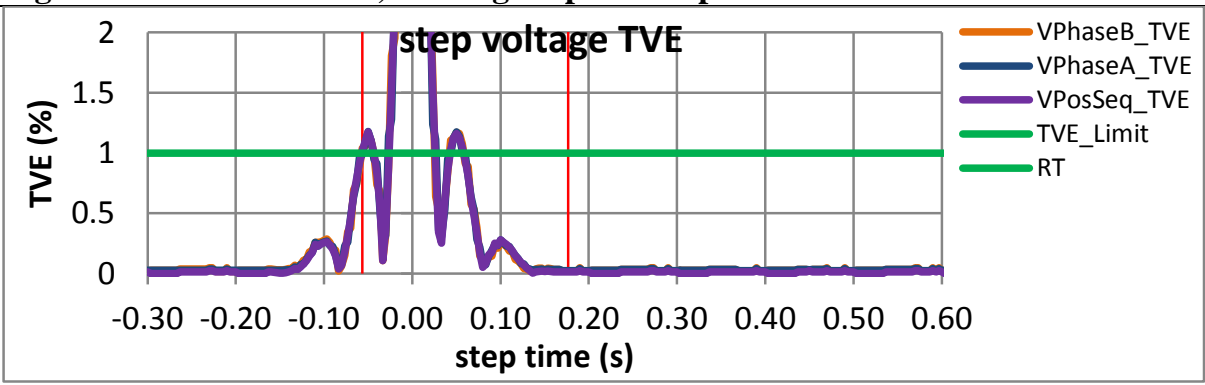

Figure 3955: Fs = 30 FPS, +10 degree phase step

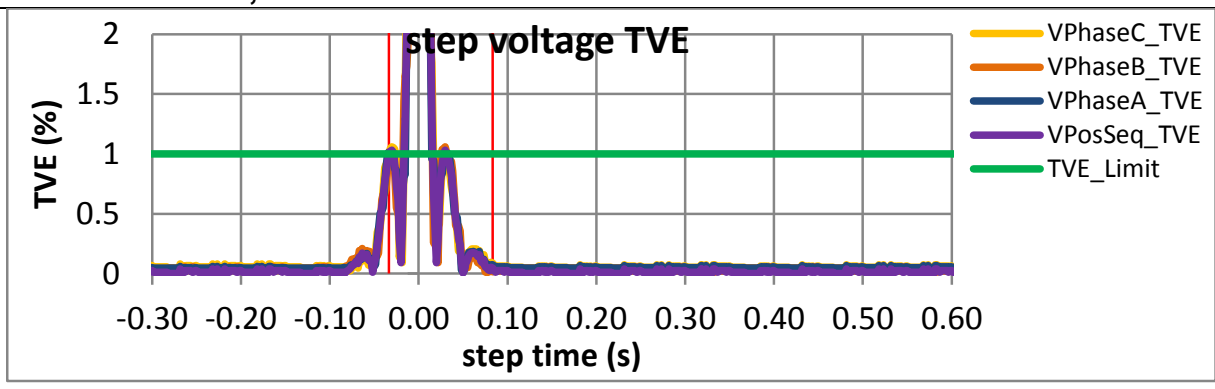

Figure 3954: Fs = 60 FPS, -10 degree phase step

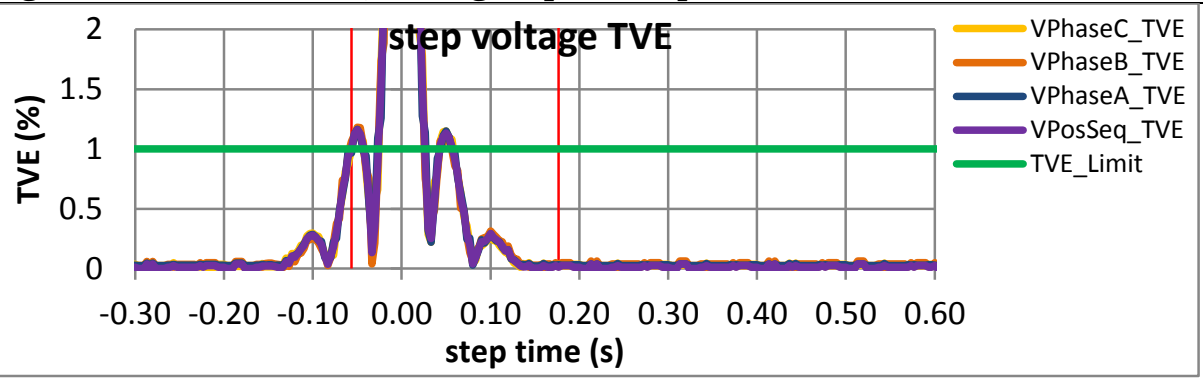

Figure 3956: Fs = 30 FPS, -10 degree phase step 


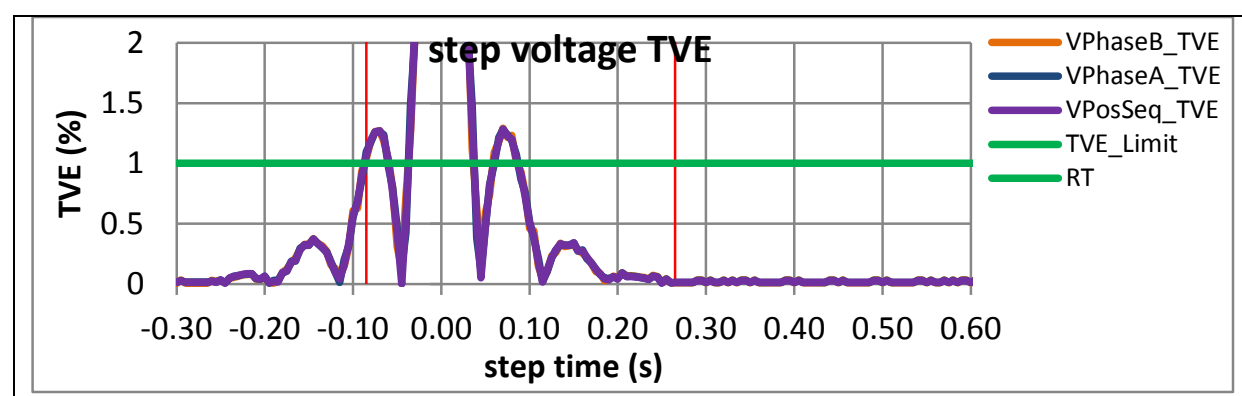

Figure 3957: Fs = 20 FPS, +10 degree phase step

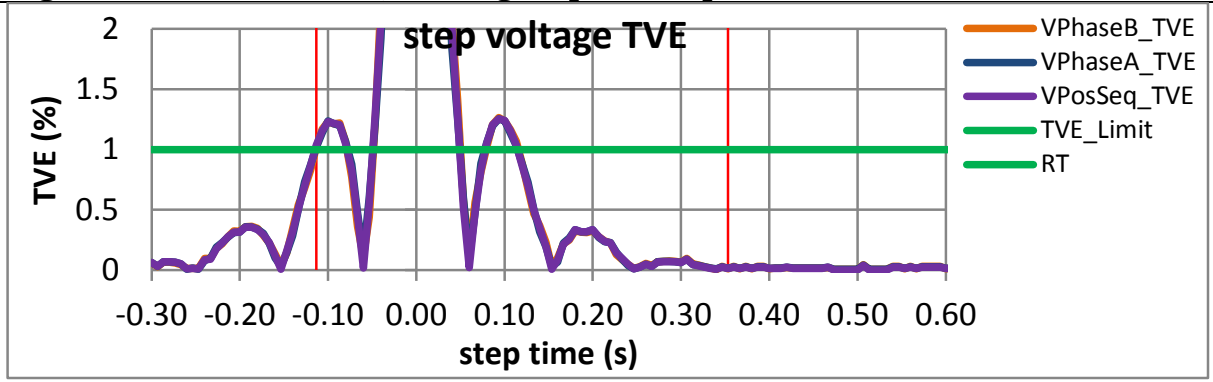

Figure 3959: Fs = 15 FPS, +10 degree phase step

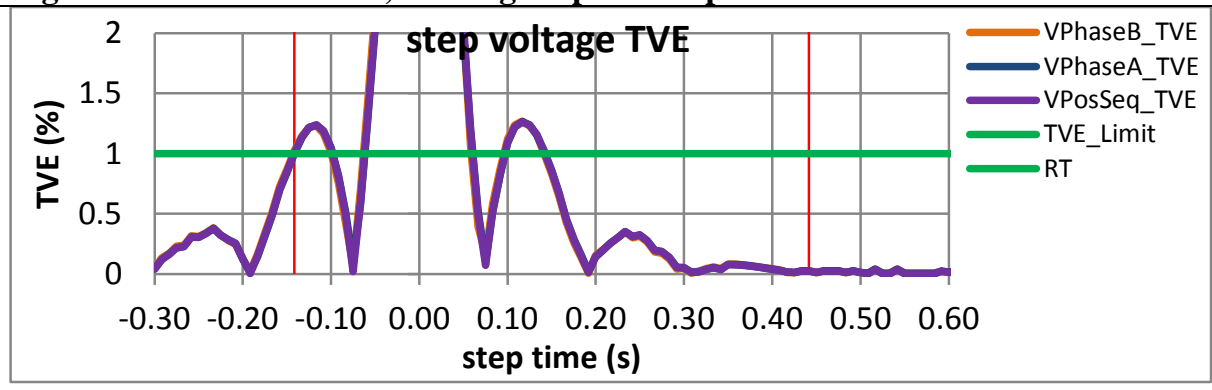

Figure 3961: Fs = 12 FPS, +10 degree phase step

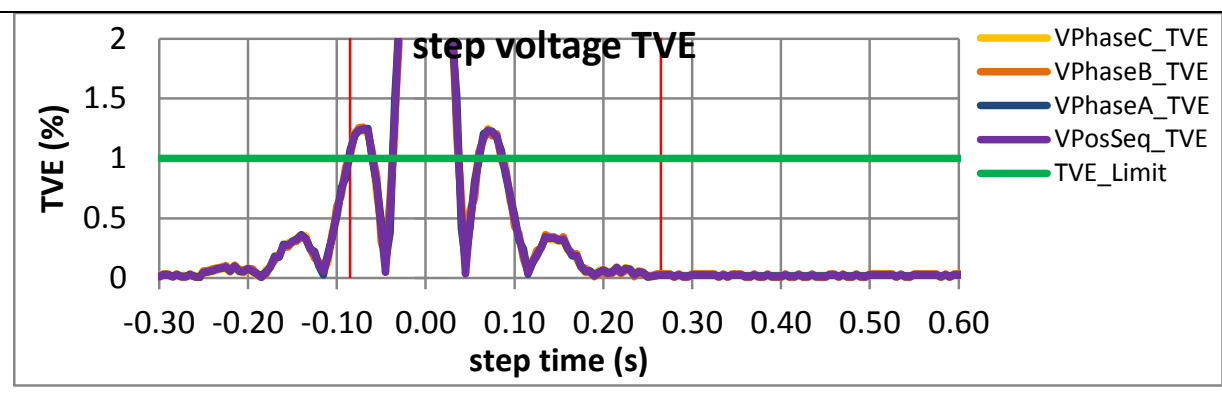

Figure 3958: Fs = 20 FPS, -10 degree phase step

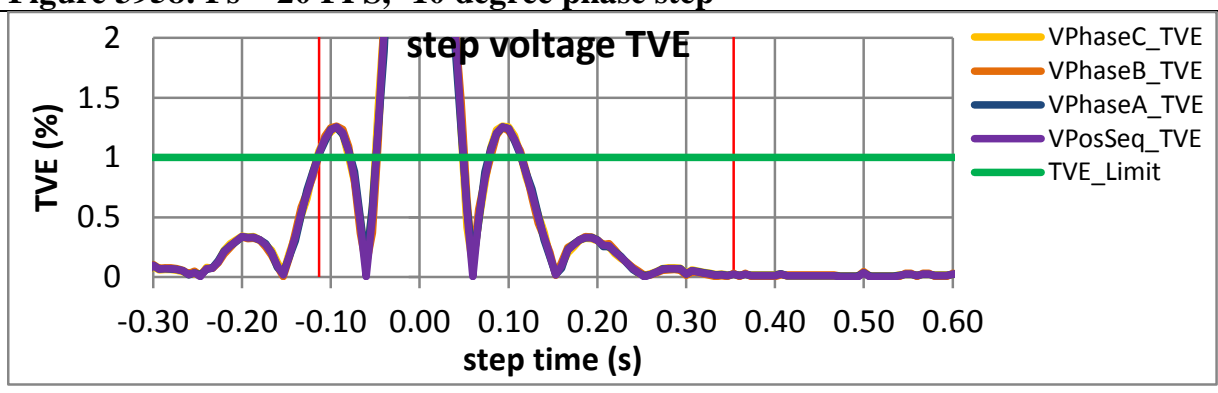

Figure 3960: Fs = 15 FPS, -10 degree phase step

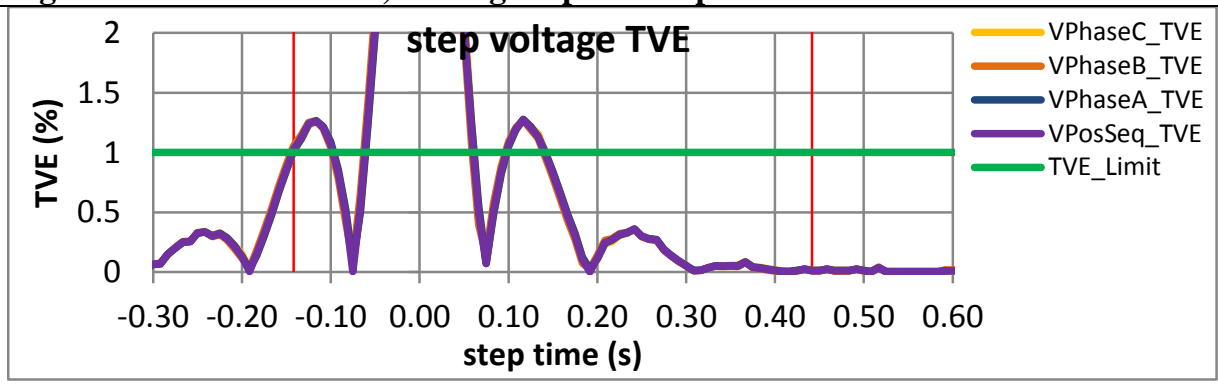

Figure 3962: Fs = 12 FPS, -10 degree phase step 


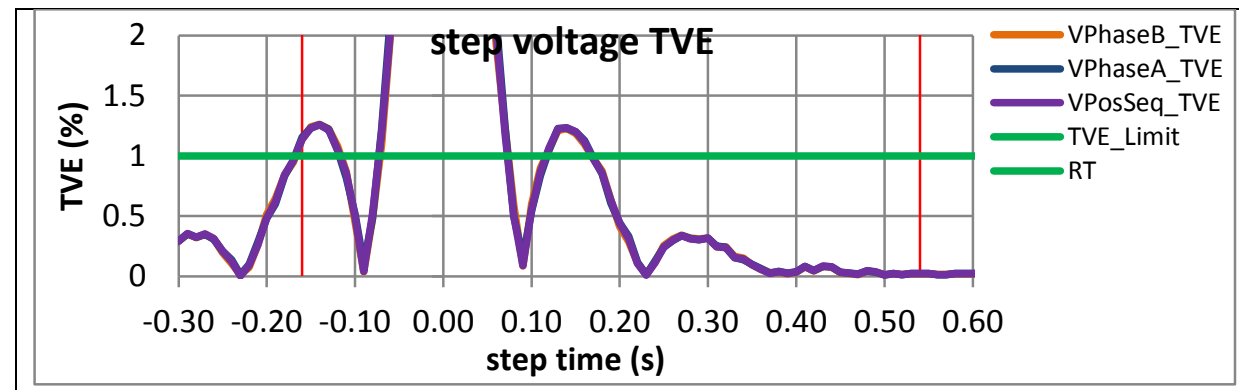

Figure 3963: Fs = 10 FPS, +10 degree phase step

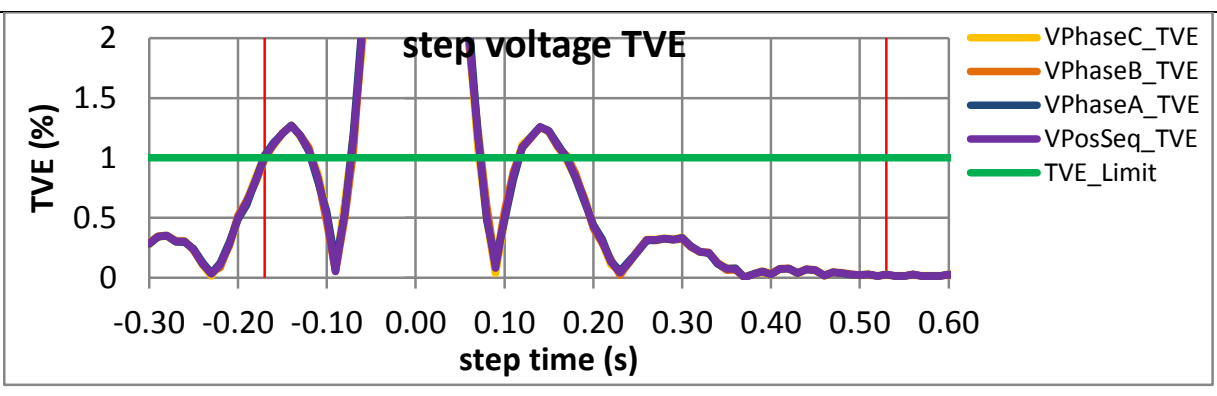

Figure 3964: Fs = 10 FPS, -10 degree phase step 
9.1.2 PMU A dynamic step change in phase voltage response time: $\mathrm{F} 0=60 \mathrm{~Hz}, \mathrm{M}$ class
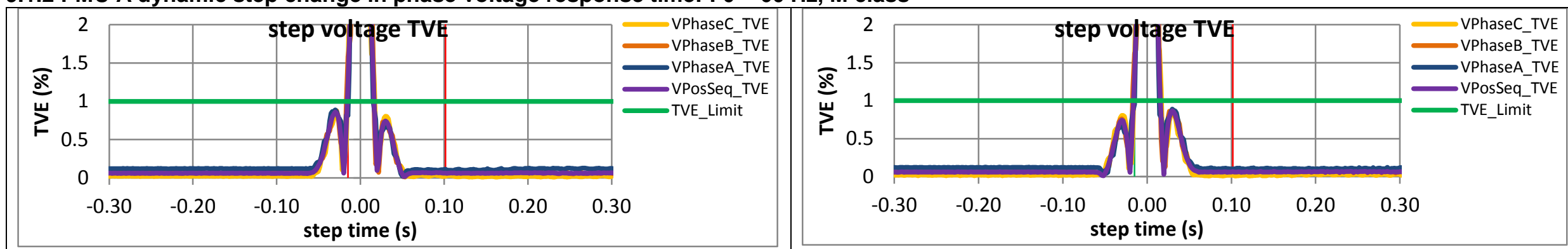

Figure 3965: Fs = 60 FPS, +10 degree phase step

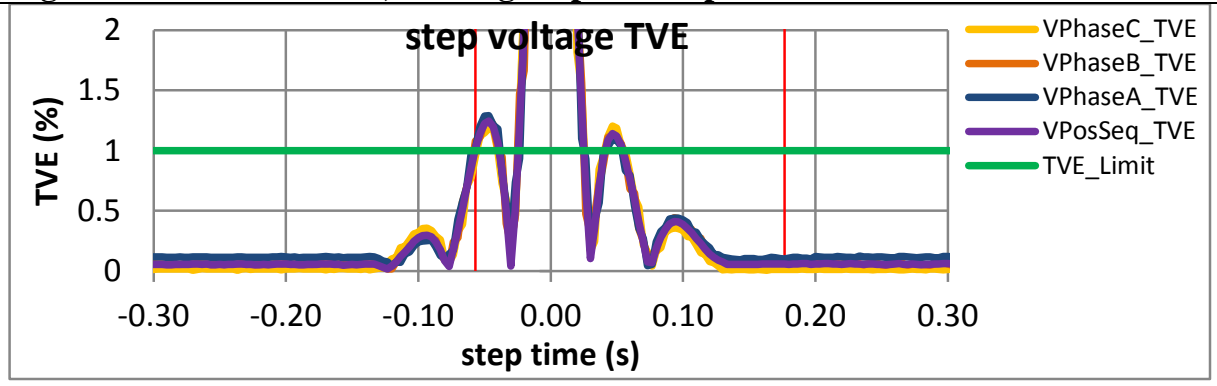

Figure 3966: Fs = 60 FPS, -10 degree phase step

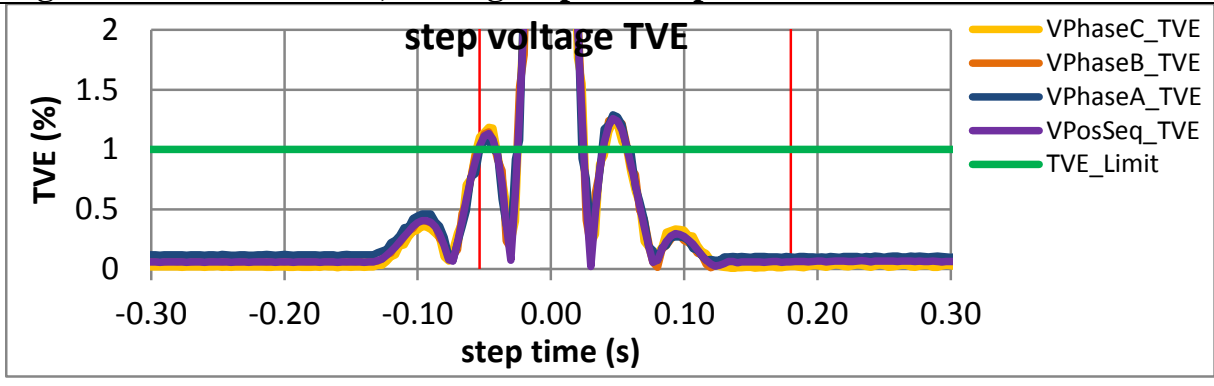

Figure 3967: Fs = 30 FPS, +10 degree phase step

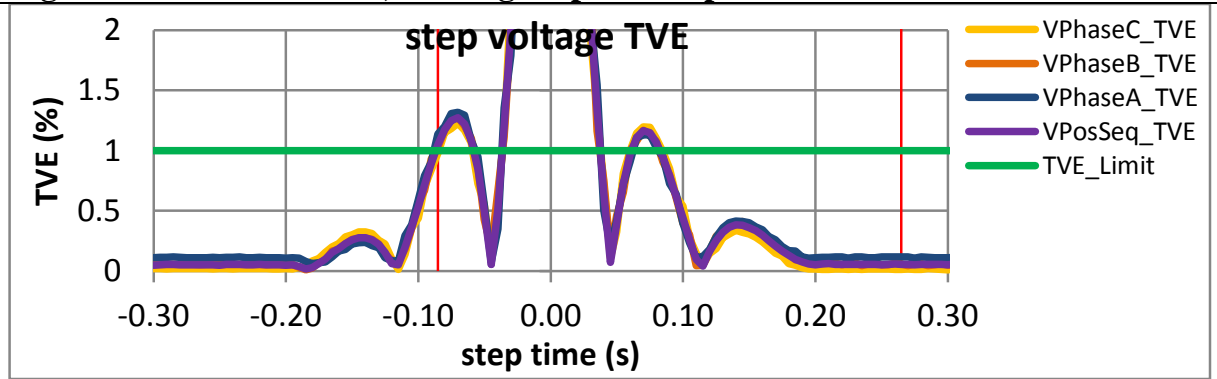

Figure 3969: Fs = 20 FPS, +10 degree phase step

Figure 3968: Fs = 30 FPS, -10 degree phase step

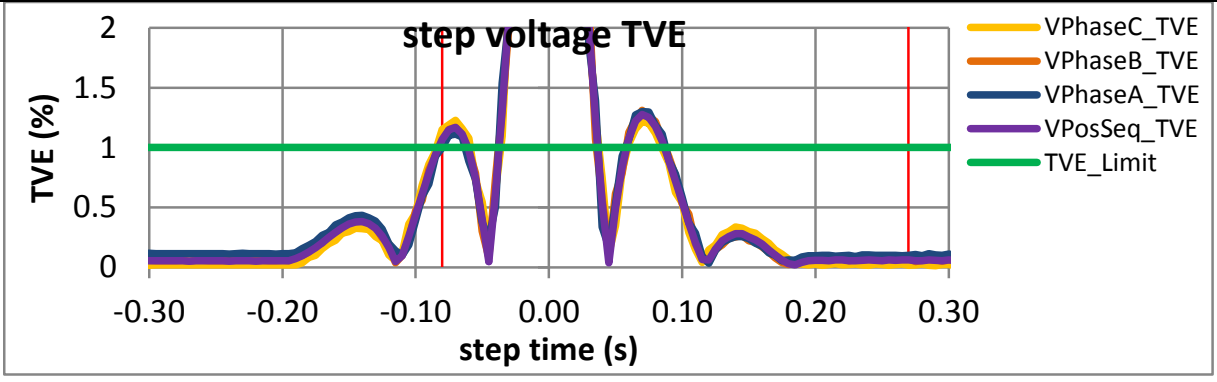

Figure 3970: Fs = 20 FPS, -10 degree phase step 


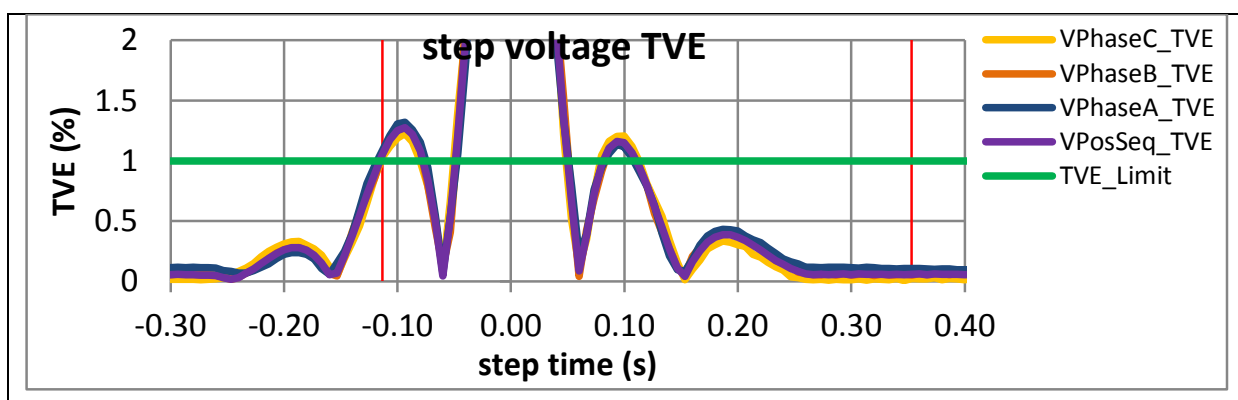

Figure 3971: Fs = 15 FPS, +10 degree phase step
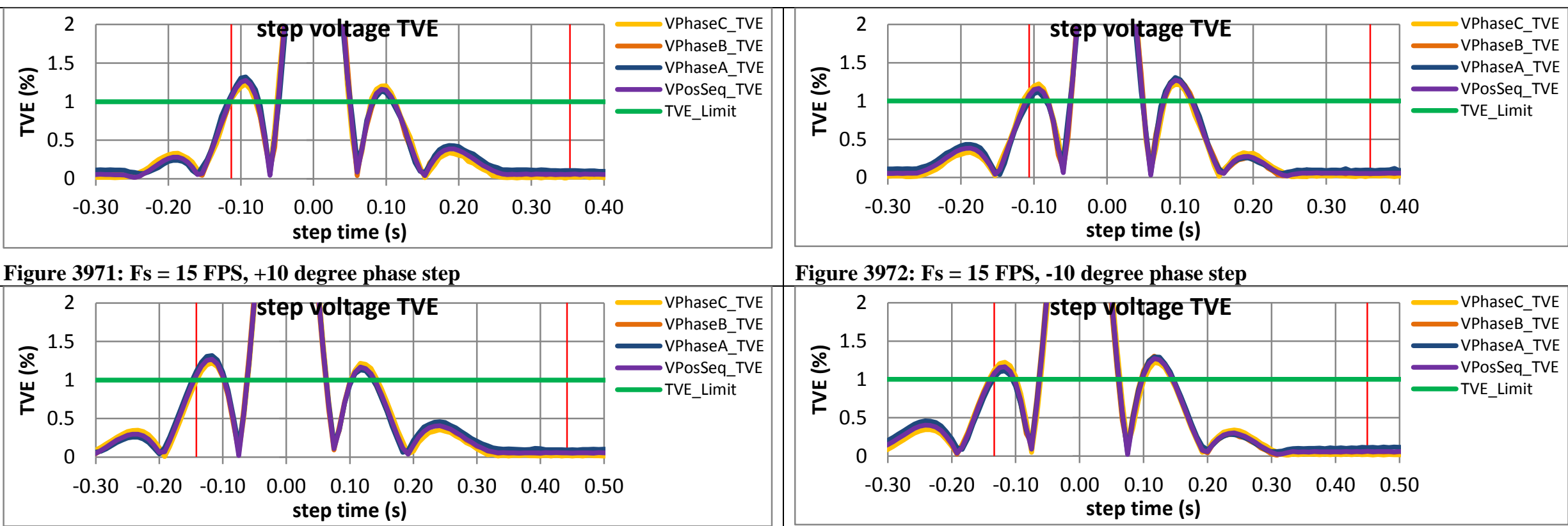

Figure 3972: Fs = 15 FPS, -10 degree phase step

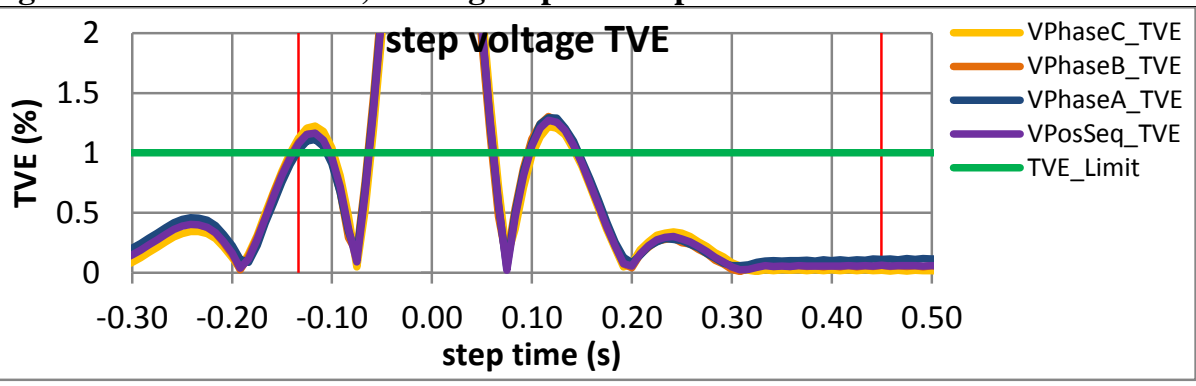

Figure 3973: Fs = 12 FPS, +10 degree phase step

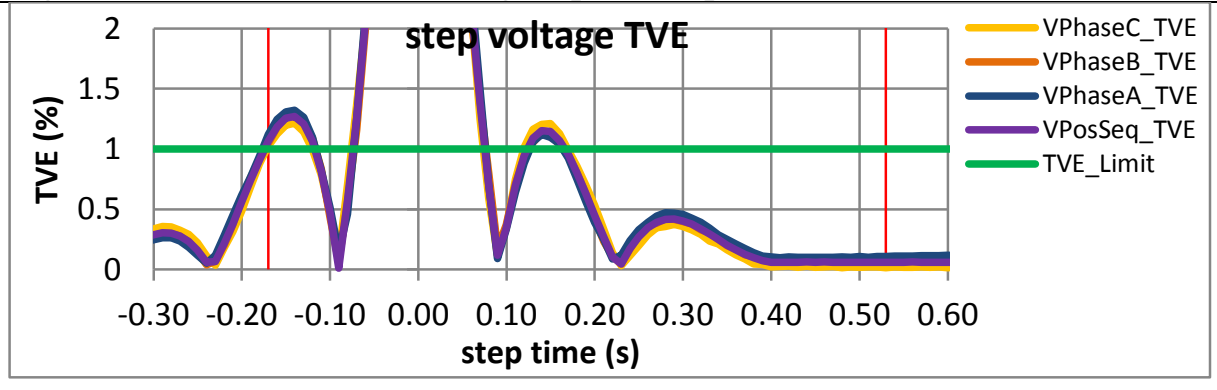

Figure 3974: Fs = 12 FPS, -10 degree phase step

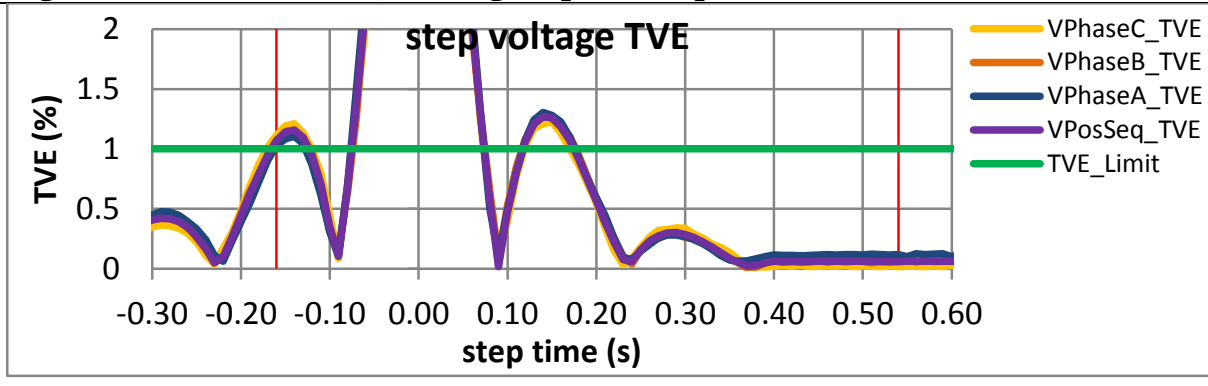

Figure 3976: Fs = 10 FPS, -10 degree phase step 
9.1.3 PMU B dynamic step change in phase voltage response time: $\mathrm{F} 0=60 \mathrm{~Hz}, \mathrm{M}$ class

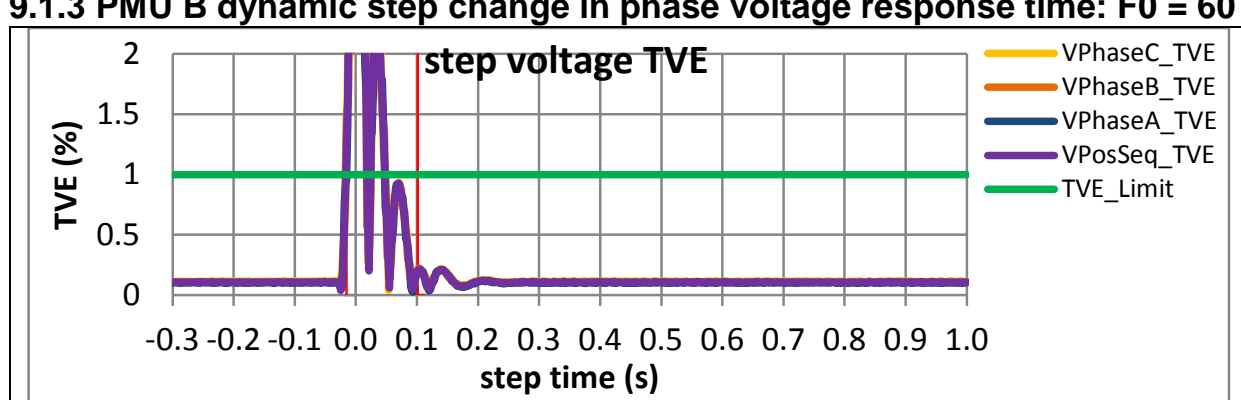

Figure 3977: Fs = 60 FPS, +10 degree phase step
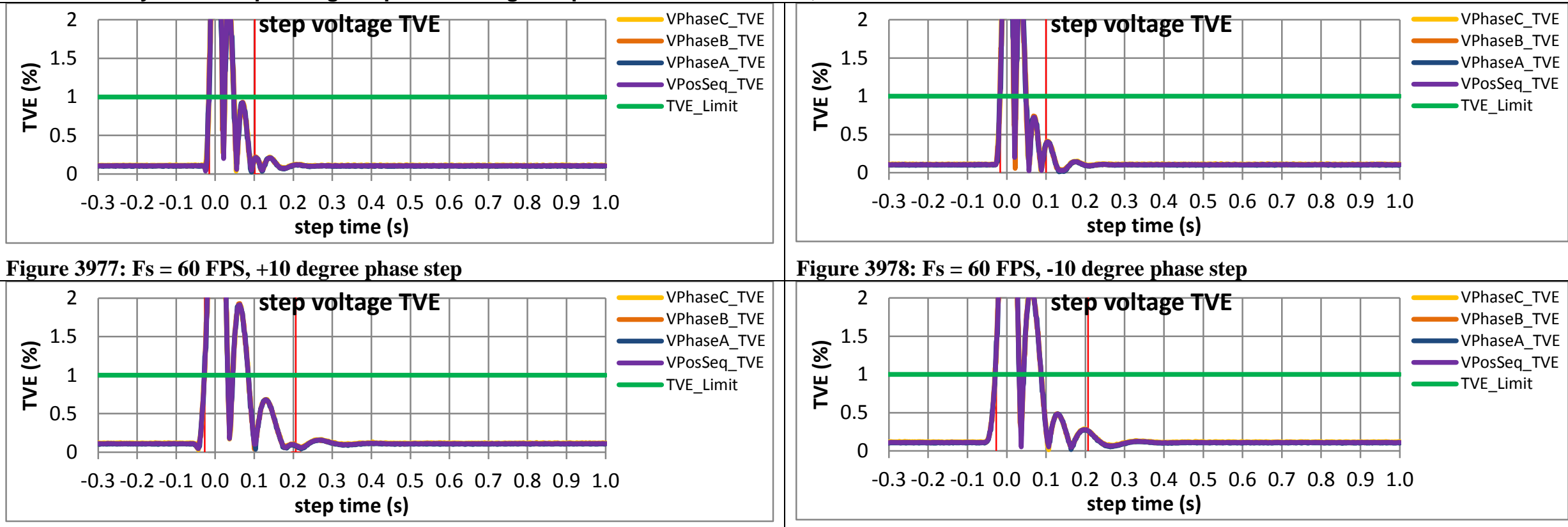

Figure 3978: Fs = 60 FPS, -10 degree phase step

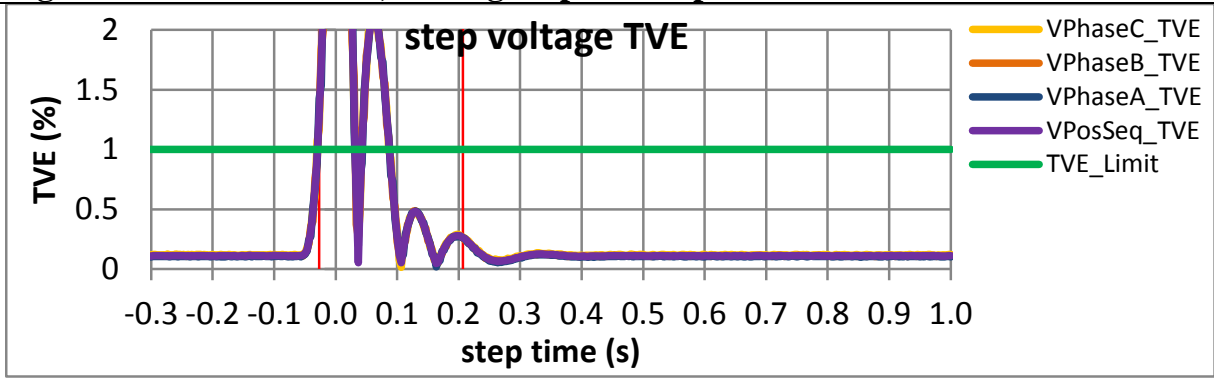

Figure 3979: Fs = 30 FPS, +10 degree phase step

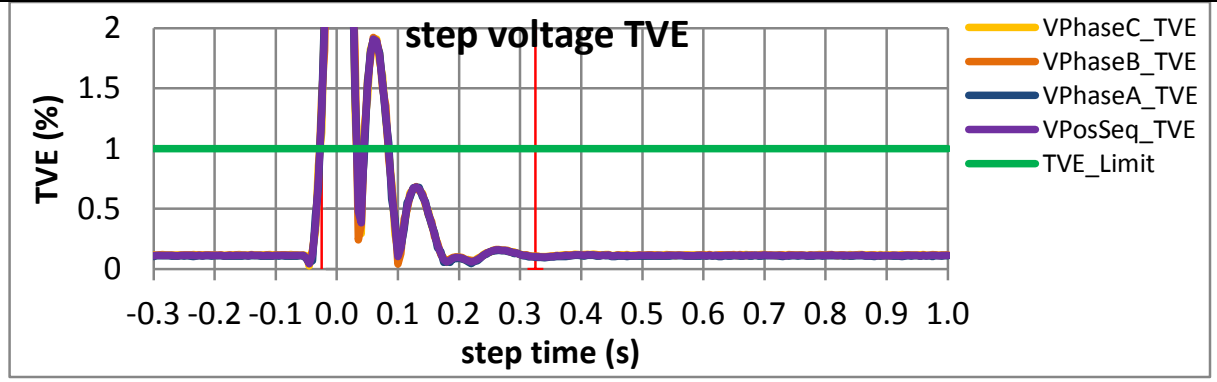

Figure 3981: Fs = 20 FPS, +10 degree phase step

Figure 3980: Fs = 30 FPS, -10 degree phase step

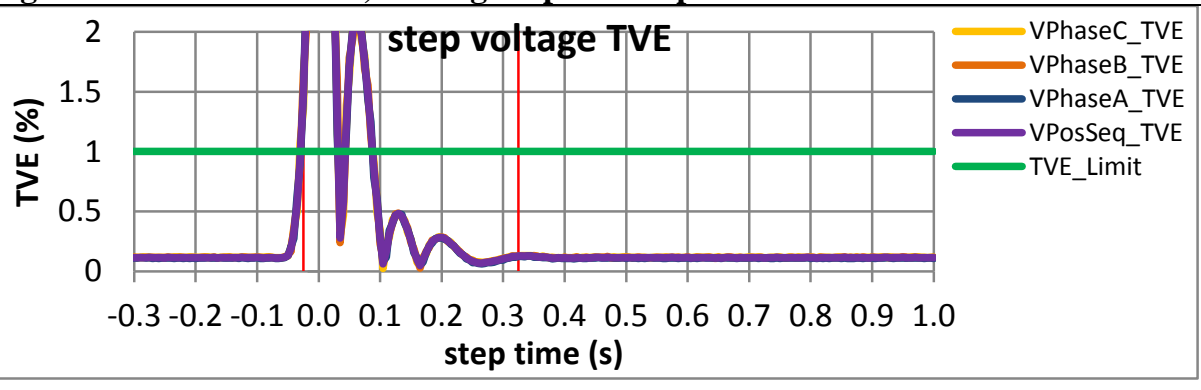

Figure 3982: Fs = 20 FPS, -10 degree phase step 


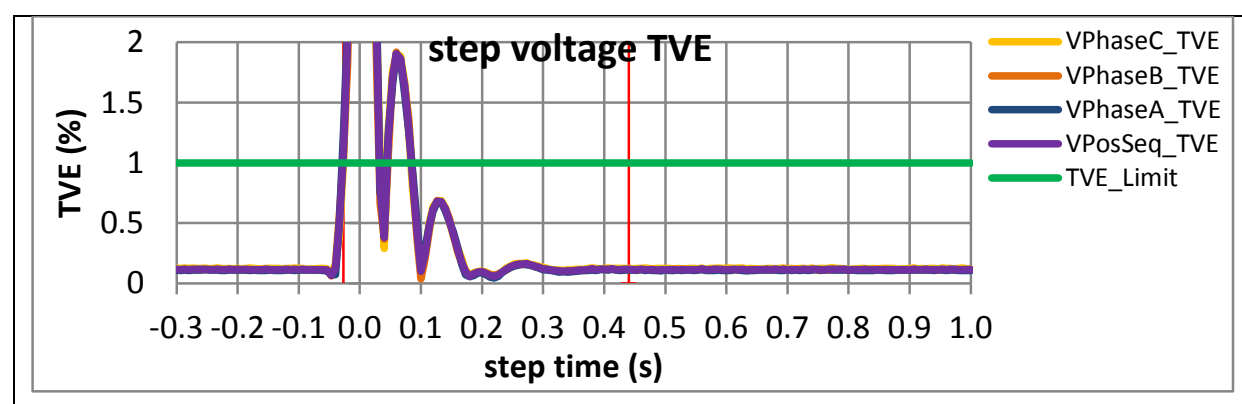

Figure 3983: Fs = 15 FPS, +10 degree phase step
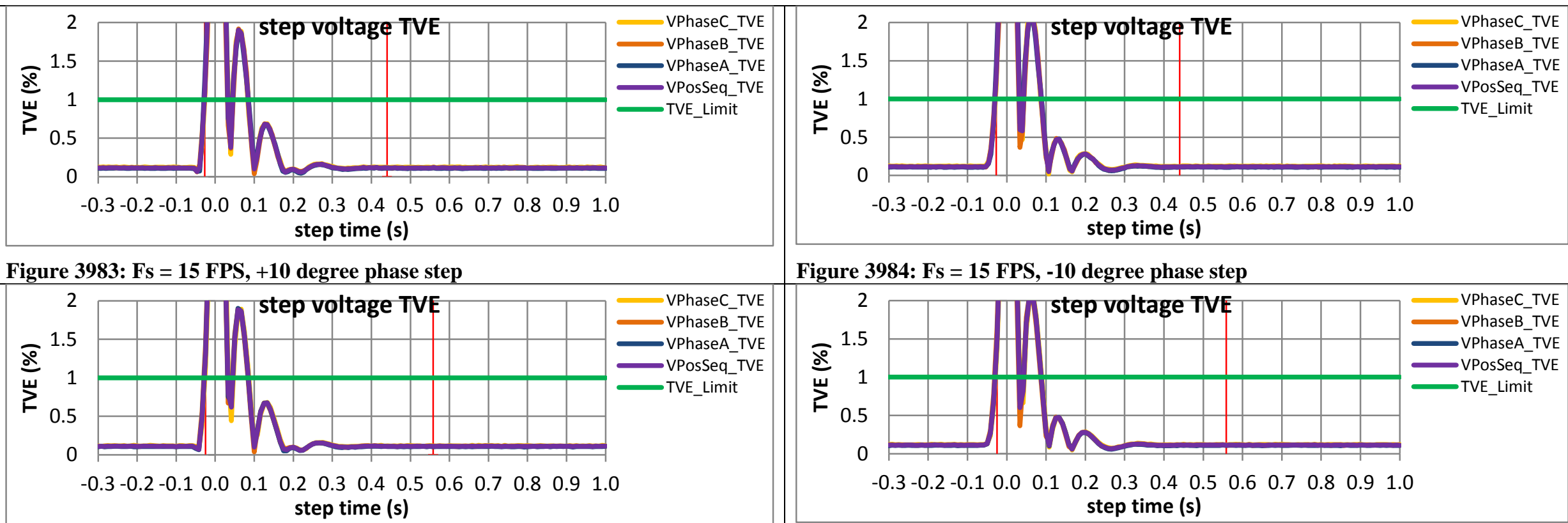

Figure 3984: Fs = 15 FPS, -10 degree phase step

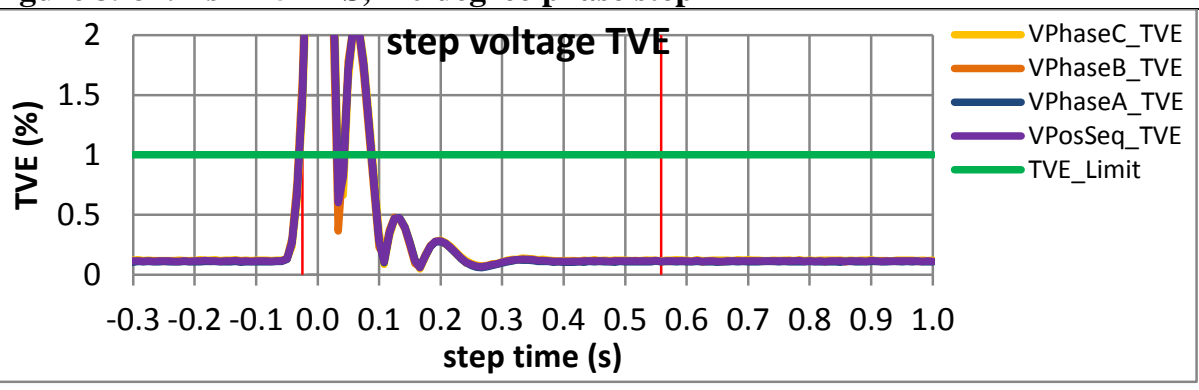

Figure 3985: Fs = 12 FPS, +10 degree phase step

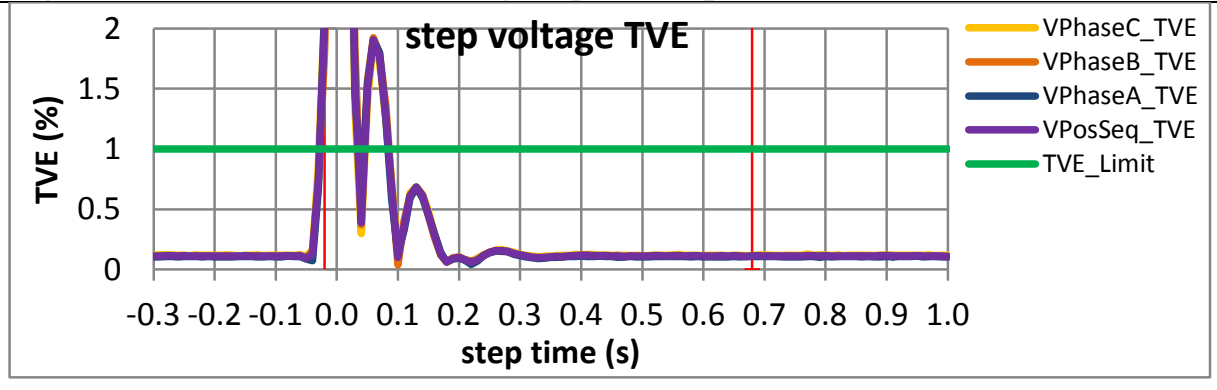

Figure 3986: Fs $=12$ FPS, -10 degree phase step

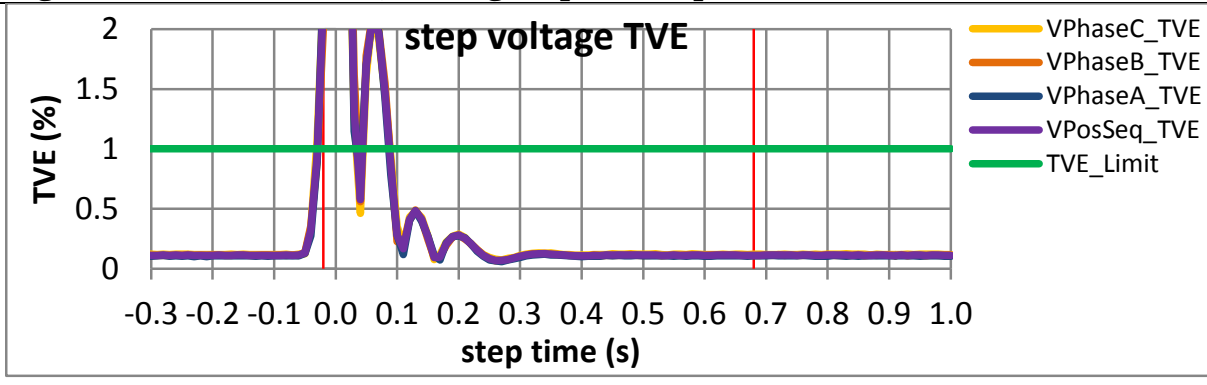

Figure 3988: Fs = 10 FPS, -10 degree phase step 
9.1.4 PMU C dynamic step change in phase voltage response time: $\mathrm{F} 0=60 \mathrm{~Hz}, \mathrm{M}$ class
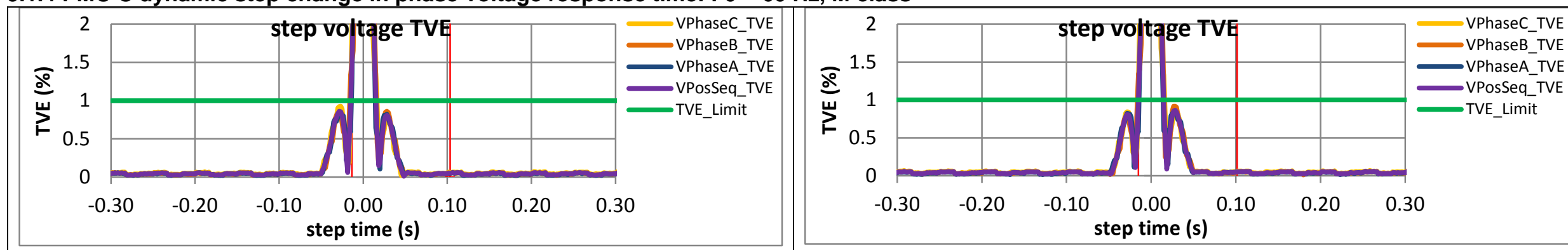

Figure 3989: Fs = 60 FPS, +10 degree phase step

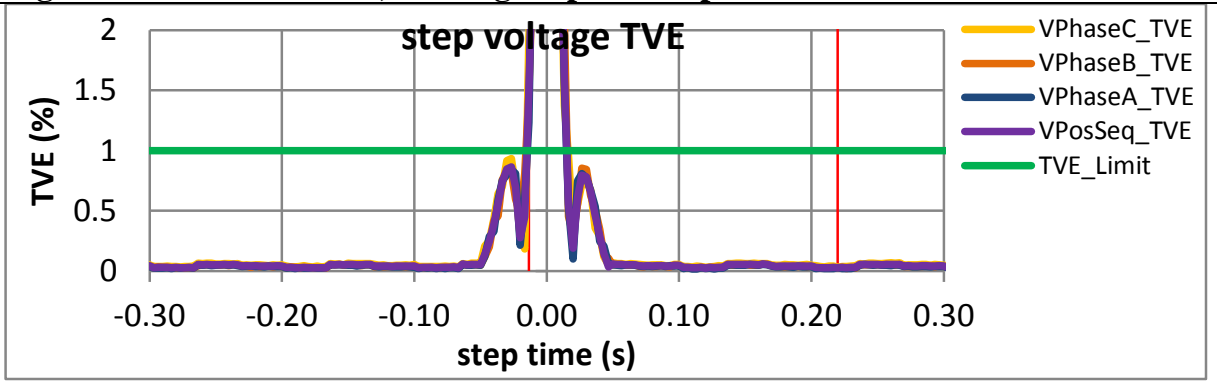

Figure 3990: Fs = 60 FPS, -10 degree phase step

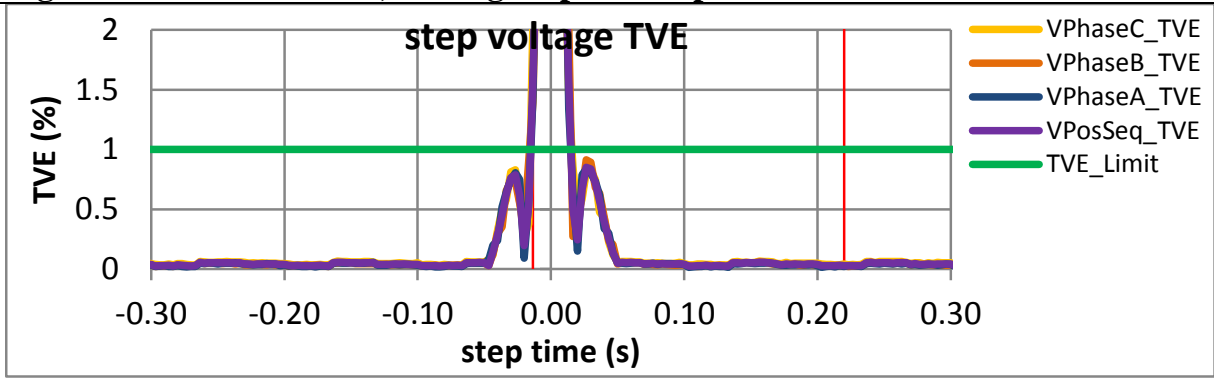

Figure 3991: Fs = 30 FPS, +10 degree phase step

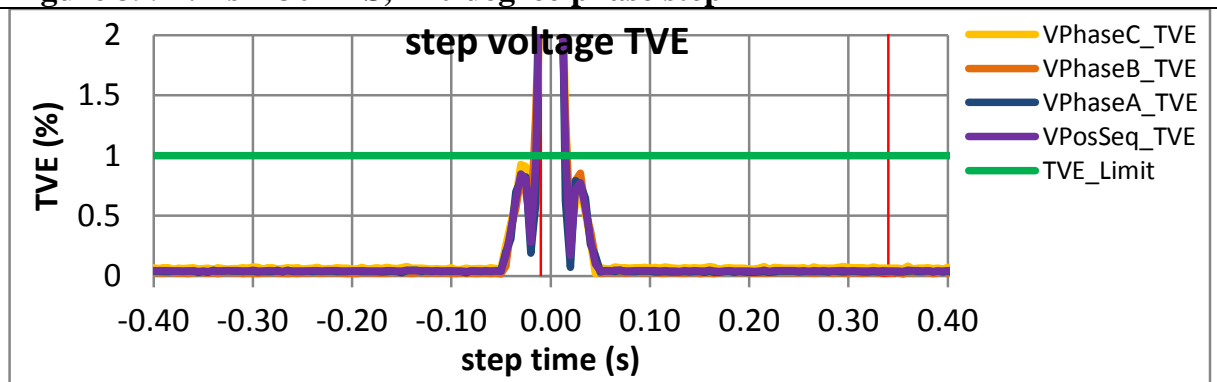

Figure 3993: Fs = 20 FPS, +10 degree phase step

Figure 3992: Fs = 30 FPS, -10 degree phase step

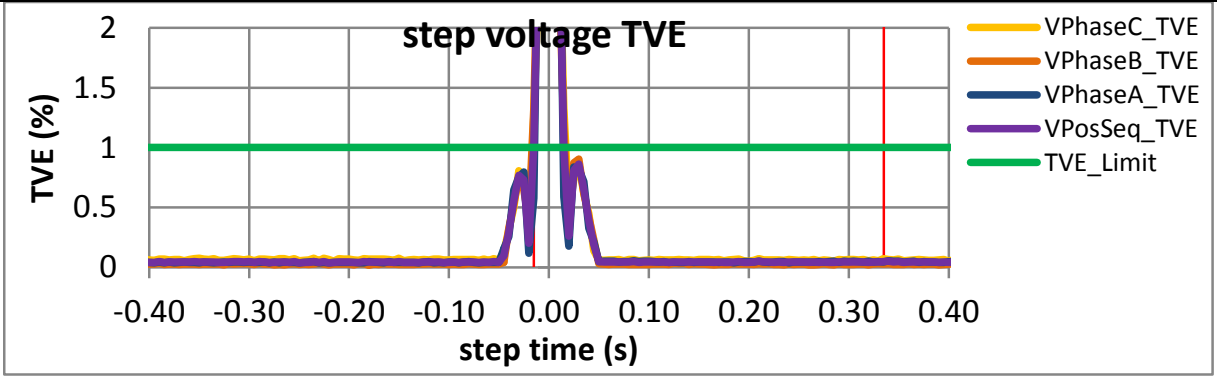

Figure 3994: Fs = 20 FPS, -10 degree phase step 


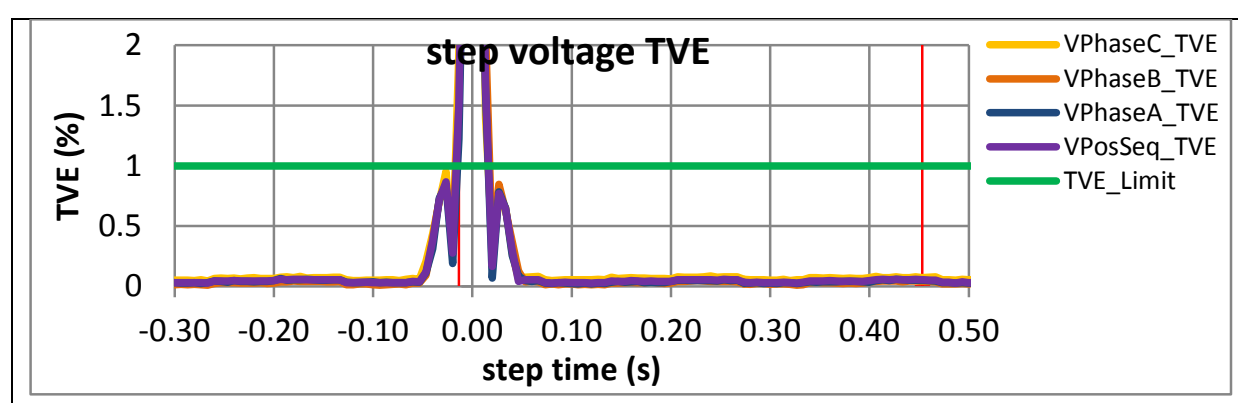

Figure 3995: Fs = 15 FPS, +10 degree phase step
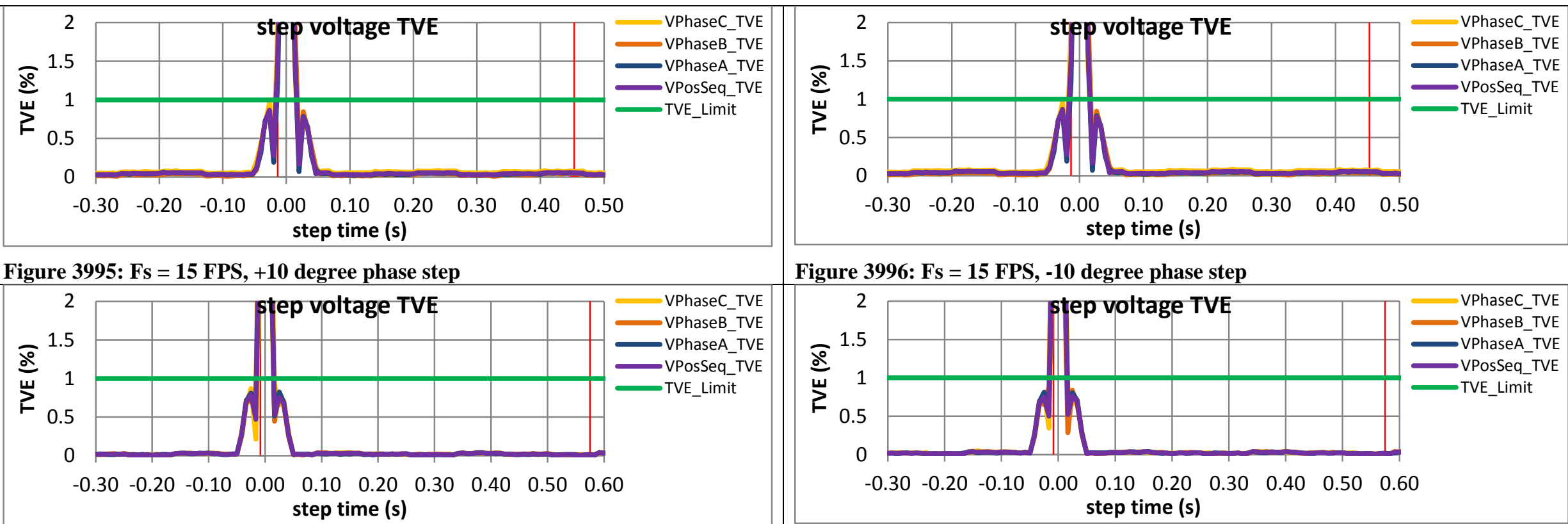

Figure 3996: Fs = 15 FPS, -10 degree phase step

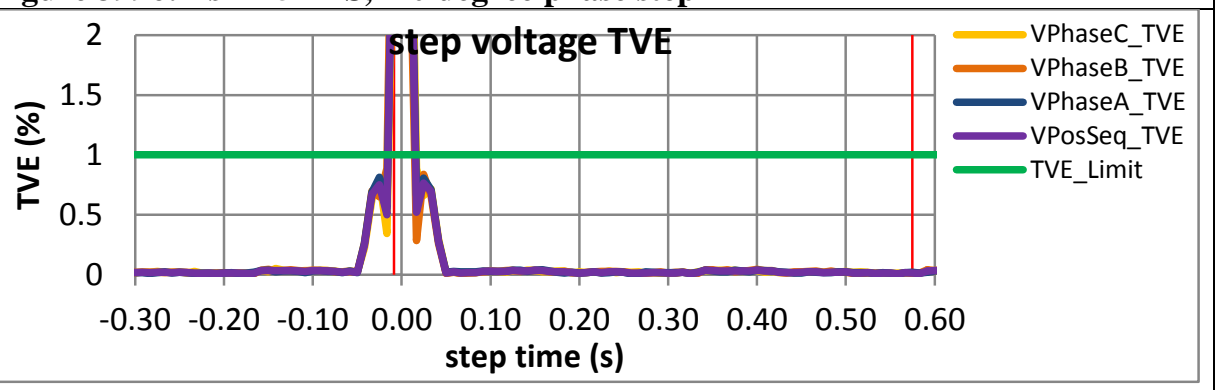

Figure 3997: Fs = 12 FPS, +10 degree phase step

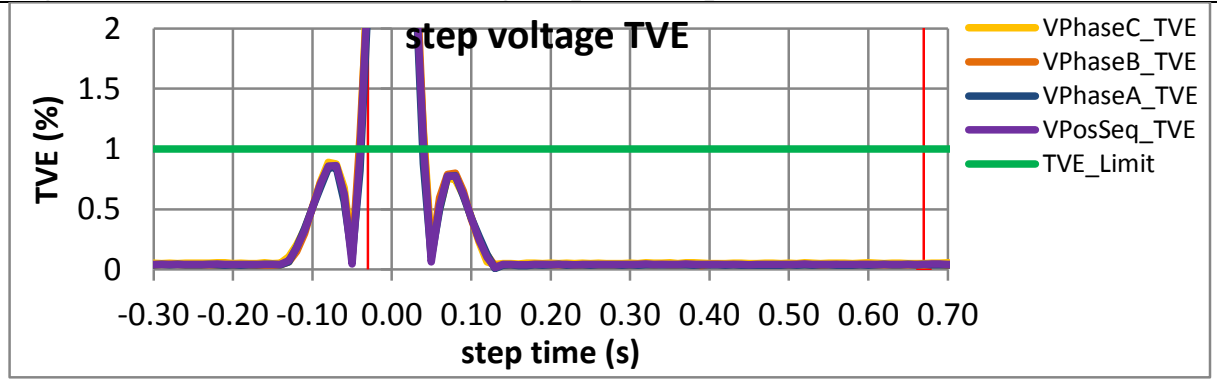

Figure 3998: Fs $=12$ FPS, -10 degree phase step

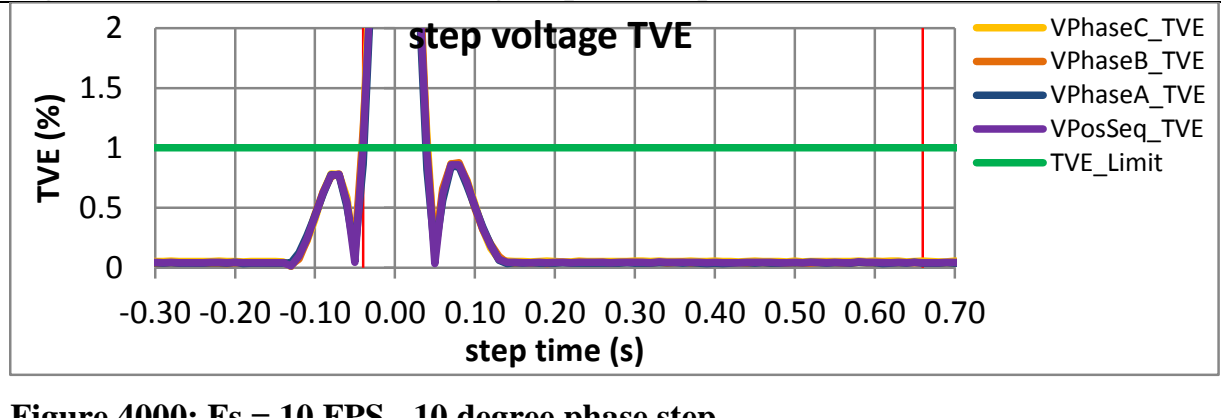

Figure 3999: Fs = 10 FPS, +10 degree phase step

Figure 4000: Fs = 10 FPS, -10 degree phase step 
9.1.5 PMU D dynamic step change in phase voltage response time: $\mathrm{F} 0=60 \mathrm{~Hz}, \mathrm{M}$ class

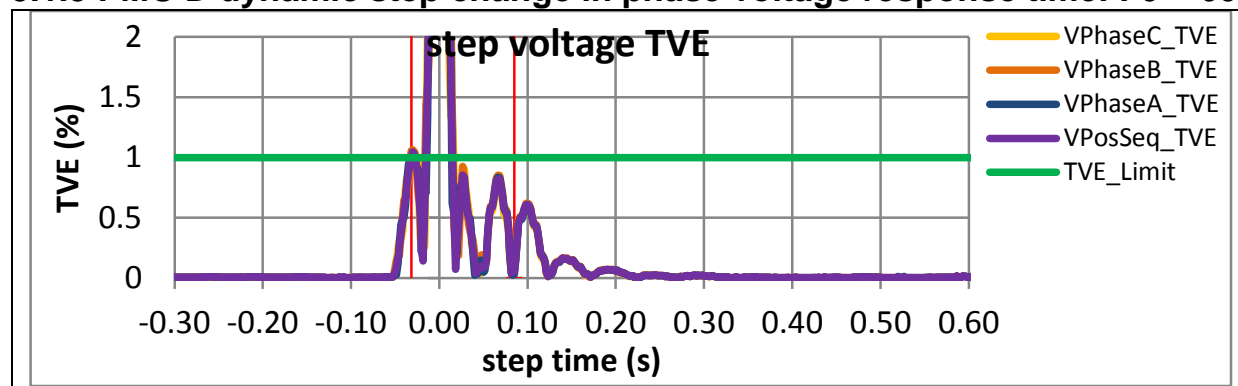

Figure 4001: Fs = 60 FPS, +10 degree phase step

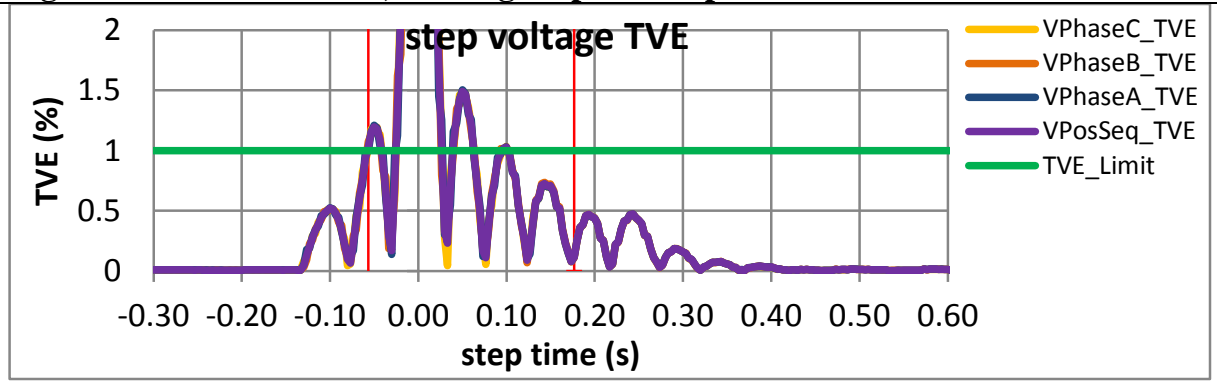

Figure 4003: Fs = 30 FPS, +10 degree phase step

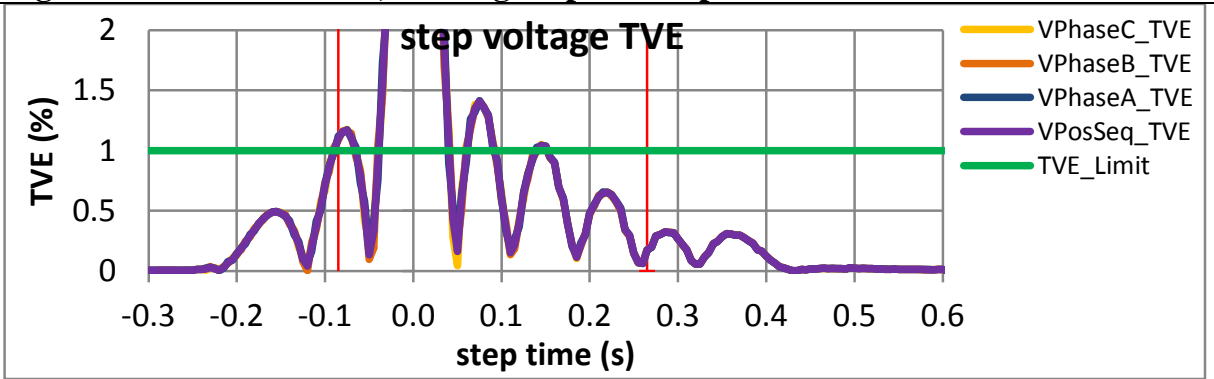

Figure 4005: Fs = 20 FPS, +10 degree phase step

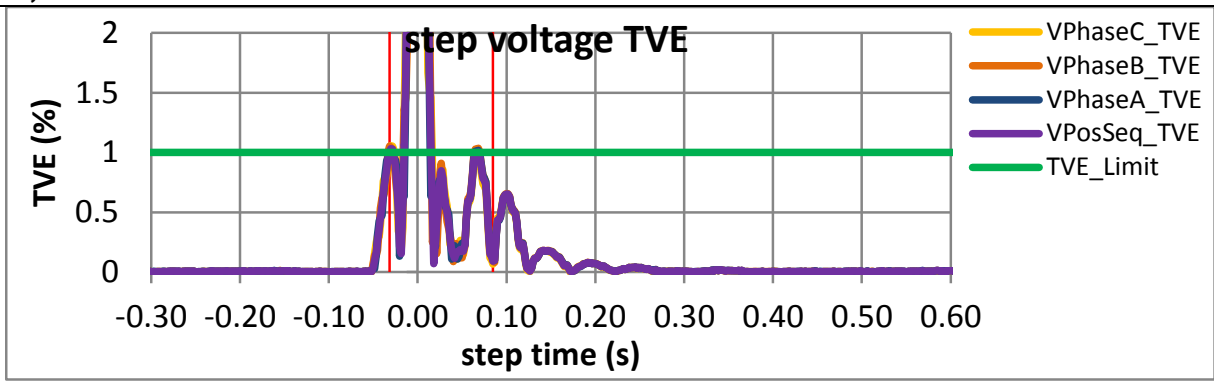

Figure 4002: Fs = 60 FPS, -10 degree phase step

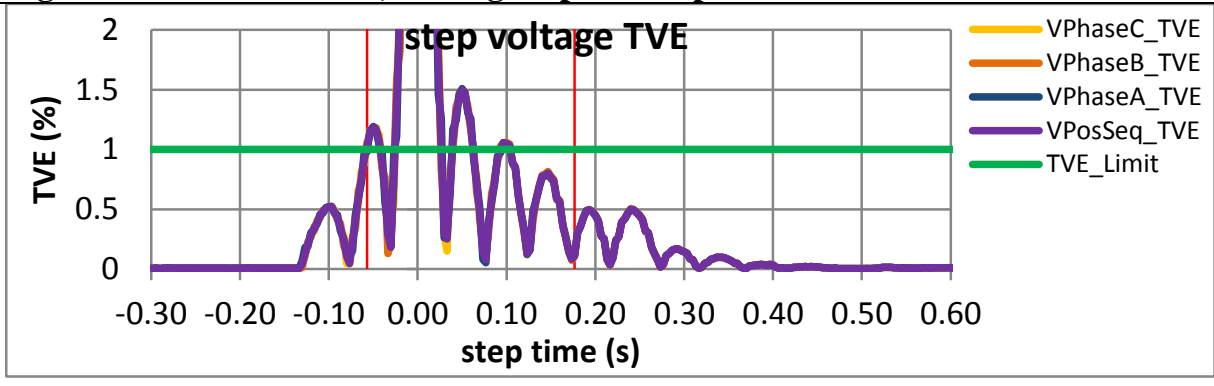

Figure 4004: Fs = 30 FPS, -10 degree phase step

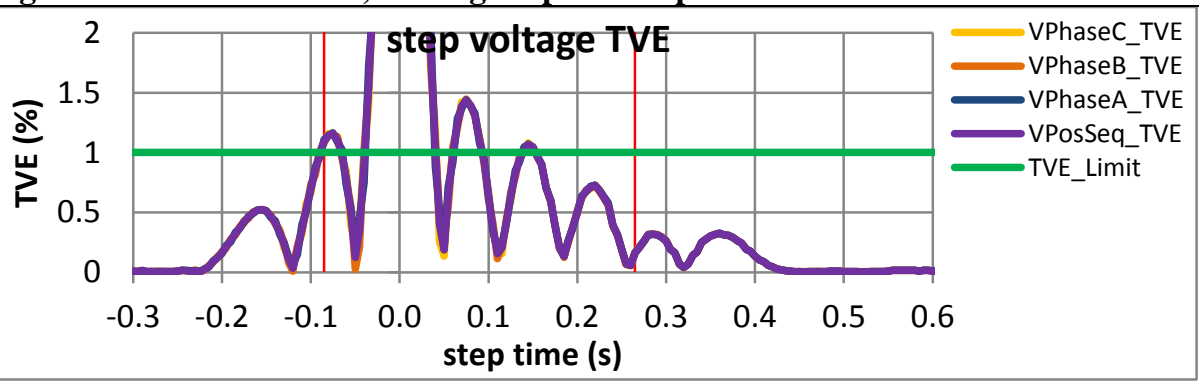

Figure 4006: Fs = 20 FPS, -10 degree phase step 


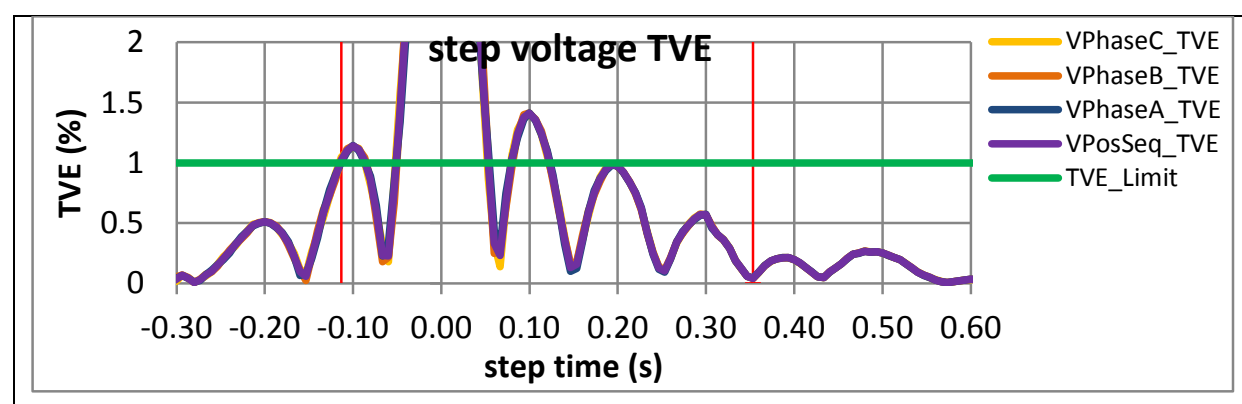

Figure 4007: Fs = 15 FPS, +10 degree phase step
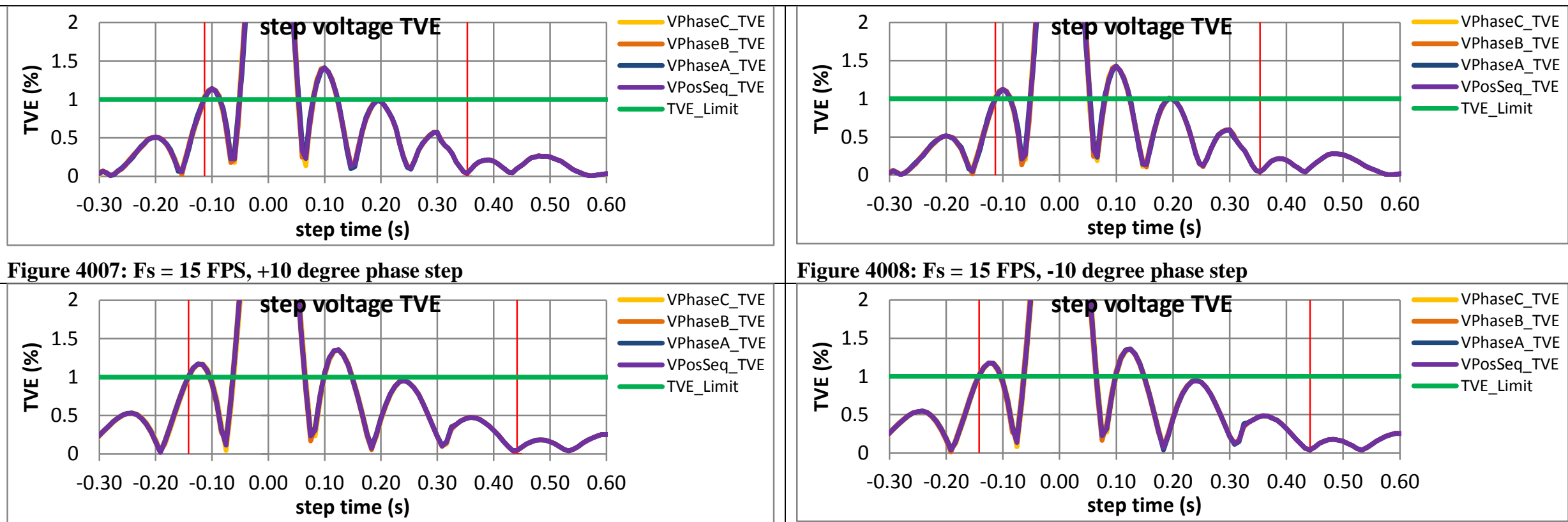

Figure 4008: Fs = 15 FPS, -10 degree phase step

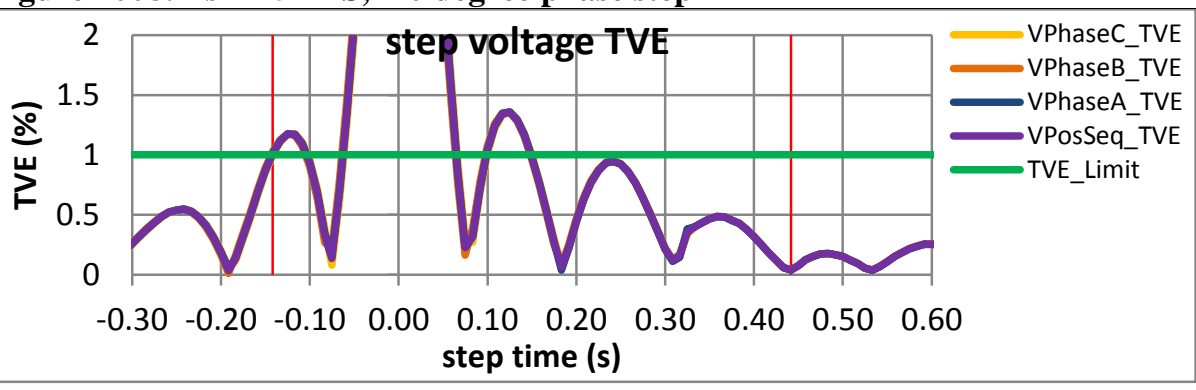

Figure 4009: Fs = 12 FPS, +10 degree phase step

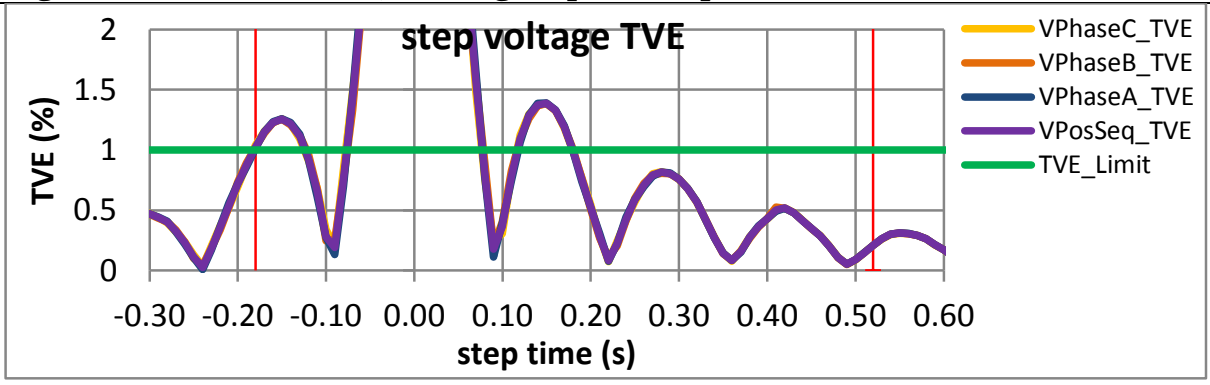

Figure 4010: Fs = 12 FPS, -10 degree phase step

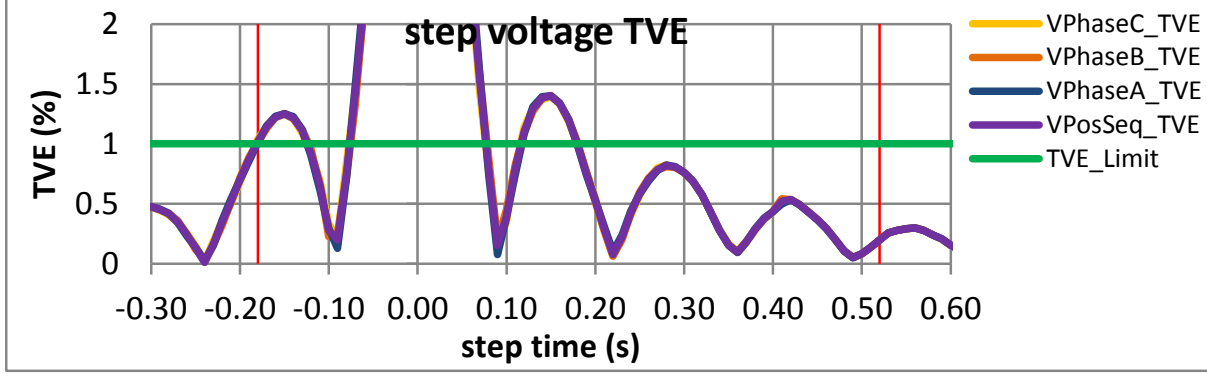

Figure 4012: Fs = 10 FPS, -10 degree phase step 
9.1.6 PMU E dynamic step change in phase voltage response time: $\mathrm{F} 0=60 \mathrm{~Hz}, \mathrm{M}$ class

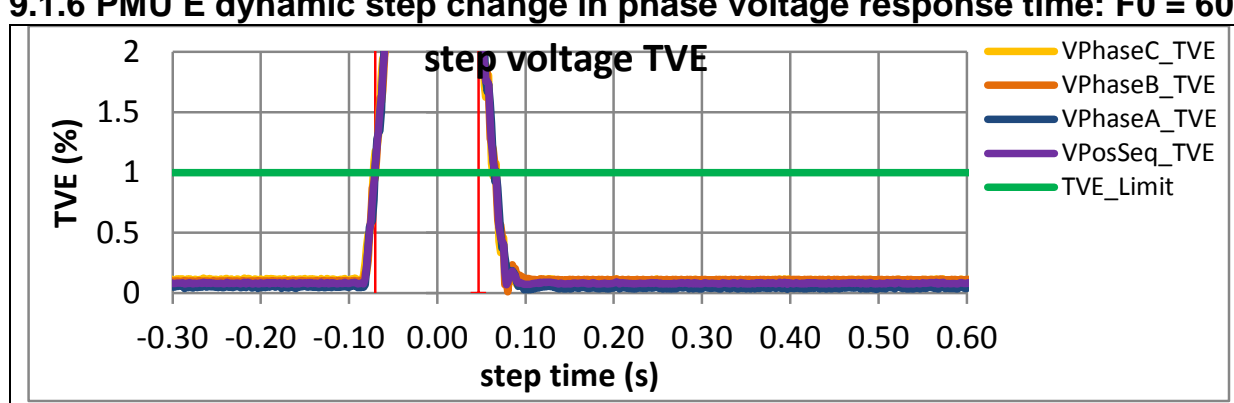

Figure 4013: Fs = 60 FPS, +10 degree phase step

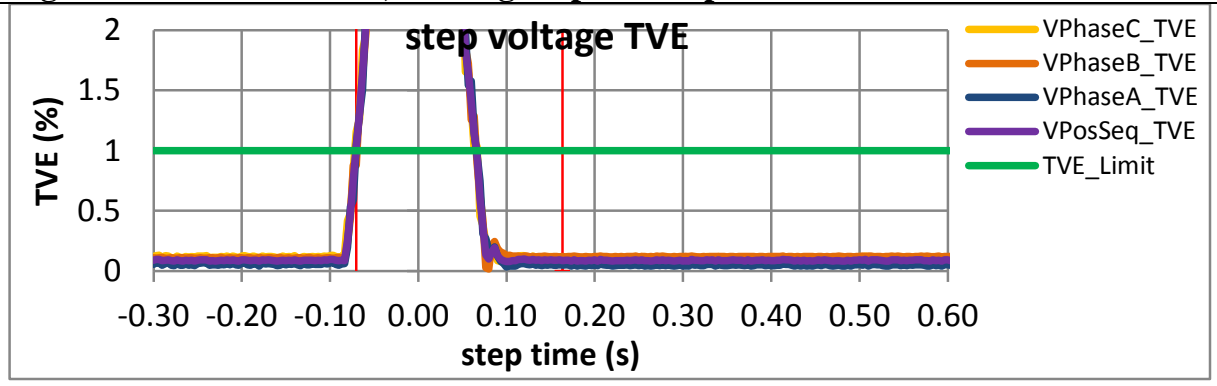

Figure 4015: Fs = 30 FPS, +10 degree phase step

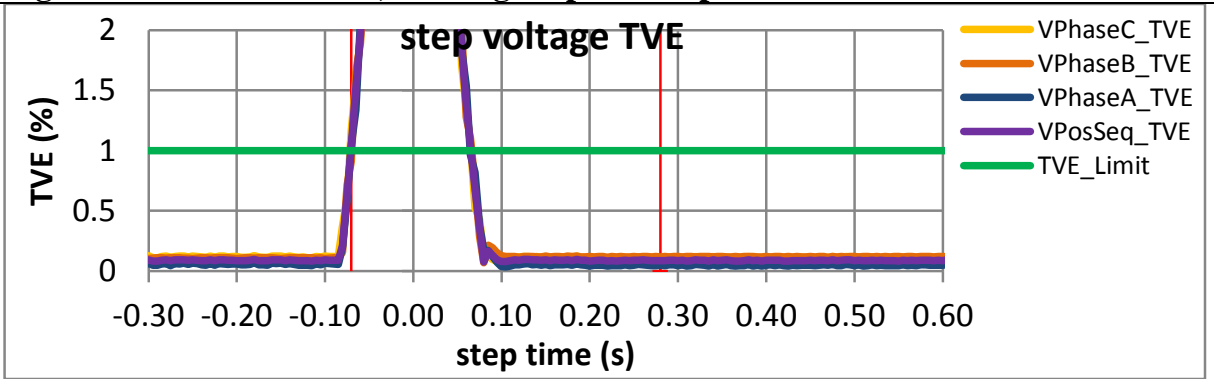

Figure 4017: Fs = 20 FPS, +10 degree phase step

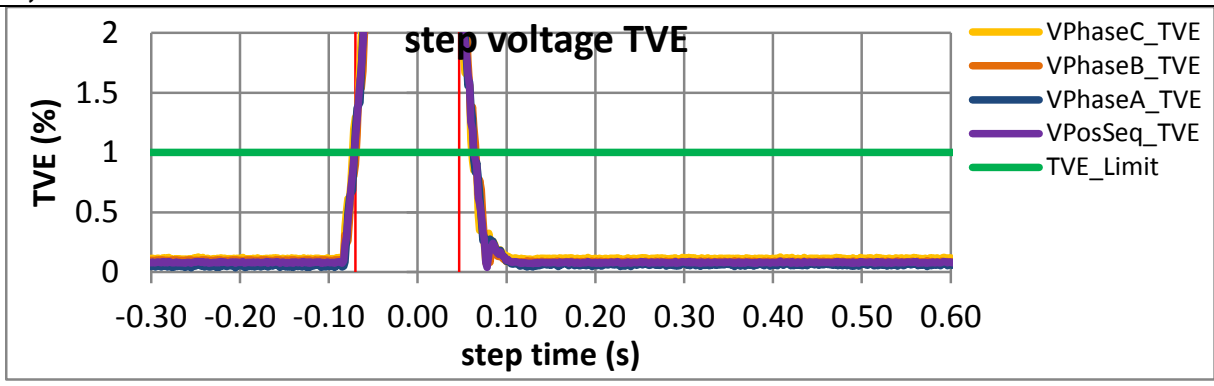

Figure 4014: Fs = 60 FPS, -10 degree phase step

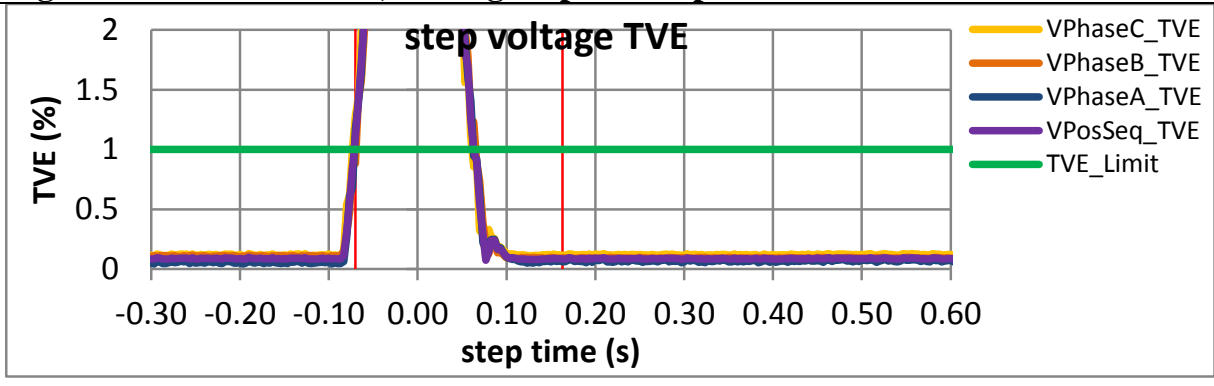

Figure 4016: Fs = 30 FPS, -10 degree phase step

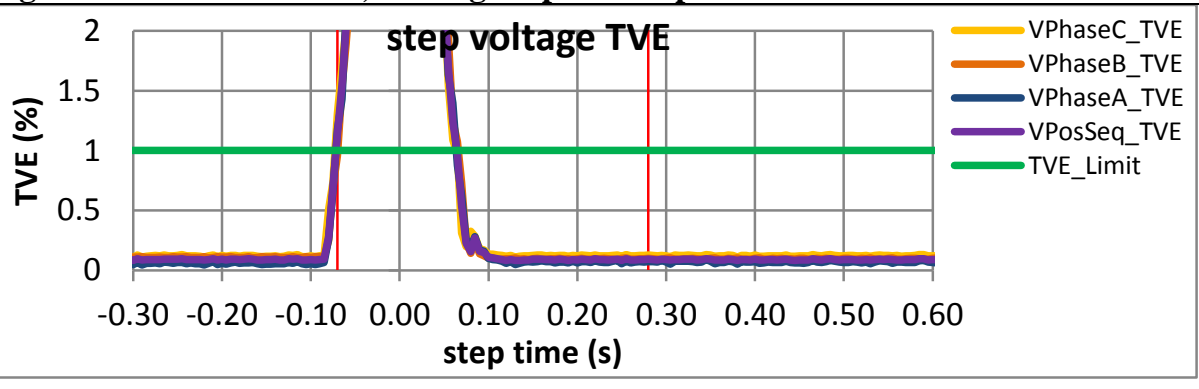

Figure 4018: Fs = 20 FPS, -10 degree phase step 


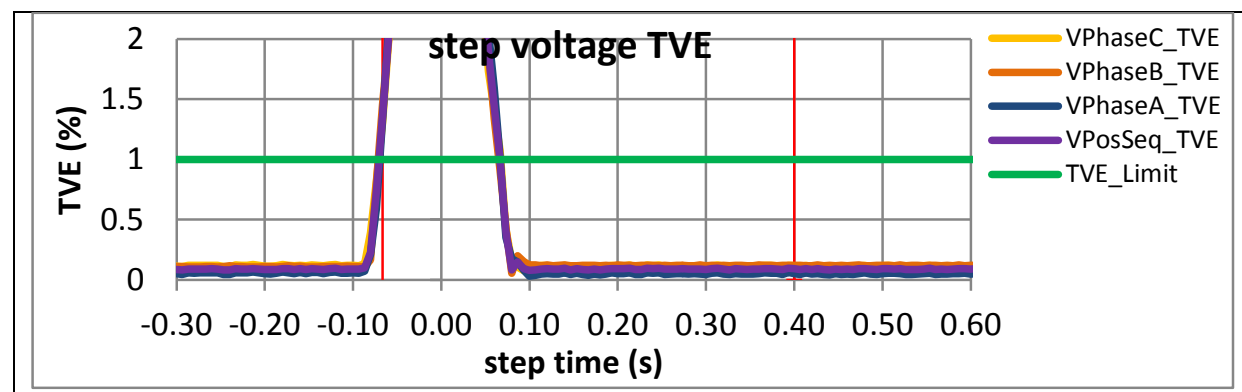

Figure 4019: Fs = 15 FPS, +10 degree phase step

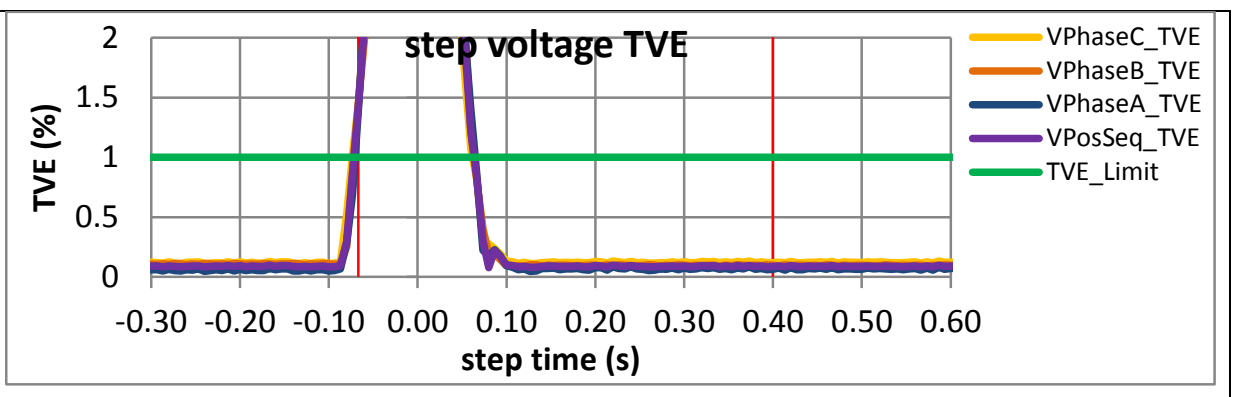

Figure 4020: Fs = 15 FPS, -10 degree phase step

Figure 4021: Fs = 12 FPS, +10 degree phase step

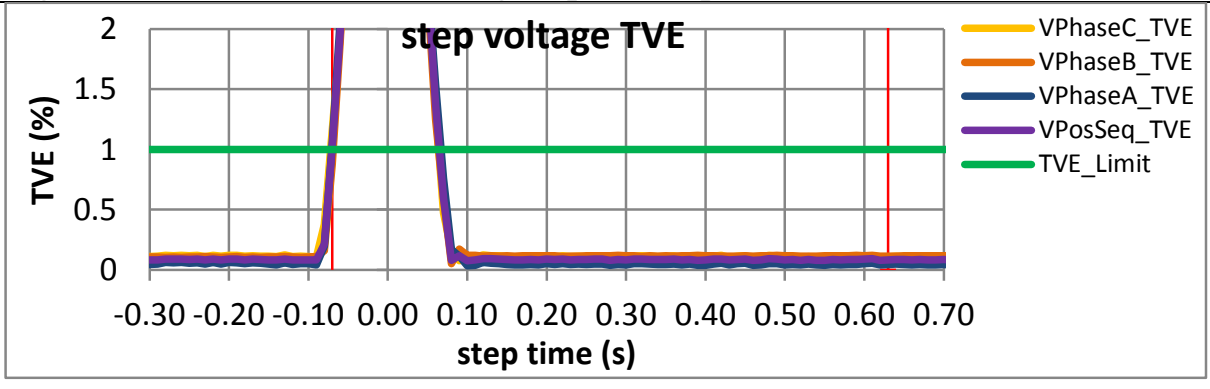

Figure 4022: Fs $=12$ FPS, -10 degree phase step

Figure 4023: Fs = 10 FPS, +10 degree phase step

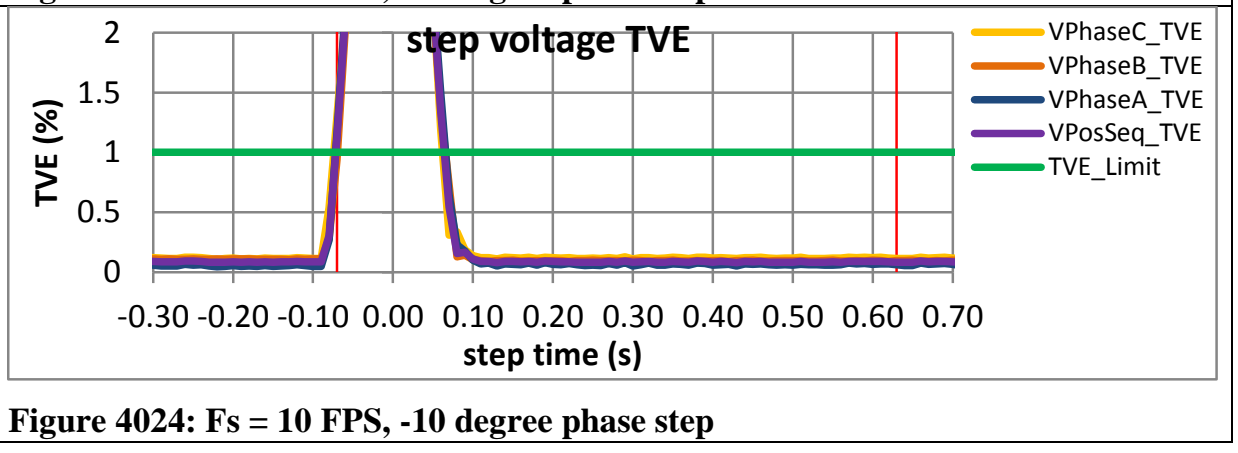


9.1.7 PMU F dynamic step change in phase voltage response time: $F 0=60 \mathrm{~Hz}, M$ class
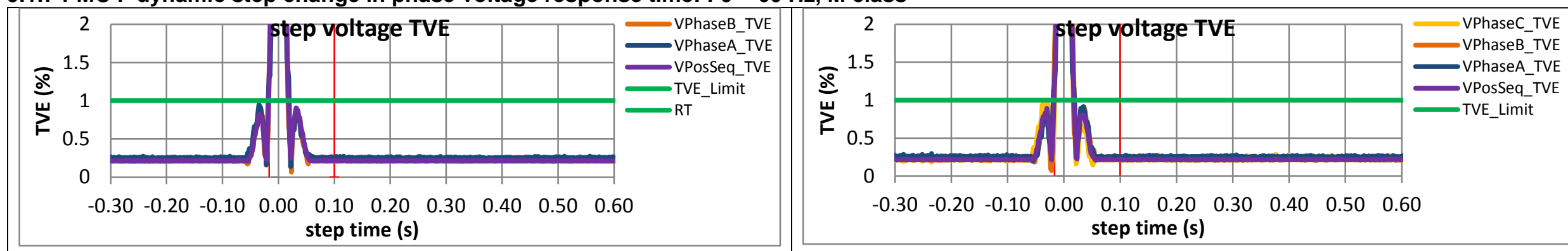

Figure 4025: Fs = 60 FPS, +10 degree phase step

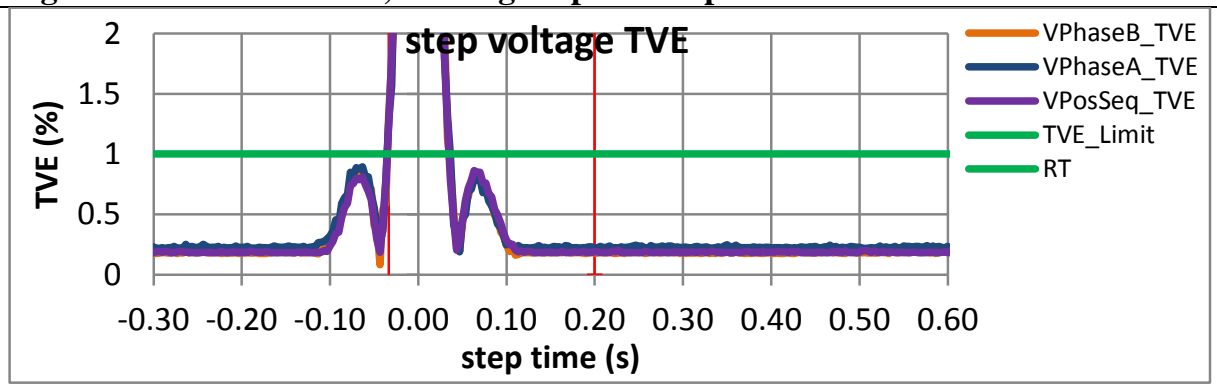

Figure 4026: Fs = 60 FPS, -10 degree phase step

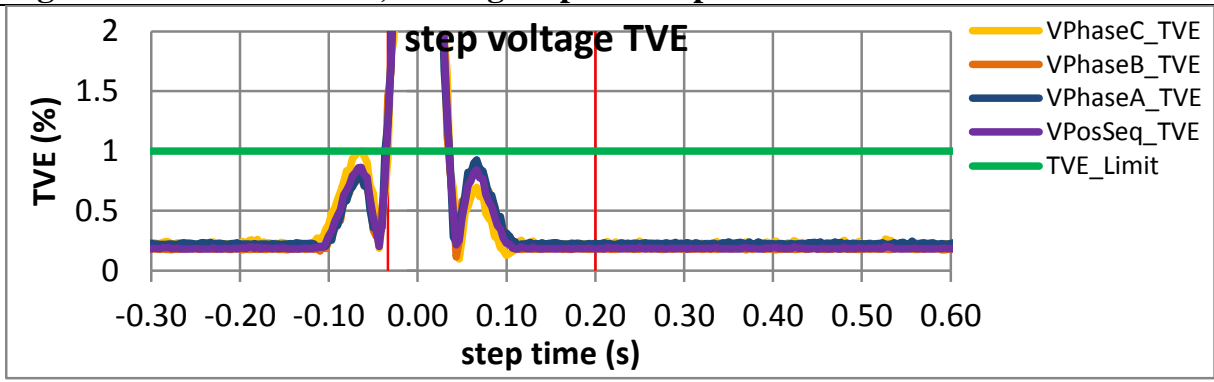

Figure 4027: Fs = 30 FPS, +10 degree phase step

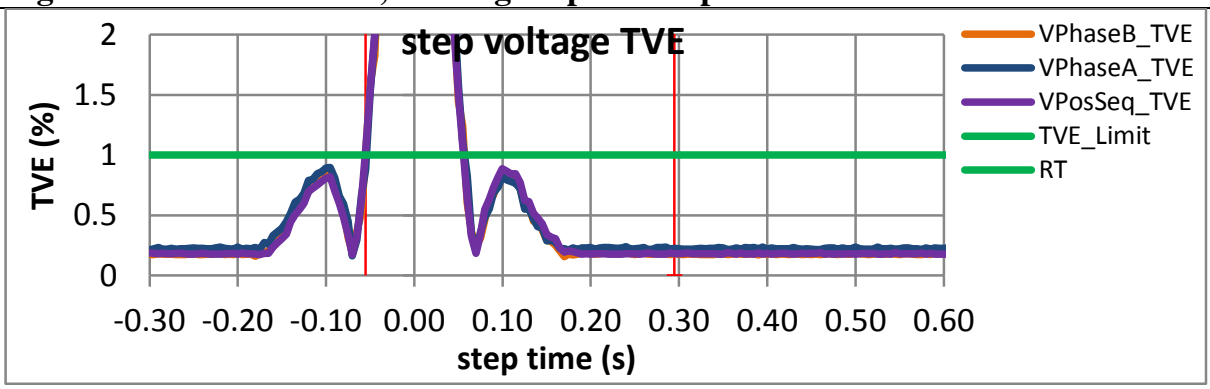

Figure 4028: Fs = 30 FPS, -10 degree phase step

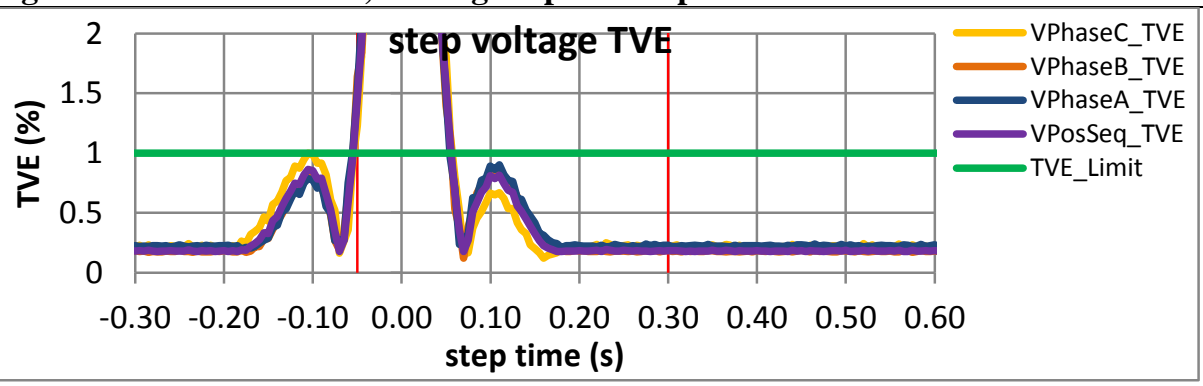

Figure 4029: Fs = 20 FPS, +10 degree phase step

Figure 4030: Fs = 20 FPS, -10 degree phase step 

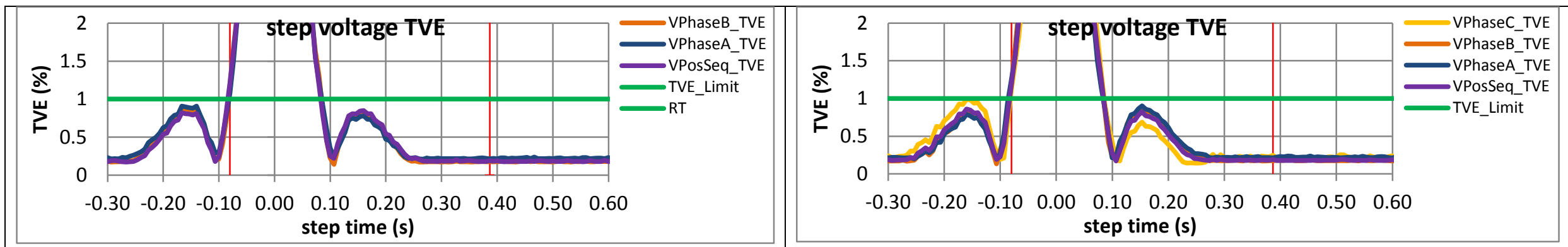

Figure 4031: Fs = 15 FPS, +10 degree phase step

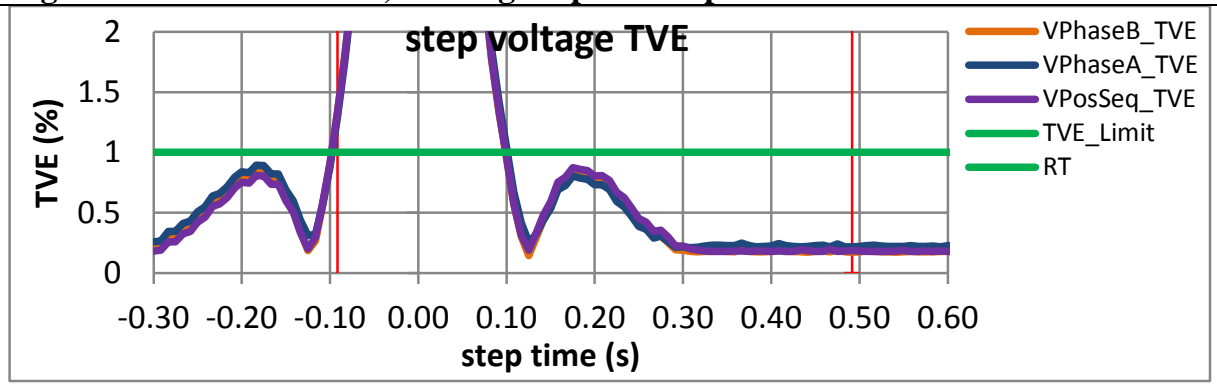

Figure 4032: Fs = 15 FPS, -10 degree phase step

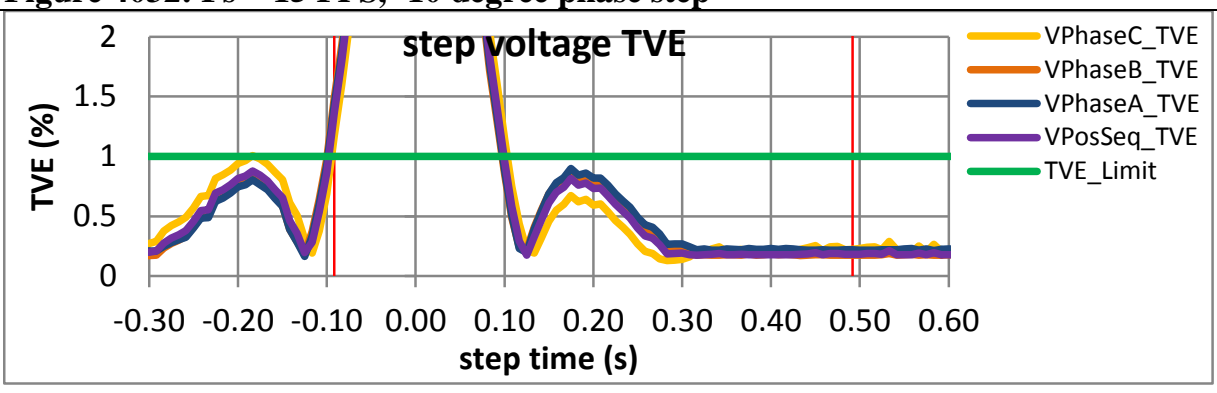

Figure 4033: Fs = 12 FPS, +10 degree phase step

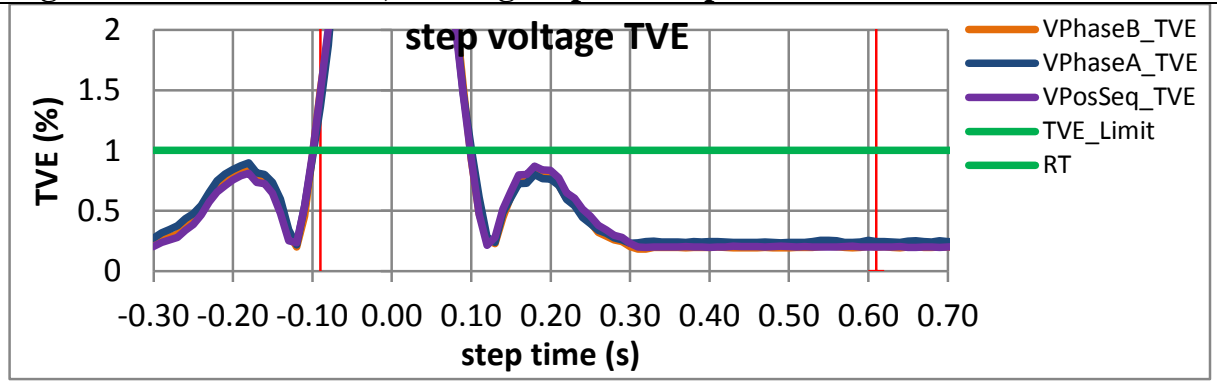

Figure 4034: Fs = 12 FPS, -10 degree phase step

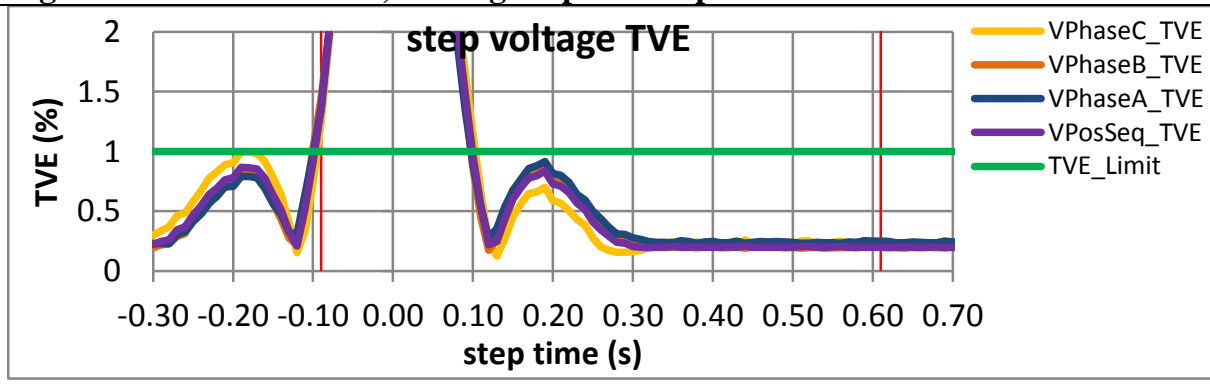

Figure 4036: Fs = 10 FPS, -10 degree phase step 


\subsubsection{PMU G dynamic step change in phase voltage response time: $F 0=60 \mathrm{~Hz}, M$ class}

Figure 4037: Fs = 60 FPS is not supported by this PMU

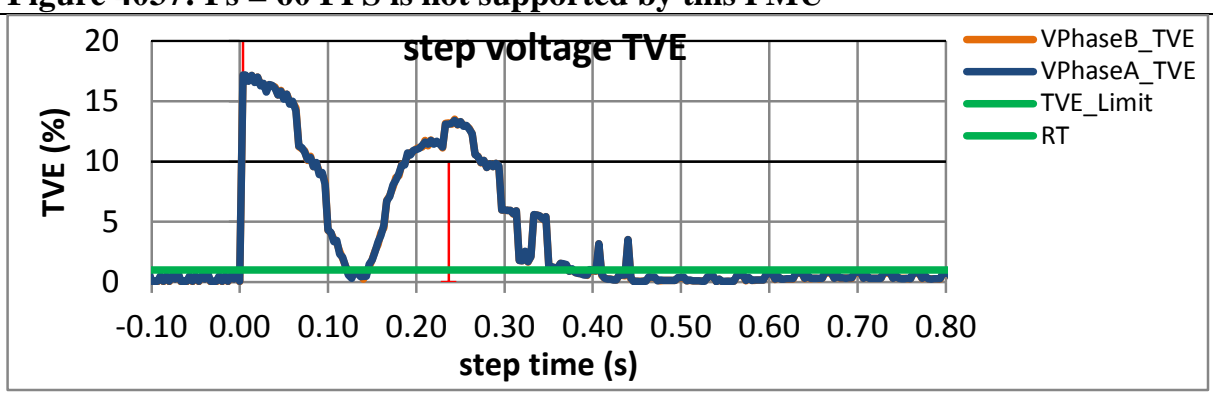

Figure 4038: Fs = 30 FPS, +10 degree phase step

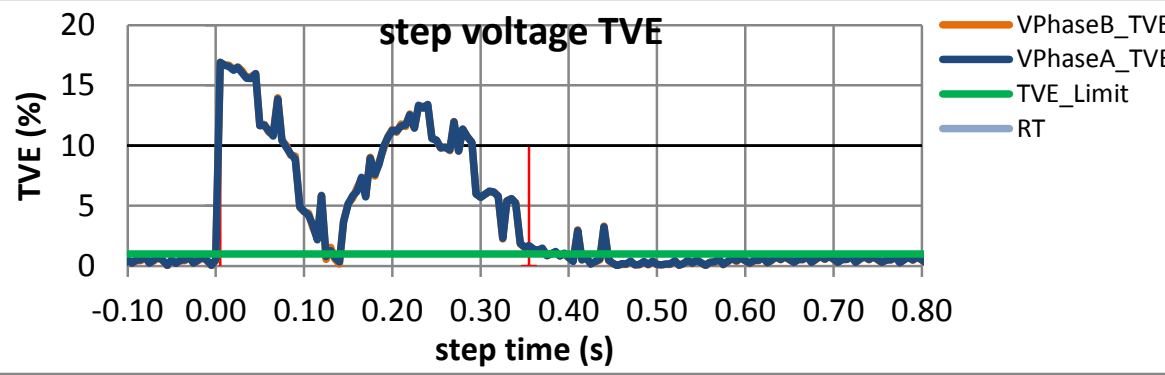

Figure 4040: Fs = 20 FPS, +10 degree phase step

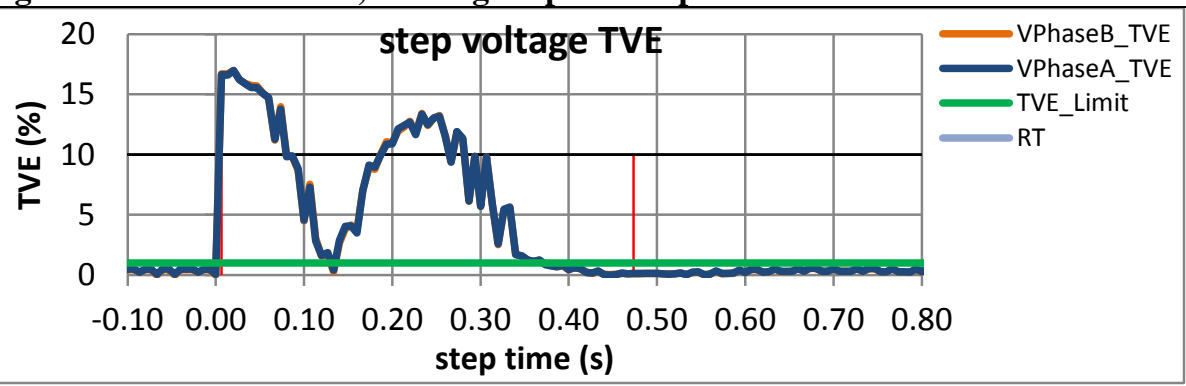

Figure 4042: Fs = 15 FPS, +10 degree phase step

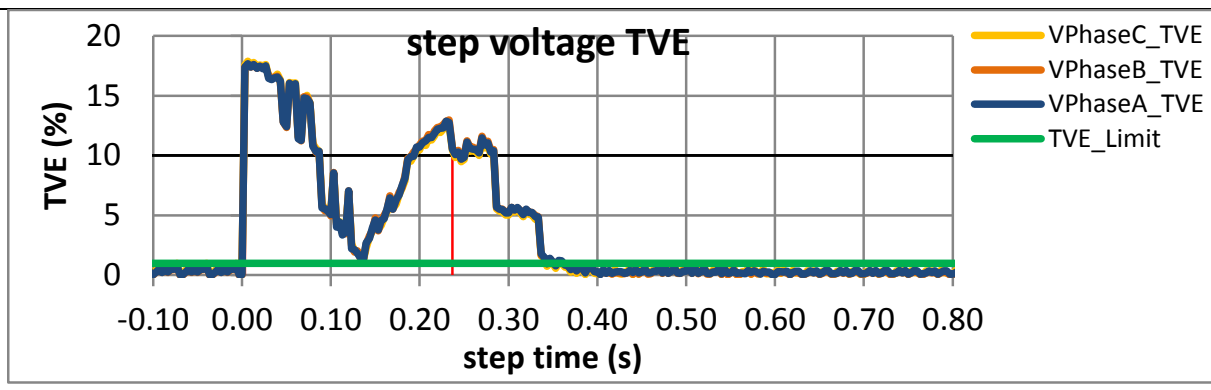

Figure 4039: Fs = 30 FPS, -10 degree phase step

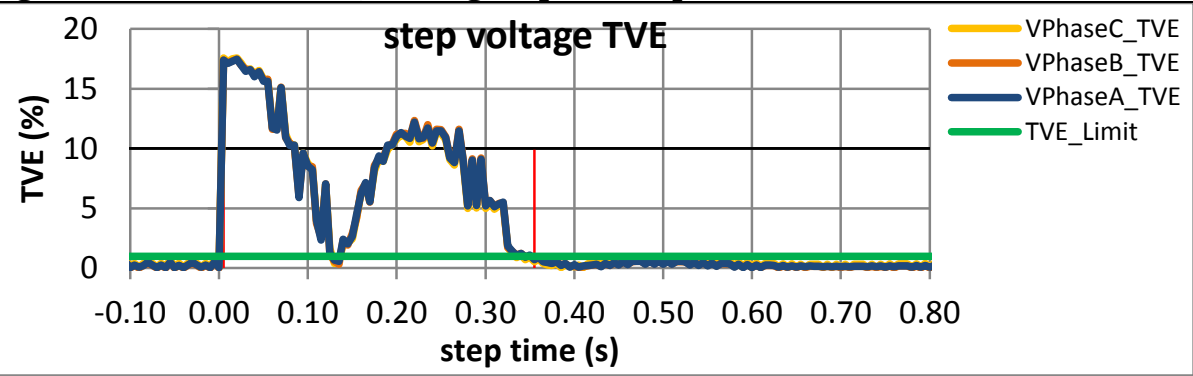

Figure 4041: Fs = 20 FPS, -10 degree phase step

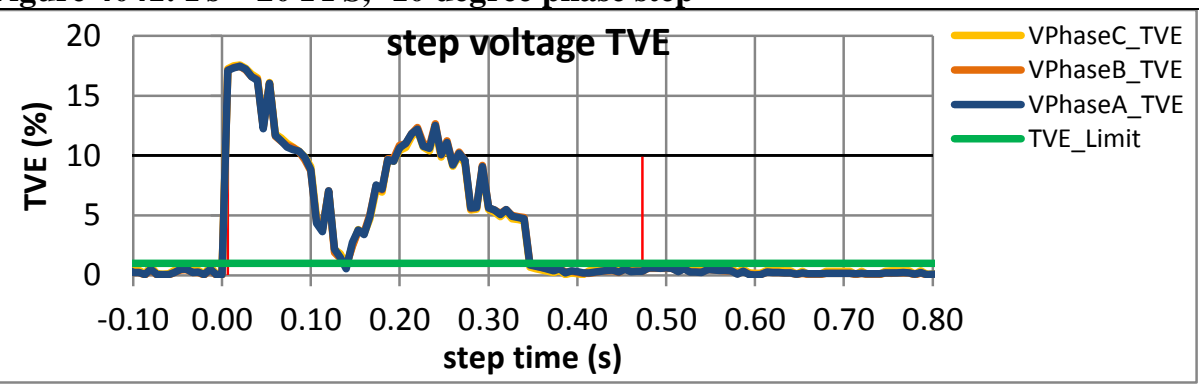

Figure 4043: Fs = 15 FPS, -10 degree phase step 

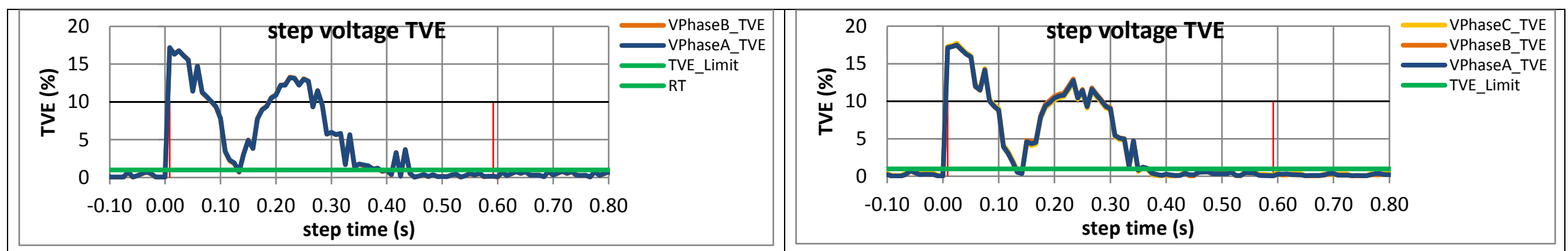

Figure 4044: Fs = 12 FPS, +10 degree phase step

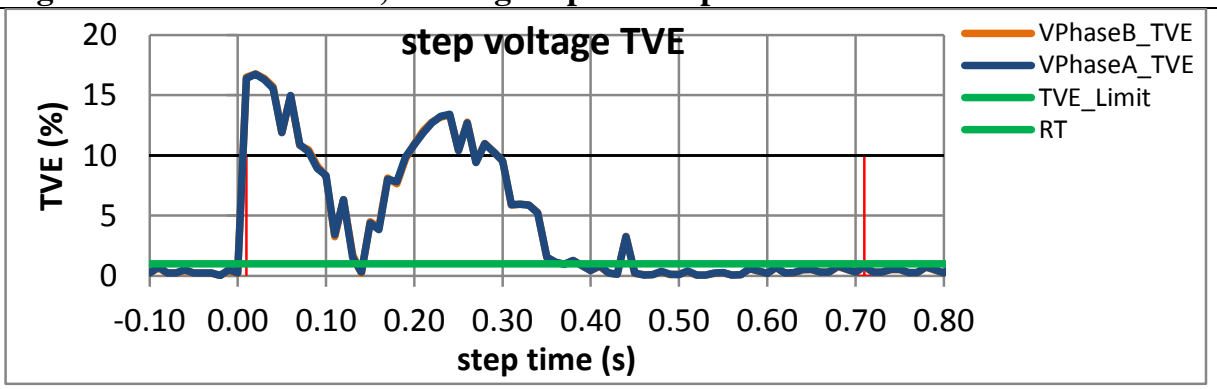

Figure 4046: Fs = 10 FPS, +10 degree phase step

Figure 4045: Fs = 12 FPS, -10 degree phase step

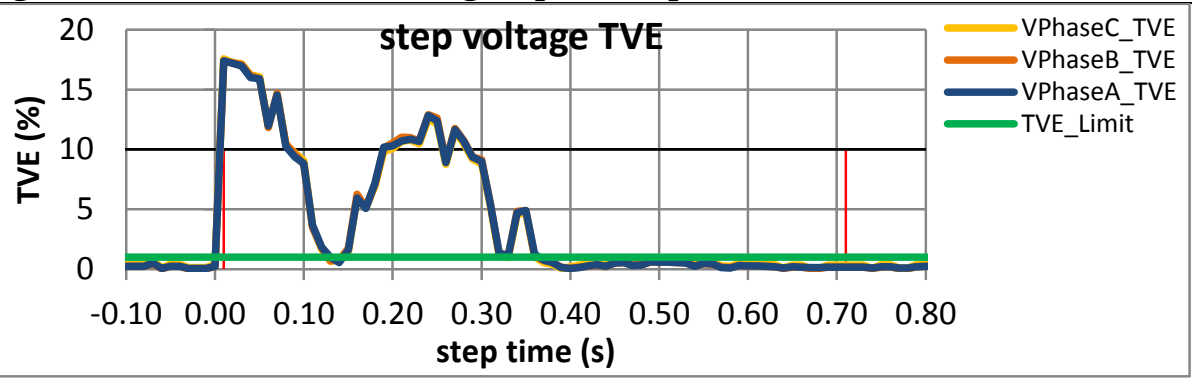

Figure 4047: Fs = 10 FPS, -10 degree phase step 
9.1.9 PMU H dynamic step change in phase voltage response time: $\mathrm{FO}=60 \mathrm{~Hz}, \mathrm{M}$ class
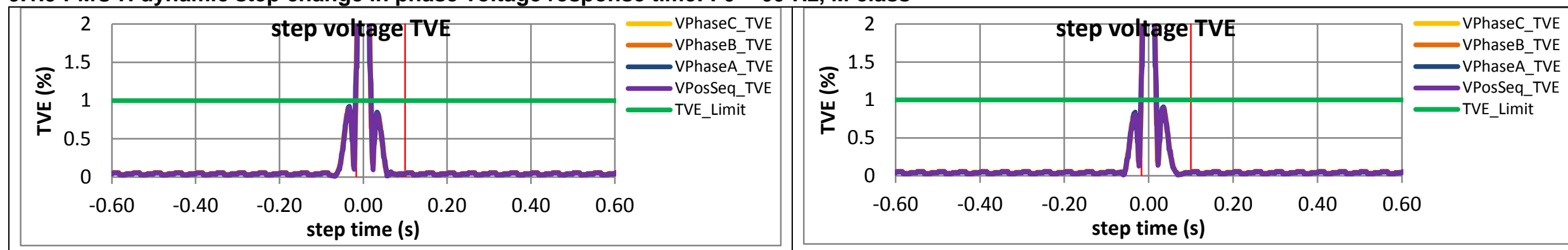

Figure 4048: Fs = 60 FPS, +10 degree phase step

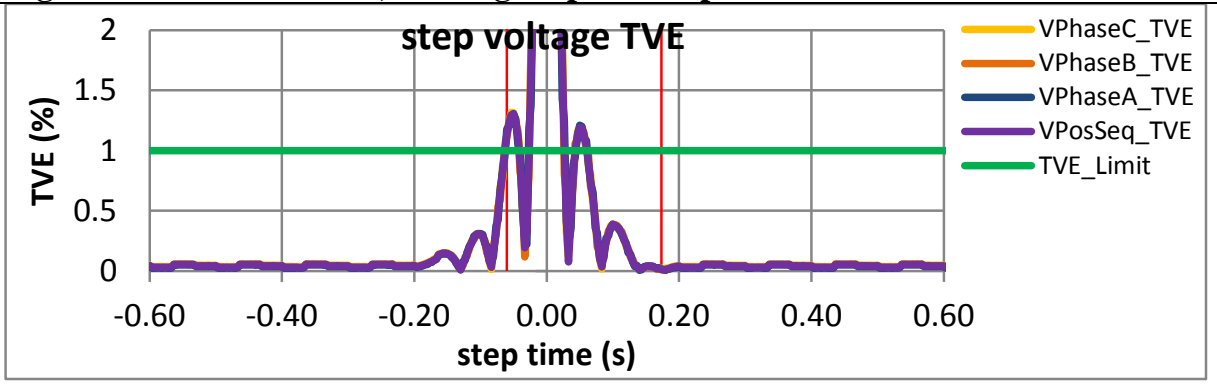

Figure 4049: Fs = 60 FPS, -10 degree phase step

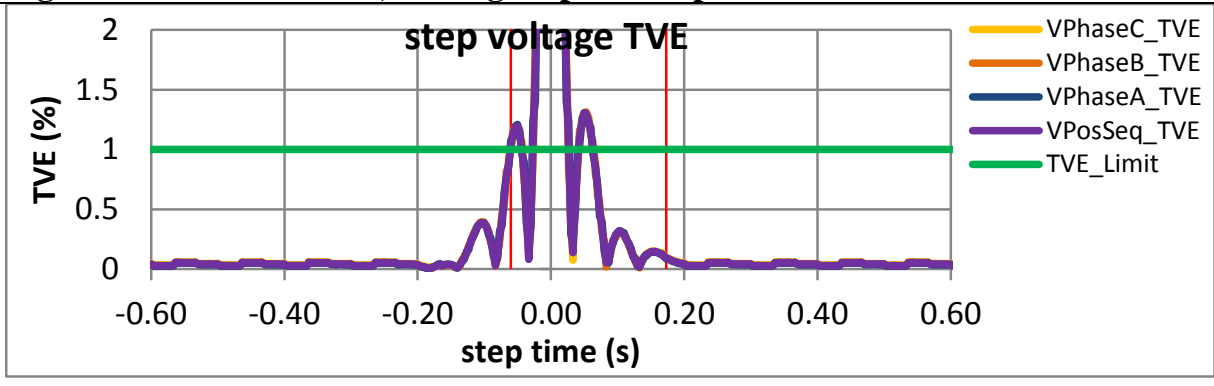

Figure 4050: Fs = 30 FPS, +10 degree phase step

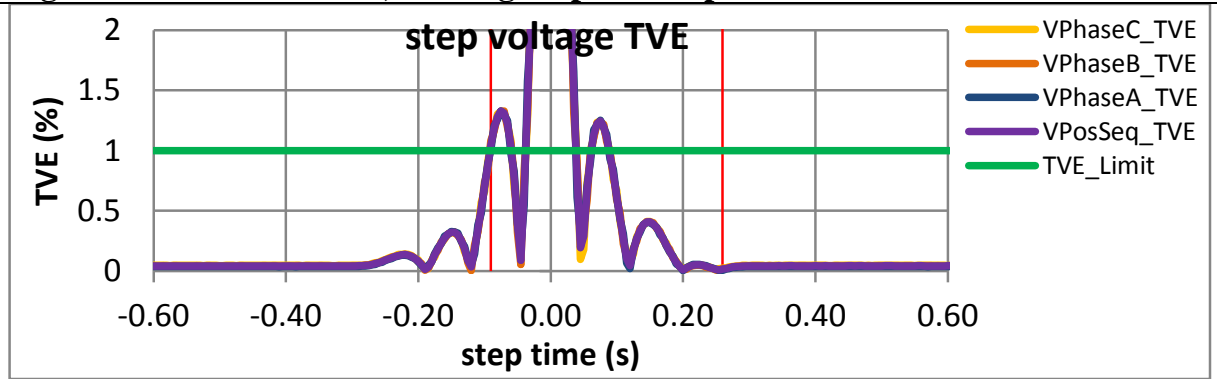

Figure 4052: Fs $=20$ FPS, +10 degree phase step

Figure 4051: Fs = 30 FPS, -10 degree phase step

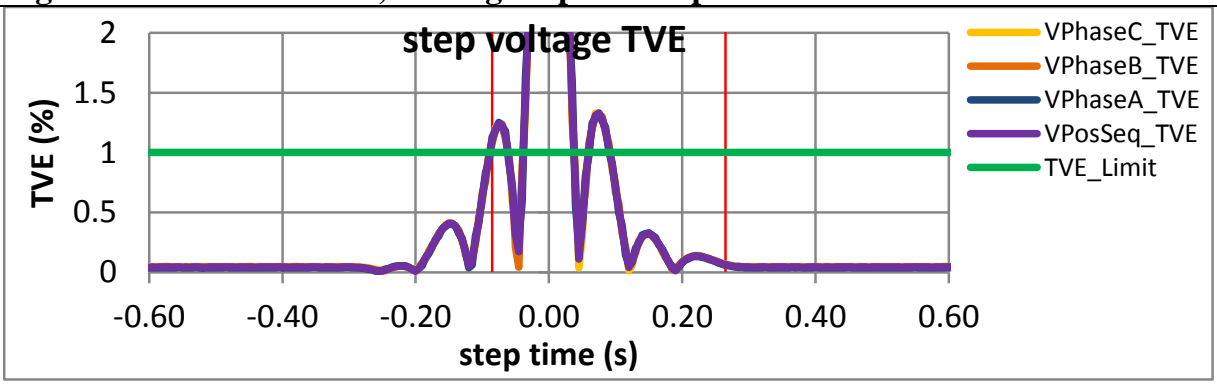

Figure 4053: Fs = 20 FPS, -10 degree phase step 


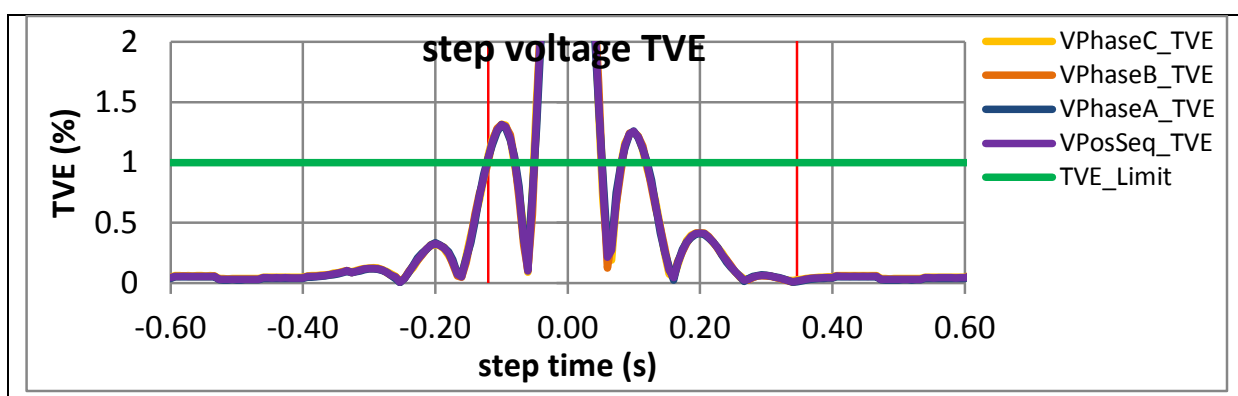

Figure 4054: Fs = 15 FPS, +10 degree phase step

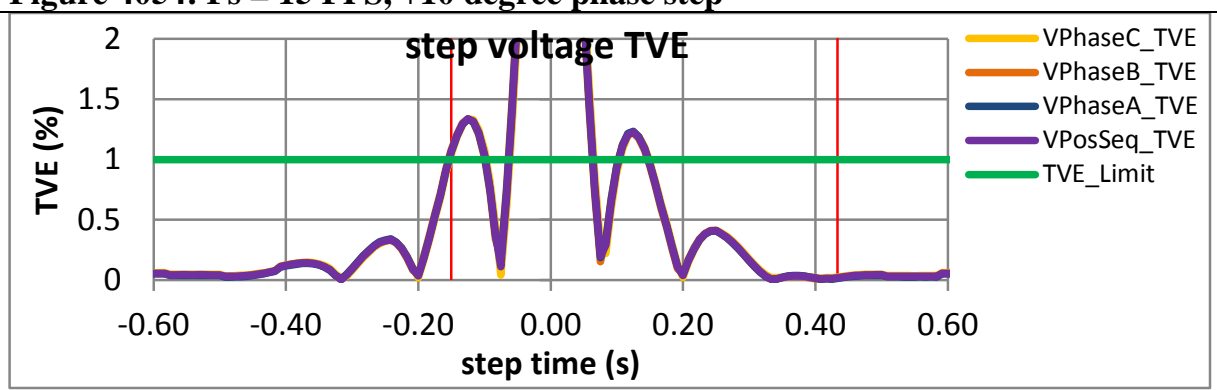

Figure 4056: Fs = 12 FPS, +10 degree phase step

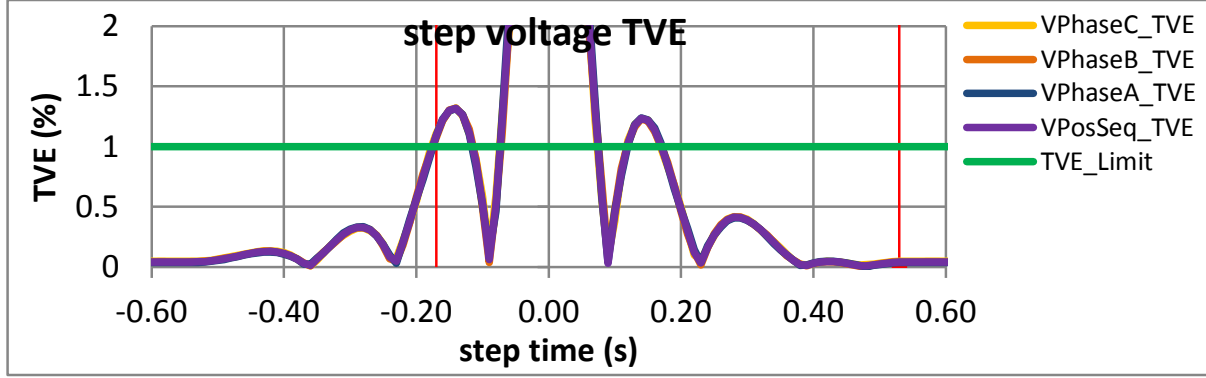

Figure 4058: Fs = 10 FPS, +10 degree phase step

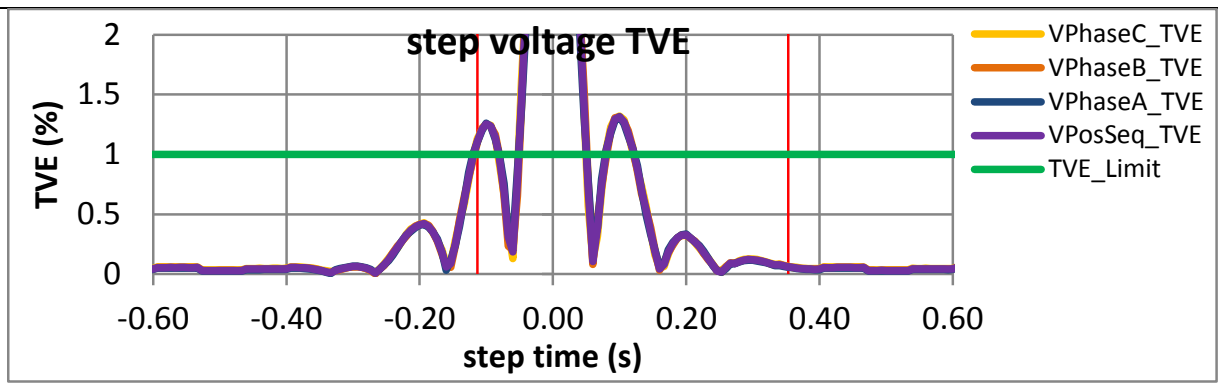

Figure 4055: Fs = 15 FPS, -10 degree phase step

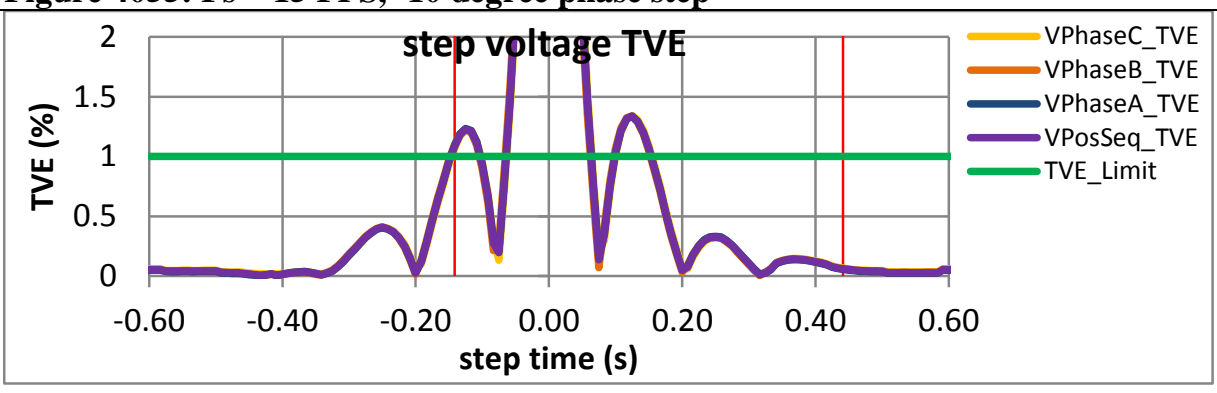

Figure 4057: Fs = 12 FPS, -10 degree phase step

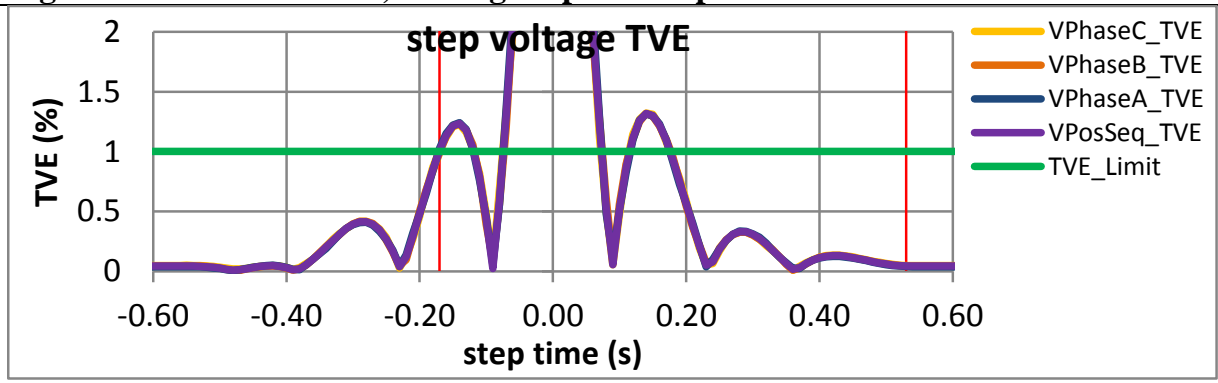

Figure 4059: Fs = 10 FPS, -10 degree phase step 
9.1.10 PMU I dynamic step change in phase voltage response time: $\mathrm{F0}=60 \mathrm{~Hz}, \mathrm{M}$ class

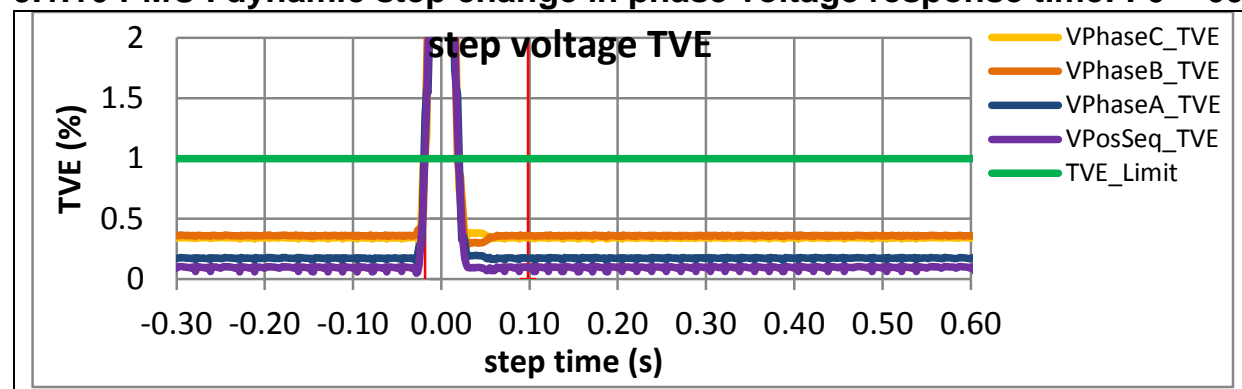

Figure 4060: Fs = 60 FPS, +10 degree phase step

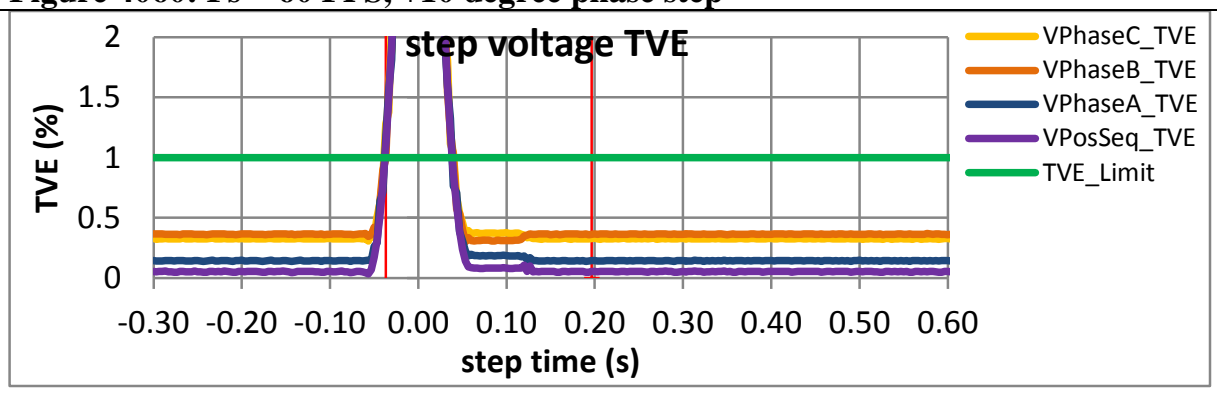

Figure 4062: Fs = 30 FPS, +10 degree phase step

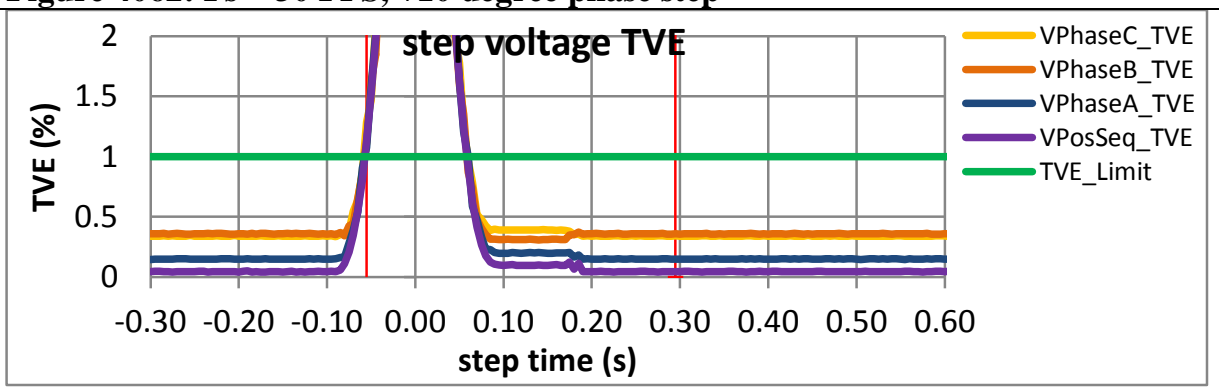

Figure 4064: Fs = 20 FPS, +10 degree phase step

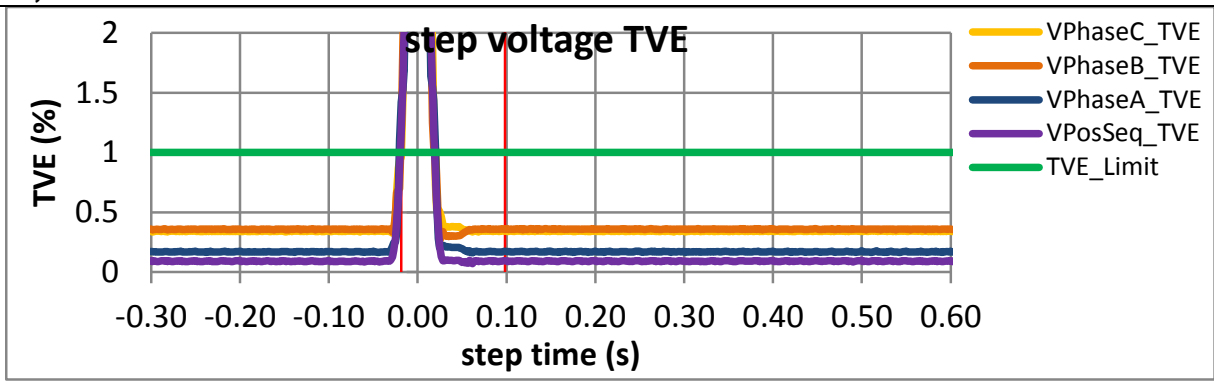

Figure 4061: Fs = 60 FPS, -10 degree phase step

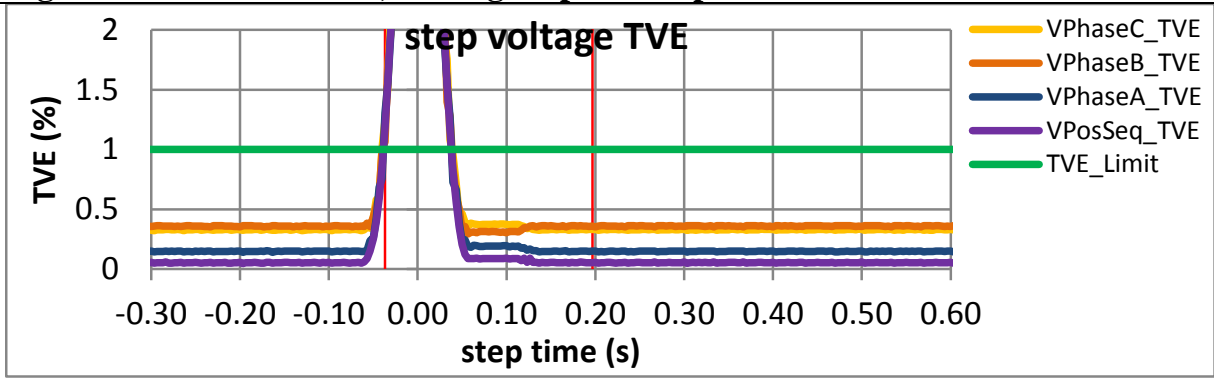

Figure 4063: Fs = 30 FPS, -10 degree phase step

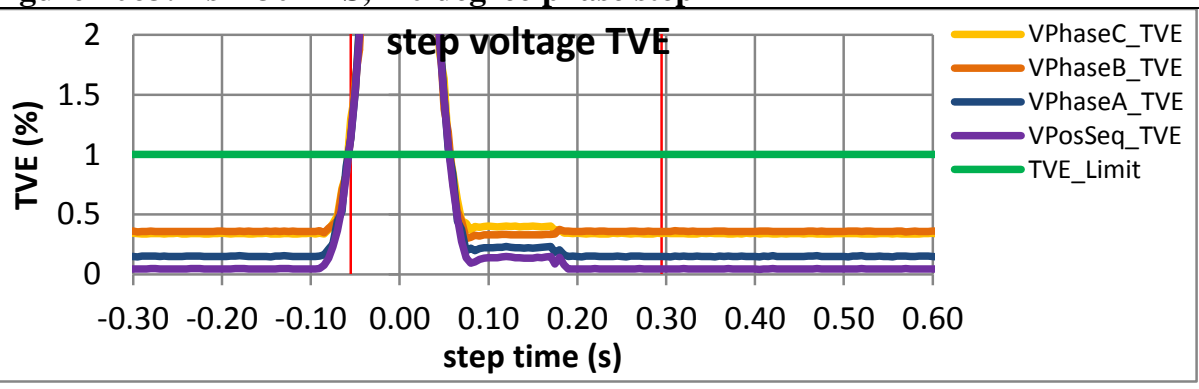

Figure 4065: Fs = 20 FPS, -10 degree phase step 


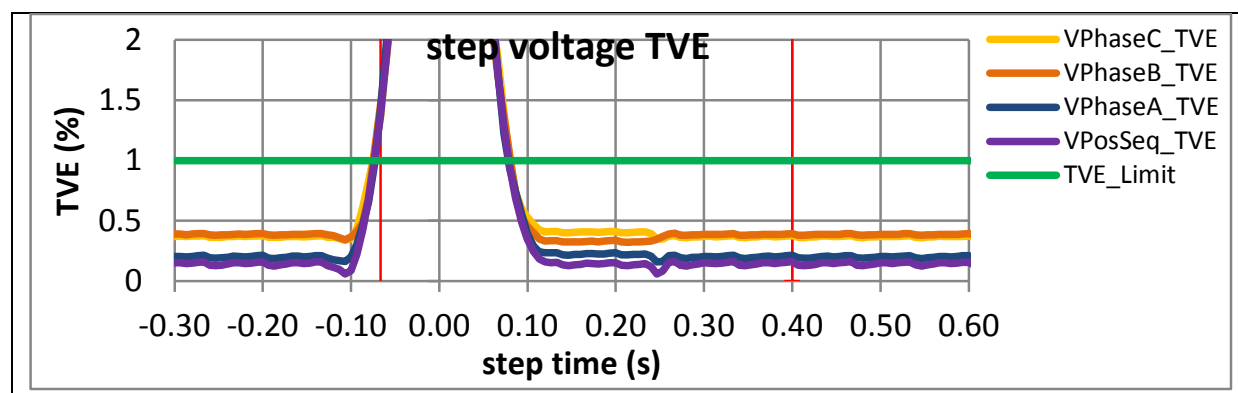

Figure 4066: Fs = 15 FPS, +10 degree phase step

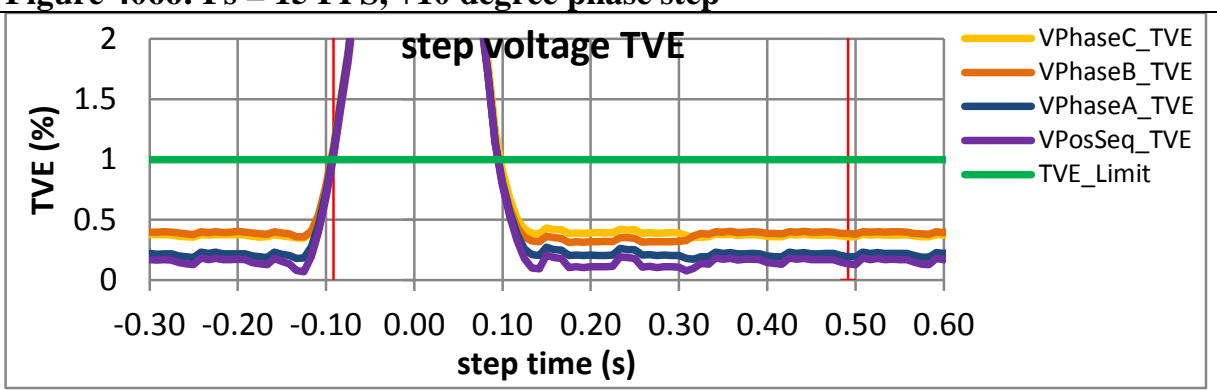

Figure 4068: Fs = 12 FPS, +10 degree phase step

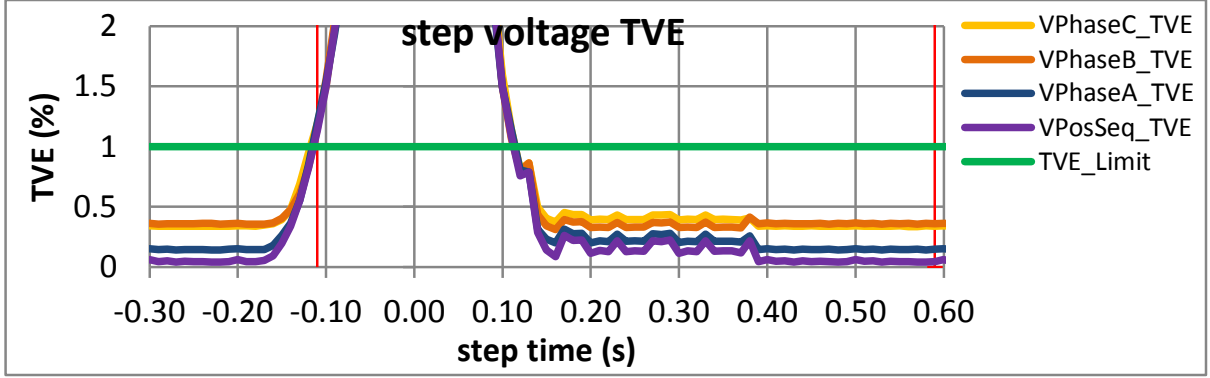

Figure 4070: Fs = 10 FPS, +10 degree phase step

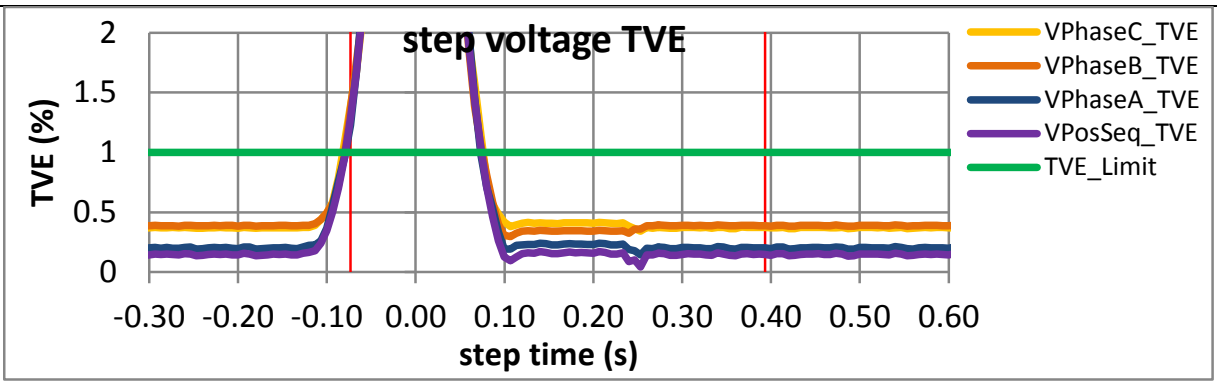

Figure 4067: Fs = 15 FPS, -10 degree phase step

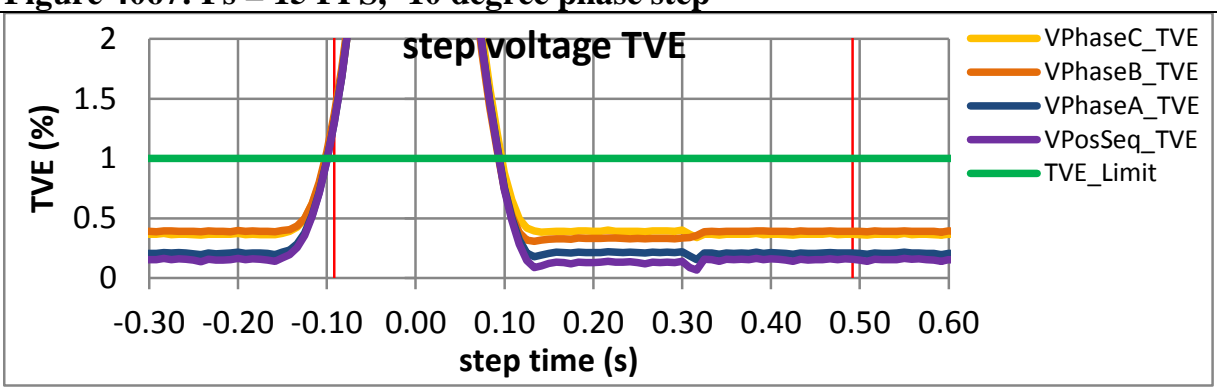

Figure 4069: Fs = 12 FPS, -10 degree phase step

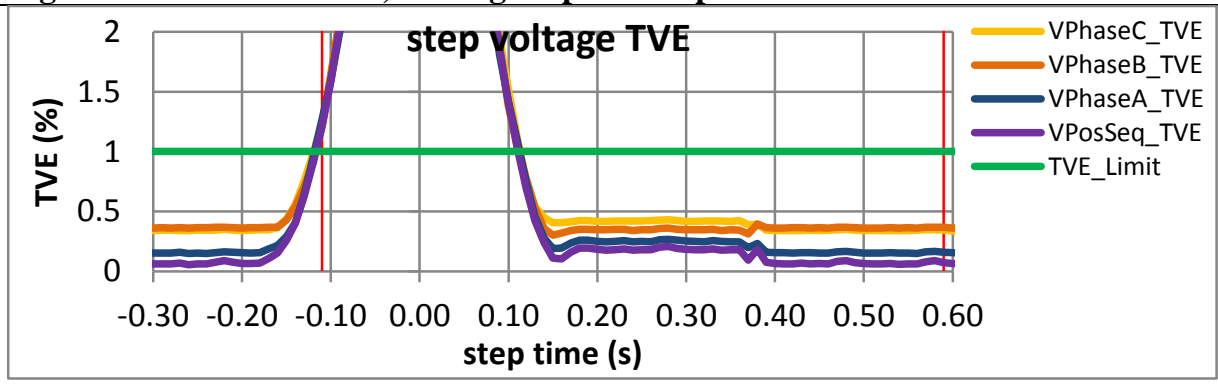

Figure 4071: Fs = 10 FPS, -10 degree phase step 
9.1.11 PMU J dynamic step change in phase voltage response time: $\mathrm{F} 0=60 \mathrm{~Hz}, \mathrm{M}$ class
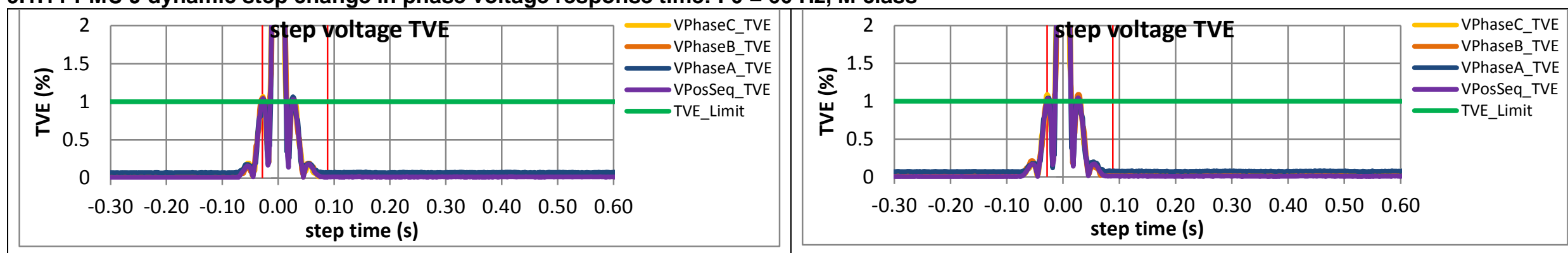

Figure 4072: Fs $=60 \mathrm{FPS},+10$ degree phase step

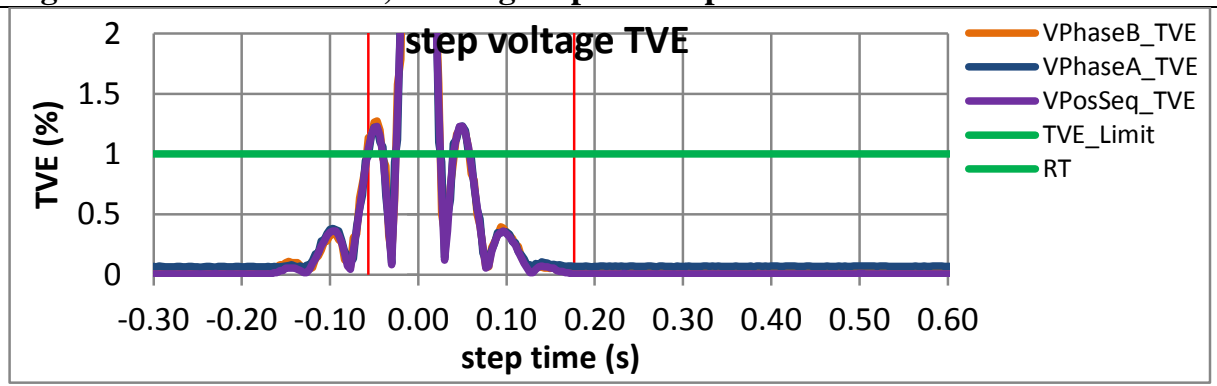

Figure 4073: Fs = 60 FPS, -10 degree phase step

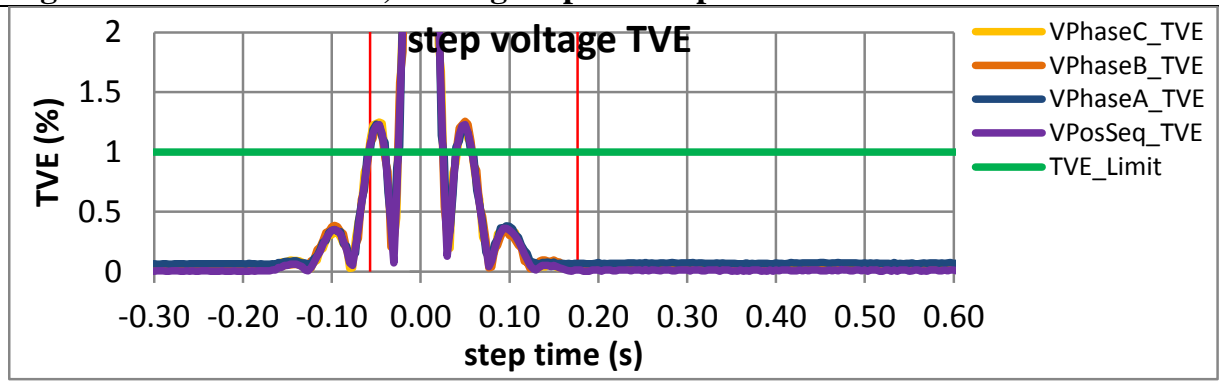

Figure 4074: Fs = 30 FPS, +10 degree phase step

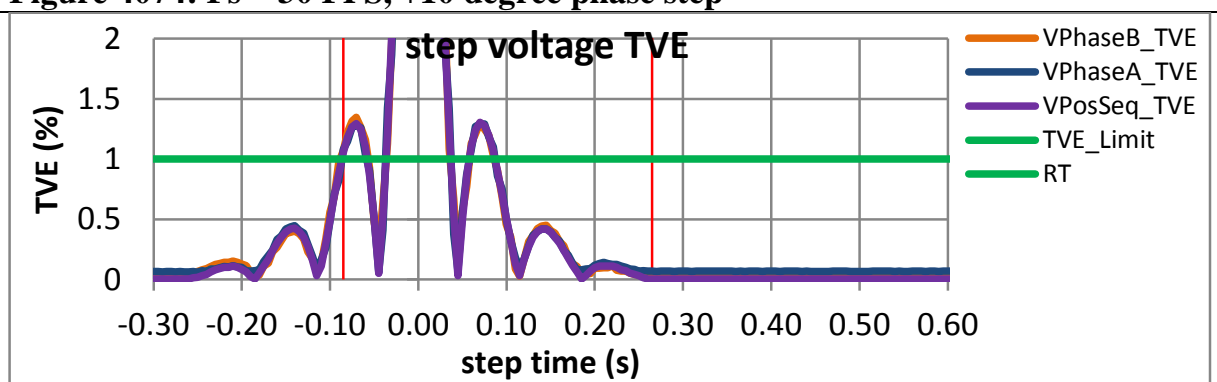

Figure 4075: Fs = 30 FPS, -10 degree phase step

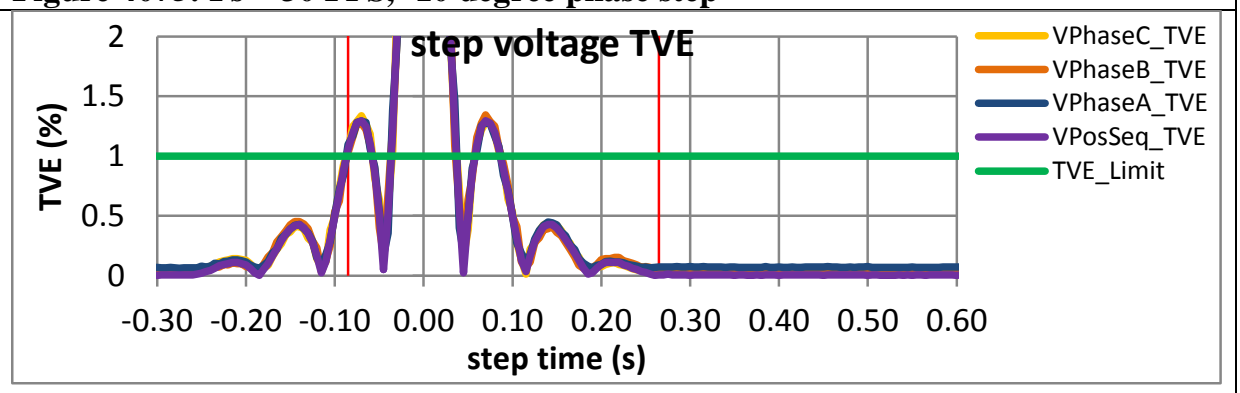

Figure 4076: Fs = 20 FPS, +10 degree phase step

Figure 4077: Fs = 20 FPS, -10 degree phase step 

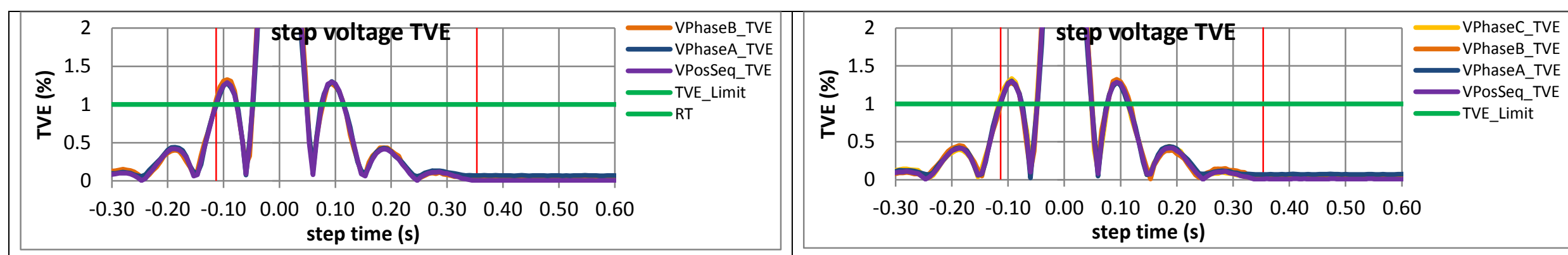

Figure 4078: Fs = 15 FPS, +10 degree phase step

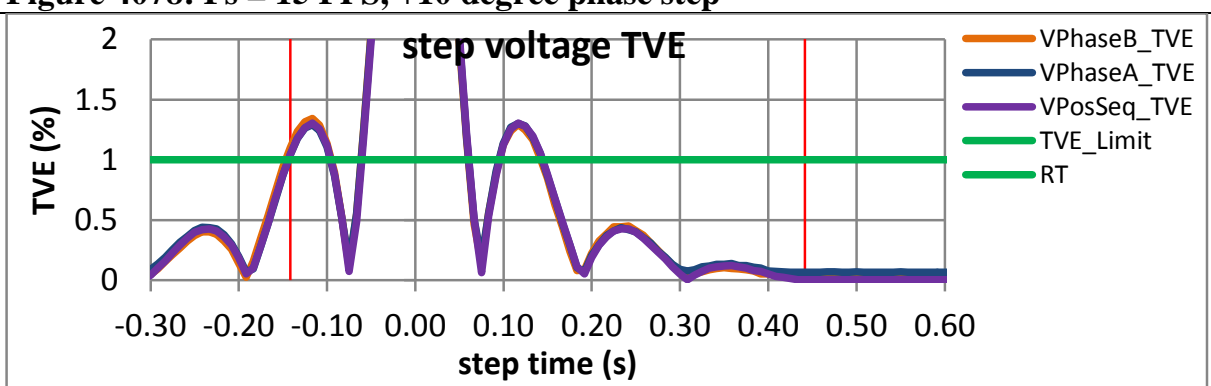

Figure 4079: Fs = 15 FPS, -10 degree phase step

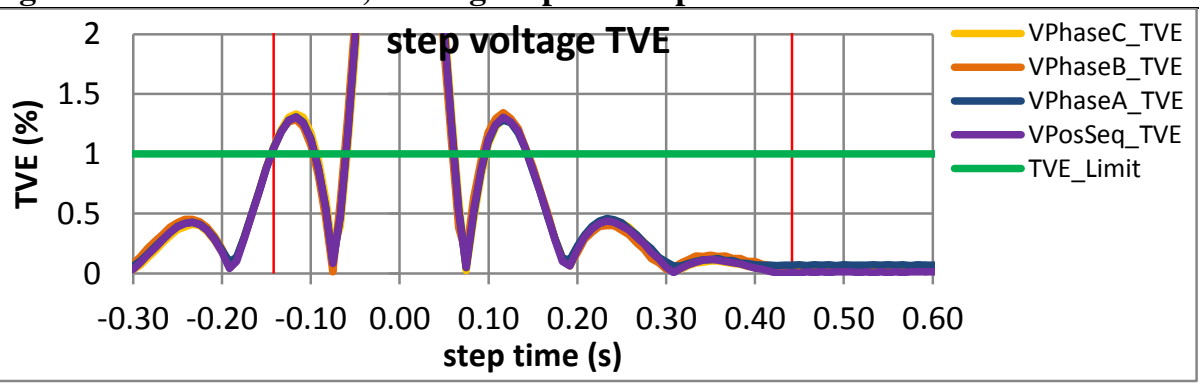

Figure 4080: Fs $=12 \mathrm{FPS},+10$ degree phase step

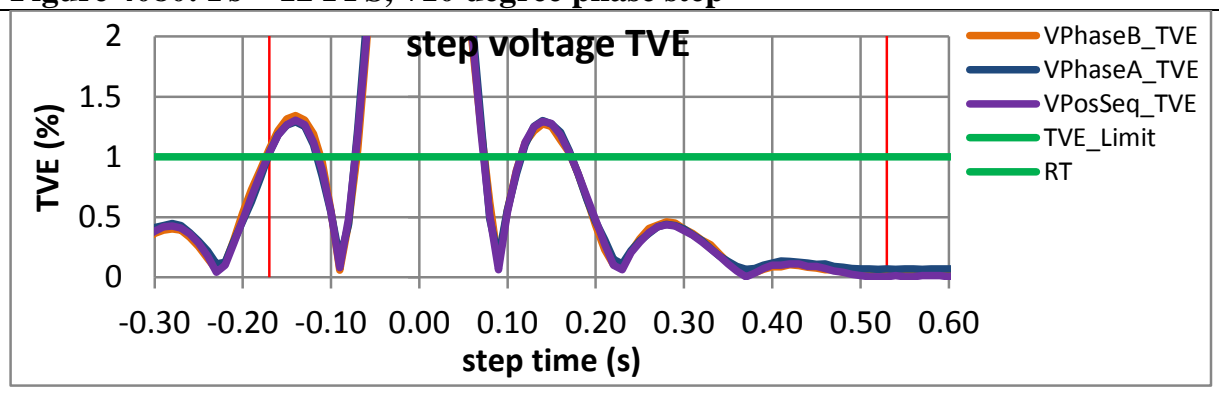

Figure 4081: Fs = 12 FPS, -10 degree phase step

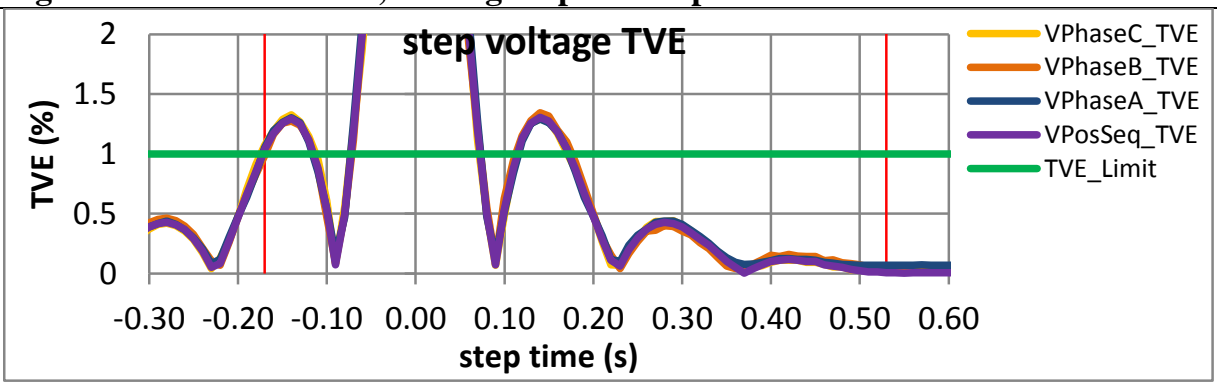

Figure 4083: Fs = 10 FPS, -10 degree phase step 


\subsection{Dynamic step change in phase: current response time}

9.2.1 C37.118.1-2011 Annex $C$ dynamic step change in phase current response time: $\mathrm{F} 0=60 \mathrm{~Hz}$, $\mathrm{M}$ class
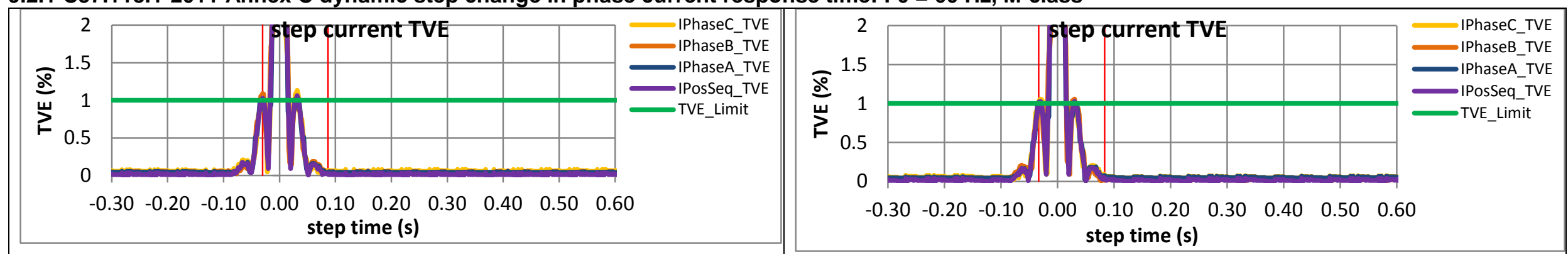

Figure 4084: Fs = $60 \mathrm{FPS},+10$ degree phase step

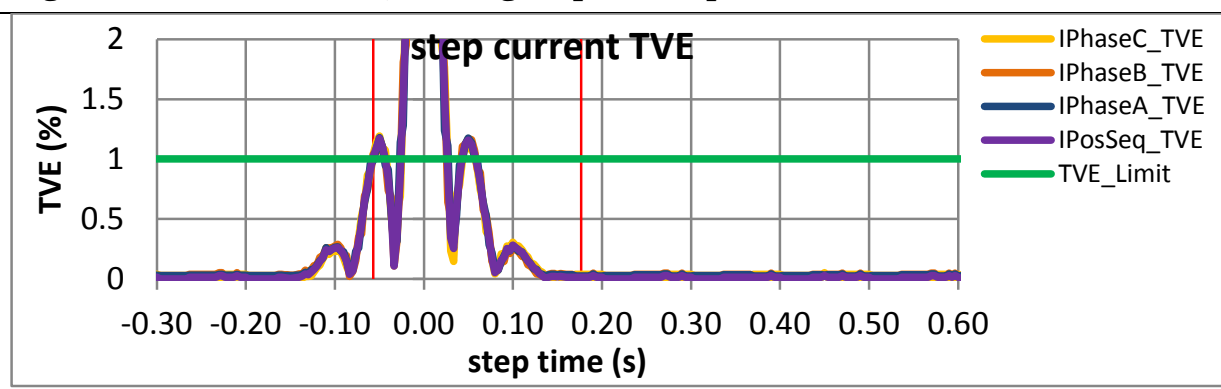

Figure 4085: Fs = 60 FPS, -10 degree phase step

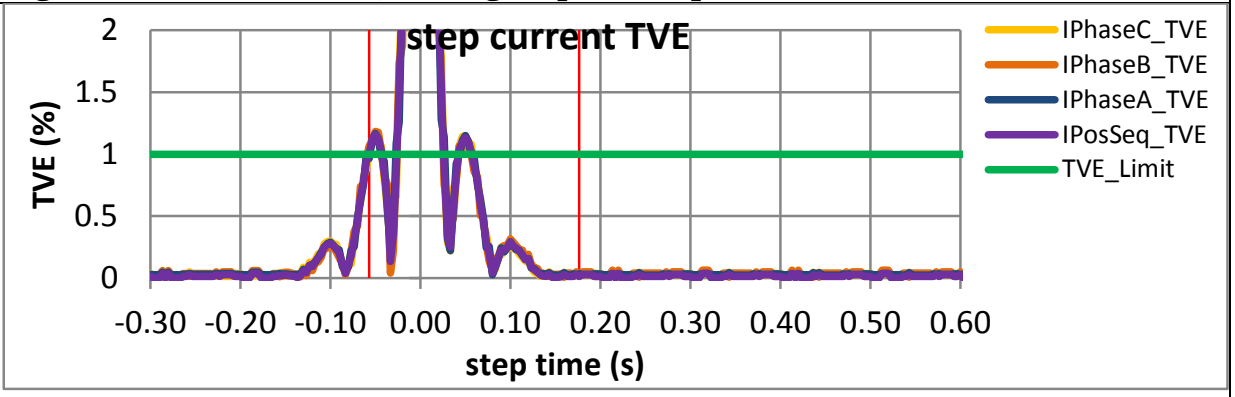

Figure 4086: Fs = 30 FPS, +10 degree phase step

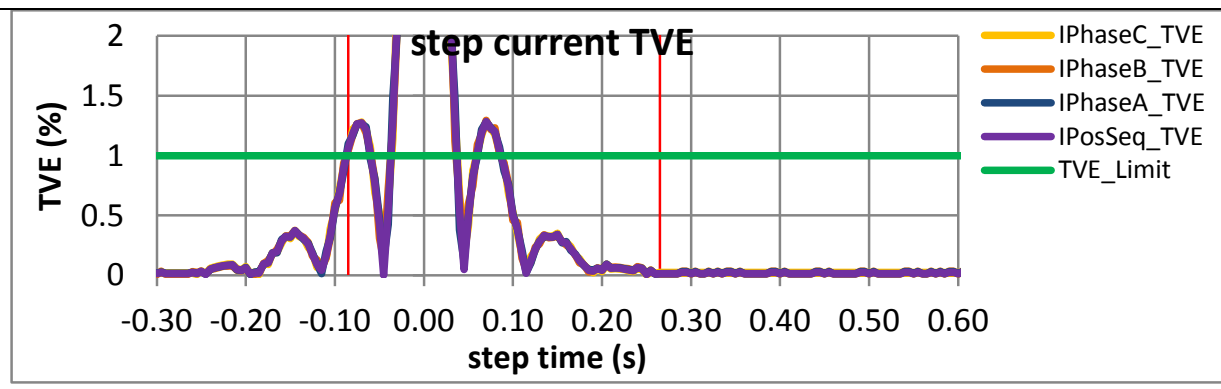

Figure 4087: Fs = 30 FPS, -10 degree phase step

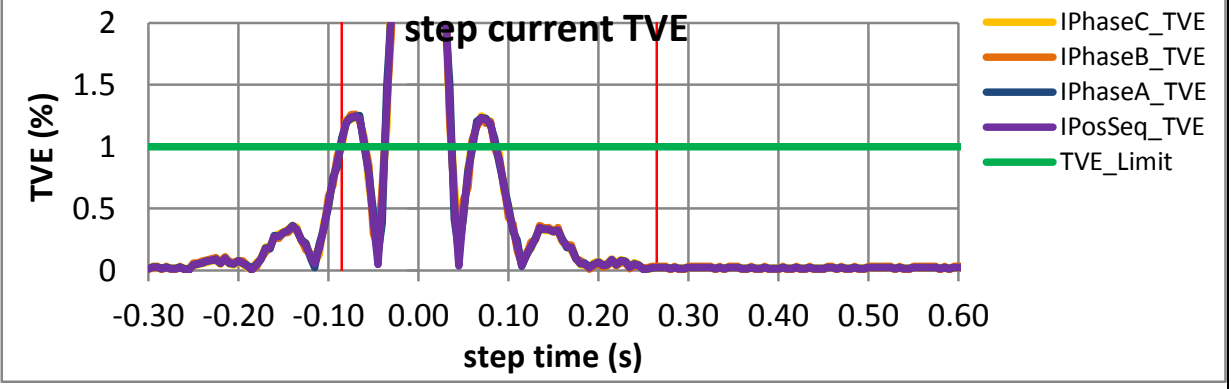

Figure 4088: Fs = 20 FPS, +10 degree phase step

Figure 4089: Fs $=20$ FPS, -10 degree phase step 

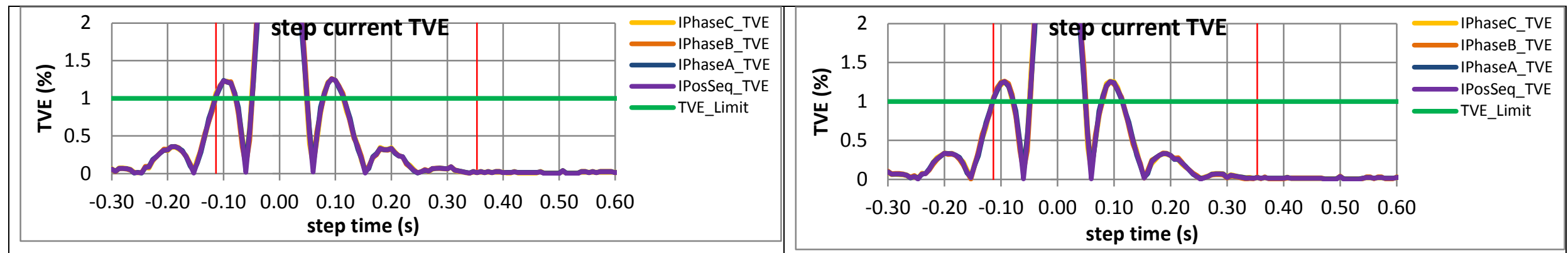

Figure 4090: Fs = 15 FPS, +10 degree phase step

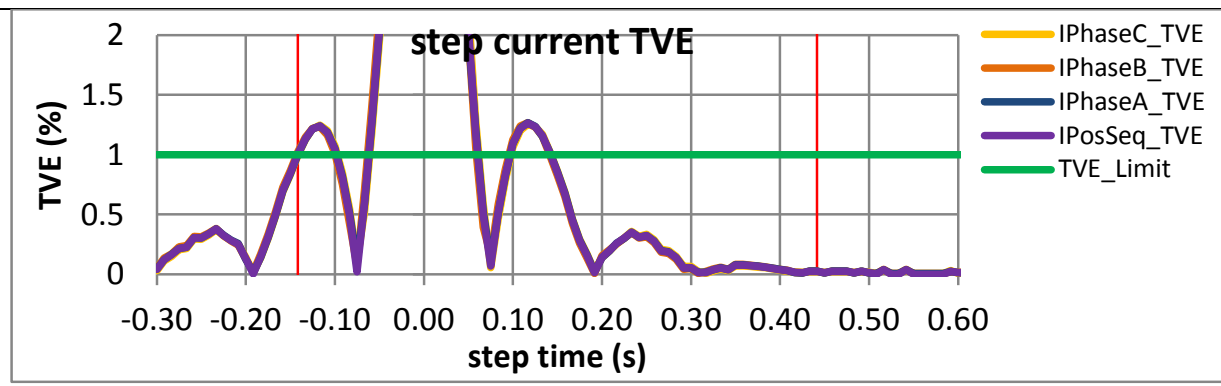

Figure 4091: Fs = 15 FPS, -10 degree phase step

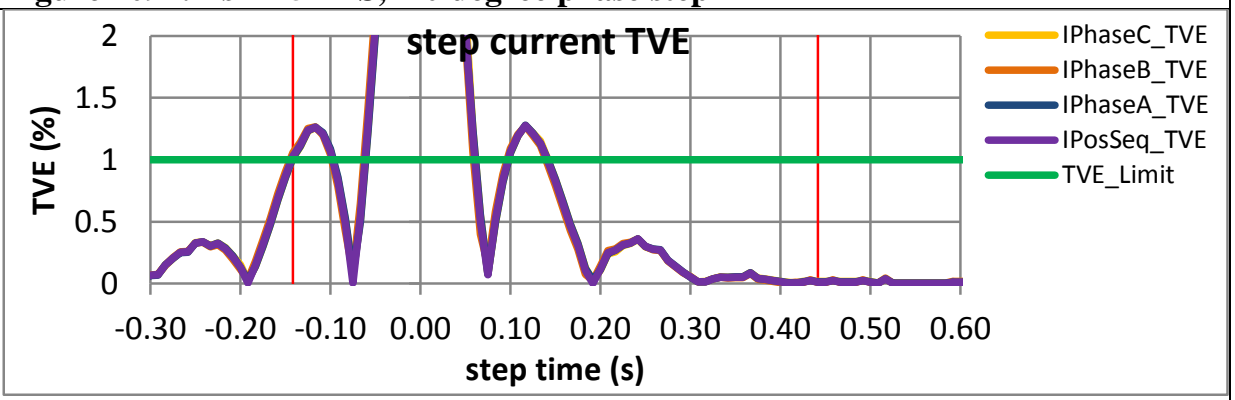

Figure 4092: Fs $=12$ FPS, +10 degree phase step

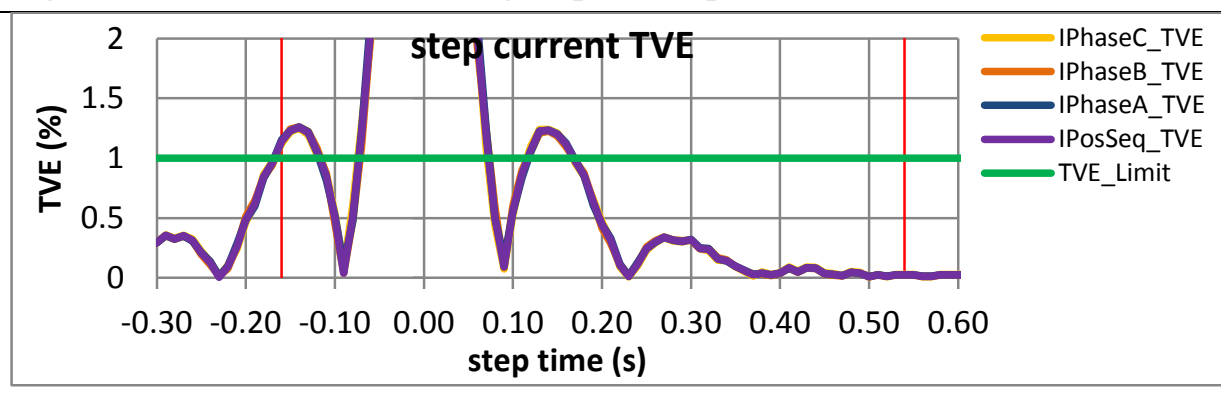

Figure 4093: Fs = 12 FPS, - 10 degree phase step

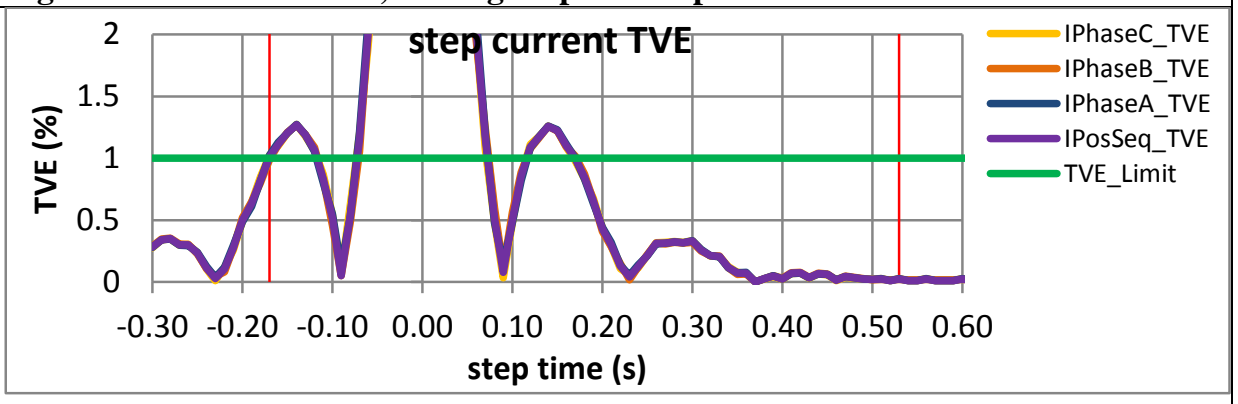

Figure 4094: Fs = 10 FPS, +10 degree phase step

Figure 4095: Fs = 10 FPS, -10 degree phase step 
9.2.2 PMU A dynamic step change in phase current response time: $\mathrm{F} 0=60 \mathrm{~Hz}, \mathrm{M}$ class
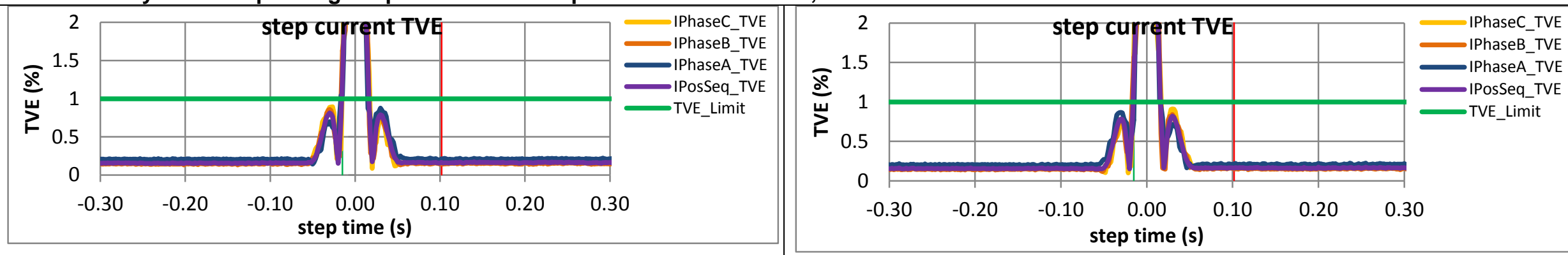

Figure 4096: Fs = 60 FPS, +10 degree phase step

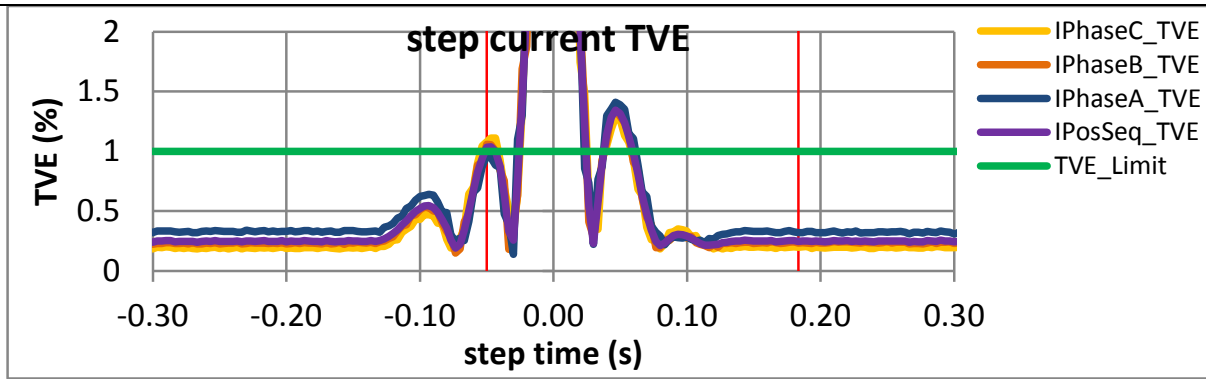

Figure 4097: Fs = 60 FPS, -10 degree phase step

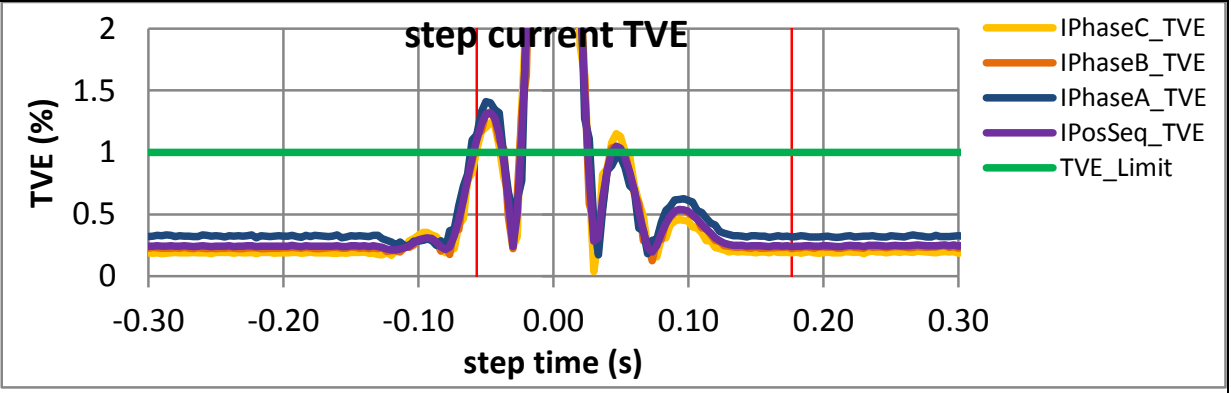

Figure 4098: Fs = 30 FPS, +10 degree phase step

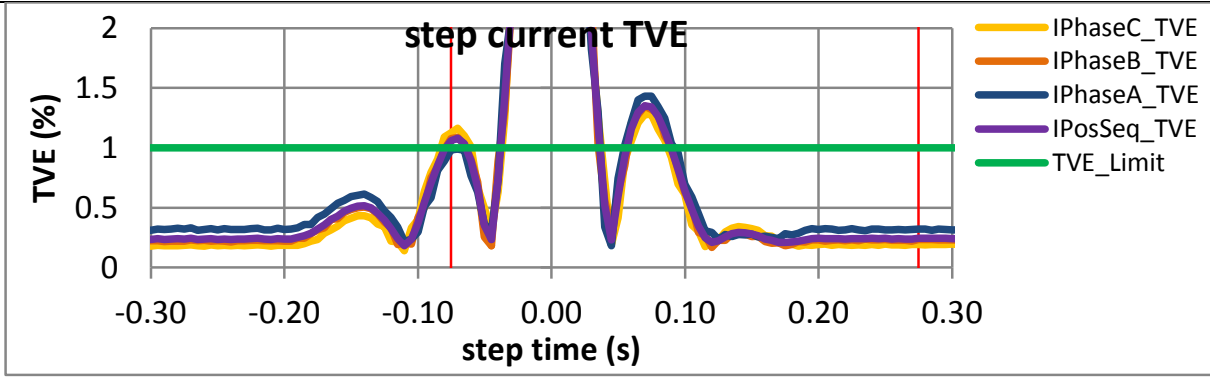

Figure 4100: Fs = 20 FPS, +10 degree phase step

Figure 4099: Fs = 30 FPS, -10 degree phase step

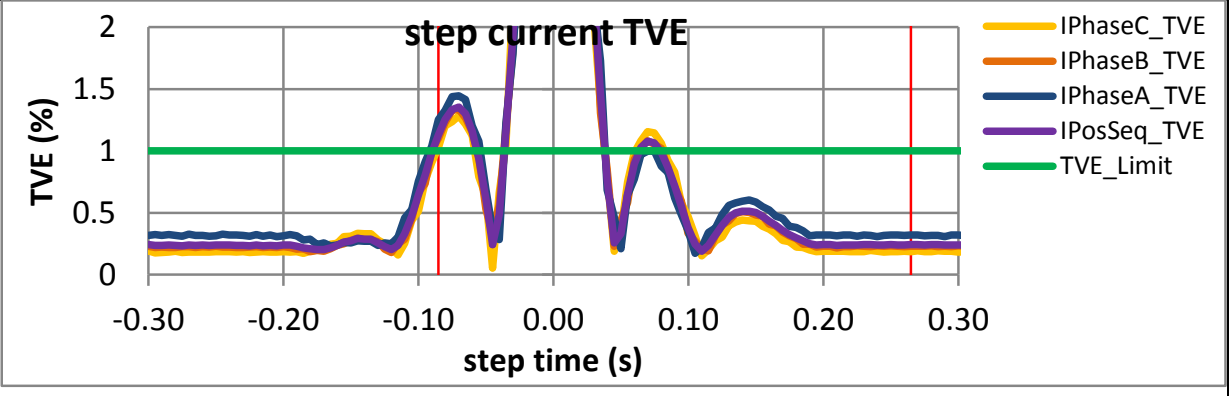

Figure 4101: Fs = 20 FPS, - 10 degree phase step 

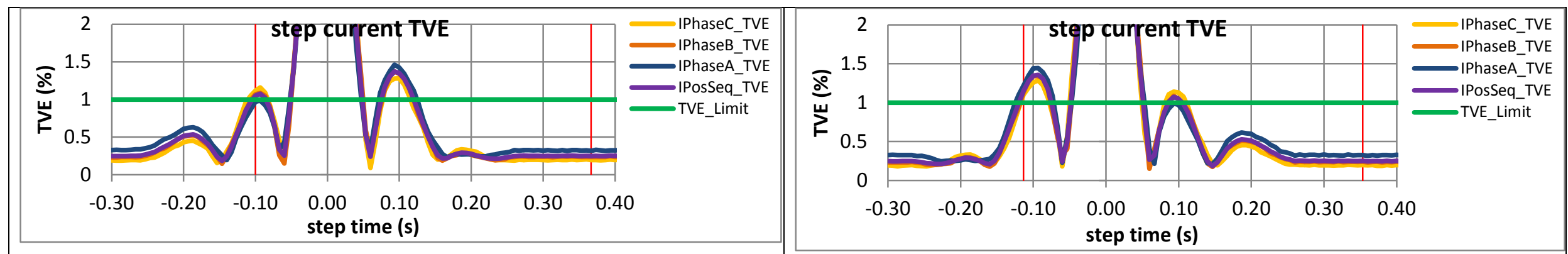

Figure 4102: Fs = 15 FPS, +10 degree phase step

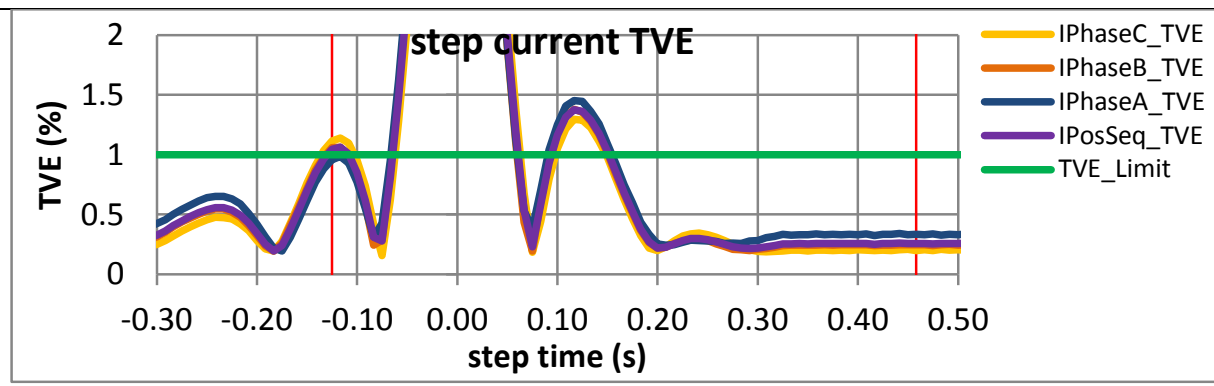

Figure 4103: Fs = 15 FPS, -10 degree phase step

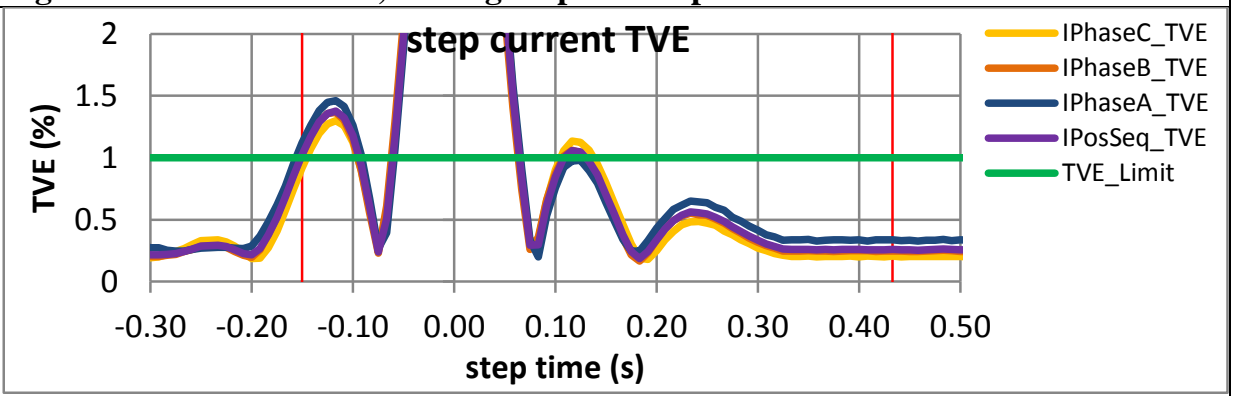

Figure 4104: Fs = 12 FPS, +10 degree phase step

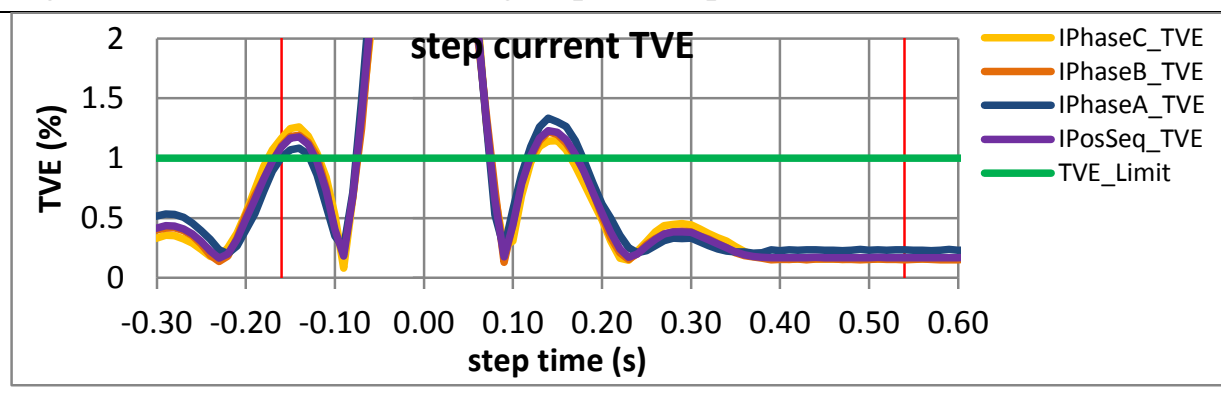

Figure 4105: Fs = 12 FPS, -10 degree phase step

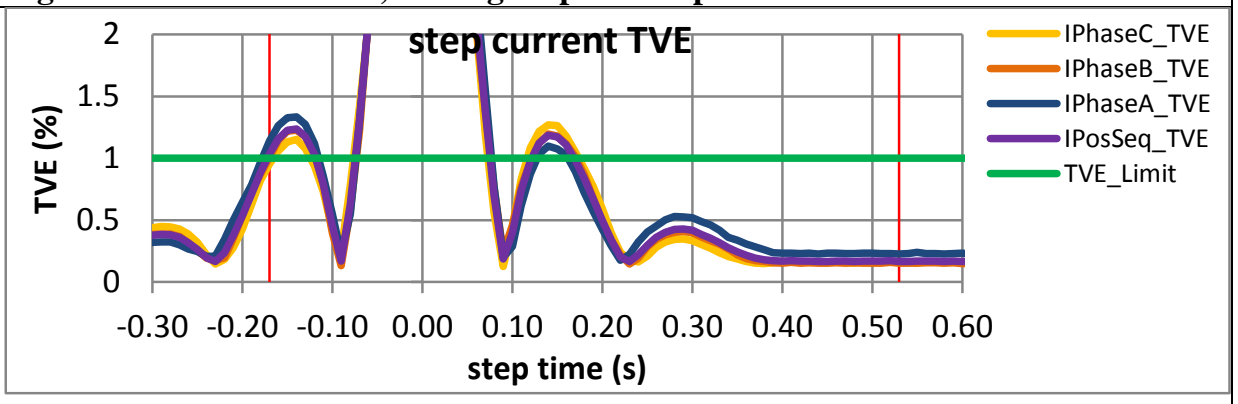

Figure 4107: Fs = 10 FPS, -10 degree phase step 
9.2.3 PMU B dynamic step change in phase current response time: $F 0=60 \mathrm{~Hz}, \mathrm{M}$ class

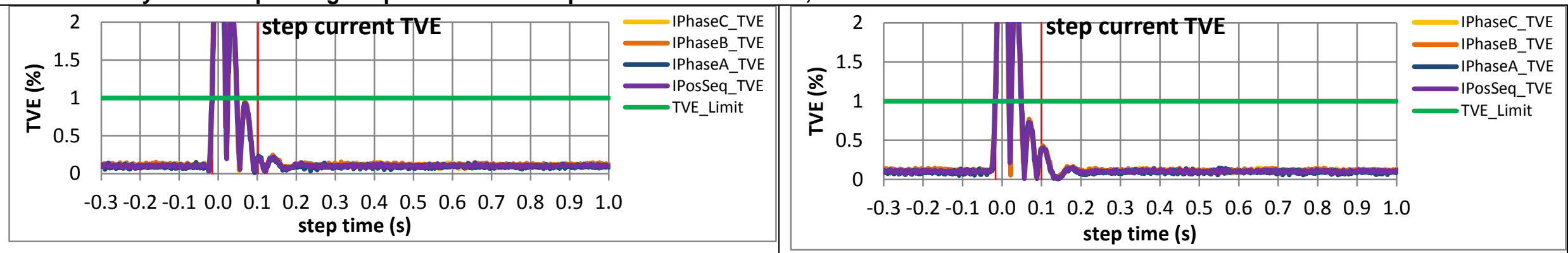

Figure 4108: Fs = 60 FPS, +10 degree phase step

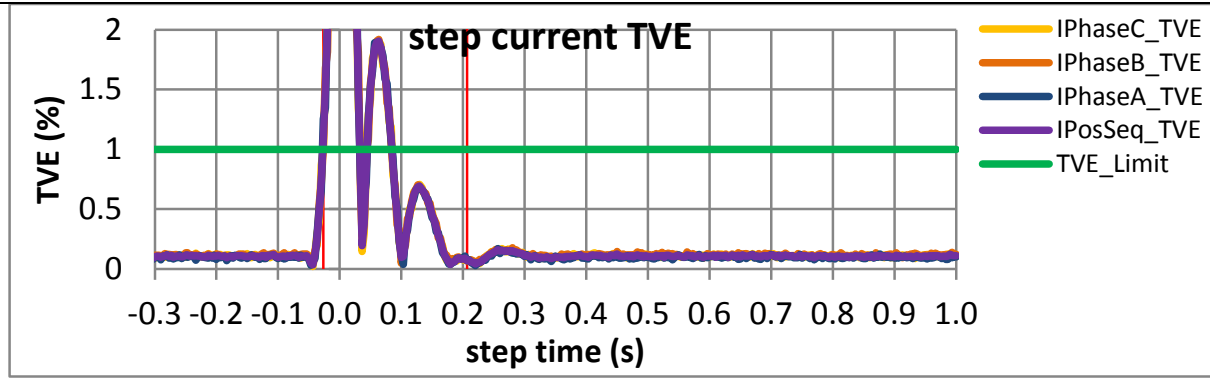

Figure 4109: Fs = 60 FPS, -10 degree phase step

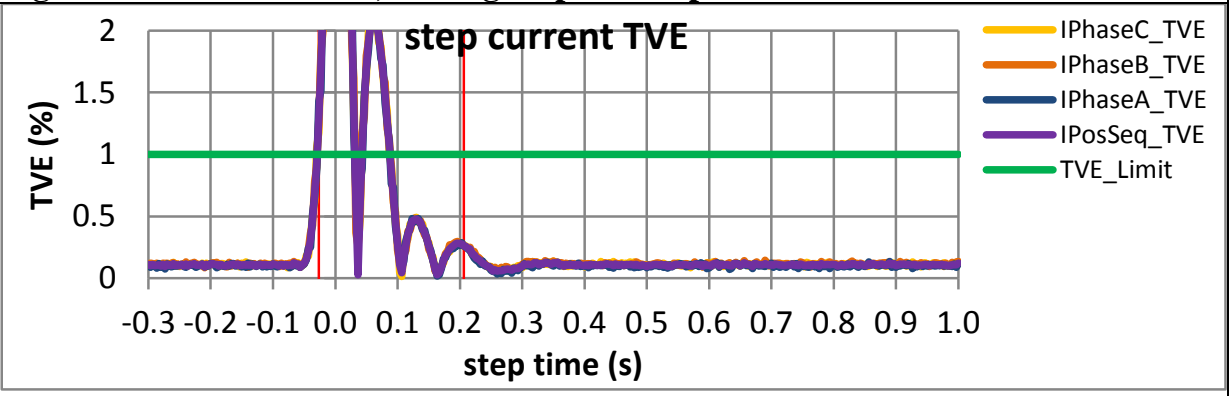

Figure 4110: Fs = 30 FPS, +10 degree phase step

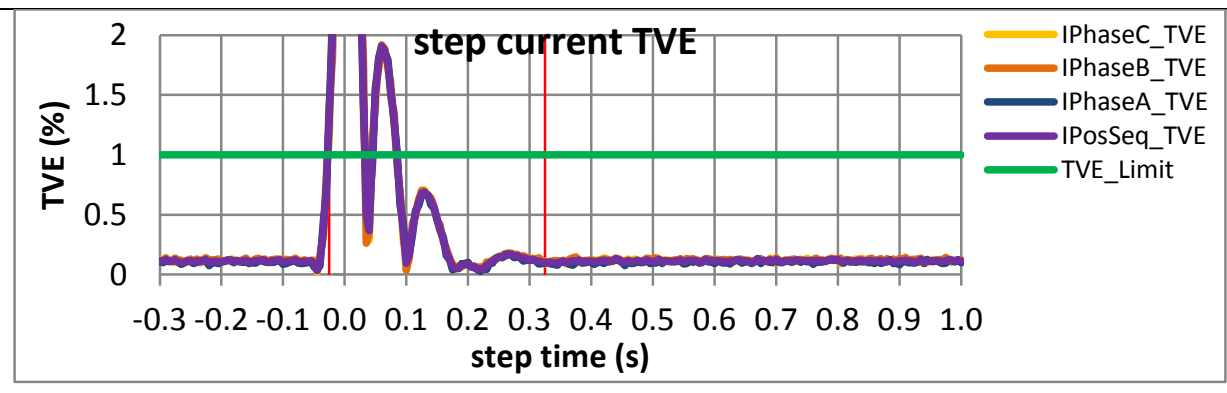

Figure 4111: Fs = 30 FPS, -10 degree phase step

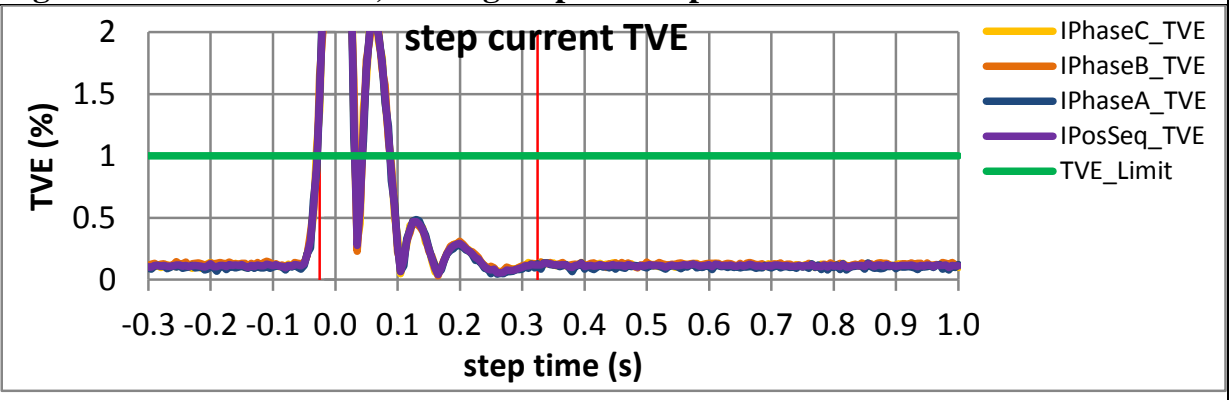

Figure 4112: Fs = 20 FPS, +10 degree phase step

Figure 4113: Fs = 20 FPS, - 10 degree phase step 

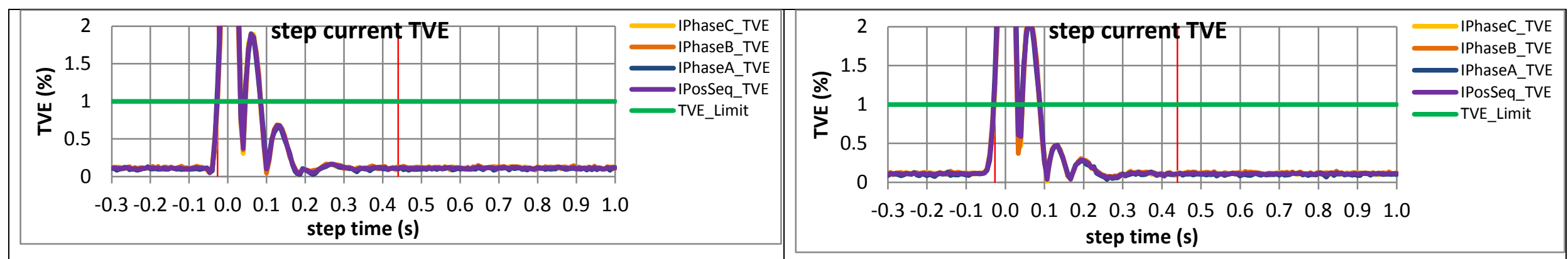

Figure 4114: Fs = 15 FPS, +10 degree phase step

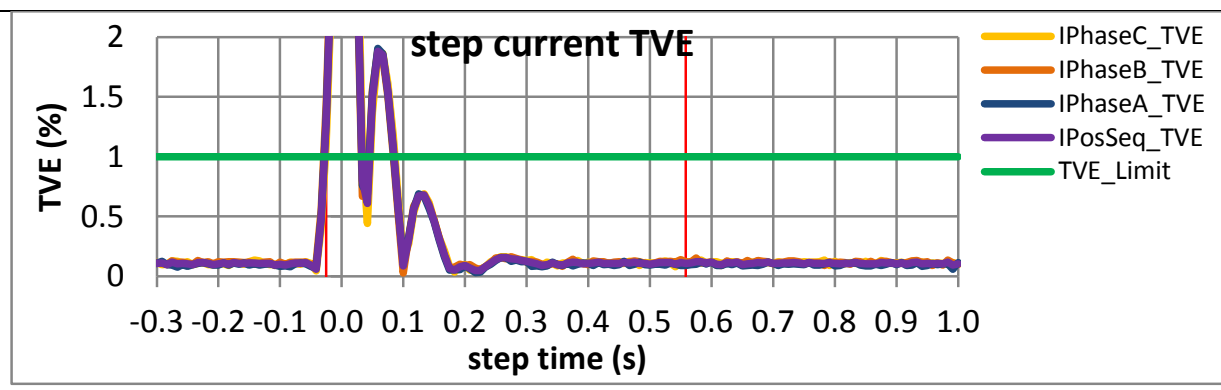

Figure 4115: Fs = 15 FPS, -10 degree phase step

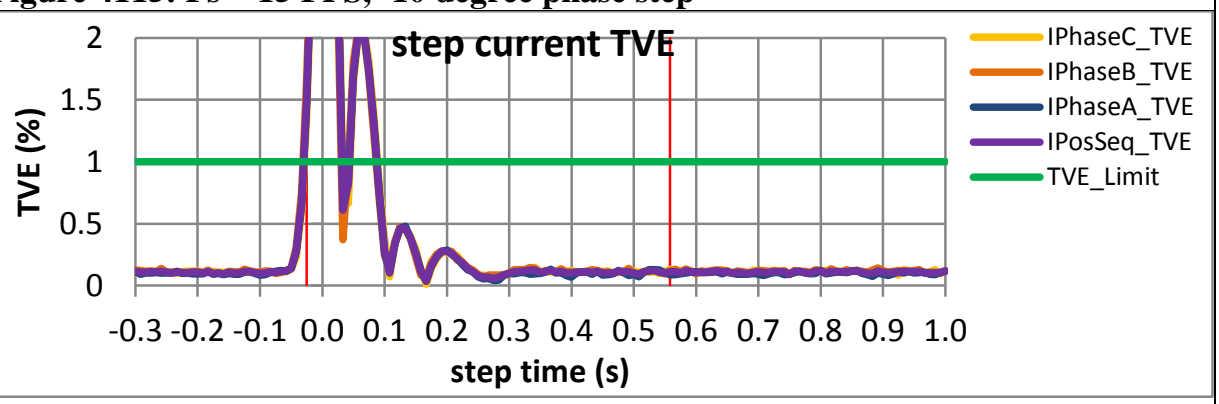

Figure 4116: Fs = 12 FPS, +10 degree phase step

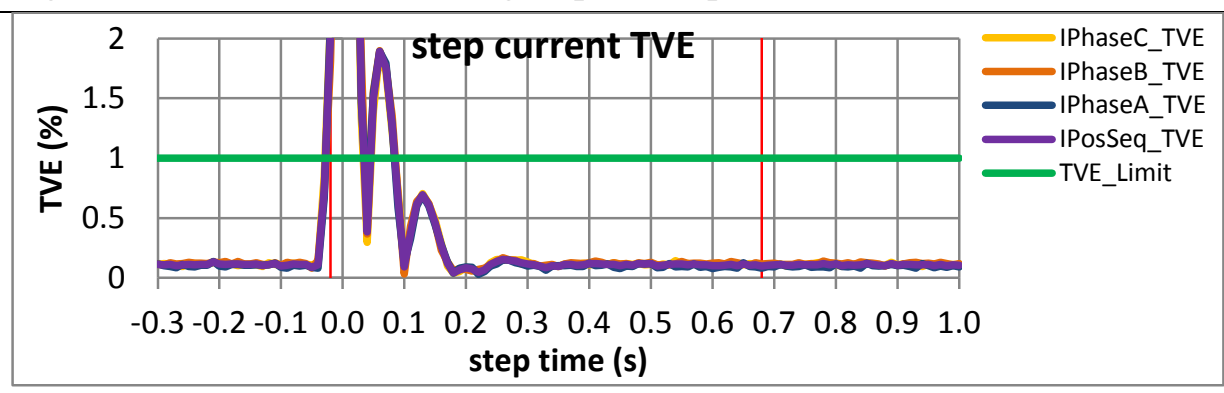

Figure 4118: Fs = 10 FPS, +10 degree phase step

Figure 4117: Fs = 12 FPS, -10 degree phase step

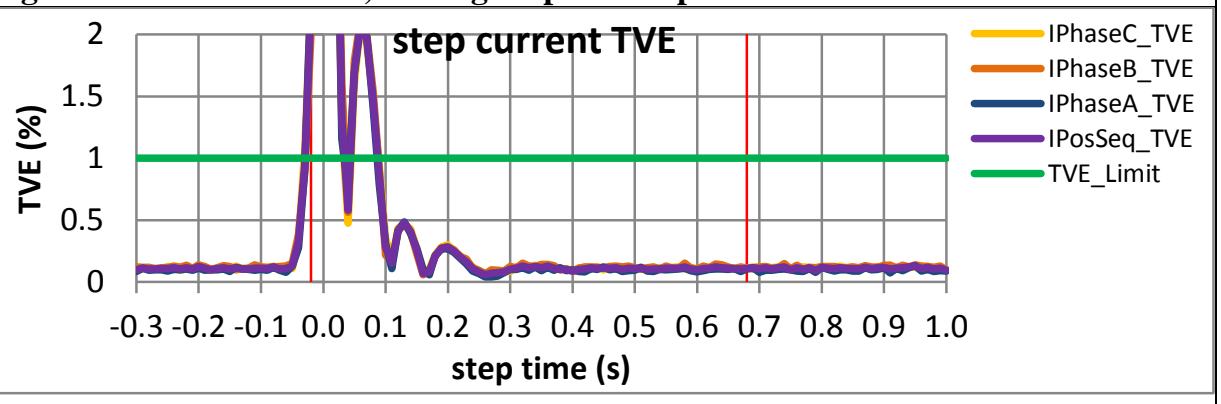

Figure 4119: Fs = 10 FPS, -10 degree phase step 
9.2.4 PMU C dynamic step change in phase current response time: $F 0=60 \mathrm{~Hz}, \mathrm{M}$ class

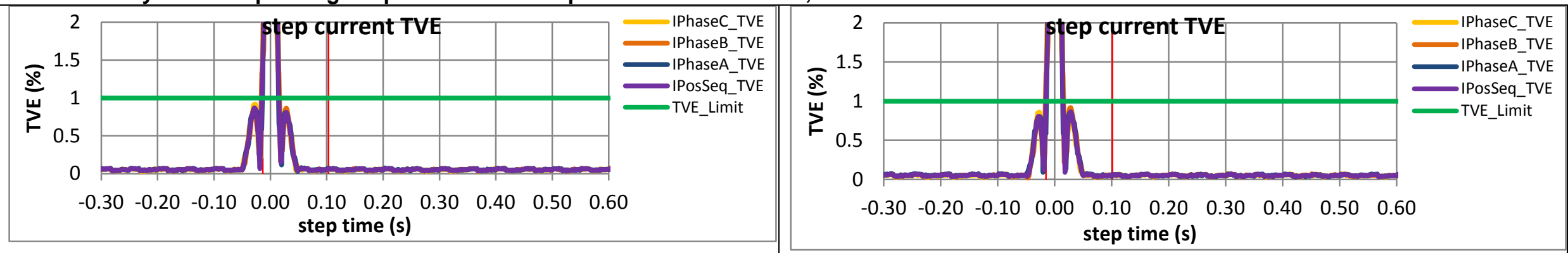

Figure 4120: Fs = 60 FPS, +10 degree phase step

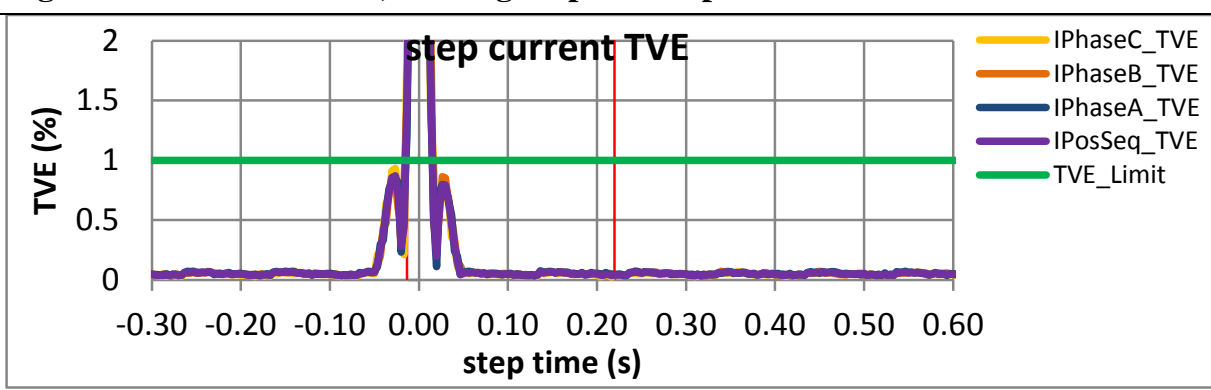

Figure 4121: Fs = 60 FPS, -10 degree phase step

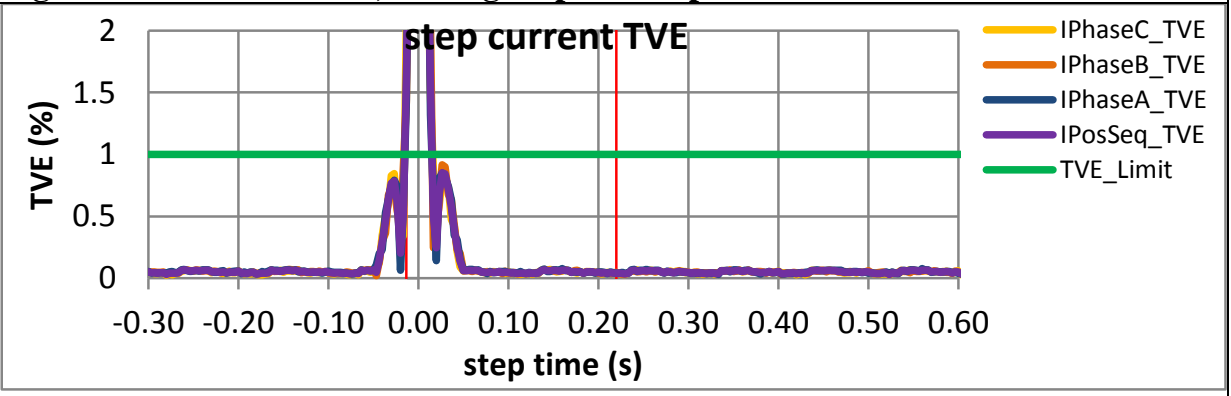

Figure 4122: Fs = 30 FPS, +10 degree phase step

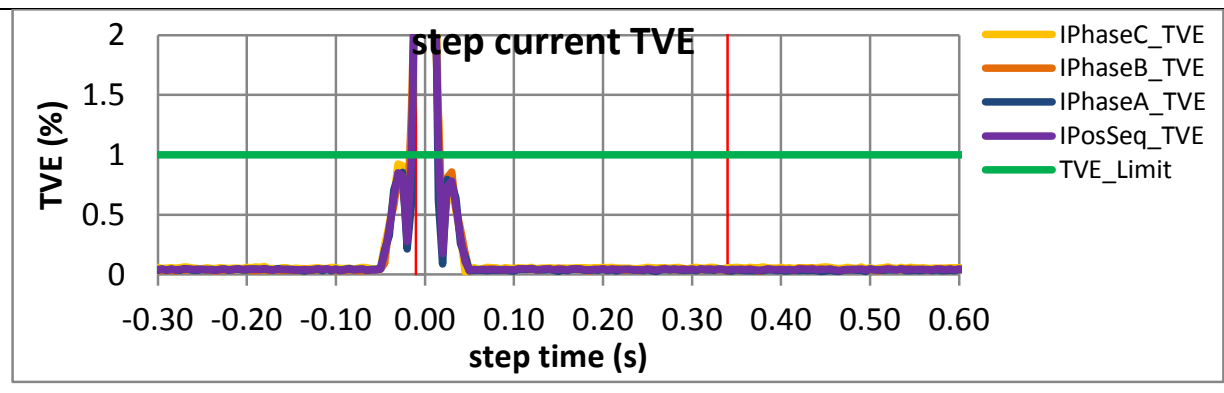

Figure 4123: Fs = 30 FPS, -10 degree phase step

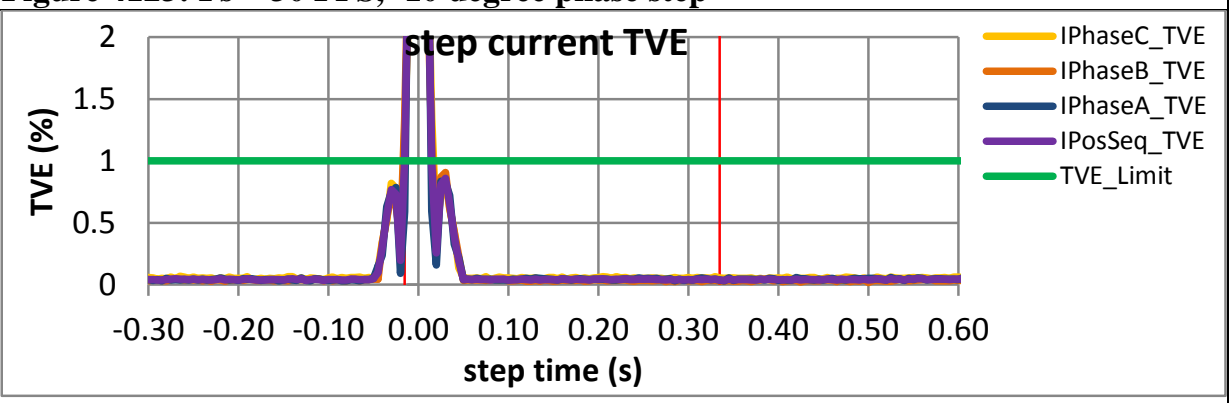

Figure 4125: Fs = 20 FPS, - 10 degree phase step 

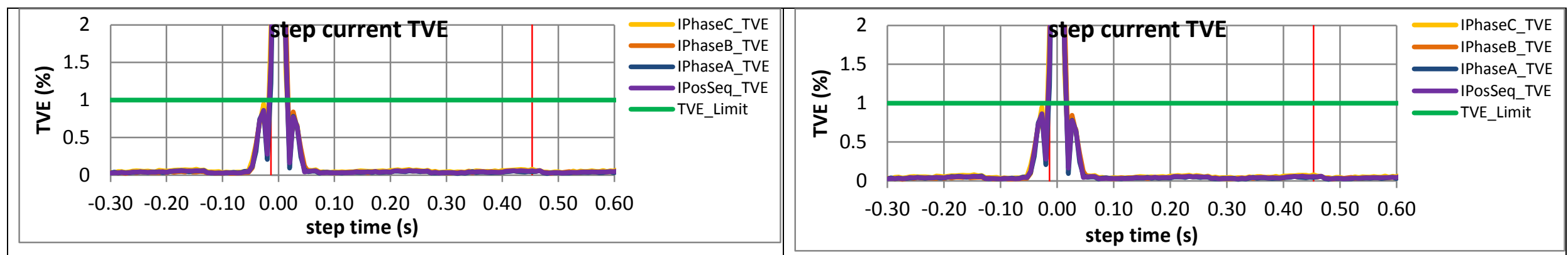

Figure 4126: Fs = 15 FPS, +10 degree phase step

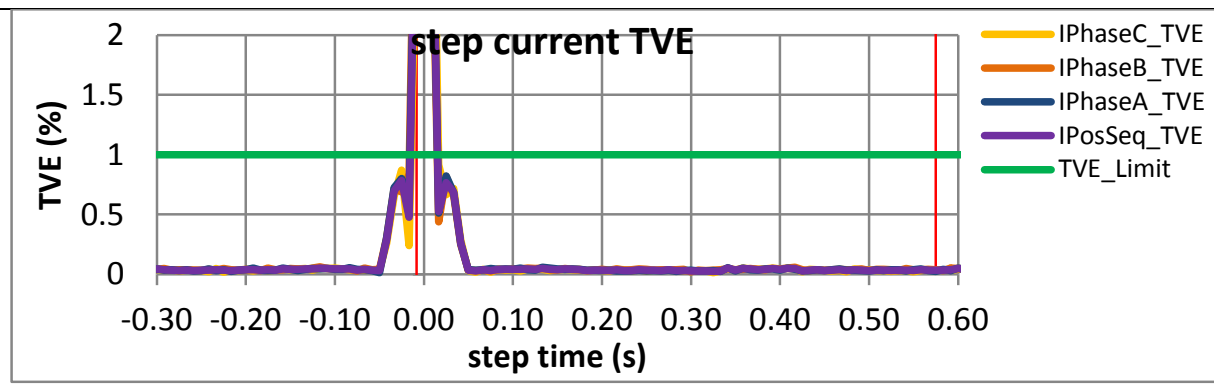

Figure 4127: Fs = 15 FPS, -10 degree phase step

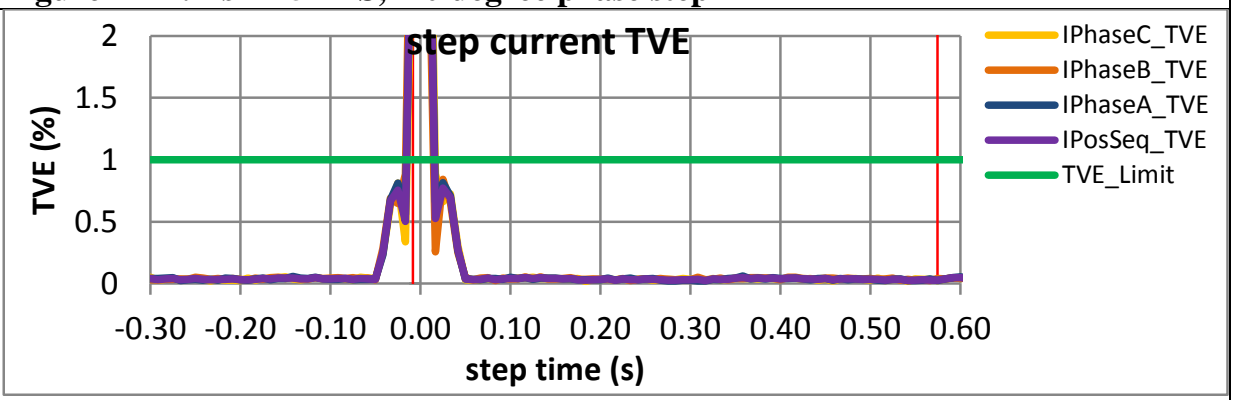

Figure 4128: Fs = 12 FPS, +10 degree phase step

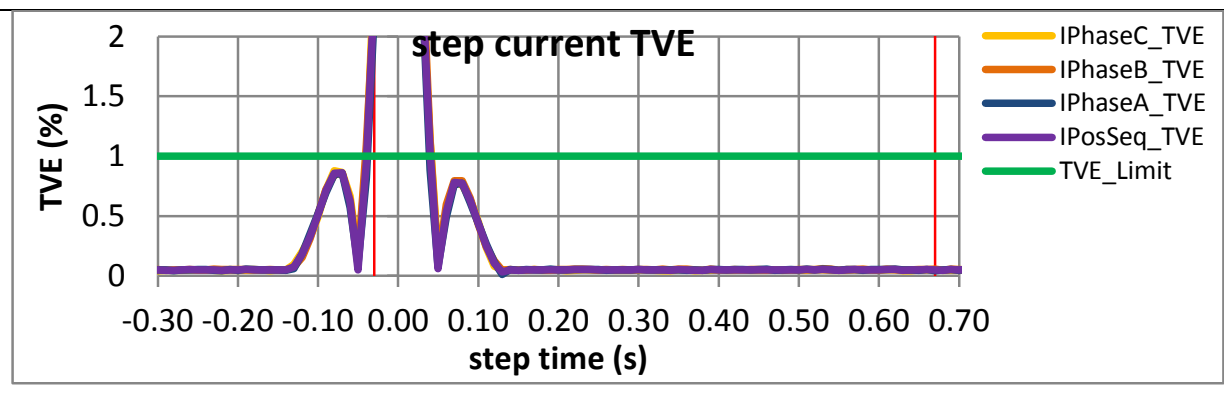

Figure 4129: Fs = 12 FPS, -10 degree phase step

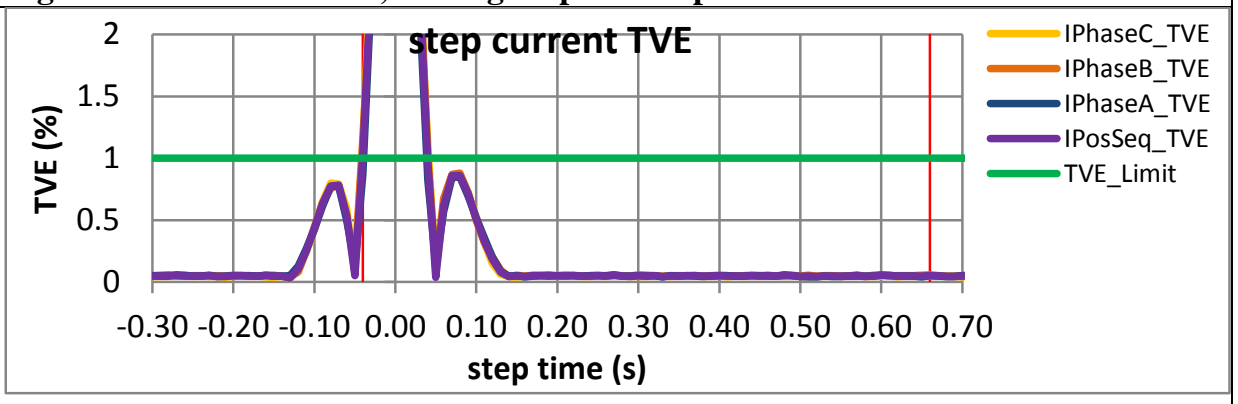

Figure 4131: Fs $=10 \mathrm{FPS},-10$ degree phase step 
9.2.5 PMU D dynamic step change in phase current response time: $F 0=60 \mathrm{~Hz}, \mathrm{M}$ class
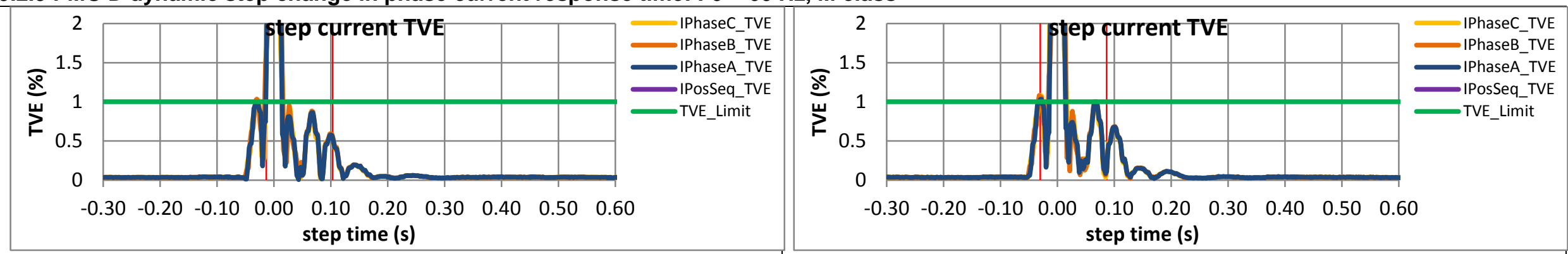

Figure 4132: Fs = 60 FPS, +10 degree phase step

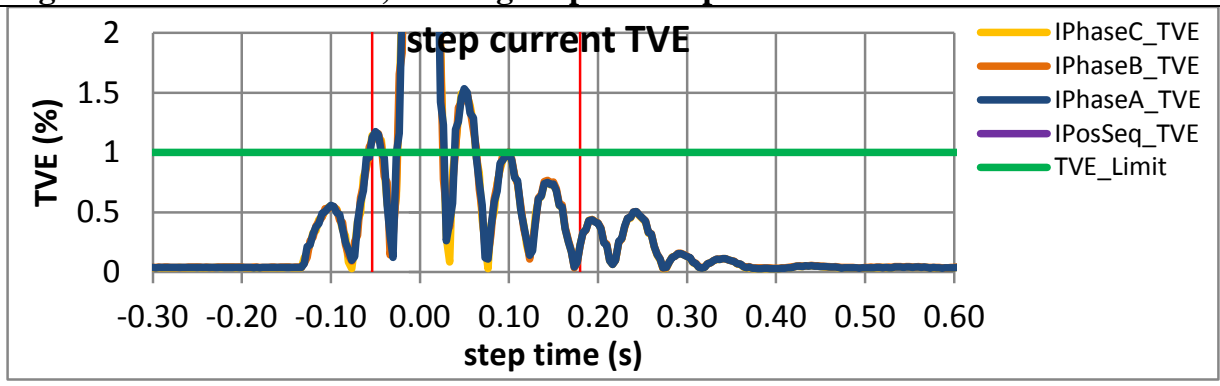

Figure 4133: Fs = 60 FPS, -10 degree phase step

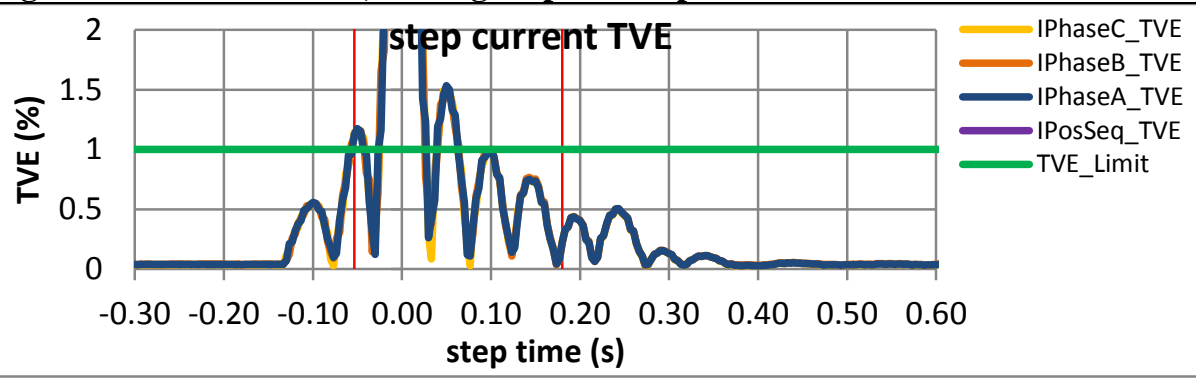

Figure 4134: Fs = 30 FPS, +10 degree phase step

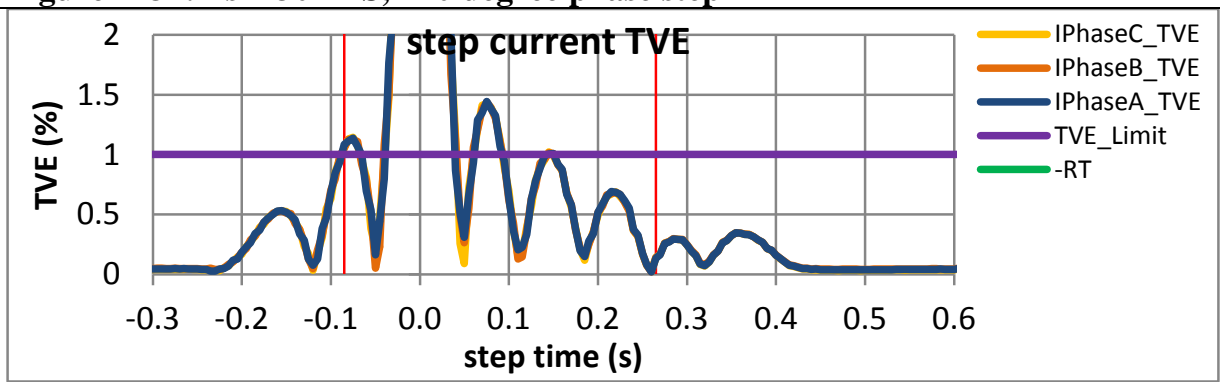

Figure 4136: Fs $=20$ FPS, +10 degree phase step

Figure 4135: Fs = 30 FPS, -10 degree phase step

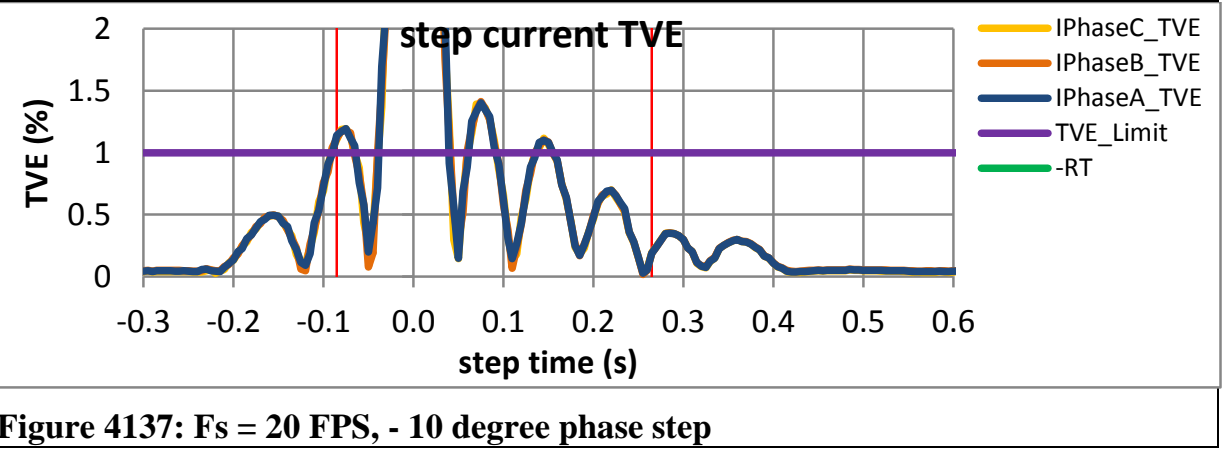




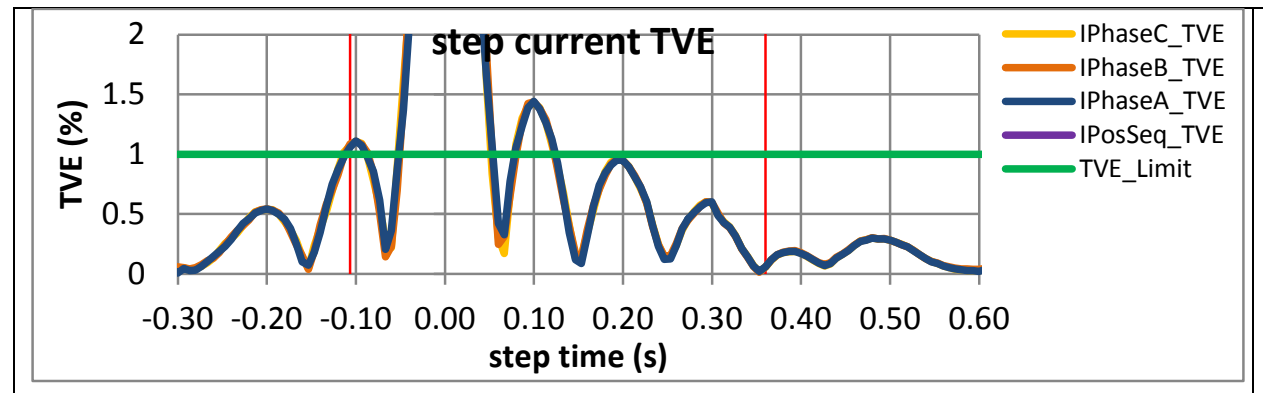

Figure 4138: Fs = 15 FPS, +10 degree phase step
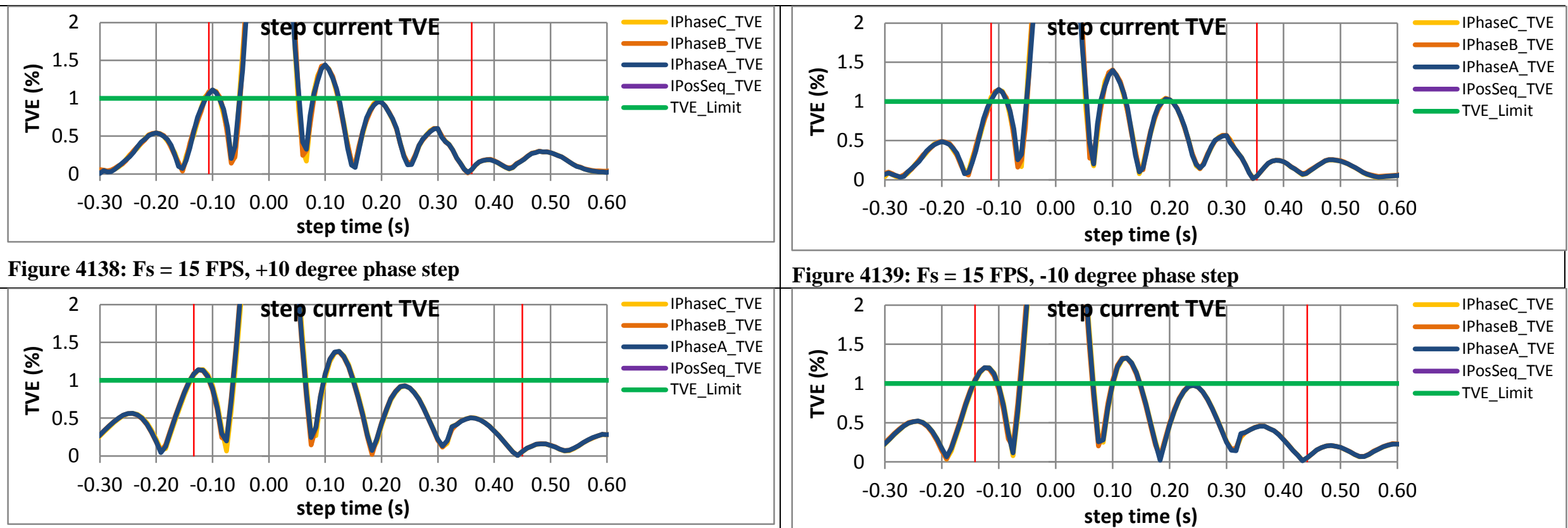

Figure 4139: Fs = 15 FPS, -10 degree phase step

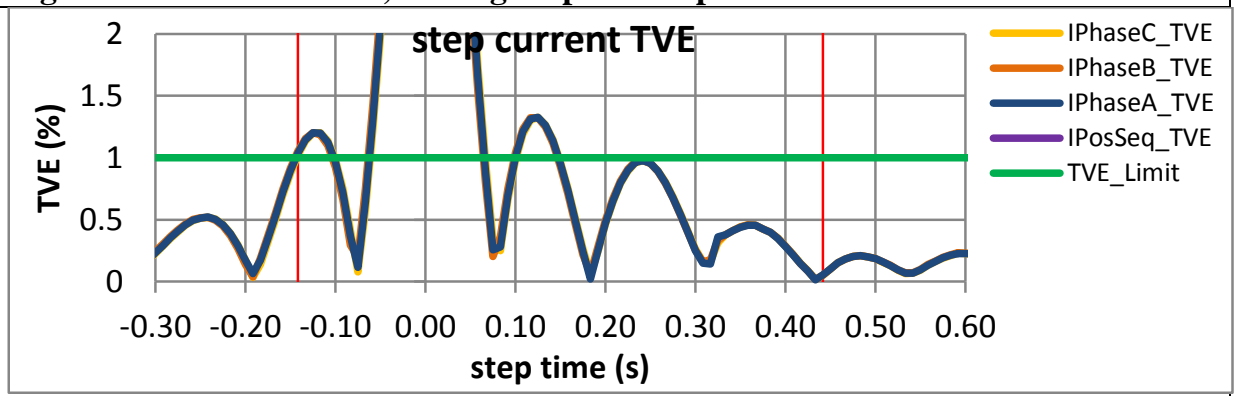

Figure 4140: Fs = 12 FPS, +10 degree phase step

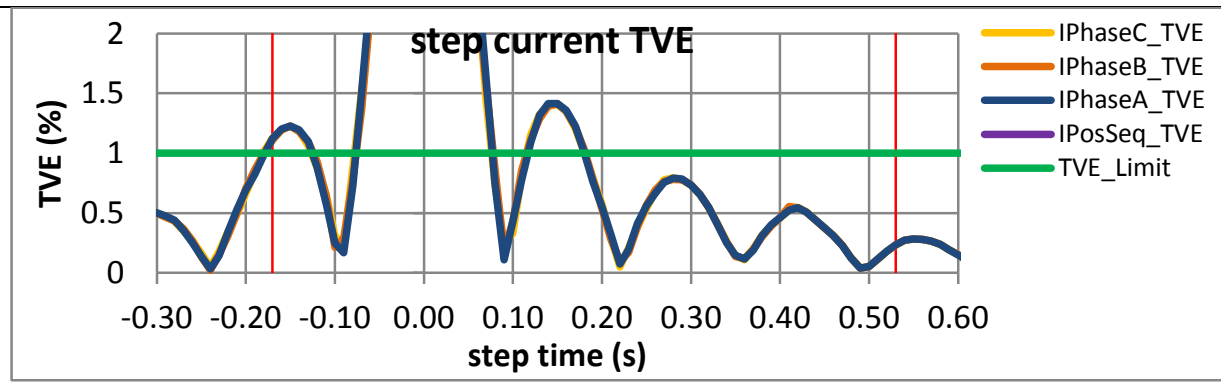

Figure 4142: Fs = 10 FPS, +10 degree phase step

Figure 4141: Fs = 12 FPS, -10 degree phase step

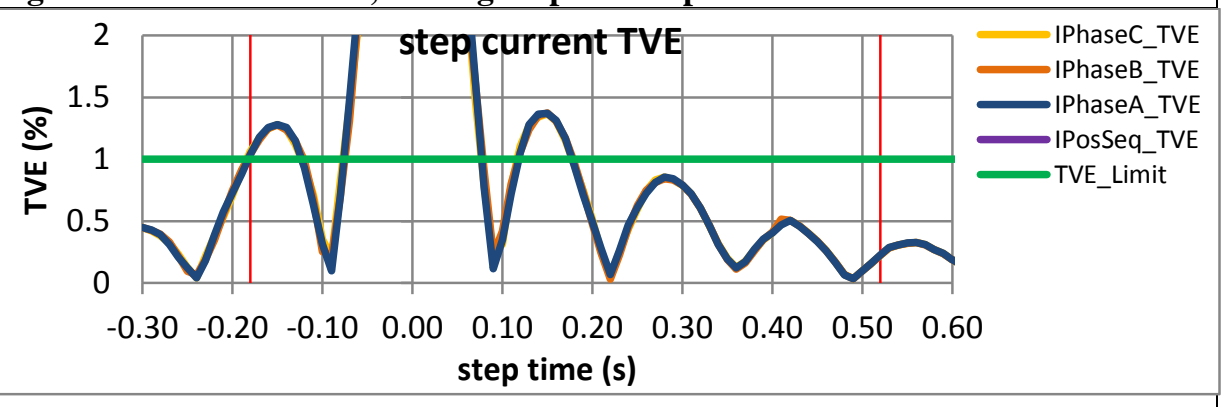

Figure 4143: Fs = 10 FPS, -10 degree phase step 
9.2.6 PMU E dynamic step change in phase current response time: $\mathrm{FO}=60 \mathrm{~Hz}, \mathrm{M}$ class

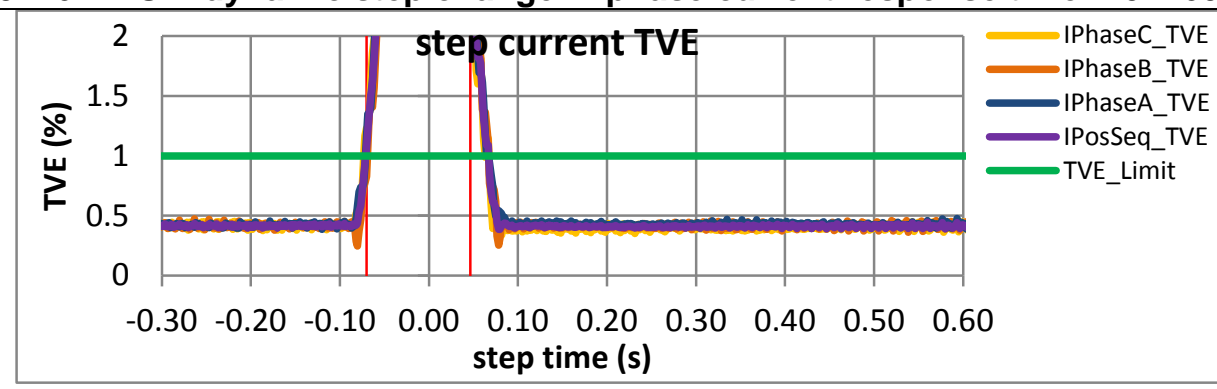

Figure 4144: Fs = 60 FPS, +10 degree phase step

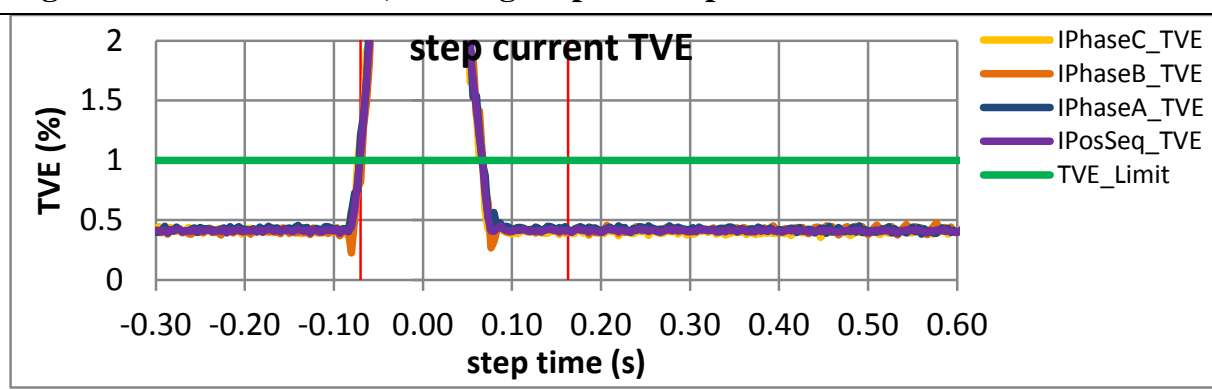

Figure 4146: Fs = 30 FPS, +10 degree phase step

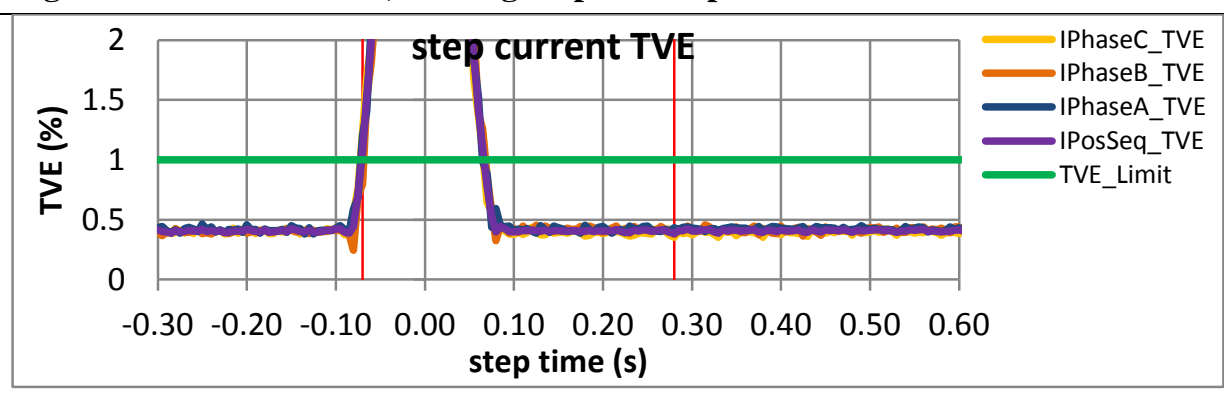

Figure 4148: Fs = 20 FPS, +10 degree phase step

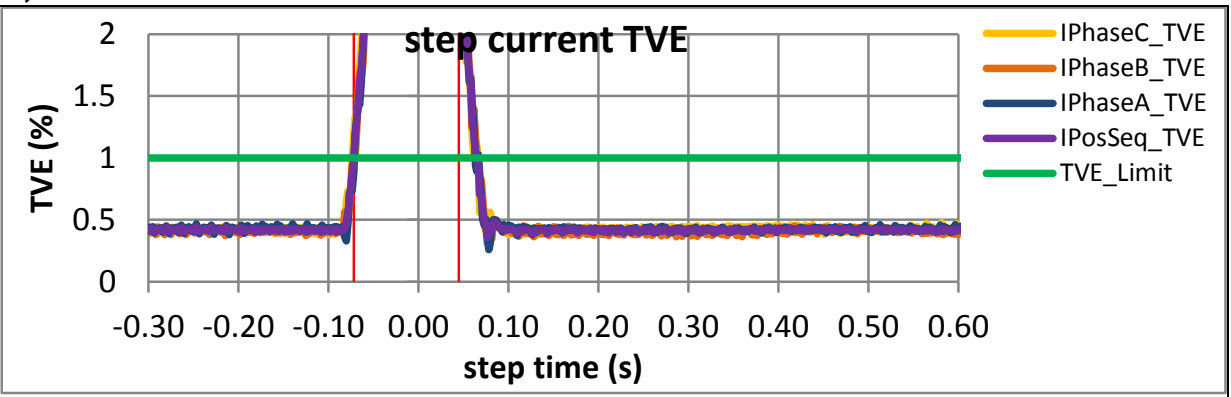

Figure 4145: Fs = 60 FPS, -10 degree phase step

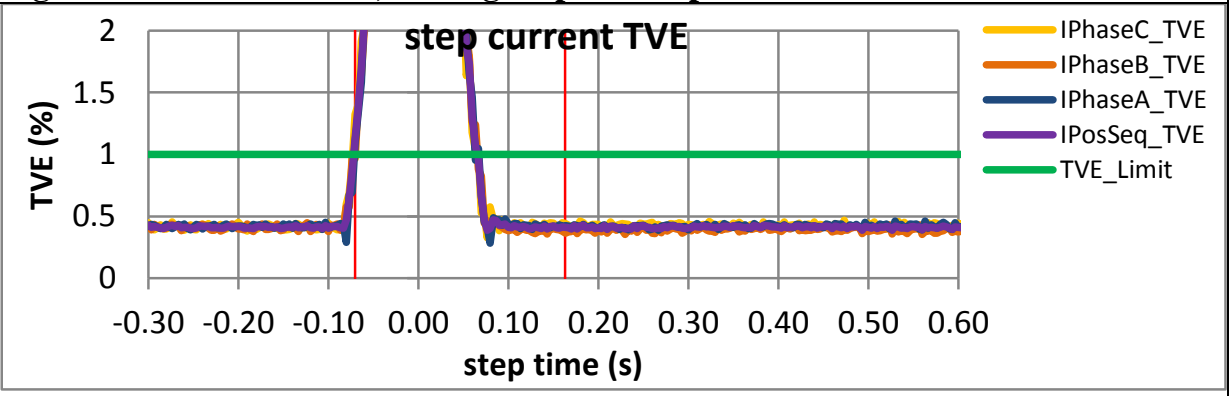

Figure 4147: Fs = 30 FPS, -10 degree phase step

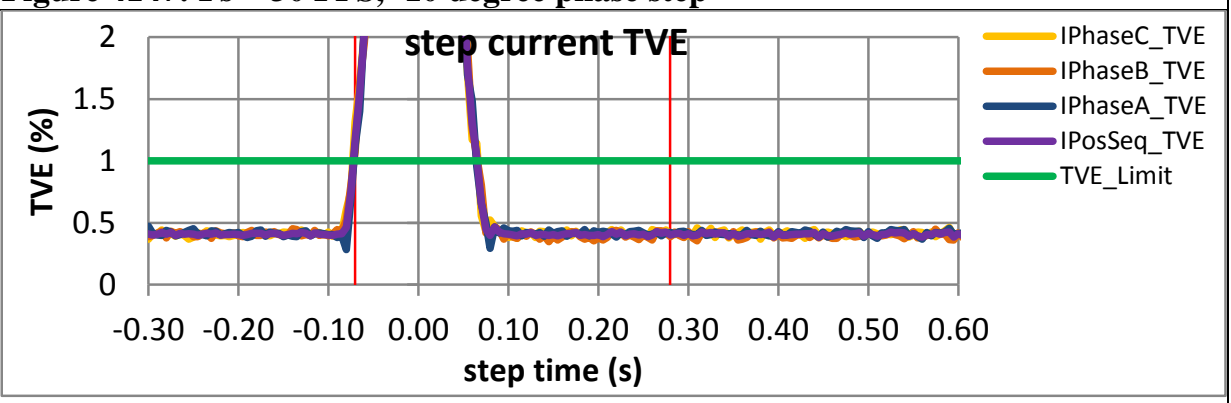

Figure 4149: Fs = 20 FPS, - 10 degree phase step 


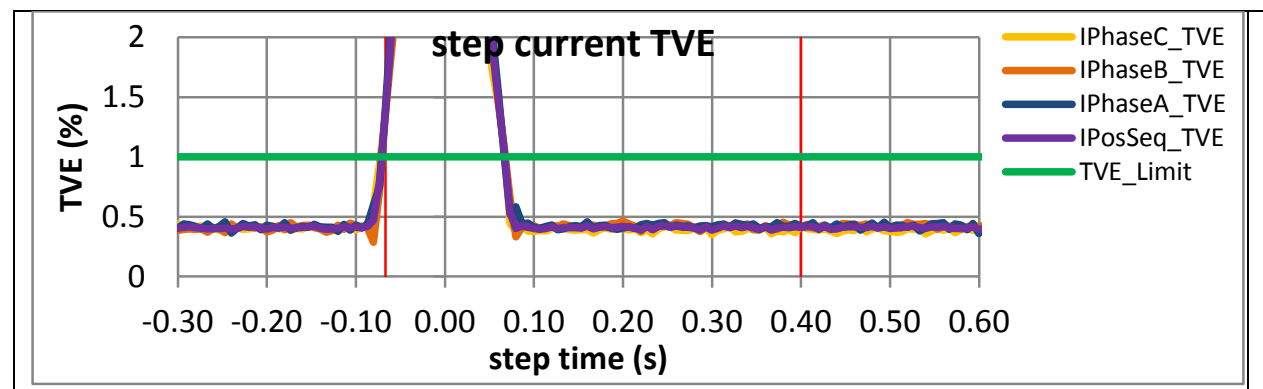

Figure 4150: Fs = 15 FPS, +10 degree phase step

Figure 4152: Fs = 12 FPS, +10 degree phase step

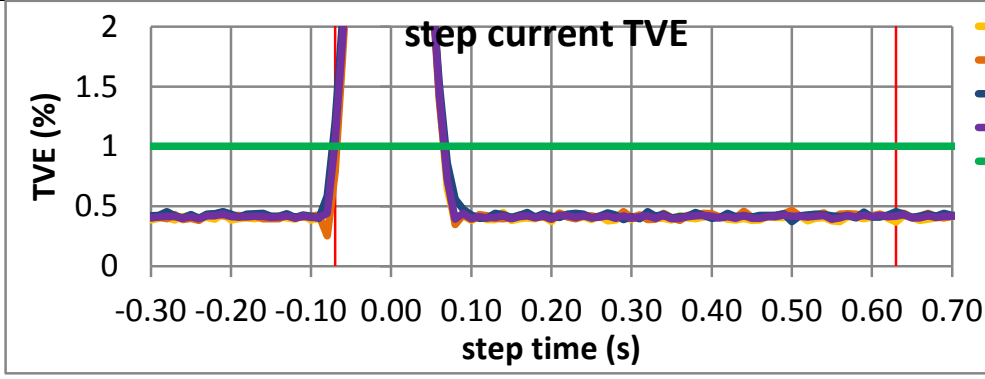

Figure 4154: Fs = 10 FPS, +10 degree phase step

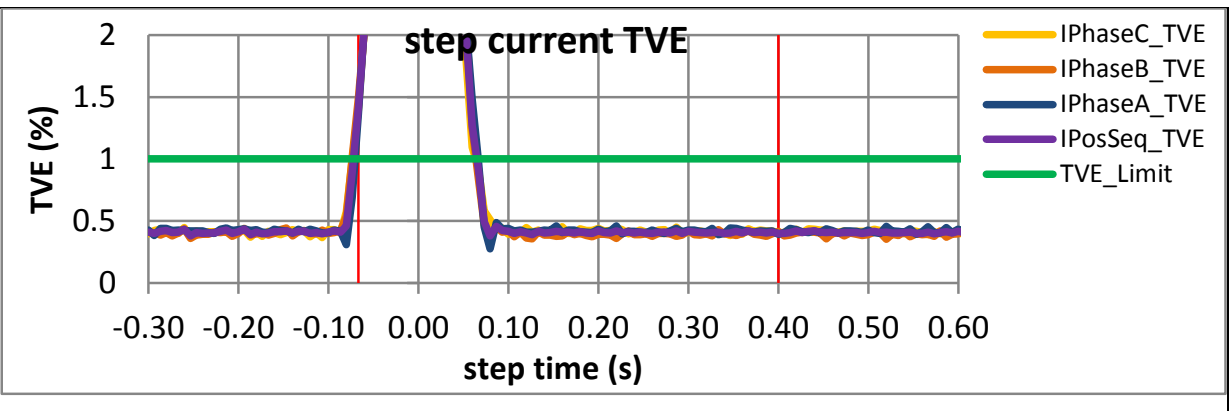

Figure 4151: Fs = 15 FPS, -10 degree phase step

Figure 4153: Fs = 12 FPS, -10 degree phase step

\begin{aligned} & IPhaseC_TVE \\ & IPhaseB_TVE \\ & IPhaseA_TVE \\ & IPosSeq_TVE \\ & \hline TVE_Limit \\ & \hline\end{aligned}

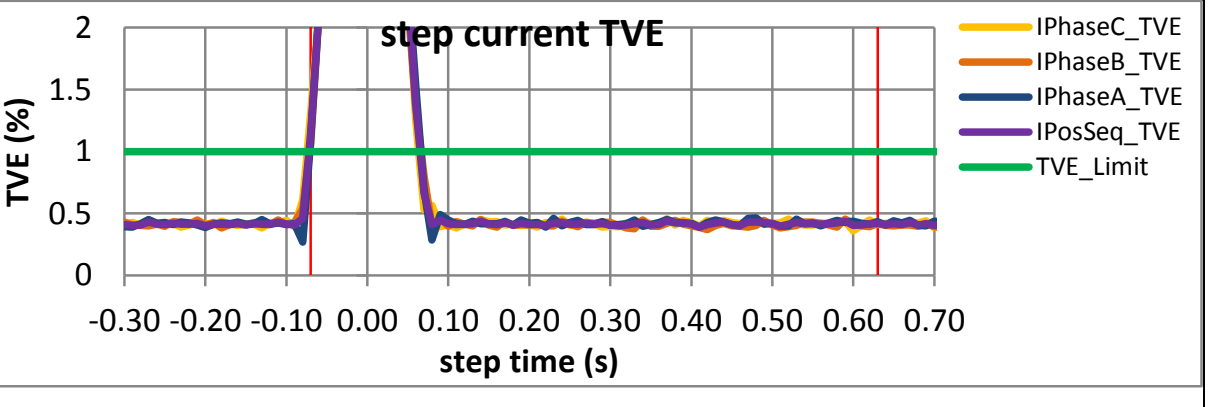

Figure 4155: Fs = 10 FPS, -10 degree phase step 
9.2.7 PMU F dynamic step change in phase current response time: $\mathrm{F} 0=60 \mathrm{~Hz}, \mathrm{M}$ class
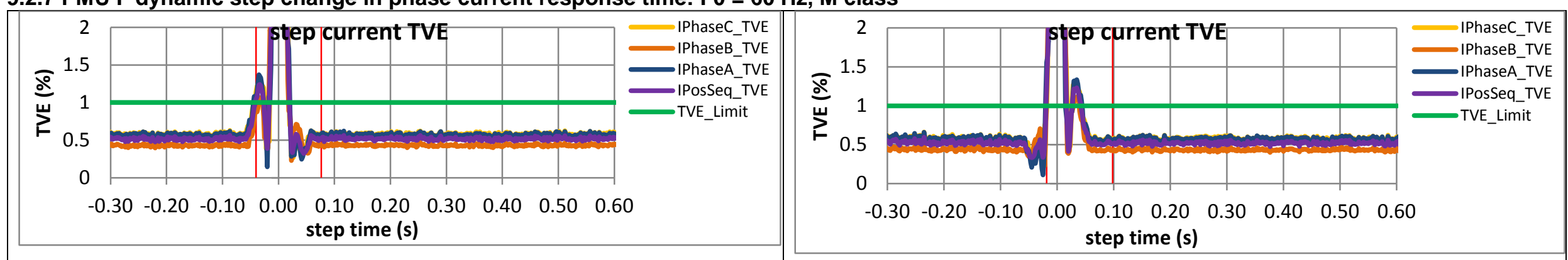

Figure 4156: Fs = $60 \mathrm{FPS},+10$ degree phase step

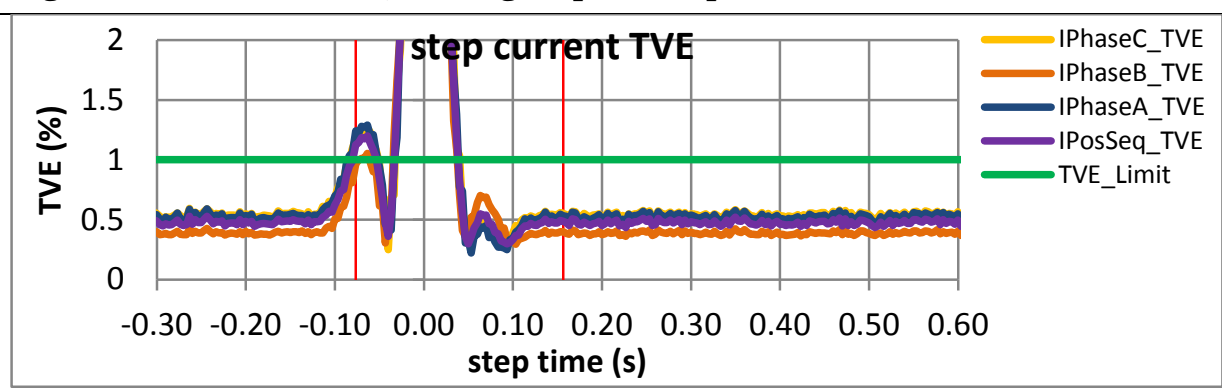

Figure 4157: Fs = 60 FPS, -10 degree phase step

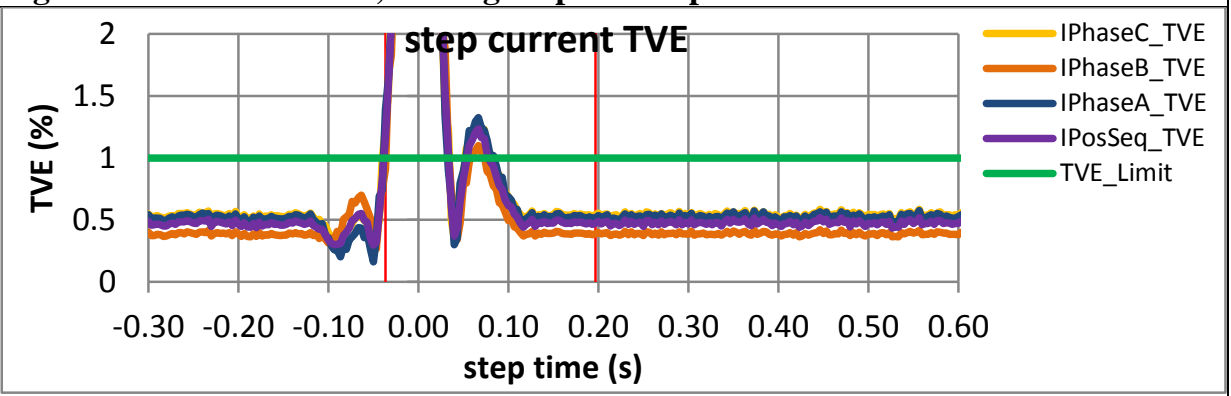

Figure 4158: Fs = 30 FPS, +10 degree phase step

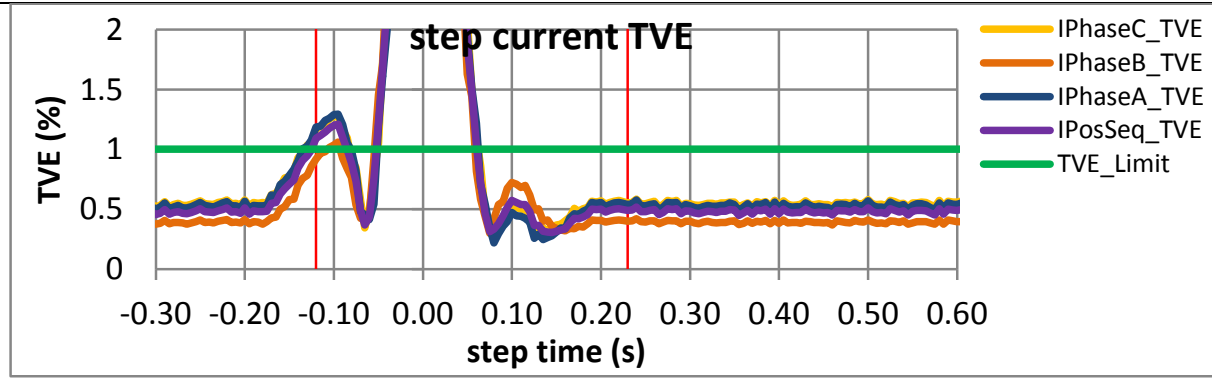

Figure 4160: Fs = 20 FPS, +10 degree phase step

Figure 4159: Fs = 30 FPS, -10 degree phase step

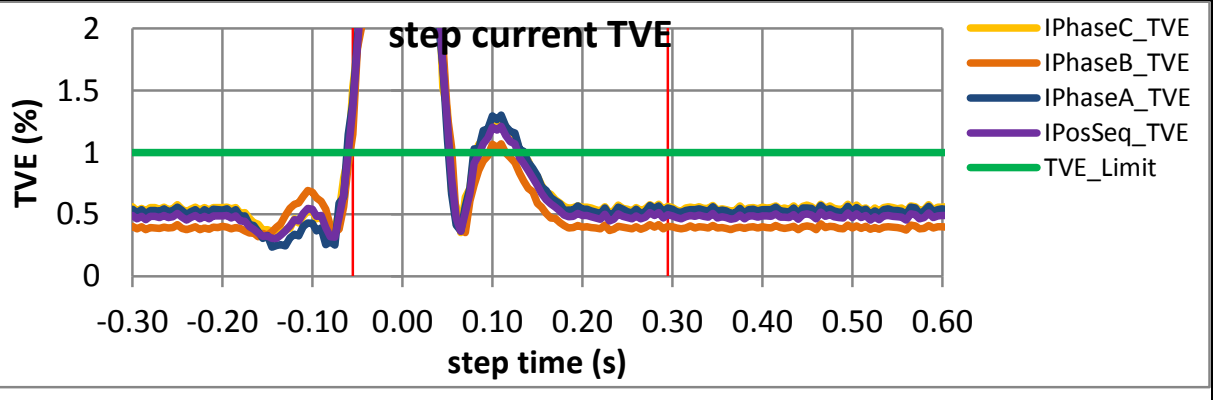

Figure 4161: Fs $=20$ FPS, -10 degree phase step 

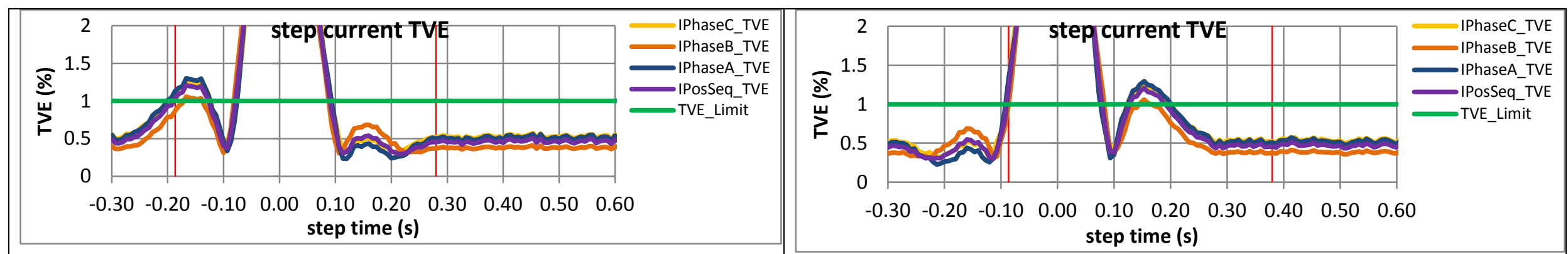

Figure 4162: Fs = 15 FPS, +10 degree phase step

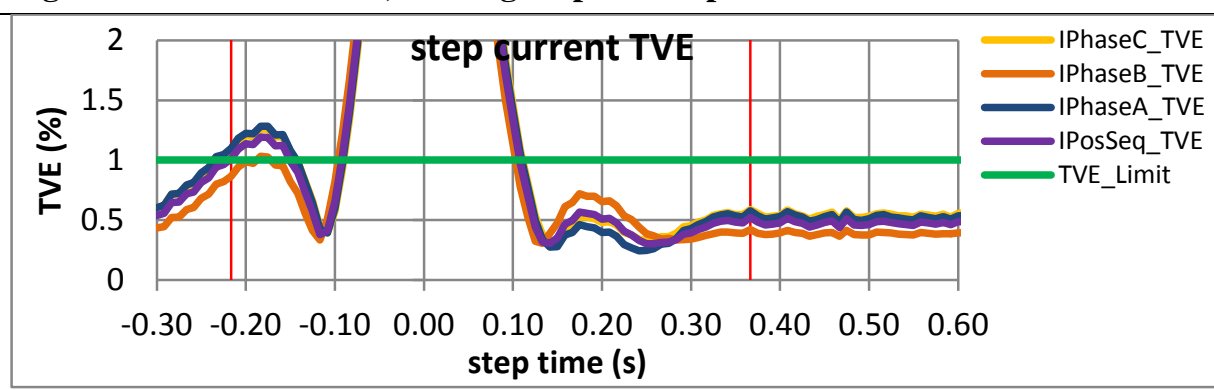

Figure 4163: Fs = 15 FPS, -10 degree phase step

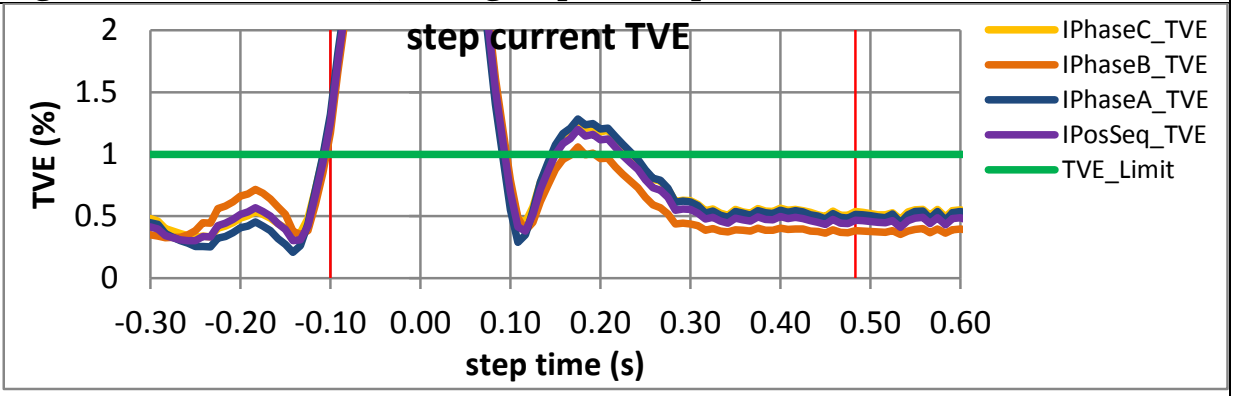

Figure 4164: Fs = 12 FPS, +10 degree phase step

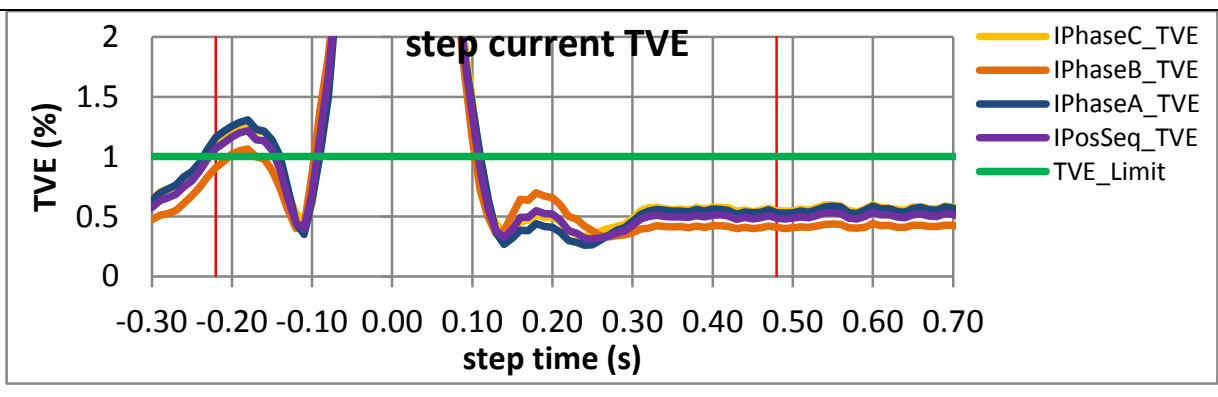

Figure 4165: Fs = 12 FPS, -10 degree phase step

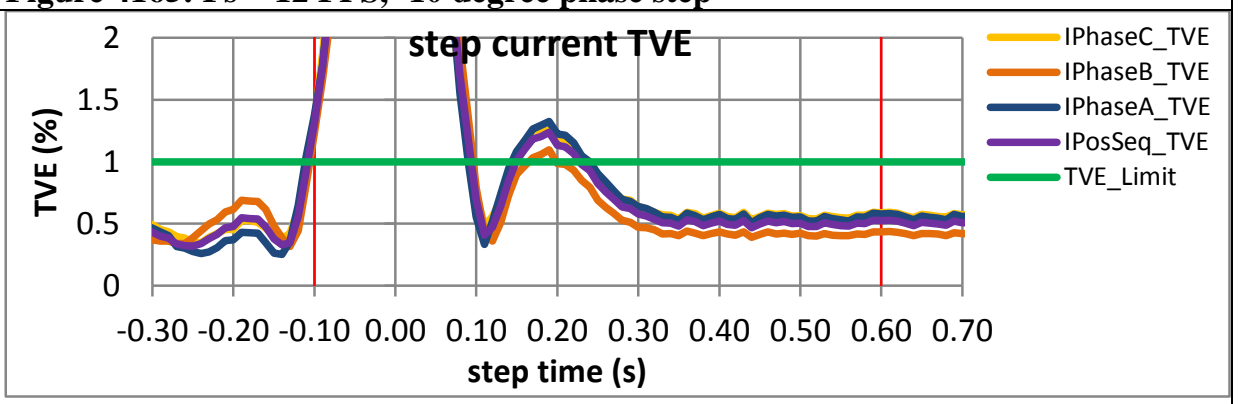

Figure 4166: Fs = 10 FPS, +10 degree phase step

Figure 4167: Fs = 10 FPS, -10 degree phase step 
9.2.8 PMU G dynamic step change in phase current response time: $\mathrm{F} 0=60 \mathrm{~Hz}, \mathrm{M}$ class

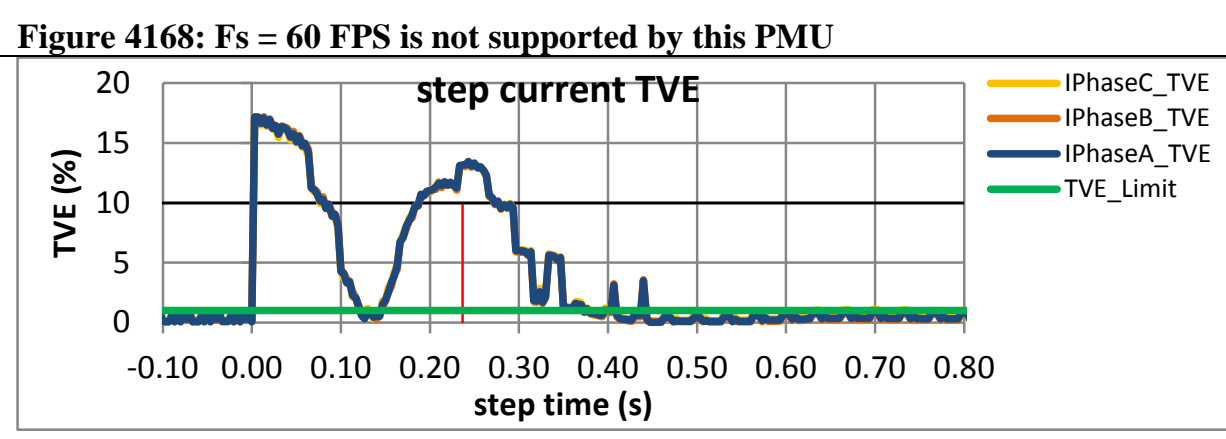

Figure 4169: Fs = 30 FPS, +10 degree phase step
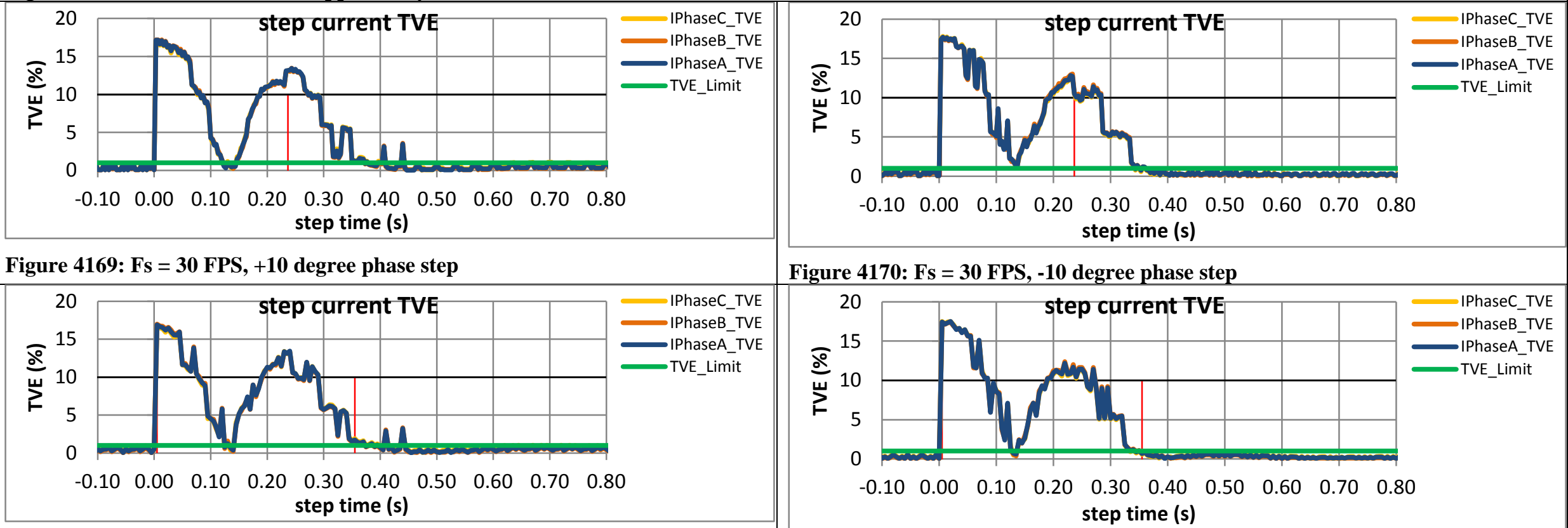

Figure 4170: Fs = 30 FPS, -10 degree phase step

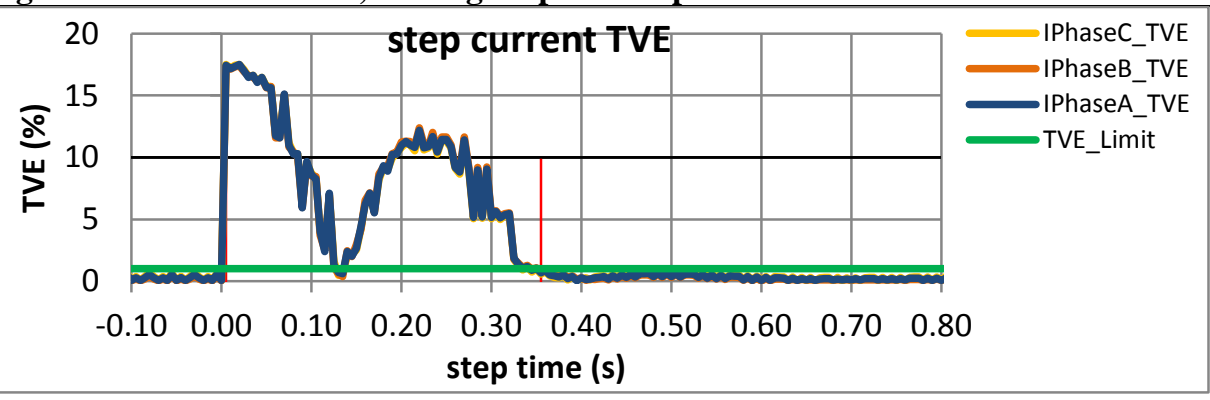

Figure 4171: Fs = 20 FPS, +10 degree phase step

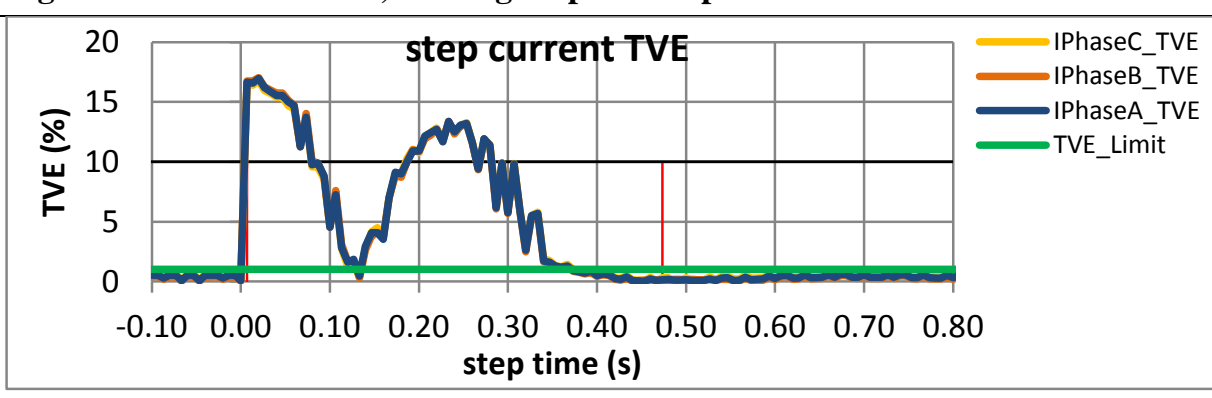

Figure 4173: Fs = 15 FPS, +10 degree phase step

Figure 4172: Fs = 20 FPS, - 10 degree phase step

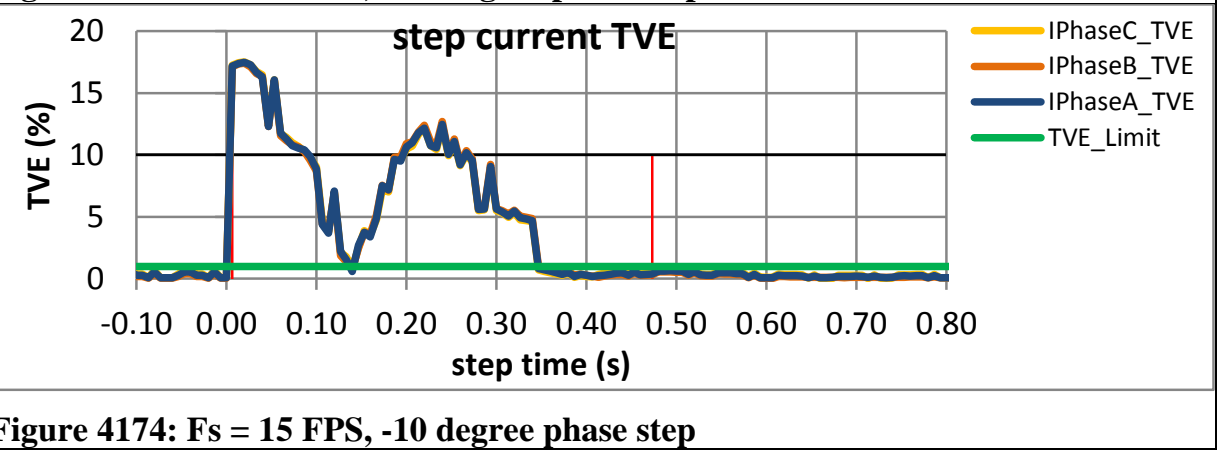



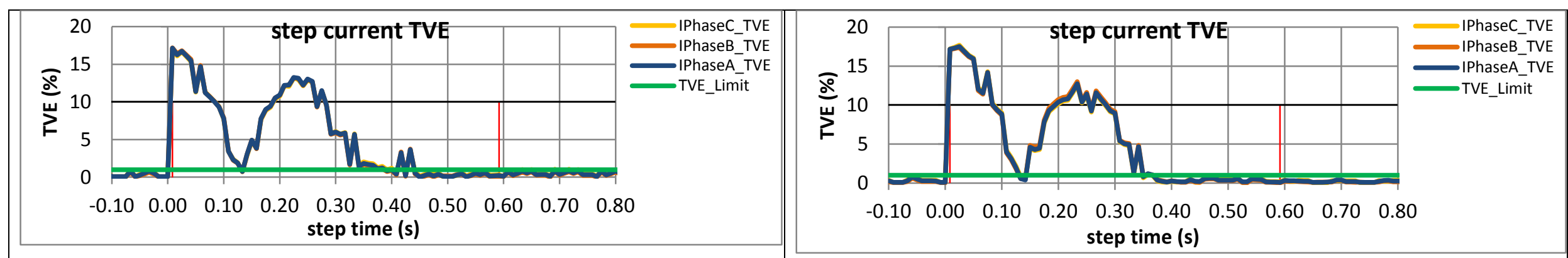

Figure 4175: Fs = 12 FPS, +10 degree phase step

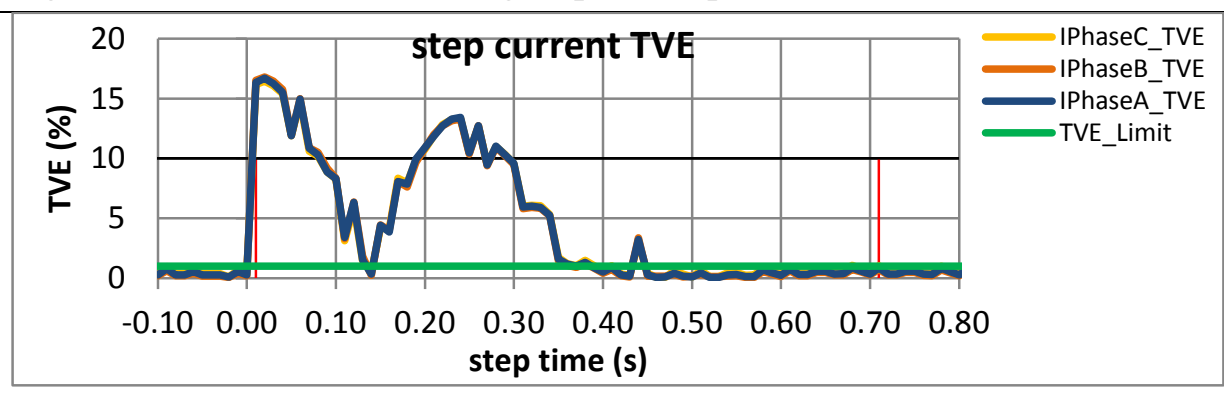

Figure 4176: Fs = 12 FPS, - 10 degree phase step

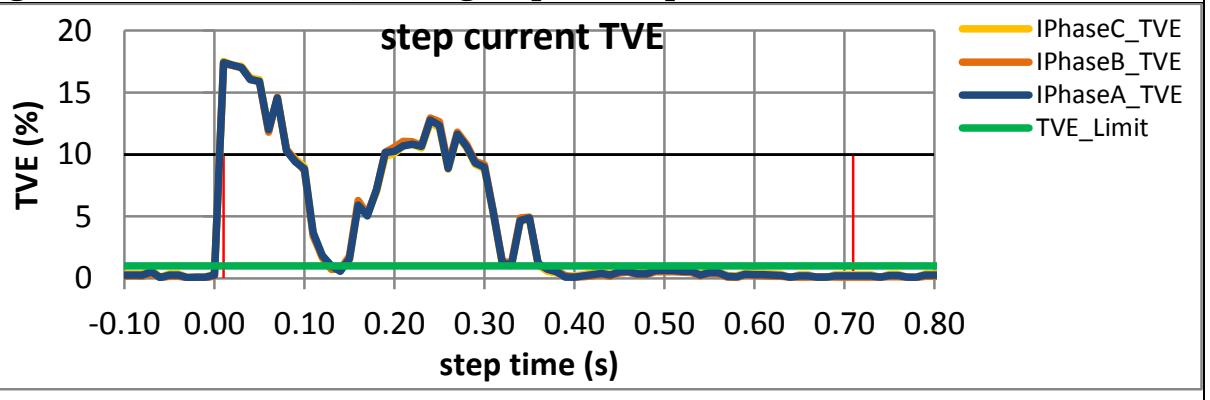

Figure 4178: Fs = 10 FPS, -10 degree phase step 
9.2.9 PMU H dynamic step change in phase current response time: $\mathrm{F} 0=60 \mathrm{~Hz}, \mathrm{M}$ class
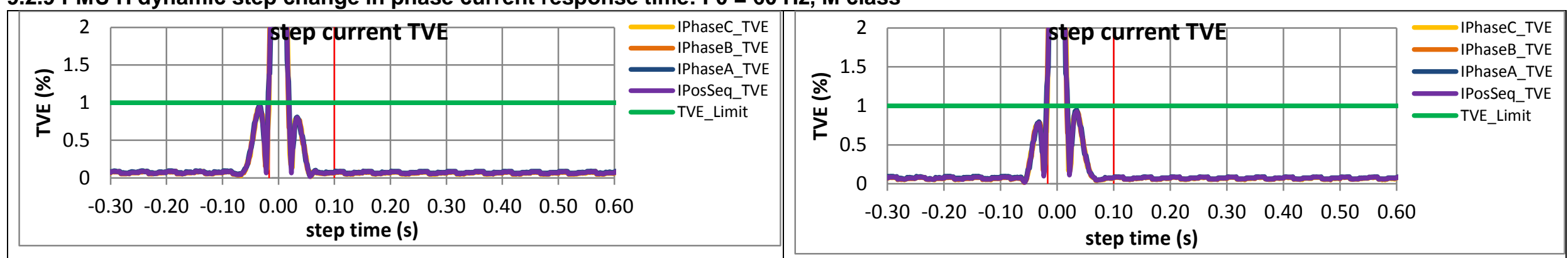

Figure 4179: Fs = 60 FPS, +10 degree phase step

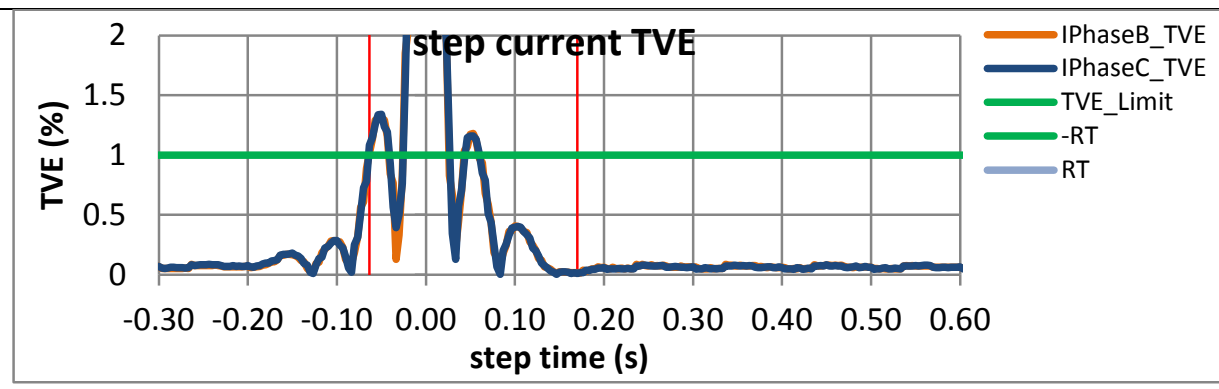

Figure 4180: Fs = 60 FPS, -10 degree phase step

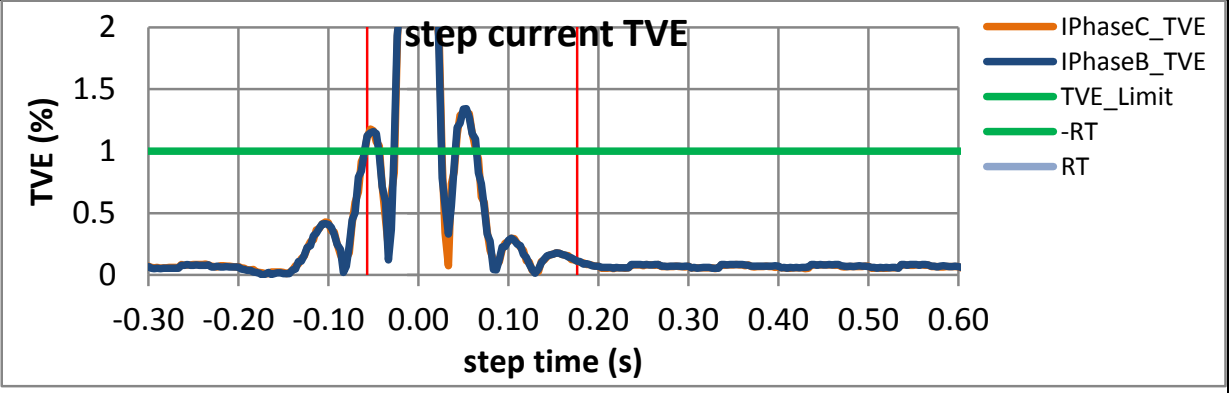

Figure 4181: Fs = 30 FPS, +10 degree phase step

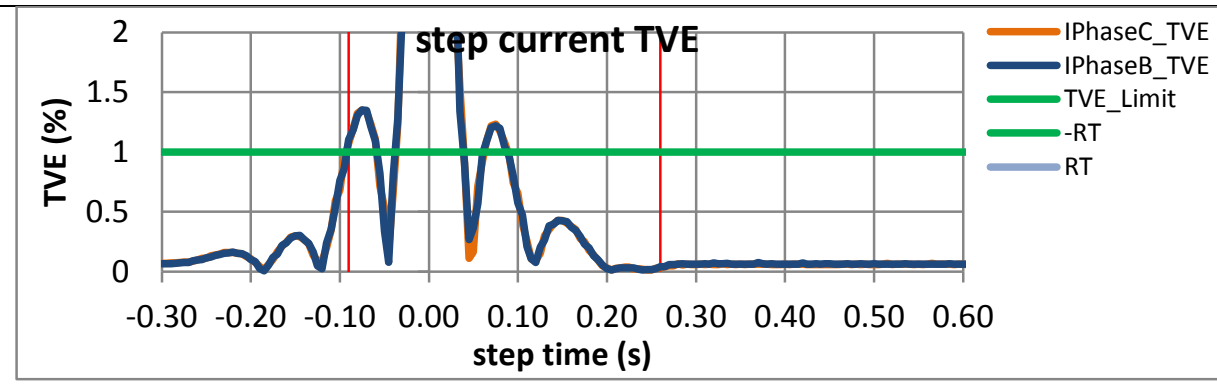

Figure 4183: Fs = 20 FPS, +10 degree phase step

Figure 4182: Fs = 30 FPS, -10 degree phase step

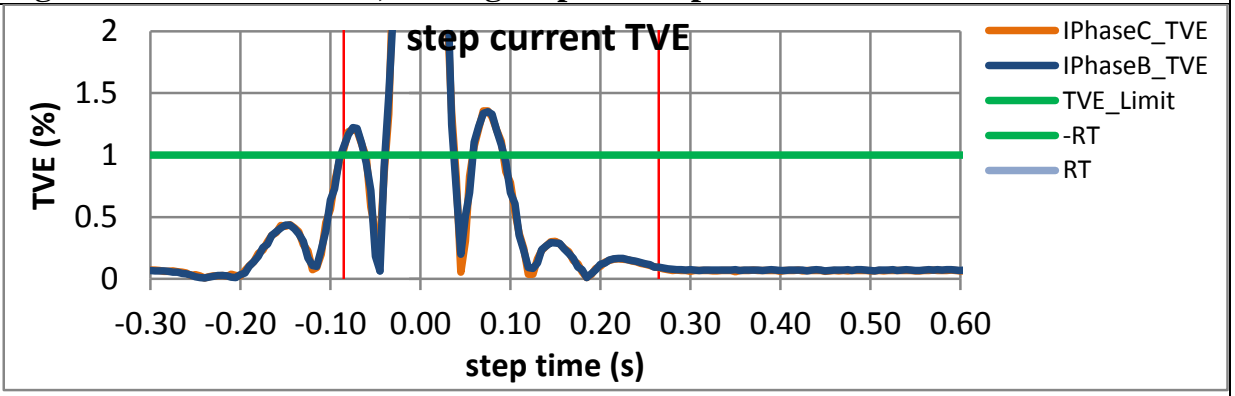

Figure 4184: Fs = 20 FPS, - 10 degree phase step 

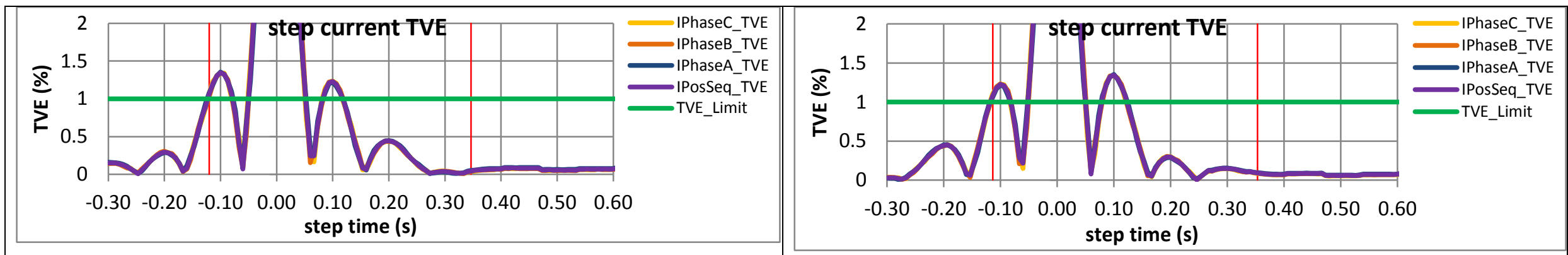

Figure 4185: Fs = 15 FPS, +10 degree phase step

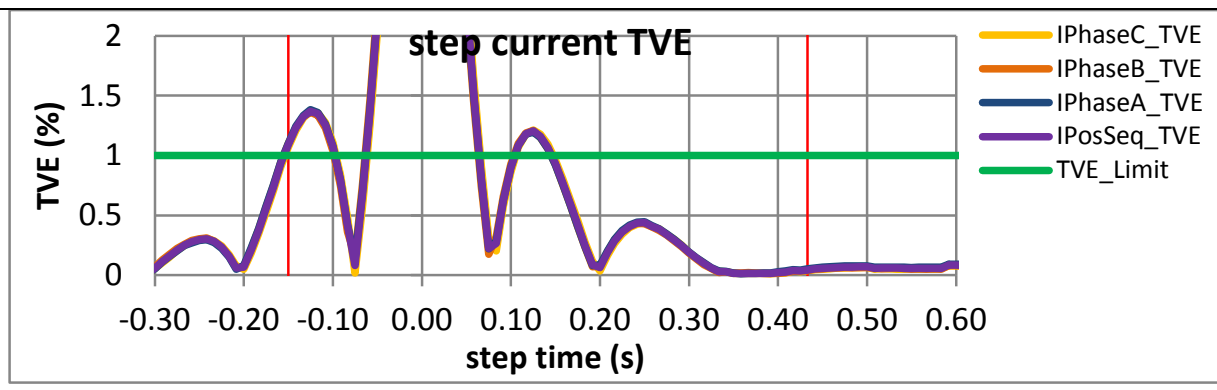

Figure 4186: Fs = 15 FPS, - 10 degree phase step

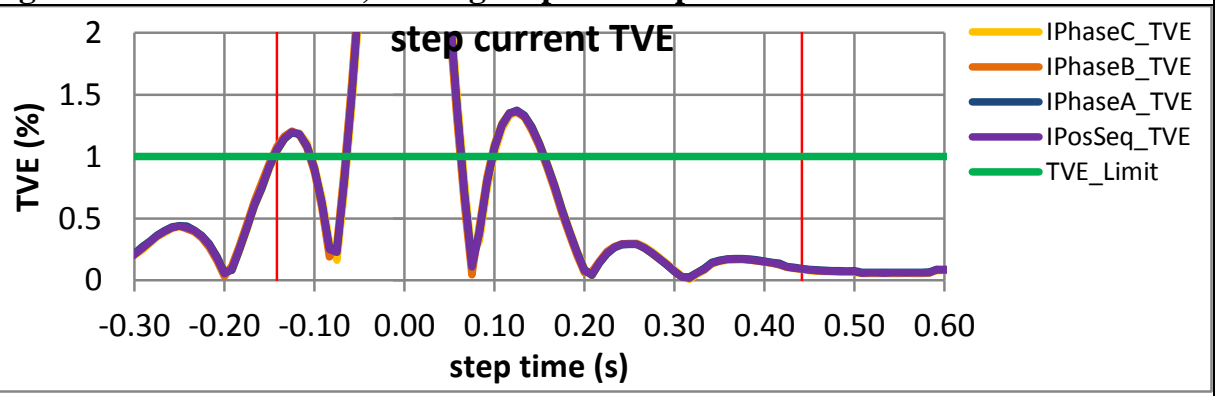

Figure 4187: Fs $=12$ FPS, +10 degree phase step

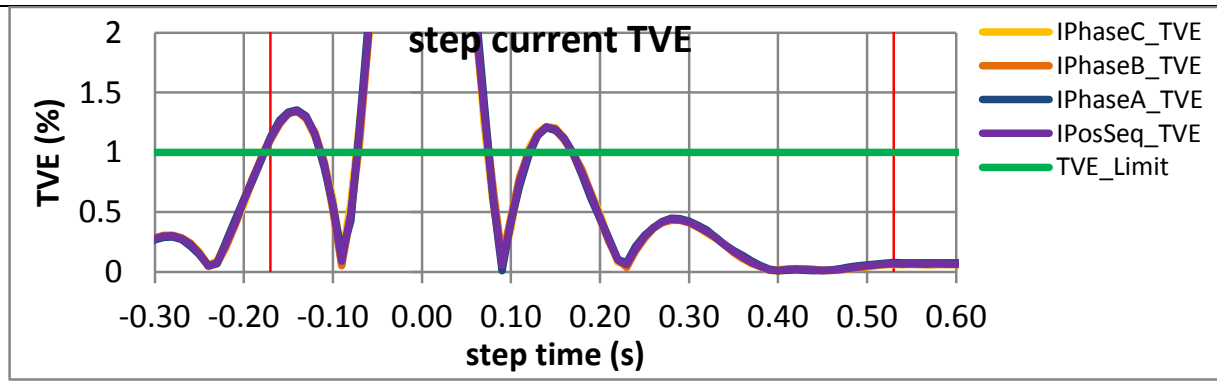

Figure 4189: Fs = 10 FPS, +10 degree phase step

Figure 4188: Fs = 12 FPS, -10 degree phase step

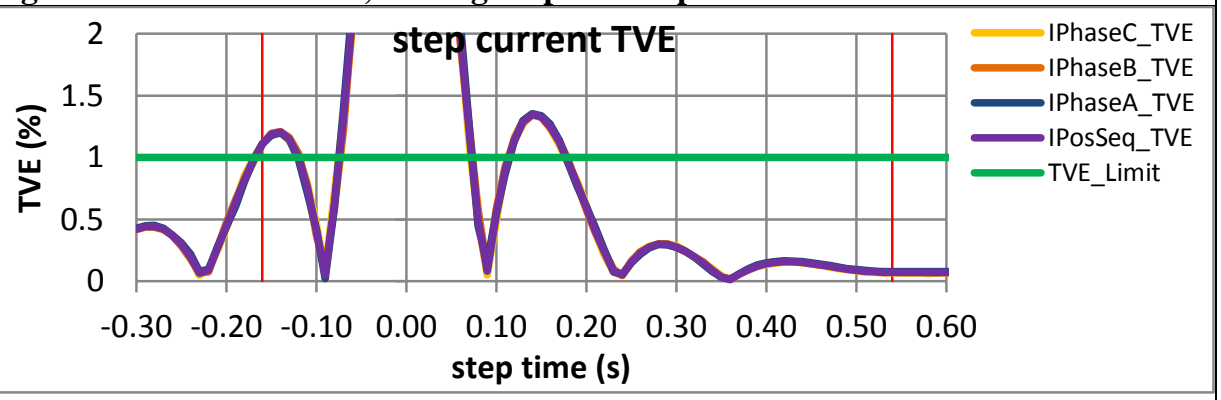

Figure 4190: Fs = 10 FPS, -10 degree phase step 
9.2.10 PMU I dynamic step change in phase current response time: $F 0=60 \mathrm{~Hz}, \mathrm{M}$ class
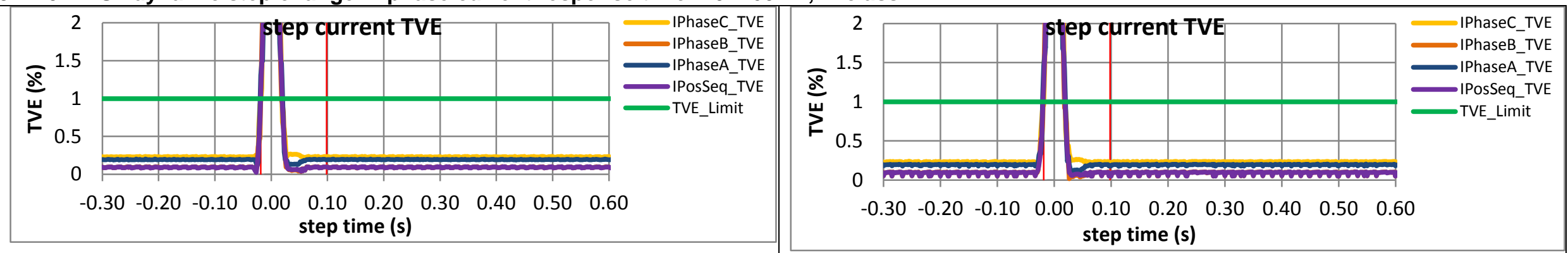

Figure 4191: Fs = 60 FPS, +10 degree phase step

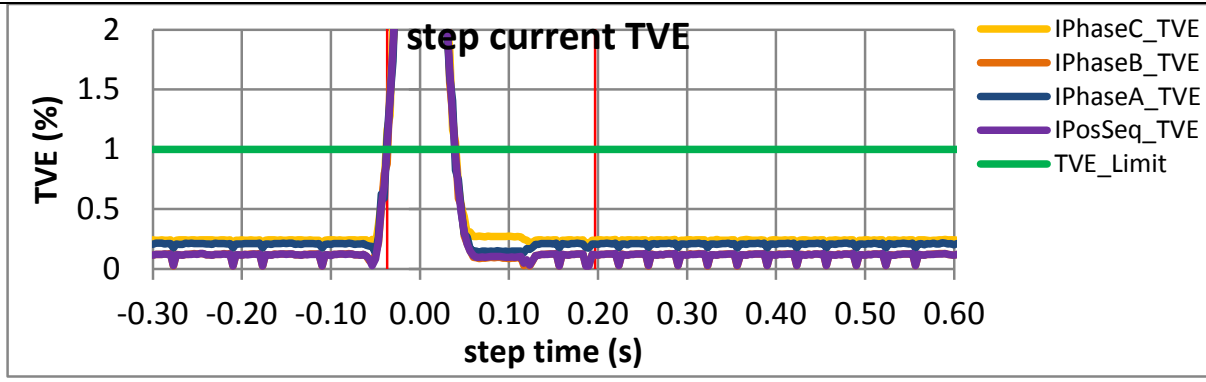

Figure 4192: Fs = 60 FPS, -10 degree phase step

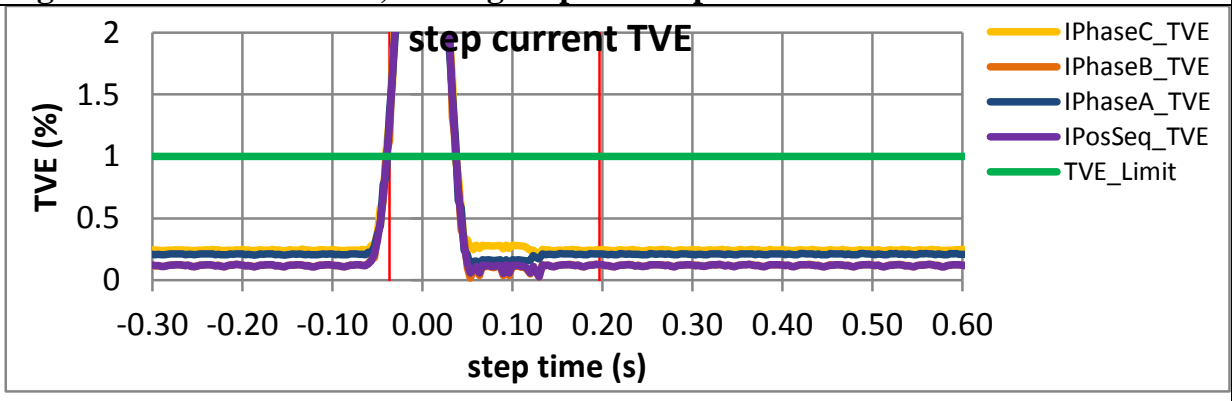

Figure 4193: Fs = 30 FPS, +10 degree phase step

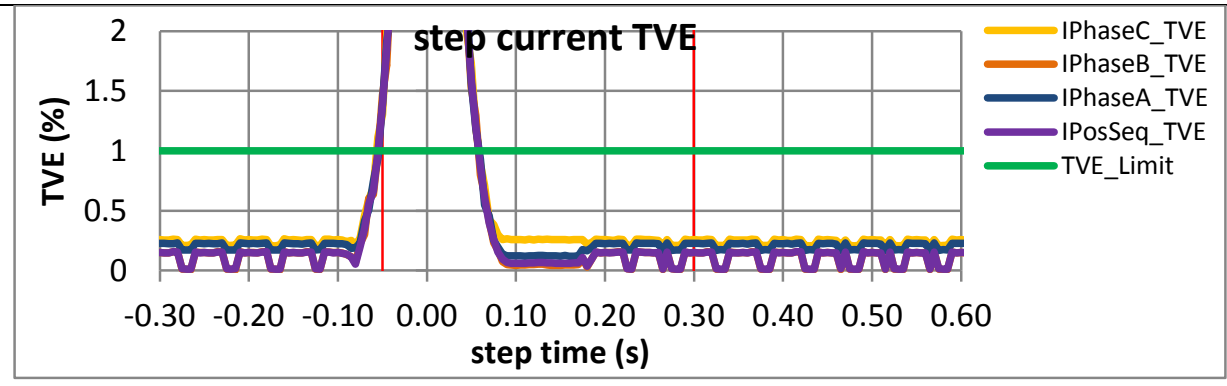

Figure 4194: Fs = 30 FPS, -10 degree phase step

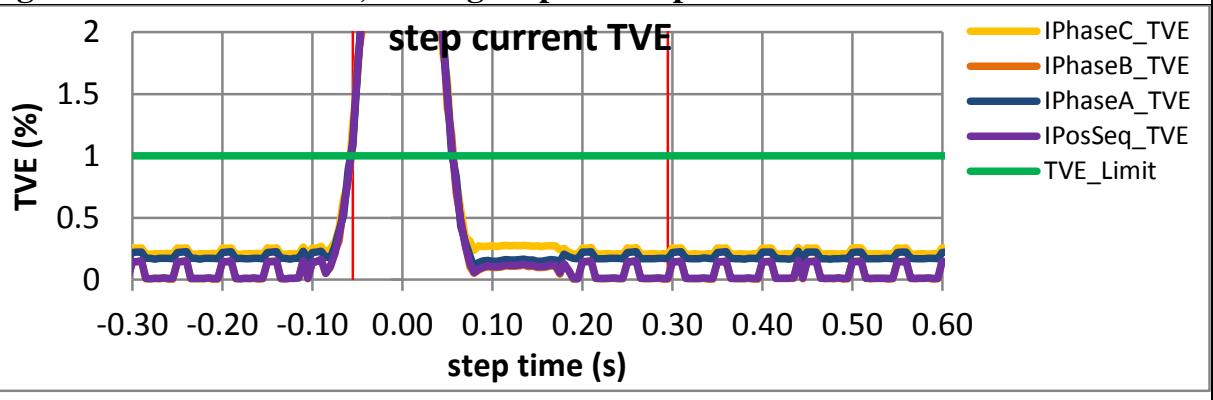

Figure 4195: Fs = 20 FPS, +10 degree phase step

Figure 4196: Fs = 20 FPS, - 10 degree phase step 

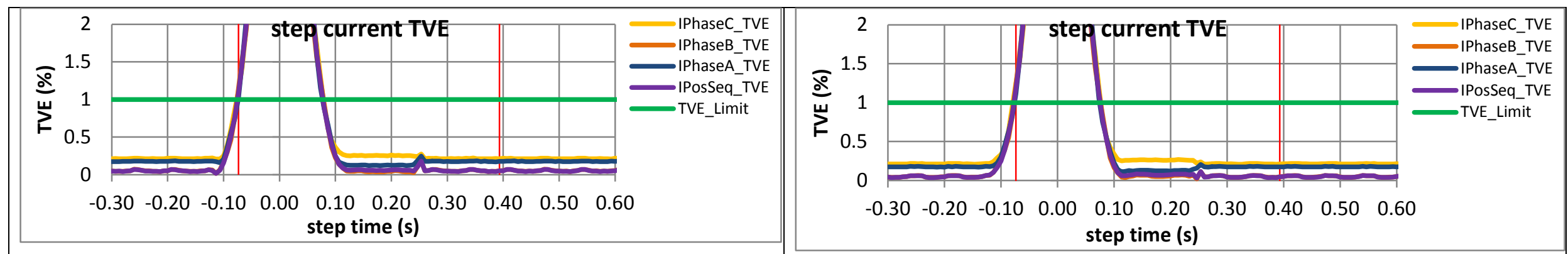

Figure 4197: Fs = 15 FPS, +10 degree phase step

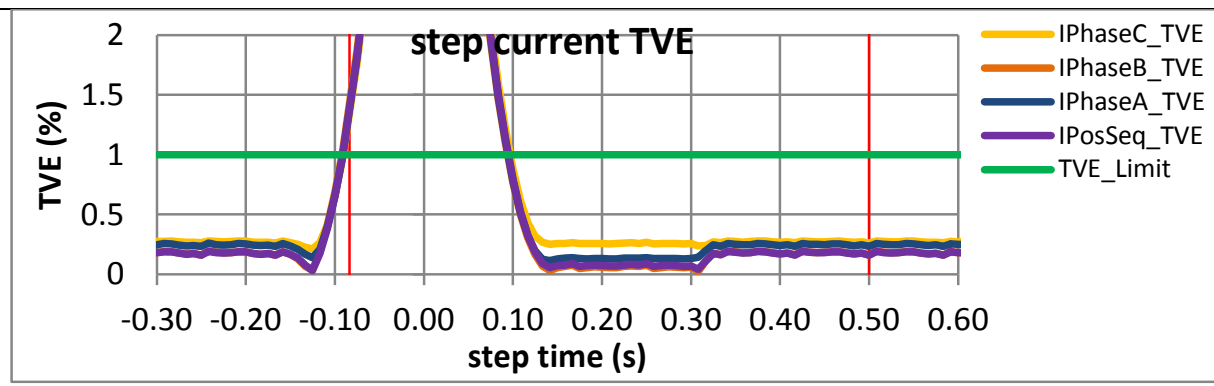

Figure 4198: Fs = 15 FPS, -10 degree phase step

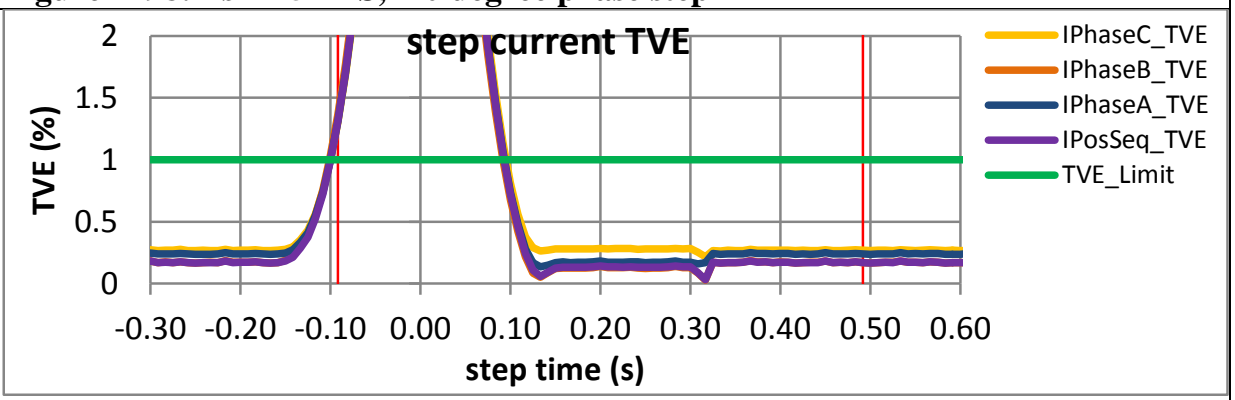

Figure 4199: Fs = 12 FPS, +10 degree phase step

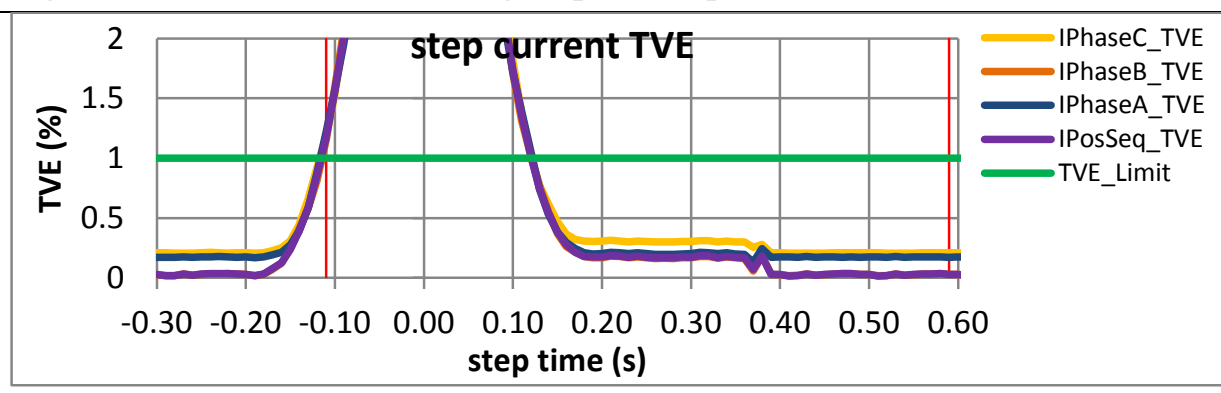

Figure 4200: Fs = 12 FPS, -10 degree phase step

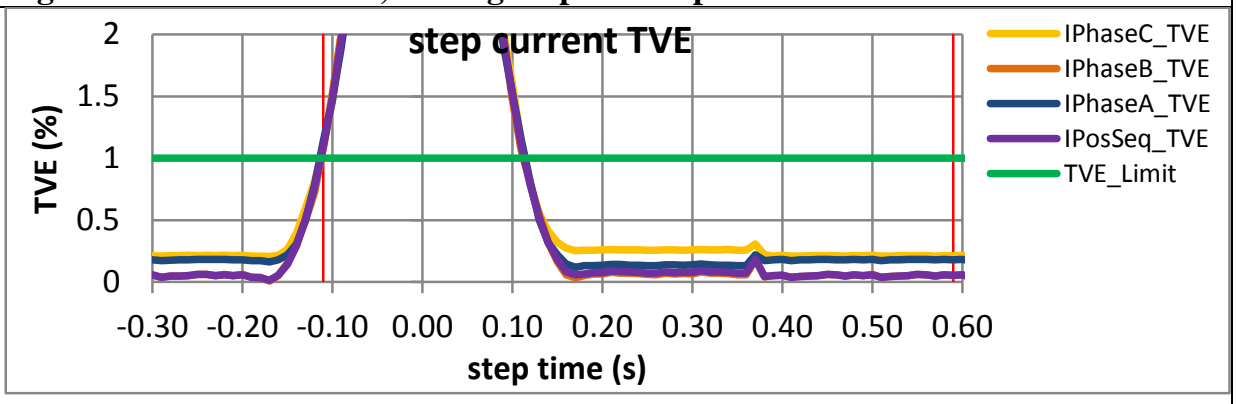

Figure 4202: Fs $=10 \mathrm{FPS},-10$ degree phase step 
9.2.11 PMU J dynamic step change in phase current response time: $\mathrm{F} 0=60 \mathrm{~Hz}, \mathrm{M}$ class

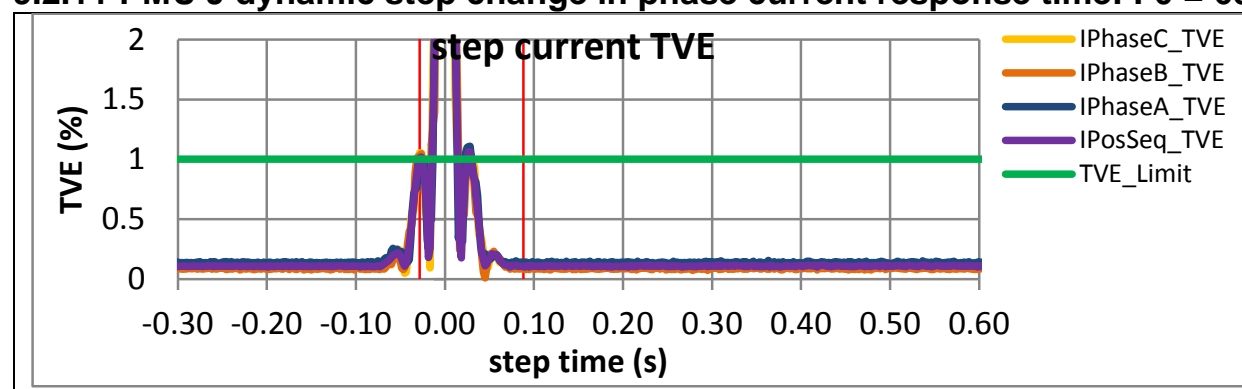

Figure 4203: Fs = $60 \mathrm{FPS},+10$ degree phase step

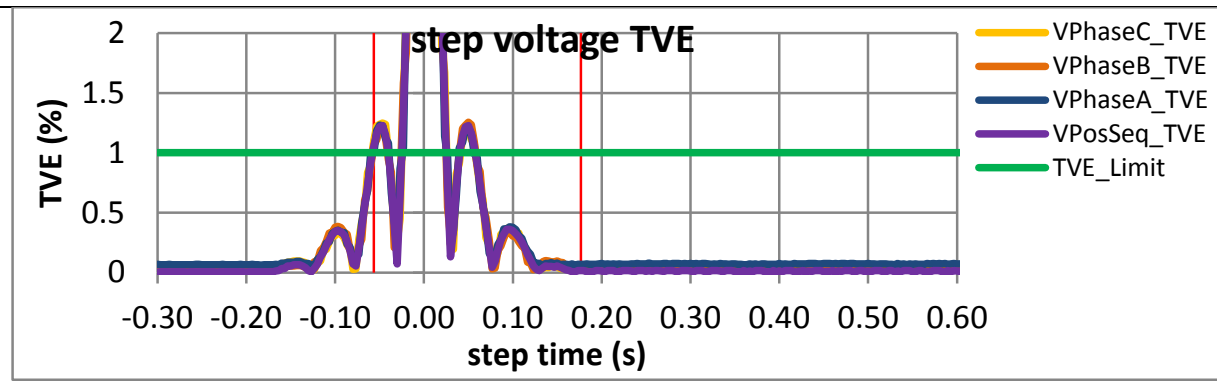

Figure 4205: Fs = 30 FPS, +10 degree phase step

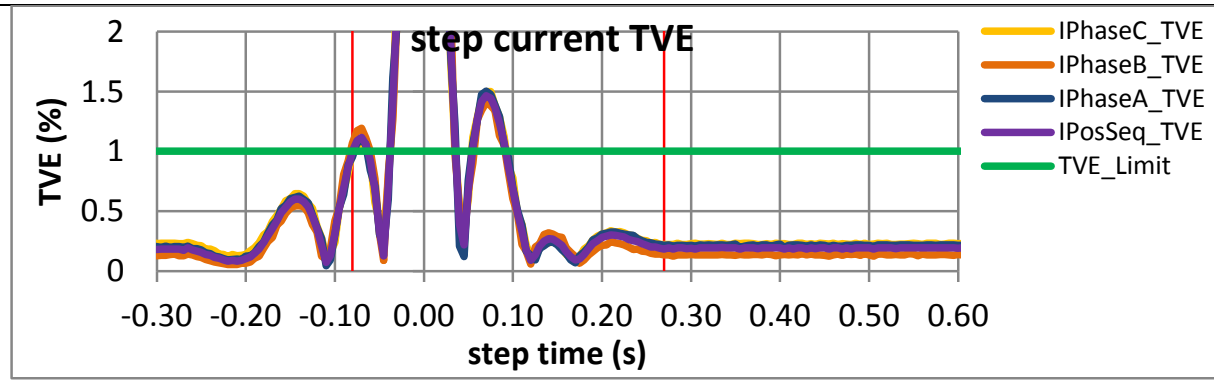

Figure 4207: Fs = 20 FPS, +10 degree phase step

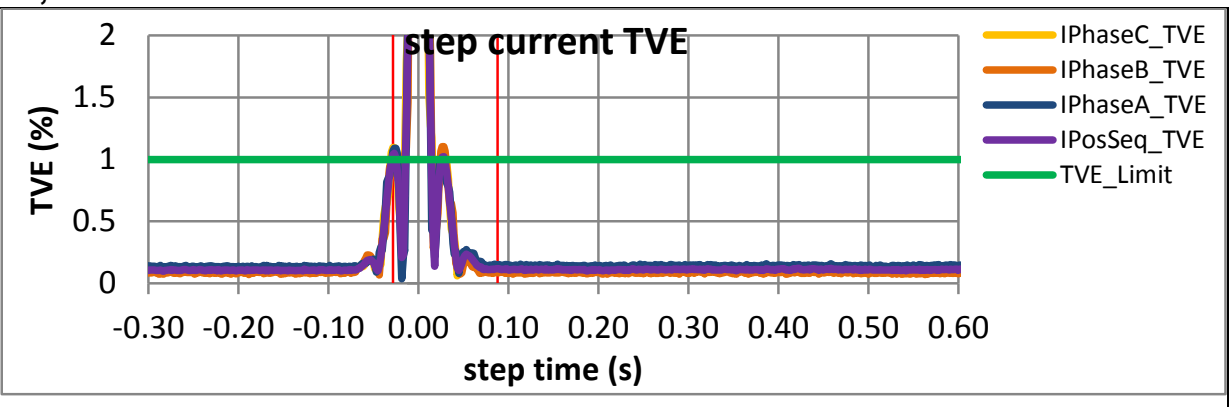

Figure 4204: Fs = 60 FPS, -10 degree phase step

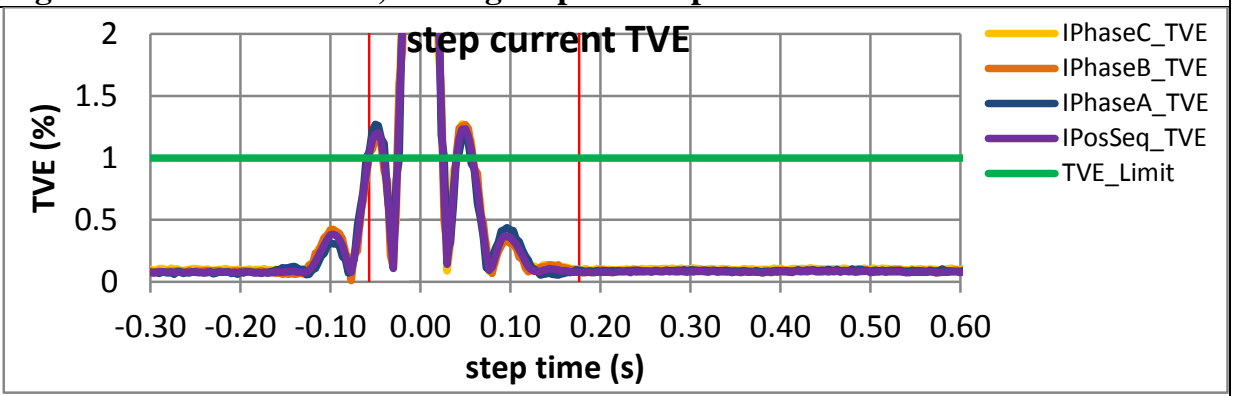

Figure 4206: Fs = 30 FPS, -10 degree phase step

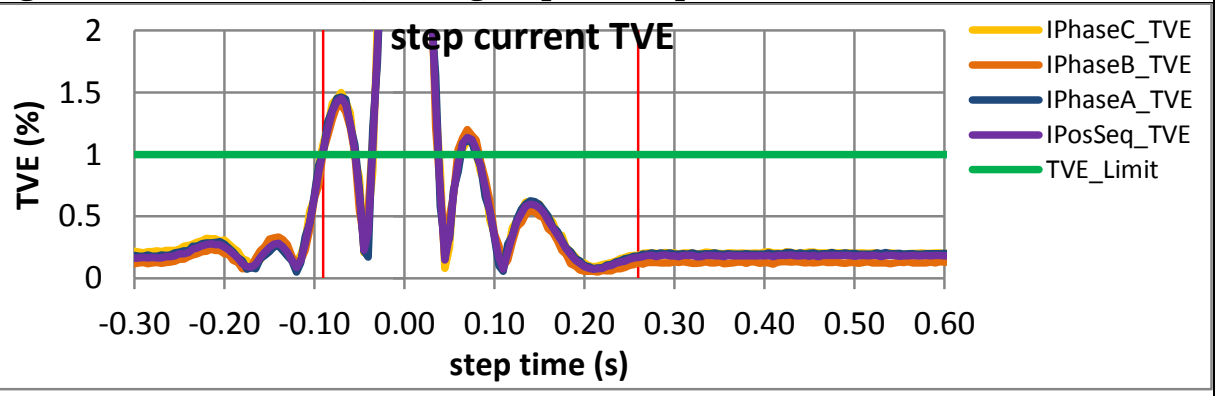

Figure 4208: Fs = 20 FPS, - 10 degree phase step 

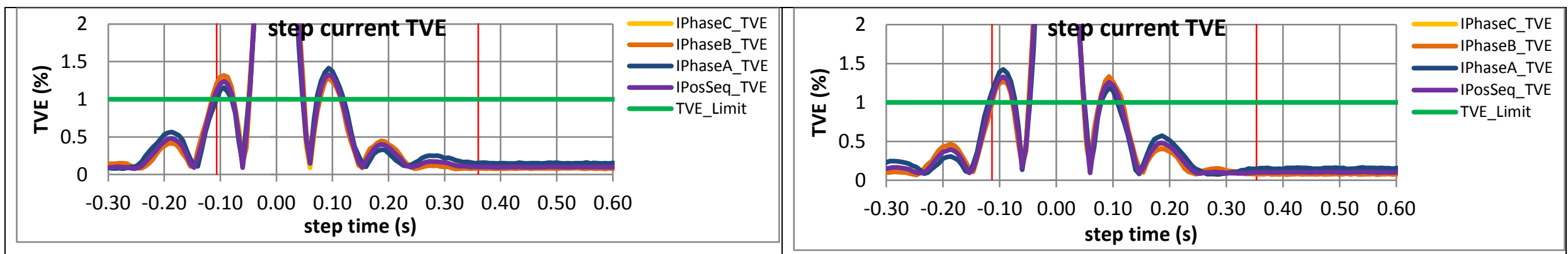

Figure 4209: Fs = 15 FPS, +10 degree phase step

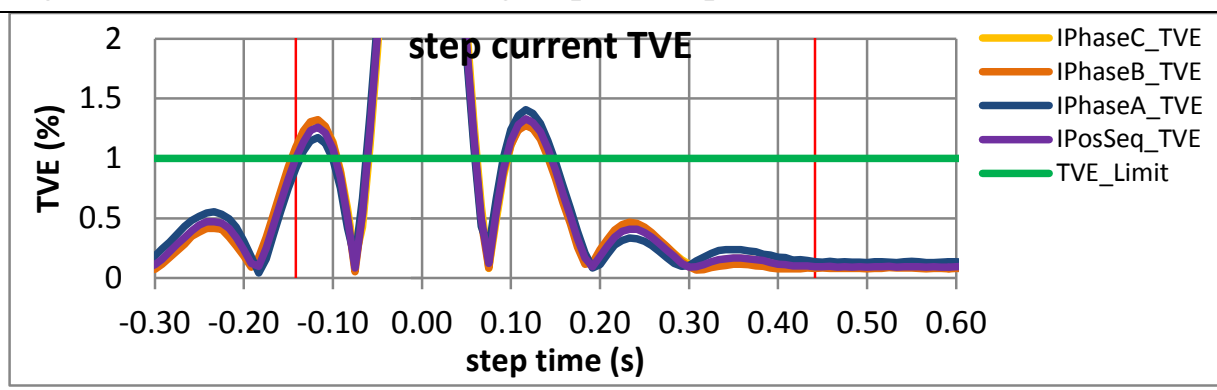

Figure 4210: Fs = 15 FPS, -10 degree phase step

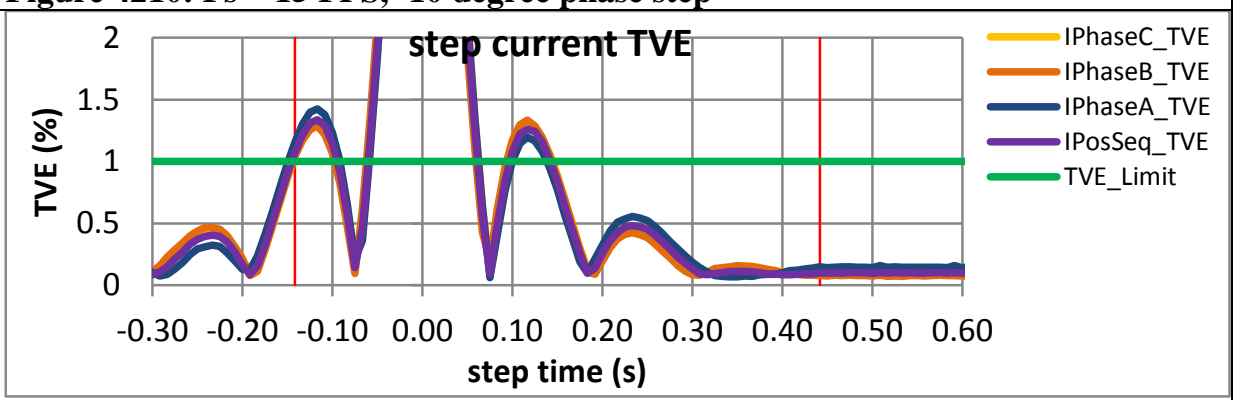

Figure 4211: Fs = 12 FPS, +10 degree phase step

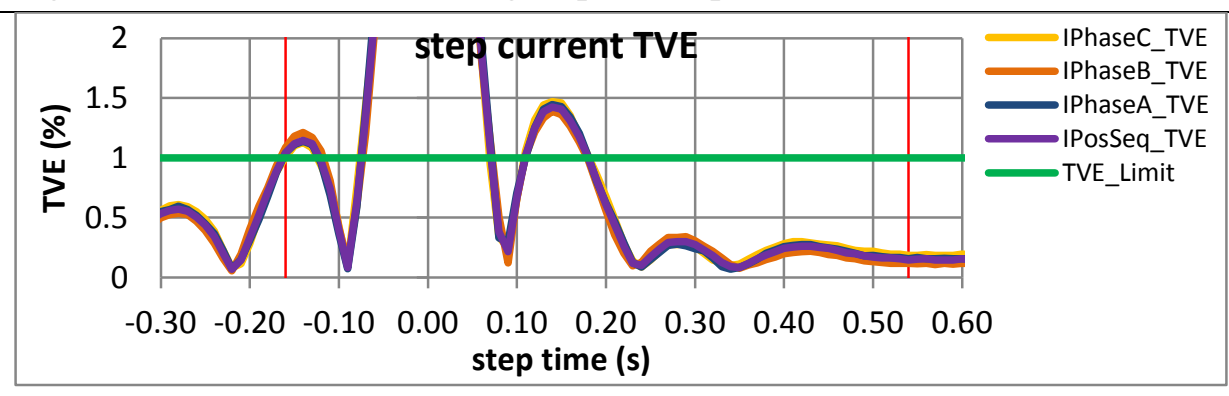

Figure 4212: Fs = 12 FPS, -10 degree phase step

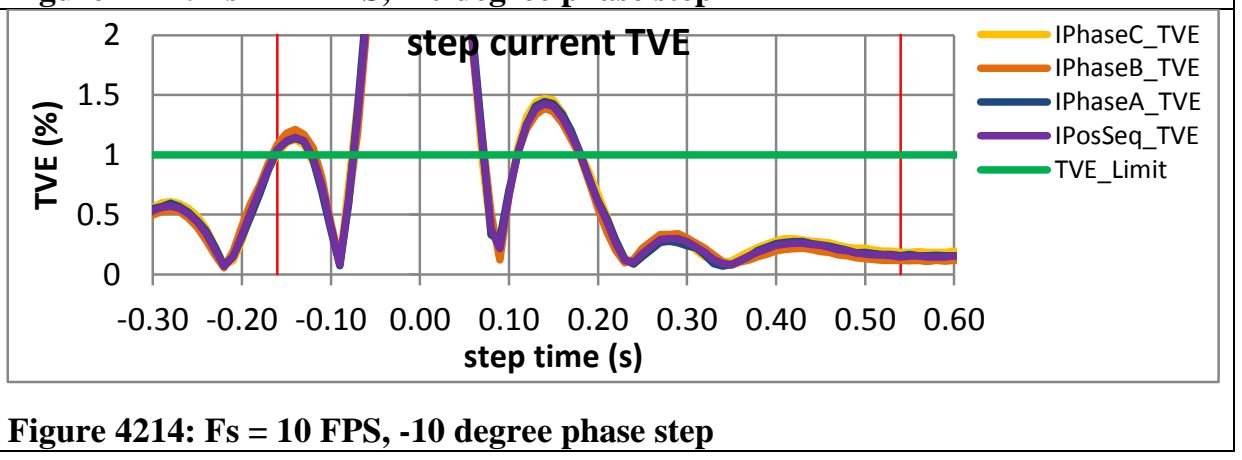

Figure 4213: Fs = 10 FPS, +10 degree phase step

Figure 4214: Fs = 10 FPS, -10 degree phase step 


\subsection{Dynamic step change in phase voltage response time: $\mathrm{F} 0=60 \mathrm{~Hz}, \mathrm{P}$ Class}

\subsubsection{C37.118.1a Annex $C$ dynamic step change in phase voltage response time: $F 0=60 \mathrm{~Hz}, \mathrm{P}$ class}
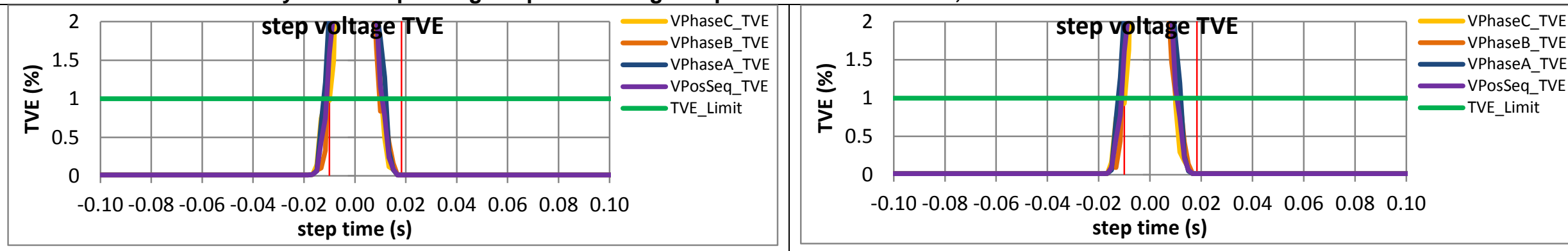

Figure 4215: Fs = 60 FPS, +10 degree phase step

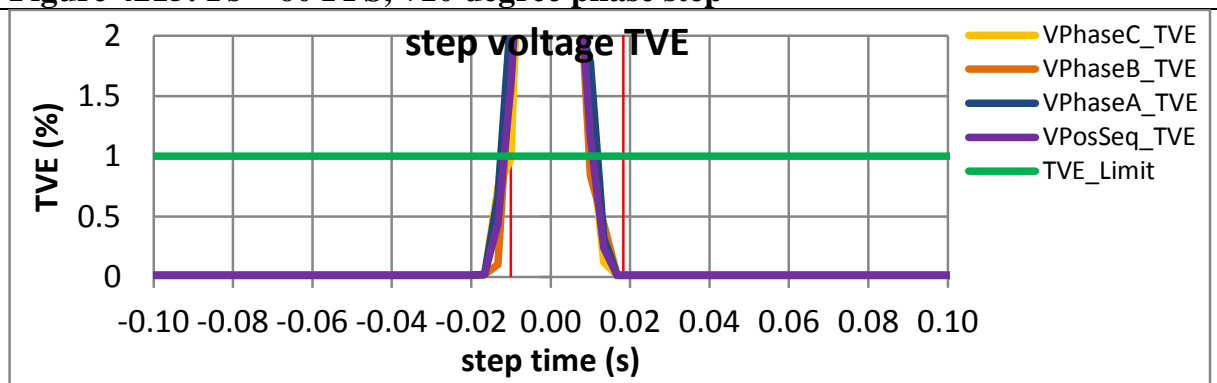

Figure 4216: Fs = 60 FPS, -10 degree phase step

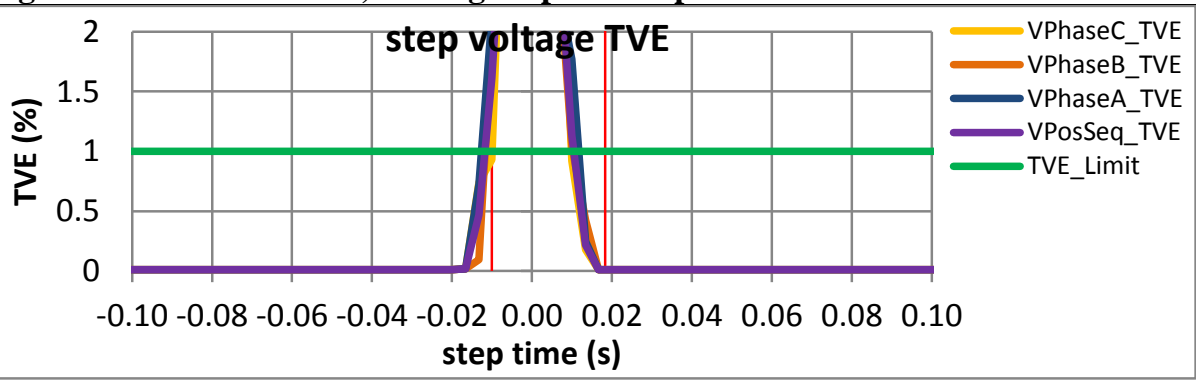

Figure 4217: Fs = 30 FPS, +10 degree phase step

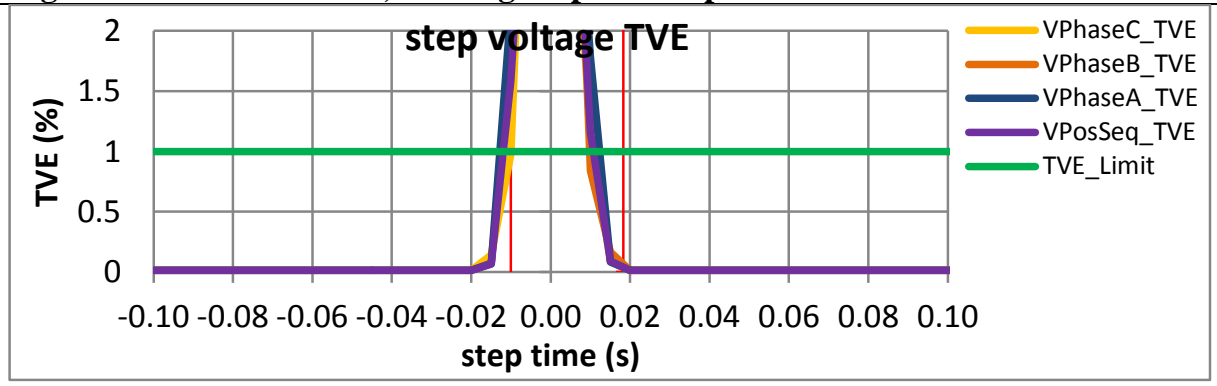

Figure 4218: Fs = 30 FPS, -10 degree phase step

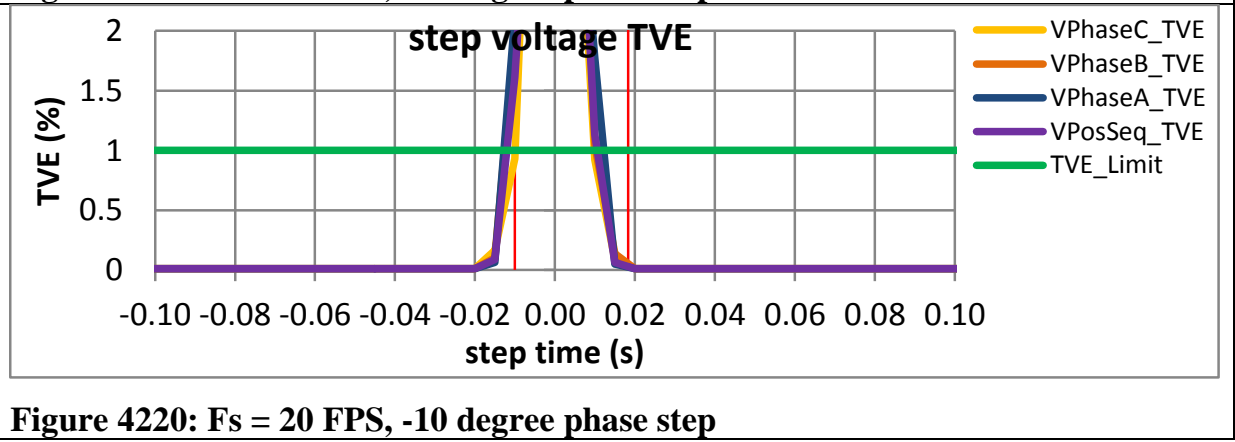

Figure 4219: Fs = 20 FPS, +10 degree phase step

Figure 4220: Fs = 20 FPS, -10 degree phase step 


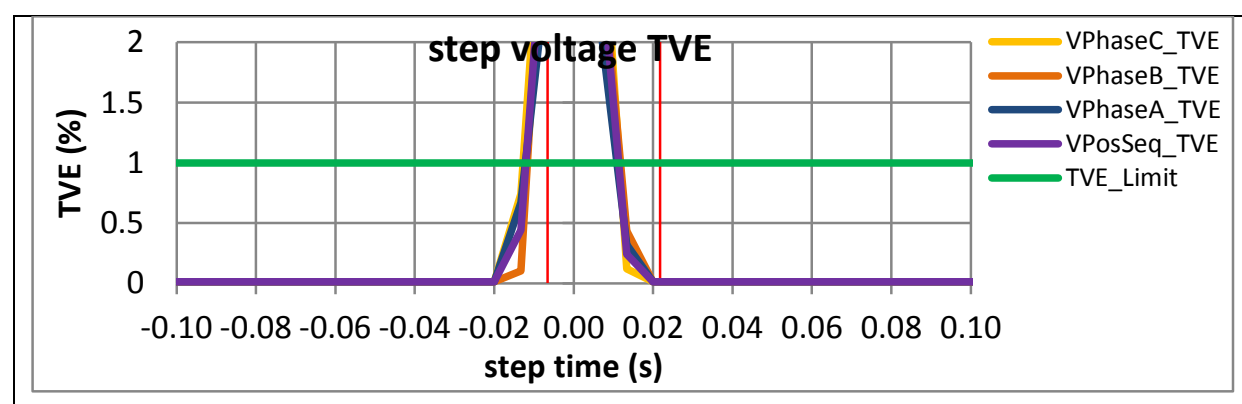

Figure 4221: Fs = 15 FPS, +10 degree phase step
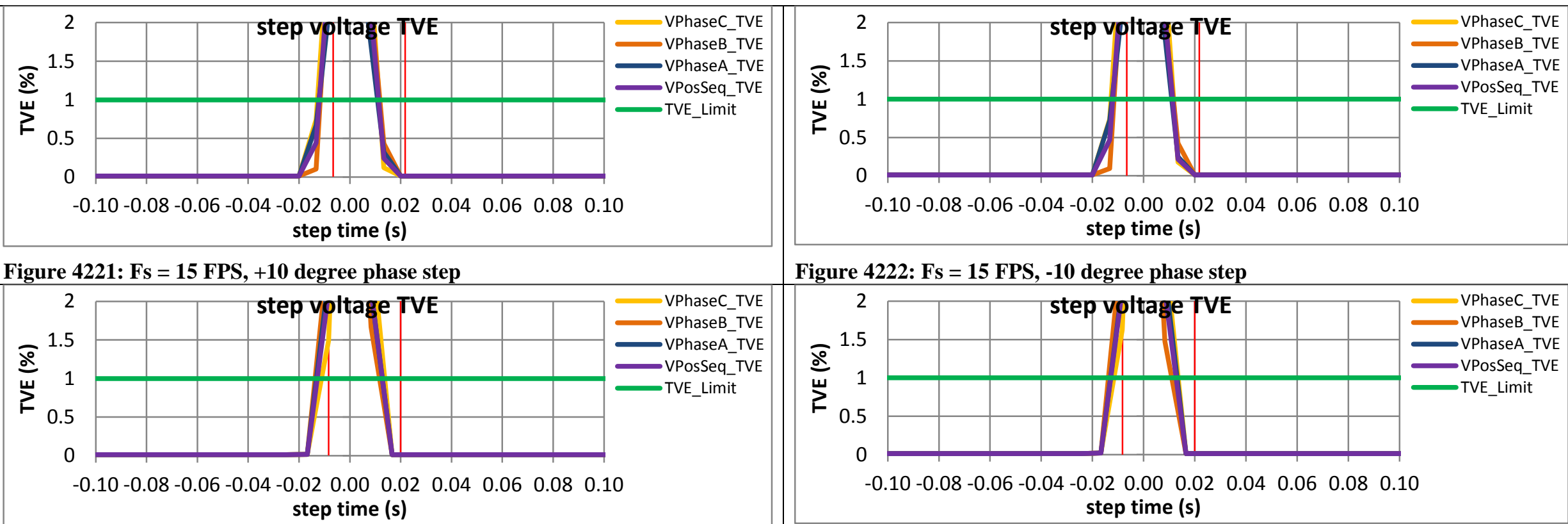

Figure 4222: Fs = 15 FPS, -10 degree phase step

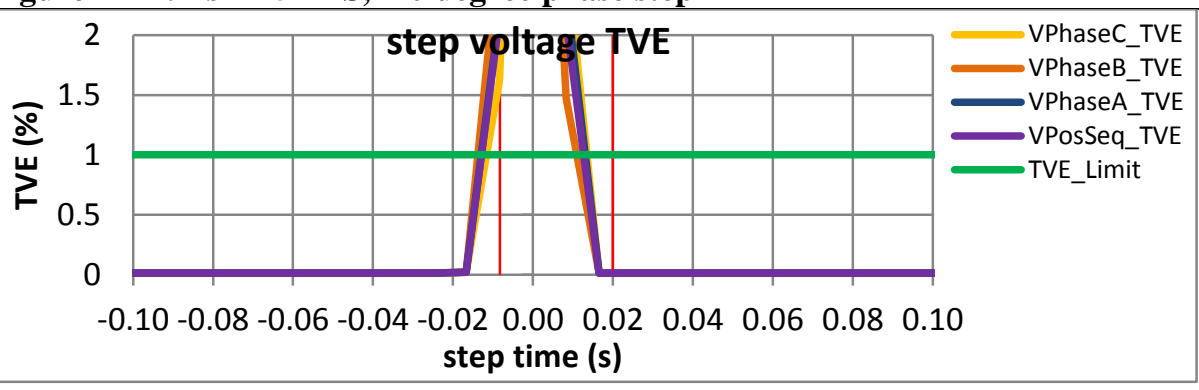

Figure 4223: Fs = 12 FPS, +10 degree phase step

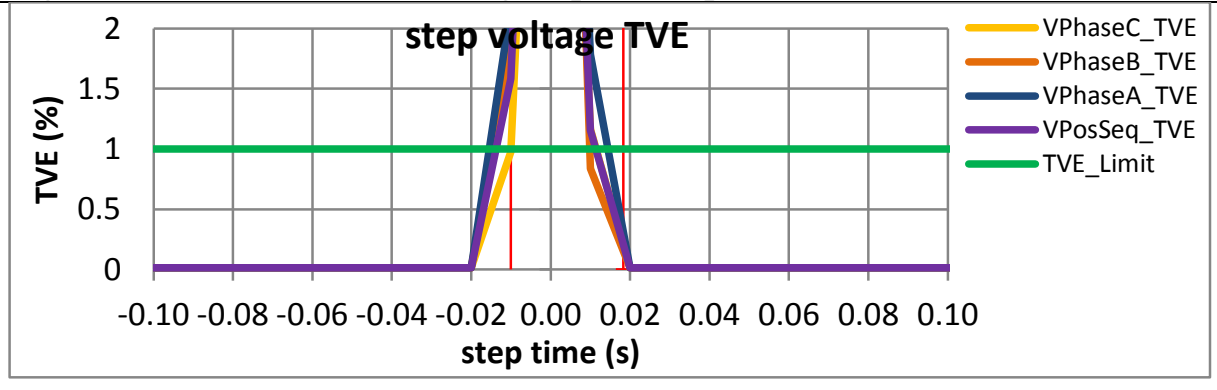

Figure 4224: Fs = 12 FPS, -10 degree phase step

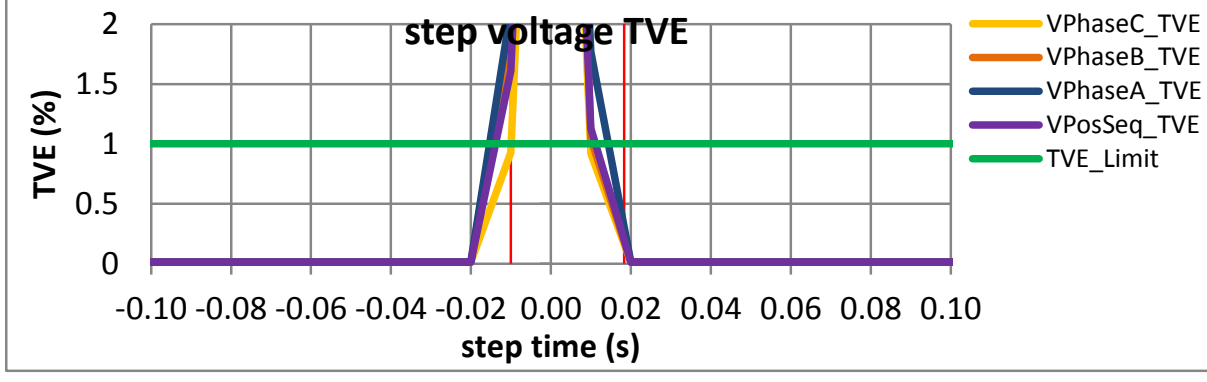

Figure 4226: Fs = 10 FPS, -10 degree phase step 
9.3.2 PMU A dynamic step change in phase voltage response time: $\mathrm{F} 0=60 \mathrm{~Hz}, \mathrm{P}$ class
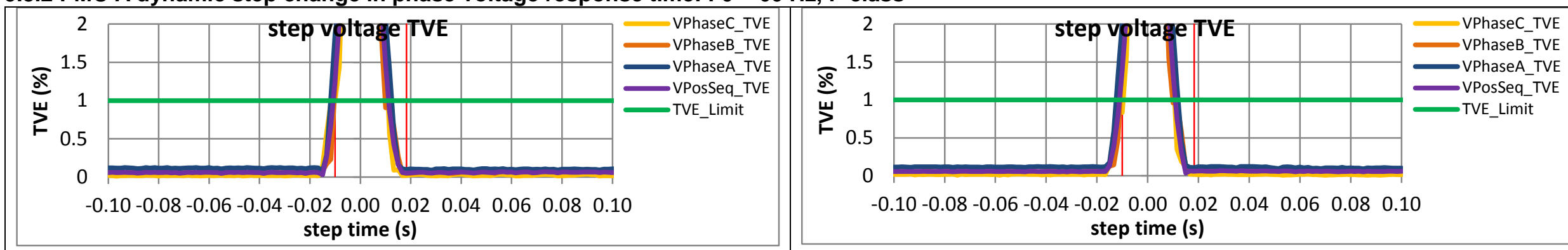

Figure 4227: Fs = 60 FPS, +10 degree phase step

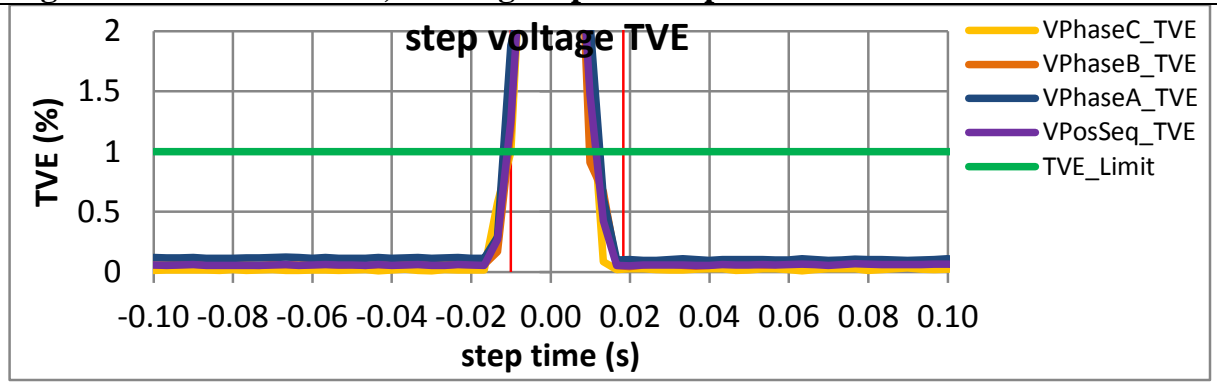

Figure 4228: Fs = 60 FPS, -10 degree phase step

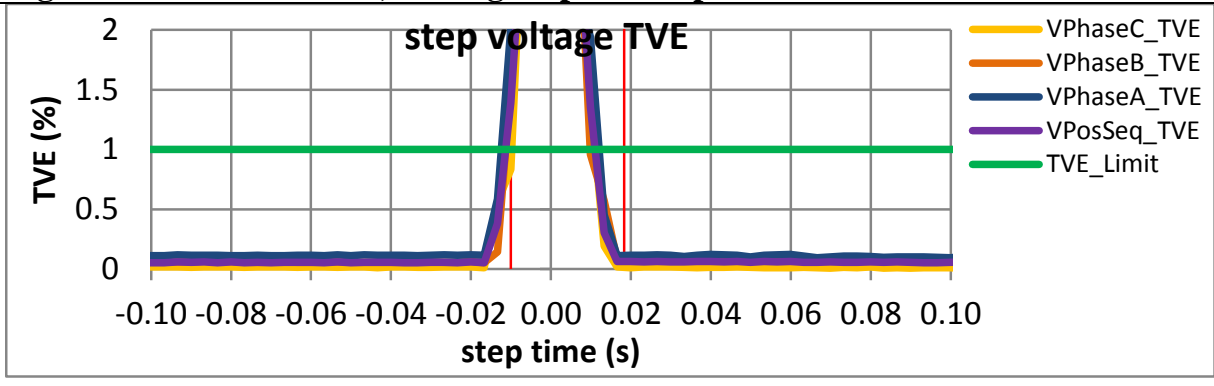

Figure 4229: Fs = 30 FPS, +10 degree phase step

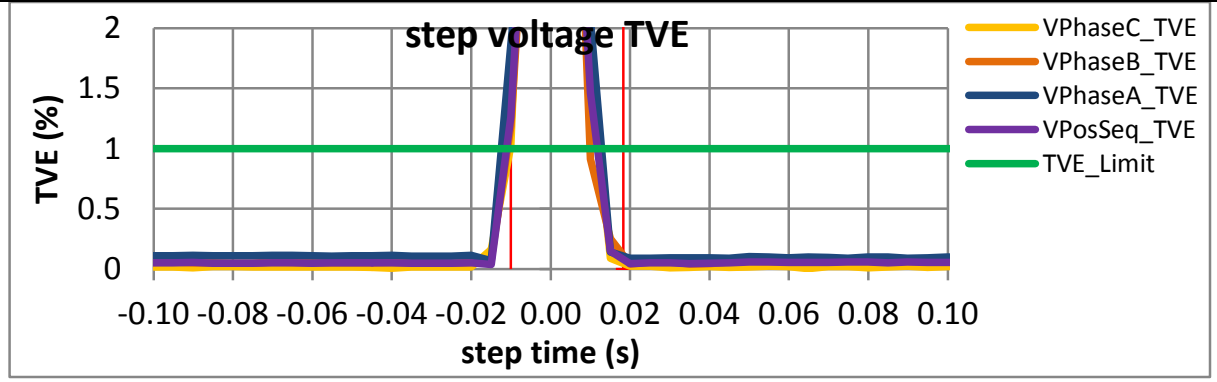

Figure 4231: Fs = 20 FPS, +10 degree phase step

Figure 4230: Fs = 30 FPS, -10 degree phase step

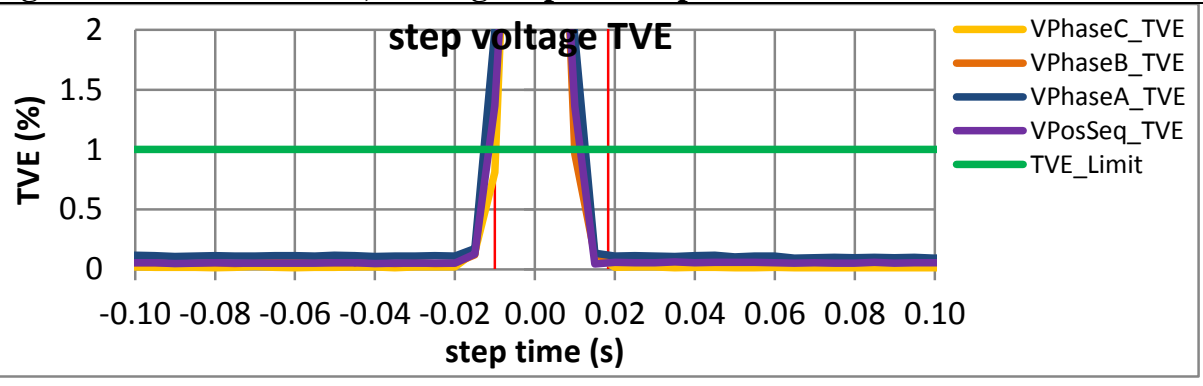

Figure 4232: Fs = 20 FPS, -10 degree phase step 


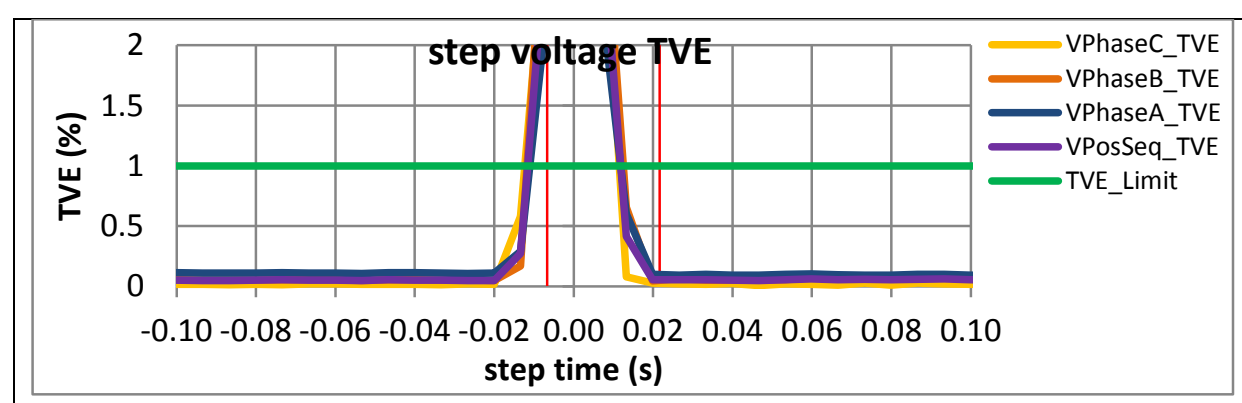

Figure 4233: Fs = 15 FPS, +10 degree phase step
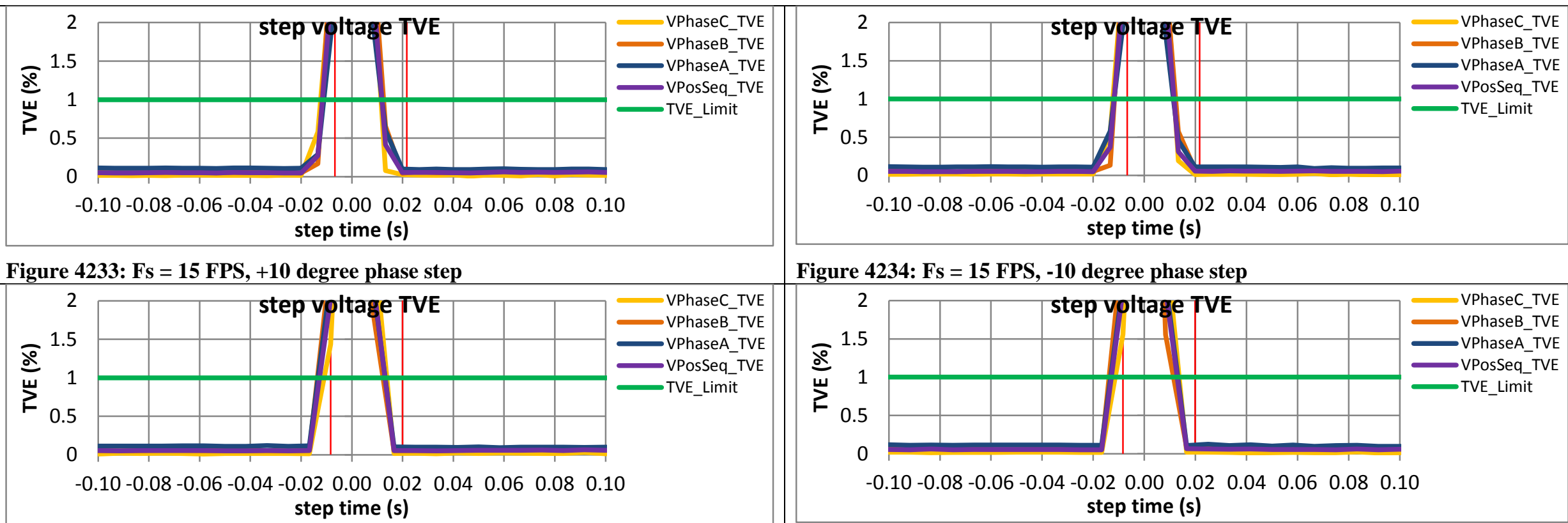

Figure 4234: Fs = 15 FPS, -10 degree phase step

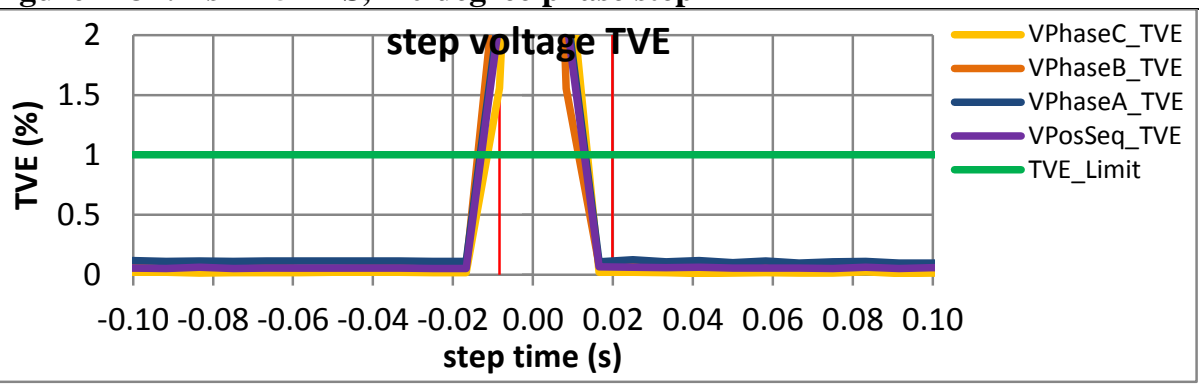

Figure 4235: Fs = 12 FPS, +10 degree phase step

Figure 4236: Fs = 12 FPS, -10 degree phase step

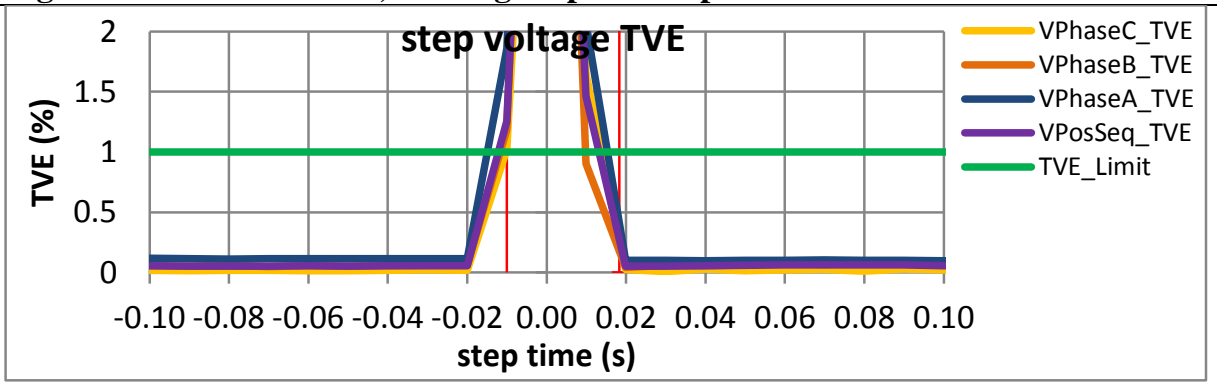

Figure 4237: Fs = 10 FPS, +10 degree phase step

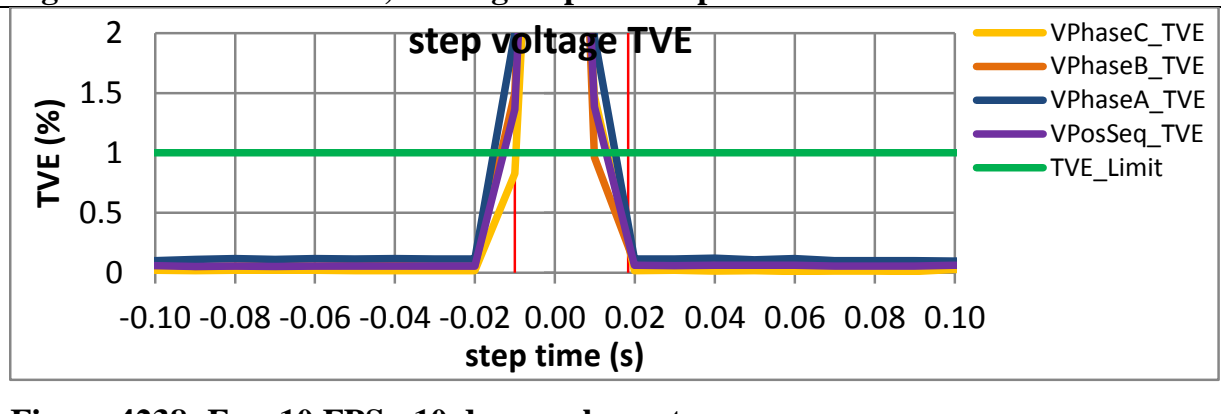

Figure 4238: Fs = 10 FPS, -10 degree phase step 
9.3.3 PMU B dynamic step change in phase voltage response time: $\mathrm{F} 0=60 \mathrm{~Hz}, \mathrm{P}$ class
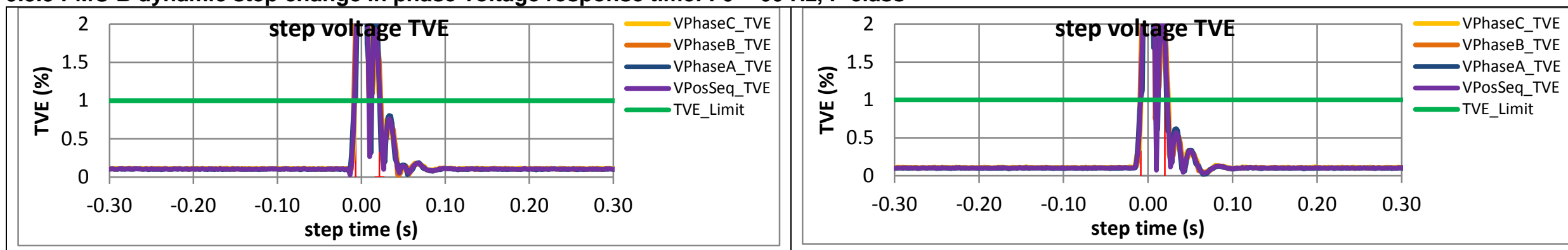

Figure 4239: Fs = 60 FPS, +10 degree phase step

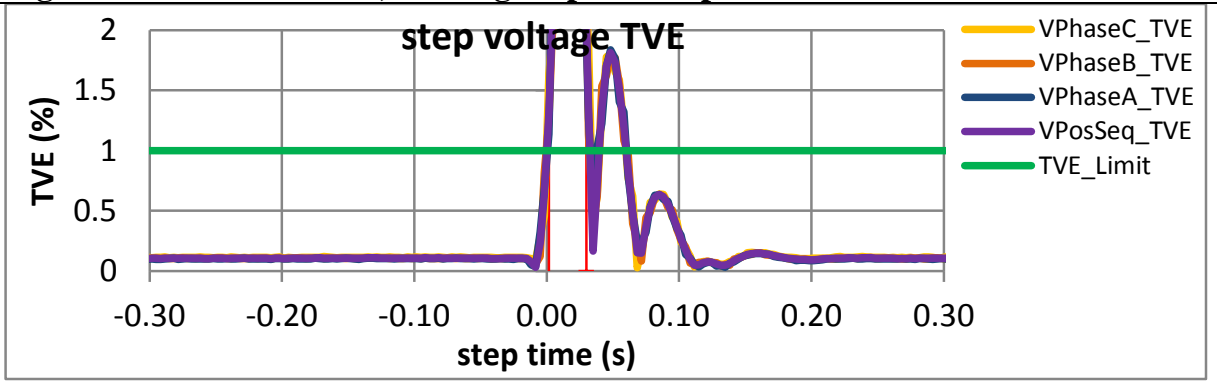

Figure 4240: Fs = 60 FPS, -10 degree phase step

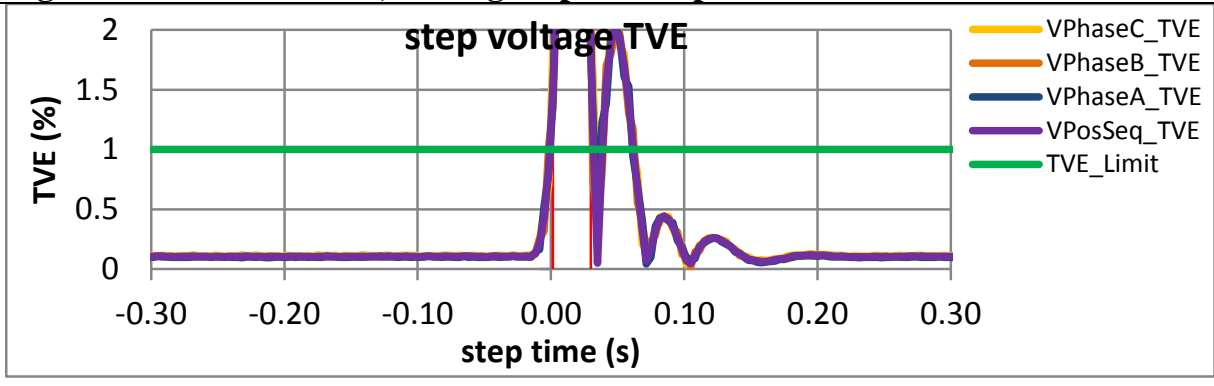

Figure 4241: Fs = 30 FPS, +10 degree phase step

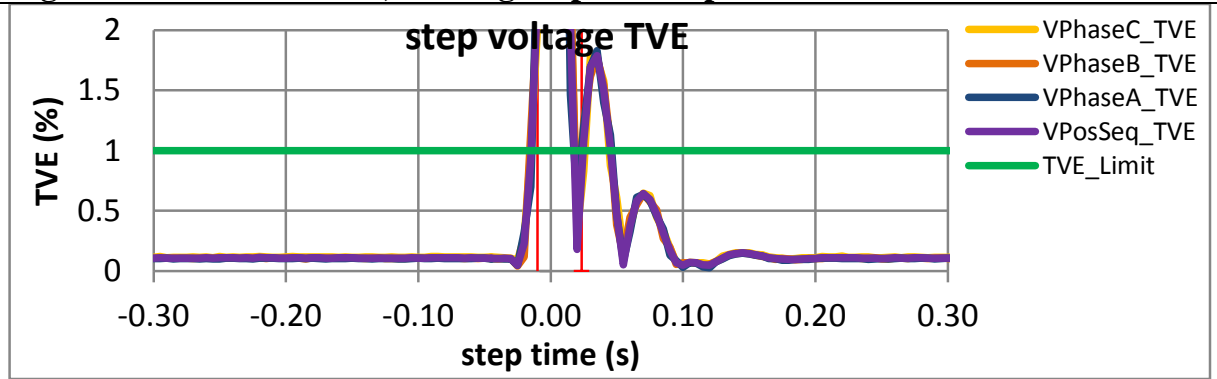

Figure 4243: Fs = 20 FPS, +10 degree phase step

Figure 4242: Fs = 30 FPS, -10 degree phase step

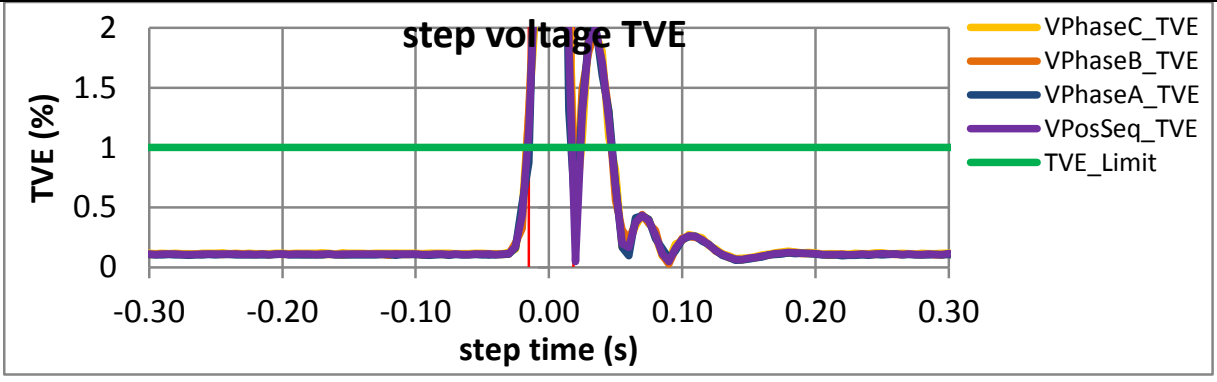

Figure 4244: Fs = 20 FPS, -10 degree phase step 

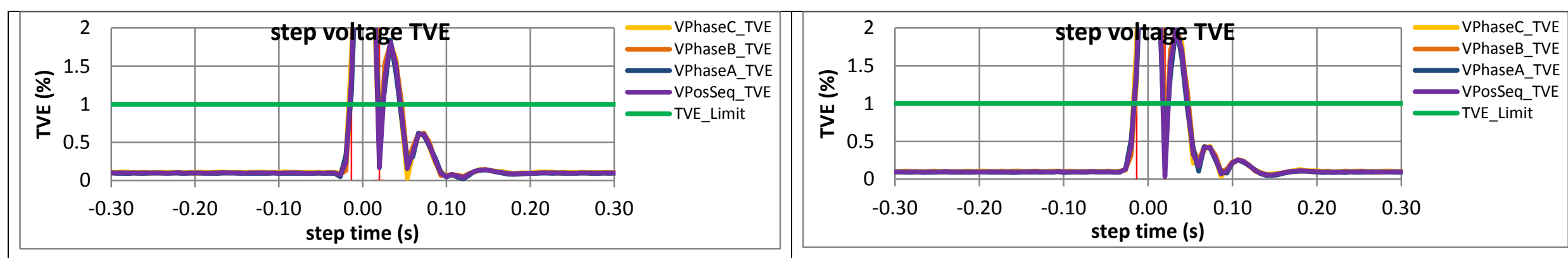

Figure 4245: Fs = 15 FPS, +10 degree phase step

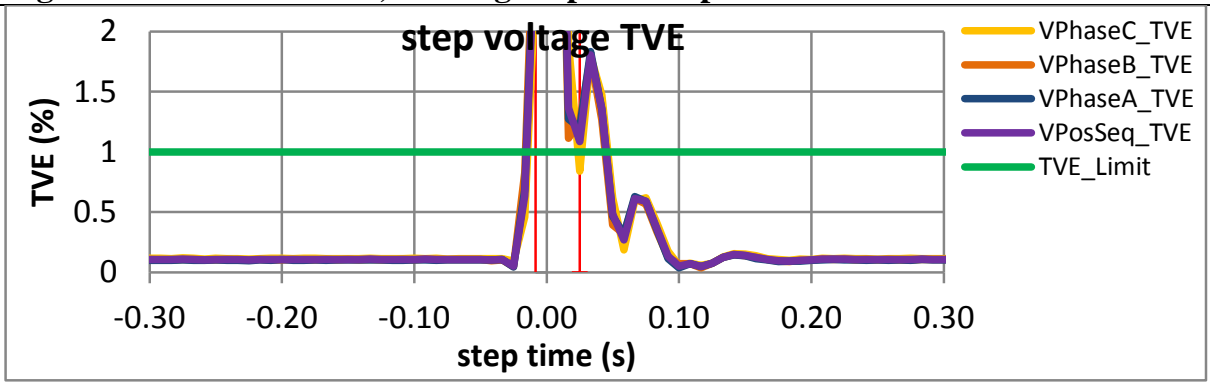

Figure 4246: Fs = 15 FPS, -10 degree phase step

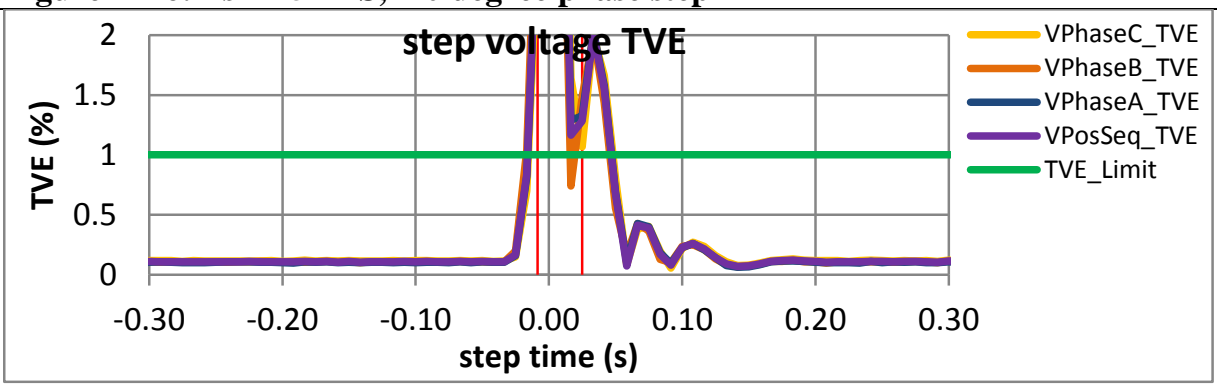

Figure 4247: Fs = 12 FPS, +10 degree phase step

Figure 4248: Fs = 12 FPS, -10 degree phase step

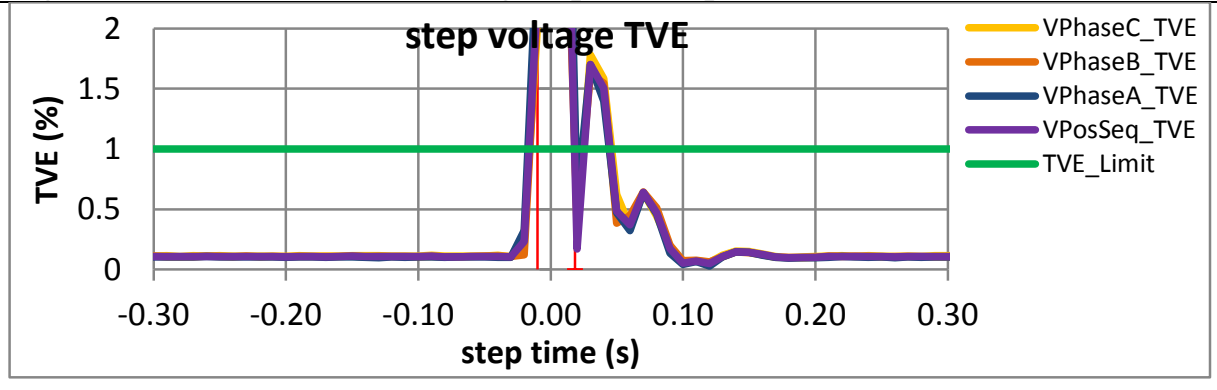

Figure 4249: Fs = 10 FPS, +10 degree phase step

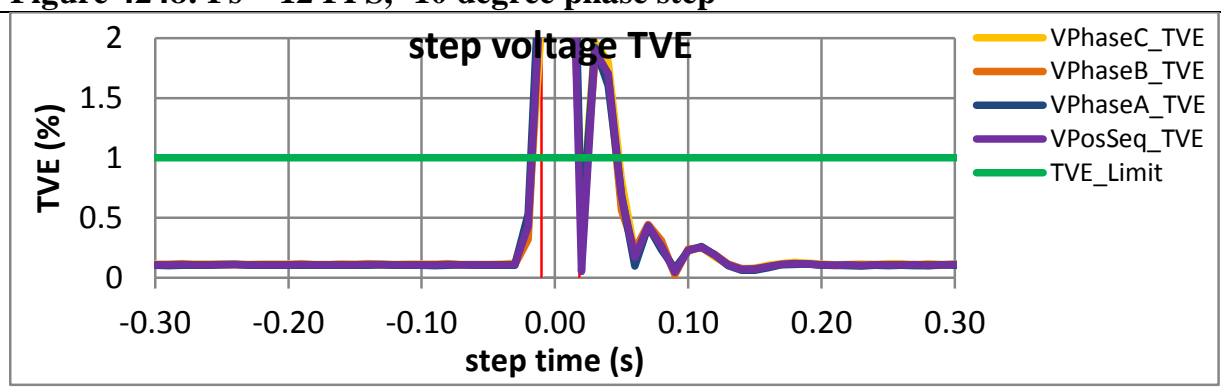

Figure 4250: Fs = 10 FPS, -10 degree phase step 
9.3.4 PMU C dynamic step change in phase voltage response time: $\mathrm{F} 0=60 \mathrm{~Hz}, \mathrm{P}$ class
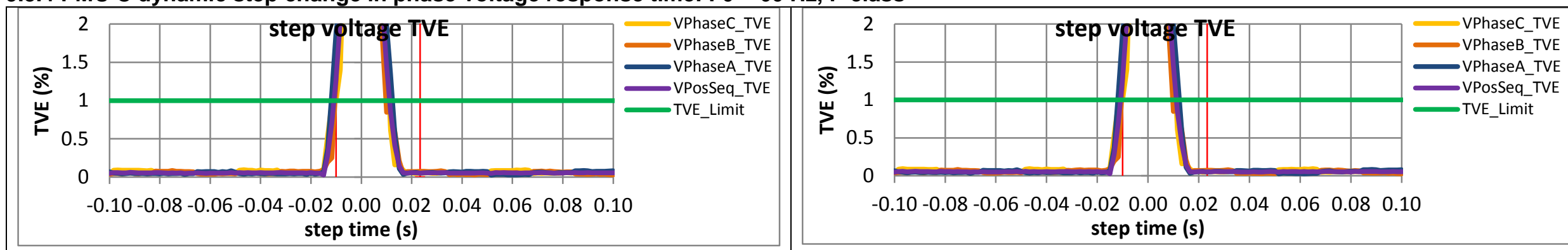

Figure 4251: Fs = 60 FPS, +10 degree phase step

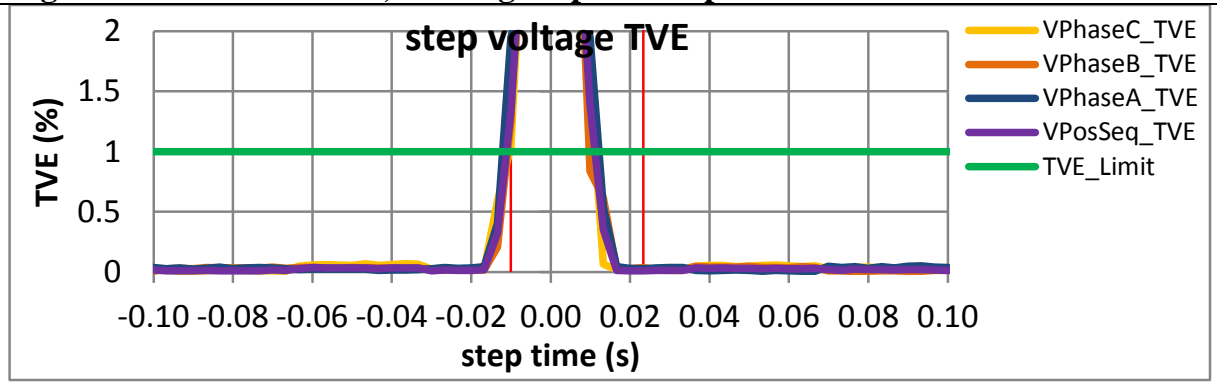

Figure 4252: Fs = 60 FPS, -10 degree phase step

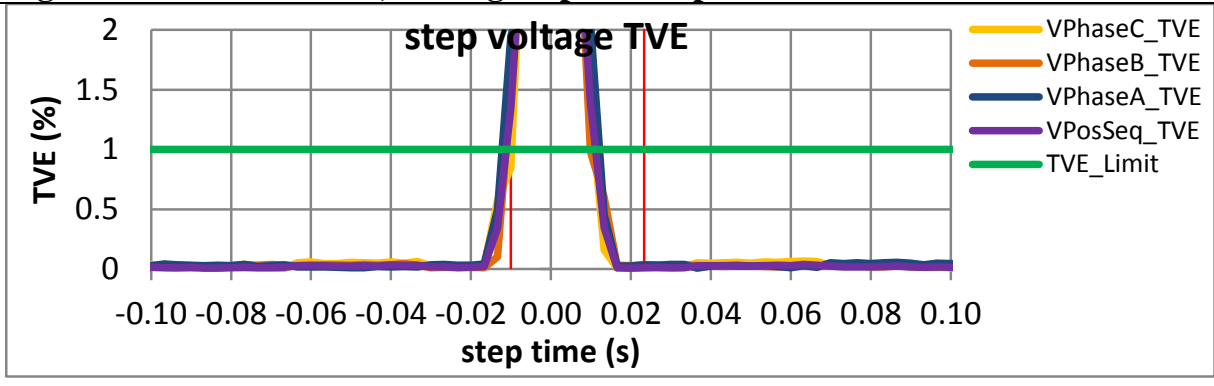

Figure 4253: Fs = 30 FPS, +10 degree phase step

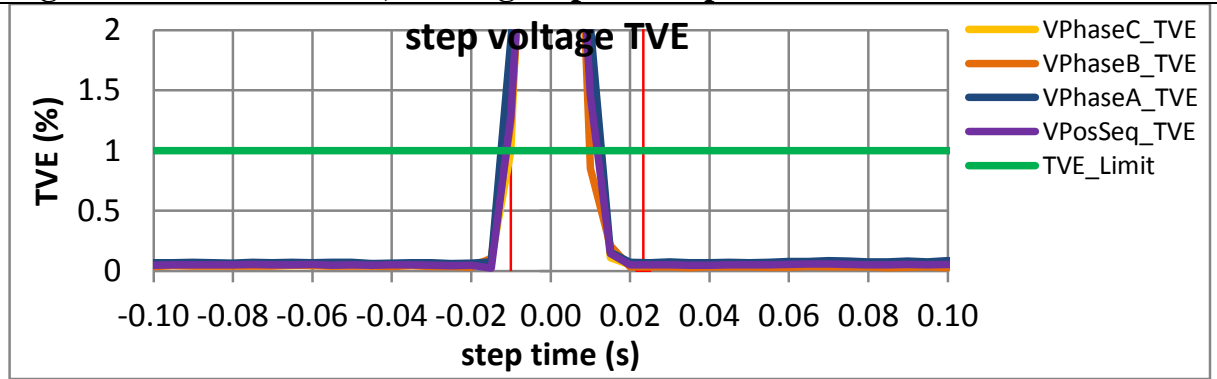

Figure 4255: Fs = 20 FPS, +10 degree phase step

Figure 4254: Fs = 30 FPS, -10 degree phase step

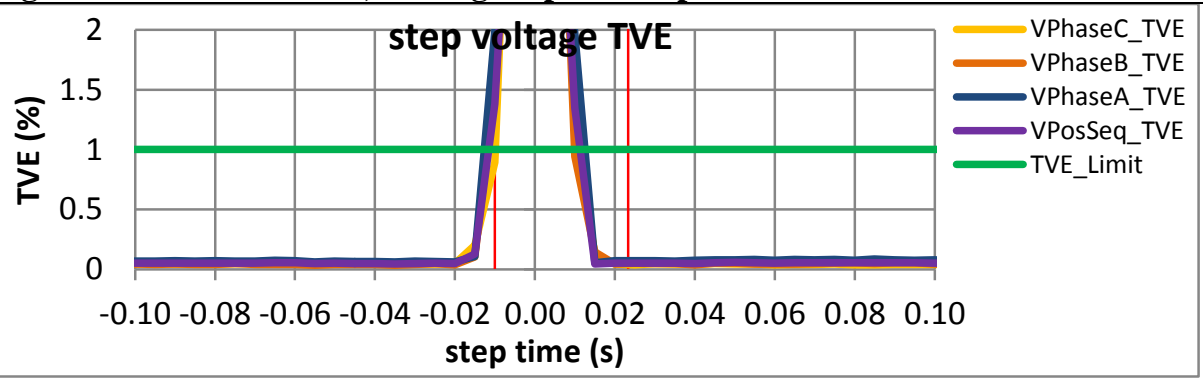

Figure 4256: Fs = 20 FPS, -10 degree phase step 


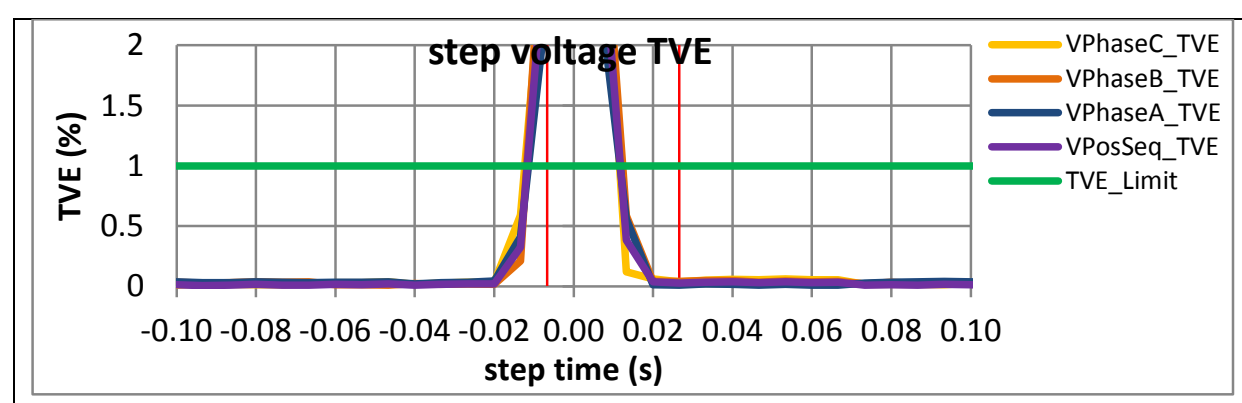

Figure 4257: Fs = 15 FPS, +10 degree phase step
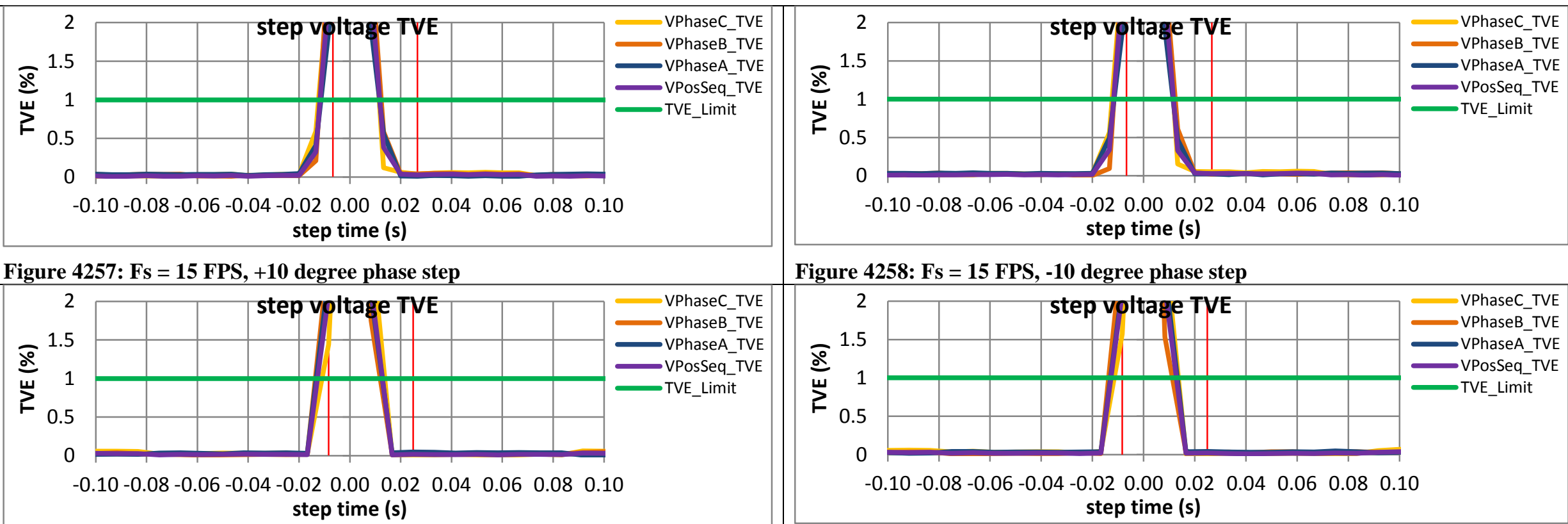

Figure 4258: Fs = 15 FPS, -10 degree phase step

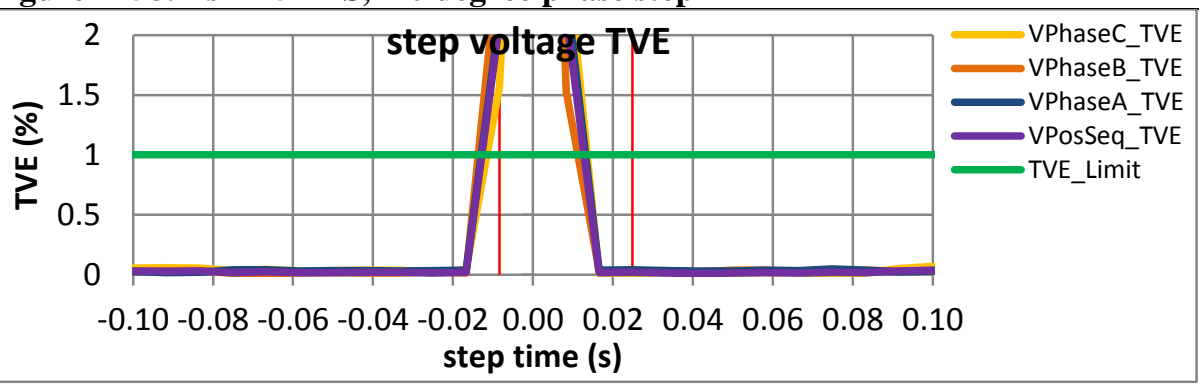

Figure 4259: Fs = 12 FPS, +10 degree phase step

Figure 4260: Fs = 12 FPS, -10 degree phase step
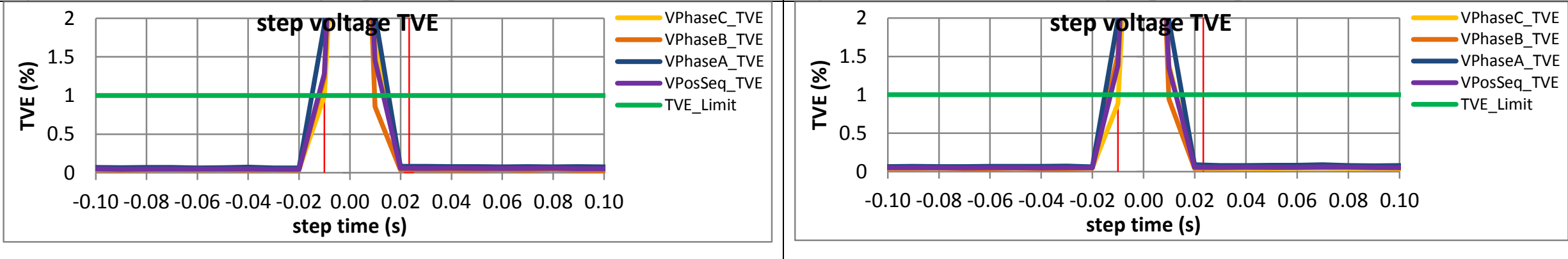

Figure 4261: Fs = 10 FPS, +10 degree phase step

Figure 4262: Fs = 10 FPS, -10 degree phase step 
9.3.5 PMU D dynamic step change in phase voltage response time: $F 0=60 \mathrm{~Hz}, \mathrm{P}$ class

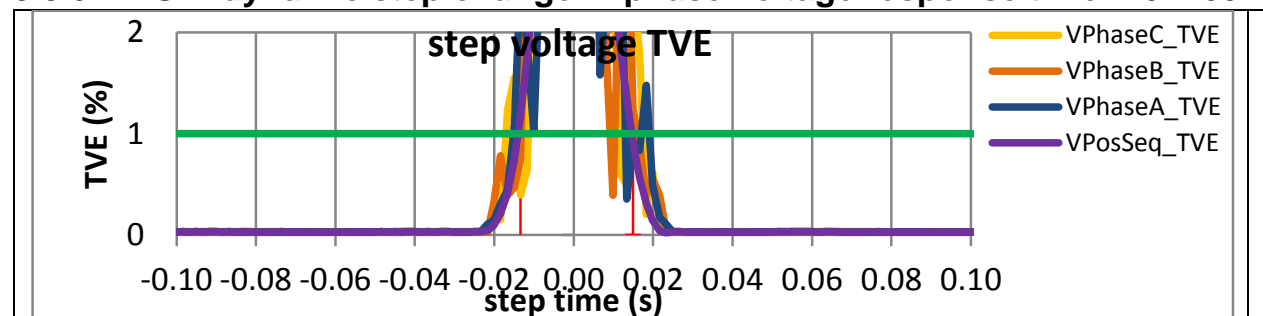

Figure 4263: Fs = 60 FPS, +10 degree phase step

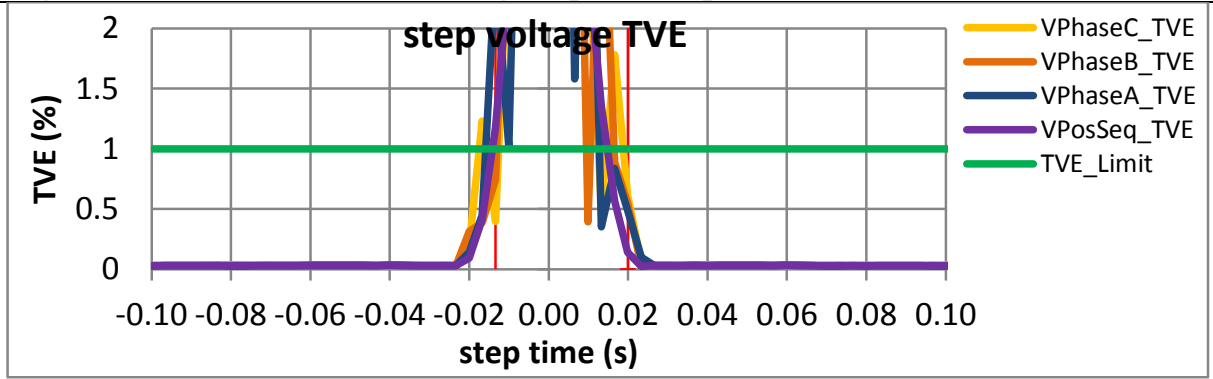

Figure 4265: Fs = 30 FPS, +10 degree phase step

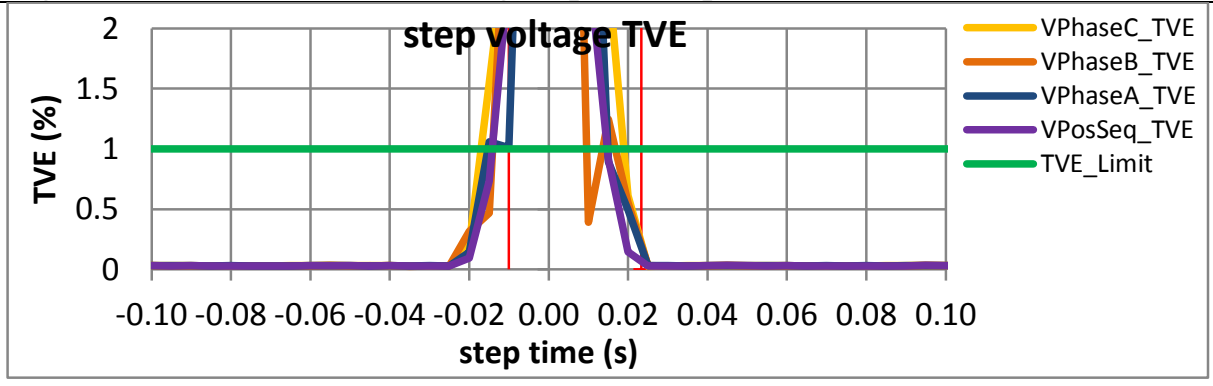

Figure 4267: Fs = 20 FPS, +10 degree phase step

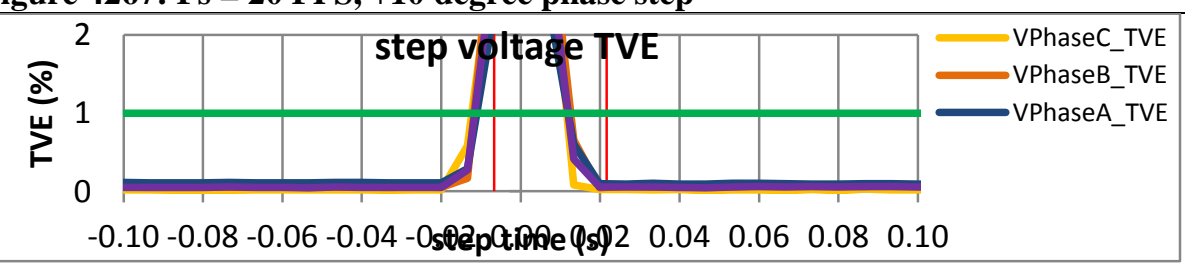

Figure 4269: Fs = 15 FPS, +10 degree phase step

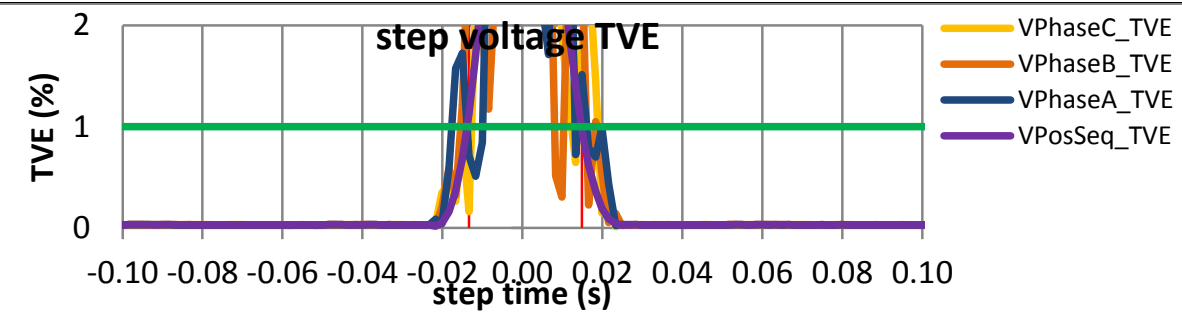

Figure 4264: Fs = 60 FPS, -10 degree phase step

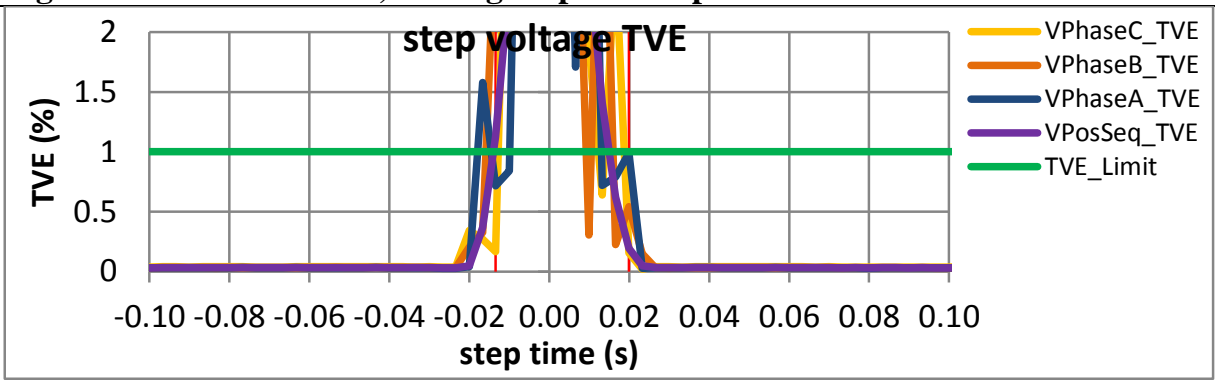

Figure 4266: Fs = 30 FPS, -10 degree phase step

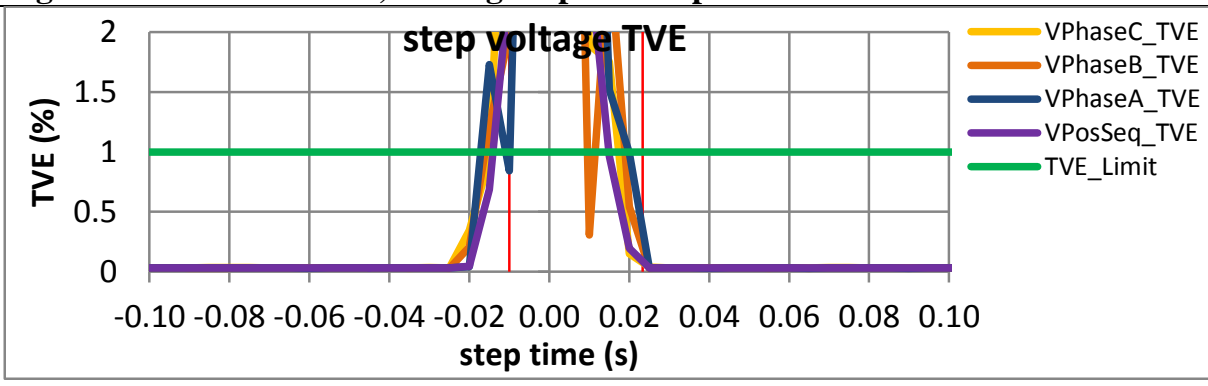

Figure 4268: Fs = 20 FPS, -10 degree phase step

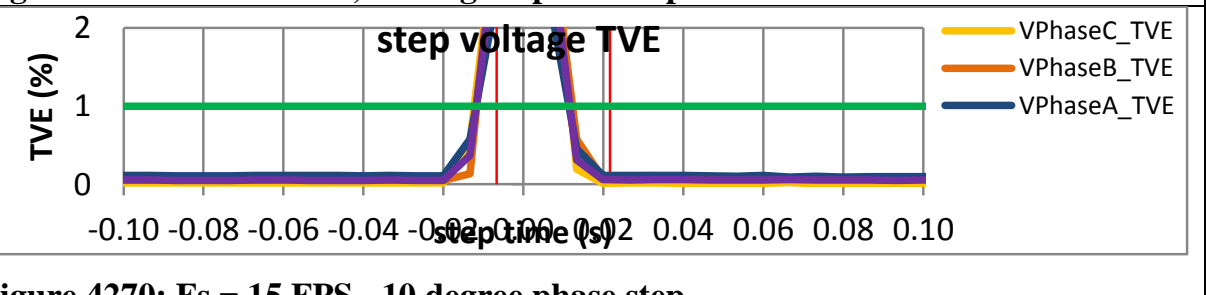

Figure 4270: Fs = 15 FPS, -10 degree phase step 

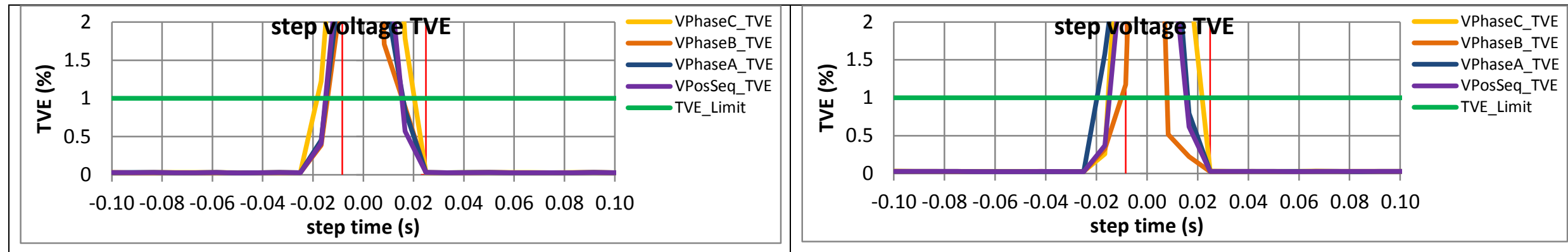

Figure 4271: Fs = 12 FPS, +10 degree phase step

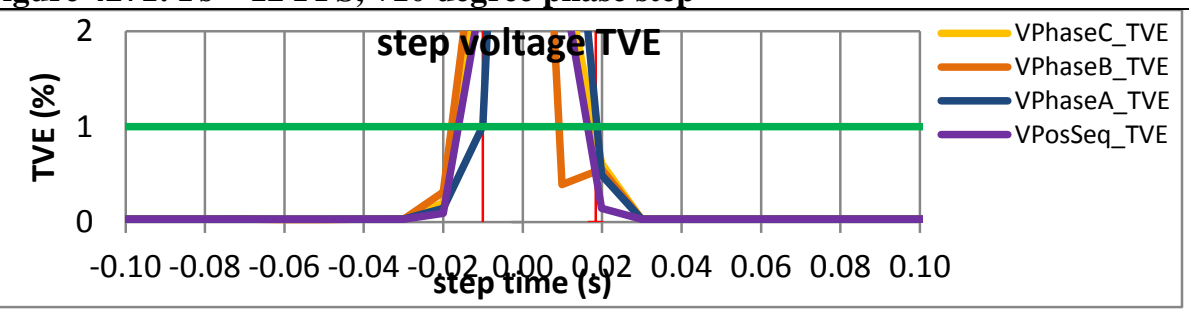

Figure 4272: Fs = 12 FPS, -10 degree phase step

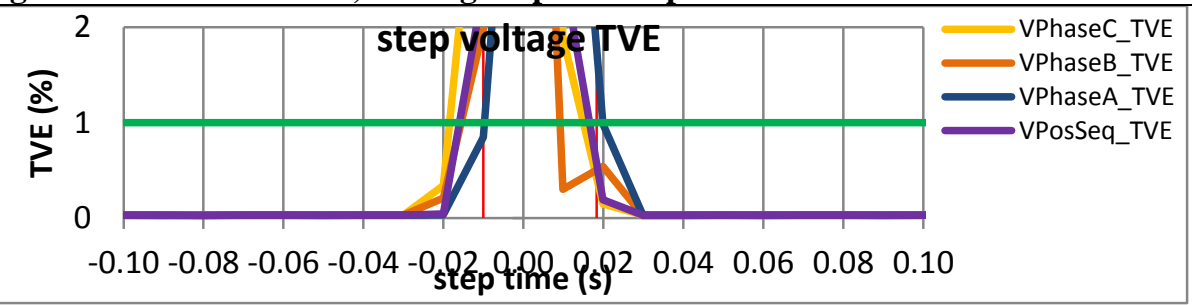

Figure 4273: Fs = 10 FPS, +10 degree phase step

Figure 4274: Fs = 10 FPS, -10 degree phase step 
9.3.6 PMU E dynamic step change in phase voltage response time: $\mathrm{F} 0=60 \mathrm{~Hz}, \mathrm{P}$ class

PMU E does not support P class.

\subsubsection{PMU F dynamic step change in phase voltage response time: $F 0=60 \mathrm{~Hz}, \mathrm{P}$ class}
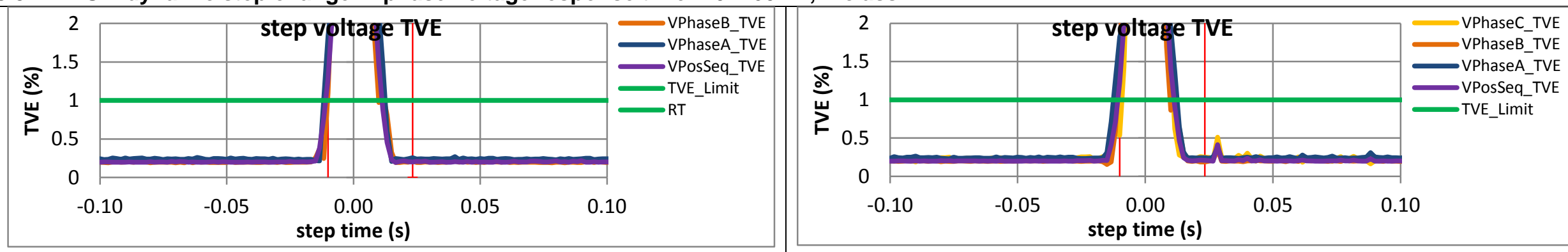

Figure 4275: Fs = 60 FPS, +10 degree phase step

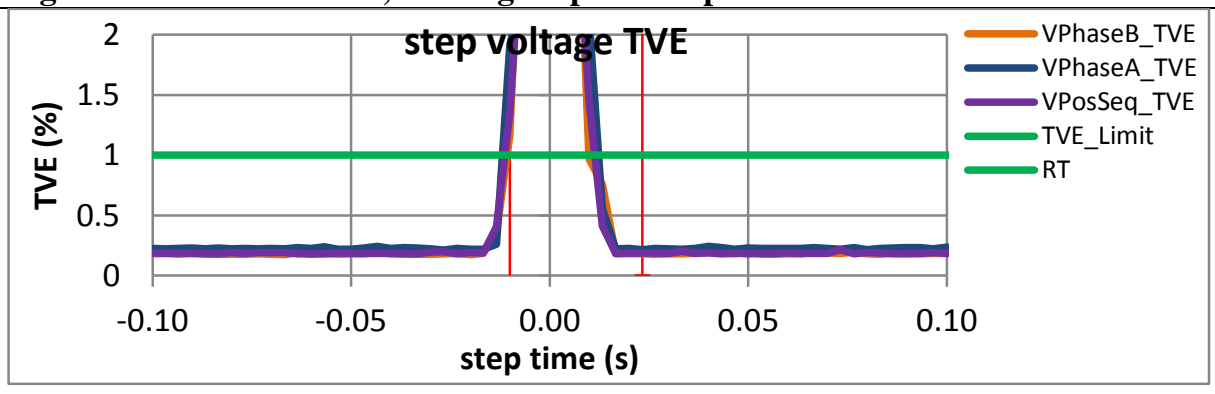

Figure 4276: Fs = 60 FPS, -10 degree phase step

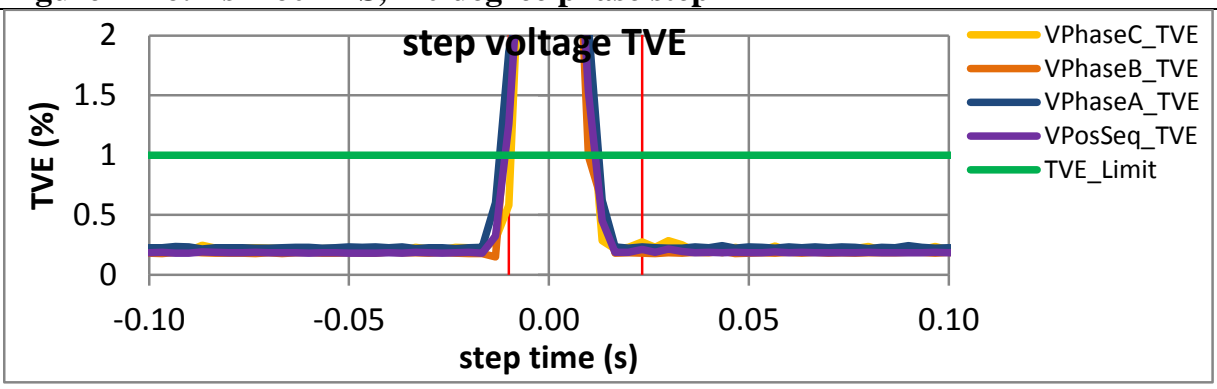

Figure 4277: Fs = 30 FPS, +10 degree phase step

Figure 4278: Fs = 30 FPS, -10 degree phase step

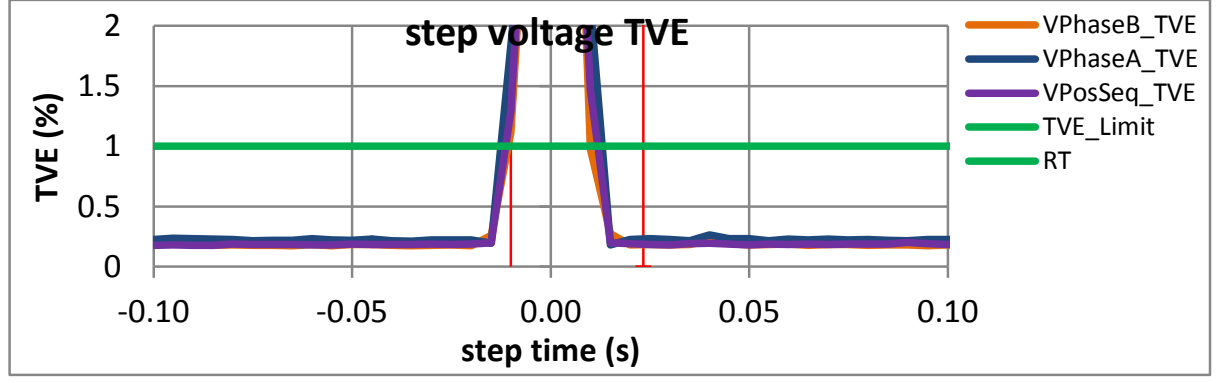

Figure 4279: Fs = 20 FPS, +10 degree phase step

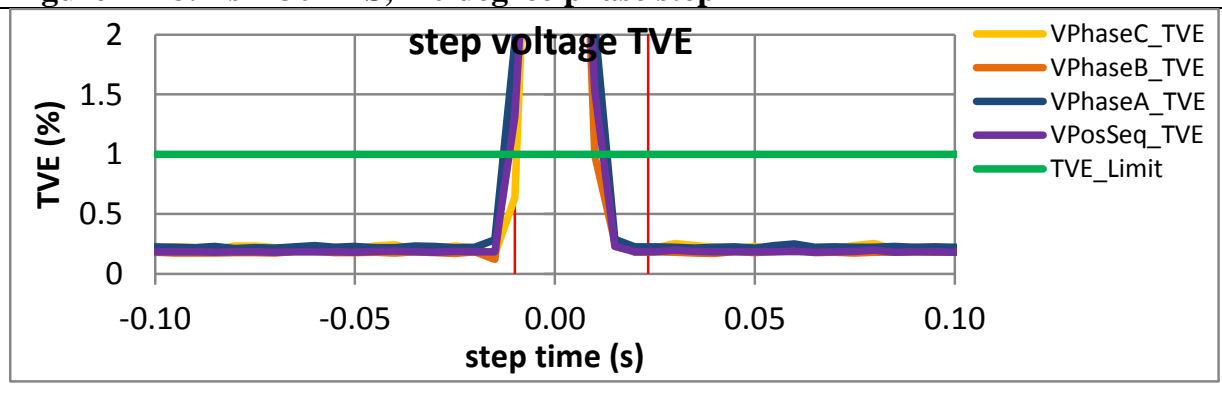

Figure 4280: Fs = 20 FPS, -10 degree phase step 


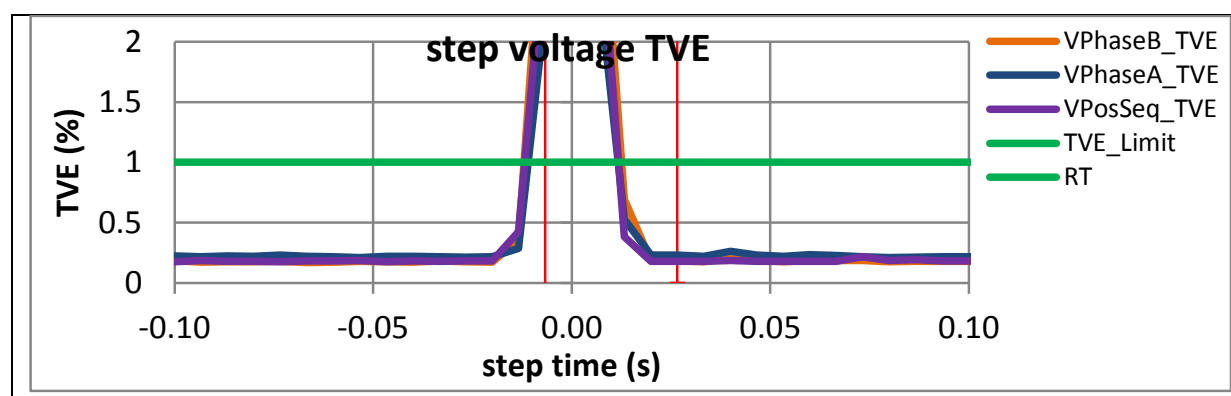

Figure 4281: Fs = 15 FPS, +10 degree phase step
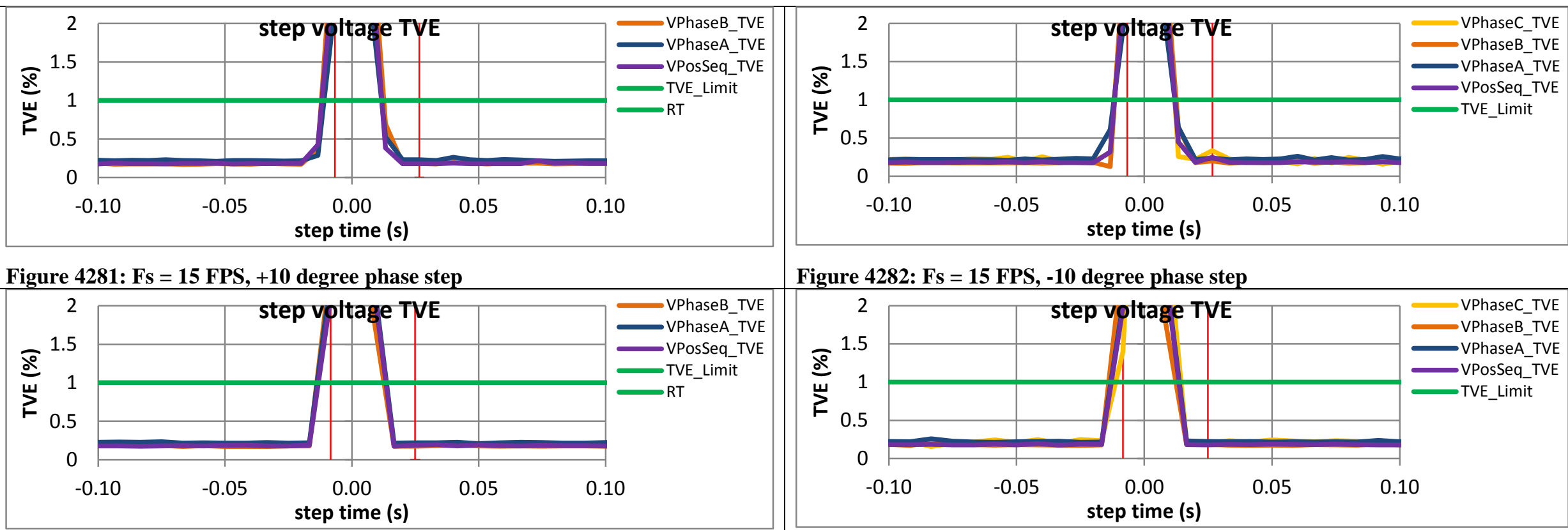

Figure 4282: Fs = 15 FPS, -10 degree phase step

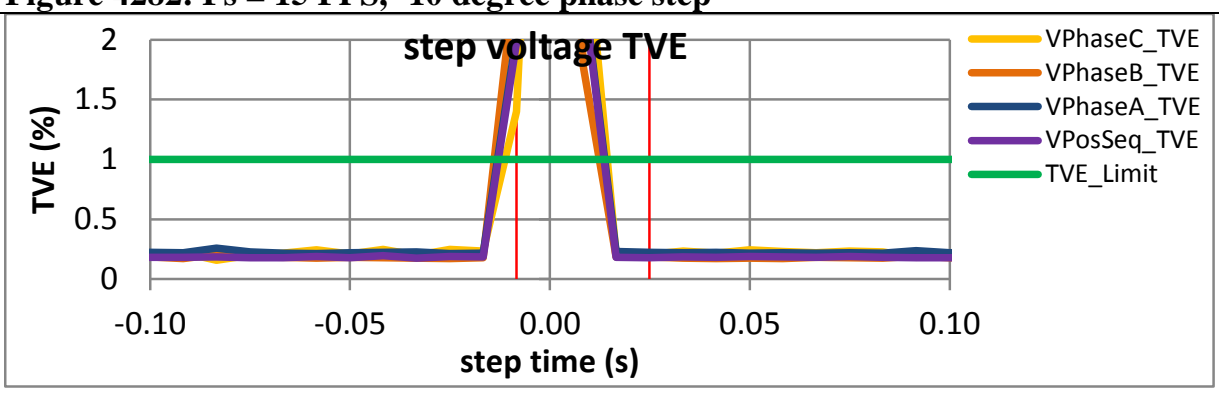

Figure 4283: Fs = 12 FPS, +10 degree phase step

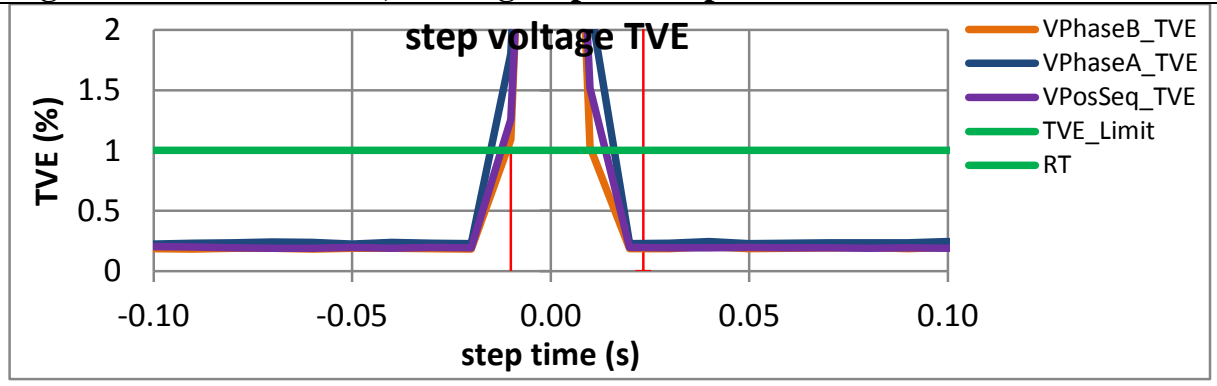

Figure 4284: Fs = 12 FPS, -10 degree phase step

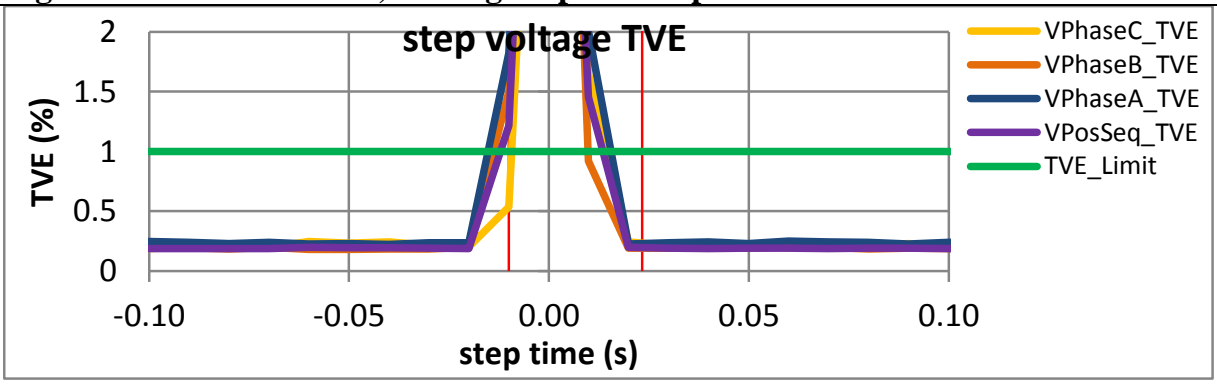

Figure 4286: Fs = 10 FPS, -10 degree phase step 
9.3.8 PMU G dynamic step change in phase voltage response time: $\mathrm{FO}=60 \mathrm{~Hz}, \mathrm{P}$ class PMU G does not support P class.

\subsubsection{PMU H dynamic step change in phase voltage response time: $\mathrm{F} 0=60 \mathrm{~Hz}, \mathrm{P}$ class}

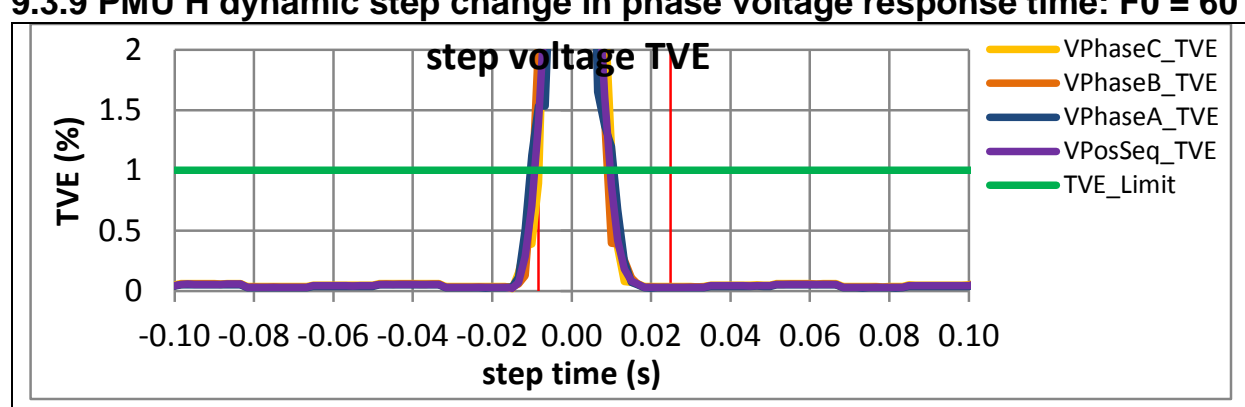

Figure 4287: Fs = 60 FPS, +10 degree phase step

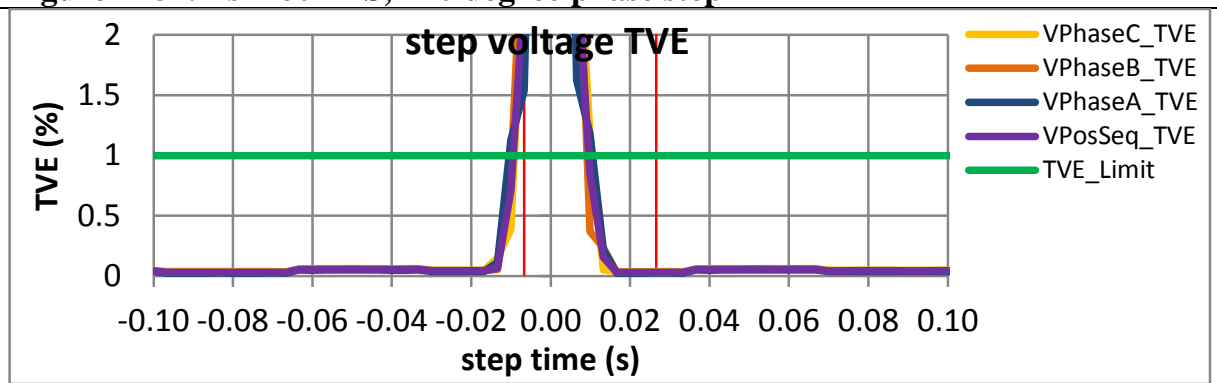

Figure 4289: Fs = 30 FPS, +10 degree phase step

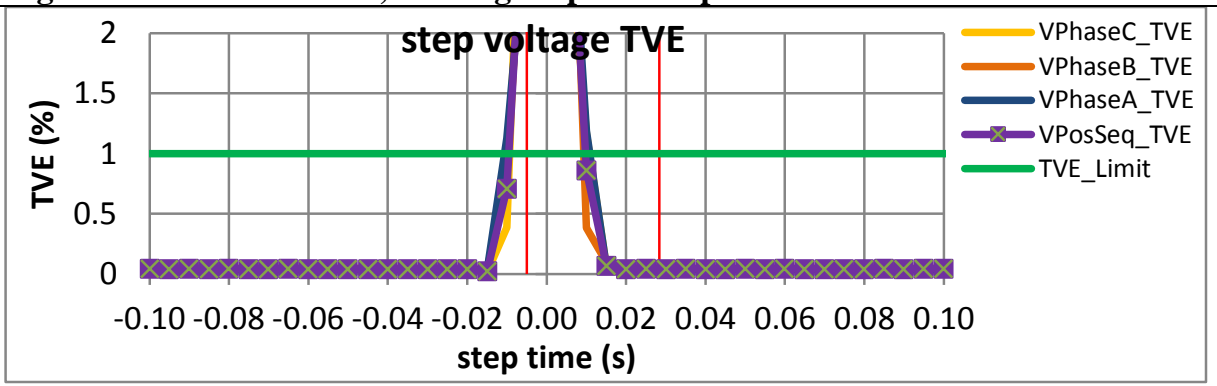

Figure 4291: Fs = 20 FPS, +10 degree phase step

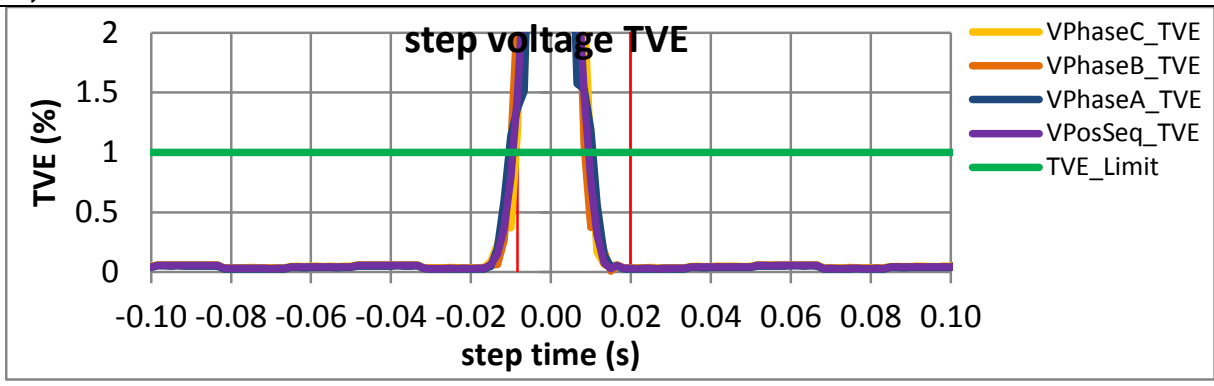

Figure 4288: Fs = 60 FPS, - 10 degree phase step

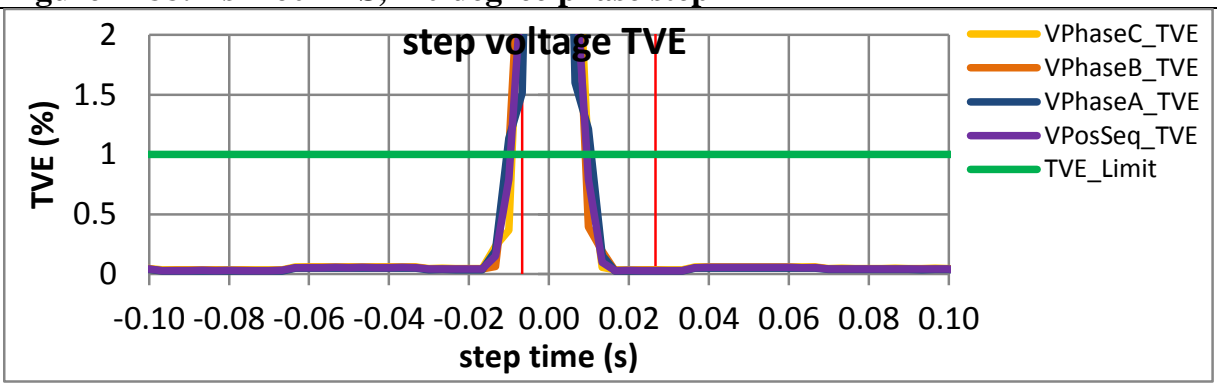

Figure 4290: Fs = 30 FPS, -10 degree phase step

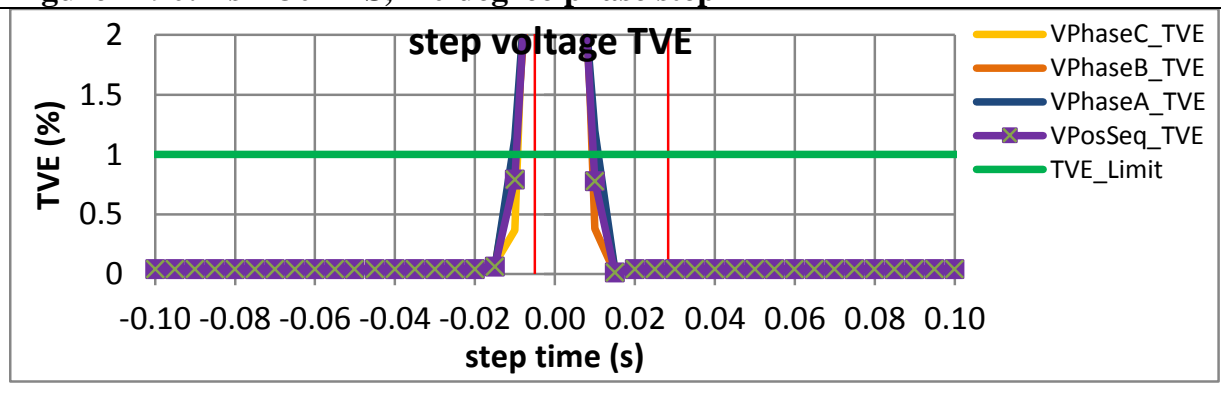

Figure 4292: Fs = 20 FPS, -10 degree phase step 


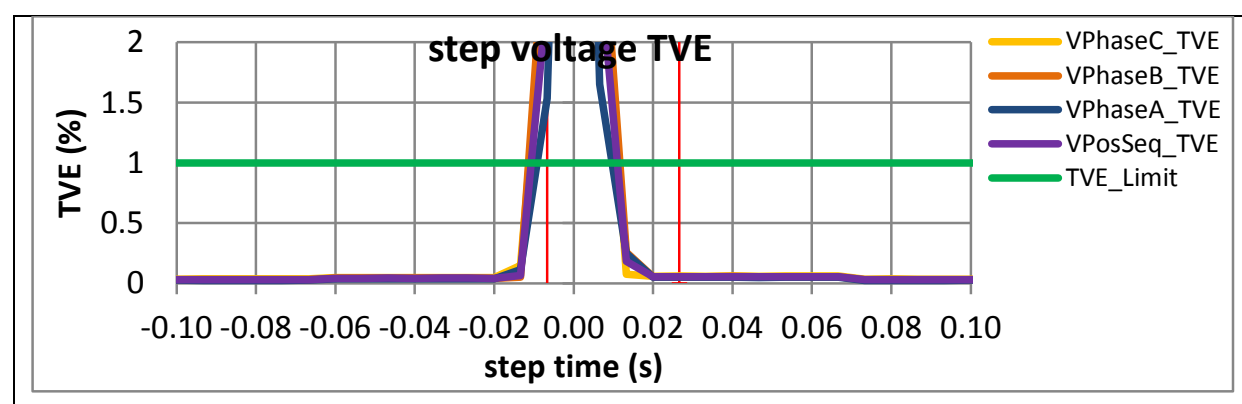

Figure 4293: Fs = 15 FPS, +10 degree phase step
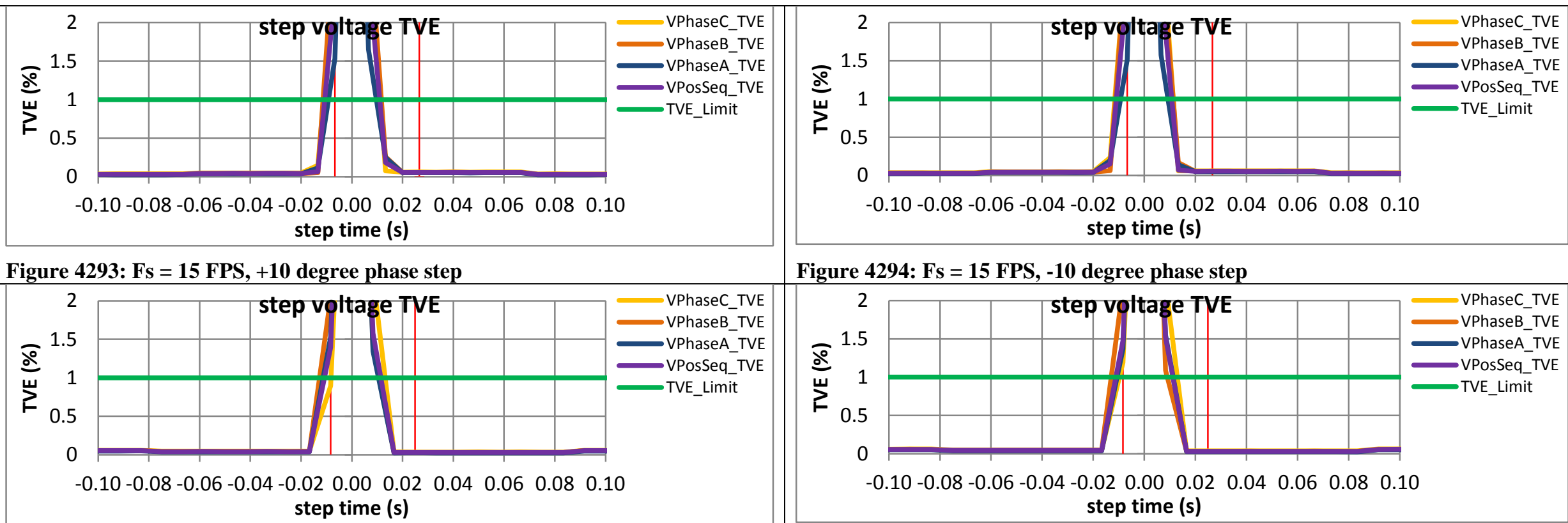

Figure 4294: Fs = 15 FPS, -10 degree phase step

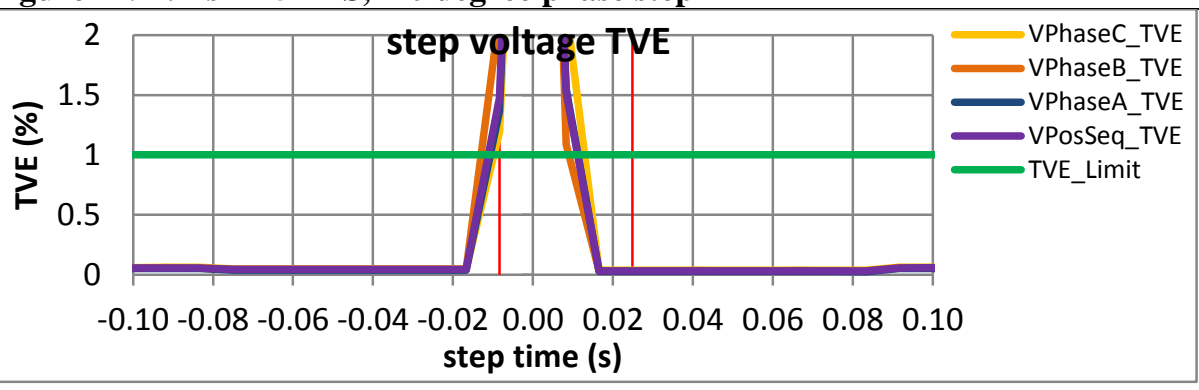

Figure 4295: Fs = 12 FPS, +10 degree phase step

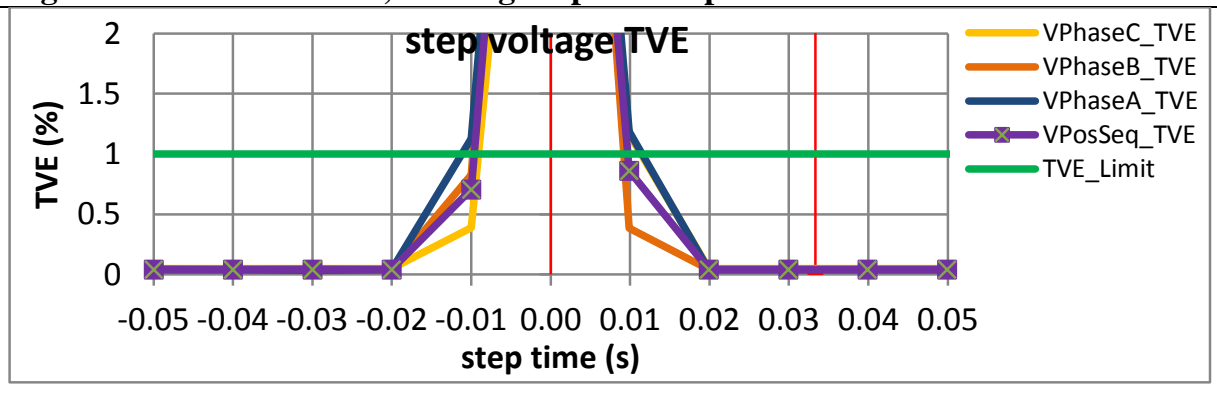

Figure 4296: Fs = 12 FPS, -10 degree phase step

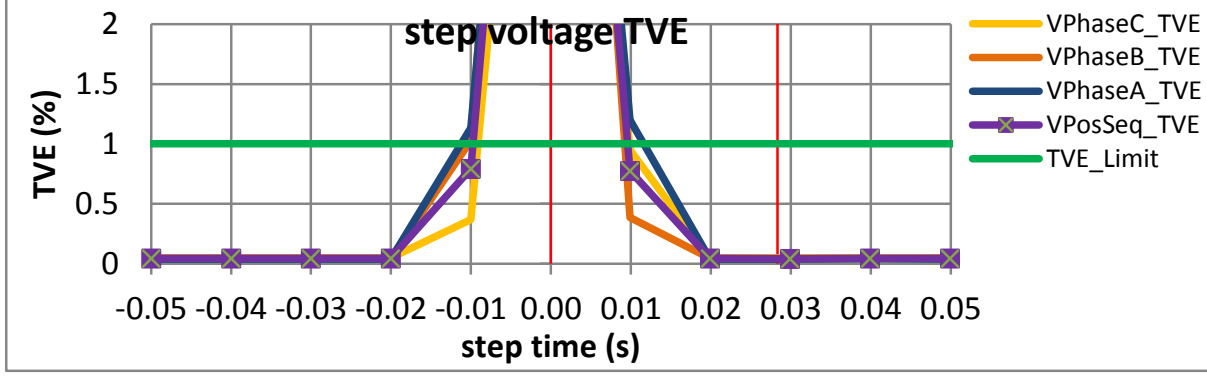

Figure 4298: Fs = 10 FPS, -10 degree phase step 
9.3.10 PMU I dynamic step change in phase voltage response time: $\mathrm{F} 0=60 \mathrm{~Hz}, \mathrm{P}$ class PMU I does not support P class

\subsubsection{PMU J dynamic step change in phase voltage response time: $\mathrm{F} 0=60 \mathrm{~Hz}, \mathrm{P}$ class}
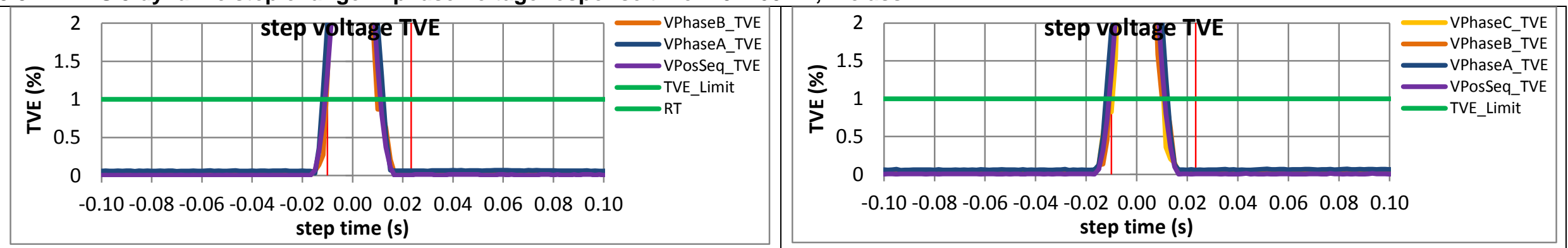

Figure 4299: Fs = $60 \mathrm{FPS},+10$ degree phase step

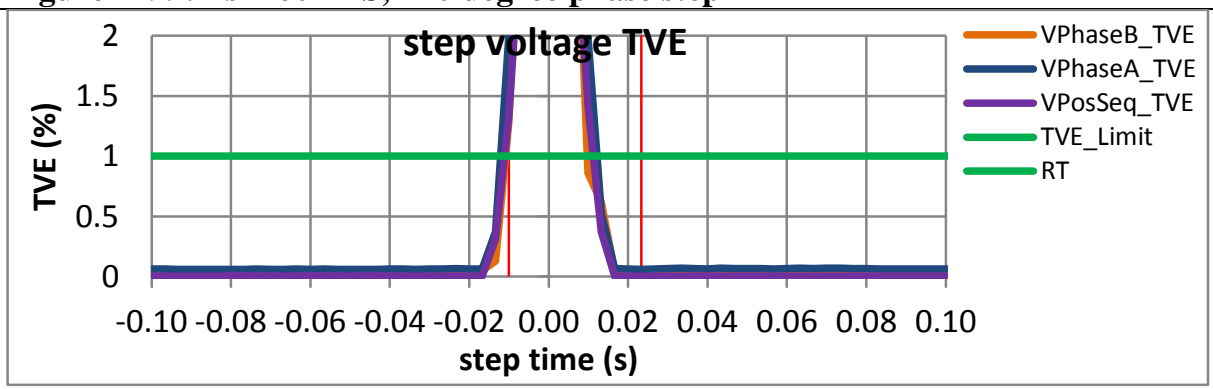

Figure 4300: Fs = 60 FPS, -10 degree phase step

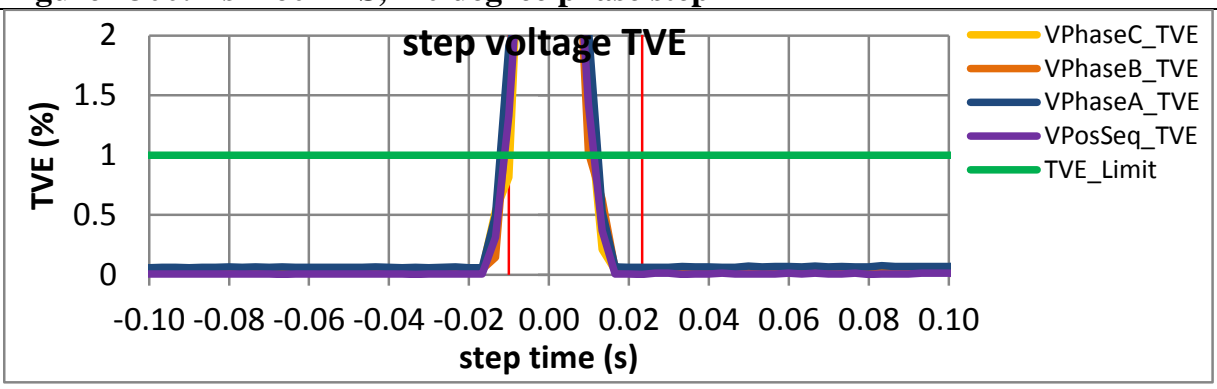

Figure 4301: Fs = 30 FPS, +10 degree phase step

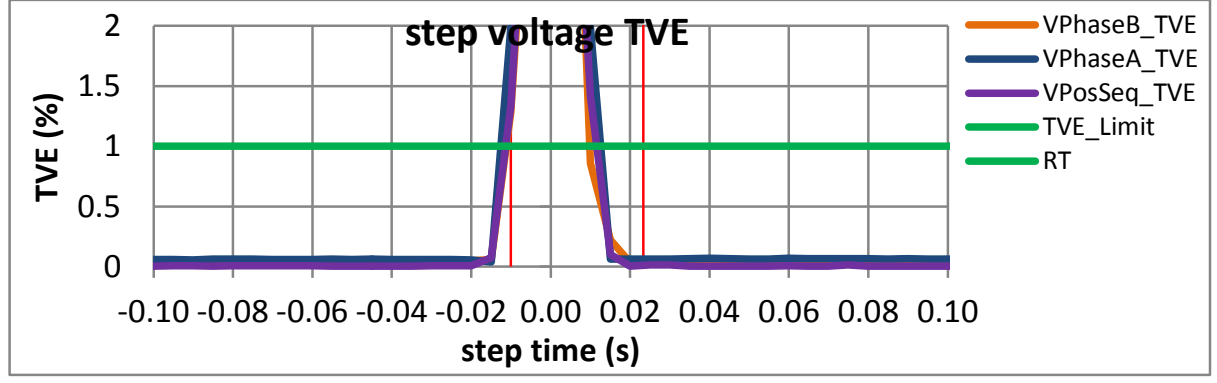

Figure 4302: Fs = 30 FPS, -10 degree phase step

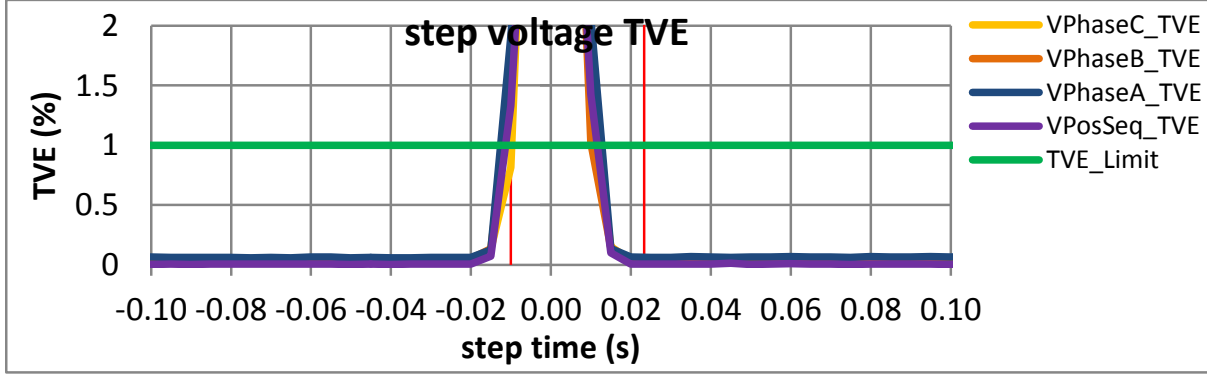

Figure 4304: Fs = 20 FPS, -10 degree phase step 

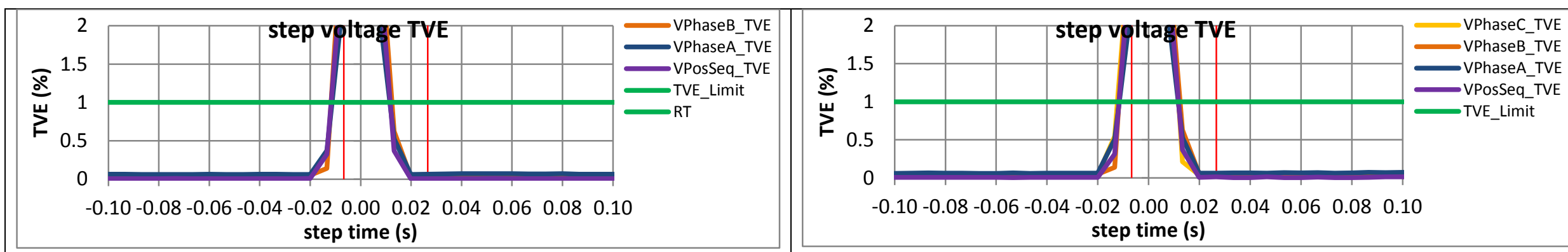

Figure 4305: Fs = 15 FPS, +10 degree phase step

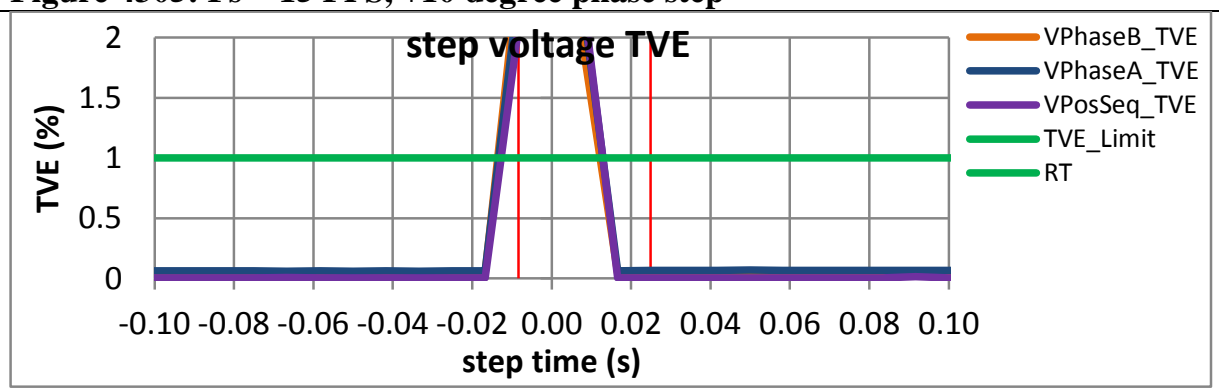

Figure 4306: Fs = 15 FPS, -10 degree phase step

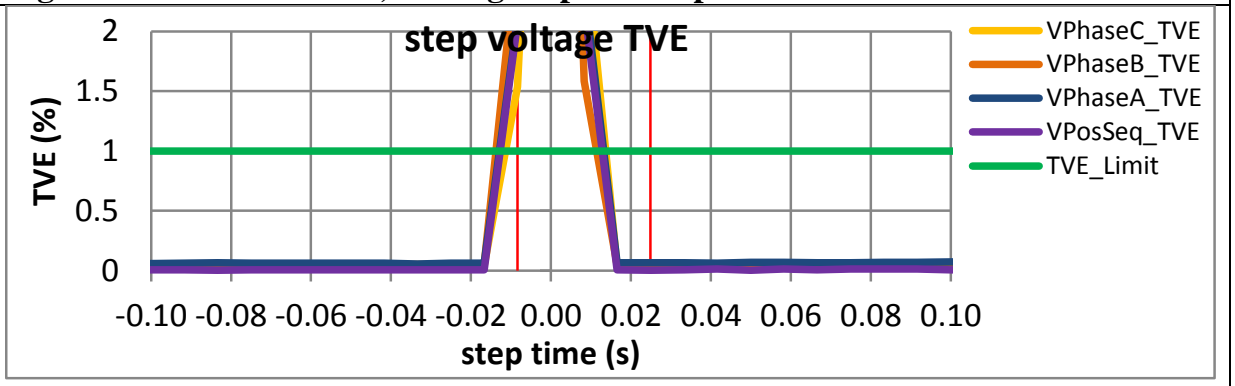

Figure 4307: Fs = 12 FPS, +10 degree phase step

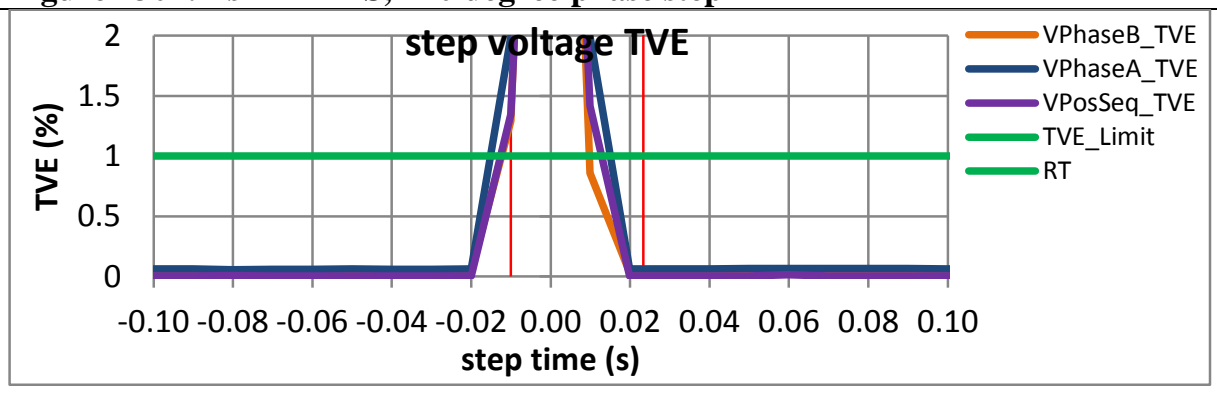

Figure 4309: Fs = 10 FPS, +10 degree phase step

Figure 4308: Fs = 12 FPS, -10 degree phase step

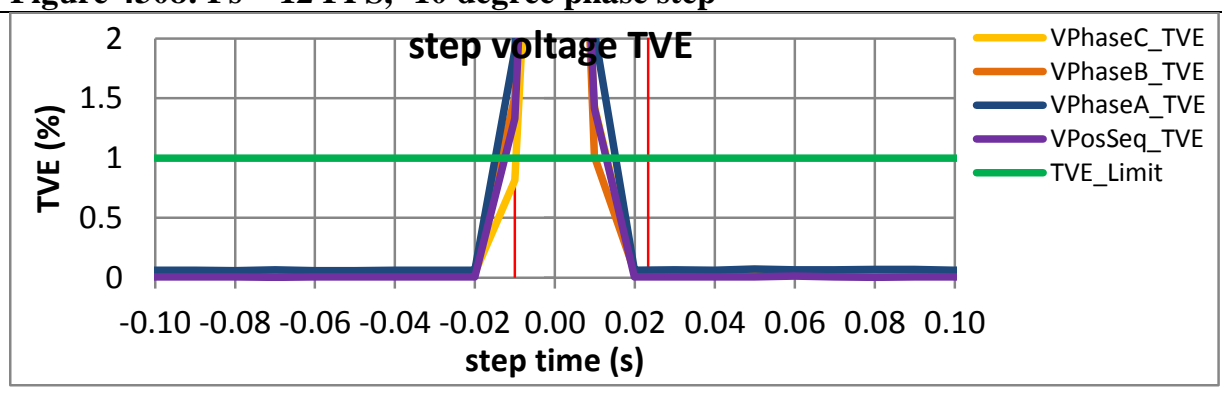

Figure 4310: Fs = 10 FPS, -10 degree phase step 
9.4 Dynamic step change in phase current response time: $F 0=60 \mathrm{~Hz}$, P Class

9.4.1 C37.118.1-2011 Annex C dynamic step change in phase current response time: $F 0=60 \mathrm{~Hz}, \mathrm{P}$ class
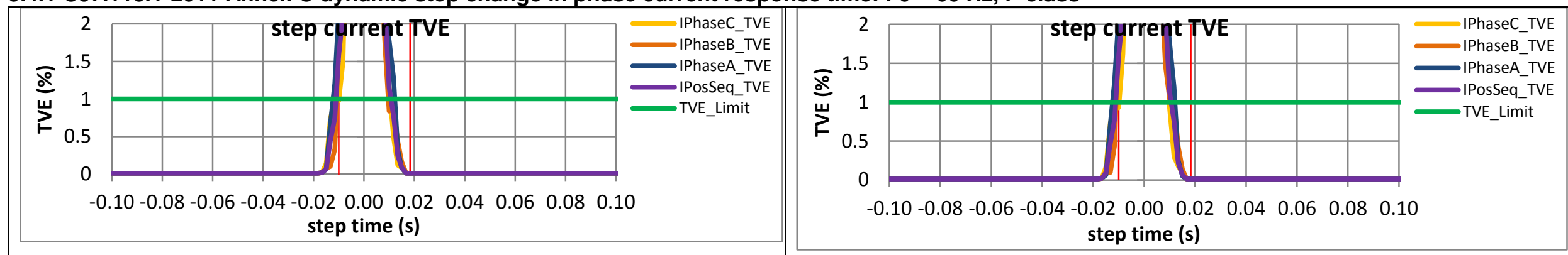

Figure 4311: Fs = 60 FPS, +10 degree phase step

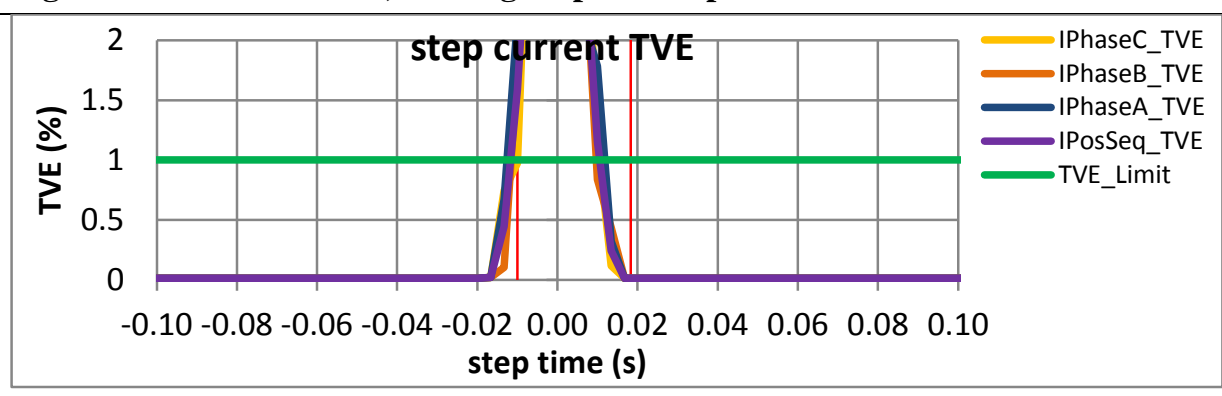

Figure 4312: Fs = 60 FPS, -10 degree phase step

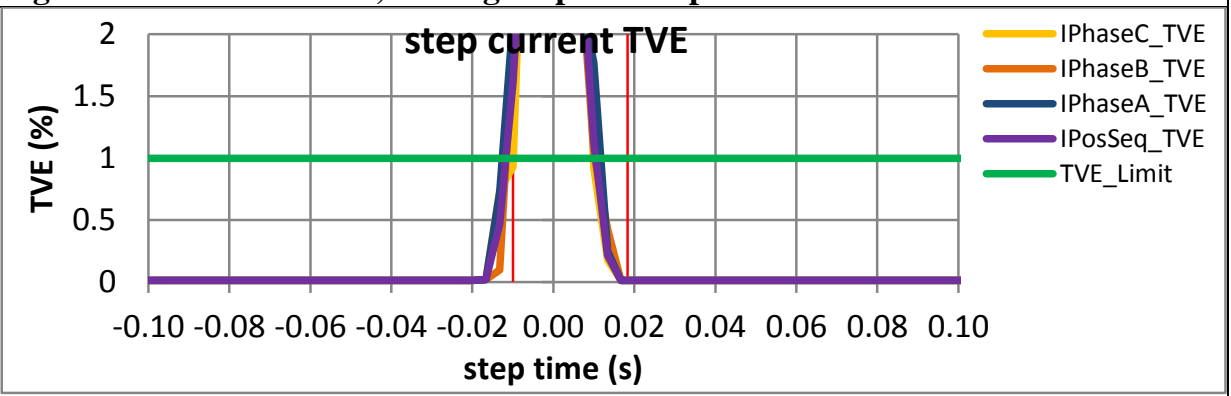

Figure 4313: Fs = 30 FPS, +10 degree phase step

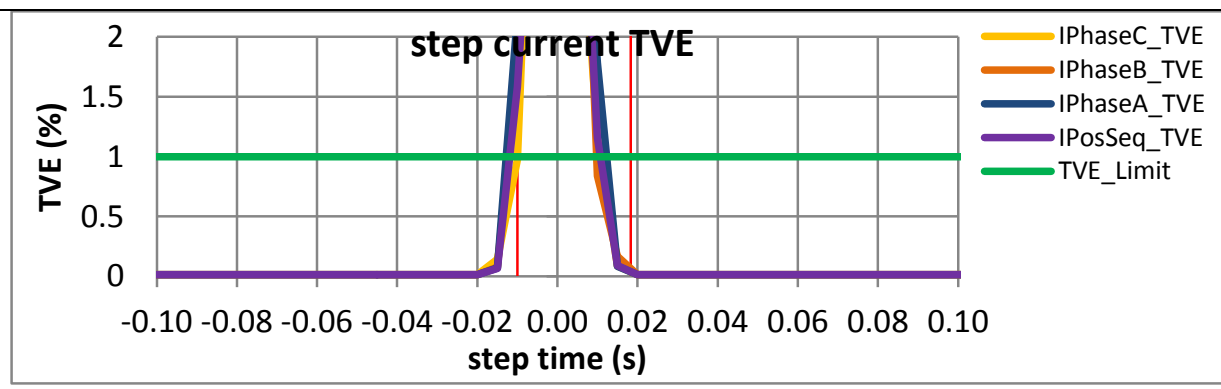

Figure 4314: Fs = 30 FPS, -10 degree phase step

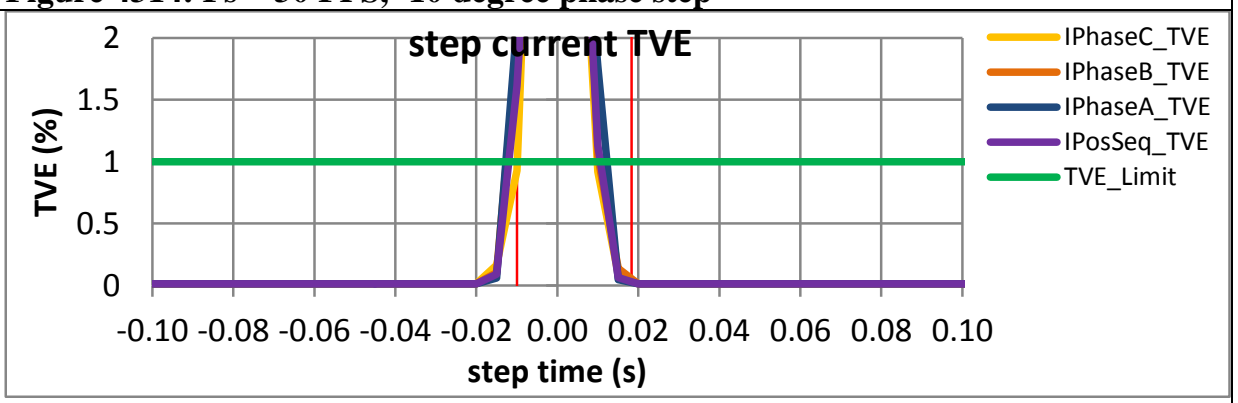

Figure 4316: Fs = 20 FPS, - 10 degree phase step 

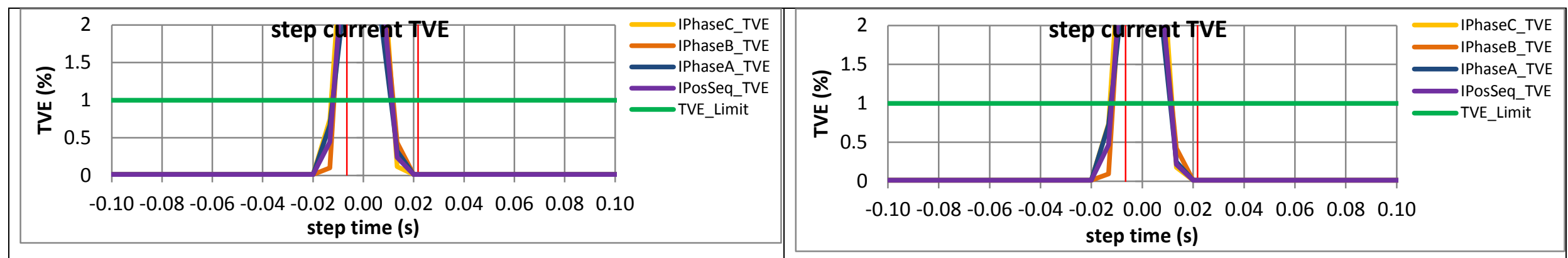

Figure 4317: Fs = 15 FPS, +10 degree phase step

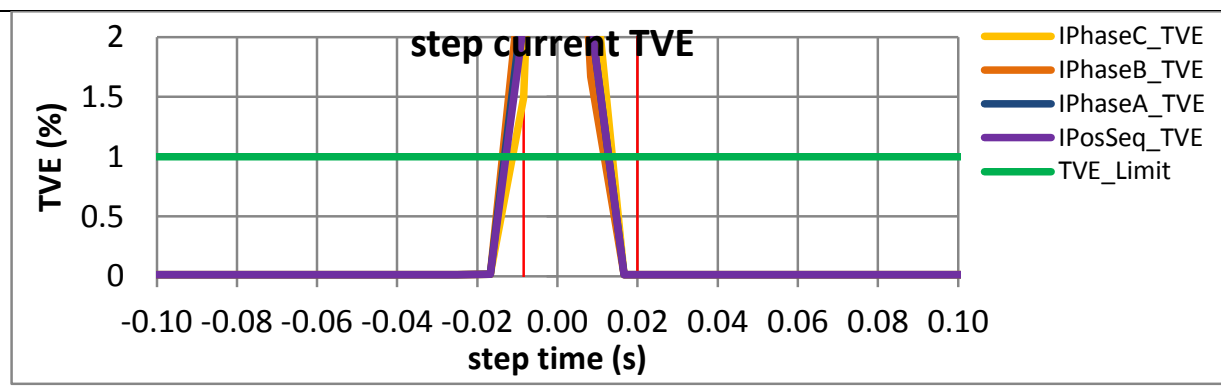

Figure 4318: Fs = 15 FPS, -10 degree phase step

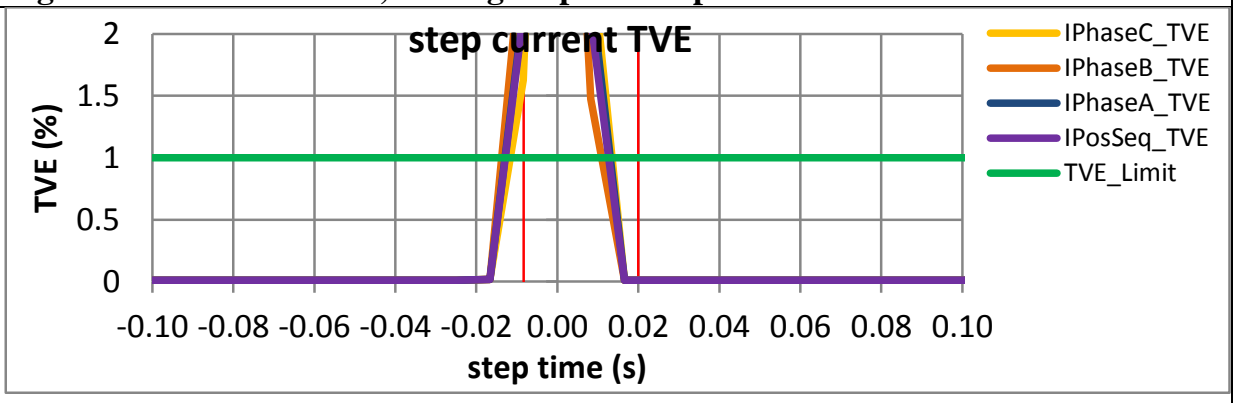

Figure 4319: Fs = 12 FPS, +10 degree phase step

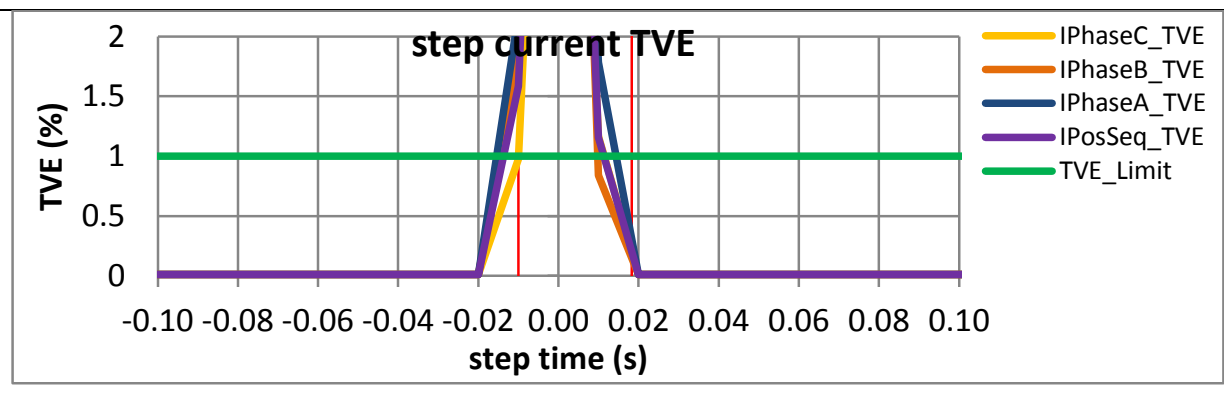

Figure 4320: Fs = 12 FPS, -10 degree phase step

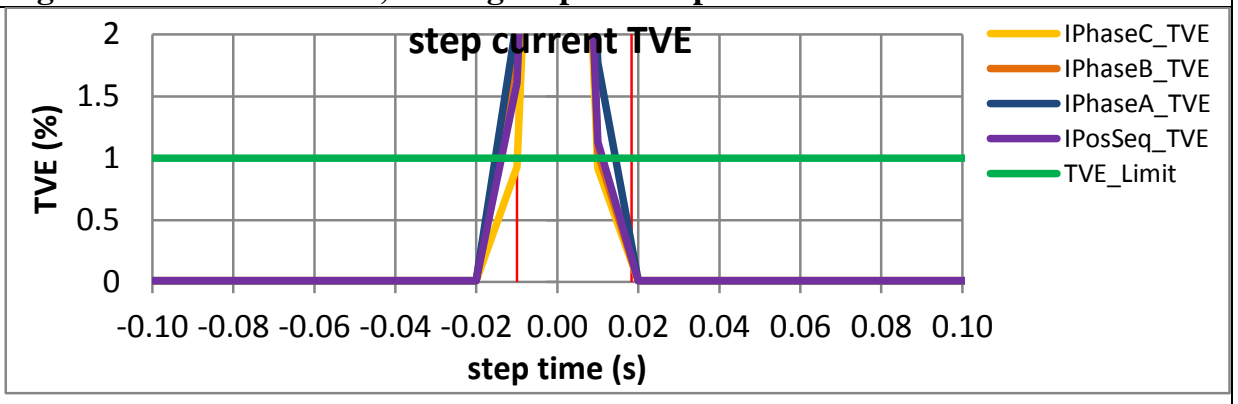

Figure 4322: Fs $=10 \mathrm{FPS},-10$ degree phase step 
9.4.2 PMU A dynamic step change in phase current response time: $\mathrm{F} 0=60 \mathrm{~Hz}, \mathrm{P}$ class

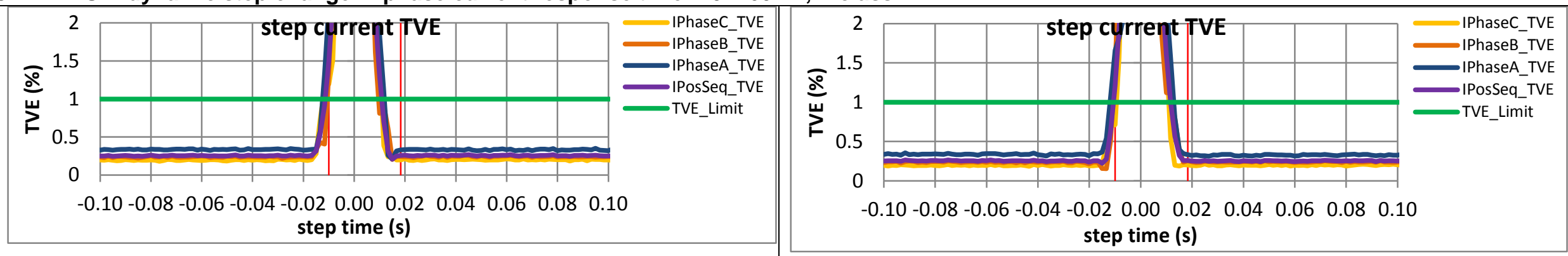

Figure 4323: Fs = 60 FPS, +10 degree phase step

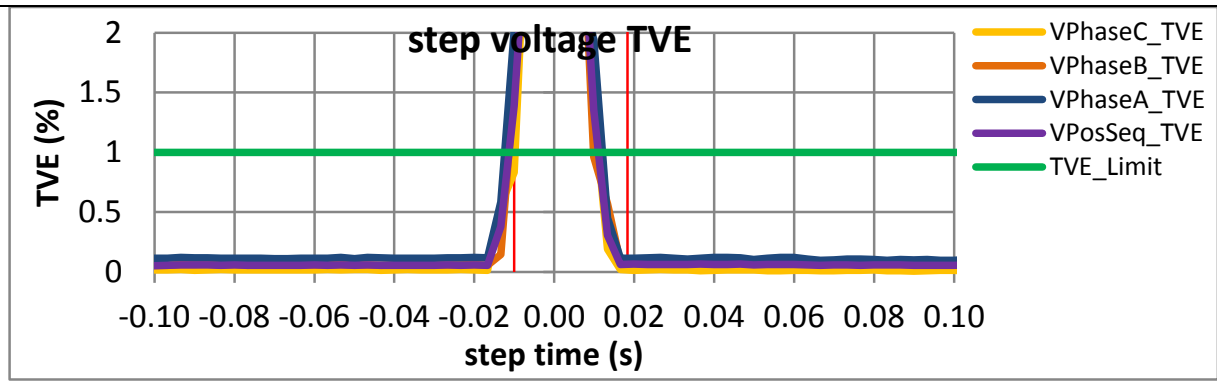

Figure 4324: Fs = 60 FPS, -10 degree phase step

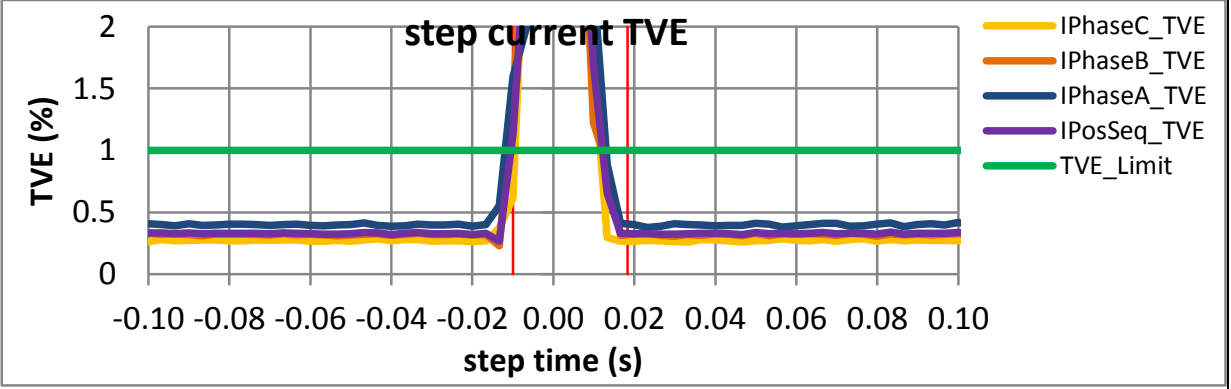

Figure 4325: Fs = 30 FPS, +10 degree phase step

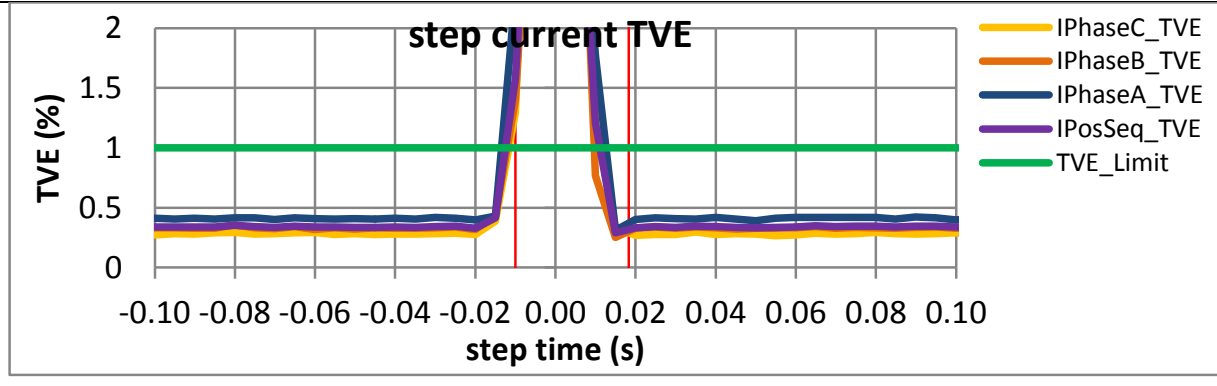

Figure 4326: Fs = 30 FPS, -10 degree phase step

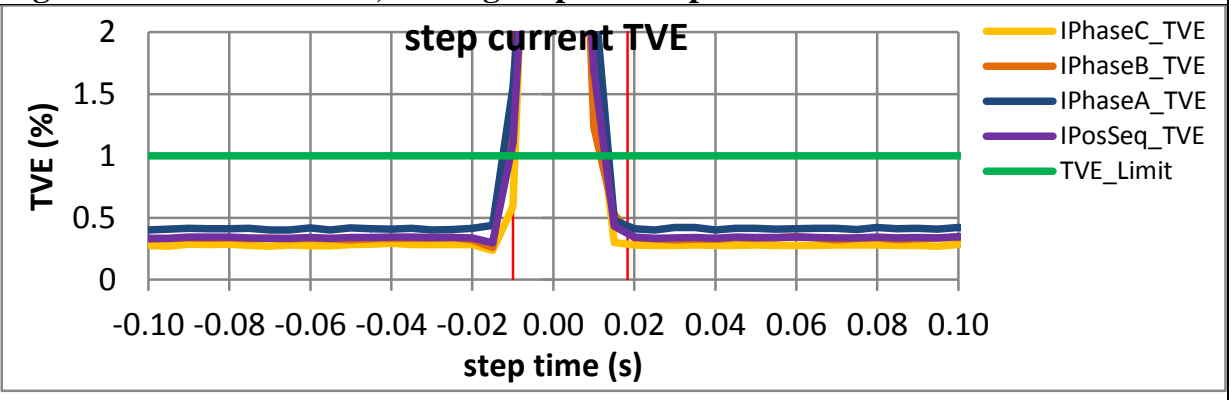

Figure 4328: Fs = 20 FPS, -10 degree phase step 

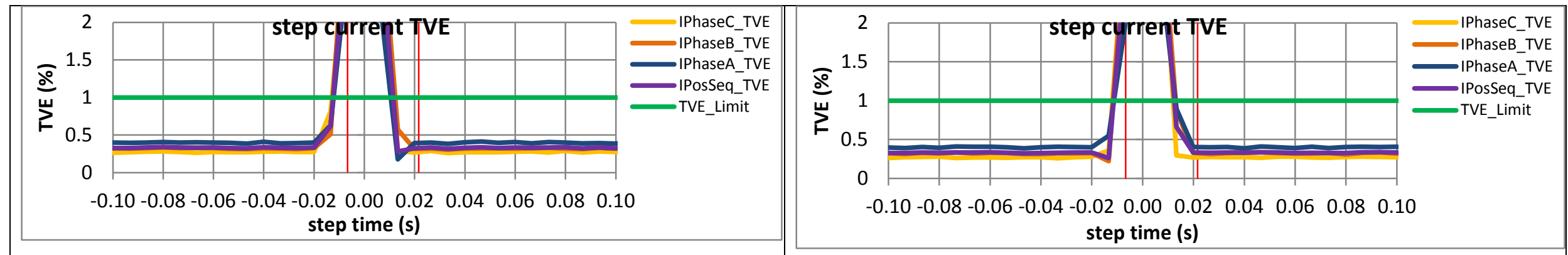

Figure 4329: Fs = 15 FPS, +10 degree phase step

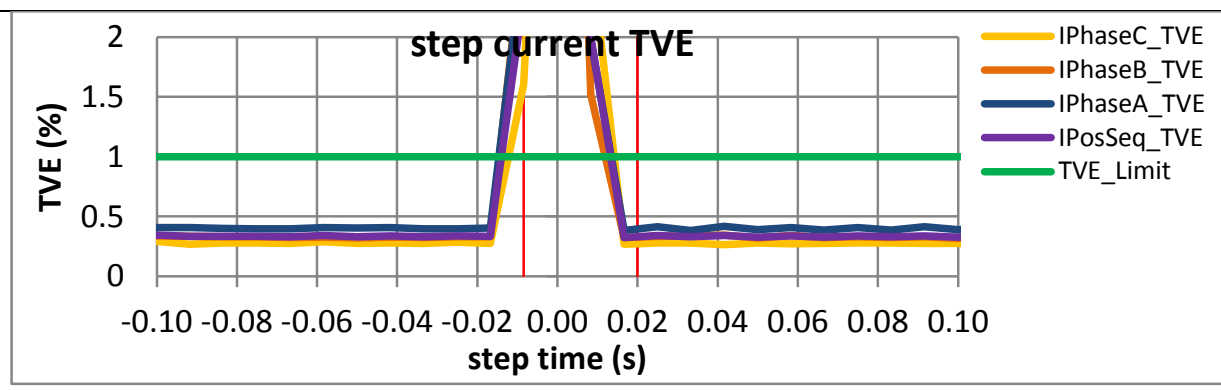

Figure 4330: Fs = 15 FPS, -10 degree phase step

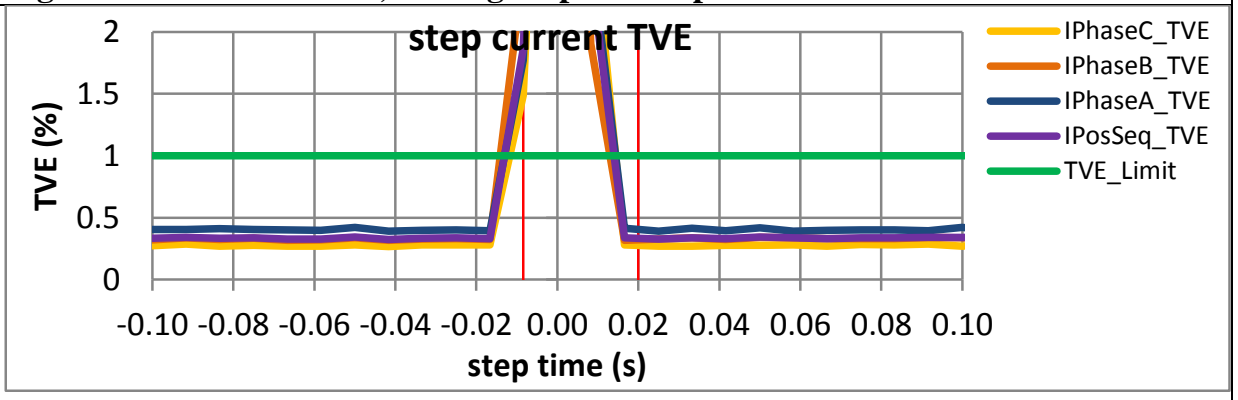

Figure 4331: Fs = 12 FPS, +10 degree phase step

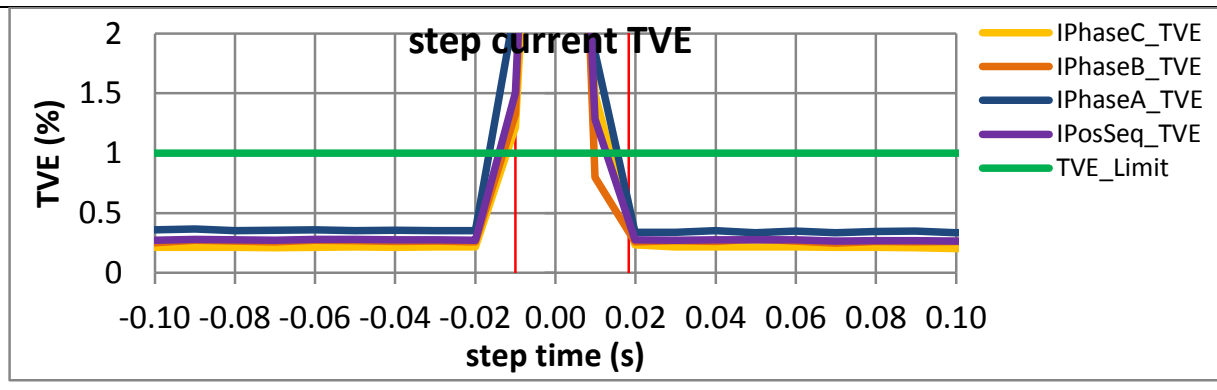

Figure 4332: Fs = 12 FPS, -10 degree phase step

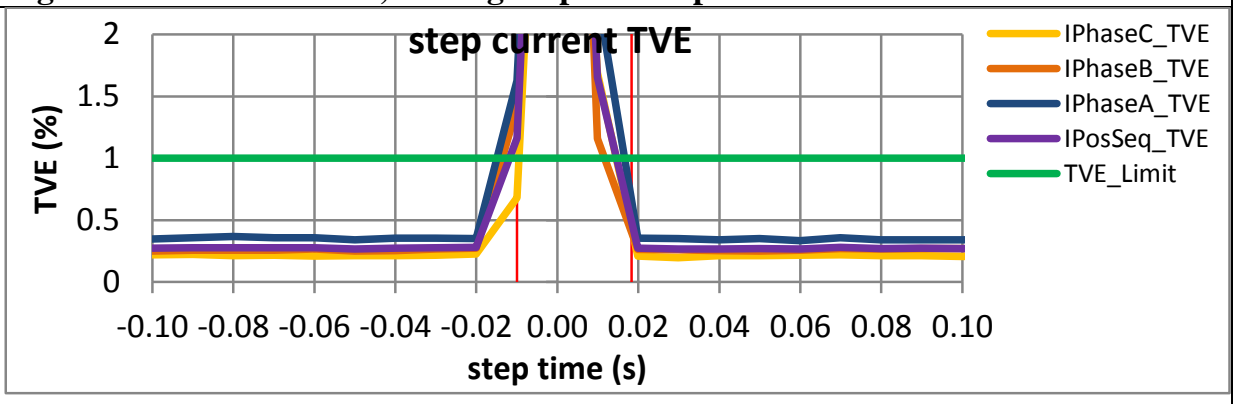

Figure 4334: Fs $=10$ FPS, -10 degree phase step 
9.4.3 PMU B dynamic step change in phase current response time: $\mathrm{F} 0=60 \mathrm{~Hz}, \mathrm{P}$ class

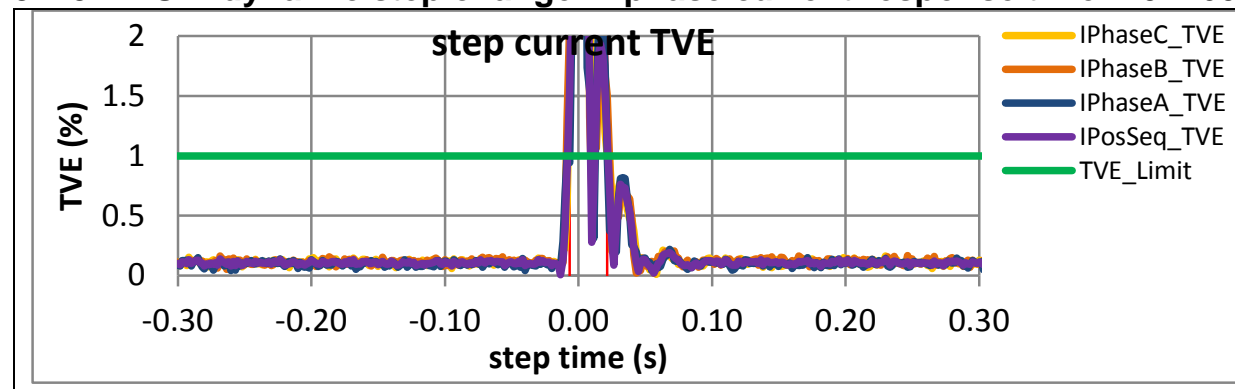

Figure 4335: Fs = 60 FPS, +10 degree phase step

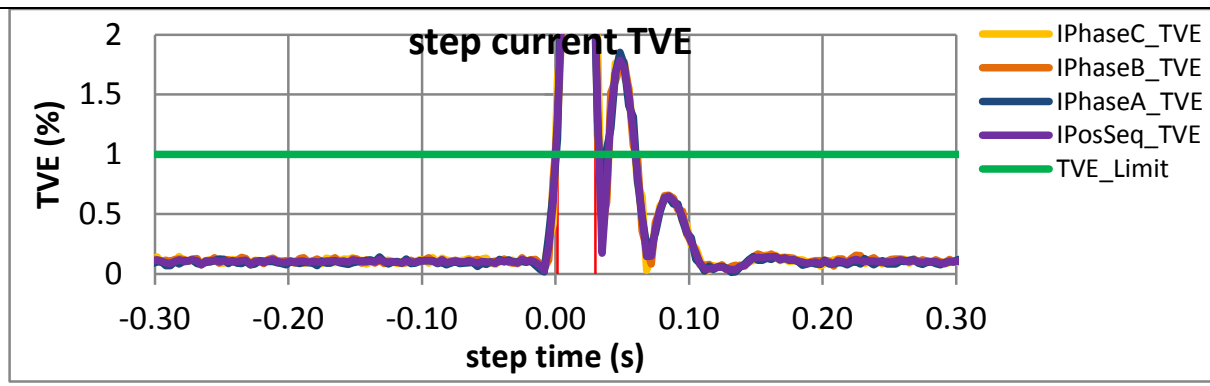

Figure 4337: Fs = 30 FPS, +10 degree phase step

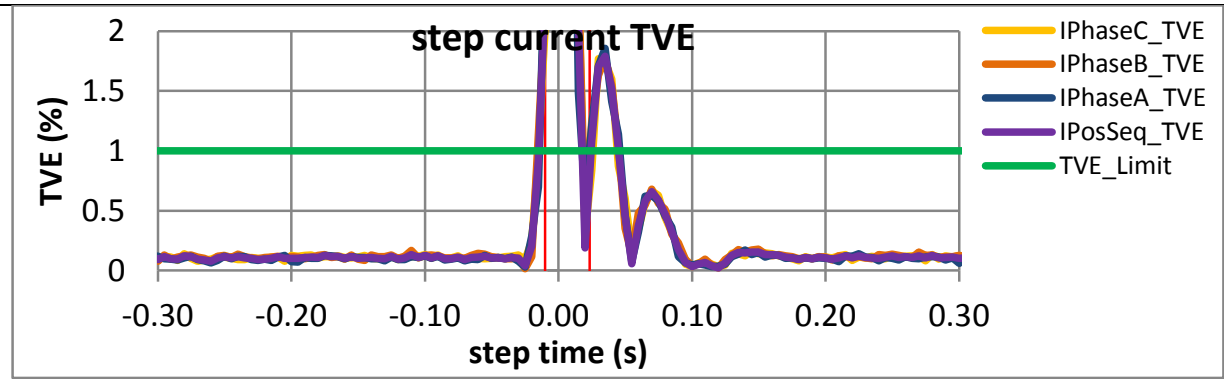

Figure 4339: Fs = 20 FPS, +10 degree phase step
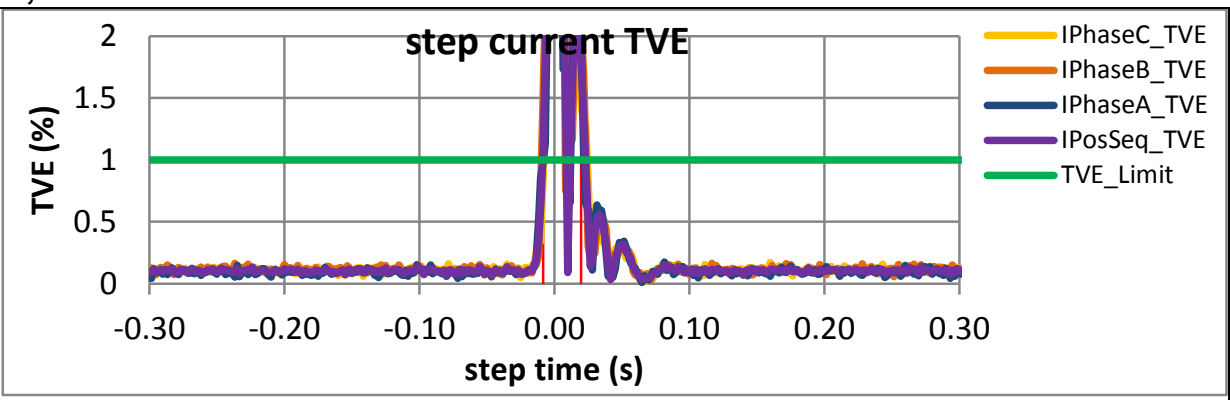

Figure 4336: Fs = 60 FPS, -10 degree phase step

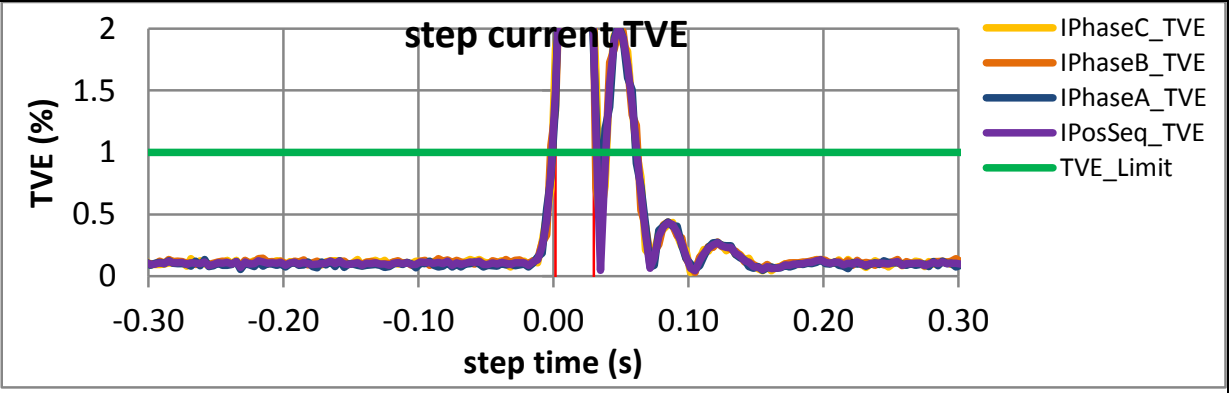

Figure 4338: Fs = 30 FPS, -10 degree phase step

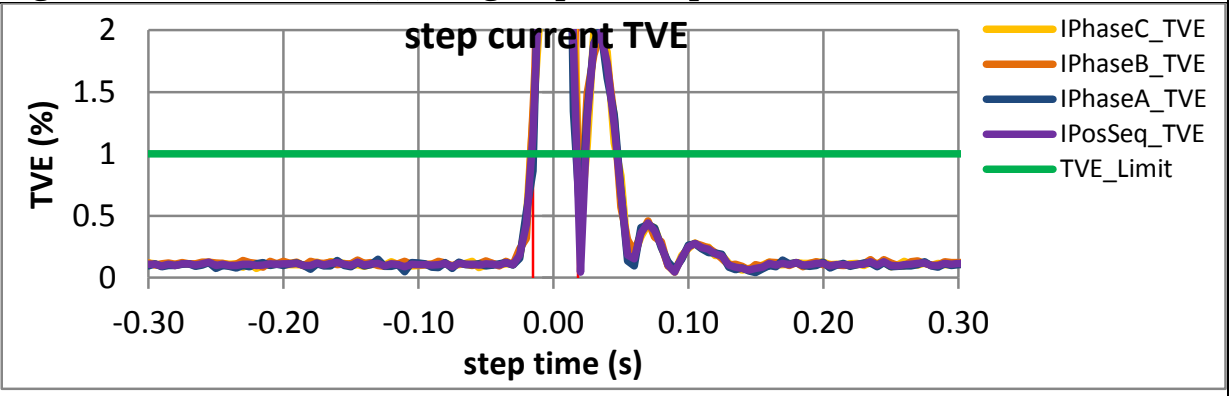

Figure 4340: Fs = 20 FPS, - 10 degree phase step 

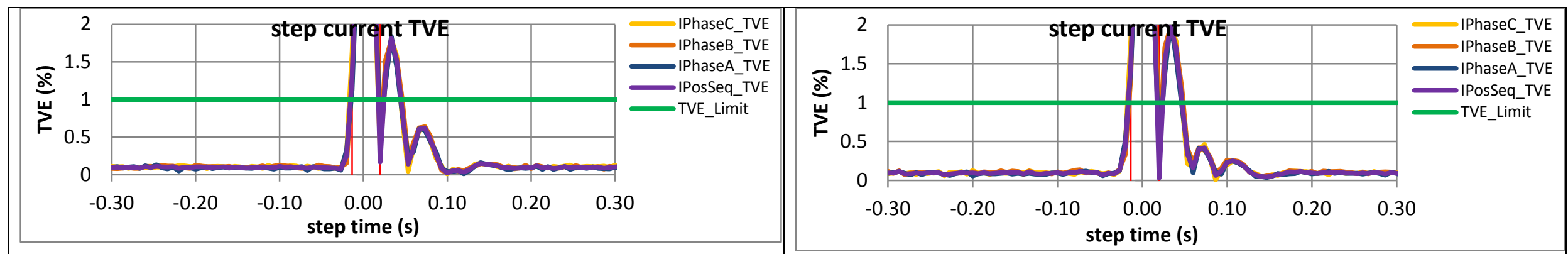

Figure 4341: Fs = 15 FPS, +10 degree phase step

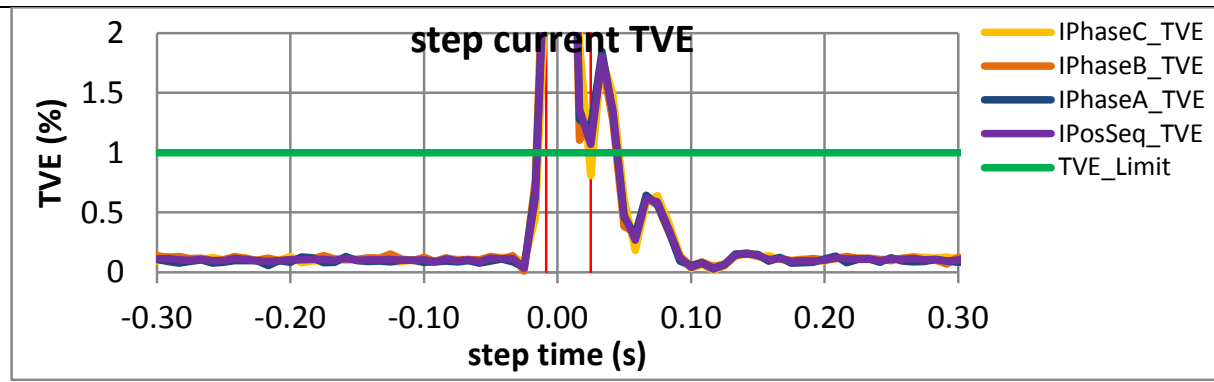

Figure 4342: Fs = 15 FPS, -10 degree phase step

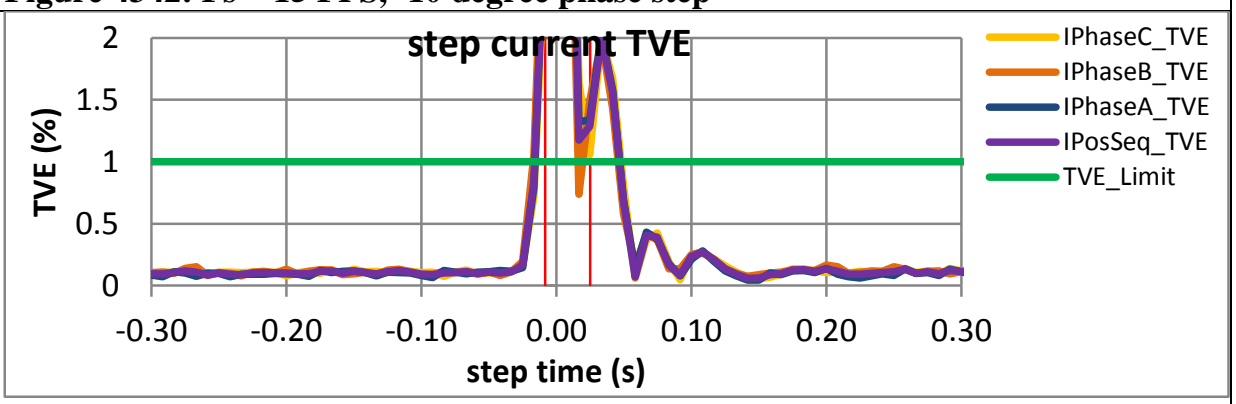

Figure 4343: Fs = 12 FPS, +10 degree phase step

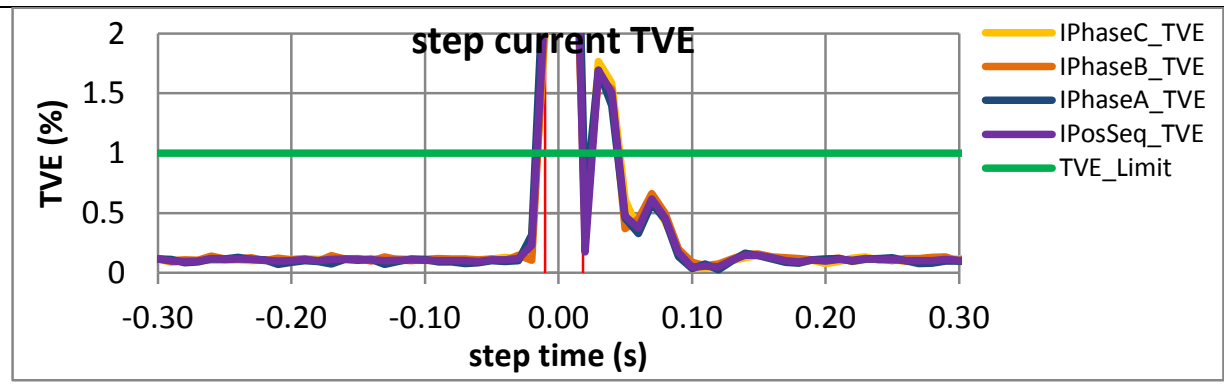

Figure 4344: Fs = 12 FPS, -10 degree phase step

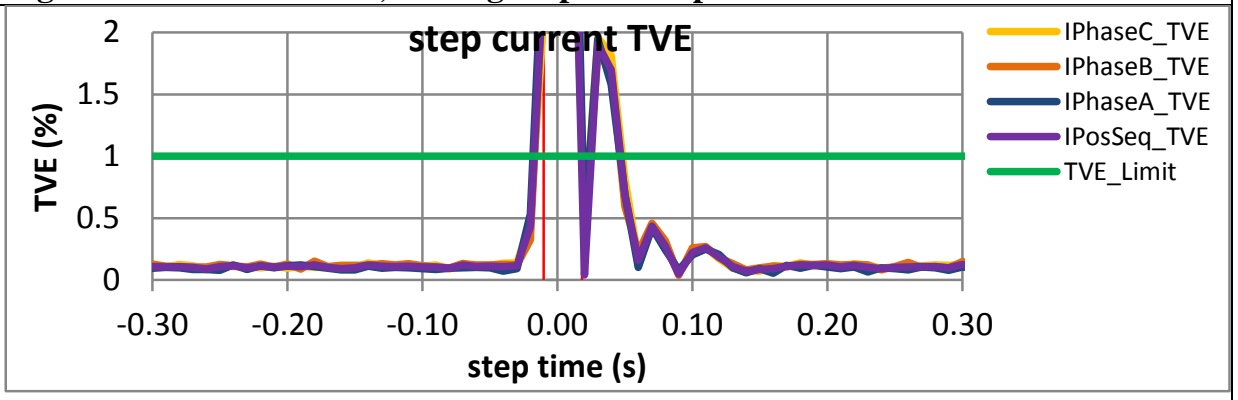

Figure 4346: Fs $=10 \mathrm{FPS},-10$ degree phase step 
9.4.4 PMU C dynamic step change in phase current response time: $F 0=60 \mathrm{~Hz}, \mathrm{P}$ class

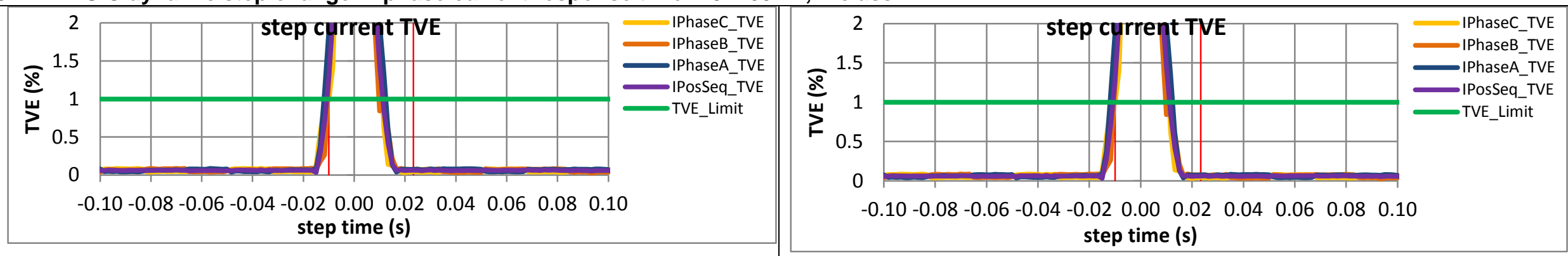

Figure 4347: Fs = 60 FPS, +10 degree phase step

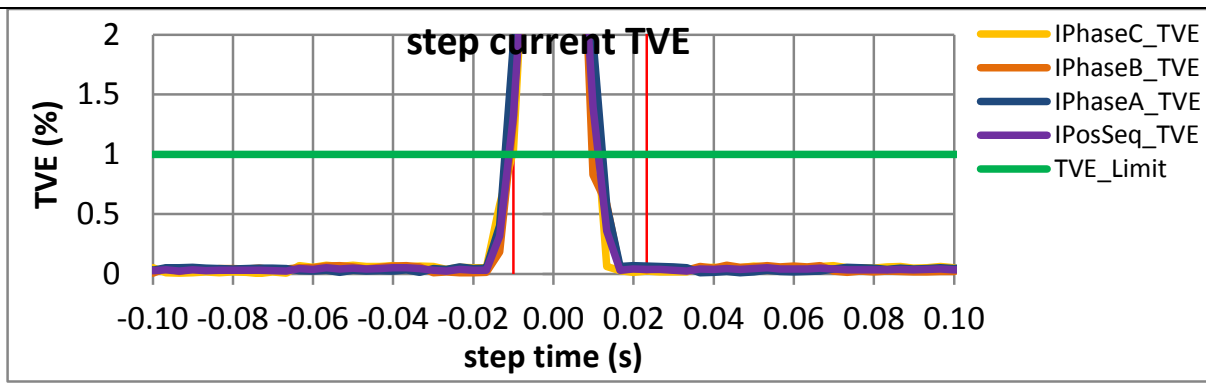

Figure 4349: Fs = 30 FPS, +10 degree phase step

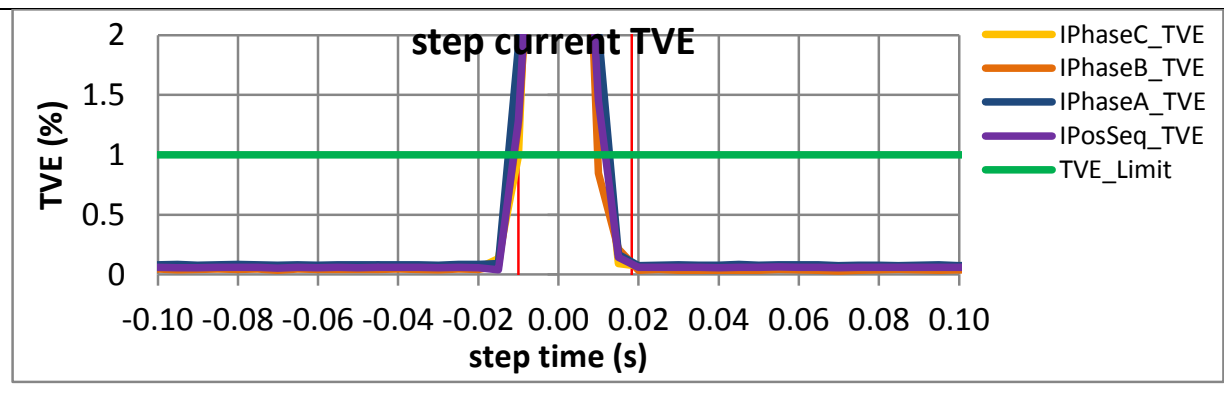

Figure 4351: Fs = 20 FPS, +10 degree phase step

Figure 4348: Fs = 60 FPS, -10 degree phase step

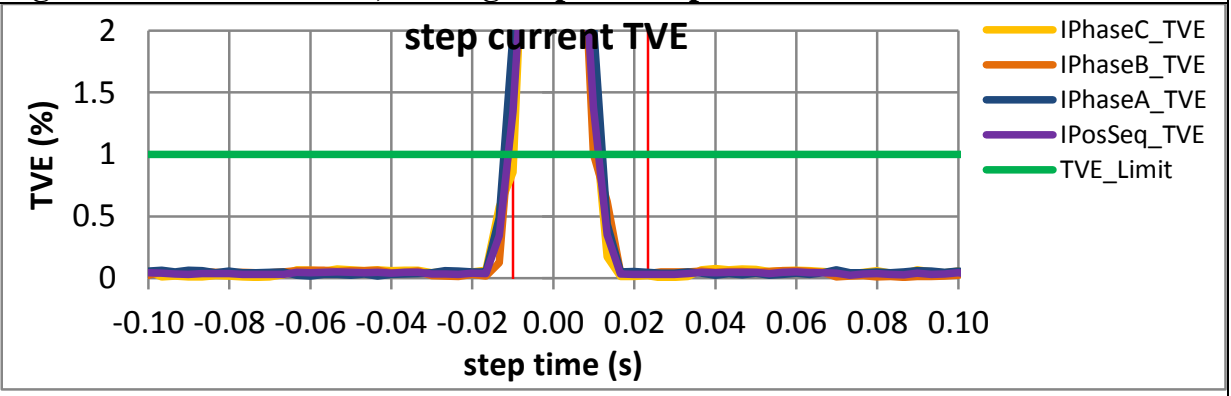

Figure 4350: Fs = 30 FPS, -10 degree phase step

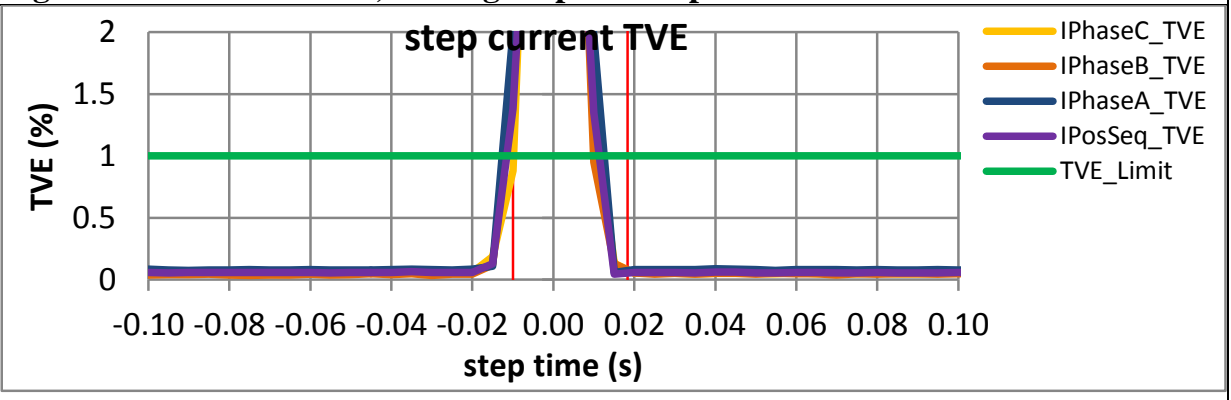

Figure 4352: Fs = 20 FPS, - 10 degree phase step 

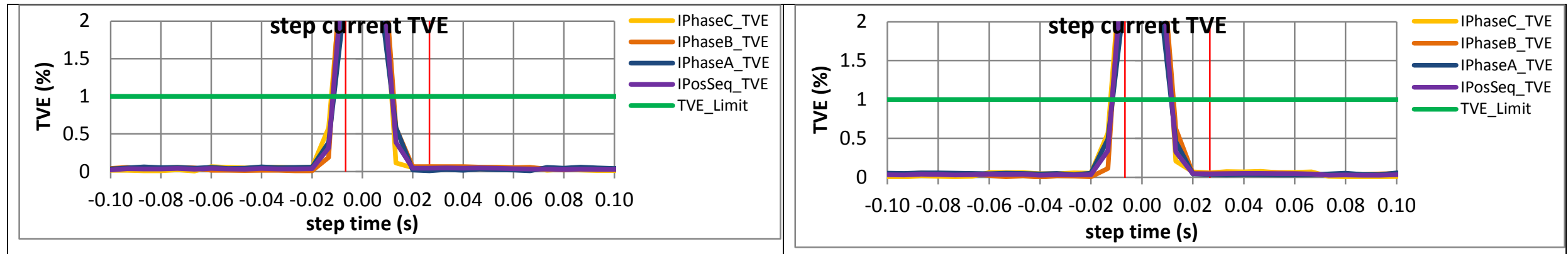

Figure 4353: Fs = 15 FPS, +10 degree phase step

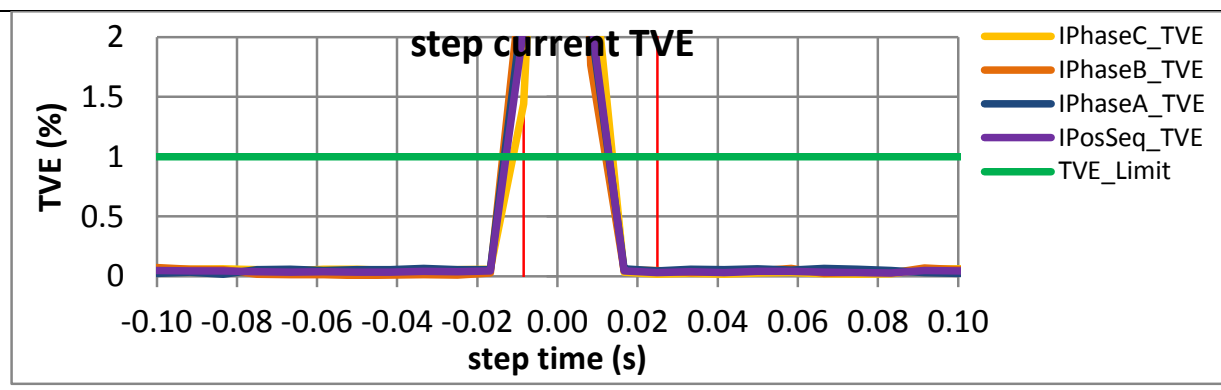

Figure 4354: Fs = 15 FPS, -10 degree phase step

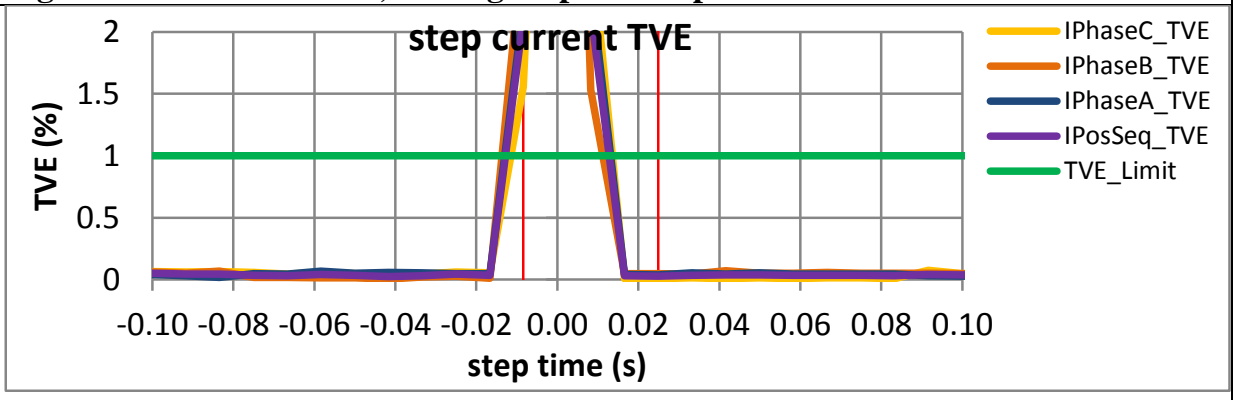

Figure 4355: Fs = 12 FPS, +10 degree phase step
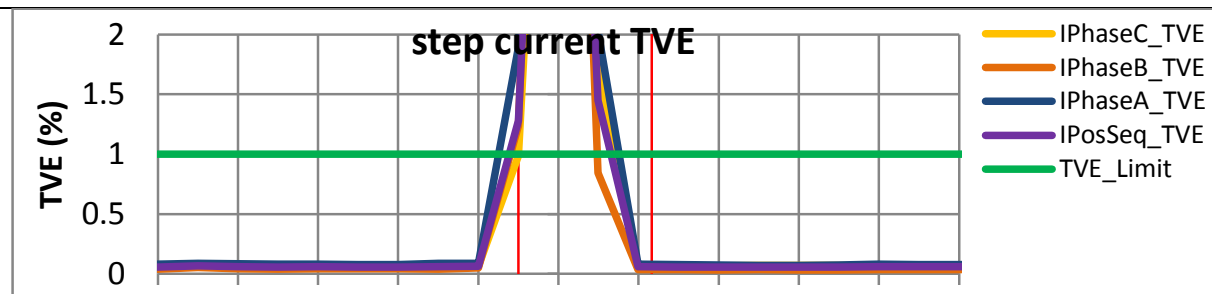

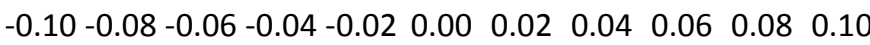
step time (s)

Figure 4357: Fs = 10 FPS, +10 degree phase step

Figure 4356: Fs = 12 FPS, -10 degree phase step

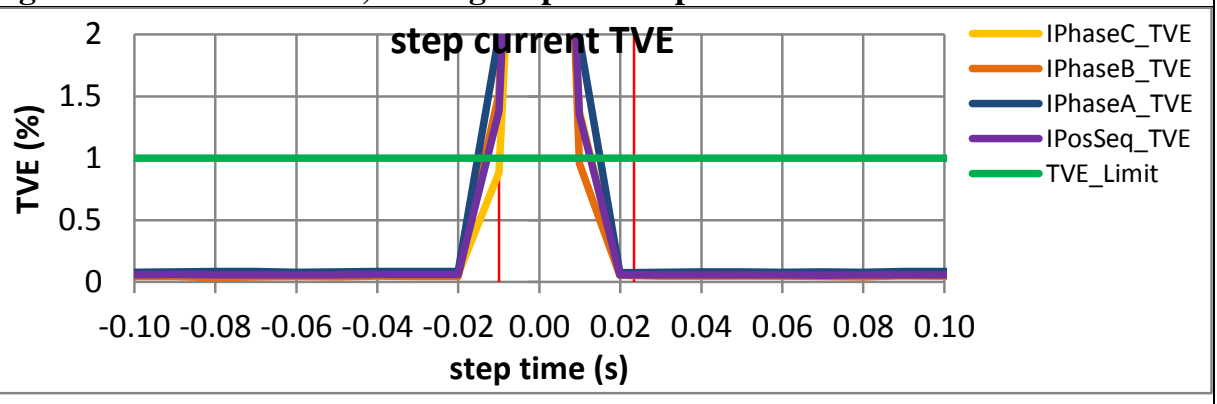

Figure 4358: Fs = 10 FPS, -10 degree phase step 
9.4.5 PMU D dynamic step change in phase current response time: $F 0=60 \mathrm{~Hz}, P$ class
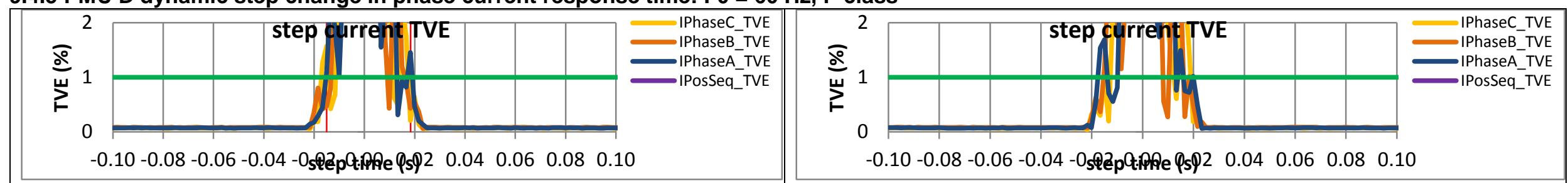

Figure 4359: Fs = 60 FPS, +10 degree phase step

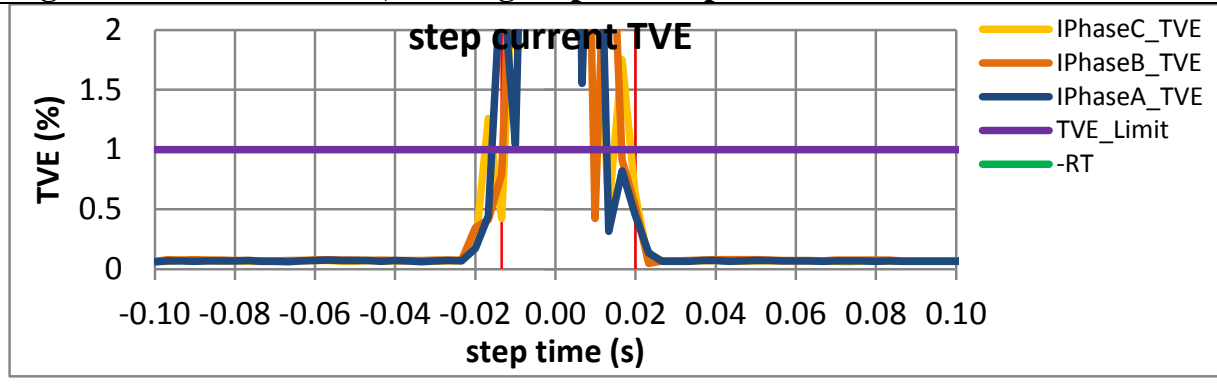

Figure 4360: Fs = 60 FPS, -10 degree phase step

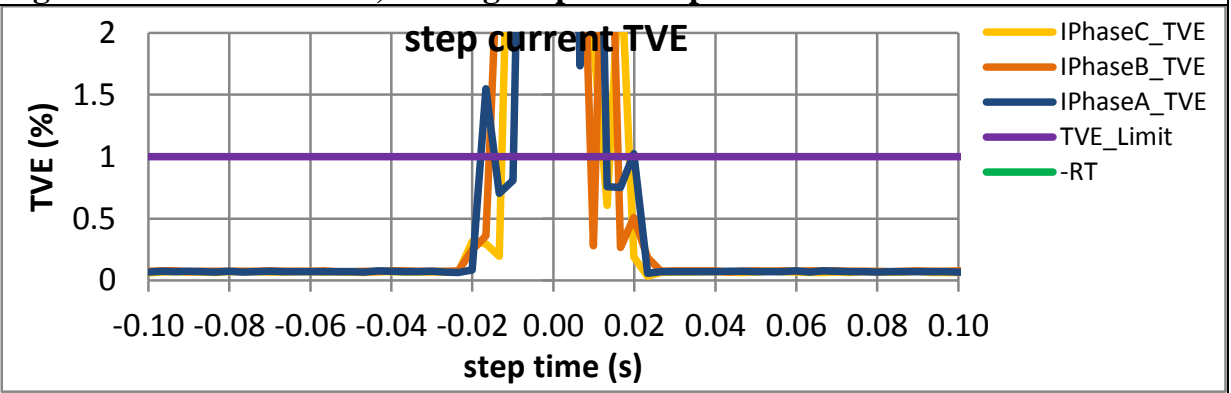

Figure 4361: Fs = 30 FPS, +10 degree phase step

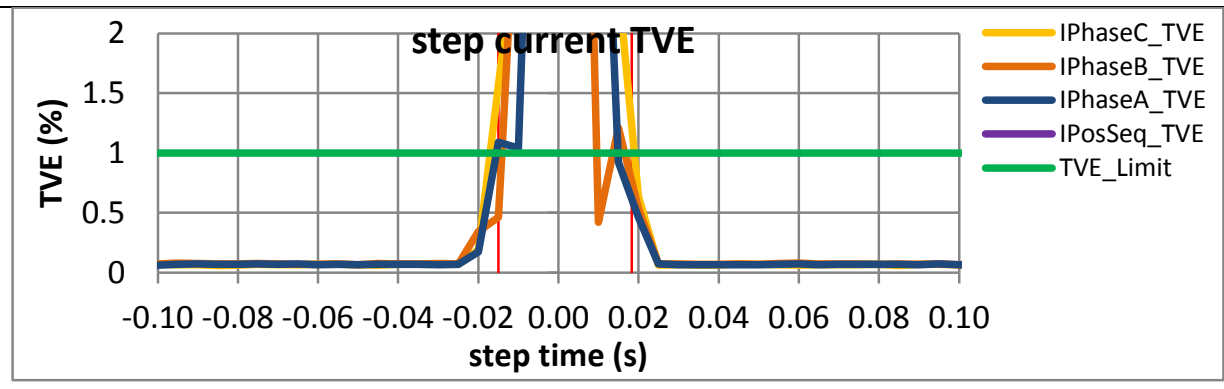

Figure 4362: Fs = 30 FPS, -10 degree phase step

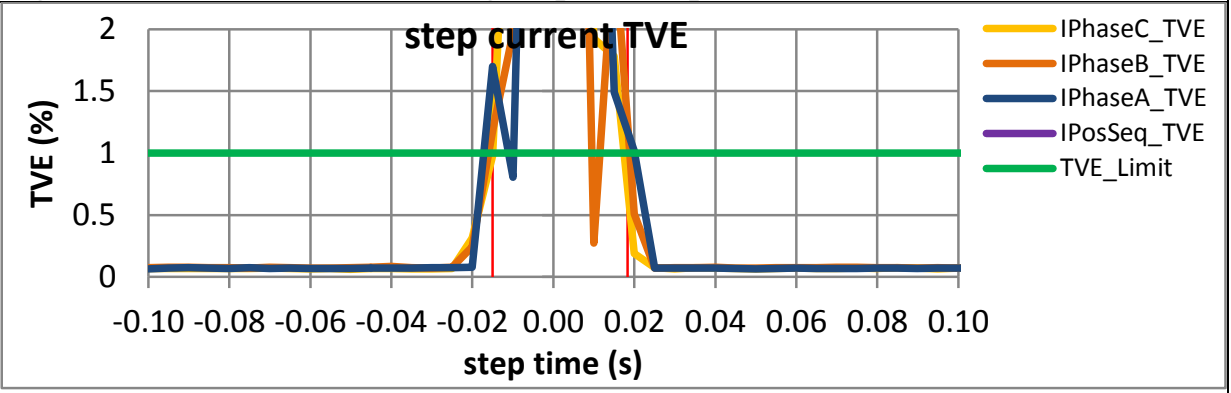

Figure 4363: Fs = 20 FPS, +10 degree phase step

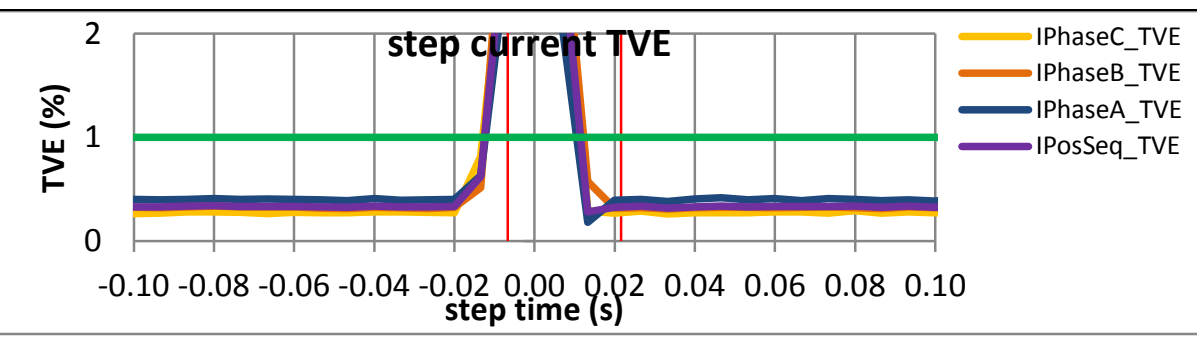

Figure 4364: Fs = 20 FPS, - 10 degree phase step

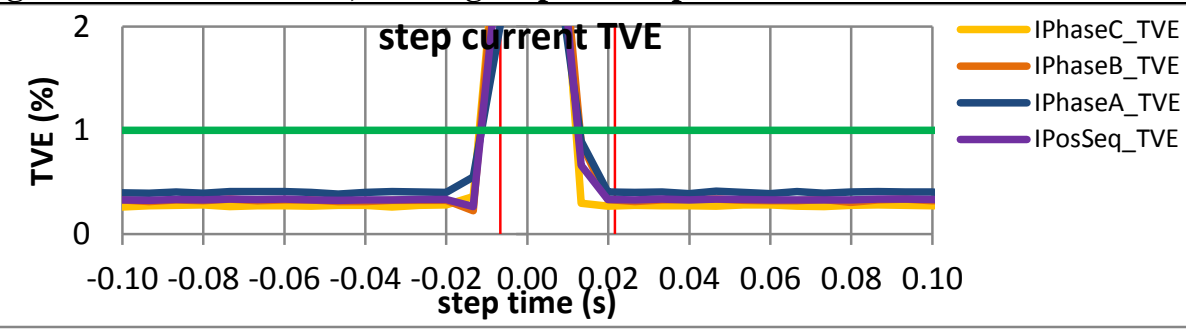

Figure 4366: Fs = 15 FPS, -10 degree phase step 


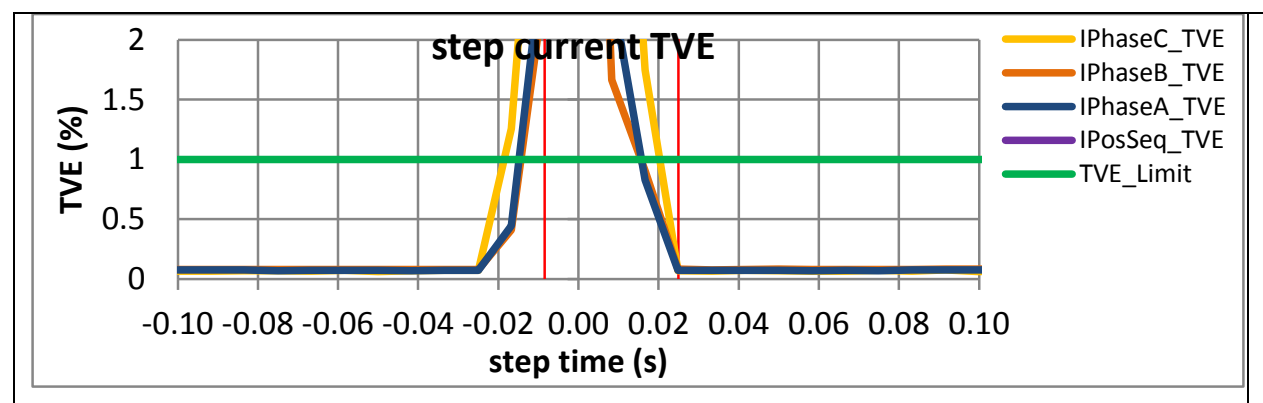

Figure 4367: Fs $=12$ FPS, +10 degree phase step

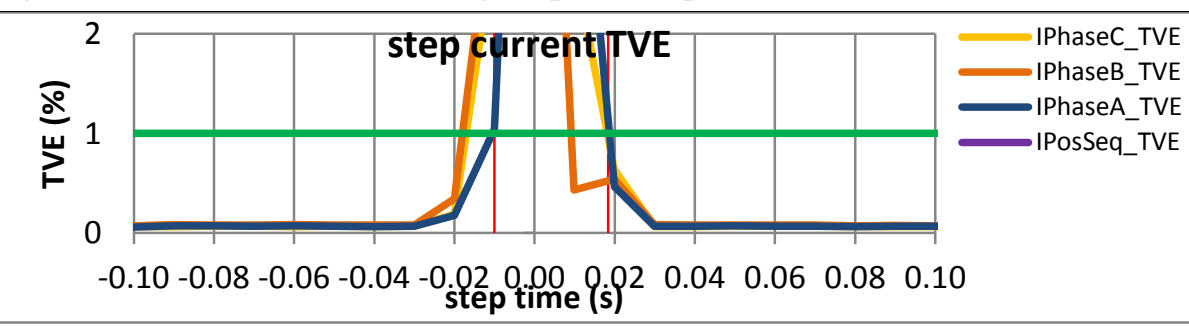

Figure 4369: Fs = 10 FPS, +10 degree phase step

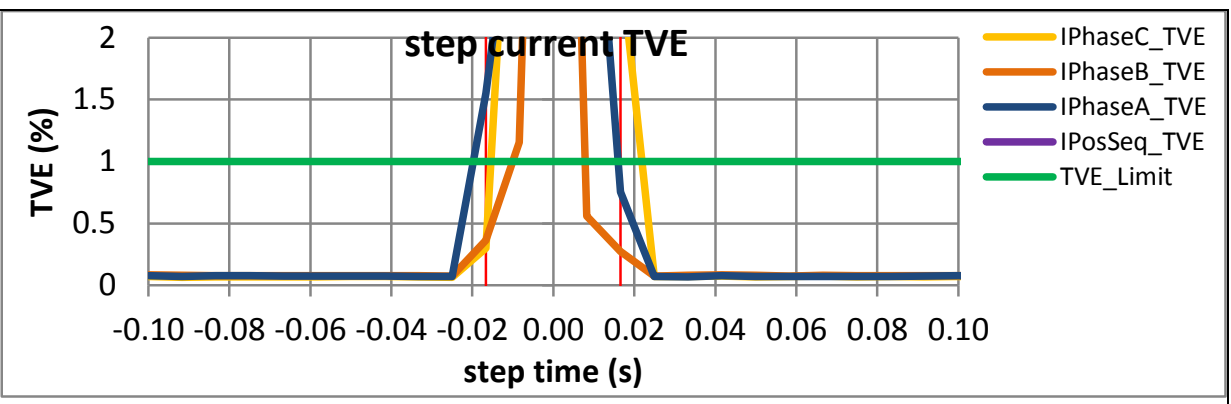

Figure 4368: Fs = 12 FPS, -10 degree phase step

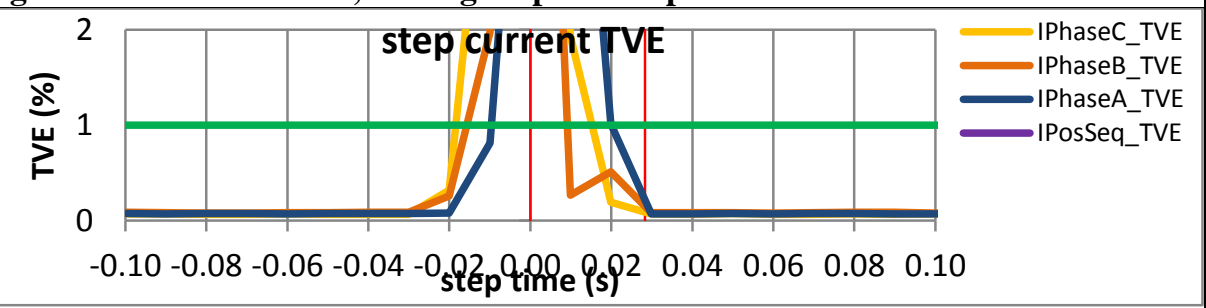

Figure 4370: Fs = 10 FPS, -10 degree phase step 
9.4.6 PMU E dynamic step change in phase current response time: $F 0=60 \mathrm{~Hz}, \mathrm{P}$ class

PMU E does not support P class.

\subsubsection{PMU F dynamic step change in phase current response time: $F 0=60 \mathrm{~Hz}, \mathrm{P}$ class}

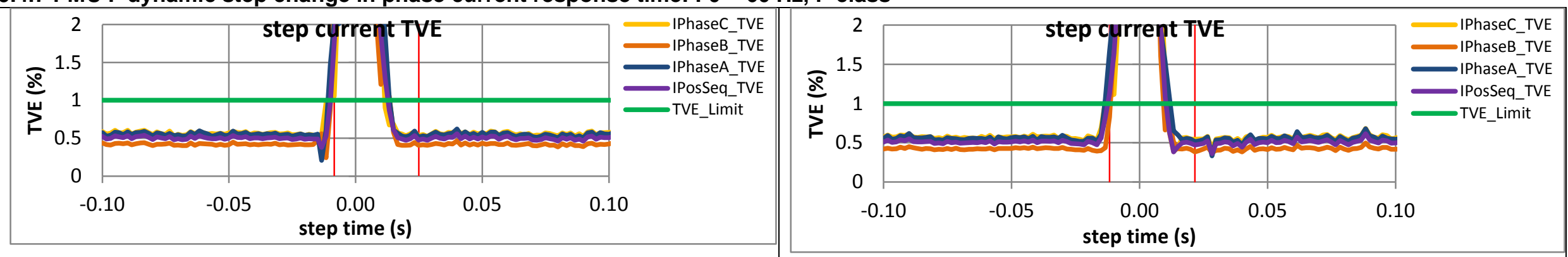

Figure 4371: Fs = 60 FPS, +10 degree phase step

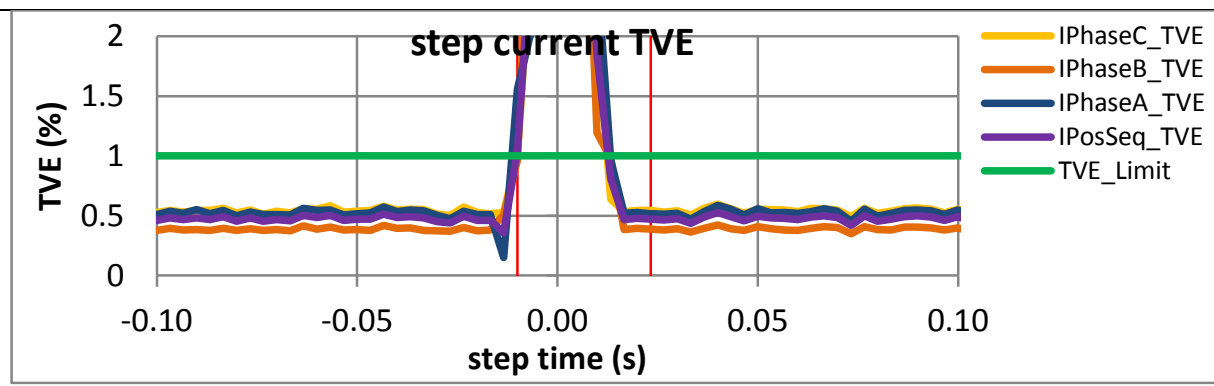

Figure 4372: Fs = 60 FPS, -10 degree phase step

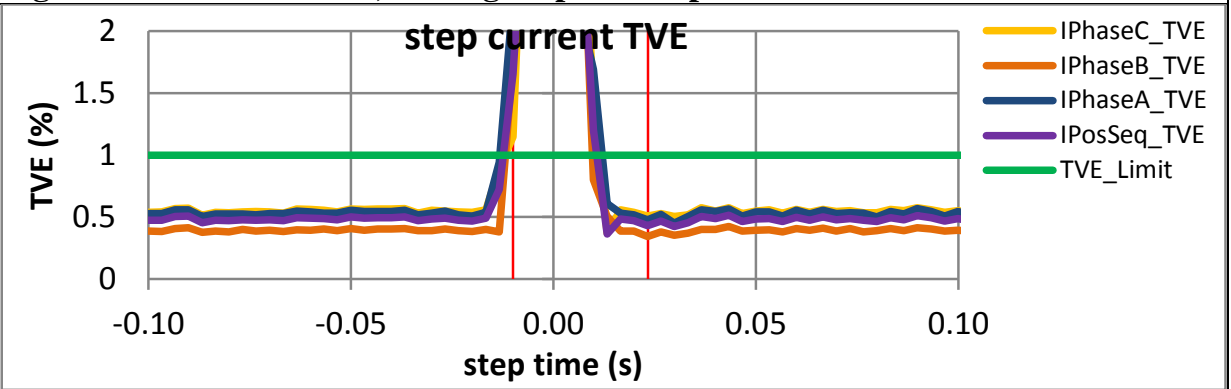

Figure 4373: Fs $=30$ FPS, +10 degree phase step

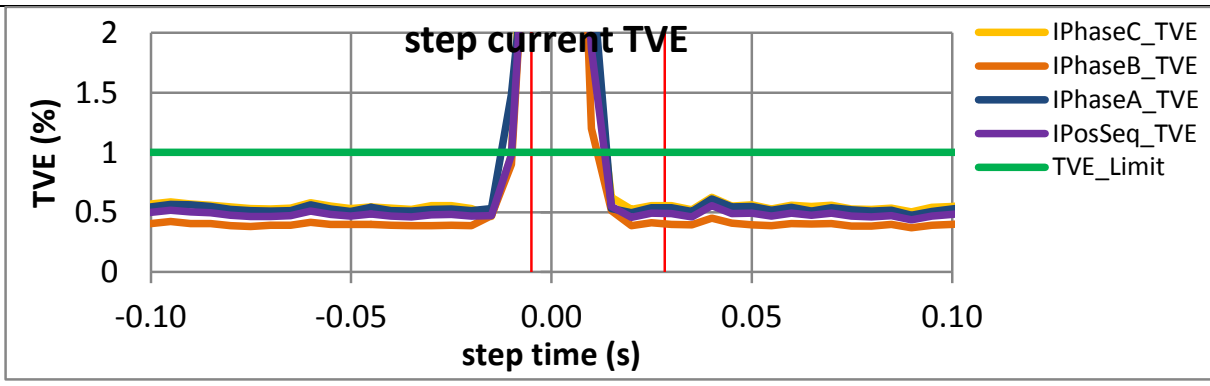

Figure 4374: Fs = 30 FPS, -10 degree phase step

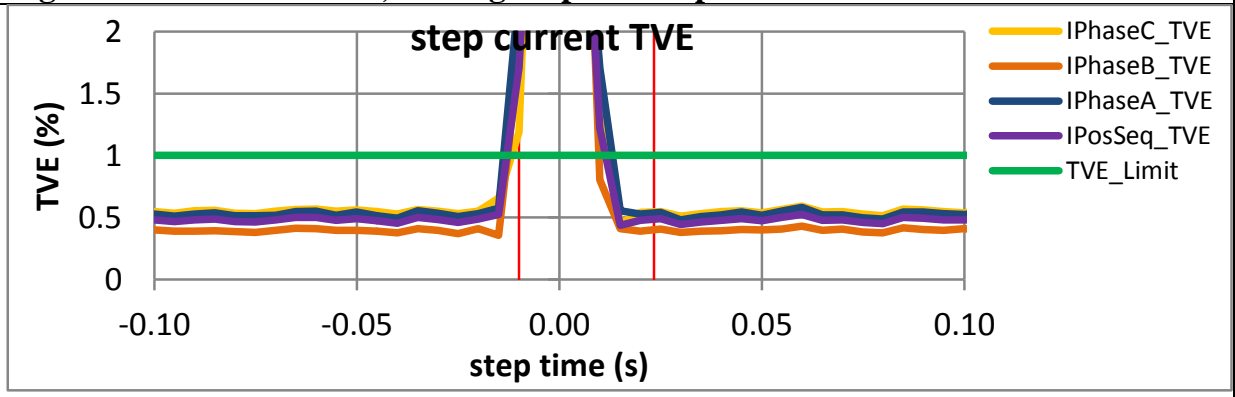

Figure 4375: Fs = 20 FPS, +10 degree phase step

Figure 4376: Fs = 20 FPS, - 10 degree phase step 


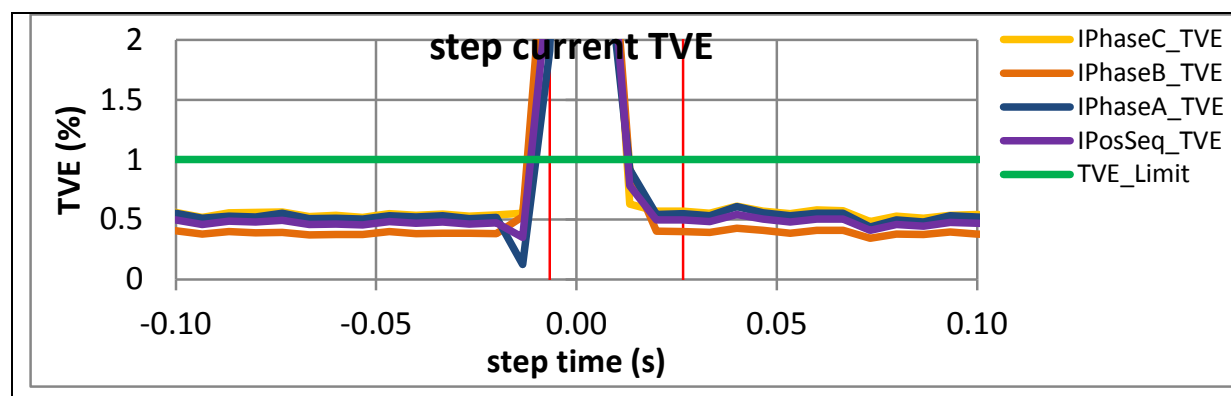

Figure 4377: Fs = 15 FPS, +10 degree phase step

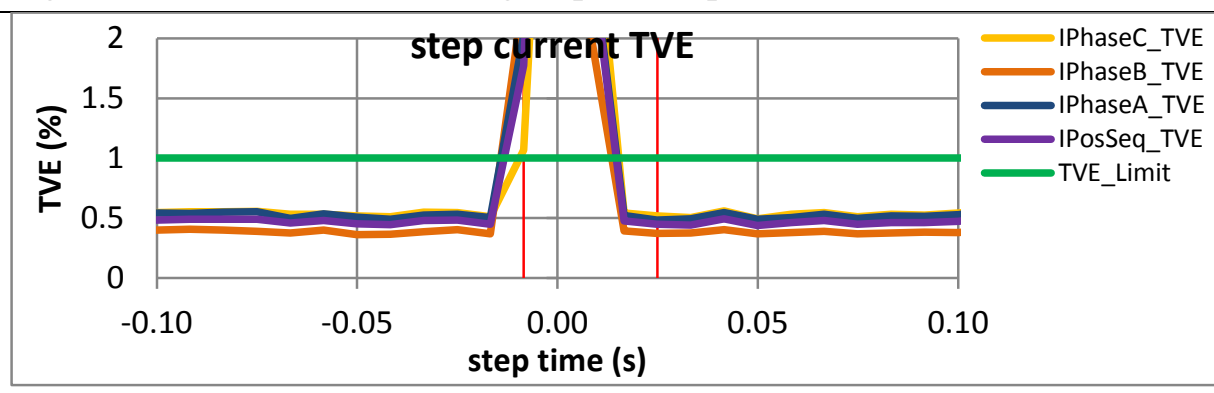

Figure 4379: Fs = 12 FPS, +10 degree phase step

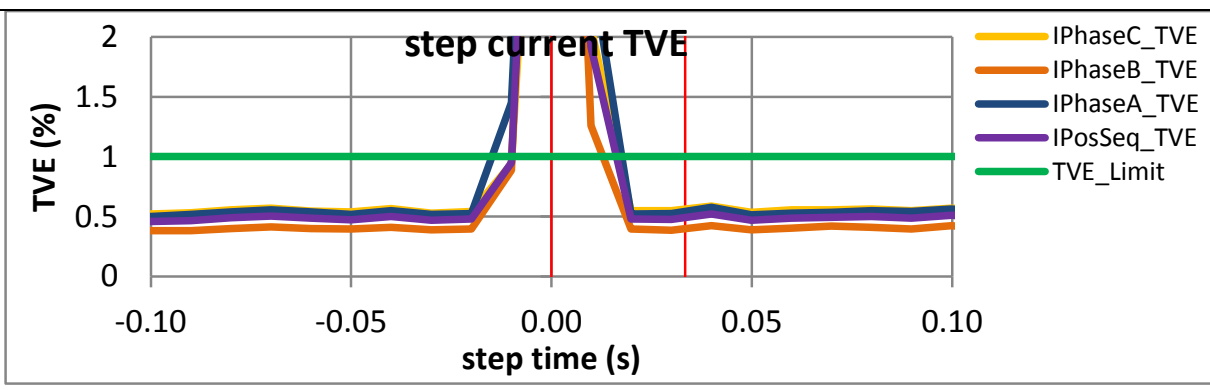

Figure 4381: Fs = 10 FPS, +10 degree phase step

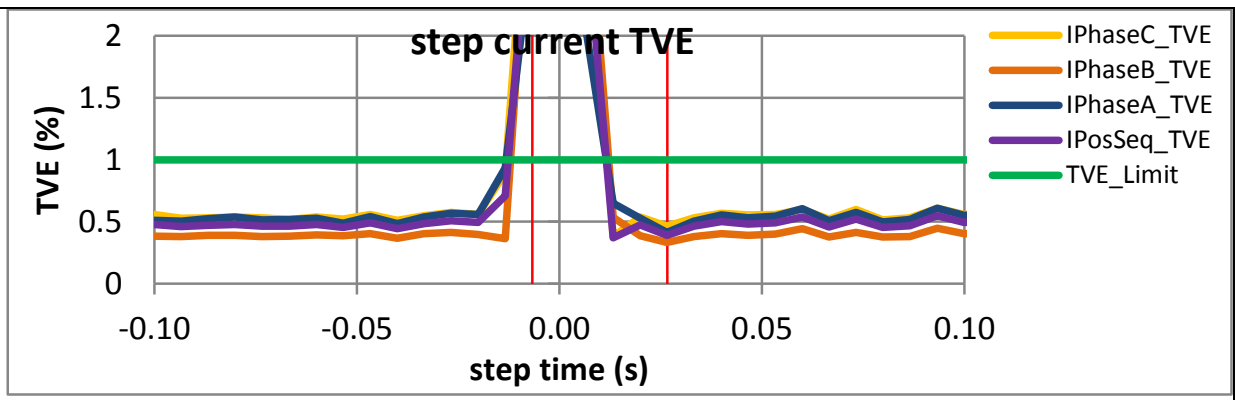

Figure 4378: Fs = 15 FPS, -10 degree phase step

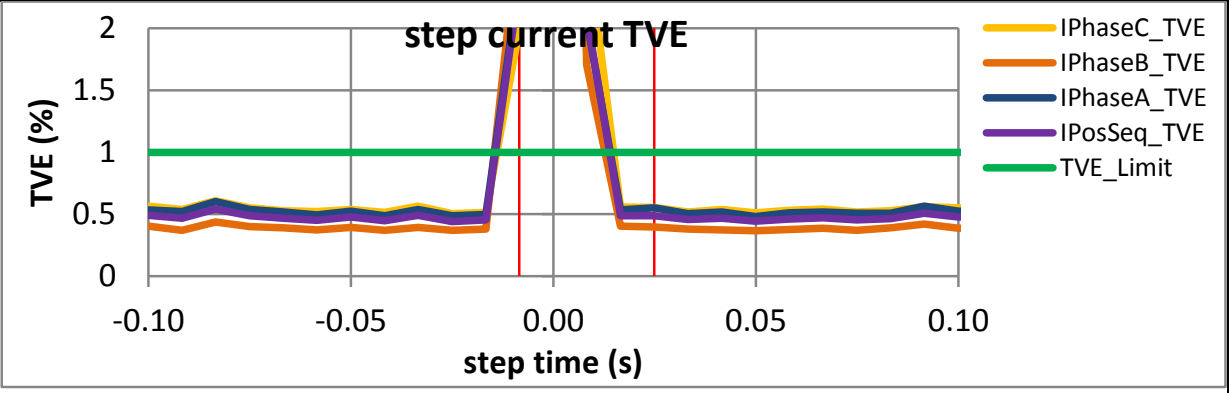

Figure 4380: Fs = 12 FPS, -10 degree phase step

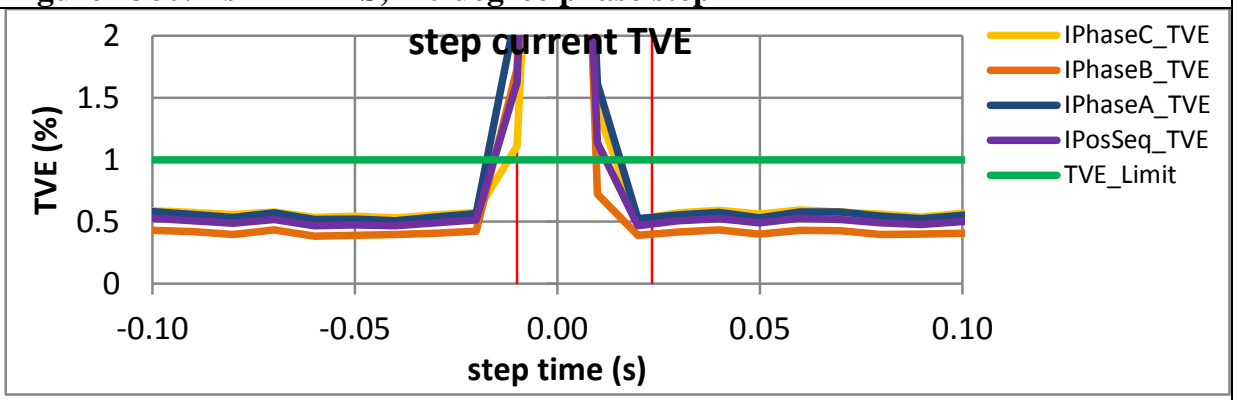

Figure 4382: Fs = 10 FPS, -10 degree phase step 
9.4.8 PMU G dynamic step change in phase current response time: $\mathrm{F} 0=60 \mathrm{~Hz}$, P class PMU G does not support P class.

\subsubsection{PMU H dynamic step change in phase current response time: $\mathrm{FO}=60 \mathrm{~Hz}, \mathrm{P}$ class}
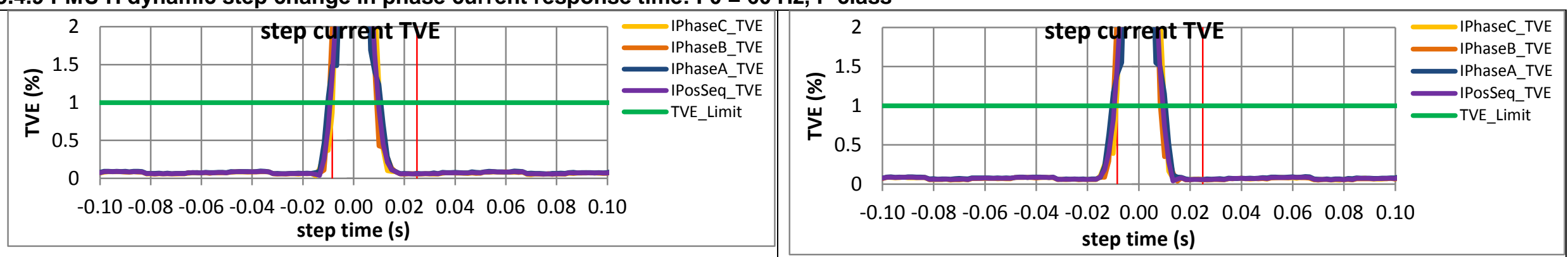

Figure 4383: Fs = 60 FPS, +10 degree phase step

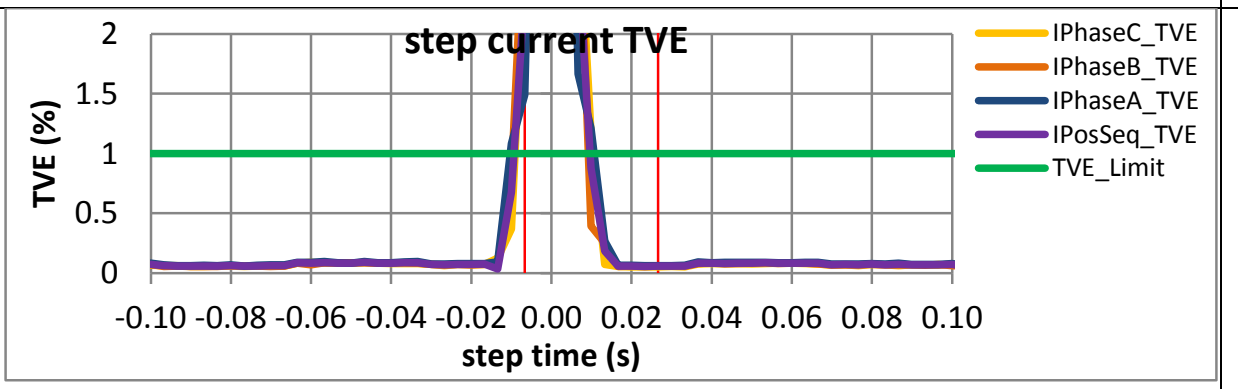

Figure 4384: Fs = 60 FPS, -10 degree phase step

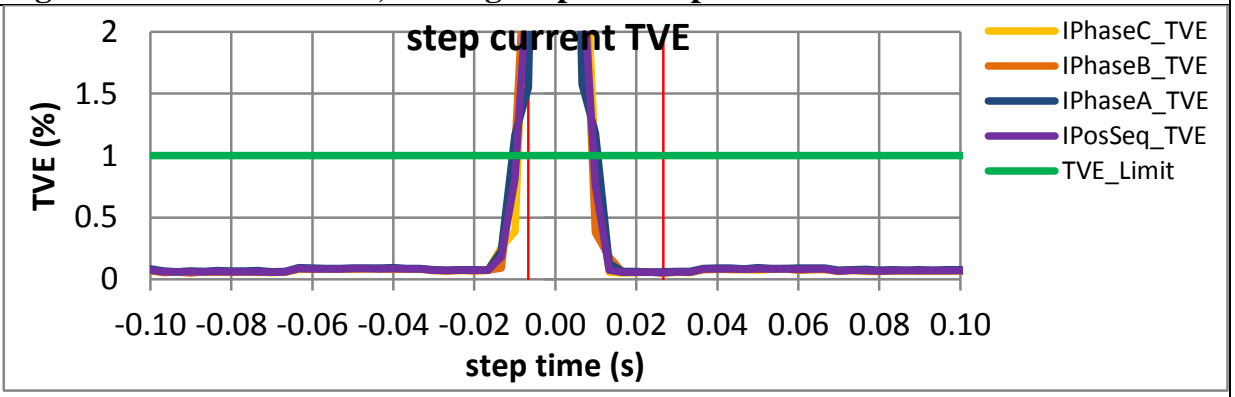

Figure 4385: Fs = 30 FPS, +10 degree phase step

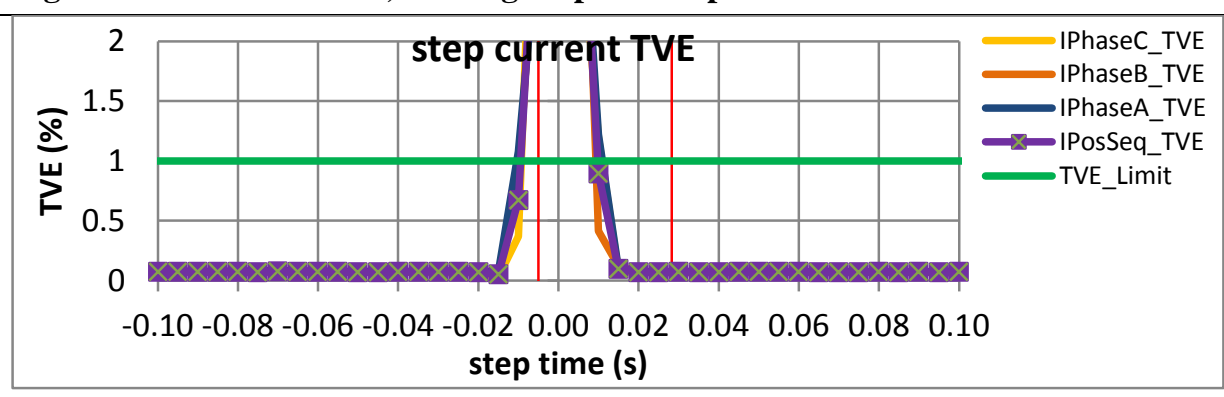

Figure 4387: Fs = 20 FPS, +10 degree phase step

Figure 4386: Fs = 30 FPS, -10 degree phase step

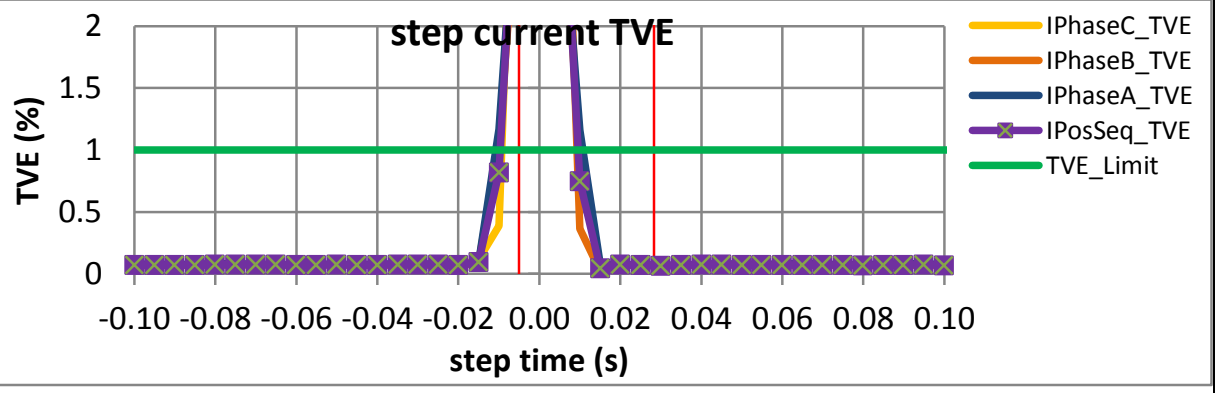

Figure 4388: Fs = 20 FPS, - 10 degree phase step 

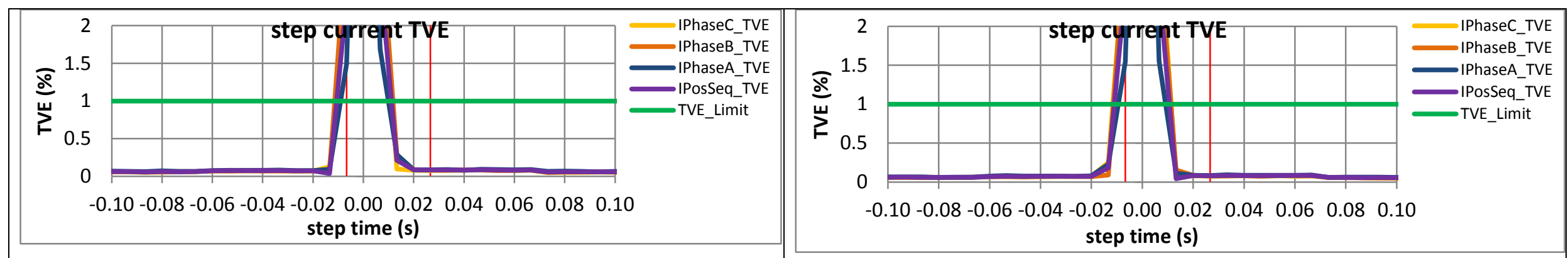

Figure 4389: Fs $=15$ FPS, +10 degree phase step

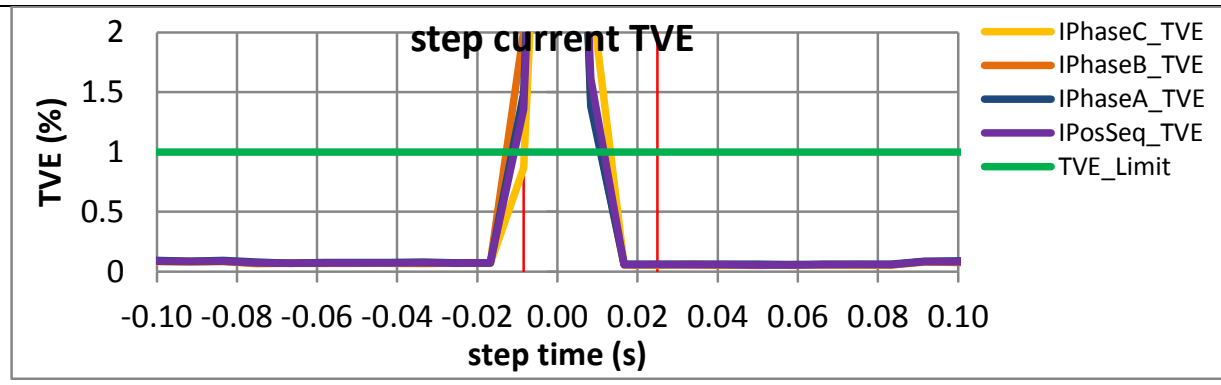

Figure 4390: Fs = 15 FPS, -10 degree phase step

Figure 4391: Fs = 12 FPS, +10 degree phase step

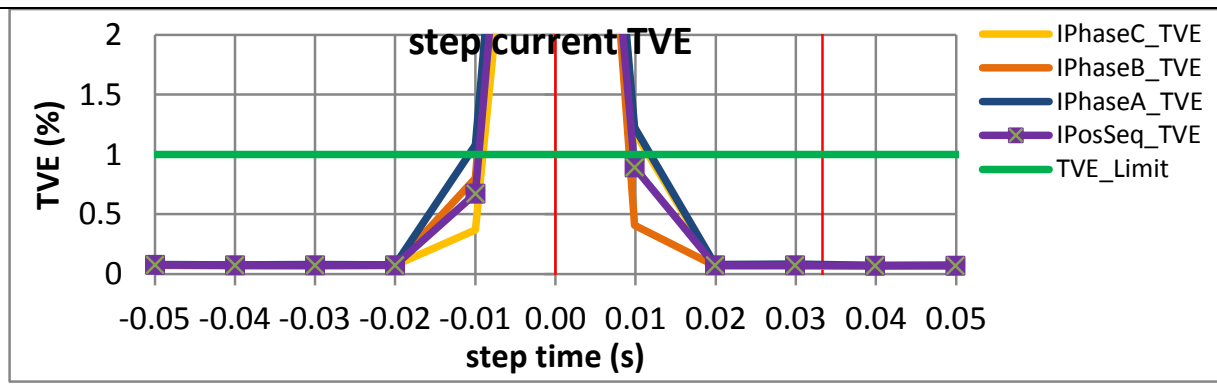

Figure 4393: Fs = 10 FPS, +10 degree phase step

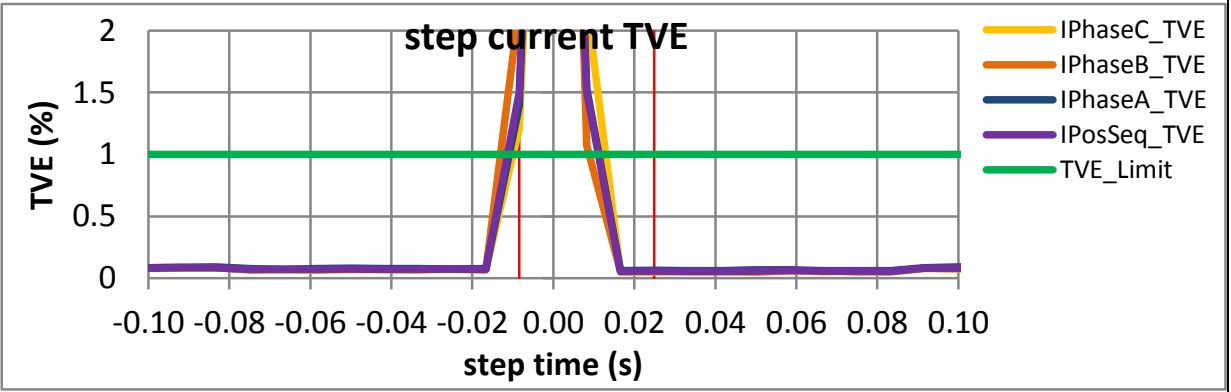

Figure 4392: Fs = 12 FPS, -10 degree phase step

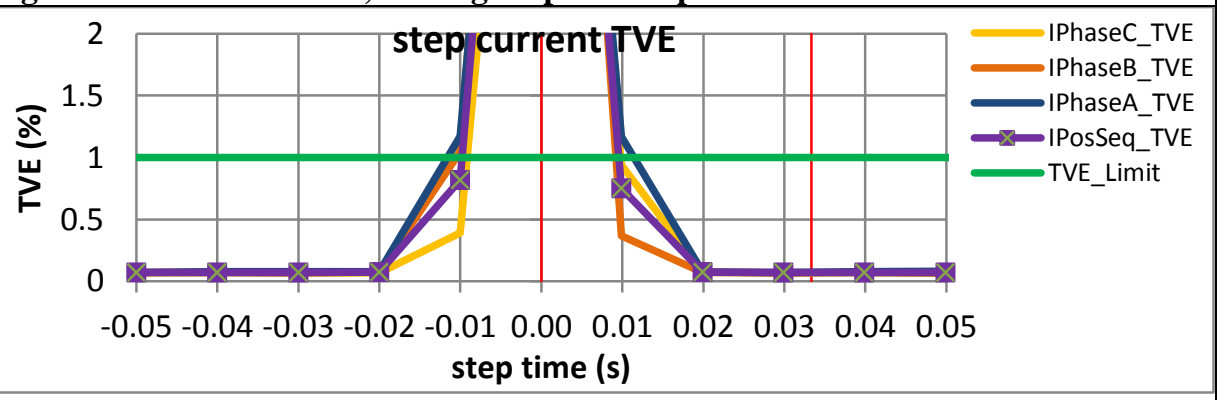

Figure 4394: Fs = 10 FPS, -10 degree phase step

9.4.10 PMU I dynamic step change in phase current response time: $\mathrm{F} 0=60 \mathrm{~Hz}, \mathrm{P}$ class

PMU I does not support P class 
9.4.11 PMU J dynamic step change in phase current response time: $\mathrm{FO}=60 \mathrm{~Hz}, \mathrm{P}$ class
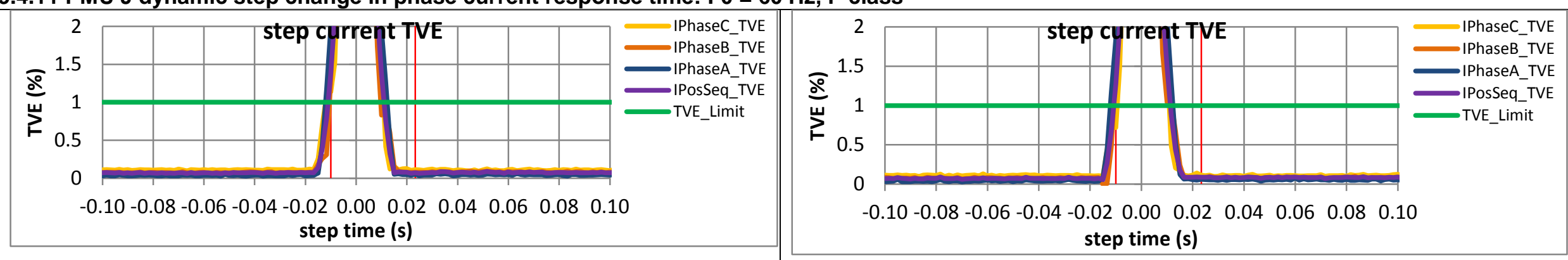

Figure 4395: Fs = 60 FPS, +10 degree phase step

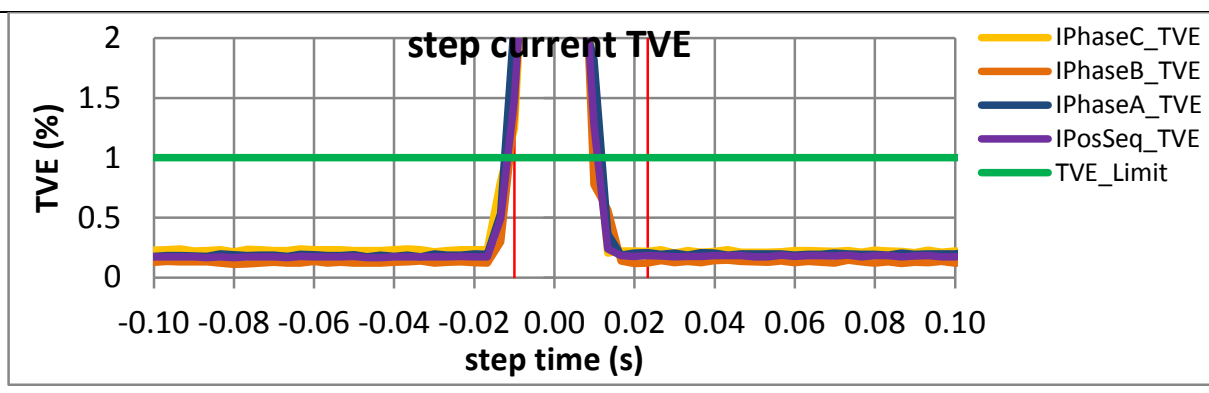

Figure 4396: Fs = $60 \mathrm{FPS},-10$ degree phase step

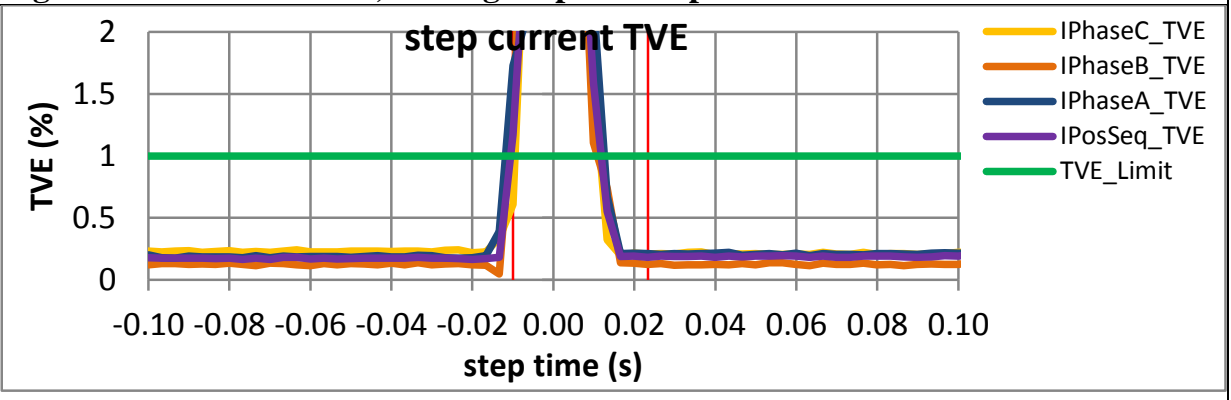

Figure 4397: Fs = 30 FPS, +10 degree phase step

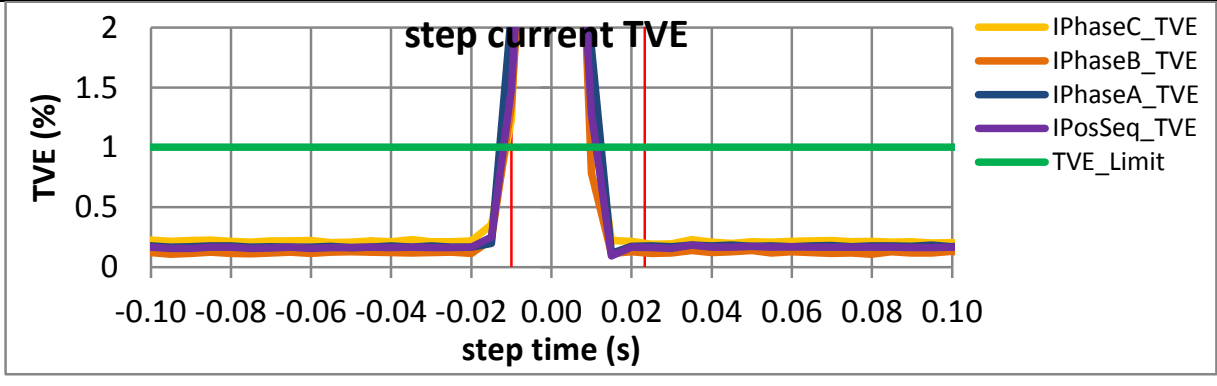

Figure 4399: Fs = 20 FPS, +10 degree phase step

Figure 4398: Fs = 30 FPS, -10 degree phase step

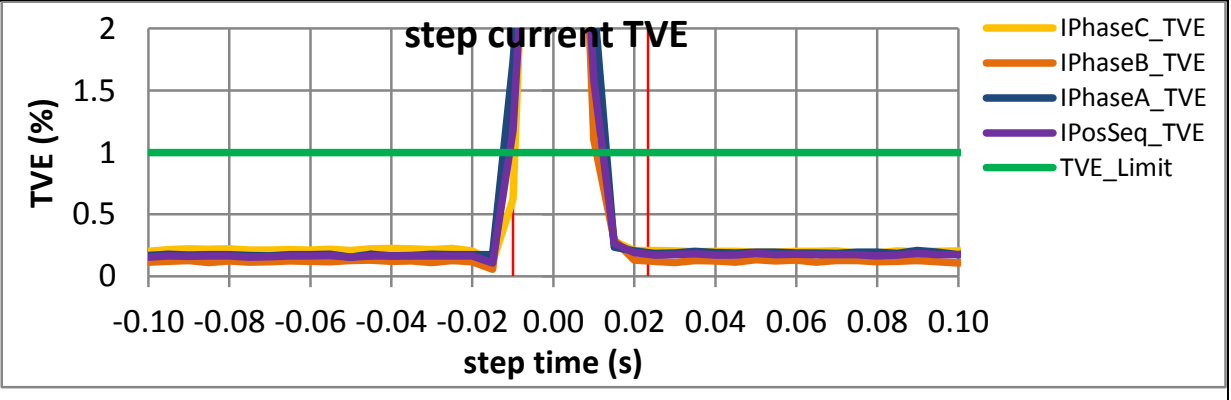

Figure 4400: Fs = $20 \mathrm{FPS},-10$ degree phase step 

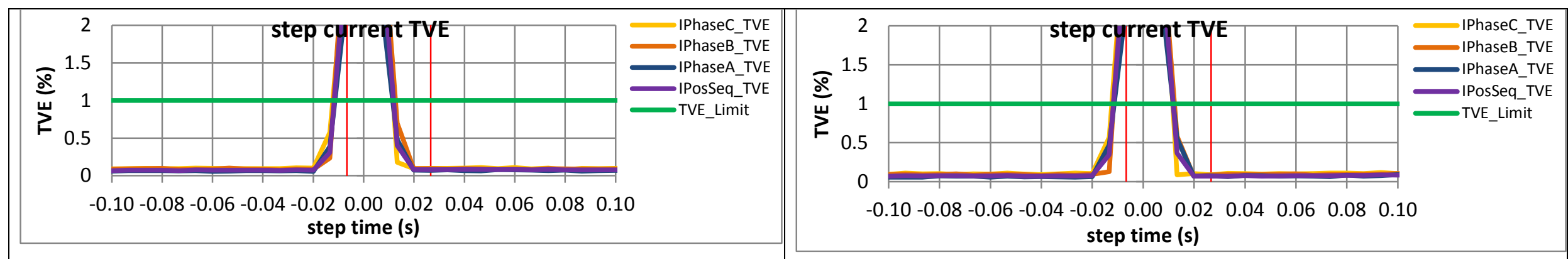

Figure 4401: Fs = 15 FPS, +10 degree phase step

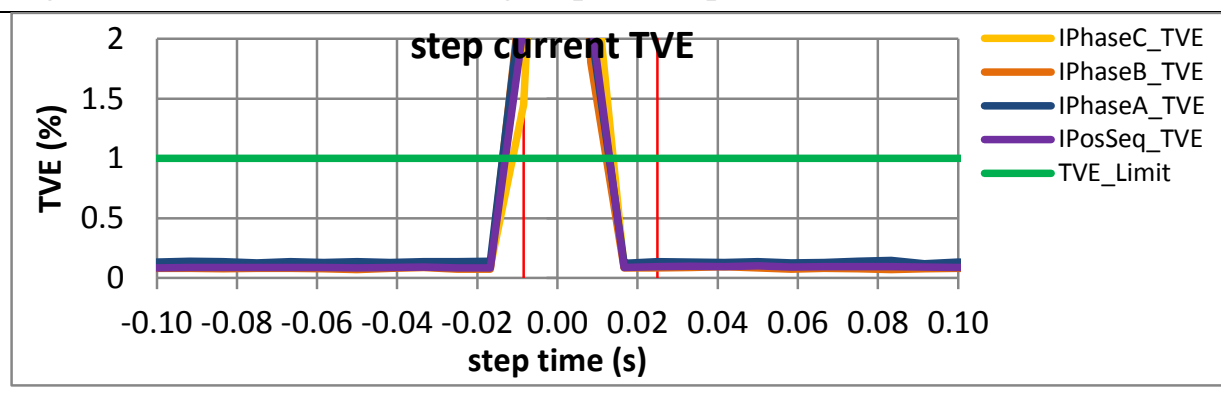

Figure 4402: Fs = 15 FPS, -10 degree phase step

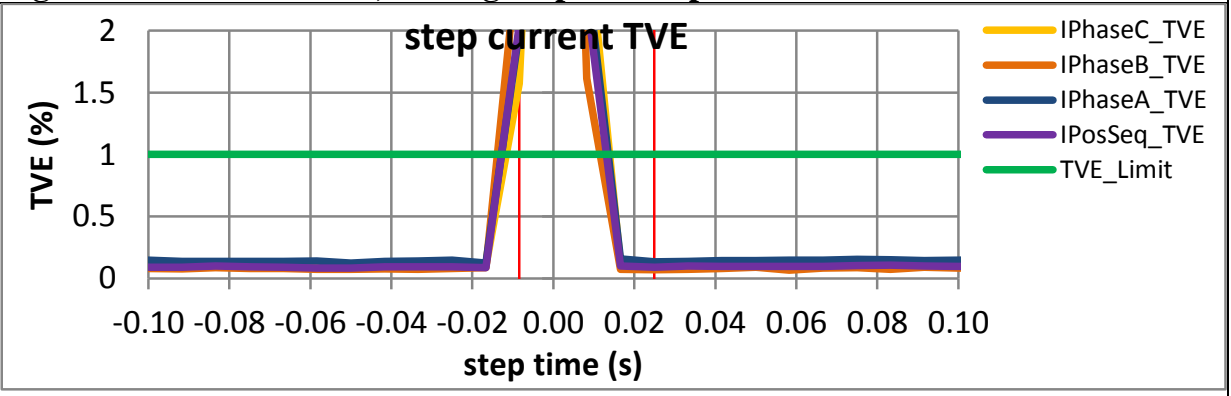

Figure 4403: Fs = 12 FPS, +10 degree phase step

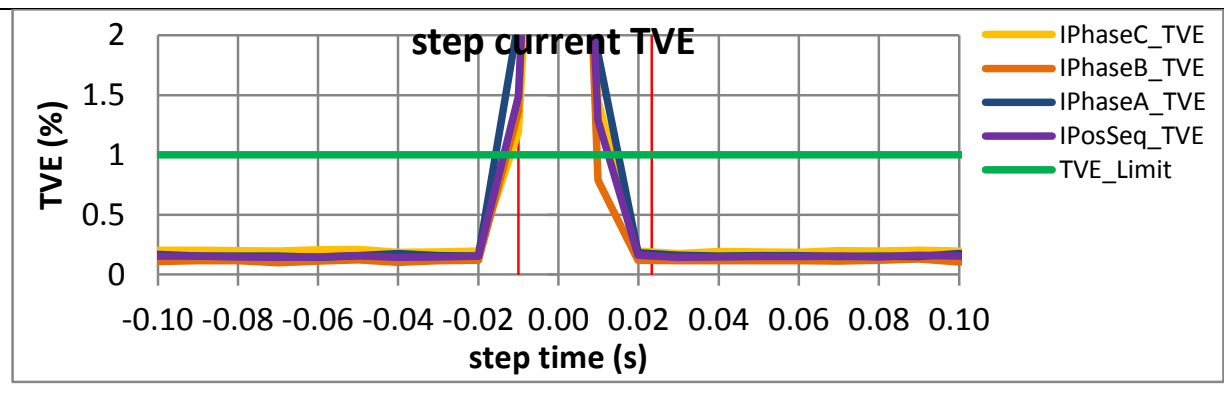

Figure 4404: Fs = 12 FPS, -10 degree phase step

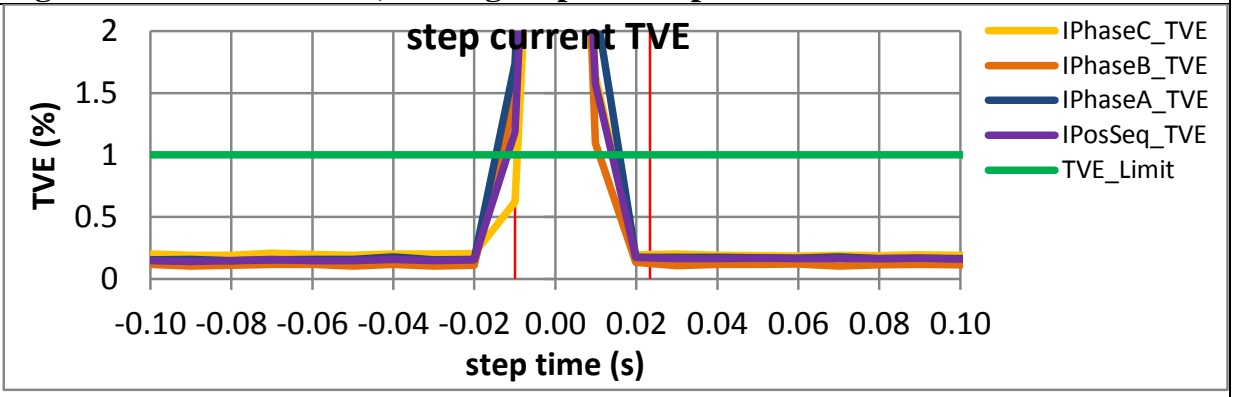

Figure 4406: Fs = 10 FPS, -10 degree phase step 
9.5 Dynamic step change in phase: frequency response time

\begin{tabular}{|c|c|c|c|c|c|c|c|c|c|c|c|c|}
\hline Fs (FPS) & $10 \mathrm{M}$ & $10 P$ & $12 \mathrm{M}$ & $12 \mathrm{P}$ & $15 \mathrm{M}$ & $15 P$ & $20 \mathrm{M}$ & $20 P$ & $30 \mathrm{M}$ & $30 P$ & $60 M$ & $60 P$ \\
\hline C37.118.1 Annex C & $\mathrm{P}$ & $P$ & $\mathrm{P}$ & $\mathrm{P}$ & $\mathrm{P}$ & $\mathrm{P}$ & $\mathrm{P}$ & $\mathrm{P}$ & $\mathrm{P}$ & $\mathrm{P}$ & $\mathrm{P}$ & $P$ \\
\hline PMU A & $\mathrm{P}$ & $\mathrm{P}$ & $\mathrm{P}$ & $\mathrm{P}$ & $\mathrm{P}$ & $\mathrm{P}$ & $\mathrm{P}$ & $\mathrm{P}$ & $\mathrm{P}$ & $\mathrm{P}$ & $\mathrm{P}$ & $\mathrm{P}$ \\
\hline PMU B & $P$ & $\mathrm{~F}$ & $P$ & $\mathrm{~F}$ & $P$ & $\mathrm{~F}$ & $P$ & $\mathrm{~F}$ & $\mathrm{P}$ & $\mathrm{F}$ & $\mathrm{P}$ & $\mathrm{F}$ \\
\hline PMU C & $\mathrm{P}$ & $\mathrm{F}$ & $\mathrm{P}$ & $\mathrm{F}$ & $\mathrm{P}$ & $\mathrm{F}$ & $\mathrm{P}$ & $\mathrm{F}$ & $\mathrm{P}$ & $\mathrm{P}$ & $\mathrm{P}$ & $\mathrm{F}$ \\
\hline PMU D & $\mathrm{P}$ & $\mathrm{P}$ & $\mathrm{P}$ & $\mathrm{P}$ & $\mathrm{P}$ & $P$ & $\mathrm{P}$ & $\mathrm{P}$ & $P$ & $P$ & $P$ & $\mathrm{P}$ \\
\hline PMU E & $\mathrm{P}$ & - & - & - & $\mathrm{P}$ & - & $\mathrm{P}$ & - & $\mathrm{P}$ & - & $\mathrm{P}$ & - \\
\hline PMU F & $\mathrm{P}$ & $\mathrm{P}$ & $\mathrm{P}$ & $\mathrm{P}$ & $\mathrm{P}$ & $\mathrm{F}$ & $\mathrm{P}$ & $\mathrm{F}$ & $\mathrm{P}$ & $\mathrm{F}$ & $\mathrm{P}$ & $\mathrm{F}$ \\
\hline PMU G & $\mathrm{P}$ & - & $\mathrm{P}$ & - & $\mathrm{P}$ & - & $\mathrm{P}$ & - & $\mathrm{P}$ & - & $\mathrm{P}$ & - \\
\hline PMU H & $P$ & $P$ & $P$ & $P$ & $P$ & $\mathrm{P}$ & $P$ & $P$ & $P$ & $P$ & $P$ & $P$ \\
\hline PMU I & $P$ & - & $P$ & - & $P$ & - & $P$ & - & $P$ & - & $P$ & - \\
\hline PMU J & $P$ & $\mathrm{P}$ & $\mathrm{P}$ & $\mathrm{P}$ & $P$ & $\mathrm{P}$ & $P$ & $\mathrm{P}$ & $\mathrm{P}$ & $\mathrm{P}$ & $\mathrm{P}$ & $P$ \\
\hline
\end{tabular}

9.5.1 C37.118.1-2011 Annex C dynamic step change in phase frequency response time: $F 0=60 \mathrm{~Hz}$, $M$ class

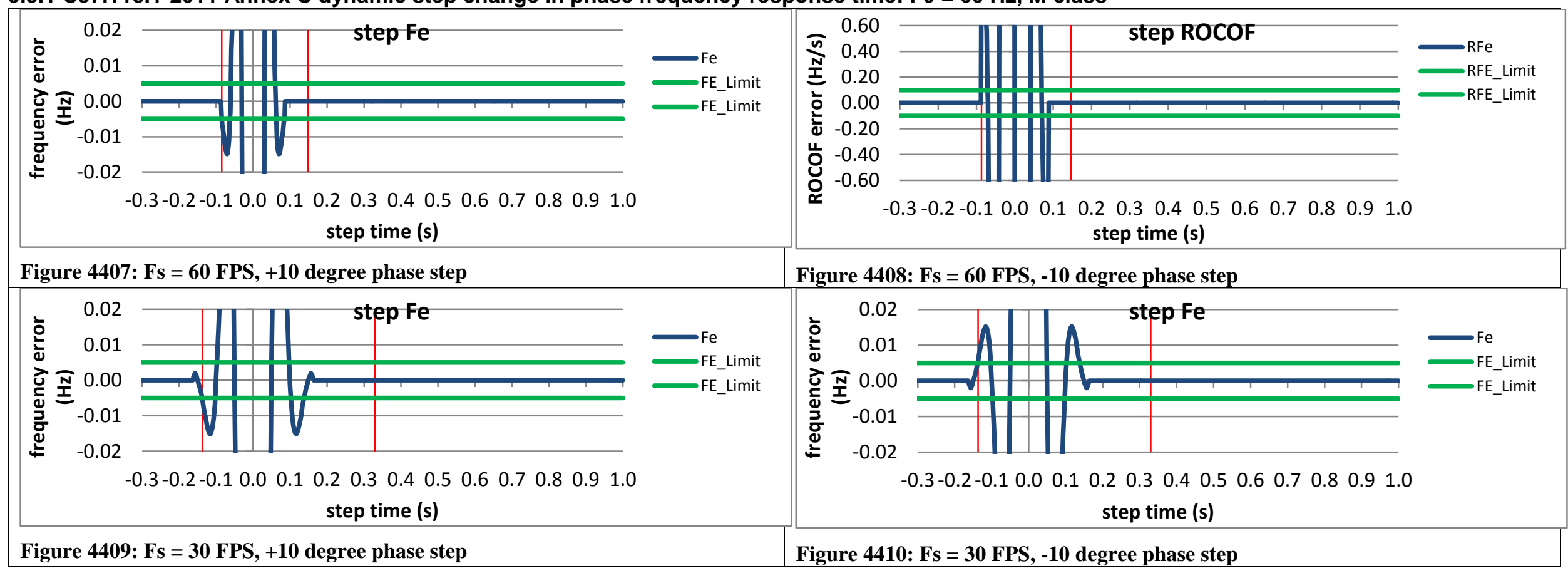



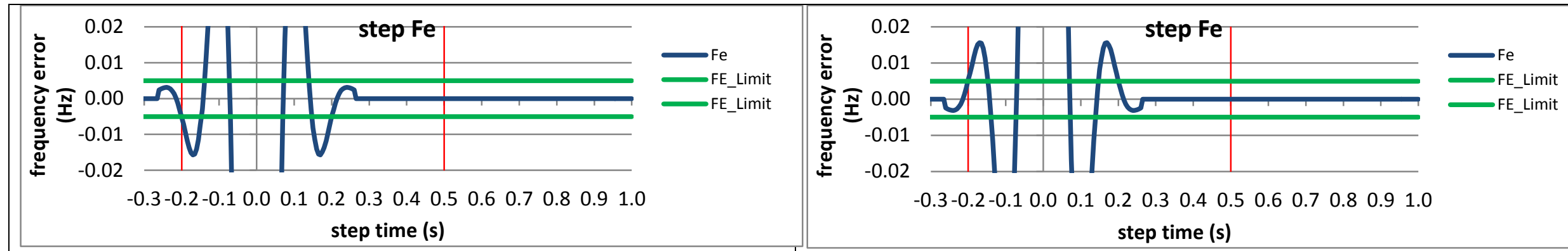

Figure 4411: Fs = 20 FPS, + 10 degree phase step

Figure 4412: Fs = 20 FPS, -10 degree phase step

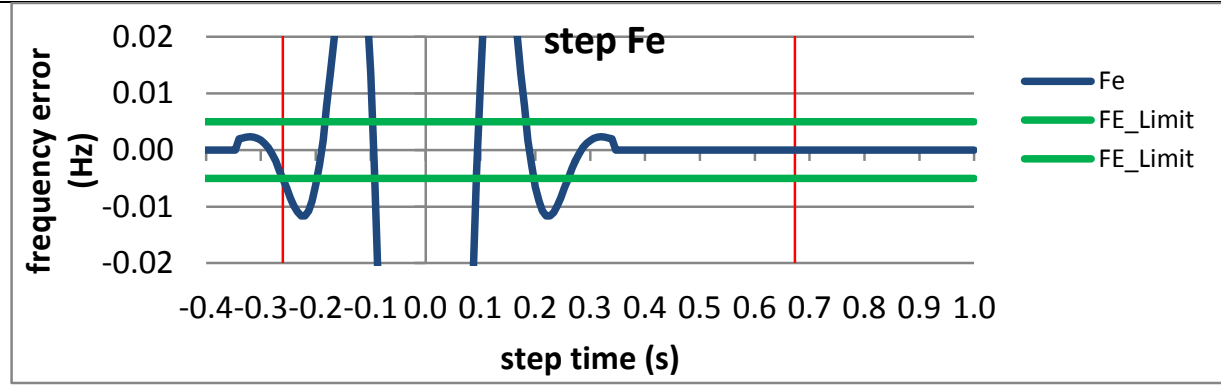

Figure 4413: Fs = 15 FPS, +10 degree phase step

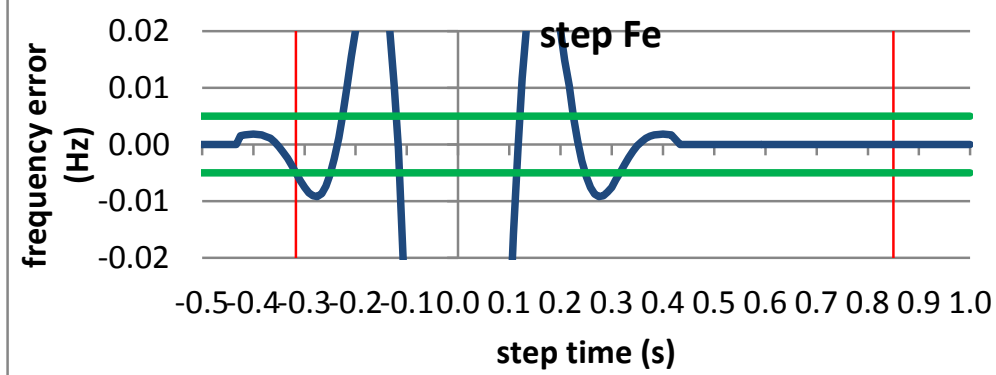

Figure 4415: Fs = 12 FPS, +10 degree phase step

$\mathrm{Fe}$
$\mathrm{FE}$ LLimit
$\mathrm{FE}$ _Limit

Figure 4416: Fs = 12 FPS, -10 degree phase step

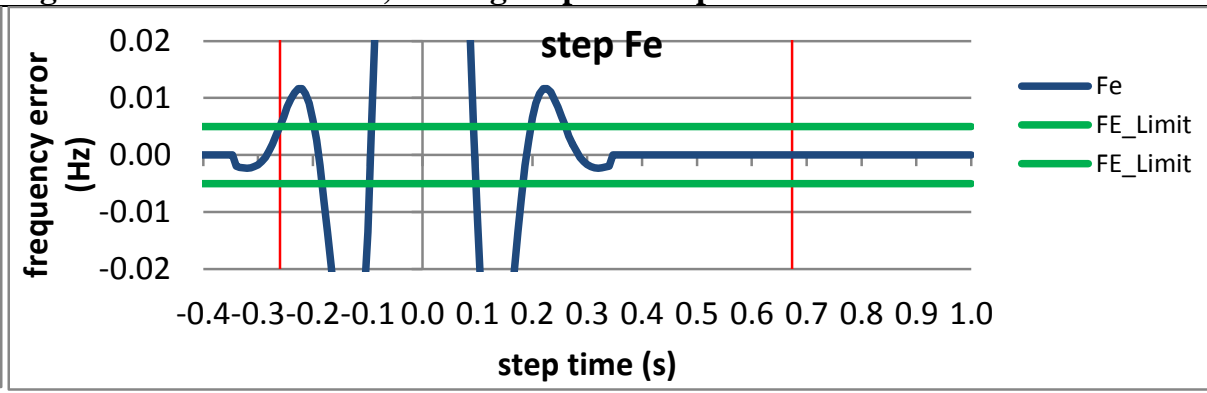

Figure 4414: Fs = 15 FPS, -10 degree phase step

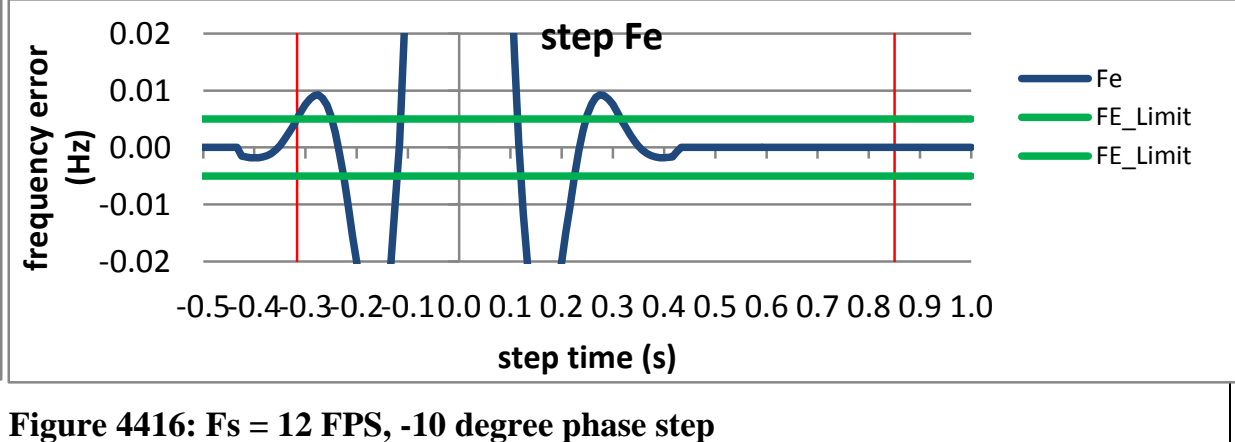




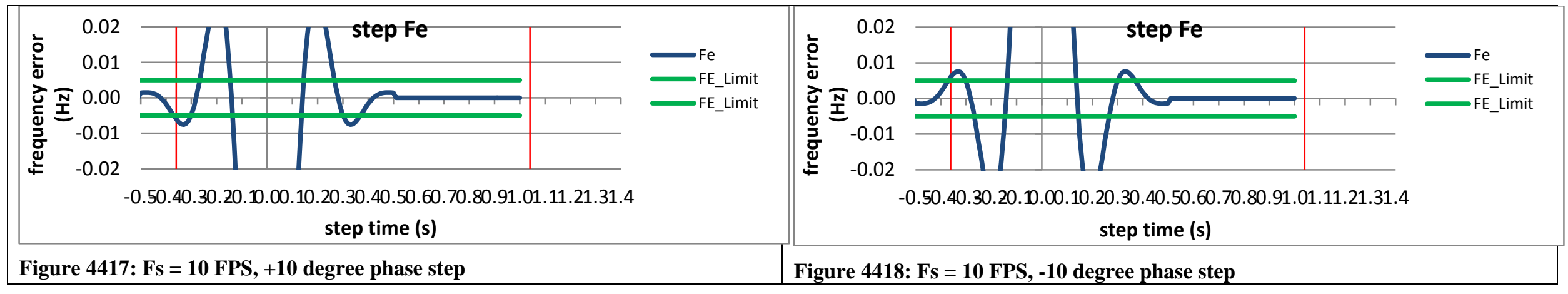


9.5.2 PMU A dynamic step change in phase frequency response time: $\mathrm{FO}=60 \mathrm{~Hz}, \mathrm{M}$ class
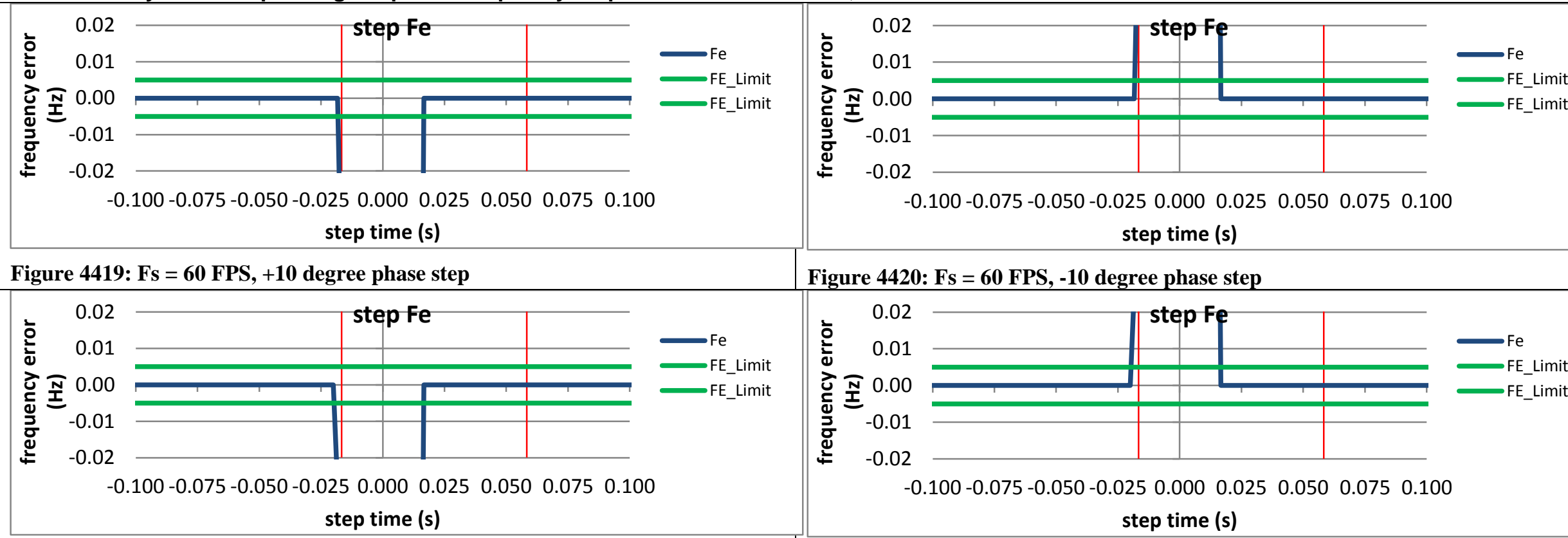

Figure 4420: Fs = 60 FPS, -10 degree phase step

Figure 4421: Fs = 30 FPS, +10 degree phase step
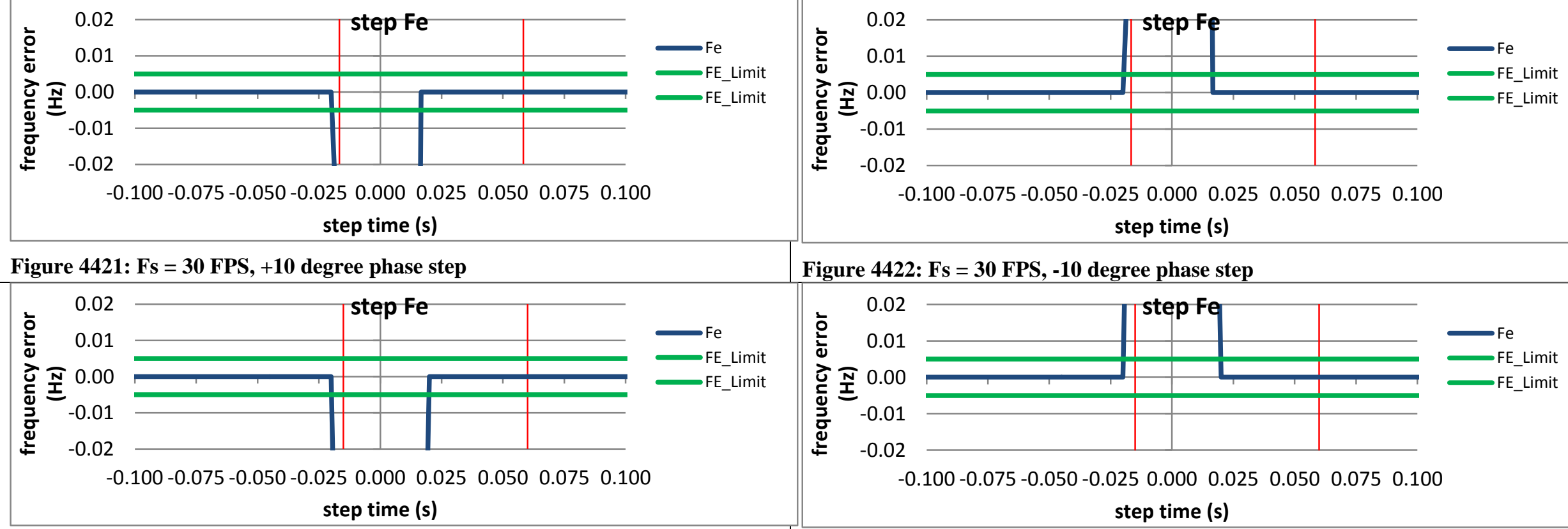

Figure 4422: Fs = 30 FPS, -10 degree phase step

Figure 4423: Fs = 20 FPS, + 10 degree phase step

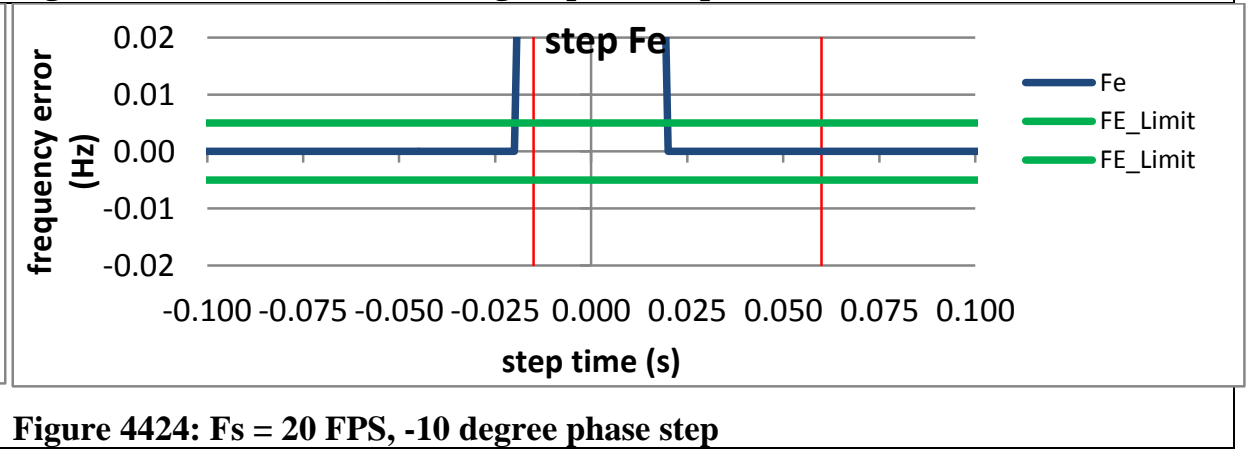




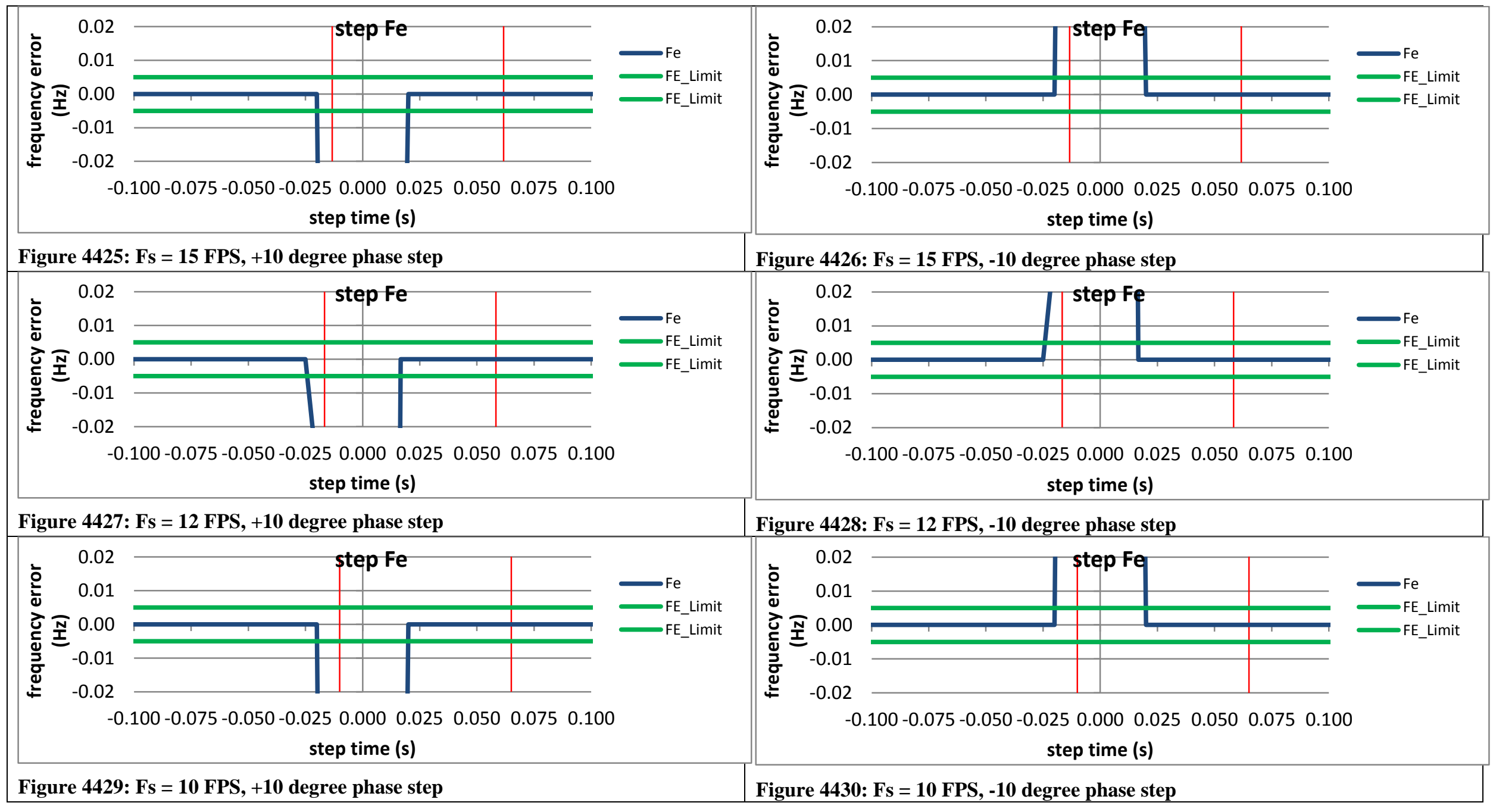


9.5.3 PMU B dynamic step change in phase frequency response time: $\mathrm{F} 0=60 \mathrm{~Hz}, \mathrm{M}$ class

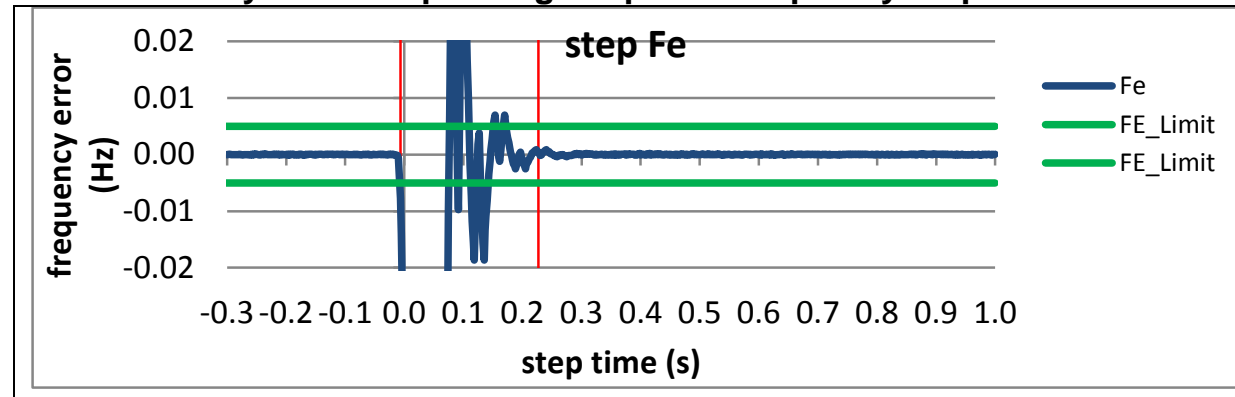

Figure 4431: Fs = 60 FPS, +10 degree phase step

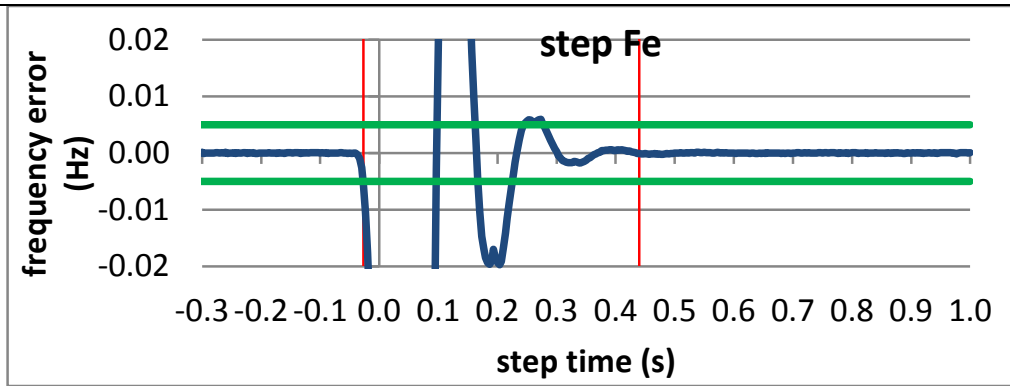

Figure 4433: Fs = 30 FPS, +10 degree phase step

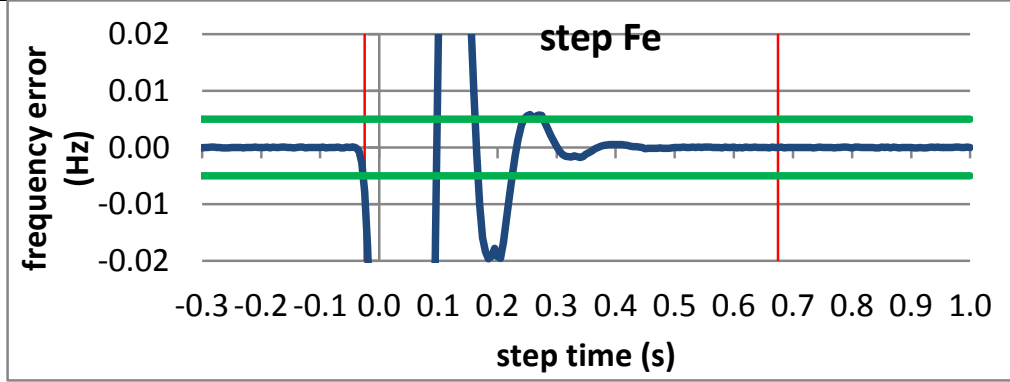

Figure 4435: Fs = 20 FPS, + 10 degree phase step

Fe
FE_Limit
FE_Limit

Fe
FE_Limit
FE_Limit

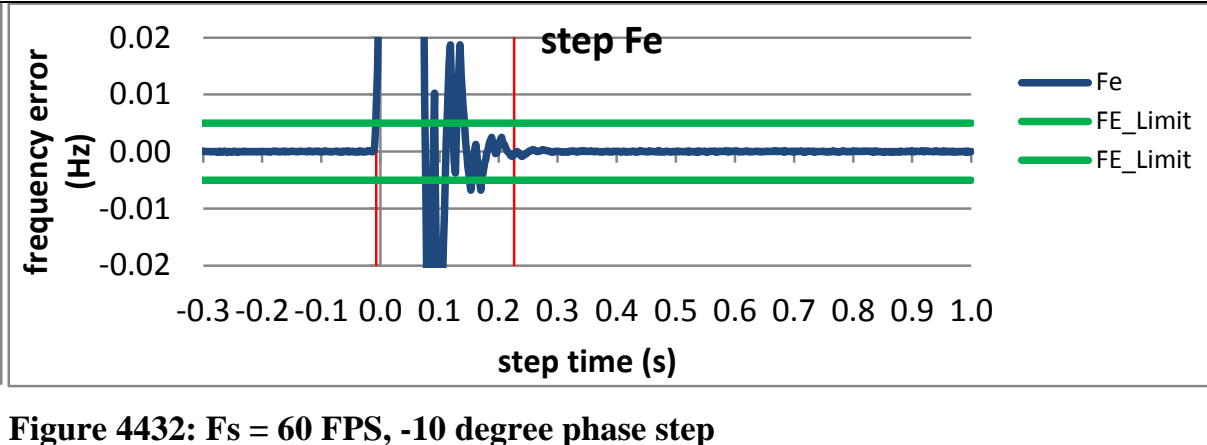

Figure 4432: Fs = 60 FPS, -10 degree phase step

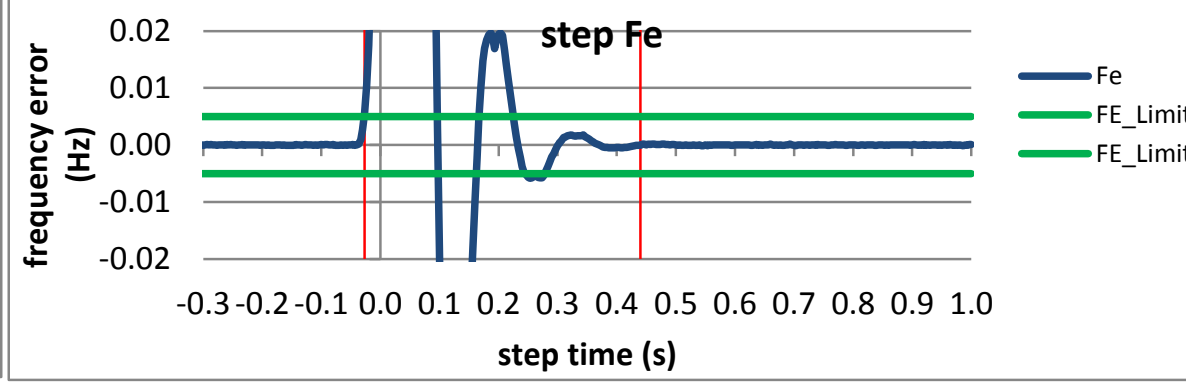

Figure 4434: Fs = 30 FPS, -10 degree phase step

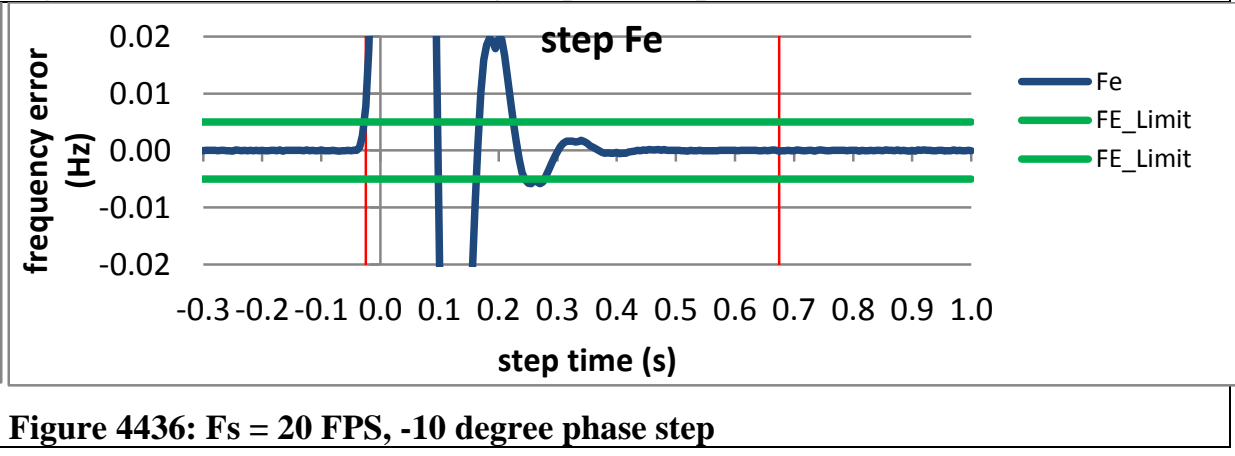




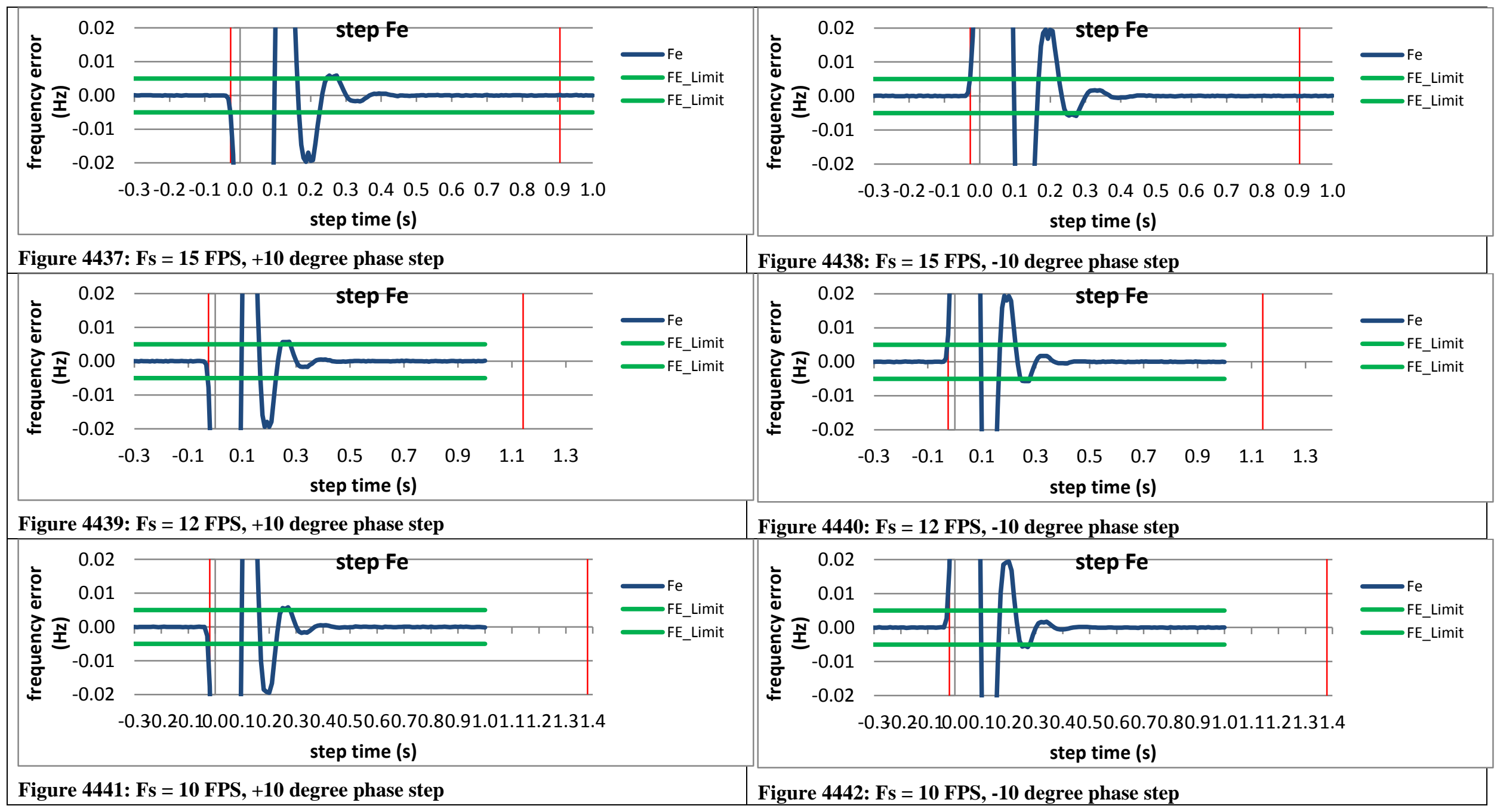


9.5.4 PMU C dynamic step change in phase frequency response time: $\mathrm{F} 0=60 \mathrm{~Hz}, \mathrm{M}$ class

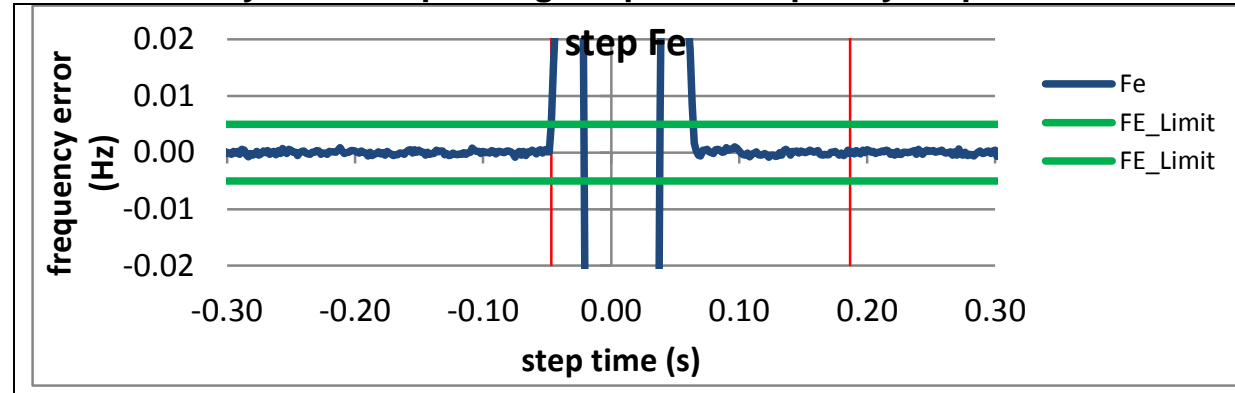

Figure 4443: Fs = 60 FPS, +10 degree phase step

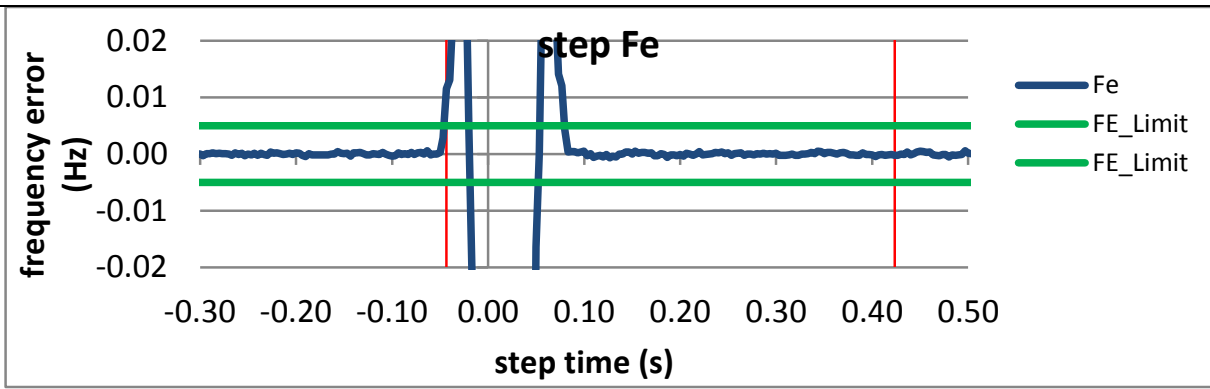

Figure 4445: Fs = 30 FPS, +10 degree phase step

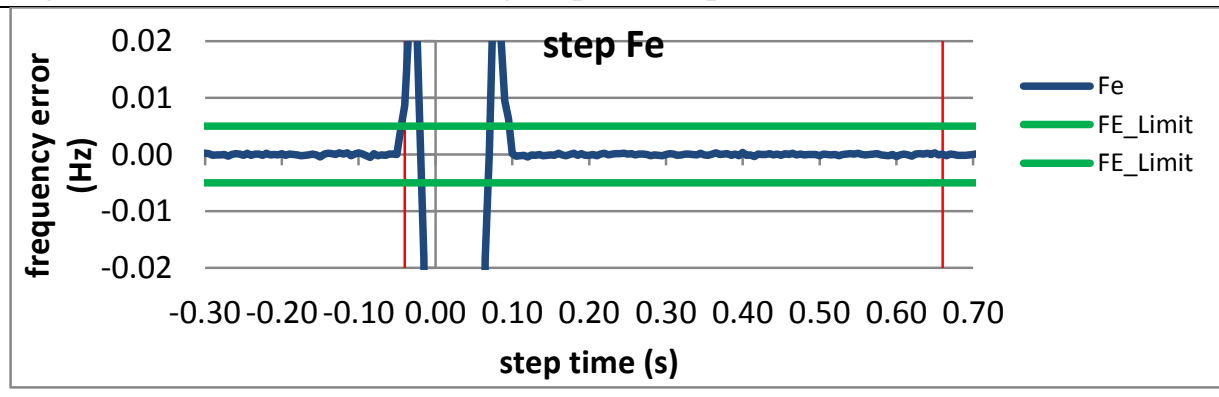

Figure 4447: Fs = 20 FPS, + 10 degree phase step

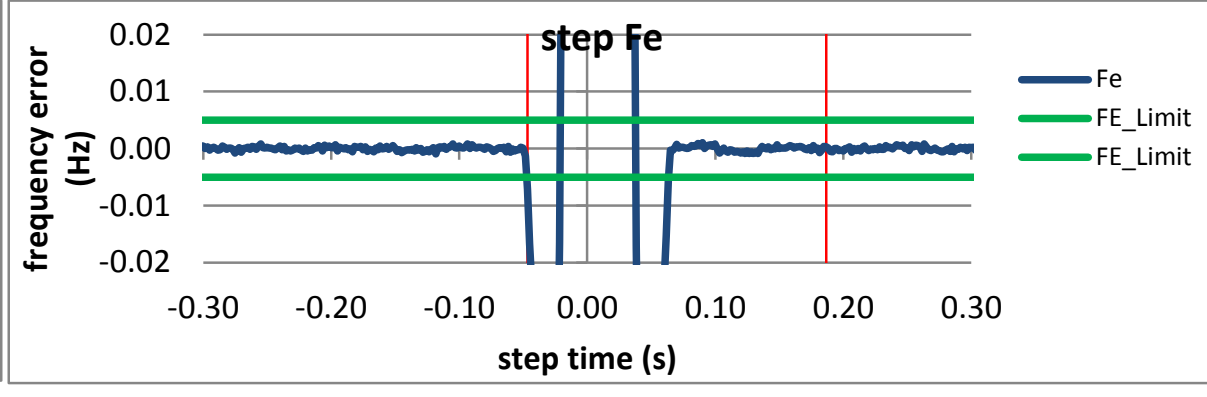

Figure 4444: Fs = 60 FPS, -10 degree phase step

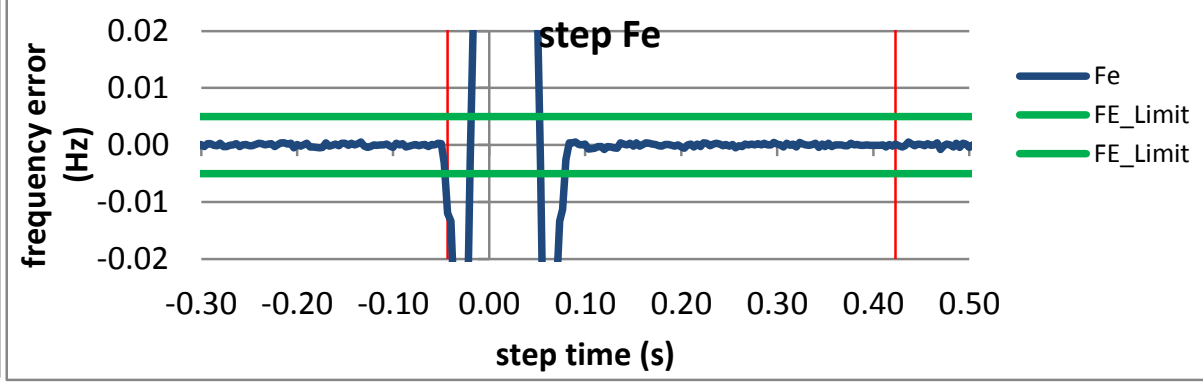

Figure 4446: Fs = 30 FPS, -10 degree phase step

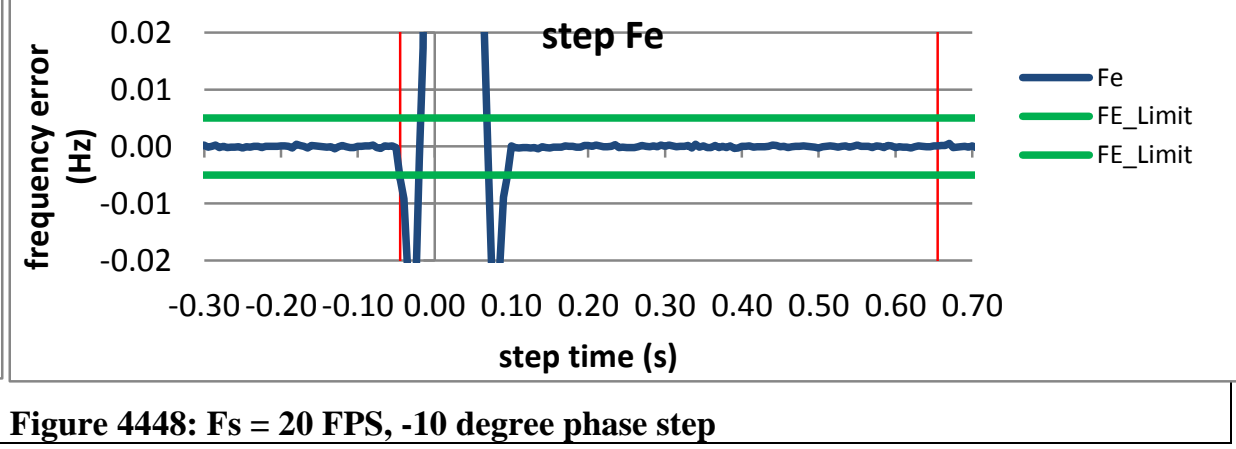




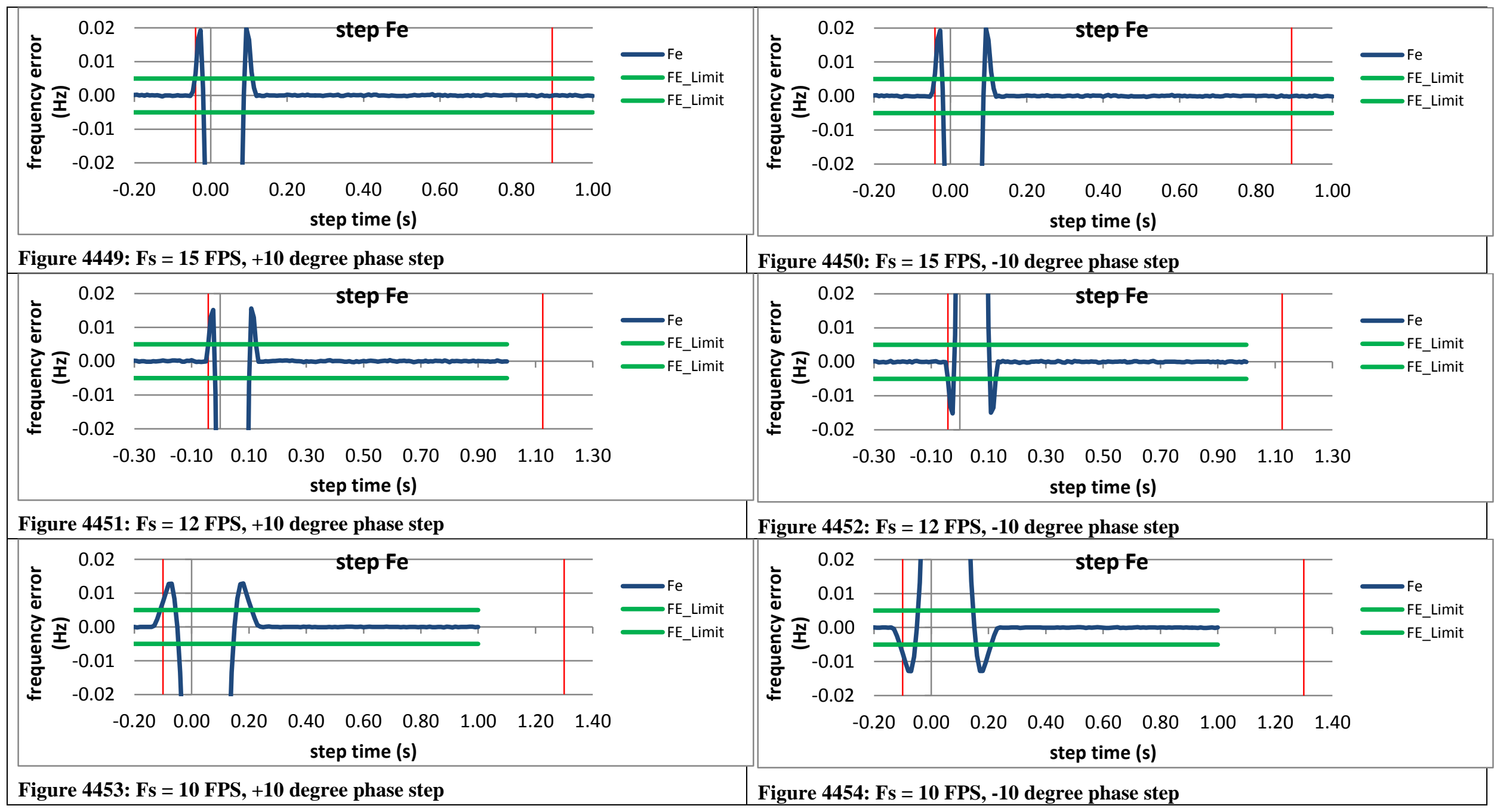


9.5.5 PMU D dynamic step change in phase frequency response time: $F 0=60 \mathrm{~Hz}, \mathrm{M}$ class

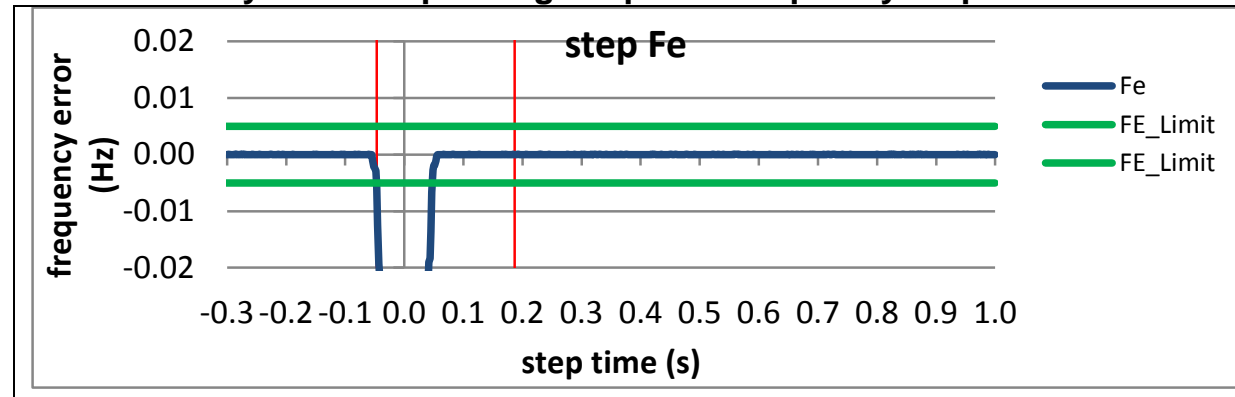

Figure 4455: Fs = 60 FPS, +10 degree phase step

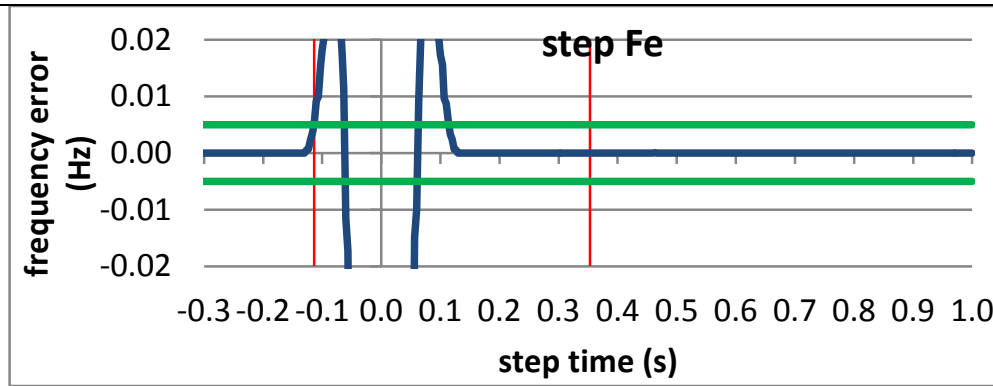

Figure 4457: Fs = 30 FPS, +10 degree phase step

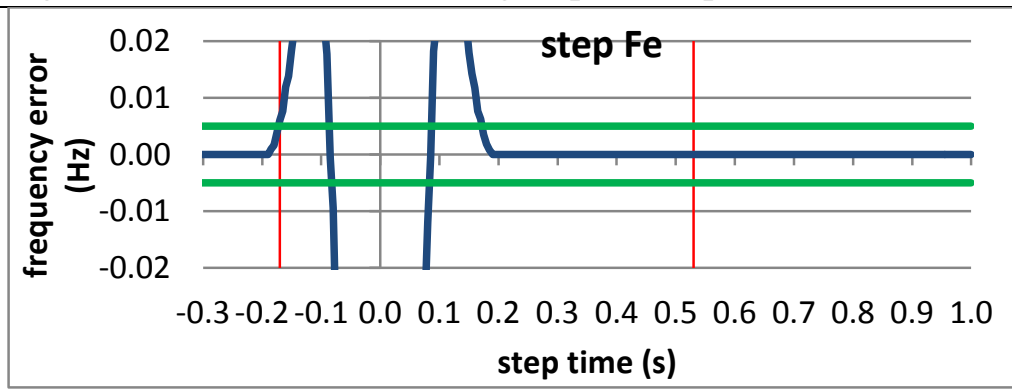

Figure 4459: Fs = 20 FPS, + 10 degree phase step

Fe
FE_Limit
FE_Limit

Fe
FE_Limit
FE_Limit

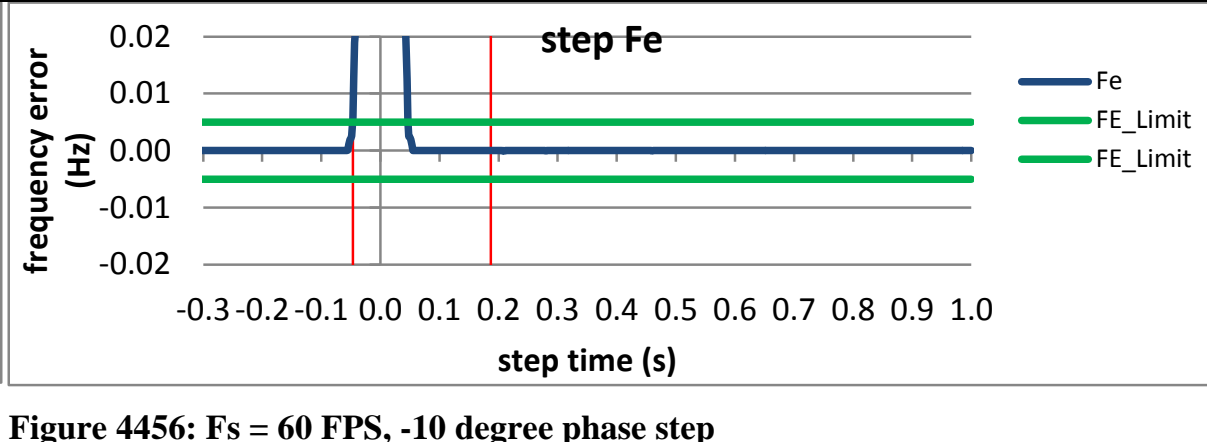

Figure 4456: Fs = 60 FPS, -10 degree phase step

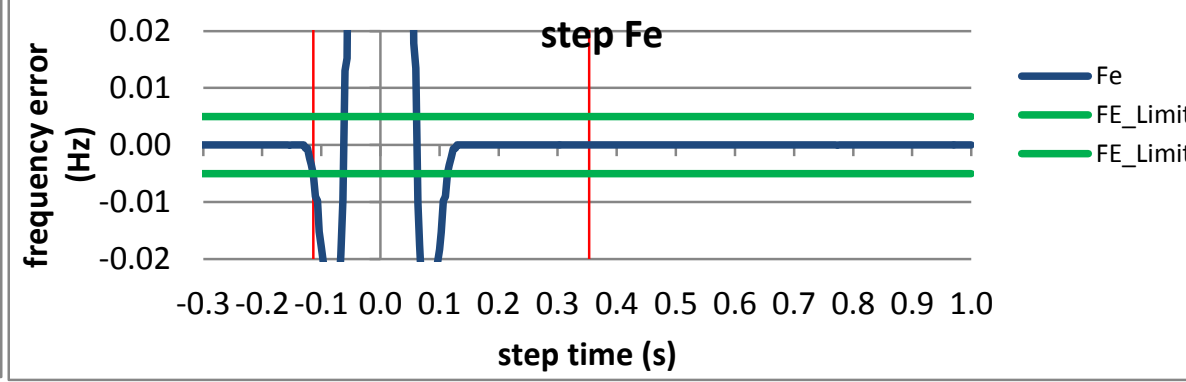

Figure 4458: Fs = 30 FPS, -10 degree phase step

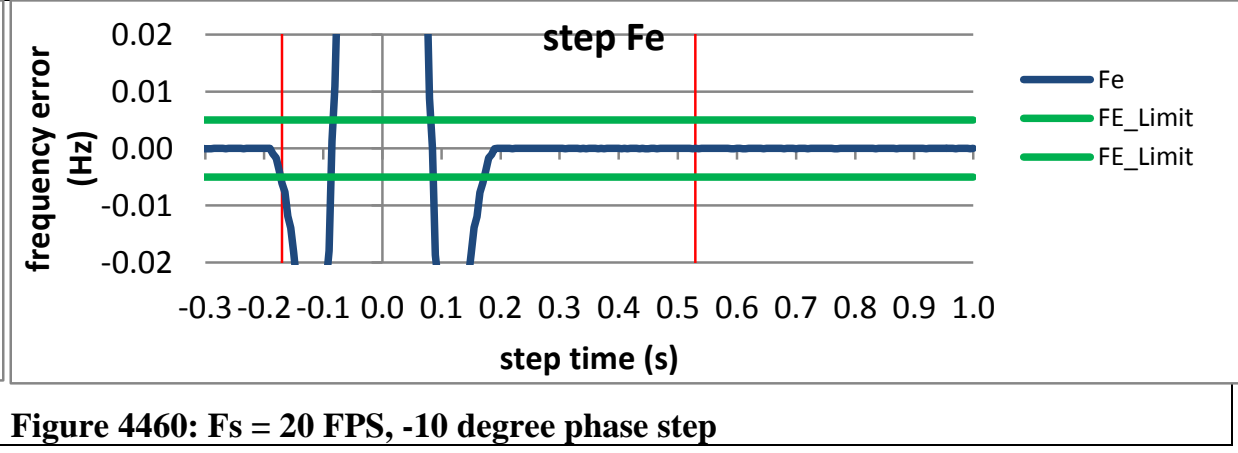




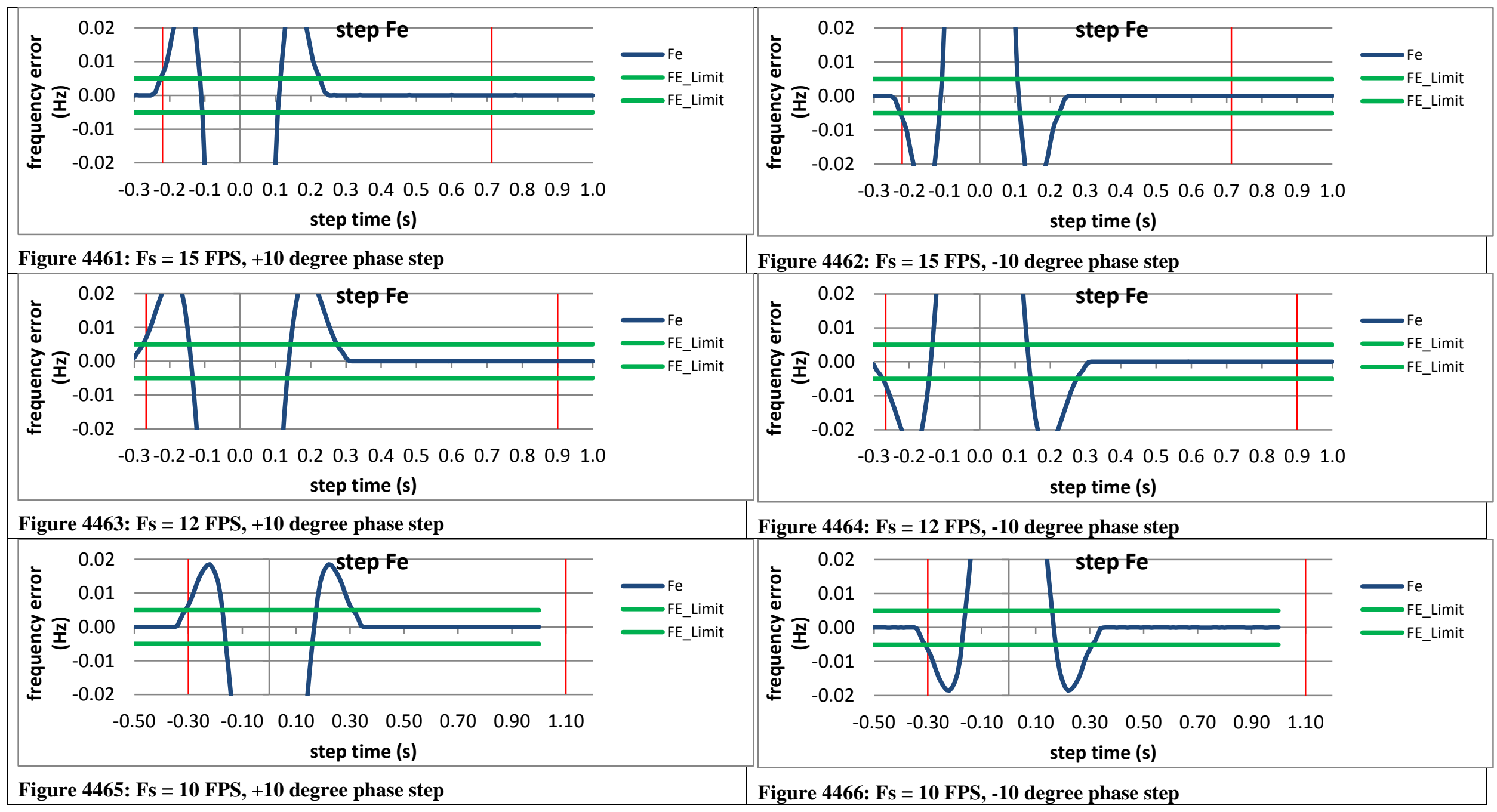


9.5.6 PMU E dynamic step change in phase frequency response time: $\mathrm{F} 0=60 \mathrm{~Hz}, \mathrm{M}$ class
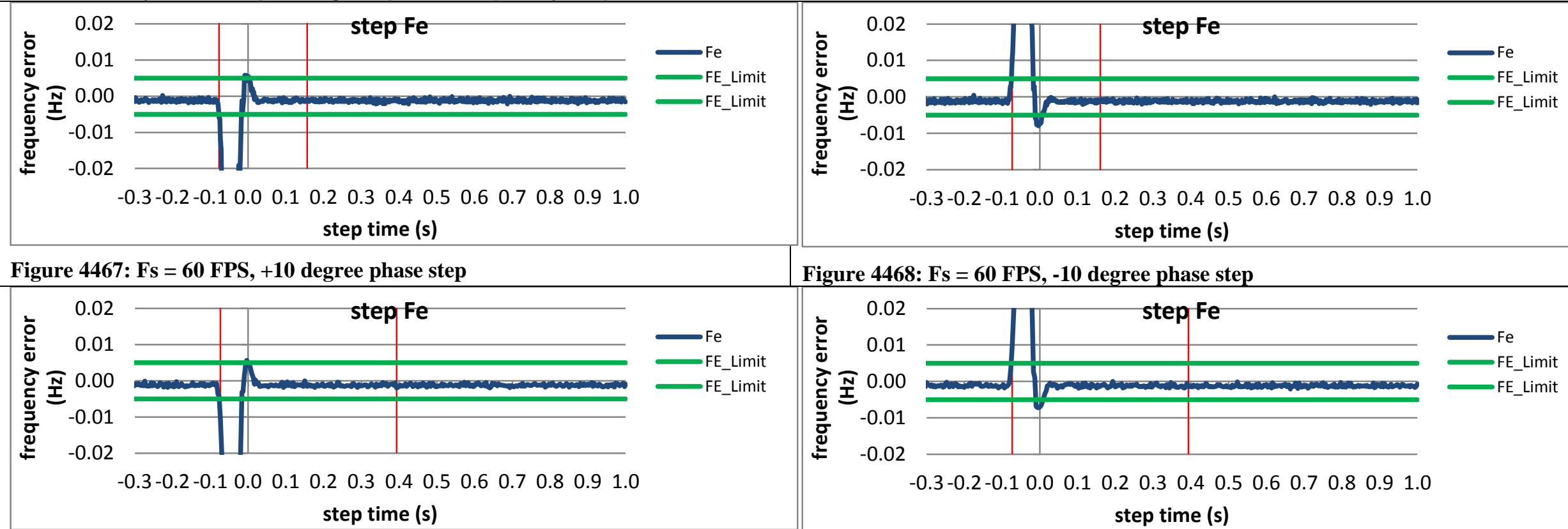

Figure 4468: Fs = 60 FPS, -10 degree phase step

Figure 4469: Fs = 30 FPS, +10 degree phase step

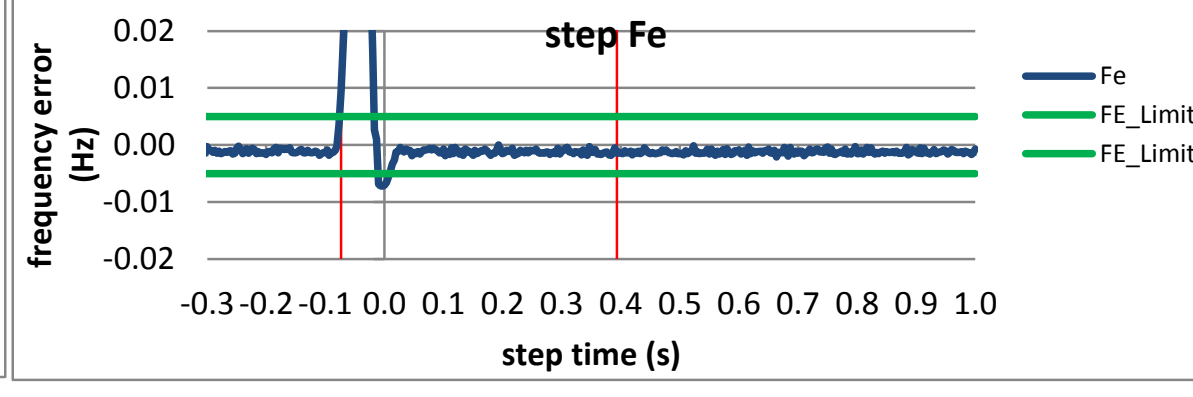

Figure 4470: Fs = 30 FPS, -10 degree phase step

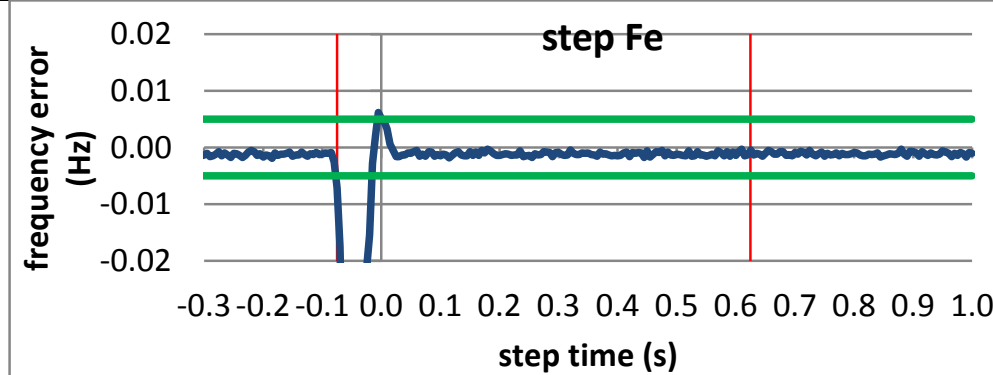

Figure 4471: Fs = 20 FPS, + 10 degree phase step

Fe
FE_Limit
FE_Limit

Fe
FE_Limit
FE_Limit

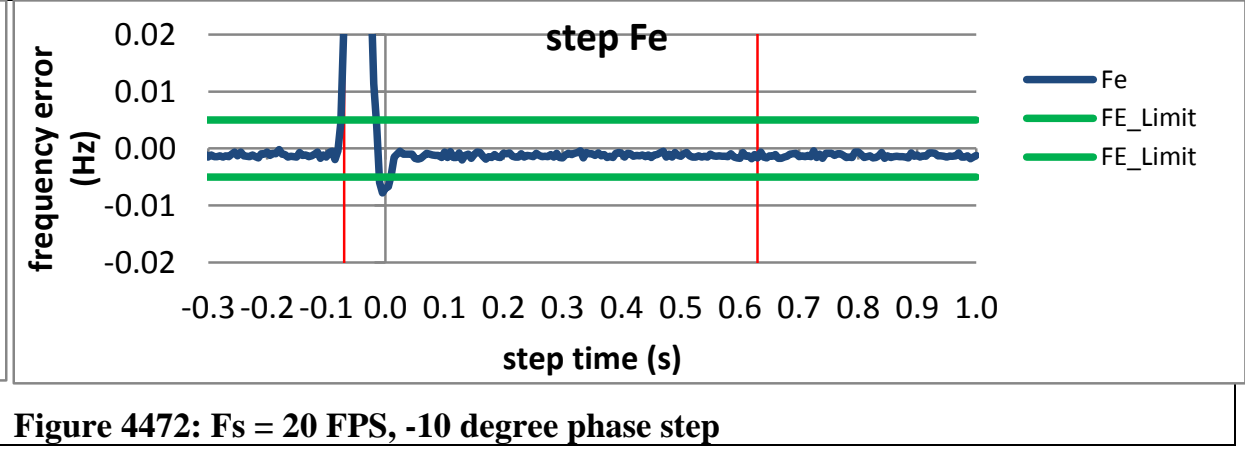




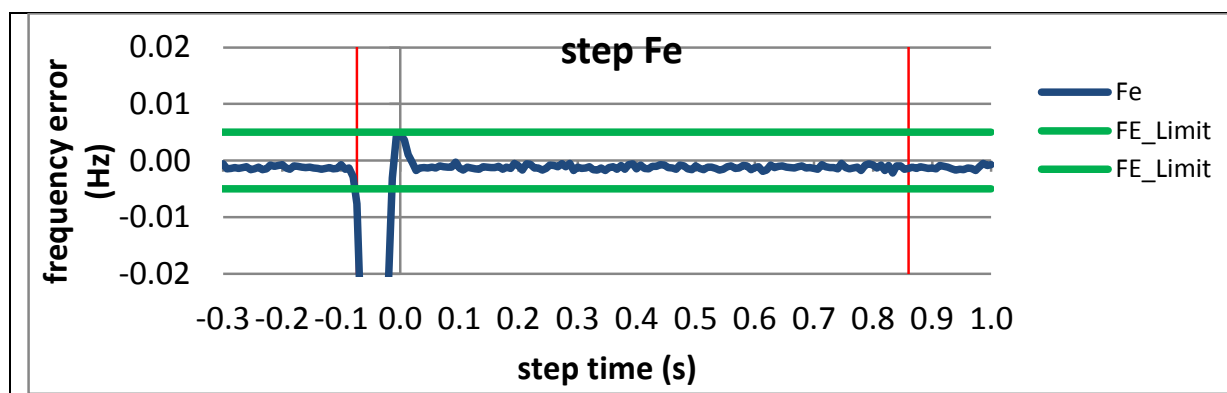

Figure 4473: Fs $=15$ FPS, +10 degree phase step

Figure 4475: Fs = 12 FPS, +10 degree phase step

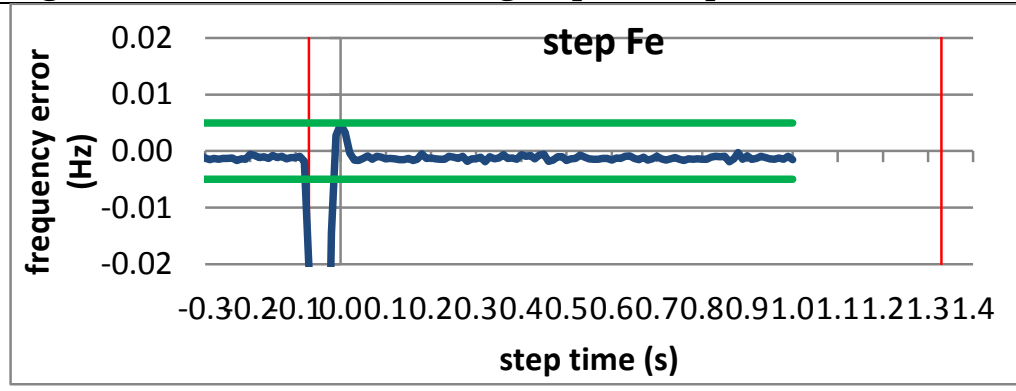

Figure 4477: Fs = 10 FPS, +10 degree phase step

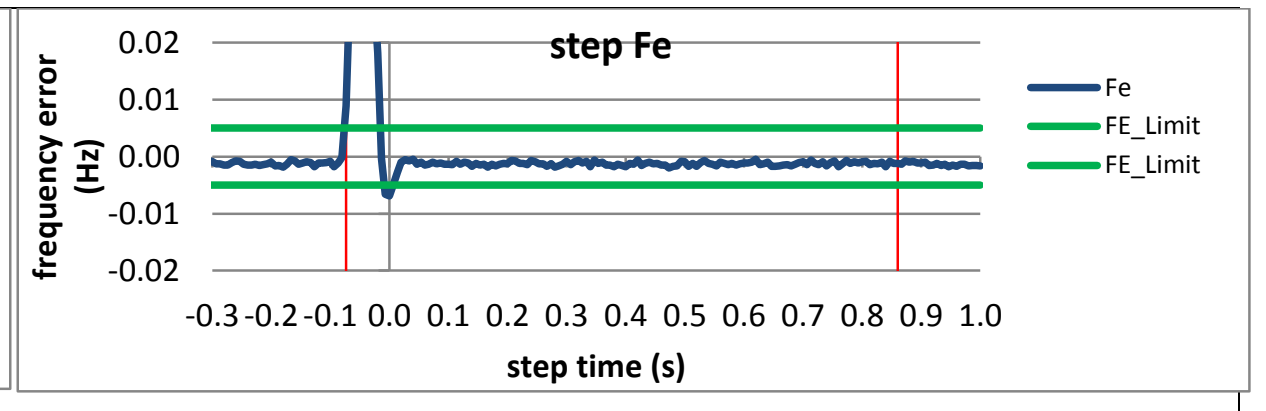

Figure 4474: Fs = 15 FPS, -10 degree phase step

Figure 4476: Fs = 12 FPS, -10 degree phase step

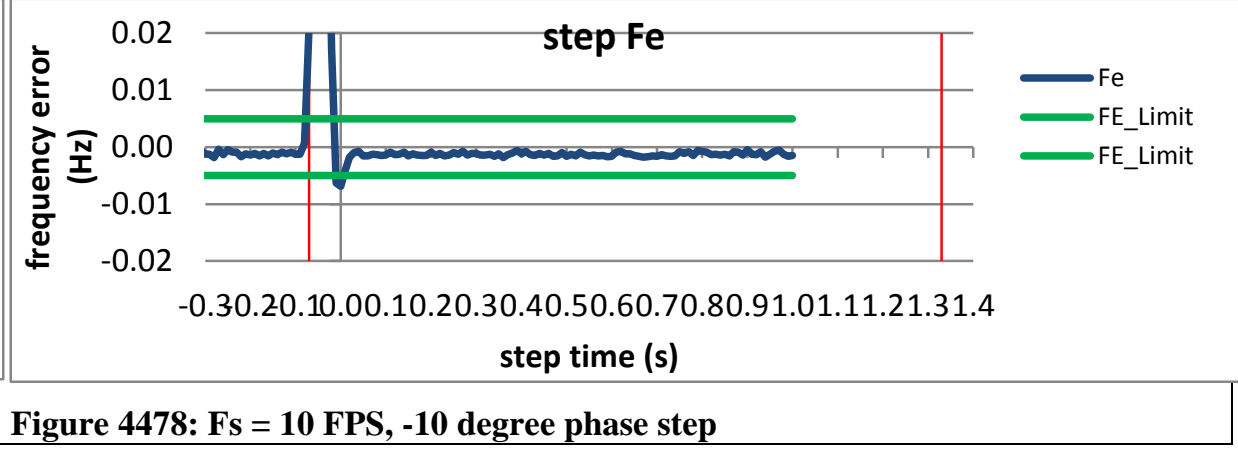


9.5.7 PMU F dynamic step change in phase frequency response time: $F 0=60 \mathrm{~Hz}, \mathrm{M}$ class
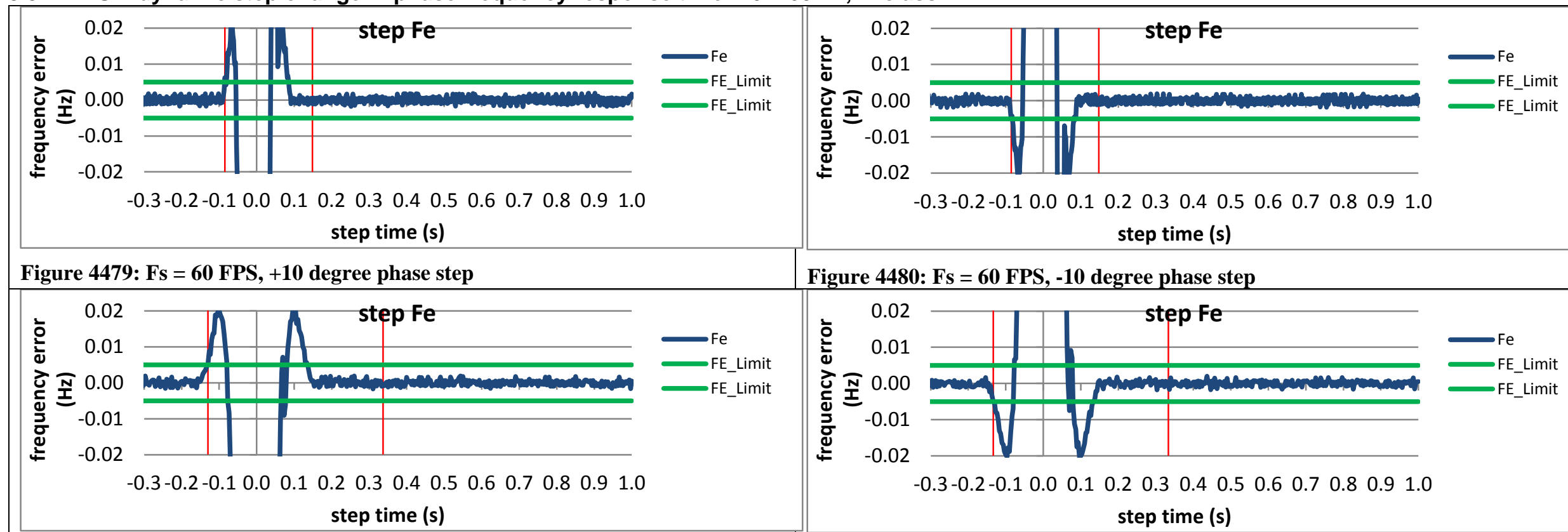

Fe
FE_Limit
FE_Limit

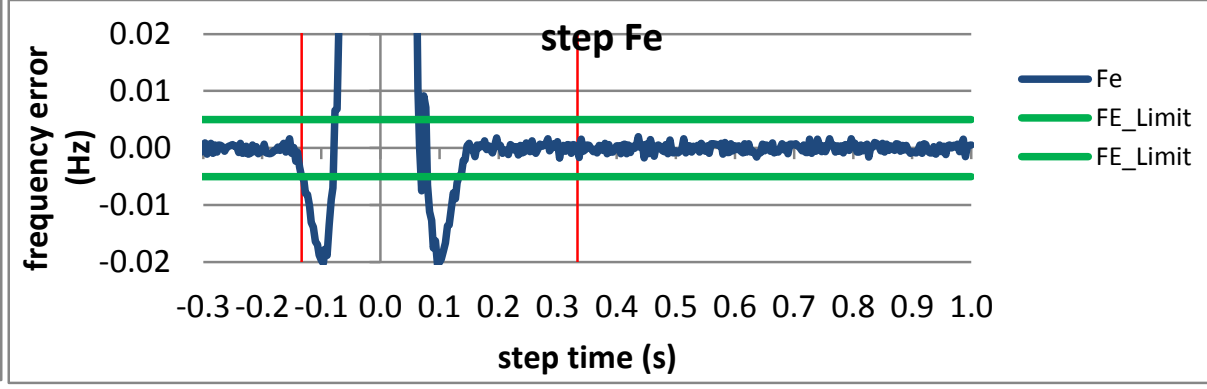

Figure 4481: Fs = 30 FPS, +10 degree phase step

Figure 4482: Fs = 30 FPS, -10 degree phase step
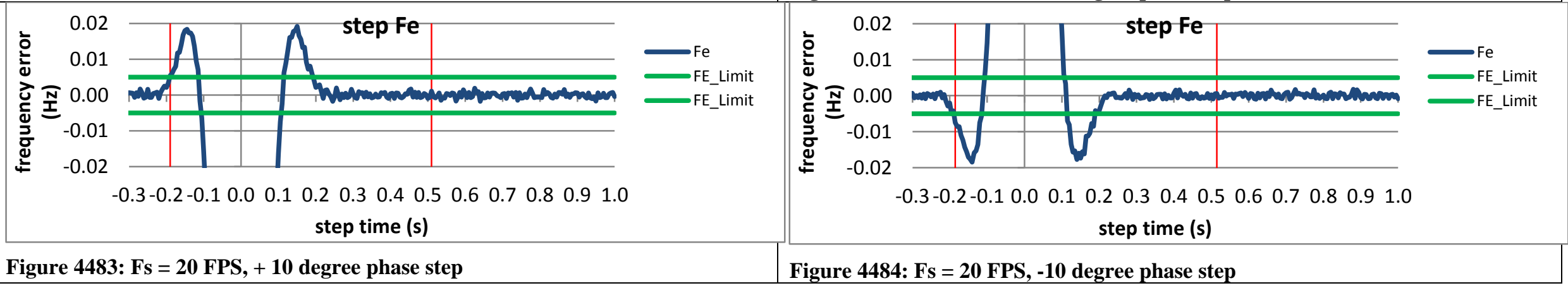

Figure 4483: Fs = 20 FPS, + 10 degree phase step

Figure 4484: Fs = 20 FPS, -10 degree phase step 

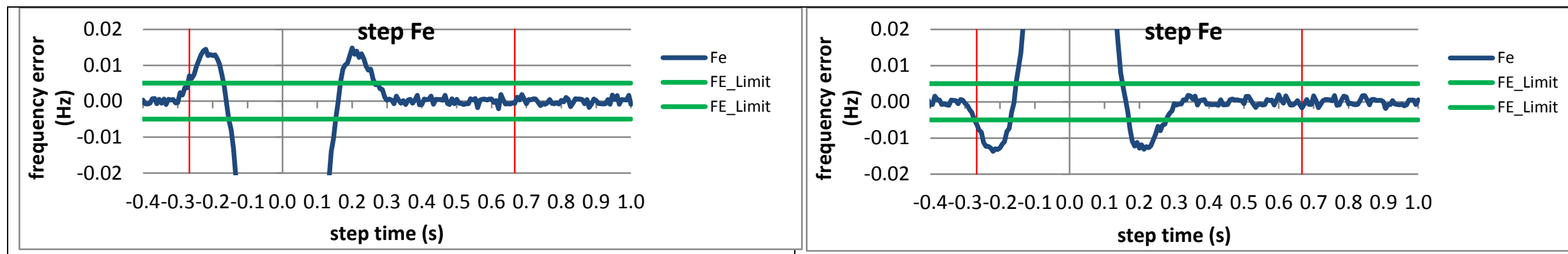

Figure 4485: Fs $=15$ FPS, +10 degree phase step

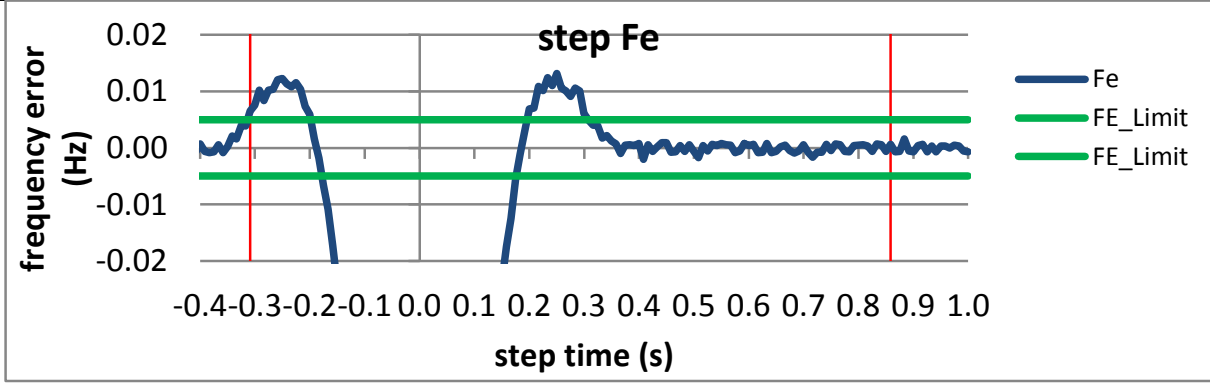

Figure 4486: Fs = 15 FPS, -10 degree phase step

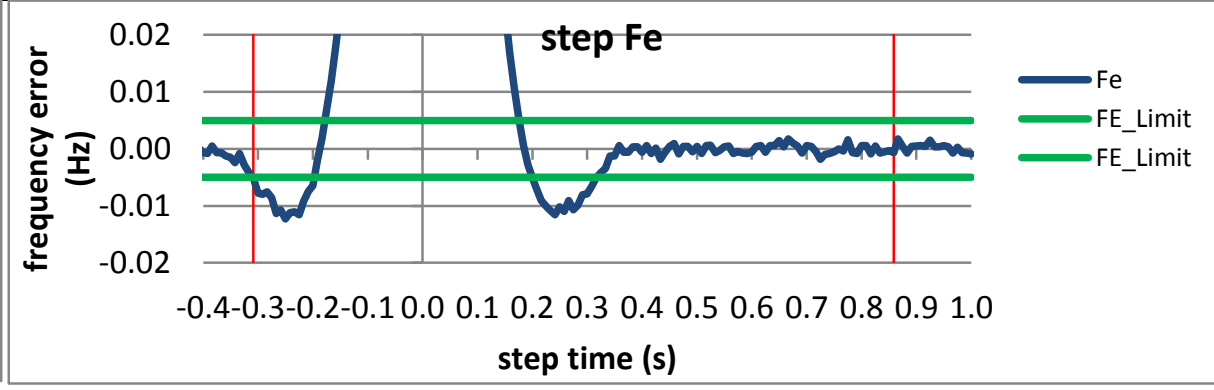

Figure 4487: Fs = 12 FPS, +10 degree phase step

Figure 4488: Fs = 12 FPS, -10 degree phase step
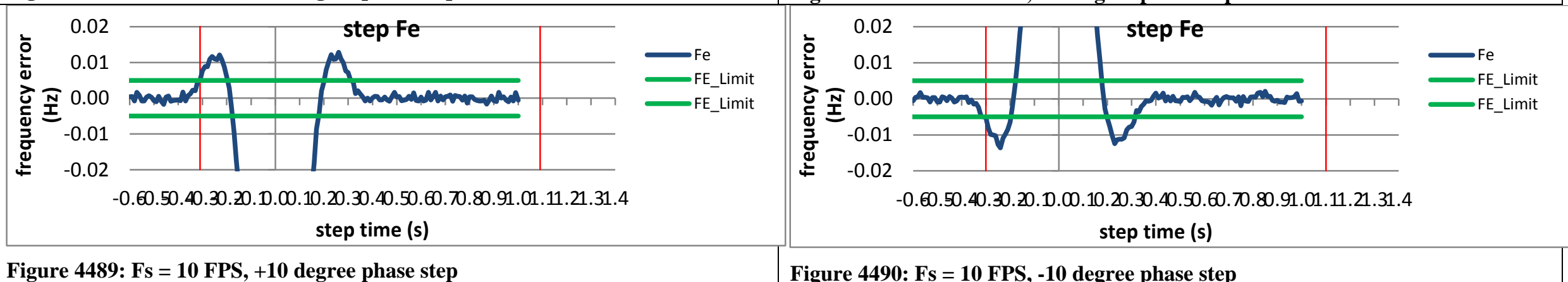

Figure 4489: Fs = 10 FPS, +10 degree phase step

Figure 4490: Fs = 10 FPS, -10 degree phase step 


\subsubsection{PMU G dynamic step change in phase frequency response time: $\mathrm{F} 0=60 \mathrm{~Hz}, \mathrm{M}$ class}

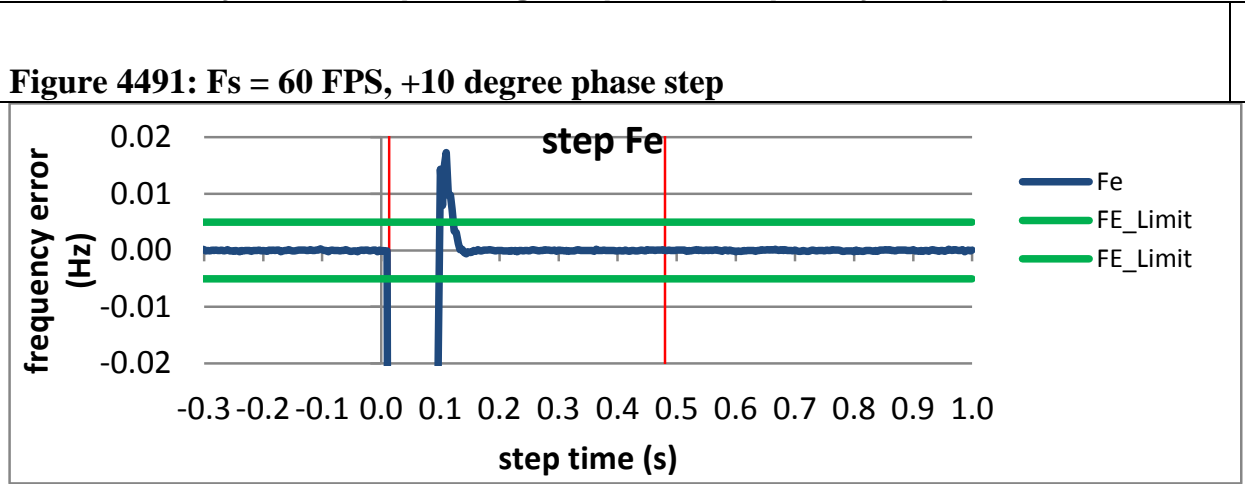

Figure 4492: Fs = 60 FPS, -10 degree phase step

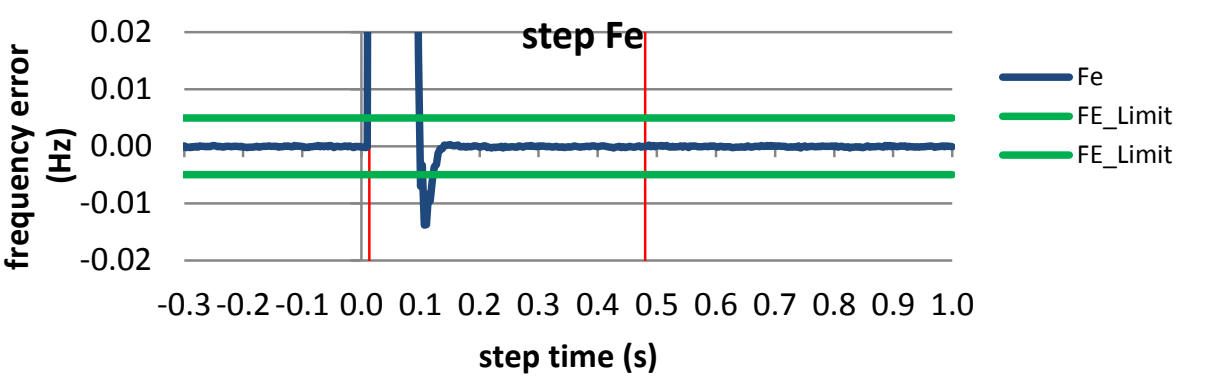

Figure 4493: Fs = 30 FPS, +10 degree phase step

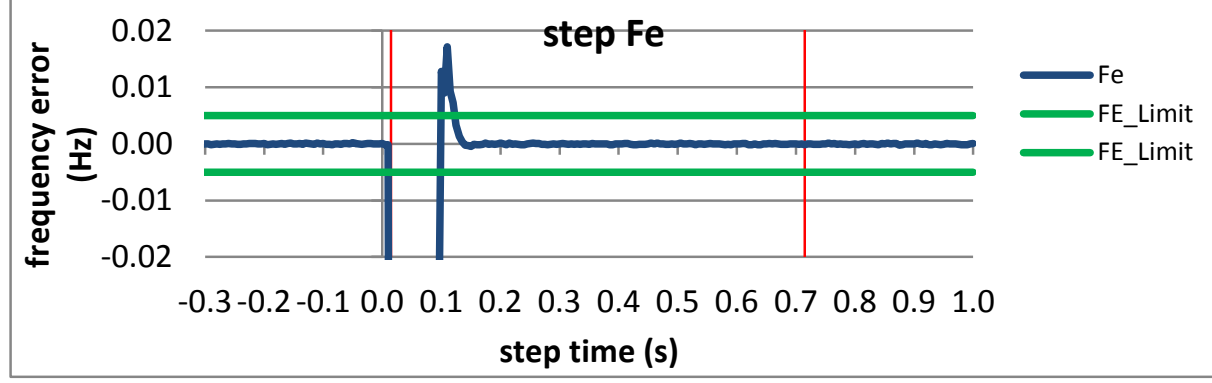

Figure 4494: Fs = 30 FPS, -10 degree phase step

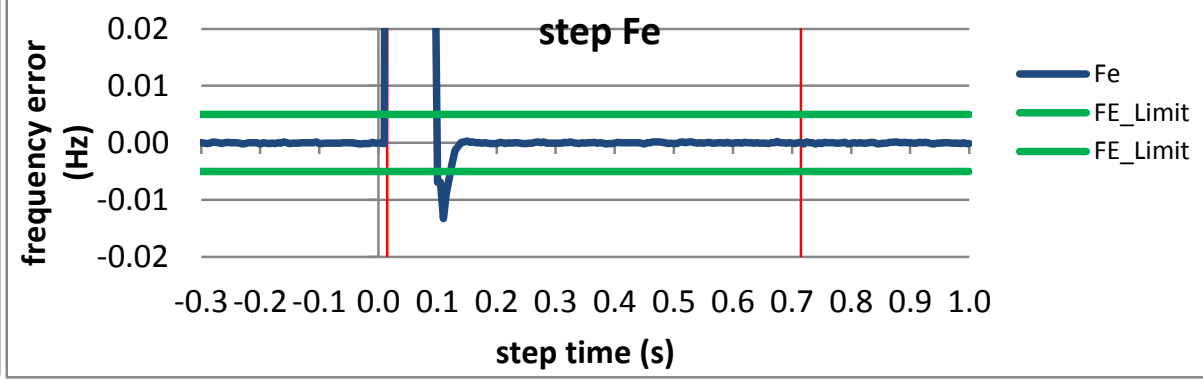

Figure 4495: Fs = 20 FPS, + 10 degree phase step

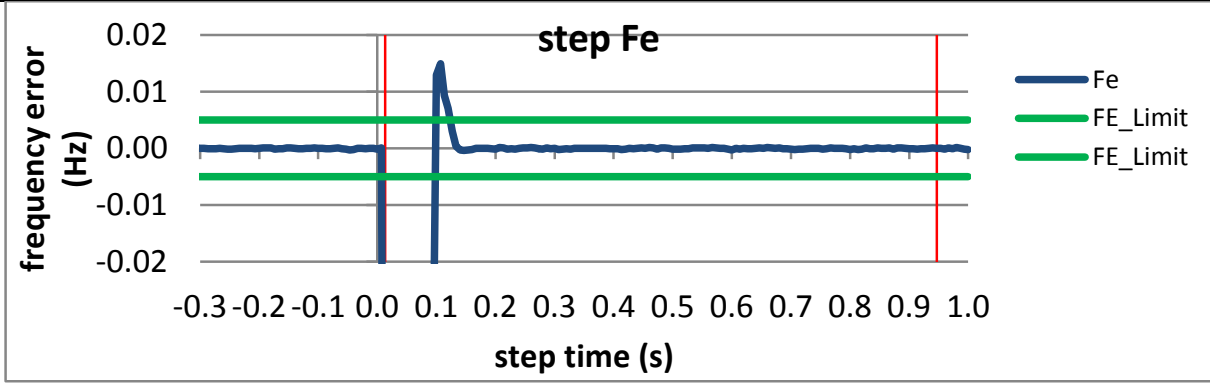

Figure 4496: Fs = 20 FPS, -10 degree phase step

Figure 4497: Fs = 15 FPS, +10 degree phase step

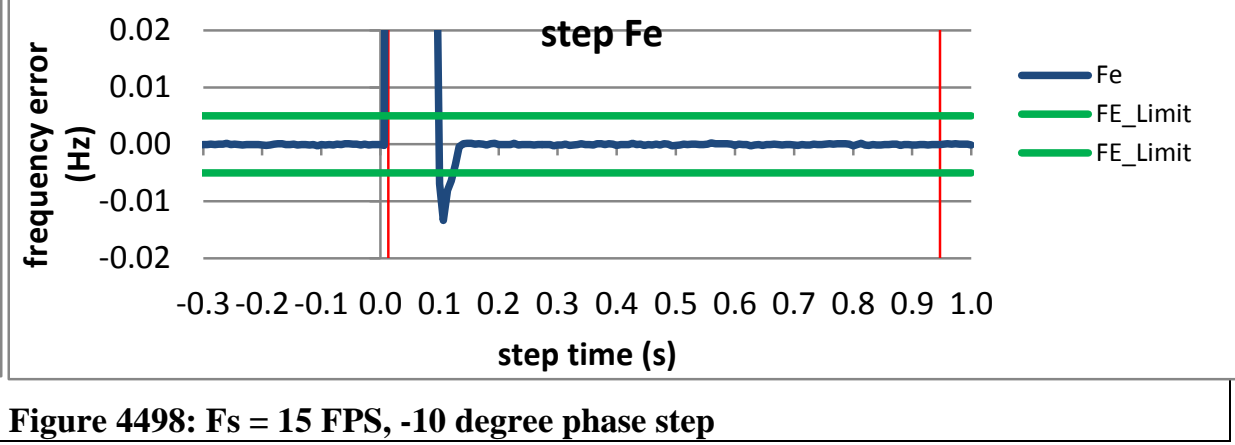




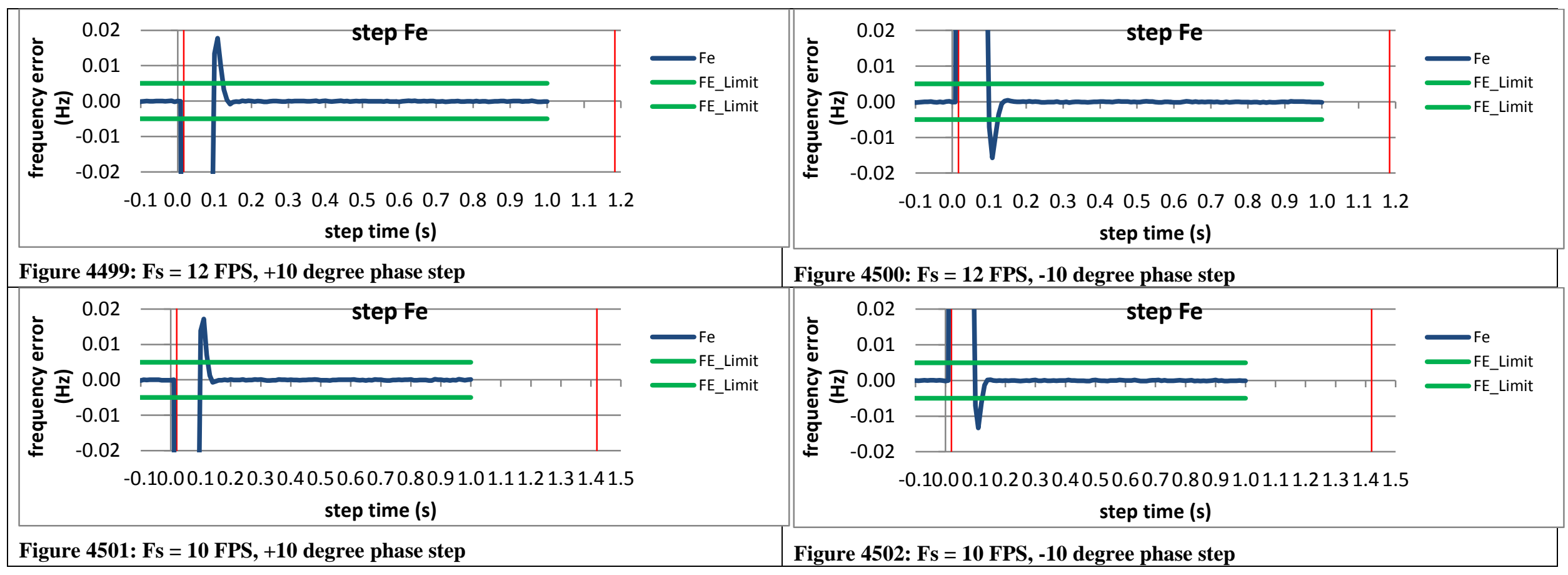




\subsubsection{PMU H dynamic step change in phase frequency response time: $F 0=60 \mathrm{~Hz}, \mathrm{M}$ class}

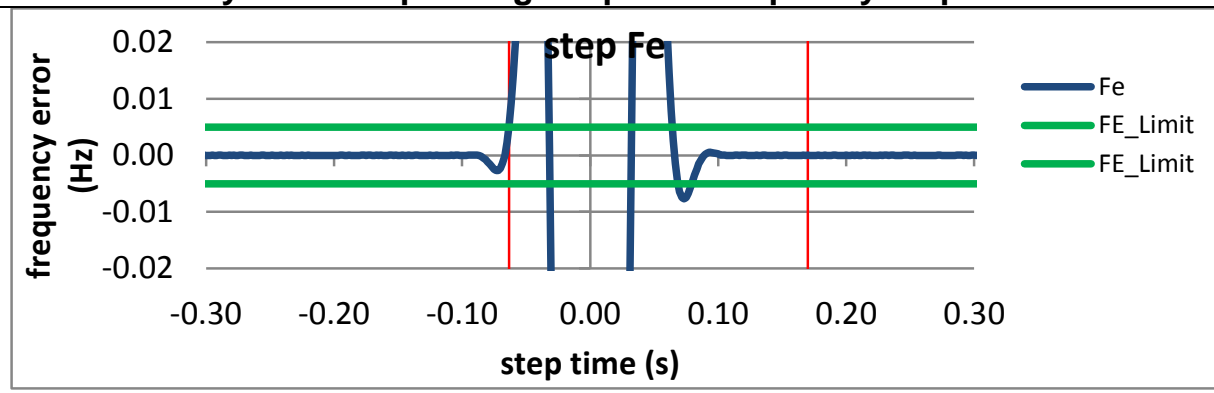

Figure 4503: Fs $=60 \mathrm{FPS},+10$ degree phase step

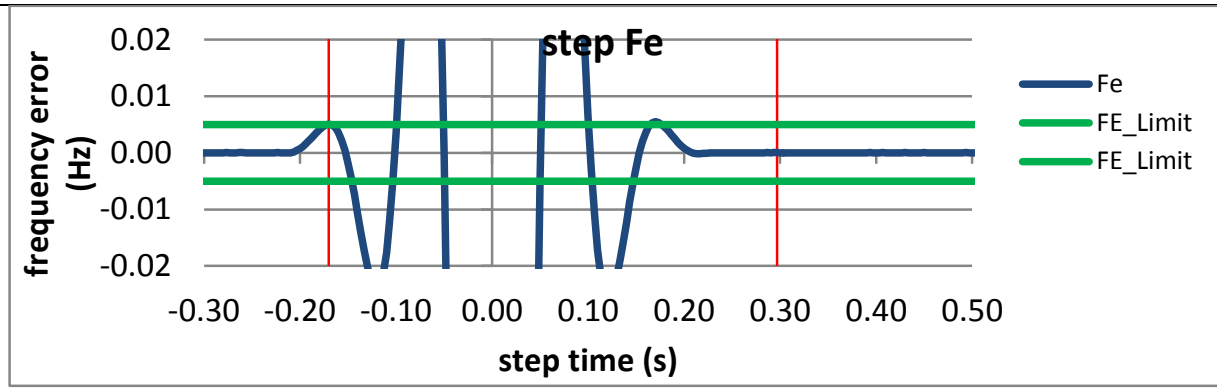

Figure 4505: Fs = 30 FPS, +10 degree phase step

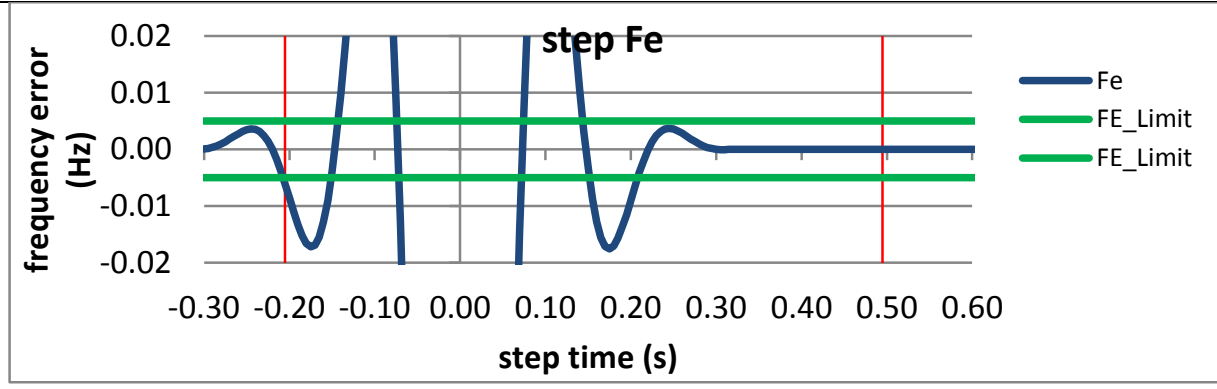

Figure 4507: Fs = 20 FPS, + 10 degree phase step

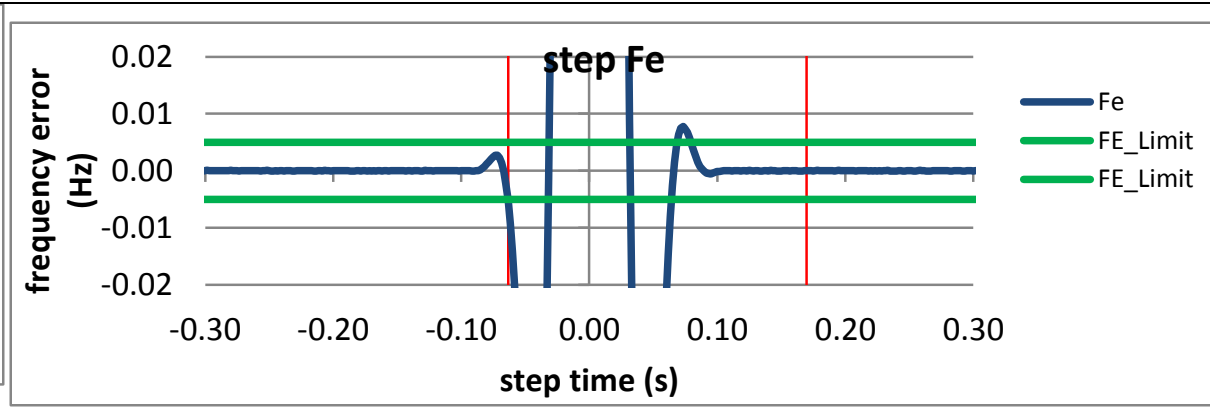

Figure 4504: Fs = 60 FPS, -10 degree phase step

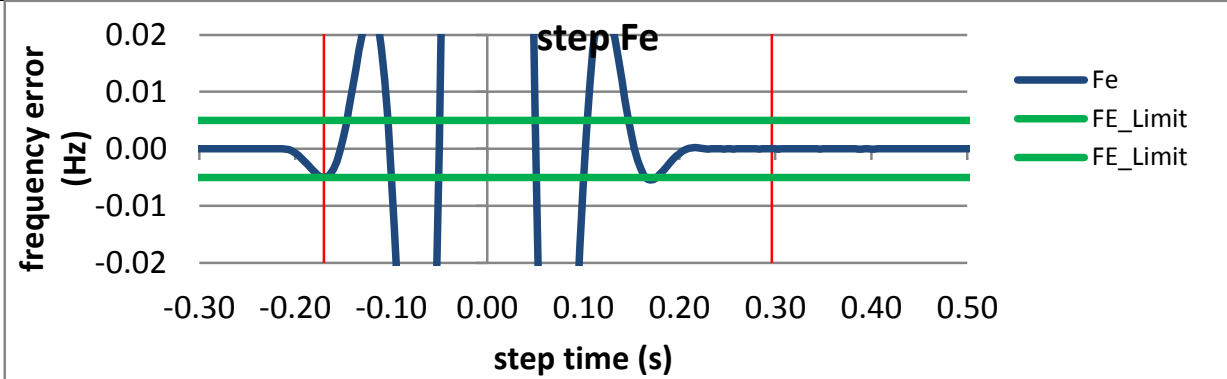

Figure 4506: Fs = 30 FPS, -10 degree phase step

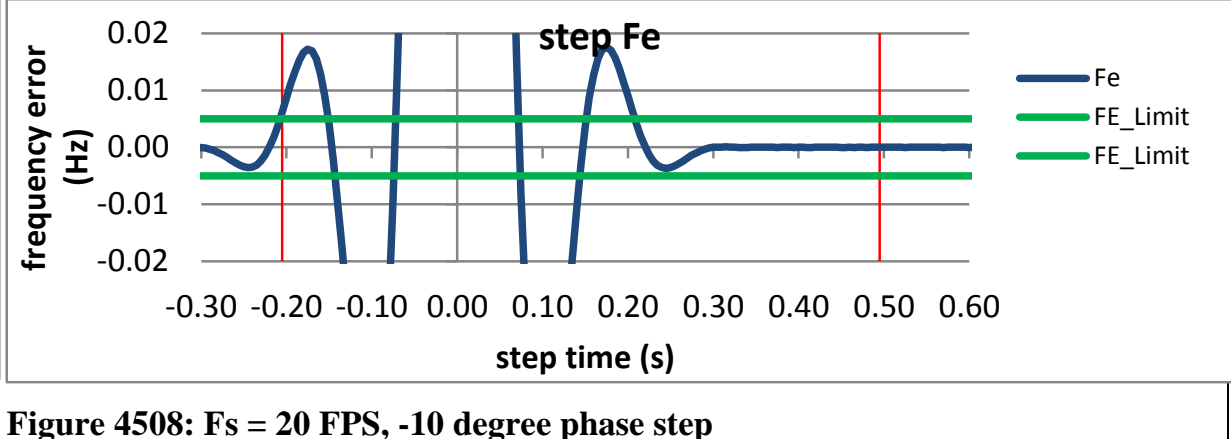




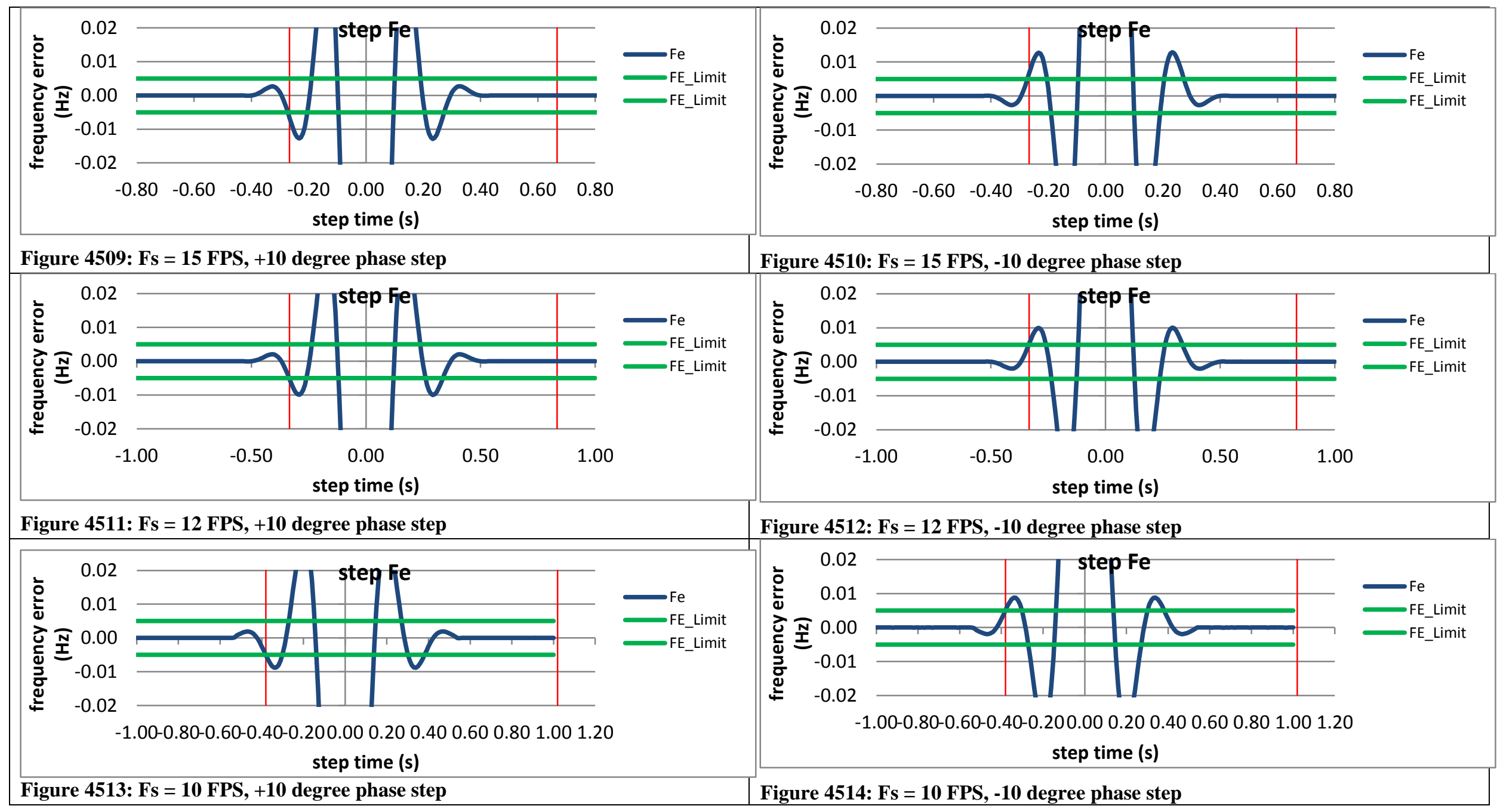


9.5.10 PMU I dynamic step change in phase frequency response time: $\mathrm{F} 0=60 \mathrm{~Hz}, \mathrm{M}$ class
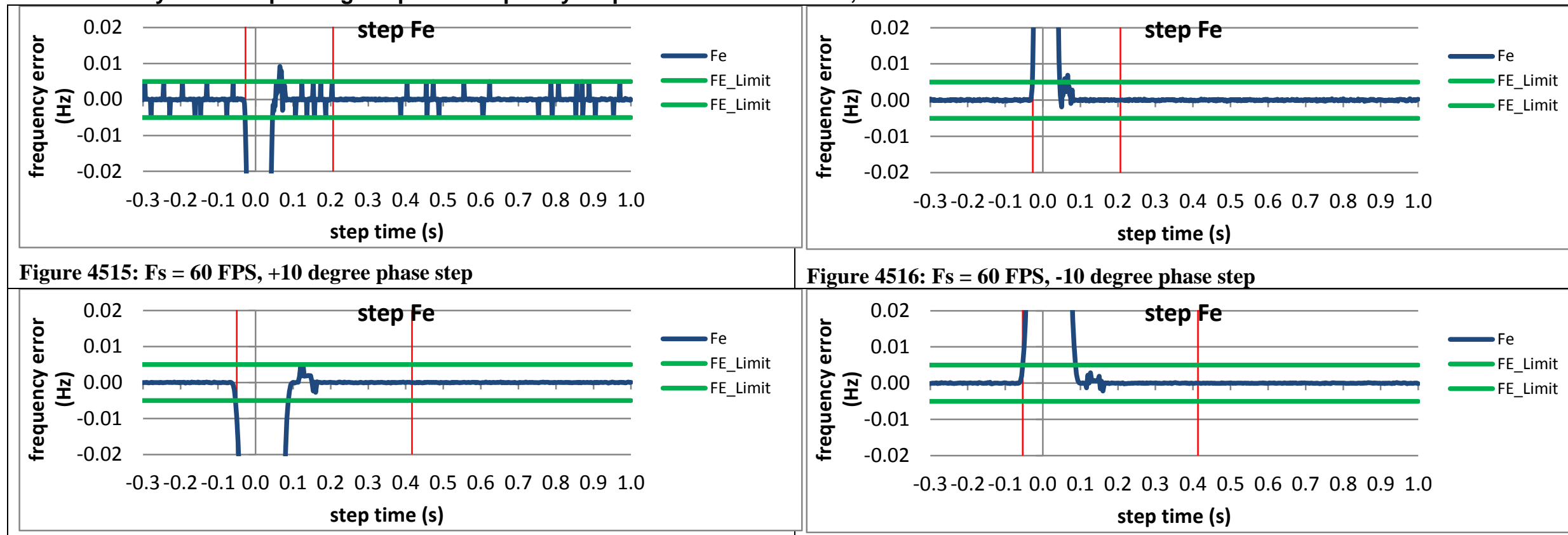

Figure 4517: Fs = 30 FPS, +10 degree phase step

Figure 4518: Fs = 30 FPS, -10 degree phase step

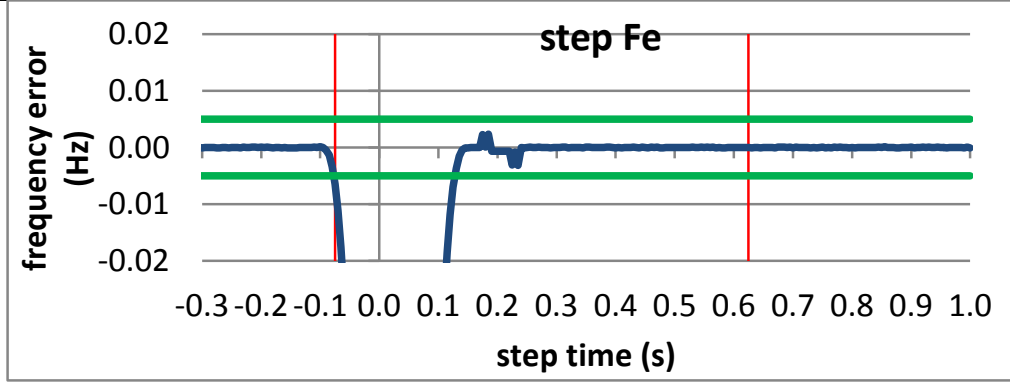

Figure 4519: Fs = 20 FPS, +10 degree phase step

Fe
FE_Limit
FE_Limit

Fe
FE_Limit
FE_Limit

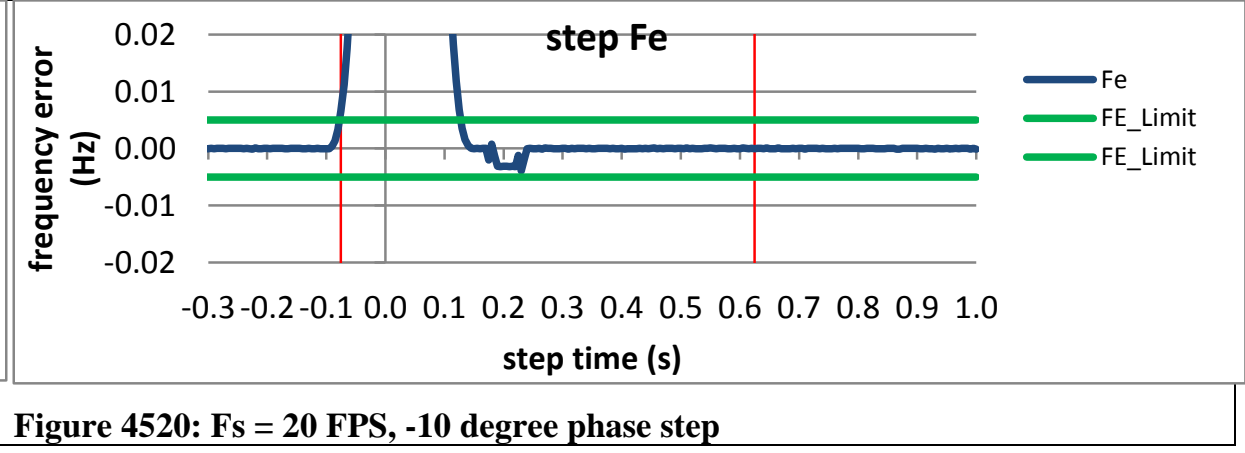




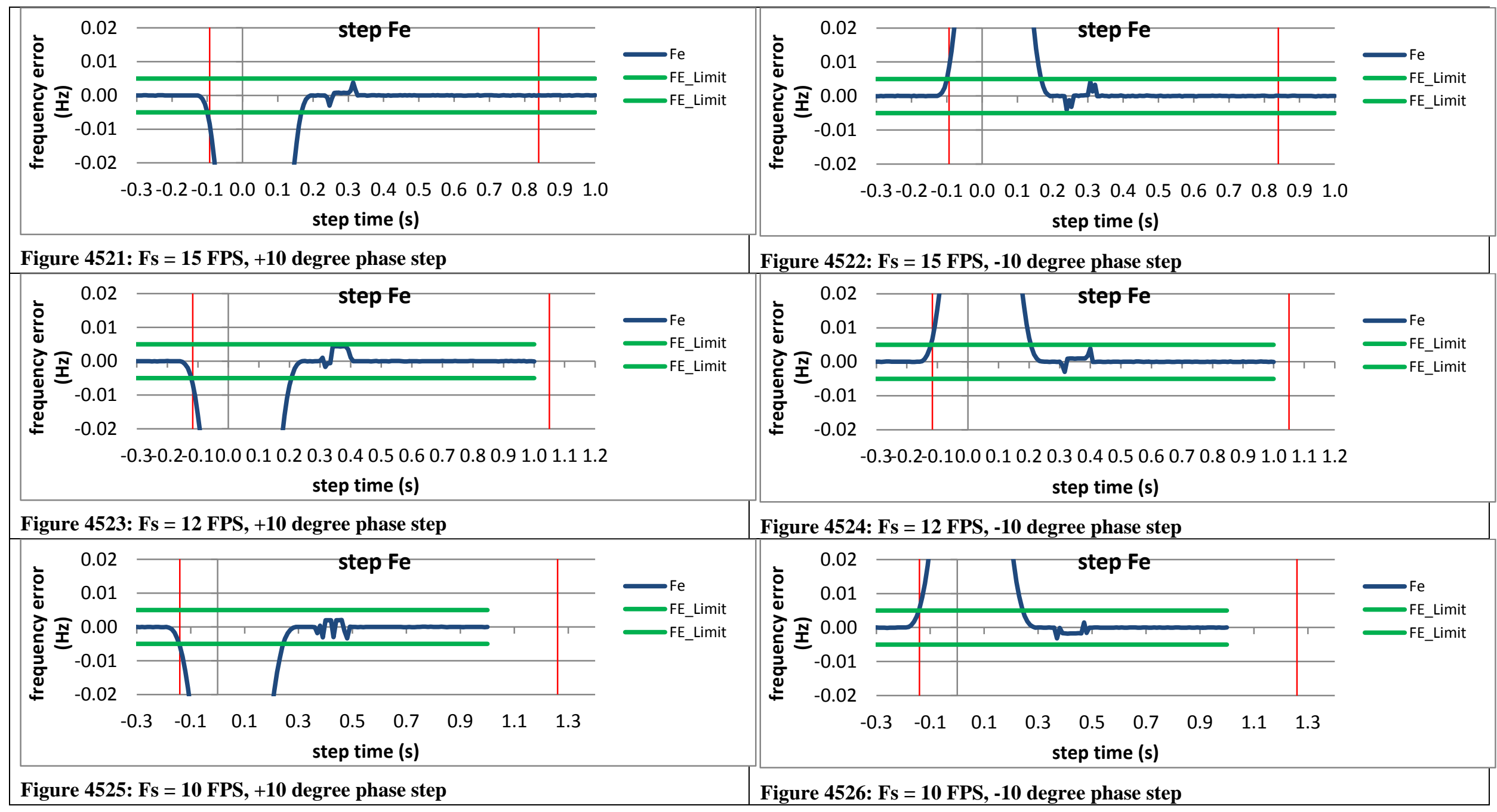


9.5.11 PMU J dynamic step change in phase frequency response time: $\mathrm{FO}=60 \mathrm{~Hz}, \mathrm{M}$ class
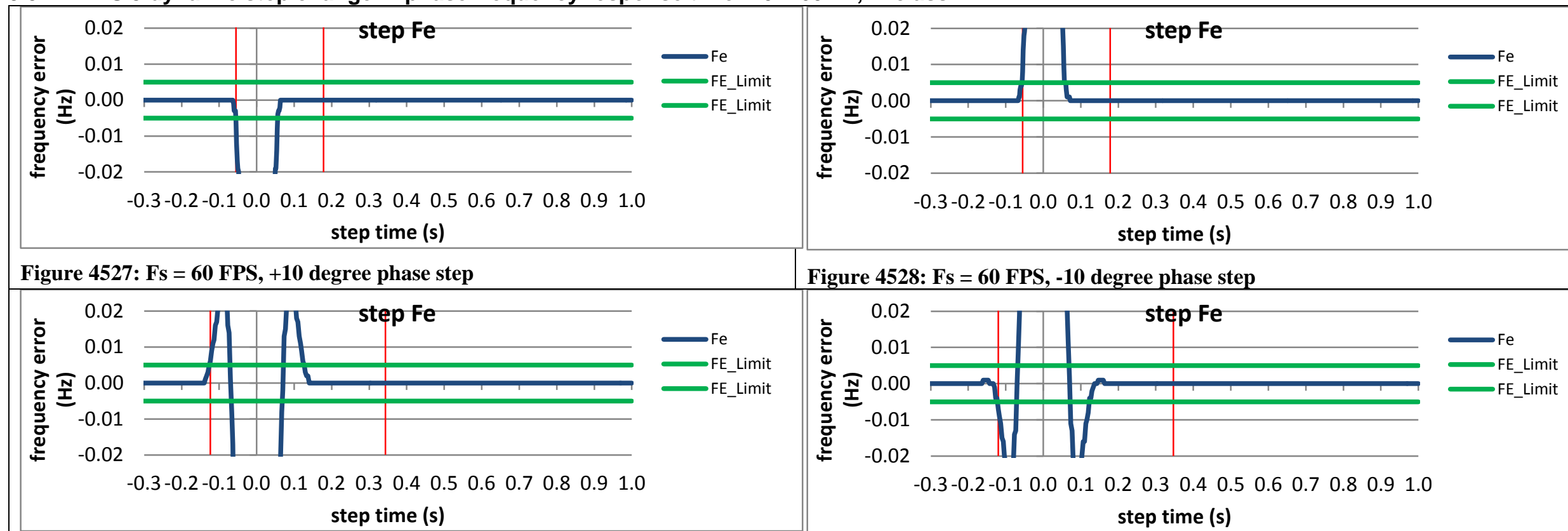

Fe
FE_Limit
FE_Limit

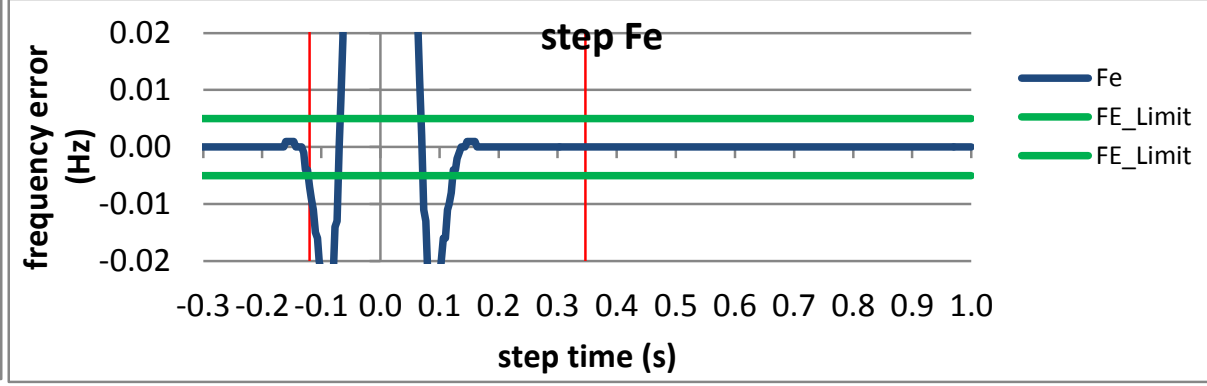

Figure 4529: Fs = 30 FPS, +10 degree phase step

Figure 4530: Fs = 30 FPS, -10 degree phase step

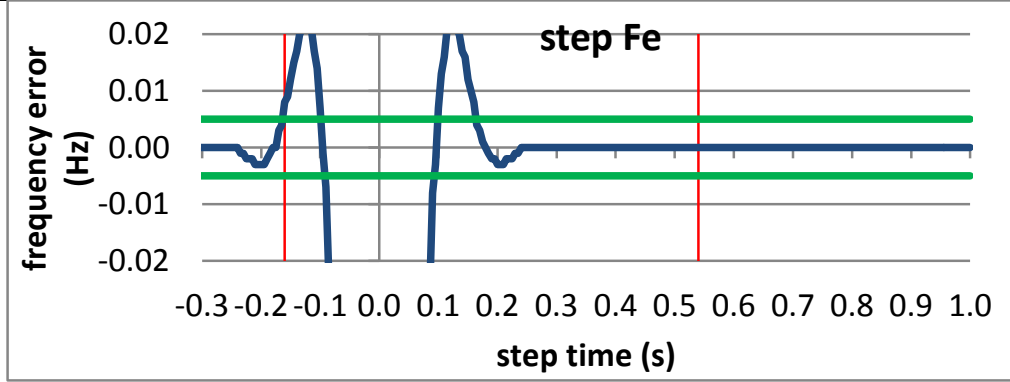

Figure 4531: Fs = 20 FPS, + 10 degree phase step

$\mathrm{Fe}$
$\mathrm{FE}$ LLimit
$\mathrm{FE}$ _Limit

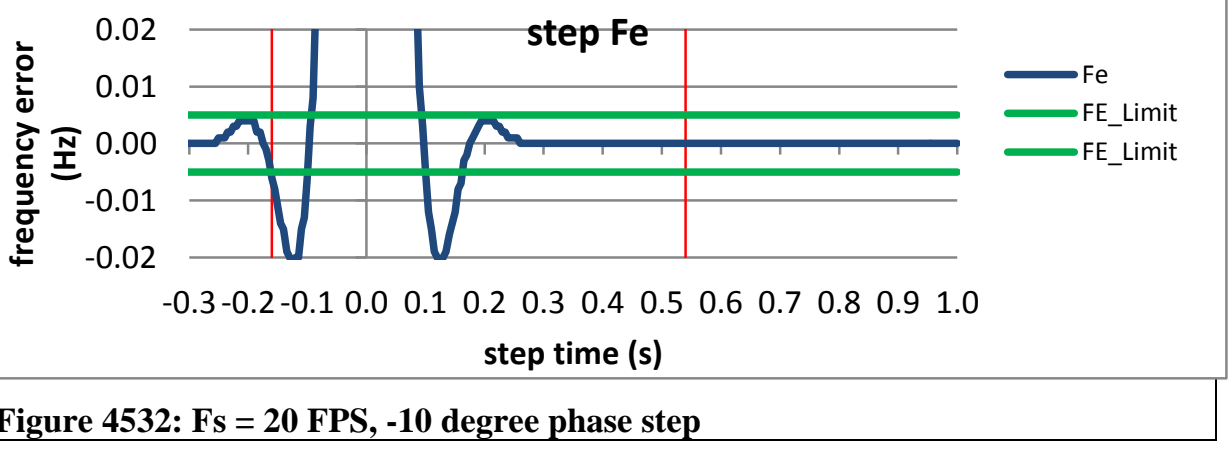



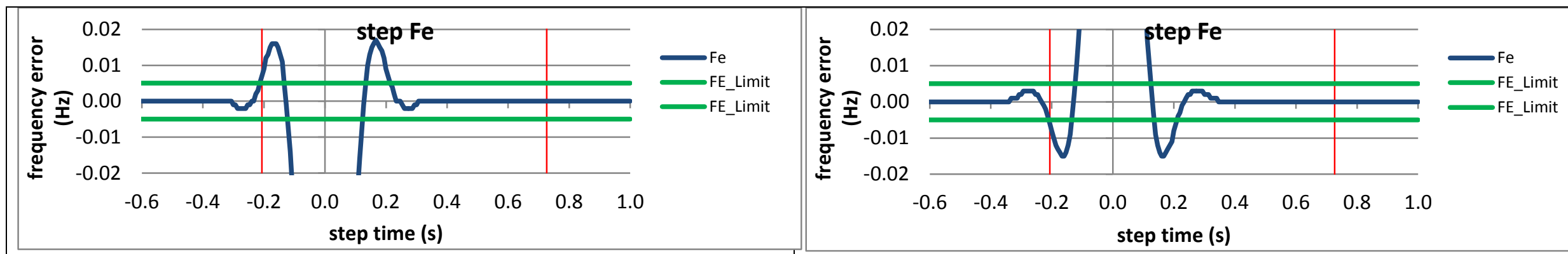

Figure 4533: Fs $=15$ FPS, +10 degree phase step

Figure 4534: Fs = 15 FPS, -10 degree phase step
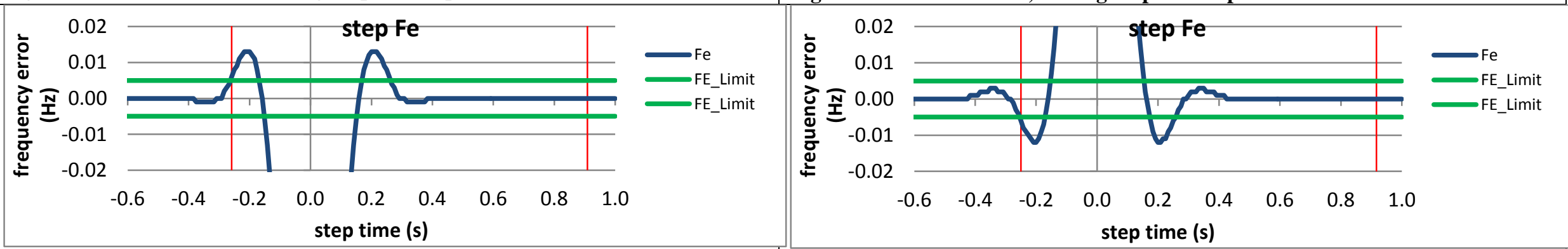

Figure 4535: Fs = 12 FPS, +10 degree phase step

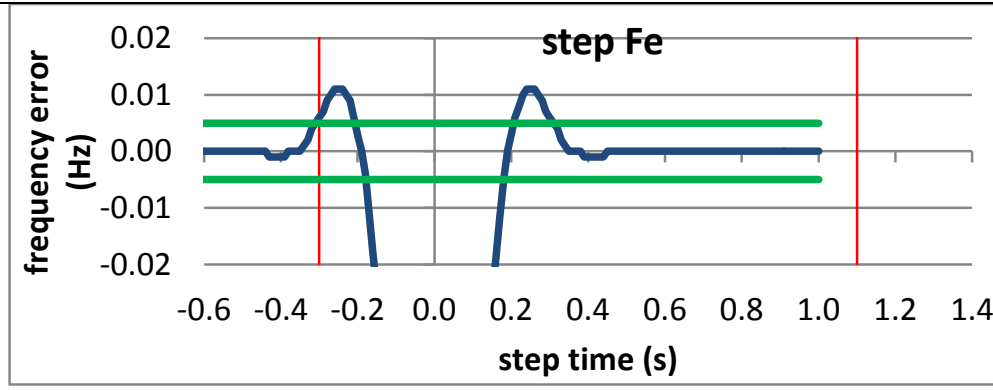

Figure 4537: Fs = 10 FPS, +10 degree phase step
Figure 4536: Fs = 12 FPS, -10 degree phase step

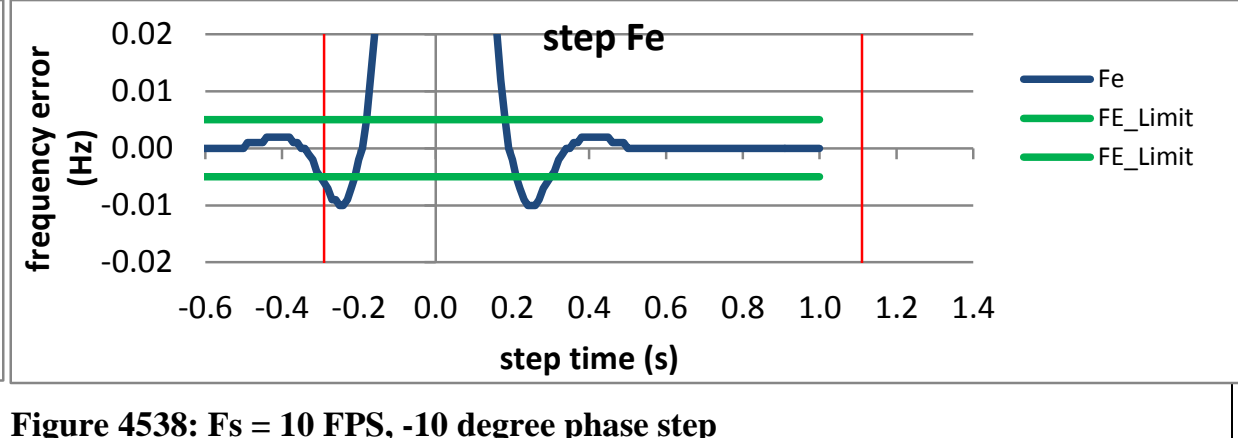


9.6 Dynamic step change in phase frequency response time: $\mathrm{FO}=60 \mathrm{~Hz}$, P Class

9.6.1 C37.118.1-2011 Annex $\mathrm{C}$ dynamic step change in phase frequency response time: $\mathrm{F} 0=60 \mathrm{~Hz}$, $\mathrm{P}$ class

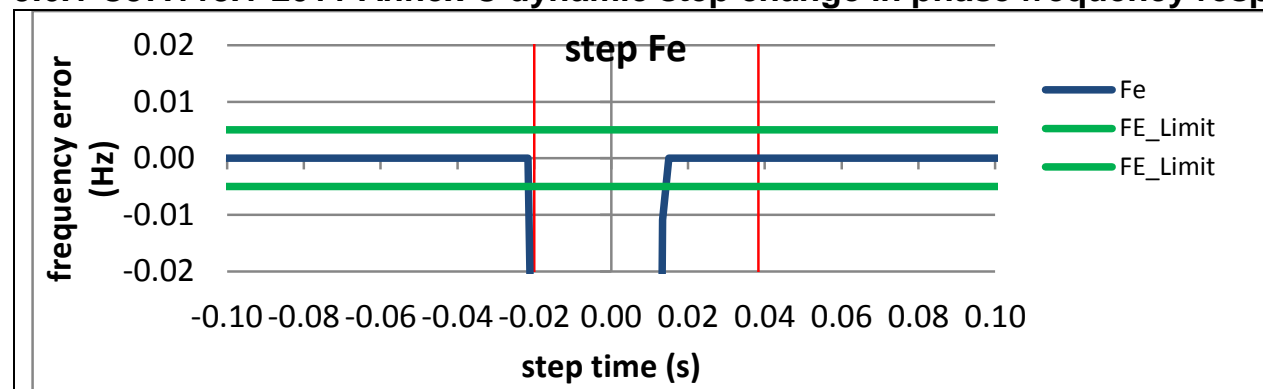

Figure 4539: Fs = 60 FPS, +10 degree phase step

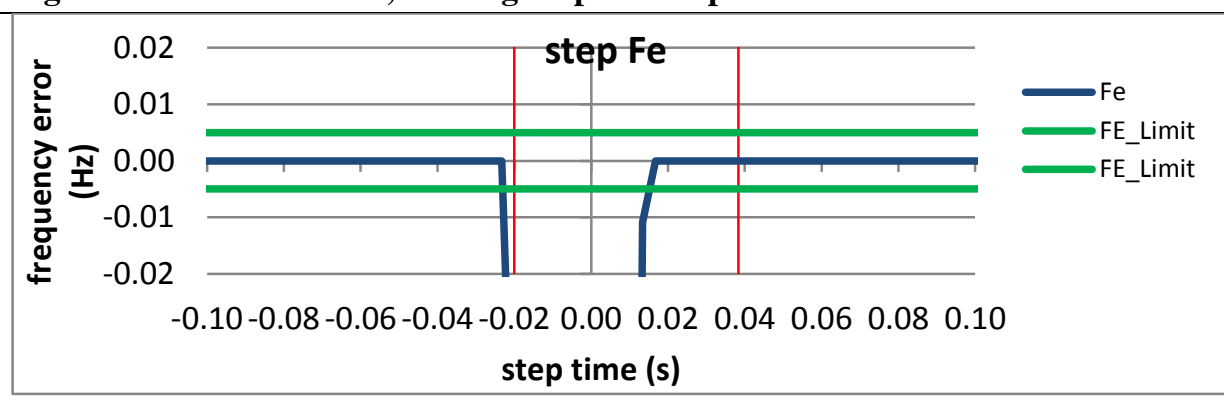

Figure 4541: Fs = 30 FPS, +10 degree phase step

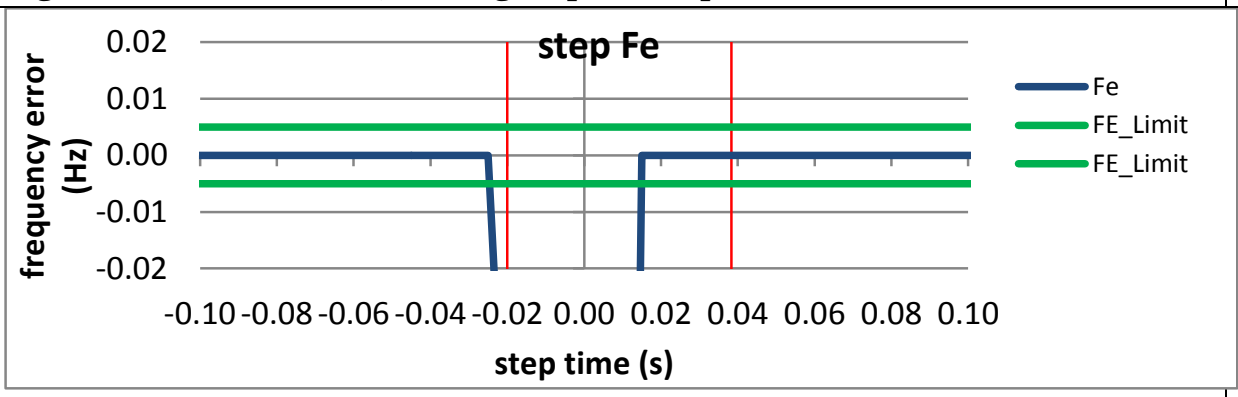

Figure 4543: Fs = 20 FPS, + 10 degree phase step

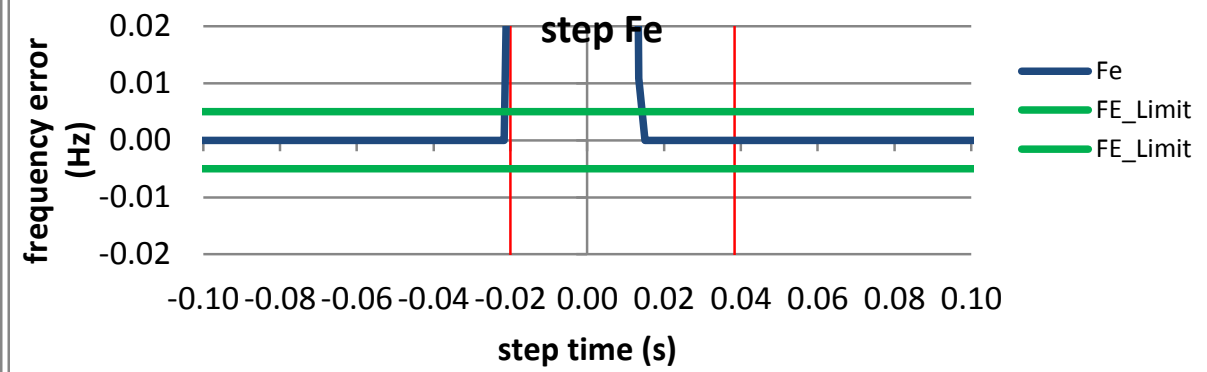

Figure 4540: Fs = 60 FPS, -10 degree phase step

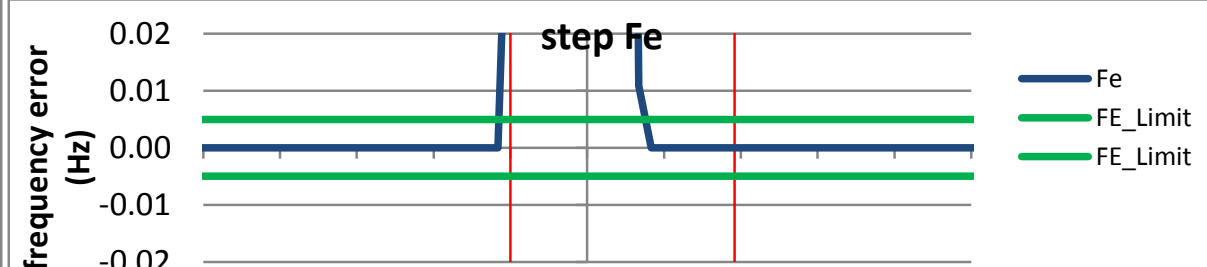

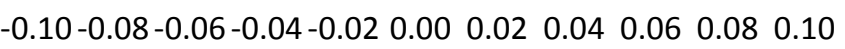
step time (s)

Figure 4542: Fs = 30 FPS, -10 degree phase step

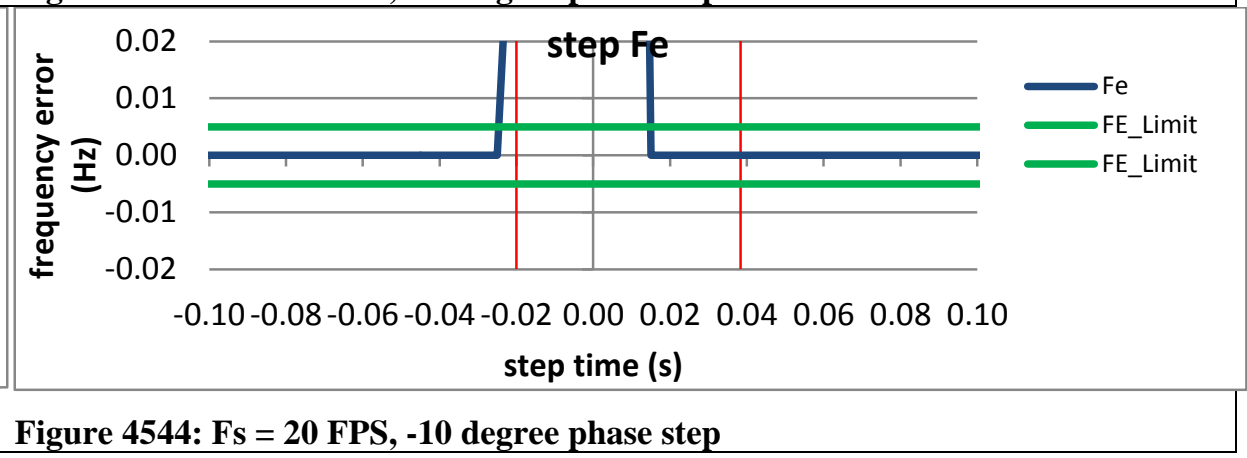




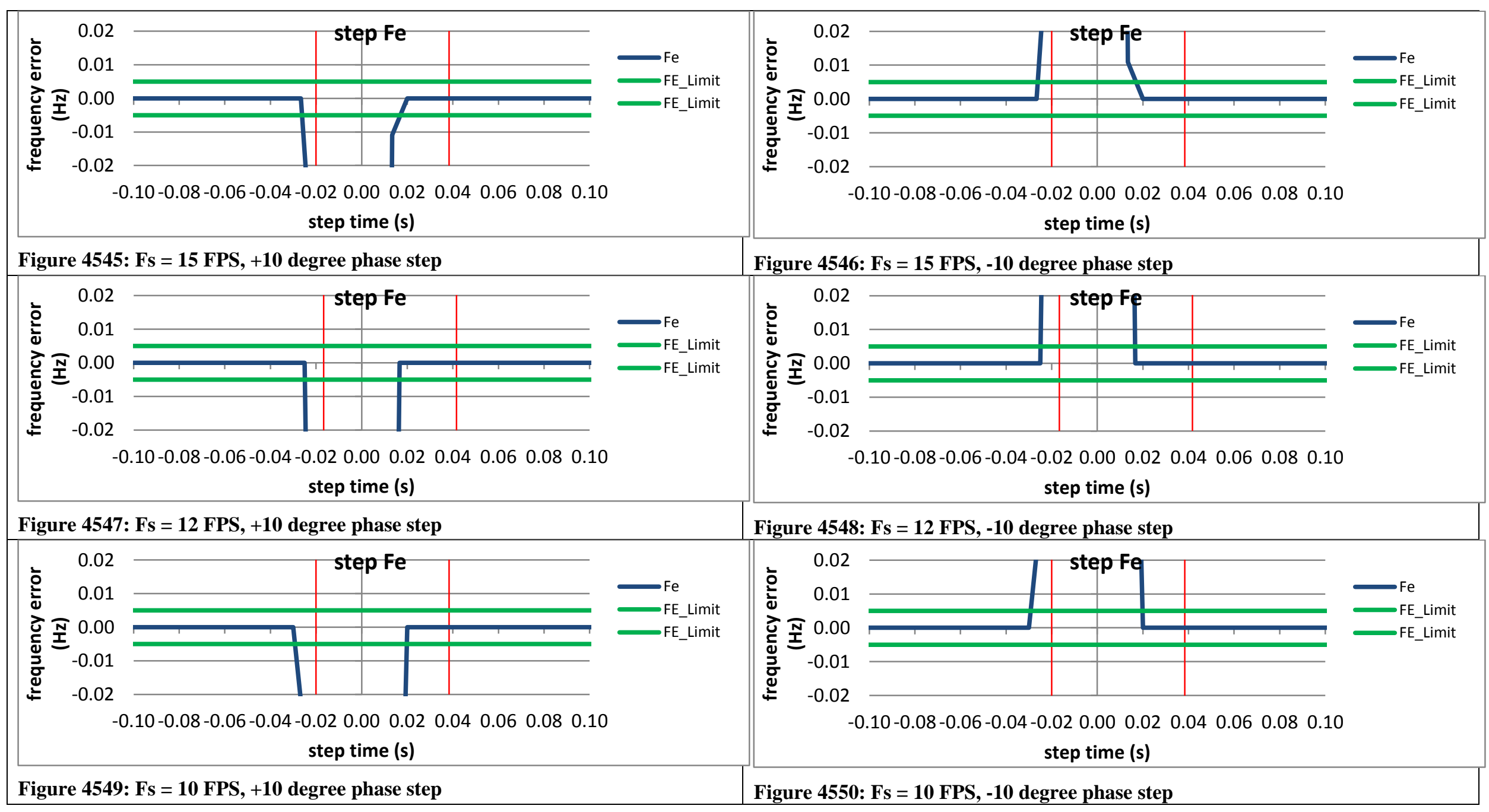


9.6.2 PMU A dynamic step change in phase frequency response time: $\mathrm{F} 0=60 \mathrm{~Hz}, \mathrm{P}$ class
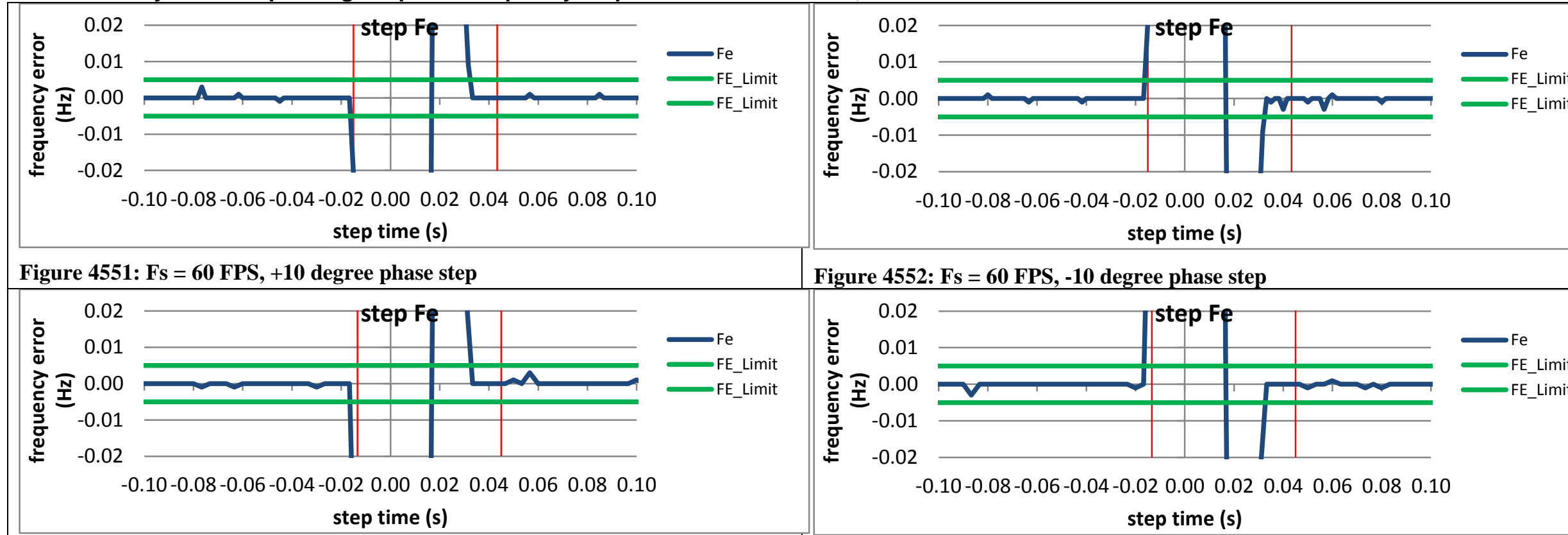

Figure 4552: Fs = 60 FPS, -10 degree phase step

Figure 4553: Fs = 30 FPS, +10 degree phase step
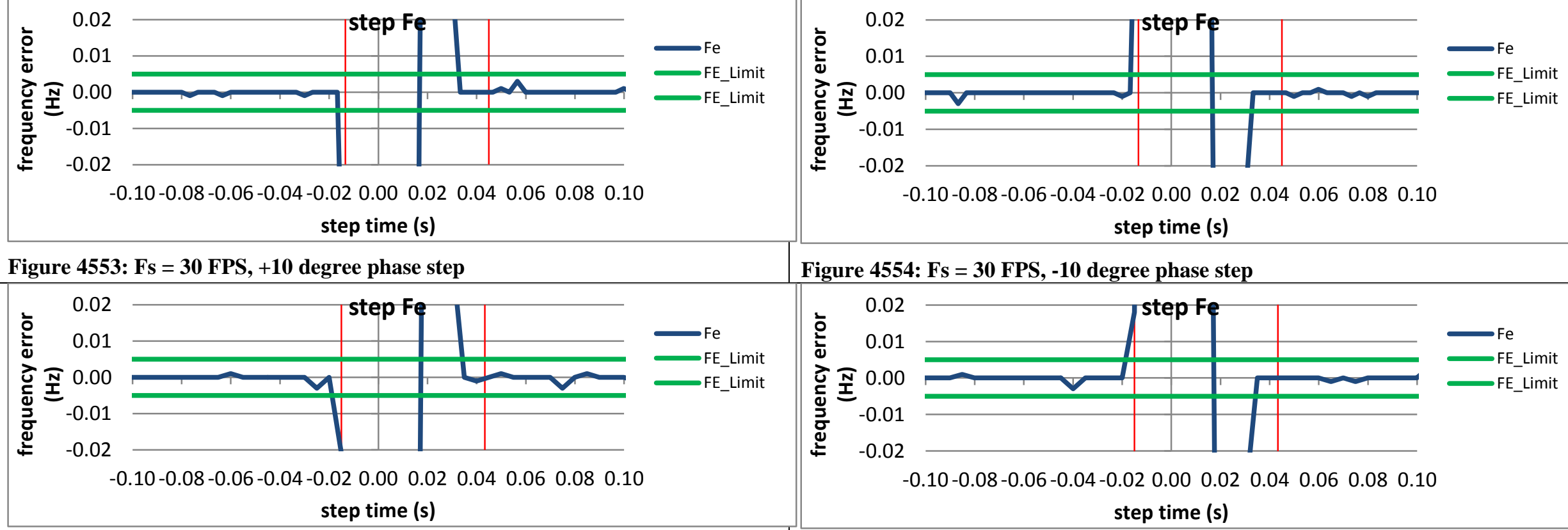

Figure 4554: Fs = 30 FPS, -10 degree phase step

Figure 4555: Fs = 20 FPS, +10 degree phase step

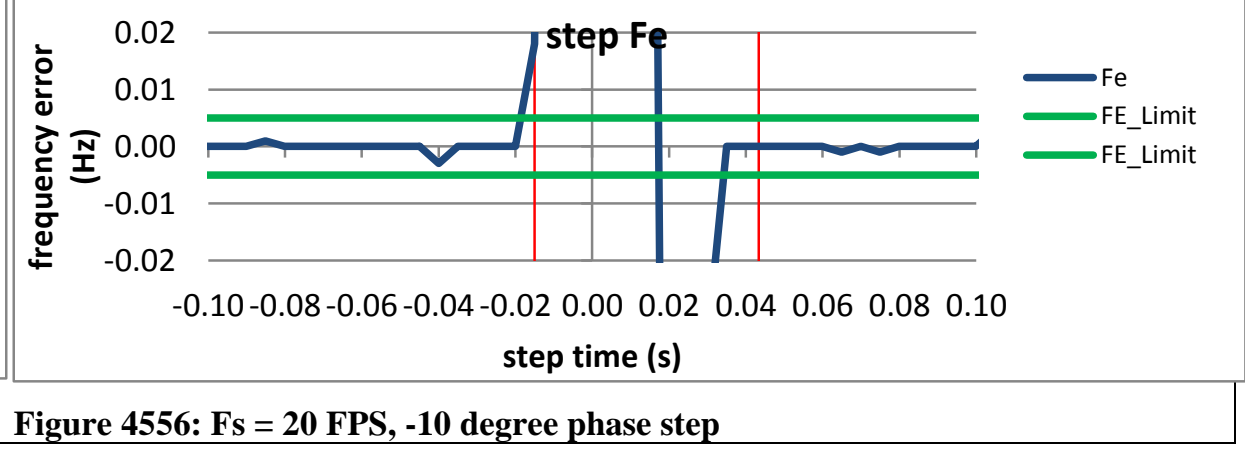




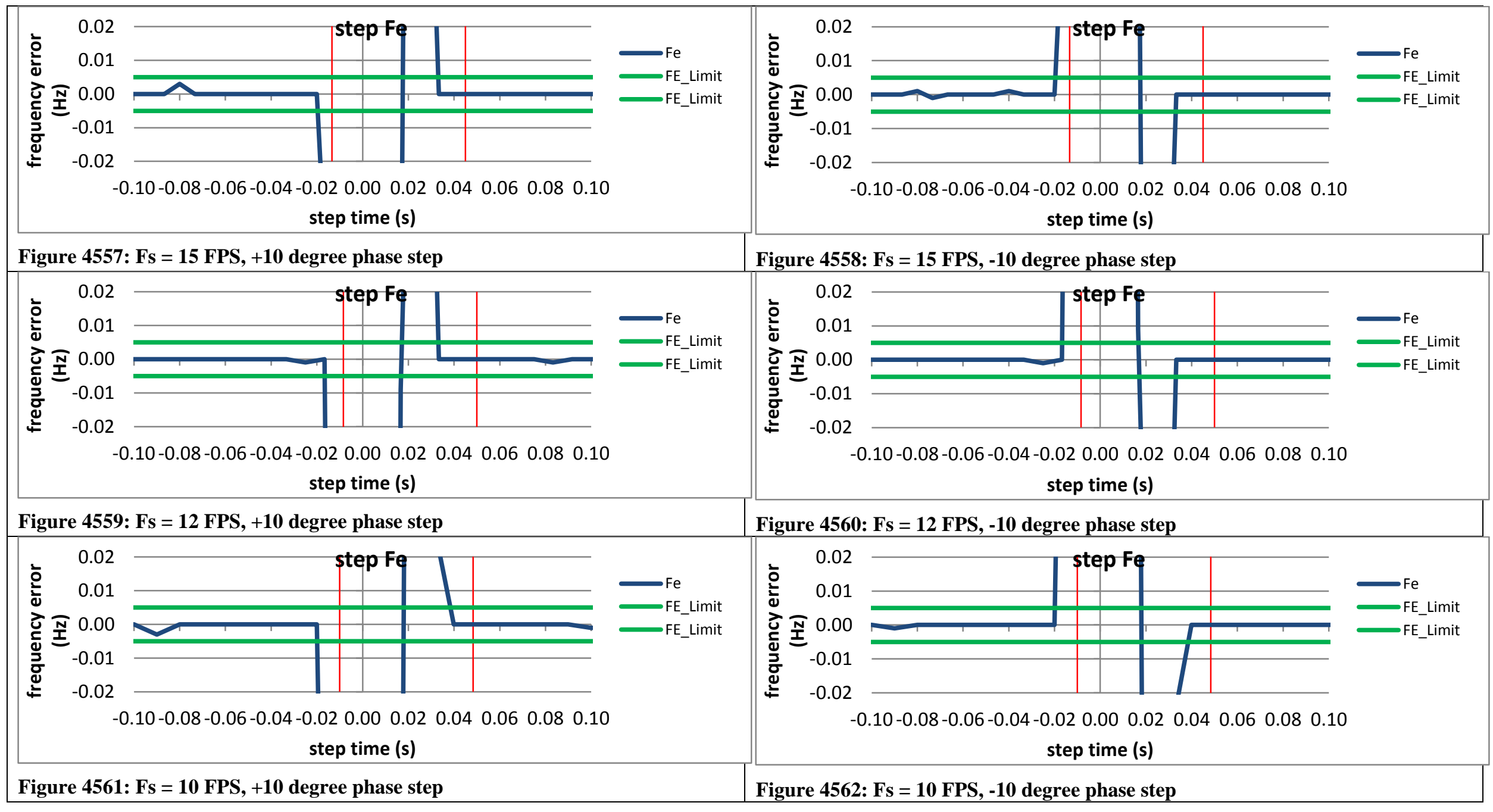


9.6.3 PMU B dynamic step change in phase frequency response time: $\mathrm{F} 0=60 \mathrm{~Hz}, \mathrm{P}$ class

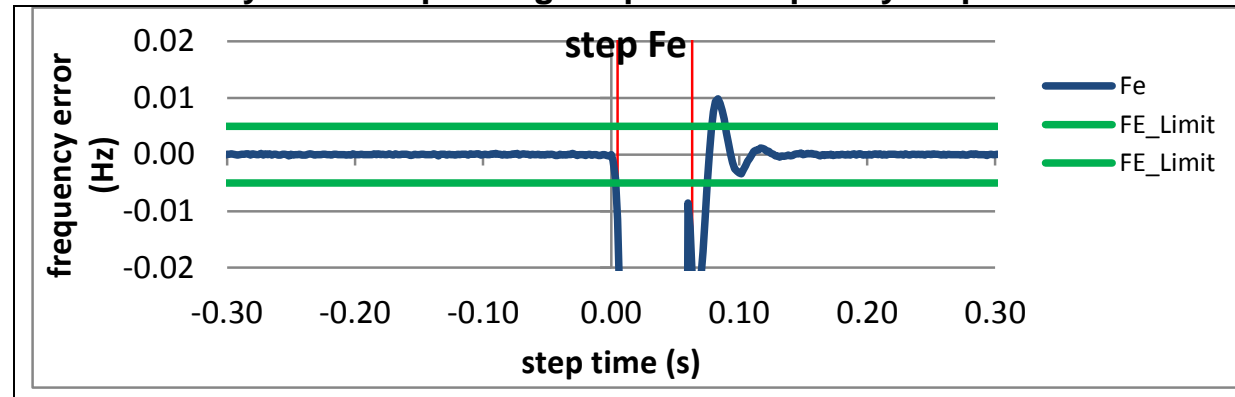

Figure 4563: Fs = 60 FPS, +10 degree phase step

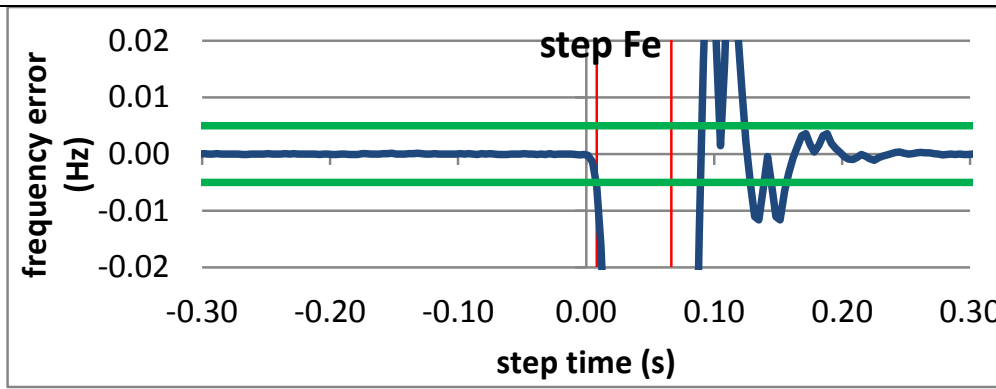

Figure 4565: Fs = 30 FPS, +10 degree phase step

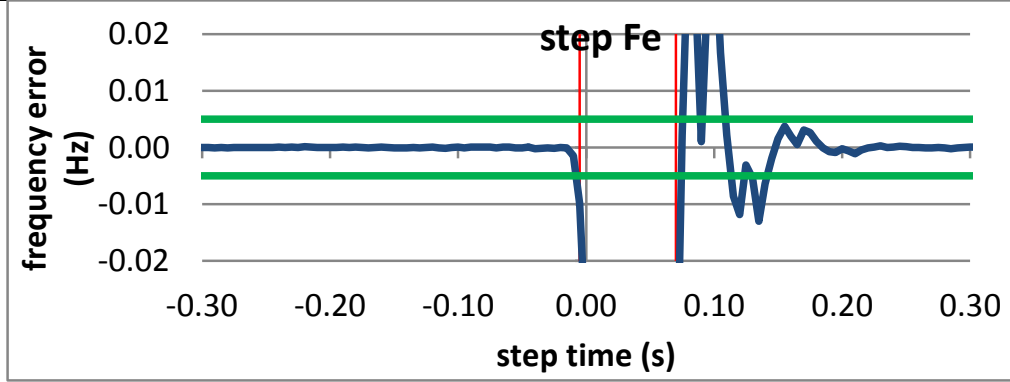

Figure 4567: Fs = 20 FPS, +10 degree phase step

Fe
FE_Limit
FE_Limit

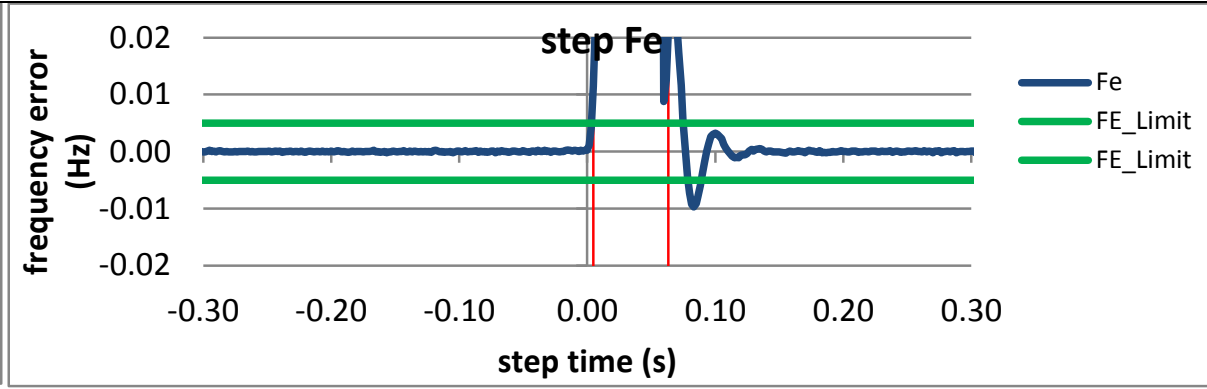

Figure 4564: Fs = 60 FPS, -10 degree phase step

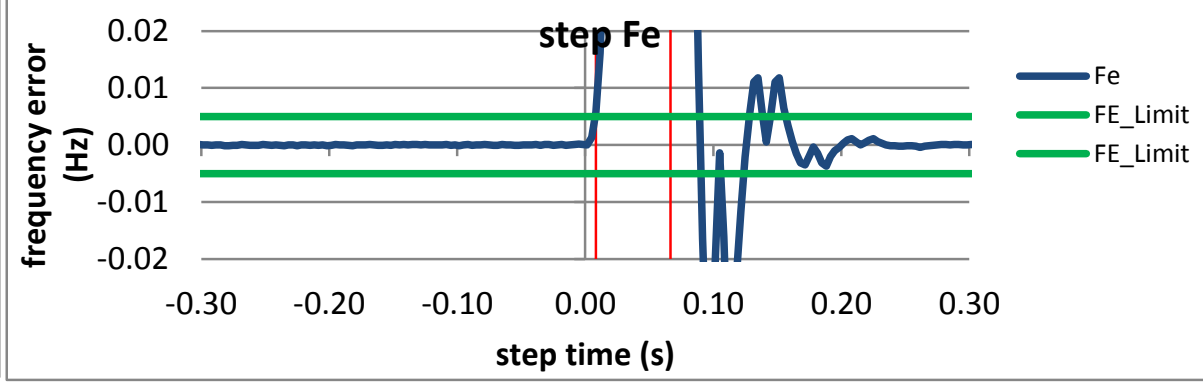

Figure 4566: Fs = 30 FPS, -10 degree phase step

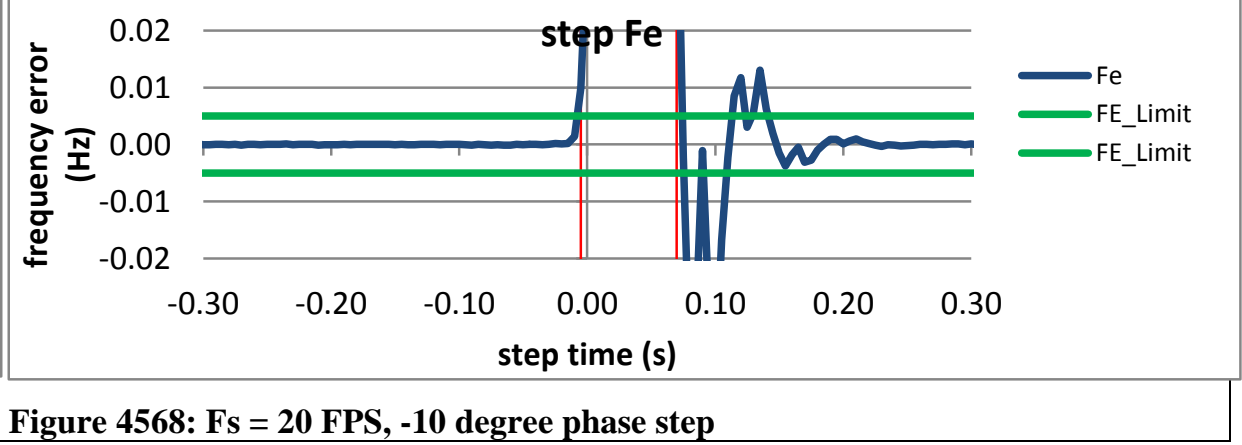



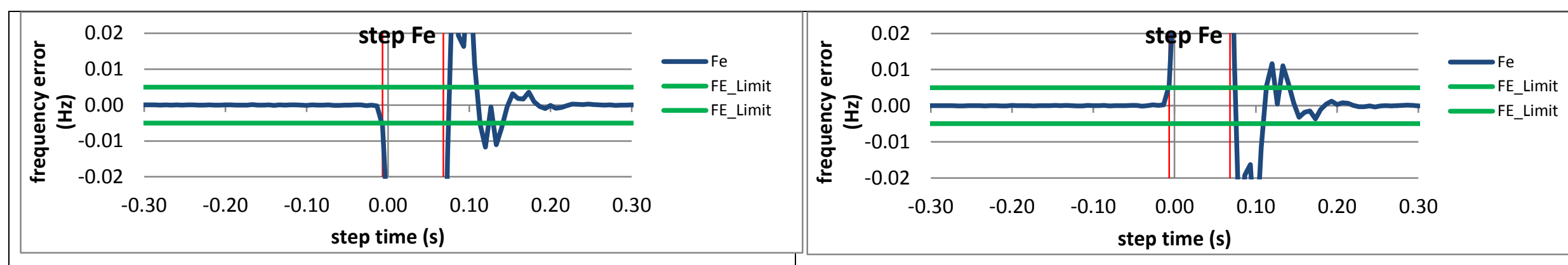

Figure 4569: Fs = 15 FPS, +10 degree phase step

Figure 4570: Fs = 15 FPS, -10 degree phase step
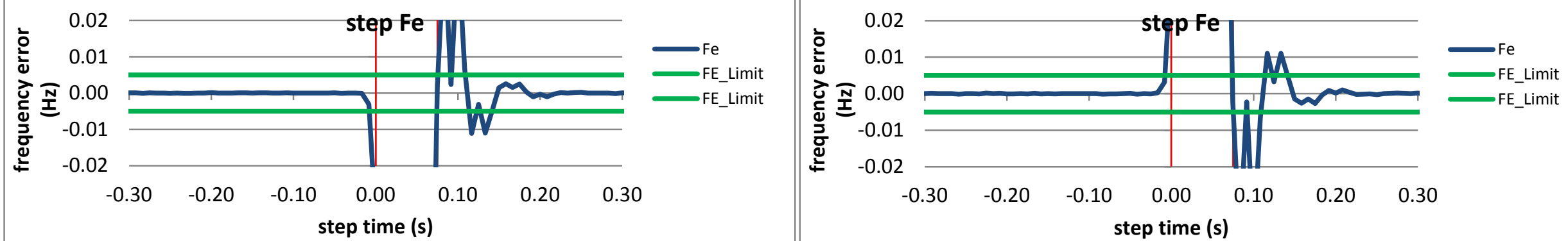

Figure 4571: Fs = 12 FPS, +10 degree phase step

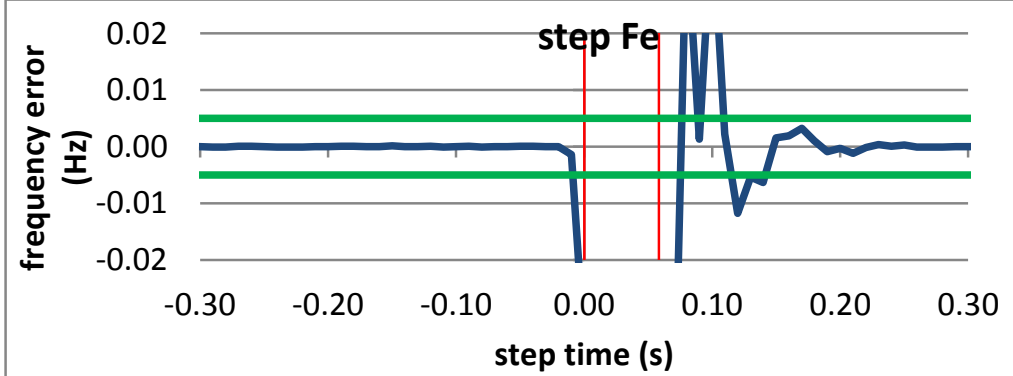

Figure 4572: Fs = 12 FPS, -10 degree phase step

Figure 4573: Fs = 10 FPS, +10 degree phase step

Fe

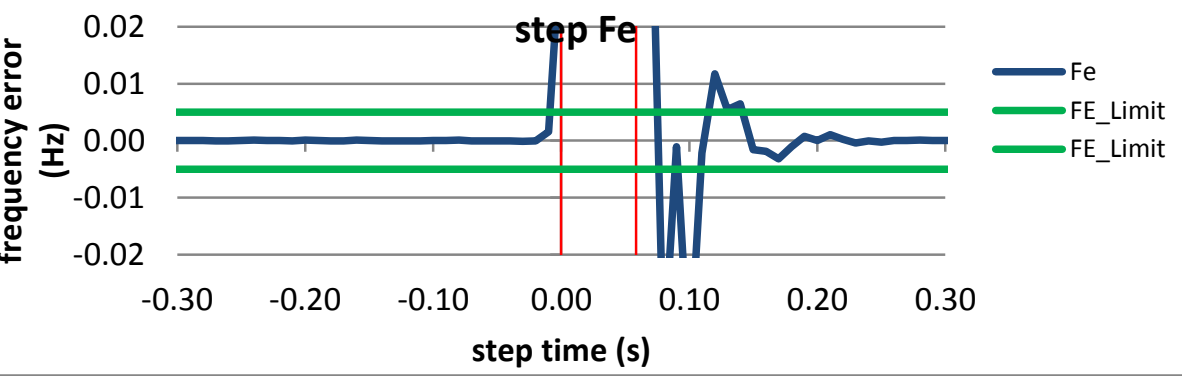

Figure 4574: Fs = 10 FPS, -10 degree phase step 
9.6.4 PMU C dynamic step change in phase frequency response time: $\mathrm{F} 0=60 \mathrm{~Hz}, \mathrm{P}$ class
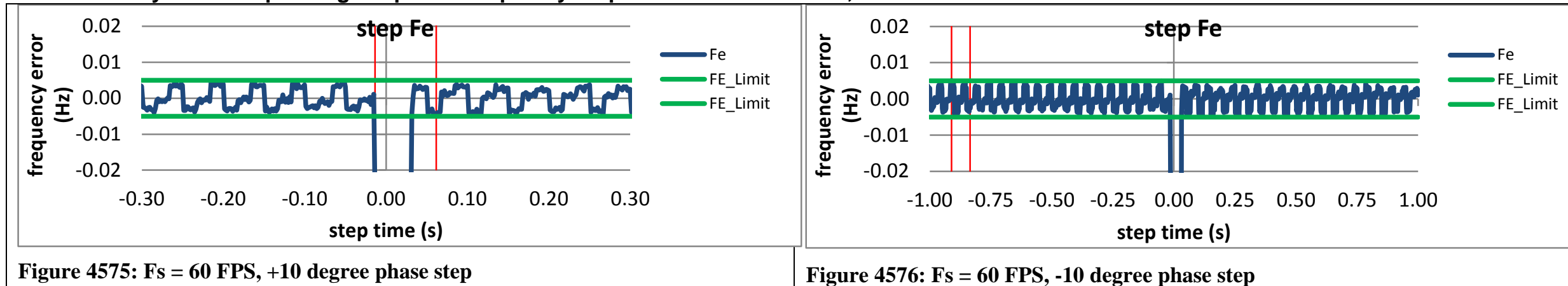

Figure 4575: Fs = 60 FPS, +10 degree phase step

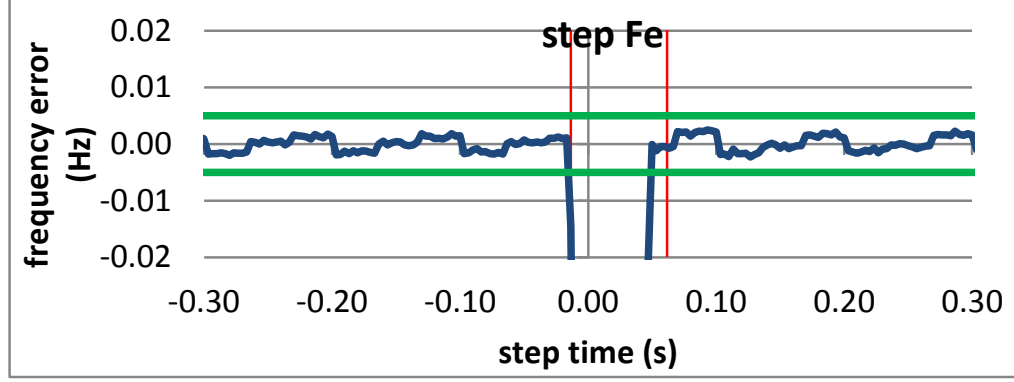

Figure 4577: Fs = 30 FPS, +10 degree phase step

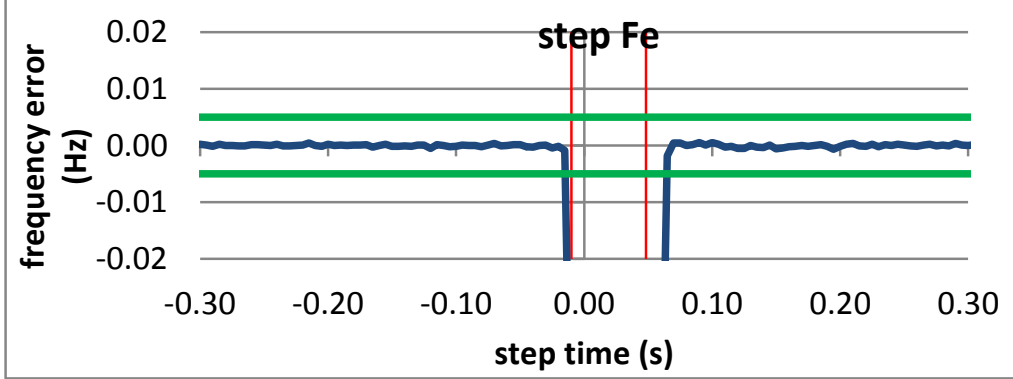

Figure 4579: Fs = 20 FPS, +10 degree phase step
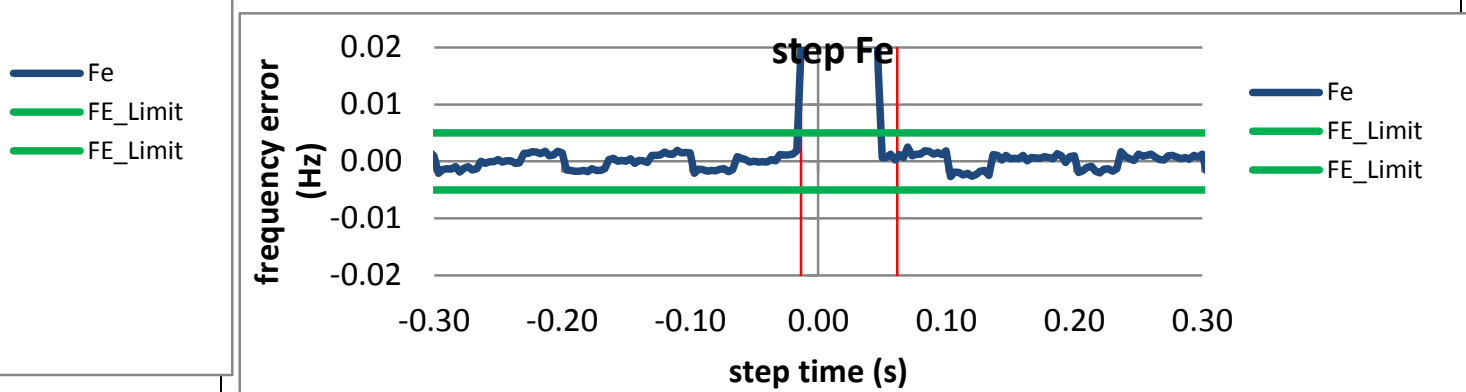

Figure 4578: Fs = 30 FPS, -10 degree phase step

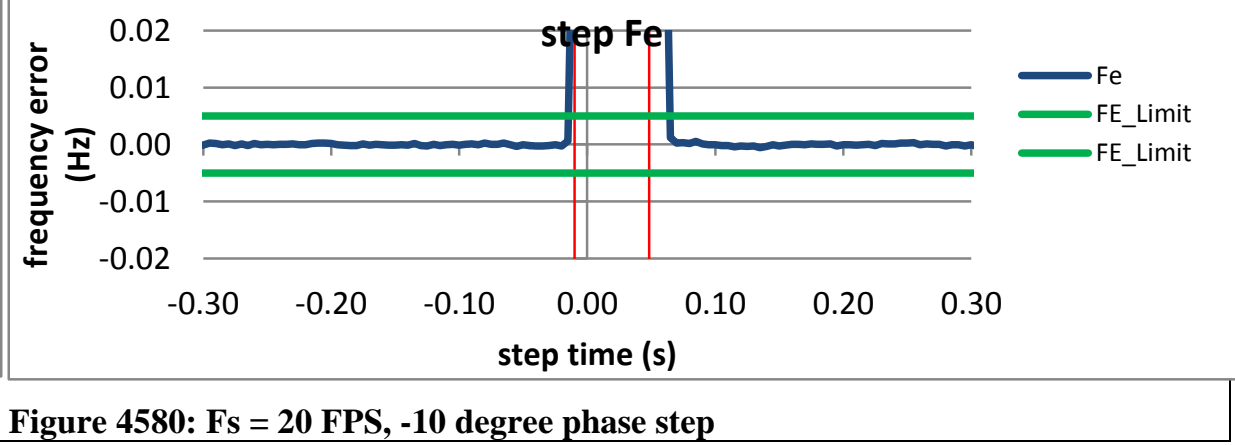




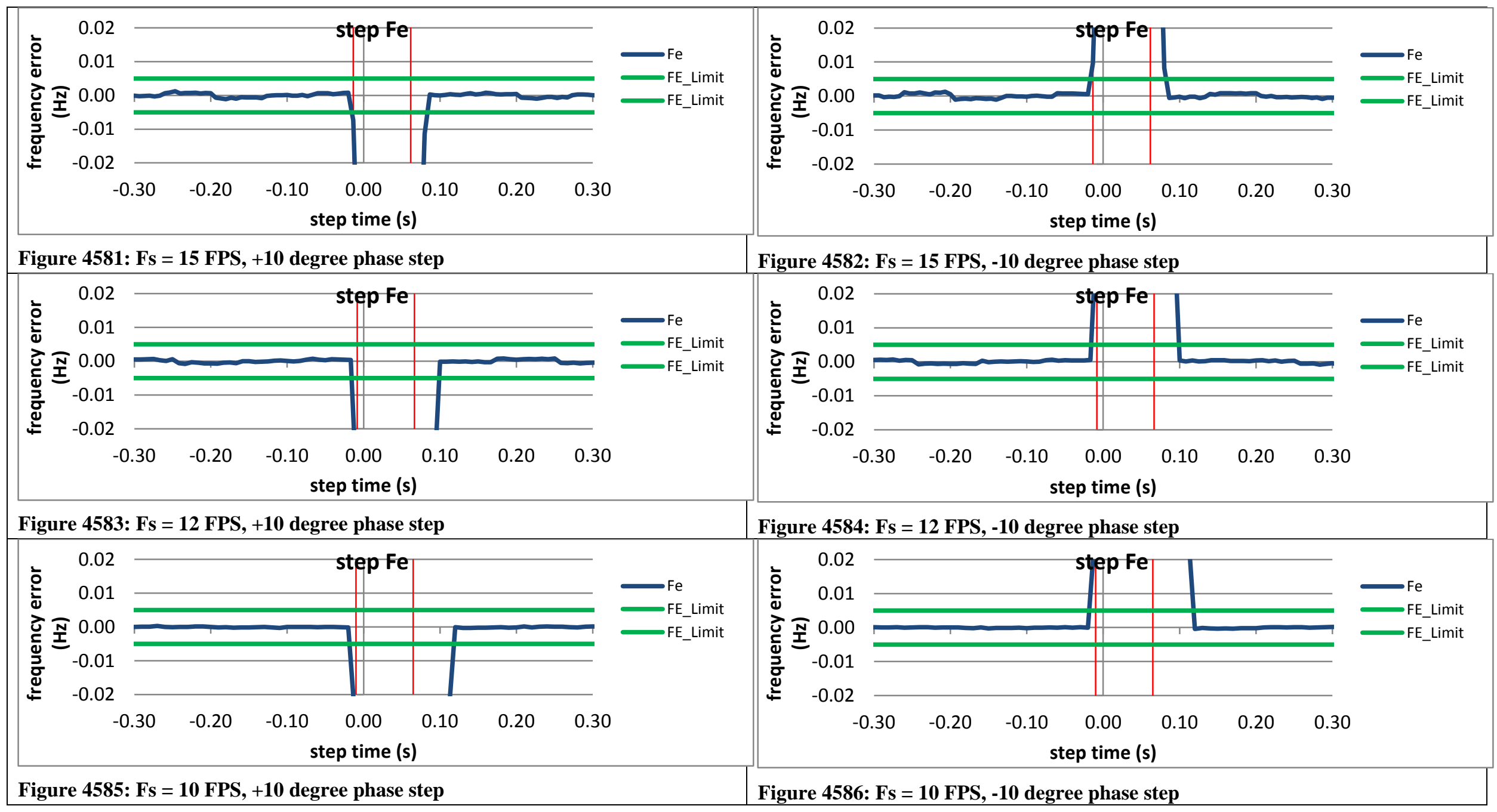


9.6.5 PMU D dynamic step change in phase frequency response time: $F 0=60 \mathrm{~Hz}, \mathrm{P}$ class

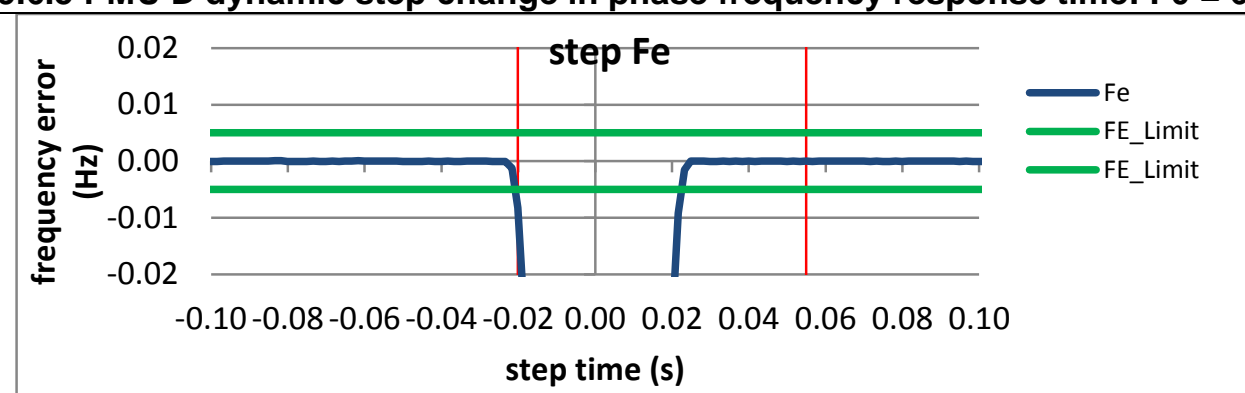

Figure 4587: Fs = 60 FPS, +10 degree phase step

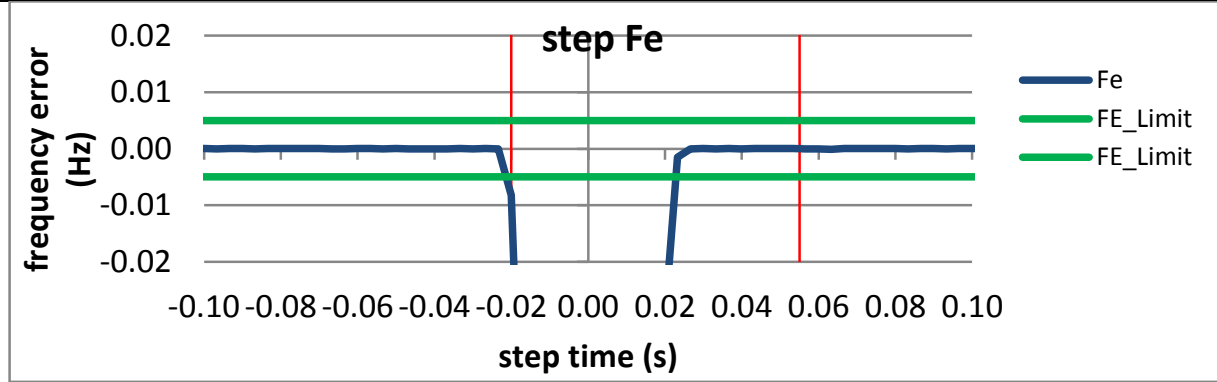

Figure 4589: Fs = 30 FPS, +10 degree phase step

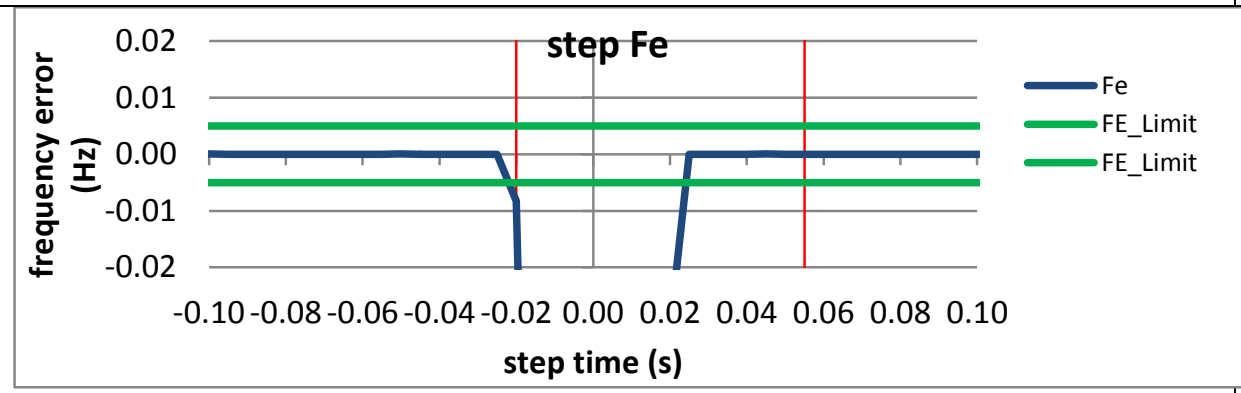

Figure 4591: Fs = 20 FPS, + 10 degree phase step

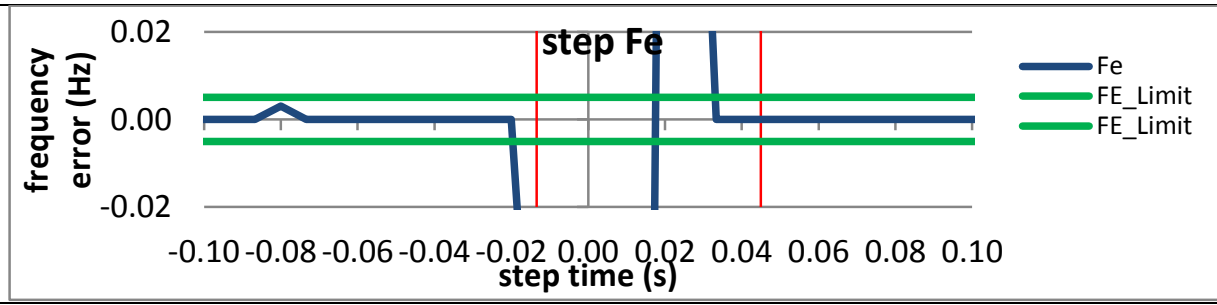

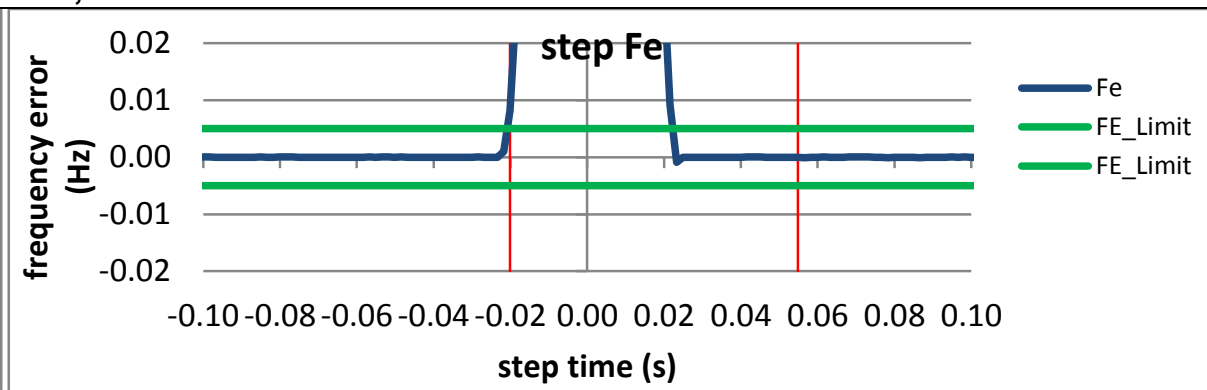

Figure 4588: Fs = 60 FPS, -10 degree phase step

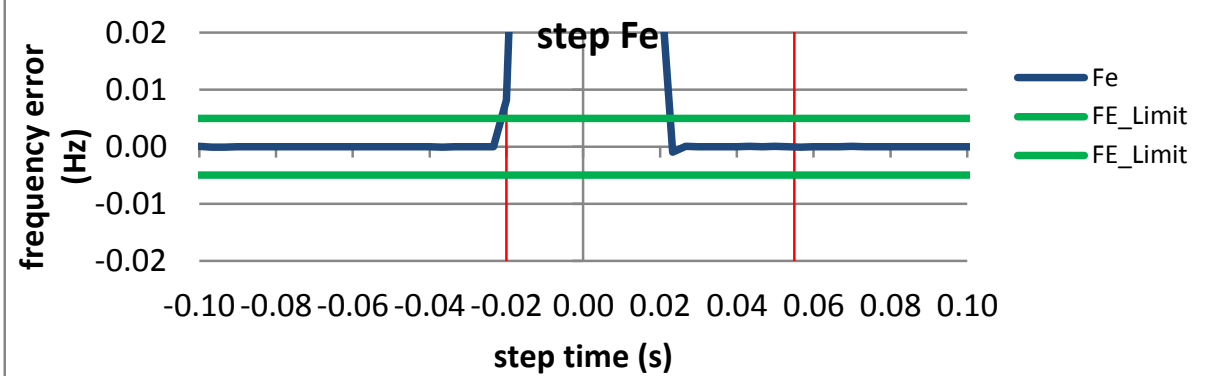

Figure 4590: Fs = 30 FPS, -10 degree phase step

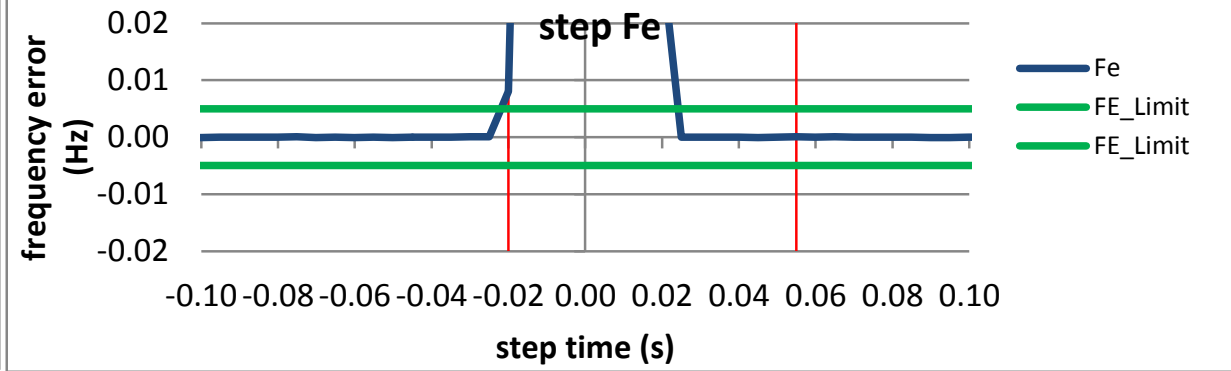

Figure 4592: Fs = 20 FPS, -10 degree phase step

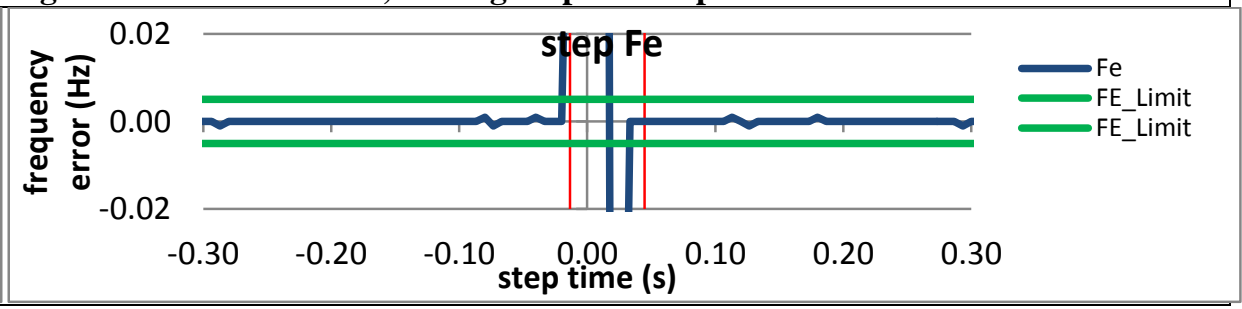




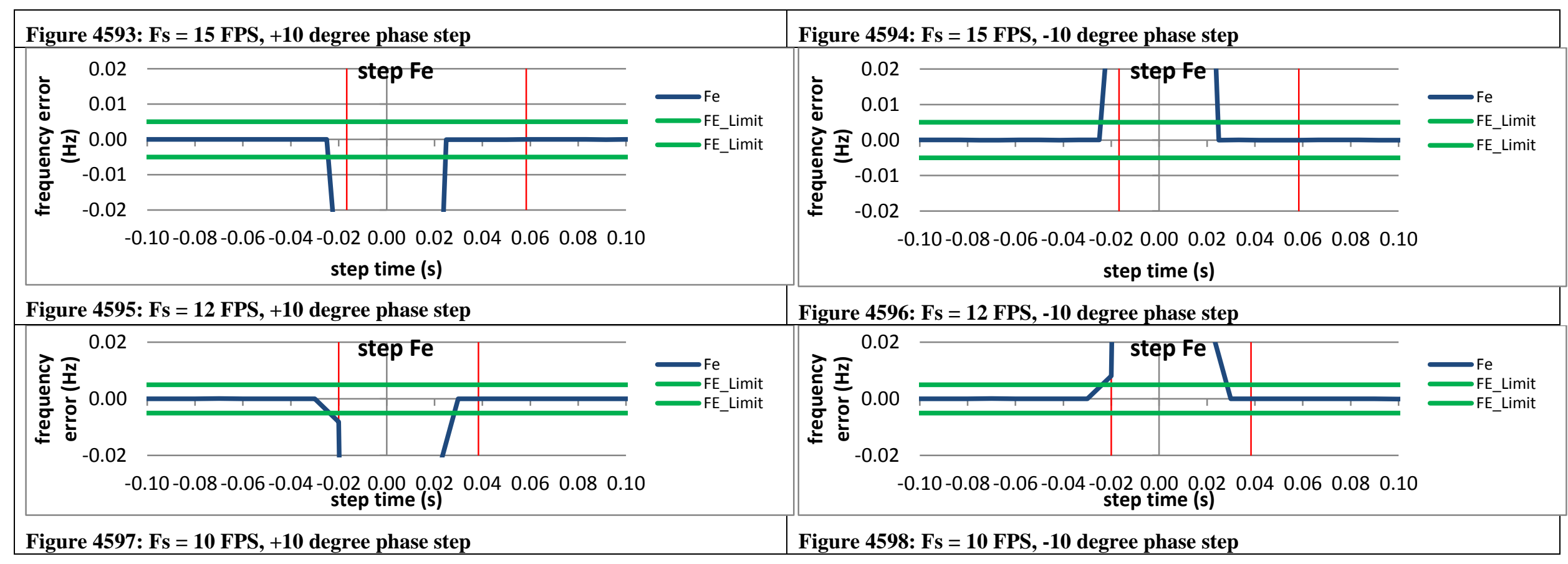


9.6.6 PMU E dynamic step change in phase frequency response time: $F 0=60 \mathrm{~Hz}, \mathrm{P}$ class PMU E does not support $P$ class

\subsubsection{PMU F dynamic step change in phase frequency response time: $\mathrm{F} 0=60 \mathrm{~Hz}, \mathrm{P}$ class}

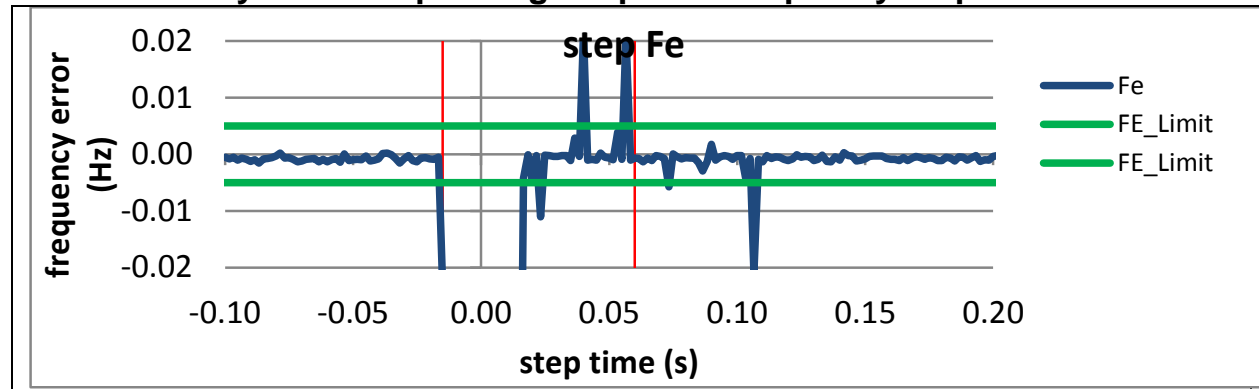

Figure 4599: Fs = 60 FPS, +10 degree phase step

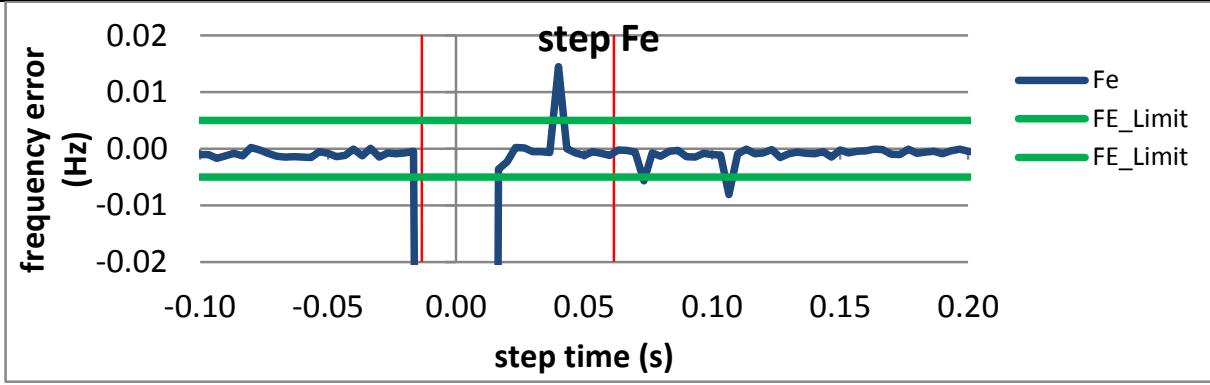

Figure 4601: Fs = 30 FPS, +10 degree phase step

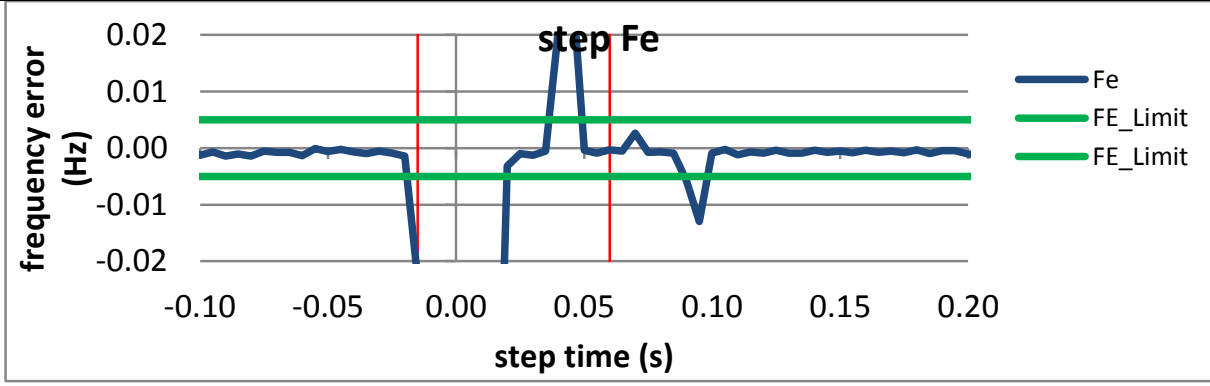

Figure 4603: Fs = 20 FPS, + 10 degree phase step

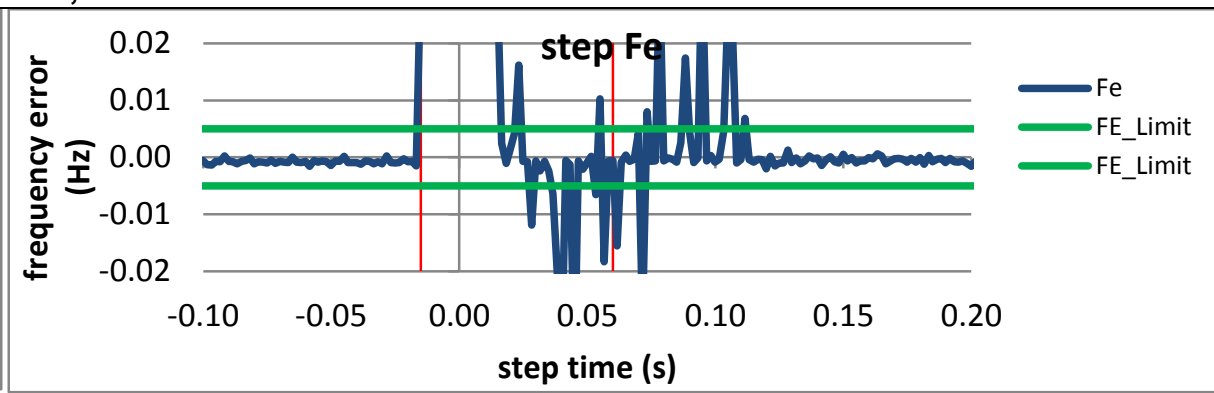

Figure 4600: Fs = 60 FPS, -10 degree phase step

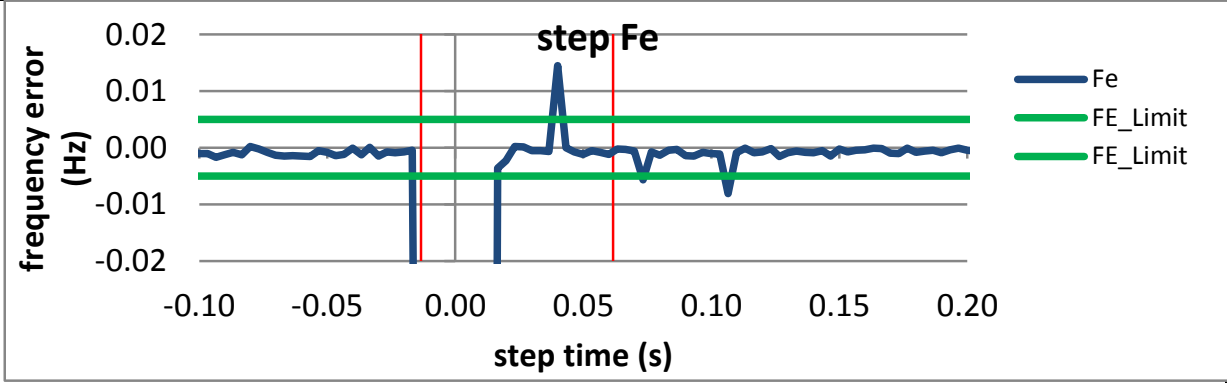

Figure 4602: Fs = 30 FPS, -10 degree phase step

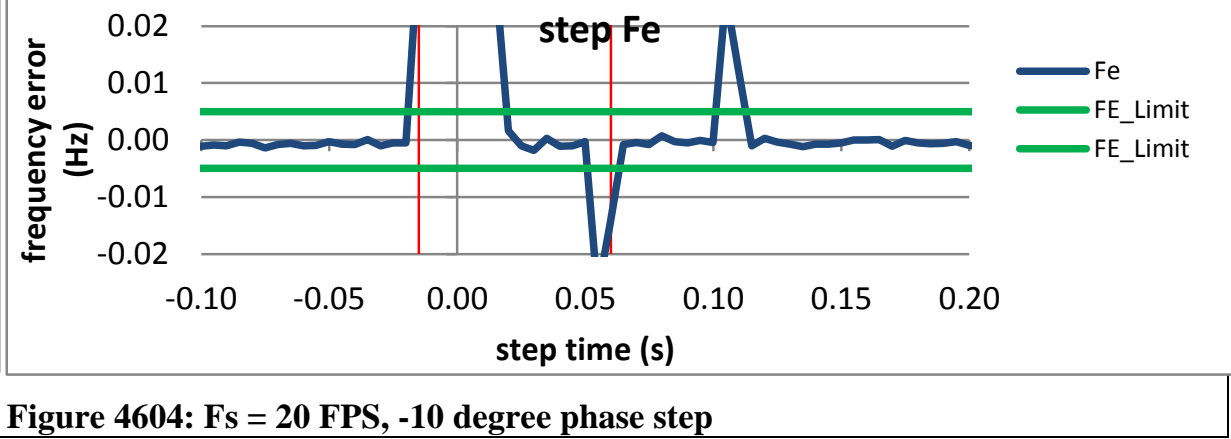



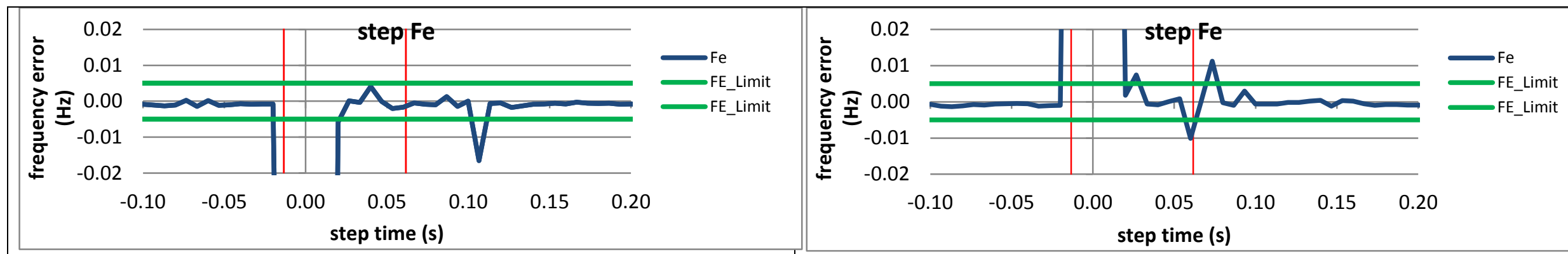

Figure 4605: Fs $=15$ FPS, +10 degree phase step

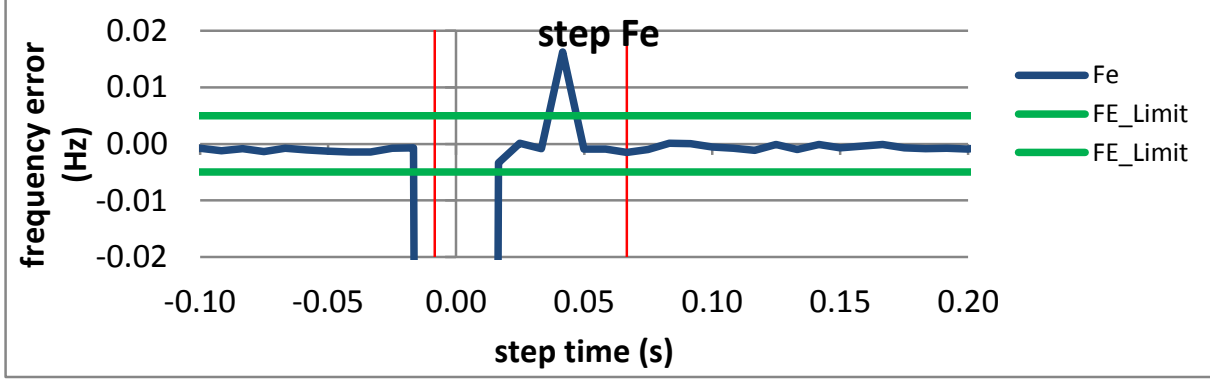

Figure 4606: Fs = 15 FPS, -10 degree phase step

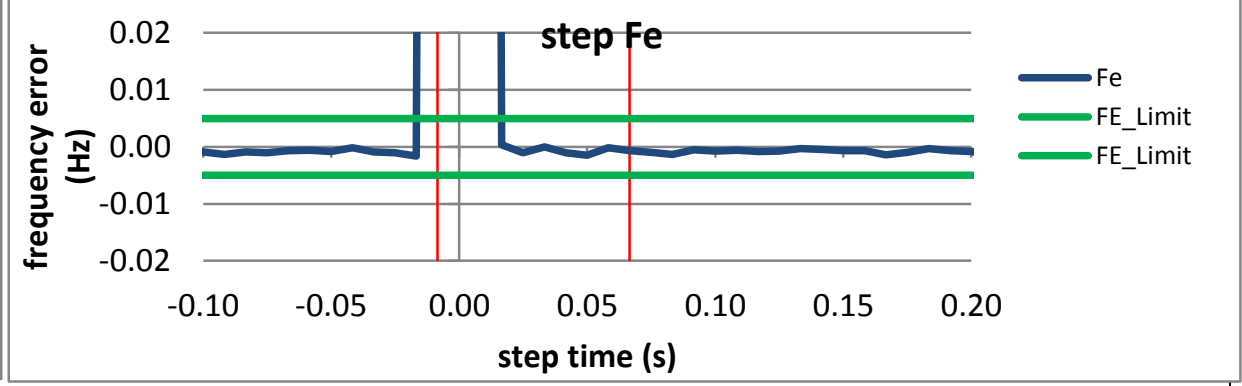

Figure 4607: Fs $=12$ FPS, +10 degree phase step

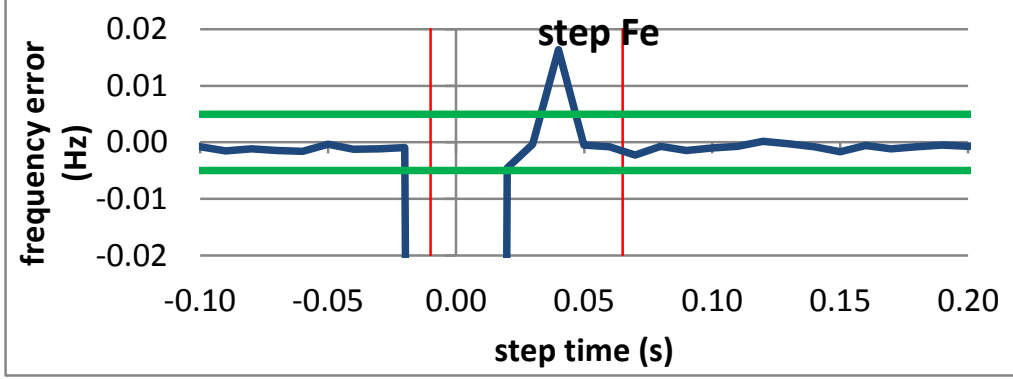

Figure 4609: Fs = 10 FPS, +10 degree phase step

Figure 4608: Fs = 12 FPS, -10 degree phase step

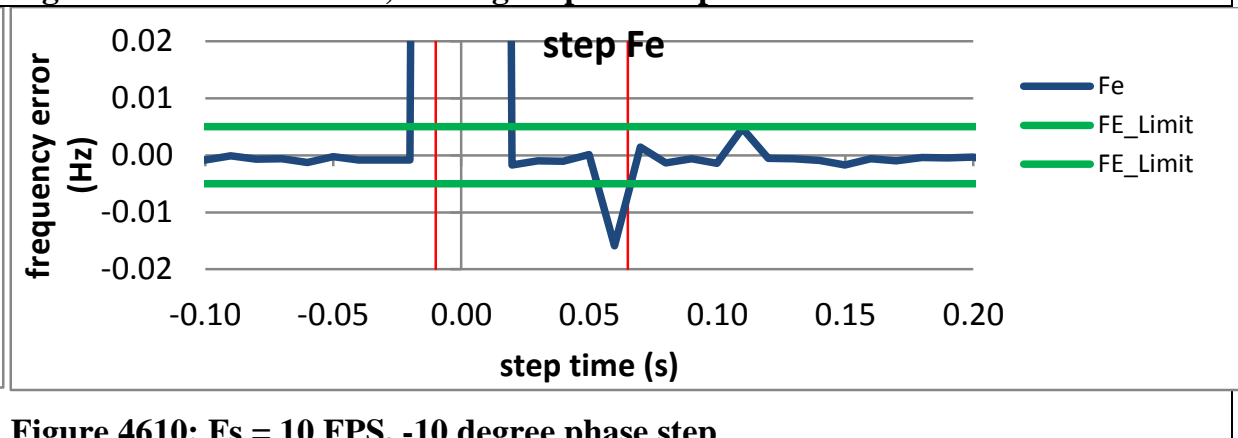


9.6.8 PMU G dynamic step change in phase frequency response time: $\mathrm{F} 0=60 \mathrm{~Hz}, \mathrm{P}$ class PMU G does not support P class.

9.6.9 PMU H dynamic step change in phase frequency response time: $\mathrm{F0}=60 \mathrm{~Hz}, \mathrm{P}$ class
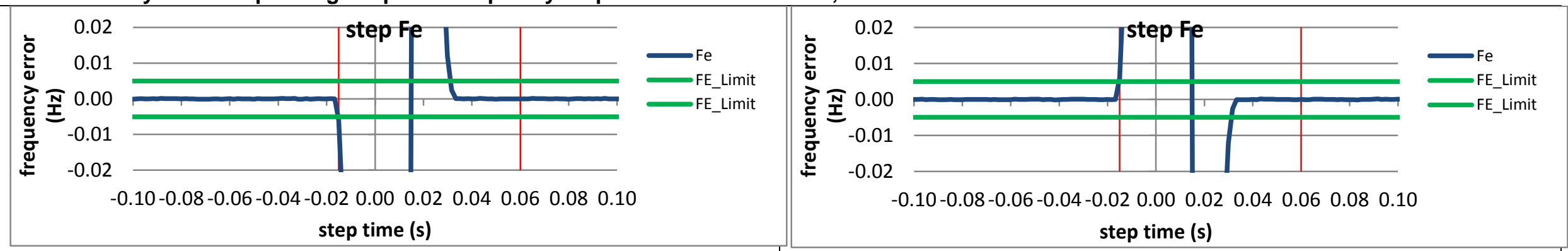

Figure 4611: Fs = 60 FPS, +10 degree phase step

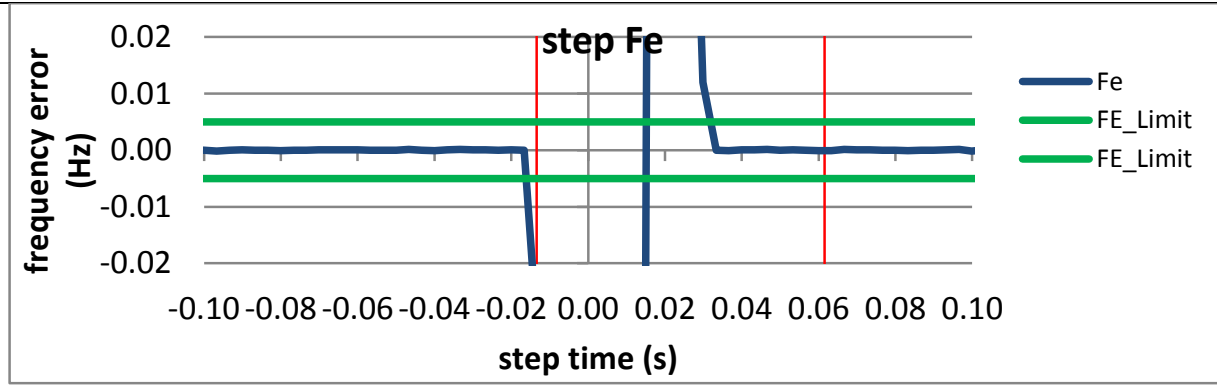

Figure 4612: Fs = 60 FPS, -10 degree phase step

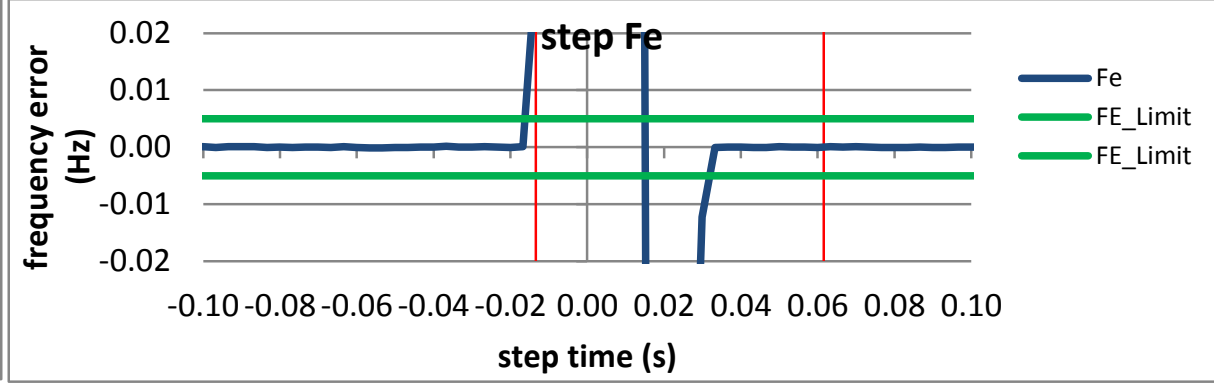

Figure 4613: Fs = 30 FPS, +10 degree phase step

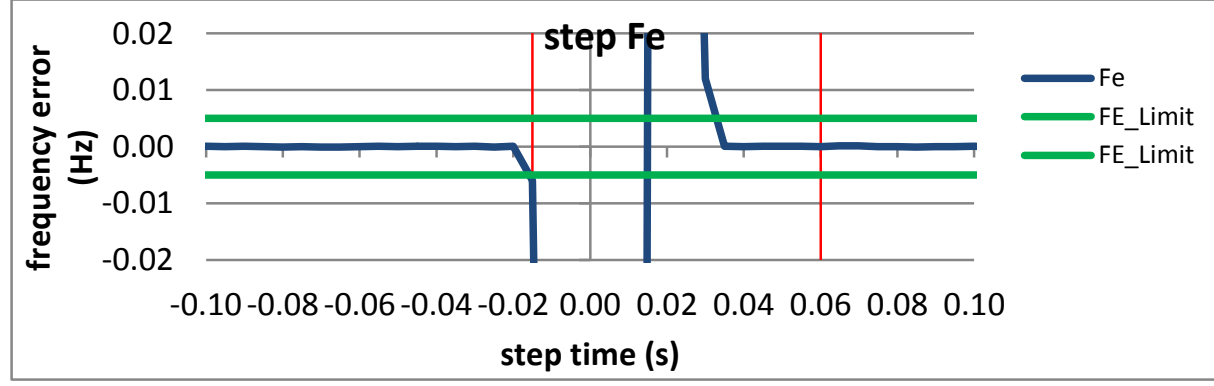

Figure 4614: Fs = 30 FPS, -10 degree phase step

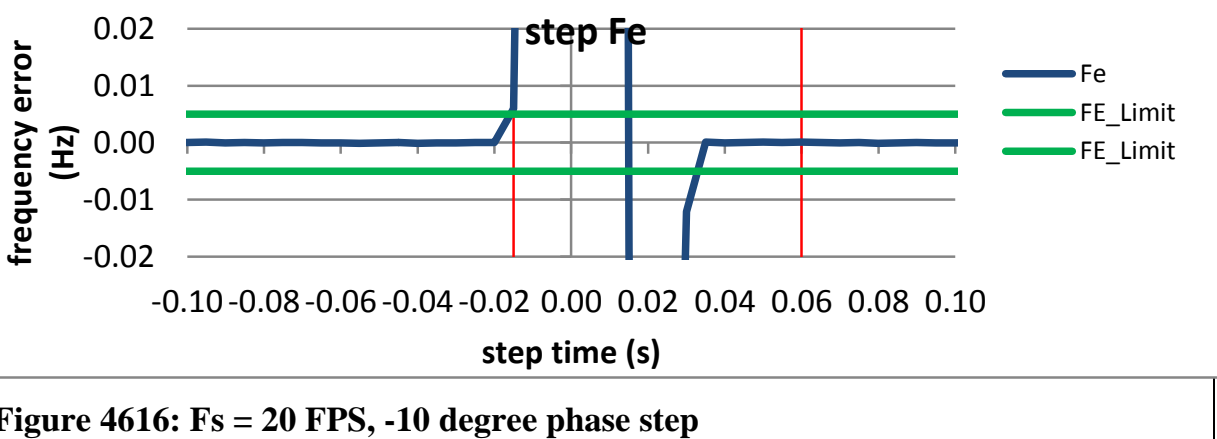

Figure 4615: Fs = 20 FPS, + 10 degree phase step

Figure 4616: Fs = 20 FPS, -10 degree phase step 


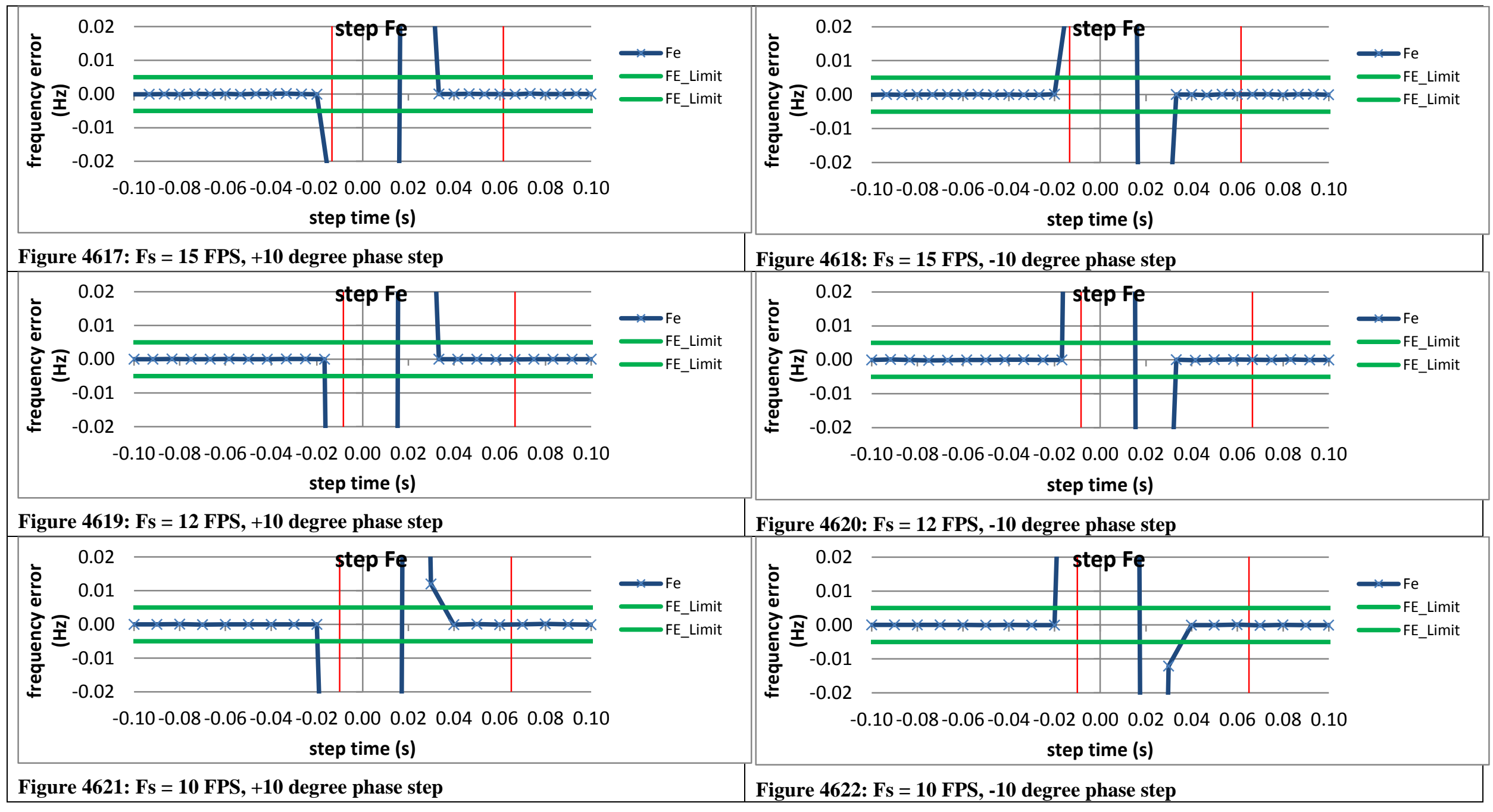


9.6.10 PMU I dynamic step change in phase frequency response time: $\mathrm{F} 0=60 \mathrm{~Hz}$, P class PMU I does not support P class

\subsubsection{PMU J dynamic step change in phase frequency response time: $\mathrm{F} 0=60 \mathrm{~Hz}, \mathrm{P}$ class}
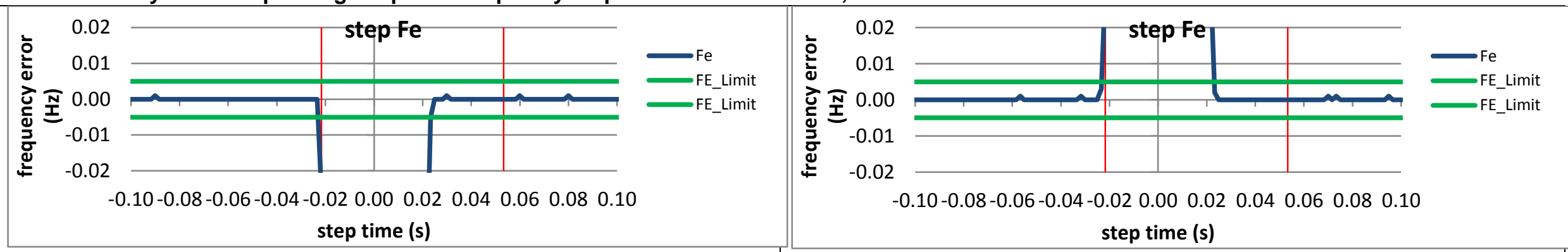

Figure 4623: Fs = 60 FPS, +10 degree phase step

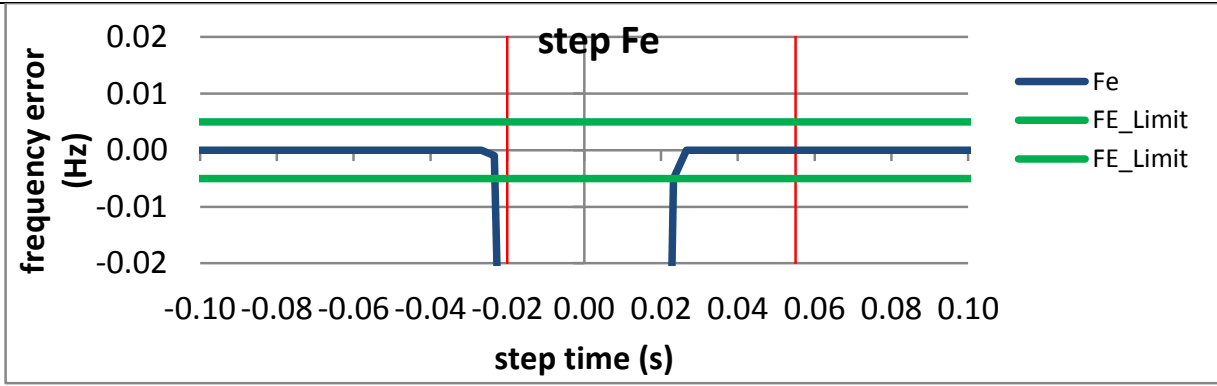

Figure 4624: Fs = 60 FPS, -10 degree phase step

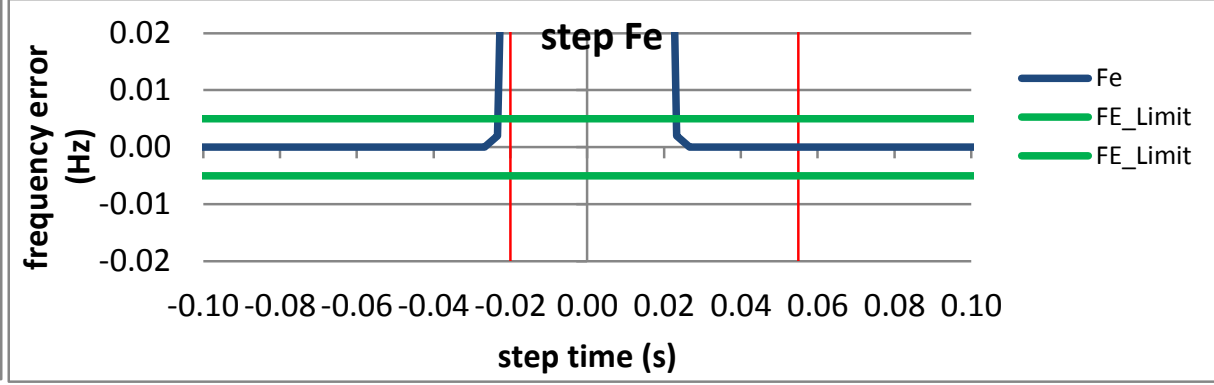

Figure 4625: Fs $=30$ FPS, +10 degree phase step

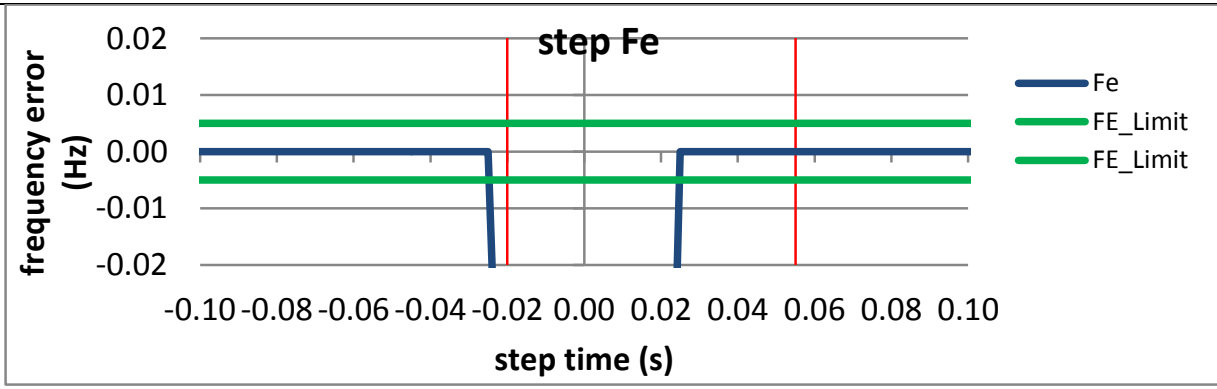

Figure 4627: Fs = 20 FPS, + 10 degree phase step

Figure 4626: Fs = 30 FPS, -10 degree phase step

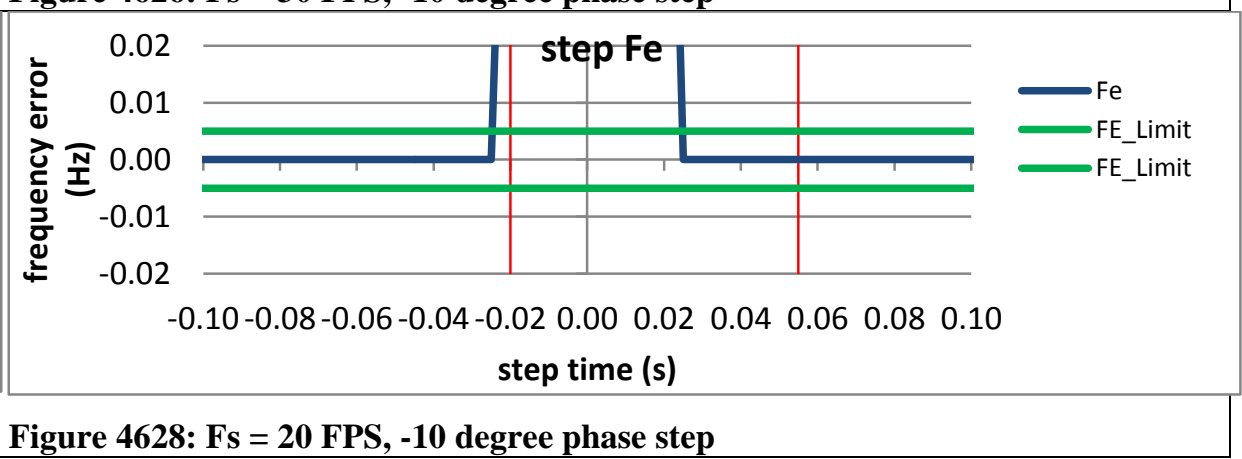




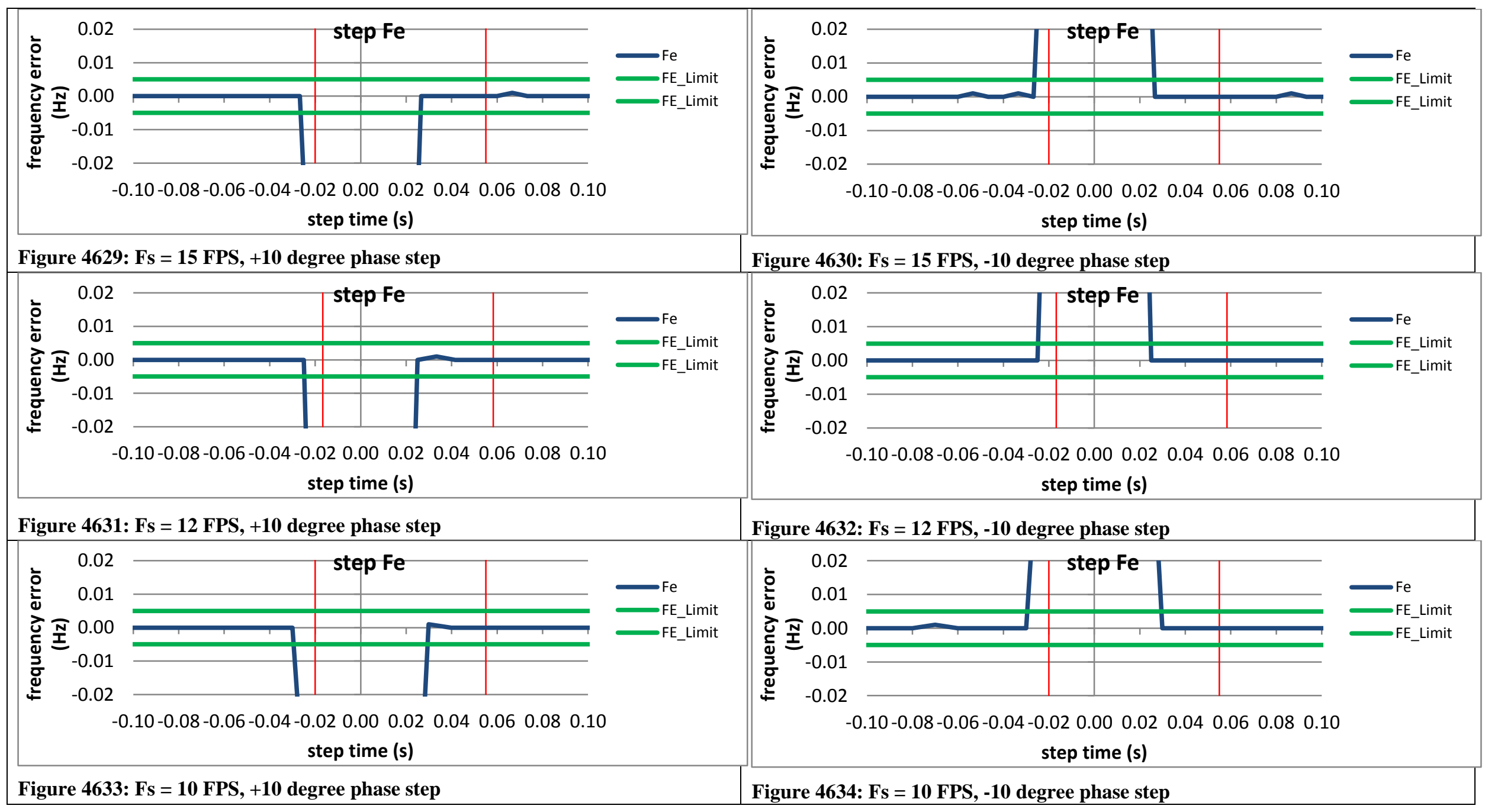


9.7 Dynamic step change in phase: ROCOF response time

\begin{tabular}{|c|c|c|c|c|c|c|c|c|c|c|c|c|}
\hline Fs (FPS) & $10 \mathrm{M}$ & $10 \mathrm{P}$ & $12 \mathrm{M}$ & $12 \mathrm{P}$ & $15 \mathrm{M}$ & $15 \mathrm{P}$ & $20 \mathrm{M}$ & $20 \mathrm{P}$ & $30 \mathrm{M}$ & $30 \mathrm{P}$ & $60 \mathrm{M}$ & $60 \mathrm{P}$ \\
\hline C37.118.1 Annex C & $\mathrm{P}$ & $\mathrm{P}$ & $\mathrm{P}$ & $\mathrm{P}$ & $\mathrm{P}$ & $\mathrm{P}$ & $\mathrm{P}$ & $\mathrm{P}$ & $\mathrm{P}$ & $\mathrm{P}$ & $\mathrm{P}$ & $\mathrm{P}$ \\
\hline PMU A & - & $\mathrm{F}$ & - & $\mathrm{F}$ & - & $\mathrm{F}$ & - & $\mathrm{F}$ & - & $\mathrm{F}$ & - & $\mathrm{F}$ \\
\hline PMU B & $\mathrm{P}$ & $\mathrm{F}$ & $\mathrm{P}$ & $\mathrm{F}$ & $\mathrm{P}$ & $\mathrm{F}$ & $\mathrm{P}$ & $\mathrm{F}$ & $\mathrm{P}$ & $\mathrm{F}$ & $\mathrm{P}$ & $\mathrm{F}$ \\
\hline $\mathrm{PMU} \mathrm{C}$ & $\mathrm{P}$ & $\mathrm{F}$ & $\mathrm{P}$ & $\mathrm{F}$ & $\mathrm{P}$ & $\mathrm{F}$ & $\mathrm{P}$ & $\mathrm{F}$ & $\mathrm{P}$ & $\mathrm{F}$ & $\mathrm{P}$ & $\mathrm{F}$ \\
\hline $\mathrm{PMU} \mathrm{D}$ & $\mathrm{P}$ & $\mathrm{P}$ & $\mathrm{P}$ & $\mathrm{P}$ & $\mathrm{P}$ & $\mathrm{P}$ & $\mathrm{P}$ & $\mathrm{P}$ & $\mathrm{P}$ & $\mathrm{P}$ & $\mathrm{P}$ & $\mathrm{P}$ \\
\hline PMU E & $\mathrm{P}$ & - & - & - & $\mathrm{P}$ & - & $\mathrm{P}$ & - & $\mathrm{P}$ & - & $\mathrm{P}$ & - \\
\hline PMU F & $\mathrm{P}$ & $\mathrm{P}$ & $\mathrm{P}$ & $\mathrm{P}$ & $\mathrm{P}$ & $\mathrm{P}$ & $\mathrm{P}$ & $\mathrm{P}$ & $\mathrm{P}$ & $\mathrm{P}$ & $\mathrm{P}$ & $\mathrm{F}$ \\
\hline PMU G* & $\mathrm{P}$ & - & $\mathrm{P}$ & - & $\mathrm{P}$ & - & $\mathrm{P}$ & - & $\mathrm{P}$ & - & $\mathrm{P}$ & - \\
\hline PMU H & $\mathrm{P}$ & $\mathrm{P}$ & $\mathrm{P}$ & $\mathrm{P}$ & $\mathrm{P}$ & $\mathrm{P}$ & $\mathrm{P}$ & $\mathrm{P}$ & $\mathrm{P}$ & $\mathrm{P}$ & $\mathrm{P}$ & $\mathrm{P}$ \\
\hline PMU I & $\mathrm{P}$ & - & $\mathrm{P}$ & - & $\mathrm{P}$ & - & $\mathrm{P}$ & - & $\mathrm{P}$ & - & $\mathrm{F}$ & - \\
\hline PMU J & $\mathrm{P}$ & $\mathrm{P}$ & $\mathrm{P}$ & $\mathrm{P}$ & $\mathrm{P}$ & $\mathrm{P}$ & $\mathrm{P}$ & $\mathrm{P}$ & $\mathrm{P}$ & $\mathrm{P}$ & $\mathrm{P}$ & $\mathrm{P}$ \\
\hline
\end{tabular}

* PMU G always outputs ROCOF $=0$

\subsubsection{C37.118.1-2011 Annex C dynamic step change in phase ROCOF error: F0 $=60 \mathrm{~Hz}, \mathrm{M}$ class}

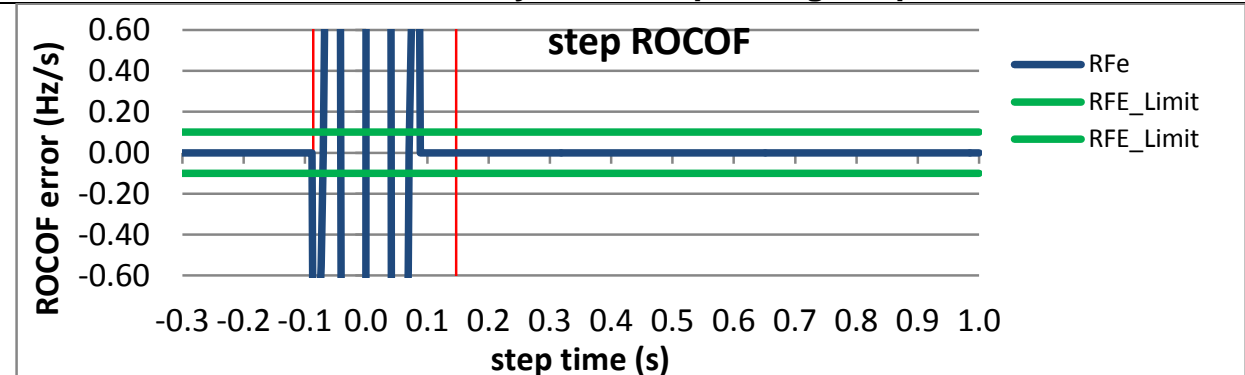

Figure 4635: Fs = 60 FPS, +10 degree phase step

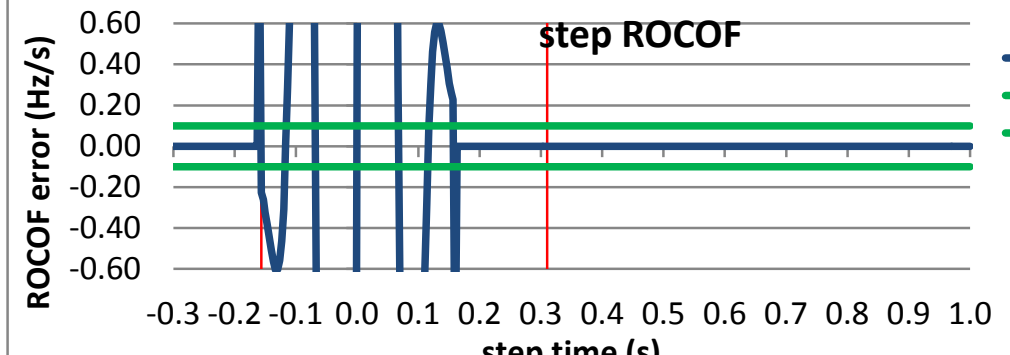
step time (s)

Figure 4637: Fs = 30 FPS, +10 degree phase step

RFe
RFE_Limit
RFE_Limit

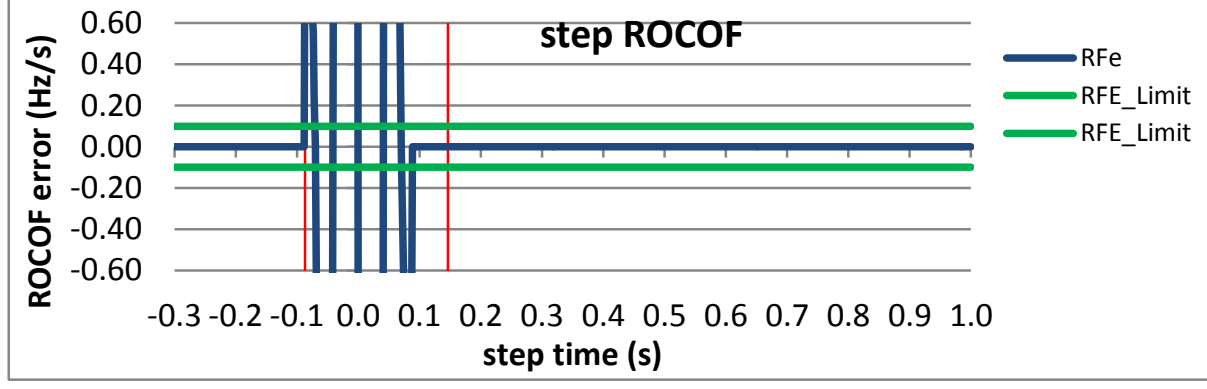

Figure 4636: Fs = 60 FPS, -10 degree phase step

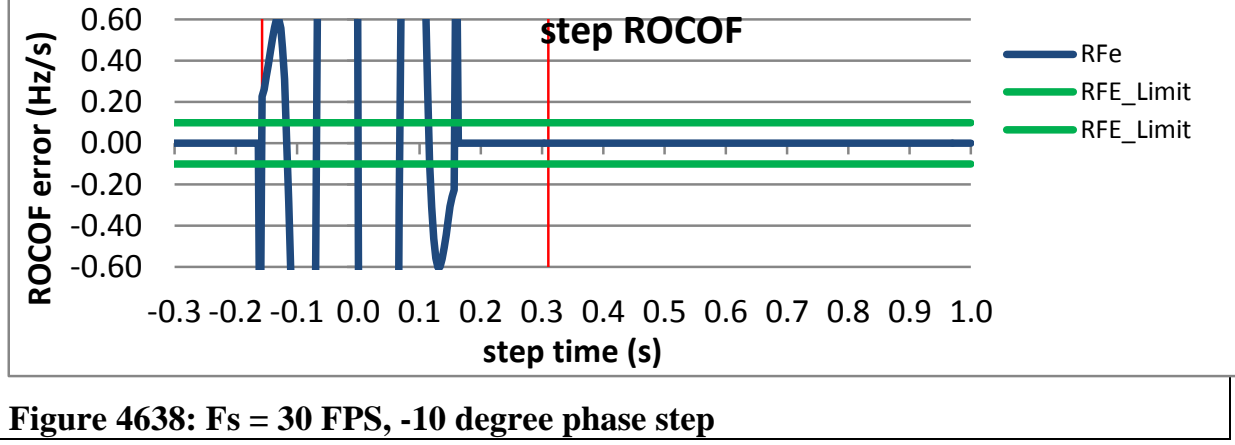



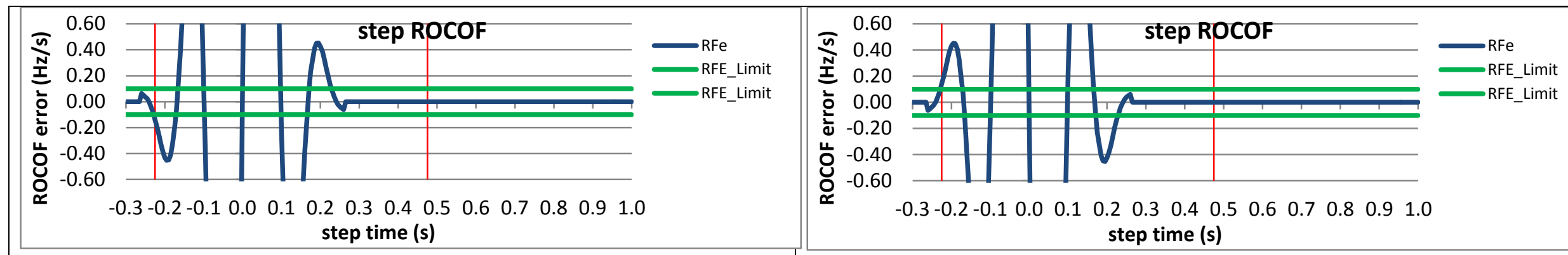

Figure 4639: Fs $=20$ FPS, +10 degree phase step

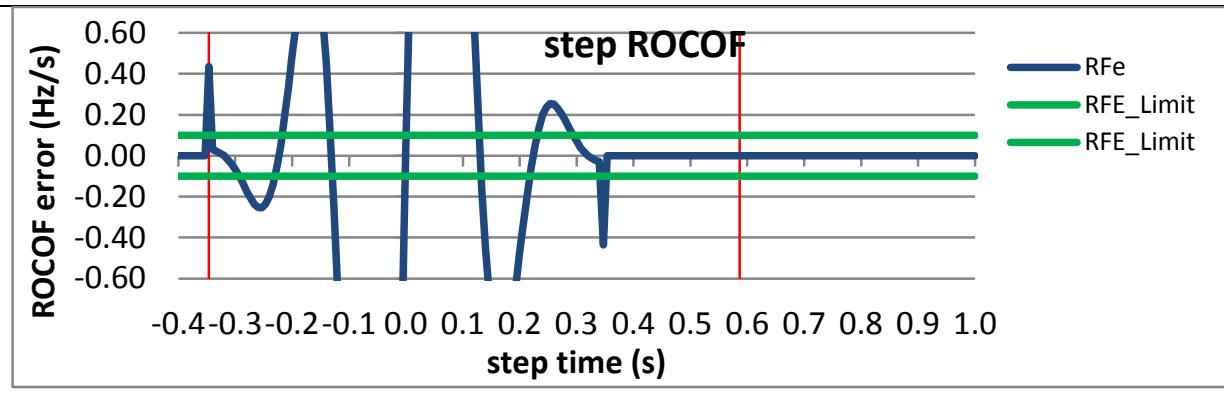

Figure 4640: Fs = 20 FPS, +10 degree phase step

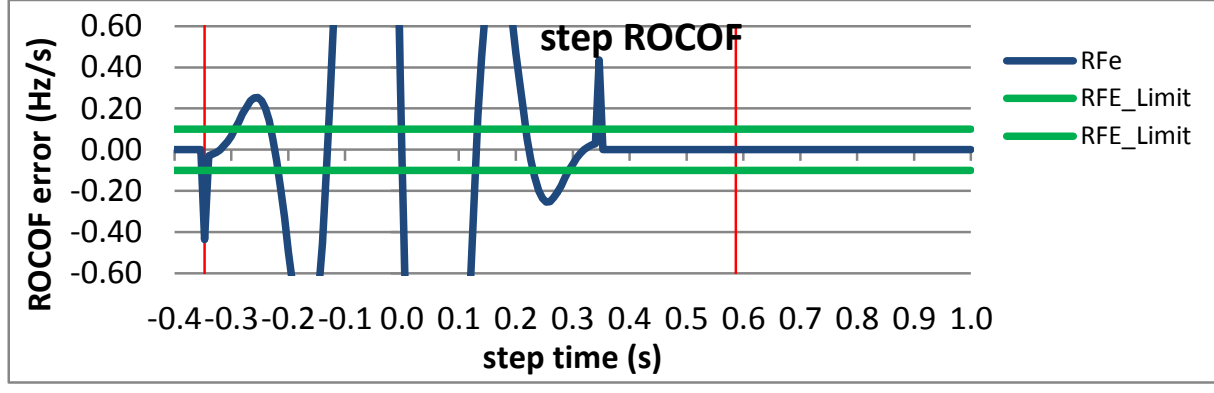

Figure 4641: Fs = 15 FPS, +10 degree phase step

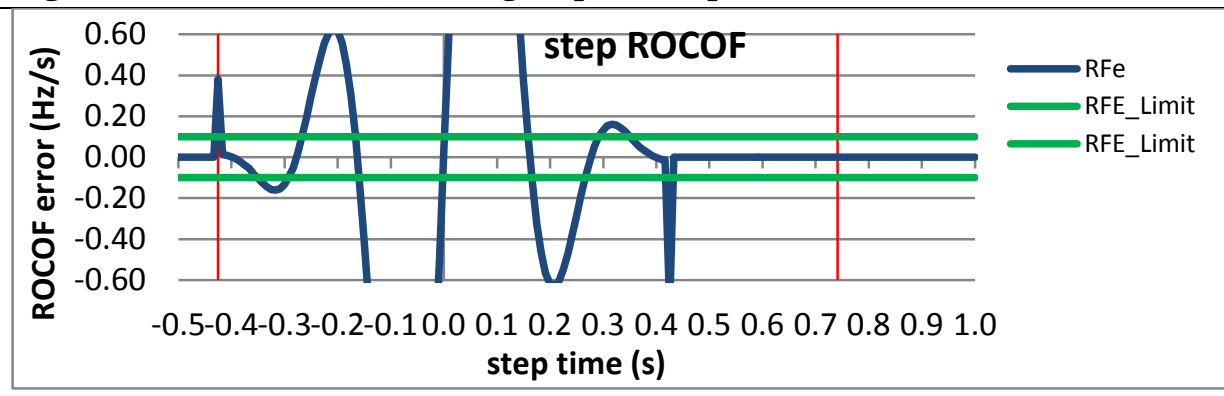

Figure 4642: Fs = 15 FPS, -10 degree phase step

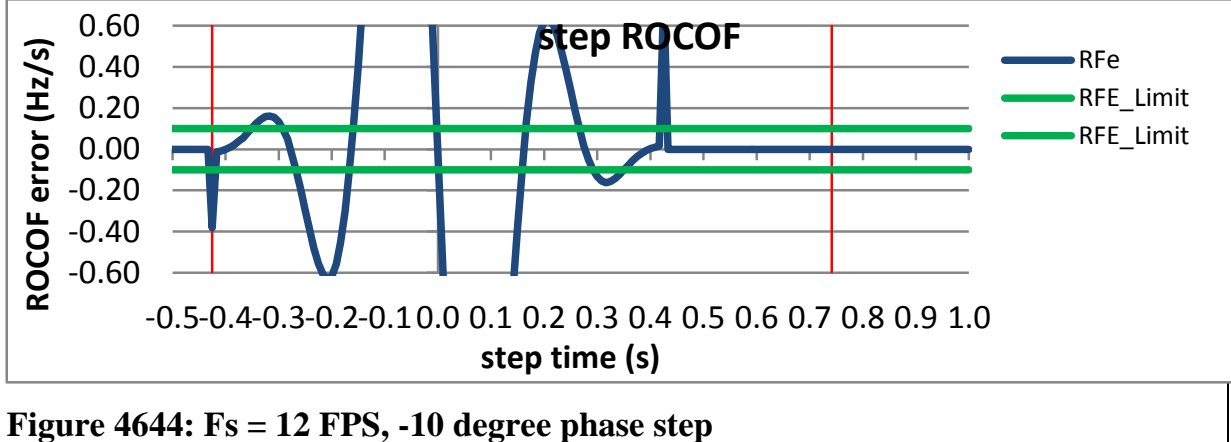

Figure 4643: Fs = 12 FPS, +10 degree phase step

Figure 4644: Fs = 12 FPS, -10 degree phase step 


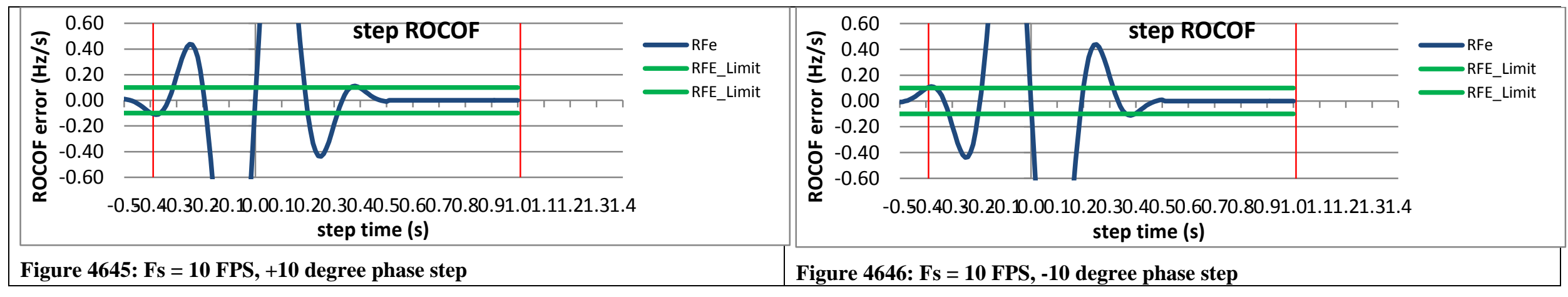


9.7.2 PMU A dynamic step change in phase ROCOF response time: $F 0=60 \mathrm{~Hz}, \mathrm{M}$ class

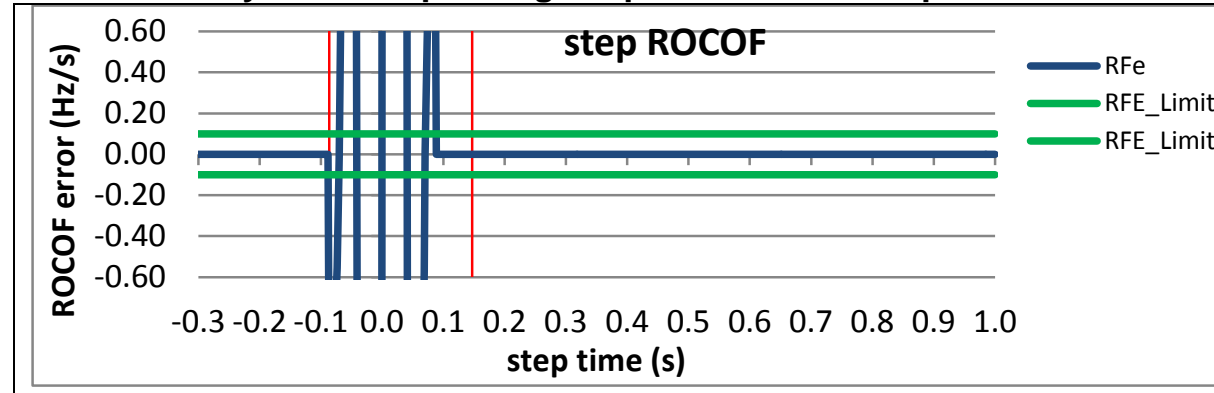

Figure 4647: Fs = 60 FPS, +10 degree phase step

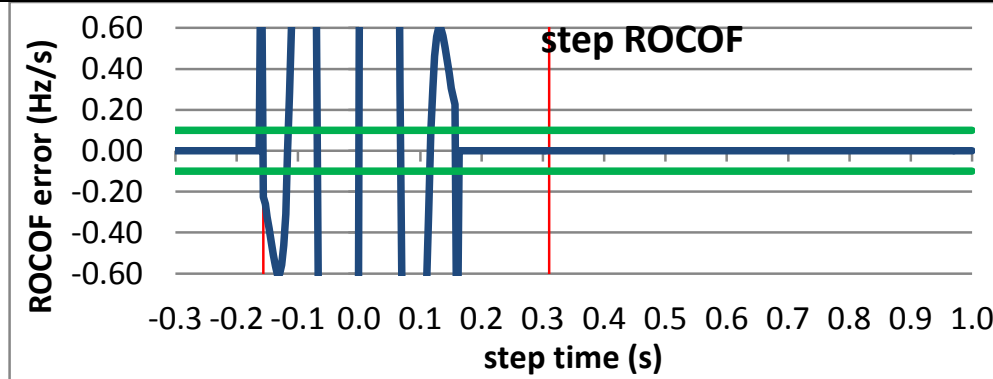

Figure 4649: Fs = 30 FPS, +10 degree phase step

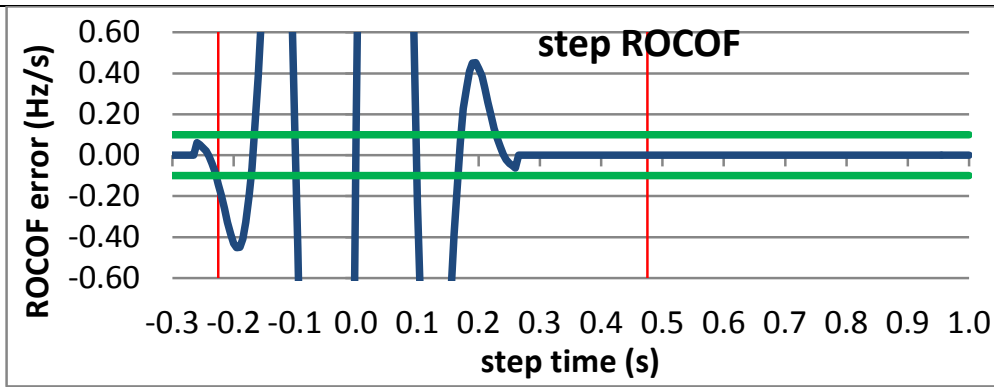

Figure 4651: Fs = 20 FPS, +10 degree phase step

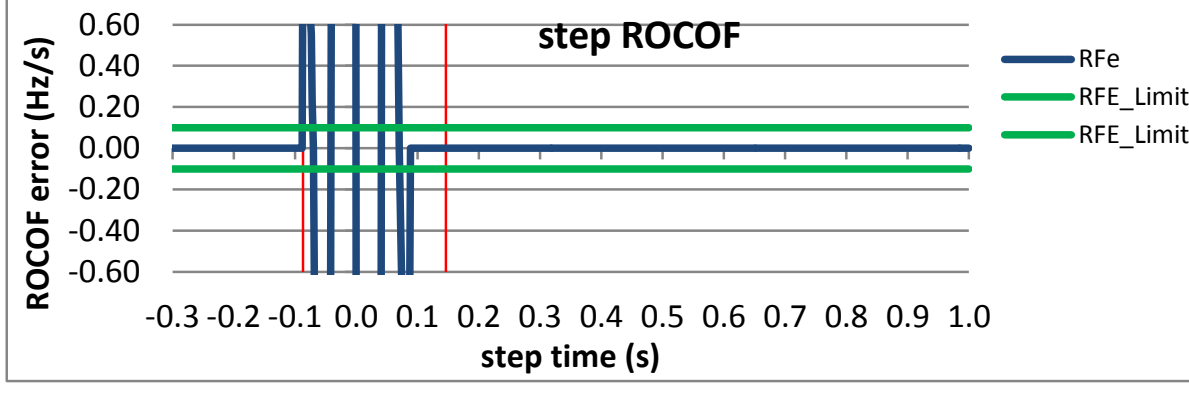

Figure 4648: Fs = 60 FPS, -10 degree phase step

RFe
RFE_Limit
RFE_Limit

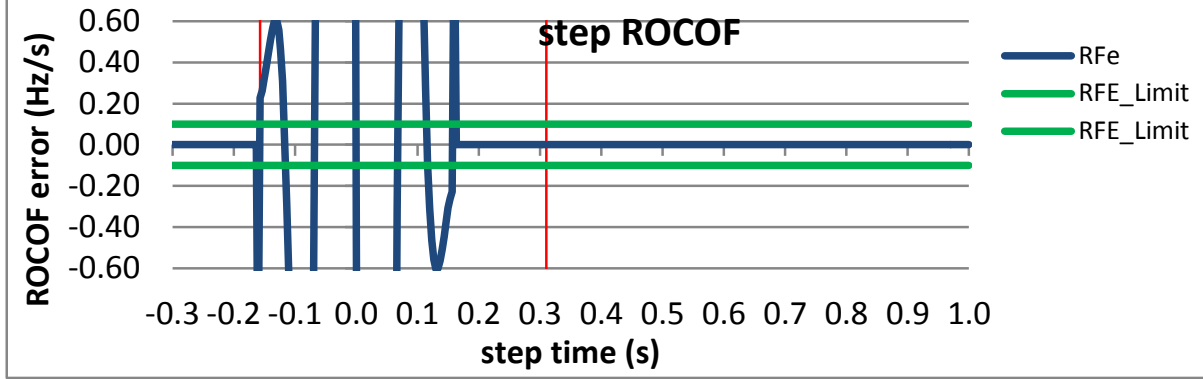

Figure 4650: Fs = 30 FPS, -10 degree phase step

RFe
RFE_Limit
RFE_Limit

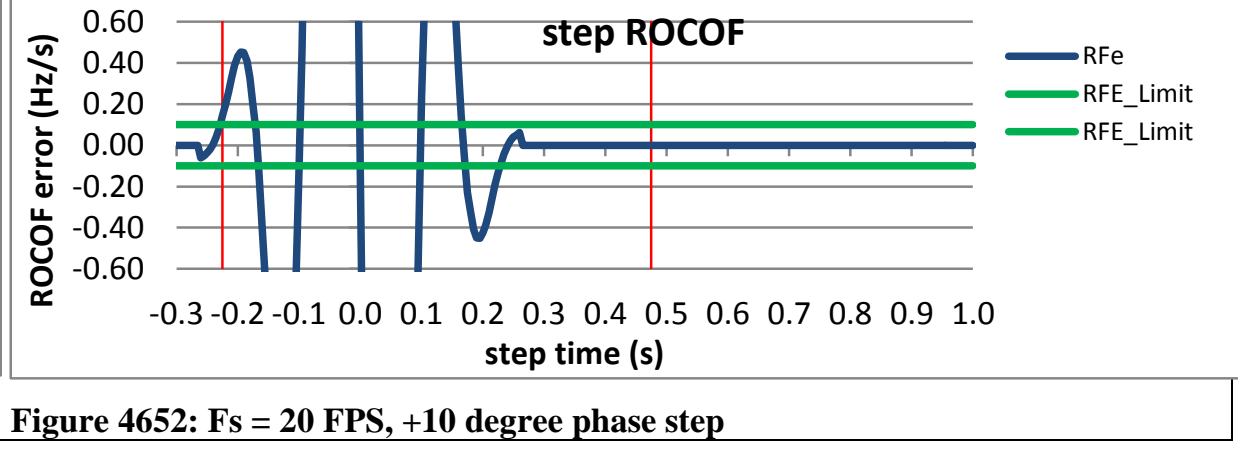



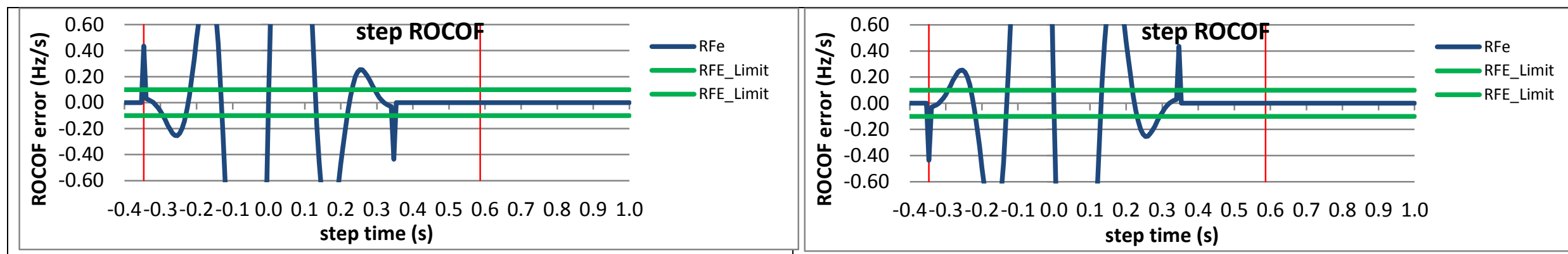

Figure 4653: Fs = 15 FPS, +10 degree phase step

Figure 4654: Fs = 15 FPS, -10 degree phase step

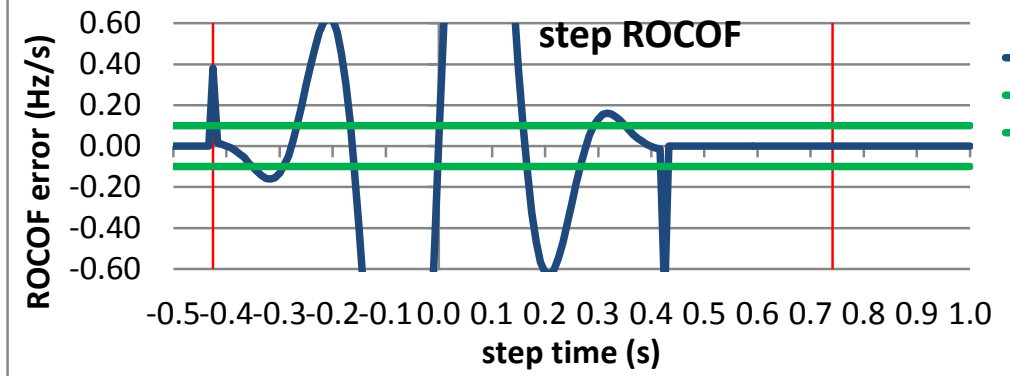

RFe
RFE_Limit
RFE_Limit

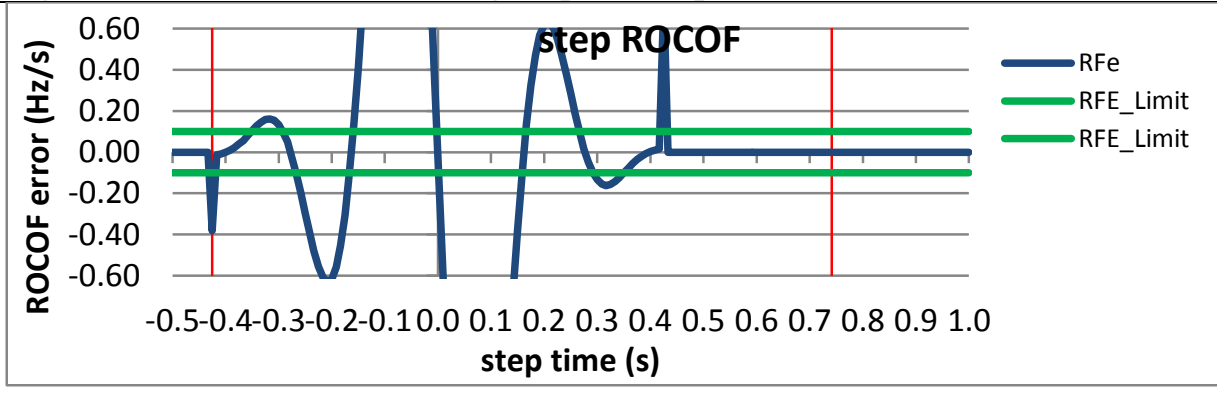

Figure 4655: Fs = 12 FPS, +10 degree phase step

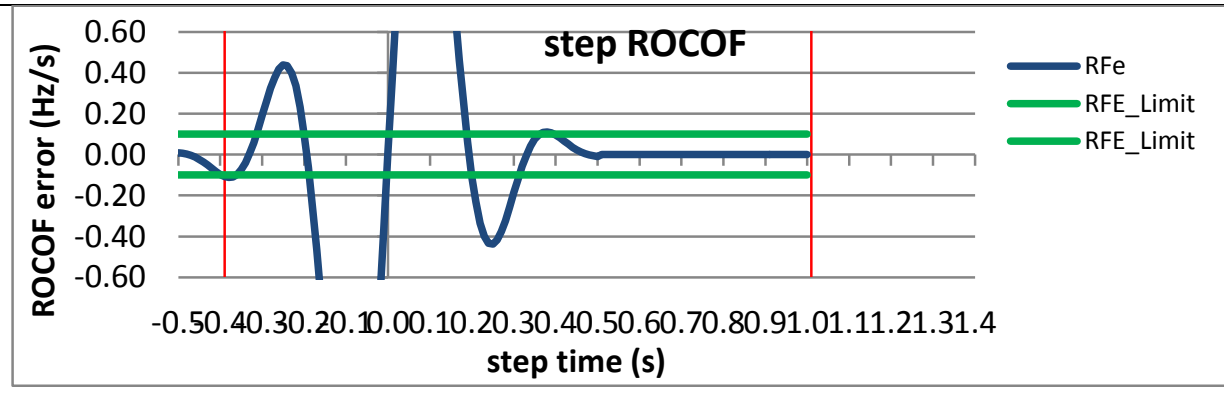

Figure 4656: Fs = 12 FPS, -10 degree phase step

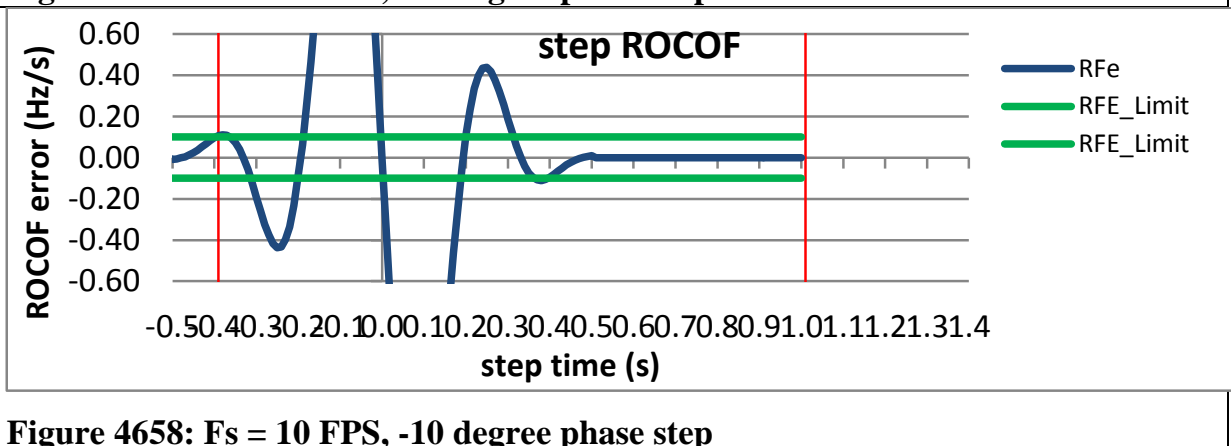

Figure 4657: Fs = 10 FPS, +10 degree phase step

Figure 4658: Fs = 10 FPS, -10 degree phase step 
9.7.3 PMU B dynamic step change in phase ROCOF response time: $F 0=60 \mathrm{~Hz}, \mathrm{M}$ class

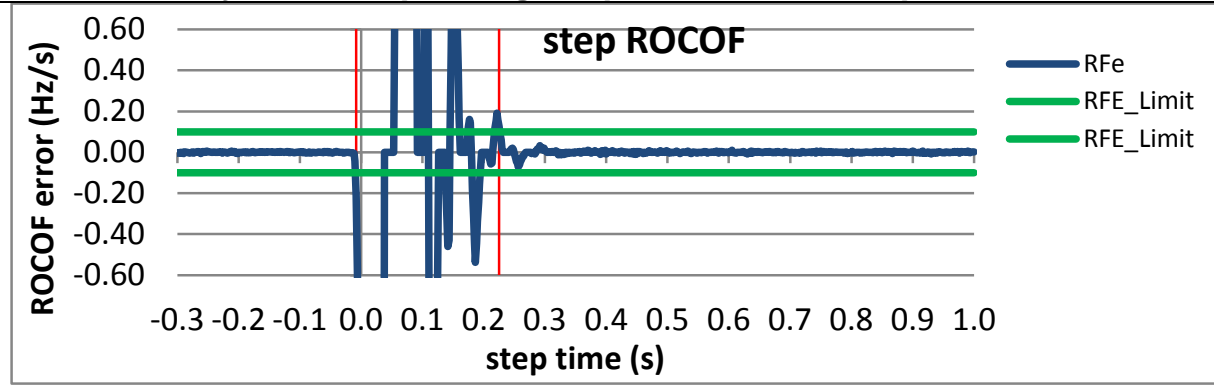

Figure 4659: Fs = 60 FPS, +10 degree phase step

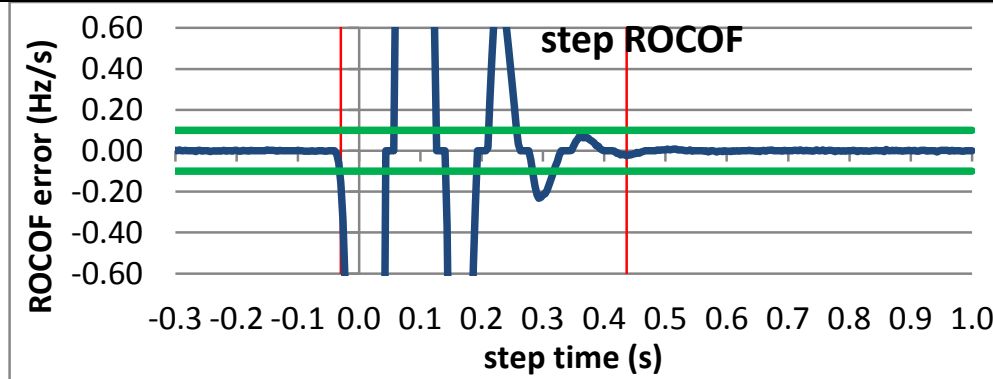

Figure 4661: Fs = 30 FPS, +10 degree phase step

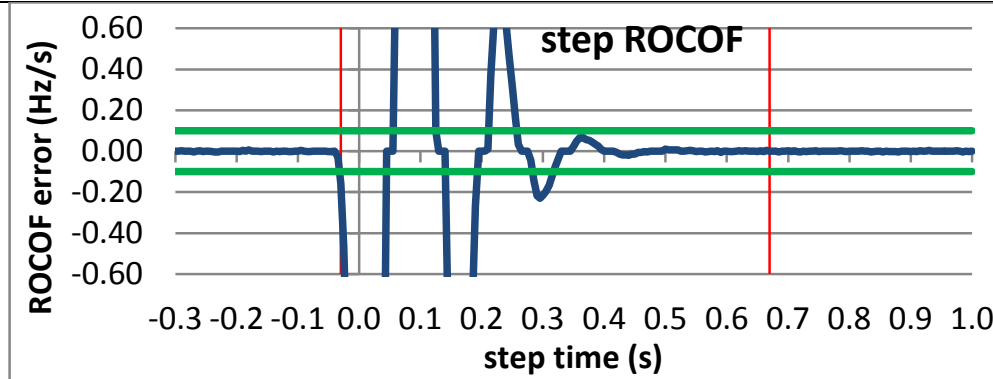

Figure 4663: Fs = 20 FPS, +10 degree phase step

RFe
RFE_Limit
RFE_Limit

RFe
RFE_Limit
RFE_Limit

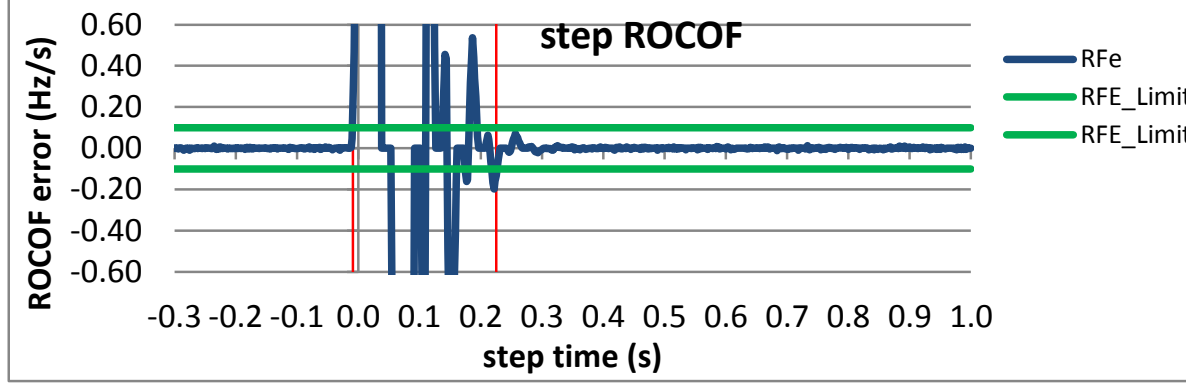

Figure 4660: Fs = 60 FPS, -10 degree phase step

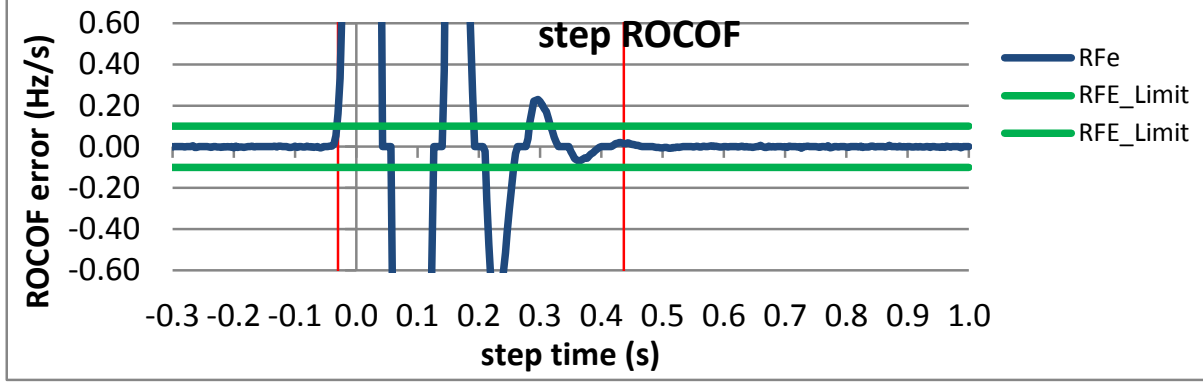

Figure 4662: Fs = 30 FPS, -10 degree phase step

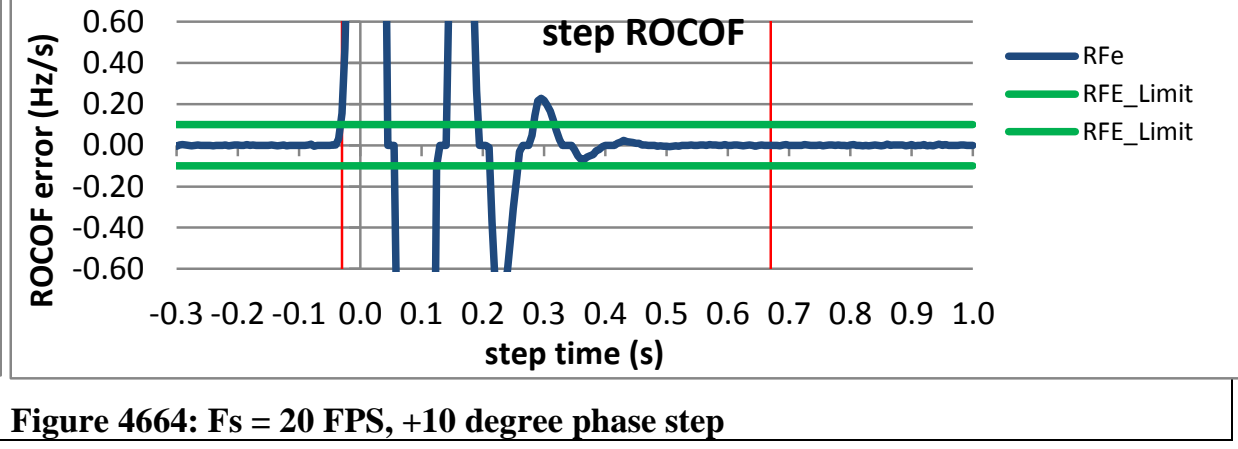



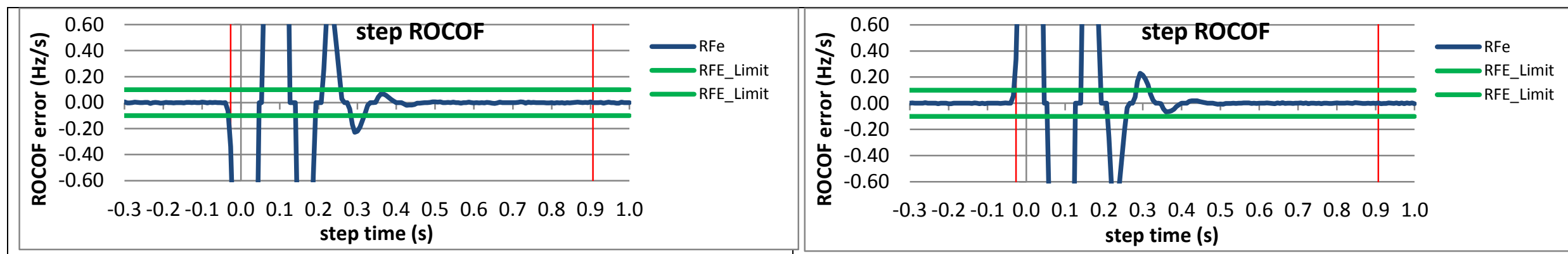

Figure 4665: Fs = 15 FPS, +10 degree phase step

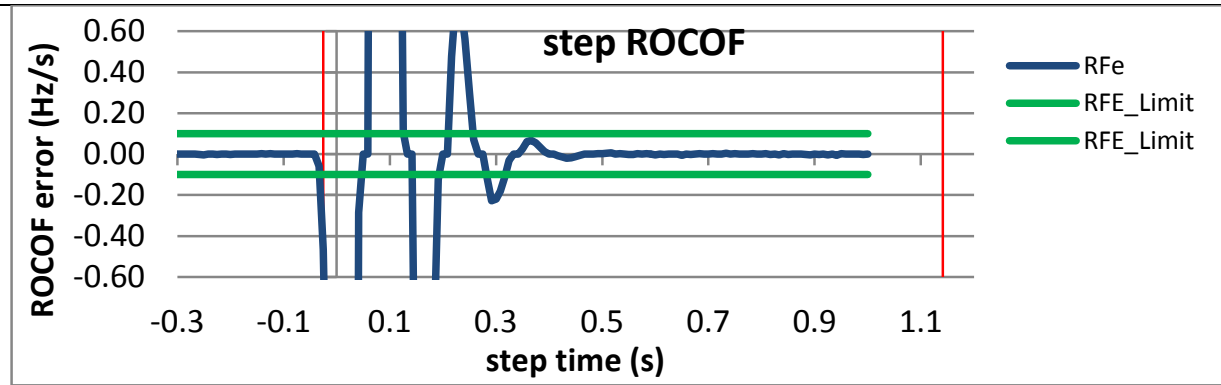

Figure 4666: Fs = 15 FPS, -10 degree phase step

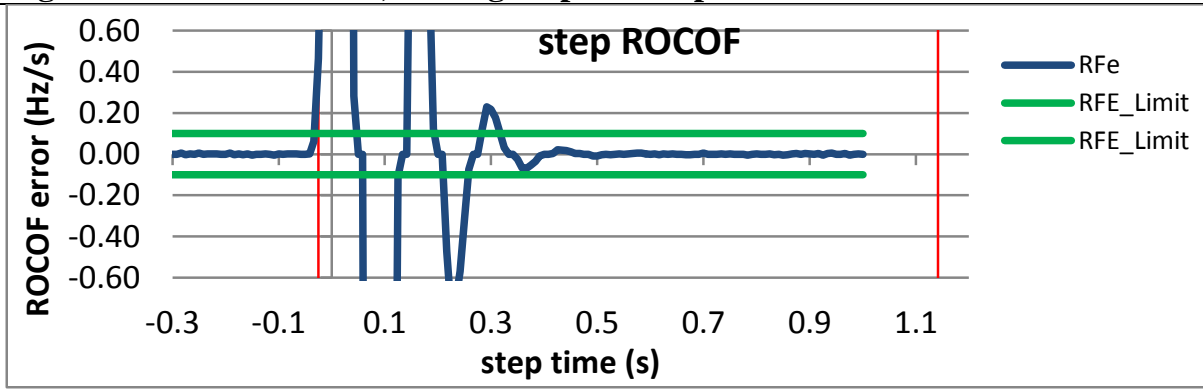

Figure 4668: Fs = 12 FPS, -10 degree phase step

Figure 4667: Fs = 12 FPS, +10 degree phase step
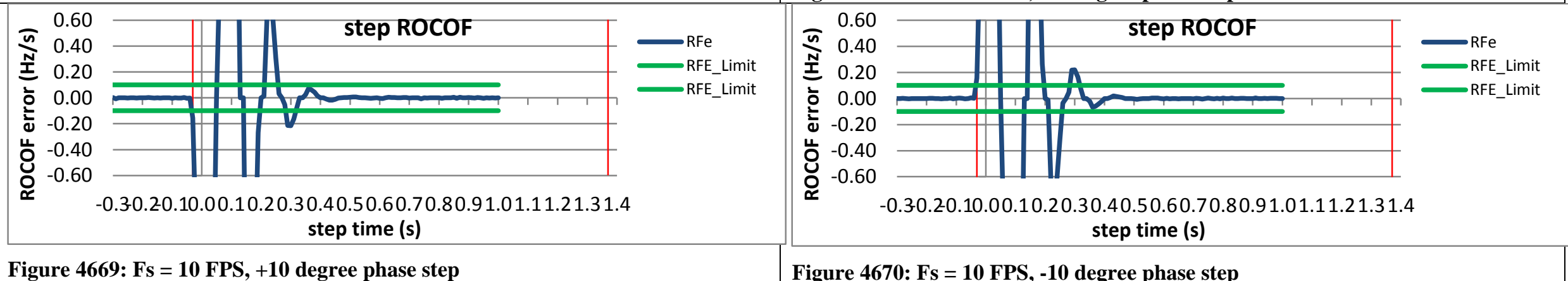

Figure 4669: Fs = 10 FPS, +10 degree phase step

Figure 4670: Fs = 10 FPS, -10 degree phase step 
9.7.4 PMU C dynamic step change in phase ROCOF response time: $\mathrm{FO}=60 \mathrm{~Hz}, \mathrm{M}$ class
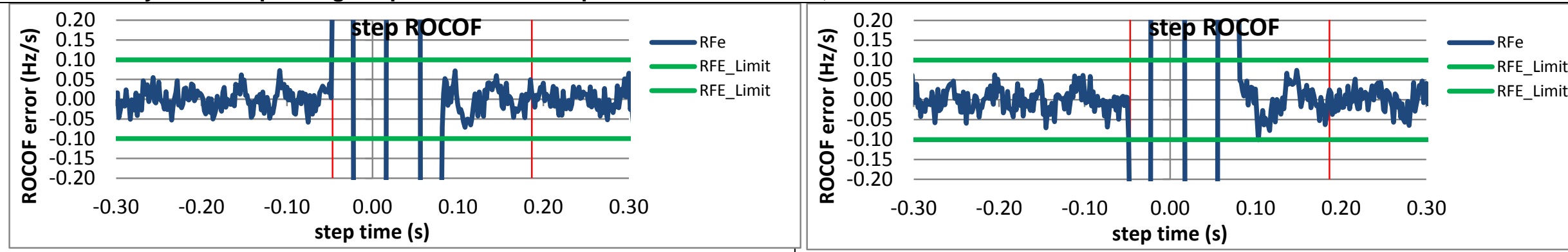

Figure 4671: Fs $=60 \mathrm{FPS},+10$ degree phase step

Figure 4672: Fs = 60 FPS, -10 degree phase step
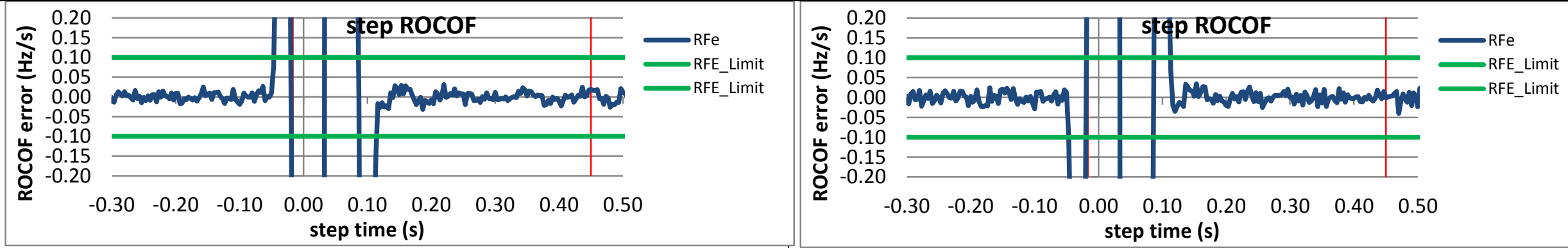

Figure 4673: Fs = 30 FPS, +10 degree phase step

Figure 4674: Fs = 30 FPS, -10 degree phase step
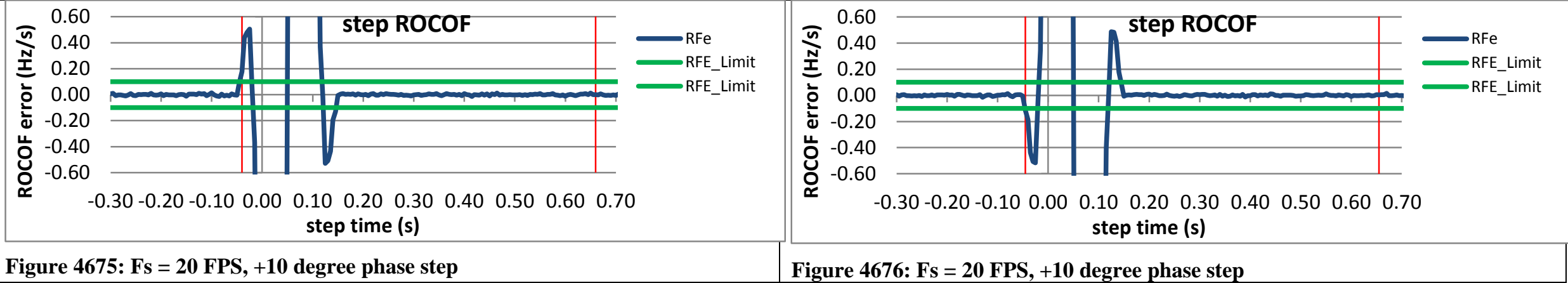

Figure 4675: Fs = 20 FPS, +10 degree phase step

Figure 4676: Fs = 20 FPS, +10 degree phase step 

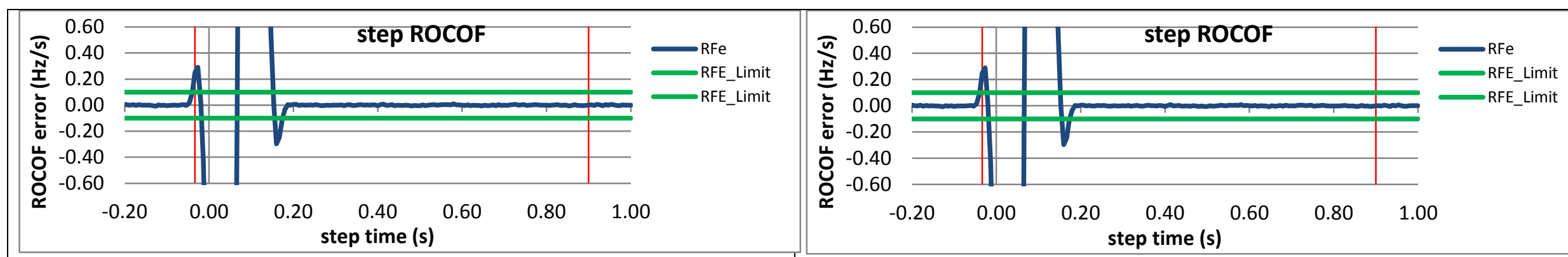

Figure 4677: Fs = 15 FPS, +10 degree phase step

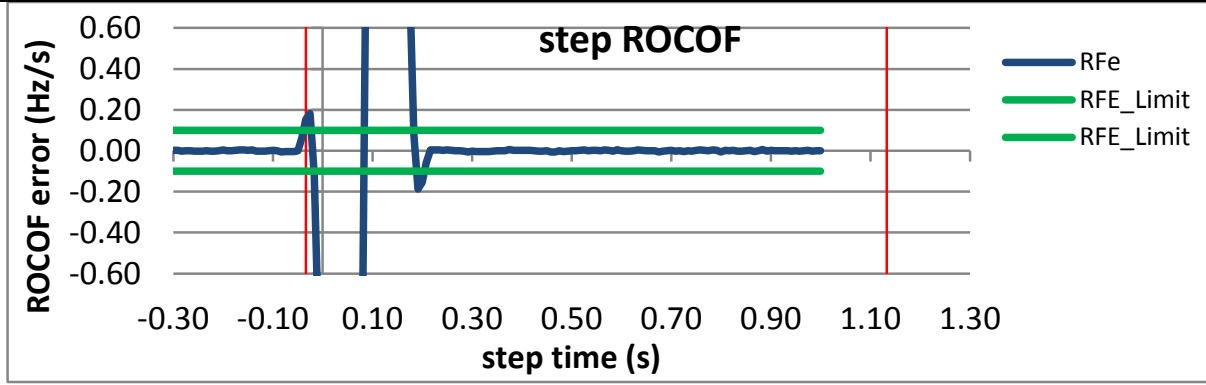

Figure 4678: Fs = 15 FPS, -10 degree phase step

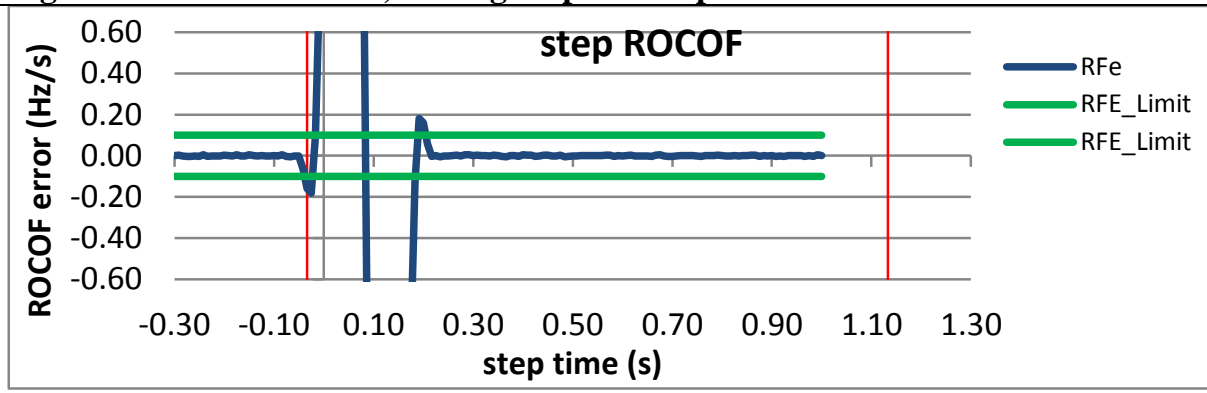

Figure 4679: Fs = 12 FPS, +10 degree phase step

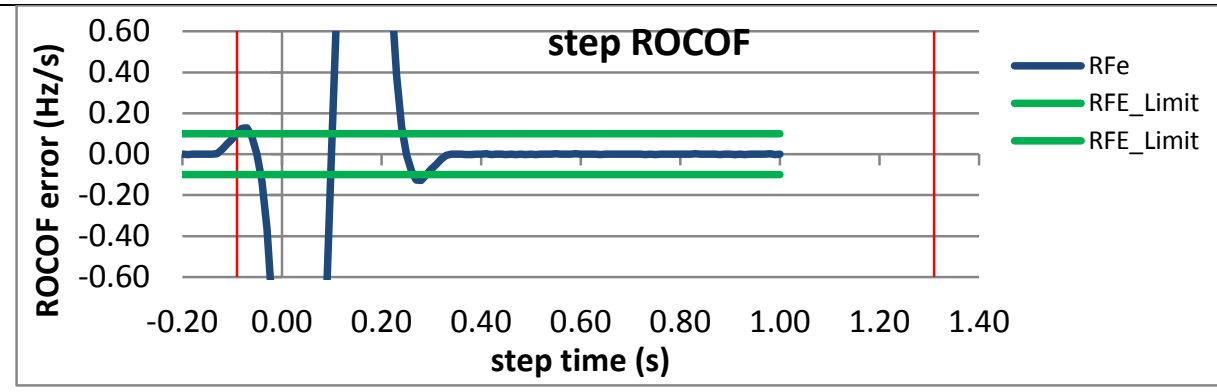

Figure 4680: Fs = 12 FPS, -10 degree phase step

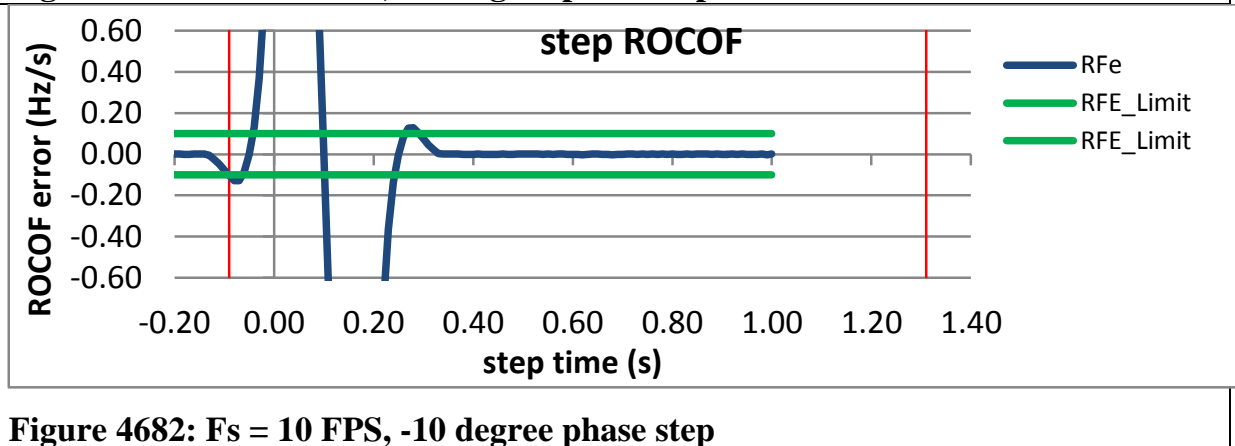

Figure 4681: Fs = 10 FPS, +10 degree phase step

Figure 4682: Fs = 10 FPS, -10 degree phase step 
9.7.5 PMU D dynamic step change in phase ROCOF response time: $F 0=60 \mathrm{~Hz}, \mathrm{M}$ class

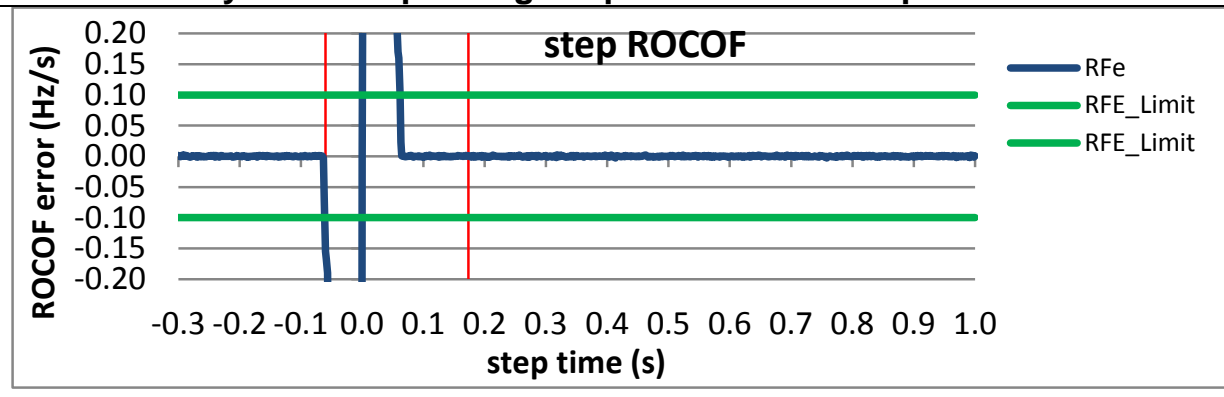

Figure 4683: Fs = 60 FPS, +10 degree phase step

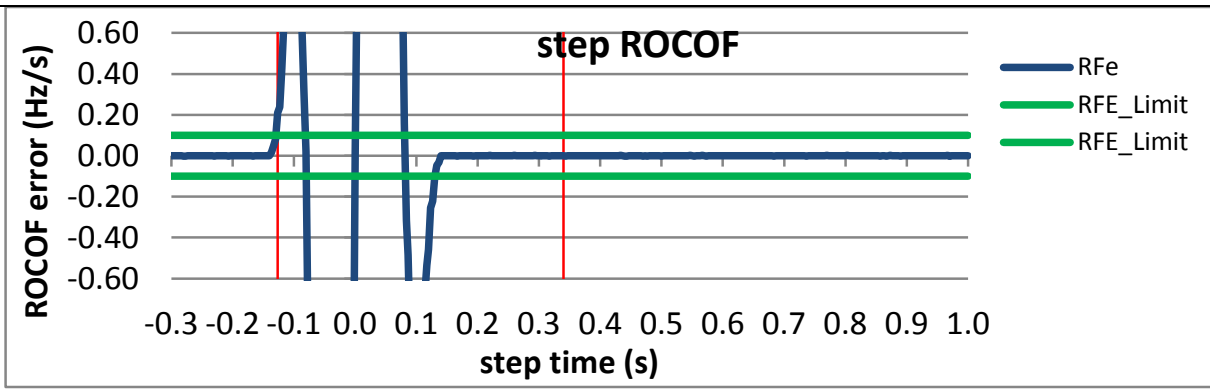

Figure 4685: Fs = 30 FPS, +10 degree phase step

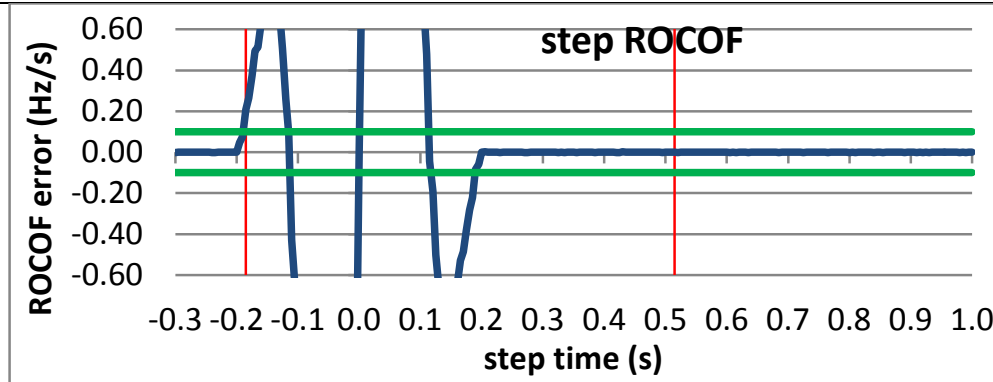

Figure 4687: Fs = 20 FPS, +10 degree phase step

RFe
RFE_Limit
RFE_Limit

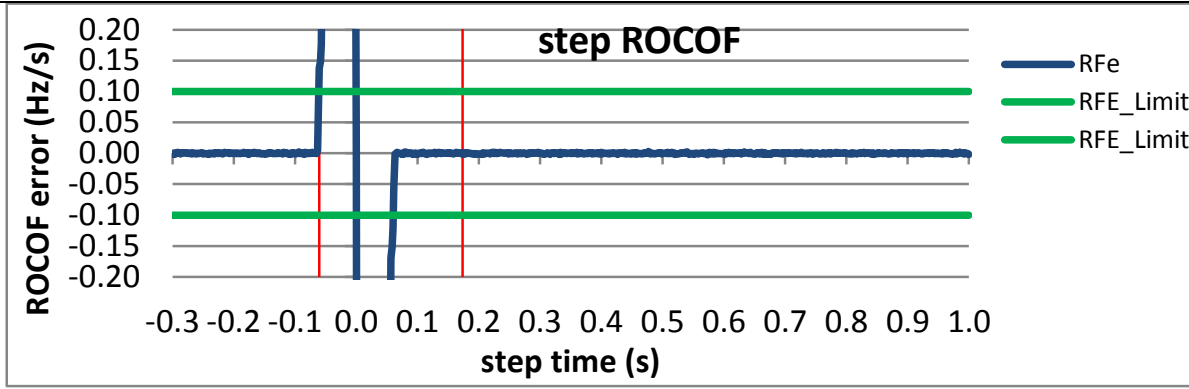

Figure 4684: Fs = 60 FPS, -10 degree phase step

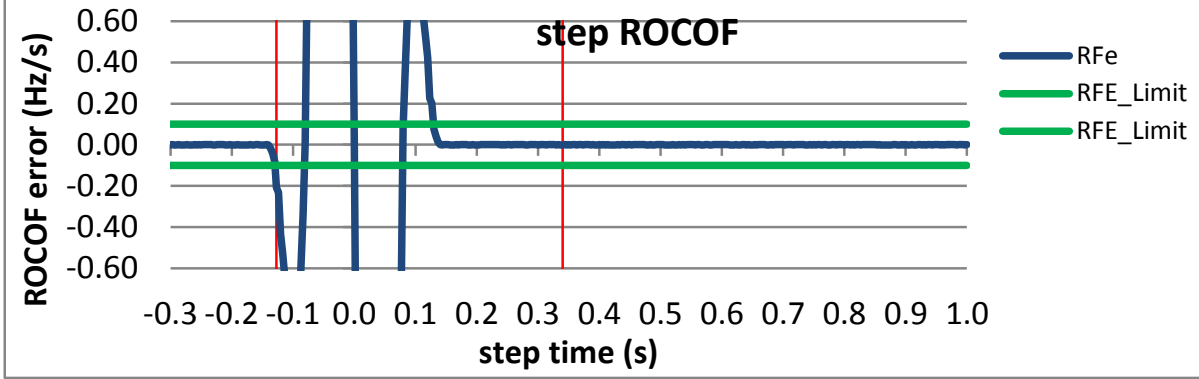

Figure 4686: Fs = 30 FPS, -10 degree phase step

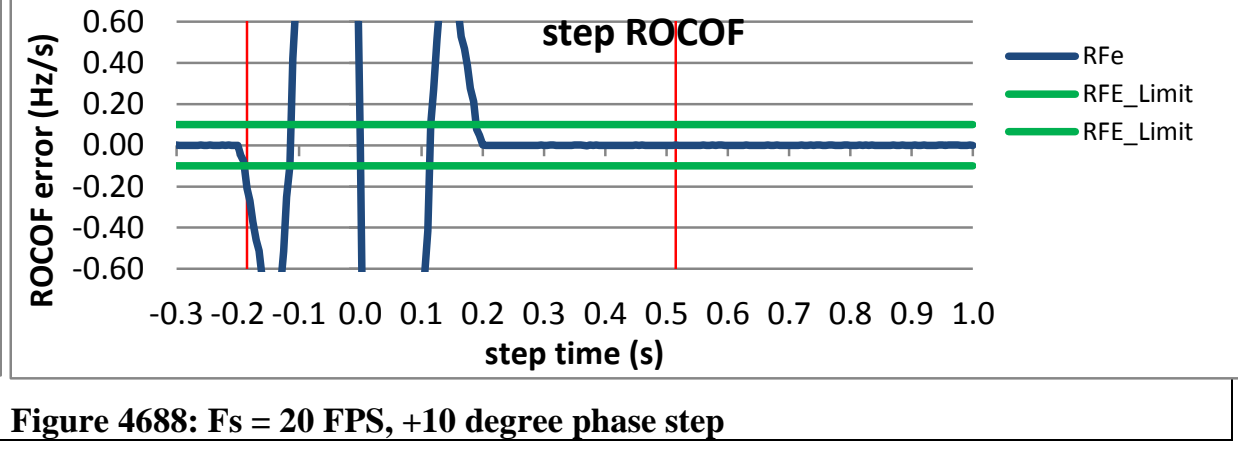



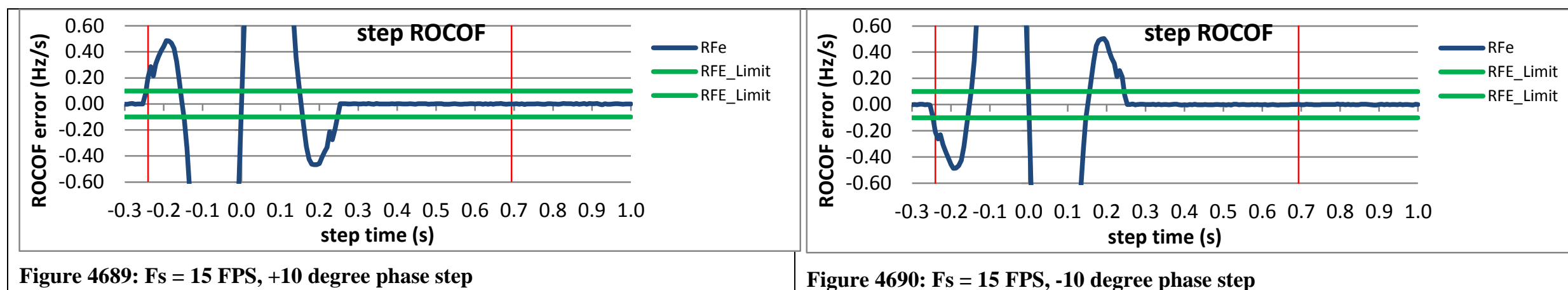

Figure 4690: Fs = 15 FPS, -10 degree phase step
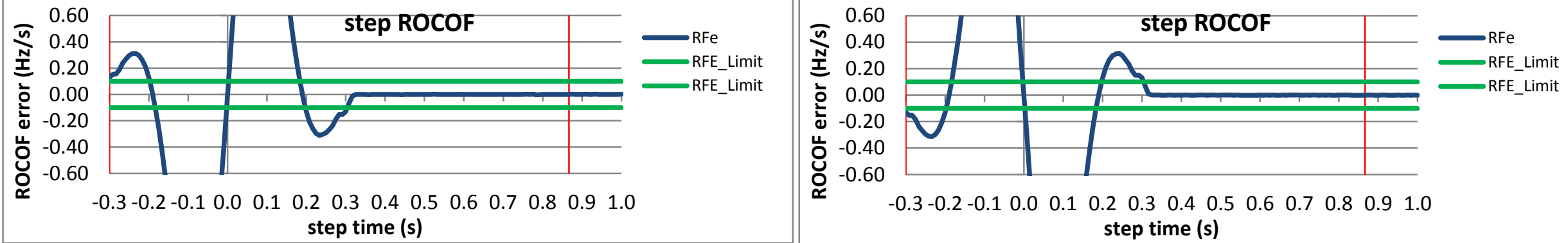

Figure 4691: Fs $=12$ FPS, +10 degree phase step

Figure 4692: Fs = 12 FPS, -10 degree phase step

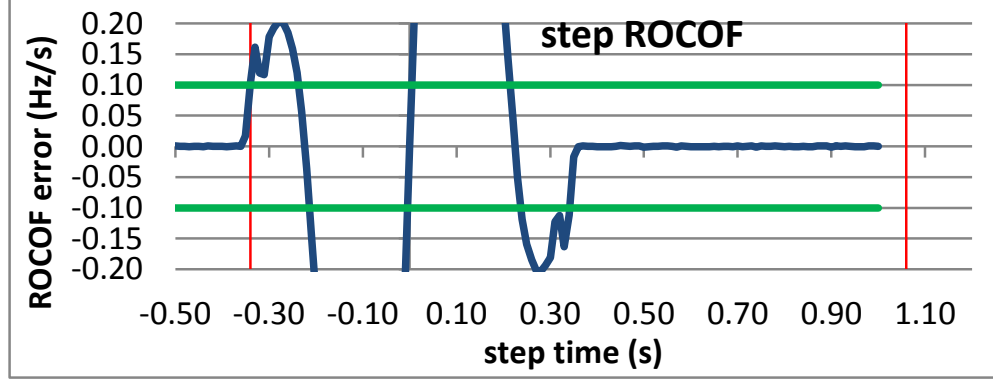

Figure 4693: Fs = 10 FPS, +10 degree phase step

RFe
RFE_Limit
RFE_Limit

RFe
RFE_Limit
RFE_Limit

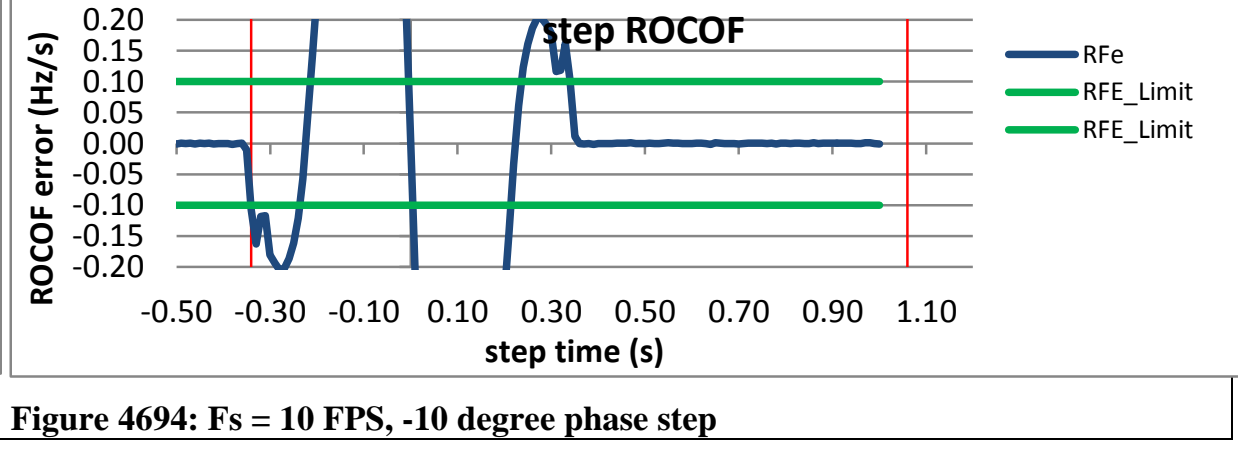


9.7.6 PMU E dynamic step change in phase ROCOF response time: $F 0=60 \mathrm{~Hz}$, M class

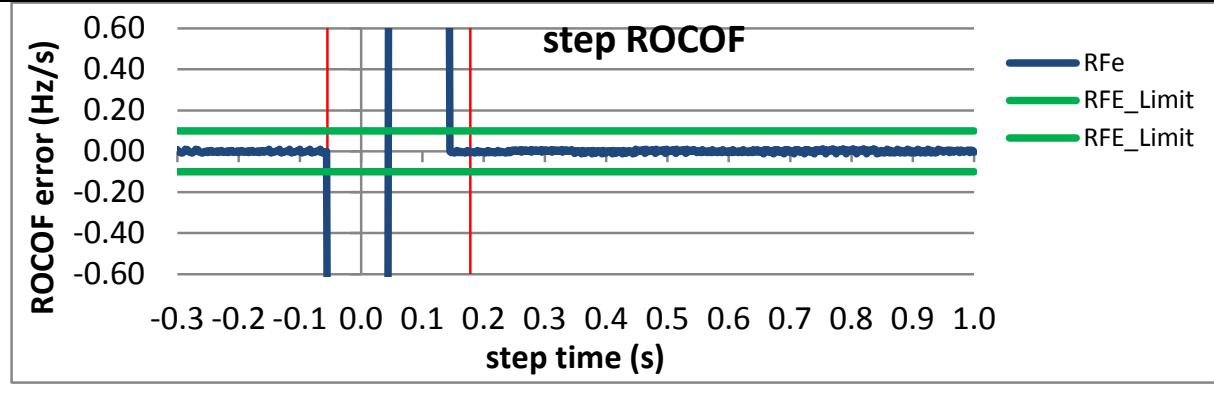

Figure 4695: Fs = 60 FPS, +10 degree phase step

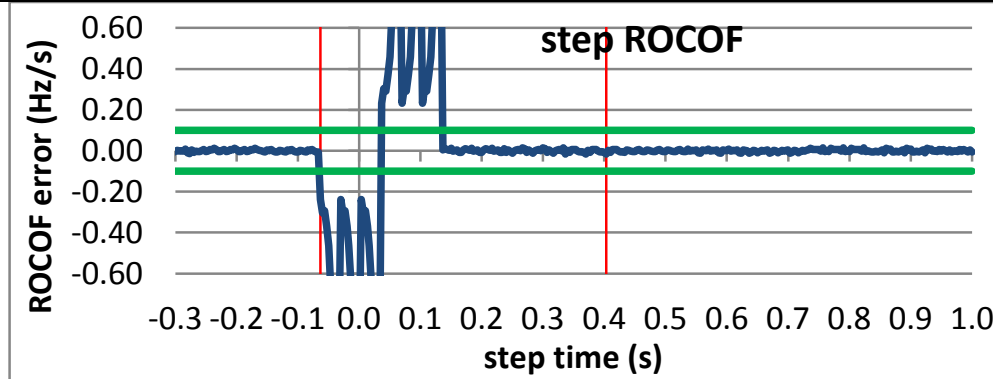

Figure 4697: Fs = 30 FPS, +10 degree phase step

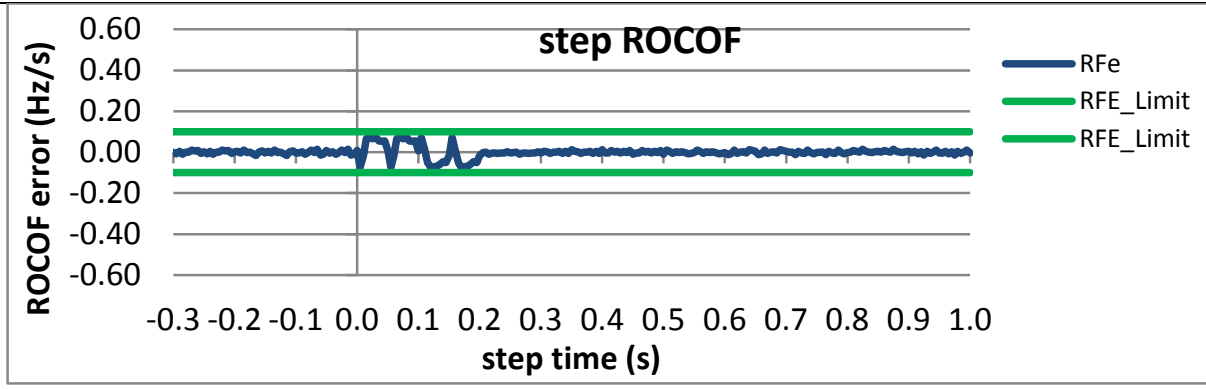

Figure 4699: Fs = 20 FPS, +10 degree phase step

RFe
RFE_Limit
RFE_Limit

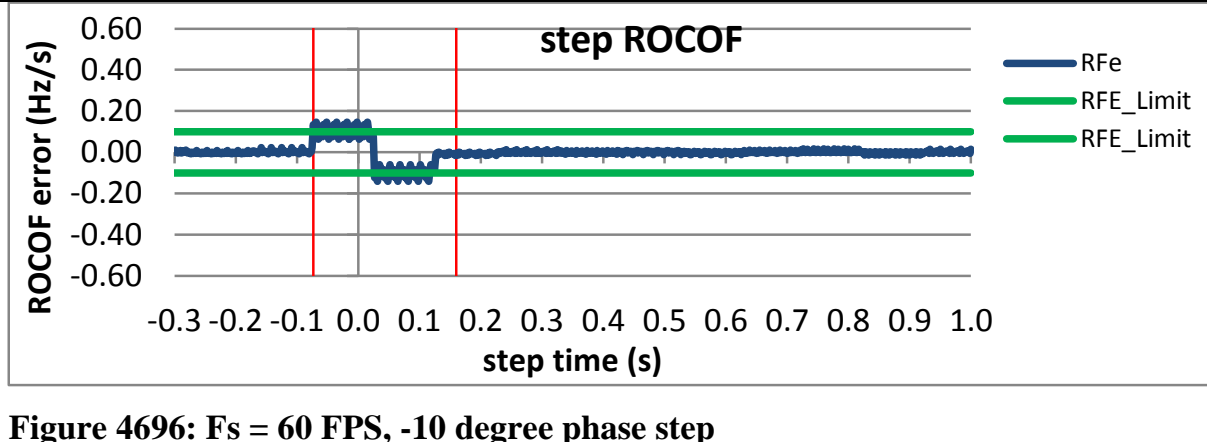

Figure 4696: Fs = 60 FPS, -10 degree phase step

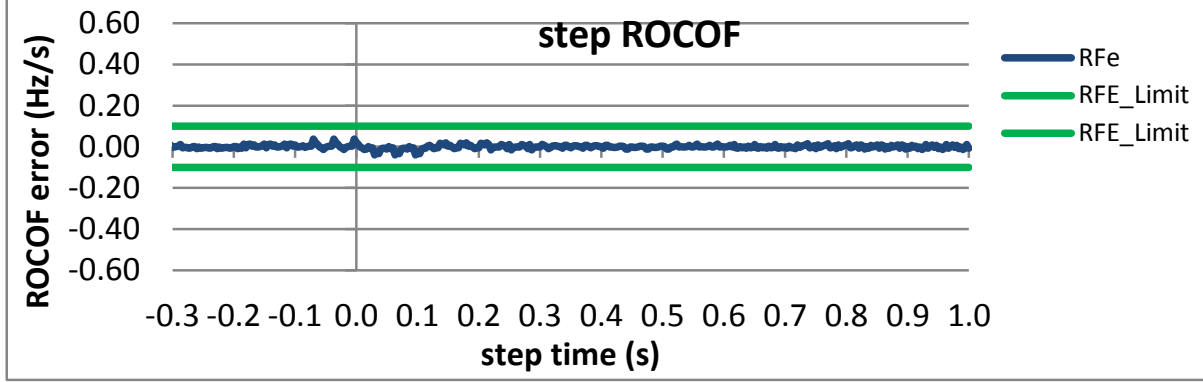

Figure 4698: Fs = 30 FPS, -10 degree phase step

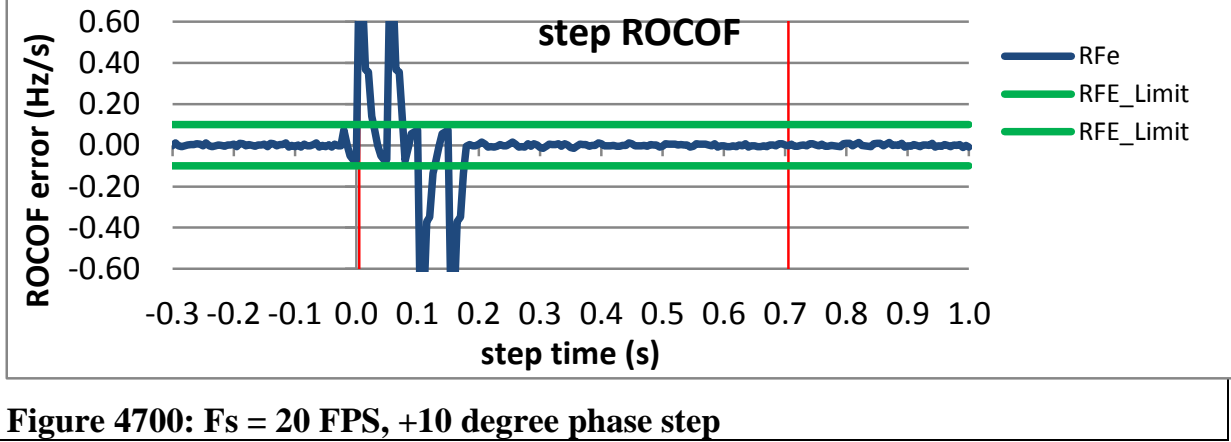




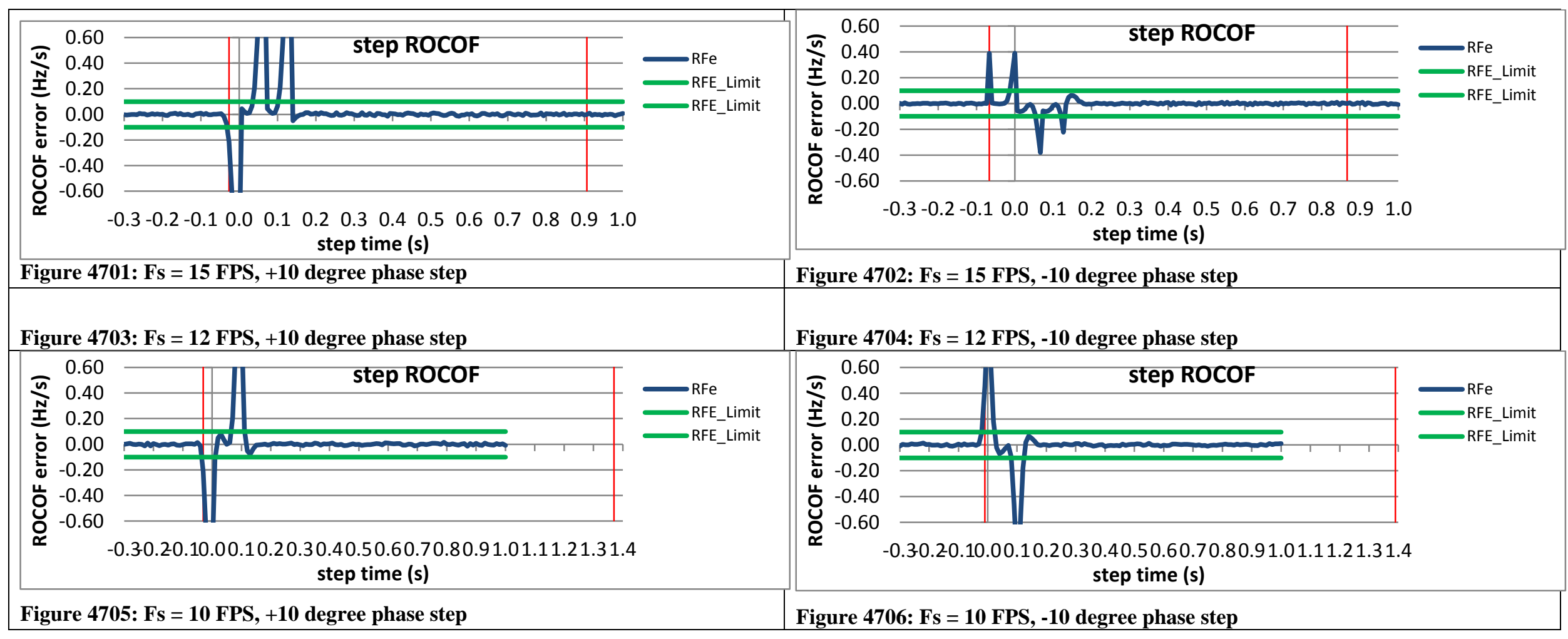


9.7.7 PMU F dynamic step change in phase ROCOF response time: $F 0=60 \mathrm{~Hz}, \mathrm{M}$ class

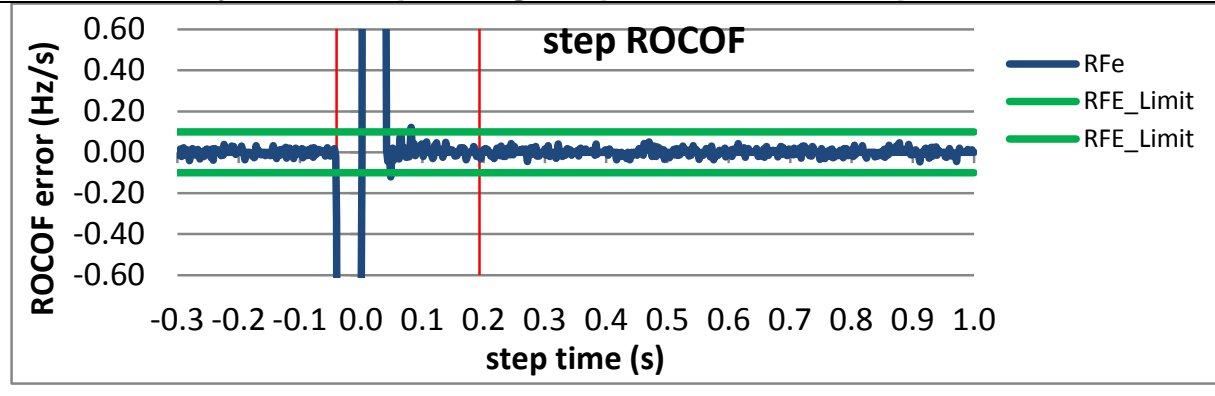

Figure 4707: Fs = 60 FPS, +10 degree phase step

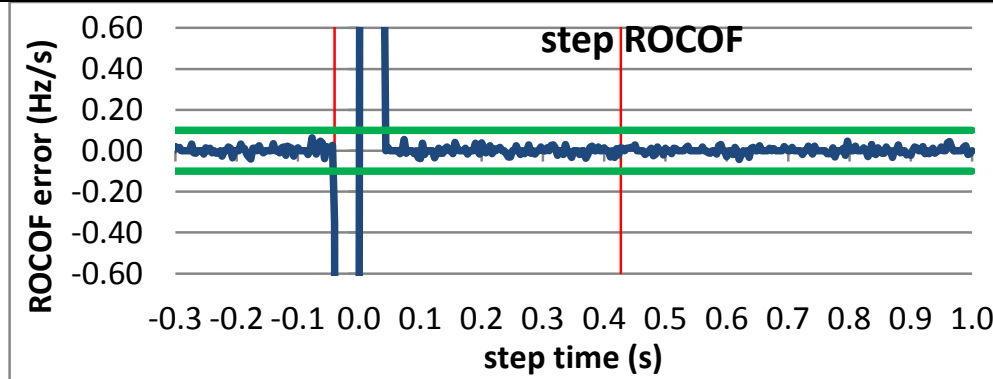

Figure 4709: Fs = 30 FPS, +10 degree phase step

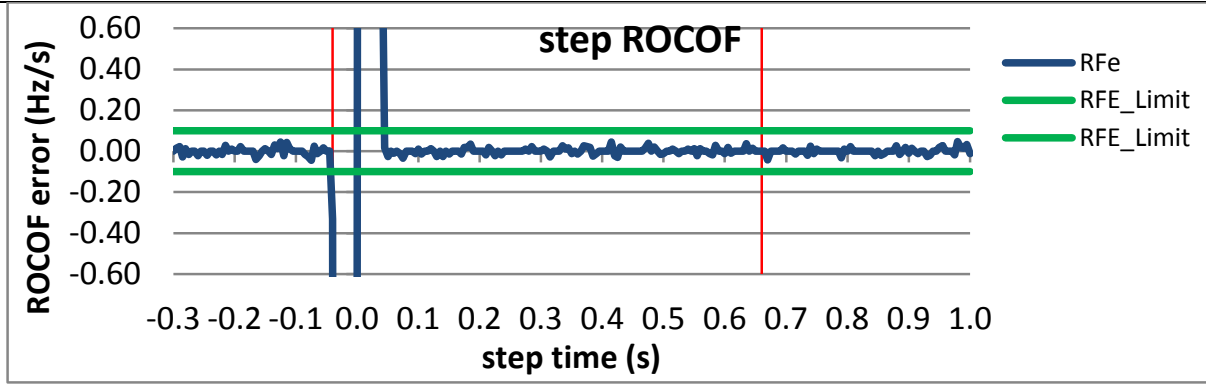

Figure 4711: Fs = 20 FPS, +10 degree phase step

RFe
RFE_Limit
RFE_Limit

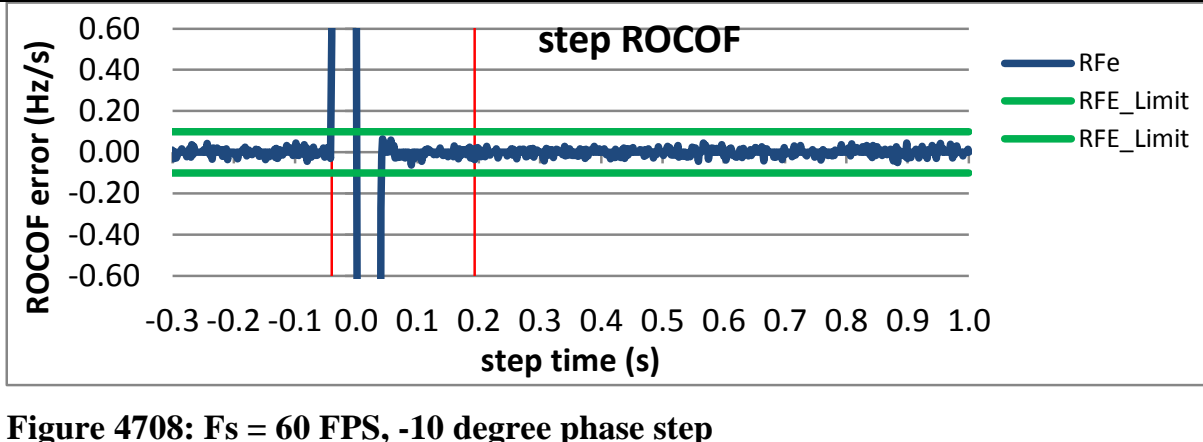

Figure 4708: Fs = $60 \mathrm{FPS},-10$ degree phase step

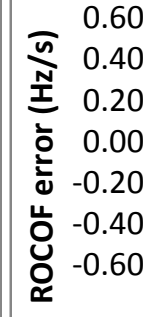
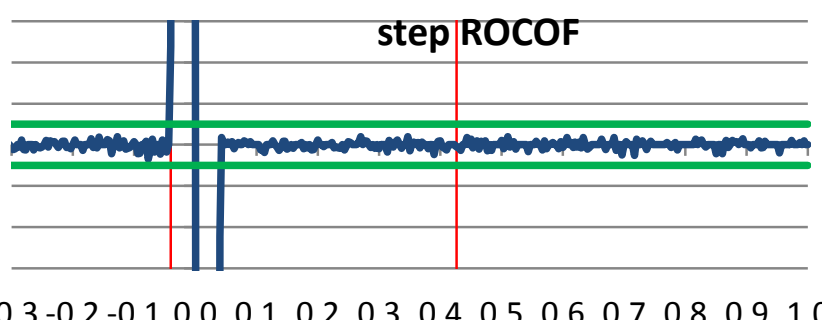

RFe

RFE_Limit

RFE_Limit

$\begin{array}{llllllllllllll}-0.3 & -0.2 & -0.1 & 0.0 & 0.1 & 0.2 & 0.3 & 0.4 & 0.5 & 0.6 & 0.7 & 0.8 & 0.9 & 1.0\end{array}$ step time (s)

Figure 4710: Fs = 30 FPS, -10 degree phase step

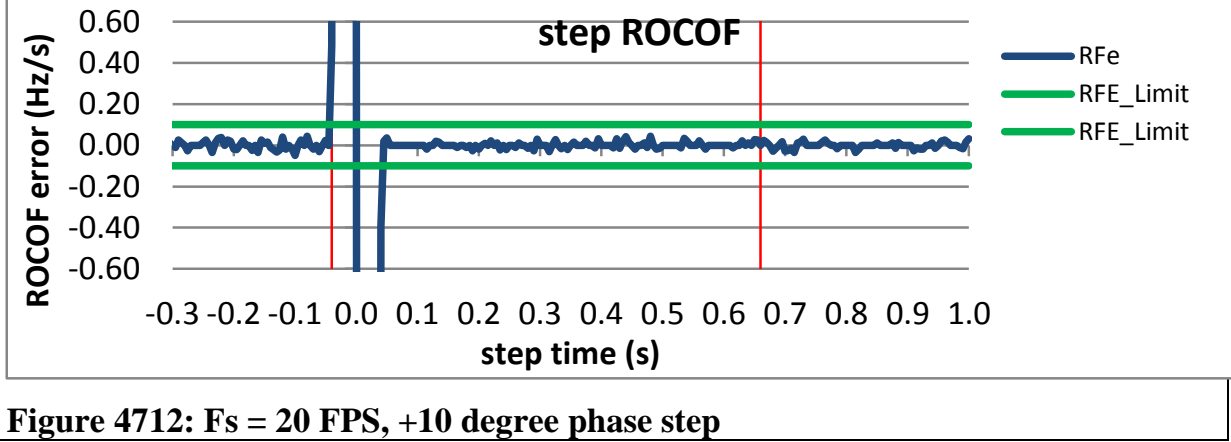




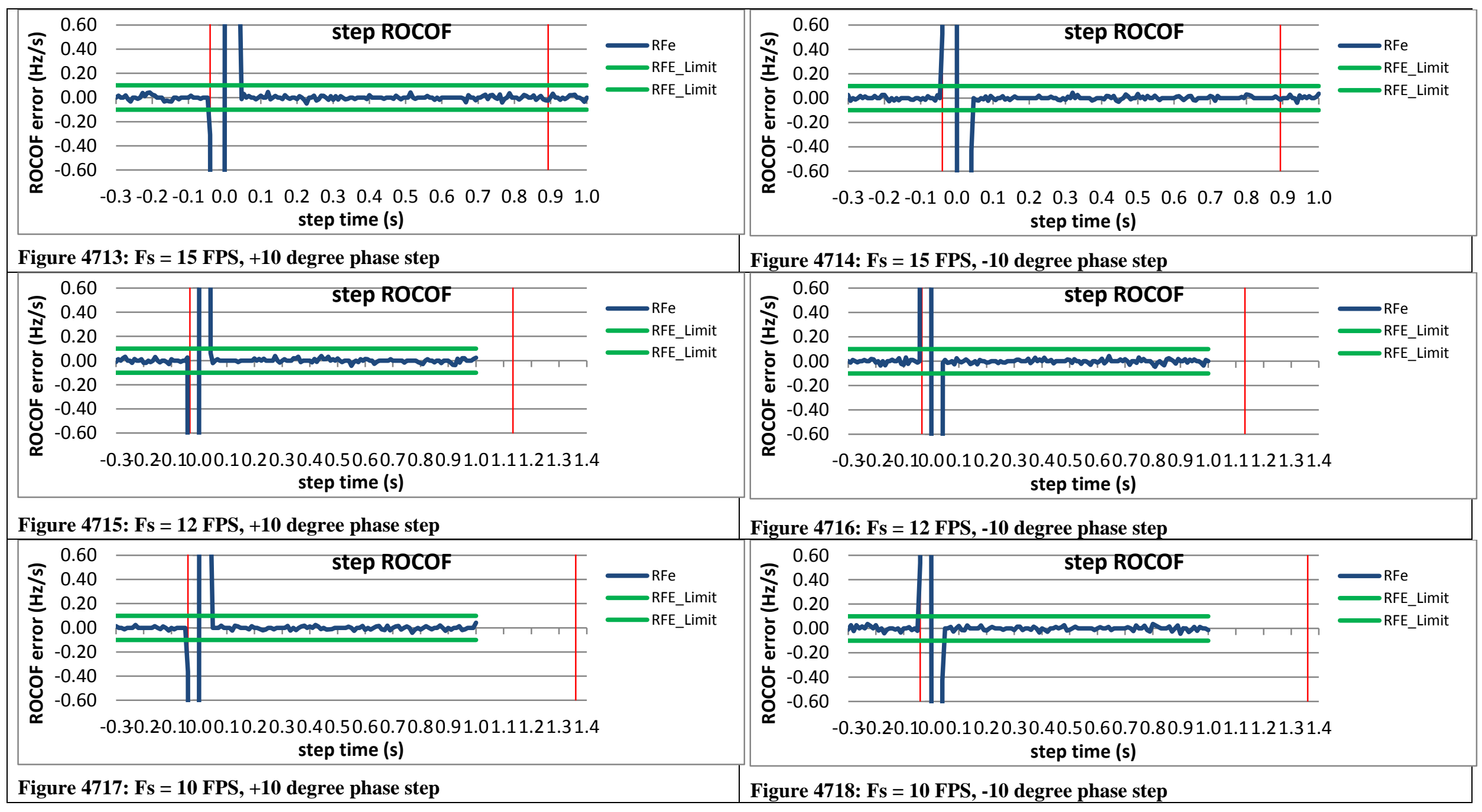


9.7.8 PMU G * dynamic step change in phase ROCOF response time: $F 0=60 \mathrm{~Hz}, \mathrm{M}$ class

Figure 4719: Fs = 60 FPS is not supported by this PMU
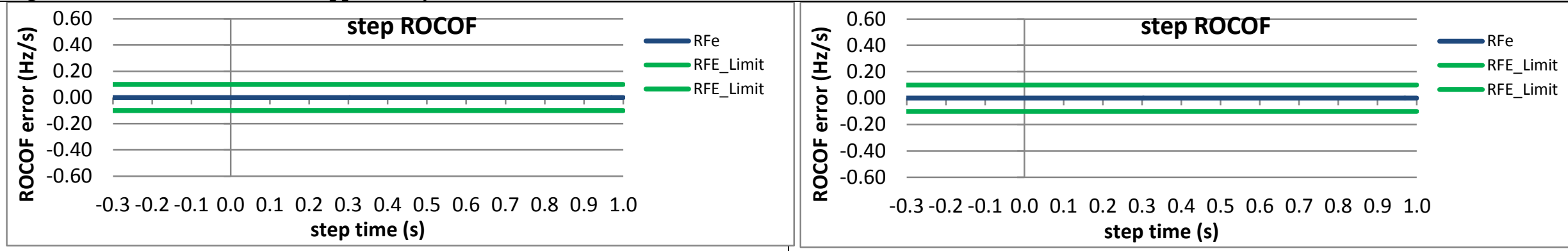

Figure 4720: Fs = 30 FPS, +10 degree phase step

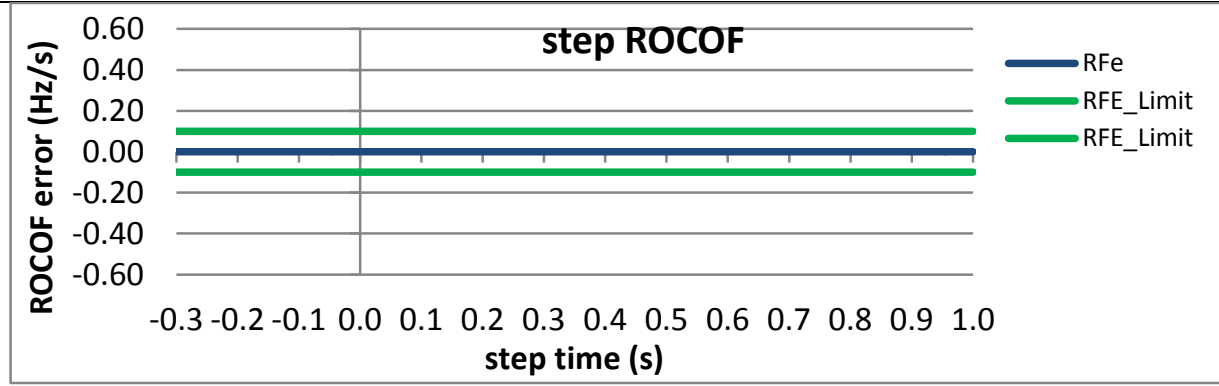

Figure 4721: Fs = 30 FPS, -10 degree phase step
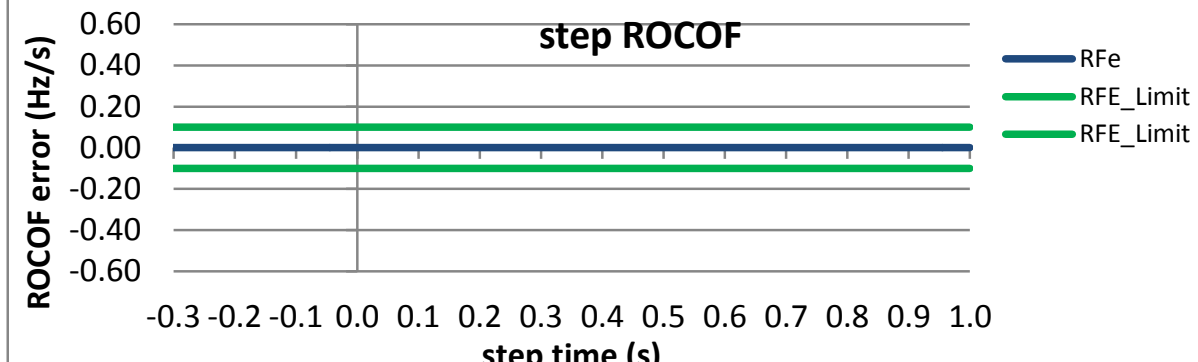
step time (s)

Figure 4722: Fs = 20 FPS, +10 degree phase step

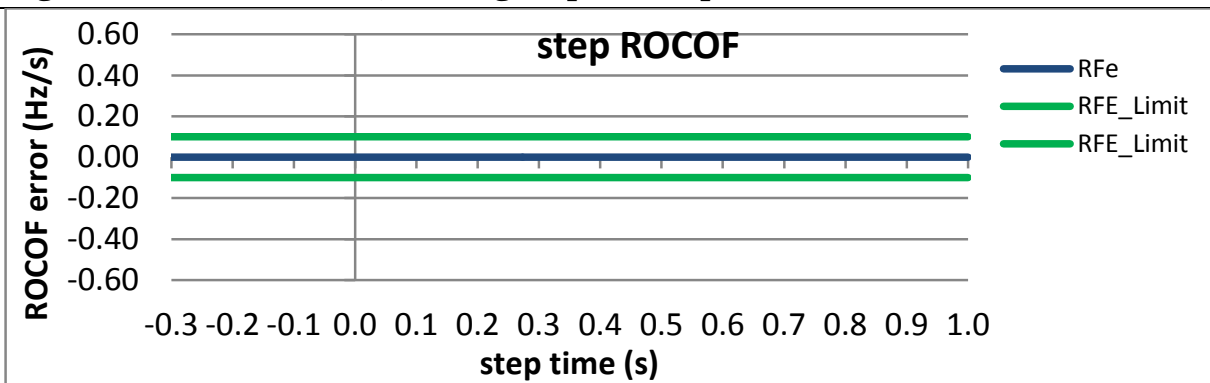

Figure 4723: Fs = 20 FPS, +10 degree phase step

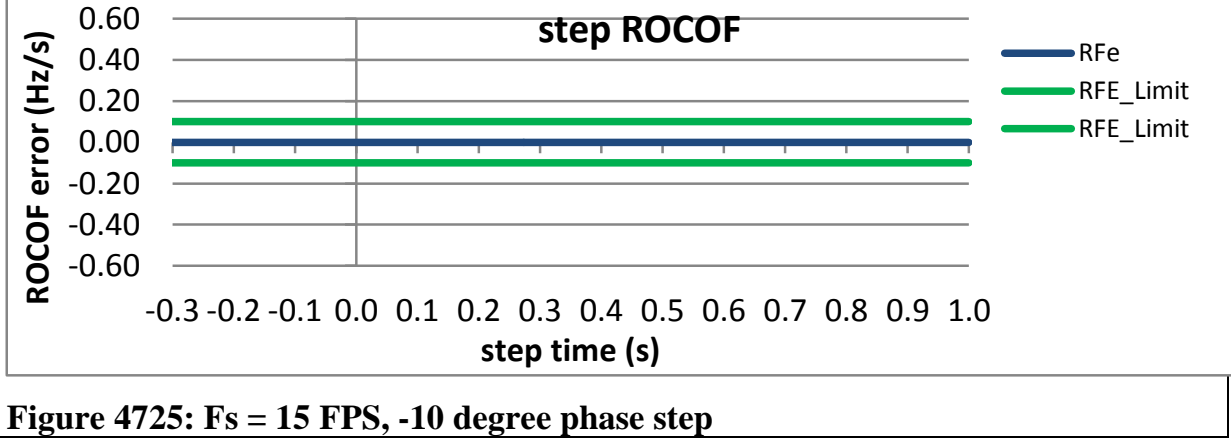




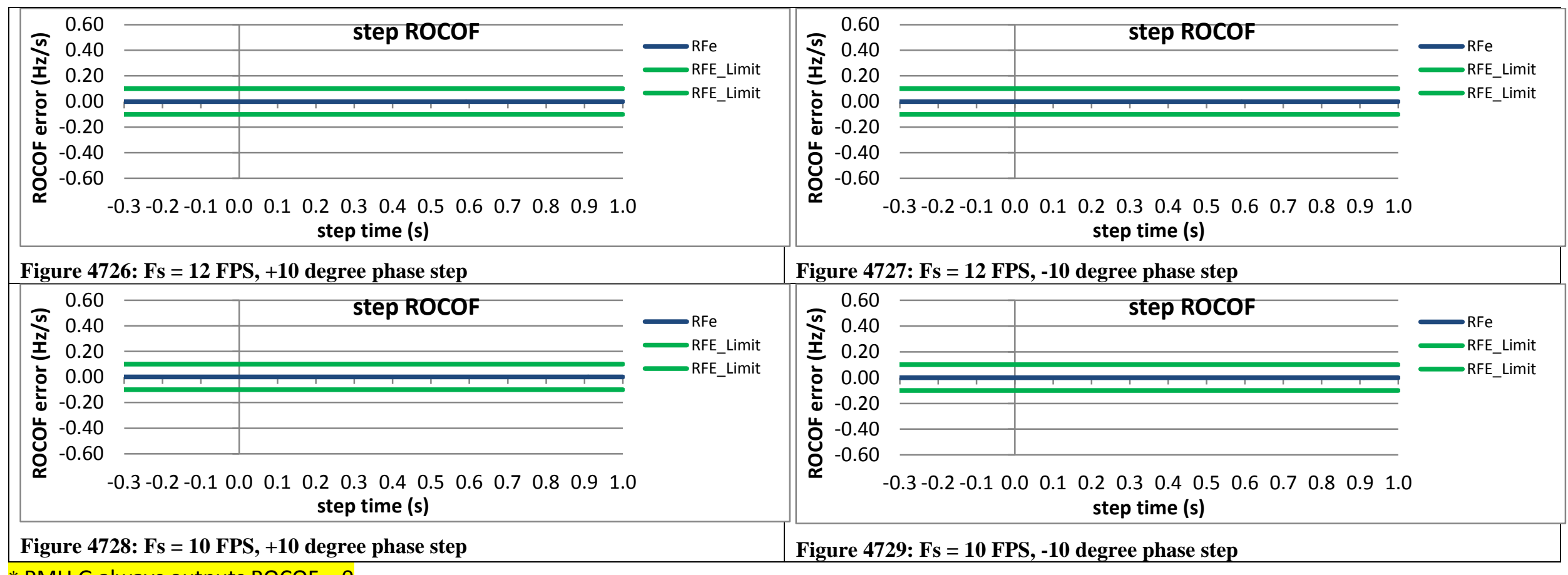

* PMU G always outputs ROCOF $=0$ 
9.7.9 PMU H dynamic step change in phase ROCOF response time: $\mathrm{FO}=60 \mathrm{~Hz}, \mathrm{M}$ class

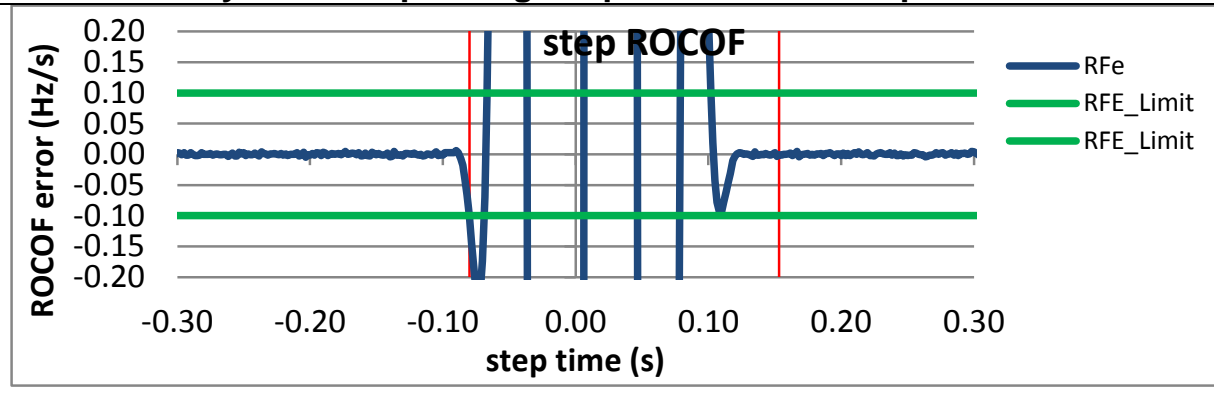

Figure 4730: Fs = 60 FPS, +10 degree phase step

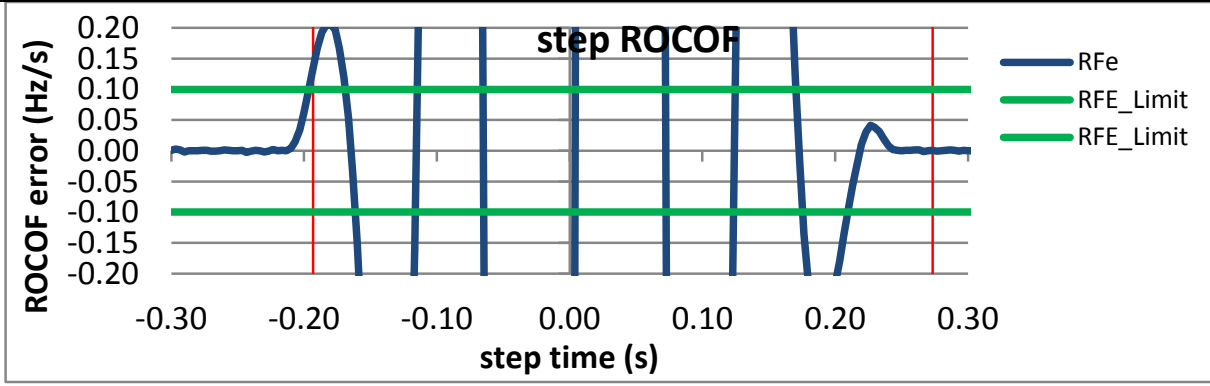

Figure 4732: Fs = 30 FPS, +10 degree phase step

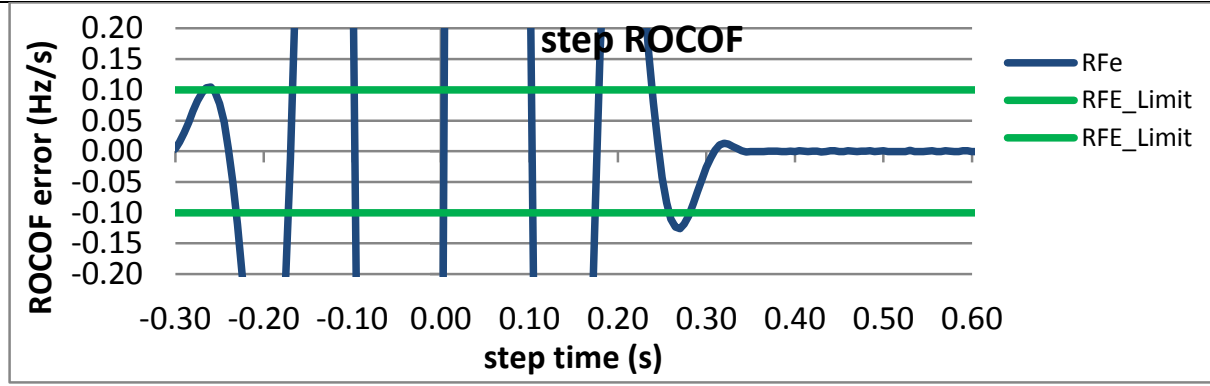

Figure 4734: Fs = 20 FPS, +10 degree phase step

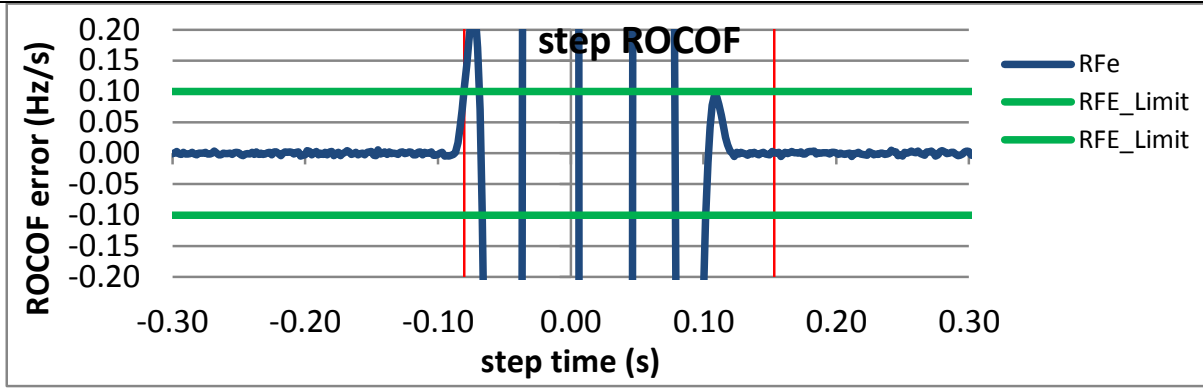

Figure 4731: Fs = 60 FPS, -10 degree phase step

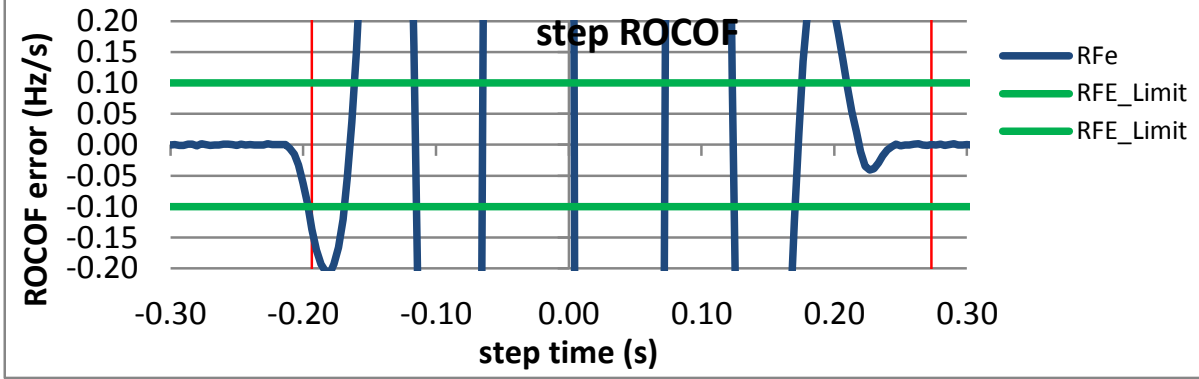

Figure 4733: Fs = 30 FPS, -10 degree phase step

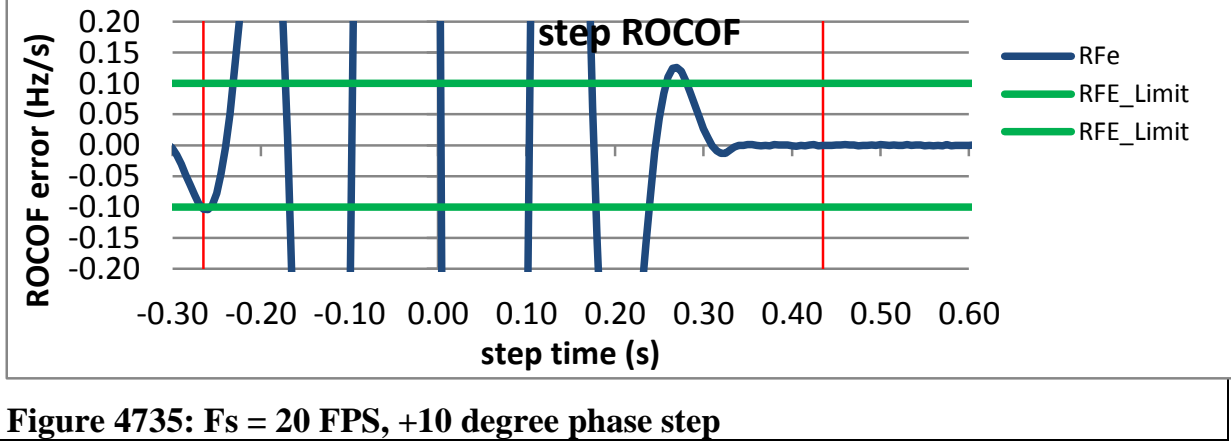



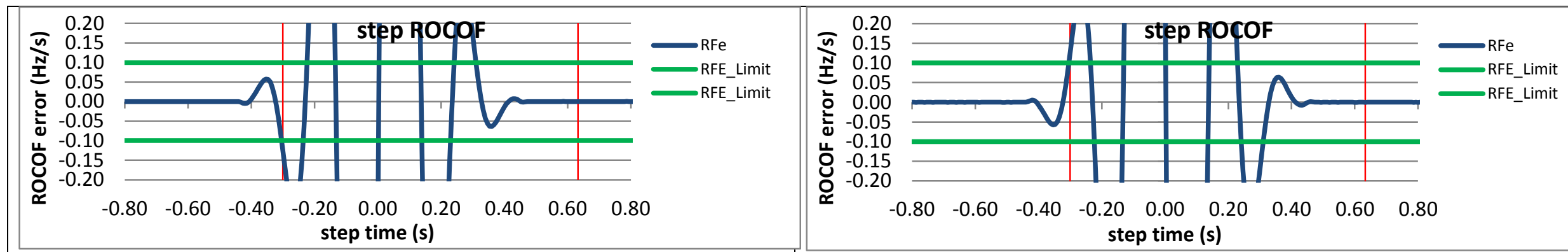

Figure 4736: Fs = 15 FPS, +10 degree phase step

Figure 4737: Fs = 15 FPS, - 10 degree phase step
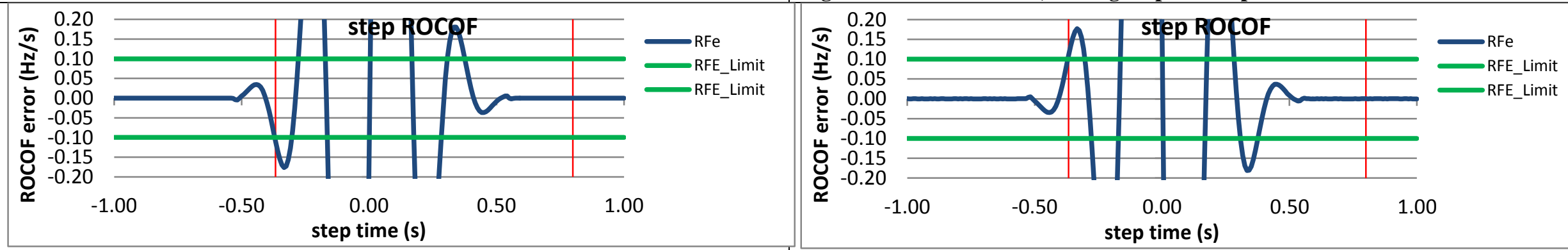

Figure 4738: Fs $=12$ FPS, +10 degree phase step

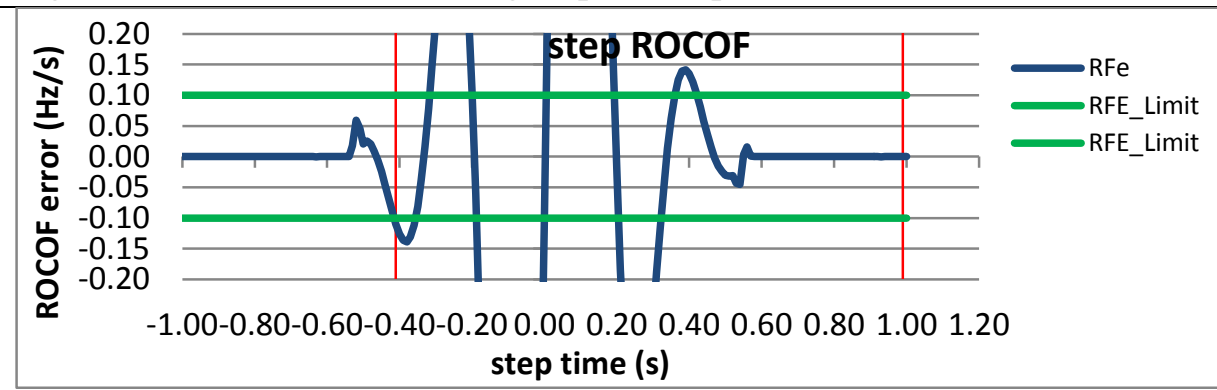

Figure 4739: Fs = 12 FPS, -10 degree phase step

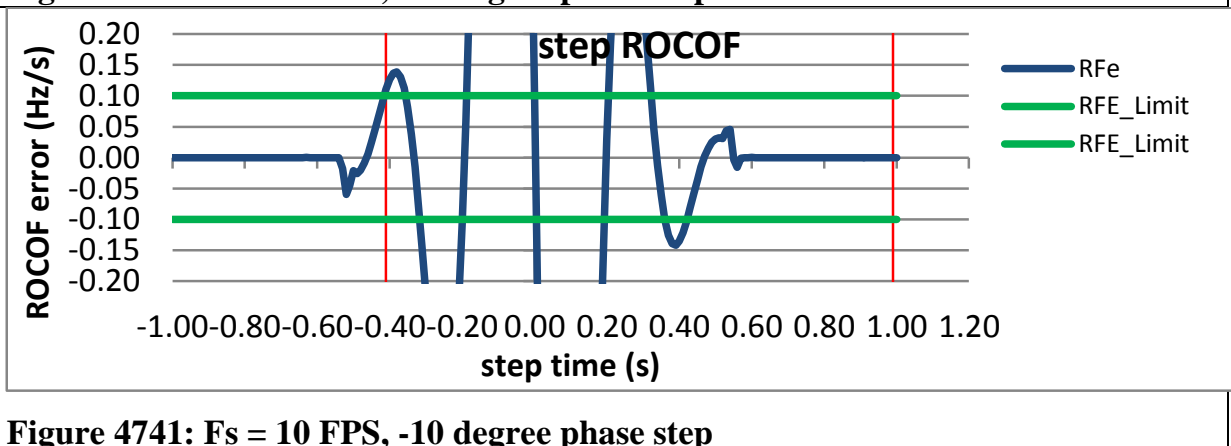

Figure 4740: Fs $=10$ FPS, +10 degree phase step

Figure 4741: Fs = 10 FPS, -10 degree phase step 
9.7.10 PMU I dynamic step change in phase ROCOF response time: $\mathrm{FO}=60 \mathrm{~Hz}$, M class

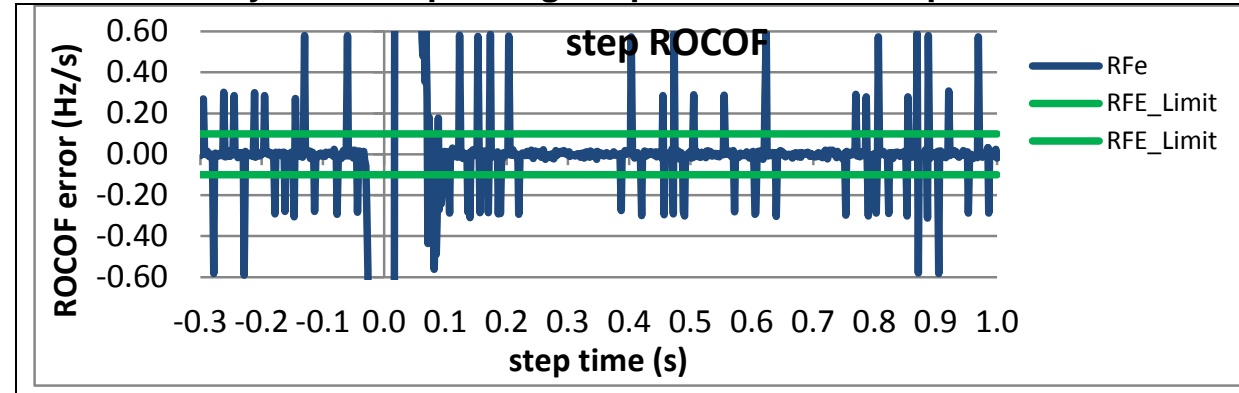

Figure 4742: Fs = 60 FPS, +10 degree phase step

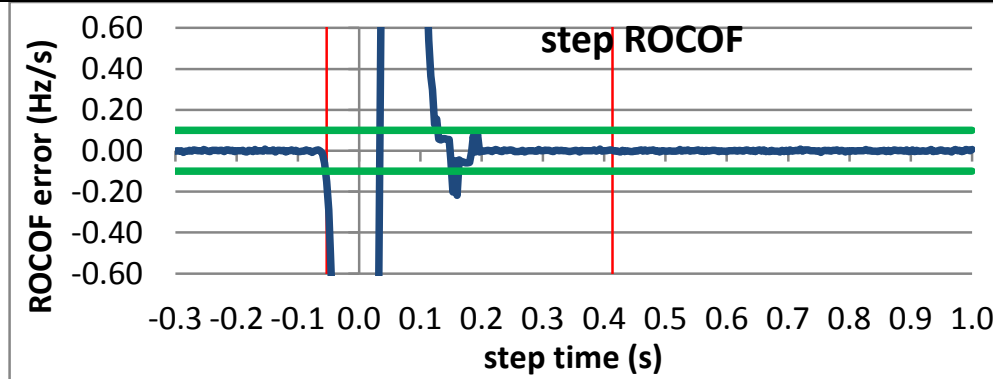

Figure 4744: Fs = 30 FPS, +10 degree phase step

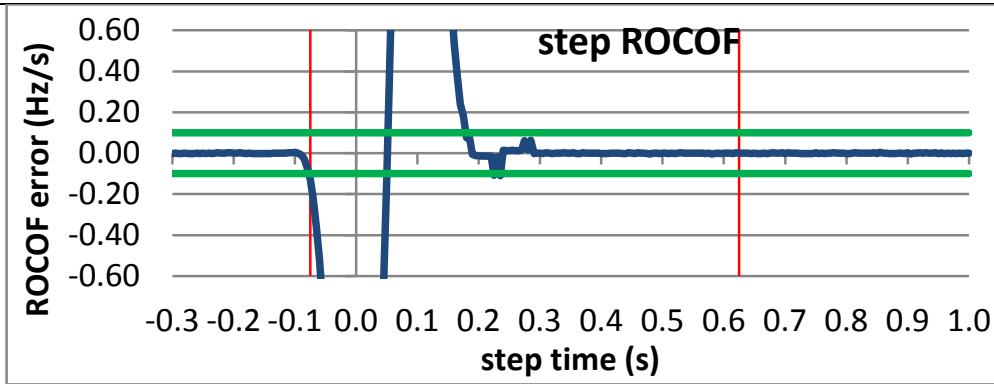

Figure 4746: Fs = 20 FPS, +10 degree phase step

RFe
RFE_Limit
RFE_Limit

RFe
RFE_Limit
RFE_Limit

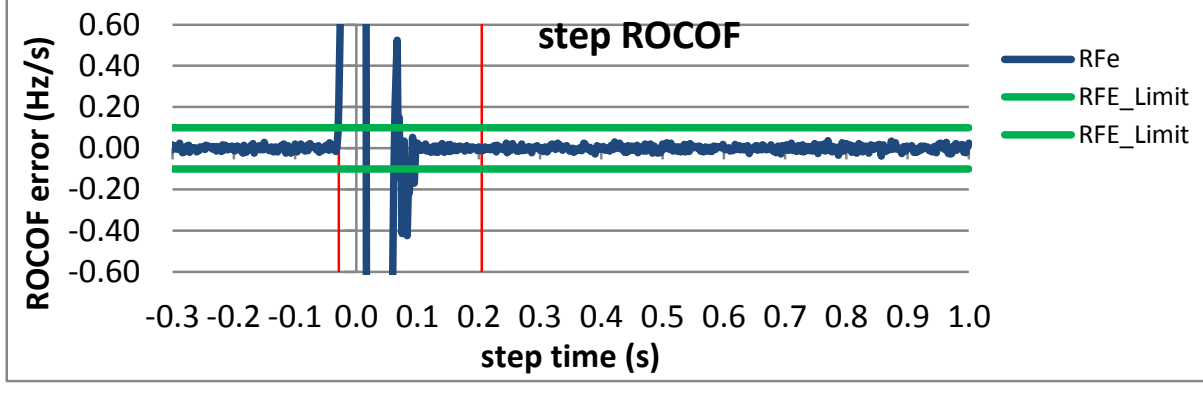

Figure 4743: Fs = 60 FPS, -10 degree phase step

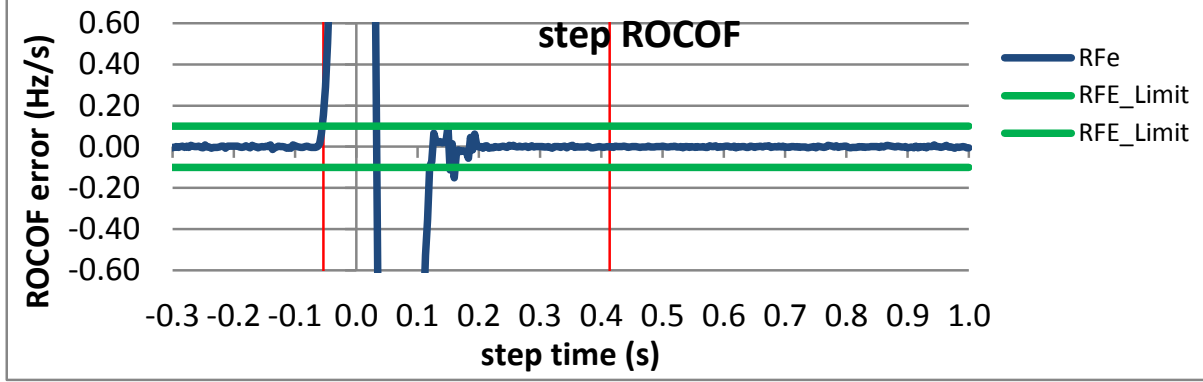

Figure 4745: Fs = 30 FPS, -10 degree phase step

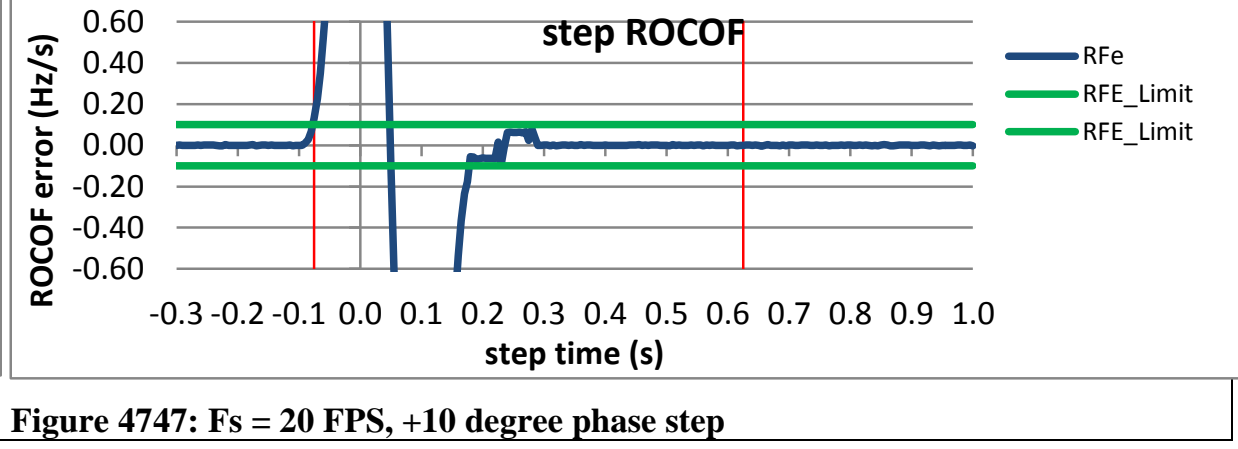



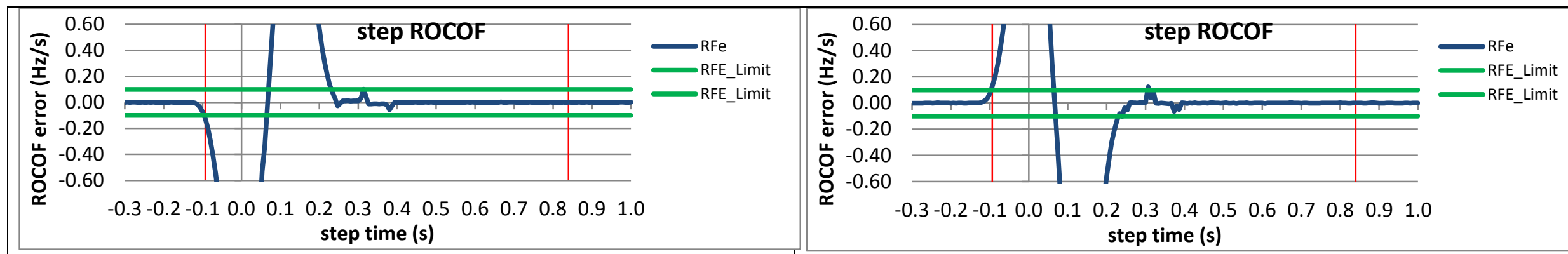

Figure 4748: Fs = 15 FPS, +10 degree phase step

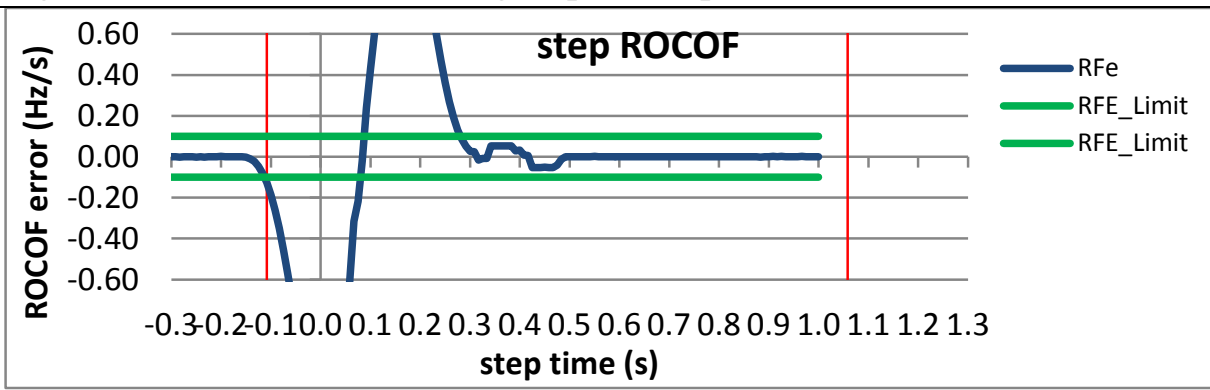

Figure 4749: Fs = 15 FPS, -10 degree phase step

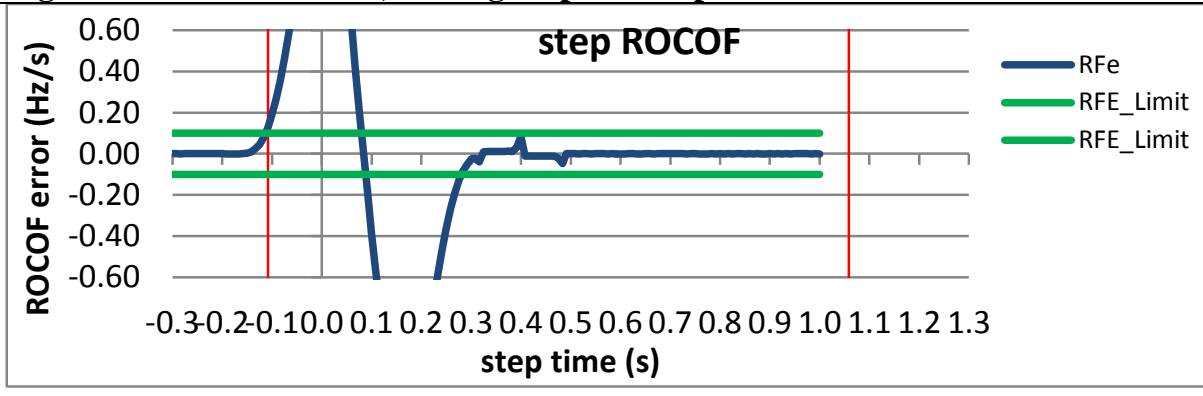

Figure 4750: Fs = 12 FPS, +10 degree phase step

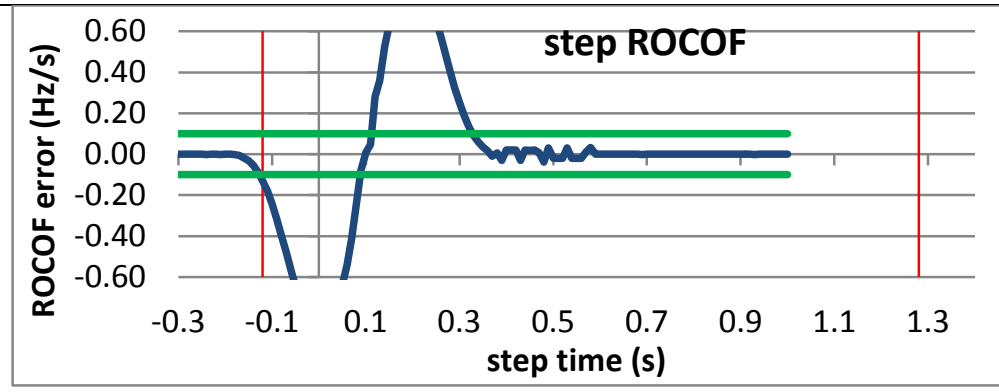

Figure 4752: Fs = 10 FPS, +10 degree phase step

RFe
RFE_Limit
RFE_Limit

Figure 4751: Fs = 12 FPS, - 10 degree phase step

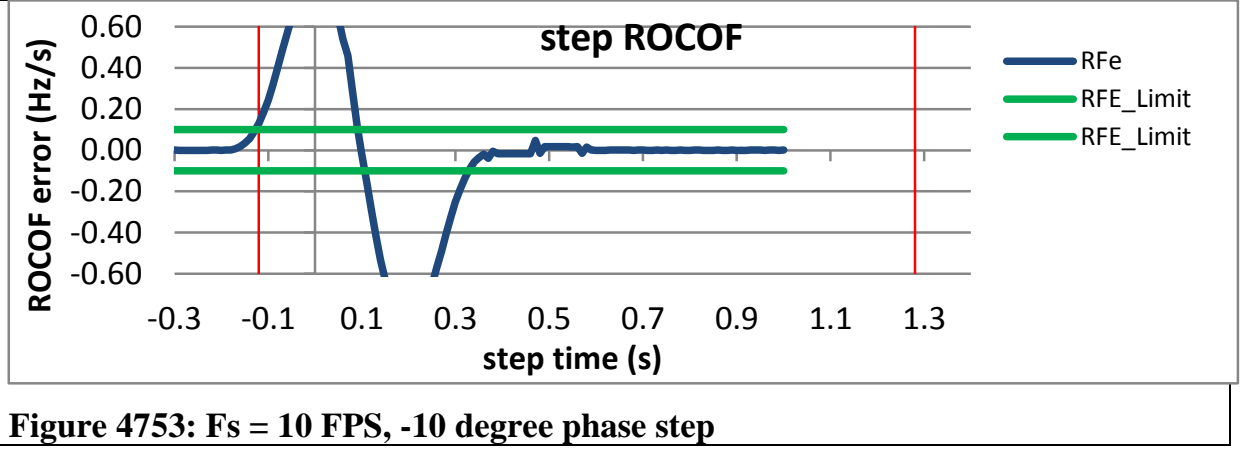


9.7.11 PMU J dynamic step change in phase ROCOF response time: $F 0=60 \mathrm{~Hz}, \mathrm{M}$ class

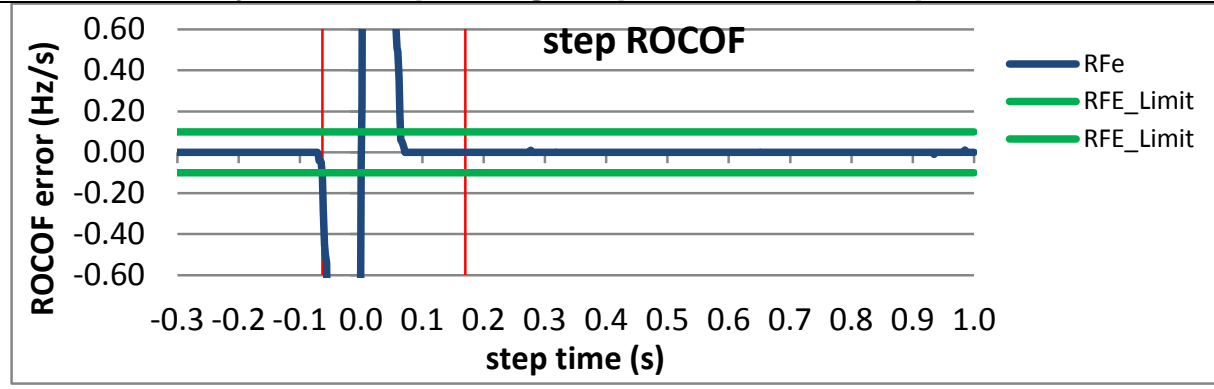

Figure 4754: Fs = 60 FPS, +10 degree phase step

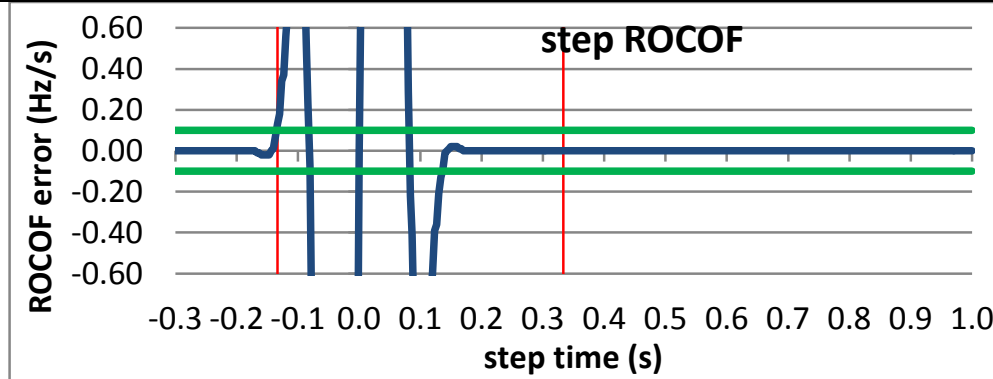

Figure 4756: Fs = 30 FPS, +10 degree phase step

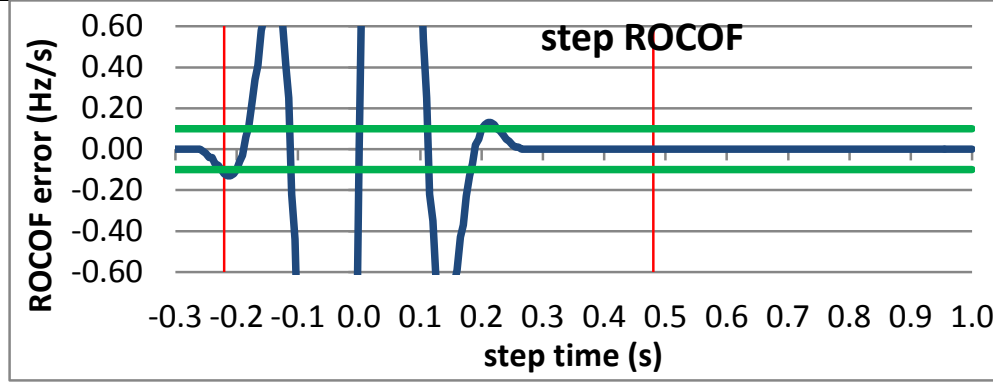

Figure 4758: Fs = 20 FPS, +10 degree phase step

RFe
RFE_Limit
RFE_Limit

RFe
RFE_Limit
RFE_Limit

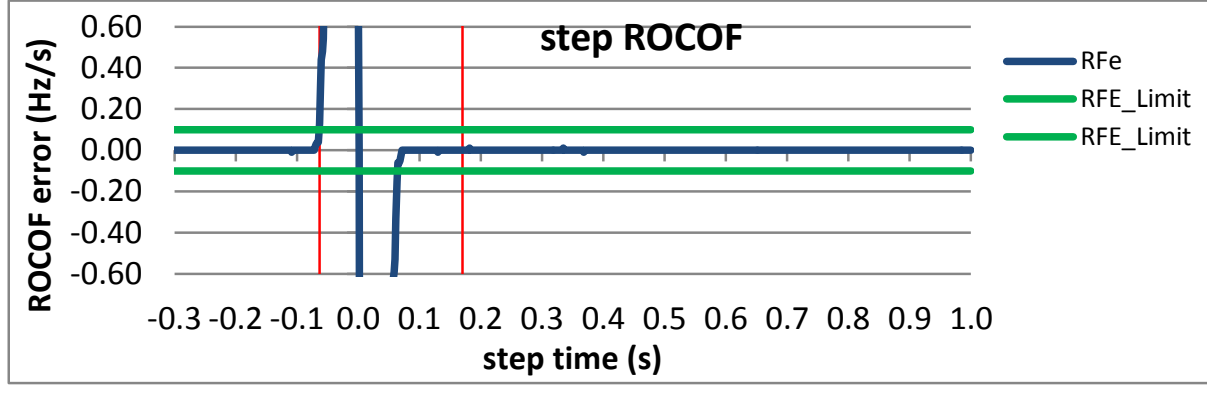

Figure 4755: Fs = 60 FPS, -10 degree phase step

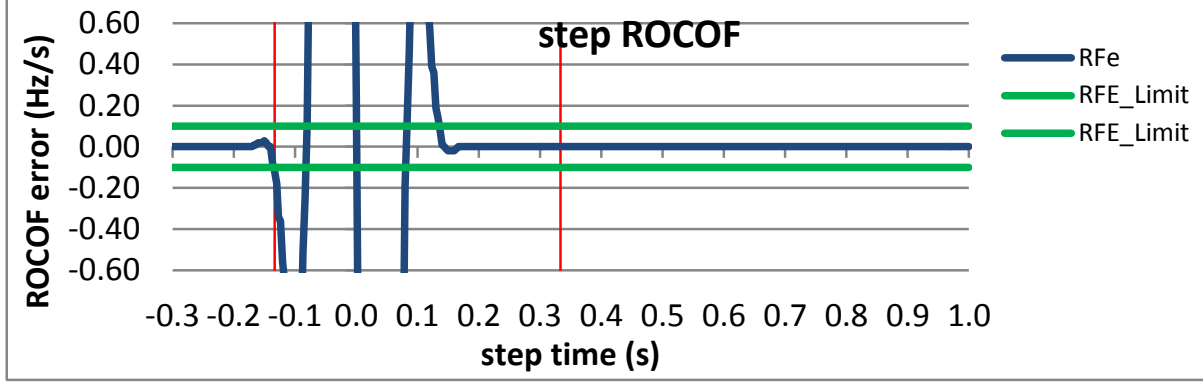

Figure 4757: Fs = 30 FPS, -10 degree phase step

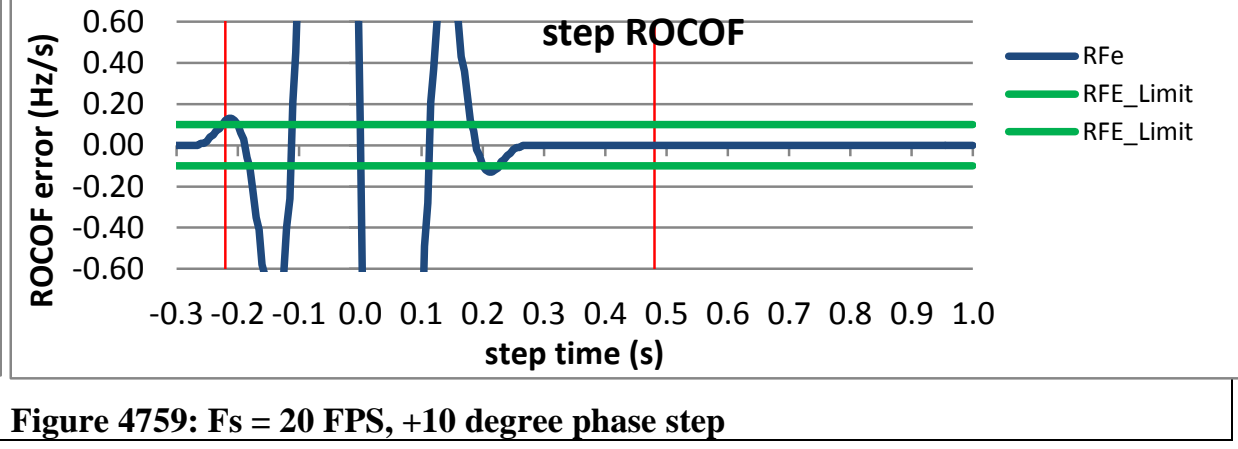



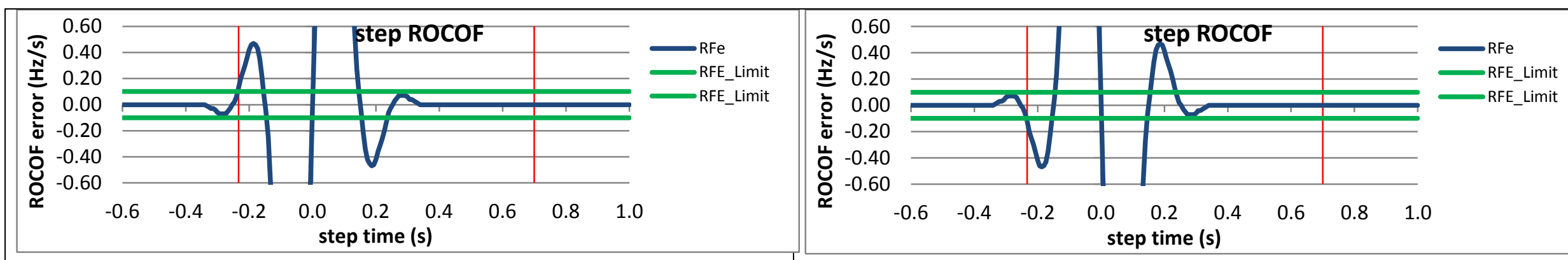

Figure 4760: Fs = 15 FPS, +10 degree phase step

Figure 4761: Fs = 15 FPS, - 10 degree phase step
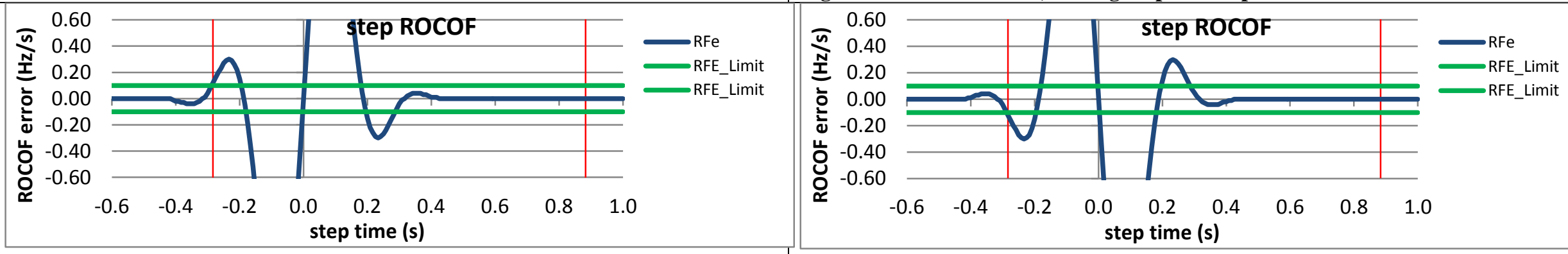

Figure 4762: Fs $=12$ FPS, +10 degree phase step

Figure 4763: Fs = 12 FPS, -10 degree phase step

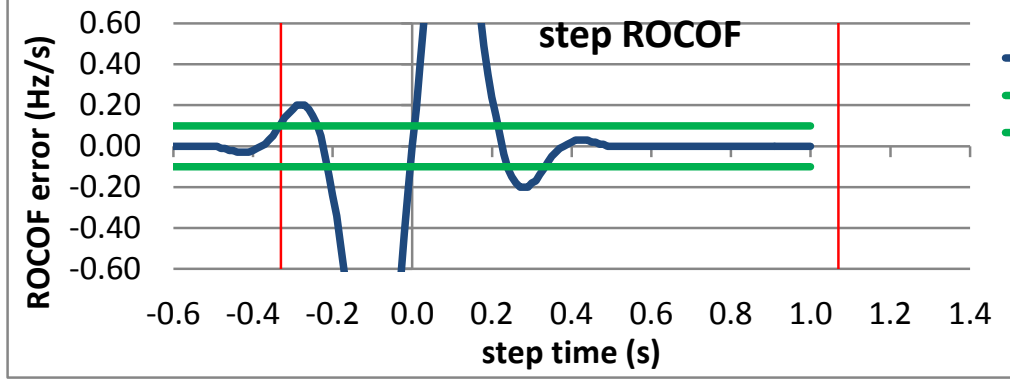

Figure 4764: Fs = 10 FPS, +10 degree phase step

\begin{tabular}{l} 
RFe \\
RFE_Limit \\
RFE_Limit \\
\hline
\end{tabular}

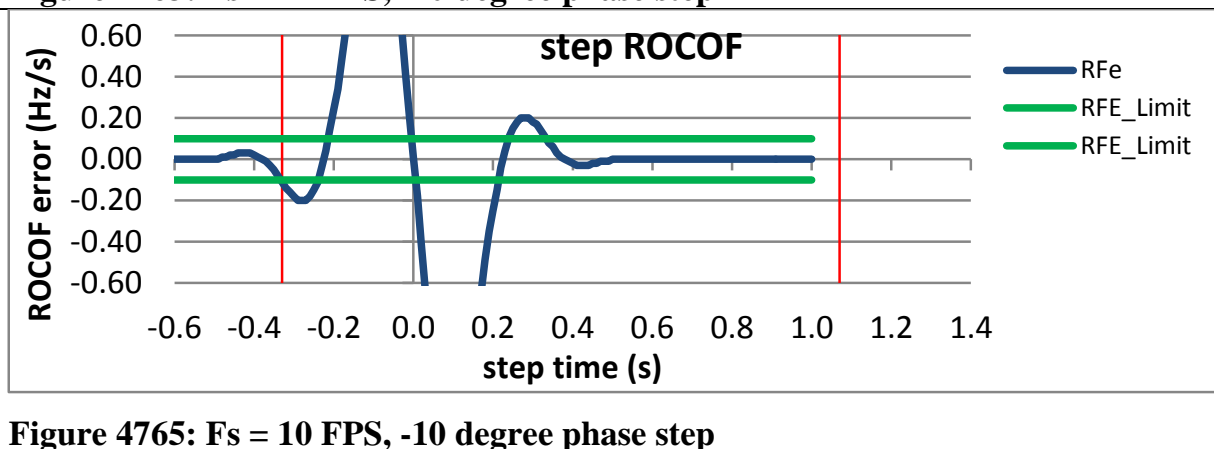


9.8 Dynamic step change in phase: ROCOF response time: $P$ class

9.8.1 C37.118.1-2011 Annex C dynamic step change in phase ROCOF error: F0 $=60 \mathrm{~Hz}, \mathrm{P}$ class
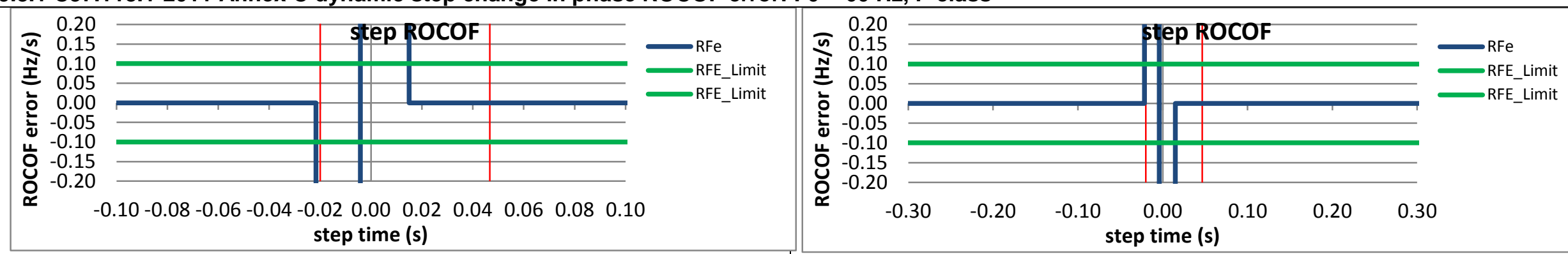

Figure 4766: Fs = 60 FPS, +10 degree phase step

Figure 4767: Fs = 60 FPS, -10 degree phase step
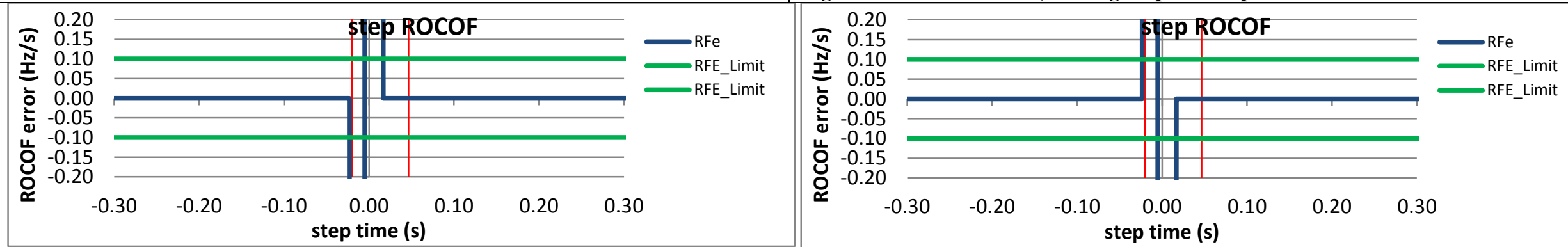

Figure 4768: Fs $=30$ FPS, +10 degree phase step

Figure 4769: Fs = 30 FPS, -10 degree phase step

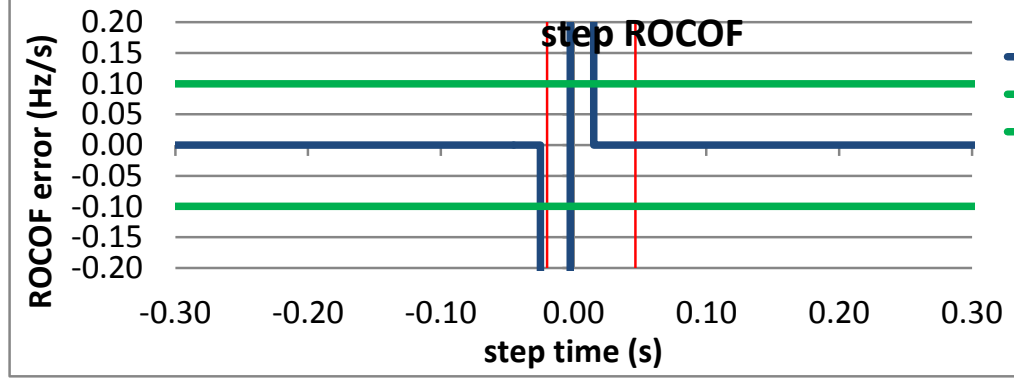

Figure 4770: Fs $=20$ FPS, +10 degree phase step

RFe
RFE_Limit
RFE_Limit

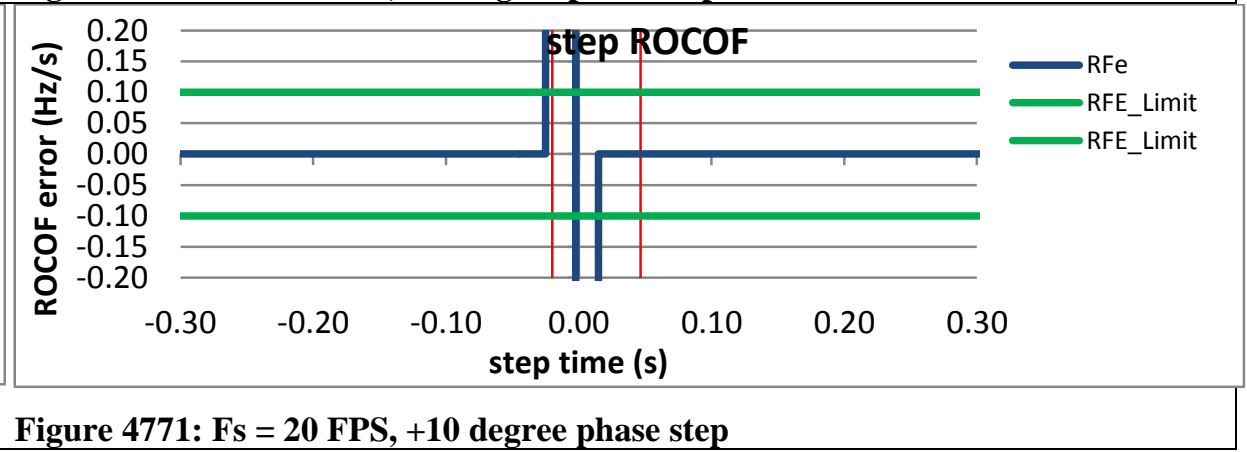



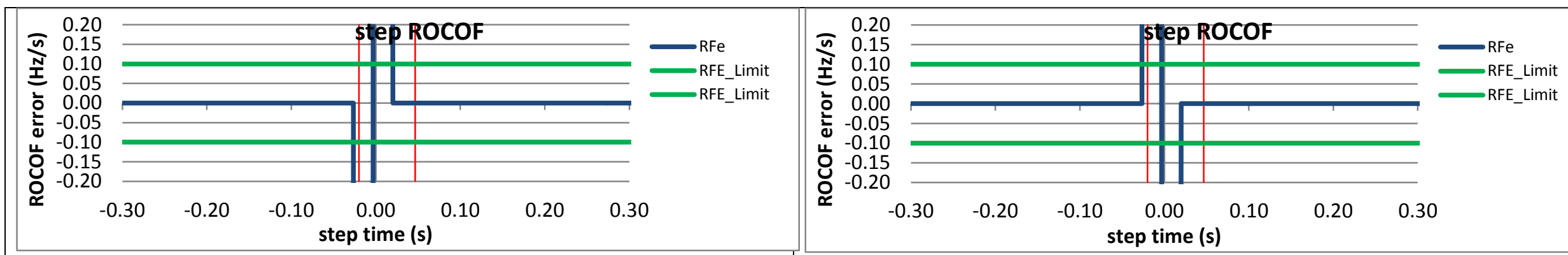

Figure 4772: Fs = 15 FPS, +10 degree phase step

Figure 4773: Fs = 15 FPS, -10 degree phase step

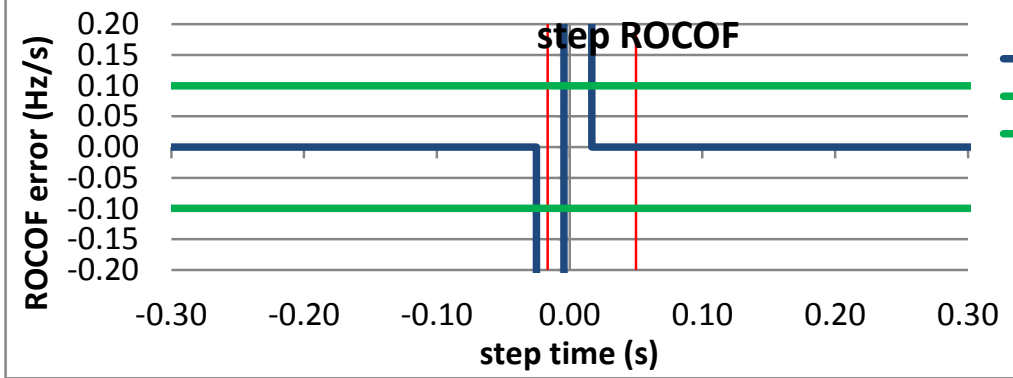

RFe
RFE_Limit
RFE_Limit

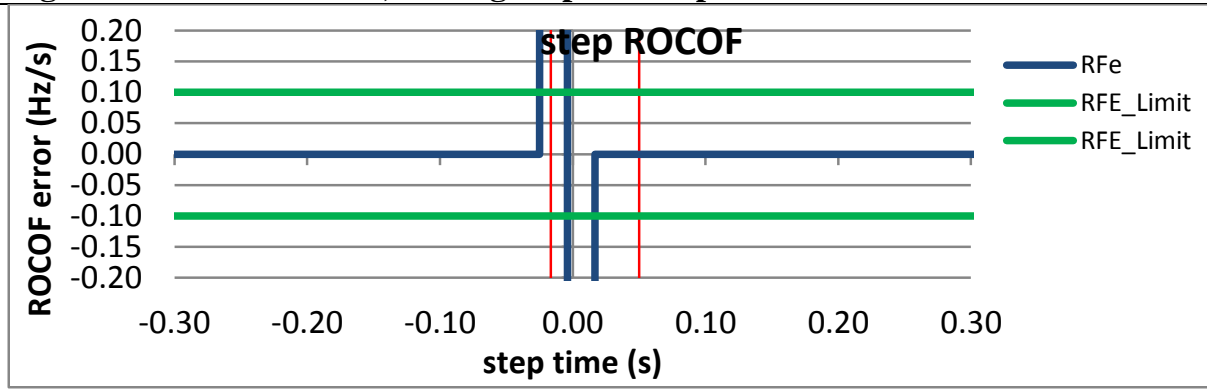

Figure 4774: Fs $=12$ FPS, +10 degree phase step

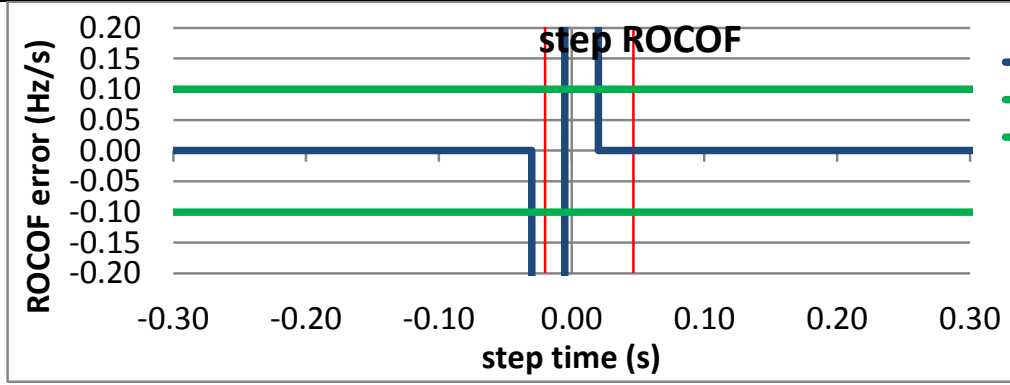

Figure 4776: Fs = 10 FPS, +10 degree phase step

RFe
RFE_Limit
RFE_Limit

Figure 4775: Fs = 12 FPS, -10 degree phase step

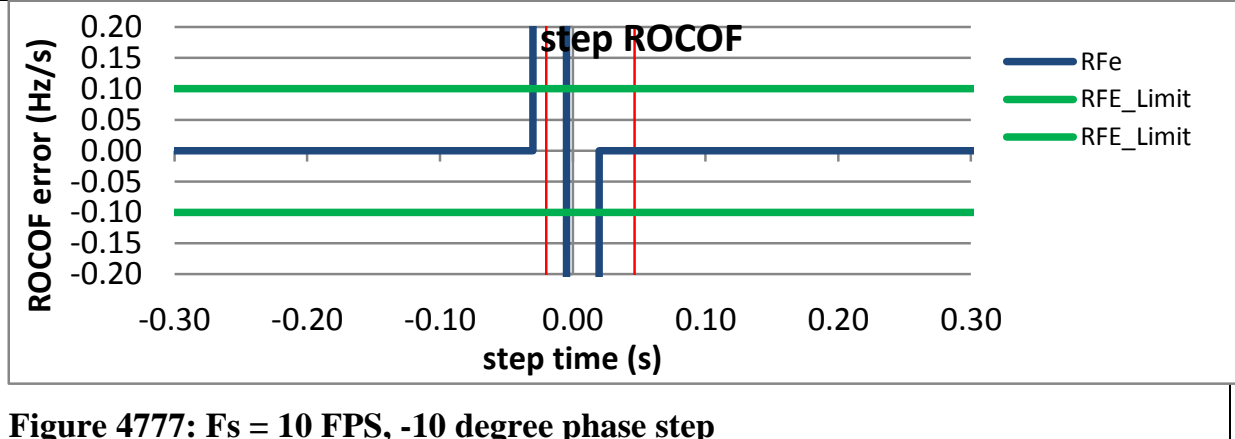


9.8.2 PMU A dynamic step change in phase ROCOF response time: $F 0=60 \mathrm{~Hz}, \mathrm{P}$ class

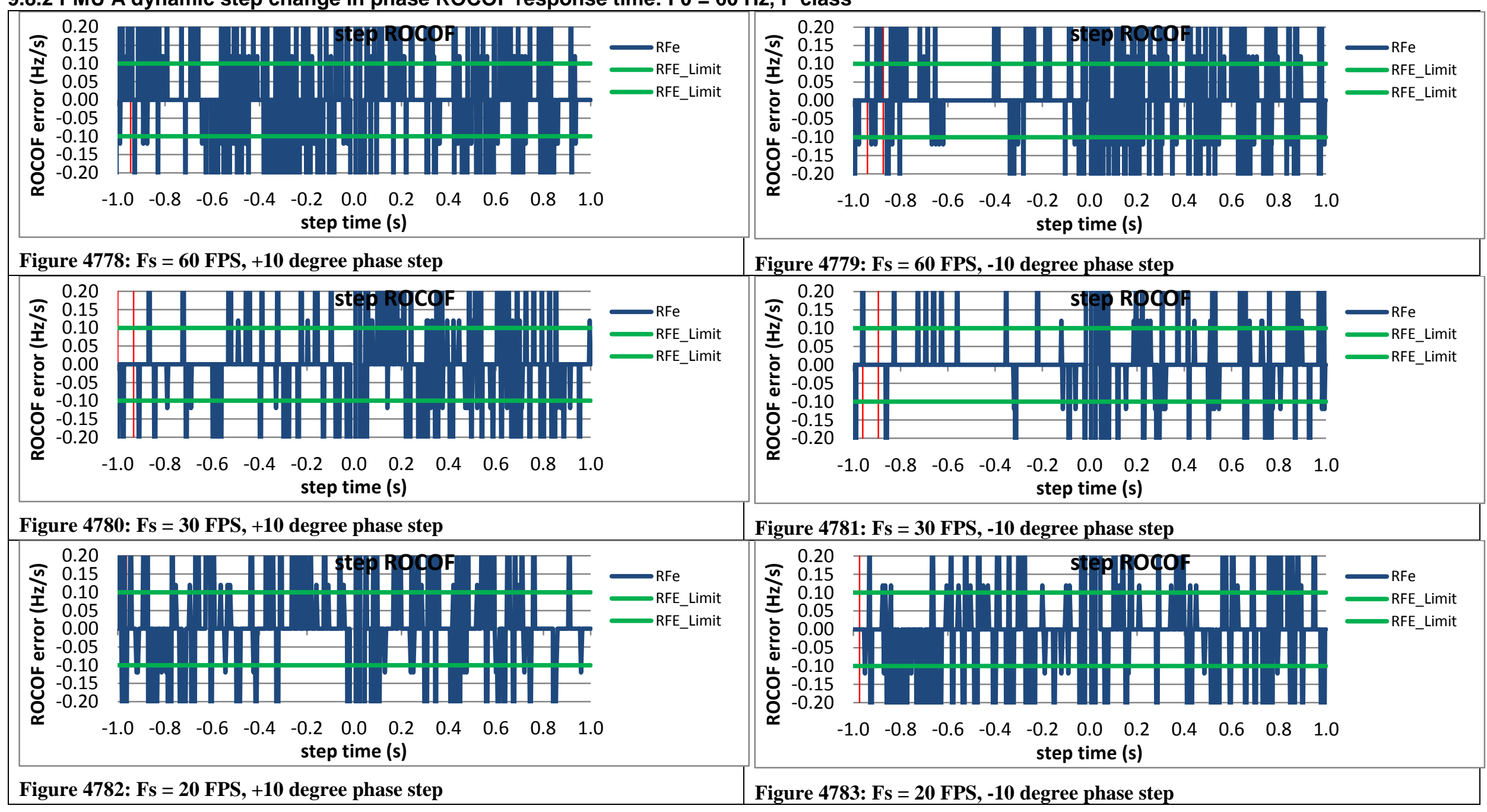




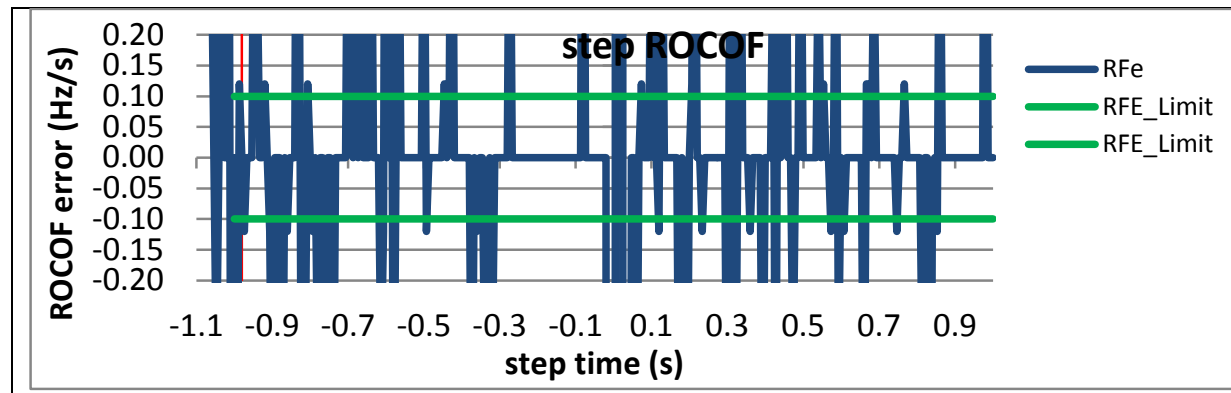

Figure 4784: Fs = 15 FPS, +10 degree phase step

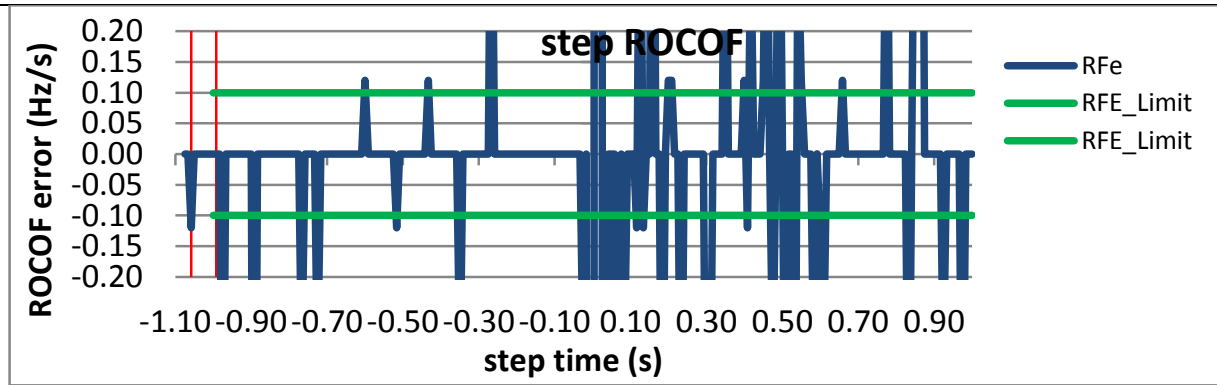

Figure 4786: Fs = 12 FPS, +10 degree phase step

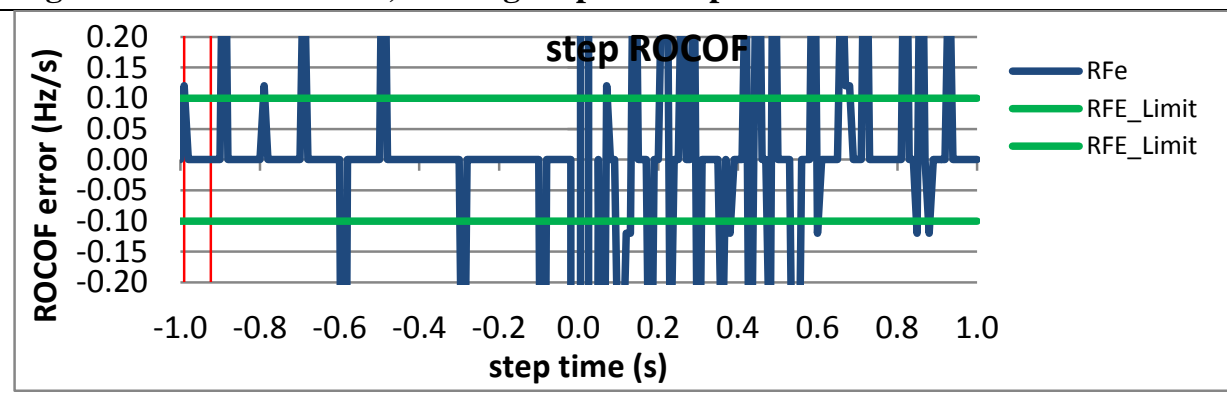

Figure 4788: Fs = 10 FPS, +10 degree phase step

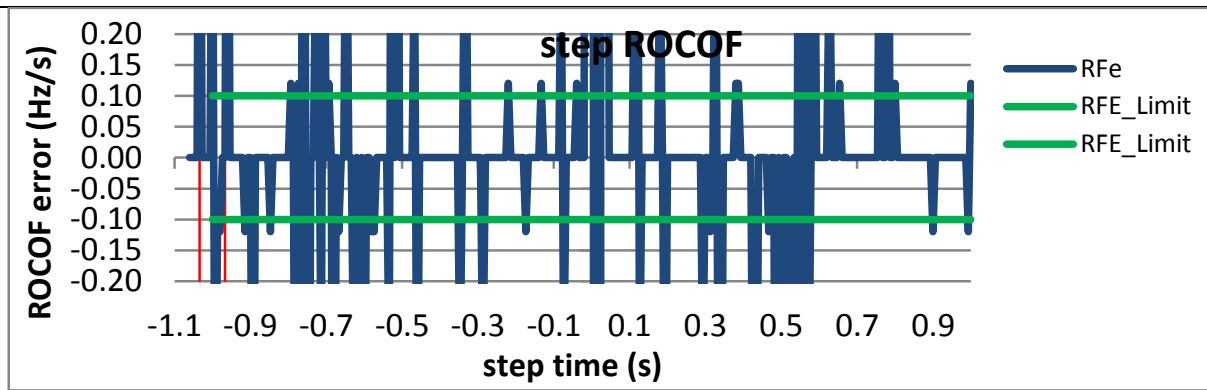

Figure 4785: Fs = 15 FPS, -10 degree phase step

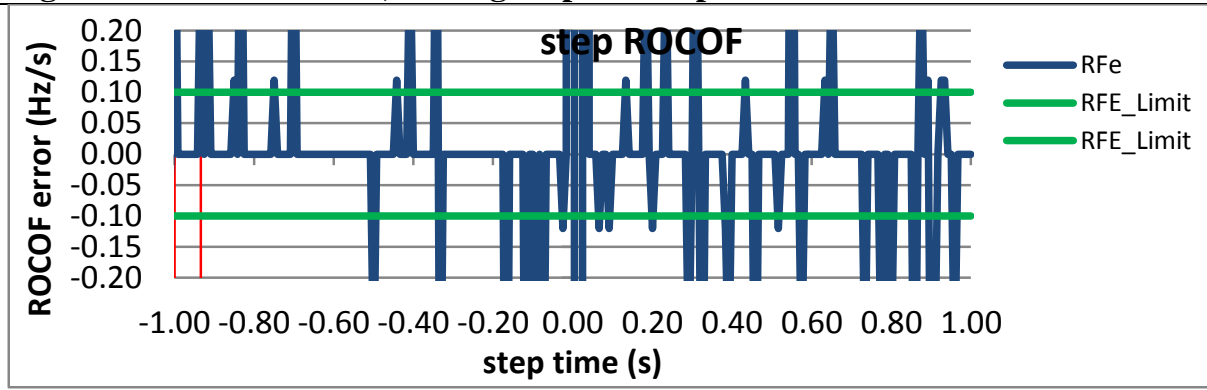

Figure 4787: Fs = 12 FPS, -10 degree phase step

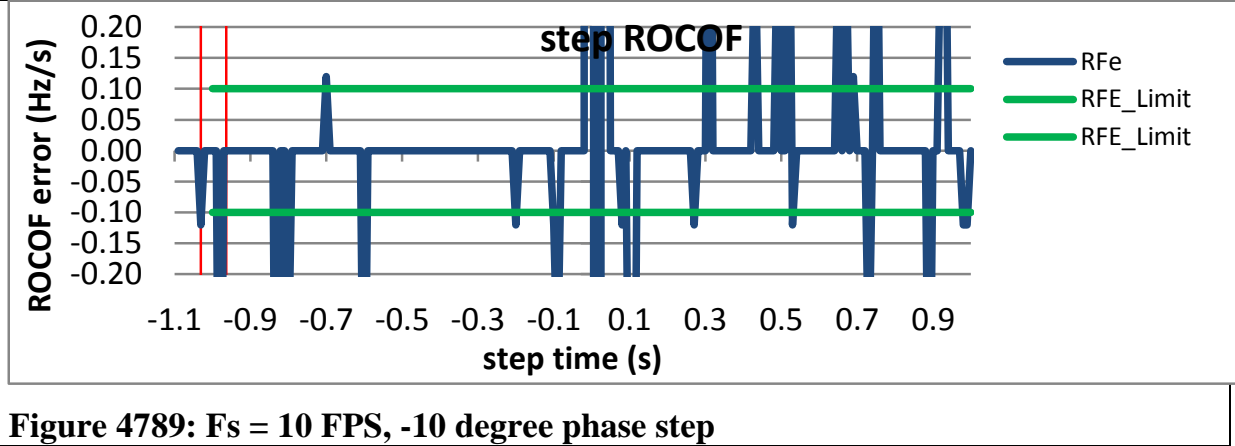


9.8.3 PMU B dynamic step change in phase ROCOF response time: $F 0=60 \mathrm{~Hz}, \mathrm{P}$ class
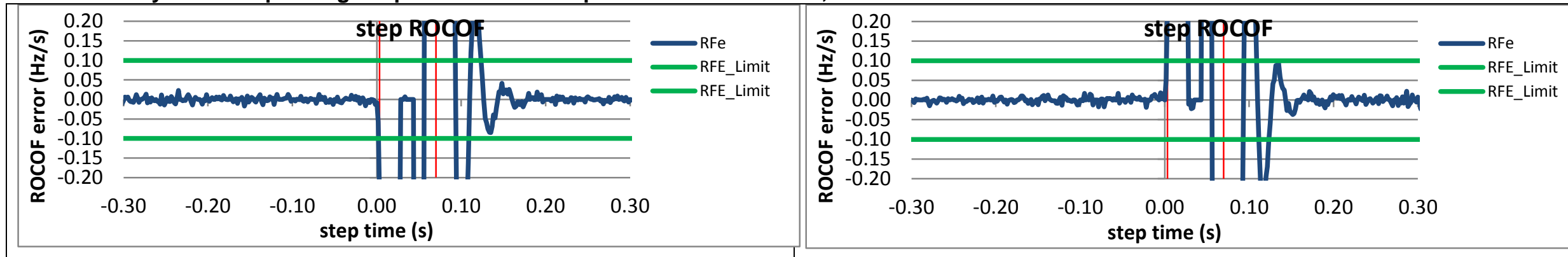

Figure 4790: Fs = 60 FPS, +10 degree phase step

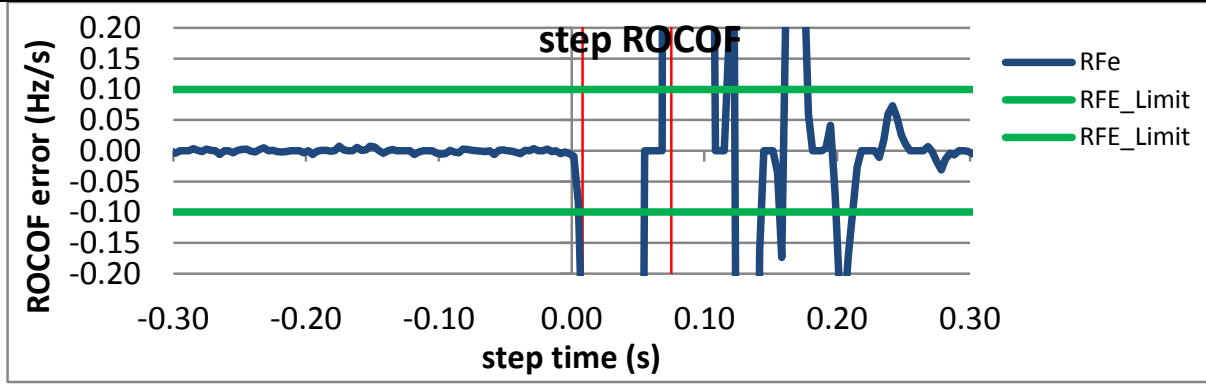

Figure 4791: Fs = 60 FPS, -10 degree phase step

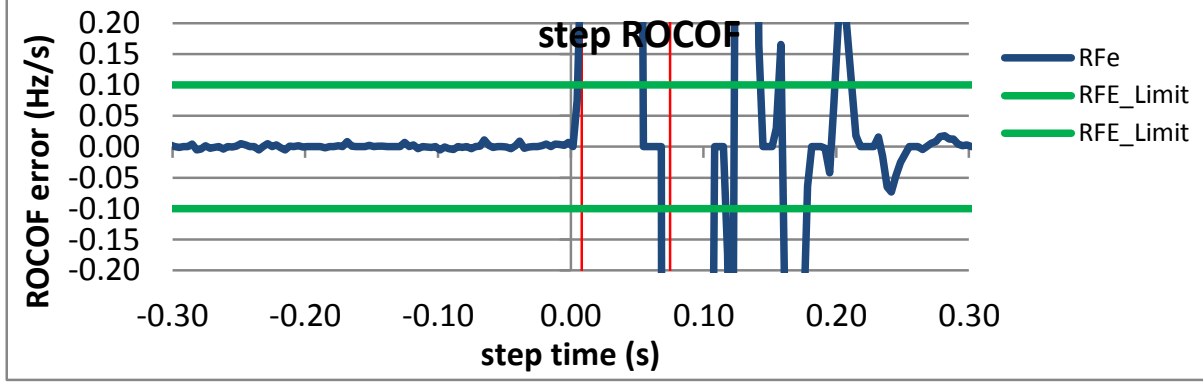

Figure 4793: Fs = 30 FPS, -10 degree phase step

Figure 4792: Fs = 30 FPS, +10 degree phase step

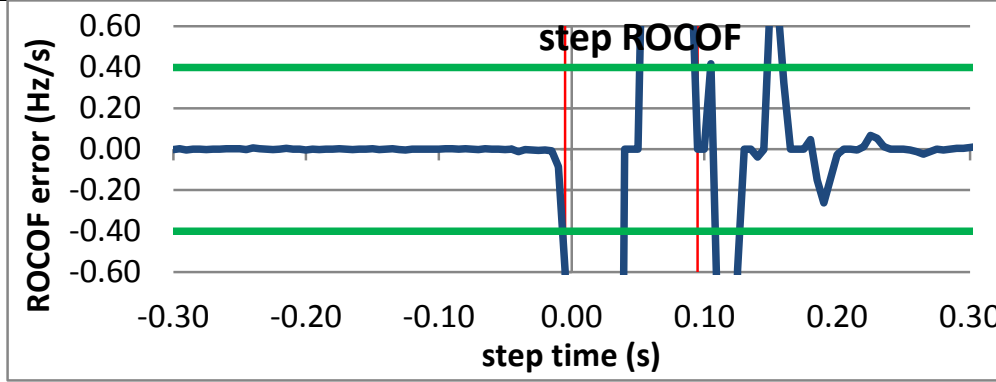

RFe
RFE_Limit
RFE_Limit

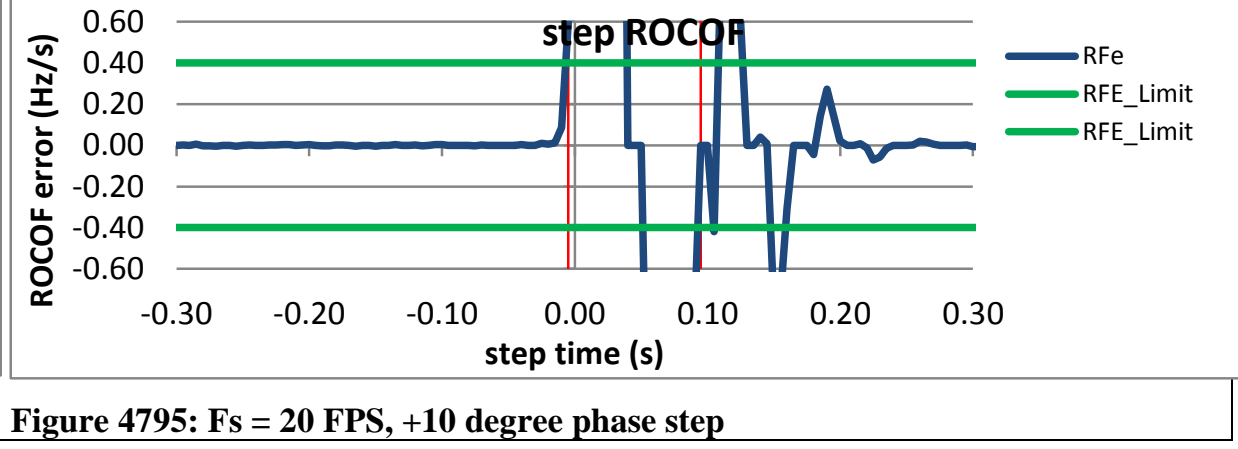

Figure 4794: Fs = 20 FPS, +10 degree phase step

Figure 4795: Fs = 20 FPS, +10 degree phase step 

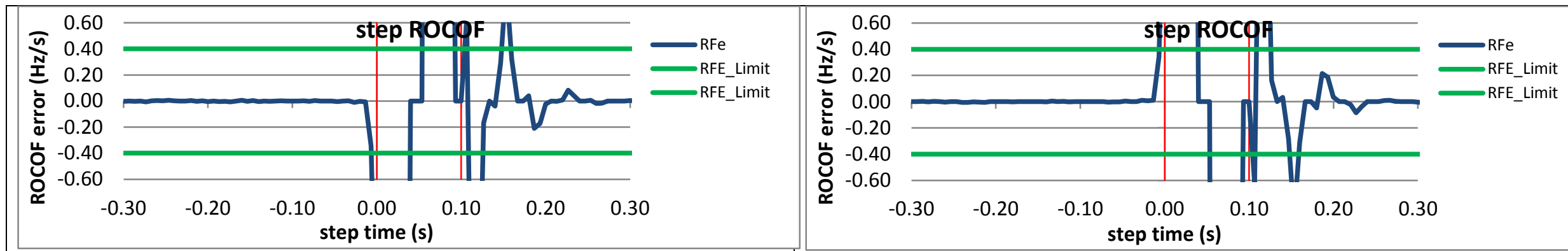

Figure 4796: $\mathrm{Fs}=15 \mathrm{FPS},+10$ degree phase step

Figure 4797: Fs = 15 FPS, - 10 degree phase step
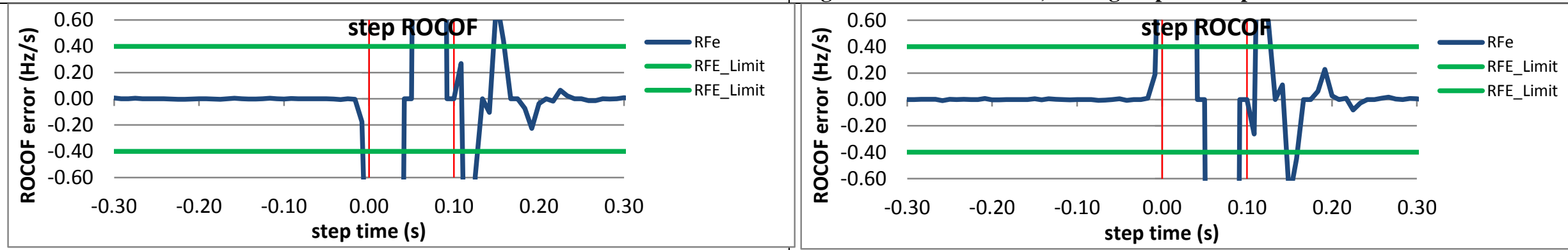

Figure 4798: Fs $=12$ FPS, +10 degree phase step

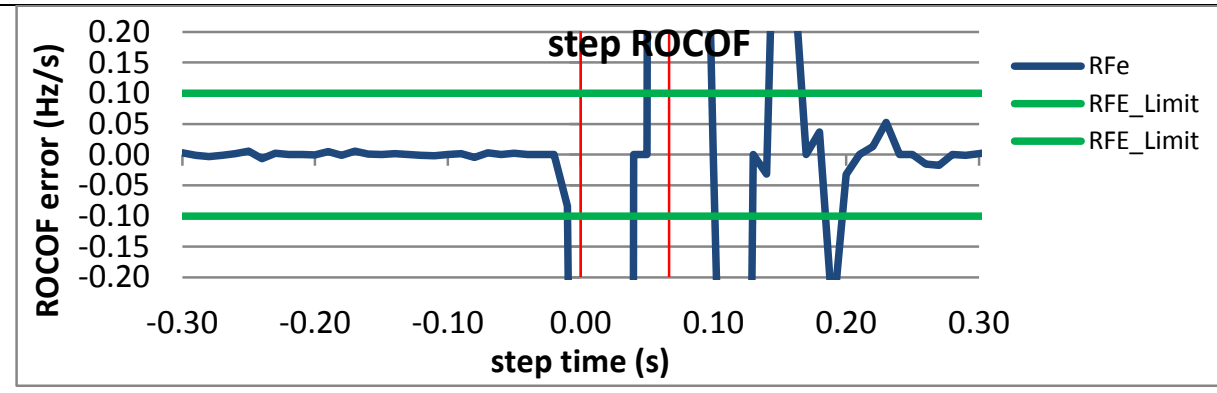

Figure 4799: Fs = 12 FPS, -10 degree phase step

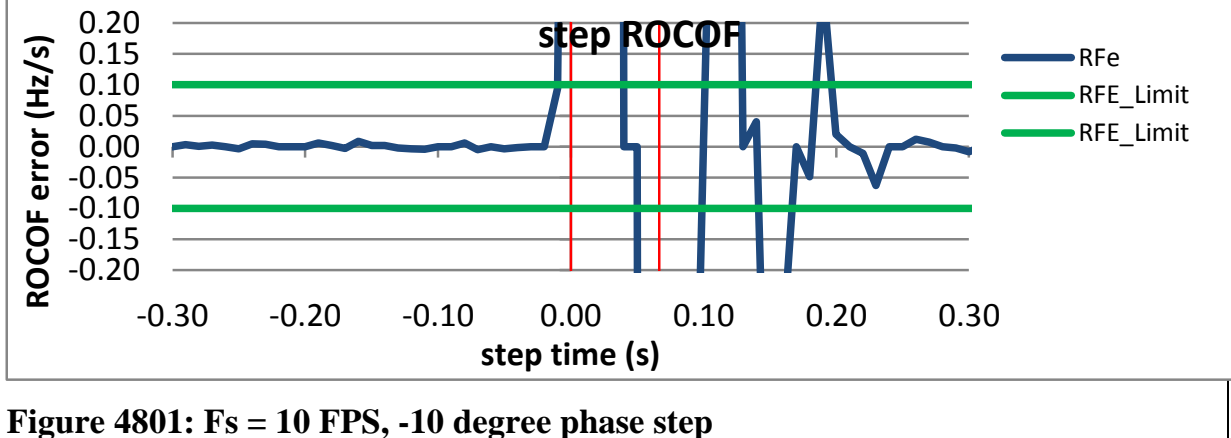

Figure 4800: Fs = 10 FPS, +10 degree phase step

Figure 4801: Fs = 10 FPS, -10 degree phase step 
9.8.4 PMU C dynamic step change in phase ROCOF response time: $F 0=60 \mathrm{~Hz}, P$ class

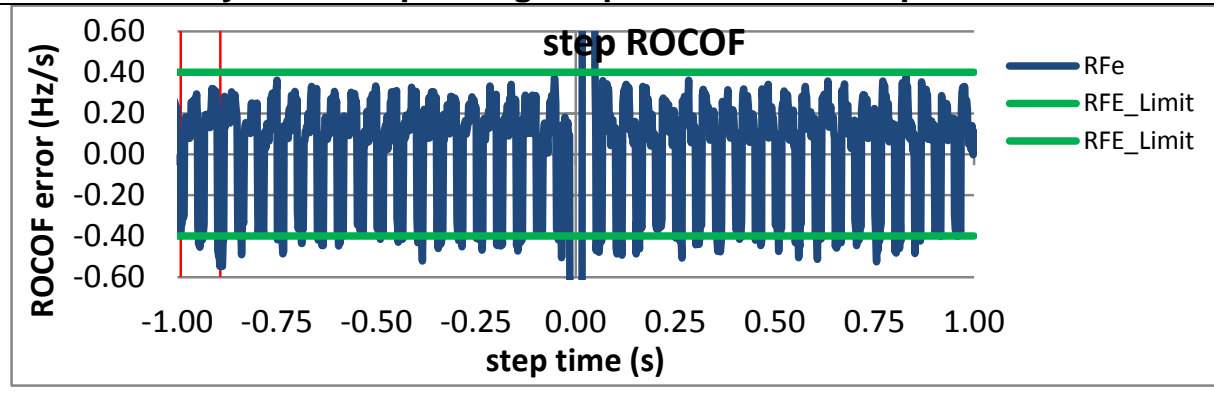

Figure 4802: Fs = 60 FPS, +10 degree phase step

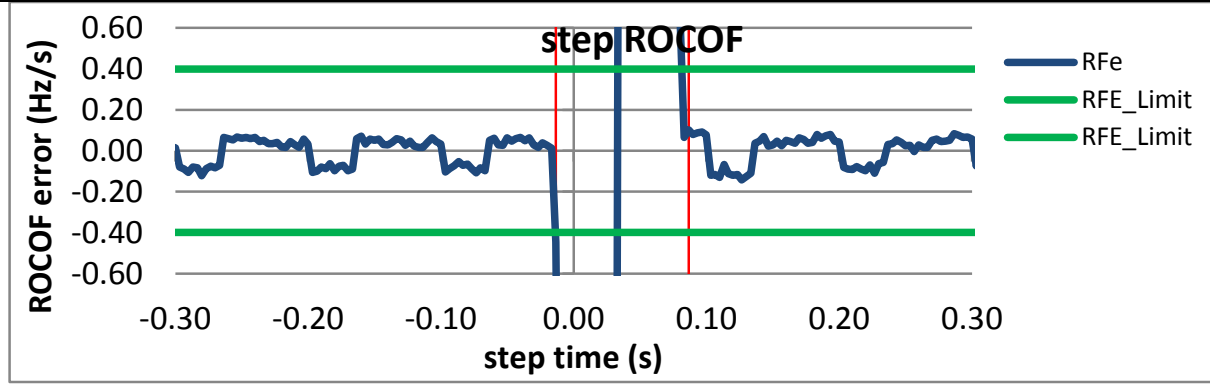

Figure 4804: Fs = 30 FPS, +10 degree phase step

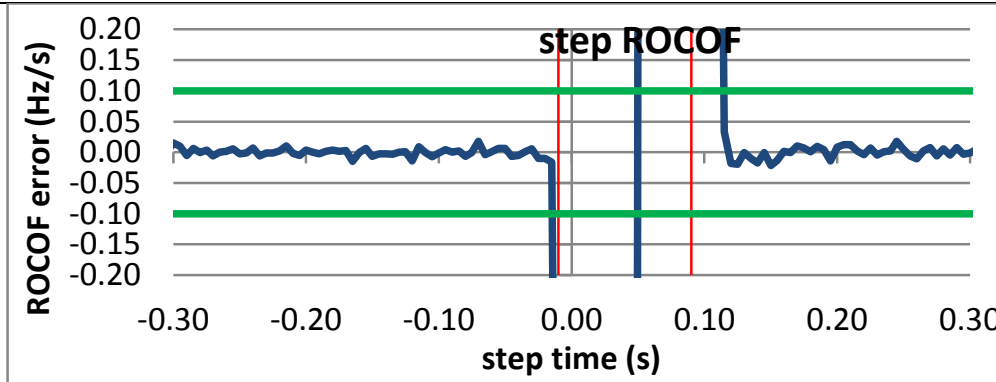

Figure 4806: Fs = 20 FPS, +10 degree phase step

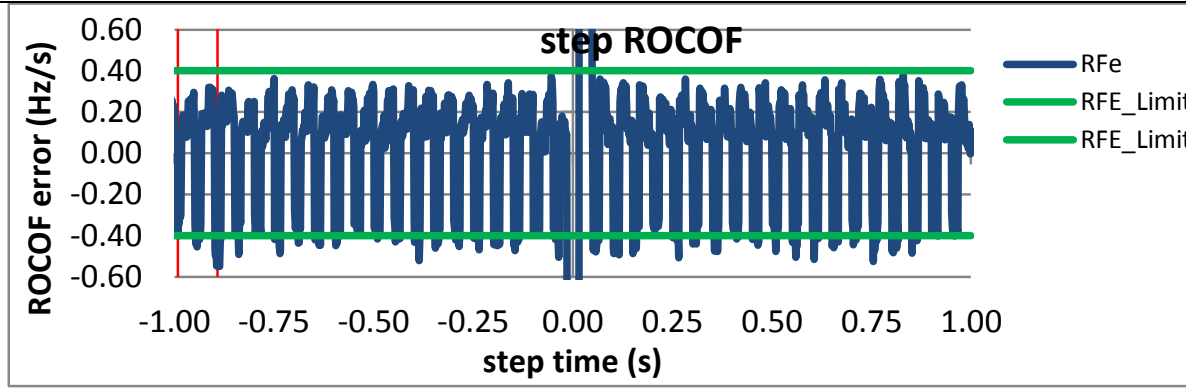

Figure 4803: Fs = 60 FPS, -10 degree phase step

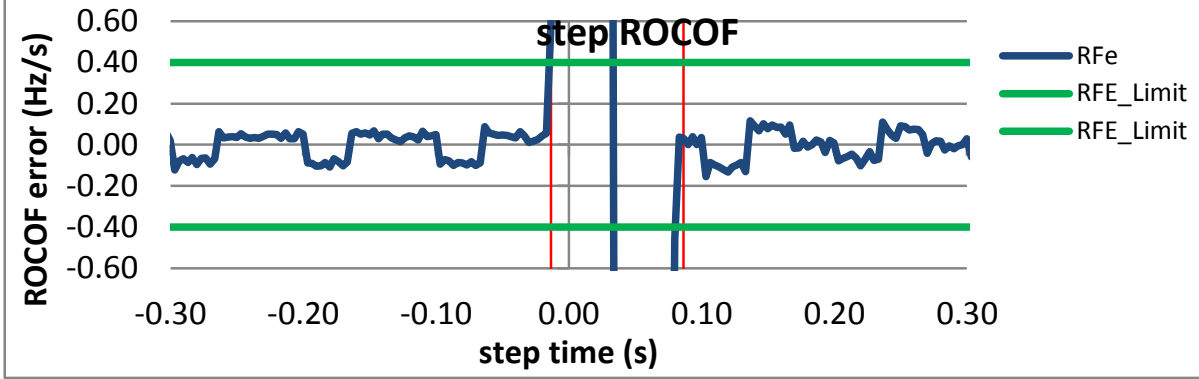

Figure 4805: Fs = 30 FPS, -10 degree phase step

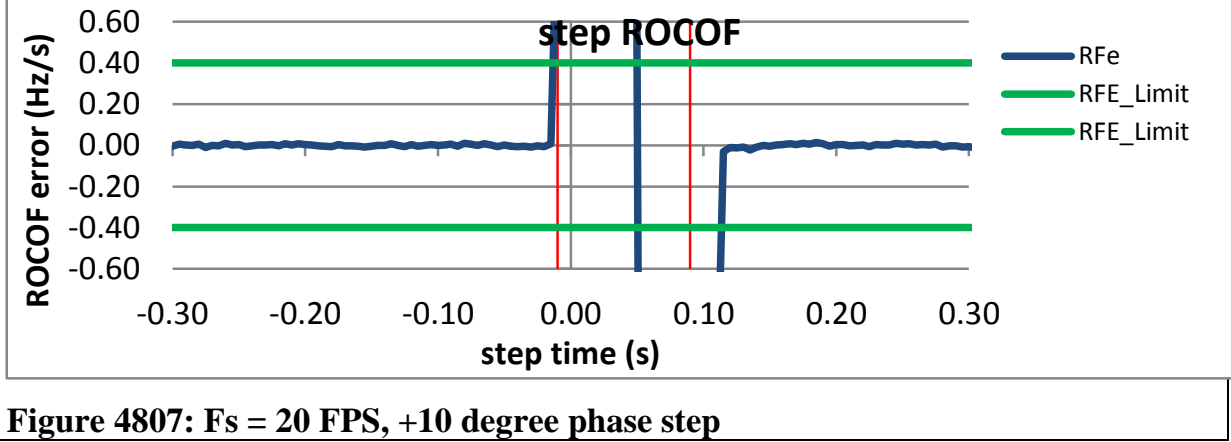



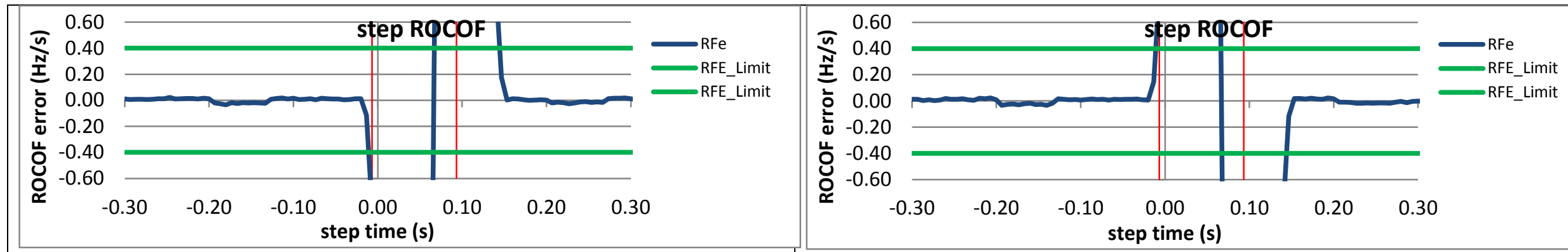

Figure 4808: Fs = 15 FPS, +10 degree phase step

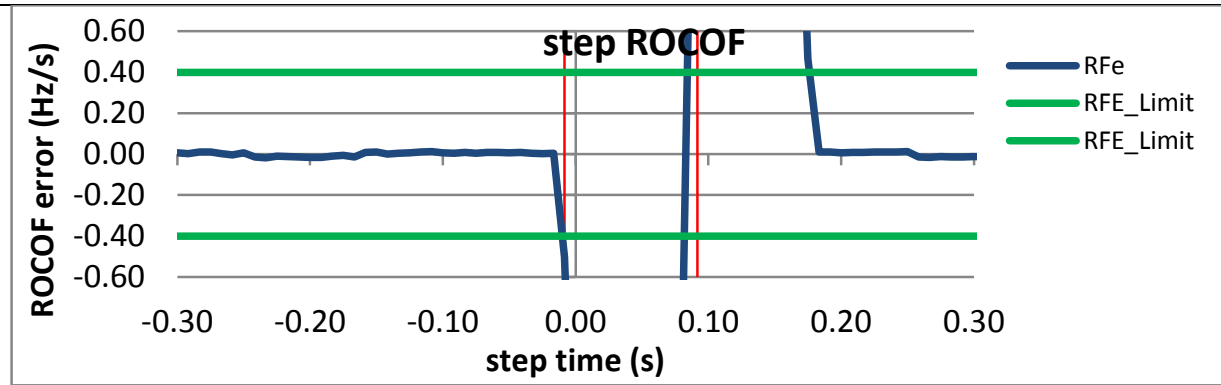

Figure 4809: Fs = 15 FPS, -10 degree phase step

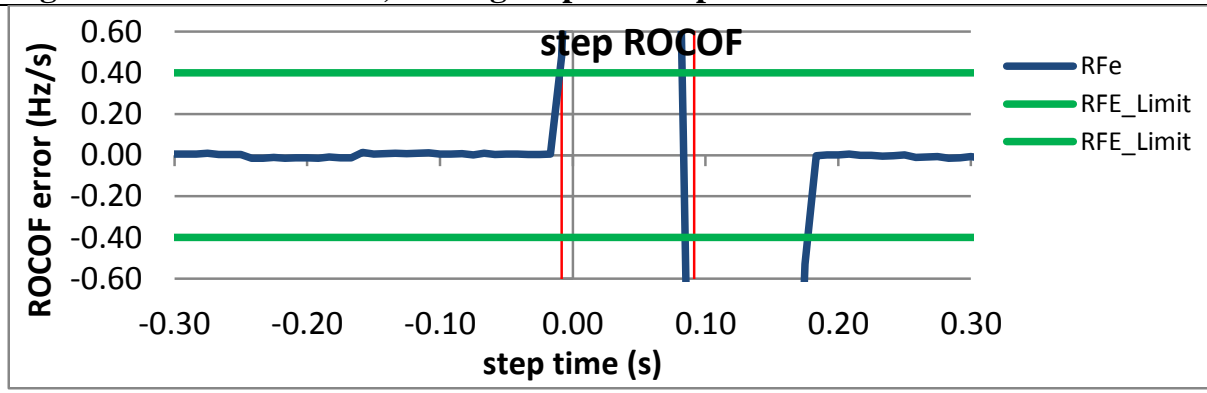

Figure 4810: Fs $=12$ FPS, +10 degree phase step

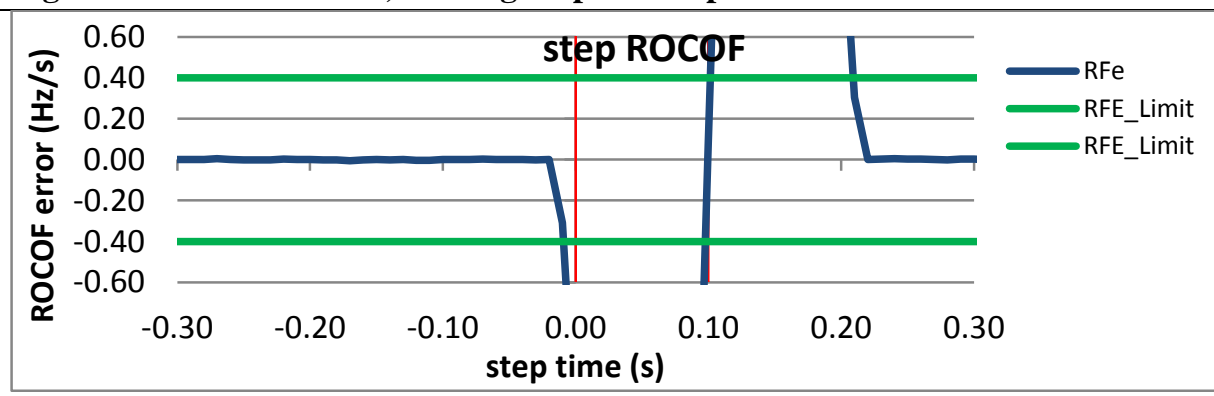

Figure 4811: Fs = 12 FPS, -10 degree phase step

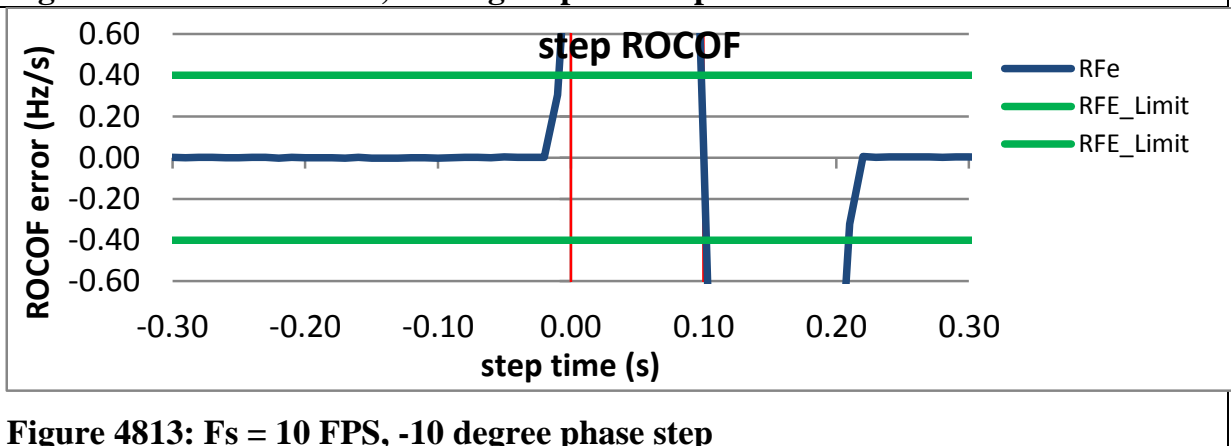

Figure 4812: Fs = 10 FPS, +10 degree phase step

Figure 4813: Fs = 10 FPS, -10 degree phase step 
9.8.5 PMU D dynamic step change in phase ROCOF response time: $F 0=60 \mathrm{~Hz}, P$ class

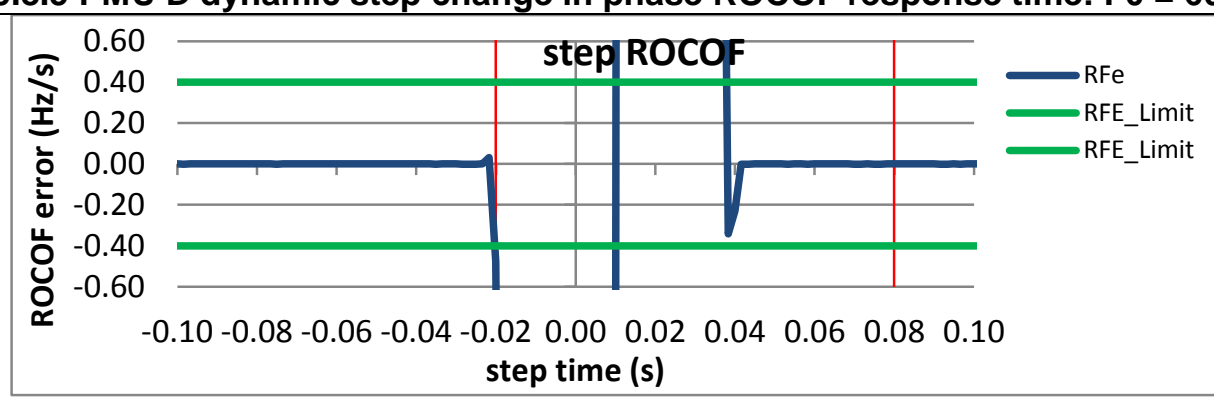

Figure 4814: Fs = 60 FPS, +10 degree phase step

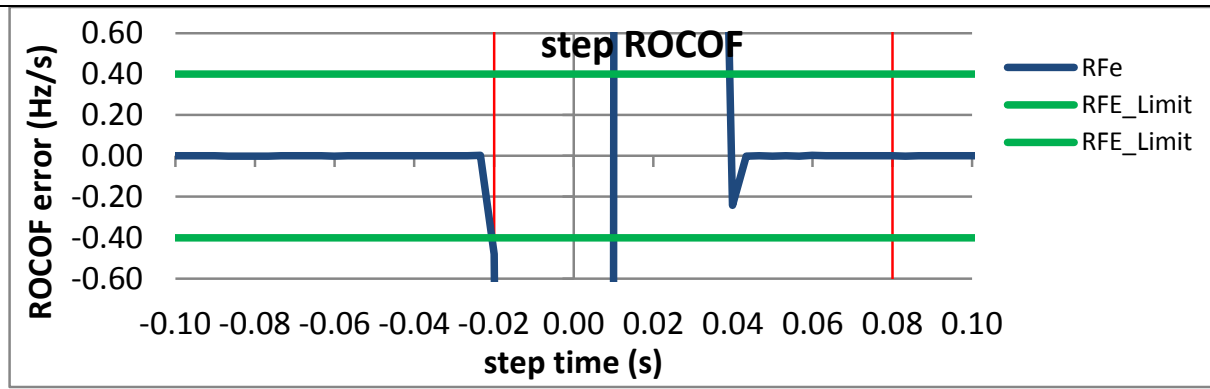

Figure 4815: Fs = 30 FPS, +10 degree phase step

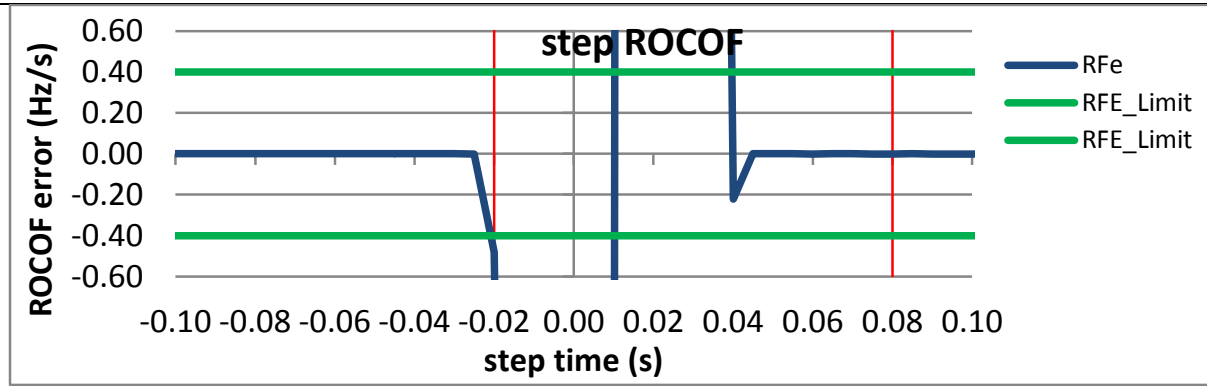

Figure 4817: Fs = 20 FPS, +10 degree phase step

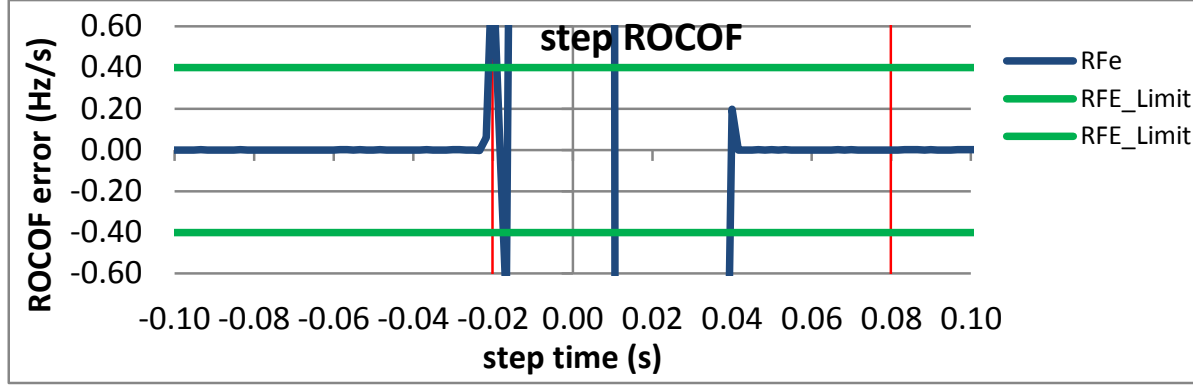

Figure 4814: Fs = 60 FPS, -10 degree phase step

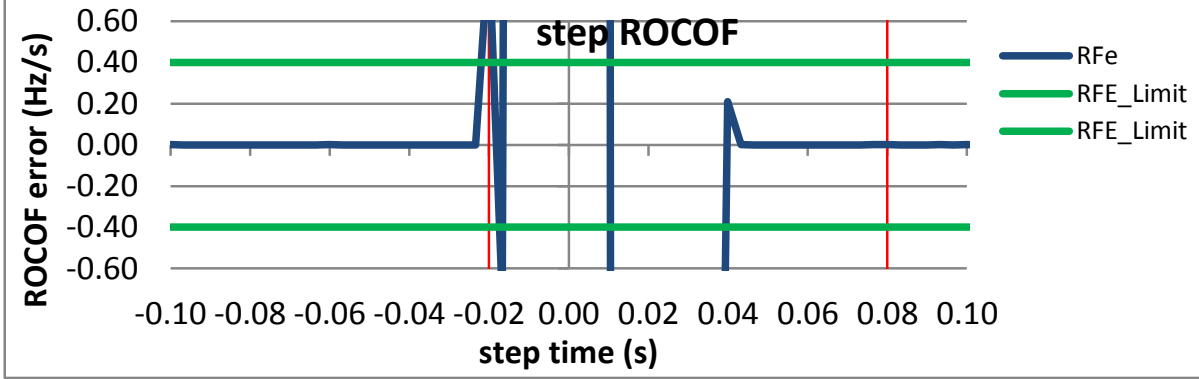

Figure 4816: Fs = 30 FPS, -10 degree phase step

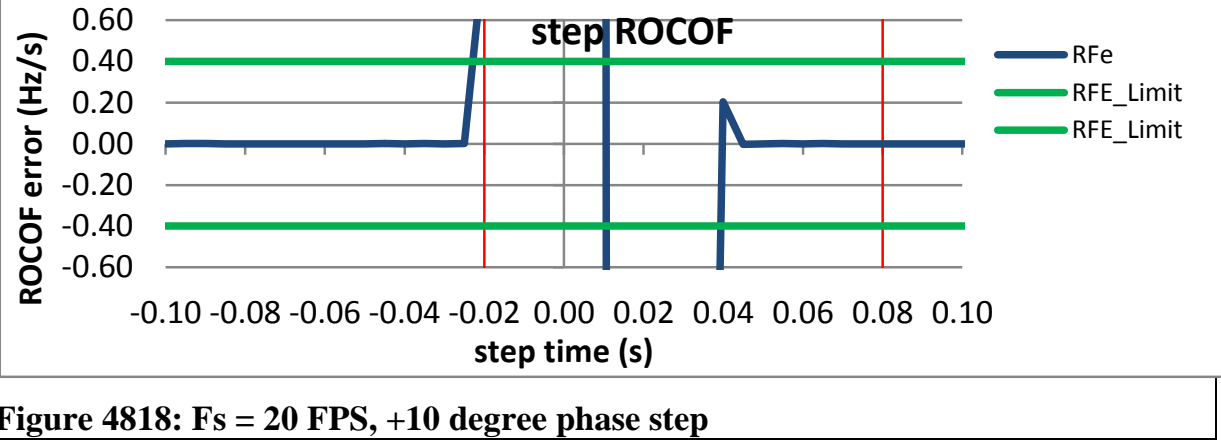




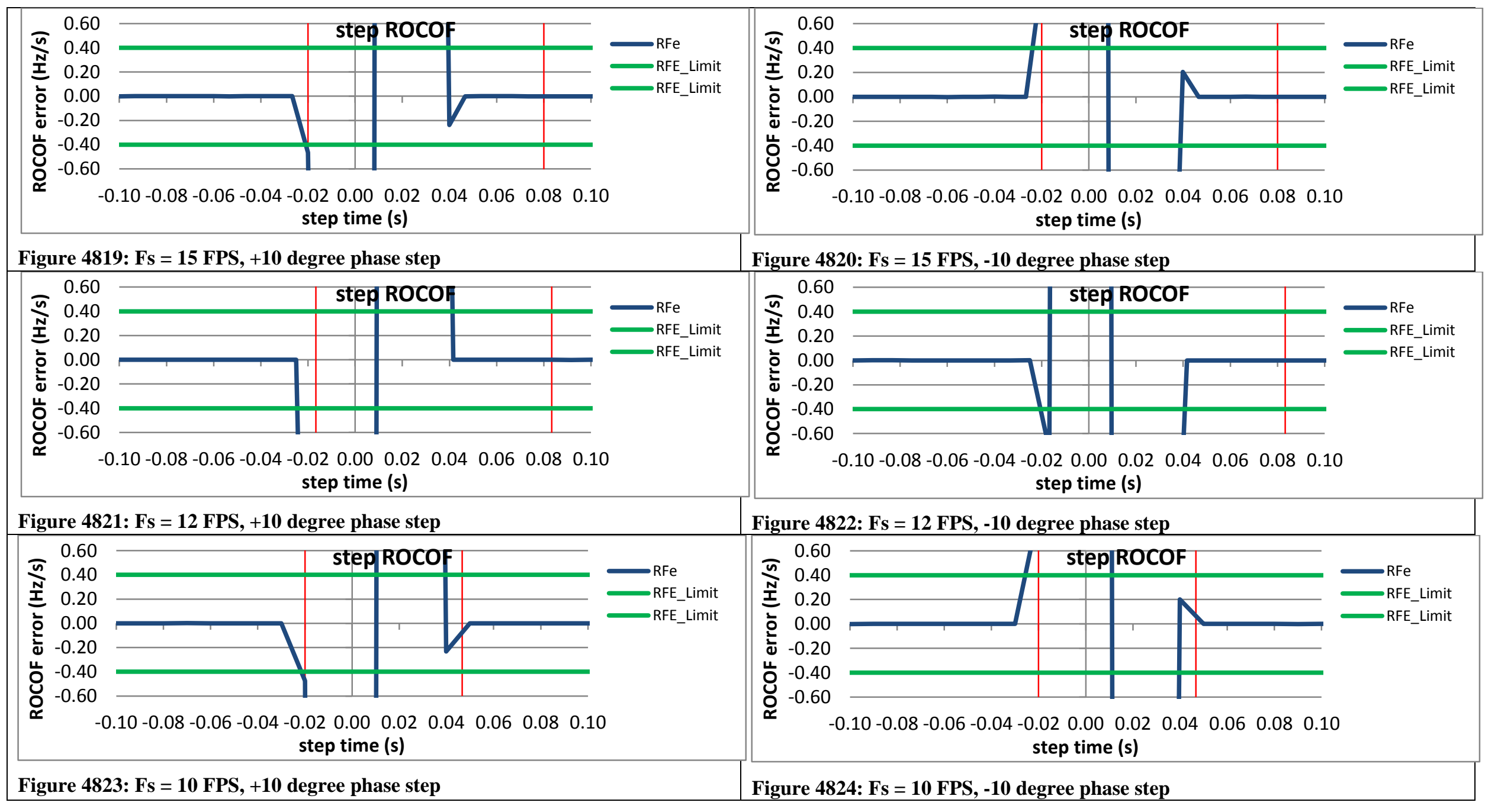


9.8.6 PMU E dynamic step change in phase ROCOF response time: $\mathrm{F} 0=60 \mathrm{~Hz}, \mathrm{P}$ class PMU E does not support P class.

9.8.7 PMU F dynamic step change in phase ROCOF response time: $\mathrm{FO}=60 \mathrm{~Hz}, \mathrm{P}$ class

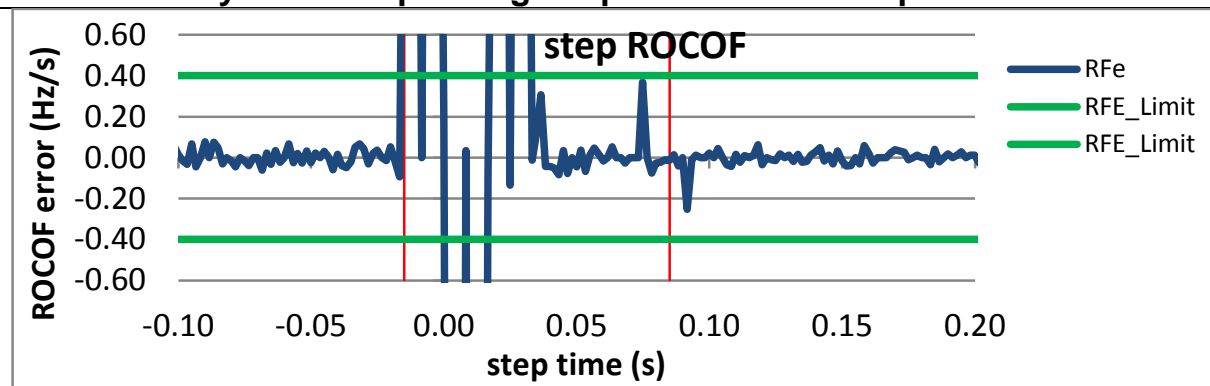

Figure 4825: Fs = 60 FPS, +10\% magnitude step

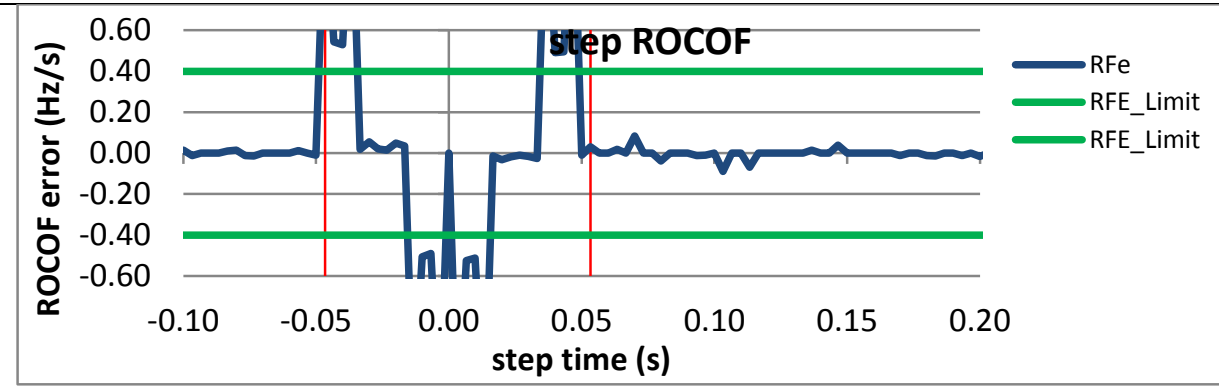

Figure 4827: Fs $=30$ FPS, $+10 \%$ magnitude step

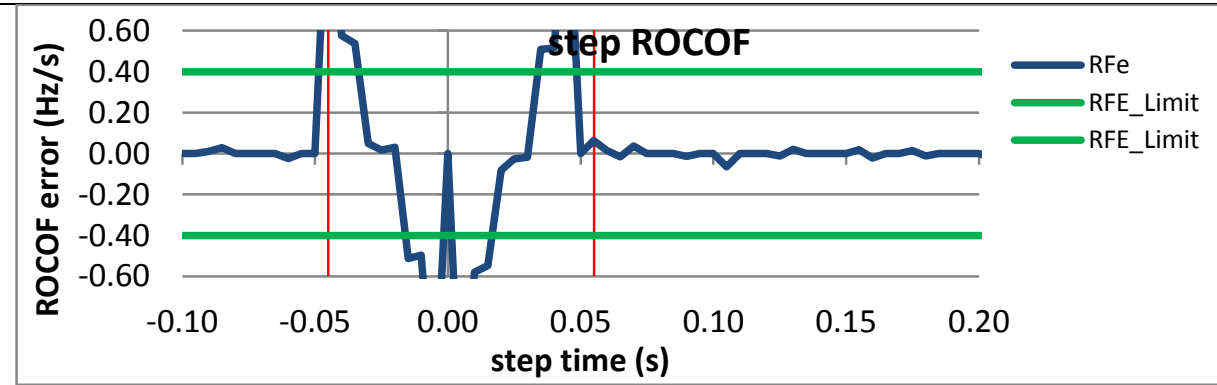

Figure 4829: Fs = 20 FPS, +10\% magnitude step

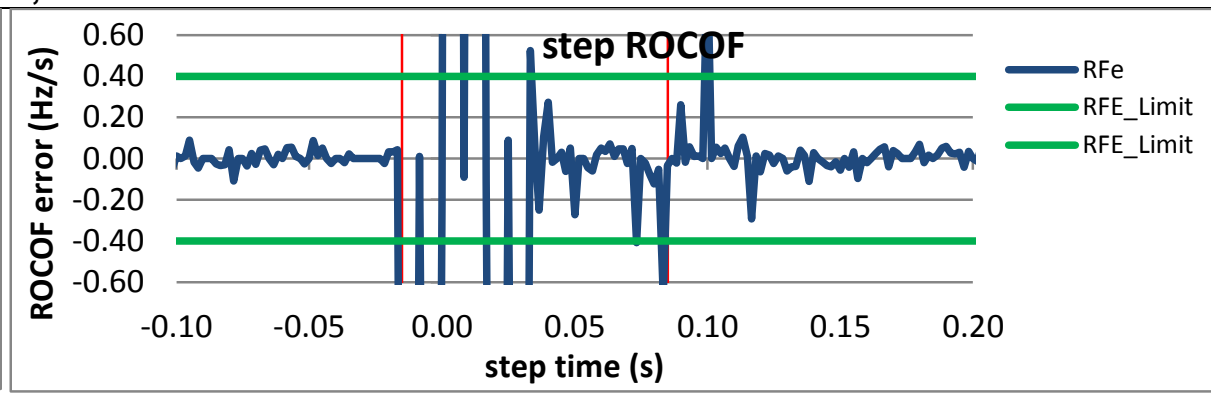

Figure 4826: Fs = 60 FPS, -10\% magnitude step

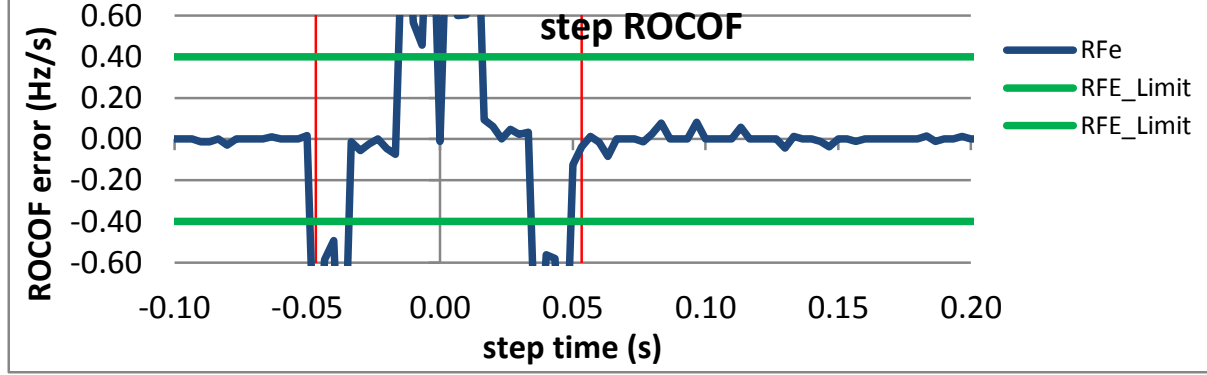

Figure 4828: Fs = 30 FPS, -10\% magnitude step

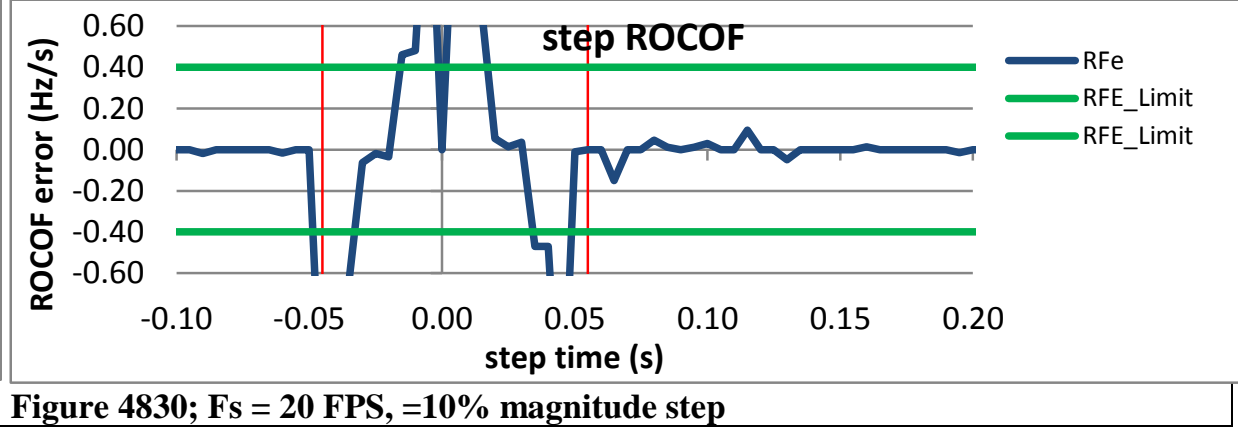



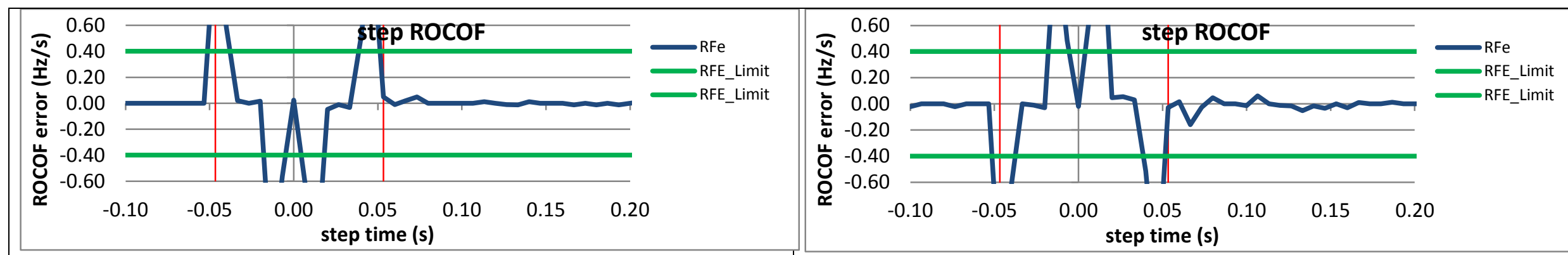

Figure 4831: Fs = 15 FPS, + 10\% magnitude step

Figure 4832: Fs = 15 FPS, - $10 \%$ magnitude step
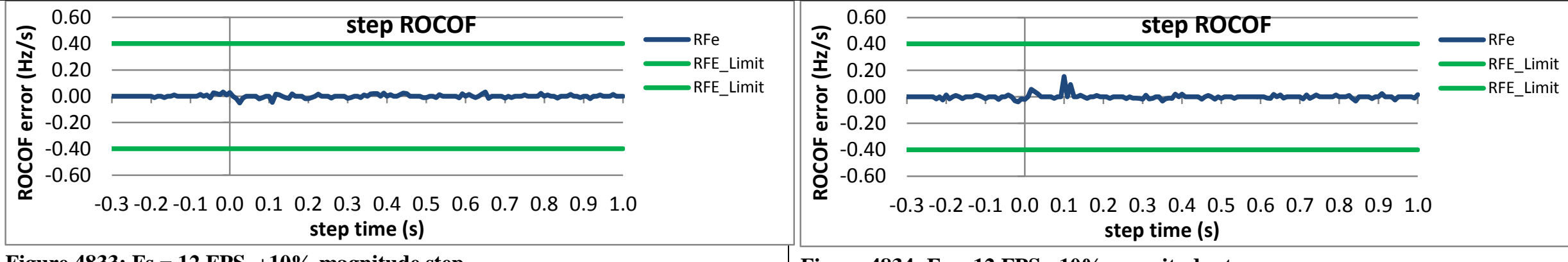

Figure 4833: Fs = 12 FPS, +10\% magnitude step

Figure 4834: Fs = 12 FPS, -10\% magnitude step

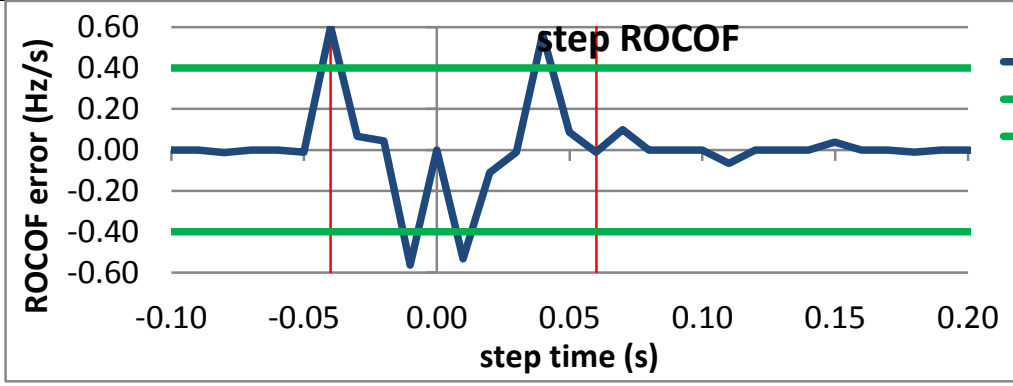

RFe
RFE_Limit
RFE_Limit

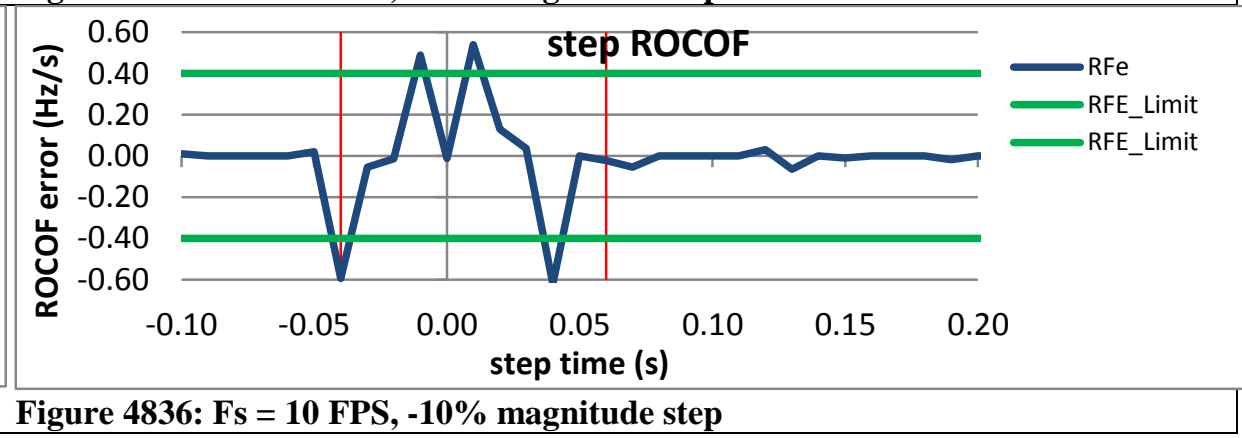

Figure 4835: Fs = 10 FPS, $+10 \%$ magnitude step

Figure 4836: Fs = 10 FPS, -10\% magnitude step 
9.8.8 PMU G dynamic step change in phase ROCOF response time: $F 0=60 \mathrm{~Hz}$, P class PMU G does not support P class.

9.8.9 PMU H dynamic step change in phase ROCOF response time: $\mathrm{F} 0=60 \mathrm{~Hz}$, P class
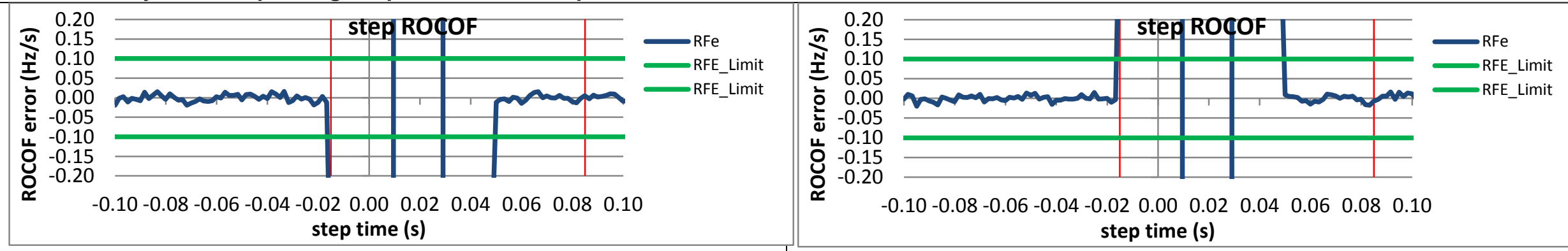

Figure 4837: Fs = 60 FPS, +10 degree phase step

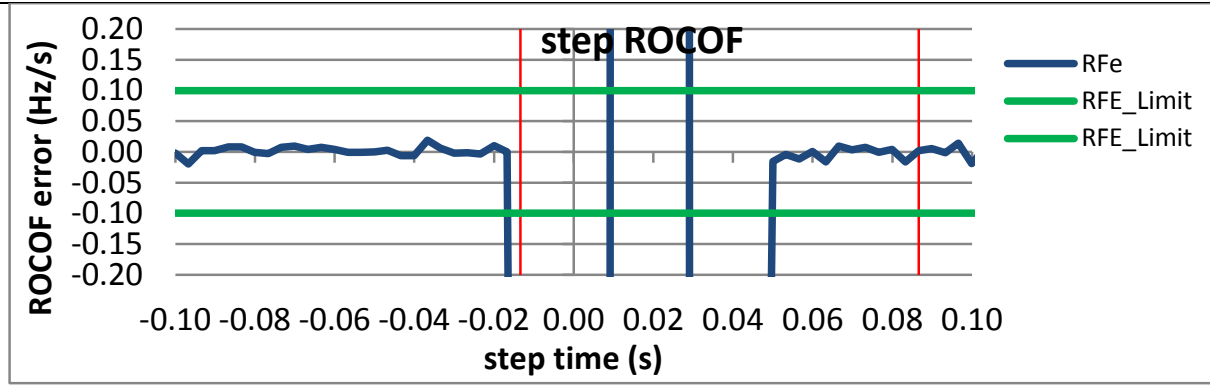

Figure 4838: Fs = 60 FPS, -10 degree phase step

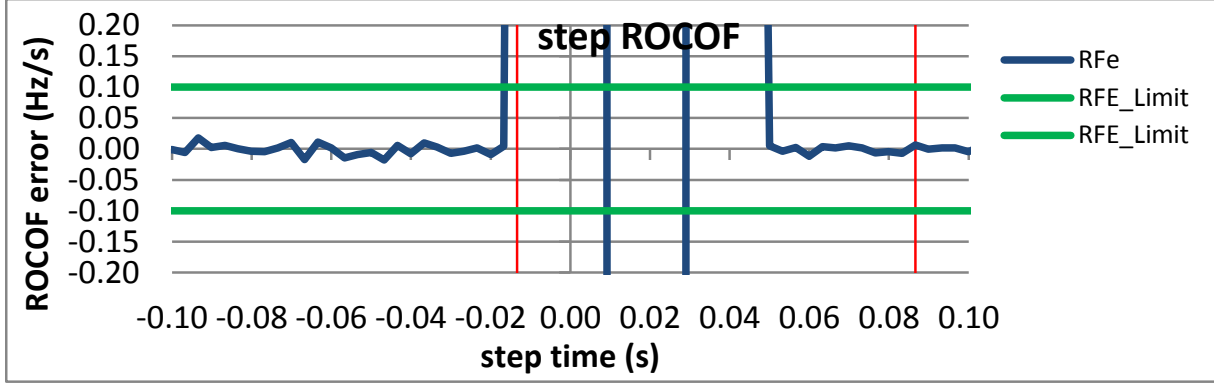

Figure 4839: Fs = 30 FPS, +10 degree phase step

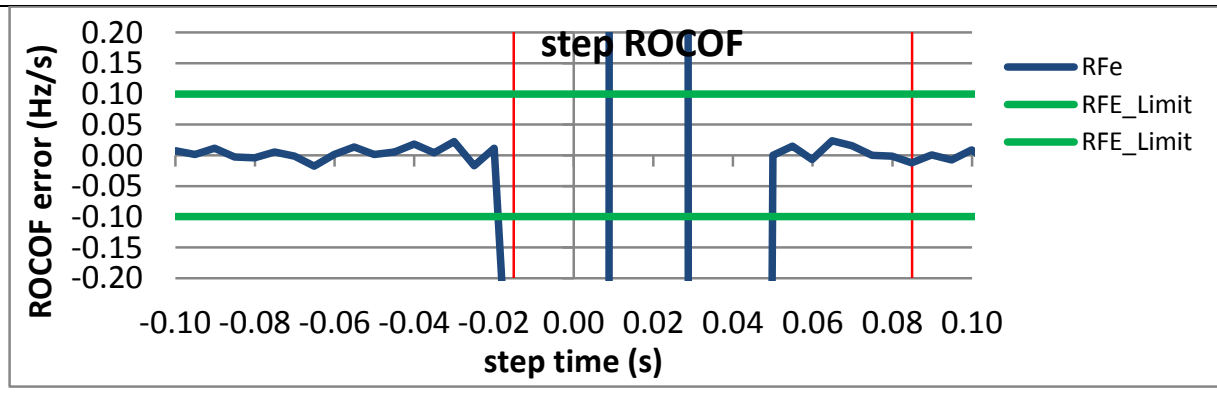

Figure 4840: Fs = 30 FPS, -10 degree phase step

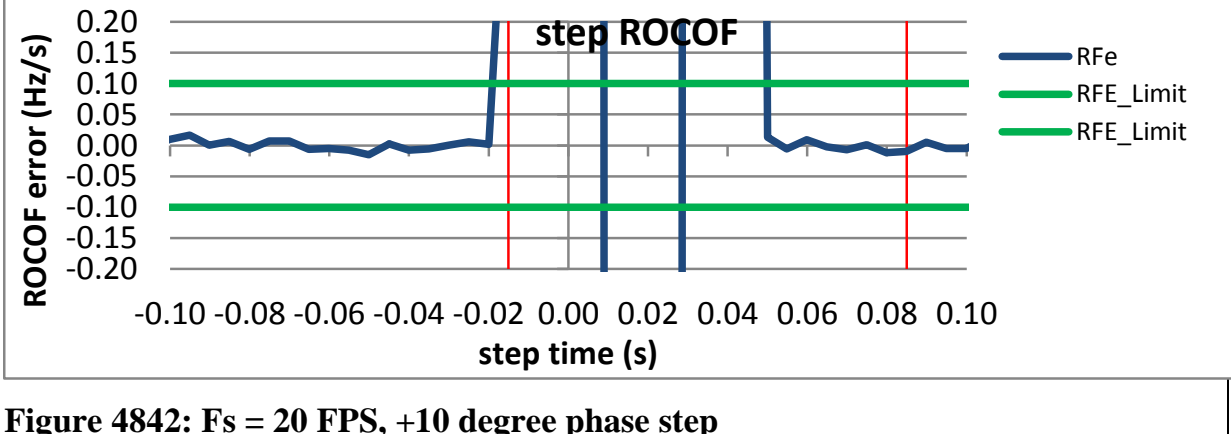

Figure 4841: Fs = 20 FPS, +10 degree phase step

Figure 4842: Fs $=20$ FPS, +10 degree phase step 


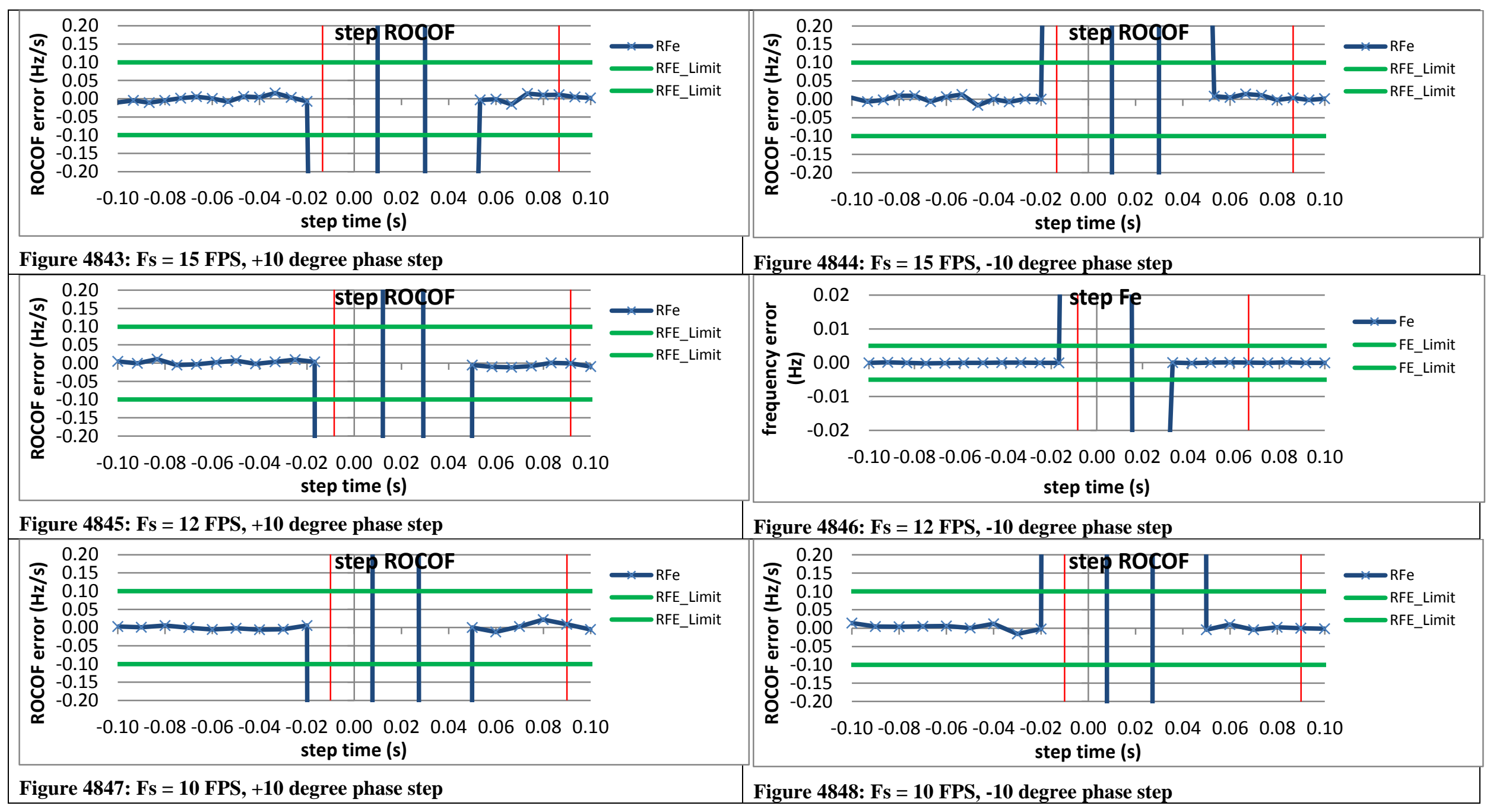

9.8.10 PMU I dynamic step change in phase ROCOF response time: $\mathrm{FO}=60 \mathrm{~Hz}, \mathrm{P}$ class

PMU I does no support P class 
9.8.11 PMU J dynamic step change in phase ROCOF response time: $\mathrm{FO}=60 \mathrm{~Hz}, \mathrm{P}$ class

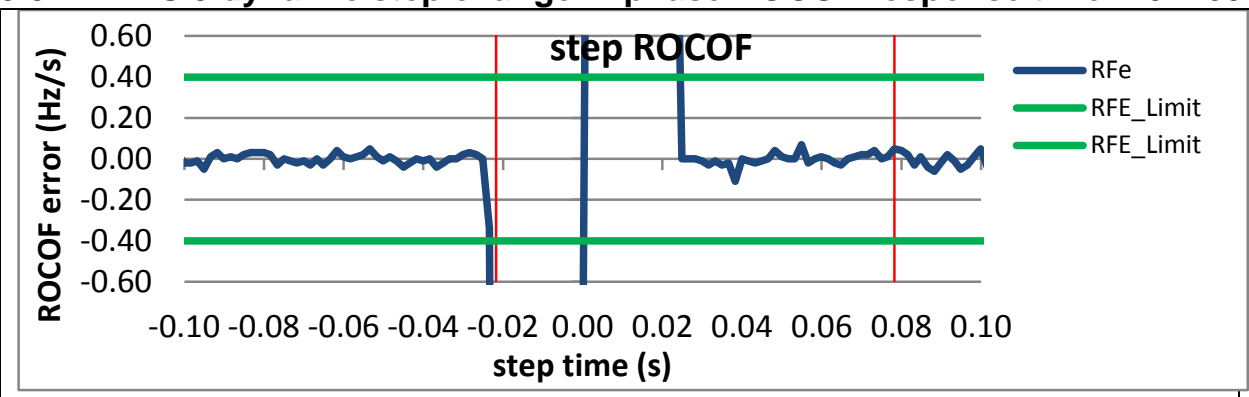

Figure 4849: Fs = 60 FPS, +10 degree phase step

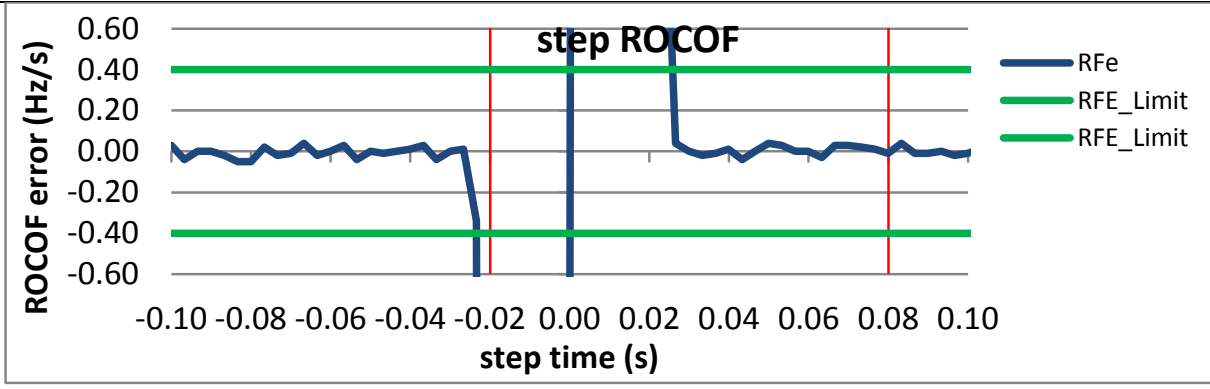

Figure 4851: Fs = 30 FPS, +10 degree phase step

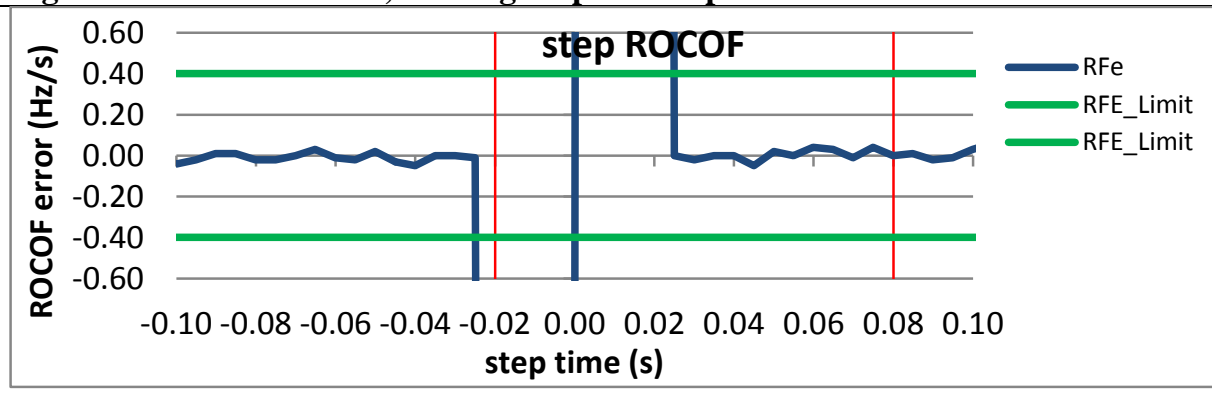

Figure 4853: Fs = 20 FPS, +10 degree phase step

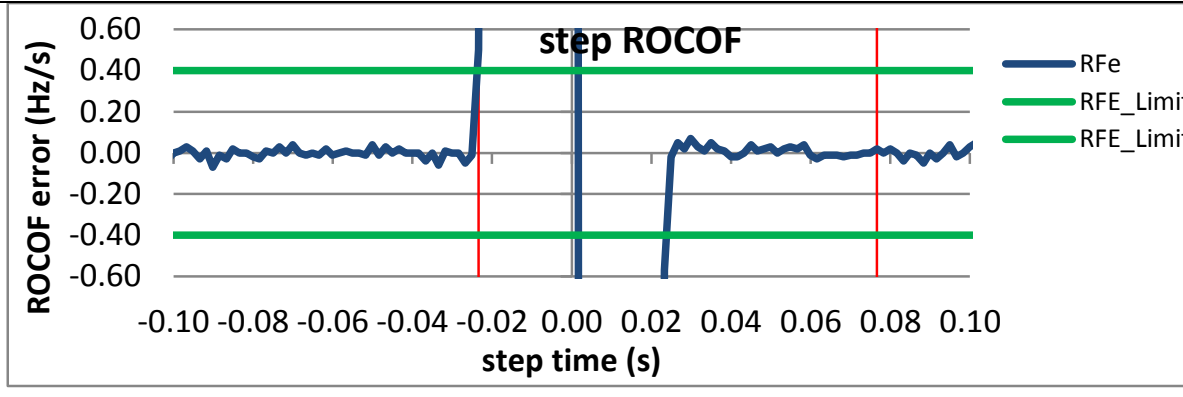

Figure 4850: Fs = 60 FPS, -10 degree phase step

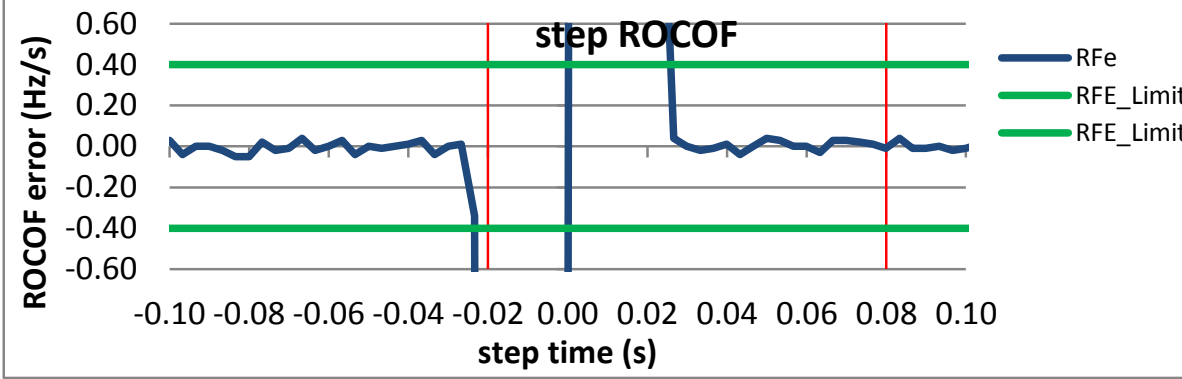

Figure 4852: Fs = 30 FPS, -10 degree phase step

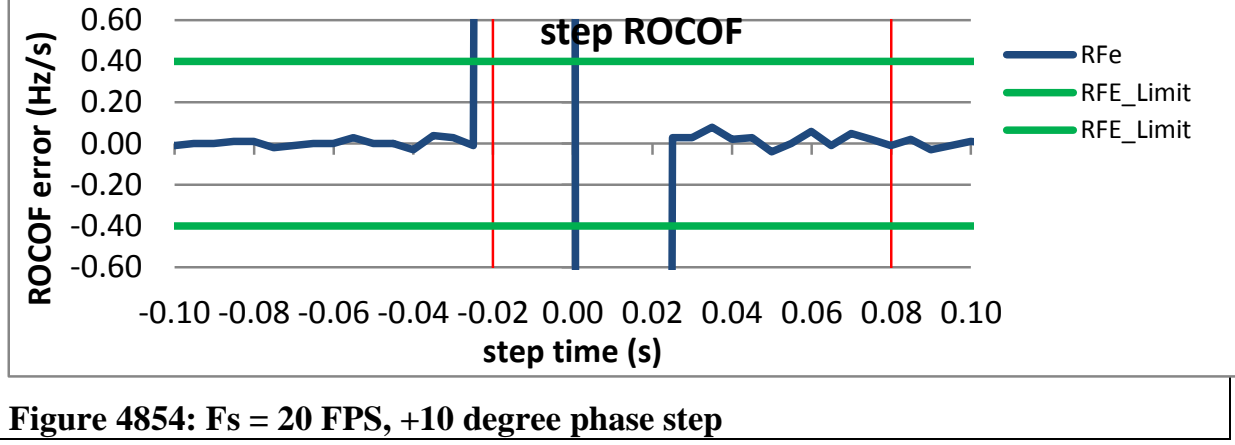



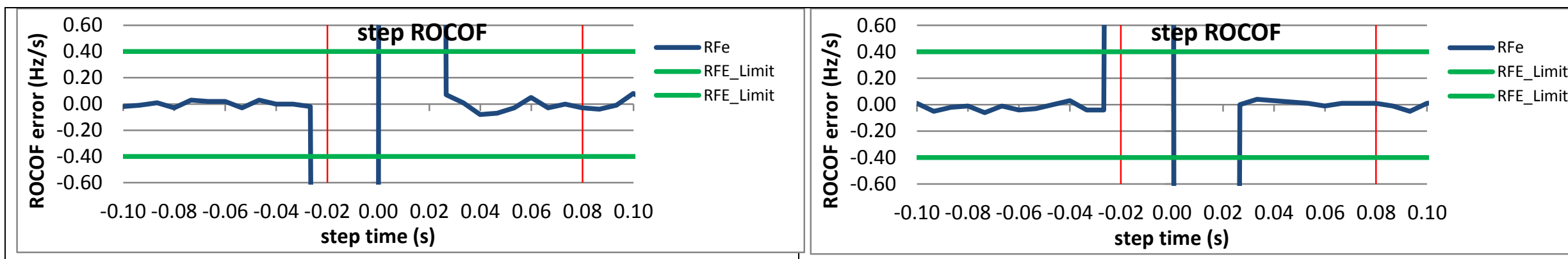

Figure 4855: Fs = 15 FPS, +10 degree phase step

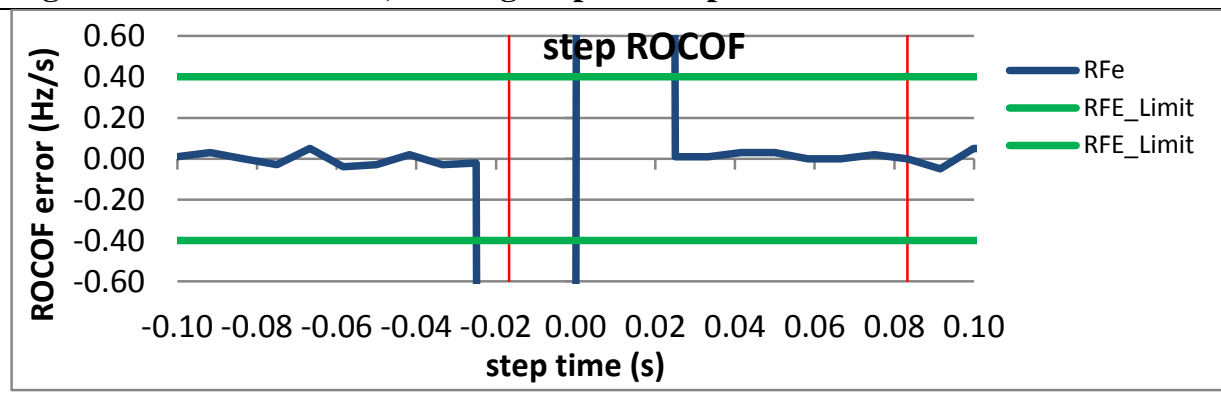

Figure 4856: Fs = 15 FPS, -10 degree phase step

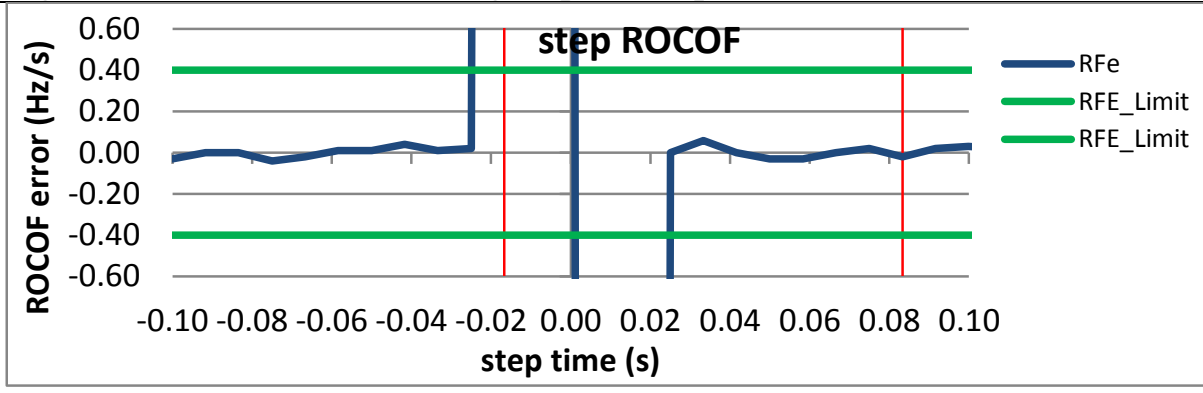

Figure 4857: Fs = 12 FPS, +10 degree phase step

Figure 4858: Fs = 12 FPS, -10 degree phase step

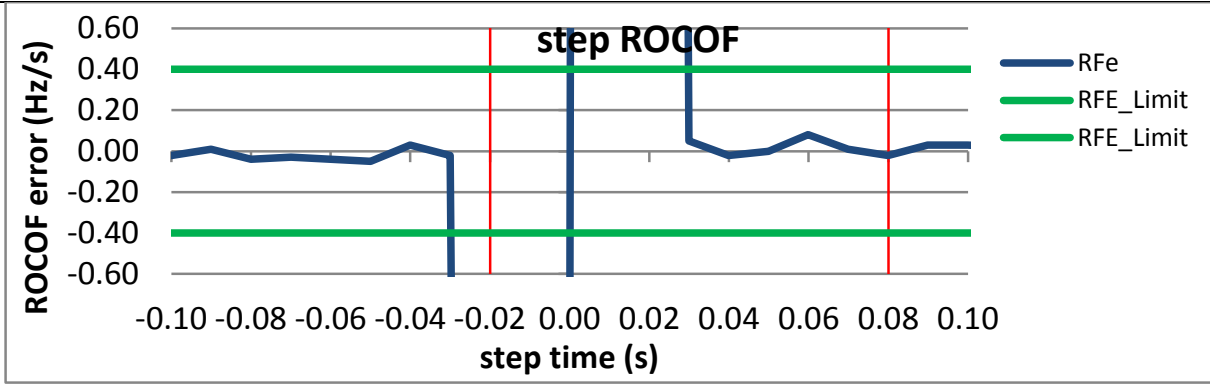

Figure 4859: Fs = 10 FPS, +10 degree phase step

\begin{tabular}{|c|c|c|c|c|c|}
\hline \multirow{2}{*}{$\begin{array}{ll} & 0.60 \\
\text { 品 } & 0.40 \\
\text { 소 } & 0.20\end{array}$} & & \multicolumn{2}{|c|}{ step ROCOF } & & $\longrightarrow \mathrm{RFe}$ \\
\hline & & & & & $\longrightarrow$ RFE_Limit \\
\hline ప 0.00 & $\sim$ & & & & RFE_Limit \\
\hline के -0.20 & & & & & \\
\hline O & & & 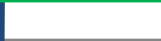 & & \\
\hline$\widetilde{x}$ & 0. & $\begin{array}{l}20.000 .02 \\
\text { tep time }(s)\end{array}$ & 0.040 .06 & $0.08 \quad 0.10$ & \\
\hline
\end{tabular}


9.9 Dynamic step change in phase: phasor overshoot: $M$ class

\begin{tabular}{|c|c|c|c|c|c|c|c|c|c|c|c|c|}
\hline Fs (FPS) & $10 \mathrm{M}$ & $10 \mathrm{P}$ & $12 \mathrm{M}$ & $12 \mathrm{P}$ & $15 \mathrm{M}$ & $15 \mathrm{P}$ & $20 \mathrm{M}$ & $20 \mathrm{P}$ & $30 \mathrm{M}$ & $30 \mathrm{P}$ & $60 \mathrm{M}$ & $60 \mathrm{P}$ \\
\hline C37.118.1 Annex C & $\mathrm{P}$ & $\mathrm{P}$ & $\mathrm{P}$ & $\mathrm{P}$ & $\mathrm{P}$ & $\mathrm{P}$ & $\mathrm{P}$ & $\mathrm{P}$ & $\mathrm{P}$ & $\mathrm{P}$ & $\mathrm{P}$ & $\mathrm{P}$ \\
\hline PMU A & $\mathrm{P}$ & $\mathrm{P}$ & & $\mathrm{P}$ & $\mathrm{P}$ & $\mathrm{P}$ & $\mathrm{P}$ & & $\mathrm{P}$ & & $\mathrm{P}$ & $\mathrm{P}$ \\
\hline PMU B & $\mathrm{F}$ & $\mathrm{F}$ & $\mathrm{F}$ & $\mathrm{F}$ & $\mathrm{F}$ & $\mathrm{F}$ & $\mathrm{F}$ & $\mathrm{F}$ & $\mathrm{F}$ & $\mathrm{F}$ & $\mathrm{F}$ & $\mathrm{F}$ \\
\hline $\mathrm{PMU} \mathrm{C}$ & $\mathrm{P}$ & $\mathrm{P}$ & $\mathrm{P}$ & $\mathrm{P}$ & $\mathrm{P}$ & $\mathrm{P}$ & $\mathrm{P}$ & $\mathrm{P}$ & $\mathrm{P}$ & $\mathrm{P}$ & $\mathrm{P}$ & $\mathrm{P}$ \\
\hline $\mathrm{PMU} \mathrm{D}$ & $\mathrm{P}$ & $\mathrm{P}$ & $\mathrm{P}$ & $\mathrm{P}$ & $\mathrm{P}$ & $\mathrm{P}$ & $\mathrm{P}$ & $\mathrm{P}$ & $\mathrm{P}$ & $\mathrm{P}$ & $\mathrm{P}$ & $\mathrm{P}$ \\
\hline PMU E & $\mathrm{P}$ & - & $\mathrm{P}$ & - & $\mathrm{P}$ & - & $\mathrm{P}$ & - & $\mathrm{P}$ & - & $\mathrm{P}$ & - \\
\hline PMU F & $\mathrm{P}$ & $\mathrm{P}$ & $\mathrm{P}$ & $\mathrm{P}$ & $\mathrm{P}$ & $\mathrm{P}$ & $\mathrm{P}$ & $\mathrm{P}$ & $\mathrm{P}$ & $\mathrm{P}$ & $\mathrm{P}$ & $\mathrm{P}$ \\
\hline PMU G & $\mathrm{F}$ & - & $\mathrm{F}$ & - & $\mathrm{F}$ & - & $\mathrm{F}$ & - & $\mathrm{F}$ & - & - & - \\
\hline PMU H & $\mathrm{P}$ & $\mathrm{P}$ & $\mathrm{P}$ & $\mathrm{P}$ & $\mathrm{P}$ & $\mathrm{P}$ & $\mathrm{P}$ & $\mathrm{P}$ & $\mathrm{P}$ & $\mathrm{P}$ & $\mathrm{P}$ & $\mathrm{P}$ \\
\hline PMU I & $\mathrm{P}$ & - & $\mathrm{P}$ & - & $\mathrm{P}$ & - & $\mathrm{P}$ & - & $\mathrm{P}$ & - & $\mathrm{P}$ & - \\
\hline PMU J & $\mathrm{P}$ & $\mathrm{P}$ & $\mathrm{P}$ & $\mathrm{P}$ & $\mathrm{P}$ & $\mathrm{P}$ & $\mathrm{P}$ & $\mathrm{P}$ & $\mathrm{P}$ & $\mathrm{P}$ & $\mathrm{P}$ & $\mathrm{P}$ \\
\hline
\end{tabular}

Dynamic step change in phase: phasor delay time M class

\begin{tabular}{|c|c|c|c|c|c|c|c|c|c|c|c|c|}
\hline Fs (FPS) & $10 \mathrm{M}$ & $10 \mathrm{P}$ & $12 \mathrm{M}$ & $12 \mathrm{P}$ & $15 \mathrm{M}$ & $15 \mathrm{P}$ & $20 \mathrm{M}$ & $20 \mathrm{P}$ & $30 \mathrm{M}$ & $30 \mathrm{P}$ & $60 \mathrm{M}$ & $60 \mathrm{P}$ \\
\hline C37.118.1 Annex C & $\mathrm{P}$ & $\mathrm{P}$ & $\mathrm{P}$ & $\mathrm{P}$ & $\mathrm{P}$ & $\mathrm{P}$ & $\mathrm{P}$ & $\mathrm{P}$ & $\mathrm{P}$ & $\mathrm{P}$ & $\mathrm{P}$ & $\mathrm{P}$ \\
\hline PMU A & $\mathrm{P}$ & $\mathrm{P}$ & $\mathrm{P}$ & $\mathrm{P}$ & $\mathrm{P}$ & $\mathrm{P}$ & $\mathrm{P}$ & $\mathrm{P}$ & $\mathrm{P}$ & $\mathrm{P}$ & $\mathrm{P}$ & $\mathrm{P}$ \\
\hline PMU B & $\mathrm{P}$ & $\mathrm{P}$ & $\mathrm{P}$ & $\mathrm{P}$ & $\mathrm{P}$ & $\mathrm{P}$ & $\mathrm{P}$ & $\mathrm{P}$ & $\mathrm{P}$ & $\mathrm{P}$ & $\mathrm{P}$ & $\mathrm{P}$ \\
\hline PMU C & $\mathrm{P}$ & $\mathrm{P}$ & $\mathrm{P}$ & $\mathrm{P}$ & $\mathrm{P}$ & $\mathrm{P}$ & $\mathrm{P}$ & $\mathrm{P}$ & $\mathrm{P}$ & $\mathrm{P}$ & $\mathrm{P}$ & $\mathrm{P}$ \\
\hline PMU D & $\mathrm{P}$ & $\mathrm{P}$ & $\mathrm{P}$ & $\mathrm{P}$ & $\mathrm{P}$ & $\mathrm{P}$ & $\mathrm{P}$ & $\mathrm{P}$ & $\mathrm{P}$ & $\mathrm{P}$ & $\mathrm{P}$ & $\mathrm{P}$ \\
\hline PMU E & $\mathrm{P}$ & - & $\mathrm{P}$ & - & $\mathrm{P}$ & - & $\mathrm{P}$ & - & $\mathrm{P}$ & - & $\mathrm{P}$ & - \\
\hline PMU F & $\mathrm{P}$ & $\mathrm{P}$ & $\mathrm{P}$ & $\mathrm{P}$ & $\mathrm{P}$ & $\mathrm{P}$ & $\mathrm{P}$ & $\mathrm{P}$ & $\mathrm{P}$ & $\mathrm{P}$ & $\mathrm{P}$ & $\mathrm{P}$ \\
\hline PMU G & $\mathrm{F}$ & - & $\mathrm{F}$ & - & $\mathrm{F}$ & - & $\mathrm{F}$ & - & $\mathrm{F}$ & - & $\mathrm{F}$ & - \\
\hline PMU H & $\mathrm{P}$ & $\mathrm{P}$ & $\mathrm{P}$ & $\mathrm{P}$ & $\mathrm{P}$ & $\mathrm{P}$ & $\mathrm{P}$ & $\mathrm{P}$ & $\mathrm{P}$ & $\mathrm{P}$ & $\mathrm{P}$ & $\mathrm{P}$ \\
\hline PMU I & $\mathrm{P}$ & - & $\mathrm{P}$ & - & $\mathrm{P}$ & - & $\mathrm{P}$ & - & $\mathrm{P}$ & - & $\mathrm{P}$ & - \\
\hline PMU J & $\mathrm{P}$ & $\mathrm{P}$ & $\mathrm{P}$ & $\mathrm{P}$ & $\mathrm{P}$ & $\mathrm{P}$ & $\mathrm{P}$ & $\mathrm{P}$ & $\mathrm{P}$ & $\mathrm{P}$ & $\mathrm{P}$ & $\mathrm{P}$ \\
\hline
\end{tabular}




\subsubsection{C37.118.1-2011 Annex C dynamic step change in phase phasor overshoot: $F 0=60 \mathrm{~Hz}, \mathrm{M}$ class}

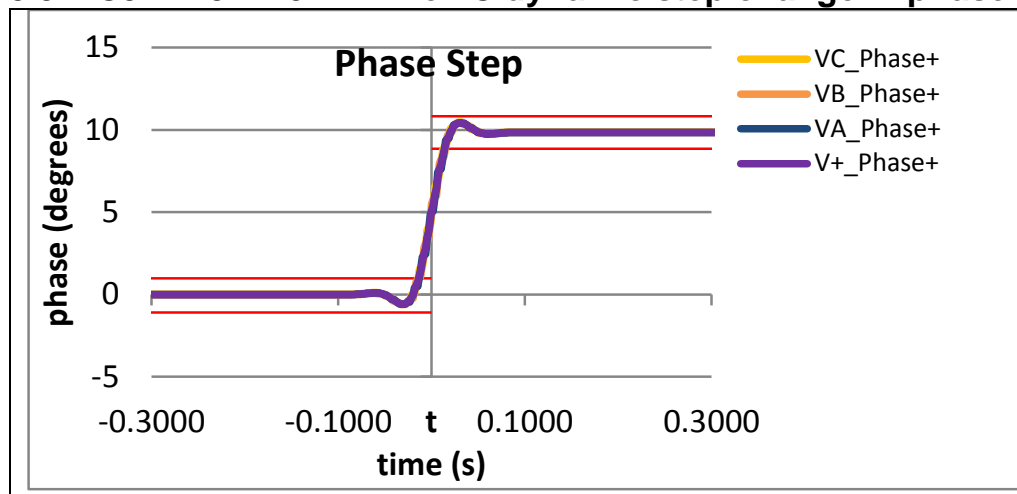

Figure 4861: Fs = 60 FPS, +10 degree phase step
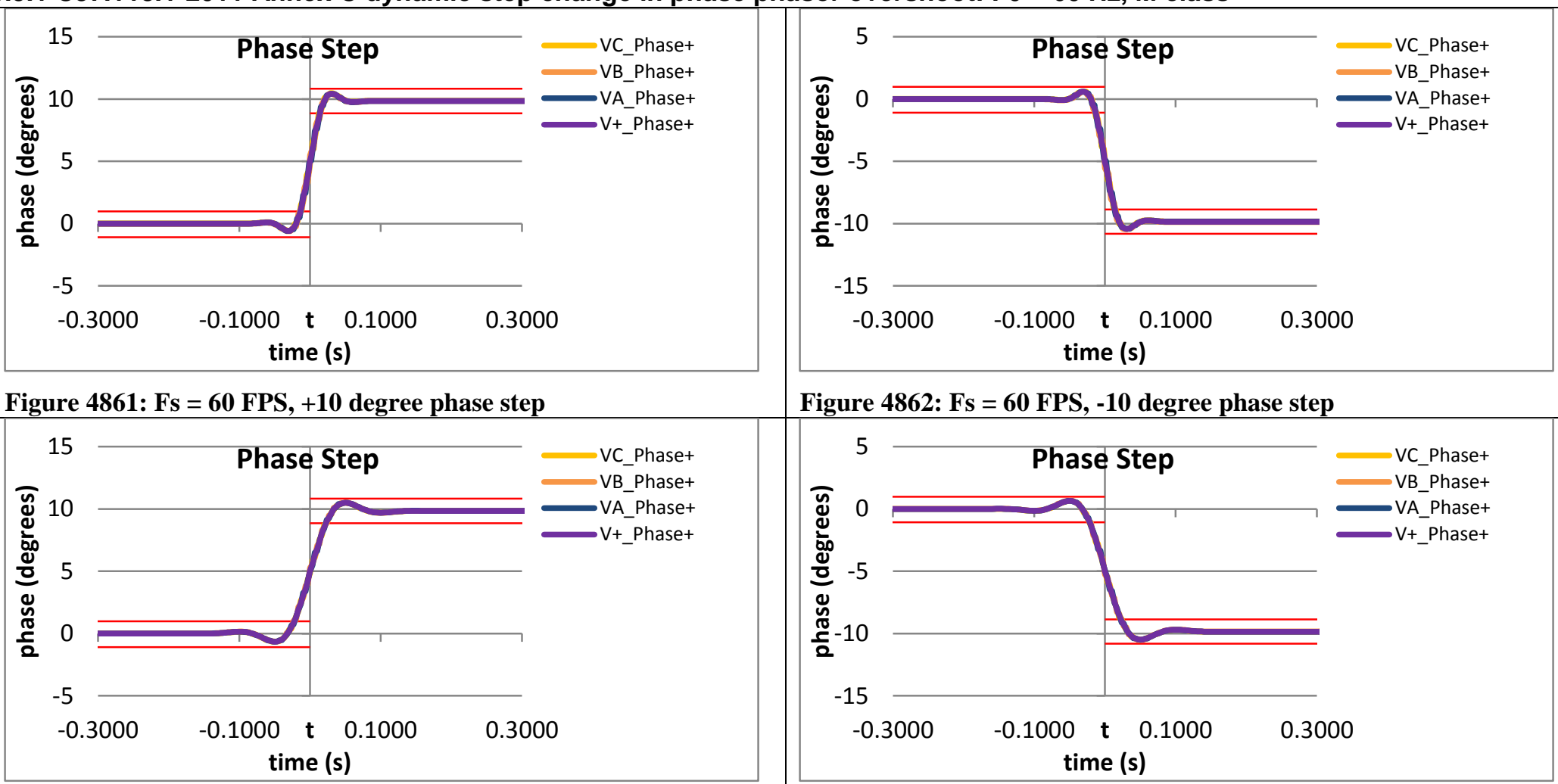

Figure 4862: Fs = 60 FPS, -10 degree phase step

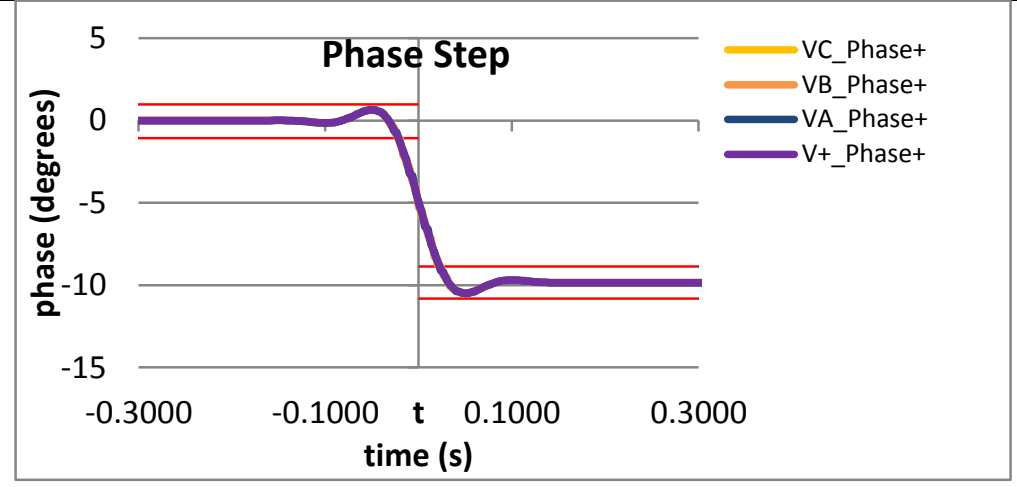

Figure 4863: Fs = 30 FPS, +10 degree phase step

Figure 4864: Fs = 30 FPS, -10 degree phase step

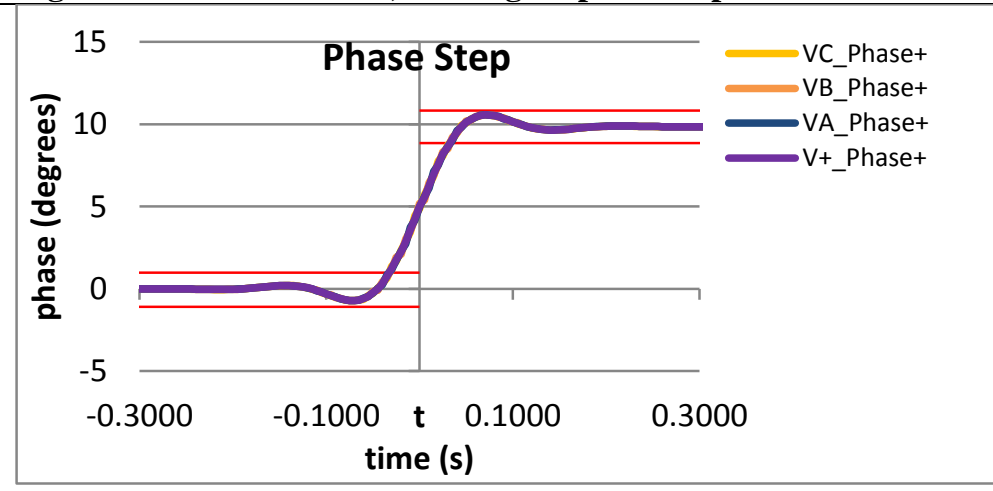

Figure 4865: Fs = 20 FPS, +10 degree phase step

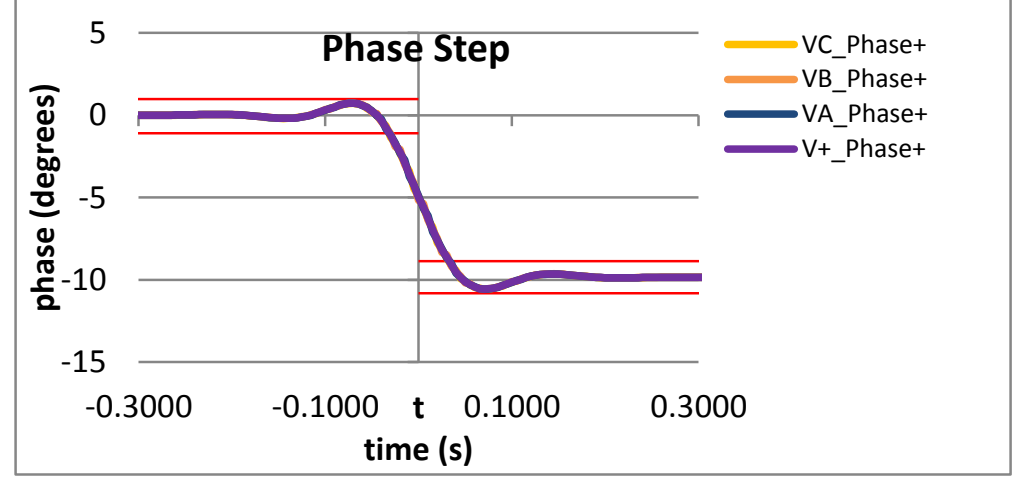

Figure 4866: Fs = 20 FPS, +10 degree phase step 


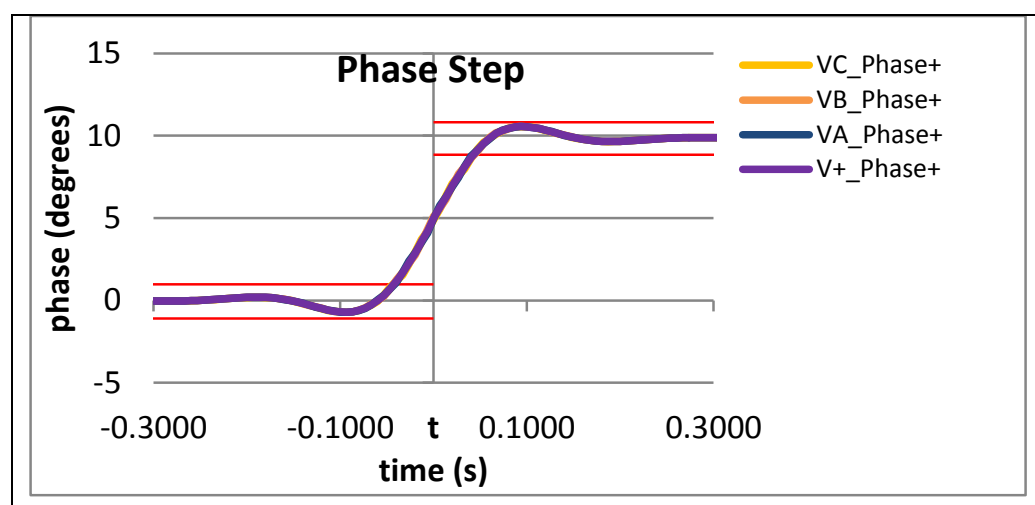

Figure 4867: Fs = 15 FPS, +10 degree phase step

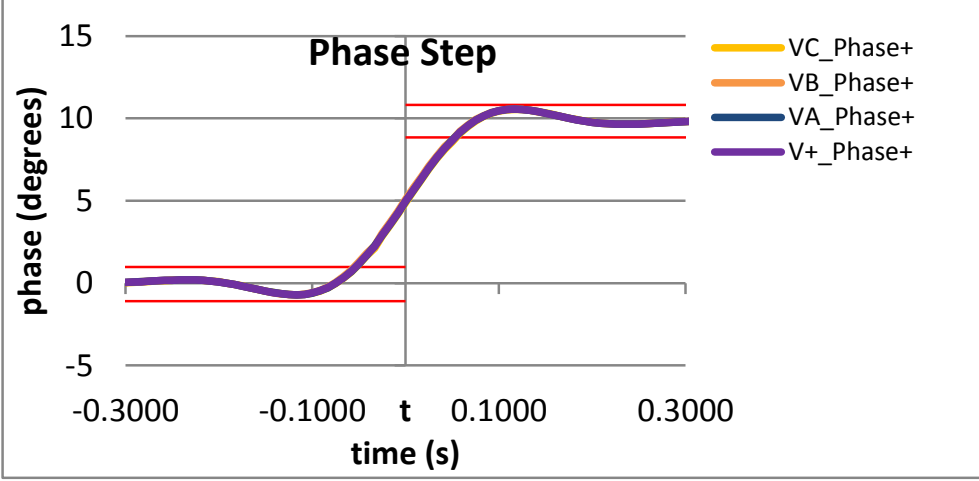

Figure 4869: Fs = 12 FPS, +10 degree phase step

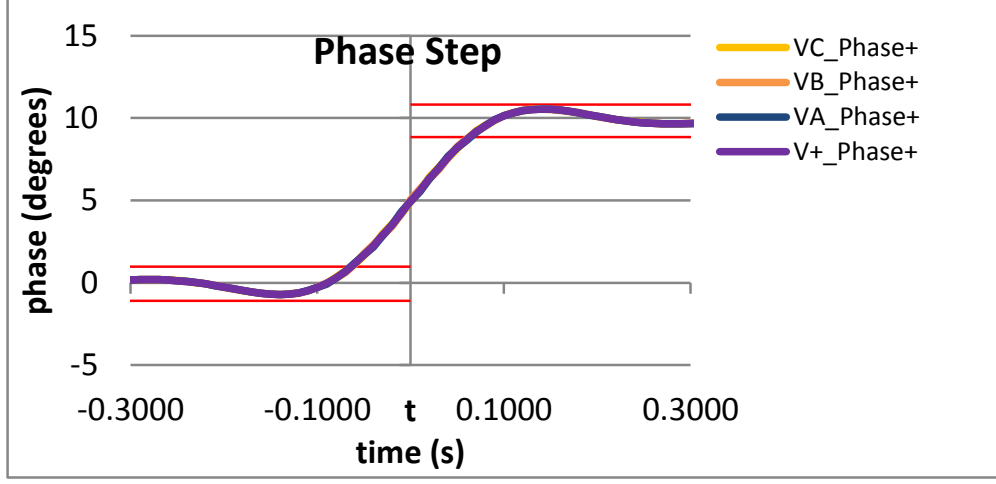

Figure 4871: Fs = 10 FPS, +10 degree phase step

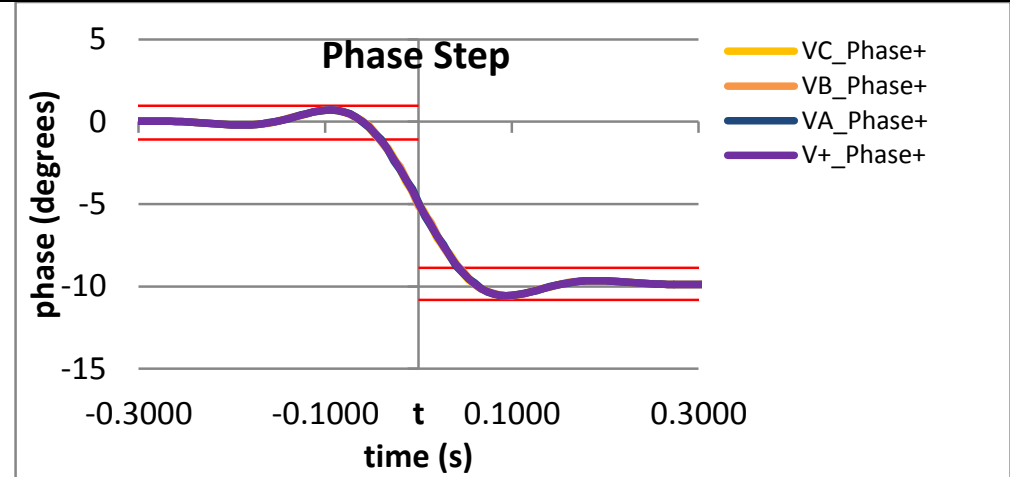

Figure 4868: Fs = 15 FPS, -10 degree phase step

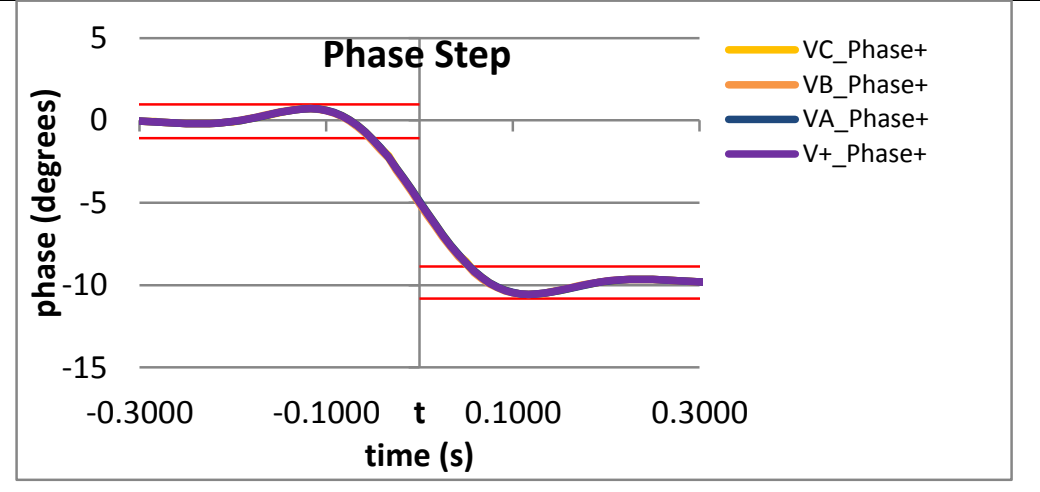

Figure 4870: Fs = 12 FPS, -10 degree phase step

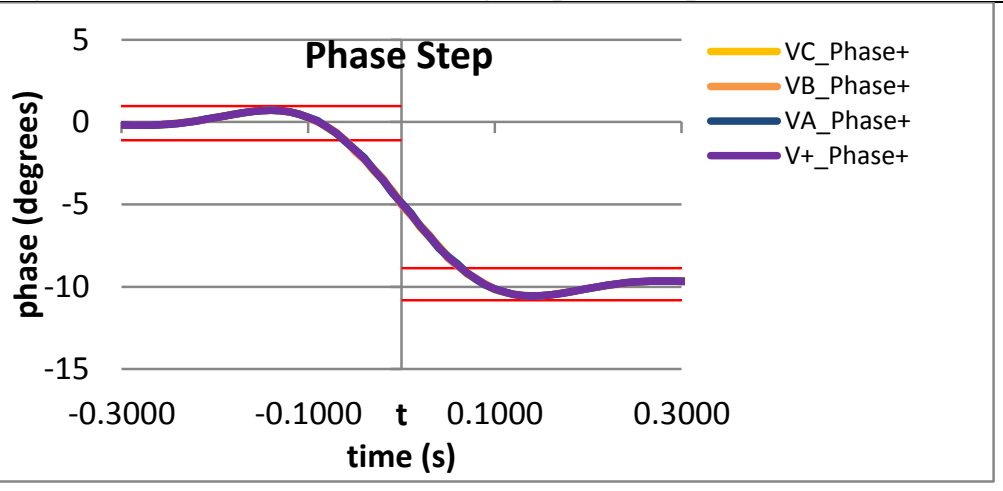

Figure 4872: Fs = 10 FPS, -10 degree phase step 


\subsubsection{PMU A dynamic step change in phase phasor overshoot: $F 0=60 \mathrm{~Hz}, \mathrm{M}$ class}
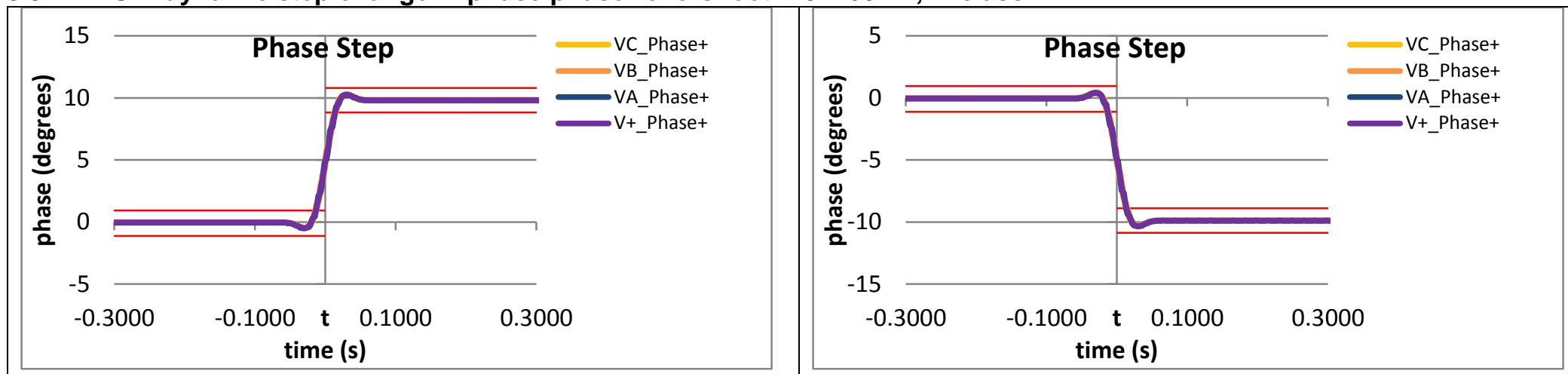

Figure 4873: Fs = 60 FPS, +10 degree phase step

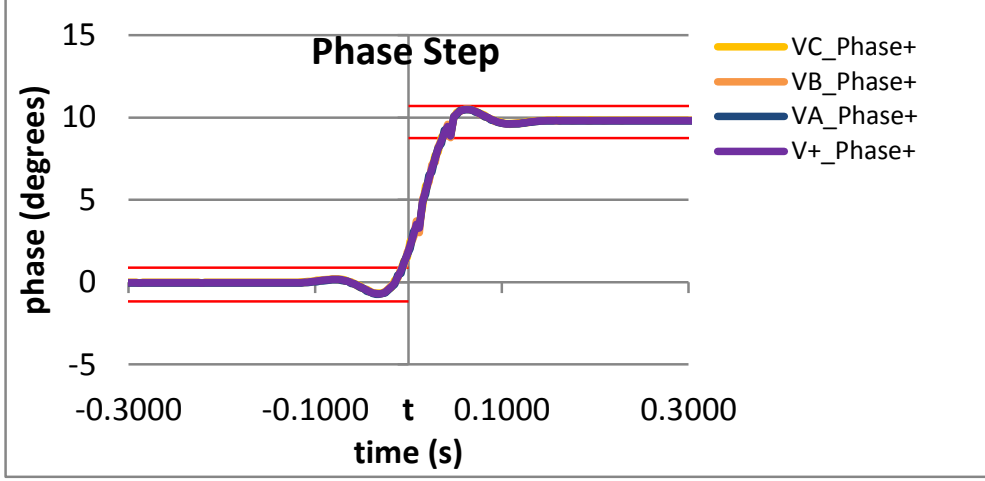

Figure 4874: Fs = 60 FPS, -10 degree phase step

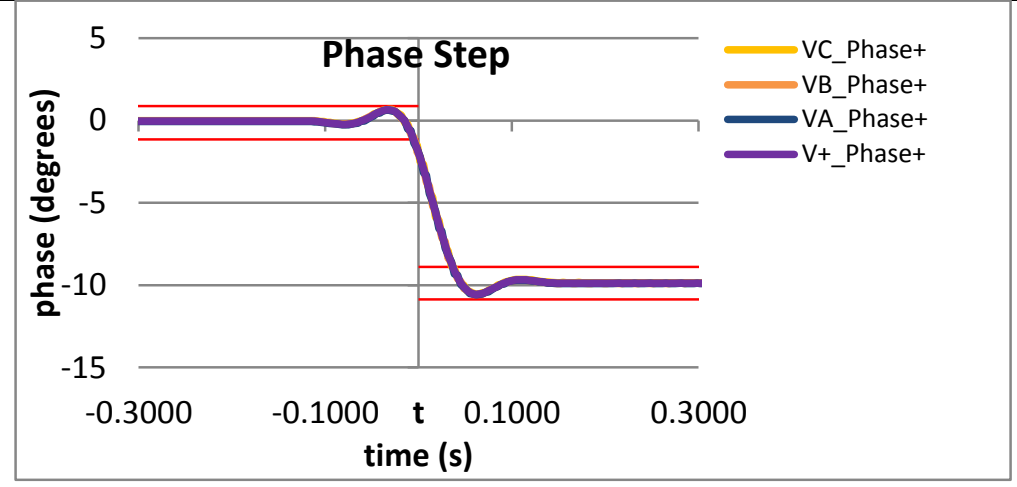

Figure 4875: Fs = 30 FPS, +10 degree phase step

Figure 4876: Fs = 30 FPS, -10 degree phase step

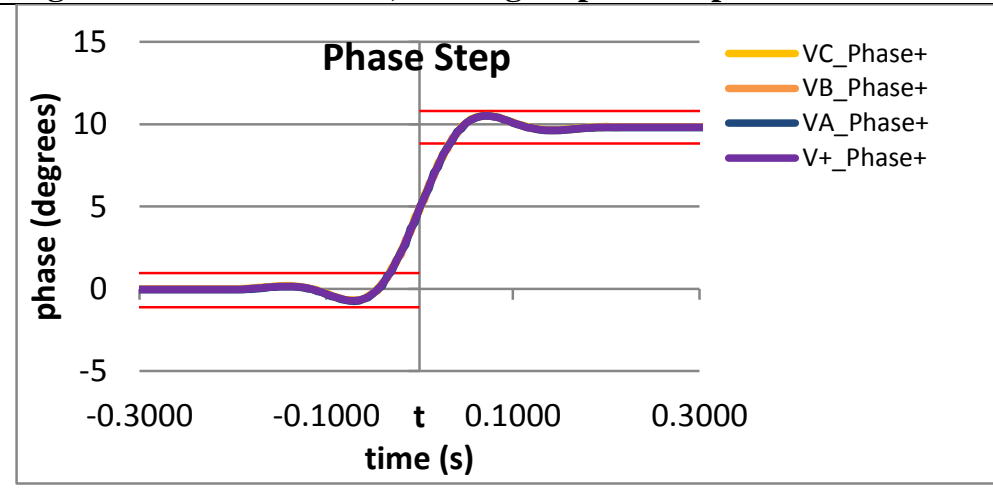

Figure 4877: Fs = 20 FPS, +10 degree phase step

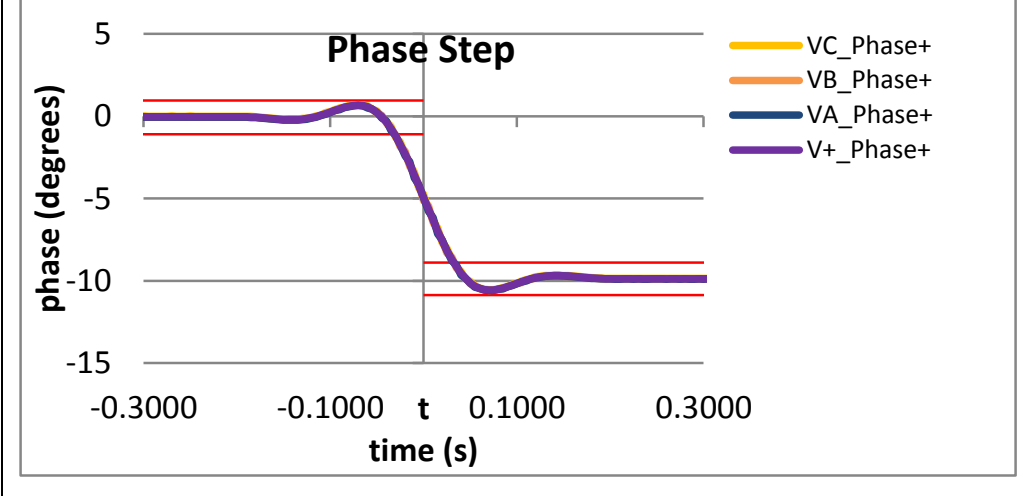

Figure 4878: Fs = 20 FPS, +10 degree phase step 


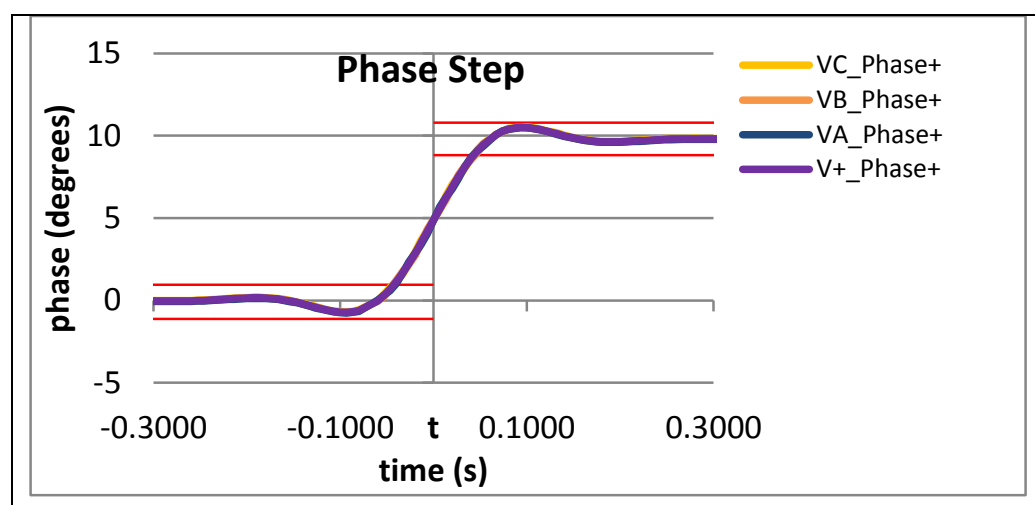

Figure 4879: Fs = 15 FPS, +10 degree phase step

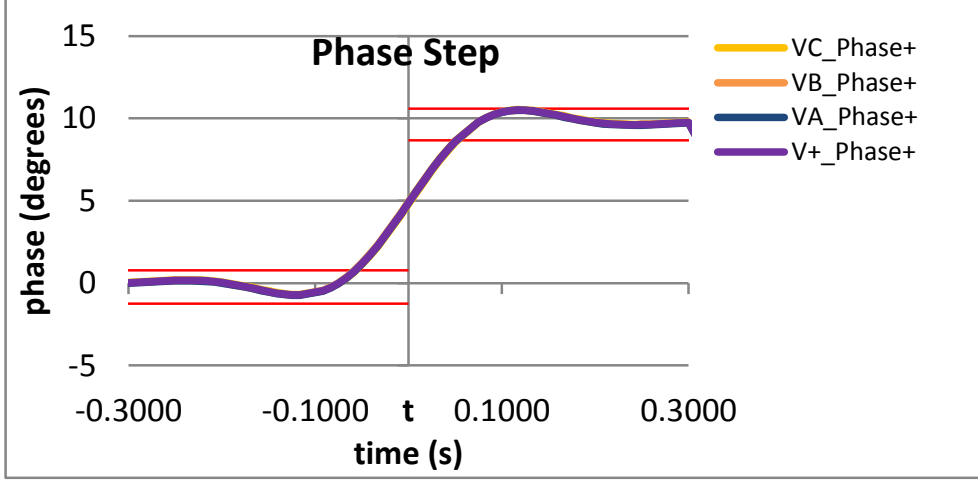

Figure 4881: Fs = 12 FPS, +10 degree phase step

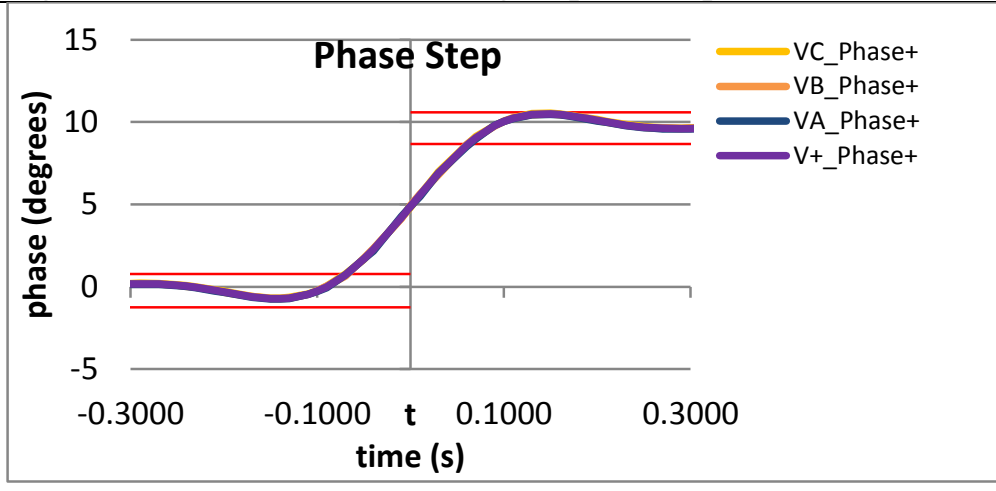

Figure 4883: Fs = 10 FPS, +10 degree phase step

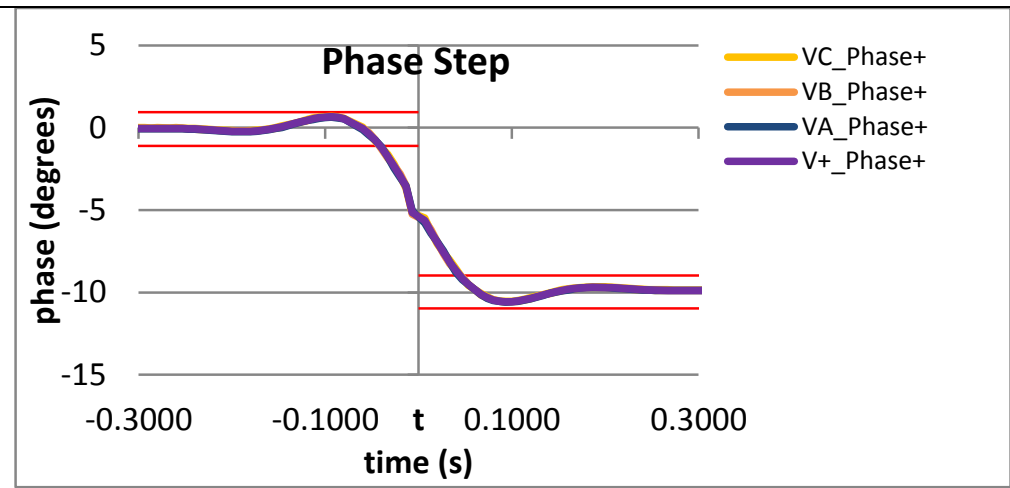

Figure 4880: Fs = 15 FPS, -10 degree phase step

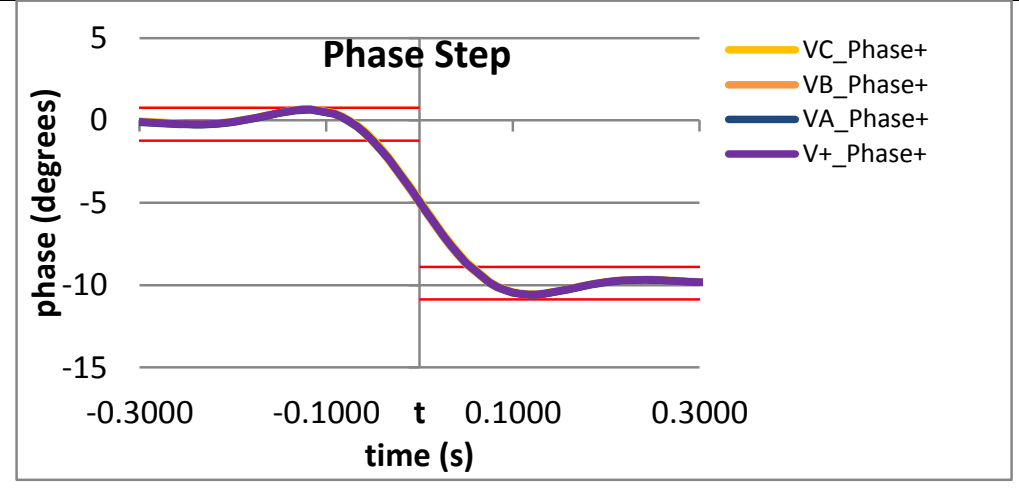

Figure 4882: Fs = 12 FPS, -10 degree phase step

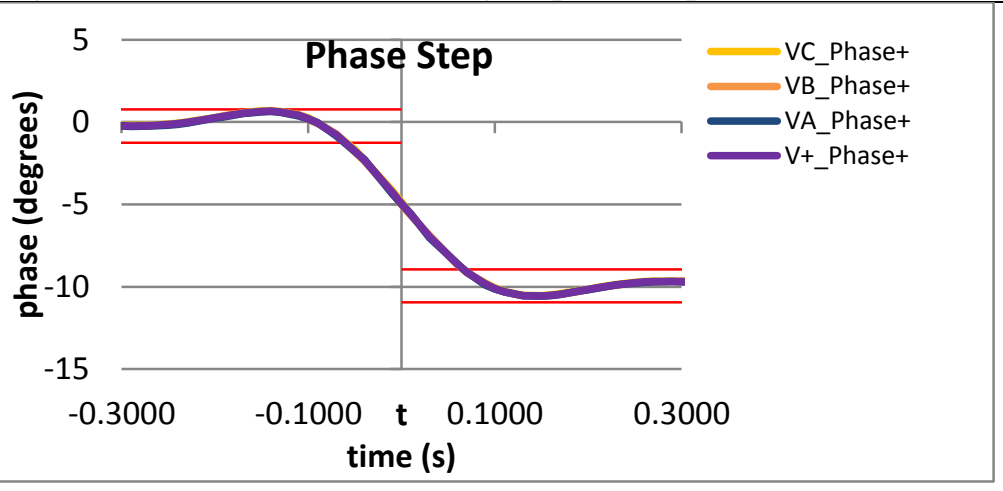

Figure 4884: Fs = 10 FPS, -10 degree phase step 
9.9.3 PMU B dynamic step change in phase phasor overshoot: $\mathrm{F} 0=60 \mathrm{~Hz}$, M class

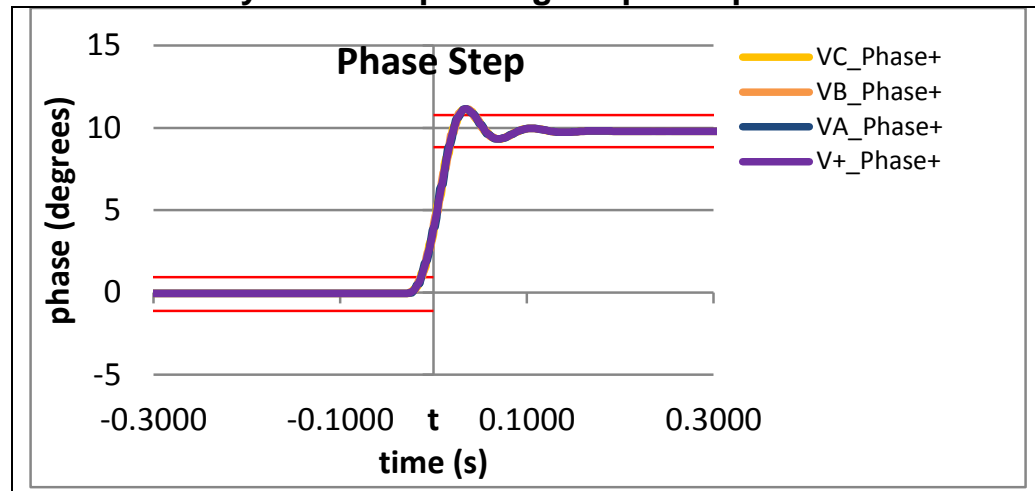

Figure 4885: Fs = 60 FPS, +10 degree phase step

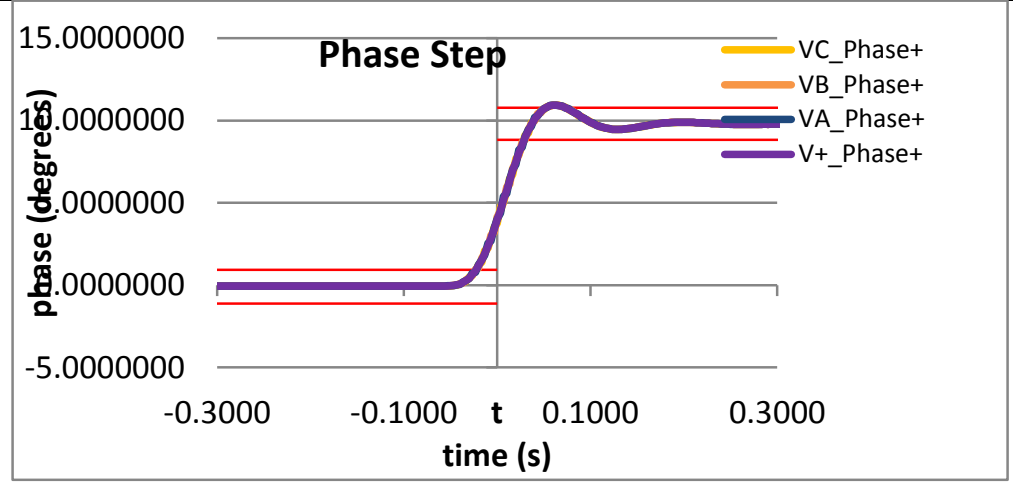

Figure 4887: Fs = 30 FPS, +10 degree phase step

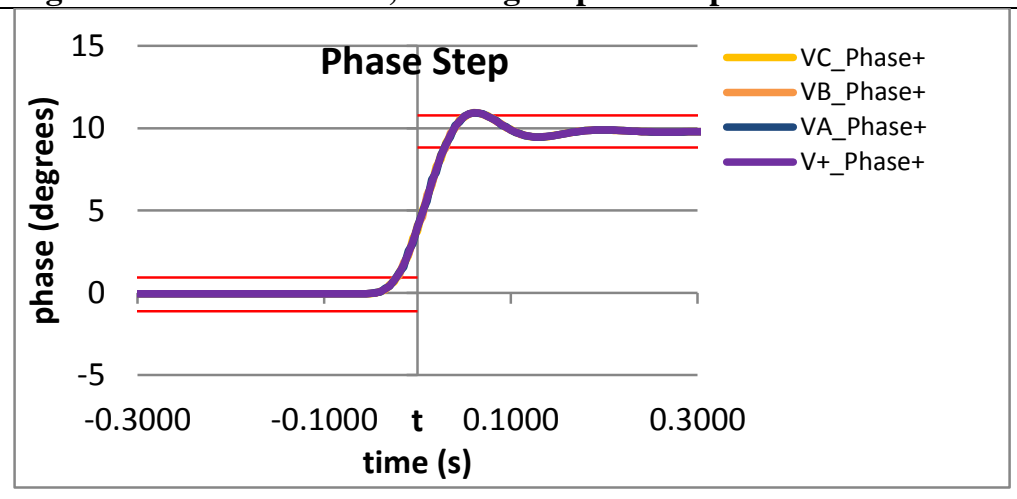

Figure 4889: Fs = 20 FPS, +10 degree phase step

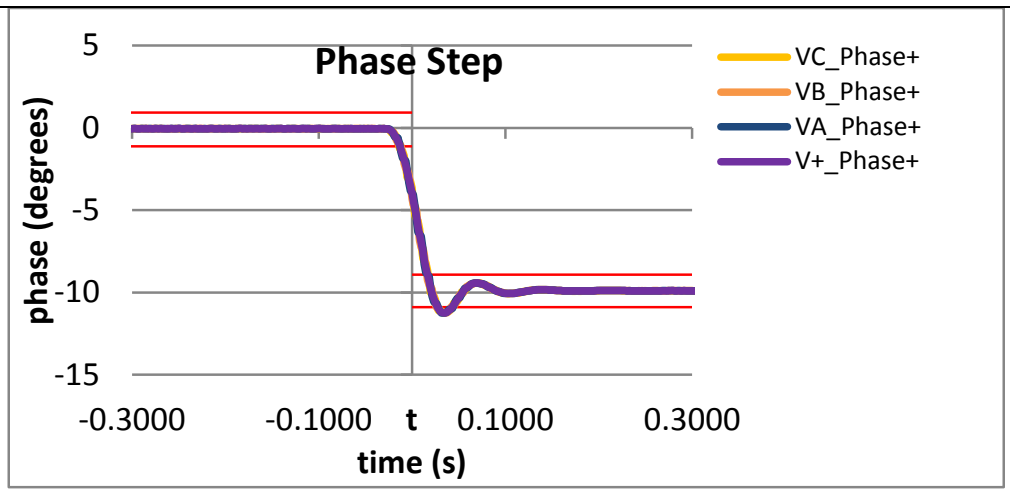

Figure 4886: Fs = 60 FPS, -10 degree phase step

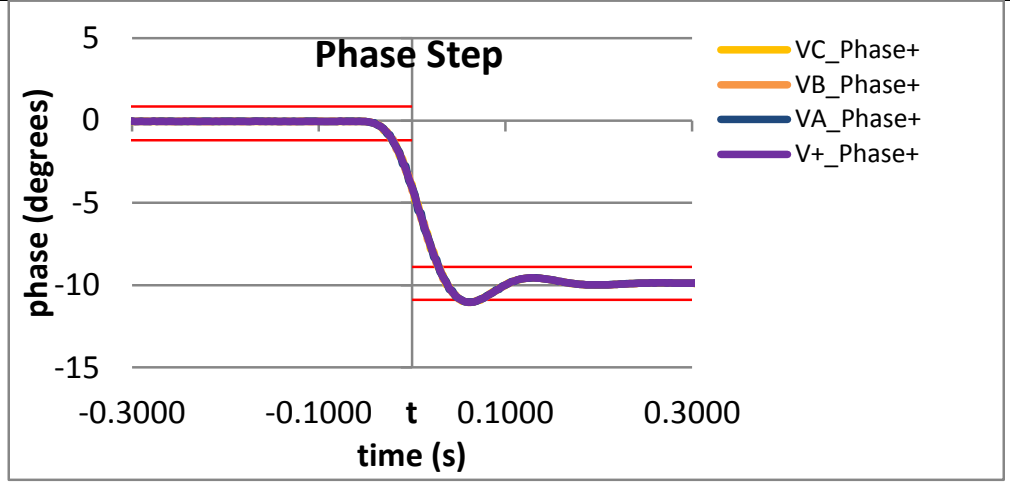

Figure 4888: Fs = 30 FPS, -10 degree phase step

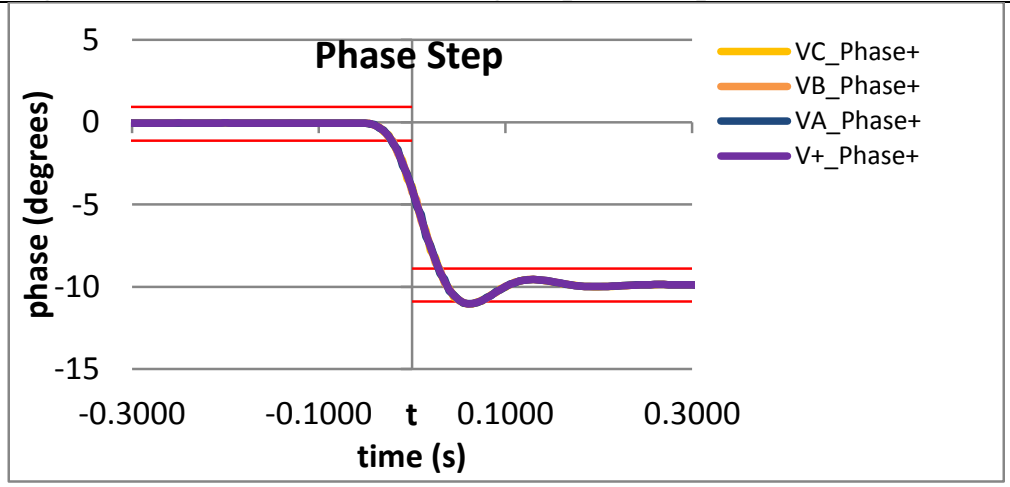

Figure 4890: Fs = 20 FPS, +10 degree phase step 
This publication is available free of charge from $\mathrm{http}: / / \mathrm{dx}$.doi.org/10.6028/NIST.IR.8106
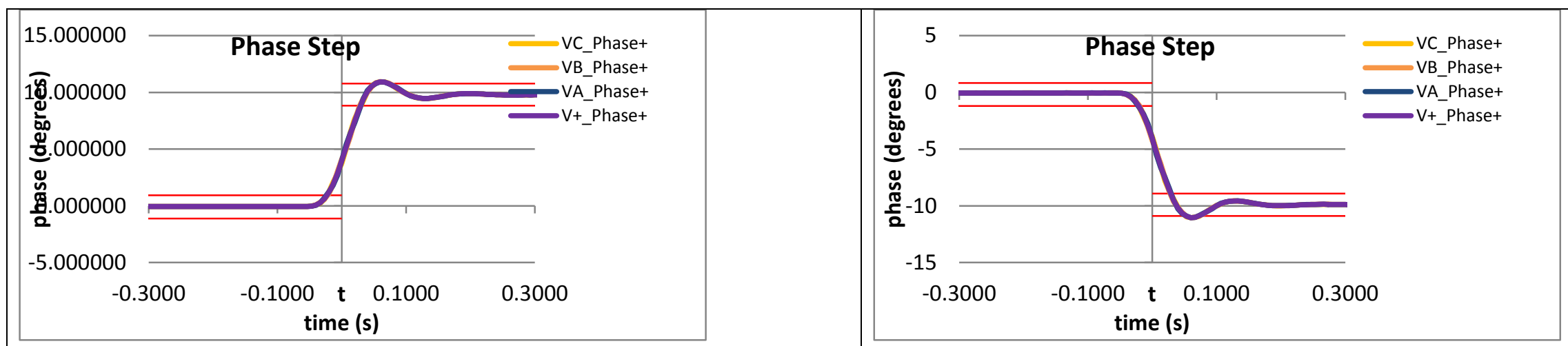

Figure 4891: Fs = 15 FPS, +10 degree phase step

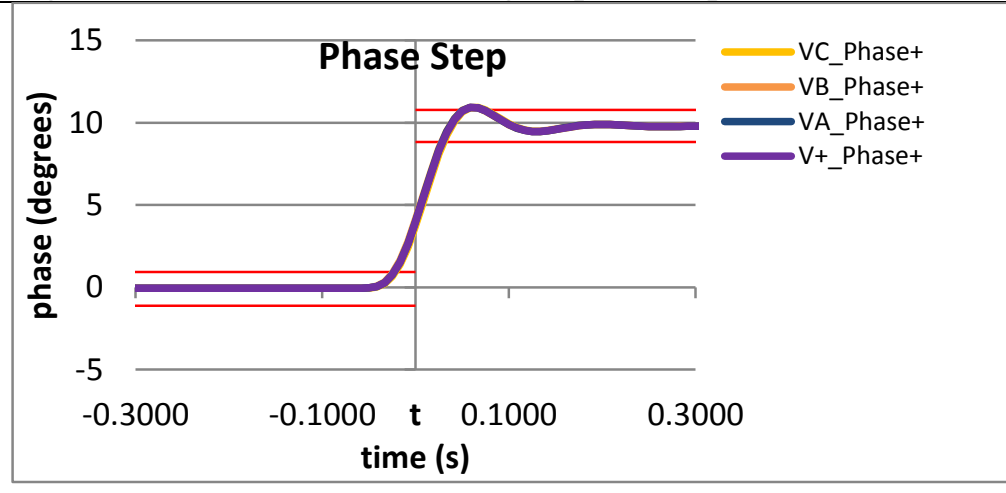

Figure 4893: Fs = 12 FPS, +10 degree phase step

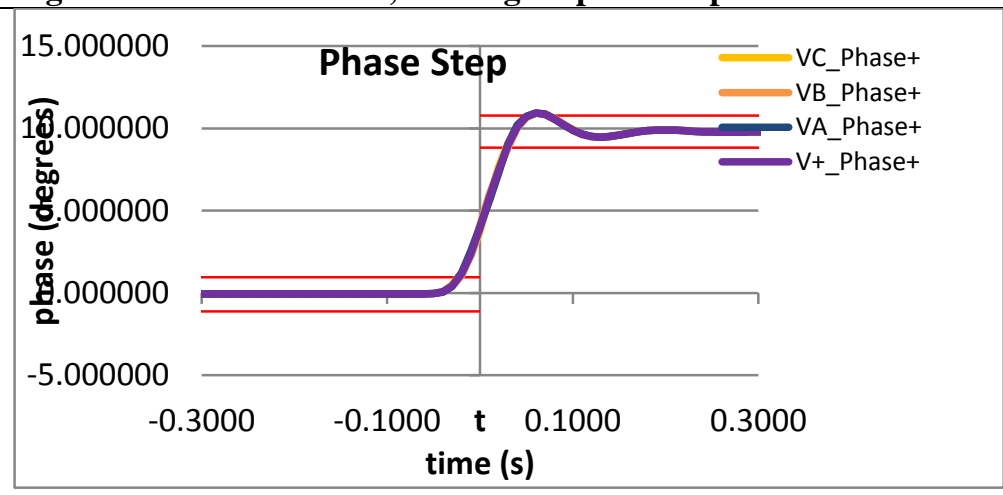

Figure 4895: Fs = 10 FPS, +10 degree phase step

Figure 4892: Fs $=15$ FPS, -10 degree phase step

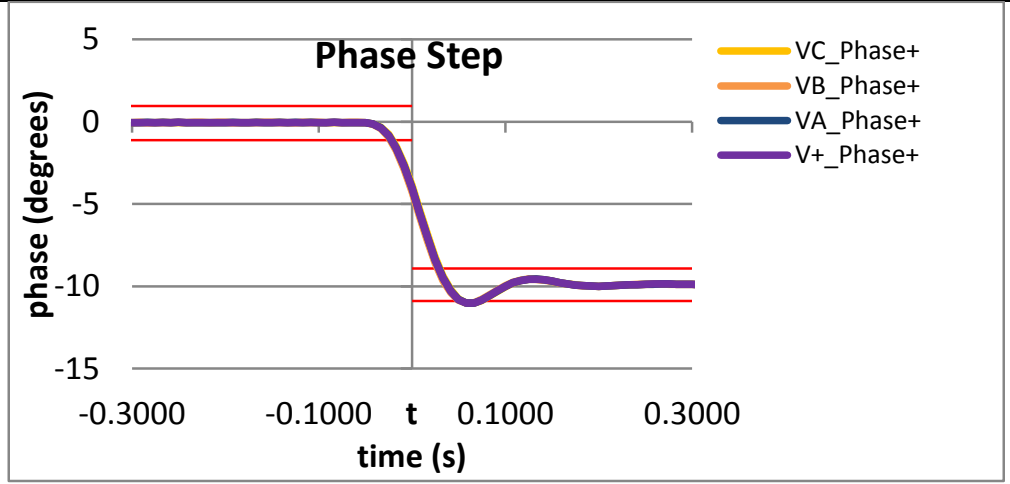

Figure 4894: Fs = 12 FPS, -10 degree phase step

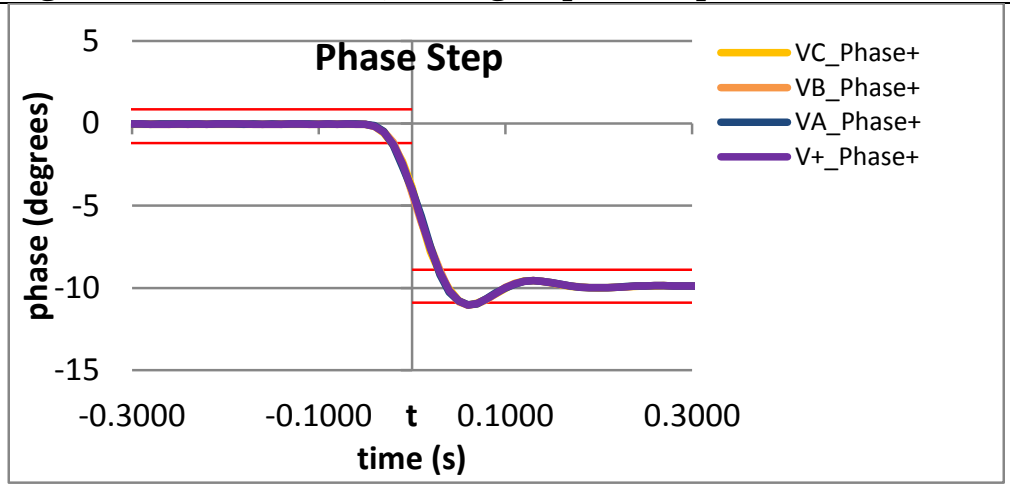

Figure 4896: Fs = 10 FPS, -10 degree phase step 
9.9.4 PMU C dynamic step change in phase phasor overshoot: $F 0=60 \mathrm{~Hz}$, M class

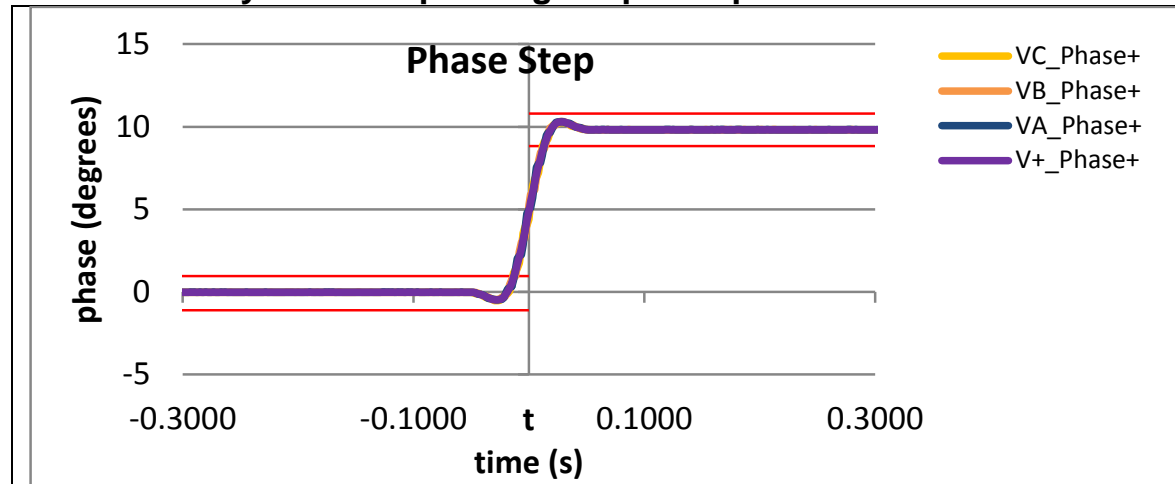

Figure 4897: Fs = $60 \mathrm{FPS},+10$ degree phase step

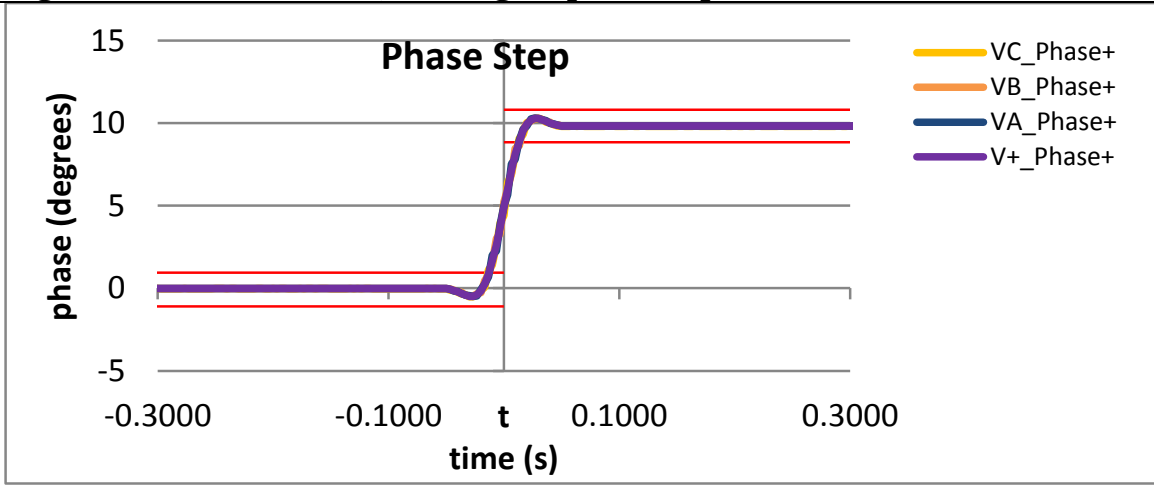

Figure 4899: Fs = 30 FPS, +10 degree phase step

Figure 4901: Fs = 20 FPS, +10 degree phase step

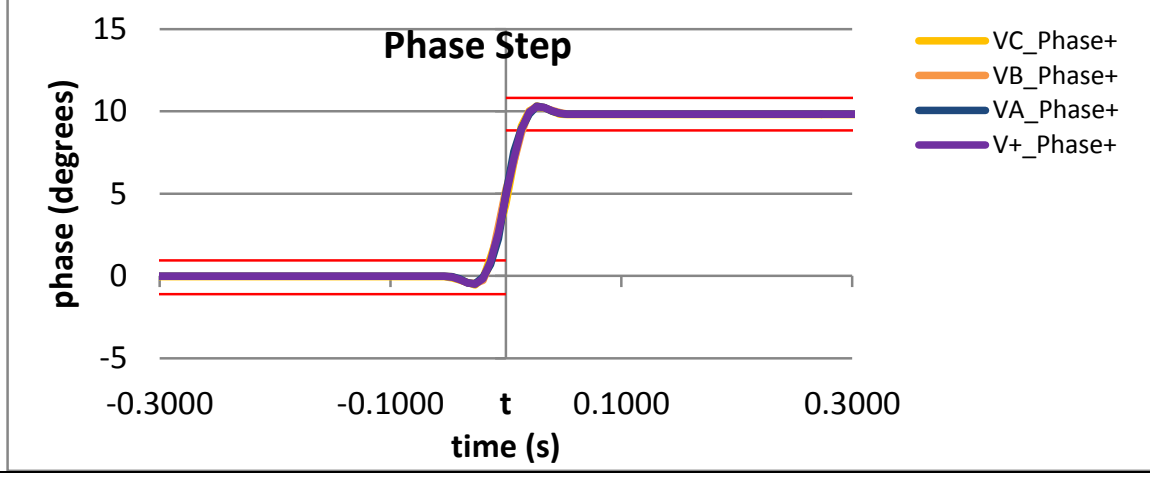

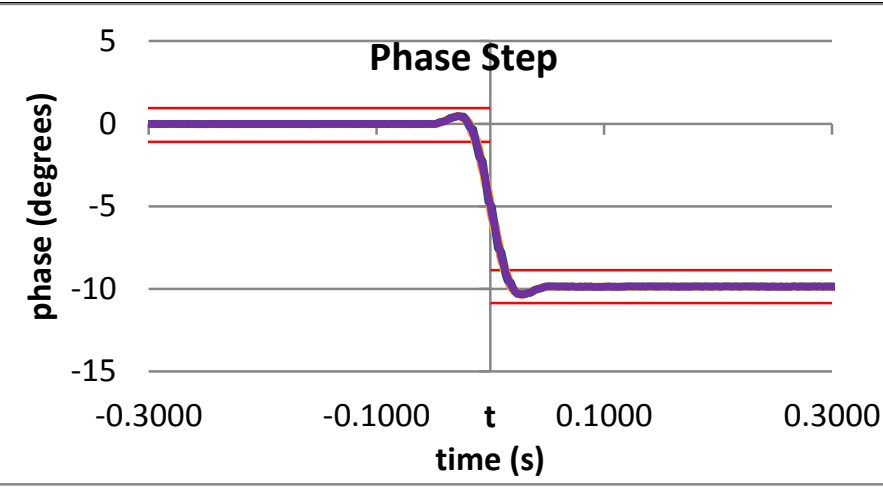

Figure 4898: Fs = 60 FPS, -10 degree phase step

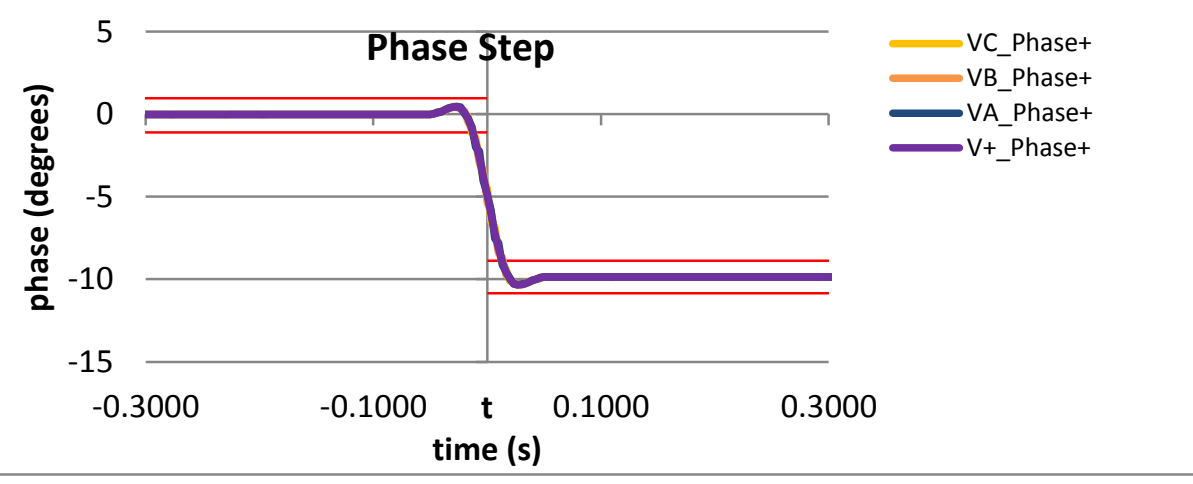

Figure 4900: Fs = 30 FPS, -10 degree phase step

Figure 4902: Fs = 20 FPS, -10 degree phase step

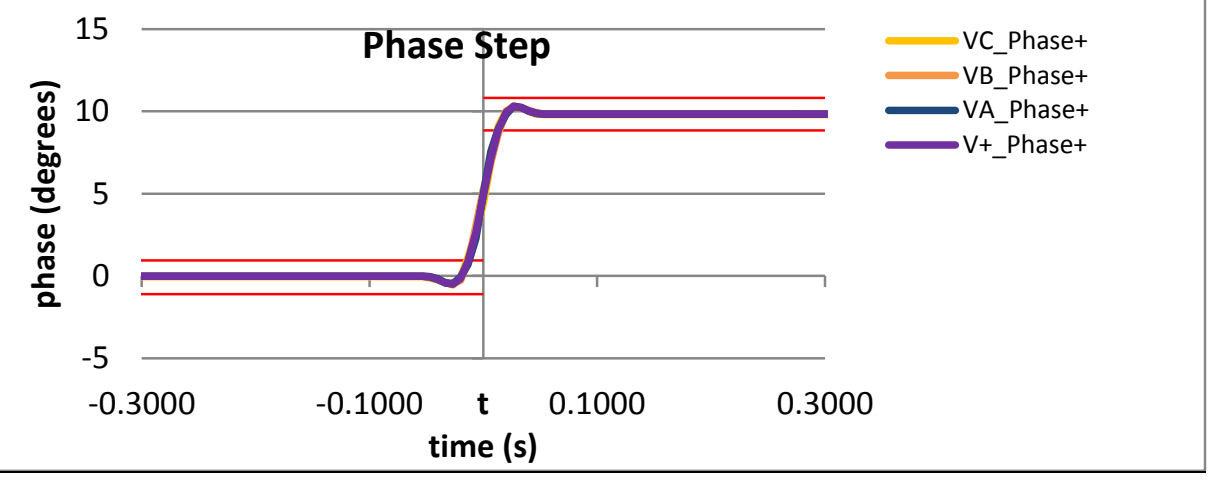




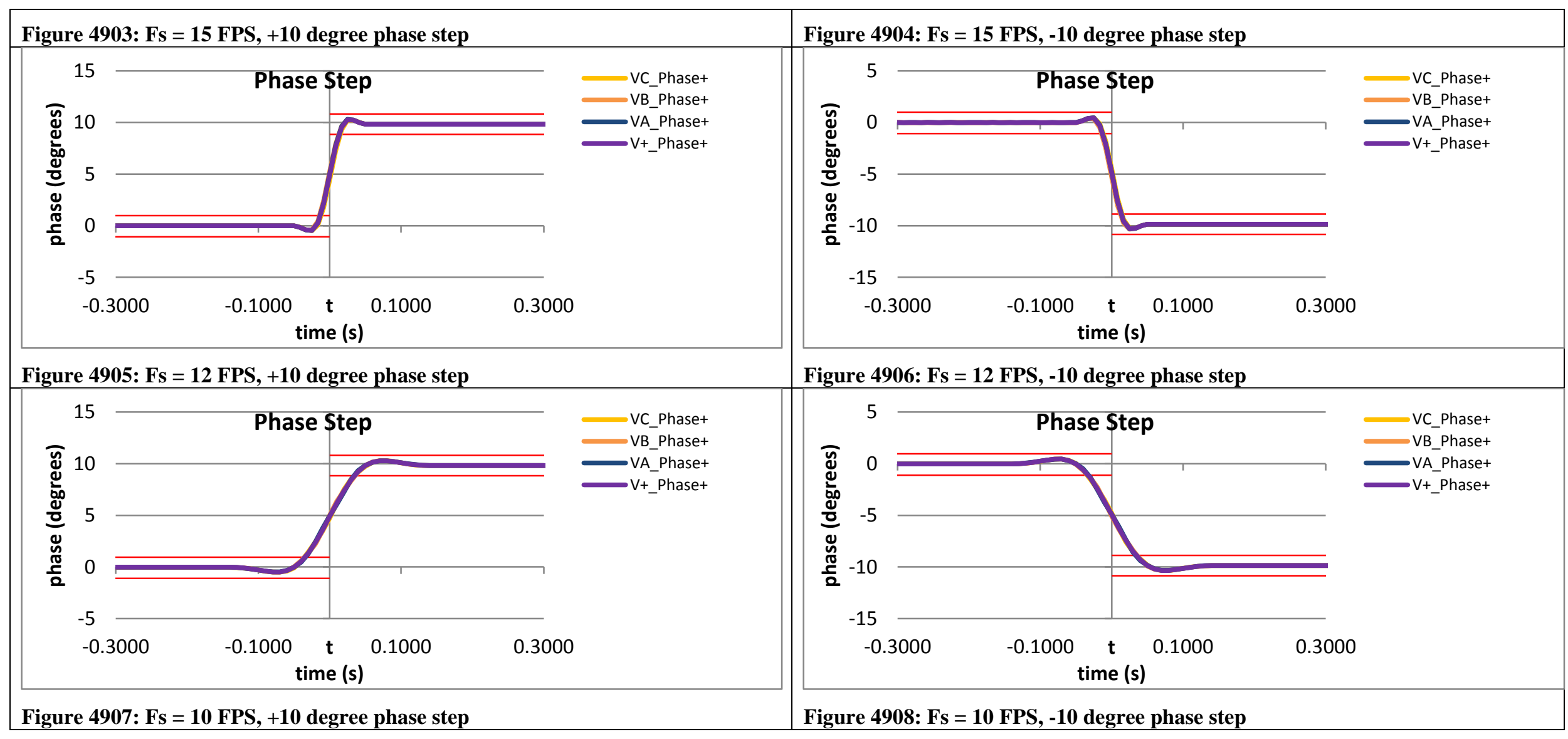


9.9.5 PMU D dynamic step change in phase phasor overshoot: $F 0=60 \mathrm{~Hz}, \mathrm{M}$ class

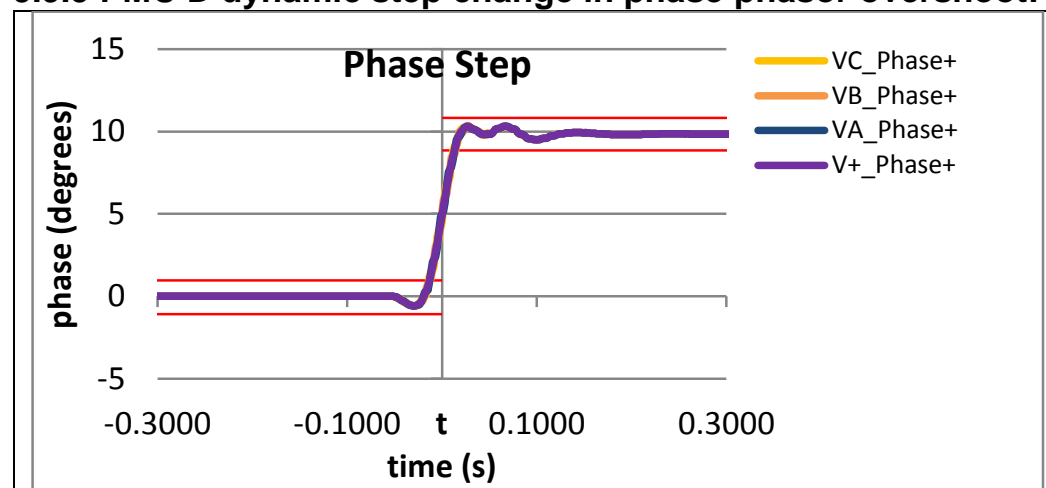

Figure 4909: Fs = 60 FPS, +10 degree phase step

Figure 4911: Fs = 30 FPS, +10 degree phase step

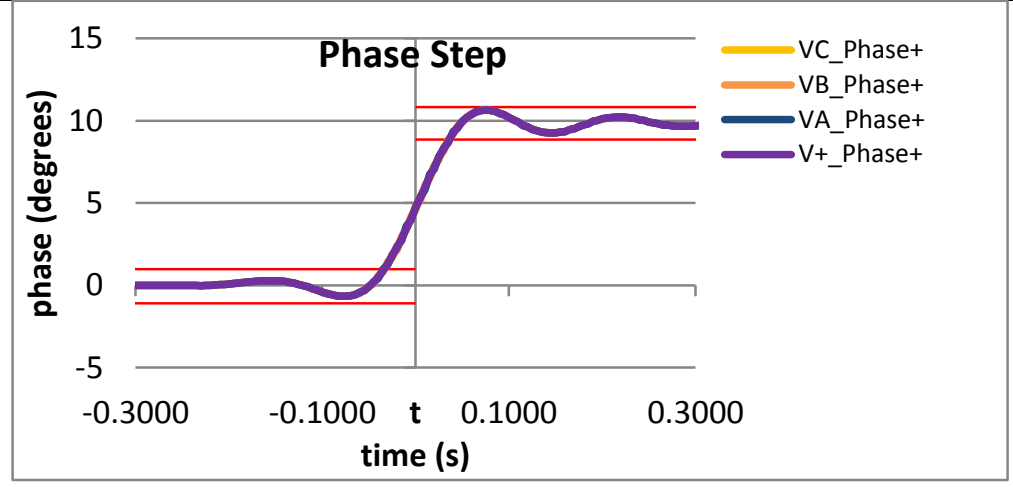

Figure 4913: Fs = 20 FPS, +10 degree phase step

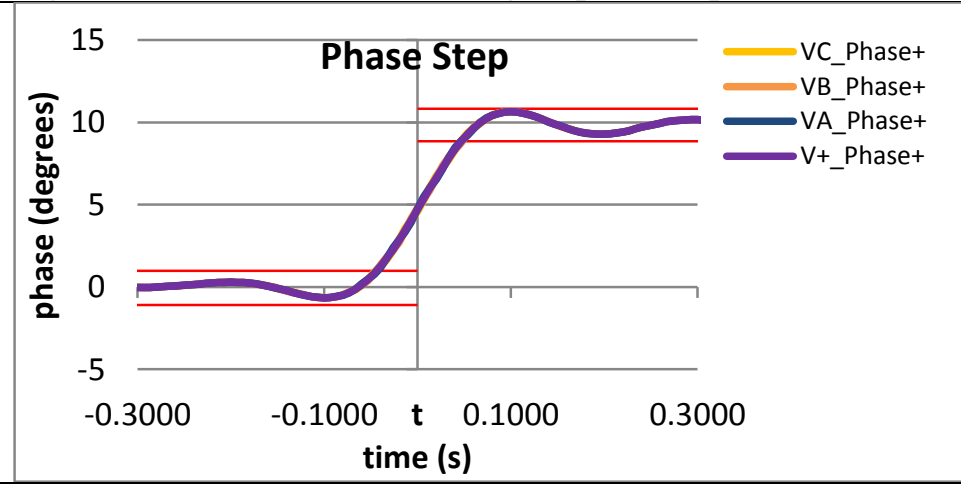

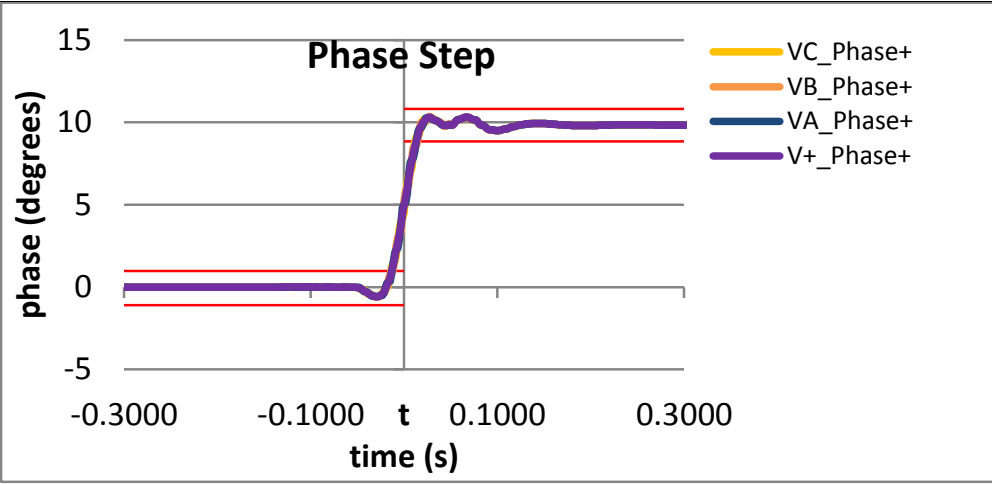

Figure 4910: Fs = 60 FPS, -10 degree phase step

Figure 4912: Fs = 30 FPS, -10 degree phase step

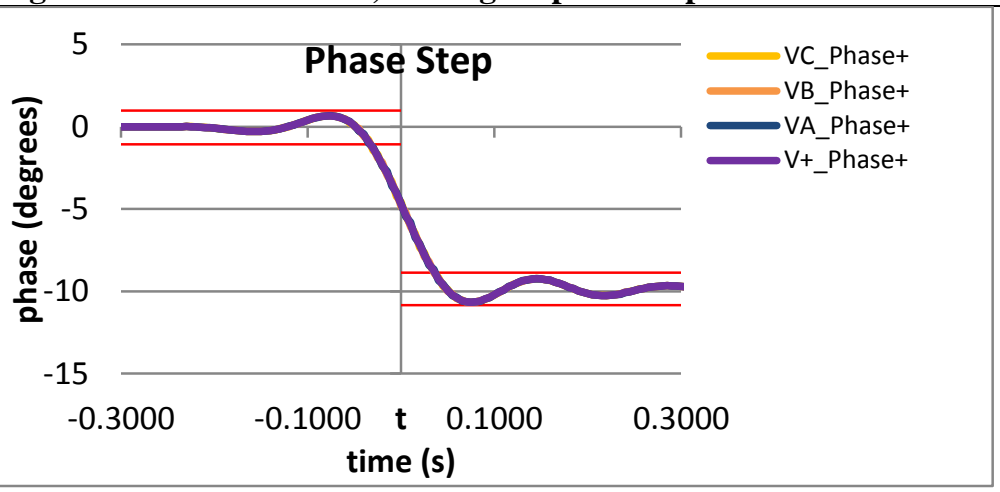

Figure 4914: Fs = 20 FPS, +10 degree phase step

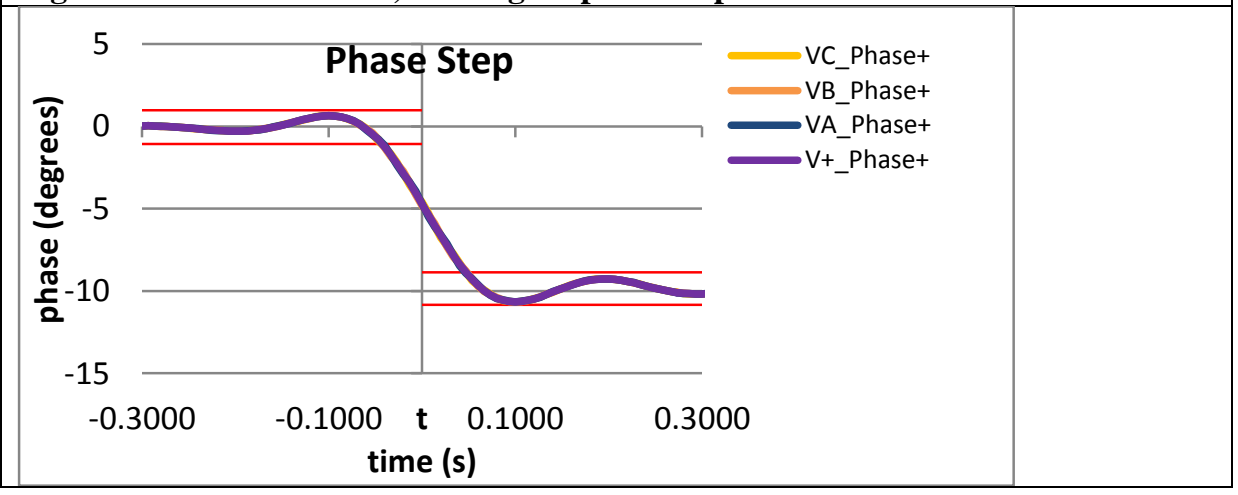




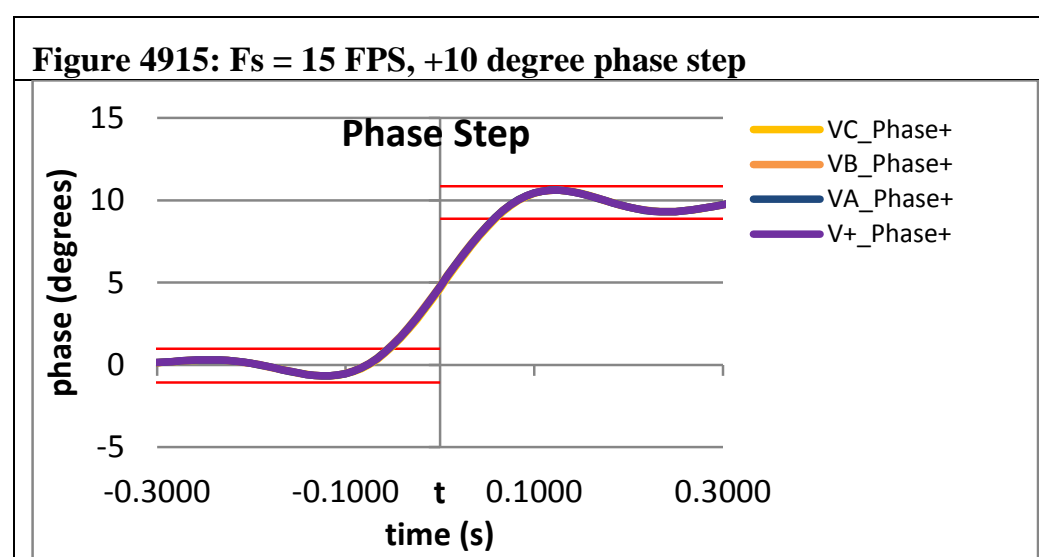

Figure 4917: Fs = 12 FPS, +10 degree phase step

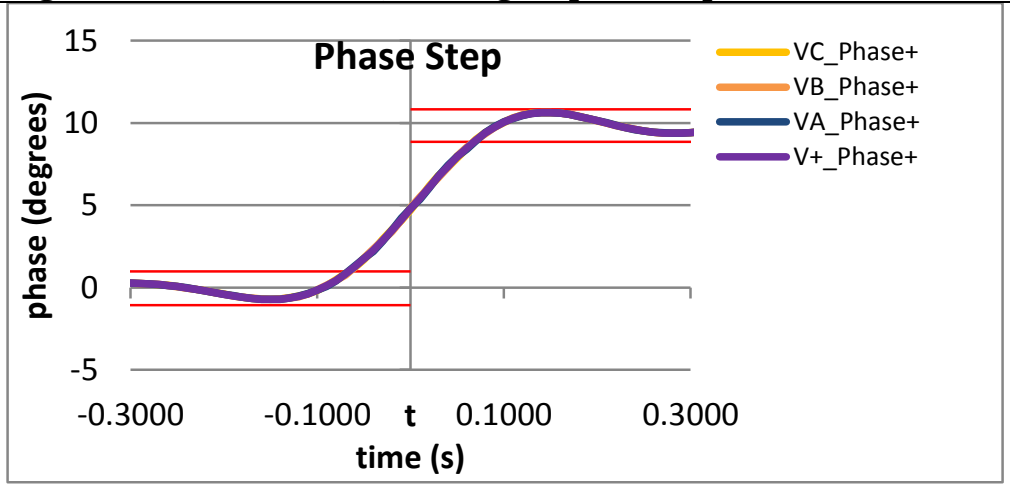

Figure 4919: Fs = 10 FPS, +10 degree phase step
Figure 4916: Fs = 15 FPS, -10 degree phase step

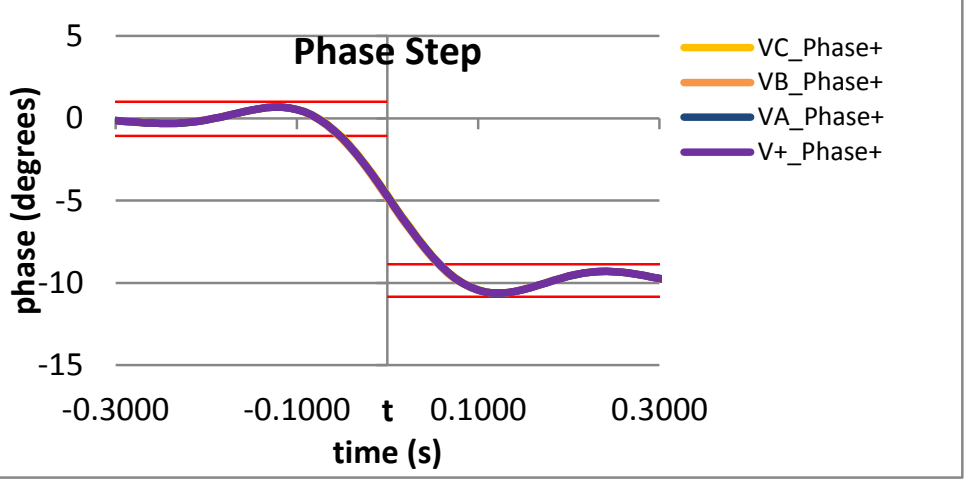

Figure 4918: Fs = 12 FPS, -10 degree phase step

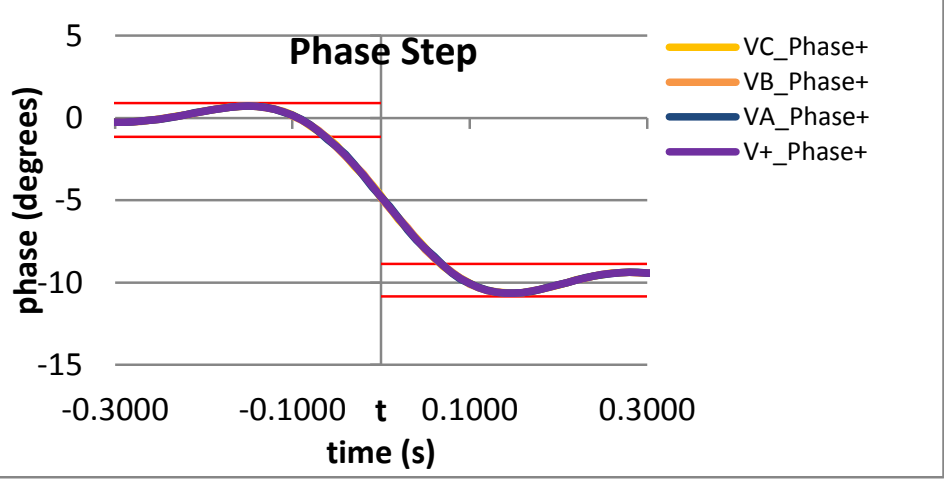

Figure 4920: Fs = 10 FPS, -10 degree phase step 
9.9.6 PMU E dynamic step change in phase phasor overshoot: $F 0=60 \mathrm{~Hz}, \mathrm{M}$ class

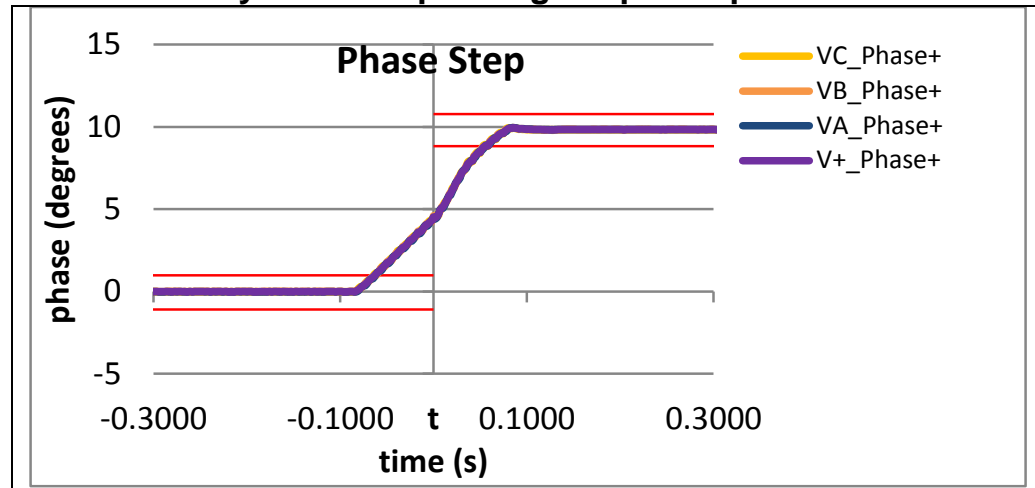

Figure 4921: Fs = 60 FPS, +10 degree phase step

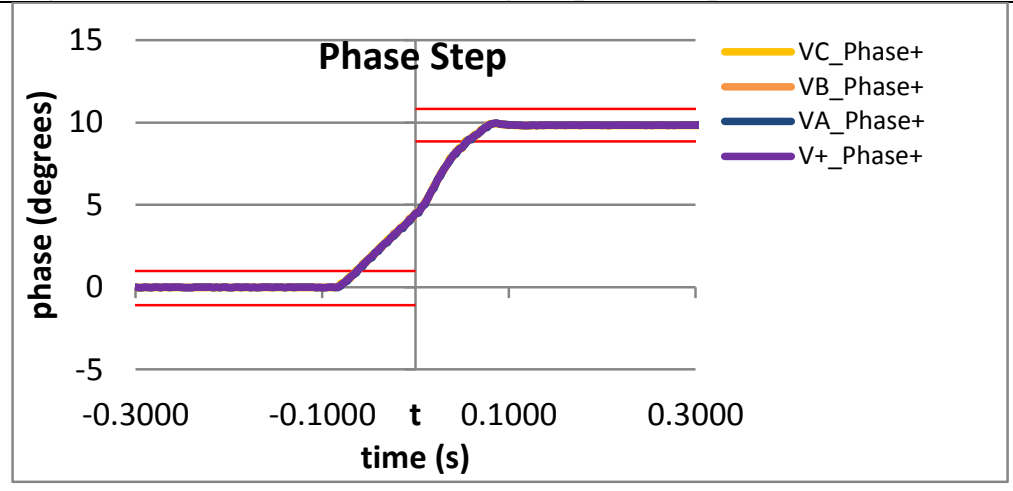

Figure 4923: Fs = 30 FPS, +10 degree phase step

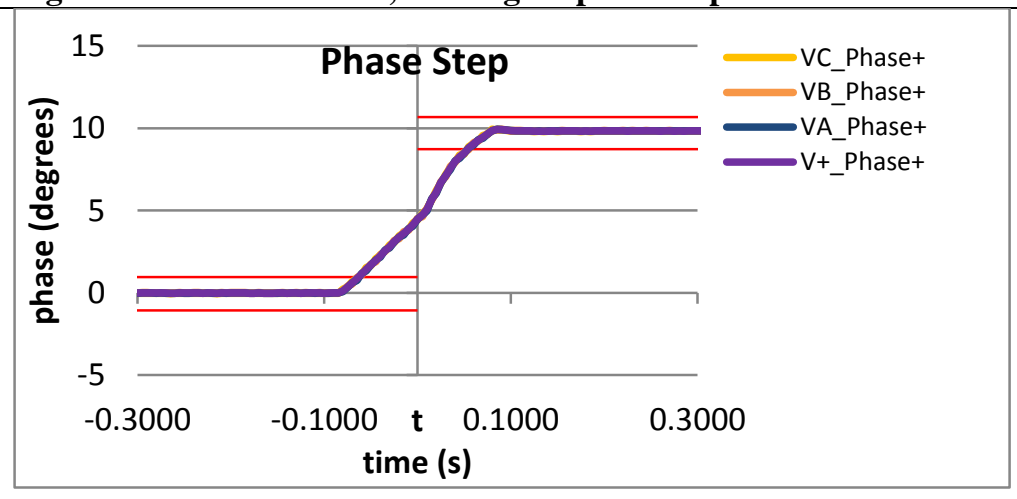

Figure 4925: Fs = 20 FPS, +10 degree phase step

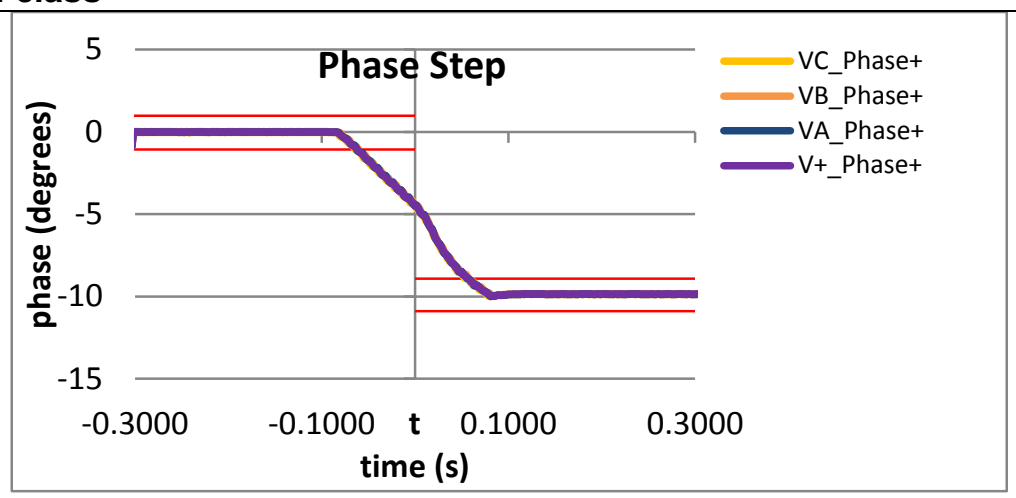

Figure 4922: Fs = 60 FPS, -10 degree phase step

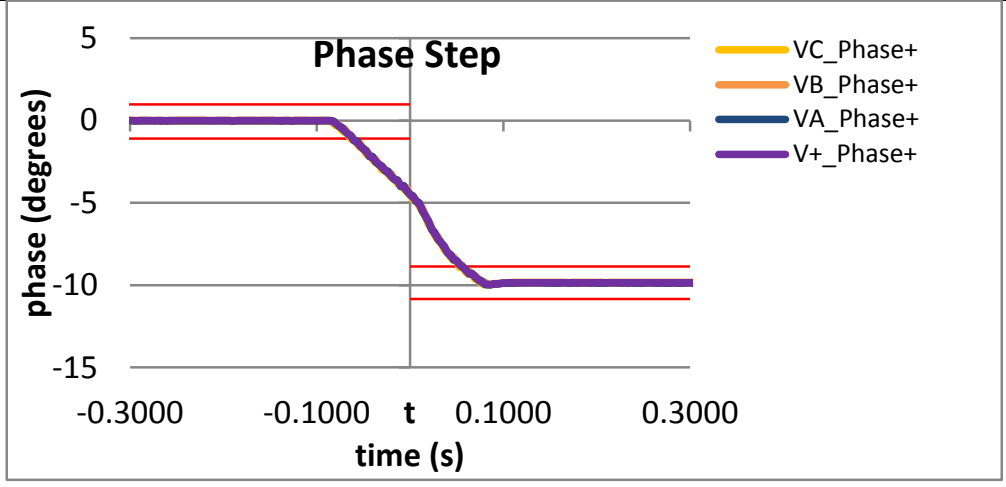

Figure 4924: Fs = 30 FPS, -10 degree phase step

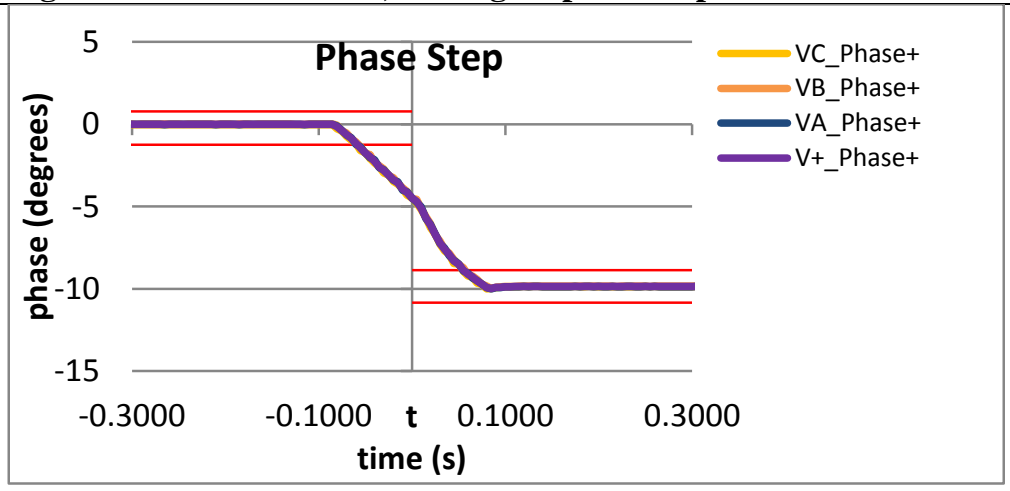

Figure 4926: Fs = 20 FPS, +10 degree phase step 


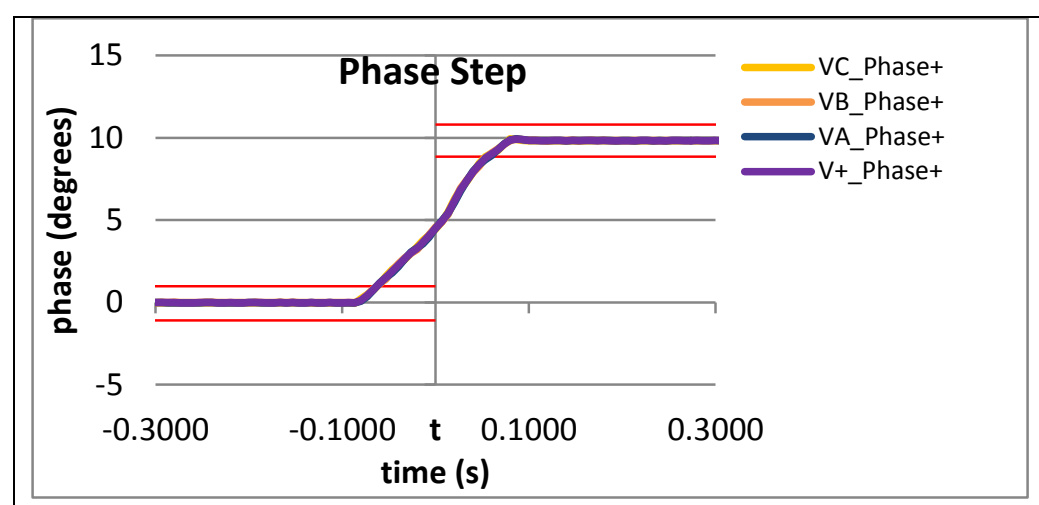

Figure 4927: Fs = 15 FPS, +10 degree phase step

Figure 4929: Fs = 12 FPS, +10 degree phase step

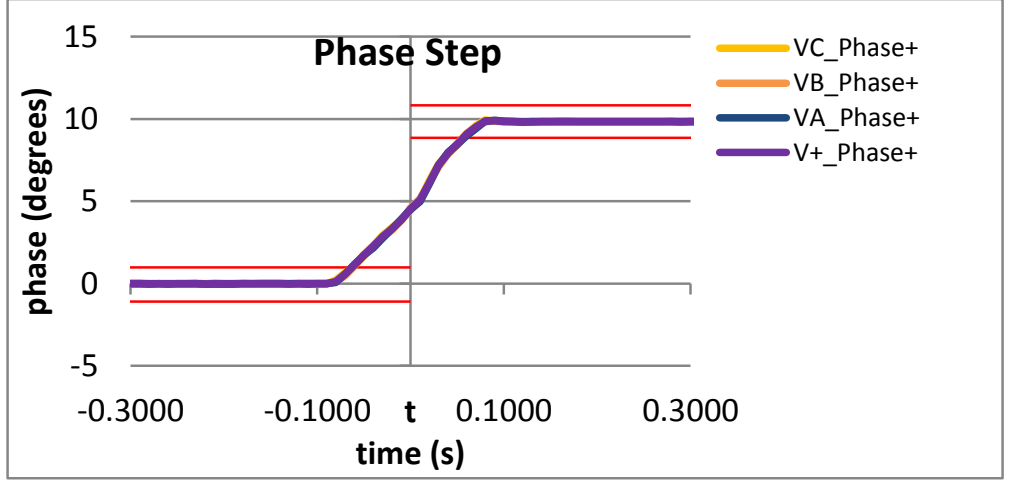

Figure 4931: Fs = 10 FPS, +10 degree phase step

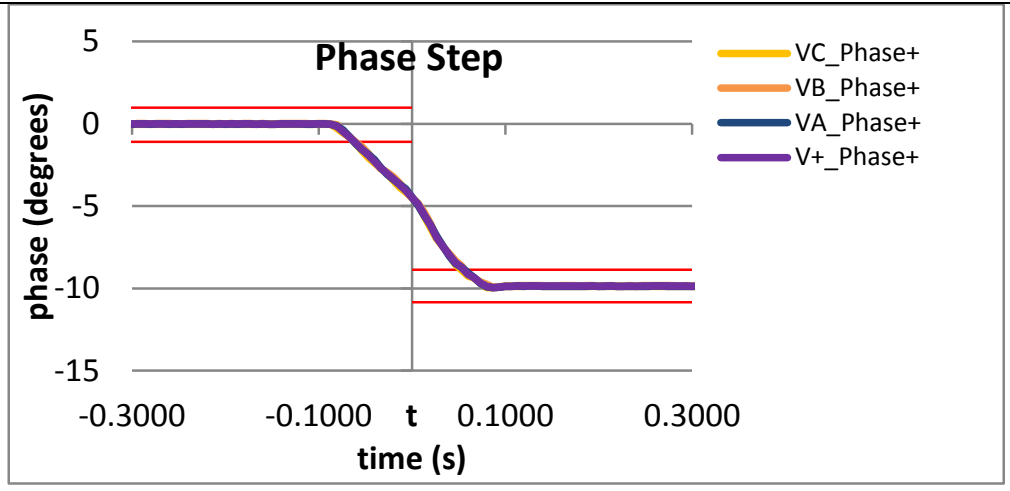

Figure 4928: Fs = 15 FPS, -10 degree phase step

Figure 4930: Fs = 12 FPS, -10 degree phase step



Figure 4932: Fs = 10 FPS, -10 degree phase step 
9.9.7 PMU F dynamic step change in phase phasor overshoot: $F 0=60 \mathrm{~Hz}, \mathrm{M}$ class

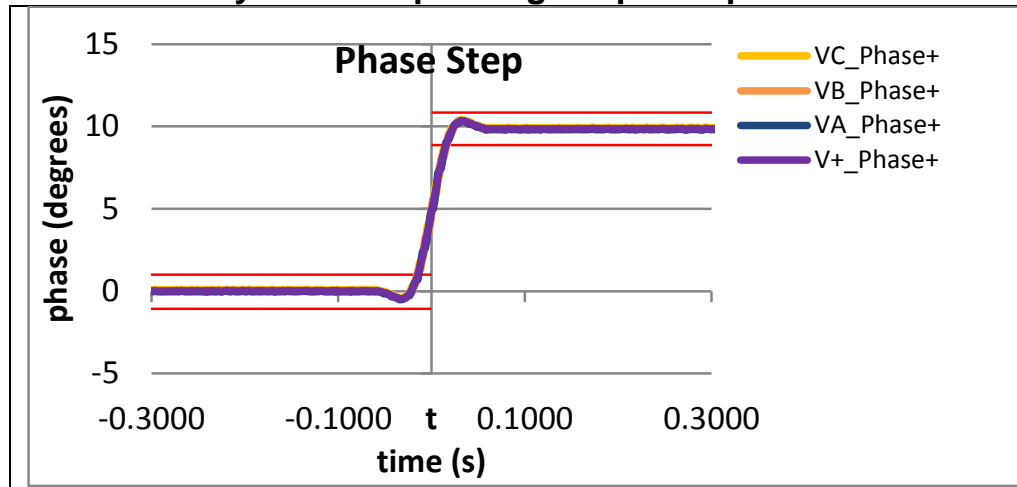

Figure 4933: Fs = 60 FPS, +10 degree phase step

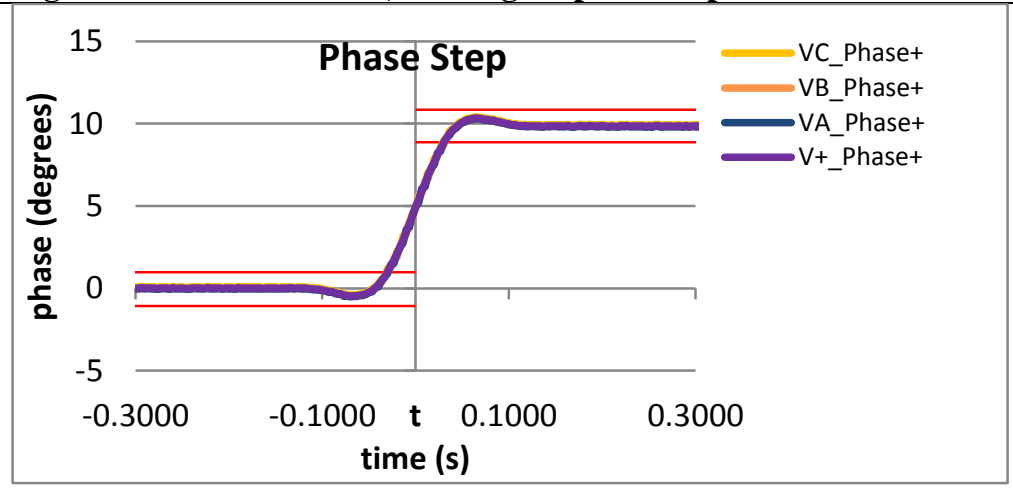

Figure 4935: Fs = 30 FPS, +10 degree phase step

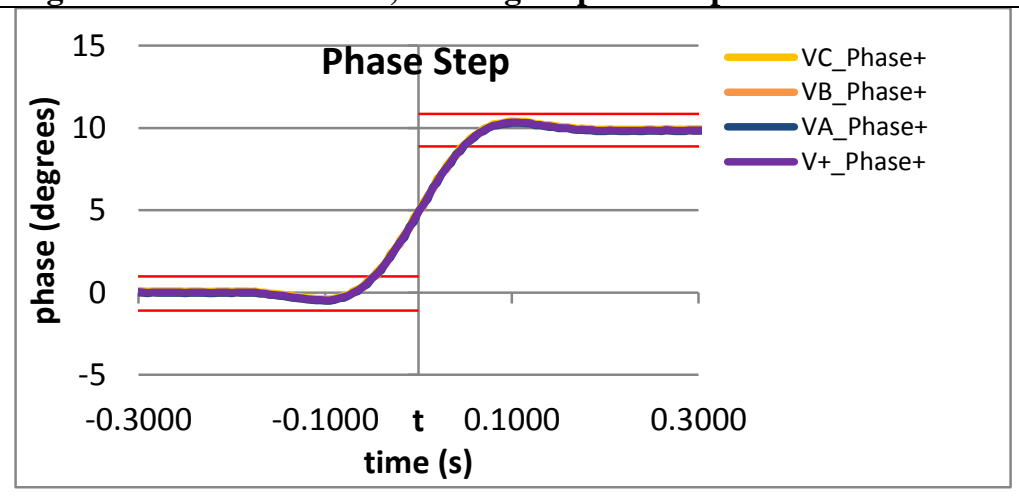

Figure 4937: Fs = 20 FPS, +10 degree phase step

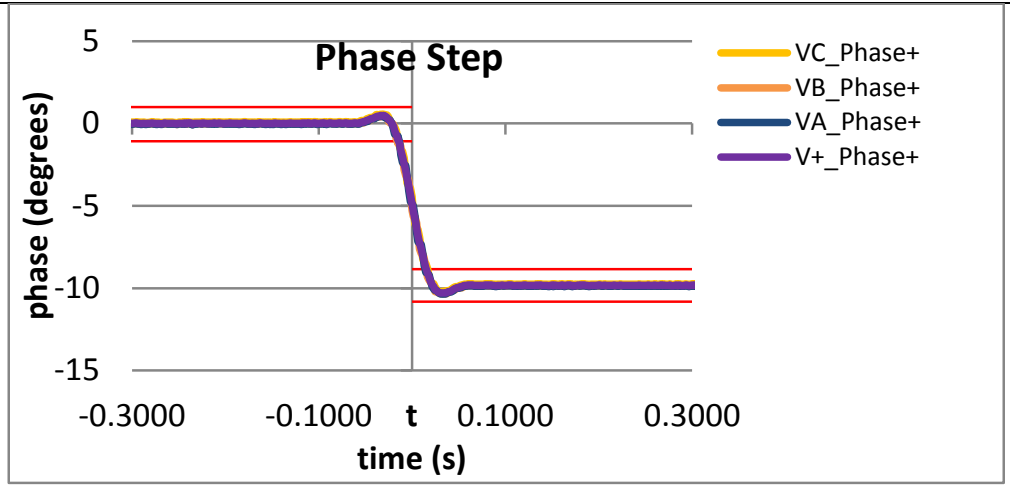

Figure 4934: Fs = 60 FPS, -10 degree phase step

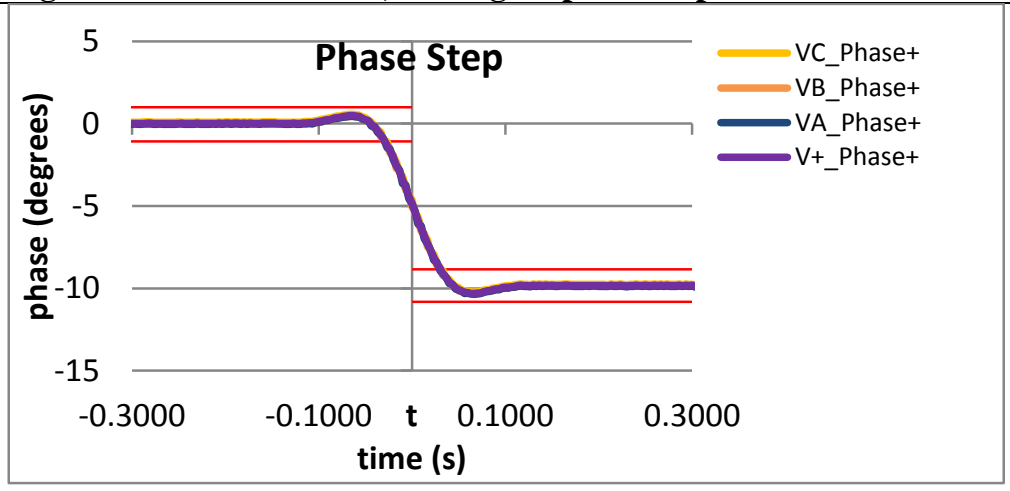

Figure 4936: Fs = 30 FPS, -10 degree phase step

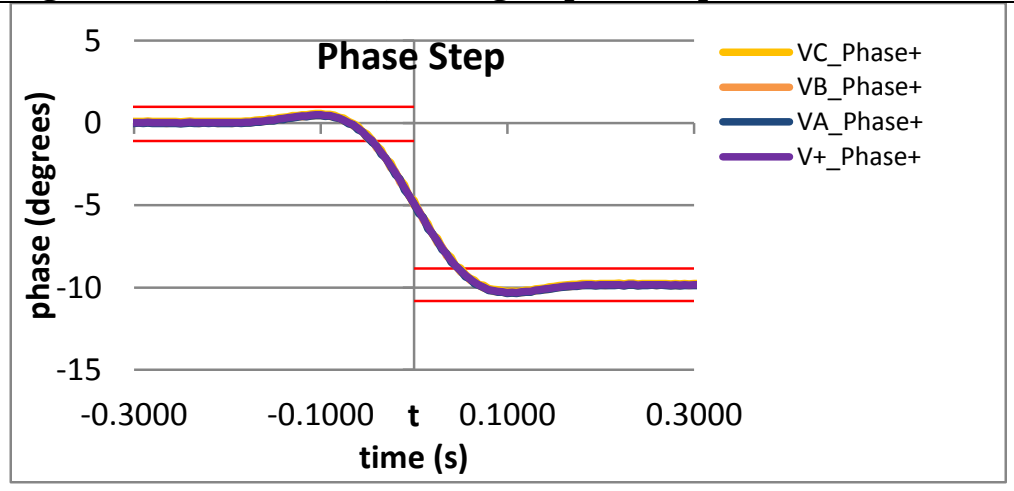

Figure 4938: Fs $=20$ FPS, +10 degree phase step 
This publication is available free of charge from $\mathrm{http}: / / \mathrm{dx}$.doi.org/10.6028/NIST.IR.8106

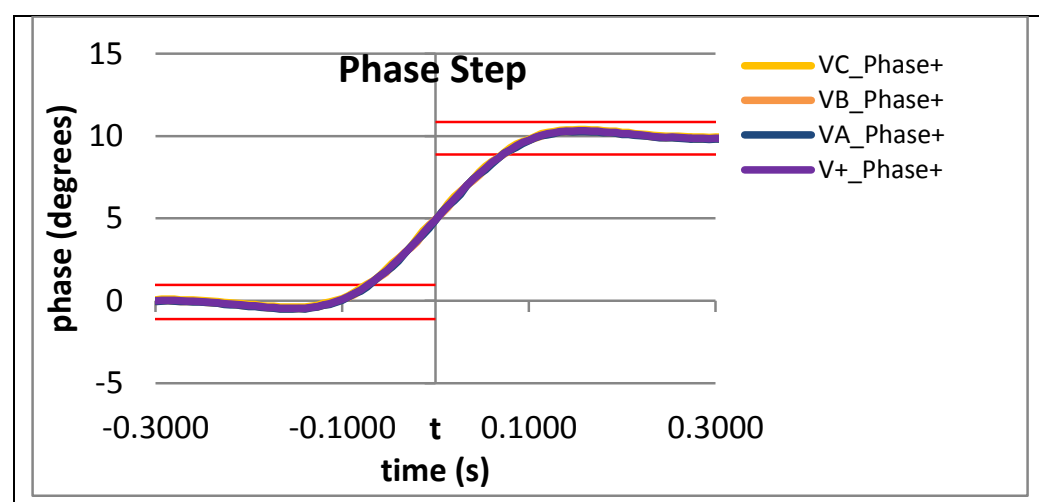

Figure 4939: Fs = 15 FPS, +10 degree phase step

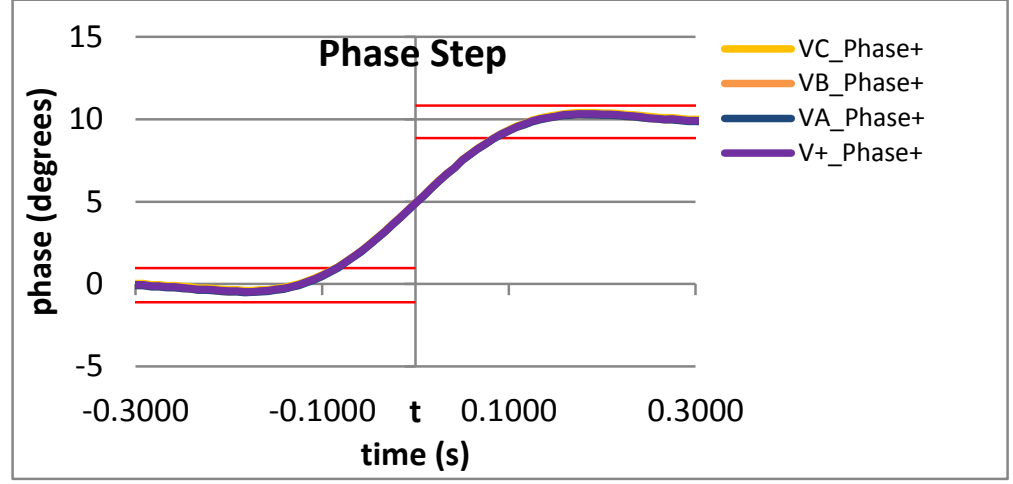

Figure 4941: Fs = 12 FPS, +10 degree phase step

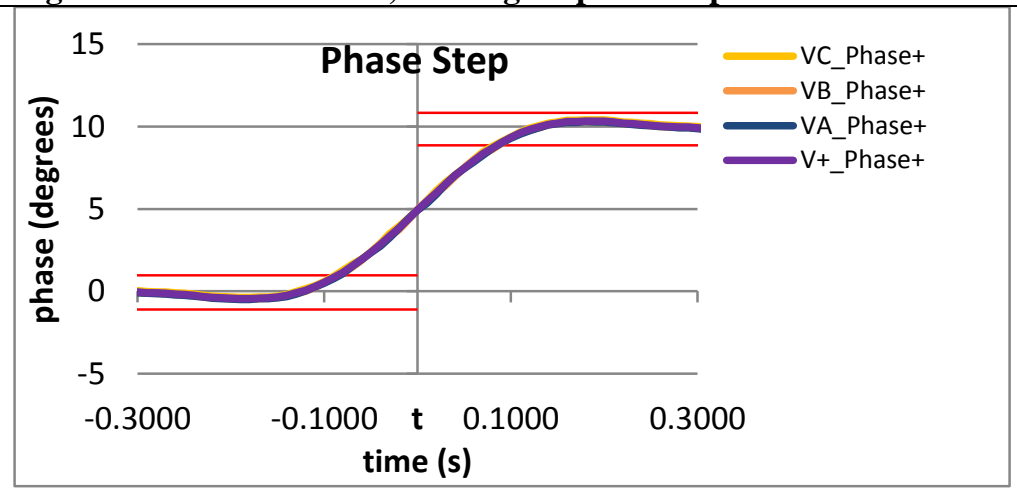

Figure 4943: Fs = 10 FPS, +10 degree phase step

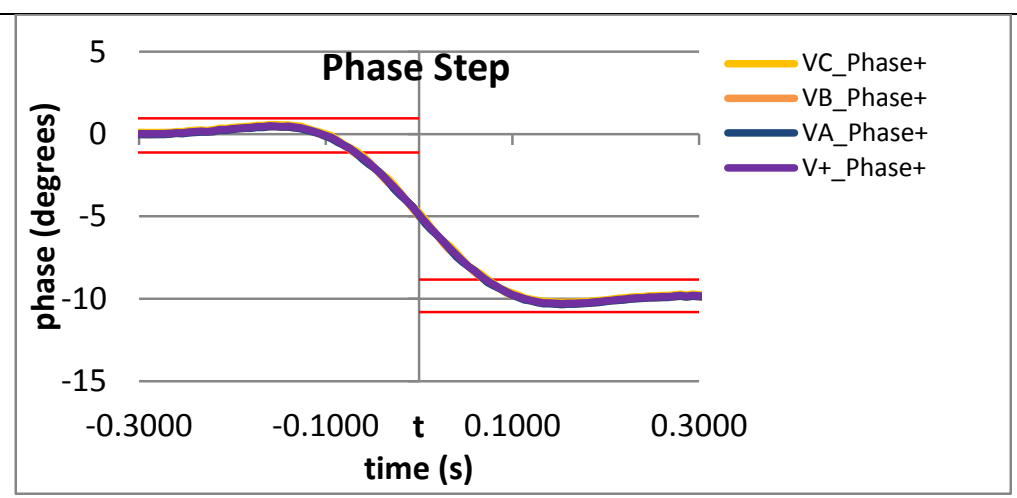

Figure 4940: Fs = 15 FPS, -10 degree phase step

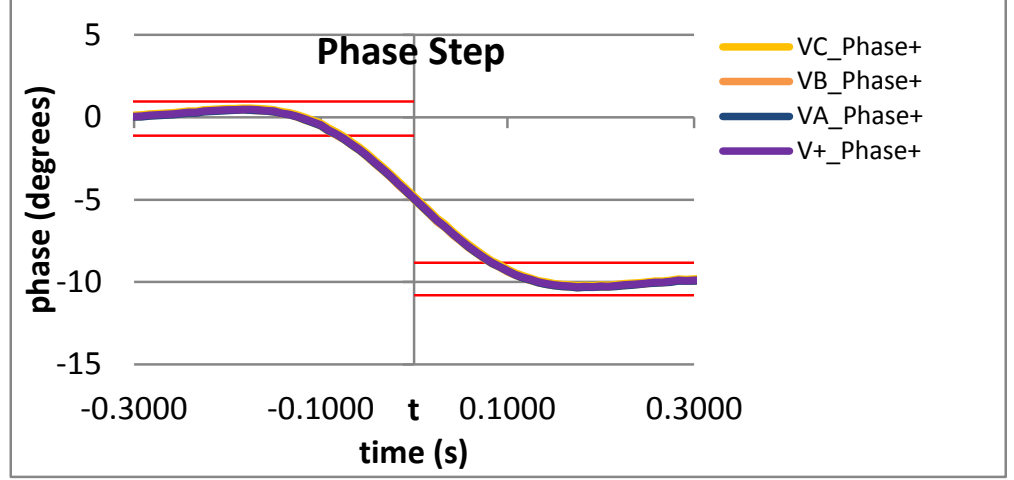

Figure 4942: Fs = 12 FPS, -10 degree phase step

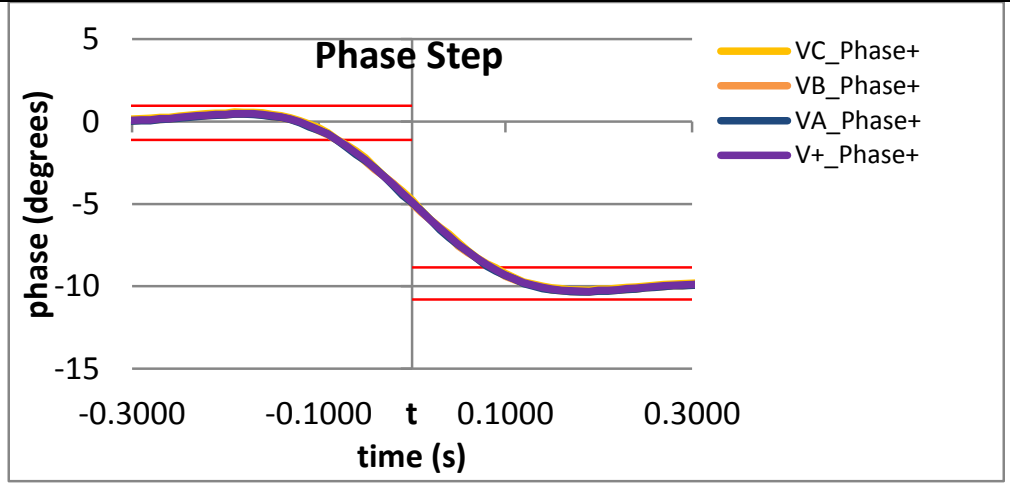

Figure 4944: Fs = 10 FPS, -10 degree phase step 
9.9.8 PMU G dynamic step change in phase phasor overshoot: $F 0=60 \mathrm{~Hz}, \mathrm{M}$ class

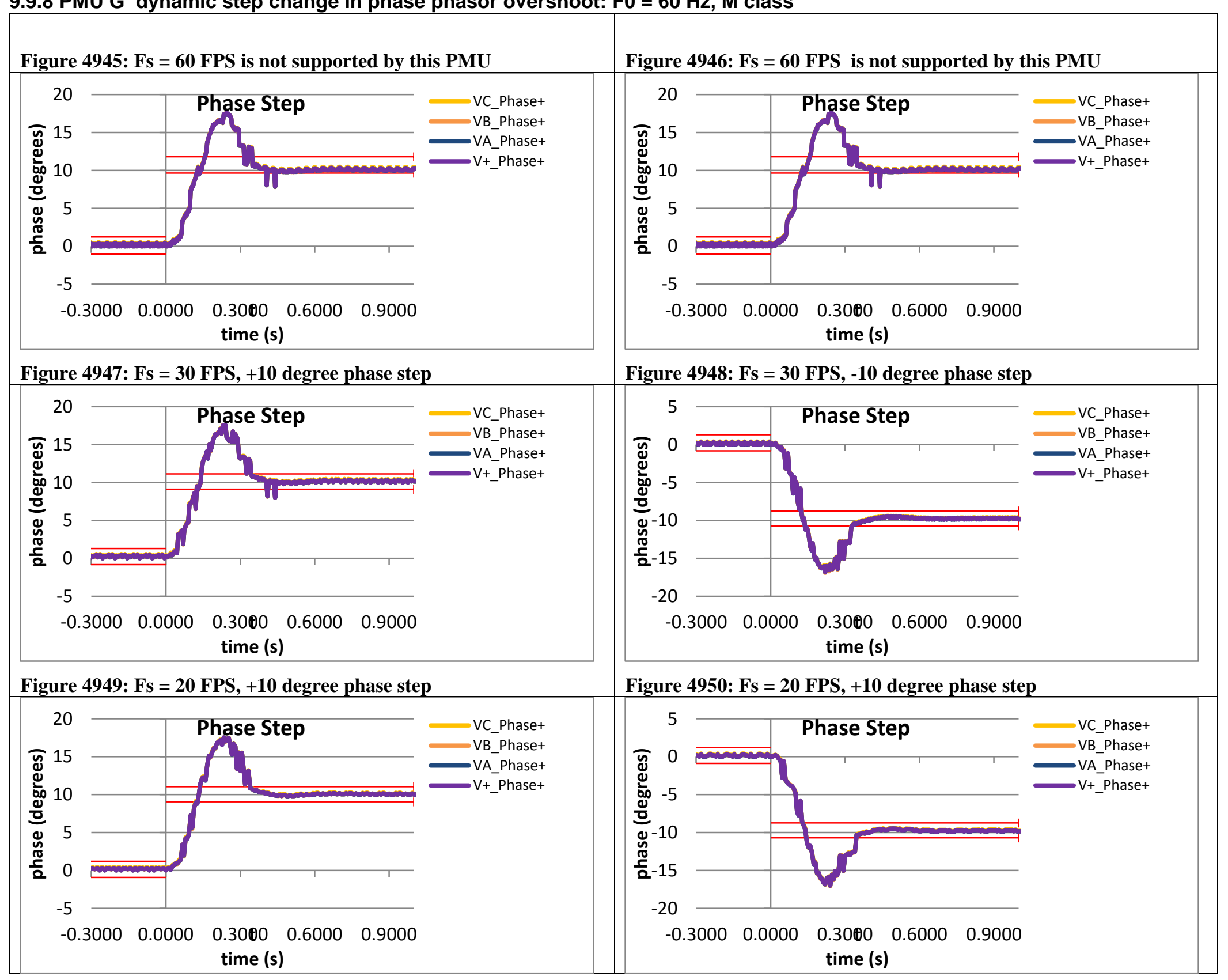




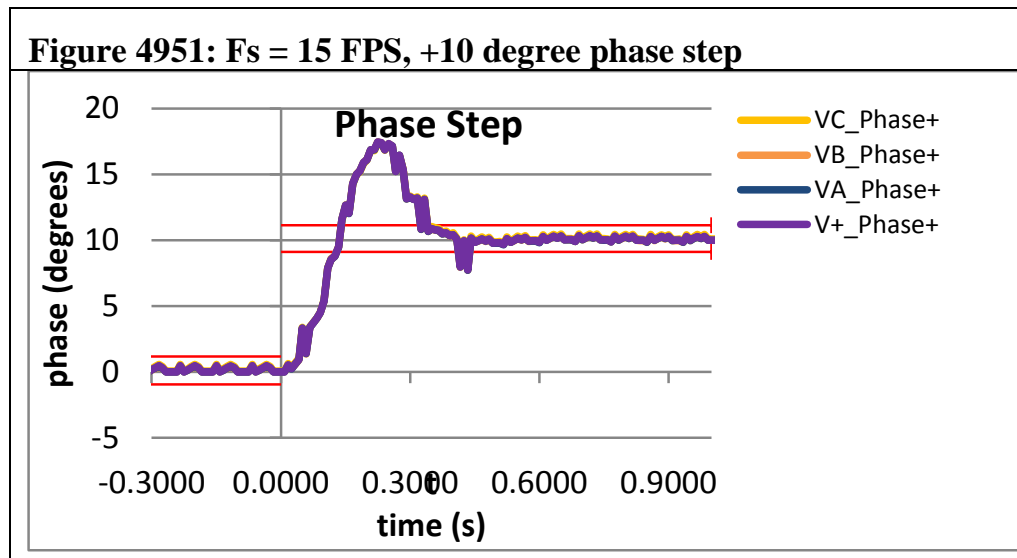

Figure 4953: Fs = 12 FPS, +10 degree phase step Figure 4952: Fs = 15 FPS, -10 degree phase step

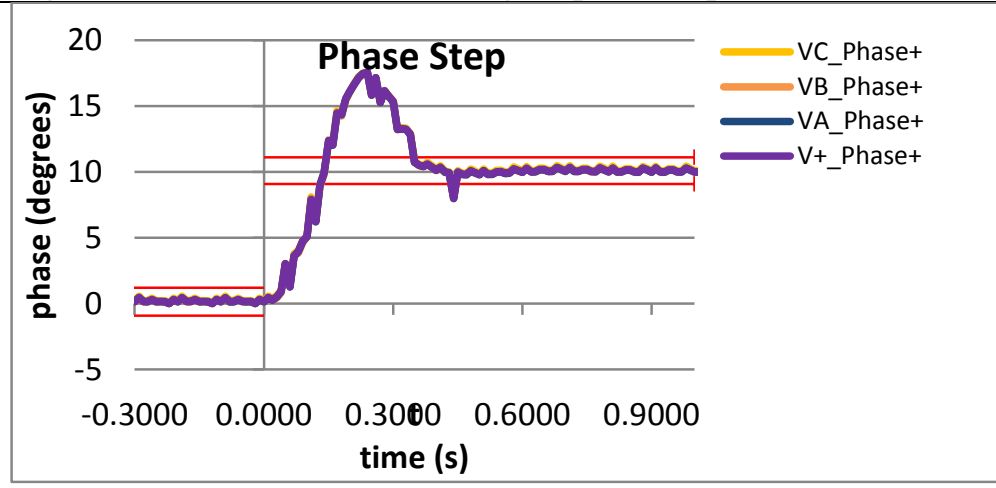

Figure 4955: Fs = 10 FPS, +10 degree phase step

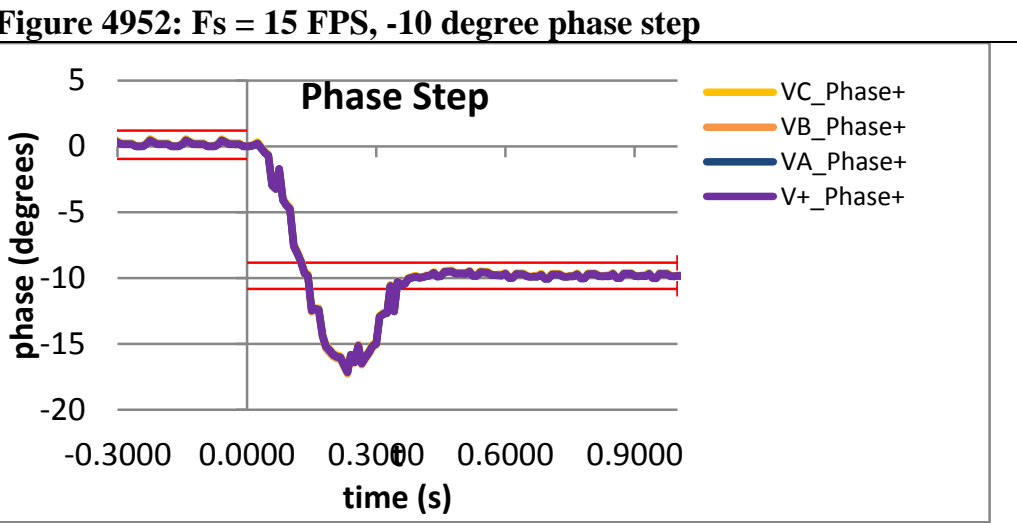

Figure 4954: Fs = 12 FPS, -10 degree phase step

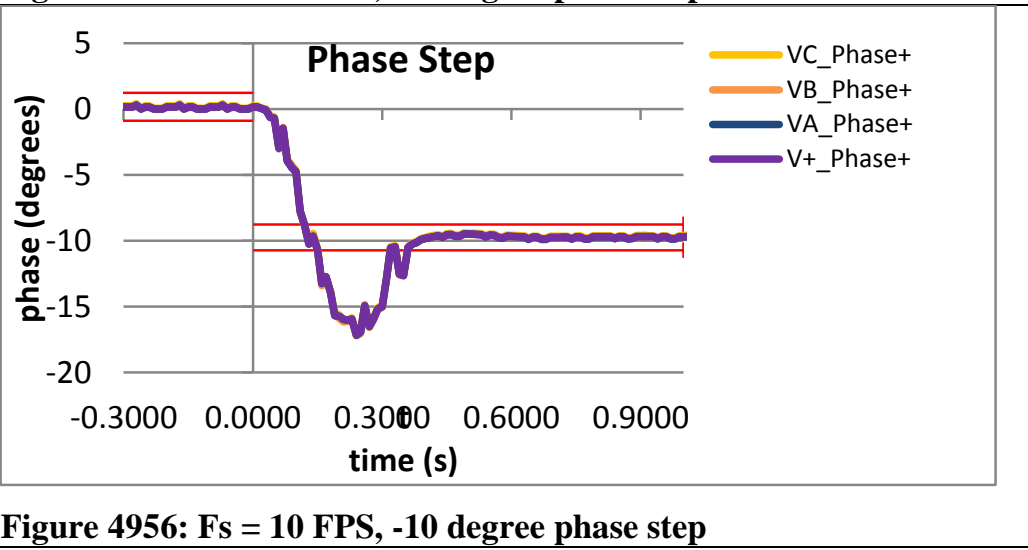


9.9.9 PMU H dynamic step change in phase phasor overshoot: $F 0=60 \mathrm{~Hz}, \mathrm{M}$ class

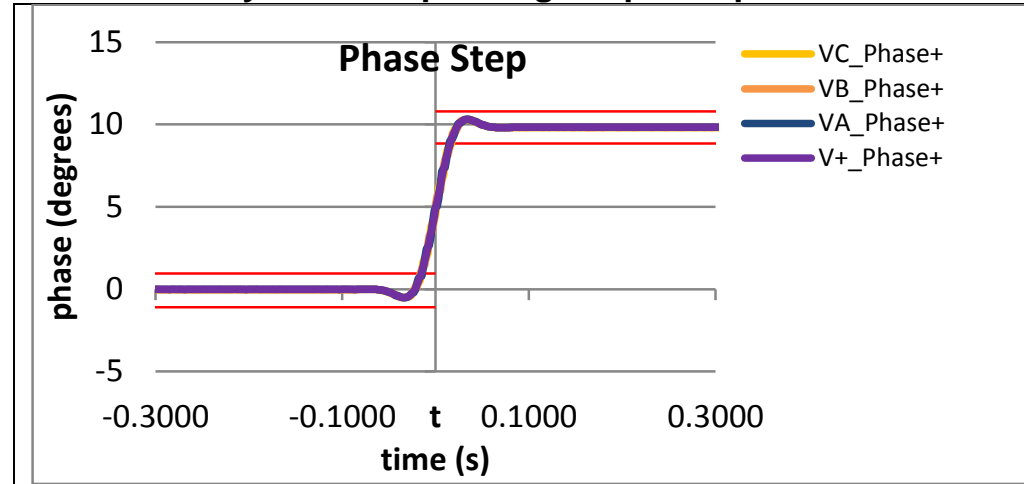

Figure 4957: Fs = 60 FPS, +10 degree phase step

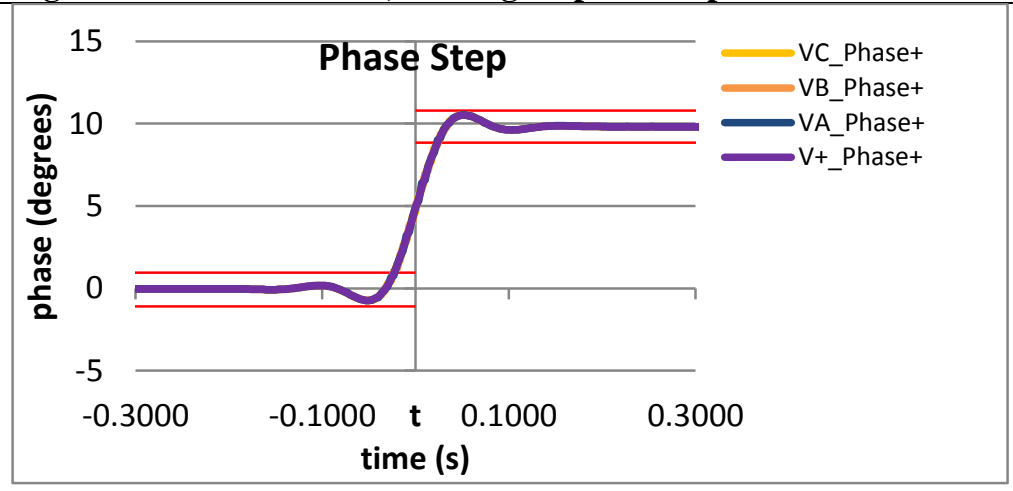

Figure 4959: Fs = 30 FPS, +10 degree phase step

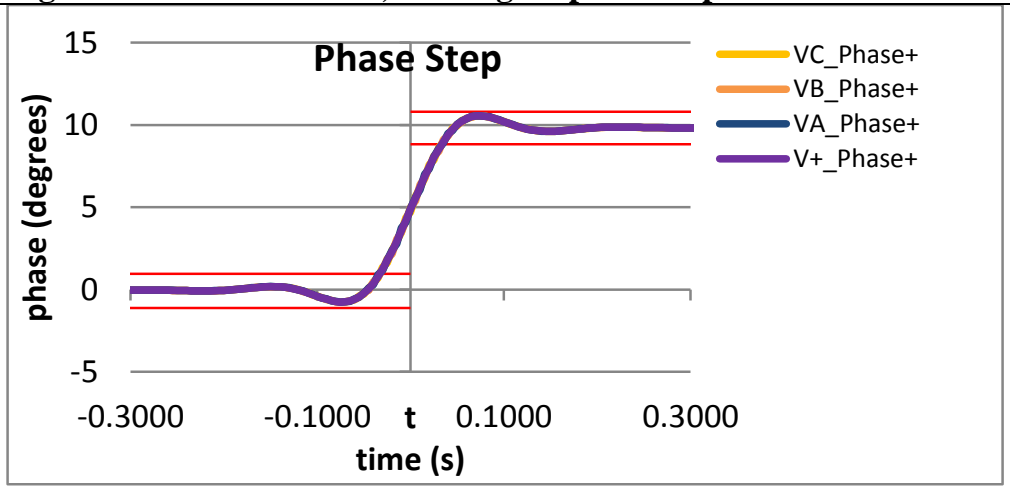

Figure 4961: Fs = 20 FPS, +10 degree phase step

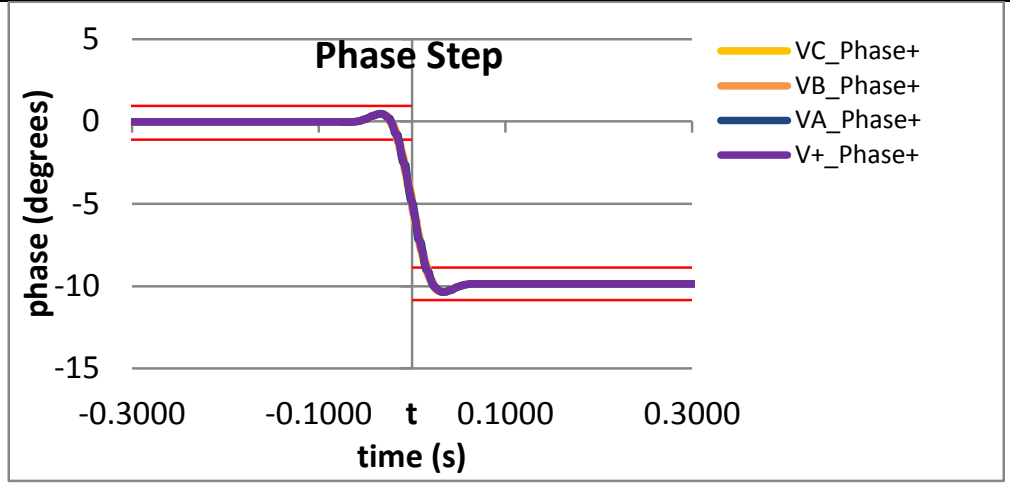

Figure 4958: Fs = 60 FPS, -10 degree phase step

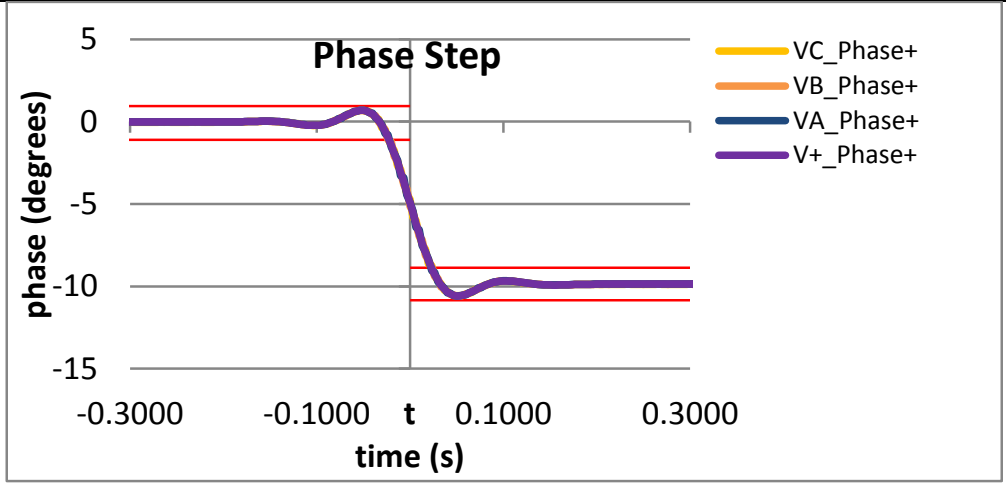

Figure 4960: Fs = 30 FPS, -10 degree phase step

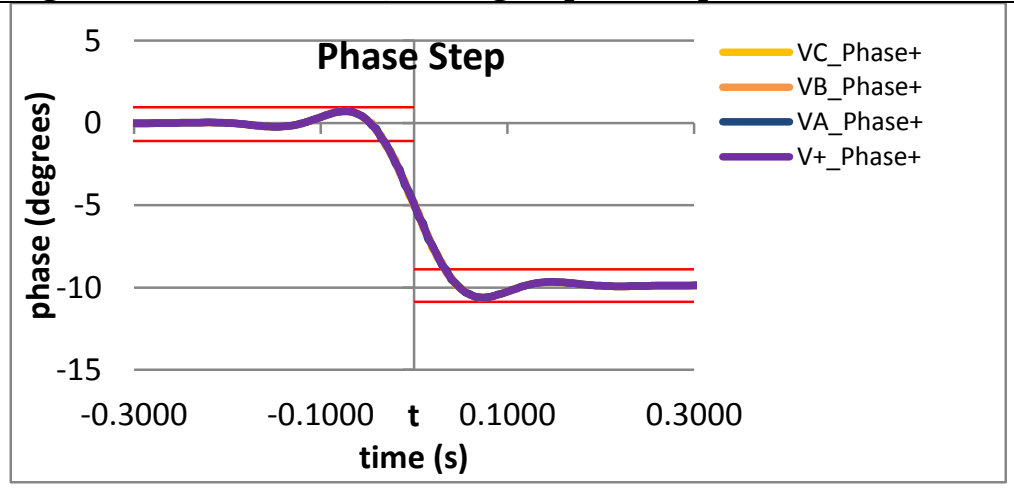

Figure 4962: Fs $=20$ FPS, +10 degree phase step 
This publication is available free of charge from $\mathrm{http}: / / \mathrm{dx}$.doi.org/10.6028/NIST.IR.8106

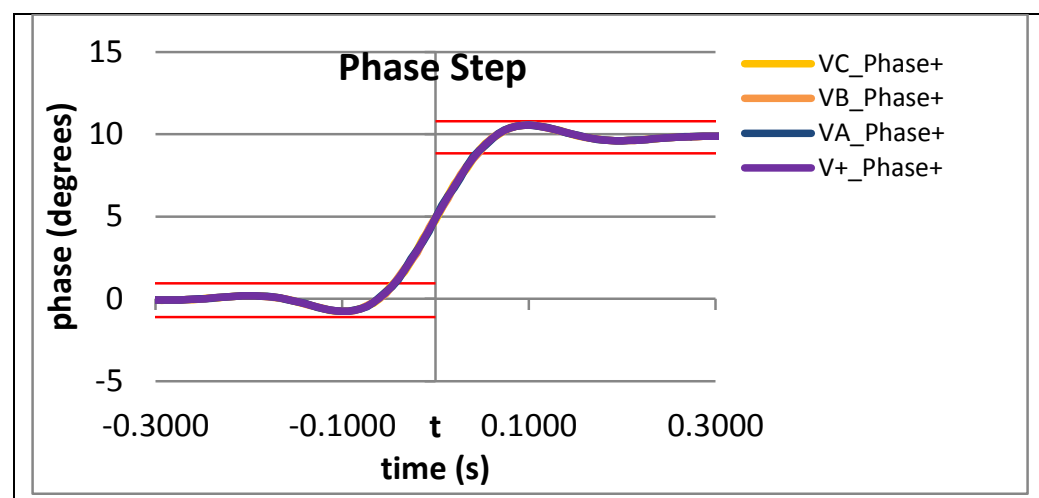

Figure 4963: Fs = 15 FPS, +10 degree phase step

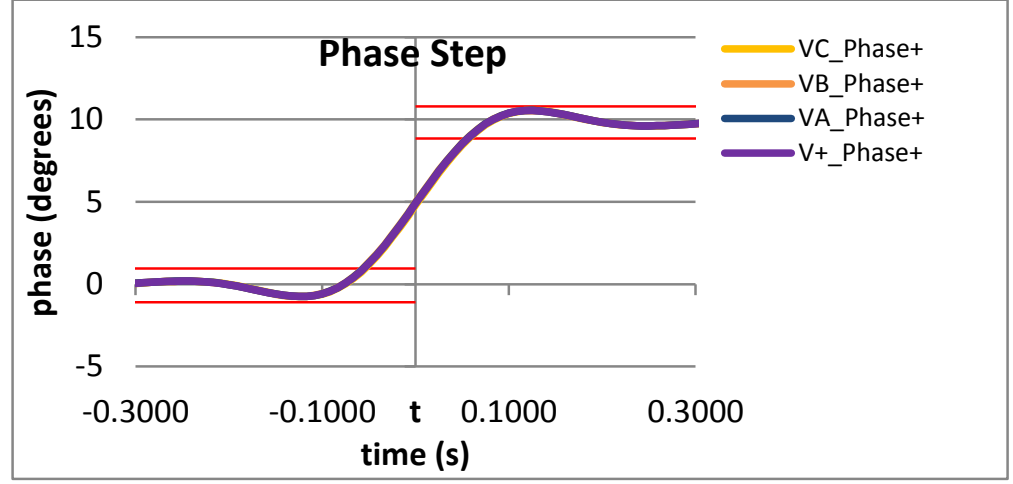

Figure 4965: Fs = 12 FPS, +10 degree phase step

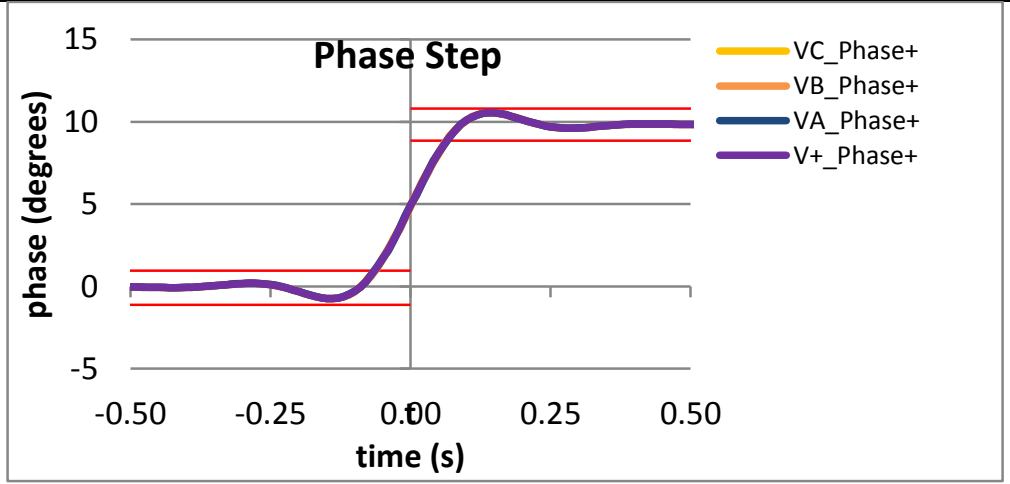

Figure 4967: Fs = 10 FPS, +10 degree phase step

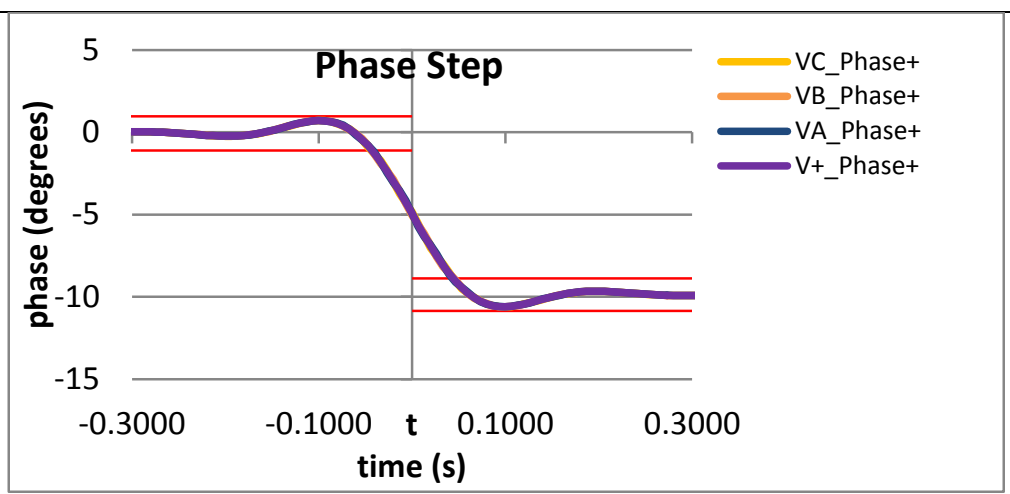

Figure 4964: Fs = 15 FPS, -10 degree phase step

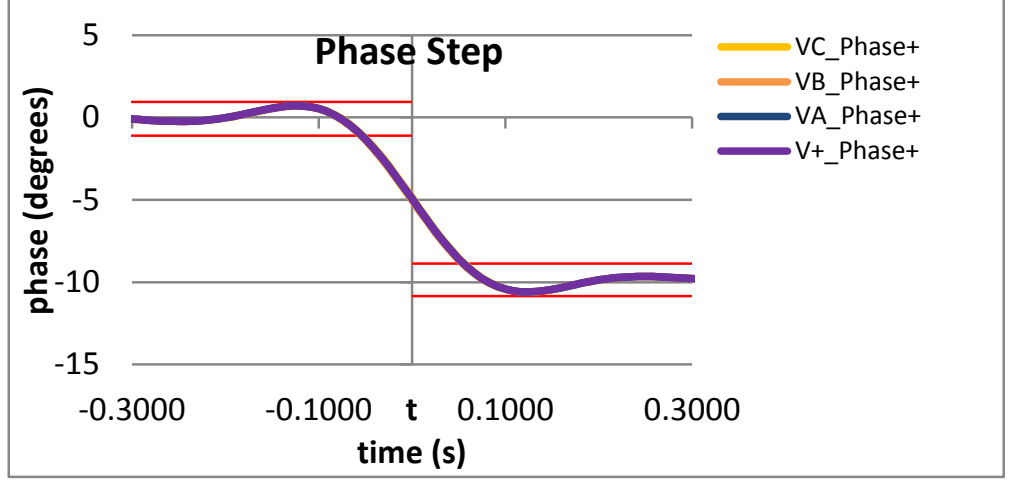

Figure 4966: Fs = 12 FPS, -10 degree phase step

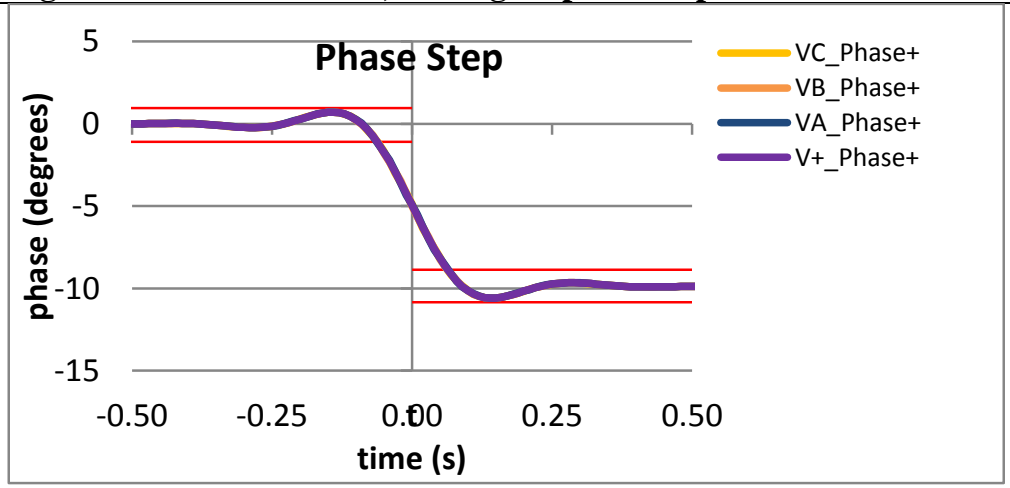

Figure 4968: Fs = 10 FPS, -10 degree phase step 
9.9.10 PMU I dynamic step change in phase phasor overshoot: $F 0=60 \mathrm{~Hz}, \mathrm{M}$ class

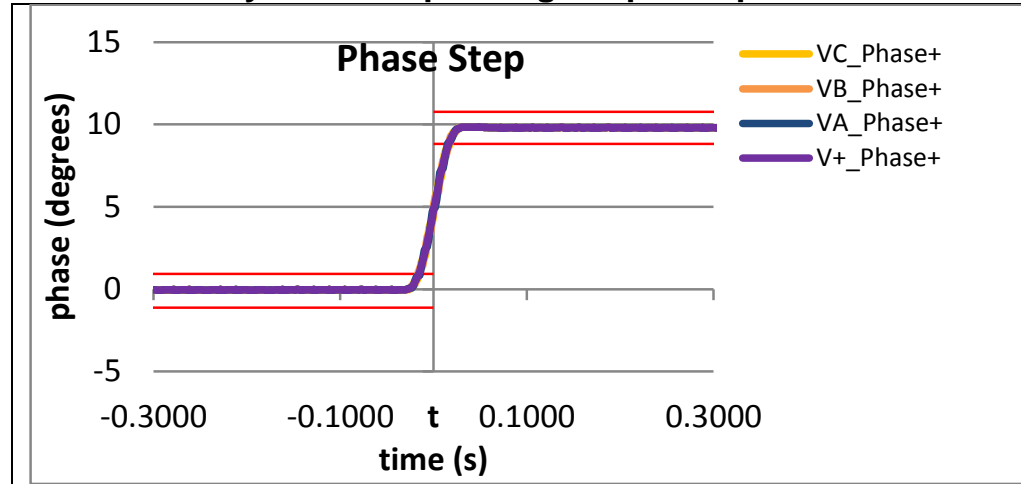

Figure 4969: Fs = 60 FPS, +10 degree phase step

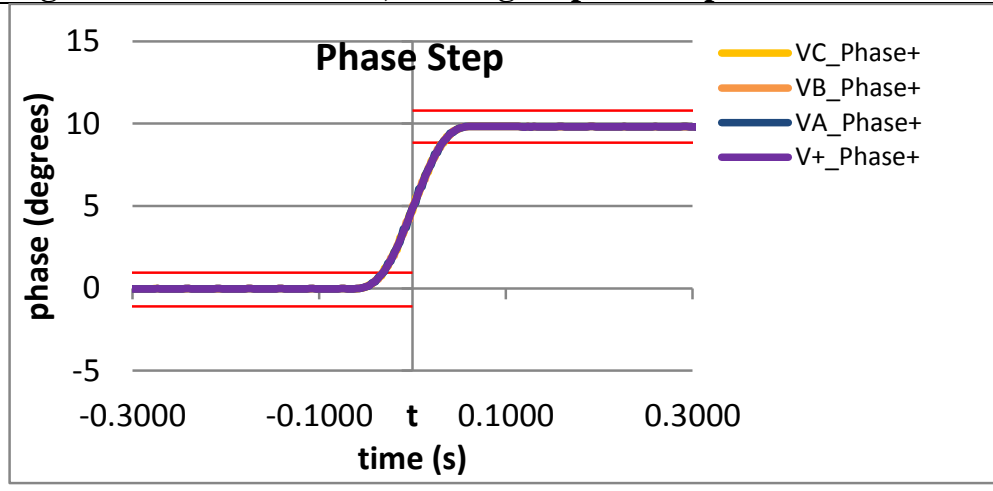

Figure 4971: Fs = 30 FPS, +10 degree phase step

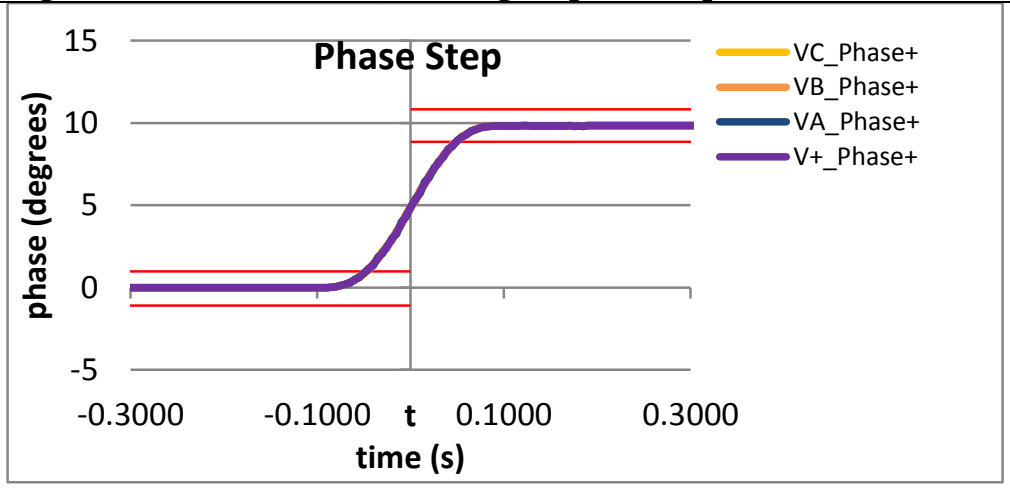

Figure 4973: Fs = 20 FPS, +10 degree phase step

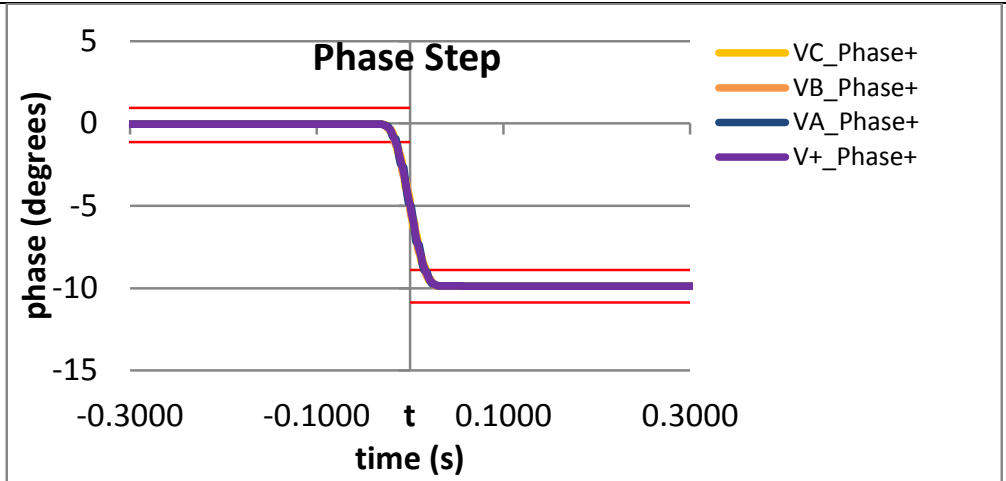

Figure 4970: Fs = 60 FPS, -10 degree phase step

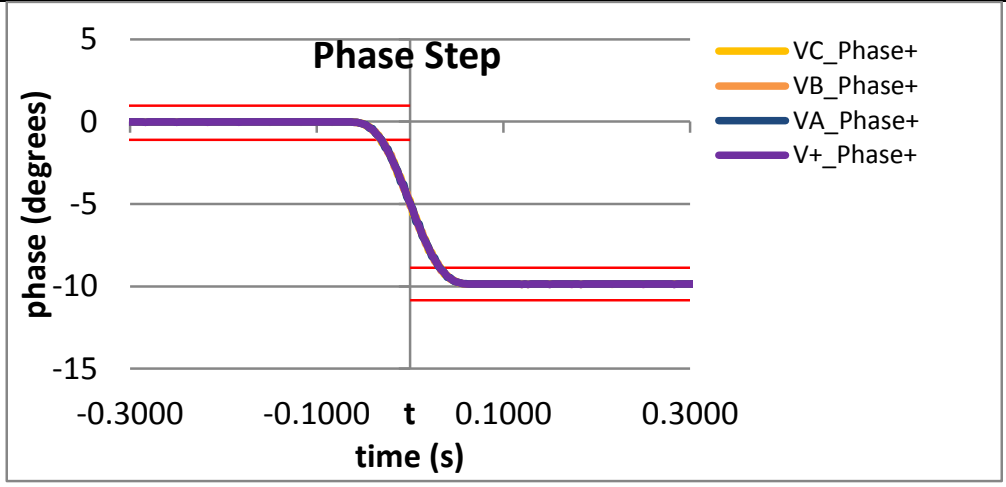

Figure 4972: Fs = 30 FPS, -10 degree phase step

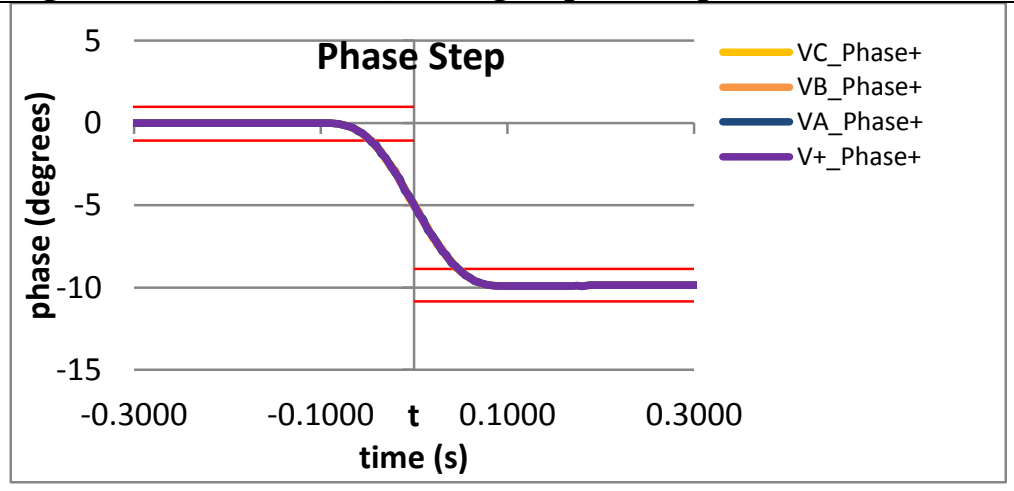

Figure 4974: Fs $=20$ FPS, +10 degree phase step 
This publication is available free of charge from $\mathrm{http}: / / \mathrm{dx}$.doi.org/10.6028/NIST.IR.8106

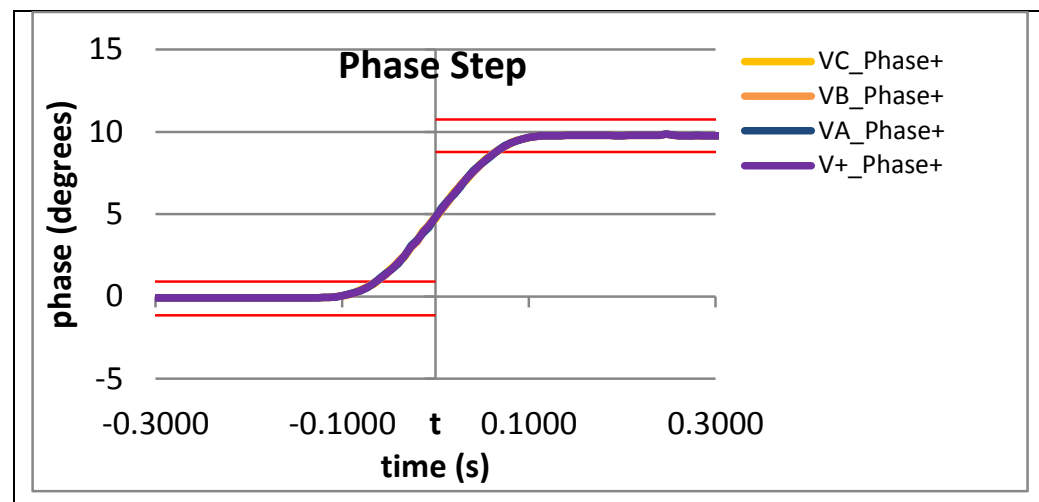

Figure 4975: Fs = 15 FPS, +10 degree phase step

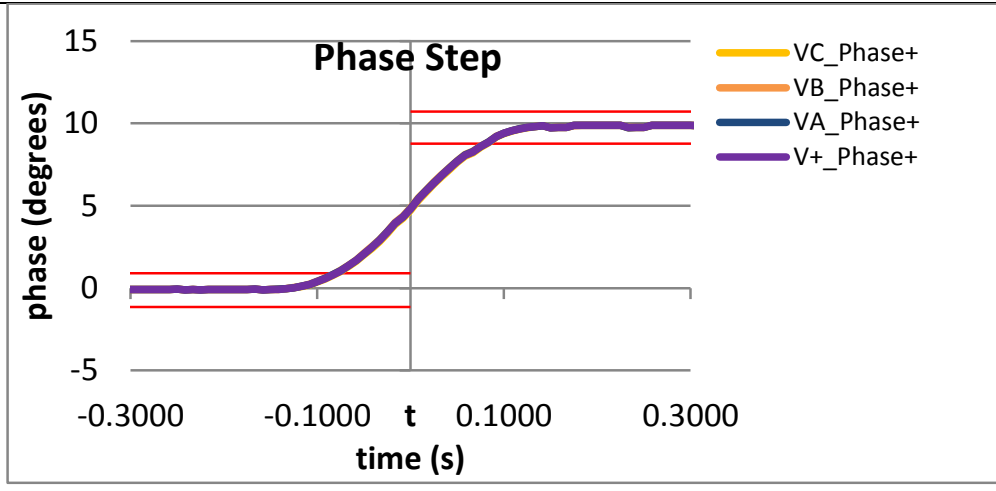

Figure 4977: Fs = 12 FPS, +10 degree phase step

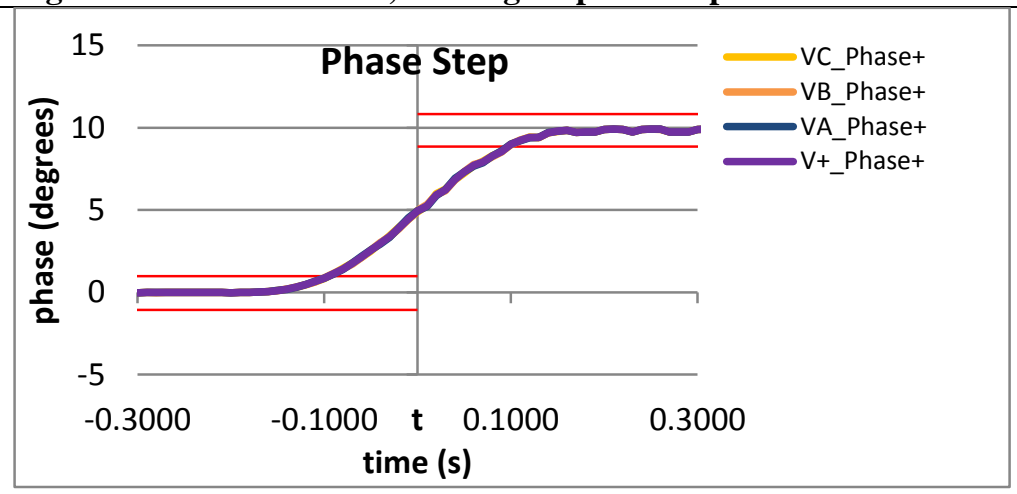

Figure 4979: Fs = 10 FPS, +10 degree phase step

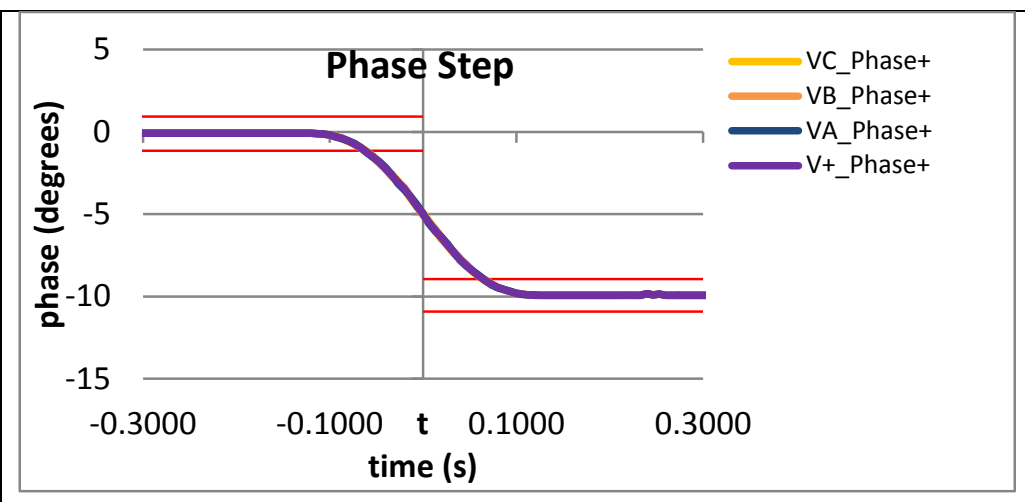

Figure 4976: Fs = 15 FPS, -10 degree phase step

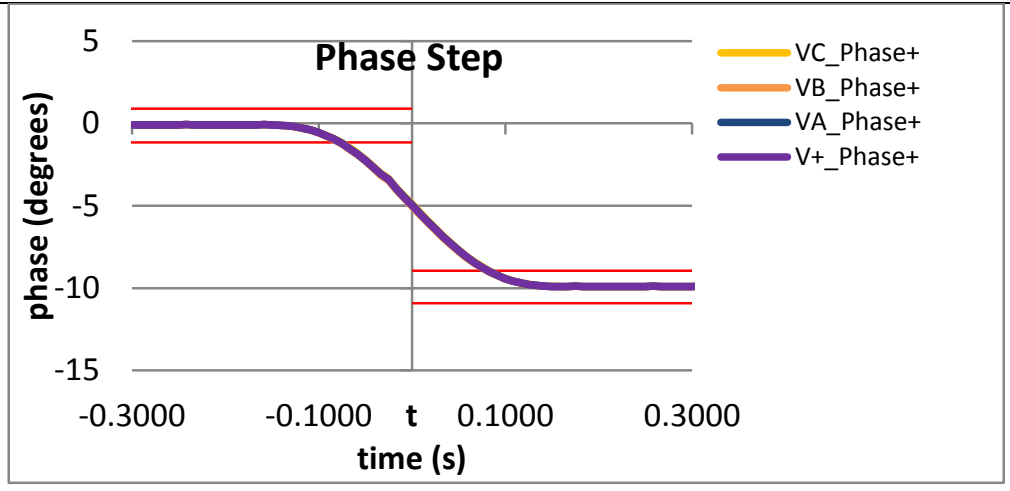

Figure 4978: Fs = 12 FPS, -10 degree phase step

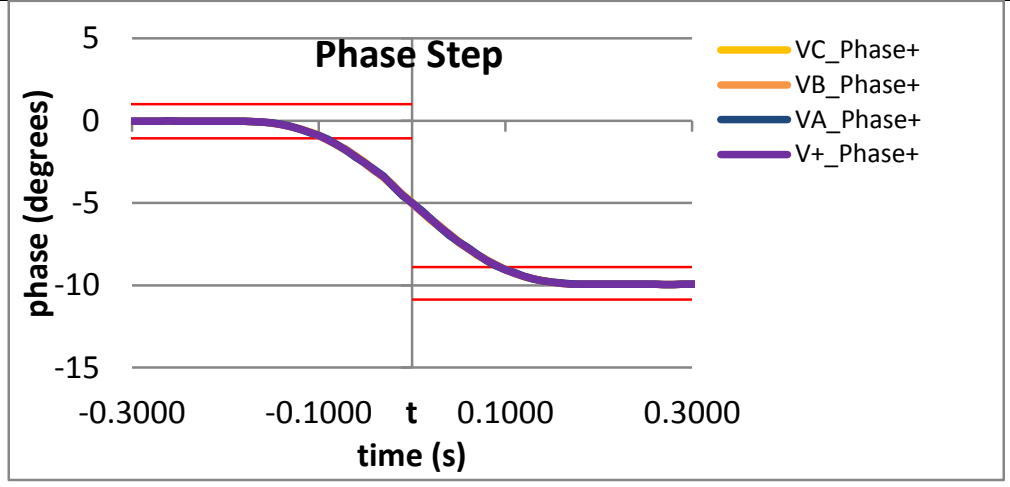

Figure 4980: Fs = 10 FPS, -10 degree phase step 
9.9.11 PMU J dynamic step change in phase phasor overshoot: $\mathrm{FO}=60 \mathrm{~Hz}, \mathrm{M}$ class

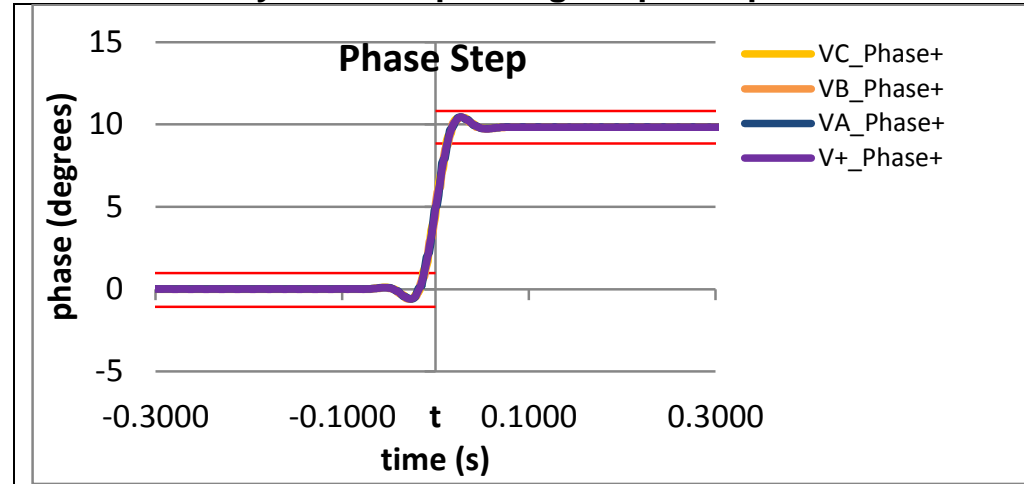

Figure 4981: Fs = 60 FPS, +10 degree phase step

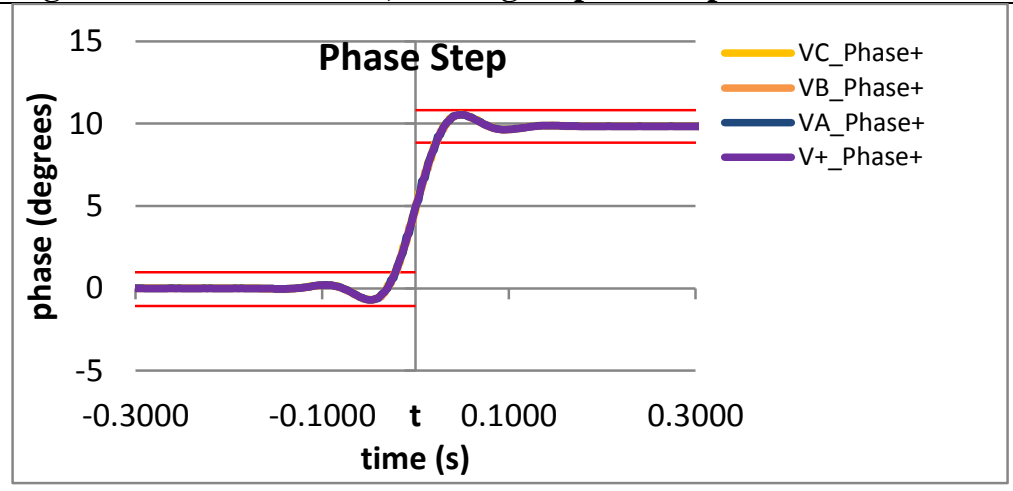

Figure 4983: Fs = 30 FPS, +10 degree phase step

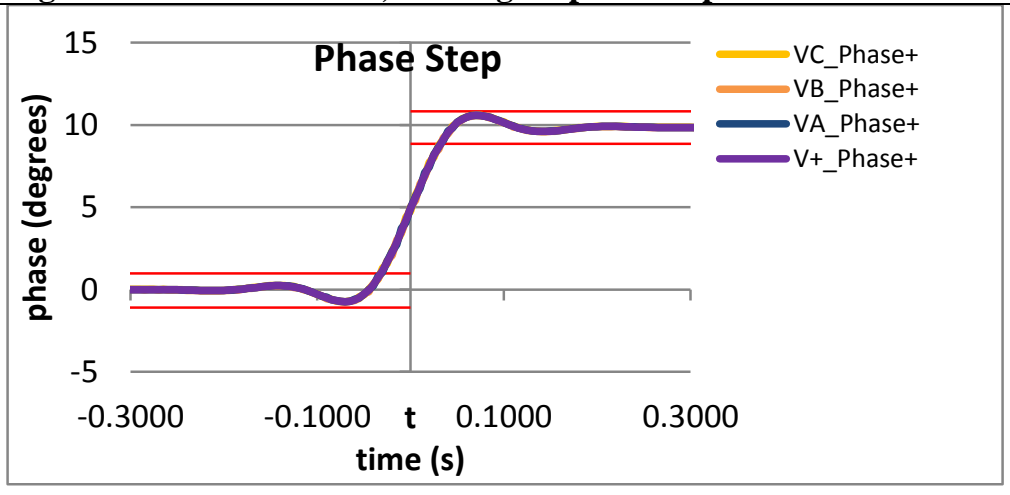

Figure 4985: Fs = 20 FPS, +10 degree phase step

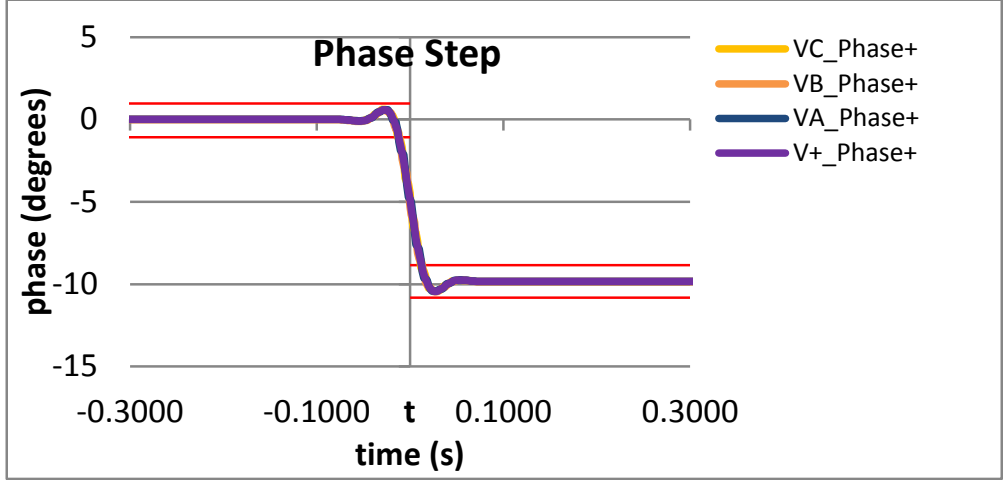

Figure 4982: Fs = 60 FPS, -10 degree phase step

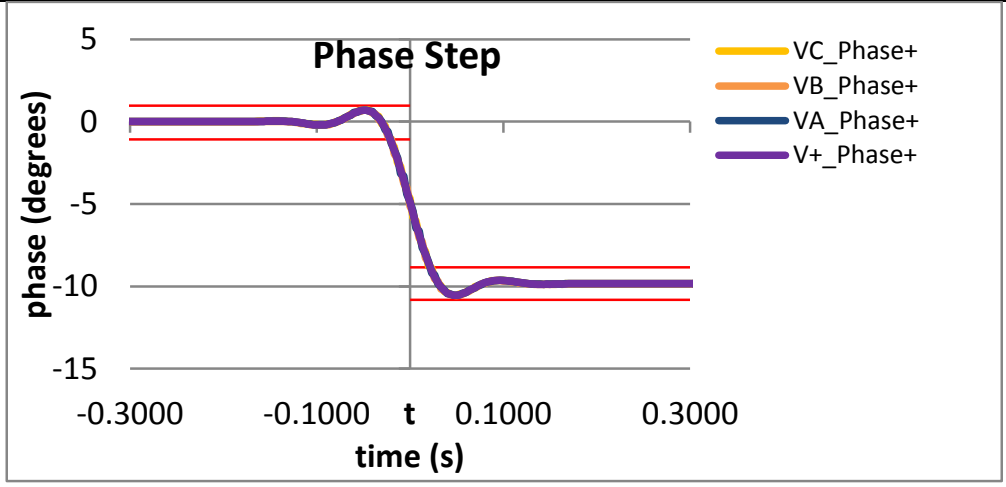

Figure 4984: Fs = 30 FPS, -10 degree phase step

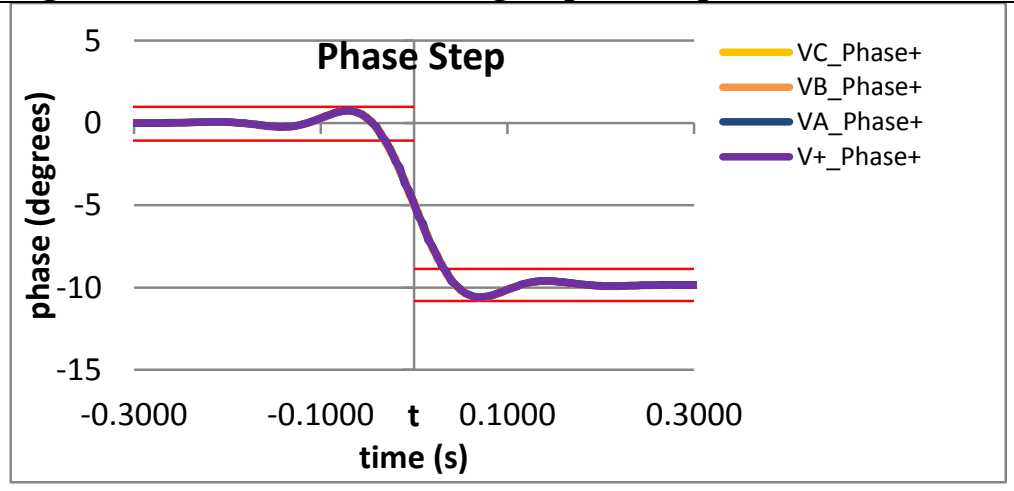

Figure 4986: Fs $=20$ FPS, +10 degree phase step 
This publication is available free of charge from $\mathrm{http}: / / \mathrm{dx}$.doi.org/10.6028/NIST.IR.8106

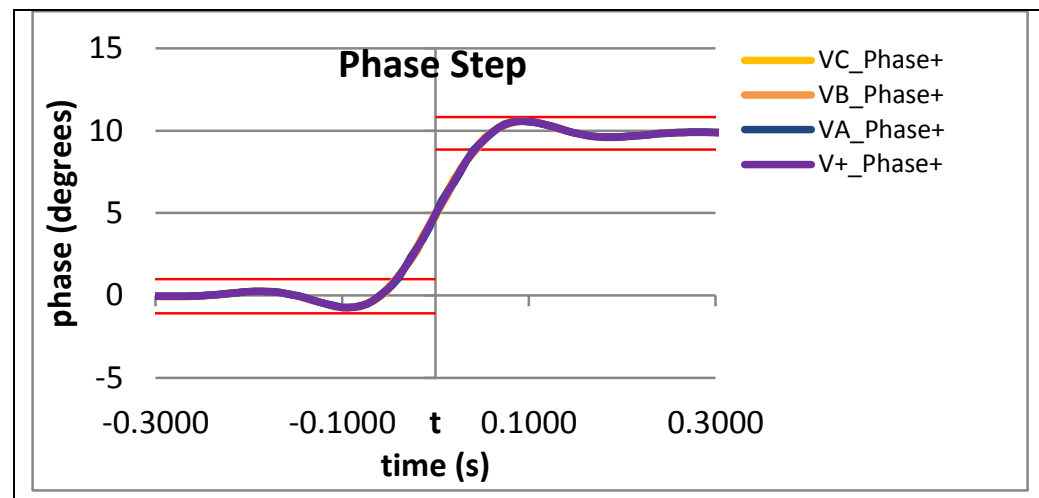

Figure 4987: Fs = 15 FPS, +10 degree phase step

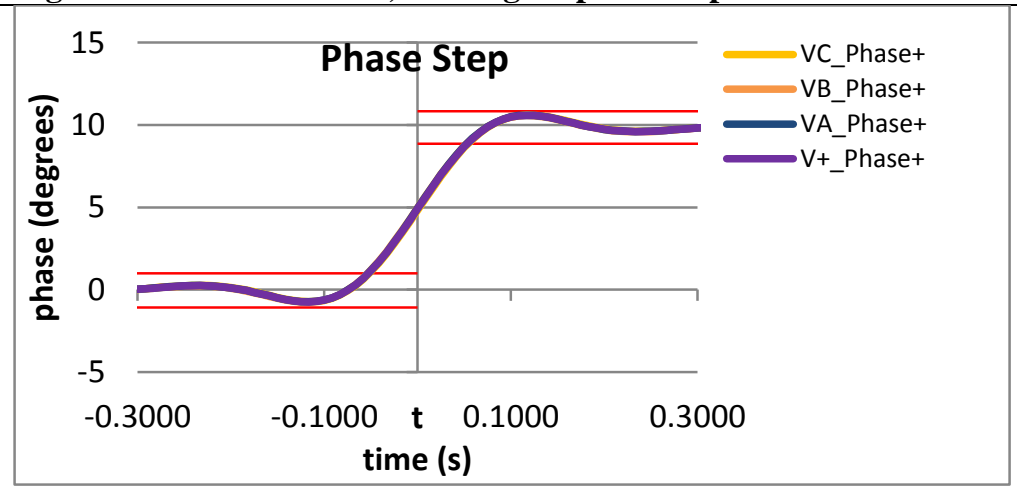

Figure 4989: Fs = 12 FPS, +10 degree phase step

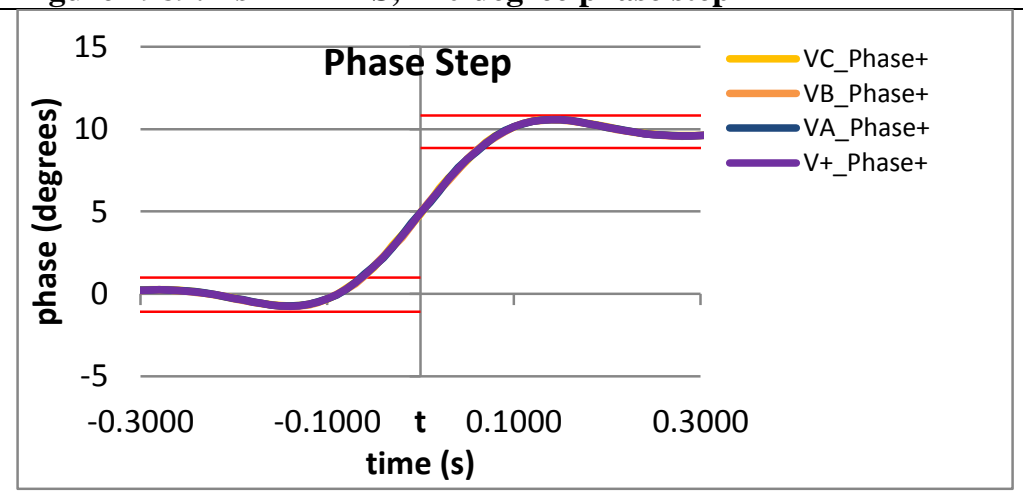

Figure 4991: Fs = 10 FPS, +10 degree phase step

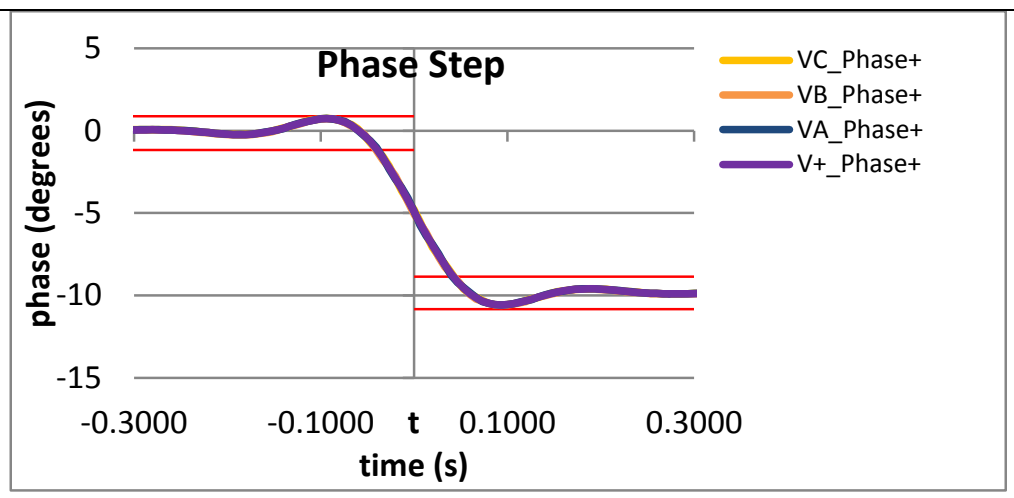

Figure 4988: Fs = 15 FPS, -10 degree phase step

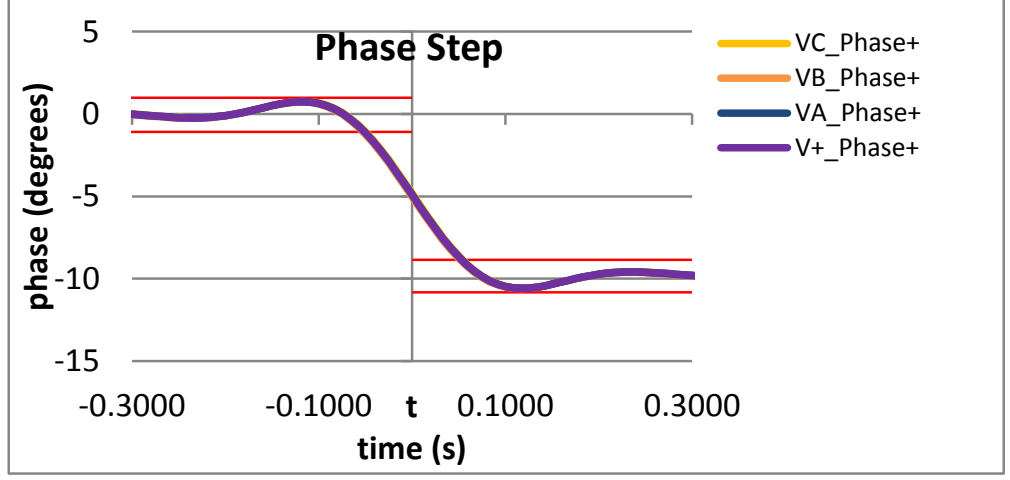

Figure 4990: Fs = 12 FPS, -10 degree phase step

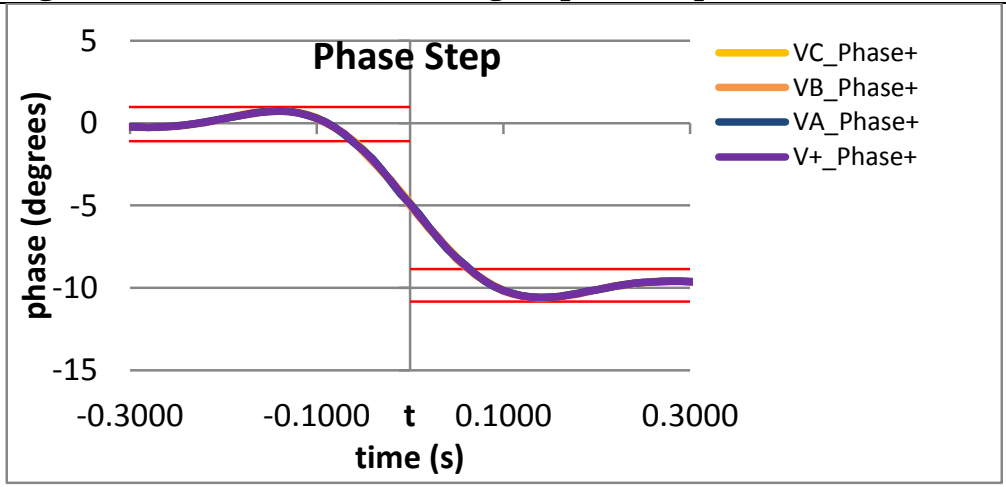

Figure 4992: Fs = 10 FPS, -10 degree phase step 
9.10 Dynamic step change in phase: phasor overshoot: $P$ class Dynamic step change in phase: phasor delay time $P$ class

9.10.1 C37.118.1-2011 Annex C dynamic step change in phase phasor overshoot: $\mathrm{F} 0=60 \mathrm{~Hz}, \mathrm{P}$ class

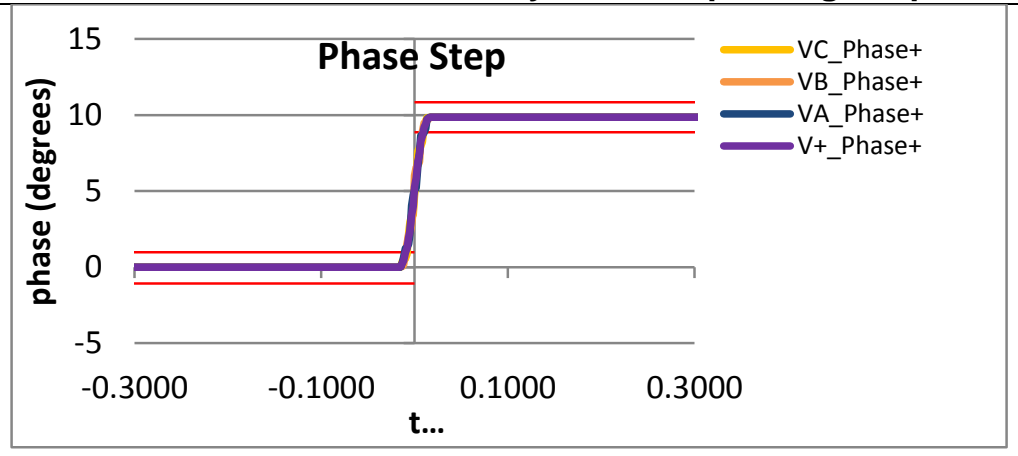

Figure 4993: Fs = 60 FPS, +10 degree phase step

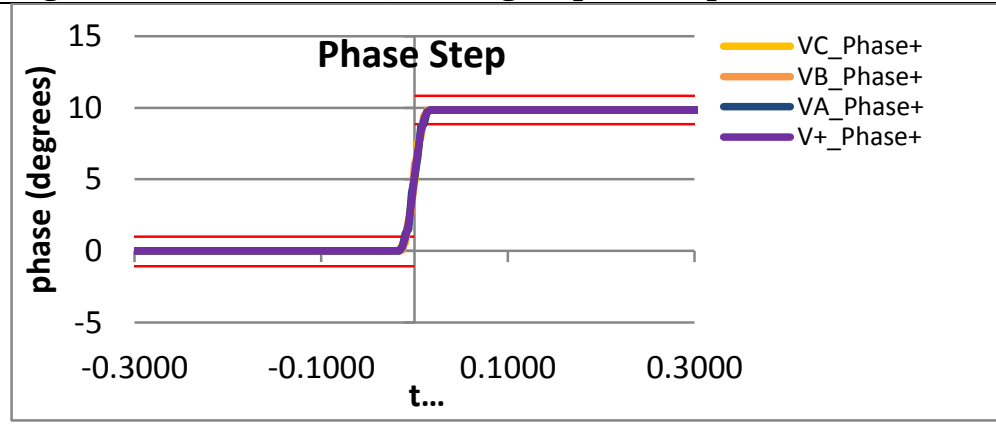

Figure 4995: Fs = 30 FPS, +10 degree phase step

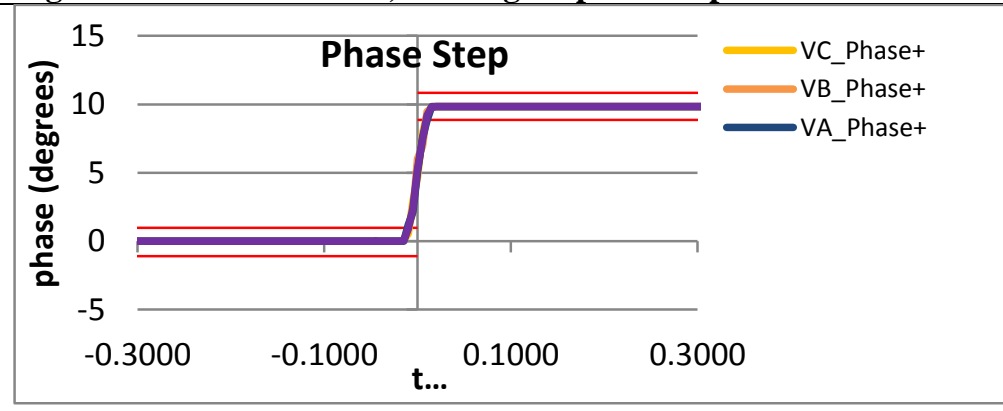

Figure 4997: Fs = 20 FPS, +10 degree phase step

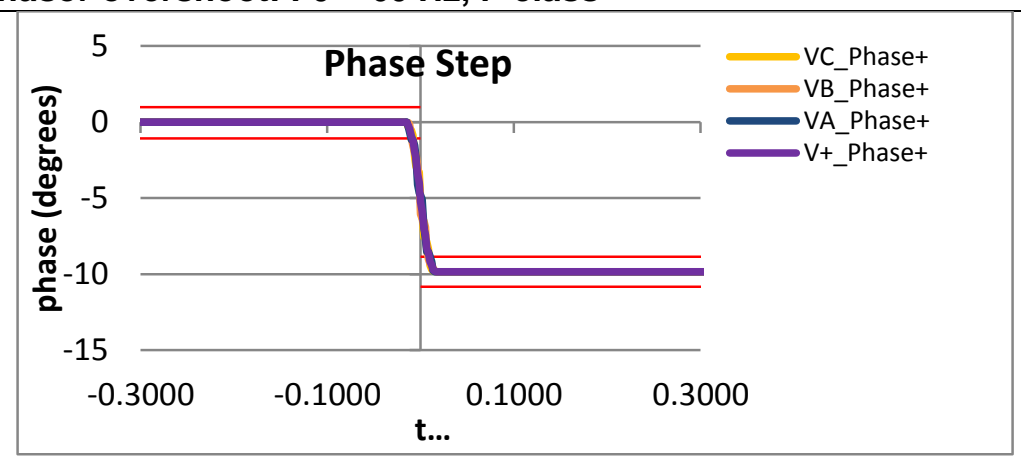

Figure 4994: Fs = 60 FPS, -10 degree phase step

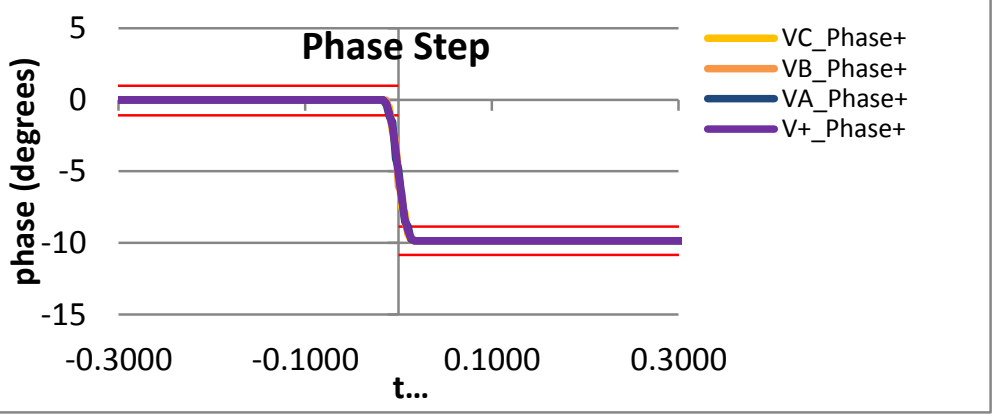

Figure 4996: Fs = 30 FPS, -10 degree phase step

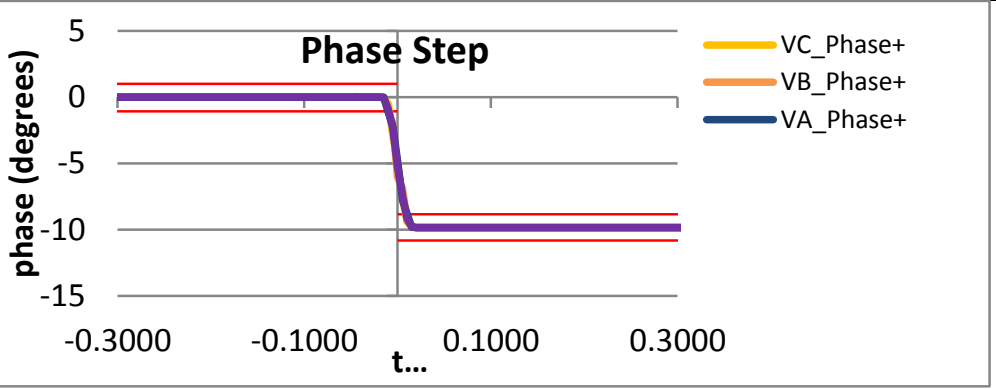

Figure 4998: Fs = 20 FPS, +10 degree phase step 

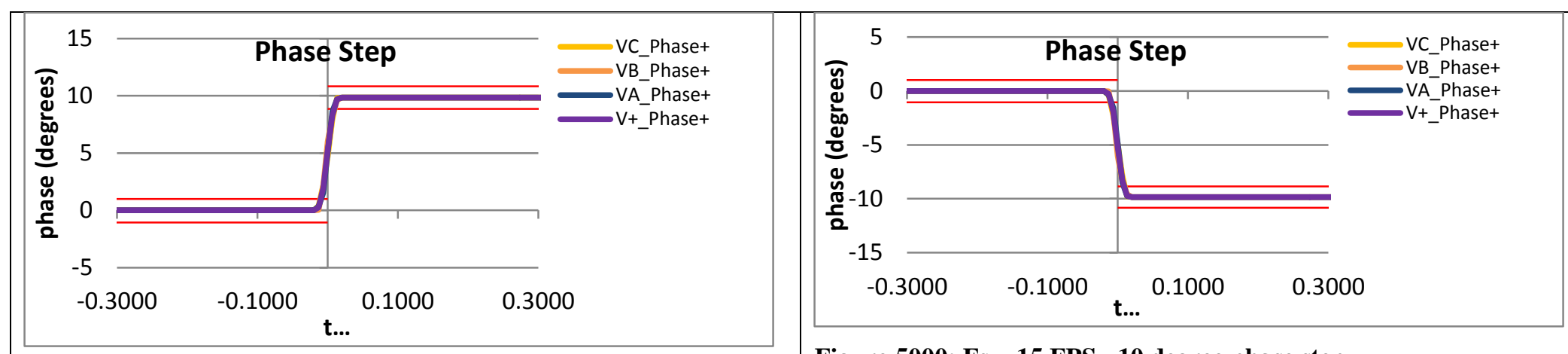

Figure 4999: Fs = 15 FPS, +10 degree phase step

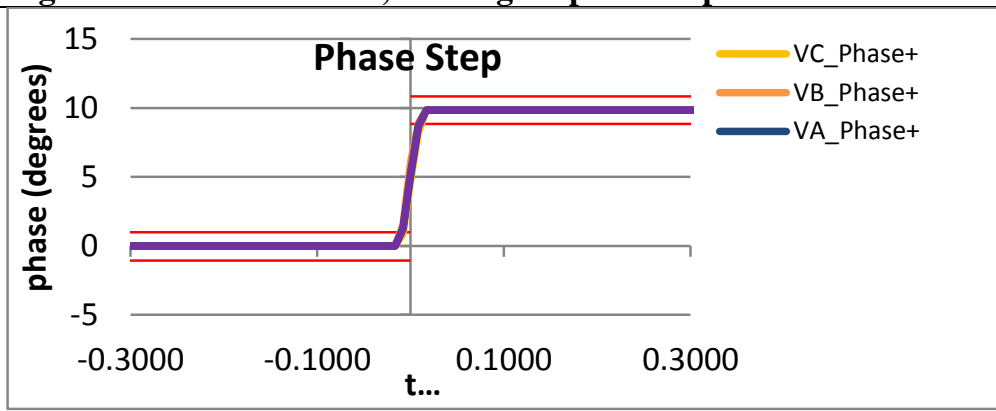

Figure 5001: Fs = 12 FPS, +10 degree phase step

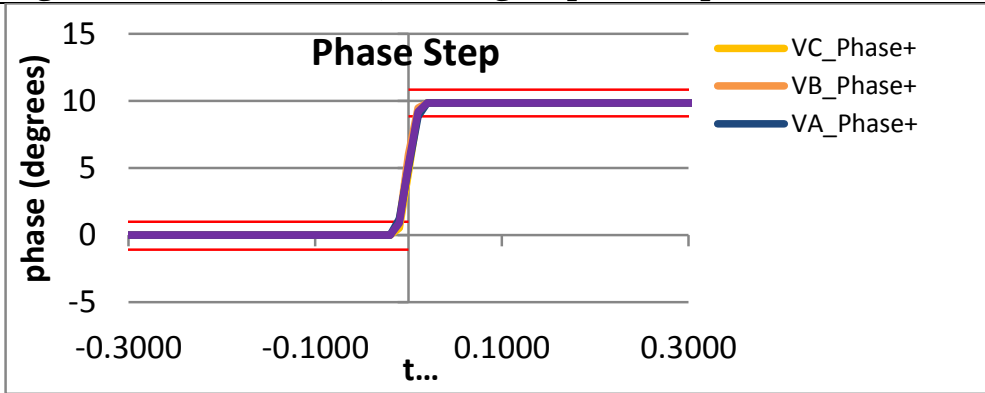

Figure 5003: Fs = 10 FPS, +10 degree phase step

Figure 5000: Fs = 15 FPS, $-\mathbf{1 0}$ degree phase step

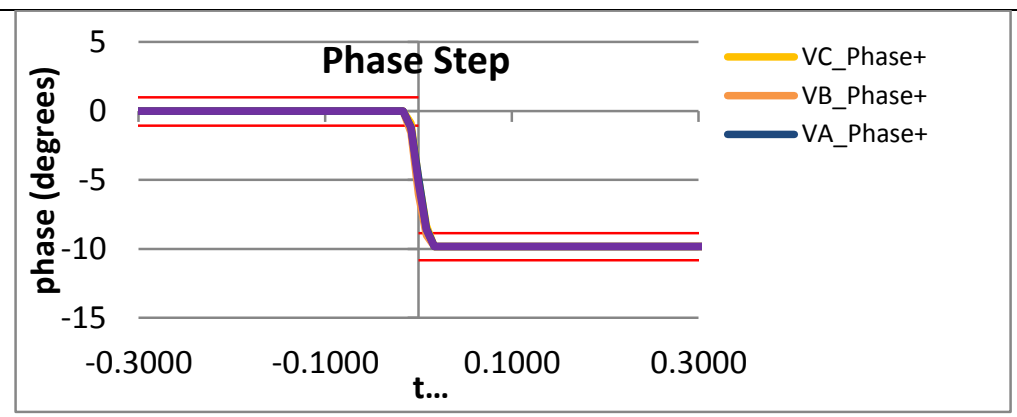

Figure 5002: Fs = 12 FPS, -10 degree phase step

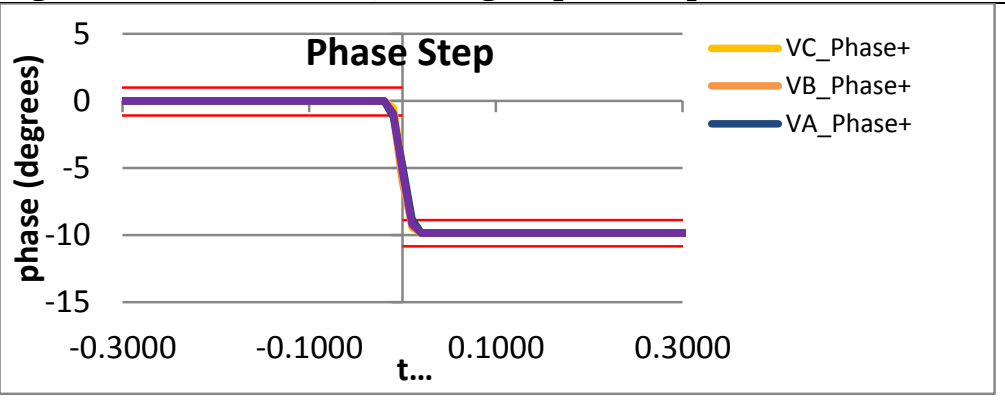

Figure 5004: Fs = 10 FPS, -10 degree phase step 


\subsubsection{PMU A dynamic step change in phase phasor overshoot: $F 0=60 \mathrm{~Hz}, P$ class}

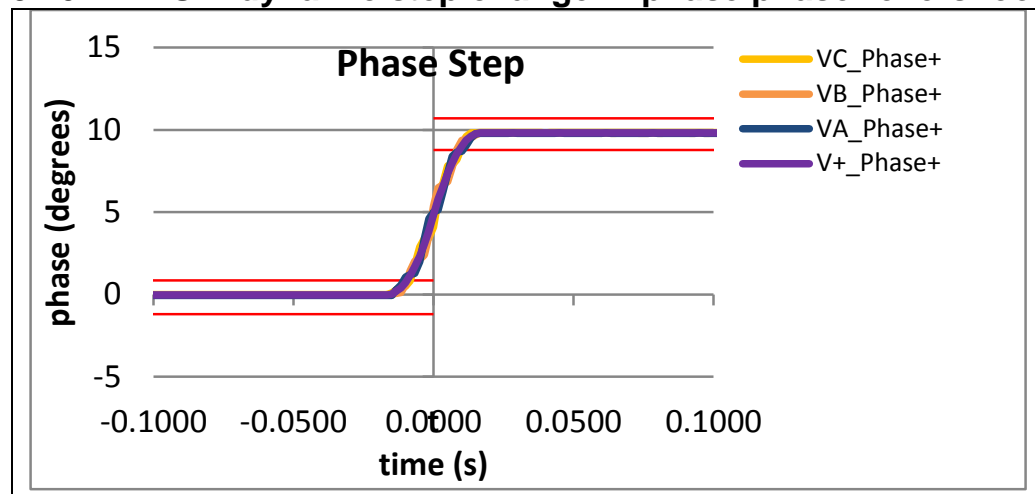

Figure 5005: Fs = 60 FPS, +10 degree phase step

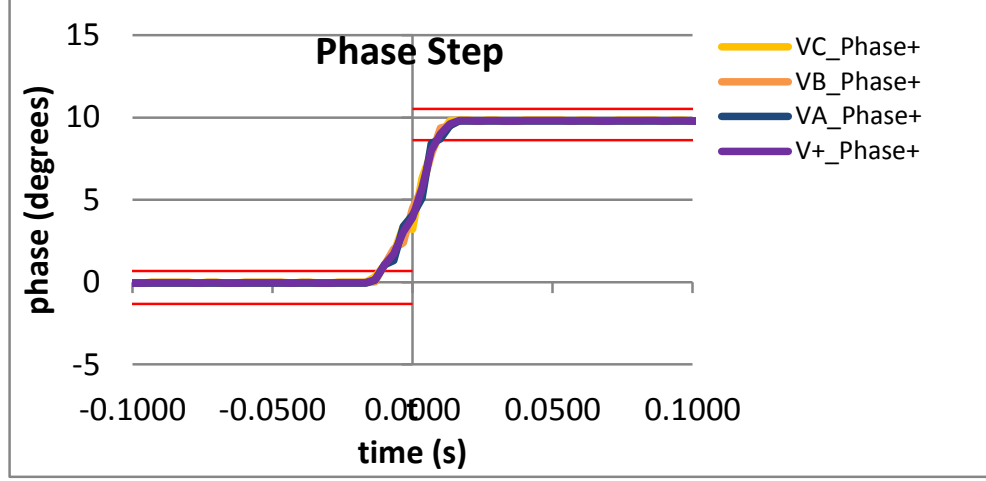

Figure 5007: Fs = 30 FPS, +10 degree phase step

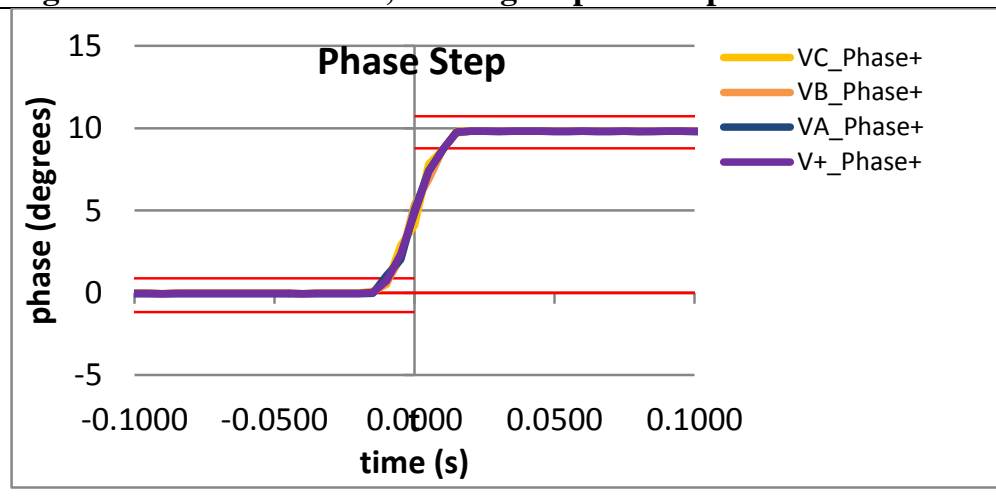

Figure 5009: Fs = 20 FPS, +10 degree phase step

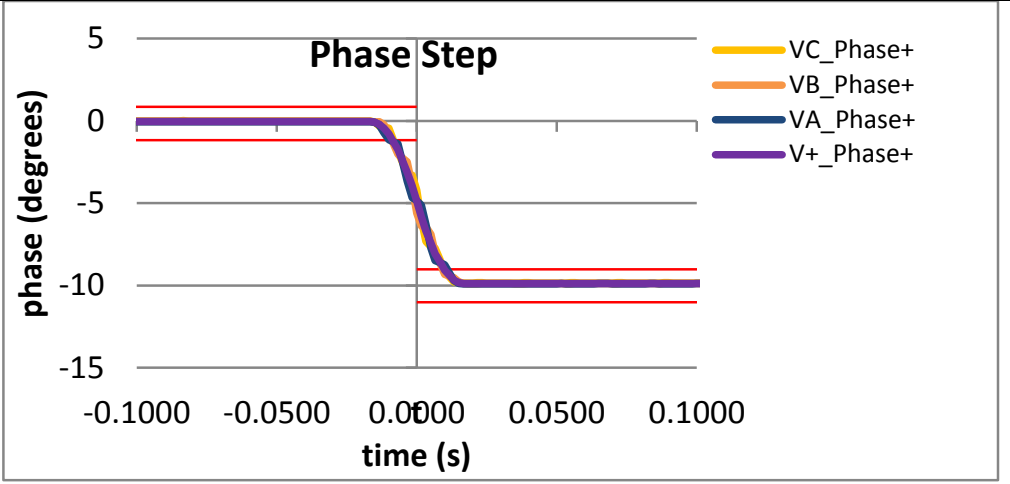

Figure 5006: Fs = 60 FPS, -10 degree phase step

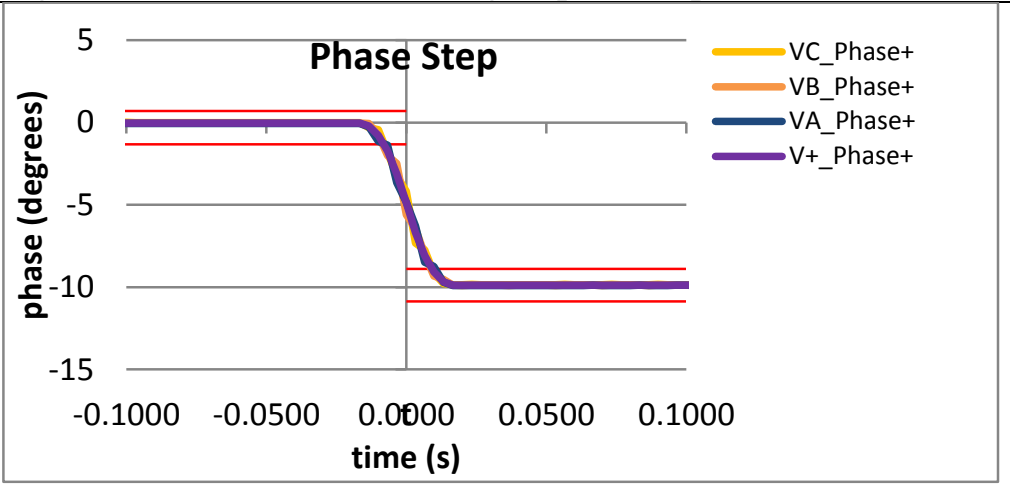

Figure 5008: Fs = 30 FPS, -10 degree phase step

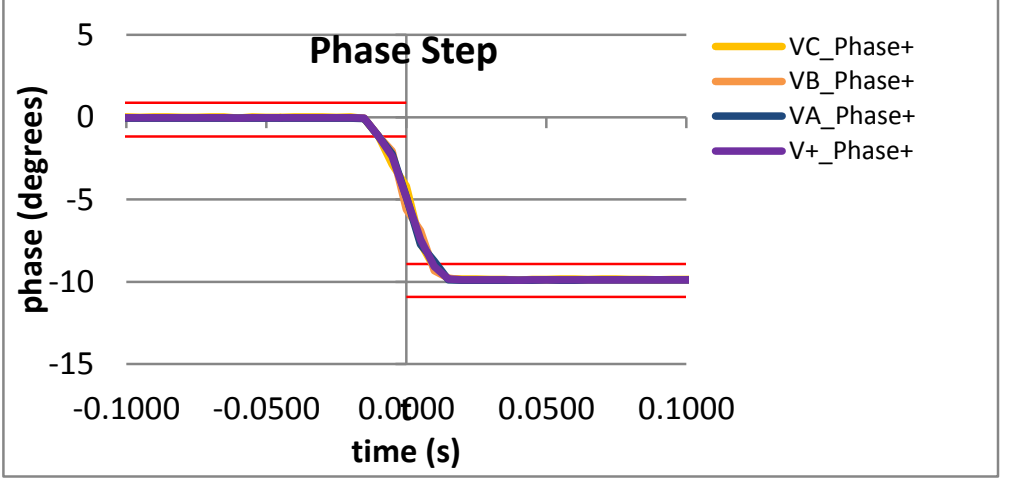

Figure 5010: Fs = 20 FPS, +10 degree phase step 


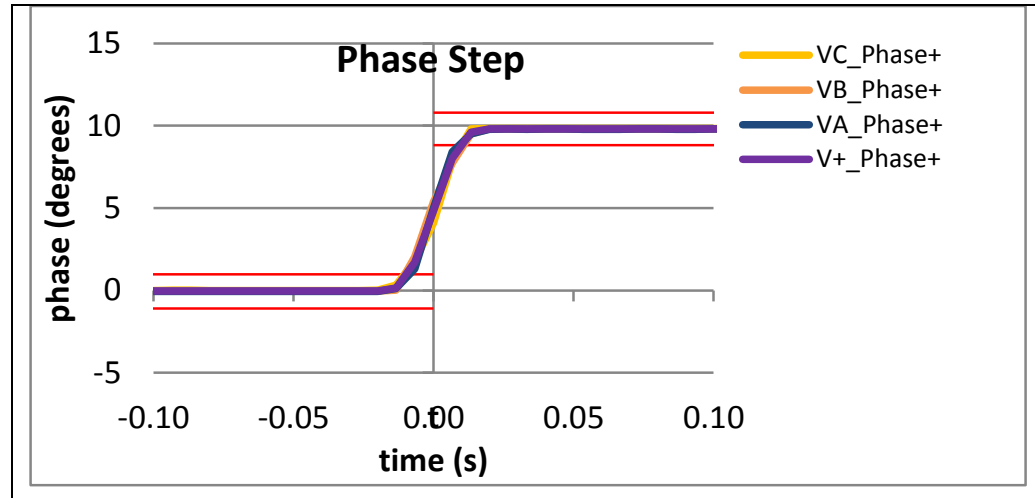

Figure 5011: Fs = 15 FPS, +10 degree phase step

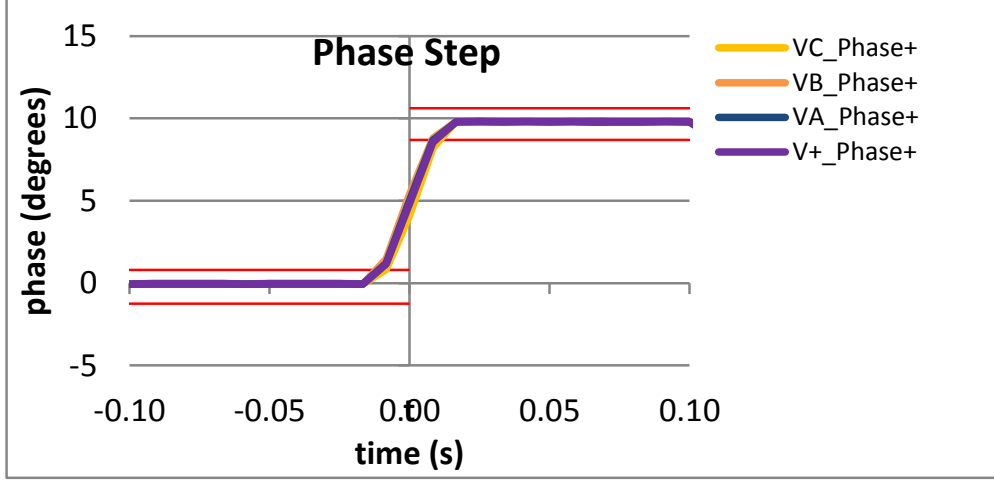

Figure 5013: Fs = 12 FPS, +10 degree phase step

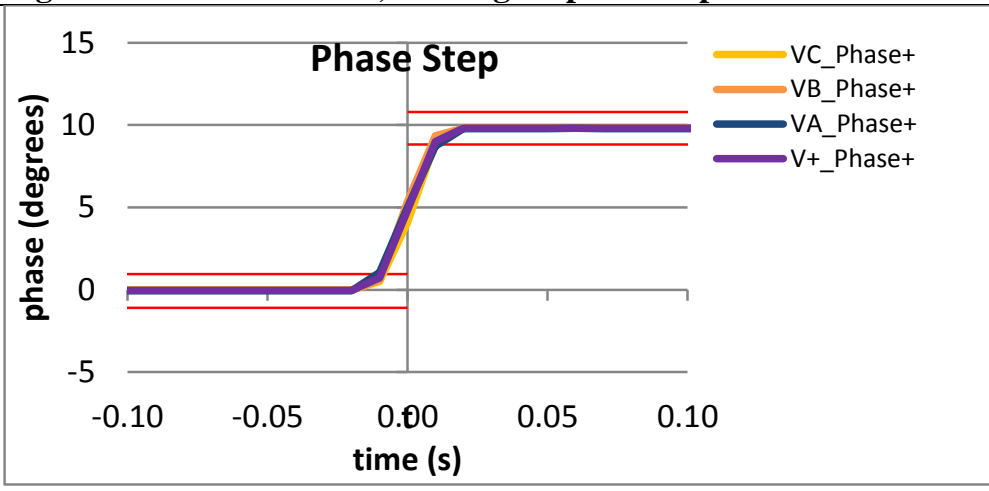

Figure 5015: Fs = 10 FPS, +10 degree phase step

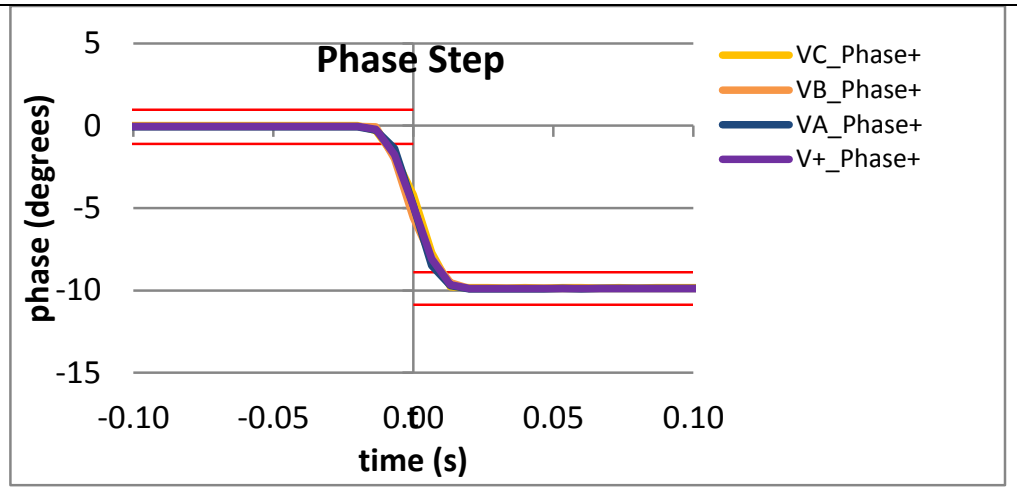

Figure 5012: Fs = 15 FPS, -10 degree phase step

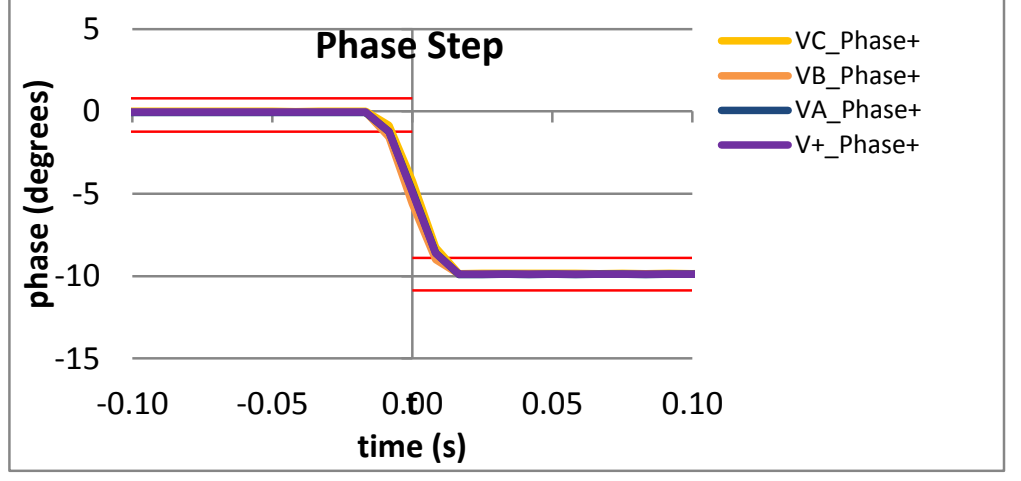

Figure 5014: Fs = 12 FPS, -10 degree phase step

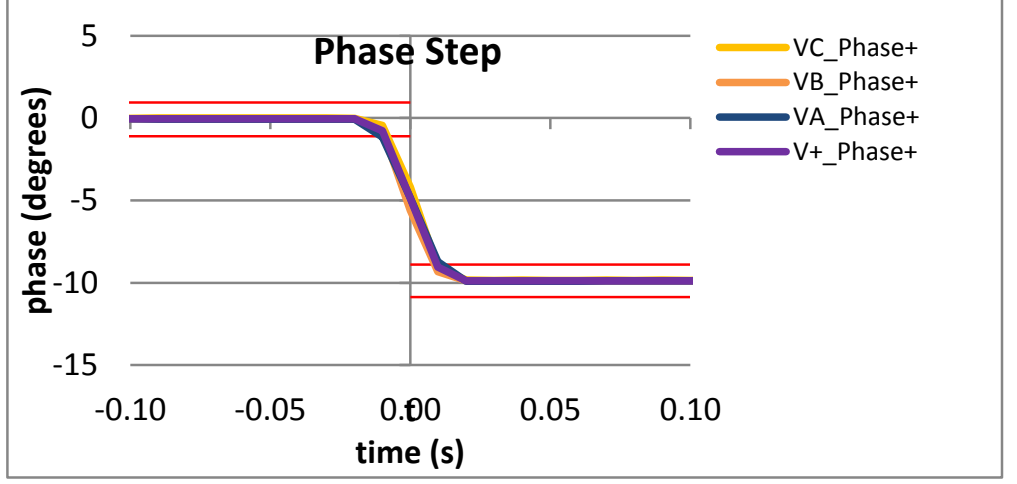

Figure 5016: Fs = 10 FPS, -10 degree phase step 
9.10.3 PMU B dynamic step change in phase phasor overshoot: $F 0=60 \mathrm{~Hz}, \mathrm{P}$ class

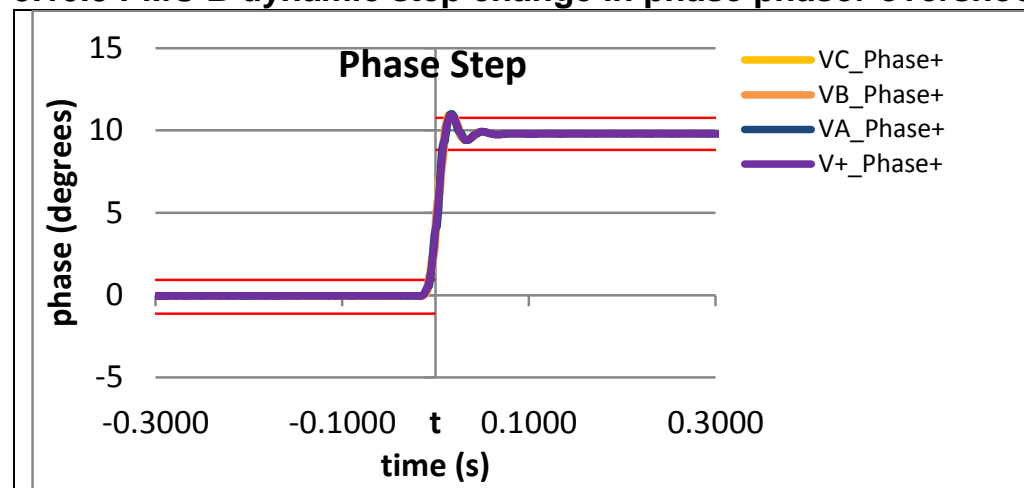

Figure 5017: Fs = 60 FPS, +10 degree phase step

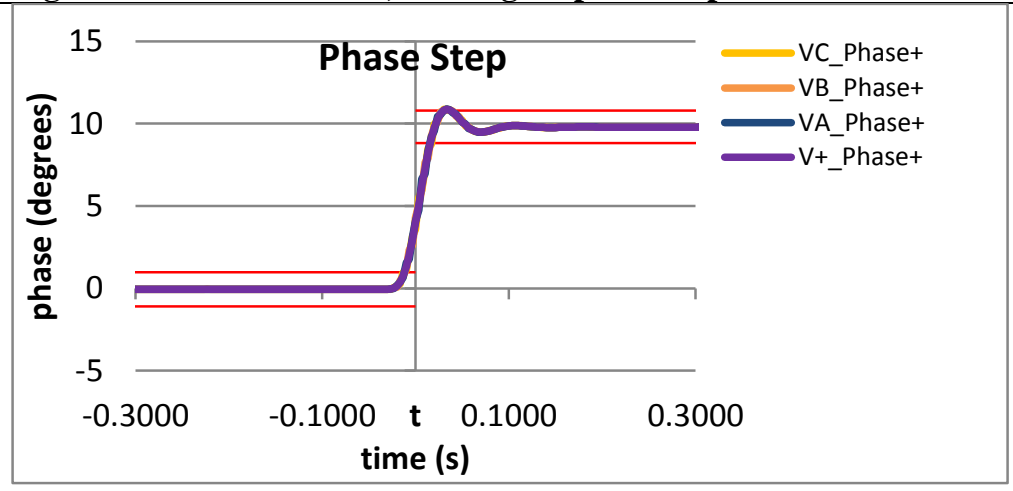

Figure 5019: Fs = 30 FPS, +10 degree phase step

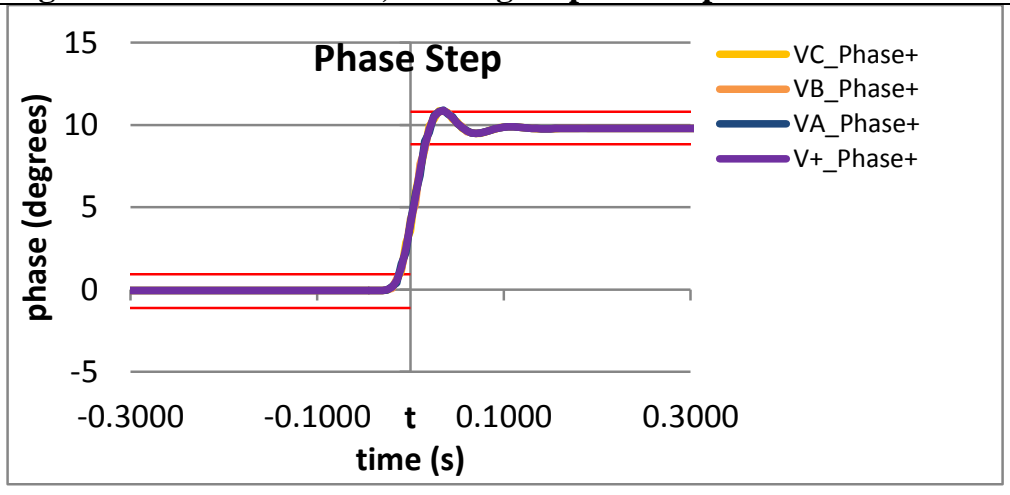

Figure 5021: Fs = 20 FPS, +10 degree phase step

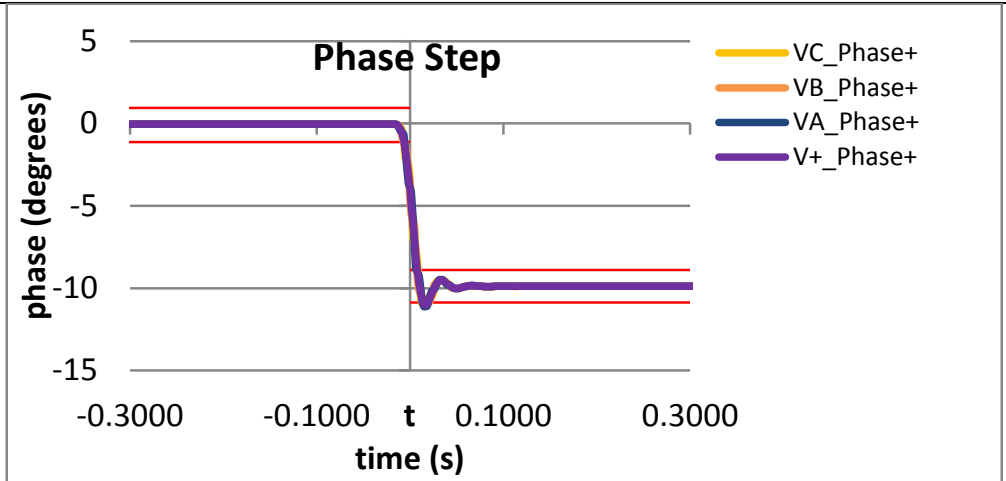

Figure 5018: Fs = 60 FPS, -10 degree phase step

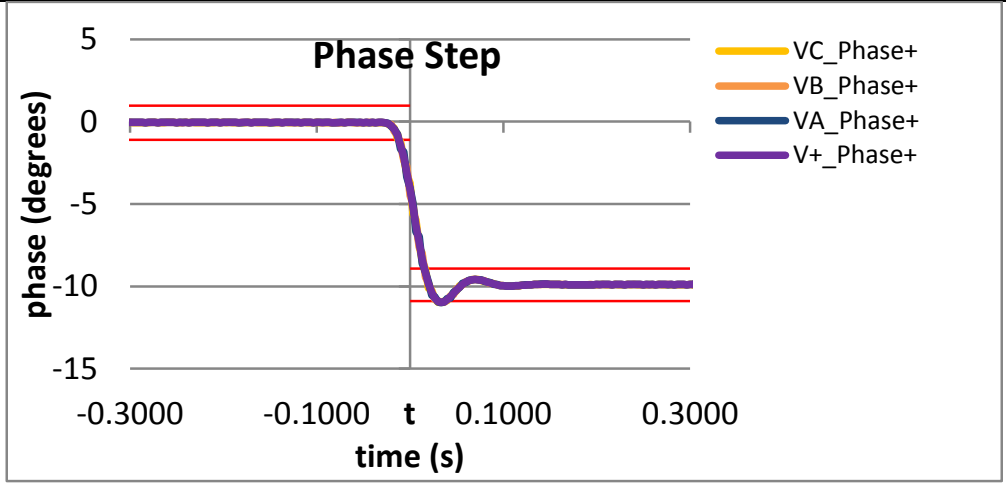

Figure 5020: Fs = 30 FPS, -10 degree phase step

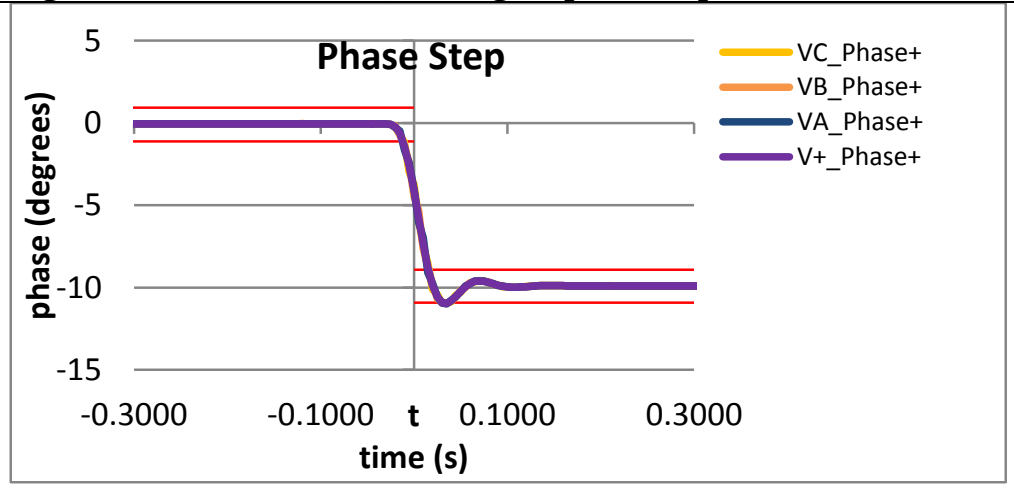

Figure 5022: Fs $=20$ FPS, +10 degree phase step 
This publication is available free of charge from $\mathrm{http}: / / \mathrm{dx}$.doi.org/10.6028/NIST.IR.8106

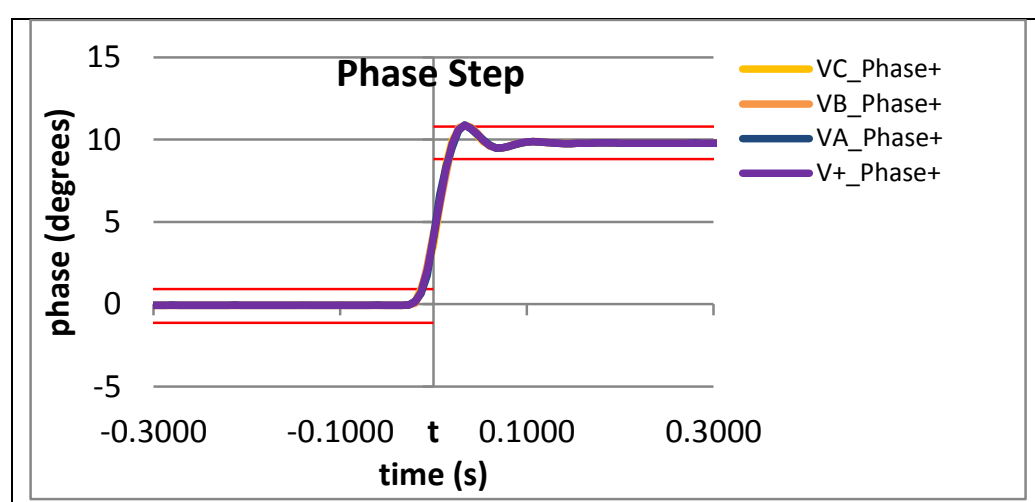

Figure 5023: Fs $=15$ FPS, +10 degree phase step

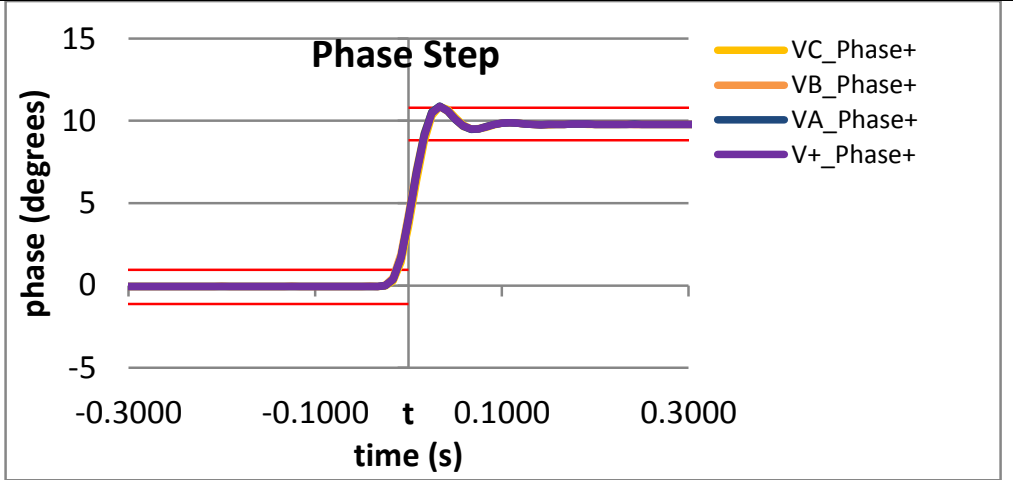

Figure 5025: Fs = 12 FPS, +10 degree phase step

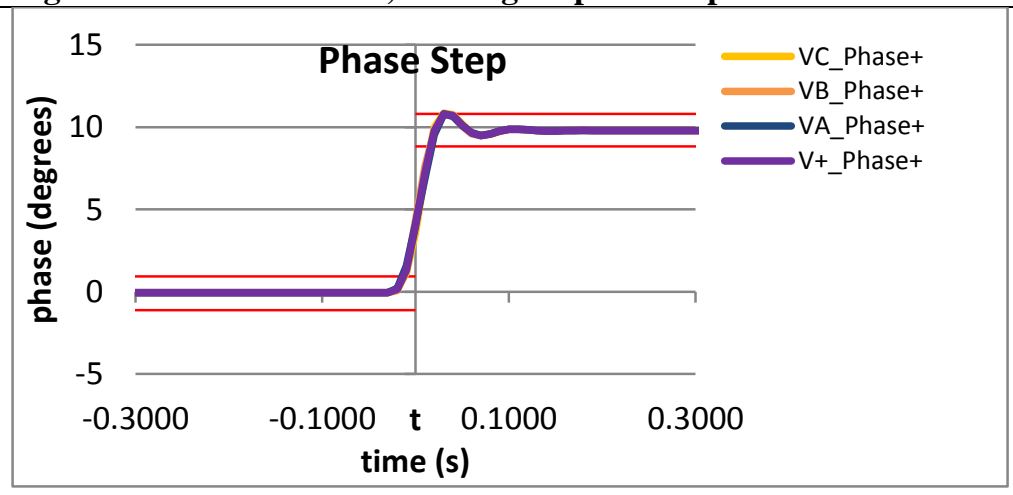

Figure 5027: Fs = 10 FPS, +10 degree phase step

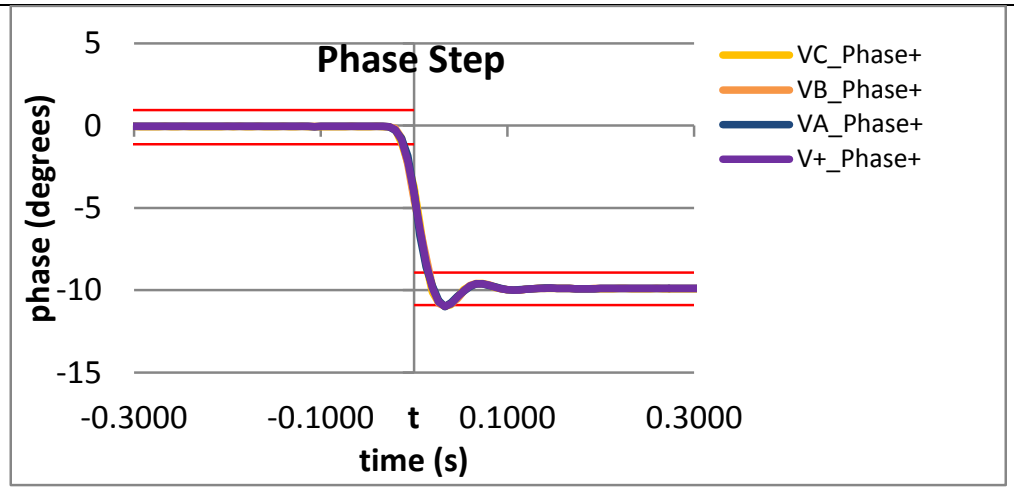

Figure 5024: Fs = 15 FPS, -10 degree phase step

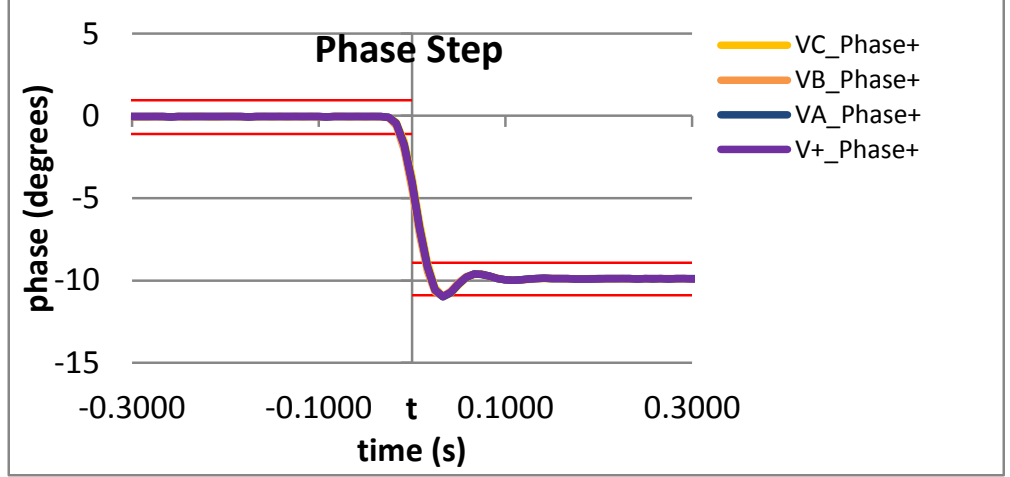

Figure 5026: Fs = 12 FPS, -10 degree phase step

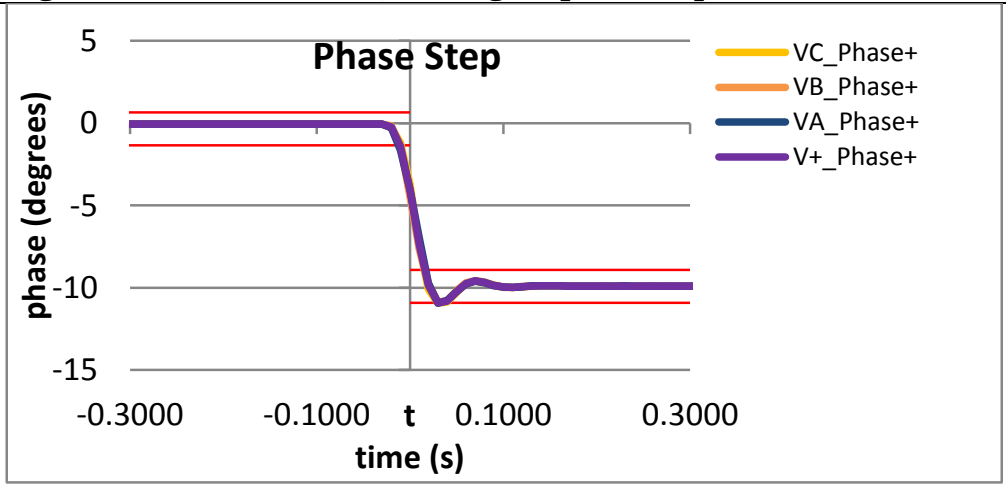

Figure 5028: Fs = 10 FPS, -10 degree phase step 
9.10.4 PMU C dynamic step change in phase phasor overshoot: $F 0=60 \mathrm{~Hz}$, $\mathrm{P}$ class

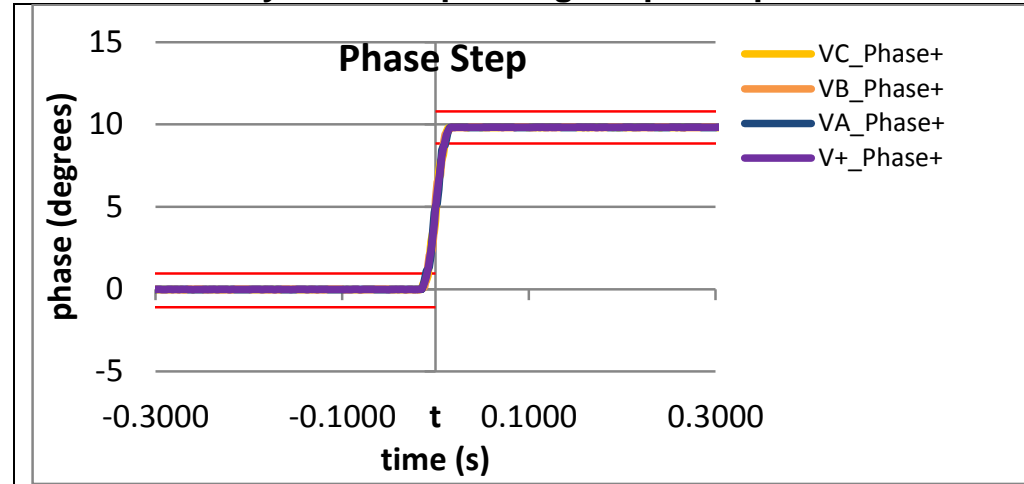

Figure 5029: Fs = 60 FPS, +10 degree phase step

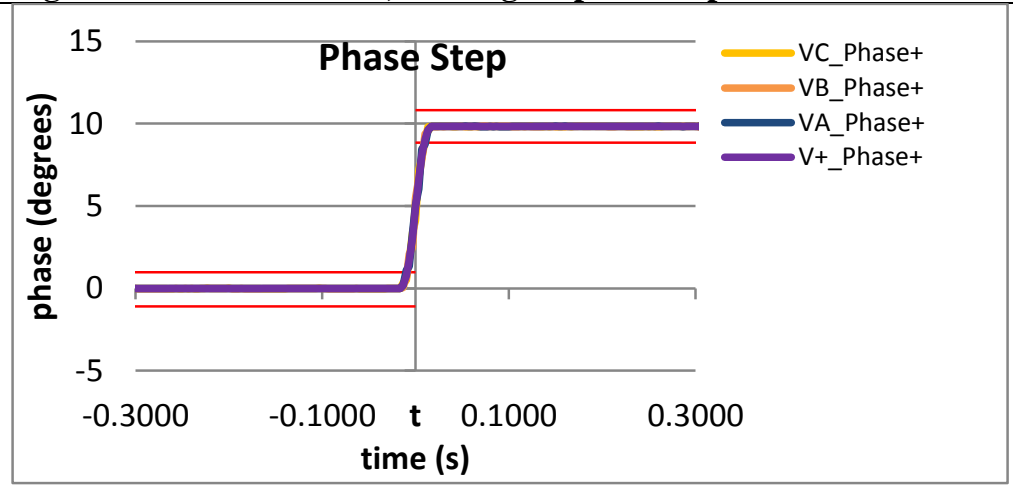

Figure 5031: Fs = 30 FPS, +10 degree phase step

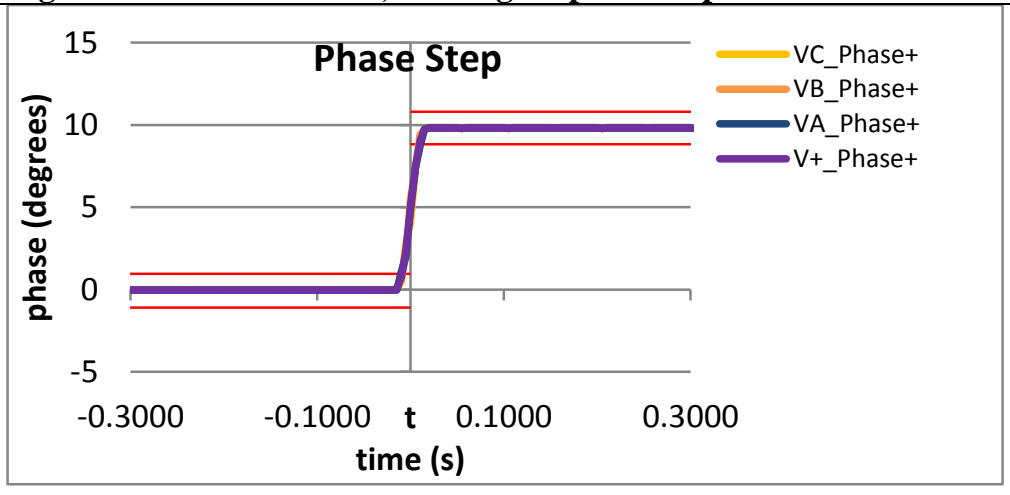

Figure 5033: Fs = 20 FPS, +10 degree phase step

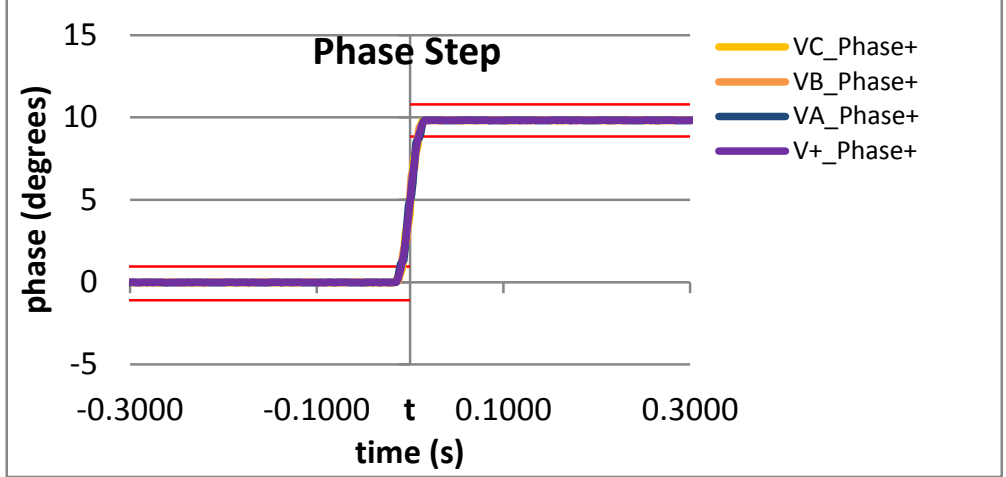

Figure 5030: Fs = 60 FPS, -10 degree phase step

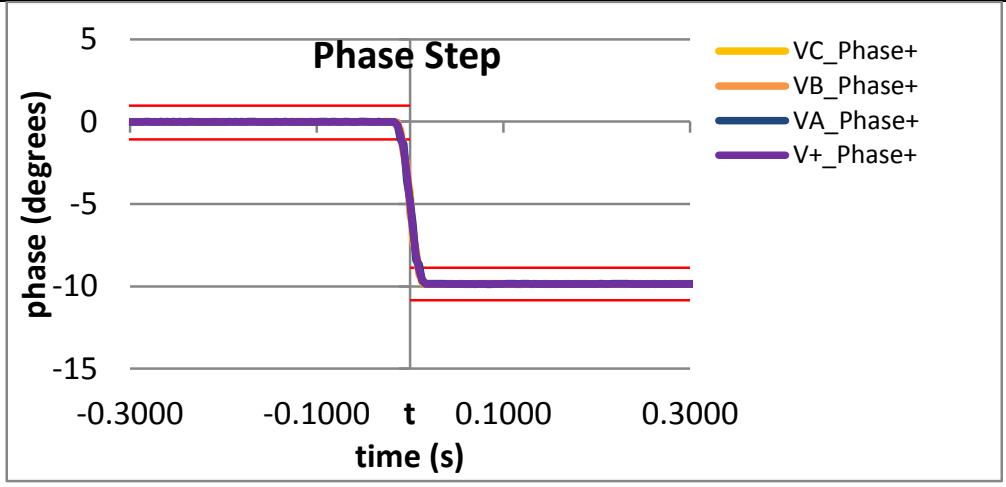

Figure 5032: Fs = 30 FPS, -10 degree phase step

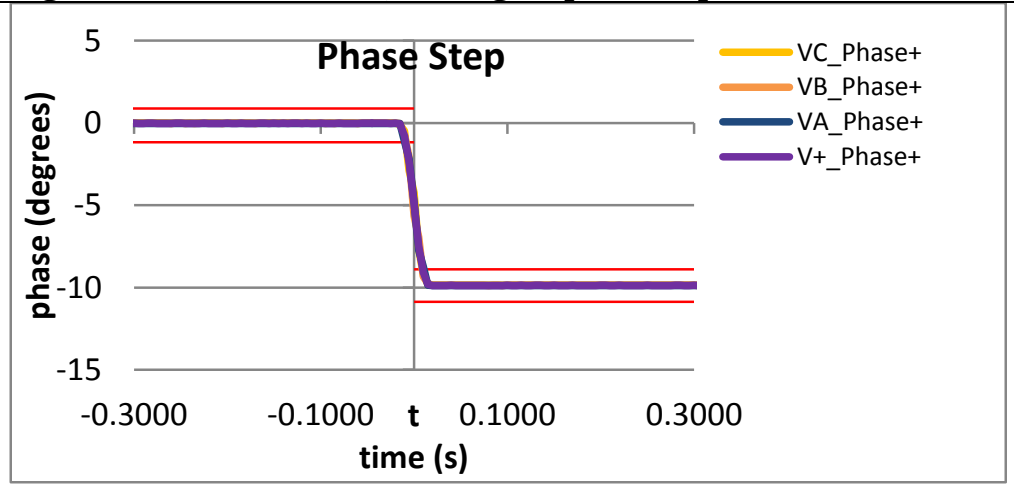

Figure 5034: Fs $=20$ FPS, +10 degree phase step 
This publication is available free of charge from $\mathrm{http}: / / \mathrm{dx}$.doi.org/10.6028/NIST.IR.8106

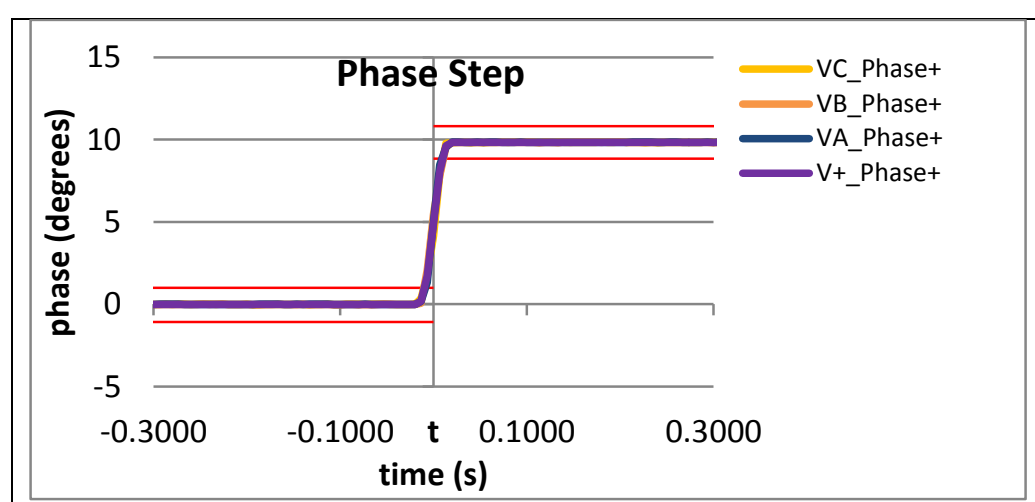

Figure 5035: Fs $=15$ FPS, +10 degree phase step

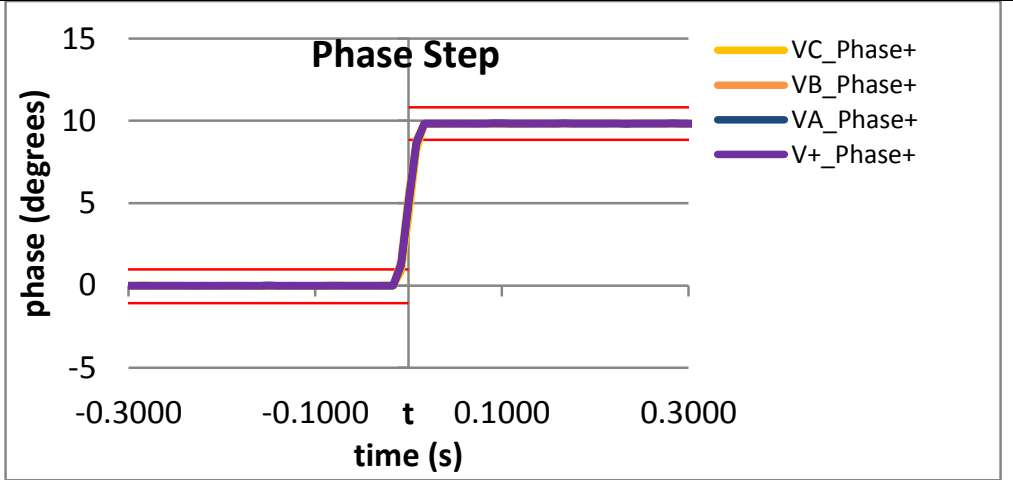

Figure 5037: Fs = 12 FPS, +10 degree phase step

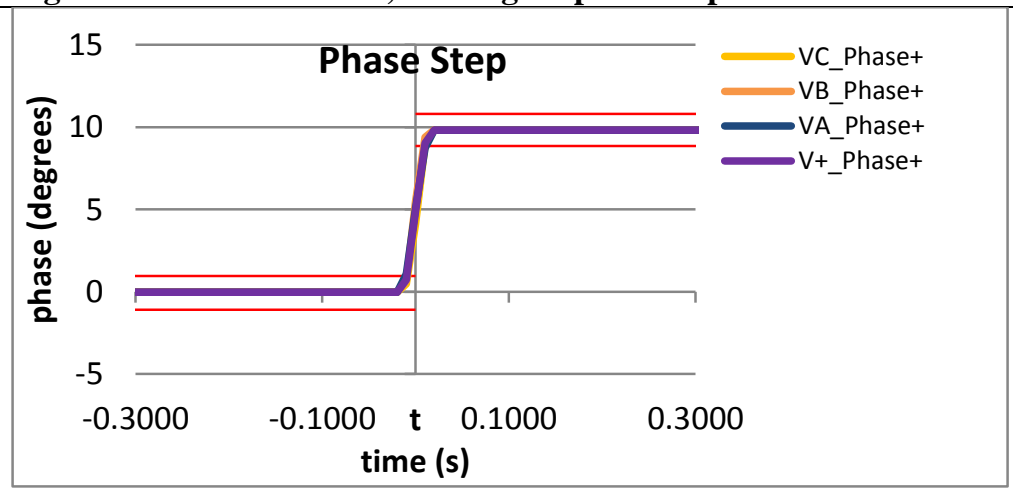

Figure 5039: Fs = 10 FPS, +10 degree phase step

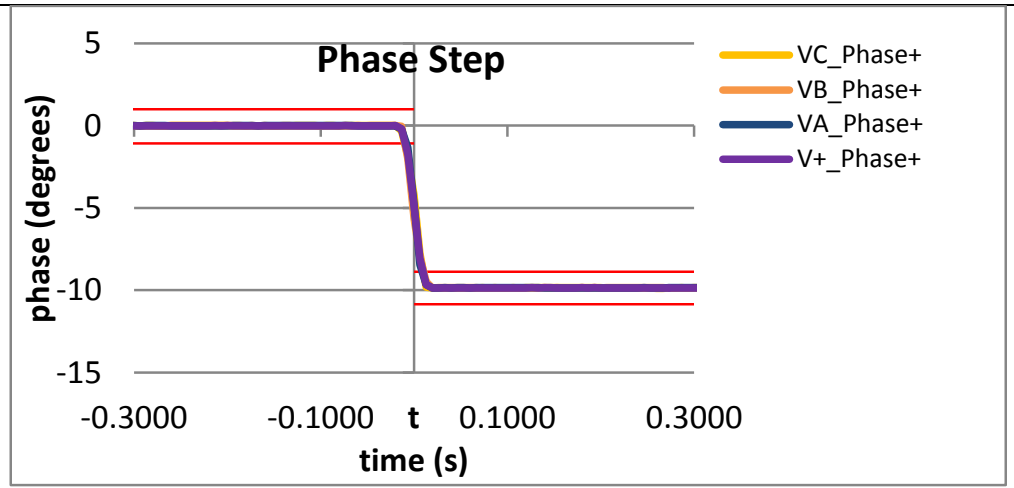

Figure 5036: Fs = 15 FPS, -10 degree phase step

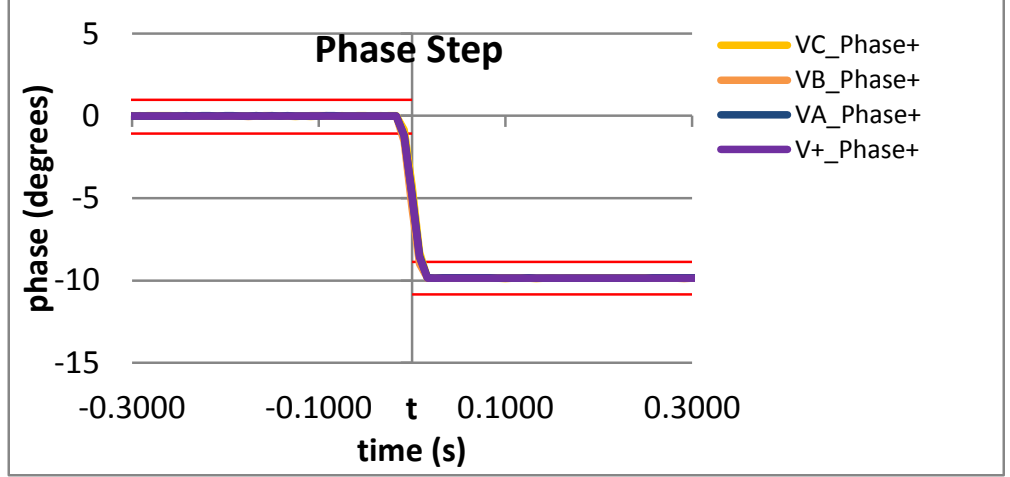

Figure 5038: Fs = 12 FPS, -10 degree phase step

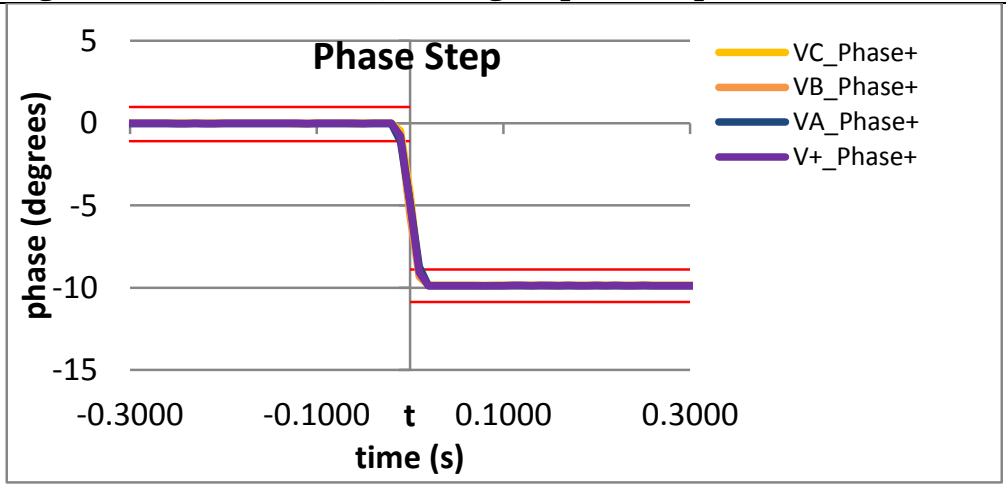

Figure 5040: Fs = 10 FPS, -10 degree phase step 
9.10.5 PMU D dynamic step change in phase phasor overshoot: $F 0=60 \mathrm{~Hz}$, P class

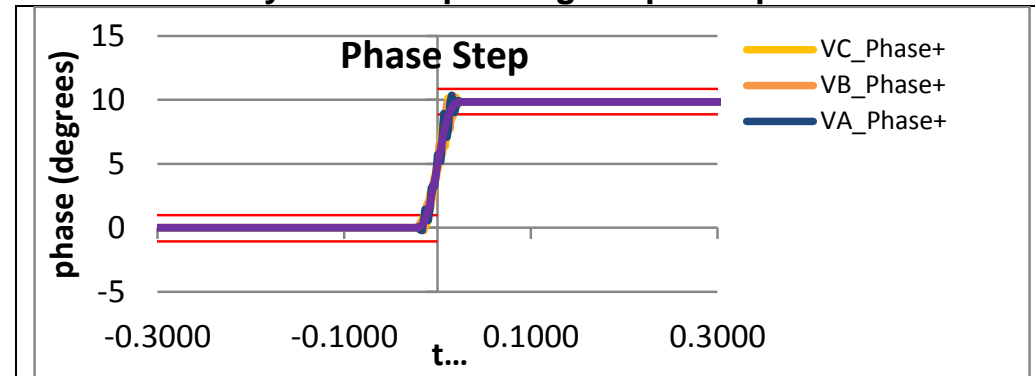

Figure 5041: Fs = 60 FPS, +10 degree phase step

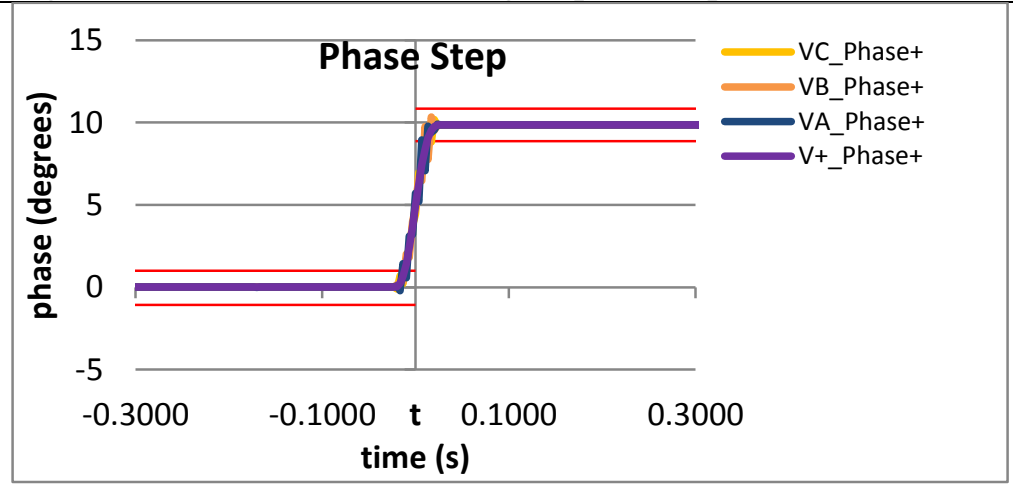

Figure 5043: Fs = 30 FPS, +10 degree phase step

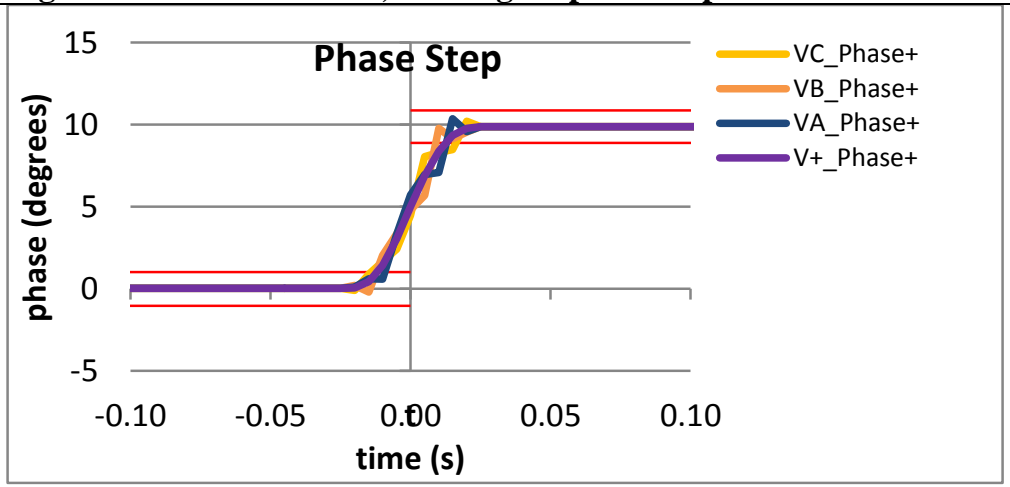

Figure 5045: Fs = 20 FPS, +10 degree phase step

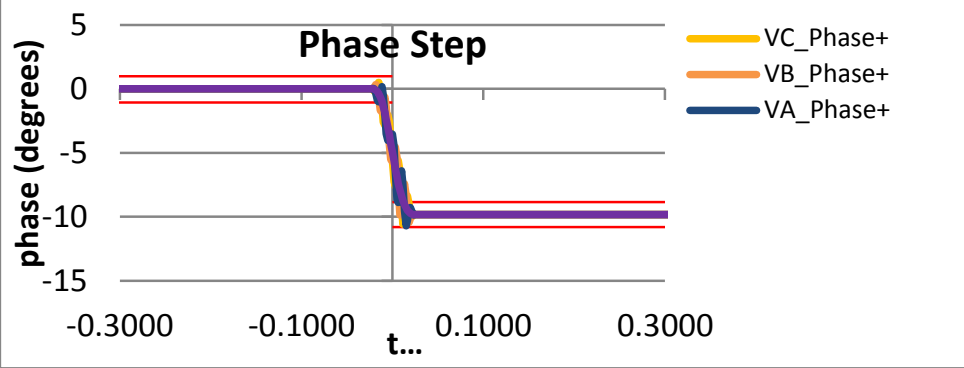

Figure 5042: Fs = 60 FPS, -10 degree phase step

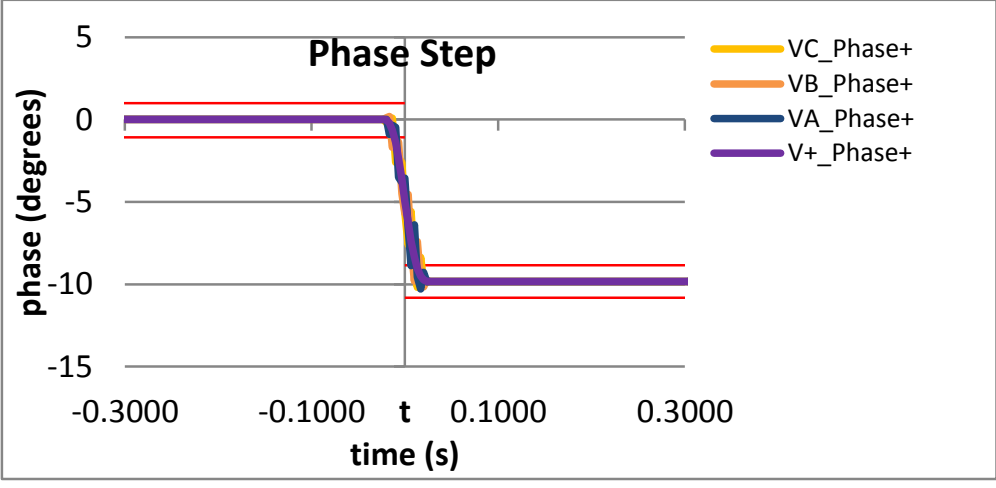

Figure 5044: Fs = 30 FPS, -10 degree phase step

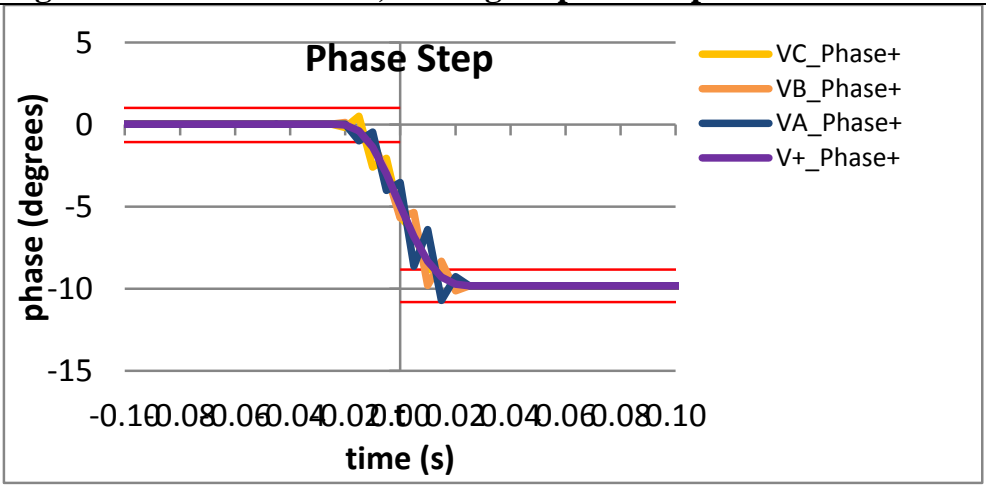

Figure 5046: Fs = 20 FPS, +10 degree phase step 
This publication is available free of charge from $\mathrm{http}: / / \mathrm{dx}$.doi.org/10.6028/NIST.IR.8106

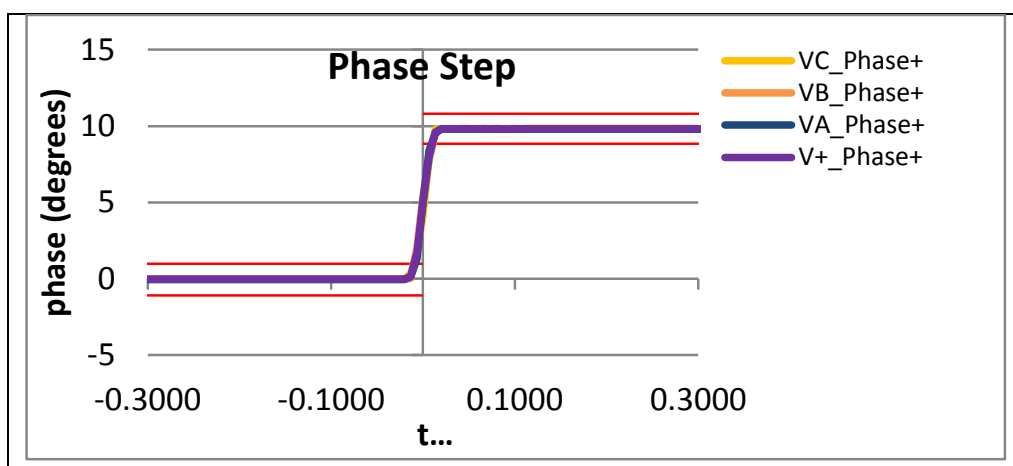

Figure 5047: Fs = 15 FPS, +10 degree phase step

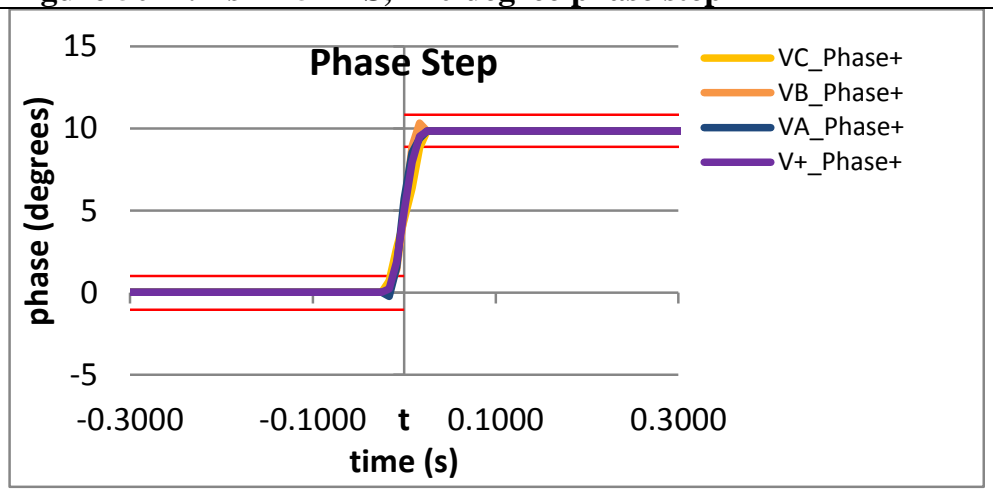

Figure 5049: Fs = 12 FPS, +10 degree phase step

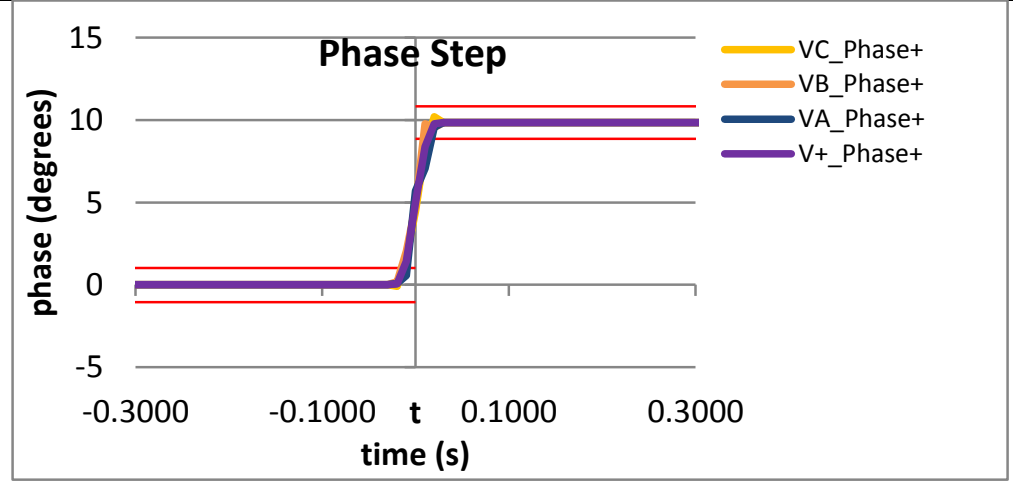

Figure 5051: Fs = 10 FPS, +10 degree phase step

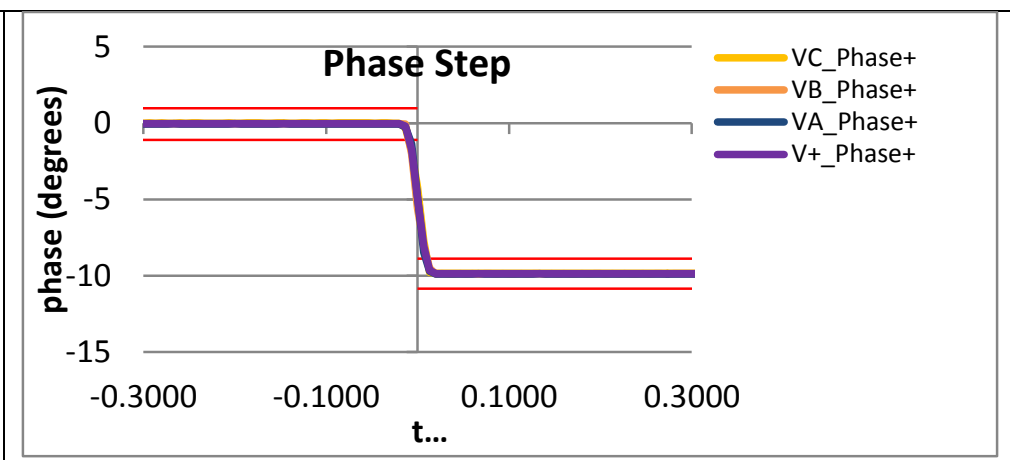

Figure 5048: Fs = 15 FPS, -10 degree phase step

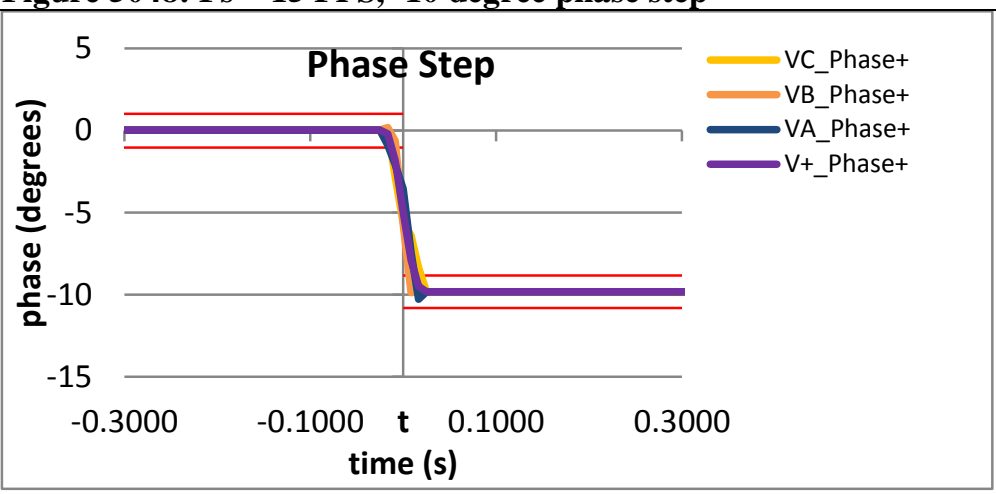

Figure 5050: Fs = 12 FPS, -10 degree phase step

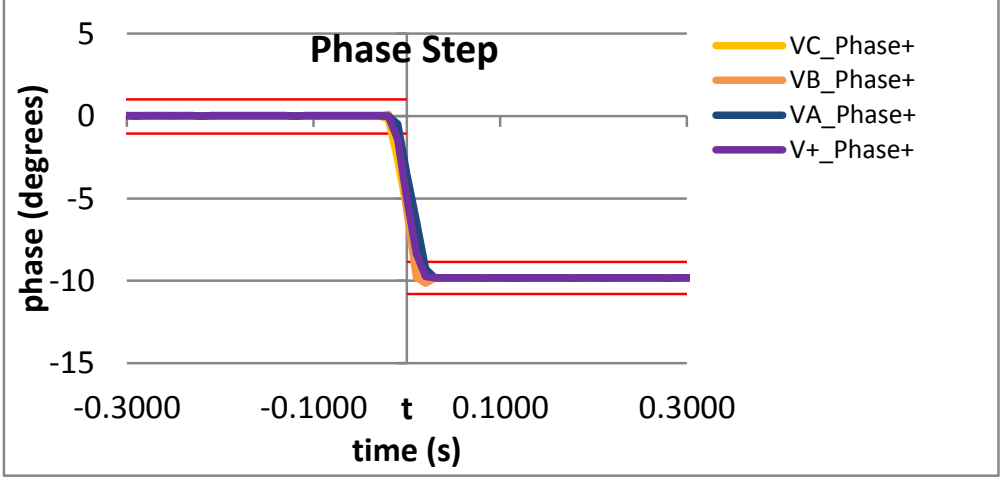

Figure 5052: Fs = 10 FPS, -10 degree phase step 
9.10.6 PMU E dynamic step change in phase phasor overshoot: $F 0=60 \mathrm{~Hz}, P$ class PMU E does not support P class.

\subsubsection{PMU F dynamic step change in phase phasor overshoot: $F 0=60 \mathrm{~Hz}, \mathrm{P}$ class}

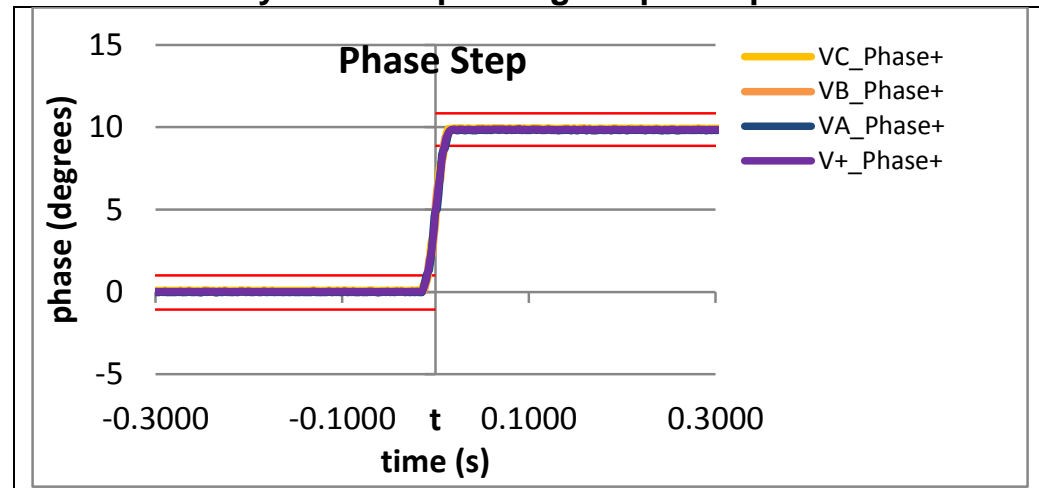

Figure 5053: Fs = 60 FPS, +10 degree phase step

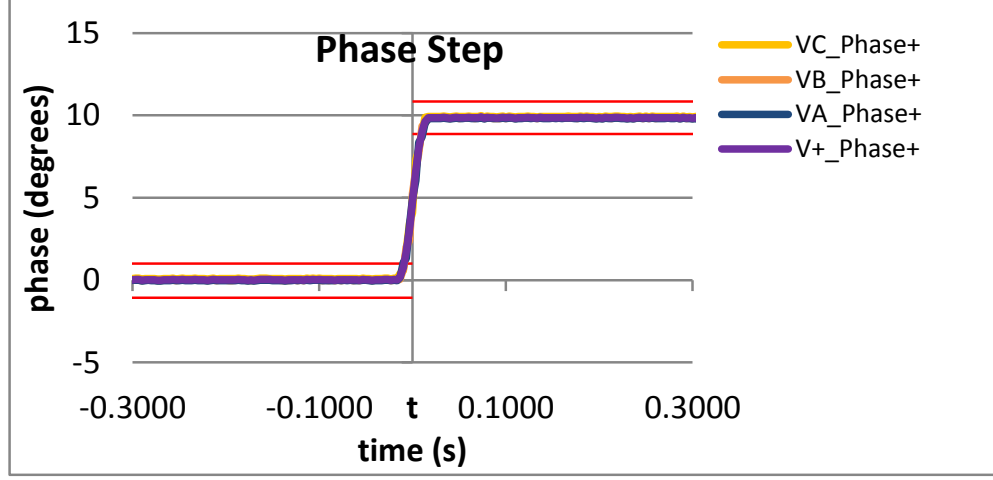

Figure 5055: Fs = 30 FPS, +10 degree phase step

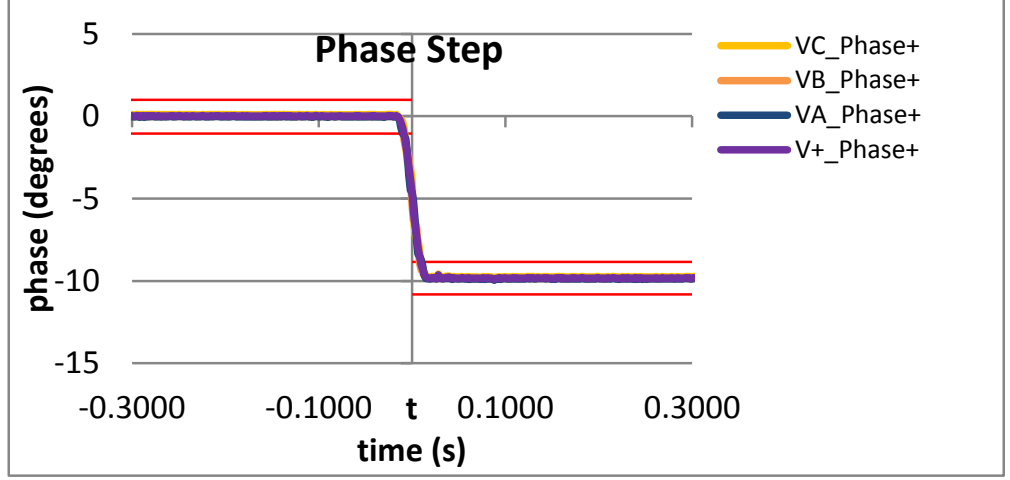

Figure 5054: Fs = 60 FPS, -10 degree phase step

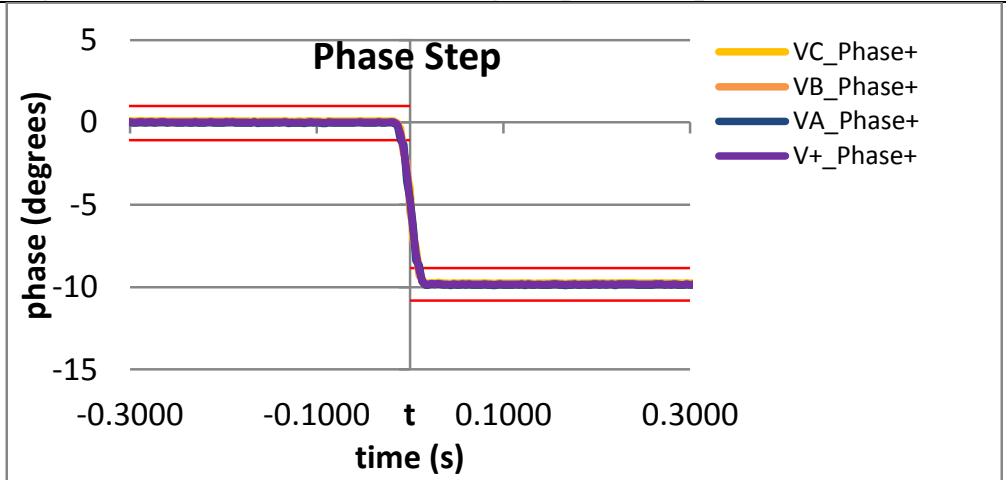

Figure 5056: Fs = 30 FPS, -10 degree phase step 


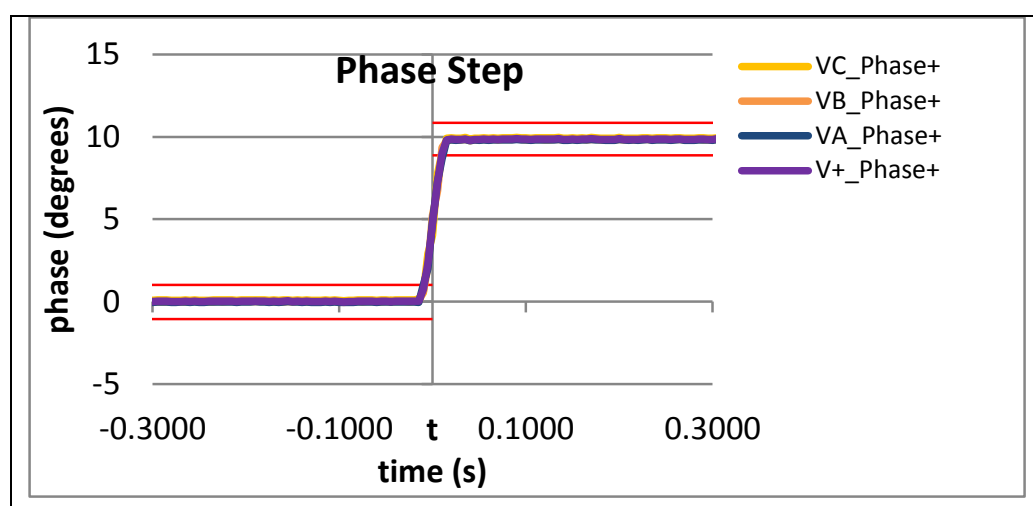

Figure 5057: Fs = 20 FPS, +10 degree phase step

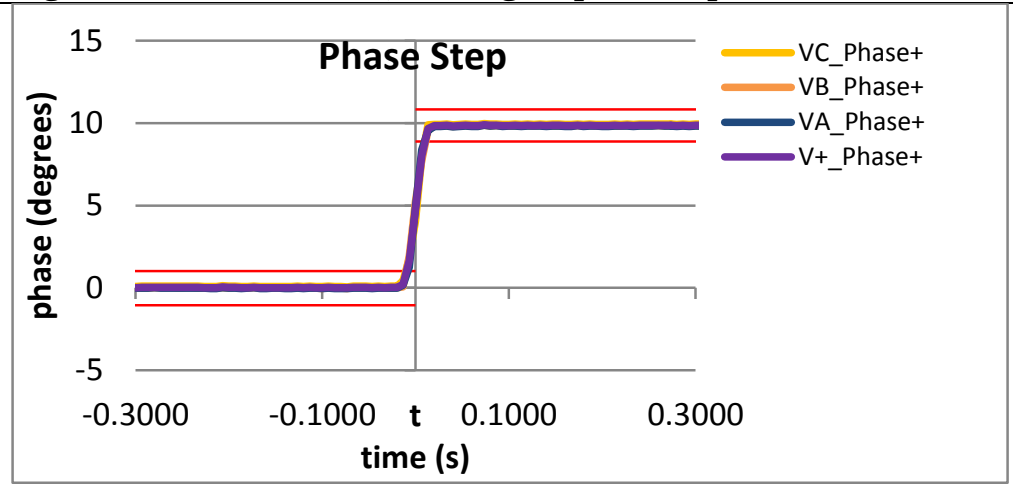

Figure 5059: Fs = 15 FPS, +10 degree phase step

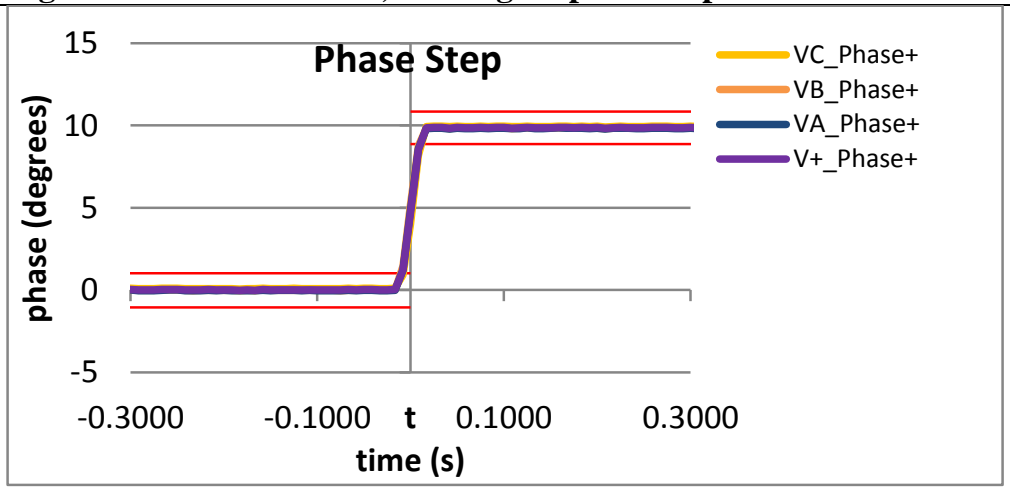

Figure 5061: Fs = 12 FPS, +10 degree phase step

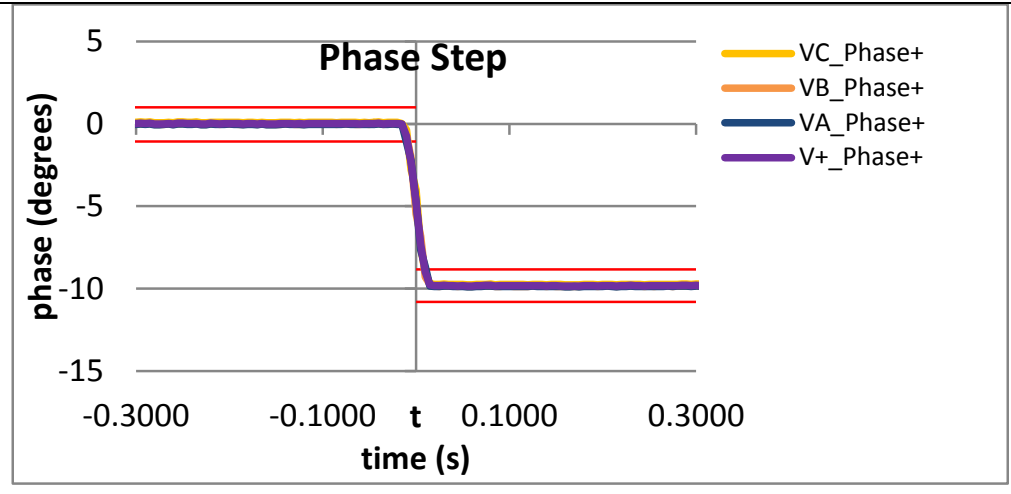

Figure 5058: Fs = 20 FPS, +10 degree phase step

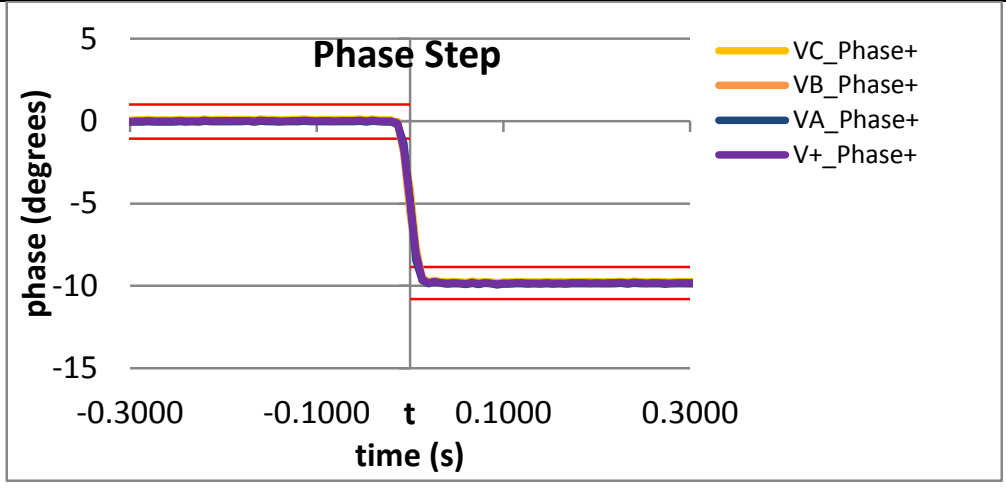

Figure 5060: Fs = 15 FPS, -10 degree phase step

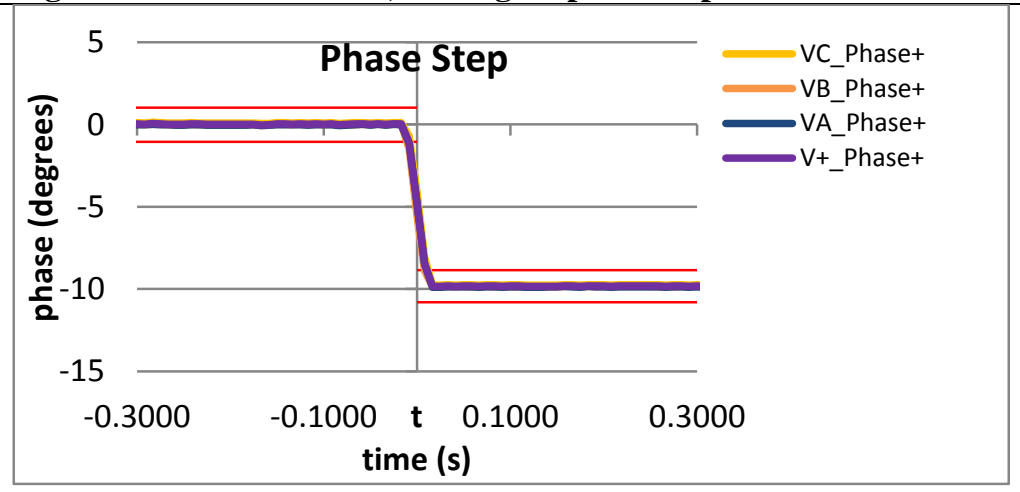

Figure 5062: Fs = 12 FPS, -10 degree phase step 


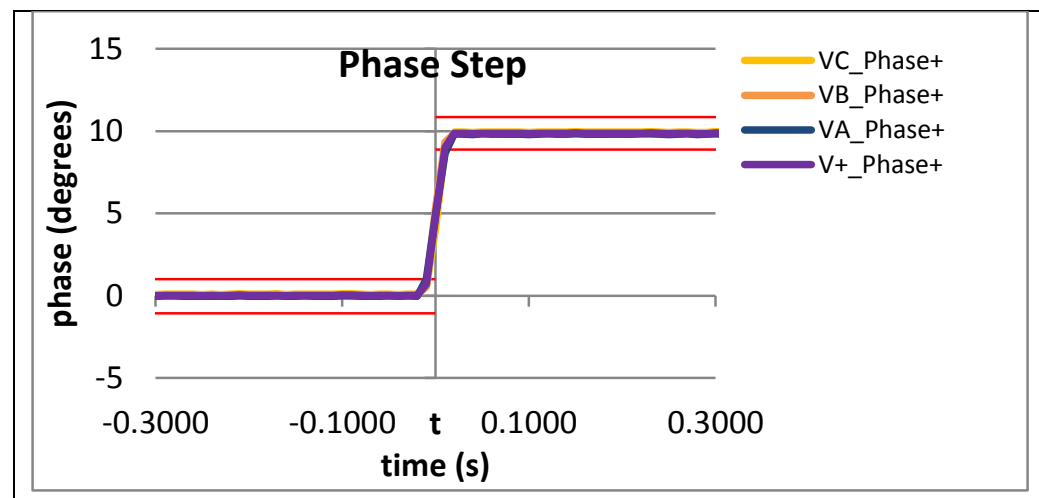

Figure 5063: Fs = 10 FPS, +10 degree phase step

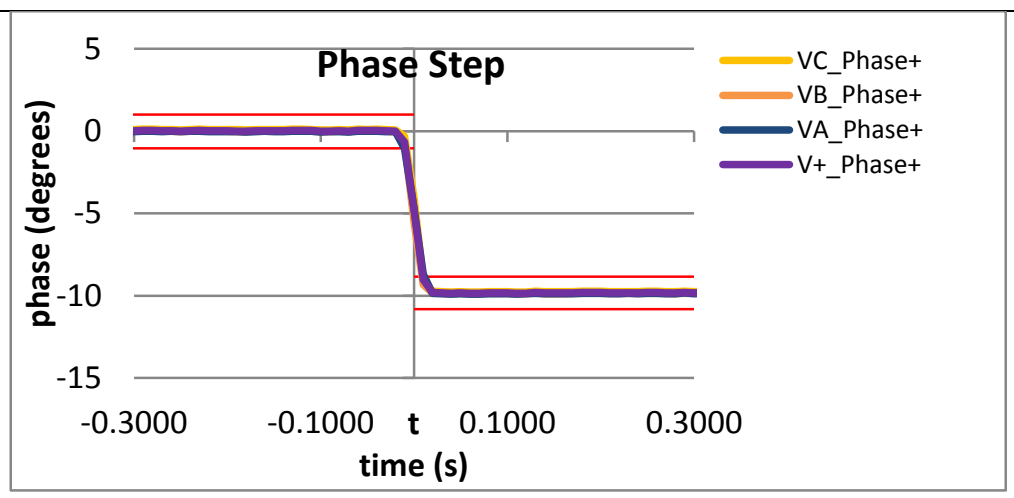

Figure 5064: Fs = 10 FPS, -10 degree phase step 
9.10.8 PMU G * dynamic step change in phase phasor overshoot: $F 0=60 \mathrm{~Hz}, \mathrm{P}$ class PMU G does not support P class.

\subsubsection{PMU H dynamic step change in phase phasor overshoot: $F 0=60 \mathrm{~Hz}$, P class}

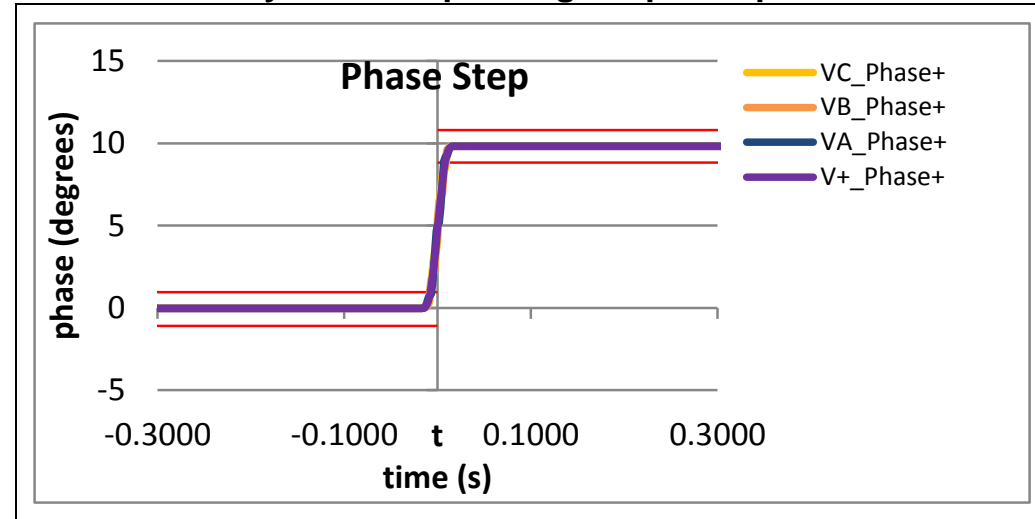

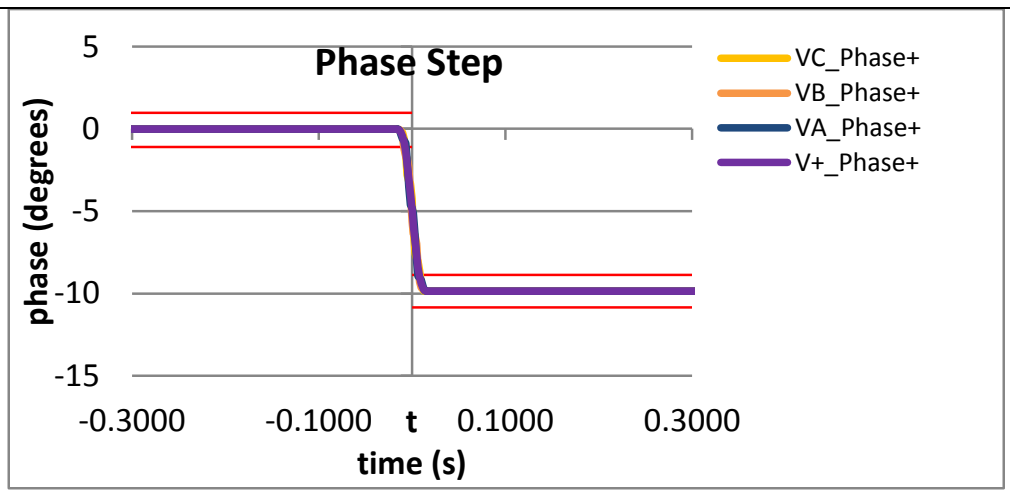

Figure 5065: Fs = 60 FPS, -10 degree phase step 


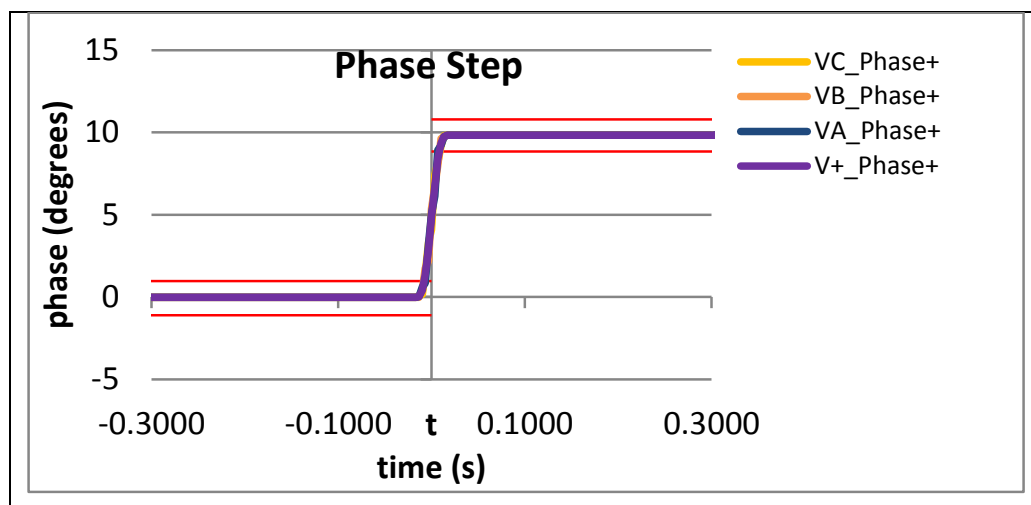

Figure 5066: Fs = 30 FPS, +10 degree phase step

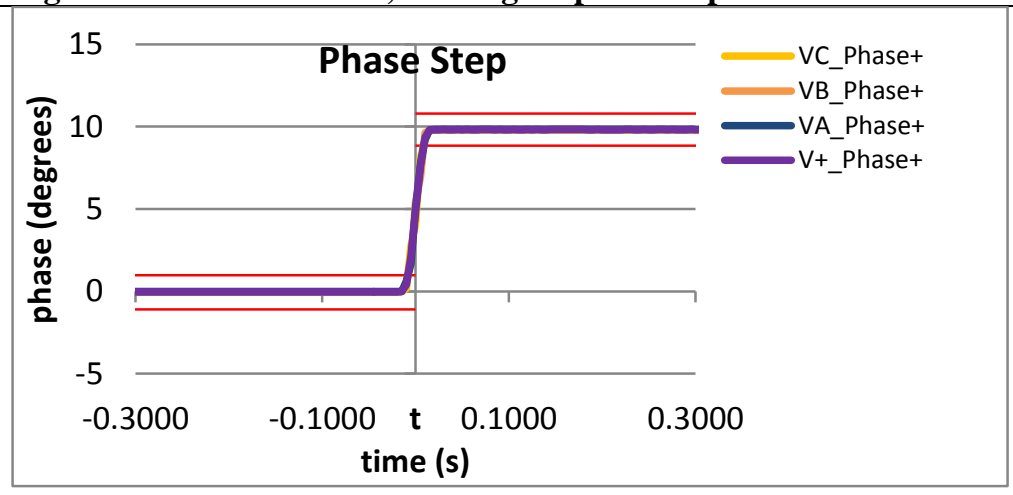

Figure 5068: Fs = 20 FPS, +10 degree phase step

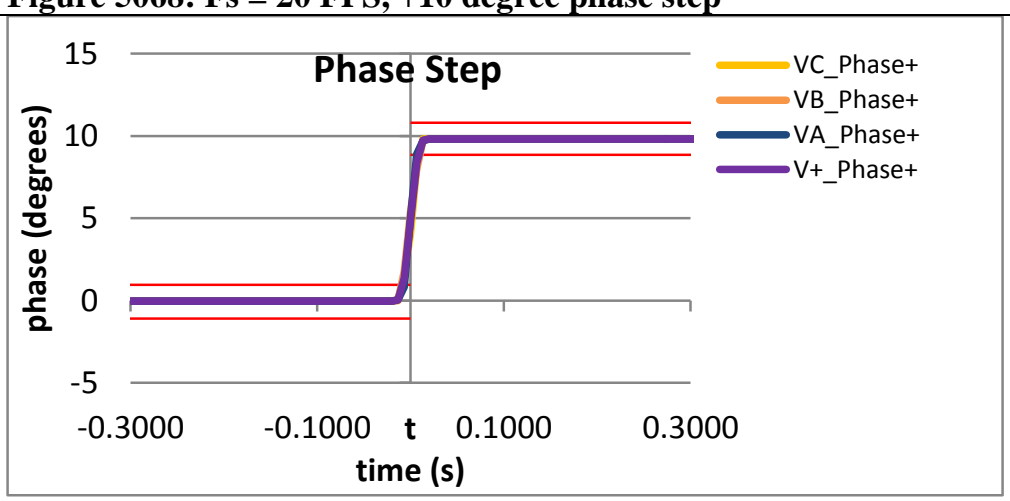

Figure 5070: Fs = 15 FPS, +10 degree phase step

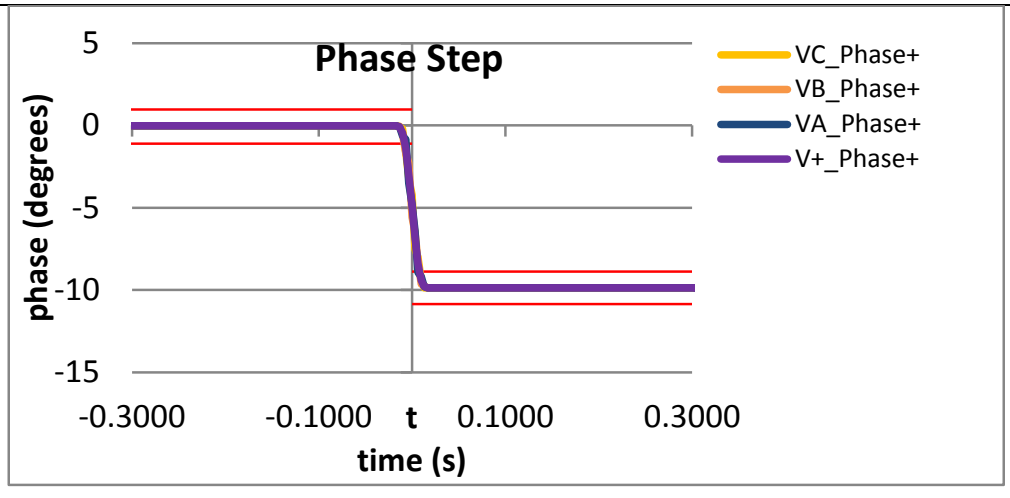

Figure 5067: Fs = 30 FPS, -10 degree phase step

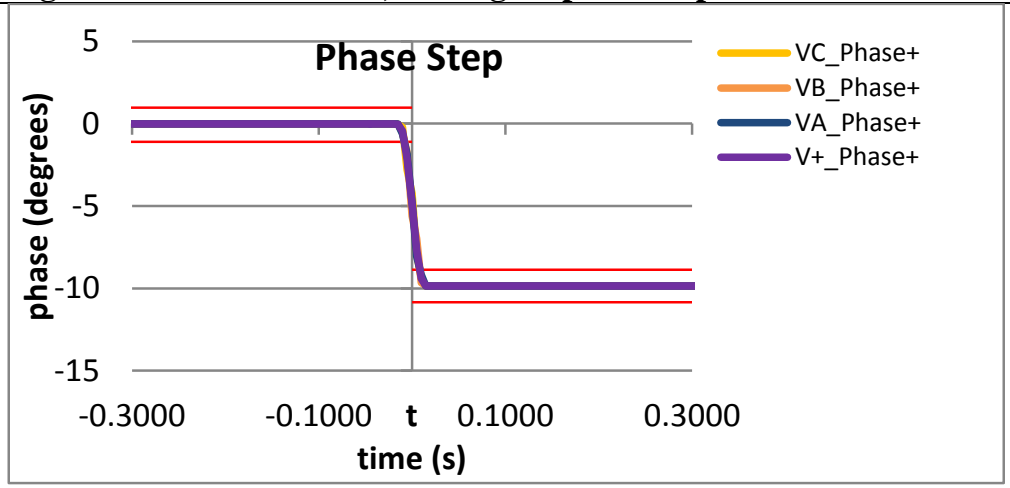

Figure 5069: Fs = 20 FPS, +10 degree phase step

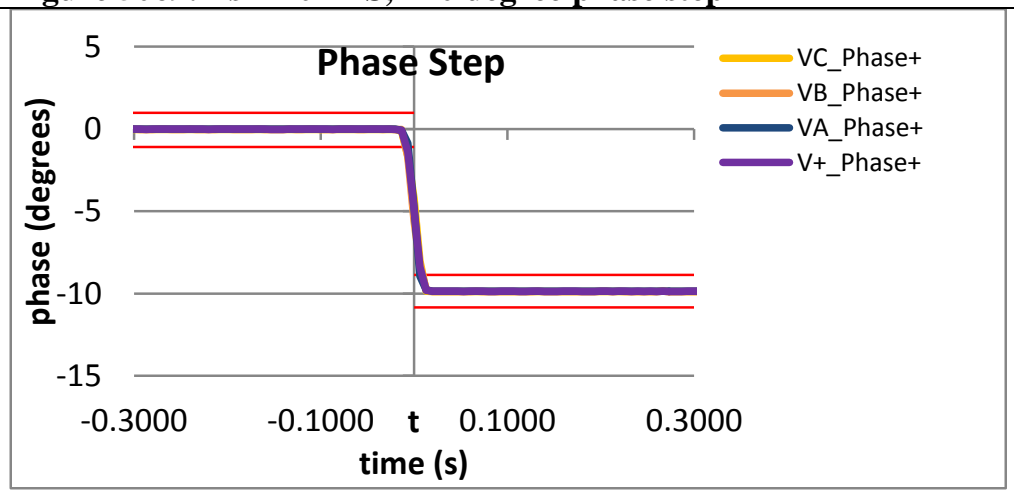

Figure 5071: Fs = 15 FPS, -10 degree phase step 


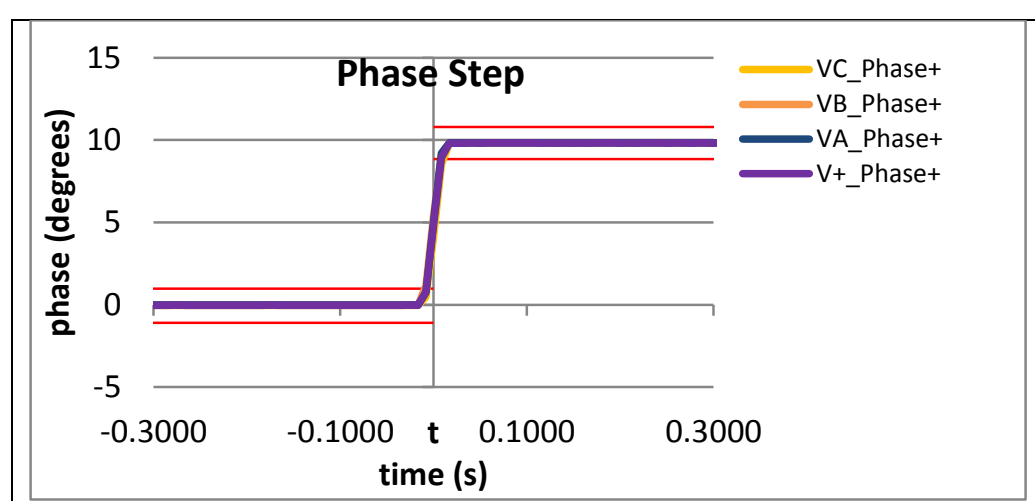

Figure 5072: Fs = 12 FPS, +10 degree phase step

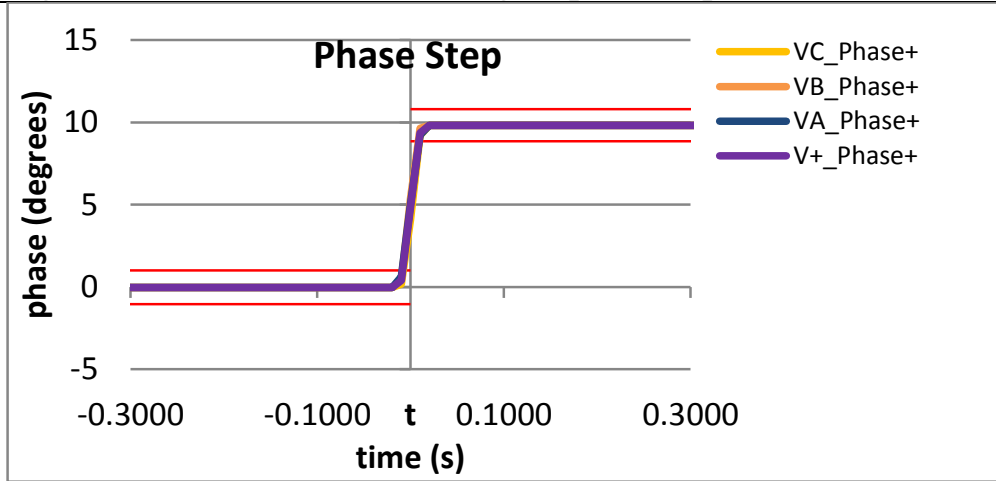

Figure 5074: Fs = 10 FPS, +10 degree phase step

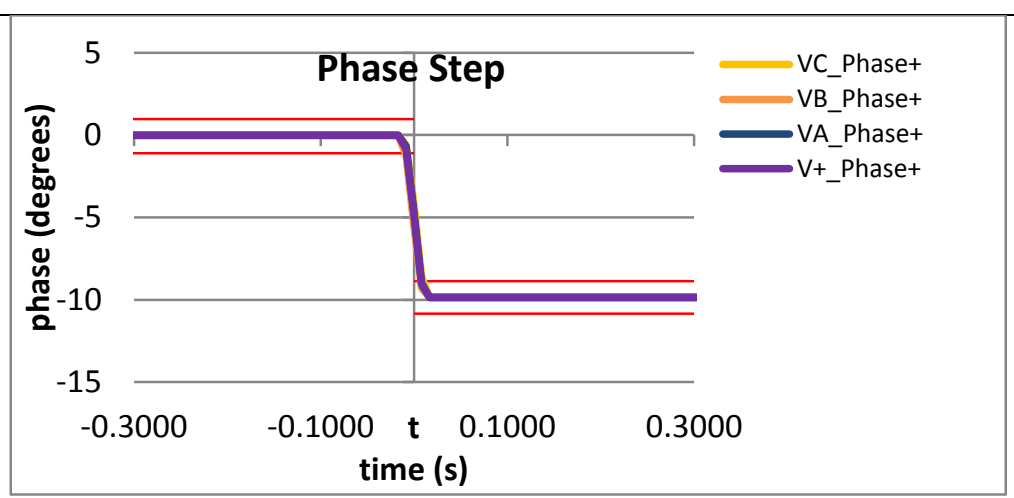

Figure 5073: Fs = 12 FPS, -10 degree phase step

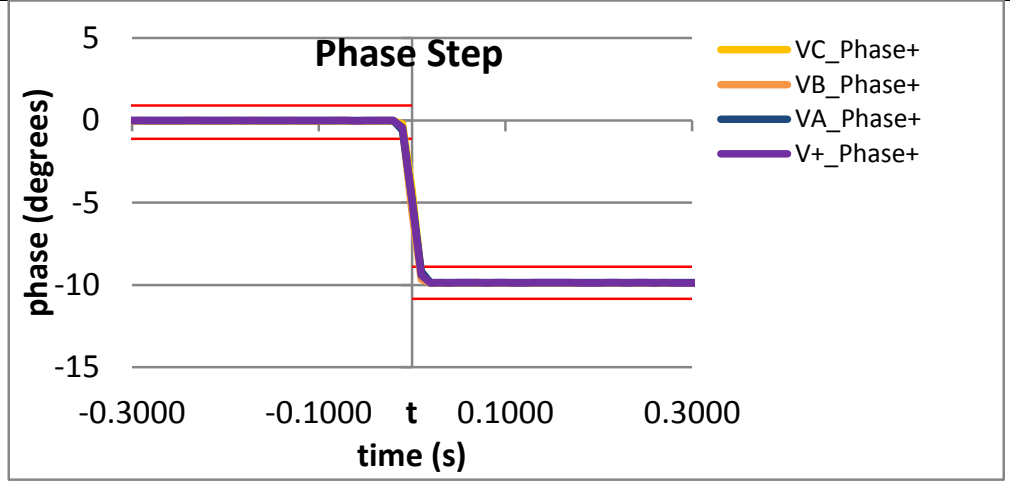

Figure 5075: Fs = 10 FPS, -10 degree phase step 
9.10.10 PMU I dynamic step change in phase phasor overshoot: $\mathrm{F} 0=60 \mathrm{~Hz}, \mathrm{P}$ class PMU I does not support P class

\subsubsection{PMU J dynamic step change in phase phasor overshoot: $F 0=60 \mathrm{~Hz}$, P class}

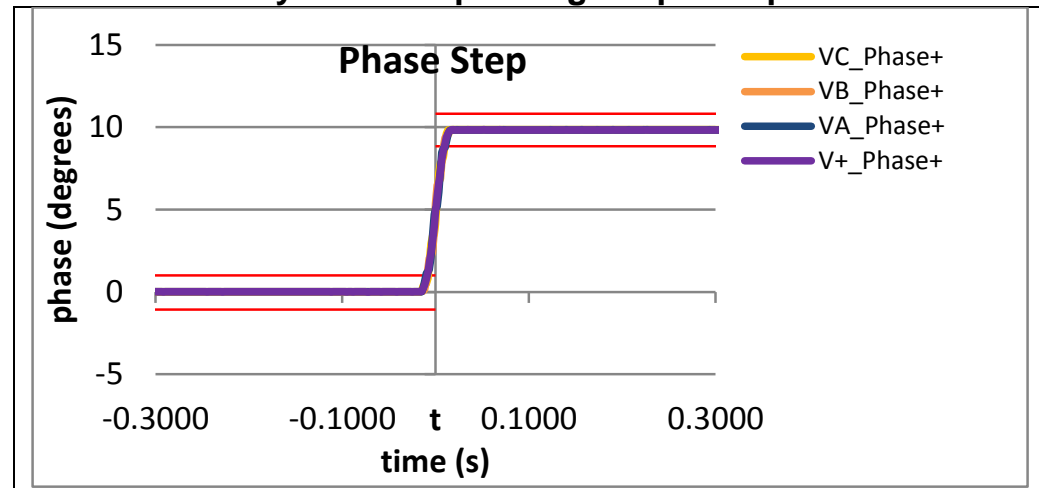

Figure 5076: Fs = 60 FPS, +10 degree phase step

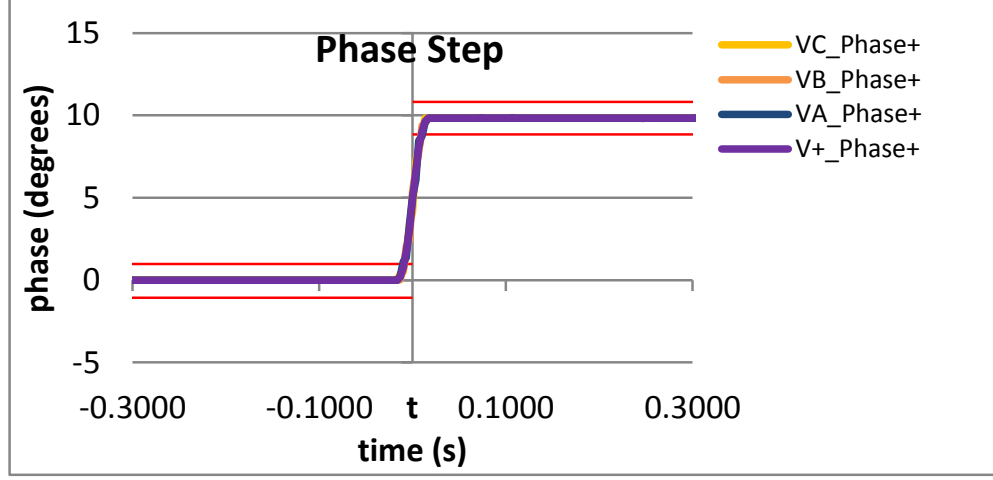

Figure 5078: Fs = 30 FPS, +10 degree phase step

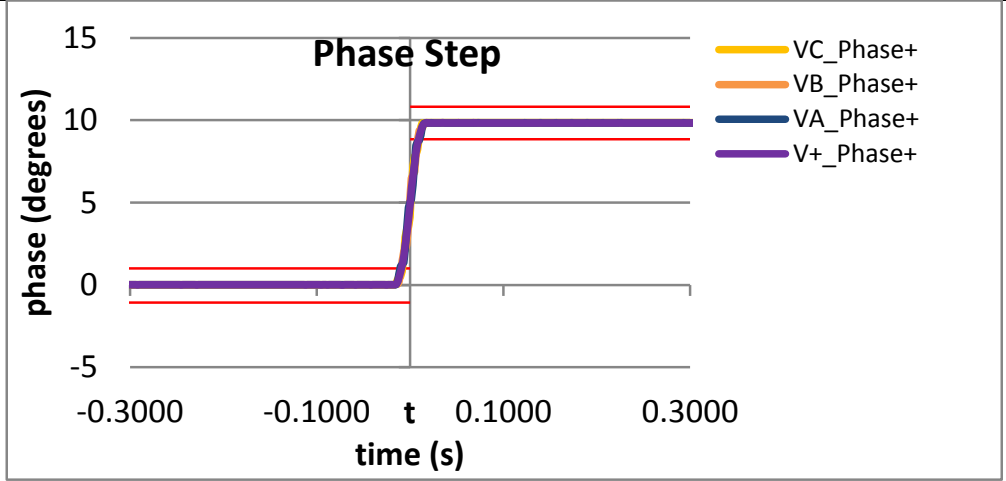

Figure 5077: Fs = 60 FPS, -10 degree phase step

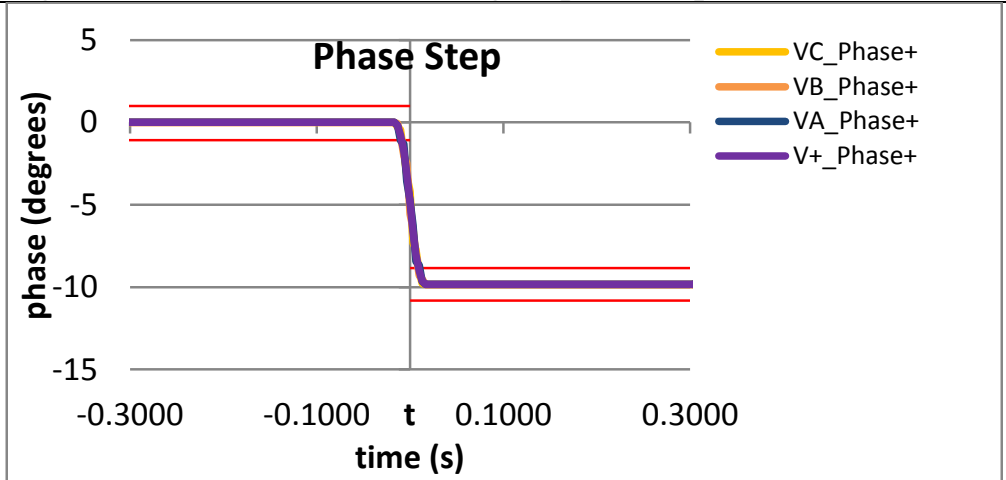

Figure 5079: Fs = 30 FPS, -10 degree phase step 


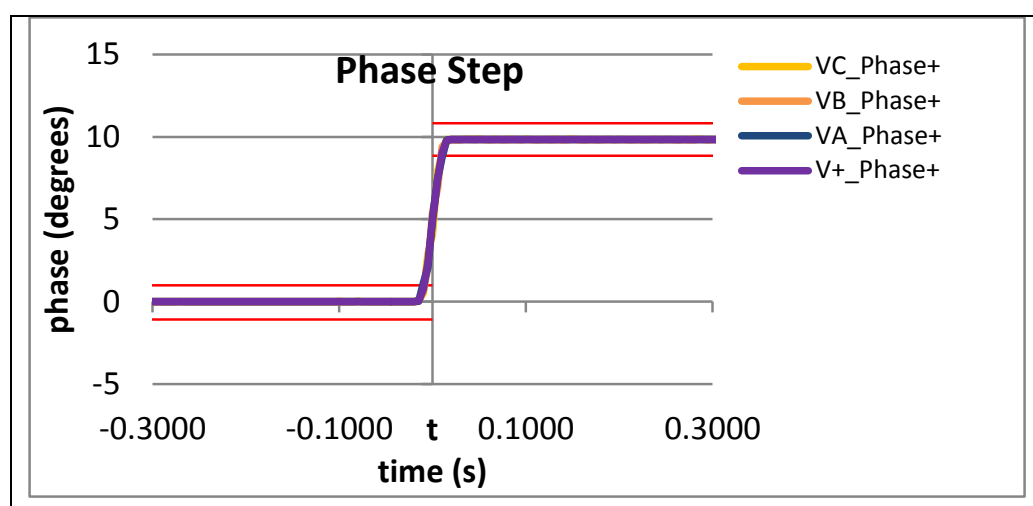

Figure 5080: Fs = 20 FPS, +10 degree phase step

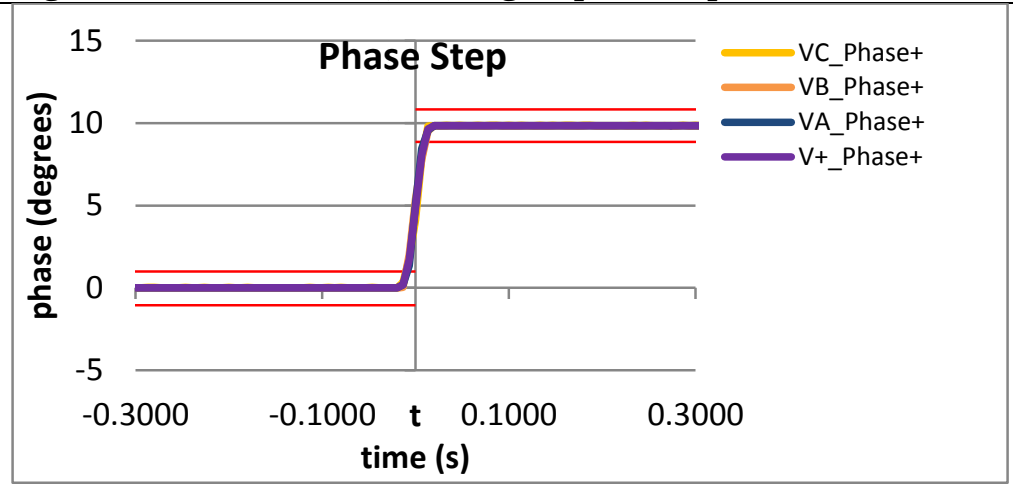

Figure 5082: Fs = 15 FPS, +10 degree phase step

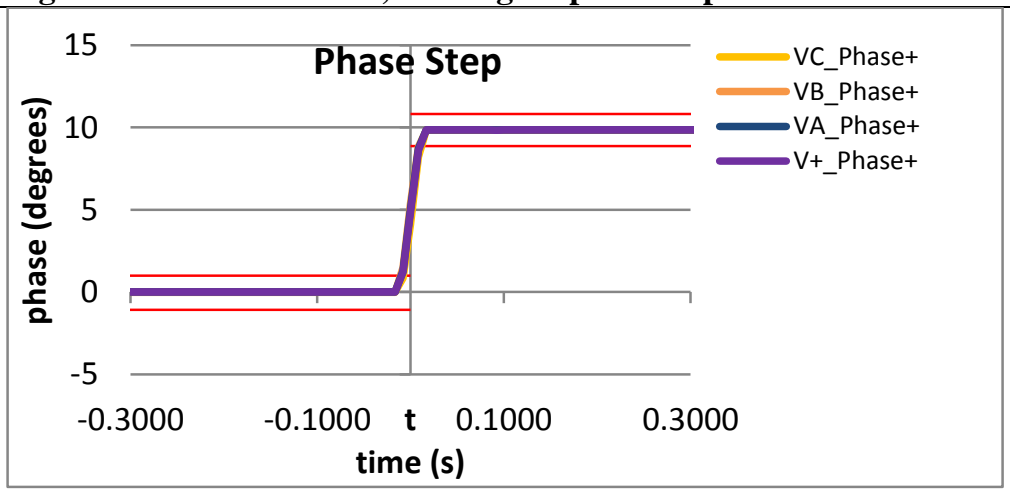

Figure 5084: Fs = 12 FPS, +10 degree phase step

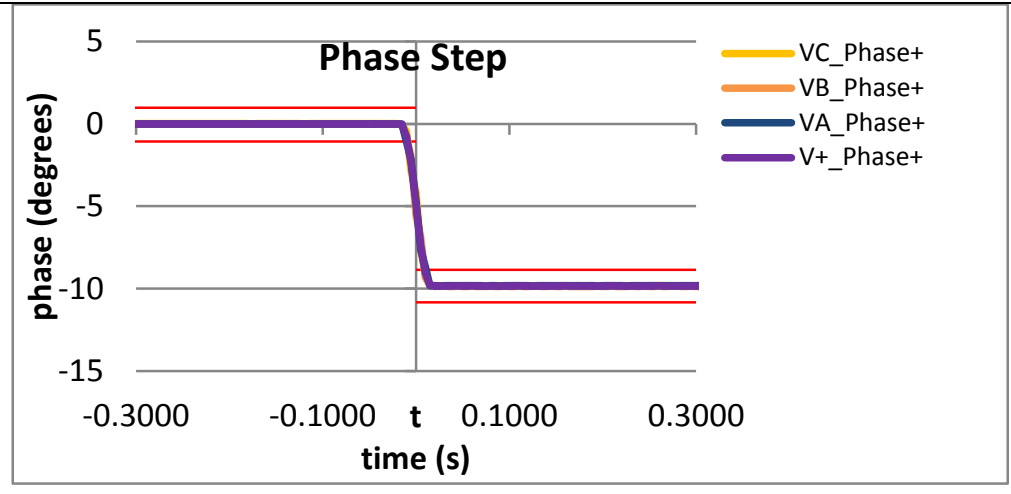

Figure 5081: Fs = 20 FPS, +10 degree phase step

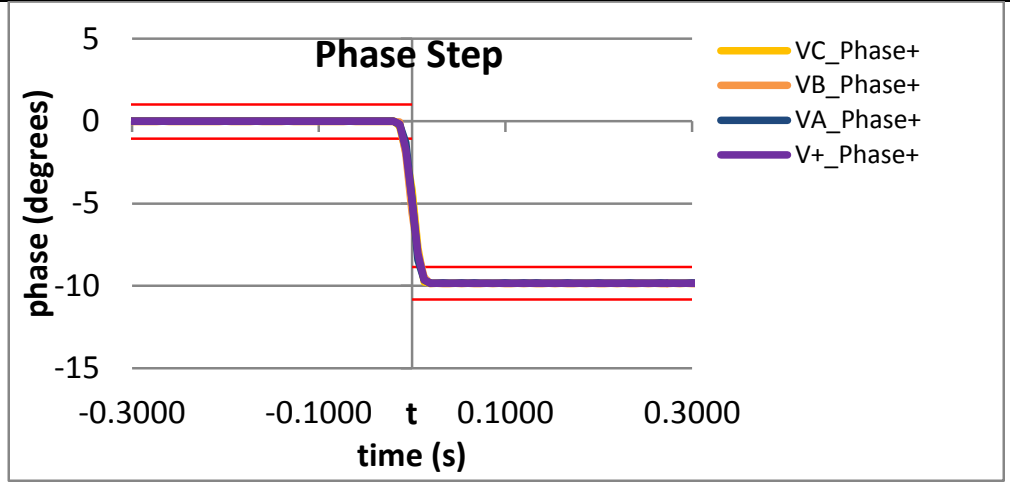

Figure 5083: Fs = 15 FPS, -10 degree phase step

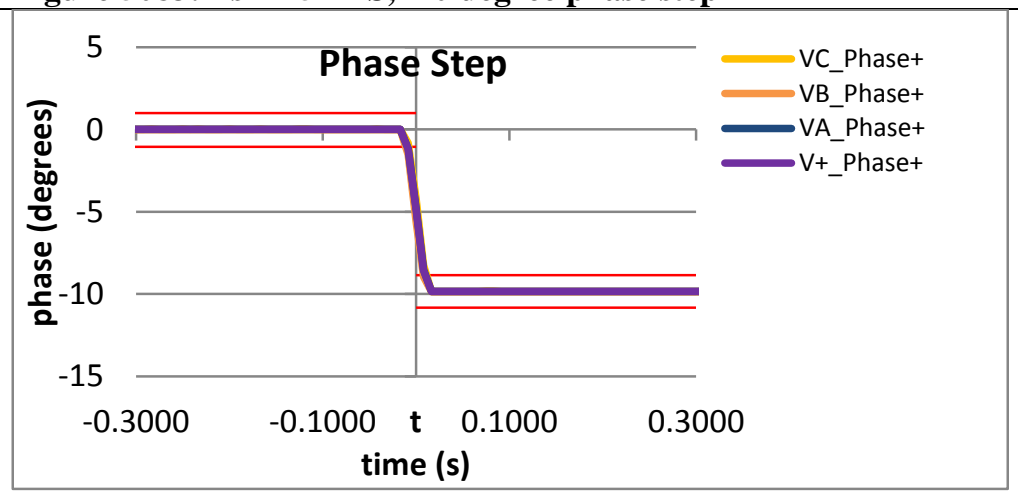

Figure 5085: Fs = 12 FPS, -10 degree phase step 


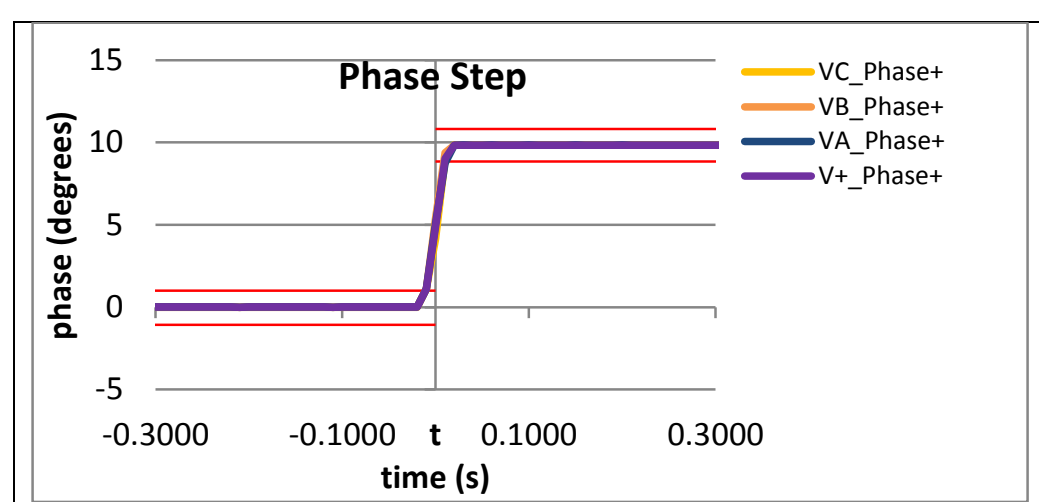

Figure 5086: Fs = 10 FPS, +10 degree phase step

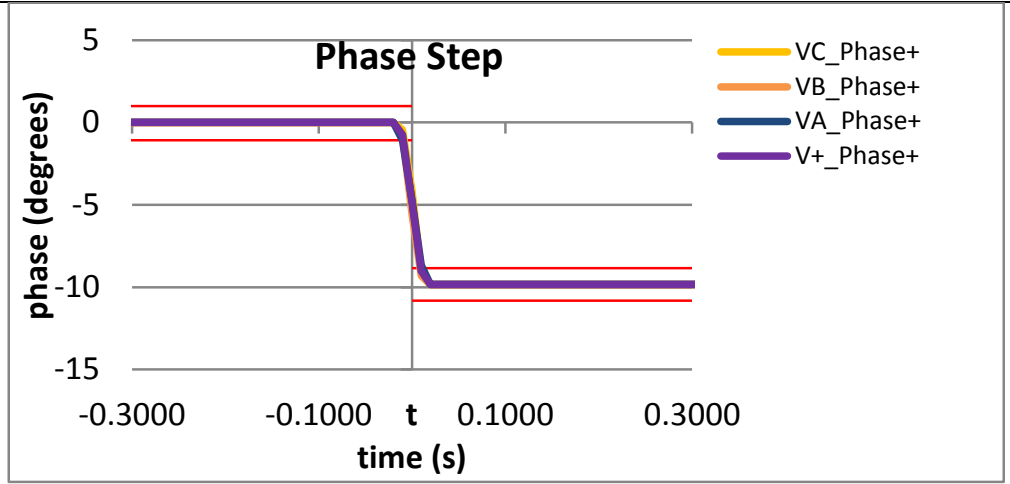

Figure 5087: Fs = 10 FPS, -10 degree phase step 


\section{Dynamic step change in magnitude}

10. Dynamic step change in magnitude.

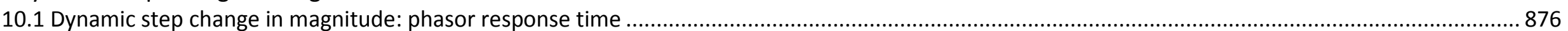

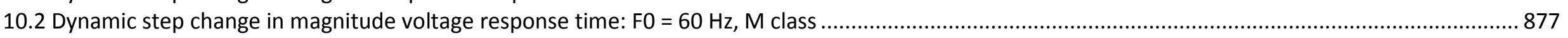

10.2.1 C37.118.1a Annex C dynamic step change in magnitude voltage response time: $\mathrm{FO}=60 \mathrm{~Hz}$, M class ............................................................................ 877

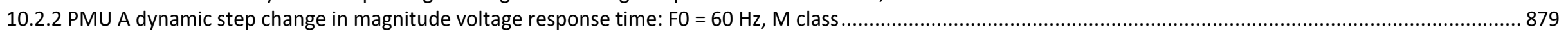

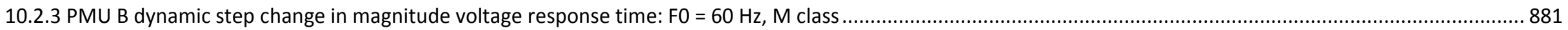

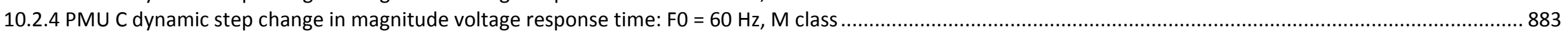

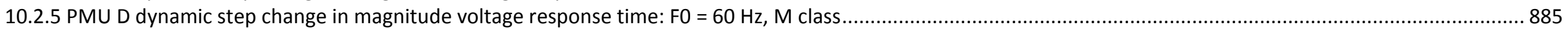

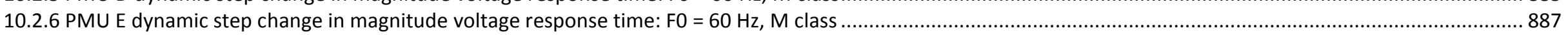

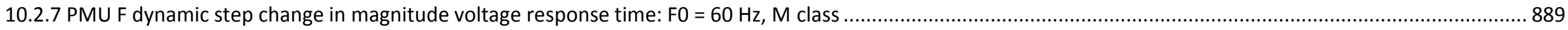

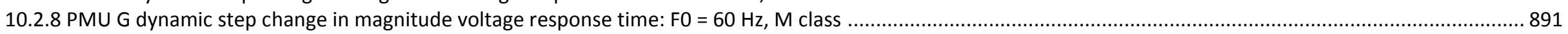

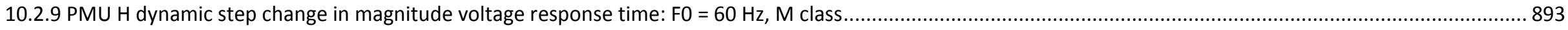

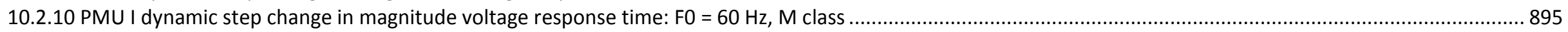

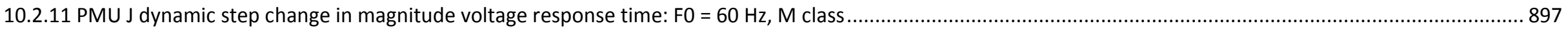

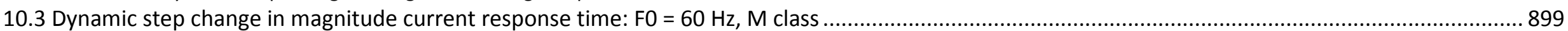

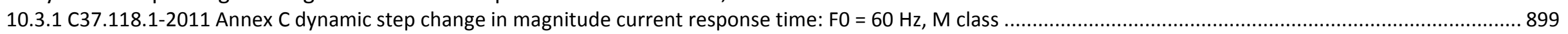

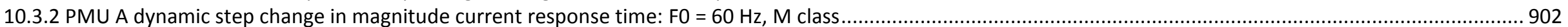

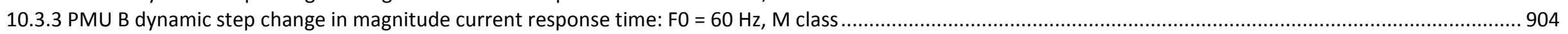

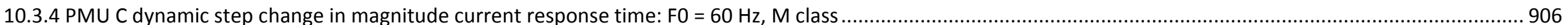

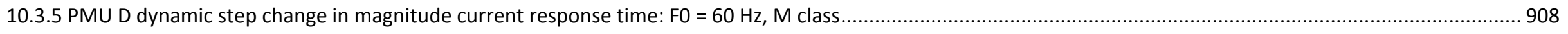

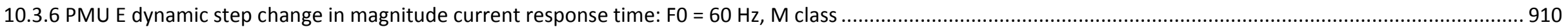

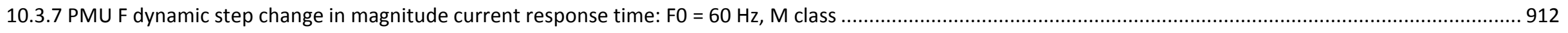

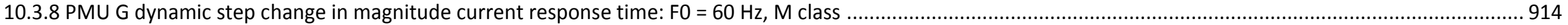

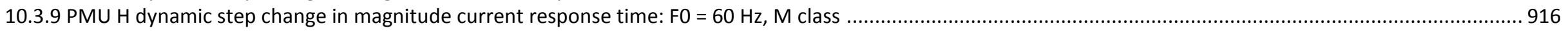

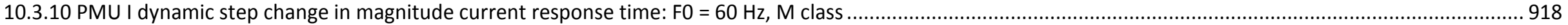

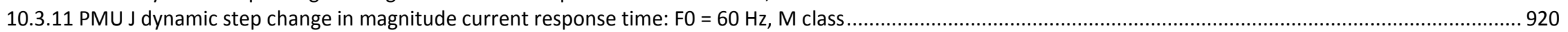

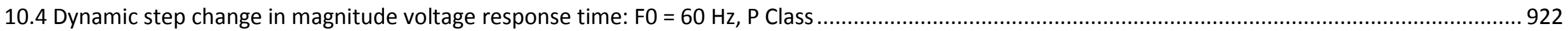

10.4.1 C37.118.1a Annex C dynamic step change in magnitude voltage response time: $\mathrm{FO}=60 \mathrm{~Hz}$, P class ............................................................................... 922

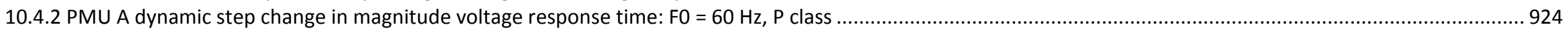

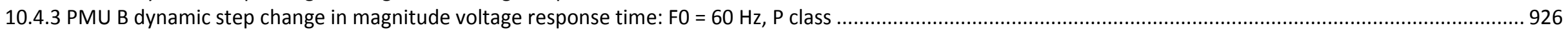

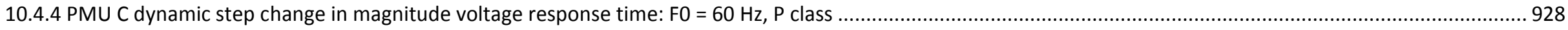

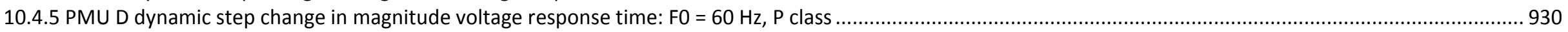

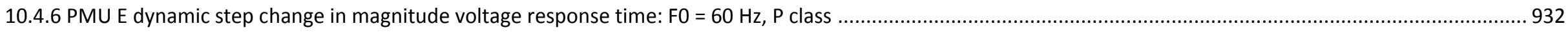

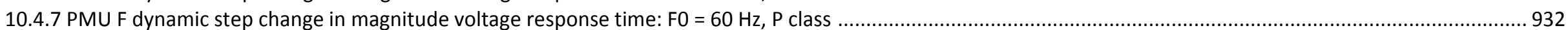

10.4.8 PMU G dynamic step change in magnitude voltage response time: $\mathrm{FO}=60 \mathrm{~Hz}$, P class .................................................................................................. 934 
10.4.9 PMU H dynamic step change in magnitude voltage response time: $\mathrm{FO}=60 \mathrm{~Hz}, \mathrm{P}$ class

10.4.10 PMU I dynamic step change in magnitude voltage response time: $\mathrm{FO}=60 \mathrm{~Hz}$, P class

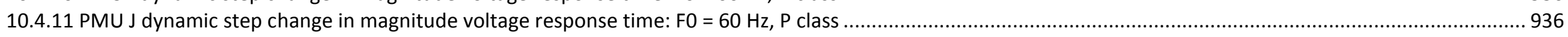

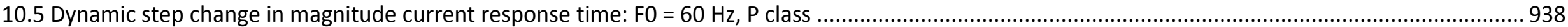

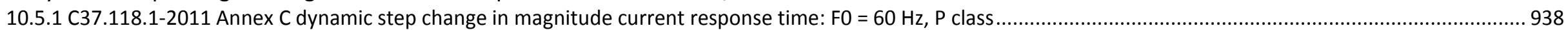

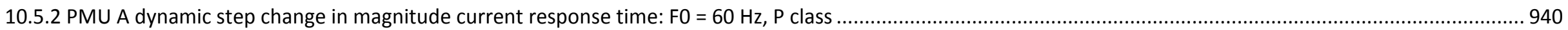

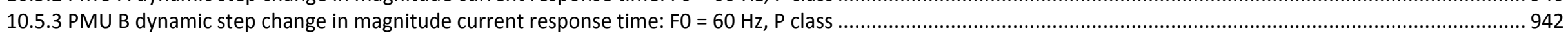

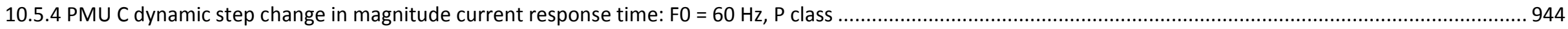

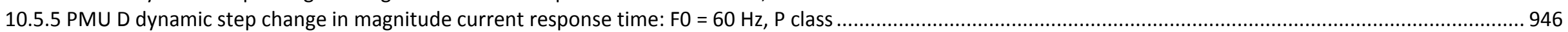

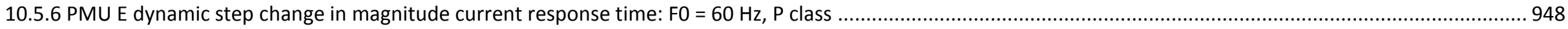

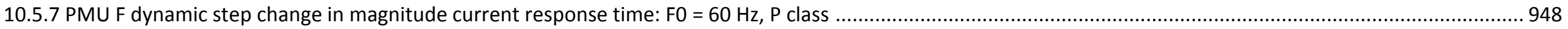

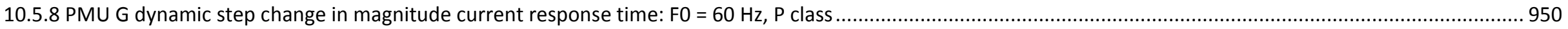

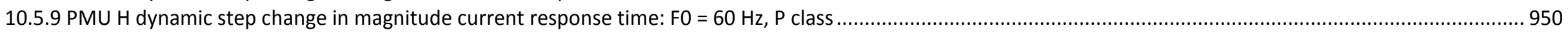

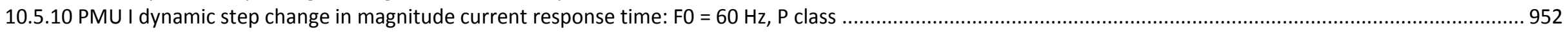

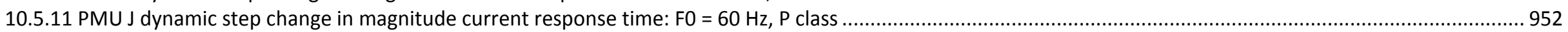

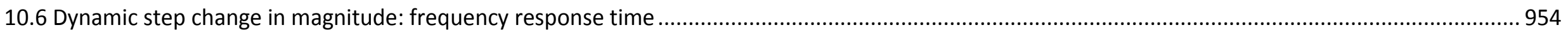

10.6.1 C37.118.1-2011 Annex C dynamic step change in magnitude frequency response time: $\mathrm{F0}=60 \mathrm{~Hz}, \mathrm{M}$ class ....................................................................... 955

10.6.2 PMU A dynamic step change in magnitude frequency response time: $\mathrm{F0}=60 \mathrm{~Hz}$, M class ……................................................................................... 957

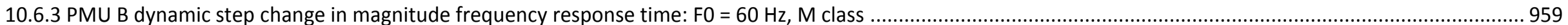

10.6.4 PMU C dynamic step change in magnitude frequency response time: $\mathrm{FO}=60 \mathrm{~Hz}$, M class .......................................................................................... 961

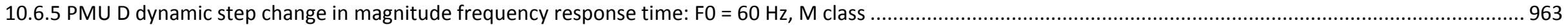

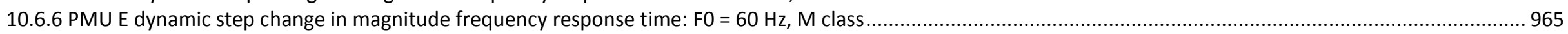

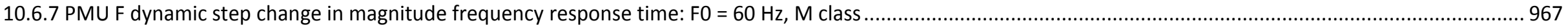

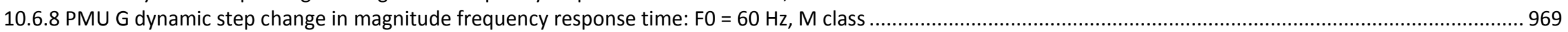

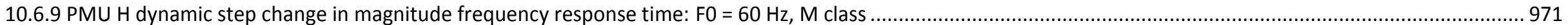

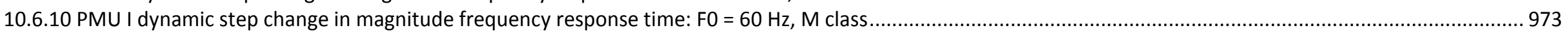

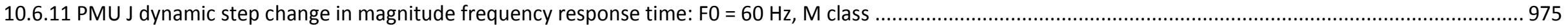

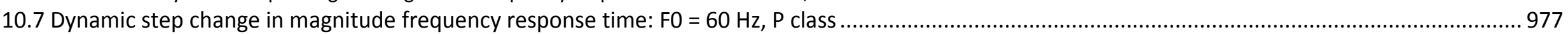

10.7.1 C37.118.1-2011 Annex C dynamic step change in magnitude frequency response time: $\mathrm{F0}=60 \mathrm{~Hz}$, P class ........................................................................ 977

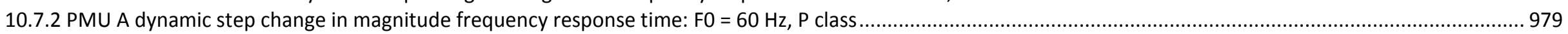

10.7.3 PMU B dynamic step change in magnitude frequency response time: $\mathrm{FO}=60 \mathrm{~Hz}$, P class ................................................................................................ 981

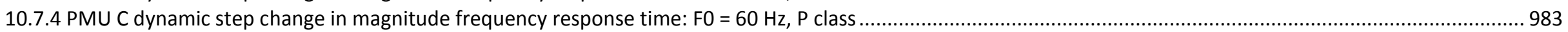

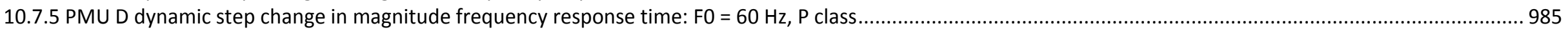

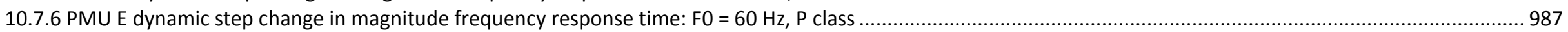

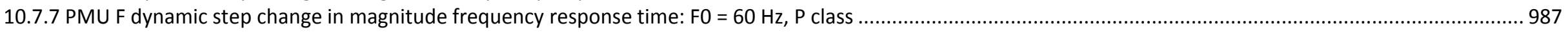

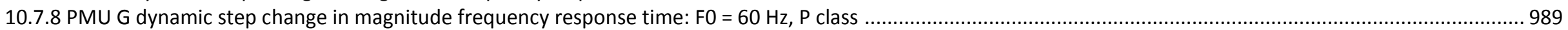

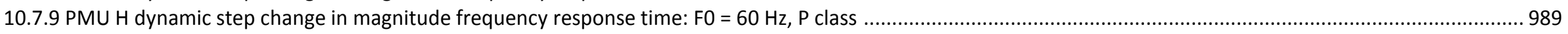

10.7.10 PMU I dynamic step change in magnitude frequency response time: $\mathrm{F} 0=60 \mathrm{~Hz}$, P class ............................................................................................ 991

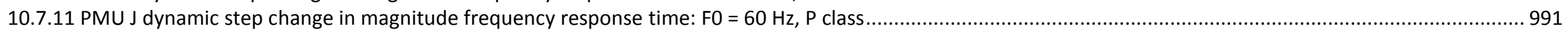


10.8 Dynamic step change in magnitude: ROCOF response time

10.9 Dynamic step change in magnitude ROCOF error: $\mathrm{FO}=60 \mathrm{~Hz}, \mathrm{M}$ class

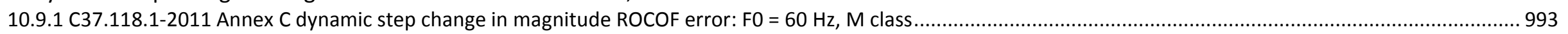

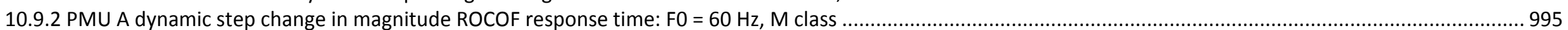

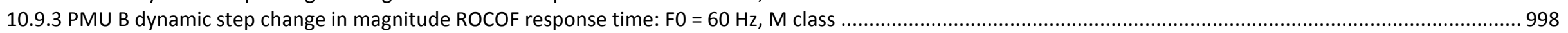

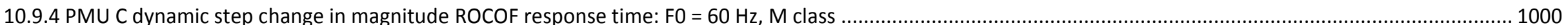

10.9.5 PMU D dynamic step change in magnitude ROCOF response time: $\mathrm{FO}=60 \mathrm{~Hz}$, M class ............................................................................................ 1002

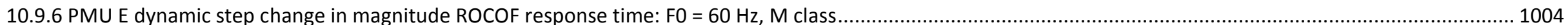

10.9.7 PMU F dynamic step change in magnitude ROCOF response time: $\mathrm{FO}=60 \mathrm{~Hz}$, M class ............................................................................................... 1006

10.9.8 PMU G * dynamic step change in magnitude ROCOF response time: $\mathrm{FO}=60 \mathrm{~Hz}$, M class .......................................................................................... 1008

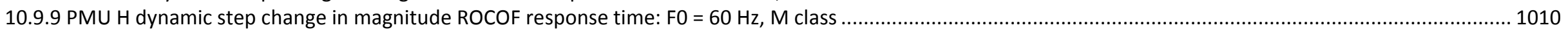

10.9.10 PMU I dynamic step change in magnitude ROCOF response time: F0 = 60 Hz, M class.................................................................................................... 1012

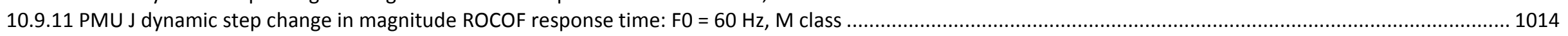

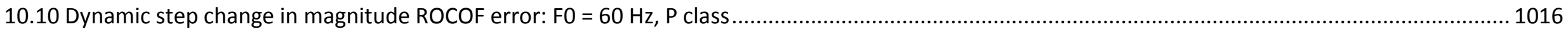

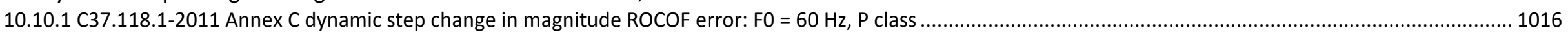

10.10.2 PMU A dynamic step change in magnitude ROCOF response time: $\mathrm{FO}=60 \mathrm{~Hz}$, P class ........................................................................................... 1018

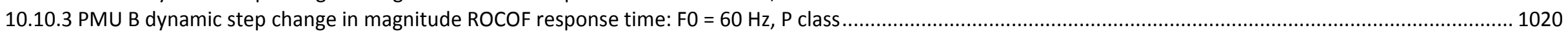

10.10.4 PMU C dynamic step change in magnitude ROCOF response time: $\mathrm{FO}=60 \mathrm{~Hz}$, P class ............................................................................................. 1022

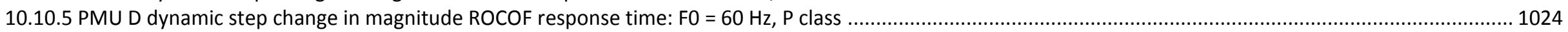

10.10.6 PMU E dynamic step change in magnitude ROCOF response time: FO = 60 Hz, P class ............................................................................................... 1026

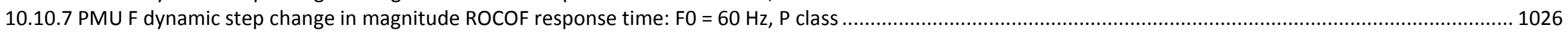

10.10.8 PMU G * dynamic step change in magnitude ROCOF response time: $\mathrm{FO}=60 \mathrm{~Hz}$, P class.............................................................................................. 1028

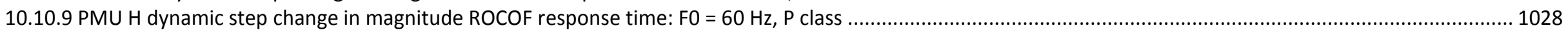

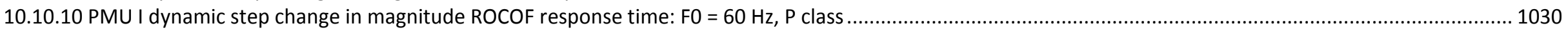

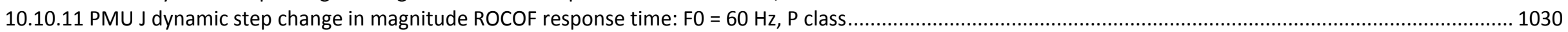

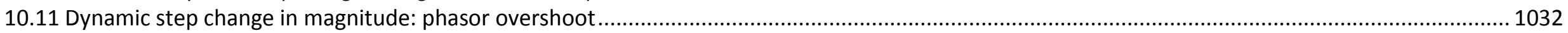

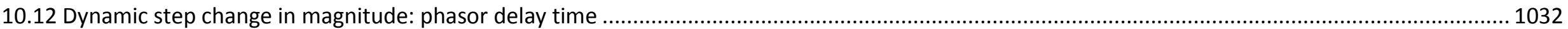

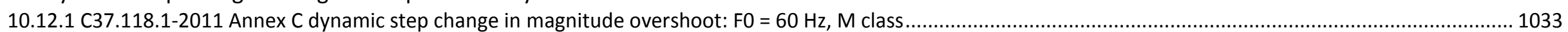

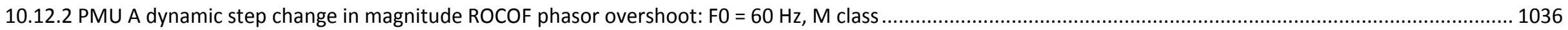

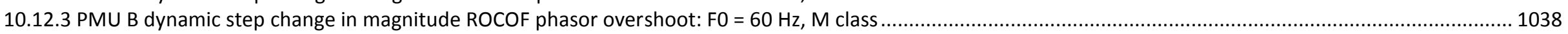

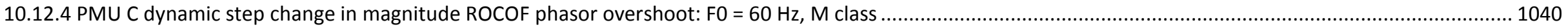

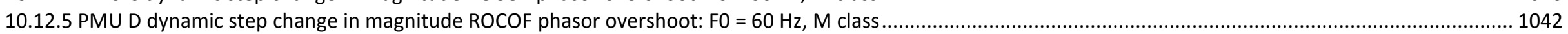

10.12.6 PMU E dynamic step change in magnitude ROCOF phasor overshoot: F0 = $60 \mathrm{~Hz}$, M class .......................................................................................... 1045

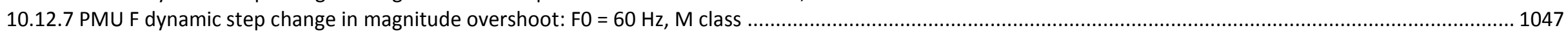

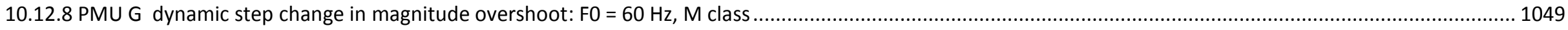

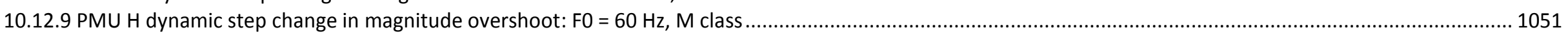

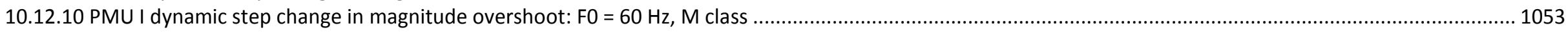

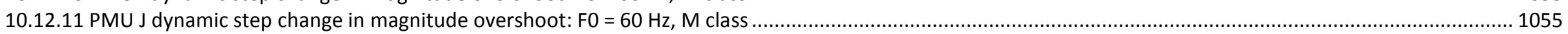


This publication is available free of charge from http://dx.doi.org/10.6028/NIST.IR.8106

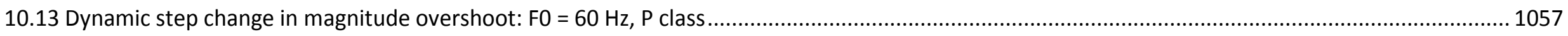

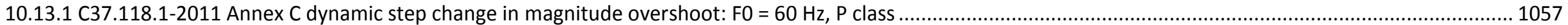

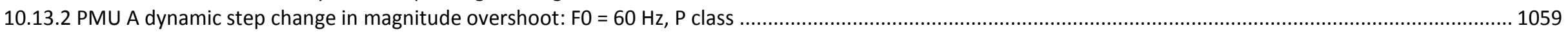

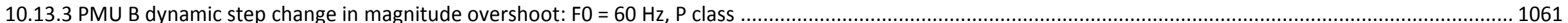

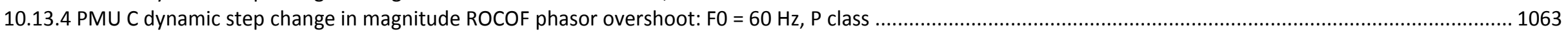

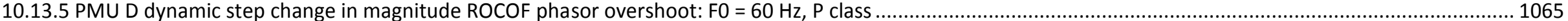

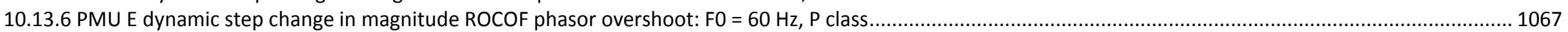

10.13.7 PMU F dynamic step change in magnitude ROCOF phasor overshoot: FO = 60 Hz, P class . .

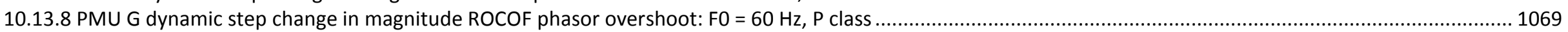

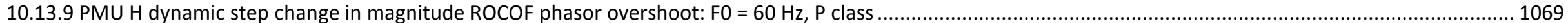

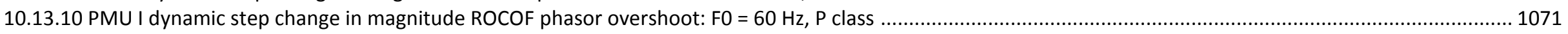


Performance during step changes in magnitude is determined by applying $\pm 10 \%$ step changes of magnitude. Response time and delay time of the phasors, frequency, and ROCOF measurements are compared to the limits. Overshoot of the phasors are compared to the limits.

A recommended practice for measuring a PMU's response to step changes in input is to run a series of $n$ step sub-tests where the occurrence of the step is displaced in time relative to the PMU report-time. During each $\left(\mathrm{n}^{\text {th }}\right)$ iteration of 10 sub-tests, the step occurs $1 / 10$ th of a reporting period further from the time of the report than the previous iteration of the subtest. The results are combined to trace an equivalent time sampled response curve. During each sub-test, the influence quantity (amplitude or phase) is stepped, the response data are gathered, and the magnitude is returned to its previous value in preparation for the next sub-test in the series. At the end of the series of sub-tests, the TVE, FE, RFE results and the PMU reported magnitude data from all sub-tests are indexed and overlaid to create smooth curves approximating the step response of the PMU. From the curves, delay time, response time, and overshoot/undershoot shall be calculated

The test plan for step change in phase and magnitude is as follows:

For the first test, let $\mathrm{n}=0$.

a) Begin with three-phase balanced input at nominal amplitude and frequency. Hold steady state for at least 1 second plus one response time period.

b) At the beginning of a reporting cycle plus $n /\left(10 \times F_{s}\right)$ (i.e., $n \times$ reporting period $\left./ 10\right)$ step the magnitude by $+10 \%$ of the nominal magnitude and hold steady state for at least 1 second plus one response time period.

c) Gather the PMU data, return the influence quantity to nominal and wait for the PMU to settle.

d) Increment $n$ by one $(n=n+1)$, then repeat step c) through step d) until $n=10-1$.

e) Index and overlay the PMU data to obtain a smooth response curve.

f) Repeat the tests for negative magnitude step.

IEEE Std. C37.118.1a-2014 sets the M class response time limit for $M$ class phasors at $7 / F_{s}$ and for frequency and ROCOF at the greater of $14 / F_{s}$ or $14 / F_{0}$. IEEE Std. C37.118.1a-2014 sets the $P$ class response time limit for phasors at $2 / F_{0}$ and the delay time limit at $1 /\left(4 F_{s}\right)$.

IEEE Std. C37.118.1a-2014 sets the $P$ class response time limit for frequency at $4.5 / F_{0}$ and for ROCOF at $6 / F_{0}$.

IEEE Std C37.118.1-2011 sets the M class phasor delay time at $1 /\left(4 \mathrm{~F}_{\mathrm{s}}\right)$ and the max overshoot at $10 \%$ of the step magnitude.

IEEE Std C37.118.1-2011 sets the P class phasor delay time at $1 /\left(4 \mathrm{~F}_{s}\right)$ and the max overshoot at $5 \%$ of the step magnitude.

The response time plots in this section show the limit of TVE, Fe, or RFe as a green horizontal line. A red vertical line appears at the response time limits. note that the limits are not interpolated, the first horizontal red line appears at the first report which is above the TVE, FE or RFE limit and the second red line is at the response time limit away from the first.

The overshoot plots in this section show red horizontal lines at the overshoot/undershoot limits around the magnitude before and after the step. 


\subsection{Dynamic step change in magnitude: phasor response time}

\begin{tabular}{|l|l|l|l|l|l|l|l|l|l|l|l|l|}
\hline Fs (FPS) & $10 M$ & $10 \mathrm{P}$ & $12 \mathrm{M}$ & $12 \mathrm{P}$ & $15 \mathrm{M}$ & $15 \mathrm{P}$ & $20 \mathrm{M}$ & $20 \mathrm{P}$ & $30 \mathrm{M}$ & $30 \mathrm{P}$ & $60 \mathrm{M}$ & $60 \mathrm{P}$ \\
\hline C37.118.1 Annex C & $\mathrm{P}$ & $\mathrm{P}$ & $\mathrm{P}$ & $\mathrm{P}$ & $\mathrm{P}$ & $\mathrm{P}$ & $\mathrm{P}$ & $\mathrm{P}$ & $\mathrm{P}$ & $\mathrm{P}$ & $\mathrm{P}$ & $\mathrm{P}$ \\
\hline PMU A & $\mathrm{P}$ & $\mathrm{P}$ & $\mathrm{P}$ & $\mathrm{P}$ & $\mathrm{P}$ & $\mathrm{P}$ & $\mathrm{P}$ & $\mathrm{P}$ & $\mathrm{P}$ & $\mathrm{P}$ & $\mathrm{P}$ & $\mathrm{P}$ \\
\hline PMU B & $\mathrm{P}$ & $\mathrm{F}$ & $\mathrm{P}$ & $\mathrm{F}$ & $\mathrm{P}$ & $\mathrm{F}$ & $\mathrm{P}$ & $\mathrm{F}$ & $\mathrm{P}$ & $\mathrm{F}$ & $\mathrm{P}$ & $\mathrm{F}$ \\
\hline PMU C & $\mathrm{P}$ & $\mathrm{P}$ & $\mathrm{P}$ & $\mathrm{P}$ & $\mathrm{P}$ & $\mathrm{P}$ & $\mathrm{P}$ & $\mathrm{P}$ & $\mathrm{P}$ & $\mathrm{P}$ & $\mathrm{P}$ & $\mathrm{P}$ \\
\hline PMU D & $\mathrm{P}$ & $\mathrm{I}$ & $\mathrm{P}$ & $\mathrm{I}$ & $\mathrm{P}$ & $\mathrm{I}$ & $\mathrm{P}$ & $\mathrm{I}$ & $\mathrm{P}$ & $\mathrm{I}$ & $\mathrm{P}$ & $\mathrm{I}$ \\
\hline PMU E & $\mathrm{P}$ & - & - & - & $\mathrm{P}$ & - & $\mathrm{P}$ & - & $\mathrm{P}$ & - & $\mathrm{F}$ & - \\
\hline PMU F & $\mathrm{P}$ & $\mathrm{P}$ & $\mathrm{P}$ & $\mathrm{P}$ & $\mathrm{P}$ & $\mathrm{P}$ & $\mathrm{P}$ & $\mathrm{P}$ & $\mathrm{P}$ & $\mathrm{P}$ & $\mathrm{P}$ & $\mathrm{P}$ \\
\hline PMU G & $\mathrm{P}$ & - & $\mathrm{P}$ & - & $\mathrm{P}$ & - & $\mathrm{F}$ & - & $\mathrm{F}$ & - & - & - \\
\hline PMU H & $\mathrm{P}$ & $\mathrm{P}$ & $\mathrm{P}$ & $\mathrm{P}$ & $\mathrm{P}$ & $\mathrm{P}$ & $\mathrm{P}$ & $\mathrm{P}$ & $\mathrm{P}$ & $\mathrm{P}$ & $\mathrm{P}$ & $\mathrm{P}$ \\
\hline PMU I & $\mathrm{P}$ & - & $\mathrm{P}$ & - & $\mathrm{P}$ & - & $\mathrm{P}$ & - & $\mathrm{P}$ & - & $\mathrm{P}$ & - \\
\hline PMU J & $\mathrm{P}$ & $\mathrm{P}$ & $\mathrm{P}$ & $\mathrm{P}$ & $\mathrm{P}$ & $\mathrm{P}$ & $\mathrm{P}$ & $\mathrm{P}$ & $\mathrm{P}$ & $\mathrm{P}$ & $\mathrm{P}$ & $\mathrm{P}$ \\
\hline
\end{tabular}

$\mathrm{P}=$ Pass, $\mathrm{F}=$ Fail $\mathrm{I}$ = indeterminate 
10.2 Dynamic step change in magnitude voltage response time: $F 0=60 \mathrm{~Hz}, M$ class

10.2.1 C37.118.1a Annex $C$ dynamic step change in magnitude voltage response time: $F 0=60 \mathrm{~Hz}$, M class

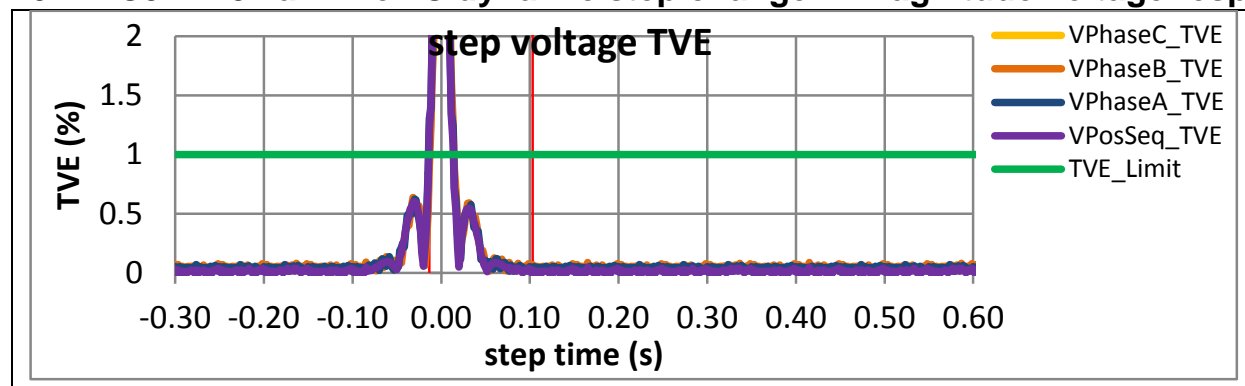

Figure 5089: Fs = 60 FPS, +10\% magnitude step

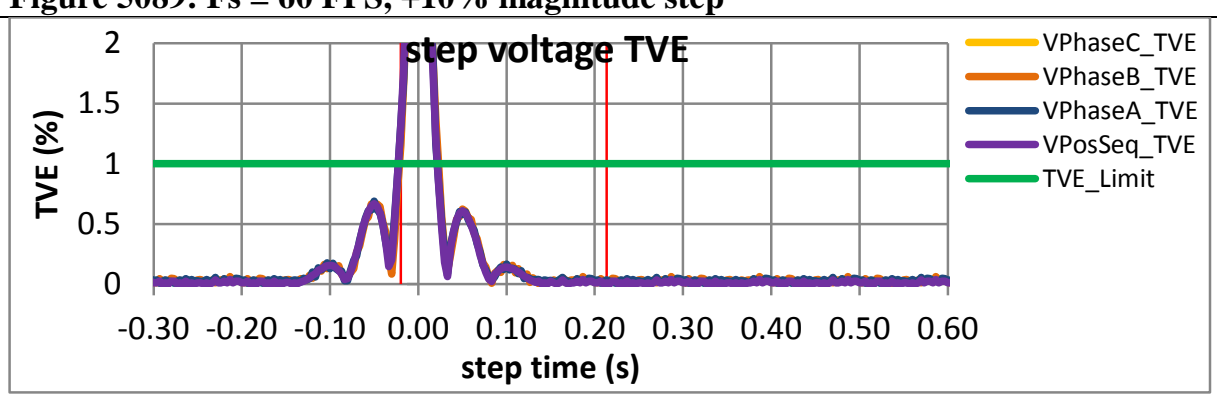

Figure 5091: Fs $=30$ FPS, $+10 \%$ magnitude step

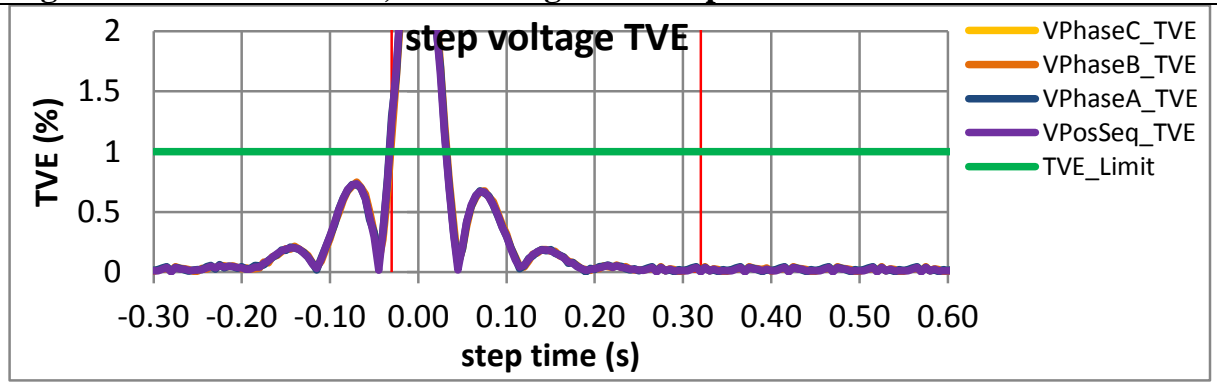

Figure 5093: Fs = 20 FPS, +10\% magnitude step

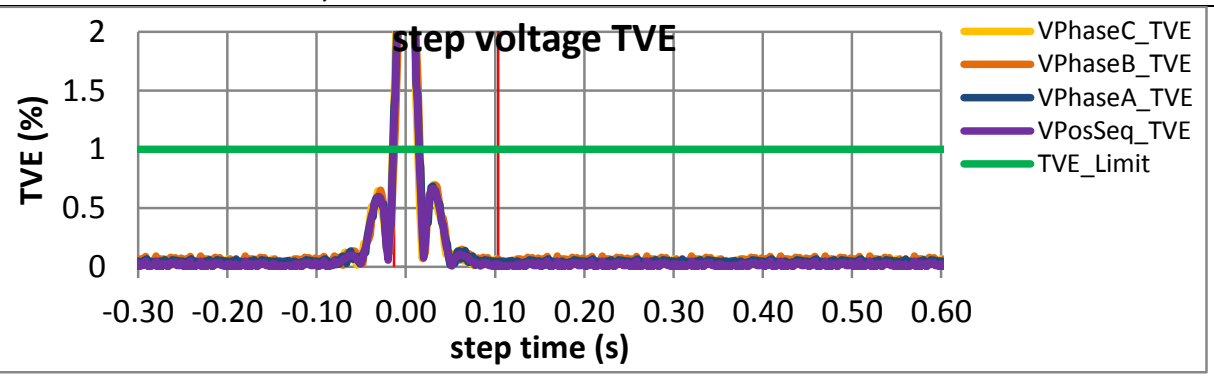

Figure 5090: Fs = 60 FPS, -10\% magnitude step

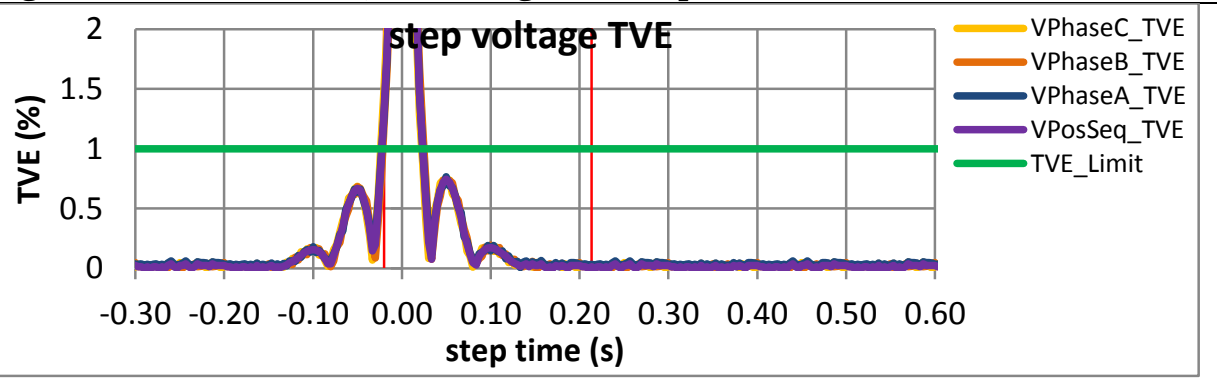

Figure 5092: Fs = 30 FPS, -10\% magnitude step

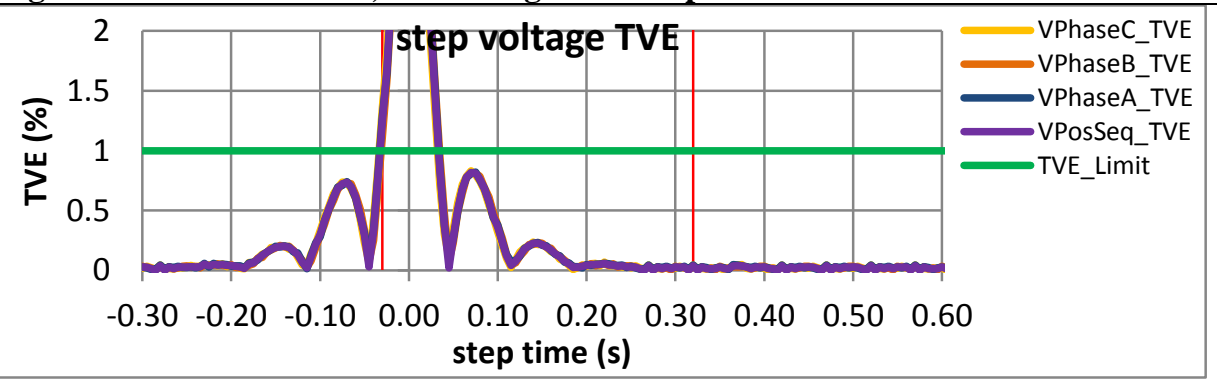

Figure 5094: Fs = 20 FPS, -10\% magnitude step 

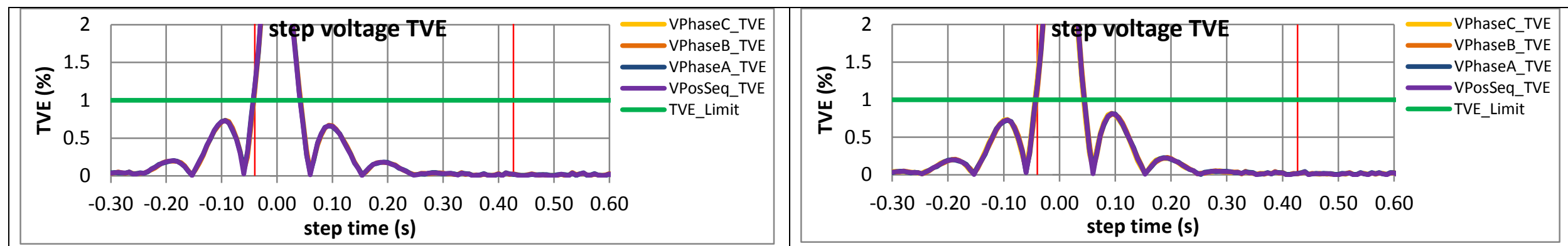

Figure 5095: Fs $=15$ FPS, $+10 \%$ magnitude step

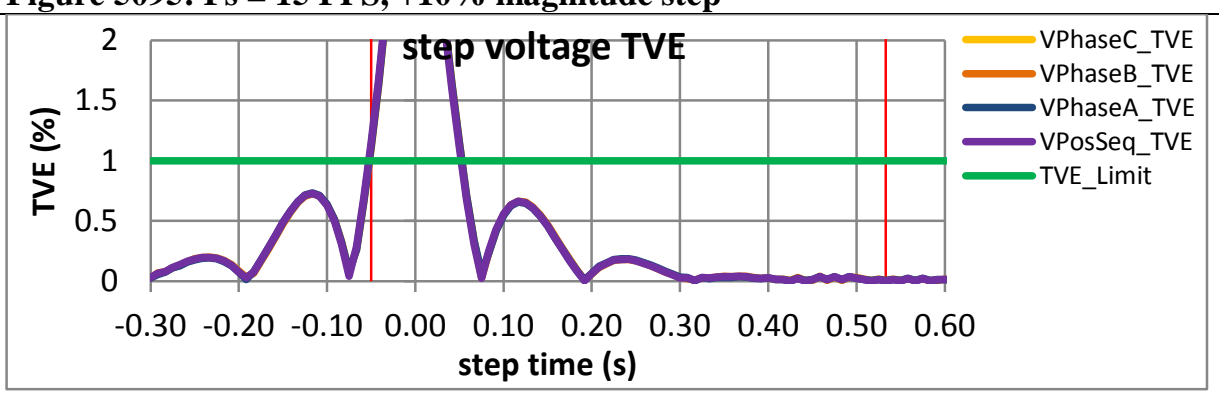

Figure 5097: Fs = 12 FPS, +10\% magnitude step

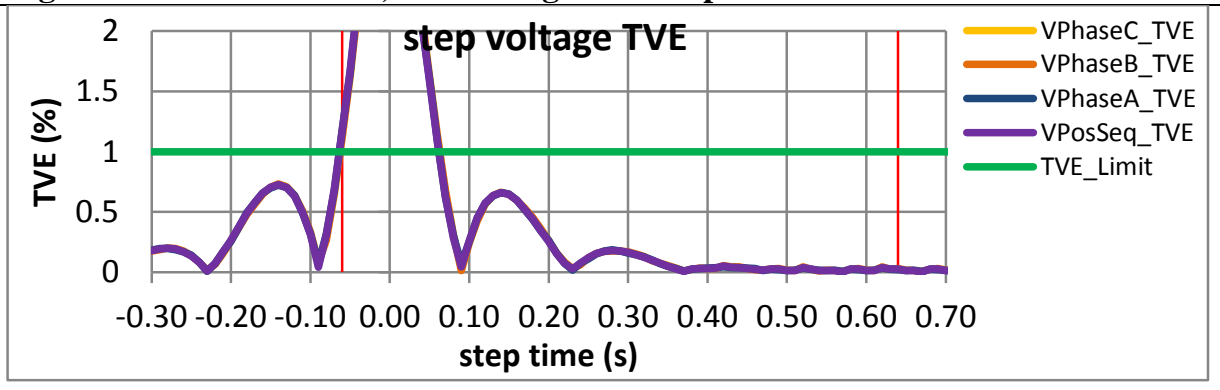

Figure 5099: Fs = 10 FPS, +10\% magnitude step

\section{Figure 5096: Fs = 15 FPS, -10\% magnitude step}

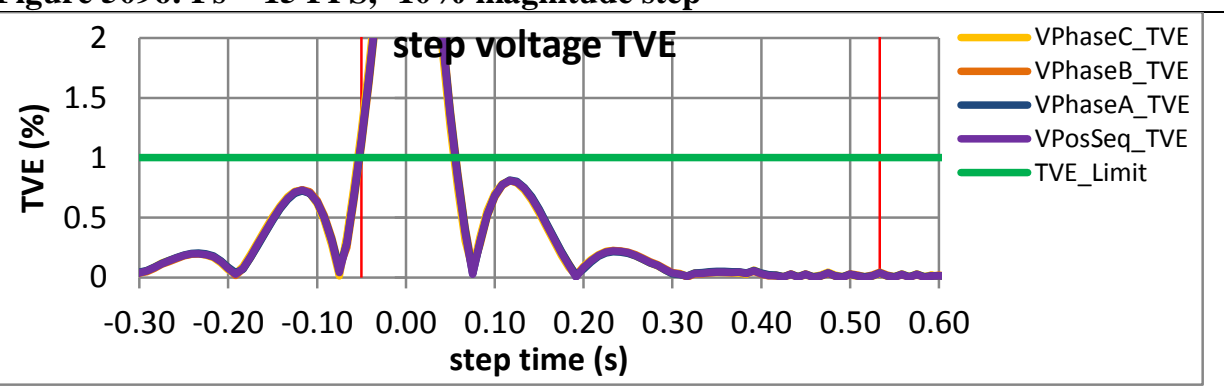

Figure 5098: Fs = 12 FPS, -10\% magnitude step

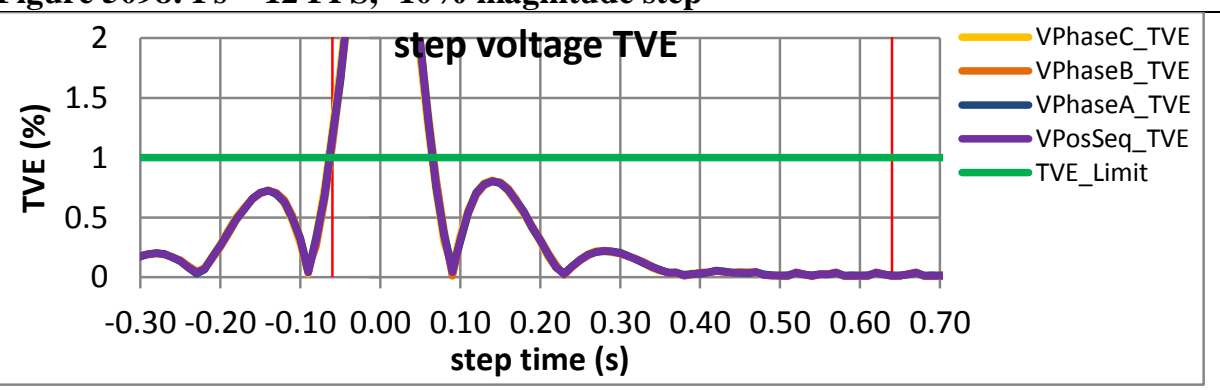

Figure 5100: Fs = 10 FPS, +10\% magnitude step 
10.2.2 PMU A dynamic step change in magnitude voltage response time: $F 0=60 \mathrm{~Hz}, \mathrm{M}$ class

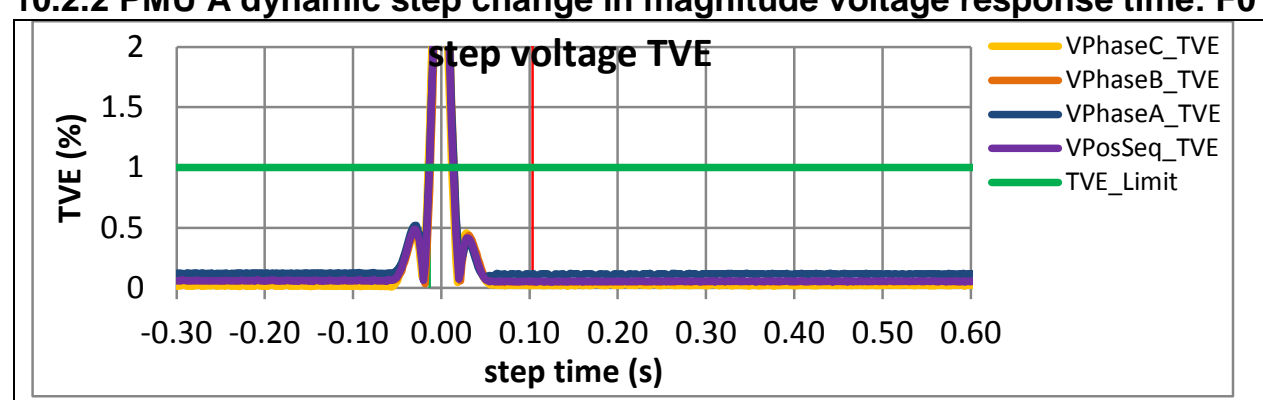

Figure 5101: Fs = 60 FPS, $+10 \%$ magnitude step

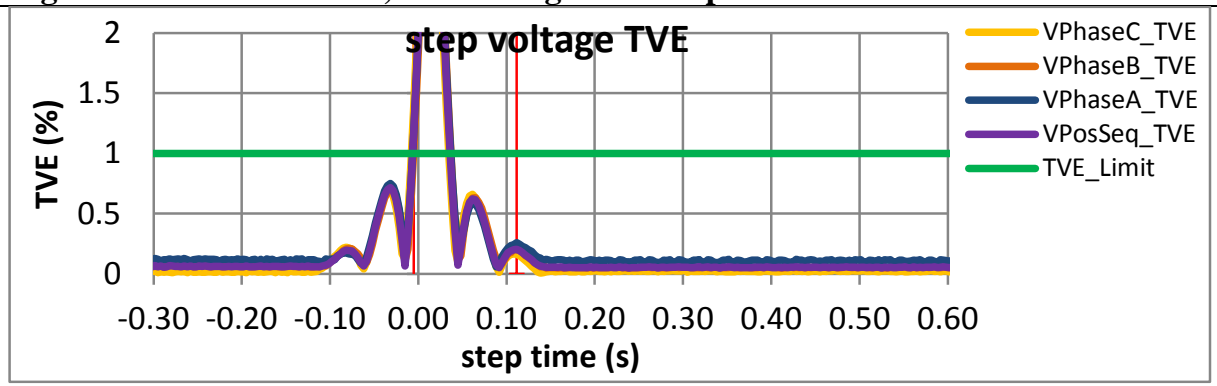

Figure 5103: Fs = 30 FPS, +10\% magnitude step

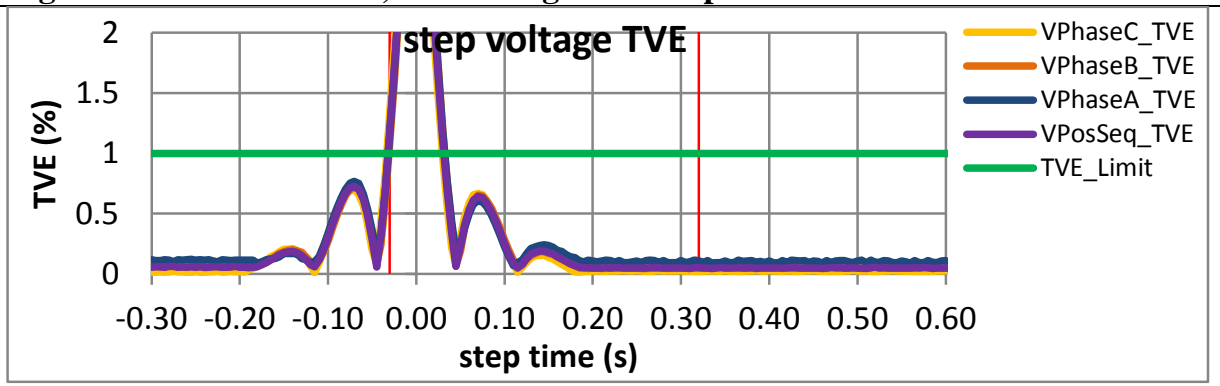

Figure 5105: Fs $=20 \mathrm{FPS},+10 \%$ magnitude step

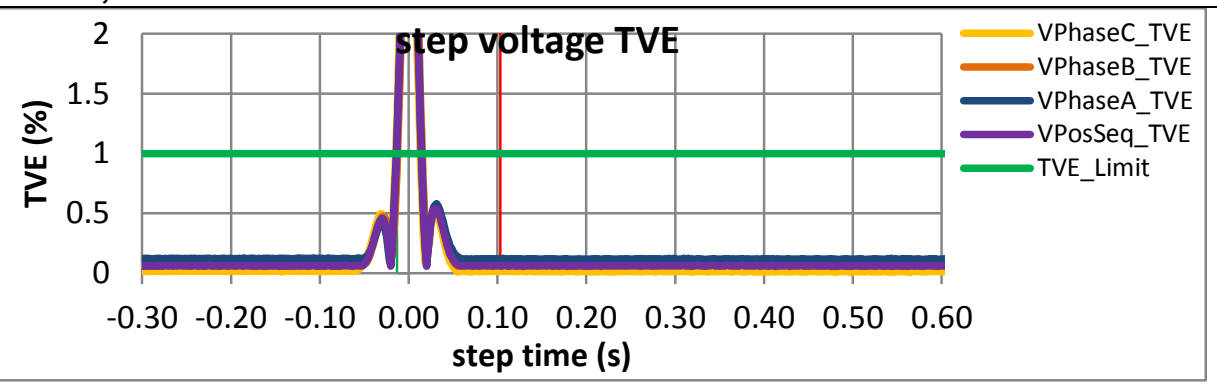

Figure 5102: Fs = 60 FPS, -10\% magnitude step

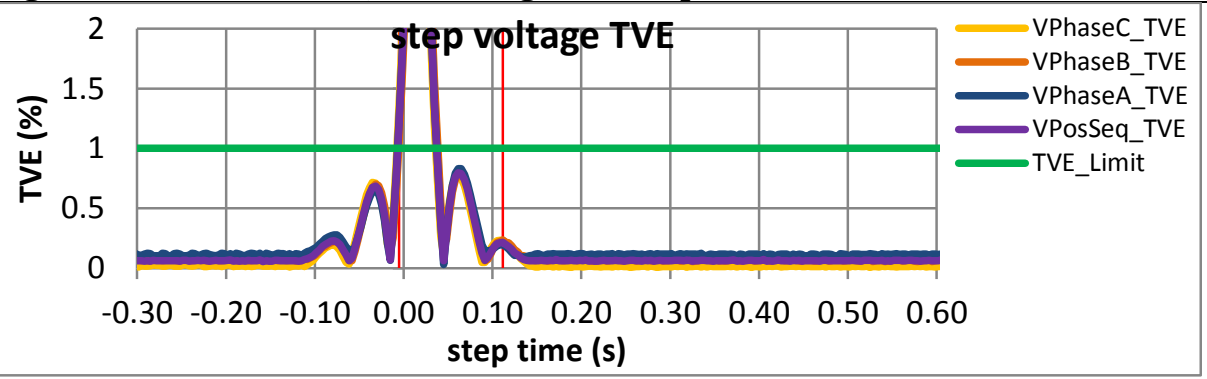

Figure 5104: Fs = 30 FPS, -10\% magnitude step

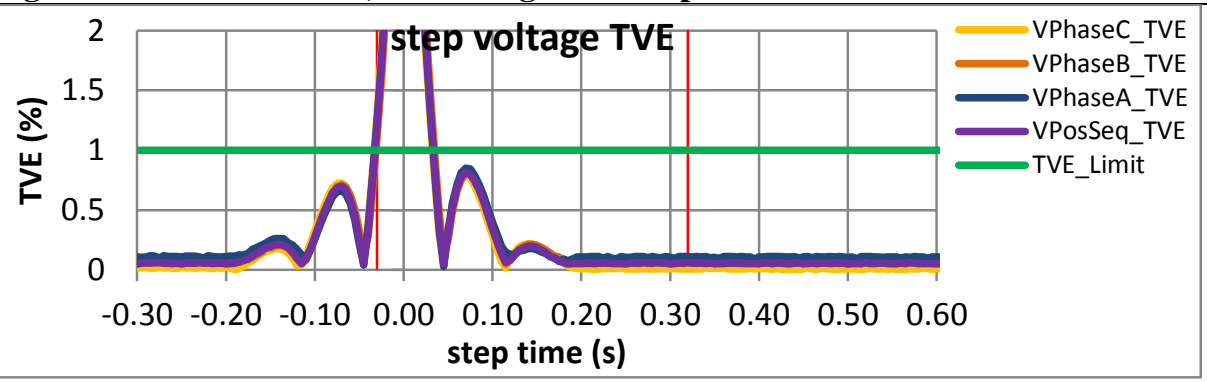

Figure 5106: Fs = 20 FPS, -10\% magnitude step 

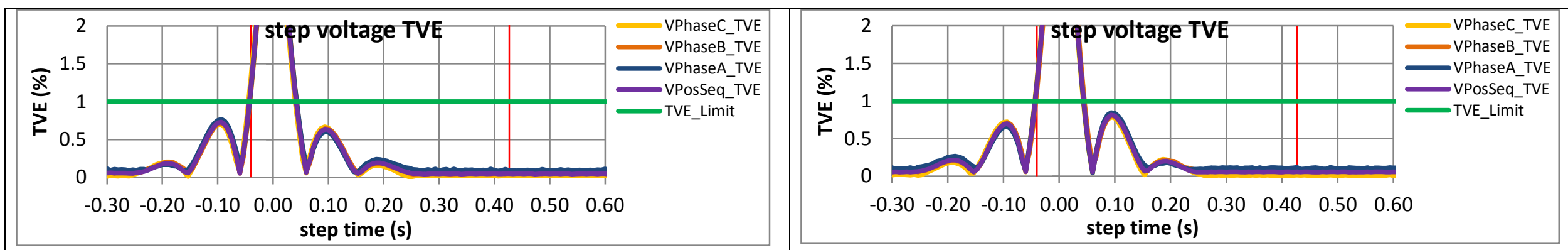

Figure 5107: Fs $=15$ FPS, $+10 \%$ magnitude step

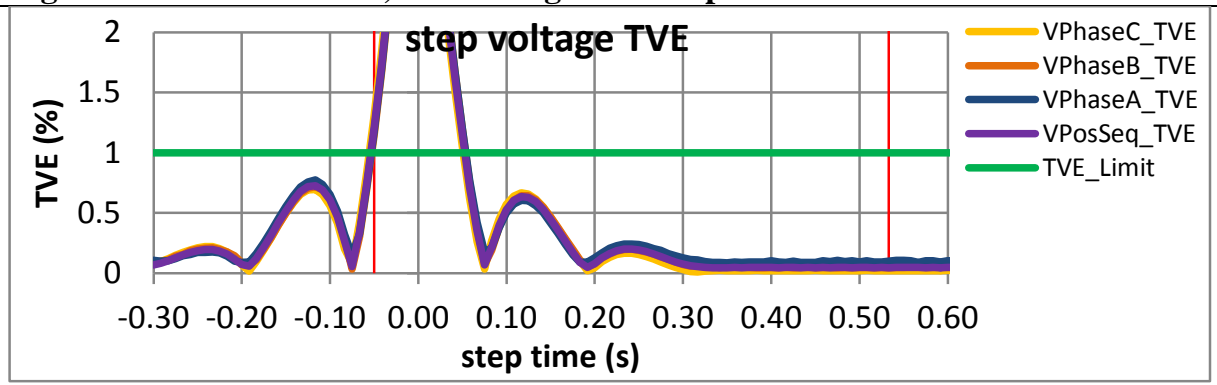

Figure 5109: Fs = 12 FPS, +10\% magnitude step

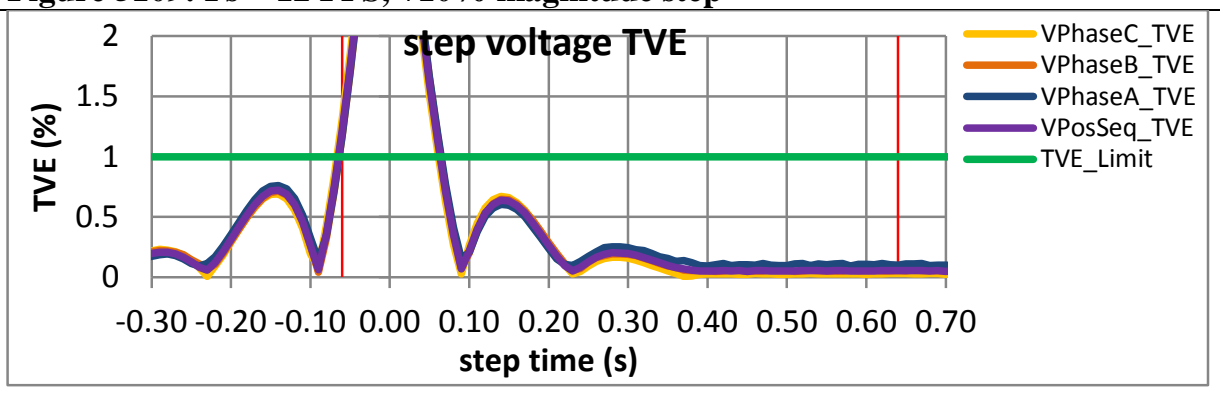

Figure 5111: Fs = 10 FPS, +10\% magnitude step

Figure 5108: Fs = 15 FPS, -10\% magnitude step

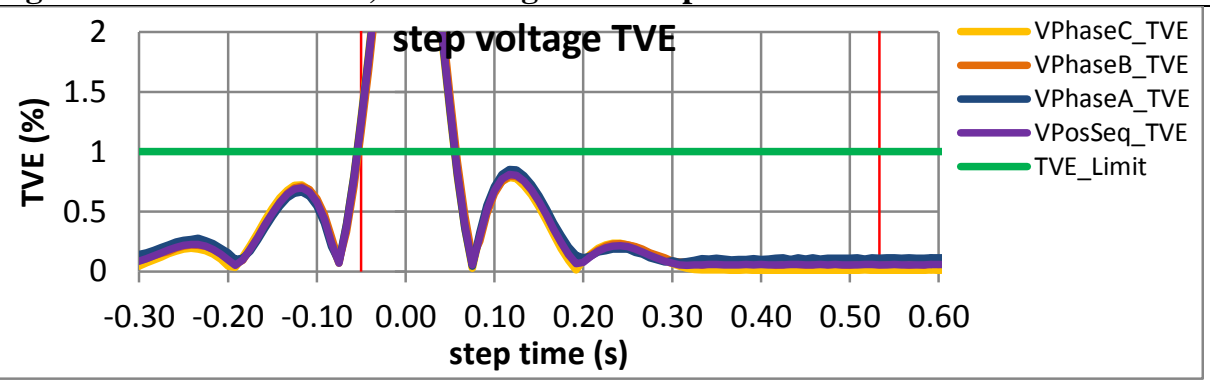

Figure 5110: Fs = 12 FPS, -10\% magnitude step

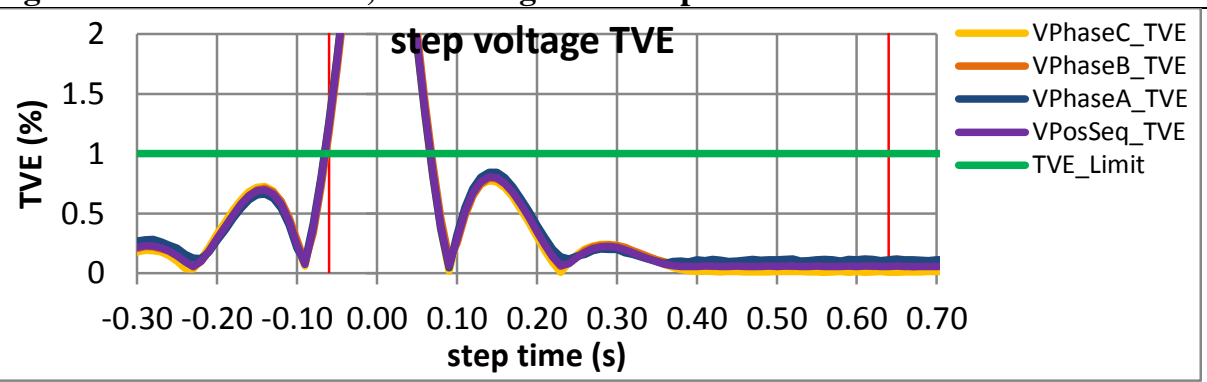

Figure 5112: Fs = 10 FPS, +10\% magnitude step 
10.2.3 PMU B dynamic step change in magnitude voltage response time: $F 0=60 \mathrm{~Hz}, \mathrm{M}$ class

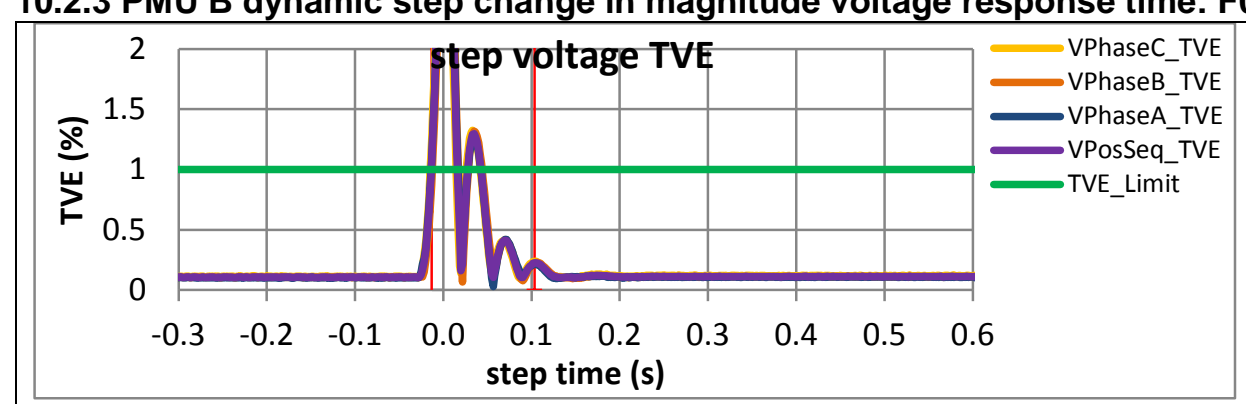

Figure 5113: Fs = 60 FPS, $+10 \%$ magnitude step

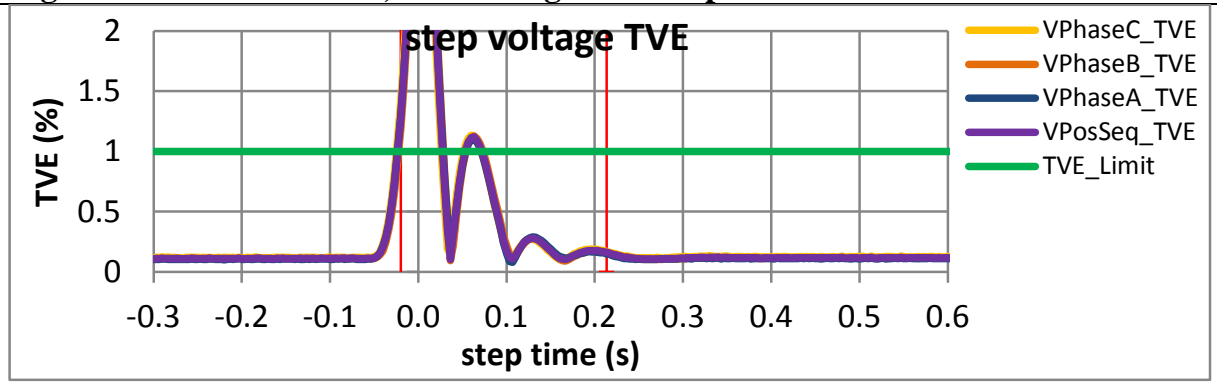

Figure 5115: Fs = 30 FPS, +10\% magnitude step

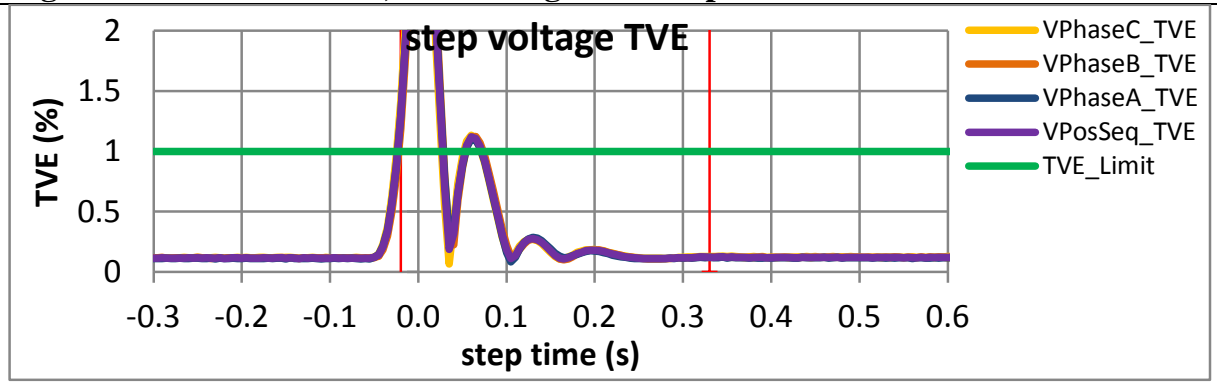

Figure 5117: Fs = 20 FPS, +10\% magnitude step

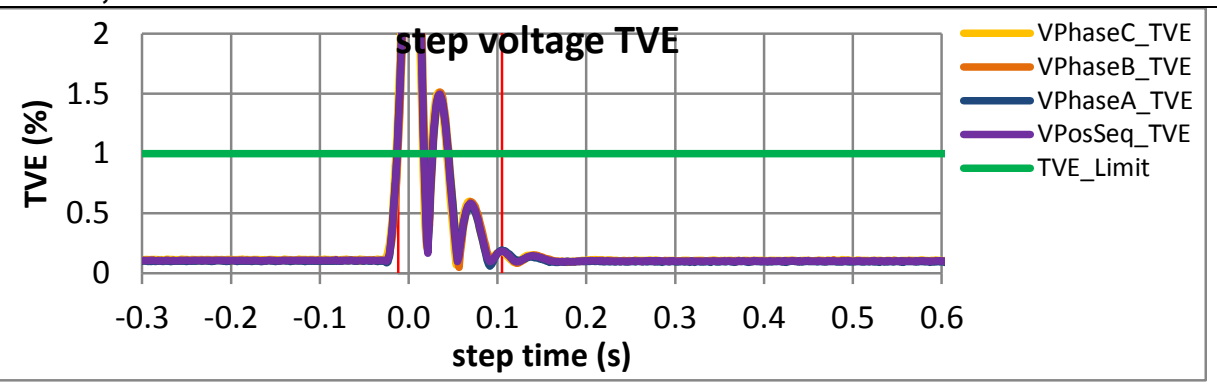

Figure 5114: Fs = 60 FPS, -10\% magnitude step

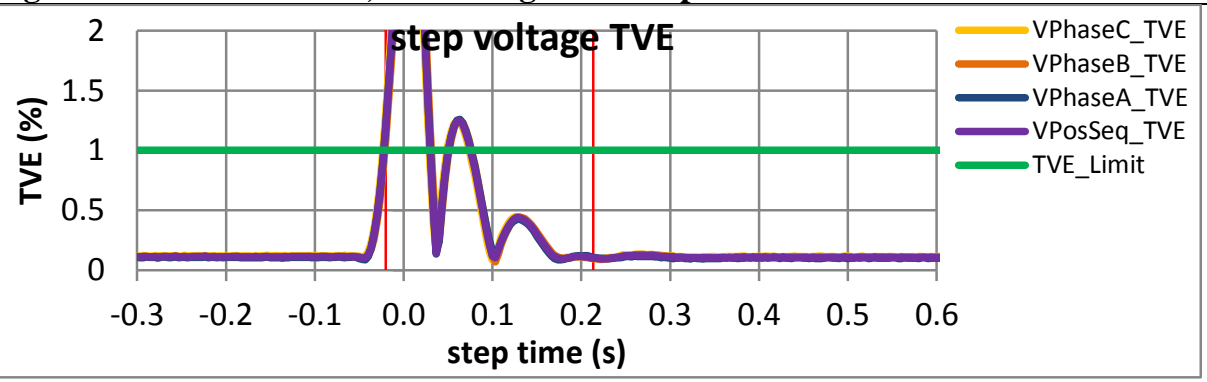

Figure 5116: Fs = 30 FPS, -10\% magnitude step

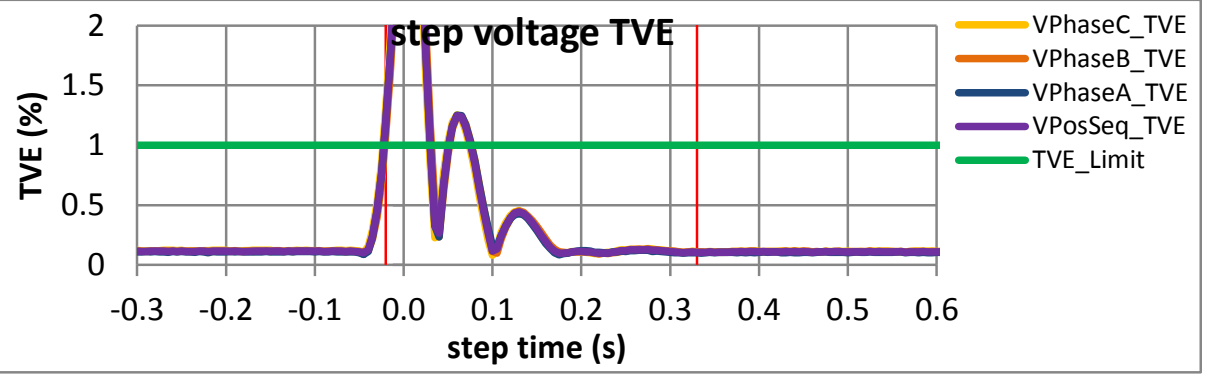

Figure 5118: Fs = 20 FPS, -10\% magnitude step 

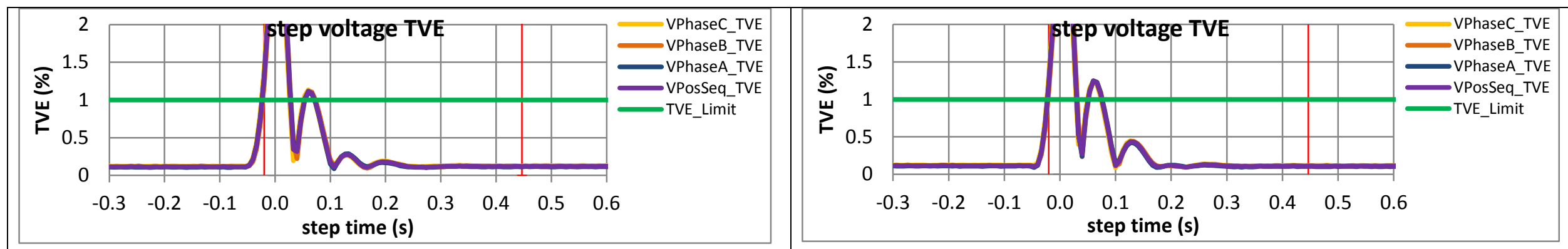

Figure 5119: Fs = 15 FPS, +10\% magnitude step

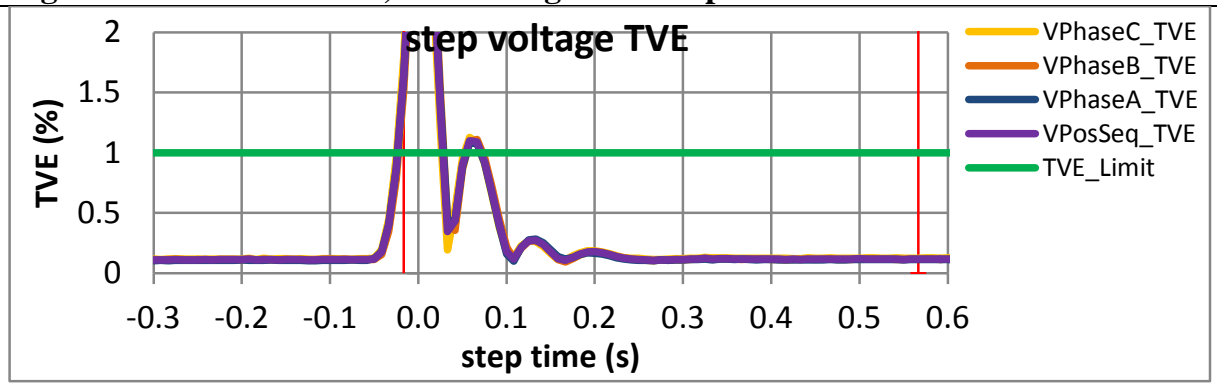

Figure 5121: Fs = 12 FPS, +10\% magnitude step

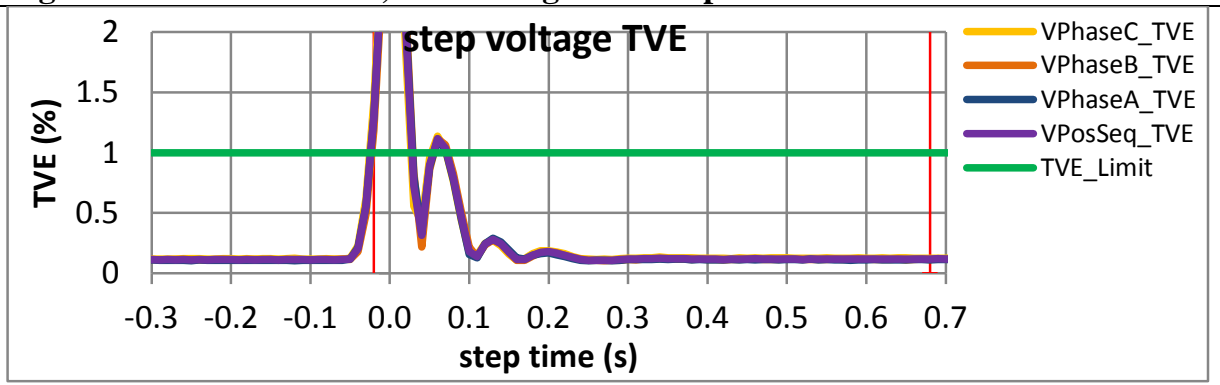

Figure 5123: Fs = 10 FPS, +10\% magnitude step

Figure 5120: Fs = 15 FPS, -10\% magnitude step

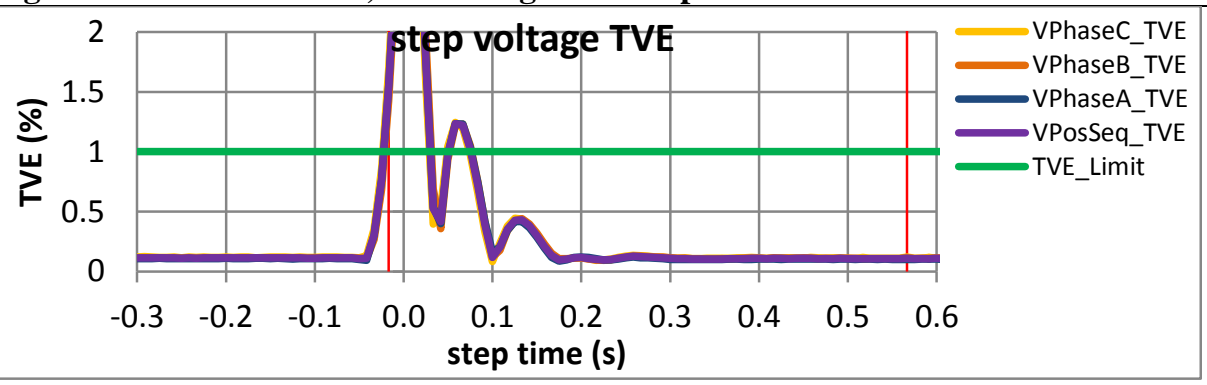

Figure 5122: Fs = 12 FPS, $-10 \%$ magnitude step

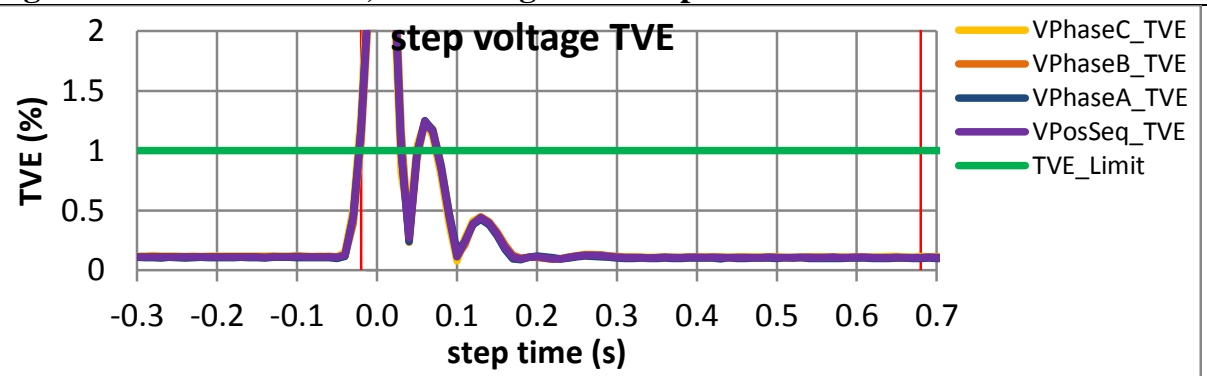

Figure 5124: Fs $=10$ FPS, $+10 \%$ magnitude step 
10.2.4 PMU C dynamic step change in magnitude voltage response time: $F 0=60 \mathrm{~Hz}, \mathrm{M}$ class

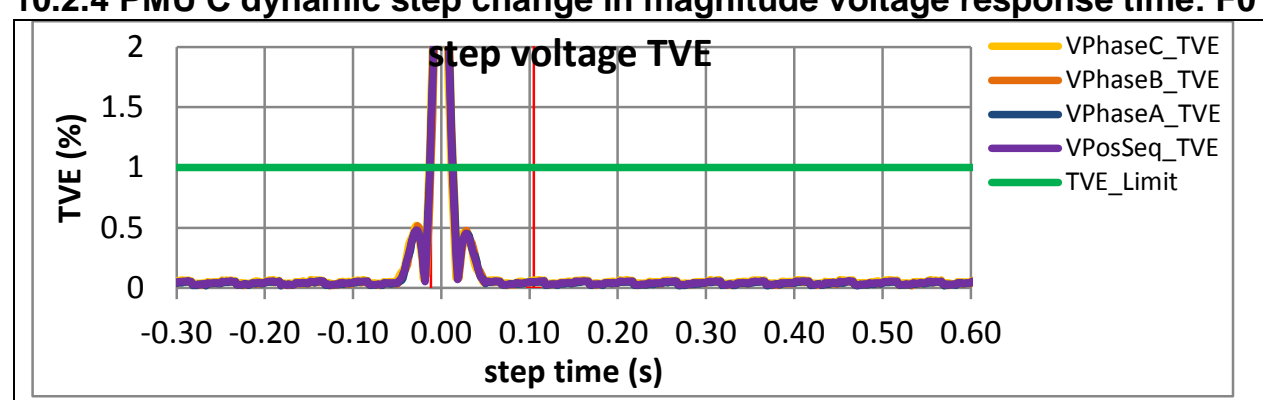

Figure 5125: Fs $=60 \mathrm{FPS},+10 \%$ magnitude step

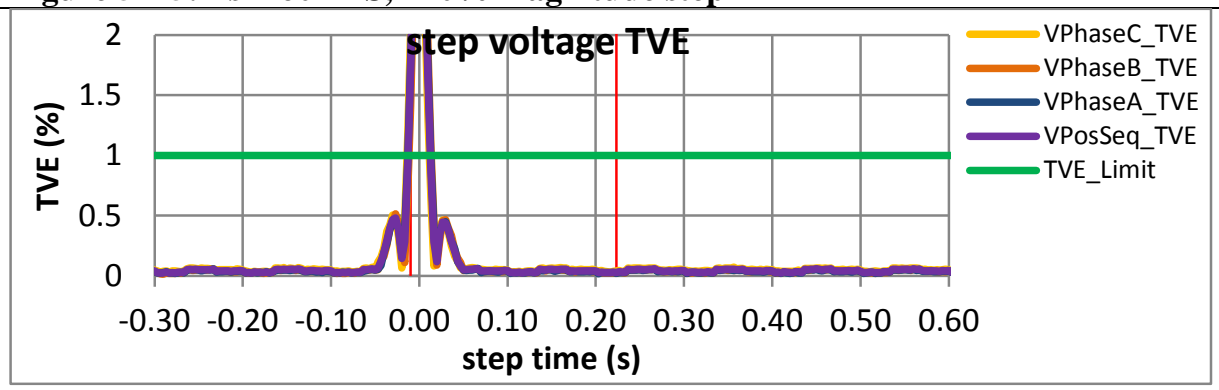

Figure 5127: Fs = 30 FPS, $+10 \%$ magnitude step

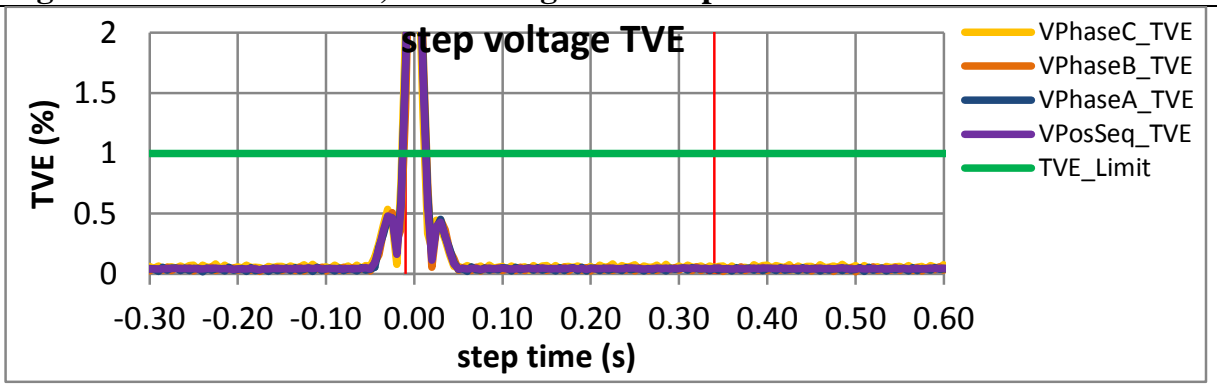

Figure 5129: Fs $=20 \mathrm{FPS},+10 \%$ magnitude step

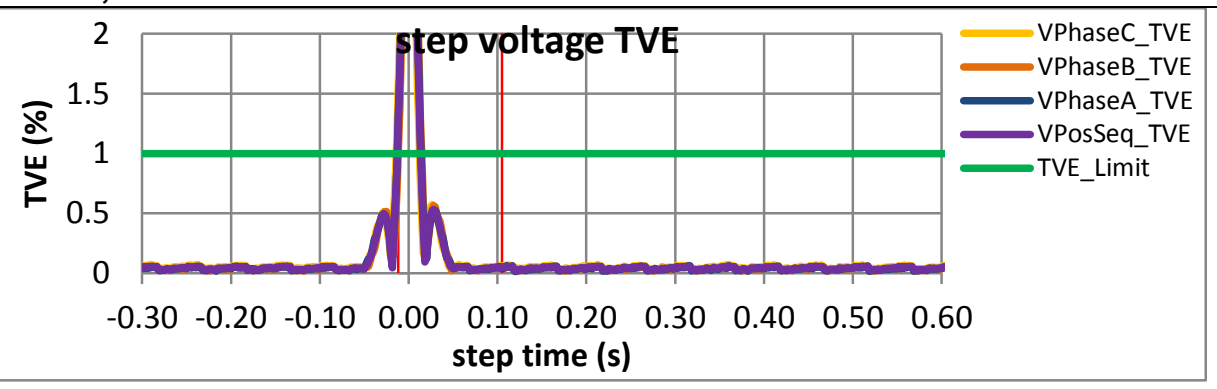

Figure 5126: Fs = 60 FPS, -10\% magnitude step

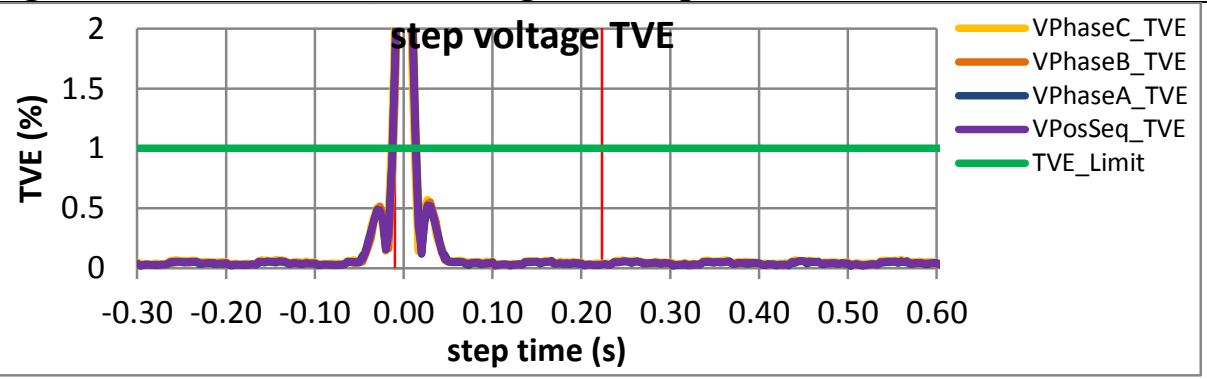

Figure 5128: Fs = 30 FPS, -10\% magnitude step

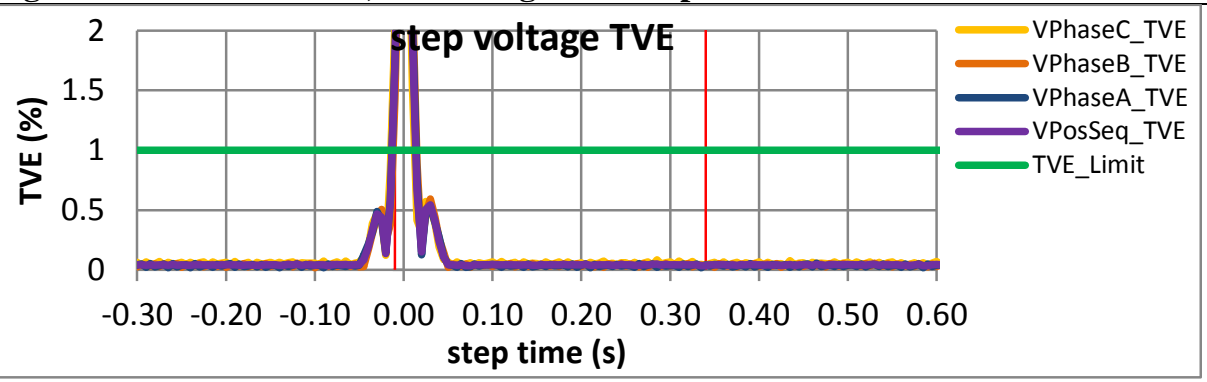

Figure 5130: Fs = 20 FPS, -10\% magnitude step 

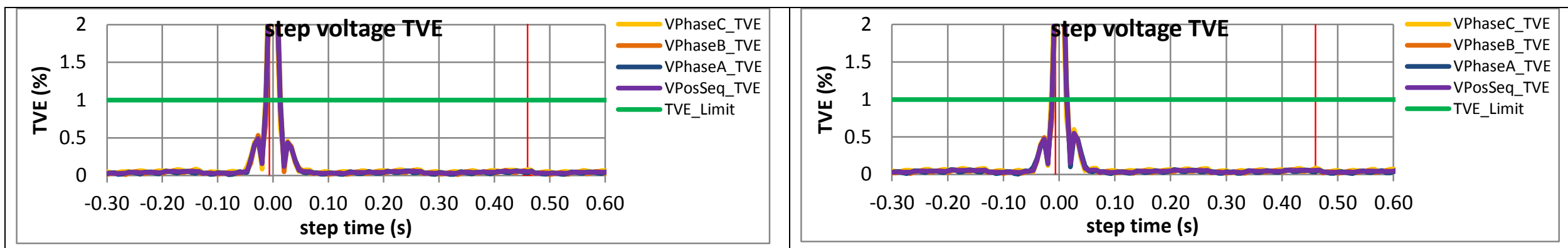

Figure 5131: Fs = 15 FPS, +10\% magnitude step

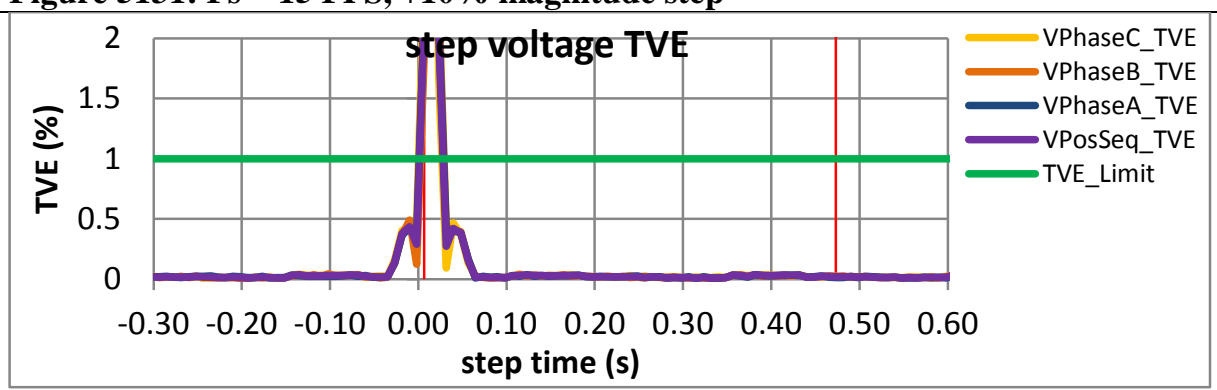

Figure 5133: Fs = 12 FPS, +10\% magnitude step

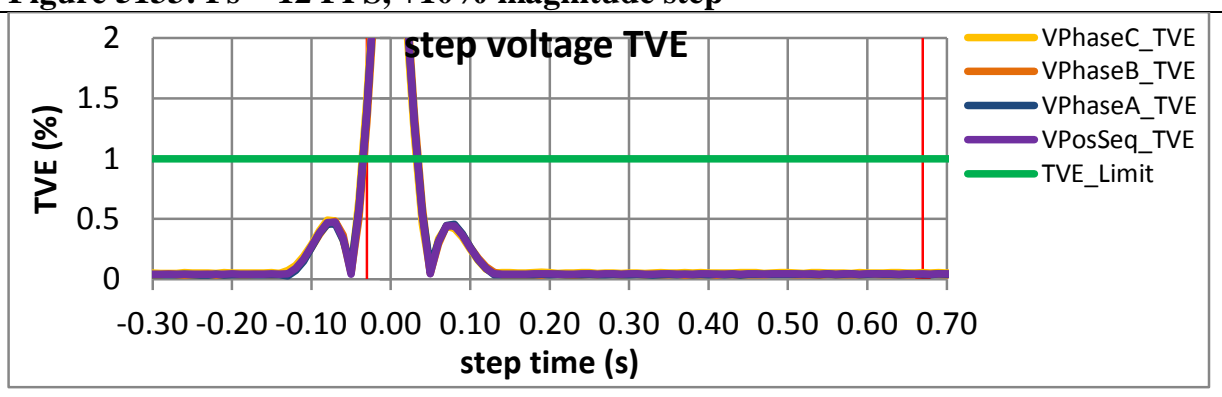

Figure 5135: Fs = 10 FPS, +10\% magnitude step

\section{Figure 5132: Fs = 15 FPS, -10\% magnitude step}

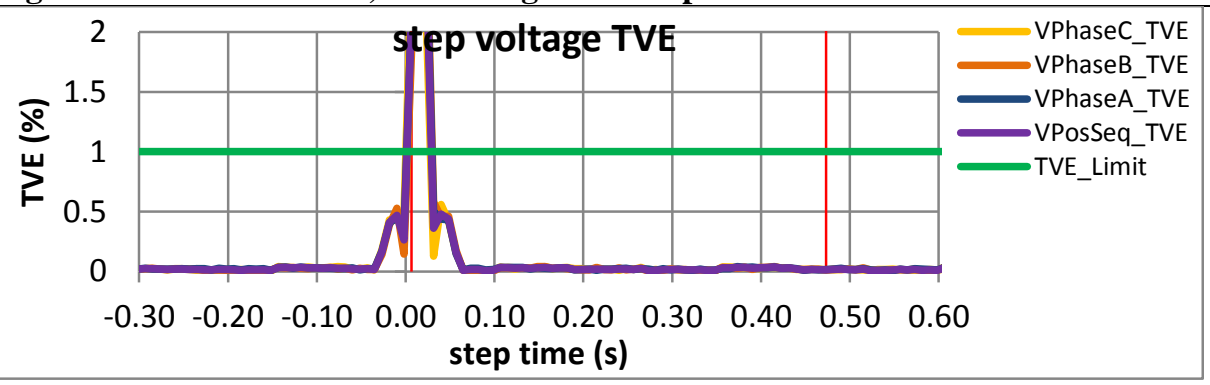

Figure 5134: Fs = 12 FPS, -10\% magnitude step

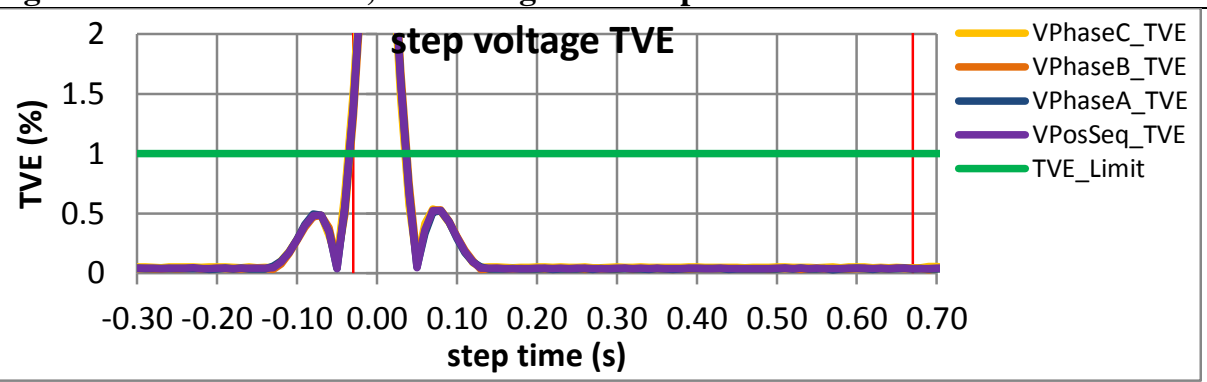

Figure 5136: Fs = 10 FPS, +10\% magnitude step 
10.2.5 PMU D dynamic step change in magnitude voltage response time: $F 0=60 \mathrm{~Hz}, \mathrm{M}$ class

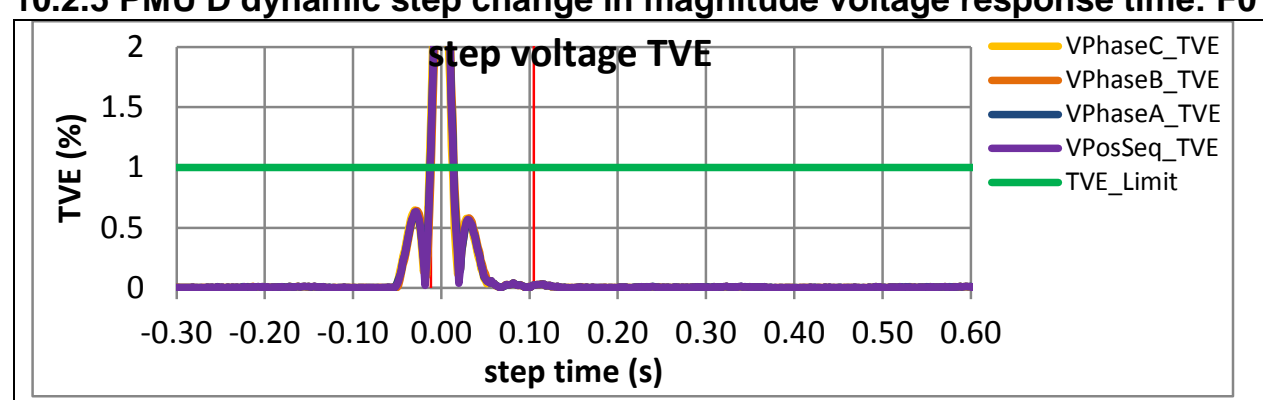

Figure 5137: Fs = 60 FPS, $+10 \%$ magnitude step

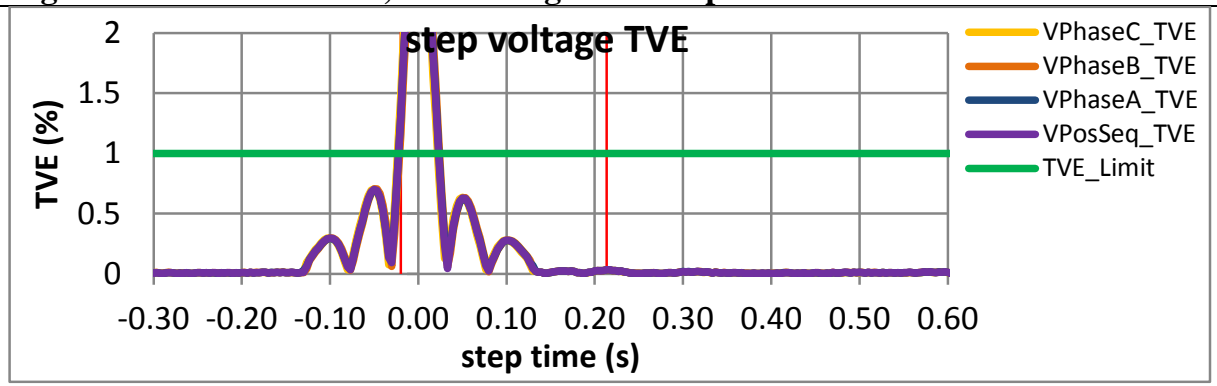

Figure 5139: Fs = 30 FPS, +10\% magnitude step

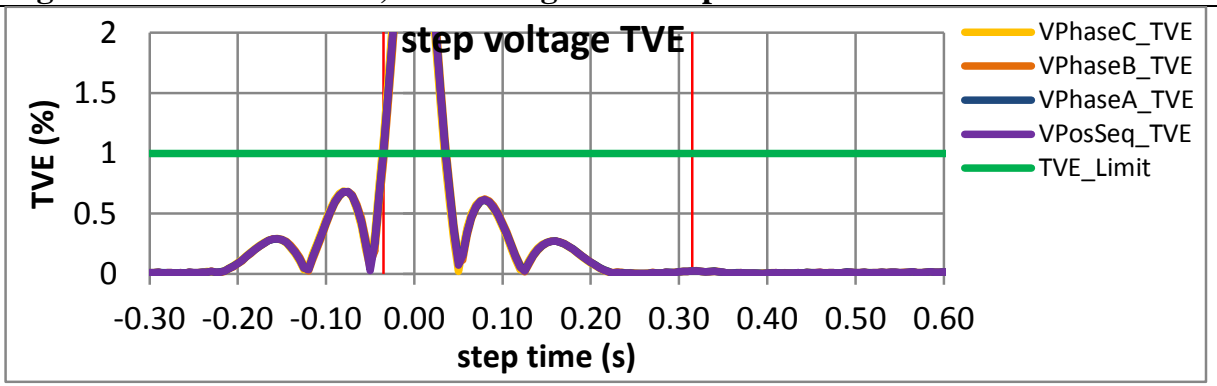

Figure 5141: Fs = 20 FPS, +10\% magnitude step

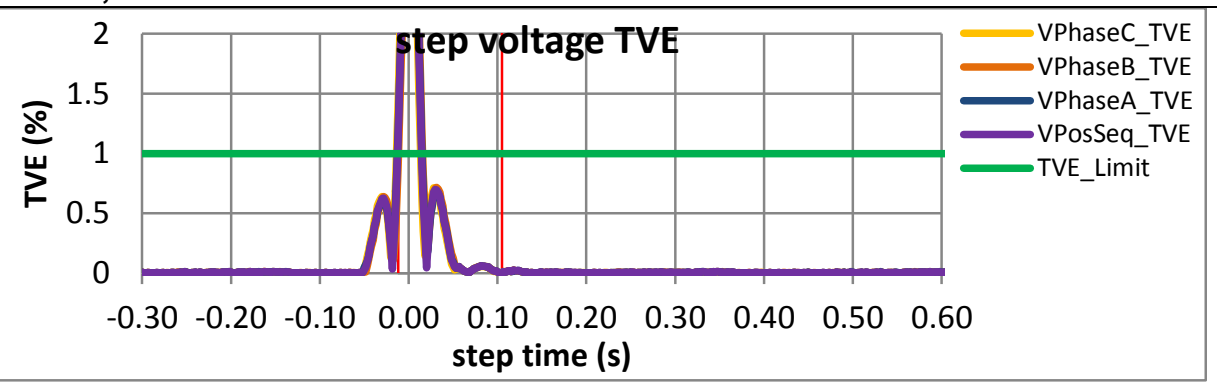

Figure 5138: Fs = 60 FPS, -10\% magnitude step

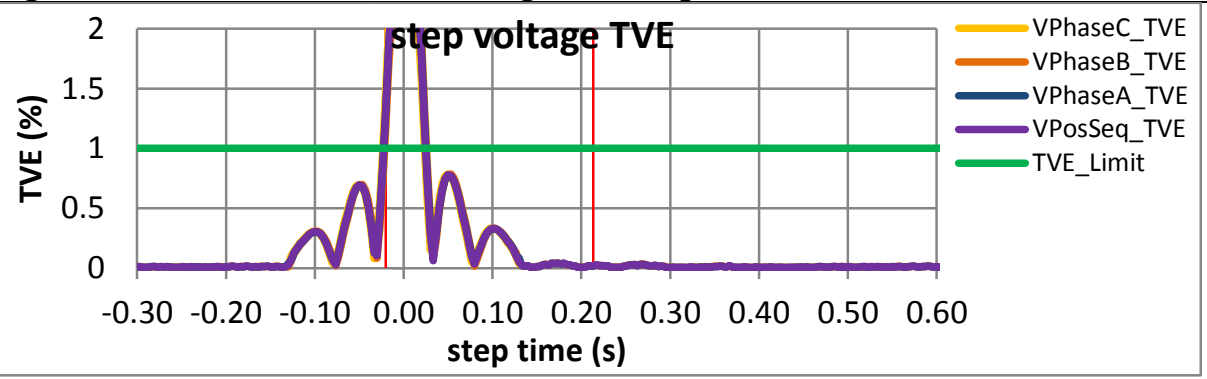

Figure 5140: Fs = 30 FPS, -10\% magnitude step

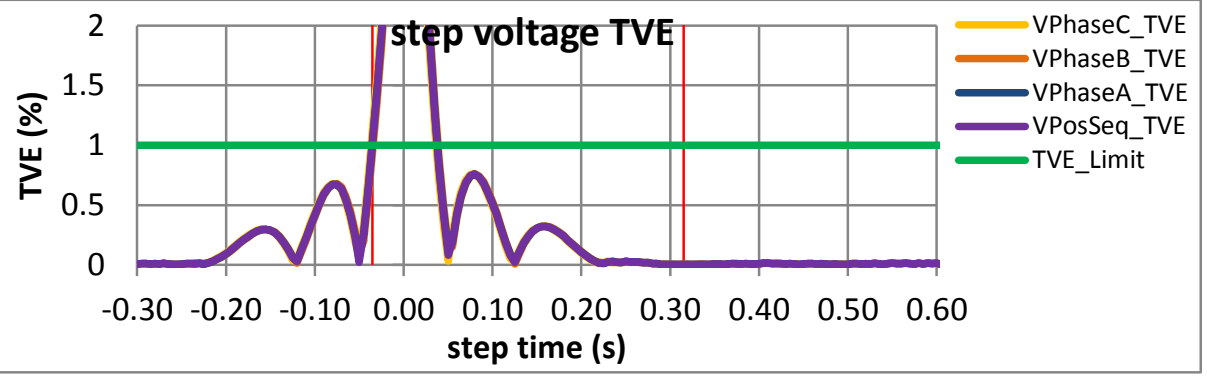

Figure 5142: Fs = 20 FPS, -10\% magnitude step 

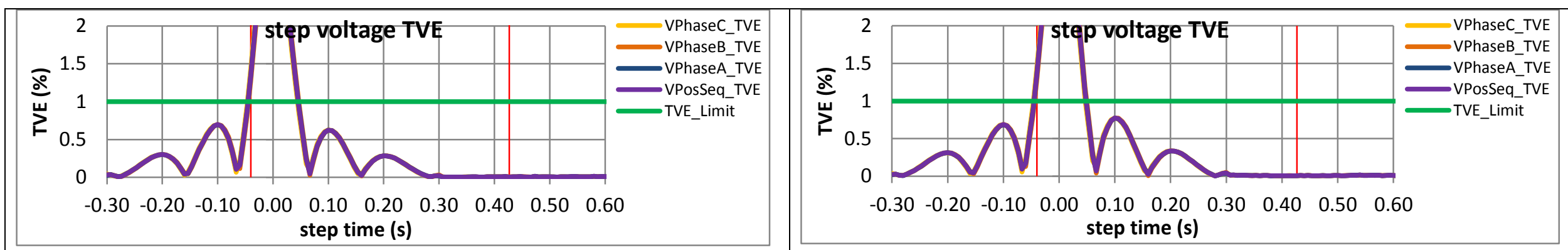

Figure 5143: Fs $=15$ FPS, $+10 \%$ magnitude step

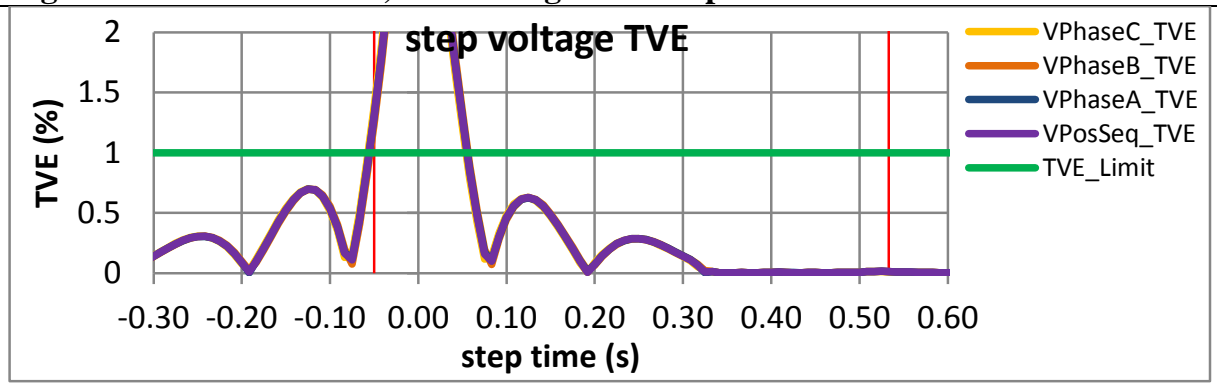

Figure 5144: Fs = 15 FPS, -10\% magnitude step

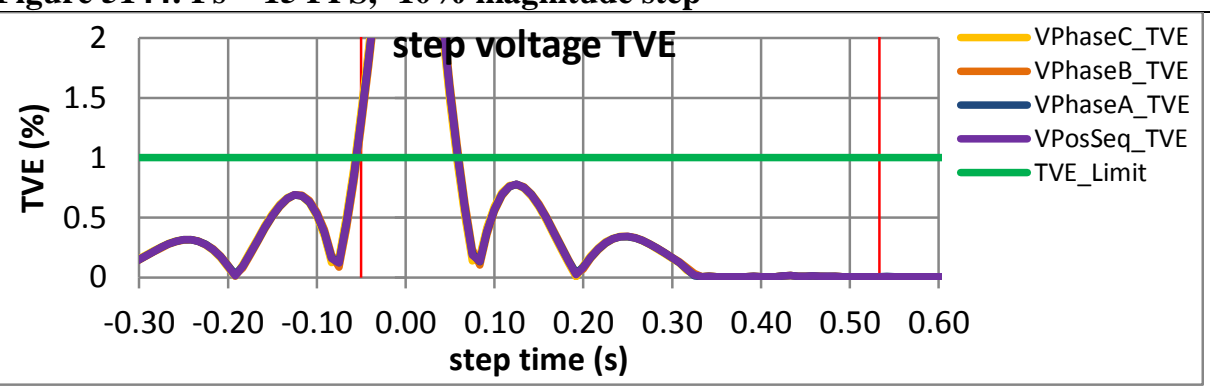

Figure 5145: Fs = 12 FPS, +10\% magnitude step

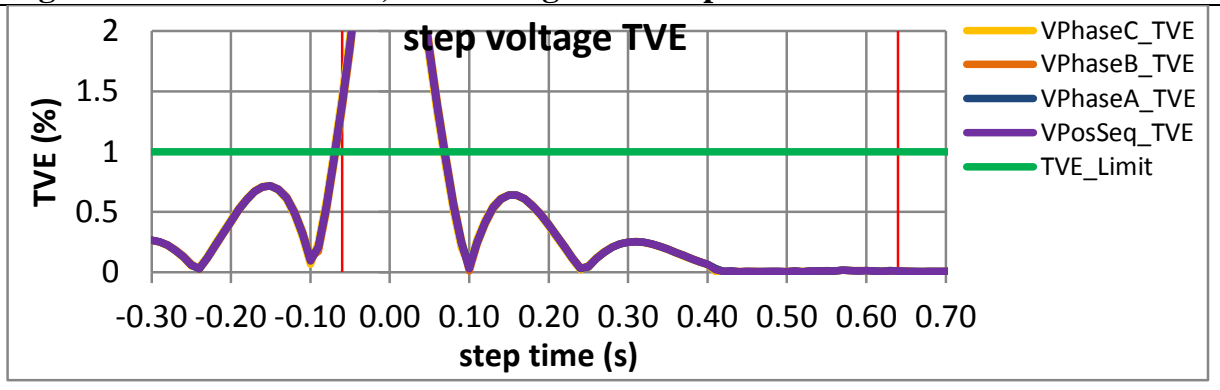

Figure 5147: Fs = 10 FPS, +10\% magnitude step

Figure 5146: Fs = 12 FPS, -10\% magnitude step

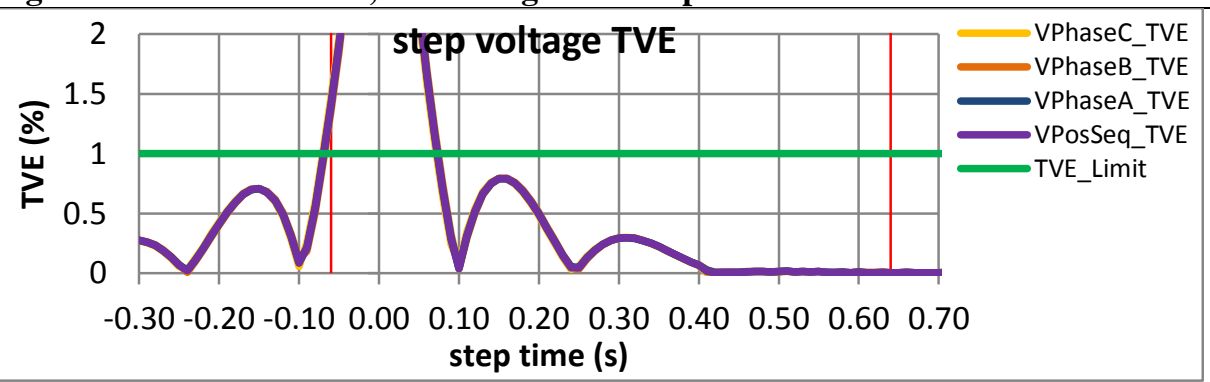

Figure 5148: Fs = 10 FPS, $+10 \%$ magnitude step 
10.2.6 PMU E dynamic step change in magnitude voltage response time: $F 0=60 \mathrm{~Hz}$, M class

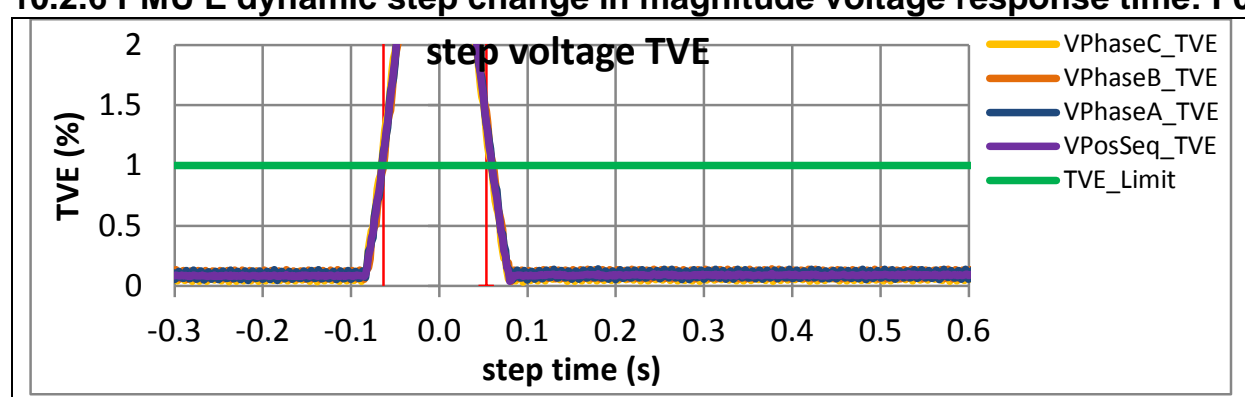

Figure 5149: Fs = 60 FPS, $+10 \%$ magnitude step
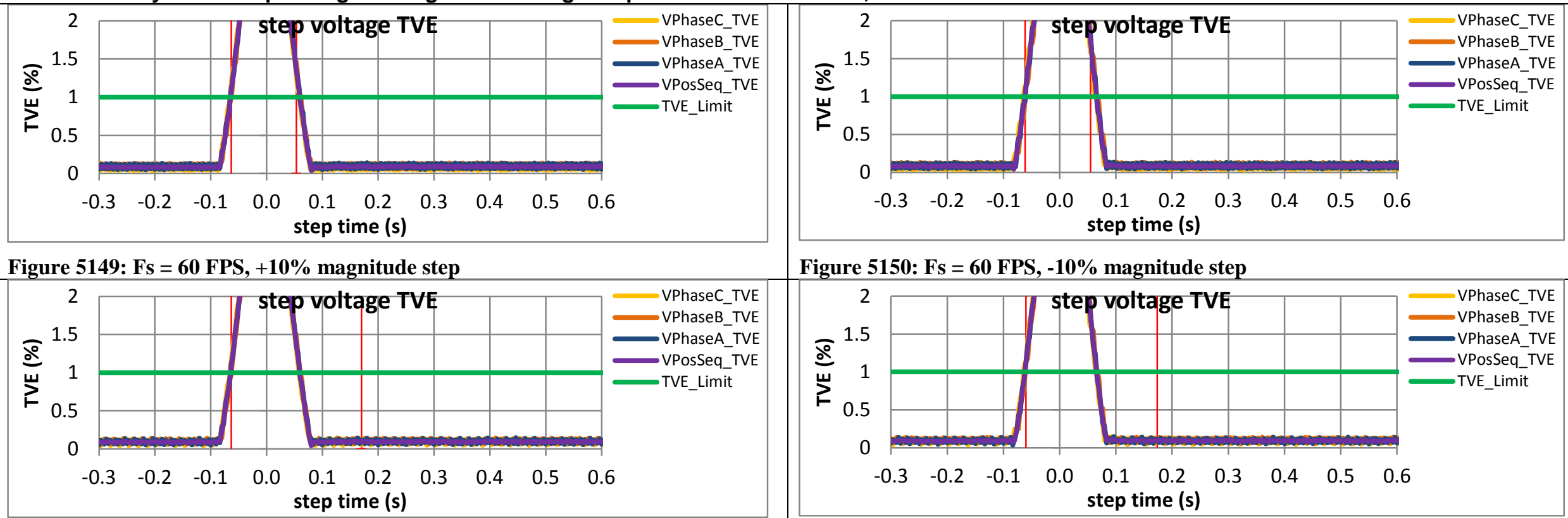

Figure 5150: Fs = 60 FPS, -10\% magnitude step

Figure 5151: Fs = 30 FPS, +10\% magnitude step
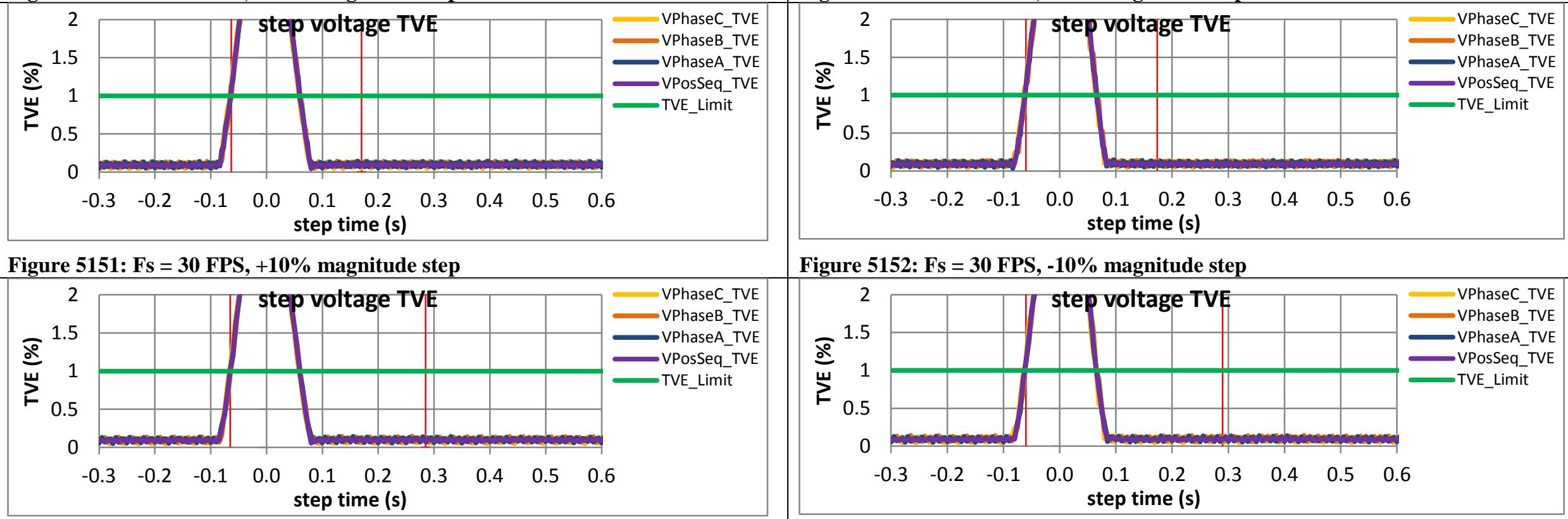

Figure 5152: Fs = 30 FPS, -10\% magnitude step

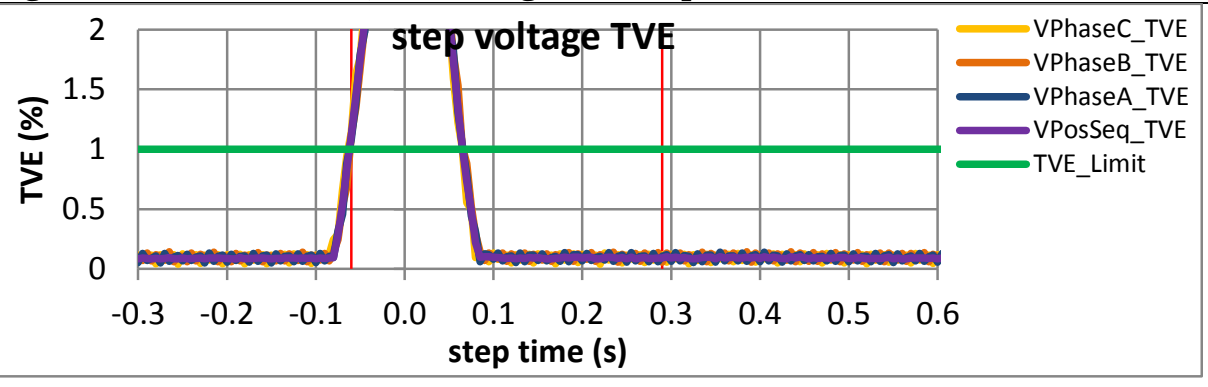

Figure 5153: Fs $=20 \mathrm{FPS},+10 \%$ magnitude step

Figure 5154: Fs = 20 FPS, -10\% magnitude step 


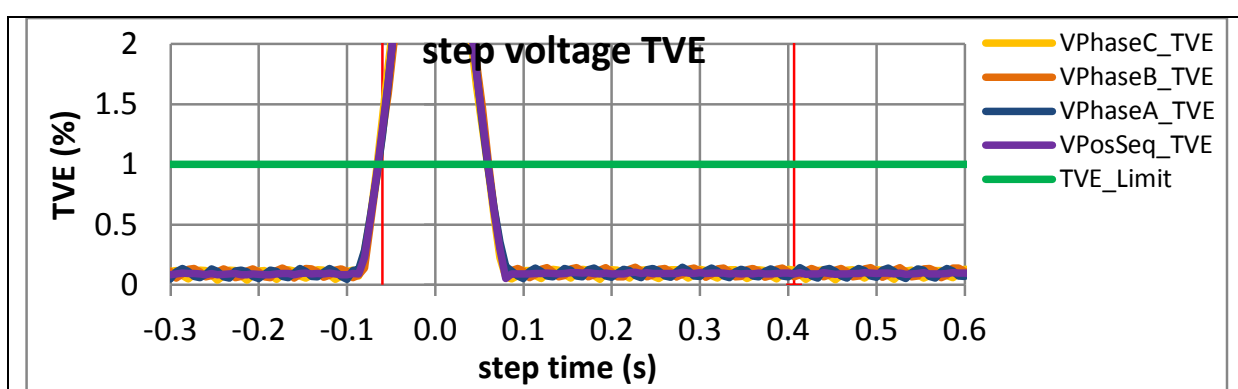

Figure 5155: Fs = 15 FPS, $+10 \%$ magnitude step

Figure 5157: Fs $=12$ FPS, $+10 \%$ magnitude step

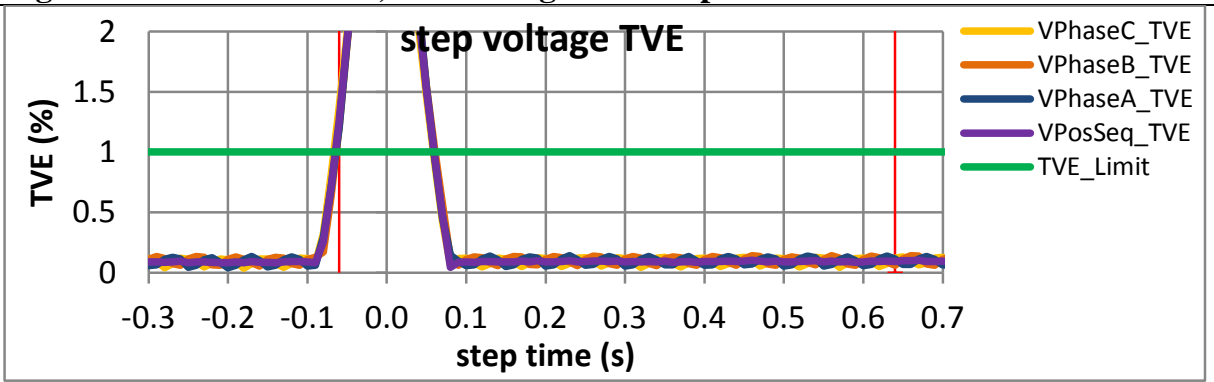

Figure 5159: Fs = 10 FPS, $+10 \%$ magnitude step

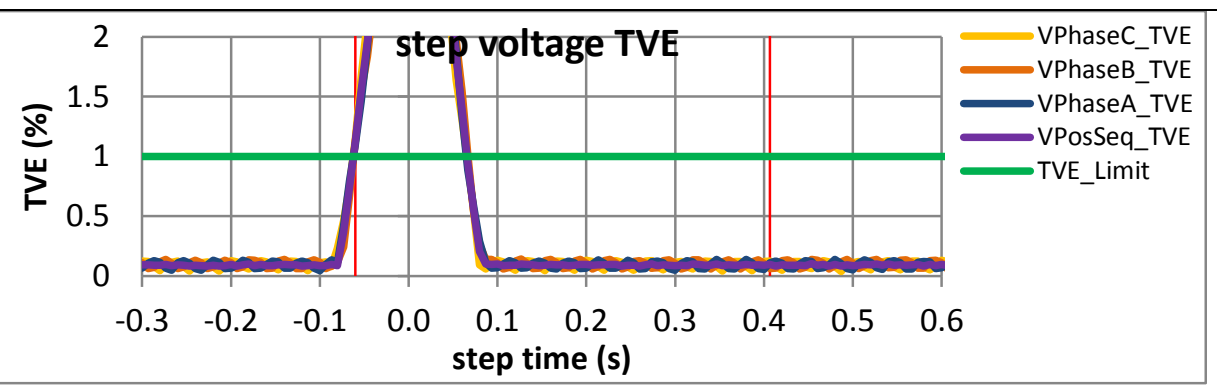

Figure 5156: Fs = 15 FPS, -10\% magnitude step

Figure 5158: Fs = 12 FPS, -10\% magnitude step

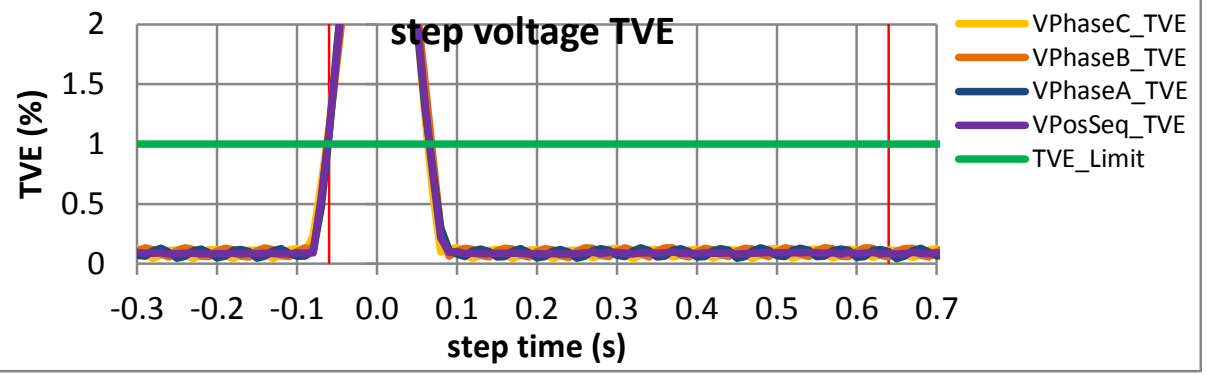

Figure 5160: Fs = 10 FPS, +10\% magnitude step 
10.2.7 PMU F dynamic step change in magnitude voltage response time: $F 0=60 \mathrm{~Hz}$, M class
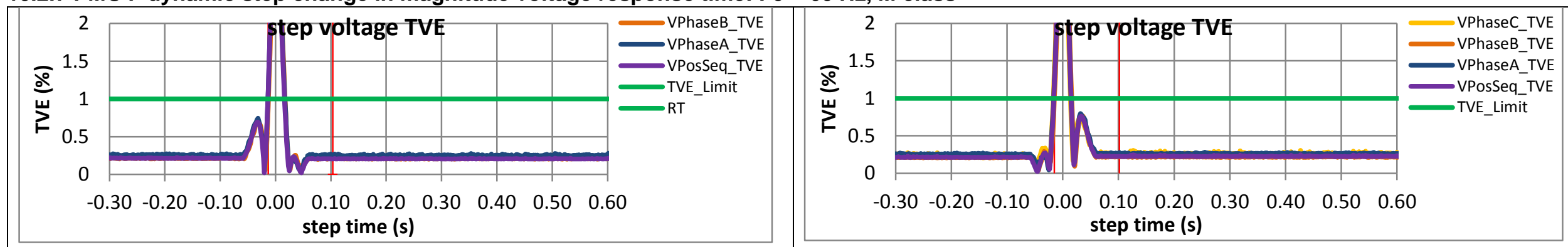

Figure 5161: Fs = 60 FPS, $+10 \%$ magnitude step

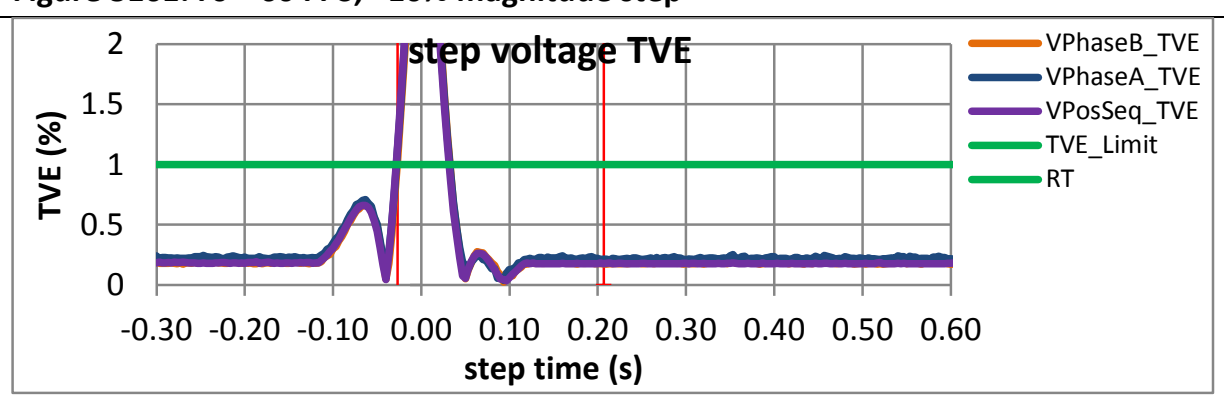

Figure 5162: Fs = $60 \mathrm{FPS},-10 \%$ magnitude step

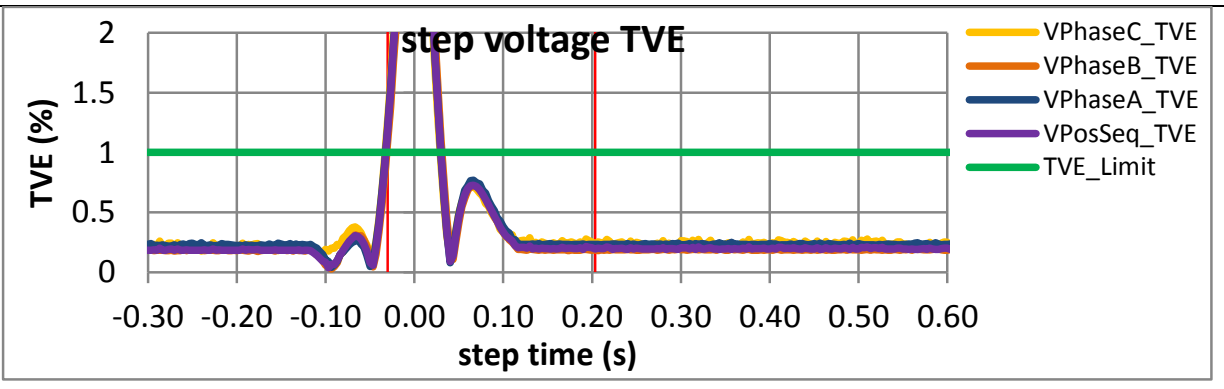

Figure 5163: $\mathrm{Fs}=30 \mathrm{FPS},+10 \%$ magnitude step

Figure 5164: Fs = 30 FPS, -10\% magnitude step
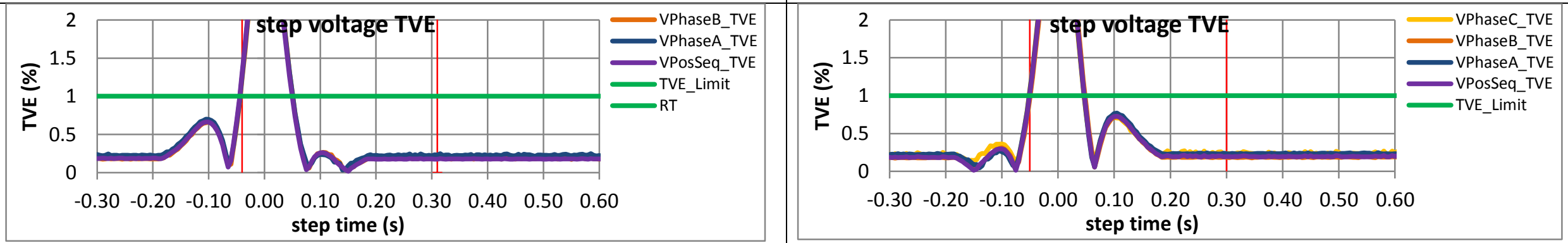

Figure 5165: Fs = 20 FPS, $+10 \%$ magnitude step

Figure 5166: Fs = 20 FPS, $-10 \%$ magnitude step 


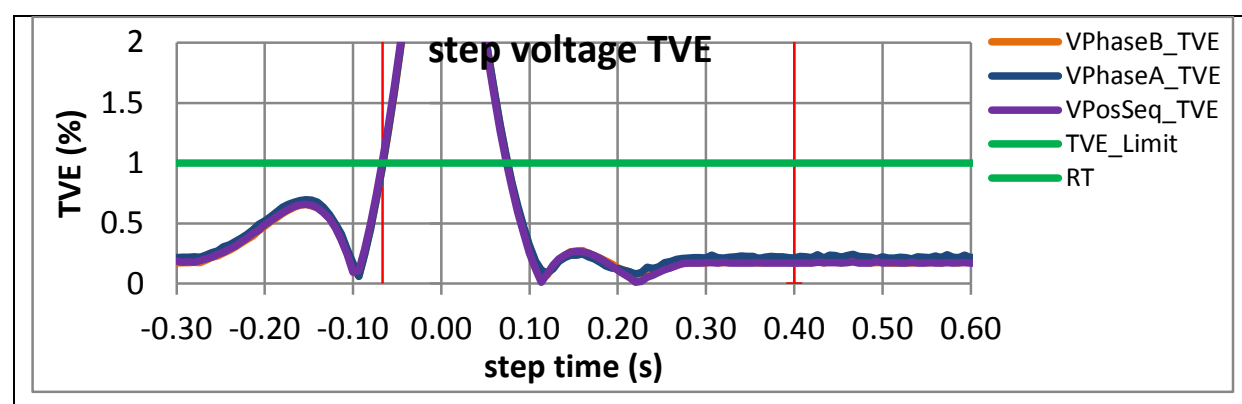

Figure 5167: Fs = 15 FPS, +10\% magnitude step

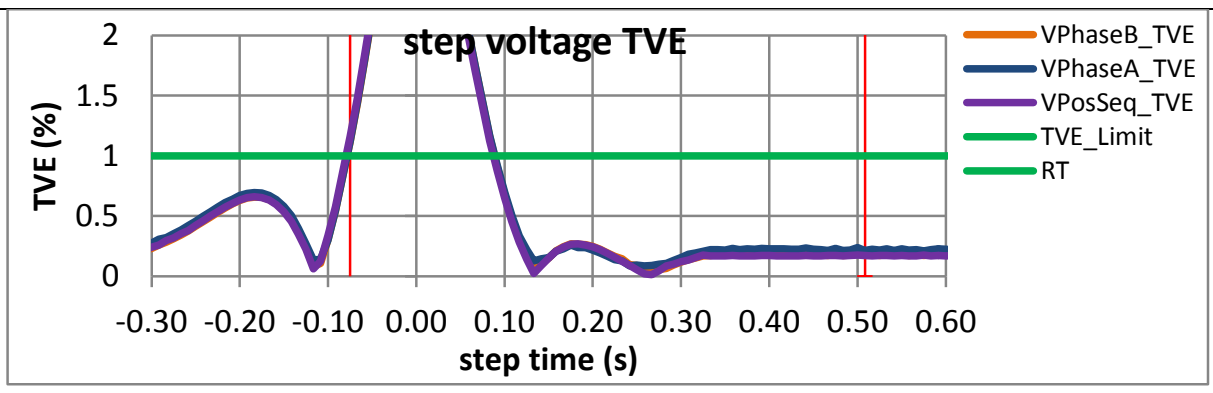

Figure 5169: Fs = 12 FPS, +10\% magnitude step

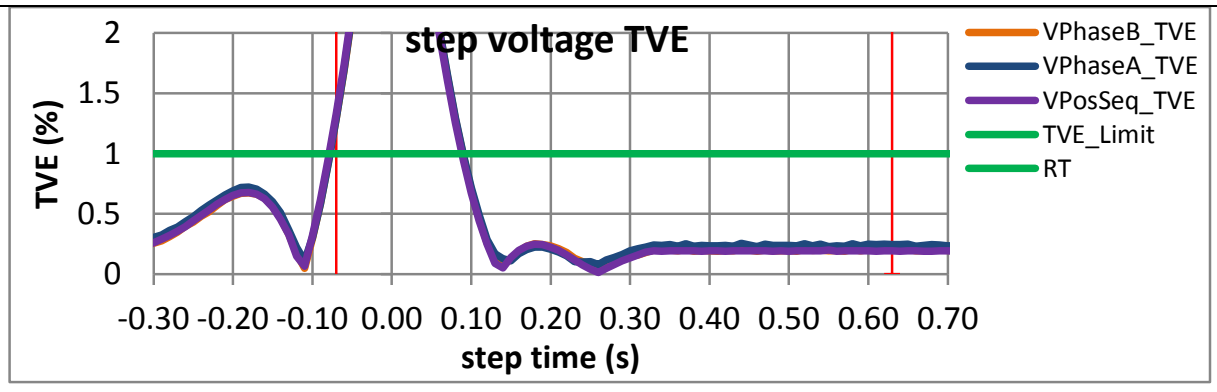

Figure 5171: Fs = 10 FPS, $+10 \%$ magnitude step

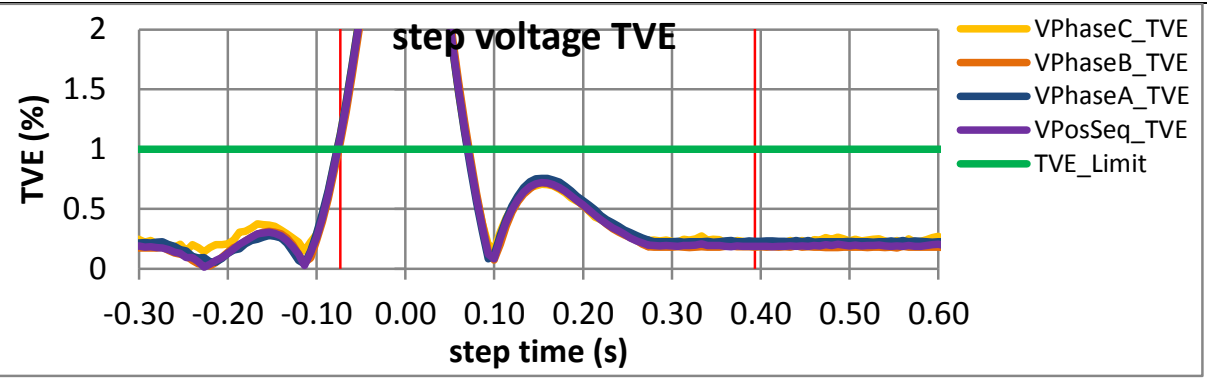

Figure 5168: Fs = 15 FPS, - $-10 \%$ magnitude step

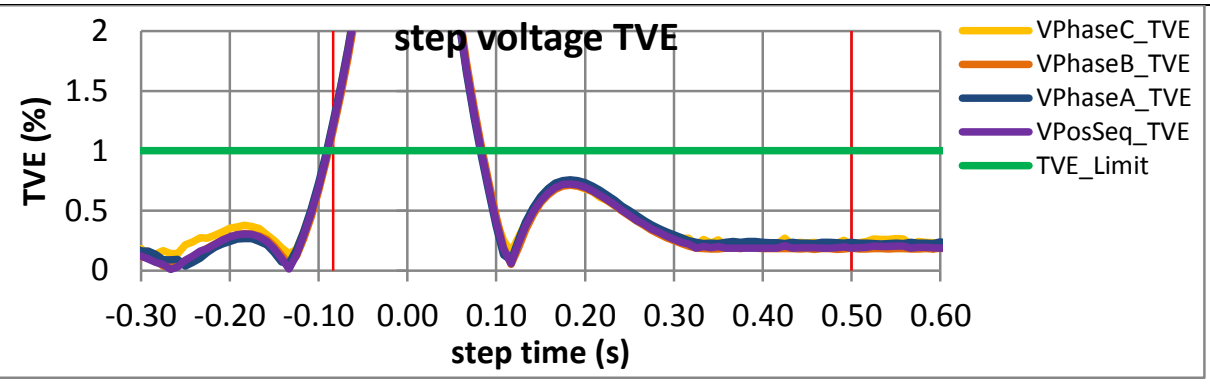

Figure 5170: Fs = $12 \mathrm{FPS},-10 \%$ magnitude step

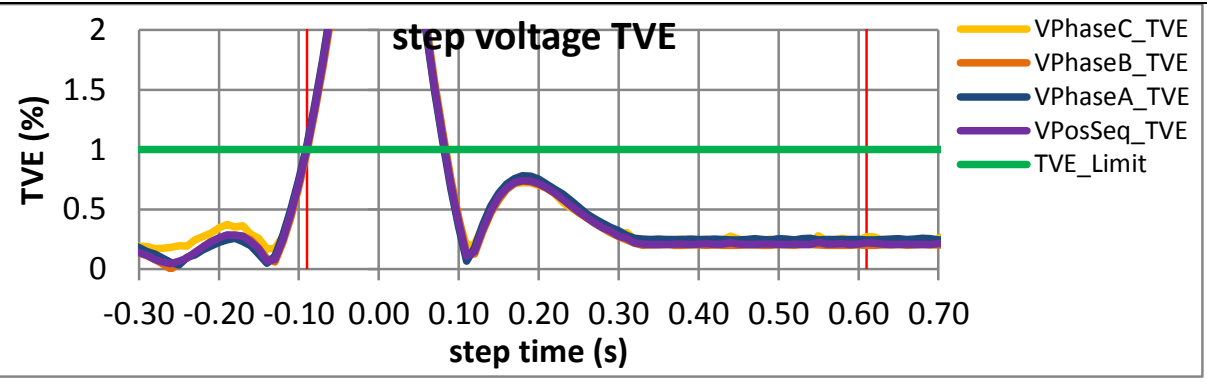

Figure 5172: Fs = $10 \mathrm{FPS},+10 \%$ magnitude step 
10.2.8 PMU G dynamic step change in magnitude voltage response time: $F 0=60 \mathrm{~Hz}, \mathrm{M}$ class

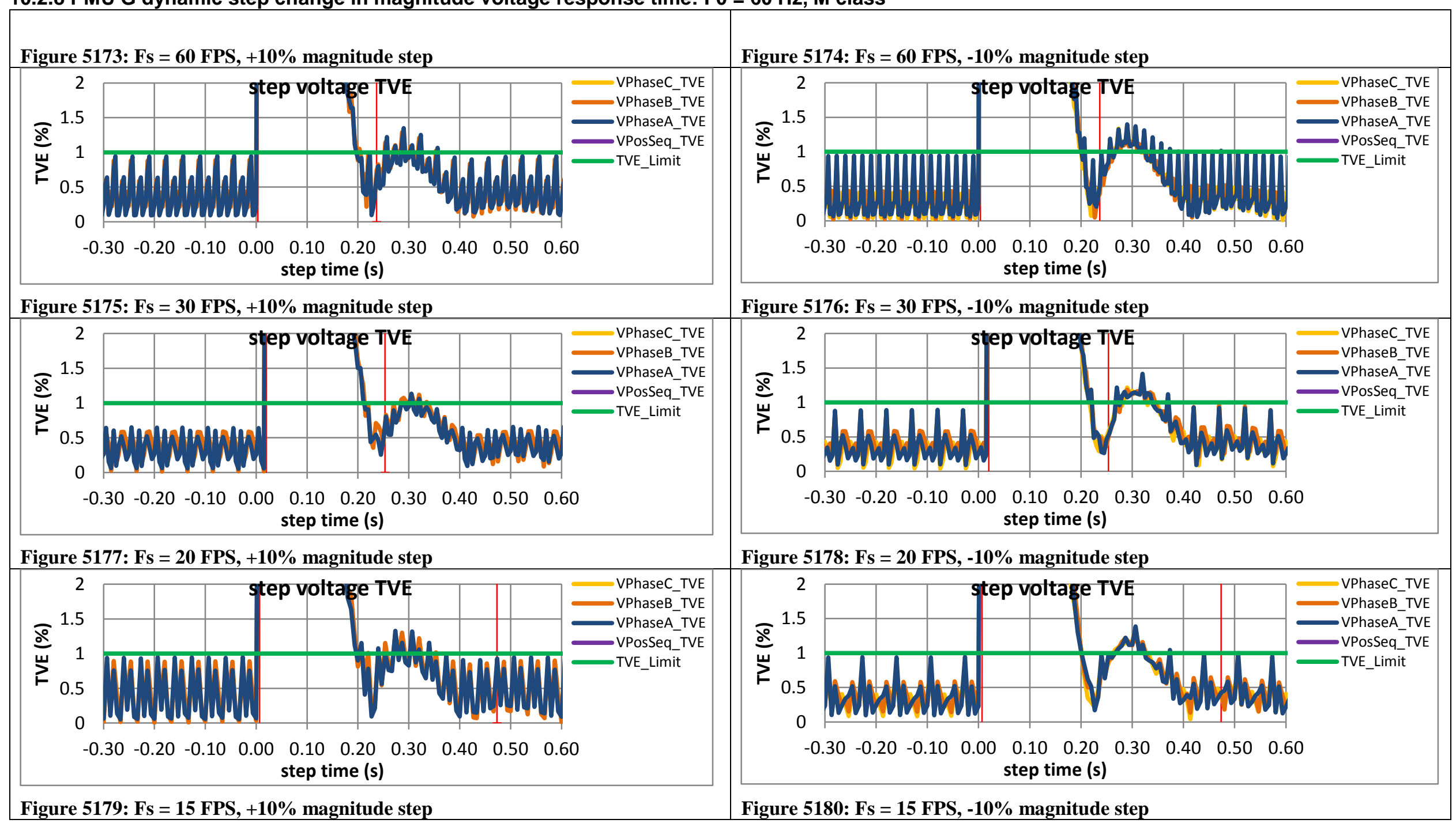



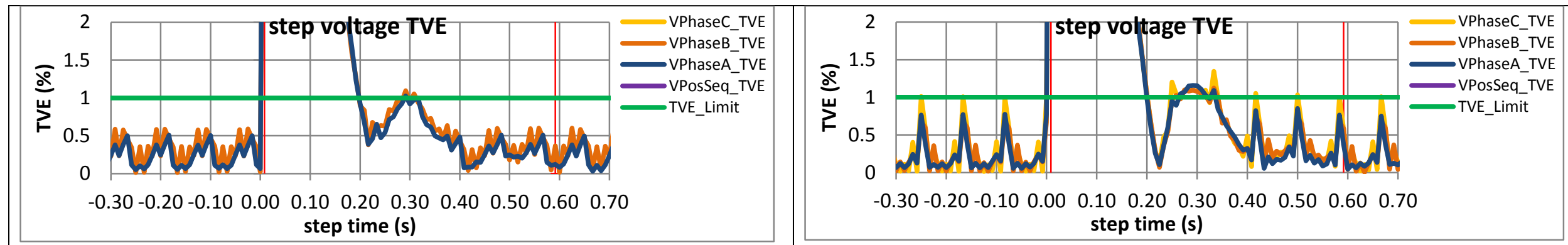

Figure 5181: Fs = 12 FPS, +10 degree magnitude step

Figure 5182: Fs = 12 FPS, -10 degree magnitude step

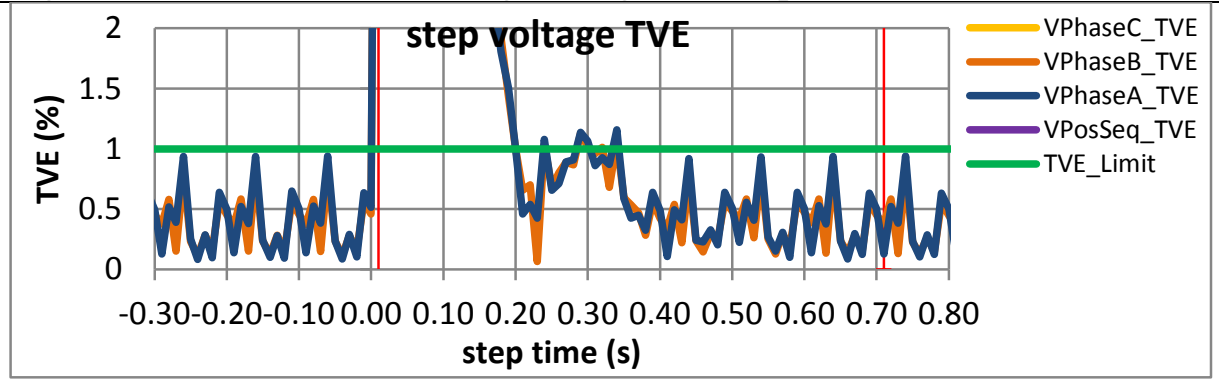

Figure 5183: Fs = 10 FPS, $+10 \%$ magnitude step

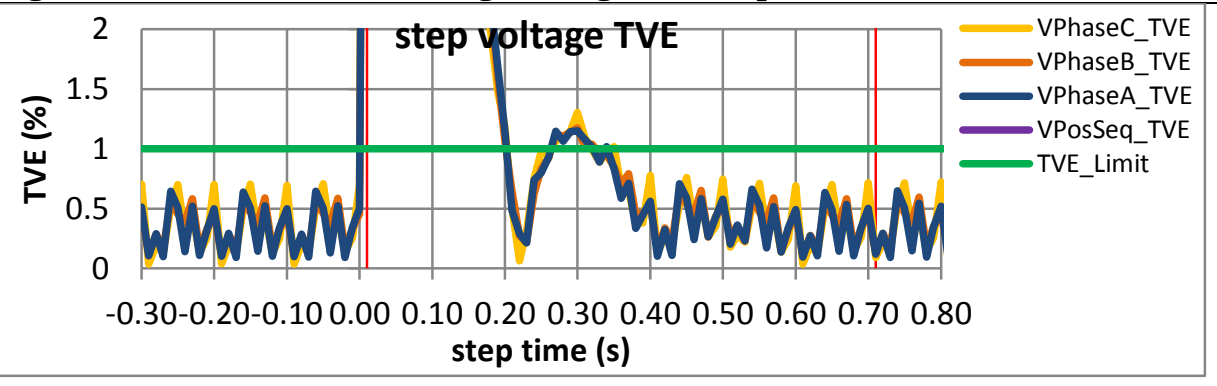

Figure 5184: Fs = 10 FPS, +10\% magnitude step 
10.2.9 PMU H dynamic step change in magnitude voltage response time: $\mathrm{F} 0=60 \mathrm{~Hz}, \mathrm{M}$ class

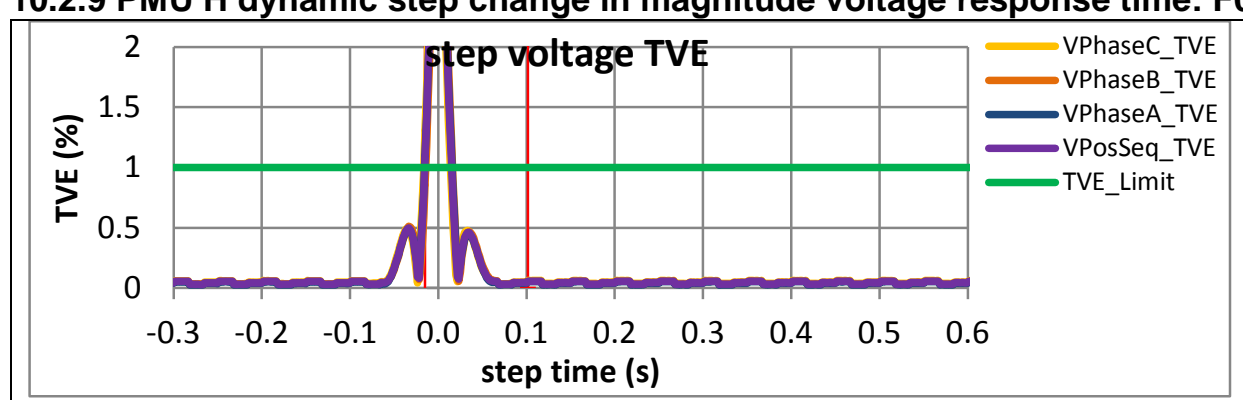

Figure 5185: Fs = 60 FPS, $+10 \%$ magnitude step

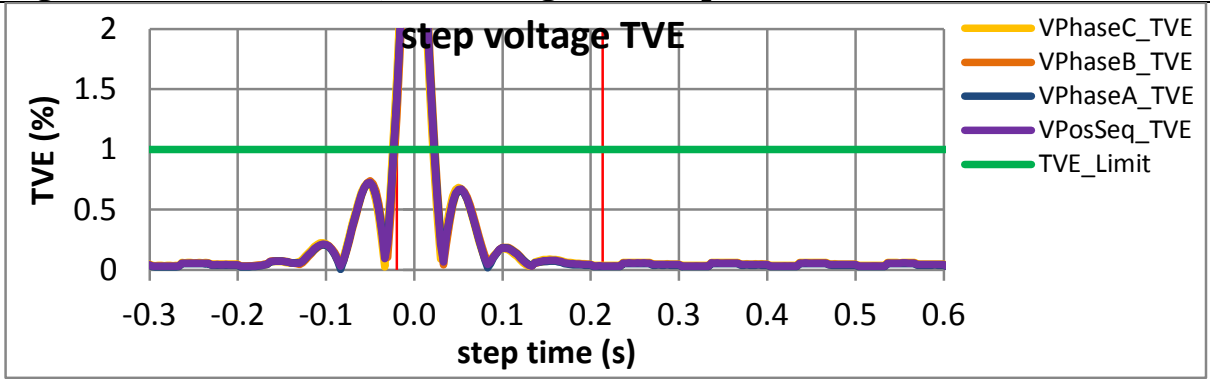

Figure 5187: Fs = 30 FPS, $+10 \%$ magnitude step

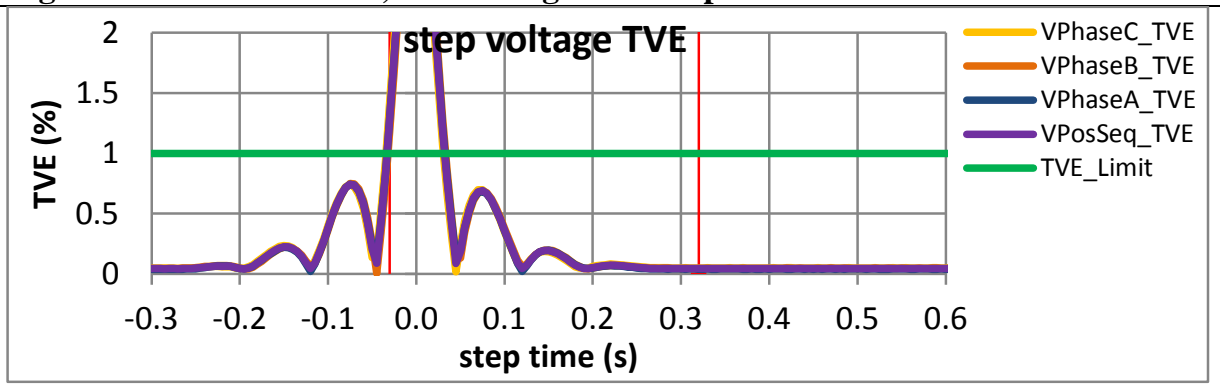

Figure 5189: Fs $=20 \mathrm{FPS},+10 \%$ magnitude step

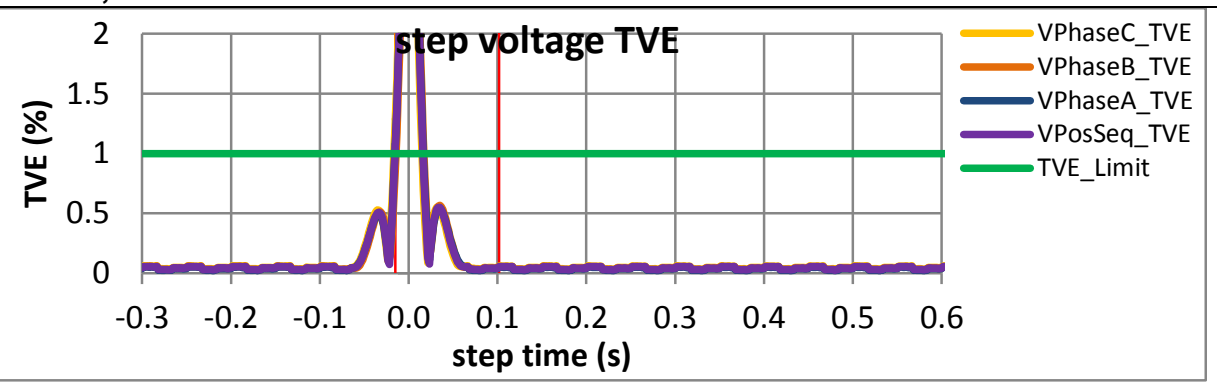

Figure 5186: Fs = 60 FPS, -10\% magnitude step

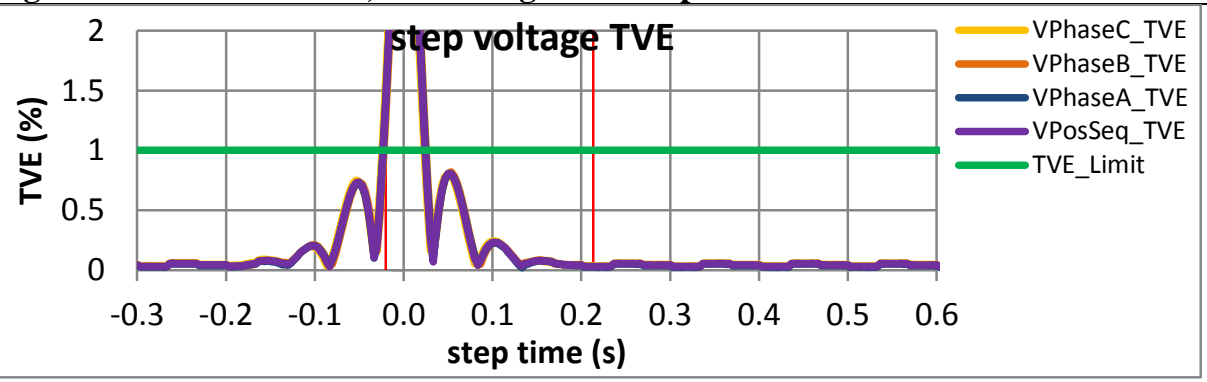

Figure 5188: Fs $=30$ FPS, $-10 \%$ magnitude step

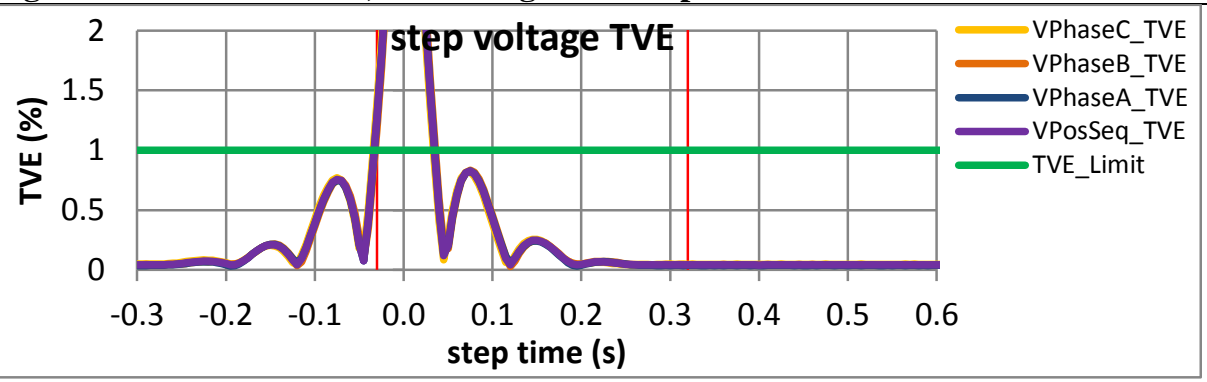

Figure 5190: Fs = 20 FPS, -10\% magnitude step 

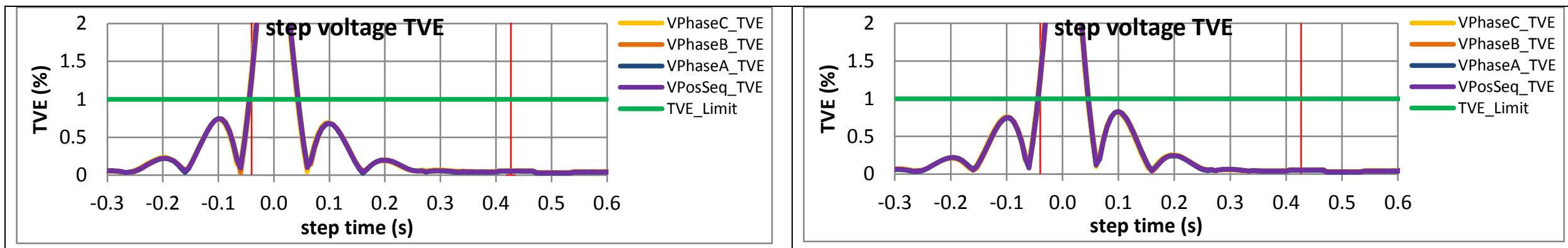

Figure 5191: Fs = 15 FPS, $+10 \%$ magnitude step

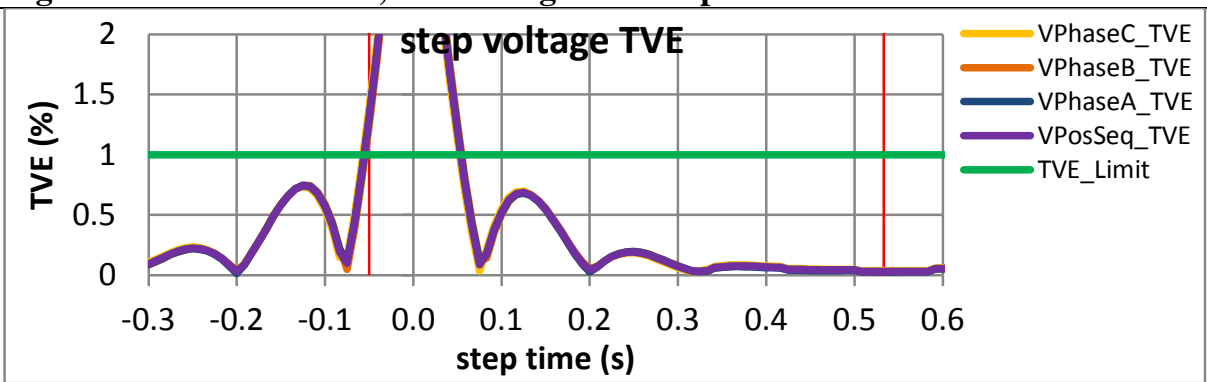

Figure 5193: Fs = 12 FPS, $+10 \%$ magnitude step

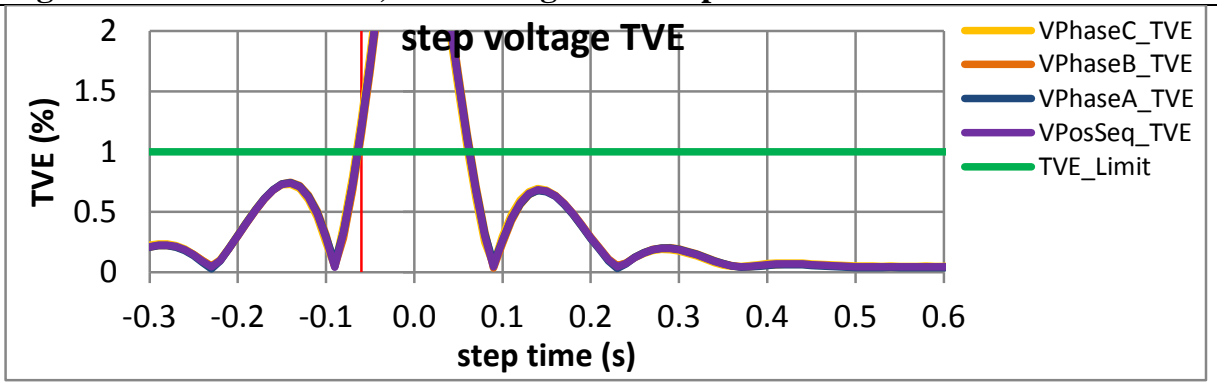

Figure 5195: Fs = 10 FPS, +10\% magnitude step

Figure 5192: Fs = 15 FPS, -10\% magnitude step

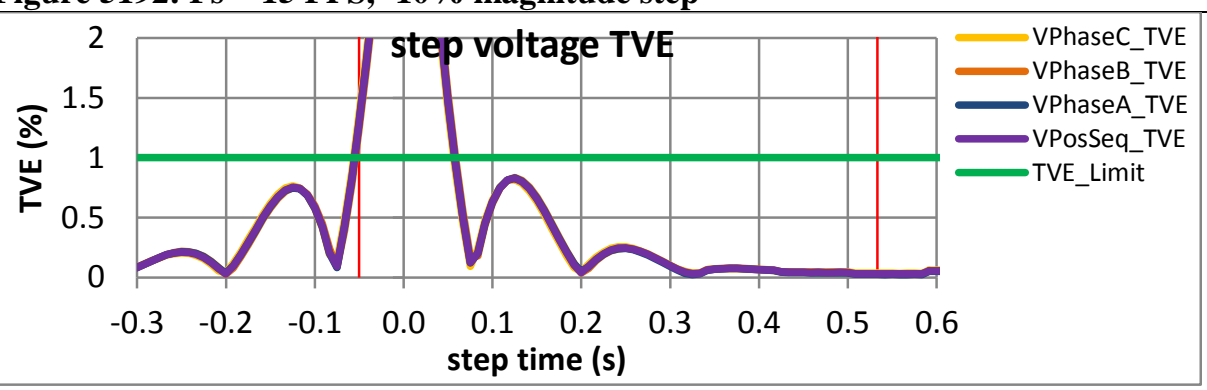

Figure 5194: Fs = 12 FPS, -10\% magnitude step

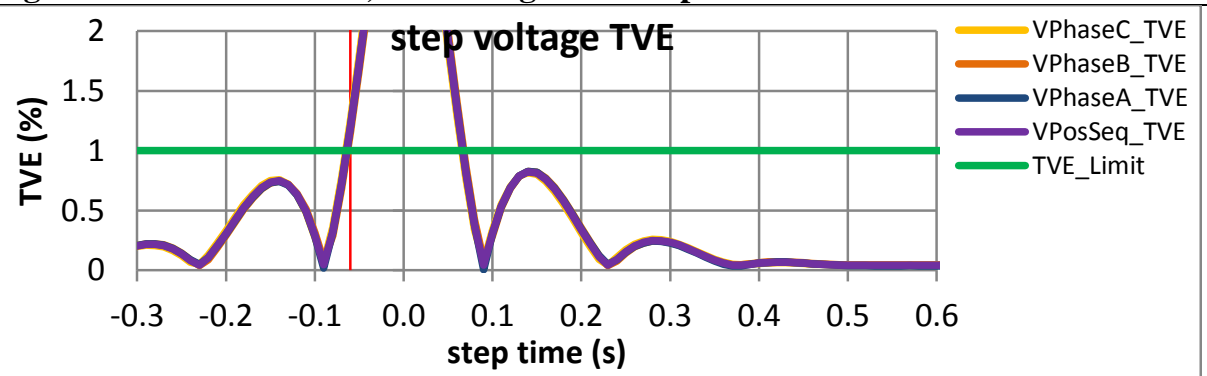

Figure 5196: Fs = 10 FPS, $+10 \%$ magnitude step 
10.2.10 PMU I dynamic step change in magnitude voltage response time: $\mathrm{FO}=60 \mathrm{~Hz}, \mathrm{M}$ class

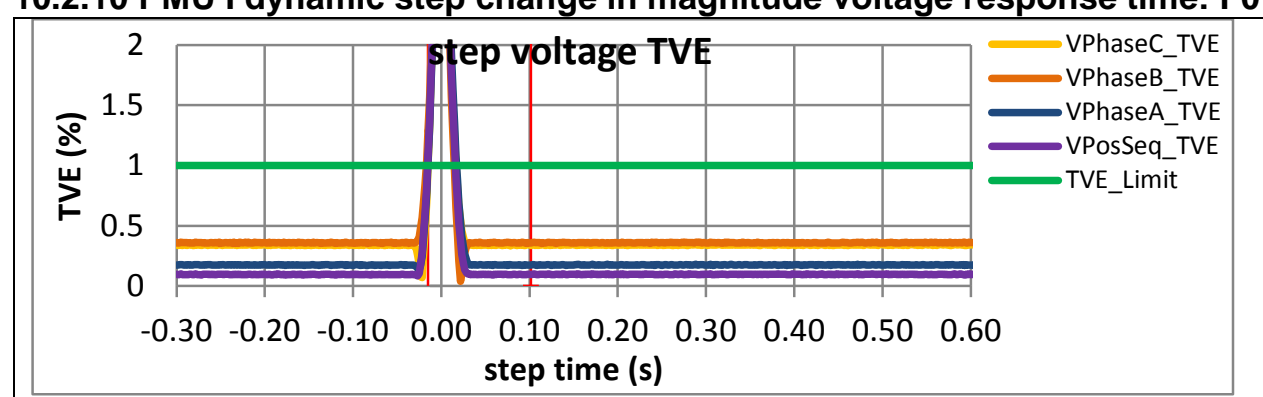

Figure 5197: Fs = 60 FPS, +10\% magnitude step

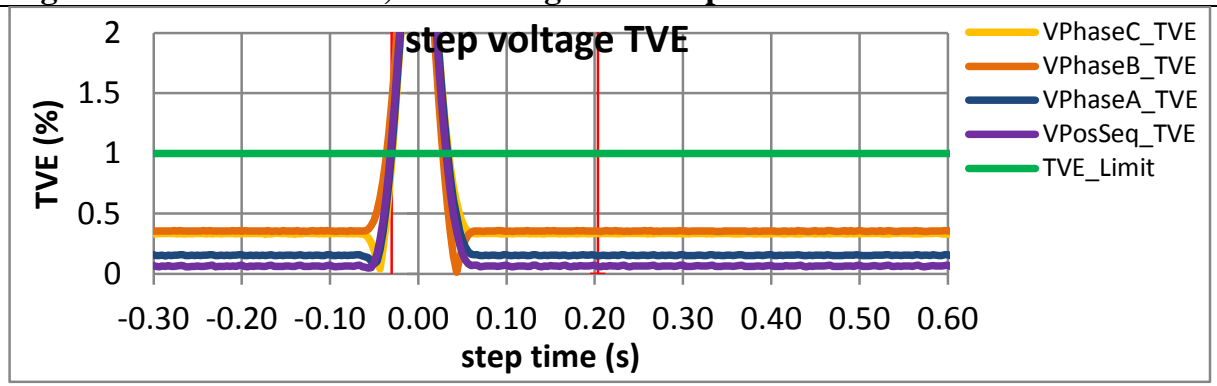

Figure 5199: Fs = 30 FPS, $+10 \%$ magnitude step

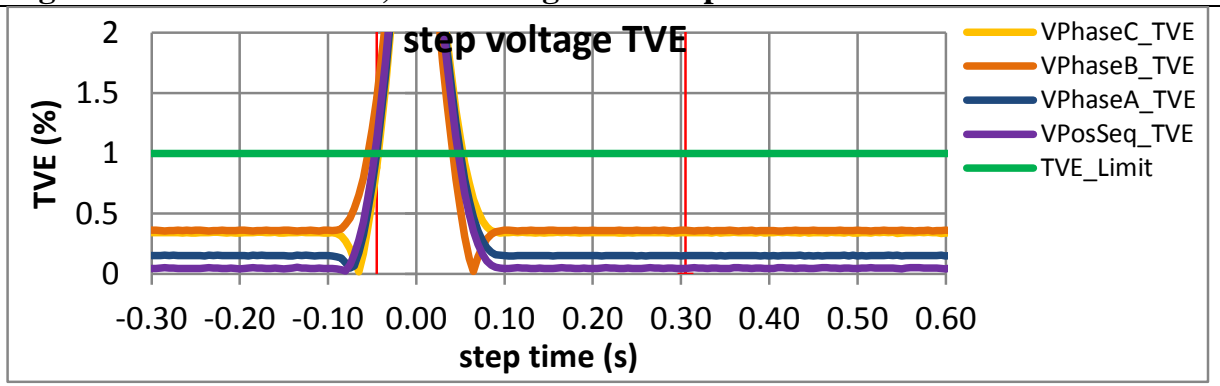

Figure 5201: Fs $=20 \mathrm{FPS},+10 \%$ magnitude step

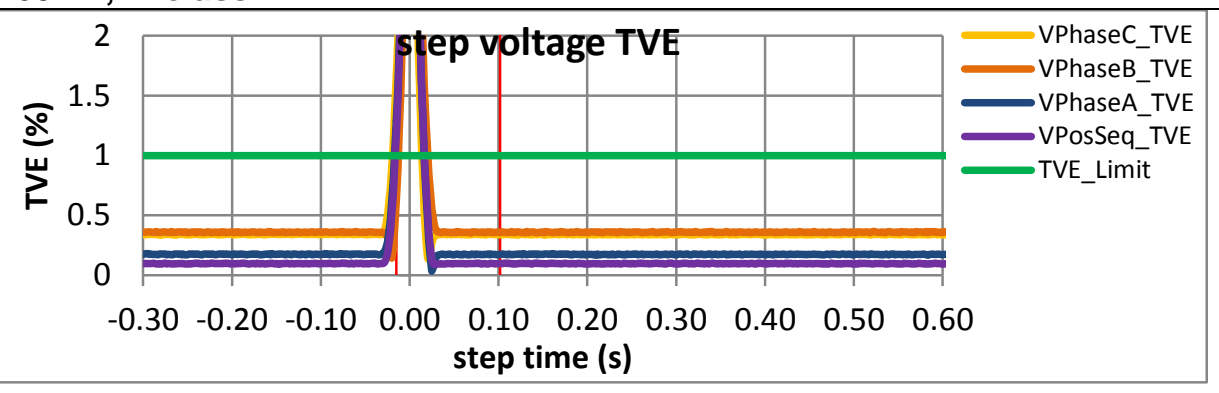

Figure 5198: Fs = 60 FPS, -10\% magnitude step

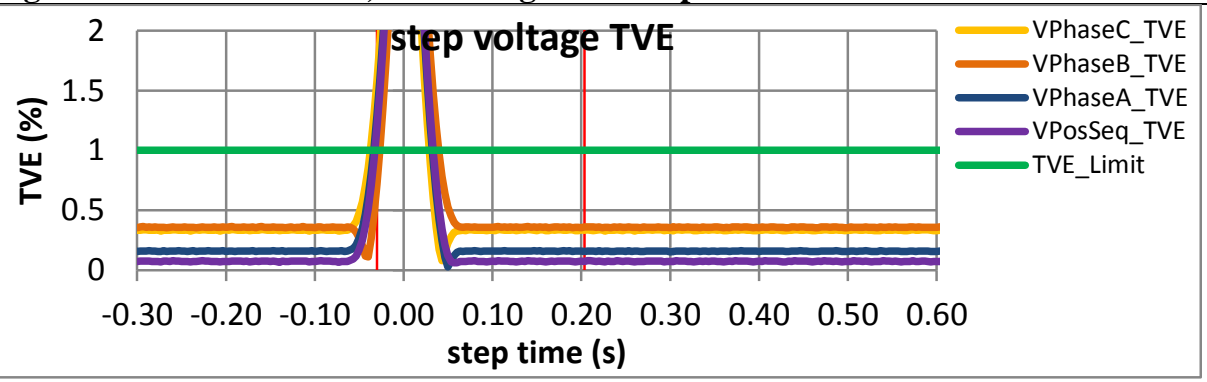

Figure 5200: Fs = 30 FPS, -10\% magnitude step

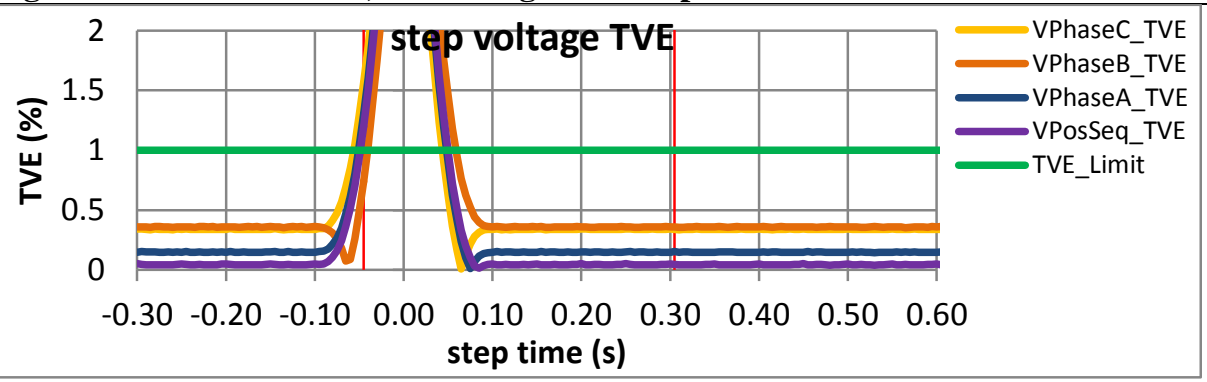

Figure 5202: Fs = 20 FPS, -10\% magnitude step 

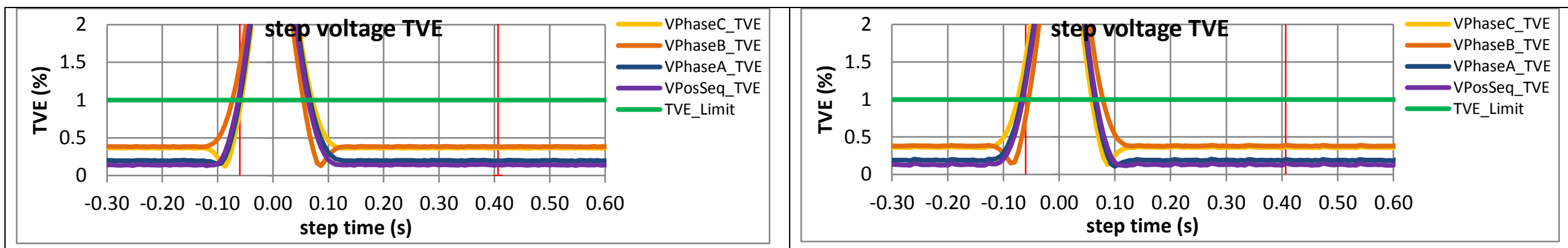

Figure 5203: Fs = 15 FPS, +10\% magnitude step

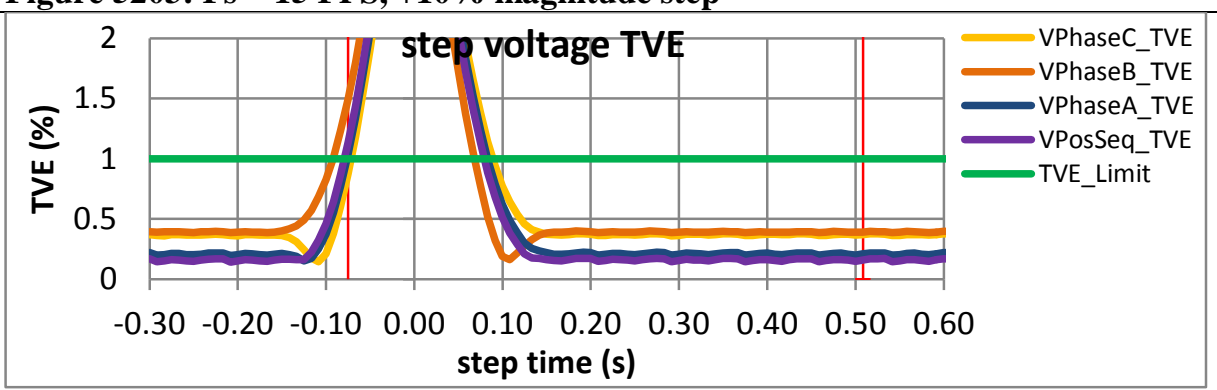

Figure 5204: Fs = 15 FPS, -10\% magnitude step

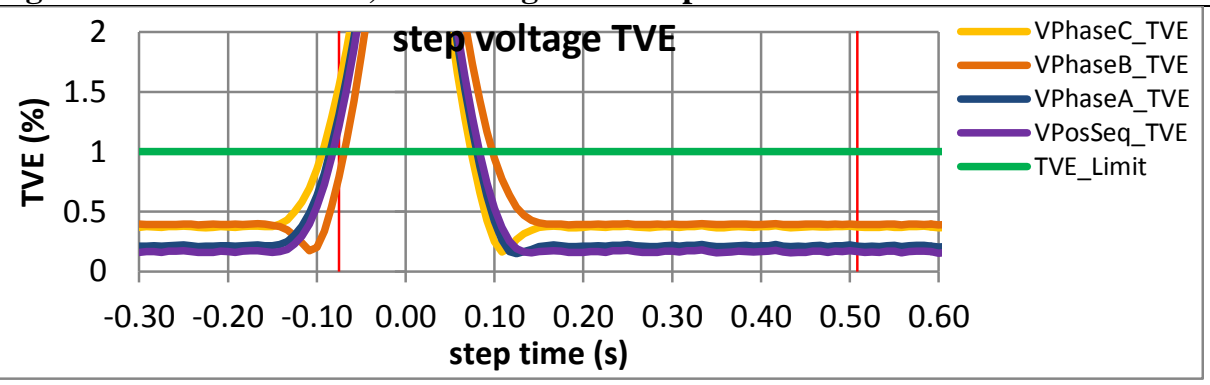

Figure 5205: Fs = 12 FPS, +10\% magnitude step

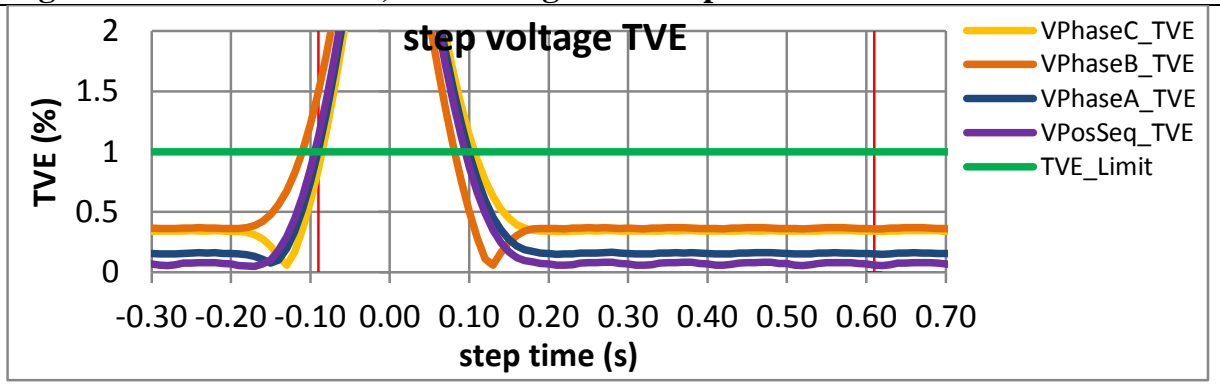

Figure 5207: Fs $=10$ FPS, +10\% magnitude step

Figure 5206: Fs = 12 FPS, -10\% magnitude step

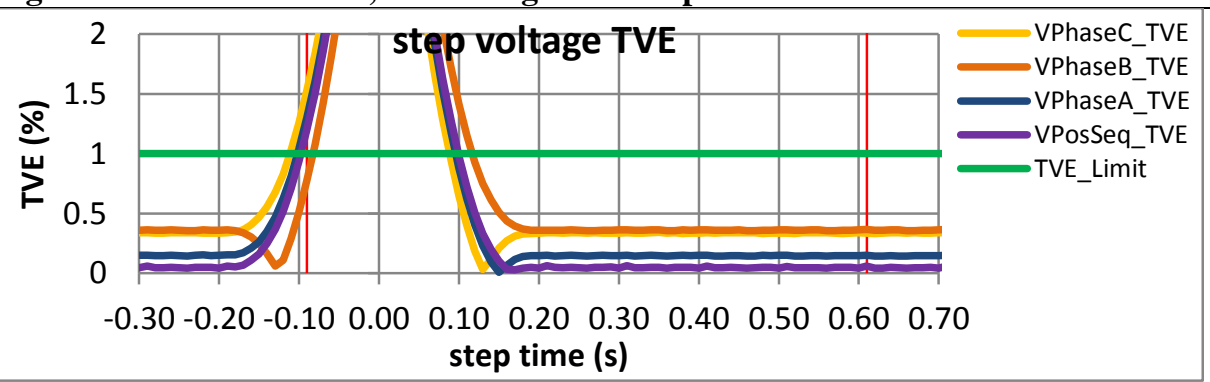

Figure 5208: Fs = 10 FPS, +10\% magnitude step 
10.2.11 PMU J dynamic step change in magnitude voltage response time: $\mathrm{F} 0=60 \mathrm{~Hz}, \mathrm{M}$ class
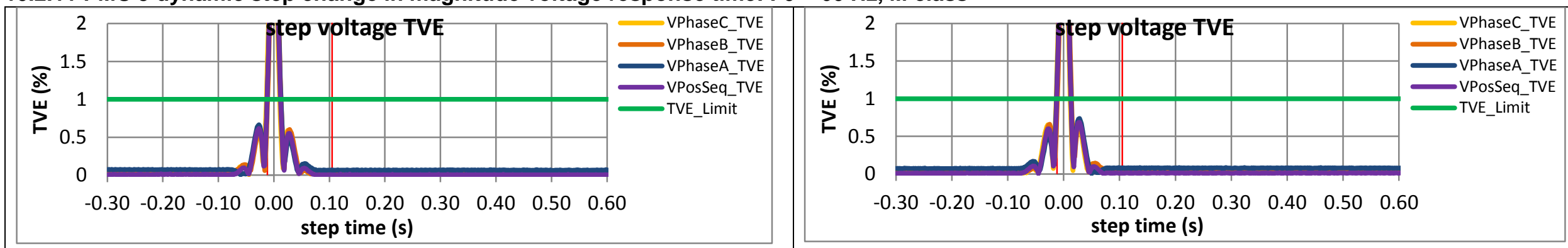

Figure 5209: Fs $=60$ FPS, $+10 \%$ magnitude step

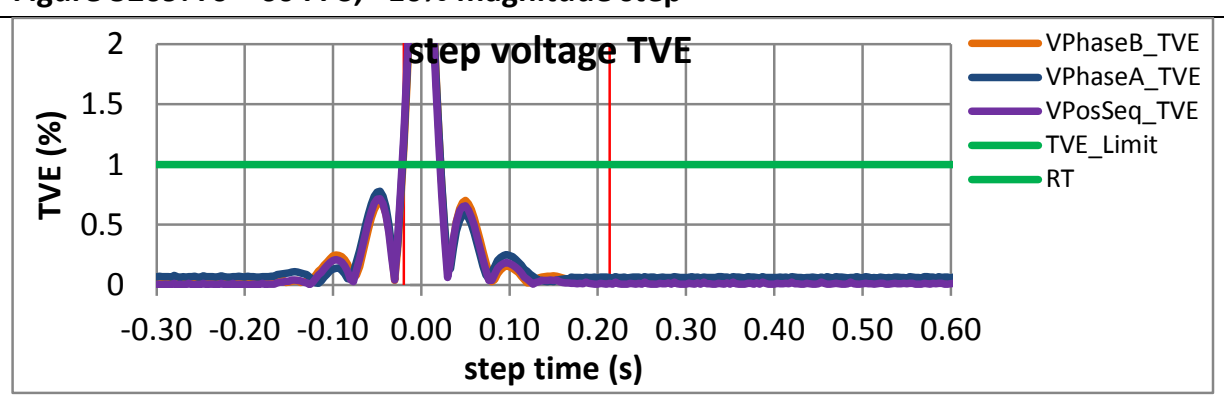

Figure 5210: Fs = $60 \mathrm{FPS},-10 \%$ magnitude step

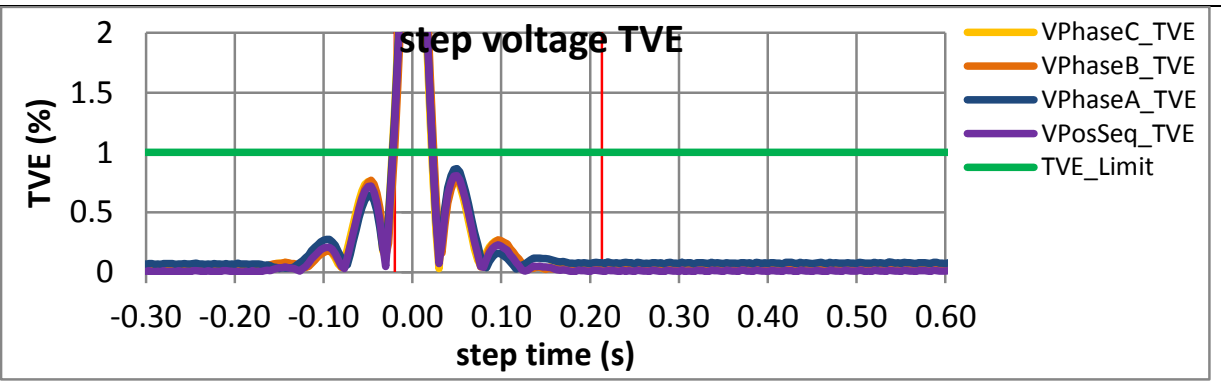

Figure 5211: Fs = 30 FPS, +10\% magnitude step

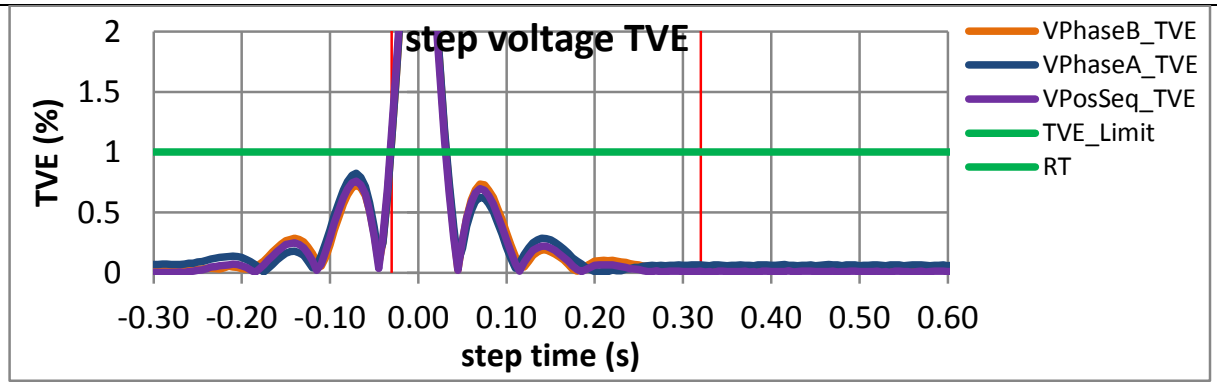

Figure 5212: Fs = 30 FPS, $-10 \%$ magnitude step

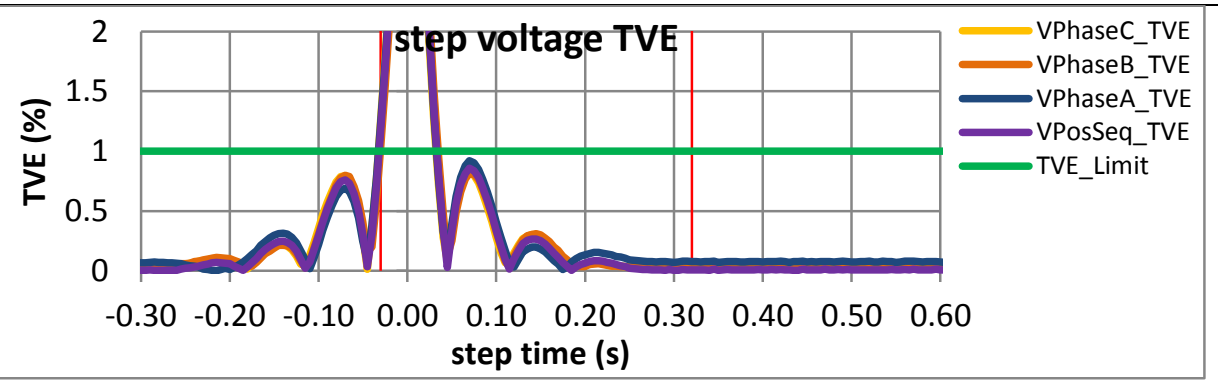

Figure 5213: Fs = 20 FPS, $+10 \%$ magnitude step

Figure 5214: Fs = 20 FPS, $-10 \%$ magnitude step 


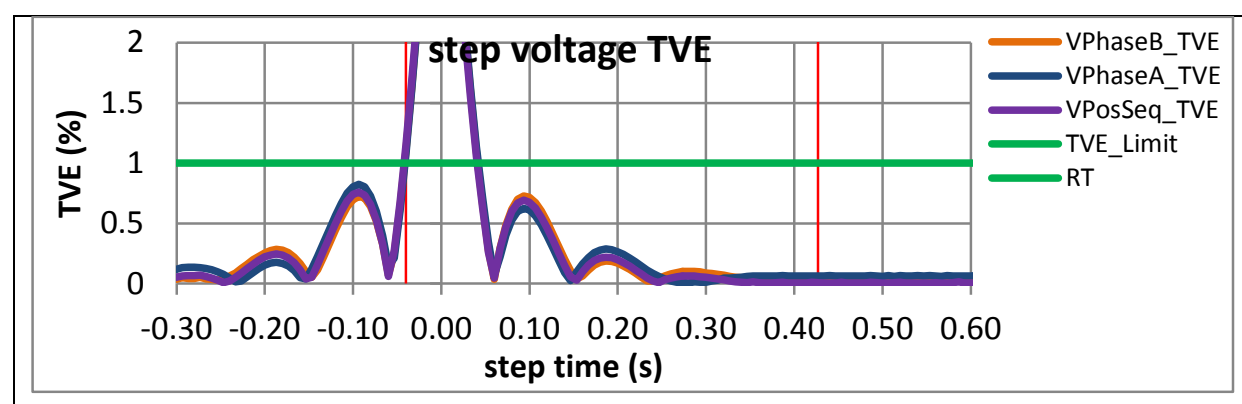

Figure 5215: Fs = 15 FPS, +10\% magnitude step

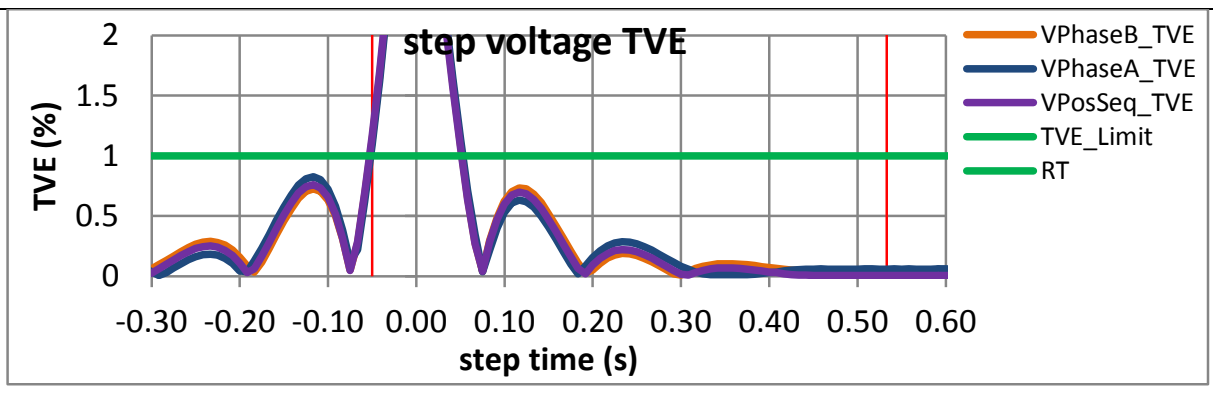

Figure 5217: Fs = 12 FPS, +10\% magnitude step

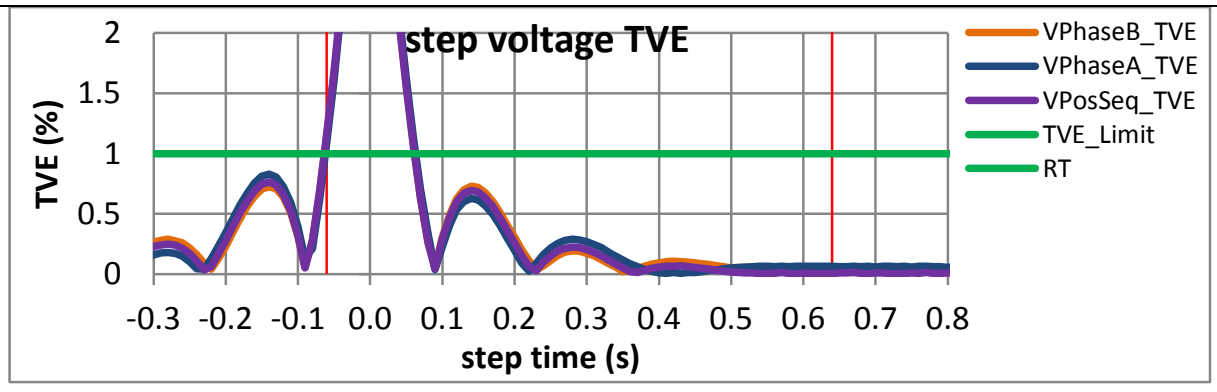

Figure 5219: Fs = 10 FPS, $+10 \%$ magnitude step

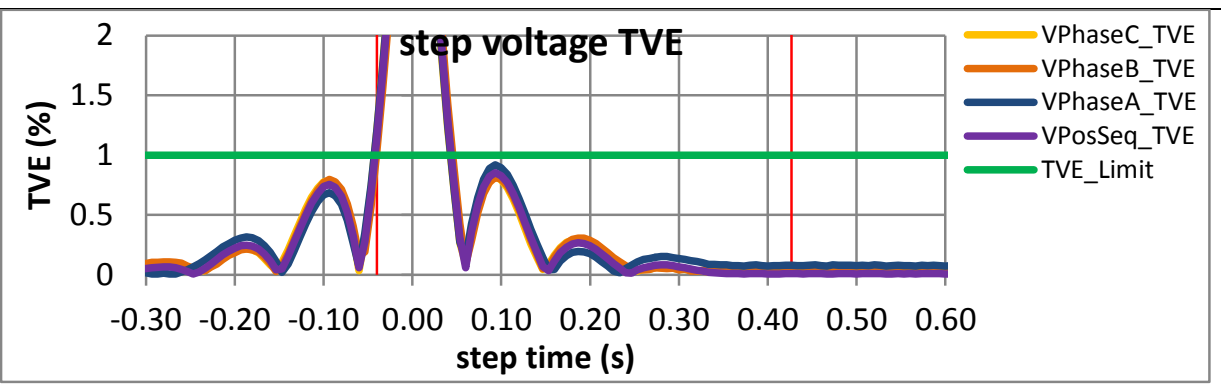

Figure 5216: Fs = $15 \mathrm{FPS},-10 \%$ magnitude step

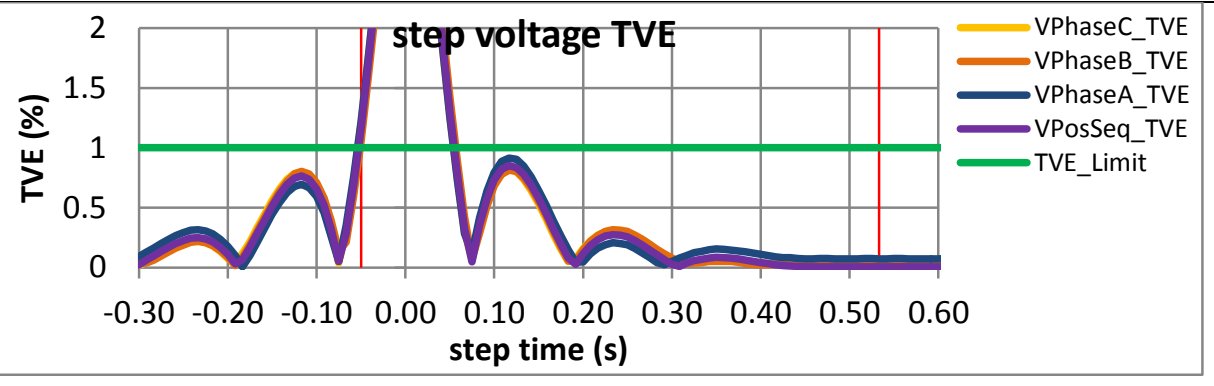

Figure 5218: Fs = 12 FPS, $-10 \%$ magnitude step

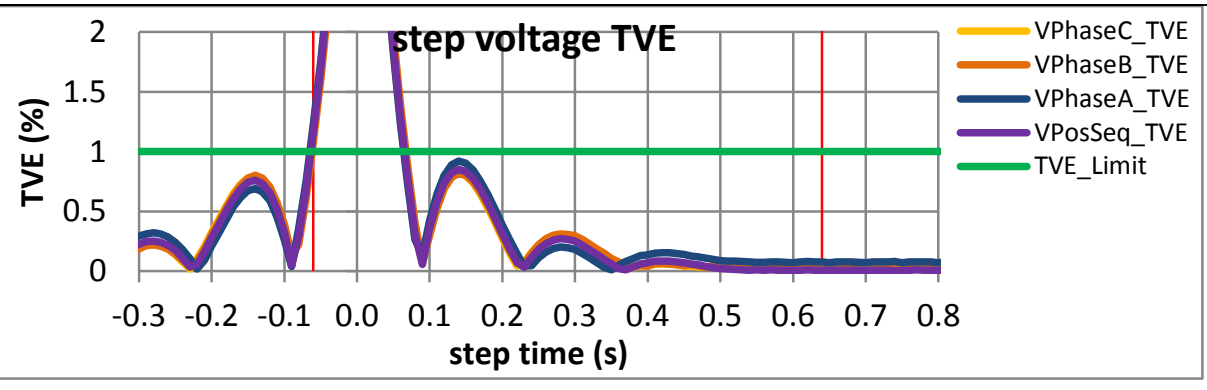

Figure 5220: Fs = $10 \mathrm{FPS},+10 \%$ magnitude step 
10.3 Dynamic step change in magnitude current response time: $F 0=60 \mathrm{~Hz}, \mathrm{M}$ class

10.3.1 C37.118.1-2011 Annex $C$ dynamic step change in magnitude current response time: $F 0=60 \mathrm{~Hz}, \mathrm{M}$ class
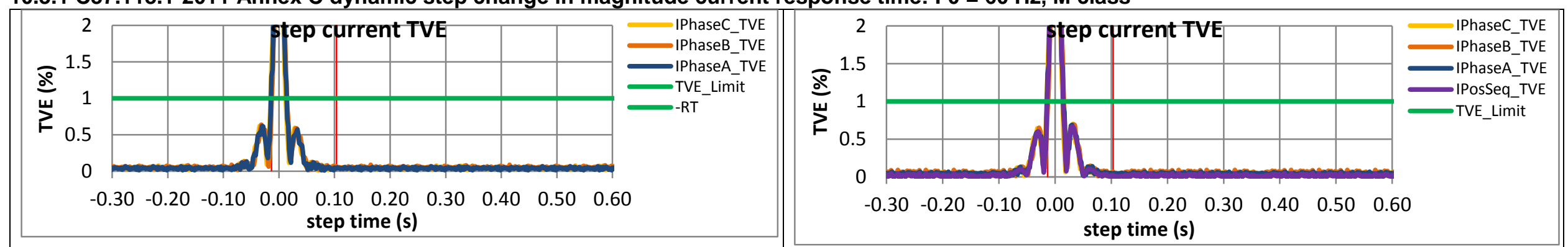

Figure 5221: Fs = 60 FPS, $+10 \%$ magnitude step

Figure 5222: Fs = 60 FPS, -10\% magnitude step

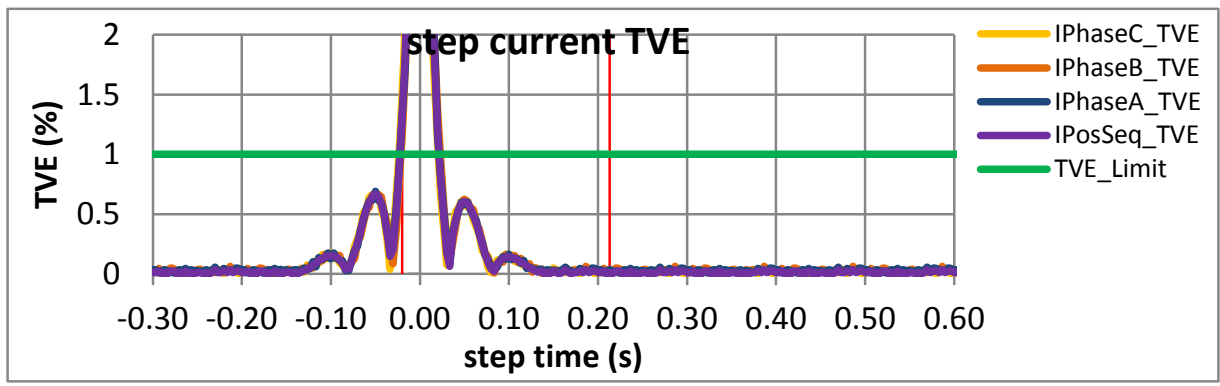

Figure 5223: Fs $=30$ FPS, $+10 \%$ magnitude step

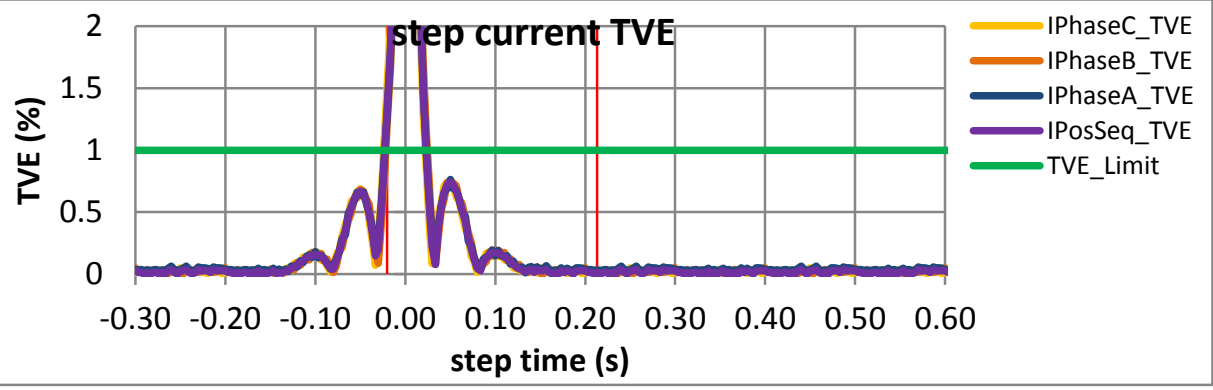

Figure 5224: Fs = 30 FPS, -10\% magnitude step 

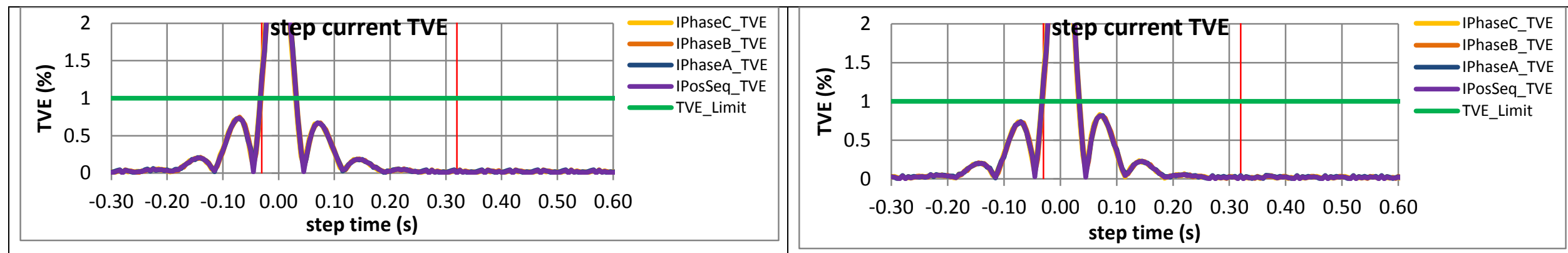

Figure 5225: Fs = 20 FPS, +10\% magnitude step

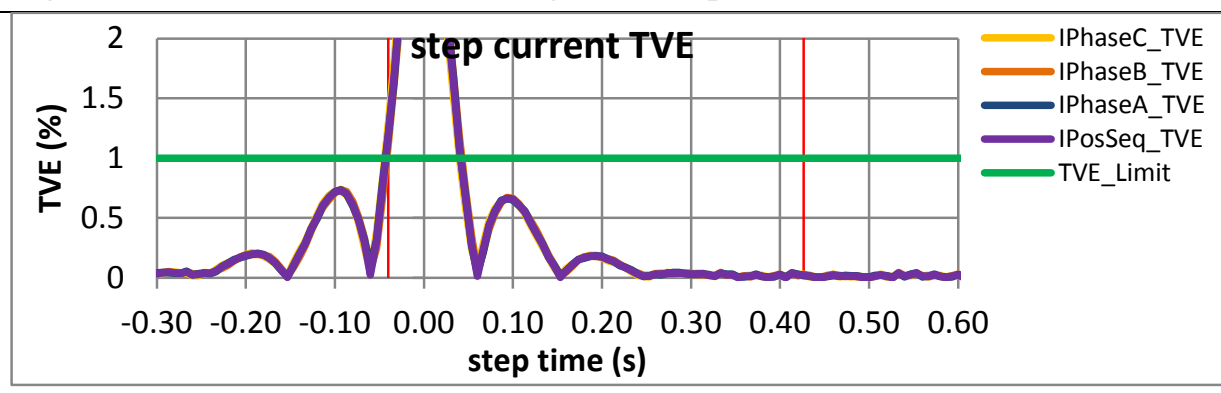

Figure 5226: Fs = 20 FPS, $+10 \%$ magnitude step

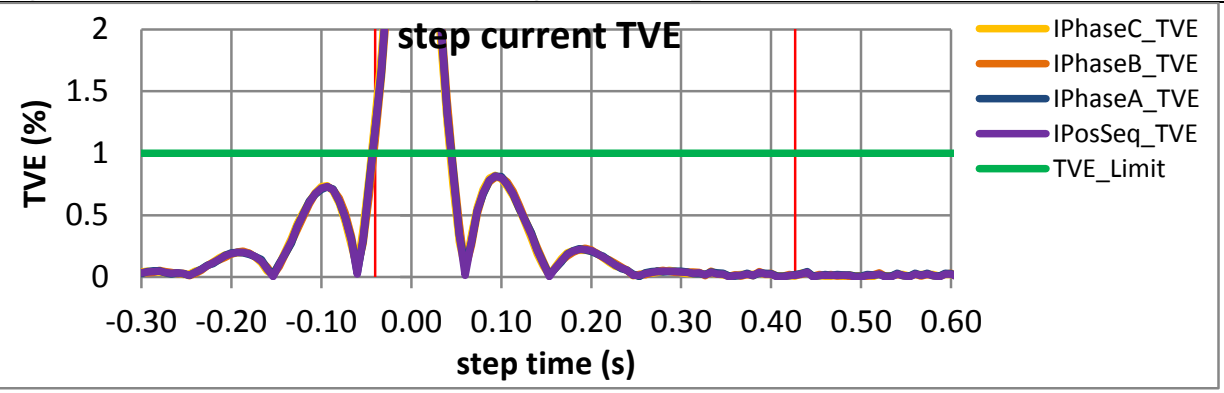

Figure 5227: Fs = 15 FPS, +10\% magnitude step

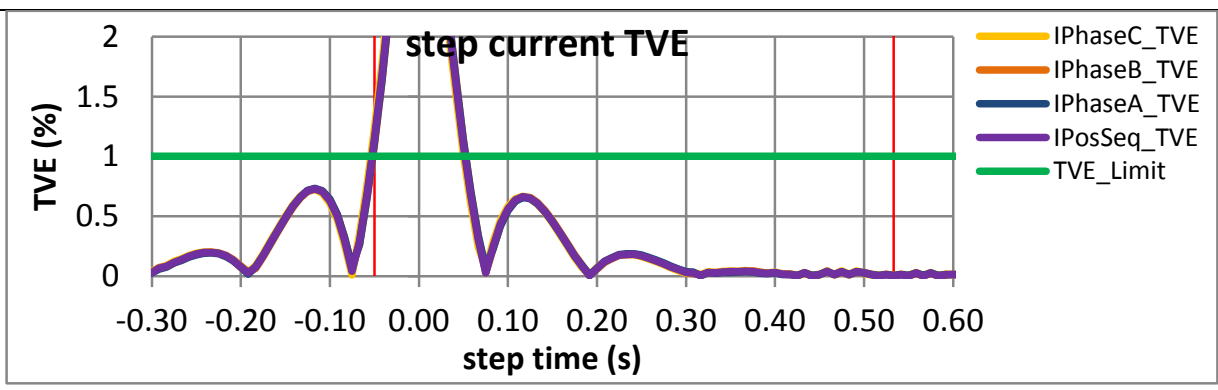

Figure 5229: Fs = 12 FPS, $+10 \%$ magnitude step

Figure 5228: Fs = 15 FPS, -10\% magnitude step

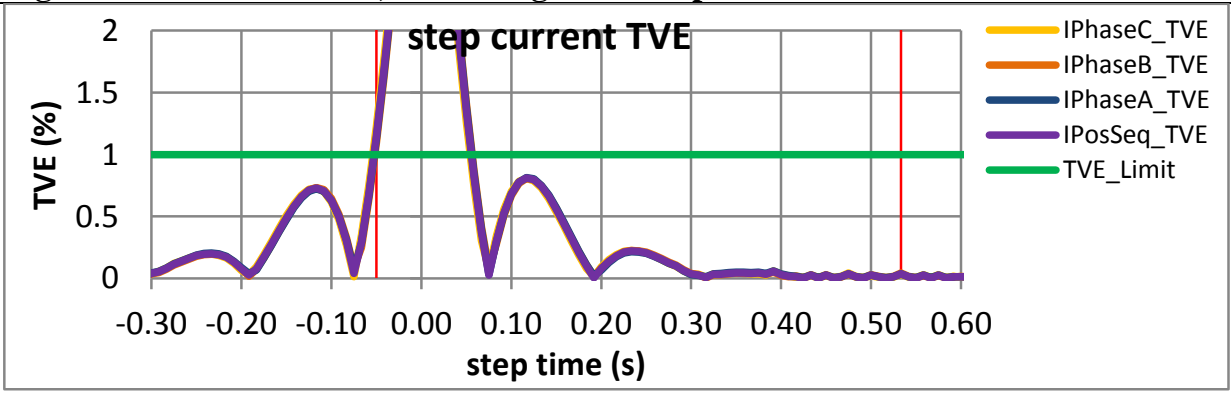

Figure 5230: Fs = 12 FPS, -10\% magnitude step 


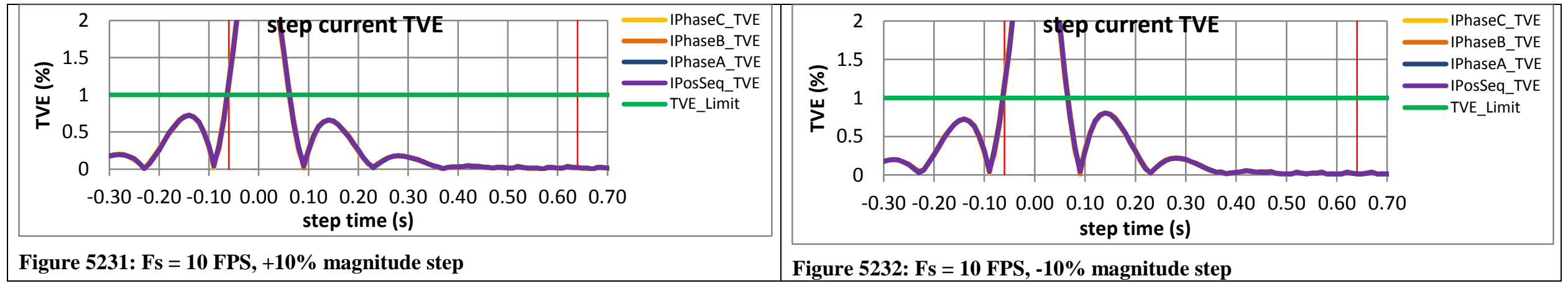


10.3.2 PMU A dynamic step change in magnitude current response time: $F 0=60 \mathrm{~Hz}, \mathrm{M}$ class
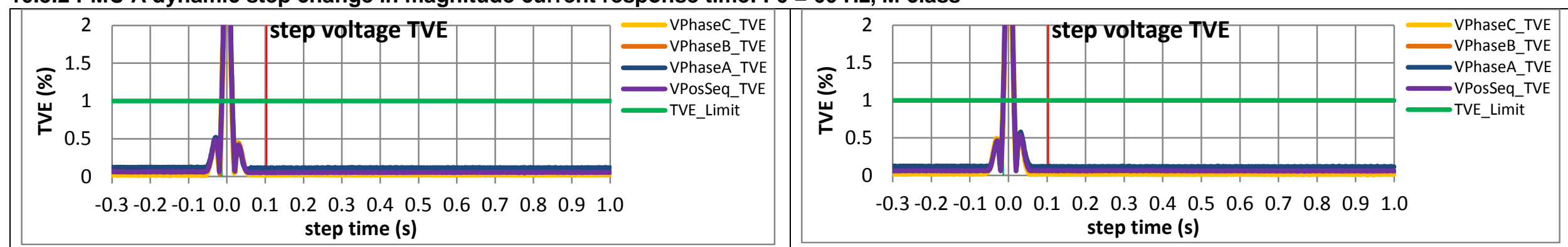

Figure 5233: Fs = 60 FPS, +10\% magnitude step

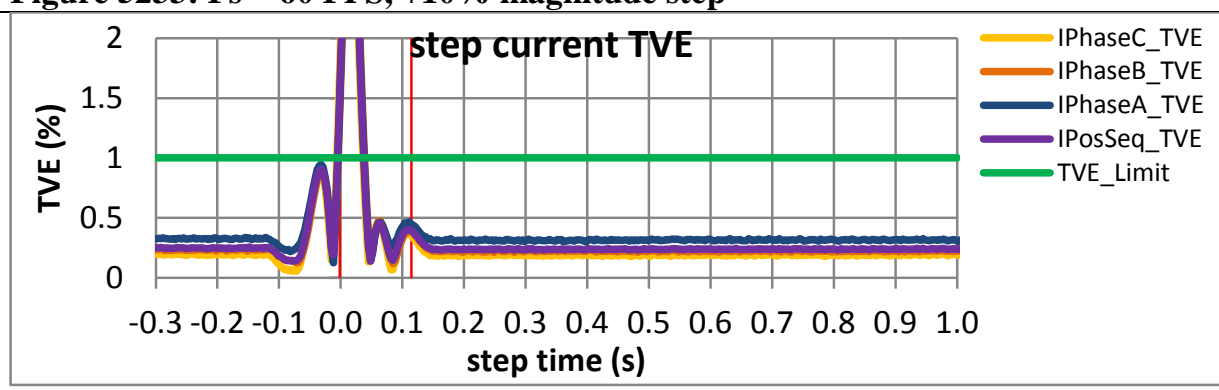

Figure 5234: Fs = 60 FPS, -10\% magnitude step

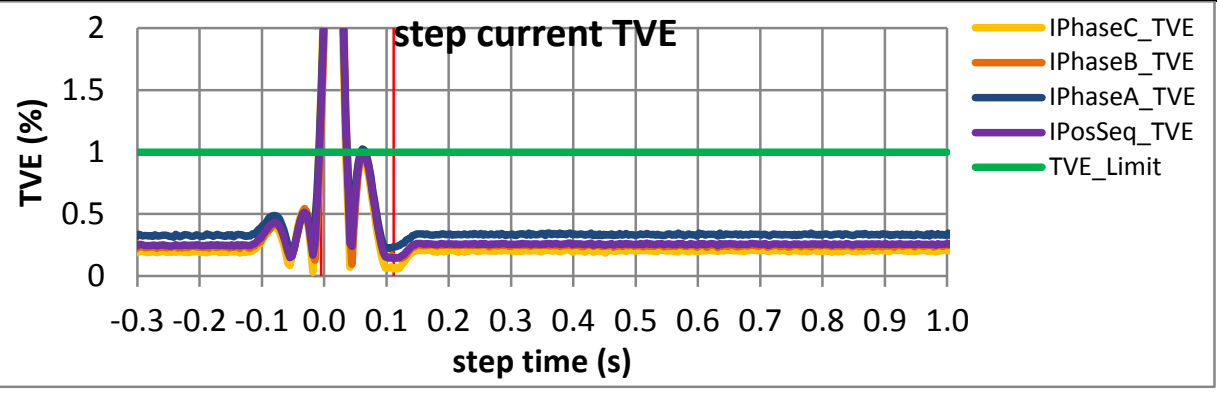

Figure 5235: Fs = 30 FPS, +10\% magnitude step

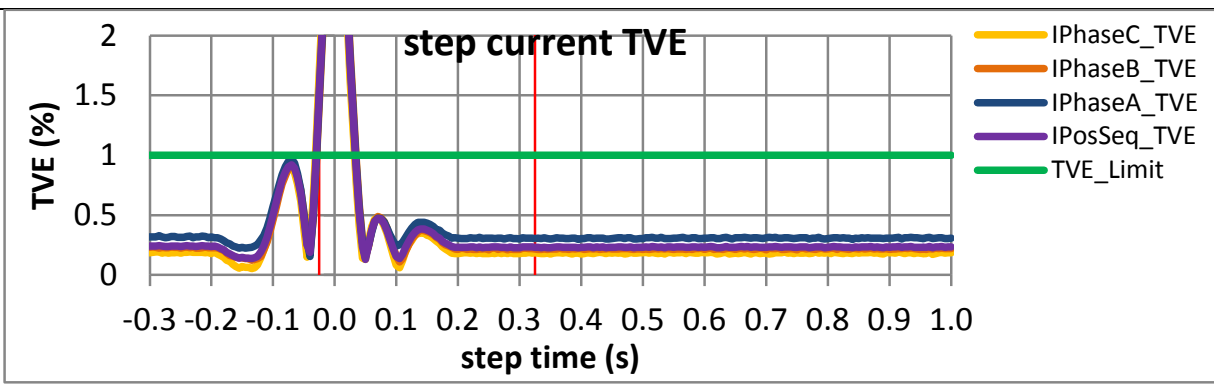

Figure 5237: Fs = 20 FPS, $+10 \%$ magnitude step

Figure 5236: Fs = 30 FPS, -10\% magnitude step

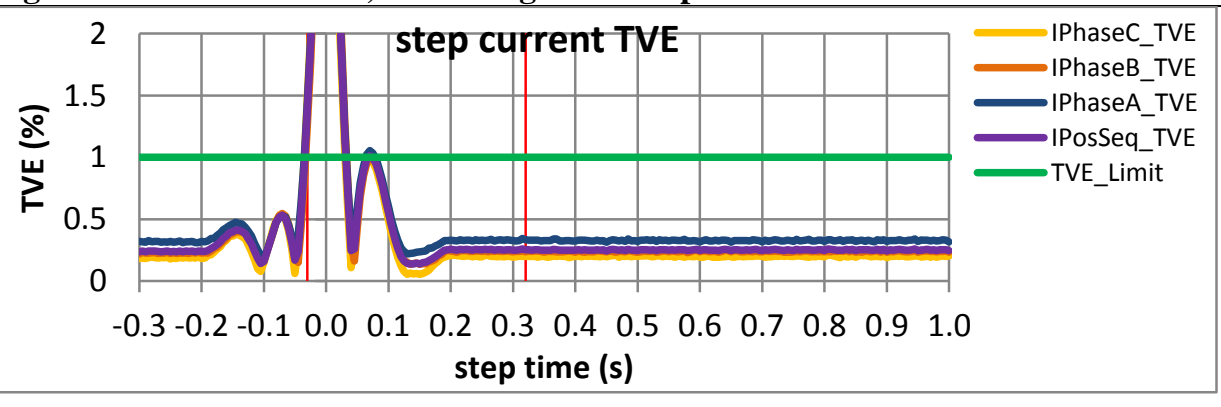

Figure 5238: Fs $=20$ FPS, $+10 \%$ magnitude step 


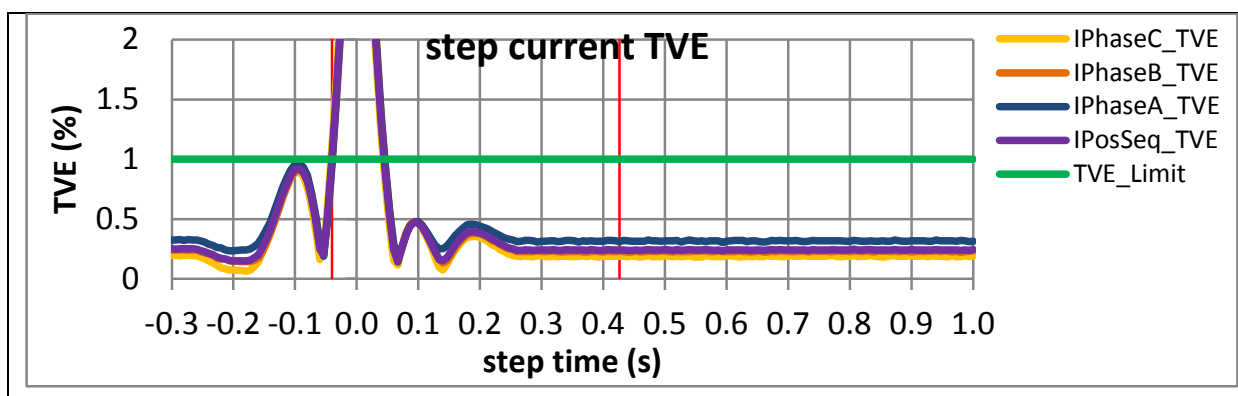

Figure 5239: Fs = 15 FPS, +10\% magnitude step
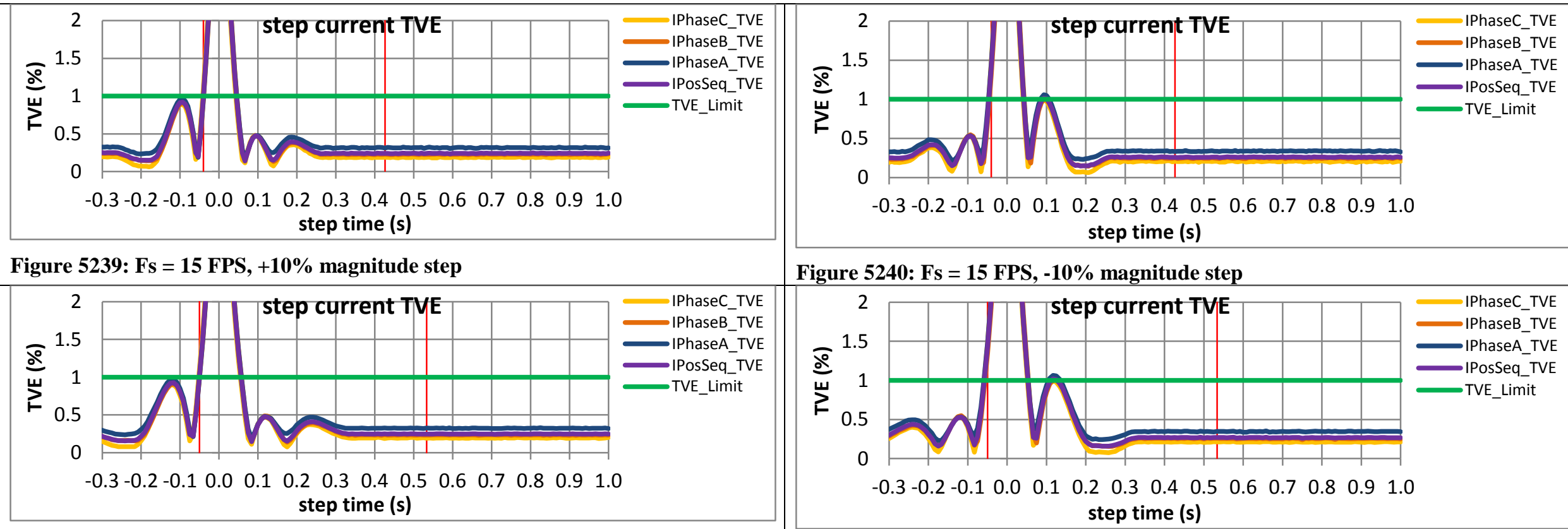

Figure 5240: Fs = 15 FPS, -10\% magnitude step

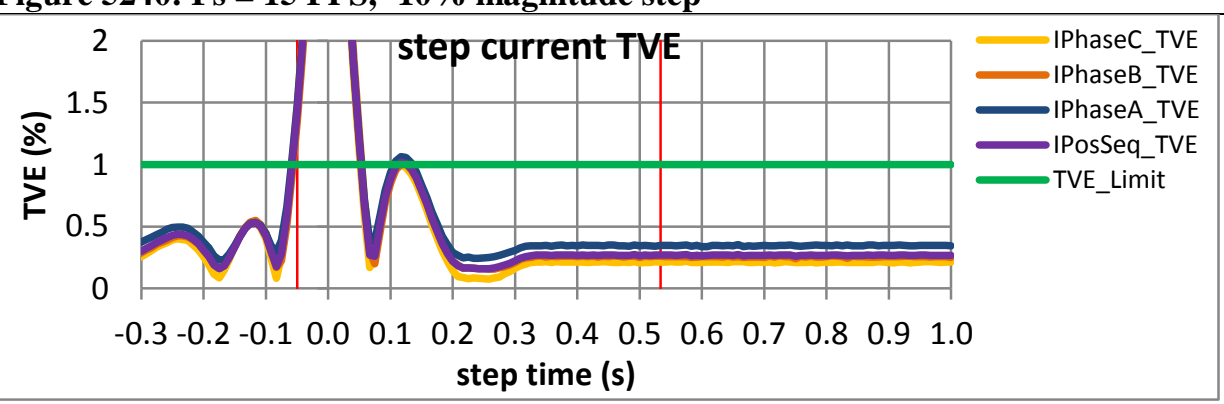

Figure 5241: Fs = 12 FPS, +10\% magnitude step

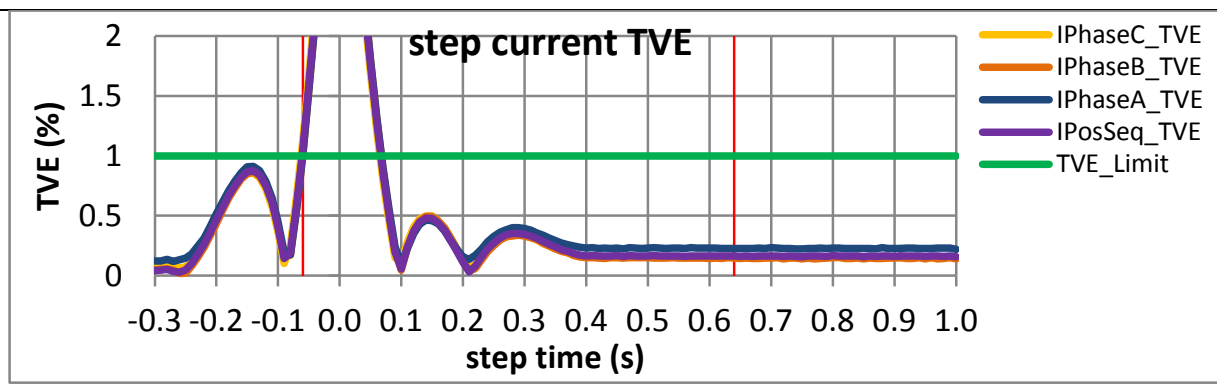

Figure 5242: Fs = 12 FPS, -10\% magnitude step

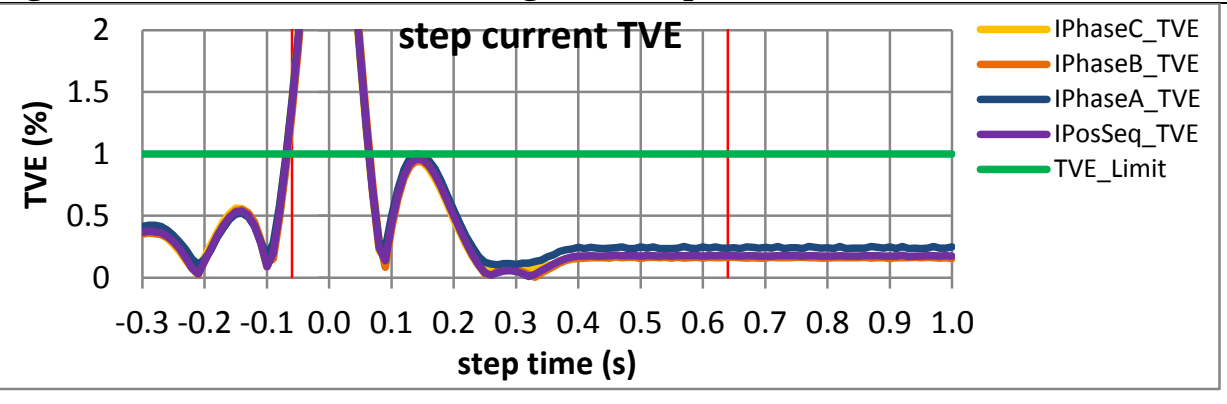

Figure 5243: Fs = 10 FPS, $+10 \%$ magnitude step

Figure 5244: Fs = 10 FPS, -10\% magnitude step 
10.3.3 PMU B dynamic step change in magnitude current response time: $F 0=60 \mathrm{~Hz}, \mathrm{M}$ class

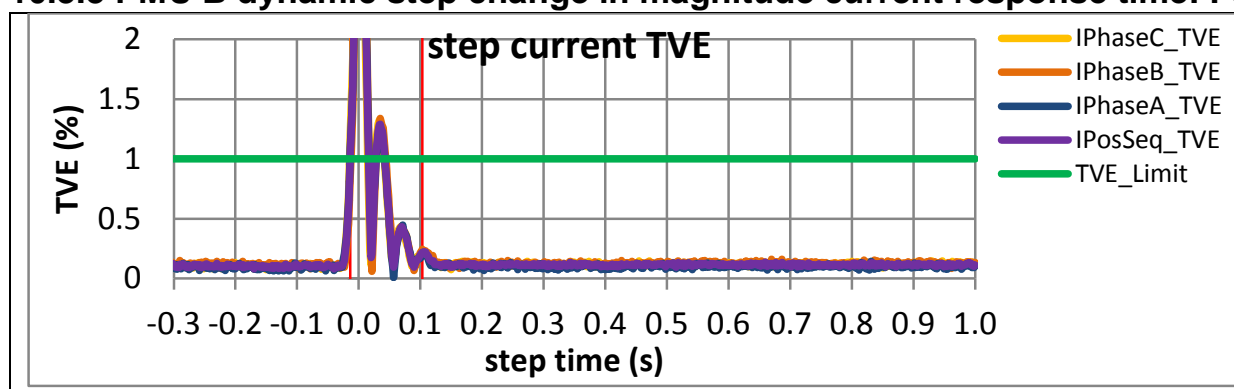

Figure 5245: Fs = 60 FPS, +10\% magnitude step

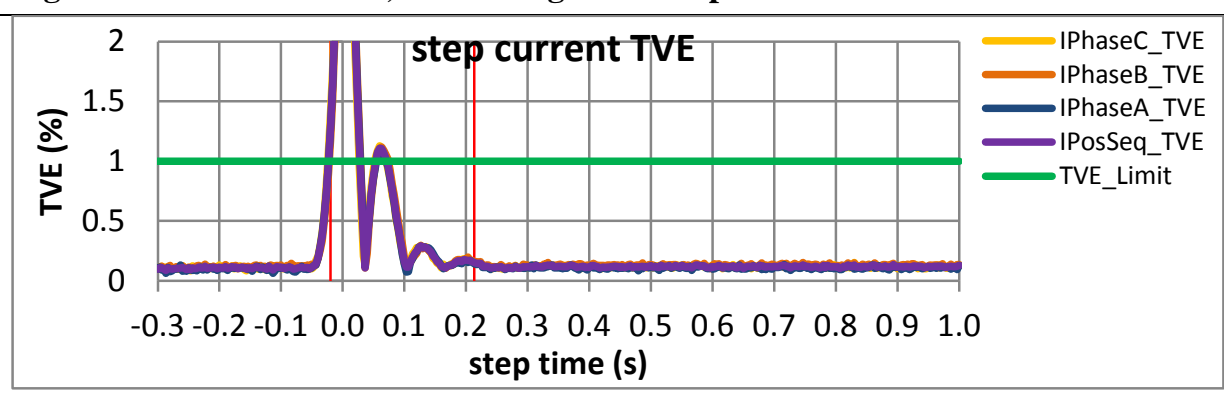

Figure 5247: Fs = 30 FPS, $+10 \%$ magnitude step

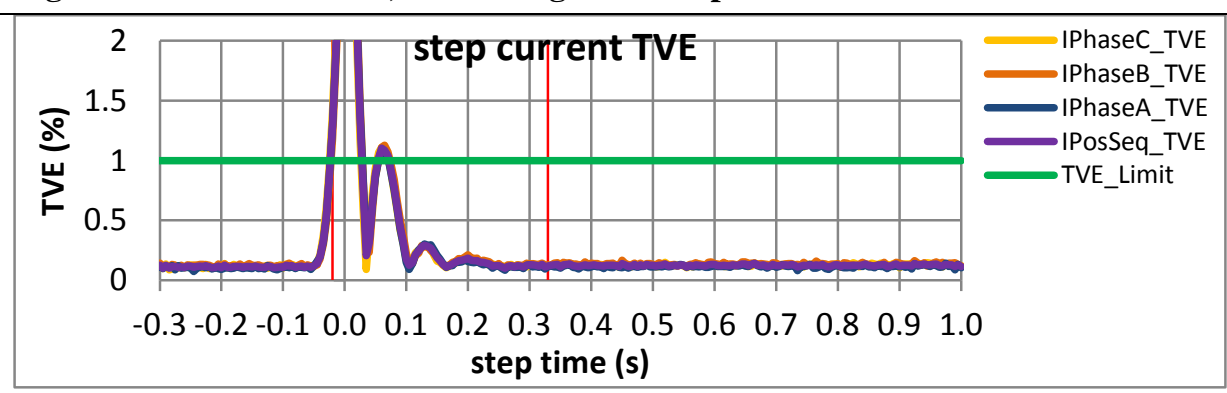

Figure 5249: Fs = 20 FPS, +10\% magnitude step

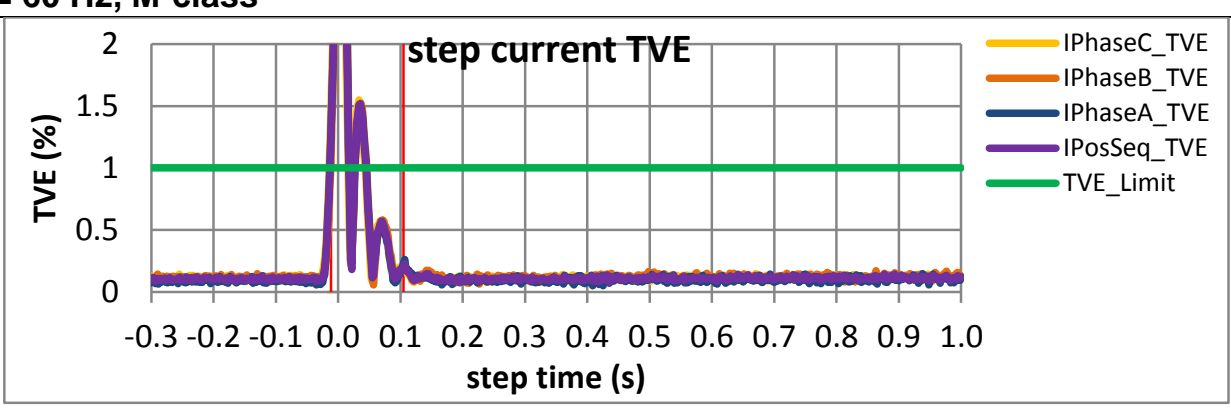

Figure 5246: Fs = 60 FPS, -10\% magnitude step

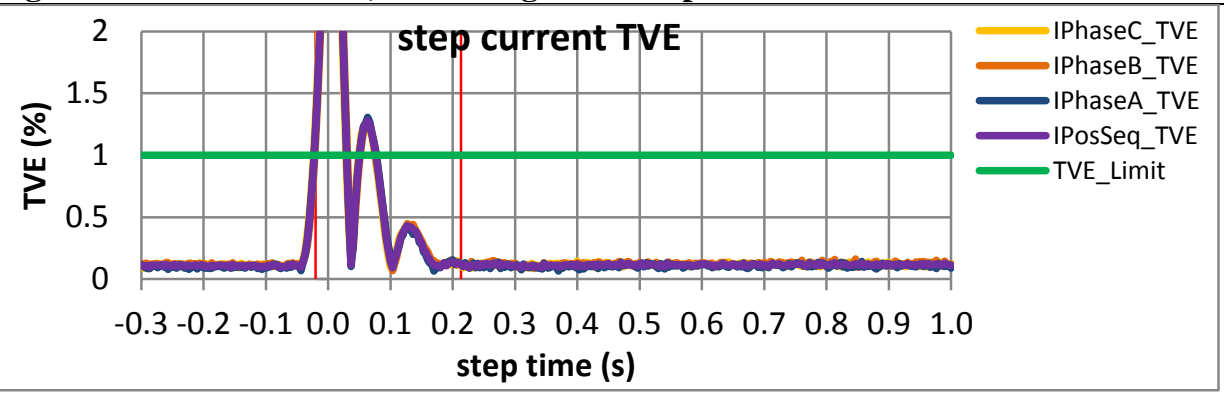

Figure 5248: Fs = 30 FPS, -10\% magnitude step

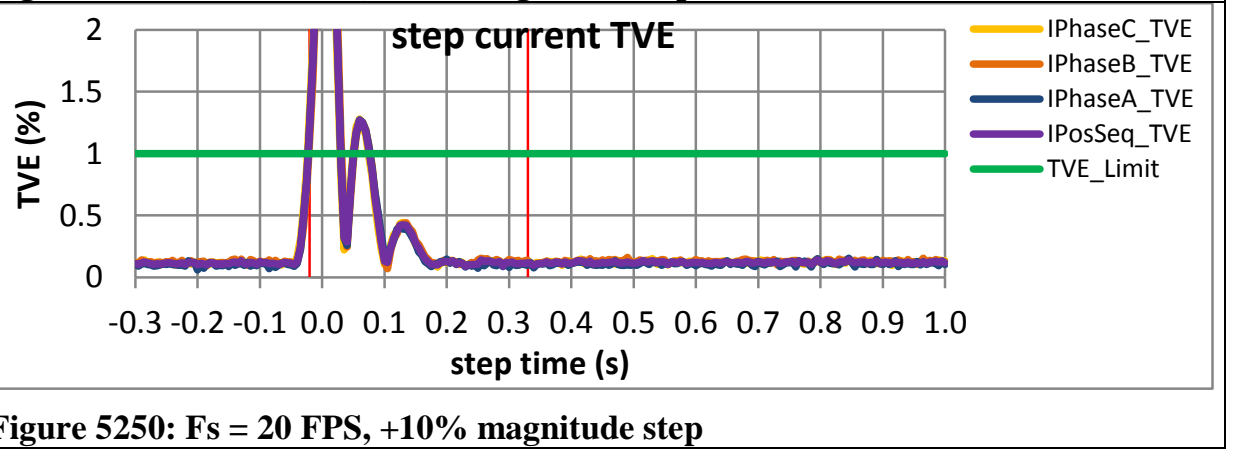




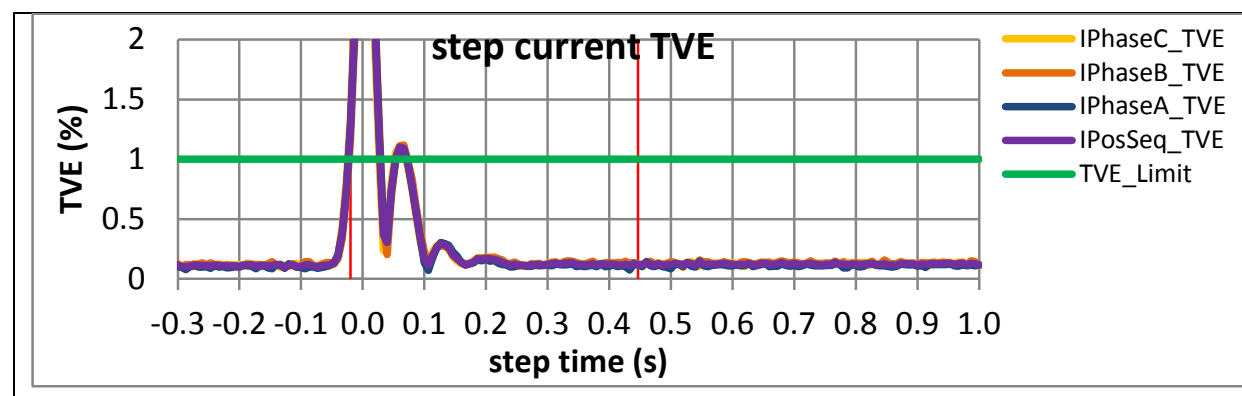

Figure 5251: Fs = 15 FPS, +10\% magnitude step
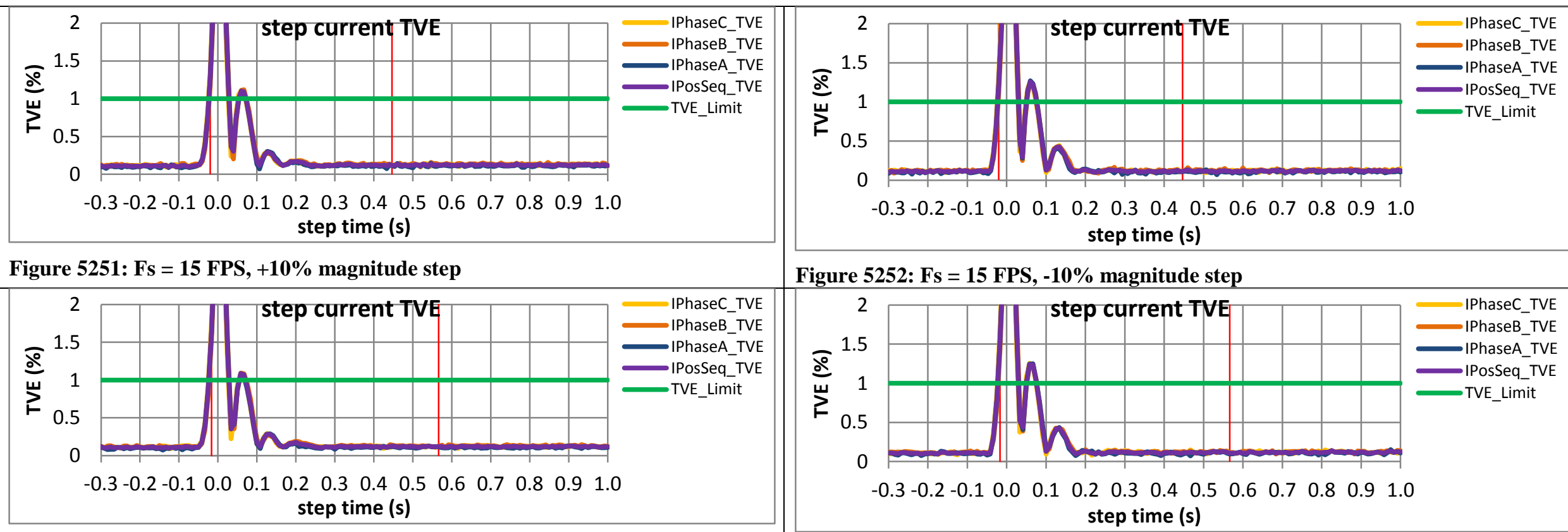

Figure 5252: Fs = 15 FPS, -10\% magnitude step

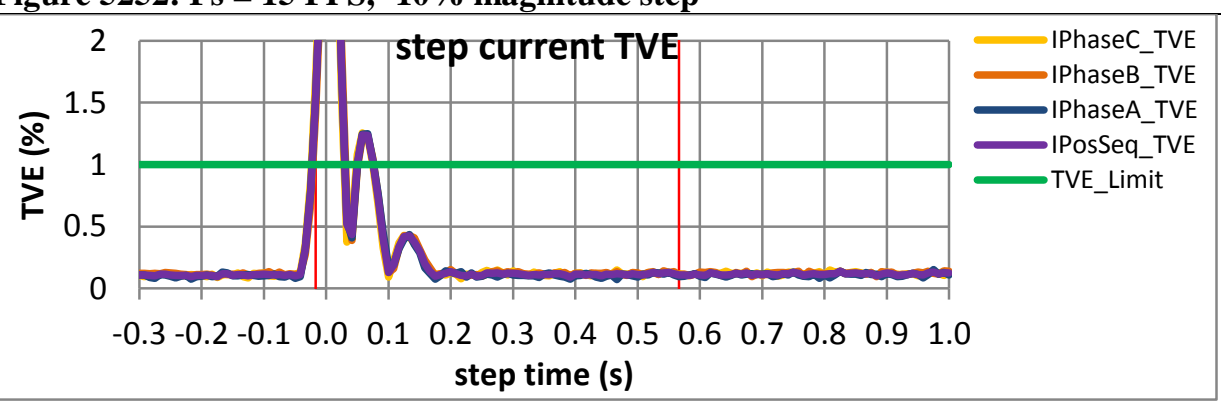

Figure 5253: Fs = 12 FPS, +10\% magnitude step

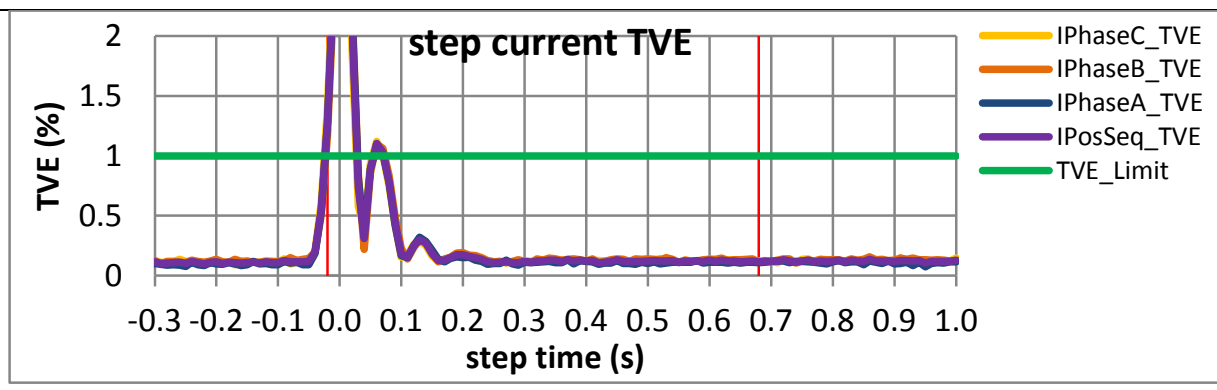

Figure 5254: Fs = 12 FPS, -10\% magnitude step

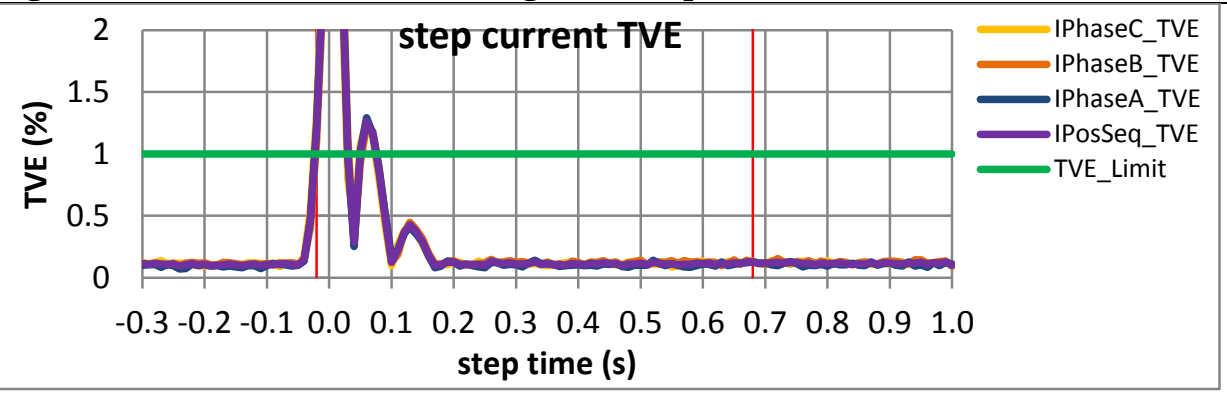

Figure 5255: Fs = 10 FPS, +10\% magnitude step

Figure 5256: Fs = 10 FPS, -10\% magnitude step 
10.3.4 PMU C dynamic step change in magnitude current response time: $F 0=60 \mathrm{~Hz}, \mathrm{M}$ class

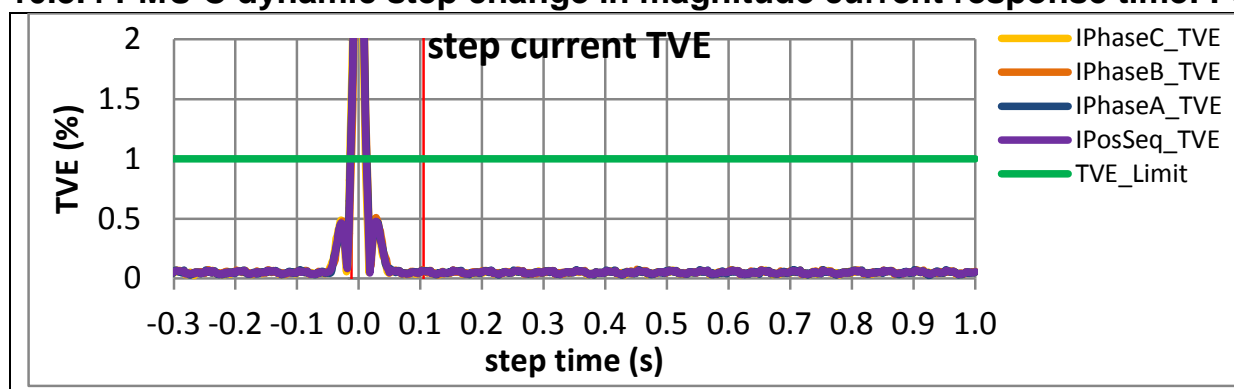

Figure 5257: Fs = 60 FPS, +10\% magnitude step

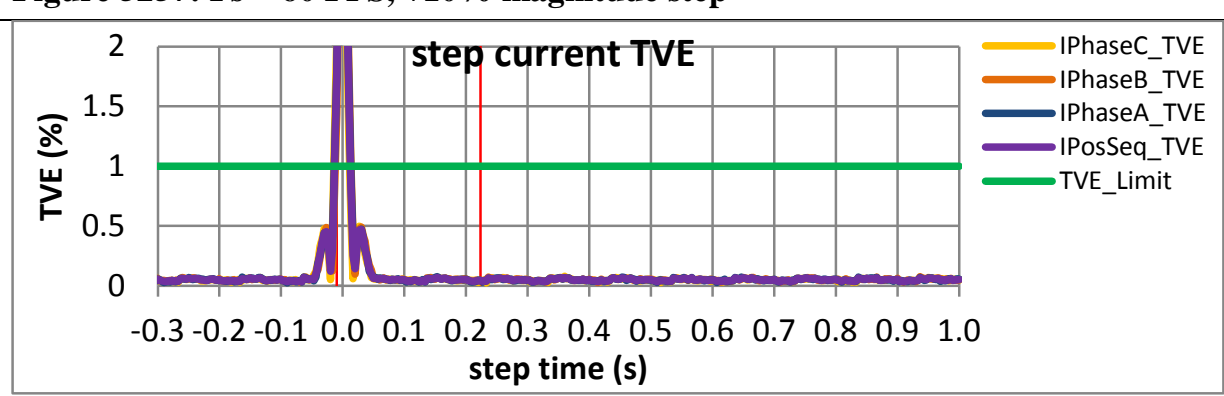

Figure 5259: Fs = 30 FPS, $+10 \%$ magnitude step

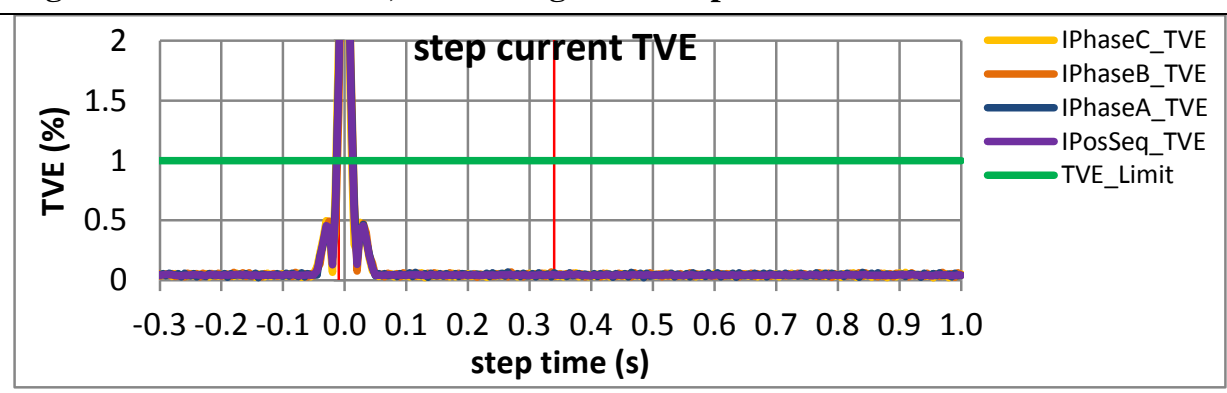

Figure 5261: Fs = 20 FPS, +10\% magnitude step

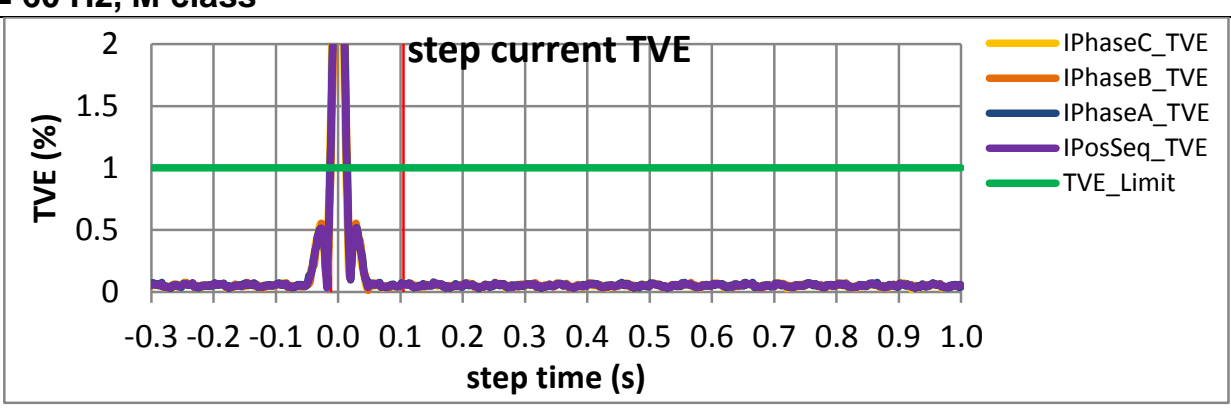

Figure 5258: Fs = 60 FPS, -10\% magnitude step

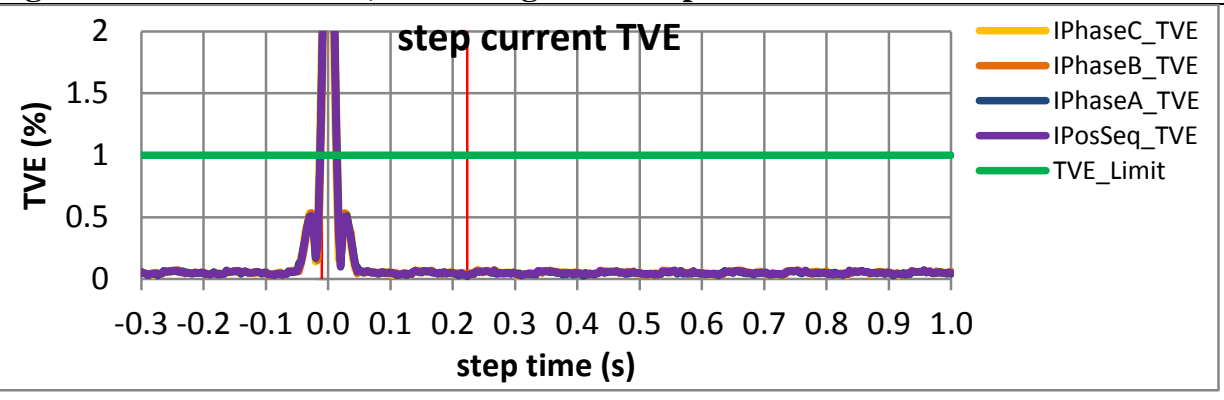

Figure 5260: Fs = 30 FPS, -10\% magnitude step

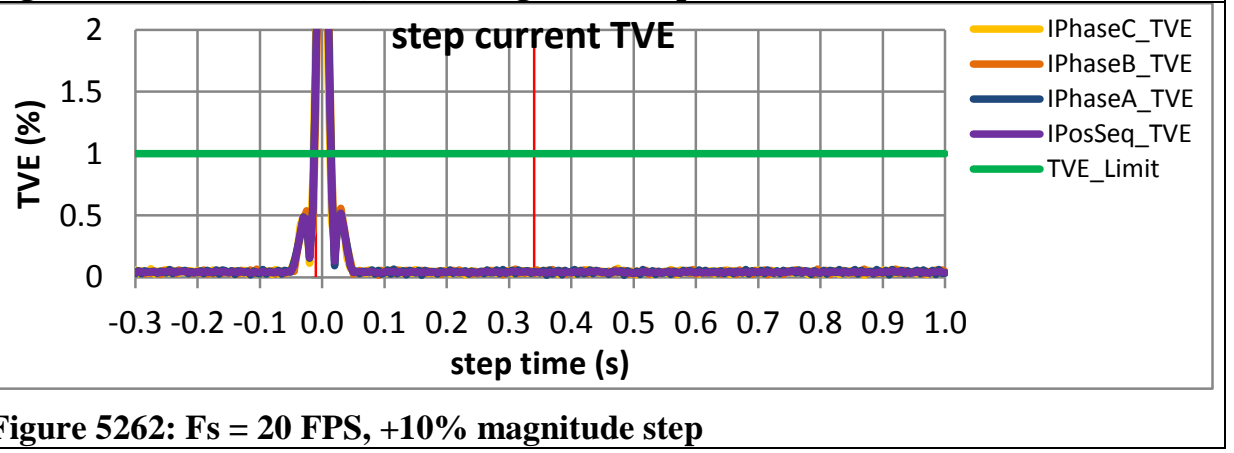




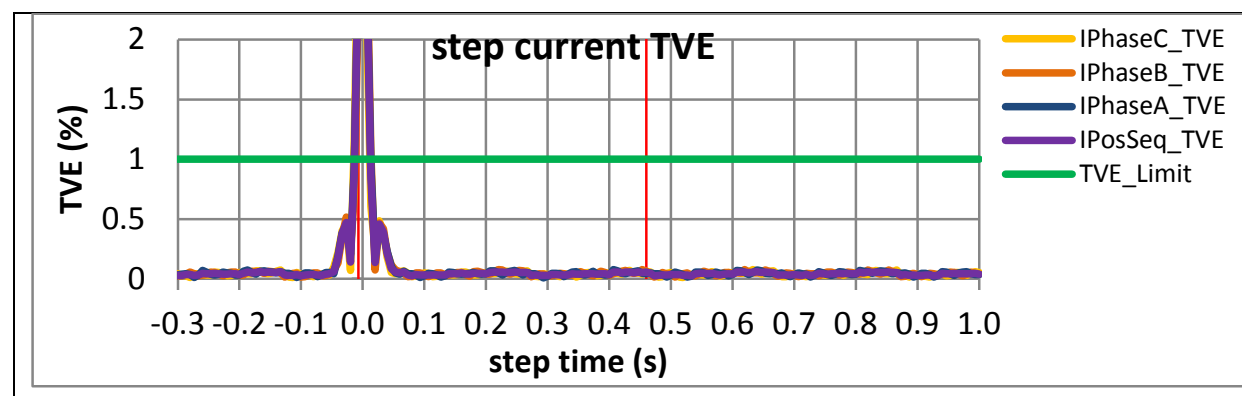

Figure 5263: Fs = 15 FPS, +10\% magnitude step
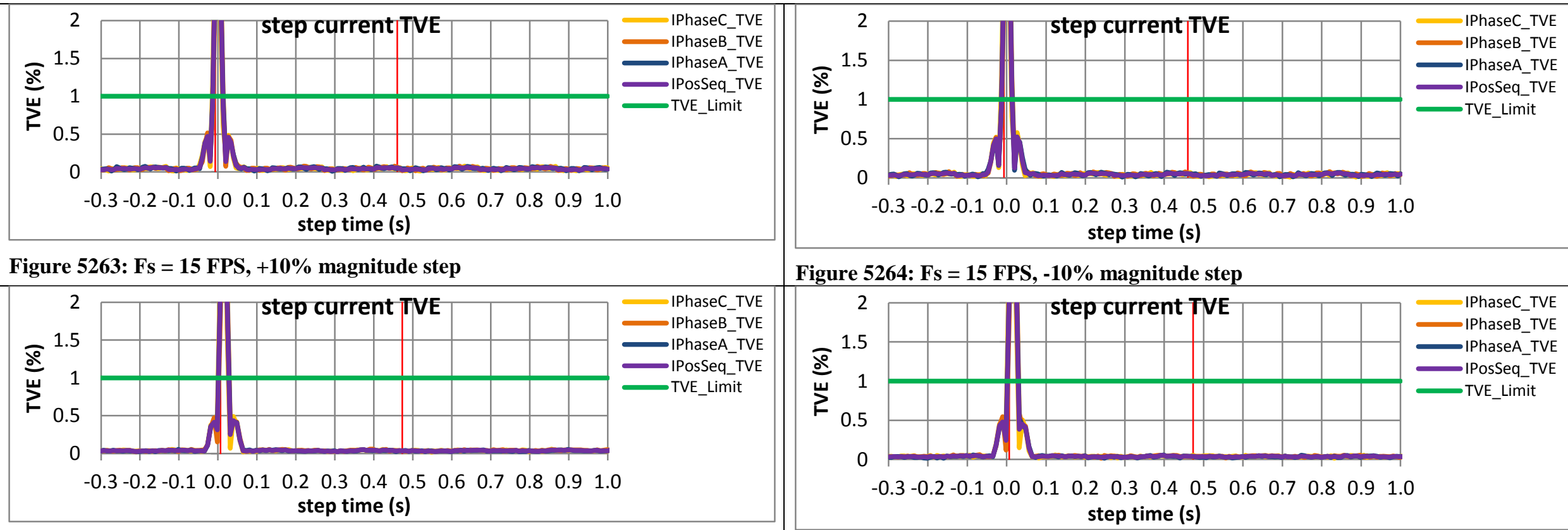

Figure 5264: Fs = 15 FPS, -10\% magnitude step

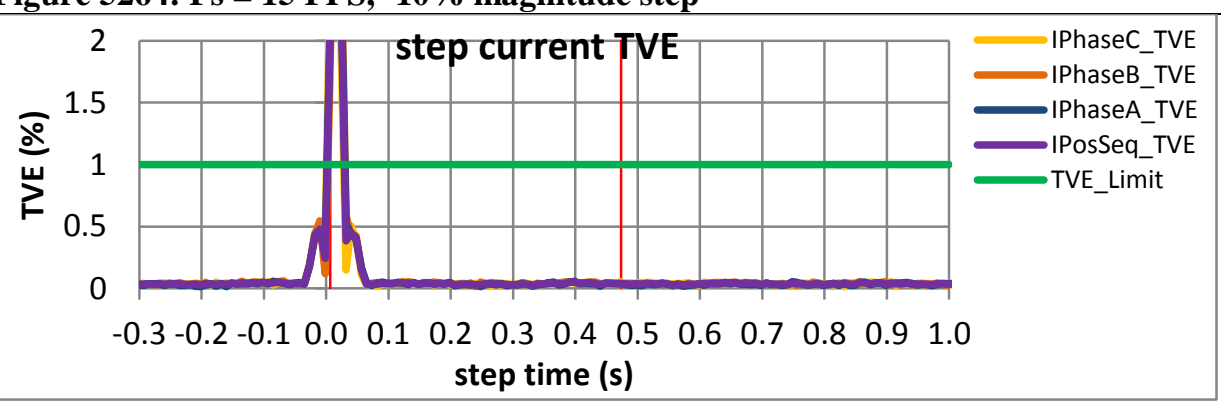

Figure 5265: Fs $=12$ FPS, $+10 \%$ magnitude step

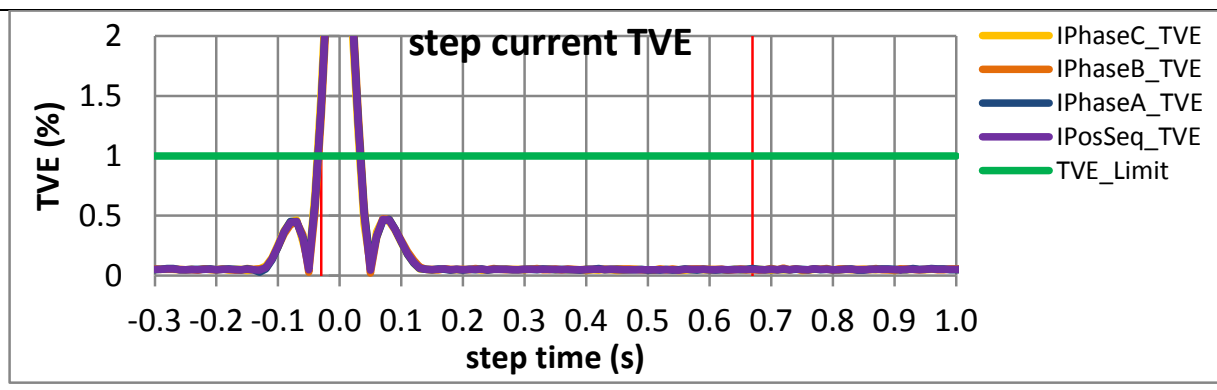

Figure 5266: Fs = 12 FPS, -10\% magnitude step

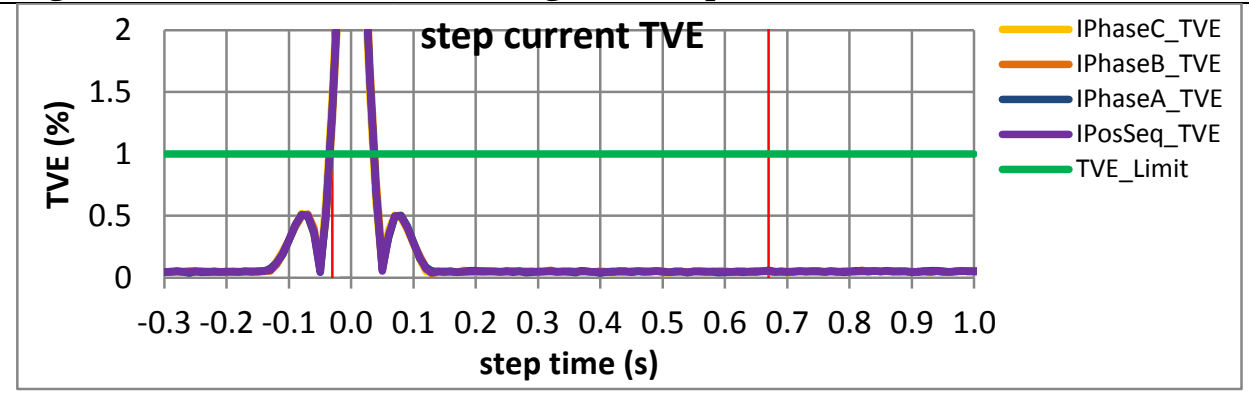

Figure 5267: Fs = 10 FPS, +10\% magnitude step

Figure 5268: Fs = 10 FPS, -10\% magnitude step 
10.3.5 PMU D dynamic step change in magnitude current response time: $F 0=60 \mathrm{~Hz}, \mathrm{M}$ class
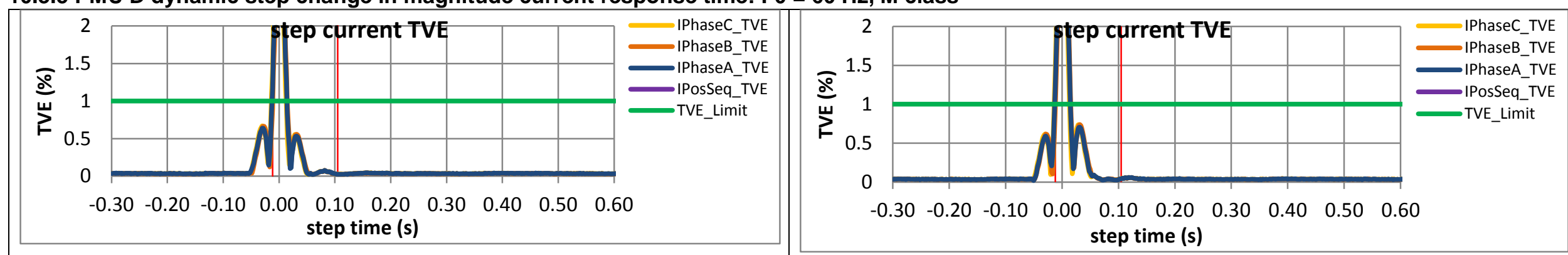

Figure 5269: Fs = 60 FPS, +10\% magnitude step

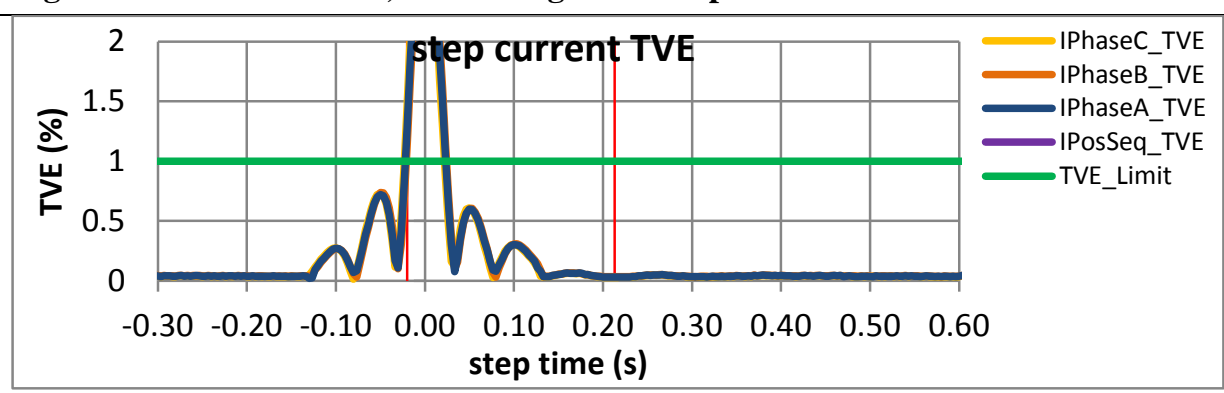

Figure 5270: Fs = 60 FPS, -10\% magnitude step

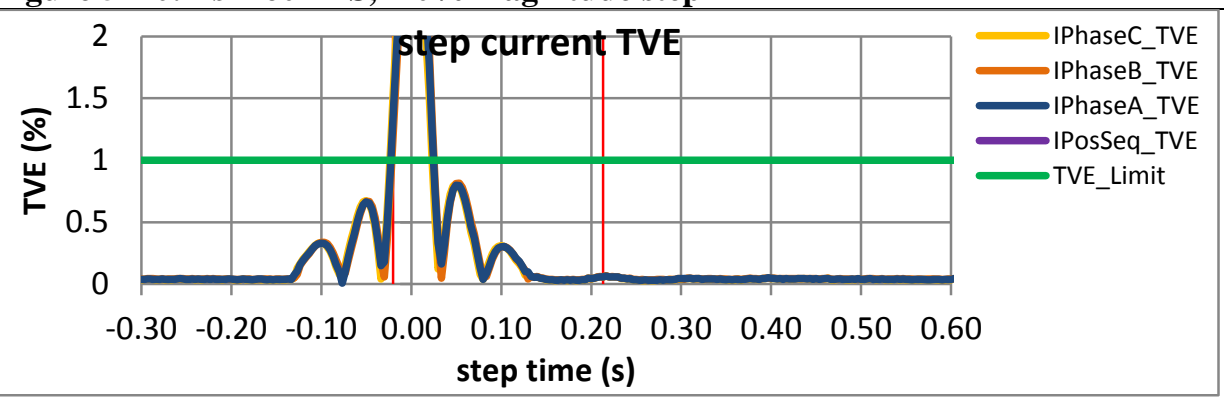

Figure 5271: Fs = 30 FPS, +10\% magnitude step

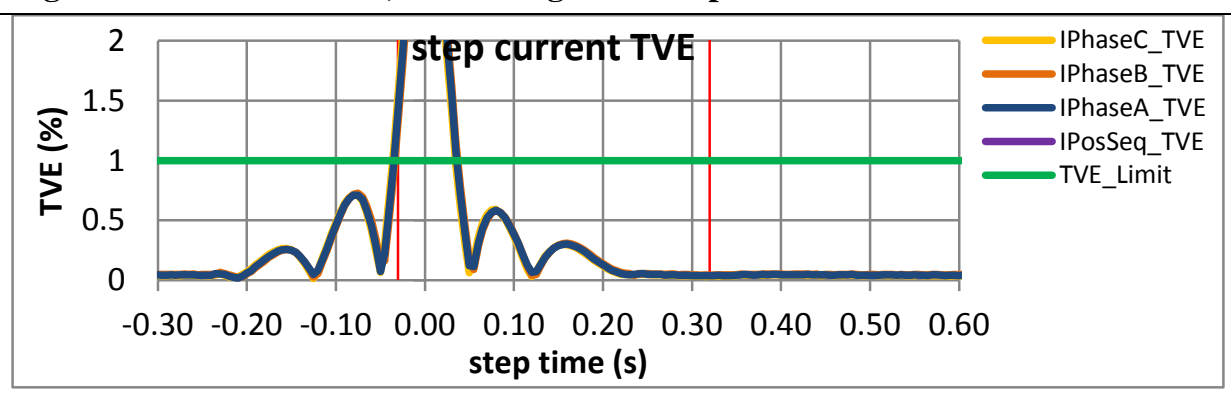

Figure 5273: Fs = 20 FPS, +10\% magnitude step

Figure 5272: Fs = 30 FPS, -10\% magnitude step

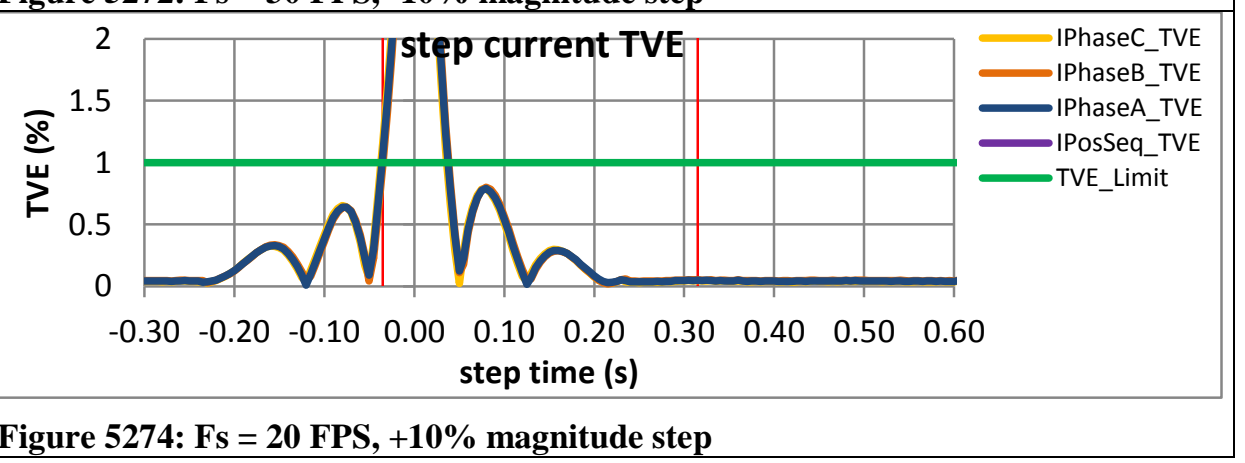




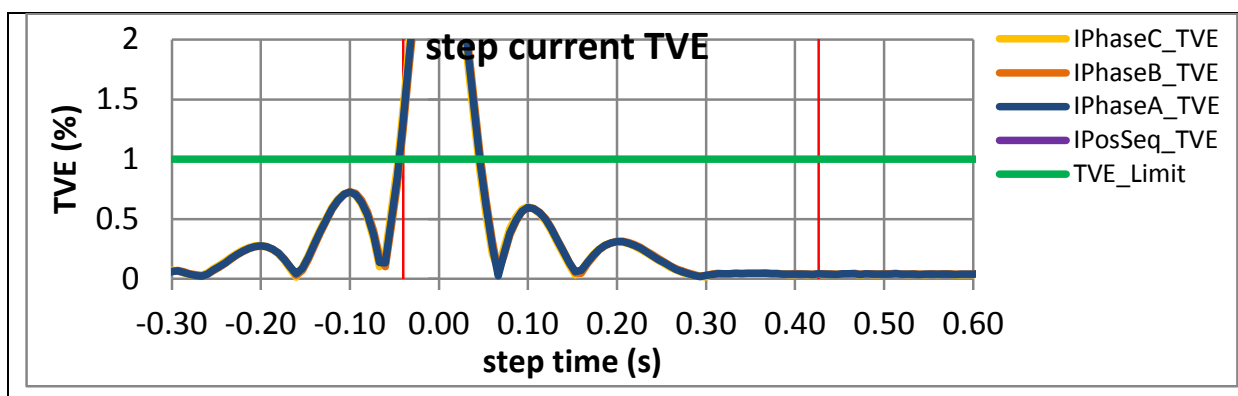

Figure 5275: Fs = 15 FPS, +10\% magnitude step
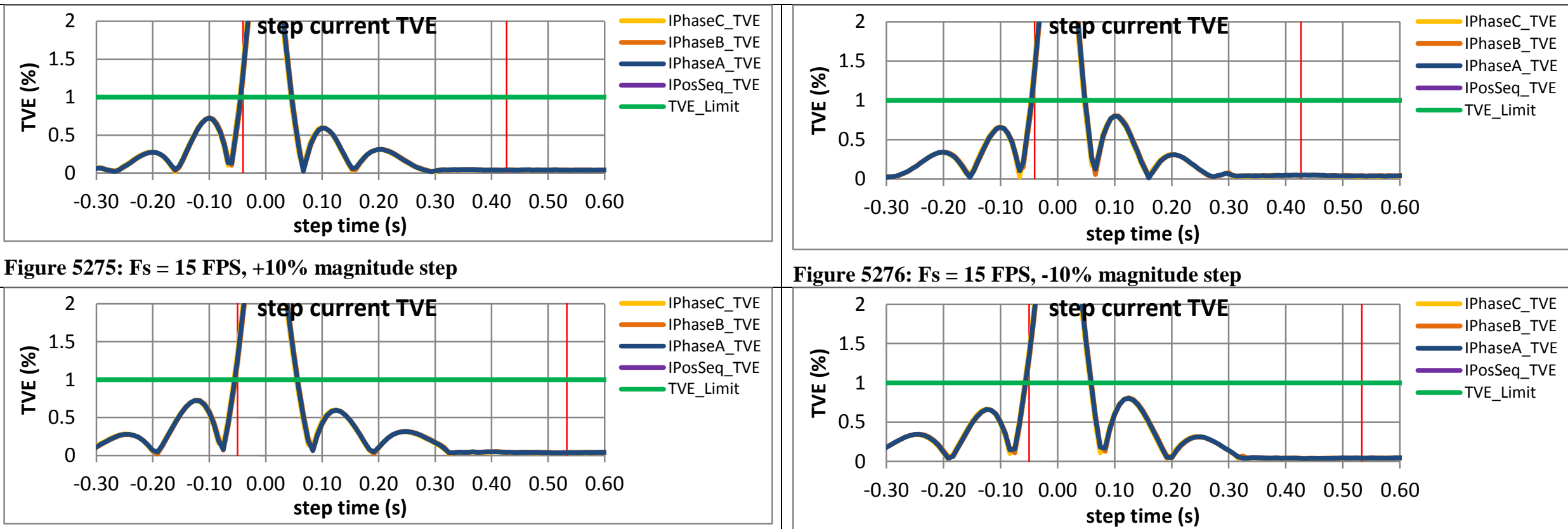

Figure 5276: Fs = 15 FPS, -10\% magnitude step

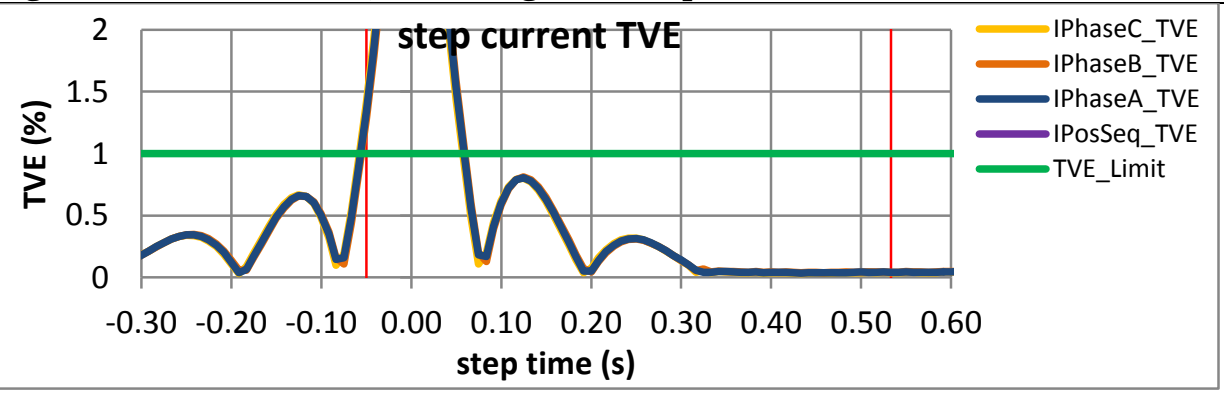

Figure 5277: Fs = 12 FPS, +10\% magnitude step

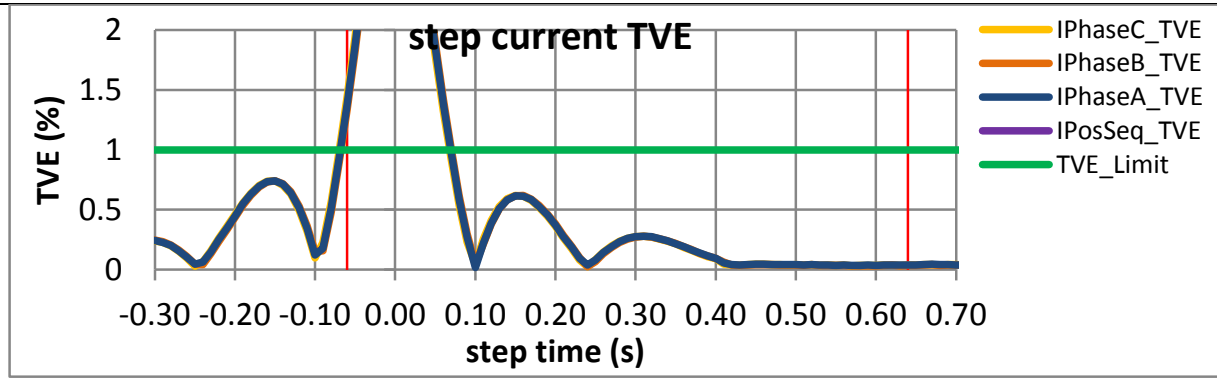

Figure 5278: Fs = 12 FPS, -10\% magnitude step

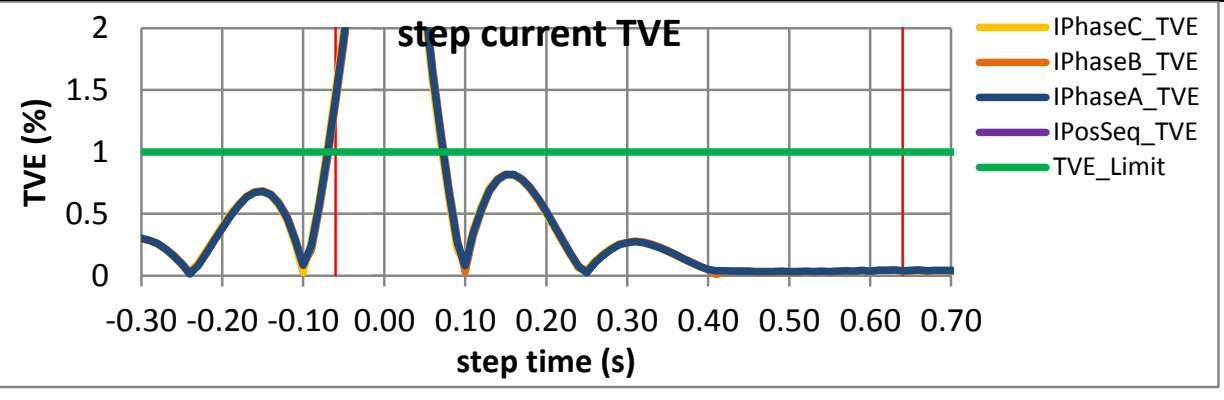

Figure 5279: Fs $=10$ FPS, $+10 \%$ magnitude step

Figure 5280: Fs = 10 FPS, -10\% magnitude step 
10.3.6 PMU E dynamic step change in magnitude current response time: $\mathrm{FO}=60 \mathrm{~Hz}, \mathrm{M}$ class
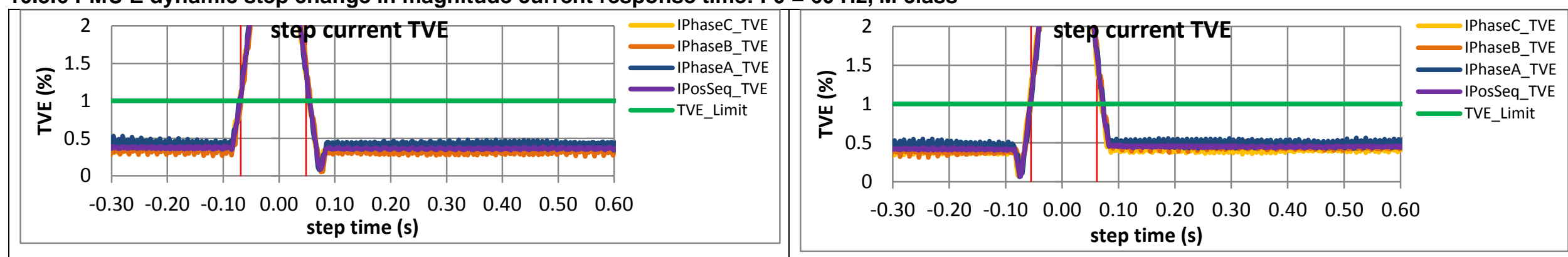

Figure 5281: Fs = 60 FPS, +10\% magnitude step

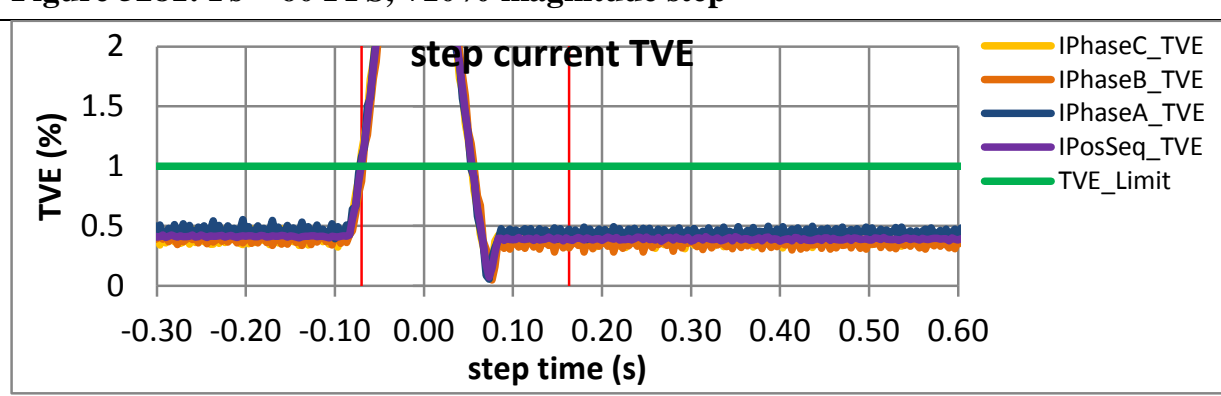

Figure 5282: Fs = 60 FPS, -10\% magnitude step

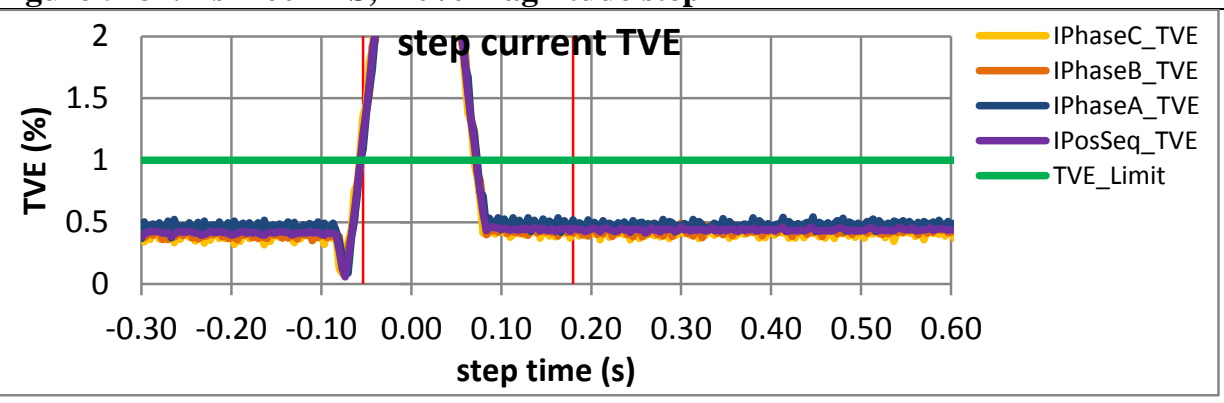

Figure 5283: Fs = 30 FPS, $+10 \%$ magnitude step

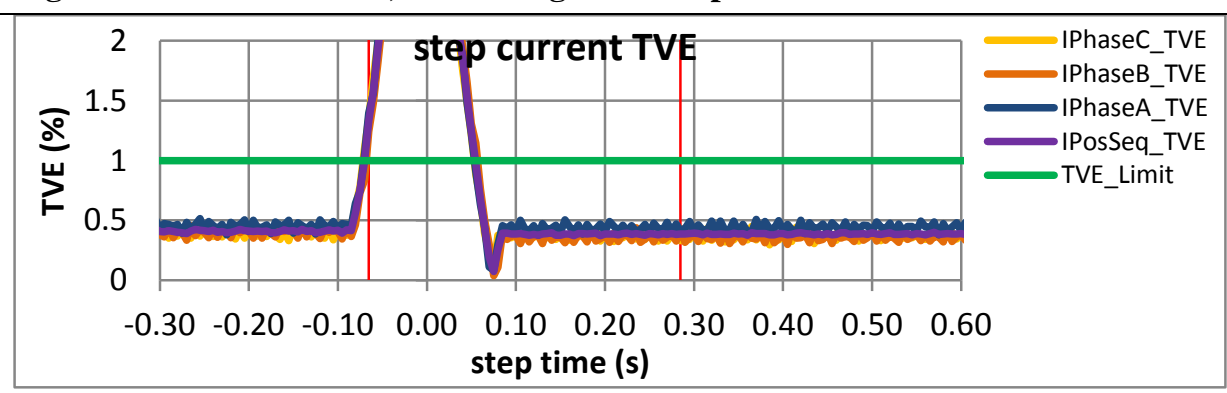

Figure 5284: Fs = 30 FPS, -10\% magnitude step

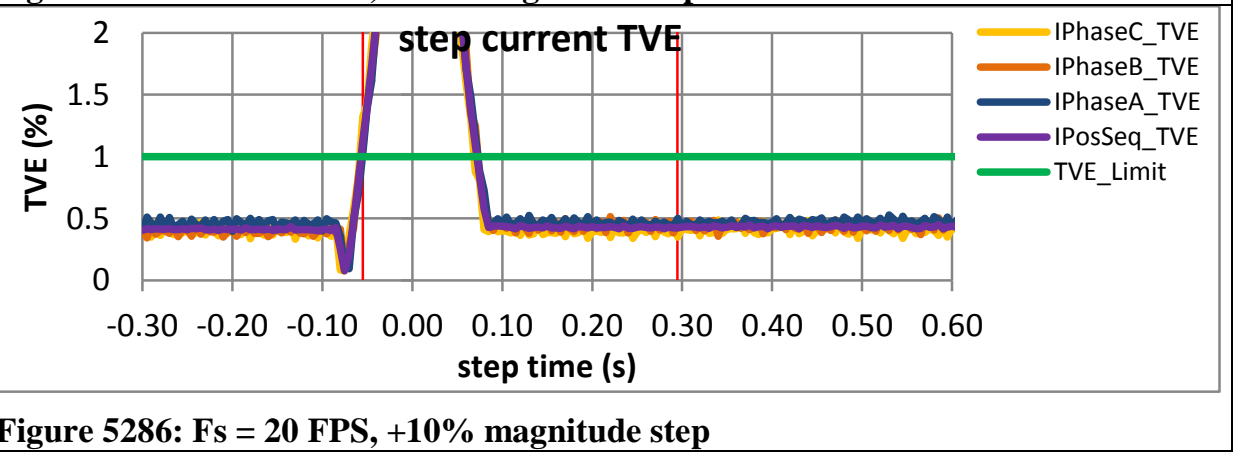

Figure 5285: Fs = 20 FPS, +10\% magnitude step

Figure 5286: Fs $=20$ FPS, $+10 \%$ magnitude step 


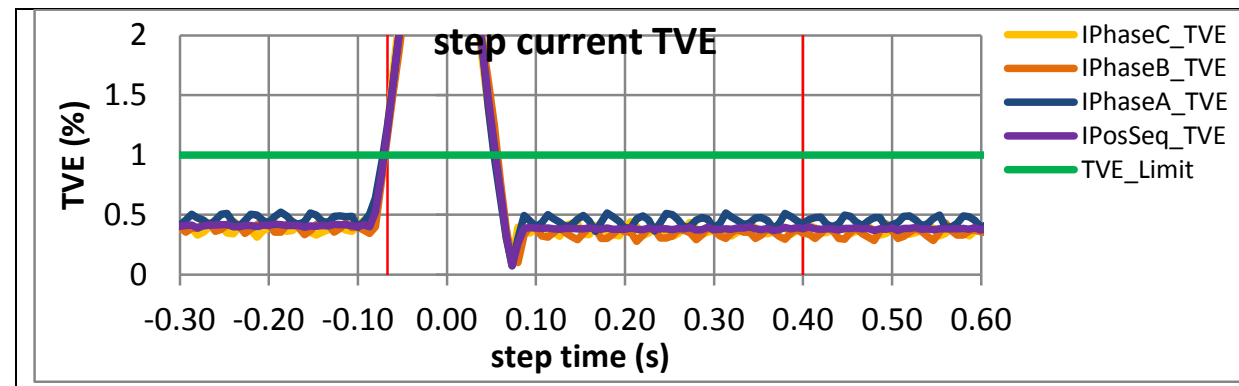

Figure 5287: Fs = 15 FPS, $+10 \%$ magnitude step

Figure 5289: Fs $=12$ FPS, $+10 \%$ magnitude step

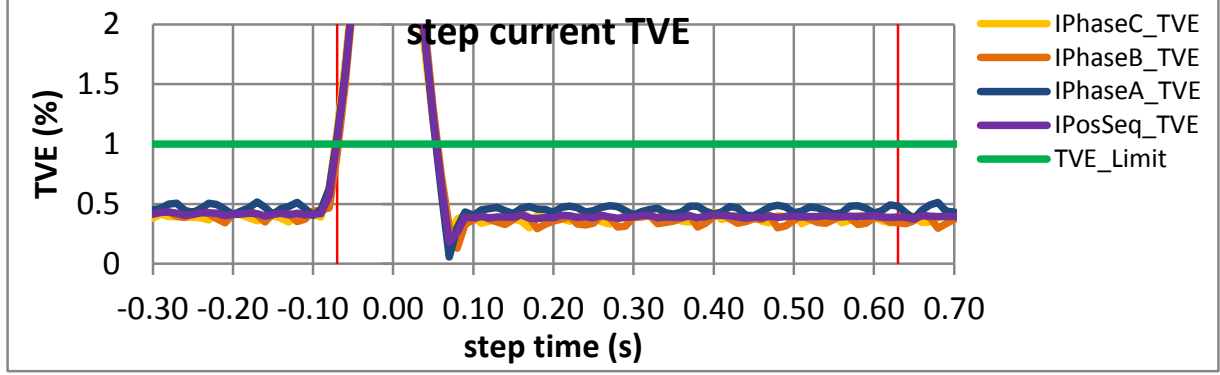

Figure 5291: Fs = 10 FPS, +10\% magnitude step

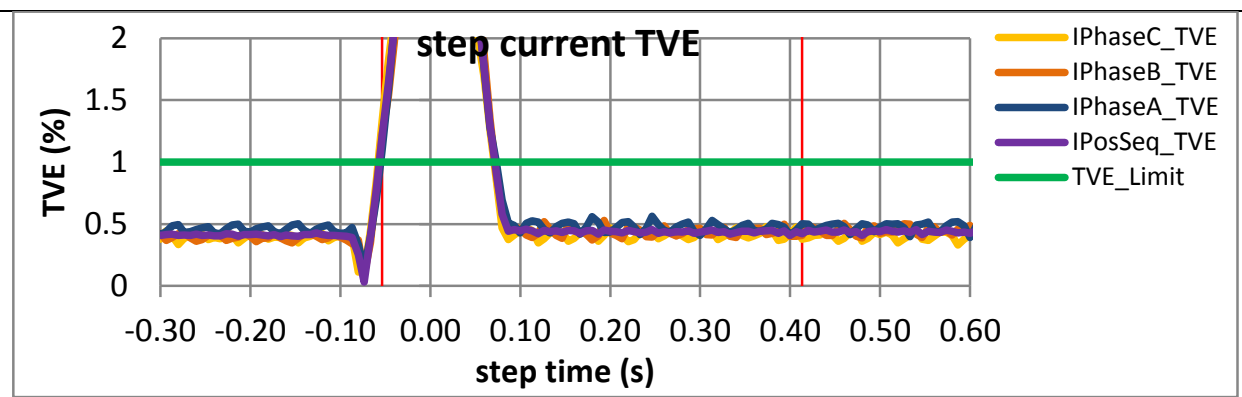

Figure 5288: Fs = 15 FPS, -10\% magnitude step

Figure 5290: Fs = 12 FPS, -10\% magnitude step

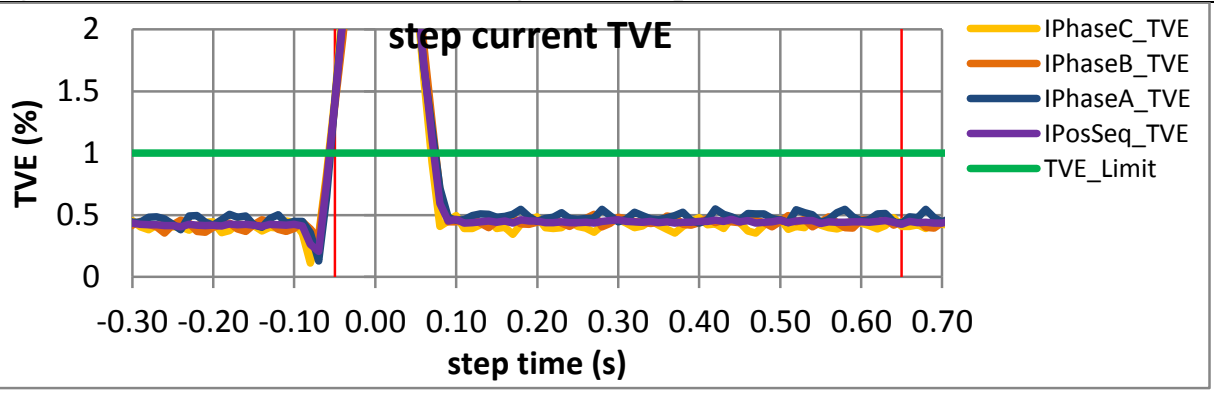

Figure 5292: Fs = 10 FPS, - $10 \%$ magnitude step 
10.3.7 PMU F dynamic step change in magnitude current response time: $F 0=60 \mathrm{~Hz}, \mathrm{M}$ class
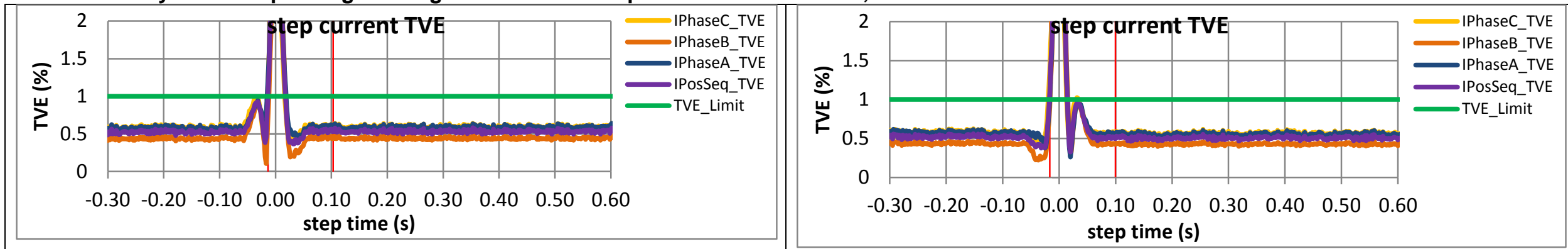

Figure 5293: Fs $=60 \mathrm{FPS},+10 \%$ magnitude step

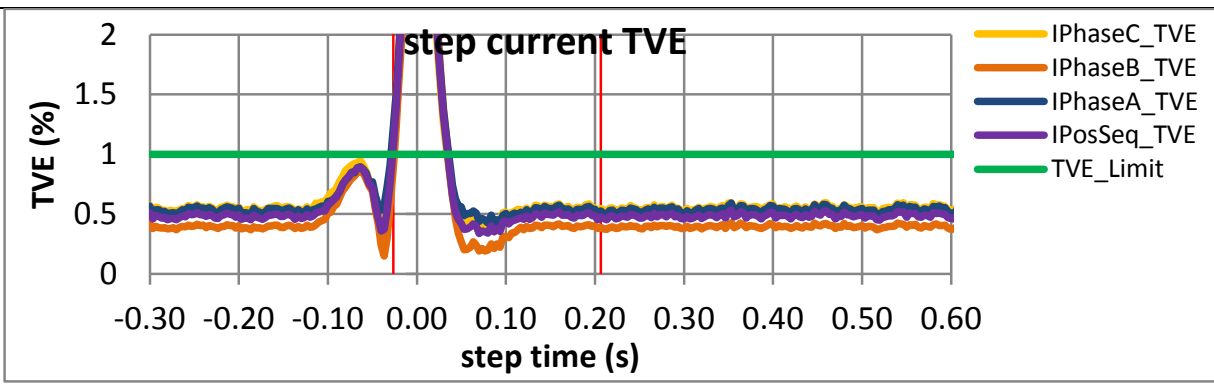

Figure 5294: Fs = 60 FPS, $-10 \%$ magnitude step

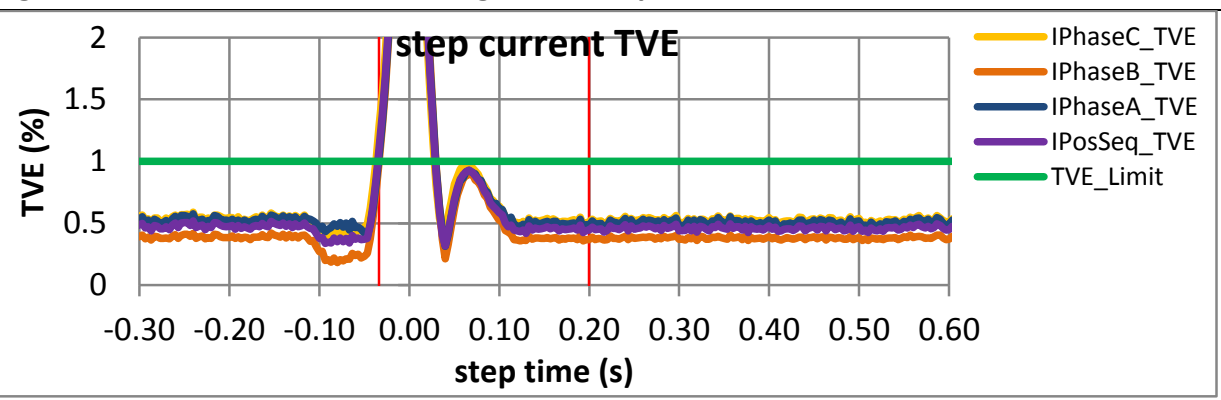

Figure 5295: Fs = 30 FPS, +10\% magnitude step

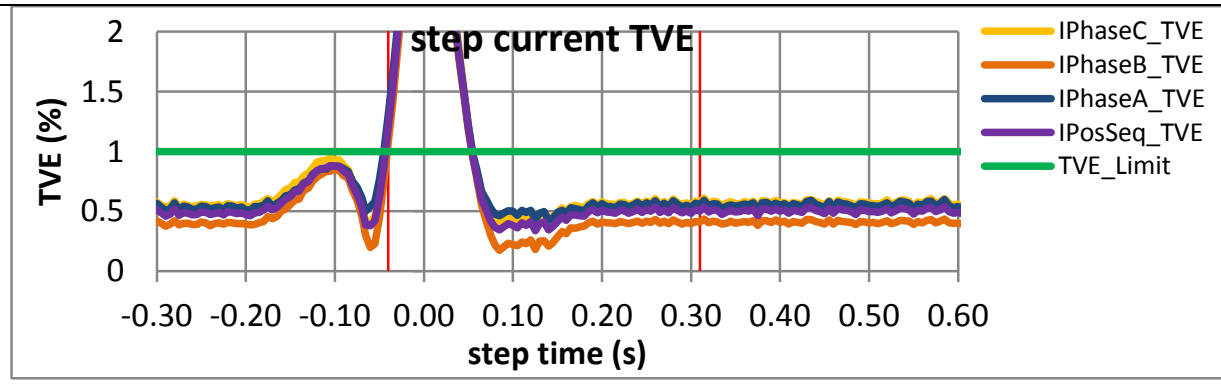

Figure 5296: Fs = 30 FPS, $-10 \%$ magnitude step

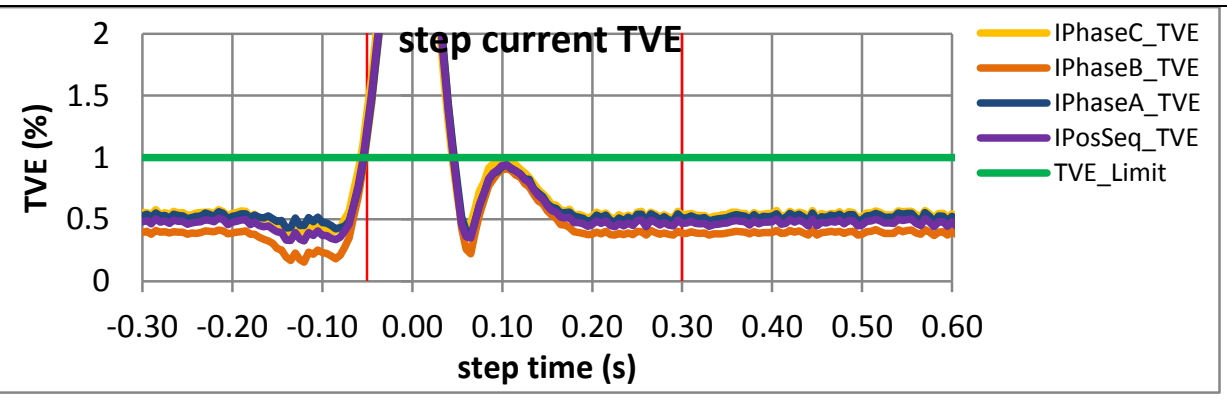

Figure 5298: Fs = 20 FPS, +10\% magnitude step 

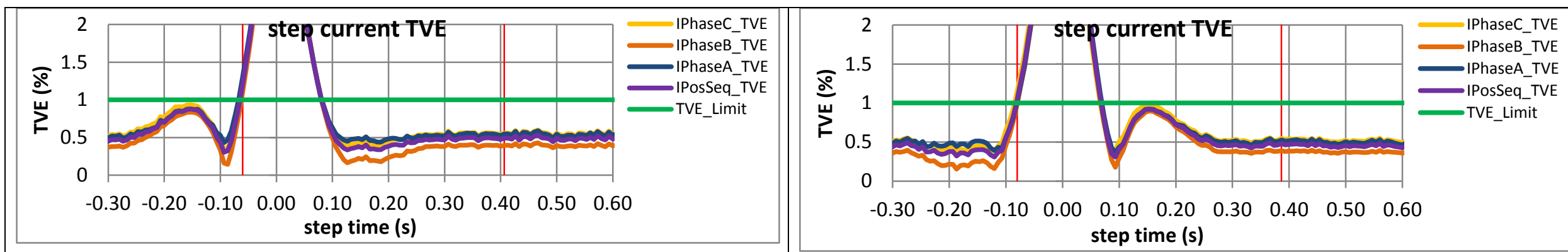

Figure 5299: Fs = $15 \mathrm{FPS},+10 \%$ magnitude step

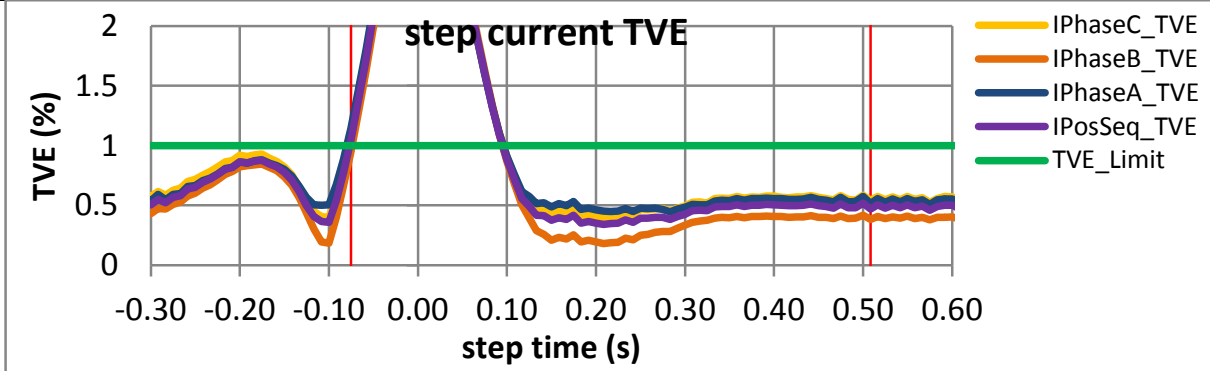

Figure 5301: Fs = 12 FPS, $+10 \%$ magnitude step

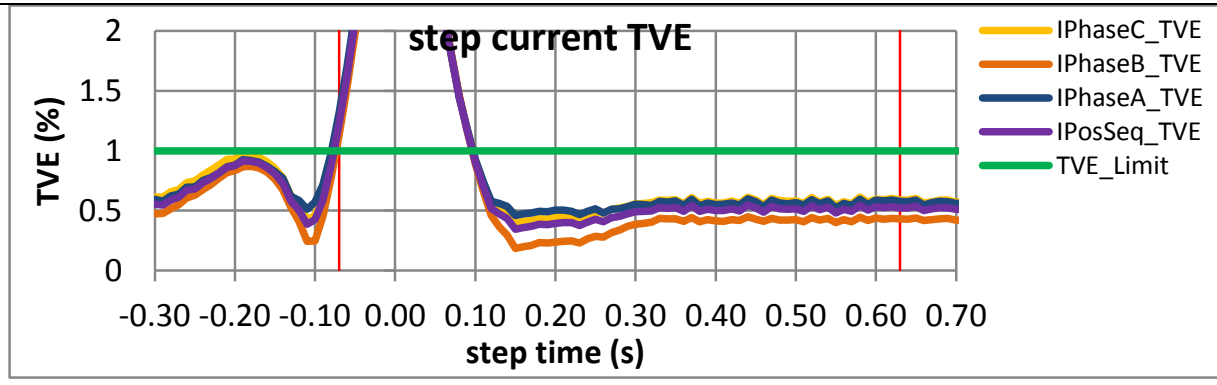

Figure 5303: Fs $=10 \mathrm{FPS},+10 \%$ magnitude step

\section{Figure 5300: Fs = $15 \mathrm{FPS},-10 \%$ magnitude step}

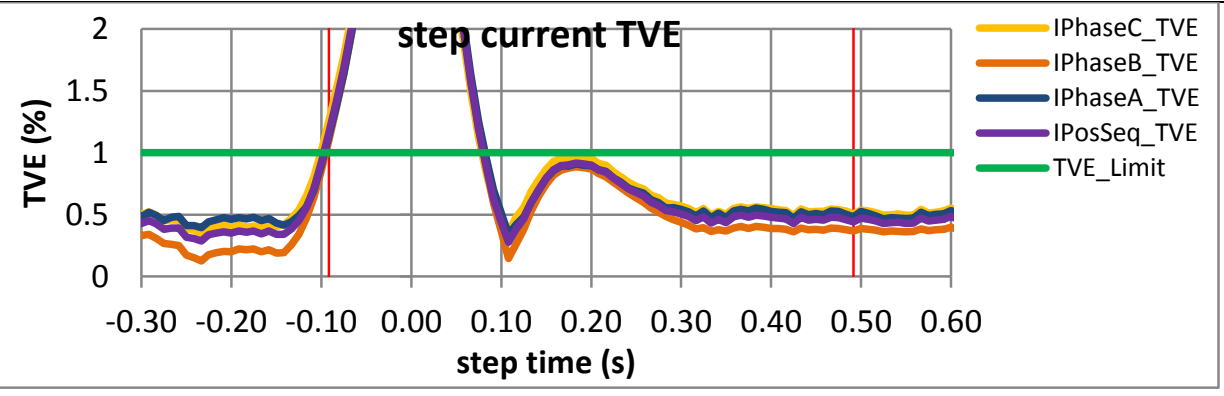

Figure 5302: Fs = 12 FPS, -10\% magnitude step

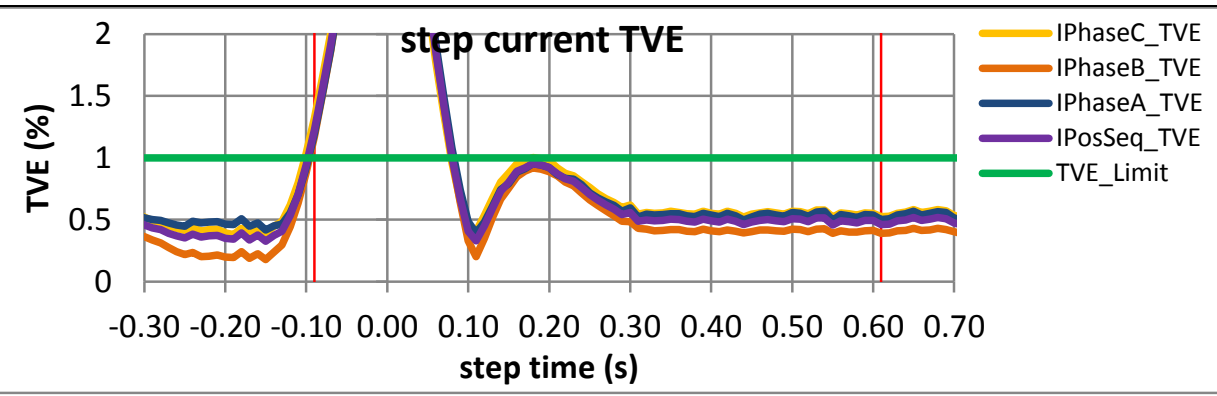

Figure 5304: Fs = $10 \mathrm{FPS},-10 \%$ magnitude step 
10.3.8 PMU G dynamic step change in magnitude current response time: $F 0=60 \mathrm{~Hz}, \mathrm{M}$ class

Figure 5305: Fs = 60 FPS is not supported by this PMU

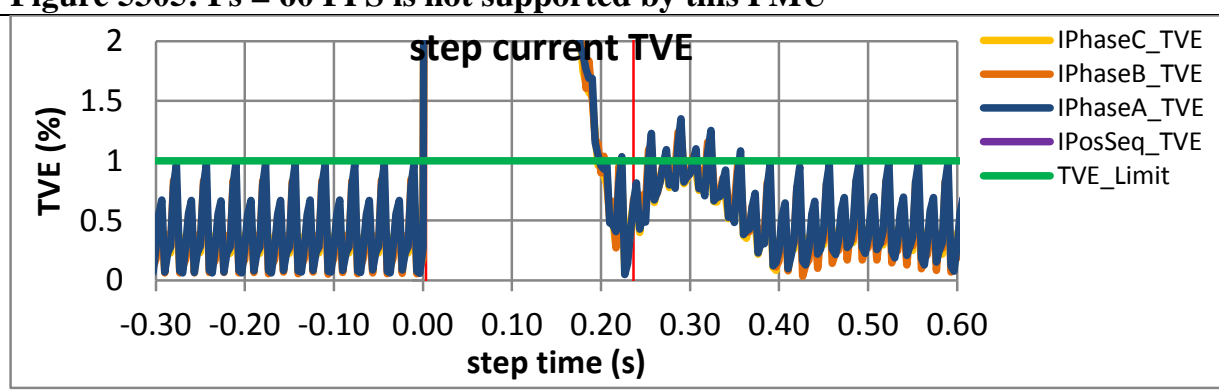

Figure 5307: Fs = 30 FPS, $+10 \%$ magnitude step

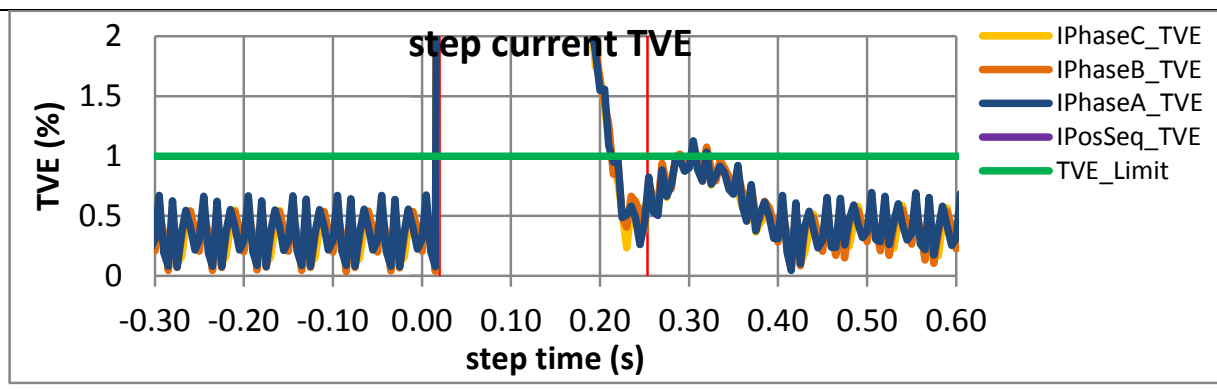

Figure 5309: Fs $=20$ FPS, $+10 \%$ magnitude step

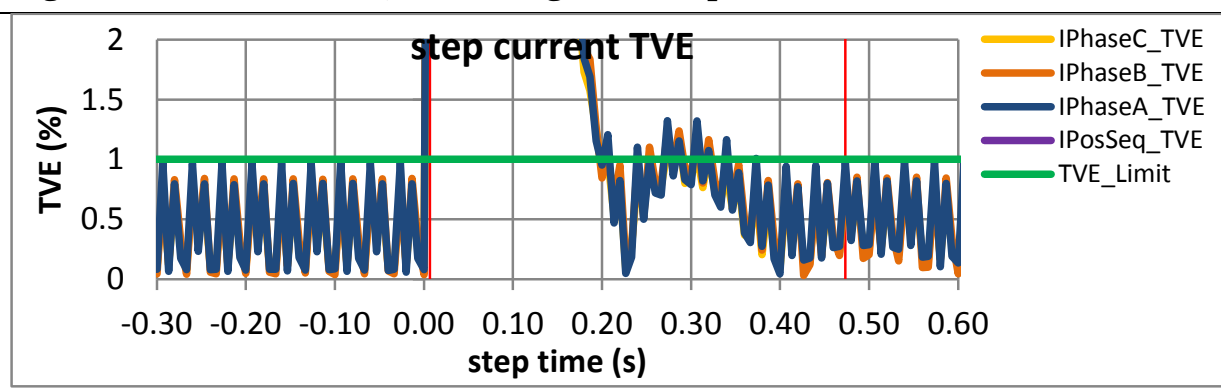

Figure 5311: Fs = 15 FPS, +10\% magnitude step
Figure 5306: Fs = 60 FPS is not supported by this PMU

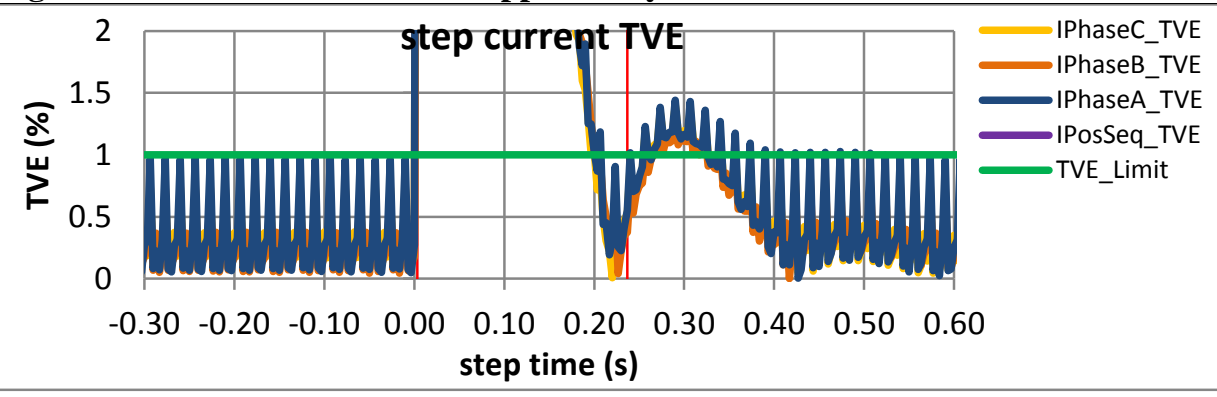

Figure 5308: Fs = 30 FPS, -10\% magnitude step

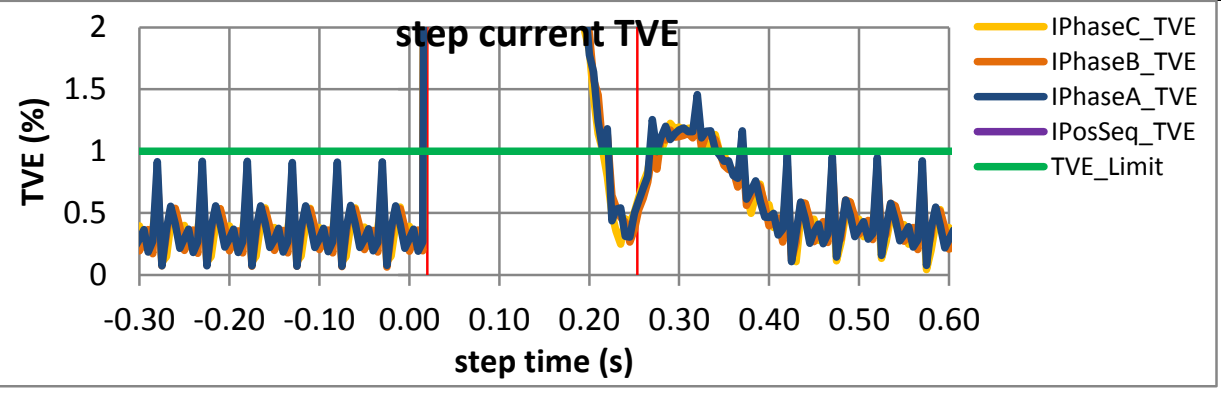

Figure 5310: Fs = 20 FPS, +10\% magnitude step

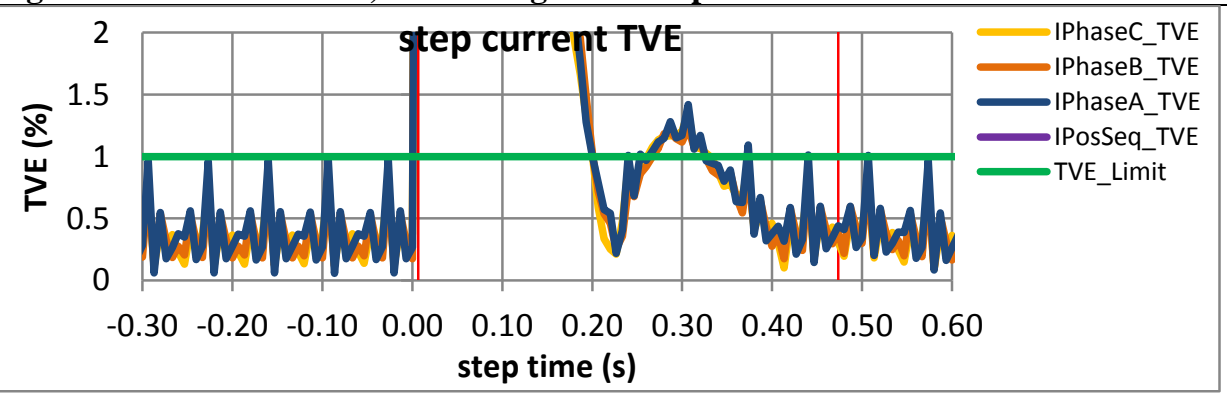

Figure 5312: Fs = 15 FPS, -10\% magnitude step 

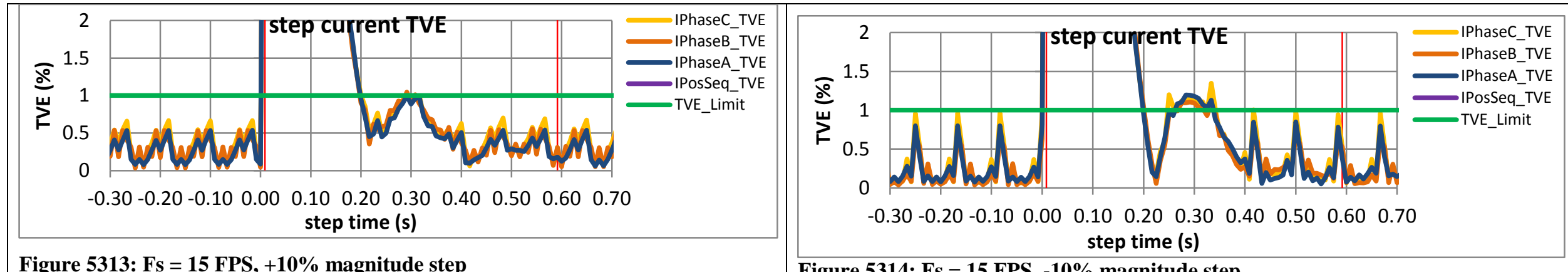

Figure 5313: Fs $=15$ FPS, +10\% magnitude step

\section{Figure 5314: Fs = 15 FPS, -10\% magnitude step}

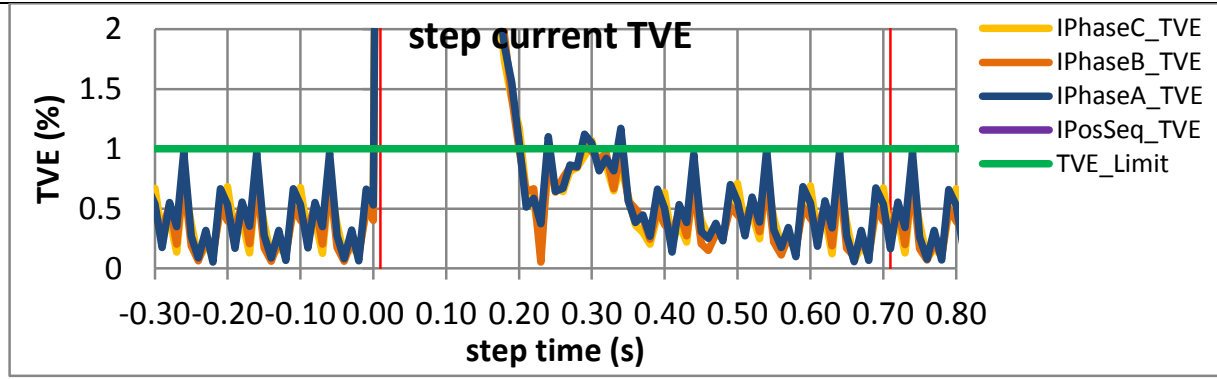

Figure 5315: Fs = 10 FPS, +10\% magnitude step

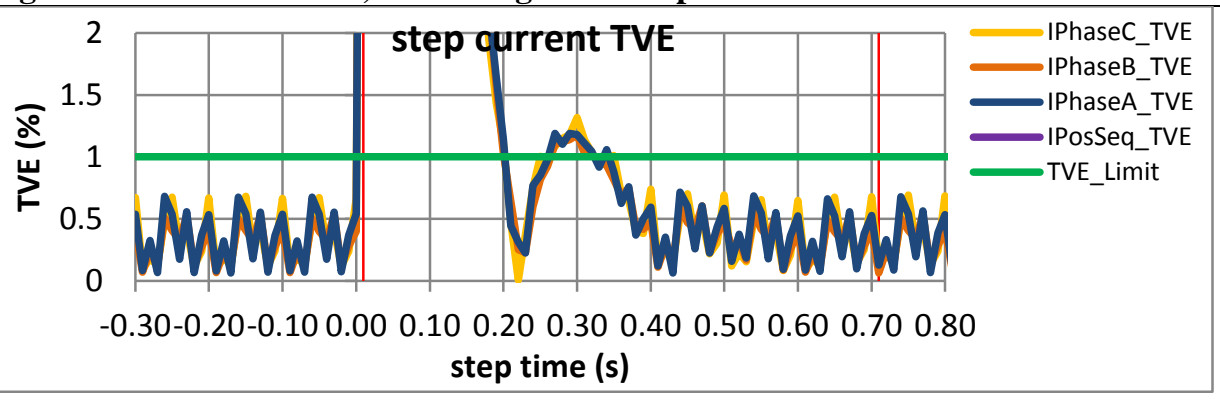

Figure 5316: Fs = 10 FPS, -10\% magnitude step 
10.3.9 PMU H dynamic step change in magnitude current response time: $\mathrm{F} 0=60 \mathrm{~Hz}, \mathrm{M}$ class

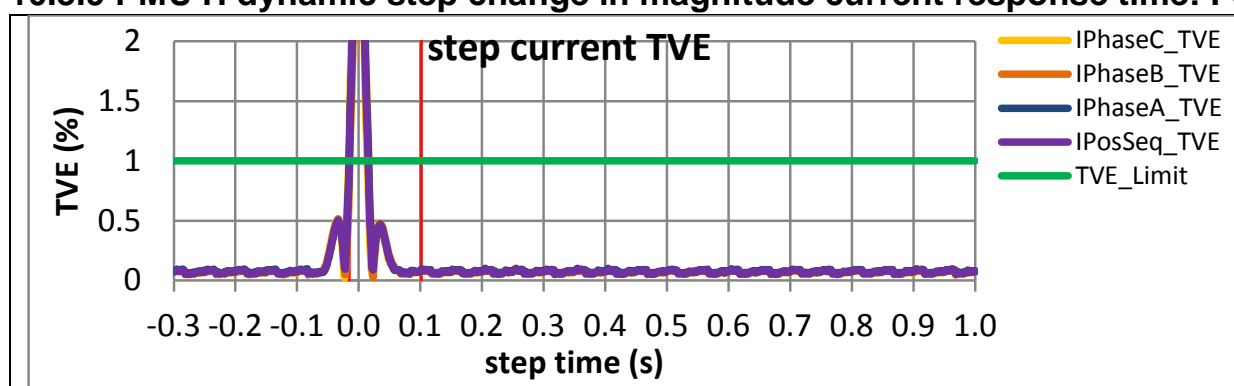

Figure 5317: Fs = 60 FPS, +10\% magnitude step

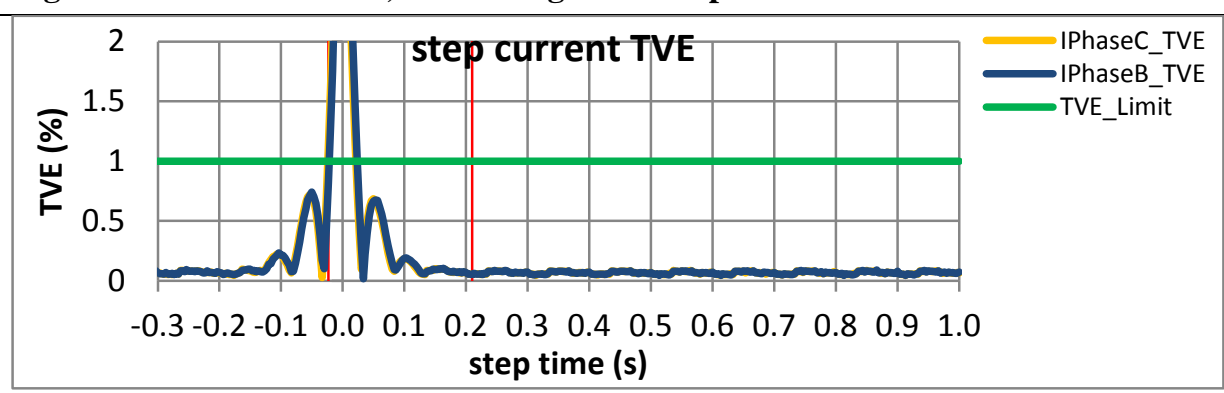

Figure 5319: Fs = 30 FPS, $+10 \%$ magnitude step

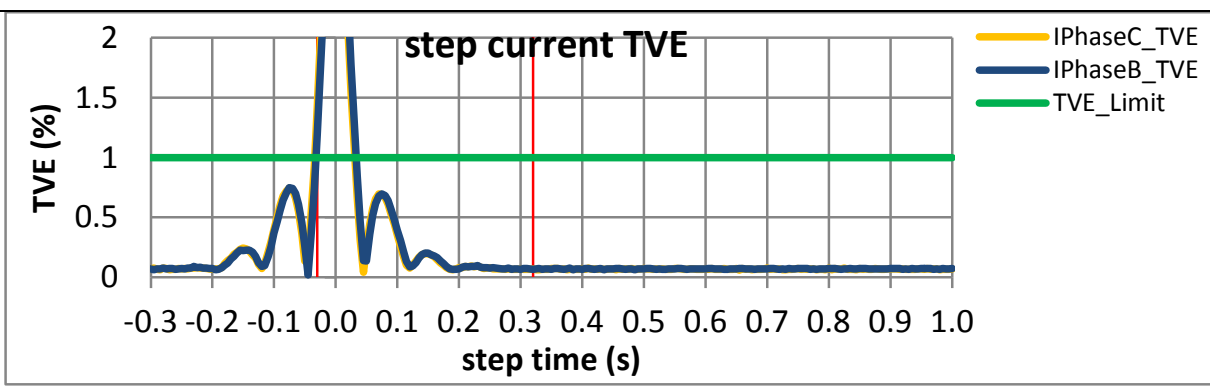

Figure 5321: Fs = 20 FPS, +10\% magnitude step

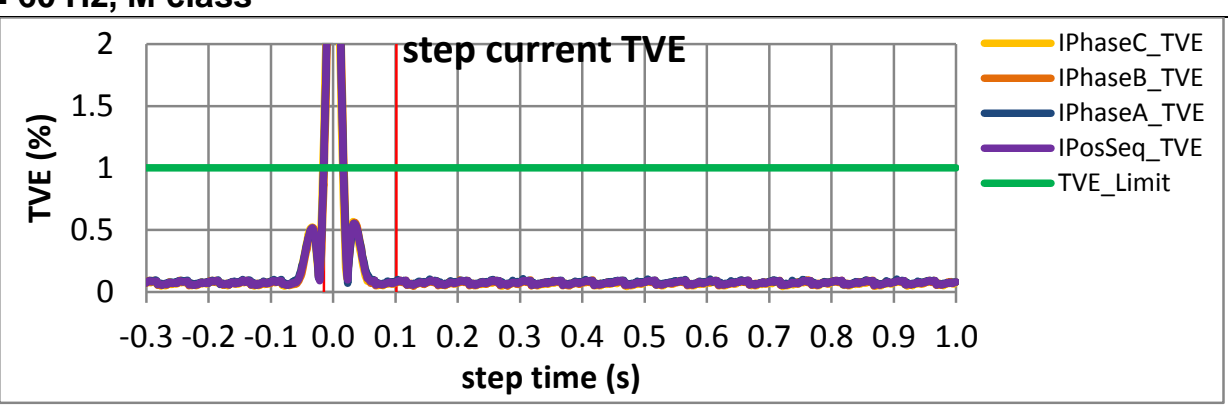

Figure 5318: Fs = 60 FPS, -10\% magnitude step

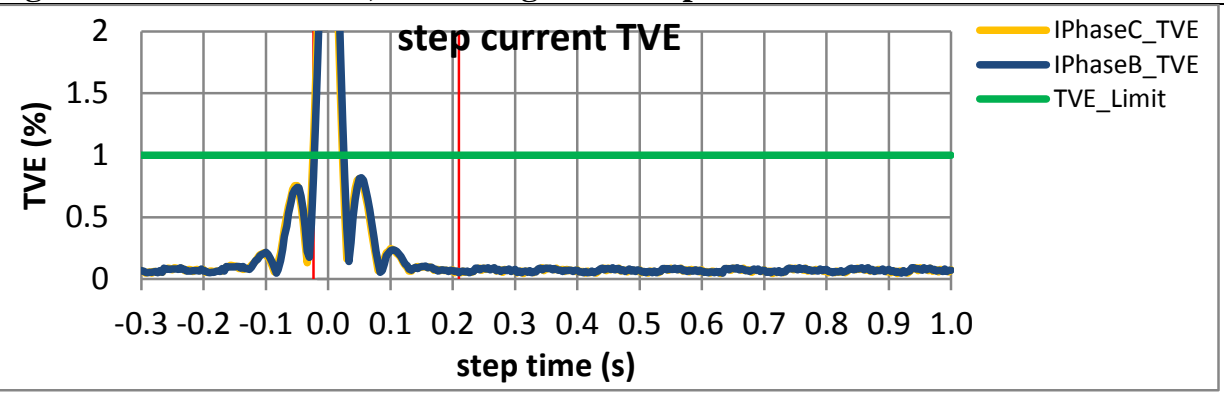

Figure 5320: Fs = 30 FPS, -10\% magnitude step

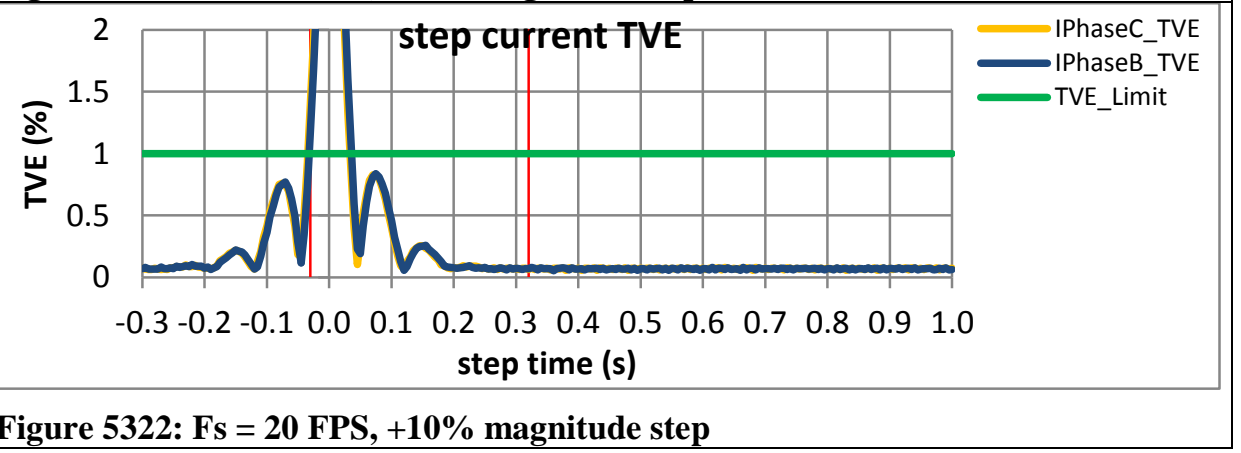




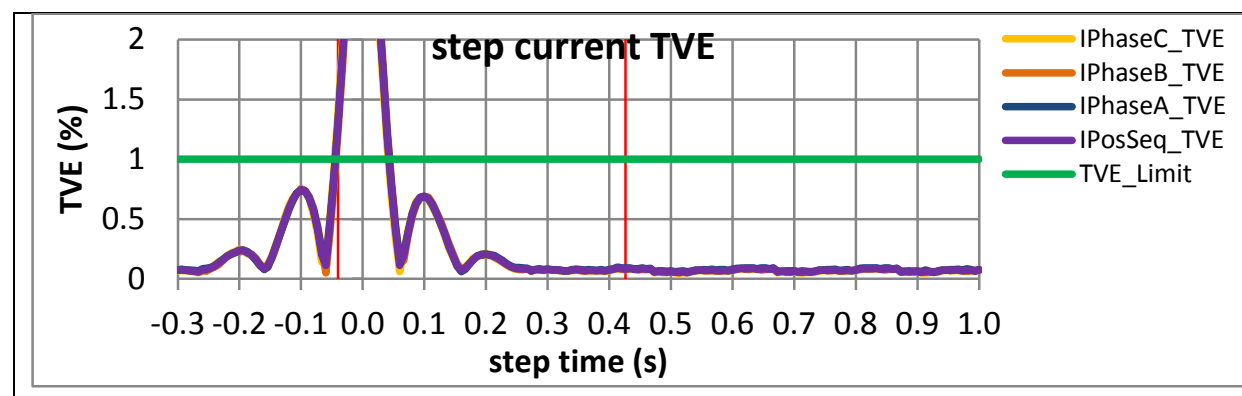

Figure 5323: Fs = 15 FPS, +10\% magnitude step
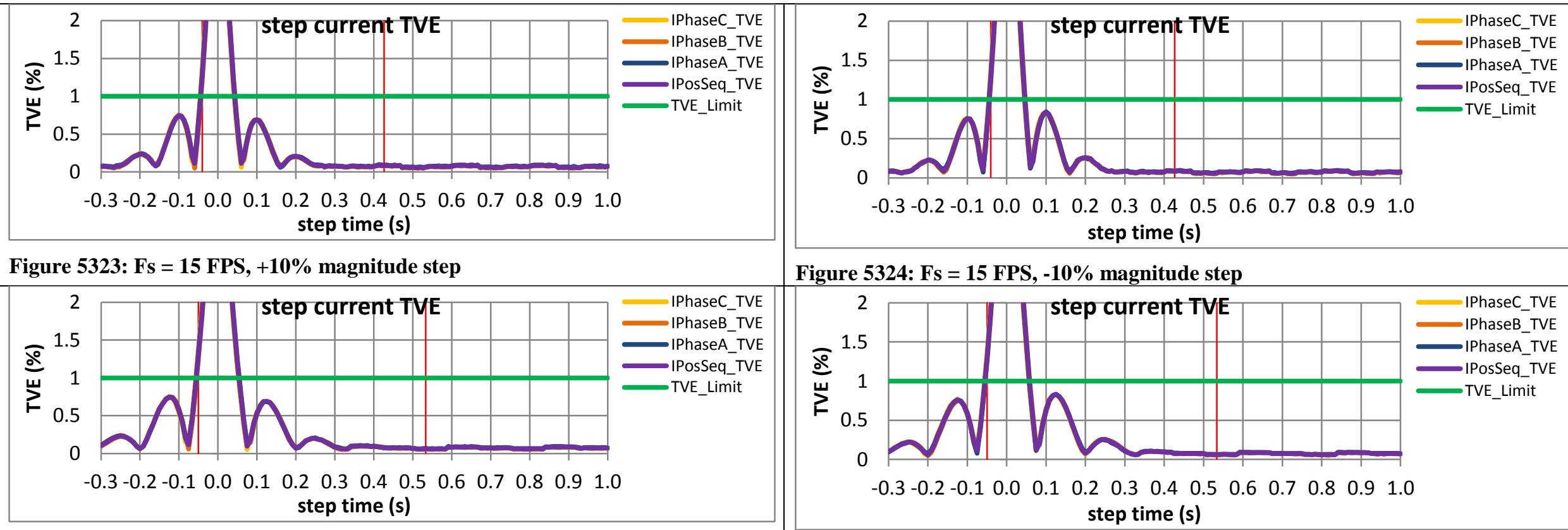

Figure 5324: Fs = 15 FPS, -10\% magnitude step

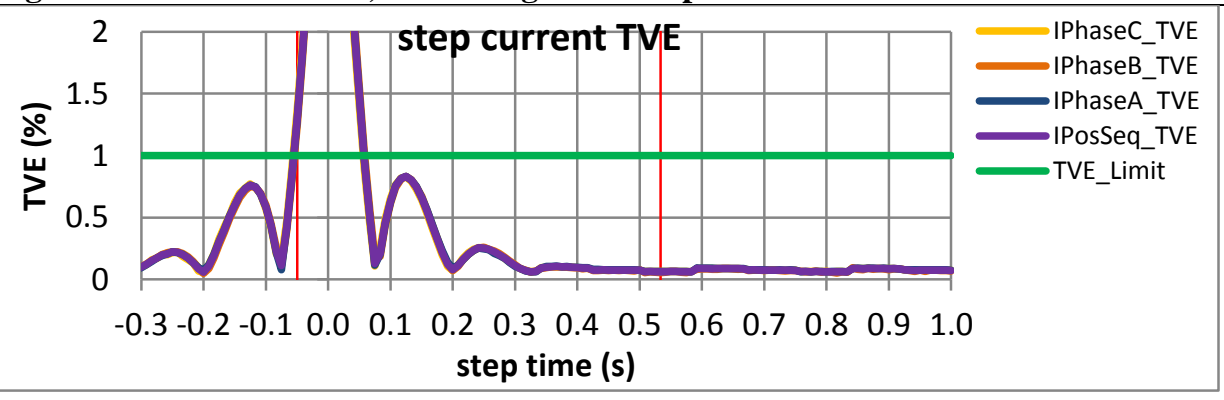

Figure 5325: Fs = 12 FPS, +10\% magnitude step

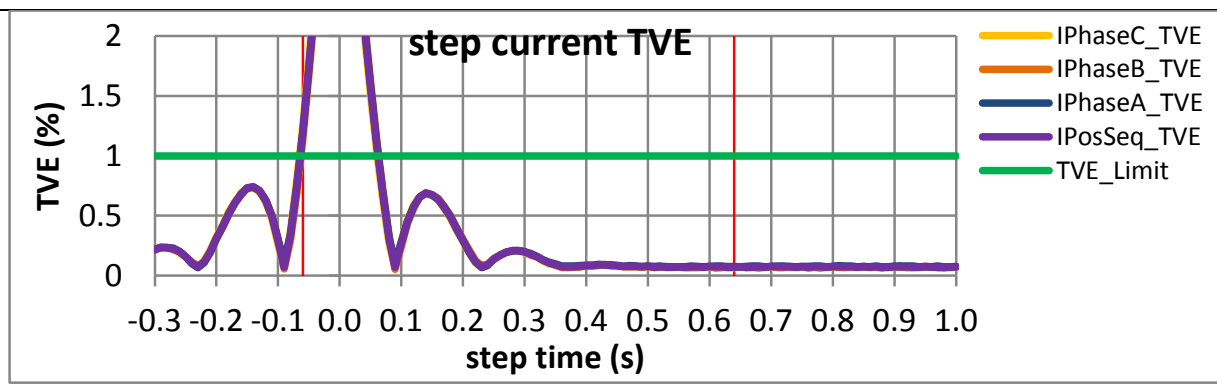

Figure 5326: Fs = 12 FPS, -10\% magnitude step

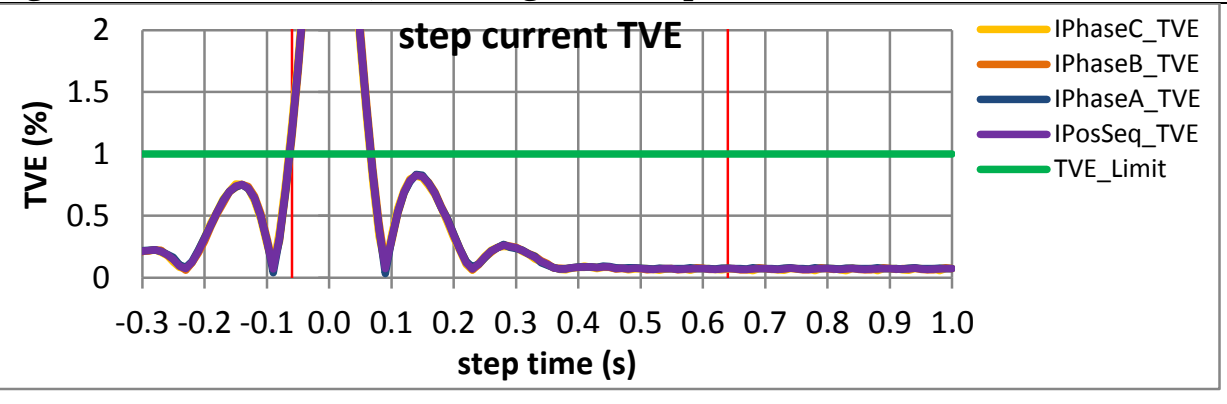

Figure 5327: Fs = 10 FPS, $+10 \%$ magnitude step

Figure 5328: Fs = 10 FPS, -10\% magnitude step 
10.3.10 PMU I dynamic step change in magnitude current response time: $F 0=60 \mathrm{~Hz}, \mathrm{M}$ class

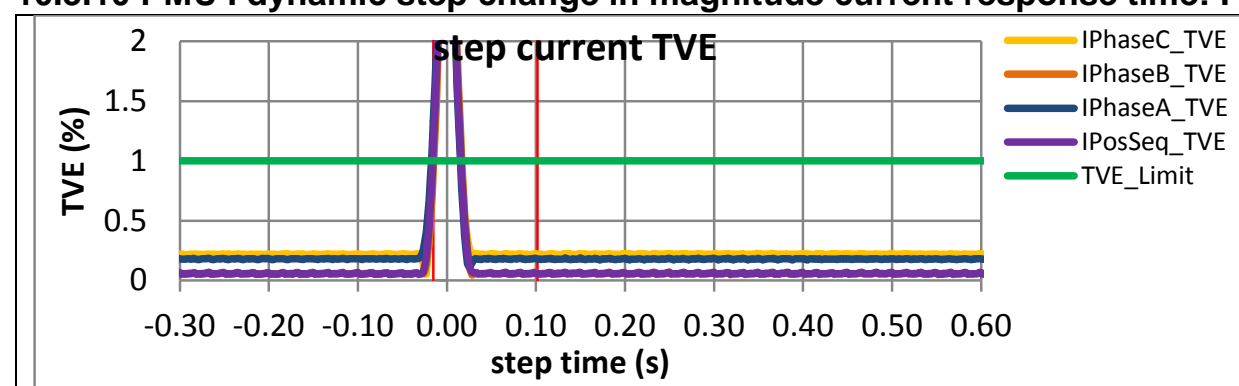

Figure 5329: Fs = 60 FPS, +10\% magnitude step

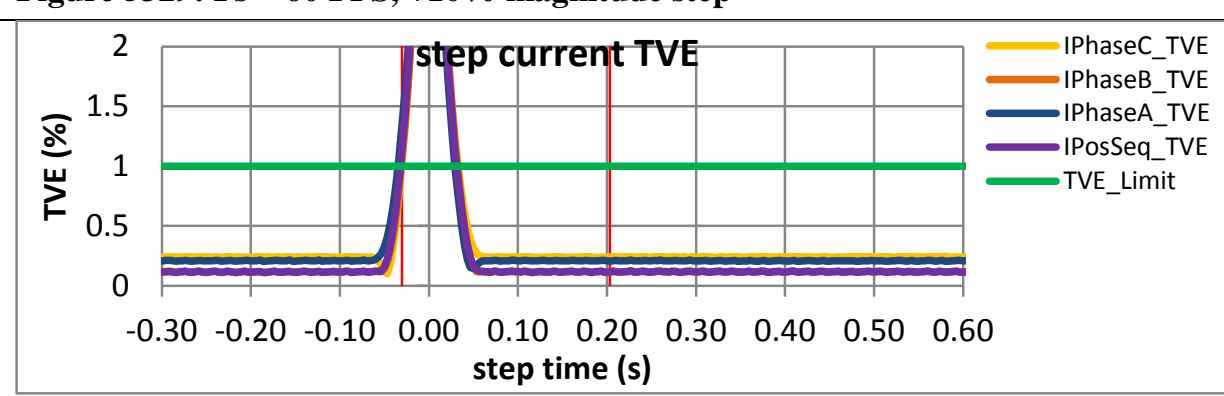

Figure 5331: Fs = 30 FPS, $+10 \%$ magnitude step

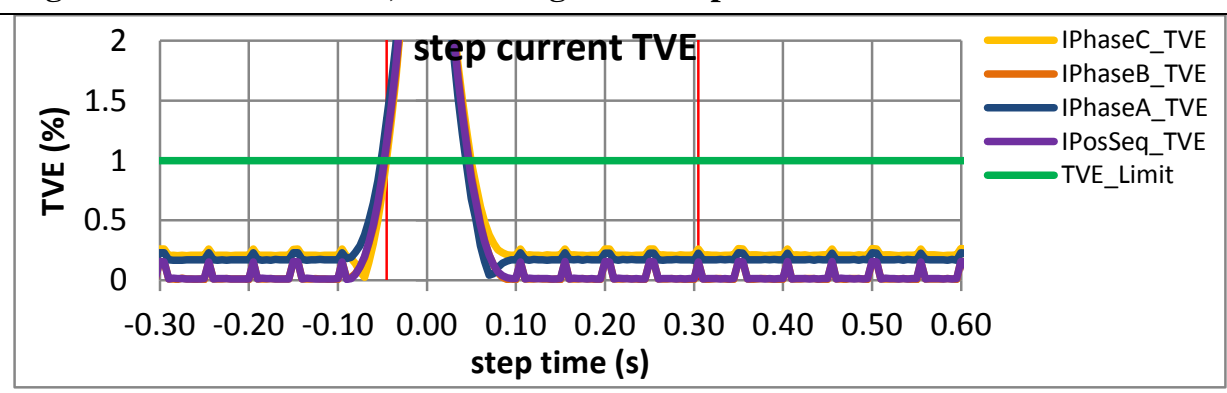

Figure 5333: Fs = 20 FPS, +10\% magnitude step

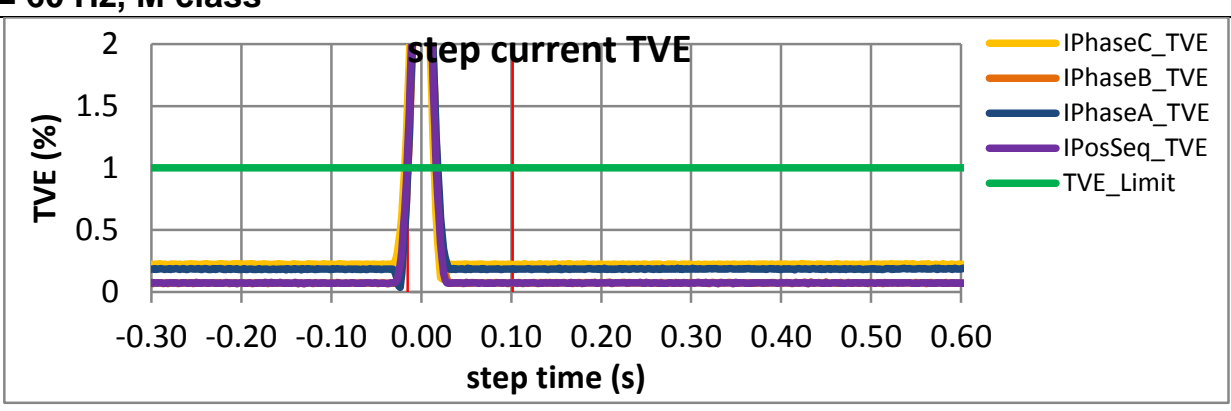

Figure 5330: Fs = 60 FPS, -10\% magnitude step

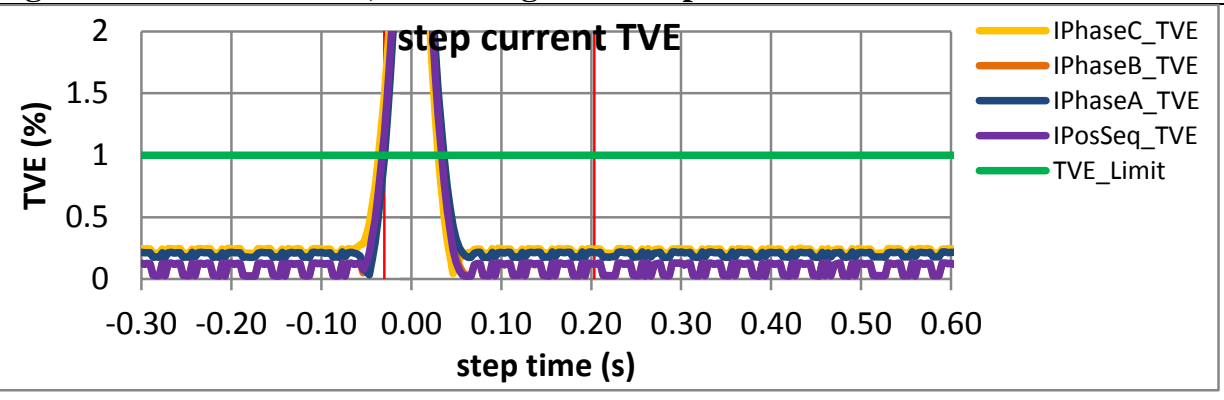

Figure 5332: Fs = 30 FPS, -10\% magnitude step

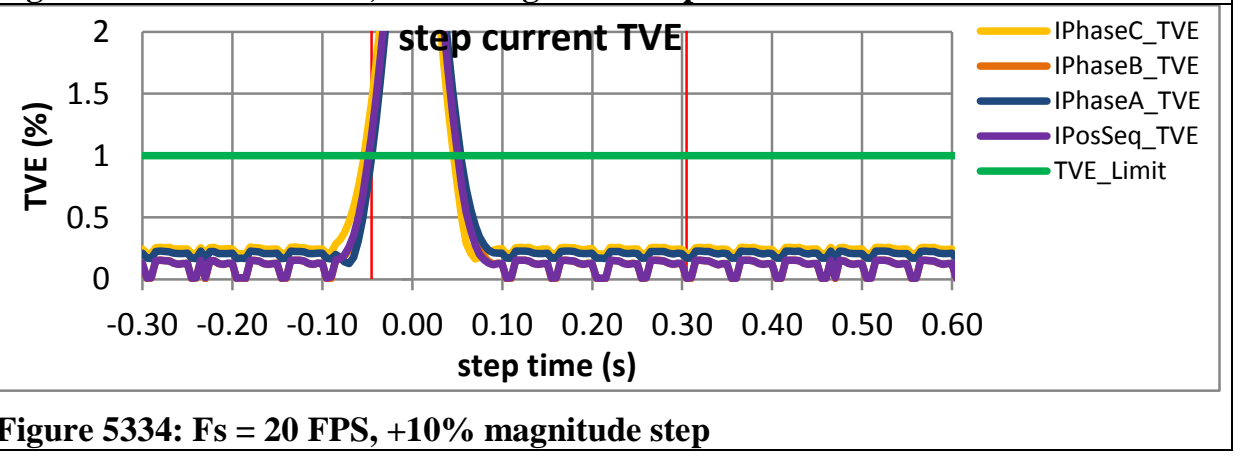




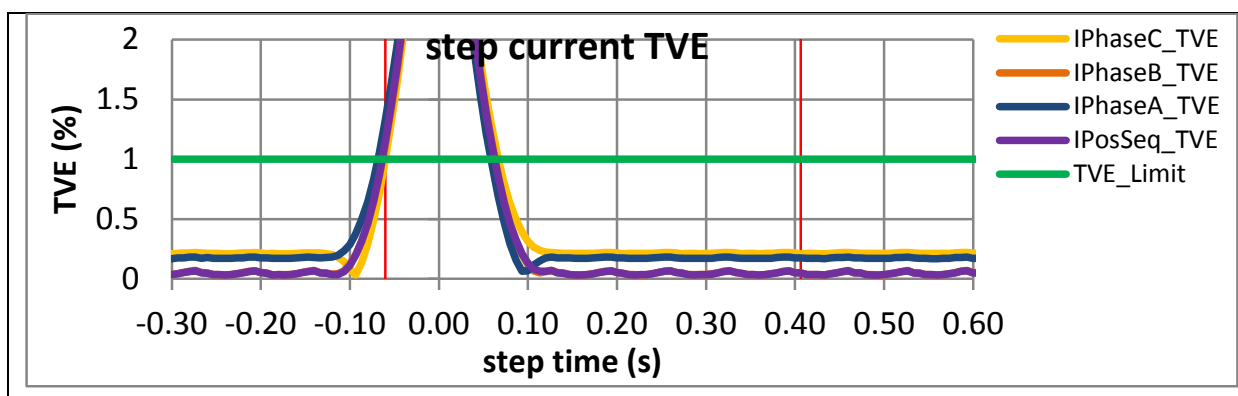

Figure 5335: Fs = 15 FPS, +10\% magnitude step
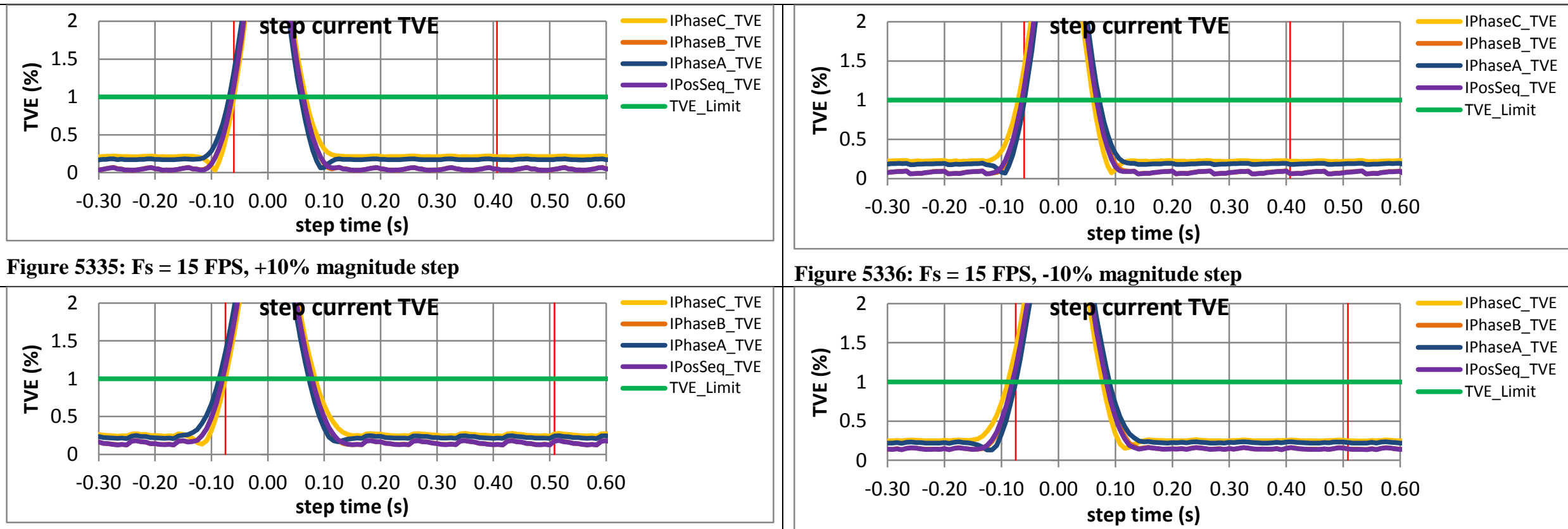

Figure 5336: Fs = 15 FPS, -10\% magnitude step

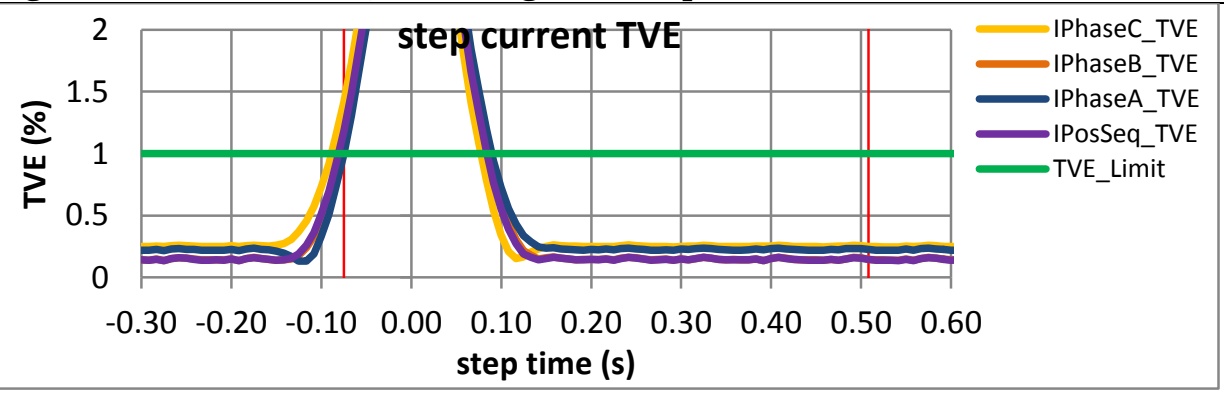

Figure 5337: Fs = 12 FPS, $+10 \%$ magnitude step

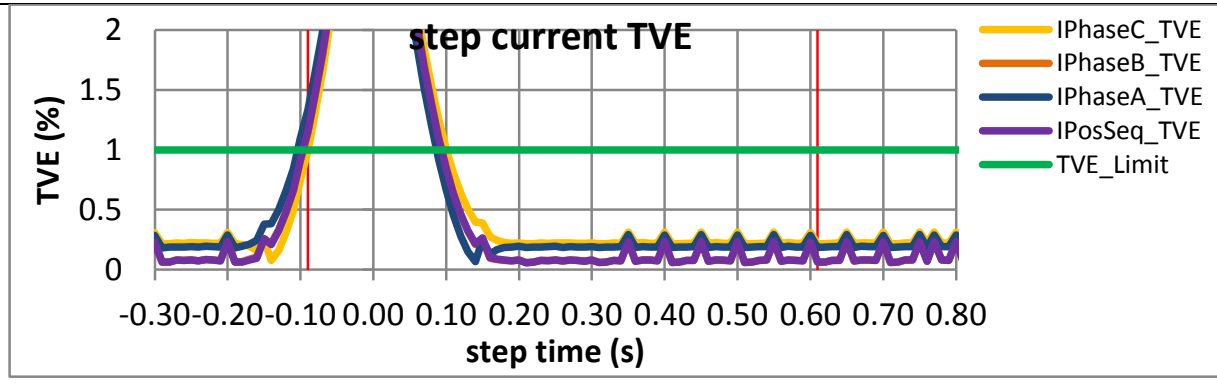

Figure 5338: Fs = 12 FPS, -10\% magnitude step

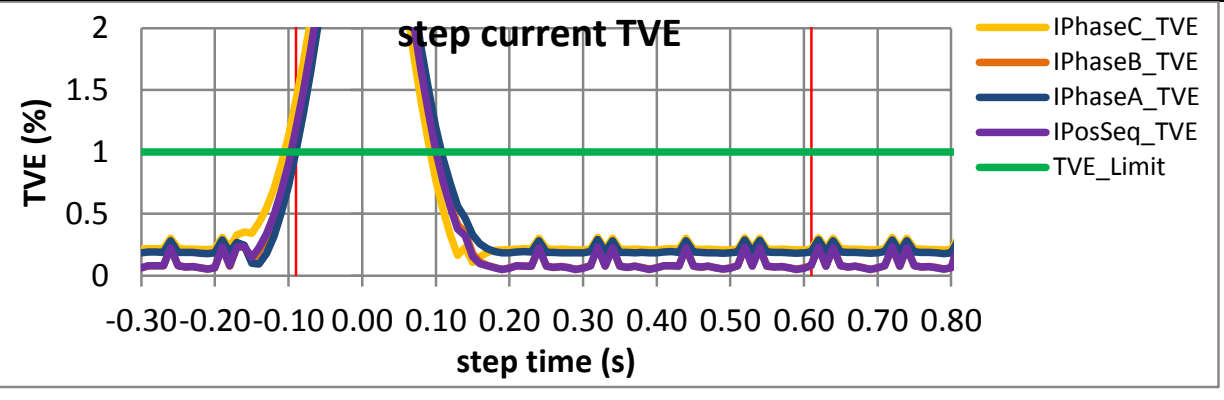

Figure 5340: Fs = 10 FPS, -10\% magnitude step 
10.3.11 PMU J dynamic step change in magnitude current response time: $\mathrm{F} 0=60 \mathrm{~Hz}, \mathrm{M}$ class

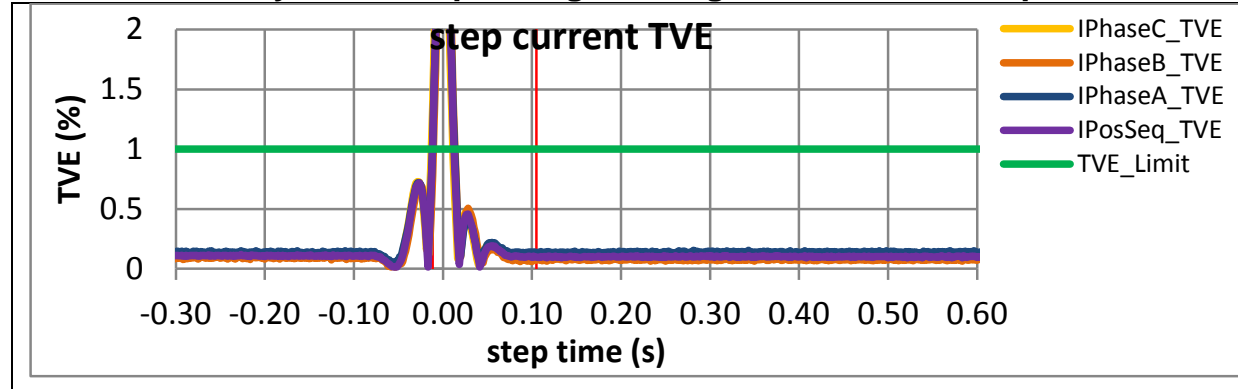

Figure 5341: Fs = 60 FPS, $+10 \%$ magnitude step

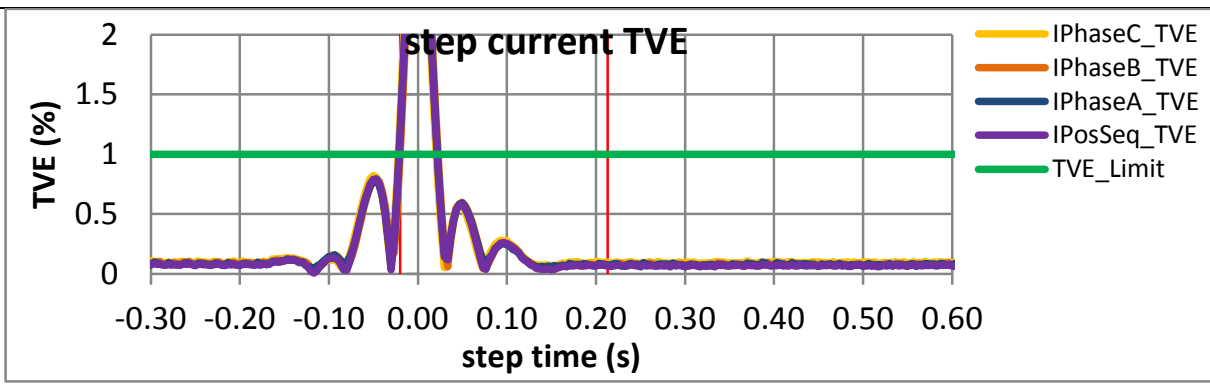

Figure 5343: Fs = 30 FPS, +10\% magnitude step

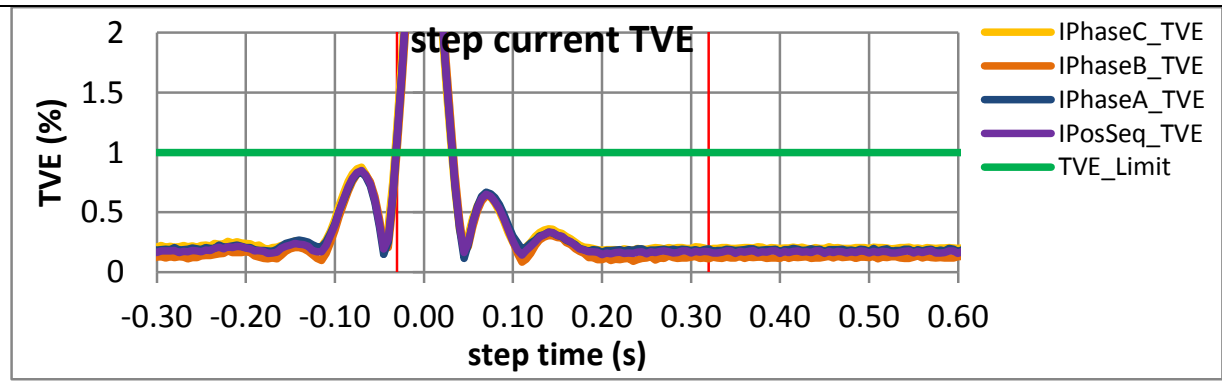

Figure 5345: Fs = 20 FPS, +10\% magnitude step

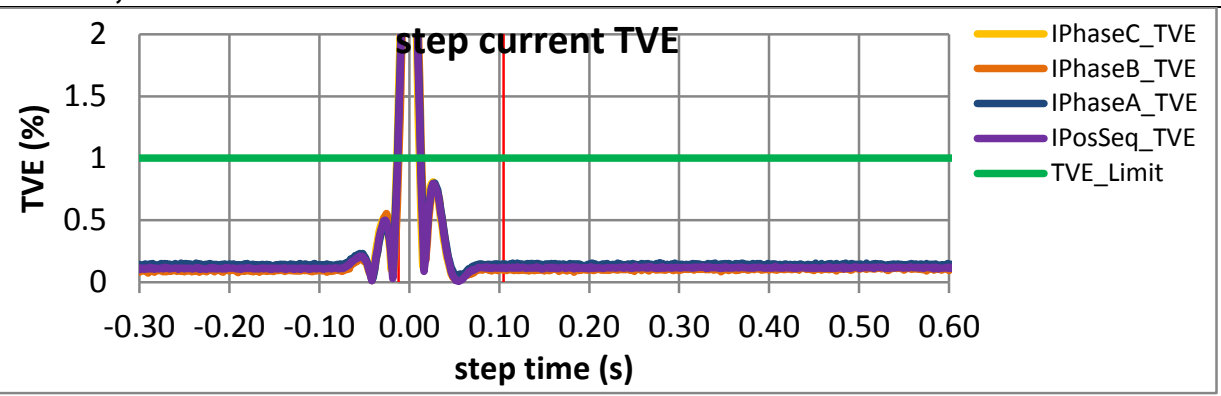

Figure 5342: Fs = 60 FPS, $-10 \%$ magnitude step

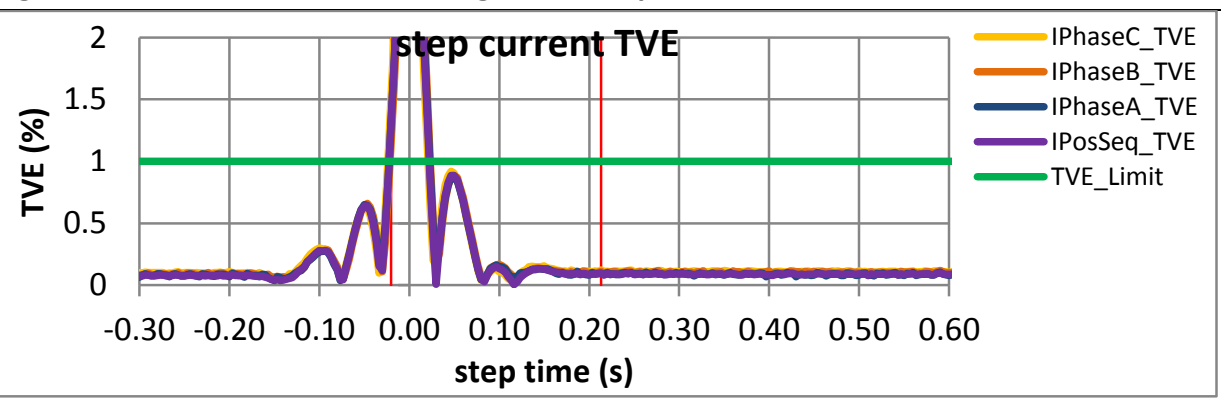

Figure 5344: Fs = 30 FPS, $-10 \%$ magnitude step

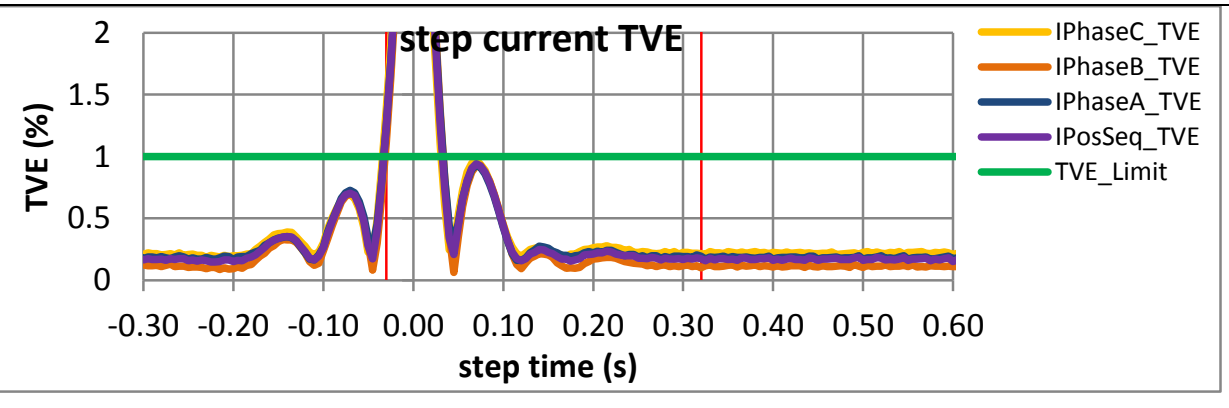

Figure 5346: Fs = 20 FPS, +10\% magnitude step 


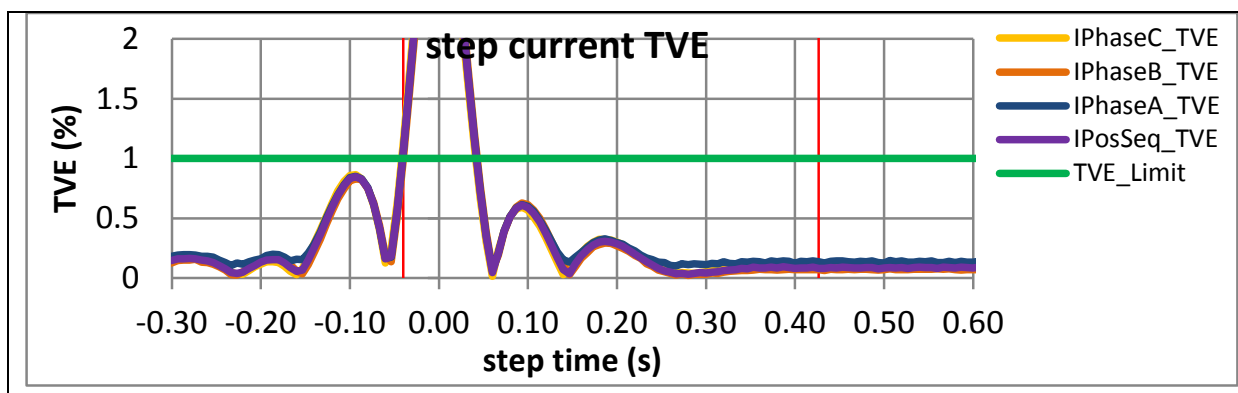

Figure 5347: Fs = $15 \mathrm{FPS},+10 \%$ magnitude step
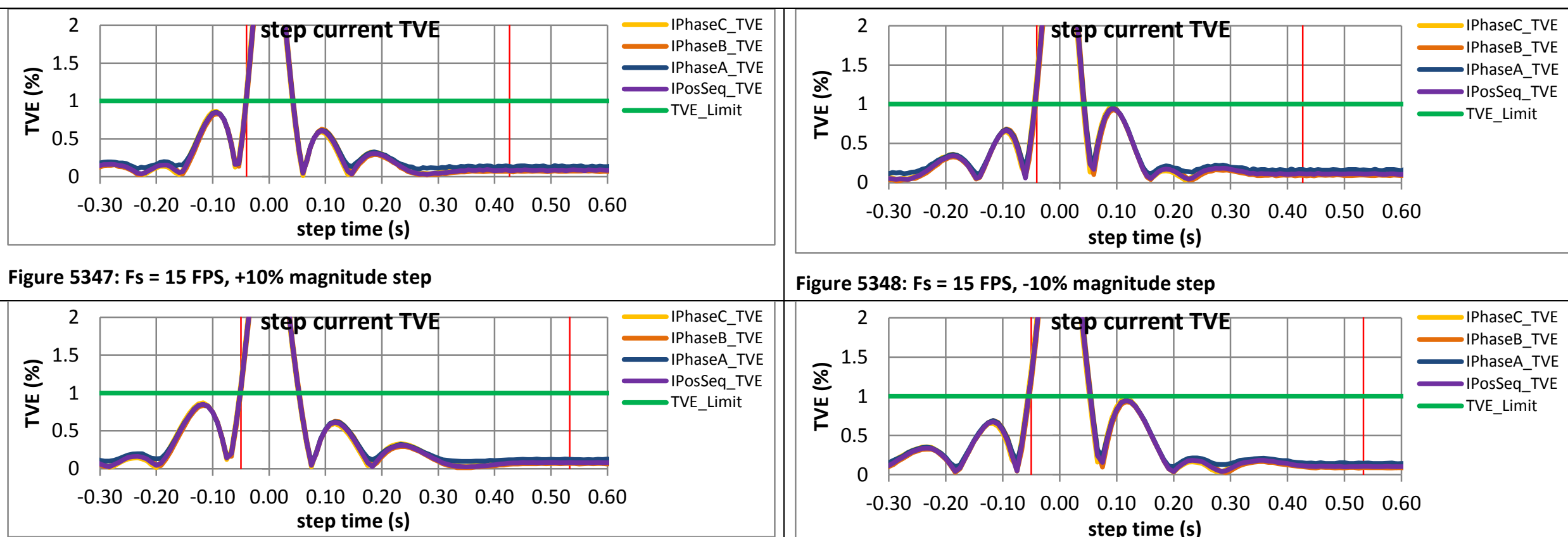

Figure 5348: $\mathrm{Fs}=15 \mathrm{FPS},-10 \%$ magnitude step

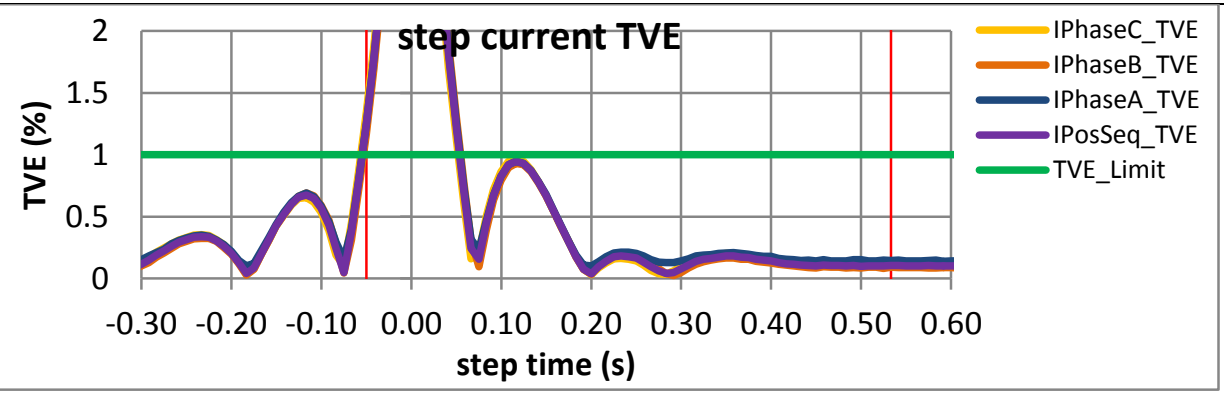

Figure 5349: Fs = 12 FPS, $+10 \%$ magnitude step

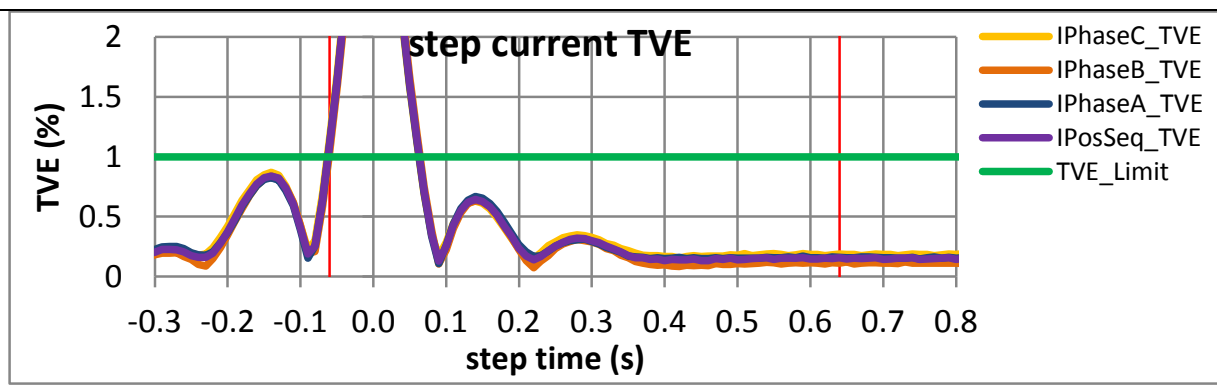

Figure 5351: Fs $=10 \mathrm{FPS},+10 \%$ magnitude step

Figure 5350: Fs = 12 FPS, $-10 \%$ magnitude step

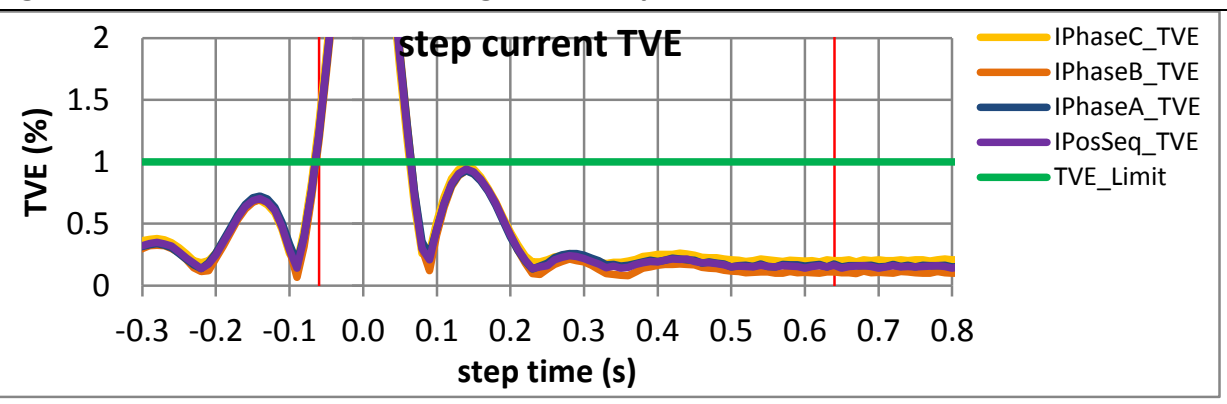

Figure 5352: Fs = $10 \mathrm{FPS},-10 \%$ magnitude step 
10.4 Dynamic step change in magnitude voltage response time: $\mathrm{F0}=60 \mathrm{~Hz}, \mathrm{P}$ Class

10.4.1 C37.118.1a Annex $C$ dynamic step change in magnitude voltage response time: $F 0=60 \mathrm{~Hz}, P$ class

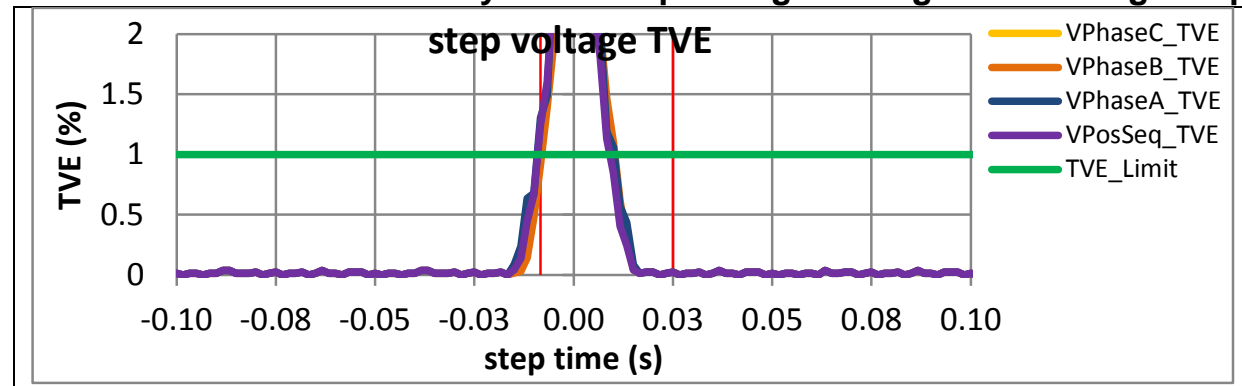

Figure 5353: Fs = 60 FPS, +10\% magnitude step
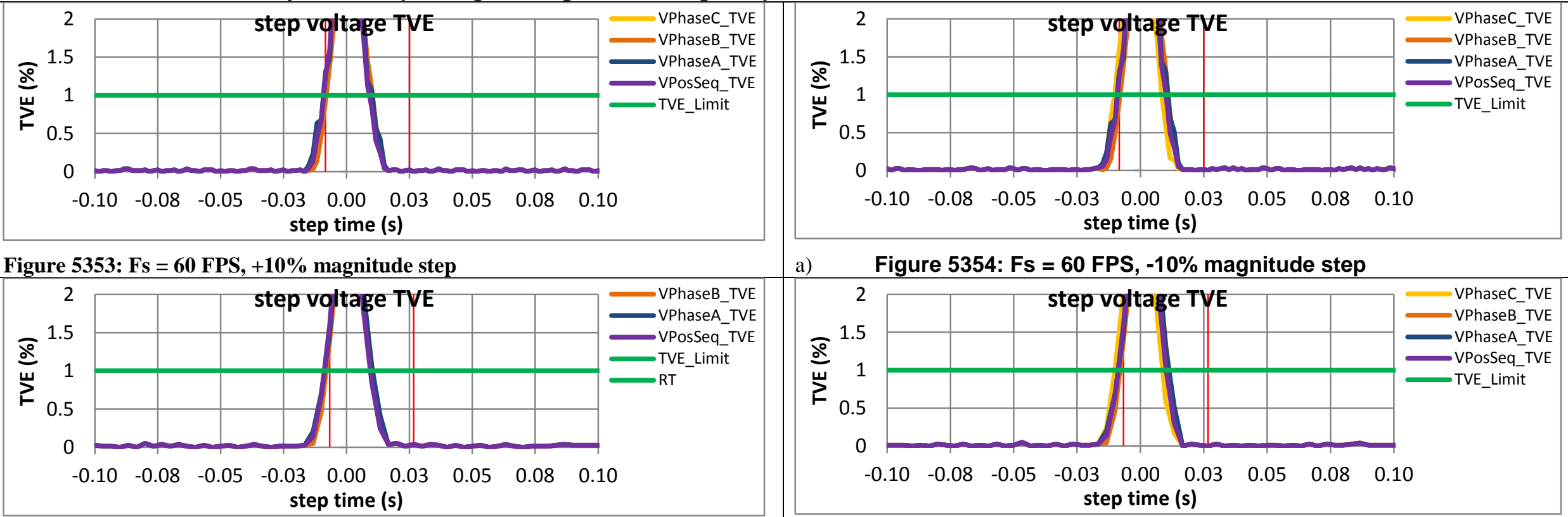

a)

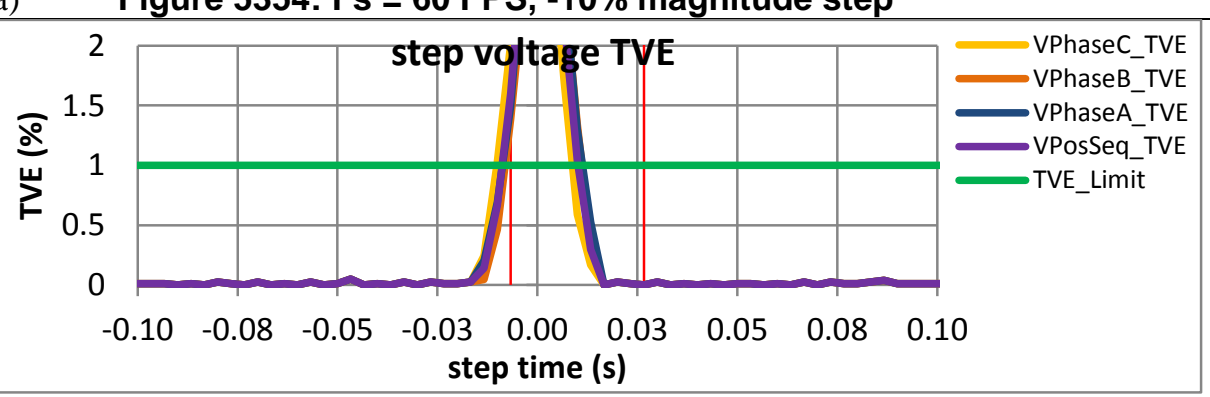

b) Figure 5355: Fs = 30 FPS, +10\% magnitude step

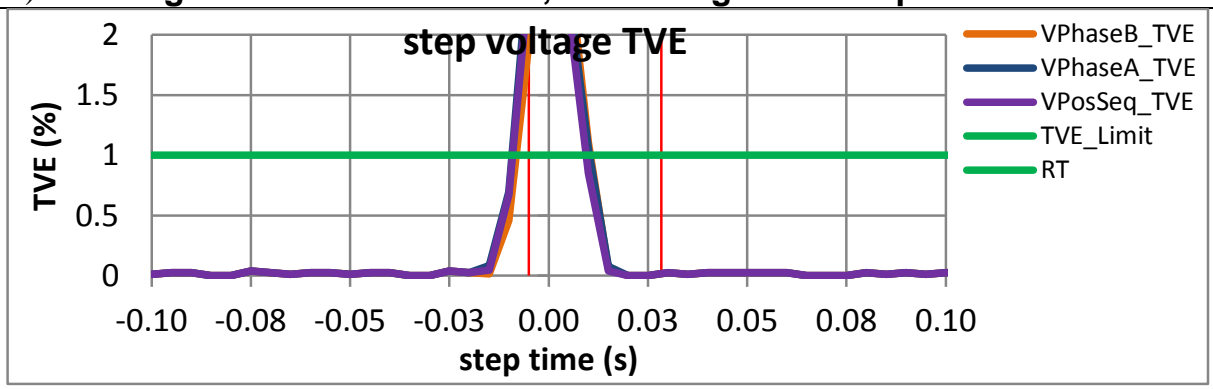

d) Figure 5357: Fs = 20 FPS, $+10 \%$ magnitude step

c)

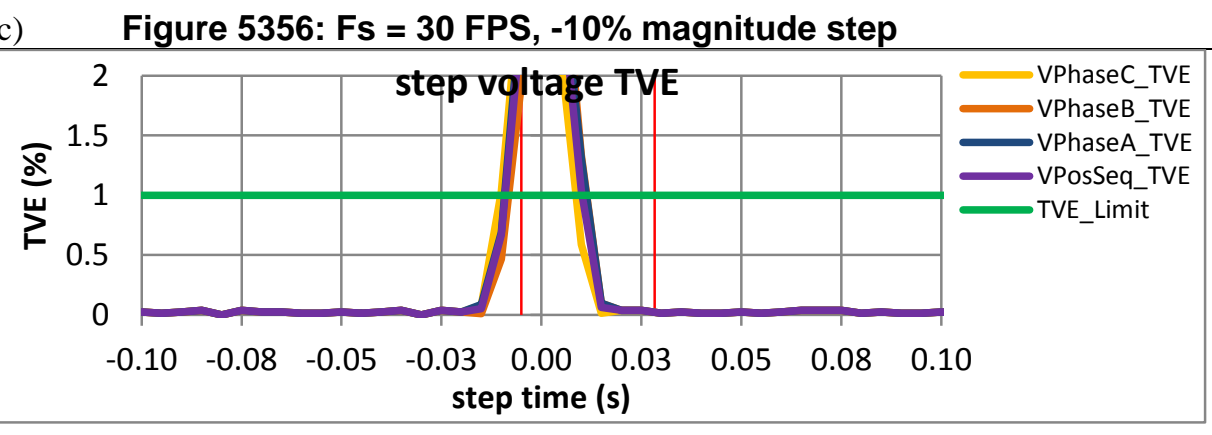

e) Figure 5358: Fs = 20 FPS, $-\mathbf{- 1 0} \%$ magnitude step 

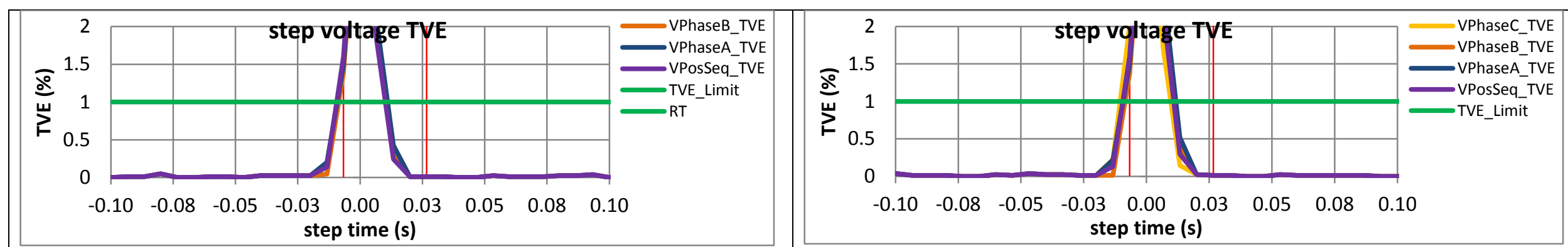

f) Figure 5359: Fs = 15 FPS, +10\% magnitude step

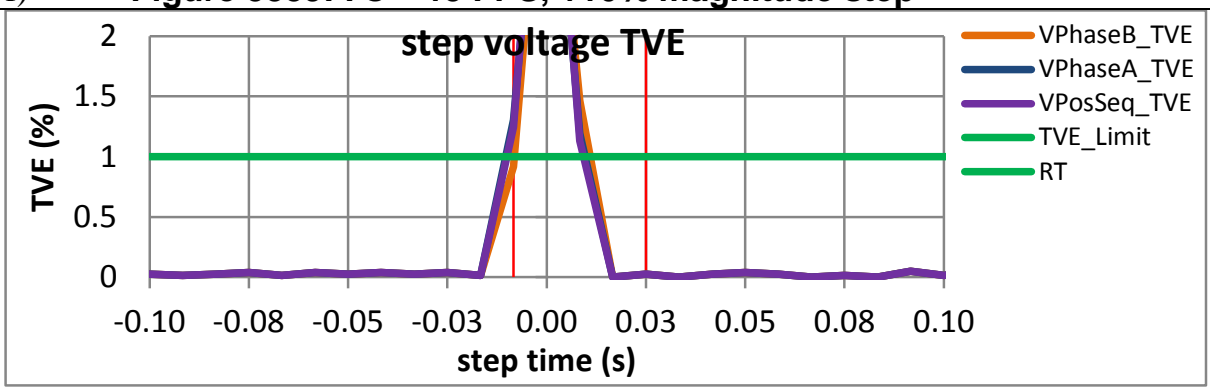

h) Figure 5361: Fs = 12 FPS, $+\mathbf{1 0} \%$ magnitude step

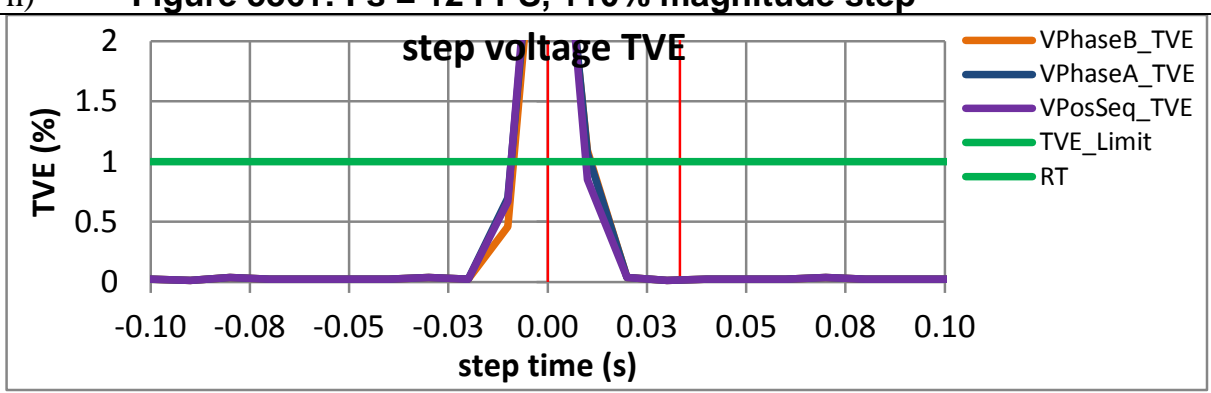

j) Figure 5363: Fs = 10 FPS, $+10 \%$ magnitude step

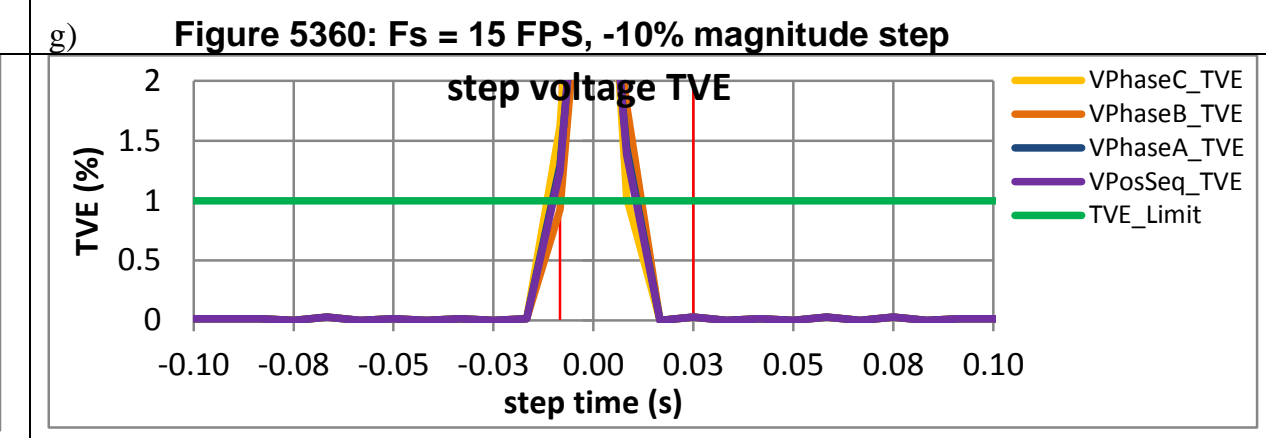

i) Figure 5362: Fs = 12 FPS, $-10 \%$ magnitude step

k)

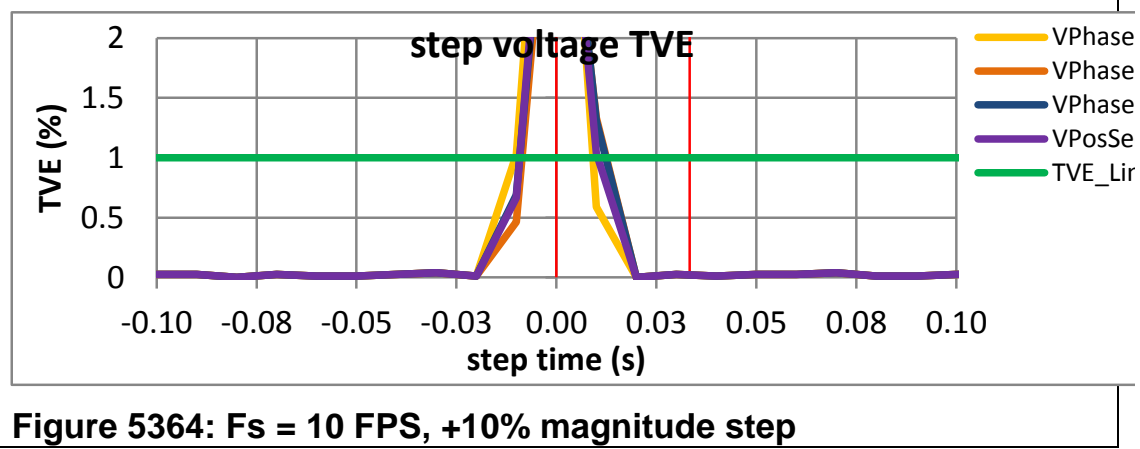


10.4.2 PMU A dynamic step change in magnitude voltage response time: $\mathrm{F} 0=60 \mathrm{~Hz}, \mathrm{P}$ class
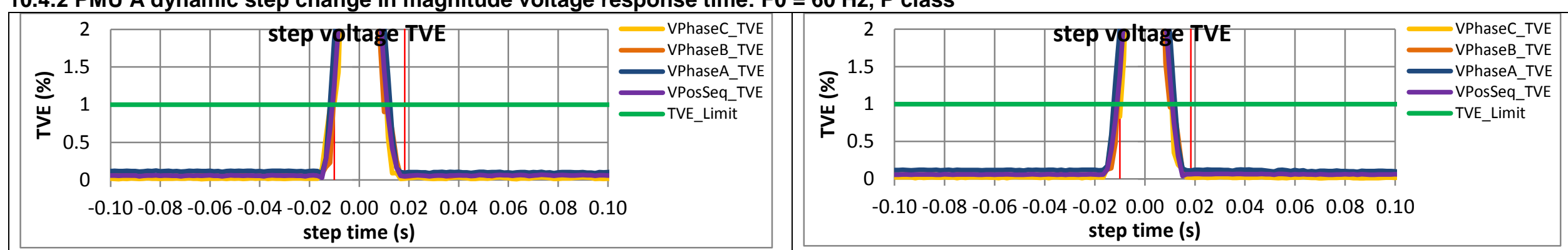

Figure 5365: Fs $=60 \mathrm{FPS},+10 \%$ magnitude step

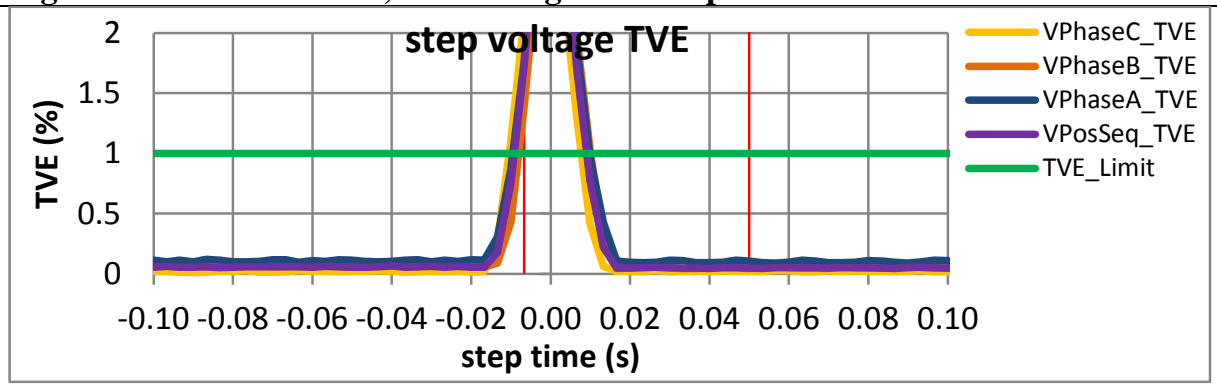

Figure 5366: Fs = 60 FPS, -10\% magnitude step

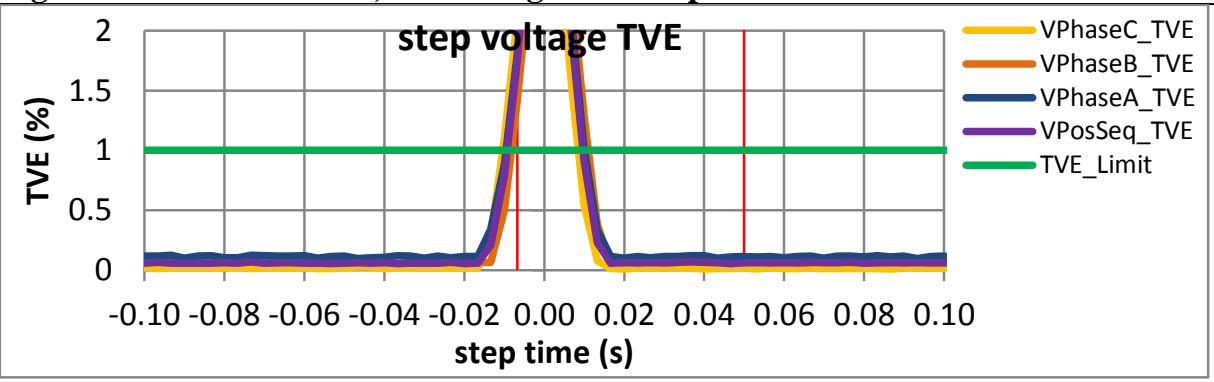

Figure 5367: Fs = 30 FPS, +10\% magnitude step

Figure 5368: Fs = 30 FPS, -10\% magnitude step

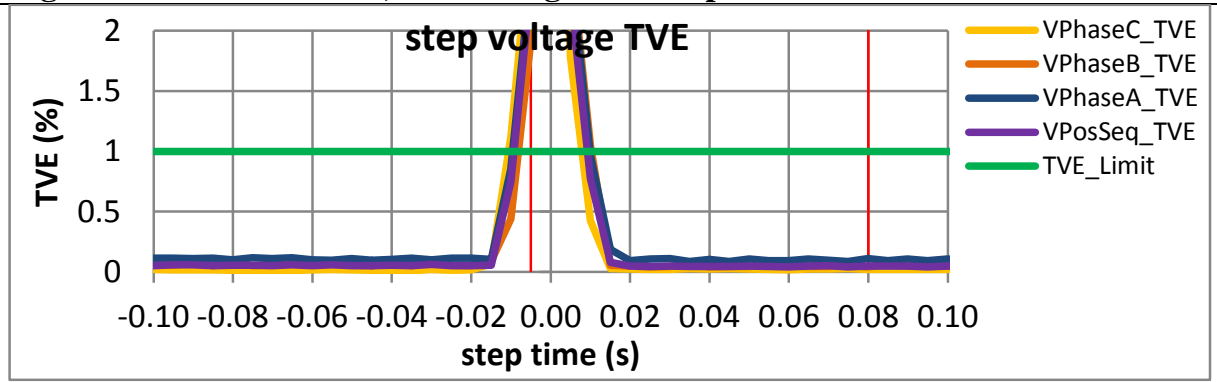

Figure 5369: Fs = 20 FPS, $+10 \%$ magnitude step

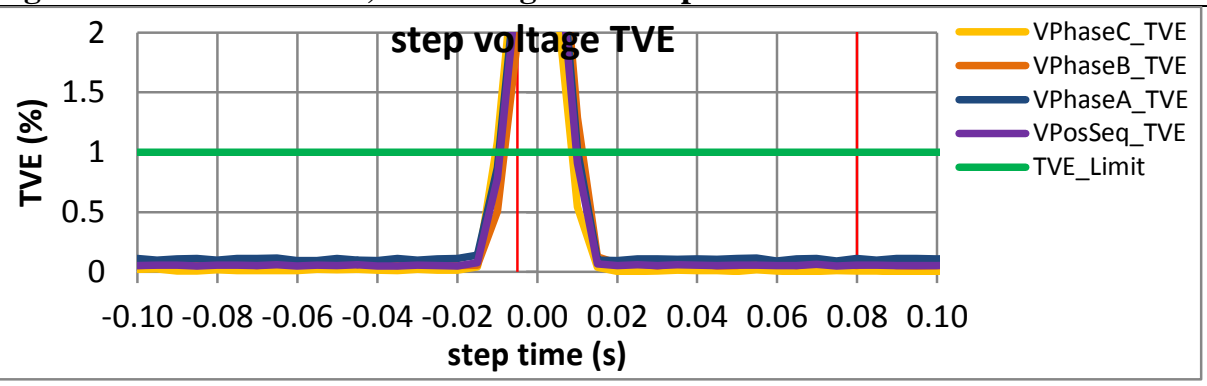

Figure 5370: Fs = 20 FPS, -10\% magnitude step 

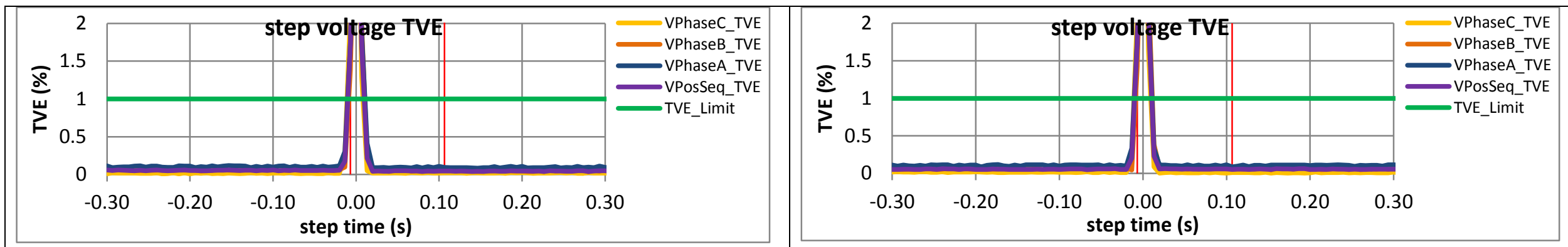

Figure 5371: Fs = 15 FPS, +10\% magnitude step

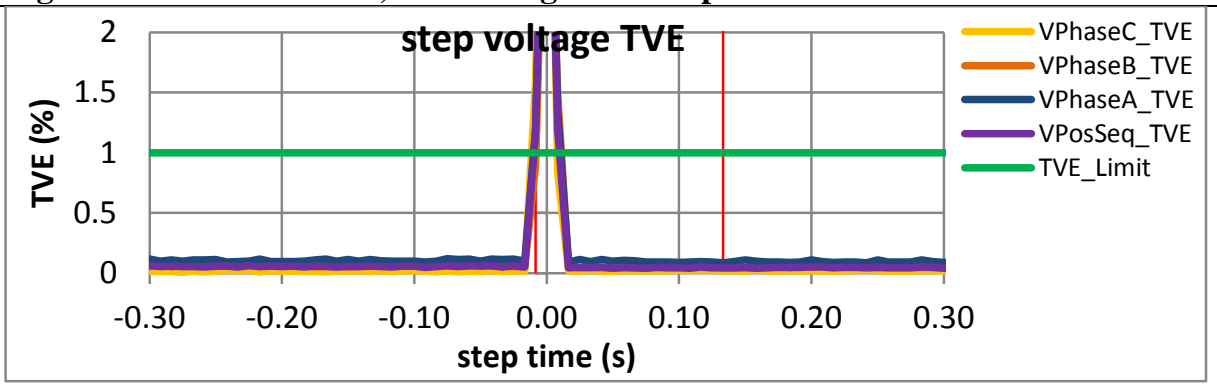

Figure 5373: Fs = 12 FPS, +10\% magnitude step

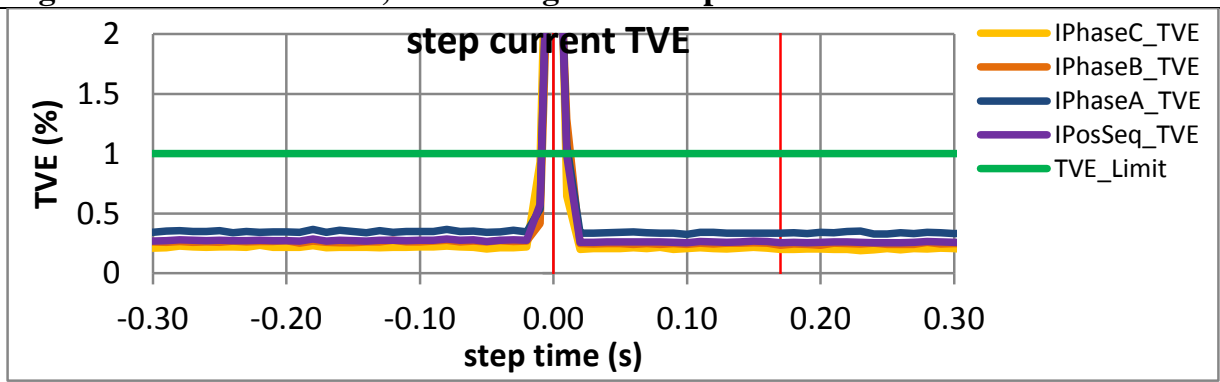

Figure 5375: Fs = 10 FPS, +10\% magnitude step

Figure 5372: Fs = 15 FPS, -10\% magnitude step

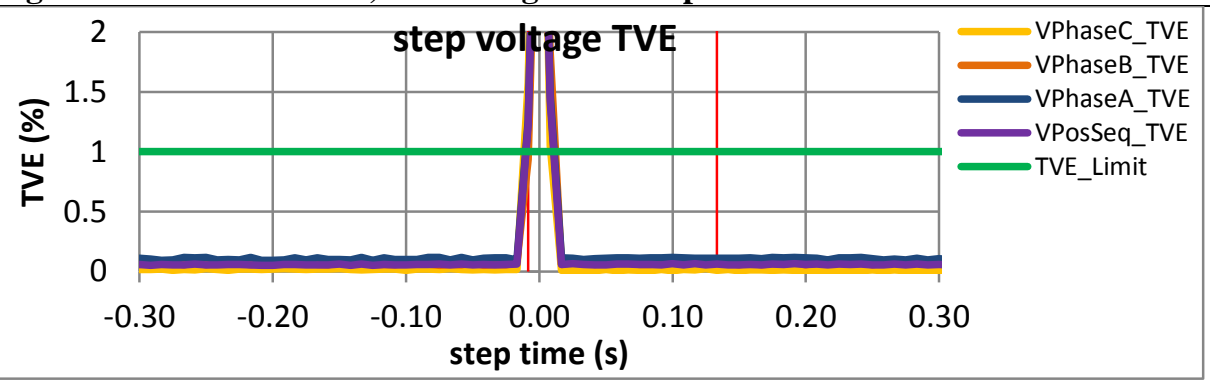

Figure 5374: Fs = 12 FPS, -10\% magnitude step

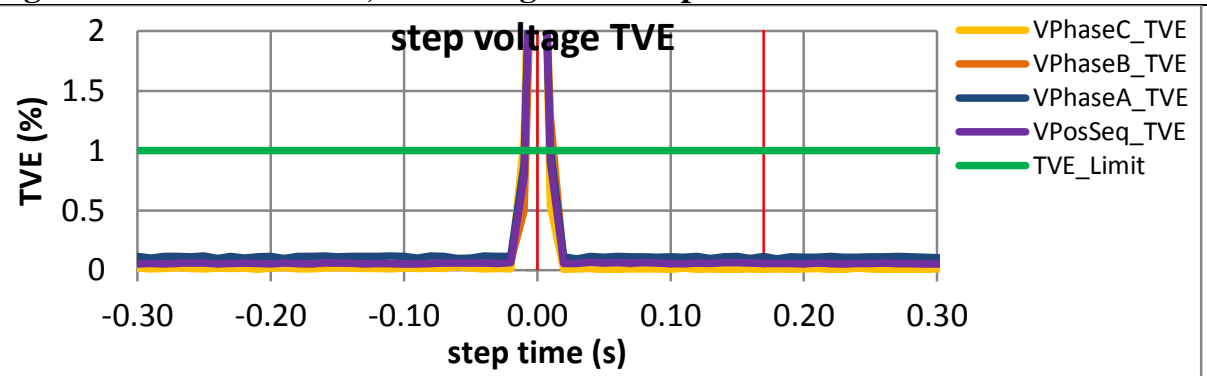

Figure 5376: Fs $=10 \mathrm{FPS},+10 \%$ magnitude step 
10.4.3 PMU B dynamic step change in magnitude voltage response time: $\mathrm{F} 0=60 \mathrm{~Hz}, \mathrm{P}$ class
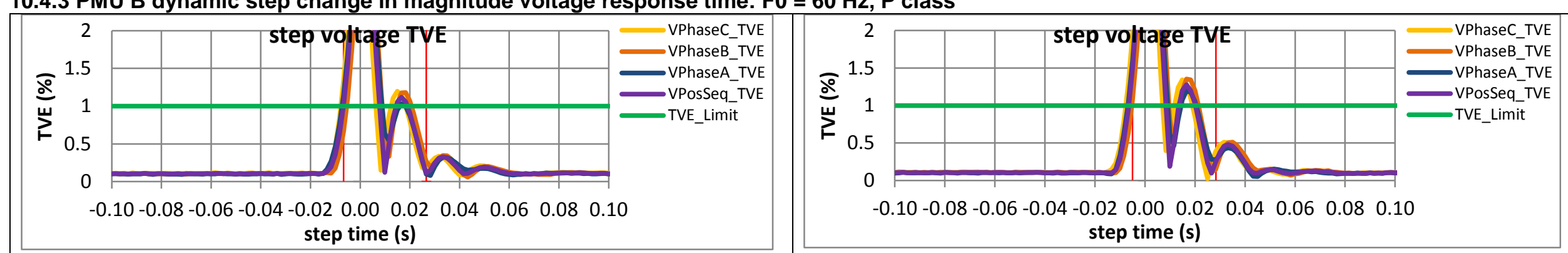

Figure 5377: Fs $=60 \mathrm{FPS},+10 \%$ magnitude step

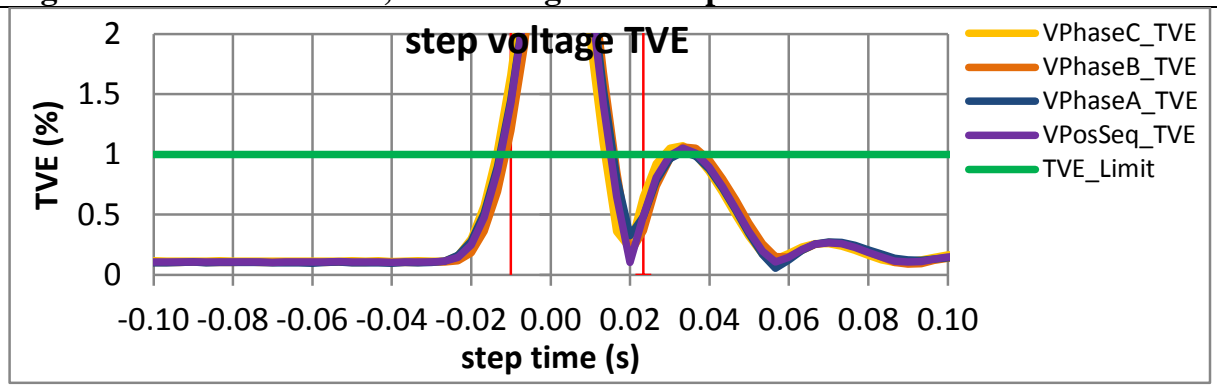

Figure 5378: Fs = 60 FPS, -10\% magnitude step

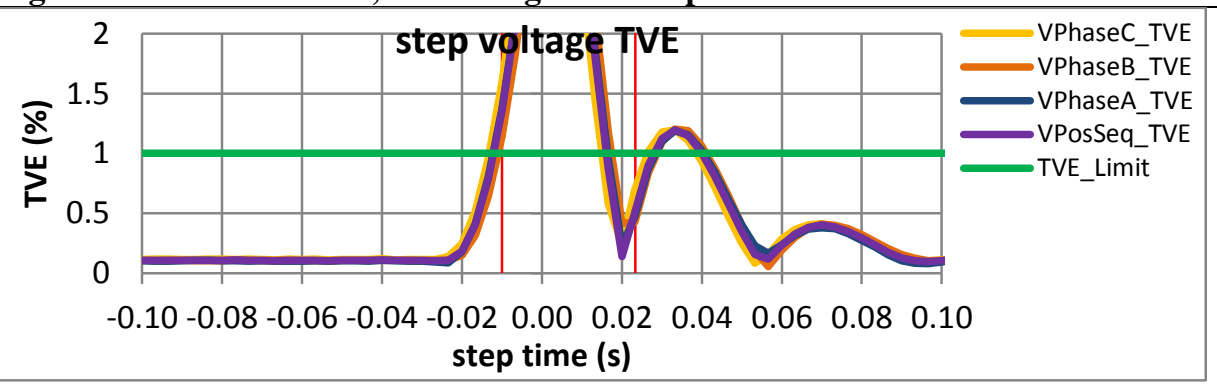

Figure 5379: Fs = 30 FPS, +10\% magnitude step

Figure 5380: Fs = 30 FPS, -10\% magnitude step

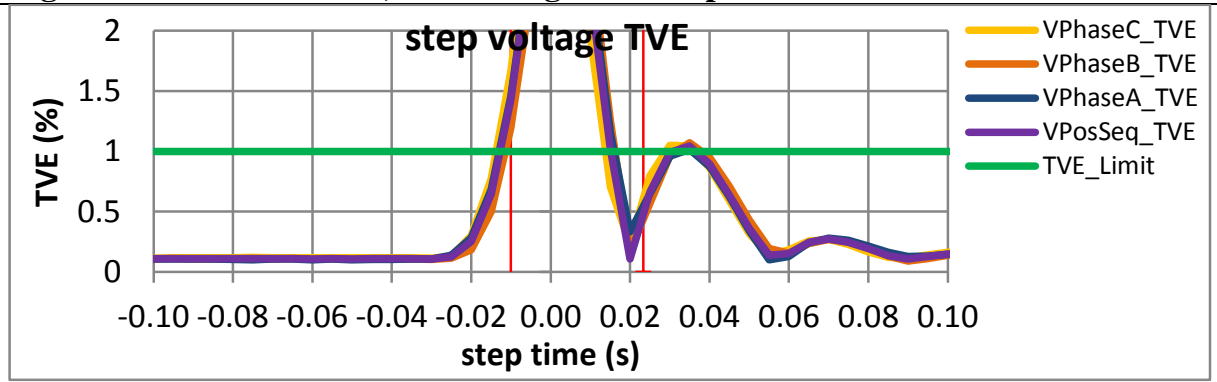

Figure 5381: Fs = 20 FPS, $+10 \%$ magnitude step

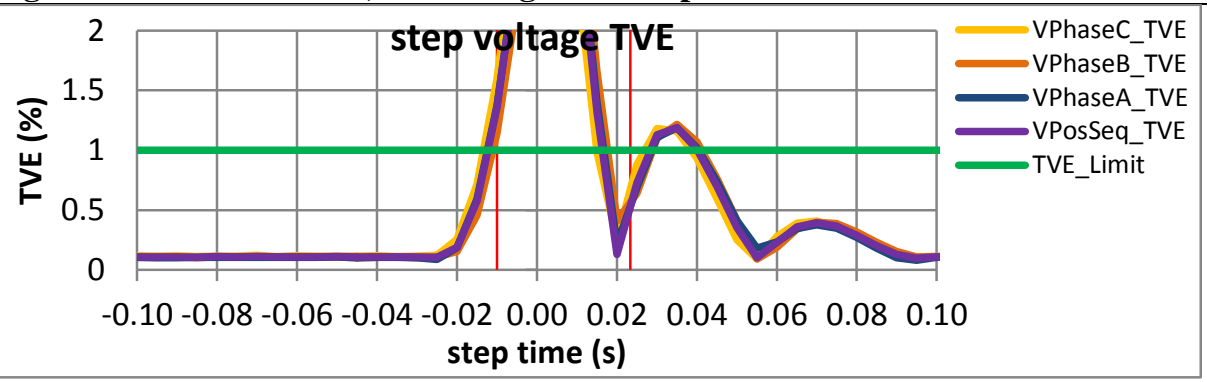

Figure 5382: Fs = 20 FPS, -10\% magnitude step 

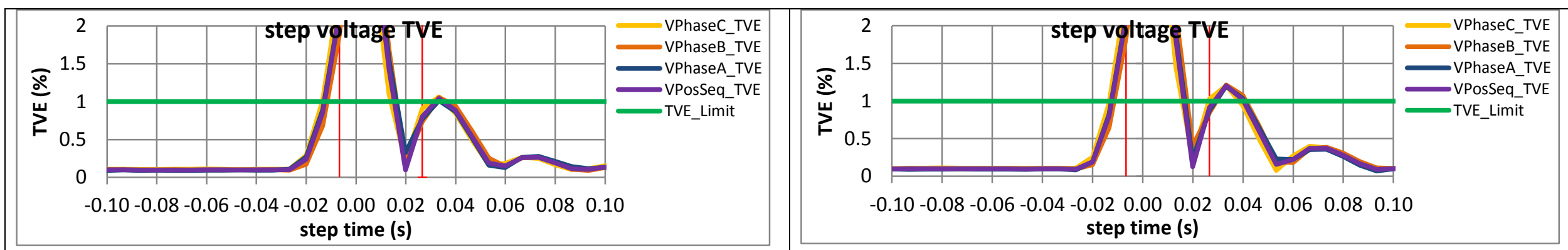

Figure 5383: Fs = 15 FPS, +10\% magnitude step

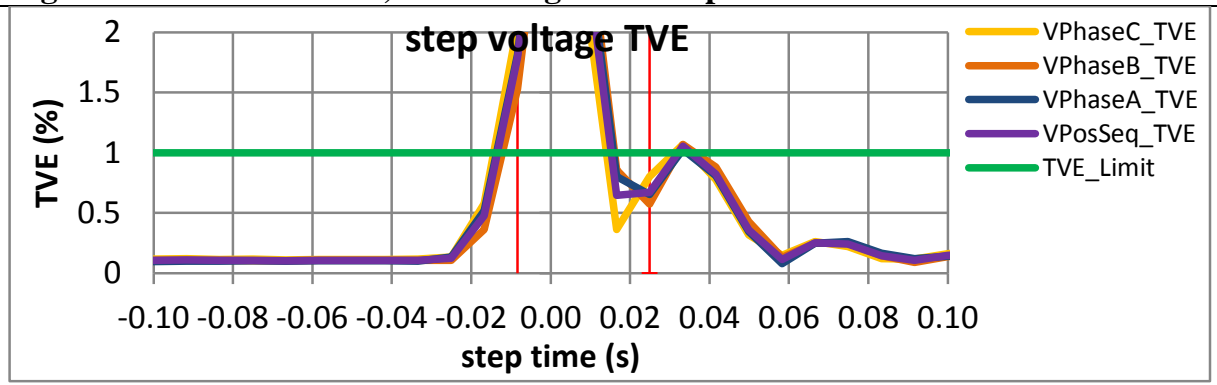

Figure 5384: Fs = 15 FPS, -10\% magnitude step

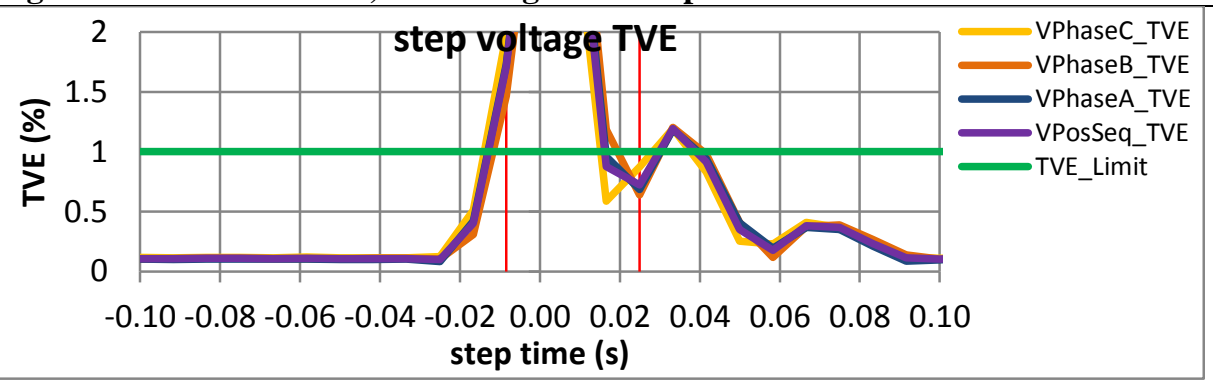

Figure 5385: Fs $=12$ FPS, $+10 \%$ magnitude step

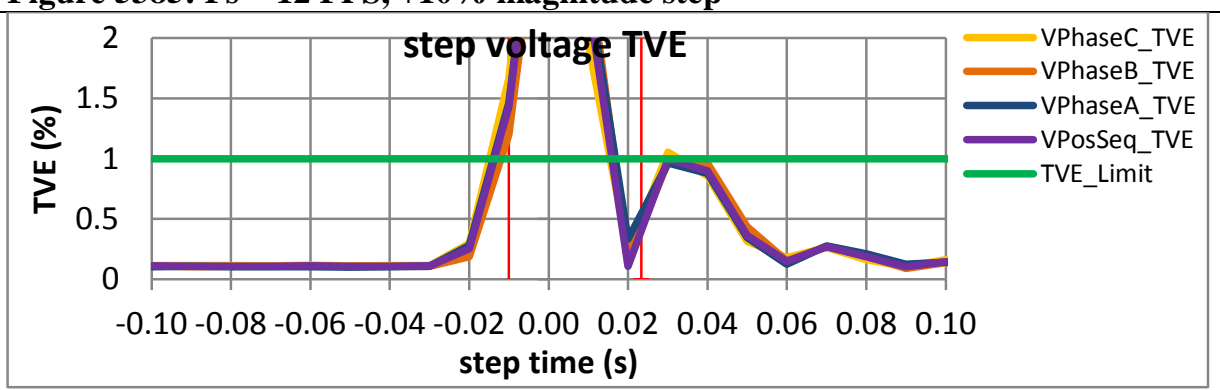

Figure 5387: Fs = 10 FPS, +10\% magnitude step

Figure 5386: Fs = 12 FPS, -10\% magnitude step

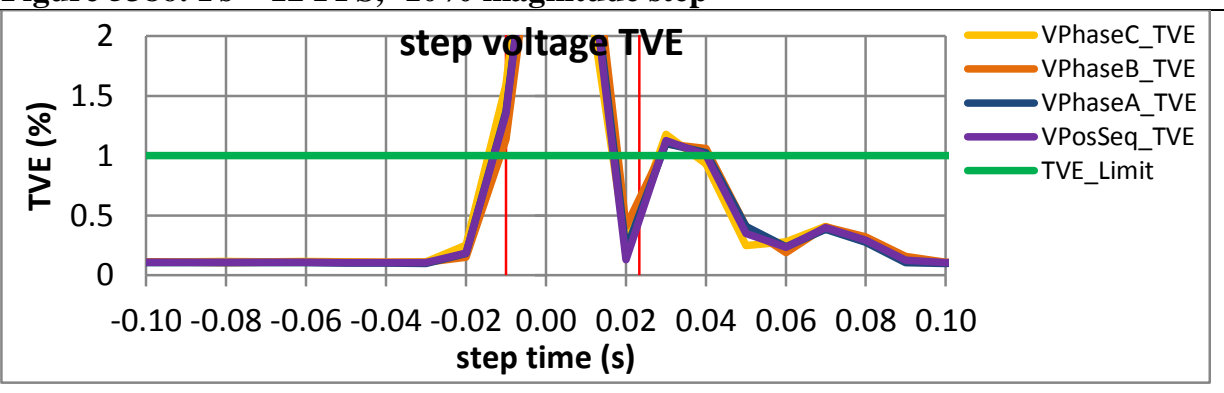

Figure 5388: Fs = 10 FPS, +10\% magnitude step 
10.4.4 PMU C dynamic step change in magnitude voltage response time: $\mathrm{F} 0=60 \mathrm{~Hz}, \mathrm{P}$ class
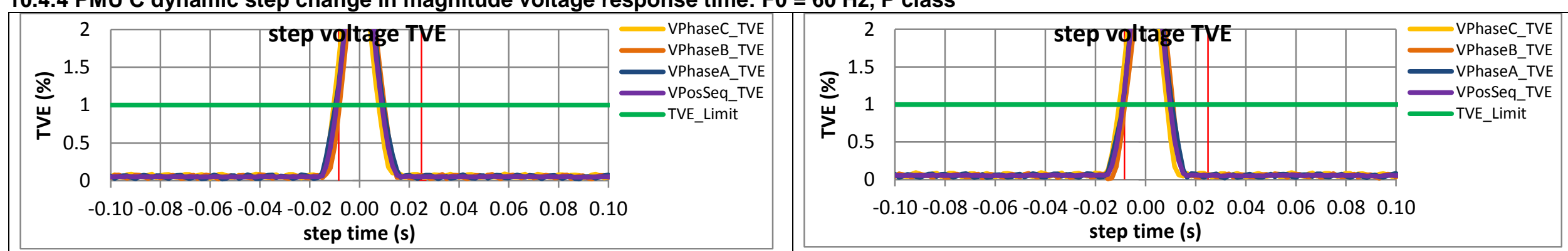

Figure 5389: Fs $=60 \mathrm{FPS},+10 \%$ magnitude step

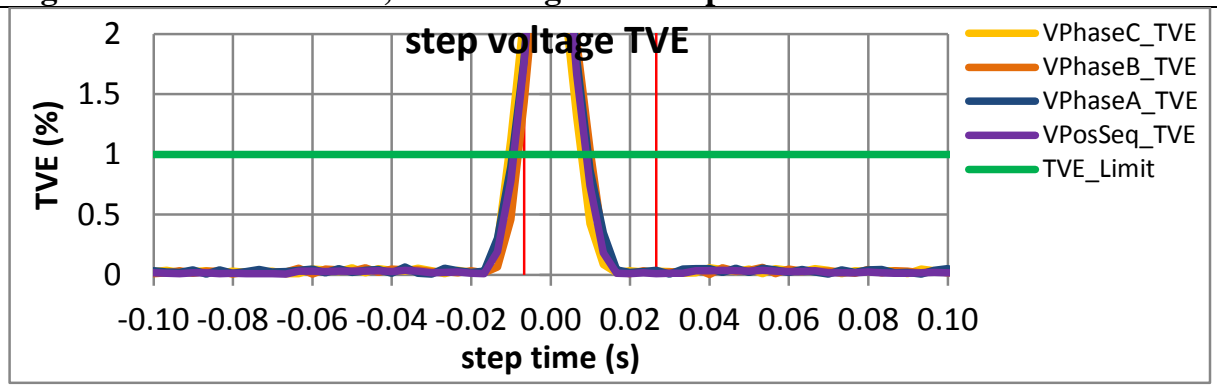

Figure 5390: Fs = 60 FPS, -10\% magnitude step

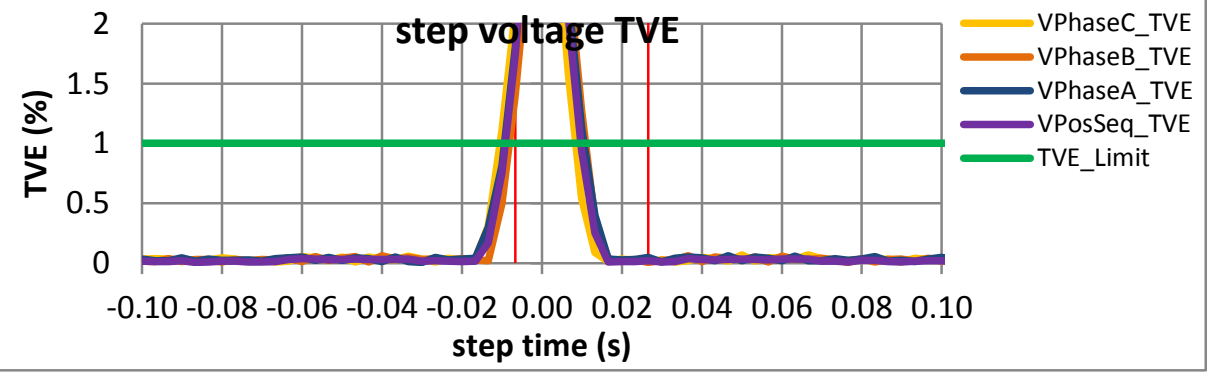

Figure 5391: Fs = 30 FPS, +10\% magnitude step

Figure 5392: Fs = 30 FPS, -10\% magnitude step

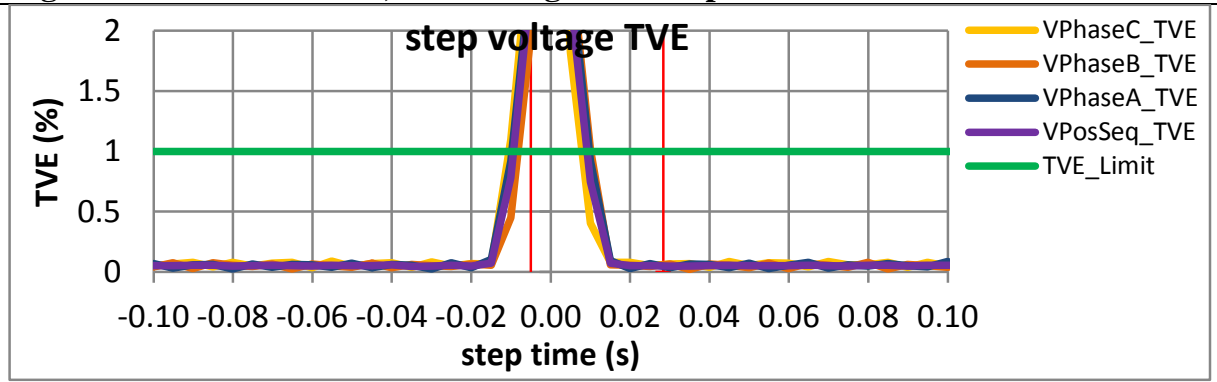

Figure 5393: Fs $=20 \mathrm{FPS},+10 \%$ magnitude step

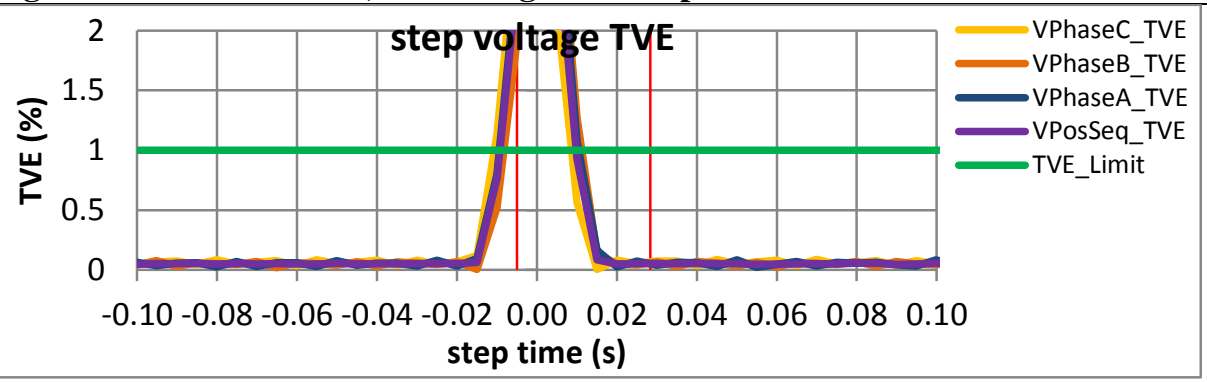

Figure 5394: Fs = 20 FPS, -10\% magnitude step 

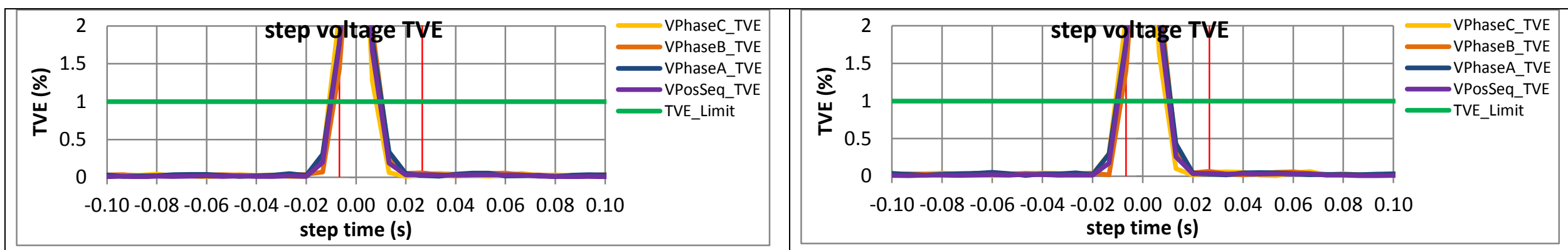

Figure 5395: Fs = 15 FPS, +10\% magnitude step

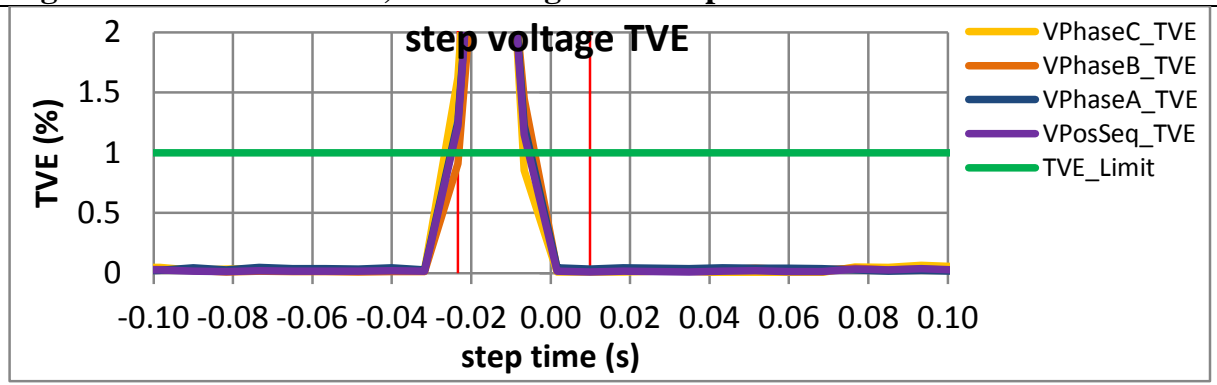

Figure 5397: Fs = 12 FPS, +10\% magnitude step

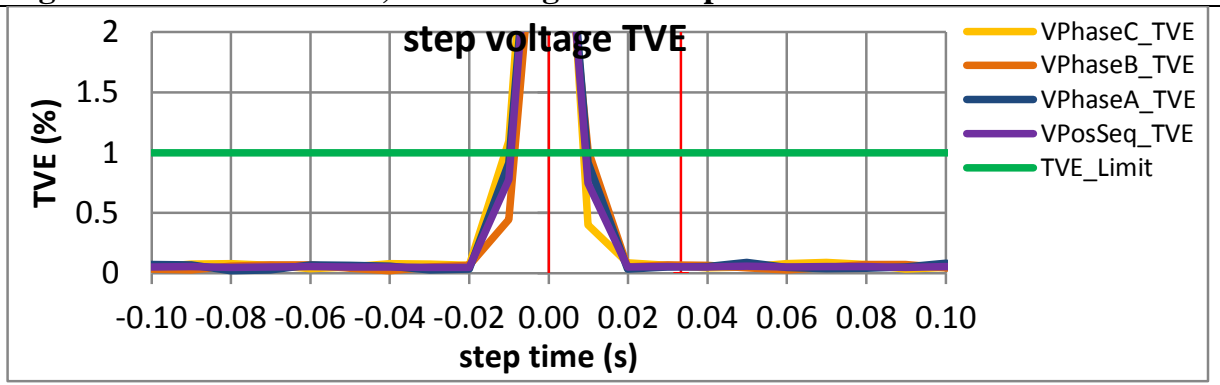

Figure 5399: Fs = 10 FPS, +10\% magnitude step

\section{Figure 5396: Fs = 15 FPS, -10\% magnitude step}

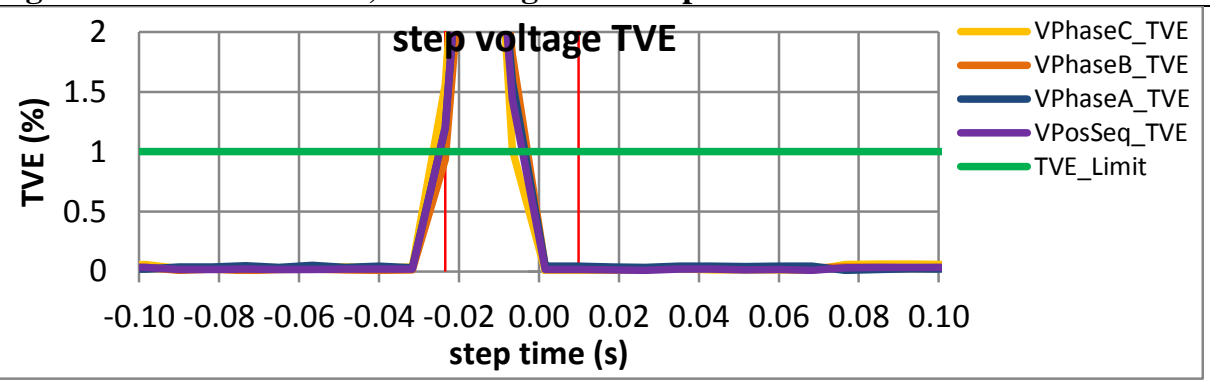

Figure 5398: Fs = 12 FPS, -10\% magnitude step

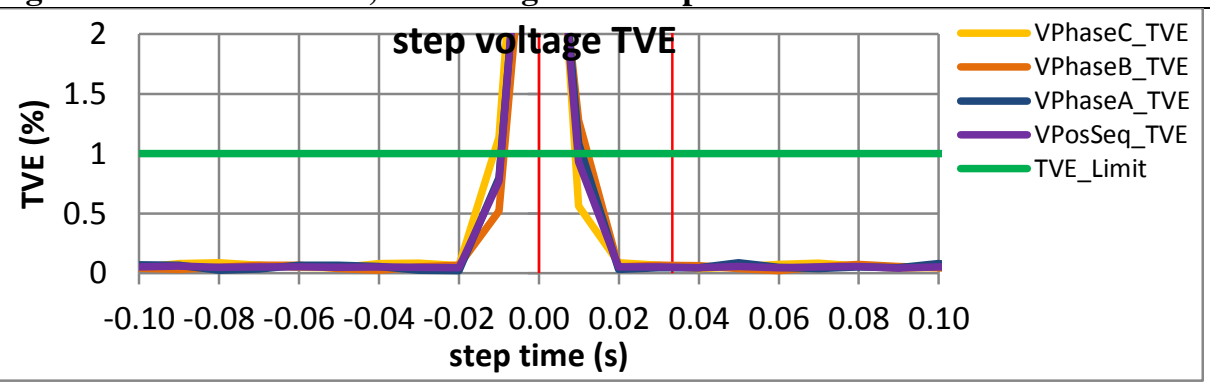

Figure 5400: Fs = 10 FPS, +10\% magnitude step 
10.4.5 PMU D dynamic step change in magnitude voltage response time: $F 0=60 \mathrm{~Hz}, \mathrm{P}$ class
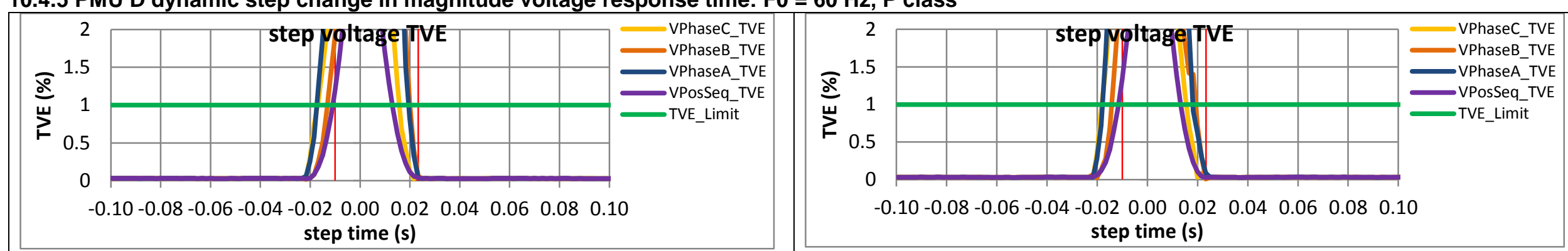

Figure 5401: Fs $=60$ FPS, $+10 \%$ magnitude step

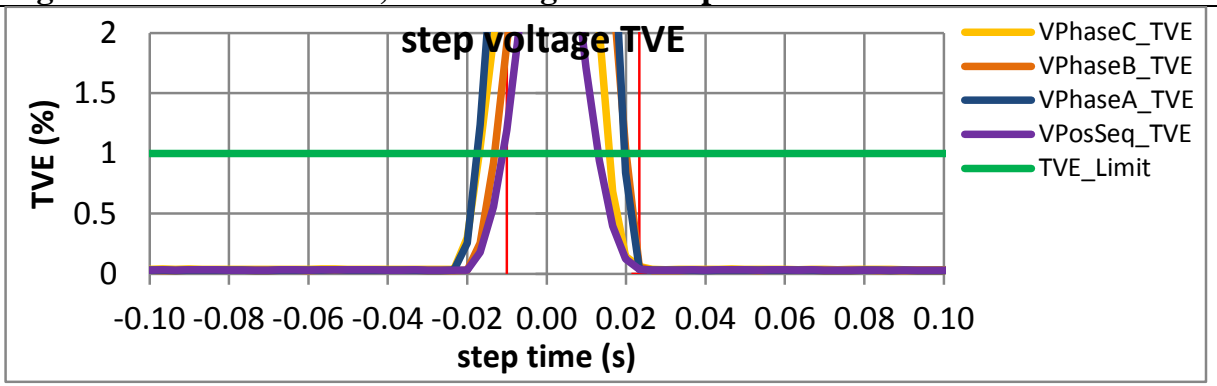

Figure 5402: Fs = $60 \mathrm{FPS},-10 \%$ magnitude step

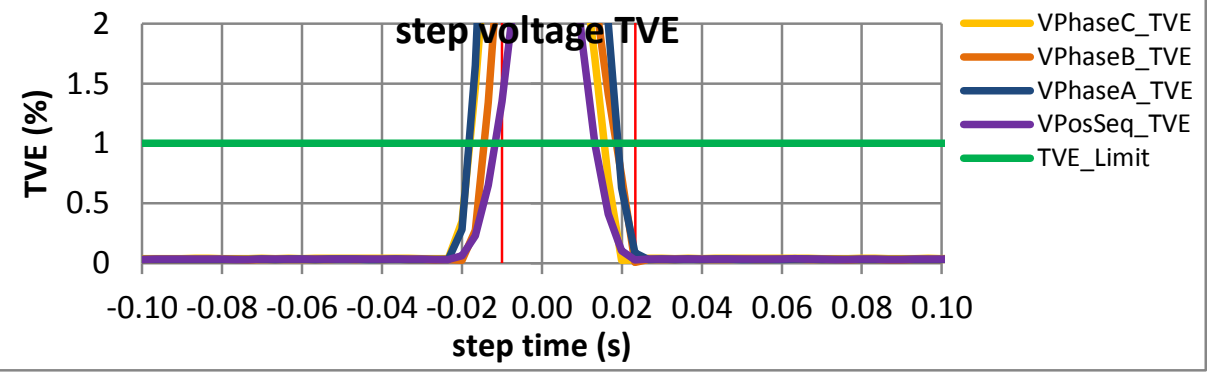

Figure 5403: Fs = 30 FPS, $+10 \%$ magnitude step

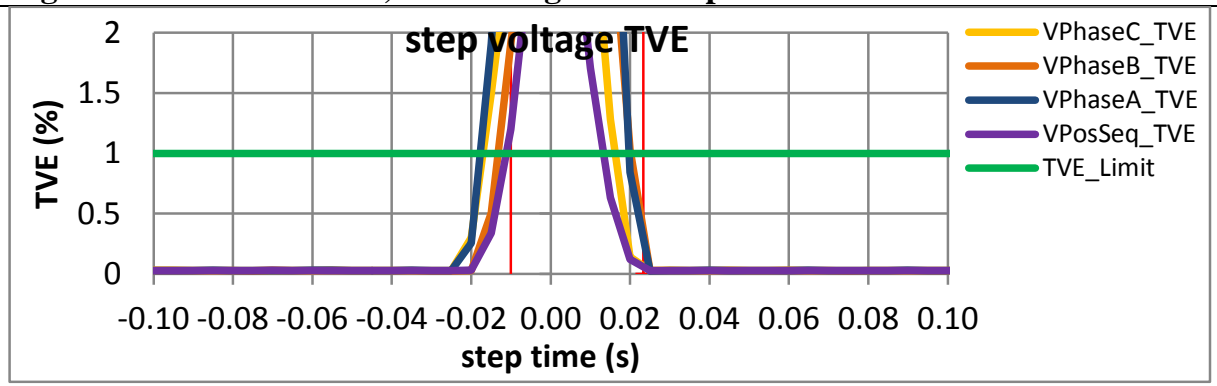

Figure 5404: Fs = 30 FPS, -10\% magnitude step

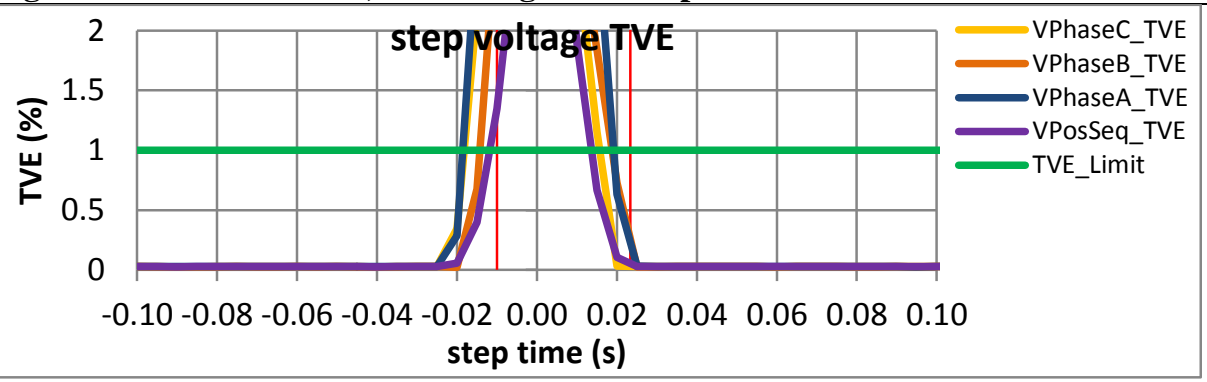

Figure 5405: Fs $=20 \mathrm{FPS},+10 \%$ magnitude step

Figure 5406: Fs = 20 FPS, -10\% magnitude step 


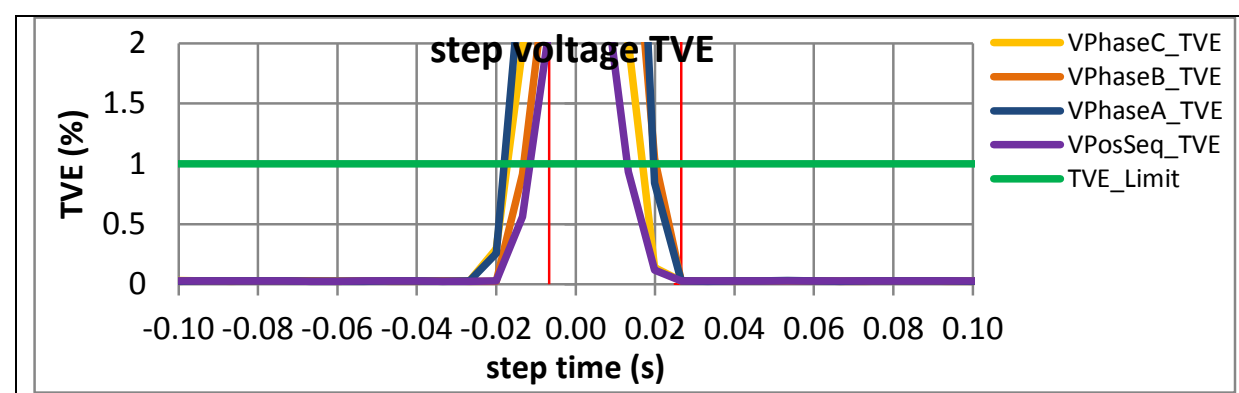

Figure 5407: Fs $=15$ FPS, +10\% magnitude step

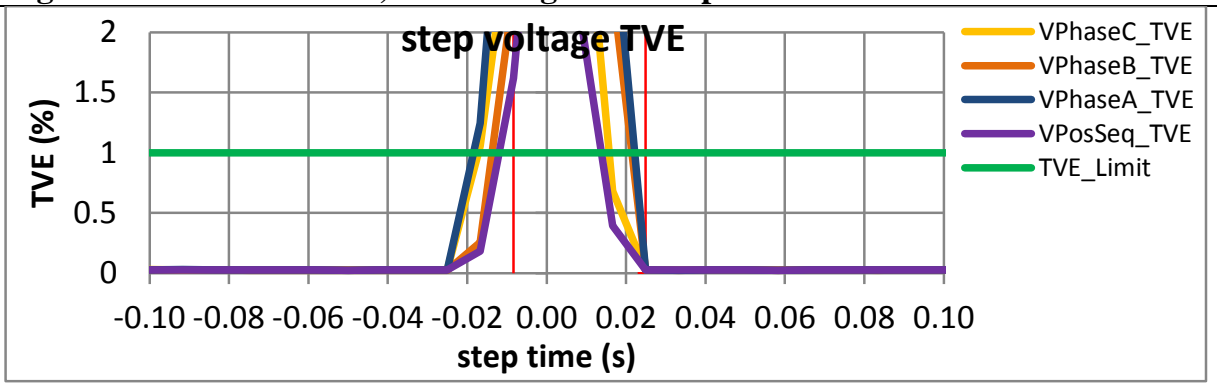

Figure 5409: Fs = 12 FPS, +10\% magnitude step

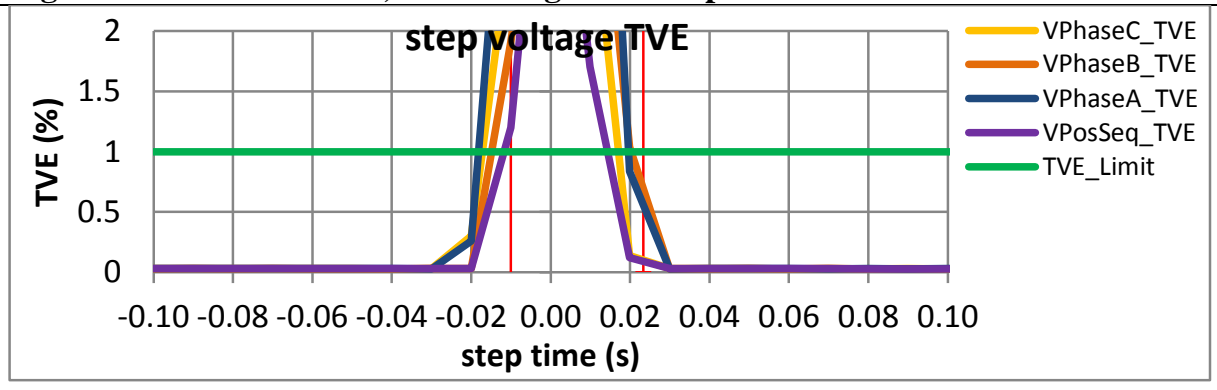

Figure 5411: Fs = 10 FPS, +10\% magnitude step

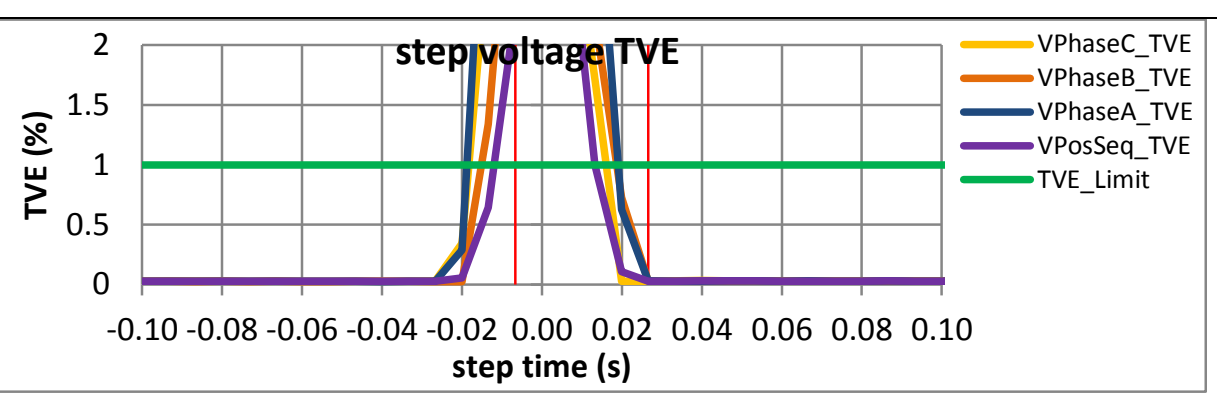

Figure 5408: Fs = 15 FPS, -10\% magnitude step

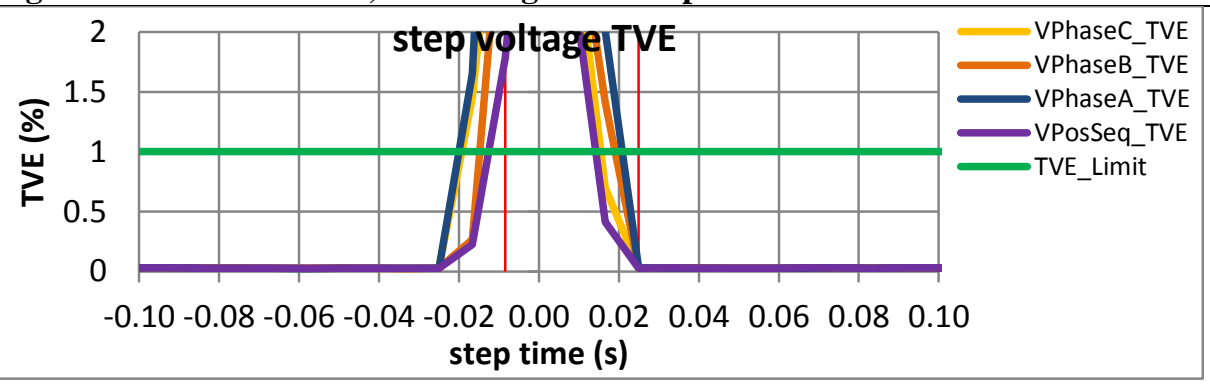

Figure 5410: Fs = 12 FPS, - $10 \%$ magnitude step

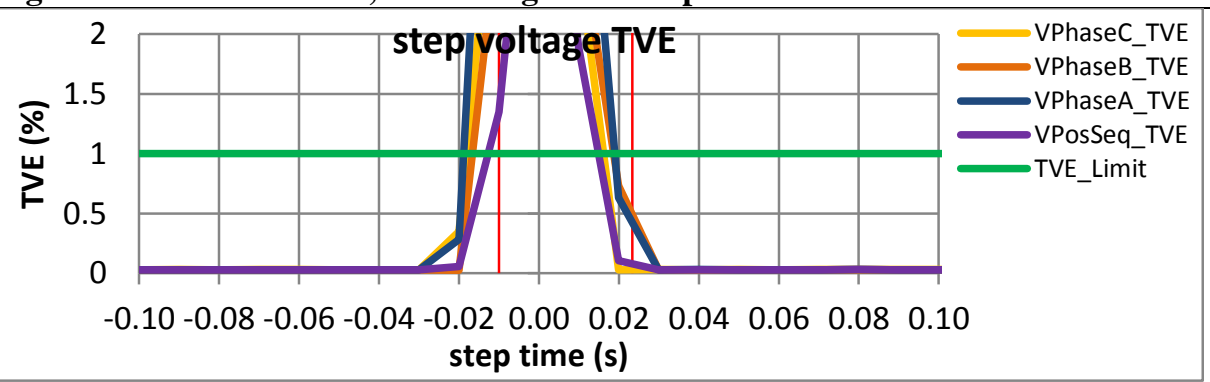

Figure 5412: Fs $=10$ FPS, $+10 \%$ magnitude step 
10.4.6 PMU E dynamic step change in magnitude voltage response time: $F 0=60 \mathrm{~Hz}, \mathrm{P}$ class PMU E does not support P class.

\subsubsection{PMU F dynamic step change in magnitude voltage response time: $F 0=60 \mathrm{~Hz}, \mathrm{P}$ class}
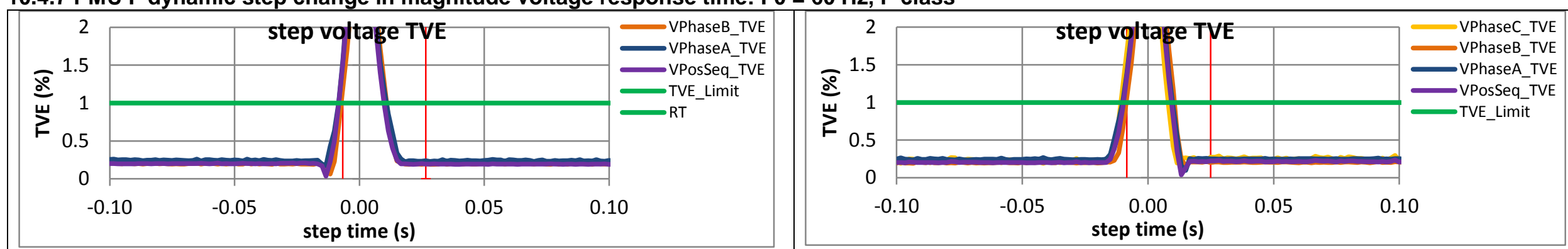

Figure 5413: Fs = $60 \mathrm{FPS},+10 \%$ magnitude step

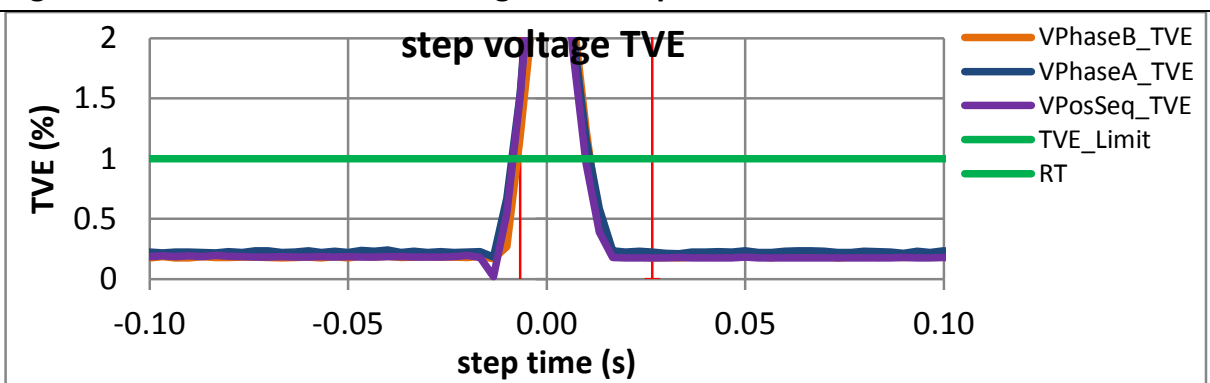

Figure 5414: Fs = 60 FPS, $-10 \%$ magnitude step

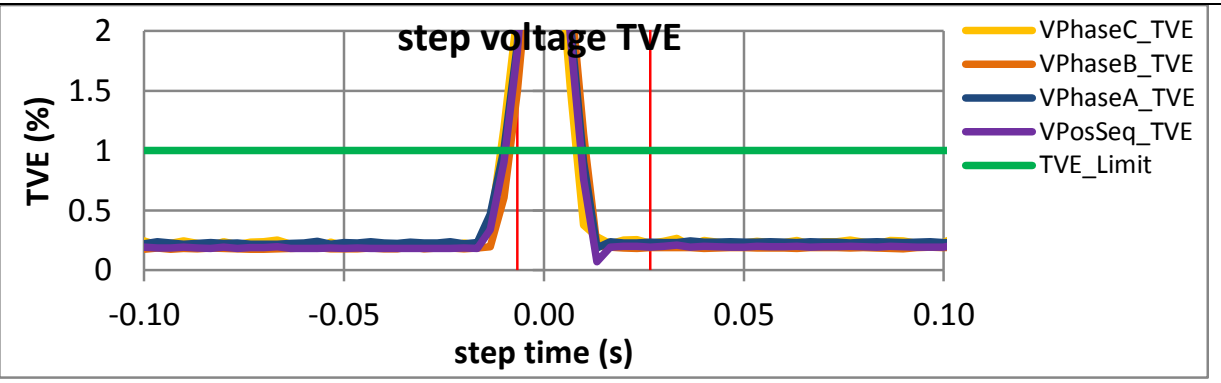

Figure 5415: Fs = 30 FPS, $+10 \%$ magnitude step

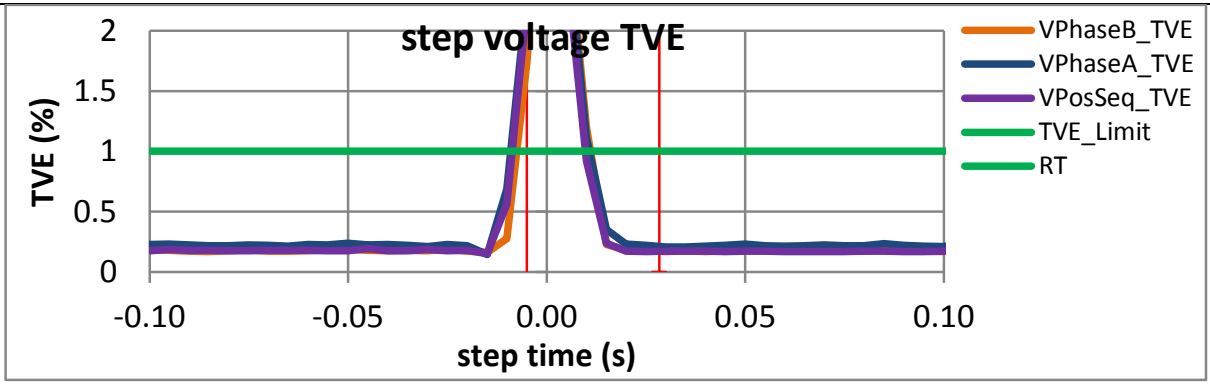

Figure 5416: Fs = $30 \mathrm{FPS},-10 \%$ magnitude step

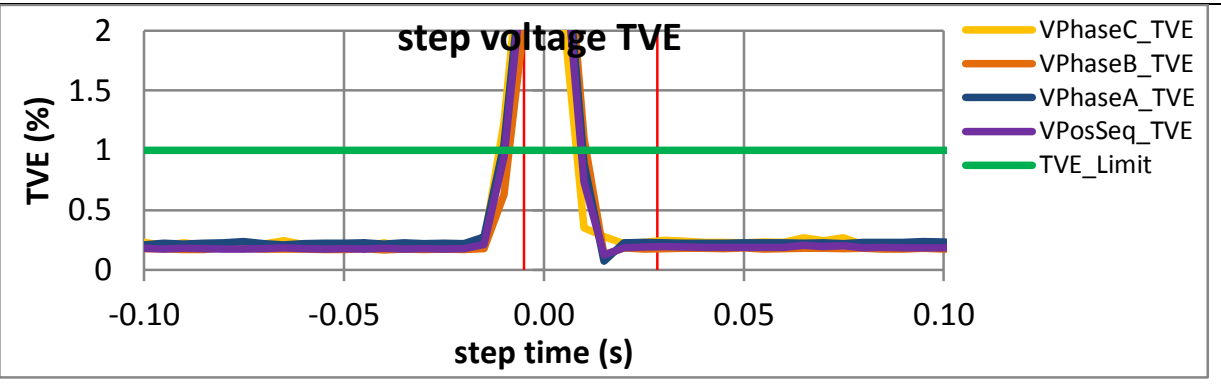

Figure 5418: Fs = 20 FPS, $-10 \%$ magnitude step 

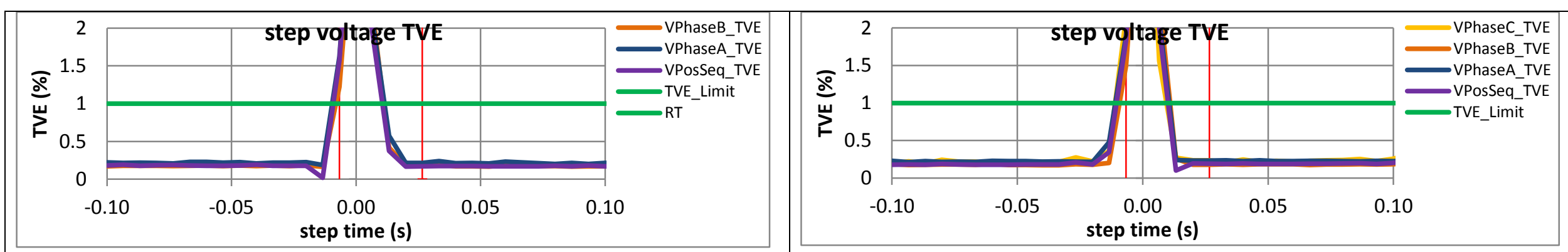

Figure 5419: Fs = $15 \mathrm{FPS},+10 \%$ magnitude step

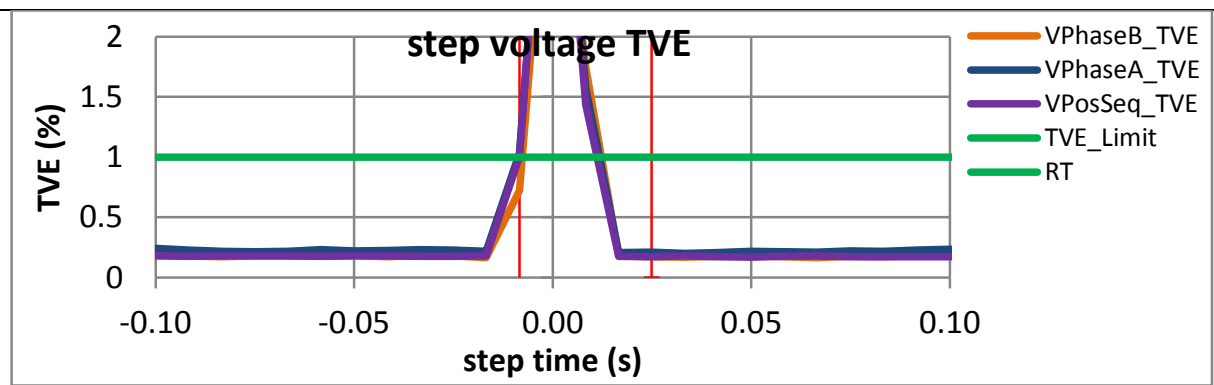

Figure 5420: Fs = 15 FPS, $-10 \%$ magnitude step

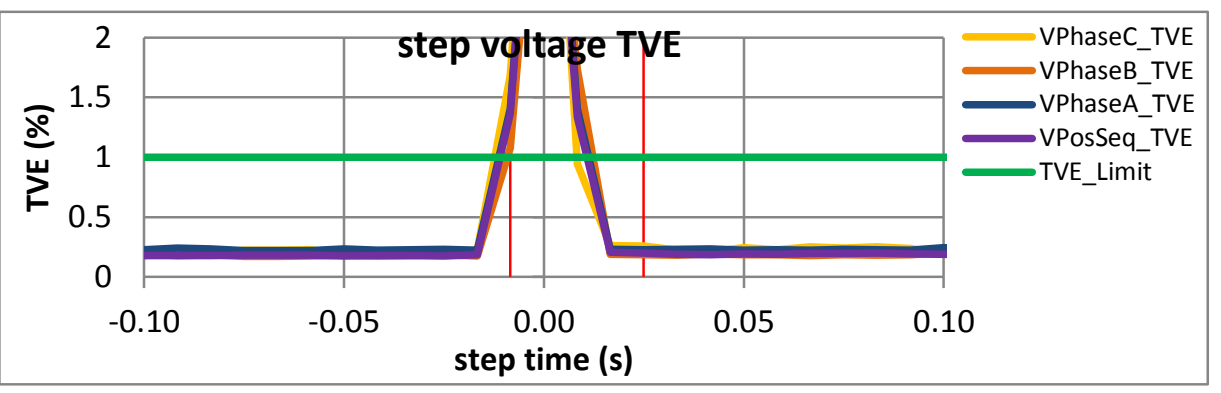

Figure 5421: Fs = 12 FPS, +10\% magnitude step

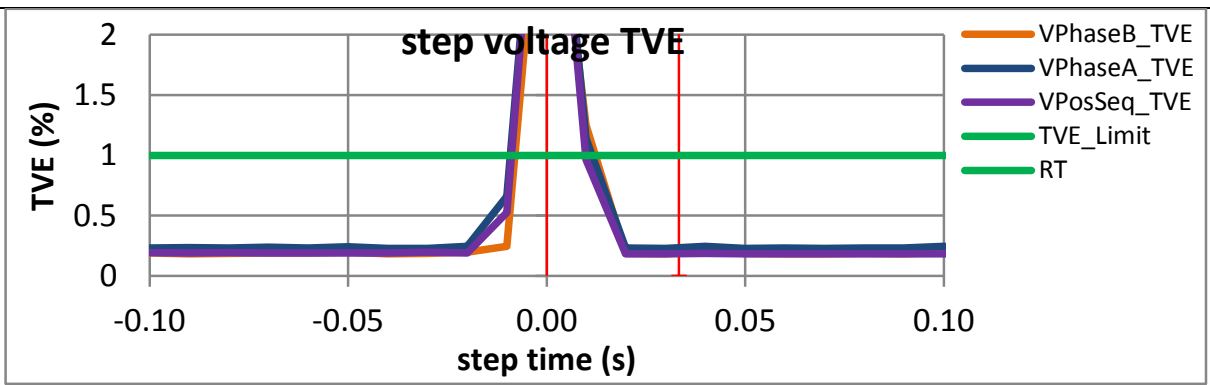

Figure 5422: Fs = 12 FPS, $-10 \%$ magnitude step

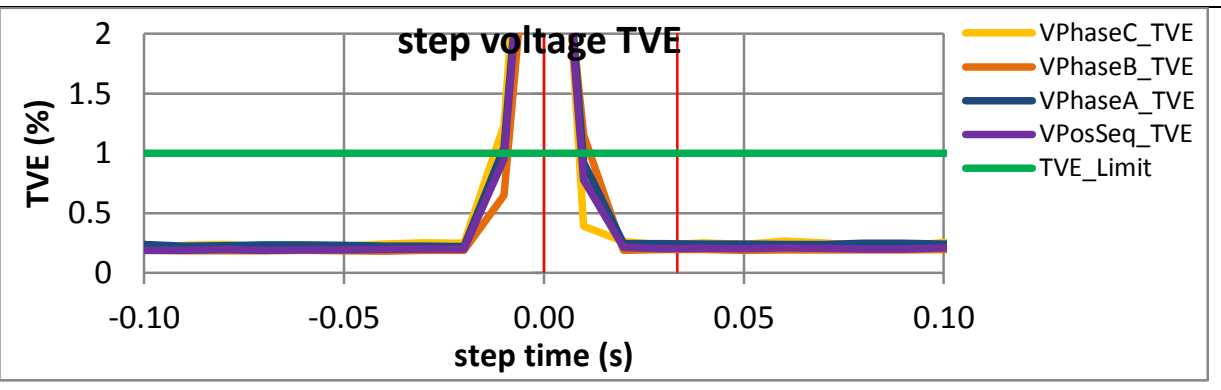

Figure 5424: Fs = 10 FPS, +10\% magnitude step 
10.4.8 PMU G dynamic step change in magnitude voltage response time: $F 0=60 \mathrm{~Hz}, \mathrm{P}$ class PMU G does not support P class.

10.4.9 PMU H dynamic step change in magnitude voltage response time: $\mathrm{F} 0=60 \mathrm{~Hz}, \mathrm{P}$ class
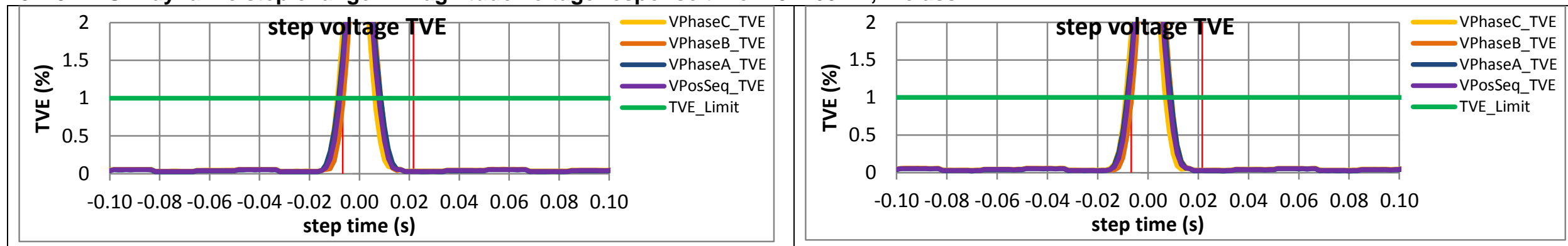

Figure 5425: Fs $=60 \mathrm{FPS},+10 \%$ magnitude step

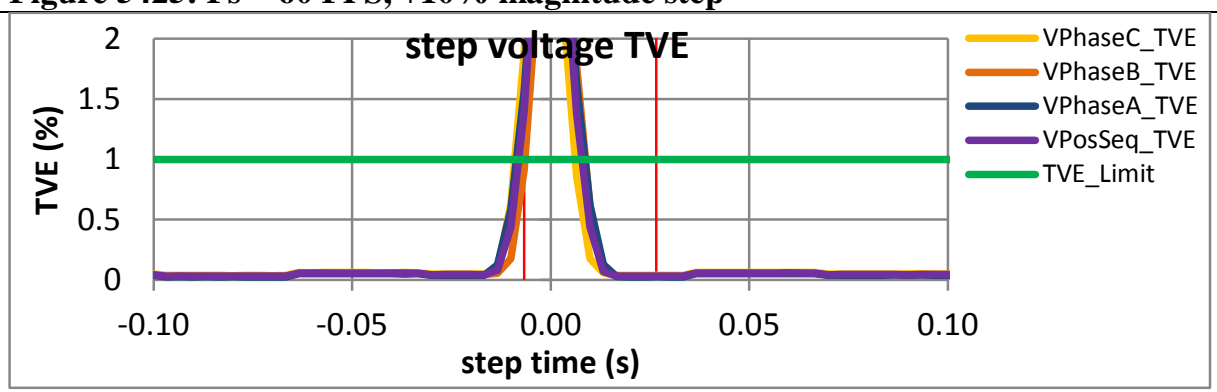

Figure 5426: Fs = 60 FPS, -10\% magnitude step

Figure 5427: Fs $=30 \mathrm{FPS},+10 \%$ magnitude step

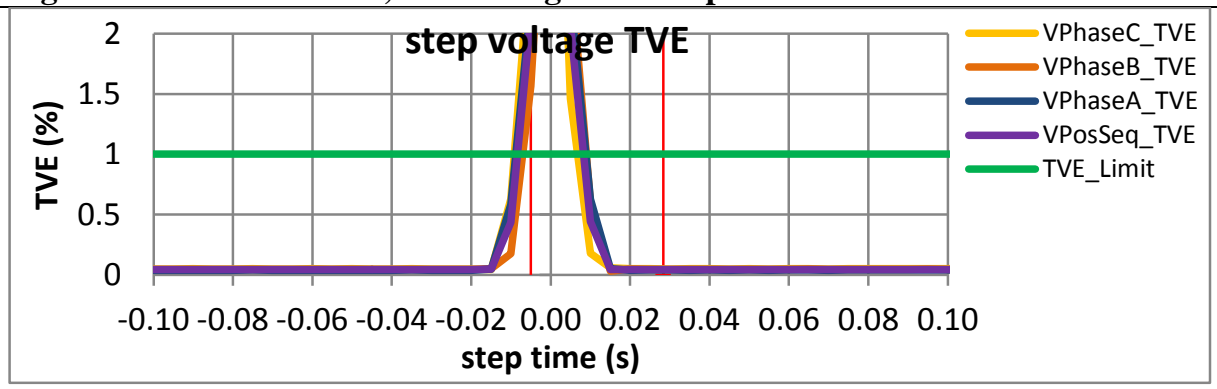

Figure 5429: Fs = 20 FPS, +10\% magnitude step

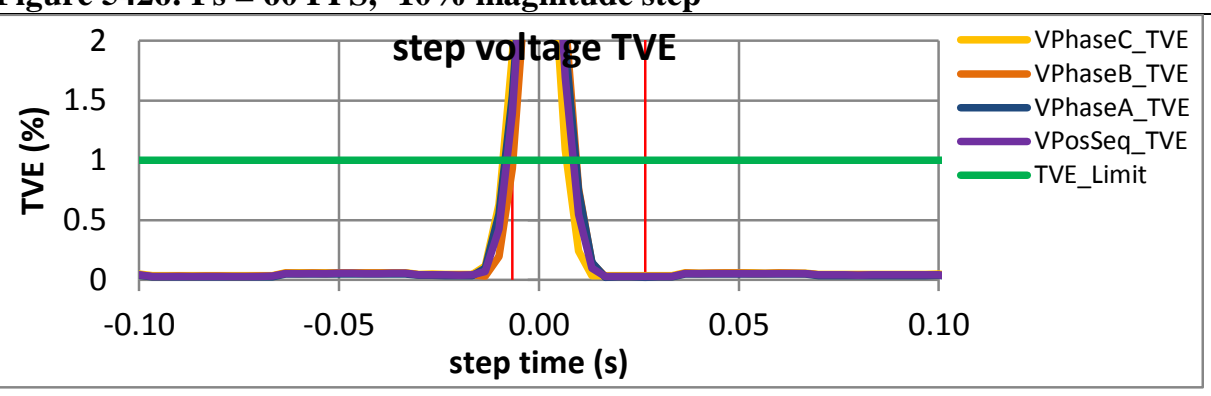

Figure 5428: Fs = 30 FPS, $-10 \%$ magnitude step

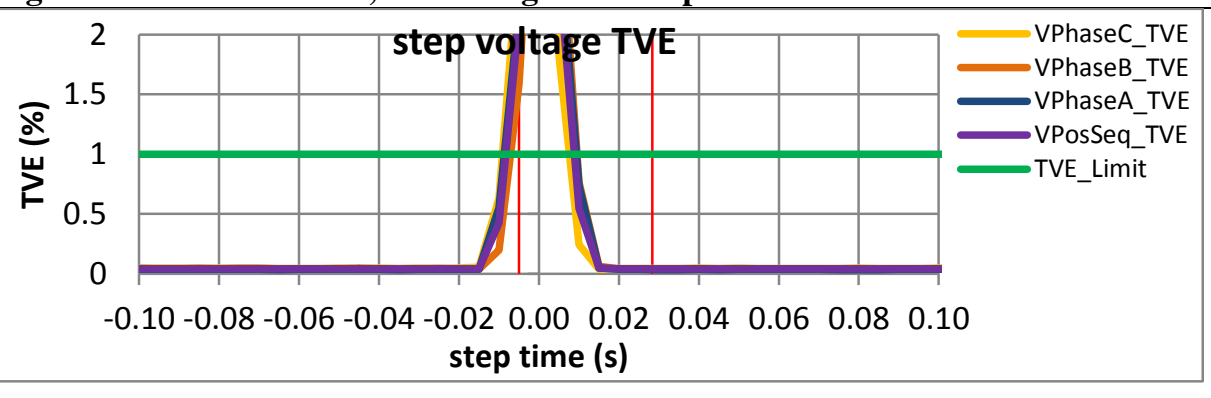

Figure 5430: Fs = 20 FPS, -10\% magnitude step 


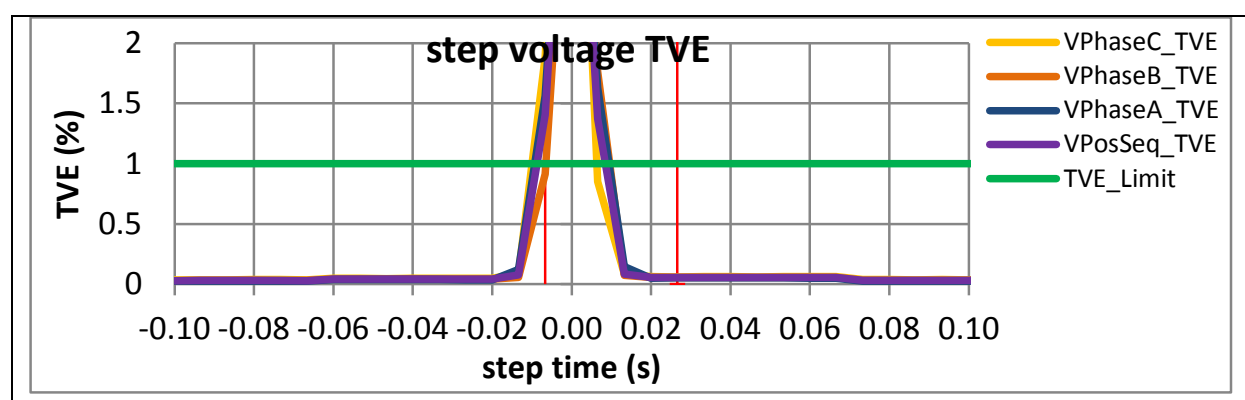

Figure 5431: Fs = 15 FPS, +10\% magnitude step
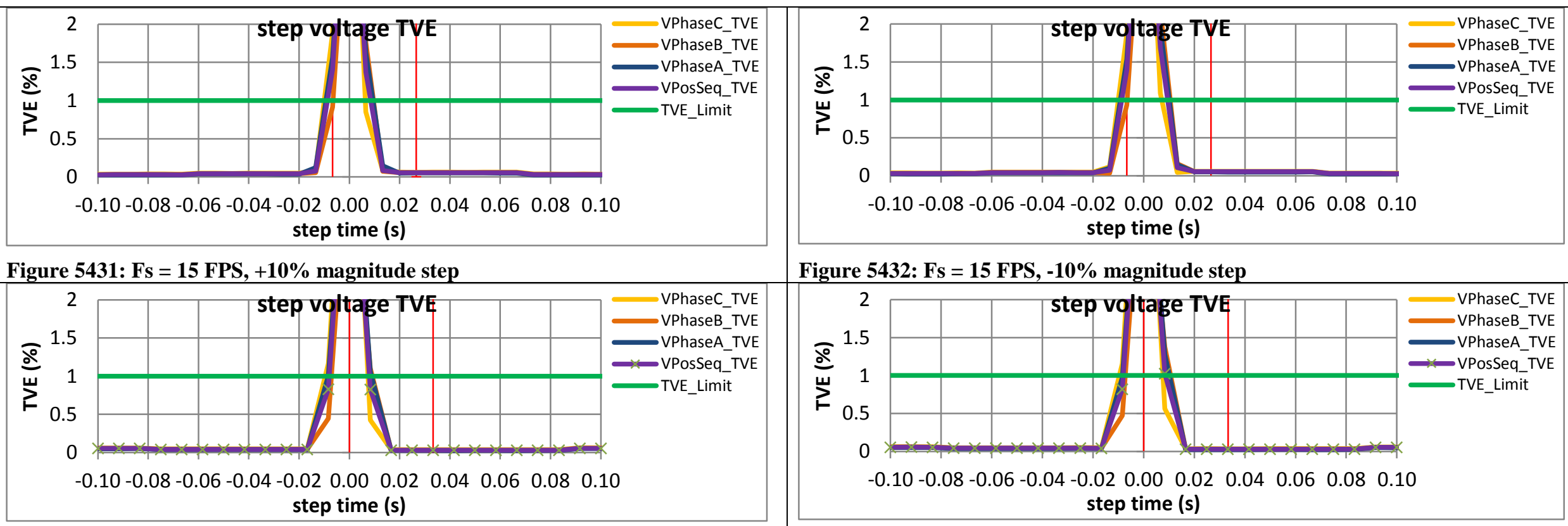

Figure 5432: Fs = 15 FPS, -10\% magnitude step

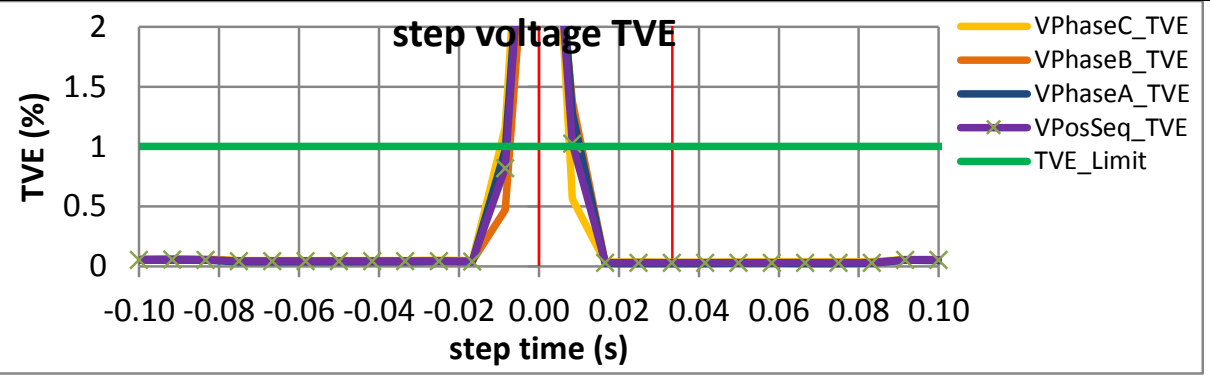

Figure 5433: Fs = 12 FPS, $+10 \%$ magnitude step

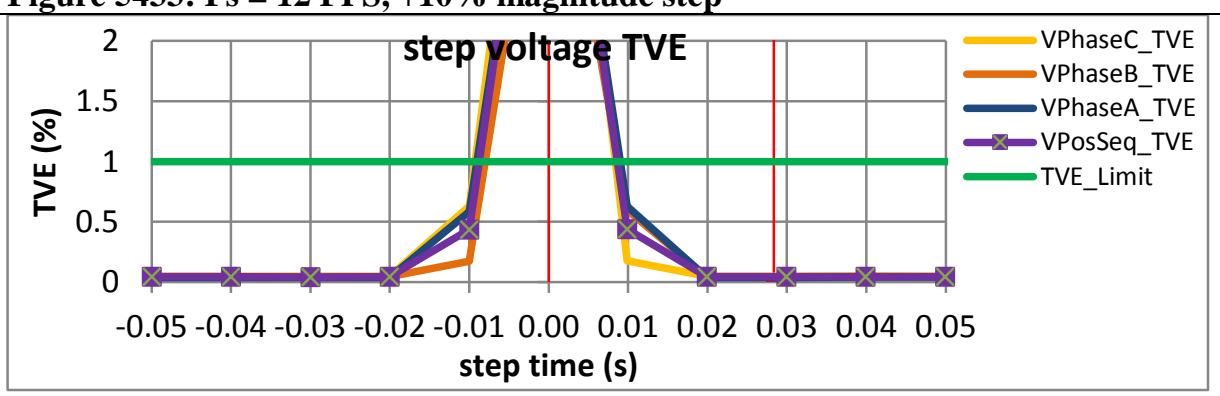

Figure 5435: Fs $=10 \mathrm{FPS},+10 \%$ magnitude step

\section{Figure 5434: Fs = 12 FPS, -10\% magnitude step}

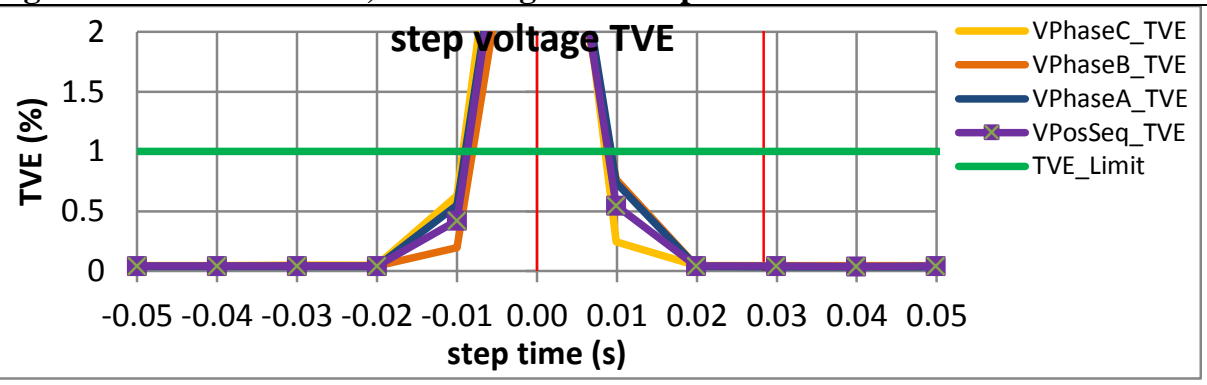

Figure 5436: Fs = 10 FPS, $+10 \%$ magnitude step 
10.4.10 PMU I dynamic step change in magnitude voltage response time: $F 0=60 \mathrm{~Hz}, \mathrm{P}$ class PMI I does not support P class

\subsubsection{PMU J dynamic step change in magnitude voltage response time: $\mathrm{F} 0=60 \mathrm{~Hz}, \mathrm{P}$ class}

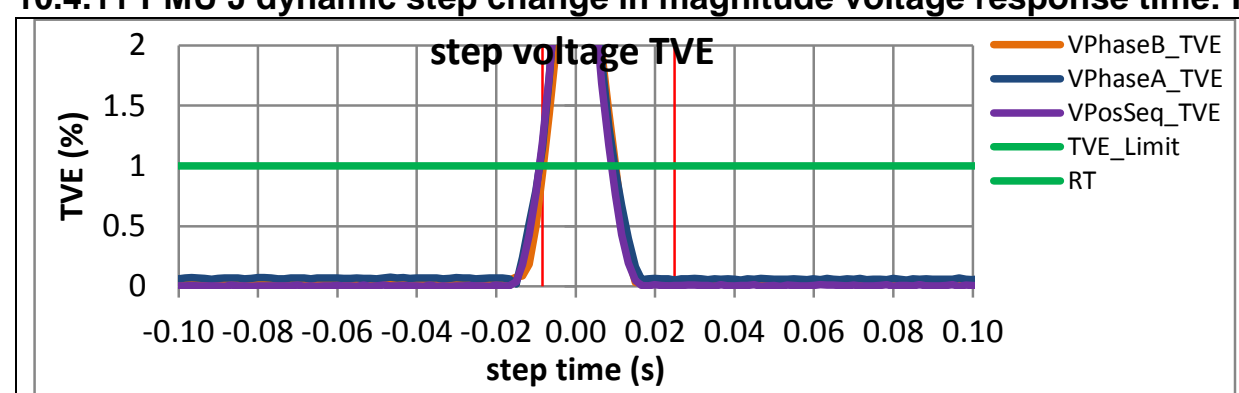

Figure 5437: Fs = 60 FPS, $+10 \%$ magnitude step

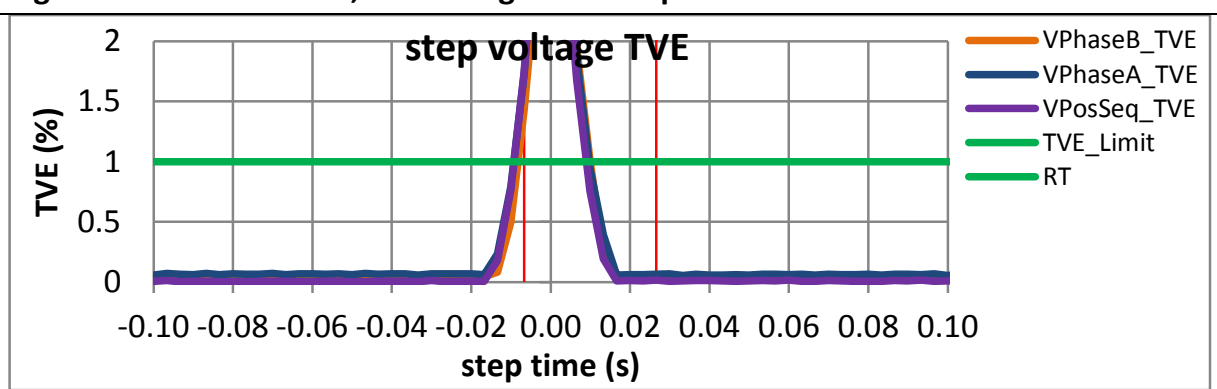

Figure 5439: Fs = 30 FPS, +10\% magnitude step

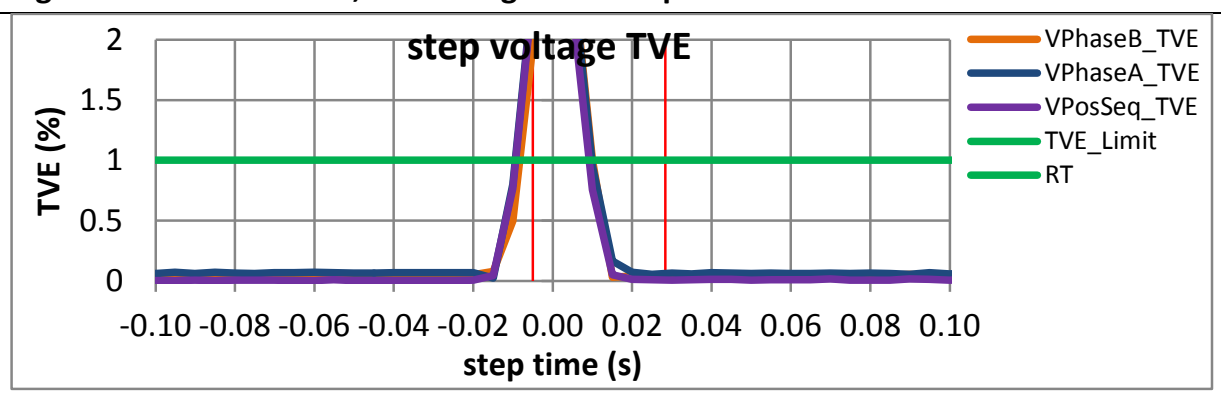

Figure 5441: Fs = 20 FPS, +10\% magnitude step

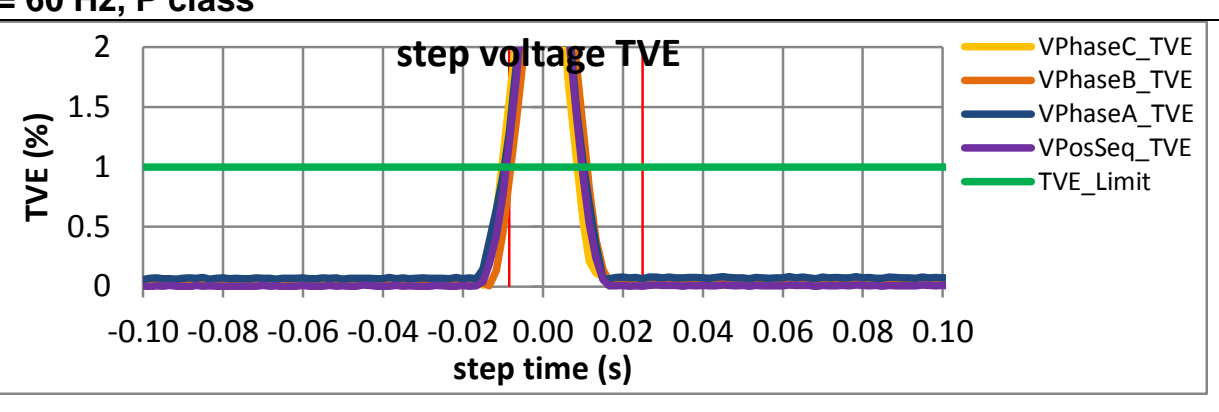

Figure 5438: Fs = 60 FPS, -10\% magnitude step

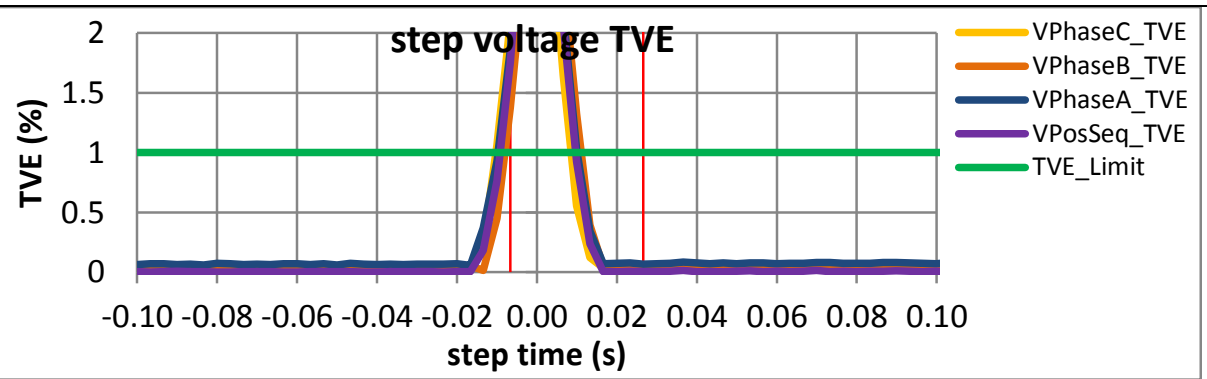

Figure 5440: Fs = 30 FPS, -10\% magnitude step

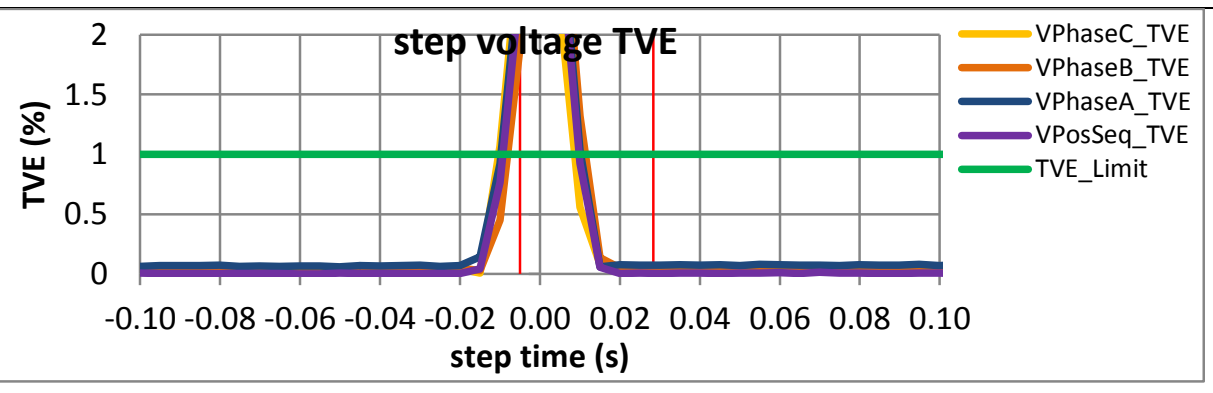

Figure 5442: Fs = 20 FPS, -10\% magnitude step 

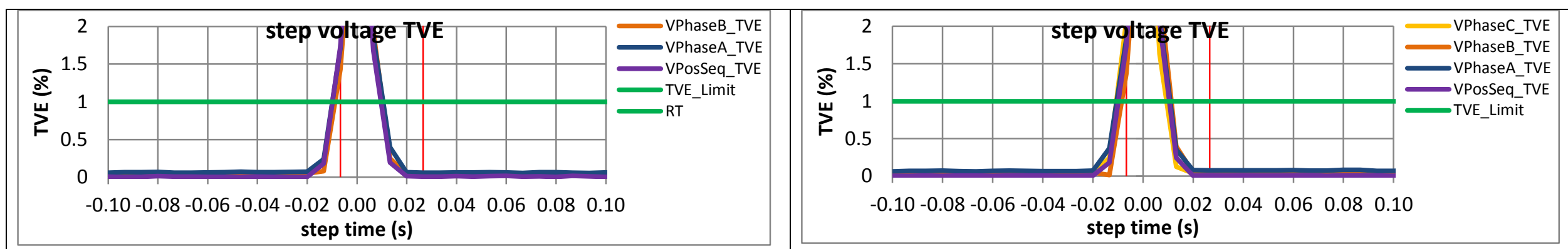

Figure 5443: Fs = $15 \mathrm{FPS},+10 \%$ magnitude step

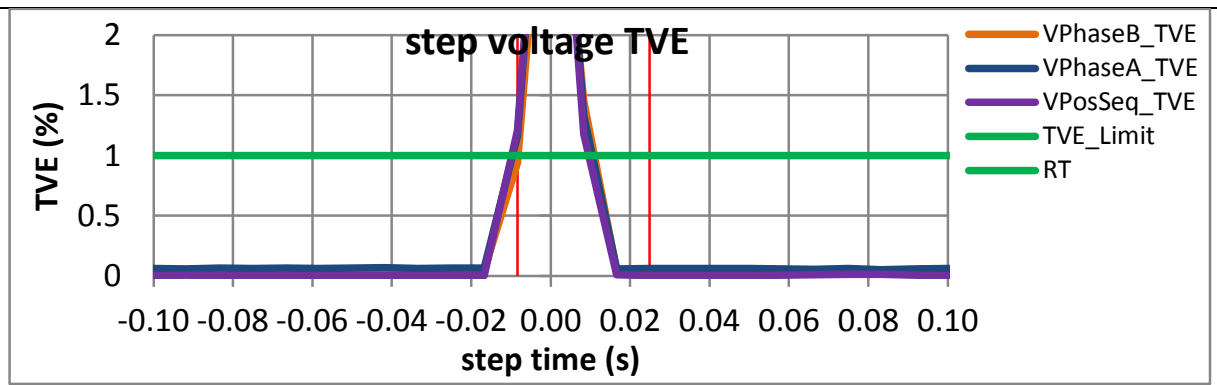

Figure 5444: Fs = 15 FPS, -10\% magnitude step

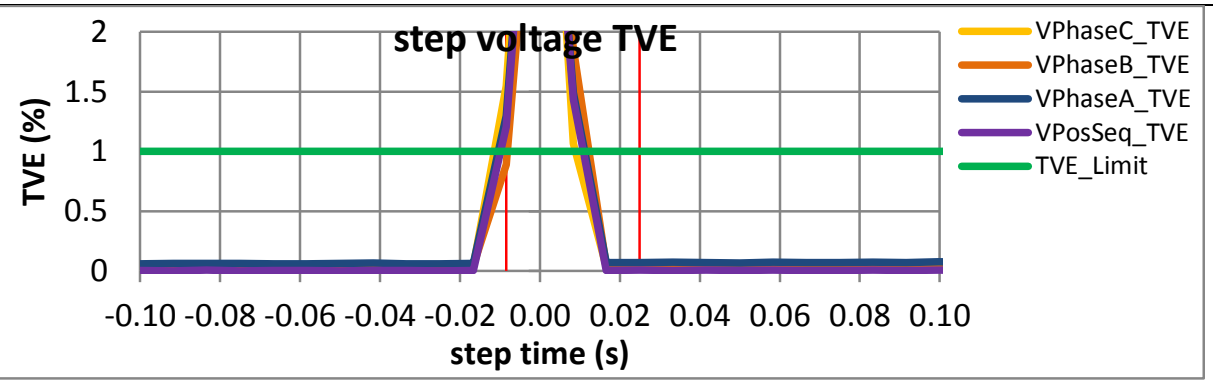

Figure 5445: Fs = 12 FPS, $+10 \%$ magnitude step

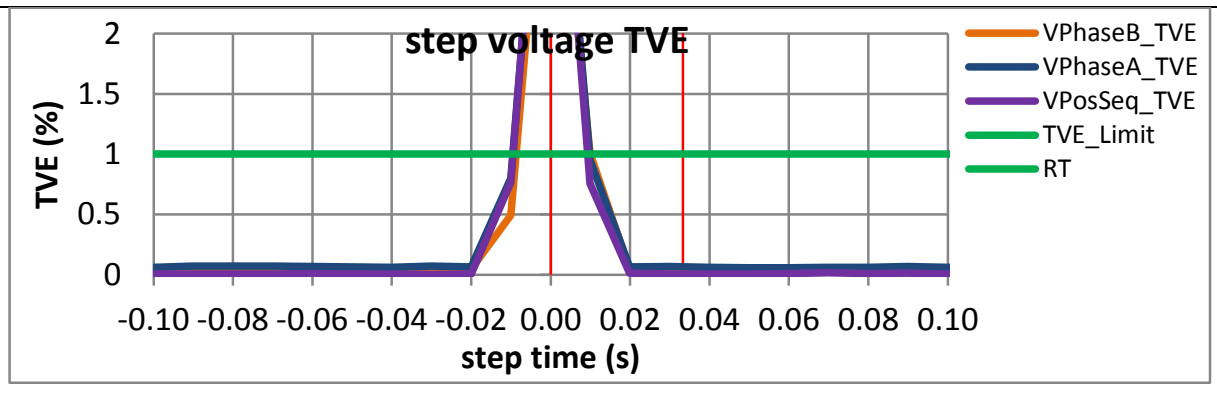

Figure 5446: Fs = 12 FPS, $-10 \%$ magnitude step

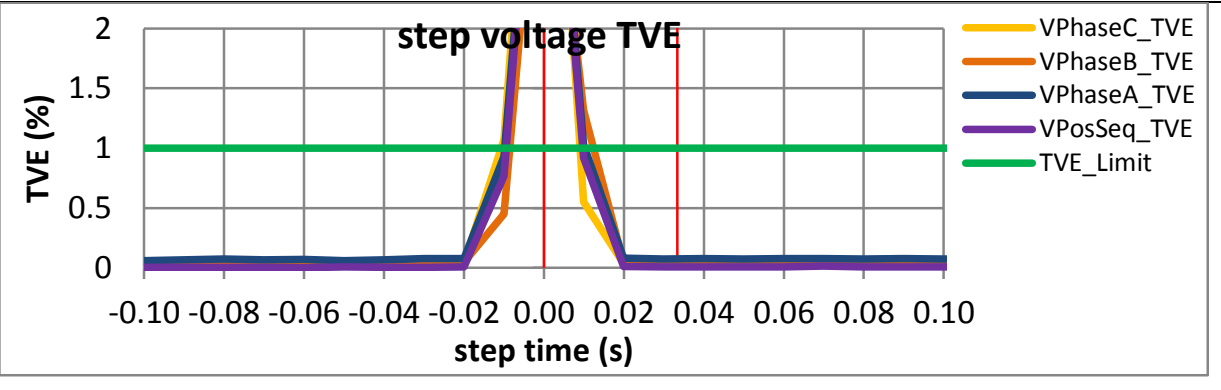

Figure 5448: Fs = 10 FPS, $+10 \%$ magnitude step 
10.5 Dynamic step change in magnitude current response time: $F 0=60 \mathrm{~Hz}$, $P$ class

10.5.1 C37.118.1-2011 Annex $C$ dynamic step change in magnitude current response time: $F 0=60 \mathrm{~Hz}$, $P$ class
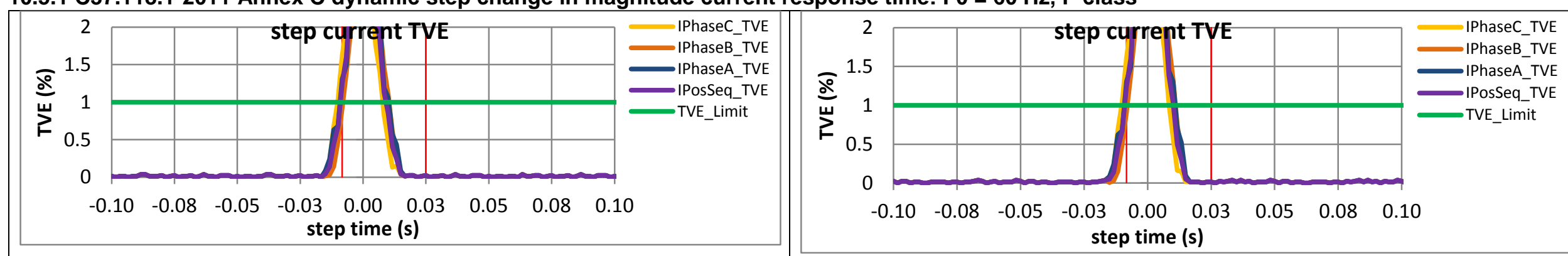

Figure 5449: Fs = 60 FPS, $+10 \%$ magnitude step

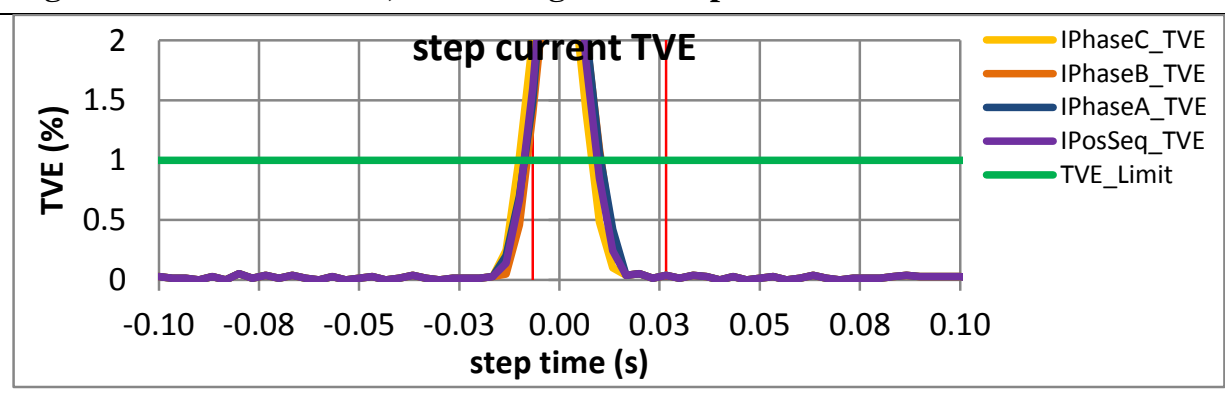

Figure 5450: Fs = 60 FPS, -10\% magnitude step

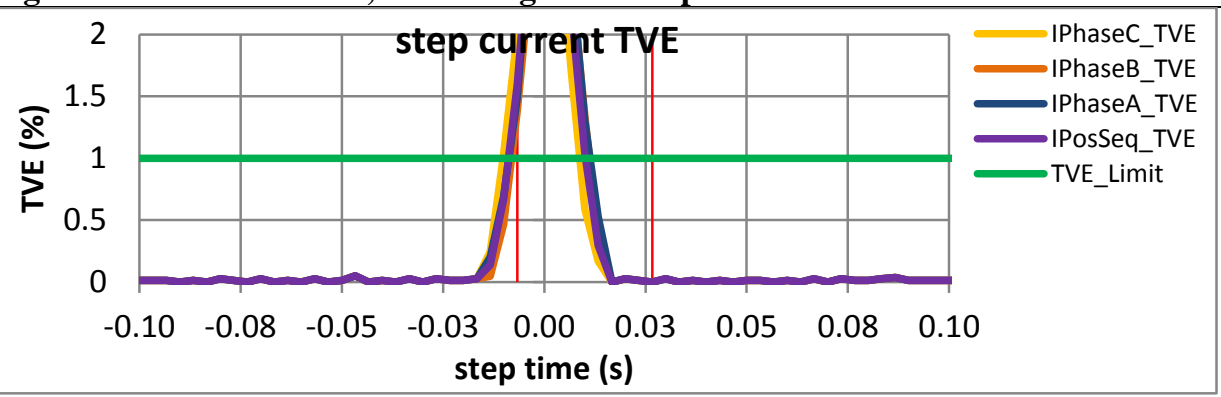

Figure 5451: Fs = 30 FPS, +10\% magnitude step

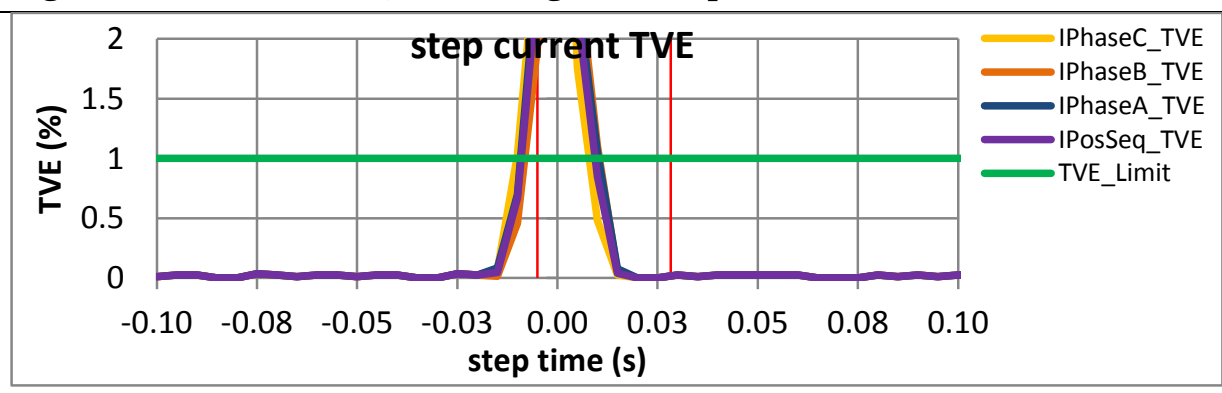

Figure 5453: Fs $=20$ FPS, $+10 \%$ magnitude step

\section{Figure 5452: Fs = 30 FPS, -10\% magnitude step}

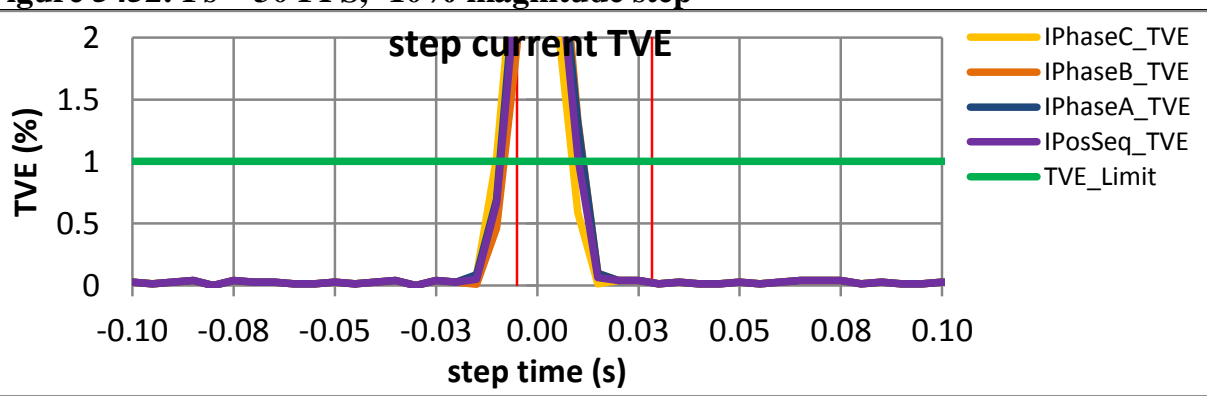

Figure 5454: Fs = 20 FPS, +10\% magnitude step 


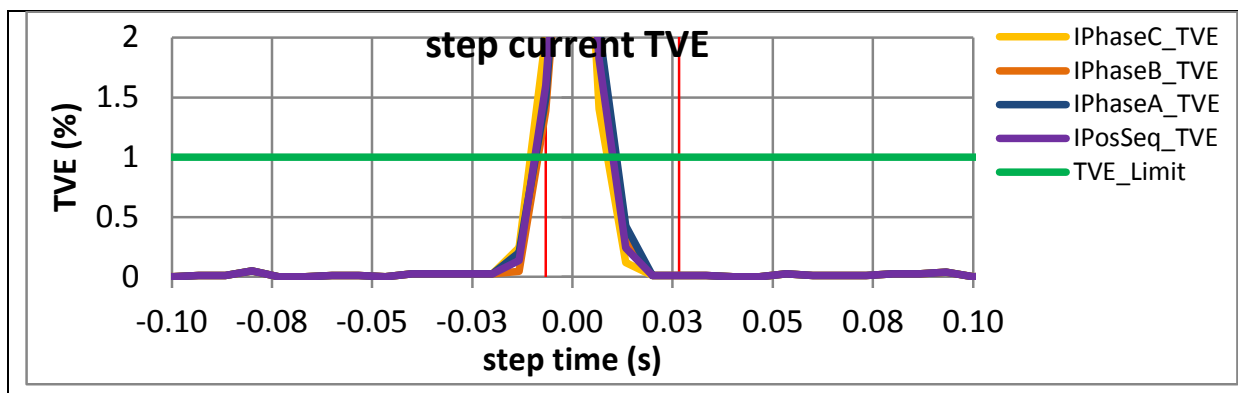

Figure 5455: Fs = 15 FPS, +10\% magnitude step

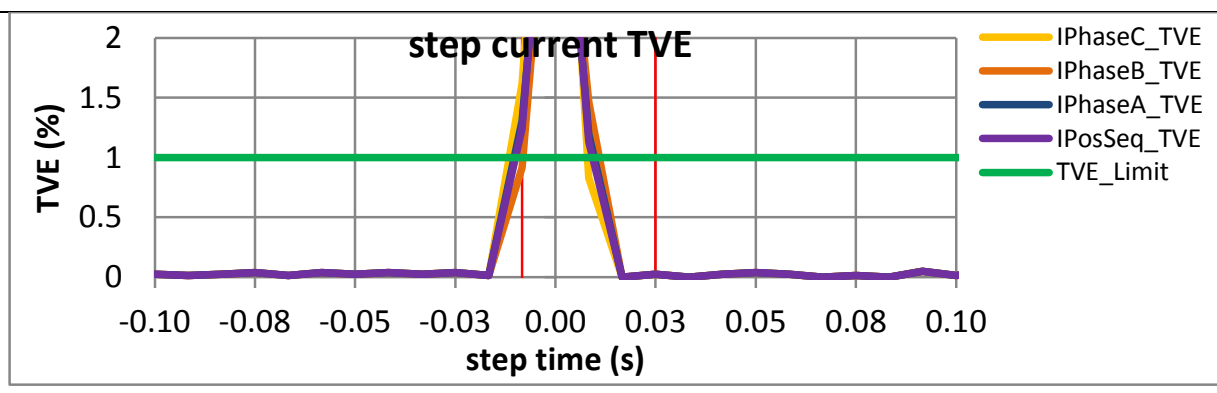

Figure 5457: Fs $=12$ FPS, $+10 \%$ magnitude step

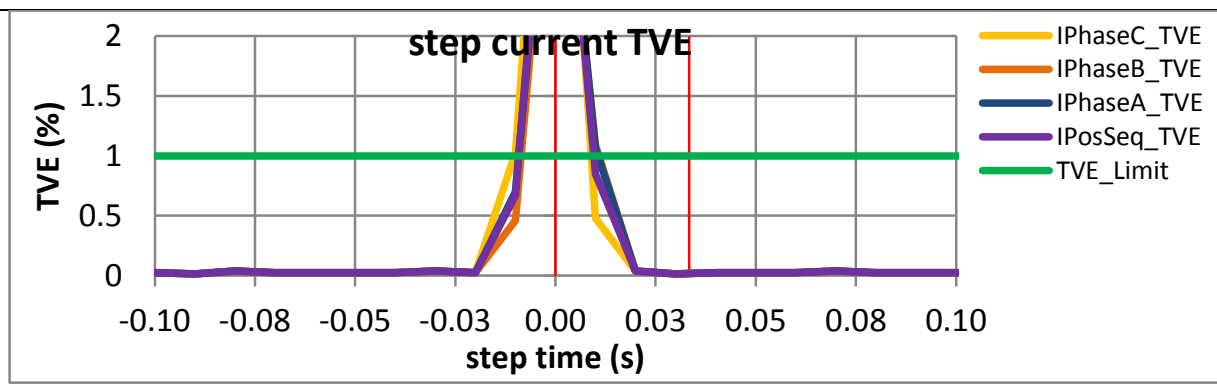

Figure 5459: Fs $=10$ FPS, $+10 \%$ magnitude step

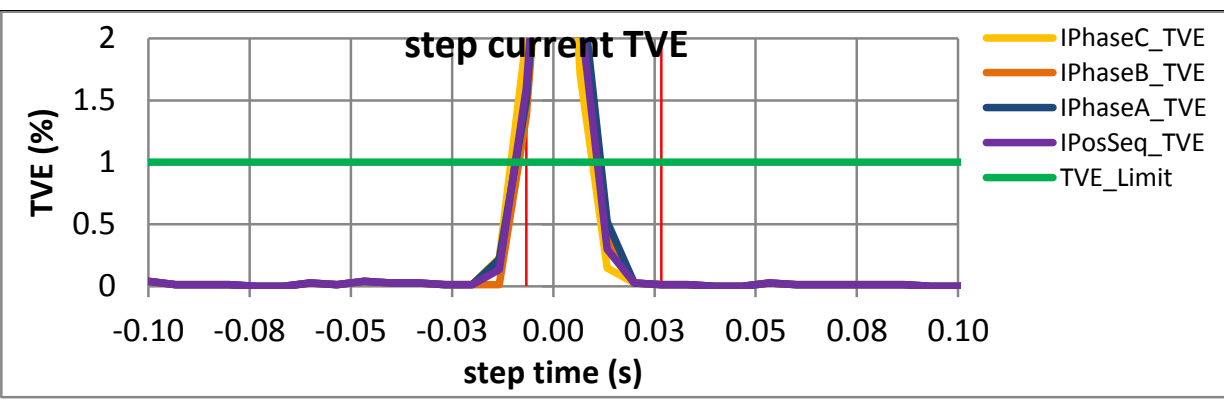

Figure 5456: Fs = 15 FPS, -10\% magnitude step

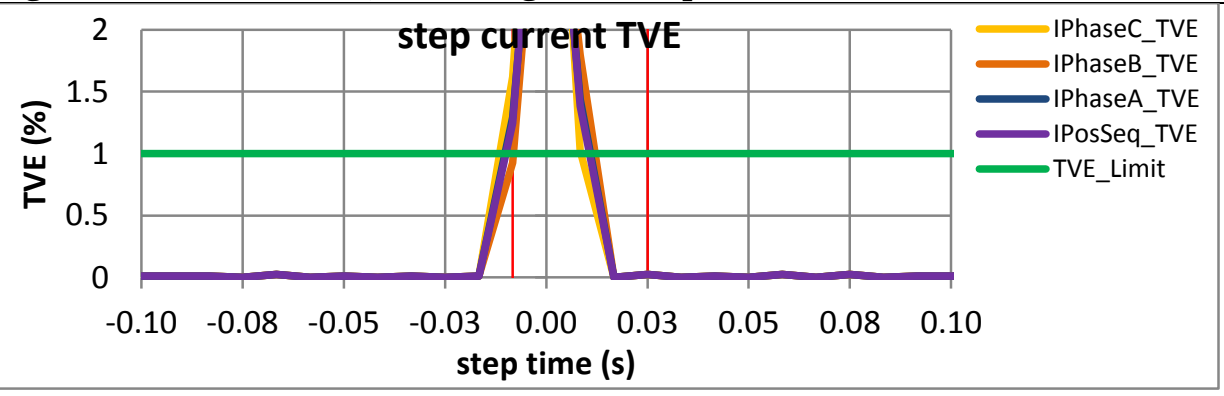

Figure 5458: Fs = 12 FPS, $-10 \%$ magnitude step

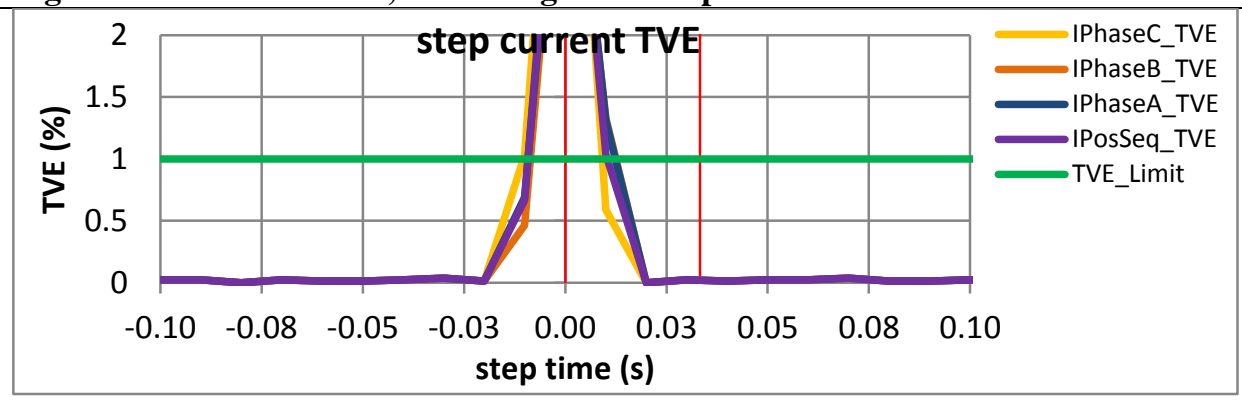

Figure 5460: Fs = 10 FPS, -10\% magnitude step 
10.5.2 PMU A dynamic step change in magnitude current response time: $F 0=60 \mathrm{~Hz}, \mathrm{P}$ class
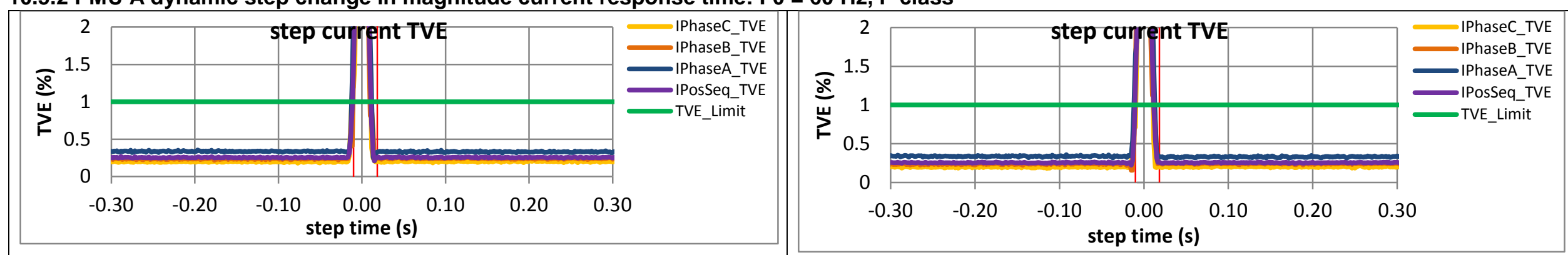

Figure 5461: Fs = 60 FPS, +10\% magnitude step

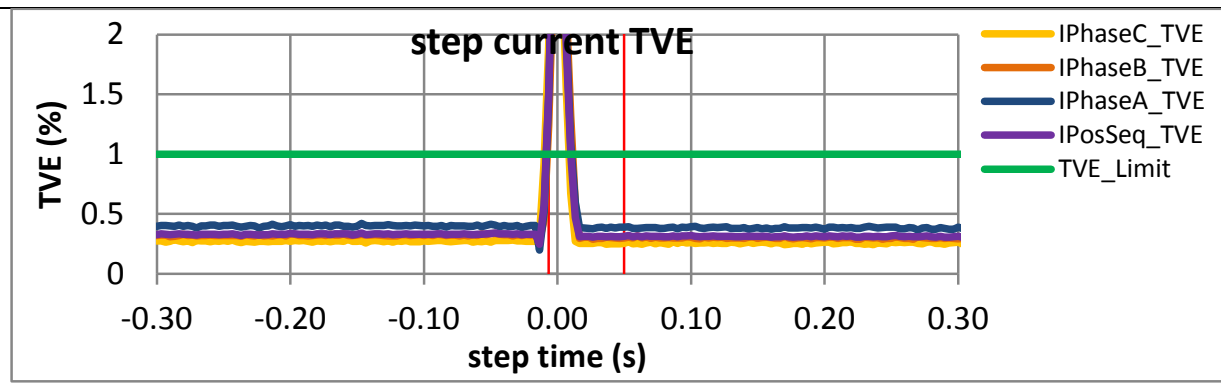

Figure 5462: Fs = 60 FPS, -10\% magnitude step

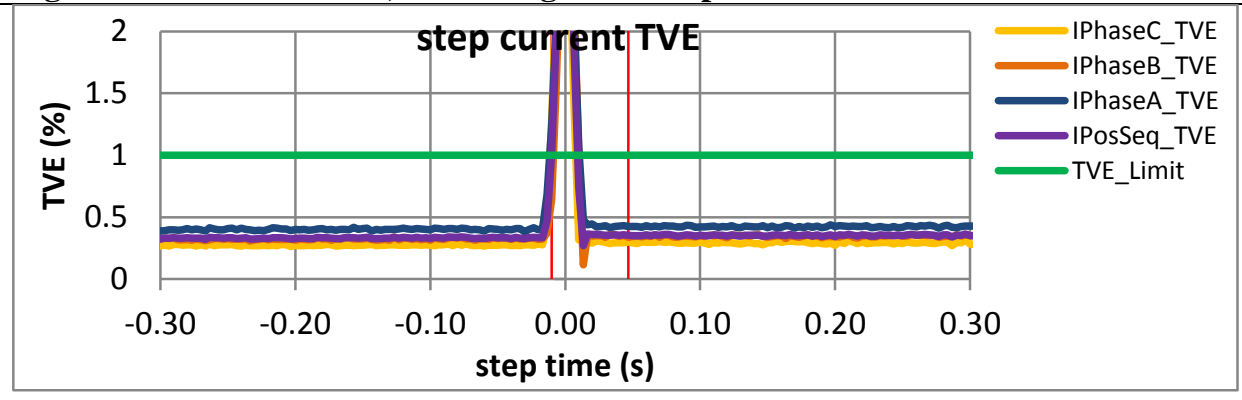

Figure 5463: Fs = 30 FPS, $+10 \%$ magnitude step

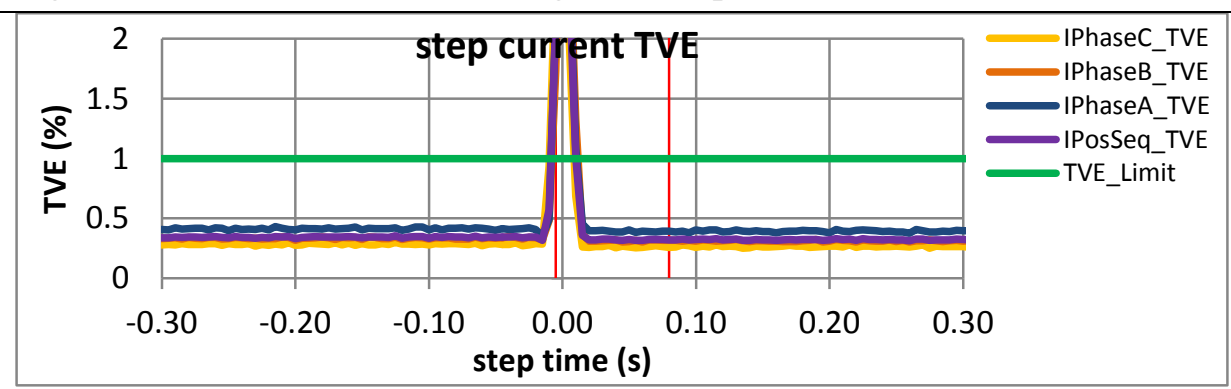

Figure 5464: Fs = 30 FPS, -10\% magnitude step

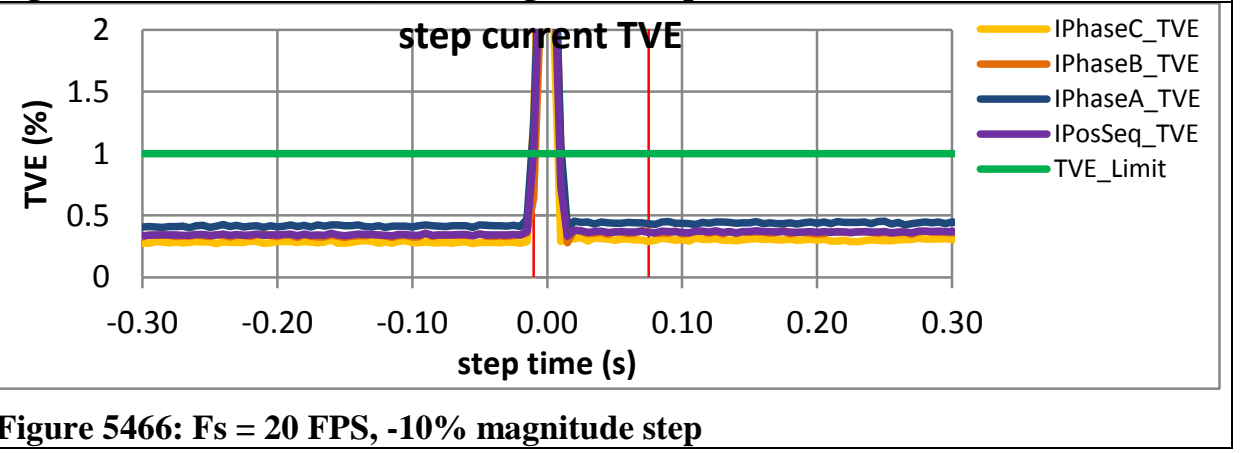

Figure 5465: Fs = 20 FPS, $+10 \%$ magnitude step

Figure 5466: Fs = 20 FPS, -10\% magnitude step 

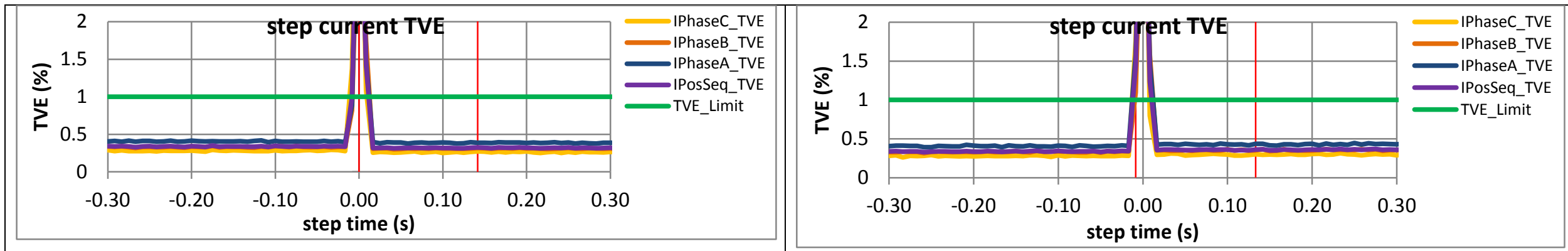

Figure 5467: Fs $=15$ FPS, $+10 \%$ magnitude step

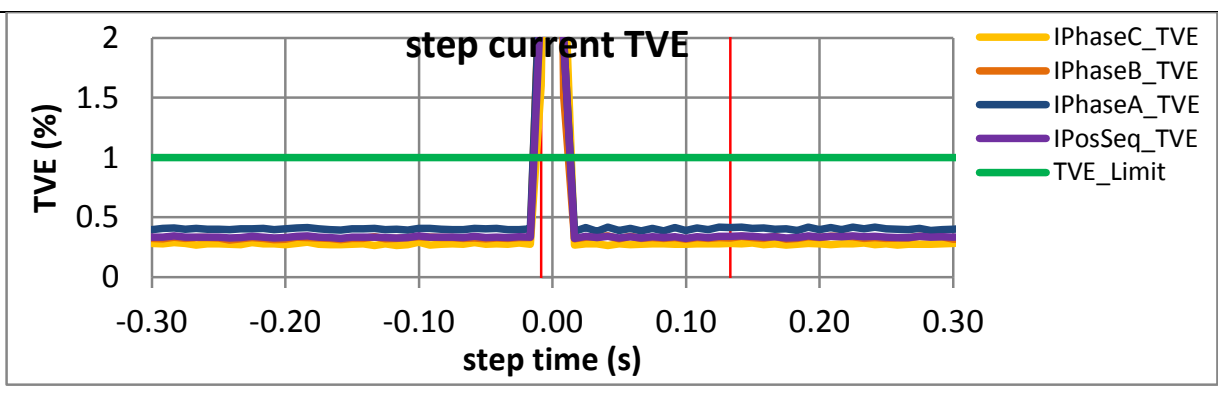

Figure 5468: Fs = 15 FPS, $-10 \%$ magnitude step

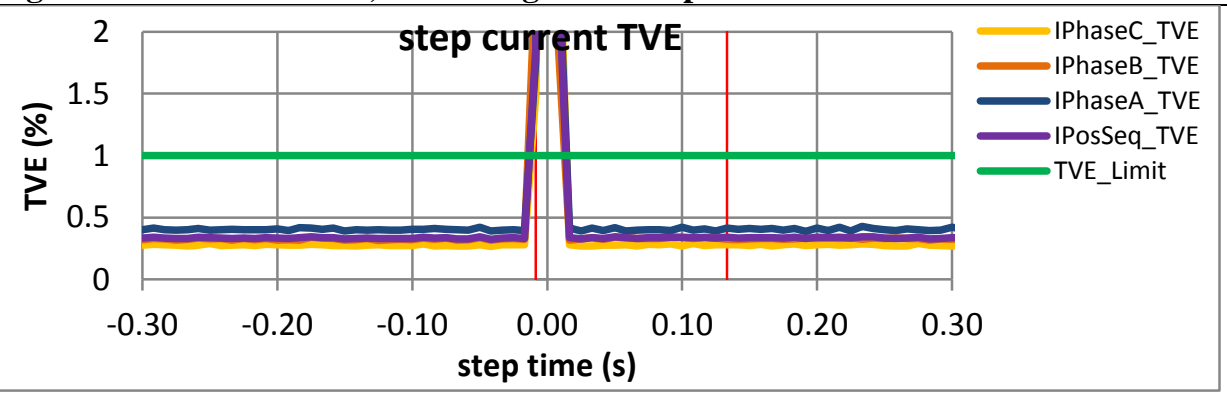

Figure 5469: Fs $=12 \mathrm{FPS},+10 \%$ magnitude step

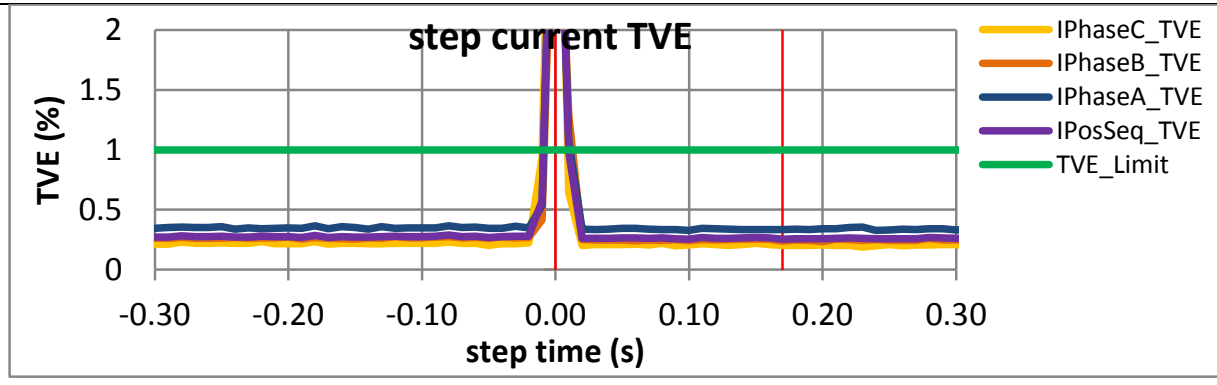

Figure 5470: Fs = 12 FPS, -10\% magnitude step

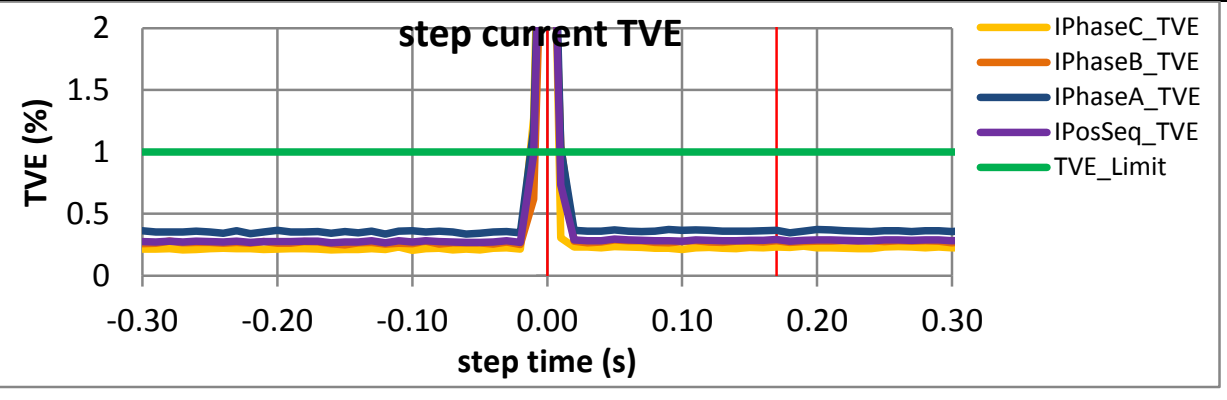

Figure 5471: Fs = 10 FPS, $+10 \%$ magnitude step

Figure 5472: Fs = 10 FPS, $+10 \%$ magnitude step 
10.5.3 PMU B dynamic step change in magnitude current response time: $F 0=60 \mathrm{~Hz}, \mathrm{P}$ class

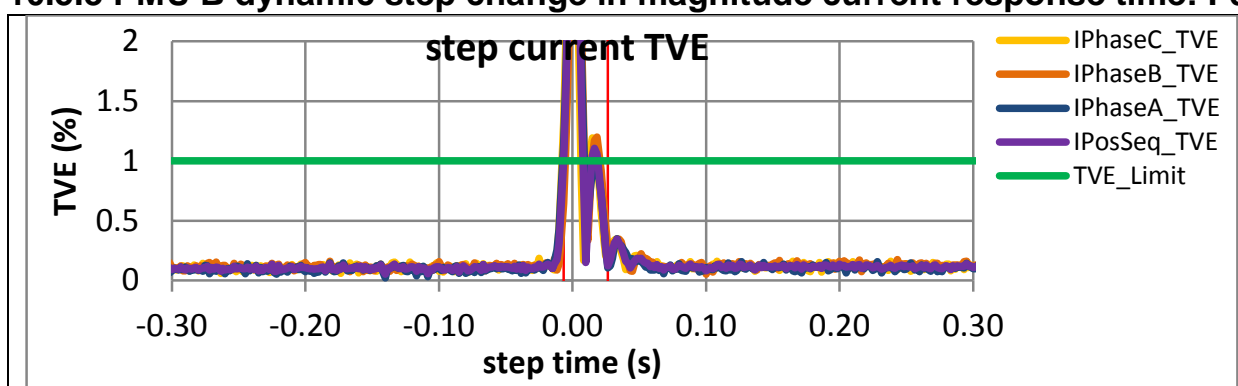

Figure 5473: Fs = 60 FPS, +10\% magnitude step

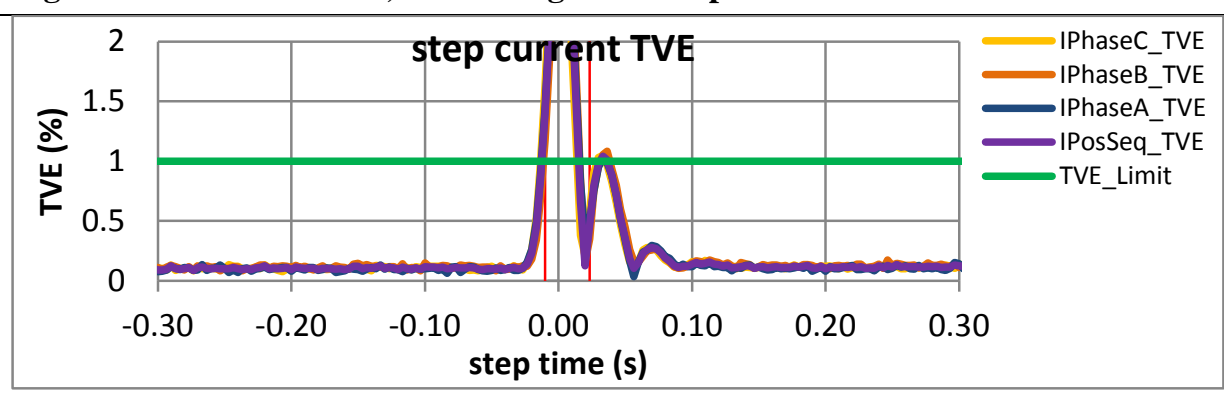

Figure 5475: Fs = 30 FPS, +10\% magnitude step

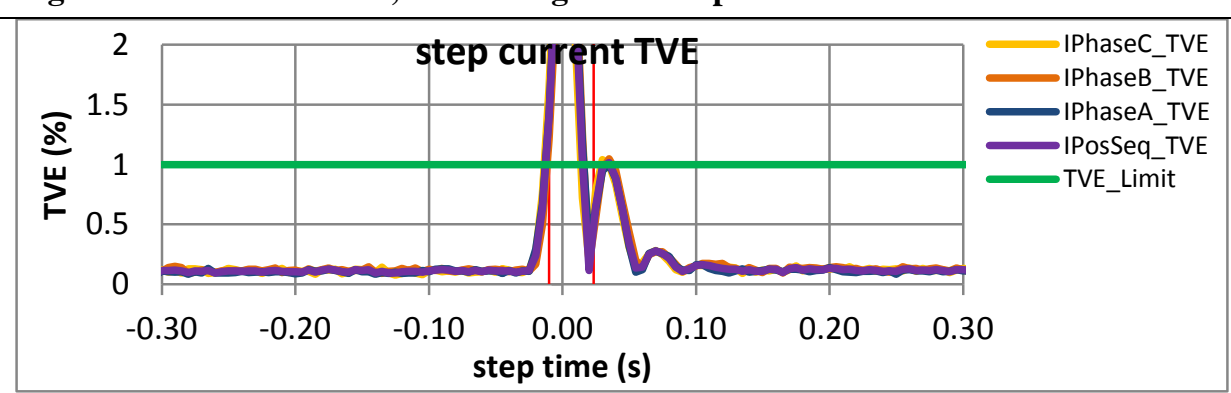

Figure 5477: Fs = 20 FPS, +10\% magnitude step

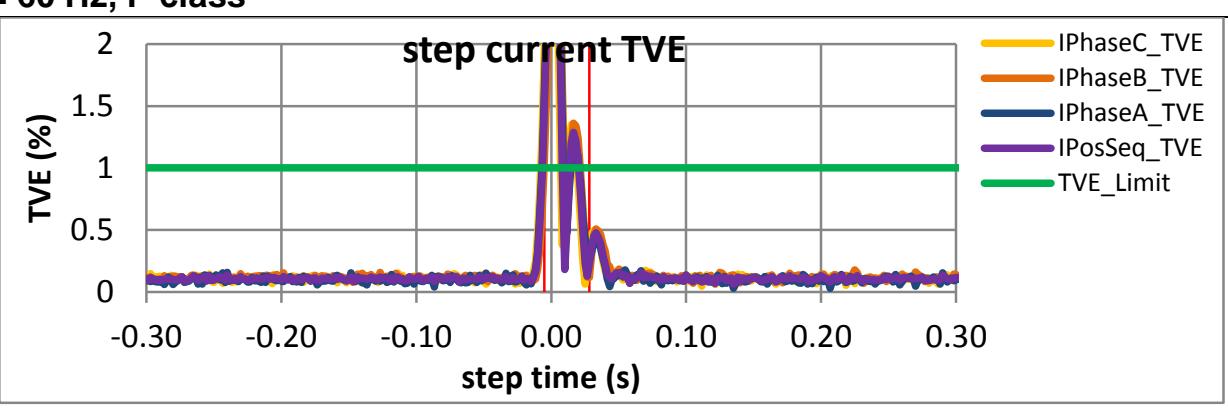

Figure 5474: Fs = 60 FPS, -10\% magnitude step

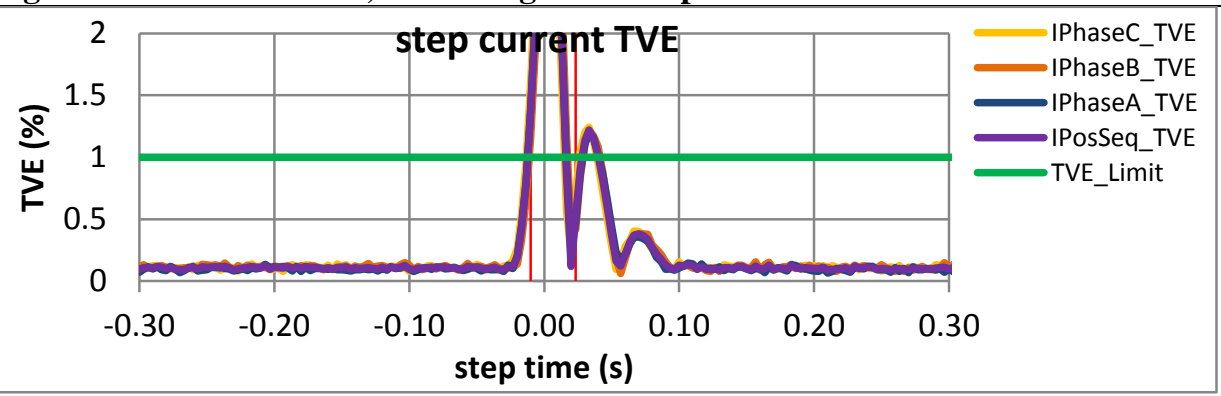

Figure 5476: Fs = 30 FPS, -10\% magnitude step

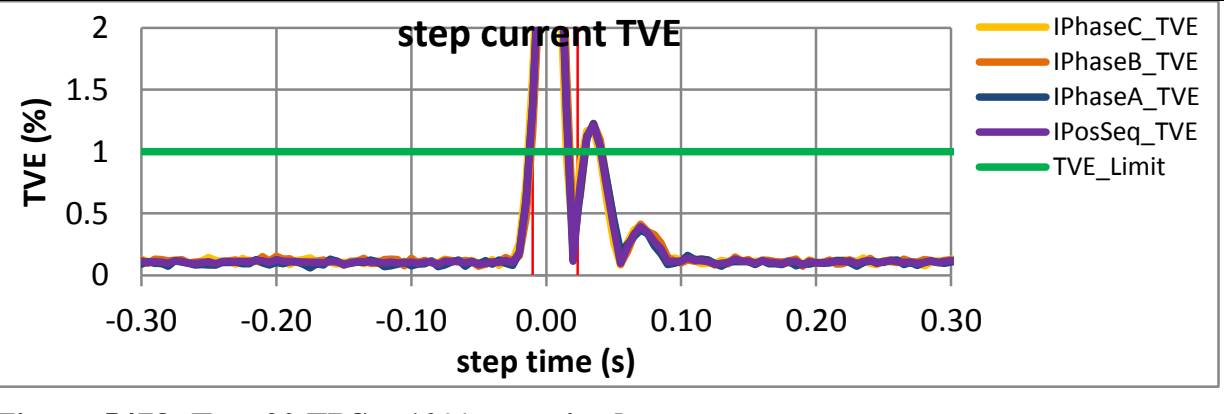

Figure 5478: Fs = 20 FPS, +10\% magnitude step 


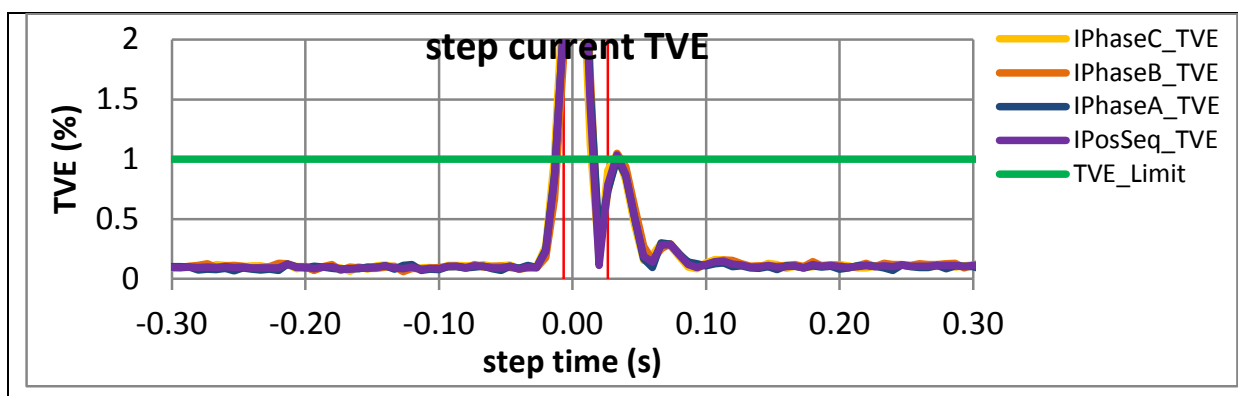

Figure 5479: Fs = 15 FPS, +10\% magnitude step

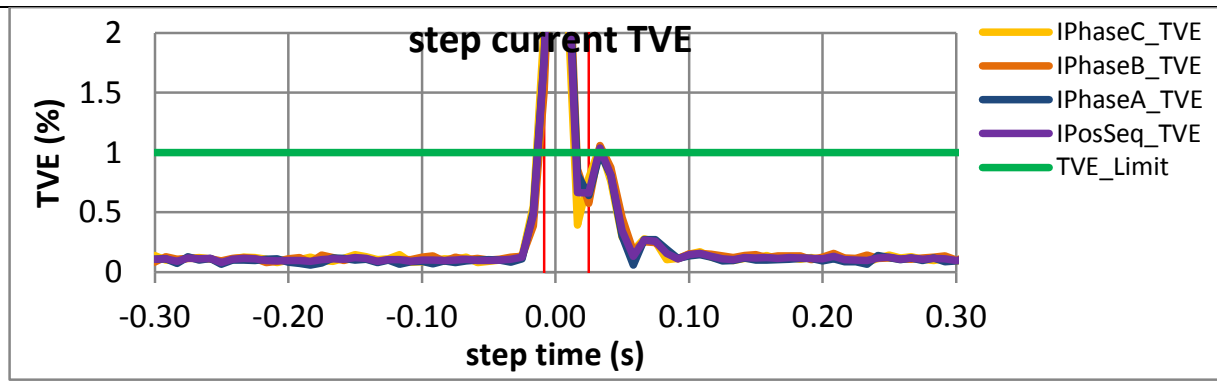

Figure 5481: Fs $=12$ FPS, $+10 \%$ magnitude step

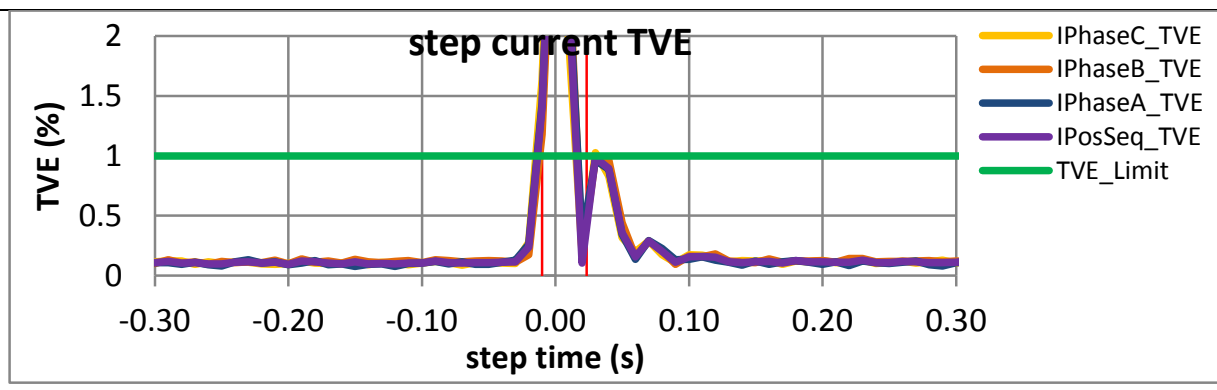

Figure 5483: Fs = 10 FPS, $+10 \%$ magnitude step

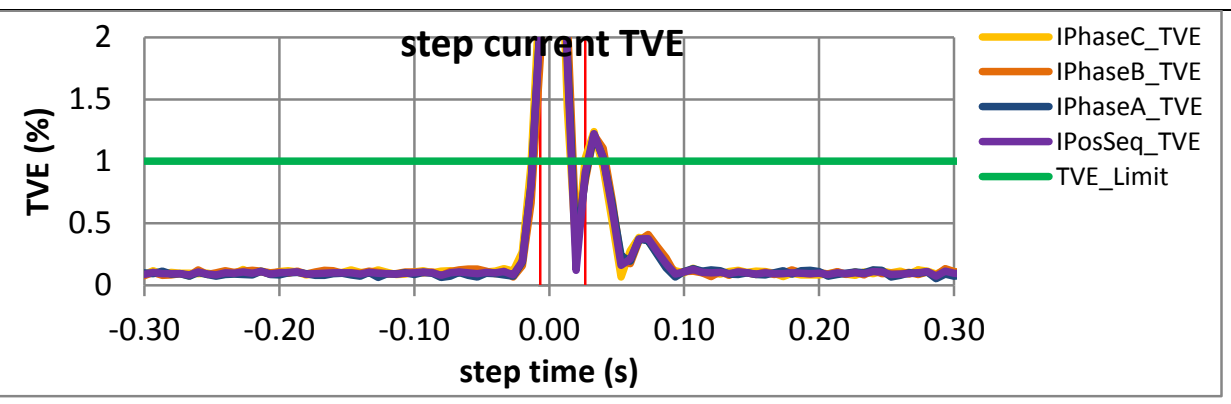

Figure 5480: Fs = 15 FPS, -10\% magnitude step

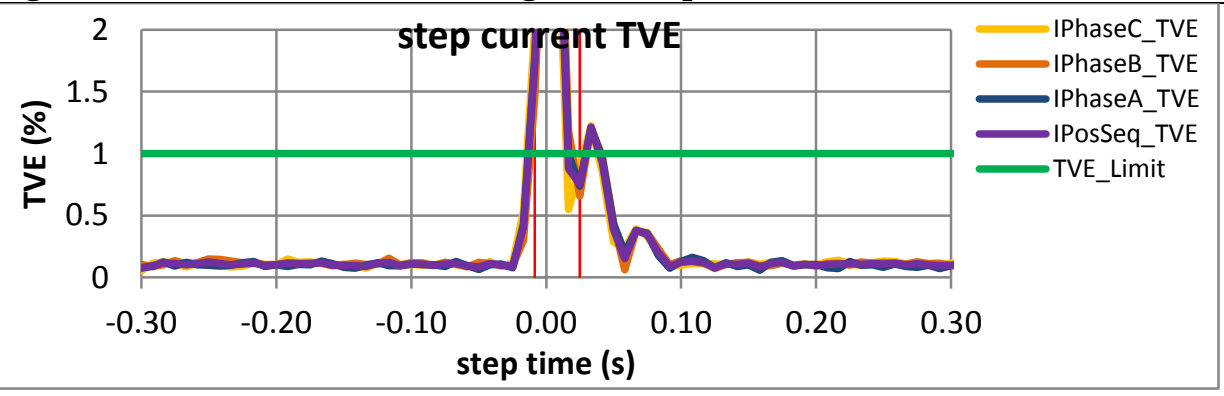

Figure 5482: Fs = 12 FPS, $-10 \%$ magnitude step

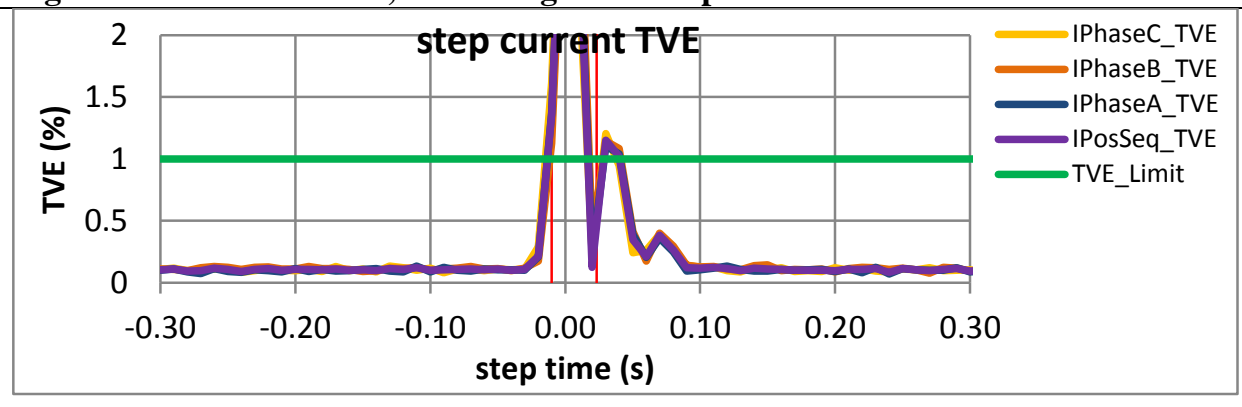

Figure 5484: Fs = 10 FPS, -10\% magnitude step 
10.5.4 PMU C dynamic step change in magnitude current response time: $\mathrm{F} 0=60 \mathrm{~Hz}, \mathrm{P}$ class

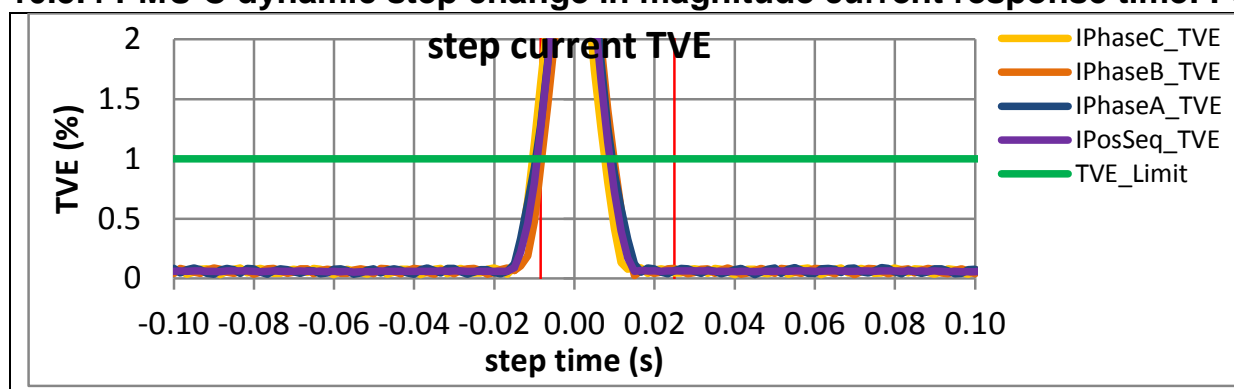

Figure 5485: Fs = 60 FPS, +10\% magnitude step

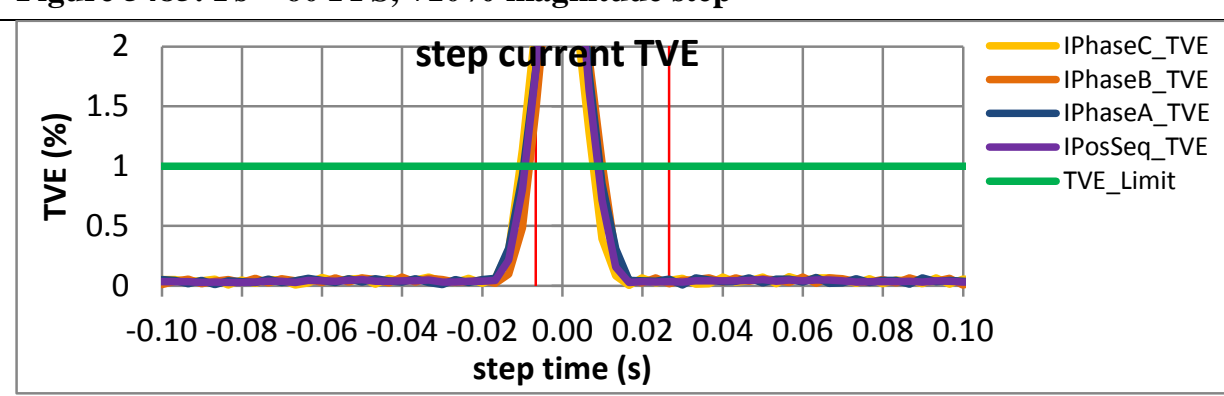

Figure 5487: Fs = 30 FPS, $+10 \%$ magnitude step

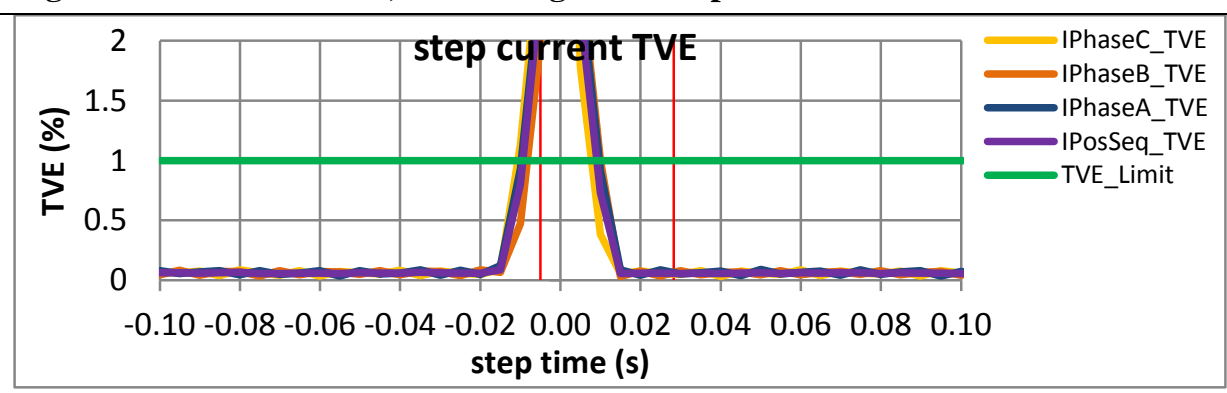

Figure 5489: Fs = 20 FPS, +10\% magnitude step

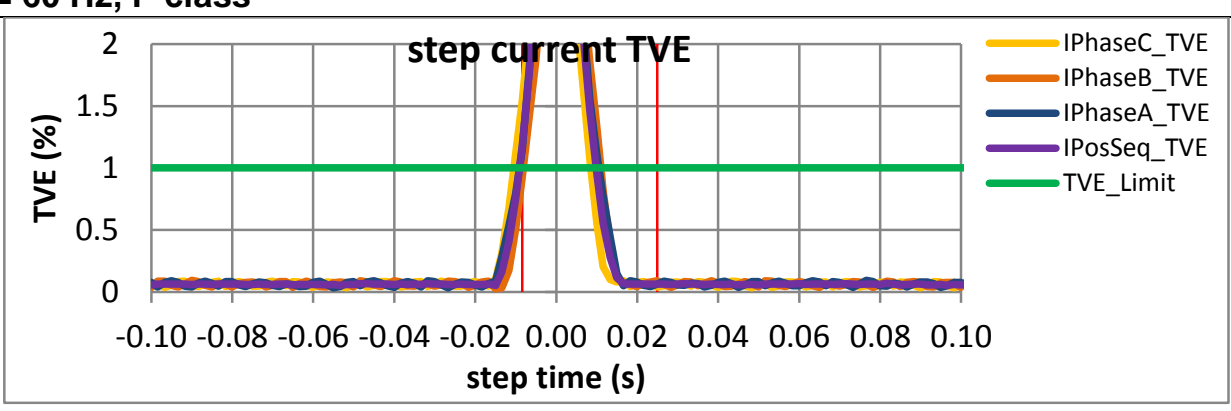

Figure 5486: Fs = 60 FPS, -10\% magnitude step

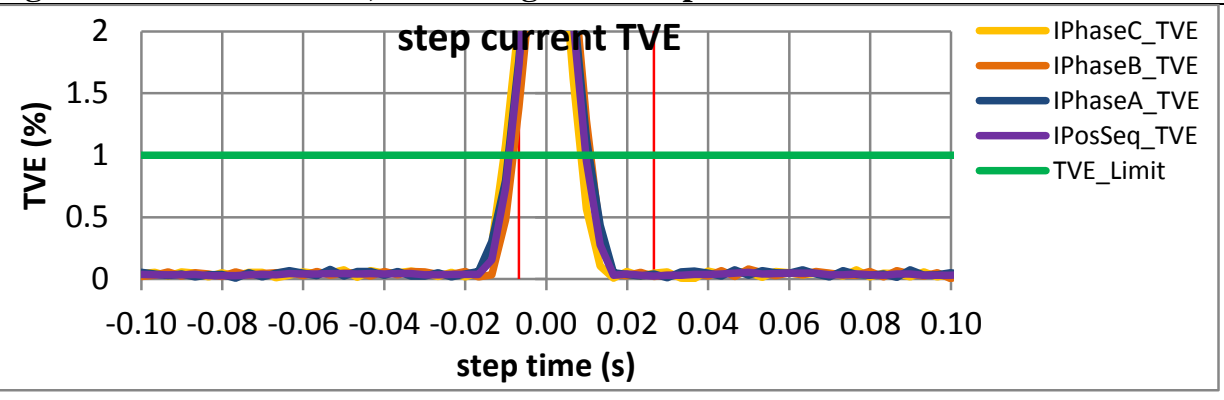

Figure 5488: Fs = 30 FPS, -10\% magnitude step

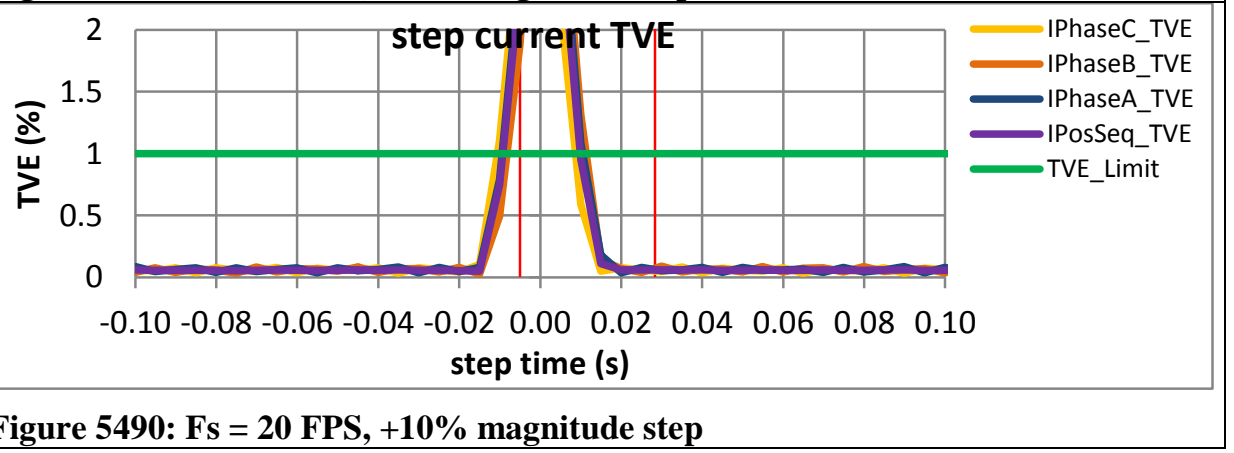




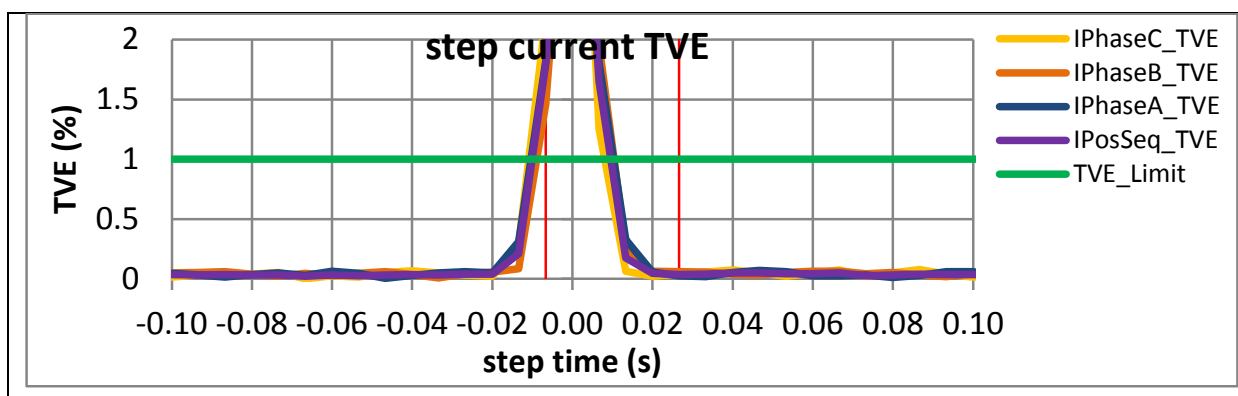

Figure 5491: Fs = 15 FPS, $+10 \%$ magnitude step
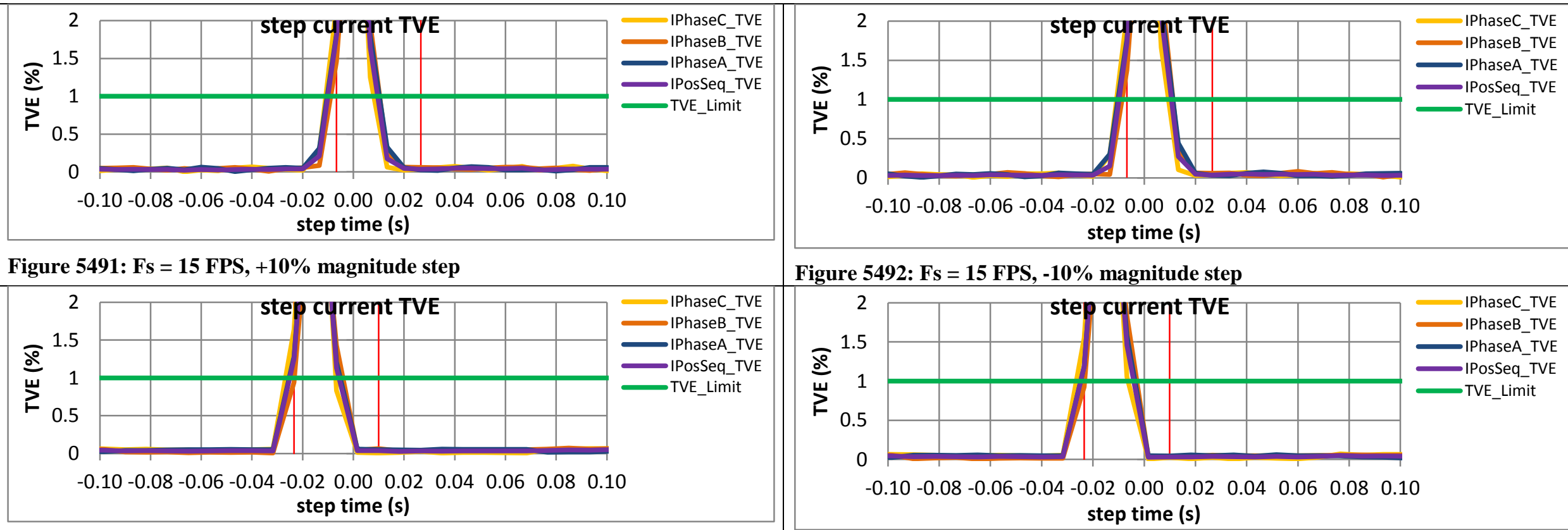

Figure 5492: Fs = 15 FPS, -10\% magnitude step

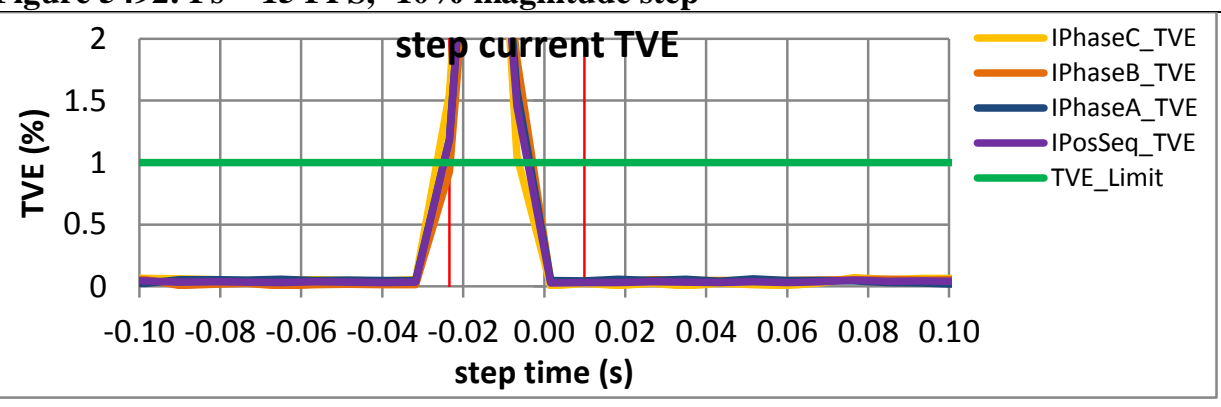

Figure 5493: Fs = 12 FPS, +10\% magnitude step

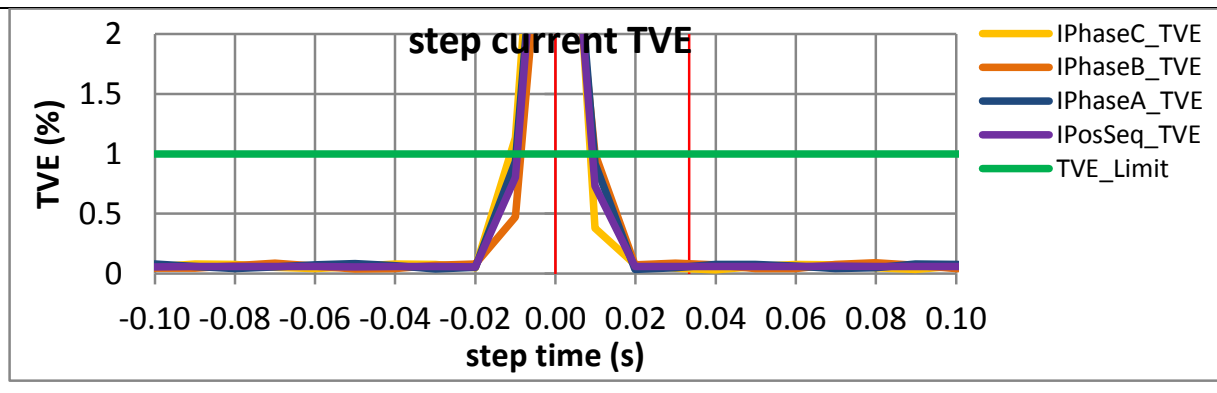

Figure 5495: Fs $=10 \mathrm{FPS},+10 \%$ magnitude step

\section{Figure 5494: Fs = 12 FPS, -10\% magnitude step}

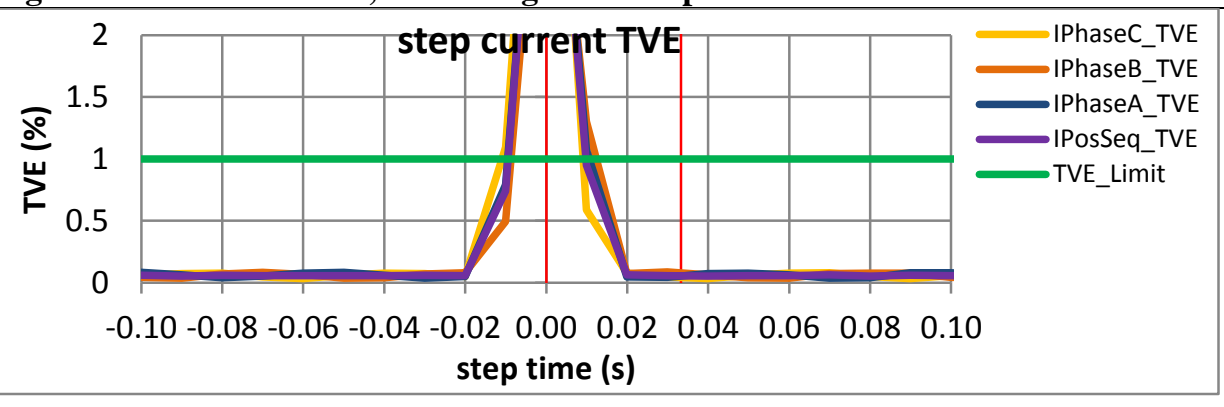

Figure 5496: Fs = 10 FPS, -10\% magnitude step 
10.5.5 PMU D dynamic step change in magnitude current response time: $F 0=60 \mathrm{~Hz}, \mathrm{P}$ class

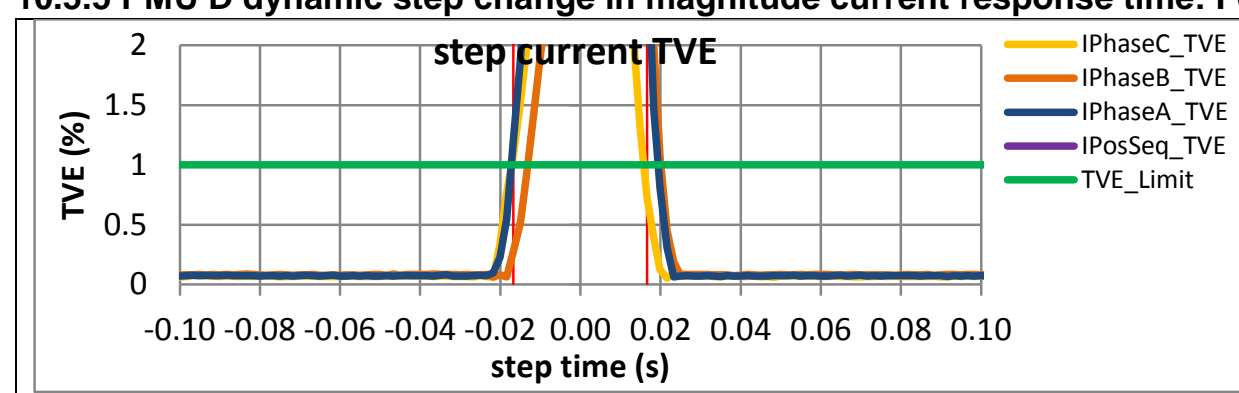

Figure 5497: Fs = 60 FPS, +10\% magnitude step

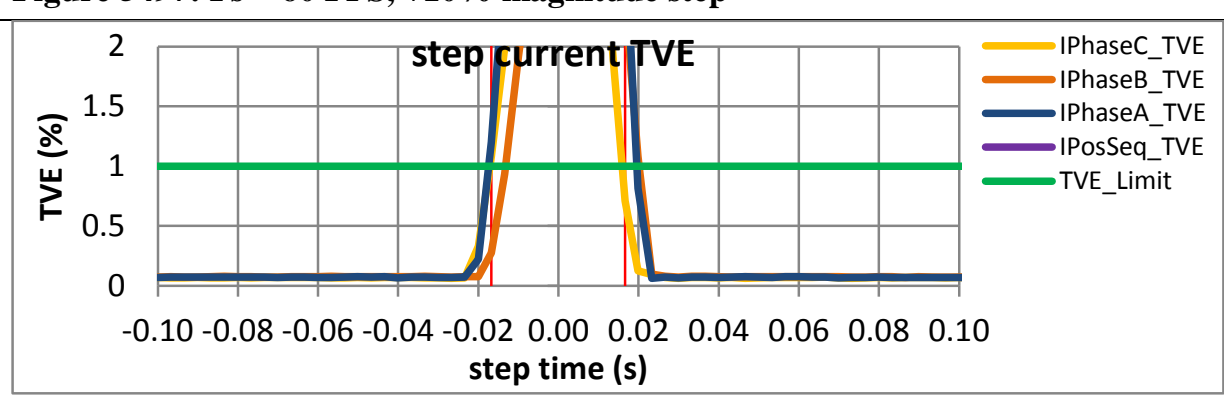

Figure 5499: Fs = 30 FPS, +10\% magnitude step

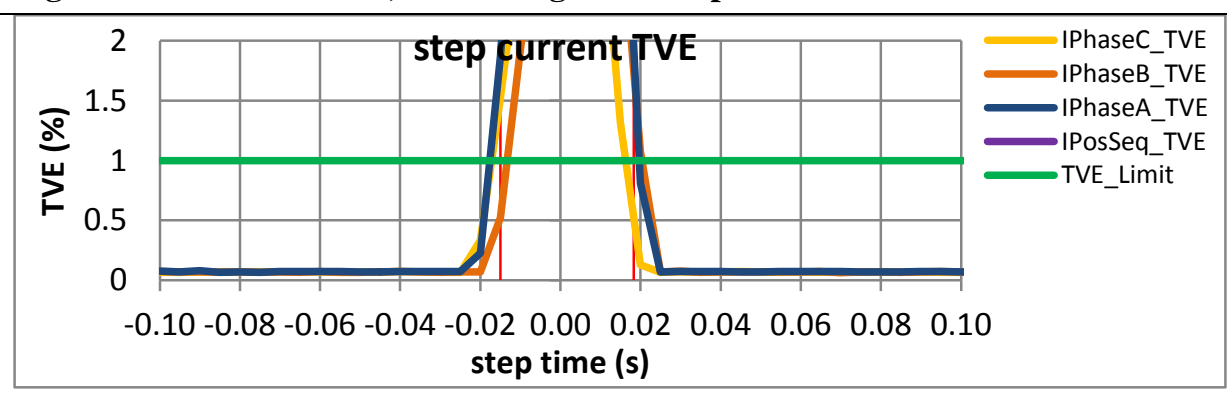

Figure 5501: Fs = 20 FPS, +10\% magnitude step

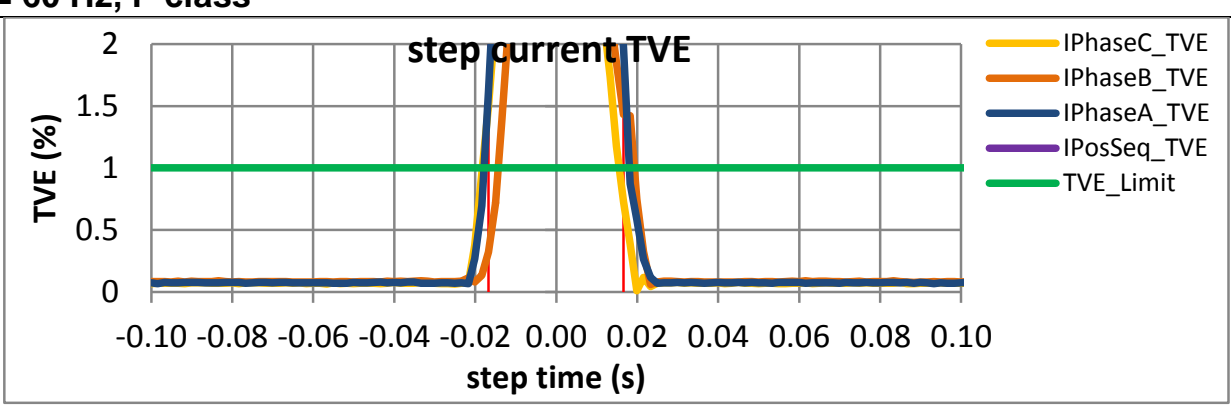

Figure 5498: Fs = 60 FPS, -10\% magnitude step

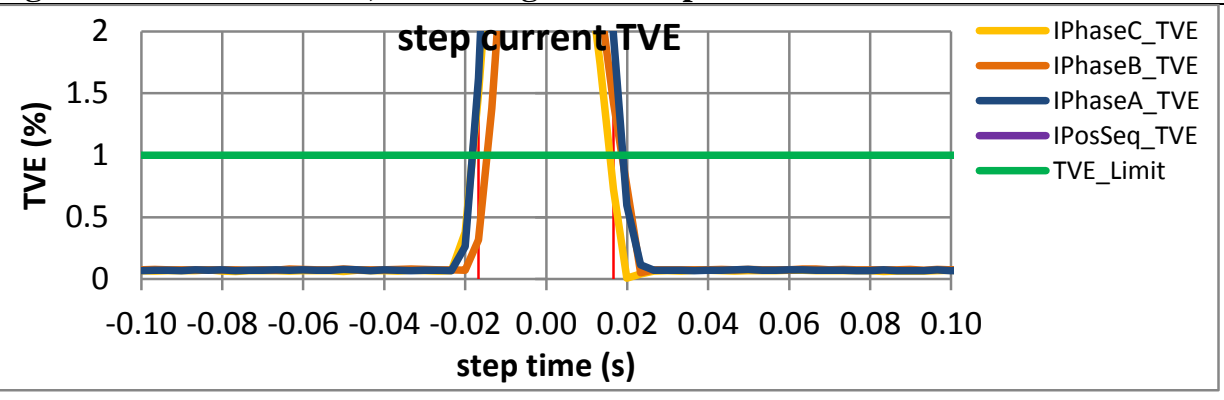

Figure 5500: Fs = 30 FPS, -10\% magnitude step

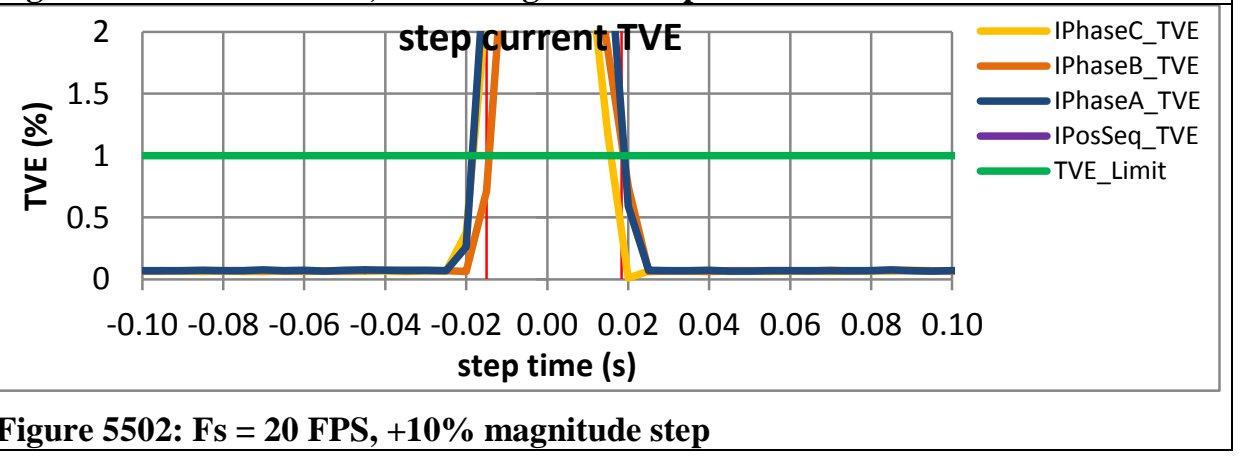




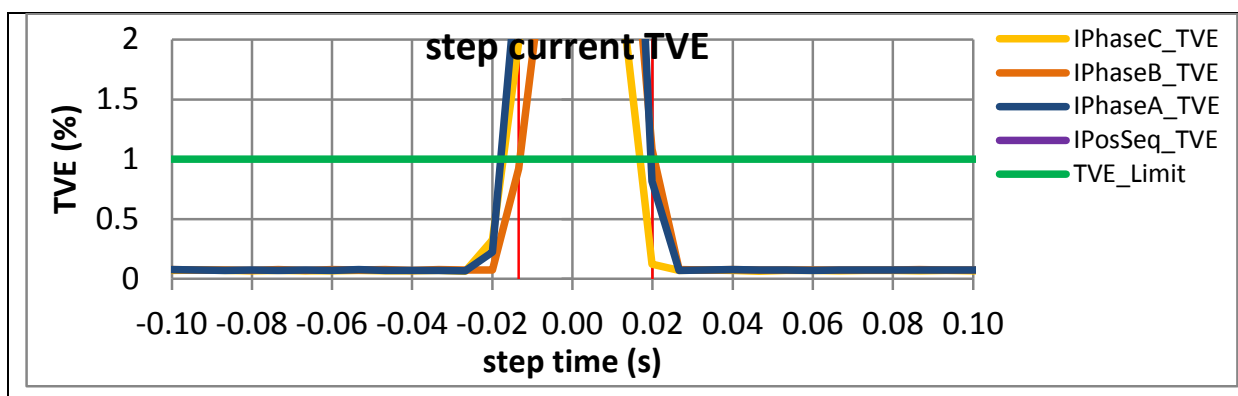

Figure 5503: Fs = 15 FPS, +10\% magnitude step
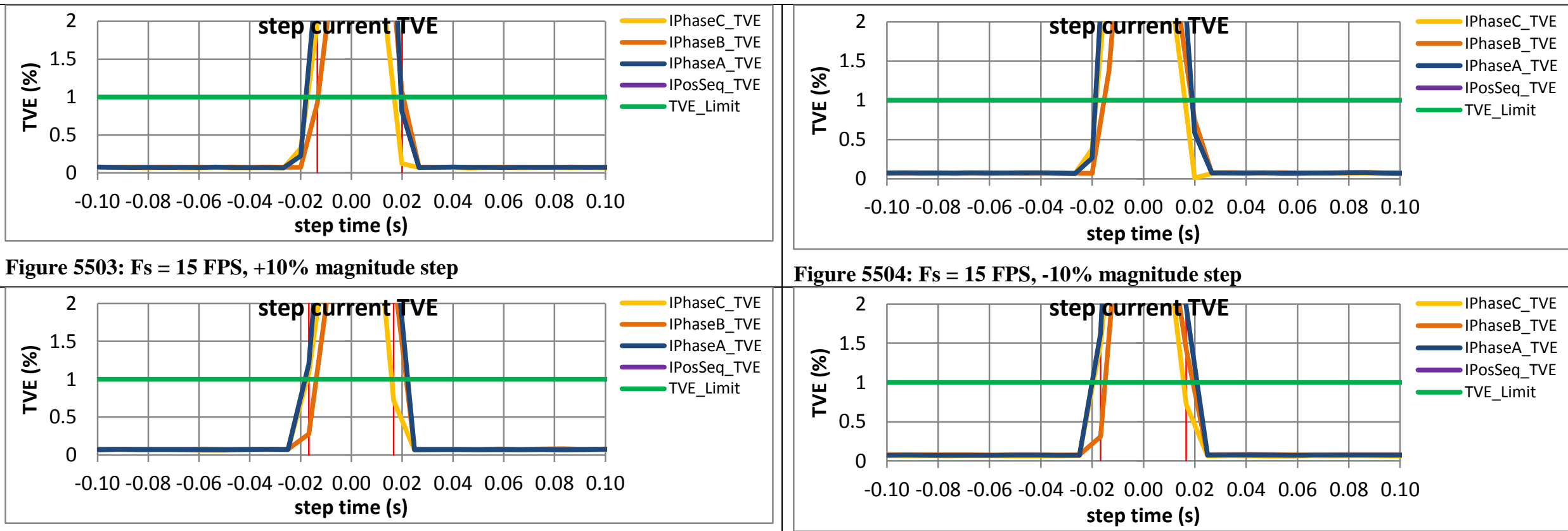

Figure 5504: Fs = 15 FPS, -10\% magnitude step

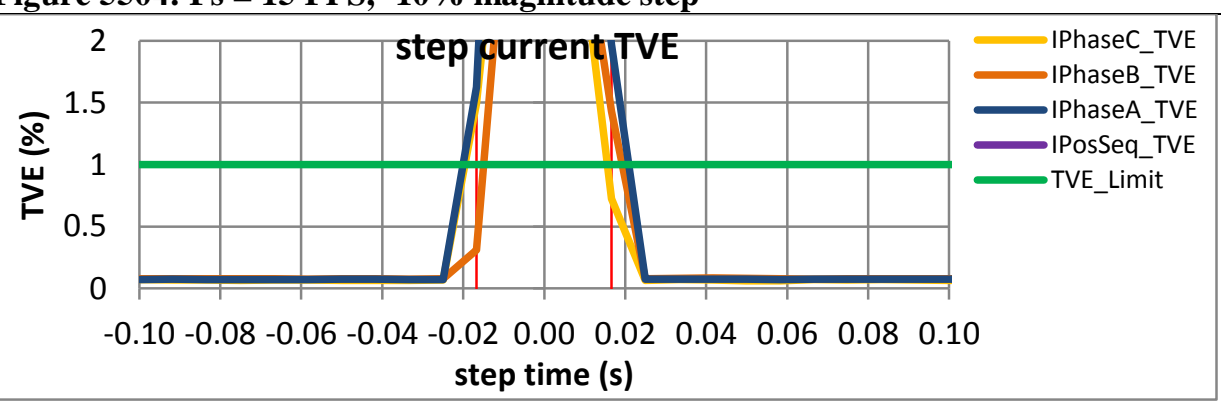

Figure 5505: Fs = 12 FPS, +10\% magnitude step

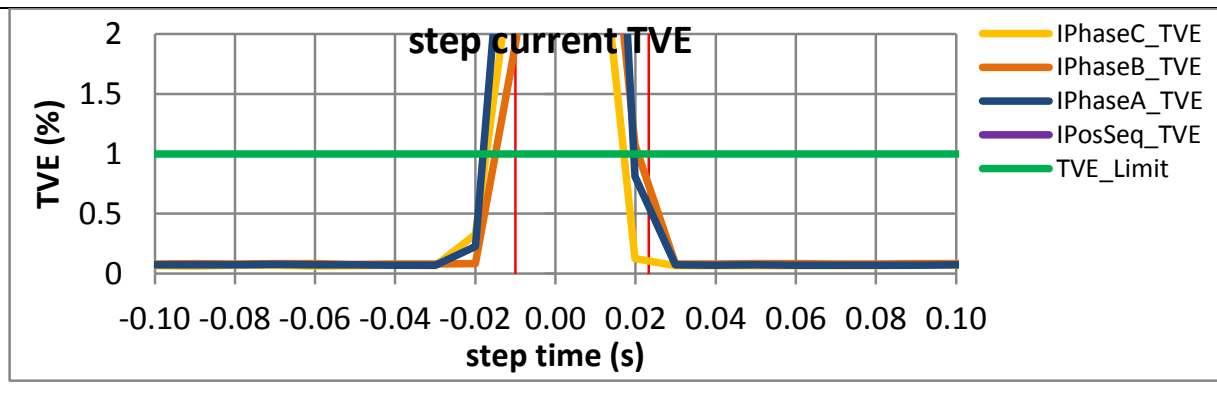

Figure 5507: Fs = 10 FPS, $+10 \%$ magnitude step

\section{Figure 5506: Fs = 12 FPS, - -10\% magnitude step}

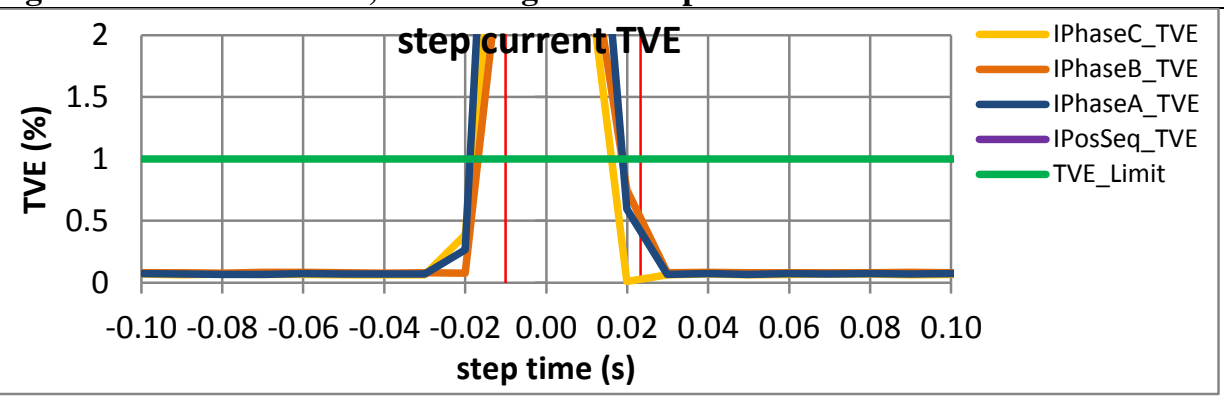

Figure 5508: Fs = 10 FPS, -10\% magnitude step 
10.5.6 PMU E dynamic step change in magnitude current response time: $\mathrm{F} 0=60 \mathrm{~Hz}, \mathrm{P}$ class PMU E does not support P class.

\subsubsection{PMU F dynamic step change in magnitude current response time: $F 0=60 \mathrm{~Hz}, \mathrm{P}$ class}

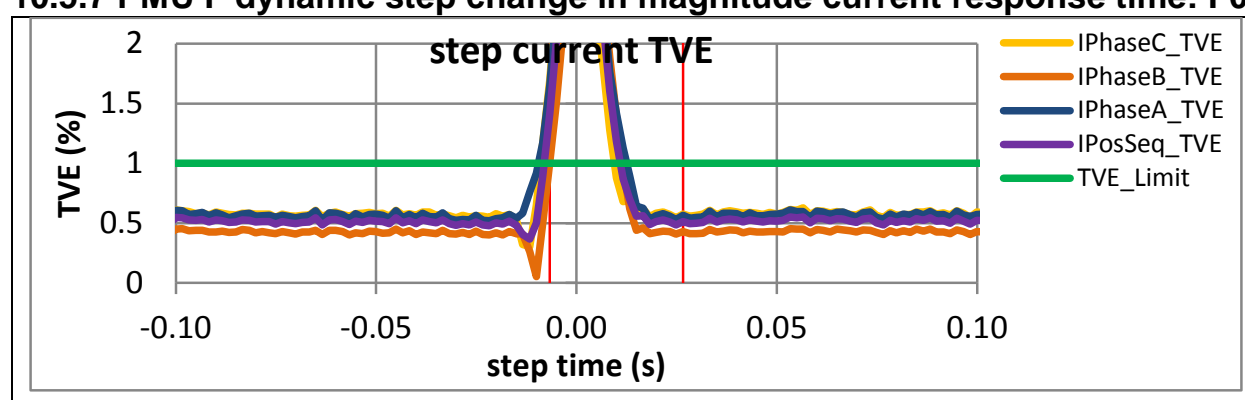

Figure 5509: Fs $=60$ FPS, $+10 \%$ magnitude step
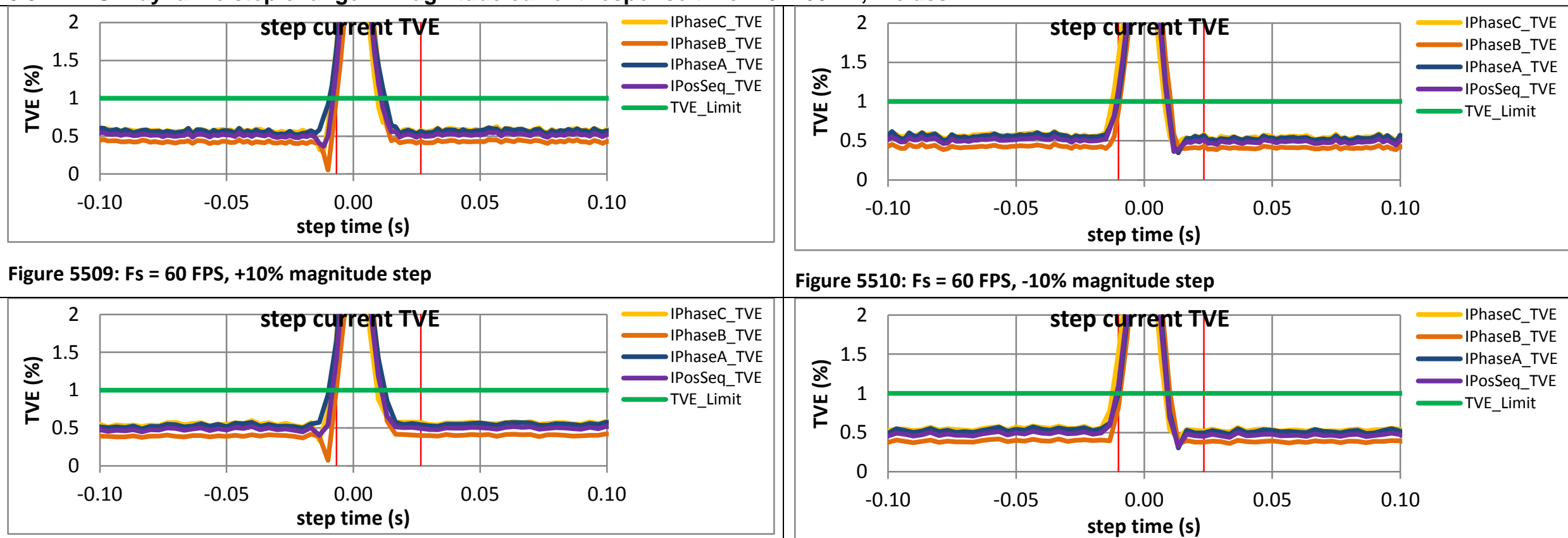

Figure 5510: Fs = 60 FPS, $-10 \%$ magnitude step

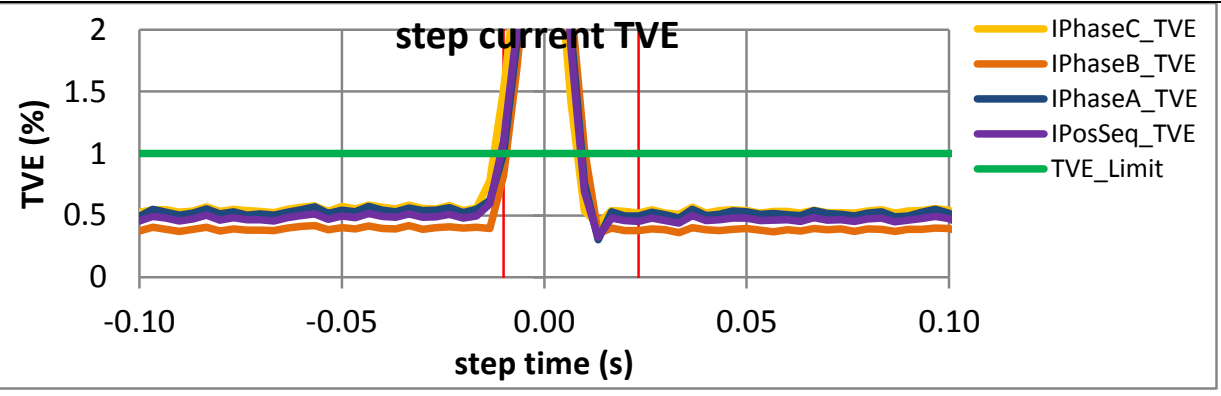

Figure 5511: Fs = 30 FPS, +10\% magnitude step

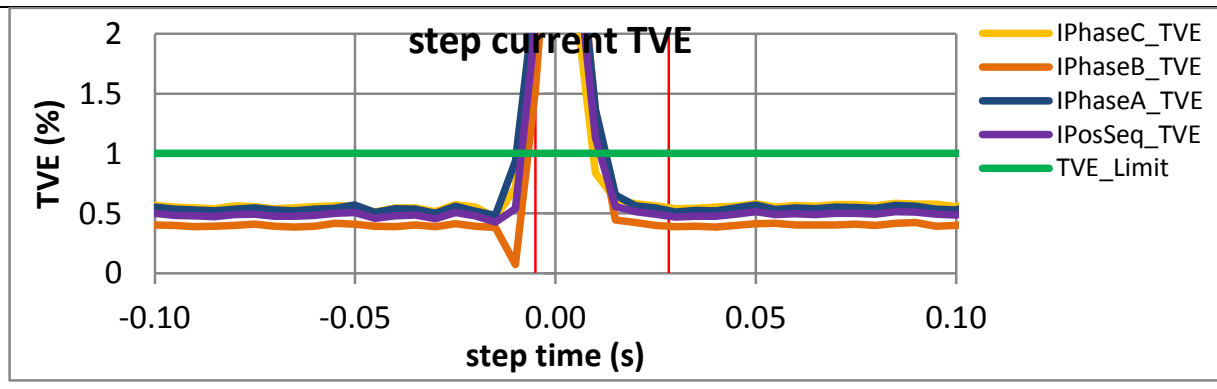

Figure 5512: Fs = 30 FPS, $-10 \%$ magnitude step

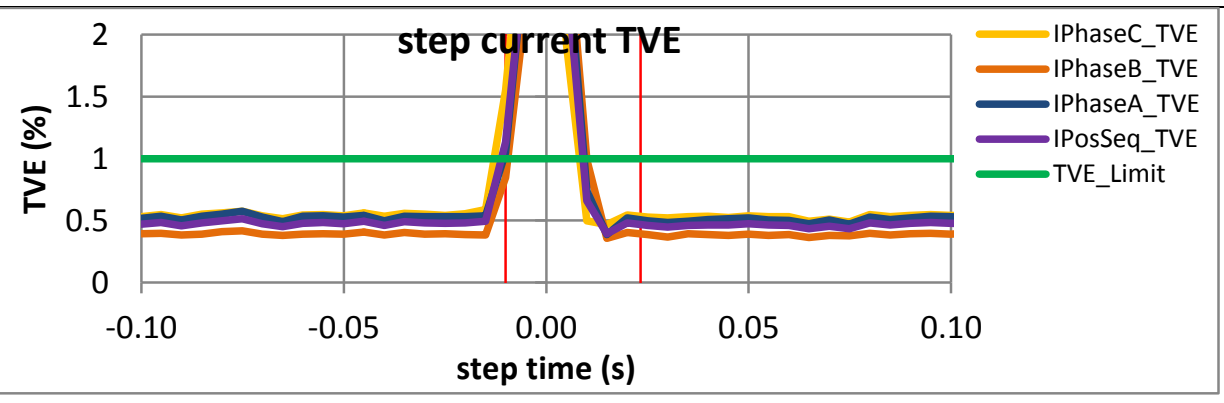

Figure 5513: Fs = 20 FPS, $+10 \%$ magnitude step

Figure 5514: Fs = 20 FPS, +10\% magnitude step 

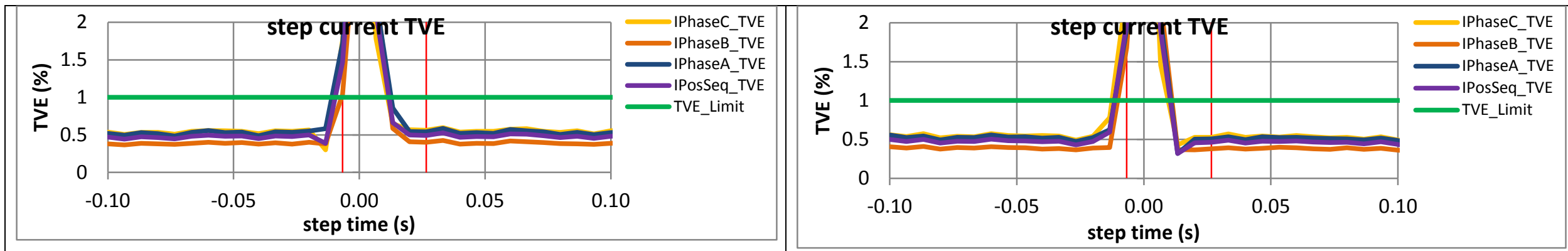

Figure 5515: Fs = 15 FPS, +10\% magnitude step

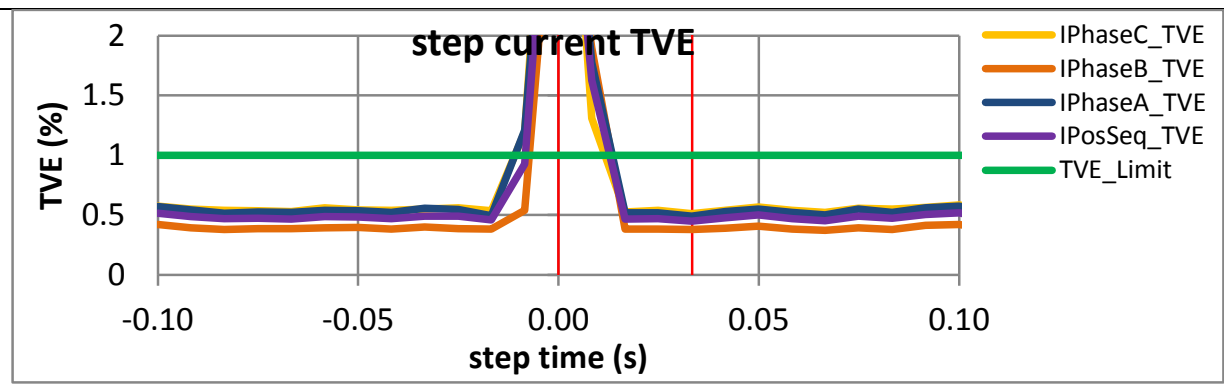

Figure 5516: Fs = 15 FPS, $-10 \%$ magnitude step

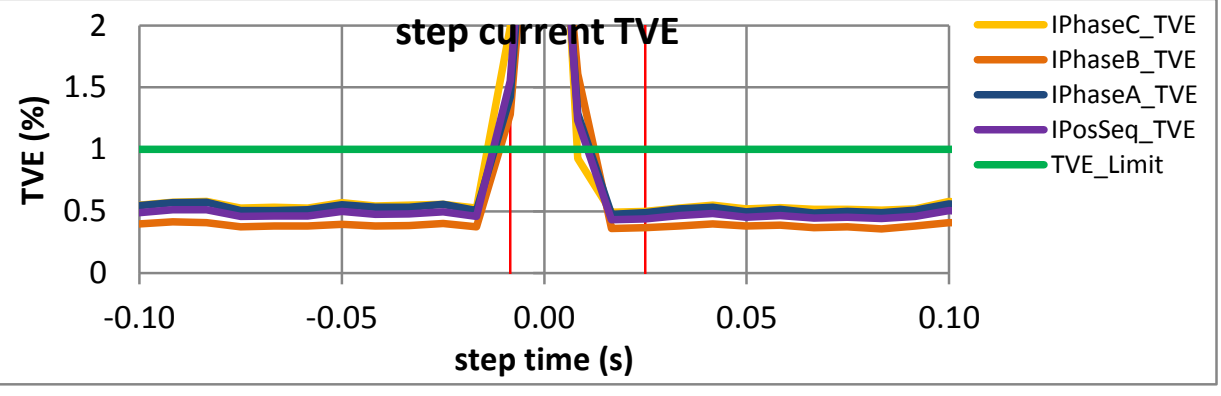

Figure 5517: Fs = 12 FPS, $+10 \%$ magnitude step

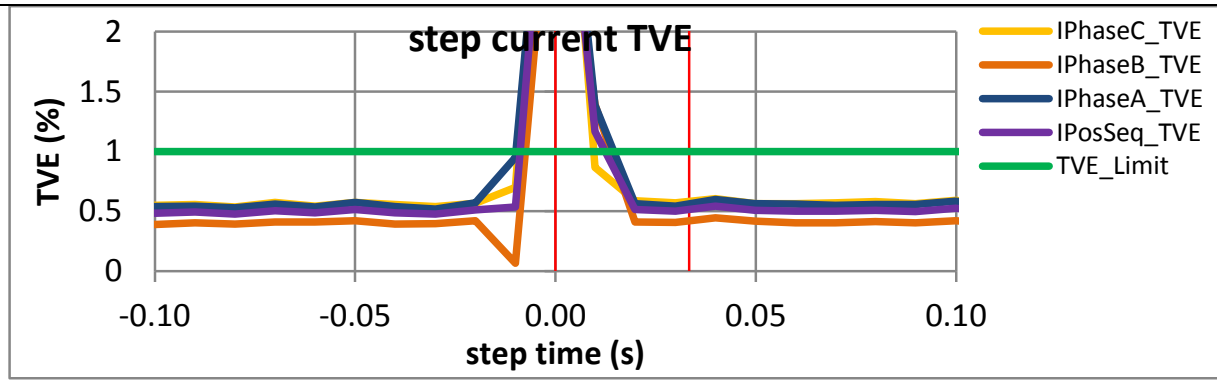

Figure 5518: Fs = 12 FPS, $-10 \%$ magnitude step

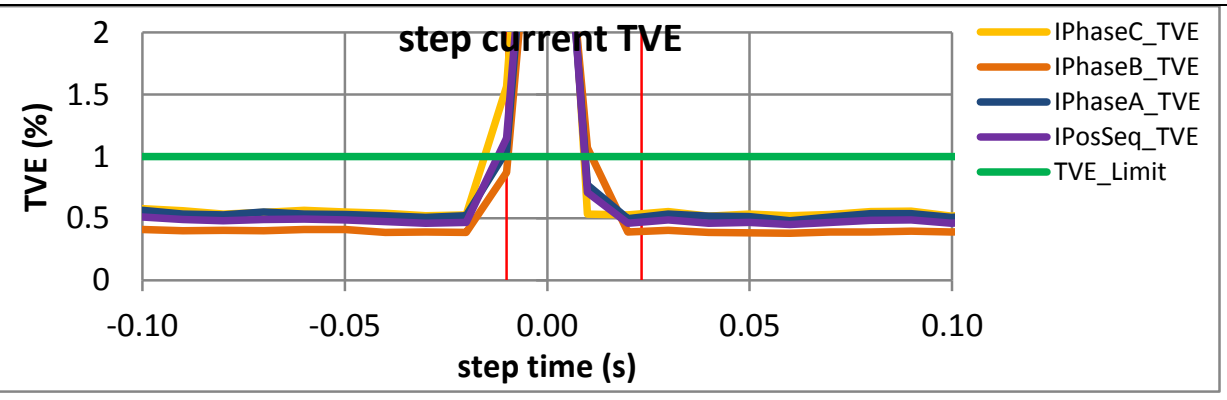

Figure 5519: Fs = $10 \mathrm{FPS},+10 \%$ magnitude step

Figure 5520: Fs = $10 \mathrm{FPS},-10 \%$ magnitude step 
10.5.8 PMU G dynamic step change in magnitude current response time: $F 0=60 \mathrm{~Hz}, \mathrm{P}$ class PMU G does not support P class.

10.5.9 PMU H dynamic step change in magnitude current response time: $\mathrm{F} 0=60 \mathrm{~Hz}, \mathrm{P}$ class
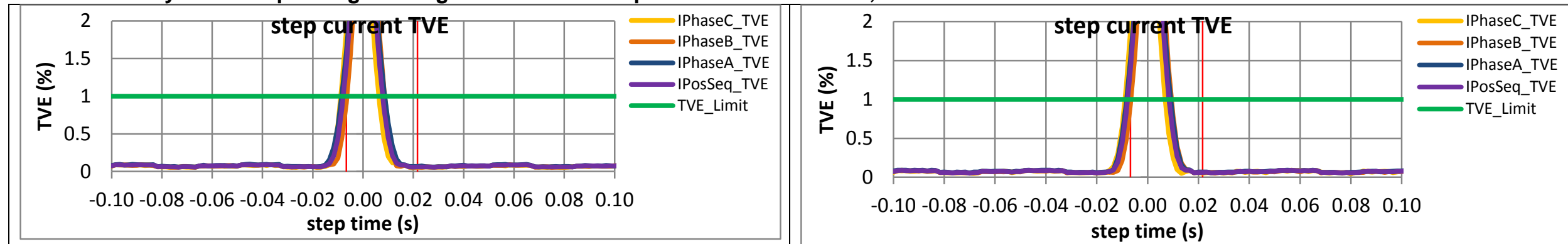

Figure 5521: Fs = 60 FPS, +10\% magnitude step

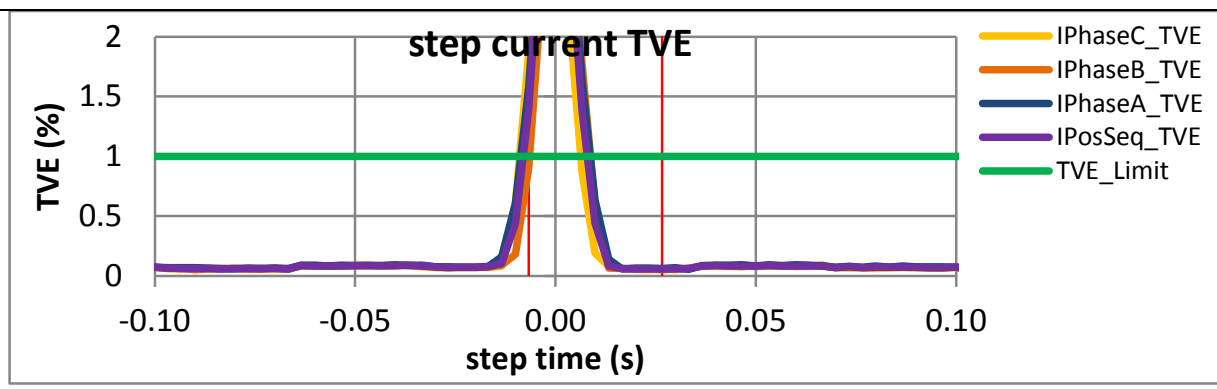

Figure 5522: Fs = 60 FPS, -10\% magnitude step

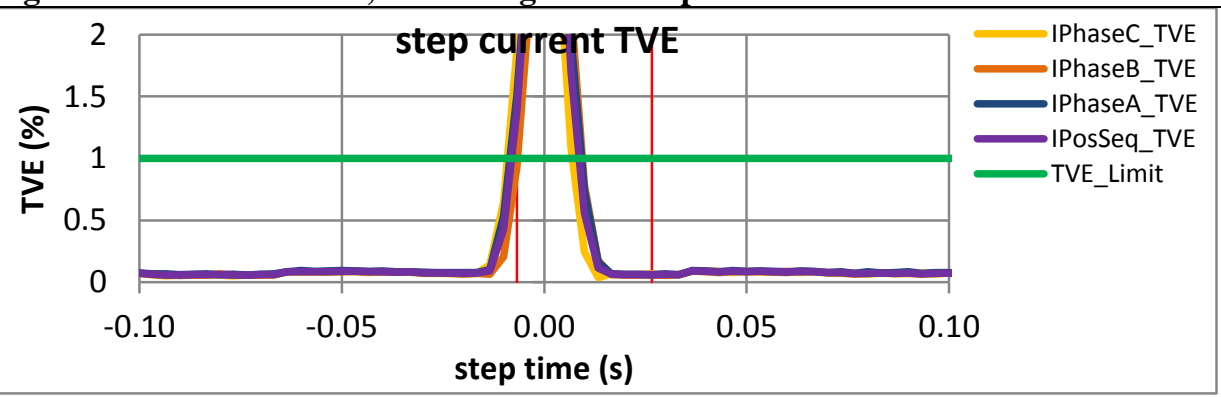

Figure 5523: Fs = 30 FPS, $+10 \%$ magnitude step

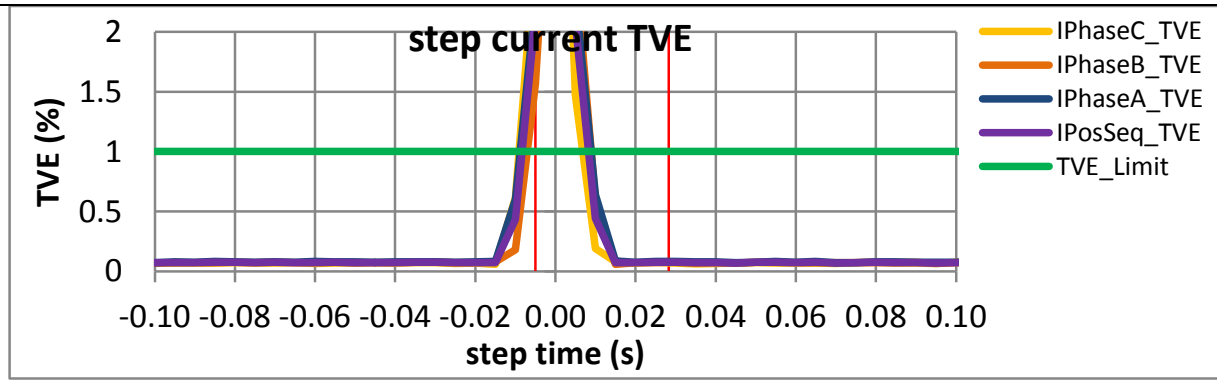

Figure 5525: Fs = 20 FPS, +10\% magnitude step

Figure 5524: Fs = 30 FPS, -10\% magnitude step

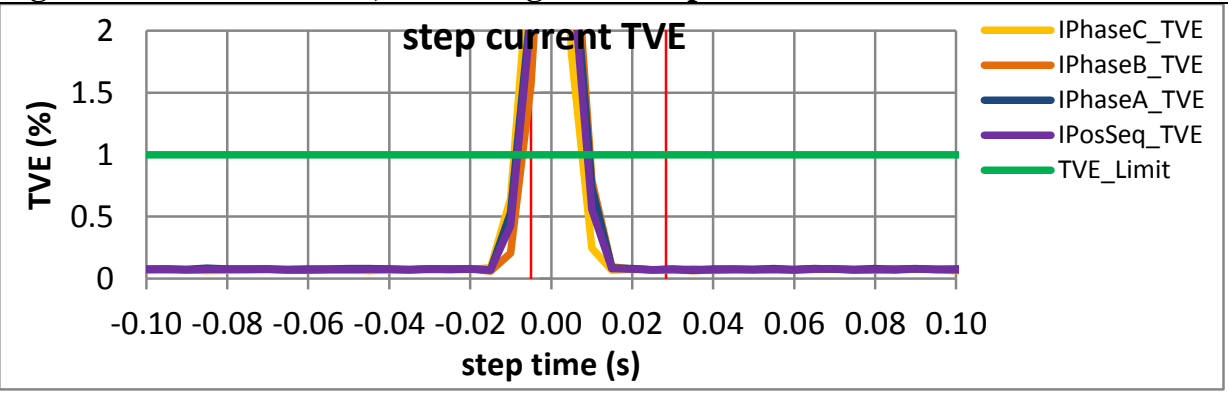

Figure 5526: Fs = 20 FPS, $+10 \%$ magnitude step 

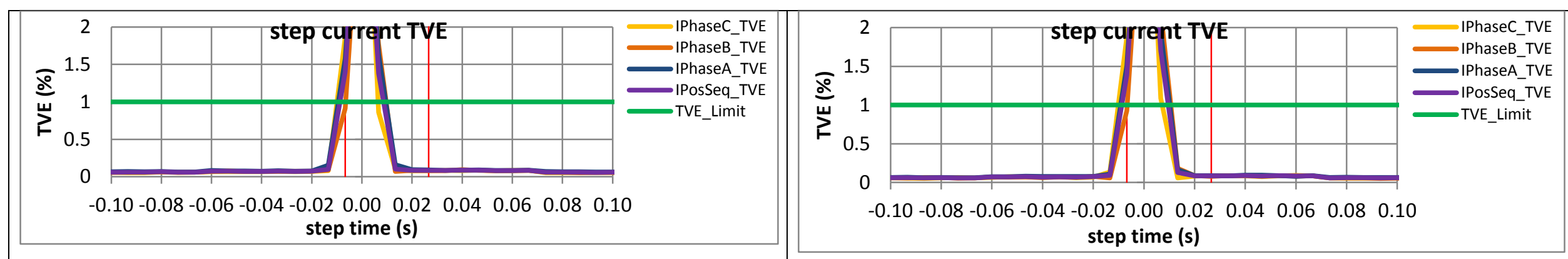

Figure 5527: Fs = 15 FPS, +10\% magnitude step

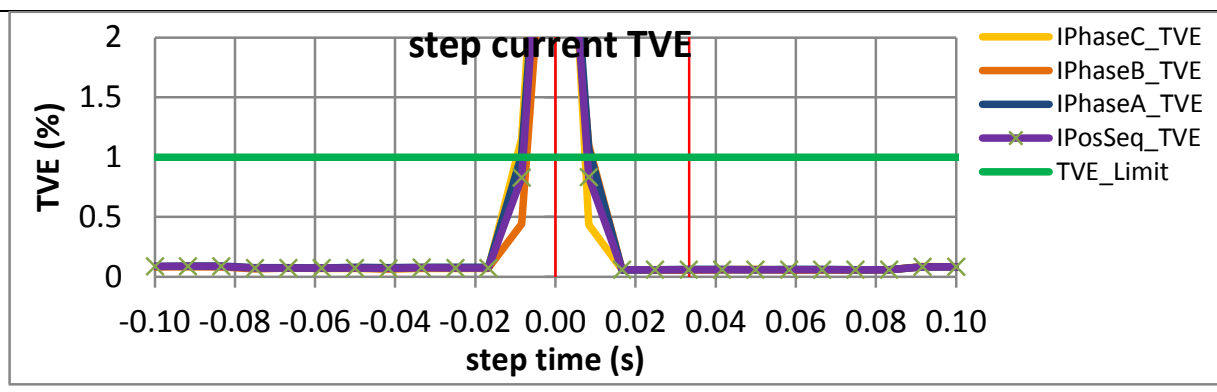

Figure 5528: Fs = 15 FPS, - -10\% magnitude step

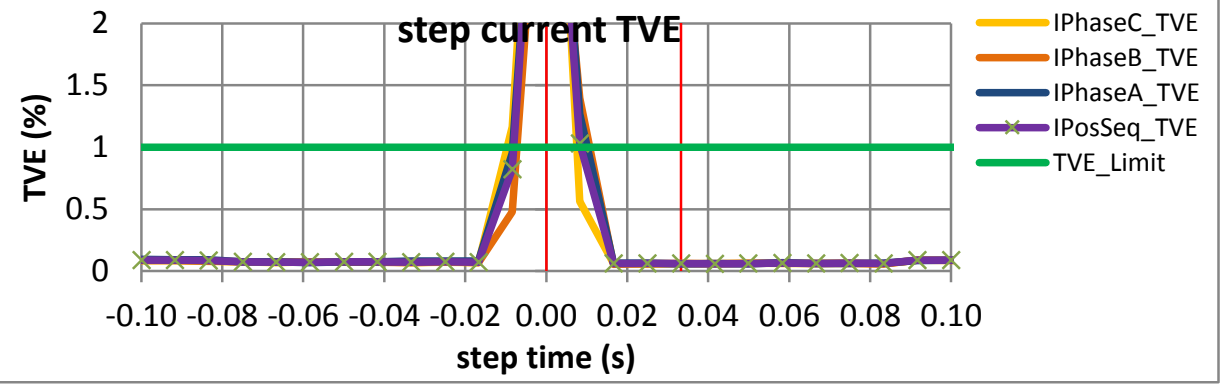

Figure 5529: Fs = 12 FPS, +10\% magnitude step

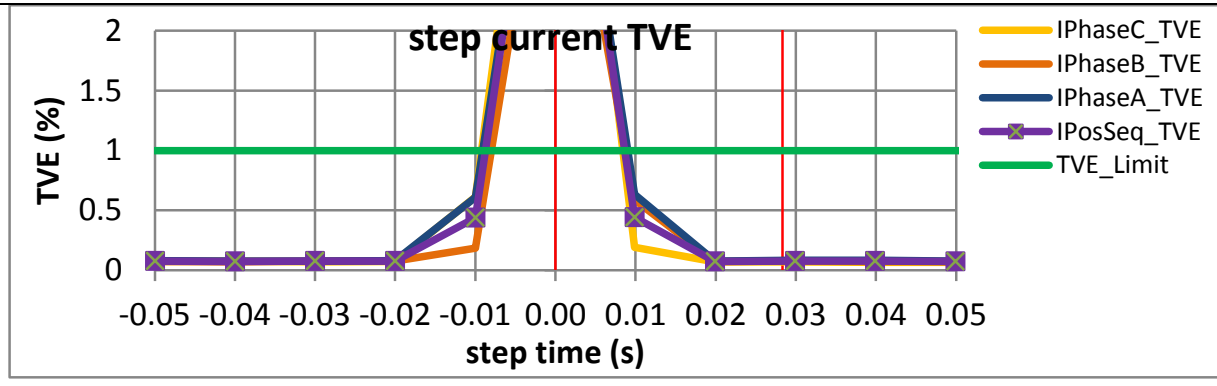

Figure 5531: Fs = 10 FPS, +10\% magnitude step

Figure 5530: Fs = 12 FPS, -10\% magnitude step

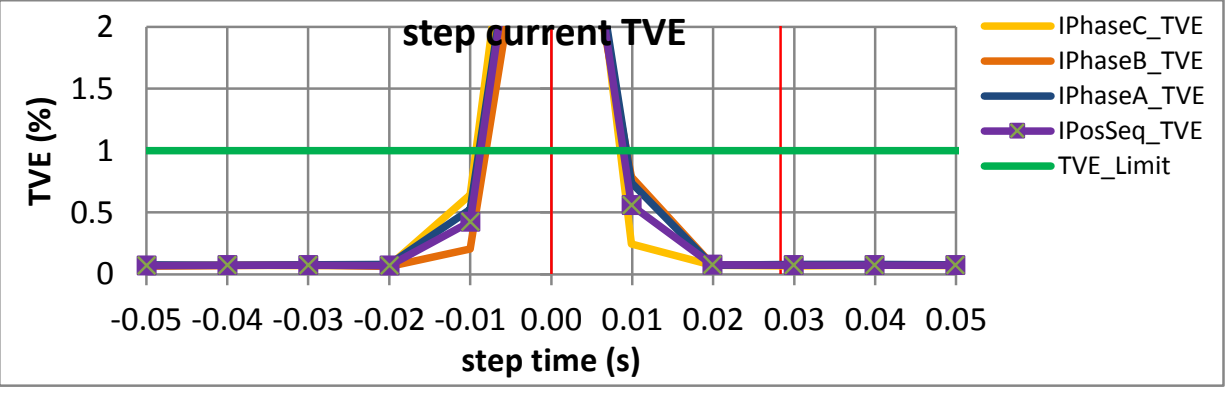

Figure 5532: Fs = 10 FPS, -10\% magnitude step 
10.5.10 PMU I dynamic step change in magnitude current response time: $F 0=60 \mathrm{~Hz}, \mathrm{P}$ class PMU I does not support P class

10.5.11 PMU J dynamic step change in magnitude current response time: $\mathrm{F} 0=60 \mathrm{~Hz}, \mathrm{P}$ class

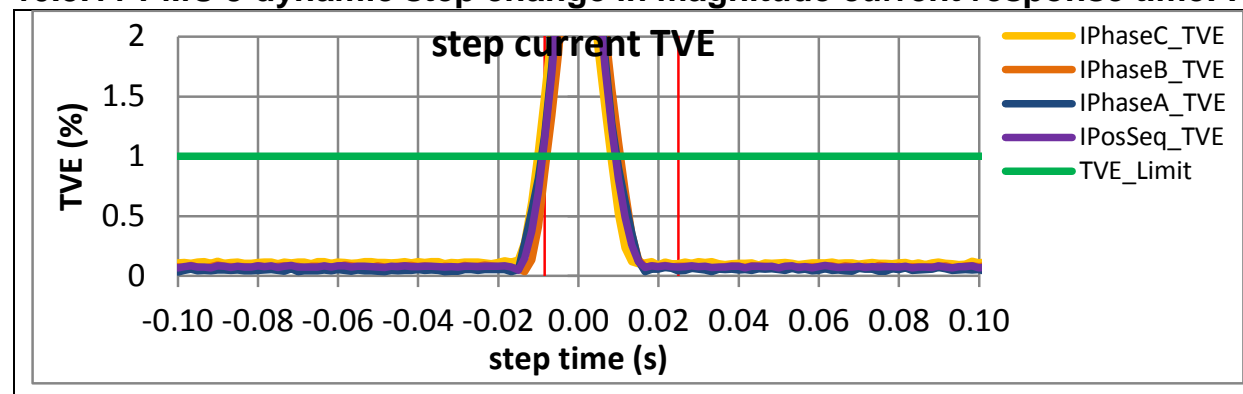

Figure 5533: Fs = $60 \mathrm{FPS},+10 \%$ magnitude step

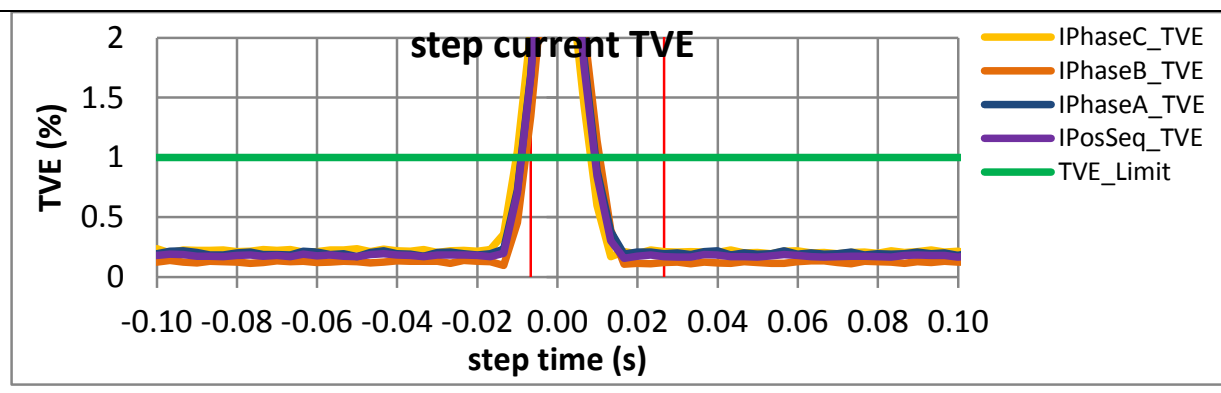

Figure 5535: Fs = $30 \mathrm{FPS},+10 \%$ magnitude step

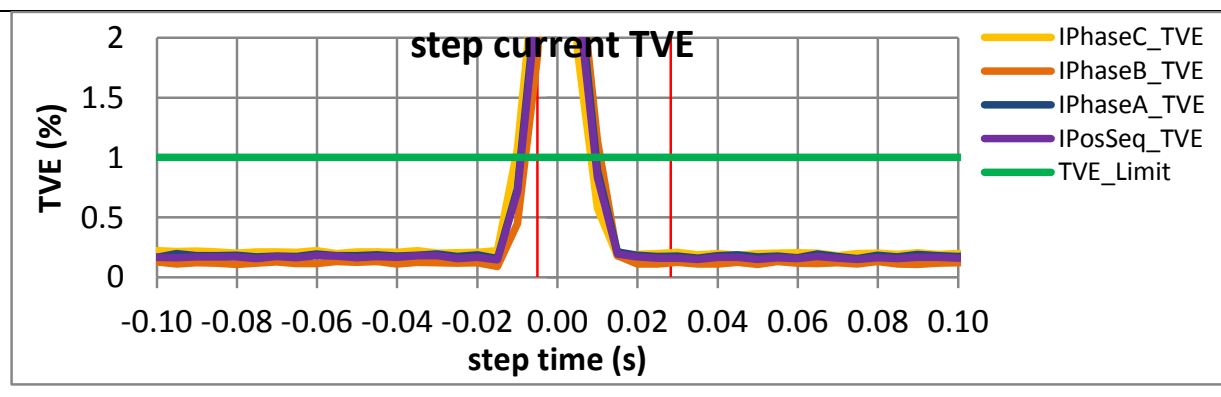

Figure 5537: Fs = 20 FPS, $+10 \%$ magnitude step

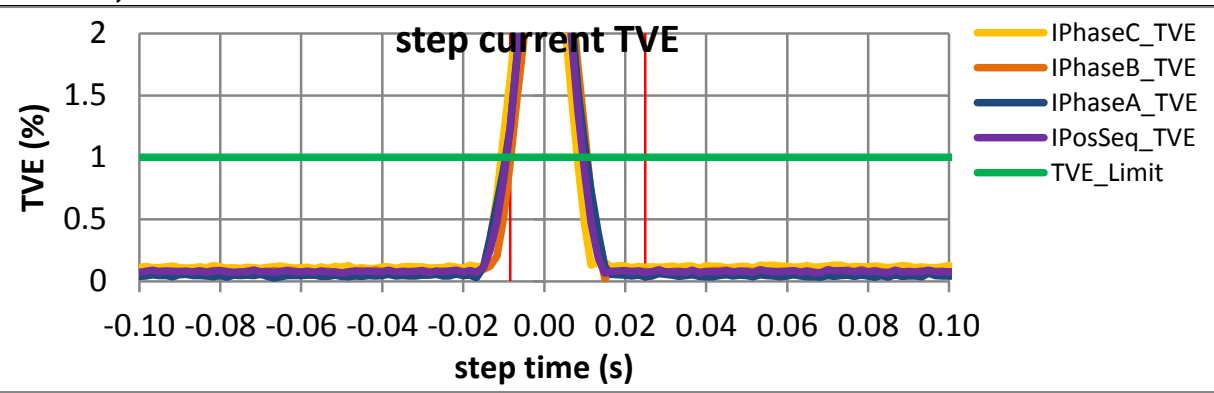

Figure 5534: Fs = 60 FPS, $-10 \%$ magnitude step

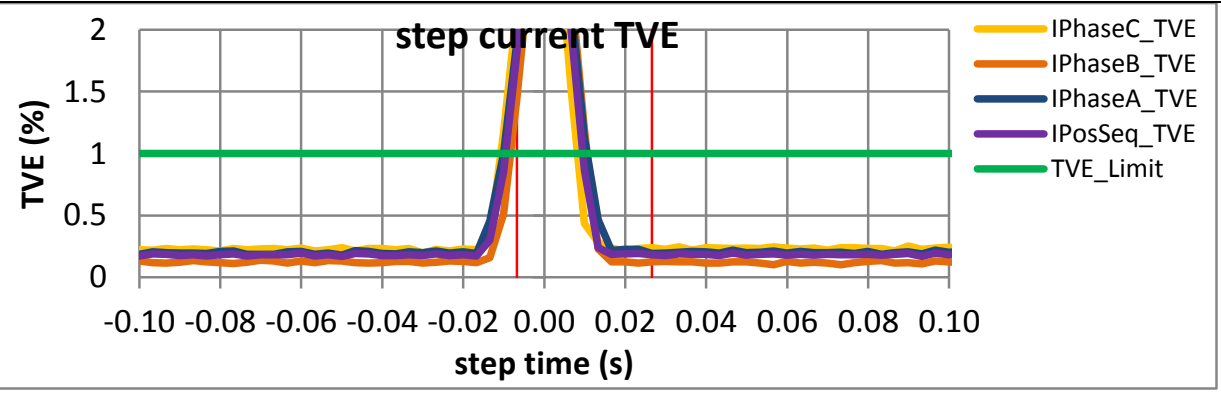

Figure 5536: Fs = 30 FPS, -10\% magnitude step

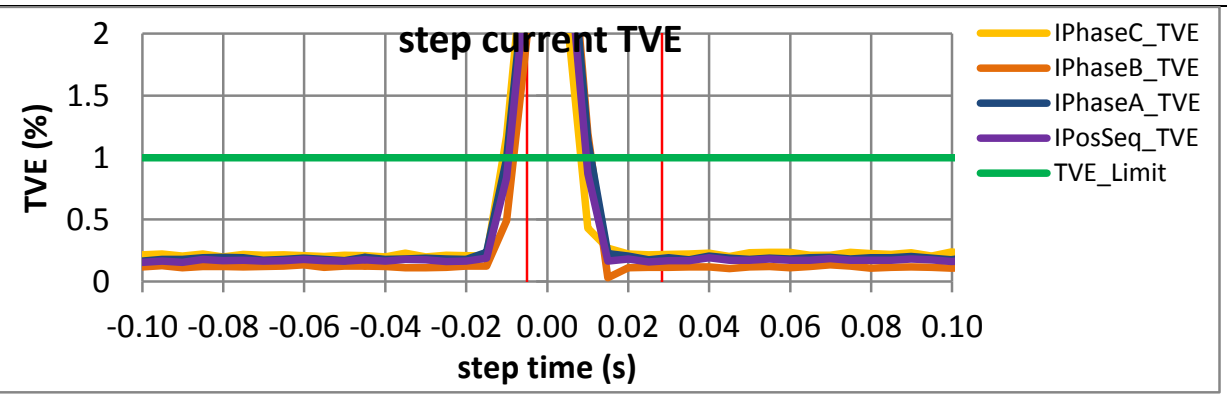

Figure 5538: Fs = 20 FPS, +10\% magnitude step 


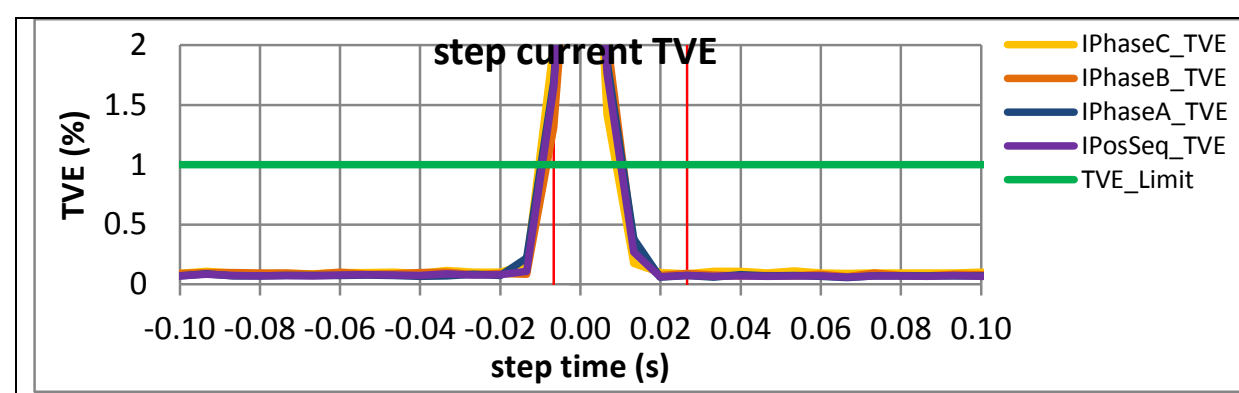

Figure 5539: Fs = 15 FPS, +10\% magnitude step

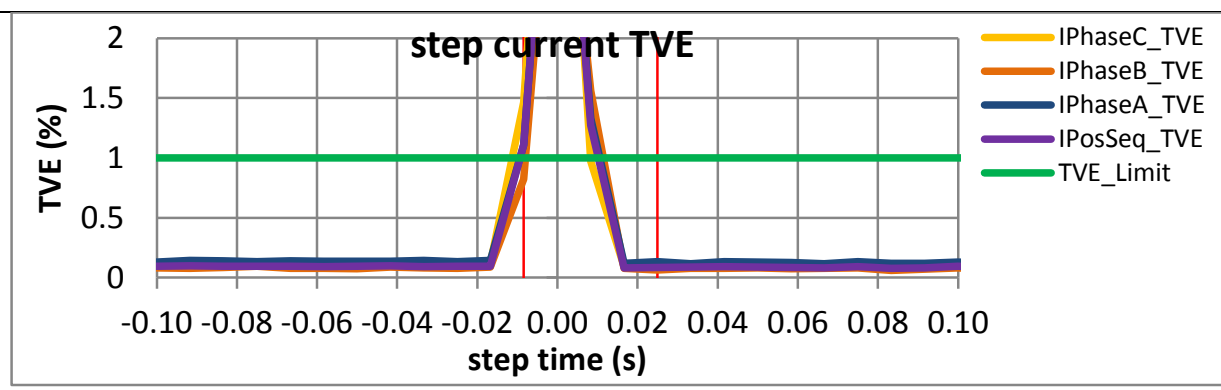

Figure 5541: Fs = 12 FPS, $+10 \%$ magnitude step

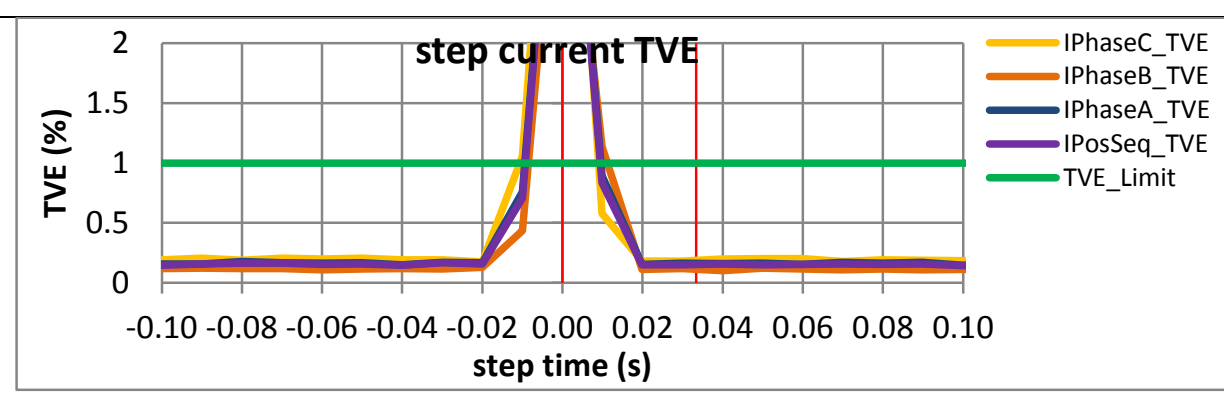

Figure 5543: Fs = $10 \mathrm{FPS},+10 \%$ magnitude step

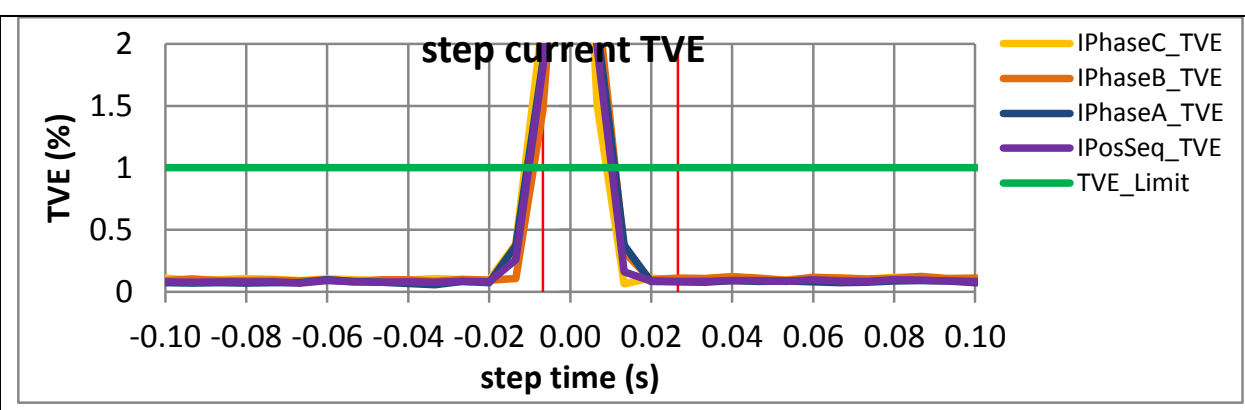

Figure 5540: Fs = 15 FPS, $-10 \%$ magnitude step

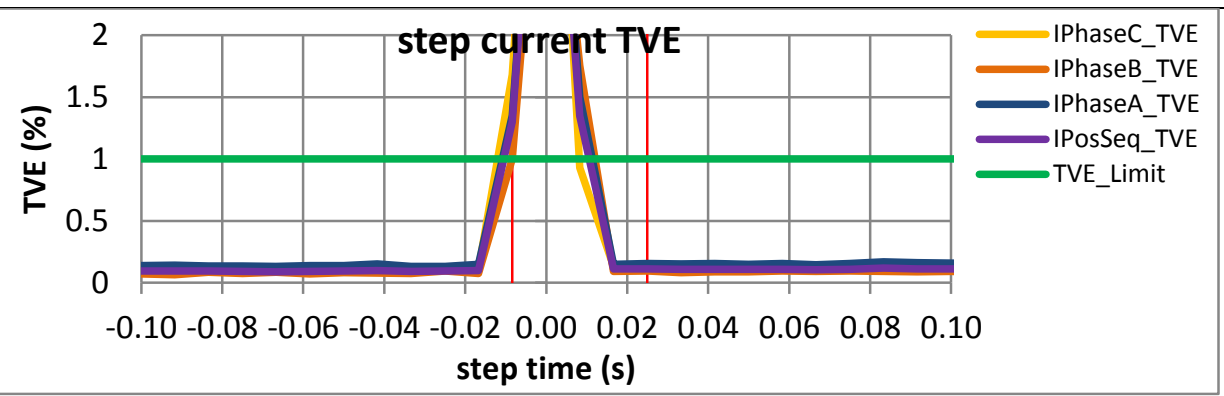

Figure 5542: Fs = 12 FPS, $-10 \%$ magnitude step

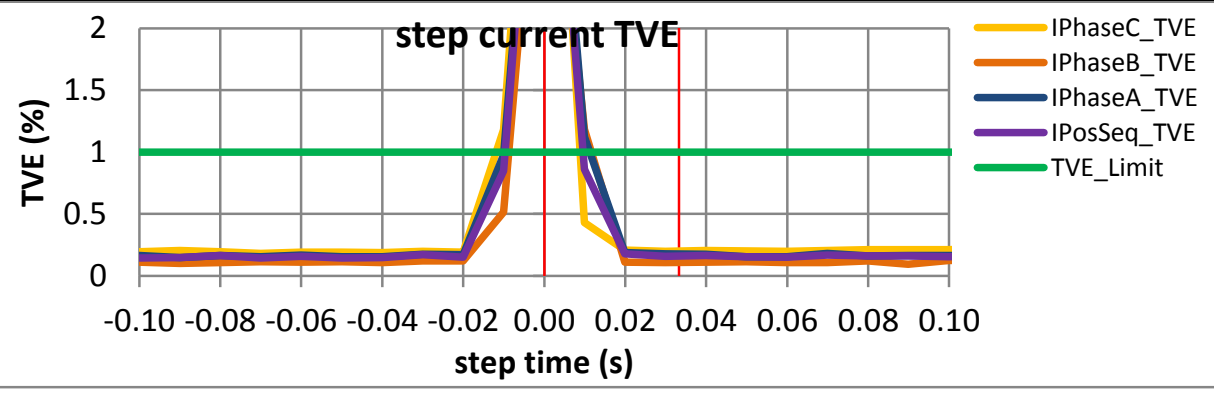

Figure 5544: Fs = 10 FPS, $-10 \%$ magnitude step 
10.6 Dynamic step change in magnitude: frequency response time

\begin{tabular}{|l|c|c|c|c|c|c|c|c|c|c|c|c|}
\hline Fs (FPS) & $10 \mathrm{M}$ & $10 \mathrm{P}$ & $12 \mathrm{M}$ & $12 \mathrm{P}$ & $15 \mathrm{M}$ & $15 \mathrm{P}$ & $20 \mathrm{M}$ & $20 \mathrm{P}$ & $30 \mathrm{M}$ & $30 \mathrm{P}$ & $60 \mathrm{M}$ & $60 \mathrm{P}$ \\
\hline C37.118.1 Annex C & $\mathrm{P}$ & $\mathrm{P}$ & $\mathrm{P}$ & $\mathrm{P}$ & $\mathrm{P}$ & $\mathrm{P}$ & $\mathrm{P}$ & $\mathrm{P}$ & $\mathrm{P}$ & $\mathrm{P}$ & $\mathrm{P}$ & $\mathrm{P}$ \\
\hline PMU A & $\mathrm{P}$ & $\mathrm{P}$ & $\mathrm{P}$ & $\mathrm{P}$ & $\mathrm{P}$ & $\mathrm{P}$ & $\mathrm{P}$ & $\mathrm{P}$ & $\mathrm{P}$ & $\mathrm{P}$ & $\mathrm{P}$ & $\mathrm{P}$ \\
\hline PMU B & $\mathrm{P}$ & $\mathrm{P}$ & $\mathrm{P}$ & $\mathrm{P}$ & $\mathrm{P}$ & $\mathrm{P}$ & $\mathrm{P}$ & $\mathrm{P}$ & $\mathrm{P}$ & $\mathrm{P}$ & $\mathrm{P}$ & $\mathrm{P}$ \\
\hline PMU C & $\mathrm{P}$ & $\mathrm{F}$ & $\mathrm{P}$ & $\mathrm{F}$ & $\mathrm{P}$ & $\mathrm{F}$ & $\mathrm{P}$ & $\mathrm{F}$ & $\mathrm{P}$ & $\mathrm{P}$ & $\mathrm{P}$ & $\mathrm{F}$ \\
\hline PMU D & $\mathrm{P}$ & $\mathrm{P}$ & $\mathrm{P}$ & $\mathrm{P}$ & $\mathrm{P}$ & $\mathrm{P}$ & $\mathrm{P}$ & $\mathrm{P}$ & $\mathrm{P}$ & $\mathrm{P}$ & $\mathrm{P}$ & $\mathrm{P}$ \\
\hline PMU E & $\mathrm{P}$ & - & - & - & $\mathrm{P}$ & - & $\mathrm{P}$ & - & $\mathrm{P}$ & - & $\mathrm{P}$ & - \\
\hline PMU F & $\mathrm{P}$ & $\mathrm{P}$ & $\mathrm{P}$ & $\mathrm{P}$ & $\mathrm{P}$ & $\mathrm{P}$ & $\mathrm{P}$ & $\mathrm{P}$ & $\mathrm{P}$ & $\mathrm{P}$ & $\mathrm{P}$ & $\mathrm{P}$ \\
\hline PMU G & $\mathrm{P}$ & - & $\mathrm{P}$ & - & $\mathrm{P}$ & - & $\mathrm{P}$ & - & $\mathrm{P}$ & - & - & - \\
\hline PMU H & $\mathrm{P}$ & $\mathrm{P}$ & $\mathrm{P}$ & $\mathrm{P}$ & $\mathrm{P}$ & $\mathrm{P}$ & $\mathrm{P}$ & $\mathrm{P}$ & $\mathrm{P}$ & $\mathrm{P}$ & $\mathrm{P}$ & $\mathrm{P}$ \\
\hline PMU I & $\mathrm{P}$ & - & $\mathrm{P}$ & - & $\mathrm{P}$ & - & $\mathrm{P}$ & - & $\mathrm{P}$ & - & $\mathrm{P}$ & - \\
\hline PMU J & $\mathrm{P}$ & $\mathrm{P}$ & $\mathrm{P}$ & $\mathrm{P}$ & $\mathrm{P}$ & $\mathrm{P}$ & $\mathrm{P}$ & $\mathrm{P}$ & $\mathrm{P}$ & $\mathrm{P}$ & $\mathrm{P}$ & $\mathrm{P}$ \\
\hline
\end{tabular}


10.6.1 C37.118.1-2011 Annex C dynamic step change in magnitude frequency response time: $\mathrm{F} 0=60 \mathrm{~Hz}$, $\mathrm{M}$ class

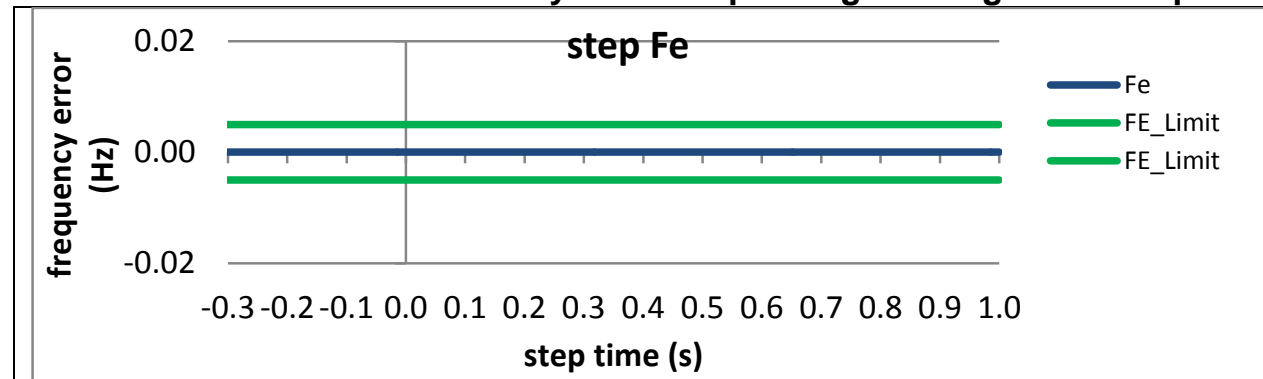

Figure 5545: Fs $=60 \mathrm{FPS},+10 \%$ magnitude step

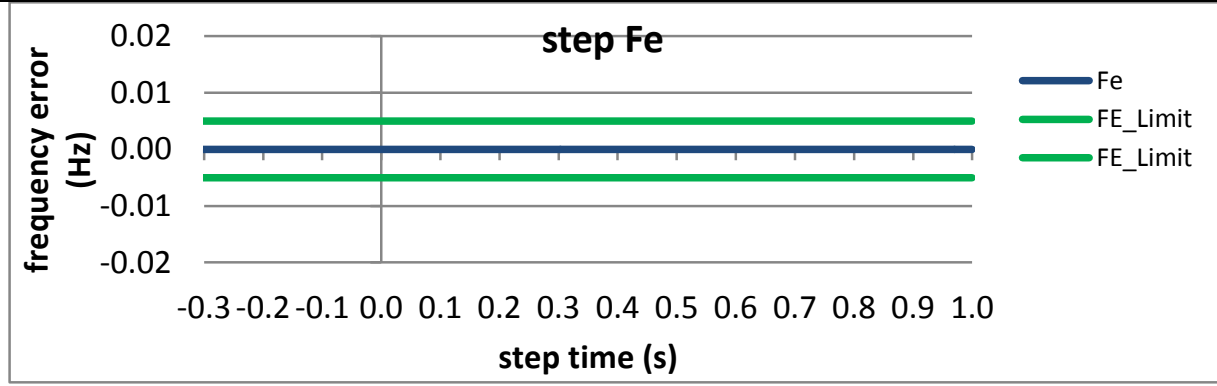

Figure 5547: Fs = 30 FPS, $+10 \%$ magnitude step

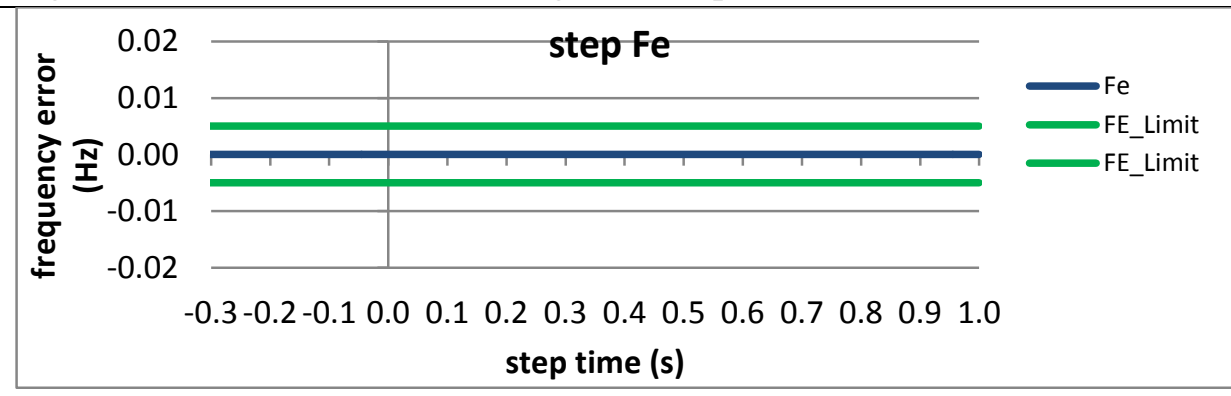

Figure 5549: Fs = 20 FPS, +10\% magnitude step

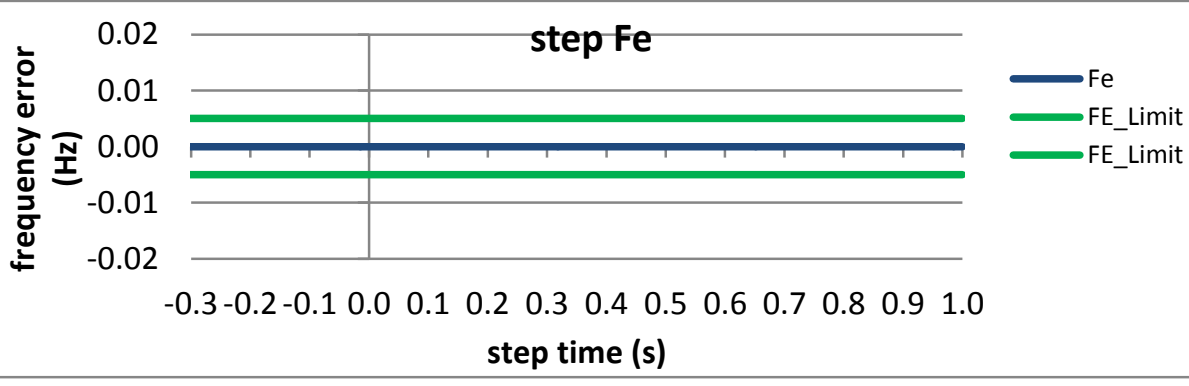

Figure 5546: Fs = 60 FPS, -10\% magnitude step

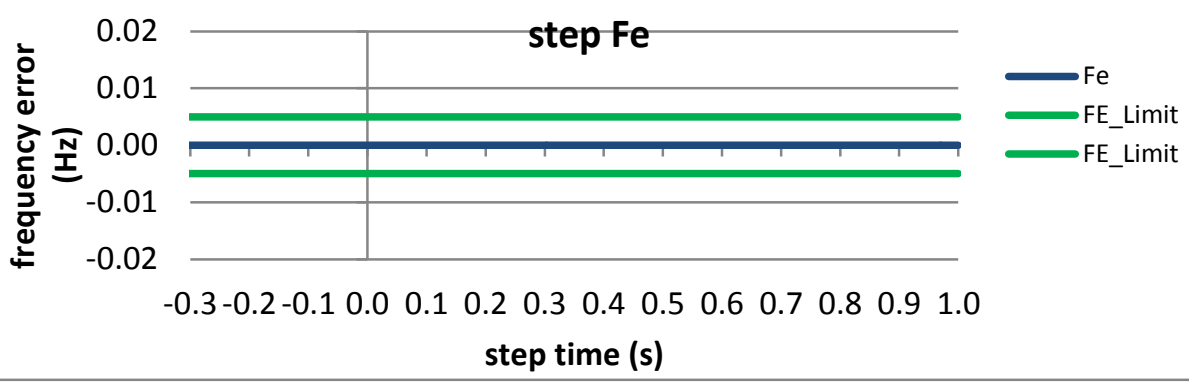

Figure 5548: Fs = 30 FPS, -10\% magnitude step

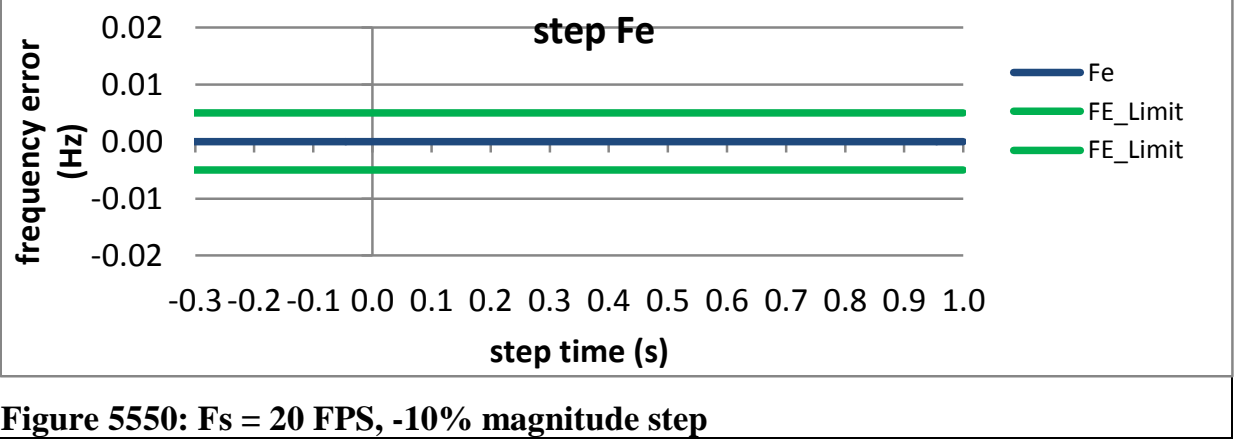




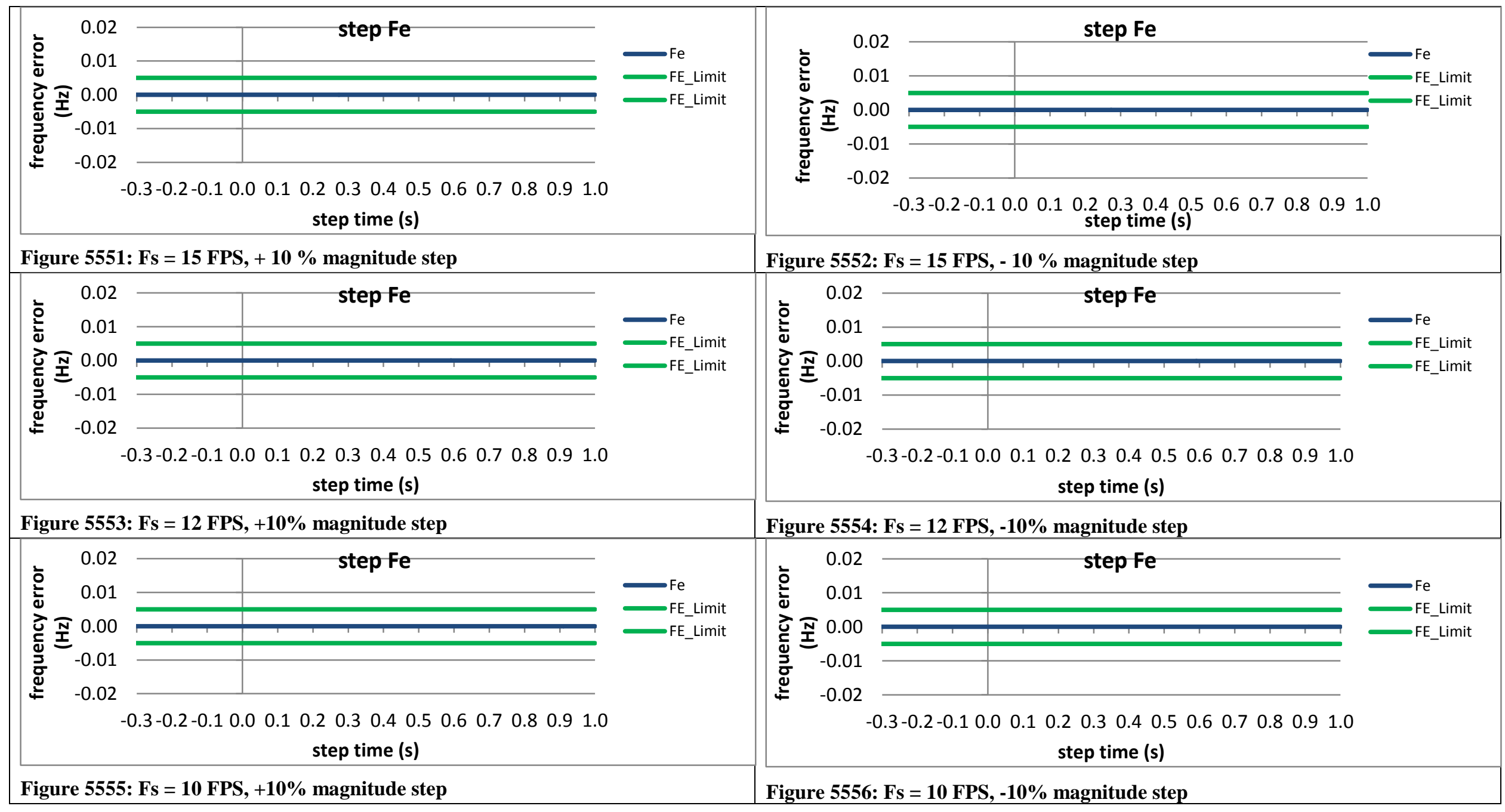


10.6.2 PMU A dynamic step change in magnitude frequency response time: $F 0=60 \mathrm{~Hz}, \mathrm{M}$ class
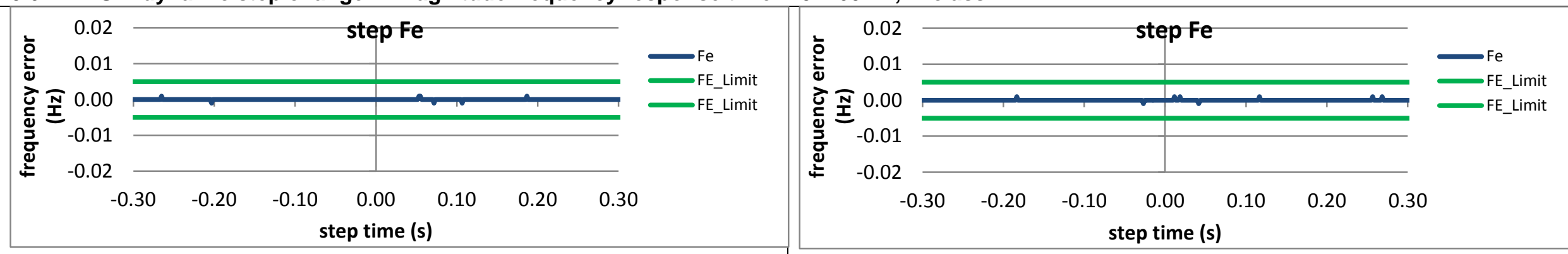

Figure 5557: Fs = 60 FPS, +10\% magnitude step

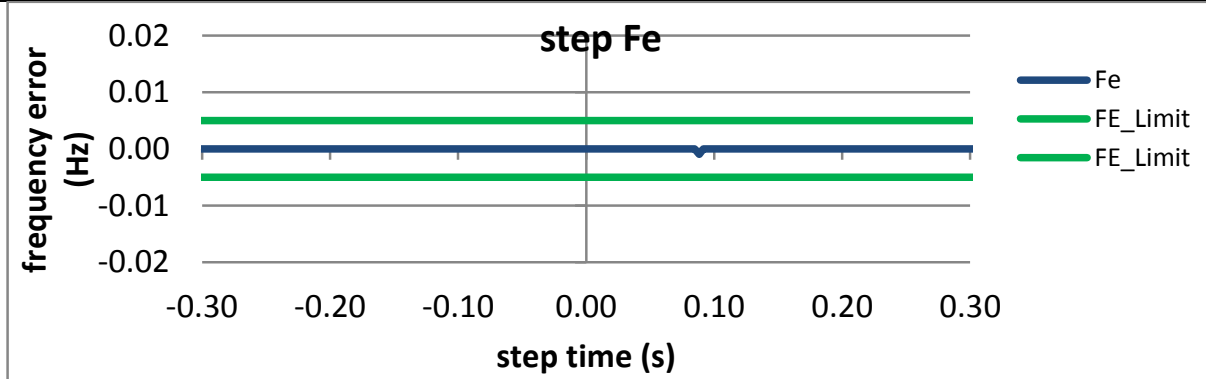

Figure 5558: Fs = 60 FPS, -10\% magnitude step

Figure 5559: Fs = 30 FPS, +10\% magnitude step
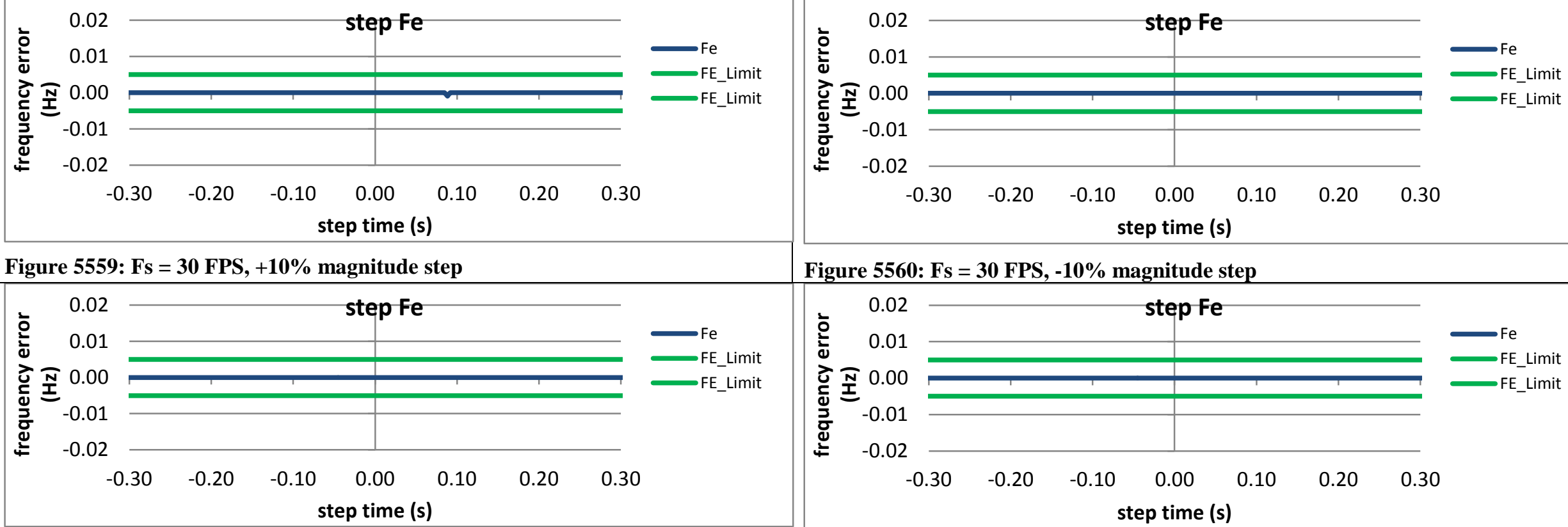

Figure 5560: Fs = 30 FPS, -10\% magnitude step

Figure 5561: Fs = 20 FPS, +10\% magnitude step

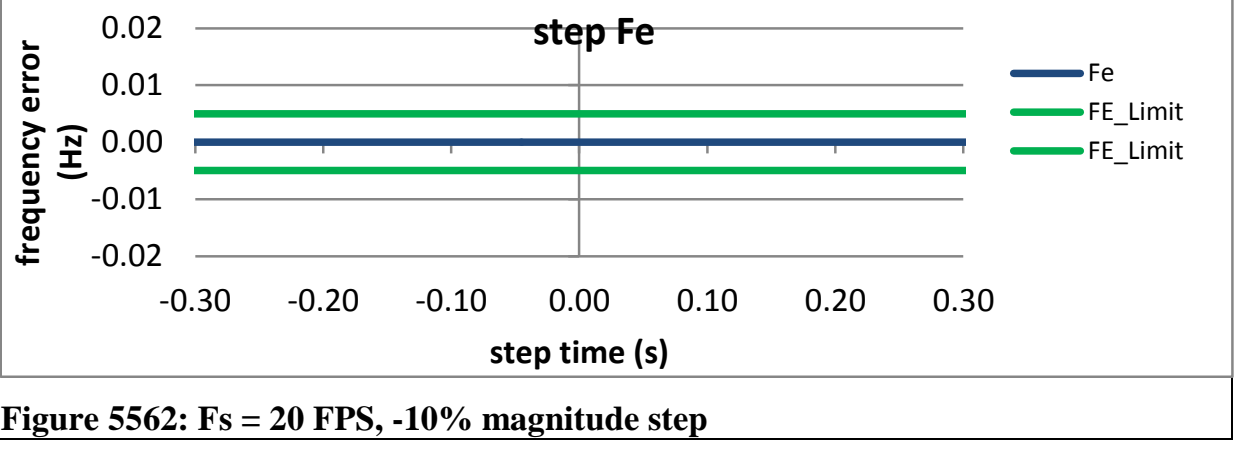



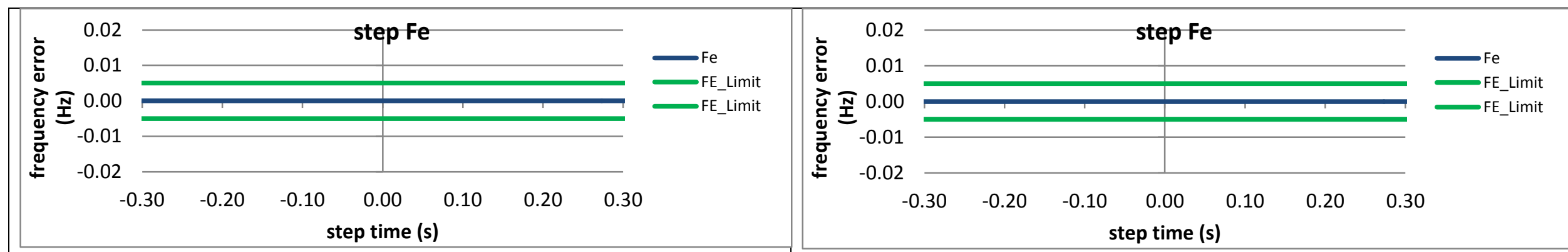

Figure 5563: Fs = 15 FPS, $+10 \%$ magnitude step

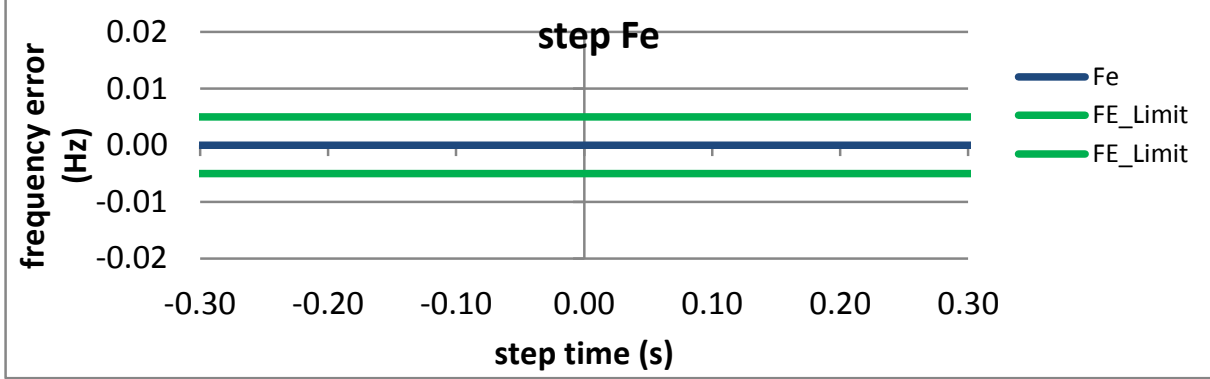

Figure 5565: Fs = 12 FPS, +10\% magnitude step

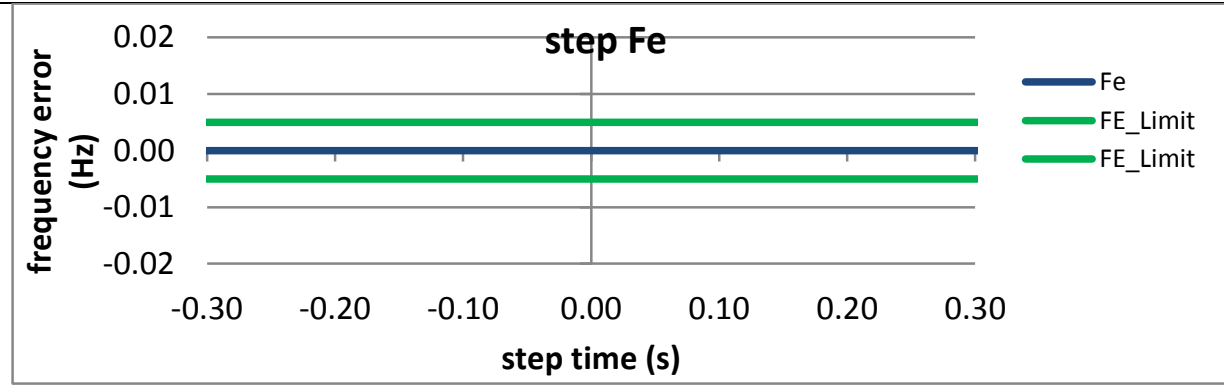

Figure 5564: Fs = 15 FPS, - $10 \%$ magnitude step

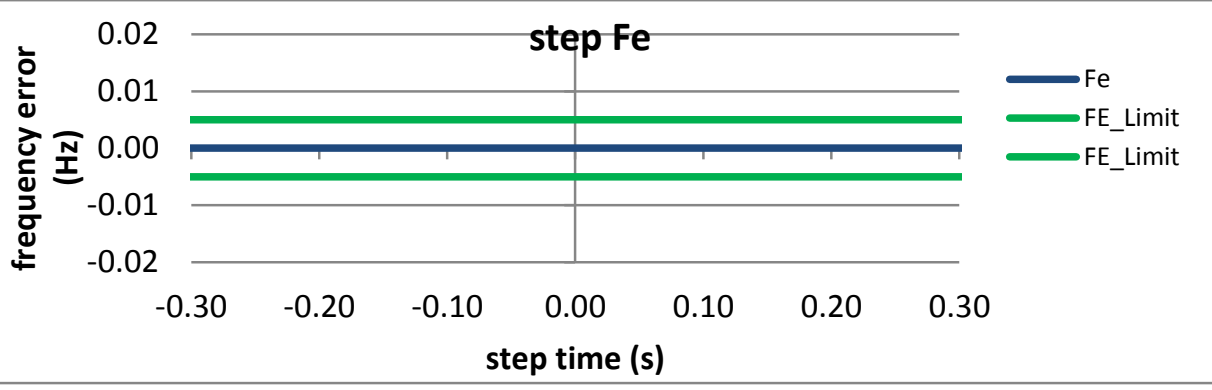

Figure 5566: Fs = 12 FPS, -10\% magnitude step

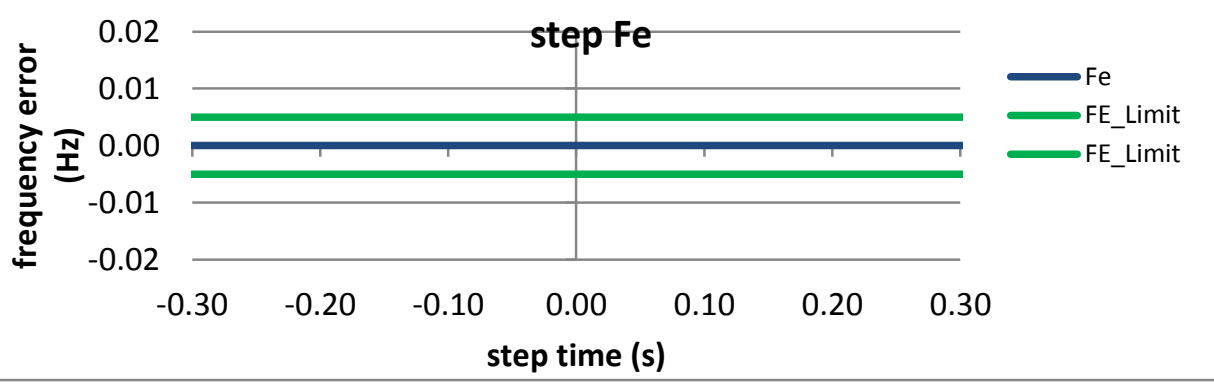

Figure 5568: Fs = 10 FPS, $-10 \%$ magnitude step 
10.6.3 PMU B dynamic step change in magnitude frequency response time: $F 0=60 \mathrm{~Hz}, \mathrm{M}$ class
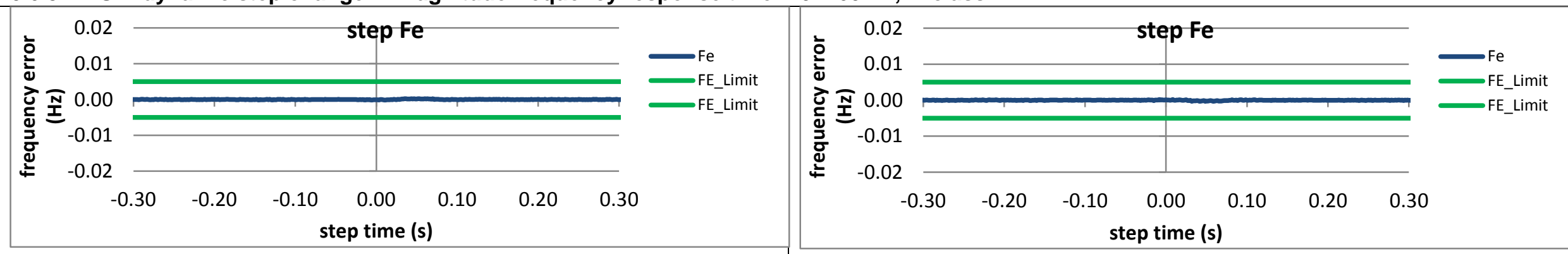

Figure 5569: Fs = 60 FPS, +10\% magnitude step

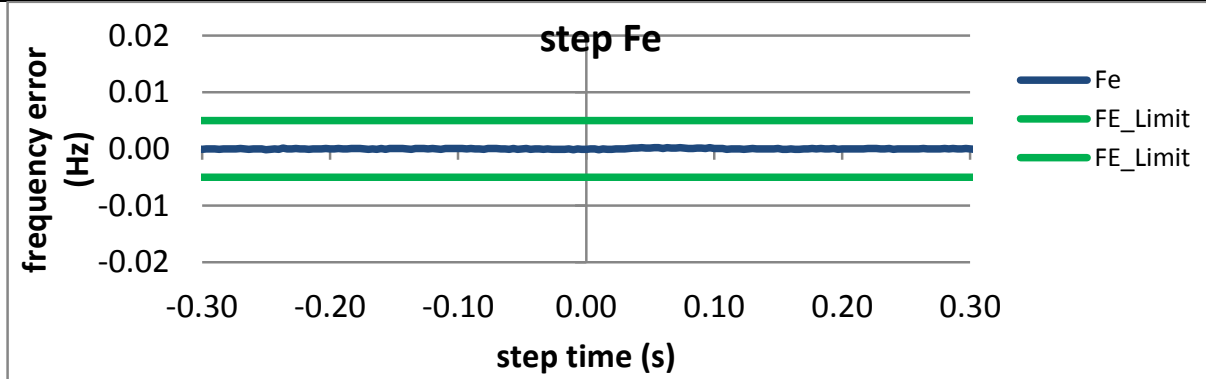

Figure 5570: Fs = 60 FPS, -10\% magnitude step

Figure 5571: Fs = 30 FPS, +10\% magnitude step
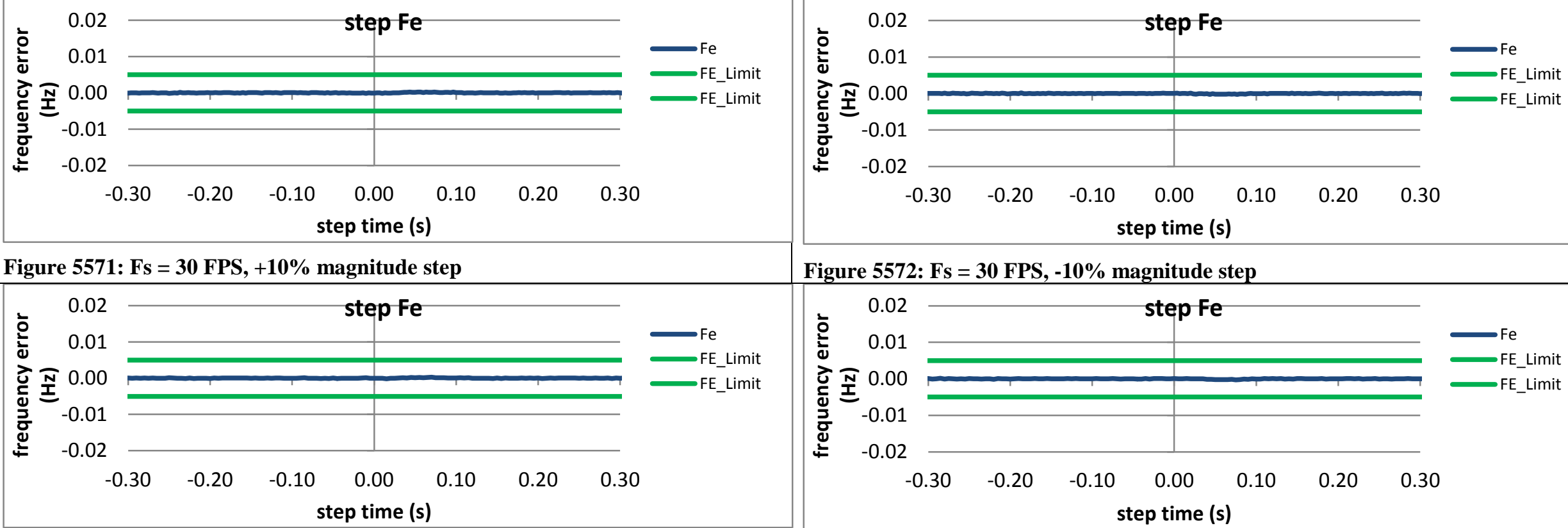

Figure 5572: Fs = 30 FPS, -10\% magnitude step

Figure 5573: Fs = 20 FPS, +10\% magnitude step

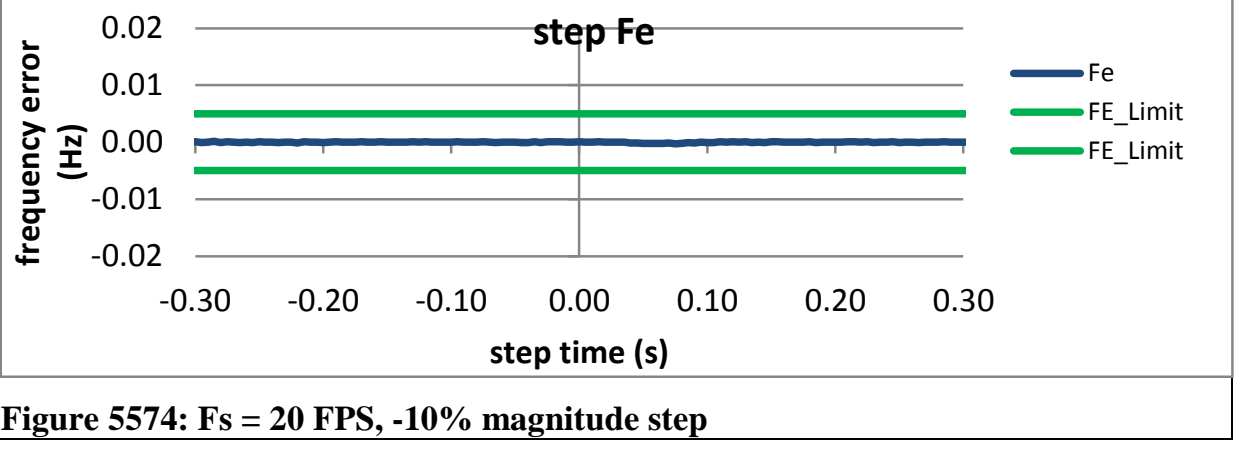



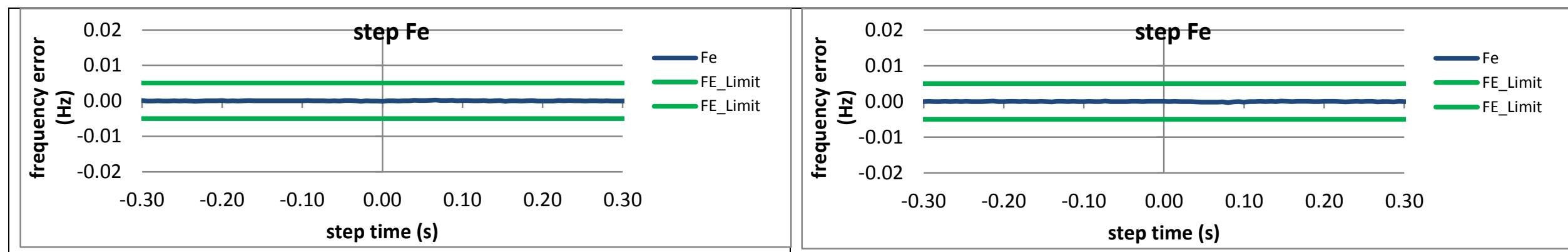

Figure 5575: Fs = 15 FPS, $+10 \%$ magnitude step

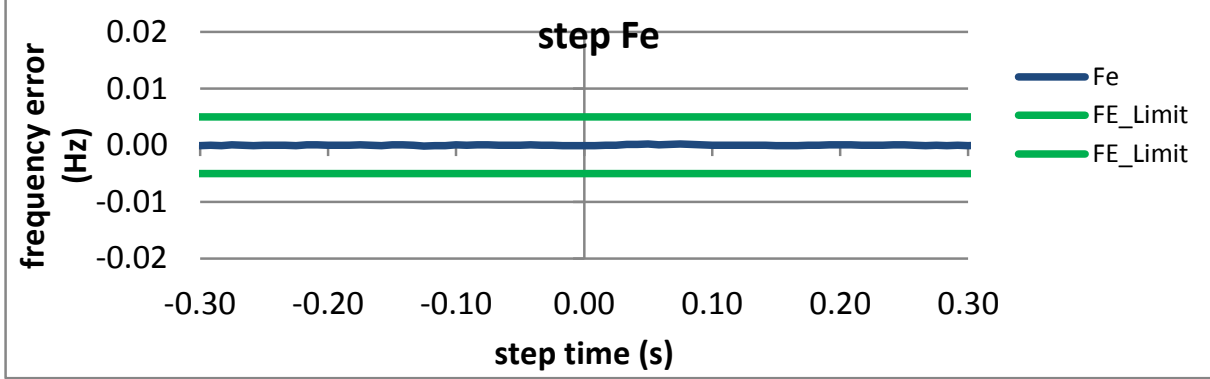

Figure 5577: Fs = 12 FPS, +10\% magnitude step

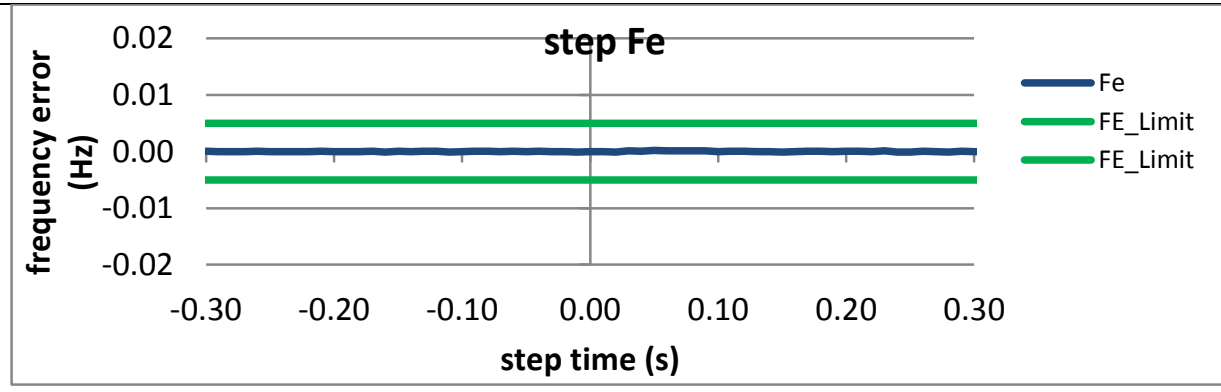

Figure 5576: Fs = 15 FPS, - $10 \%$ magnitude step

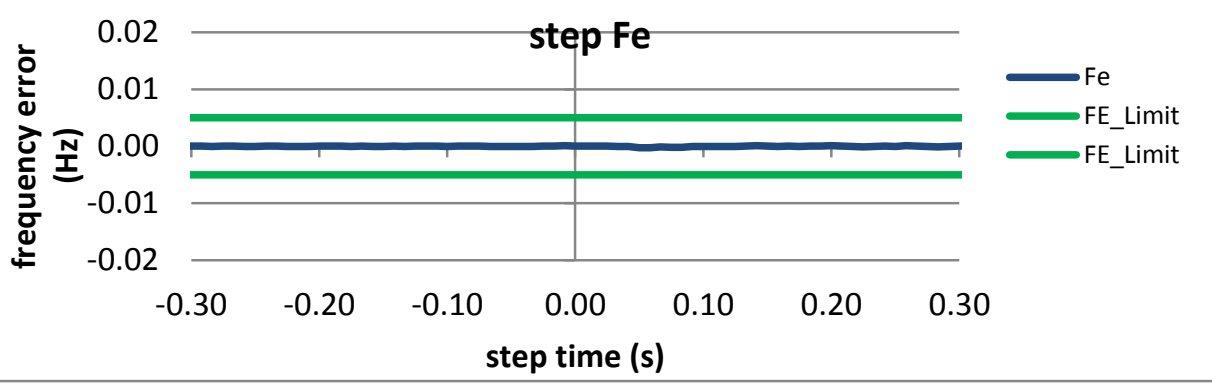

Figure 5578: Fs = 12 FPS, -10\% magnitude step

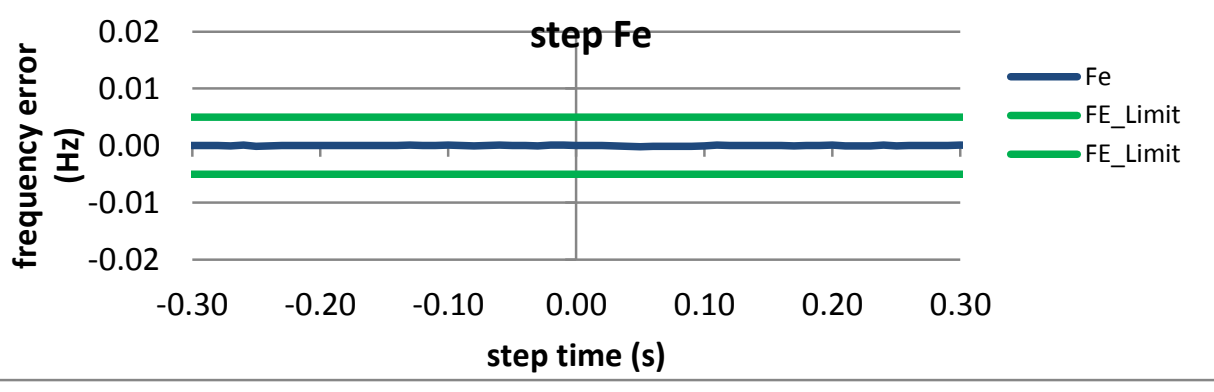

Figure 5580: Fs = 10 FPS, $-10 \%$ magnitude step 
10.6.4 PMU C dynamic step change in magnitude frequency response time: $F 0=60 \mathrm{~Hz}, \mathrm{M}$ class

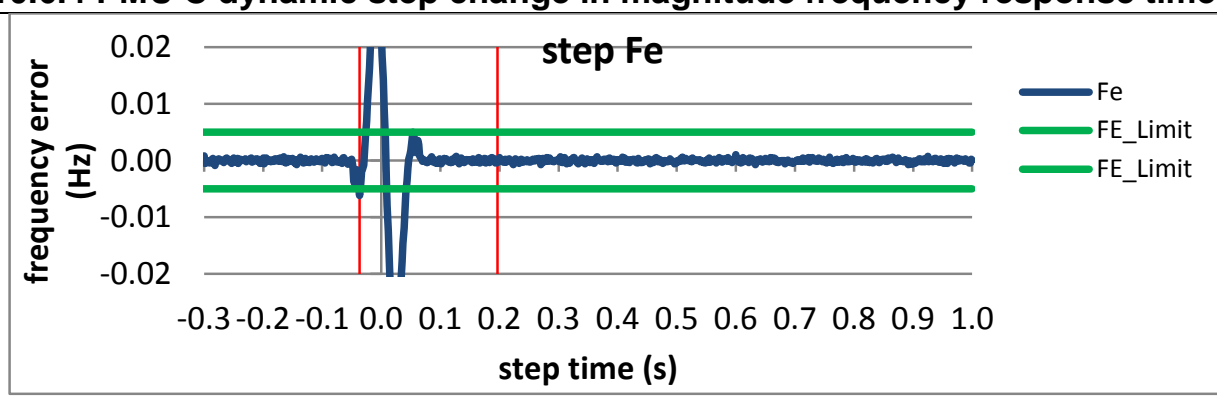

Figure 5581: Fs = 60 FPS, +10\% magnitude step

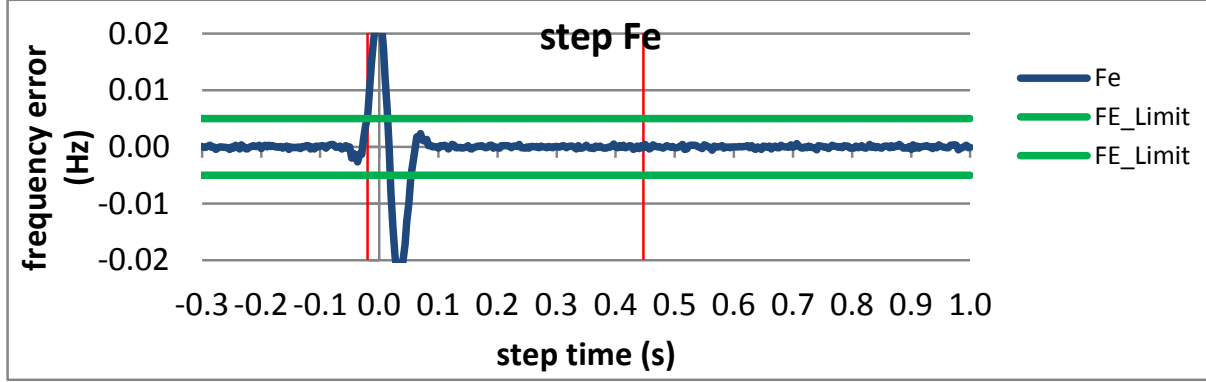

Figure 5583: Fs $=30$ FPS, $+10 \%$ magnitude step

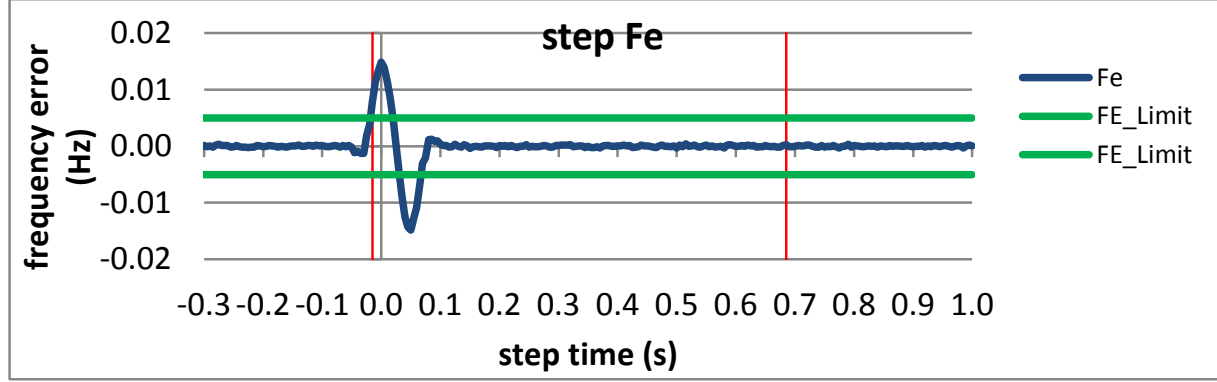

Figure 5585: Fs $=20$ FPS, $+10 \%$ magnitude step

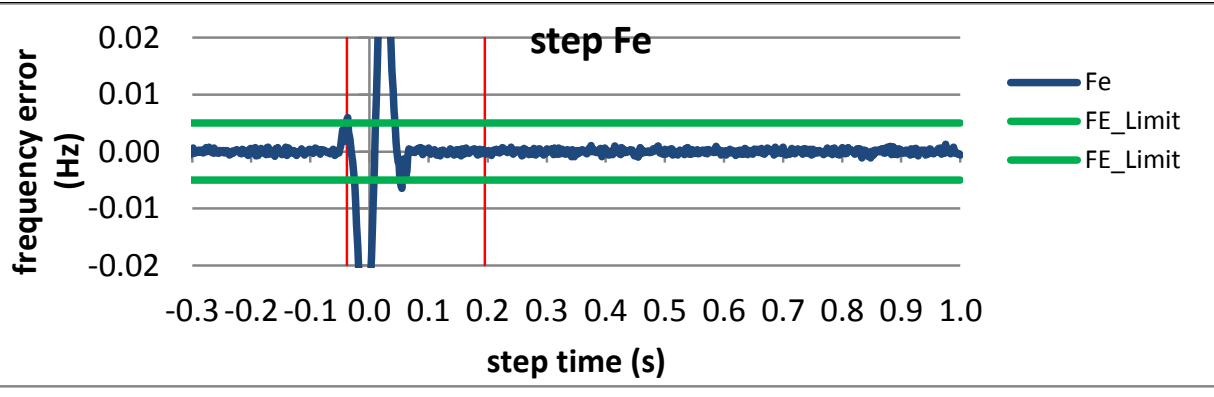

Figure 5582: Fs = 60 FPS, -10\% magnitude step

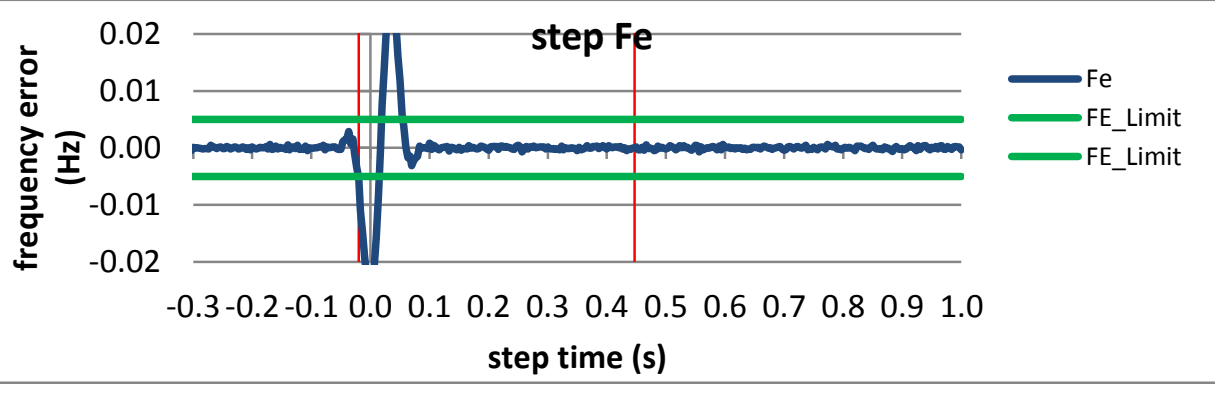

Figure 5584: Fs = 30 FPS, -10\% magnitude step

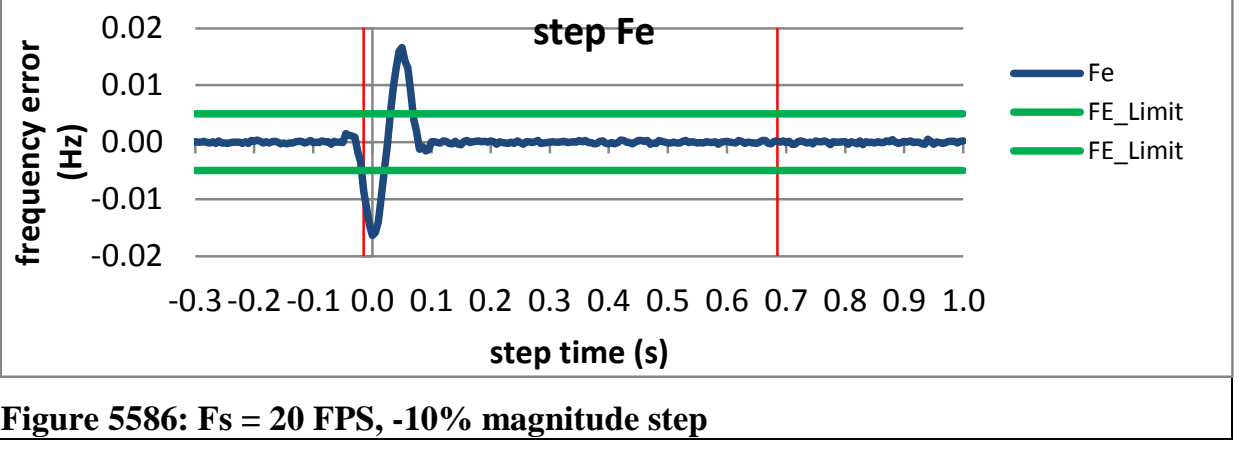



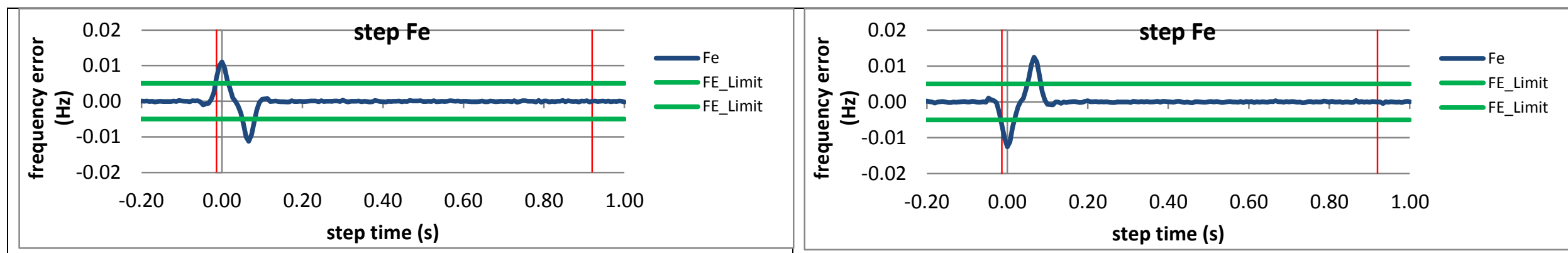

Figure 5587: Fs = 15 FPS, $+10 \%$ magnitude step

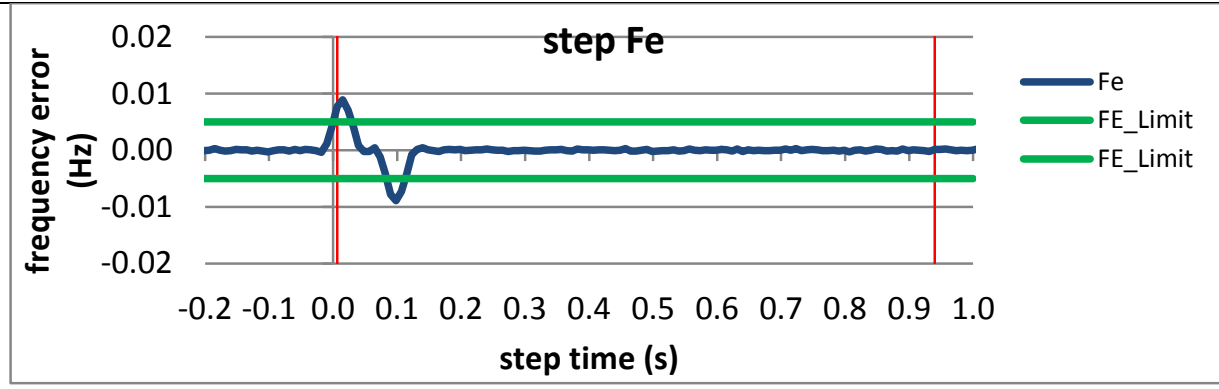

Figure 5588: Fs = 15 FPS, - $10 \%$ magnitude step

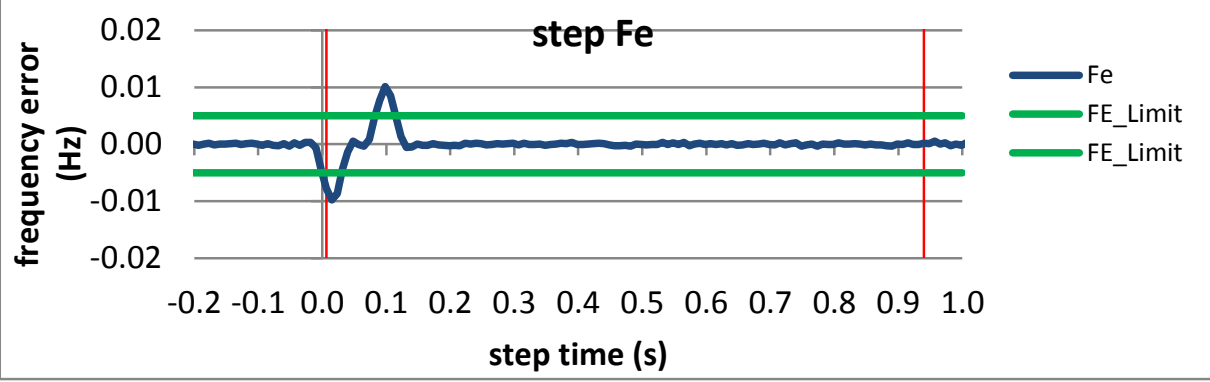

Figure 5589: Fs = 12 FPS, $+10 \%$ magnitude step

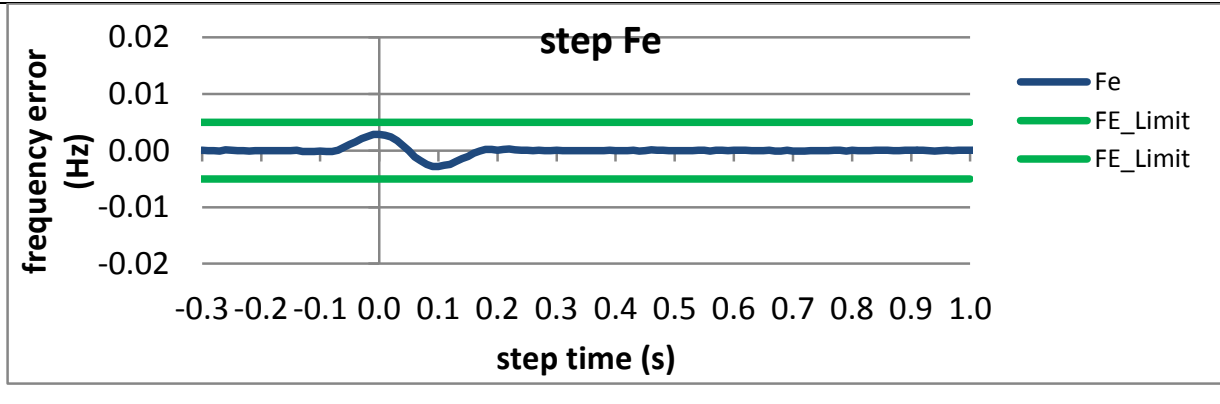

Figure 5590: Fs = 12 FPS, -10\% magnitude step

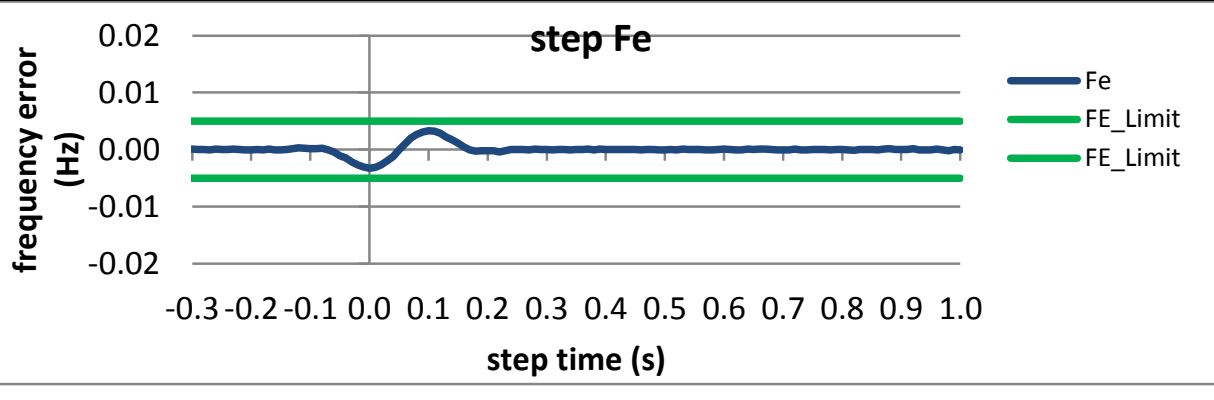

Figure 5592: Fs = 10 FPS, -10\% magnitude step 
10.6.5 PMU D dynamic step change in magnitude frequency response time: $F 0=60 \mathrm{~Hz}, \mathrm{M}$ class

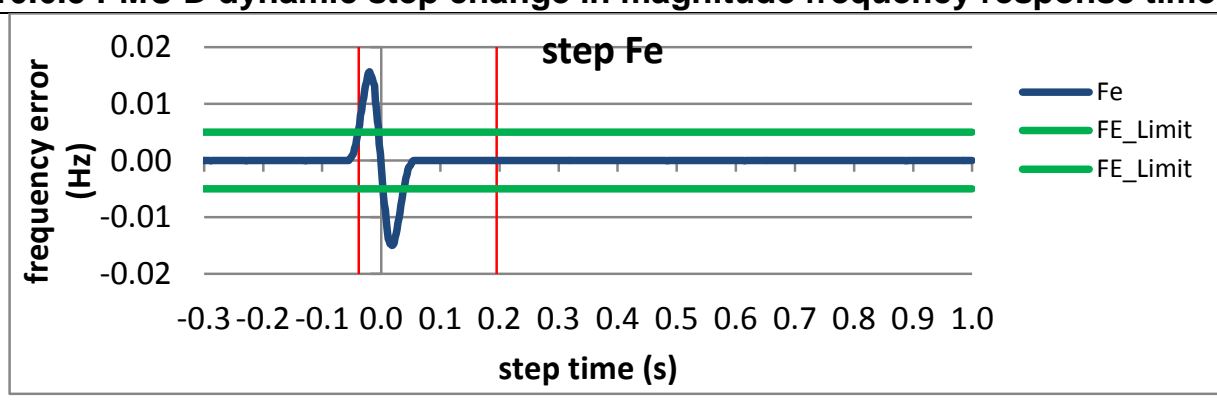

Figure 5593: Fs = 60 FPS, +10\% magnitude step

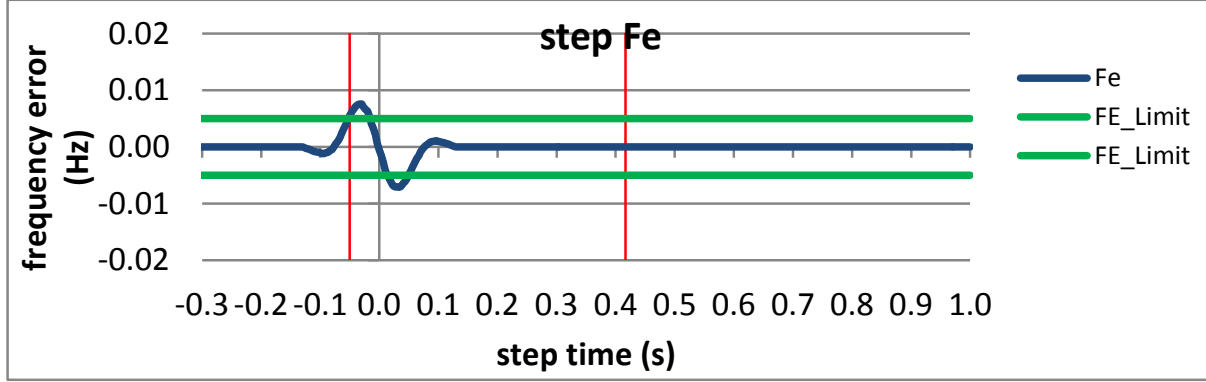

Figure 5595: Fs $=30$ FPS, $+10 \%$ magnitude step

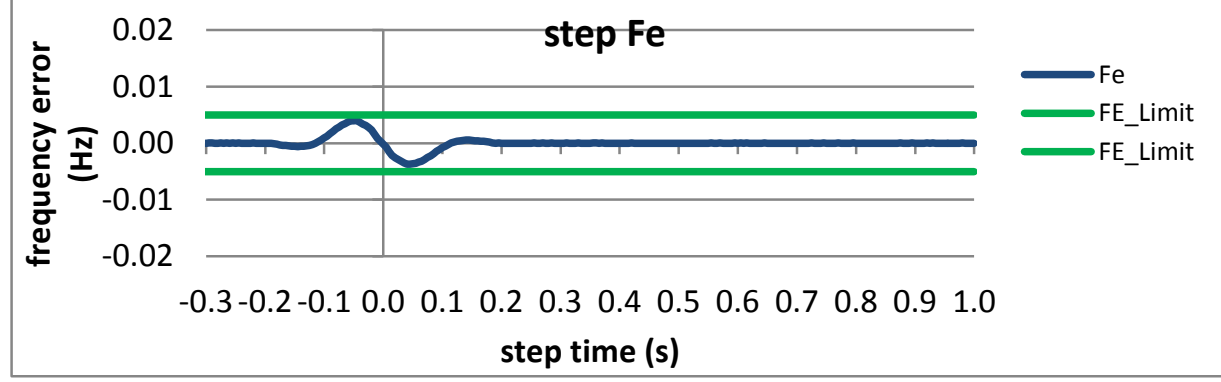

Figure 5597: Fs = 20 FPS, +10\% magnitude step

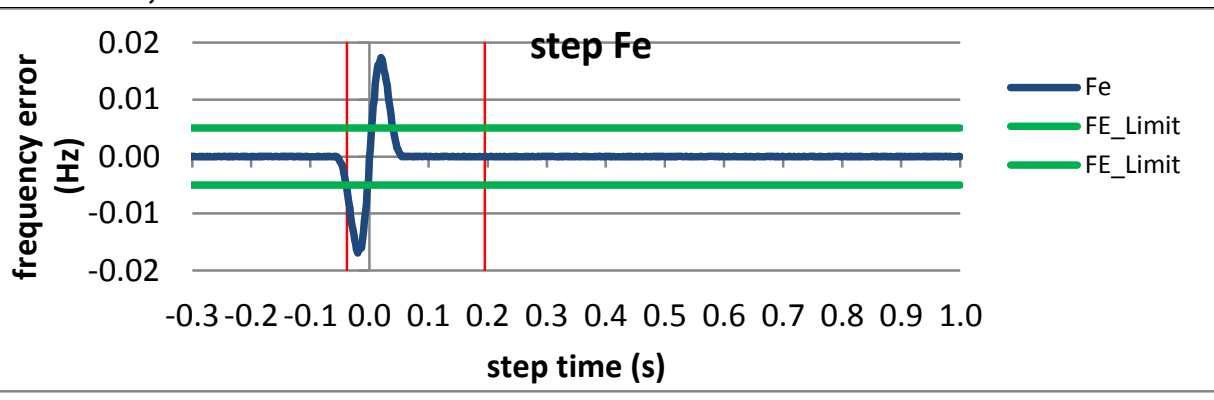

Figure 5594: Fs = 60 FPS, -10\% magnitude step

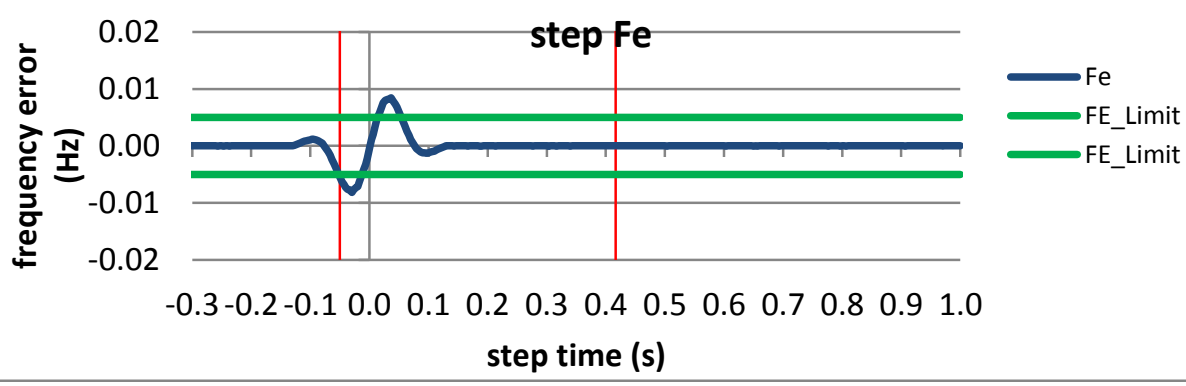

Figure 5596: Fs = 30 FPS, -10\% magnitude step

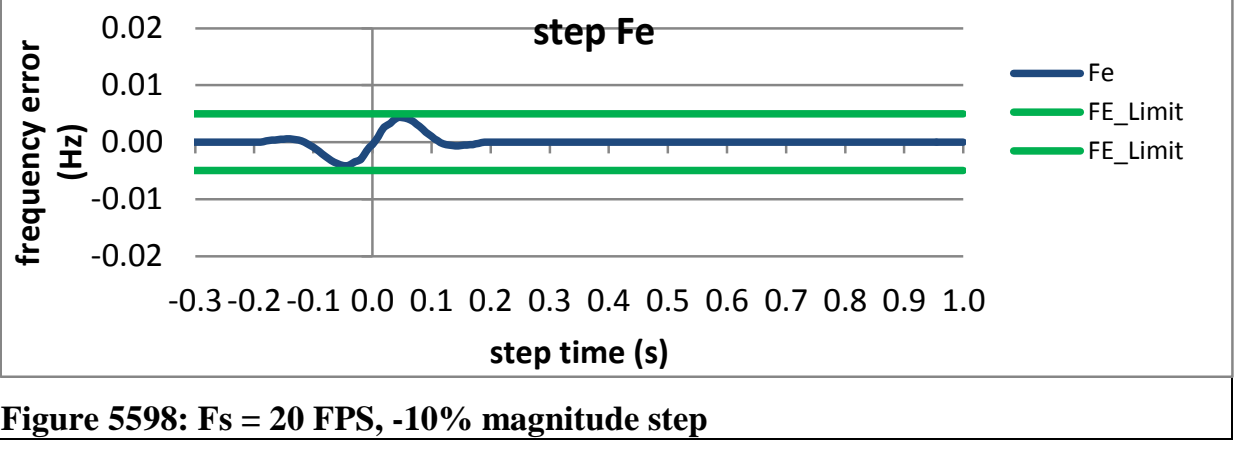



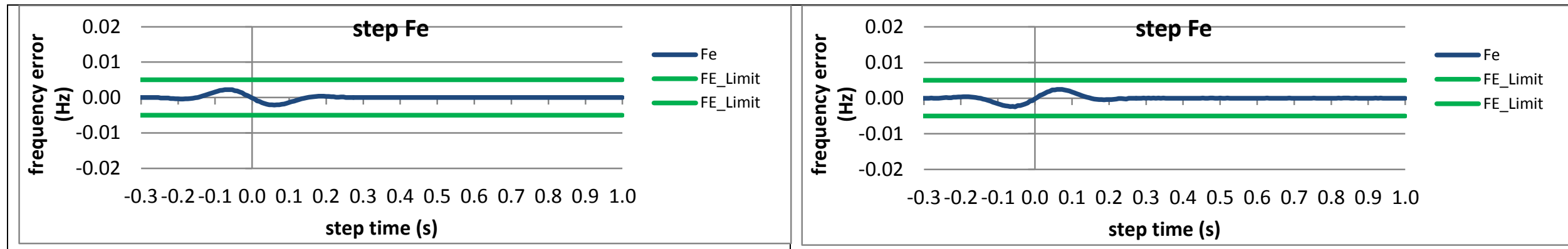

Figure 5599: Fs = 15 FPS, $+10 \%$ magnitude step

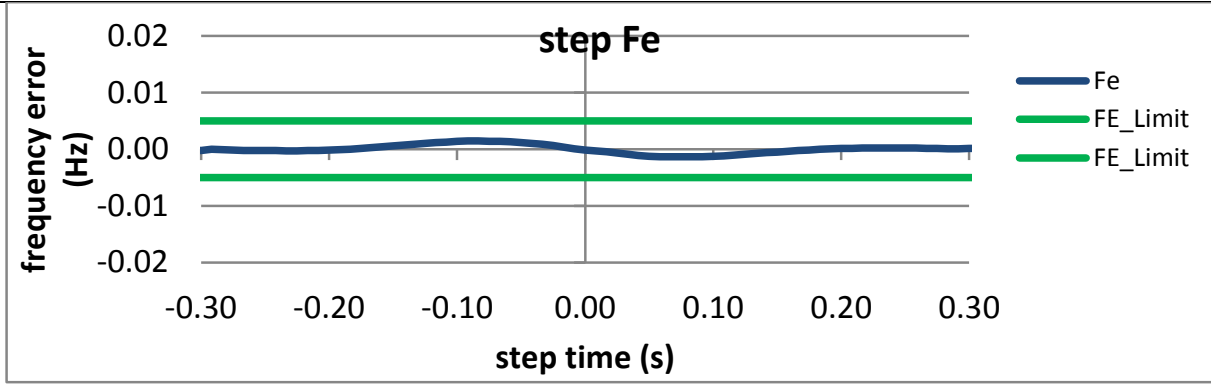

Figure 5601: Fs = 12 FPS, $+10 \%$ magnitude step

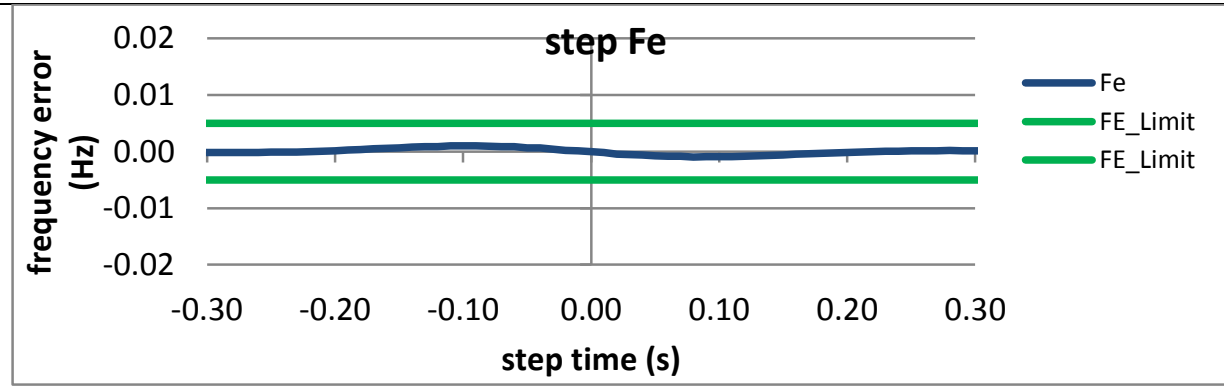

Figure 5600: Fs = 15 FPS, - $10 \%$ magnitude step

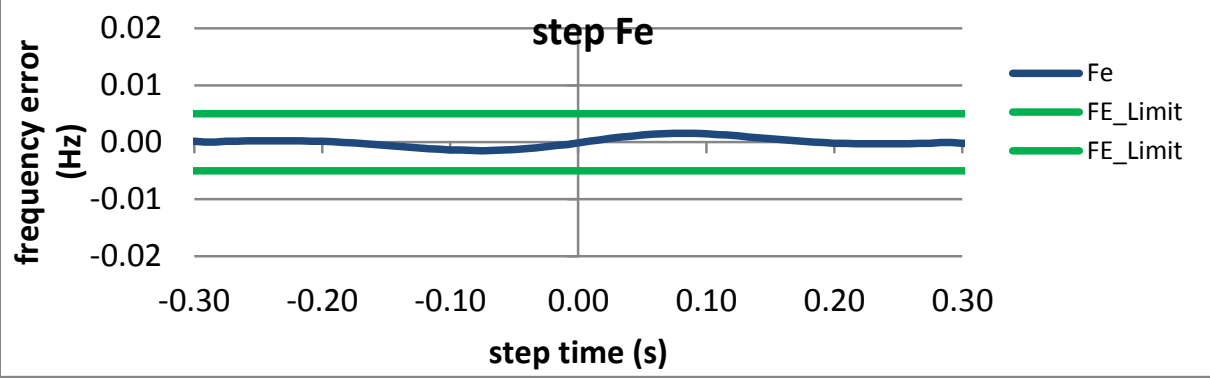

Figure 5602: Fs = 12 FPS, -10\% magnitude step

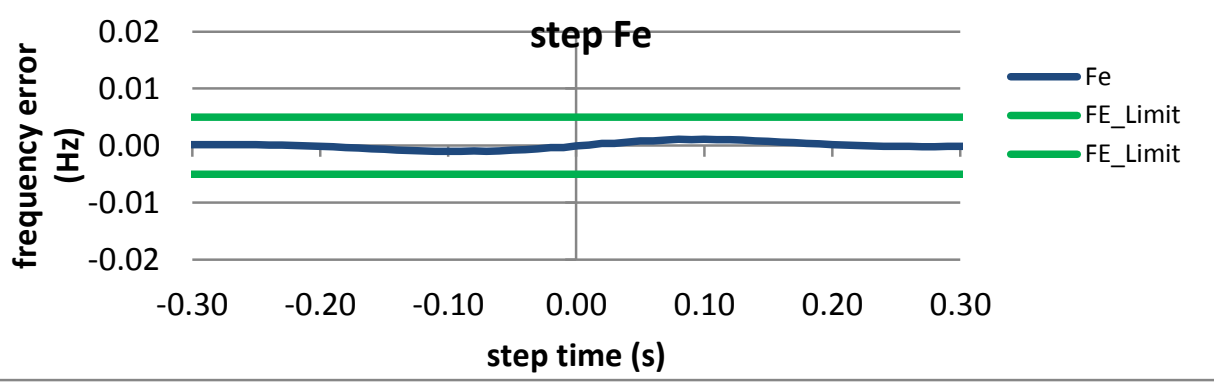

Figure 5603: Fs = 10 FPS, $+10 \%$ magnitude step

Figure 5604: Fs = 10 FPS, $-10 \%$ magnitude step 
10.6.6 PMU E dynamic step change in magnitude frequency response time: $F 0=60 \mathrm{~Hz}, \mathrm{M}$ class
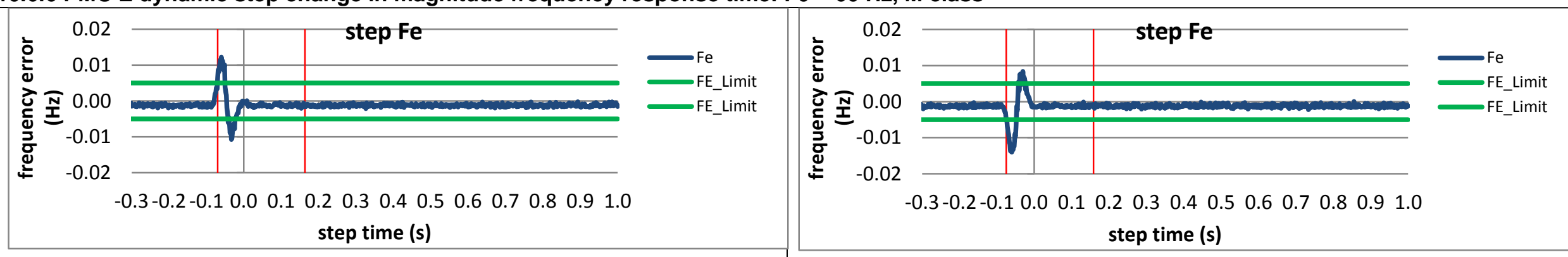

Figure 5605: Fs = 60 FPS, +10\% magnitude step

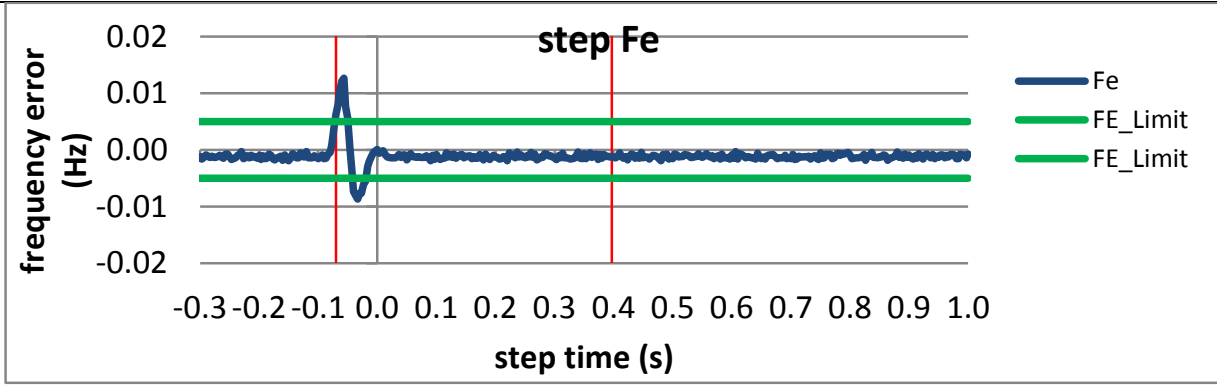

Figure 5606: Fs = 60 FPS, -10\% magnitude step

Figure 5607: Fs = 30 FPS, +10\% magnitude step
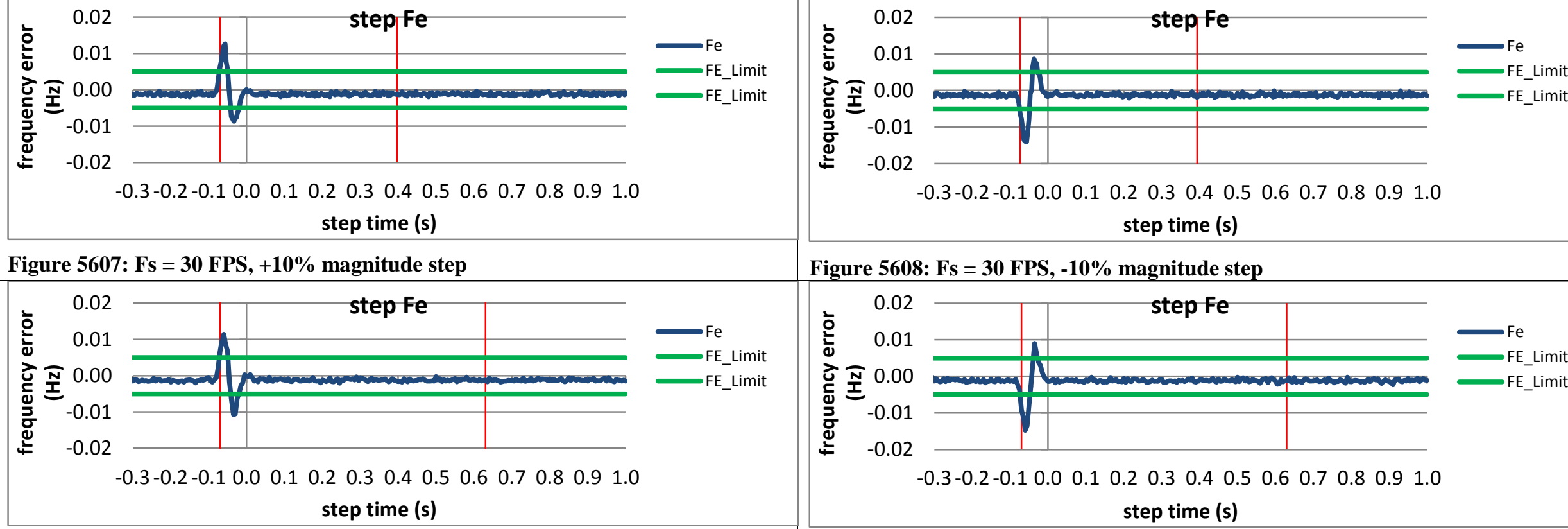

Figure 5608: Fs = 30 FPS, -10\% magnitude step

Figure 5609: Fs $=20$ FPS, +10\% magnitude step

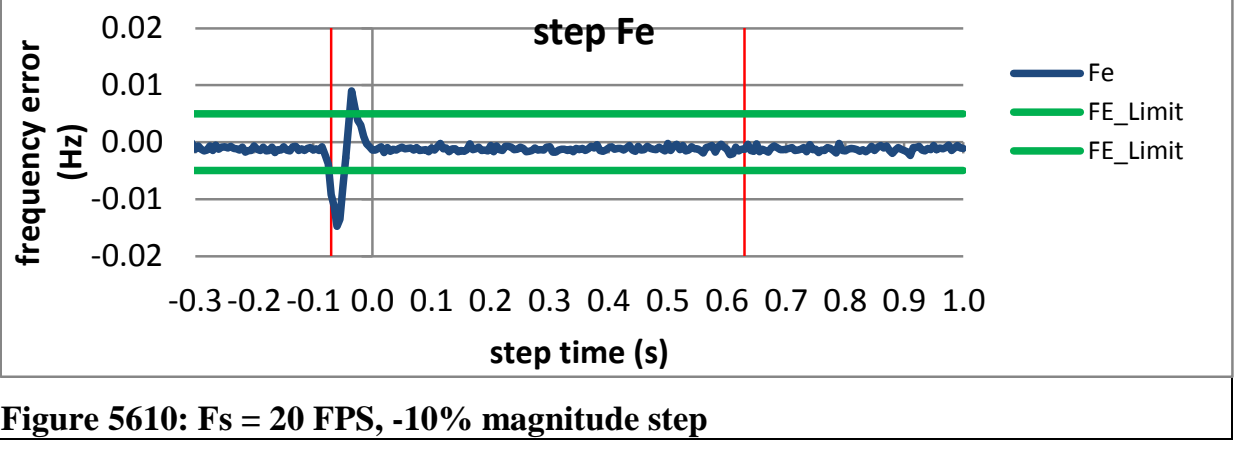




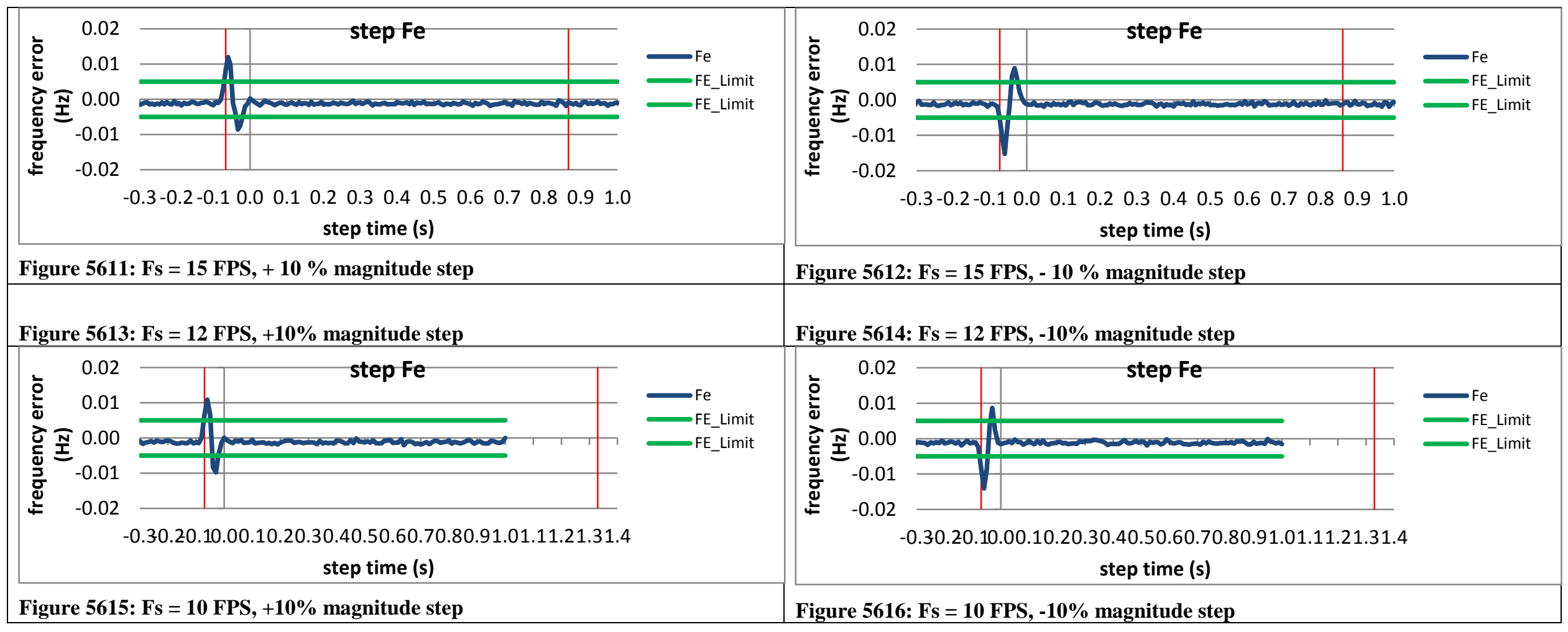


10.6.7 PMU F dynamic step change in magnitude frequency response time: $\mathrm{F} 0=60 \mathrm{~Hz}, \mathrm{M}$ class

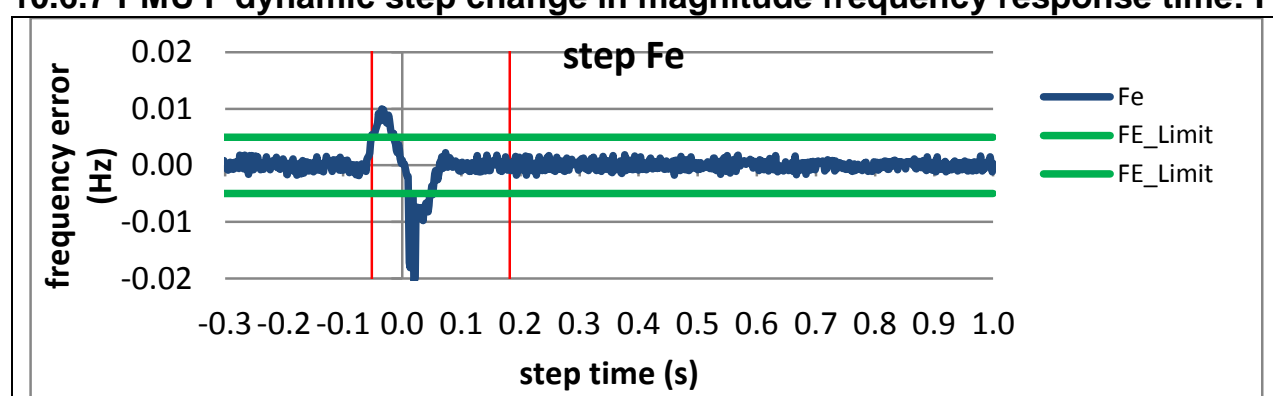

Figure 5617: Fs = 60 FPS, $+10 \%$ magnitude step

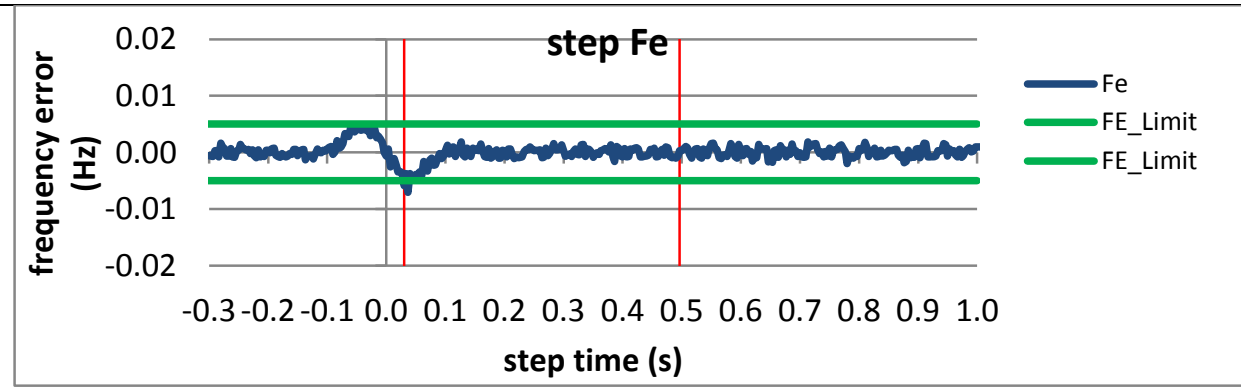

Figure 5619: Fs = 30 FPS, $+10 \%$ magnitude step

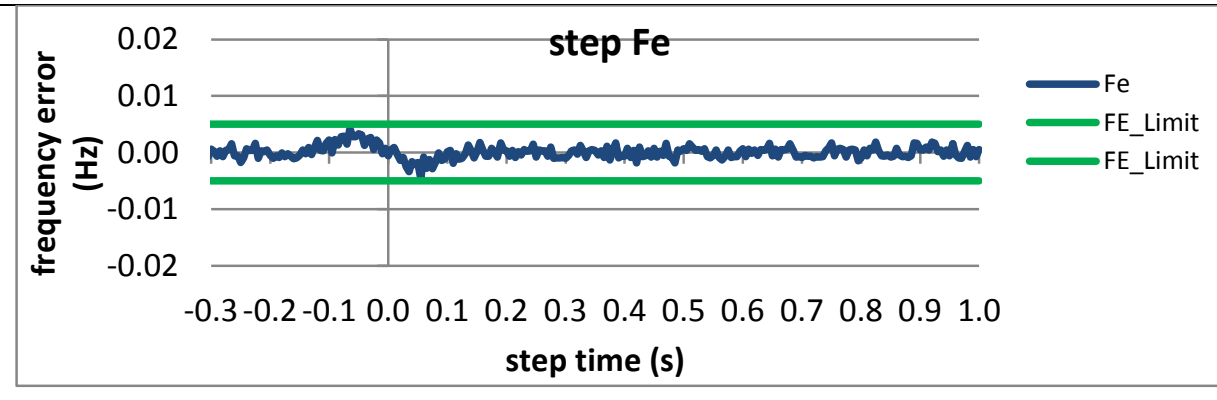

Figure 5621: Fs = 20 FPS, $+10 \%$ magnitude step

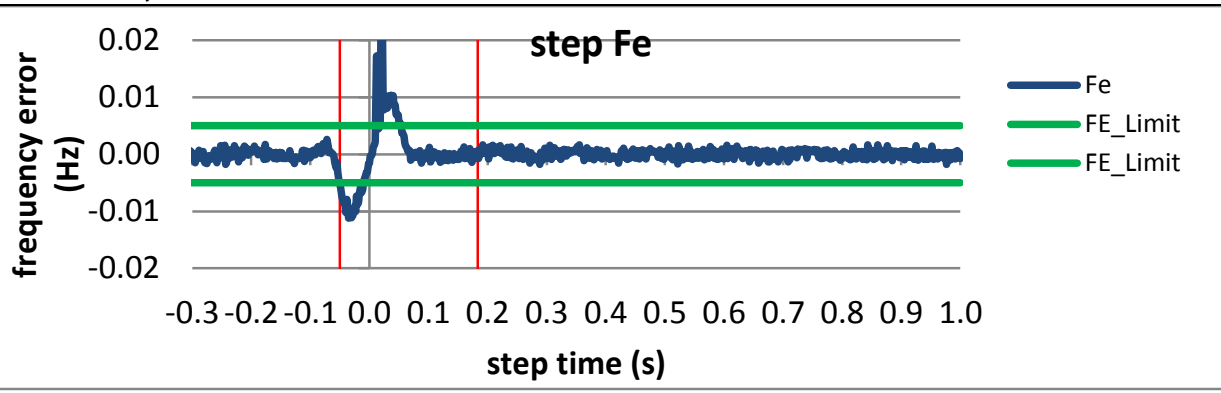

Figure 5618: Fs = 60 FPS, $-10 \%$ magnitude step

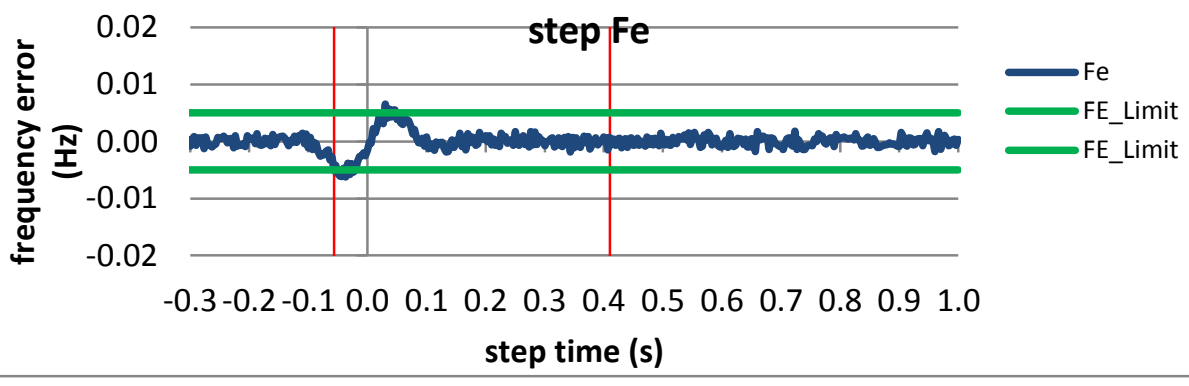

Figure 5620: Fs = 30 FPS, $-10 \%$ magnitude step

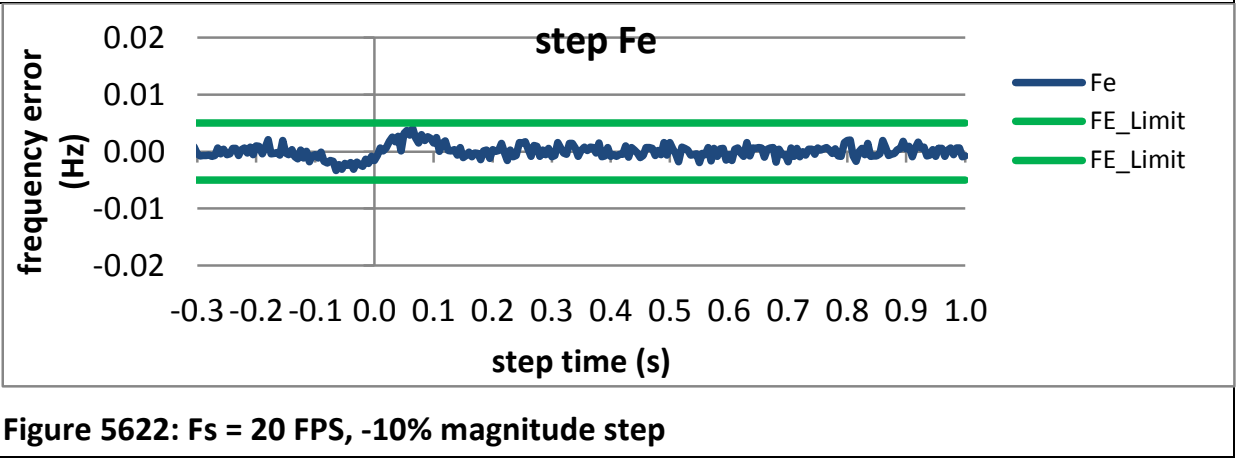



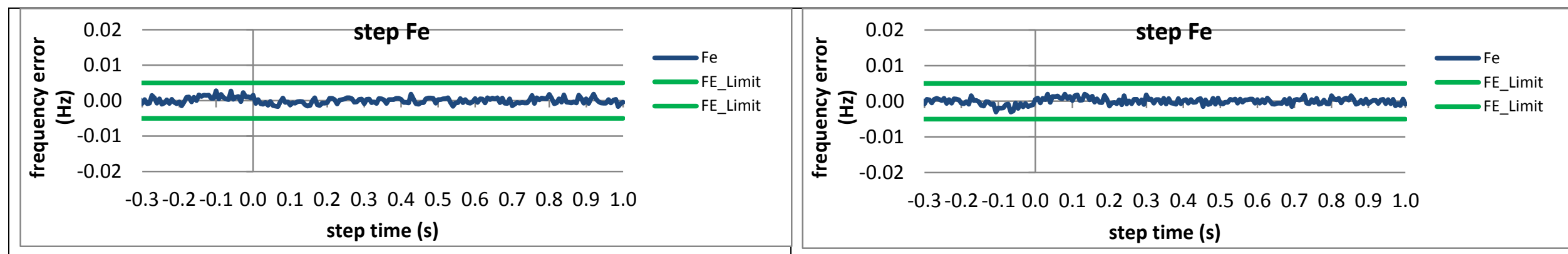

Figure 5623: Fs = $15 \mathrm{FPS},+10 \%$ magnitude step

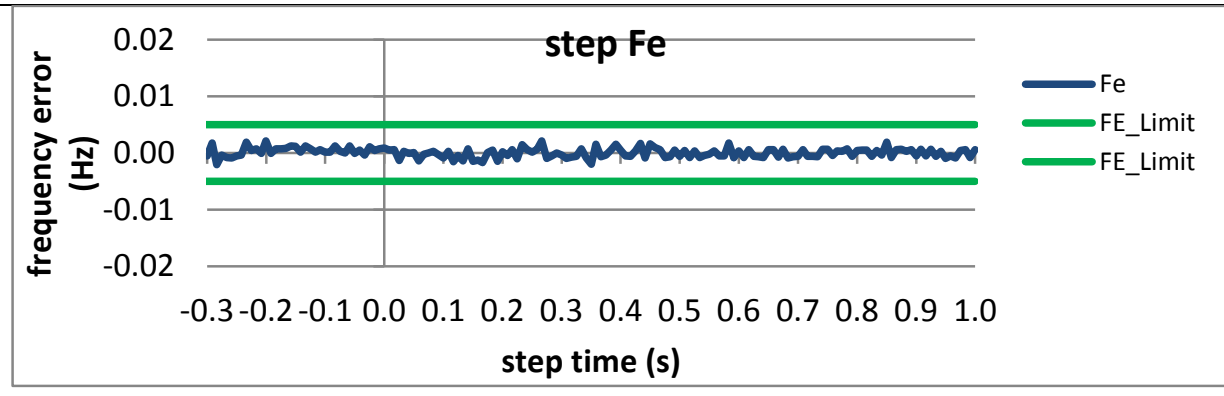

Figure 5624: Fs = 15 FPS, - $10 \%$ magnitude step

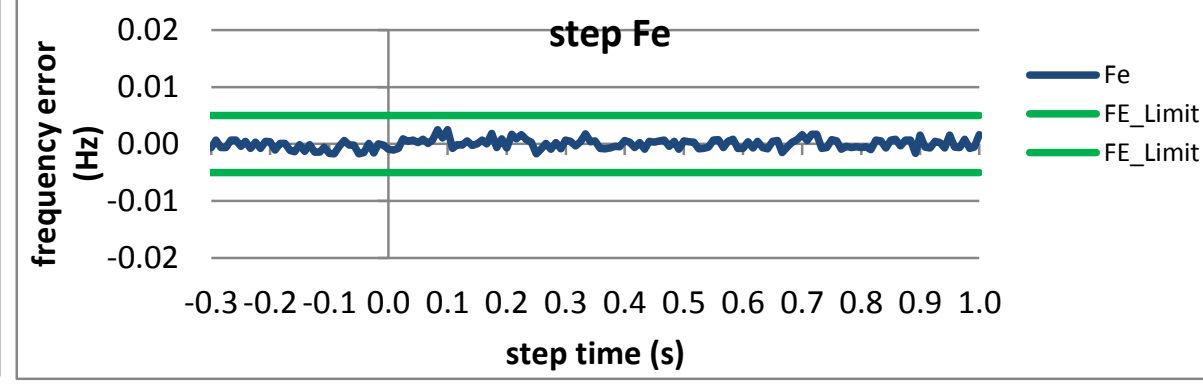

Figure 5625: Fs = $12 \mathrm{FPS},+10 \%$ magnitude step

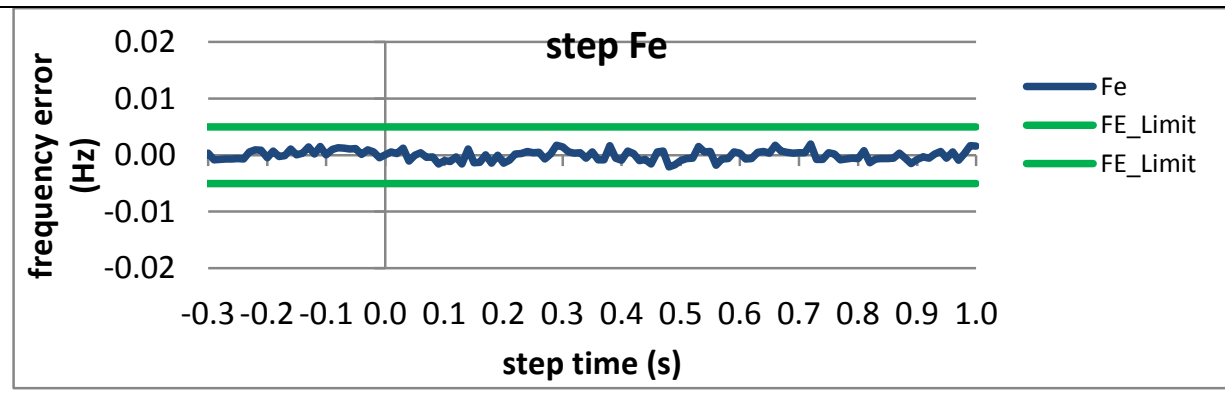

Figure 5626: Fs = 12 FPS, -10\% magnitude step

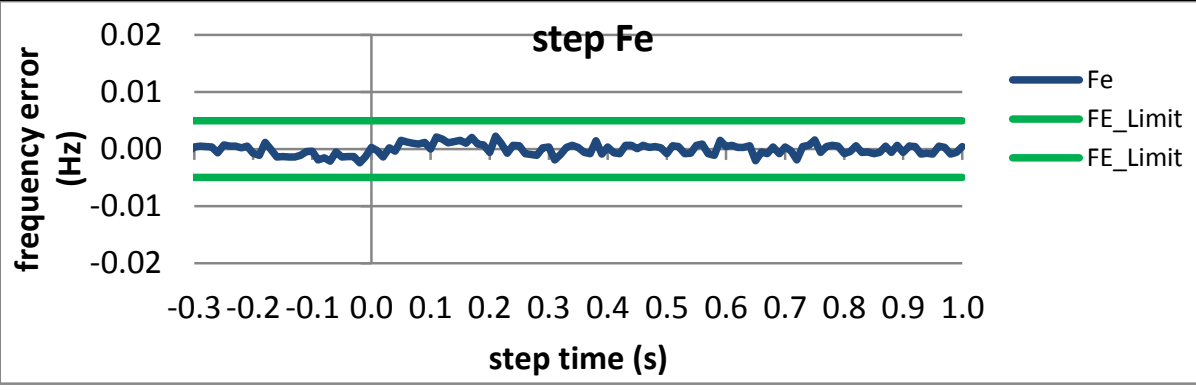

Figure 5627: Fs = 10 FPS, $+10 \%$ magnitude step

Figure 5628: Fs = 10 FPS, $-10 \%$ magnitude step 
10.6.8 PMU G dynamic step change in magnitude frequency response time: $F 0=60 \mathrm{~Hz}, \mathrm{M}$ class

Figure 5629: Fs = 60 FPS is not supported by this PMU

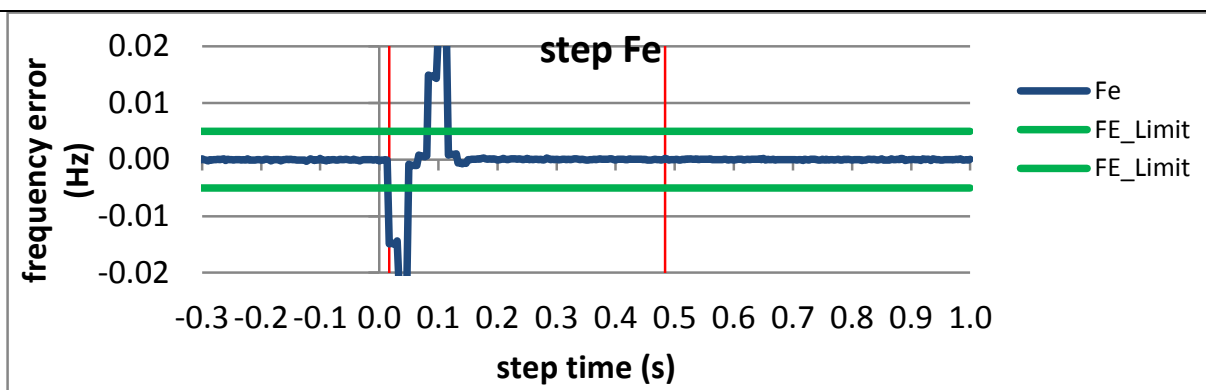

Figure 5630: $\mathrm{Fs}=30 \mathrm{FPS},+\mathbf{1 0} \%$ magnitude step

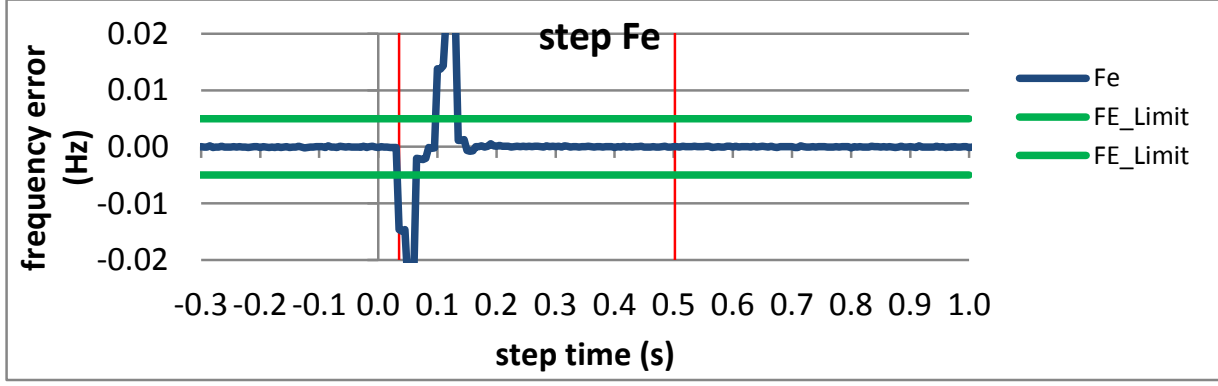

Figure 5632: Fs = 20 FPS, +10\% magnitude step

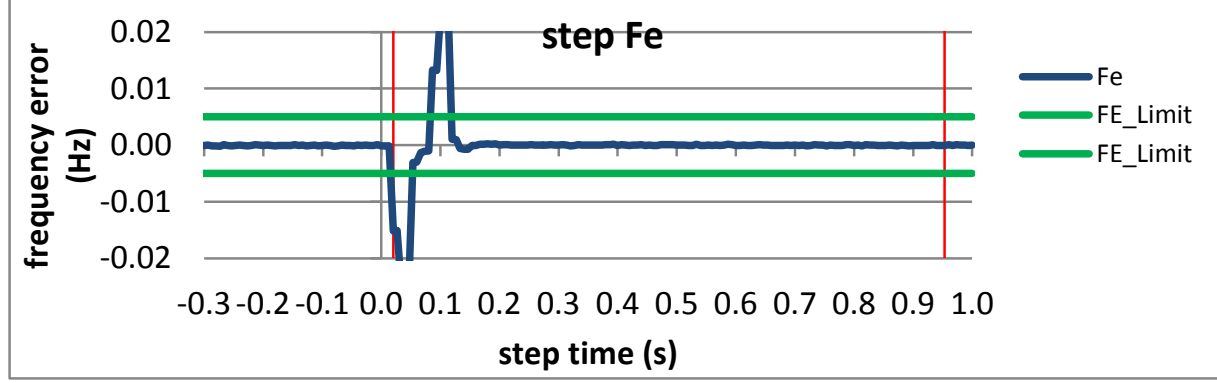

Figure 5634: Fs $=15$ FPS, $+10 \%$ magnitude step

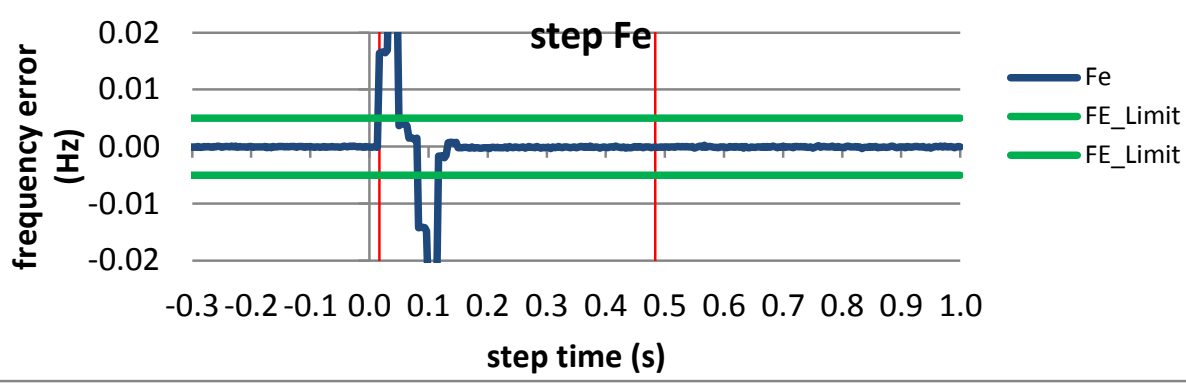

Figure 5631: Fs = 30 FPS, -10\% magnitude step

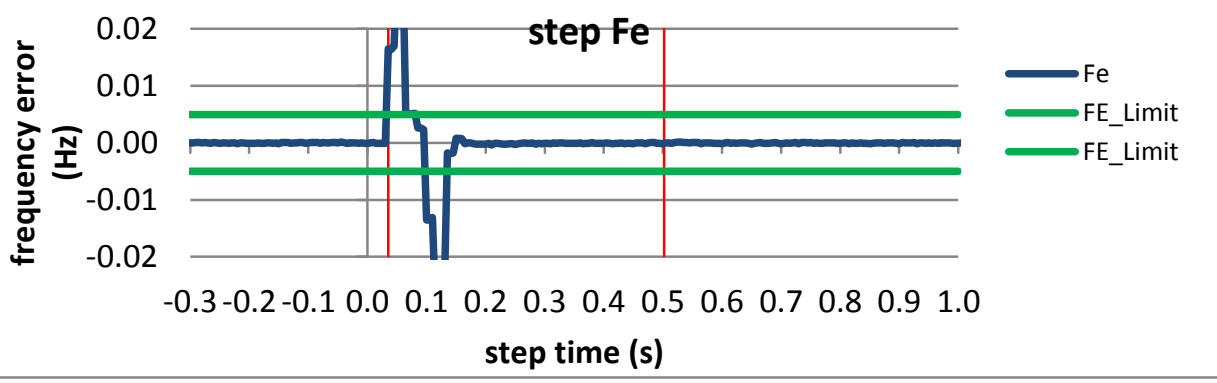

Figure 5633: Fs = 20 FPS, -10\% magnitude step

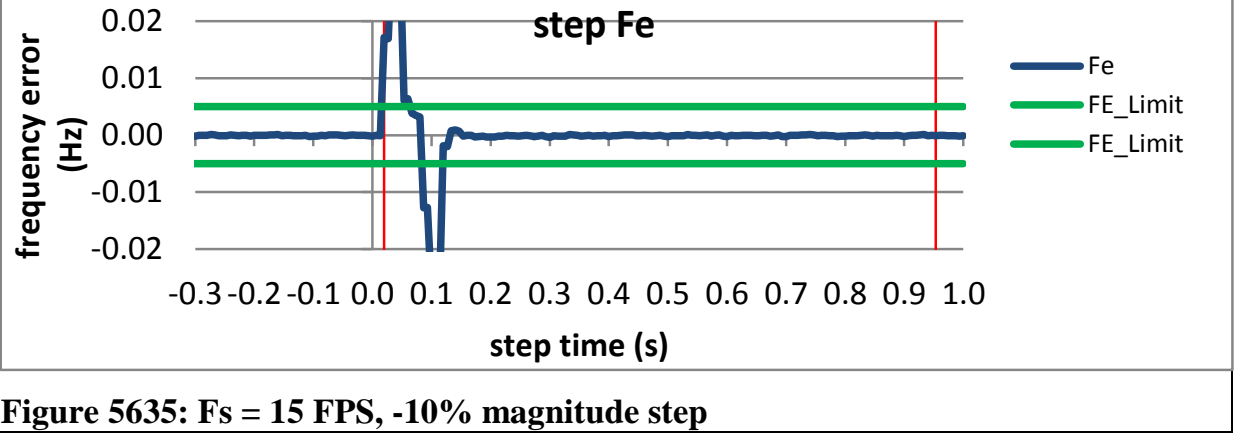



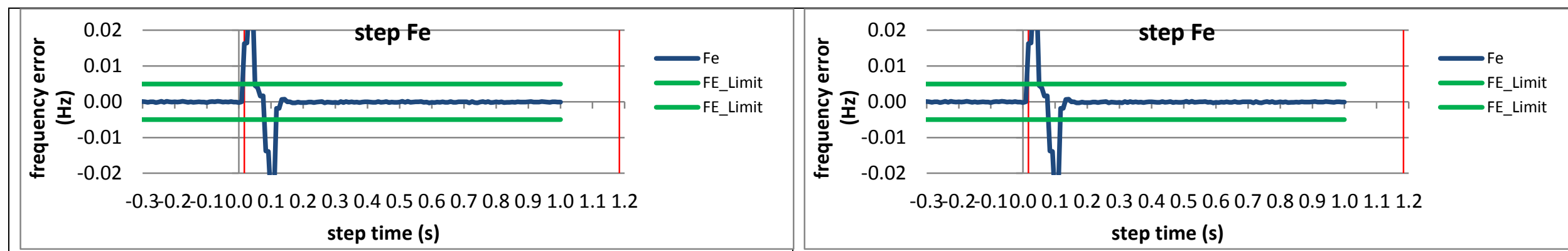

Figure 5636: Fs $=15$ FPS, $+10 \%$ magnitude step

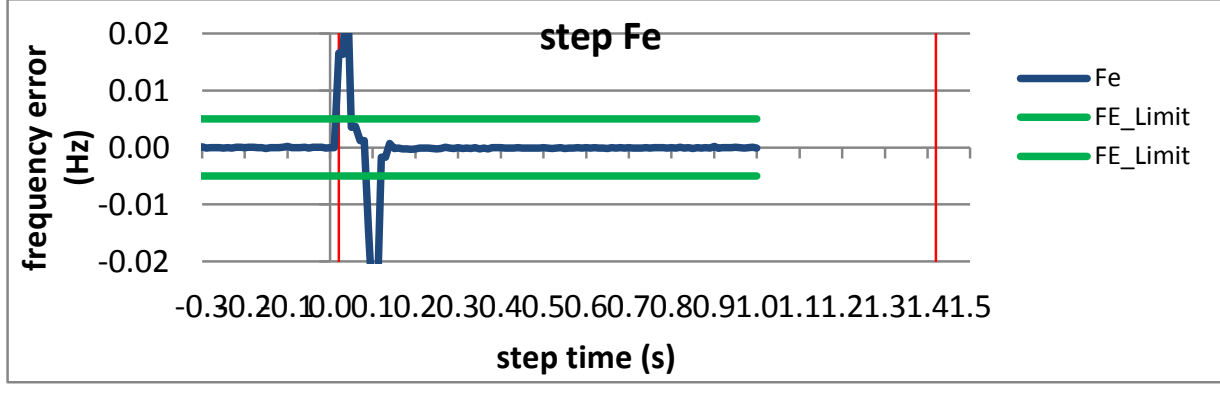

Figure 5637: Fs = 15 FPS, -10\% magnitude step

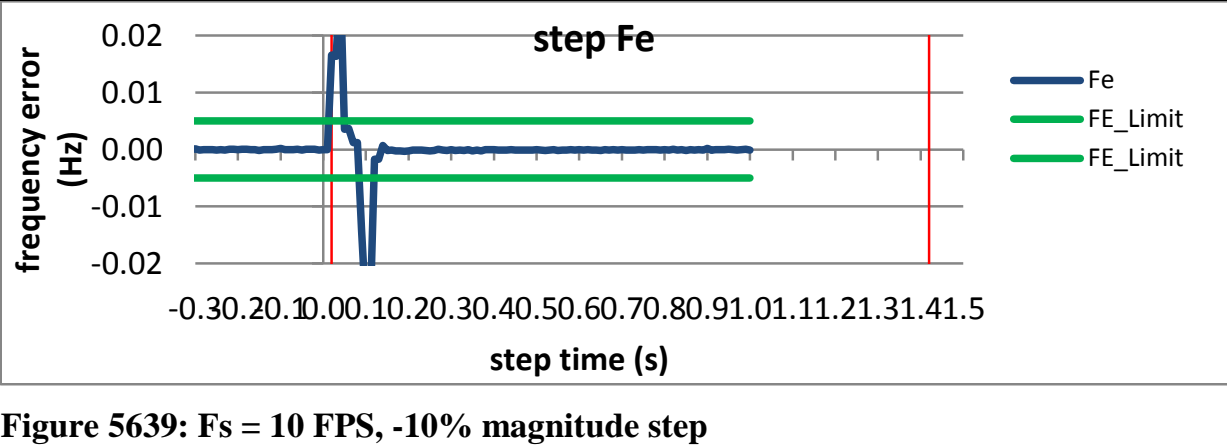

Figure 5638: Fs = 10 FPS, $+10 \%$ magnitude step

Figure 5639: Fs = 10 FPS, -10\% magnitude step 
10.6.9 PMU H dynamic step change in magnitude frequency response time: $\mathrm{F} 0=60 \mathrm{~Hz}, \mathrm{M}$ class
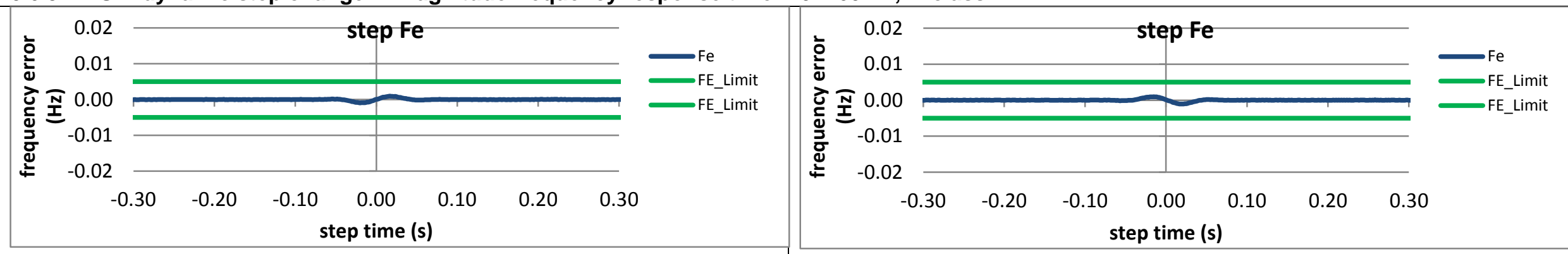

Figure 5640: Fs = 60 FPS, +10\% magnitude step

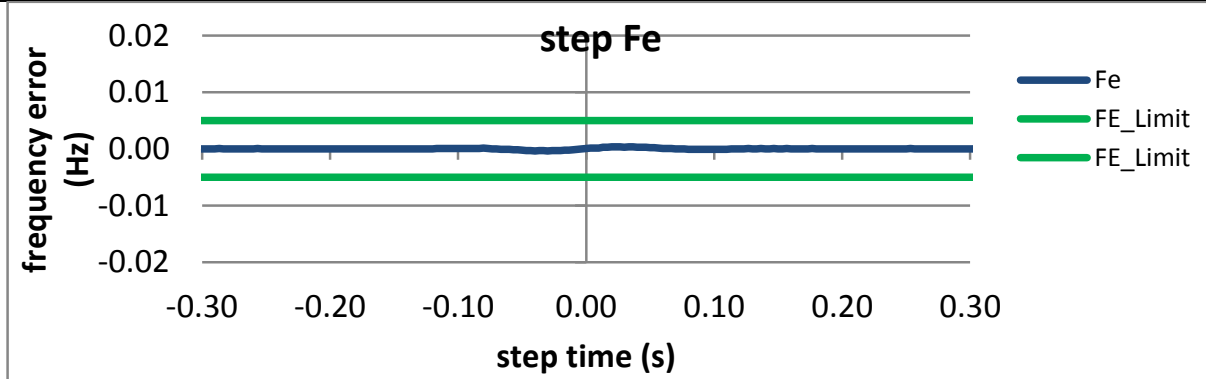

Figure 5641: Fs = 60 FPS, -10\% magnitude step

Figure 5642: Fs = 30 FPS, +10\% magnitude step
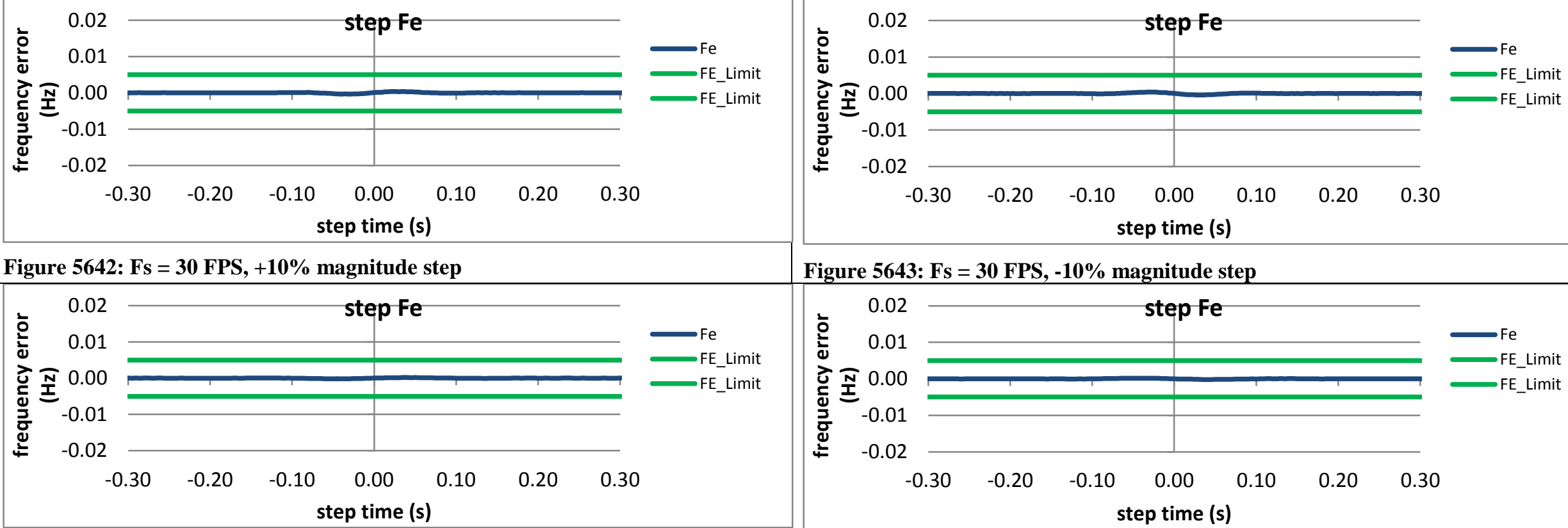

Figure 5643: Fs = 30 FPS, -10\% magnitude step

Figure 5644: Fs = 20 FPS, +10\% magnitude step

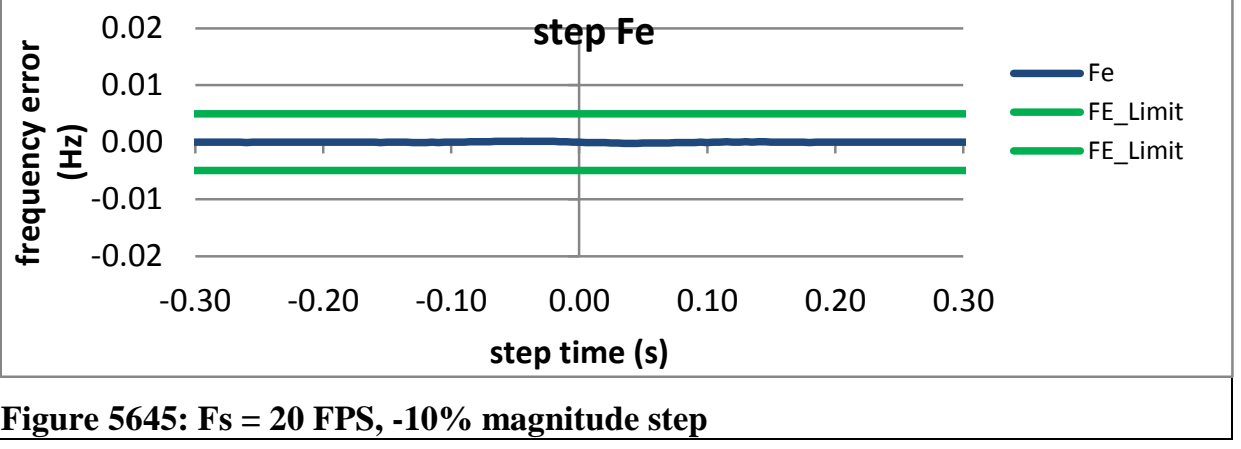



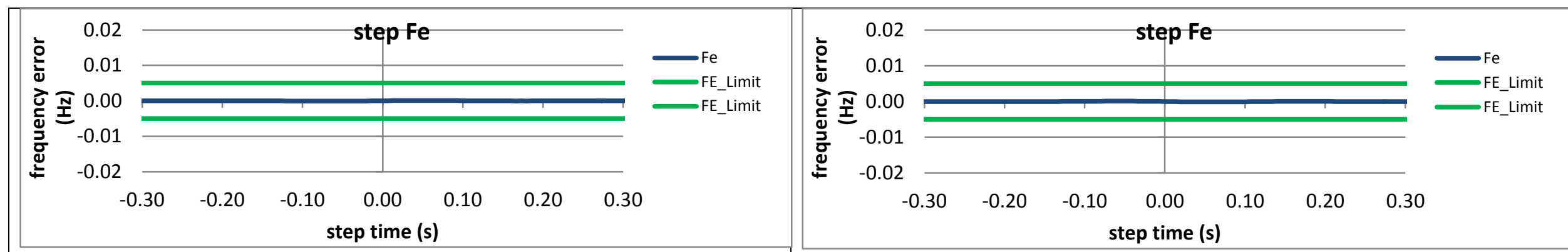

Figure 5646: Fs $=15$ FPS, $+10 \%$ magnitude step

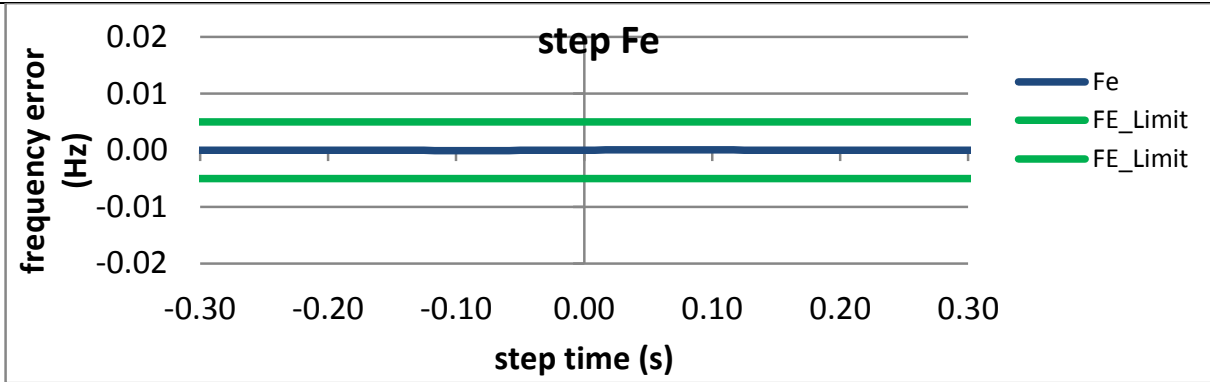

Figure 5648: Fs = 12 FPS, +10\% magnitude step

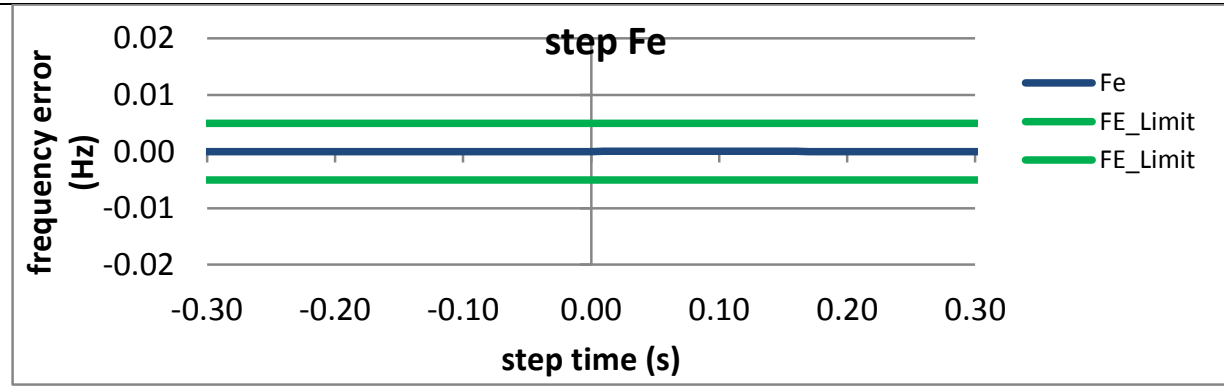

Figure 5647: Fs = 15 FPS, - $10 \%$ magnitude step

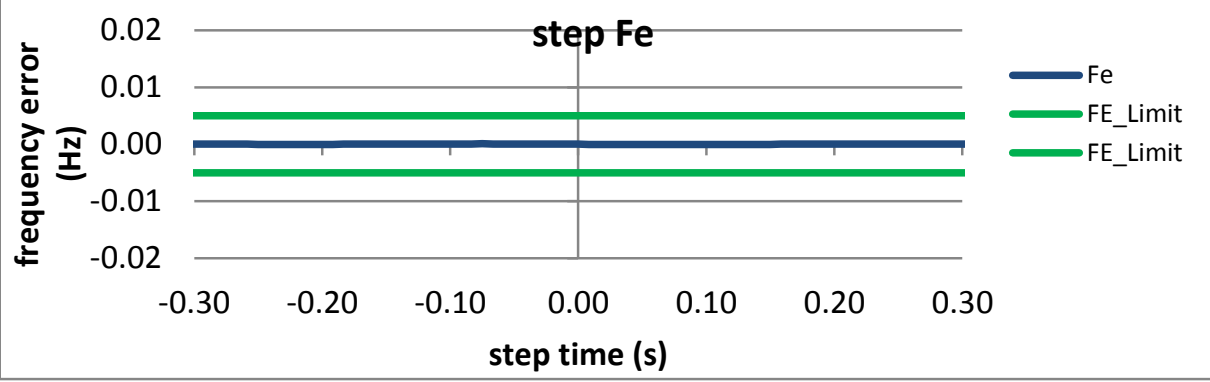

Figure 5649: Fs = 12 FPS, -10\% magnitude step

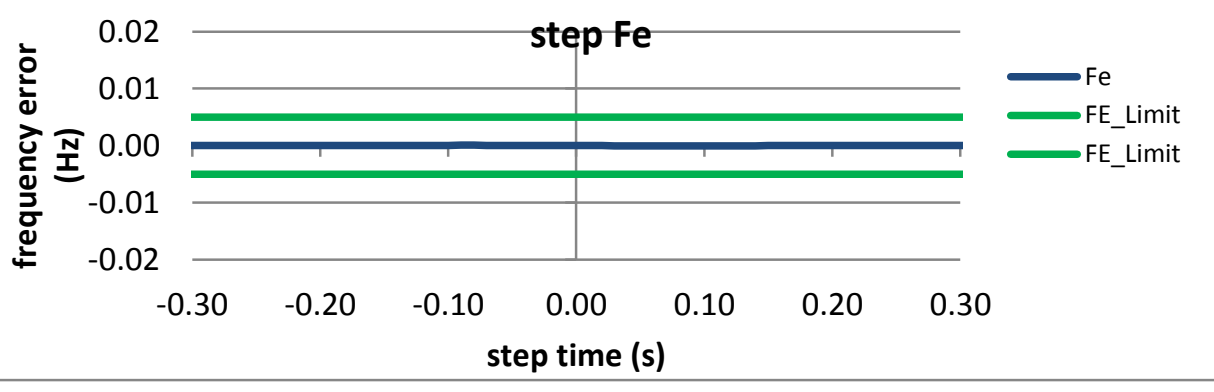

Figure 5651: Fs = 10 FPS, -10\% magnitude step 
10.6.10 PMU I dynamic step change in magnitude frequency response time: $\mathrm{F} 0=60 \mathrm{~Hz}, \mathrm{M}$ class

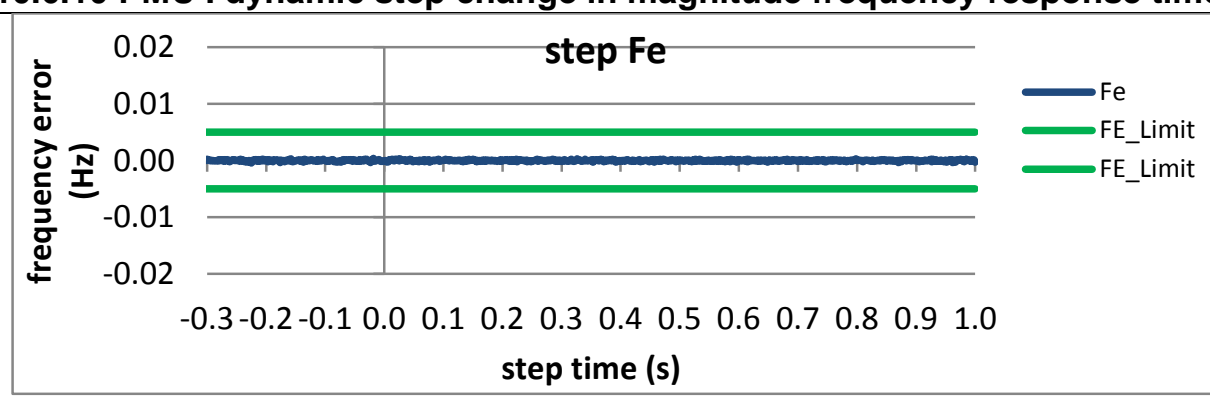

Figure 5652: Fs = 60 FPS, +10\% magnitude step

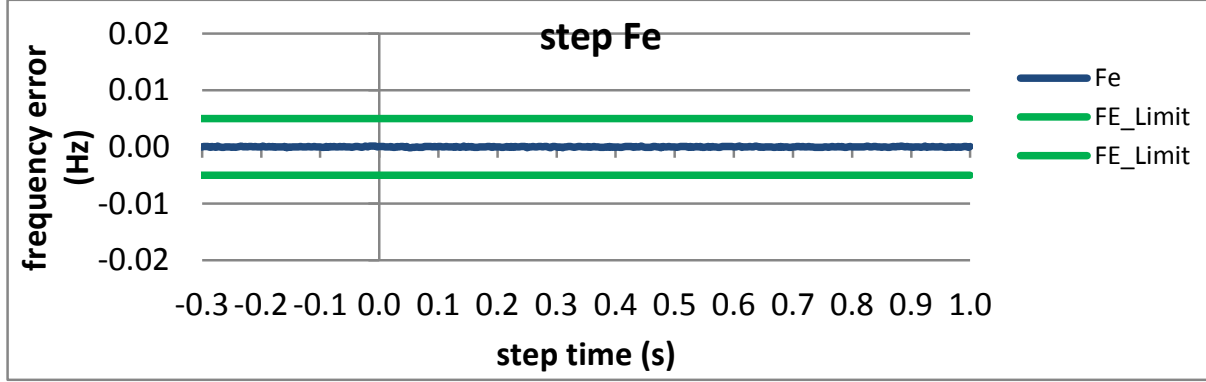

Figure 5654: Fs $=30$ FPS, $+10 \%$ magnitude step

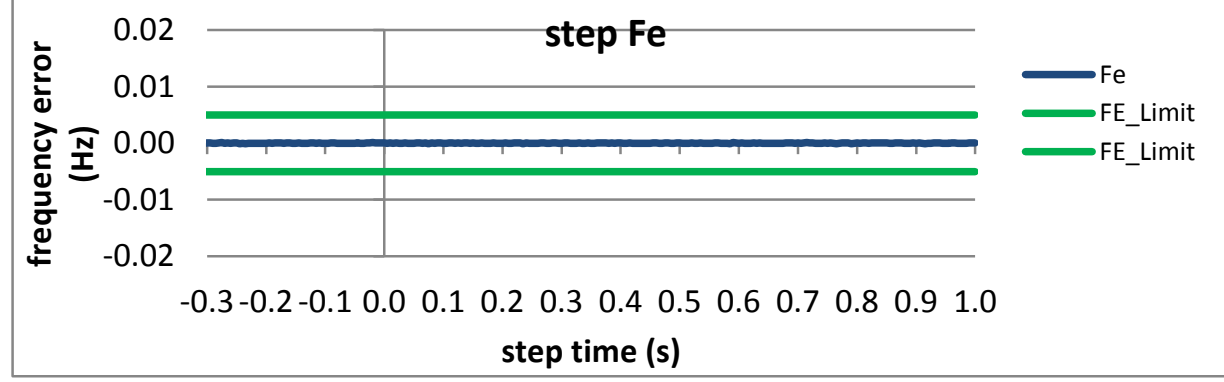

Figure 5656: Fs = 20 FPS, +10\% magnitude step

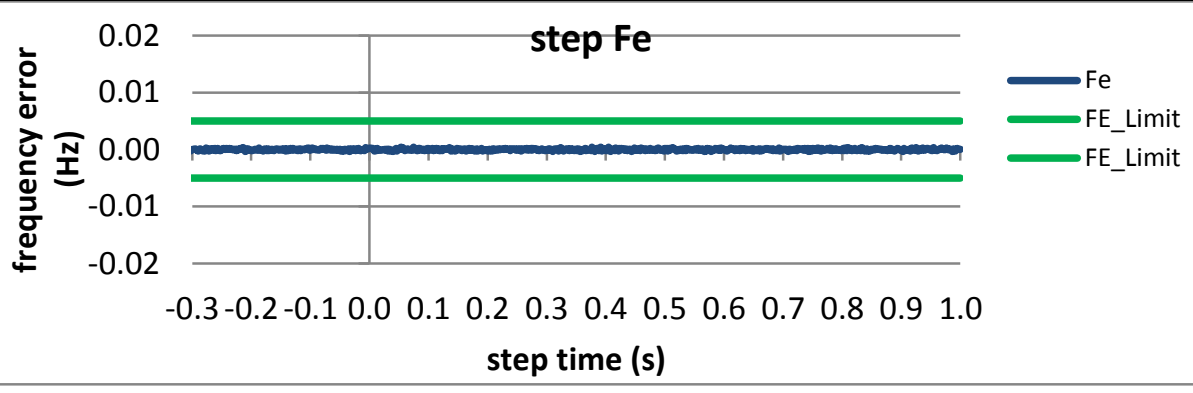

Figure 5653: Fs = 60 FPS, -10\% magnitude step

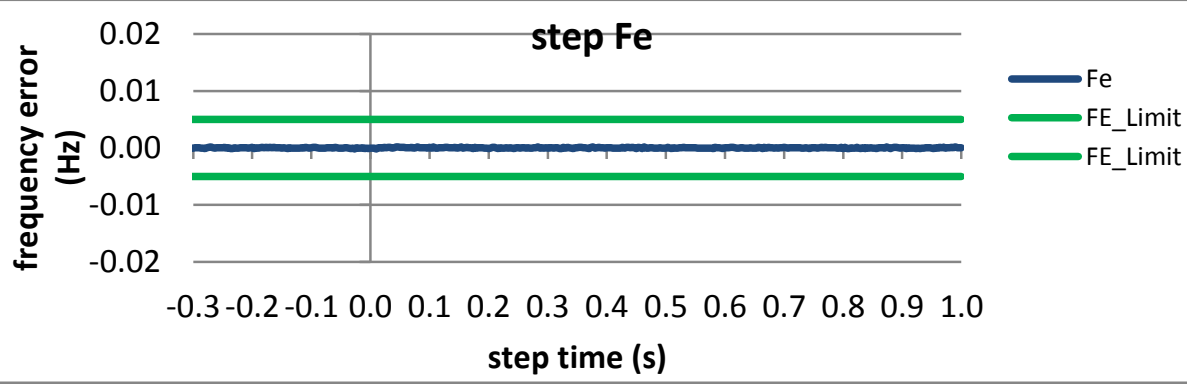

Figure 5655: Fs = 30 FPS, -10\% magnitude step

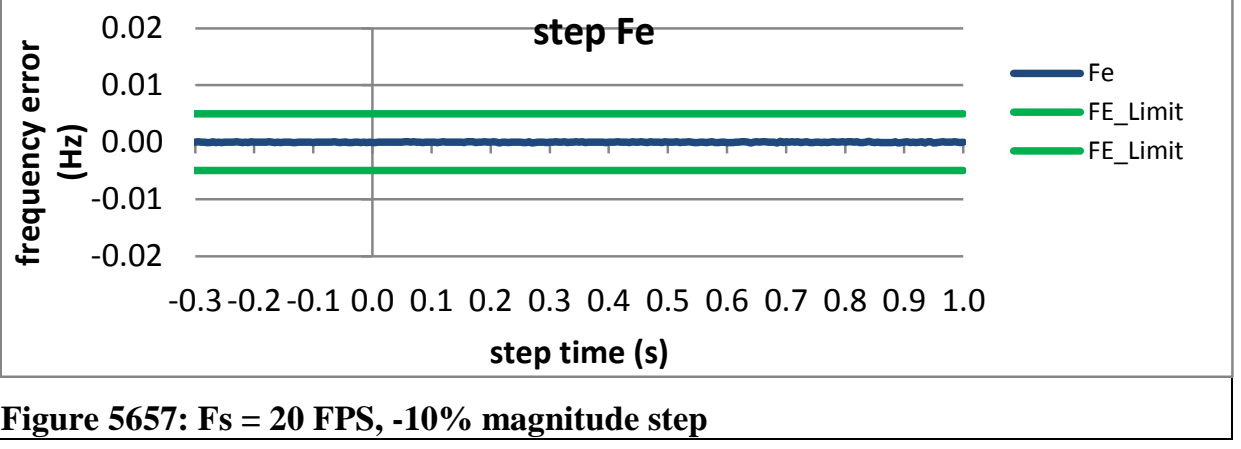



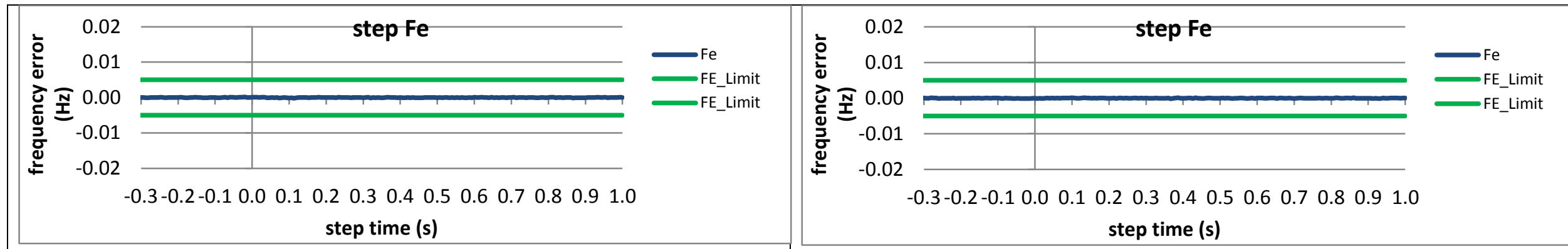

Figure 5658: Fs $=15$ FPS, $+10 \%$ magnitude step

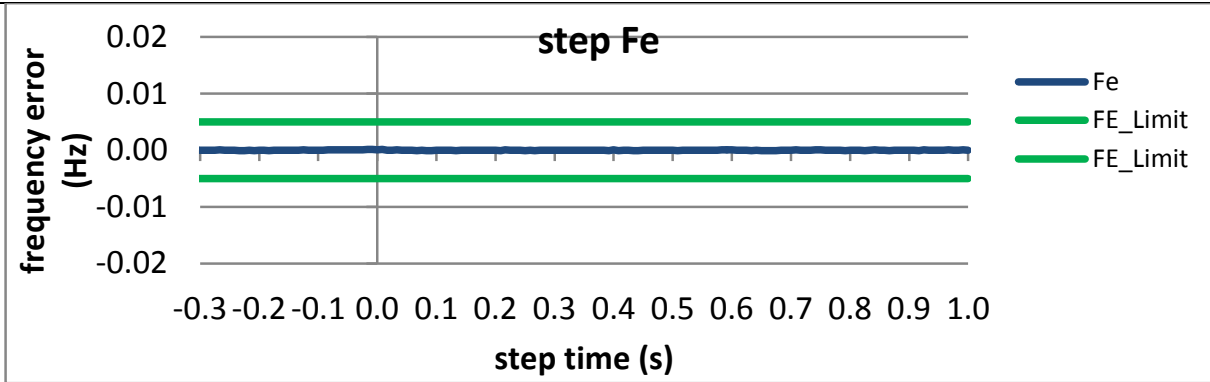

Figure 5659: Fs = 15 FPS, - $10 \%$ magnitude step

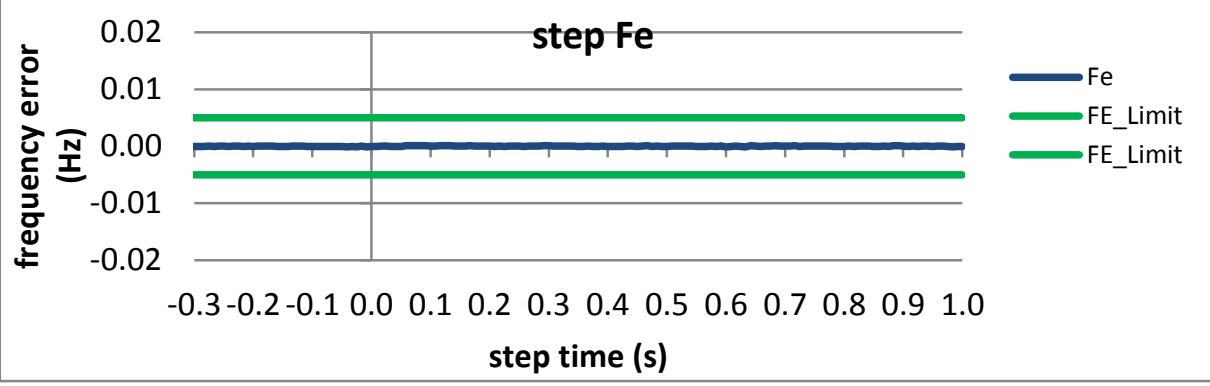

Figure 5660: Fs $=12$ FPS, +10\% magnitude step

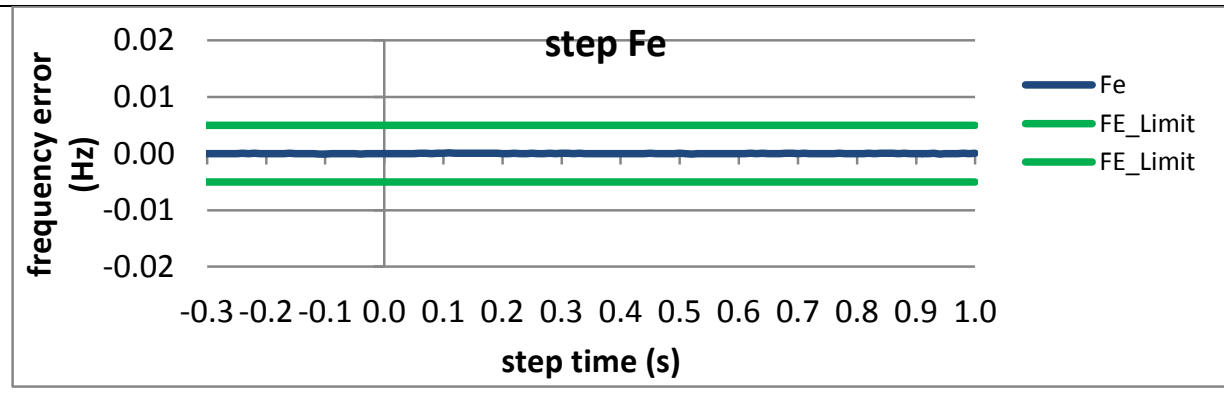

Figure 5661: Fs = 12 FPS, -10\% magnitude step

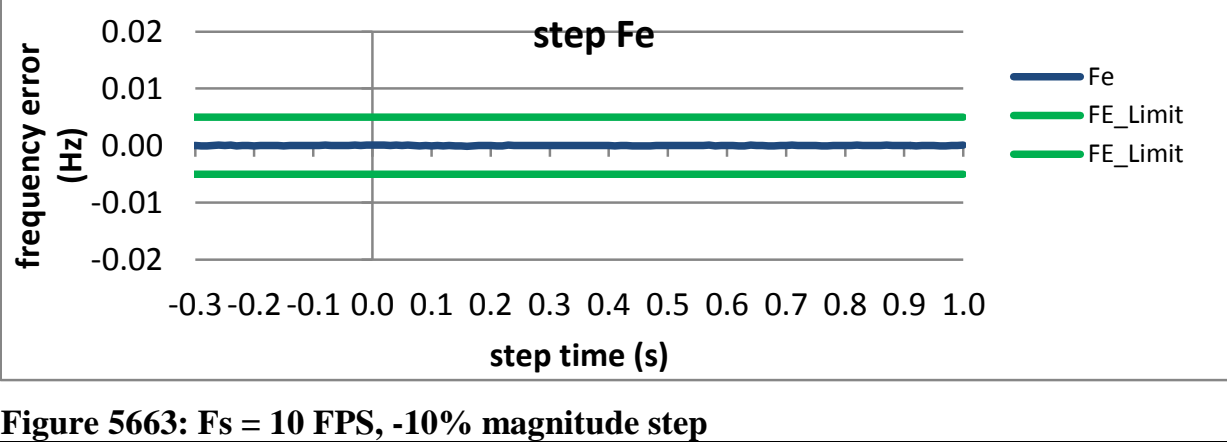

Figure 5662: Fs = 10 FPS, +10\% magnitude step

Figure 5663: Fs = 10 FPS, -10\% magnitude step 
10.6.11 PMU J dynamic step change in magnitude frequency response time: $\mathrm{F} 0=60 \mathrm{~Hz}, \mathrm{M}$ class

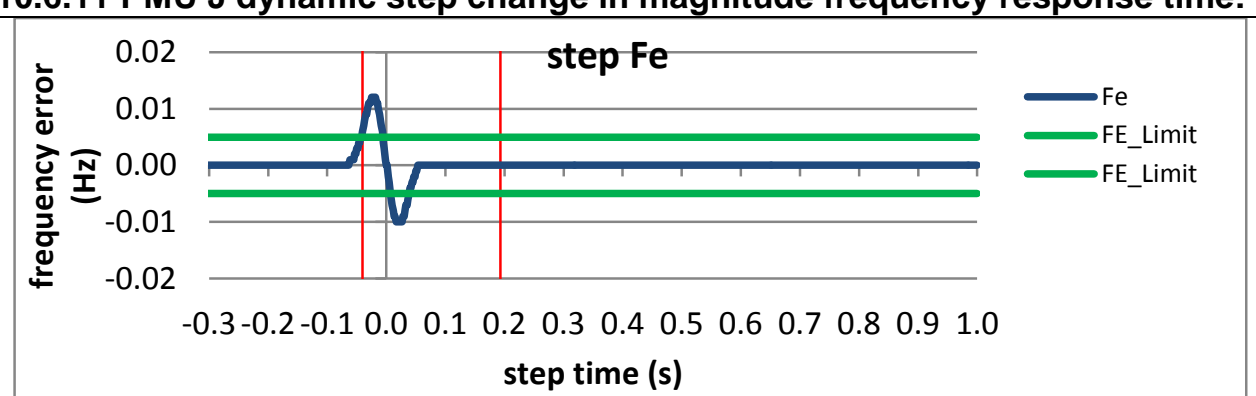

Figure 5664: Fs = $60 \mathrm{FPS},+10 \%$ magnitude step

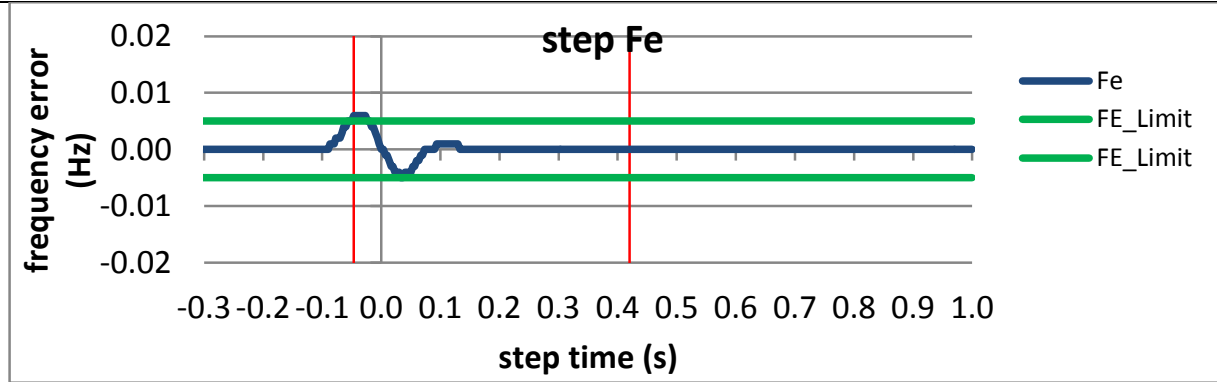

Figure 5666: Fs = 30 FPS, $+10 \%$ magnitude step

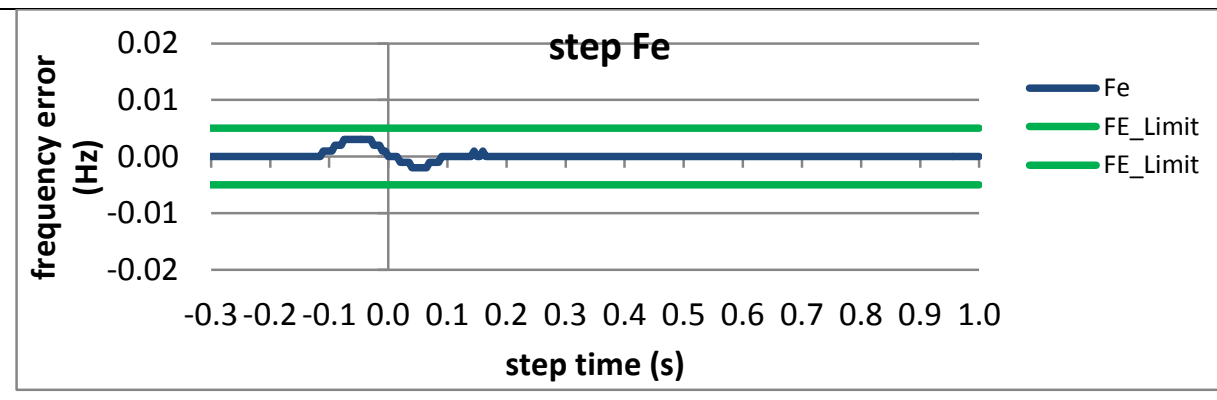

Figure 5668: Fs = 20 FPS, $+10 \%$ magnitude step

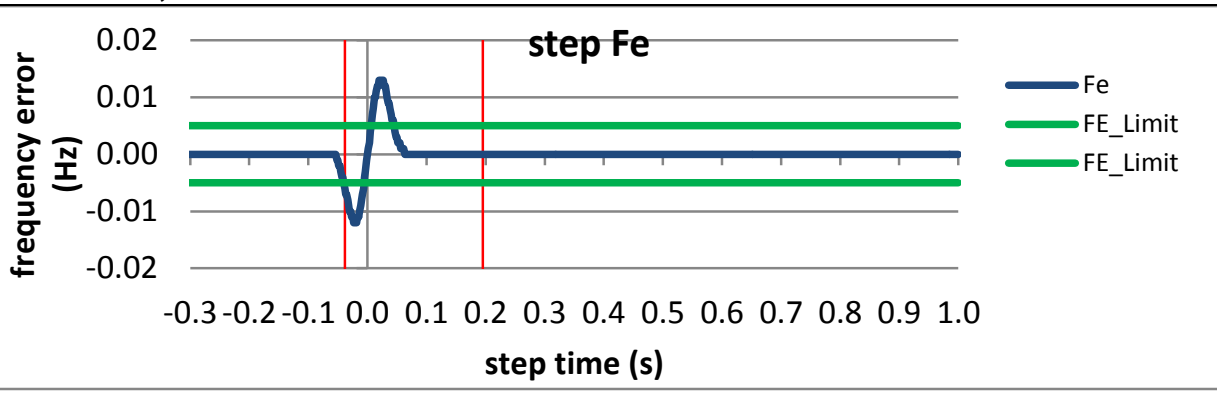

Figure 5665: Fs = 60 FPS, -10\% magnitude step

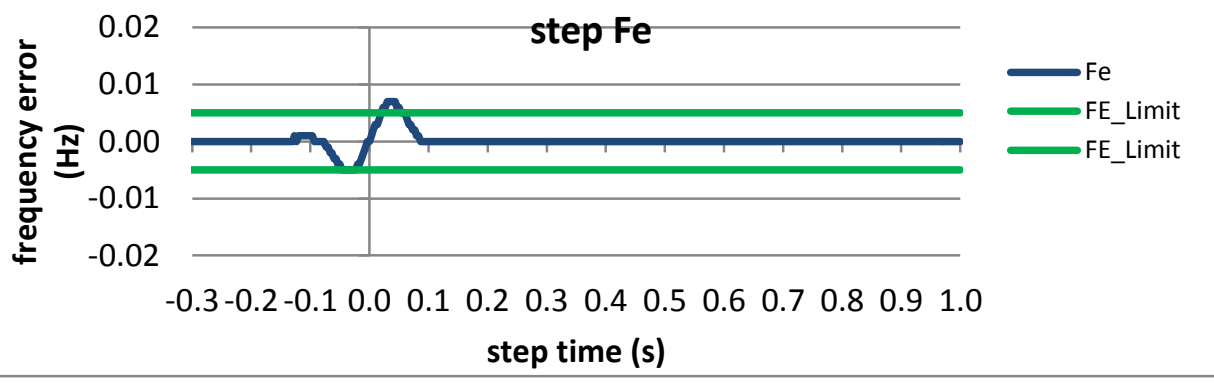

Figure 5667: Fs = 30 FPS, -10\% magnitude step

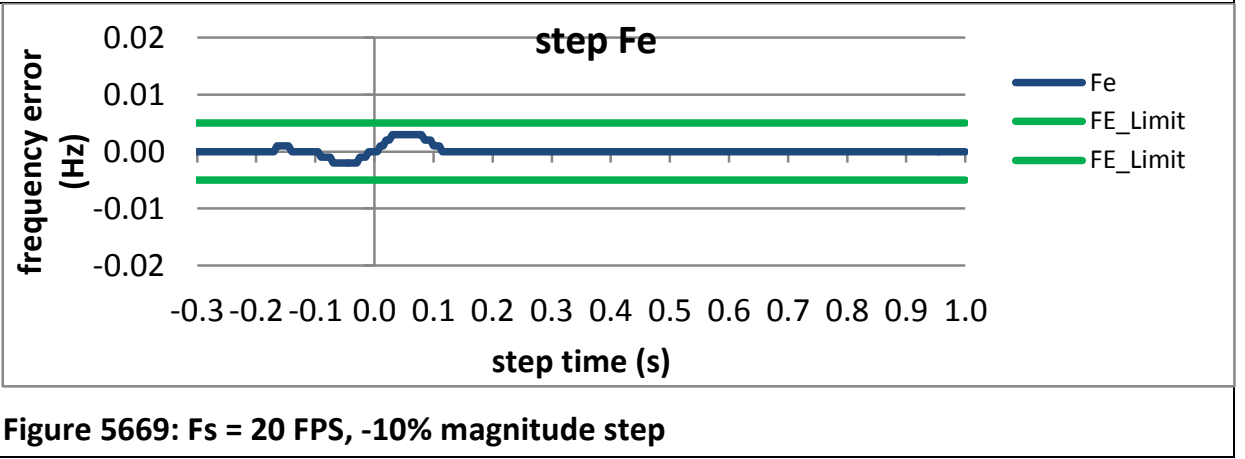



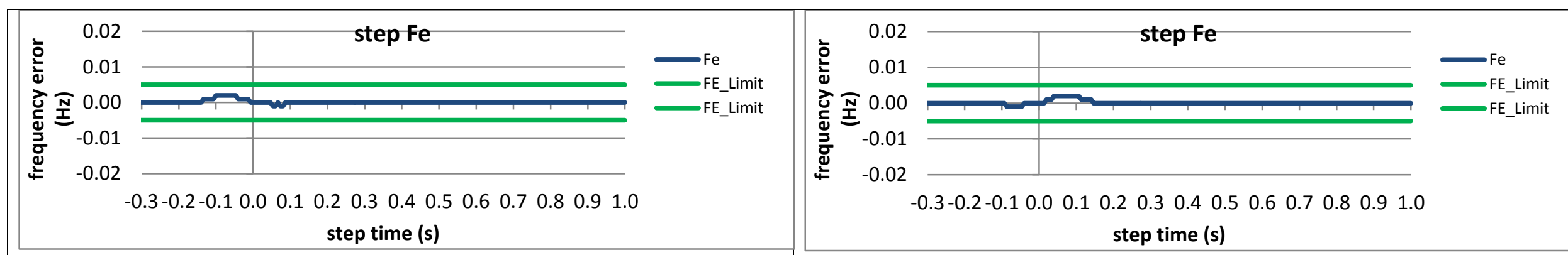

Figure 5670: Fs = $15 \mathrm{FPS},+10 \%$ magnitude step

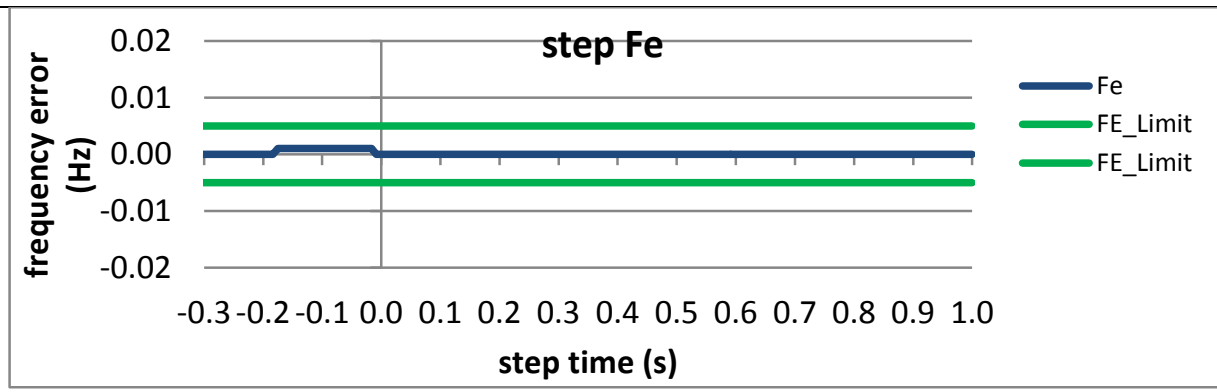

Figure 5671: Fs = 15 FPS, - $10 \%$ magnitude step

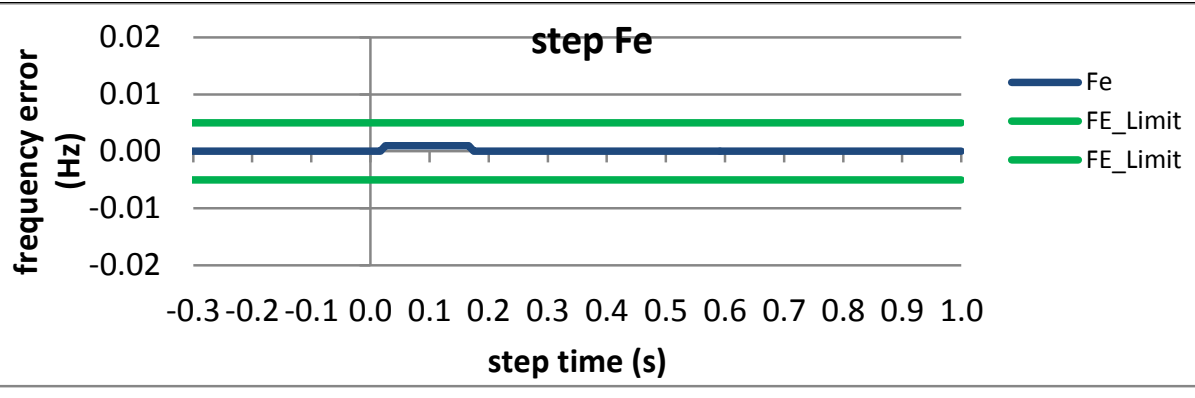

Figure 5672: Fs = 12 FPS, $+10 \%$ magnitude step

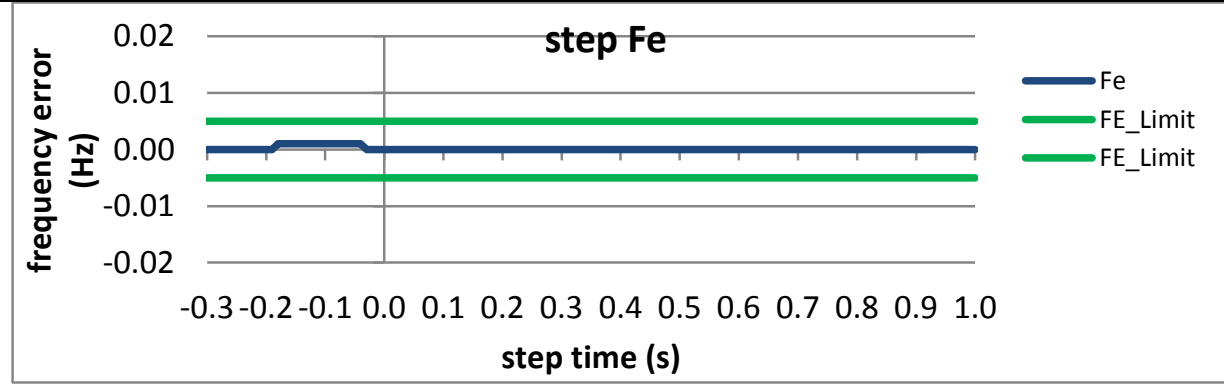

Figure 5673: Fs = 12 FPS, $-10 \%$ magnitude step

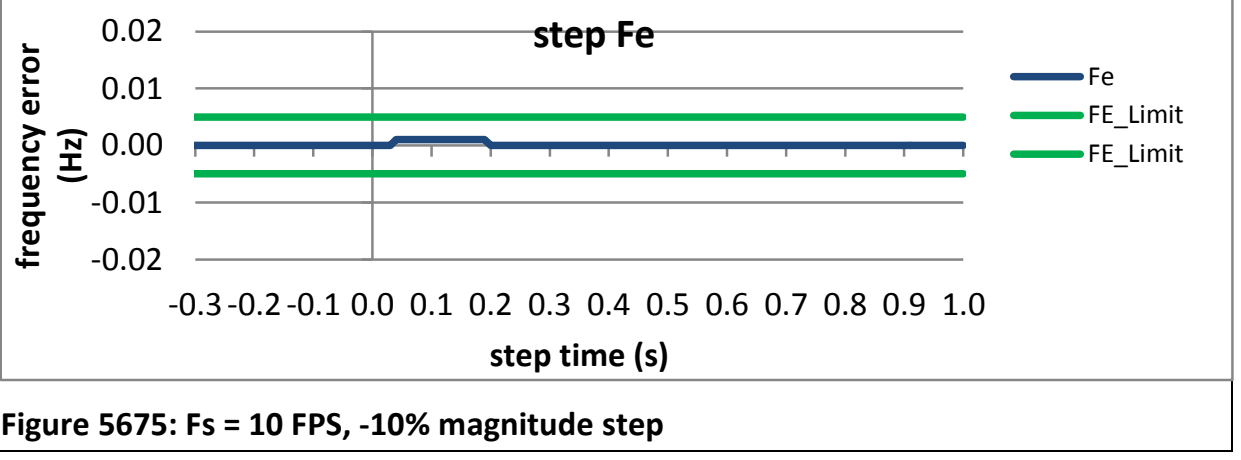

Figure 5674: Fs = 10 FPS, $+10 \%$ magnitude step 


\subsection{Dynamic step change in magnitude frequency response time: $\mathrm{F} 0=60 \mathrm{~Hz}, \mathrm{P}$ class}

\subsubsection{C37.118.1-2011 Annex C dynamic step change in magnitude frequency response time: $\mathrm{F} 0=60 \mathrm{~Hz}, \mathrm{P}$ class}

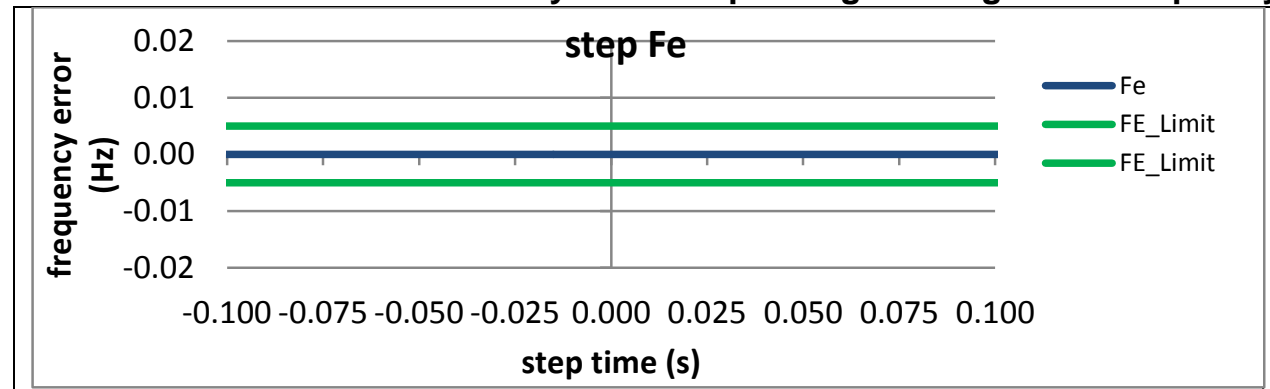

Figure 5676: Fs = 60 FPS, $+10 \%$ magnitude step

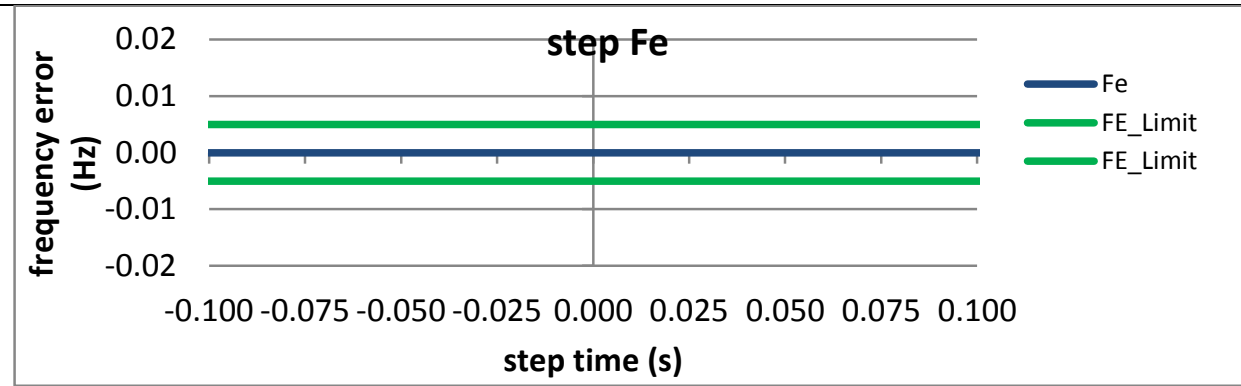

Figure 5678: Fs = 30 FPS, $+10 \%$ magnitude step

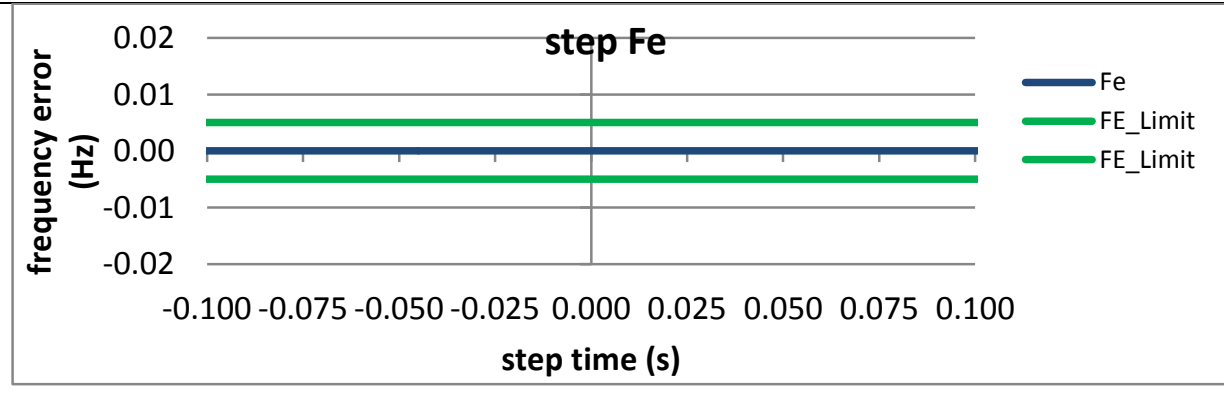

Figure 5680: Fs = 20 FPS, $+10 \%$ magnitude step

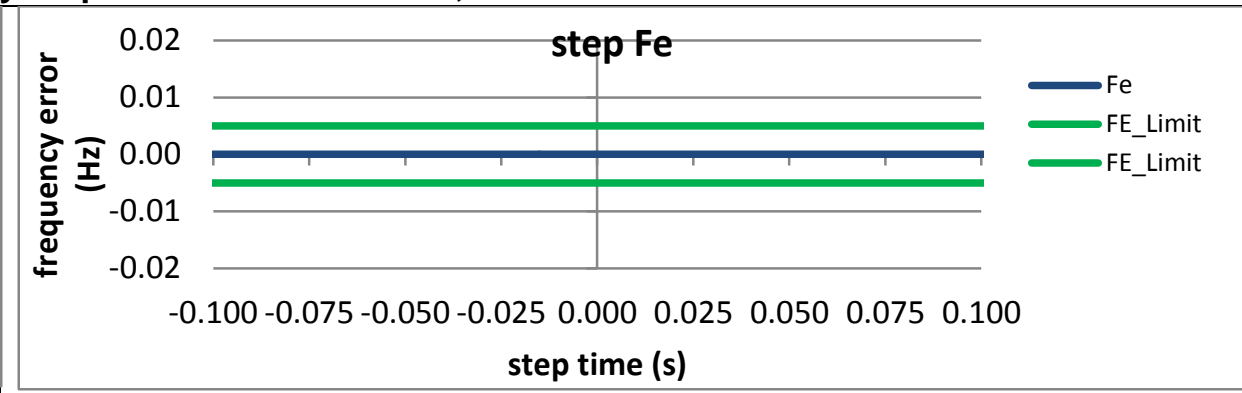

Figure 5677: Fs = 60 FPS, -10\% magnitude step

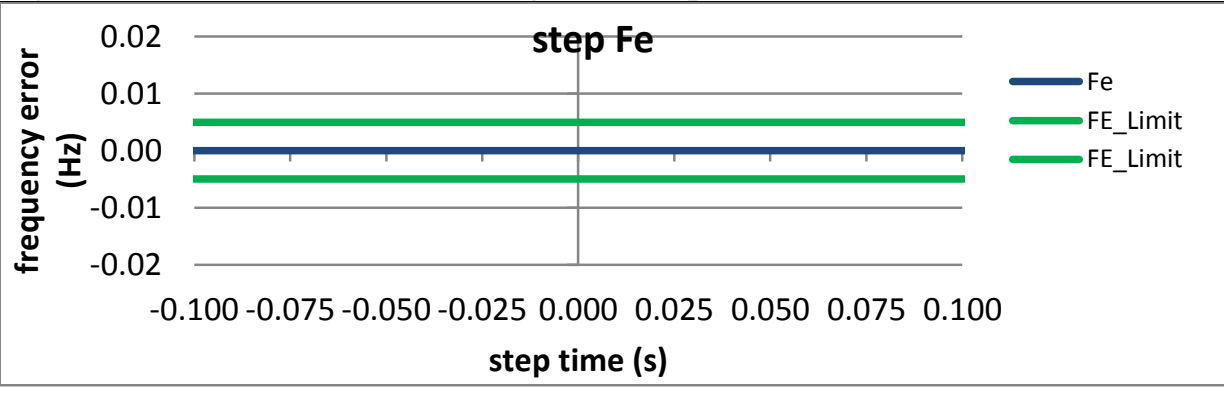

Figure 5679: Fs = 30 FPS, -10\% magnitude step

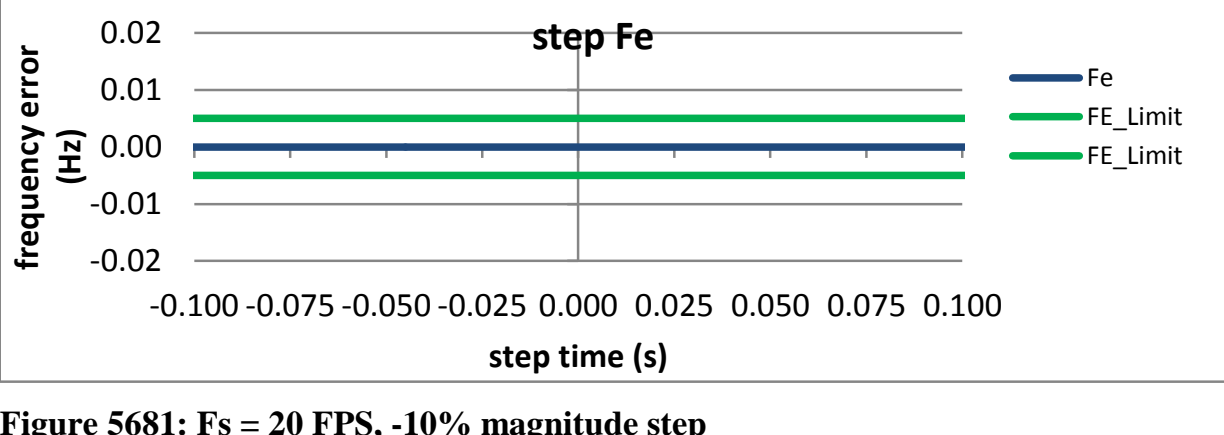




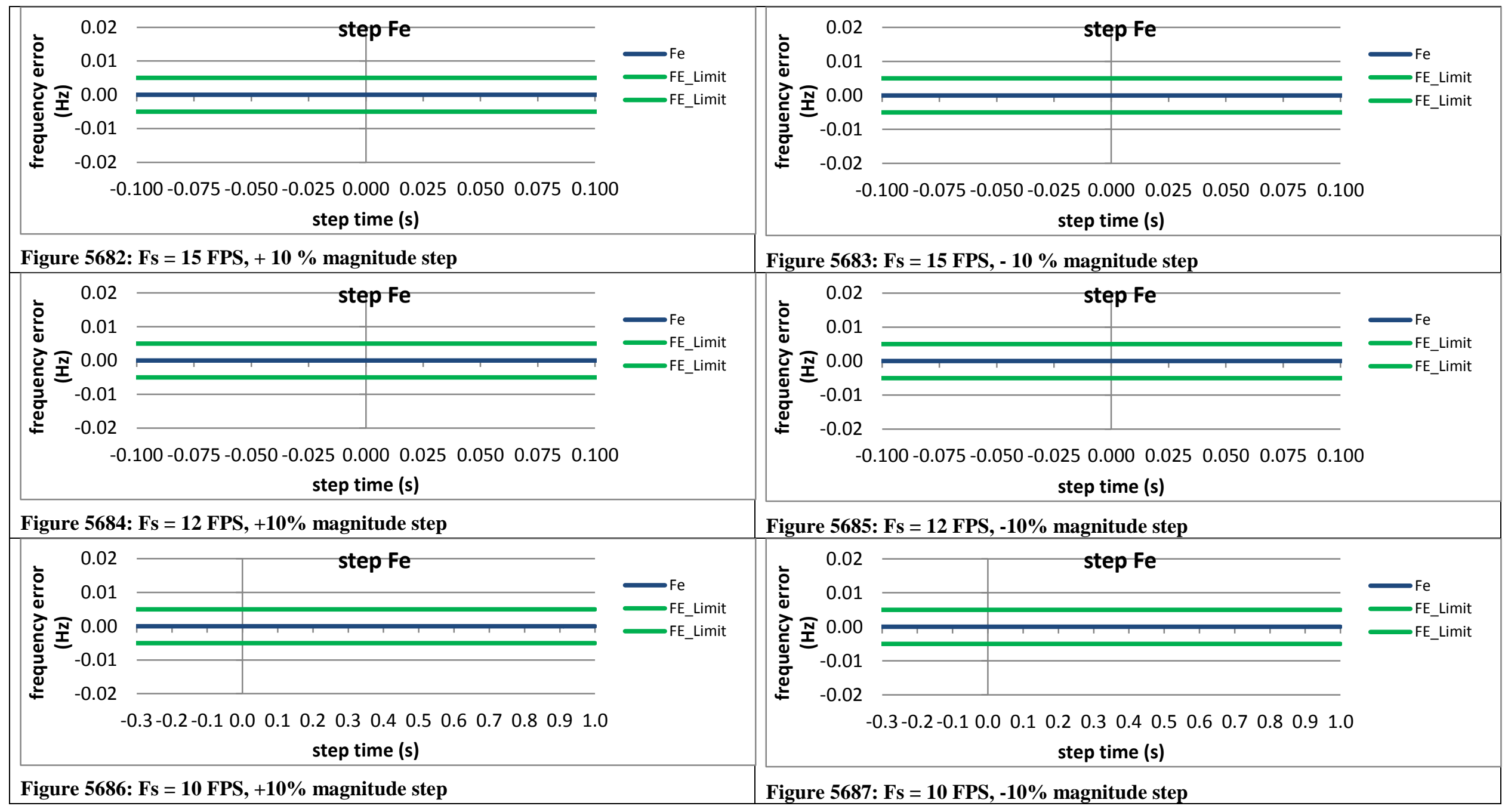


10.7.2 PMU A dynamic step change in magnitude frequency response time: $F 0=60 \mathrm{~Hz}, \mathrm{P}$ class

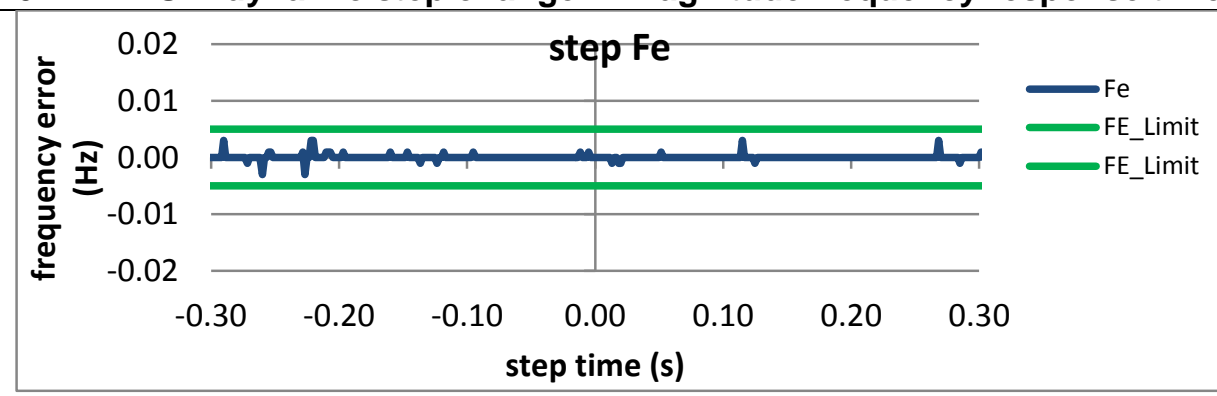

Figure 5688: Fs = 60 FPS, +10\% magnitude step

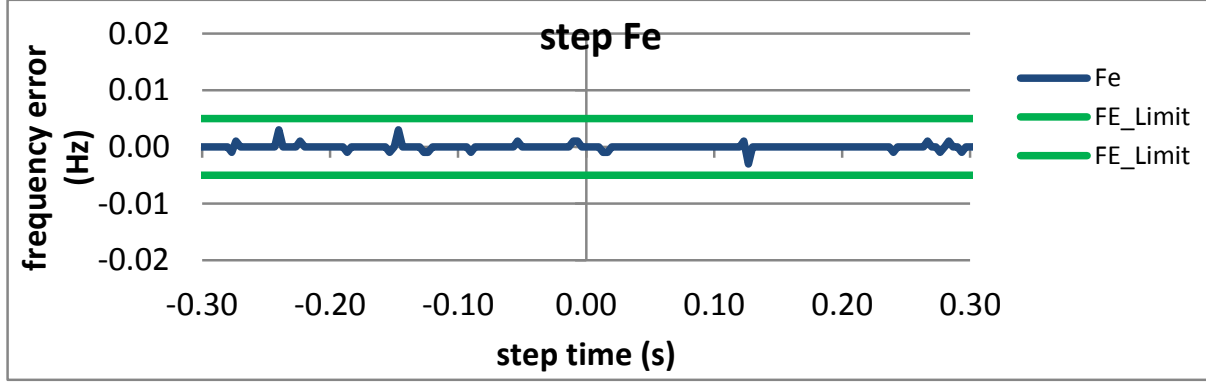

Figure 5690: Fs $=30 \mathrm{FPS},+10 \%$ magnitude step

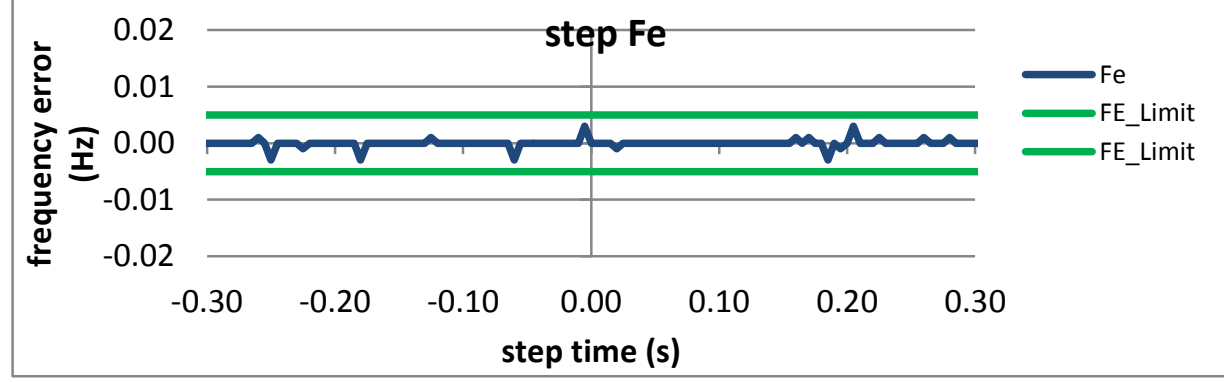

Figure 5692: Fs $=20$ FPS, $+10 \%$ magnitude step

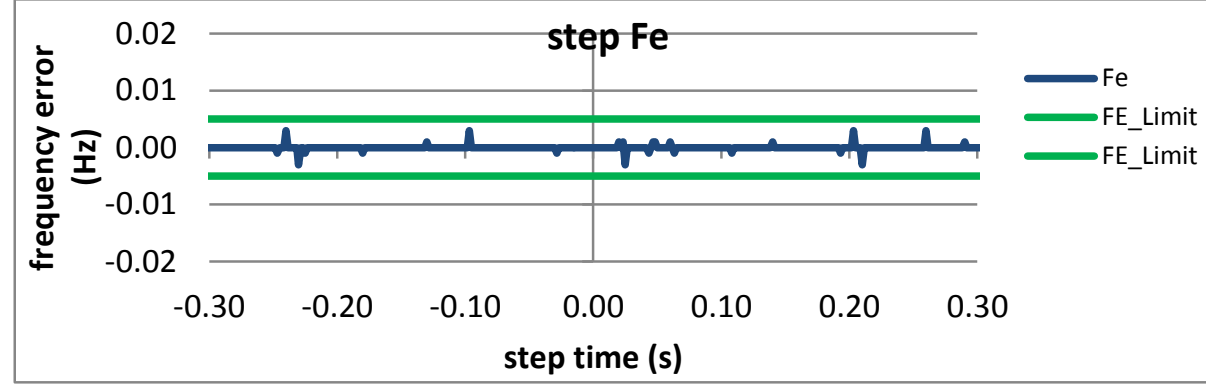

Figure 5689: Fs = 60 FPS, -10\% magnitude step

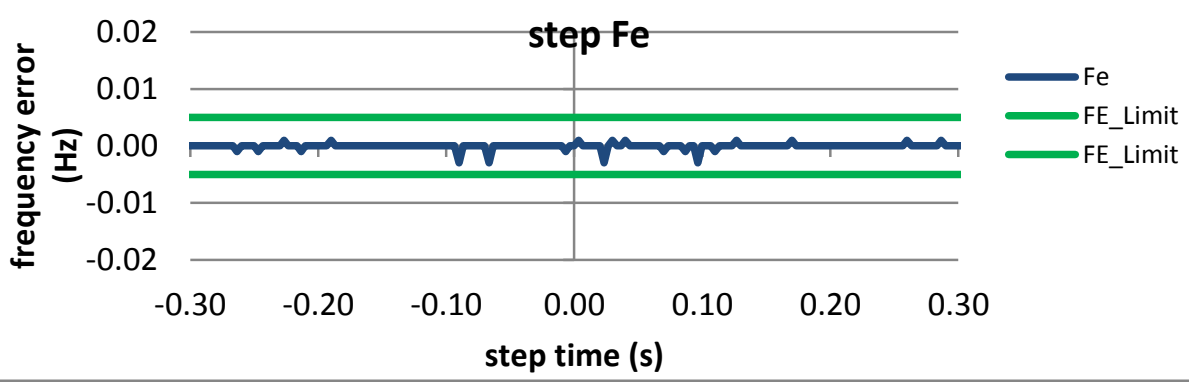

Figure 5691: Fs = 30 FPS, -10\% magnitude step

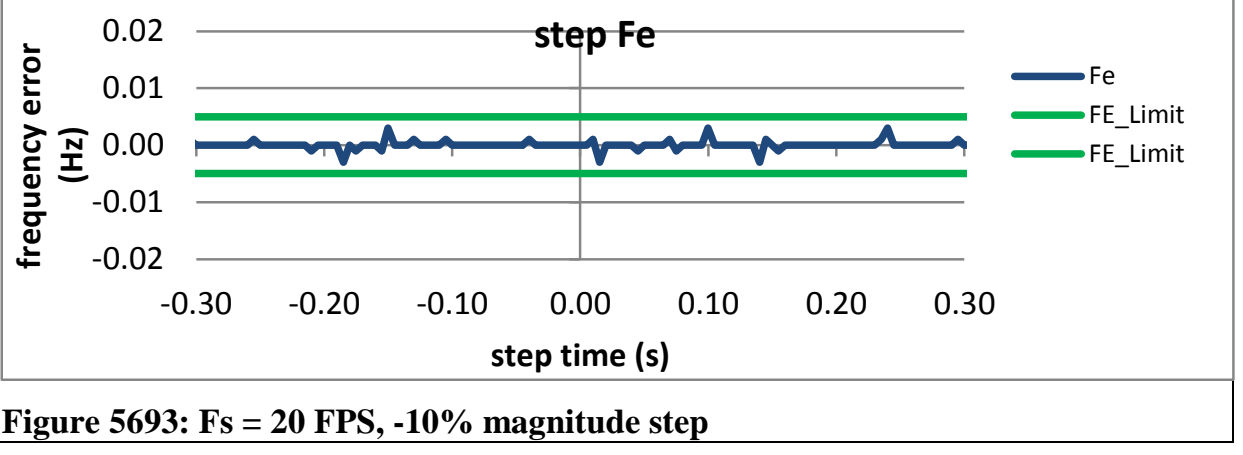



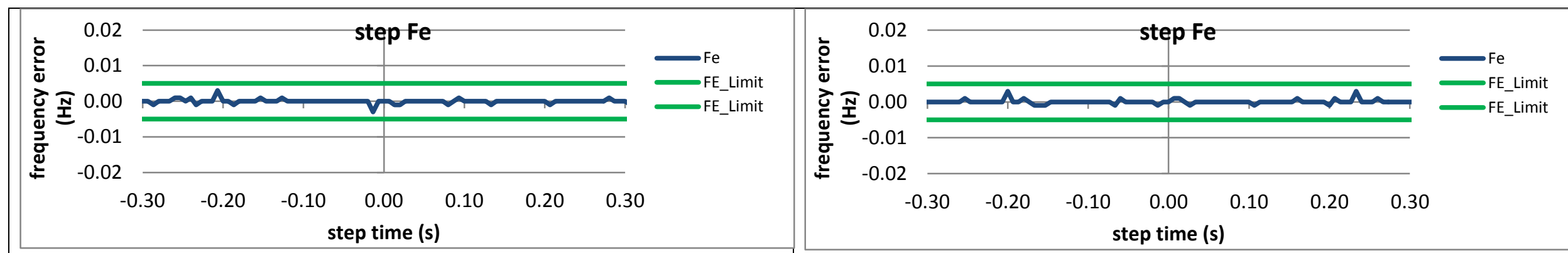

Figure 5694: Fs $=15$ FPS, $+10 \%$ magnitude step

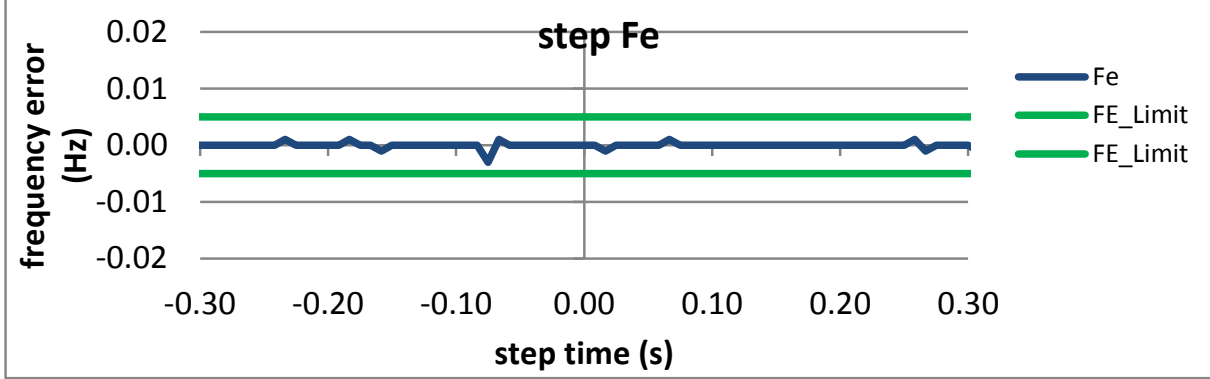

Figure 5696: Fs = 12 FPS, +10\% magnitude step

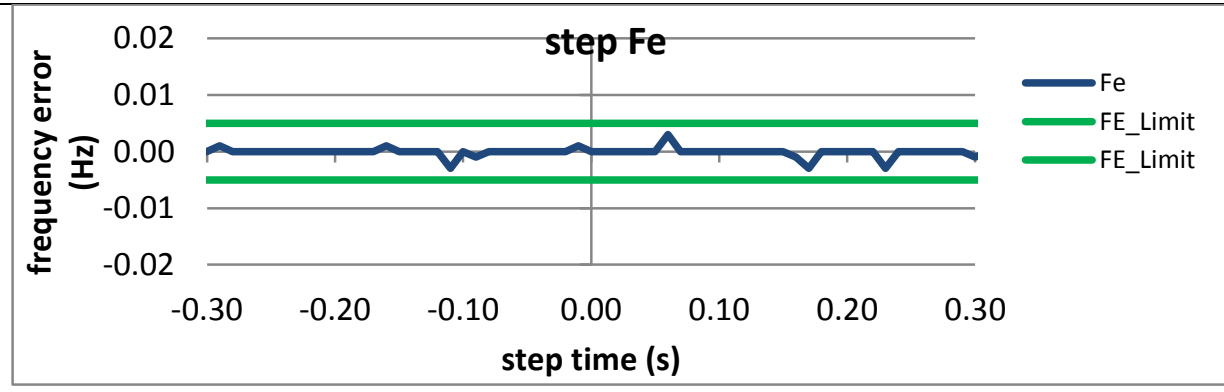

Figure 5698: Fs = 10 FPS, $+10 \%$ magnitude step

Figure 5695: Fs = 15 FPS, $-10 \%$ magnitude step

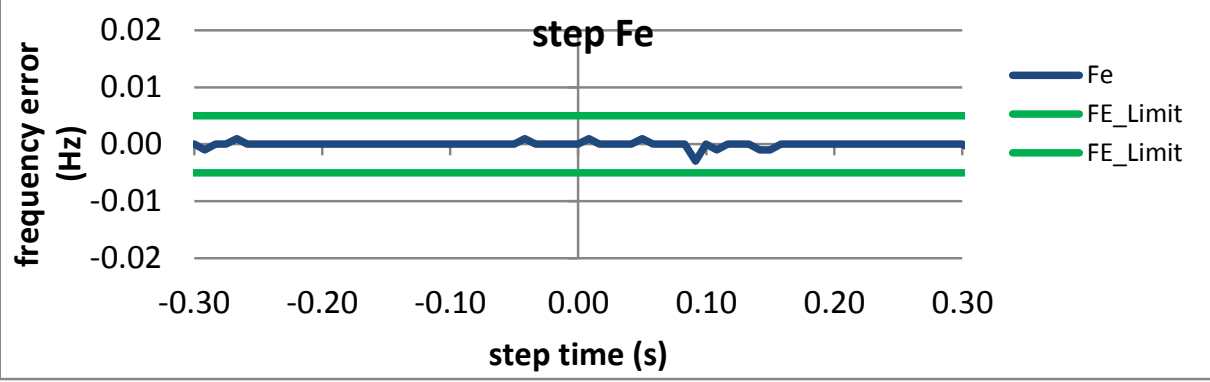

Figure 5697: Fs = 12 FPS, -10\% magnitude step

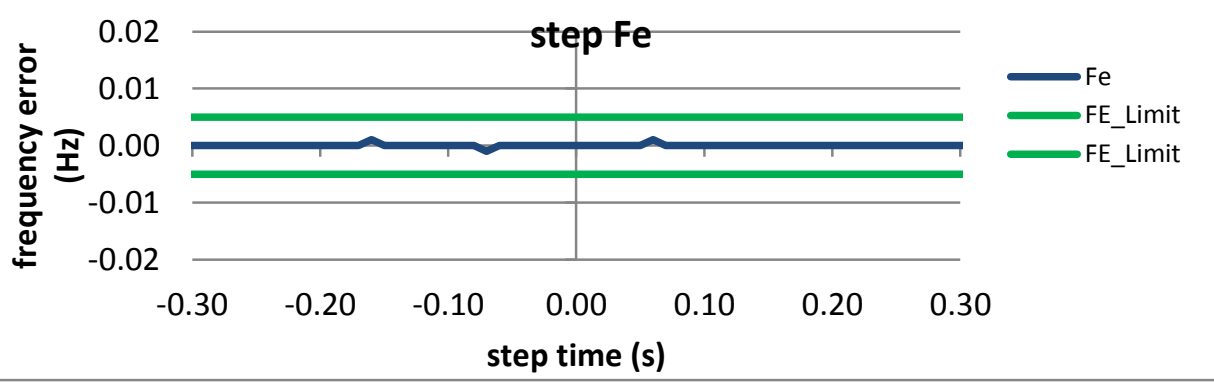

Figure 5699: Fs = 10 FPS, $+10 \%$ magnitude step 
10.7.3 PMU B dynamic step change in magnitude frequency response time: $F 0=60 \mathrm{~Hz}, \mathrm{P}$ class

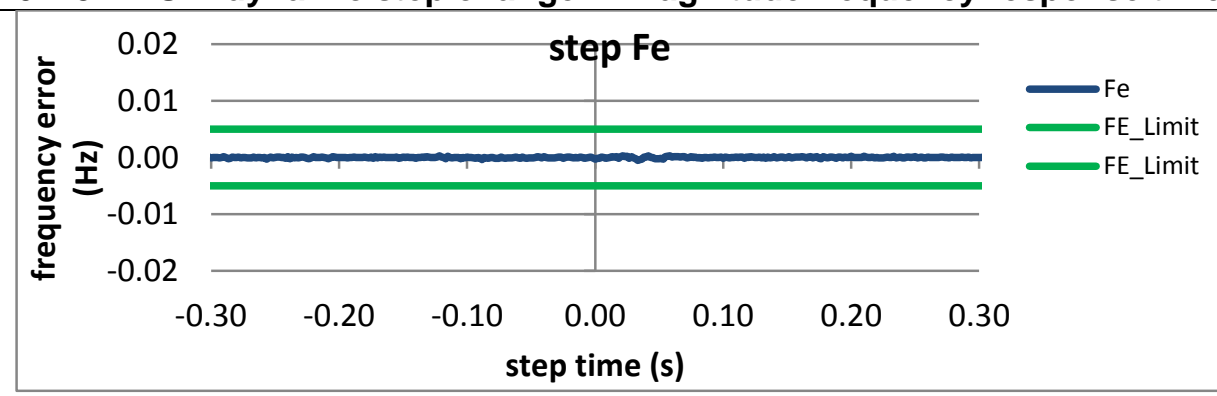

Figure 5700: Fs = 60 FPS, +10\% magnitude step

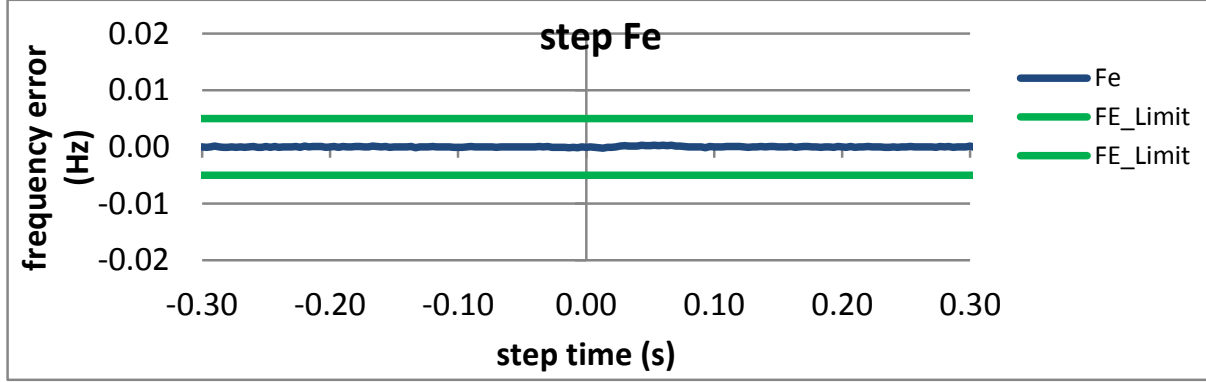

Figure 5702: Fs $=30$ FPS, $+10 \%$ magnitude step

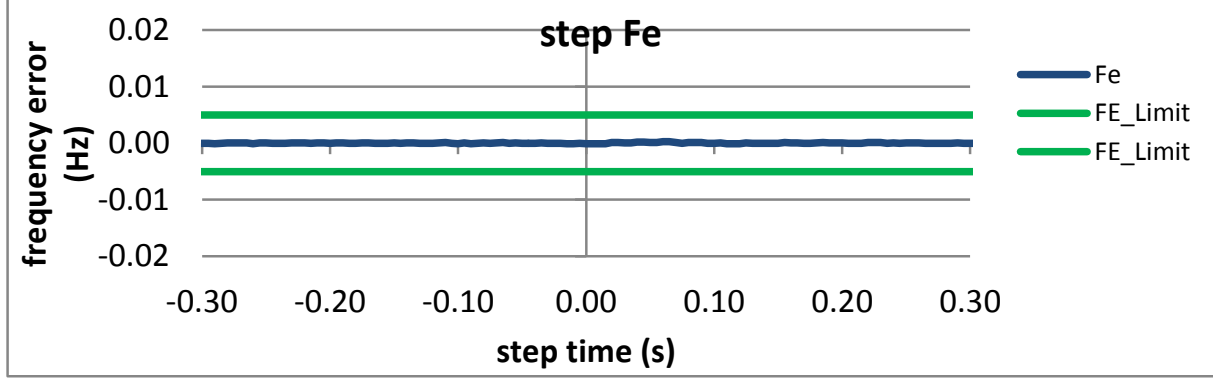

Figure 5704: Fs $=20$ FPS, $+10 \%$ magnitude step

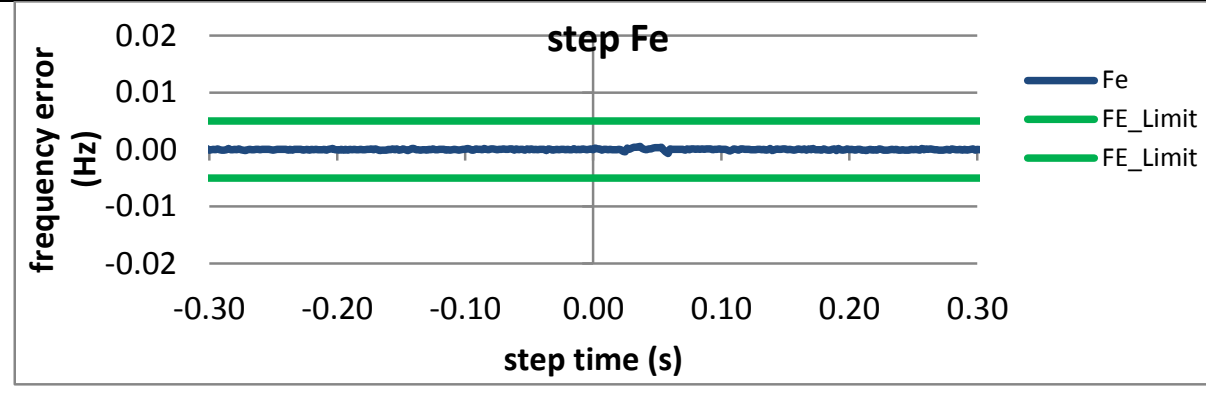

Figure 5701: Fs = 60 FPS, -10\% magnitude step

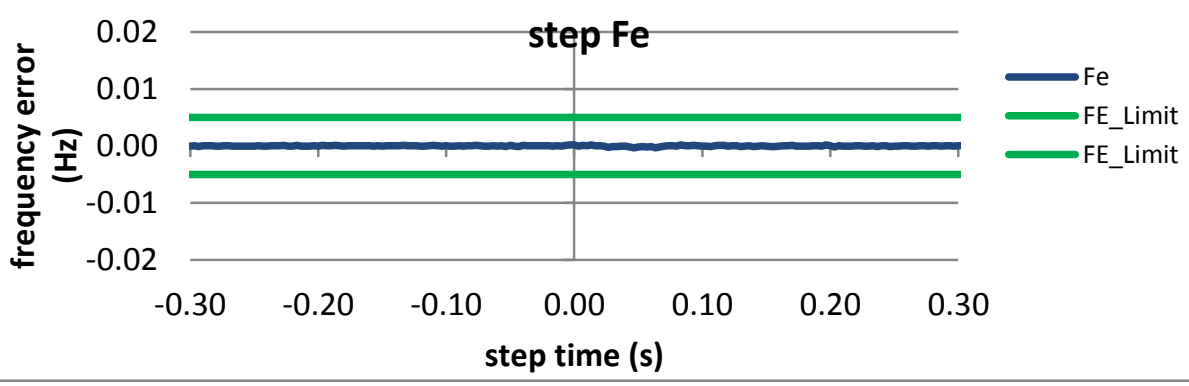

Figure 5703: Fs = 30 FPS, -10\% magnitude step

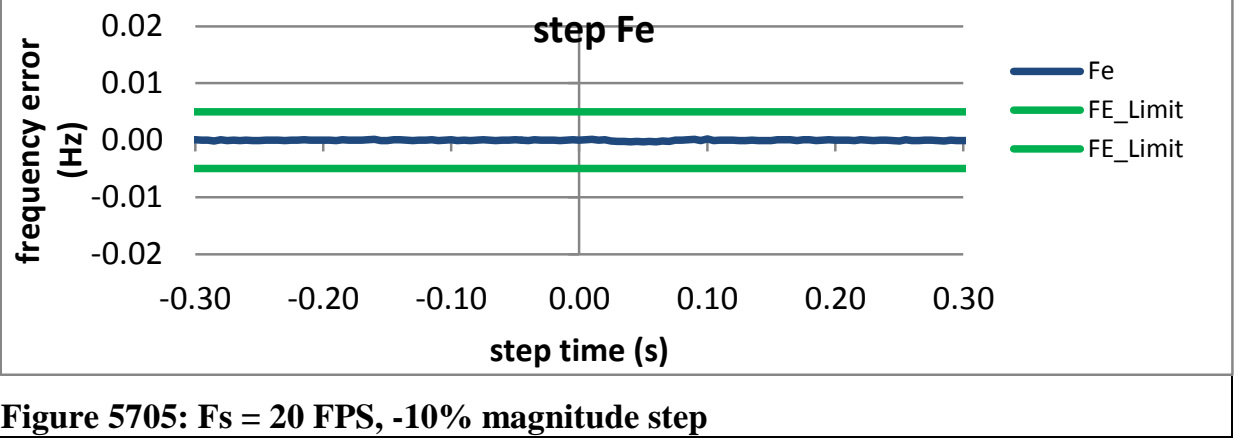



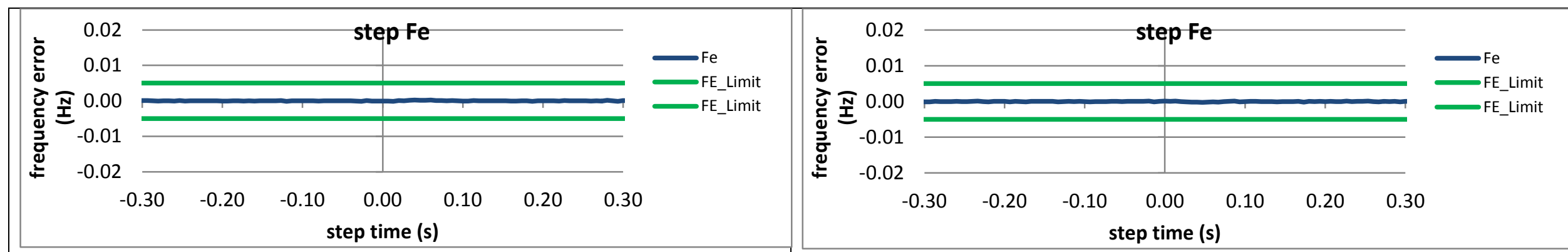

Figure 5706: Fs $=15$ FPS, $+10 \%$ magnitude step

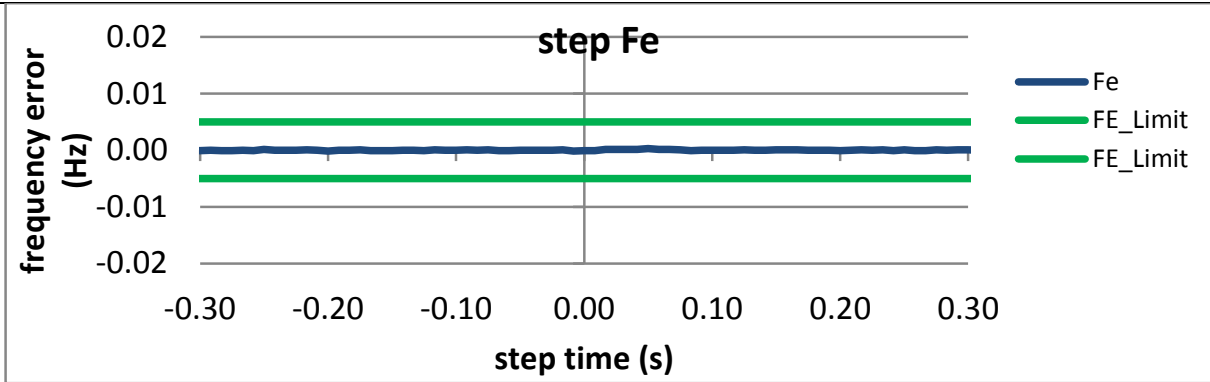

Figure 5708: Fs = 12 FPS, $+10 \%$ magnitude step

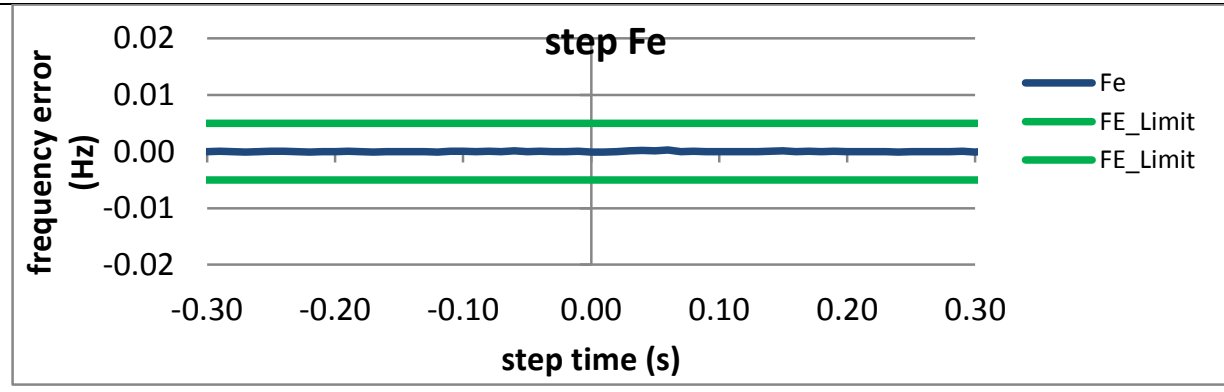

Figure 5707: Fs = 15 FPS, - $10 \%$ magnitude step

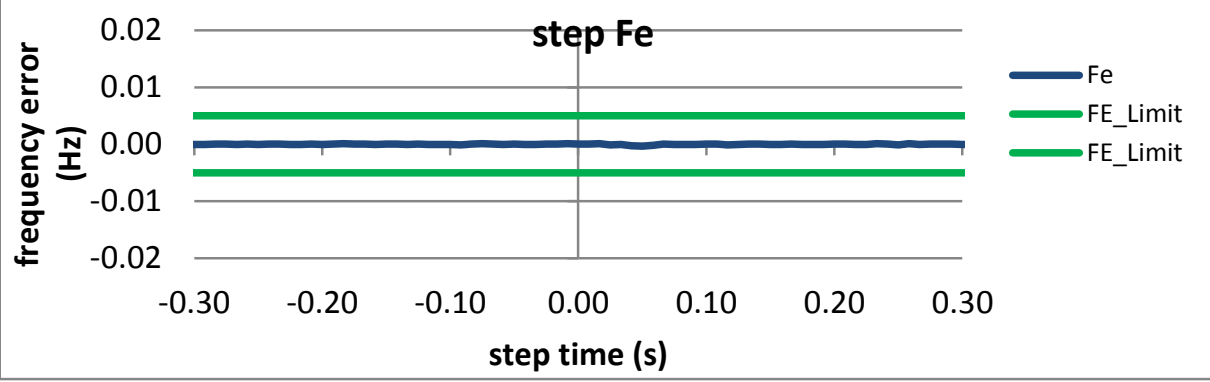

Figure 5709: Fs = 12 FPS, -10\% magnitude step

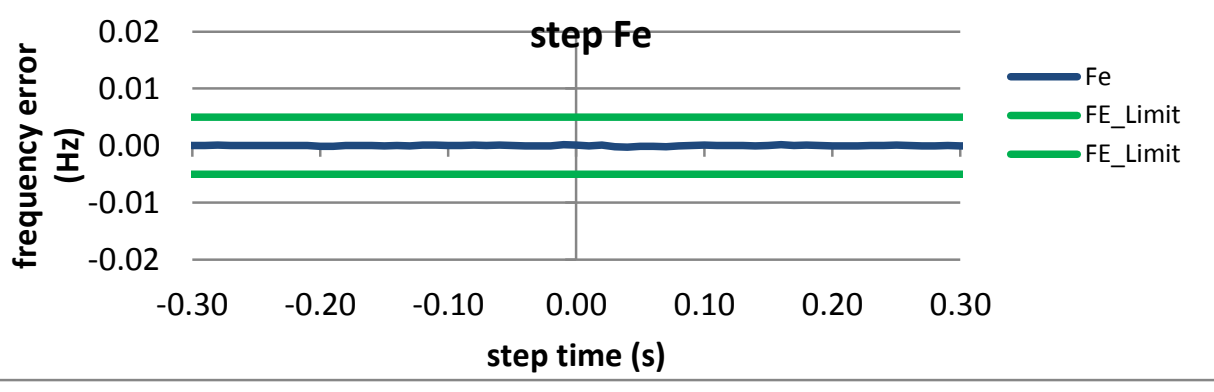

Figure 5711: Fs = 10 FPS, -10\% magnitude step 
10.7.4 PMU C dynamic step change in magnitude frequency response time: $F 0=60 \mathrm{~Hz}, \mathrm{P}$ class
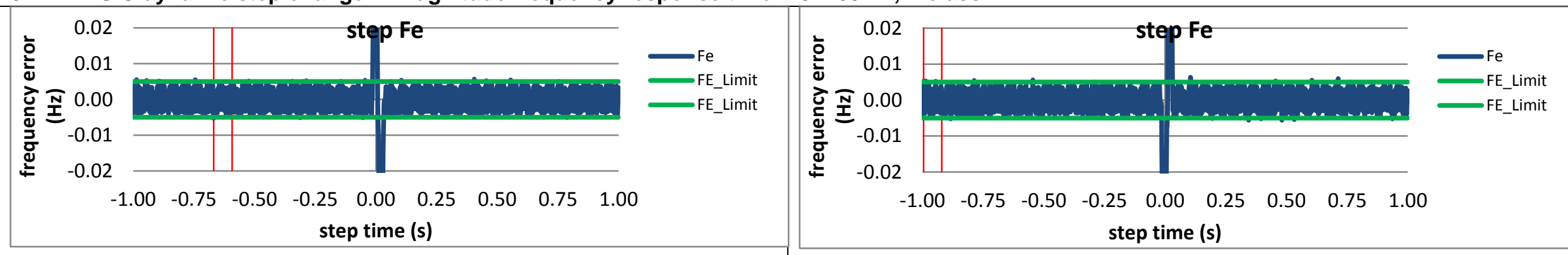

Figure 5712: Fs $=60$ FPS, +10\% magnitude step

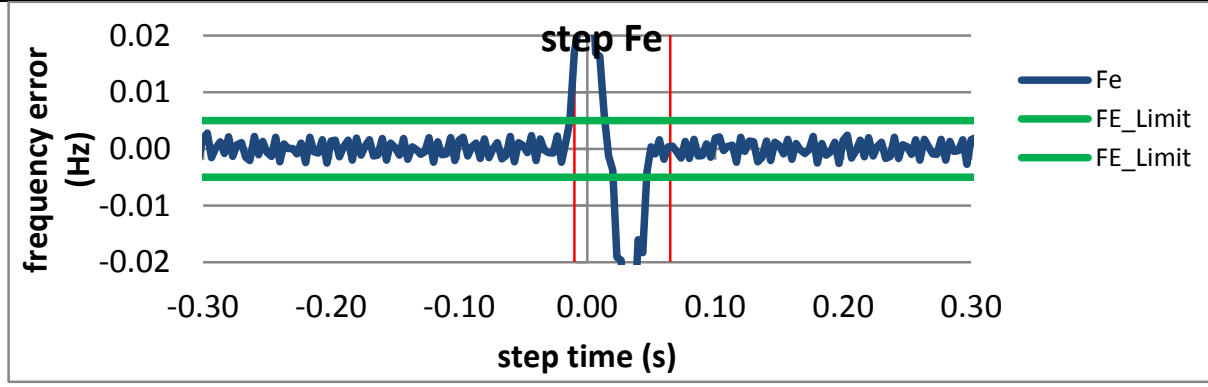

Figure 5713: Fs = 60 FPS, -10\% magnitude step

Figure 5714: Fs = 30 FPS, +10\% magnitude step

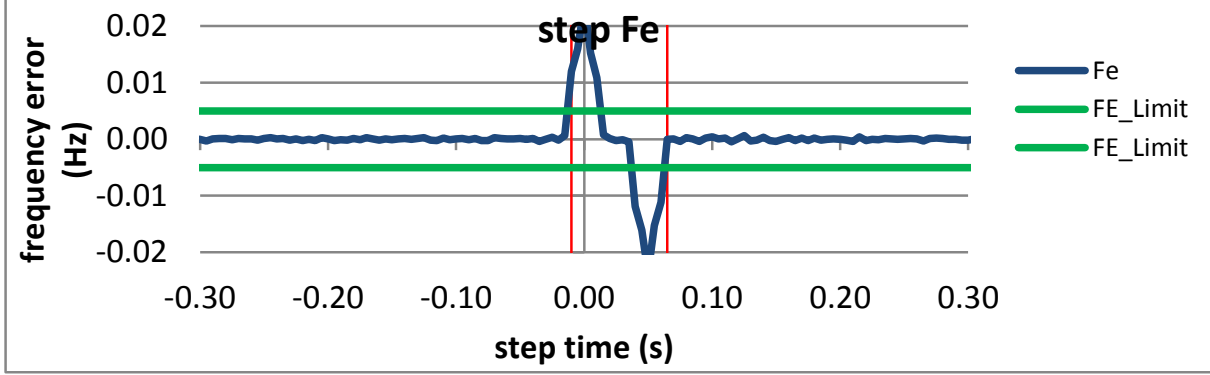

Figure 5716: Fs = 20 FPS, +10\% magnitude step

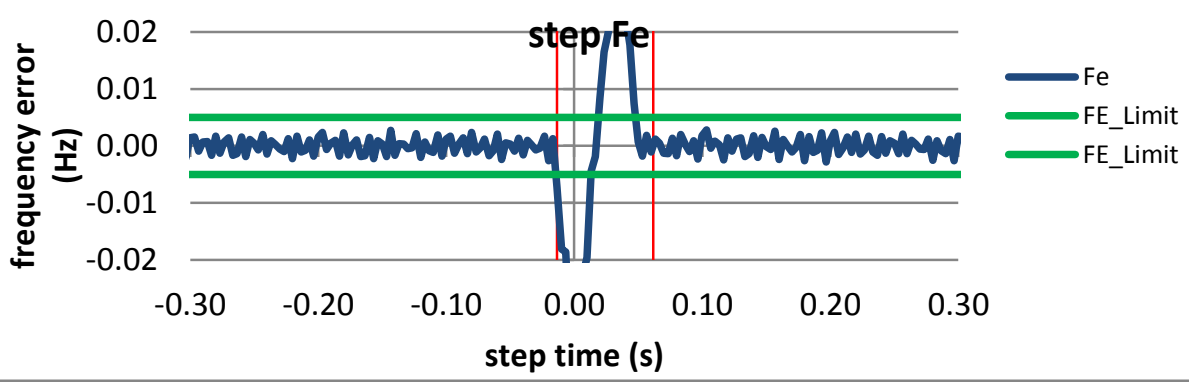

Figure 5715: Fs = 30 FPS, -10\% magnitude step

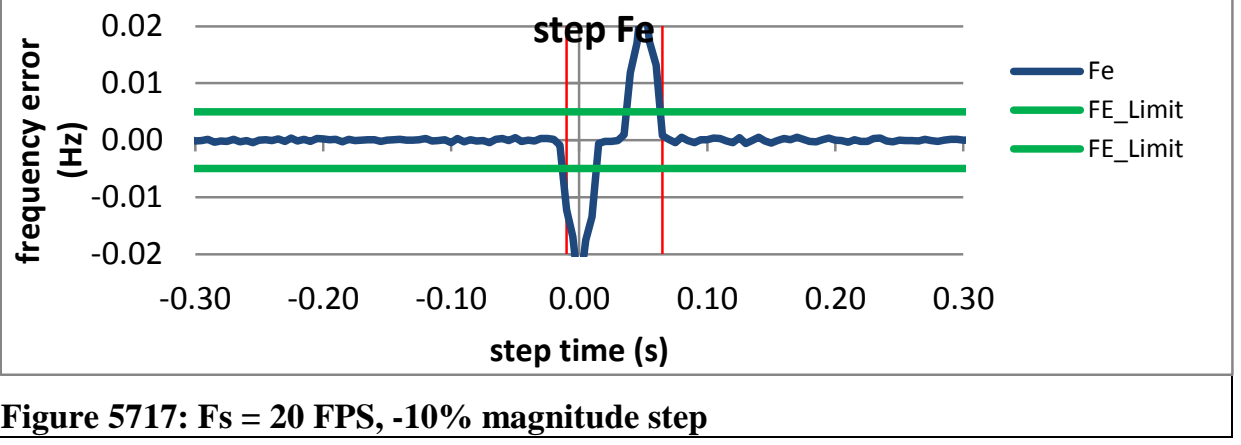



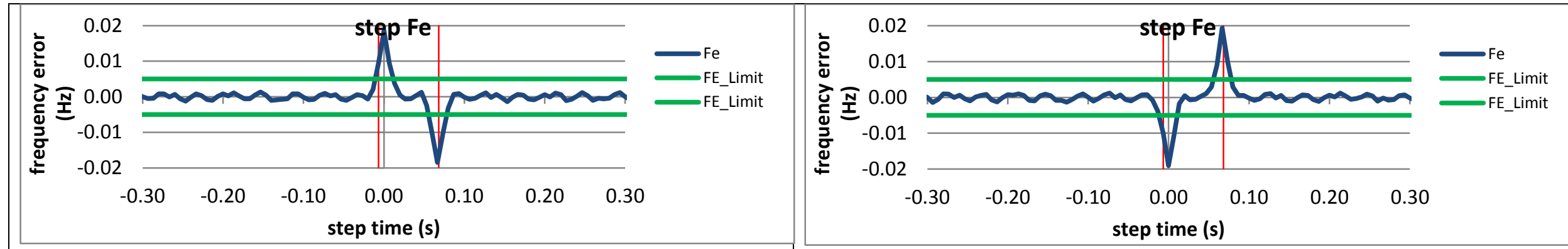

Figure 5718: Fs = 15 FPS, $+10 \%$ magnitude step

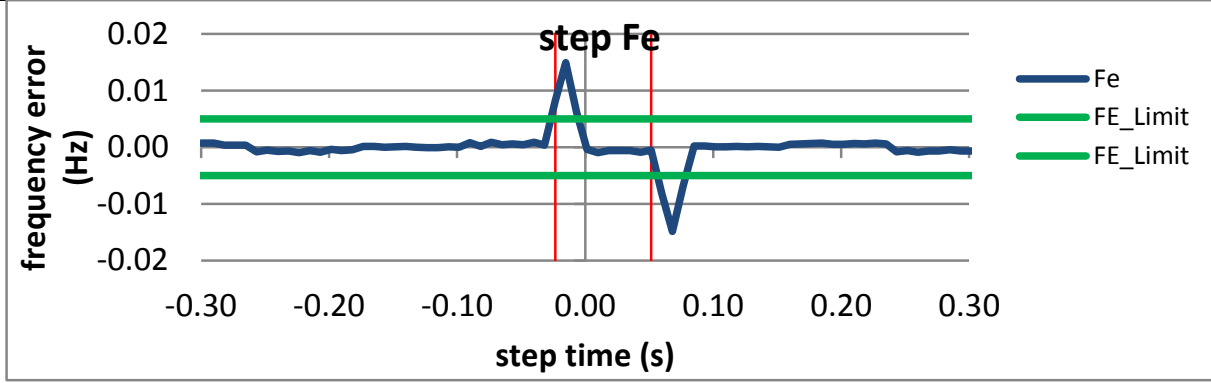

Figure 5720: Fs = 12 FPS, +10\% magnitude step

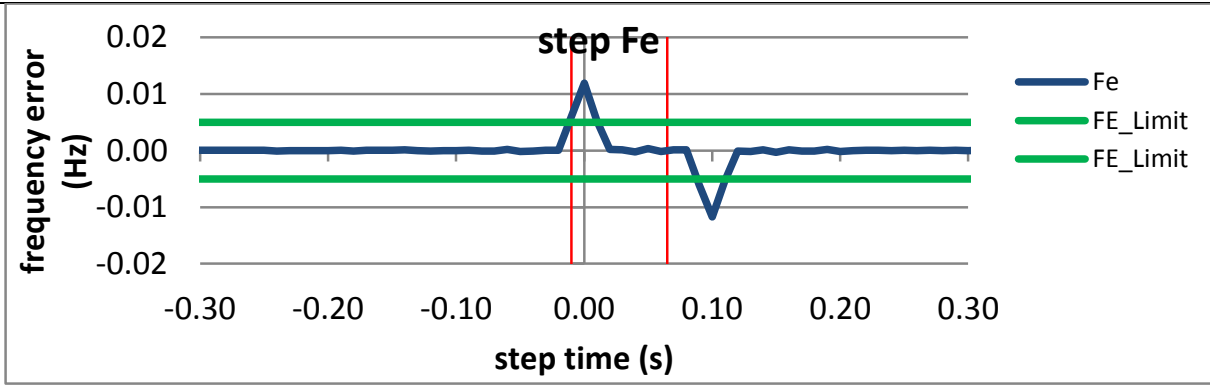

Figure 5722: Fs = 10 FPS, $+10 \%$ magnitude step

Figure 5719: Fs = 15 FPS, - $10 \%$ magnitude step

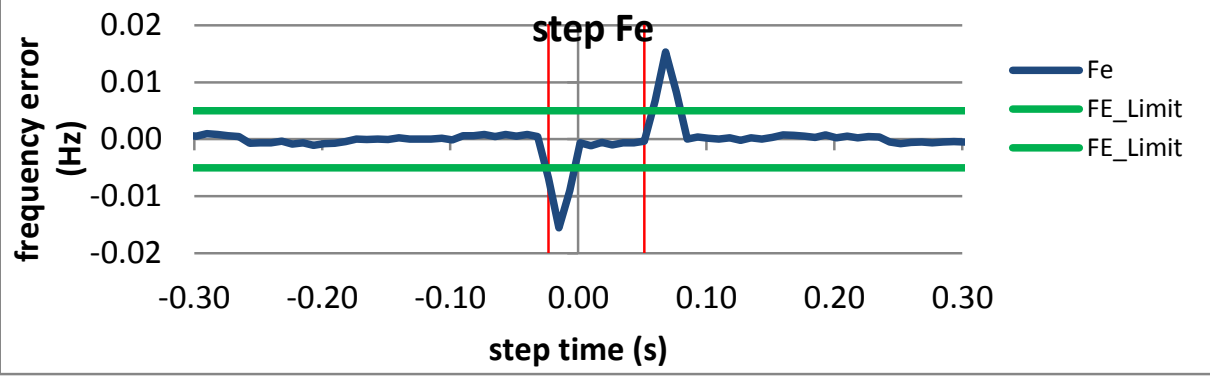

Figure 5721: Fs = 12 FPS, -10\% magnitude step

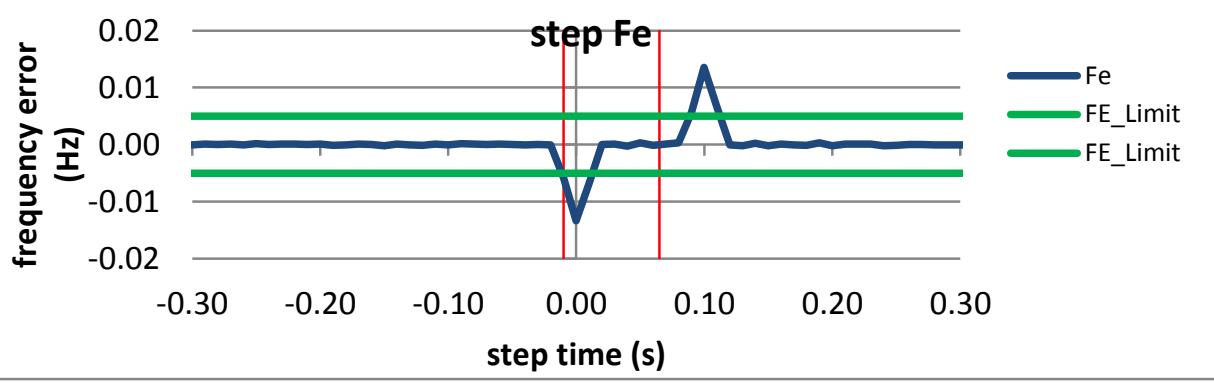

Figure 5723: Fs = 10 FPS, -10\% magnitude step 
10.7.5 PMU D dynamic step change in magnitude frequency response time: $F 0=60 \mathrm{~Hz}, \mathrm{P}$ class

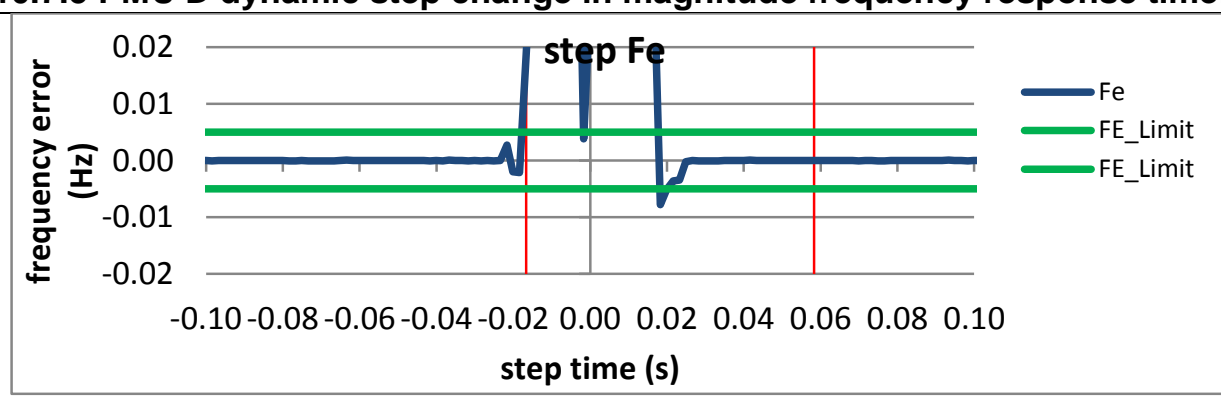

Figure 5724: Fs = 60 FPS, +10\% magnitude step

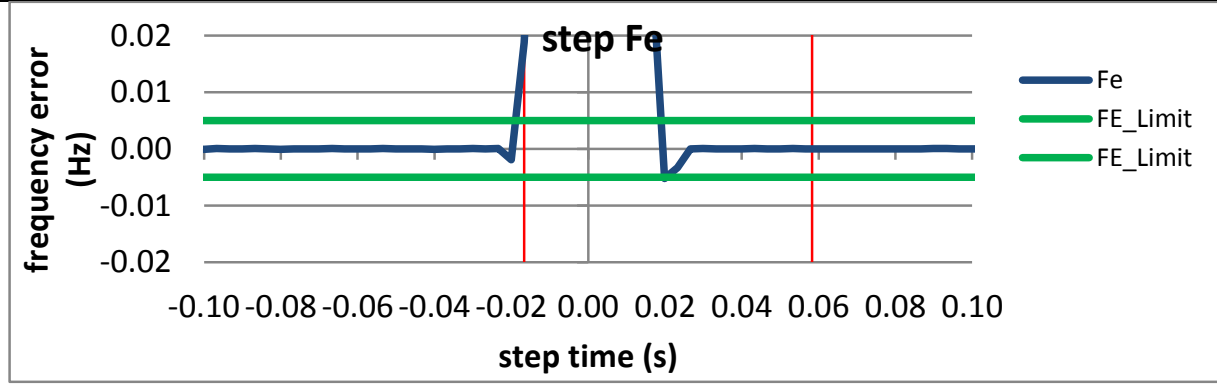

Figure 5726: Fs = 30 FPS, +10\% magnitude step

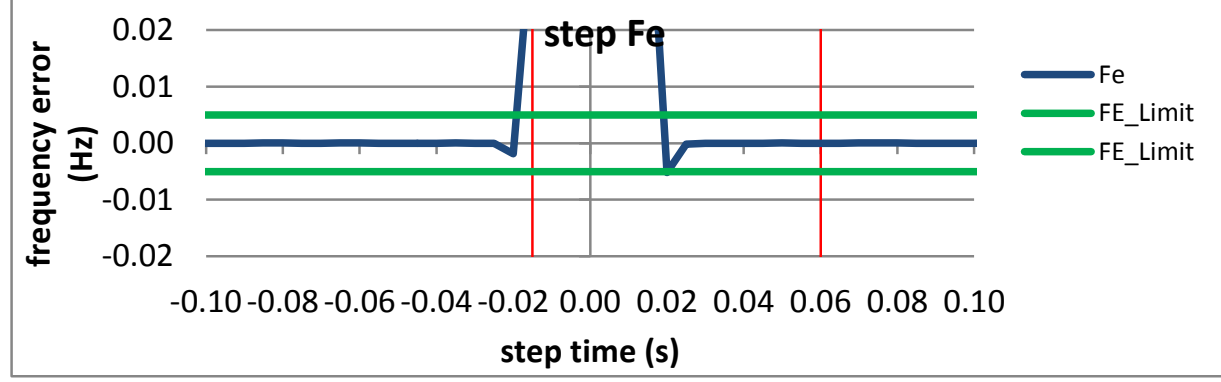

Figure 5728: Fs = 20 FPS, +10\% magnitude step

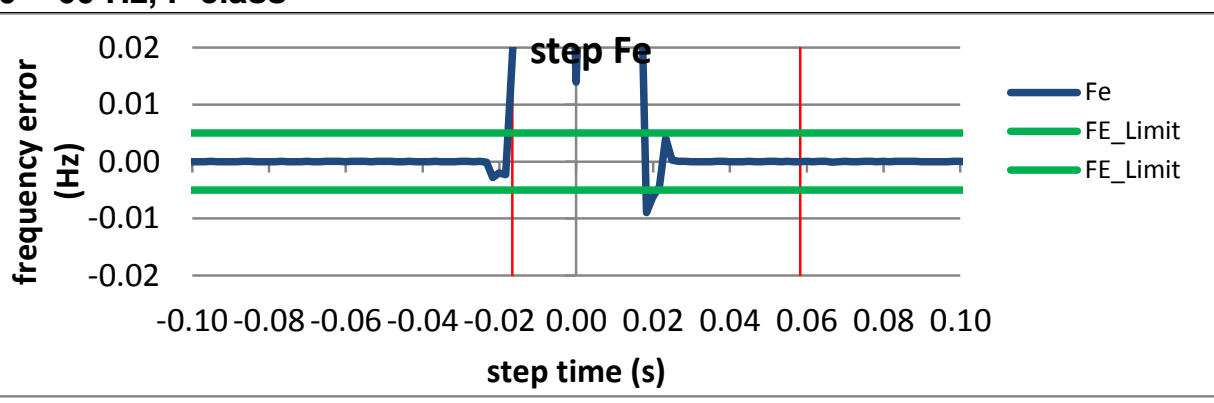

Figure 5725: Fs = 60 FPS, -10\% magnitude step

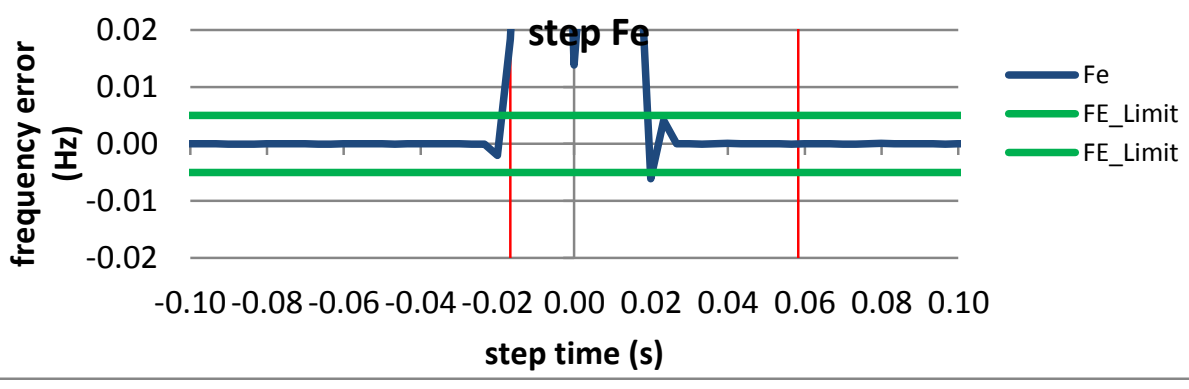

Figure 5727: Fs = 30 FPS, -10\% magnitude step

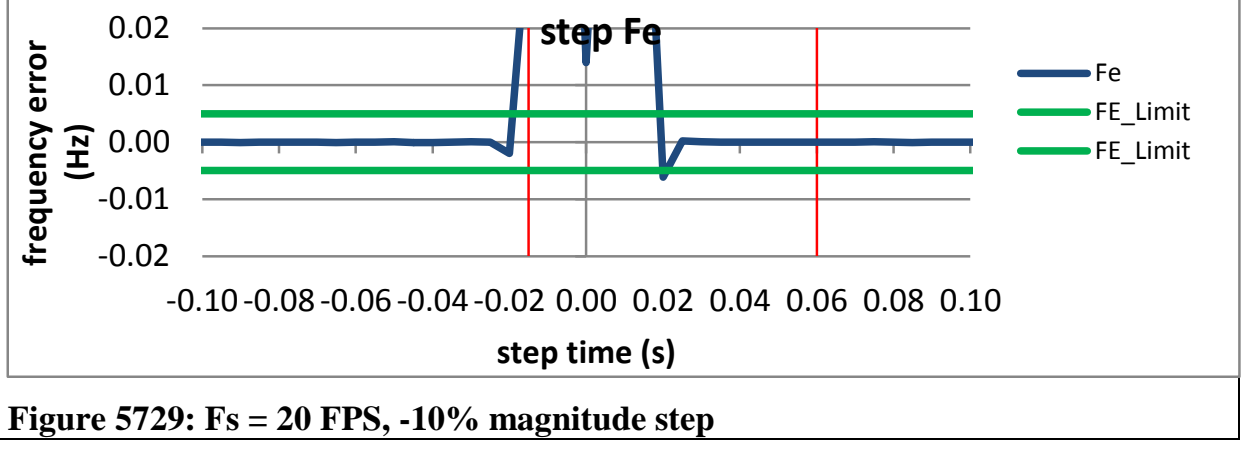



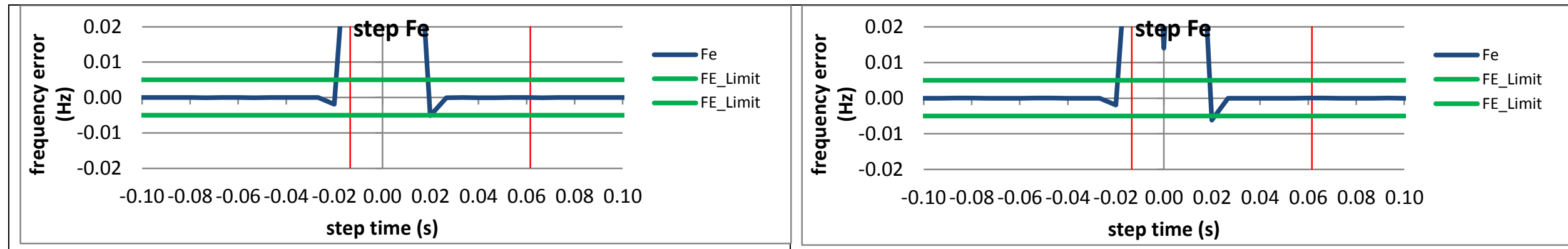

Figure 5730: Fs = 15 FPS, $+10 \%$ magnitude step

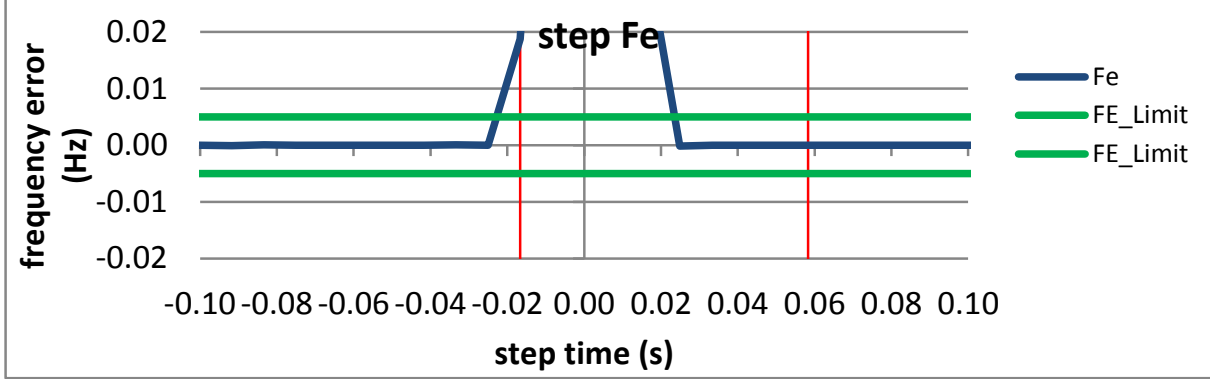

Figure 5732: Fs = 12 FPS, +10\% magnitude step

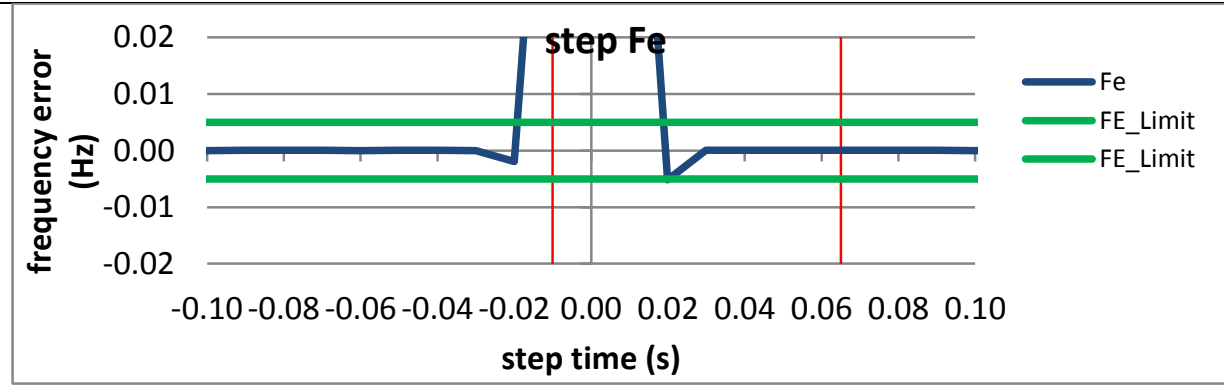

Figure 5734: Fs = 10 FPS, $+10 \%$ magnitude step

Figure 5731: Fs = 15 FPS, - $10 \%$ magnitude step

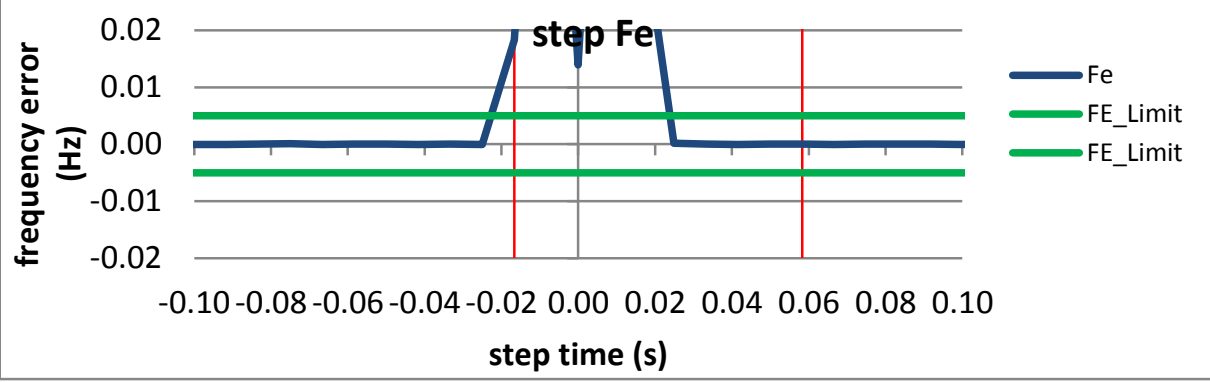

Figure 5733: Fs = 12 FPS, -10\% magnitude step

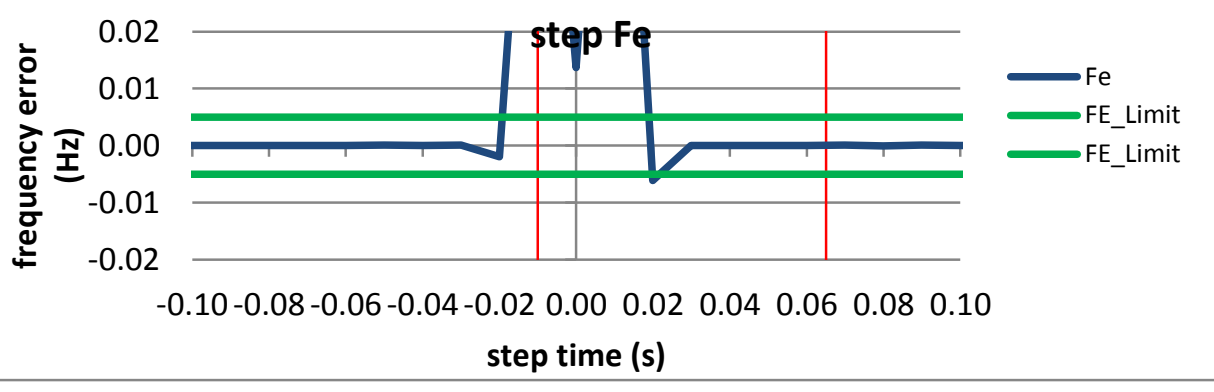

Figure 5735: Fs = 10 FPS, -10\% magnitude step 
10.7.6 PMU E dynamic step change in magnitude frequency response time: $F 0=60 \mathrm{~Hz}, \mathrm{P}$ class PMU E does not support P class

\subsubsection{PMU F dynamic step change in magnitude frequency response time: $F 0=60 \mathrm{~Hz}, \mathrm{P}$ class}
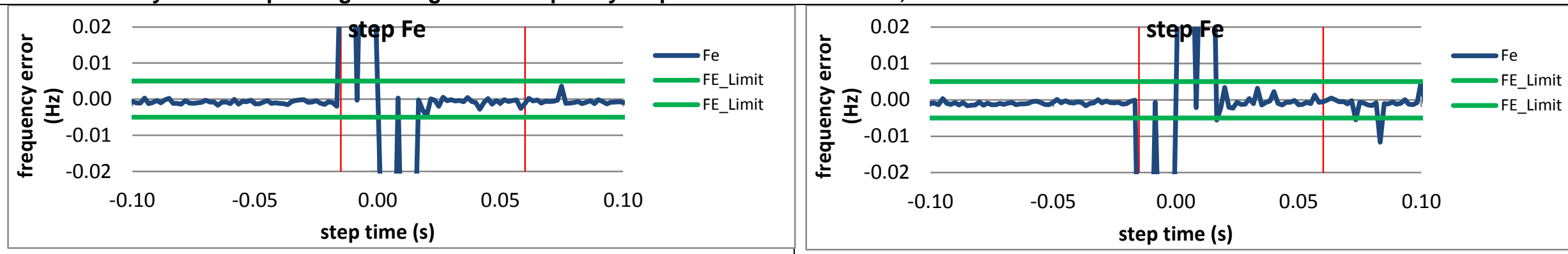

Figure 5736: Fs $=60 \mathrm{FPS},+10 \%$ magnitude step

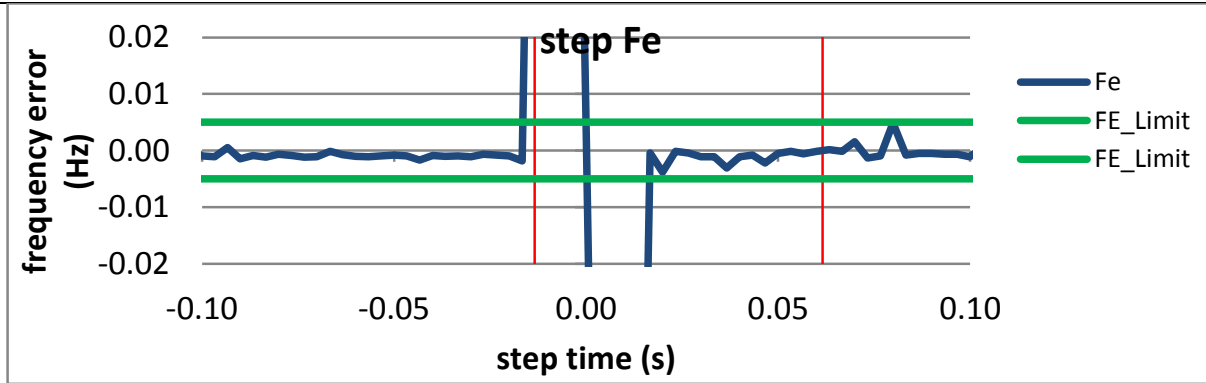

Figure 5737: Fs = 60 FPS, $-10 \%$ magnitude step

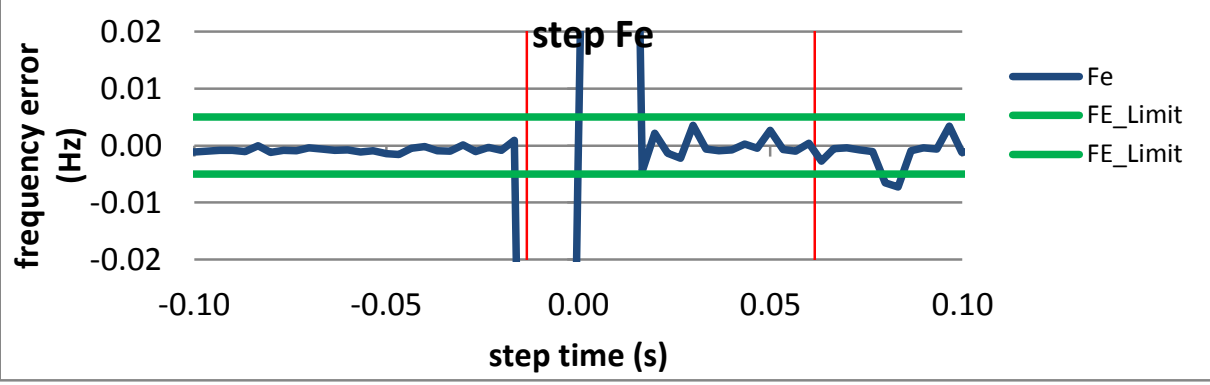

Figure 5738: $\mathrm{Fs}=30 \mathrm{FPS},+10 \%$ magnitude step

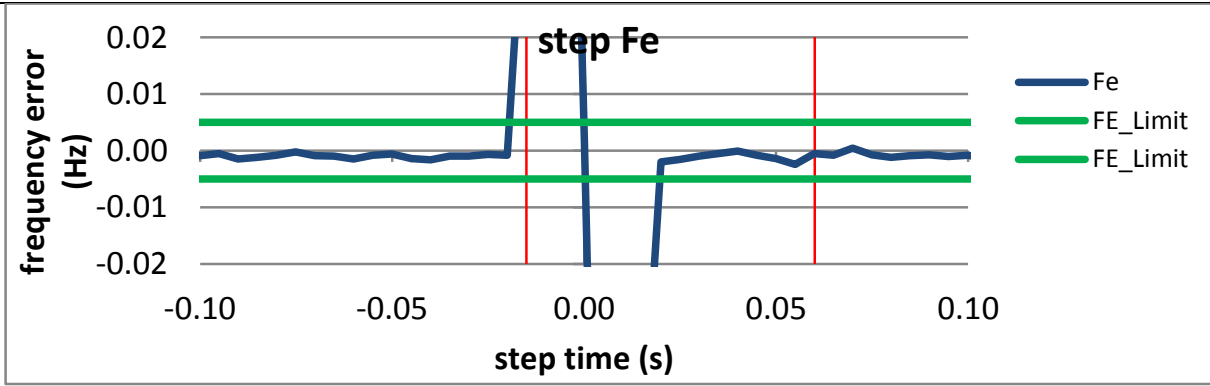

Figure 5739: Fs = 30 FPS, $-10 \%$ magnitude step

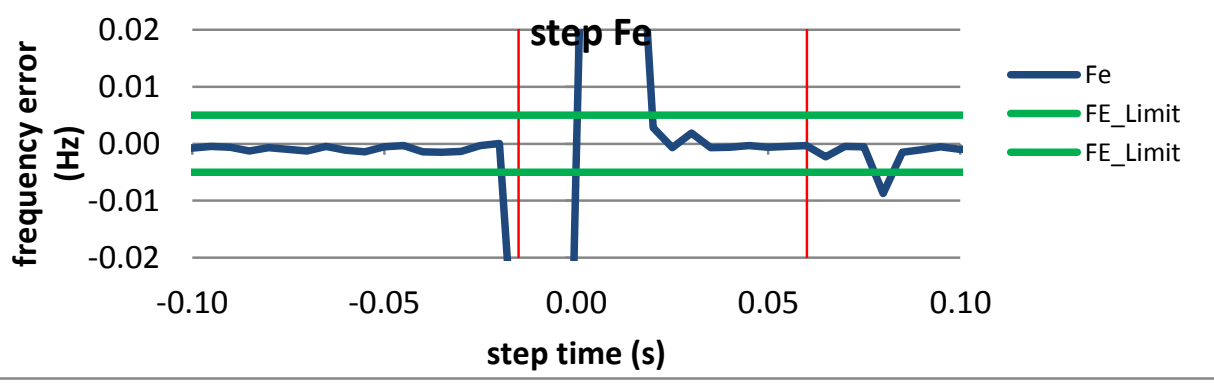

Figure 5740: Fs $=20 \mathrm{FPS},+10 \%$ magnitude step

Figure 5741: Fs = $20 \mathrm{FPS},-10 \%$ magnitude step 

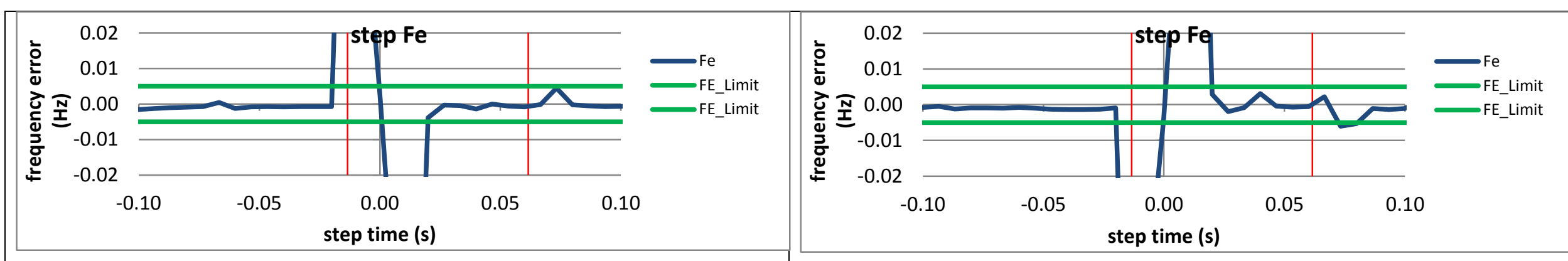

Figure 5742: Fs = $15 \mathrm{FPS},+10 \%$ magnitude step

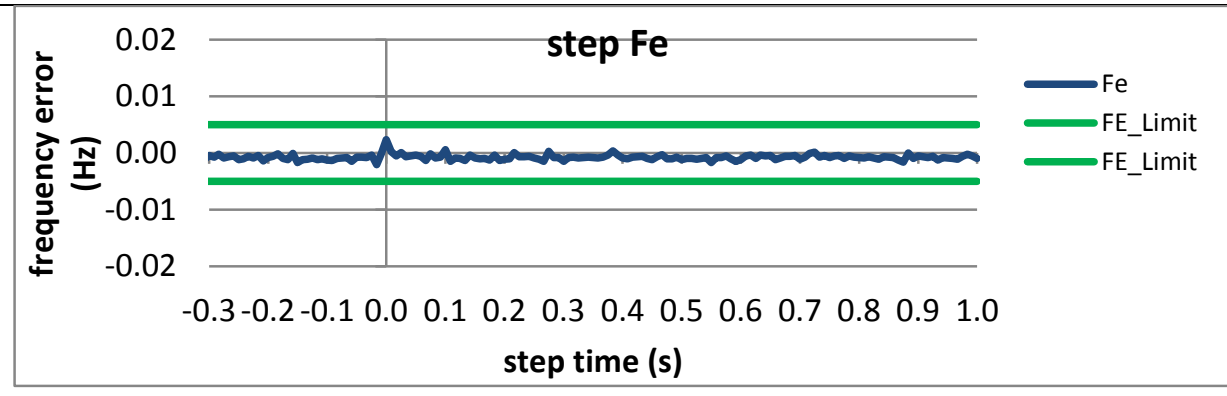

Figure 5743: Fs = 15 FPS, - $10 \%$ magnitude step

\section{Figure 5744: Fs = 12 FPS, $+10 \%$ magnitude step}

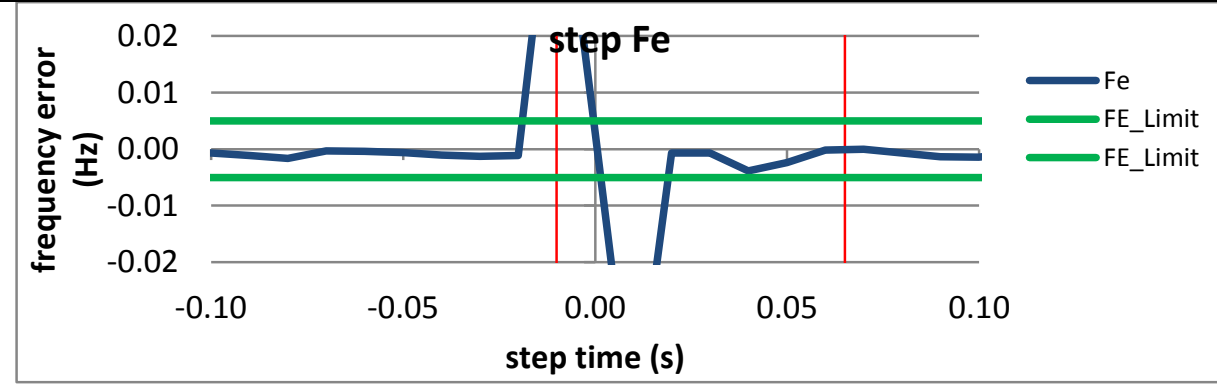

Figure 5746: $\mathrm{Fs}=10 \mathrm{FPS},+10 \%$ magnitude step

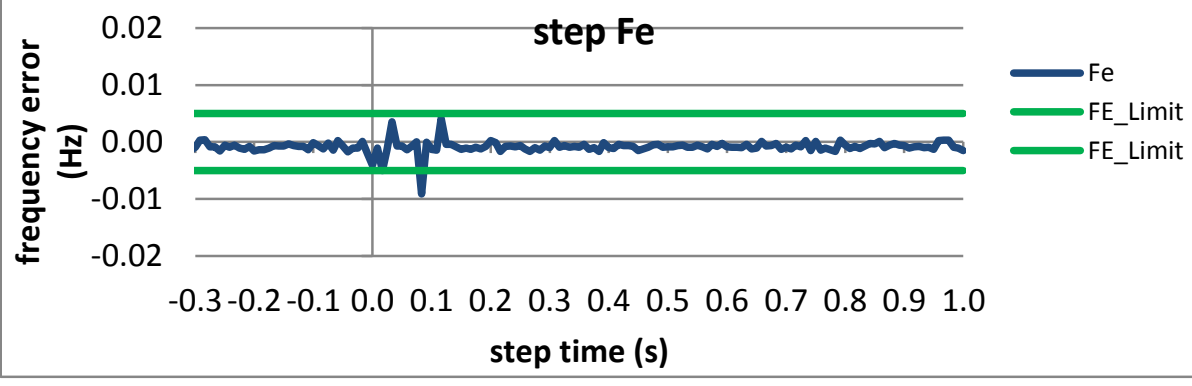

Figure 5745: Fs = 12 FPS, $-10 \%$ magnitude step

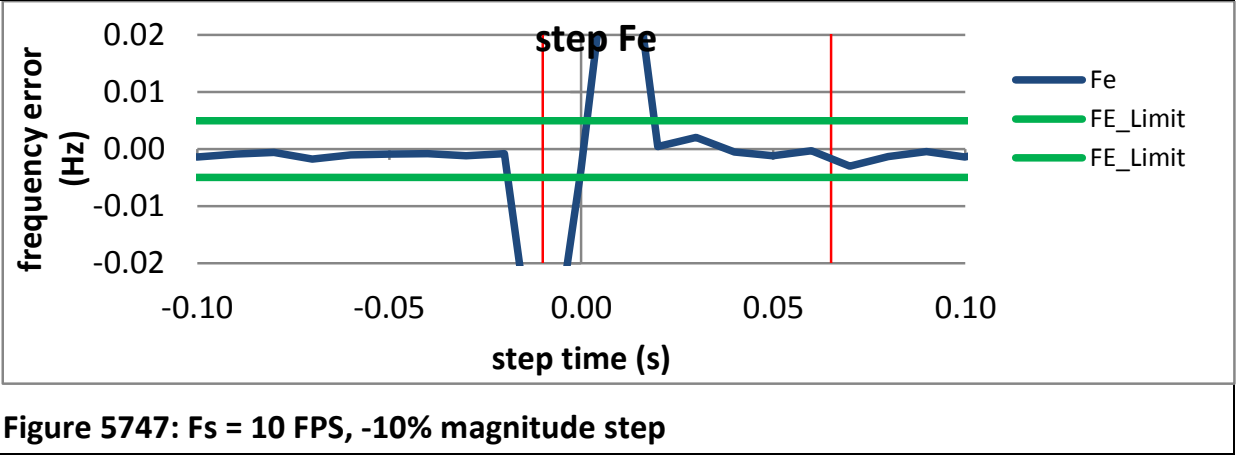


10.7.8 PMU G dynamic step change in magnitude frequency response time: $F 0=60 \mathrm{~Hz}, \mathrm{P}$ class

PMU G does not support P class

\subsubsection{PMU H dynamic step change in magnitude frequency response time: $\mathrm{F} 0=60 \mathrm{~Hz}, \mathrm{P}$ class}

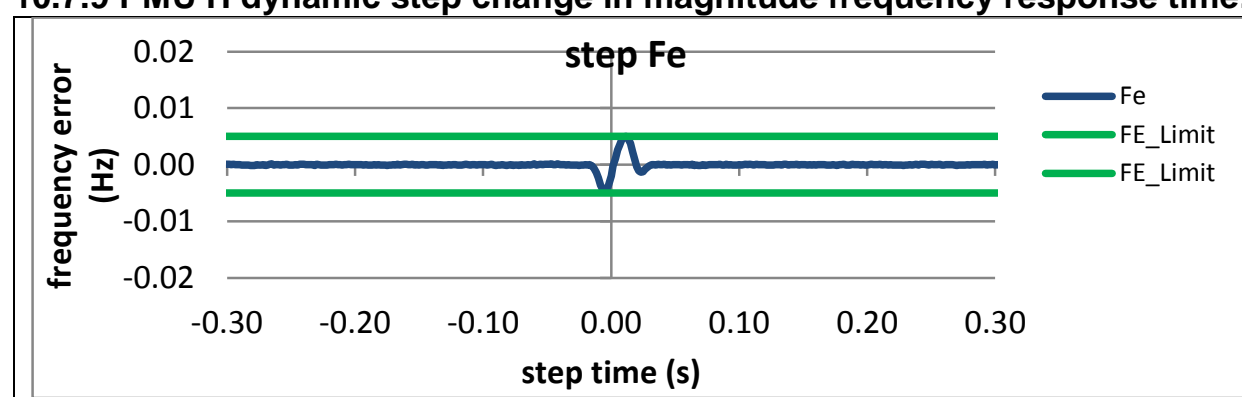

Figure 5748: Fs = 60 FPS, $+10 \%$ magnitude step

Figure 5750: Fs = 30 FPS, $+10 \%$ magnitude step

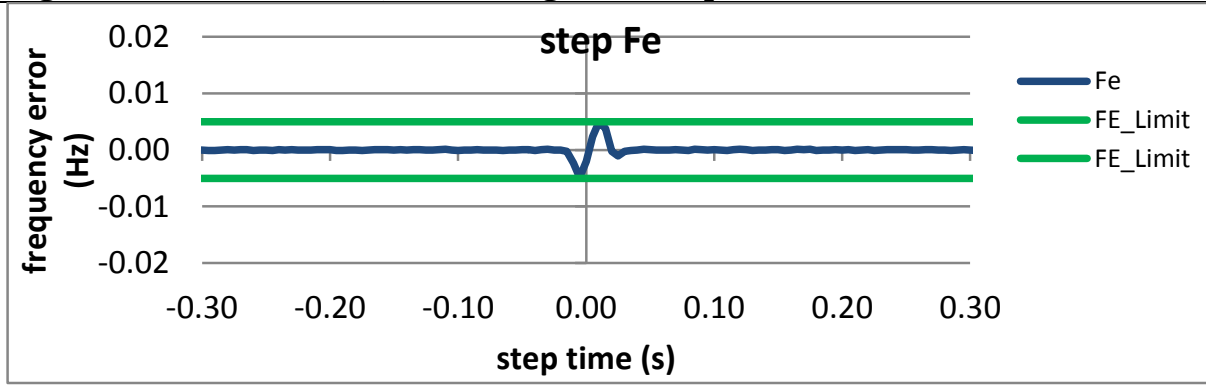

Figure 5752: Fs = 20 FPS, +10\% magnitude step

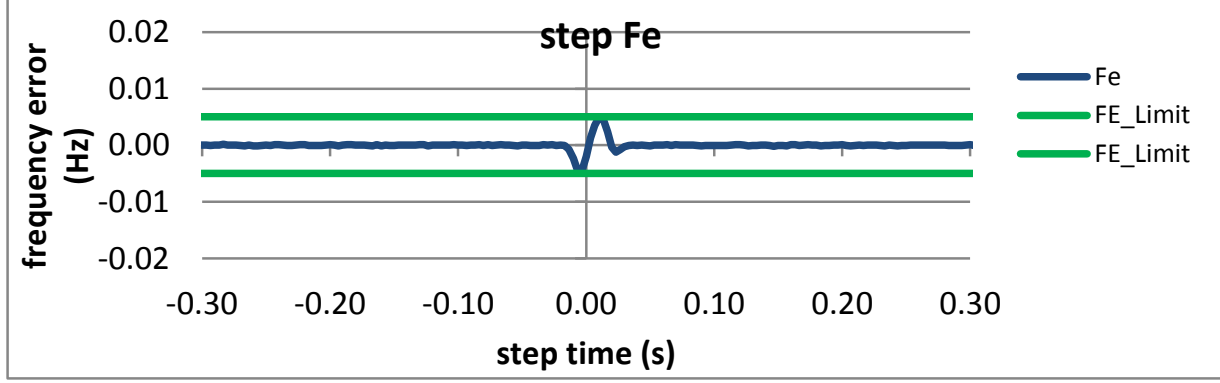

Figure 5754: Fs = 15 FPS, $+10 \%$ magnitude step

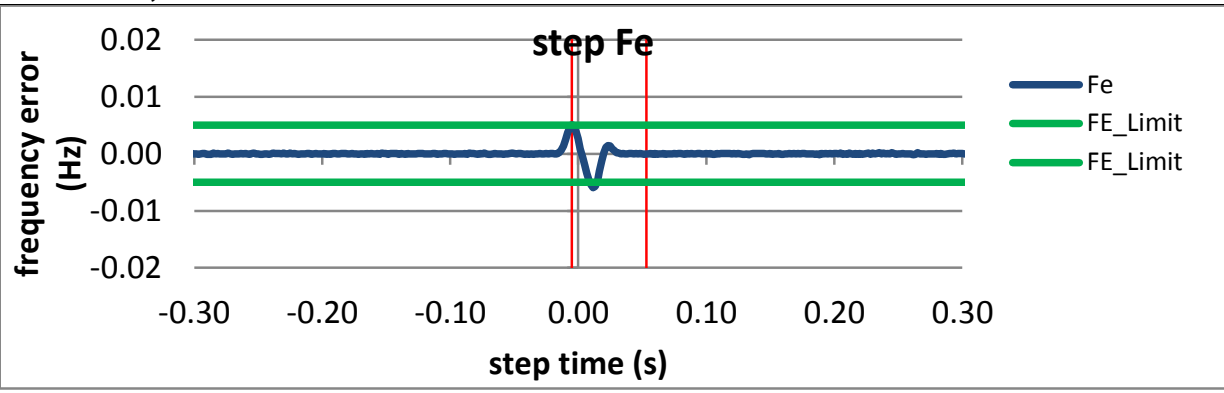

Figure 5749: Fs = 60 FPS, -10\% magnitude step

Figure 5751: Fs = 30 FPS, -10\% magnitude step

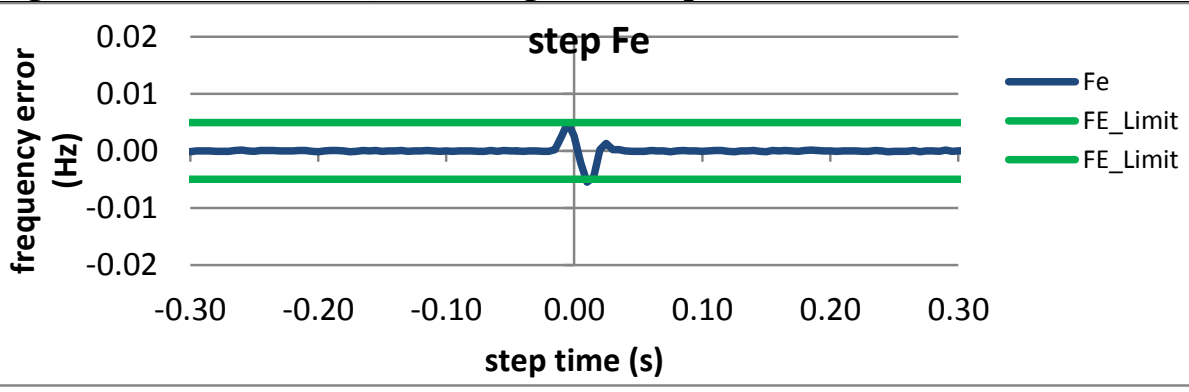

Figure 5753: Fs = 20 FPS, -10\% magnitude step

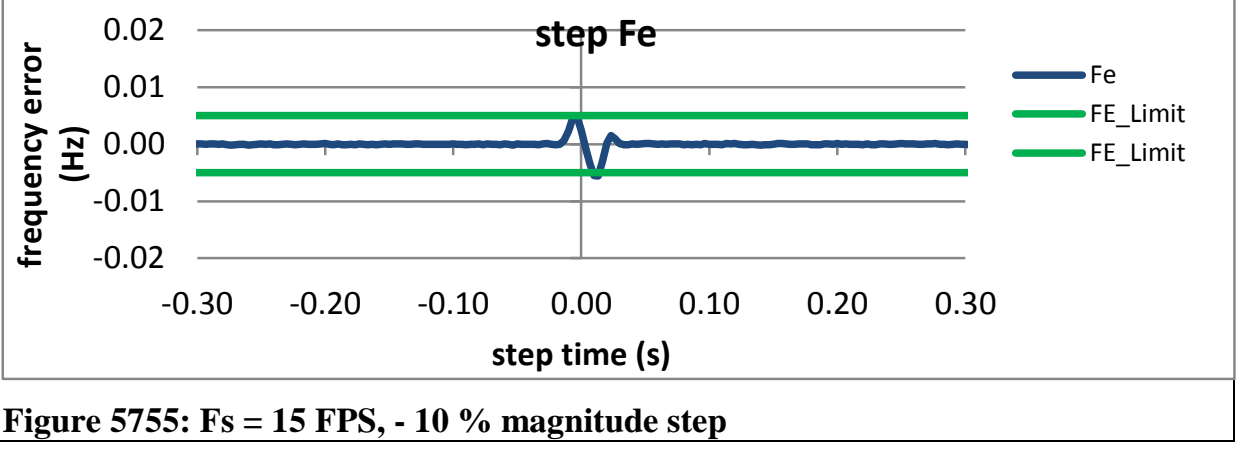



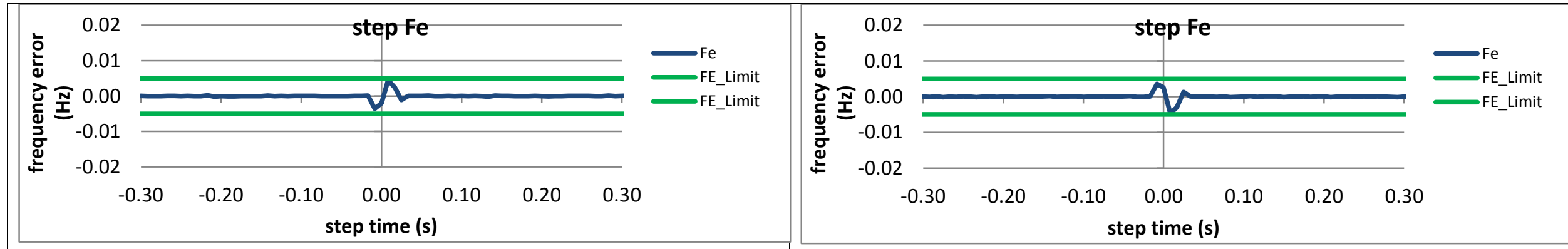

Figure 5756: Fs $=12$ FPS, $+10 \%$ magnitude step

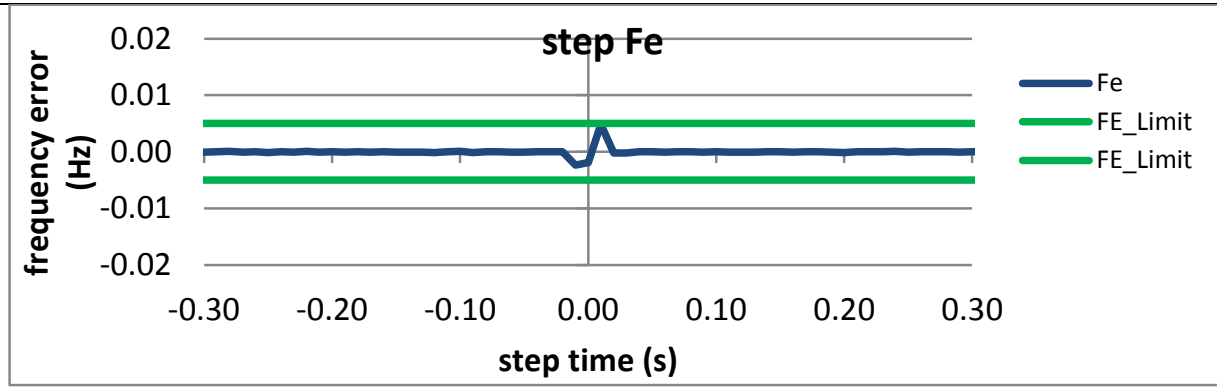

Figure 5758: Fs = 10 FPS, $+10 \%$ magnitude step

Figure 5757: Fs = 12 FPS, -10\% magnitude step

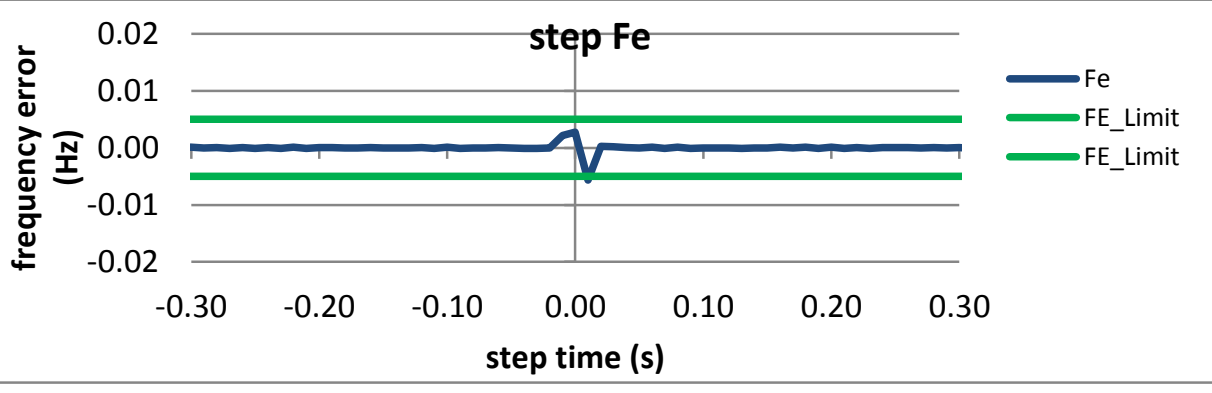

Figure 5759: Fs = 10 FPS, -10\% magnitude step 
10.7.10 PMU I dynamic step change in magnitude frequency response time: $F 0=60 \mathrm{~Hz}, \mathrm{P}$ class PMU I does not support P class

\subsubsection{PMU J dynamic step change in magnitude frequency response time: $F 0=60 \mathrm{~Hz}, \mathrm{P}$ class}
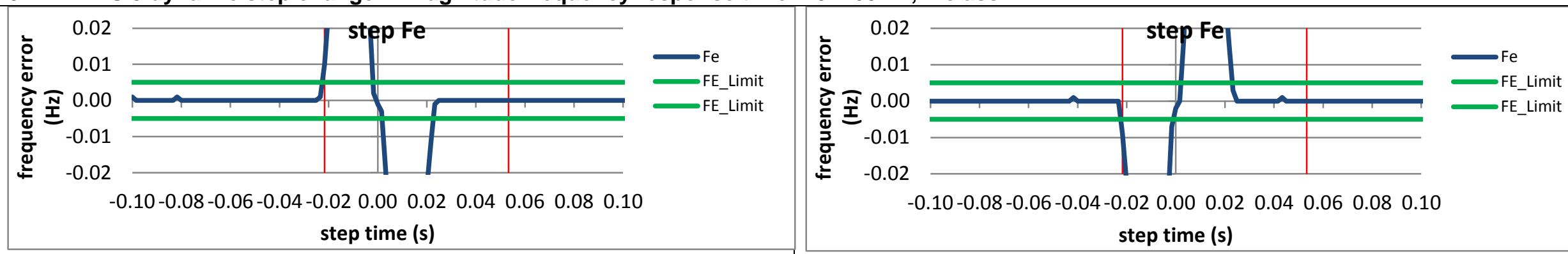

Figure 5760: Fs $=60 \mathrm{FPS},+10 \%$ magnitude step

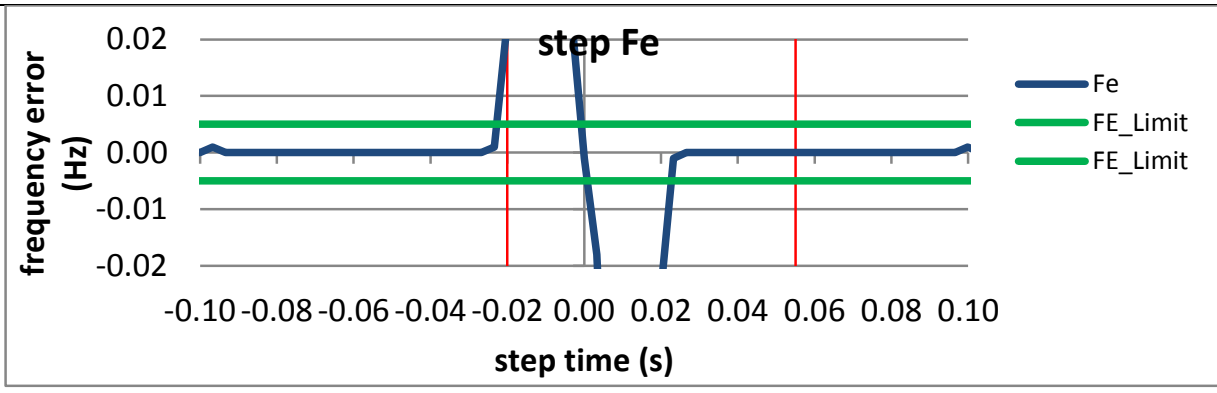

Figure 5761: Fs = $60 \mathrm{FPS},-10 \%$ magnitude step

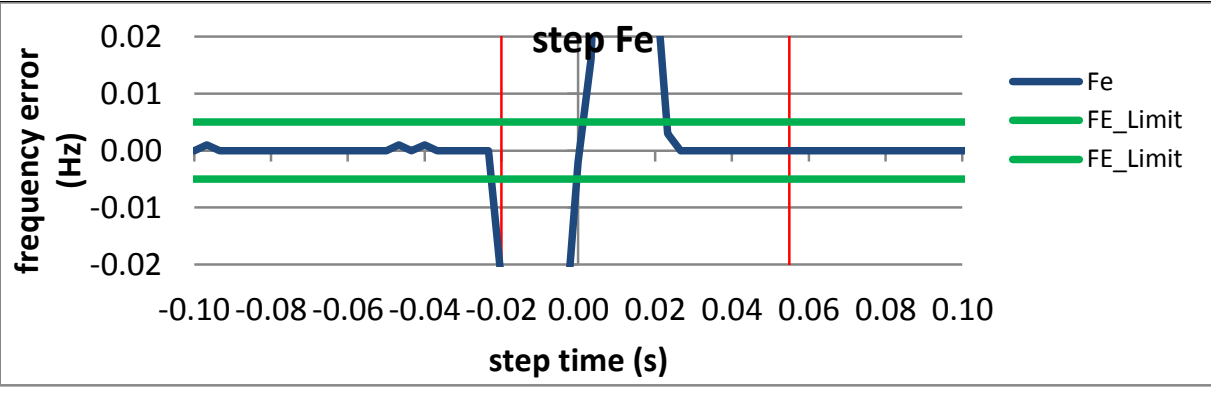

Figure 5762: Fs $=30 \mathrm{FPS},+10 \%$ magnitude step

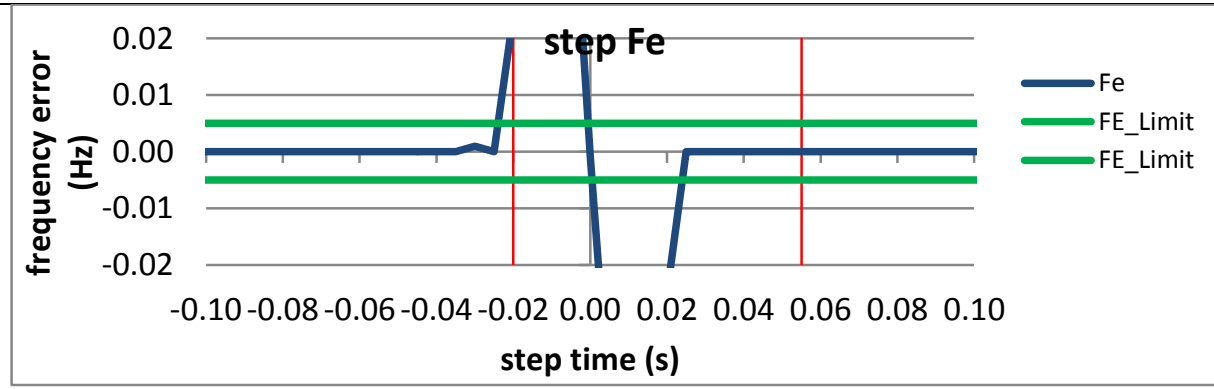

Figure 5763: Fs = 30 FPS, $-10 \%$ magnitude step

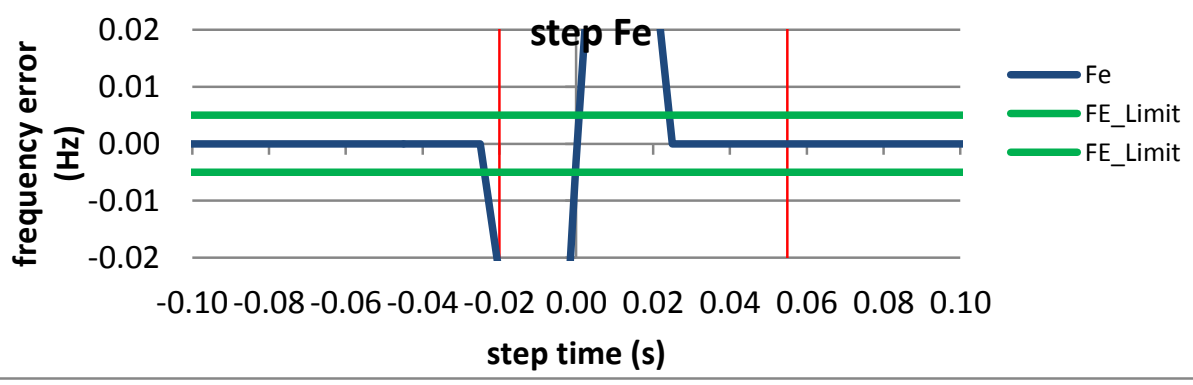

Figure 5764: Fs $=20$ FPS, $+10 \%$ magnitude step

Figure 5765: Fs = $20 \mathrm{FPS},-10 \%$ magnitude step 


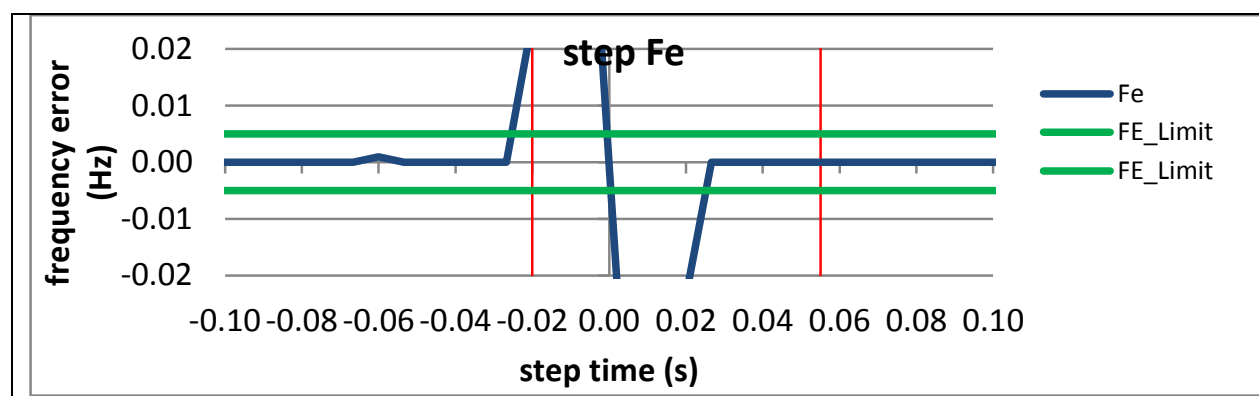

Figure 5766: Fs = $15 \mathrm{FPS},+10 \%$ magnitude step

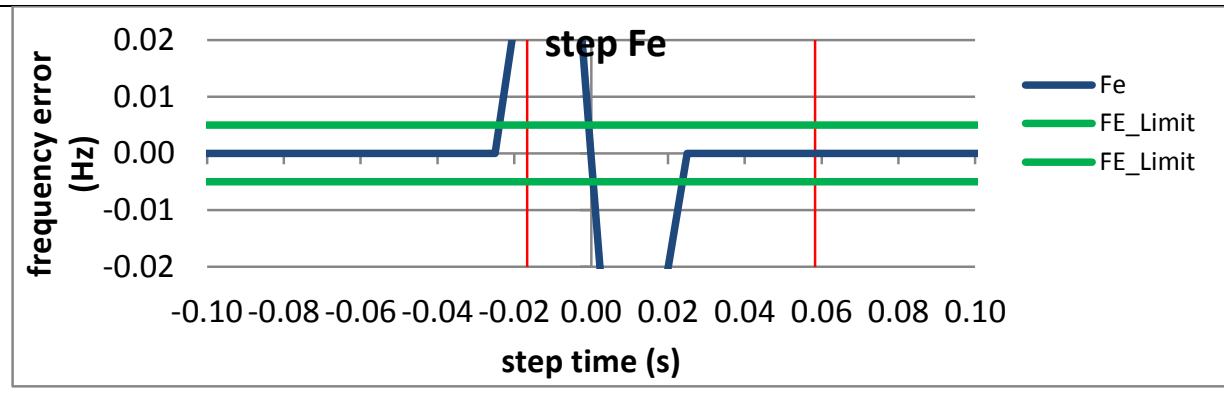

Figure 5768: Fs = 12 FPS, $+10 \%$ magnitude step

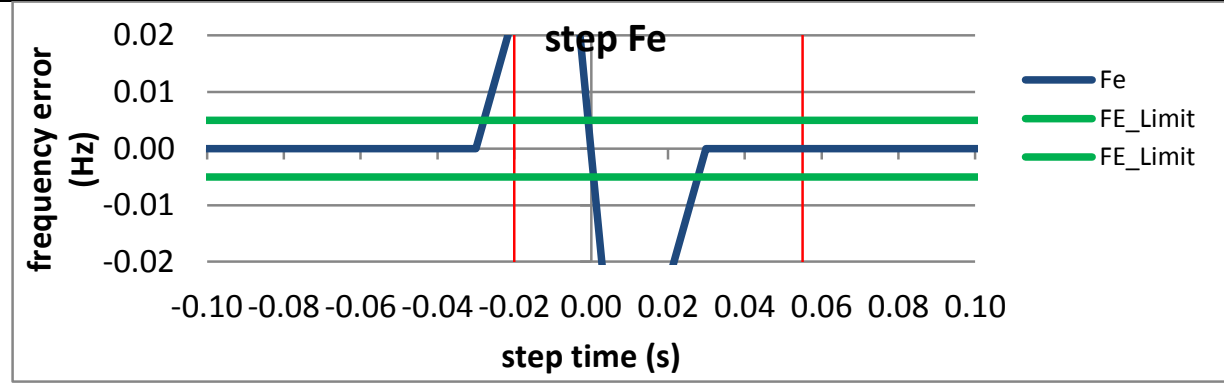

Figure 5770: Fs = 10 FPS, +10\% magnitude step

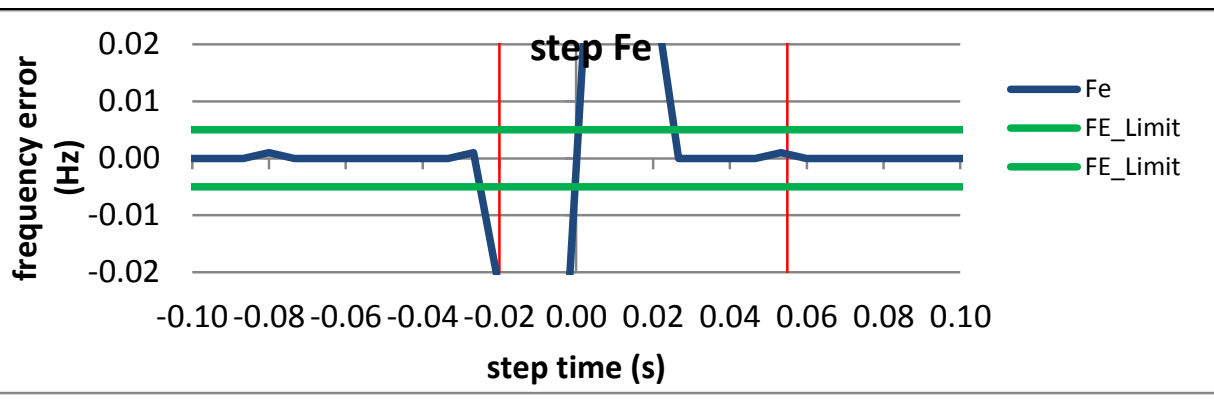

Figure 5767: Fs = 15 FPS, - $10 \%$ magnitude step

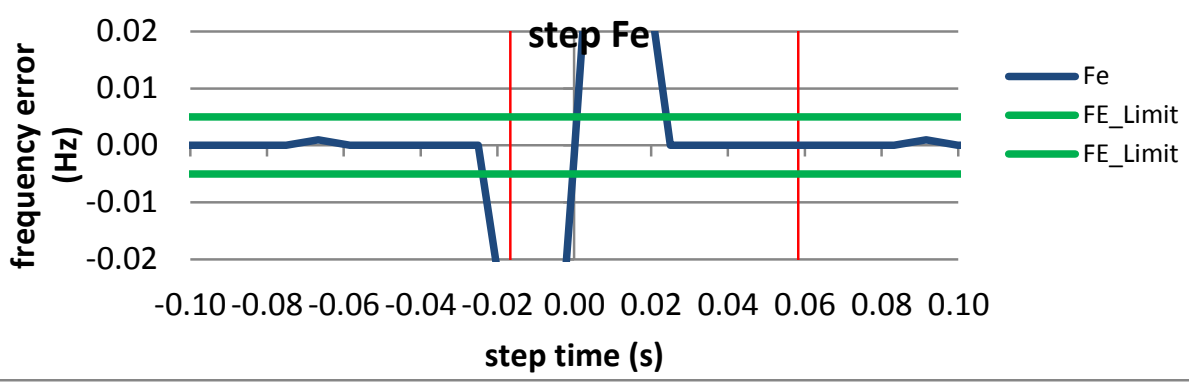

Figure 5769: Fs = 12 FPS, -10\% magnitude step

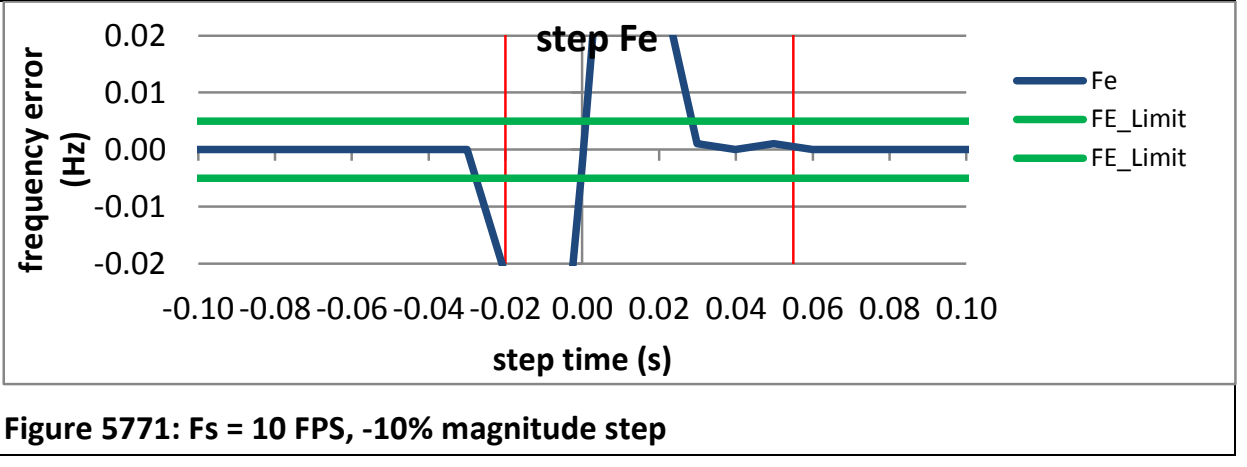


10.8 Dynamic step change in magnitude: ROCOF response time

\begin{tabular}{|l|c|c|c|c|c|c|c|c|c|c|c|c|}
\hline Fs (FPS) & $10 \mathrm{M}$ & $10 \mathrm{P}$ & $12 \mathrm{M}$ & $12 \mathrm{P}$ & $15 \mathrm{M}$ & $15 \mathrm{P}$ & $20 \mathrm{M}$ & $20 \mathrm{P}$ & $30 \mathrm{M}$ & $30 \mathrm{P}$ & $60 \mathrm{M}$ & $60 \mathrm{P}$ \\
\hline C37.118.1 Annex C & $\mathrm{P}$ & $\mathrm{P}$ & $\mathrm{P}$ & $\mathrm{P}$ & $\mathrm{P}$ & $\mathrm{P}$ & $\mathrm{P}$ & $\mathrm{P}$ & $\mathrm{P}$ & $\mathrm{P}$ & $\mathrm{P}$ & $\mathrm{P}$ \\
\hline PMU A & $\mathrm{P}$ & $\mathrm{F}$ & $\mathrm{P}$ & $\mathrm{F}$ & $\mathrm{P}$ & $\mathrm{F}$ & $\mathrm{P}$ & $\mathrm{F}$ & $\mathrm{P}$ & $\mathrm{F}$ & $\mathrm{F}$ & $\mathrm{F}$ \\
\hline PMU B & $\mathrm{P}$ & $\mathrm{P}$ & $\mathrm{P}$ & $\mathrm{P}$ & $\mathrm{P}$ & $\mathrm{P}$ & $\mathrm{P}$ & $\mathrm{P}$ & $\mathrm{P}$ & $\mathrm{P}$ & $\mathrm{P}$ & $\mathrm{P}$ \\
\hline PMU C & $\mathrm{P}$ & $\mathrm{P}$ & $\mathrm{P}$ & $\mathrm{P}$ & $\mathrm{P}$ & $\mathrm{P}$ & $\mathrm{P}$ & $\mathrm{I}$ & $\mathrm{P}$ & $\mathrm{P}$ & $\mathrm{P}$ & $\mathrm{P}$ \\
\hline PMU D & $\mathrm{P}$ & $\mathrm{P}$ & $\mathrm{P}$ & $\mathrm{P}$ & $\mathrm{P}$ & $\mathrm{P}$ & $\mathrm{P}$ & $\mathrm{P}$ & $\mathrm{P}$ & $\mathrm{P}$ & $\mathrm{P}$ & $\mathrm{P}$ \\
\hline PMU E & $\mathrm{P}$ & - & - & - & $\mathrm{P}$ & - & $\mathrm{P}$ & - & $\mathrm{P}$ & - & $\mathrm{P}$ & - \\
\hline PMU F & $\mathrm{P}$ & $\mathrm{P}$ & $\mathrm{P}$ & $\mathrm{P}$ & $\mathrm{P}$ & $\mathrm{P}$ & $\mathrm{P}$ & $\mathrm{P}$ & $\mathrm{P}$ & $\mathrm{P}$ & $\mathrm{P}$ & $\mathrm{P}$ \\
\hline PMU G* & $\mathrm{P}$ & - & $\mathrm{P}$ & - & $\mathrm{P}$ & - & $\mathrm{P}$ & - & $\mathrm{P}$ & - & - & - \\
\hline PMU H & $\mathrm{P}$ & $\mathrm{P}$ & $\mathrm{P}$ & $\mathrm{P}$ & $\mathrm{P}$ & $\mathrm{P}$ & $\mathrm{P}$ & $\mathrm{P}$ & $\mathrm{P}$ & $\mathrm{P}$ & $\mathrm{P}$ & $\mathrm{P}$ \\
\hline PMU I & $\mathrm{P}$ & - & $\mathrm{P}$ & - & $\mathrm{P}$ & - & $\mathrm{P}$ & - & $\mathrm{P}$ & - & $\mathrm{P}$ & - \\
\hline PMU J & $\mathrm{P}$ & $\mathrm{P}$ & $\mathrm{P}$ & $\mathrm{P}$ & $\mathrm{P}$ & $\mathrm{P}$ & $\mathrm{P}$ & $\mathrm{P}$ & $\mathrm{P}$ & $\mathrm{P}$ & $\mathrm{P}$ & $\mathrm{P}$ \\
\hline
\end{tabular}

$\mathrm{P}=$ Pass, $\mathrm{F}=$ Fail, $\mathrm{I}=$ Indeterminate

* PMU G always outputs ROCOF $=0$.

10.9 Dynamic step change in magnitude ROCOF error: $F 0=60 \mathrm{~Hz}$, M class

10.9.1 C37.118.1-2011 Annex $C$ dynamic step change in magnitude ROCOF error: $F 0=60 \mathrm{~Hz}$, M class
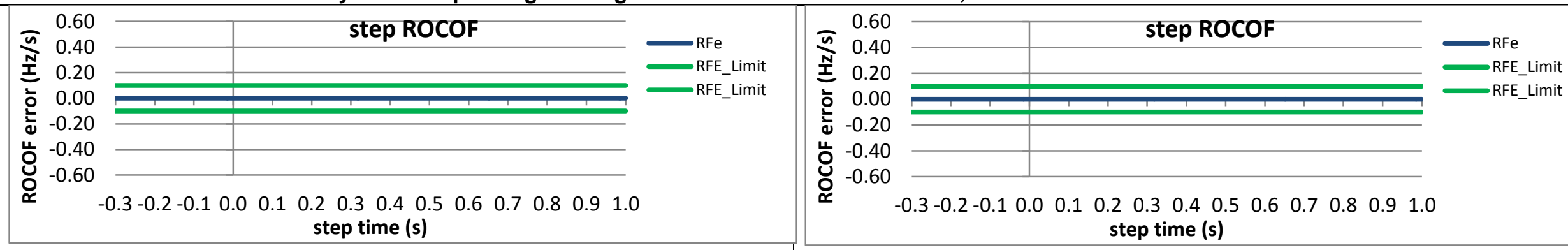

Figure 5772: Fs $=60 \mathrm{FPS},+10 \%$ magnitude step

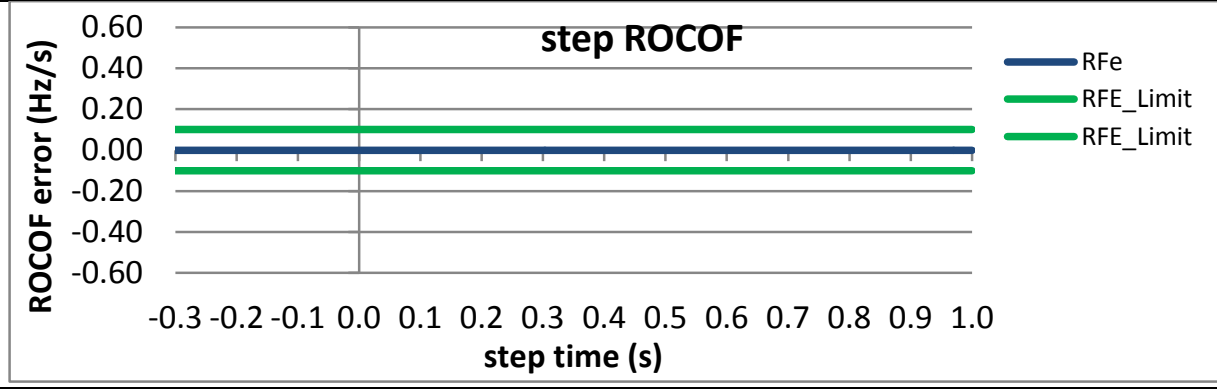

Figure 5773: Fs = 60 FPS, -10\% magnitude step

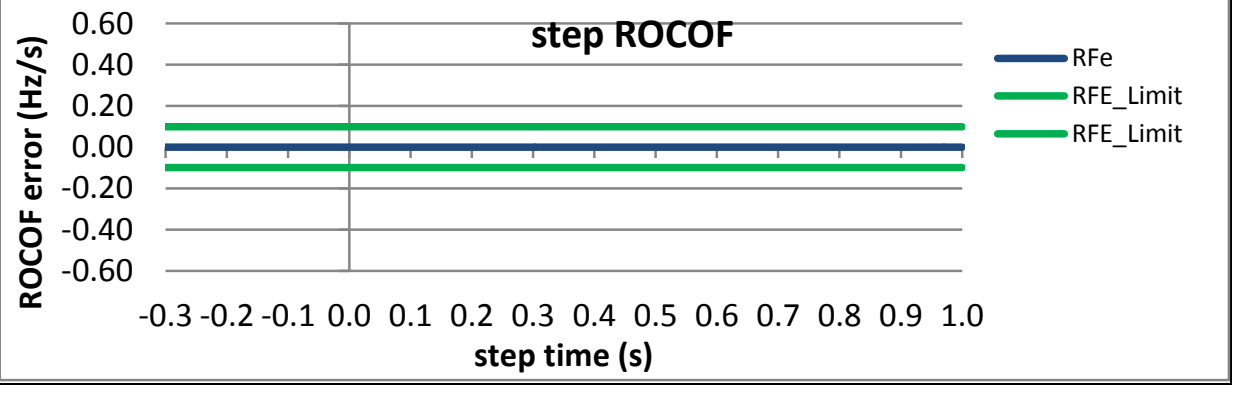




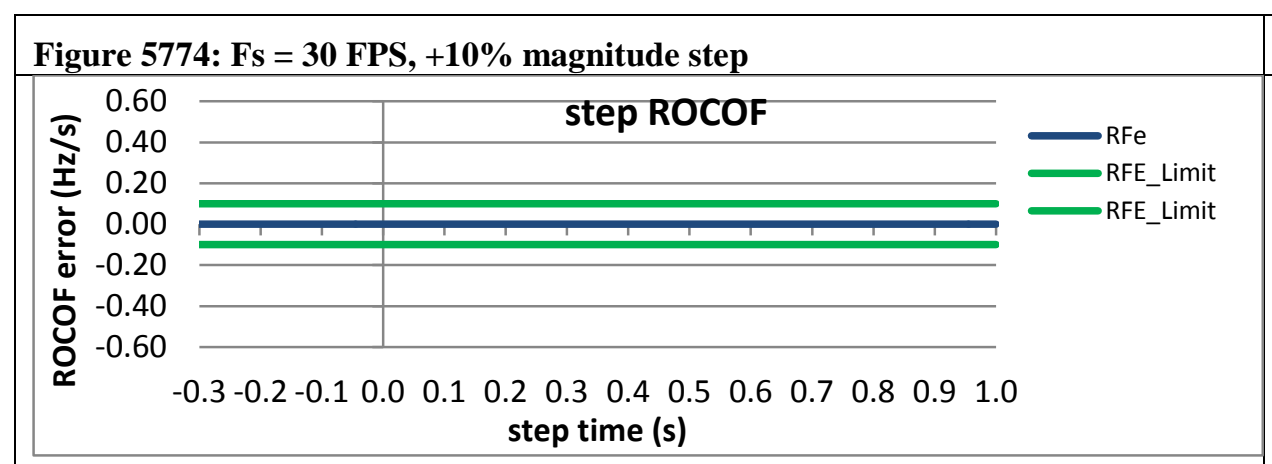

Figure 5775: Fs = 30 FPS, -10\% magnitude step

Figure 5776: Fs $=20$ FPS, $+\mathbf{1 0} \%$ magnitude step
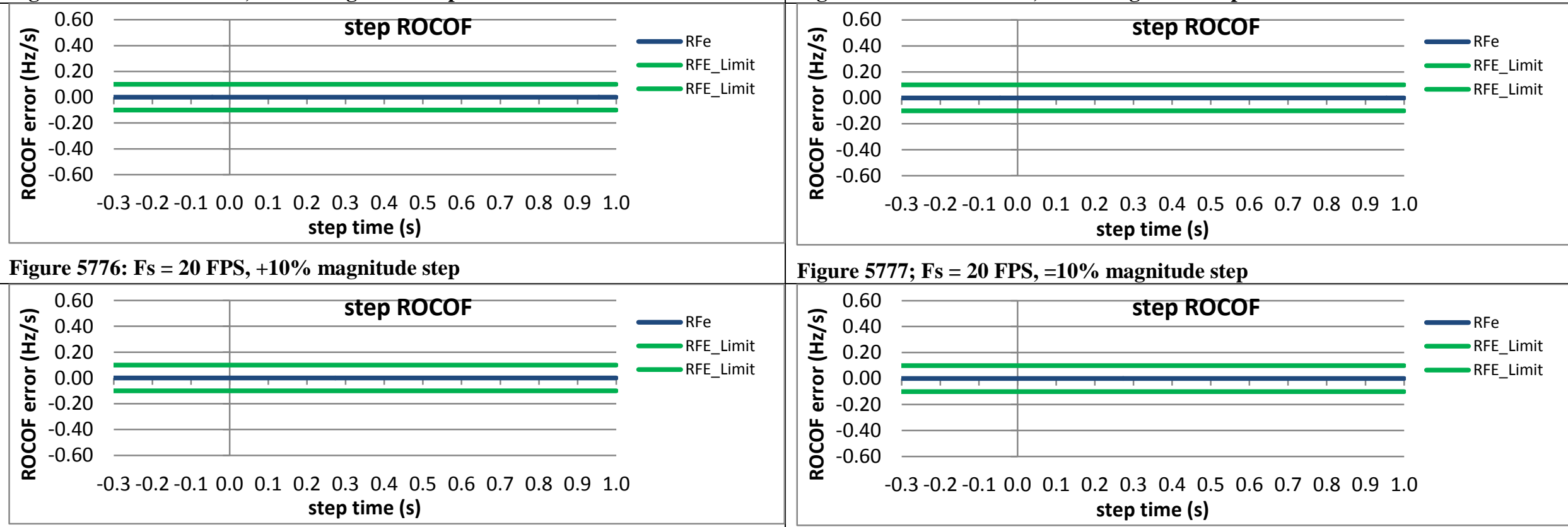

Figure 5777; Fs = 20 FPS, =10\% magnitude step

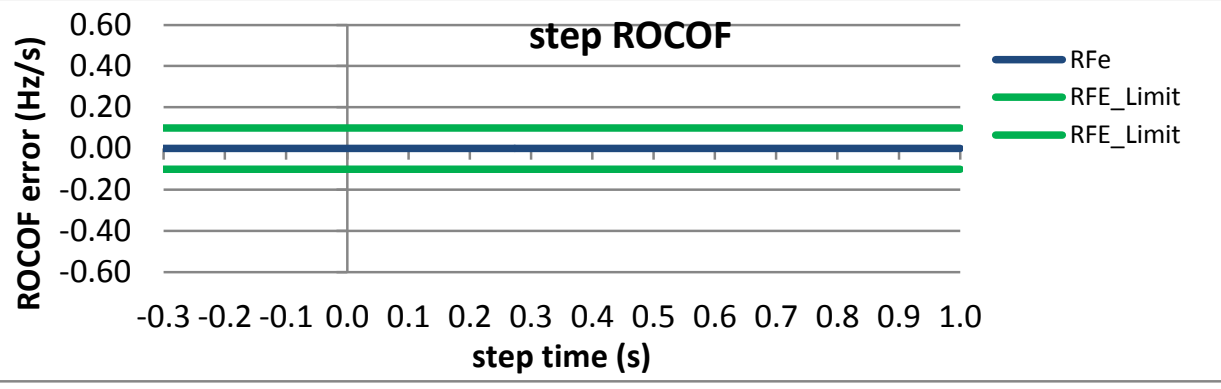

Figure 5778: Fs = 15 FPS, $+10 \%$ magnitude step

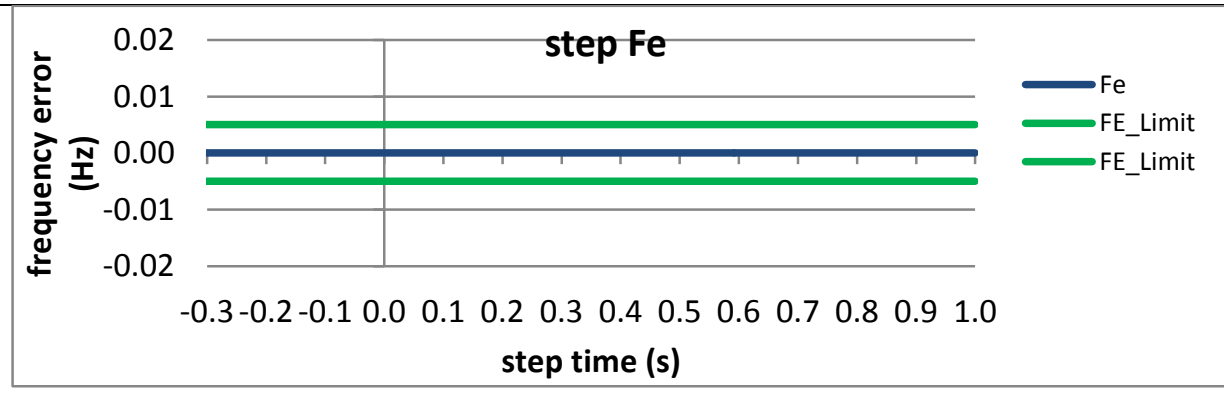

Figure 5779: Fs = 15 FPS, - $10 \%$ magnitude step

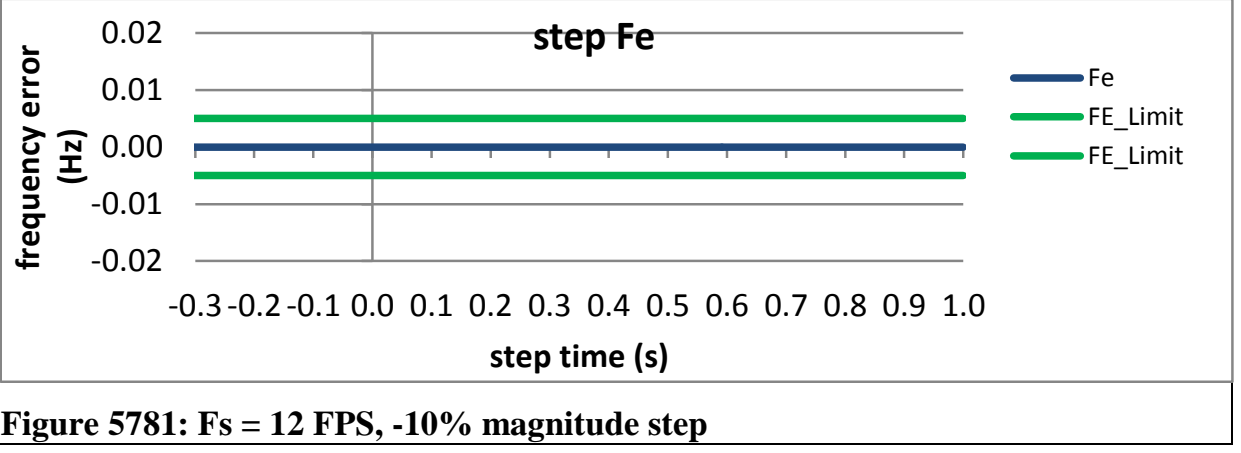




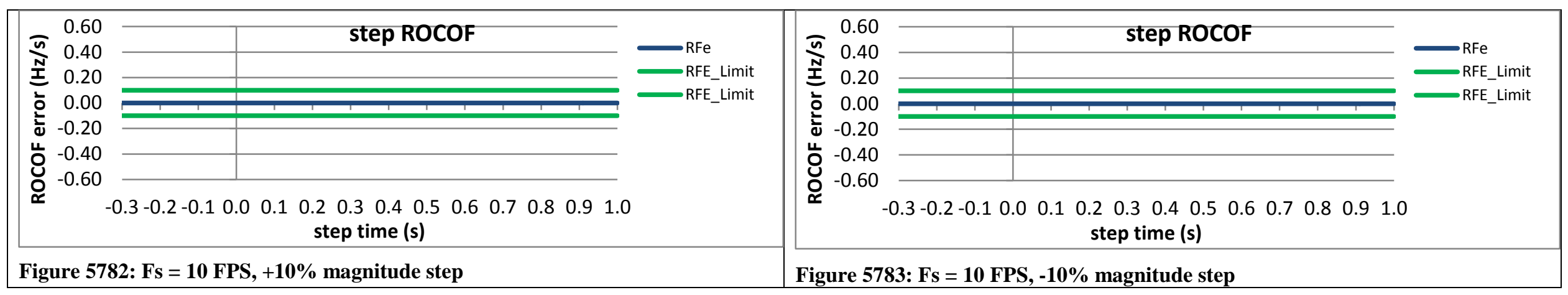

10.9.2 PMU A dynamic step change in magnitude ROCOF response time: $F 0=60 \mathrm{~Hz}, \mathrm{M}$ class

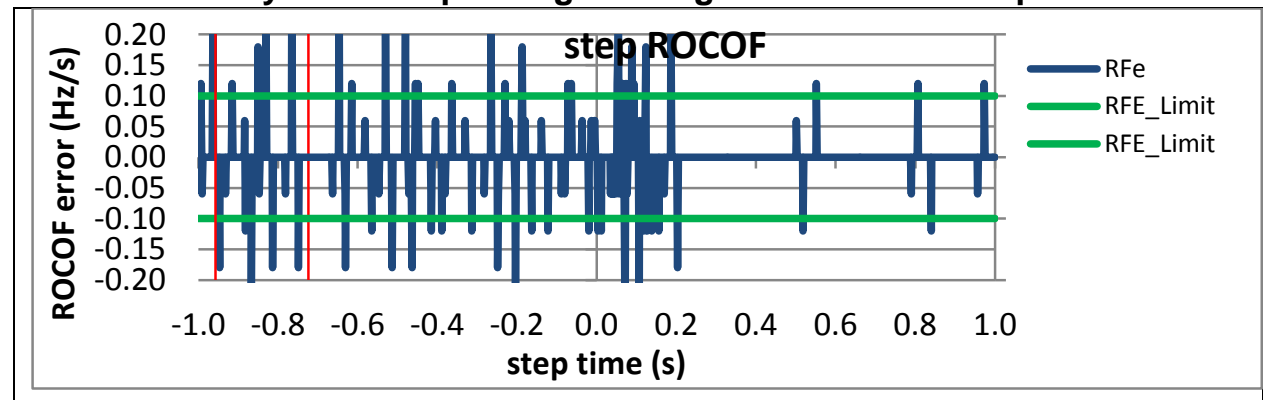

Figure 5784: Fs = 60 FPS, $+10 \%$ magnitude step

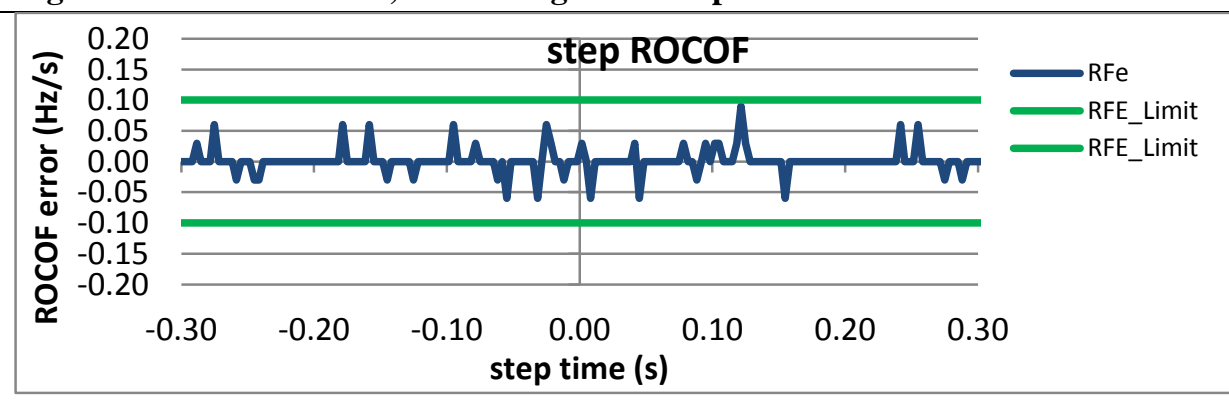

Figure 5786: Fs = 30 FPS, $+10 \%$ magnitude step

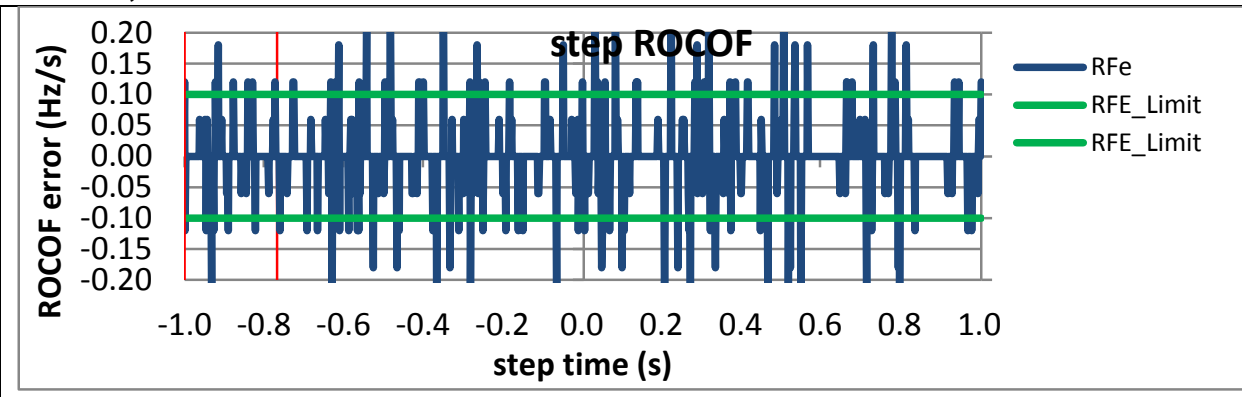

Figure 5785: Fs = 60 FPS, -10\% magnitude step

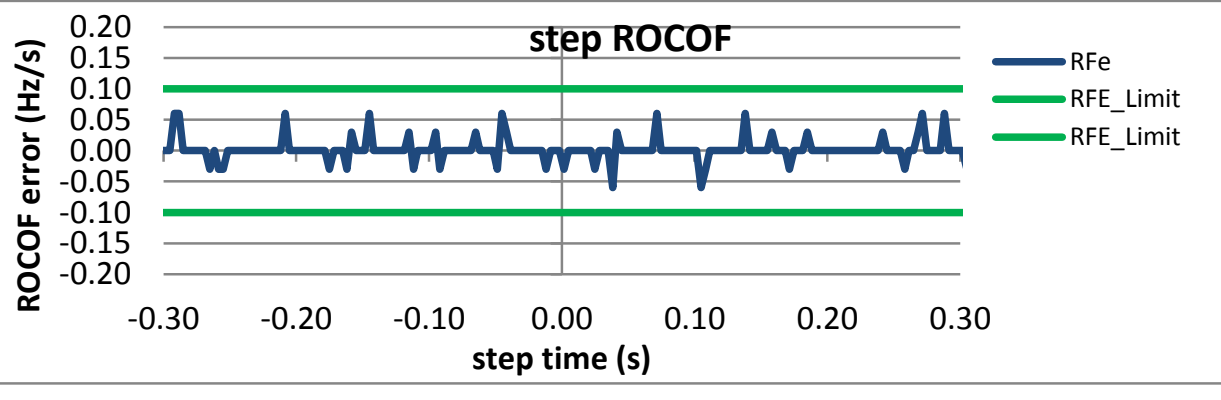

Figure 5787: Fs = 30 FPS, -10\% magnitude step 

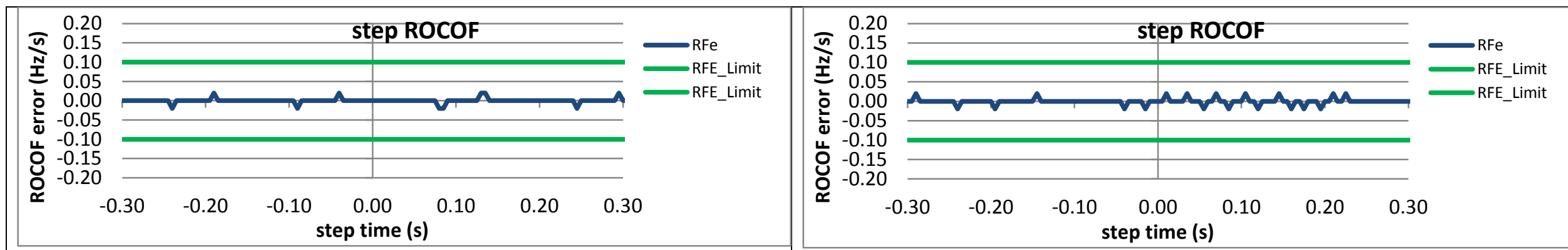

Figure 5788: Fs $=20$ FPS, $+10 \%$ magnitude step

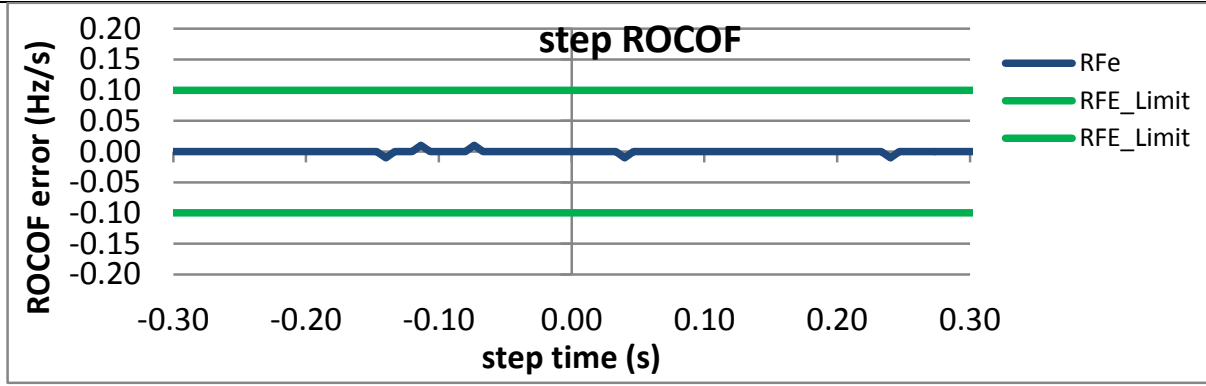

Figure 5789; Fs = 20 FPS, =10\% magnitude step

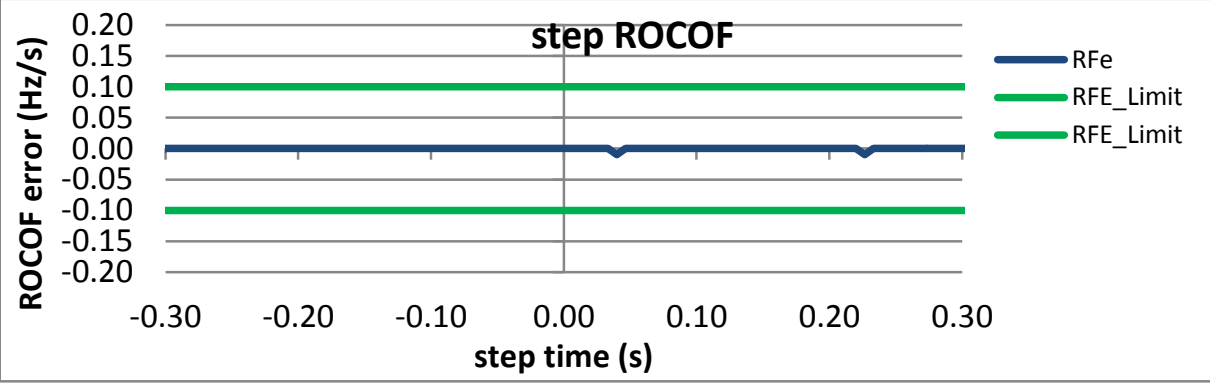

Figure 5790: Fs $=15$ FPS, $+10 \%$ magnitude step

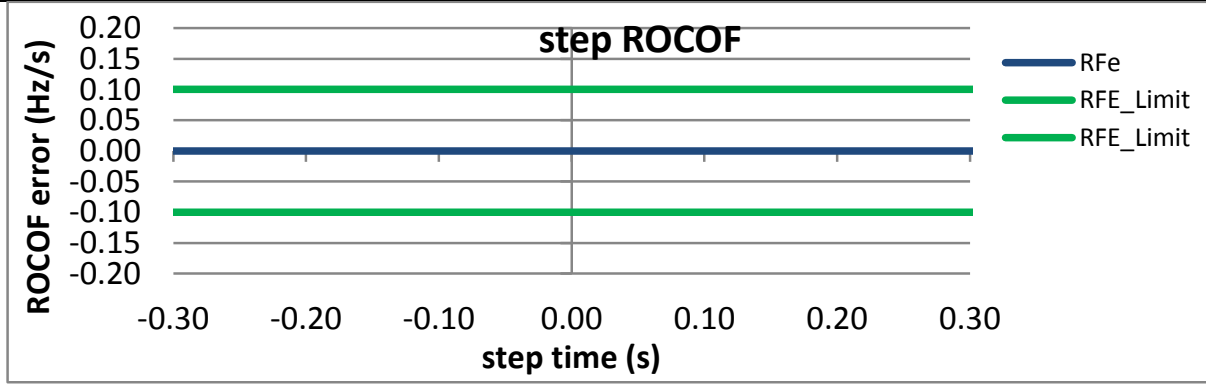

Figure 5791: Fs = 15 FPS, - $10 \%$ magnitude step

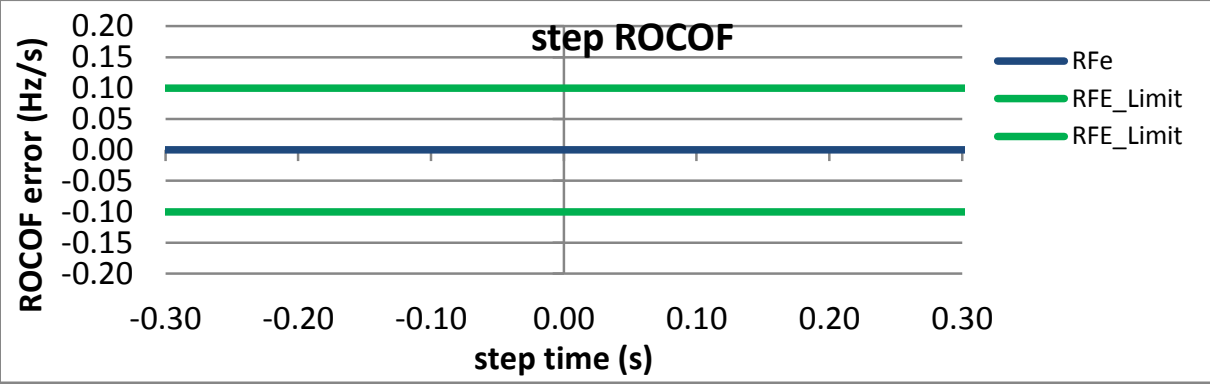

Figure 5793: Fs = 12 FPS, -10\% magnitude step 


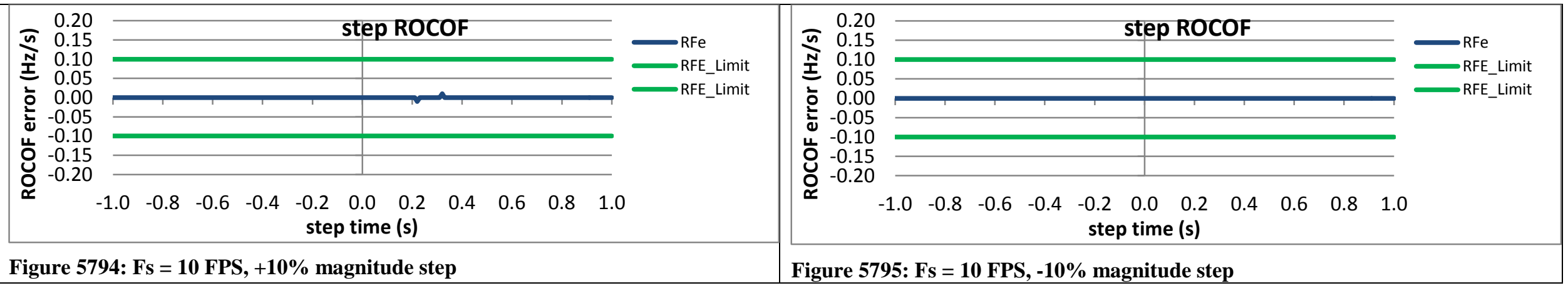


10.9.3 PMU B dynamic step change in magnitude ROCOF response time: $F 0=60 \mathrm{~Hz}, \mathrm{M}$ class
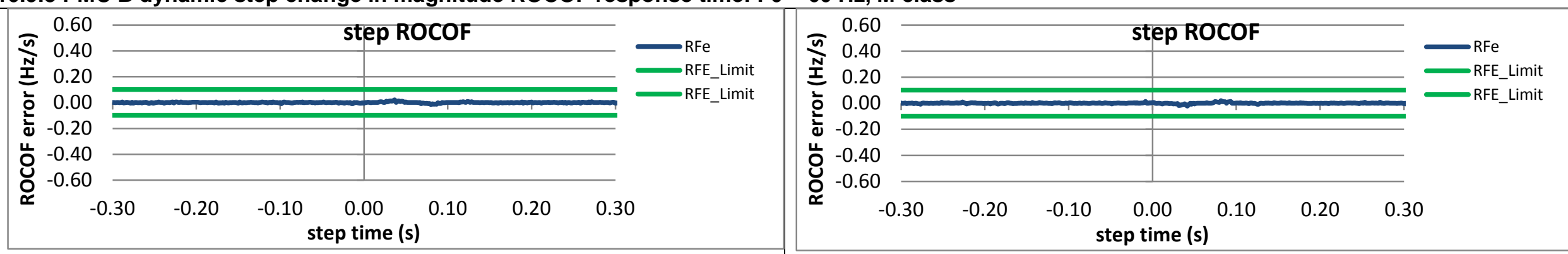

Figure 5796: Fs = 60 FPS, +10\% magnitude step

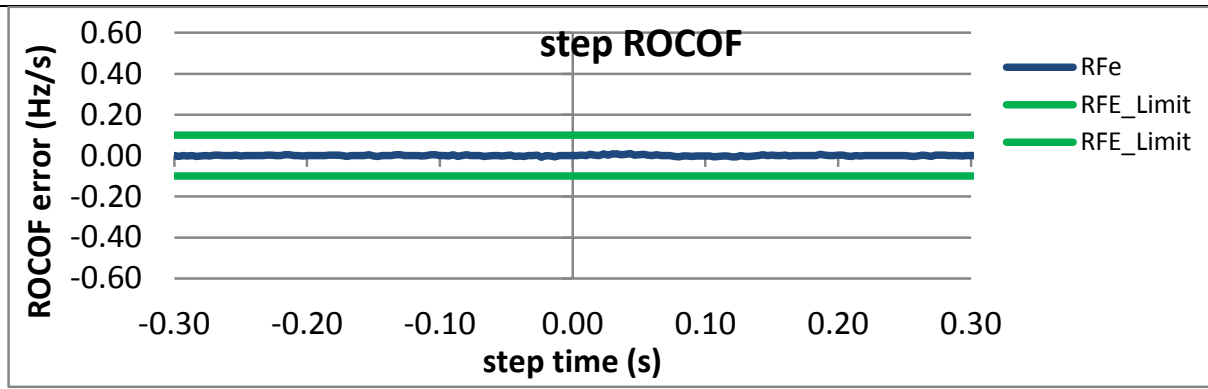

Figure 5797: Fs = 60 FPS, -10\% magnitude step

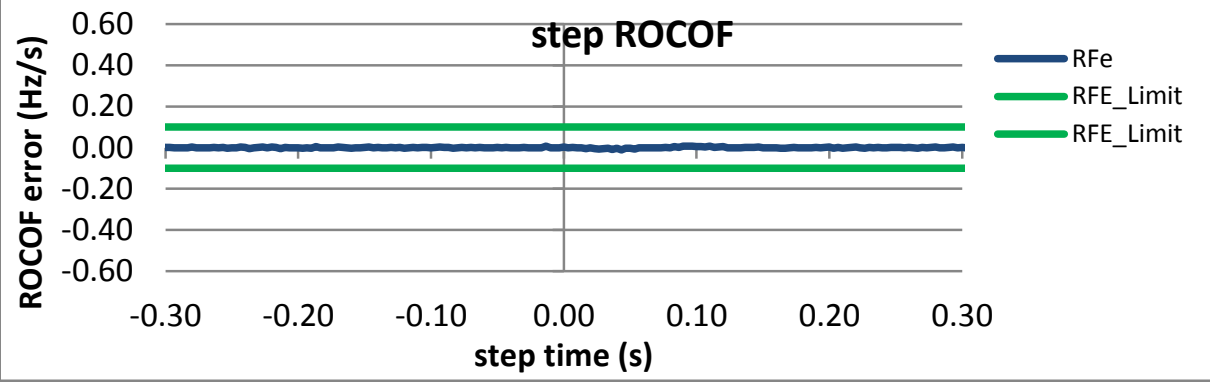

Figure 5798: Fs $=30$ FPS, $+10 \%$ magnitude step

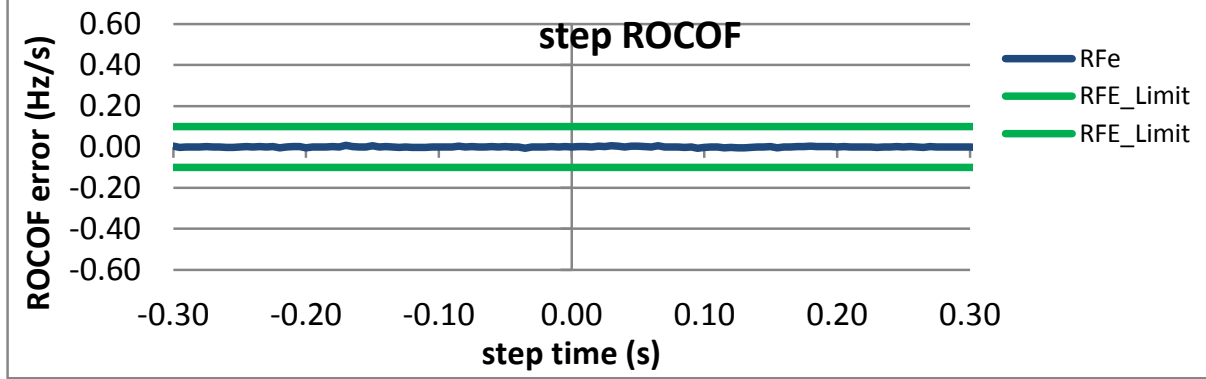

Figure 5799: Fs = 30 FPS, -10\% magnitude step

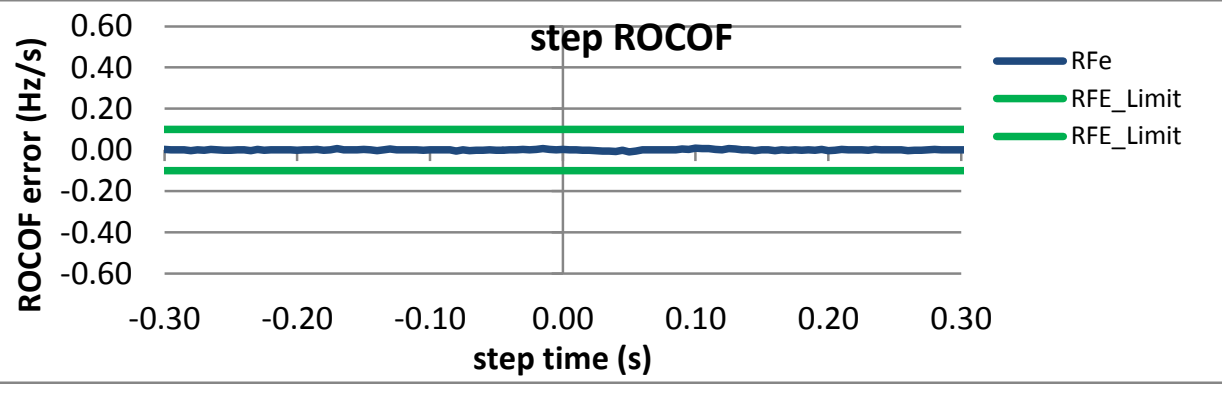

Figure 5800: Fs $=20$ FPS, $+10 \%$ magnitude step

Figure 5801; Fs = 20 FPS, =10\% magnitude step 

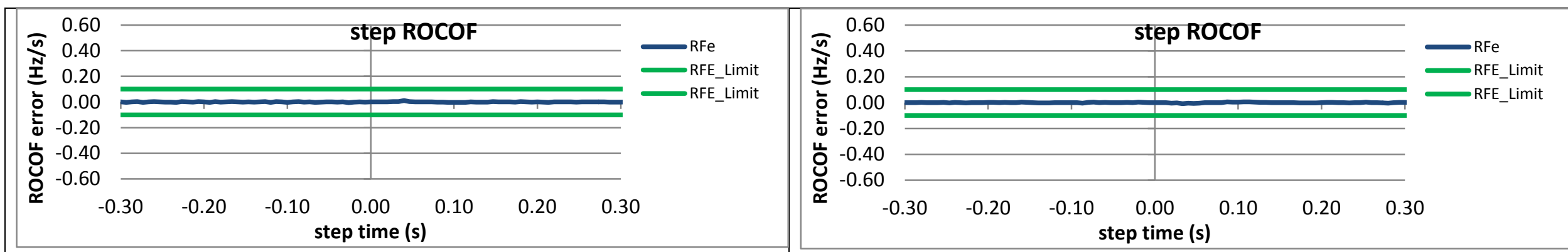

Figure 5802: Fs = 15 FPS, + 10\% magnitude step

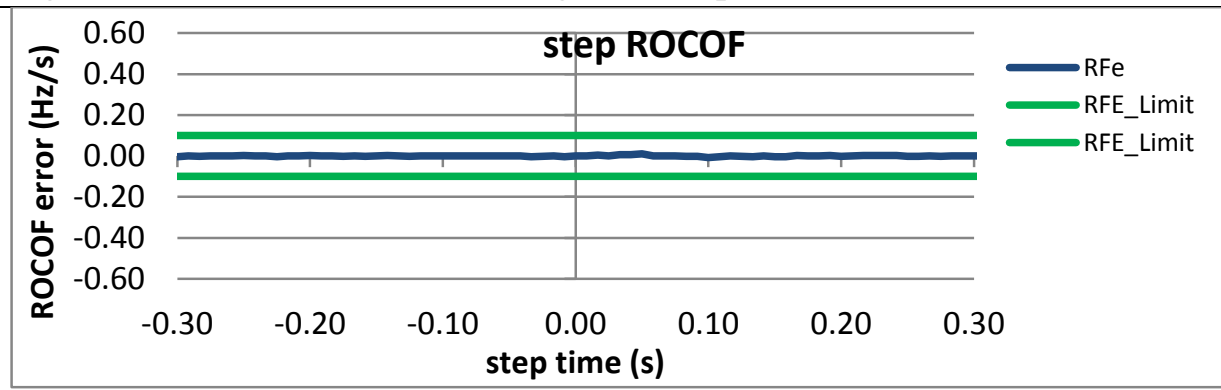

Figure 5803: Fs = 15 FPS, - $10 \%$ magnitude step

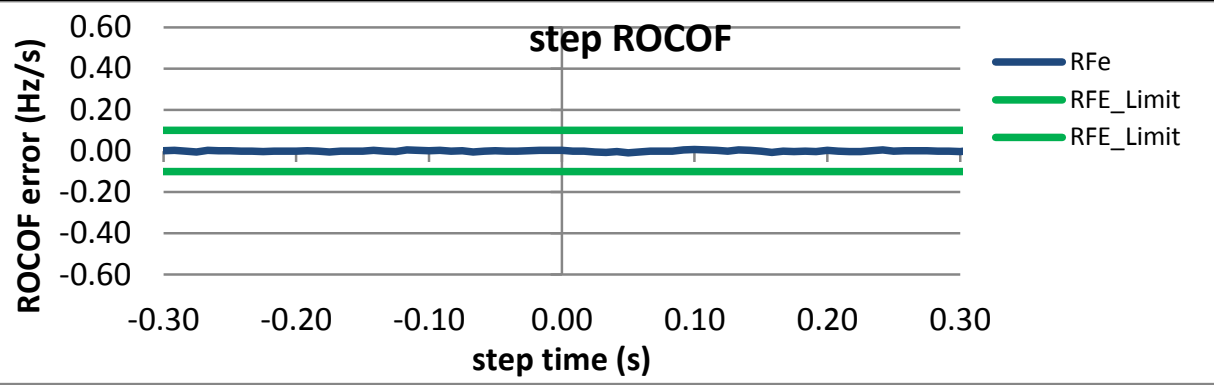

Figure 5804: Fs = 12 FPS, +10\% magnitude step

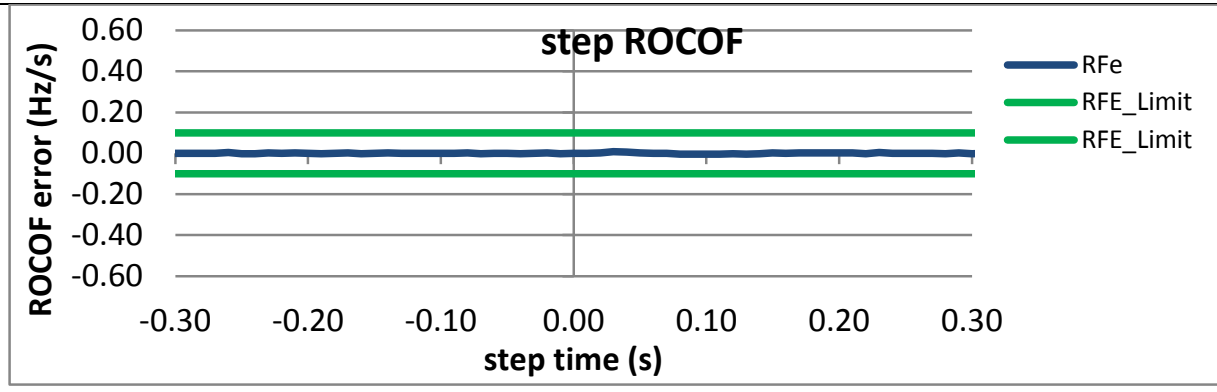

Figure 5805: Fs = 12 FPS, - $10 \%$ magnitude step

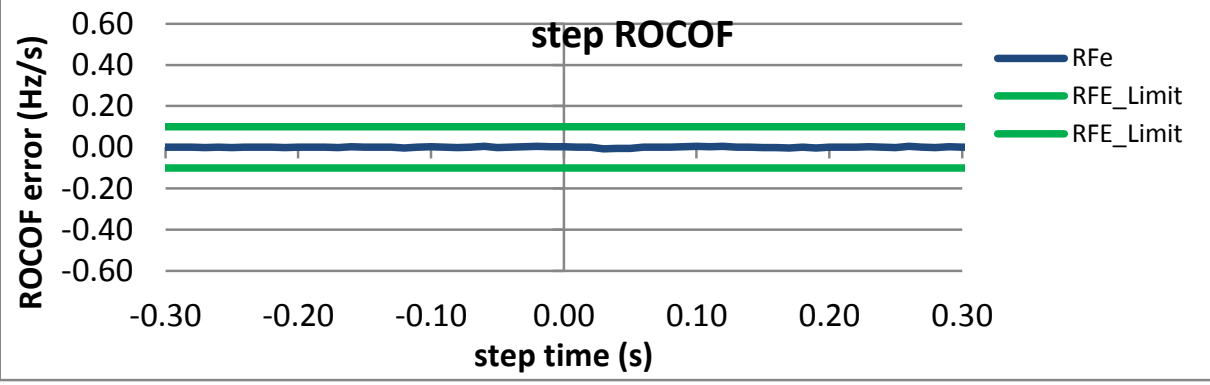

Figure 5807: Fs = 10 FPS, -10\% magnitude step 
10.9.4 PMU C dynamic step change in magnitude ROCOF response time: $\mathrm{F} 0=60 \mathrm{~Hz}, \mathrm{M}$ class
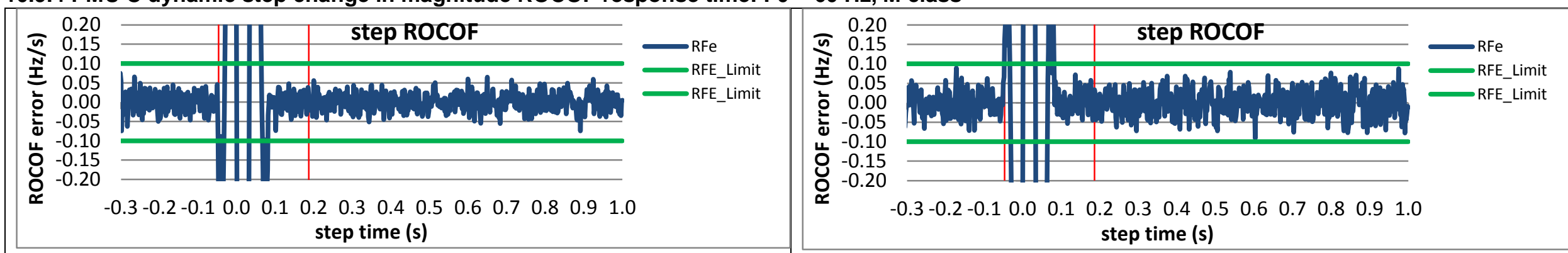

Figure 5808: Fs = 60 FPS, +10\% magnitude step

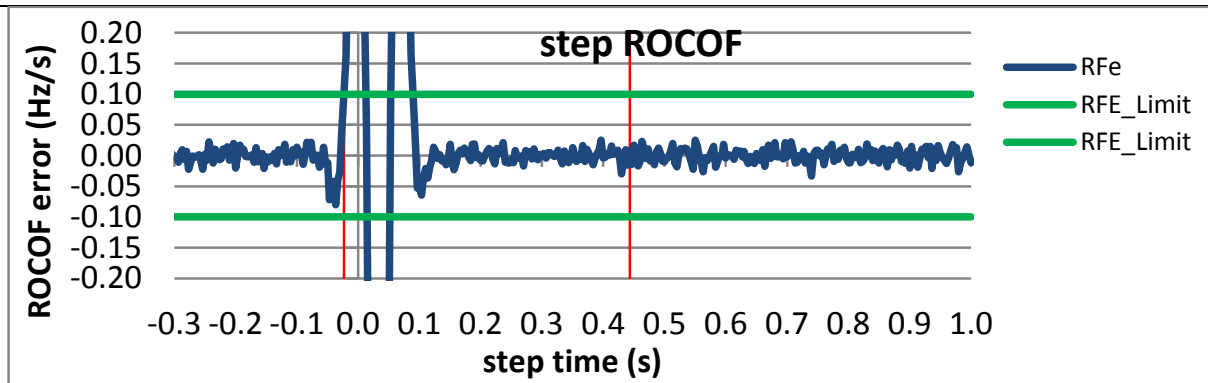

Figure 5809: Fs = 60 FPS, -10\% magnitude step

Figure 5810: Fs = 30 FPS, +10\% magnitude step
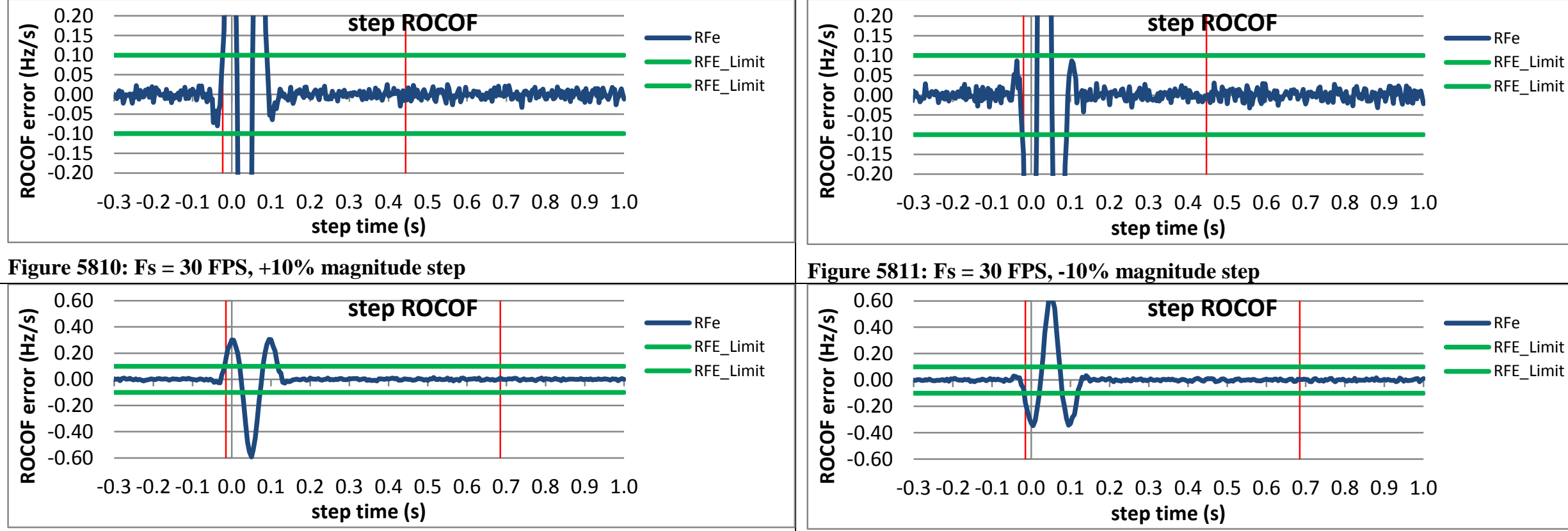

Figure 5811: Fs = 30 FPS, -10\% magnitude step

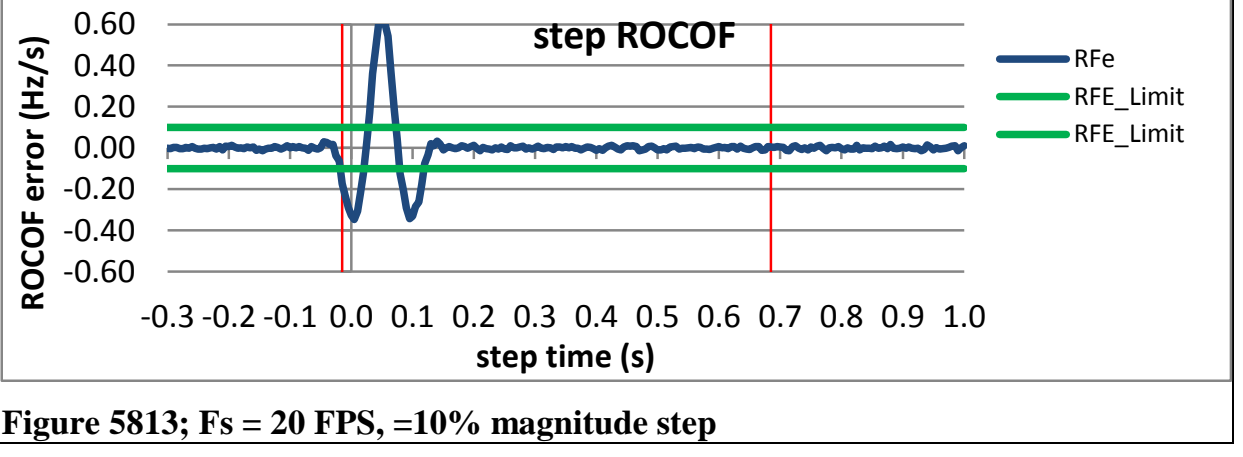

Figure 5812: Fs = 20 FPS, +10\% magnitude step

Figure 5813; Fs = 20 FPS, =10\% magnitude step 

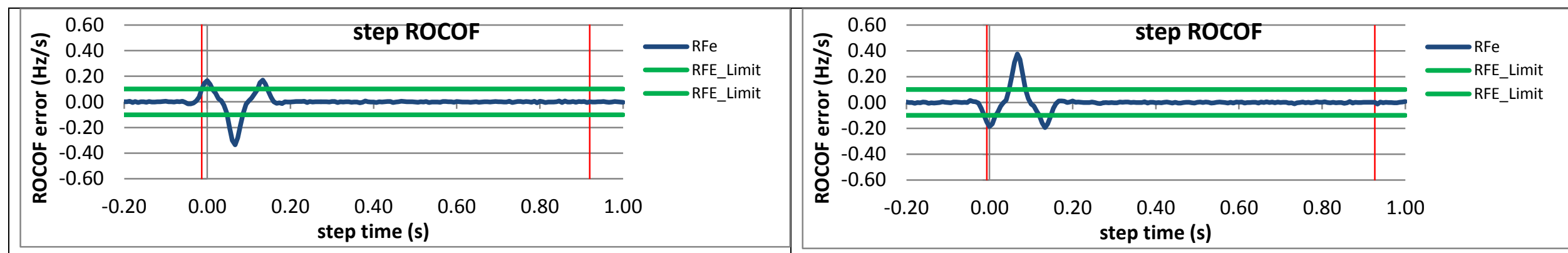

Figure 5814: Fs = 15 FPS, + 10\% magnitude step

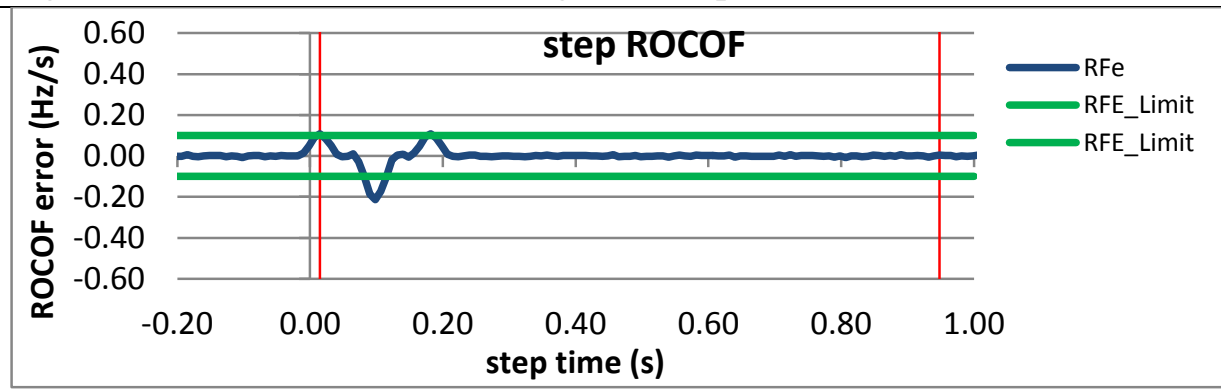

Figure 5815: Fs = 15 FPS, - $10 \%$ magnitude step

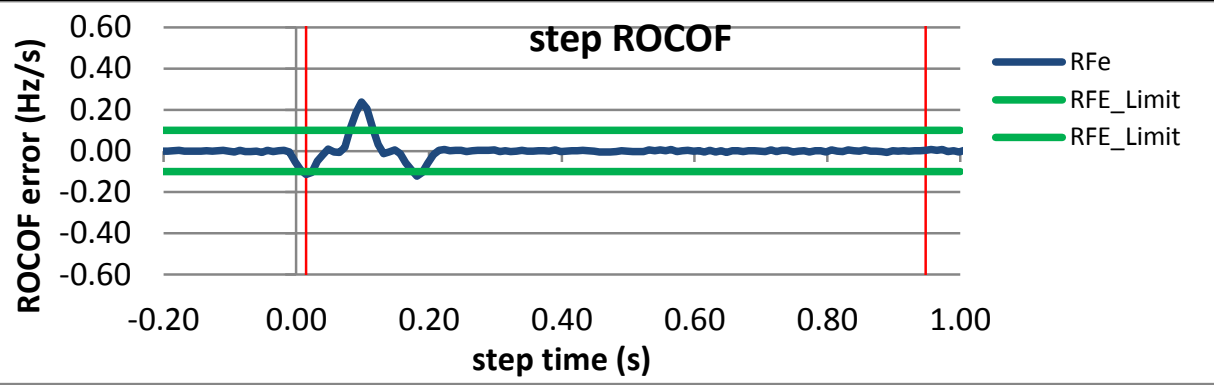

Figure 5816: Fs $=12$ FPS, $+10 \%$ magnitude step

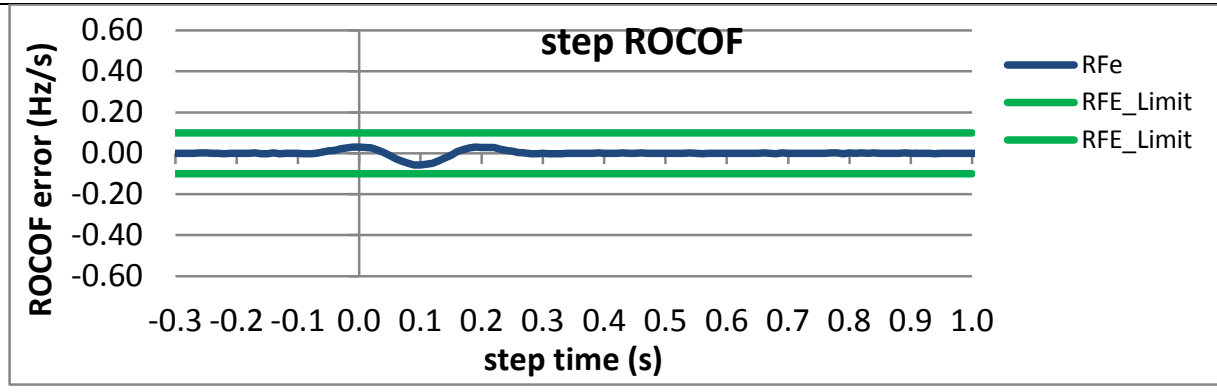

Figure 5817: Fs = 12 FPS, - $10 \%$ magnitude step

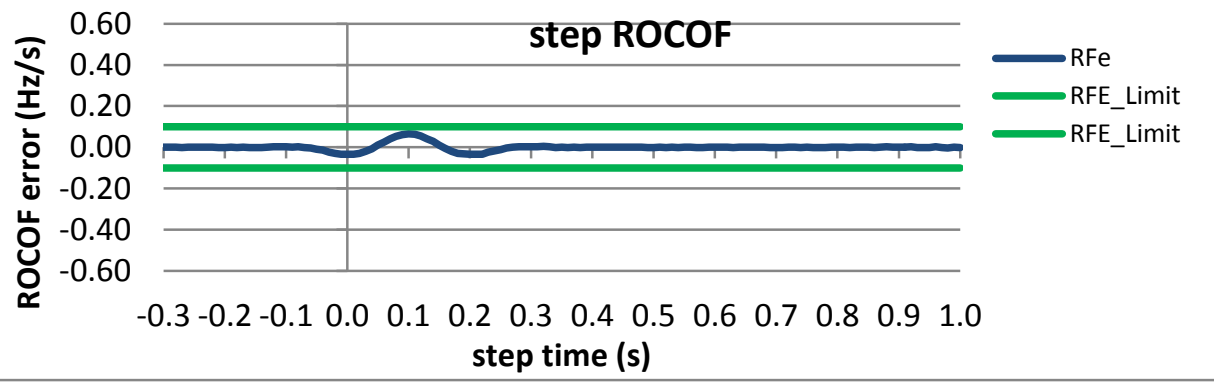

Figure 5819: Fs = 10 FPS, -10\% magnitude step 
10.9.5 PMU D dynamic step change in magnitude ROCOF response time: $F 0=60 \mathrm{~Hz}, \mathrm{M}$ class
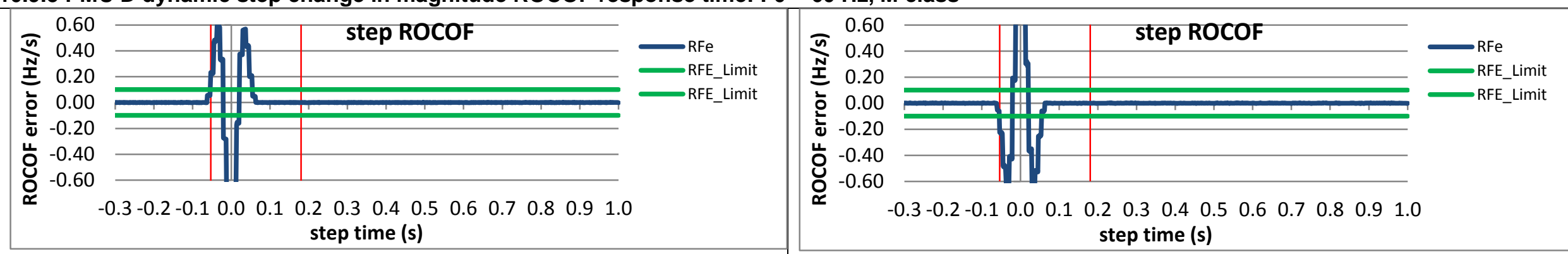

Figure 5820: Fs = 60 FPS, +10\% magnitude step

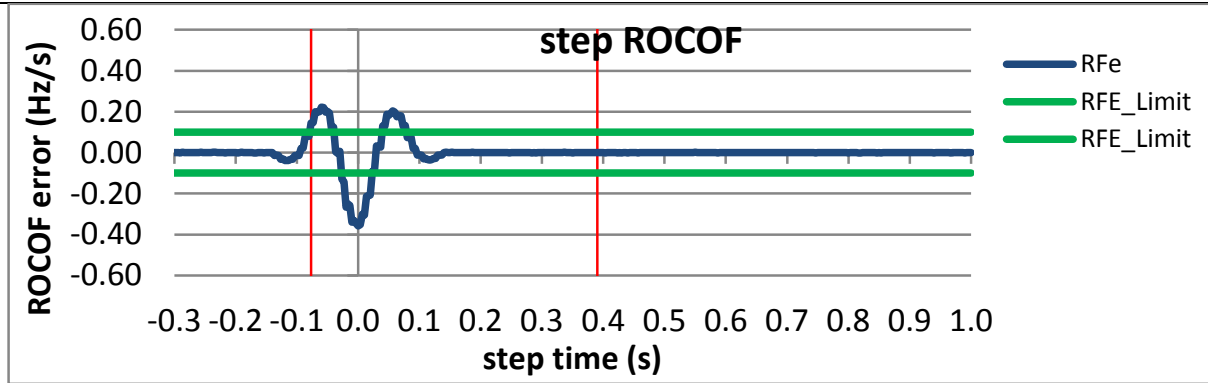

Figure 5821: Fs = 60 FPS, -10\% magnitude step
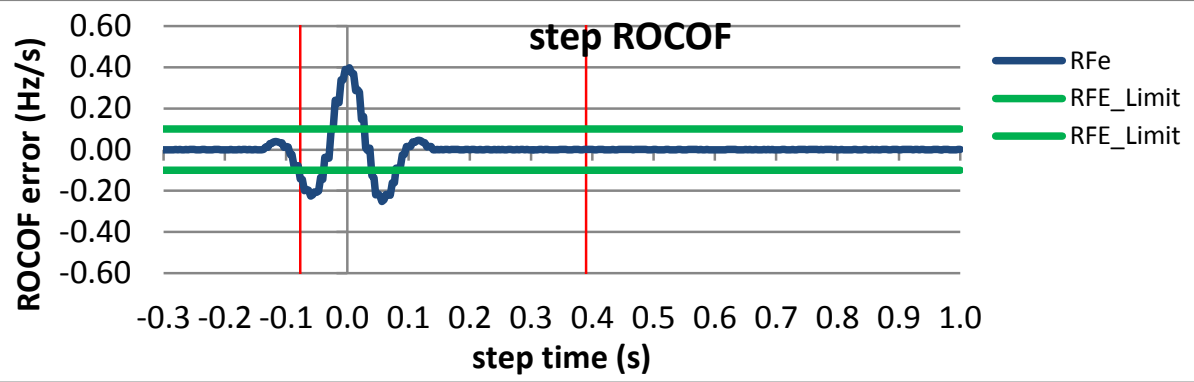

Figure 5822: Fs $=30$ FPS, +10\% magnitude step

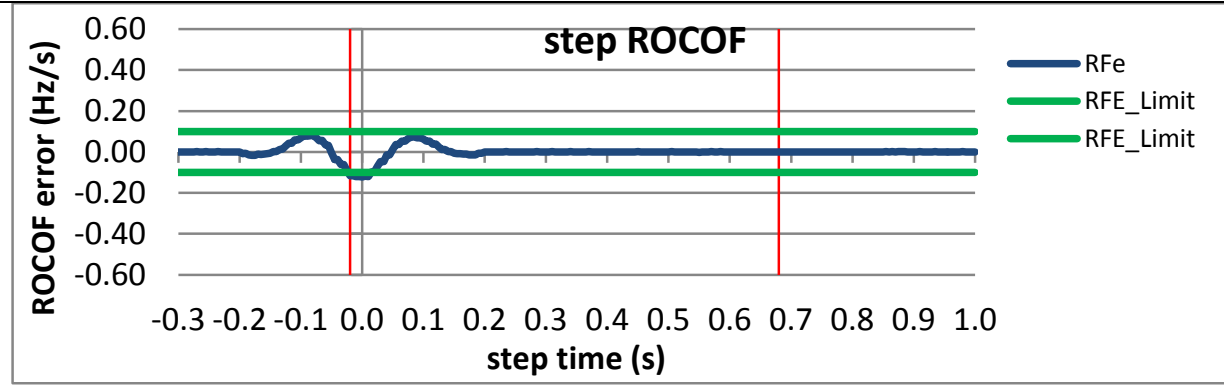

Figure 5823: Fs = 30 FPS, -10\% magnitude step

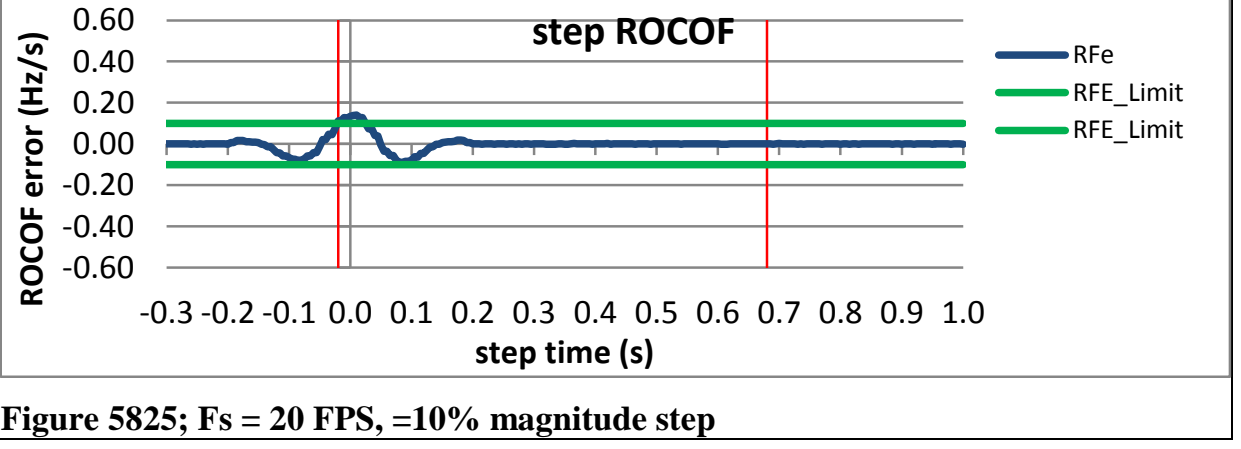

Figure 5824: Fs = 20 FPS, +10\% magnitude step

Figure 5825; Fs $=20$ FPS, $=10 \%$ magnitude step 

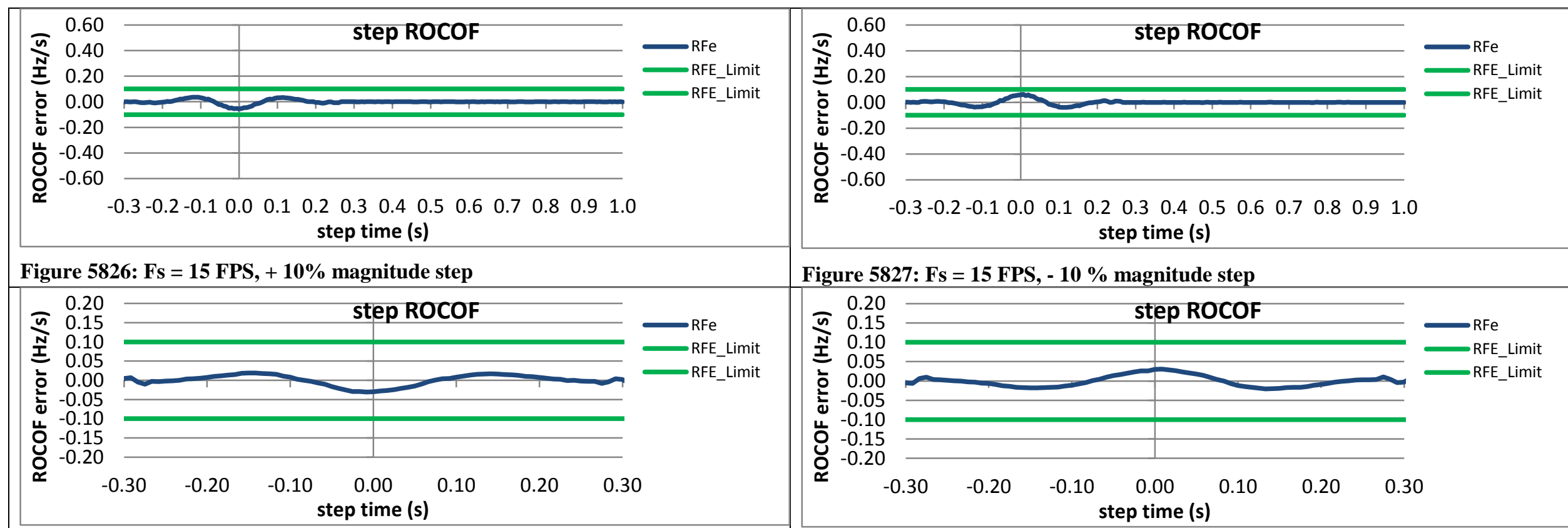

Figure 5827: Fs = 15 FPS, - $10 \%$ magnitude step

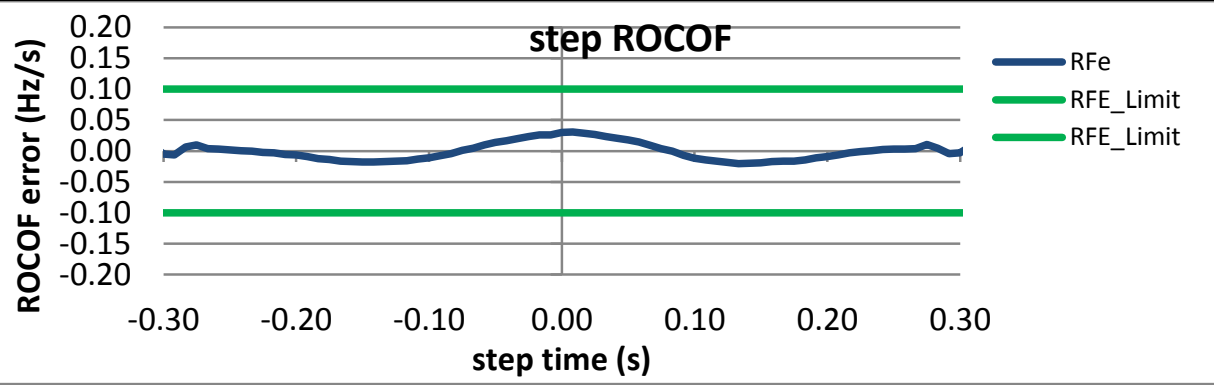

Figure 5828: Fs = 12 FPS, $+10 \%$ magnitude step

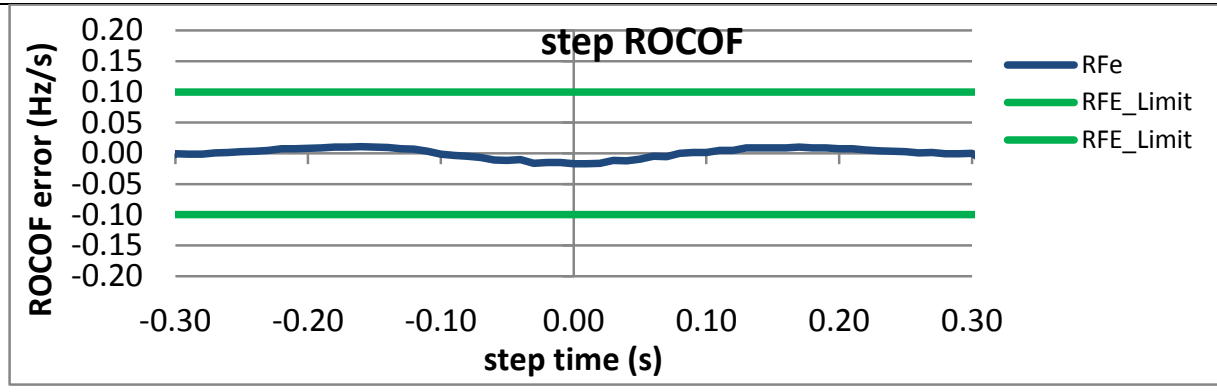

Figure 5829: Fs = 12 FPS, -10\% magnitude step

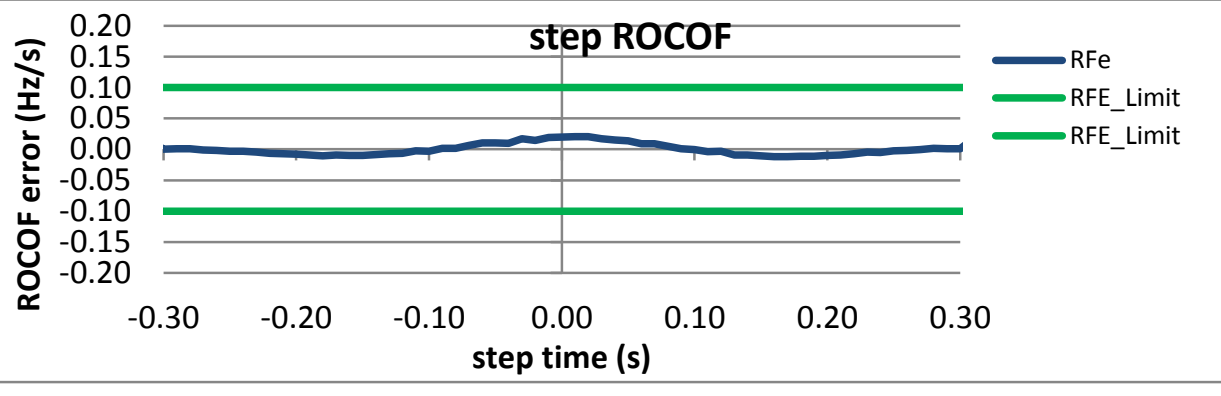

Figure 5831: Fs = 10 FPS, -10\% magnitude step 
10.9.6 PMU E dynamic step change in magnitude ROCOF response time: $\mathrm{FO}=60 \mathrm{~Hz}, \mathrm{M}$ class
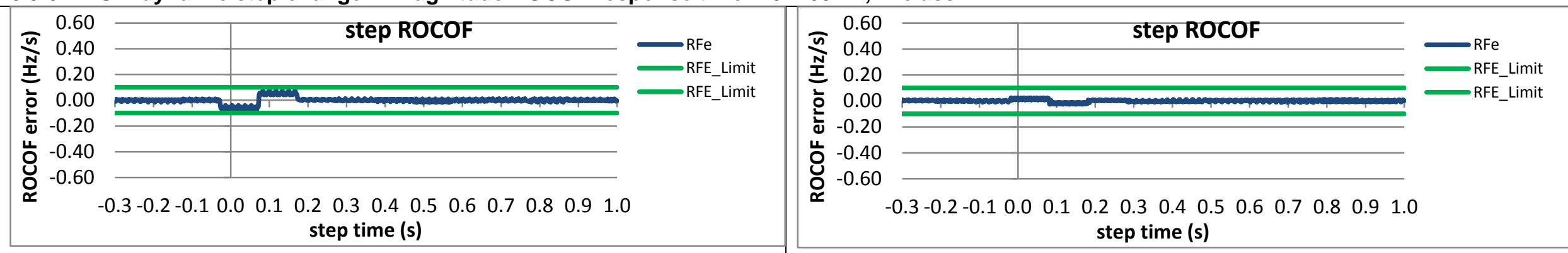

Figure 5832: Fs $=60$ FPS, $+10 \%$ magnitude step

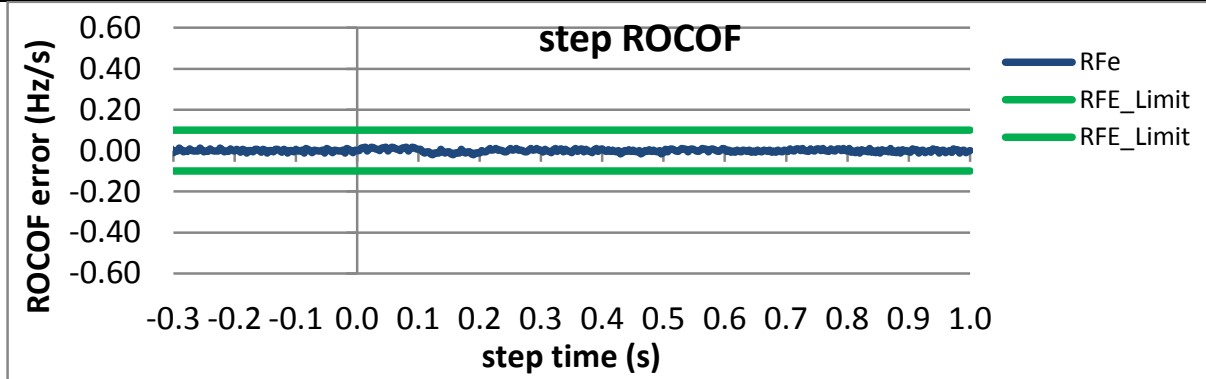

Figure 5833: Fs = 60 FPS, -10\% magnitude step
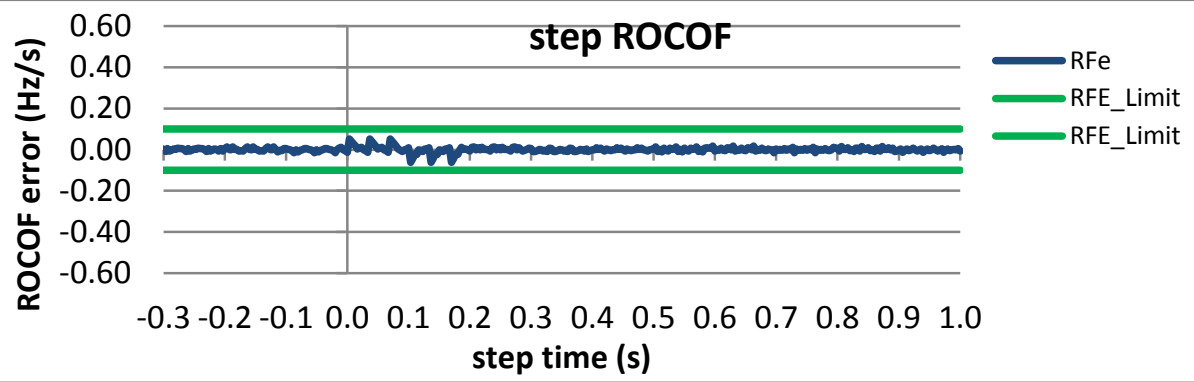

Figure 5834: Fs = 30 FPS, +10\% magnitude step

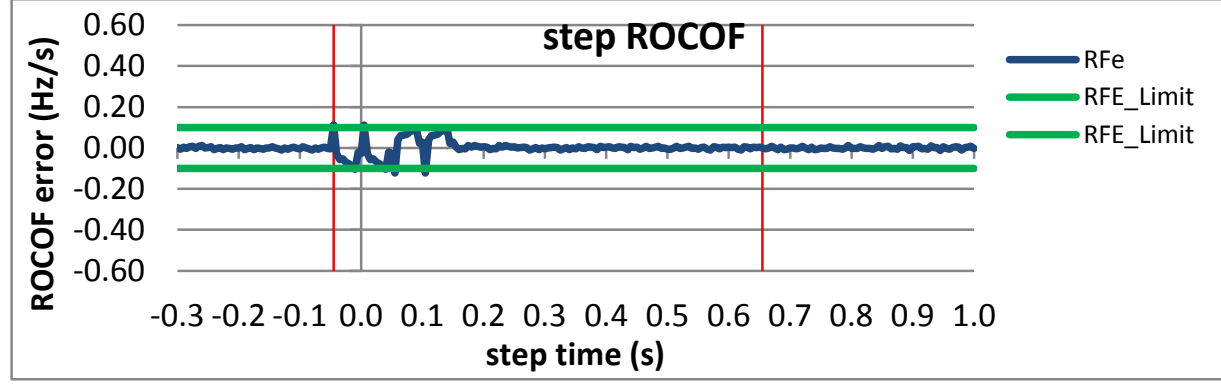

Figure 5835: Fs = 30 FPS, -10\% magnitude step

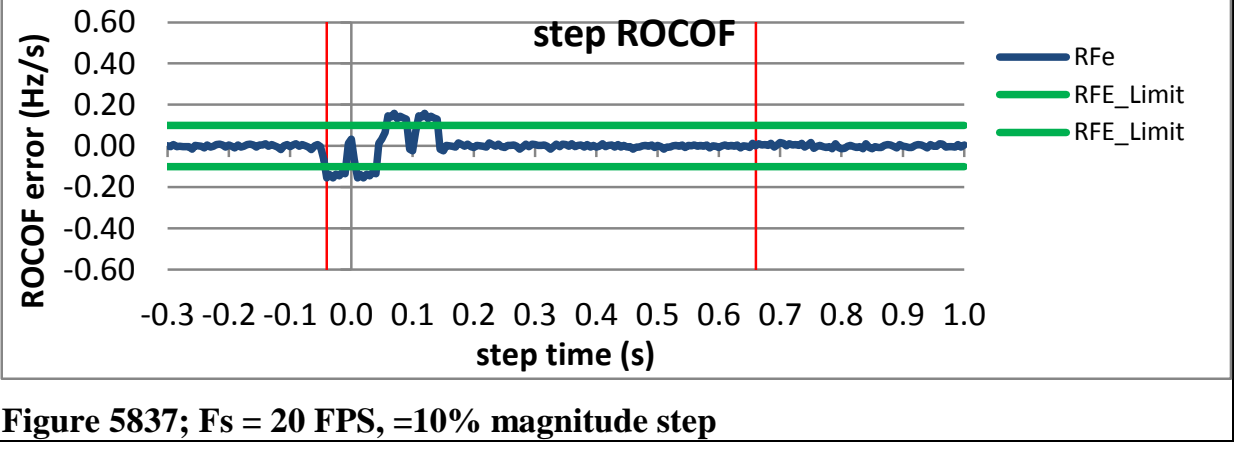

Figure 5836: Fs $=20$ FPS, $+10 \%$ magnitude step

Figure 5837; Fs $=20$ FPS, $=10 \%$ magnitude step 


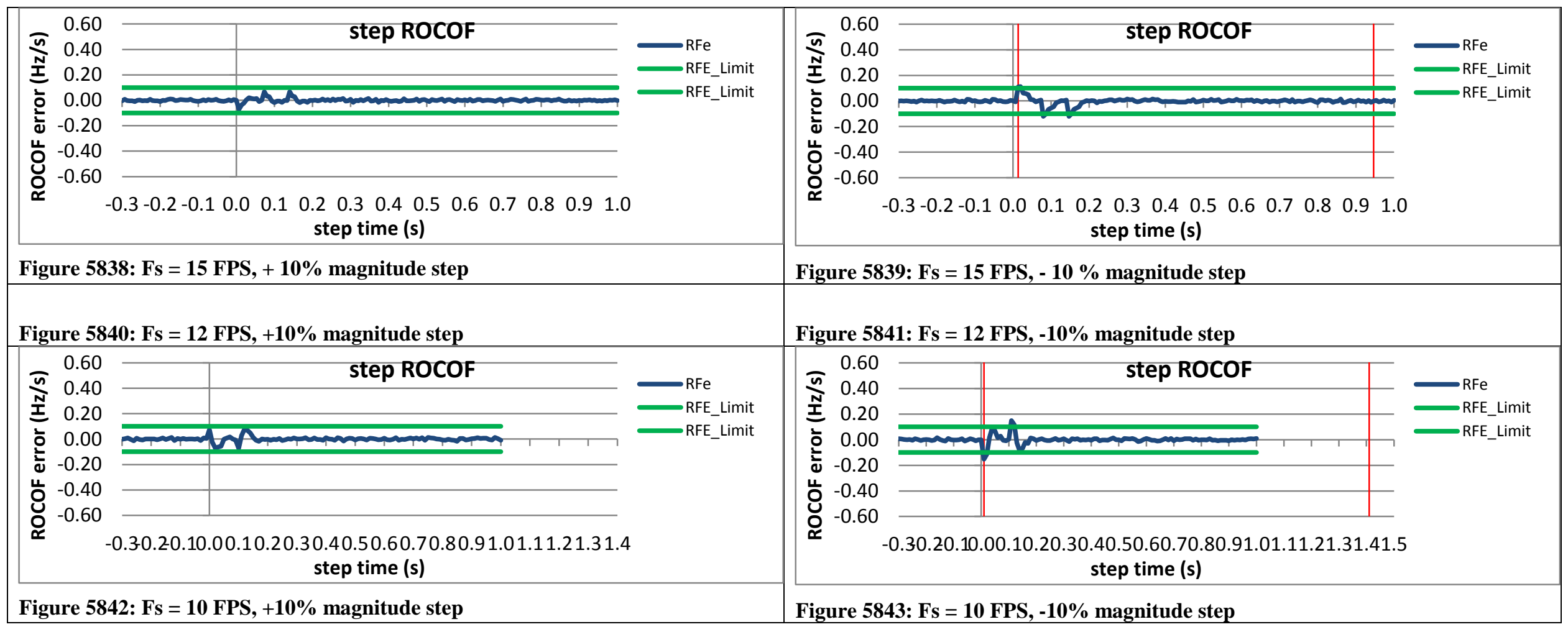


10.9.7 PMU F dynamic step change in magnitude ROCOF response time: $F 0=60 \mathrm{~Hz}, \mathrm{M}$ class

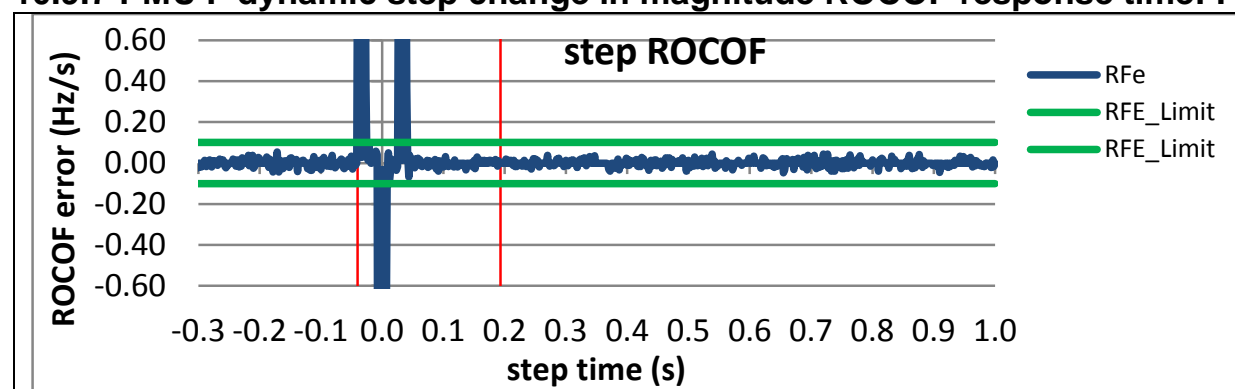

Figure 5844: Fs $=60 \mathrm{FPS},+10 \%$ magnitude step

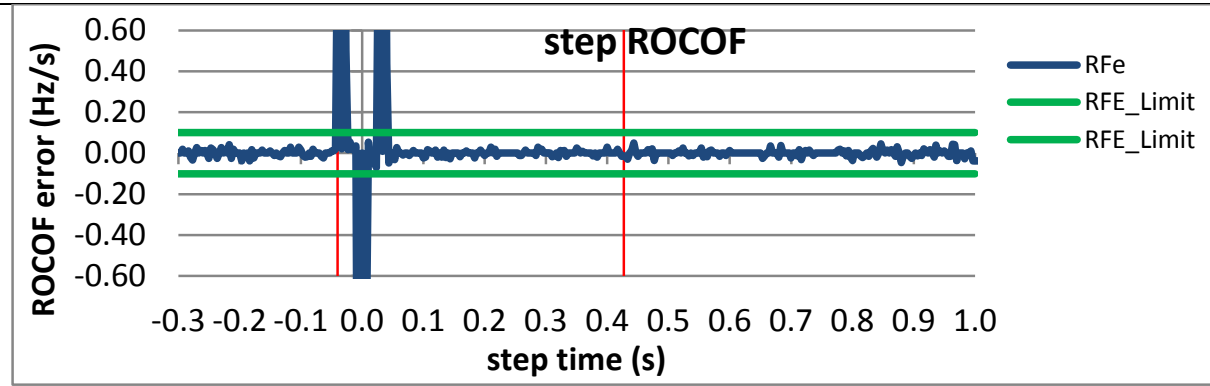

Figure 5846: Fs = $30 \mathrm{FPS},+10 \%$ magnitude step

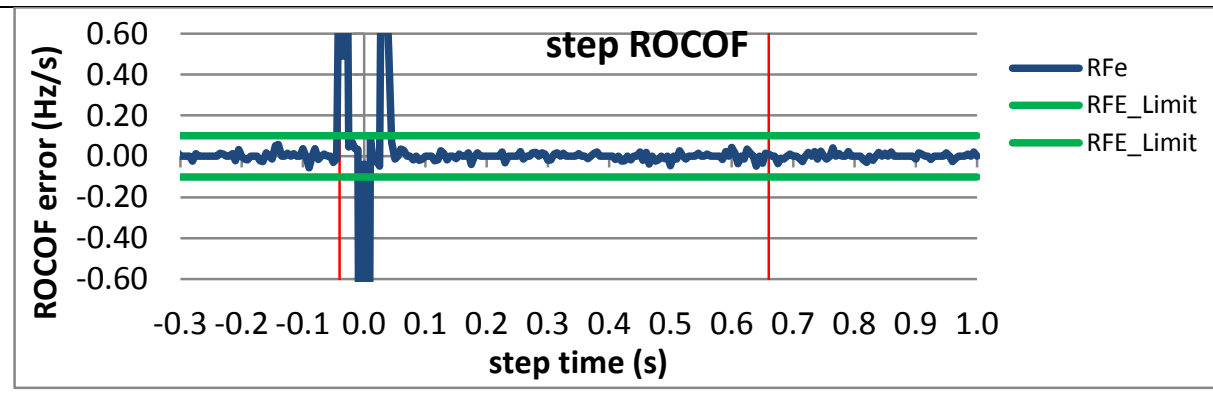

Figure 5848: Fs = 20 FPS, $+10 \%$ magnitude step

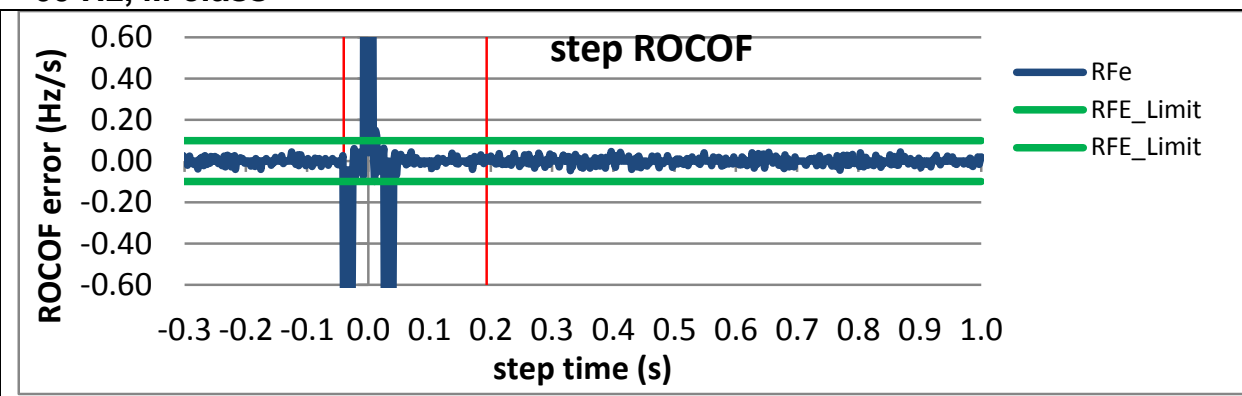

Figure 5845: Fs = 60 FPS, -10\% magnitude step

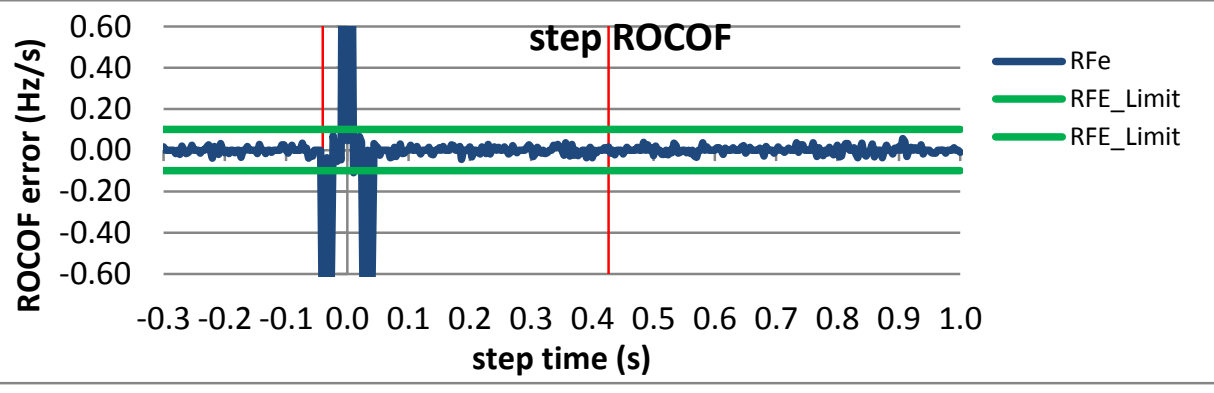

Figure 5847: Fs = 30 FPS, $-10 \%$ magnitude step

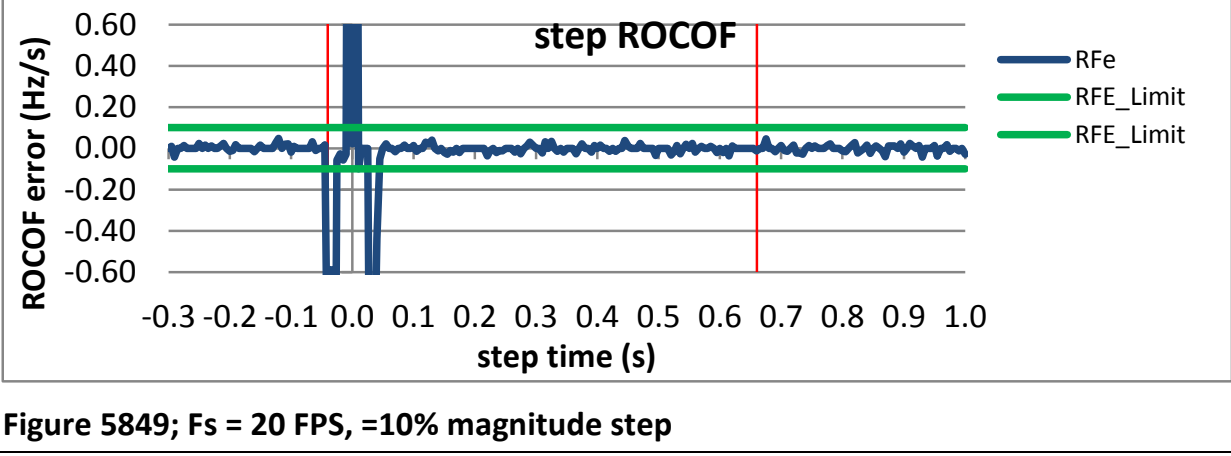




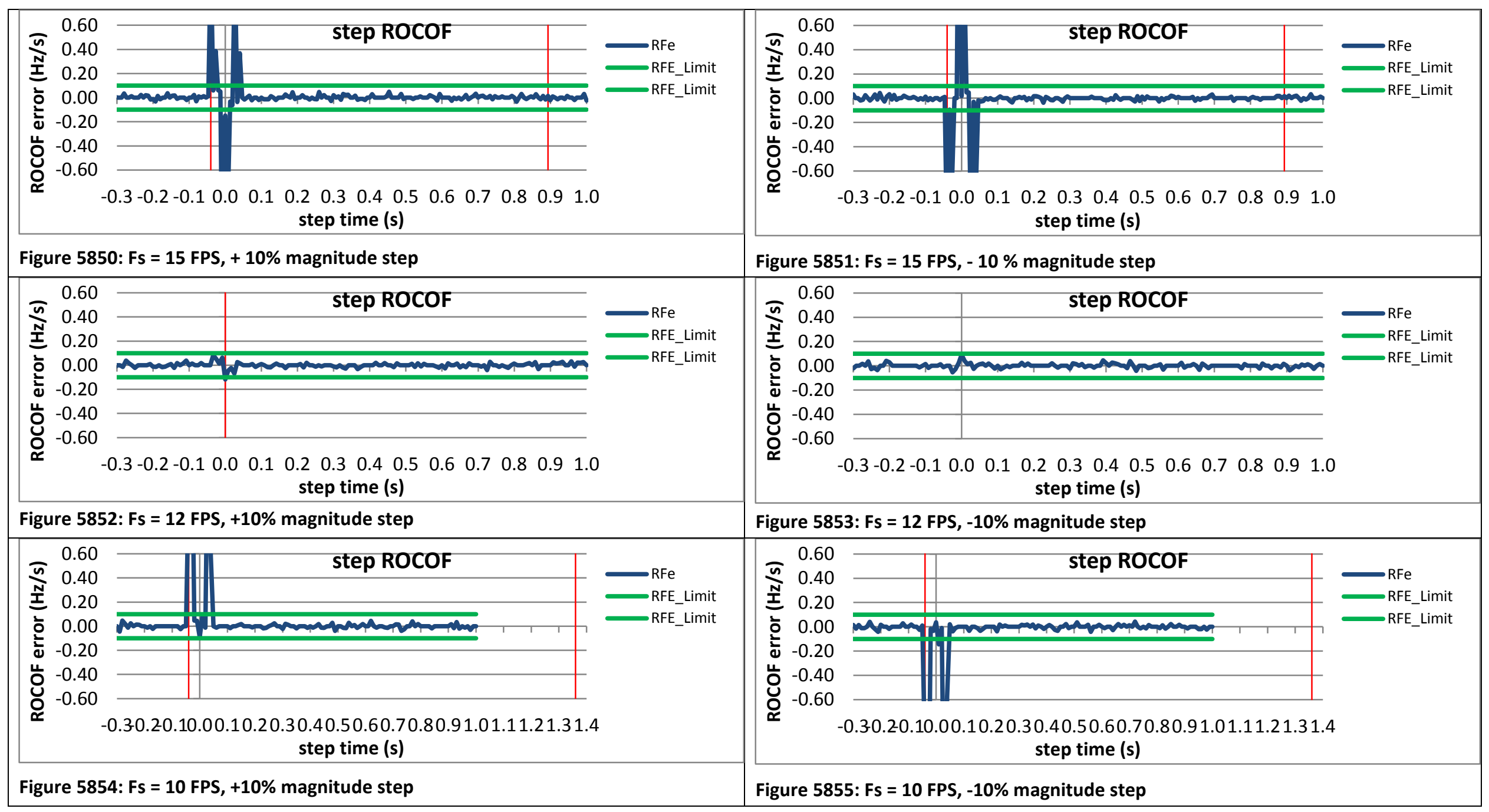


10.9.8 PMU G * dynamic step change in magnitude ROCOF response time: $F 0=60 \mathrm{~Hz}, \mathrm{M}$ class

Figure 5856: Fs = 60 FPS is not supported by this PMU
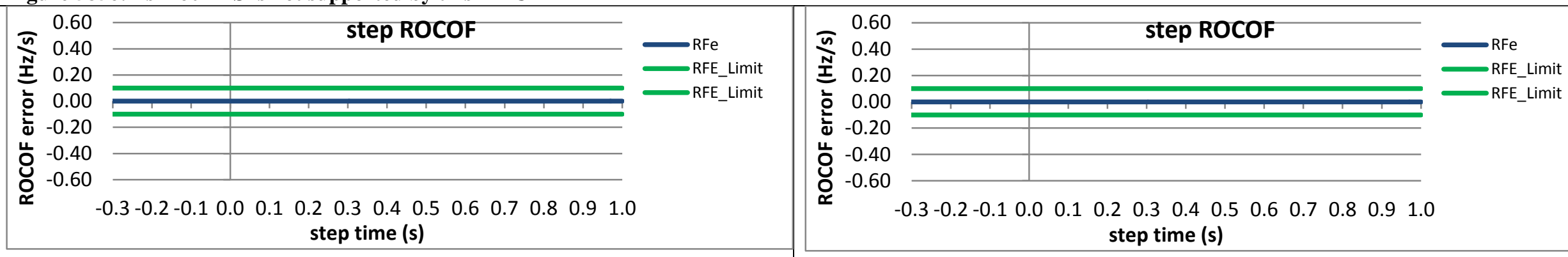

Figure 5857: Fs $=30$ FPS, $+\mathbf{1 0 \%}$ magnitude step

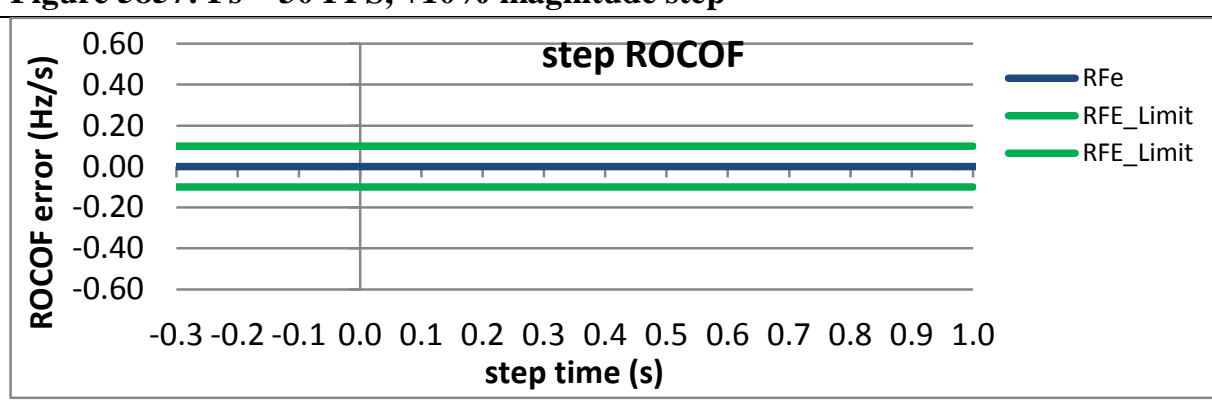

Figure 5858: Fs = 30 FPS, -10\% magnitude step

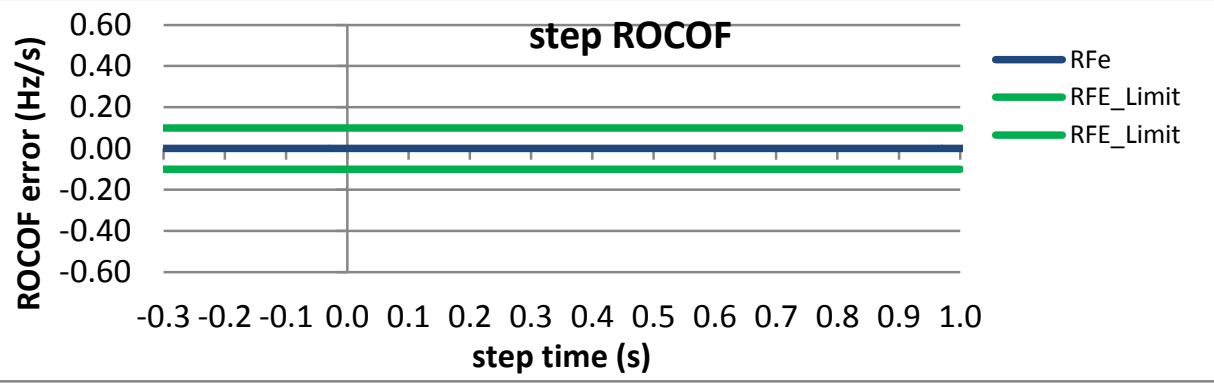

Figure 5859: Fs $=20$ FPS, $+10 \%$ magnitude step

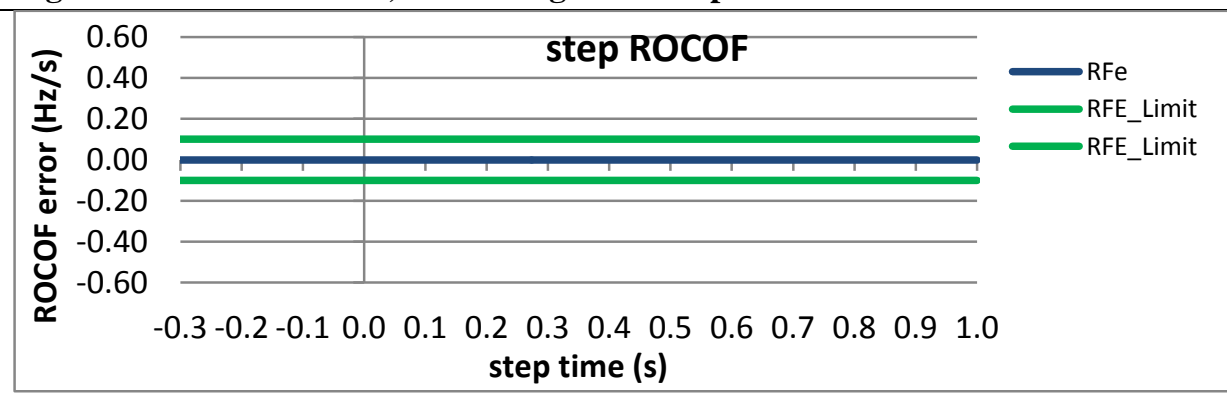

Figure 5860; Fs = 20 FPS, =10\% magnitude step

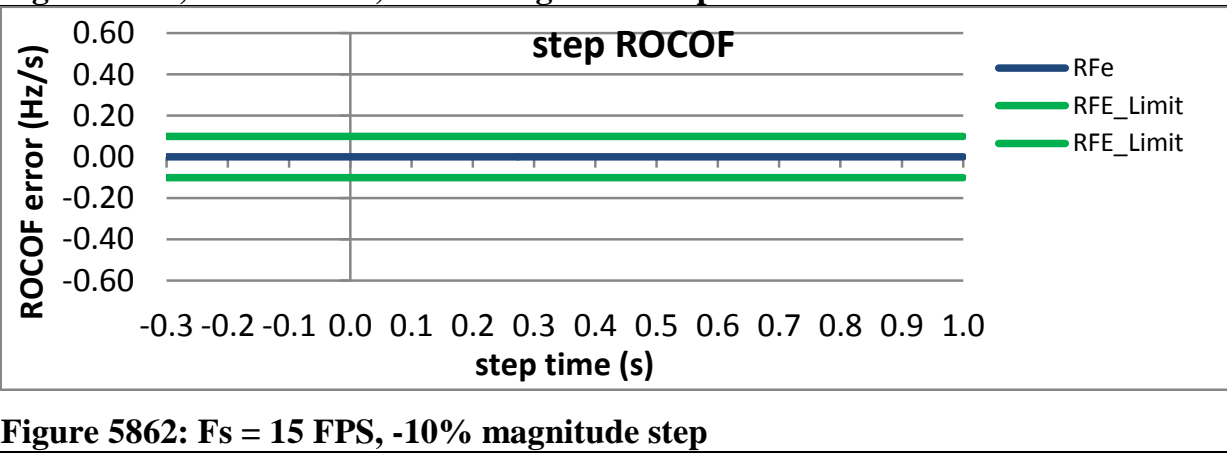



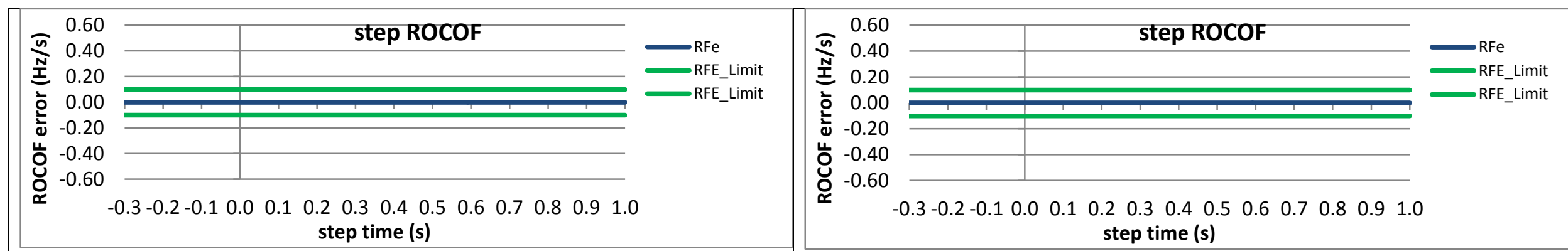

Figure 5863: Fs = 15 FPS, $+10 \%$ magnitude step

Figure 5864: Fs = 15 FPS, -10\% magnitude step
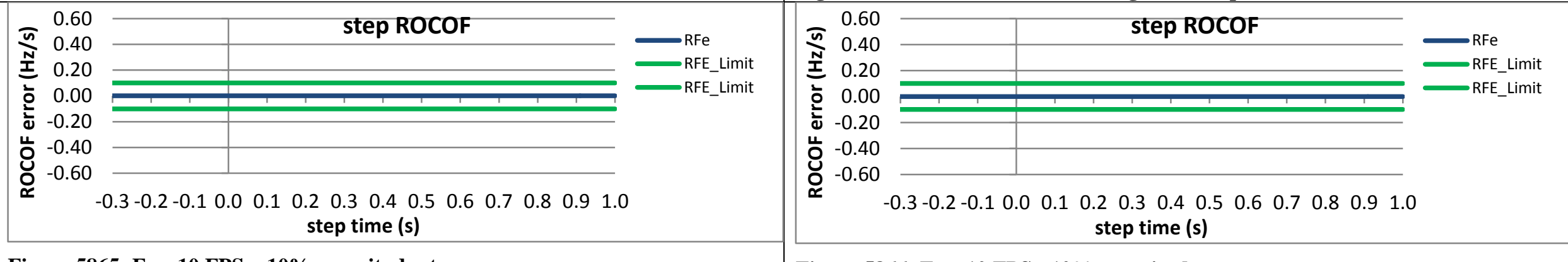

Figure 5865: Fs = 10 FPS, +10\% magnitude step

* PMU G always outputs ROCOF $=0$

Figure 5866: Fs = 10 FPS, -10\% magnitude step 
10.9.9 PMU H dynamic step change in magnitude ROCOF response time: $\mathrm{FO}=60 \mathrm{~Hz}, \mathrm{M}$ class
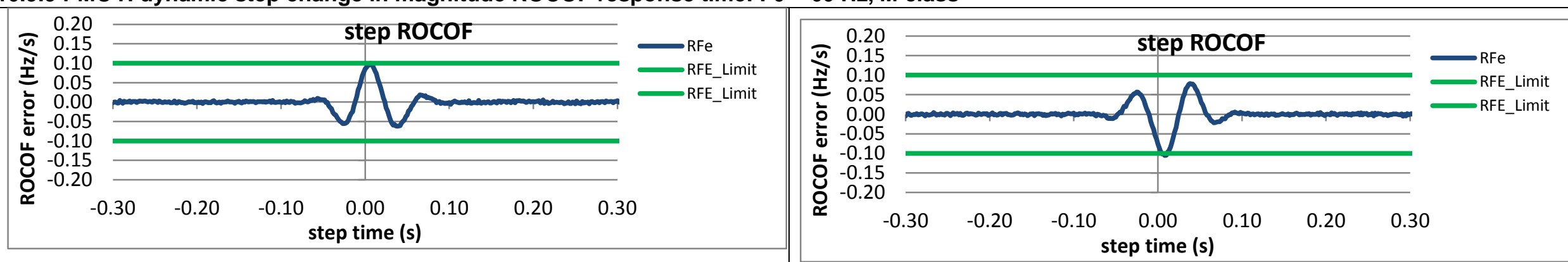

Figure 5867: Fs = 60 FPS, +10\% magnitude step

Figure 5868: Fs = 60 FPS, -10\% magnitude step

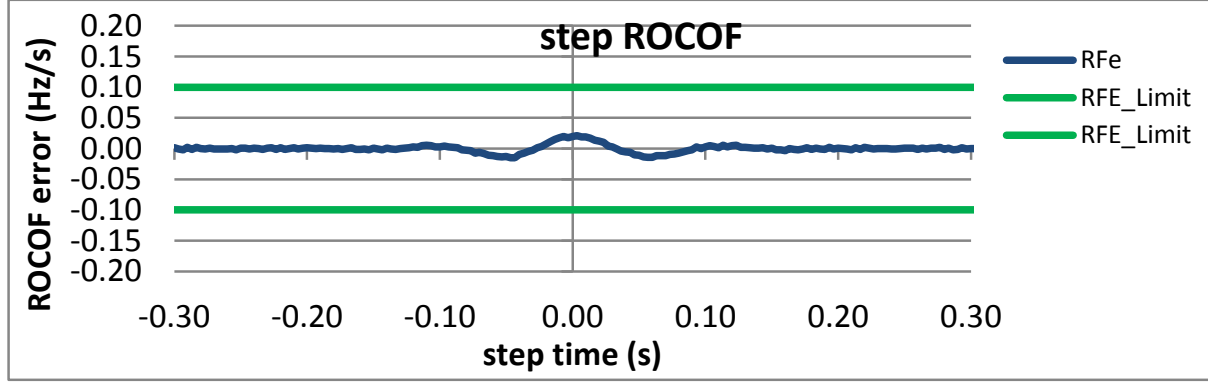

Figure 5869: Fs $=30$ FPS, $+10 \%$ magnitude step
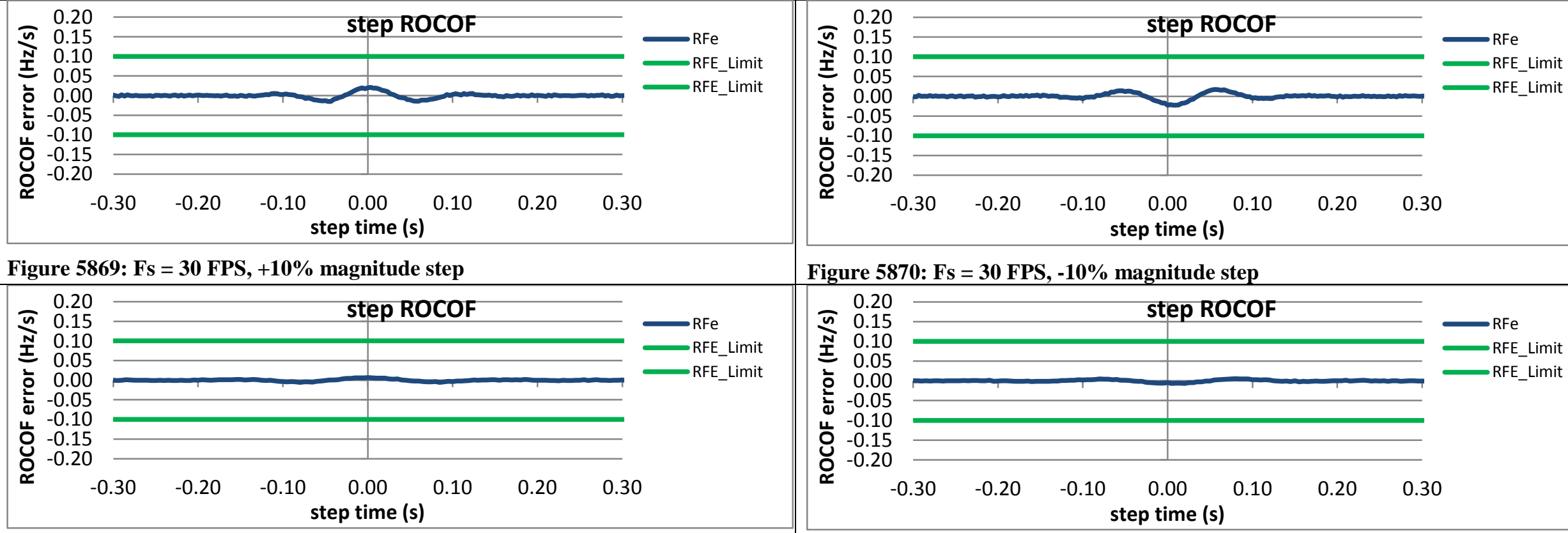

Figure 5870: Fs = 30 FPS, -10\% magnitude step

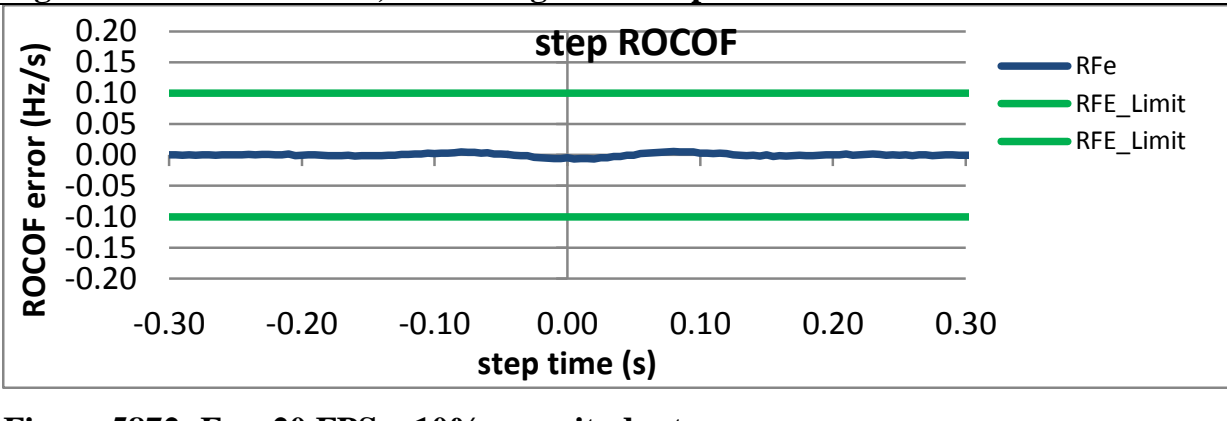

Figure 5871: Fs = 20 FPS, +10\% magnitude step

Figure 5872; Fs = 20 FPS, $=10 \%$ magnitude step 

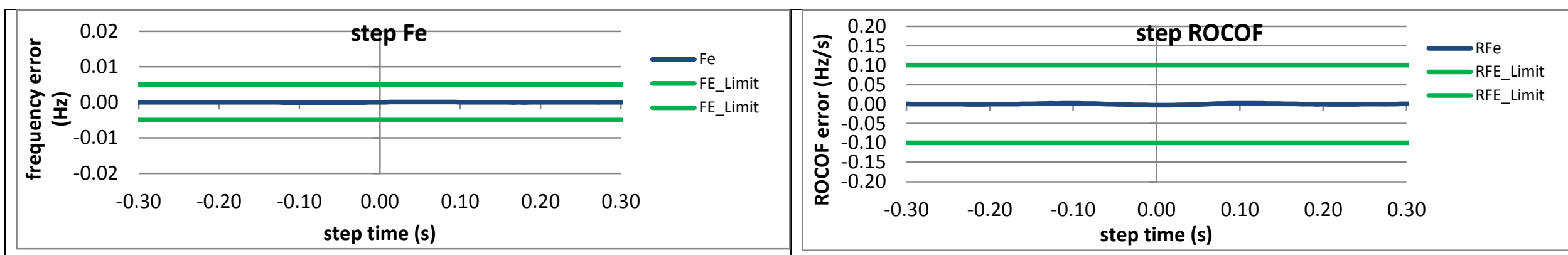

Figure 5873: Fs = 15 FPS, + 10\% magnitude step

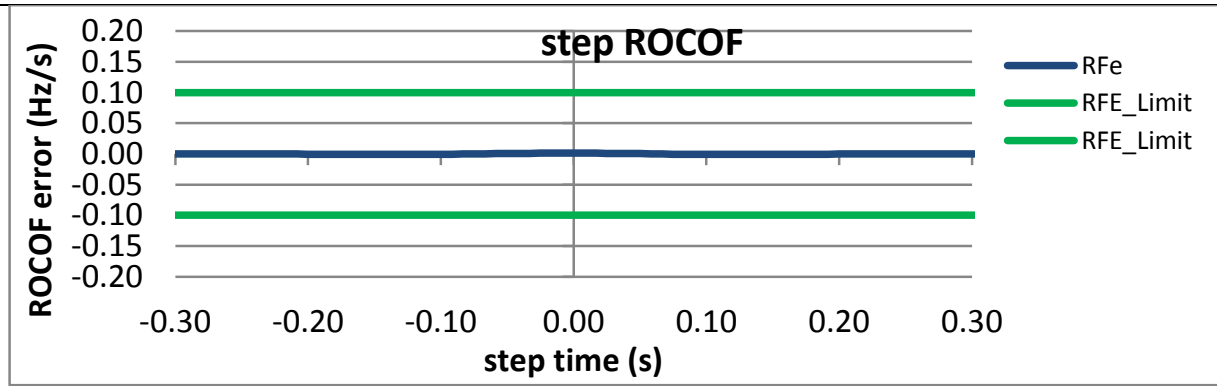

Figure 5874: Fs = 15 FPS, - $10 \%$ magnitude step

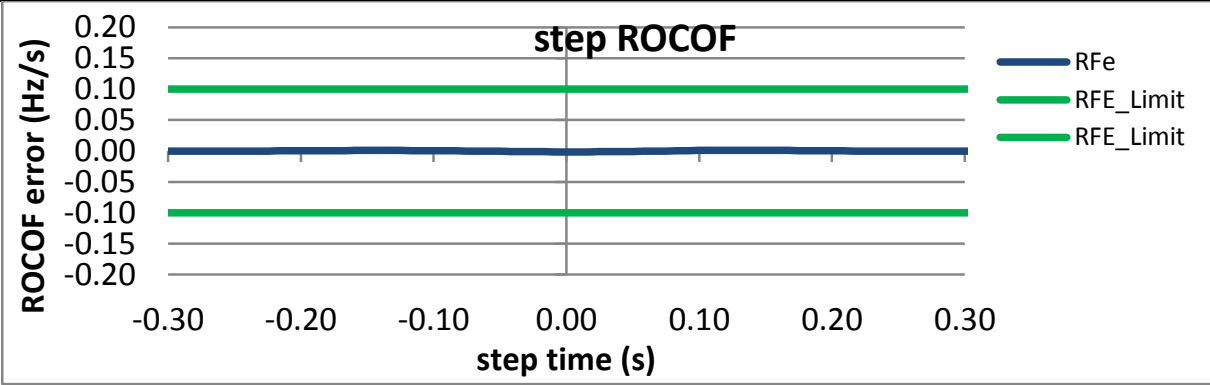

Figure 5875: Fs = 12 FPS, $+10 \%$ magnitude step

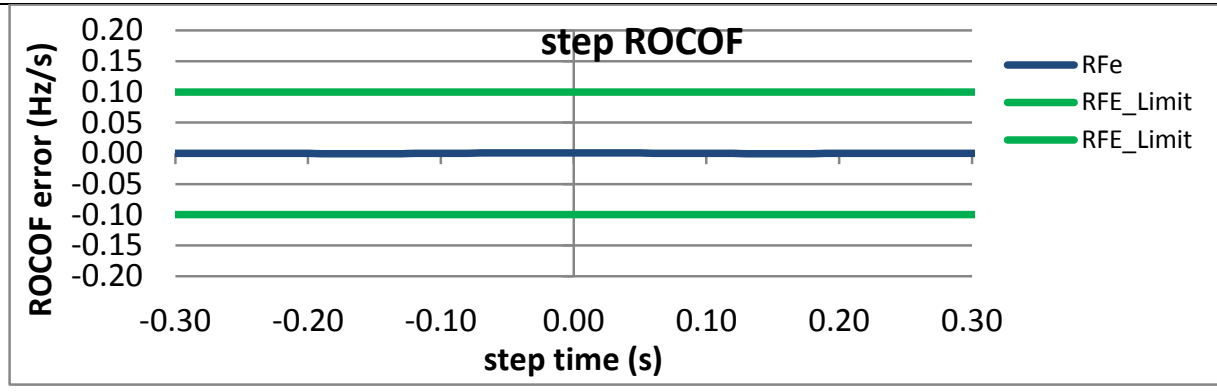

Figure 5876: Fs = 12 FPS, $-10 \%$ magnitude step

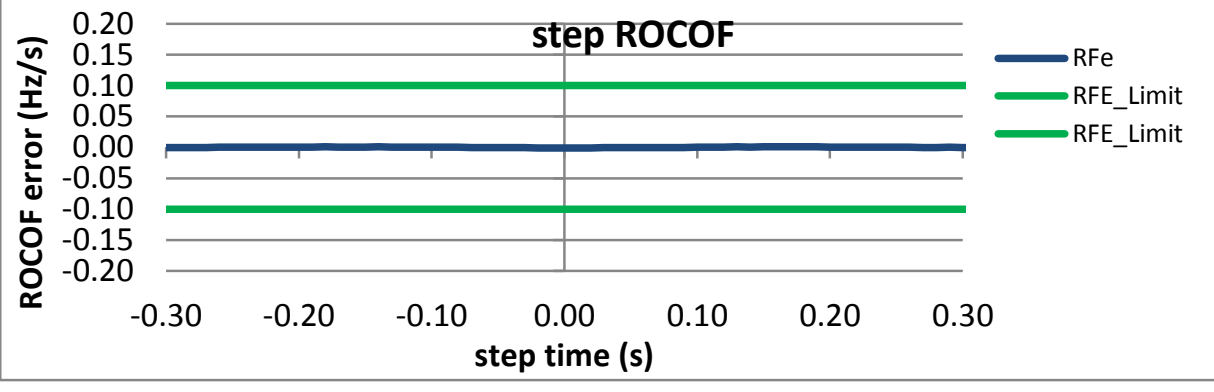

Figure 5878: Fs = 10 FPS, -10\% magnitude step 
10.9.10 PMU I dynamic step change in magnitude ROCOF response time: $F 0=60 \mathrm{~Hz}, \mathrm{M}$ class
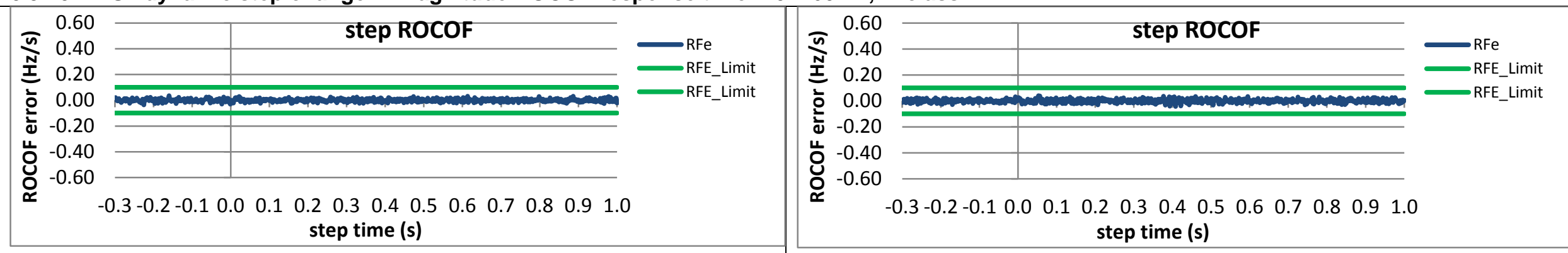

Figure 5879: Fs = 60 FPS, $+10 \%$ magnitude step

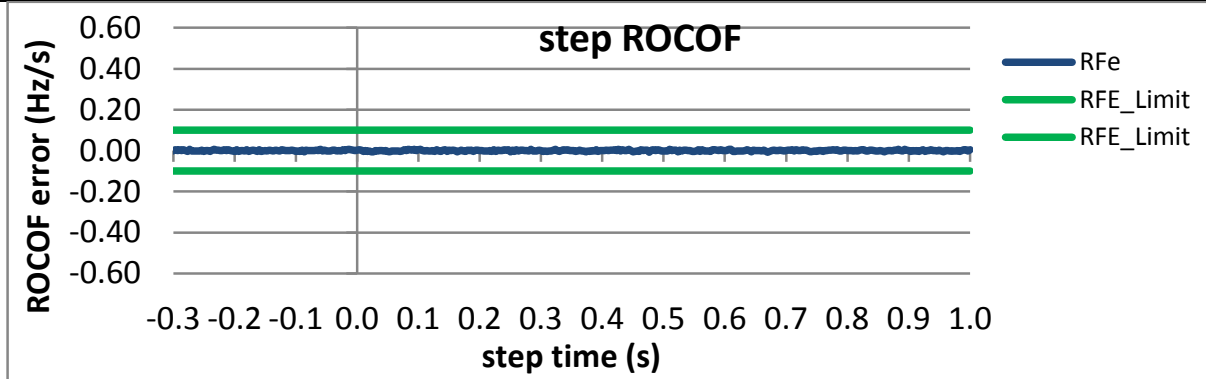

Figure 5880: Fs = 60 FPS, -10\% magnitude step

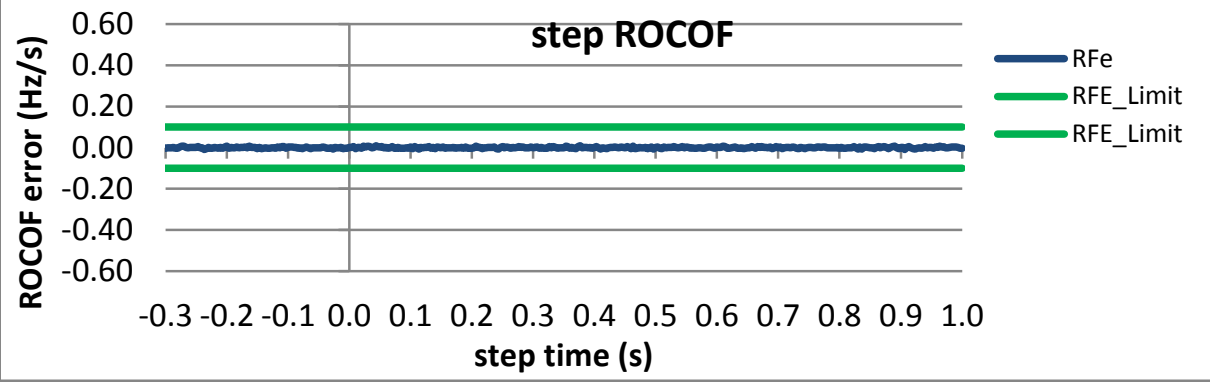

Figure 5881: Fs = 30 FPS, +10\% magnitude step

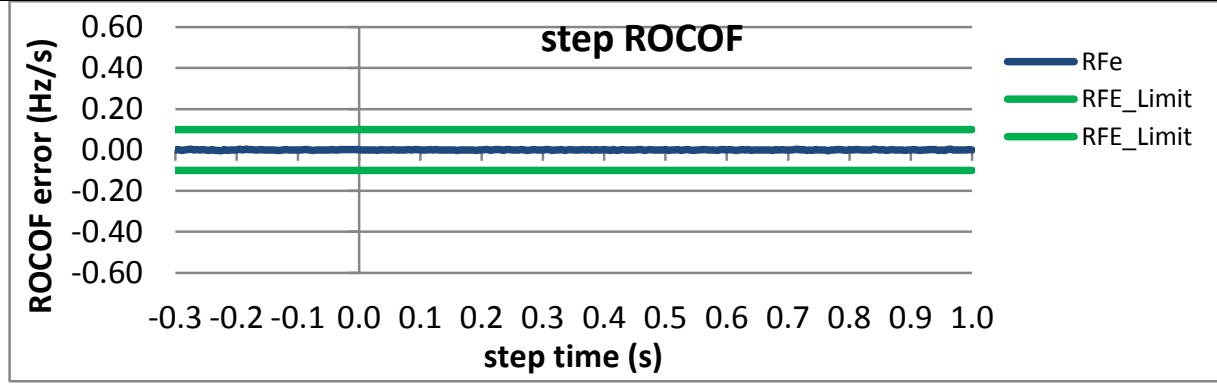

Figure 5882: Fs = 30 FPS, -10\% magnitude step

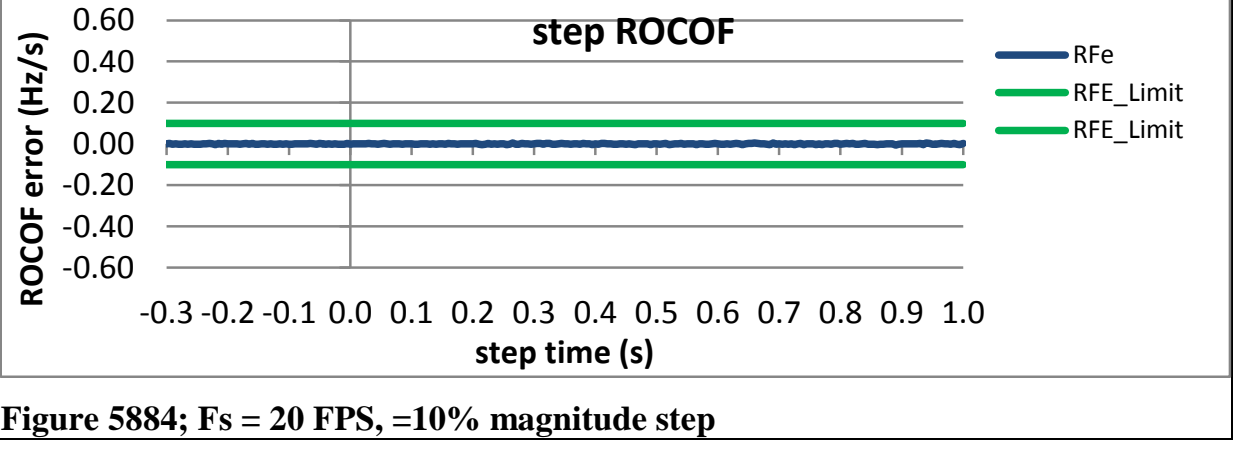

Figure 5883: Fs $=20$ FPS, $+10 \%$ magnitude step

Figure 5884; Fs = 20 FPS, =10\% magnitude step 

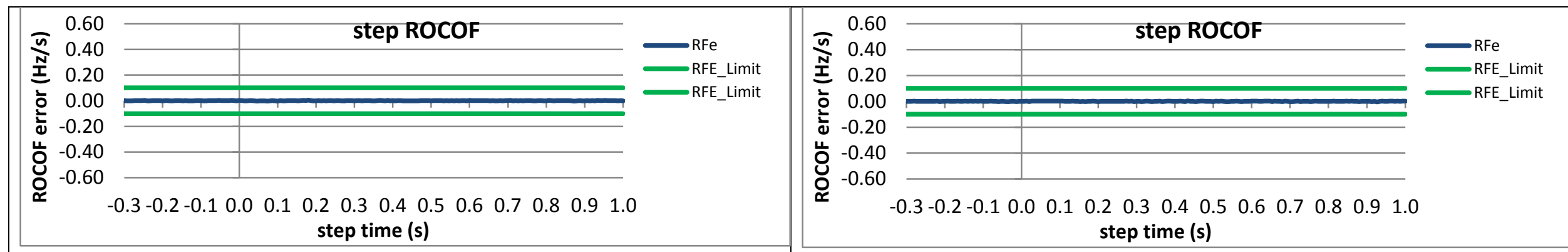

Figure 5885: Fs = 15 FPS, + 10\% magnitude step

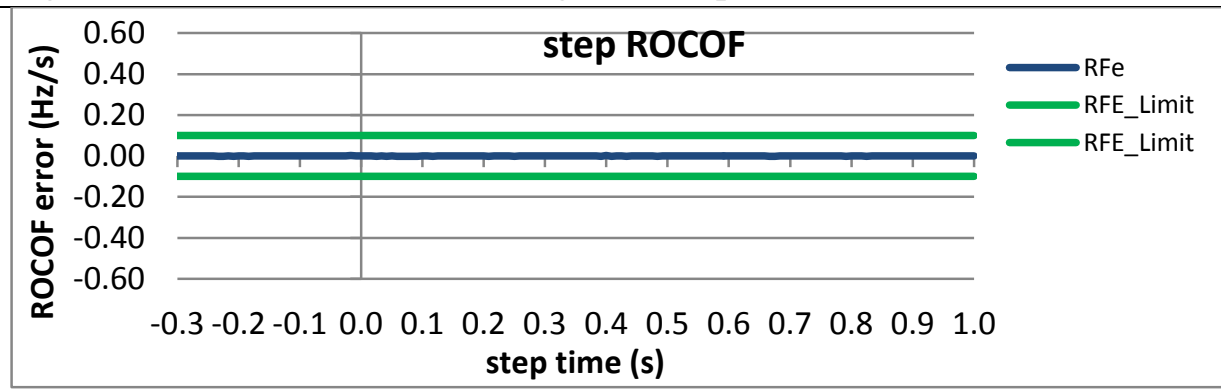

Figure 5886: Fs = 15 FPS, - $10 \%$ magnitude step

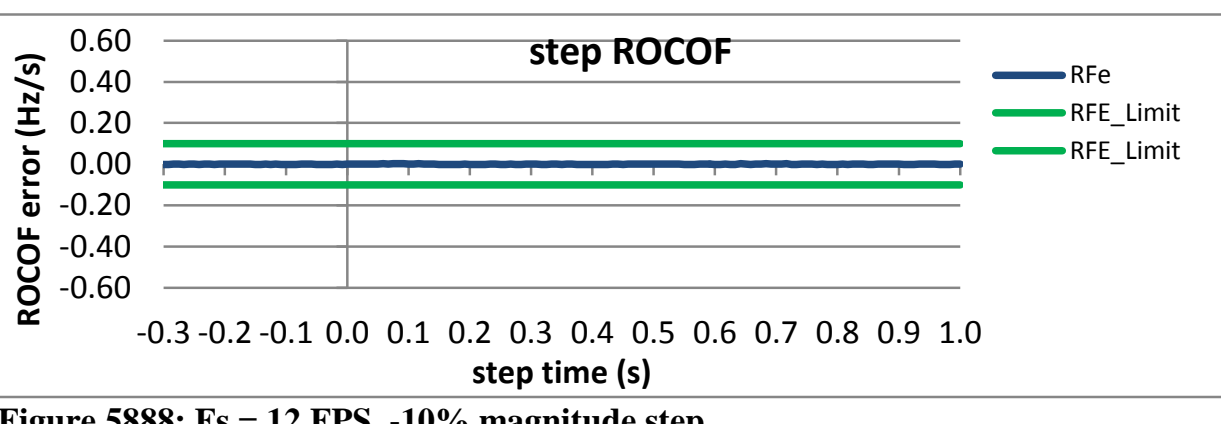

Figure 5887: Fs = 12 FPS, $+10 \%$ magnitude step

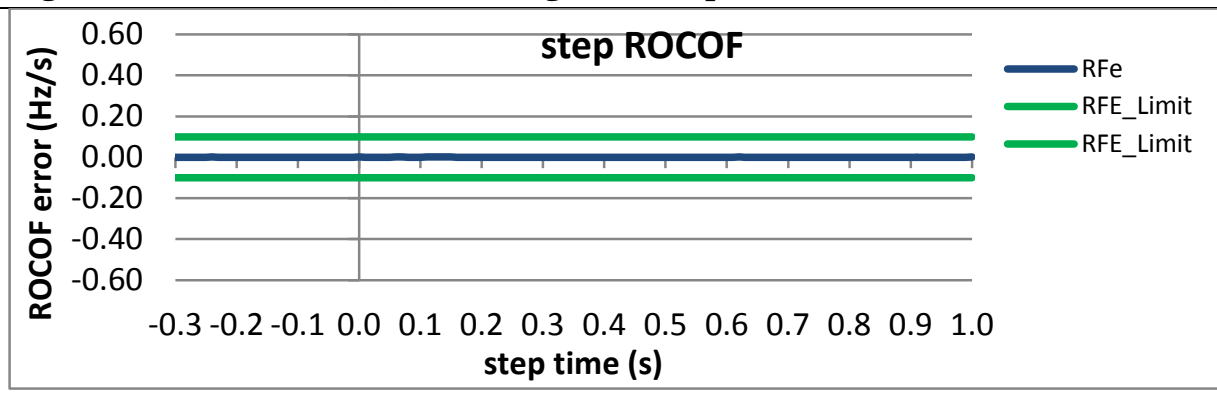

Figure 5888: Fs = 12 FPS, -10\% magnitude step

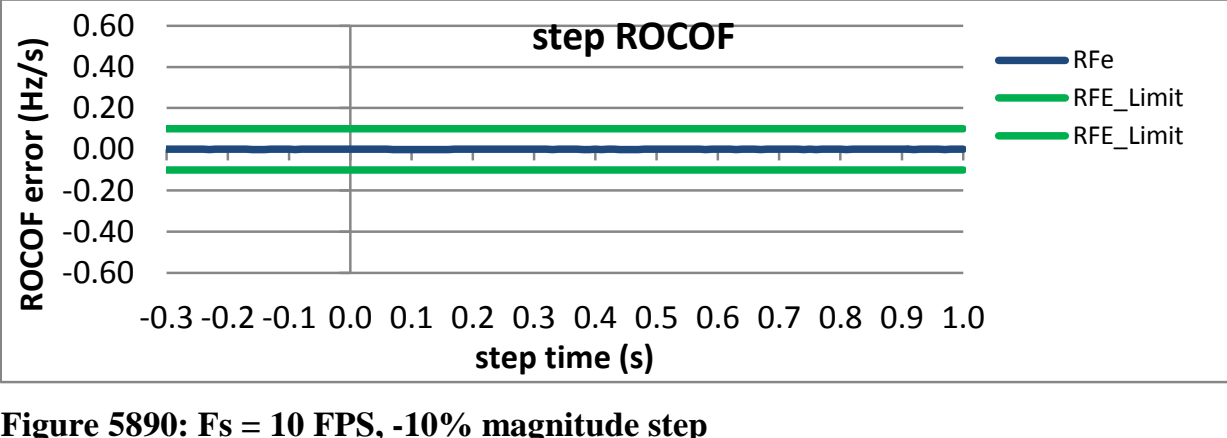

Figure 5889: Fs = 10 FPS, +10\% magnitude step

Figure 5890: Fs = 10 FPS, -10\% magnitude step 
10.9.11 PMU J dynamic step change in magnitude ROCOF response time: $F 0=60 \mathrm{~Hz}, \mathrm{M}$ class

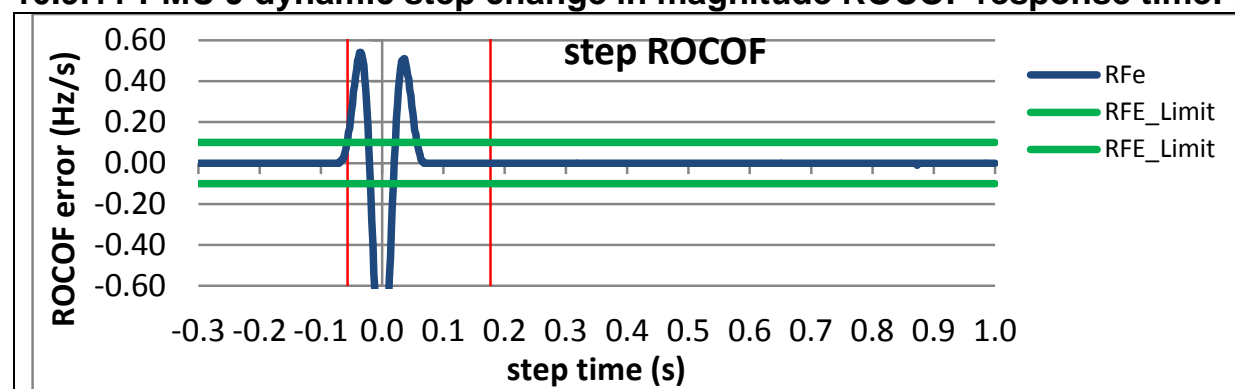

Figure 5891: Fs = $60 \mathrm{FPS},+10 \%$ magnitude step

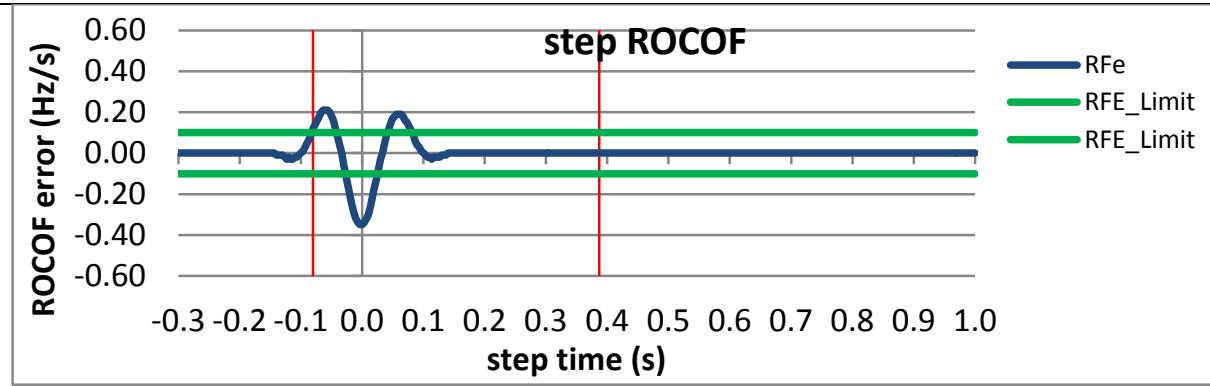

Figure 5893: Fs = $30 \mathrm{FPS},+10 \%$ magnitude step

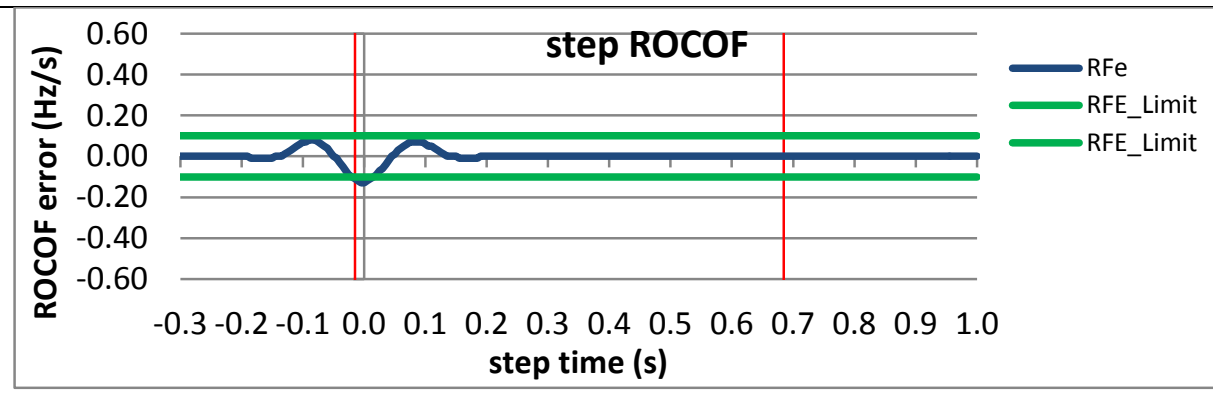

Figure 5895: Fs = 20 FPS, $+10 \%$ magnitude step

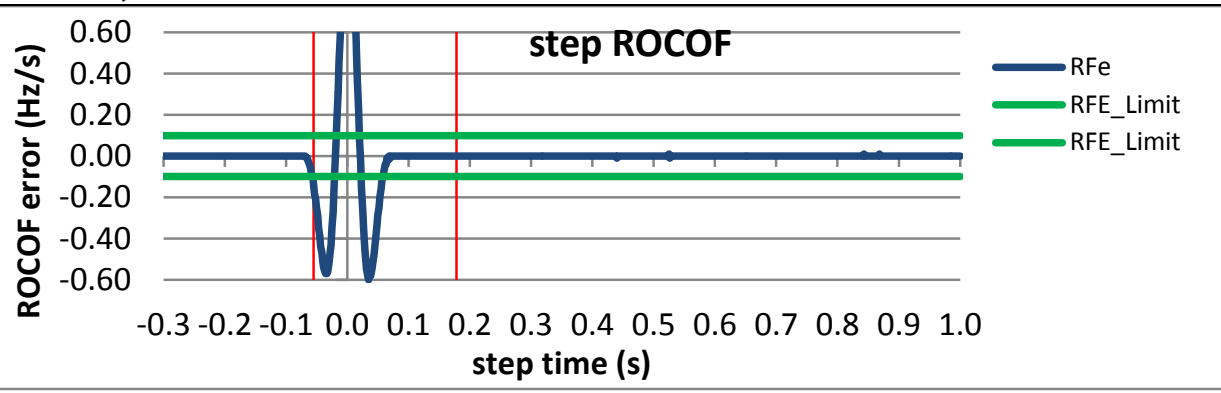

Figure 5892: Fs = 60 FPS, -10\% magnitude step

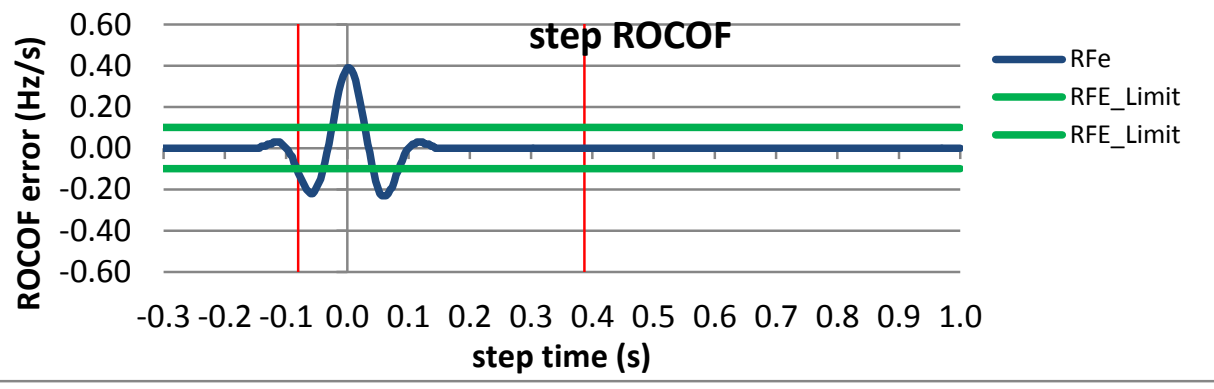

Figure 5894: Fs = 30 FPS, $-10 \%$ magnitude step

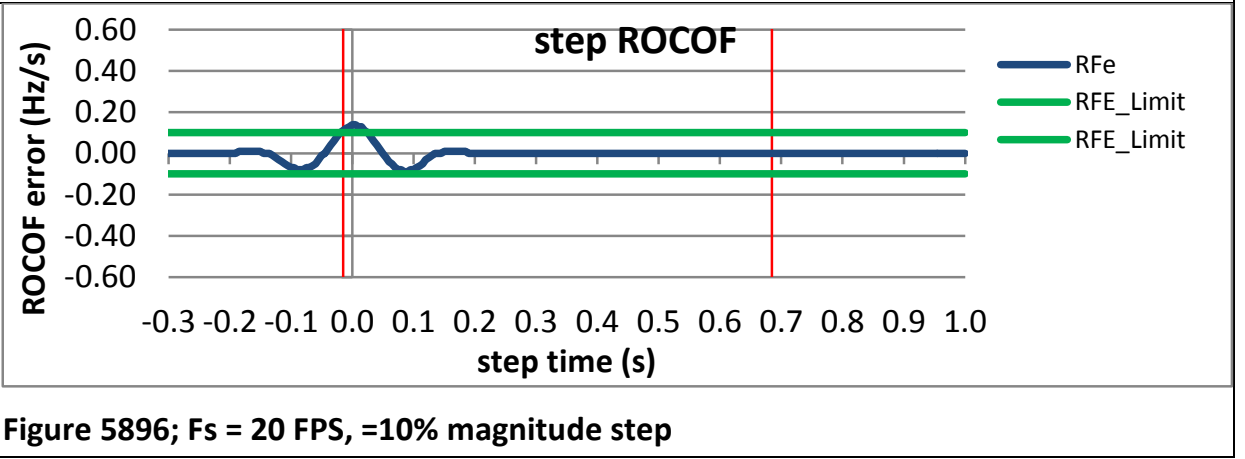



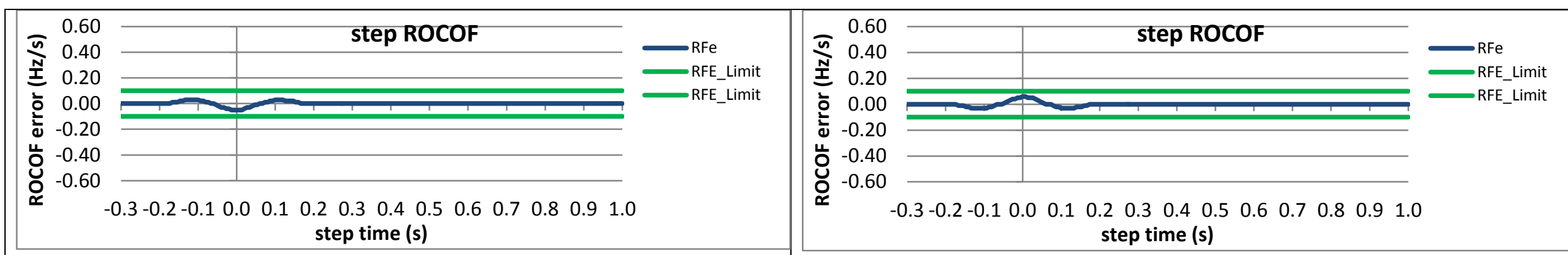

Figure 5897: Fs = 15 FPS, + 10\% magnitude step

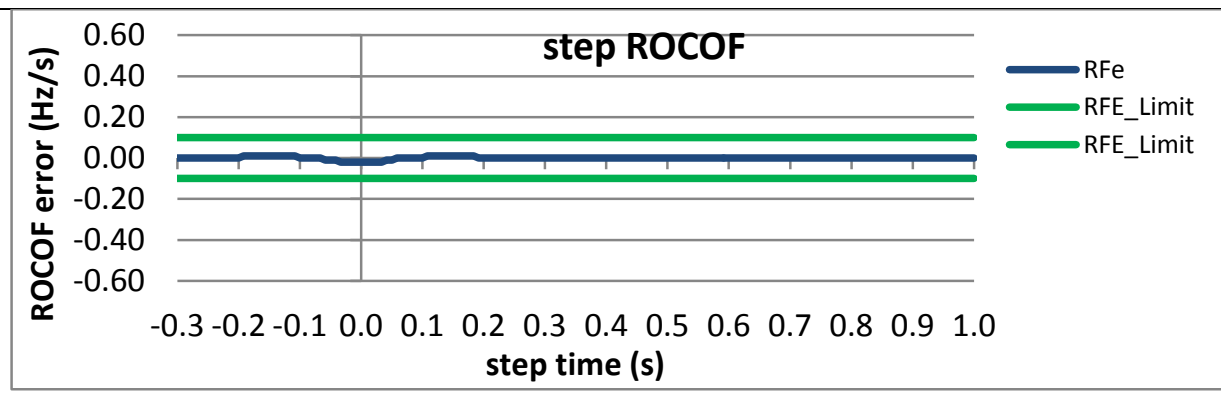

Figure 5898: Fs = 15 FPS, - $10 \%$ magnitude step

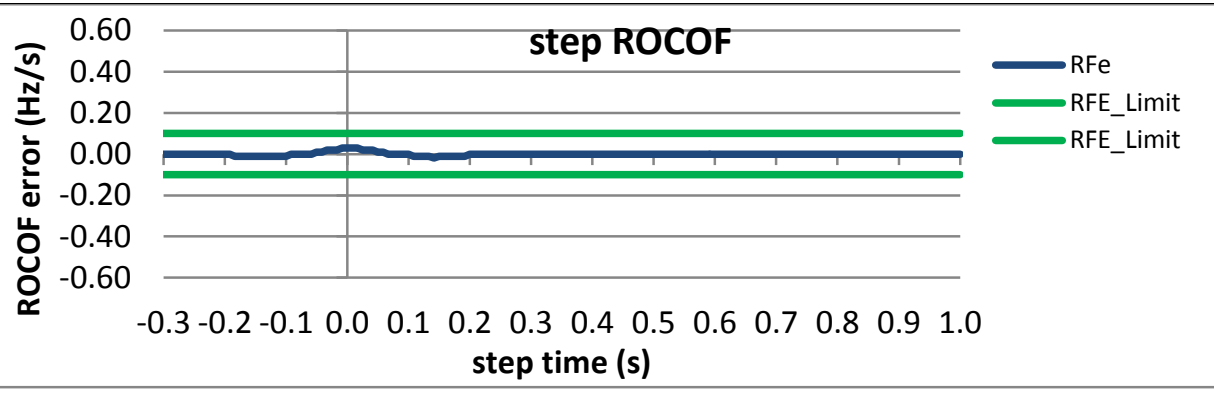

Figure 5899: $\mathrm{Fs}=12 \mathrm{FPS},+10 \%$ magnitude step

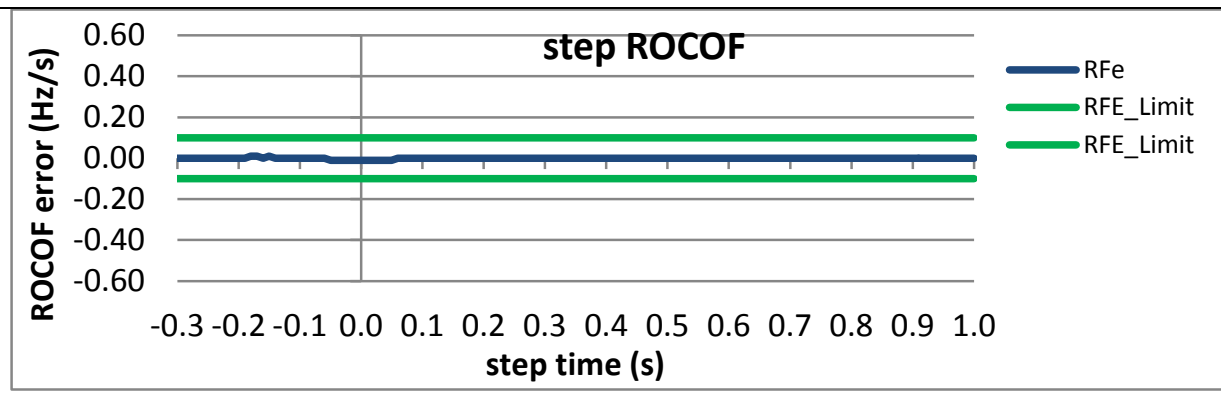

Figure 5900: Fs = 12 FPS, $-10 \%$ magnitude step

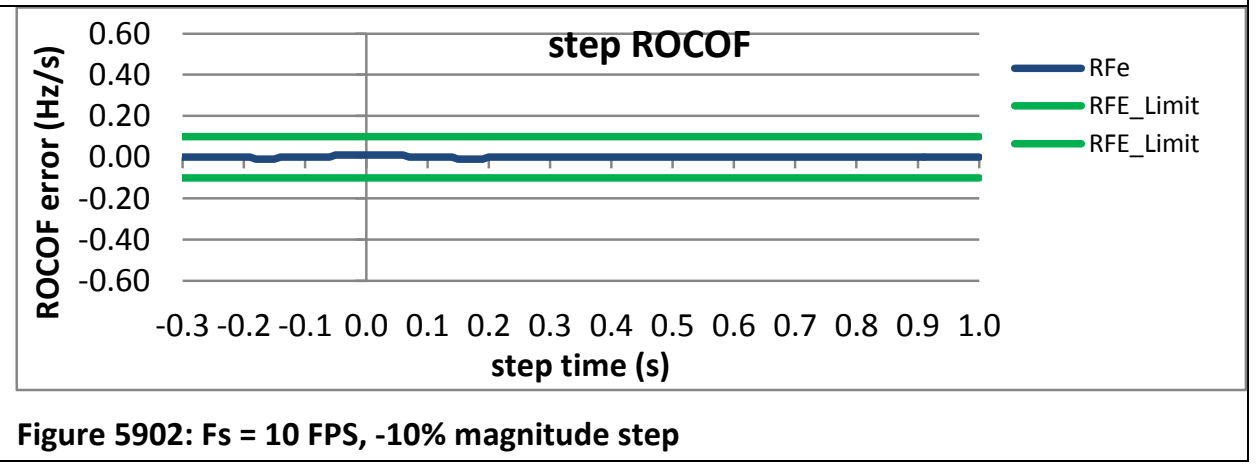


10.10 Dynamic step change in magnitude ROCOF error: $F 0=60 \mathrm{~Hz}$, P class

10.10.1 C37.118.1-2011 Annex C dynamic step change in magnitude ROCOF error: $F 0=60 \mathrm{~Hz}$, P class

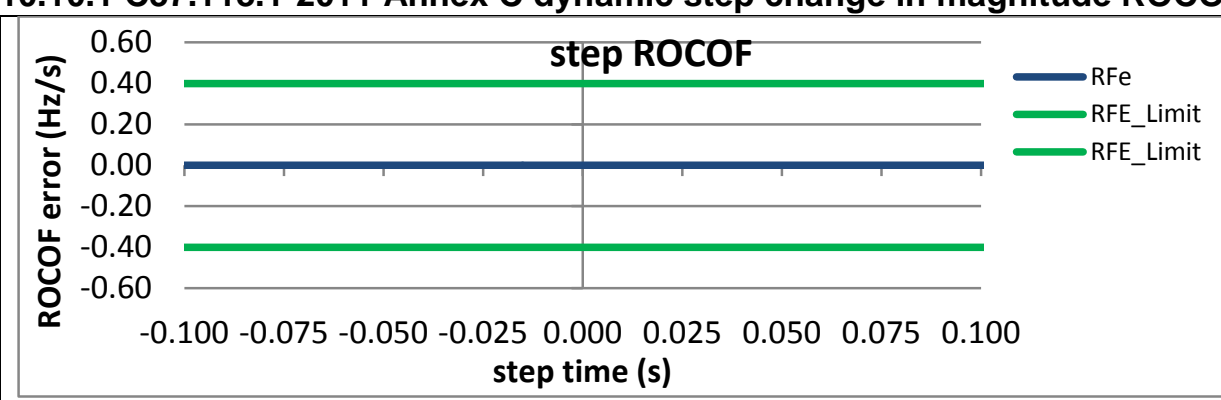

Figure 5903: Fs = 60 FPS, $+10 \%$ magnitude step
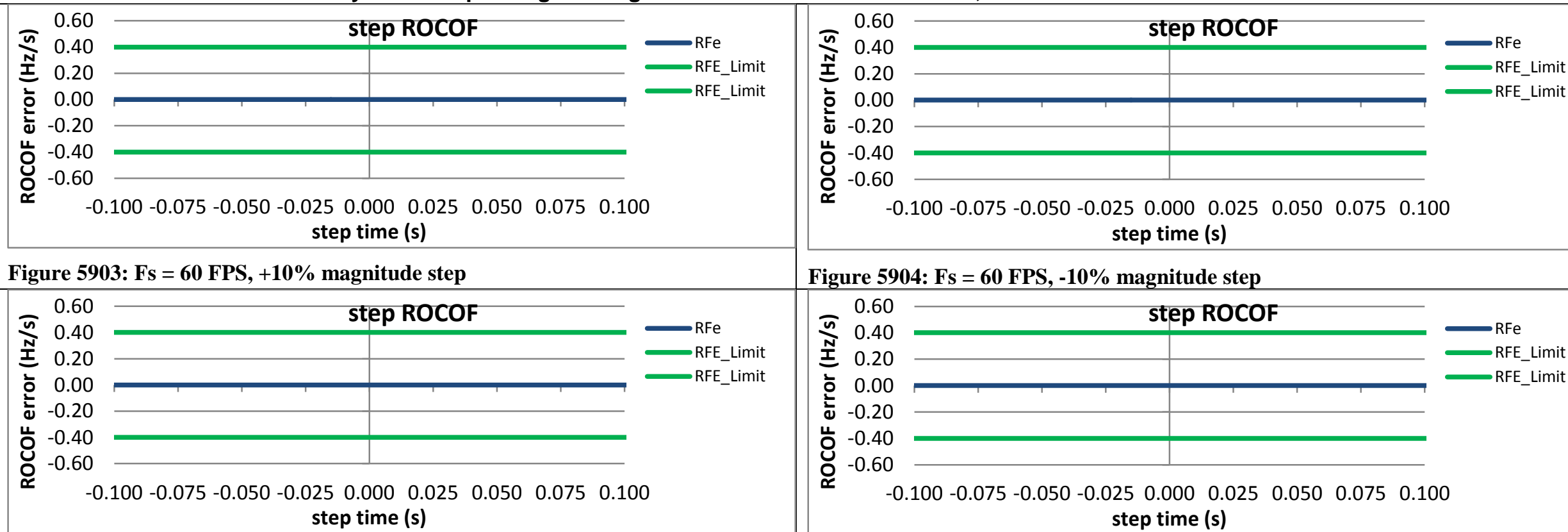

Figure 5904: Fs = 60 FPS, -10\% magnitude step

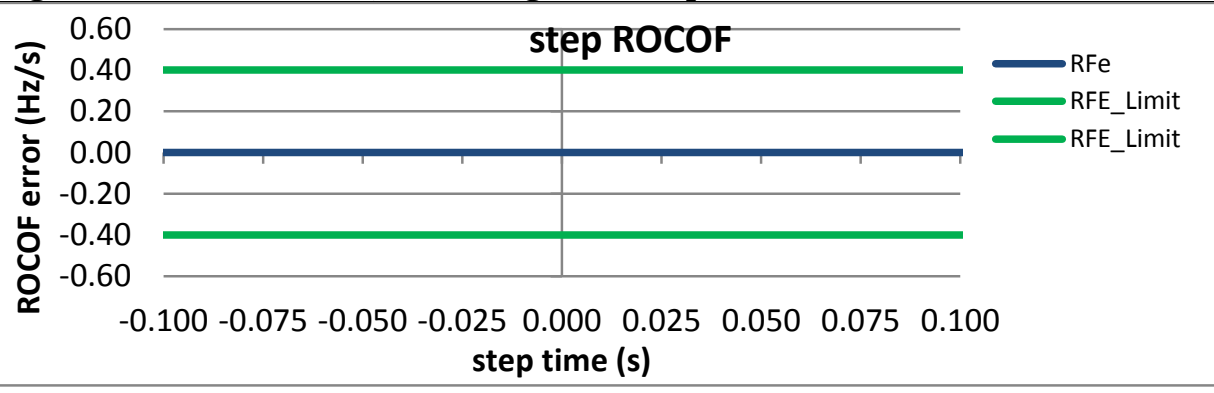

Figure 5905: Fs = 30 FPS, $+10 \%$ magnitude step

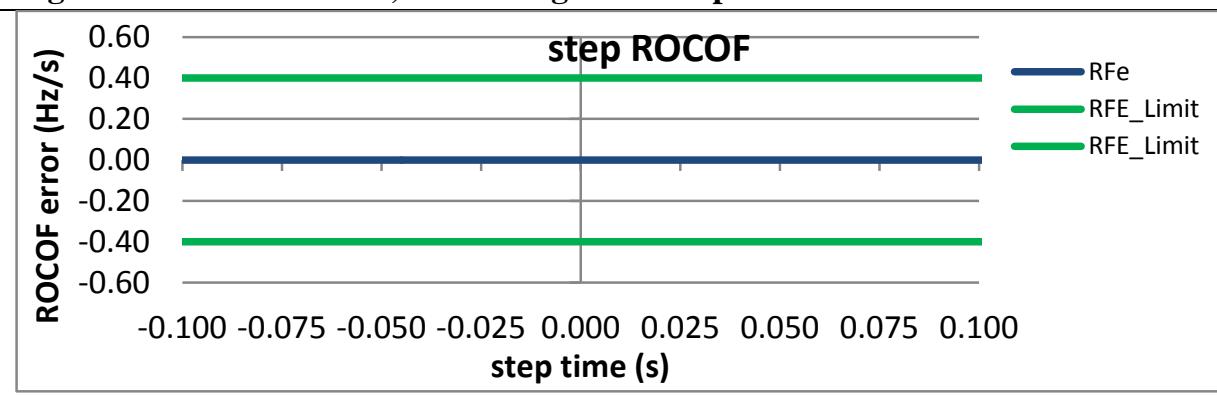

Figure 5906: Fs = 30 FPS, -10\% magnitude step

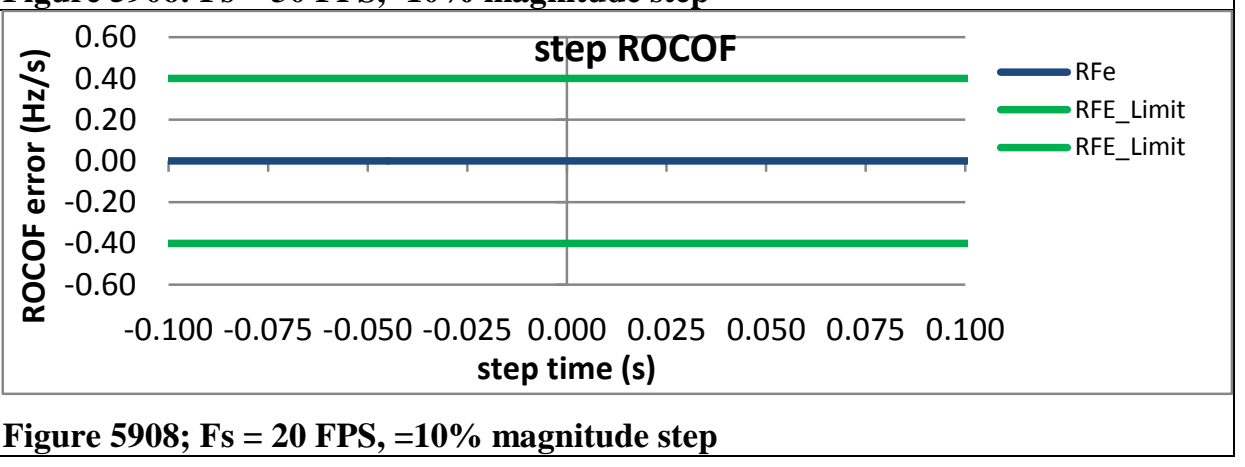

Figure 5907: Fs = 20 FPS, +10\% magnitude step 


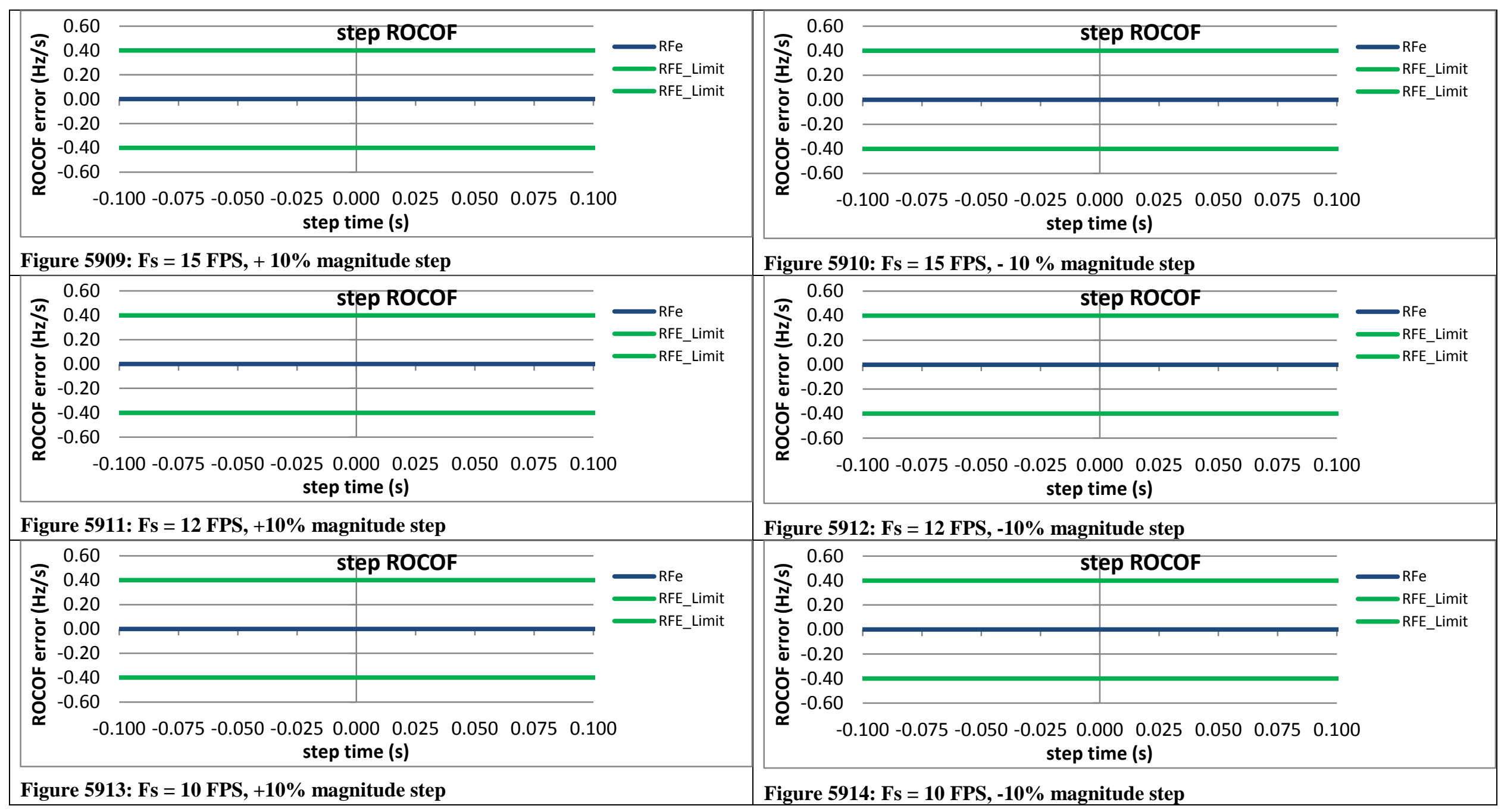


10.10.2 PMU A dynamic step change in magnitude ROCOF response time: $\mathrm{F} 0=60 \mathrm{~Hz}, \mathrm{P}$ class

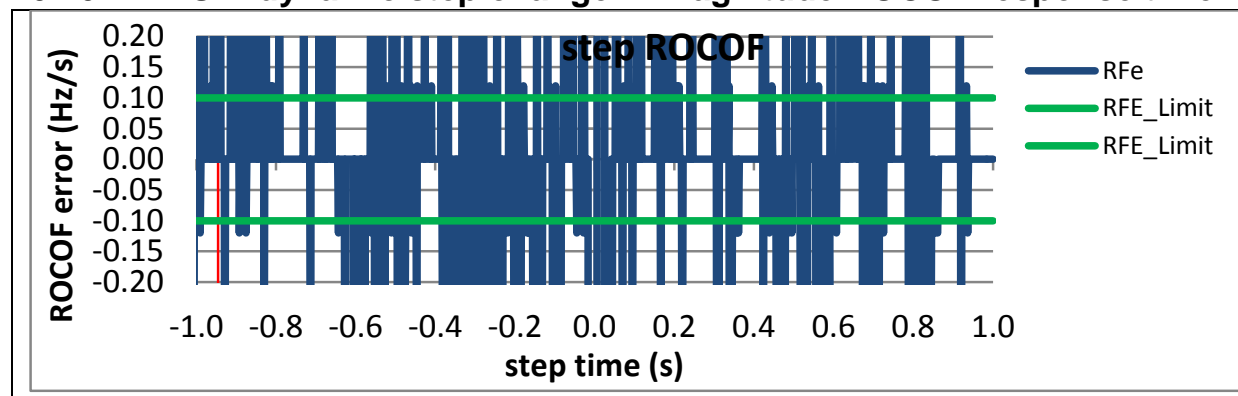

Figure 5915: Fs = 60 FPS, +10 degree phase step

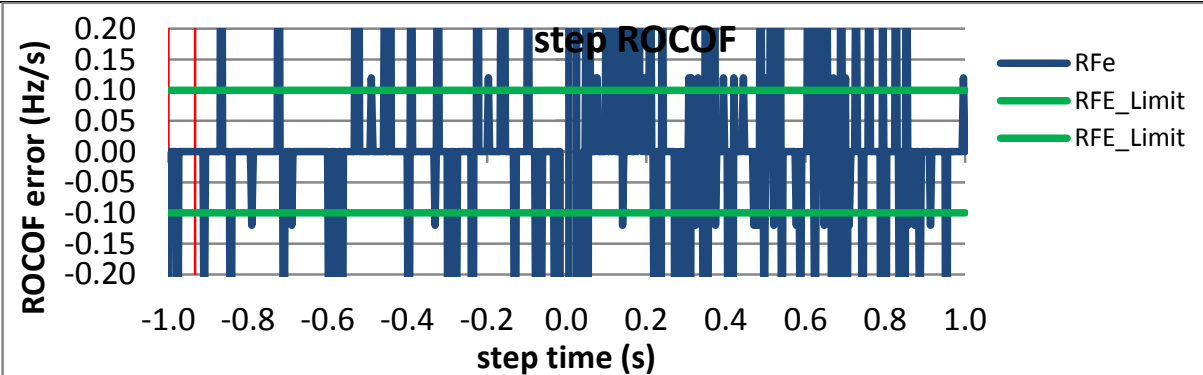

Figure 5917: Fs = 30 FPS, +10 degree phase step

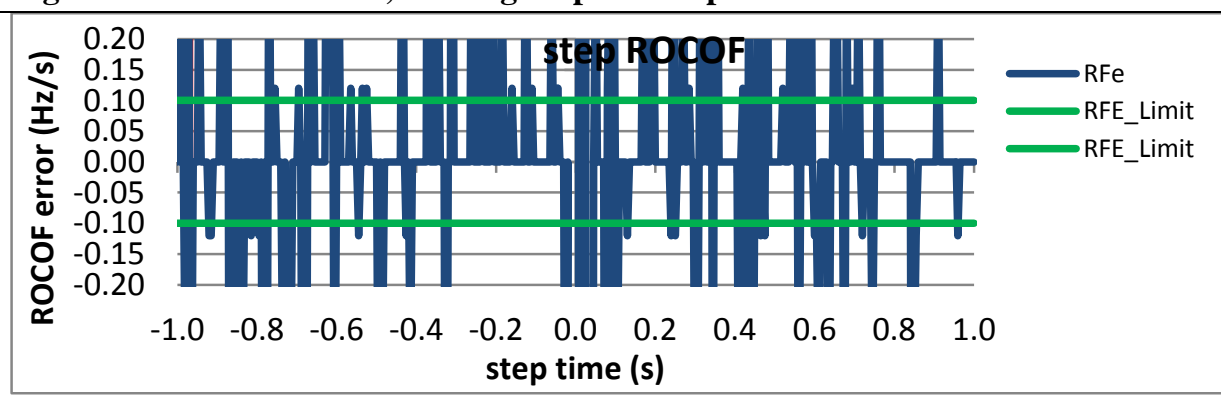

Figure 5919: Fs = 20 FPS, +10 degree phase step

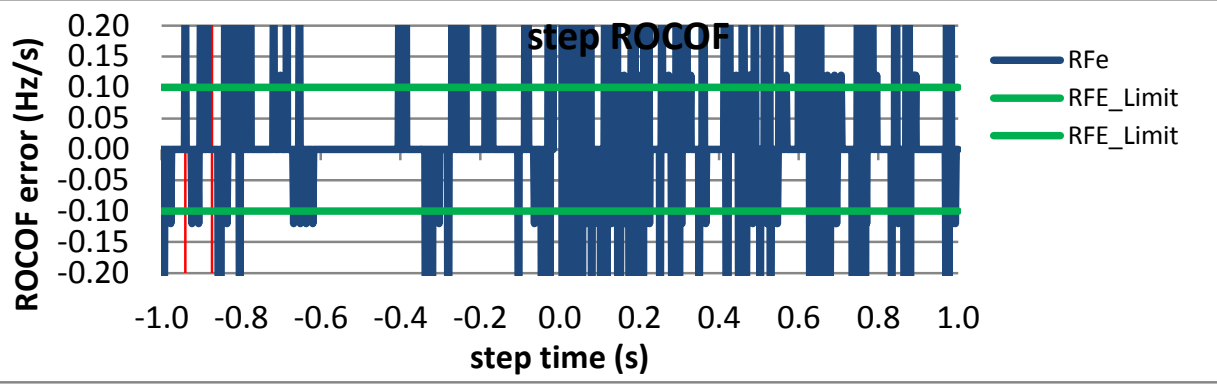

Figure 5916: Fs = 60 FPS, -10 degree phase step

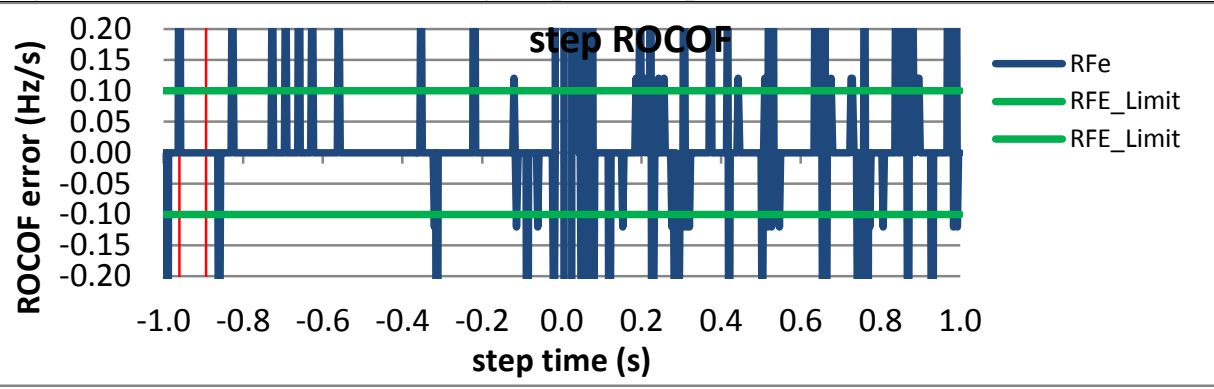

Figure 5918: Fs = 30 FPS, -10 degree phase step

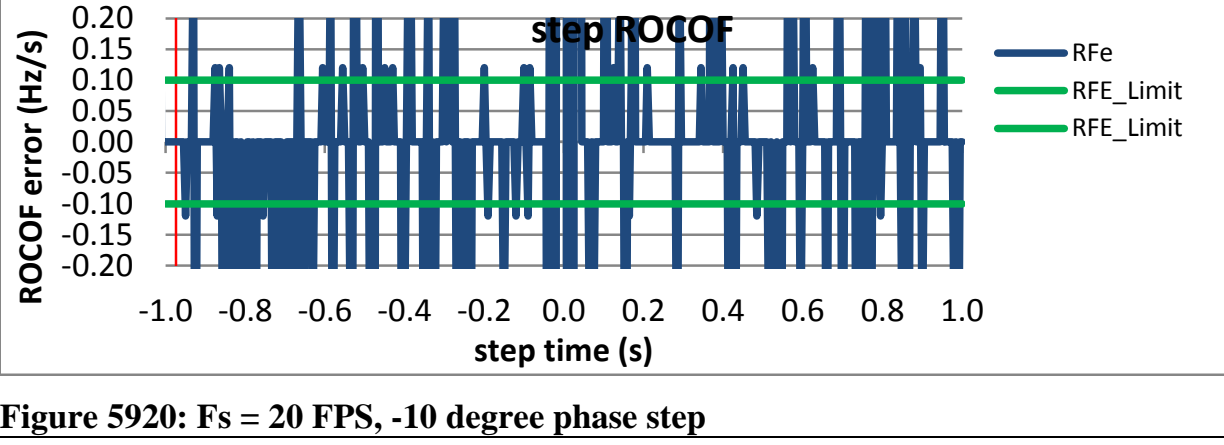




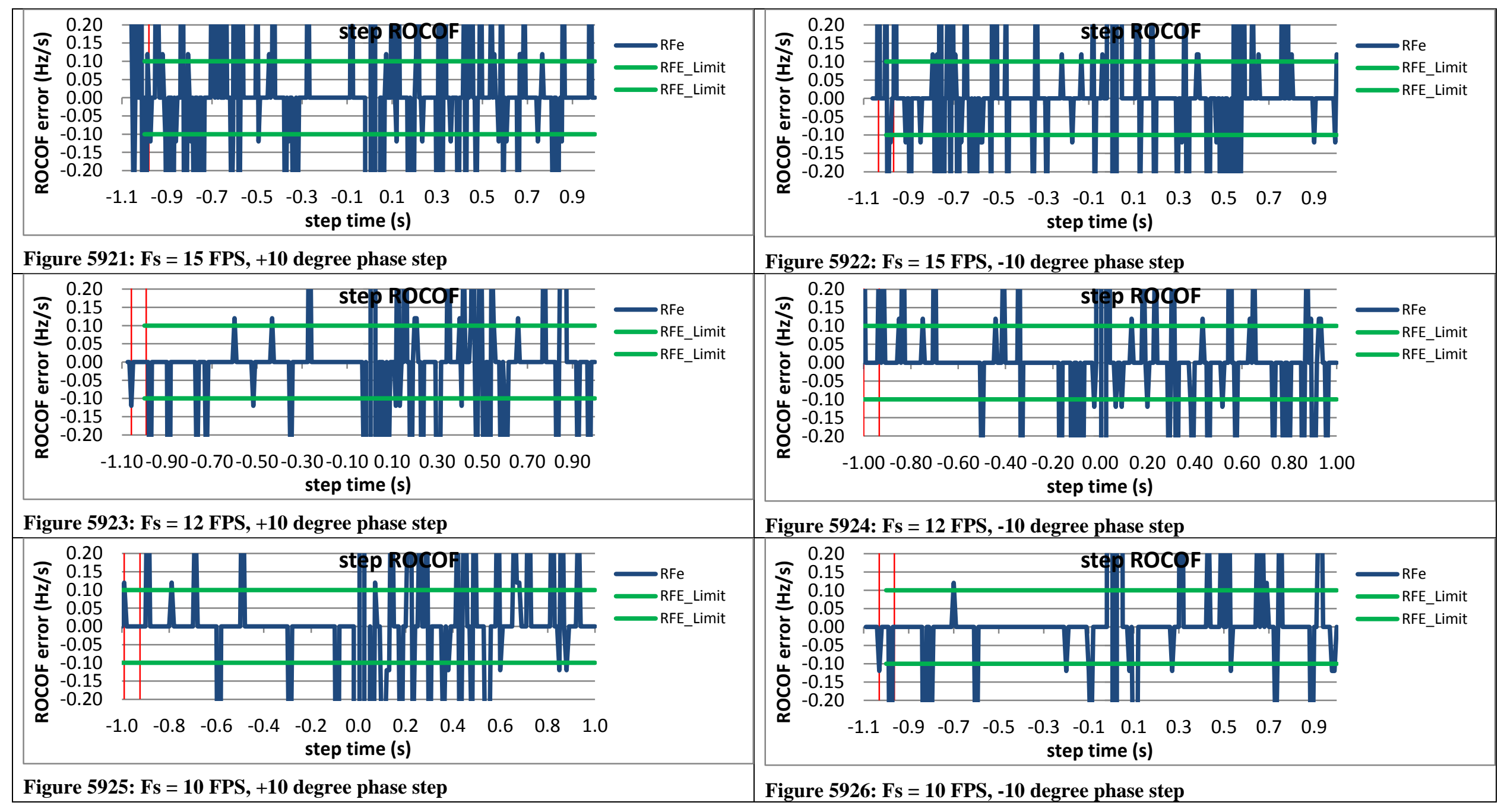


10.10.3 PMU B dynamic step change in magnitude ROCOF response time: $F 0=60 \mathrm{~Hz}, P$ class
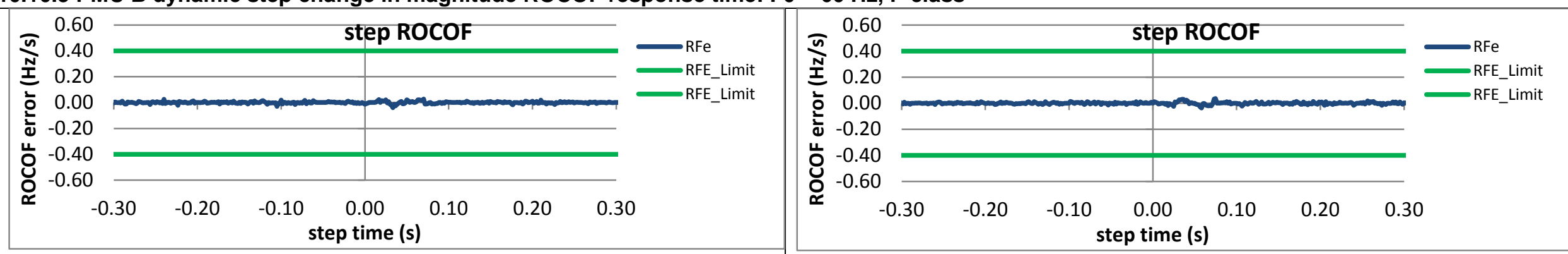

Figure 5927: Fs = 60 FPS, +10\% magnitude step

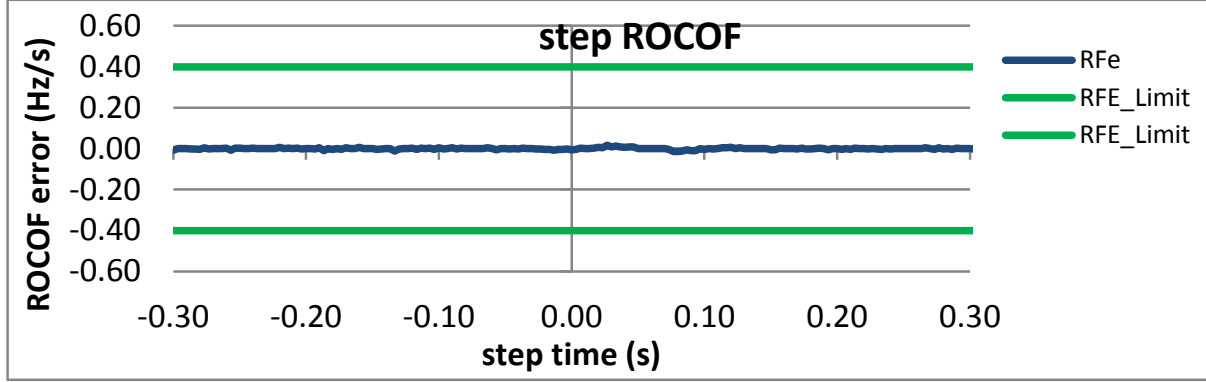

Figure 5928: Fs = 60 FPS, -10\% magnitude step

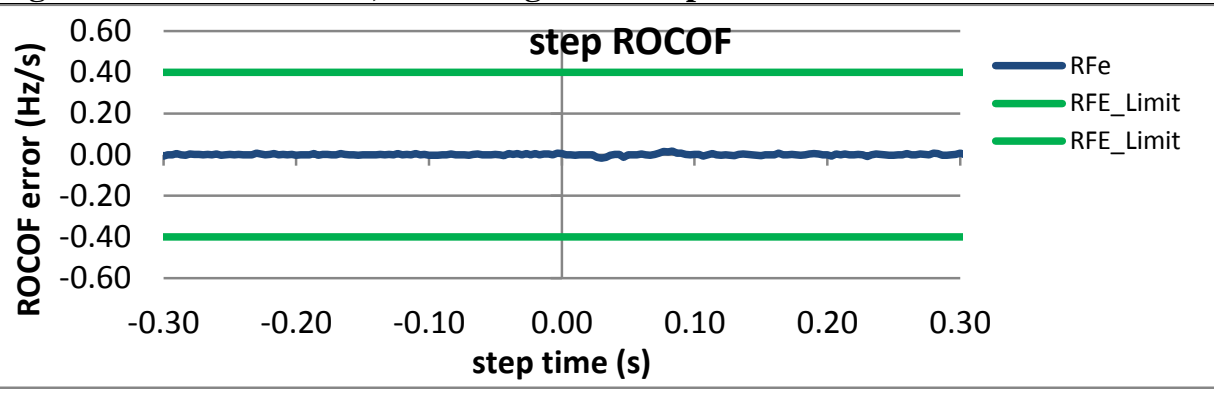

Figure 5929: Fs $=30$ FPS, $+10 \%$ magnitude step

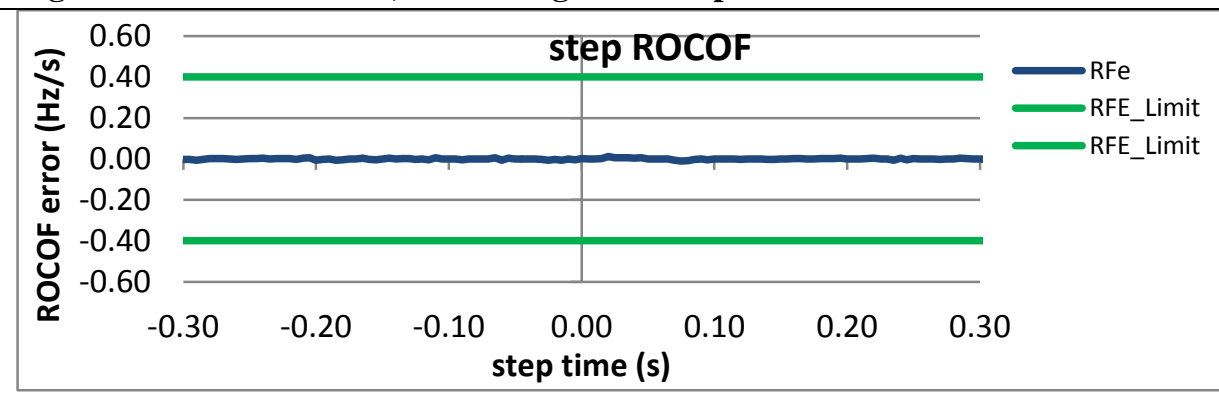

Figure 5930: Fs = 30 FPS, -10\% magnitude step

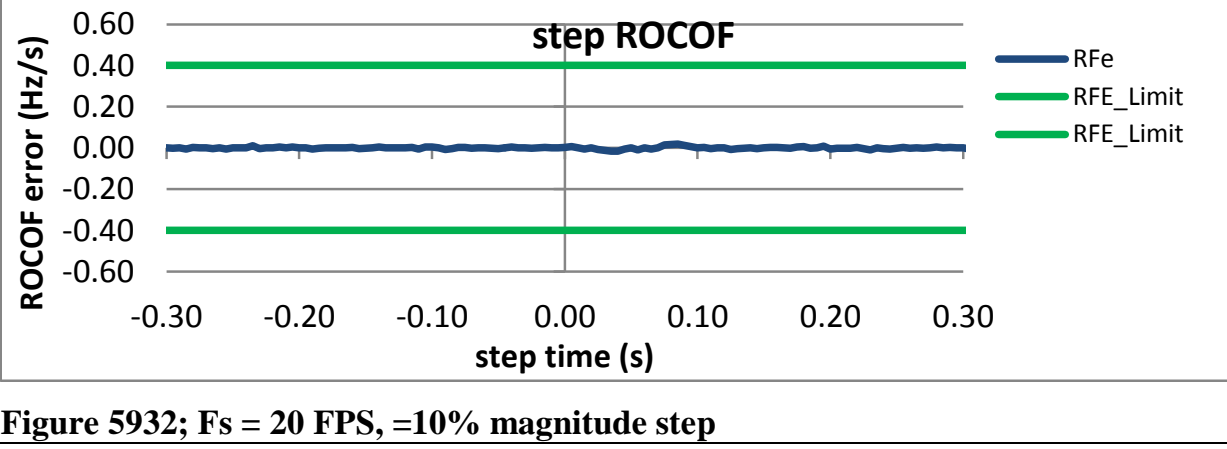

Figure 5931: Fs $=20$ FPS, $+10 \%$ magnitude step

Figure 5932; Fs $=20$ FPS, $=10 \%$ magnitude step 

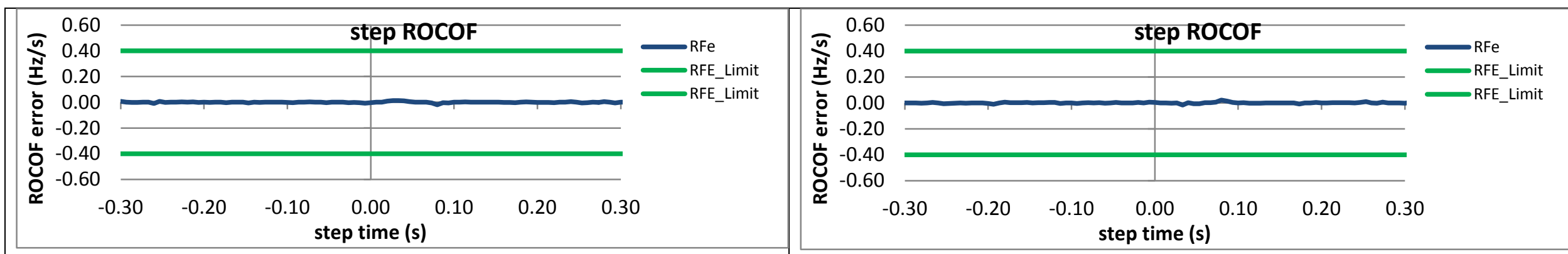

Figure 5933: Fs = 15 FPS, $+10 \%$ magnitude step

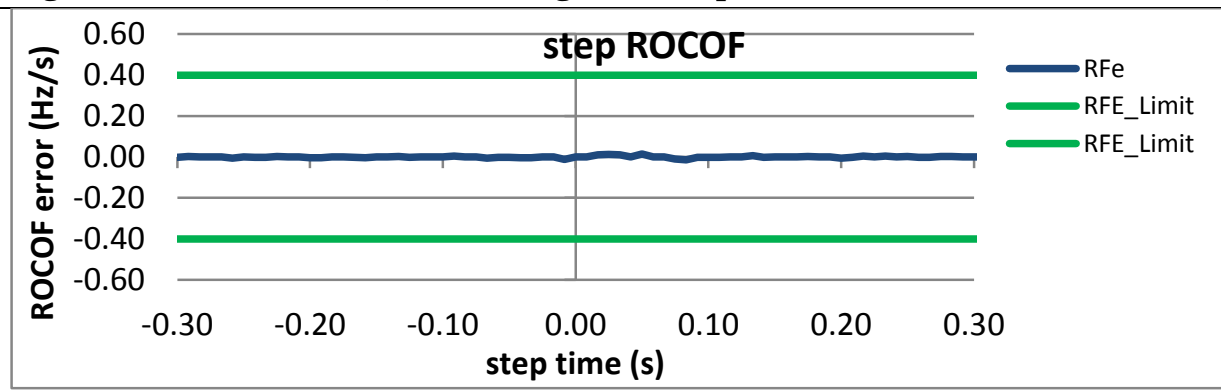

Figure 5934: Fs = 15 FPS, - $10 \%$ magnitude step

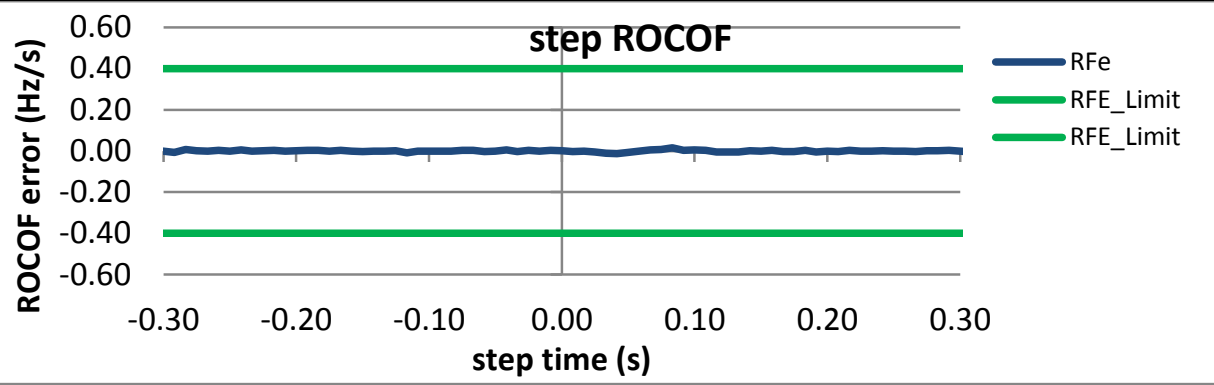

Figure 5935: Fs $=12$ FPS, $+10 \%$ magnitude step

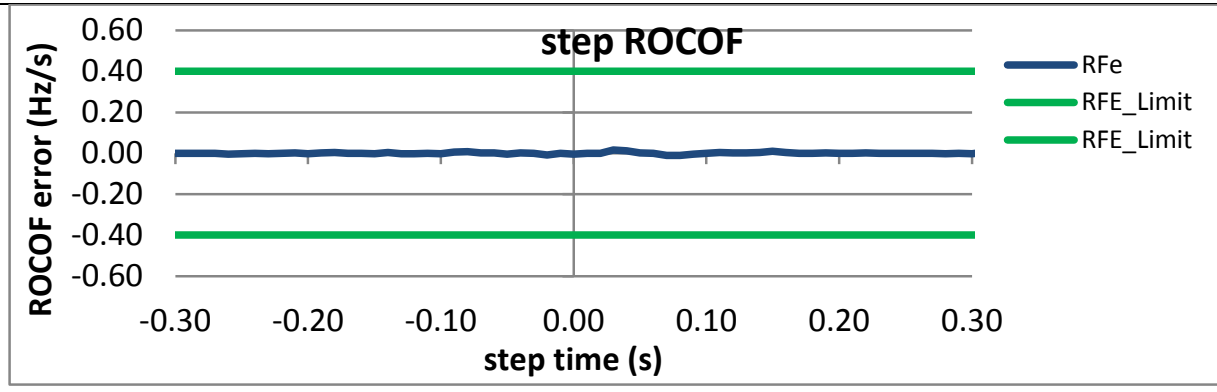

Figure 5936: Fs = 12 FPS, $-\mathbf{- 1 0} \%$ magnitude step

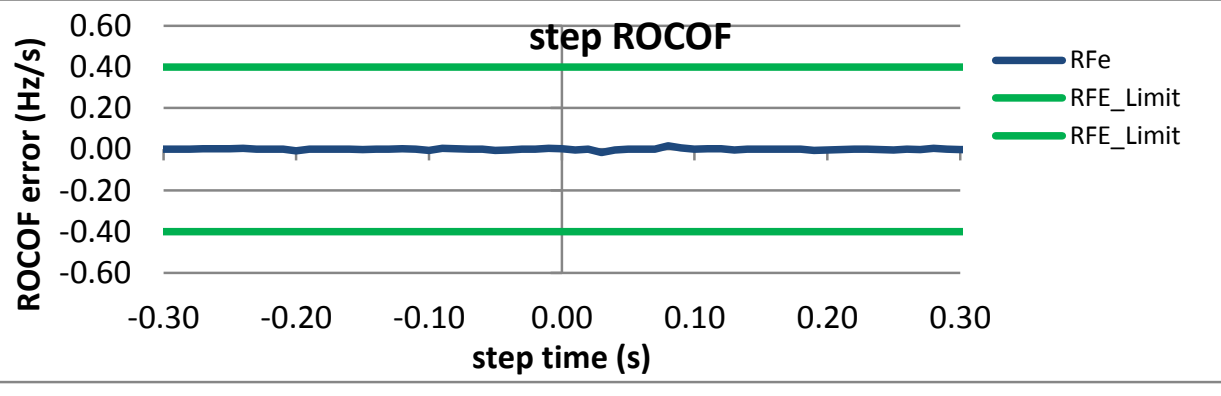

Figure 5938: Fs = 10 FPS, -10\% magnitude step 
10.10.4 PMU C dynamic step change in magnitude ROCOF response time: $F 0=60 \mathrm{~Hz}, \mathrm{P}$ class
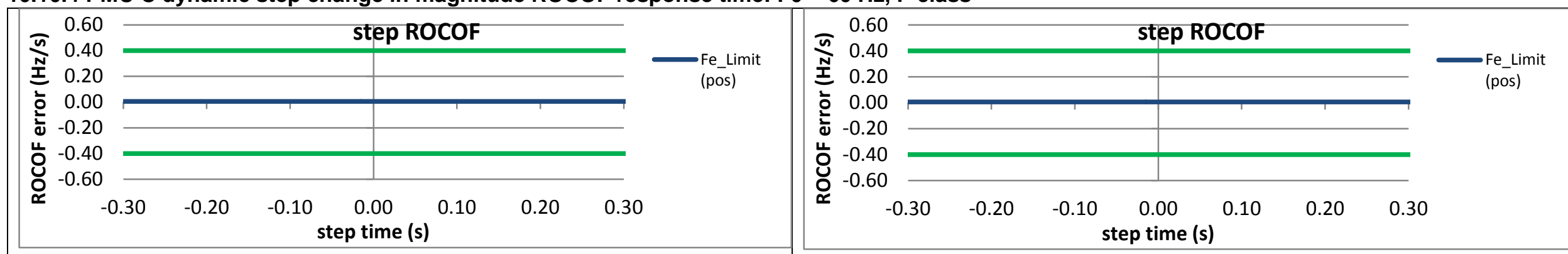

Figure 5939: Fs = 60 FPS, +10\% magnitude step

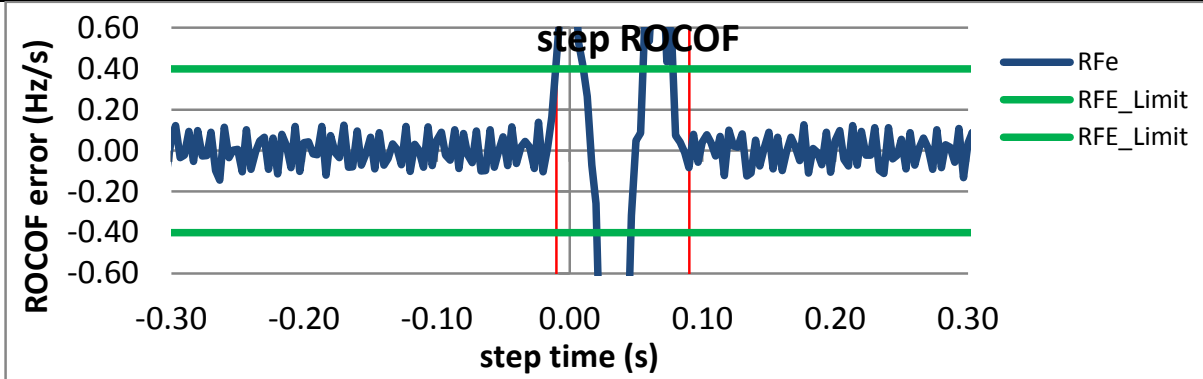

Figure 5940: Fs = 60 FPS, -10\% magnitude step

Figure 5941: Fs = 30 FPS, + 10\% magnitude step

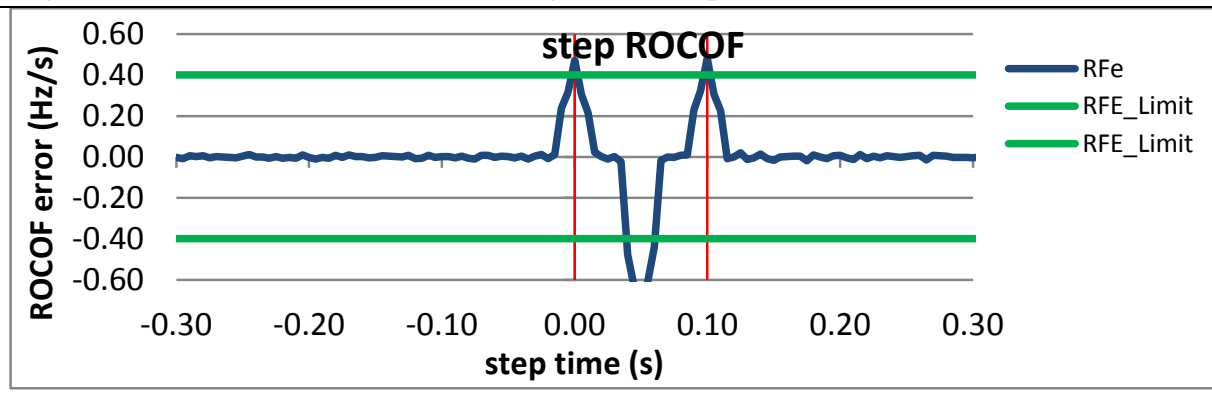

Figure 5943: Fs = 20 FPS, +10\% magnitude step

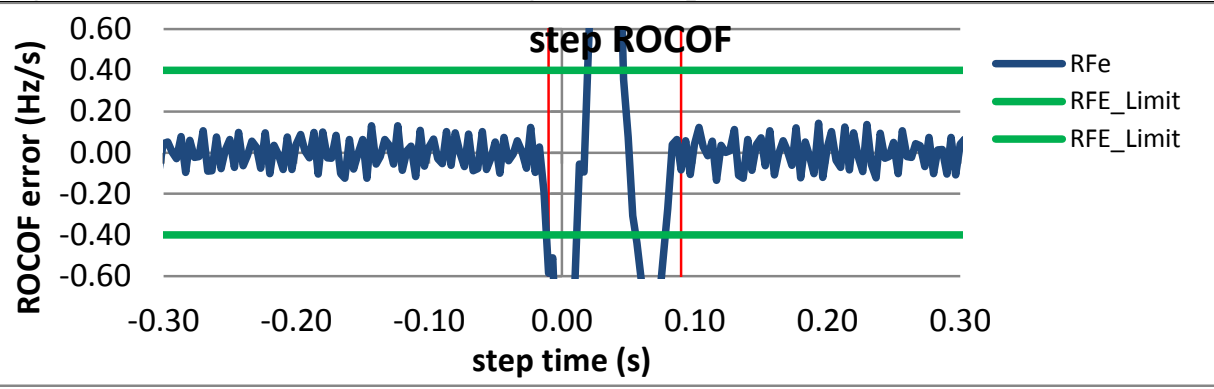

Figure 5942: Fs = 30 FPS, -10\% magnitude step

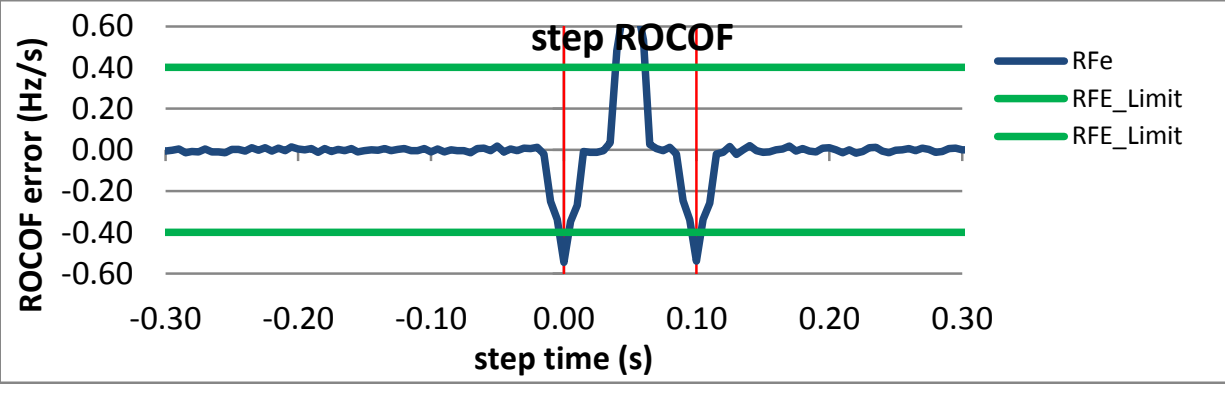

Figure 5944; Fs = 20 FPS, $=10 \%$ magnitude step 

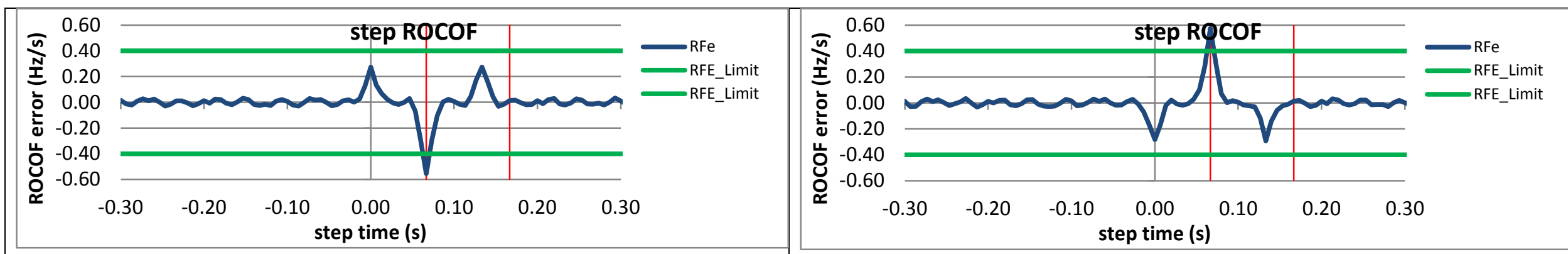

Figure 5945: Fs = 15 FPS, + 10\% magnitude step

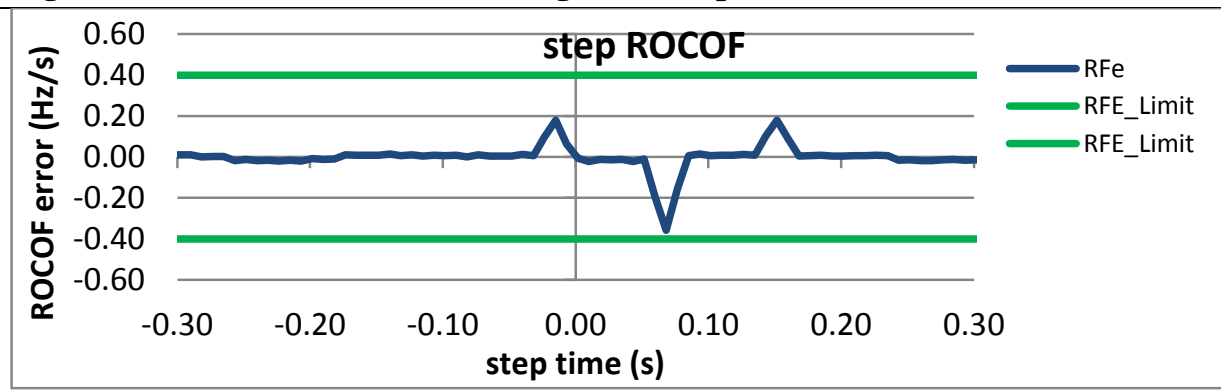

Figure 5946: Fs = 15 FPS, - $10 \%$ magnitude step

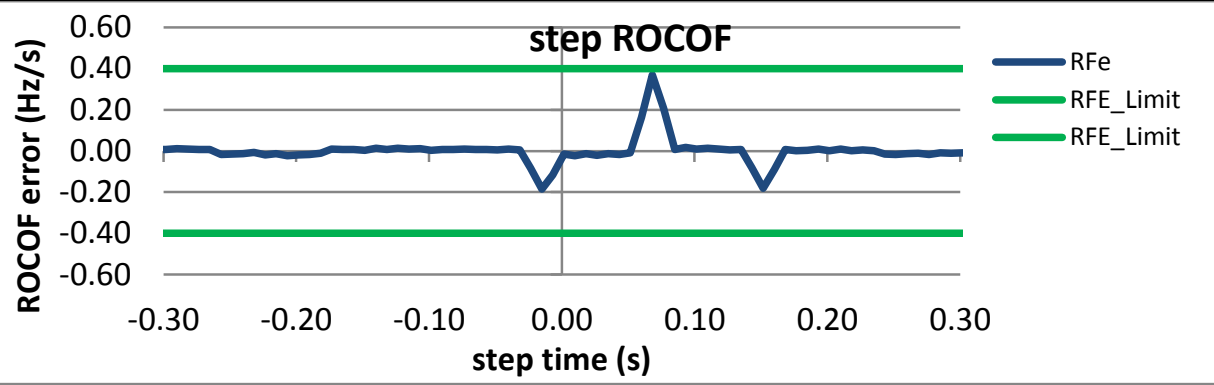

Figure 5947: Fs $=12$ FPS, $+10 \%$ magnitude step

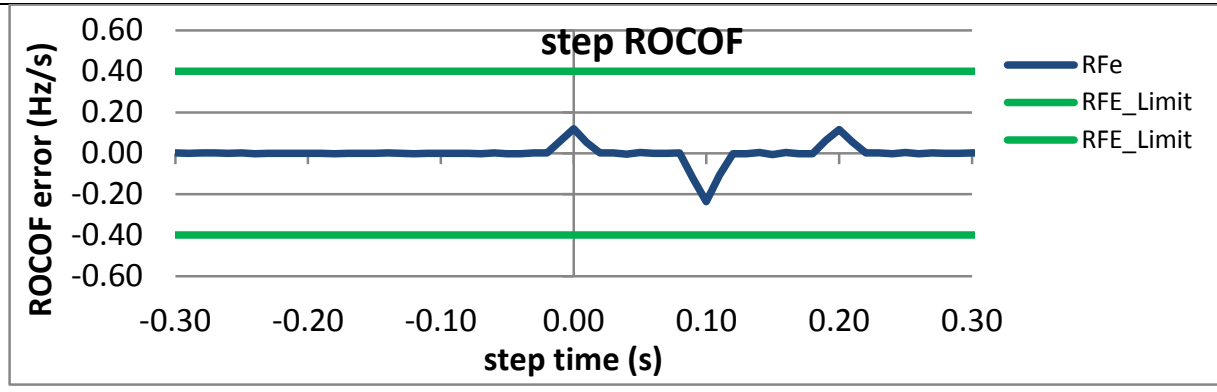

Figure 5948: Fs = 12 FPS, - $10 \%$ magnitude step

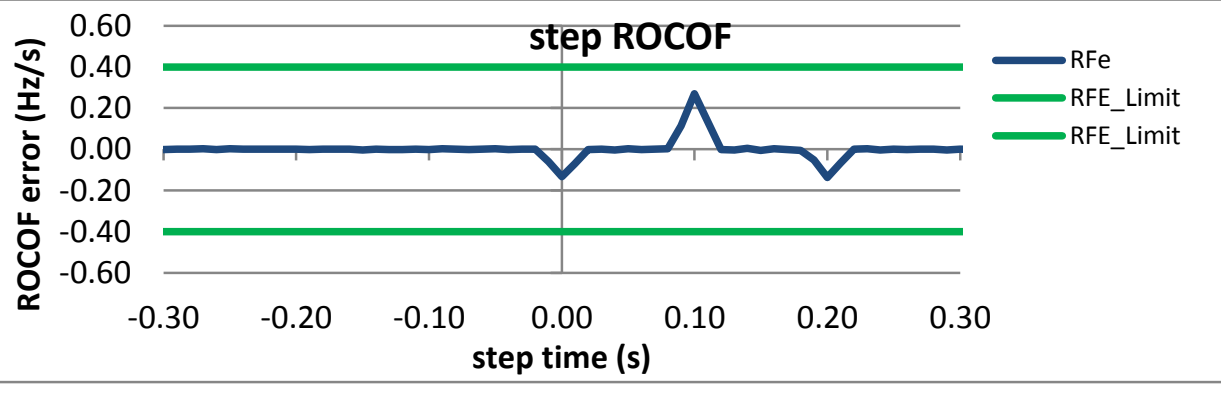

Figure 5950: Fs = 10 FPS, -10\% magnitude step 
10.10.5 PMU D dynamic step change in magnitude ROCOF response time: $\mathrm{F} 0=60 \mathrm{~Hz}, \mathrm{P}$ class

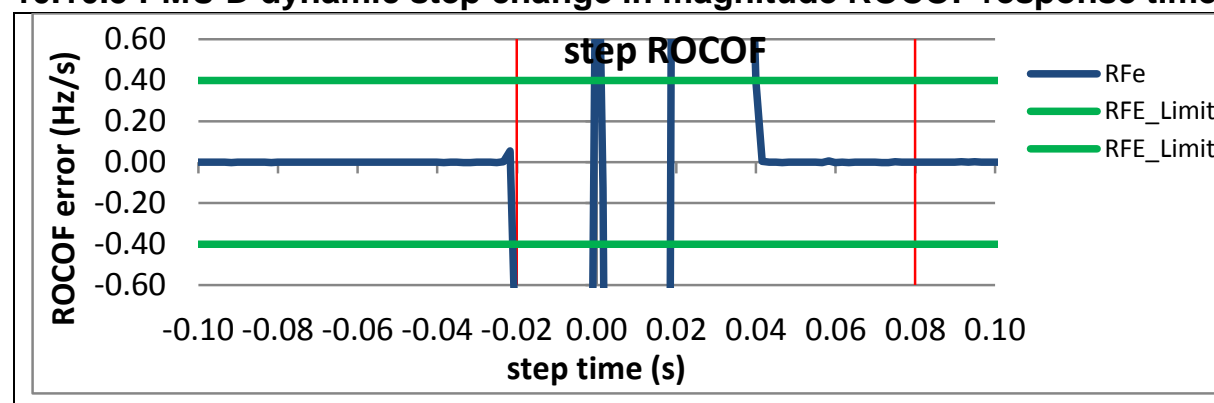

Figure 5951: Fs = 60 FPS, +10\% magnitude step

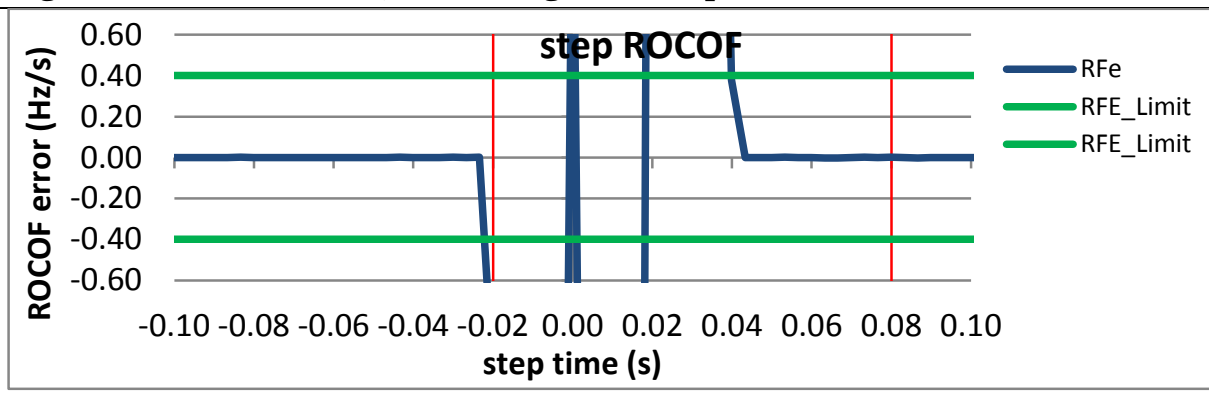

Figure 5953: Fs $=30$ FPS, $+10 \%$ magnitude step

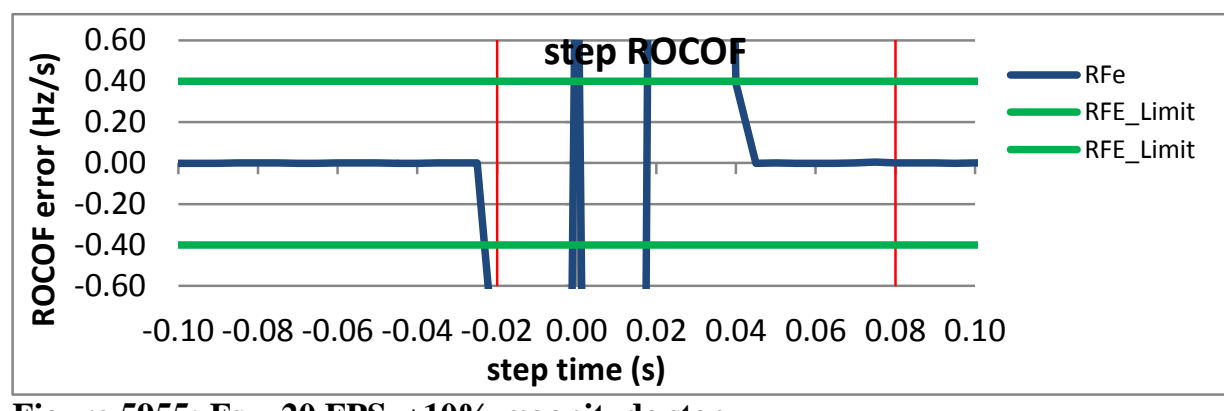

Figure 5955: Fs = 20 FPS, +10\% magnitude step

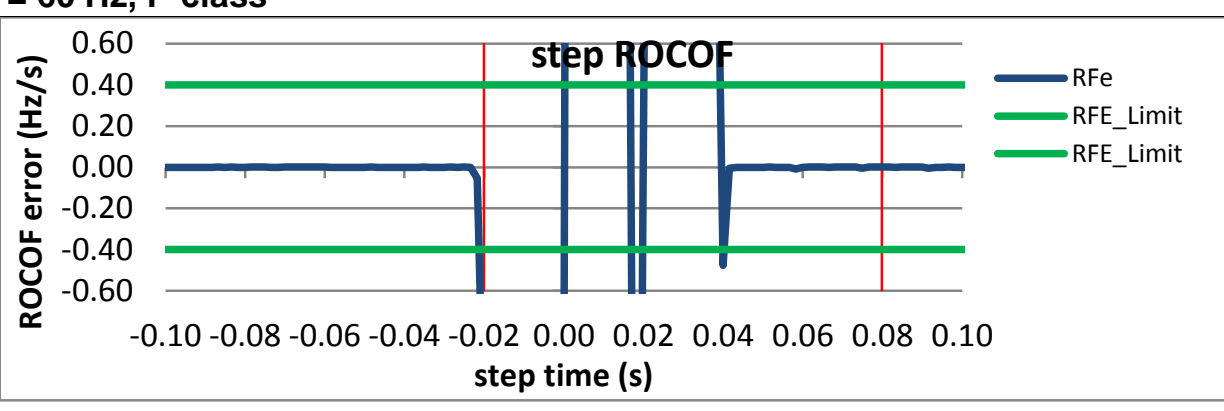

Figure 5952: Fs = 60 FPS, -10\% magnitude step

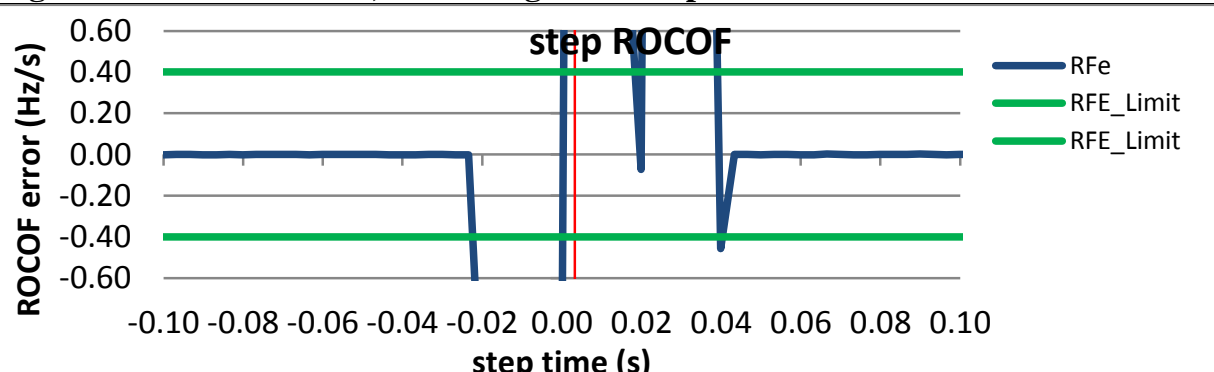
step time (s)

Figure 5954: Fs = 30 FPS, -10\% magnitude step

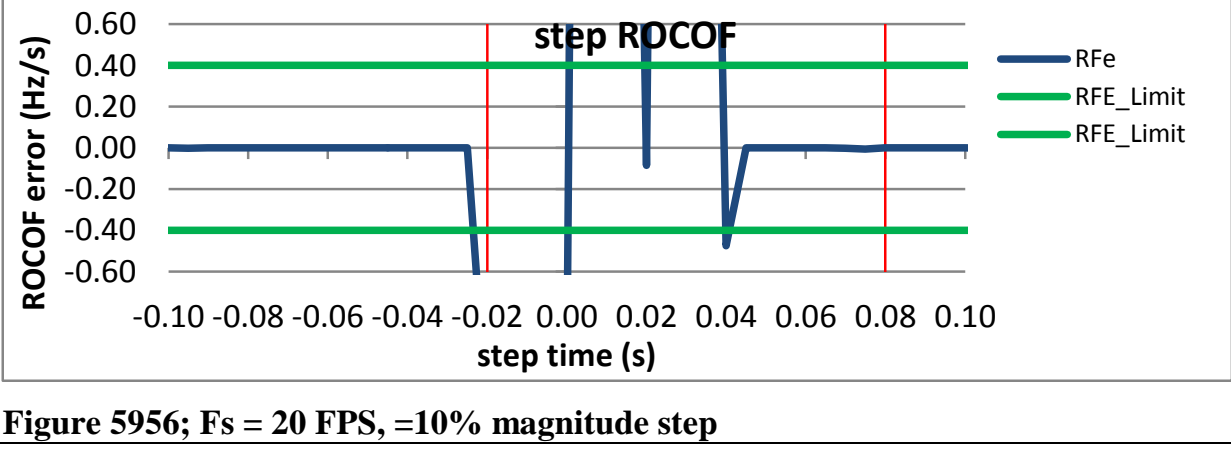



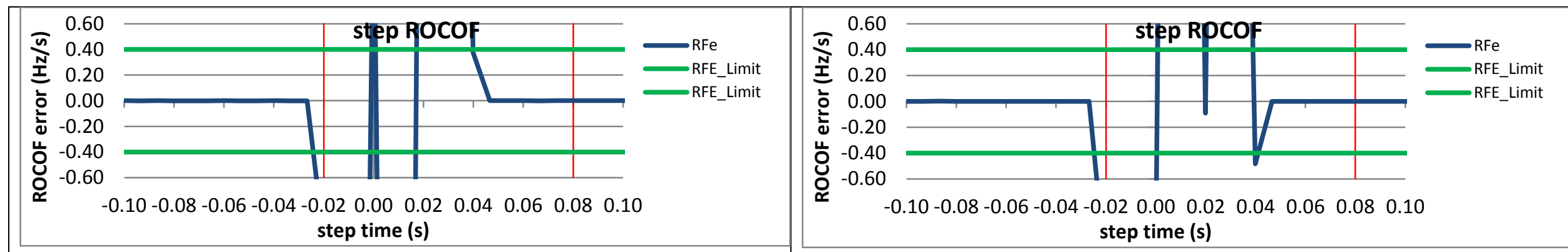

Figure 5957: Fs = 15 FPS, $+10 \%$ magnitude step

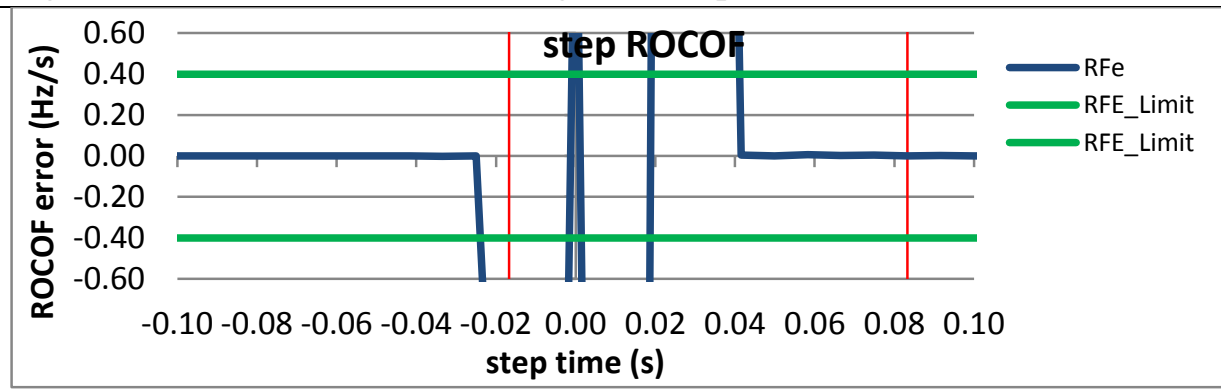

Figure 5958: Fs = 15 FPS, - $10 \%$ magnitude step

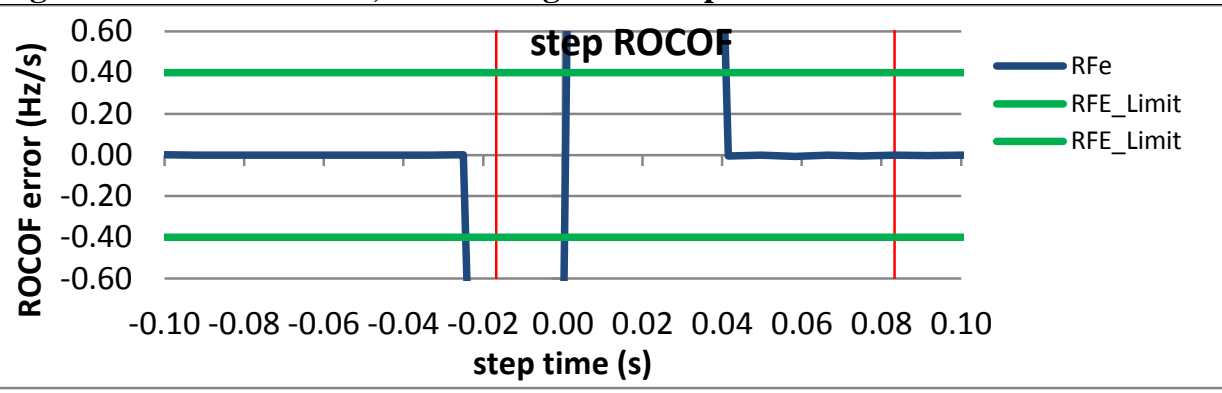

Figure 5959: Fs $=12$ FPS, +10\% magnitude step

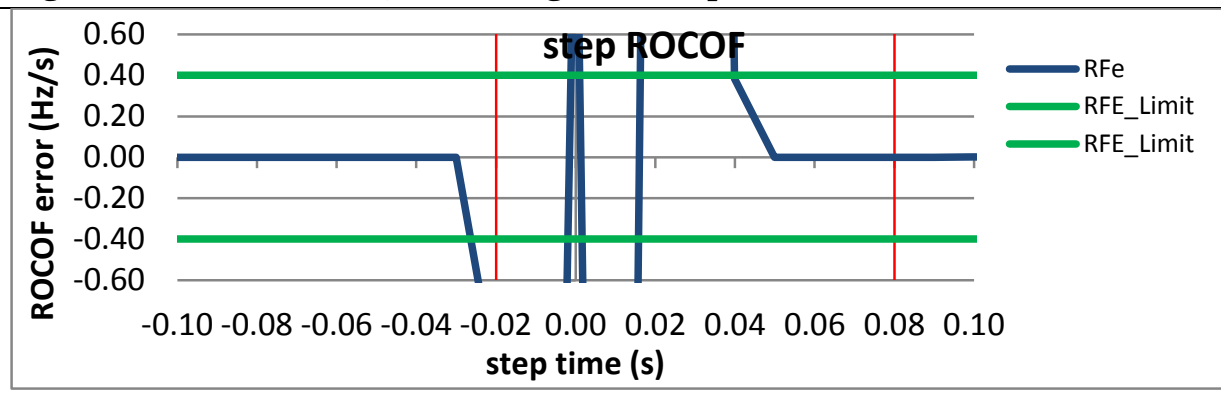

Figure 5960: Fs = 12 FPS, -10\% magnitude step

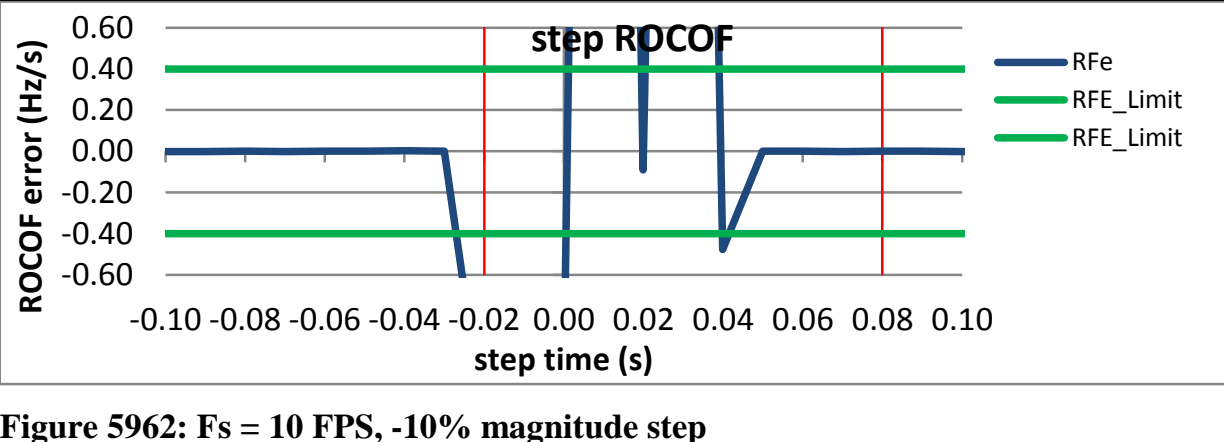

Figure 5961: Fs = 10 FPS, $+10 \%$ magnitude step

Figure 5962: Fs = 10 FPS, -10\% magnitude step 
10.10.6 PMU E dynamic step change in magnitude ROCOF response time: $\mathrm{F} 0=60 \mathrm{~Hz}$, $\mathrm{P}$ class PMU E does not support $P$ class.

\subsubsection{PMU F dynamic step change in magnitude ROCOF response time: $F 0=60 \mathrm{~Hz}, \mathrm{P}$ class}
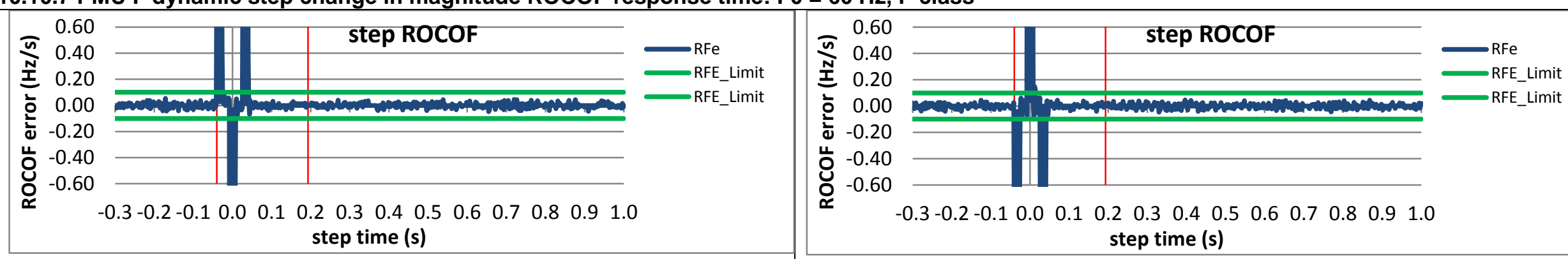

Figure 5963: Fs $=60 \mathrm{FPS},+10 \%$ magnitude step

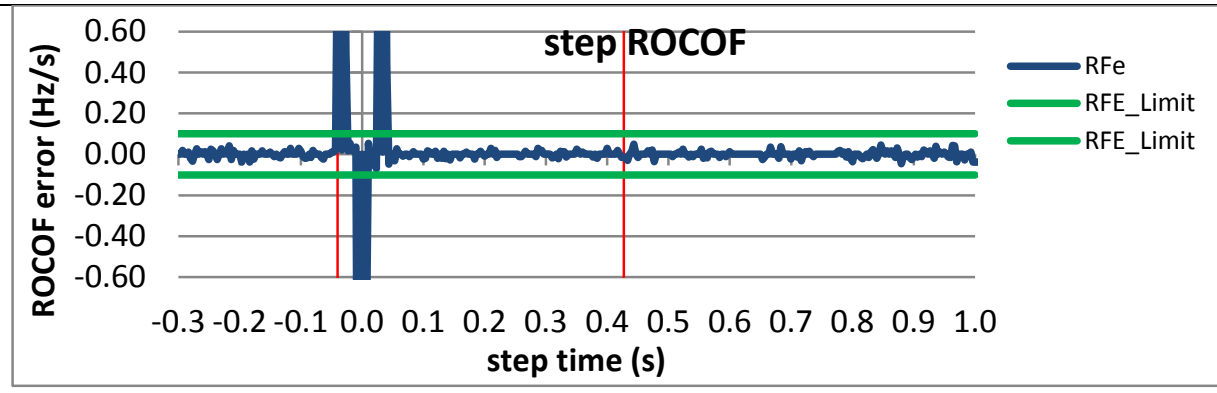

Figure 5964: Fs = $60 \mathrm{FPS},-10 \%$ magnitude step

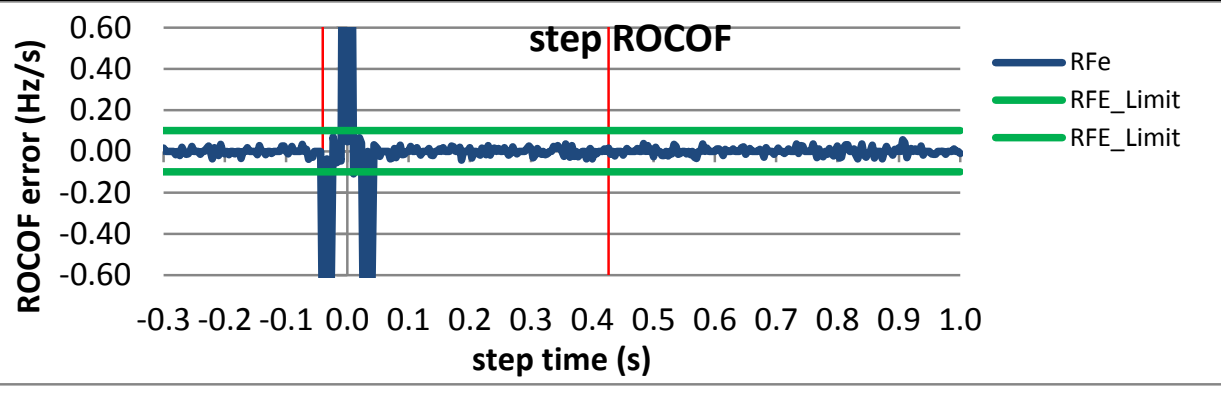

Figure 5965: Fs = 30 FPS, $+10 \%$ magnitude step

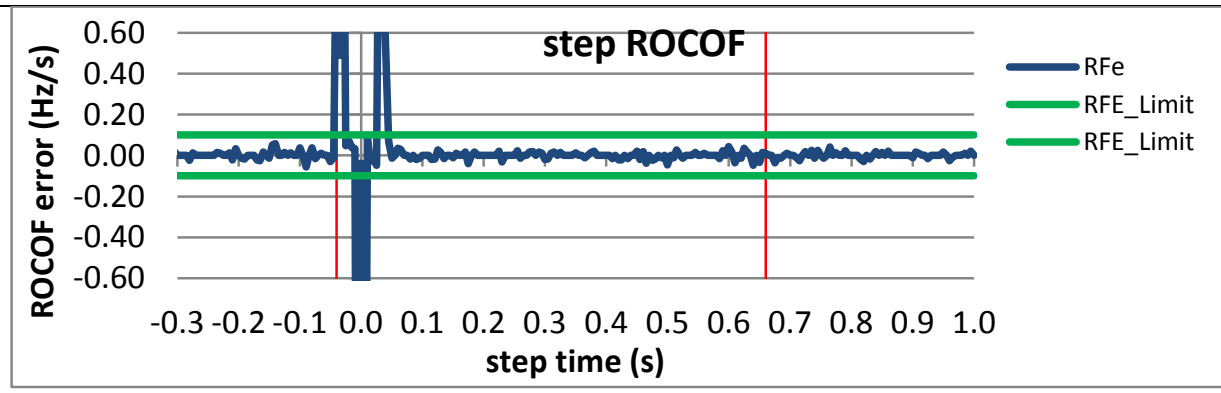

Figure 5966: Fs = $30 \mathrm{FPS},-10 \%$ magnitude step

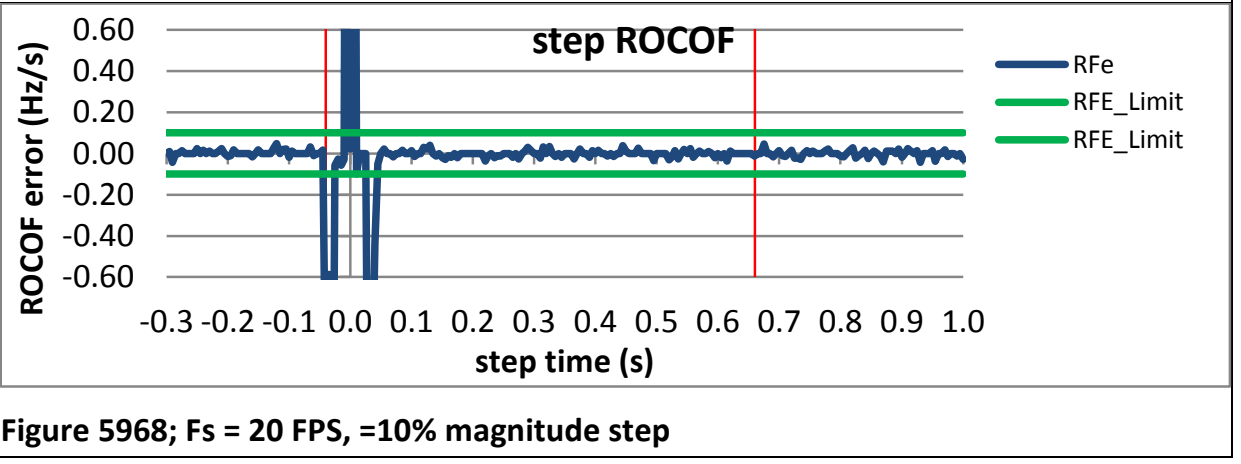




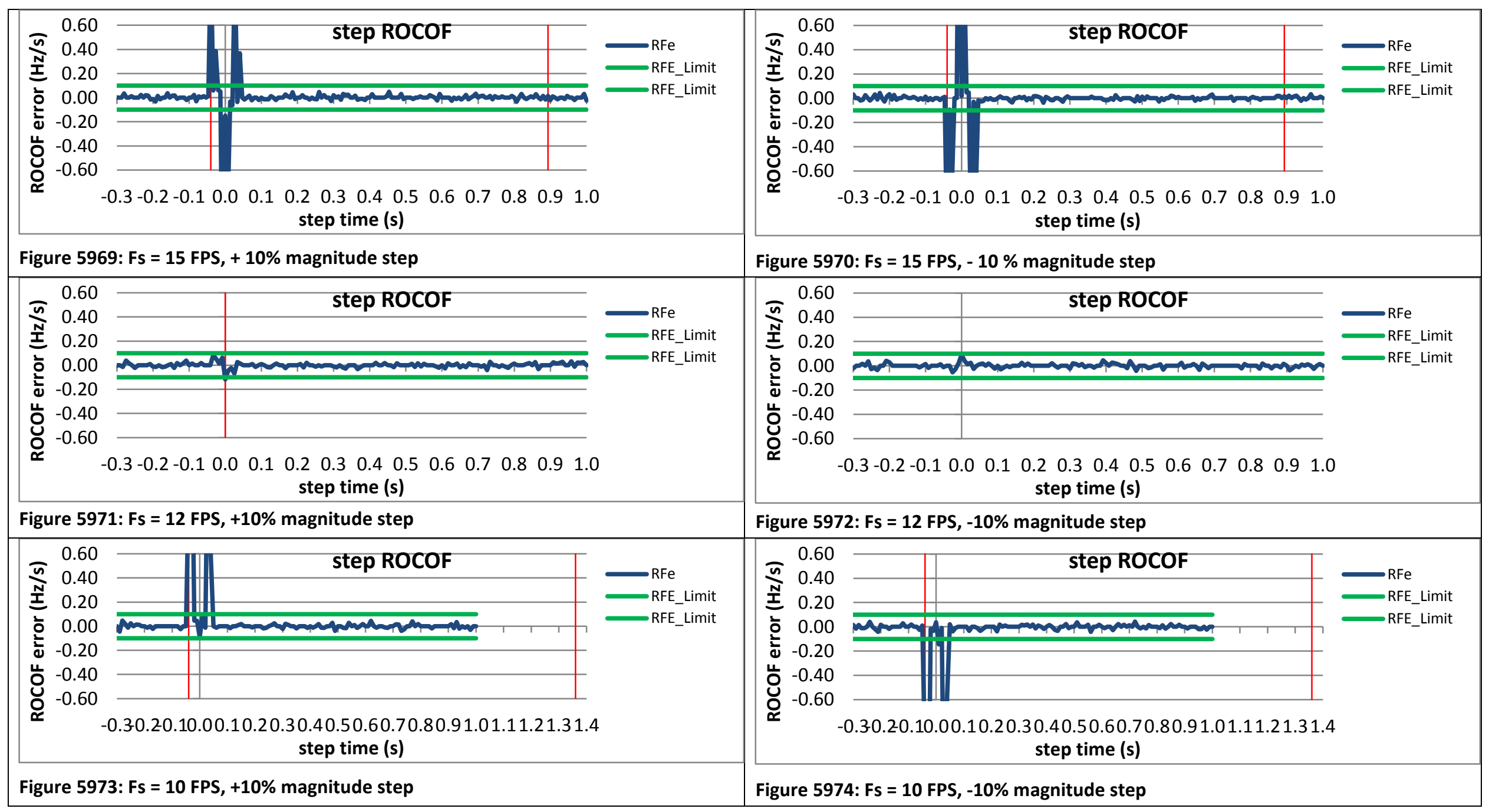


10.10.8 PMU G * dynamic step change in magnitude ROCOF response time: $\mathrm{FO}=60 \mathrm{~Hz}, \mathrm{P}$ class PMU G does not support P class.

\subsubsection{PMU H dynamic step change in magnitude ROCOF response time: $F 0=60 \mathrm{~Hz}, \mathrm{P}$ class}
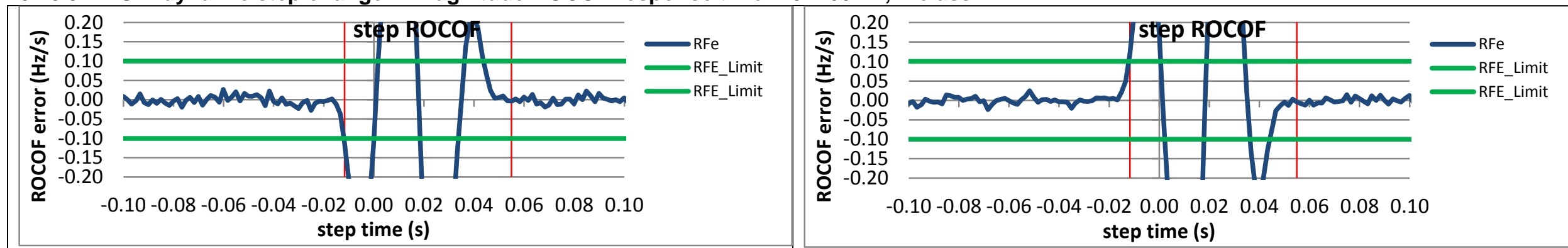

Figure 5975: Fs $=60$ FPS, $+10 \%$ magnitude step

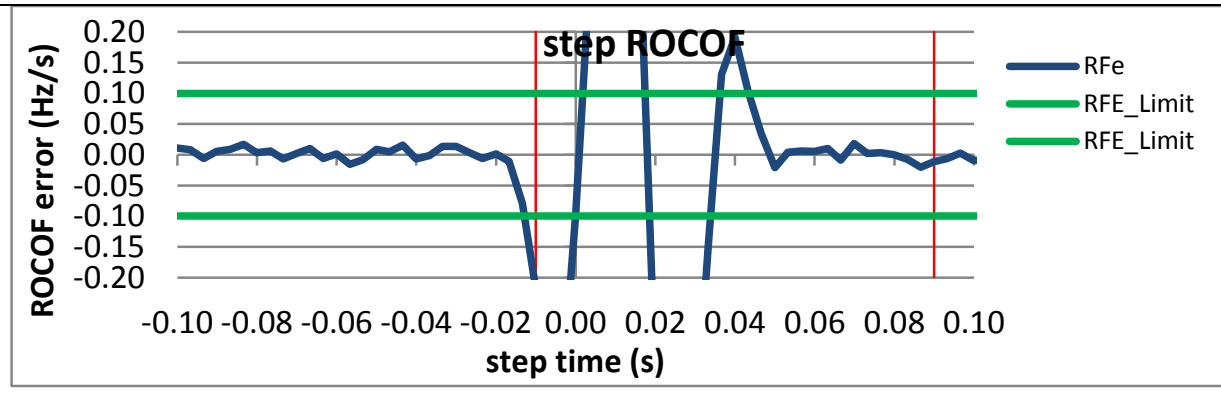

Figure 5976: Fs = 60 FPS, -10\% magnitude step

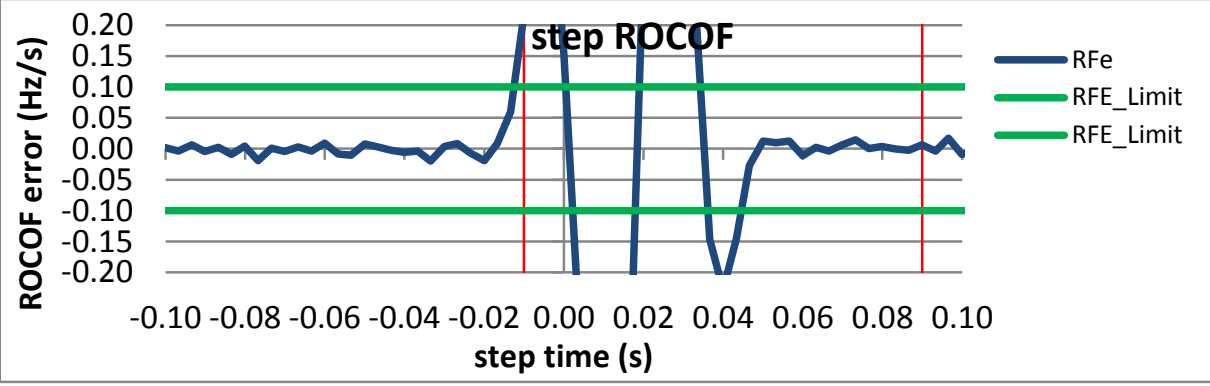

Figure 5977: Fs = 30 FPS, +10\% magnitude step

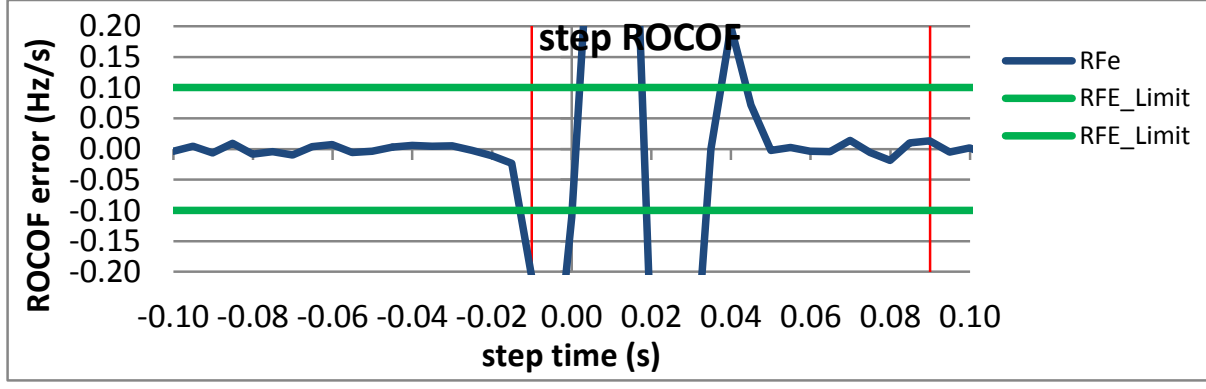

Figure 5979: Fs = 20 FPS, +10\% magnitude step

Figure 5978: Fs = 30 FPS, -10\% magnitude step

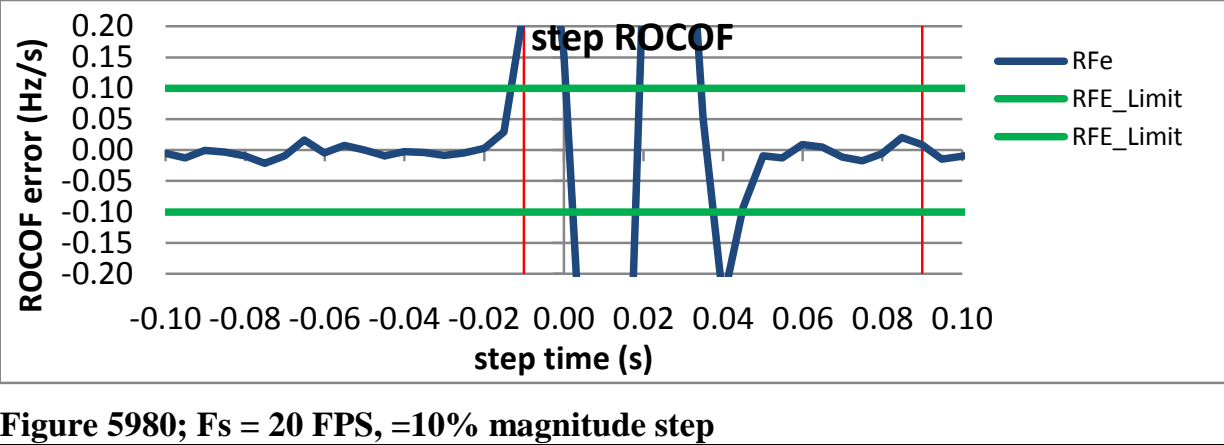



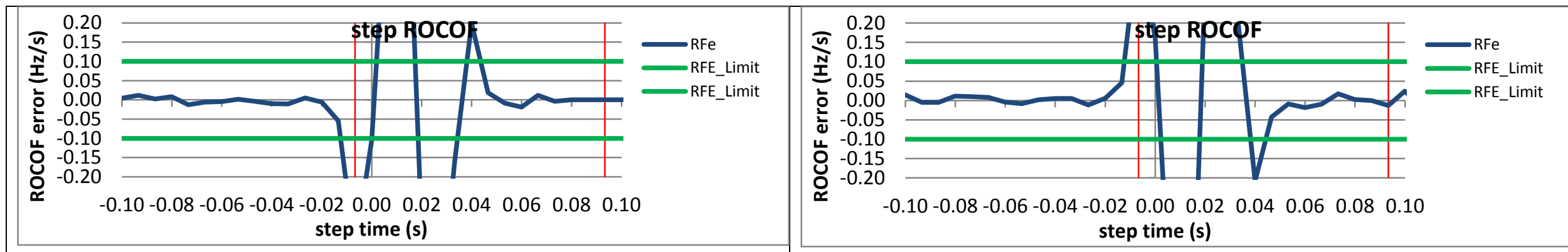

Figure 5981: Fs = 15 FPS, + 10\% magnitude step

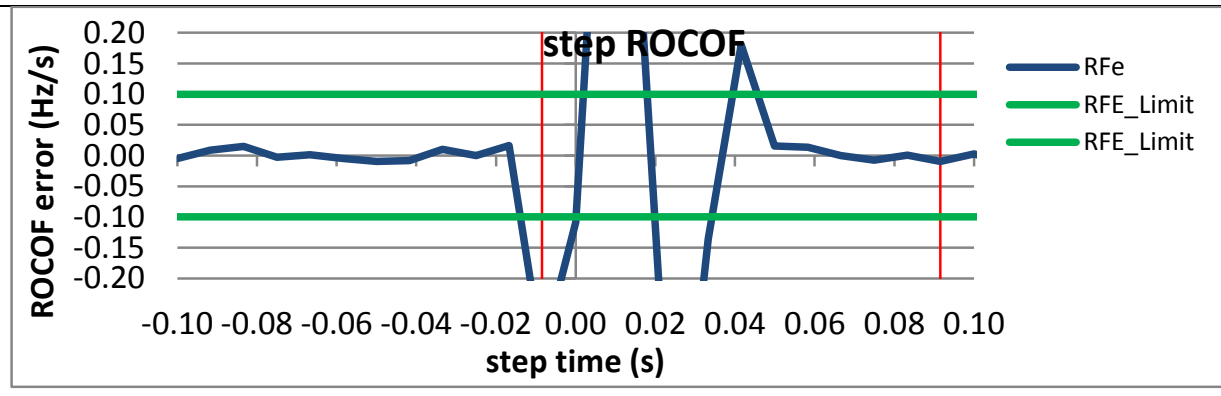

Figure 5982: Fs = 15 FPS, - $10 \%$ magnitude step

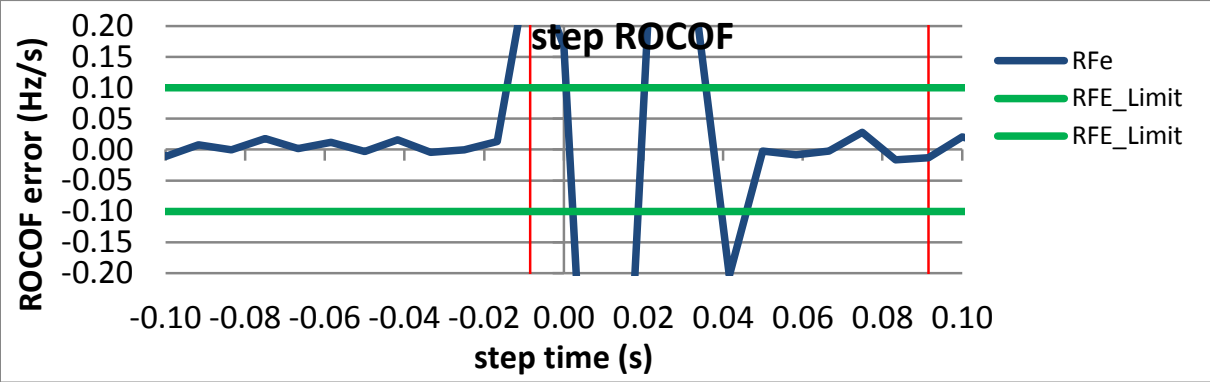

Figure 5983: Fs = 12 FPS, +10\% magnitude step

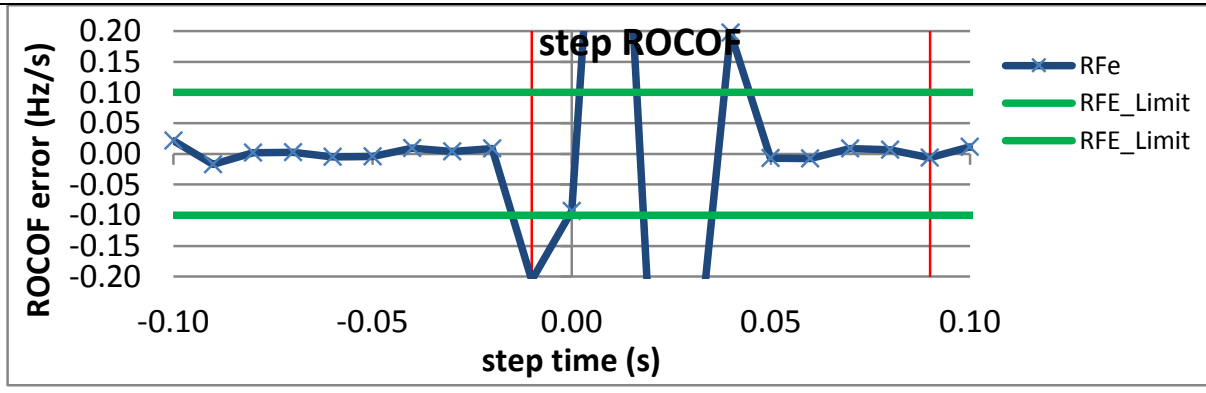

Figure 5985: Fs = 10 FPS, +10\% magnitude step

Figure 5984: Fs = 12 FPS, -10\% magnitude step

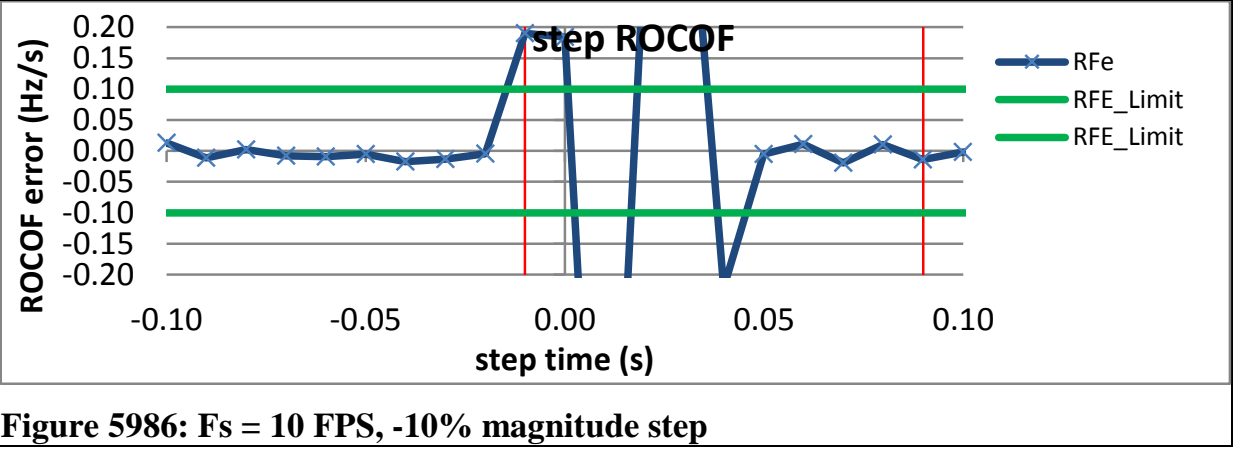


10.10.10 PMU I dynamic step change in magnitude ROCOF response time: $F 0=60 \mathrm{~Hz}, P$ class PMU I does not support P class

\subsubsection{PMU J dynamic step change in magnitude ROCOF response time: $\mathrm{F} 0=60 \mathrm{~Hz}, \mathrm{P}$ class}
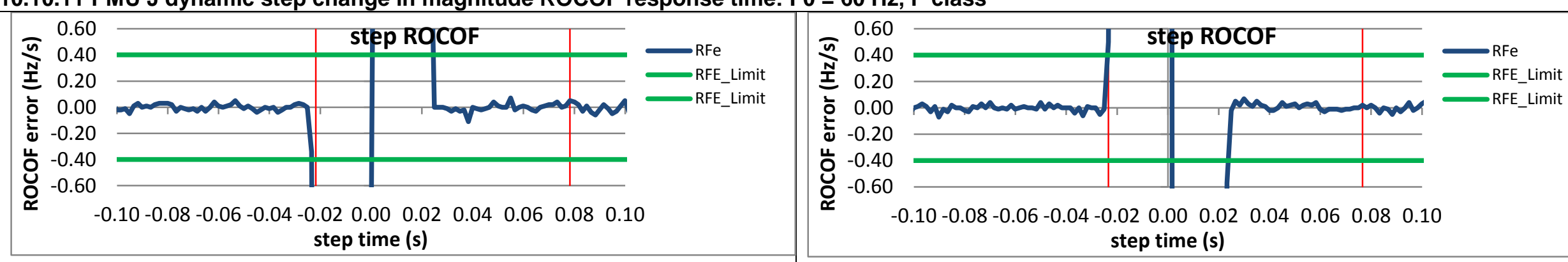

Figure 5987: Fs = 60 FPS, +10 degree phase step

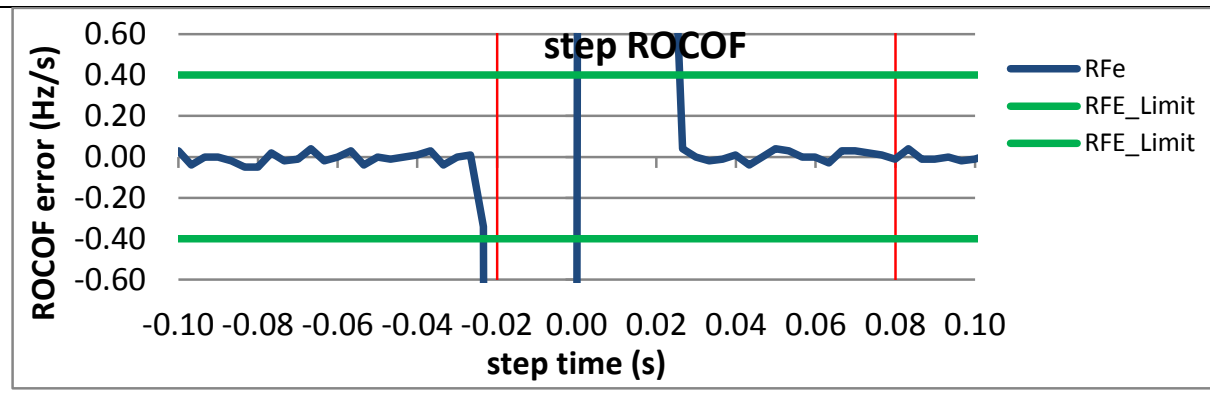

Figure 5988: Fs = 60 FPS, -10 degree phase step

Figure 5989: Fs = 30 FPS, +10 degree phase step

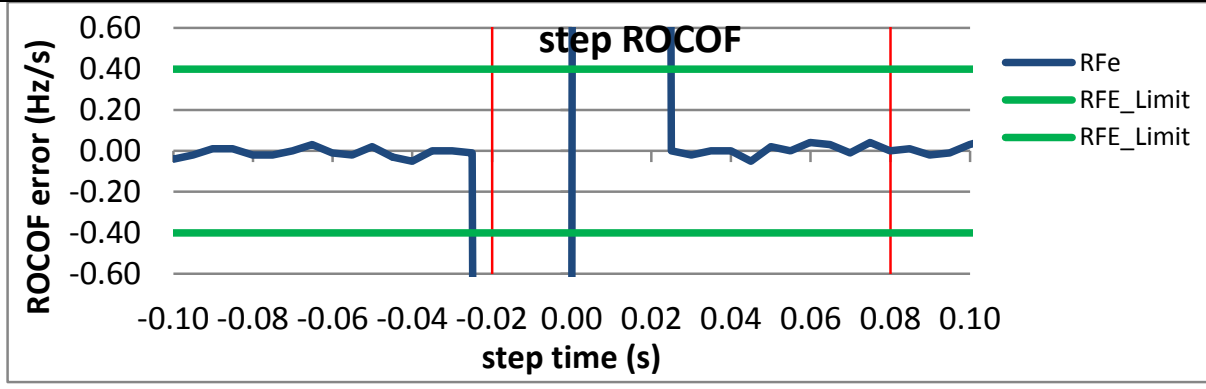

Figure 5991: Fs = 20 FPS, +10 degree phase step

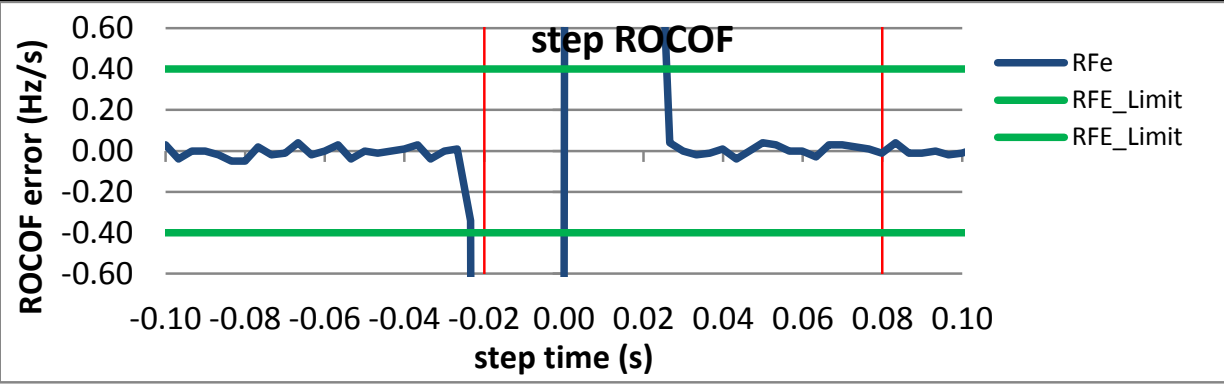

Figure 5990: Fs = $30 \mathrm{FPS},-10$ degree phase step

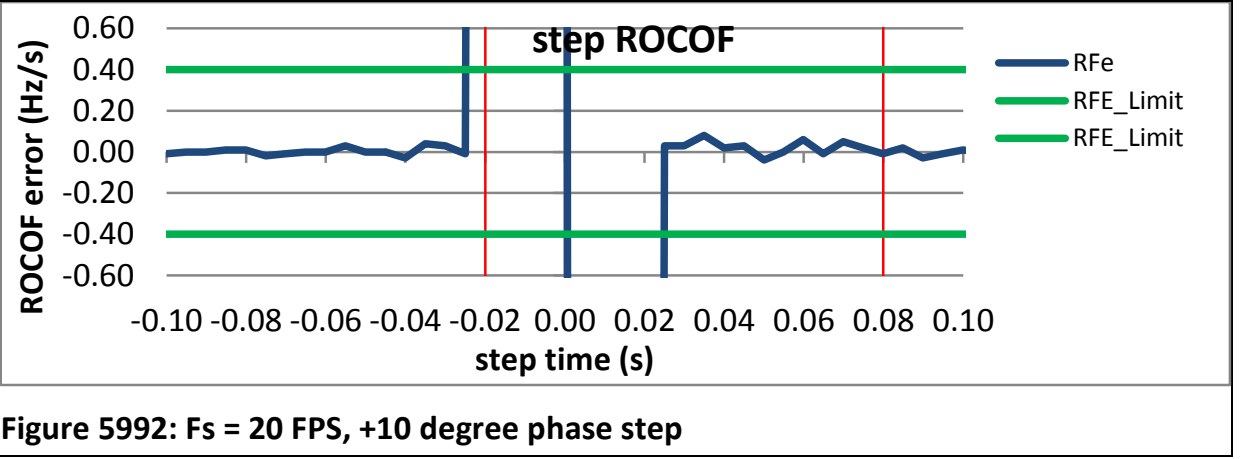




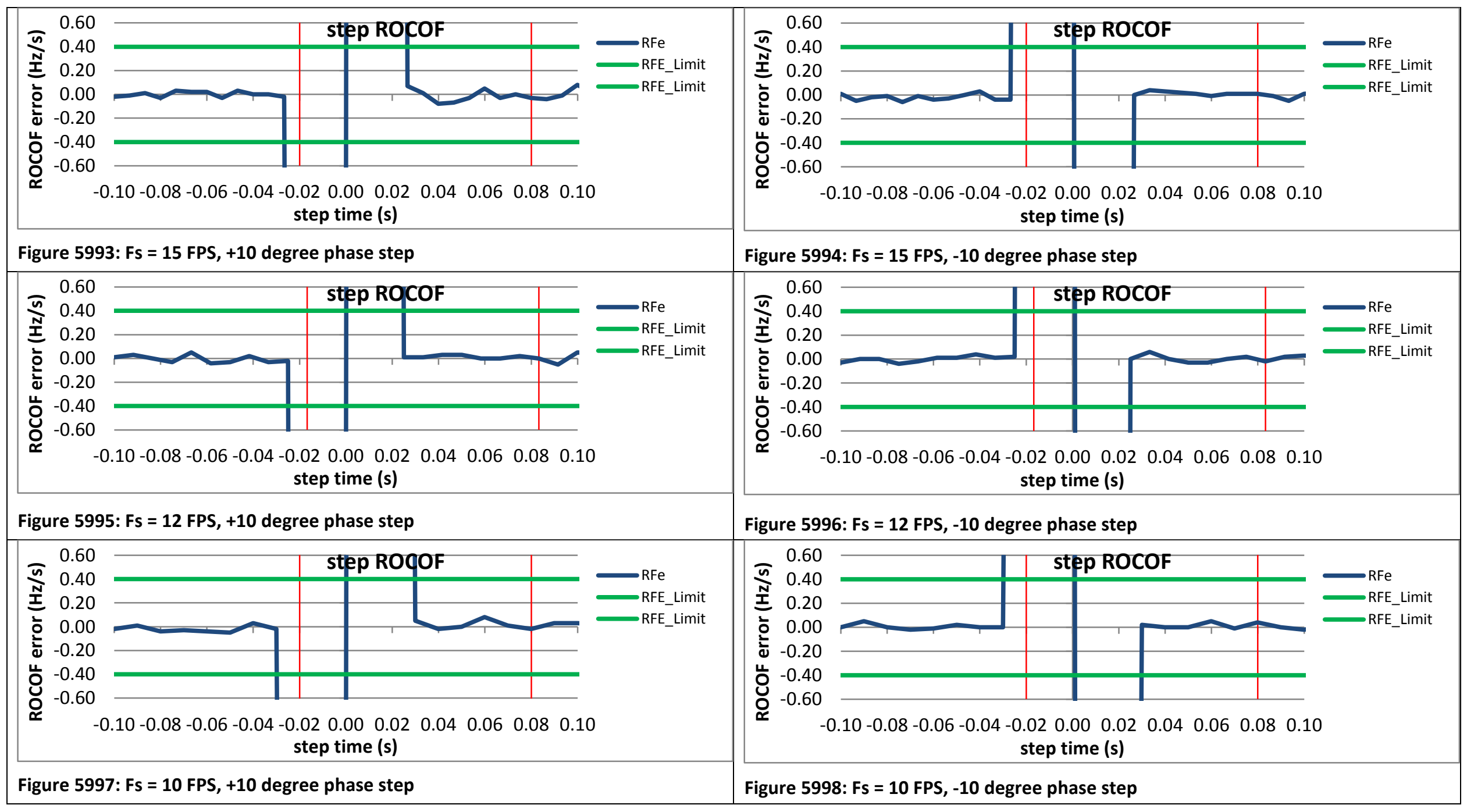


10.11 Dynamic step change in magnitude: phasor overshoot

\begin{tabular}{|l|c|c|c|c|c|c|c|c|c|c|c|c|}
\hline Fs (FPS) & $10 M$ & $10 \mathrm{P}$ & $12 \mathrm{M}$ & $12 \mathrm{P}$ & $15 \mathrm{M}$ & $15 \mathrm{P}$ & $20 \mathrm{M}$ & $20 \mathrm{P}$ & $30 \mathrm{M}$ & $30 \mathrm{P}$ & $60 \mathrm{M}$ & $60 \mathrm{P}$ \\
\hline C37.118.1 Annex C & $\mathrm{P}$ & $\mathrm{P}$ & $\mathrm{P}$ & $\mathrm{P}$ & $\mathrm{P}$ & $\mathrm{P}$ & $\mathrm{P}$ & $\mathrm{P}$ & $\mathrm{P}$ & $\mathrm{P}$ & $\mathrm{P}$ & $\mathrm{P}$ \\
\hline PMU A & $\mathrm{P}$ & $\mathrm{P}$ & $\mathrm{P}$ & $\mathrm{P}$ & $\mathrm{P}$ & $\mathrm{P}$ & $\mathrm{P}$ & $\mathrm{P}$ & $\mathrm{P}$ & $\mathrm{P}$ & $\mathrm{P}$ & $\mathrm{P}$ \\
\hline PMU B & $\mathrm{F}$ & $\mathrm{F}$ & $\mathrm{F}$ & $\mathrm{F}$ & $\mathrm{F}$ & $\mathrm{F}$ & $\mathrm{F}$ & $\mathrm{F}$ & $\mathrm{F}$ & $\mathrm{F}$ & $\mathrm{F}$ & $\mathrm{F}$ \\
\hline PMU C & $\mathrm{P}$ & $\mathrm{P}$ & $\mathrm{P}$ & $\mathrm{P}$ & $\mathrm{P}$ & $\mathrm{P}$ & $\mathrm{P}$ & $\mathrm{P}$ & $\mathrm{P}$ & $\mathrm{P}$ & $\mathrm{P}$ & $\mathrm{P}$ \\
\hline PMU D & $\mathrm{P}$ & $\mathrm{P}$ & $\mathrm{P}$ & $\mathrm{P}$ & $\mathrm{P}$ & $\mathrm{P}$ & $\mathrm{P}$ & $\mathrm{P}$ & $\mathrm{P}$ & $\mathrm{P}$ & $\mathrm{P}$ & $\mathrm{P}$ \\
\hline PMU E & $\mathrm{P}$ & - & - & - & $\mathrm{P}$ & - & $\mathrm{P}$ & - & $\mathrm{P}$ & - & $\mathrm{P}$ & - \\
\hline PMU F & $\mathrm{P}$ & $\mathrm{P}$ & $\mathrm{P}$ & $\mathrm{P}$ & $\mathrm{P}$ & $\mathrm{P}$ & $\mathrm{P}$ & $\mathrm{P}$ & $\mathrm{P}$ & $\mathrm{P}$ & $\mathrm{P}$ & $\mathrm{P}$ \\
\hline PMU G & $\mathrm{P}$ & - & $\mathrm{I}$ & - & $\mathrm{I}$ & - & $\mathrm{I}$ & - & $\mathrm{I}$ & - & - & - \\
\hline PMU H & $\mathrm{P}$ & $\mathrm{P}$ & $\mathrm{P}$ & $\mathrm{P}$ & $\mathrm{P}$ & $\mathrm{P}$ & $\mathrm{P}$ & $\mathrm{P}$ & $\mathrm{P}$ & $\mathrm{P}$ & $\mathrm{P}$ & $\mathrm{P}$ \\
\hline PMU I & $\mathrm{P}$ & - & $\mathrm{P}$ & - & $\mathrm{P}$ & - & $\mathrm{P}$ & - & $\mathrm{P}$ & - & $\mathrm{P}$ & - \\
\hline PMU J & $\mathrm{P}$ & $\mathrm{P}$ & $\mathrm{P}$ & $\mathrm{P}$ & $\mathrm{P}$ & $\mathrm{P}$ & $\mathrm{P}$ & $\mathrm{P}$ & $\mathrm{P}$ & $\mathrm{P}$ & $\mathrm{P}$ & $\mathrm{P}$ \\
\hline
\end{tabular}

\subsection{Dynamic step change in magnitude: phasor delay time}

\begin{tabular}{|l|c|c|c|c|c|c|c|c|c|c|c|c|}
\hline Fs (FPS) & $10 \mathrm{M}$ & $10 \mathrm{P}$ & $12 \mathrm{M}$ & $12 \mathrm{P}$ & $15 \mathrm{M}$ & $15 \mathrm{P}$ & $20 \mathrm{M}$ & $20 \mathrm{P}$ & $30 \mathrm{M}$ & $30 \mathrm{P}$ & $60 \mathrm{M}$ & $60 \mathrm{P}$ \\
\hline C37.118.1 Annex C & $\mathrm{P}$ & $\mathrm{P}$ & $\mathrm{P}$ & $\mathrm{P}$ & $\mathrm{P}$ & $\mathrm{P}$ & $\mathrm{P}$ & $\mathrm{P}$ & $\mathrm{P}$ & $\mathrm{P}$ & $\mathrm{P}$ & $\mathrm{P}$ \\
\hline PMU A & $\mathrm{P}$ & $\mathrm{P}$ & $\mathrm{P}$ & $\mathrm{P}$ & $\mathrm{P}$ & $\mathrm{P}$ & $\mathrm{P}$ & $\mathrm{P}$ & $\mathrm{P}$ & $\mathrm{P}$ & $\mathrm{P}$ & $\mathrm{P}$ \\
\hline PMU B & $\mathrm{P}$ & $\mathrm{P}$ & $\mathrm{P}$ & $\mathrm{P}$ & $\mathrm{P}$ & $\mathrm{P}$ & $\mathrm{P}$ & $\mathrm{P}$ & $\mathrm{P}$ & $\mathrm{P}$ & $\mathrm{P}$ & $\mathrm{P}$ \\
\hline PMU C & $\mathrm{P}$ & $\mathrm{P}$ & $\mathrm{P}$ & $\mathrm{P}$ & $\mathrm{P}$ & $\mathrm{P}$ & $\mathrm{P}$ & $\mathrm{P}$ & $\mathrm{P}$ & $\mathrm{P}$ & $\mathrm{P}$ & $\mathrm{P}$ \\
\hline PMU D & $\mathrm{P}$ & $\mathrm{P}$ & $\mathrm{P}$ & $\mathrm{P}$ & $\mathrm{P}$ & $\mathrm{P}$ & $\mathrm{P}$ & $\mathrm{P}$ & $\mathrm{P}$ & $\mathrm{P}$ & $\mathrm{P}$ & $\mathrm{P}$ \\
\hline PMU E & $\mathrm{P}$ & - & - & - & $\mathrm{P}$ & - & $\mathrm{P}$ & - & $\mathrm{P}$ & - & $\mathrm{P}$ & - \\
\hline PMU F & $\mathrm{P}$ & $\mathrm{P}$ & $\mathrm{P}$ & $\mathrm{P}$ & $\mathrm{P}$ & $\mathrm{P}$ & $\mathrm{P}$ & $\mathrm{P}$ & $\mathrm{P}$ & $\mathrm{P}$ & $\mathrm{P}$ & $\mathrm{P}$ \\
\hline PMU G & $\mathrm{F}$ & - & $\mathrm{F}$ & - & $\mathrm{F}$ & - & $\mathrm{F}$ & - & $\mathrm{F}$ & - & - & - \\
\hline PMU H & $\mathrm{P}$ & $\mathrm{P}$ & $\mathrm{P}$ & $\mathrm{P}$ & $\mathrm{P}$ & $\mathrm{P}$ & $\mathrm{P}$ & $\mathrm{P}$ & $\mathrm{P}$ & $\mathrm{P}$ & $\mathrm{P}$ & $\mathrm{P}$ \\
\hline PMU I & $\mathrm{P}$ & - & $\mathrm{P}$ & - & $\mathrm{P}$ & - & $\mathrm{P}$ & - & $\mathrm{P}$ & - & $\mathrm{P}$ & - \\
\hline PMU J & $\mathrm{P}$ & $\mathrm{P}$ & $\mathrm{P}$ & $\mathrm{P}$ & $\mathrm{P}$ & $\mathrm{P}$ & $\mathrm{P}$ & $\mathrm{P}$ & $\mathrm{P}$ & $\mathrm{P}$ & $\mathrm{P}$ & $\mathrm{P}$ \\
\hline
\end{tabular}


10.12.1 C37.118.1-2011 Annex $C$ dynamic step change in magnitude overshoot: $F 0=60 \mathrm{~Hz}$, $M$ class
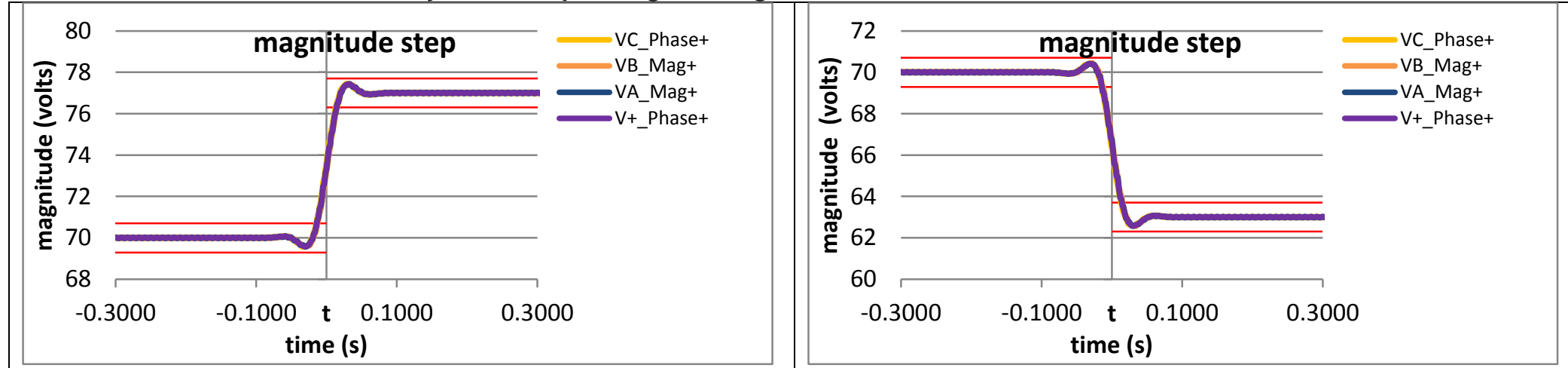

Figure 5999: Fs = 60 FPS, +10\% magnitude step

Figure 6000: Fs = 60 FPS, -10\% magnitude step
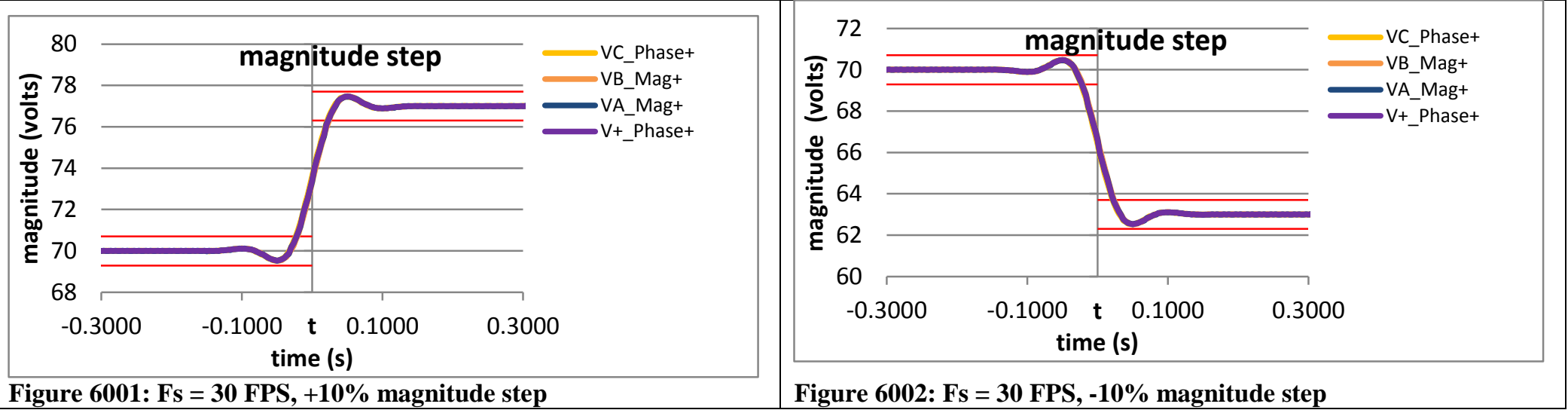

Figure 6001: Fs = 30 FPS, +10\% magnitude step

Figure 6002: Fs = 30 FPS, - $-10 \%$ magnitude step 


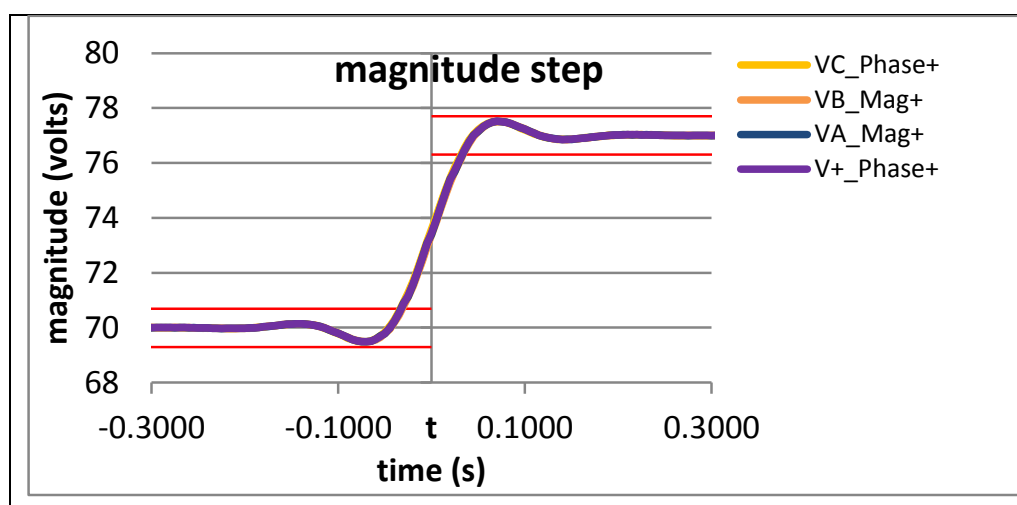

Figure 6003: Fs $=20 \mathrm{FPS},+10 \%$ magnitude step

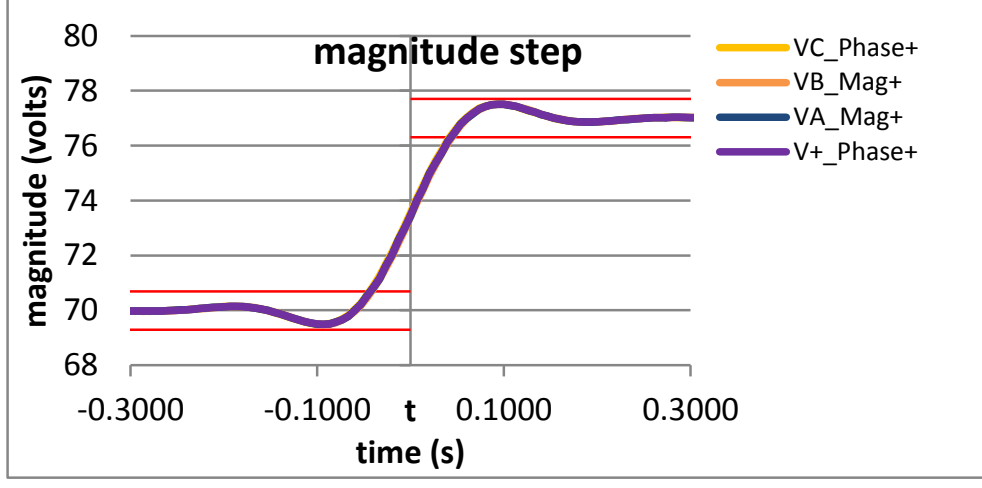

Figure 6005: Fs = 15 FPS, + 10\% magnitude step

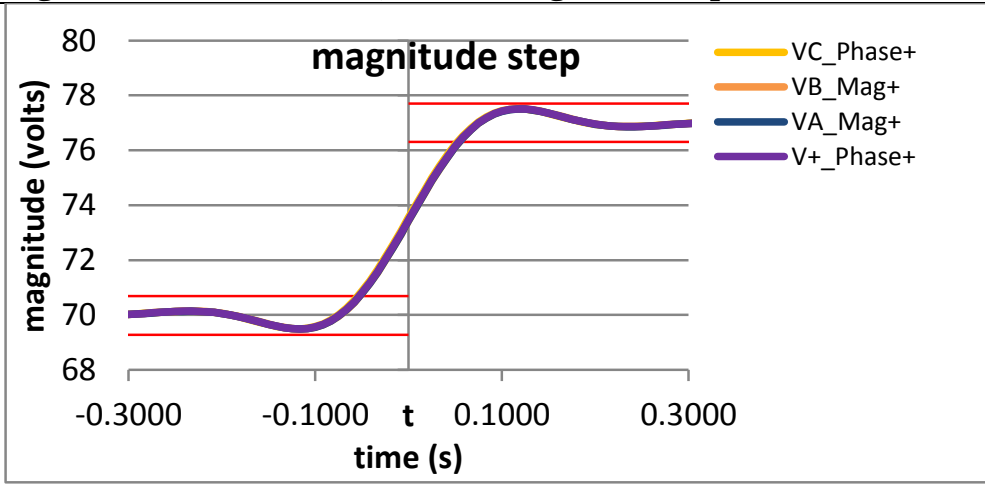

Figure 6007: Fs = 12 FPS, $+10 \%$ magnitude step

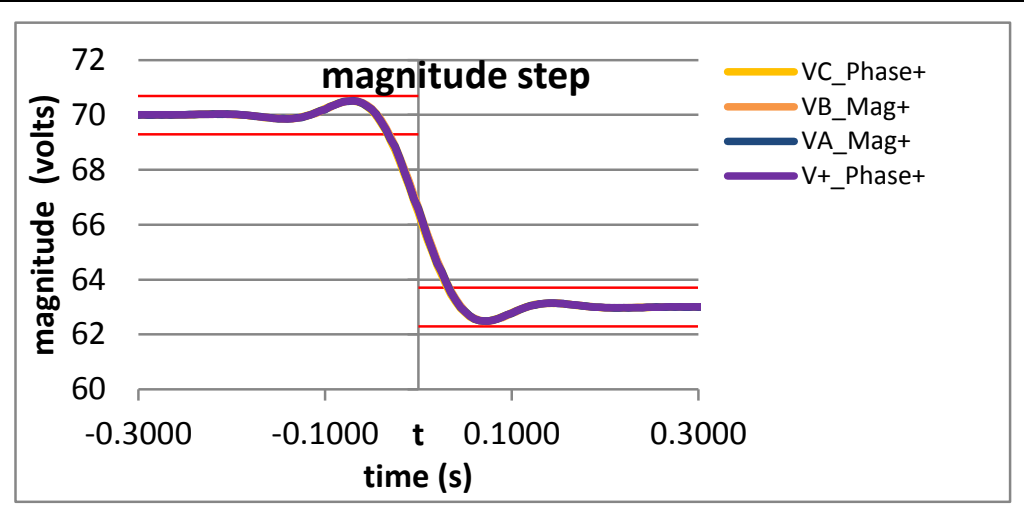

Figure 6004; Fs = 20 FPS, =10\% magnitude step

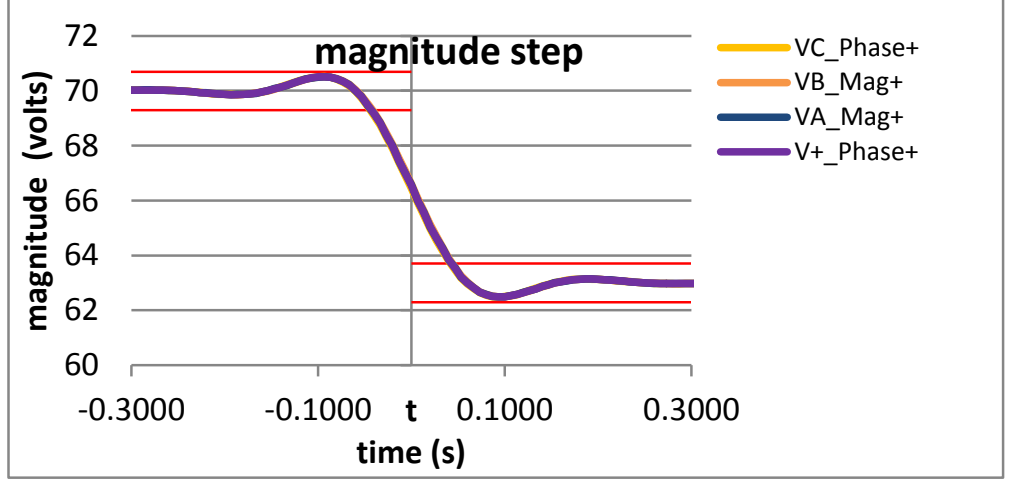

Figure 6006: Fs = 15 FPS, - $10 \%$ magnitude step

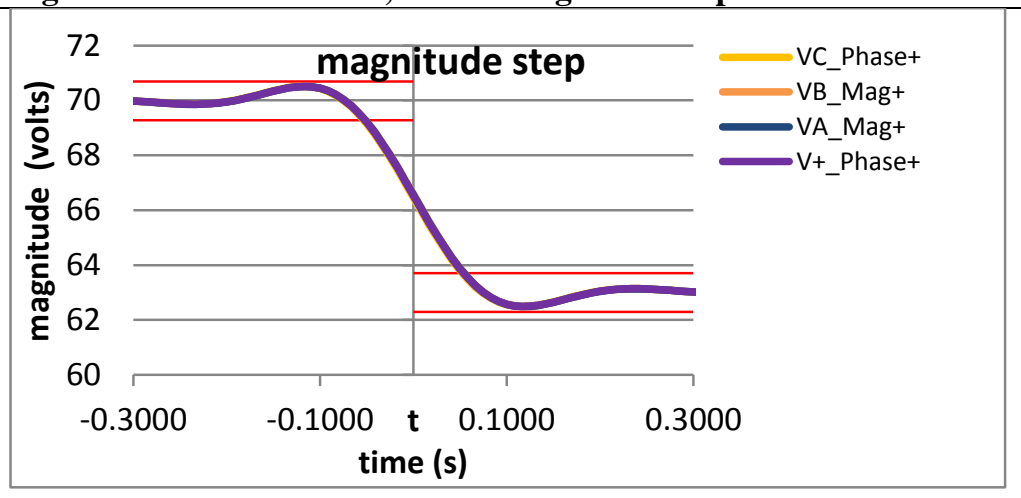

Figure 6008: Fs = 12 FPS, $-10 \%$ magnitude step 


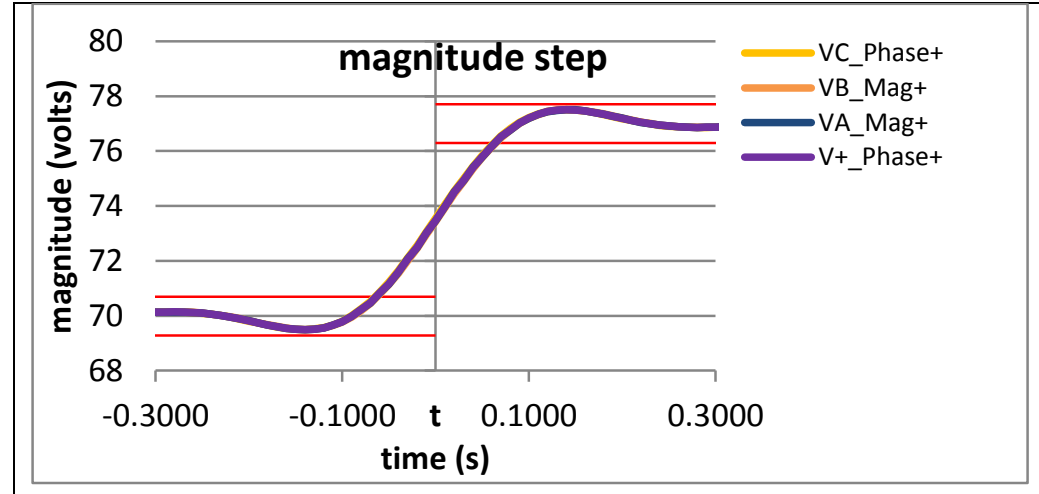

Figure 6009: Fs = 10 FPS, $+10 \%$ magnitude step

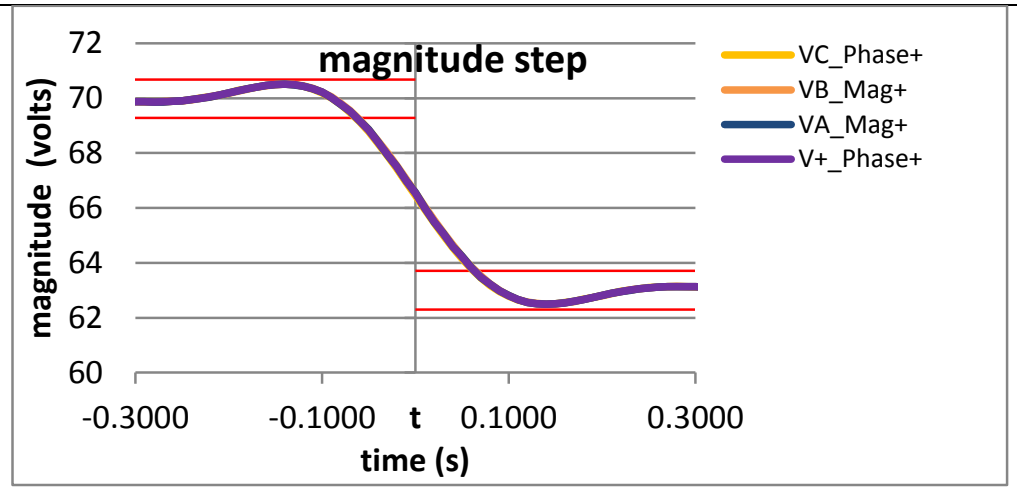

Figure 6010: Fs = 10 FPS, -10\% magnitude step 
10.12.2 PMU A dynamic step change in magnitude ROCOF phasor overshoot: $F 0=60 \mathrm{~Hz}$, M class
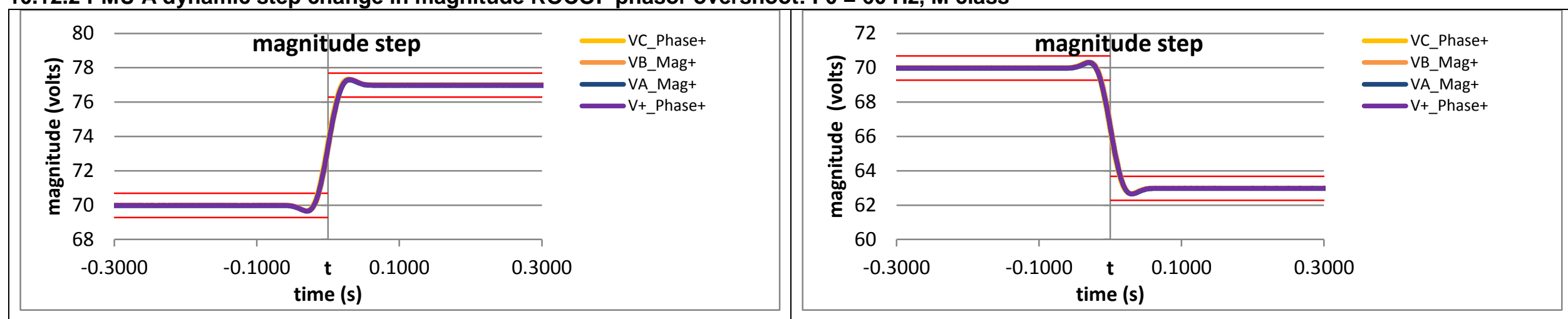

Figure 6011: Fs $=60 \mathrm{FPS},+10 \%$ magnitude step

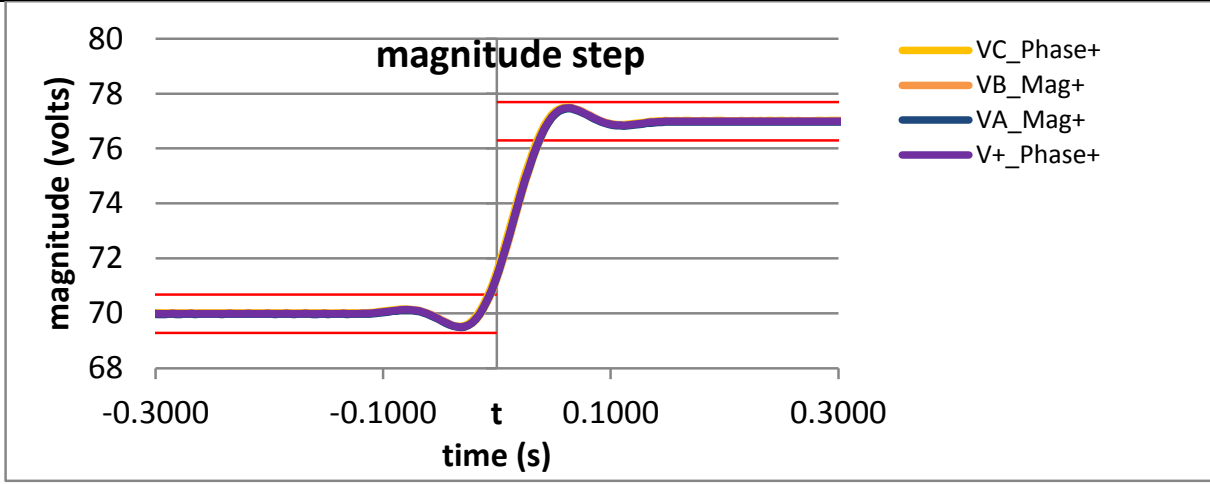

Figure 6012: Fs = 60 FPS, -10\% magnitude step

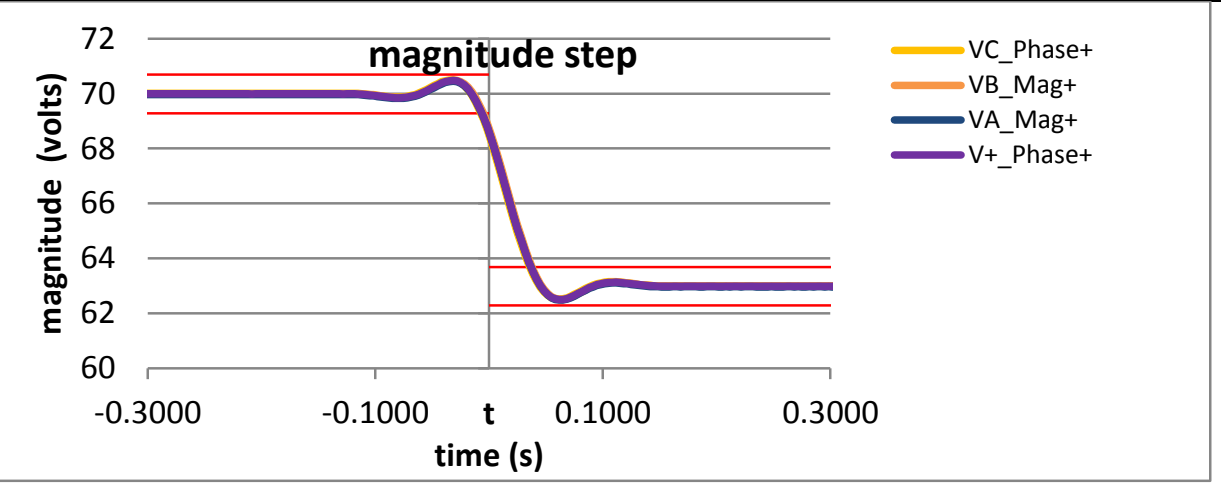

Figure 6013: Fs = 30 FPS, +10\% magnitude step

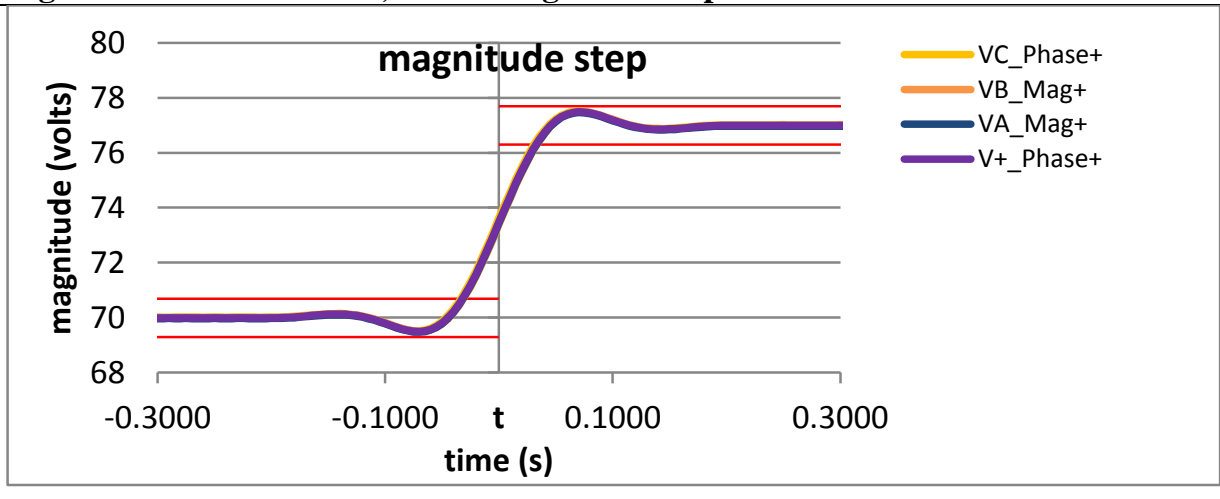

Figure 6015: Fs = 20 FPS, +10\% magnitude step

Figure 6014: Fs = 30 FPS, -10\% magnitude step

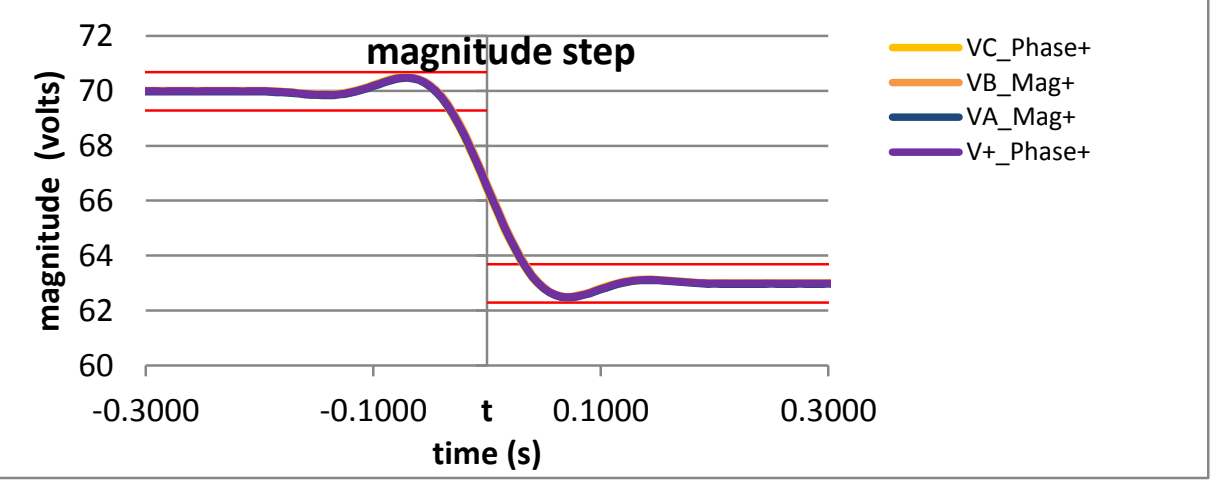

Figure 6016; Fs = 20 FPS, =10\% magnitude step 

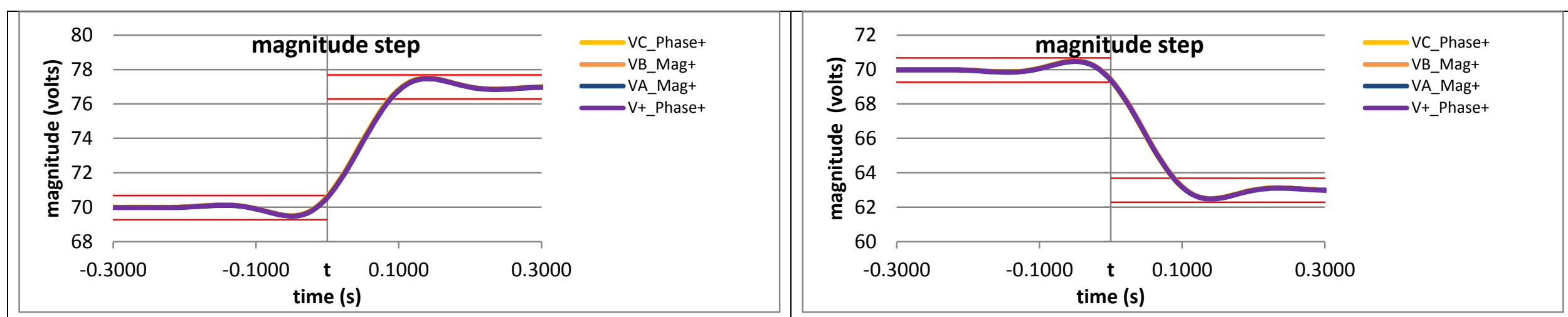

Figure 6017: Fs = 15 FPS, + 10\% magnitude step

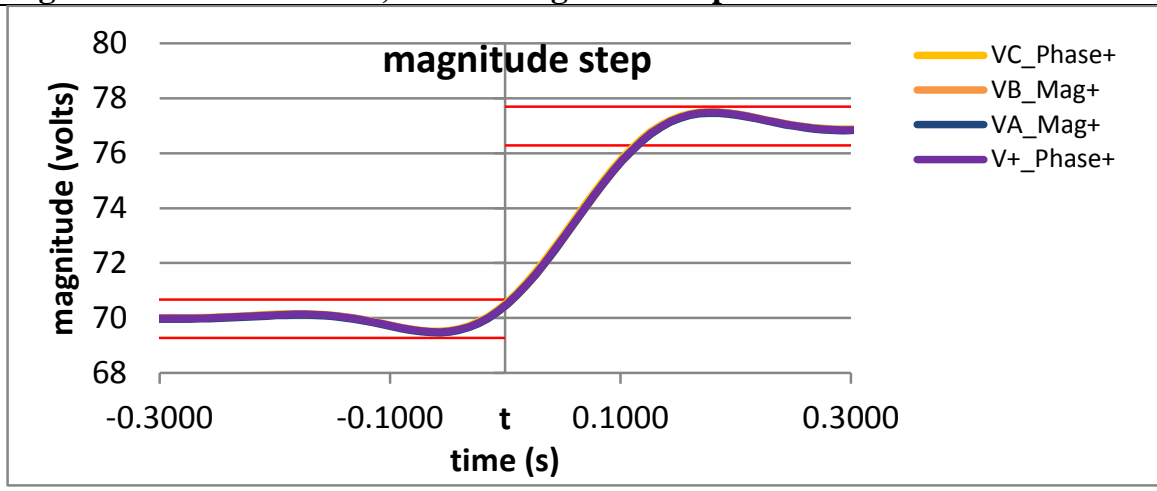

Figure 6019: Fs = 12 FPS, $+10 \%$ magnitude step

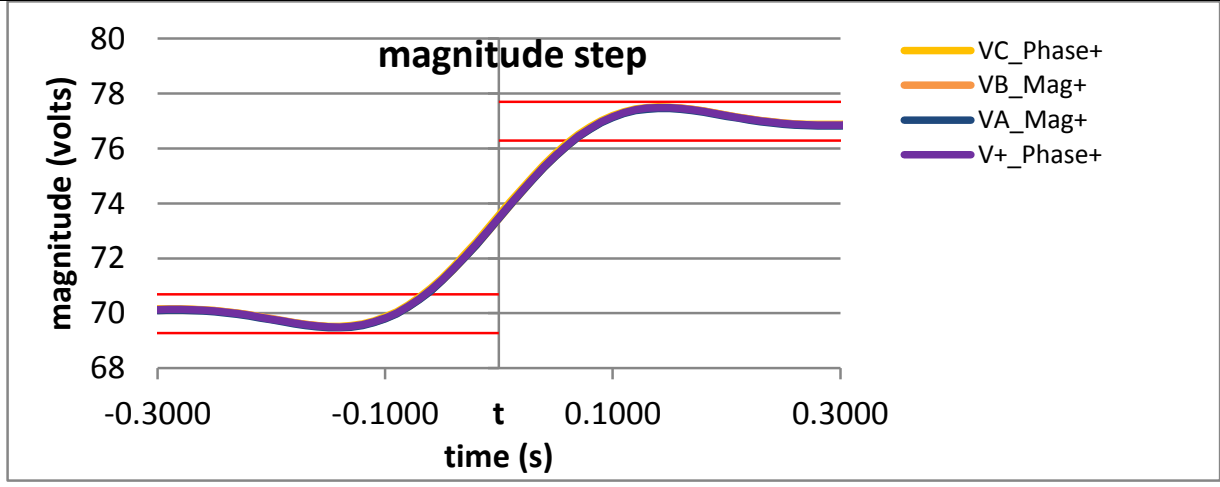

Figure 6018: Fs = 15 FPS, - $10 \%$ magnitude step

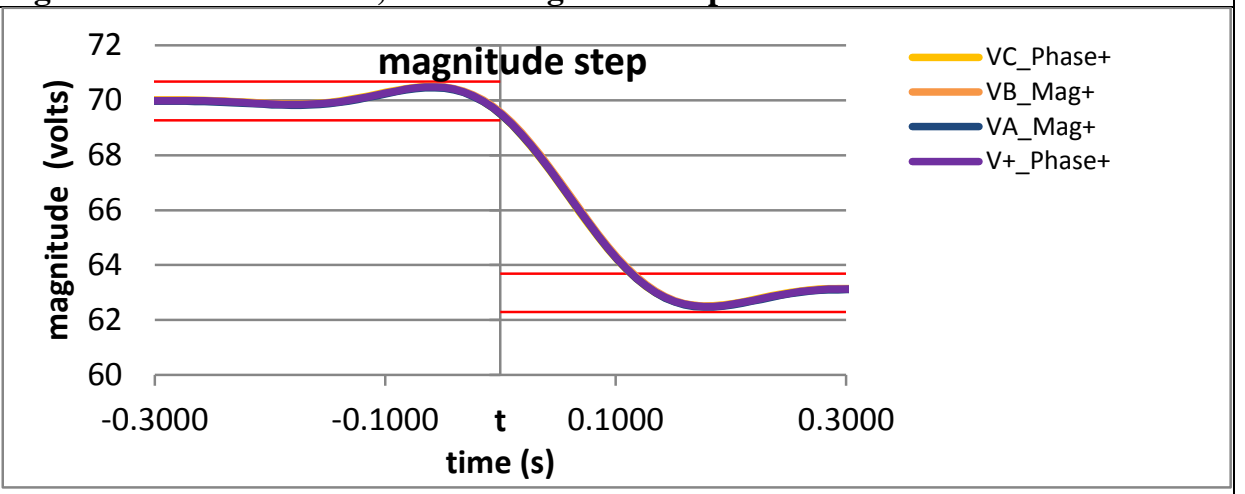

Figure 6020: Fs = 12 FPS, -10\% magnitude step

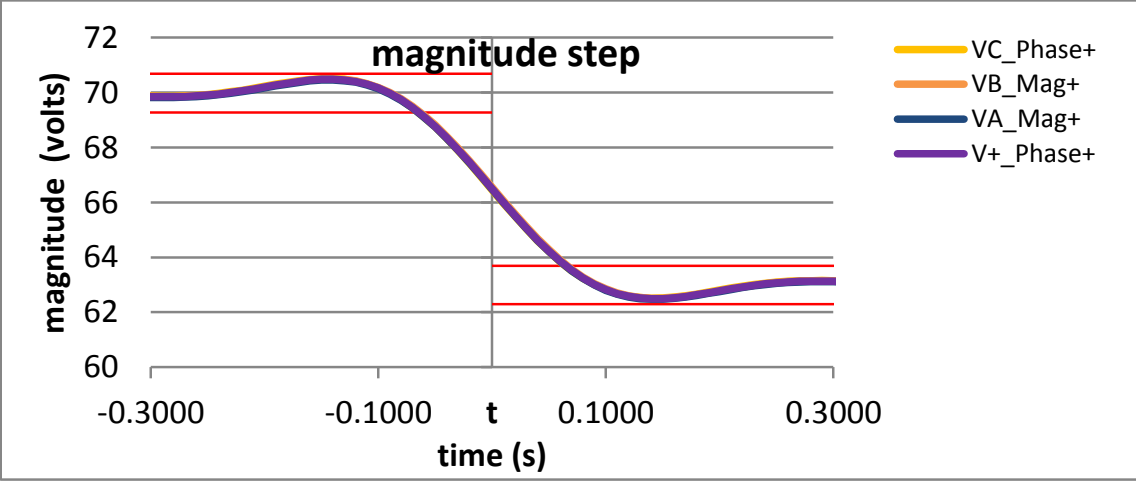

Figure 6021: Fs = 10 FPS, +10\% magnitude step

Figure 6022: Fs = 10 FPS, -10\% magnitude step 
10.12.3 PMU B dynamic step change in magnitude ROCOF phasor overshoot: $F 0=60 \mathrm{~Hz}$, M class

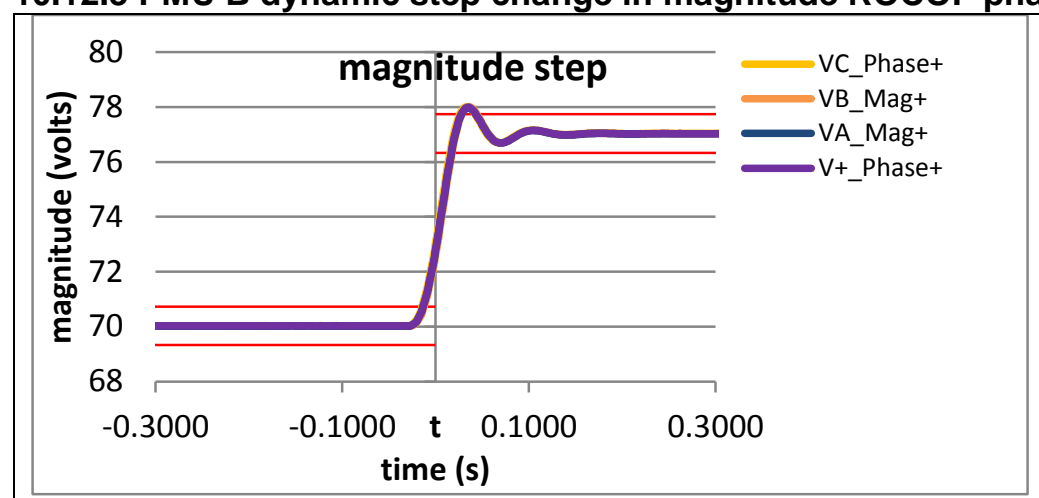

Figure 6023: Fs = 60 FPS, $+10 \%$ magnitude step

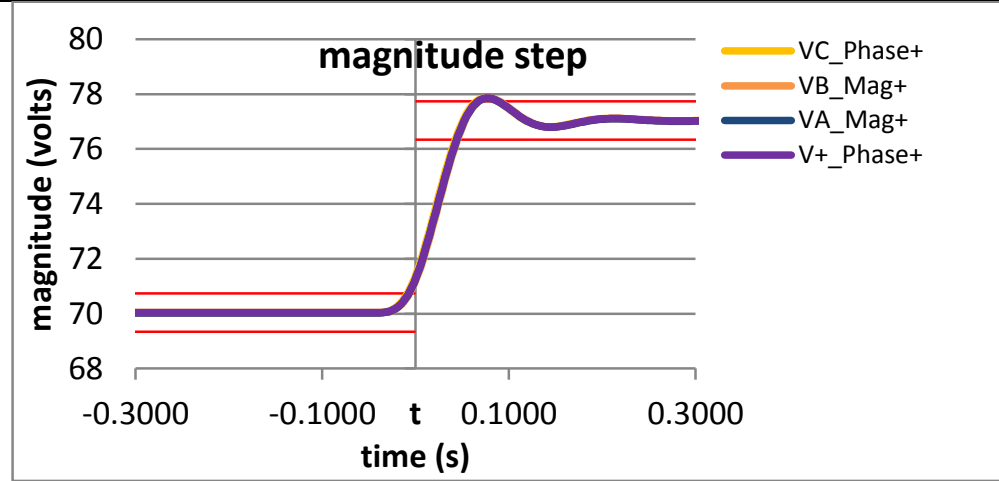

Figure 6025: Fs = 30 FPS, +10\% magnitude step

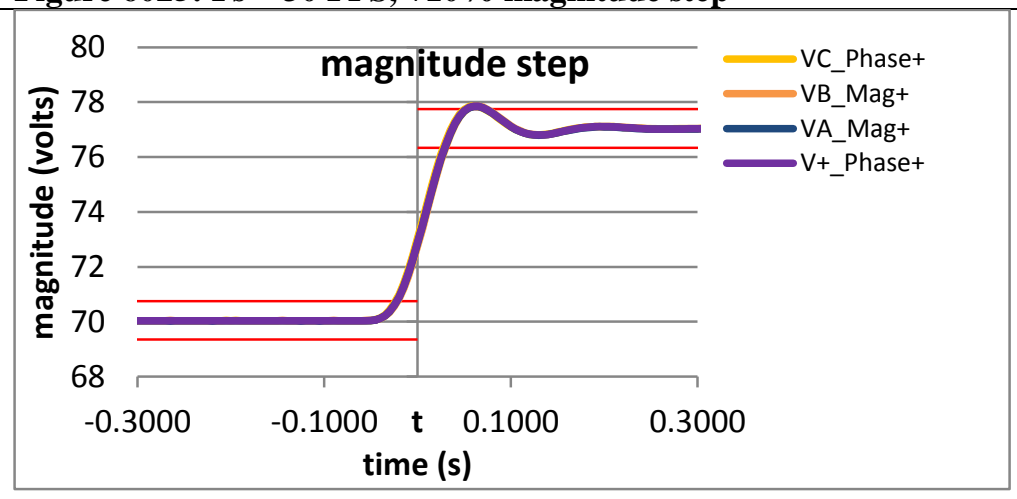

Figure 6027: Fs $=20$ FPS, $+10 \%$ magnitude step

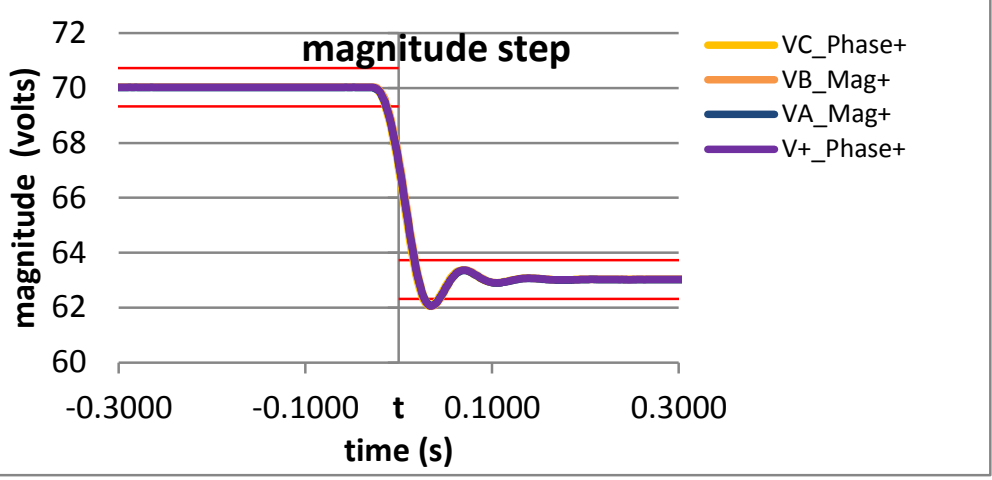

Figure 6024: Fs = 60 FPS, -10\% magnitude step

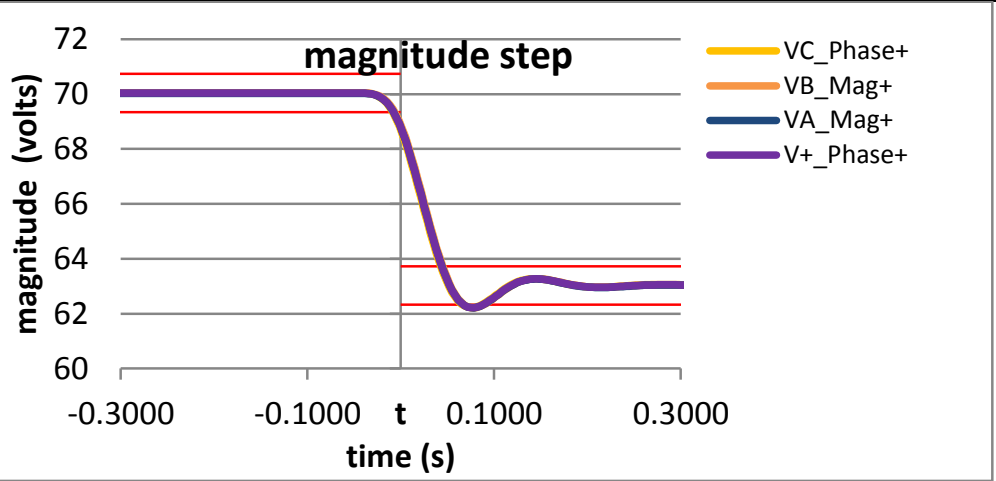

Figure 6026: Fs = 30 FPS, -10\% magnitude step

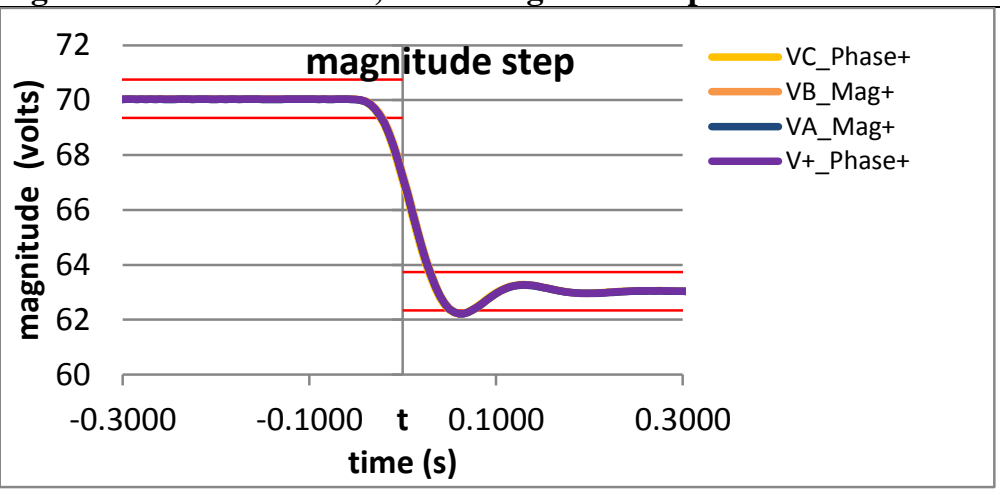

Figure 6028; Fs = 20 FPS, =10\% magnitude step 


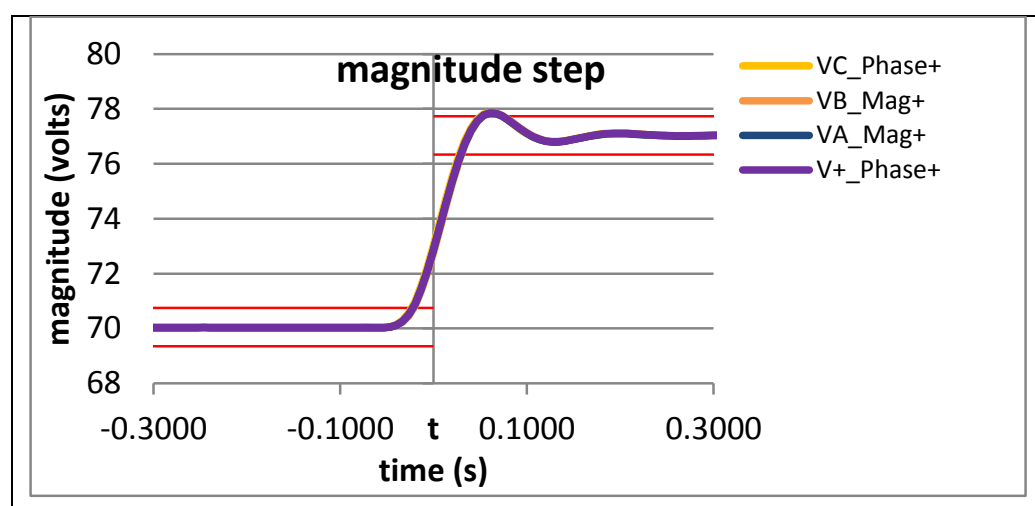

Figure 6029: Fs = 15 FPS, + 10\% magnitude step

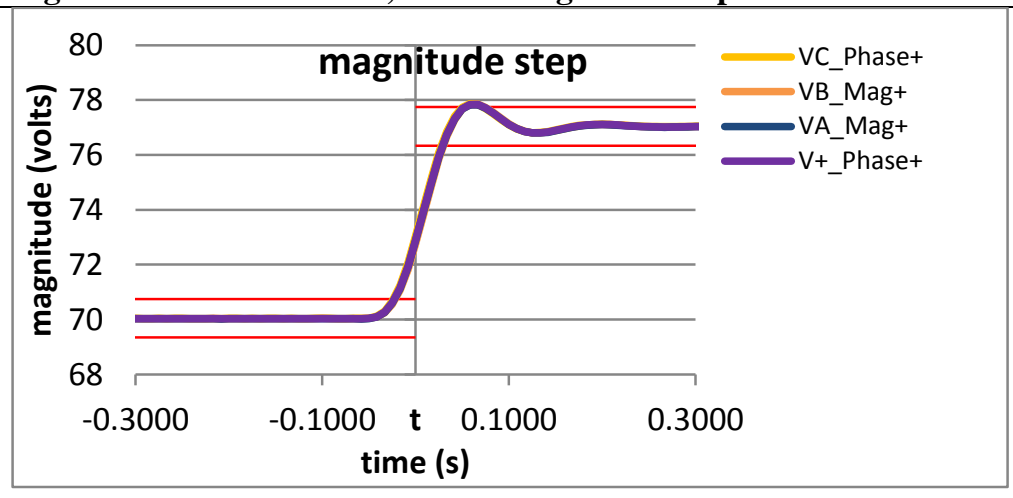

Figure 6031: Fs = 12 FPS, +10\% magnitude step

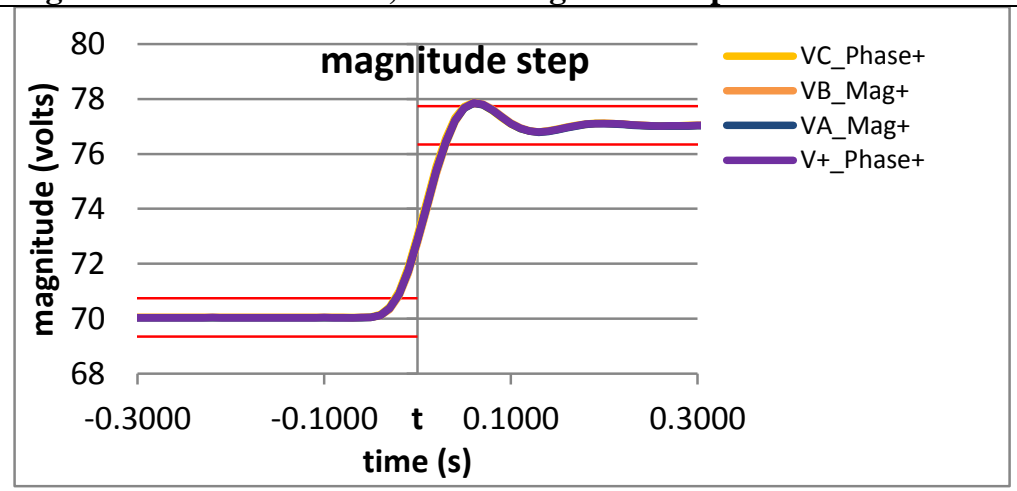

Figure 6033: Fs = 10 FPS, $+10 \%$ magnitude step

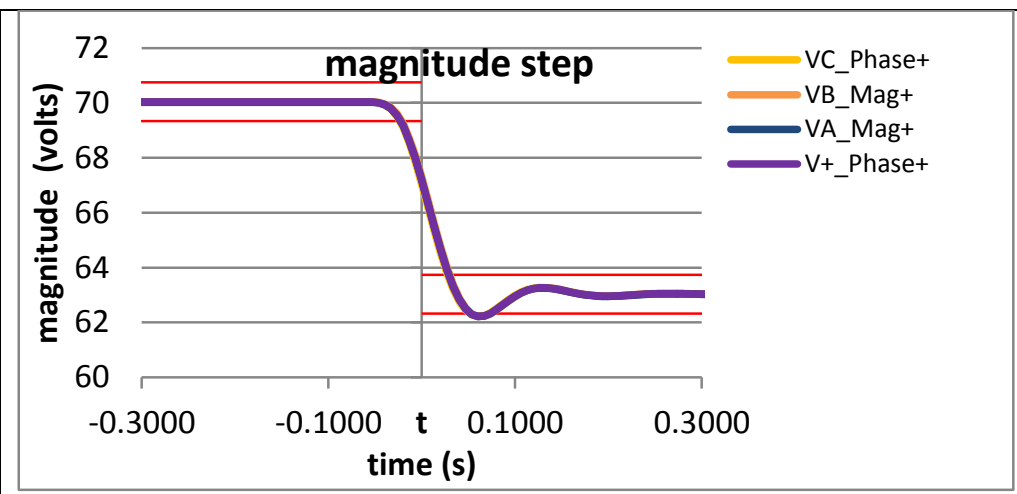

Figure 6030: Fs $=15$ FPS, - $10 \%$ magnitude step

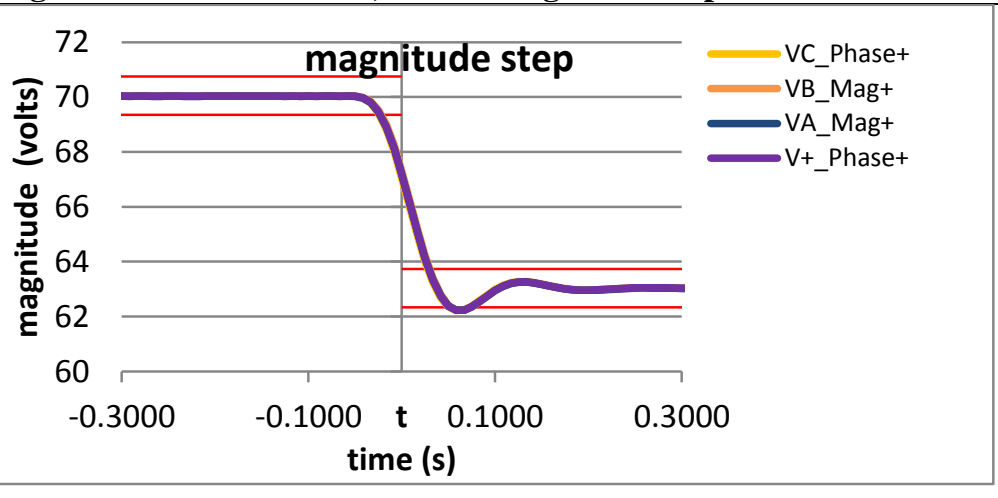

Figure 6032: Fs = 12 FPS, -10\% magnitude step

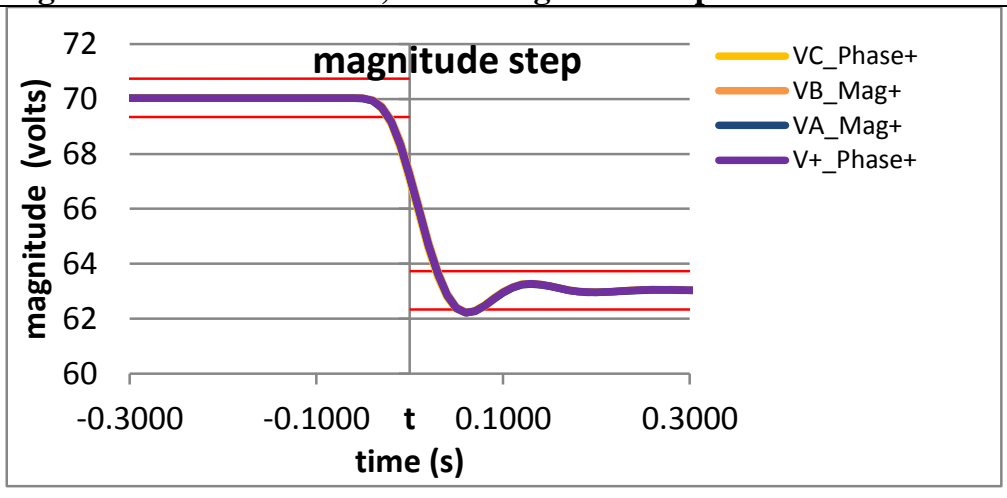

Figure 6034: Fs = 10 FPS, -10\% magnitude step 
10.12.4 PMU C dynamic step change in magnitude ROCOF phasor overshoot: $F 0=60 \mathrm{~Hz}$, M class
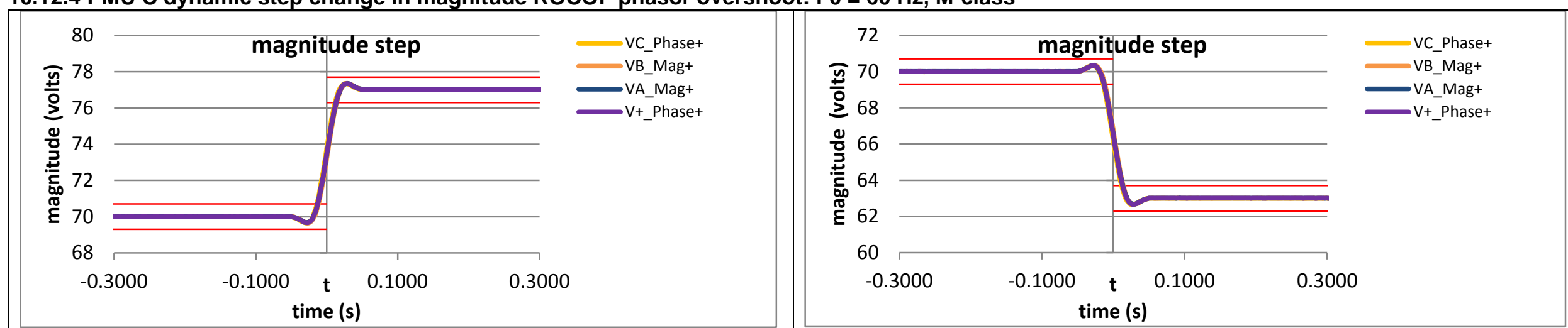

Figure 6035: Fs = 60 FPS, $+10 \%$ magnitude step

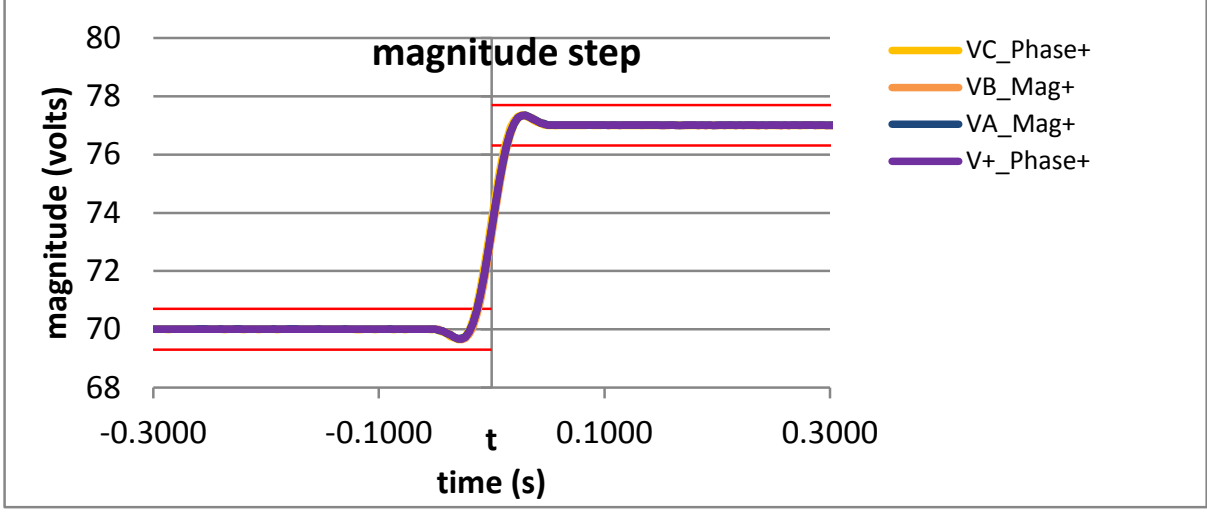

Figure 6036: Fs = 60 FPS, -10\% magnitude step

Figure 6037: Fs = 30 FPS, +10\% magnitude step
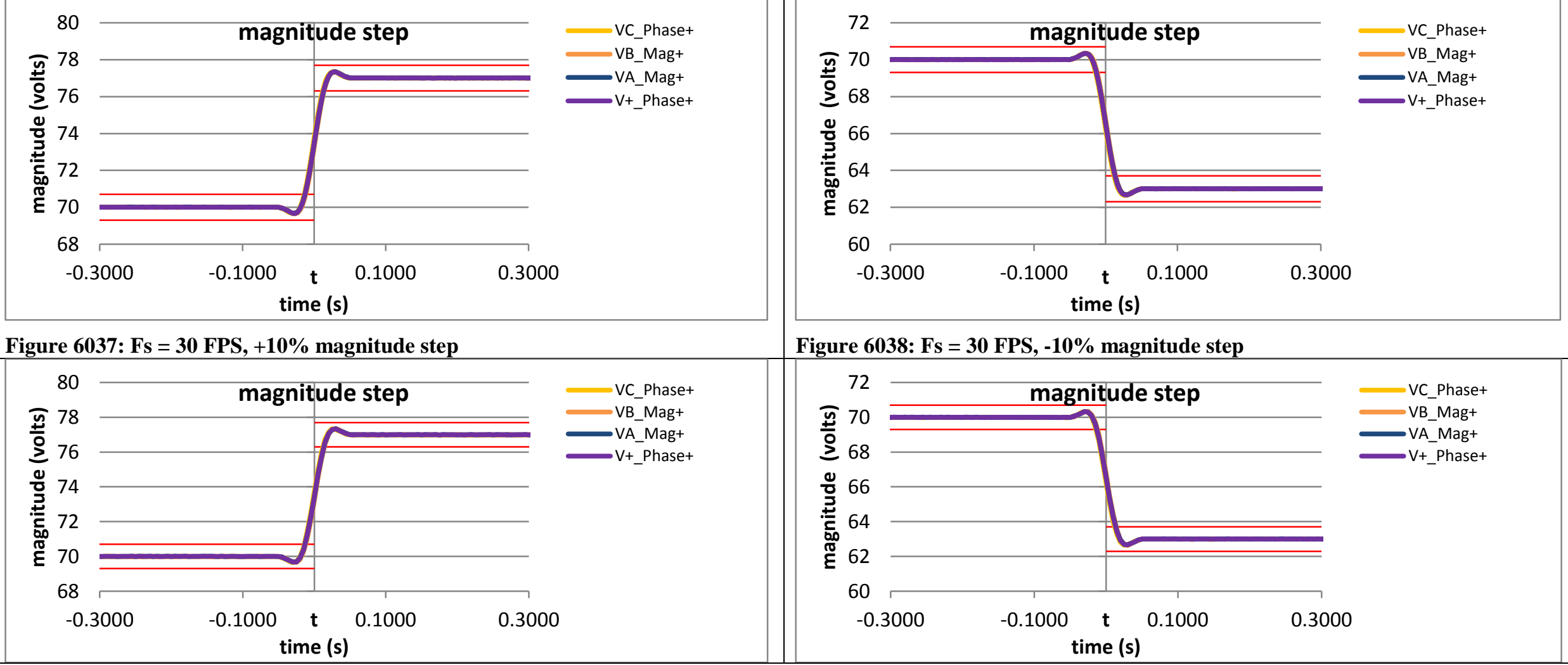

Figure 6038: Fs = 30 FPS, -10\% magnitude step

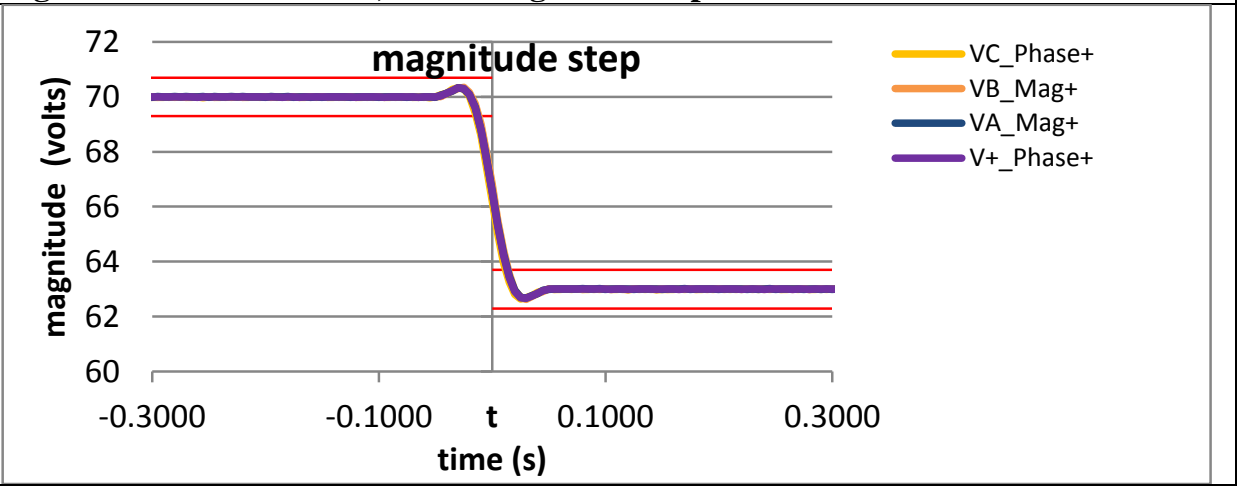




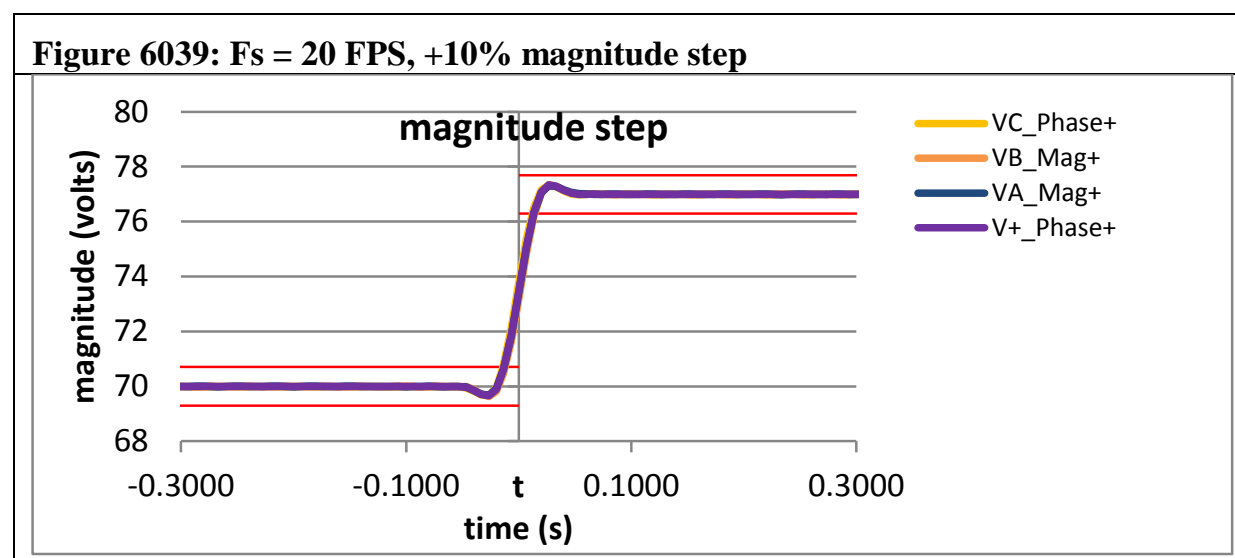

Figure 6040; Fs = 20 FPS, =10\% magnitude step

Figure 6041: Fs = 15 FPS, + 10\% magnitude step

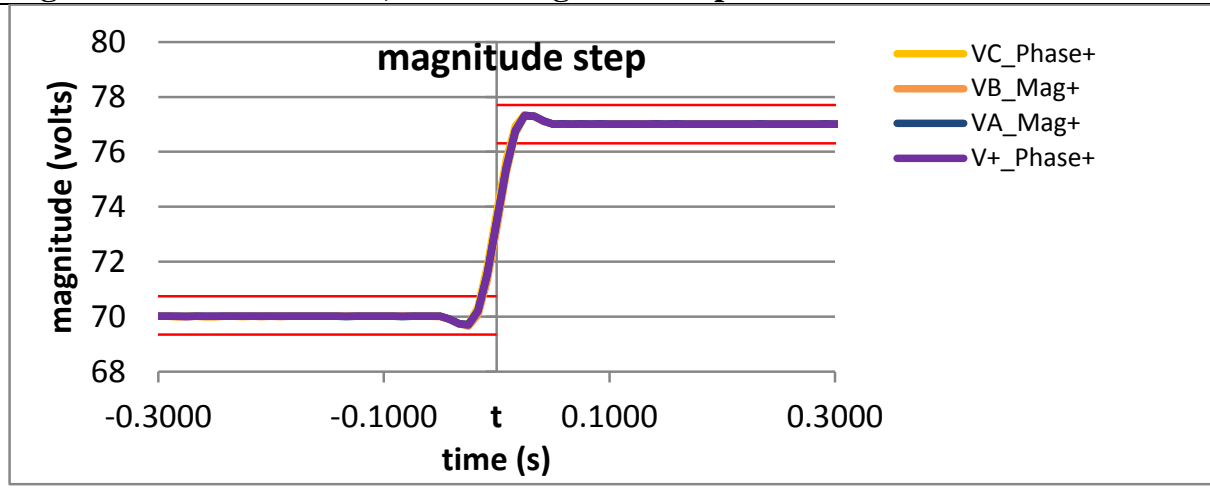

Figure 6043: Fs = 12 FPS, +10\% magnitude step

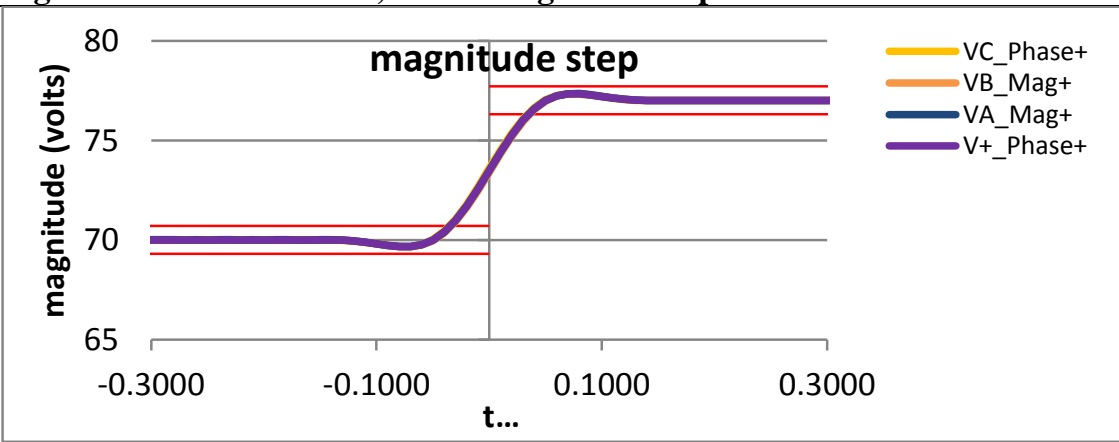

Figure 6045: Fs = 10 FPS, +10\% magnitude step

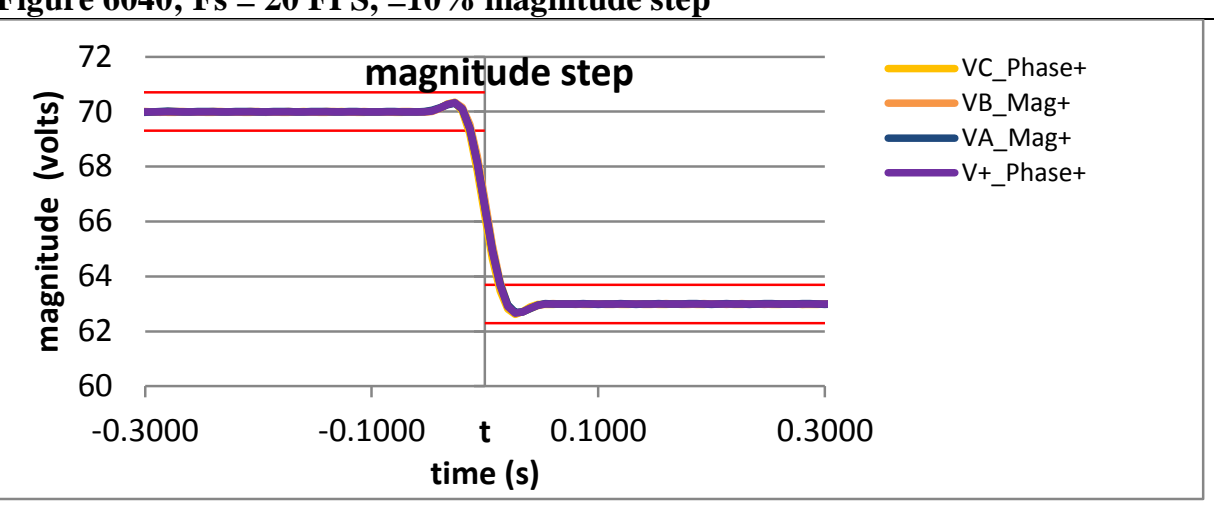

Figure 6042: Fs = 15 FPS, - $10 \%$ magnitude step

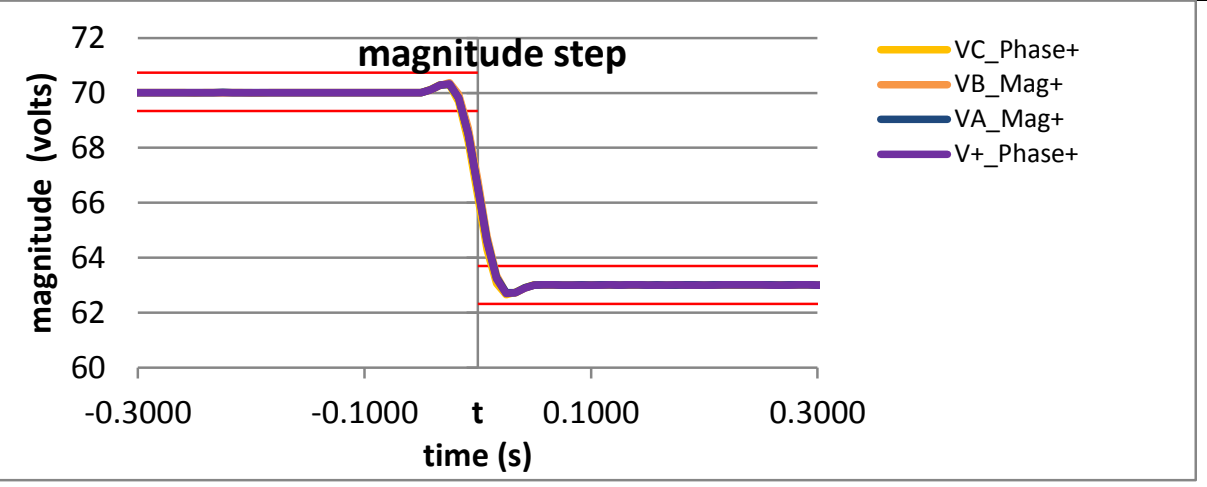

Figure 6044: Fs = 12 FPS, -10\% magnitude step

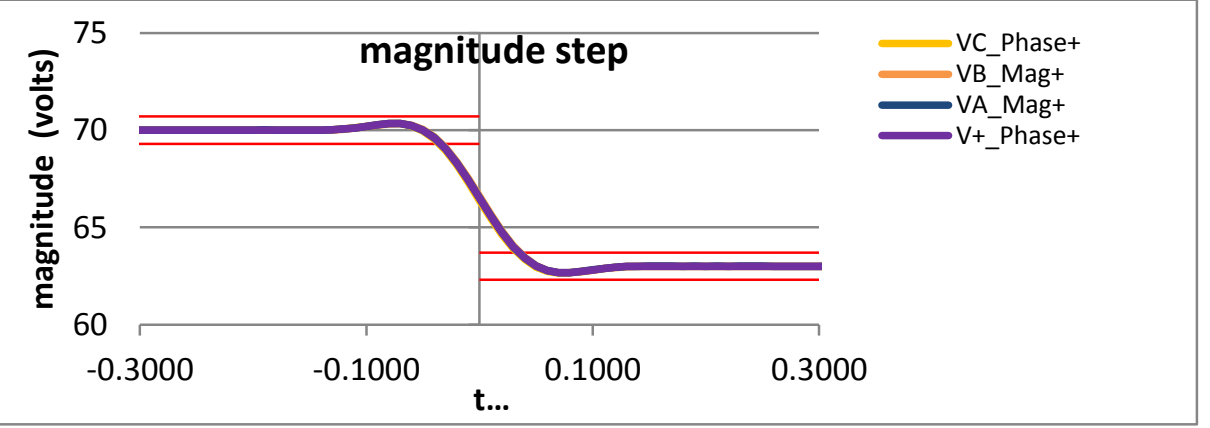

Figure 6046: Fs = $10 \mathrm{FPS},-10 \%$ magnitude step 
10.12.5 PMU D dynamic step change in magnitude ROCOF phasor overshoot: $F 0=60 \mathrm{~Hz}, \mathrm{M}$ class

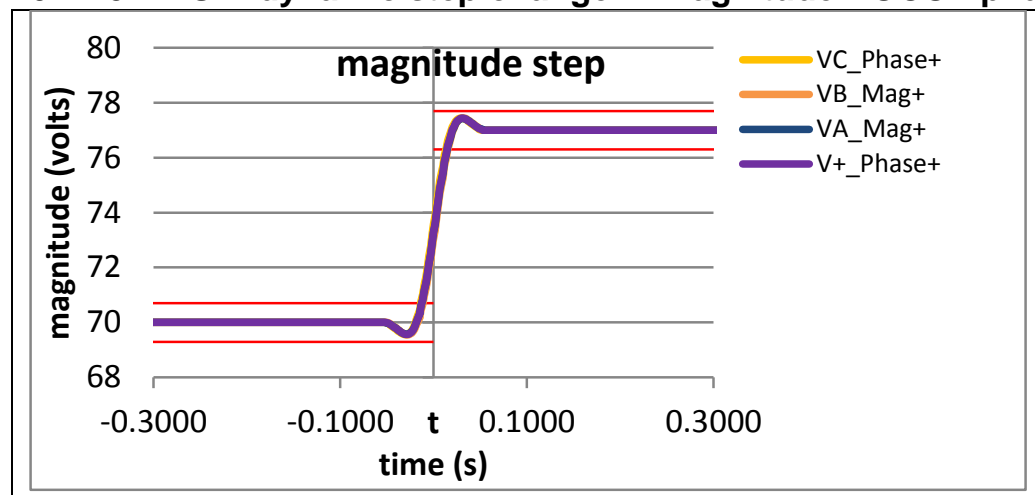

Figure 6047: Fs = 60 FPS, $+10 \%$ magnitude step

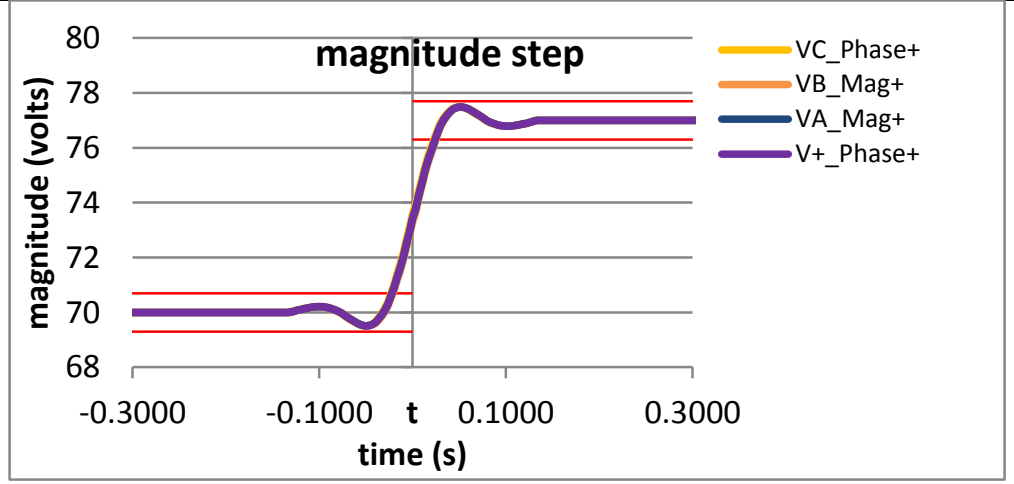

Figure 6049: Fs = 30 FPS, +10\% magnitude step

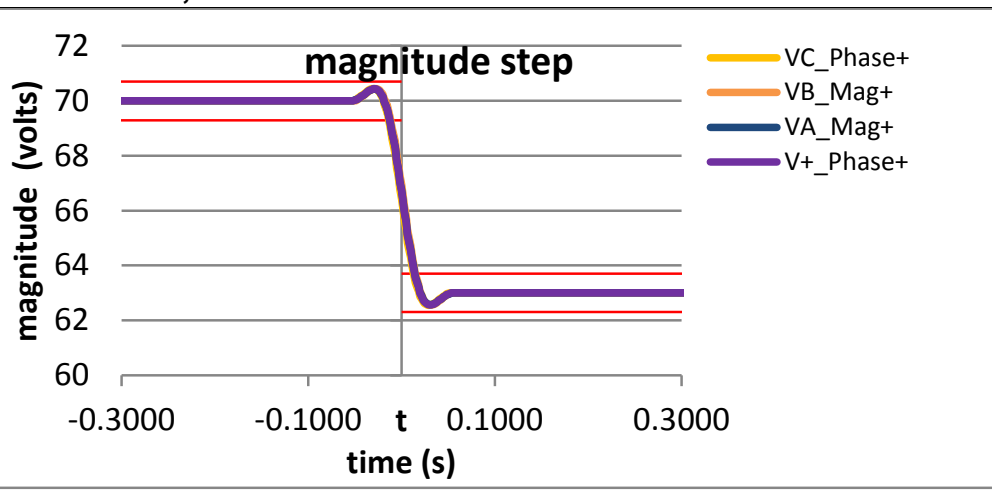

Figure 6048: Fs = 60 FPS, -10\% magnitude step

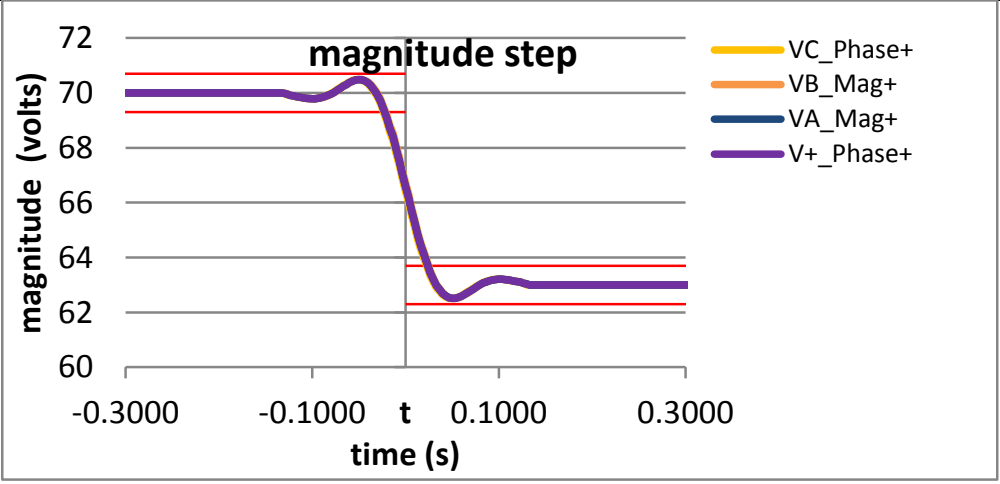

Figure 6050: Fs = 30 FPS, -10\% magnitude step 


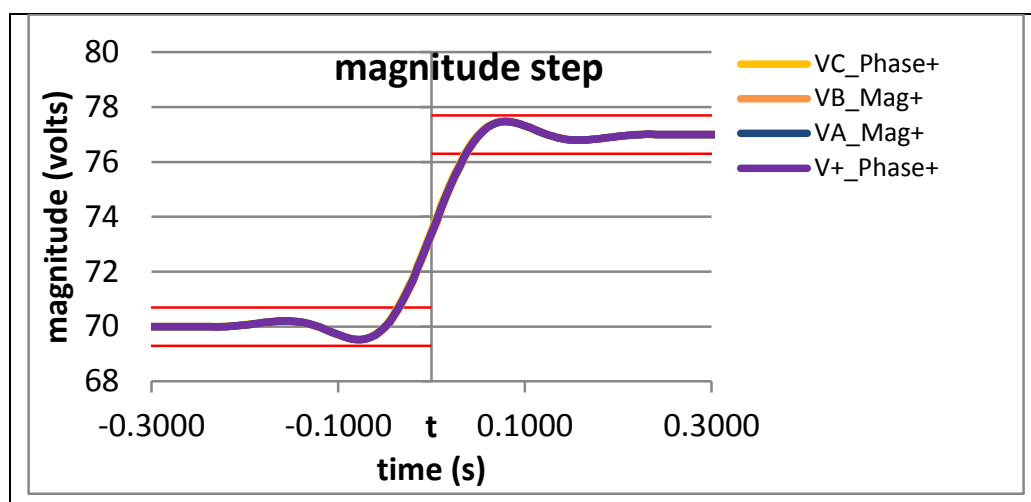

Figure 6051: Fs = 20 FPS, +10\% magnitude step
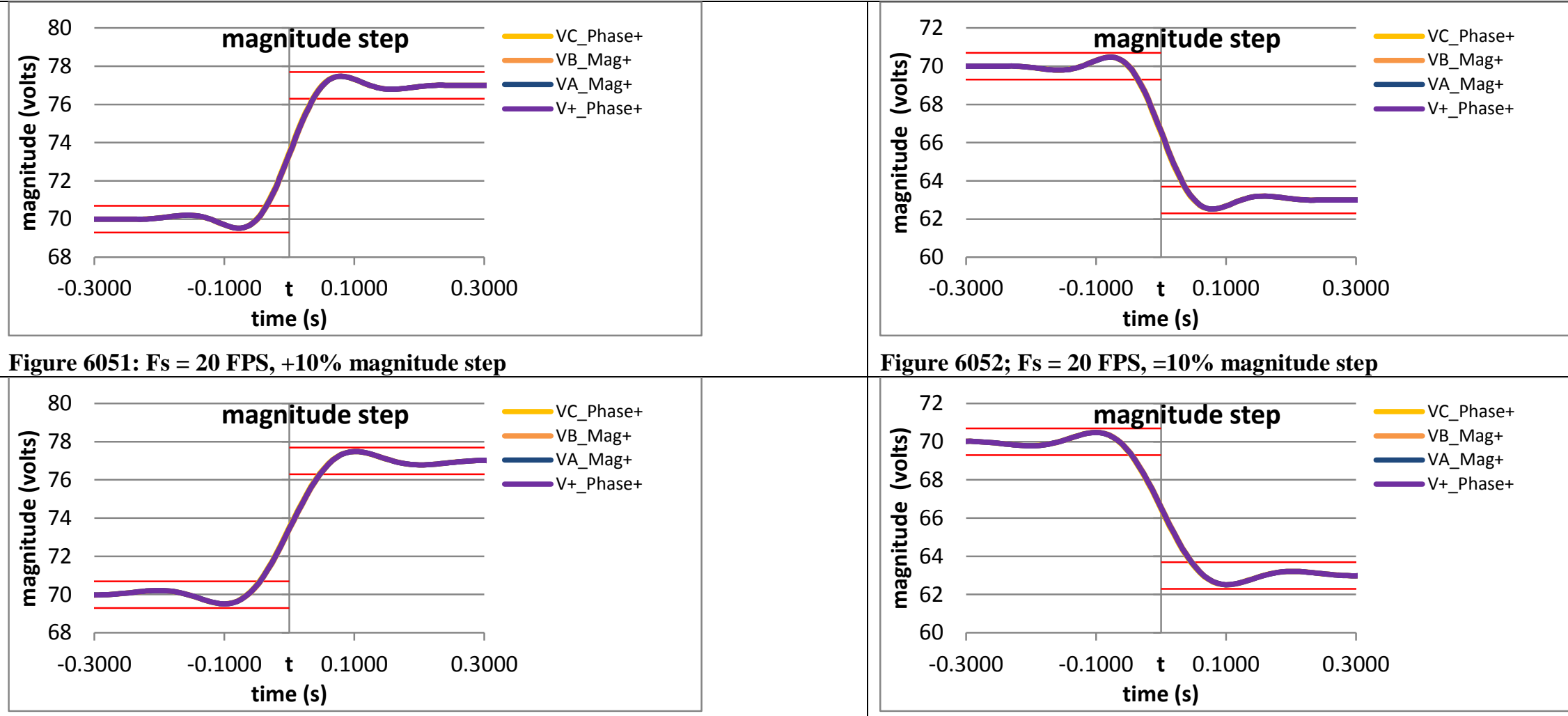

Figure 6052; Fs = 20 FPS, =10\% magnitude step

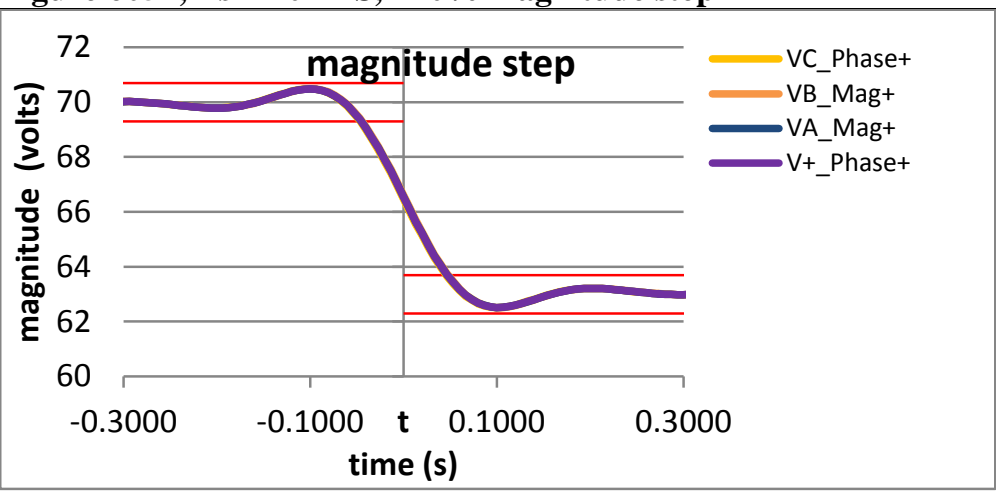

Figure 6053: Fs = 15 FPS, + 10\% magnitude step

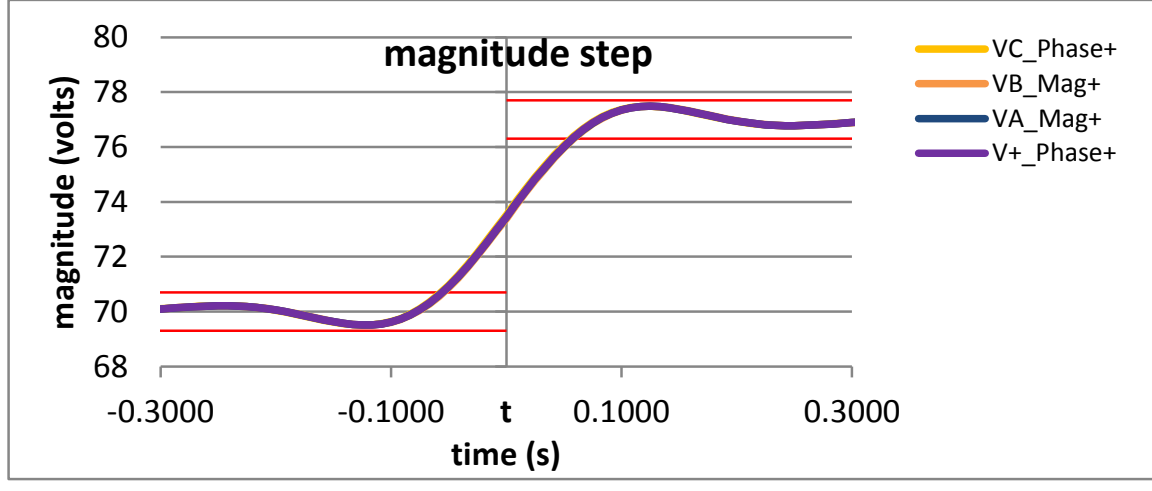

Figure 6055: Fs = 12 FPS, +10\% magnitude step

Figure 6054: Fs = 15 FPS, - $10 \%$ magnitude step

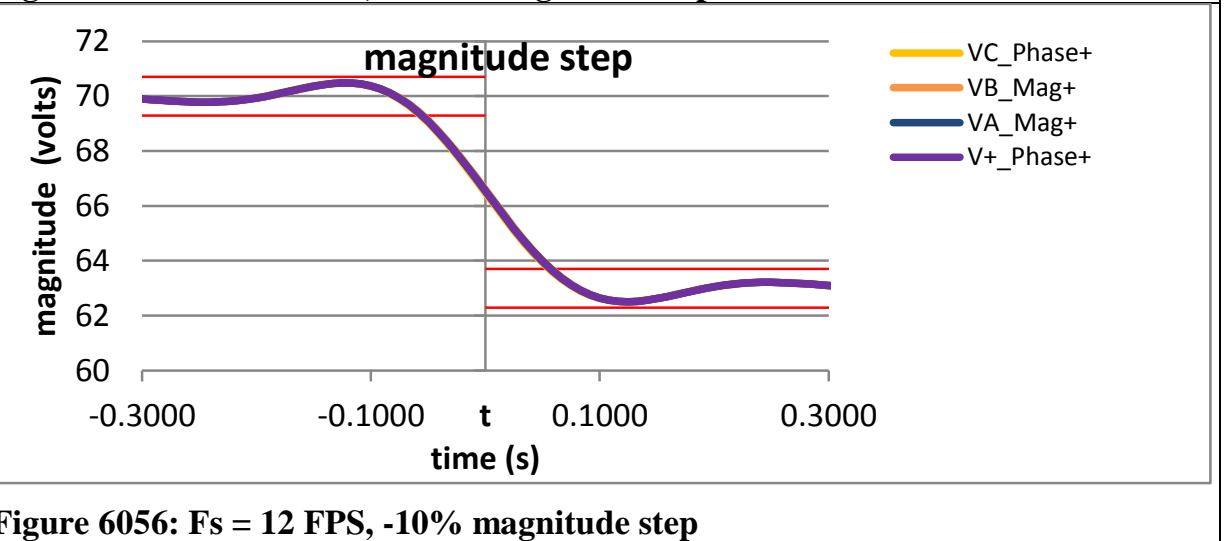




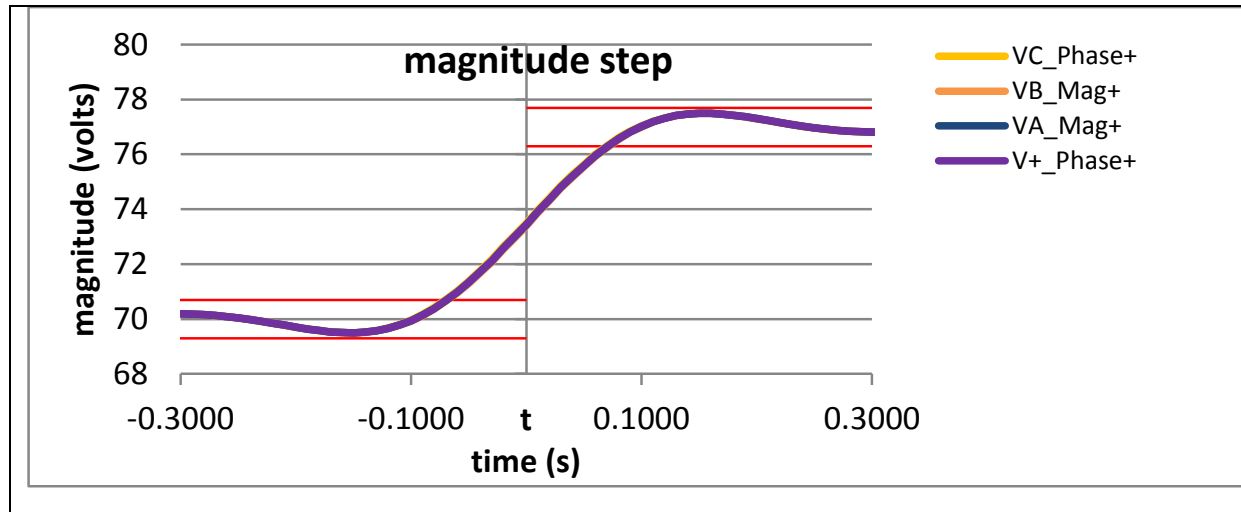

Figure 6057: Fs = 10 FPS, +10\% magnitude step

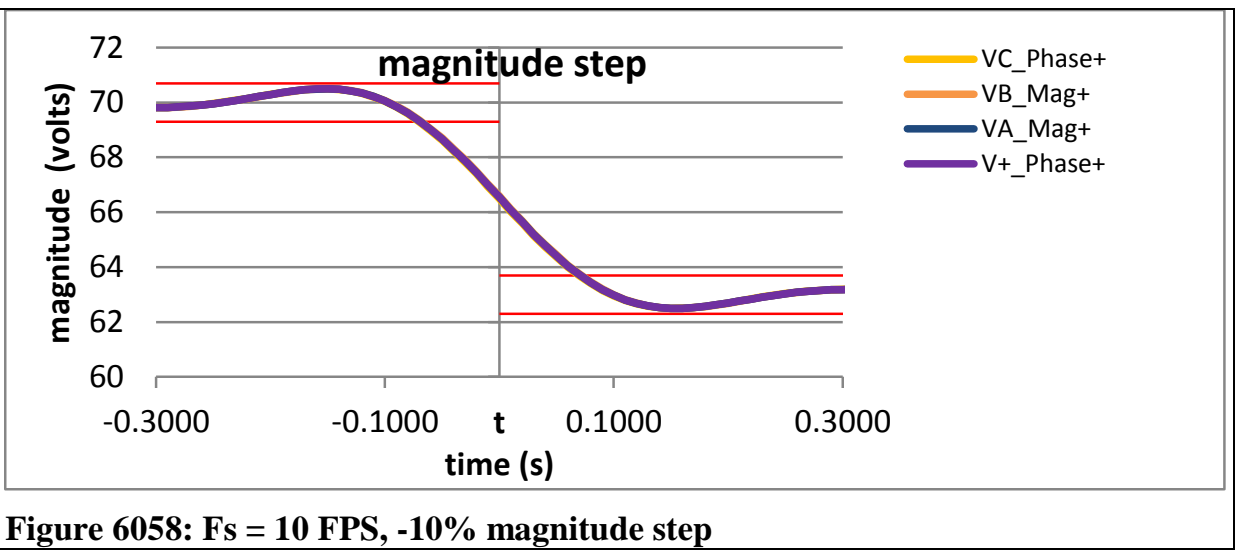


10.12.6 PMU E dynamic step change in magnitude ROCOF phasor overshoot: $F 0=60 \mathrm{~Hz}$, M class

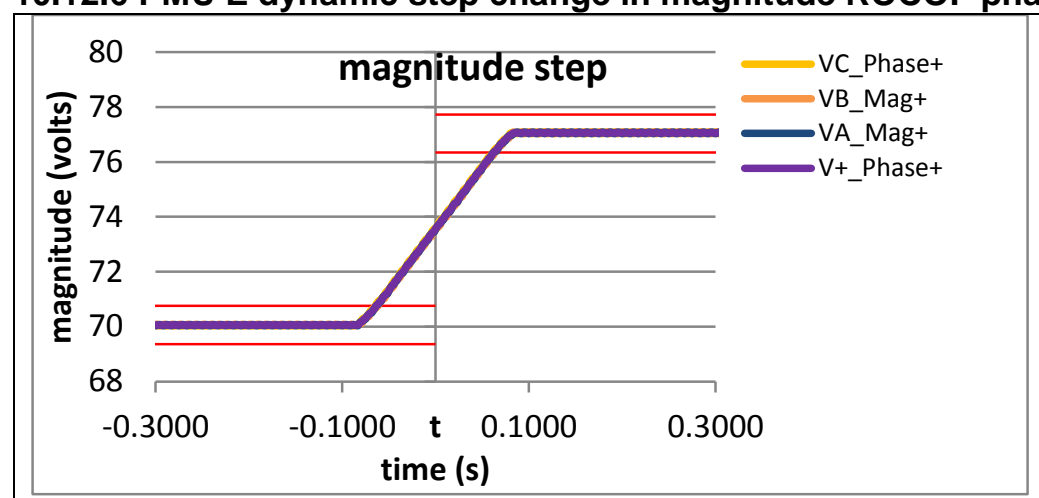

Figure 6059: Fs = 60 FPS, $+10 \%$ magnitude step

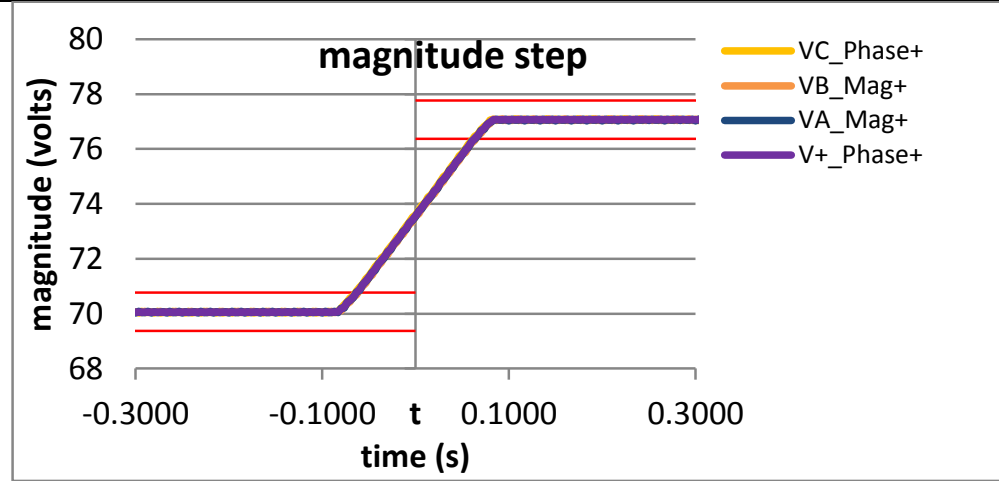

Figure 6061: Fs = 30 FPS, +10\% magnitude step

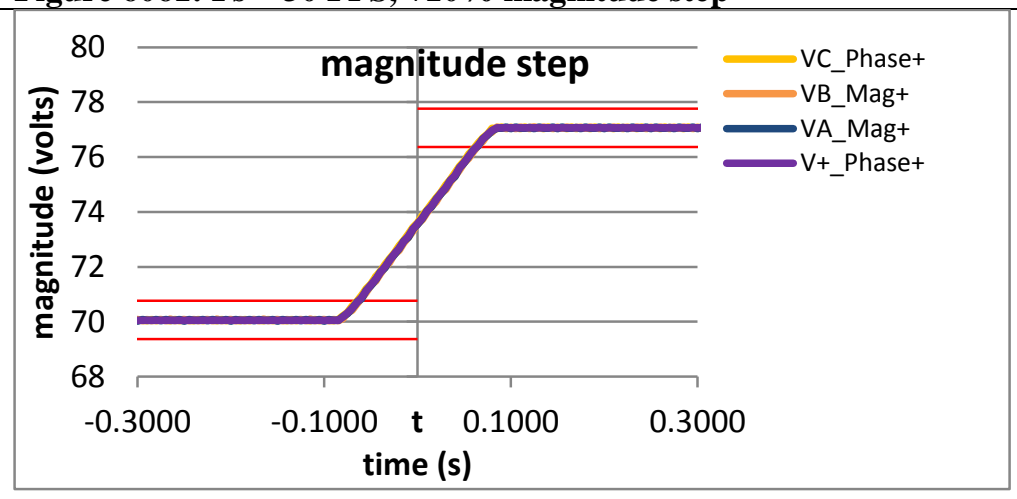

Figure 6063: Fs $=20$ FPS, $+10 \%$ magnitude step

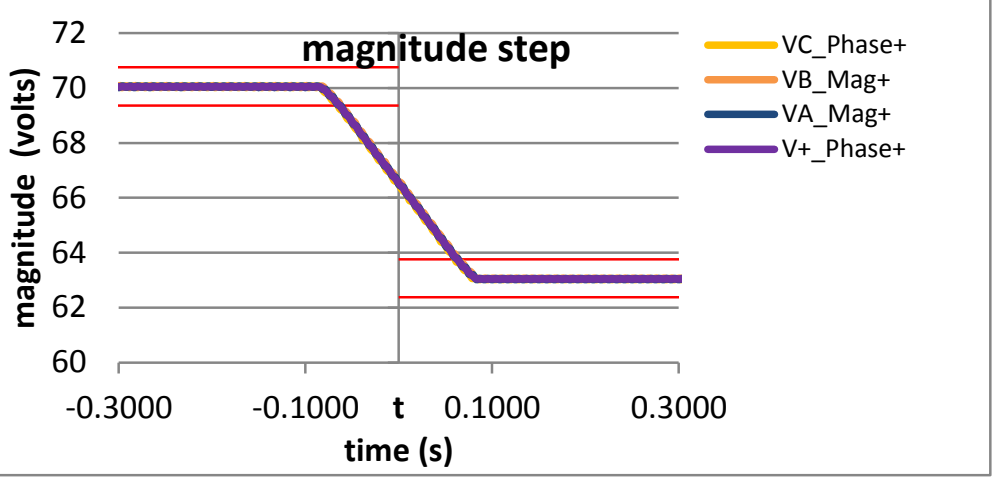

Figure 6060: Fs $=60$ FPS, $-10 \%$ magnitude step

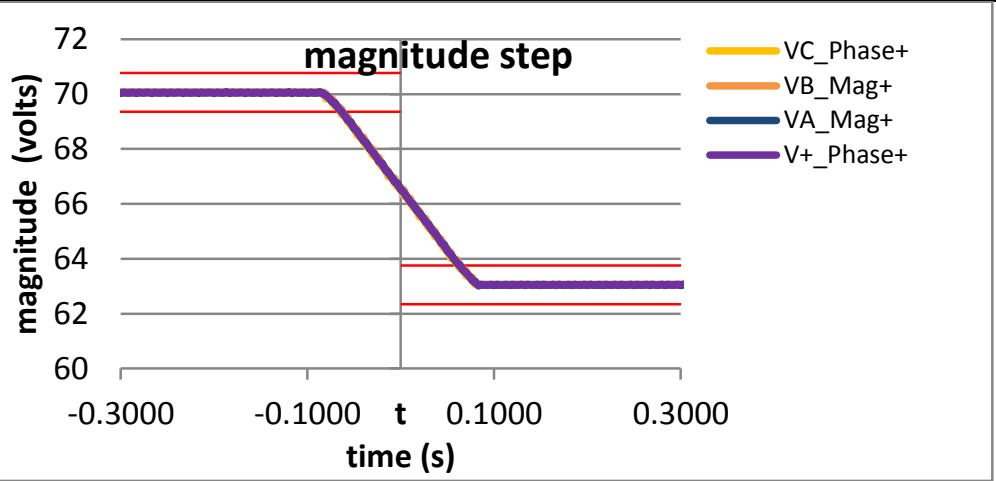

Figure 6062: Fs = 30 FPS, -10\% magnitude step

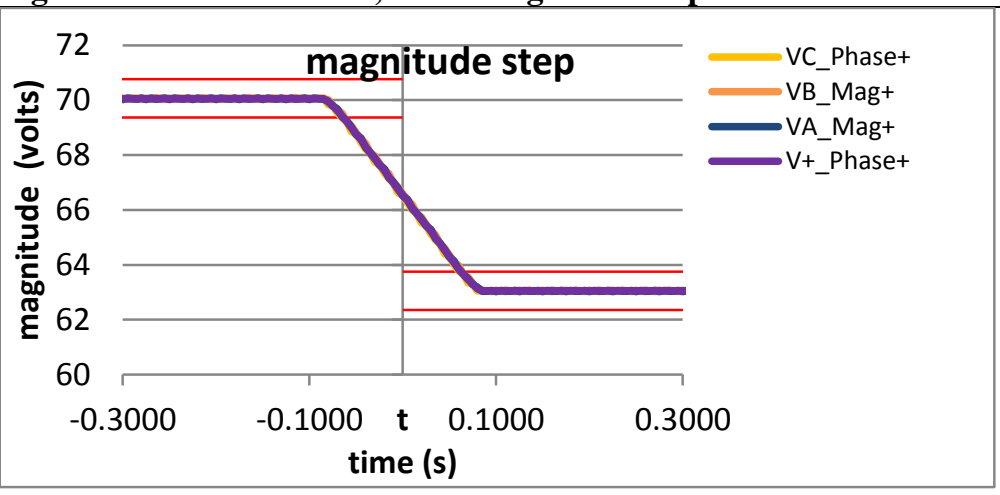

Figure 6064; Fs = 20 FPS, $=10 \%$ magnitude step 


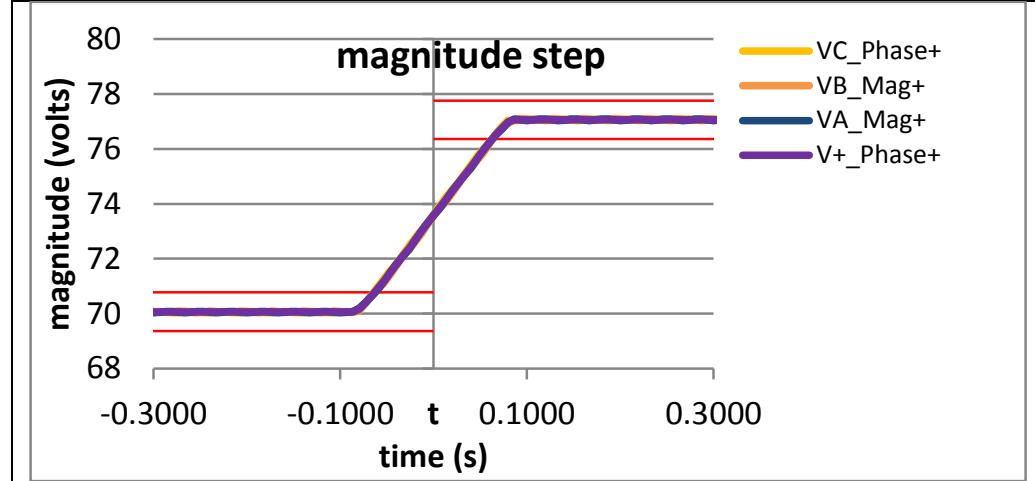

Figure 6065: Fs = 15 FPS, + 10\% magnitude step

Figure 6067: Fs = 12 FPS, $+10 \%$ magnitude step

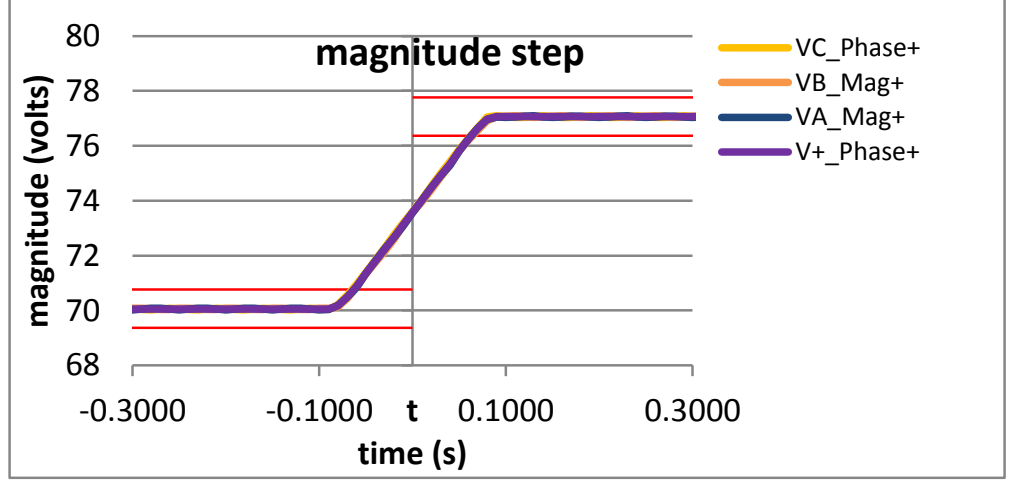

Figure 6069: Fs $=10 \mathrm{FPS},+10 \%$ magnitude step

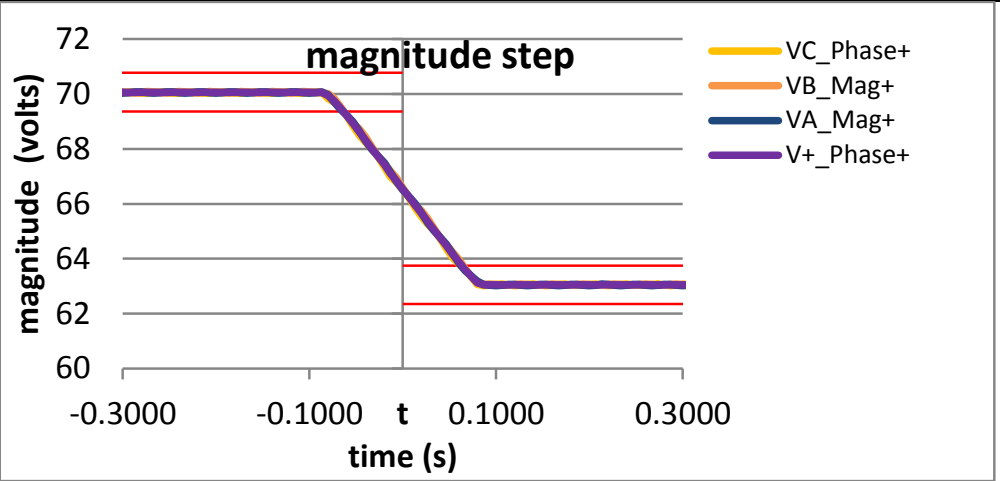

Figure 6066: Fs = 15 FPS, - $10 \%$ magnitude step

Figure 6068: Fs = 12 FPS, $-10 \%$ magnitude step

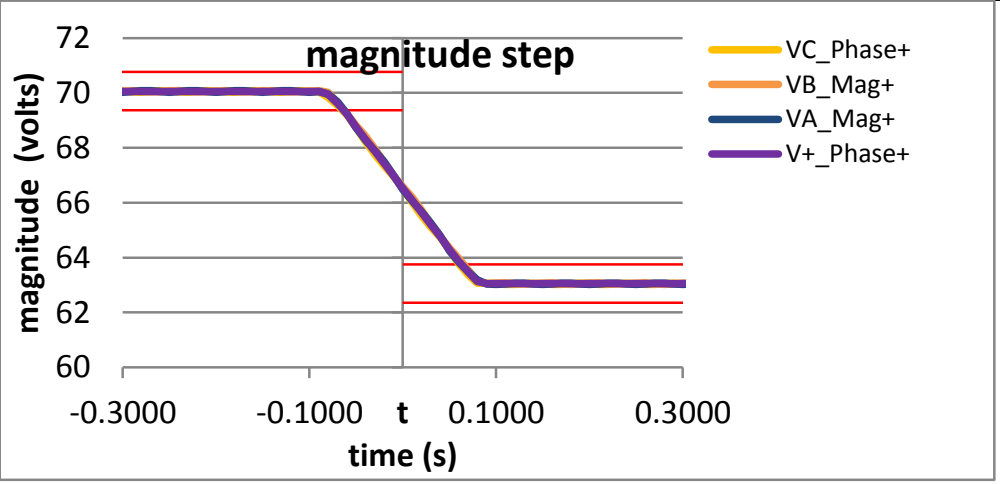

Figure 6070: Fs = 10 FPS, -10\% magnitude step 
10.12.7 PMU F dynamic step change in magnitude overshoot: $F 0=60 \mathrm{~Hz}$, M class

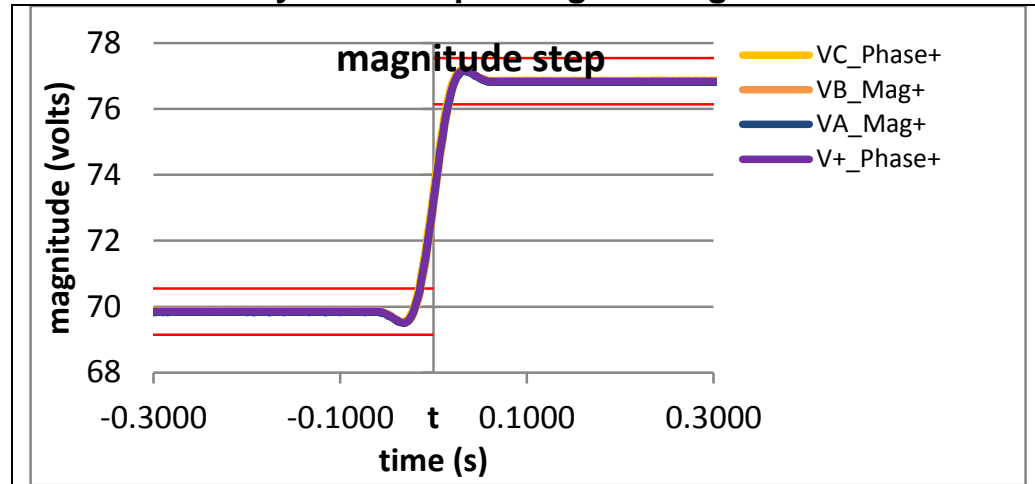

Figure 6071: Fs = 60 FPS, +10\% magnitude step

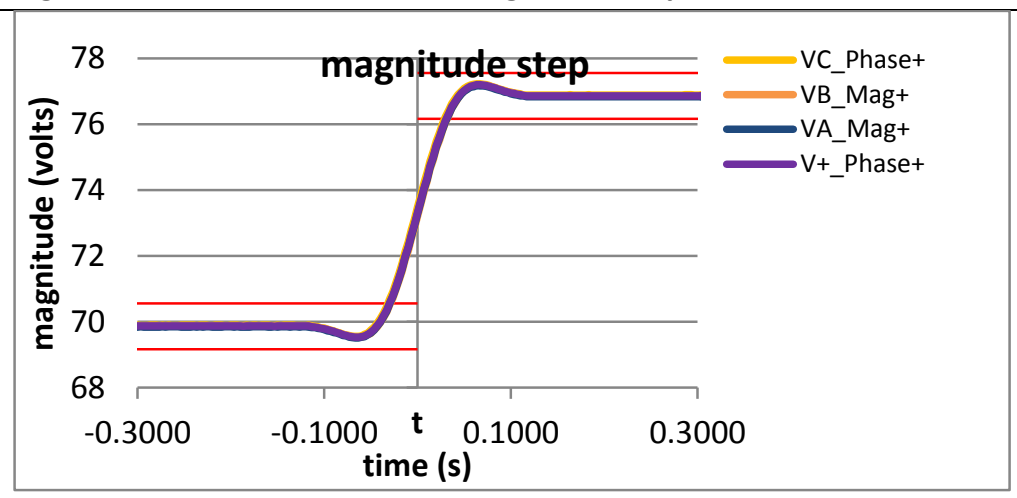

Figure 6073: Fs = 30 FPS, $+10 \%$ magnitude step

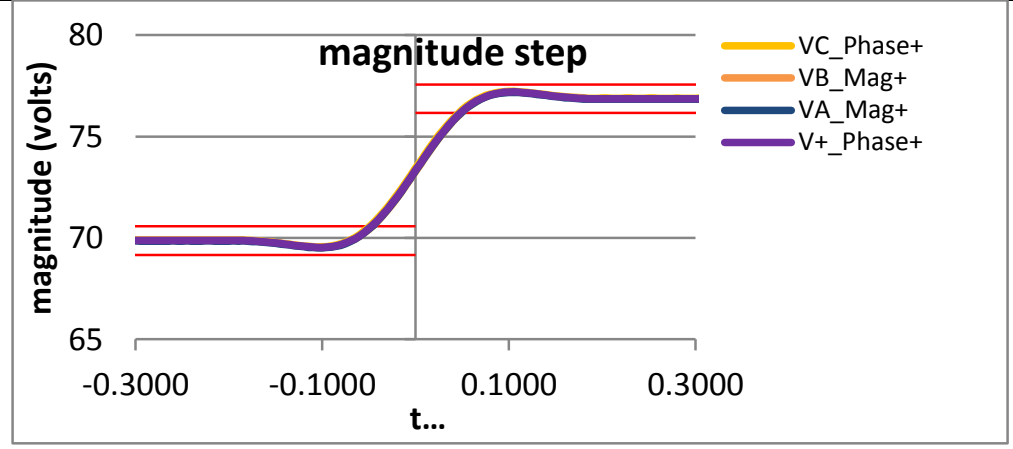

Figure 6075: Fs = 20 FPS, +10\% magnitude step

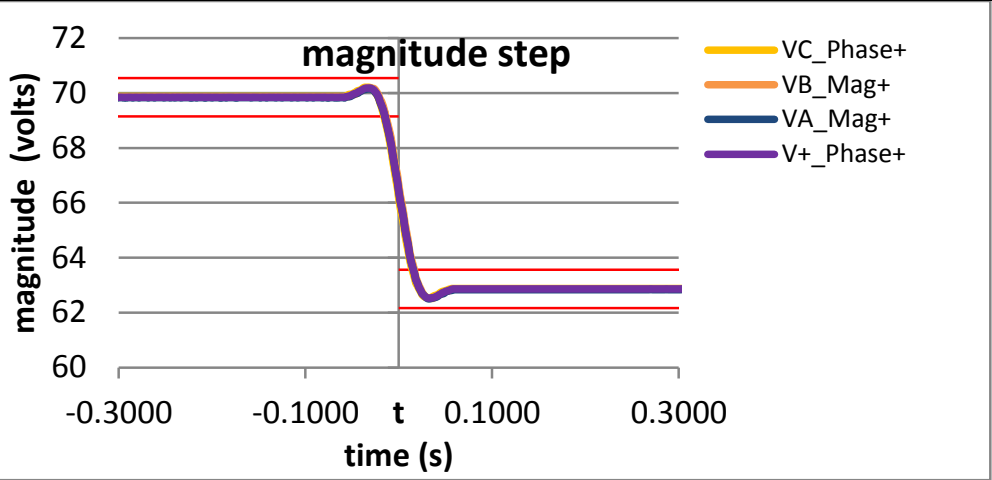

Figure 6072: Fs = 60 FPS, $-10 \%$ magnitude step

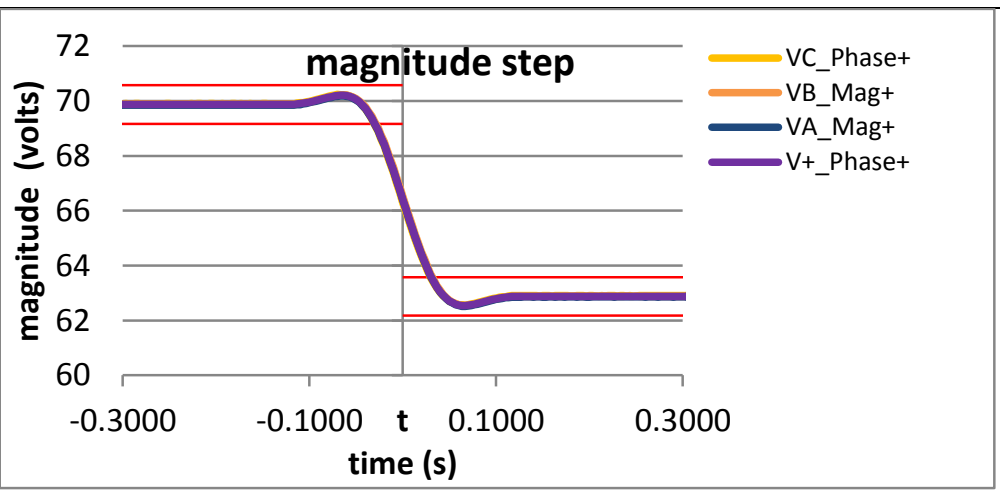

Figure 6074: Fs = 30 FPS, -10\% magnitude step

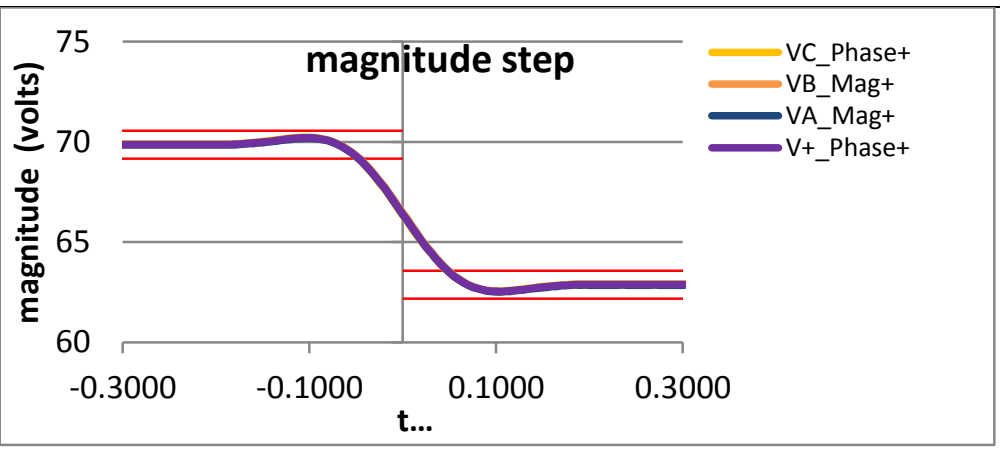

Figure 6076; Fs = 20 FPS, =10\% magnitude step 


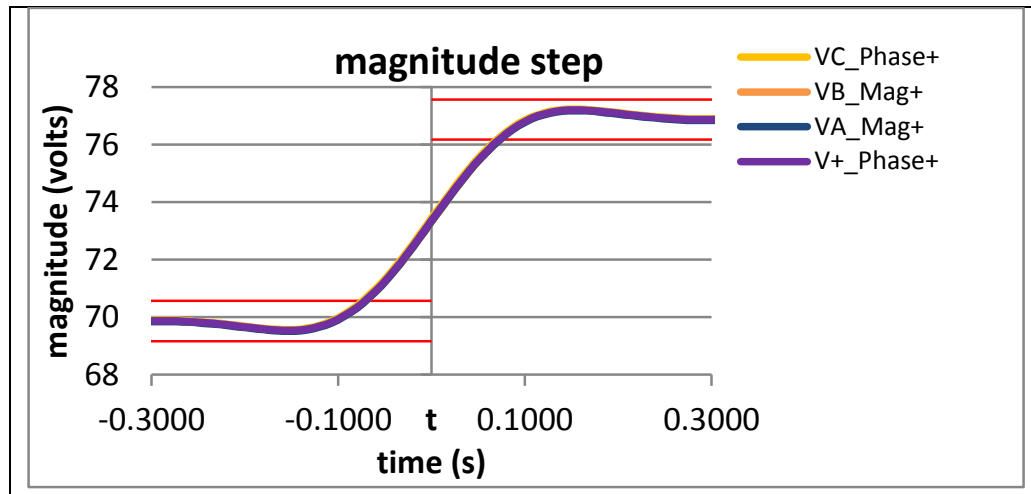

Figure 6077: Fs = 15 FPS, $+10 \%$ magnitude step

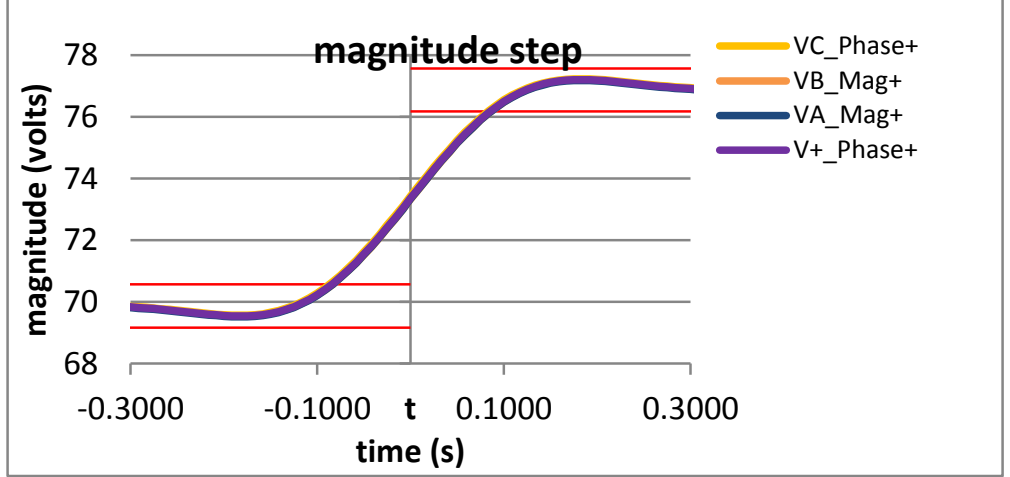

Figure 6079: Fs = 12 FPS, $+10 \%$ magnitude step

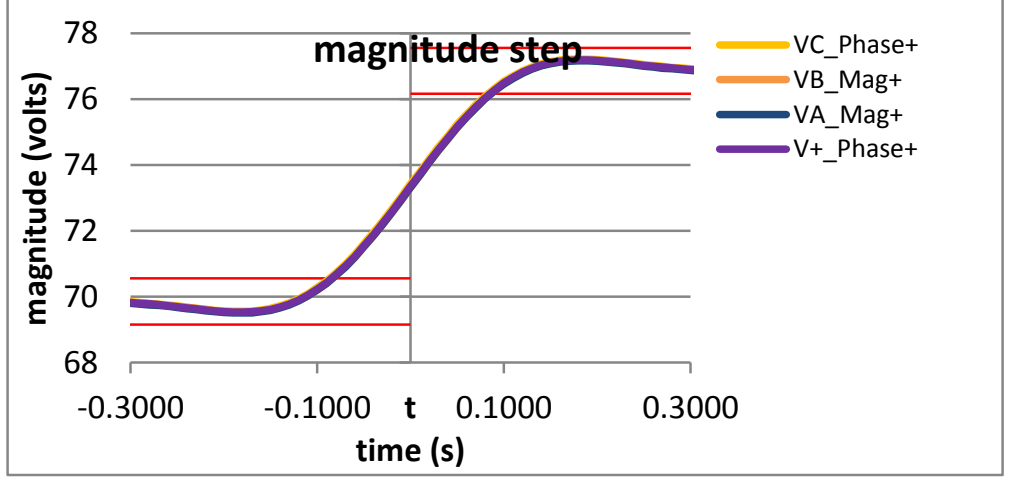

Figure 6081: Fs = 10 FPS, $+10 \%$ magnitude step

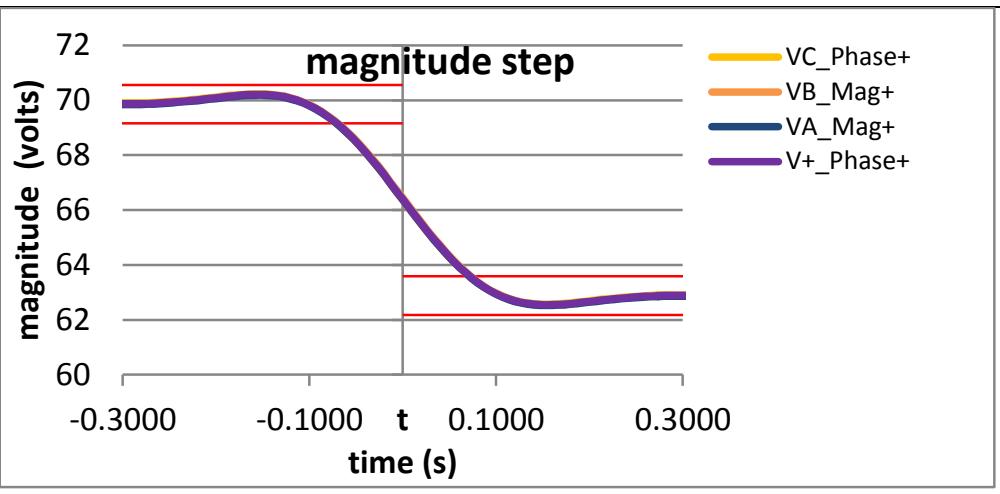

Figure 6078: Fs = 15 FPS, - $10 \%$ magnitude step

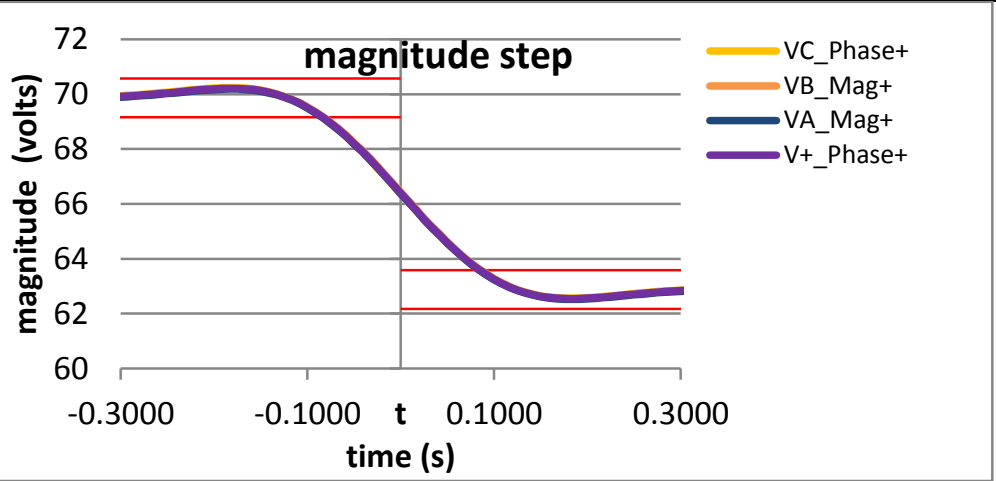

Figure 6080: Fs = 12 FPS, $-10 \%$ magnitude step

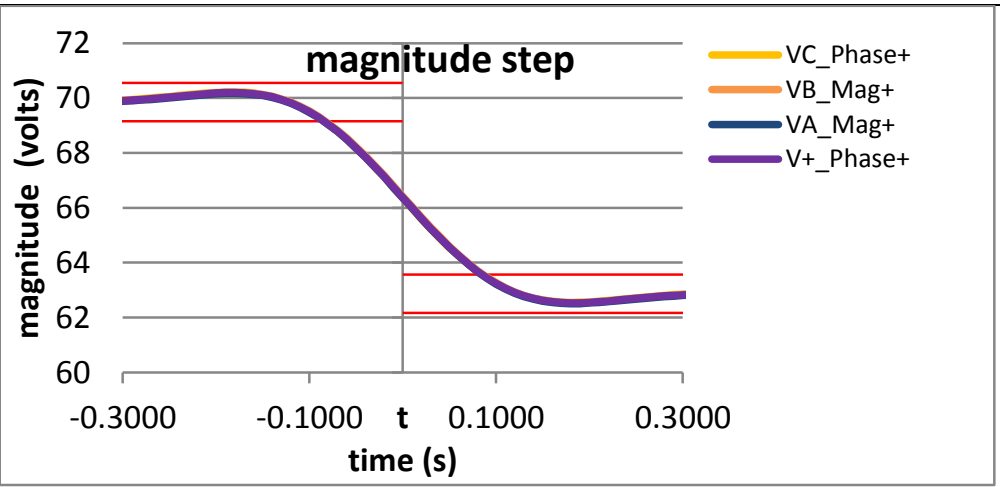

Figure 6082: Fs = $10 \mathrm{FPS},-10 \%$ magnitude step 
10.12.8 PMU G dynamic step change in magnitude overshoot: $F 0=60 \mathrm{~Hz}$, M class

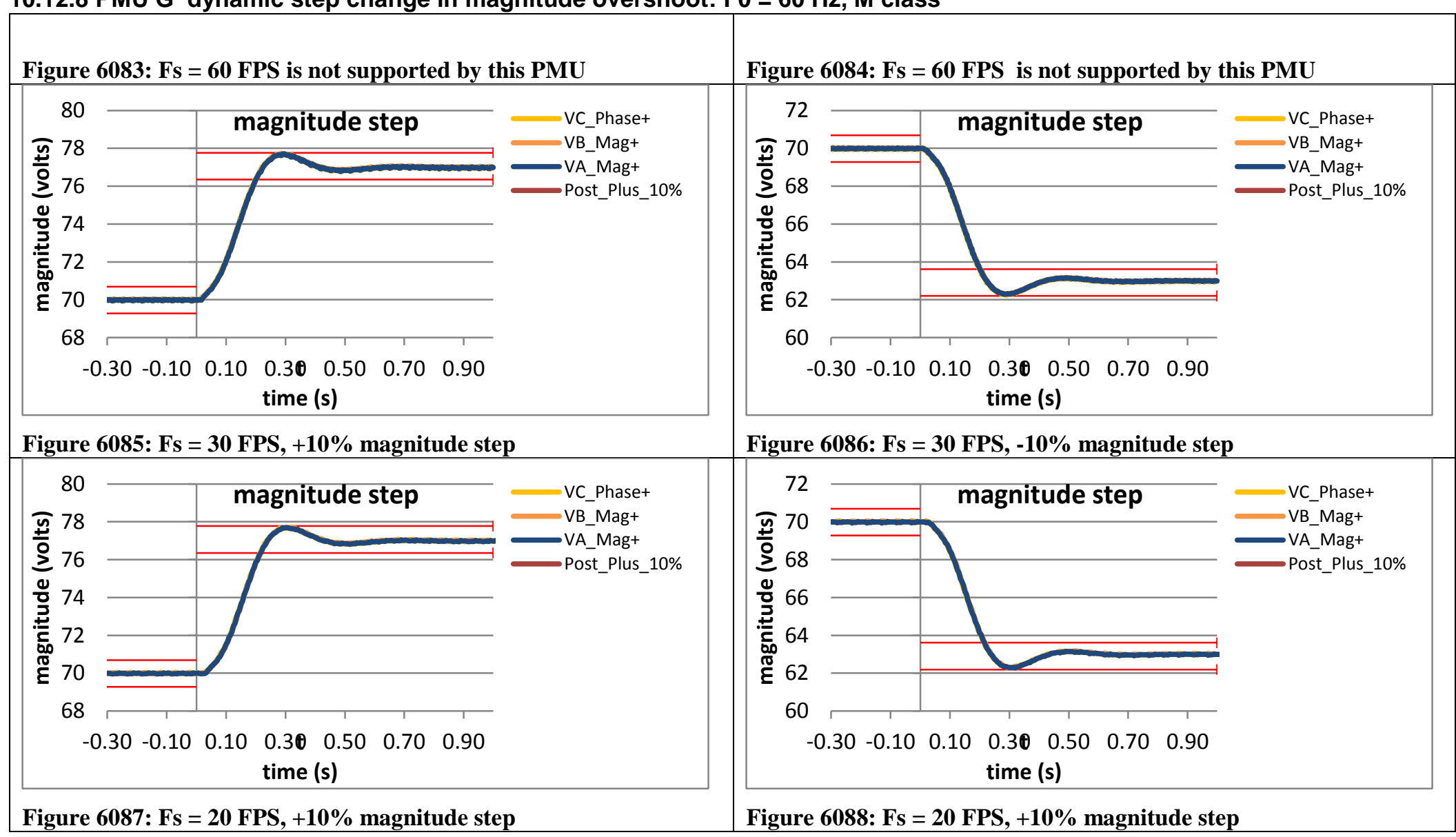




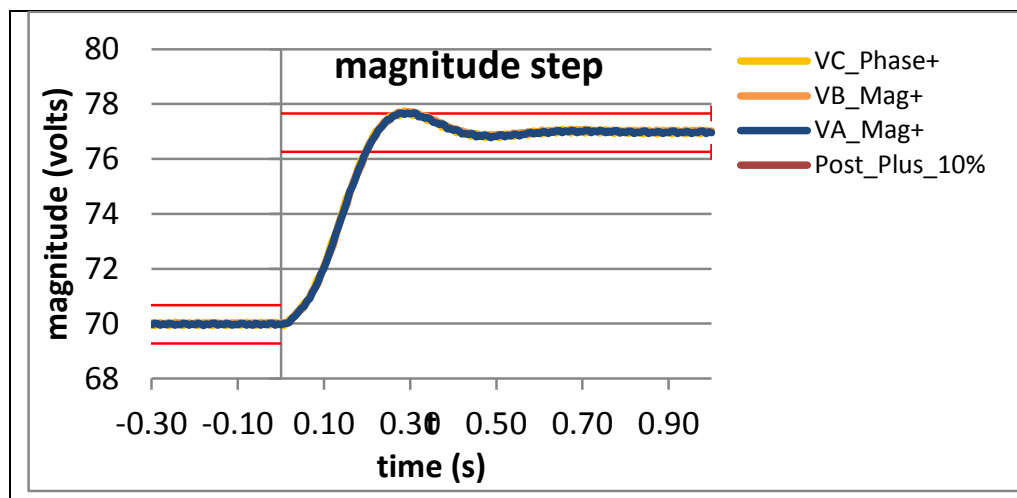

Figure 6089: Fs = 15 FPS, +10\% magnitude step

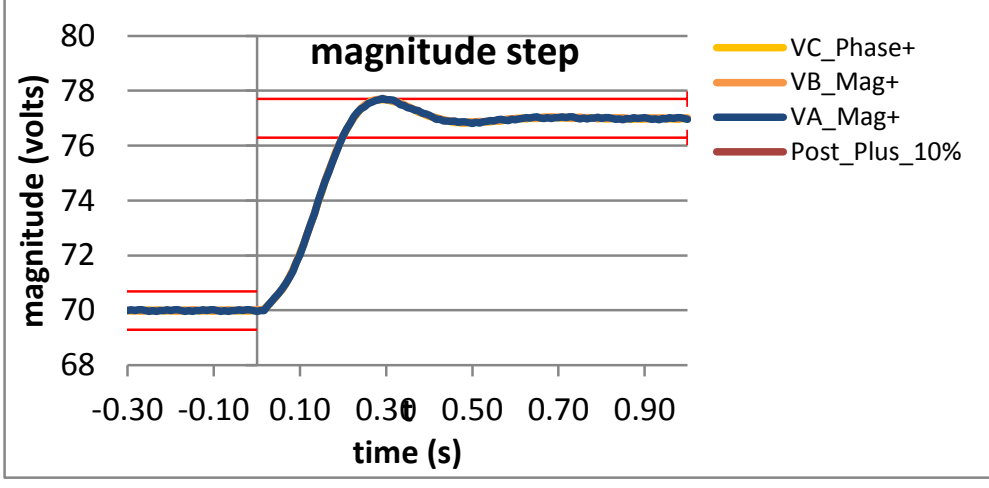

Figure 6091: Fs $=12$ FPS, $+10 \%$ magnitude step

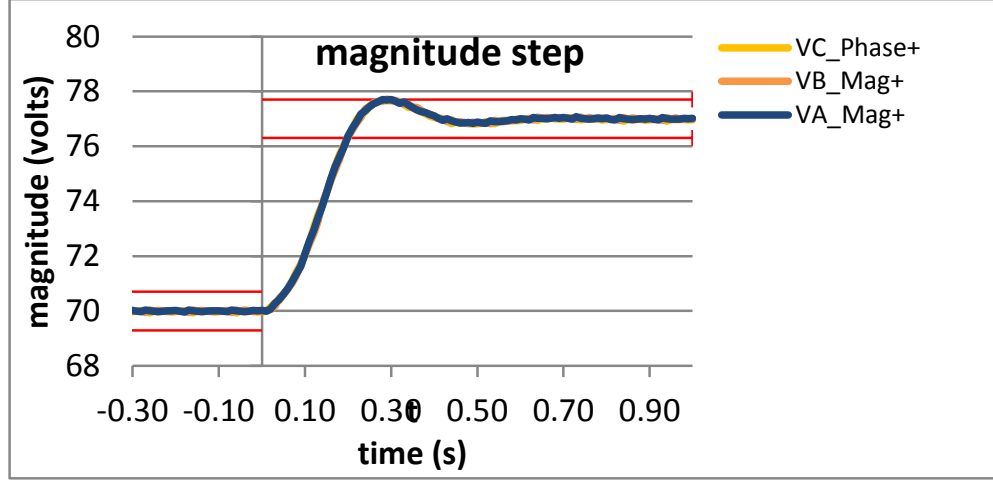

Figure 6093: Fs = 10 FPS, $+10 \%$ magnitude step

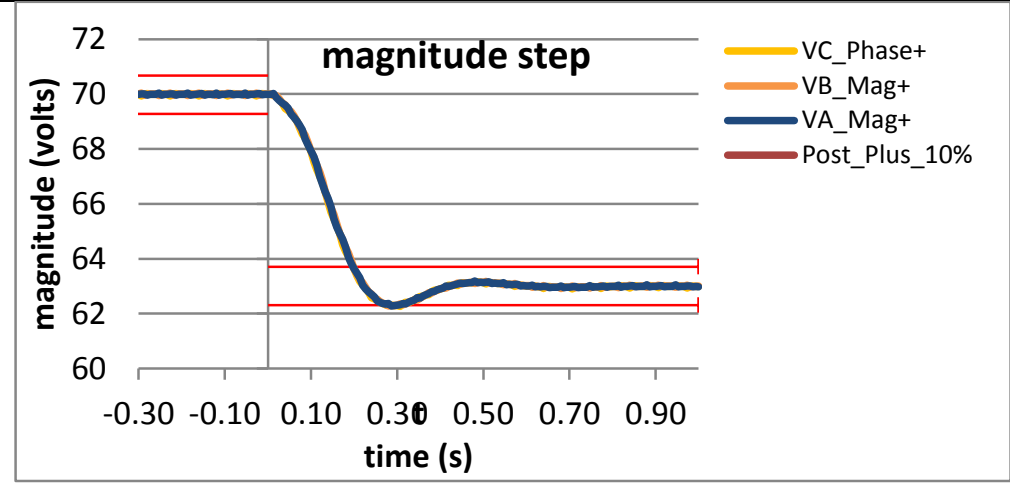

Figure 6090: Fs = 15 FPS, -10\% magnitude step

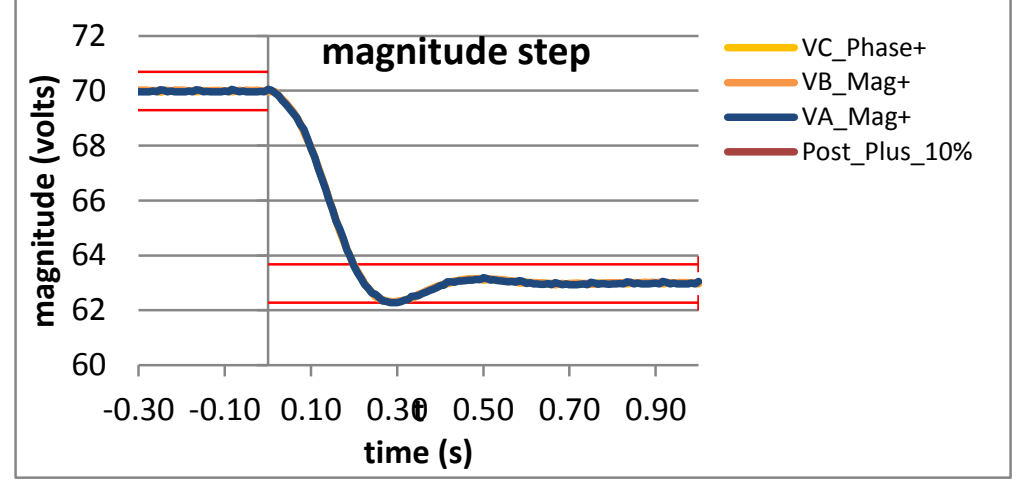

Figure 6092: Fs = 12 FPS, -10\% magnitude step

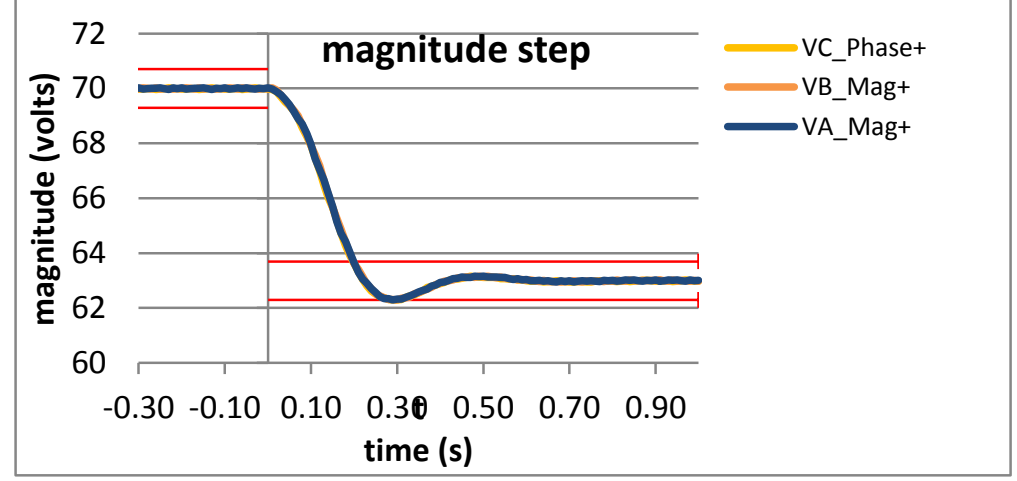

Figure 6094: Fs = 10 FPS, -10\% magnitude step 
10.12.9 PMU H dynamic step change in magnitude overshoot: $\mathrm{FO}=60 \mathrm{~Hz}, \mathrm{M}$ class

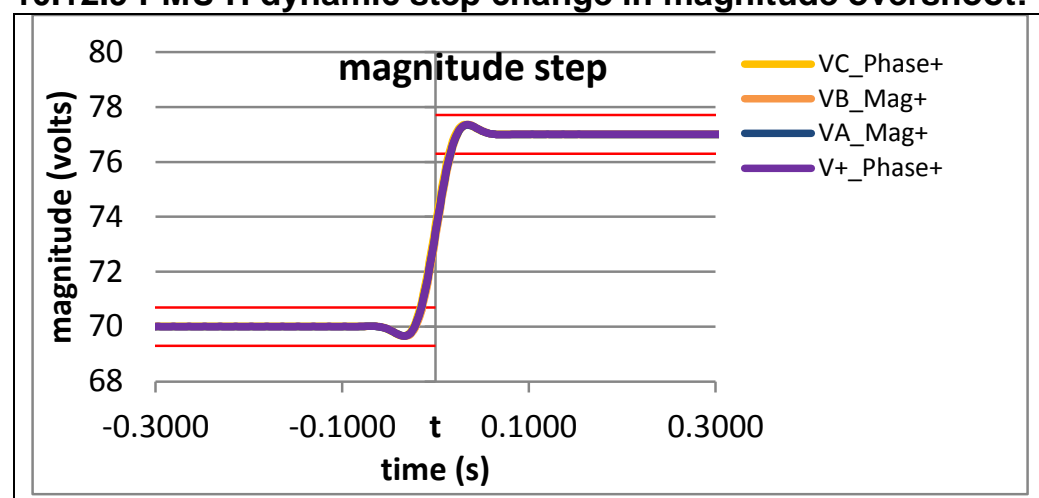

Figure 6095: Fs = 60 FPS, +10\% magnitude step

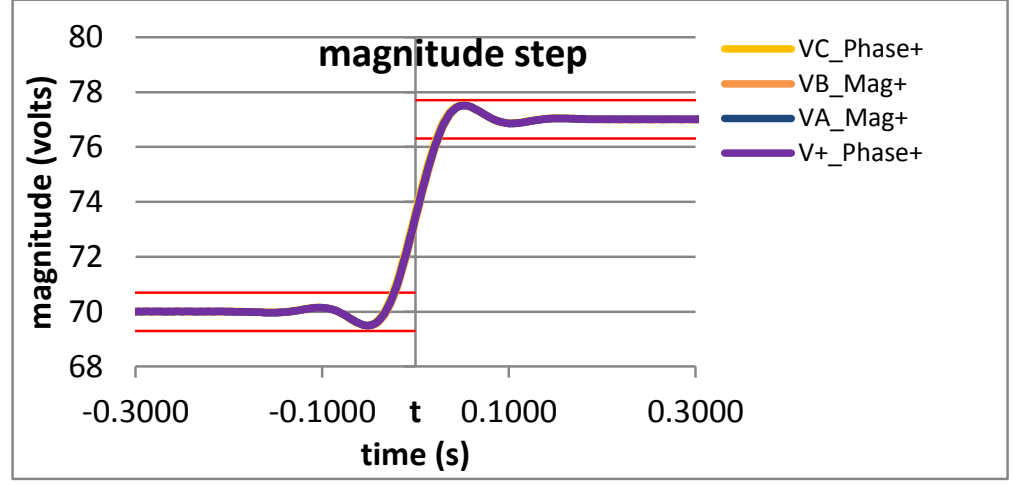

Figure 6097: Fs = 30 FPS, +10\% magnitude step

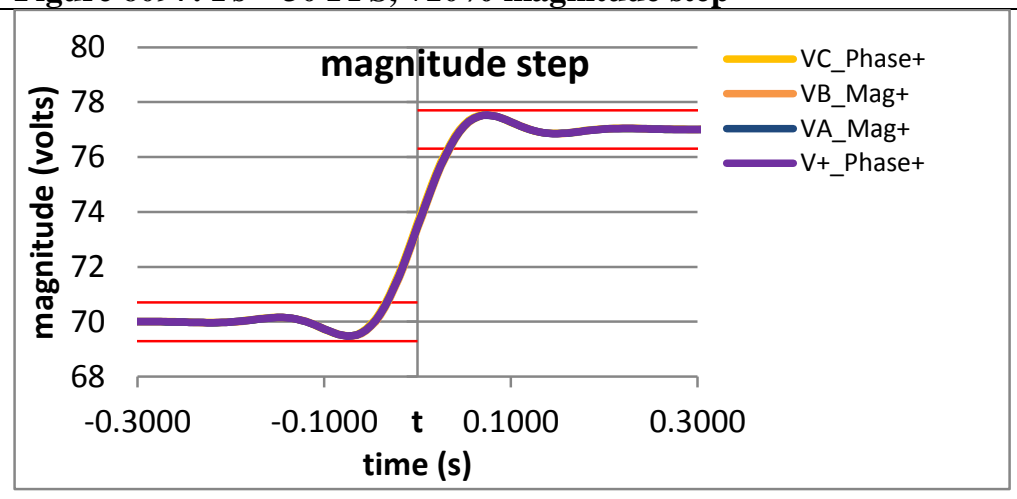

Figure 6099: Fs $=20$ FPS, $+10 \%$ magnitude step

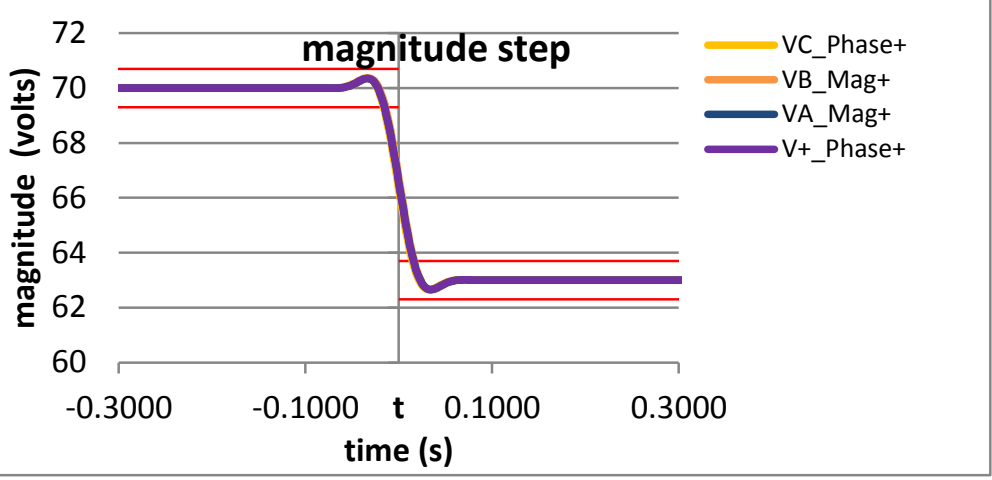

Figure 6096: Fs $=60$ FPS, $-10 \%$ magnitude step

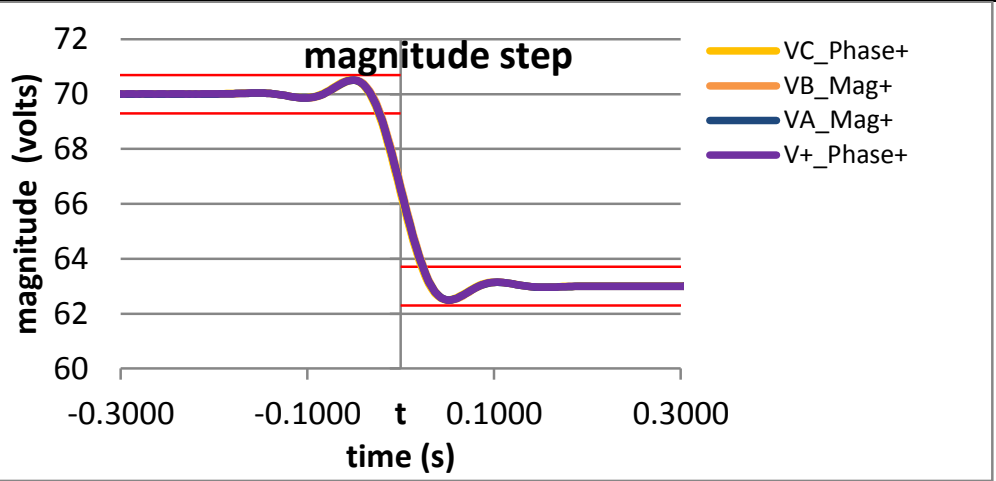

Figure 6098: Fs = 30 FPS, -10\% magnitude step

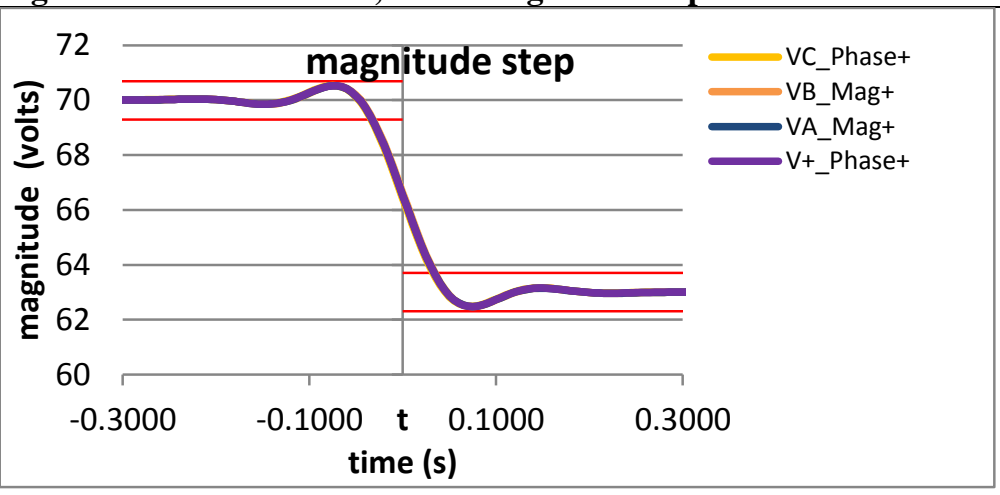

Figure 6100; Fs $=20$ FPS, $=10 \%$ magnitude step 


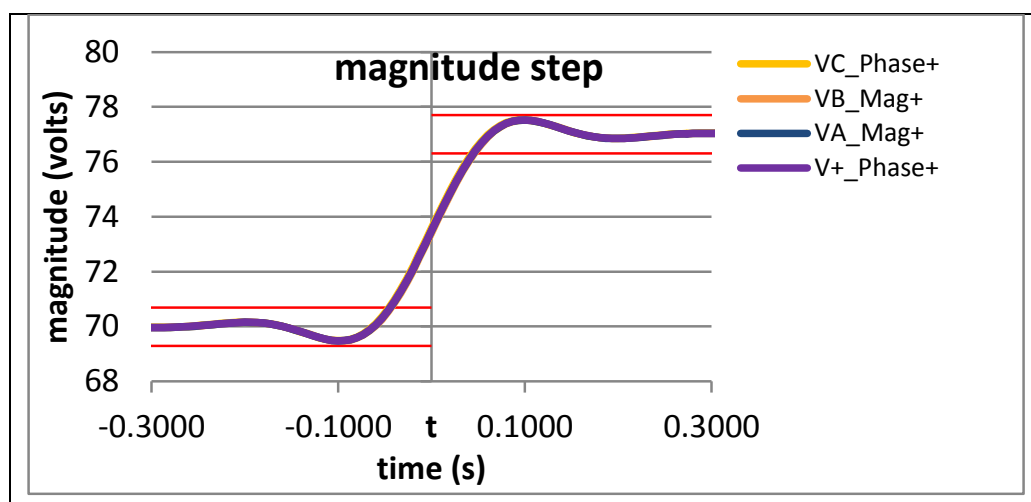

Figure 6101: Fs = 15 FPS, + 10\% magnitude step

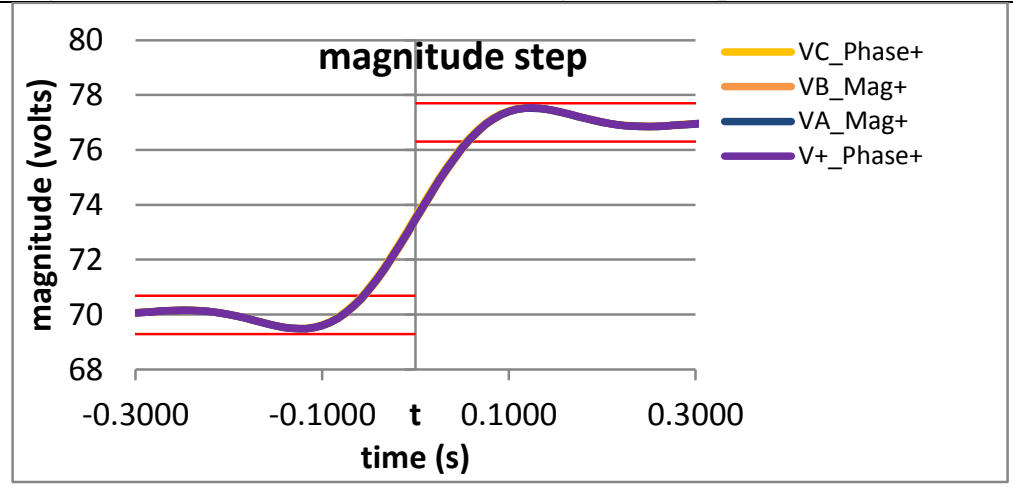

Figure 6103: Fs = 12 FPS, +10\% magnitude step

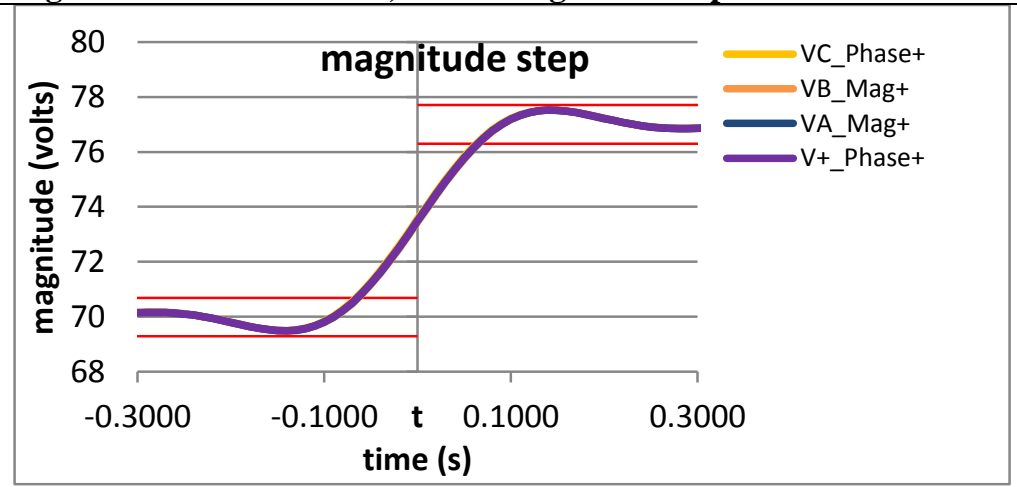

Figure 6105: Fs = 10 FPS, +10\% magnitude step

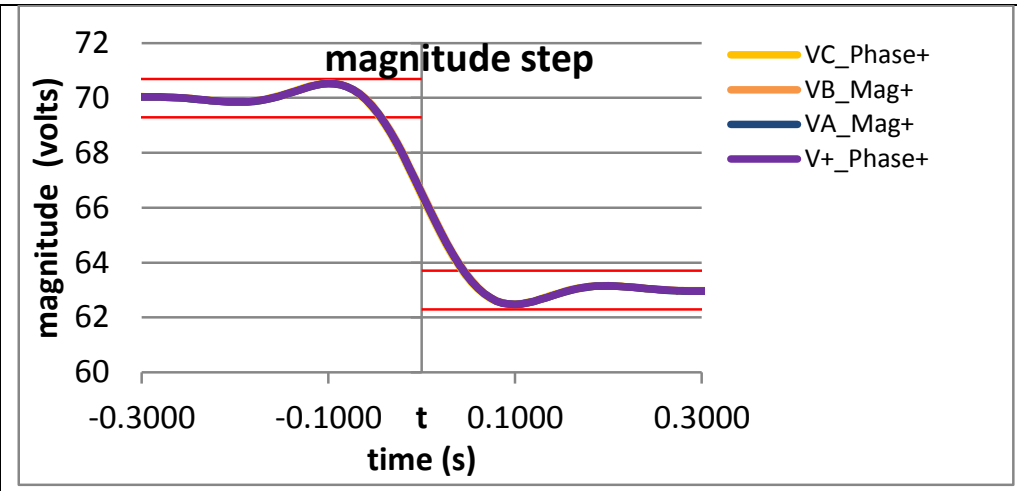

Figure 6102: Fs = 15 FPS, - $10 \%$ magnitude step

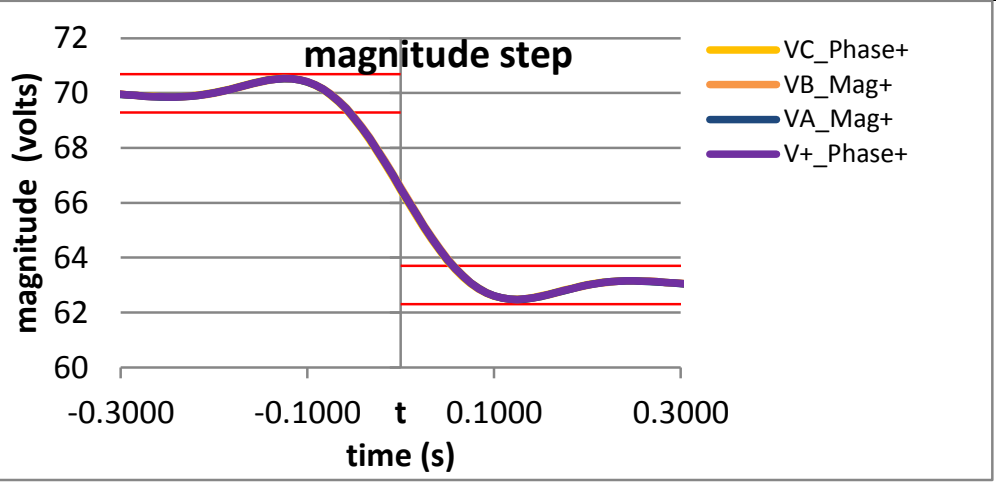

Figure 6104: Fs = 12 FPS, -10\% magnitude step

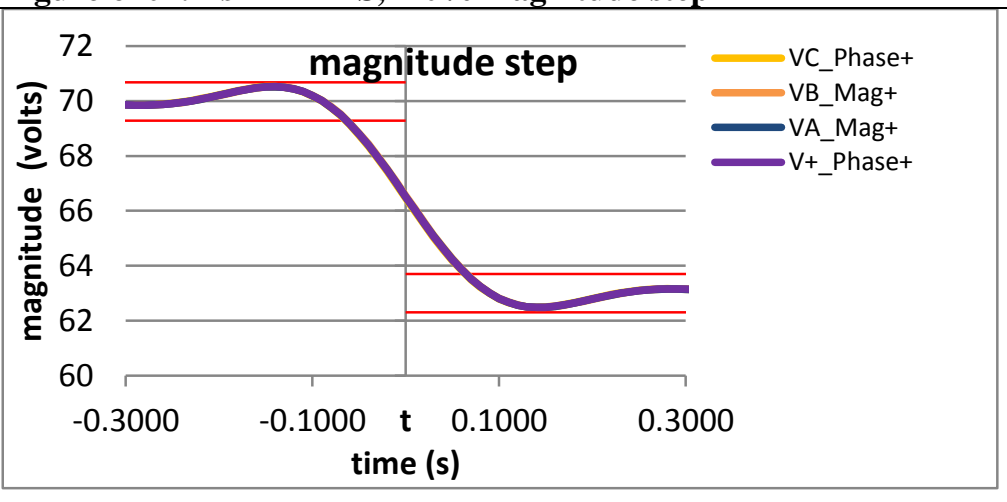

Figure 6106: Fs = 10 FPS, -10\% magnitude step 
10.12.10 PMU I dynamic step change in magnitude overshoot: $F 0=60 \mathrm{~Hz}$, M class

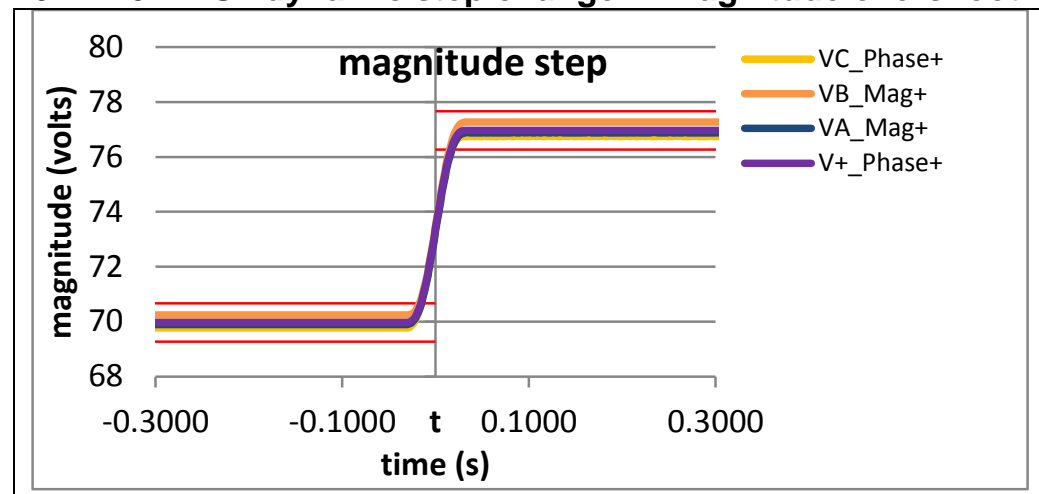

Figure 6107: Fs = 60 FPS, +10\% magnitude step

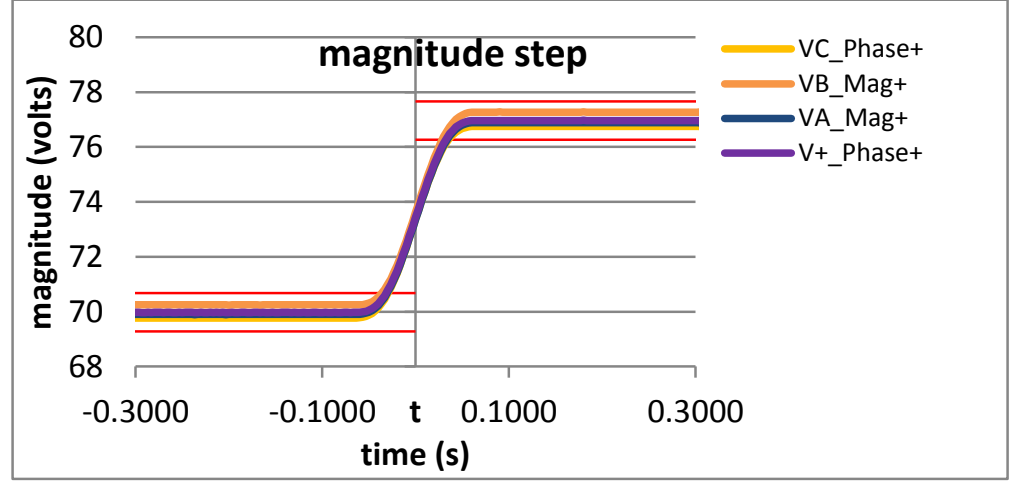

Figure 6109: Fs = 30 FPS, +10\% magnitude step

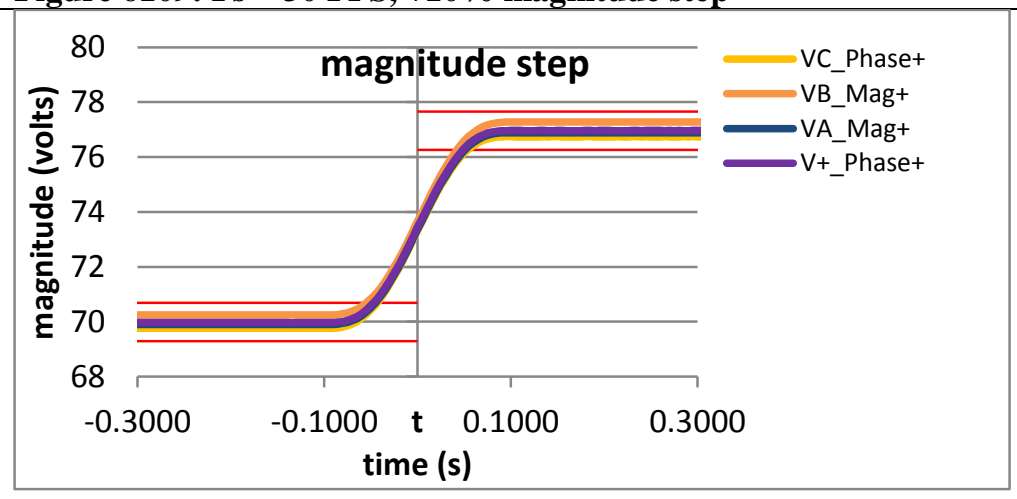

Figure 6111: Fs $=20$ FPS, $+10 \%$ magnitude step

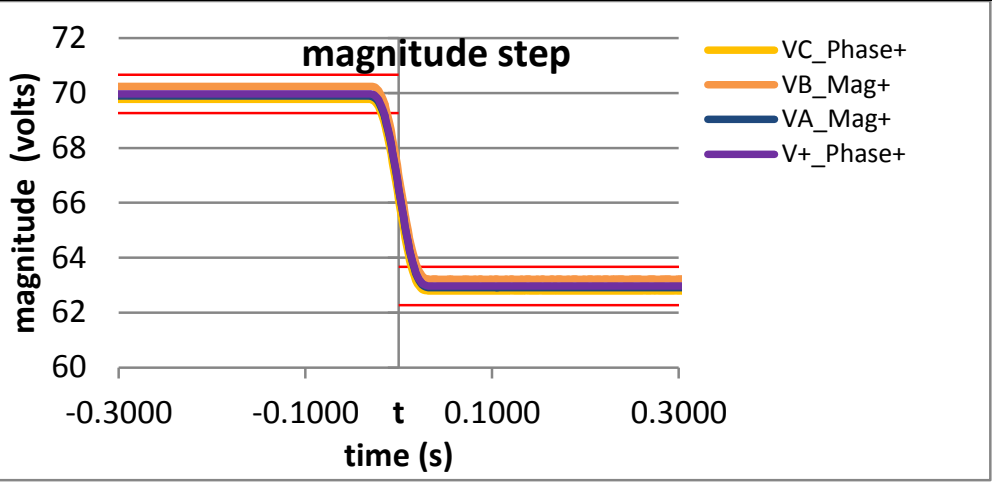

Figure 6108: Fs $=60$ FPS, $-10 \%$ magnitude step

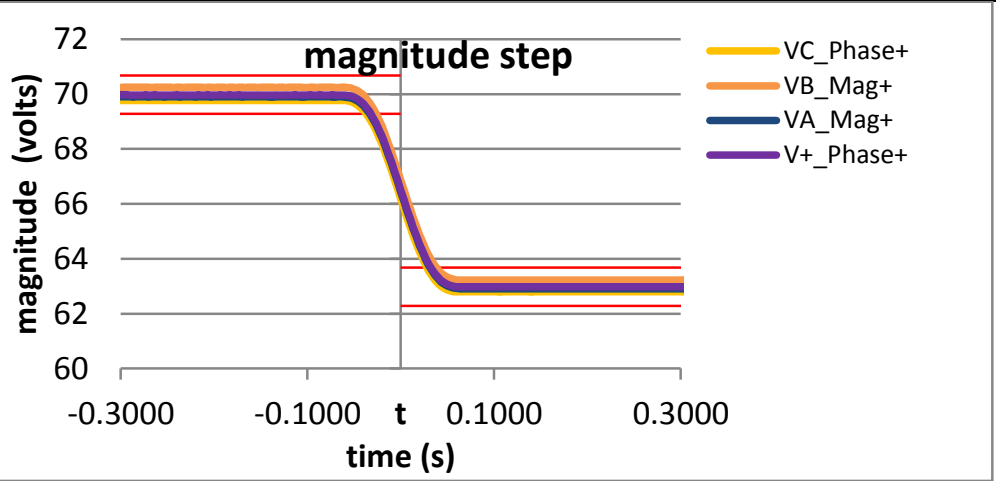

Figure 6110: Fs = 30 FPS, -10\% magnitude step

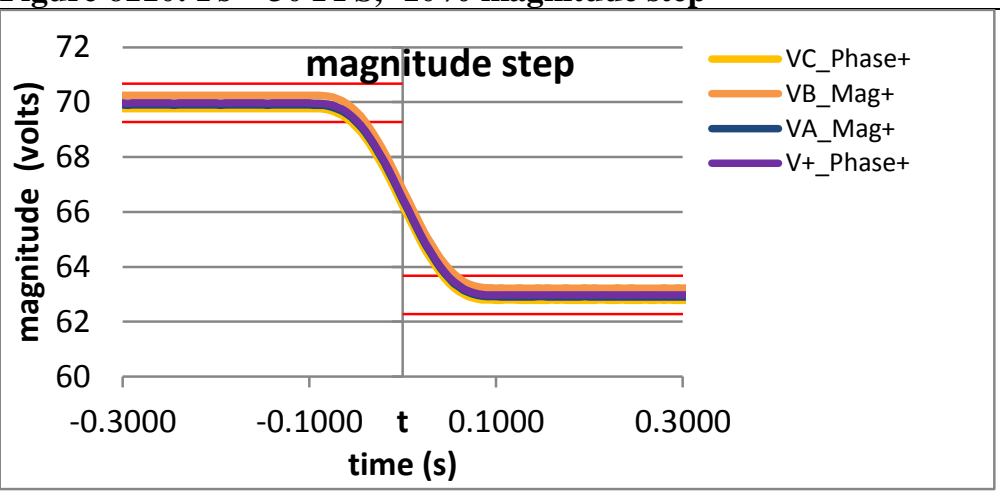

Figure 6112; Fs $=20$ FPS, $=10 \%$ magnitude step 


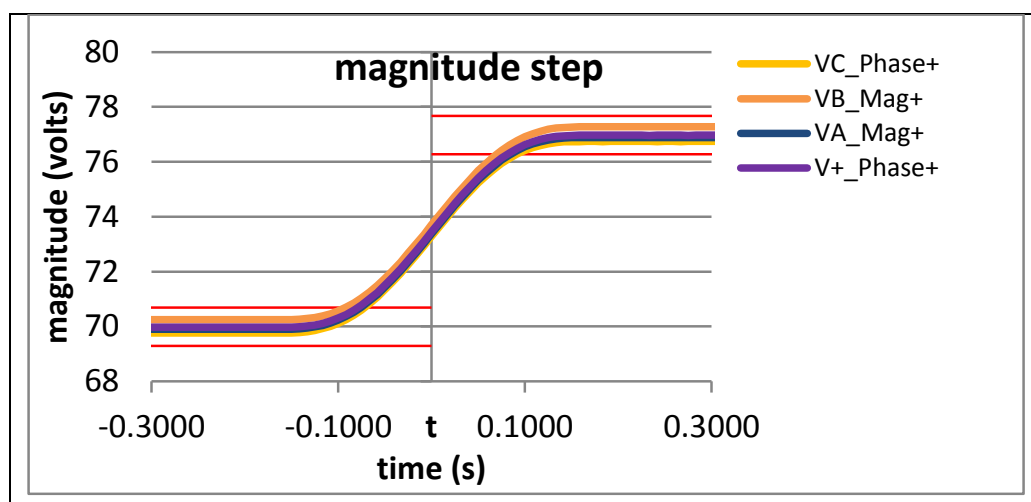

Figure 6113: Fs = 15 FPS, + 10\% magnitude step

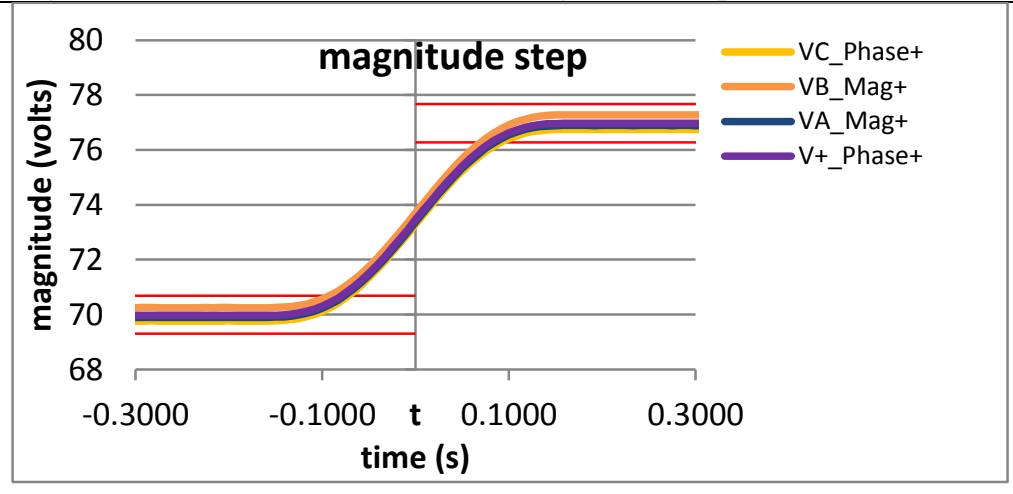

Figure 6115: Fs = 12 FPS, +10\% magnitude step

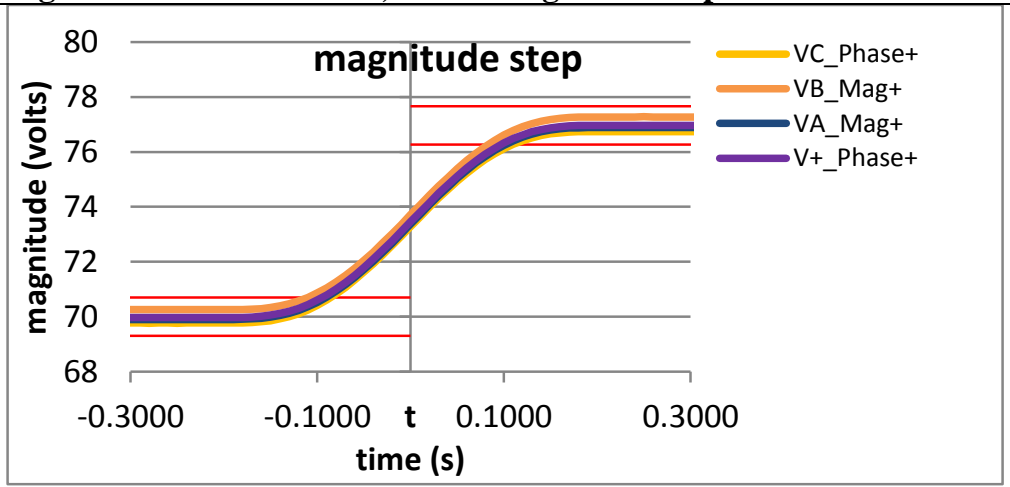

Figure 6117: Fs = 10 FPS, $+10 \%$ magnitude step

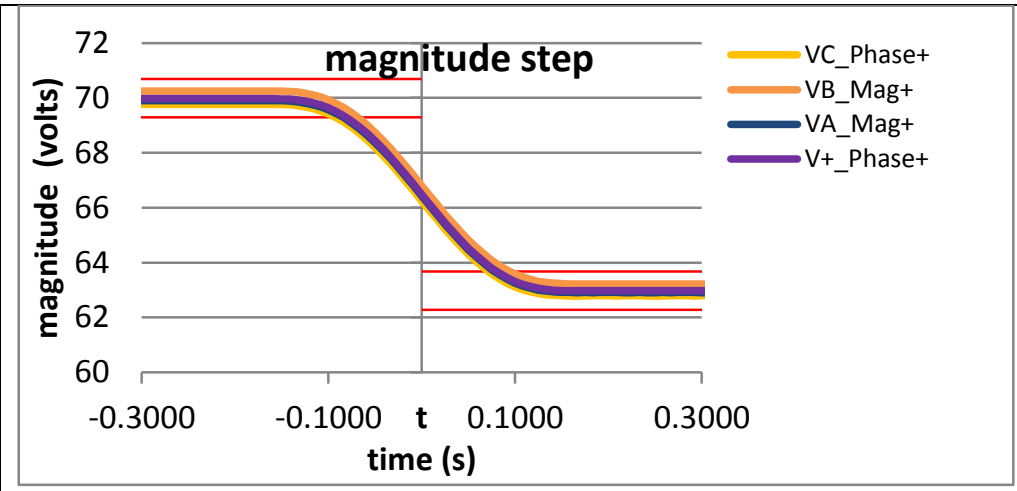

Figure 6114: Fs = 15 FPS, - $10 \%$ magnitude step

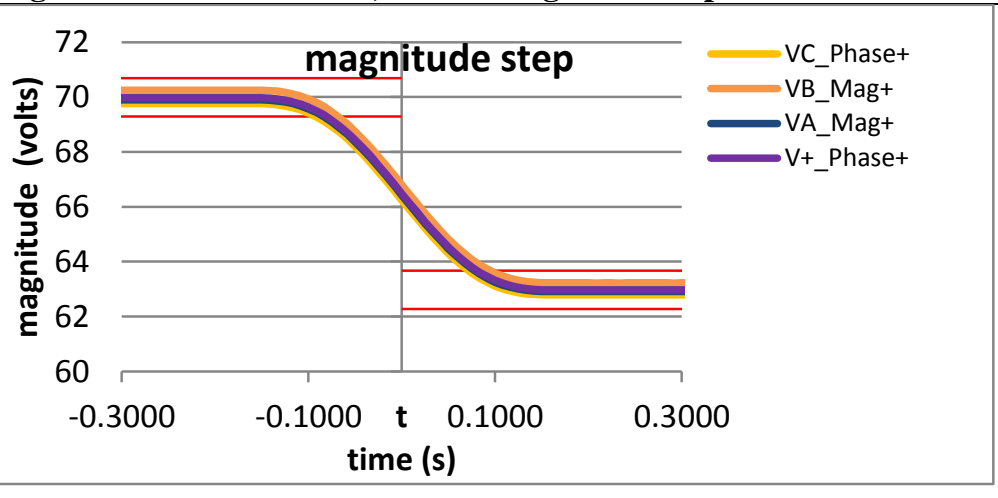

Figure 6116: Fs = 12 FPS, -10\% magnitude step

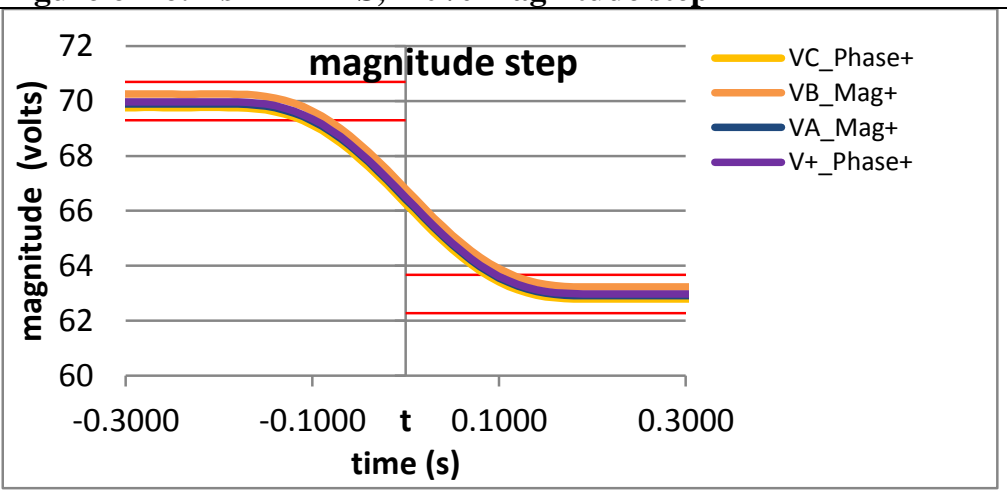

Figure 6118: Fs = 10 FPS, -10\% magnitude step 
10.12.11 PMU J dynamic step change in magnitude overshoot: $F 0=60 \mathrm{~Hz}, \mathrm{M}$ class

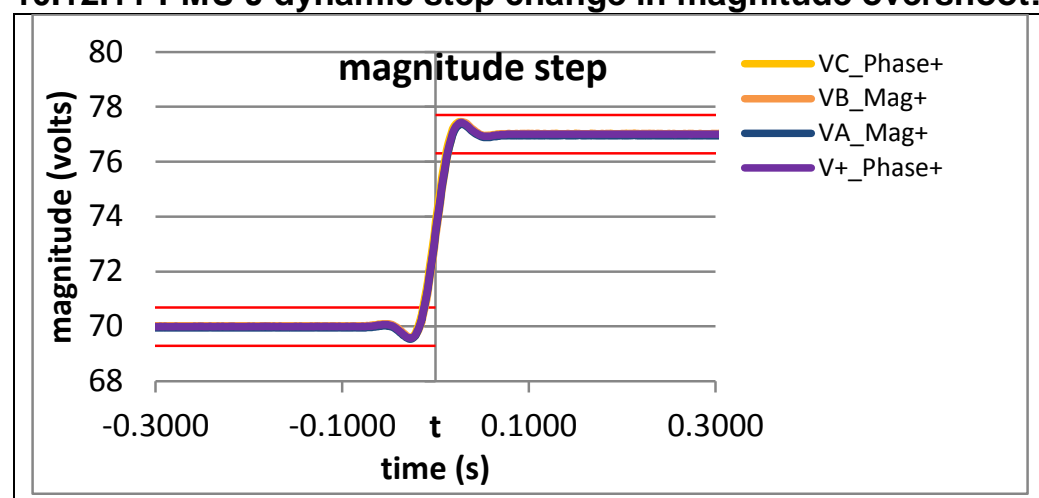

Figure 6119: Fs = $60 \mathrm{FPS},+10 \%$ magnitude step

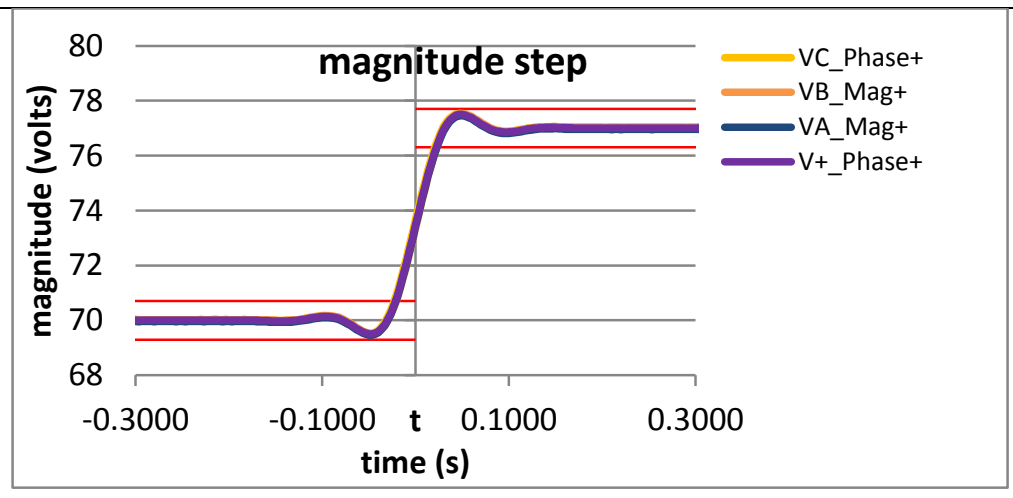

Figure 6121: Fs = 30 FPS, $+10 \%$ magnitude step

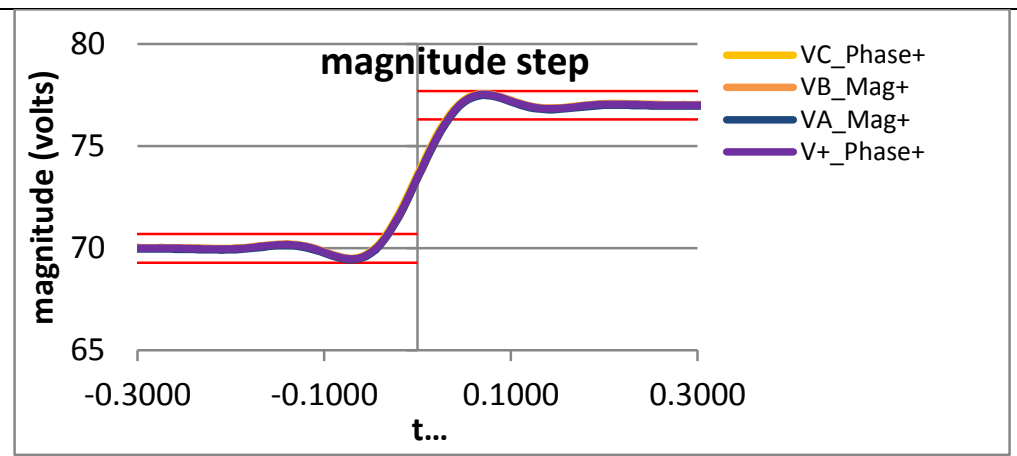

Figure 6123: Fs $=20$ FPS, $+10 \%$ magnitude step

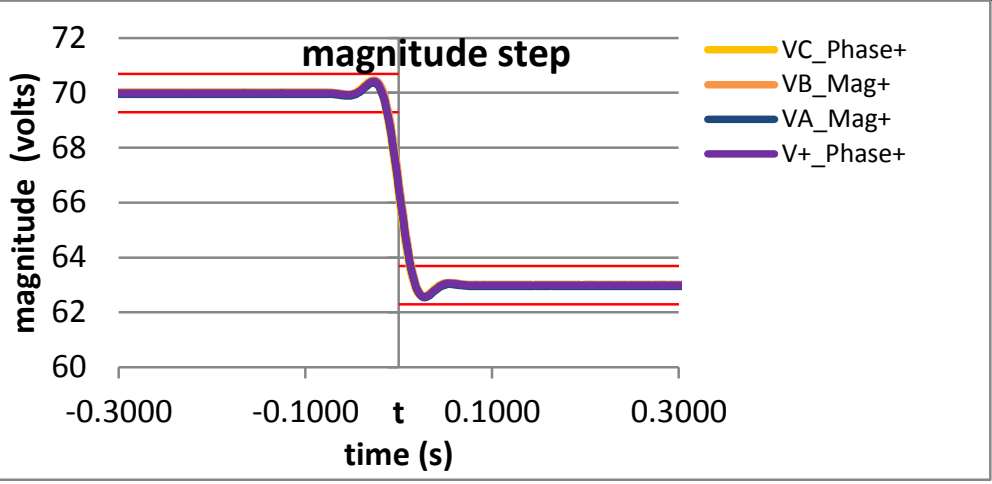

Figure 6120: Fs = $60 \mathrm{FPS},-10 \%$ magnitude step

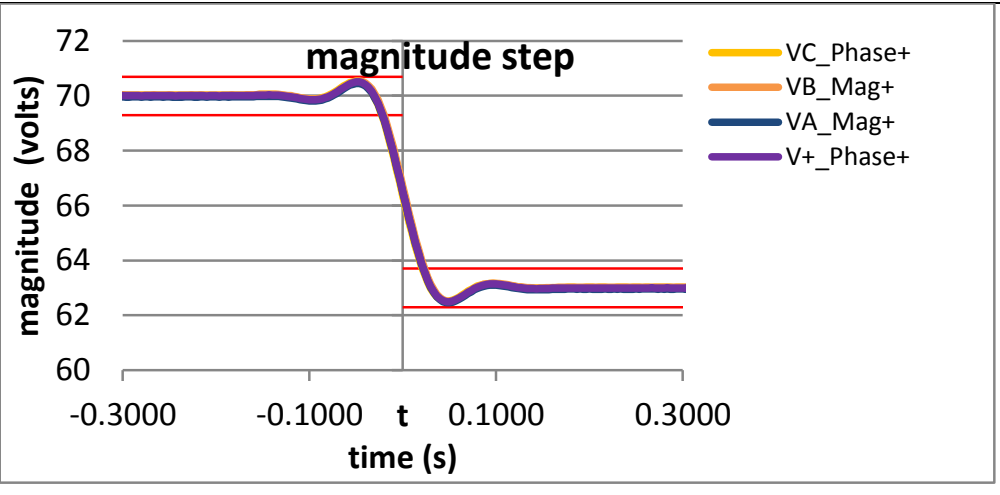

Figure 6122: Fs = $30 \mathrm{FPS},-10 \%$ magnitude step

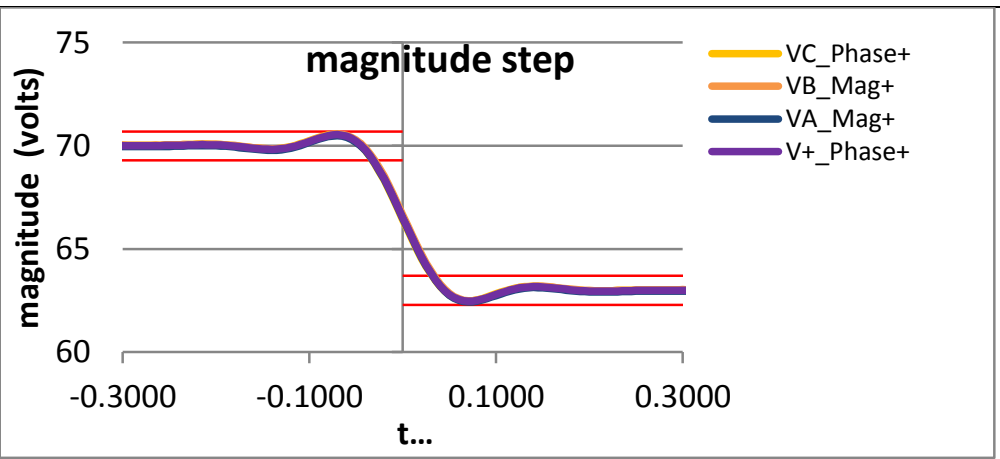

Figure 6124; Fs = 20 FPS, =10\% magnitude step 


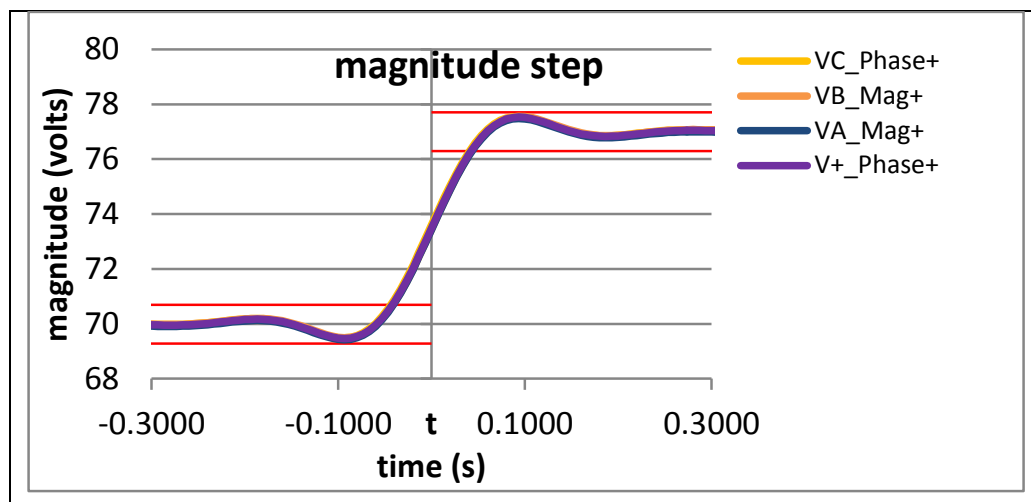

Figure 6125: Fs = $15 \mathrm{FPS},+10 \%$ magnitude step

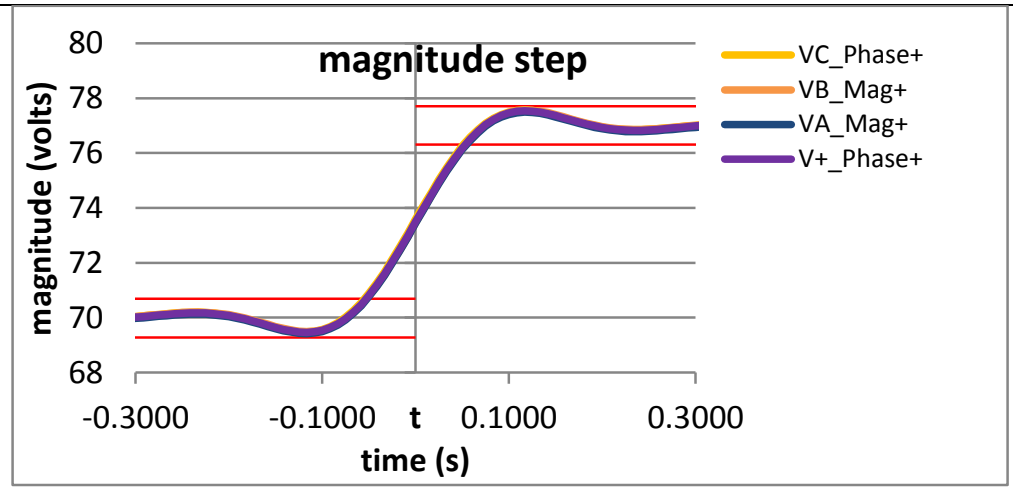

Figure 6127: Fs = 12 FPS, $+10 \%$ magnitude step

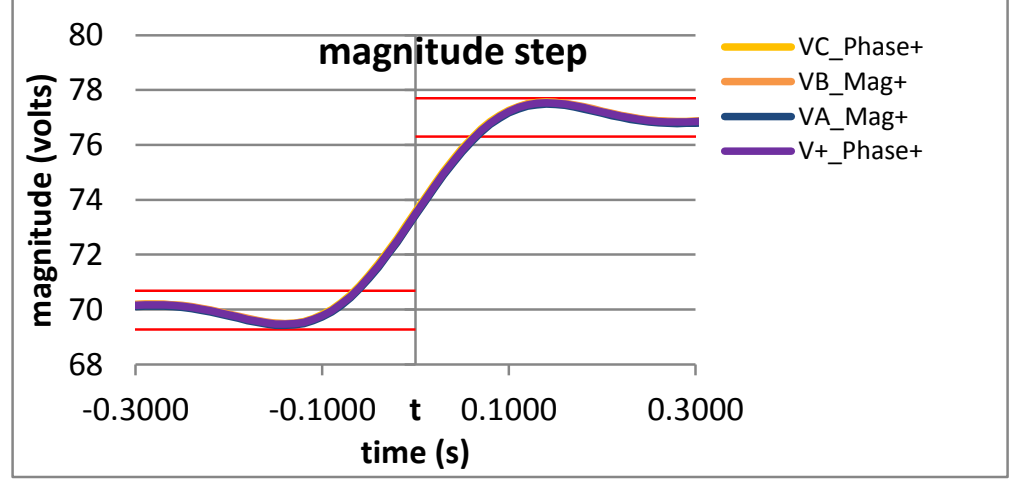

Figure 6129: $\mathrm{Fs}=10 \mathrm{FPS},+10 \%$ magnitude step

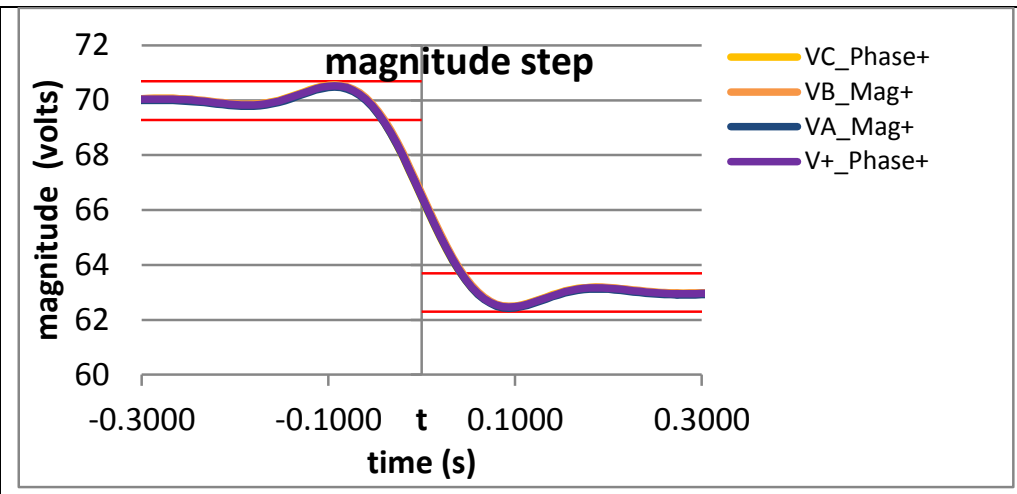

Figure 6126: Fs = $15 \mathrm{FPS},-10 \%$ magnitude step

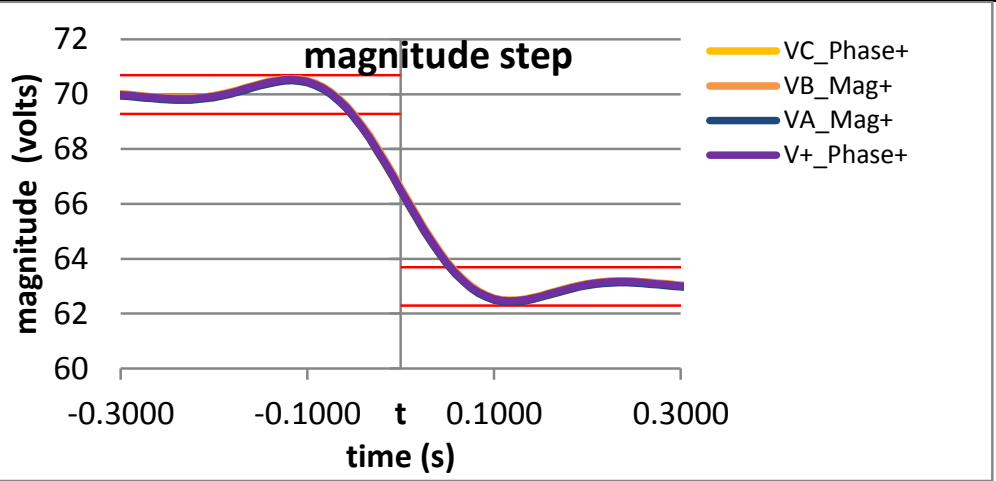

Figure 6128: Fs = 12 FPS, $-10 \%$ magnitude step

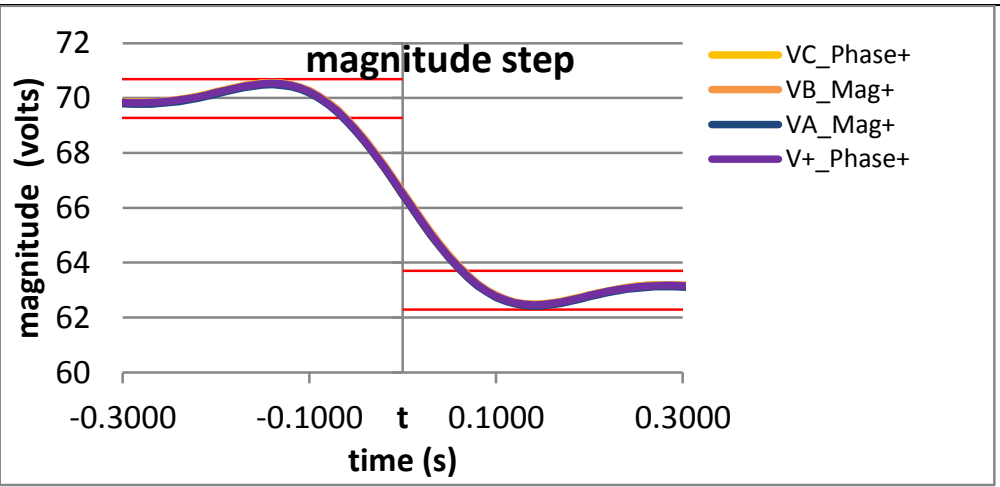

Figure 6130: Fs = $10 \mathrm{FPS},-10 \%$ magnitude step 
10.13 Dynamic step change in magnitude overshoot: $F 0=60 \mathrm{~Hz}$, $P$ class

10.13.1 C37.118.1-2011 Annex $C$ dynamic step change in magnitude overshoot: $F 0=60 \mathrm{~Hz}$, P class

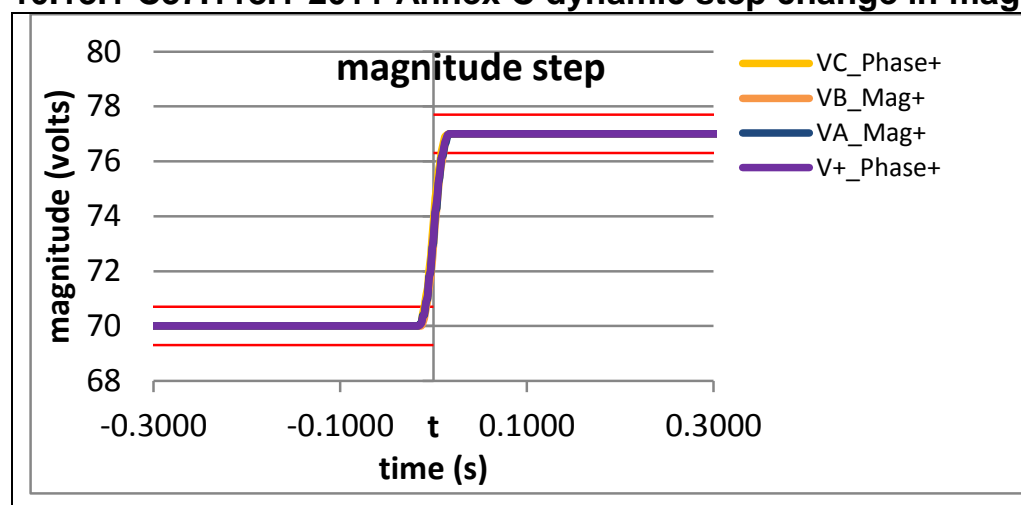

Figure 6131: Fs = 60 FPS, $+10 \%$ magnitude step

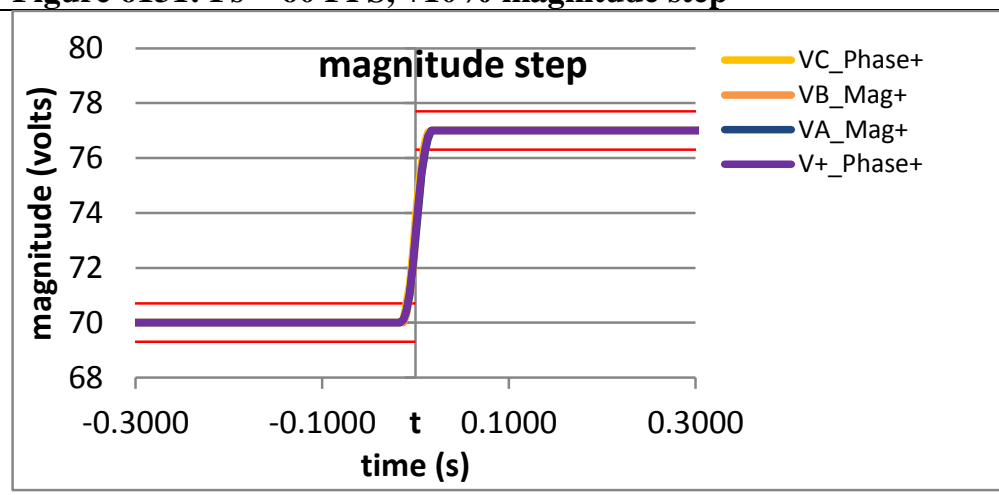

Figure 6133: Fs = 30 FPS, +10\% magnitude step

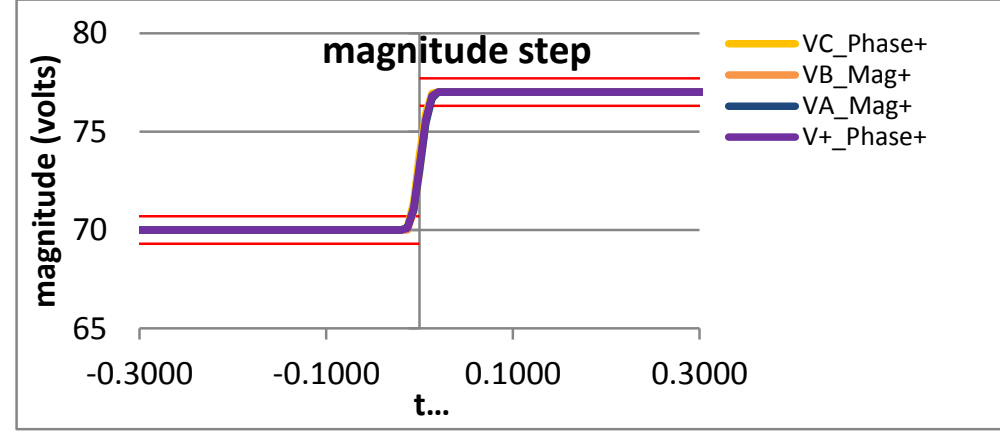

Figure 6135: Fs = 20 FPS, +10\% magnitude step

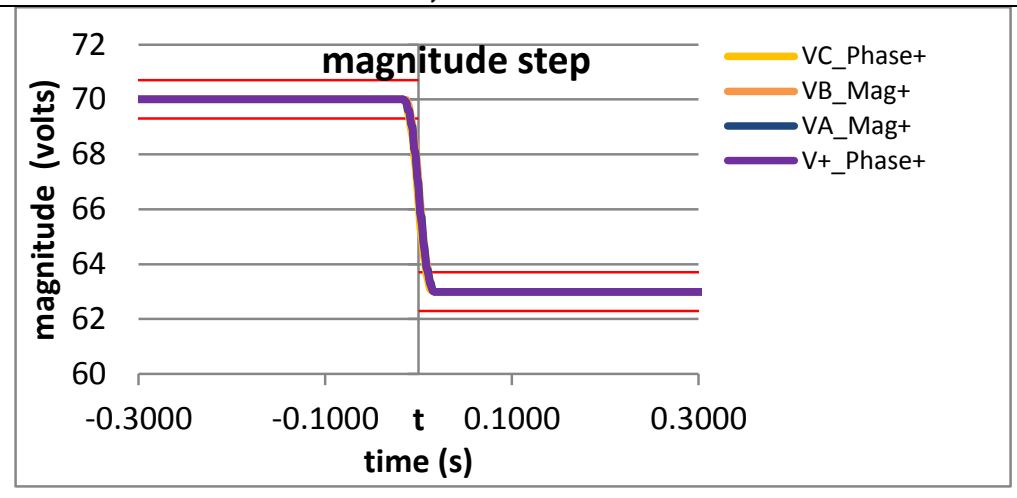

Figure 6132: Fs = 60 FPS, -10\% magnitude step

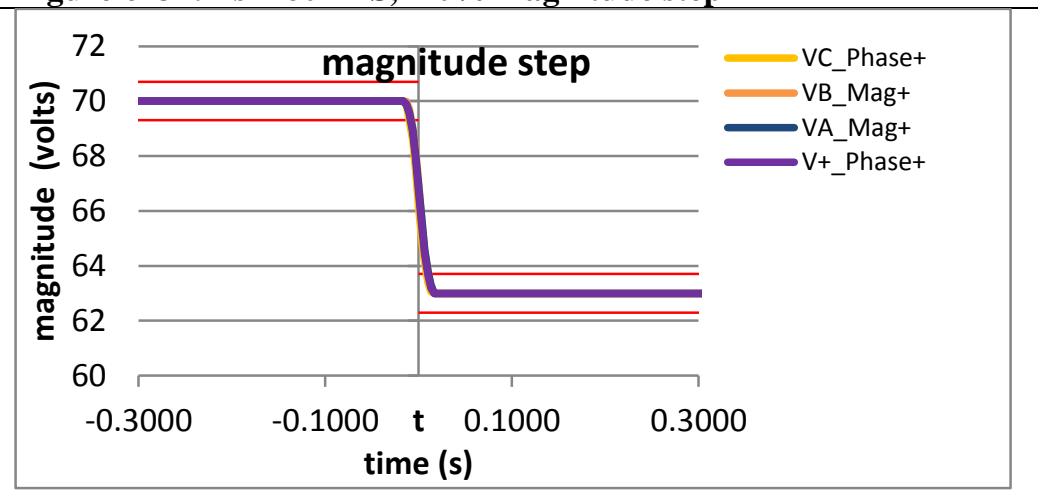

Figure 6134: Fs = 30 FPS, -10\% magnitude step

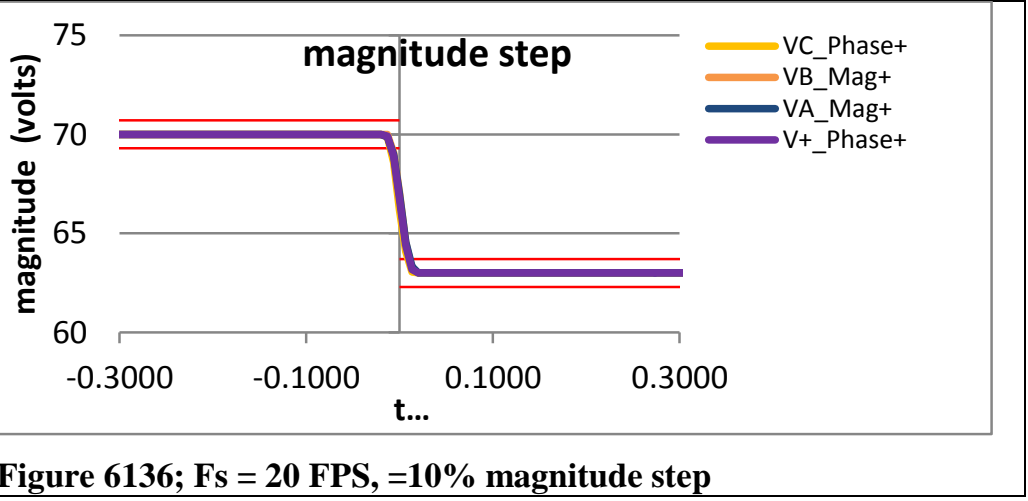




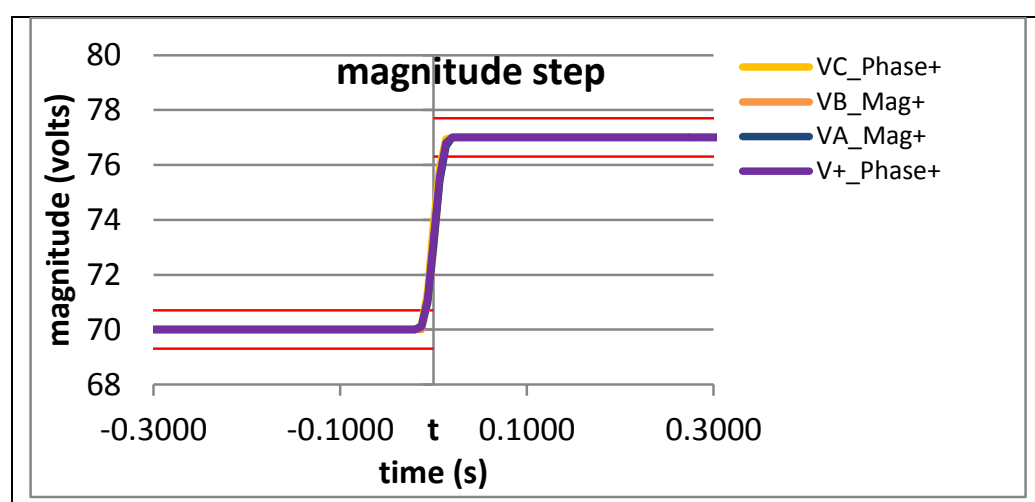

Figure 6137: Fs = 15 FPS, $+10 \%$ magnitude step
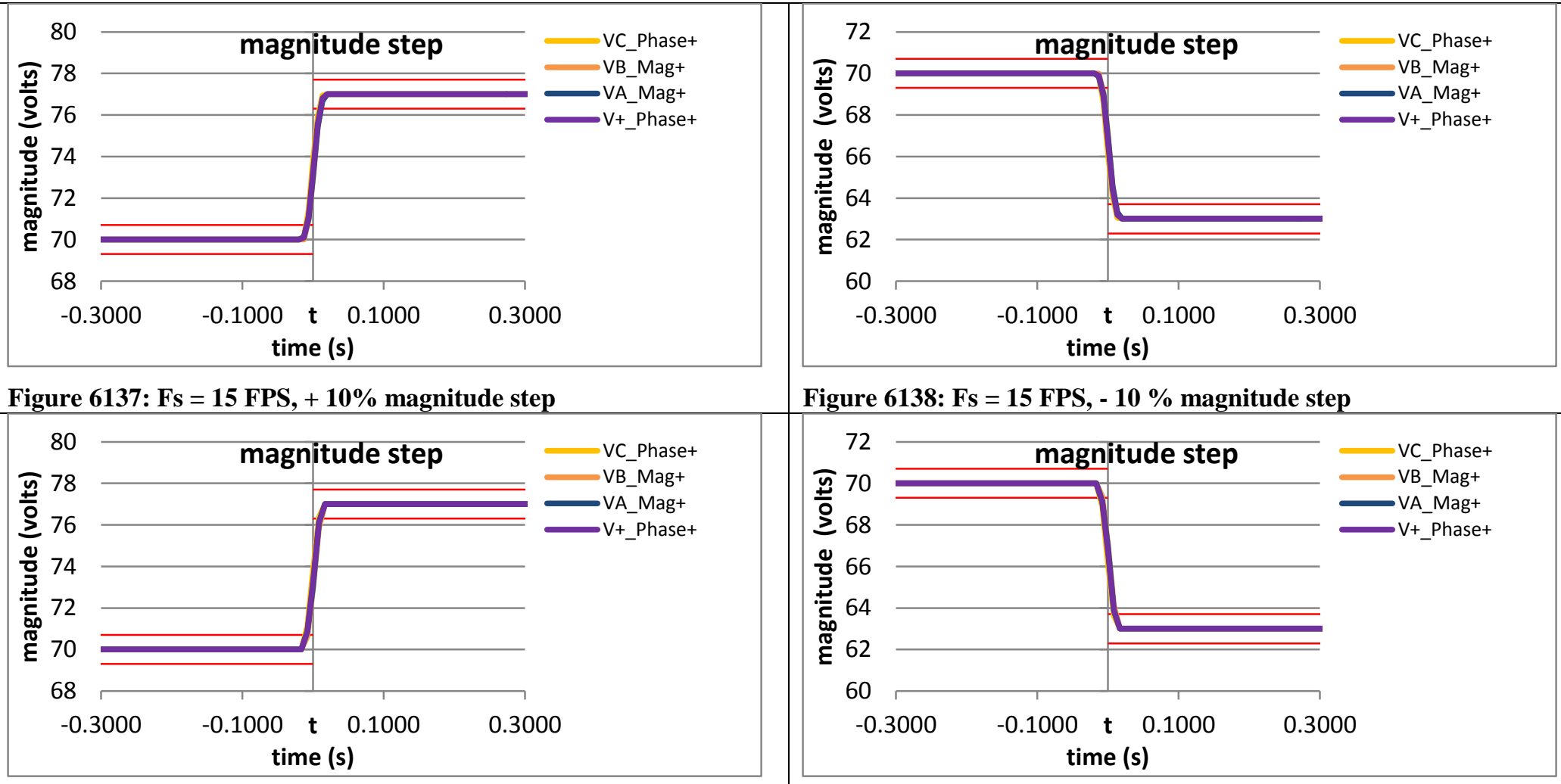

Figure 6138: Fs = 15 FPS, - $10 \%$ magnitude step

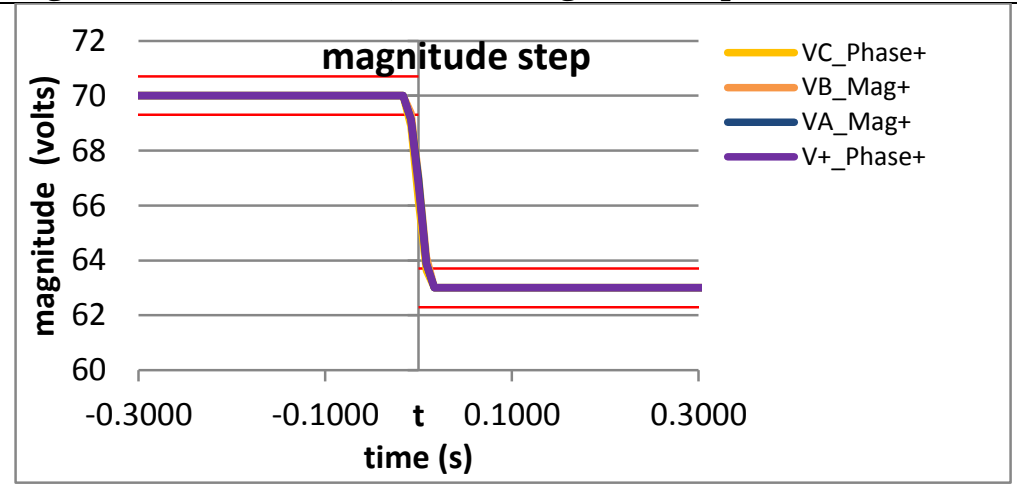

Figure 6139: Fs = 12 FPS, +10\% magnitude step

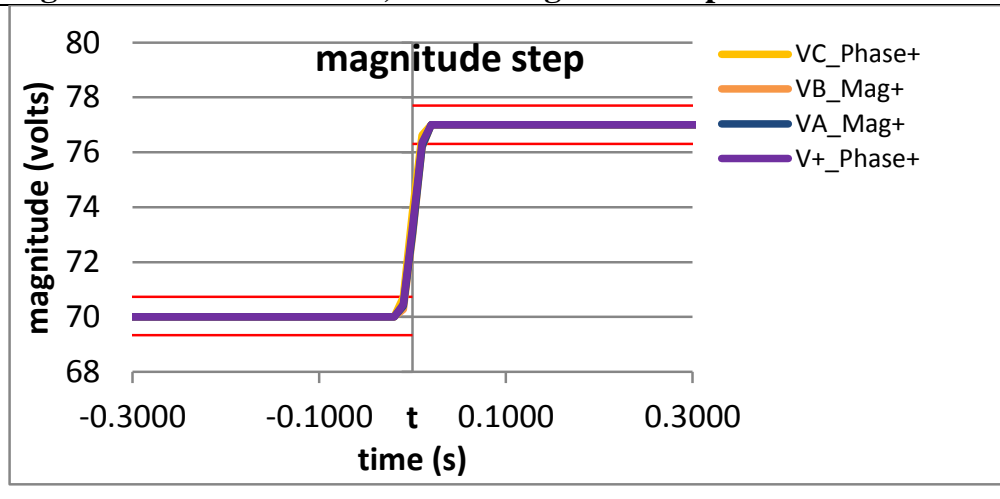

Figure 6140: Fs = 12 FPS, -10\% magnitude step

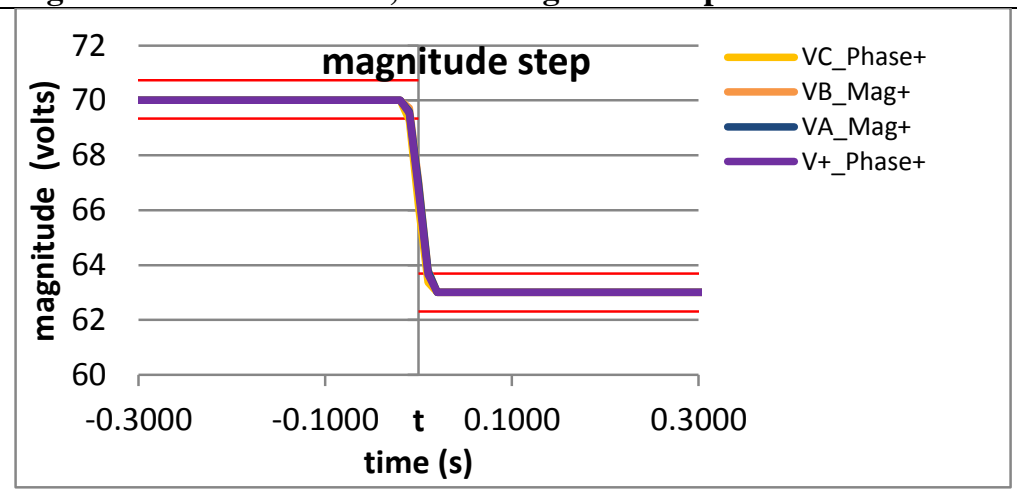

Figure 6141: Fs $=10$ FPS, $+10 \%$ magnitude step

Figure 6142: Fs = 10 FPS, -10\% magnitude step 
10.13.2 PMU A dynamic step change in magnitude overshoot: $F 0=60 \mathrm{~Hz}, \mathrm{P}$ class

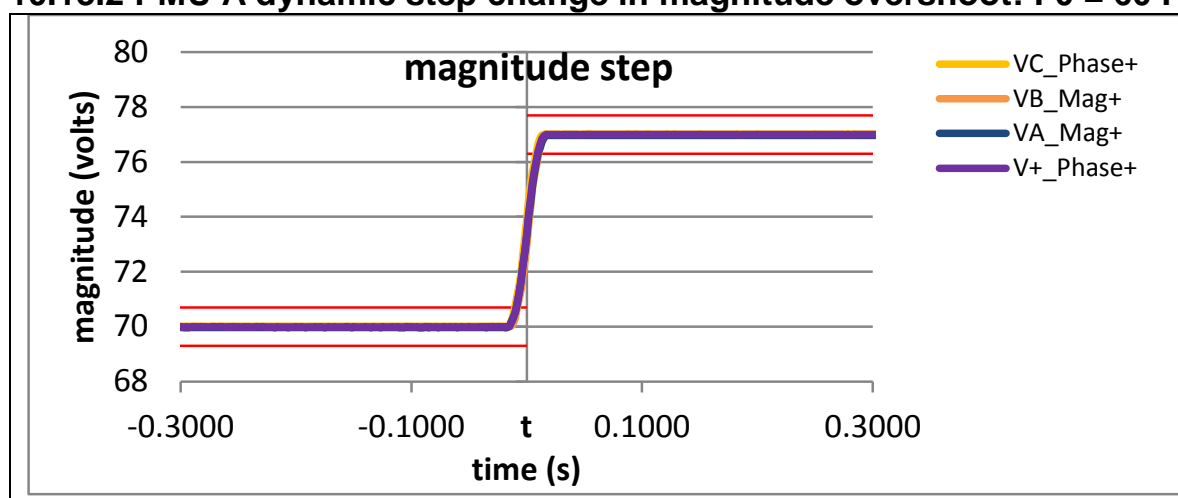

Figure 6143: Fs = 60 FPS, $+10 \%$ magnitude step

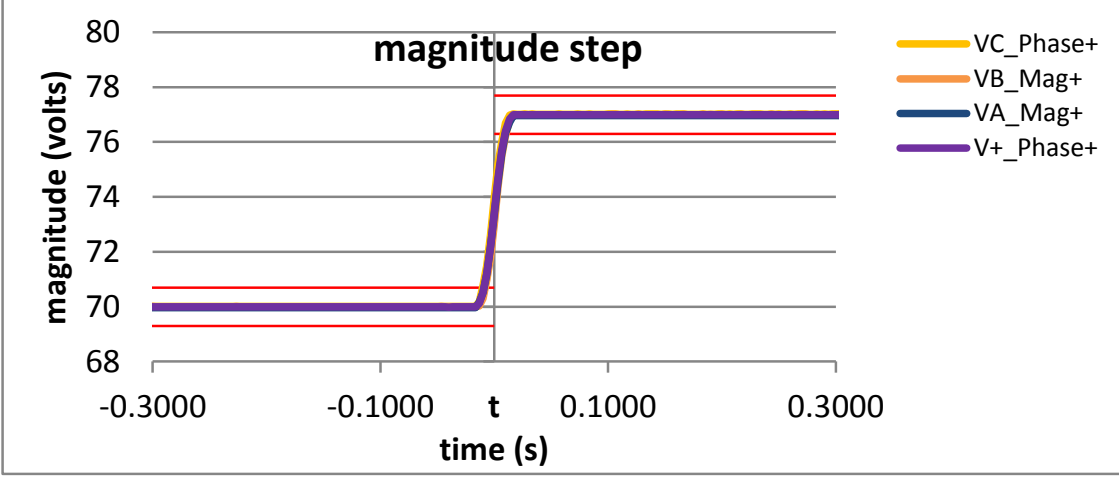

Figure 6145: Fs = 30 FPS, +10\% magnitude step

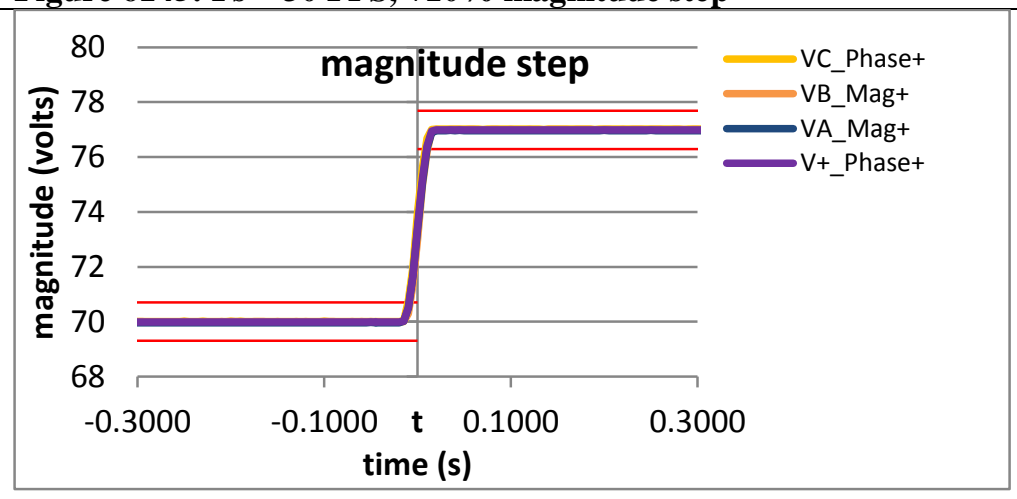

Figure 6147: Fs $=20$ FPS, $+10 \%$ magnitude step

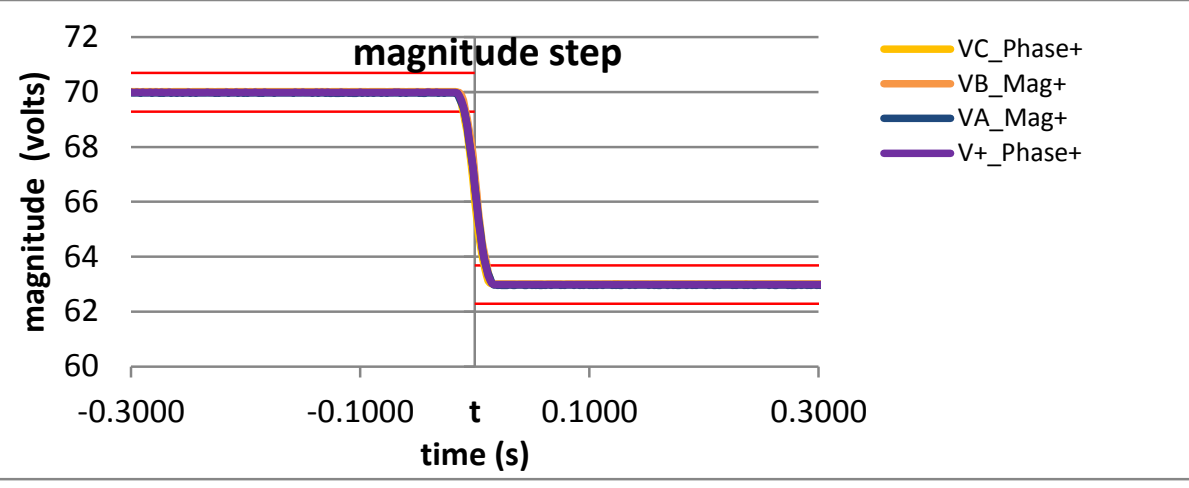

Figure 6144: Fs = 60 FPS, $-10 \%$ magnitude step

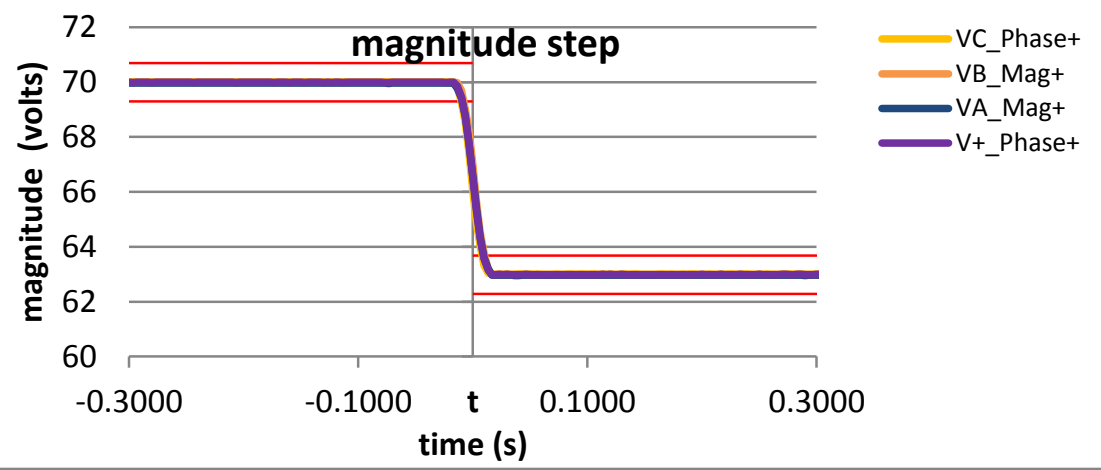

Figure 6146: Fs = 30 FPS, -10\% magnitude step

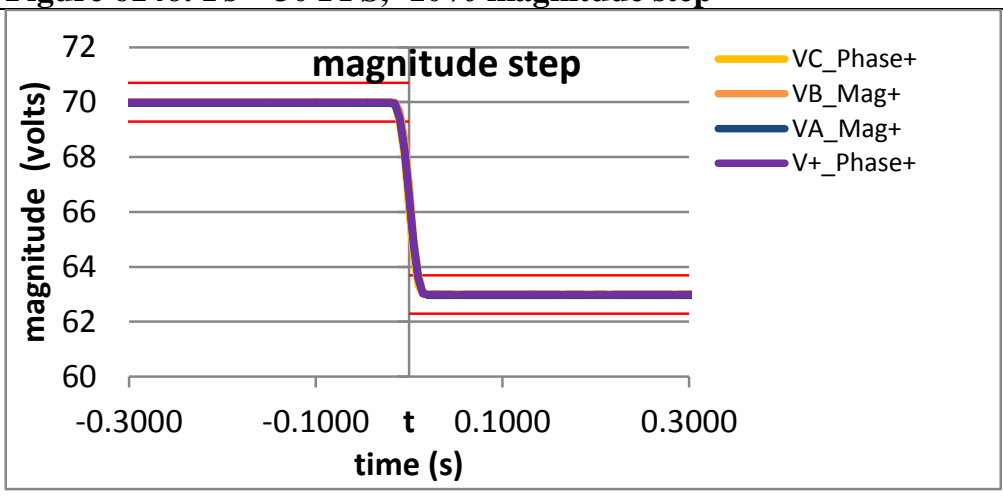

Figure 6148; Fs = 20 FPS, =10\% magnitude step 


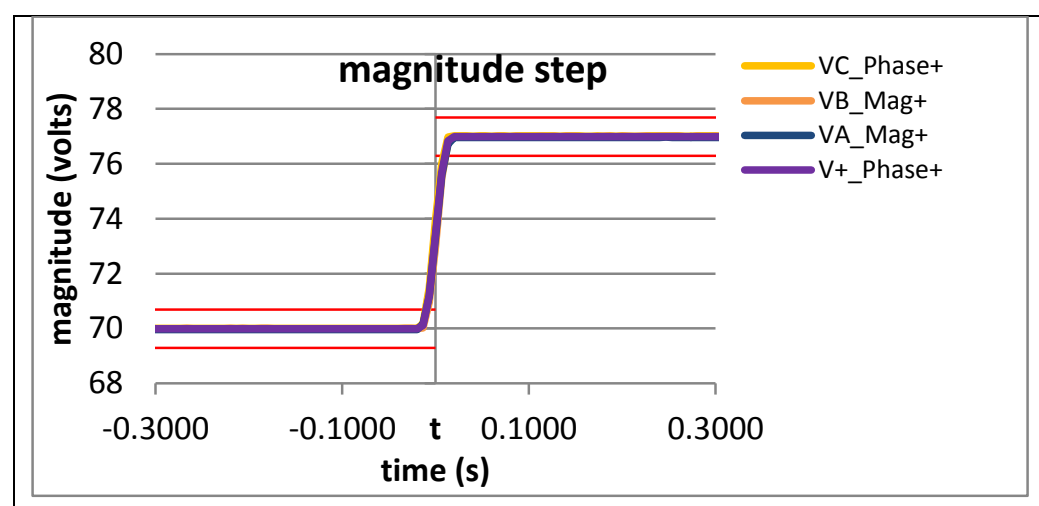

Figure 6149: Fs = 15 FPS, + 10\% magnitude step

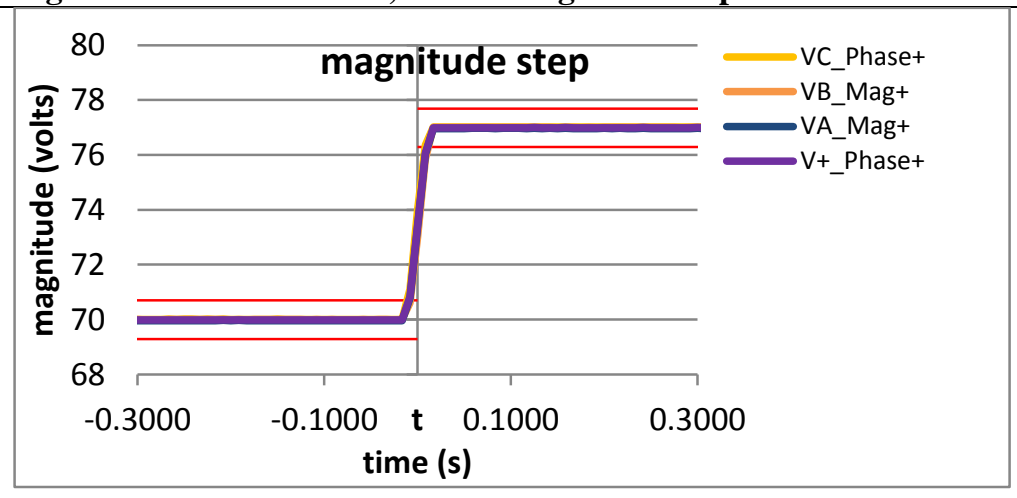

Figure 6151: Fs $=12$ FPS, $+10 \%$ magnitude step

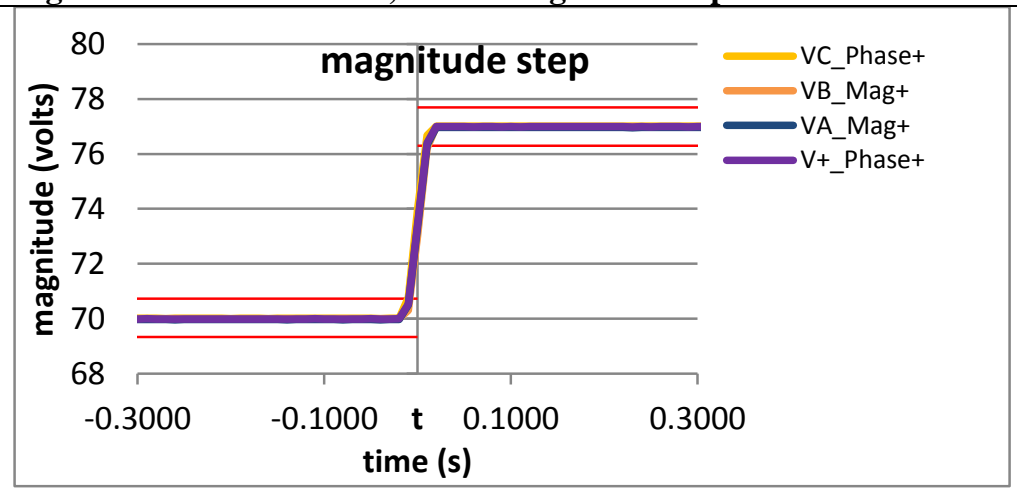

Figure 6153: Fs = 10 FPS, $+10 \%$ magnitude step

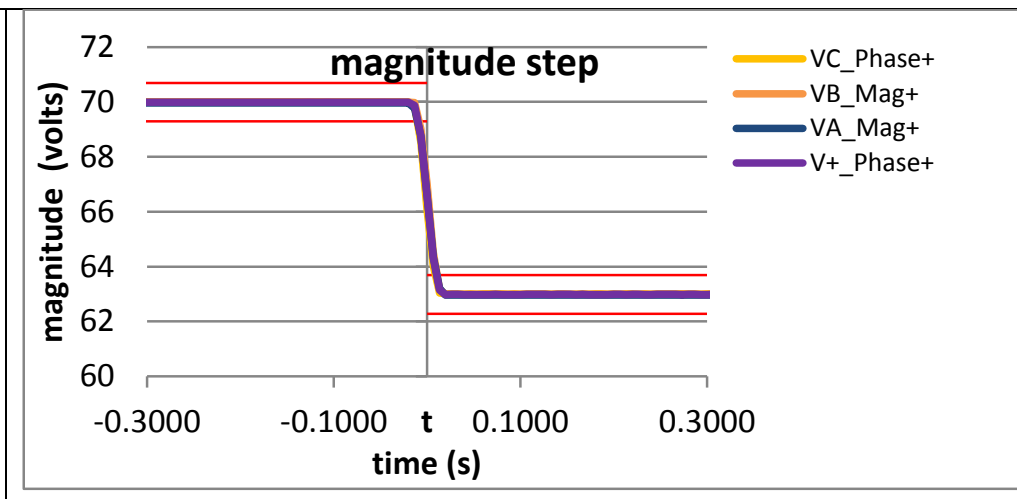

Figure 6150: Fs = 15 FPS, - $10 \%$ magnitude step

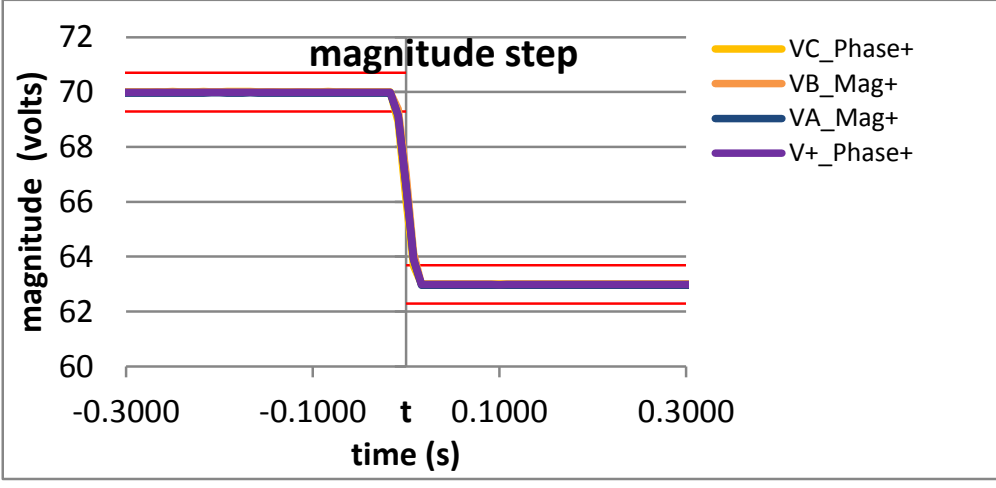

Figure 6152: Fs = 12 FPS, -10\% magnitude step

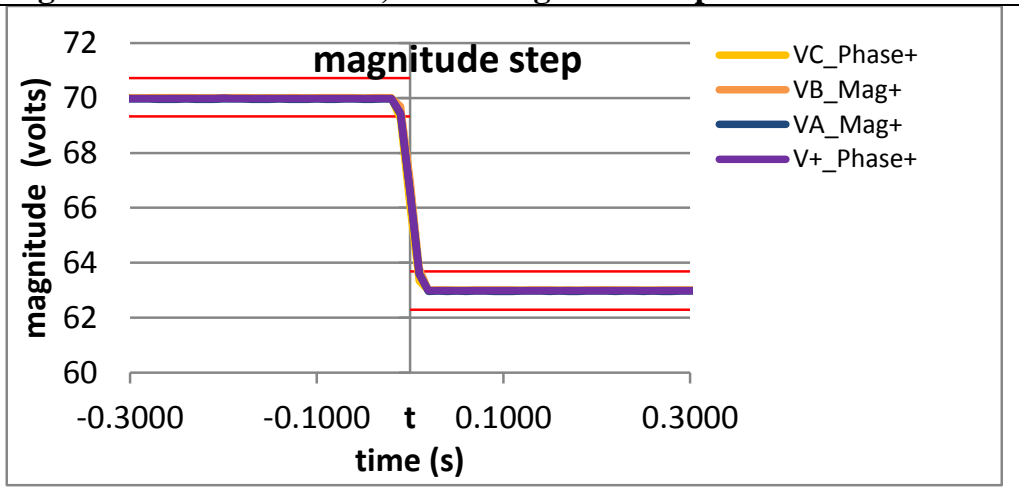

Figure 6154: Fs = 10 FPS, -10\% magnitude step 
10.13.3 PMU B dynamic step change in magnitude overshoot: $F 0=60 \mathrm{~Hz}, P$ class

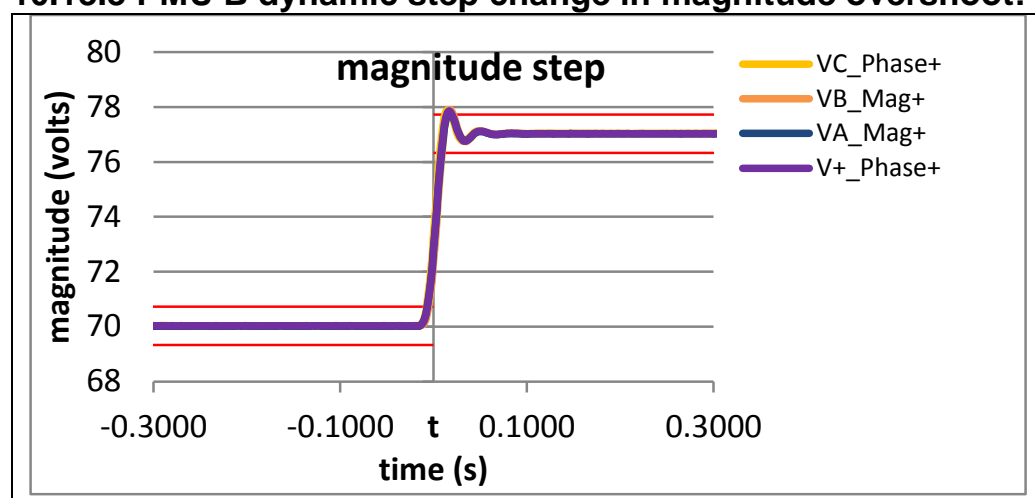

Figure 6155: Fs $=60 \mathrm{FPS},+10 \%$ magnitude step

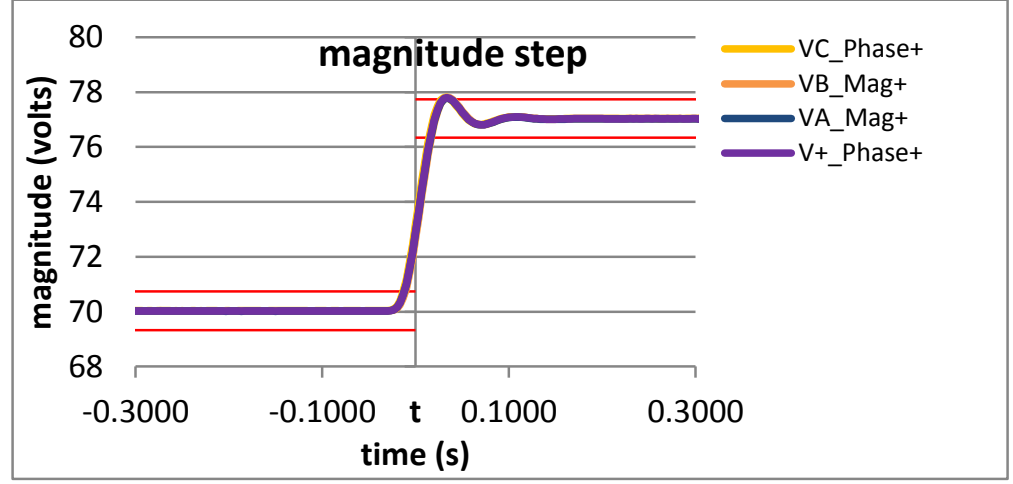

Figure 6157: Fs = 30 FPS, +10\% magnitude step

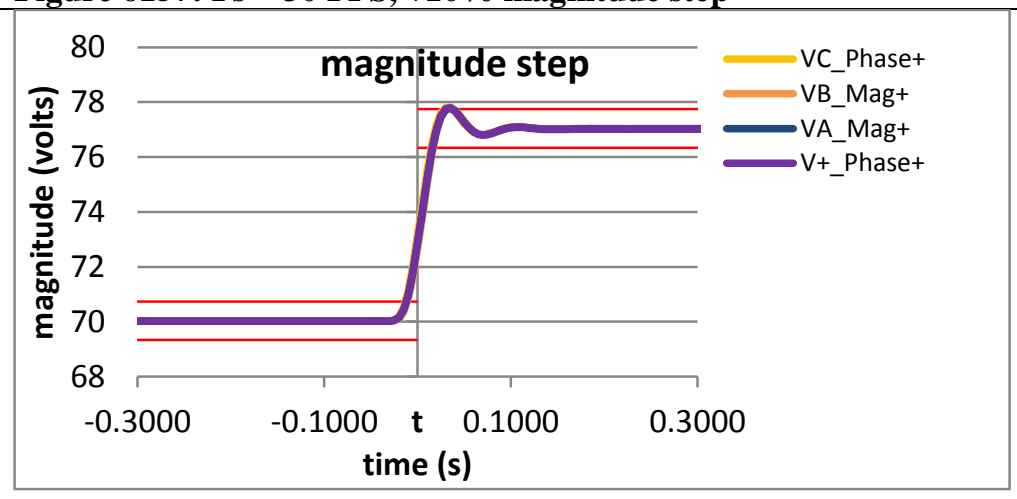

Figure 6159: Fs $=20$ FPS, $+10 \%$ magnitude step

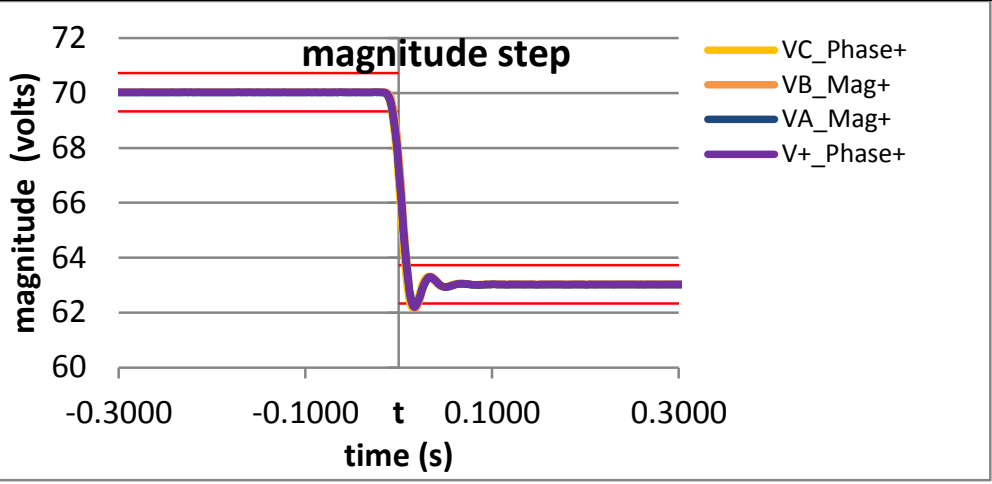

Figure 6156: Fs $=60$ FPS, $-10 \%$ magnitude step

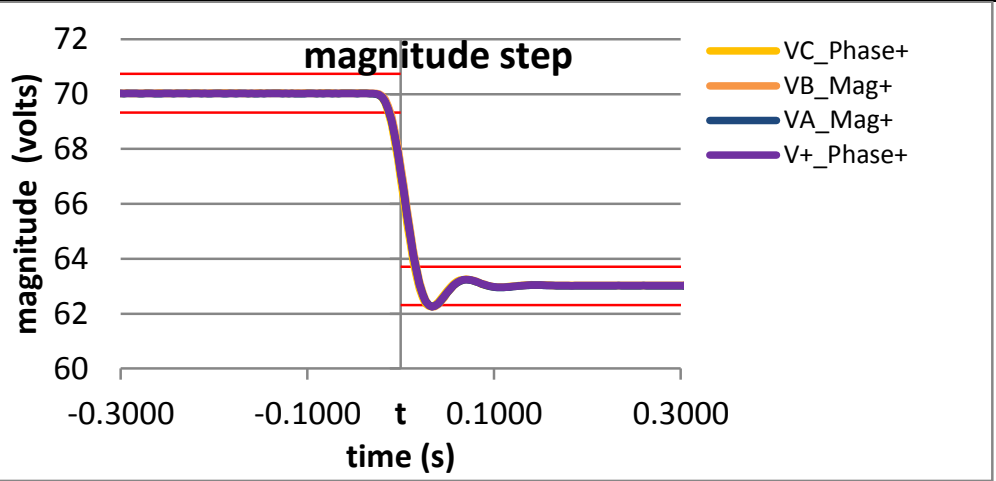

Figure 6158: Fs = 30 FPS, -10\% magnitude step

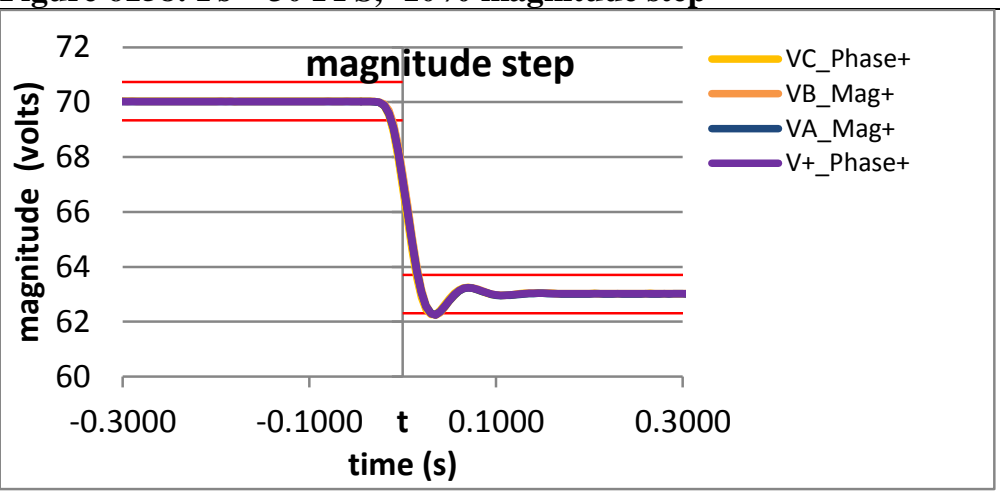

Figure 6160; Fs $=20$ FPS, $=10 \%$ magnitude step 


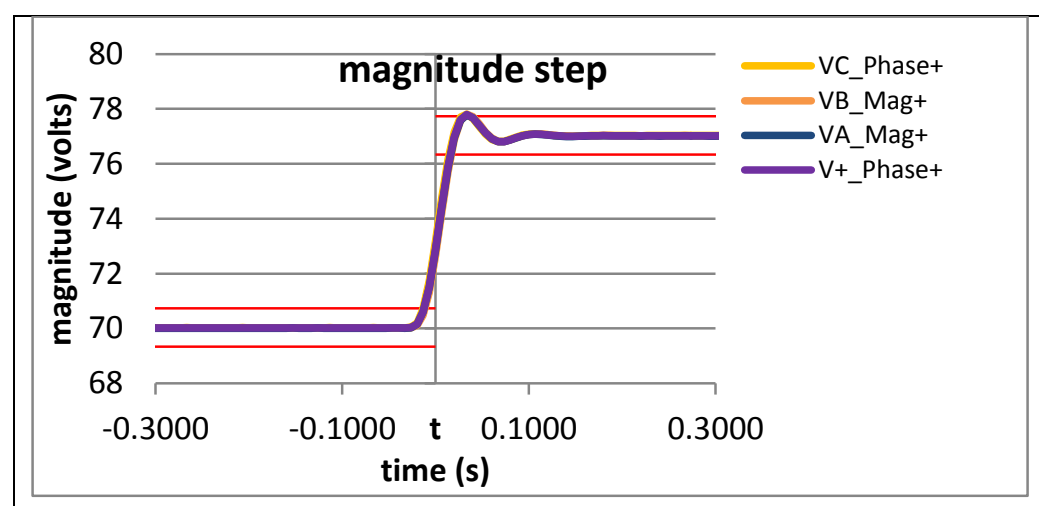

Figure 6161: Fs = 15 FPS, + 10\% magnitude step

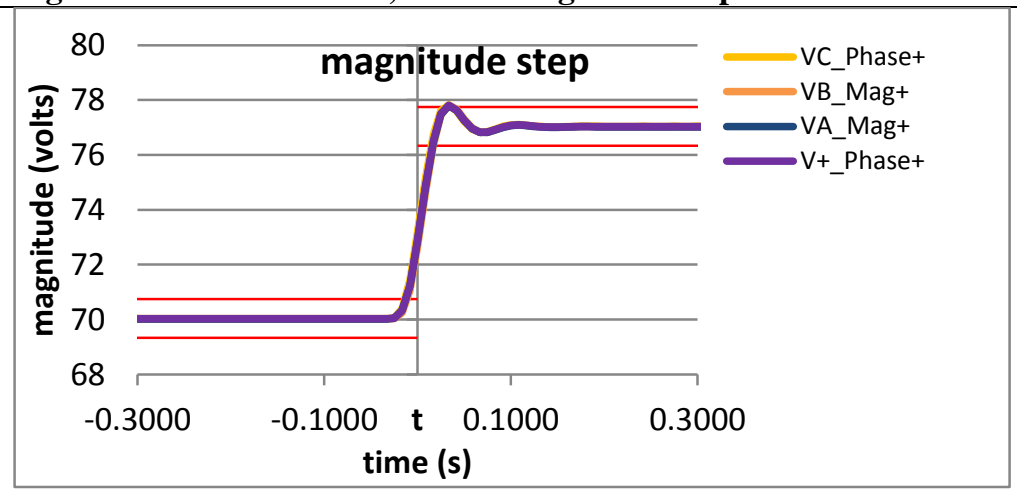

Figure 6163: Fs $=12$ FPS, $+10 \%$ magnitude step

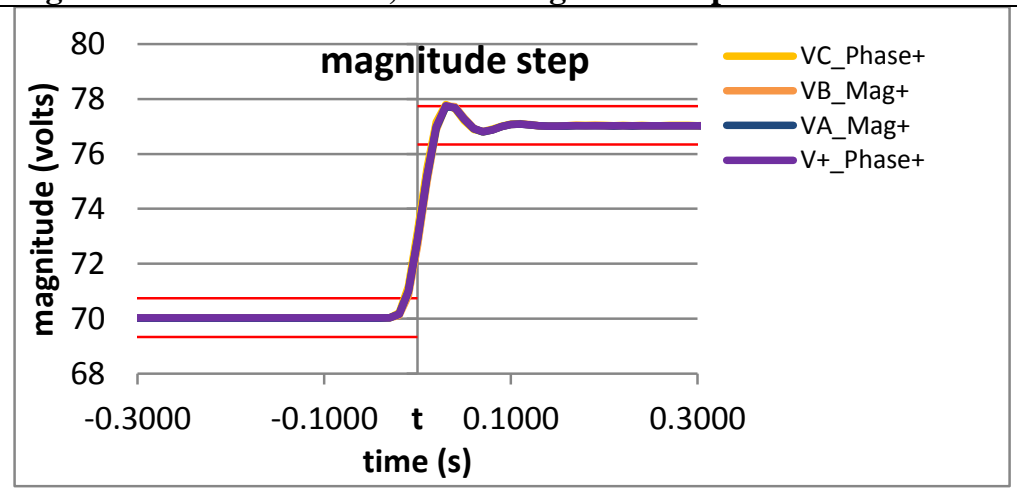

Figure 6165: Fs = 10 FPS, +10\% magnitude step

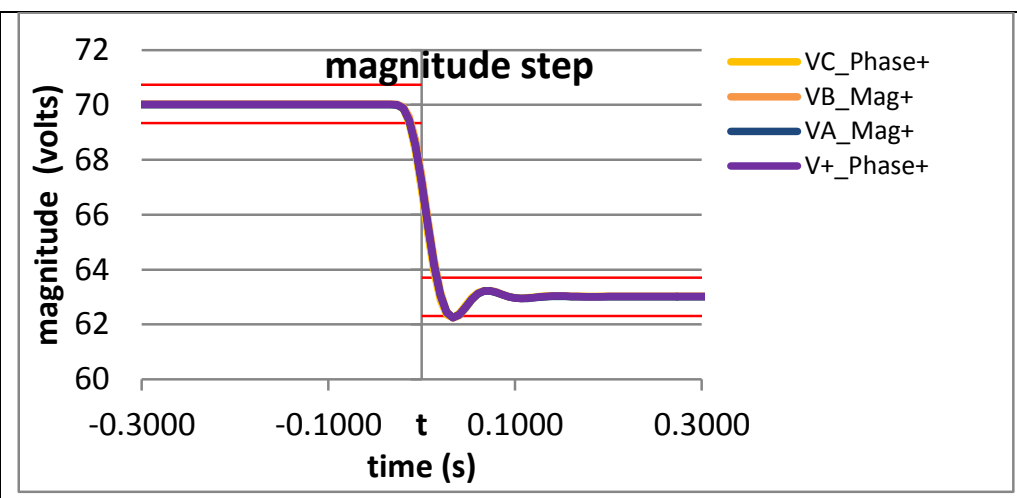

Figure 6162: Fs = 15 FPS, - $10 \%$ magnitude step

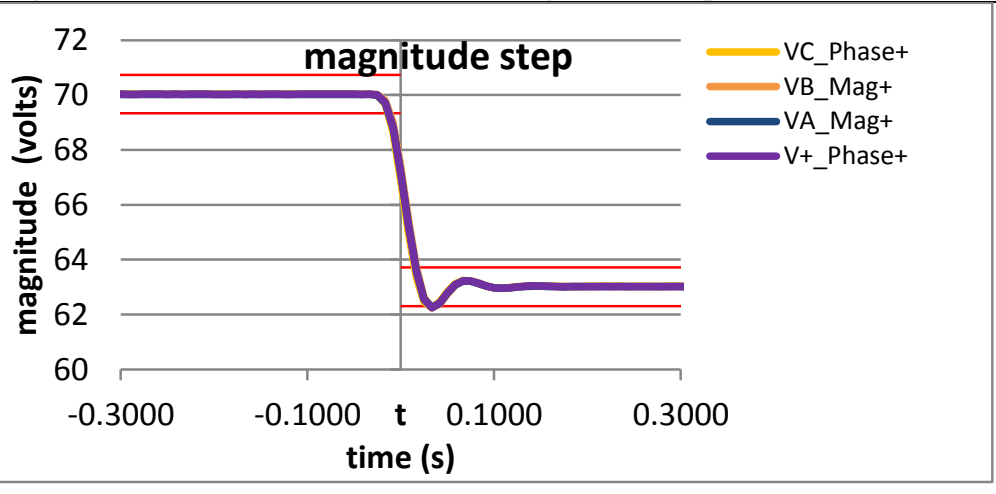

Figure 6164: Fs = 12 FPS, -10\% magnitude step

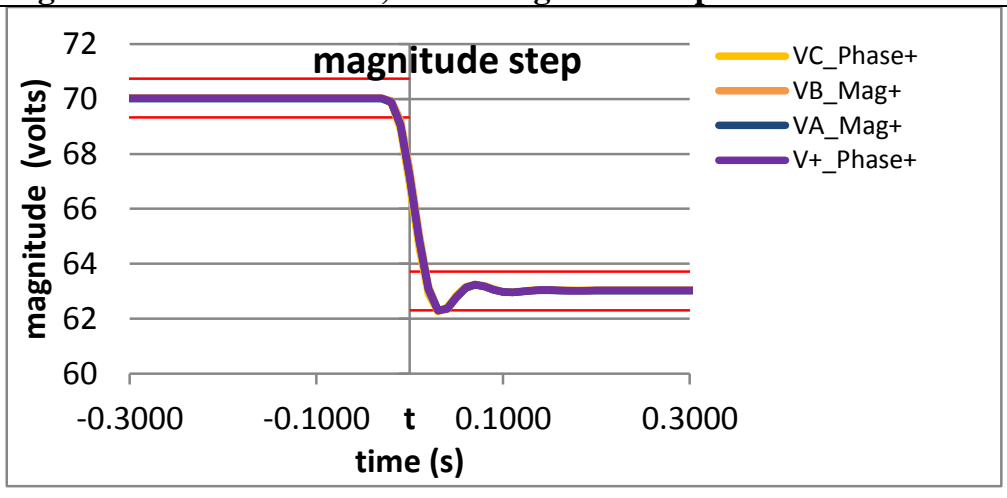

Figure 6166: Fs = 10 FPS, $-10 \%$ magnitude step 
10.13.4 PMU C dynamic step change in magnitude ROCOF phasor overshoot: $F 0=60 \mathrm{~Hz}$, $\mathrm{P}$ class

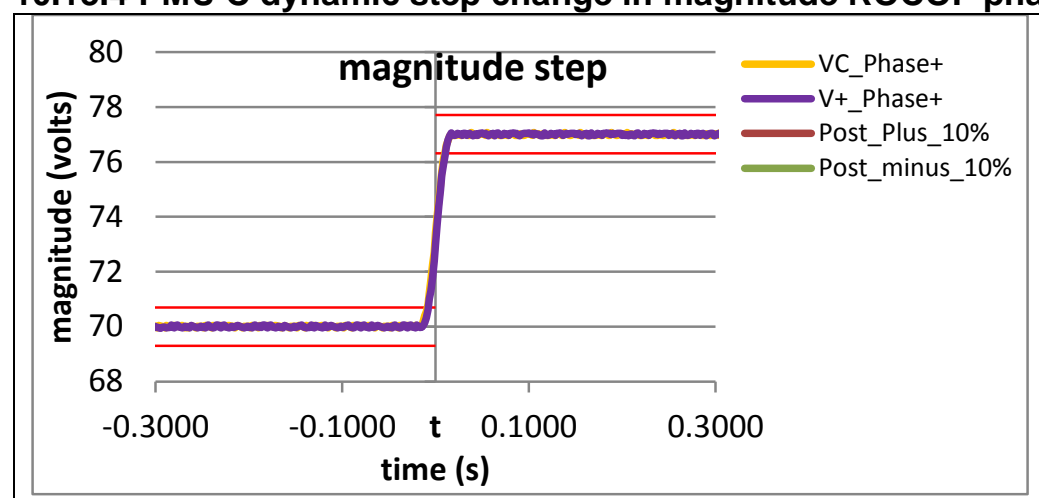

Figure 6167: Fs $=60$ FPS, $+10 \%$ magnitude step

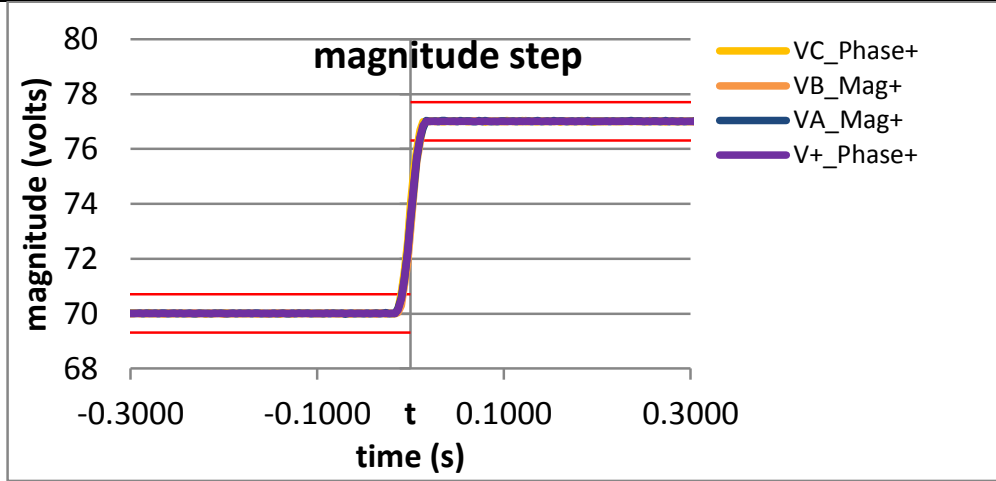

Figure 6169: Fs = 30 FPS, +10\% magnitude step

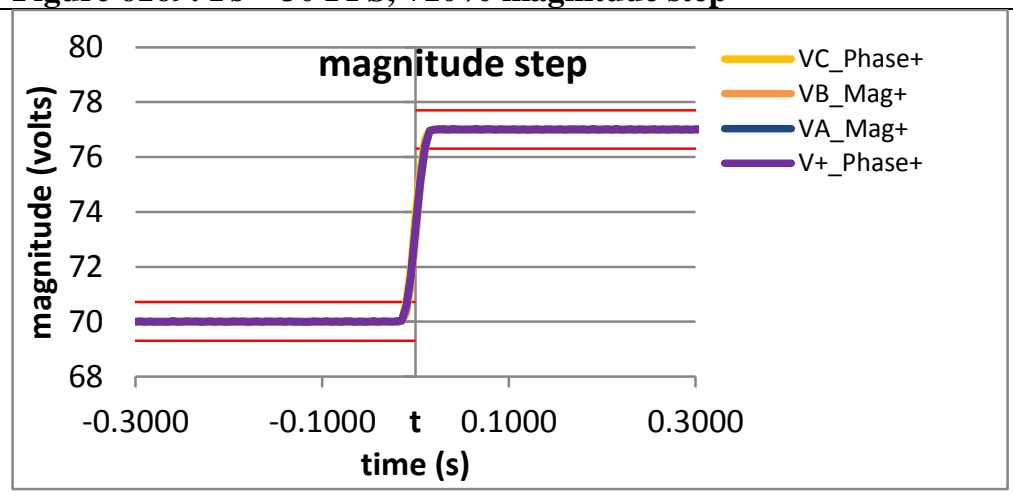

Figure 6171: Fs $=20$ FPS, $+10 \%$ magnitude step

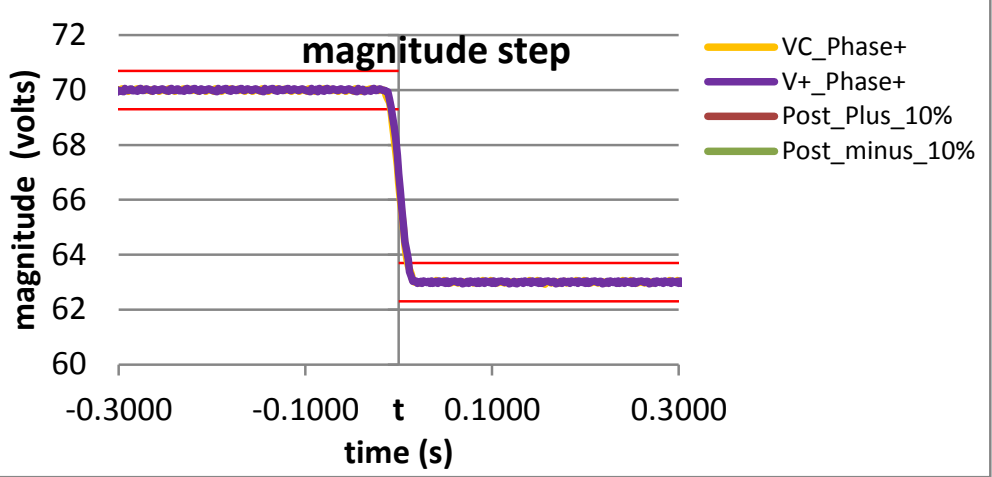

Figure 6168: Fs $=60$ FPS, $-10 \%$ magnitude step

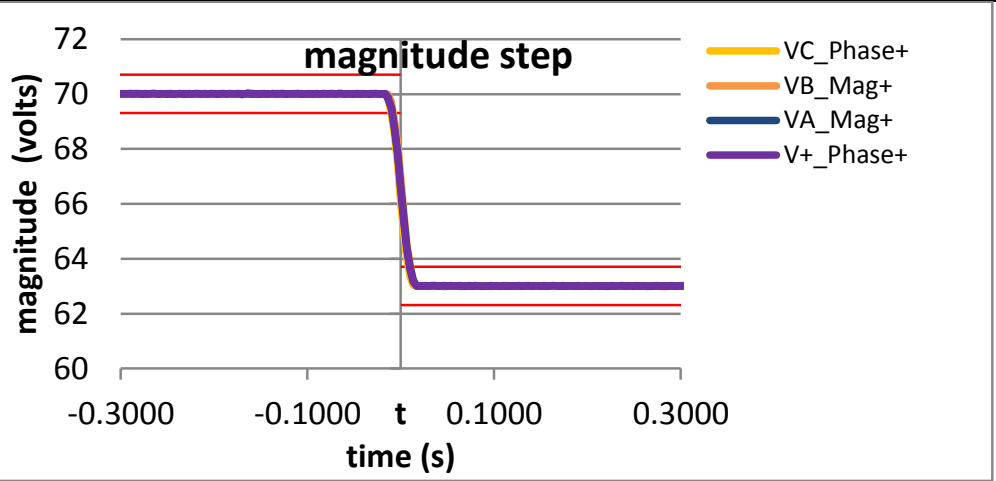

Figure 6170: Fs = 30 FPS, -10\% magnitude step

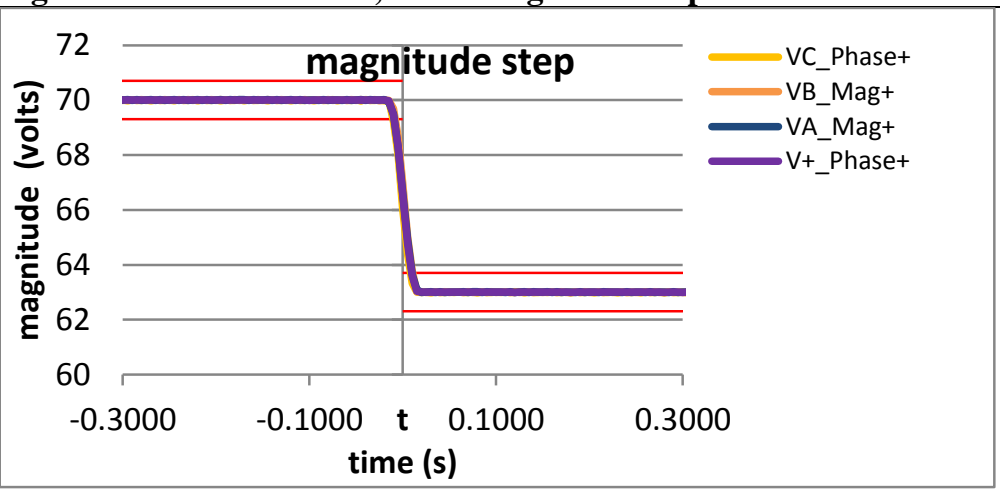

Figure 6172; Fs = 20 FPS, $=10 \%$ magnitude step 


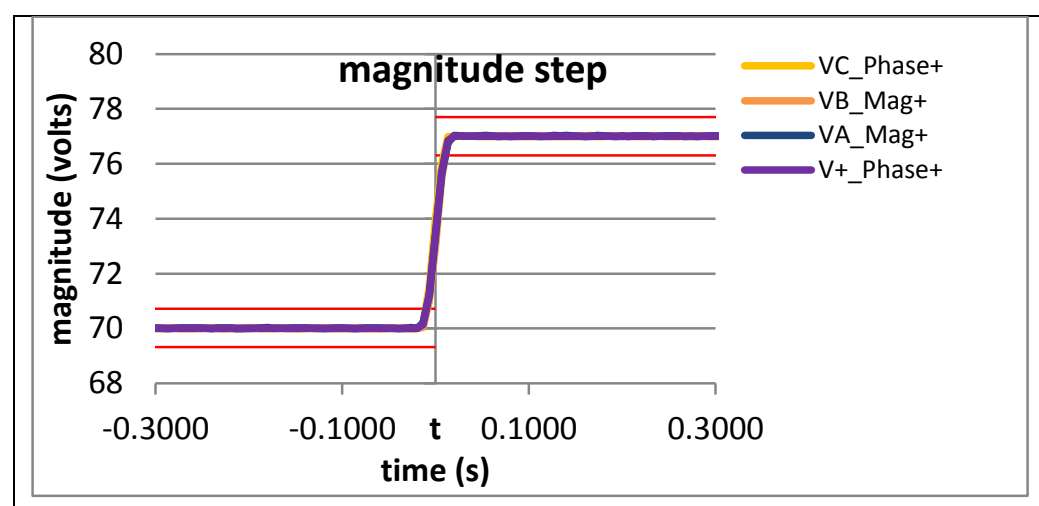

Figure 6173: Fs = 15 FPS, + 10\% magnitude step

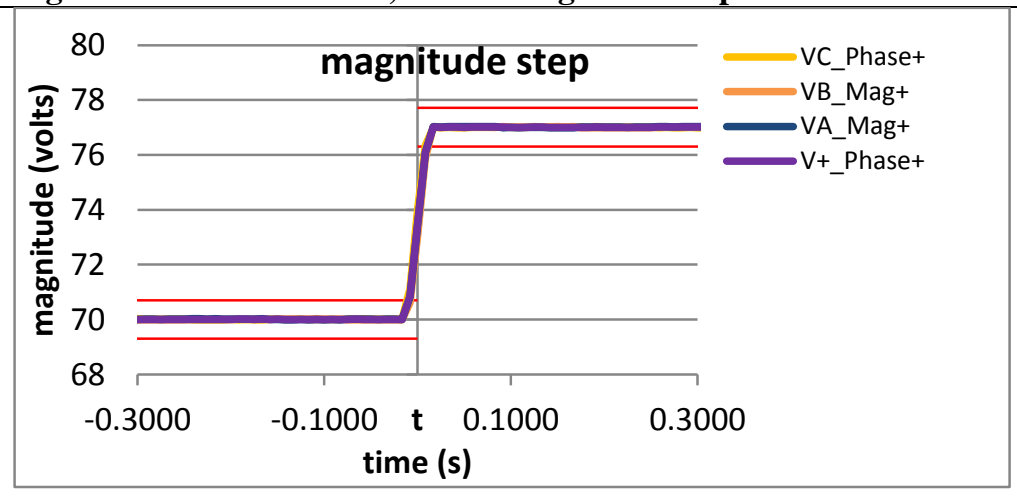

Figure 6175: Fs = 12 FPS, +10\% magnitude step

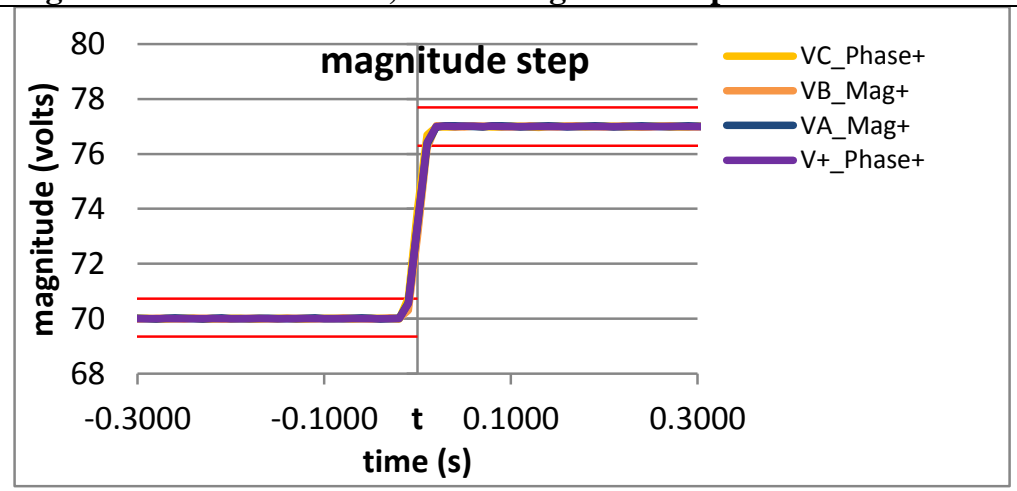

Figure 6177: Fs = 10 FPS, $+10 \%$ magnitude step

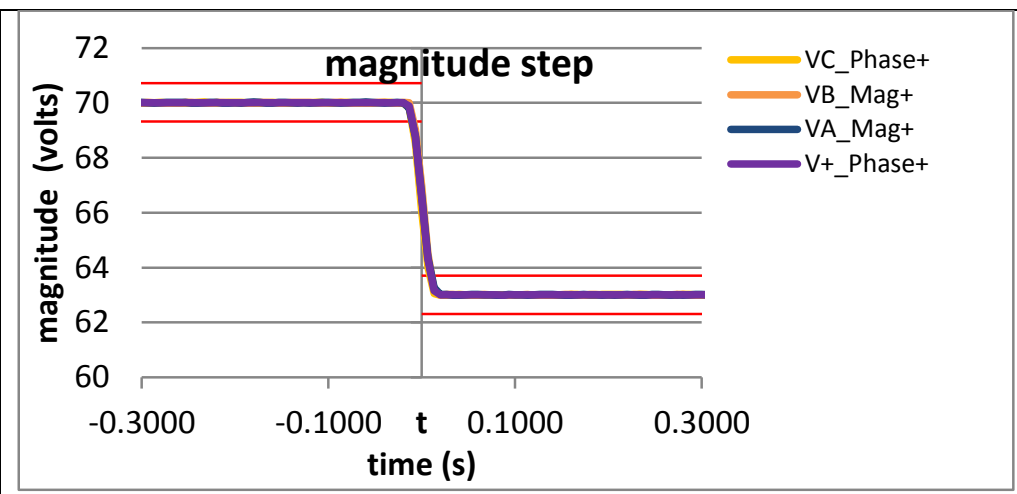

Figure 6174: Fs = 15 FPS, - $10 \%$ magnitude step

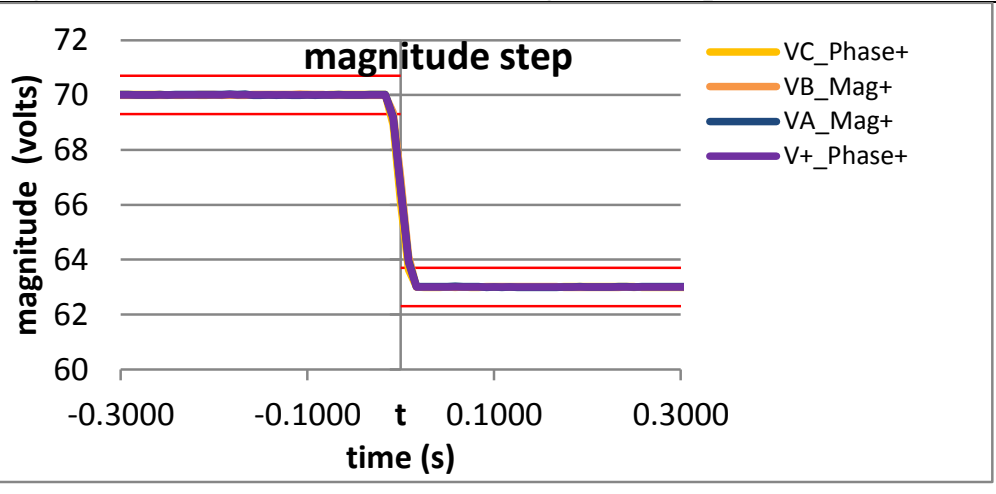

Figure 6176: Fs = 12 FPS, -10\% magnitude step

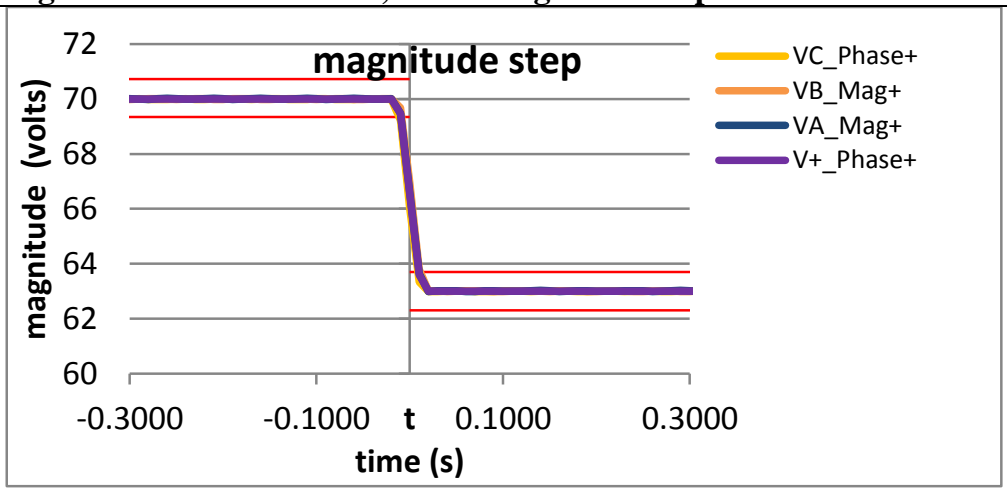

Figure 6178: Fs = 10 FPS, $-10 \%$ magnitude step 
10.13.5 PMU D dynamic step change in magnitude ROCOF phasor overshoot: $F 0=60 \mathrm{~Hz}$, $P$ class

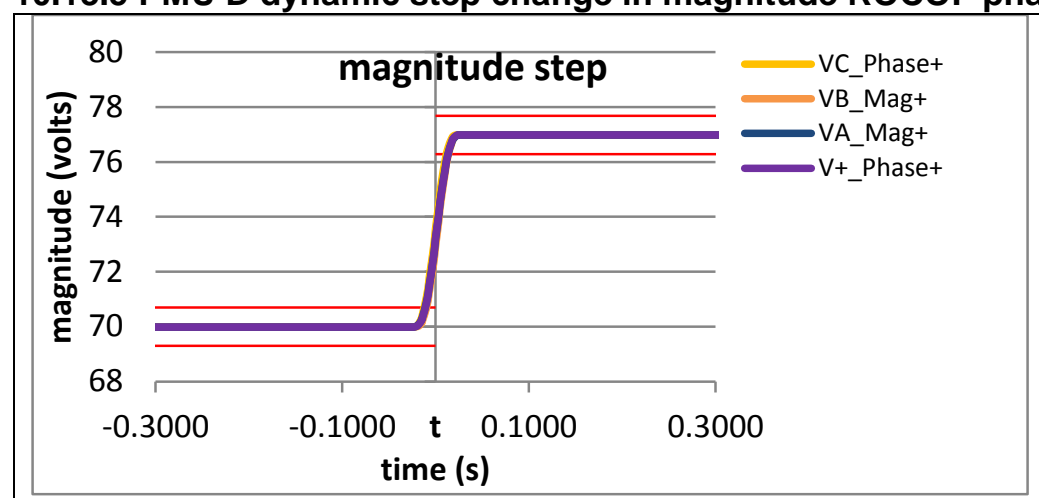

Figure 6179: Fs = 60 FPS, $+10 \%$ magnitude step

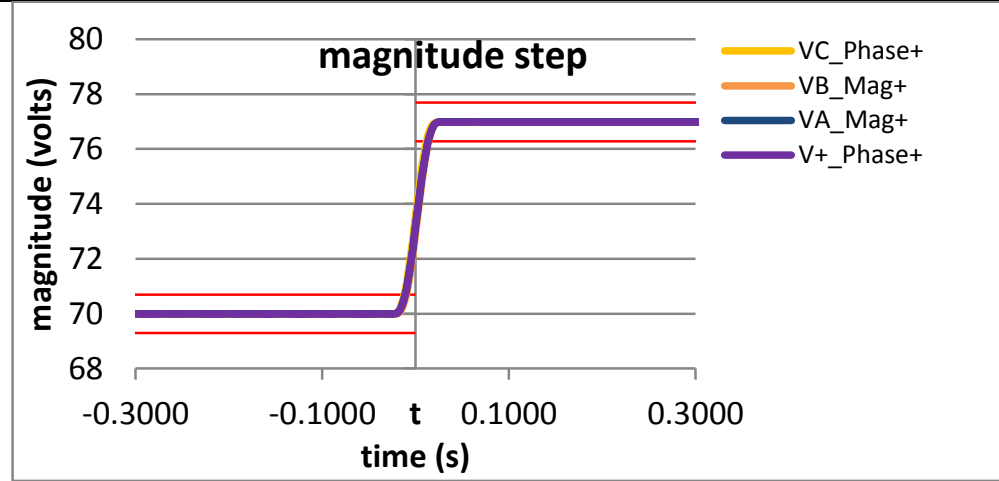

Figure 6181: Fs = 30 FPS, +10\% magnitude step

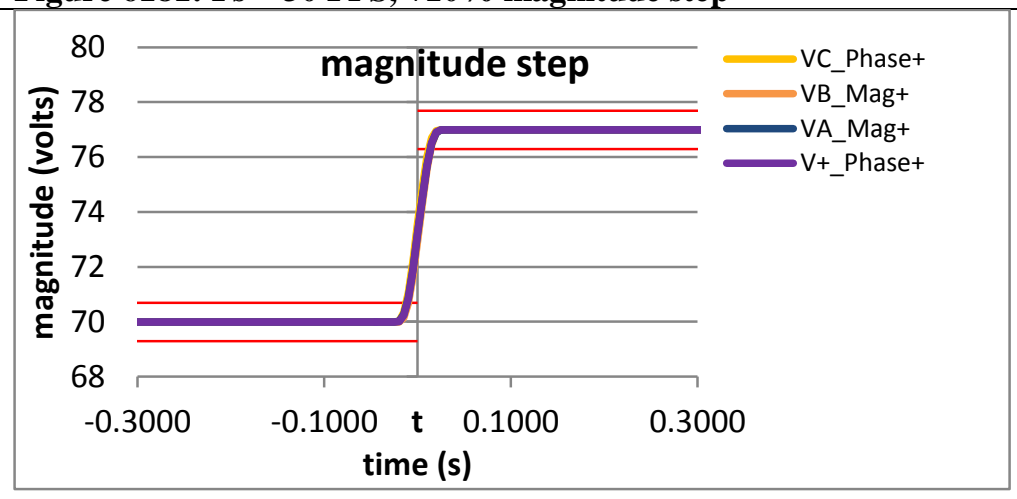

Figure 6183: Fs $=20$ FPS, $+10 \%$ magnitude step

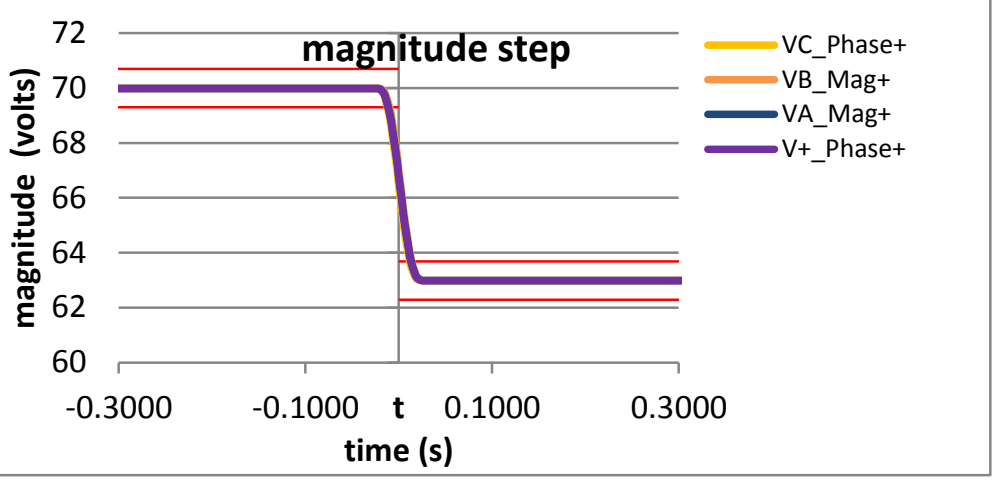

Figure 6180: Fs $=60$ FPS, $-10 \%$ magnitude step

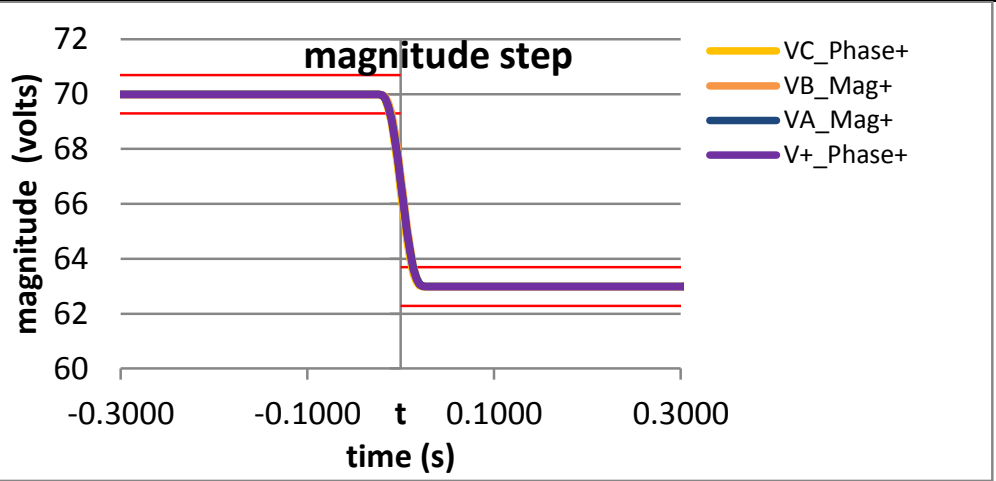

Figure 6182: Fs = 30 FPS, -10\% magnitude step

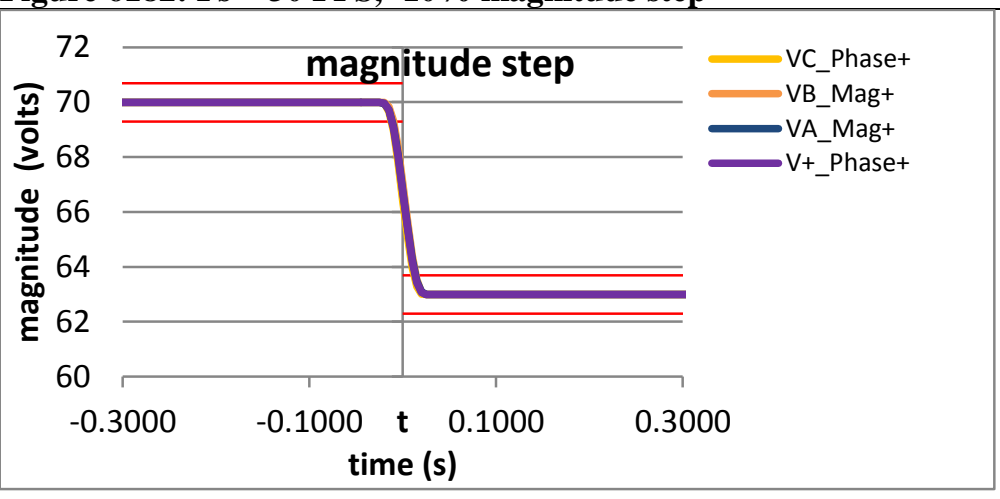

Figure 6184; Fs = 20 FPS, =10\% magnitude step 


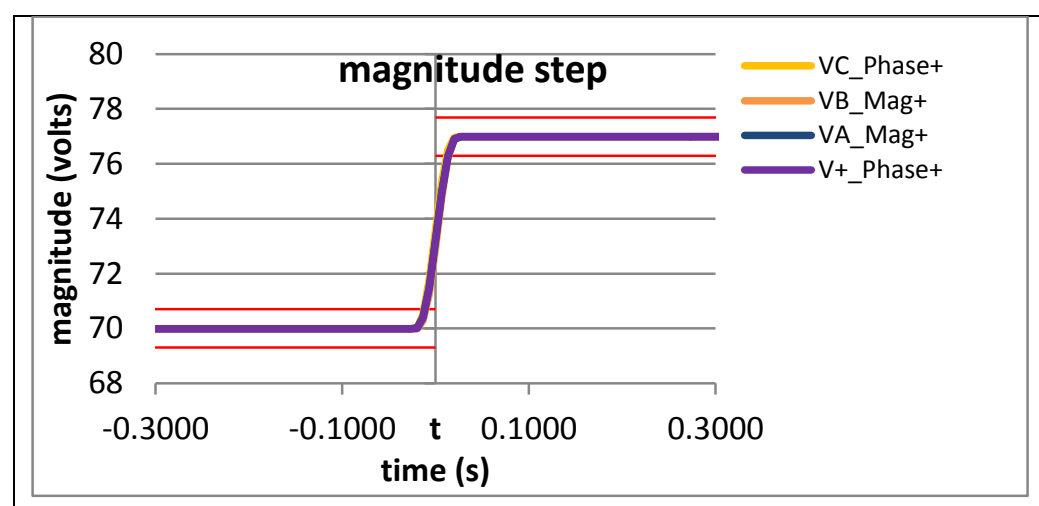

Figure 6185: Fs = 15 FPS, + 10\% magnitude step

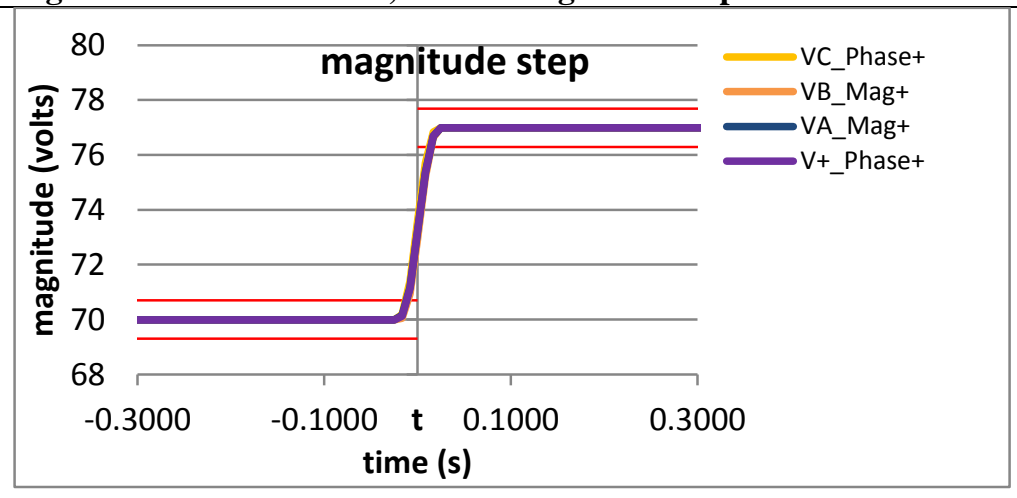

Figure 6187: Fs $=12$ FPS, $+10 \%$ magnitude step

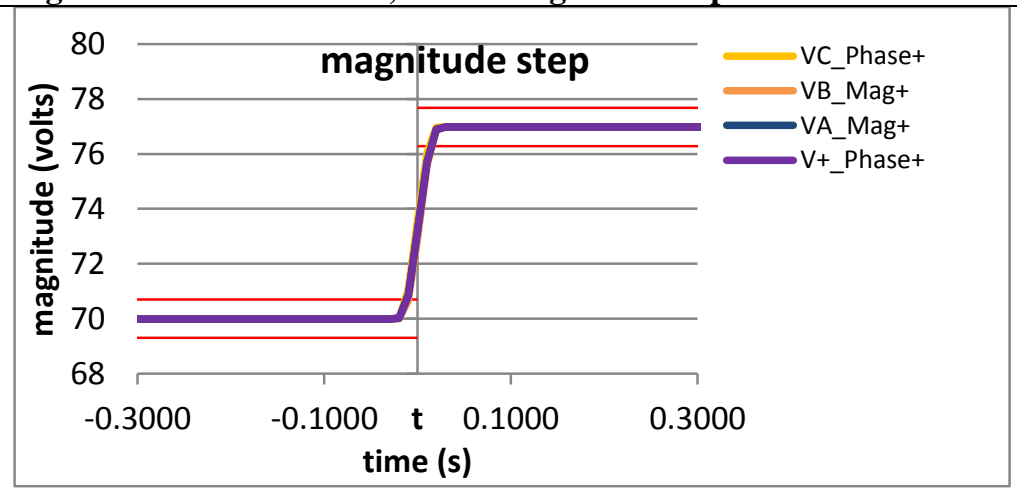

Figure 6189: Fs = 10 FPS, $+10 \%$ magnitude step

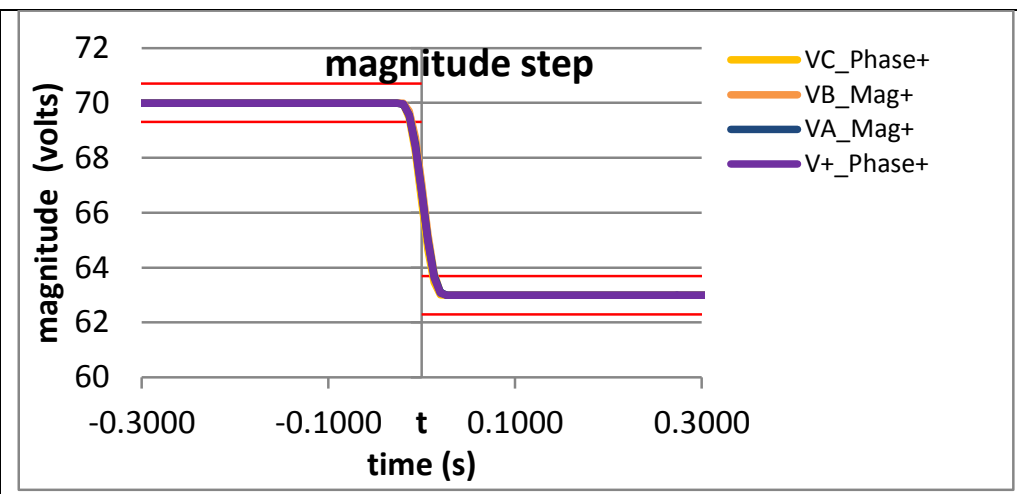

Figure 6186: Fs = 15 FPS, - $10 \%$ magnitude step

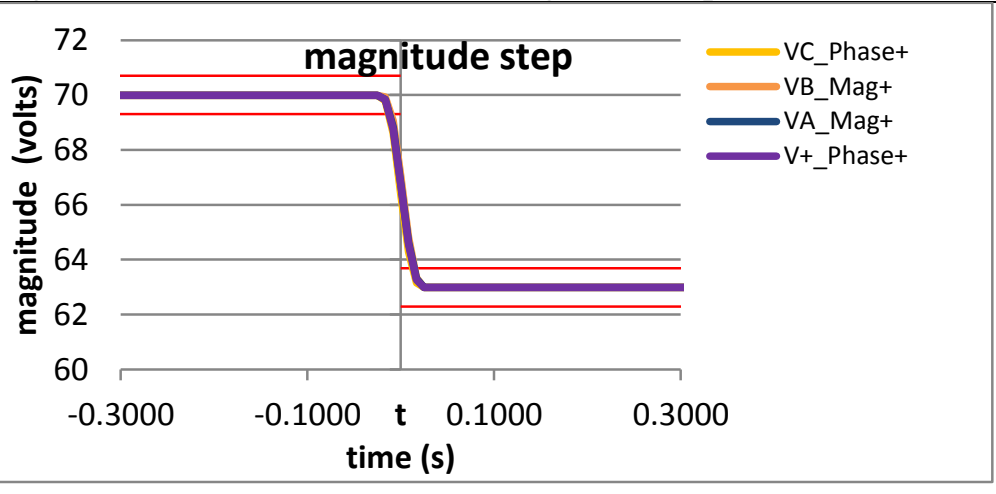

Figure 6188: Fs = 12 FPS, -10\% magnitude step

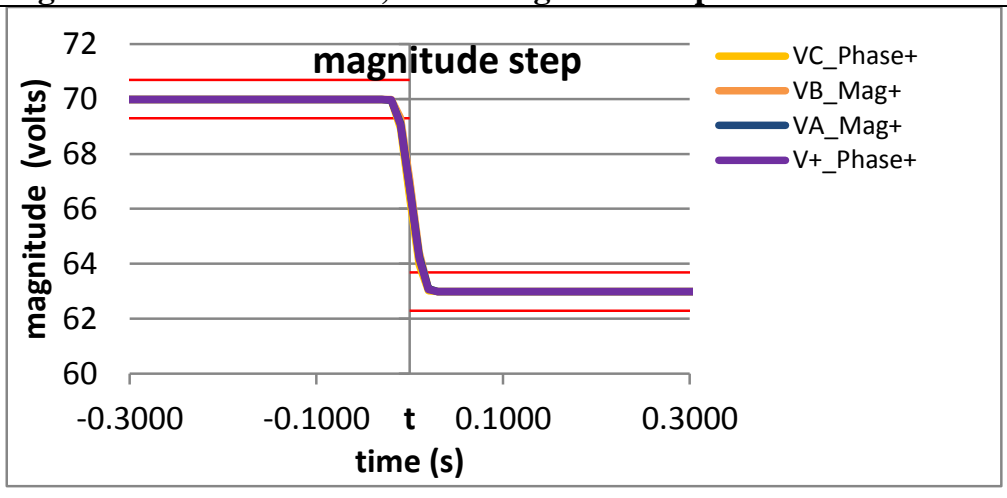

Figure 6190: Fs = 10 FPS, -10\% magnitude step 
10.13.6 PMU E dynamic step change in magnitude ROCOF phasor overshoot: $F 0=60 \mathrm{~Hz}, P$ class PMU E does not support P class.

\subsubsection{PMU F dynamic step change in magnitude ROCOF phasor overshoot: $F 0=60 \mathrm{~Hz}$, $P$ class}

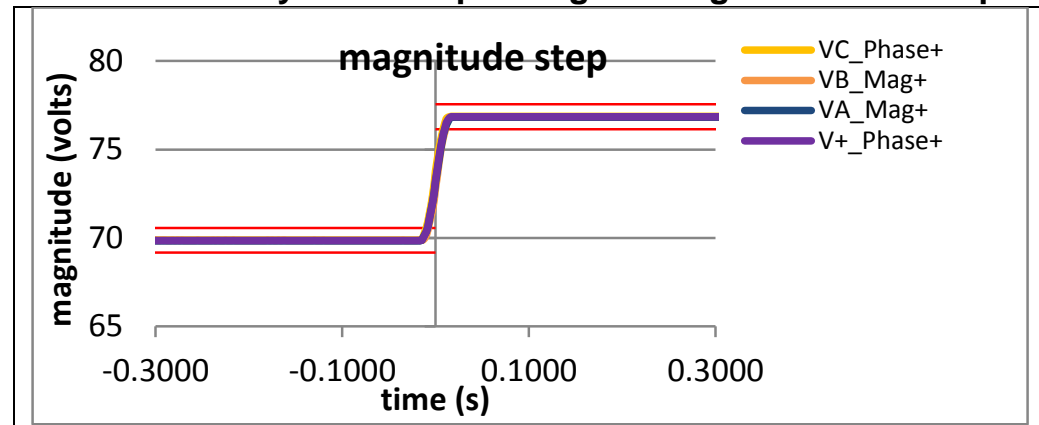

Figure 6191: Fs = 60 FPS, +10\% magnitude step

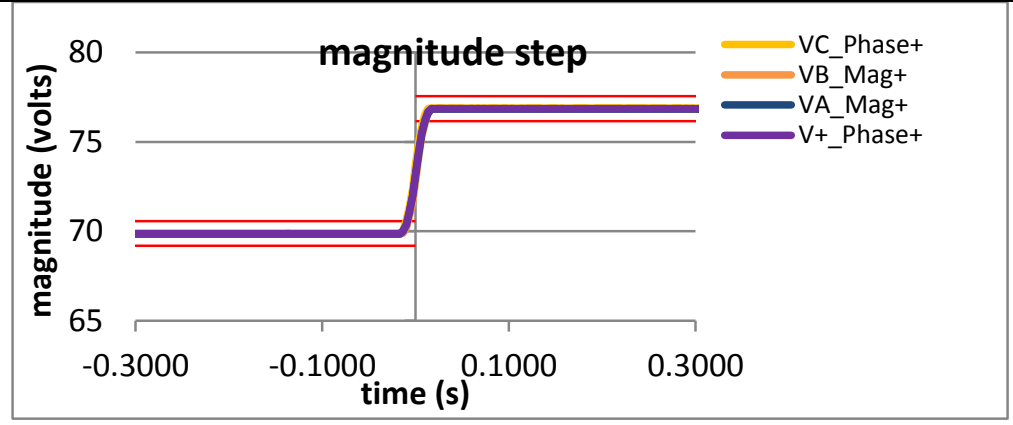

Figure 6193: $\mathrm{Fs}=30 \mathrm{FPS},+10 \%$ magnitude step

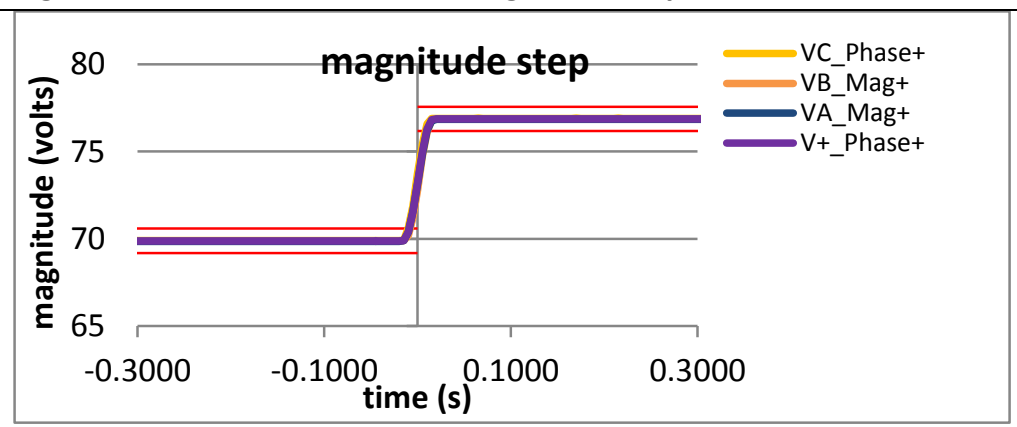

Figure 6195: Fs = 20 FPS, +10\% magnitude step

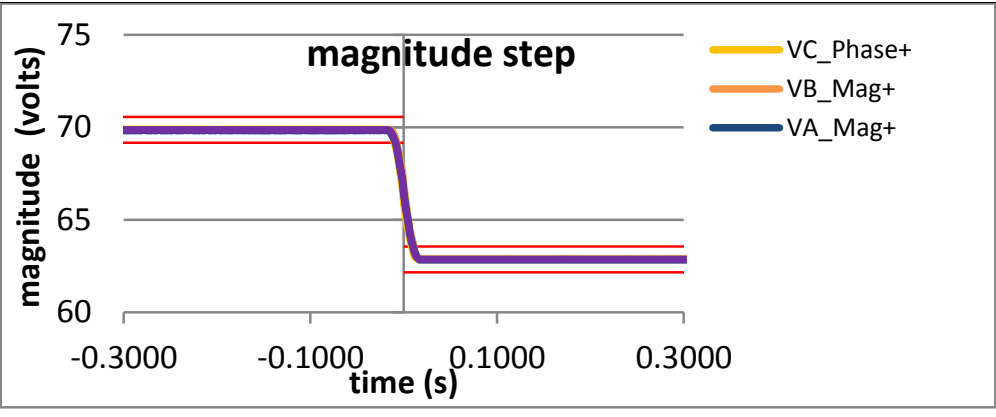

Figure 6192: Fs = 60 FPS, -10\% magnitude step

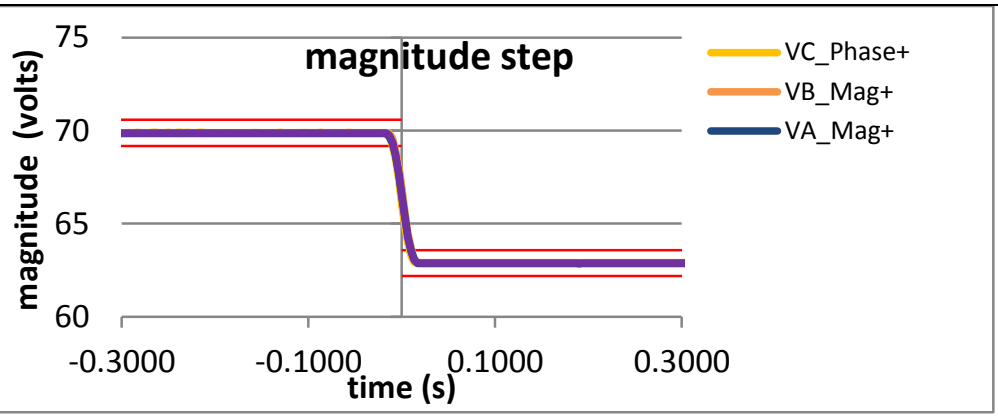

Figure 6194: Fs = 30 FPS, $-10 \%$ magnitude step

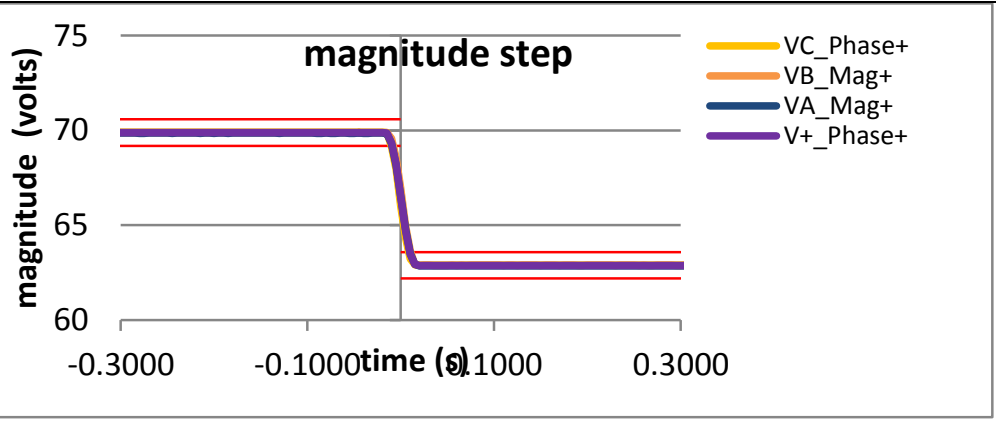

Figure 6196; Fs = 20 FPS, =10\% magnitude step 


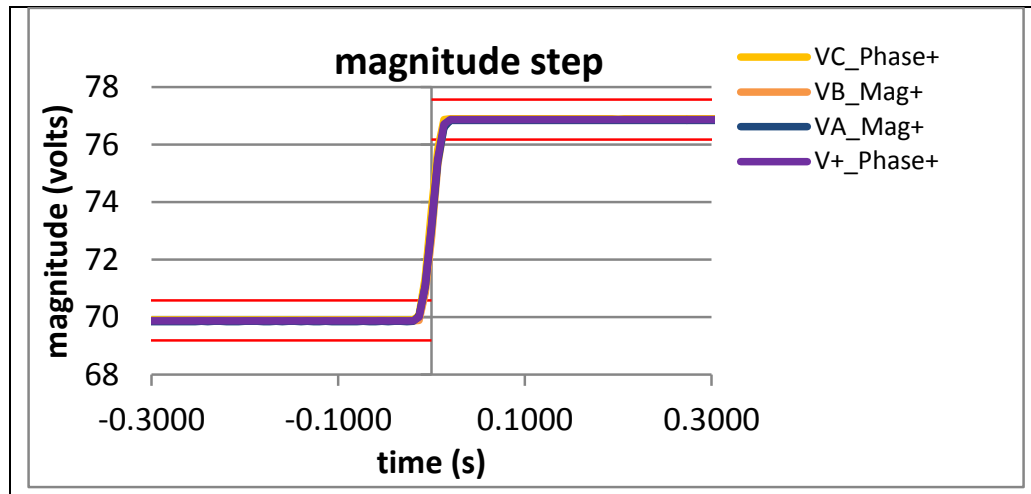

Figure 6197: Fs = 15 FPS, + 10\% magnitude step

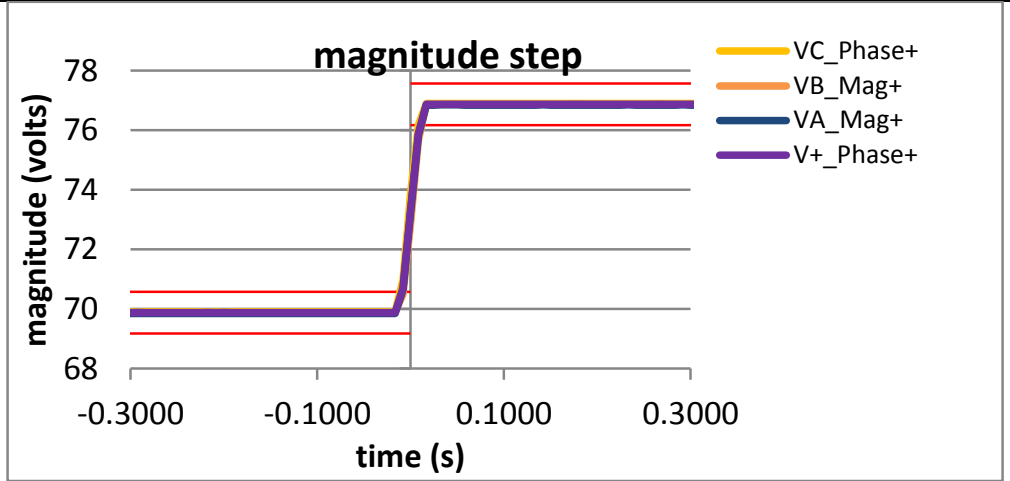

Figure 6199: Fs = 12 FPS, +10\% magnitude step

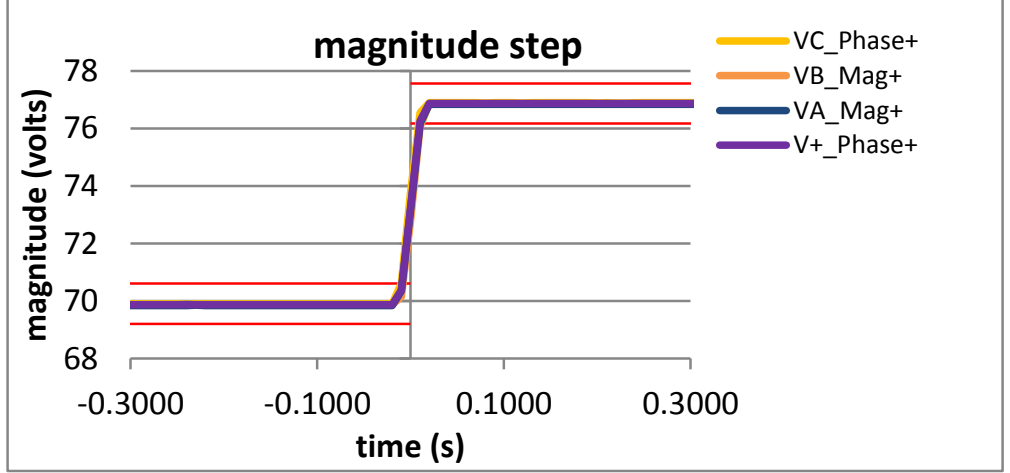

Figure 6201: Fs = $10 \mathrm{FPS},+10 \%$ magnitude step

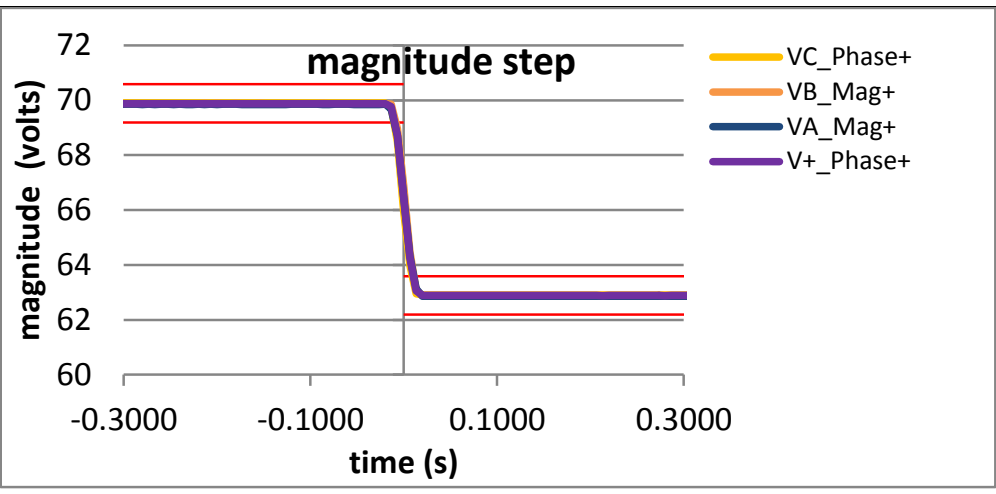

Figure 6198: Fs = 15 FPS, - $10 \%$ magnitude step

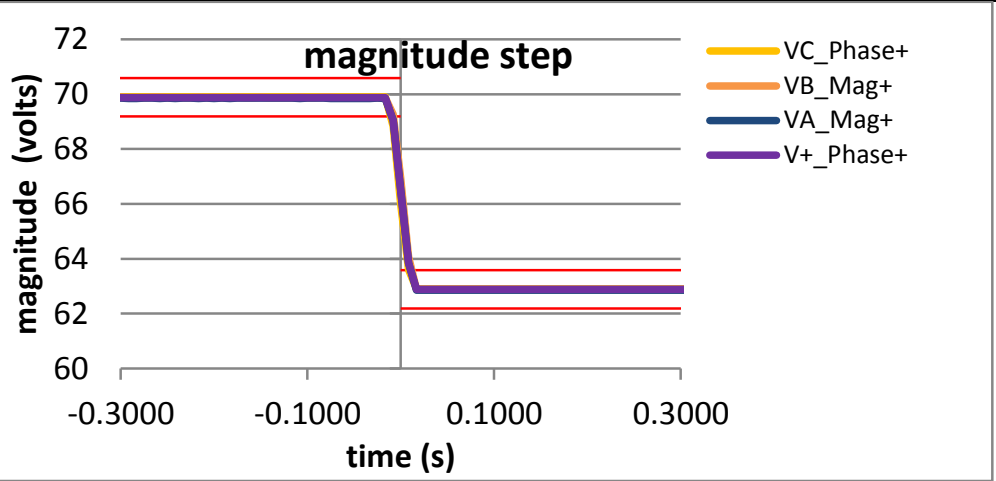

Figure 6200: Fs = 12 FPS, -10\% magnitude step

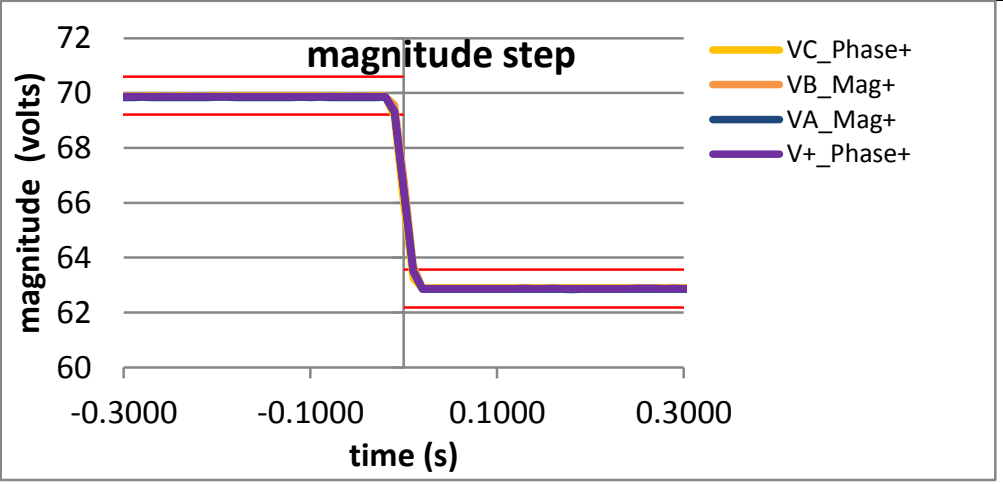

Figure 6202: Fs = 10 FPS, $-10 \%$ magnitude step 
10.13.8 PMU G dynamic step change in magnitude ROCOF phasor overshoot: $F 0=60 \mathrm{~Hz}, \mathrm{P}$ class PMU G does not support P class.

\subsubsection{PMU H dynamic step change in magnitude ROCOF phasor overshoot: $\mathrm{FO}=60 \mathrm{~Hz}$, P class}

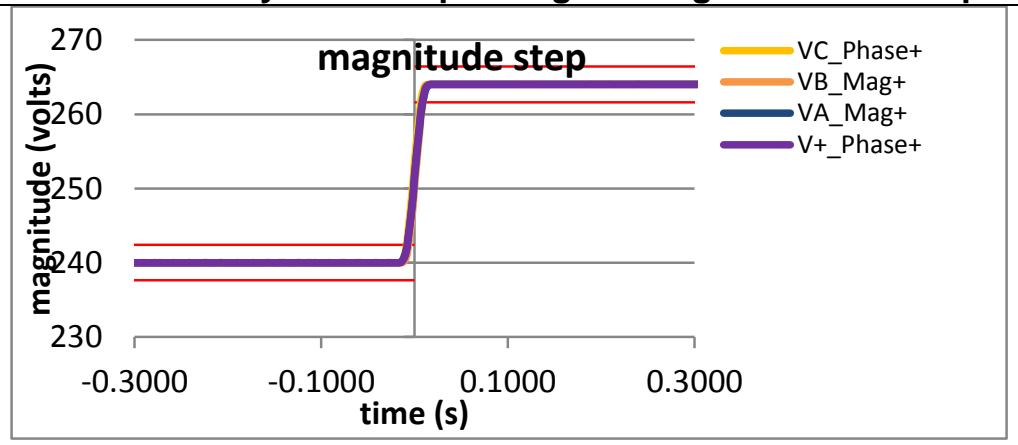

Figure 6203: Fs = 60 FPS, $+10 \%$ magnitude step

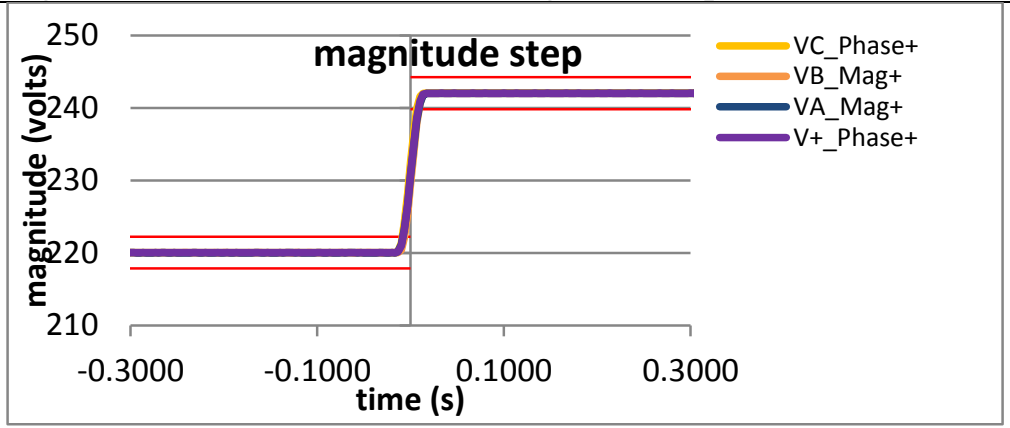

Figure 6205: Fs = 30 FPS, $+10 \%$ magnitude step

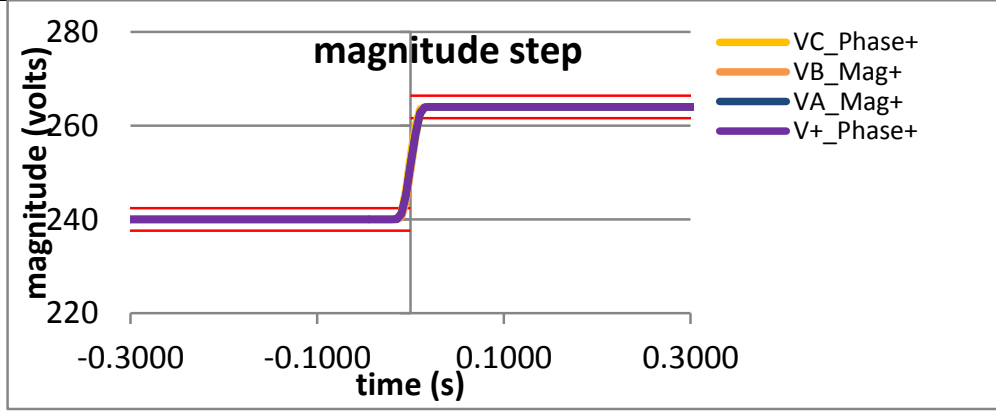

Figure 6207: Fs = 20 FPS, $+10 \%$ magnitude step

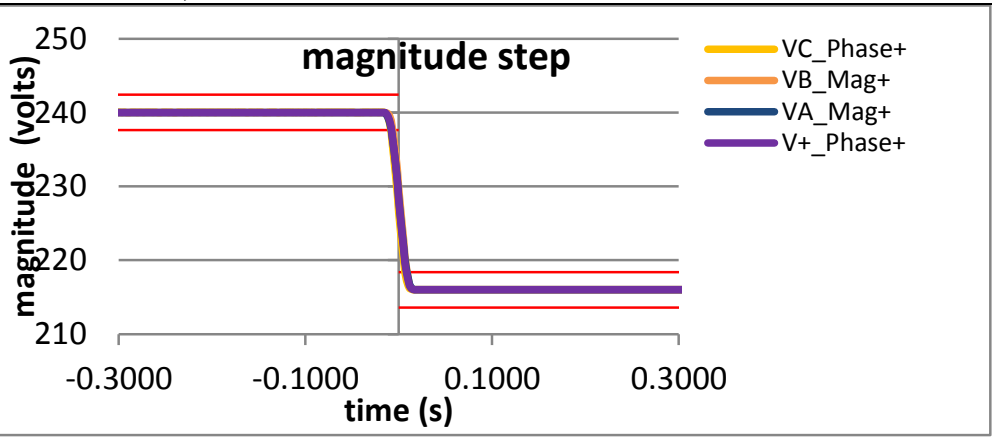

Figure 6204: Fs = 60 FPS, -10\% magnitude step

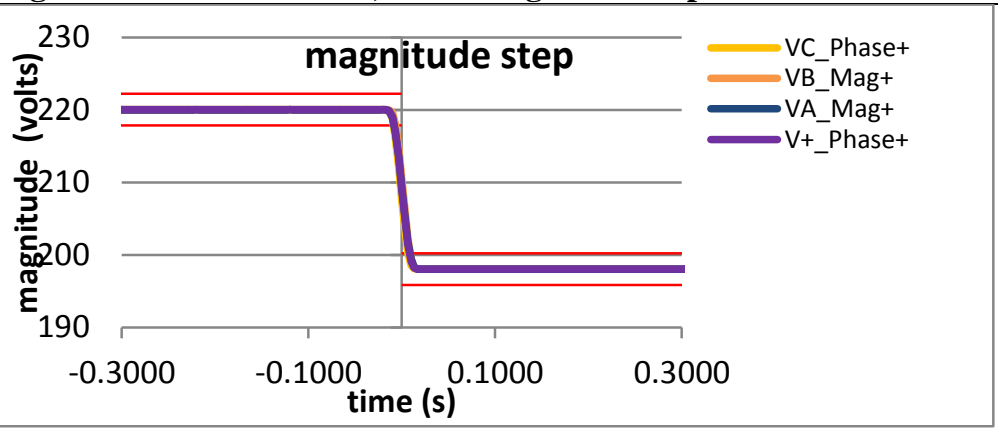

Figure 6206: Fs = 30 FPS, -10\% magnitude step

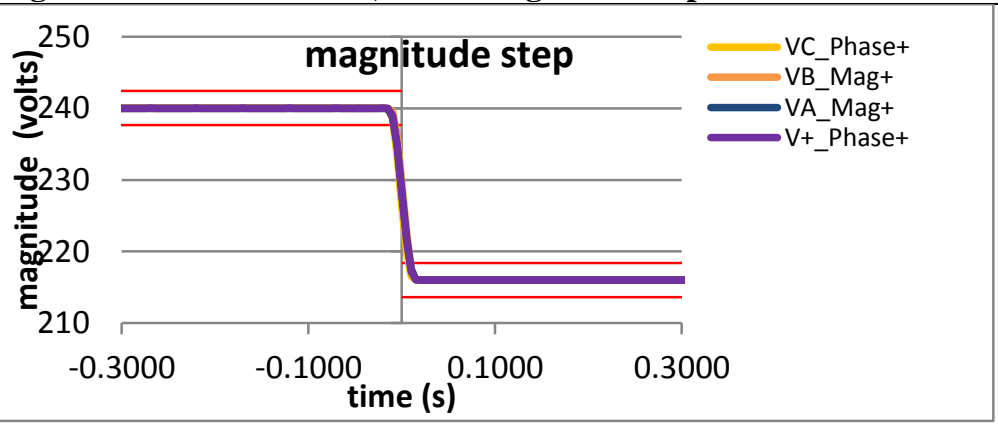

Figure 6208; Fs = 20 FPS, =10\% magnitude step 


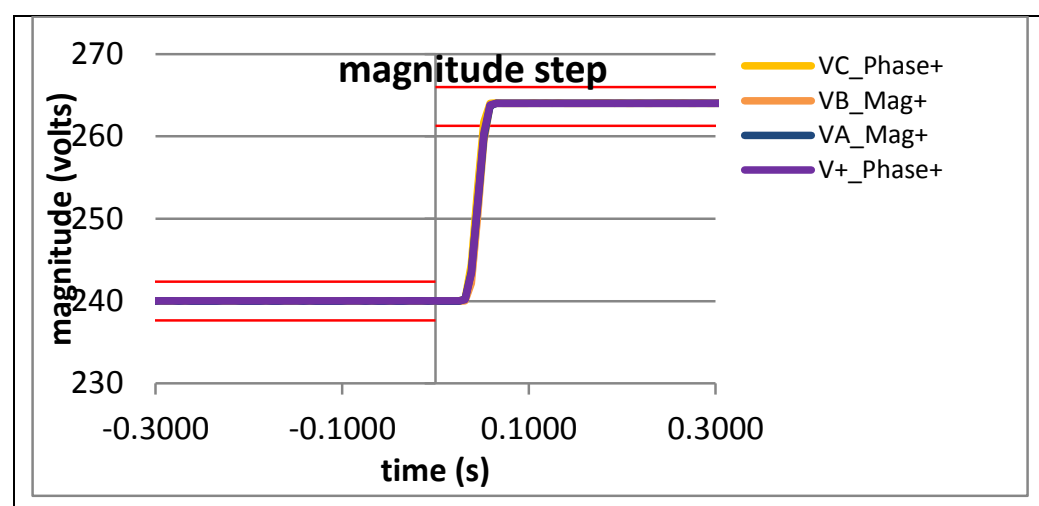

Figure 6209: Fs $=15$ FPS, + 10\% magnitude step

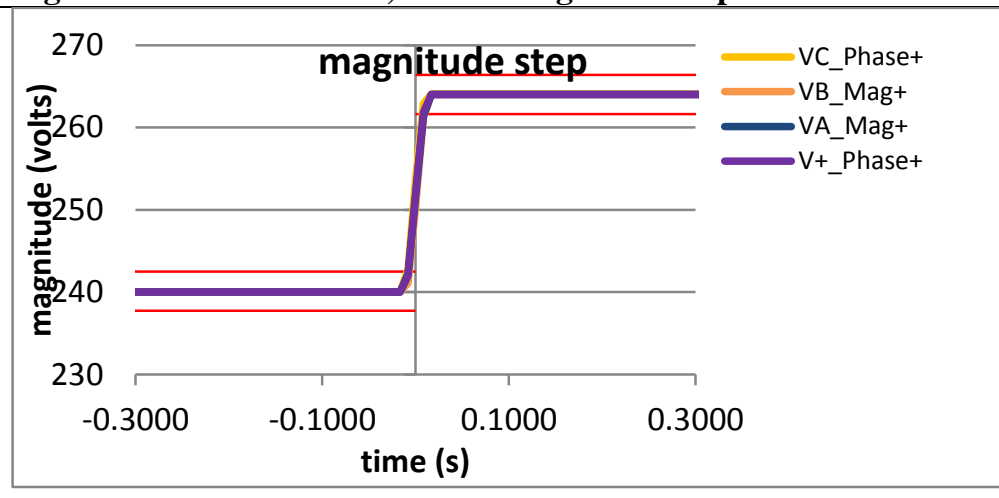

Figure 6211: Fs = 12 FPS, +10\% magnitude step

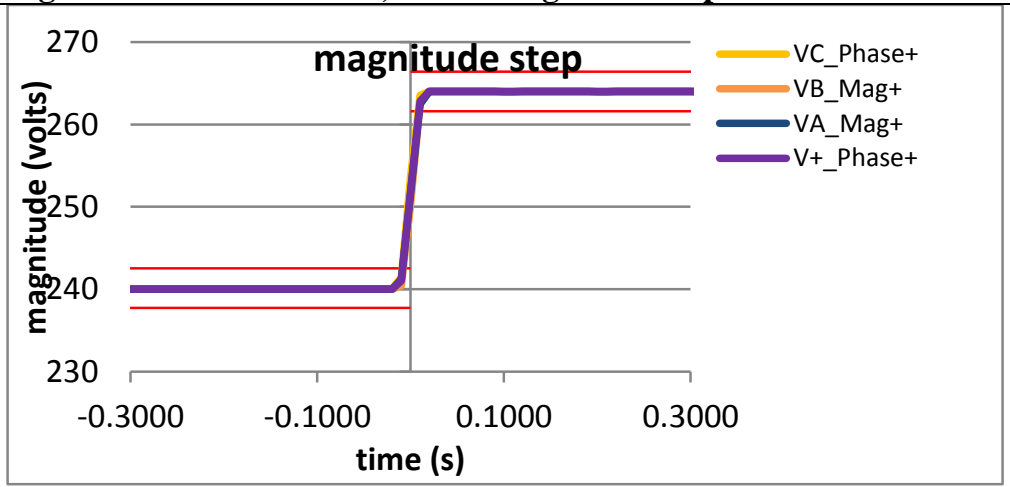

Figure 6213: Fs = 10 FPS, $+10 \%$ magnitude step

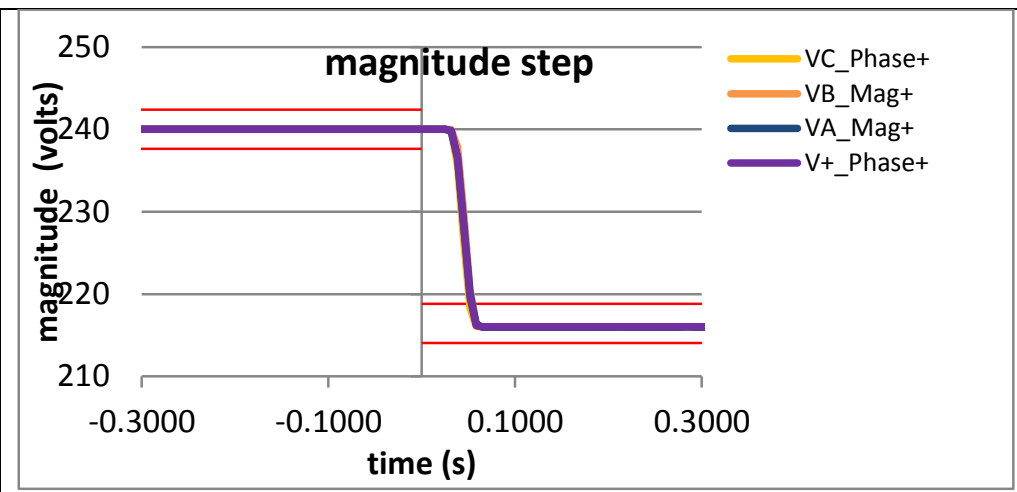

Figure 6210: Fs = 15 FPS, - $10 \%$ magnitude step

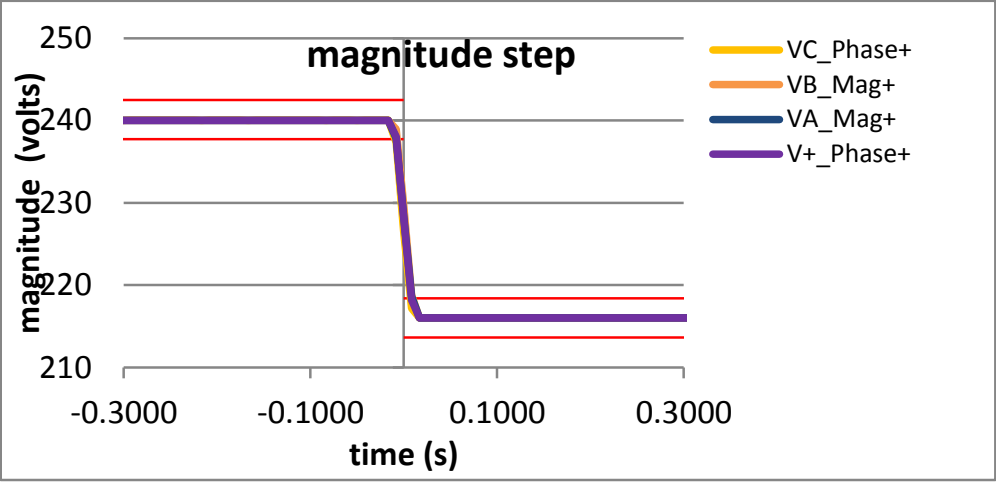

Figure 6212: Fs = 12 FPS, -10\% magnitude step

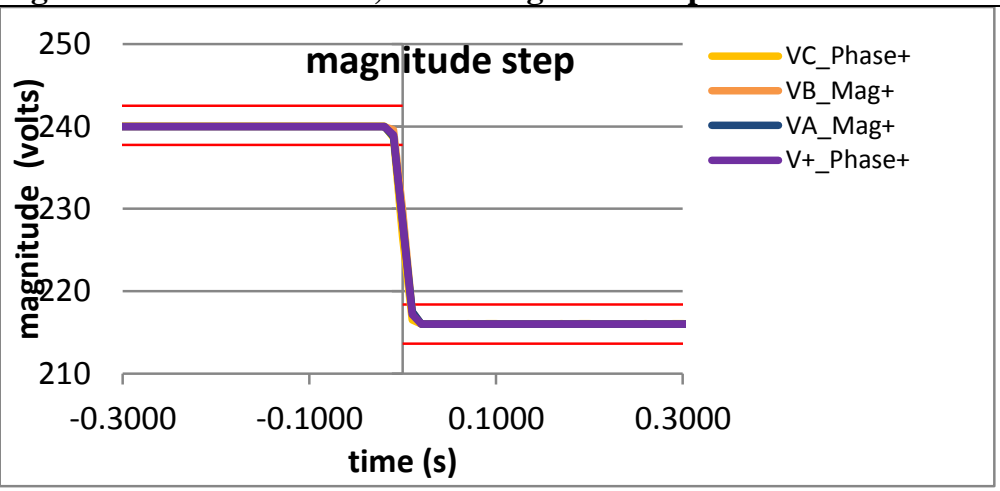

Figure 6214: Fs = 10 FPS, -10\% magnitude step 
10.13.10 PMU I dynamic step change in magnitude ROCOF phasor overshoot: $F 0=60 \mathrm{~Hz}$, P class PMU I does not support $P$ class

PMU J dynamic step change in magnitude ROCOF phasor overshoot: $F 0=60 \mathrm{~Hz}, \mathrm{P}$ class

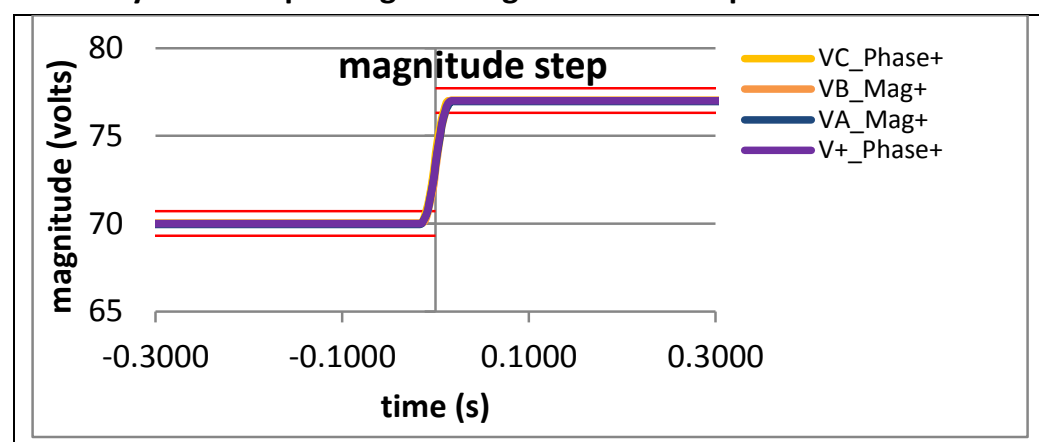

Figure 6215: Fs = 60 FPS, $+10 \%$ magnitude step

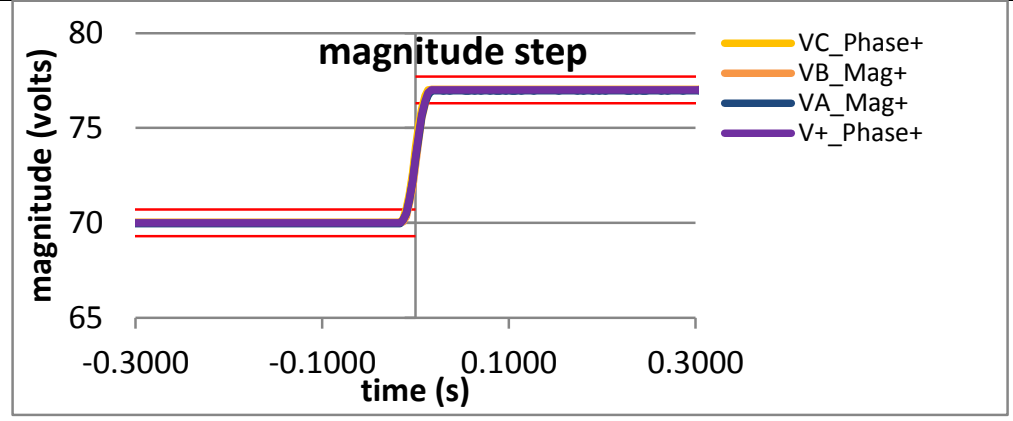

Figure 6217: Fs = $30 \mathrm{FPS},+10 \%$ magnitude step

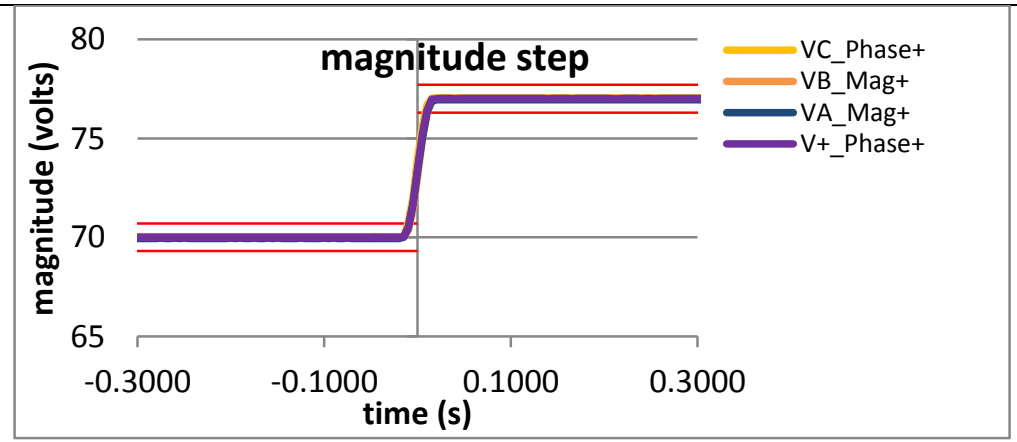

Figure 6219: Fs = 20 FPS, +10\% magnitude step

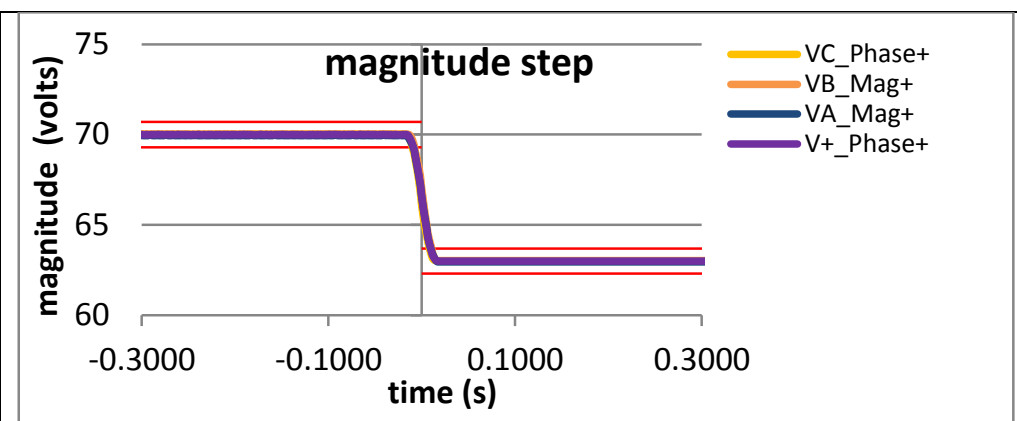

Figure 6216: Fs = 60 FPS, -10\% magnitude step

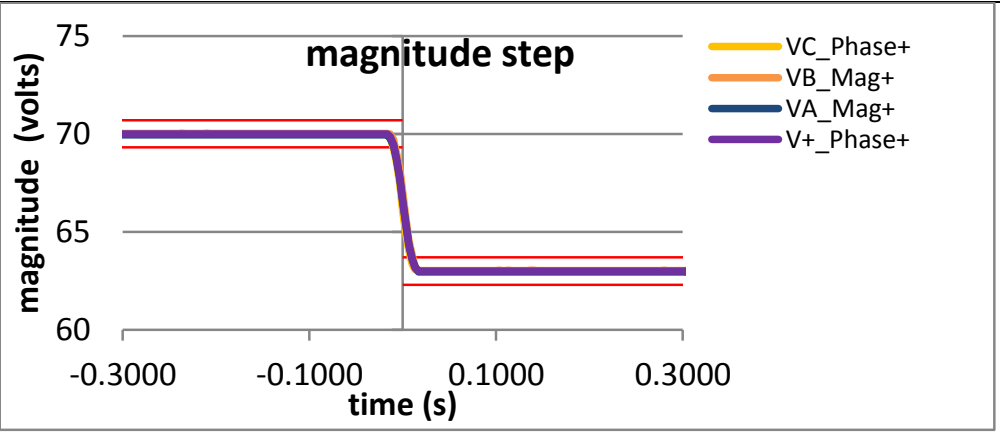

Figure 6218: Fs = 30 FPS, -10\% magnitude step

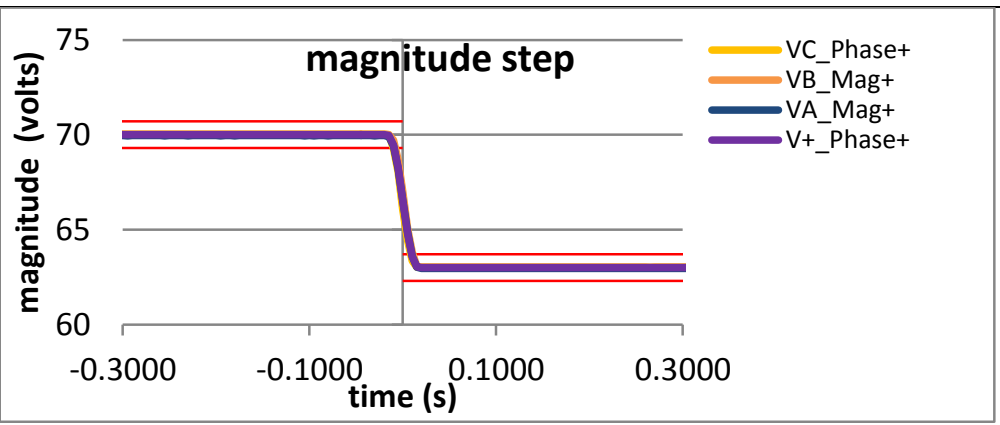

Figure 6220; Fs = 20 FPS, =10\% magnitude step 


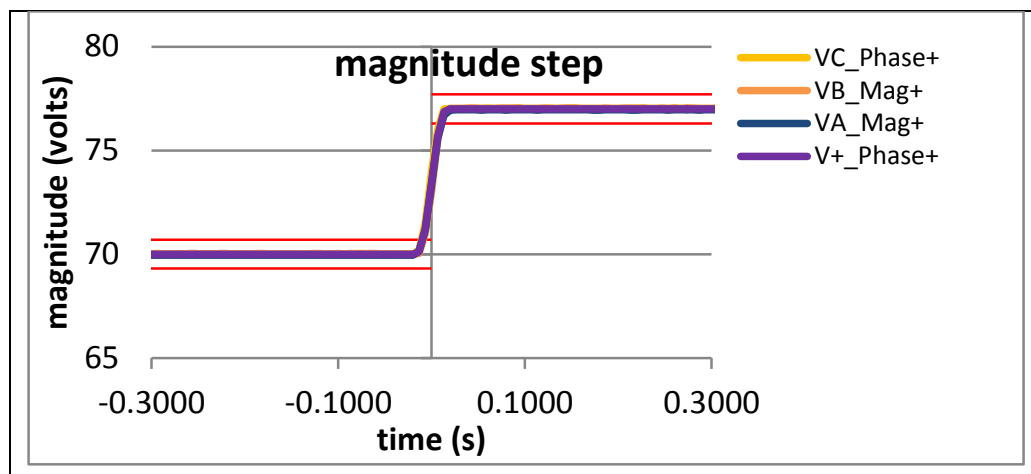

Figure 6221: Fs = 15 FPS, + 10\% magnitude step

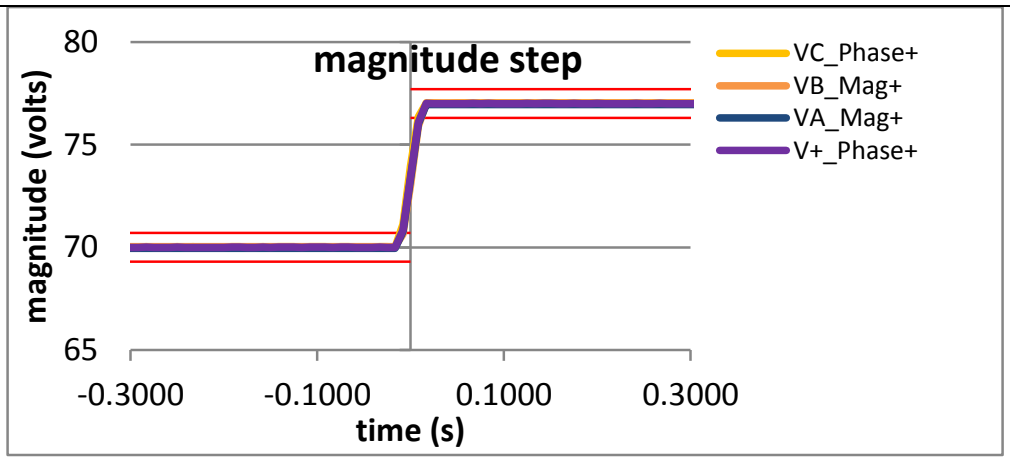

Figure 6223: Fs = 12 FPS, $+10 \%$ magnitude step

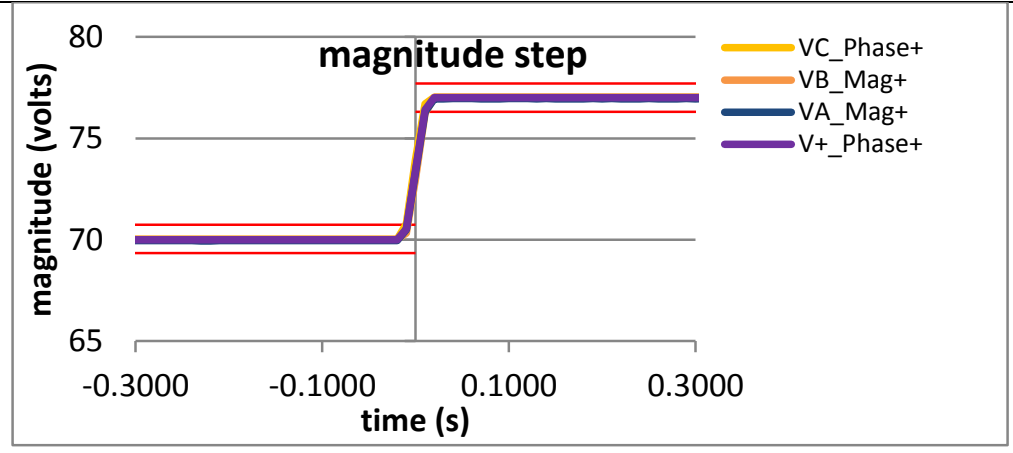

Figure 6225: Fs = 10 FPS, +10\% magnitude step

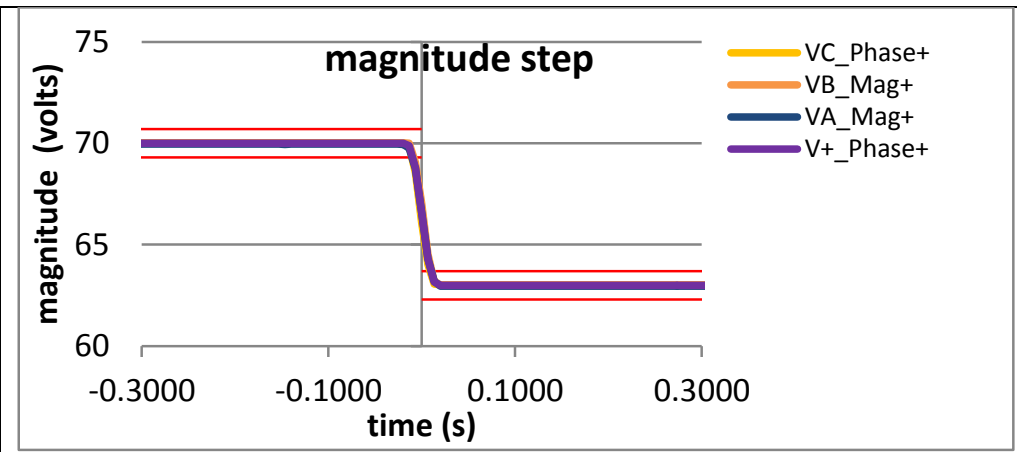

Figure 6222: Fs = 15 FPS, - 10 \% magnitude step

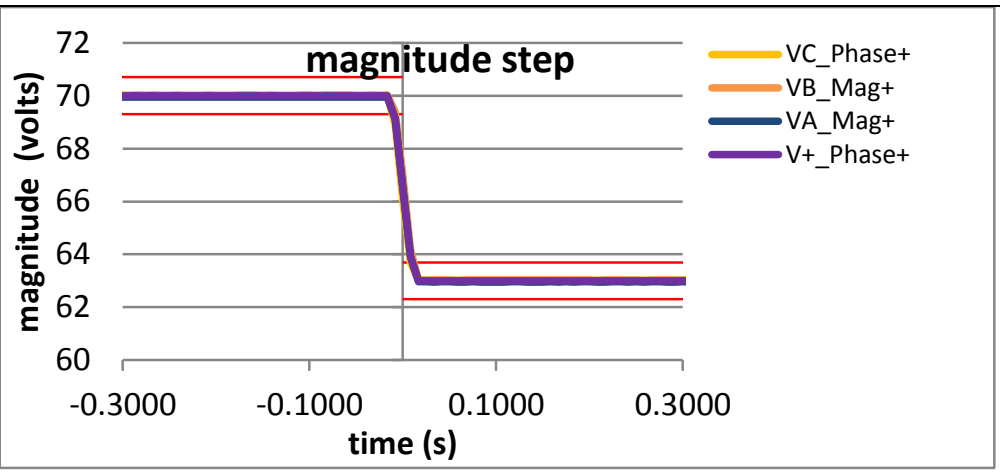

Figure 6224: Fs = 12 FPS, -10\% magnitude step

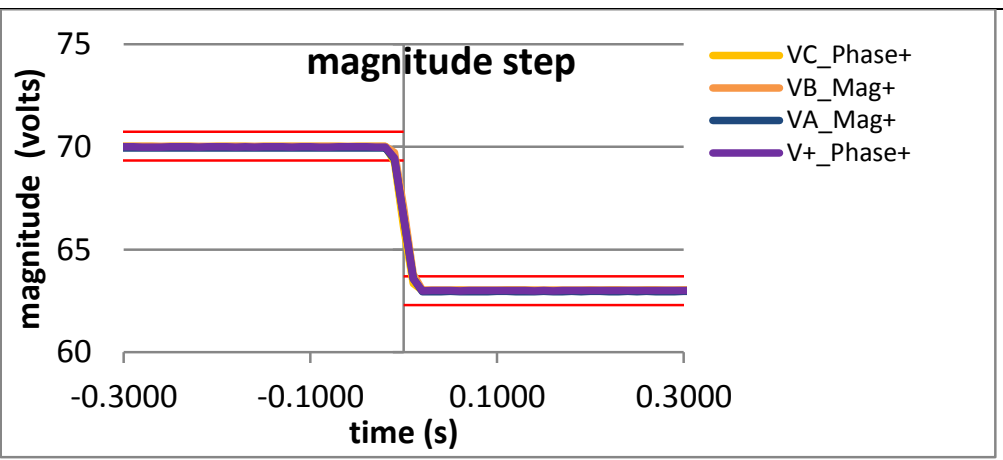

Figure 6226: Fs = 10 FPS, $-10 \%$ magnitude step 


\section{Conclusion}

11. Conclusion

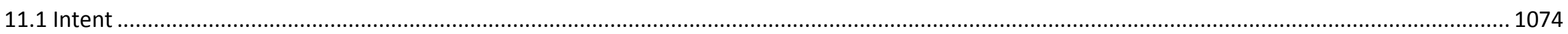

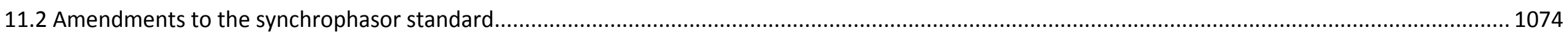

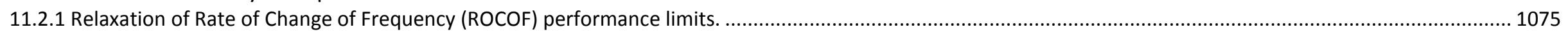

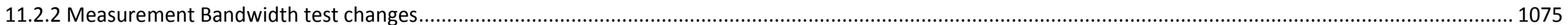

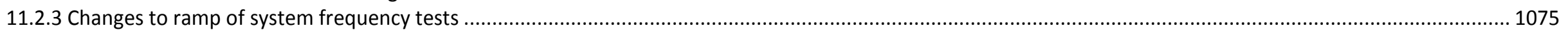

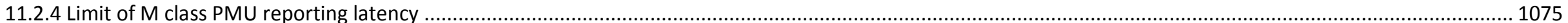

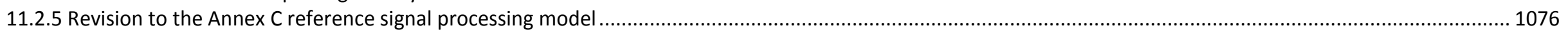

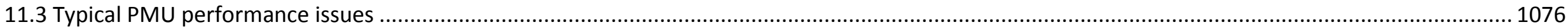

11.3.1 Offset in the time of the frequency estimate

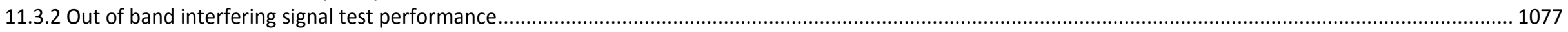

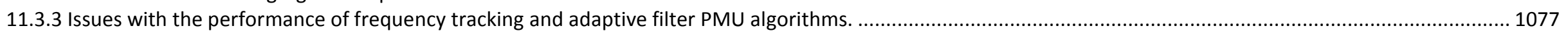

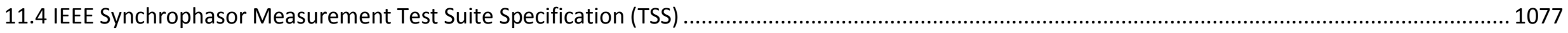

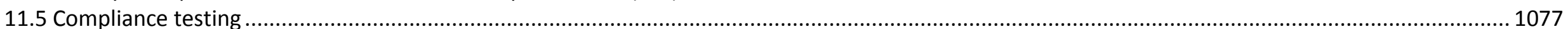




\subsection{Intent}

The intent of this assessment of PMU performance is multi-fold:

- Invite all PMU vendors to participate in an independent assessment of the performance of their PMU products

- Provide the participating vendors with test data

- Allow for vendors to compare independent test results with their in-house testing

- Vendors may revise their PMU designs and submit for re-testing

- This report contains the latest re-test results

- Provide test results (vendor name and PMU model obscured) to the synchrophasor standards setting organizations

- Test results were used to validate test methods and resulted in:

- Amendment to IEEE Std. C37.118.1-2011 published as C37.118.1a-2014

- Drafting and publication of IEEE Synchrophasor Measurement Test Suite Specification

- Detailed test data to be used in the evaluation of requirements for applications which use PMU data as input

- Help NIST and other organizations gain an understanding of common performance issues in PMUs as well as some issues only seen in one make and model of PMU

- Produce this report:

- Intended to be used by

- Utilities to aid in understanding that PMU performance from model to model varies significantly

- Designers of applications which use PMU data as their inputs

- Test labs to provide examples of test results of different models of PMU

- Students and professors as examples of the performance of PMU models and principles of test reporting

- Governments and regulatory agencies to provide examples of the range of performance of PMUs currently used in electrical power systems

\subsection{Amendments to the synchrophasor standard}

Several lessons were learned over the course of the NIST PMU assessment that lead directly to the 2014 amendment of IEEE C37.118.1-2011. All the limits shown in this assessment report were based upon the IEEE Std. C37.118.1a-2014 amendment. The following sections describe these changes. 


\subsubsection{Relaxation of Rate of Change of Frequency (ROCOF) performance limits.}

The NIST PMU assessment provides information indicating that many PMUs installed in power systems were susceptible to internal noise from analog to digital conversion processes and digital algorithms. The performance limits for ROCOF had been determined through the use of digital simulations of the C37.118.1a Annex C Signal Processing Model that did not implement this noise. The standards setting organization determined that the ROCOF limits could become a limiting factor in the design of PMUs and that ROCOF performance did not need to impose such stringent limits so they were relaxed for all tests, and removed for out-of-band interfering signals tests and for $M$ class harmonic distortion testing.

\subsubsection{Measurement Bandwidth test changes}

Analysis of the assessment data for the combined amplitude and phase modulation tests showed that little useful information about the root cause of PMU performance issues could be attained from the test results. The combined modulation test was removed from the standard and replaced with an amplitudemodulation-only test to complement the existing phase modulation test.

The performance limits for frequency and ROCOF for the measurement bandwidth phase modulation test were found by NIST to be so high for some reporting rates, that a PMU that reported ' 0 ' for all frequency and ROCOF would still pass the tests. Frequency and ROCOF limits for measurement bandwidth tests were revised so that PMUs reporting ' 0 ' frequency or ROCOF would not pass the test.

\subsubsection{Changes to ramp of system frequency tests}

During NIST's implementation of the ramp of system frequency test, several ambiguities were found in the standard's description of the test. NISTrecommended descriptive changes that were adopted in the amendment for clarification. Some PMUs tested by NIST were unable to provide meaningful synchrophasors beyond the standard-specified bandwidth limits, depending on PMU class and reporting rate, and required a long period of time to once again begin providing meaningful information. NIST recommended that the test prohibit operation beyond the specified bandwidth. NIST found that the concept of "transition time" in the standard was ambiguous and difficult to implement. The amendment revised the "transition time" to a "measurement exclusion interval". The test now requires that the frequency ramp begin and end at the specified synchrophasor bandwidth limits and that an interval of reports at the beginning and end of the ramp shall not be included in the measurement. This is intended to remove the response of the PMU to the step in ROCOF which occurs at the beginning and end of the test.

\subsubsection{Limit of M class PMU reporting latency}

Through NISTs work with several vendors and after several PMU revisions from one vendor, it was determined that some implementations would not be able to meet the maximum PMU reporting latency specified by the standard. The issue was brought up and discussed by the standards setting organization, and the body decided from what was known to date about the needs of applications using M class PMU data, reporting latency did not need to be a critical factor behind 
$\mathrm{M}$ class designs. The reporting latency was relaxed from five reports to seven reports. Research into the performance needs of applications which use PMU data is ongoing.

\subsubsection{Revision to the Annex $C$ reference signal processing model}

Part of NIST's assessment included assessment of the C37.118.1-2011 Annex C reference signal processing model. Some ambiguities in the model descriptions were found and revisions were proposed. An error in the $\mathrm{M}$ class filter parameter table was found and one filter reference frequency and filter order was amended.

\subsection{Typical PMU performance issues}

Some of the PMUs assessed by NIST exhibited some typical issues. Several of the vendors were able to correct for these issues and submitted revisions for retesting.

\subsubsection{Offset in the time of the frequency estimate}

Some PMUs do not estimate their frequency at the precise time of the timestamp in the report. This offset in the time of the frequency estimate is only exhibited by the results of the ramp of system frequency or measurement bandwidth phase modulation tests. In the ramp of system frequency test, it is exhibited by a constant offset in frequency error which is directly proportional to the difference between the time of PMU report timestamp and the actual time of the frequency estimate. Since the frequency ramp rate of the test is specified to be $1 \mathrm{~Hz} / \mathrm{s}$, the frequency error (FE) offset will be $1 \mathrm{~Hz}$ for each second of time offset. NIST pointed out that the definition of frequency error in the PMU standard had an inverted sign inside the absolute value marks so that if frequency error were measured as defined by the standard but without the absolute value being taken, the FE offset would be the opposite sign of the time offset. the new draft PMU standard being considered at the time of this writing removes the absolute value marks and negates the difference so a signed frequency error has the correct sign value.

\subsubsection{Offset in the time of the ROCOF estimate}

Because ROCOF is defined by the PMU standard as the derivative in frequency, those PMUs that have an offset in the time of the frequency estimate will also have an offset in the time of the ROCOF estimate. The magnitude of the offset may be greater for the ROCOF estimate since multiple frequency estimates are used to determine a ROCOF estimate. The offset in the time of the ROCOF estimate can only be seen in the results of the measurement bandwidth phase modulation tests and not in any other test. 


\subsubsection{Out-of-band interfering signal test performance}

Many of the PMUs as they were first submitted to NIST failed to pass the limits for out-of-band interfering signals testing. This test exposes the PMU to interfering signals (a single interharmonic) from $10 \mathrm{~Hz}$ up to the nominal frequency minus the Nyquist frequency of the reporting rate, and from the nominal frequency plus the reporting rate Nyquist frequency up to the second harmonic of the nominal frequency. The test verifies the performance of the PMUs antialiasing filters since any signal not properly filtered out would be aliased and appear as a signal frequency within the PMU bandwidth as a false frequency component that does not actually exist in the input signal. Many of the PMU vendors revised their PMU performance and submitted for re-testing.

\subsubsection{Issues with the performance of frequency tracking and adaptive filter PMU algorithms.}

The 2005 PMU standard did not require any testing under dynamic conditions. Some PMU vendors used frequency tracking or adaptive filtering algorithms in order to improve the steady state performance of their products. However some of these PMUs were unable to perform within the limits for the 2011 dynamic tests. There were various root causes such as the time constant of the adaptive algorithms not being fast enough to keep up with dynamic signal changes. Several of the vendors revised their PMUs and submitted for re-test which subsequently passed the tests.

\subsection{IEEE Synchrophasor Measurement Test Suite Specification (TSS)}

PMU assessment testing at NIST was carried out prior to and during development of the IEEE Synchrophasor Measurement Test Suite Specification. Much of what was learned, during the assessment, about implementing PMU tests in accordance with the synchrophasor standard supported the drafting of the TSS.

\subsection{Compliance testing}

Assessing PMUs models for conformity to the latest standards can ensure the best performance and interoperability under dynamically-changing grid conditions. Since PMUs have many configuration options and may be included in multi-function devices, end users can additionally have their PMUs tested using the configuration with which they will be installed. 